\title{
Electrostatic Basis for Enantioselective Brønsted-Acid Catalyzed Asymmetric Ring Openings of meso-Epoxides
}

\author{
Trevor J. Seguin and Steven E. Wheeler \\ Department of Chemistry, Texas A\&M University, College Station, TX 77842 \\ E-mail:wheeler@chem.tamu.edu
}

\section{Additional Computational Details}

\section{Conformational searches for lowest-lying transition states}

For each reaction/catalyst combination, we considered all reasonable conformations in order to identify the lowest-lying $(\mathrm{R}, \mathrm{R})$ and $(\mathrm{S}, \mathrm{S})$ transition states.

First, for each of the catalysts there are a number of conformational possibilities. For instance, PA-2 exhibits in different conformations depending on whether the hydrogenated aromatic rings on the BINOL scaffold exist in either a twisted or boat-like conformation. There are two possible twisted and two possible boat conformations for each ring, resulting in 16 total possible PA-2 conformations. For PA-1A, a number of conformations arise from rotations of the sigma bond to the three isopropyl groups. In general, we found that the 2and 6-isopropyl groups favor rotamers in which the C-C-C-H dihedral angle from the 1position of the phenyl ring to the hydrogen bound to the secondary carbon of the isopropyl group is close to zero. The orientation of the 4-isopropyl group varied for each transition state, and we present results for the lowest-energy conformer located in each case.

Similarly, the 5-OMe substituent of the nucleophile in Reaction 1 can exist in two conformations, both of which were considered for each transition state for each catalyst.

Finally, there are a number of ways of orientating the nucleophile and electrophile within the binding site of the catalyst. That is, in addition to the most favorable orientation depicted in Figure 2 of the main paper, additional TS(S,S) configurations were considered in which the substrates were rotated 90 degrees to take advantage of the same electrostatic stabilization discussed in the paper. However, these orientations were always less favorable.

\section{Comparison of predicted er values from different DFT methods}

Data presented in the main paper were computed at the B97-D3/def2-TZVP//PCM-B97D/def2-TZVP level of theory using quasi-RRHO free energy corrections. However, for comparisons, we also predicted er values for the reaction/catalyst combinations for which experimental data are available using wB97X-D/6-311+G(d,p)//wB97X-D/6-31G(d) and M06-2X/6-311+G(d,p)//M06-2X/6-31G(d) using both RRHO and quasi-RRHO free energy corrections. The corresponding data is listed below, where "B97D" denotes B97-D3/def2TZVP//PCM-B97-D/def2-TZVP, “wB97XD” denotes wB97X-D/6-311+G(d,p)//wB97X$\mathrm{D} / 6-31 \mathrm{G}(\mathrm{d})$, and "M06-2X" denotes M06-2X/6-311+G(d,p)//M06-2X/6-31G(d). All computations used PCM to account for solvent effects. 
Table S1. Comparison of predicted vs experimental er values for 6 reaction/catalyst combinations using B97D, wB97XD, and M06-2X paired with either RRHO or quasi-RRHO based thermochemical corrections.

\begin{tabular}{cccccc}
\hline Eq & Cat & $\boldsymbol{e r}(\mathbf{E x p )}$ & $\boldsymbol{e r}$ (B97D) & $\boldsymbol{e r}$ (wB97XD) & $\boldsymbol{e r}$ (M06-2X) \\
\hline \multicolumn{5}{c}{ RRHO (298.15K) } \\
\hline 1 & $1 \mathrm{a}$ & $80.5: 19.5$ & $94.5: 5.5$ & $95.5: 4.5$ & $81.4: 18.6$ \\
1 & $1 \mathrm{~b}$ & $54.5: 45.5$ & $22.8: 77.2$ & $92.5: 7.5$ & $25.3: 74.7$ \\
2a & $1 \mathrm{a}$ & $78.5: 21.5$ & $35.5: 64.5$ & $74.4: 25.6$ & $78.1: 21.9$ \\
2a & 2 & $91.5: 8.5$ & $97.9: 2.1$ & $90.9: 9.1$ & $79.3: 20.7$ \\
2b & $1 \mathrm{a}$ & $80.5: 19.5$ & $64: 36$ & $66.8: 33.2$ & $23.3: 76.7$ \\
2b & 2 & $90.0: 10.0$ & $94.7: 5.3$ & $95: 5$ & $93.1: 6.9$ \\
\hline \multicolumn{5}{c}{ Quasi-RRHO (298.15K) } \\
\hline 1 & $1 \mathrm{a}$ & $80.5: 19.5$ & $84.1: 15.9$ & $92.9: 7.1$ & $80.8: 19.2$ \\
1 & $1 \mathrm{~b}$ & $54.5: 45.5$ & $36.4: 63.6$ & $89.6: 10.4$ & $40.8: 59.2$ \\
2a & $1 \mathrm{a}$ & $78.5: 21.5$ & $70.6: 29.4$ & $82.7: 17.3$ & $77: 23$ \\
2a & 2 & $91.5: 8.5$ & $94.5: 5.5$ & $93: 7$ & $83.1: 16.9$ \\
2b & $1 \mathrm{a}$ & $80.5: 19.5$ & $80.8: 19.2$ & $65.4: 34.6$ & $26: 74$ \\
2b & 2 & $90.0: 10.0$ & $91.4: 8.6$ & $93: 7$ & $91.7: 8.3$ \\
\hline \multicolumn{5}{c}{}
\end{tabular}

\section{Exploration of impact of $\mathrm{CH}^{\cdots} \mathrm{O}$ interaction on barrier height differences}

The $\mathrm{CH}^{\cdots} \mathrm{O}$ distance is consistently shorter in $\mathrm{TS}(\mathrm{R}, \mathrm{R})$ than in $\mathrm{TS}(\mathrm{S}, \mathrm{S})$ for all reaction/catalyst combinations studied, suggesting that this $\mathrm{CH}^{\cdots} \mathrm{O}$ interaction is responsible for the preferential stabilization of TS(R,R) over TS(S,S). To find out whether this $\mathrm{CH}^{\cdots} \mathrm{O}$ interaction is the cause of the energy difference, or simply a common effect of other causes, we examined $\mathrm{H}_{3} \mathrm{PO}_{4}$ as a model catalyst for Reaction 1 . This simple model catalyst results in a free energy difference between $\mathrm{TS}(\mathrm{S}, \mathrm{S})$ and $\mathrm{TS}(\mathrm{R}, \mathrm{R})$ of $0.6 \mathrm{kcal} \mathrm{mol}^{-1}$. Moreover, the $\mathrm{CH}^{\cdots} \mathrm{O}$ distance is $0.13 \AA$ longer in $\mathrm{TS}(\mathrm{S}, \mathrm{S})$ than in TS(R,R), consistent with the distance difference for the real catalysts (see Figure $4 \mathrm{~b}$ in the main paper). However, re-optimization of $\mathrm{TS}(\mathrm{S}, \mathrm{S})$ with the $\mathrm{CH}^{\cdots} \mathrm{O}$ distance constrained to match that in $\mathrm{TS}(\mathrm{R}, \mathrm{R})$ results in $a$ slight increase in relative free energy. Apparently, the difference in $\mathrm{CH}^{\cdots} \mathrm{O}$ distances do not cause the preferential stabilization of TS(R,R) over TS(S,S), but instead reflect other differences between these two TS structures. As noted in the main paper, we attribute this difference to the different electrostatic environments of the two $\mathrm{CH}$ groups of the epoxide. 


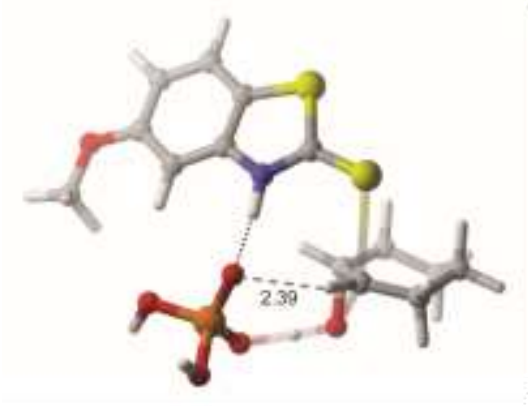

$\mathrm{TS}(R, R)$

$\Delta \Delta G^{*}=0.0$

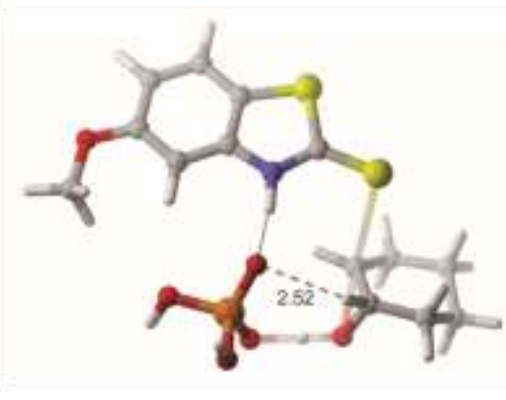

$\mathrm{TS}(S, S)$

$\Delta \Delta G^{*}=0.6$

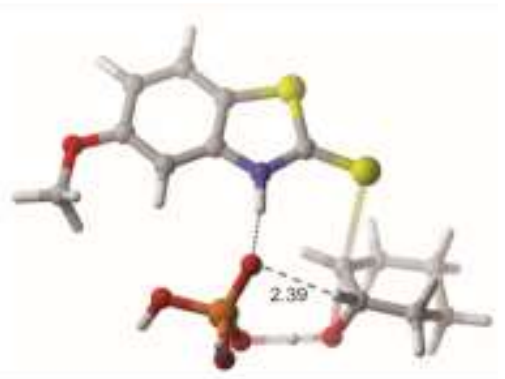

$\mathrm{TS}(S, S)$

Constrained

$\Delta \Delta \mathrm{G}^{+}=0.8$

Figure S1. Computed TS structures for $\mathrm{H}_{3} \mathrm{PO}_{4}$ as a model catalyst for reaction 1 . In the rightmost structure, the $\mathrm{CH}^{\cdots} \mathrm{O}$ distance in $\mathrm{TS}(\mathrm{S}, \mathrm{S})$ is constrained to match that in $\mathrm{TS}(\mathrm{R}, \mathrm{R})$. Relative free energies are provided in kcal mol-1; $\mathrm{CH}^{\cdots} \mathrm{O}$ distances are provide in Angstroms.

\section{Absolute Energies for Computed Transition States}

Table S1. Absolute free energies (298K, in hartrees) at the B97-D/def2-TZVP and B97D3/def2-TZVP//B97-D/def2-TZVP levels of theory for all transition states for reaction 1 catalyzed by PA-1A with free energies relative to the lowest-lying $(R, R)$ transition state $(\mathrm{kcal} / \mathrm{mol})$. The standard RRHO model was used to compute the free energies.

\begin{tabular}{ccccc}
\hline TS Name & G[B97-D/def2-TZVP] & $\mathbf{G}_{\text {rel }}$ & $\begin{array}{c}\text { G[B97-D3/def2-TZVP// } \\
\text { B97-D/def2-TZVP] }\end{array}$ & $\mathbf{G}_{\text {rel }}$ \\
\hline (R,R) Transition States & & & & 0.3 \\
\hline TS1(R,R) & -4125.394143 & 0.0 & -4125.658091 & 0.0 \\
TS2(R,R) & -4125.394032 & 0.1 & -4125.658530 & 0.6 \\
TS3(R,R) & -4125.393240 & 0.6 & -4125.657533 & 1.0 \\
TS4(R,R) & -4125.392888 & 0.8 & -4125.656868 & 1.0 \\
TS5(R,R) & -4125.392699 & 0.9 & -4125.656958 & 1.5 \\
TS6(R,R) & -4125.392011 & 1.3 & -4125.656202 & 1.2 \\
TS7(R,R) & -4125.391991 & 1.4 & -4125.656552 & 2.4 \\
TS8(R,R) & -4125.390938 & 2.0 & -4125.654630 & 2.0 \\
TS9(R,R) & -4125.390793 & 2.1 & -4125.655351 & 6.6 \\
TS10(R,R) & -4125.385166 & 5.6 & -4125.648052 & \\
\hline (S,S) Transition States & & & & 1.7 \\
\hline TS11(S,S) & -4125.391927 & 1.4 & -4125.655845 & 1.9 \\
TS12(S,S) & -4125.390918 & 2.0 & -4125.655500 & 2.1 \\
TS13(S,S) & -4125.390898 & 2.0 & -4125.655225 & 1.8 \\
TS14(S,S) & -4125.390764 & 2.1 & -4125.655705 & 1.7 \\
TS15(S,S) & -4125.390761 & 2.1 & -4125.655777 & 2.2 \\
TS16(S,S) & -4125.390738 & 2.1 & -4125.655025 & 2.8 \\
TS17(S,S) & -4125.390446 & 2.3 & -4125.654024 & -4.0 \\
TS18(S,S) & -4125.390029 & 2.6 & -4125.654677 & 2.3 \\
TS19(S,S) & -4125.389917 & 2.7 & -4125.654746 & \\
TS20(S,S) & -4125.389850 & 2.7 & -4125.653711 & \\
TS21(S,S) & -4125.389128 & 3.1 & & \\
& & & & \\
\end{tabular}


TS22(S,S)

TS23(S,S)

TS24(S,S)

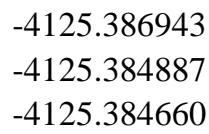

$-4125.386943$

$-4125.384660$
4.5

5.8

6.0
$-4125.651850$

4.2

6.7

6.9

Table S2. Absolute free energies (298K, in hartrees) at the B97-D/def2-TZVP and B97D3/def2-TZVP//B97-D/def2-TZVP levels of theory for all transition states for reaction 1 catalyzed by PA-1A with free energies relative to the lowest-lying $(\mathrm{R}, \mathrm{R})$ transition state (kcal/mol). The Quasi-RRHO model was used to compute the free energies. The lowest-lying $(\mathrm{R}, \mathrm{R})$ and $(\mathrm{S}, \mathrm{S})$ transition states at the B97-D3/def2-TZVP//B97-D/def2-TZVP level are in bold.

\begin{tabular}{ccccc}
\hline TS Name & G[B97-D/def2-TZVP] & $\mathbf{G}_{\text {rel }}$ & $\begin{array}{c}\text { G[B97-D3/def2-TZVP/I } \\
\text { B97-D/def2-TZVP] }\end{array}$ & $\mathbf{G}_{\text {rel }}$ \\
\hline (R,R) Transition States & & & & \\
\hline TS1(R,R) & -4125.377291 & 0.0 & -4125.641239 & 0.1 \\
TS5(R,R) & -4125.376912 & 0.2 & -4125.641170 & 0.1 \\
TS2(R,R) & $\mathbf{- 4 1 2 5 . 3 7 6 8 9 4}$ & $\mathbf{0 . 2}$ & $\mathbf{- 4 1 2 5 . 6 4 1 3 9 2}$ & $\mathbf{0 . 0}$ \\
TS3(R,R) & -4125.376471 & 0.5 & -4125.640763 & 0.4 \\
TS6(R,R) & -4125.376429 & 0.5 & -4125.640620 & 0.5 \\
TS4(R,R) & -4125.376305 & 0.6 & -4125.640285 & 0.7 \\
TS8(R,R) & -4125.374654 & 1.7 & -4125.638346 & 1.9 \\
TS7(R,R) & -4125.374599 & 1.7 & -4125.639160 & 1.4 \\
TS9(R,R) & -4125.374069 & 2.0 & -4125.638628 & 1.7 \\
TS10(R,R) & -4125.369451 & 4.9 & -4125.632337 & 5.7 \\
\hline (S,S) Transition States & & & & \\
\hline TS12(S,S) & $-\mathbf{4 1 2 5 . 3 7 5 2 4 0}$ & $\mathbf{1 . 3}$ & $\mathbf{- 4 1 2 5 . 6 3 9 8 2 2}$ & 1.7 \\
TS11(S,S) & -4125.374772 & 1.6 & -4125.638690 & 1.2 \\
TS18(S,S) & -4125.374762 & 1.6 & -4125.639410 & 1.5 \\
TS13(S,S) & -4125.374722 & 1.6 & -4125.639049 & 1.6 \\
TS16(S,S) & -4125.374601 & 1.7 & -4125.638888 & 1.2 \\
TS14(S,S) & -4125.374480 & 1.8 & -4125.639421 & 2.3 \\
TS17(S,S) & -4125.374085 & 2.0 & -4125.637663 & 1.6 \\
TS19(S,S) & -4125.373858 & 2.2 & -4125.638806 & 2.1 \\
TS21(S,S) & -4125.373537 & 2.4 & -4125.638120 & 1.8 \\
TS15(S,S) & -4125.373493 & 2.4 & -4125.638509 & 2.2 \\
TS20(S,S) & -4125.372956 & 2.7 & -4125.637852 & 3.2 \\
TS22(S,S) & -4125.371311 & 3.8 & -4125.636218 & 5.9 \\
TS23(S,S) & -4125.369013 & 5.2 & -4125.631961 & 6.0 \\
TS24(S,S) & -4125.368988 & 5.2 & -4125.631863 &
\end{tabular}

Table S3. Absolute free energies (195K, in hartrees) at the B97-D/def2-TZVP and B97D3/def2-TZVP//B97-D/def2-TZVP levels of theory for all transition states for reaction 1 catalyzed by PA-1A with free energies relative to the lowest-lying $(\mathrm{R}, \mathrm{R})$ transition state $(\mathrm{kcal} / \mathrm{mol})$. The standard RRHO model was used to compute the free energies.

\begin{tabular}{ccccc}
\hline TS Name & G[B97-D/def2-TZVP] & $\mathbf{G}_{\text {rel }}$ & $\begin{array}{c}\text { G[B97-D3/def2-TZVP// } \\
\text { B97-D/def2-TZVP] }\end{array}$ & $\mathbf{G}_{\text {rel }}$ \\
\hline (R,R) Transition States & & & & \\
\hline TS1(R,R) & -4125.338067 & 0.0 & -4125.602015 & 0.1 \\
TS2(R,R) & -4125.337733 & 0.2 & -4125.602231 & 0.0 \\
TS5(R,R) & -4125.337456 & 0.4 & -4125.601714 & 0.3
\end{tabular}




\begin{tabular}{ccccc} 
TS3(R,R) & -4125.337185 & 0.6 & -4125.601477 & 0.5 \\
TS4(R,R) & -4125.337037 & 0.6 & -4125.601017 & 0.8 \\
TS6(R,R) & -4125.336869 & 0.8 & -4125.601060 & 0.7 \\
TS7(R,R) & -4125.335524 & 1.6 & -4125.600085 & 1.3 \\
TS8(R,R) & -4125.335338 & 1.7 & -4125.599030 & 2.0 \\
TS9(R,R) & -4125.334727 & 2.1 & -4125.599286 & 1.8 \\
TS10(R,R) & -4125.330071 & 5.0 & -4125.592957 & 5.8 \\
\hline (S,S) Transition States & & & & \\
\hline TS11(S,S) & -4125.335777 & 1.4 & -4125.599695 & 1.6 \\
TS12(S,S) & -4125.335695 & 1.5 & -4125.600277 & 1.6 \\
TS13(S,S) & -4125.335345 & 1.7 & -4125.599672 & 1.7 \\
TS16(S,S) & -4125.335206 & 1.8 & -4125.599493 & 1.5 \\
TS18(S,S) & -4125.335168 & 1.8 & -4125.599816 & 1.4 \\
TS14(S,S) & -4125.335014 & 1.9 & -4125.599955 & 2.3 \\
TS17(S,S) & -4125.334936 & 2.0 & -4125.598514 & 1.8 \\
TS15(S,S) & -4125.334352 & 2.3 & -4125.599368 & 1.8 \\
TS19(S,S) & -4125.334348 & 2.3 & -4125.599296 & 2.2 \\
TS21(S,S) & -4125.334068 & 2.5 & -4125.598651 & 2.3 \\
TS20(S,S) & -4125.333704 & 2.7 & -4125.598600 & 3.5 \\
TS22(S,S) & -4125.331673 & 4.0 & -4125.596580 & 6.0 \\
TS23(S,S) & -4125.329651 & 5.3 & -4125.592599 & 6.1 \\
TS24(S,S) & -4125.329612 & 5.3 & -4125.592487 &
\end{tabular}

Table S4. Absolute free energies (195K, in hartrees) at the B97-D/def2-TZVP and B97D3/def2-TZVP//B97-D/def2-TZVP levels of theory for all transition states for reaction 1 catalyzed by PA-1A with free energies relative to the lowest-lying $(R, R)$ transition state $(\mathrm{kcal} / \mathrm{mol})$. The Quasi-RRHO model was used to compute the free energies. The lowest-lying $(\mathrm{R}, \mathrm{R})$ and $(\mathrm{S}, \mathrm{S})$ transition states at the B97-D3/def2-TZVP//B97-D/def2-TZVP level are in bold.

\begin{tabular}{ccccc}
\hline TS Name & G[B97-D/def2-TZVP] & $\mathbf{G}_{\text {rel }}$ & $\begin{array}{c}\text { G[B97-D3/def2-TZVP// } \\
\text { B97-D/def2-TZVP] }\end{array}$ & $\mathbf{G}_{\text {rel }}$ \\
\hline (R,R) Transition States & & & & 0.2 \\
\hline TS1(R,R) & -4125.330709 & 0.0 & -4125.594657 & $\mathbf{0 . 0}$ \\
TS5(R,R) & $\mathbf{- 4 1 2 5 . 3 3 0 6 7 2}$ & $\mathbf{0 . 0}$ & $-\mathbf{4 1 2 5 . 5 9 4 9 3 0}$ & 0.1 \\
TS2(R,R) & -4125.330210 & 0.3 & -4125.594708 & 0.3 \\
TS6(R,R) & -4125.330209 & 0.3 & -4125.594400 & 0.5 \\
TS3(R,R) & -4125.329870 & 0.5 & -4125.594162 & 0.7 \\
TS4(R,R) & -4125.329839 & 0.5 & -4125.593819 & 1.9 \\
TS8(R,R) & -4125.328276 & 1.5 & -4125.591968 & 1.6 \\
TS7(R,R) & -4125.327847 & 1.8 & -4125.592408 & 1.8 \\
TS9(R,R) & -4125.327439 & 2.1 & -4125.591998 & 5.5 \\
TS10(R,R) & -4125.323261 & 4.7 & -4125.586147 & \\
\hline (S,S) Transition States & & & & $\mathbf{0 . 9}$ \\
\hline TS12(S,S) & -4125.328972 & $\mathbf{1 . 1}$ & $-\mathbf{4 1 2 5 . 5 9 3 5 5 4}$ & 1.0 \\
TS18(S,S) & -4125.328697 & 1.3 & -4125.593345 & 1.4 \\
TS13(S,S) & -4125.328362 & 1.5 & -4125.592689 & 1.5 \\
TS16(S,S) & -4125.328243 & 1.5 & -4125.592530 & 1.8 \\
TS11(S,S) & -4125.328157 & 1.6 & -4125.592075 & 1.3 \\
TS14(S,S) & -4125.327996 & 1.7 & -4125.592937 & 2.2 \\
TS17(S,S) & -4125.327831 & 1.8 & -4125.591409 & 1.6 \\
TS19(S,S) & -4125.327453 & 2.0 & -4125.592401 & \\
& & & & \\
\end{tabular}




$\begin{array}{lllll}\text { TS21(S,S) } & -4125.327394 & 2.1 & -4125.591977 & 1.9 \\ \text { TS15(S,S) } & -4125.326735 & 2.5 & -4125.591751 & 2.0 \\ \text { TS20(S,S) } & -4125.326326 & 2.8 & -4125.591222 & 2.3 \\ \text { TS22(S,S) } & -4125.325011 & 3.6 & -4125.589918 & 3.1 \\ \text { TS24(S,S) } & -4125.322857 & 4.9 & -4125.585732 & 5.8 \\ \text { TS23(S,S) } & -4125.322748 & 5.0 & -4125.585696 & 5.8\end{array}$

Table S5. Absolute free energies (298K, in hartrees) at the wB97XD/6-31G(d) and wB97XD/6-311+G(d,p)// wB97XD/6-31G(d) levels of theory for all transition states for reaction 1 catalyzed by $P A-1 A$ with free energies relative to the lowest-lying $(R, R)$ transition state $(\mathrm{kcal} / \mathrm{mol})$. The standard RRHO model was used to compute the free energies.

\begin{tabular}{|c|c|c|c|c|}
\hline TS Name & G[wB97XD/6-31G(d)] & $\mathbf{G}_{\text {rel }}$ & $\begin{array}{c}\text { G[wB97XD/6-311+G(d,p)// } \\
\text { wB97XD/6-31G(d)] }\end{array}$ & $\mathbf{G}_{\text {rel }}$ \\
\hline \multicolumn{5}{|c|}{$(\mathbf{R}, \mathbf{R})$ Transition States } \\
\hline TS5(R,R) & -4125.322072 & 0.0 & -4126.116023 & 0.0 \\
\hline TS6(R,R) & -4125.321855 & 0.1 & -4126.115768 & 0.2 \\
\hline TS2(R,R) & -4125.319953 & 1.3 & -4126.113366 & 1.7 \\
\hline TS1(R,R) & -4125.319776 & 1.4 & -4126.113486 & 1.6 \\
\hline TS4(R,R) & -4125.319003 & 1.9 & -4126.112720 & 2.1 \\
\hline TS3(R,R) & -4125.318531 & 2.2 & -4126.111891 & 2.6 \\
\hline TS8(R,R) & -4125.318521 & 2.2 & -4126.112154 & 2.4 \\
\hline TS9(R,R) & -4125.317275 & 3.0 & -4126.110247 & 3.6 \\
\hline TS7(R,R) & -4125.314842 & 4.5 & -4126.107712 & 5.2 \\
\hline $\mathrm{TS} 10(\mathrm{R}, \mathrm{R})$ & -4125.311202 & 6.8 & -4126.104669 & 7.1 \\
\hline \multicolumn{5}{|c|}{ (S,S) Transition States } \\
\hline $\mathrm{TS} 12(\mathrm{~S}, \mathrm{~S})$ & -4125.318943 & 2.0 & -4126.113133 & 1.8 \\
\hline $\mathrm{TS} 21(\mathrm{~S}, \mathrm{~S})$ & -4125.318843 & 2.0 & -4126.112916 & 1.9 \\
\hline $\mathrm{TS} 18(\mathrm{~S}, \mathrm{~S})$ & -4125.318531 & 2.2 & -4126.112793 & 2.0 \\
\hline TS16(S,S) & -4125.317721 & 2.7 & -4126.111499 & 2.8 \\
\hline $\mathrm{TS} 14(\mathrm{~S}, \mathrm{~S})$ & -4125.317486 & 2.9 & -4126.111253 & 3.0 \\
\hline $\mathrm{TS} 17(\mathrm{~S}, \mathrm{~S})$ & -4125.317160 & 3.1 & -4126.111290 & 3.0 \\
\hline $\mathrm{TS} 13(\mathrm{~S}, \mathrm{~S})$ & -4125.317091 & 3.1 & -4126.110988 & 3.2 \\
\hline TS20(S,S) & -4125.316179 & 3.7 & -4126.109357 & 4.2 \\
\hline $\mathrm{TS} 11(\mathrm{~S}, \mathrm{~S})$ & -4125.316175 & 3.7 & -4126.109796 & 3.9 \\
\hline TS19(S,S) & -4125.315582 & 4.1 & -4126.108972 & 4.4 \\
\hline $\mathrm{TS} 15(\mathrm{~S}, \mathrm{~S})$ & -4125.315458 & 4.2 & -4126.109366 & 4.2 \\
\hline $\mathrm{TS} 22(\mathrm{~S}, \mathrm{~S})$ & -4125.313128 & 5.6 & -4126.106510 & 6.0 \\
\hline $\mathrm{TS} 24(\mathrm{~S}, \mathrm{~S})$ & -4125.311535 & 6.6 & -4126.105686 & 6.5 \\
\hline TS23(S,S) & -4125.309014 & 8.2 & -4126.102615 & 8.4 \\
\hline
\end{tabular}

Table S6. Absolute free energies (298K, in hartrees) at the wB97XD/6-31G(d) and wB97XD/6-311+G(d,p)// wB97XD/6-31G(d) levels of theory for all transition states for reaction 1 catalyzed by PA-1A with free energies relative to the lowest-lying $(R, R)$ transition state $(\mathrm{kcal} / \mathrm{mol})$. The Quasi-RRHO model was used to compute the free energies.

\begin{tabular}{ccccc}
\hline TS Name & G[wB97XD/6-31G(d)] & $\mathbf{G}_{\text {rel }}$ & $\begin{array}{c}\text { G[wB97XD/6-311+G(d,p)// } \\
\text { wB97XD/6-31G(d)] }\end{array}$ & $\mathbf{G}_{\text {rel }}$ \\
\hline (R,R) Transition States & & & & \\
\hline TS5(R,R) & -4125.307419 & 0.0 & -4126.101370 & 0.0 \\
TS6(R,R) & -4125.307328 & 0.1 & -4126.101241 & 0.1
\end{tabular}




\begin{tabular}{ccccc} 
TS1(R,R) & -4125.305208 & 1.4 & -4126.098918 & 1.5 \\
TS2(R,R) & -4125.304982 & 1.5 & -4126.098395 & 1.9 \\
TS4(R,R) & -4125.304738 & 1.7 & -4126.098455 & 1.8 \\
TS8(R,R) & -4125.303869 & 2.2 & -4126.097502 & 2.4 \\
TS3(R,R) & -4125.303831 & 2.3 & -4126.097191 & 2.6 \\
TS9(R,R) & -4125.302301 & 3.2 & -4126.095274 & 3.8 \\
TS7(R,R) & -4125.300420 & 4.4 & -4126.093290 & 5.1 \\
TS10(R,R) & -4125.297711 & 6.1 & -4126.091178 & 6.4 \\
\hline (S,S) Transition States & & & & 1.5 \\
\hline TS18(S,S) & -4125.304678 & 1.7 & -4126.098941 & 1.6 \\
TS12(S,S) & -4125.304655 & 1.7 & -4126.098845 & 1.7 \\
TS21(S,S) & -4125.304510 & 1.8 & -4126.098583 & 2.7 \\
TS16(S,S) & -4125.303360 & 2.5 & -4126.097138 & 2.9 \\
TS13(S,S) & -4125.302916 & 2.8 & -4126.096813 & 3.2 \\
TS14(S,S) & -4125.302554 & 3.1 & -4126.096320 & 3.6 \\
TS17(S,S) & -4125.301510 & 3.7 & -4126.095640 & 4.1 \\
TS19(S,S) & -4125.301428 & 3.8 & -4126.094818 & 4.2 \\
TS11(S,S) & -4125.301092 & 4.0 & -4126.094712 & 4.8 \\
TS20(S,S) & -4125.300568 & 4.3 & -4126.093746 & 4.7 \\
TS15(S,S) & -4125.299903 & 4.7 & -4126.093812 & 5.3 \\
TS22(S,S) & -4125.299505 & 5.0 & -4126.092887 & 6.4 \\
TS24(S,S) & -4125.297037 & 6.5 & -4126.091188 & 7.7 \\
TS23(S,S) & -4125.295456 & 7.5 & -4126.089056 &
\end{tabular}

Table S7. Absolute free energies (298K, in hartrees) at the M06-2X/6-31G(d) and M062X/6-311+G(d,p)// M06-2X /6-31G(d) levels of theory for all transition states for reaction 1 catalyzed by PA-1A with free energies relative to the lowest-lying $(R, R)$ transition state $(\mathrm{kcal} / \mathrm{mol})$. The standard RRHO model was used to compute the free energies.

\begin{tabular}{ccccc}
\hline TS Name & G[M06-2X/6-31G(d)] & $\mathbf{G}_{\text {rel }}$ & $\begin{array}{c}\text { G[M06-2X/6-311+G(d,p)// } \\
\text { M06-2X/6-31G(d)] }\end{array}$ & $\mathbf{G}_{\text {rel }}$ \\
\hline (R,R) Transition States & & & & 0.0 \\
\hline TS5(R,R) & -4124.884053 & 0.0 & -4125.767799 & 0.6 \\
TS6(R,R) & -4124.883243 & 0.5 & -4125.766856 & 1.0 \\
TS1(R,R) & -4124.882783 & 0.8 & -4125.766262 & 1.8 \\
TS4(R,R) & -4124.881554 & 1.6 & -4125.764995 & 2.9 \\
TS7(R,R) & -4124.881063 & 1.9 & -4125.763239 & 2.7 \\
TS2(R,R) & -4124.881014 & 1.9 & -4125.763526 & 2.8 \\
TS3(R,R) & -4124.880832 & 2.0 & -4125.763284 & 3.1 \\
TS9(R,R) & -4124.880564 & 2.2 & -4125.762806 & 2.6 \\
TS8(R,R) & -4124.880371 & 2.3 & -4125.763633 & 5.8 \\
TS10(R,R) & -4124.875402 & 5.4 & -4125.758633 & \\
\hline (S,S) Transition States & & & & \\
\hline TS18(S,S) & -4124.882187 & 1.2 & -4125.766405 & \\
TS12(S,S) & -4124.881854 & 1.4 & -4125.766039 & 1.1 \\
TS17(S,S) & -4124.881535 & 1.6 & -4125.765180 & 1.6 \\
TS13(S,S) & -4124.881003 & 1.9 & -4125.764597 & 2.0 \\
TS11(S,S) & -4124.880714 & 2.1 & -4125.763570 & 2.7 \\
TS16(S,S) & -4124.880380 & 2.3 & -4125.764033 & 2.4 \\
TS21(S,S) & -4124.880282 & 2.4 & -4125.764269 & 2.2 \\
TS14(S,S) & -4124.879255 & 3.0 & -4125.762196 & -4125.761998 \\
TS19(S,S) & -4124.879003 & 3.2 & & 3.6 \\
& & & & \\
\end{tabular}




$\begin{array}{lllll}\text { TS22(S,S) } & -4124.876034 & 5.0 & -4125.758678 & 5.7 \\ \text { TS15(S,S) } & -4124.875450 & 5.4 & -4125.758087 & 6.1 \\ \text { TS23(S,S) } & -4124.875146 & 5.6 & -4125.758842 & 5.6 \\ \text { TS24(S,S) } & -4124.874582 & 5.9 & -4125.758197 & 6.0 \\ \text { TS20(S,S) } & -4124.874411 & 6.1 & -4125.757101 & 6.7\end{array}$

Table S8. Absolute free energies (298K, in hartrees) at the M06-2X/6-31G(d) and M062X/6-311+G(d,p)// M06-2X /6-31G(d) levels of theory for all transition states for reaction 1 catalyzed by PA-1A with free energies relative to the lowest-lying $(R, R)$ transition state (kcal/mol). The Quasi-RRHO model was used to compute the free energies.

\begin{tabular}{|c|c|c|c|c|}
\hline TS Name & G[M06-2X/6-31G(d)] & $\mathbf{G}_{\text {rel }}$ & $\begin{array}{c}\text { G[M06-2X/6-311+G(d,p)// } \\
\text { M06-2X/6-31G(d)] }\end{array}$ & $\mathbf{G}_{\text {rel }}$ \\
\hline \multicolumn{5}{|c|}{$(\mathbf{R}, \mathbf{R})$ Transition States } \\
\hline TS5(R,R) & -4124.869273 & 0.0 & -4125.753020 & 0.0 \\
\hline TS6(R,R) & -4124.868827 & 0.3 & -4125.752439 & 0.4 \\
\hline $\mathrm{TS} 1(\mathrm{R}, \mathrm{R})$ & -4124.867486 & 1.1 & -4125.750965 & 1.3 \\
\hline TS4(R,R) & -4124.866762 & 1.6 & -4125.750203 & 1.8 \\
\hline $\mathrm{TS} 2(\mathrm{R}, \mathrm{R})$ & -4124.866156 & 2.0 & -4125.748667 & 2.7 \\
\hline TS8(R,R) & -4124.865995 & 2.1 & -4125.749257 & 2.4 \\
\hline $\mathrm{TS} 3(\mathrm{R}, \mathrm{R})$ & -4124.865976 & 2.1 & -4125.748429 & 2.9 \\
\hline TS7(R,R) & -4124.865182 & 2.6 & -4125.747357 & 3.6 \\
\hline $\mathrm{TS} 9(\mathrm{R}, \mathrm{R})$ & -4124.865005 & 2.7 & -4125.747247 & 3.6 \\
\hline TS10(R,R) & -4124.861795 & 4.7 & -4125.745027 & 5.0 \\
\hline \multicolumn{5}{|c|}{ (S,S) Transition States } \\
\hline TS18(S,S) & -4124.867446 & 1.1 & -4125.751664 & 0.9 \\
\hline $\mathrm{TS} 12(\mathrm{~S}, \mathrm{~S})$ & -4124.867260 & 1.3 & -4125.751446 & 1.0 \\
\hline $\mathrm{TS} 13(\mathrm{~S}, \mathrm{~S})$ & -4124.865799 & 2.2 & -4125.749393 & 2.3 \\
\hline $\mathrm{TS} 17(\mathrm{~S}, \mathrm{~S})$ & -4124.865757 & 2.2 & -4125.749403 & 2.3 \\
\hline $\mathrm{TS} 21(\mathrm{~S}, \mathrm{~S})$ & -4124.865649 & 2.3 & -4125.749636 & 2.1 \\
\hline $\mathrm{TS} 16(\mathrm{~S}, \mathrm{~S})$ & -4124.865525 & 2.4 & -4125.749178 & 2.4 \\
\hline $\mathrm{TS} 11(\mathrm{~S}, \mathrm{~S})$ & -4124.865289 & 2.5 & -4125.748144 & 3.1 \\
\hline $\mathrm{TS} 14(\mathrm{~S}, \mathrm{~S})$ & -4124.864335 & 3.1 & -4125.747276 & 3.6 \\
\hline TS19(S,S) & -4124.864146 & 3.2 & -4125.747141 & 3.7 \\
\hline $\mathrm{TS} 22(\mathrm{~S}, \mathrm{~S})$ & -4124.861589 & 4.8 & -4125.744234 & 5.5 \\
\hline $\mathrm{TS} 23(\mathrm{~S}, \mathrm{~S})$ & -4124.861104 & 5.1 & -4125.744800 & 5.2 \\
\hline $\mathrm{TS} 15(\mathrm{~S}, \mathrm{~S})$ & -4124.860775 & 5.3 & -4125.743412 & 6.0 \\
\hline $\mathrm{TS} 24(\mathrm{~S}, \mathrm{~S})$ & -4124.860187 & 5.7 & -4125.743802 & 5.8 \\
\hline $\mathrm{TS} 20(\mathrm{~S}, \mathrm{~S})$ & -4124.860117 & 5.7 & -4125.742806 & 6.4 \\
\hline
\end{tabular}

Table S9. Absolute free energies (298K, in hartrees) at the B97-D/def2-TZVP and B97D3/def2-TZVP//B97-D/def2-TZVP levels of theory for all transition states for reaction 1 catalyzed by PA-1B with free energies relative to the lowest-lying $(\mathrm{R}, \mathrm{R})$ transition state $(\mathrm{kcal} / \mathrm{mol})$. The standard RRHO model was used to compute the free energies.

\begin{tabular}{lcccc}
\hline TS Name & G[B97-D/def2-TZVP] & $\mathbf{G}_{\text {rel }}$ & $\begin{array}{c}\text { G[B97-D3/def2-TZVP// } \\
\text { B97-D/def2-TZVP] }\end{array}$ & $\mathbf{G}_{\text {rel }}$ \\
\hline (R,R) Transition States & & & & 0.0 \\
\hline TS25(R,R) & -3918.079799 & 0.0 & -3918.354673 & \\
\hline (S,S) Transition States & & & & \\
\hline
\end{tabular}


Table S10. Absolute free energies (298K, in hartrees) at the B97-D/def2-TZVP and B97D3/def2-TZVP//B97-D/def2-TZVP levels of theory for all transition states for reaction 1 catalyzed by PA-1B with free energies relative to the lowest-lying $(\mathrm{R}, \mathrm{R})$ transition state $(\mathrm{kcal} / \mathrm{mol})$. The Quasi-RRHO model was used to compute the free energies. The lowest-lying $(\mathrm{R}, \mathrm{R})$ and $(\mathrm{S}, \mathrm{S})$ transition states at the B97-D3/def2-TZVP//B97-D/def2-TZVP level are in bold.

\begin{tabular}{ccccc}
\hline TS Name & G[B97-D/def2-TZVP] & $\mathbf{G}_{\text {rel }}$ & $\begin{array}{c}\text { G[B97-D3/def2-TZVP// } \\
\text { B97-D/def2-TZVP] }\end{array}$ & $\mathbf{G}_{\text {rel }}$ \\
\hline (R,R) Transition States & & & & \\
\hline TS25(R,R) & $\mathbf{- 3 9 1 8 . 0 6 7 0 7}$ & $\mathbf{0 . 0}$ & $\mathbf{- 3 9 1 8 . 3 4 1 9 5}$ & $\mathbf{0 . 0}$ \\
\hline (S,S) Transition States & & & & \\
\hline TS26(S,S) & $\mathbf{- 3 9 1 8 . 0 6 7 4 2}$ & $\mathbf{- 0 . 2}$ & $\mathbf{- 3 9 1 8 . 3 4 2 4 7}$ & $\mathbf{- 0 . 3}$ \\
TS27(S,S) & -3918.06275 & 2.7 & -3918.33723 & 3.0
\end{tabular}

Table S11. Absolute free energies (298K, in hartrees) at the wB97XD/6-31G(d) and wB97XD/6-311+G(d,p)// wB97XD/6-31G(d) levels of theory for all transition states for reaction 1 catalyzed by $\mathrm{PA}-1 \mathrm{~B}$ with free energies relative to the lowest-lying $(\mathrm{R}, \mathrm{R})$ transition state $(\mathrm{kcal} / \mathrm{mol})$. The standard RRHO model was used to compute the free energies.

\begin{tabular}{ccccc}
\hline TS Name & G[wB97XD/6-31G(d)] & $\mathbf{G}_{\text {rel }}$ & $\begin{array}{c}\text { G[wB97XD/6-311+G(d,p)// } \\
\mathbf{w B 9 7 X D / 6 - 3 1 G ( d ) ]}\end{array}$ & $\mathbf{G}_{\text {rel }}$ \\
\hline (R,R) Transition States & & & & \\
\hline TS25(R,R) & -3918.022802 & 0.0 & -3918.742447 & 0.0 \\
\hline (S,S) Transition States & & & & 1.5 \\
\hline TS26(S,S) & -3918.020296 & 1.6 & -3918.740076 & 4.4 \\
TS27(S,S) & -3918.016072 & 4.2 & -3918.735467 &
\end{tabular}

Table S12. Absolute free energies (298K, in hartrees) at the wB97XD/6-31G(d) and wB97XD/6-311+G(d,p)// wB97XD/6-31G(d) levels of theory for all transition states for reaction 1 catalyzed by PA-1B with free energies relative to the lowest-lying $(R, R)$ transition state $(\mathrm{kcal} / \mathrm{mol})$. The Quasi-RRHO model was used to compute the free energies.

\begin{tabular}{ccccc}
\hline TS Name & G[wB97XD/6-31G(d)] & $\mathbf{G}_{\text {rel }}$ & $\begin{array}{c}\text { G[wB97XD/6-311+G(d,p)// } \\
\mathbf{w B 9 7 X D / 6 - 3 1 G ( d ) ]}\end{array}$ & $\mathbf{G}_{\text {rel }}$ \\
\hline (R,R) Transition States & & & & \\
\hline TS25(R,R) & -3918.011141 & 0.0 & -3918.730786 & 0.0 \\
\hline (S,S) Transition States & & & & \\
\hline TS26(S,S) & -3918.008971 & 1.4 & -3918.728751 & 1.3 \\
TS27(S,S) & -3918.003814 & 4.6 & -3918.723209 & 4.8
\end{tabular}

Table S13. Absolute free energies (298K, in hartrees) at the M06-2X/6-31G(d) and M062X/6-311+G(d,p)// M06-2X/6-31G(d) levels of theory for all transition states for reaction 1 catalyzed by PA-1B with free energies relative to the lowest-lying $(R, R)$ transition state $(\mathrm{kcal} / \mathrm{mol})$. The standard RRHO model was used to compute the free energies. 


\begin{tabular}{ccccc}
\hline TS Name & G[M06-2X/6-31G(d)] & $\mathbf{G}_{\text {rel }}$ & $\begin{array}{c}\text { G[M06-2X/6-311+G(d,p)// } \\
\text { M06-2X/6-31G(d)] }\end{array}$ & $\mathbf{G}_{\text {rel }}$ \\
\hline (R,R) Transition States & & & & \\
\hline TS25(R,R) & -3917.747393 & 0.0 & -3918.547422 & 0.0 \\
\hline (S,S) Transition States & & & & -0.6 \\
\hline TS26(S,S) & -3917.748198 & -0.5 & -3918.548442 & 1.4 \\
TS27(S,S) & -3917.744935 & 1.5 & -3918.545111 &
\end{tabular}

Table S14. Absolute free energies (298K, in hartrees) at the M06-2X/6-31G(d) and M062X/6-311+G(d,p)// M06-2X/6-31G(d) levels of theory for all transition states for reaction 1 catalyzed by PA-1B with free energies relative to the lowest-lying $(\mathrm{R}, \mathrm{R})$ transition state $(\mathrm{kcal} / \mathrm{mol})$. The Quasi-RRHO model was used to compute the free energies.

\begin{tabular}{ccccc}
\hline TS Name & G[M06-2X/6-31G(d)] & $\mathbf{G}_{\text {rel }}$ & $\begin{array}{c}\text { G[M06-2X/6-311+G(d,p)// } \\
\text { M06-2X/6-31G(d)] }\end{array}$ & $\mathbf{G}_{\text {rel }}$ \\
\hline (R,R) Transition States & & & & \\
\hline TS25(R,R) & -3917.736216 & 0.0 & -3918.536245 & 0.0 \\
\hline (S,S) Transition States & & & & -0.2 \\
\hline TS26(S,S) & -3917.736351 & -0.1 & -3918.536595 & 2.1 \\
TS27(S,S) & -3917.7327 & 2.2 & -3918.532876 &
\end{tabular}

Table S15. Absolute free energies (298K, in hartrees) at the B97-D/def2-TZVP and B97D3/def2-TZVP//B97-D/def2-TZVP levels of theory for all transition states for reaction 1 catalyzed by PA-2 with free energies relative to the lowest-lying $(\mathrm{R}, \mathrm{R})$ transition state $(\mathrm{kcal} / \mathrm{mol})$. The standard RRHO model was used to compute the free energies. TS74(S,S) has two imaginary frequencies.

\begin{tabular}{ccccc}
\hline TS Name & G[B97-D/def2-TZVP] & $\mathbf{G}_{\text {rel }}$ & $\begin{array}{c}\text { G[B97-D3/def2-TZVP// } \\
\text { B97-D/def2-TZVP] }\end{array}$ & $\mathbf{G}_{\text {rel }}$ \\
\hline (R,R) Transition States & & & & \\
\hline TS28(R,R) & -4356.205891 & 0.0 & -4356.496213 & 0.3 \\
TS29(R,R) & -4356.205622 & 0.2 & -4356.495011 & 1.1 \\
TS30(R,R) & -4356.205603 & 0.2 & -4356.494991 & 1.1 \\
TS31(R,R) & -4356.205553 & 0.2 & -4356.496700 & 0.0 \\
TS32(R,R) & -4356.205551 & 0.2 & -4356.496698 & 0.0 \\
TS33(R,R) & -4356.205092 & 0.5 & -4356.495776 & 0.6 \\
TS34(R,R) & -4356.205091 & 0.5 & -4356.495775 & 0.6 \\
TS35(R,R) & -4356.204969 & 0.6 & -4356.494558 & 1.3 \\
TS36(R,R) & -4356.204803 & 0.7 & -4356.494400 & 1.4 \\
TS37(R,R) & -4356.204767 & 0.7 & -4356.495801 & 0.6 \\
TS38(R,R) & -4356.204764 & 0.7 & -4356.495795 & 0.6 \\
TS39(R,R) & -4356.204725 & 0.7 & -4356.495309 & 0.9 \\
TS40(R,R) & -4356.204684 & 0.8 & -4356.494399 & 1.4 \\
TS41(R,R) & -4356.204670 & 0.8 & -4356.494385 & 1.5 \\
TS42(R,R) & -4356.204549 & 0.8 & -4356.494975 & 1.1 \\
TS43(R,R) & -4356.204418 & 0.9 & -4356.495088 & 1.0 \\
TS44(R,R) & -4356.204205 & 1.1 & -4356.493785 & 1.8 \\
TS45(R,R) & -4356.204146 & 1.1 & -4356.494859 & 1.2 \\
TS46(R,R) & -4356.204145 & 1.1 & -4356.494857 & 1.2
\end{tabular}




\begin{tabular}{|c|c|c|c|c|}
\hline TS47(R,R) & -4356.204009 & 1.2 & -4356.494300 & 1.5 \\
\hline TS48(R,R) & -4356.203741 & 1.3 & -4356.494946 & 1.1 \\
\hline TS49(R,R) & -4356.203726 & 1.4 & -4356.494931 & 1.1 \\
\hline TS50(R,R) & -4356.203587 & 1.4 & -4356.493839 & 1.8 \\
\hline TS51(R,R) & -4356.202330 & 2.2 & -4356.491488 & 3.3 \\
\hline TS52(R,R) & -4356.202329 & 2.2 & -4356.491486 & 3.3 \\
\hline TS53(R,R) & -4356.202253 & 2.3 & -4356.492003 & 2.9 \\
\hline TS54(R,R) & -4356.202253 & 2.3 & -4356.492003 & 2.9 \\
\hline TS55(R,R) & -4356.201895 & 2.5 & -4356.491112 & 3.5 \\
\hline TS56(R,R) & -4356.201895 & 2.5 & -4356.491112 & 3.5 \\
\hline TS57(R,R) & -4356.201862 & 2.5 & -4356.491018 & 3.6 \\
\hline TS58(R,R) & -4356.201588 & 2.7 & -4356.489803 & 4.3 \\
\hline TS59(R,R) & -4356.201422 & 2.8 & -4356.491125 & 3.5 \\
\hline \multicolumn{5}{|c|}{$(\mathbf{S , S})$ Transition States } \\
\hline TS60(S,S) & -4356.206312 & -0.3 & -4356.496854 & $\overline{-0.1}$ \\
\hline TS61(S,S) & -4356.205963 & 0.0 & -4356.496256 & 0.3 \\
\hline TS62(S,S) & -4356.205630 & 0.2 & -4356.496168 & 0.3 \\
\hline TS63(S,S) & -4356.205478 & 0.3 & -4356.495830 & 0.5 \\
\hline TS64(S,S) & -4356.205478 & 0.3 & -4356.495830 & 0.5 \\
\hline TS65(S,S) & -4356.204691 & 0.8 & -4356.494252 & 1.5 \\
\hline TS66(S,S) & -4356.204555 & 0.8 & -4356.494098 & 1.6 \\
\hline TS67(S,S) & -4356.204096 & 1.1 & -4356.494535 & 1.4 \\
\hline TS68(S,S) & -4356.204001 & 1.2 & -4356.494410 & 1.4 \\
\hline TS69(S,S) & -4356.203934 & 1.2 & -4356.494513 & 1.4 \\
\hline TS70(S,S) & -4356.203749 & 1.3 & -4356.494166 & 1.6 \\
\hline TS71(S,S) & -4356.203746 & 1.3 & -4356.493434 & 2.0 \\
\hline TS72(S,S) & -4356.203745 & 1.3 & -4356.493433 & 2.0 \\
\hline TS73(S,S) & -4356.203421 & 1.5 & -4356.493950 & 1.7 \\
\hline TS74(S,S) & -4356.202716 & 2.0 & -4356.492758 & 2.5 \\
\hline TS75(S,S) & -4356.202611 & 2.1 & -4356.492230 & 2.8 \\
\hline TS76(S,S) & -4356.202611 & 2.1 & -4356.492230 & 2.8 \\
\hline TS77(S,S) & -4356.202575 & 2.1 & -4356.492151 & 2.9 \\
\hline TS78(S,S) & -4356.202563 & 2.1 & -4356.492138 & 2.9 \\
\hline TS79(S,S) & -4356.202523 & 2.1 & -4356.493444 & 2.0 \\
\hline TS80(S,S) & -4356.202216 & 2.3 & -4356.491941 & 3.0 \\
\hline TS81(S,S) & -4356.202005 & 2.4 & -4356.490806 & 3.7 \\
\hline TS82(S,S) & -4356.201999 & 2.4 & -4356.490799 & 3.7 \\
\hline TS83(S,S) & -4356.201934 & 2.5 & -4356.492783 & 2.5 \\
\hline TS84(S,S) & -4356.201933 & 2.5 & -4356.492784 & 2.5 \\
\hline TS85(S,S) & -4356.201773 & 2.6 & -4356.490105 & 4.1 \\
\hline TS86(S,S) & -4356.201735 & 2.6 & -4356.491295 & 3.4 \\
\hline TS87(S,S) & -4356.201732 & 2.6 & -4356.491292 & 3.4 \\
\hline TS88(S,S) & -4356.201441 & 2.8 & -4356.492060 & 2.9 \\
\hline TS89(S,S) & -4356.201441 & 2.8 & -4356.492060 & 2.9 \\
\hline TS90(S,S) & -4356.200535 & 3.4 & -4356.490987 & 3.6 \\
\hline TS91(S,S) & -4356.198812 & 4.4 & -4356.488017 & 5.4 \\
\hline
\end{tabular}

Table S16. Absolute free energies (298K, in hartrees) at the B97-D/def2-TZVP and B97D3/def2-TZVP//B97-D/def2-TZVP levels of theory for all transition states for reaction 1 catalyzed by PA- 2 with free energies relative to the lowest-lying $(R, R)$ transition state $(\mathrm{kcal} / \mathrm{mol})$. The Quasi-RRHO model was used to compute the free energies. TS74(S,S) has two imaginary frequencies. The lowest-lying $(\mathrm{R}, \mathrm{R})$ and $(\mathrm{S}, \mathrm{S})$ transition states at the B97D3/def2-TZVP//B97-D/def2-TZVP level are in bold. 


\begin{tabular}{|c|c|c|c|c|}
\hline TS Name & G[B97-D/def2-TZVP] & $\mathbf{G}_{\text {rel }}$ & $\begin{array}{c}\text { G[B97-D3/def2-TZVP// } \\
\text { B97-D/def2-TZVP] }\end{array}$ & $\mathbf{G}_{\text {rel }}$ \\
\hline \multicolumn{5}{|c|}{$(\mathbf{R}, \mathbf{R})$ Transition States } \\
\hline TS35(R,R) & -4356.191014 & 0.0 & -4356.480603 & 0.4 \\
\hline TS36(R,R) & -4356.190952 & 0.0 & -4356.480548 & 0.5 \\
\hline TS28(R,R) & -4356.190942 & $\mathbf{0 . 0}$ & -4356.481265 & $\mathbf{0 . 0}$ \\
\hline TS29(R,R) & -4356.190779 & 0.1 & -4356.480168 & 0.7 \\
\hline TS30(R,R) & -4356.190767 & 0.2 & -4356.480155 & 0.7 \\
\hline TS40(R,R) & -4356.190331 & 0.4 & -4356.480046 & 0.8 \\
\hline TS44(R,R) & -4356.190326 & 0.4 & -4356.479906 & 0.9 \\
\hline TS41(R,R) & -4356.190324 & 0.4 & -4356.480039 & 0.8 \\
\hline TS34(R,R) & -4356.190263 & 0.5 & -4356.480946 & 0.2 \\
\hline TS33(R,R) & -4356.190263 & 0.5 & -4356.480946 & 0.2 \\
\hline TS43(R,R) & -4356.1902 & 0.5 & -4356.48087 & 0.2 \\
\hline TS42(R,R) & -4356.190179 & 0.5 & -4356.480605 & 0.4 \\
\hline TS39(R,R) & -4356.19017 & 0.5 & -4356.480753 & 0.3 \\
\hline TS31(R,R) & -4356.19001 & 0.6 & -4356.481158 & 0.1 \\
\hline TS32(R,R) & -4356.190009 & 0.6 & -4356.481156 & 0.1 \\
\hline TS37(R,R) & -4356.18969 & 0.8 & -4356.480724 & 0.3 \\
\hline TS38(R,R) & -4356.18969 & 0.8 & -4356.480721 & 0.3 \\
\hline TS47(R,R) & -4356.189685 & 0.8 & -4356.479976 & 0.8 \\
\hline TS50(R,R) & -4356.189506 & 0.9 & -4356.479758 & 0.9 \\
\hline TS46(R,R) & -4356.189269 & 1.1 & -4356.479981 & 0.8 \\
\hline TS45(R,R) & -4356.189269 & 1.1 & -4356.479982 & 0.8 \\
\hline TS53(R,R) & -4356.188802 & 1.4 & -4356.478552 & 1.7 \\
\hline TS54(R,R) & -4356.188802 & 1.4 & -4356.478552 & 1.7 \\
\hline TS56(R,R) & -4356.188527 & 1.6 & -4356.477744 & 2.2 \\
\hline TS55(R,R) & -4356.188527 & 1.6 & -4356.477744 & 2.2 \\
\hline TS52(R,R) & -4356.188384 & 1.7 & -4356.477541 & 2.3 \\
\hline TS51(R,R) & -4356.188384 & 1.7 & -4356.477542 & 2.3 \\
\hline TS48(R,R) & -4356.188333 & 1.7 & -4356.479538 & 1.1 \\
\hline TS49(R,R) & -4356.188324 & 1.7 & -4356.479529 & 1.1 \\
\hline TS58(R,R) & -4356.187833 & 2.0 & -4356.476048 & 3.3 \\
\hline TS57(R,R) & -4356.187341 & 2.3 & -4356.476497 & 3.0 \\
\hline TS59(R,R) & -4356.187092 & 2.5 & -4356.476795 & 2.8 \\
\hline \multicolumn{5}{|c|}{$(\mathrm{S}, \mathrm{S})$ Transition States } \\
\hline TS65(S,S) & -4356.190339 & 0.4 & -4356.4799 & 0.9 \\
\hline TS66(S,S) & -4356.190246 & 0.5 & -4356.479788 & 0.9 \\
\hline TS61(S,S) & -4356.190238 & 0.5 & -4356.48053 & 0.5 \\
\hline TS60(S,S) & -4356.190058 & 0.6 & -4356.480601 & 0.4 \\
\hline TS63(S,S) & -4356.189993 & 0.6 & -4356.480345 & 0.6 \\
\hline TS64(S,S) & -4356.189993 & 0.6 & -4356.480345 & 0.6 \\
\hline TS67(S,S) & -4356.189743 & 0.8 & -4356.480182 & 0.7 \\
\hline TS62(S,S) & -4356.189713 & 0.8 & -4356.480251 & 0.6 \\
\hline TS69(S,S) & -4356.18953 & 0.9 & -4356.48011 & 0.7 \\
\hline TS68(S,S) & -4356.189506 & 0.9 & -4356.479915 & 0.8 \\
\hline TS74(S,S) & -4356.189333 & 1.1 & -4356.479374 & 1.2 \\
\hline TS70(S,S) & -4356.189258 & 1.1 & -4356.479676 & 1.0 \\
\hline $\mathrm{TS} 72(\mathrm{~S}, \mathrm{~S})$ & -4356.189111 & 1.2 & -4356.4788 & 1.5 \\
\hline TS71(S,S) & -4356.189111 & 1.2 & -4356.4788 & 1.5 \\
\hline TS73(S,S) & -4356.189105 & 1.2 & -4356.479634 & 1.0 \\
\hline TS75(S,S) & -4356.188801 & 1.4 & -4356.47842 & 1.8 \\
\hline
\end{tabular}




$\begin{array}{lllll}\text { TS76(S,S) } & -4356.188801 & 1.4 & -4356.47842 & 1.8 \\ \text { TS77(S,S) } & -4356.18849 & 1.6 & -4356.478066 & 2.0 \\ \text { TS78(S,S) } & -4356.188483 & 1.6 & -4356.478058 & 2.0 \\ \text { TS86(S,S) } & -4356.187869 & 2.0 & -4356.477429 & 2.4 \\ \text { TS87(S,S) } & -4356.187867 & 2.0 & -4356.477427 & 2.4 \\ \text { TS81(S,S) } & -4356.18762 & 2.1 & -4356.476421 & 3.0 \\ \text { TS82(S,S) } & -4356.187615 & 2.1 & -4356.476415 & 3.0 \\ \text { TS83(S,S) } & -4356.187613 & 2.1 & -4356.478462 & 1.8 \\ \text { TS84(S,S) } & -4356.187612 & 2.1 & -4356.478463 & 1.8 \\ \text { TS85(S,S) } & -4356.187488 & 2.2 & -4356.47582 & 3.4 \\ \text { TS79(S,S) } & -4356.186949 & 2.6 & -4356.477869 & 2.1 \\ \text { TS89(S,S) } & -4356.18687 & 2.6 & -4356.477489 & 2.4 \\ \text { TS88(S,S) } & -4356.186869 & 2.6 & -4356.477487 & 3.0 \\ \text { TS80(S,S) } & -4356.186838 & 2.6 & -4356.476563 & 3.2 \\ \text { TS90(S,S) } & -4356.185682 & 3.3 & -4356.476133 & 4.4 \\ \text { TS91(S,S) } & -4356.185106 & 3.7 & -4356.474311 & \end{array}$

Table S17. Absolute free energies (298K, in hartrees) at the B97-D/def2-TZVP and B97D3/def2-TZVP//B97-D/def2-TZVP levels of theory for all transition states for reaction 2a catalyzed by PA-1A with free energies relative to the lowest-lying $(\mathrm{R}, \mathrm{R})$ transition state $(\mathrm{kcal} / \mathrm{mol})$. The standard RRHO model was used to compute the free energies. TS116(R,R) has two imaginary frequencies.

\begin{tabular}{ccccc}
\hline TS Name & G[B97-D/def2-TZVP] & $\mathbf{G}_{\text {rel }}$ & $\begin{array}{c}\text { G[B97-D3/def2-TZVP// } \\
\text { B97-D/def2-TZVP] }\end{array}$ & $\mathbf{G}_{\text {rel }}$ \\
\hline (R,R) Transition States & & & & \\
\hline TS92(R,R) & -3310.754535 & 0.0 & -3311.001018 & 0.0 \\
TS93(R,R) & -3310.754388 & 0.1 & -3311.001054 & 0.0 \\
TS94(R,R) & -3310.754246 & 0.2 & -3311.000968 & 0.1 \\
TS95(R,R) & -3310.754223 & 0.2 & -3311.001047 & 0.0 \\
TS96(R,R) & -3310.753983 & 0.3 & -3311.000125 & 0.6 \\
TS97(R,R) & -3310.753199 & 0.8 & -3310.999860 & 0.7 \\
TS98(R,R) & -3310.753158 & 0.9 & -3311.000345 & 0.4 \\
TS99(R,R) & -3310.752810 & 1.1 & -3311.000458 & 0.4 \\
TS100(R,R) & -3310.752495 & 1.3 & -3310.998960 & 1.3 \\
TS101(R,R) & -3310.752355 & 1.4 & -3310.999972 & 0.7 \\
TS102(R,R) & -3310.752355 & 1.4 & -3310.999972 & 0.7 \\
TS103(R,R) & -3310.752107 & 1.5 & -3310.999135 & 1.2 \\
TS104(R,R) & -3310.752093 & 1.5 & -3310.999375 & 1.1 \\
TS105(R,R) & -3310.751801 & 1.7 & -3310.998034 & 1.9 \\
TS106(R,R) & -3310.751710 & 1.8 & -3310.997931 & 2.0 \\
TS107(R,R) & -3310.751651 & 1.8 & -3310.997717 & 2.1 \\
TS108(R,R) & -3310.751477 & 1.9 & -3310.998272 & 1.7 \\
TS109(R,R) & -3310.751448 & 1.9 & -3310.998453 & 1.6 \\
TS110(R,R) & -3310.751117 & 2.1 & -3310.997725 & 2.1 \\
TS111(R,R) & -3310.751108 & 2.2 & -3310.998375 & 1.7 \\
TS112(R,R) & -3310.750922 & 2.3 & -3310.997233 & 2.4 \\
TS113(R,R) & -3310.750727 & 2.4 & -3310.997591 & 2.2 \\
TS114(R,R) & -3310.750423 & 2.6 & -3310.997432 & 2.3 \\
TS115(R,R) & -3310.750304 & 2.7 & -3310.997264 & 2.4 \\
TS116(R,R) & -3310.749685 & 3.0 & -3310.996241 & 3.0 \\
\hline (S,S) Transition States & & & & \\
\hline
\end{tabular}




$\begin{array}{lllll} & & & & \\ \text { TS117(S,S) } & -3310.755010 & -0.3 & -3311.000896 & 0.1 \\ \text { TS118(S,S) } & -3310.753855 & 0.4 & -3311.001619 & -0.4 \\ \text { TS119(S,S) } & -3310.753388 & 0.7 & -3310.999360 & 1.1 \\ \text { TS120(S,S) } & -3310.752991 & 1.0 & -3310.998781 & 1.4 \\ \text { TS121(S,S) } & -3310.752790 & 1.1 & -3310.998547 & 1.6 \\ \text { TS122(S,S) } & -3310.752642 & 1.2 & -3310.998919 & 1.3 \\ \text { TS123(S,S) } & -3310.752412 & 1.3 & -3310.998711 & 1.5 \\ \text { TS124(S,S) } & -3310.751963 & 1.6 & -3310.997737 & 2.1 \\ \text { TS125(S,S) } & -3310.751127 & 2.1 & -3310.996746 & 2.7 \\ \text { TS126(S,S) } & -3310.751107 & 2.2 & -3310.997317 & 2.3 \\ \text { TS127(S,S) } & -3310.750339 & 2.6 & -3310.995344 & 3.6 \\ \text { TS128(S,S) } & -3310.750154 & 2.7 & -3310.997149 & 2.5 \\ \text { TS129(S,S) } & -3310.750087 & 2.8 & -3310.996723 & 2.7 \\ \text { TS130(S,S) } & -3310.750078 & 2.8 & -3310.996743 & 2.7 \\ \text { TS131(S,S) } & -3310.750047 & 2.8 & -3310.994920 & 3.8 \\ \text { TS132(S,S) } & -3310.749235 & 3.3 & -3310.996703 & 2.7 \\ \text { TS133(S,S) } & -3310.749202 & 3.3 & -3310.993937 & 4.5 \\ \text { TS134(S,S) } & -3310.748890 & 3.5 & -3310.996047 & 3.1 \\ \text { TS135(S,S) } & -3310.748883 & 3.5 & -3310.996489 & 2.9 \\ \text { TS136(S,S) } & -3310.748828 & 3.6 & -3310.994386 & 4.2 \\ \text { TS137(S,S) } & -3310.748734 & 3.6 & -3310.996279 & 3.0 \\ \text { TS138(S,S) } & -3310.748718 & 3.7 & -3310.995129 & 3.7 \\ \text { TS139(S,S) } & -3310.748390 & 3.9 & -3310.993142 & 5.0 \\ \text { TS140(S,S) } & -3310.748264 & 3.9 & -3310.994694 & 4.0 \\ \text { TS141(S,S) } & -3310.747852 & 4.2 & -3310.994408 & 4.2 \\ \text { TS142(S,S) } & -3310.747831 & 4.2 & -3310.995355 & 3.6 \\ \text { TS143(S,S) } & -3310.746529 & 5.0 & -3310.992458 & 5.4 \\ \text { TS144(S,S) } & -3310.746104 & 5.3 & -3310.991172 & 6.2 \\ & & & & \end{array}$

Table S18. Absolute free energies (298K, in hartrees) at the B97-D/def2-TZVP and B97D3/def2-TZVP//B97-D/def2-TZVP levels of theory for all transition states for reaction 2a catalyzed by PA-1A with free energies relative to the lowest-lying $(R, R)$ transition state (kcal/mol). The Quasi-RRHO model was used to compute the free energies. TS116(R,R) has two imaginary frequencies. The lowest-lying $(\mathrm{R}, \mathrm{R})$ and $(\mathrm{S}, \mathrm{S})$ transition states at the B97D3/def2-TZVP//B97-D/def2-TZVP level are in bold.

\begin{tabular}{ccccc}
\hline TS Name & G[B97-D/def2-TZVP] & $\mathbf{G}_{\text {rel }}$ & $\begin{array}{c}\text { G[B97-D3/def2-TZVP// } \\
\text { B97-D/def2-TZVP] }\end{array}$ & $\mathbf{G}_{\text {rel }}$ \\
\hline (R,R) Transition States & & & & \\
\hline TS95(R,R) & $\mathbf{- 3 3 1 0 . 7 3 6 5 4}$ & $\mathbf{0 . 0}$ & $\mathbf{- 3 3 1 0 . 9 8 3 3 7}$ & $\mathbf{0 . 0}$ \\
TS92(R,R) & -3310.73633 & 0.1 & -3310.98282 & 0.3 \\
TS96(R,R) & -3310.7361 & 0.3 & -3310.98224 & 0.7 \\
TS94(R,R) & -3310.73574 & 0.5 & -3310.98247 & 0.6 \\
TS93(R,R) & -3310.73573 & 0.5 & -3310.9824 & 0.6 \\
TS99(R,R) & -3310.73554 & 0.6 & -3310.98319 & 0.1 \\
TS102(R,R) & -3310.73554 & 0.6 & -3310.98315 & 0.1 \\
TS101(R,R) & -3310.73554 & 0.6 & -3310.98315 & 0.1 \\
TS98(R,R) & -3310.73532 & 0.8 & -3310.98251 & 0.5 \\
TS97(R,R) & -3310.73532 & 0.8 & -3310.98198 & 0.9 \\
TS106(R,R) & -3310.73512 & 0.9 & -3310.98134 & 1.3 \\
TS108(R,R) & -3310.73504 & 0.9 & -3310.98184 & 1.0 \\
TS104(R,R) & -3310.73503 & 0.9 & -3310.98232 & 0.7
\end{tabular}




\begin{tabular}{|c|c|c|c|c|}
\hline $\mathrm{TS} 100(\mathrm{R}, \mathrm{R})$ & -3310.73503 & 1.0 & -3310.98149 & 1.2 \\
\hline TS107(R,R) & -3310.73502 & 1.0 & -3310.98108 & 1.4 \\
\hline TS112(R,R) & -3310.73472 & 1.1 & -3310.98103 & 1.5 \\
\hline TS103(R,R) & -3310.73461 & 1.2 & -3310.98164 & 1.1 \\
\hline TS105(R,R) & -3310.73458 & 1.2 & -3310.98081 & 1.6 \\
\hline TS109(R,R) & -3310.73454 & 1.3 & -3310.98155 & 1.1 \\
\hline TS111(R,R) & -3310.73434 & 1.4 & -3310.98161 & 1.1 \\
\hline TS110(R,R) & -3310.73423 & 1.5 & -3310.98084 & 1.6 \\
\hline TS113(R,R) & -3310.73403 & 1.6 & -3310.9809 & 1.5 \\
\hline TS114(R,R) & -3310.73392 & 1.6 & -3310.98093 & 1.5 \\
\hline TS115(R,R) & -3310.73379 & 1.7 & -3310.98075 & 1.6 \\
\hline TS116(R,R) & -3310.73367 & 1.8 & -3310.98022 & 2.0 \\
\hline \multicolumn{5}{|c|}{$(\mathbf{S}, \mathbf{S})$ Transition States } \\
\hline TS117(S,S) & -3310.73649 & 0.0 & -3310.98238 & 0.6 \\
\hline TS119(S,S) & -3310.73571 & 0.5 & -3310.98168 & 1.1 \\
\hline $\mathrm{TS} 120(\mathrm{~S}, \mathrm{~S})$ & -3310.73531 & 0.8 & -3310.9811 & 1.4 \\
\hline TS121(S,S) & -3310.7353 & 0.8 & -3310.98105 & 1.5 \\
\hline TS118(S,S) & -3310.73477 & 1.1 & -3310.98254 & 0.5 \\
\hline TS122(S,S) & -3310.73471 & 1.2 & -3310.98099 & 1.5 \\
\hline TS124(S,S) & -3310.73471 & 1.2 & -3310.98048 & 1.8 \\
\hline TS123(S,S) & -3310.73459 & 1.2 & -3310.98089 & 1.6 \\
\hline TS127(S,S) & -3310.73446 & 1.3 & -3310.97946 & 2.4 \\
\hline $\mathrm{TS} 125(\mathrm{~S}, \mathrm{~S})$ & -3310.73416 & 1.5 & -3310.97978 & 2.3 \\
\hline TS133(S,S) & -3310.73388 & 1.7 & -3310.97862 & 3.0 \\
\hline TS126(S,S) & -3310.73386 & 1.7 & -3310.98007 & 2.1 \\
\hline TS130(S,S) & -3310.73356 & 1.9 & -3310.98022 & 2.0 \\
\hline TS128(S,S) & -3310.73345 & 1.9 & -3310.98045 & 1.8 \\
\hline TS131(S,S) & -3310.73336 & 2.0 & -3310.97823 & 3.2 \\
\hline TS136(S,S) & -3310.73302 & 2.2 & -3310.97858 & 3.0 \\
\hline TS129(S,S) & -3310.73257 & 2.5 & -3310.9792 & 2.6 \\
\hline TS139(S,S) & -3310.73246 & 2.6 & -3310.97721 & 3.9 \\
\hline TS132(S,S) & -3310.73228 & 2.7 & -3310.97975 & 2.3 \\
\hline TS135(S,S) & -3310.73205 & 2.8 & -3310.97965 & 2.3 \\
\hline TS134(S,S) & -3310.73174 & 3.0 & -3310.9789 & 2.8 \\
\hline TS138(S,S) & -3310.73169 & 3.0 & -3310.9781 & 3.3 \\
\hline TS142(S,S) & -3310.73164 & 3.1 & -3310.97916 & 2.6 \\
\hline TS137(S,S) & -3310.73161 & 3.1 & -3310.97916 & 2.6 \\
\hline TS140(S,S) & -3310.73149 & 3.2 & -3310.97792 & 3.4 \\
\hline TS141(S,S) & -3310.73117 & 3.4 & -3310.97773 & 3.5 \\
\hline TS144(S,S) & -3310.7307 & 3.7 & -3310.97577 & 4.8 \\
\hline TS143(S,S) & -3310.72966 & 4.3 & -3310.97559 & 4.9 \\
\hline
\end{tabular}

Table S19. Absolute free energies (298K, in hartrees) at the wB97XD/6-31G(d) and wB97XD/6-311+G(d,p)//wB97XD/6-31G(d) levels of theory for all transition states for reaction 2a catalyzed by PA-1A with free energies relative to the lowest-lying $(R, R)$ transition state $(\mathrm{kcal} / \mathrm{mol})$. The standard RRHO model was used to compute the free energies. TS133(S,S) has two imaginary frequencies.

\begin{tabular}{ccccc}
\hline TS Name & G[wB97XD/6-31G(d)] & Grel $_{\text {r }}$ & $\begin{array}{c}\text { G[wB97XD/6-311+G(d,p)// } \\
\text { wB97XD/6-31G(d)] }\end{array}$ & $\mathbf{G}_{\text {rel }}$ \\
\hline (R,R) Transition States & & & & \\
\hline TS106(R,R $)$ & -3310.758794 & 0.0 & -3311.504127 & 0.0
\end{tabular}




\begin{tabular}{|c|c|c|c|c|}
\hline TS110(R,R) & -3310.758758 & 0.0 & -3311.50409 & 0.0 \\
\hline TS105(R,R) & -3310.757311 & 0.9 & -3311.503166 & 0.6 \\
\hline TS100(R,R) & -3310.757089 & 1.1 & -3311.502686 & 0.9 \\
\hline TS112(R,R) & -3310.756742 & 1.3 & -3311.501064 & 1.9 \\
\hline TS116(R,R) & -3310.756723 & 1.3 & -3311.50229 & 1.2 \\
\hline TS108(R,R) & -3310.756706 & 1.3 & -3311.501993 & 1.3 \\
\hline TS95(R,R) & -3310.756673 & 1.3 & -3311.501983 & 1.3 \\
\hline TS96(R,R) & -3310.756486 & 1.4 & -3311.502343 & 1.1 \\
\hline TS92(R,R) & -3310.756396 & 1.5 & -3311.502304 & 1.1 \\
\hline TS97(R,R) & -3310.756177 & 1.6 & -3311.502258 & 1.2 \\
\hline TS104(R,R) & -3310.756032 & 1.7 & -3311.501285 & 1.8 \\
\hline TS101(R,R) & -3310.755925 & 1.8 & -3311.501073 & 1.9 \\
\hline TS102(R,R) & -3310.75588 & 1.8 & -3311.501028 & 1.9 \\
\hline TS99(R,R) & -3310.755617 & 2.0 & -3311.500798 & 2.1 \\
\hline TS98(R,R) & -3310.755345 & 2.2 & -3311.50062 & 2.2 \\
\hline TS94(R,R) & -3310.755298 & 2.2 & -3311.501371 & 1.7 \\
\hline TS93(R,R) & -3310.755282 & 2.2 & -3311.501355 & 1.7 \\
\hline TS107(R,R) & -3310.754525 & 2.7 & -3311.499859 & 2.7 \\
\hline TS103(R,R) & -3310.754004 & 3.0 & -3311.499508 & 2.9 \\
\hline TS111(R,R) & -3310.753089 & 3.6 & -3311.498554 & 3.5 \\
\hline TS109(R,R) & -3310.753063 & 3.6 & -3311.498528 & 3.5 \\
\hline TS113(R,R) & -3310.753046 & 3.6 & -3311.498514 & 3.5 \\
\hline TS114(R,R) & -3310.752023 & 4.2 & -3311.49751 & 4.2 \\
\hline TS115(R,R) & -3310.751806 & 4.4 & -3311.497292 & 4.3 \\
\hline \multicolumn{5}{|c|}{ (S,S) Transition States } \\
\hline TS119(S,S) & -3310.757176 & 1.0 & -3311.50312 & $\overline{0.6}$ \\
\hline $\mathrm{TS} 124(\mathrm{~S}, \mathrm{~S})$ & -3310.756558 & 1.4 & -3311.502586 & 1.0 \\
\hline $\mathrm{TS} 121(\mathrm{~S}, \mathrm{~S})$ & -3310.756355 & 1.5 & -3311.502258 & 1.2 \\
\hline $\mathrm{TS} 120(\mathrm{~S}, \mathrm{~S})$ & -3310.756346 & 1.5 & -3311.502249 & 1.2 \\
\hline $\mathrm{TS} 117(\mathrm{~S}, \mathrm{~S})$ & -3310.756309 & 1.6 & -3311.502212 & 1.2 \\
\hline $\mathrm{TS} 123(\mathrm{~S}, \mathrm{~S})$ & -3310.756029 & 1.7 & -3311.502337 & 1.1 \\
\hline $\mathrm{TS} 122(\mathrm{~S}, \mathrm{~S})$ & -3310.75601 & 1.7 & -3311.502319 & 1.1 \\
\hline $\mathrm{TS} 126(\mathrm{~S}, \mathrm{~S})$ & -3310.75596 & 1.8 & -3311.50226 & 1.2 \\
\hline $\mathrm{TS} 130(\mathrm{~S}, \mathrm{~S})$ & -3310.755356 & 2.2 & -3311.501051 & 1.9 \\
\hline TS129(S,S) & -3310.755173 & 2.3 & -3311.500755 & 2.1 \\
\hline $\mathrm{TS} 128(\mathrm{~S}, \mathrm{~S})$ & -3310.754759 & 2.5 & -3311.500168 & 2.5 \\
\hline $\mathrm{TS} 132(\mathrm{~S}, \mathrm{~S})$ & -3310.754706 & 2.6 & -3311.499952 & 2.6 \\
\hline $\mathrm{TS} 142(\mathrm{~S}, \mathrm{~S})$ & -3310.754703 & 2.6 & -3311.499948 & 2.6 \\
\hline $\mathrm{TS} 125(\mathrm{~S}, \mathrm{~S})$ & -3310.754391 & 2.8 & -3311.500667 & 2.2 \\
\hline TS136(S,S) & -3310.75371 & 3.2 & -3311.499973 & 2.6 \\
\hline $\mathrm{TS} 141(\mathrm{~S}, \mathrm{~S})$ & -3310.753018 & 3.6 & -3311.498716 & 3.4 \\
\hline $\mathrm{TS} 144(\mathrm{~S}, \mathrm{~S})$ & -3310.752555 & 3.9 & -3311.496513 & 4.8 \\
\hline TS143(S,S) & -3310.752505 & 3.9 & -3311.498744 & 3.4 \\
\hline $\mathrm{TS} 127(\mathrm{~S}, \mathrm{~S})$ & -3310.752357 & 4.0 & -3311.497168 & 4.4 \\
\hline TS137(S,S) & -3310.752356 & 4.0 & -3311.497691 & 4.0 \\
\hline $\mathrm{TS} 135(\mathrm{~S}, \mathrm{~S})$ & -3310.751895 & 4.3 & -3311.49714 & 4.4 \\
\hline $\mathrm{TS} 118(\mathrm{~S}, \mathrm{~S})$ & -3310.751174 & 4.8 & -3311.496517 & 4.8 \\
\hline TS138(S,S) & -3310.751155 & 4.8 & -3311.496874 & 4.6 \\
\hline TS139(S,S) & -3310.750794 & 5.0 & -3311.495944 & 5.1 \\
\hline $\mathrm{TS} 131(\mathrm{~S}, \mathrm{~S})$ & -3310.75027 & 5.3 & -3311.495133 & 5.6 \\
\hline $\mathrm{TS} 140(\mathrm{~S}, \mathrm{~S})$ & -3310.750176 & 5.4 & -3311.495892 & 5.2 \\
\hline $\mathrm{TS} 134(\mathrm{~S}, \mathrm{~S})$ & -3310.749959 & 5.5 & -3311.495373 & 5.5 \\
\hline TS133(S,S) & -3310.749485 & 5.8 & -3311.494223 & 6.2 \\
\hline
\end{tabular}


Table S20. Absolute free energies (298K, in hartrees) at the wB97XD/6-31G(d) and wB97XD/6-311+G(d,p)//wB97XD/6-31G(d) levels of theory for all transition states for reaction 2a catalyzed by PA-1A with free energies relative to the lowest-lying $(R, R)$ transition state $(\mathrm{kcal} / \mathrm{mol})$. The standard RRHO model was used to compute the free energies. TS133(S,S) has two imaginary frequencies.

\begin{tabular}{|c|c|c|c|c|}
\hline TS Name & G[wB97XD/6-31G(d)] & $\mathbf{G}_{\text {rel }}$ & $\begin{array}{c}\text { G[wB97XD/6-311+G(d,p)// } \\
\text { wB97XD/6-31G(d)] }\end{array}$ & $\mathbf{G}_{\text {rel }}$ \\
\hline \multicolumn{5}{|c|}{$(\mathbf{R}, \mathbf{R})$ Transition States } \\
\hline TS106(R,R) & -3310.743306 & 0.0 & -3311.488638 & 0.0 \\
\hline TS110(R,R) & -3310.743287 & 0.0 & -3311.488619 & 0.0 \\
\hline $\mathrm{TS} 112(\mathrm{R}, \mathrm{R})$ & -3310.74208 & 0.8 & -3311.486403 & 1.4 \\
\hline TS95(R,R) & -3310.741818 & 0.9 & -3311.487128 & 0.9 \\
\hline TS108(R,R) & -3310.741679 & 1.0 & -3311.486966 & 1.0 \\
\hline TS101(R,R) & -3310.741676 & 1.0 & -3311.486824 & 1.1 \\
\hline TS102(R,R) & -3310.741652 & 1.0 & -3311.4868 & 1.2 \\
\hline TS116(R,R) & -3310.741321 & 1.2 & -3311.486887 & 1.1 \\
\hline TS105(R,R) & -3310.741318 & 1.2 & -3311.487174 & 0.9 \\
\hline TS96(R,R) & -3310.741314 & 1.2 & -3311.487171 & 0.9 \\
\hline TS92(R,R) & -3310.741299 & 1.3 & -3311.487208 & 0.9 \\
\hline TS104(R,R) & -3310.741245 & 1.3 & -3311.486499 & 1.3 \\
\hline TS100(R,R) & -3310.741132 & 1.4 & -3311.486729 & 1.2 \\
\hline TS99(R,R) & -3310.741126 & 1.4 & -3311.486307 & 1.5 \\
\hline TS97(R,R) & -3310.740467 & 1.8 & -3311.486548 & 1.3 \\
\hline TS98(R,R) & -3310.740407 & 1.8 & -3311.485682 & 1.9 \\
\hline TS107(R,R) & -3310.740204 & 1.9 & -3311.485538 & 1.9 \\
\hline TS94(R,R) & -3310.739851 & 2.2 & -3311.485924 & 1.7 \\
\hline TS93(R,R) & -3310.73984 & 2.2 & -3311.485913 & 1.7 \\
\hline TS103(R,R) & -3310.739435 & 2.4 & -3311.484939 & 2.3 \\
\hline TS111(R,R) & -3310.738956 & 2.7 & -3311.484421 & 2.6 \\
\hline TS109(R,R) & -3310.738945 & 2.7 & -3311.48441 & 2.7 \\
\hline TS113(R,R) & -3310.738861 & 2.8 & -3311.484329 & 2.7 \\
\hline TS114(R,R) & -3310.738189 & 3.2 & -3311.483675 & 3.1 \\
\hline $\mathrm{TS} 115(\mathrm{R}, \mathrm{R})$ & -3310.738085 & 3.3 & -3311.483571 & 3.2 \\
\hline \multicolumn{5}{|c|}{$(\mathbf{S , S})$ Transition States } \\
\hline TS119(S,S) & -3310.741214 & 1.3 & -3311.487159 & 0.9 \\
\hline TS124(S,S) & -3310.740468 & 1.8 & -3311.486496 & 1.3 \\
\hline TS130(S,S) & -3310.740406 & 1.8 & -3311.486101 & 1.6 \\
\hline $\mathrm{TS} 121(\mathrm{~S}, \mathrm{~S})$ & -3310.7402 & 1.9 & -3311.486104 & 1.6 \\
\hline TS120(S,S) & -3310.740197 & 2.0 & -3311.4861 & 1.6 \\
\hline $\mathrm{TS} 117(\mathrm{~S}, \mathrm{~S})$ & -3310.740171 & 2.0 & -3311.486074 & 1.6 \\
\hline TS126(S,S) & -3310.739885 & 2.1 & -3311.486185 & 1.5 \\
\hline TS128(S,S) & -3310.73985 & 2.2 & -3311.485259 & 2.1 \\
\hline TS123(S,S) & -3310.73971 & 2.3 & -3311.486019 & 1.6 \\
\hline TS122(S,S) & -3310.739702 & 2.3 & -3311.486011 & 1.6 \\
\hline TS129(S,S) & -3310.739239 & 2.6 & -3311.484821 & 2.4 \\
\hline TS132(S,S) & -3310.739036 & 2.7 & -3311.484282 & 2.7 \\
\hline TS142(S,S) & -3310.739034 & 2.7 & -3311.484279 & 2.7 \\
\hline $\mathrm{TS} 125(\mathrm{~S}, \mathrm{~S})$ & -3310.739001 & 2.7 & -3311.485277 & 2.1 \\
\hline TS136(S,S) & -3310.73856 & 3.0 & -3311.484823 & 2.4 \\
\hline $\mathrm{TS} 127(\mathrm{~S}, \mathrm{~S})$ & -3310.738246 & 3.2 & -3311.483057 & 3.5 \\
\hline TS144(S,S) & -3310.738103 & 3.3 & -3311.482061 & 4.1 \\
\hline TS141(S,S) & -3310.737986 & 3.3 & -3311.483684 & 3.1 \\
\hline
\end{tabular}




$\begin{array}{lllll}\text { TS135(S,S) } & -3310.737668 & 3.5 & -3311.482913 & 3.6 \\ \text { TS137(S,S) } & -3310.737264 & 3.8 & -3311.482598 & 3.8 \\ \text { TS118(S,S) } & -3310.737075 & 3.9 & -3311.482418 & 3.9 \\ \text { TS143(S,S) } & -3310.736696 & 4.1 & -3311.482935 & 3.6 \\ \text { TS133(S,S) } & -3310.736554 & 4.2 & -3311.481292 & 4.6 \\ \text { TS131(S,S) } & -3310.736382 & 4.3 & -3311.481245 & 4.6 \\ \text { TS138(S,S) } & -3310.736332 & 4.4 & -3311.482051 & 4.1 \\ \text { TS139(S,S) } & -3310.736252 & 4.4 & -3311.481402 & 4.5 \\ \text { TS140(S,S) } & -3310.73597 & 4.6 & -3311.481686 & 4.4 \\ \text { TS134(S,S) } & -3310.735791 & 4.7 & -3311.481206 & 4.7\end{array}$

Table S21. Absolute free energies (298K, in hartrees) at the M06-2X/6-31G(d) and M062X/6-311+G(d,p)//M06-2X/6-31G(d) levels of theory for all transition states for reaction 2a catalyzed by PA-1A with free energies relative to the lowest-lying $(\mathrm{R}, \mathrm{R})$ transition state $(\mathrm{kcal} / \mathrm{mol})$. The standard RRHO model was used to compute the free energies.

\begin{tabular}{|c|c|c|c|c|}
\hline TS Name & G[M06-2X/6-31G(d)] & $\mathbf{G}_{\text {rel }}$ & $\begin{array}{c}\text { G[M06-2X/6-311+G(d,p)// } \\
\text { M06-2X/6-31G(d)] }\end{array}$ & $\mathbf{G}_{\text {rel }}$ \\
\hline \multicolumn{5}{|c|}{ (R,R) Transition States } \\
\hline $\mathrm{TS} 110(\mathrm{R}, \mathrm{R})$ & -3310.355067 & 0.0 & -3311.180072 & 0.0 \\
\hline TS116(R,R) & -3310.355058 & 0.0 & -3311.180062 & 0.0 \\
\hline TS92(R,R) & -3310.354565 & 0.3 & -3311.179525 & 0.3 \\
\hline TS93(R,R) & -3310.354504 & 0.4 & -3311.179465 & 0.4 \\
\hline TS94(R,R) & -3310.354465 & 0.4 & -3311.179426 & 0.4 \\
\hline TS100(R,R) & -3310.35424 & 0.5 & -3311.179162 & 0.6 \\
\hline TS105(R,R) & -3310.354233 & 0.5 & -3311.179157 & 0.6 \\
\hline TS97(R,R) & -3310.353566 & 0.9 & -3311.178553 & 1.0 \\
\hline TS96(R,R) & -3310.353562 & 0.9 & -3311.178548 & 1.0 \\
\hline TS95(R,R) & -3310.352734 & 1.5 & -3311.177708 & 1.5 \\
\hline $\mathrm{TS} 112(\mathrm{R}, \mathrm{R})$ & -3310.352437 & 1.7 & -3311.177433 & 1.7 \\
\hline TS99(R,R) & -3310.352105 & 1.9 & -3311.176979 & 1.9 \\
\hline TS107(R,R) & -3310.351911 & 2.0 & -3311.176766 & 2.1 \\
\hline TS108(R,R) & -3310.351908 & 2.0 & -3311.176765 & 2.1 \\
\hline TS106(R,R) & -3310.351369 & 2.3 & -3311.176413 & 2.3 \\
\hline TS98(R,R) & -3310.351343 & 2.3 & -3311.176359 & 2.3 \\
\hline TS101(R,R) & -3310.35093 & 2.6 & -3311.175681 & 2.8 \\
\hline TS102(R,R) & -3310.350929 & 2.6 & -3311.175681 & 2.8 \\
\hline TS111(R,R) & -3310.35072 & 2.7 & -3311.175516 & 2.9 \\
\hline TS109(R,R) & -3310.350254 & 3.0 & -3311.175302 & 3.0 \\
\hline TS114(R,R) & -3310.350253 & 3.0 & -3311.175302 & 3.0 \\
\hline TS103(R,R) & -3310.350111 & 3.1 & -3311.17536 & 3.0 \\
\hline TS104(R,R) & -3310.349884 & 3.3 & -3311.174834 & 3.3 \\
\hline TS113(R,R) & -3310.349392 & 3.6 & -3311.174389 & 3.6 \\
\hline $\mathrm{TS} 115(\mathrm{R}, \mathrm{R})$ & -3310.349384 & 3.6 & -3311.174381 & 3.6 \\
\hline \multicolumn{5}{|c|}{$(\mathbf{S , S})$ Transition States } \\
\hline $\mathrm{TS} 126(\mathrm{~S}, \mathrm{~S})$ & -3310.353784 & 0.8 & -3311.178871 & 0.8 \\
\hline $\mathrm{TS} 123(\mathrm{~S}, \mathrm{~S})$ & -3310.353365 & 1.1 & -3311.178815 & 0.8 \\
\hline TS122(S,S) & -3310.353364 & 1.1 & -3311.178815 & 0.8 \\
\hline TS119(S,S) & -3310.352923 & 1.3 & -3311.177852 & 1.4 \\
\hline $\mathrm{TS} 121(\mathrm{~S}, \mathrm{~S})$ & -3310.352438 & 1.6 & -3311.177283 & 1.7 \\
\hline TS120(S,S) & -3310.352437 & 1.7 & -3311.177281 & 1.8 \\
\hline $\mathrm{TS} 117(\mathrm{~S}, \mathrm{~S})$ & -3310.352436 & 1.7 & -3311.177281 & 1.8 \\
\hline
\end{tabular}




$\begin{array}{lllll}\text { TS124(S,S) } & -3310.352435 & 1.7 & -3311.17728 & 1.8 \\ \text { TS138(S,S) } & -3310.35243 & 1.7 & -3311.177275 & 1.8 \\ \text { TS125(S,S) } & -3310.351946 & 2.0 & -3311.176484 & 2.3 \\ \text { TS136(S,S) } & -3310.351521 & 2.2 & -3311.17613 & 2.5 \\ \text { TS130(S,S) } & -3310.350895 & 2.6 & -3311.17603 & 2.5 \\ \text { TS133(S,S) } & -3310.35073 & 2.7 & -3311.174582 & 3.4 \\ \text { TS127(S,S) } & -3310.350628 & 2.8 & -3311.174785 & 3.3 \\ \text { TS131(S,S) } & -3310.350342 & 3.0 & -3311.17461 & 3.4 \\ \text { TS140(S,S) } & -3310.350223 & 3.0 & -3311.175812 & 2.7 \\ \text { TS128(S,S) } & -3310.350126 & 3.1 & -3311.17521 & 3.1 \\ \text { TS129(S,S) } & -3310.349216 & 3.7 & -3311.174444 & 3.5 \\ \text { TS139(S,S) } & -3310.349082 & 3.8 & -3311.173704 & 4.0 \\ \text { TS144(S,S) } & -3310.34861 & 4.1 & -3311.172091 & 5.0 \\ \text { TS132(S,S) } & -3310.348483 & 4.1 & -3311.173349 & 4.2 \\ \text { TS137(S,S) } & -3310.348253 & 4.3 & -3311.173062 & 4.4 \\ \text { TS141(S,S) } & -3310.34823 & 4.3 & -3311.173404 & 4.2 \\ \text { TS142(S,S) } & -3310.348077 & 4.4 & -3311.172797 & 4.6 \\ \text { TS143(S,S) } & -3310.347937 & 4.5 & -3311.173306 & 4.6 \\ \text { TS118(S,S) } & -3310.347898 & 4.5 & -3311.172706 & 4.7 \\ \text { TS135(S,S) } & -3310.347796 & 4.6 & -3311.172554 & 4.9 \\ \text { TS134(S,S) } & -3310.347479 & 4.8 & -3311.172341 & \end{array}$

Table S22. Absolute free energies (298K, in hartrees) at the M06-2X/6-31G(d) and M062X/6-311+G(d,p)//M06-2X/6-31G(d) levels of theory for all transition states for reaction $2 \mathrm{a}$ catalyzed by PA-1A with free energies relative to the lowest-lying $(R, R)$ transition state ( $\mathrm{kcal} / \mathrm{mol})$. The Quasi-RRHO model was used to compute the free energies.

\begin{tabular}{ccccc}
\hline TS Name & G[M06-2X/6-31G(d)] & $\mathbf{G}_{\text {rel }}$ & $\begin{array}{c}\text { G[M06-2X/6-311+G(d,p)// } \\
\text { M06-2X/6-31G(d)] }\end{array}$ & $\mathbf{G}_{\text {rel }}$ \\
\hline (R,R) Transition States & & & & \\
\hline TS92(R,R) & -3310.338438 & 0.0 & -3311.163398 & 0.0 \\
TS93(R,R) & -3310.338408 & 0.0 & -3311.163369 & 0.0 \\
TS94(R,R) & -3310.338388 & 0.0 & -3311.163349 & 0.0 \\
TS110(R,R) & -3310.338074 & 0.2 & -3311.163078 & 0.2 \\
TS116(R,R) & -3310.338069 & 0.2 & -3311.163073 & 0.2 \\
TS97(R,R) & -3310.337855 & 0.4 & -3311.162841 & 0.3 \\
TS96(R,R) & -3310.337852 & 0.4 & -3311.162838 & 0.4 \\
TS100(R,R) & -3310.337725 & 0.4 & -3311.162647 & 0.5 \\
TS105(R,R) & -3310.337721 & 0.5 & -3311.162644 & 0.5 \\
TS95(R,R) & -3310.337066 & 0.9 & -3311.16204 & 0.9 \\
TS112(R,R) & -3310.336835 & 1.0 & -3311.161831 & 1.0 \\
TS107(R,R) & -3310.336759 & 1.1 & -3311.161614 & 1.1 \\
TS108(R,R) & -3310.336758 & 1.1 & -3311.161615 & 1.1 \\
TS99(R,R) & -3310.336559 & 1.2 & -3311.161432 & 1.4 \\
TS106(R,R) & -3310.33617 & 1.4 & -3311.161214 & 1.6 \\
TS101(R,R) & -3310.336083 & 1.5 & -3311.160834 & 1.6 \\
TS102(R,R) & -3310.336082 & 1.5 & -3311.160833 & 1.8 \\
TS98(R,R) & -3310.335458 & 1.9 & -3311.160474 & 2.2 \\
TS111(R,R) & -3310.33514 & 2.1 & -3311.159936 & 2.1 \\
TS109(R,R) & -3310.335034 & 2.1 & -3311.160082 & 2.1 \\
TS114(R,R) & -3310.335034 & 2.1 & -3311.160082 & 2.3 \\
TS104(R,R) & -3310.334814 & 2.3 & -3311.159764 & 2.2 \\
TS103(R,R) & -3310.334646 & 2.4 & -3311.159894 &
\end{tabular}




\begin{tabular}{|c|c|c|c|c|}
\hline TS113(R,R) & -3310.334609 & 2.4 & -3311.159606 & 2.4 \\
\hline $\mathrm{TS} 115(\mathrm{R}, \mathrm{R})$ & -3310.334605 & 2.4 & -3311.159602 & 2.4 \\
\hline \multicolumn{5}{|c|}{$(\mathrm{S}, \mathrm{S})$ Transition States } \\
\hline TS126(S,S) & -3310.336966 & 0.9 & -3311.162054 & 0.8 \\
\hline TS119(S,S) & -3310.336943 & 0.9 & -3311.161872 & 1.0 \\
\hline $\mathrm{TS} 122(\mathrm{~S}, \mathrm{~S})$ & -3310.336808 & 1.0 & -3311.162259 & 0.7 \\
\hline TS123(S,S) & -3310.336808 & 1.0 & -3311.162258 & 0.7 \\
\hline $\mathrm{TS} 121(\mathrm{~S}, \mathrm{~S})$ & -3310.336667 & 1.1 & -3311.161512 & 1.2 \\
\hline TS120(S,S) & -3310.336666 & 1.1 & -3311.161511 & 1.2 \\
\hline $\mathrm{TS} 117(\mathrm{~S}, \mathrm{~S})$ & -3310.336665 & 1.1 & -3311.16151 & 1.2 \\
\hline TS124(S,S) & -3310.336665 & 1.1 & -3311.16151 & 1.2 \\
\hline TS138(S,S) & -3310.336663 & 1.1 & -3311.161508 & 1.2 \\
\hline $\mathrm{TS} 133(\mathrm{~S}, \mathrm{~S})$ & -3310.336091 & 1.5 & -3311.159944 & 2.2 \\
\hline $\mathrm{TS} 127(\mathrm{~S}, \mathrm{~S})$ & -3310.33597 & 1.5 & -3311.160127 & 2.1 \\
\hline $\mathrm{TS} 125(\mathrm{~S}, \mathrm{~S})$ & -3310.335968 & 1.5 & -3311.160506 & 1.8 \\
\hline TS136(S,S) & -3310.335726 & 1.7 & -3311.160335 & 1.9 \\
\hline $\mathrm{TS} 130(\mathrm{~S}, \mathrm{~S})$ & -3310.335414 & 1.9 & -3311.16055 & 1.8 \\
\hline TS128(S,S) & -3310.334967 & 2.2 & -3311.160051 & 2.1 \\
\hline TS131(S,S) & -3310.334586 & 2.4 & -3311.158854 & 2.9 \\
\hline TS144(S,S) & -3310.334313 & 2.6 & -3311.157794 & 3.5 \\
\hline $\mathrm{TS} 140(\mathrm{~S}, \mathrm{~S})$ & -3310.333963 & 2.8 & -3311.159551 & 2.4 \\
\hline TS139(S,S) & -3310.333714 & 3.0 & -3311.158335 & 3.2 \\
\hline TS129(S,S) & -3310.333454 & 3.1 & -3311.158682 & 3.0 \\
\hline $\mathrm{TS} 142(\mathrm{~S}, \mathrm{~S})$ & -3310.332903 & 3.5 & -3311.157622 & 3.6 \\
\hline $\mathrm{TS} 141(\mathrm{~S}, \mathrm{~S})$ & -3310.332856 & 3.5 & -3311.15803 & 3.4 \\
\hline TS132(S,S) & -3310.332854 & 3.5 & -3311.157719 & 3.6 \\
\hline TS135(S,S) & -3310.332796 & 3.5 & -3311.157554 & 3.7 \\
\hline TS118(S,S) & -3310.332636 & 3.6 & -3311.157444 & 3.7 \\
\hline $\mathrm{TS} 137(\mathrm{~S}, \mathrm{~S})$ & -3310.332323 & 3.8 & -3311.157132 & 3.9 \\
\hline TS143(S,S) & -3310.33209 & 4.0 & -3311.157459 & 3.7 \\
\hline TS134(S,S) & -3310.331845 & 4.1 & -3311.156706 & 4.2 \\
\hline
\end{tabular}

Table S23. Absolute free energies (298K, in hartrees) at the B97-D/def2-TZVP and B97D3/def2-TZVP//B97-D/def2-TZVP levels of theory for all transition states for reaction 2a catalyzed by PA-1B with free energies relative to the lowest-lying $(R, R)$ transition state $(\mathrm{kcal} / \mathrm{mol})$. The standard RRHO model was used to compute the free energies.

\begin{tabular}{ccccc}
\hline TS Name & G[B97-D/def2-TZVP] & $\mathbf{G}_{\text {rel }}$ & $\begin{array}{c}\text { G[B97-D3/def2-TZVP// } \\
\text { B97-D/def2-TZVP] }\end{array}$ & $\mathbf{G}_{\text {rel }}$ \\
\hline (R,R) Transition States & & & & \\
\hline TS145(R,R) & -3217.918980 & 0.0 & -3218.178704 & 0.0 \\
\hline (S,S) Transition States & & & & 1.2 \\
\hline TS146(S,S) & -3217.918128 & 0.5 & -3218.176738 & 0.7 \\
TS147(S,S) & -3217.917606 & 0.9 & -3218.177555 &
\end{tabular}


Table S24. Absolute free energies (298K, in hartrees) at the B97-D/def2-TZVP and B97D3/def2-TZVP//B97-D/def2-TZVP levels of theory for all transition states for reaction 2a catalyzed by PA-1B with free energies relative to the lowest-lying $(\mathrm{R}, \mathrm{R})$ transition state $(\mathrm{kcal} / \mathrm{mol})$. The Quasi-RRHO model was used to compute the free energies. The lowest-lying $(\mathrm{R}, \mathrm{R})$ and $(\mathrm{S}, \mathrm{S})$ transition states at the B97-D3/def2-TZVP//B97-D/def2-TZVP level are in bold.

\begin{tabular}{ccccc}
\hline TS Name & G[B97-D/def2-TZVP] & $\mathbf{G}_{\text {rel }}$ & $\begin{array}{c}\text { G[B97-D3/def2-TZVP// } \\
\text { B97-D/def2-TZVP] }\end{array}$ & $\mathbf{G}_{\text {rel }}$ \\
\hline (R,R) Transition States & & & & \\
\hline TS145(R,R) & $\mathbf{- 3 2 1 7 . 9 0 5 5 1 8}$ & $\mathbf{0 . 0}$ & $\mathbf{- 3 2 1 8 . 1 6 5 2 4 3}$ & $\mathbf{0 . 0}$ \\
\hline (S,S) Transition States & & & & \\
\hline TS147(S,S) & $\mathbf{- 3 2 1 7 . 9 0 4 1 0 7}$ & $\mathbf{0 . 9}$ & $\mathbf{- 3 2 1 8 . 1 6 4 0 5 6}$ & $\mathbf{0 . 7}$ \\
TS146(S,S) & -3217.904083 & 0.9 & -3218.162693 & 1.6
\end{tabular}

Table S25. Absolute free energies (298K, in hartrees) at the B97-D/def2-TZVP and B97D3/def2-TZVP//B97-D/def2-TZVP levels of theory for all transition states for reaction 2a catalyzed by PA-2 with free energies relative to the lowest-lying $(R, R)$ transition state $(\mathrm{kcal} / \mathrm{mol})$. The standard RRHO model was used to compute the free energies.

\begin{tabular}{ccccc}
\hline TS Name & G[B97-D/def2-TZVP] & $\mathbf{G}_{\text {rel }}$ & $\begin{array}{c}\text { G[B97-D3/def2-TZVP// } \\
\text { B97-D/def2-TZVP] }\end{array}$ & $\mathbf{G}_{\text {rel }}$ \\
\hline (R,R) Transition States & & & & \\
\hline TS148(R,R) & -3541.577722 & 0.0 & -3541.851038 & 0.0 \\
TS149(R,R) & -3541.575978 & 1.1 & -3541.849320 & 1.1 \\
TS150(R,R) & -3541.575907 & 1.1 & -3541.849231 & 1.1 \\
TS151(R,R) & -3541.575647 & 1.3 & -3541.848794 & 1.4 \\
TS152(R,R) & -3541.575322 & 1.5 & -3541.849005 & 1.3 \\
TS153(R,R) & -3541.575201 & 1.6 & -3541.848218 & 1.8 \\
TS154(R,R) & -3541.575003 & 1.7 & -3541.847945 & 1.9 \\
TS155(R,R) & -3541.574894 & 1.8 & -3541.847746 & 2.1 \\
TS156(R,R) & -3541.574156 & 2.2 & -3541.847047 & 2.5 \\
TS157(R,R) & -3541.574094 & 2.3 & -3541.847173 & 2.4 \\
TS158(R,R) & -3541.573606 & 2.6 & -3541.846885 & 2.6 \\
TS159(R,R) & -3541.573541 & 2.6 & -3541.846368 & 2.9 \\
TS160(R,R) & -3541.572906 & 3.0 & -3541.845489 & 3.5 \\
TS161(R,R) & -3541.572449 & 3.3 & -3541.845541 & 3.4 \\
TS162(R,R) & -3541.571383 & 4.0 & -3541.844234 & 4.3 \\
TS163(R,R) & -3541.570669 & 4.4 & -3541.842427 & 5.4 \\
\hline (S,S) Transition States & & & & \\
\hline TS164(S,S) & -3541.573821 & 2.4 & -3541.847400 & 2.3 \\
TS165(S,S) & -3541.573588 & 2.6 & -3541.846442 & 2.9 \\
TS166(S,S) & -3541.573490 & 2.7 & -3541.846822 & 2.6 \\
TS167(S,S) & -3541.573135 & 2.9 & -3541.846398 & 2.9 \\
TS168(S,S) & -3541.573112 & 2.9 & -3541.846325 & 3.0 \\
TS169(S,S) & -3541.573073 & 2.9 & -3541.845971 & 3.2 \\
TS170(S,S) & -3541.572826 & 3.1 & -3541.845891 & 3.2 \\
TS171(S,S) & -3541.572750 & 3.1 & -3541.845730 & 3.3 \\
TS172(S,S) & -3541.572570 & 3.2 & -3541.845741 & 3.3 \\
TS173(S,S) & -3541.572233 & 3.4 & -3541.845238 & -3.9 \\
TS174(S,S) & -3541.572060 & 3.6 & -3541.844795 & \\
& & & & \\
\end{tabular}




$\begin{array}{lllll}\text { TS175(S,S) } & -3541.571915 & 3.6 & -3541.844712 & 4.0 \\ \text { TS176(S,S) } & -3541.571777 & 3.7 & -3541.845204 & 3.7 \\ \text { TS177(S,S) } & -3541.571748 & 3.7 & -3541.844867 & 3.9 \\ \text { TS178(S,S) } & -3541.571724 & 3.8 & -3541.844486 & 4.1 \\ \text { TS179(S,S) } & -3541.571716 & 3.8 & -3541.844749 & 3.9 \\ \text { TS180(S,S) } & -3541.571689 & 3.8 & -3541.844525 & 4.1 \\ \text { TS181(S,S) } & -3541.571669 & 3.8 & -3541.844615 & 4.0 \\ \text { TS182(S,S) } & -3541.571573 & 3.9 & -3541.844169 & 4.3 \\ \text { TS183(S,S) } & -3541.571564 & 3.9 & -3541.844400 & 4.2 \\ \text { TS184(S,S) } & -3541.571217 & 4.1 & -3541.843971 & 4.4 \\ \text { TS185(S,S) } & -3541.571005 & 4.2 & -3541.843940 & 4.5 \\ \text { TS186(S,S) } & -3541.570512 & 4.5 & -3541.843336 & 4.8 \\ \text { TS187(S,S) } & -3541.570124 & 4.8 & -3541.842647 & 5.3 \\ \text { TS188(S,S) } & -3541.570049 & 4.8 & -3541.842733 & 5.2 \\ \text { TS189(S,S) } & -3541.570038 & 4.8 & -3541.842637 & 5.3 \\ \text { TS190(S,S) } & -3541.569741 & 5.0 & -3541.842229 & 5.5 \\ \text { TS191(S,S) } & -3541.569055 & 5.4 & -3541.841469 & 6.0 \\ \text { TS192(S,S) } & -3541.568937 & 5.5 & -3541.841263 & 6.1 \\ \text { TS193(S,S) } & -3541.568765 & 5.6 & -3541.841352 & 6.1 \\ \text { TS194(S,S) } & -3541.568677 & 5.7 & -3541.839883 & 7.0 \\ \text { TS195(S,S) } & -3541.568529 & 5.8 & -3541.840201 & 6.8\end{array}$

Table S26. Absolute free energies (298K, in hartrees) at the B97-D/def2-TZVP and B97D3/def2-TZVP//B97-D/def2-TZVP levels of theory for all transition states for reaction 2a catalyzed by PA-2 with free energies relative to the lowest-lying $(R, R)$ transition state $(\mathrm{kcal} / \mathrm{mol})$. The Quasi-RRHO model was used to compute the free energies. The lowest-lying $(\mathrm{R}, \mathrm{R})$ and $(\mathrm{S}, \mathrm{S})$ transition states at the B97-D3/def2-TZVP//B97-D/def2-TZVP level are in bold.

\begin{tabular}{|c|c|c|c|c|}
\hline TS Name & G[B97-D/def2-TZVP] & $\mathbf{G}_{\text {rel }}$ & $\begin{array}{c}\text { G[B97-D3/def2-TZVP// } \\
\text { B97-D/def2-TZVP] }\end{array}$ & $\mathbf{G}_{\text {rel }}$ \\
\hline \multicolumn{5}{|c|}{$(\mathbf{R}, \mathbf{R})$ Transition States } \\
\hline TS148(R,R) & -3541.56117 & 0.0 & -3541.83448 & $\mathbf{0 . 0}$ \\
\hline TS149(R,R) & -3541.56018 & 0.6 & -3541.83353 & 0.6 \\
\hline TS150(R,R) & -3541.56006 & 0.7 & -3541.83338 & 0.7 \\
\hline TS152(R,R) & -3541.55965 & 1.0 & -3541.83334 & 0.7 \\
\hline TS153(R,R) & -3541.55935 & 1.1 & -3541.83236 & 1.3 \\
\hline TS151(R,R) & -3541.5592 & 1.2 & -3541.83235 & 1.3 \\
\hline TS155(R,R) & -3541.55881 & 1.5 & -3541.83166 & 1.8 \\
\hline TS157(R,R) & -3541.55877 & 1.5 & -3541.83185 & 1.7 \\
\hline TS154(R,R) & -3541.55876 & 1.5 & -3541.8317 & 1.7 \\
\hline TS156(R,R) & -3541.55849 & 1.7 & -3541.83138 & 1.9 \\
\hline TS159(R,R) & -3541.55816 & 1.9 & -3541.83099 & 2.2 \\
\hline TS158(R,R) & -3541.55807 & 1.9 & -3541.83135 & 2.0 \\
\hline TS161(R,R) & -3541.55705 & 2.6 & -3541.83014 & 2.7 \\
\hline TS160(R,R) & -3541.55692 & 2.7 & -3541.8295 & 3.1 \\
\hline TS162(R,R) & -3541.55636 & 3.0 & -3541.82921 & 3.3 \\
\hline TS163(R,R) & -3541.55584 & 3.3 & -3541.8276 & 4.3 \\
\hline \multicolumn{5}{|c|}{$(\mathbf{S}, \mathbf{S})$ Transition States } \\
\hline TS164(S,S) & -3541.55822 & 1.9 & -3541.8318 & 1.7 \\
\hline TS166(S,S) & -3541.55809 & 1.9 & -3541.83143 & 1.9 \\
\hline TS167(S,S) & -3541.55795 & 2.0 & -3541.83122 & 2.0 \\
\hline
\end{tabular}




$\begin{array}{lllll}\text { TS165(S,S) } & -3541.55779 & 2.1 & -3541.83064 & 2.4 \\ \text { TS168(S,S) } & -3541.55772 & 2.2 & -3541.83093 & 2.2 \\ \text { TS171(S,S) } & -3541.55758 & 2.3 & -3541.83056 & 2.5 \\ \text { TS169(S,S) } & -3541.55753 & 2.3 & -3541.83043 & 2.5 \\ \text { TS172(S,S) } & -3541.55752 & 2.3 & -3541.8307 & 2.4 \\ \text { TS177(S,S) } & -3541.5571 & 2.6 & -3541.83021 & 2.7 \\ \text { TS176(S,S) } & -3541.55696 & 2.6 & -3541.83038 & 2.6 \\ \text { TS170(S,S) } & -3541.55685 & 2.7 & -3541.82991 & 2.9 \\ \text { TS178(S,S) } & -3541.55683 & 2.7 & -3541.82959 & 3.1 \\ \text { TS173(S,S) } & -3541.55669 & 2.8 & -3541.8297 & 3.0 \\ \text { TS174(S,S) } & -3541.55664 & 2.8 & -3541.82938 & 3.2 \\ \text { TS184(S,S) } & -3541.55652 & 2.9 & -3541.82928 & 3.3 \\ \text { TS180(S,S) } & -3541.55645 & 3.0 & -3541.82929 & 3.3 \\ \text { TS175(S,S) } & -3541.5564 & 3.0 & -3541.8292 & 3.3 \\ \text { TS181(S,S) } & -3541.55621 & 3.1 & -3541.82915 & 3.3 \\ \text { TS183(S,S) } & -3541.5561 & 3.2 & -3541.82894 & 3.5 \\ \text { TS179(S,S) } & -3541.55605 & 3.2 & -3541.82908 & 3.4 \\ \text { TS185(S,S) } & -3541.55592 & 3.3 & -3541.82885 & 3.5 \\ \text { TS186(S,S) } & -3541.55584 & 3.3 & -3541.82867 & 3.6 \\ \text { TS182(S,S) } & -3541.5556 & 3.5 & -3541.82819 & 3.9 \\ \text { TS189(S,S) } & -3541.55541 & 3.6 & -3541.828 & 4.1 \\ \text { TS188(S,S) } & -3541.55521 & 3.7 & -3541.82789 & 4.1 \\ \text { TS190(S,S) } & -3541.55507 & 3.8 & -3541.82756 & 4.3 \\ \text { TS187(S,S) } & -3541.55472 & 4.0 & -3541.82725 & 4.5 \\ \text { TS193(S,S) } & -3541.55467 & 4.1 & -3541.82725 & 4.5 \\ \text { TS191(S,S) } & -3541.55426 & 4.3 & -3541.82668 & 4.9 \\ \text { TS192(S,S) } & -3541.55406 & 4.5 & -3541.82638 & 5.1 \\ \text { TS195(S,S) } & -3541.55397 & 4.5 & -3541.82564 & 5.5 \\ \text { TS194(S,S) } & -3541.55382 & 4.6 & -3541.82503 & \end{array}$

Table S27. Absolute free energies (233K, in hartrees) at the B97-D/def2-TZVP and B97D3/def2-TZVP//B97-D/def2-TZVP levels of theory for all transition states for reaction 2a catalyzed by PA-2 with free energies relative to the lowest-lying $(R, R)$ transition state $(\mathrm{kcal} / \mathrm{mol})$. The standard RRHO model was used to compute the free energies.

\begin{tabular}{ccccc}
\hline TS Name & G[B97-D/def2-TZVP] & $\mathbf{G}_{\text {rel }}$ & $\begin{array}{c}\text { G[B97-D3/def2-TZVP// } \\
\text { B97-D/def2-TZVP] }\end{array}$ & $\mathbf{G}_{\text {rel }}$ \\
\hline (R,R) Transition States & & & & \\
\hline TS148(R,R) & -3541.543481 & 0.0 & -3541.816797 & 0.0 \\
TS149(R,R) & -3541.542048 & 0.9 & -3541.815390 & 0.9 \\
TS150(R,R) & -3541.541995 & 0.9 & -3541.815319 & 0.9 \\
TS151(R,R) & -3541.541431 & 1.3 & -3541.814577 & 1.4 \\
TS152(R,R) & -3541.541421 & 1.3 & -3541.815104 & 1.1 \\
TS153(R,R) & -3541.541208 & 1.4 & -3541.814225 & 1.6 \\
TS154(R,R) & -3541.540860 & 1.6 & -3541.813802 & 1.9 \\
TS155(R,R) & -3541.540808 & 1.7 & -3541.813659 & 2.0 \\
TS157(R,R) & -3541.540306 & 2.0 & -3541.813385 & 2.1 \\
TS156(R,R) & -3541.540229 & 2.0 & -3541.813120 & 2.3 \\
TS158(R,R) & -3541.539750 & 2.3 & -3541.813030 & 2.4 \\
TS159(R,R) & -3541.539729 & 2.4 & -3541.812556 & 2.7 \\
TS160(R,R) & -3541.538865 & 2.9 & -3541.811449 & 3.4 \\
TS161(R,R) & -3541.538599 & 3.1 & -3541.811691 & 3.2 \\
TS162(R,R) & -3541.537656 & 3.7 & -3541.810507 & 3.9 \\
& & & &
\end{tabular}




\begin{tabular}{|c|c|c|c|c|}
\hline TS163(R,R) & -3541.537071 & 4.0 & -3541.808829 & 5.0 \\
\hline \multicolumn{5}{|c|}{ (S,S) Transition States } \\
\hline TS164(S,S) & -3541.539923 & 2.2 & -3541.813501 & 2.1 \\
\hline TS166(S,S) & -3541.539735 & 2.4 & -3541.813067 & 2.3 \\
\hline TS165(S,S) & -3541.539570 & 2.5 & -3541.812425 & 2.7 \\
\hline TS167(S,S) & -3541.539475 & 2.5 & -3541.812739 & 2.5 \\
\hline TS168(S,S) & -3541.539415 & 2.6 & -3541.812628 & 2.6 \\
\hline TS169(S,S) & -3541.539186 & 2.7 & -3541.812084 & 3.0 \\
\hline TS171(S,S) & -3541.539174 & 2.7 & -3541.812154 & 2.9 \\
\hline TS172(S,S) & -3541.538981 & 2.8 & -3541.812153 & 2.9 \\
\hline TS170(S,S) & -3541.538769 & 3.0 & -3541.811834 & 3.1 \\
\hline TS173(S,S) & -3541.538370 & 3.2 & -3541.811375 & 3.4 \\
\hline TS177(S,S) & -3541.538367 & 3.2 & -3541.811486 & 3.3 \\
\hline TS176(S,S) & -3541.538271 & 3.3 & -3541.811698 & 3.2 \\
\hline TS174(S,S) & -3541.538252 & 3.3 & -3541.810987 & 3.6 \\
\hline TS178(S,S) & -3541.538130 & 3.4 & -3541.810893 & 3.7 \\
\hline TS175(S,S) & -3541.538106 & 3.4 & -3541.810903 & 3.7 \\
\hline TS180(S,S) & -3541.538020 & 3.4 & -3541.810857 & 3.7 \\
\hline TS181(S,S) & -3541.537891 & 3.5 & -3541.810837 & 3.7 \\
\hline TS184(S,S) & -3541.537750 & 3.6 & -3541.810504 & 3.9 \\
\hline TS183(S,S) & -3541.537698 & 3.6 & -3541.810534 & 3.9 \\
\hline TS179(S,S) & -3541.537693 & 3.6 & -3541.810726 & 3.8 \\
\hline TS182(S,S) & -3541.537484 & 3.8 & -3541.810080 & 4.2 \\
\hline TS185(S,S) & -3541.537364 & 3.8 & -3541.810299 & 4.1 \\
\hline TS186(S,S) & -3541.537021 & 4.1 & -3541.809844 & 4.4 \\
\hline TS189(S,S) & -3541.536653 & 4.3 & -3541.809252 & 4.7 \\
\hline TS188(S,S) & -3541.536586 & 4.3 & -3541.809270 & 4.7 \\
\hline TS190(S,S) & -3541.536341 & 4.5 & -3541.808829 & 5.0 \\
\hline TS187(S,S) & -3541.536288 & 4.5 & -3541.808812 & 5.0 \\
\hline TS193(S,S) & -3541.535587 & 5.0 & -3541.808174 & 5.4 \\
\hline TS191(S,S) & -3541.535501 & 5.0 & -3541.807915 & 5.6 \\
\hline TS192(S,S) & -3541.535397 & 5.1 & -3541.807723 & 5.7 \\
\hline TS194(S,S) & -3541.535134 & 5.2 & -3541.806340 & 6.6 \\
\hline TS195(S,S) & -3541.535046 & 5.3 & -3541.806718 & 6.3 \\
\hline
\end{tabular}

Table S28. Absolute free energies (233K, in hartrees) at the B97-D/def2-TZVP and B97D3/def2-TZVP//B97-D/def2-TZVP levels of theory for all transition states for reaction 2a catalyzed by PA-2 with free energies relative to the lowest-lying $(R, R)$ transition state $(\mathrm{kcal} / \mathrm{mol})$. The Quasi-RRHO model was used to compute the free energies. The lowest-lying $(\mathrm{R}, \mathrm{R})$ and $(\mathrm{S}, \mathrm{S})$ transition states at the B97-D3/def2-TZVP//B97-D/def2-TZVP level are in bold.

\begin{tabular}{ccccc}
\hline TS Name & G[B97-D/def2-TZVP] & $\mathbf{G}_{\text {rel }}$ & $\begin{array}{c}\text { G[B97-D3/def2-TZVP// } \\
\text { B97-D/def2-TZVP] }\end{array}$ & $\mathbf{G}_{\text {rel }}$ \\
\hline (R,R) Transition States & & & & \\
\hline TS148(R,R) & $\mathbf{- 3 5 4 1 . 5 3 2 8 4}$ & $\mathbf{0 . 0}$ & $\mathbf{- 3 5 4 1 . 8 0 6 1 5}$ & $\mathbf{0 . 0}$ \\
TS149(R,R) & -3541.53198 & 0.5 & -3541.80532 & 0.5 \\
TS150(R,R) & -3541.53188 & 0.6 & -3541.80521 & 0.6 \\
TS152(R,R) & -3541.53147 & 0.9 & -3541.80515 & 0.6 \\
TS153(R,R) & -3541.53112 & 1.1 & -3541.80413 & 1.3 \\
TS151(R,R) & -3541.53089 & 1.2 & -3541.80403 & 1.3 \\
TS157(R,R) & -3541.53059 & 1.4 & -3541.80367 & 1.6
\end{tabular}




\begin{tabular}{|c|c|c|c|c|}
\hline TS155(R,R) & -3541.53053 & 1.4 & -3541.80338 & 1.7 \\
\hline TS154(R,R) & -3541.53047 & 1.5 & -3541.80341 & 1.7 \\
\hline TS156(R,R) & -3541.53025 & 1.6 & -3541.80314 & 1.9 \\
\hline TS159(R,R) & -3541.52996 & 1.8 & -3541.80279 & 2.1 \\
\hline TS158(R,R) & -3541.52986 & 1.9 & -3541.80314 & 1.9 \\
\hline TS161(R,R) & -3541.52886 & 2.5 & -3541.80195 & 2.6 \\
\hline TS160(R,R) & -3541.52868 & 2.6 & -3541.80127 & 3.1 \\
\hline TS162(R,R) & -3541.52818 & 2.9 & -3541.80103 & 3.2 \\
\hline TS163(R,R) & -3541.52772 & 3.2 & -3541.79948 & 4.2 \\
\hline \multicolumn{5}{|c|}{ (S,S) Transition States } \\
\hline TS164(S,S) & -3541.53002 & 1.8 & -3541.8036 & 1.6 \\
\hline TS166(S,S) & -3541.52997 & 1.8 & -3541.8033 & 1.8 \\
\hline TS167(S,S) & -3541.52987 & 1.9 & -3541.80313 & 1.9 \\
\hline TS168(S,S) & -3541.52966 & 2.0 & -3541.80287 & 2.1 \\
\hline TS171(S,S) & -3541.52958 & 2.0 & -3541.80256 & 2.3 \\
\hline TS165(S,S) & -3541.52951 & 2.1 & -3541.80237 & 2.4 \\
\hline TS172(S,S) & -3541.52947 & 2.1 & -3541.80264 & 2.2 \\
\hline TS169(S,S) & -3541.52933 & 2.2 & -3541.80222 & 2.5 \\
\hline TS177(S,S) & -3541.52916 & 2.3 & -3541.80228 & 2.4 \\
\hline TS176(S,S) & -3541.52897 & 2.4 & -3541.8024 & 2.4 \\
\hline TS178(S,S) & -3541.52875 & 2.6 & -3541.80152 & 2.9 \\
\hline $\mathrm{TS} 170(\mathrm{~S}, \mathrm{~S})$ & -3541.52858 & 2.7 & -3541.80164 & 2.8 \\
\hline TS184(S,S) & -3541.52852 & 2.7 & -3541.80127 & 3.1 \\
\hline TS173(S,S) & -3541.5285 & 2.7 & -3541.80151 & 2.9 \\
\hline $\mathrm{TS} 174(\mathrm{~S}, \mathrm{~S})$ & -3541.52844 & 2.8 & -3541.80117 & 3.1 \\
\hline $\mathrm{TS} 180(\mathrm{~S}, \mathrm{~S})$ & -3541.52839 & 2.8 & -3541.80123 & 3.1 \\
\hline $\mathrm{TS} 175(\mathrm{~S}, \mathrm{~S})$ & -3541.52822 & 2.9 & -3541.80102 & 3.2 \\
\hline TS181(S,S) & -3541.52808 & 3.0 & -3541.80103 & 3.2 \\
\hline TS183(S,S) & -3541.52792 & 3.1 & -3541.80076 & 3.4 \\
\hline TS185(S,S) & -3541.52781 & 3.2 & -3541.80075 & 3.4 \\
\hline TS186(S,S) & -3541.52779 & 3.2 & -3541.80061 & 3.5 \\
\hline TS179(S,S) & -3541.52776 & 3.2 & -3541.8008 & 3.4 \\
\hline TS189(S,S) & -3541.52745 & 3.4 & -3541.80005 & 3.8 \\
\hline TS182(S,S) & -3541.52732 & 3.5 & -3541.79992 & 3.9 \\
\hline TS188(S,S) & -3541.52722 & 3.5 & -3541.79991 & 3.9 \\
\hline TS190(S,S) & -3541.52711 & 3.6 & -3541.79959 & 4.1 \\
\hline TS193(S,S) & -3541.52678 & 3.8 & -3541.79937 & 4.3 \\
\hline TS187(S,S) & -3541.52653 & 4.0 & -3541.79905 & 4.5 \\
\hline TS191(S,S) & -3541.52621 & 4.2 & -3541.79862 & 4.7 \\
\hline TS192(S,S) & -3541.52603 & 4.3 & -3541.79835 & 4.9 \\
\hline TS195(S,S) & -3541.52589 & 4.4 & -3541.79756 & 5.4 \\
\hline TS194(S,S) & -3541.52575 & 4.4 & -3541.79696 & 5.8 \\
\hline
\end{tabular}


Table S29. Absolute free energies (298K, in hartrees) at the wB97XD/6-31G(d) and wB97XD/6-311+G(d,p)//wB97XD/6-31G(d) levels of theory for all transition states for reaction $2 \mathrm{a}$ catalyzed by $\mathrm{PA}-2$ with free energies relative to the lowest-lying $(\mathrm{R}, \mathrm{R})$ transition state $(\mathrm{kcal} / \mathrm{mol})$. The standard RRHO model was used to compute the free energies.

\begin{tabular}{|c|c|c|c|c|}
\hline TS Name & G[wB97XD/6-31G(d)] & $\mathbf{G}_{\text {rel }}$ & $\begin{array}{c}\text { G[wB97XD/6-311+G(d,p)// } \\
\text { wB97XD/6-31G(d)] }\end{array}$ & $\mathbf{G}_{\text {rel }}$ \\
\hline \multicolumn{5}{|c|}{$(\mathbf{R}, \mathbf{R})$ Transition States } \\
\hline TS150(R,R) & -3541.695518 & 0.0 & -3542.472902 & 0.0 \\
\hline TS153(R,R) & -3541.695303 & 0.1 & -3542.472848 & 0.0 \\
\hline TS149(R,R) & -3541.695297 & 0.1 & -3542.472821 & 0.1 \\
\hline TS152(R,R) & -3541.695271 & 0.2 & -3542.472874 & 0.0 \\
\hline TS148(R,R) & -3541.694978 & 0.3 & -3542.472553 & 0.2 \\
\hline TS159(R,R) & -3541.694468 & 0.7 & -3542.471989 & 0.6 \\
\hline TS151(R,R) & -3541.694161 & 0.9 & -3542.471728 & 0.7 \\
\hline TS154(R,R) & -3541.694059 & 0.9 & -3542.47148 & 0.9 \\
\hline TS157(R,R) & -3541.693627 & 1.2 & -3542.471097 & 1.1 \\
\hline TS161(R,R) & -3541.693471 & 1.3 & -3542.471044 & 1.2 \\
\hline TS156(R,R) & -3541.693308 & 1.4 & -3542.470715 & 1.4 \\
\hline TS155(R,R) & -3541.693216 & 1.4 & -3542.470782 & 1.3 \\
\hline TS158(R,R) & -3541.692852 & 1.7 & -3542.470315 & 1.6 \\
\hline TS160(R,R) & -3541.692712 & 1.8 & -3542.470277 & 1.6 \\
\hline TS162(R,R) & -3541.692621 & 1.8 & -3542.470109 & 1.8 \\
\hline $\mathrm{TS} 163(\mathrm{R}, \mathrm{R})$ & -3541.690967 & 2.9 & -3542.468416 & 2.8 \\
\hline \multicolumn{5}{|c|}{ (S,S) Transition States } \\
\hline TS164(S,S) & -3541.692652 & 1.8 & -3542.470726 & 1.4 \\
\hline TS176(S,S) & -3541.692531 & 1.9 & -3542.468629 & 2.7 \\
\hline $\mathrm{TS} 167(\mathrm{~S}, \mathrm{~S})$ & -3541.692368 & 2.0 & -3542.470344 & 1.6 \\
\hline TS166(S,S) & -3541.692218 & 2.1 & -3542.470354 & 1.6 \\
\hline $\mathrm{TS} 172(\mathrm{~S}, \mathrm{~S})$ & -3541.691973 & 2.2 & -3542.469836 & 1.9 \\
\hline $\mathrm{TS} 165(\mathrm{~S}, \mathrm{~S})$ & -3541.691429 & 2.6 & -3542.469403 & 2.2 \\
\hline $\mathrm{TS} 173(\mathrm{~S}, \mathrm{~S})$ & -3541.691364 & 2.6 & -3542.46929 & 2.3 \\
\hline TS186(S,S) & -3541.691317 & 2.6 & -3542.469298 & 2.3 \\
\hline TS169(S,S) & -3541.691292 & 2.7 & -3542.4693 & 2.3 \\
\hline $\mathrm{TS} 174(\mathrm{~S}, \mathrm{~S})$ & -3541.691037 & 2.8 & -3542.469114 & 2.4 \\
\hline $\mathrm{TS} 178(\mathrm{~S}, \mathrm{~S})$ & -3541.690821 & 2.9 & -3542.46673 & 3.9 \\
\hline TS177(S,S) & -3541.6908 & 3.0 & -3542.466875 & 3.8 \\
\hline TS168(S,S) & -3541.690697 & 3.0 & -3542.466784 & 3.8 \\
\hline TS170(S,S) & -3541.690391 & 3.2 & -3542.46838 & 2.8 \\
\hline TS190(S,S) & -3541.690337 & 3.3 & -3542.466444 & 4.1 \\
\hline $\mathrm{TS} 171(\mathrm{~S}, \mathrm{~S})$ & -3541.690175 & 3.4 & -3542.466154 & 4.2 \\
\hline TS175(S,S) & -3541.689974 & 3.5 & -3542.467875 & 3.2 \\
\hline TS184(S,S) & -3541.689942 & 3.5 & -3542.465947 & 4.4 \\
\hline TS189(S,S) & -3541.689439 & 3.8 & -3542.465418 & 4.7 \\
\hline TS185(S,S) & -3541.68904 & 4.1 & -3542.466941 & 3.7 \\
\hline TS179(S,S) & -3541.688992 & 4.1 & -3542.466978 & 3.7 \\
\hline TS193(S,S) & -3541.688976 & 4.1 & -3542.465061 & 4.9 \\
\hline TS181(S,S) & -3541.688822 & 4.2 & -3542.464742 & 5.1 \\
\hline TS187(S,S) & -3541.688667 & 4.3 & -3542.466605 & 4.0 \\
\hline TS188(S,S) & -3541.688559 & 4.4 & -3542.464592 & 5.2 \\
\hline TS180(S,S) & -3541.688384 & 4.5 & -3542.464425 & 5.3 \\
\hline TS195(S,S) & -3541.68828 & 4.5 & -3542.46621 & 4.2 \\
\hline TS182(S,S) & -3541.688225 & 4.6 & -3542.466075 & 4.3 \\
\hline
\end{tabular}




$\begin{array}{lllll}\text { TS191(S,S) } & -3541.687739 & 4.9 & -3542.46367 & 5.8 \\ \text { TS183(S,S) } & -3541.687714 & 4.9 & -3542.463739 & 5.8 \\ \text { TS192(S,S) } & -3541.686203 & 5.8 & -3542.46225 & 6.7 \\ \text { TS194(S,S) } & -3541.686186 & 5.9 & -3542.462154 & 6.7\end{array}$

Table S30. Absolute free energies (298K, in hartrees) at the wB97XD/6-31G(d) and wB97XD/6-311+G(d,p)//wB97XD/6-31G(d) levels of theory for all transition states for reaction 2a catalyzed by PA-2 with free energies relative to the lowest-lying $(R, R)$ transition state $(\mathrm{kcal} / \mathrm{mol})$. The Quasi-RRHO model was used to compute the free energies.

\begin{tabular}{|c|c|c|c|c|}
\hline TS Name & G[wB97XD/6-31G(d)] & $\mathbf{G}_{\text {rel }}$ & $\begin{array}{c}\text { G[wB97XD/6-311+G(d,p)// } \\
\text { wB97XD/6-31G(d)] }\end{array}$ & $\mathbf{G}_{\text {rel }}$ \\
\hline \multicolumn{5}{|c|}{$(\mathbf{R}, \mathbf{R})$ Transition States } \\
\hline $\mathrm{TS} 150(\mathrm{R}, \mathrm{R})$ & -3541.681478 & 0.0 & -3542.458862 & 0.1 \\
\hline TS149(R,R) & -3541.681452 & 0.0 & -3542.458976 & 0.0 \\
\hline TS152(R,R) & -3541.681211 & 0.2 & -3542.458814 & 0.1 \\
\hline $\mathrm{TS} 148(\mathrm{R}, \mathrm{R})$ & -3541.681168 & 0.2 & -3542.458743 & 0.1 \\
\hline TS153(R,R) & -3541.680985 & 0.3 & -3542.458529 & 0.3 \\
\hline TS159(R,R) & -3541.68055 & 0.6 & -3542.458072 & 0.6 \\
\hline TS157(R,R) & -3541.680037 & 0.9 & -3542.457507 & 0.9 \\
\hline TS154(R,R) & -3541.679908 & 1.0 & -3542.457329 & 1.0 \\
\hline TS151(R,R) & -3541.6798 & 1.1 & -3542.457367 & 1.0 \\
\hline TS156(R,R) & -3541.679554 & 1.2 & -3542.456961 & 1.3 \\
\hline TS155(R,R) & -3541.679479 & 1.3 & -3542.457045 & 1.2 \\
\hline TS158(R,R) & -3541.679104 & 1.5 & -3542.456567 & 1.5 \\
\hline TS161(R,R) & -3541.678879 & 1.6 & -3542.456452 & 1.6 \\
\hline TS160(R,R) & -3541.678499 & 1.9 & -3542.456065 & 1.8 \\
\hline TS162(R,R) & -3541.678479 & 1.9 & -3542.455967 & 1.9 \\
\hline TS163(R,R) & -3541.677182 & 2.7 & -3542.45463 & 2.7 \\
\hline \multicolumn{5}{|c|}{$(\mathbf{S , S})$ Transition States } \\
\hline TS167(S,S) & -3541.678489 & 1.9 & -3542.456465 & 1.6 \\
\hline TS164(S,S) & -3541.678457 & 1.9 & -3542.456532 & 1.5 \\
\hline TS166(S,S) & -3541.678167 & 2.1 & -3542.456303 & 1.7 \\
\hline TS172(S,S) & -3541.677996 & 2.2 & -3542.455859 & 2.0 \\
\hline $\mathrm{TS} 176(\mathrm{~S}, \mathrm{~S})$ & -3541.67788 & 2.3 & -3542.453978 & 3.1 \\
\hline TS169(S,S) & -3541.677354 & 2.6 & -3542.455362 & 2.3 \\
\hline $\mathrm{TS} 165(\mathrm{~S}, \mathrm{~S})$ & -3541.677261 & 2.6 & -3542.455235 & 2.3 \\
\hline TS177(S,S) & -3541.677133 & 2.7 & -3542.453209 & 3.6 \\
\hline TS186(S,S) & -3541.677114 & 2.7 & -3542.455094 & 2.4 \\
\hline $\mathrm{TS} 173(\mathrm{~S}, \mathrm{~S})$ & -3541.677084 & 2.8 & -3542.455009 & 2.5 \\
\hline TS168(S,S) & -3541.676895 & 2.9 & -3542.452982 & 3.8 \\
\hline TS178(S,S) & -3541.676735 & 3.0 & -3542.452644 & 4.0 \\
\hline $\mathrm{TS} 174(\mathrm{~S}, \mathrm{~S})$ & -3541.676725 & 3.0 & -3542.454802 & 2.6 \\
\hline TS171(S,S) & -3541.676466 & 3.1 & -3542.452445 & 4.1 \\
\hline TS170(S,S) & -3541.676269 & 3.3 & -3542.454258 & 3.0 \\
\hline TS184(S,S) & -3541.676176 & 3.3 & -3542.452181 & 4.3 \\
\hline TS190(S,S) & -3541.676116 & 3.4 & -3542.452223 & 4.2 \\
\hline TS175(S,S) & -3541.675868 & 3.5 & -3542.453769 & 3.3 \\
\hline TS189(S,S) & -3541.675426 & 3.8 & -3542.451405 & 4.8 \\
\hline TS185(S,S) & -3541.675388 & 3.8 & -3542.453289 & 3.6 \\
\hline TS193(S,S) & -3541.675085 & 4.0 & -3542.45117 & 4.9 \\
\hline TS181(S,S) & -3541.674989 & 4.1 & -3542.45091 & 5.1 \\
\hline
\end{tabular}




$\begin{array}{lllll}\text { TS188(S,S }) & -3541.67492 & 4.1 & -3542.450953 & 5.0 \\ \text { TS180(S,S) } & -3541.674899 & 4.1 & -3542.450941 & 5.0 \\ \text { TS179(S,S) } & -3541.674709 & 4.2 & -3542.452696 & 3.9 \\ \text { TS187(S,S) } & -3541.674388 & 4.4 & -3542.452326 & 4.2 \\ \text { TS182(S,S) } & -3541.674198 & 4.6 & -3542.452048 & 4.3 \\ \text { TS195(S,S) } & -3541.674156 & 4.6 & -3542.452085 & 4.3 \\ \text { TS183(S,S) } & -3541.674126 & 4.6 & -3542.450151 & 5.5 \\ \text { TS191(S,S) } & -3541.673702 & 4.9 & -3542.449633 & 5.9 \\ \text { TS194(S,S) } & -3541.672865 & 5.4 & -3542.448833 & 6.4 \\ \text { TS192(S,S) } & -3541.672859 & 5.4 & -3542.448906 & 6.3\end{array}$

Table S31. Absolute free energies (298K, in hartrees) at the M06-2X/6-31G(d) and M062X/6-311+G(d,p)//M06-2X/6-31G(d) levels of theory for all transition states for reaction $2 \mathrm{a}$ catalyzed by PA-2 with free energies relative to the lowest-lying $(\mathrm{R}, \mathrm{R})$ transition state $(\mathrm{kcal} / \mathrm{mol})$. The standard RRHO model was used to compute the free energies.

\begin{tabular}{|c|c|c|c|c|}
\hline TS Name & G[M06-2X/6-31G(d)] & $\mathbf{G}_{\text {rel }}$ & $\begin{array}{c}\text { G[M06-2X/6-311+G(d,p)// } \\
\text { M06-2X/6-31G(d)] }\end{array}$ & $\mathbf{G}_{\text {rel }}$ \\
\hline \multicolumn{5}{|c|}{ (R,R) Transition States } \\
\hline TS158(R,R) & -3541.257885 & 0.0 & -3542.124019 & 0.0 \\
\hline TS152(R,R) & -3541.257723 & 0.1 & -3542.123771 & 0.2 \\
\hline TS149(R,R) & -3541.257143 & 0.5 & -3542.123314 & 0.4 \\
\hline $\mathrm{TS} 148(\mathrm{R}, \mathrm{R})$ & -3541.257089 & 0.5 & -3542.123219 & 0.5 \\
\hline TS150(R,R) & -3541.256893 & 0.6 & -3542.122901 & 0.7 \\
\hline TS157(R,R) & -3541.256498 & 0.9 & -3542.122643 & 0.9 \\
\hline TS153(R,R) & -3541.255974 & 1.2 & -3542.122108 & 1.2 \\
\hline TS151(R,R) & -3541.255342 & 1.6 & -3542.121439 & 1.6 \\
\hline TS159(R,R) & -3541.254586 & 2.1 & -3542.120751 & 2.1 \\
\hline $\mathrm{TS} 155(\mathrm{R}, \mathrm{R})$ & -3541.254563 & 2.1 & -3542.120766 & 2.0 \\
\hline TS156(R,R) & -3541.254491 & 2.1 & -3542.120586 & 2.2 \\
\hline TS161(R,R) & -3541.254309 & 2.2 & -3542.120601 & 2.1 \\
\hline TS154(R,R) & -3541.254248 & 2.3 & -3542.120391 & 2.3 \\
\hline TS162(R,R) & -3541.253567 & 2.7 & -3542.119744 & 2.7 \\
\hline TS160(R,R) & -3541.253451 & 2.8 & -3542.119587 & 2.8 \\
\hline $\mathrm{TS} 163(\mathrm{R}, \mathrm{R})$ & -3541.252296 & 3.5 & -3542.118398 & 3.5 \\
\hline \multicolumn{5}{|c|}{ (S,S) Transition States } \\
\hline $\mathrm{TS} 166(\mathrm{~S}, \mathrm{~S})$ & -3541.25605 & 1.2 & -3542.122752 & 0.8 \\
\hline TS167(S,S) & -3541.255525 & 1.5 & -3542.12217 & 1.2 \\
\hline $\mathrm{TS} 172(\mathrm{~S}, \mathrm{~S})$ & -3541.255402 & 1.6 & -3542.121935 & 1.3 \\
\hline TS164(S,S) & -3541.255087 & 1.8 & -3542.121605 & 1.5 \\
\hline $\mathrm{TS} 165(\mathrm{~S}, \mathrm{~S})$ & -3541.254707 & 2.0 & -3542.121339 & 1.7 \\
\hline $\mathrm{TS} 169(\mathrm{~S}, \mathrm{~S})$ & -3541.254601 & 2.1 & -3542.121265 & 1.7 \\
\hline $\mathrm{TS} 176(\mathrm{~S}, \mathrm{~S})$ & -3541.254577 & 2.1 & -3542.118556 & 3.4 \\
\hline $\mathrm{TS} 171(\mathrm{~S}, \mathrm{~S})$ & -3541.254483 & 2.1 & -3542.118611 & 3.4 \\
\hline TS168(S,S) & -3541.254317 & 2.2 & -3542.118495 & 3.5 \\
\hline $\mathrm{TS} 178(\mathrm{~S}, \mathrm{~S})$ & -3541.254139 & 2.4 & -3542.118337 & 3.6 \\
\hline $\mathrm{TS} 177(\mathrm{~S}, \mathrm{~S})$ & -3541.254087 & 2.4 & -3542.118343 & 3.6 \\
\hline $\mathrm{TS} 170(\mathrm{~S}, \mathrm{~S})$ & -3541.254045 & 2.4 & -3542.120682 & 2.1 \\
\hline TS184(S,S) & -3541.253827 & 2.5 & -3542.118034 & 3.8 \\
\hline $\mathrm{TS} 173(\mathrm{~S}, \mathrm{~S})$ & -3541.253792 & 2.6 & -3542.120437 & 2.2 \\
\hline $\mathrm{TS} 179(\mathrm{~S}, \mathrm{~S})$ & -3541.25366 & 2.7 & -3542.120493 & 2.2 \\
\hline TS186(S,S) & -3541.252839 & 3.2 & -3542.119479 & 2.8 \\
\hline
\end{tabular}




$\begin{array}{lcccc}\text { TS181(S,S) } & -3541.252756 & 3.2 & -3542.116904 & 4.5 \\ \text { TS185(S,S) } & -3541.25266 & 3.3 & -3542.119267 & 3.0 \\ \text { TS180(S,S) } & -3541.252578 & 3.3 & -3542.116827 & 4.5 \\ \text { TS174(S,S) } & -3541.252297 & 3.5 & -3542.119068 & 3.1 \\ \text { TS183(S,S) } & -3541.252259 & 3.5 & -3542.116656 & 4.6 \\ \text { TS190(S,S) } & -3541.251882 & 3.8 & -3542.116067 & 5.0 \\ \text { TS175(S,S) } & -3541.251869 & 3.8 & -3542.118571 & 3.4 \\ \text { TS188(S,S) } & -3541.251603 & 3.9 & -3542.115745 & 5.2 \\ \text { TS187(S,S) } & -3541.251538 & 4.0 & -3542.118301 & 3.6 \\ \text { TS182(S,S) } & -3541.251516 & 4.0 & -3542.118209 & 3.6 \\ \text { TS193(S,S) } & -3541.251305 & 4.1 & -3542.11549 & 5.4 \\ \text { TS189(S,S) } & -3541.251298 & 4.1 & -3542.115502 & 5.3 \\ \text { TS192(S,S) } & -3541.250672 & 4.5 & -3542.114938 & 5.7 \\ \text { TS191(S,S) } & -3541.250492 & 4.6 & -3542.114743 & 5.8 \\ \text { TS195(S,S) } & -3541.250316 & 4.7 & -3542.116957 & 4.4 \\ \text { TS194(S,S) } & -3541.249904 & 5.0 & -3542.114116 & 6.2\end{array}$

Table S32. Absolute free energies (298K, in hartrees) at the M06-2X/6-31G(d) and M062X/6-311+G(d,p)//M06-2X/6-31G(d) levels of theory for all transition states for reaction $2 \mathrm{a}$ catalyzed by PA-2 with free energies relative to the lowest-lying $(\mathrm{R}, \mathrm{R})$ transition state (kcal/mol). The Quasi-RRHO model was used to compute the free energies.

\begin{tabular}{|c|c|c|c|c|}
\hline TS Name & G[M06-2X/6-31G(d)] & $\mathbf{G}_{\text {rel }}$ & $\begin{array}{c}\text { G[M06-2X/6-311+G(d,p)// } \\
\text { M06-2X/6-31G(d)] }\end{array}$ & $\mathbf{G}_{\text {rel }}$ \\
\hline \multicolumn{5}{|c|}{$(\mathbf{R}, \mathbf{R})$ Transition States } \\
\hline $\mathrm{TS} 152(\mathrm{R}, \mathrm{R})$ & -3541.243345 & 0.0 & -3542.109393 & 0.0 \\
\hline TS149(R,R) & -3541.243209 & 0.1 & -3542.10938 & 0.0 \\
\hline $\mathrm{TS} 148(\mathrm{R}, \mathrm{R})$ & -3541.243198 & 0.1 & -3542.109328 & 0.0 \\
\hline $\mathrm{TS} 150(\mathrm{R}, \mathrm{R})$ & -3541.243047 & 0.2 & -3542.109055 & 0.2 \\
\hline $\mathrm{TS} 158(\mathrm{R}, \mathrm{R})$ & -3541.242519 & 0.5 & -3542.108653 & 0.5 \\
\hline TS157(R,R) & -3541.242327 & 0.6 & -3542.108472 & 0.6 \\
\hline $\mathrm{TS} 153(\mathrm{R}, \mathrm{R})$ & -3541.242085 & 0.8 & -3542.108219 & 0.7 \\
\hline $\mathrm{TS} 151(\mathrm{R}, \mathrm{R})$ & -3541.241253 & 1.3 & -3542.10735 & 1.3 \\
\hline $\mathrm{TS} 155(\mathrm{R}, \mathrm{R})$ & -3541.240846 & 1.6 & -3542.107049 & 1.5 \\
\hline $\mathrm{TS} 159(\mathrm{R}, \mathrm{R})$ & -3541.240841 & 1.6 & -3542.107005 & 1.5 \\
\hline TS156(R,R) & -3541.240793 & 1.6 & -3542.106888 & 1.6 \\
\hline $\mathrm{TS} 154(\mathrm{R}, \mathrm{R})$ & -3541.2406 & 1.7 & -3542.106743 & 1.7 \\
\hline $\mathrm{TS} 161(\mathrm{R}, \mathrm{R})$ & -3541.240102 & 2.0 & -3542.106394 & 1.9 \\
\hline TS162(R,R) & -3541.239406 & 2.5 & -3542.105583 & 2.4 \\
\hline TS160(R,R) & -3541.239402 & 2.5 & -3542.105537 & 2.4 \\
\hline $\mathrm{TS} 163(\mathrm{R}, \mathrm{R})$ & -3541.238836 & 2.8 & -3542.104938 & 2.8 \\
\hline \multicolumn{5}{|c|}{$(\mathbf{S}, \mathbf{S})$ Transition States } \\
\hline TS166(S,S) & -3541.24119 & 1.4 & -3542.107892 & 0.9 \\
\hline TS167(S,S) & -3541.240925 & 1.5 & -3542.10757 & 1.1 \\
\hline TS172(S,S) & -3541.240832 & 1.6 & -3542.107365 & 1.3 \\
\hline $\mathrm{TS} 171(\mathrm{~S}, \mathrm{~S})$ & -3541.240501 & 1.8 & -3542.10463 & 3.0 \\
\hline TS164(S,S) & -3541.24048 & 1.8 & -3542.106999 & 1.5 \\
\hline TS168(S,S) & -3541.24035 & 1.9 & -3542.104528 & 3.1 \\
\hline $\mathrm{TS} 177(\mathrm{~S}, \mathrm{~S})$ & -3541.240293 & 1.9 & -3542.104549 & 3.0 \\
\hline TS176(S,S) & -3541.240234 & 2.0 & -3542.104212 & 3.3 \\
\hline TS165(S,S) & -3541.239844 & 2.2 & -3542.106476 & 1.8 \\
\hline TS178(S,S) & -3541.239826 & 2.2 & -3542.104024 & 3.4 \\
\hline
\end{tabular}




$\begin{array}{lllll}\text { TS169(S,S) } & -3541.239807 & 2.2 & -3542.106472 & 1.8 \\ \text { TS184(S,S) } & -3541.239717 & 2.3 & -3542.103924 & 3.4 \\ \text { TS170(S,S) } & -3541.238974 & 2.7 & -3542.105611 & 2.4 \\ \text { TS173(S,S) } & -3541.238896 & 2.8 & -3542.105541 & 2.4 \\ \text { TS180(S,S) } & -3541.238608 & 3.0 & -3542.102857 & 4.1 \\ \text { TS181(S,S) } & -3541.238595 & 3.0 & -3542.102742 & 4.2 \\ \text { TS186(S,S) } & -3541.238443 & 3.1 & -3542.105083 & 2.7 \\ \text { TS185(S,S) } & -3541.238334 & 3.1 & -3542.104941 & 2.8 \\ \text { TS174(S,S) } & -3541.238239 & 3.2 & -3542.10501 & 2.8 \\ \text { TS190(S,S) } & -3541.238223 & 3.2 & -3542.102408 & 4.4 \\ \text { TS179(S,S) } & -3541.238122 & 3.3 & -3542.104955 & 2.8 \\ \text { TS188(S,S) } & -3541.23808 & 3.3 & -3542.102222 & 4.5 \\ \text { TS189(S,S) } & -3541.237996 & 3.4 & -3542.102201 & 4.5 \\ \text { TS183(S,S) } & -3541.237954 & 3.4 & -3542.102351 & 4.4 \\ \text { TS175(S,S) } & -3541.237936 & 3.4 & -3542.104638 & 3.0 \\ \text { TS193(S,S) } & -3541.237918 & 3.4 & -3542.102103 & 4.6 \\ \text { TS187(S,S) } & -3541.236905 & 4.0 & -3542.103668 & 3.6 \\ \text { TS192(S,S) } & -3541.236889 & 4.1 & -3542.101154 & 5.2 \\ \text { TS182(S,S) } & -3541.236809 & 4.1 & -3542.103503 & 3.7 \\ \text { TS191(S,S) } & -3541.23678 & 4.1 & -3542.101032 & 5.2 \\ \text { TS194(S,S) } & -3541.23647 & 4.3 & -3542.100682 & 5.5 \\ \text { TS195(S,S) } & -3541.23632 & 4.4 & -3542.102961 & 4.0\end{array}$

Table S33. Absolute free energies (298K, in hartrees) at the B97-D/def2-TZVP and B97D3/def2-TZVP//B97-D/def2-TZVP levels of theory for all transition states for reaction $2 b$ catalyzed by PA-1A with free energies relative to the lowest-lying $(R, R)$ transition state $(\mathrm{kcal} / \mathrm{mol})$. The standard RRHO model was used to compute the free energies.

\begin{tabular}{lllll}
\hline TS Name & G[B97-D/def2-TZVP] & $\mathbf{G}_{\text {rel }}$ & $\begin{array}{c}\text { G[B97-D3/def2-TZVP// } \\
\text { B97-D/def2-TZVP] }\end{array}$ & $\mathbf{G}_{\text {rel }}$ \\
\hline (R,R) Transition States & & & & 0.3 \\
\hline TS196(R,R) & -3633.751531 & 0.0 & -3634.004070 & 0.0 \\
TS197(R,R) & -3633.751285 & 0.2 & -3634.004599 & 0.1 \\
TS198(R,R) & -3633.750983 & 0.3 & -3634.004366 & 0.9 \\
TS199(R,R) & -3633.750835 & 0.4 & -3634.003215 & 0.4 \\
TS200(R,R) & -3633.750757 & 0.5 & -3634.003891 & 1.1 \\
TS201(R,R) & -3633.750507 & 0.6 & -3634.002869 & 0.8 \\
TS202(R,R) & -3633.750451 & 0.7 & -3634.003318 & 1.0 \\
TS203(R,R) & -3633.750421 & 0.7 & -3634.003005 & 0.8 \\
TS204(R,R) & -3633.750236 & 0.8 & -3634.003370 & 0.9 \\
TS205(R,R) & -3633.750232 & 0.8 & -3634.003137 & 1.6 \\
TS206(R,R) & -3633.749920 & 1.0 & -3634.002107 & 1.3 \\
TS207(R,R) & -3633.749875 & 1.0 & -3634.002563 & 1.3 \\
TS208(R,R) & -3633.749830 & 1.1 & -3634.002559 & 1.6 \\
TS209(R,R) & -3633.749806 & 1.1 & -3634.002094 & 1.3 \\
TS210(R,R) & -3633.749491 & 1.3 & -3634.002556 & 1.1 \\
TS211(R,R) & -3633.749423 & 1.3 & -3634.002812 & 1.3 \\
TS212(R,R) & -3633.749324 & 1.4 & -3634.002480 & 1.3 \\
TS213(R,R) & -3633.749230 & 1.4 & -3634.002515 & 1.5 \\
TS214(R,R) & -3633.749225 & 1.4 & -3634.002270 & 1.4 \\
TS215(R,R) & -3633.749157 & 1.5 & -3634.002411 & 2.1 \\
TS216(R,R) & -3633.749148 & 1.5 & -3634.001303 & 1.5 \\
TS217(R,R) & -3633.749084 & 1.5 & -3634.002136 & \\
\end{tabular}




\begin{tabular}{|c|c|c|c|c|}
\hline TS218(R,R) & -3633.749067 & 1.5 & -3634.002732 & 1.2 \\
\hline TS219(R,R) & -3633.748892 & 1.7 & -3634.000949 & 2.3 \\
\hline TS220(R,R) & -3633.748808 & 1.7 & -3634.001836 & 1.7 \\
\hline TS221(R,R) & -3633.748263 & 2.1 & -3634.001460 & 2.0 \\
\hline TS222(R,R) & -3633.748079 & 2.2 & -3634.001222 & 2.1 \\
\hline TS223(R,R) & -3633.747899 & 2.3 & -3634.000879 & 2.3 \\
\hline \multicolumn{5}{|c|}{$(\mathbf{S}, \mathbf{S})$ Transition States } \\
\hline TS224(S,S) & -3633.751266 & 0.2 & -3634.003494 & 0.7 \\
\hline TS225(S,S) & -3633.751150 & 0.2 & -3634.002992 & 1.0 \\
\hline TS226(S,S) & -3633.751065 & 0.3 & -3634.004056 & 0.3 \\
\hline TS227(S,S) & -3633.750985 & 0.3 & -3634.002531 & 1.3 \\
\hline TS228(S,S) & -3633.750936 & 0.4 & -3634.002857 & 1.1 \\
\hline TS229(S,S) & -3633.750215 & 0.8 & -3634.003271 & 0.8 \\
\hline $\mathrm{TS} 230(\mathrm{~S}, \mathrm{~S})$ & -3633.749986 & 1.0 & -3634.002713 & 1.2 \\
\hline $\mathrm{TS} 231(\mathrm{~S}, \mathrm{~S})$ & -3633.749758 & 1.1 & -3634.002791 & 1.1 \\
\hline TS232(S,S) & -3633.749658 & 1.2 & -3634.001114 & 2.2 \\
\hline TS233(S,S) & -3633.749586 & 1.2 & -3634.002862 & 1.1 \\
\hline TS234(S,S) & -3633.749504 & 1.3 & -3634.002130 & 1.5 \\
\hline TS235(S,S) & -3633.749249 & 1.4 & -3634.002500 & 1.3 \\
\hline TS236(S,S) & -3633.749093 & 1.5 & -3634.002581 & 1.3 \\
\hline TS237(S,S) & -3633.748852 & 1.7 & -3633.999085 & 3.5 \\
\hline TS238(S,S) & -3633.748798 & 1.7 & -3634.002122 & 1.6 \\
\hline TS239(S,S) & -3633.748651 & 1.8 & -3633.998867 & 3.6 \\
\hline TS240(S,S) & -3633.748437 & 1.9 & -3634.001276 & 2.1 \\
\hline TS241(S,S) & -3633.748389 & 2.0 & -3634.000549 & 2.5 \\
\hline TS242(S,S) & -3633.748319 & 2.0 & -3634.001206 & 2.1 \\
\hline TS243(S,S) & -3633.748229 & 2.1 & -3634.001093 & 2.2 \\
\hline TS244(S,S) & -3633.748122 & 2.1 & -3634.001179 & 2.1 \\
\hline TS245(S,S) & -3633.747940 & 2.3 & -3633.998050 & 4.1 \\
\hline TS246(S,S) & -3633.747324 & 2.6 & -3633.997513 & 4.4 \\
\hline TS247(S,S) & -3633.747145 & 2.8 & -3633.997021 & 4.8 \\
\hline TS248(S,S) & -3633.747066 & 2.8 & -3633.998668 & 3.7 \\
\hline TS249(S,S) & -3633.746756 & 3.0 & -3633.998287 & 4.0 \\
\hline TS250(S,S) & -3633.742975 & 5.4 & -3633.995301 & 5.8 \\
\hline TS251(S,S) & -3633.742672 & 5.6 & -3633.994856 & 6.1 \\
\hline
\end{tabular}

Table S34. Absolute free energies (298K, in hartrees) at the B97-D/def2-TZVP and B97D3/def2-TZVP//B97-D/def2-TZVP levels of theory for all transition states for reaction $2 b$ catalyzed by PA-1A with free energies relative to the lowest-lying $(\mathrm{R}, \mathrm{R})$ transition state $(\mathrm{kcal} / \mathrm{mol})$. The Quasi-RRHO model was used to compute the free energies. The lowest-lying $(\mathrm{R}, \mathrm{R})$ and $(\mathrm{S}, \mathrm{S})$ transition states at the B97-D3/def2-TZVP//B97-D/def2-TZVP level are in bold.

\begin{tabular}{ccccc}
\hline TS Name & G[B97-D/def2-TZVP] & $\mathbf{G}_{\text {rel }}$ & $\begin{array}{c}\text { G[B97-D3/def2-TZVP// } \\
\text { B97-D/def2-TZVP] }\end{array}$ & $\mathbf{G}_{\text {rel }}$ \\
\hline (R,R) Transition States & & & & \\
\hline TS198(R,R) & $\mathbf{- 3 6 3 3 . 7 3 3 8 6 1}$ & $\mathbf{0 . 0}$ & $\mathbf{- 3 6 3 3 . 9 8 7 2 4 4}$ & $\mathbf{0 . 0}$ \\
TS196(R,R) & -3633.733546 & 0.2 & -3633.986085 & 0.7 \\
TS200(R,R) & -3633.733458 & 0.3 & -3633.986592 & 0.4 \\
TS204(R,R) & -3633.733458 & 0.3 & -3633.986592 & 0.4 \\
TS197(R,R) & -3633.733293 & 0.4 & -3633.986608 & 0.4 \\
TS202(R,R) & -3633.733197 & 0.4 & -3633.986064 & 0.7
\end{tabular}




\begin{tabular}{|c|c|c|c|c|}
\hline TS199(R,R) & -3633.733141 & 0.5 & -3633.985521 & 11 \\
\hline $\mathrm{TS} 211(\mathrm{R}, \mathrm{R})$ & -3633.733062 & 0.5 & -3633.986451 & 0.5 \\
\hline TS203(R,R) & -3633.732998 & 0.5 & -3633.985582 & 1.0 \\
\hline TS208(R,R) & -3633.732970 & 0.6 & -3633.985699 & 1.0 \\
\hline TS207(R,R) & -3633.732932 & 0.6 & -3633.985621 & 1.0 \\
\hline TS201(R,R) & -3633.732890 & 0.6 & -3633.985252 & 1.3 \\
\hline TS210(R,R) & -3633.732749 & 0.7 & -3633.985814 & 0.9 \\
\hline TS215(R,R) & -3633.732728 & 0.7 & -3633.985982 & 0.8 \\
\hline $\mathrm{TS} 214(\mathrm{R}, \mathrm{R})$ & -3633.732692 & 0.7 & -3633.985738 & 0.9 \\
\hline TS205(R,R) & -3633.732658 & 0.8 & -3633.985562 & 1.1 \\
\hline TS209(R,R) & -3633.732652 & 0.8 & -3633.984940 & 1.4 \\
\hline TS213(R,R) & -3633.732639 & 0.8 & -3633.985923 & 0.8 \\
\hline $\mathrm{TS} 212(\mathrm{R}, \mathrm{R})$ & -3633.732628 & 0.8 & -3633.985784 & 0.9 \\
\hline $\mathrm{TS} 217(\mathrm{R}, \mathrm{R})$ & -3633.732609 & 0.8 & -3633.985661 & 1.0 \\
\hline $\mathrm{TS} 220(\mathrm{R}, \mathrm{R})$ & -3633.732485 & 0.9 & -3633.985513 & 1.1 \\
\hline TS206(R,R) & -3633.732459 & 0.9 & -3633.984646 & 1.6 \\
\hline TS216(R,R) & -3633.732385 & 0.9 & -3633.984540 & 1.7 \\
\hline TS219(R,R) & -3633.732239 & 1.0 & -3633.984296 & 1.9 \\
\hline TS218(R,R) & -3633.731946 & 1.2 & -3633.985610 & 1.0 \\
\hline TS221(R,R) & -3633.731823 & 1.3 & -3633.985021 & 1.4 \\
\hline TS222(R,R) & -3633.731818 & 1.3 & -3633.984961 & 1.4 \\
\hline $\mathrm{TS} 223(\mathrm{R}, \mathrm{R})$ & -3633.731499 & 1.5 & -3633.984479 & 1.7 \\
\hline \multicolumn{5}{|c|}{ (S,S) Transition States } \\
\hline $\mathrm{TS} 225(\mathrm{~S}, \mathrm{~S})$ & -3633.733694 & 0.1 & -3633.985536 & 1.1 \\
\hline TS228(S,S) & -3633.733444 & 0.3 & -3633.985364 & 1.2 \\
\hline TS224(S,S) & -3633.733282 & 0.4 & -3633.985510 & 1.1 \\
\hline $\mathrm{TS} 227(\mathrm{~S}, \mathrm{~S})$ & -3633.733265 & 0.4 & -3633.984811 & 1.5 \\
\hline $\mathrm{TS} 239(\mathrm{~S}, \mathrm{~S})$ & -3633.732988 & 0.5 & -3633.983204 & 2.5 \\
\hline $\mathrm{TS} 230(\mathrm{~S}, \mathrm{~S})$ & -3633.732892 & 0.6 & -3633.985618 & 1.0 \\
\hline TS229(S,S) & -3633.732830 & 0.6 & -3633.985886 & 0.9 \\
\hline $\mathrm{TS} 226(\mathrm{~S}, \mathrm{~S})$ & -3633.732614 & 0.8 & -3633.985605 & 1.0 \\
\hline $\mathrm{TS} 237(\mathrm{~S}, \mathrm{~S})$ & -3633.732596 & 0.8 & -3633.982829 & 2.8 \\
\hline $\mathrm{TS} 238(\mathrm{~S}, \mathrm{~S})$ & -3633.732459 & 0.9 & -3633.985783 & 0.9 \\
\hline $\mathrm{TS} 232(\mathrm{~S}, \mathrm{~S})$ & -3633.732397 & 0.9 & -3633.983853 & 2.1 \\
\hline $\mathrm{TS} 242(\mathrm{~S}, \mathrm{~S})$ & -3633.732393 & 0.9 & -3633.985280 & 1.2 \\
\hline $\mathrm{TS} 234(\mathrm{~S}, \mathrm{~S})$ & -3633.732378 & 0.9 & -3633.985004 & 1.4 \\
\hline $\mathrm{TS} 244(\mathrm{~S}, \mathrm{~S})$ & -3633.732253 & 1.0 & -3633.985310 & 1.2 \\
\hline TS235(S,S) & -3633.732137 & 1.1 & -3633.985387 & 1.2 \\
\hline TS240(S,S) & -3633.732002 & 1.2 & -3633.984841 & 1.5 \\
\hline TS243(S,S) & -3633.731868 & 1.3 & -3633.984731 & 1.6 \\
\hline TS247(S,S) & -3633.731860 & 1.3 & -3633.981735 & 3.5 \\
\hline TS231(S,S) & -3633.731816 & 1.3 & -3633.984849 & 1.5 \\
\hline TS233(S,S) & -3633.731788 & 1.3 & -3633.985064 & 1.4 \\
\hline TS245(S,S) & -3633.731437 & 1.5 & -3633.981548 & 3.6 \\
\hline $\mathrm{TS} 241(\mathrm{~S}, \mathrm{~S})$ & -3633.731388 & 1.6 & -3633.983548 & 2.3 \\
\hline TS236(S,S) & -3633.731305 & 1.6 & -3633.984793 & 1.5 \\
\hline $\mathrm{TS} 248(\mathrm{~S}, \mathrm{~S})$ & -3633.731088 & 1.7 & -3633.982690 & 2.9 \\
\hline TS249(S,S) & -3633.730993 & 1.8 & -3633.982524 & 3.0 \\
\hline $\mathrm{TS} 246(\mathrm{~S}, \mathrm{~S})$ & -3633.730941 & 1.8 & -3633.981130 & 3.8 \\
\hline $\mathrm{TS} 250(\mathrm{~S}, \mathrm{~S})$ & -3633.727149 & 4.2 & -3633.979475 & 4.9 \\
\hline $\mathrm{TS} 251(\mathrm{~S}, \mathrm{~S})$ & -3633.726963 & 4.3 & -3633.979146 & 5.1 \\
\hline
\end{tabular}


Table S35. Absolute free energies (298K, in hartrees) at the wB97XD/6-31G(d) and wB97XD/6-311+G(d,p) // wB97XD/6-31G(d) levels of theory for all transition states for reaction $2 \mathrm{~b}$ catalyzed by PA-1A with free energies relative to the lowest-lying $(R, R)$ transition state $(\mathrm{kcal} / \mathrm{mol})$. The standard RRHO model was used to compute the free energies. TS209(R,R) has two imaginary frequencies.

\begin{tabular}{|c|c|c|c|c|}
\hline TS Name & G[wB97XD/6-31G(d)] & $\mathbf{G}_{\text {rel }}$ & $\begin{array}{c}\text { G[wB97XD/6-311+G(d,p)// } \\
\text { wB97XD/6-31G(d)] }\end{array}$ & $\mathbf{G}_{\text {rel }}$ \\
\hline \multicolumn{5}{|c|}{$(\mathbf{R}, \mathbf{R})$ Transition States } \\
\hline $\mathrm{TS} 201(\mathrm{R}, \mathrm{R})$ & -3633.719478 & 0.0 & -3634.468926 & 0.0 \\
\hline TS222(R,R) & -3633.718843 & 0.4 & -3634.468134 & 0.5 \\
\hline TS197(R,R) & -3633.718639 & 0.5 & -3634.467889 & 0.7 \\
\hline TS210(R,R) & -3633.718627 & 0.5 & -3634.467583 & 0.8 \\
\hline TS220(R,R) & -3633.718518 & 0.6 & -3634.467473 & 0.9 \\
\hline TS217(R,R) & -3633.718508 & 0.6 & -3634.467463 & 0.9 \\
\hline $\mathrm{TS} 218(\mathrm{R}, \mathrm{R})$ & -3633.718449 & 0.6 & -3634.467373 & 1.0 \\
\hline TS213(R,R) & -3633.718421 & 0.7 & -3634.467097 & 1.1 \\
\hline TS204(R,R) & -3633.718395 & 0.7 & -3634.466946 & 1.2 \\
\hline TS206(R,R) & -3633.718359 & 0.7 & -3634.467674 & 0.8 \\
\hline TS214(R,R) & -3633.718332 & 0.7 & -3634.467054 & 1.2 \\
\hline TS211(R,R) & -3633.718326 & 0.7 & -3634.467179 & 1.1 \\
\hline $\mathrm{TS} 212(\mathrm{R}, \mathrm{R})$ & -3633.718318 & 0.7 & -3634.467040 & 1.2 \\
\hline TS223(R,R) & -3633.717869 & 1.0 & -3634.466553 & 1.5 \\
\hline TS205(R,R) & -3633.717802 & 1.1 & -3634.466947 & 1.2 \\
\hline TS207(R,R) & -3633.717754 & 1.1 & -3634.466883 & 1.3 \\
\hline TS208(R,R) & -3633.717751 & 1.1 & -3634.466880 & 1.3 \\
\hline TS221(R,R) & -3633.717695 & 1.1 & -3634.466607 & 1.5 \\
\hline TS199(R,R) & -3633.717643 & 1.2 & -3634.467095 & 1.1 \\
\hline TS203(R,R) & -3633.717626 & 1.2 & -3634.467151 & 1.1 \\
\hline TS196(R,R) & -3633.717600 & 1.2 & -3634.467052 & 1.2 \\
\hline TS198(R,R) & -3633.717195 & 1.4 & -3634.466283 & 1.7 \\
\hline TS215(R,R) & -3633.717011 & 1.5 & -3634.465966 & 1.9 \\
\hline TS200(R,R) & -3633.717007 & 1.6 & -3634.465662 & 2.0 \\
\hline TS216(R,R) & -3633.716338 & 2.0 & -3634.465455 & 2.2 \\
\hline TS219(R,R) & -3633.716331 & 2.0 & -3634.465448 & 2.2 \\
\hline TS209(R,R) & -3633.715988 & 2.2 & -3634.465465 & 2.2 \\
\hline TS202(R,R) & -3633.715459 & 2.5 & -3634.464357 & 2.9 \\
\hline \multicolumn{5}{|c|}{$(\mathbf{S , S})$ Transition States } \\
\hline $\mathrm{TS} 228(\mathrm{~S}, \mathrm{~S})$ & -3633.718946 & 0.3 & -3634.468266 & 0.4 \\
\hline $\mathrm{TS} 225(\mathrm{~S}, \mathrm{~S})$ & -3633.717967 & 0.9 & -3634.467335 & 1.0 \\
\hline $\mathrm{TS} 240(\mathrm{~S}, \mathrm{~S})$ & -3633.717937 & 1.0 & -3634.467166 & 1.1 \\
\hline $\mathrm{TS} 244(\mathrm{~S}, \mathrm{~S})$ & -3633.717860 & 1.0 & -3634.467278 & 1.0 \\
\hline $\mathrm{TS} 235(\mathrm{~S}, \mathrm{~S})$ & -3633.717710 & 1.1 & -3634.467178 & 1.1 \\
\hline $\mathrm{TS} 230(\mathrm{~S}, \mathrm{~S})$ & -3633.717422 & 1.3 & -3634.466544 & 1.5 \\
\hline TS243(S,S) & -3633.717245 & 1.4 & -3634.466605 & 1.5 \\
\hline $\mathrm{TS} 232(\mathrm{~S}, \mathrm{~S})$ & -3633.717121 & 1.5 & -3634.466207 & 1.7 \\
\hline TS227(S,S) & -3633.716709 & 1.7 & -3634.465765 & 2.0 \\
\hline $\mathrm{TS} 229(\mathrm{~S}, \mathrm{~S})$ & -3633.716670 & 1.8 & -3634.466047 & 1.8 \\
\hline $\mathrm{TS} 241(\mathrm{~S}, \mathrm{~S})$ & -3633.716567 & 1.8 & -3634.466110 & 1.8 \\
\hline $\mathrm{TS} 242(\mathrm{~S}, \mathrm{~S})$ & -3633.716200 & 2.1 & -3634.465394 & 2.2 \\
\hline TS233(S,S) & -3633.716073 & 2.1 & -3634.465503 & 2.1 \\
\hline $\mathrm{TS} 249(\mathrm{~S}, \mathrm{~S})$ & -3633.715695 & 2.4 & -3634.463689 & 3.3 \\
\hline TS248(S,S) & -3633.715685 & 2.4 & -3634.463564 & 3.4 \\
\hline
\end{tabular}




$\begin{array}{lllll}\text { TS234(S,S) } & -3633.715556 & 2.5 & -3634.464614 & 2.7 \\ \text { TS224(S,S) } & -3633.715328 & 2.6 & -3634.464631 & 2.7 \\ \text { TS237(S,S) } & -3633.715128 & 2.7 & -3634.463341 & 3.5 \\ \text { TS238(S,S) } & -3633.715078 & 2.8 & -3634.464457 & 2.8 \\ \text { TS236(S,S) } & -3633.714797 & 2.9 & -3634.463662 & 3.3 \\ \text { TS231(S,S) } & -3633.714193 & 3.3 & -3634.463735 & 3.3 \\ \text { TS239(S,S) } & -3633.714172 & 3.3 & -3634.462899 & 3.8 \\ \text { TS226(S,S) } & -3633.713975 & 3.5 & -3634.463451 & 3.4 \\ \text { TS247(S,S) } & -3633.713523 & 3.7 & -3634.461818 & 4.5 \\ \text { TS251(S,S) } & -3633.712589 & 4.3 & -3634.460282 & 5.4 \\ \text { TS245(S,S) } & -3633.711635 & 4.9 & -3634.460209 & 5.5 \\ \text { TS246(S,S) } & -3633.711352 & 5.1 & -3634.459709 & 5.8 \\ \text { TS250(S,S) } & -3633.711132 & 5.2 & -3634.458843 & 6.3\end{array}$

Table S36. Absolute free energies (298K, in hartrees) at the wB97XD/6-31G(d) and wB97XD/6-311+G(d,p) // wB97XD/6-31G(d) levels of theory for all transition states for reaction $2 \mathrm{~b}$ catalyzed by PA-1A with free energies relative to the lowest-lying $(R, R)$ transition state $(\mathrm{kcal} / \mathrm{mol})$. The Quasi-RRHO model was used to compute the free energies. TS209(R,R) has two imaginary frequencies.

\begin{tabular}{|c|c|c|c|c|}
\hline TS Name & G[wB97XD/6-31G(d)] & $\mathbf{G}_{\text {rel }}$ & $\begin{array}{c}\text { G[wB97XD/6-311+G(d,p)// } \\
\text { wB97XD/6-31G(d)] }\end{array}$ & $\mathbf{G}_{\text {rel }}$ \\
\hline \multicolumn{5}{|c|}{$(\mathbf{R}, \mathbf{R})$ Transition States } \\
\hline TS204(R,R) & -3633.704004 & 0.0 & -3634.452555 & 0.4 \\
\hline TS222(R,R) & -3633.703948 & 0.0 & -3634.453239 & 0.0 \\
\hline TS214(R,R) & -3633.703817 & 0.1 & -3634.452539 & 0.4 \\
\hline TS212(R,R) & -3633.703806 & 0.1 & -3634.452528 & 0.4 \\
\hline TS211(R,R) & -3633.703754 & 0.2 & -3634.452607 & 0.4 \\
\hline TS218(R,R) & -3633.703725 & 0.2 & -3634.452649 & 0.4 \\
\hline TS213(R,R) & -3633.703642 & 0.2 & -3634.452317 & 0.6 \\
\hline TS210(R,R) & -3633.703180 & 0.5 & -3634.452136 & 0.7 \\
\hline TS221(R,R) & -3633.703147 & 0.5 & -3634.452059 & 0.7 \\
\hline TS223(R,R) & -3633.703128 & 0.5 & -3634.451812 & 0.9 \\
\hline TS220(R,R) & -3633.703128 & 0.5 & -3634.452083 & 0.7 \\
\hline TS217(R,R) & -3633.703127 & 0.6 & -3634.452082 & 0.7 \\
\hline TS197(R,R) & -3633.703062 & 0.6 & -3634.452312 & 0.6 \\
\hline TS198(R,R) & -3633.702896 & 0.7 & -3634.451984 & 0.8 \\
\hline TS201(R,R) & -3633.702891 & 0.7 & -3634.452339 & 0.6 \\
\hline TS205(R,R) & -3633.702650 & 0.8 & -3634.451795 & 0.9 \\
\hline TS207(R,R) & -3633.702449 & 1.0 & -3634.451578 & 1.0 \\
\hline TS208(R,R) & -3633.702448 & 1.0 & -3634.451577 & 1.0 \\
\hline TS215(R,R) & -3633.702387 & 1.0 & -3634.451343 & 1.2 \\
\hline TS200(R,R) & -3633.702380 & 1.0 & -3634.451035 & 1.4 \\
\hline TS203(R,R) & -3633.702063 & 1.2 & -3634.451588 & 1.0 \\
\hline TS206(R,R) & -3633.702060 & 1.2 & -3634.451375 & 1.2 \\
\hline TS216(R,R) & -3633.701788 & 1.4 & -3634.450904 & 1.5 \\
\hline TS219(R,R) & -3633.701780 & 1.4 & -3634.450897 & 1.5 \\
\hline TS199(R,R) & -3633.701714 & 1.4 & -3634.451166 & 1.3 \\
\hline TS196(R,R) & -3633.701688 & 1.5 & -3634.451140 & 1.3 \\
\hline TS209(R,R) & -3633.701241 & 1.7 & -3634.450718 & 1.6 \\
\hline TS202(R,R) & -3633.701141 & 1.8 & -3634.450039 & 2.0 \\
\hline
\end{tabular}




\begin{tabular}{|c|c|c|c|c|}
\hline TS228(S,S) & -3633.703317 & 0.4 & -3634.452637 & 0.4 \\
\hline $\mathrm{TS} 235(\mathrm{~S}, \mathrm{~S})$ & -3633.702710 & 0.8 & -3634.452177 & 0.7 \\
\hline $\mathrm{TS} 243(\mathrm{~S}, \mathrm{~S})$ & -3633.702628 & 0.9 & -3634.451988 & 0.8 \\
\hline TS244(S,S) & -3633.702586 & 0.9 & -3634.452004 & 0.8 \\
\hline TS230(S,S) & -3633.702576 & 0.9 & -3634.451698 & 1.0 \\
\hline $\mathrm{TS} 240(\mathrm{~S}, \mathrm{~S})$ & -3633.702524 & 0.9 & -3634.451753 & 0.9 \\
\hline $\mathrm{TS} 225(\mathrm{~S}, \mathrm{~S})$ & -3633.702345 & 1.0 & -3634.451713 & 1.0 \\
\hline TS242(S,S) & -3633.702186 & 1.1 & -3634.451380 & 1.2 \\
\hline TS229(S,S) & -3633.701834 & 1.4 & -3634.451211 & 1.3 \\
\hline TS234(S,S) & -3633.701521 & 1.6 & -3634.450579 & 1.7 \\
\hline $\mathrm{TS} 232(\mathrm{~S}, \mathrm{~S})$ & -3633.701354 & 1.7 & -3634.450440 & 1.8 \\
\hline TS249(S,S) & -3633.701162 & 1.8 & -3634.449156 & 2.6 \\
\hline TS238(S,S) & -3633.701105 & 1.8 & -3634.450484 & 1.7 \\
\hline TS227(S,S) & -3633.701103 & 1.8 & -3634.450159 & 1.9 \\
\hline $\mathrm{TS} 237(\mathrm{~S}, \mathrm{~S})$ & -3633.701095 & 1.8 & -3634.449307 & 2.5 \\
\hline $\mathrm{TS} 248(\mathrm{~S}, \mathrm{~S})$ & -3633.700940 & 1.9 & -3634.448819 & 2.8 \\
\hline TS241(S,S) & -3633.700364 & 2.3 & -3634.449907 & 2.1 \\
\hline TS224(S,S) & -3633.700224 & 2.4 & -3634.449526 & 2.3 \\
\hline $\mathrm{TS} 247(\mathrm{~S}, \mathrm{~S})$ & -3633.700217 & 2.4 & -3634.448512 & 3.0 \\
\hline TS233(S,S) & -3633.700110 & 2.4 & -3634.449541 & 2.3 \\
\hline TS236(S,S) & -3633.699842 & 2.6 & -3634.448707 & 2.8 \\
\hline TS239(S,S) & -3633.699720 & 2.7 & -3634.448447 & 3.0 \\
\hline TS226(S,S) & -3633.699374 & 2.9 & -3634.448851 & 2.8 \\
\hline TS231(S,S) & -3633.699319 & 2.9 & -3634.448861 & 2.7 \\
\hline TS251(S,S) & -3633.697885 & 3.8 & -3634.445578 & 4.8 \\
\hline TS246(S,S) & -3633.697211 & 4.3 & -3634.445568 & 4.8 \\
\hline $\mathrm{TS} 245(\mathrm{~S}, \mathrm{~S})$ & -3633.697102 & 4.3 & -3634.445676 & 4.7 \\
\hline $\mathrm{TS} 250(\mathrm{~S}, \mathrm{~S})$ & -3633.697053 & 4.4 & -3634.444763 & 5.3 \\
\hline
\end{tabular}

Table S37. Absolute free energies (298K, in hartrees) at the M06-2X/6-31G(d) and M06$2 \mathrm{X} / 6-311+\mathrm{G}(\mathrm{d}, \mathrm{p}) / / \mathrm{M} 06-2 \mathrm{X} / 6-31 \mathrm{G}(\mathrm{d})$ levels of theory for all transition states for reaction $2 \mathrm{~b}$ catalyzed by PA-1A with free energies relative to the lowest-lying $(\mathrm{R}, \mathrm{R})$ transition state (kcal/mol). The standard RRHO model was used to compute the free energies.

\begin{tabular}{ccccc}
\hline TS Name & G[M06-2X/6-31G(d)] & $\mathbf{G}_{\text {rel }}$ & $\begin{array}{c}\text { G[M06-2X/6-311+G(d,p)// } \\
\text { M06-2X/6-31G(d)] }\end{array}$ & $\mathbf{G}_{\text {rel }}$ \\
\hline (R,R) Transition States & & & & 0.0 \\
\hline TS199(R,R) & -3633.306823 & 0.0 & -3634.139032 & 0.0 \\
TS196(R,R) & -3633.306812 & 0.0 & -3634.139022 & 0.0 \\
TS209(R,R) & -3633.306810 & 0.0 & -3634.138984 & 0.0 \\
TS203(R,R) & -3633.306804 & 0.0 & -3634.138977 & 0.5 \\
TS201(R,R) & -3633.306259 & 0.4 & -3634.138258 & 0.6 \\
TS206(R,R) & -3633.306059 & 0.5 & -3634.138029 & 1.4 \\
TS216(R,R) & -3633.305117 & 1.1 & -3634.136783 & 1.5 \\
TS204(R,R) & -3633.305020 & 1.1 & -3634.136706 & 2.0 \\
TS215(R,R) & -3633.304233 & 1.6 & -3634.135784 & 1.8 \\
TS211(R,R) & -3633.304168 & 1.7 & -3634.136128 & 1.8 \\
TS218(R,R) & -3633.304038 & 1.7 & -3634.136226 & 1.6 \\
TS197(R,R) & -3633.304023 & 1.8 & -3634.136517 & 2.2 \\
TS213(R,R) & -3633.303954 & 1.8 & -3634.135584 & 2.3 \\
TS205(R,R) & -3633.303644 & 2.0 & -3634.135361 & 2.3 \\
TS200(R,R) & -3633.303633 & 2.0 & -3634.135324 & 2.5 \\
TS207(R,R) & -3633.303497 & 2.1 & -3634.135062 &
\end{tabular}




\begin{tabular}{|c|c|c|c|c|}
\hline TS208(R,R) & -3633.303497 & 2.1 & -3634.135062 & 2.5 \\
\hline TS219(R,R) & -3633.303440 & 2.1 & -3634.134977 & 2.5 \\
\hline TS210(R,R) & -3633.303404 & 2.1 & -3634.135038 & 2.5 \\
\hline TS217(R,R) & -3633.303403 & 2.1 & -3634.135034 & 2.5 \\
\hline TS220(R,R) & -3633.303397 & 2.1 & -3634.135030 & 2.5 \\
\hline TS202(R,R) & -3633.303142 & 2.3 & -3634.134687 & 2.7 \\
\hline TS214(R,R) & -3633.302924 & 2.4 & -3634.134661 & 2.7 \\
\hline TS212(R,R) & -3633.302923 & 2.4 & -3634.134660 & 2.7 \\
\hline TS221(R,R) & -3633.302917 & 2.5 & -3634.134935 & 2.6 \\
\hline TS198(R,R) & -3633.302754 & 2.6 & -3634.134935 & 2.6 \\
\hline TS223(R,R) & -3633.302574 & 2.7 & -3634.134357 & 2.9 \\
\hline TS222(R,R) & -3633.302522 & 2.7 & -3634.134705 & 2.7 \\
\hline \multicolumn{5}{|c|}{$(\mathbf{S}, \mathbf{S})$ Transition States } \\
\hline TS225(S,S) & -3633.308242 & -0.9 & -3634.140155 & -0.7 \\
\hline $\mathrm{TS} 228(\mathrm{~S}, \mathrm{~S})$ & -3633.307126 & -0.2 & -3634.139006 & 0.0 \\
\hline $\mathrm{TS} 241(\mathrm{~S}, \mathrm{~S})$ & -3633.306056 & 0.5 & -3634.138102 & 0.6 \\
\hline TS224(S,S) & -3633.305920 & 0.6 & -3634.138052 & 0.6 \\
\hline TS232(S,S) & -3633.305815 & 0.6 & -3634.137529 & 0.9 \\
\hline TS247(S,S) & -3633.304403 & 1.5 & -3634.135218 & 2.4 \\
\hline TS227(S,S) & -3633.304299 & 1.6 & -3634.136038 & 1.9 \\
\hline TS238(S,S) & -3633.303861 & 1.9 & -3634.136733 & 1.4 \\
\hline TS229(S,S) & -3633.303686 & 2.0 & -3634.136386 & 1.7 \\
\hline TS237(S,S) & -3633.303608 & 2.0 & -3634.134178 & 3.0 \\
\hline TS234(S,S) & -3633.303247 & 2.2 & -3634.135461 & 2.2 \\
\hline TS242(S,S) & -3633.303137 & 2.3 & -3634.135723 & 2.1 \\
\hline TS230(S,S) & -3633.303128 & 2.3 & -3634.135466 & 2.2 \\
\hline TS239(S,S) & -3633.302986 & 2.4 & -3634.134432 & 2.9 \\
\hline TS231(S,S) & -3633.302736 & 2.6 & -3634.134899 & 2.6 \\
\hline TS226(S,S) & -3633.302651 & 2.6 & -3634.134752 & 2.7 \\
\hline TS240(S,S) & -3633.302642 & 2.6 & -3634.135174 & 2.4 \\
\hline TS244(S,S) & -3633.302634 & 2.6 & -3634.135231 & 2.4 \\
\hline TS243(S,S) & -3633.302568 & 2.7 & -3634.135224 & 2.4 \\
\hline TS249(S,S) & -3633.302470 & 2.7 & -3634.133211 & 3.7 \\
\hline TS235(S,S) & -3633.302381 & 2.8 & -3634.135310 & 2.3 \\
\hline TS248(S,S) & -3633.302330 & 2.8 & -3634.132860 & 3.9 \\
\hline TS233(S,S) & -3633.302322 & 2.8 & -3634.134390 & 2.9 \\
\hline TS246(S,S) & -3633.300617 & 3.9 & -3634.131133 & 5.0 \\
\hline TS245(S,S) & -3633.300229 & 4.1 & -3634.131996 & 4.4 \\
\hline TS236(S,S) & -3633.300184 & 4.2 & -3634.131830 & 4.5 \\
\hline TS251(S,S) & -3633.299620 & 4.5 & -3634.129491 & 6.0 \\
\hline TS250(S,S) & -3633.298671 & 5.1 & -3634.128428 & 6.7 \\
\hline
\end{tabular}

Table S38. Absolute free energies (298K, in hartrees) at the M06-2X/6-31G(d) and M062X/6-311+G(d,p) // M06-2X/6-31G(d) levels of theory for all transition states for reaction $2 \mathrm{~b}$ catalyzed by PA-1A with free energies relative to the lowest-lying $(\mathrm{R}, \mathrm{R})$ transition state (kcal/mol). The Quasi-RRHO model was used to compute the free energies.

\begin{tabular}{ccccc}
\hline TS Name & \multirow{2}{*}{ G[M06-2X/6-31G(d)] } & G & $\begin{array}{c}\text { G[M06-2X/6-311+G(d,p)// } \\
\text { M06-2X/6-31G(d)] }\end{array}$ & G $_{\text {rel }}$ \\
\hline (R,R) Transition States & & & & \\
\hline TS199(R,R) & -3633.290163 & 0.0 & -3634.122372 & 0.0 \\
TS196(R,R) & -3633.290157 & 0.0 & -3634.122367 & 0.0
\end{tabular}




\begin{tabular}{|c|c|c|c|c|}
\hline TS209(R,R) & -3633290127 & 0 & -3634122301 & 0 \\
\hline TS203(R,R) & -3633.290123 & 0.0 & -3634.122297 & 0.0 \\
\hline TS201(R,R) & -3633.289902 & 0.2 & -3634.121901 & 0.3 \\
\hline TS206(R,R) & -3633.289691 & 0.3 & -3634.121661 & 0.4 \\
\hline TS204(R,R) & -3633.289209 & 0.6 & -3634.120895 & 0.9 \\
\hline TS216(R,R) & -3633.289004 & 0.7 & -3634.120670 & 1.1 \\
\hline TS211(R,R) & -3633.288733 & 0.9 & -3634.120693 & 1.1 \\
\hline TS215(R,R) & -3633.288567 & 1.0 & -3634.120118 & 1.4 \\
\hline TS218(R,R) & -3633.288560 & 1.0 & -3634.120748 & 1.0 \\
\hline TS213(R,R) & -3633.288444 & 1.1 & -3634.120073 & 1.4 \\
\hline TS197(R,R) & -3633.288302 & 1.2 & -3634.120796 & 1.0 \\
\hline TS200(R,R) & -3633.288193 & 1.2 & -3634.119884 & 1.6 \\
\hline TS205(R,R) & -3633.288102 & 1.3 & -3634.119819 & 1.6 \\
\hline TS208(R,R) & -3633.288069 & 1.3 & -3634.119634 & 1.7 \\
\hline TS207(R,R) & -3633.288069 & 1.3 & -3634.119634 & 1.7 \\
\hline $\mathrm{TS} 210(\mathrm{R}, \mathrm{R})$ & -3633.288017 & 1.3 & -3634.119650 & 1.7 \\
\hline TS217(R,R) & -3633.288016 & 1.3 & -3634.119647 & 1.7 \\
\hline TS220(R,R) & -3633.288013 & 1.3 & -3634.119646 & 1.7 \\
\hline TS214(R,R) & -3633.287997 & 1.4 & -3634.119734 & 1.7 \\
\hline $\mathrm{TS} 212(\mathrm{R}, \mathrm{R})$ & -3633.287997 & 1.4 & -3634.119734 & 1.7 \\
\hline TS219(R,R) & -3633.287996 & 1.4 & -3634.119533 & 1.8 \\
\hline TS221(R,R) & -3633.287986 & 1.4 & -3634.120005 & 1.5 \\
\hline TS202(R,R) & -3633.287868 & 1.4 & -3634.119413 & 1.9 \\
\hline TS198(R,R) & -3633.287842 & 1.5 & -3634.120023 & 1.5 \\
\hline TS223(R,R) & -3633.287700 & 1.5 & -3634.119483 & 1.8 \\
\hline $\mathrm{TS} 222(\mathrm{R}, \mathrm{R})$ & -3633.287604 & 1.6 & -3634.119787 & 1.6 \\
\hline \multicolumn{5}{|c|}{ (S,S) Transition States } \\
\hline $\mathrm{TS} 225(\mathrm{~S}, \mathrm{~S})$ & -3633.291447 & -0.8 & -3634.123360 & $\overline{-0.6}$ \\
\hline $\mathrm{TS} 228(\mathrm{~S}, \mathrm{~S})$ & -3633.290848 & -0.4 & -3634.122728 & -0.2 \\
\hline $\mathrm{TS} 224(\mathrm{~S}, \mathrm{~S})$ & -3633.289837 & 0.2 & -3634.121969 & 0.3 \\
\hline $\mathrm{TS} 241(\mathrm{~S}, \mathrm{~S})$ & -3633.289827 & 0.2 & -3634.121872 & 0.3 \\
\hline $\mathrm{TS} 232(\mathrm{~S}, \mathrm{~S})$ & -3633.289667 & 0.3 & -3634.121381 & 0.6 \\
\hline $\mathrm{TS} 247(\mathrm{~S}, \mathrm{~S})$ & -3633.289393 & 0.5 & -3634.120208 & 1.4 \\
\hline $\mathrm{TS} 237(\mathrm{~S}, \mathrm{~S})$ & -3633.289002 & 0.7 & -3634.119572 & 1.8 \\
\hline $\mathrm{TS} 227(\mathrm{~S}, \mathrm{~S})$ & -3633.288852 & 0.8 & -3634.120591 & 1.1 \\
\hline $\mathrm{TS} 234(\mathrm{~S}, \mathrm{~S})$ & -3633.288582 & 1.0 & -3634.120796 & 1.0 \\
\hline TS239(S,S) & -3633.288515 & 1.0 & -3634.119960 & 1.5 \\
\hline $\mathrm{TS} 230(\mathrm{~S}, \mathrm{~S})$ & -3633.288344 & 1.1 & -3634.120682 & 1.1 \\
\hline $\mathrm{TS} 238(\mathrm{~S}, \mathrm{~S})$ & -3633.287958 & 1.4 & -3634.120830 & 1.0 \\
\hline $\mathrm{TS} 229(\mathrm{~S}, \mathrm{~S})$ & -3633.287949 & 1.4 & -3634.120649 & 1.1 \\
\hline $\mathrm{TS} 242(\mathrm{~S}, \mathrm{~S})$ & -3633.287946 & 1.4 & -3634.120532 & 1.2 \\
\hline $\mathrm{TS} 249(\mathrm{~S}, \mathrm{~S})$ & -3633.287888 & 1.4 & -3634.118628 & 2.3 \\
\hline $\mathrm{TS} 248(\mathrm{~S}, \mathrm{~S})$ & -3633.287737 & 1.5 & -3634.118267 & 2.6 \\
\hline $\mathrm{TS} 243(\mathrm{~S}, \mathrm{~S})$ & -3633.287476 & 1.7 & -3634.120132 & 1.4 \\
\hline $\mathrm{TS} 244(\mathrm{~S}, \mathrm{~S})$ & -3633.287338 & 1.8 & -3634.119935 & 1.5 \\
\hline $\mathrm{TS} 240(\mathrm{~S}, \mathrm{~S})$ & -3633.287319 & 1.8 & -3634.119851 & 1.6 \\
\hline $\mathrm{TS} 235(\mathrm{~S}, \mathrm{~S})$ & -3633.286972 & 2.0 & -3634.119901 & 1.6 \\
\hline $\mathrm{TS} 231(\mathrm{~S}, \mathrm{~S})$ & -3633.286877 & 2.1 & -3634.119039 & 2.1 \\
\hline TS226(S,S) & -3633.286806 & 2.1 & -3634.118907 & 2.2 \\
\hline TS246(S,S) & -3633.286064 & 2.6 & -3634.116580 & 3.6 \\
\hline TS233(S,S) & -3633.285983 & 2.6 & -3634.118051 & 2.7 \\
\hline $\mathrm{TS} 245(\mathrm{~S}, \mathrm{~S})$ & -3633.285670 & 2.8 & -3634.117437 & 3.1 \\
\hline TS251(S,S) & -3633.285545 & 2.9 & -3634.115416 & 4.4 \\
\hline TS236(S.S) & -3633.285063 & 3.2 & -3634.116710 & 3.6 \\
\hline
\end{tabular}


Table S39. Absolute free energies (298K, in hartrees) at the B97-D/def2-TZVP and B97D3/def2-TZVP//B97-D/def2-TZVP levels of theory for all transition states for reaction $2 b$ catalyzed by PA-1B with free energies relative to the lowest-lying $(R, R)$ transition state $(\mathrm{kcal} / \mathrm{mol})$. The standard RRHO model was used to compute the free energies.

\begin{tabular}{ccccc}
\hline TS Name & G[B97-D/def2-TZVP] & $\mathbf{G}_{\text {rel }}$ & $\begin{array}{c}\text { G[B97-D3/def2-TZVP// } \\
\text { B97-D/def2-TZVP] }\end{array}$ & $\mathbf{G}_{\text {rel }}$ \\
\hline (R,R) Transition States & & & & \\
\hline TS252(R,R) & -3540.909813 & 0.0 & -3541.176112 & 0.0 \\
TS253(R,R) & -3540.909775 & 0.0 & -3541.176073 & 0.0 \\
\hline (S,S) Transition States & & & & \\
\hline TS254(S,S) & -3540.909917 & -0.1 & -3541.175800 & 0.2 \\
TS255(S,S) & -3540.909444 & 0.2 & -3541.174744 & 0.9 \\
TS256(S,S) & -3540.908136 & 1.1 & -3541.174442 & 1.0
\end{tabular}

Table S40. Absolute free energies (298K, in hartrees) at the B97-D/def2-TZVP and B97D3/def2-TZVP//B97-D/def2-TZVP levels of theory for all transition states for reaction $2 b$ catalyzed by PA-1B with free energies relative to the lowest-lying $(R, R)$ transition state (kcal/mol). The Quasi-RRHO model was used to compute the free energies. The lowest-lying $(\mathrm{R}, \mathrm{R})$ and $(\mathrm{S}, \mathrm{S})$ transition states at the B97-D3/def2-TZVP//B97-D/def2-TZVP level are in bold.

\begin{tabular}{ccccc}
\hline TS Name & G[B97-D/def2-TZVP] & $\mathbf{G}_{\text {rel }}$ & $\begin{array}{c}\text { G[B97-D3/def2-TZVP// } \\
\text { B97-D/def2-TZVP] }\end{array}$ & $\mathbf{G}_{\text {rel }}$ \\
\hline (R,R) Transition States & & & & \\
\hline TS252(R,R) & $\mathbf{- 3 5 4 0 . 8 9 5 8 2 7}$ & $\mathbf{0 . 0}$ & $\mathbf{- 3 5 4 1 . 1 6 2 1 2 6}$ & $\mathbf{0 . 0}$ \\
TS253(R,R) & -3540.895808 & 0.0 & -3541.162106 & 0.0 \\
\hline (S,S) Transition States & & & & \\
\hline TS254(S,S) & $\mathbf{- 3 5 4 0 . 8 9 5 7 5 1}$ & $\mathbf{0 . 0}$ & $\mathbf{- 3 5 4 1 . 1 6 1 6 3 4}$ & $\mathbf{0 . 3}$ \\
TS255(S,S) & -3540.895392 & 0.3 & -3541.160693 & 0.9 \\
TS256(S,S) & -3540.894434 & 0.9 & -3541.160740 & 0.9
\end{tabular}

Table S41. Absolute free energies (298K, in hartrees) at the B97-D/def2-TZVP and B97D3/def2-TZVP//B97-D/def2-TZVP levels of theory for all transition states for reaction $2 \mathrm{~b}$ catalyzed by PA- 2 with free energies relative to the lowest-lying $(R, R)$ transition state $(\mathrm{kcal} / \mathrm{mol})$. The standard RRHO model was used to compute the free energies.

\begin{tabular}{ccccr}
\hline TS Name & G[B97-D/def2-TZVP] & G $_{\text {rel }}$ & $\begin{array}{c}\text { G[B97-D3/def2-TZVP// } \\
\text { B97-D/def2-TZVP] }\end{array}$ & G $_{\text {rel }}$ \\
\hline (R,R) Transition States & & & & \\
\hline TS257(R,R) & -3864.567330 & 0.0 & -3864.847507 & 0.0 \\
TS258(R,R) & -3864.566972 & 0.2 & -3864.846689 & 0.5 \\
TS259(R,R) & -3864.566961 & 0.2 & -3864.847269 & 0.1 \\
TS260(R,R) & -3864.566755 & 0.4 & -3864.846634 & 0.5 \\
TS261(R,R) & -3864.566740 & 0.4 & -3864.846751 & 0.5 \\
TS262(R,R) & -3864.566324 & 0.6 & -3864.846138 & 0.9 \\
TS263(R,R) & -3864.565740 & 1.0 & -3864.846275 & 0.8
\end{tabular}




\begin{tabular}{|c|c|c|c|c|}
\hline TS264(R,R) & -3864.565635 & 1.1 & -3864.846093 & 0.9 \\
\hline $\mathrm{TS} 265(\mathrm{R}, \mathrm{R})$ & -3864.565358 & 1.2 & -3864.845485 & 1.3 \\
\hline TS266(R,R) & -3864.564747 & 1.6 & -3864.844056 & 2.2 \\
\hline TS267(R,R) & -3864.564630 & 1.7 & -3864.844538 & 1.9 \\
\hline TS268(R,R) & -3864.564098 & 2.0 & -3864.843426 & 2.6 \\
\hline TS269(R,R) & -3864.564056 & 2.1 & -3864.843437 & 2.6 \\
\hline $\mathrm{TS} 270(\mathrm{R}, \mathrm{R})$ & -3864.563858 & 2.2 & -3864.843193 & 2.7 \\
\hline TS271(R,R) & -3864.563721 & 2.3 & -3864.843169 & 2.7 \\
\hline $\mathrm{TS} 272(\mathrm{R}, \mathrm{R})$ & -3864.562479 & 3.0 & -3864.840807 & 4.2 \\
\hline \multicolumn{5}{|c|}{$(\mathbf{S}, \mathbf{S})$ Transition States } \\
\hline $\mathrm{TS} 273(\mathrm{~S}, \mathrm{~S})$ & -3864.565187 & 1.3 & -3864.844780 & 1.7 \\
\hline $\mathrm{TS} 274(\mathrm{~S}, \mathrm{~S})$ & -3864.564992 & 1.5 & -3864.843392 & 2.6 \\
\hline $\mathrm{TS} 275(\mathrm{~S}, \mathrm{~S})$ & -3864.564947 & 1.5 & -3864.843621 & 2.4 \\
\hline TS276(S,S) & -3864.564802 & 1.6 & -3864.843889 & 2.3 \\
\hline $\mathrm{TS} 277(\mathrm{~S}, \mathrm{~S})$ & -3864.564640 & 1.7 & -3864.844482 & 1.9 \\
\hline $\mathrm{TS} 278(\mathrm{~S}, \mathrm{~S})$ & -3864.564632 & 1.7 & -3864.843379 & 2.6 \\
\hline $\mathrm{TS} 279(\mathrm{~S}, \mathrm{~S})$ & -3864.564358 & 1.9 & -3864.842948 & 2.9 \\
\hline TS280(S,S) & -3864.564153 & 2.0 & -3864.843638 & 2.4 \\
\hline TS281(S,S) & -3864.564061 & 2.1 & -3864.843417 & 2.6 \\
\hline $\mathrm{TS} 282(\mathrm{~S}, \mathrm{~S})$ & -3864.563927 & 2.1 & -3864.843630 & 2.4 \\
\hline $\mathrm{TS} 283(\mathrm{~S}, \mathrm{~S})$ & -3864.563812 & 2.2 & -3864.843352 & 2.6 \\
\hline TS284(S,S) & -3864.563745 & 2.2 & -3864.842053 & 3.4 \\
\hline TS285(S,S) & -3864.563723 & 2.3 & -3864.843242 & 2.7 \\
\hline TS286(S,S) & -3864.563703 & 2.3 & -3864.842239 & 3.3 \\
\hline TS287(S,S) & -3864.563396 & 2.5 & -3864.843091 & 2.8 \\
\hline TS288(S,S) & -3864.563183 & 2.6 & -3864.841661 & 3.7 \\
\hline TS289(S,S) & -3864.563105 & 2.7 & -3864.841643 & 3.7 \\
\hline TS290(S,S) & -3864.562939 & 2.8 & -3864.842198 & 3.3 \\
\hline TS291(S,S) & -3864.562742 & 2.9 & -3864.841413 & 3.8 \\
\hline TS292(S,S) & -3864.562741 & 2.9 & -3864.841836 & 3.6 \\
\hline TS293(S,S) & -3864.562651 & 2.9 & -3864.840901 & 4.1 \\
\hline TS294(S,S) & -3864.562587 & 3.0 & -3864.841996 & 3.5 \\
\hline TS295(S,S) & -3864.562563 & 3.0 & -3864.840501 & 4.4 \\
\hline TS296(S,S) & -3864.562392 & 3.1 & -3864.841587 & 3.7 \\
\hline TS297(S,S) & -3864.562188 & 3.2 & -3864.840396 & 4.5 \\
\hline TS298(S,S) & -3864.562070 & 3.3 & -3864.840162 & 4.6 \\
\hline TS299(S,S) & -3864.561791 & 3.5 & -3864.840942 & 4.1 \\
\hline TS300(S,S) & -3864.561644 & 3.6 & -3864.839835 & 4.8 \\
\hline TS301(S,S) & -3864.561325 & 3.8 & -3864.840564 & 4.4 \\
\hline TS302(S,S) & -3864.561158 & 3.9 & -3864.839291 & 5.2 \\
\hline TS303(S,S) & -3864.560128 & 4.5 & -3864.838938 & 5.4 \\
\hline TS304(S,S) & -3864.559966 & 4.6 & -3864.837097 & 6.5 \\
\hline
\end{tabular}


Table S42. Absolute free energies (298K, in hartrees) at the B97-D/def2-TZVP and B97D3/def2-TZVP//B97-D/def2-TZVP levels of theory for all transition states for reaction $2 \mathrm{~b}$ catalyzed by PA-2 with free energies relative to the lowest-lying $(\mathrm{R}, \mathrm{R})$ transition state $(\mathrm{kcal} / \mathrm{mol})$. The Quasi-RRHO model was used to compute the free energies. The lowest-lying $(\mathrm{R}, \mathrm{R})$ and $(\mathrm{S}, \mathrm{S})$ transition states at the B97-D3/def2-TZVP//B97-D/def2-TZVP level are in bold.

\begin{tabular}{|c|c|c|c|c|}
\hline TS Name & G[B97-D/def2-TZVP] & $\mathbf{G}_{\text {rel }}$ & $\begin{array}{c}\text { G[B97-D3/def2-TZVP// } \\
\text { B97-D/def2-TZVP] }\end{array}$ & $\mathbf{G}_{\text {rel }}$ \\
\hline \multicolumn{5}{|c|}{$(\mathbf{R}, \mathbf{R})$ Transition States } \\
\hline TS259(R,R) & -3864.5513 & 0.0 & -3864.83161 & 0.0 \\
\hline TS258(R,R) & -3864.55117 & 0.1 & -3864.83089 & 0.5 \\
\hline TS260(R,R) & -3864.5511 & 0.1 & -3864.83098 & 0.4 \\
\hline TS261(R,R) & -3864.55108 & 0.1 & -3864.83109 & 0.3 \\
\hline TS263(R,R) & -3864.55056 & 0.5 & -3864.8311 & 0.3 \\
\hline TS262(R,R) & -3864.55042 & 0.6 & -3864.83024 & 0.9 \\
\hline TS257(R,R) & -3864.55041 & 0.6 & -3864.83059 & 0.6 \\
\hline TS264(R,R) & -3864.55014 & 0.7 & -3864.8306 & 0.6 \\
\hline TS266(R,R) & -3864.54915 & 1.3 & -3864.82846 & 2.0 \\
\hline TS265(R,R) & -3864.54911 & 1.4 & -3864.82924 & 1.5 \\
\hline TS267(R,R) & -3864.54902 & 1.4 & -3864.82893 & 1.7 \\
\hline TS270(R,R) & -3864.54872 & 1.6 & -3864.82805 & 2.2 \\
\hline TS269(R,R) & -3864.54867 & 1.7 & -3864.82805 & 2.2 \\
\hline $\mathrm{TS} 271(\mathrm{R}, \mathrm{R})$ & -3864.54846 & 1.8 & -3864.82791 & 2.3 \\
\hline TS268(R,R) & -3864.548 & 2.1 & -3864.82732 & 2.7 \\
\hline $\mathrm{TS} 272(\mathrm{R}, \mathrm{R})$ & -3864.54708 & 2.6 & -3864.82541 & 3.9 \\
\hline \multicolumn{5}{|c|}{ (S,S) Transition States } \\
\hline $\mathrm{TS} 275(\mathrm{~S}, \mathrm{~S})$ & -3864.54987 & 0.9 & -3864.82855 & 1.9 \\
\hline $\mathrm{TS} 278(\mathrm{~S}, \mathrm{~S})$ & -3864.54963 & 1.0 & -3864.82838 & 2.0 \\
\hline $\mathrm{TS} 276(\mathrm{~S}, \mathrm{~S})$ & -3864.54963 & 1.1 & -3864.82871 & 1.8 \\
\hline TS277(S,S) & -3864.54953 & 1.1 & -3864.82937 & 1.4 \\
\hline TS274(S,S) & -3864.54953 & 1.1 & -3864.82793 & 2.3 \\
\hline TS279(S,S) & -3864.54953 & 1.1 & -3864.82812 & 2.2 \\
\hline TS280(S,S) & -3864.54925 & 1.3 & -3864.82874 & 1.8 \\
\hline $\mathrm{TS} 281(\mathrm{~S}, \mathrm{~S})$ & -3864.54918 & 1.3 & -3864.82853 & 1.9 \\
\hline TS282(S,S) & -3864.54912 & 1.4 & -3864.82882 & 1.7 \\
\hline $\mathrm{TS} 285(\mathrm{~S}, \mathrm{~S})$ & -3864.54896 & 1.5 & -3864.82848 & 2.0 \\
\hline TS288(S,S) & -3864.54857 & 1.7 & -3864.82705 & 2.9 \\
\hline $\mathrm{TS} 286(\mathrm{~S}, \mathrm{~S})$ & -3864.54846 & 1.8 & -3864.827 & 2.9 \\
\hline TS287(S,S) & -3864.54846 & 1.8 & -3864.82815 & 2.2 \\
\hline TS284(S,S) & -3864.5484 & 1.8 & -3864.82671 & 3.1 \\
\hline $\mathrm{TS} 273(\mathrm{~S}, \mathrm{~S})$ & -3864.5484 & 1.8 & -3864.82799 & 2.3 \\
\hline $\mathrm{TS} 290(\mathrm{~S}, \mathrm{~S})$ & -3864.54815 & 2.0 & -3864.82741 & 2.6 \\
\hline TS289(S,S) & -3864.54786 & 2.2 & -3864.8264 & 3.3 \\
\hline TS283(S,S) & -3864.54784 & 2.2 & -3864.82738 & 2.7 \\
\hline TS291(S,S) & -3864.54781 & 2.2 & -3864.82648 & 3.2 \\
\hline TS297(S,S) & -3864.54754 & 2.4 & -3864.82575 & 3.7 \\
\hline TS298(S,S) & -3864.5475 & 2.4 & -3864.82559 & 3.8 \\
\hline TS294(S,S) & -3864.5474 & 2.4 & -3864.82681 & 3.0 \\
\hline TS292(S,S) & -3864.54737 & 2.5 & -3864.82647 & 3.2 \\
\hline TS295(S,S) & -3864.54723 & 2.6 & -3864.82517 & 4.0 \\
\hline TS293(S,S) & -3864.54722 & 2.6 & -3864.82547 & 3.9 \\
\hline
\end{tabular}




$\begin{array}{lllll}\text { TS300(S,S) } & -3864.54721 & 2.6 & -3864.8254 & 3.9 \\ \text { TS296(S,S) } & -3864.54713 & 2.6 & -3864.82632 & 3.3 \\ \text { TS299(S,S) } & -3864.54695 & 2.7 & -3864.8261 & 3.5 \\ \text { TS301(S,S) } & -3864.54652 & 3.0 & -3864.82576 & 3.7 \\ \text { TS302(S,S) } & -3864.54575 & 3.5 & -3864.82388 & 4.8 \\ \text { TS304(S,S) } & -3864.54566 & 3.5 & -3864.82279 & 5.5 \\ \text { TS303(S,S) } & -3864.54515 & 3.9 & -3864.82396 & 4.8\end{array}$

Table S43. Absolute free energies (233K, in hartrees) at the B97-D/def2-TZVP and B97D3/def2-TZVP//B97-D/def2-TZVP levels of theory for all transition states for reaction $2 b$ catalyzed by PA-2 with free energies relative to the lowest-lying $(\mathrm{R}, \mathrm{R})$ transition state $(\mathrm{kcal} / \mathrm{mol})$. The standard RRHO model was used to compute the free energies.

\begin{tabular}{|c|c|c|c|c|}
\hline TS Name & G[B97-D/def2-TZVP] & $\mathbf{G}_{\text {rel }}$ & $\begin{array}{c}\text { G[B97-D3/def2-TZVP// } \\
\text { B97-D/def2-TZVP] }\end{array}$ & $\mathbf{G}_{\text {rel }}$ \\
\hline \multicolumn{5}{|c|}{$(\mathbf{R}, \mathbf{R})$ Transition States } \\
\hline TS258(R,R) & -3864.532916 & 0.0 & -3864.812633 & 0.3 \\
\hline TS259(R,R) & -3864.532865 & 0.0 & -3864.813173 & 0.0 \\
\hline TS260(R,R) & -3864.532734 & 0.1 & -3864.812614 & 0.4 \\
\hline TS261(R,R) & -3864.532722 & 0.1 & -3864.812733 & 0.3 \\
\hline TS257(R,R) & -3864.532666 & 0.2 & -3864.812843 & 0.2 \\
\hline TS262(R,R) & -3864.532169 & 0.5 & -3864.811982 & 0.7 \\
\hline TS263(R,R) & -3864.531898 & 0.6 & -3864.812434 & 0.5 \\
\hline TS264(R,R) & -3864.531629 & 0.8 & -3864.812087 & 0.7 \\
\hline TS265(R,R) & -3864.530992 & 1.2 & -3864.811120 & 1.3 \\
\hline TS266(R,R) & -3864.530747 & 1.4 & -3864.810056 & 2.0 \\
\hline TS267(R,R) & -3864.530634 & 1.4 & -3864.810542 & 1.7 \\
\hline TS269(R,R) & -3864.530169 & 1.7 & -3864.809550 & 2.3 \\
\hline TS270(R,R) & -3864.530054 & 1.8 & -3864.809389 & 2.4 \\
\hline TS271(R,R) & -3864.529897 & 1.9 & -3864.809346 & 2.4 \\
\hline TS268(R,R) & -3864.529810 & 1.9 & -3864.809138 & 2.5 \\
\hline $\mathrm{TS} 272(\mathrm{R}, \mathrm{R})$ & -3864.528504 & 2.8 & -3864.806832 & 4.0 \\
\hline \multicolumn{5}{|c|}{ (S,S) Transition States } \\
\hline $\mathrm{TS} 275(\mathrm{~S}, \mathrm{~S})$ & -3864.531233 & 1.1 & -3864.809908 & 2.0 \\
\hline $\mathrm{TS} 274(\mathrm{~S}, \mathrm{~S})$ & -3864.531033 & 1.2 & -3864.809433 & 2.3 \\
\hline TS276(S,S) & -3864.530967 & 1.2 & -3864.810053 & 2.0 \\
\hline $\mathrm{TS} 278(\mathrm{~S}, \mathrm{~S})$ & -3864.530938 & 1.2 & -3864.809685 & 2.2 \\
\hline $\mathrm{TS} 277(\mathrm{~S}, \mathrm{~S})$ & -3864.530817 & 1.3 & -3864.810659 & 1.6 \\
\hline $\mathrm{TS} 279(\mathrm{~S}, \mathrm{~S})$ & -3864.530740 & 1.4 & -3864.809330 & 2.4 \\
\hline $\mathrm{TS} 273(\mathrm{~S}, \mathrm{~S})$ & -3864.530594 & 1.5 & -3864.810187 & 1.9 \\
\hline $\mathrm{TS} 280(\mathrm{~S}, \mathrm{~S})$ & -3864.530476 & 1.5 & -3864.809961 & 2.0 \\
\hline $\mathrm{TS} 281(\mathrm{~S}, \mathrm{~S})$ & -3864.530401 & 1.6 & -3864.809756 & 2.1 \\
\hline $\mathrm{TS} 282(\mathrm{~S}, \mathrm{~S})$ & -3864.530194 & 1.7 & -3864.809897 & 2.1 \\
\hline $\mathrm{TS} 285(\mathrm{~S}, \mathrm{~S})$ & -3864.530104 & 1.8 & -3864.809622 & 2.2 \\
\hline TS284(S,S) & -3864.529891 & 1.9 & -3864.808199 & 3.1 \\
\hline TS286(S,S) & -3864.529872 & 1.9 & -3864.808408 & 3.0 \\
\hline $\mathrm{TS} 287(\mathrm{~S}, \mathrm{~S})$ & -3864.529633 & 2.1 & -3864.809328 & 2.4 \\
\hline TS288(S,S) & -3864.529552 & 2.1 & -3864.808029 & 3.2 \\
\hline TS283(S,S) & -3864.529547 & 2.1 & -3864.809087 & 2.6 \\
\hline TS290(S,S) & -3864.529223 & 2.3 & -3864.808482 & 2.9 \\
\hline TS289(S,S) & -3864.529176 & 2.3 & -3864.807714 & 3.4 \\
\hline TS291(S,S) & -3864.529022 & 2.4 & -3864.807693 & 3.4 \\
\hline
\end{tabular}




$\begin{array}{lllll}\text { TS292(S,S) } & -3864.528859 & 2.5 & -3864.807953 & 3.3 \\ \text { TS294(S,S) } & -3864.528751 & 2.6 & -3864.808160 & 3.1 \\ \text { TS293(S,S) } & -3864.528658 & 2.7 & -3864.806908 & 3.9 \\ \text { TS295(S,S) } & -3864.528641 & 2.7 & -3864.806579 & 4.1 \\ \text { TS297(S,S) } & -3864.528620 & 2.7 & -3864.806828 & 4.0 \\ \text { TS296(S,S) } & -3864.528564 & 2.7 & -3864.807759 & 3.4 \\ \text { TS298(S,S) } & -3864.528538 & 2.7 & -3864.806630 & 4.1 \\ \text { TS300(S,S) } & -3864.528182 & 3.0 & -3864.806373 & 4.3 \\ \text { TS299(S,S) } & -3864.528133 & 3.0 & -3864.807283 & 3.7 \\ \text { TS301(S,S) } & -3864.527712 & 3.3 & -3864.806951 & 3.9 \\ \text { TS302(S,S) } & -3864.527155 & 3.6 & -3864.805288 & 4.9 \\ \text { TS304(S,S) } & -3864.526478 & 4.0 & -3864.803609 & 6.0 \\ \text { TS303(S,S) } & -3864.526368 & 4.1 & -3864.805178 & 5.0\end{array}$

Table S44. Absolute free energies (233K, in hartrees) at the B97-D/def2-TZVP and B97D3/def2-TZVP//B97-D/def2-TZVP levels of theory for all transition states for reaction $2 b$ catalyzed by PA-2 with free energies relative to the lowest-lying $(R, R)$ transition state $(\mathrm{kcal} / \mathrm{mol})$. The Quasi-RRHO model was used to compute the free energies. The lowest-lying $(\mathrm{R}, \mathrm{R})$ and $(\mathrm{S}, \mathrm{S})$ transition states at the B97-D3/def2-TZVP//B97-D/def2-TZVP level are in bold.

\begin{tabular}{|c|c|c|c|c|}
\hline TS Name & G[B97-D/def2-TZVP] & $\mathbf{G}_{\text {rel }}$ & $\begin{array}{c}\text { G[B97-D3/def2-TZVP// } \\
\text { B97-D/def2-TZVP] } \\
\end{array}$ & $\mathbf{G}_{\text {rel }}$ \\
\hline \multicolumn{5}{|c|}{$(\mathbf{R}, \mathbf{R})$ Transition States } \\
\hline $\operatorname{TS259}(\mathbf{R}, \mathbf{R})$ & -3864.52296 & $\mathbf{0 . 0}$ & -3864.80326 & 0.0 \\
\hline TS258(R,R) & -3864.52286 & 0.1 & -3864.80257 & 0.4 \\
\hline TS260(R,R) & -3864.52279 & 0.1 & -3864.80267 & 0.4 \\
\hline TS261(R,R) & -3864.52278 & 0.1 & -3864.80279 & 0.3 \\
\hline TS263(R,R) & -3864.52234 & 0.4 & -3864.80287 & 0.2 \\
\hline TS262(R,R) & -3864.52208 & 0.5 & -3864.8019 & 0.9 \\
\hline TS257(R,R) & -3864.52185 & 0.7 & -3864.80203 & 0.8 \\
\hline TS264(R,R) & -3864.52184 & 0.7 & -3864.80229 & 0.6 \\
\hline TS266(R,R) & -3864.52087 & 1.3 & -3864.80017 & 1.9 \\
\hline TS267(R,R) & -3864.52075 & 1.4 & -3864.80066 & 1.6 \\
\hline TS265(R,R) & -3864.52067 & 1.4 & -3864.8008 & 1.5 \\
\hline TS270(R,R) & -3864.52051 & 1.5 & -3864.79984 & 2.1 \\
\hline TS269(R,R) & -3864.52044 & 1.6 & -3864.79982 & 2.2 \\
\hline TS271(R,R) & -3864.52025 & 1.7 & -3864.7997 & 2.2 \\
\hline TS268(R,R) & -3864.51959 & 2.1 & -3864.79892 & 2.7 \\
\hline $\mathrm{TS} 272(\mathrm{R}, \mathrm{R})$ & -3864.51876 & 2.6 & -3864.79709 & 3.9 \\
\hline \multicolumn{5}{|c|}{$(\mathrm{S}, \mathrm{S})$ Transition States } \\
\hline TS275(S,S) & -3864.52173 & 0.8 & -3864.8004 & 1.8 \\
\hline TS278(S,S) & -3864.52148 & 0.9 & -3864.80022 & 1.9 \\
\hline TS276(S,S) & -3864.52141 & 1.0 & -3864.8005 & 1.7 \\
\hline TS279(S,S) & -3864.5214 & 1.0 & -3864.79999 & 2.1 \\
\hline TS277(S,S) & -3864.5213 & 1.0 & -3864.80115 & 1.3 \\
\hline TS274(S,S) & -3864.52124 & 1.1 & -3864.79964 & 2.3 \\
\hline TS280(S,S) & -3864.52109 & 1.2 & -3864.80058 & 1.7 \\
\hline TS281(S,S) & -3864.52103 & 1.2 & -3864.80039 & 1.8 \\
\hline TS282(S,S) & -3864.52091 & 1.3 & -3864.80062 & 1.7 \\
\hline TS285(S,S) & -3864.52082 & 1.3 & -3864.80034 & 1.8 \\
\hline TS288(S,S) & -3864.5204 & 1.6 & -3864.79888 & 2.8 \\
\hline
\end{tabular}




$\begin{array}{lllll}\text { TS287(S,S) } & -3864.52025 & 1.7 & -3864.79995 & 2.1 \\ \text { TS286(S,S) } & -3864.52025 & 1.7 & -3864.79879 & 2.8 \\ \text { TS284(S,S) } & -3864.52018 & 1.7 & -3864.79848 & 3.0 \\ \text { TS290(S,S) } & -3864.51995 & 1.9 & -3864.79921 & 2.5 \\ \text { TS273(S,S) } & -3864.51982 & 2.0 & -3864.79942 & 2.4 \\ \text { TS291(S,S) } & -3864.51962 & 2.1 & -3864.79829 & 3.1 \\ \text { TS289(S,S) } & -3864.51958 & 2.1 & -3864.79812 & 3.2 \\ \text { TS297(S,S) } & -3864.51942 & 2.2 & -3864.79763 & 3.5 \\ \text { TS298(S,S) } & -3864.5194 & 2.2 & -3864.79749 & 3.6 \\ \text { TS283(S,S) } & -3864.5194 & 2.2 & -3864.79894 & 2.7 \\ \text { TS294(S,S) } & -3864.51918 & 2.4 & -3864.79858 & 2.9 \\ \text { TS300(S,S) } & -3864.51914 & 2.4 & -3864.79733 & 3.7 \\ \text { TS292(S,S) } & -3864.51913 & 2.4 & -3864.79823 & 3.2 \\ \text { TS295(S,S) } & -3864.51898 & 2.5 & -3864.79691 & 4.0 \\ \text { TS296(S,S) } & -3864.51891 & 2.5 & -3864.79811 & 3.2 \\ \text { TS293(S,S) } & -3864.5189 & 2.5 & -3864.79715 & 3.8 \\ \text { TS299(S,S) } & -3864.51879 & 2.6 & -3864.79794 & 3.3 \\ \text { TS301(S,S) } & -3864.51839 & 2.9 & -3864.79763 & 3.5 \\ \text { TS304(S,S) } & -3864.51752 & 3.4 & -3864.79466 & 5.4 \\ \text { TS302(S,S) } & -3864.51741 & 3.5 & -3864.79554 & 4.8 \\ \text { TS303(S,S) } & -3864.51695 & 3.8 & -3864.79576 & 4.7\end{array}$

Table S45. Absolute free energies (195K, in hartrees) at the B97-D/def2-TZVP and B97D3/def2-TZVP//B97-D/def2-TZVP levels of theory for all transition states for reaction $2 b$ catalyzed by PA-2 with free energies relative to the lowest-lying $(R, R)$ transition state $(\mathrm{kcal} / \mathrm{mol})$. The standard RRHO model was used to compute the free energies.

\begin{tabular}{ccccc}
\hline TS Name & G[B97-D/def2-TZVP] & $\mathbf{G}_{\text {rel }}$ & $\begin{array}{c}\text { G[B97-D3/def2-TZVP// } \\
\text { B97-D/def2-TZVP] }\end{array}$ & $\mathbf{G}_{\text {rel }}$ \\
\hline (R,R) Transition States & & & & 0.3 \\
\hline TS258(R,R) & -3864.515849 & 0.0 & -3864.795566 & 0.0 \\
TS259(R,R) & -3864.515778 & 0.0 & -3864.796086 & 0.3 \\
TS260(R,R) & -3864.515688 & 0.1 & -3864.795568 & 0.2 \\
TS261(R,R) & -3864.515678 & 0.1 & -3864.795689 & 0.4 \\
TS257(R,R) & -3864.515251 & 0.4 & -3864.795428 & 0.8 \\
TS262(R,R) & -3864.515045 & 0.5 & -3864.794858 & 0.4 \\
TS263(R,R) & -3864.514957 & 0.6 & -3864.795493 & 0.6 \\
TS264(R,R) & -3864.514595 & 0.8 & -3864.795053 & 1.4 \\
TS265(R,R) & -3864.513751 & 1.3 & -3864.793879 & 1.9 \\
TS266(R,R) & -3864.513714 & 1.3 & -3864.793023 & 1.6 \\
TS267(R,R) & -3864.513601 & 1.4 & -3864.793509 & 2.2 \\
TS269(R,R) & -3864.513201 & 1.7 & -3864.792582 & 2.3 \\
TS270(R,R) & -3864.513134 & 1.7 & -3864.792469 & 2.3 \\
TS271(R,R) & -3864.512964 & 1.8 & -3864.792413 & 2.6 \\
TS268(R,R) & -3864.512611 & 2.0 & -3864.791939 & 3.9 \\
TS272(R,R) & -3864.511483 & 2.7 & -3864.789811 & \\
\hline (S,S) Transition States & & & & 2.9 \\
\hline TS275(S,S) & -3864.514361 & 0.9 & -3864.793036 & -3864.792823 \\
TS278(S,S) & -3864.514077 & 1.1 & -3864.793115 & \\
TS276(S,S) & -3864.514029 & 1.1 & -3864.792419 & \\
TS274(S,S) & -3864.514019 & 1.1 & & 2.9 \\
\hline
\end{tabular}




\begin{tabular}{|c|c|c|c|c|}
\hline TS279(S,S) & -3864.513924 & 1.2 & -3864.792514 & 2.2 \\
\hline TS277(S,S) & -3864.513888 & 1.2 & -3864.793730 & 1.5 \\
\hline TS280(S,S) & -3864.513629 & 1.4 & -3864.793114 & 1.9 \\
\hline TS281(S,S) & -3864.513563 & 1.4 & -3864.792918 & 2.0 \\
\hline TS282(S,S) & -3864.513318 & 1.6 & -3864.793021 & 1.9 \\
\hline TS285(S,S) & -3864.513290 & 1.6 & -3864.792808 & 2.1 \\
\hline TS273(S,S) & -3864.513219 & 1.7 & -3864.792812 & 2.1 \\
\hline TS284(S,S) & -3864.512938 & 1.8 & -3864.791246 & 3.0 \\
\hline TS286(S,S) & -3864.512932 & 1.8 & -3864.791468 & 2.9 \\
\hline TS287(S,S) & -3864.512739 & 2.0 & -3864.792434 & 2.3 \\
\hline TS288(S,S) & -3864.512729 & 2.0 & -3864.791206 & 3.1 \\
\hline TS283(S,S) & -3864.512361 & 2.2 & -3864.791901 & 2.6 \\
\hline TS290(S,S) & -3864.512355 & 2.2 & -3864.791614 & 2.8 \\
\hline TS289(S,S) & -3864.512181 & 2.3 & -3864.790719 & 3.4 \\
\hline TS291(S,S) & -3864.512147 & 2.3 & -3864.790818 & 3.3 \\
\hline TS292(S,S) & -3864.511893 & 2.5 & -3864.790987 & 3.2 \\
\hline TS297(S,S) & -3864.511833 & 2.5 & -3864.790041 & 3.8 \\
\hline TS294(S,S) & -3864.511813 & 2.5 & -3864.791222 & 3.1 \\
\hline TS298(S,S) & -3864.511771 & 2.6 & -3864.789863 & 3.9 \\
\hline TS295(S,S) & -3864.511649 & 2.6 & -3864.789587 & 4.1 \\
\hline TS296(S,S) & -3864.511629 & 2.6 & -3864.790824 & 3.3 \\
\hline TS293(S,S) & -3864.511626 & 2.7 & -3864.789876 & 3.9 \\
\hline TS300(S,S) & -3864.511455 & 2.8 & -3864.789646 & 4.0 \\
\hline TS299(S,S) & -3864.511296 & 2.9 & -3864.790446 & 3.5 \\
\hline TS301(S,S) & -3864.510900 & 3.1 & -3864.790139 & 3.7 \\
\hline TS302(S,S) & -3864.510121 & 3.6 & -3864.788254 & 4.9 \\
\hline TS304(S,S) & -3864.509736 & 3.8 & -3864.786867 & 5.8 \\
\hline TS303(S,S) & -3864.509471 & 4.0 & -3864.788281 & 4.9 \\
\hline
\end{tabular}

Table S46. Absolute free energies (195K, in hartrees) at the B97-D/def2-TZVP and B97D3/def2-TZVP//B97-D/def2-TZVP levels of theory for all transition states for reaction $2 \mathrm{~b}$ catalyzed by PA-2 with free energies relative to the lowest-lying $(\mathrm{R}, \mathrm{R})$ transition state $(\mathrm{kcal} / \mathrm{mol})$. The Quasi-RRHO model was used to compute the free energies. The lowest-lying $(\mathrm{R}, \mathrm{R})$ and $(\mathrm{S}, \mathrm{S})$ transition states at the B97-D3/def2-TZVP//B97-D/def2-TZVP level are in bold.

\begin{tabular}{ccccc}
\hline TS Name & G[B97-D/def2-TZVP] & $\mathbf{G}_{\text {rel }}$ & $\begin{array}{c}\text { G[B97-D3/def2-TZVP// } \\
\text { B97-D/def2-TZVP] }\end{array}$ & $\mathbf{G}_{\text {rel }}$ \\
\hline (R,R) Transition States & & & & $\mathbf{- 3 8 6 4 . 7 8 9 1 9}$ \\
\hline TS259(R,R) & $\mathbf{- 3 8 6 4 . 5 0 8 8 8}$ & $\mathbf{0 . 0}$ & -3864.78852 & 0.0 \\
TS258(R,R) & -3864.50881 & 0.0 & -3864.78875 & 0.3 \\
TS261(R,R) & -3864.50874 & 0.1 & -3864.78862 & 0.4 \\
TS260(R,R) & -3864.50874 & 0.1 & -3864.78887 & 0.2 \\
TS263(R,R) & -3864.50834 & 0.3 & -3864.78782 & 0.9 \\
TS262(R,R) & -3864.50801 & 0.5 & -3864.78824 & 0.6 \\
TS264(R,R) & -3864.50779 & 0.7 & -3864.78782 & 0.9 \\
TS257(R,R) & -3864.50765 & 0.8 & -3864.78613 & 1.9 \\
TS266(R,R) & -3864.50683 & 1.3 & -3864.78663 & 1.6 \\
TS267(R,R) & -3864.50672 & 1.4 & -3864.78667 & 1.6 \\
TS265(R,R) & -3864.50654 & 1.5 & -3864.78585 & 2.1 \\
TS270(R,R) & -3864.50651 & 1.5 & -3864.78581 & 2.1 \\
TS269(R,R) & -3864.50643 & 1.5 & &
\end{tabular}




\begin{tabular}{|c|c|c|c|c|}
\hline TS271(R,R) & -3864.50626 & 1.6 & -3864.78571 & 2.2 \\
\hline TS268(R,R) & -3864.50548 & 2.1 & -3864.78481 & 2.7 \\
\hline $\mathrm{TS} 272(\mathrm{R}, \mathrm{R})$ & -3864.5047 & 2.6 & -3864.78303 & 3.9 \\
\hline \multicolumn{5}{|c|}{ (S,S) Transition States } \\
\hline $\mathrm{TS} 275(\mathrm{~S}, \mathrm{~S})$ & -3864.50777 & 0.7 & -3864.78644 & 1.7 \\
\hline $\mathrm{TS} 278(\mathrm{~S}, \mathrm{~S})$ & -3864.50751 & 0.9 & -3864.78626 & 1.8 \\
\hline $\mathrm{TS} 279(\mathrm{~S}, \mathrm{~S})$ & -3864.50745 & 0.9 & -3864.78604 & 2.0 \\
\hline $\mathrm{TS} 276(\mathrm{~S}, \mathrm{~S})$ & -3864.50741 & 0.9 & -3864.7865 & 1.7 \\
\hline TS277(S,S) & -3864.5073 & 1.0 & -3864.78715 & 1.3 \\
\hline $\mathrm{TS} 274(\mathrm{~S}, \mathrm{~S})$ & -3864.50719 & 1.1 & -3864.78559 & 2.3 \\
\hline $\mathrm{TS} 280(\mathrm{~S}, \mathrm{~S})$ & -3864.50713 & 1.1 & -3864.78661 & 1.6 \\
\hline TS281(S,S) & -3864.50708 & 1.1 & -3864.78643 & 1.7 \\
\hline $\mathrm{TS} 282(\mathrm{~S}, \mathrm{~S})$ & -3864.50692 & 1.2 & -3864.78663 & 1.6 \\
\hline $\mathrm{TS} 285(\mathrm{~S}, \mathrm{~S})$ & -3864.50687 & 1.3 & -3864.78639 & 1.8 \\
\hline TS288(S,S) & -3864.50642 & 1.5 & -3864.7849 & 2.7 \\
\hline $\mathrm{TS} 287(\mathrm{~S}, \mathrm{~S})$ & -3864.50626 & 1.6 & -3864.78596 & 2.0 \\
\hline TS286(S,S) & -3864.50625 & 1.6 & -3864.78479 & 2.8 \\
\hline $\mathrm{TS} 284(\mathrm{~S}, \mathrm{~S})$ & -3864.50617 & 1.7 & -3864.78448 & 3.0 \\
\hline TS290(S,S) & -3864.50596 & 1.8 & -3864.78522 & 2.5 \\
\hline TS291(S,S) & -3864.50564 & 2.0 & -3864.78431 & 3.1 \\
\hline $\mathrm{TS} 273(\mathrm{~S}, \mathrm{~S})$ & -3864.50561 & 2.0 & -3864.78521 & 2.5 \\
\hline $\mathrm{TS} 289(\mathrm{~S}, \mathrm{~S})$ & -3864.50554 & 2.1 & -3864.78407 & 3.2 \\
\hline TS297(S,S) & -3864.50548 & 2.1 & -3864.78369 & 3.5 \\
\hline TS298(S,S) & -3864.50547 & 2.1 & -3864.78356 & 3.5 \\
\hline $\mathrm{TS} 283(\mathrm{~S}, \mathrm{~S})$ & -3864.50527 & 2.3 & -3864.78481 & 2.7 \\
\hline $\mathrm{TS} 300(\mathrm{~S}, \mathrm{~S})$ & -3864.50523 & 2.3 & -3864.78342 & 3.6 \\
\hline TS294(S,S) & -3864.50517 & 2.3 & -3864.78458 & 2.9 \\
\hline TS292(S,S) & -3864.50512 & 2.4 & -3864.78422 & 3.1 \\
\hline TS295(S,S) & -3864.50495 & 2.5 & -3864.78289 & 4.0 \\
\hline TS296(S,S) & -3864.50492 & 2.5 & -3864.78411 & 3.2 \\
\hline TS293(S,S) & -3864.50484 & 2.5 & -3864.78309 & 3.8 \\
\hline $\mathrm{TS} 299(\mathrm{~S}, \mathrm{~S})$ & -3864.50483 & 2.5 & -3864.78398 & 3.3 \\
\hline TS301(S,S) & -3864.50445 & 2.8 & -3864.78369 & 3.4 \\
\hline $\mathrm{TS} 304(\mathrm{~S}, \mathrm{~S})$ & -3864.50358 & 3.3 & -3864.78071 & 5.3 \\
\hline $\mathrm{TS} 302(\mathrm{~S}, \mathrm{~S})$ & -3864.50334 & 3.5 & -3864.78147 & 4.8 \\
\hline TS303(S,S) & -3864.50296 & 3.7 & -3864.78177 & 4.7 \\
\hline
\end{tabular}

Table S47. Absolute free energies (298K, in hartrees) at the wB97XD/6-31G(d) and wB97XD/6-311+G(d,p) //wB97XD/6-31G(d) levels of theory for all transition states for reaction $2 \mathrm{~b}$ catalyzed by $\mathrm{PA}-2$ with free energies relative to the lowest-lying $(\mathrm{R}, \mathrm{R})$ transition state $(\mathrm{kcal} / \mathrm{mol})$. The standard RRHO model was used to compute the free energies.

TS276(S,S) has two imaginary frequencies.

\begin{tabular}{ccccc}
\hline TS Name & G[wB97XD/6-31G(d)] & $\mathbf{G}_{\text {rel }}$ & $\begin{array}{c}\text { G[wB97XD/6-311+G(d,p)// } \\
\text { wB97XD/6-31G(d)] }\end{array}$ & $\mathbf{G}_{\text {rel }}$ \\
\hline (R,R) Transition States & & & & 0.0 \\
\hline TS263(R,R) & -3864.649276 & 0.0 & -3865.430268 & 0.5 \\
TS261(R,R) & -3864.648579 & 0.4 & -3865.42954 & 0.5 \\
TS260(R,R) & -3864.648548 & 0.5 & -3865.429454 & 0.9 \\
TS258(R,R) & -3864.648038 & 0.8 & -3865.42889 & 1.3 \\
TS262(R,R) & -3864.647443 & 1.2 & -3865.428189 & 1.4 \\
TS259(R,R) & -3864.647316 & 1.2 & -3865.428065 &
\end{tabular}




\begin{tabular}{|c|c|c|c|c|}
\hline TS264(R,R) & -3864.647034 & 1.4 & -3865.427767 & 1.6 \\
\hline TS269(R,R) & -3864.646688 & 1.6 & -3865.427502 & 1.7 \\
\hline TS266(R,R) & -3864.646121 & 2.0 & -3865.427035 & 2.0 \\
\hline TS270(R,R) & -3864.645851 & 2.1 & -3865.426682 & 2.3 \\
\hline TS257(R,R) & -3864.645797 & 2.2 & -3865.426593 & 2.3 \\
\hline TS267(R,R) & -3864.645551 & 2.3 & -3865.426299 & 2.5 \\
\hline TS271(R,R) & -3864.645114 & 2.6 & -3865.425841 & 2.8 \\
\hline TS268(R,R) & -3864.645068 & 2.6 & -3865.425908 & 2.7 \\
\hline TS272(R,R) & -3864.644925 & 2.7 & -3865.425744 & 2.8 \\
\hline $\mathrm{TS} 265(\mathrm{R}, \mathrm{R})$ & -3864.642485 & 4.3 & -3865.423155 & 4.5 \\
\hline \multicolumn{5}{|c|}{$(\mathrm{S}, \mathrm{S})$ Transition States } \\
\hline $\mathrm{TS} 275(\mathrm{~S}, \mathrm{~S})$ & -3864.647566 & 1.1 & -3865.427157 & 2.0 \\
\hline $\mathrm{TS} 278(\mathrm{~S}, \mathrm{~S})$ & -3864.647348 & 1.2 & -3865.427047 & 2.0 \\
\hline $\mathrm{TS} 279(\mathrm{~S}, \mathrm{~S})$ & -3864.647218 & 1.3 & -3865.426984 & 2.1 \\
\hline TS286(S,S) & -3864.646505 & 1.7 & -3865.426207 & 2.5 \\
\hline $\mathrm{TS} 280(\mathrm{~S}, \mathrm{~S})$ & -3864.646356 & 1.8 & -3865.427487 & 1.7 \\
\hline TS298(S,S) & -3864.645617 & 2.3 & -3865.425348 & 3.1 \\
\hline $\mathrm{TS} 274(\mathrm{~S}, \mathrm{~S})$ & -3864.645614 & 2.3 & -3865.425338 & 3.1 \\
\hline TS300(S,S) & -3864.645453 & 2.4 & -3865.425031 & 3.3 \\
\hline TS277(S,S) & -3864.64476 & 2.8 & -3865.426082 & 2.6 \\
\hline TS297(S,S) & -3864.644495 & 3.0 & -3865.424217 & 3.8 \\
\hline TS281(S,S) & -3864.644381 & 3.1 & -3865.425419 & 3.0 \\
\hline TS296(S,S) & -3864.644297 & 3.1 & -3865.425385 & 3.1 \\
\hline TS285(S,S) & -3864.644034 & 3.3 & -3865.42517 & 3.2 \\
\hline TS287(S,S) & -3864.643761 & 3.5 & -3865.424738 & 3.5 \\
\hline TS276(S,S) & -3864.643729 & 3.5 & -3865.423485 & 4.3 \\
\hline TS288(S,S) & -3864.643727 & 3.5 & -3865.423324 & 4.4 \\
\hline TS299(S,S) & -3864.643688 & 3.5 & -3865.424878 & 3.4 \\
\hline TS284(S,S) & -3864.643656 & 3.5 & -3865.423312 & 4.4 \\
\hline TS301(S,S) & -3864.643355 & 3.7 & -3865.424505 & 3.6 \\
\hline TS293(S,S) & -3864.643153 & 3.8 & -3865.422761 & 4.7 \\
\hline TS282(S,S) & -3864.643003 & 3.9 & -3865.424002 & 3.9 \\
\hline TS292(S,S) & -3864.642549 & 4.2 & -3865.423743 & 4.1 \\
\hline TS289(S,S) & -3864.642531 & 4.2 & -3865.422216 & 5.1 \\
\hline TS294(S,S) & -3864.642404 & 4.3 & -3865.423529 & 4.2 \\
\hline TS291(S,S) & -3864.642379 & 4.3 & -3865.42196 & 5.2 \\
\hline TS290(S,S) & -3864.642288 & 4.4 & -3865.423496 & 4.2 \\
\hline TS295(S,S) & -3864.64161 & 4.8 & -3865.421313 & 5.6 \\
\hline TS304(S,S) & -3864.641484 & 4.9 & -3865.421124 & 5.7 \\
\hline TS302(S,S) & -3864.641256 & 5.0 & -3865.42242 & 4.9 \\
\hline TS283(S,S) & -3864.641238 & 5.0 & -3865.422281 & 5.0 \\
\hline TS303(S,S) & -3864.641047 & 5.2 & -3865.422256 & 5.0 \\
\hline TS273(S,S) & -3864.639376 & 6.2 & -3865.420373 & 6.2 \\
\hline
\end{tabular}

Table S48. Absolute free energies (298K, in hartrees) at the wB97XD/6-31G(d) and wB97XD/6-311+G(d,p) //wB97XD/6-31G(d) levels of theory for all transition states for reaction $2 \mathrm{~b}$ catalyzed by $\mathrm{PA}-2$ with free energies relative to the lowest-lying $(\mathrm{R}, \mathrm{R})$ transition state (kcal/mol). The Quasi-RRHO model was used to compute the free energies. TS276(S,S) has two imaginary frequencies.

\begin{tabular}{lcccc}
\hline TS Name & G[wB97XD/6-31G(d)] & $G_{\text {rel }}$ & $\begin{array}{c}\text { G[wB97XD/6-311+G(d,p)// } \\
\text { wB97XD/6-31G(d) }\end{array}$ & $G_{\text {rel }}$ \\
\hline
\end{tabular}




\begin{tabular}{|c|c|c|c|c|}
\hline \multicolumn{5}{|c|}{$(\mathbf{R}, \mathbf{R})$ Transition States } \\
\hline TS263(R,R) & -3864.634739 & 0.0 & -3865.415731 & 0.0 \\
\hline $\mathrm{TS} 260(\mathrm{R}, \mathrm{R})$ & -3864.634579 & 0.1 & -3865.415485 & 0.2 \\
\hline $\mathrm{TS} 261(\mathrm{R}, \mathrm{R})$ & -3864.634405 & 0.2 & -3865.415366 & 0.2 \\
\hline $\mathrm{TS} 258(\mathrm{R}, \mathrm{R})$ & -3864.63409 & 0.4 & -3865.414942 & 0.5 \\
\hline $\mathrm{TS} 259(\mathrm{R}, \mathrm{R})$ & -3864.634008 & 0.5 & -3865.414756 & 0.6 \\
\hline $\mathrm{TS} 264(\mathrm{R}, \mathrm{R})$ & -3864.633604 & 0.7 & -3865.414336 & 0.9 \\
\hline $\mathrm{TS} 262(\mathrm{R}, \mathrm{R})$ & -3864.632997 & 1.1 & -3865.413744 & 1.2 \\
\hline $\mathrm{TS} 269(\mathrm{R}, \mathrm{R})$ & -3864.632522 & 1.4 & -3865.413337 & 1.5 \\
\hline $\mathrm{TS} 266(\mathrm{R}, \mathrm{R})$ & -3864.632108 & 1.7 & -3865.413022 & 1.7 \\
\hline $\mathrm{TS} 257(\mathrm{R}, \mathrm{R})$ & -3864.631912 & 1.8 & -3865.412708 & 1.9 \\
\hline $\mathrm{TS} 270(\mathrm{R}, \mathrm{R})$ & -3864.631846 & 1.8 & -3865.412678 & 1.9 \\
\hline $\mathrm{TS} 267(\mathrm{R}, \mathrm{R})$ & -3864.631494 & 2.0 & -3865.412242 & 2.2 \\
\hline $\mathrm{TS} 271(\mathrm{R}, \mathrm{R})$ & -3864.631393 & 2.1 & -3865.412119 & 2.3 \\
\hline $\mathrm{TS} 272(\mathrm{R}, \mathrm{R})$ & -3864.630745 & 2.5 & -3865.411564 & 2.6 \\
\hline $\mathrm{TS} 268(\mathrm{R}, \mathrm{R})$ & -3864.630487 & 2.7 & -3865.411327 & 2.8 \\
\hline $\mathrm{TS} 265(\mathrm{R}, \mathrm{R})$ & -3864.629768 & 3.1 & -3865.410438 & 3.3 \\
\hline \multicolumn{5}{|c|}{ (S,S) Transition States } \\
\hline $\mathrm{TS} 275(\mathrm{~S}, \mathrm{~S})$ & -3864.632877 & 1.2 & -3865.412468 & 2.0 \\
\hline $\mathrm{TS} 278(\mathrm{~S}, \mathrm{~S})$ & -3864.632671 & 1.3 & -3865.412369 & 2.1 \\
\hline $\mathrm{TS} 279(\mathrm{~S}, \mathrm{~S})$ & -3864.632612 & 1.3 & -3865.412378 & 2.1 \\
\hline $\mathrm{TS} 280(\mathrm{~S}, \mathrm{~S})$ & -3864.632158 & 1.6 & -3865.413289 & 1.5 \\
\hline $\mathrm{TS} 274(\mathrm{~S}, \mathrm{~S})$ & -3864.631275 & 2.2 & -3865.410999 & 3.0 \\
\hline $\mathrm{TS} 286(\mathrm{~S}, \mathrm{~S})$ & -3864.631113 & 2.3 & -3865.410814 & 3.1 \\
\hline $\mathrm{TS} 277(\mathrm{~S}, \mathrm{~S})$ & -3864.630961 & 2.4 & -3865.412282 & 2.2 \\
\hline TS298(S,S) & -3864.63081 & 2.5 & -3865.410541 & 3.3 \\
\hline $\mathrm{TS} 276(\mathrm{~S}, \mathrm{~S})$ & -3864.630789 & 2.5 & -3865.410544 & 3.3 \\
\hline $\mathrm{TS} 300(\mathrm{~S}, \mathrm{~S})$ & -3864.630772 & 2.5 & -3865.41035 & 3.4 \\
\hline $\mathrm{TS} 281(\mathrm{~S}, \mathrm{~S})$ & -3864.630754 & 2.5 & -3865.411792 & 2.5 \\
\hline $\mathrm{TS} 285(\mathrm{~S}, \mathrm{~S})$ & -3864.630498 & 2.7 & -3865.411635 & 2.6 \\
\hline $\mathrm{TS} 282(\mathrm{~S}, \mathrm{~S})$ & -3864.63043 & 2.7 & -3865.41143 & 2.7 \\
\hline $\mathrm{TS} 287(\mathrm{~S}, \mathrm{~S})$ & -3864.630424 & 2.7 & -3865.411401 & 2.7 \\
\hline $\mathrm{TS} 297(\mathrm{~S}, \mathrm{~S})$ & -3864.630272 & 2.8 & -3865.409994 & 3.6 \\
\hline $\mathrm{TS} 288(\mathrm{~S}, \mathrm{~S})$ & -3864.630038 & 3.0 & -3865.409635 & 3.8 \\
\hline $\mathrm{TS} 299(\mathrm{~S}, \mathrm{~S})$ & -3864.629908 & 3.0 & -3865.411098 & 2.9 \\
\hline TS296(S,S) & -3864.629877 & 3.1 & -3865.410965 & 3.0 \\
\hline $\mathrm{TS} 284(\mathrm{~S}, \mathrm{~S})$ & -3864.629737 & 3.1 & -3865.409393 & 4.0 \\
\hline $\mathrm{TS} 301(\mathrm{~S}, \mathrm{~S})$ & -3864.62932 & 3.4 & -3865.41047 & 3.3 \\
\hline $\mathrm{TS} 290(\mathrm{~S}, \mathrm{~S})$ & -3864.629057 & 3.6 & -3865.410265 & 3.4 \\
\hline $\mathrm{TS} 292(\mathrm{~S}, \mathrm{~S})$ & -3864.629013 & 3.6 & -3865.410207 & 3.5 \\
\hline $\mathrm{TS} 293(\mathrm{~S}, \mathrm{~S})$ & -3864.628888 & 3.7 & -3865.408496 & 4.5 \\
\hline $\mathrm{TS} 294(\mathrm{~S}, \mathrm{~S})$ & -3864.628846 & 3.7 & -3865.409971 & 3.6 \\
\hline $\mathrm{TS} 291(\mathrm{~S}, \mathrm{~S})$ & -3864.628743 & 3.8 & -3865.408324 & 4.6 \\
\hline $\mathrm{TS} 289(\mathrm{~S}, \mathrm{~S})$ & -3864.628363 & 4.0 & -3865.408047 & 4.8 \\
\hline $\mathrm{TS} 283(\mathrm{~S}, \mathrm{~S})$ & -3864.628308 & 4.0 & -3865.409351 & 4.0 \\
\hline $\mathrm{TS} 304(\mathrm{~S}, \mathrm{~S})$ & -3864.627723 & 4.4 & -3865.407362 & 5.3 \\
\hline $\mathrm{TS} 295(\mathrm{~S}, \mathrm{~S})$ & -3864.627704 & 4.4 & -3865.407406 & 5.2 \\
\hline $\mathrm{TS} 303(\mathrm{~S}, \mathrm{~S})$ & -3864.626898 & 4.9 & -3865.408107 & 4.8 \\
\hline
\end{tabular}


Table S49. Absolute free energies (298K, in hartrees) at the M06-2X/6-31G(d) and M06$2 \mathrm{X} / 6-311+\mathrm{G}(\mathrm{d}, \mathrm{p}) / / \mathrm{M} 06-2 \mathrm{X} / 6-31 \mathrm{G}(\mathrm{d})$ levels of theory for all transition states for reaction $2 \mathrm{~b}$ catalyzed by PA- 2 with free energies relative to the lowest-lying $(R, R)$ transition state $(\mathrm{kcal} / \mathrm{mol})$. The standard RRHO model was used to compute the free energies.

\begin{tabular}{|c|c|c|c|c|}
\hline TS Name & G[M06-2X/6-31G(d)] & $\mathbf{G}_{\text {rel }}$ & $\begin{array}{c}\text { G[M06-2X/6-311+G(d,p)// } \\
\text { M06-2X/6-31G(d)] }\end{array}$ & $\mathbf{G}_{\text {rel }}$ \\
\hline \multicolumn{5}{|c|}{$(\mathbf{R}, \mathbf{R})$ Transition States } \\
\hline $\mathrm{TS} 261(\mathrm{R}, \mathrm{R})$ & -3864.20196 & 0.0 & -3865.07379 & 0.0 \\
\hline TS260(R,R) & -3864.201752 & 0.1 & -3865.073633 & 0.1 \\
\hline TS258(R,R) & -3864.201673 & 0.2 & -3865.073441 & 0.2 \\
\hline TS263(R,R) & -3864.201643 & 0.2 & -3865.073394 & 0.2 \\
\hline TS264(R,R) & -3864.200961 & 0.6 & -3865.072887 & 0.6 \\
\hline TS259(R,R) & -3864.200922 & 0.7 & -3865.07286 & 0.6 \\
\hline TS262(R,R) & -3864.200253 & 1.1 & -3865.072063 & 1.1 \\
\hline TS267(R,R) & -3864.199455 & 1.6 & -3865.071233 & 1.6 \\
\hline TS270(R,R) & -3864.199204 & 1.7 & -3865.071061 & 1.7 \\
\hline TS266(R,R) & -3864.19918 & 1.7 & -3865.071026 & 1.7 \\
\hline TS269(R,R) & -3864.199119 & 1.8 & -3865.070953 & 1.8 \\
\hline TS271(R,R) & -3864.198984 & 1.9 & -3865.070791 & 1.9 \\
\hline TS257(R,R) & -3864.198502 & 2.2 & -3865.07054 & 2.0 \\
\hline TS265(R,R) & -3864.197897 & 2.5 & -3865.069829 & 2.5 \\
\hline TS268(R,R) & -3864.197819 & 2.6 & -3865.069698 & 2.6 \\
\hline $\mathrm{TS} 272(\mathrm{R}, \mathrm{R})$ & -3864.196734 & 3.3 & -3865.068538 & 3.3 \\
\hline \multicolumn{5}{|c|}{$(\mathrm{S}, \mathrm{S})$ Transition States } \\
\hline $\mathrm{TS} 279(\mathrm{~S}, \mathrm{~S})$ & -3864.20009 & 1.2 & -3865.070504 & 2.1 \\
\hline TS288(S,S) & -3864.199778 & 1.4 & -3865.070102 & 2.3 \\
\hline $\mathrm{TS} 275(\mathrm{~S}, \mathrm{~S})$ & -3864.199417 & 1.6 & -3865.069713 & 2.6 \\
\hline $\mathrm{TS} 276(\mathrm{~S}, \mathrm{~S})$ & -3864.199196 & 1.7 & -3865.069317 & 2.8 \\
\hline $\mathrm{TS} 285(\mathrm{~S}, \mathrm{~S})$ & -3864.198881 & 1.9 & -3865.071335 & 1.5 \\
\hline TS291(S,S) & -3864.198809 & 2.0 & -3865.069127 & 2.9 \\
\hline TS278(S,S) & -3864.198592 & 2.1 & -3865.068949 & 3.0 \\
\hline $\mathrm{TS} 274(\mathrm{~S}, \mathrm{~S})$ & -3864.198245 & 2.3 & -3865.068614 & 3.2 \\
\hline TS280(S,S) & -3864.19824 & 2.3 & -3865.07072 & 1.9 \\
\hline TS290(S,S) & -3864.198073 & 2.4 & -3865.070465 & 2.1 \\
\hline TS281(S,S) & -3864.198052 & 2.5 & -3865.070445 & 2.1 \\
\hline TS296(S,S) & -3864.197957 & 2.5 & -3865.070394 & 2.1 \\
\hline $\mathrm{TS} 282(\mathrm{~S}, \mathrm{~S})$ & -3864.197856 & 2.6 & -3865.070221 & 2.2 \\
\hline $\mathrm{TS} 277(\mathrm{~S}, \mathrm{~S})$ & -3864.19779 & 2.6 & -3865.070204 & 2.3 \\
\hline TS286(S,S) & -3864.197729 & 2.7 & -3865.068152 & 3.5 \\
\hline TS287(S,S) & -3864.197633 & 2.7 & -3865.069999 & 2.4 \\
\hline TS289(S,S) & -3864.197612 & 2.7 & -3865.068167 & 3.5 \\
\hline TS297(S,S) & -3864.197573 & 2.8 & -3865.067904 & 3.7 \\
\hline TS292(S,S) & -3864.197441 & 2.8 & -3865.069908 & 2.4 \\
\hline TS299(S,S) & -3864.197309 & 2.9 & -3865.069723 & 2.6 \\
\hline TS294(S,S) & -3864.197277 & 2.9 & -3865.069657 & 2.6 \\
\hline TS301(S,S) & -3864.196984 & 3.1 & -3865.069352 & 2.8 \\
\hline TS284(S,S) & -3864.196887 & 3.2 & -3865.067266 & 4.1 \\
\hline TS283(S,S) & -3864.196761 & 3.3 & -3865.069289 & 2.8 \\
\hline TS298(S,S) & -3864.19667 & 3.3 & -3865.066979 & 4.3 \\
\hline TS300(S,S) & -3864.196621 & 3.4 & -3865.066903 & 4.3 \\
\hline TS293(S,S) & -3864.195855 & 3.8 & -3865.066273 & 4.7 \\
\hline TS303(S,S) & -3864.195805 & 3.9 & -3865.068279 & 3.5 \\
\hline
\end{tabular}




$\begin{array}{lllll}\text { TS295(S,S) } & -3864.195694 & 3.9 & -3865.066132 & 4.8 \\ \text { TS273(S,S) } & -3864.195548 & 4.0 & -3865.068032 & 3.6 \\ \text { TS302(S,S) } & -3864.195526 & 4.0 & -3865.067953 & 3.7 \\ \text { TS304(S,S) } & -3864.195008 & 4.4 & -3865.065363 & 5.3\end{array}$

Table S50. Absolute free energies (298K, in hartrees) at the M06-2X/6-31G(d) and M06$2 \mathrm{X} / 6-311+\mathrm{G}(\mathrm{d}, \mathrm{p}) / / \mathrm{M} 06-2 \mathrm{X} / 6-31 \mathrm{G}(\mathrm{d})$ levels of theory for all transition states for reaction $2 \mathrm{~b}$ catalyzed by PA- 2 with free energies relative to the lowest-lying $(R, R)$ transition state (kcal/mol). The Quasi-RRHO model was used to compute the free energies.

\begin{tabular}{|c|c|c|c|c|}
\hline TS Name & G[M06-2X/6-31G(d)] & $\mathbf{G}_{\text {rel }}$ & $\begin{array}{c}\text { G[M06-2X/6-311+G(d,p)// } \\
\text { M06-2X/6-31G(d)] }\end{array}$ & $\mathbf{G}_{\text {rel }}$ \\
\hline \multicolumn{5}{|c|}{$(\mathbf{R}, \mathbf{R})$ Transition States } \\
\hline TS261(R,R) & -3864.187455 & 0.0 & -3865.059285 & 0.0 \\
\hline $\mathrm{TS} 260(\mathrm{R}, \mathrm{R})$ & -3864.187409 & 0.0 & -3865.05929 & 0.0 \\
\hline TS258(R,R) & -3864.187323 & 0.1 & -3865.059092 & 0.1 \\
\hline $\mathrm{TS} 263(\mathrm{R}, \mathrm{R})$ & -3864.187136 & 0.2 & -3865.058888 & 0.3 \\
\hline TS259(R,R) & -3864.18635 & 0.7 & -3865.058288 & 0.6 \\
\hline $\mathrm{TS} 262(\mathrm{R}, \mathrm{R})$ & -3864.185999 & 0.9 & -3865.057809 & 0.9 \\
\hline TS264(R,R) & -3864.185963 & 0.9 & -3865.057889 & 0.9 \\
\hline $\mathrm{TS} 267(\mathrm{R}, \mathrm{R})$ & -3864.185048 & 1.5 & -3865.056826 & 1.5 \\
\hline TS270(R,R) & -3864.185043 & 1.5 & -3865.0569 & 1.5 \\
\hline $\mathrm{TS} 266(\mathrm{R}, \mathrm{R})$ & -3864.184973 & 1.6 & -3865.05682 & 1.6 \\
\hline TS269(R,R) & -3864.18493 & 1.6 & -3865.056764 & 1.6 \\
\hline $\mathrm{TS} 271(\mathrm{R}, \mathrm{R})$ & -3864.18483 & 1.6 & -3865.056636 & 1.7 \\
\hline TS257(R,R) & -3864.183882 & 2.2 & -3865.05592 & 2.1 \\
\hline $\mathrm{TS} 265(\mathrm{R}, \mathrm{R})$ & -3864.183436 & 2.5 & -3865.055368 & 2.5 \\
\hline TS268(R,R) & -3864.183388 & 2.6 & -3865.055266 & 2.5 \\
\hline $\mathrm{TS} 272(\mathrm{R}, \mathrm{R})$ & -3864.182916 & 2.8 & -3865.054719 & 2.9 \\
\hline \multicolumn{5}{|c|}{$(\mathrm{S}, \mathrm{S})$ Transition States } \\
\hline $\mathrm{TS} 279(\mathrm{~S}, \mathrm{~S})$ & -3864.185768 & 1.1 & -3865.056182 & 2.0 \\
\hline $\mathrm{TS} 275(\mathrm{~S}, \mathrm{~S})$ & -3864.185393 & 1.3 & -3865.055689 & 2.3 \\
\hline $\mathrm{TS} 288(\mathrm{~S}, \mathrm{~S})$ & -3864.185165 & 1.4 & -3865.055489 & 2.4 \\
\hline TS276(S,S) & -3864.185036 & 1.5 & -3865.055156 & 2.6 \\
\hline $\mathrm{TS} 278(\mathrm{~S}, \mathrm{~S})$ & -3864.18484 & 1.6 & -3865.055197 & 2.6 \\
\hline TS285(S,S) & -3864.18457 & 1.8 & -3865.057024 & 1.4 \\
\hline $\mathrm{TS} 280(\mathrm{~S}, \mathrm{~S})$ & -3864.184458 & 1.9 & -3865.056938 & 1.5 \\
\hline TS274(S,S) & -3864.184367 & 1.9 & -3865.054736 & 2.9 \\
\hline $\mathrm{TS} 281(\mathrm{~S}, \mathrm{~S})$ & -3864.184231 & 2.0 & -3865.056624 & 1.7 \\
\hline TS291(S,S) & -3864.184075 & 2.1 & -3865.054393 & 3.1 \\
\hline $\mathrm{TS} 277(\mathrm{~S}, \mathrm{~S})$ & -3864.183942 & 2.2 & -3865.056356 & 1.8 \\
\hline TS282(S,S) & -3864.183637 & 2.4 & -3865.056002 & 2.1 \\
\hline $\mathrm{TS} 286(\mathrm{~S}, \mathrm{~S})$ & -3864.183628 & 2.4 & -3865.054051 & 3.3 \\
\hline TS290(S,S) & -3864.183627 & 2.4 & -3865.056019 & 2.1 \\
\hline TS297(S,S) & -3864.18352 & 2.5 & -3865.053852 & 3.4 \\
\hline TS284(S,S) & -3864.183225 & 2.7 & -3865.053604 & 3.6 \\
\hline TS296(S,S) & -3864.183138 & 2.7 & -3865.055575 & 2.3 \\
\hline TS287(S,S) & -3864.183107 & 2.7 & -3865.055473 & 2.4 \\
\hline TS289(S,S) & -3864.18307 & 2.8 & -3865.053624 & 3.6 \\
\hline TS298(S,S) & -3864.183041 & 2.8 & -3865.05335 & 3.7 \\
\hline $\mathrm{TS} 300(\mathrm{~S}, \mathrm{~S})$ & -3864.182943 & 2.8 & -3865.053225 & 3.8 \\
\hline TS299(S,S) & -3864.182875 & 2.9 & -3865.055289 & 2.5 \\
\hline
\end{tabular}




$\begin{array}{lllll}\text { TS292(S,S) } & -3864.182844 & 2.9 & -3865.055312 & 2.5 \\ \text { TS294(S,S) } & -3864.182655 & 3.0 & -3865.055035 & 2.7 \\ \text { TS301(S,S) } & -3864.182564 & 3.1 & -3865.054932 & 2.7 \\ \text { TS293(S,S) } & -3864.181873 & 3.5 & -3865.052292 & 4.4 \\ \text { TS283(S,S) } & -3864.181799 & 3.5 & -3865.054327 & 3.1 \\ \text { TS295(S,S) } & -3864.181716 & 3.6 & -3865.052153 & 4.5 \\ \text { TS304(S,S) } & -3864.181535 & 3.7 & -3865.05189 & 4.6 \\ \text { TS302(S,S) } & -3864.181033 & 4.0 & -3865.05346 & 3.7 \\ \text { TS273(S,S) } & -3864.181021 & 4.0 & -3865.053505 & 3.6 \\ \text { TS303(S,S) } & -3864.181018 & 4.0 & -3865.053492 & 3.6\end{array}$

Table S51. Absolute free energies (298K, in hartrees) at the wB97XD/6$311+\mathrm{G}(\mathrm{d}, \mathrm{p}) / / \mathrm{wB} 97 \mathrm{XD} / 6-31 \mathrm{G}(\mathrm{d})$ level of theory for the thiocarboxylysis/trans-esterification organocascade in which cyclohexene oxide $(1 \mathrm{~b})$ is the epoxide. Free energies are given relative to $1 \mathrm{a}+1 \mathrm{~b}(\mathrm{kcal} / \mathrm{mol})$. The Quasi-RRHO model was used to compute the free energies.

\begin{tabular}{ccc}
\hline Name & $\begin{array}{c}\mathbf{G}[\mathbf{w B 9 7 X D / 6 - 3 1 1 + G ( d , p ) / /} \\
\mathbf{w B 9 7 X D / 6 - 3 1 G ( d ) ]}\end{array}$ & $\mathbf{G}_{\text {rel }}$ \\
\hline $1 \mathrm{a}$ & -2546.168237 & \\
$1 \mathrm{~b}$ & -309.717533 & \\
$1 \mathrm{a}+1 \mathrm{~b}$ & -2855.885770 & 0.0 \\
2 & -2855.880455 & 3.3 \\
$\mathrm{TS} 1$ & -2855.860346 & 16.0 \\
3 & -2855.920742 & -21.9 \\
4 & -2855.918220 & -20.4 \\
$\mathrm{TS} 2$ & -2855.890435 & -2.9 \\
5 & -2855.906316 & -12.9 \\
6 & -2855.905820 & -12.6 \\
$\mathrm{TS} 3$ & -2855.892939 & -4.5 \\
7 & -2855.918262 & -20.4 \\
8 & -2855.930322 & -28.0
\end{tabular}


Table S52. Absolute free energies (298K, in hartrees) at the wB97XD/6$311+\mathrm{G}(\mathrm{d}, \mathrm{p}) / / \mathrm{wB} 97 \mathrm{XD} / 6-31 \mathrm{G}(\mathrm{d})$ level of theory for the thiocarboxylysis/trans-esterification organocascade in which cyclopentene oxide (1c) is the epoxide. Free energies are given relative to $1 \mathrm{a}+1 \mathrm{c}(\mathrm{kcal} / \mathrm{mol})$. The Quasi-RRHO model was used to compute the free energies.

\begin{tabular}{ccc}
\hline Name & $\begin{array}{c}\text { G[wB97XD/6-311+G(d,p)// } \\
\mathbf{w B 9 7 X D / 6 - 3 1 G ( d ) ]}\end{array}$ & $\mathbf{G}_{\text {rel }}$ \\
\hline 1a & -2546.168237 & \\
1c & -270.428251 & \\
1a+1c & -2816.596488 & 0.0 \\
2 & -2816.590534 & 3.7 \\
TS1 & -2816.567579 & 18.1 \\
3 & -2816.627276 & -19.3 \\
4 & -2816.623062 & -16.7 \\
TS2 & -2816.586693 & 6.1 \\
5 & -2816.602454 & -3.7 \\
6 & -2816.598180 & -1.1 \\
TS3 & -2816.590501 & 3.8 \\
7 & -2816.621945 & -16.0 \\
8 & -2816.635263 & -24.3
\end{tabular}


B97-D/def2-TZVP Optimized Cartesion Coordinates $(\AA)$ and absolute solution phase free energies $(\mathrm{G})$ in hartrees. Reported free energies were computed using the RRHO model at the B97-

D/def2-TZVP level of theory. Free energies computed with the Quasi-RRHO model and/or at the B97-D3/def2-TZVP//B97-

$\mathrm{D} /$ def2-TZVP level are given in the appropriate tables above.

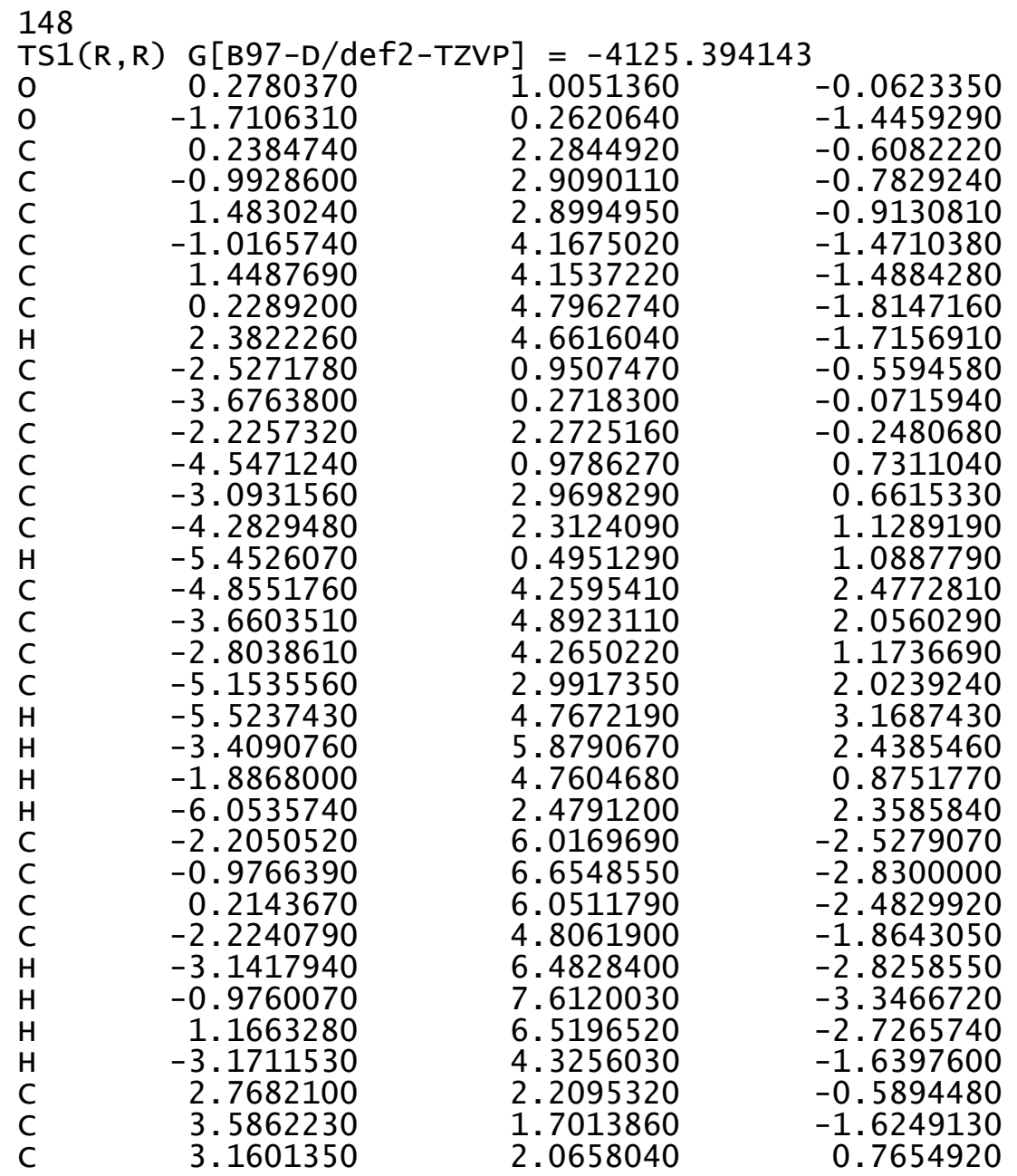

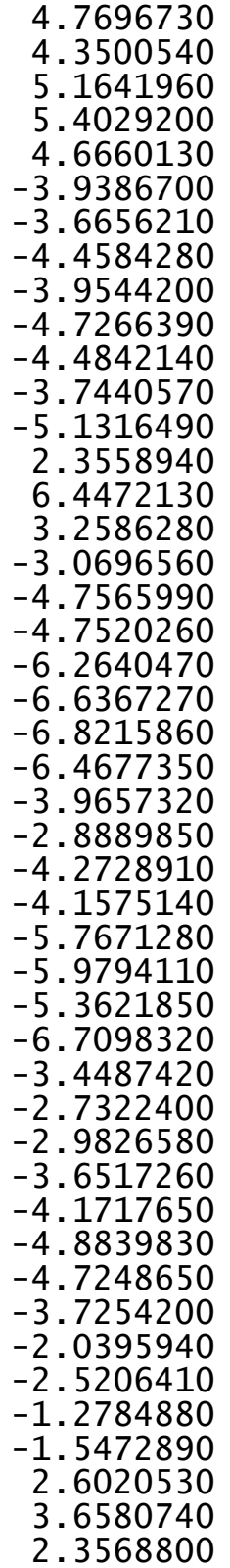

1. 0332440

1.3870550

0.8581370

0.6431710

1.2633610

$-1.1551010$

$-2.1784160$

$-1.4767580$

$-3.5009720$

$-2.8190860$

$-3.8437100$

$-4.2929500$

$-3.0725360$

2. 7129470

0.1264050

1. 9175770

$-1.8700960$

$-5.2942890$

$-0.4157200$

$-0.3255710$

$-1.2761480$

$-0.1014060$

0.4643030

$-0.6741440$

$-0.7171620$

$-1.6235700$

0.1301430

$-5.9390170$

$-6.9732540$

$-5.9580470$

$-5.3774070$

$-6.1100920$

$-5.6742060$

$-6.1227740$

$-7.1475860$

$-1.7163040$

$-0.9285150$

$-2.6594400$

$-1.4667280$

$-2.9130410$

$-3.8713440$

$-3.0769460$

$-2.5536070$

4.2377700

4.4403420

4. 6971230
$-1.2832530$

1.0499070

0.0443500

$-2.0765210$

2. 0825800

$-0.4277950$

0.5121990

$-1.6975220$

0.1647270

$-1.9988960$

$-1.0841980$

0.8783630

$-2.9775570$

1.8906530

0.3956500

$-3.0978290$

1.8812990

$-1.4410080$

$-2.7494030$

$-3.0323850$

$-3.4361070$

$-2.1137570$

$-3.7678290$

$-4.0486750$

$-3.8473730$

$-4.5059210$

$-4.7713070$

$-0.4735400$

$-0.7755240$

0.5469580

$-0.4572090$

$-1.4754560$

$-2.1833110$

$-0.4813050$

$-1.7731140$

2.9479330

2. 6799820

3.0538410

3. 9203210

2. 3480330

2. 5826420

1. 5795780

3. 2594730

1. 9101170

2. 1347990

0.9470490 


$\begin{array}{rrrr}\text { H } & 1.9869550 & 4.7089700 & 2.6887920 \\ \mathrm{C} & 2.6362560 & 2.1390420 & 3.2868520 \\ \mathrm{H} & 2.5367650 & 1.0496330 & 3.3074320 \\ \mathrm{H} & 3.6469830 & 2.4034630 & 3.6254250 \\ \mathrm{H} & 1.9226240 & 2.5616810 & 4.0031210 \\ \mathrm{C} & 7.6800410 & 0.8258150 & -0.2078060 \\ \mathrm{H} & 8.5994180 & 0.3061440 & 0.0931260 \\ \mathrm{H} & 7.6258880 & 0.8169630 & -1.3044010 \\ \mathrm{H} & 7.7420890 & 1.8701470 & 0.1235640 \\ \mathrm{C} & 6.3930080 & -1.3470150 & -0.0478160 \\ \mathrm{H} & 5.5254160 & -1.8593420 & 0.3866300 \\ \mathrm{H} & 6.3172510 & -1.4121390 & -1.1405300 \\ \mathrm{H} & 7.3043550 & -1.8750690 & 0.2631660 \\ \mathrm{C} & 3.4673430 & 0.6682020 & -3.9697290 \\ \mathrm{H} & 4.5239660 & 0.3743070 & -4.0075520 \\ \mathrm{H} & 2.8817060 & -0.1743350 & -3.5952550 \\ \mathrm{H} & 3.1459280 & 0.8823210 & -4.9972580 \\ \mathrm{C} & 4.1017940 & 3.0840440 & -3.6547430 \\ \mathrm{H} & 5.1697360 & 2.8310380 & -3.6082470 \\ \mathrm{H} & 3.8377830 & 3.2787060 & -4.7029660 \\ \mathrm{H} & 3.9467920 & 4.0035470 & -3.0786970 \\ \mathrm{P} & -0.2286670 & -0.2430510 & -0.9931880 \\ \mathrm{O} & 0.5505840 & -0.3208730 & -2.2826260 \\ \mathrm{O} & -0.2744190 & -1.4291730 & -0.0845030 \\ \mathrm{H} & 2.2021510 & 2.1932300 & -3.1774550 \\ \mathrm{H} & 6.5476380 & 0.1471610 & 1.4900590 \\ \mathrm{H} & 1.2917800 & 2.5528750 & 1.6827140 \\ \mathrm{H} & -4.4340380 & 0.5581680 & -2.3627050 \\ \mathrm{H} & -5.1931720 & -5.3099110 & -2.4497490 \\ \mathrm{H} & -2.5430580 & -0.9125520 & 1.8017300 \\ \mathrm{C} & 2.8848620 & -2.4532780 & -1.9638250 \\ \mathrm{C} & 1.8579770 & -3.3883420 & -1.4960490 \\ \mathrm{H} & 2.9981400 & -1.5271820 & -1.3955090 \\ \mathrm{H} & 1.0374790 & -2.9860550 & -0.9113790 \\ \mathrm{O} & 1.8199250 & -2.3356050 & -2.9766670 \\ \mathrm{C} & 2.4229140 & -2.8459680 & 2.1746970 \\ \mathrm{C} & 1.8147190 & -1.5324180 & 4.2483880 \\ \mathrm{C} & 1.0649790 & -1.2020640 & 3.1076340 \\ \mathrm{~N} & 1.4357320 & -1.9399350 & 1.9958430 \\ \mathrm{H} & 0.9126420 & -1.8194970 & 1.1034960 \\ \mathrm{~S} & 2.9783870 & -2.7774850 & 3.8450280 \\ \mathrm{~S} & 3.0606550 & -3.9099870 & 1.0438030 \\ \mathrm{H} & 1.2332530 & -1.4913880 & -2.6912760 \\ \mathrm{C} & 0.5568720 & 0.0743700 & 5.5091780 \\ \mathrm{C} & -0.1839740 & 0.4098940 & 4.3530620 \\ \mathrm{C} & 0.0620520 & -0.2305330 & 3.1345930 \\ & & & \\ & & & \end{array}$

$\begin{array}{rrr}1.5577070 & -0.8893440 & 5.4642870 \\ 0.3384520 & 0.5970470 & 6.4363080 \\ -0.4763420 & 0.0020930 & 2.2257430 \\ 2.1261680 & -1.1316600 & 6.3577080 \\ 3.9754500 & -4.3608040 & -3.2331500 \\ 3.2345390 & -5.3512540 & -2.3208040 \\ 1.8438670 & -4.8225410 & -1.9262080 \\ 4.1747950 & -3.0115030 & -2.5218300 \\ 4.9534520 & -4.7672200 & -3.5162910 \\ 3.1227100 & -6.3233840 & -2.8145970 \\ 1.1498260 & -4.8995580 & -2.7759490 \\ 4.8530650 & -3.1366910 & -1.6685680 \\ 4.6212390 & -2.2653610 & -3.1899210 \\ 3.4013850 & -4.2081250 & -4.1566120 \\ 3.8270850 & -5.5119510 & -1.4095750 \\ 1.3990900 & -5.4119620 & -1.1165210 \\ -1.1174610 & 1.3876410 & 4.5166530 \\ -1.8269610 & 1.8165420 & 3.3426310 \\ -1.1274230 & 2.1856530 & 2.5827130 \\ -2.4175660 & 0.9938920 & 2.9201240 \\ -2.4892740 & 2.6223070 & 3.6632550\end{array}$

TS2 $(R, R)$ G $[B 97-D /$ def2-TZVP $]=-4125.394032$

$\begin{array}{lrcr}\text { TS2 }(\mathrm{R}, \mathrm{R}) & \mathrm{G}[\mathrm{B} 97-\mathrm{D} / \mathrm{def}-\mathrm{TZVP}]=-4125.394032 & \\ \mathrm{O} & 0.4619870 & 0.7453470 & -0.2299560 \\ \mathrm{O} & -1.6127160 & 0.1518710 & -1.5718950 \\ \mathrm{C} & 0.5195940 & 2.0110490 & -0.7994260 \\ \mathrm{C} & -0.6570030 & 2.7365560 & -0.9580000 \\ \mathrm{C} & 1.8039620 & 2.5204030 & -1.1329620 \\ \mathrm{C} & -0.5898670 & 3.9864520 & -1.6604930 \\ \mathrm{C} & 1.8636010 & 3.7708210 & -1.7161910 \\ \mathrm{C} & 0.6968030 & 4.5097390 & -2.0281260 \\ \mathrm{H} & 2.8327360 & 4.1942880 & -1.9674780 \\ \mathrm{C} & -2.3682640 & 0.9331530 & -0.7043890 \\ \mathrm{C} & -3.5946800 & 0.3890530 & -0.2343260 \\ \mathrm{C} & -1.9339140 & 2.2188950 & -0.4000980 \\ \mathrm{C} & -4.3988400 & 1.1977510 & 0.5435040 \\ \mathrm{C} & -2.7317310 & 3.0107420 & 0.4945680 \\ \mathrm{C} & -4.0001690 & 2.4989650 & 0.9333810 \\ \mathrm{H} & -5.3562070 & 0.8170760 & 0.8912080 \\ \mathrm{C} & -4.4049380 & 4.5370560 & 2.2057860 \\ \mathrm{C} & -3.1278400 & 5.0174880 & 1.8272000 \\ \mathrm{C} & -2.3137860 & 4.2741270 & 0.9963330 \\ \mathrm{C} & -4.8235120 & 3.2956790 & 1.7747750 \\ \mathrm{H} & -5.0410330 & 5.1370560 & 2.8527820 \\ \mathrm{H} & -2.7818780 & 5.9797520 & 2.1983190 \\ \mathrm{H} & -1.3373560 & 4.6585110 & 0.7219010\end{array}$




-5.7872630
-1.6391900
-0.3676860
0.7749330
-1.7468290
-2.5395680
-0.2967640
1.7584410
-2.7268010
3.0432480
3.8217080
3.4489220
4.9861220
4.6084980
5.3894650
5.5947770
4.9169010
-3.9983470
-3.9872580
-4.3996790
-4.3985680
-4.7937670
-4.8011600
-4.3939370
-5.1071860
2.6944800
6.6764730
3.4678120
-3.5463650
-5.2162480
-4.4599030
-5.9194550
-6.3718620
-6.5216280
-5.9582080
-3.6020220
-2.5607070
-3.9820760
-3.6306780
-4.2436760
-4.5294020
-4.2639950
-3.2150980
-6.6613080
-7.3659740
-6.7495670

2.8953810

5.9157350

6.4505260

5.7559130

4.7172860

6.4515920

7.3995350

6.1433550

4.3171480

1.7384220

1.2310800

1. 5248130

0.5127840

0.7813700

0.2619730

0.1245400

0.6053880

$-1.0093420$

$-1.9974530$

$-1.3425280$

$-3.2966890$

$-2.6551180$

$-3.6480860$

$-4.0641130$

$-2.9076140$

2. 1665460

$-0.5014730$

1. 4688130

$-1.6985200$

$-5.0709280$

$-0.3126570$

$-0.0586420$

$-0.9788000$

0.2824000

0.7074390

$-0.7289700$

$-0.8870550$

$-1.6568810$

0.0550370

$-5.7106930$

$-6.7518750$

$-5.1591500$

$-5.6948630$

$-5.1329820$

$-4.7087220$

$-4.5644240$

2.0847620
-2.7238140
-3.0473630
-2.7086340
-2.0466690
-3.0162450
-3.5741050
-2.9689630
-1.8071650
-0.8472290
-1.9123670
0.4948440
-1.6108610
0.7379950
-0.2997820
-2.4252910
1.7629190
-0.5633250
0.4477390
-1.8768950
0.1217960
-2.1519740
-1.1676790
0.8938610
-3.1628290
1.6560080
-0.0361590
-3.3758720
1.8757920
-1.4978160
-2.9976500
-3.4236620
-3.8163910
-2.5716050
-4.2097560
-4.2064720
-3.9047230
-4.6534740
-4.9749270
-2.5079890
-2.7091870
-3.4573310
-2.1260350
-2.0282720
-1.3017660
-2.9633040

\begin{abstract}
$-6.9498730$
$-4.7621550$

$-5.4619720$

$-5.2997690$

$-4.4337850$

$-2.5429180$

$-3.0115600$

$-1.6888890$

$-2.1779810$

3.0446270

4.1135800

2.8240190

2.4671700

2.9398750

2.8096220

3.9554920

2. 2307960

6.6396150

7.5410030

6.6145530

5.7574720

7.8704220

7.9331280

7.7535400

8.8120580

3.5103230

4.5248550

2. 8403710

3.1942070

4.4059470

5.4433030

4.1255520

4.3656350

$-0.1991570$

0.5595280

$-0.3709310$

2. 4446450

6.8312480

1.6217520

$-4.0629240$

$-5.1700420$

$-3.0423300$

3.0737420

2. 1111840

2. 9895640

1. 1608020
\end{abstract}

-6.1728030
-1.5924320
-0.8199310
-2.5496610
-1.3413150
-2.7328970
-3.7183610
-2.8275500
-2.4109930
3.6677950
3.7894780
4.1855160
4.1425590
1.4977770
0.4131180
1.7002850
1.8934520
-1.3542220
-1.9771630
-0.7186960
-2.0064040
0.4754960
1.0550180
1.1781830
-0.0732180
0.1860930
-0.2294370
-0.5761890
0.4152800
2.5326720
2.1720360
2.7402640
3.4705290
-0.4885630
-0.7514200
-1.5903870
1.8538700
-1.1761570
2.0915720
0.6368320
-5.6530160
-0.7279550
-2.4652870
-3.4711780
-1.4643930
-3.1190010

2.8167050

2.4760220

2.8537460

3.8345680

2. 4161390

2.5343420

1.7384560

3.3995060

1.7461890

1.9688860

0.8064110

2.5510890

3.0161720

2. 9586680

3.3813320

3.7522520

1.2422730

1. 3012030

2. 1369350

1.2683200

0.0044330

$-0.9250930$

0.8408110

0.1419370

$-4.2250250$

$-4.2736870$

$-3.8179320$

$-5.2511030$

$-3.9813560$

$-3.9680550$

$-5.0226960$

$-3.4145240$

$-1.0810080$

$-2.3574850$

$-0.0828050$

$-3.4252790$

$-0.8900480$

1.4536320

$-2.6253560$

$-0.5664860$

1.8813590

$-1.3612650$

$-0.8986600$

$-0.9338740$

$-0.5109030$ 


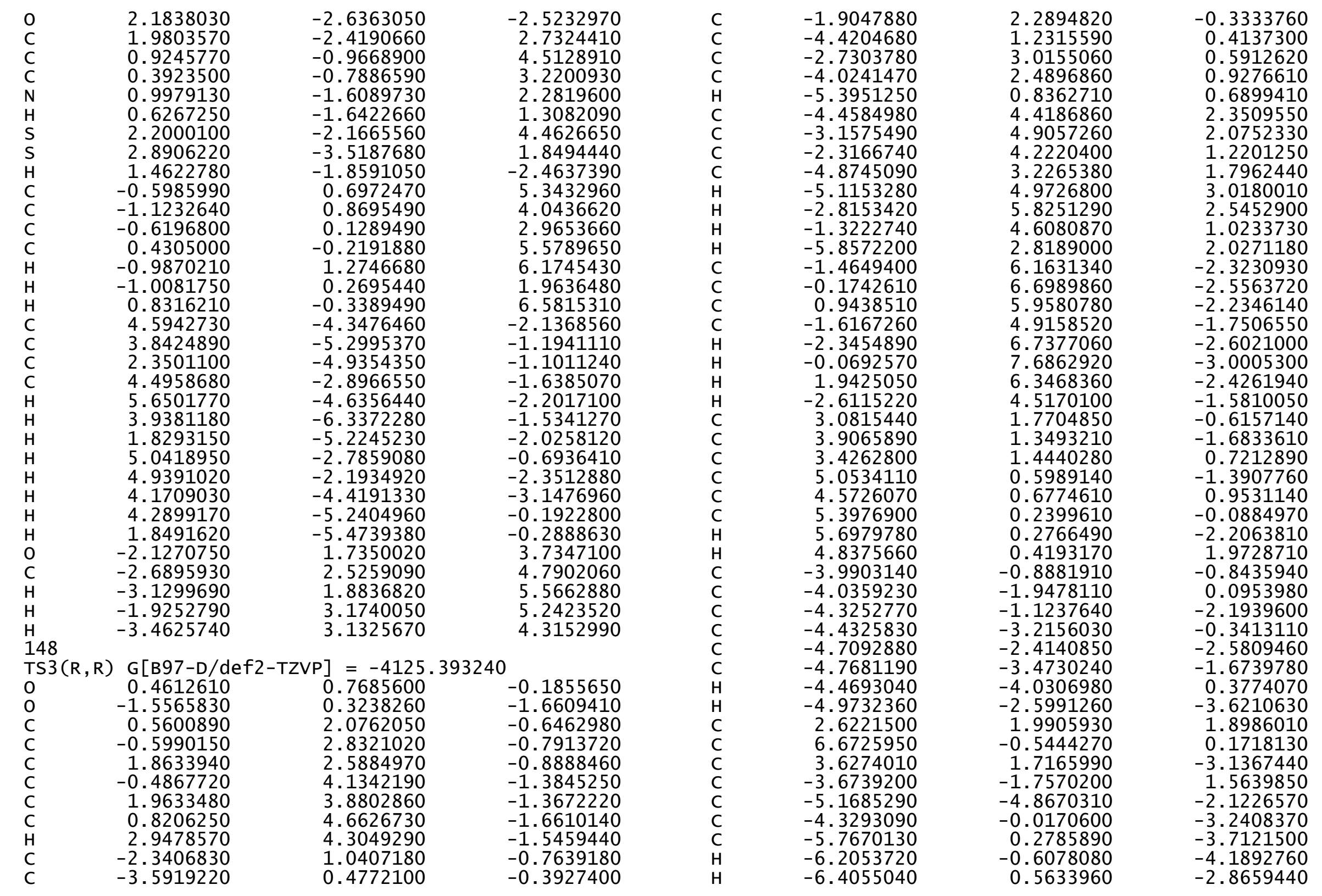




-5.7660560
-3.4206200
-2.3939660
-3.7833590
-3.4136030
-6.4324590
-6.7242530
-6.2451550
-7.2706140
-4.0119710
-3.1127670
-3.7619200
-4.2979710
-4.9410220
-5.6279870
-5.4700470
-4.6728720
-2.6897600
-3.1537090
-1.7995820
-2.3812950
2.9598860
4.0208020
2.7631310
2.3549750
2.8133290
2.6930730
3.8108600
2.0701140
6.5635070
7.4667770
6.4740820
5.6896730
7.8518920
7.9725590
7.6711940
8.7872740
3.6939540
4.6972920
2.9753170
3.4586060
4.6102140
5.6402080
4.3820230
4.5576860
-0.1723850

1.0985530 $-0.3533410$ $-0.5381510$ $-1.2453020$ 0.4828440 $-5.3577010$ $-6.3516720$ $-5.4309940$ $-4.6665680$ $-5.8672920$ $-5.5357200$ $-5.9593580$ $-6.8601190$ $-1.7372210$ $-0.9413340$ $-2.6966490$ $-1.5734330$ $-2.8251690$ - 3.8198200 $-2.8601760$ $-2.5810710$ 3. 4802060 3. 5880610 4.0722150 3. 8841430 1. 2135490

0.1368500

1. 3880730 1. 5444260 $-1.5297570$ $-2.1507950$ $-0.9940690$ $-2.1854610$ 0.4252140 1. 1018070 1. 0328790 $-0.1328810$ 0.5094000 0.0649730 $-0.2606880$ 0.8343100 2. 8102950 2. 4301370 3. 1124180 3. 6968840 $-0.3781990$

-4.4429170
-4.4375400
-4.1027870
-4.9645790
-5.1494030
-1.3910610
-1.7556350
-0.3116570
-1.5469030
-1.9292470
-2.4636790
-0.8639910
-2.3017490
2.4407440
2.1284210
2.3626160
3.4934250
2.0740030
2.0871630
1.4383830
3.0981350
2.1233820
2.3872830
1.2229500
2.9465630
3.2088300
3.0565560
3.6337690
3.9433230
1.3468970
1.3971030
2.3007130
1.2471970
0.3973610
-0.4579140
1.2943990
0.5396530
-4.0884300
-4.1017250
-3.7953440
-5.1103370
-3.6034450
-3.5747490
-4.6343710
-2.9601000
-1.1700520

0.6380050 $-0.3978790$

2. 6143860

6.8876670

1. 5589880

$-3.9438970$

$-5.3962630$

$-3.1819530$ 3. 1049970 2. 1214370 3. 0086240 1.1588440 2. 2601100 1.8364520 0.7236610 0.2431810 0.8785340

0.5454760 1. 9905220 2. 7686140 1. 5402980 $-0.8200350$ $-1.2950610$ $-0.7529750$ 0.1946700 $-1.2361460$ $-1.1023800$ 0.5575080 4.6436240 3. 8540840 2. 3608410 4. 5348310 5.6996980 3. 9587230 1.8749960 5. 0480630 5.0065160 4.2553980 4.2631330 1.8264700 $-2.2866590$ $-2.8842020$ $-3.3530110$ $-2.1345840$ $-3.6387160$
-0.5380000
-1.5638360
2.1251310
-1.1221260
1.9297750
0.9002000
-4.8152610
-0.7867310
-2.3528780
-3.3888050
-1.3922050
-3.0649020
-2.4152950
-2.6581660
-1.3350310
-1.0528370
-1.8055790
-1.7591830
-2.5411850
-3.6995970
-1.6409320
0.2739430
0.5482960
-0.1095510
-0.6659680
0.7894900
0.1102900
-0.8643240
-4.1702140
-5.1959430
-4.8308270
-2.7688460
-4.4598190
-6.2007750
-5.0296330
-2.7483360
-2.0077510
-4.1471800
-5.2262400
-5.4380100
1.4382080
2.1500280
1.4534990
2.7582780
2.7946890

$-2.4319050$ $-0.2851940$ $-3.2033530$ $-0.7381840$ 1.6476230 $-2.7848370$ $-3.1968530$ 1.6748360 $-1.4831210$ $-1.1474260$ $-0.9735010$ $-0.7656280$ $-2.6885260$

2. 5459250

4. 3908280

3. 0963610

2. 1218770

1. 1354010

4. 2974290

1.6165650 $-2.5912200$

5. 2885440

3.9873450

2.8749010

5.4916000

6.1465710

1.8731920

6.4962540

$-2.3698020$

$-1.5423780$

$-1.4729840$

$-1.7466310$

$-2.4237850$

$-1.9676330$

$-2.4395490$

$-0.7776150$

$-2.3770390$

$-3.3967720$

$-0.5230730$

$-0.7339050$

3. 7089170

4.8009140

5.5107570

5. 3269500

4. 3469750 


$\begin{array}{lrrr}\text { TS4 (R, R) } & \text { G }[\mathrm{B} 97-\mathrm{D} / \mathrm{de} \text { ef }-\mathrm{TZVP}]=-4125.392888 & \\ \mathrm{O} & 0.3004210 & 0.9847840 & -0.0983050 \\ \mathrm{O} & -1.7412040 & 0.1880150 & -1.3711220 \\ \mathrm{C} & 0.2598530 & 2.2281660 & -0.7218470 \\ \mathrm{C} & -0.9683320 & 2.8589890 & -0.8963480 \\ \mathrm{C} & 1.5009660 & 2.8077560 & -1.0999720 \\ \mathrm{C} & -0.9988640 & 4.0733030 & -1.6594700 \\ \mathrm{C} & 1.4637410 & 4.0221710 & -1.7556100 \\ \mathrm{C} & 0.2423090 & 4.6606310 & -2.0831520 \\ \mathrm{H} & 2.3964690 & 4.4989230 & -2.0457000 \\ \mathrm{C} & -2.5204800 & 0.9419130 & -0.5048940 \\ \mathrm{C} & -3.6622060 & 0.3111380 & 0.0601950 \\ \mathrm{C} & -2.1912700 & 2.2749750 & -0.2846740 \\ \mathrm{C} & -4.4968120 & 1.0792230 & 0.8451470 \\ \mathrm{C} & -3.0178640 & 3.0386990 & 0.6089210 \\ \mathrm{C} & -4.2010760 & 2.4301540 & 1.1530980 \\ \mathrm{H} & -5.3956680 & 0.6324690 & 1.2622940 \\ \mathrm{C} & -4.6982090 & 4.4631950 & 2.4011320 \\ \mathrm{C} & -3.5086970 & 5.0493930 & 1.9043430 \\ \mathrm{C} & -2.6919640 & 4.3564230 & 1.0336320 \\ \mathrm{C} & -5.0306650 & 3.1760660 & 2.0339930 \\ \mathrm{H} & -5.3353980 & 5.0219330 & 3.0827650 \\ \mathrm{H} & -3.2295720 & 6.0524670 & 2.2191650 \\ \mathrm{H} & -1.7769070 & 4.8170620 & 0.6781330 \\ \mathrm{H} & -5.9263320 & 2.6992860 & 2.4280550 \\ \mathrm{C} & -2.1980140 & 5.8716750 & -2.7900280 \\ \mathrm{C} & -0.9723910 & 6.4707300 & -3.1723990 \\ \mathrm{C} & 0.2216170 & 5.8717420 & -2.8277450 \\ \mathrm{C} & -2.2104220 & 4.7050140 & -2.0515800 \\ \mathrm{H} & -3.1382100 & 6.3323270 & -3.0852630 \\ \mathrm{H} & -0.9768640 & 7.3937560 & -3.7477860 \\ \mathrm{H} & 1.1708620 & 6.3101330 & -3.1307310 \\ \mathrm{H} & -3.1558160 & 4.2536230 & -1.7677700 \\ \mathrm{C} & 2.7884130 & 2.1262260 & -0.7695980 \\ \mathrm{C} & 3.5769100 & 1.5559900 & -1.7954110 \\ \mathrm{C} & 3.2131160 & 2.0525020 & 0.5814610 \\ \mathrm{C} & 4.7612920 & 0.8929250 & -1.4468580 \\ \mathrm{C} & 4.4026000 & 1.3761460 & 0.8728670 \\ \mathrm{C} & 5.1851140 & 0.7823000 & -0.1217770 \\ \mathrm{H} & 5.3702650 & 0.4530320 & -2.2329400 \\ \mathrm{H} & 4.7424380 & 1.3033840 & 1.9027640 \\ \mathrm{C} & -3.9466100 & -1.1325410 & -0.1943160 \\ \mathrm{C} & -3.6976660 & -2.0889410 & 0.8163460 \\ \mathrm{C} & -4.4608830 & -1.5381930 & -1.4450390 \\ \mathrm{C} & -4.0070020 & -3.4309150 & -1.6556070 \\ \mathrm{C} & -4.7420060 & -2.8918790 & \end{array}$

-4.5269430
-3.8217630
-5.1391410
2.4417080
6.4636080
3.2173770
-3.1043610
-4.8171840
-4.7430090
-6.2540430
-6.6322670
-6.8118820
-6.4515050
-3.9552880
-2.8799570
-4.2697010
-4.1374640
-3.8827610
-4.0738680
-4.0515040
-2.8298460
-6.2913030
-6.9629890
-6.5341630
-6.4830950
-4.2072070
-4.9327220
-4.7445450
-3.7639950
-2.0550280
-2.5199270
-1.2977640
-1.5608060
2.6726540
3.7345120
2.3832450
2.0847550
2.7737800
2.6824480
3.7940580
2.0824610
7.6942060
8.6110960
7.6151530
7.7821300
6.3723320

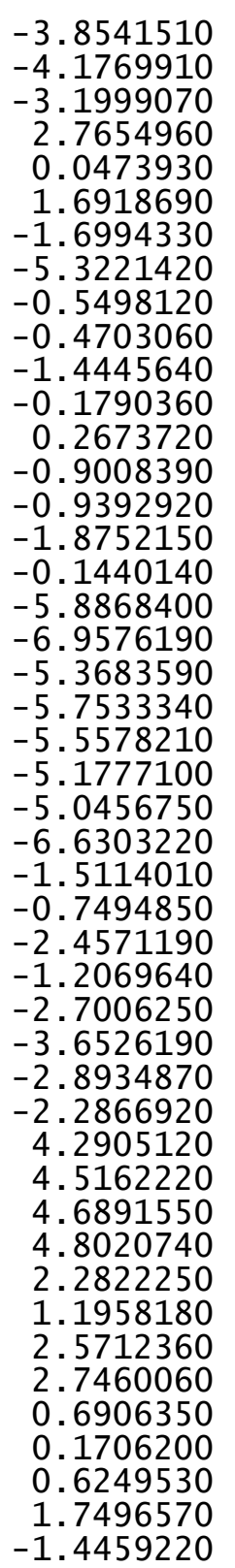

$-0.6653760$

1. 3299110

$-2.6206100$

1.6902720

0.2387810

$-3.2704610$

2. 1657980

$-0.9227150$

$-2.5690780$

$-2.8613800$

$-3.1978530$

$-1.9619540$

$-3.6507100$

$-3.8450640$

$-3.6367250$

$-4.2412720$

$-4.6196370$

$-2.0109740$

$-2.1631490$

$-2.9641360$

$-1.7313440$

$-1.3009060$

$-0.5207160$

$-2.2411690$

$-1.4379560$

3. 2260880

2. 9206390

3. 3789600

4.1841740

2. 6789810

2. 9655610

1. 9136500

3. 5662780

1.6077510

1.7755970

0.6302480

2. 3819150

3. 1089990

3. 2009890

3. 3947150

3. 8215470

$-0.4282280$

$-0.1203340$

$-1.5213610$

$-0.1542210$ 


$\begin{array}{rrrr}\text { H } & 5.5080430 & -1.9191140 & \\ \text { H } & 6.2654870 & -1.5648570 & -1.2112580 \\ \text { H } & 7.2827460 & -1.9738990 & 0.1879710 \\ \mathrm{C} & 3.3546260 & 0.3799530 & -4.0607310 \\ \mathrm{H} & 4.3968110 & 0.0385960 & -4.0984670 \\ \mathrm{H} & 2.7423410 & -0.4084590 & -3.6171830 \\ \mathrm{H} & 3.0193160 & 0.5377950 & -5.0940790 \\ \mathrm{C} & 4.0848470 & 2.7883910 & -3.9233800 \\ \mathrm{H} & 5.1451280 & 2.5041260 & -3.8838140 \\ \mathrm{H} & 3.8015810 & 2.9214930 & -4.9762180 \\ \mathrm{H} & 3.9722580 & 3.7495800 & -3.4079550 \\ \mathrm{P} & -0.2536900 & -0.3121010 & -0.9313090 \\ \mathrm{O} & 0.4828400 & -0.4836080 & -2.2365340 \\ \mathrm{O} & -0.2899280 & -1.4366480 & 0.0527940 \\ \mathrm{H} & 2.1708460 & 2.0036690 & -3.3424600 \\ \mathrm{H} & 6.5897540 & 0.1231660 & 1.3280590 \\ \mathrm{H} & 1.3727590 & 2.5834590 & 1.5300250 \\ \mathrm{H} & -4.4211020 & 0.4469010 & -2.2500090 \\ \mathrm{H} & -4.6157400 & -5.8656030 & 0.0113970 \\ \mathrm{H} & -2.5959260 & -0.7376560 & 2.0369310 \\ \mathrm{C} & 2.7968220 & -2.6203310 & -1.8577290 \\ \mathrm{C} & 1.7779050 & -3.5164410 & -1.3039680 \\ \mathrm{H} & 2.9318820 & -1.6627000 & -1.3498710 \\ \mathrm{H} & 0.9768980 & -3.0709520 & -0.7234650 \\ \mathrm{O} & 1.7040480 & -2.5552500 & -2.8451680 \\ \mathrm{C} & 2.4510970 & -2.7492780 & 2.3143020 \\ \mathrm{C} & 1.9302270 & -1.2986110 & 4.3190120 \\ \mathrm{C} & 1.1453450 & -1.0303610 & 3.1853490 \\ \mathrm{~N} & 1.4674840 & -1.8443520 & 2.1119620 \\ \mathrm{H} & 0.9194850 & -1.7704340 & 1.2291540 \\ \mathrm{~S} & 3.0645900 & -2.5827450 & 3.9570090 \\ \mathrm{~S} & 3.0394040 & -3.8910920 & 1.2332020 \\ \mathrm{H} & 1.1349470 & -1.6891480 & -2.5944340 \\ \mathrm{C} & 0.7376610 & 0.4031510 & 5.5163860 \\ \mathrm{C} & -0.0374140 & 0.6769100 & 4.3664570 \\ \mathrm{C} & 0.1553410 & -0.0449390 & 3.1842450 \\ \mathrm{C} & 1.7231410 & -0.5769480 & 5.4997710 \\ \mathrm{H} & 0.5585540 & 0.9872740 & 6.4148310 \\ \mathrm{H} & -0.4125710 & 0.1379460 & 2.2817620 \\ \mathrm{H} & 2.3187300 & -0.7711080 & 6.3873070 \\ \mathrm{C} & 3.8401980 & -4.6122460 & -3.0351870 \\ \mathrm{C} & 3.1205880 & -5.5365940 & -2.0403070 \\ \mathrm{C} & 1.7440620 & -4.9744980 & -1.6422130 \\ \mathrm{C} & 4.0664020 & -3.2211400 & -2.4186360 \\ \mathrm{H} & 4.8077440 & -5.0426980 & -3.3187070 \\ \mathrm{H} & 2.9901530 & -6.5378240 & -2.4668240 \\ & & & \\ & & & \end{array}$

$\begin{array}{rr}1.0263280 & -5.1005150 \\ 4.7717630 & -3.2937400 \\ 4.4934180 & -2.5220760 \\ 3.2408030 & -4.5161260 \\ 3.7383840 & -5.6418740 \\ 1.3196230 & -5.5082570 \\ -0.9496440 & 1.6792980 \\ -1.6867190 & 2.0515740 \\ -1.0032590 & 2.3579250 \\ -2.3070540 & 1.2183240 \\ -2.3225770 & 2.8898700\end{array}$

$-2.4660240$

$-1.5815660$

$-3.1475520$

$-3.9501230$

$-1.1377380$

$-0.7843790$

4.4990790

3.3230090

2. 5216310

2.9695060

3.6122360

148

$\operatorname{TS} 5(R, R) \quad G[B 97-D /$ def2-TZVP $]=-4125.392699$

$\begin{array}{lrlr}\text { O } & 0.2262430 & 1.0208710 & -0.0411450 \\ \mathrm{O} & -1.7600500 & 0.2511620 & -1.4084940 \\ \mathrm{C} & 0.1145140 & 2.3219720 & -0.5234900 \\ \mathrm{C} & -1.1478750 & 2.8982580 & -0.6262510 \\ \mathrm{C} & 1.3204590 & 3.0039340 & -0.8450580 \\ \mathrm{C} & -1.2496330 & 4.1908460 & -1.2420680 \\ \mathrm{C} & 1.2123460 & 4.2876960 & -1.3400380 \\ \mathrm{C} & -0.0444600 & 4.8947040 & -1.5838480 \\ \mathrm{H} & 2.1139610 & 4.8460890 & -1.5766900 \\ \mathrm{C} & -2.5859090 & 0.8599000 & -0.4738050 \\ \mathrm{C} & -3.6979760 & 0.1090400 & -0.0062550 \\ \mathrm{C} & -2.3359040 & 2.1763920 & -0.0984540 \\ \mathrm{C} & -4.5860810 & 0.7358540 & 0.8424840 \\ \mathrm{C} & -3.2162660 & 2.7864360 & 0.8606100 \\ \mathrm{C} & -4.3704650 & 2.0562710 & 1.3079420 \\ \mathrm{H} & -5.4661800 & 0.1975250 & 1.1857150 \\ \mathrm{C} & -5.0010940 & 3.9022340 & 2.7679430 \\ \mathrm{C} & -3.8401580 & 4.6060600 & 2.3652090 \\ \mathrm{C} & -2.9728860 & 4.0631500 & 1.4385090 \\ \mathrm{C} & -5.2539450 & 2.6493670 & 2.2502040 \\ \mathrm{H} & -5.6787200 & 4.3431560 & 3.4954080 \\ \mathrm{H} & -3.6229860 & 5.5803080 & 2.7974130 \\ \mathrm{H} & -2.0818040 & 4.6122050 & 1.1553580 \\ \mathrm{H} & -6.1266610 & 2.0822840 & 2.5689680 \\ \mathrm{C} & -2.5491910 & 6.0386260 & -2.1631430 \\ \mathrm{C} & -1.3600760 & 6.7489680 & -2.4603530 \\ \mathrm{C} & -0.1339350 & 6.1824840 & -2.1795330 \\ \mathrm{C} & -2.4951770 & 4.7936840 & -1.5680900 \\ \mathrm{H} & -3.5141170 & 6.4758430 & -2.4103470 \\ \mathrm{H} & -1.4173660 & 7.7321010 & -2.9221070 \\ \mathrm{H} & 0.7890610 & 6.7057080 & -2.4232380 \\ \mathrm{H} & -3.4133780 & 4.2587720 & -1.3470210 \\ \mathrm{C} & 2.6377770 & 2.3283990 & -0.6469870 \\ & & & \end{array}$




3.4033770
3.0955050
4.5824250
4.2723720
5.0180910
5.1758820
4.6217200
-3.9083720
-3.5563300
-4.4589350
-3.8032060
-4.6791360
-4.3633390
-3.5369940
-5.1050470
2.3630660
6.2930530
3.0260990
-2.9228250
-4.5982310
-4.8255730
-6.3389560
-6.6482480
-6.9126200
-6.5914030
-4.0177010
-2.9419890
-4.2505680
-4.2660640
-5.5776580
-5.7646350
-5.1585390
-6.5355100
-3.2707170
-2.5768700
-2.7883960
-3.4522120
-3.9920560
-4.7268580
-4.5237500
-3.5186310
-1.8609450
-2.3186400
-1.1210490
-1.3468320
2.6143730

1.9238080

2.0554000

1.1973670

1. 3214510

0.8618560

0.8720890

1.0937250

$-1.3067260$

$-2.3662990$

$-1.5804470$

$-3.6791770$

$-2.9148350$

$-3.9760310$

$-4.4991910$

$-3.1313790$

2.6224330

0.0625950

2. 3012490

$-2.1035840$

$-5.4153840$

$-0.4764880$

$-0.4687450$

$-1.4121830$

$-0.3426860$

0.3530010

$-0.5957820$

$-0.5702090$

$-1.5360140$

0.2351610

$-6.1309660$

$-7.1567720$

$-6.1821160$

$-5.5979160$

$-6.1937930$

$-5.7081620$

$-6.2397690$

$-7.2216500$

$-2.0140750$

$-1.2307020$

$-2.9715080$

$-1.7942350$

$-3.1446700$

$-4.1194480$

$-3.2672610$

$-2.8119090$

4.1431710

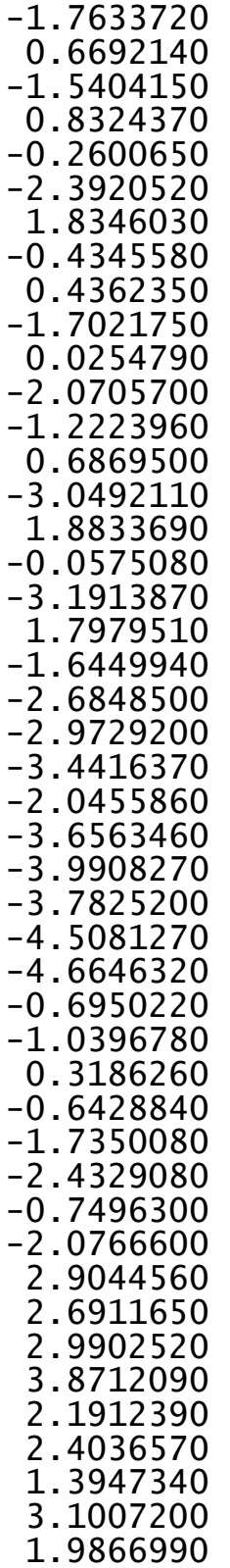

3.6830960
2.3102440
2.0508680
2.7296020
2.6268130
3.7619090
2.0657700
6.1003590
7.0235320
5.8630890
5.2850170
7.4356380
7.6076390
7.1827260
8.3666070
3.2136970
4.2721320
2.6581800
2.8481720
3.8472660
4.9159150
3.5429100
3.7161190
-0.2448340
0.4962190
-0.2082250
1.9671120
6.5815790
1.2875610
-4.5755170
-5.0491500
-2.4153980
3.0593480
2.1440660
2.9993910
1.2332110
2.0717620
2.5266950
1.9326210
1.1578610
1.5227970
0.9840610
3.1103440
3.1612500
1.3558220
0.6720640
4. 3321060

4.6630830

4.5618920

1.9596410

0.8714510

2. 2005310

2.3346650

$-1.1155260$

$-1.7063720$

$-0.7516180$

$-1.7760330$

0.9773310

1.7988440

1.4118850

0.4046770

1. 1647740

0.8970160

0.2724820

1.4921900

3.5307420

3.2776720

3.8496600

4. 3735920

$-0.2060890$

$-0.1801620$

$-1.4380740$

2.5778270

$-0.3456080$

2.4755740

0.4895620

$-5.3963910$

$-1.1339020$

$-2.0378270$

$-3.1536100$

$-1.1800060$

$-2.9403720$

$-1.9385890$

$-2.9203090$

$-1.7040220$

$-1.3408140$

$-2.0245900$

$-1.8745430$

$-2.9101470$

$-3.9277750$

-1.2054280
-0.1693290
2.1568970

1.0725610

2.8314930

3.2184950

3.1749010

3.5059980

4.0056390

0.9141440

0.9783660

1.9220900

0.5953570

0.4278340

$-0.2790930$

1.4042540

0.5360270

$-4.2099750$

$-4.3240380$

$-3.9126720$

$-5.1918590$

$-3.6354960$

$-3.6611980$

$-4.6415660$

$-2.9473170$

$-1.0184810$

$-2.3323170$

$-0.1727030$

$-3.2053710$

$-1.0364420$

1.7308160

$-2.2332050$

$-2.6473480$

1.7406880

$-1.9737820$

$-1.7121810$

$-1.3009220$

$-1.1629880$

$-3.0604730$

1. 9939760

4.1281130

3.0143860

1.8670590

0.9882200

3.6548990

0.8110760

$-2.7498260$

5.4732180 


$\begin{array}{rrr}-0.0969360 & 0.1966020 & 4.3448740 \\ 0.1388730 & -0.3897230 & 3.0974750 \\ 1.6877480 & -1.1131790 & 5.3726330 \\ 0.4611440 & 0.3117420 & 6.4243050 \\ -0.4169460 & -0.1285000 & 2.2070410 \\ 2.2762420 & -1.3806770 & 6.2456890 \\ 4.5117800 & -3.6015460 & -3.3455190 \\ 3.8445900 & -4.7917290 & -2.6378380 \\ 2.3652770 & -4.5060060 & -2.3197690 \\ 4.4567530 & -2.3399210 & -2.4672670 \\ 5.5576000 & -3.8370440 & -3.5749980 \\ 3.9096820 & -5.6939860 & -3.2569170 \\ 1.7631920 & -4.5511170 & -3.2386280 \\ 5.0833750 & -2.4746950 & -1.5756700 \\ 4.8341020 & -1.4609780 & -3.0021610 \\ 4.0012630 & -3.4059030 & -4.2977660 \\ 4.3797520 & -4.9980390 & -1.7008590 \\ 1.9444110 & -5.2564220 & -1.6415260 \\ -1.0481520 & 1.1456460 & 4.5638840 \\ -1.8002500 & 1.5904820 & 3.4229840 \\ -1.1323360 & 2.0115120 & 2.6613830 \\ -2.3712360 & 0.7622370 & 2.9843960 \\ -2.4821200 & 2.3601760 & 3.7878540\end{array}$

148

\begin{tabular}{lrrr} 
TS6 (R, R) & \multicolumn{2}{c}{ G $[\mathrm{B} 97-\mathrm{D} / \mathrm{def}-\mathrm{TZVP}]=-4125.392011$} \\
$\mathrm{O}$ & 0.2604140 & 1.0113290 & -0.0457770 \\
$\mathrm{O}$ & -1.7639370 & 0.2065130 & -1.3317770 \\
$\mathrm{C}$ & 0.1577230 & 2.2848400 & -0.5988560 \\
$\mathrm{C}$ & -1.0970040 & 2.8781040 & -0.6988660 \\
$\mathrm{C}$ & 1.3644200 & 2.9222530 & -0.9984620 \\
$\mathrm{C}$ & -1.1942480 & 4.1378800 & -1.3793770 \\
$\mathrm{C}$ & 1.2647070 & 4.1812690 & -1.5552020 \\
$\mathrm{C}$ & 0.0125610 & 4.8005510 & -1.7909350 \\
$\mathrm{H}$ & 2.1686830 & 4.7063220 & -1.8515450 \\
$\mathrm{C}$ & -2.5590500 & 0.8736070 & -0.4098210 \\
$\mathrm{C}$ & -3.6686410 & 0.1638440 & 0.1234430 \\
$\mathrm{C}$ & -2.2817980 & 2.2031560 & -0.1064030 \\
$\mathrm{C}$ & -4.5235270 & 0.8441140 & 0.9653700 \\
$\mathrm{C}$ & -3.1279720 & 2.8731680 & 0.8430370 \\
$\mathrm{C}$ & -4.2782310 & 2.1827420 & 1.3584670 \\
$\mathrm{H}$ & -5.3997750 & 0.3349280 & 1.3588290 \\
$\mathrm{C}$ & -4.8446450 & 4.1088030 & 2.7390790 \\
$\mathrm{C}$ & -3.6867520 & 4.7751100 & 2.2692570 \\
$\mathrm{C}$ & -2.8521380 & 4.1739380 & 1.3486570 \\
$\mathrm{C}$ & -5.1273040 & 2.8346770 & 2.2936090 \\
$\mathrm{H}$ & -5.4960540 & 4.5957020 & 3.4611690
\end{tabular}

-3.4456030
-1.9616630
-5.9974590
-2.4872090
-1.2950380
-0.0713790
-2.4378510
-3.4511180
-1.3486570
0.8533450
-3.3587200
2.6736350
3.3833870
3.1808900
4.5587690
4.3507640
5.0444050
5.1099320
4.7382750
-3.9127290
-3.5826760
-4.4837040
-3.8746770
-4.7493600
-4.4586110
-3.6279700
-5.1923650
2.5107380
6.3199840
2.9548980
-2.9266210
-4.7519610
-4.8333990
-6.3560230
-6.7303750
-6.8811020
-6.5986660
-4.0926740
-3.0086290
-4.4012270
-4.3246370
-3.9462920
-4.1358560
-4.2388710
-2.8698330
-6.2582540

5.766920

4.6950250

2. 2960980

5.9596030

6.6322100

6.0580220

4.7457090

6.4009400

7. 5913470

6. 5503630

4. 2391110

2. 2256500

1.7581390

1.9981280

1.0211400

1.2492000

0.7340240

0.6505970

1.0578760

$-1.2676200$

$-2.2853160$

$-1.5970160$

$-3.6135270$

$-2.9391990$

$-3.9623640$

$-4.4072710$

$-3.1895860$

2.6301060

$-0.0696540$

2. 0811140

$-1.9701790$

$-5.4134970$

$-0.5394240$

$-0.4715660$

$-1.4278090$

$-0.2542590$

0.3144360

$-0.7873760$

$-0.8123590$

$-1.7429910$

0.0119510

$-5.8778120$

$-6.9403630$

$-5.3022200$

-5.7362720
-5.6476050
2. 6451710

1.0153990

2. 6644990

$-2.3597630$

2.7249620

$-2.4491490$

$-1.7033670$

$-2.6035970$

$-3.2350890$

$-2.7449930$

$-1.4329310$

$-0.8237240$

$-1.9528410$

0.4823230

$-1.7480090$

0.6279650

$-0.4736450$

$-2.6095880$

1.6234950

$-0.2267150$

0.6969440

$-1.4747080$

0.3615190

$-1.7649310$

$-0.8587230$

1.0634160

$-2.7265730$

1.7000590

$-0.2927440$

$-3.3800140$

2. 0364710

$-1.1954720$

$-2.5135190$

$-2.7391120$

$-3.1275950$

$-1.7999760$

$-3.4668060$

$-3.8412400$

$-3.6805060$

$-4.2845900$

$-4.5576320$

$-2.4243240$

$-2.6270710$

$-3.3124500$

$-2.2645680$

-1. 4187080 


$\begin{array}{rr}\mathrm{H} & -6.8378040 \\ \mathrm{H} & -6.6126870 \\ \mathrm{H} & -6.4547480 \\ \mathrm{C} & -3.9779140 \\ \mathrm{H} & -4.7116000 \\ \mathrm{H} & -4.5133160 \\ \mathrm{H} & -3.4887530 \\ \mathrm{C} & -1.8596450 \\ \mathrm{H} & -2.3146960 \\ \mathrm{H} & -1.1339050 \\ \mathrm{H} & -1.3289250 \\ \mathrm{C} & 2.7949440 \\ \mathrm{H} & 3.8731490 \\ \mathrm{H} & 2.4623380 \\ \mathrm{H} & 2.2753310 \\ \mathrm{C} & 2.9205730 \\ \mathrm{H} & 2.7972620 \\ \mathrm{H} & 3.9681220 \\ \mathrm{H} & 2.2967310 \\ \mathrm{C} & 6.1795750 \\ \mathrm{H} & 7.0920990 \\ \mathrm{H} & 6.0298650 \\ \mathrm{H} & 5.3276500 \\ \mathrm{C} & 7.4994460 \\ \mathrm{H} & 7.6335280 \\ \mathrm{H} & 7.3115160 \\ \mathrm{H} & 8.4309570 \\ \mathrm{C} & 3.0706760 \\ \mathrm{H} & 4.1147830 \\ \mathrm{H} & 2.4959580 \\ \mathrm{H} & 2.6829250 \\ \mathrm{C} & 3.7864300 \\ \mathrm{H} & 4.8459990 \\ \mathrm{H} & 3.4450000 \\ \mathrm{H} & 3.7086970 \\ \mathrm{P} & -0.2486980 \\ \mathrm{O} & 0.4694350 \\ \mathrm{O} & -0.2179480 \\ \mathrm{H} & 1.9032360 \\ \mathrm{H} & 6.5420440 \\ \mathrm{H} & 1.4272700 \\ \mathrm{H} & -4.5159020 \\ \mathrm{H} & -4.4377560 \\ \mathrm{H} & -2.4182180 \\ \mathrm{C} & 3.0208540 \\ \mathrm{C} & 2.0732160 \\ & \end{array}$

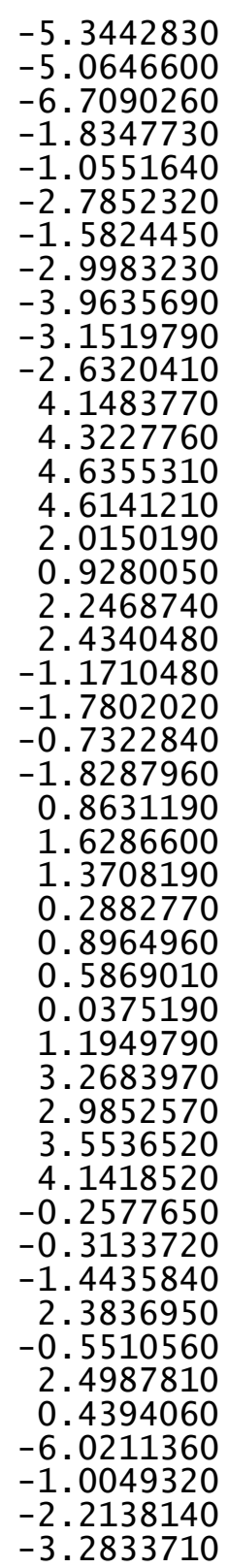

-0.5375400
-2.2790930
-1.6206630
3.1553700
2.9241270
3.2840130
4.1062440
2.4509990
2.7075590
1.6469470
3.3379880
1.7278580
1.8455420
0.8054670
2.5761980
3.0454320
3.0499180
3.2805800
3.8429510
0.7725490
0.8078320
1.7672640
0.5625510
0.0508320
-0.7239050
1.0065570
0.1425380
-4.3534990
-4.4903610
-3.9996000
-5.3362190
-3.9115790
-3.9739390
-4.9157960
-3.2540560
-0.9468100
-2.2722110
-0.0373980
-3.3642530
-1.2554470
1.5992250
-2.1391020
-0.3349390
1.9340110
-1.8619320
-1.5321970

\begin{abstract}
3.0099990
1. 1811010

2. 0167100

2. 5287860

2. 0198930

1. 2208600

1.5391670

0.9824870

3. 1591240

3. 1095140

1. 3219320

0.8289800

0.0334300

0.2221610

1.8232190

0.6541080

$-0.3551670$

2. 4318960

4. 3866780

3. 6938930

2.2337510

4.3952040

5.4185950

3.7120220

1.6080020

5.0327040

4.7953160

3.8638330

4.2435720

1.8028310

$-0.8971350$

$-1.6829780$

$-1.0372000$

$-2.2745350$

$-2.3463790$
\end{abstract}

H

TS7 $(R, R) \quad G[B 97-D / d e f 2-T Z V P]=-4125.391991$

$\begin{array}{lrrr}\text { TS7 }(\mathrm{R}, \mathrm{R}) & \mathrm{G}[\mathrm{B} 97-\mathrm{D} / \mathrm{def}-\mathrm{TZVP}] & =-4125.391991 & \\ \mathrm{O} & -0.4554730 & -0.7773830 & -0.3010980 \\ \mathrm{O} & 1.6489590 & -0.1287200 & -1.5703090 \\ \mathrm{C} & -0.5195290 & -1.9953330 & -0.9672020 \\ \mathrm{C} & 0.6484570 & -2.7217250 & -1.1735150 \\ \mathrm{C} & -1.8077130 & -2.4687310 & -1.3336120 \\ \mathrm{C} & 0.5737520 & -3.9030500 & -1.9835050 \\ \mathrm{C} & -1.8767550 & -3.6492810 & -2.0451430 \\ \mathrm{C} & -0.7138680 & -4.3649410 & -2.4239970 \\ \mathrm{H} & -2.8492850 & -4.0445790 & -2.3273780\end{array}$

$-1.2260100$

$-0.9773730$

2. 1477650

4. 2246310

3.1136430

1.9975290

1.1244080

3.7890220

1.0094340

$-2.6448400$

5.5148990

4. 3898990

3.1709030

5.4397710

6.4428090

2.2832240

6.3096730

$-3.1921350$

$-2.4178520$

$-2.0804850$

$-2.3689080$

$-3.4309740$

$-2.9968020$

$-2.9822280$

$-1.4835260$

$-2.9471270$

$-1.4845810$

$-1.3585110$

4.5841090

3.4450080

2. 6395160

3.0728600

3. 7869990
$-2.9333920$

$-4.1423510$

2. 1559970

0.9395010

2. 5816380

$-4.0445790$

$-2.3273780$ 


$\begin{array}{rr}\mathrm{C} & 2.3709660 \\ \mathrm{C} & 3.5701520 \\ \mathrm{C} & 1.9175420 \\ \mathrm{C} & 4.3287060 \\ \mathrm{C} & 2.6671910 \\ \mathrm{C} & 3.9068940 \\ \mathrm{H} & 5.2656590 \\ \mathrm{C} & 4.2305550 \\ \mathrm{C} & 2.9839010 \\ \mathrm{C} & 2.2228890 \\ \mathrm{C} & 4.6746510 \\ \mathrm{H} & 4.8235460 \\ \mathrm{H} & 2.6182500 \\ \mathrm{H} & 1.2672760 \\ \mathrm{H} & 5.6163970 \\ \mathrm{C} & 1.6138850 \\ \mathrm{C} & 0.3412320 \\ \mathrm{C} & -0.7972300 \\ \mathrm{C} & 1.7267130 \\ \mathrm{H} & 2.5108420 \\ \mathrm{H} & 0.2663080 \\ \mathrm{H} & -1.7808880 \\ \mathrm{H} & 2.7070750 \\ \mathrm{C} & -3.0397650 \\ \mathrm{C} & -3.8392390 \\ \mathrm{C} & -3.4131680 \\ \mathrm{C} & -5.0005420 \\ \mathrm{C} & -4.5843420 \\ \mathrm{C} & -5.3920770 \\ \mathrm{H} & -5.6202230 \\ \mathrm{H} & -4.8906240 \\ \mathrm{C} & 3.9847800 \\ \mathrm{C} & 3.9163290 \\ \mathrm{C} & 4.4421850 \\ \mathrm{C} & 4.3297660 \\ \mathrm{C} & 4.8328430 \\ \mathrm{C} & 4.7852130 \\ \mathrm{H} & 4.2809250 \\ \mathrm{H} & 5.1872360 \\ \mathrm{C} & -2.6103700 \\ \mathrm{C} & -6.6661260 \\ \mathrm{C} & -3.5137990 \\ \mathrm{C} & 3.4132430 \\ \mathrm{C} & 5.1784970 \\ \mathrm{C} & 4.5663890 \\ \mathrm{C} & 6.0466810 \\ & \end{array}$

$\begin{array}{rr}-0.9727050 & -0.7325400 \\ -0.4667340 & -0.1623670 \\ -2.2725940 & -0.5434960 \\ -1.3322820 & 0.5999350 \\ -3.1341160 & 0.3270000 \\ -2.6560410 & 0.8746700 \\ -0.9831880 & 1.0265370 \\ -4.7734150 & 2.0364470 \\ -5.2277150 & 1.5407750 \\ -4.4291140 & 0.7107670 \\ -3.5080960 & 1.7144640 \\ -5.4152590 & 2.6842190 \\ -6.2128360 & 1.8223190 \\ -4.7907690 & 0.3467930 \\ -3.1324250 & 2.1106970 \\ -5.7449610 & -3.1981780 \\ -6.2176280 & -3.6037090 \\ -5.5365690 & -3.2245190 \\ -4.6191860 & -2.4065560 \\ -6.2710340 & -3.5177690 \\ -7.1093660 & -4.2220230 \\ -5.8785050 & -3.5418100 \\ -4.2633820 & -2.1054200 \\ -1.7468500 & -0.8939990 \\ -1.0493240 & -1.8268970 \\ -1.7936220 & 0.4728430 \\ -0.4076060 & -1.3757690 \\ -1.1390880 & 0.8685010 \\ -0.4434770 & -0.0360510 \\ 0.1239460 & -2.0939340 \\ -1.1711500 & 1.9110000 \\ 0.9511560 & -0.3719500 \\ 1.8689410 & 0.7018260 \\ 1.3755780 & -1.6400120 \\ 3.1896020 & 0.4824010 \\ 2.7071910 & -1.8089320 \\ 3.6312640 & -0.7613710 \\ 3.9048250 & 1.3014060 \\ 3.0289510 & -2.7858170 \\ -2.6080000 & 1.4851420 \\ 0.2363890 & 0.4345540 \\ -1.0334380 & -3.3160850 \\ 1.4708100 & 2.0849250 \\ 5.0815420 & -0.9781950 \\ 0.4254970 & -2.8244280 \\ 0.2207070 & -3.2024460\end{array}$

6.4999960

6.6177710

6.1318500

3.7529680

2. 6980580

4. 1360190

3. 8245880

4.1711110

4.4323500

4.1801160

3. 1519500

6.6092680

7.3373770

6.6882680

6.8749840

4.5902490

5. 2778080

5.1555820

4. 2166490

2. 4255380

2. 9265900

1. 6107970

1.9994720

$-2.9321040$

$-3.9915120$

$-2.7309370$

$-2.3228510$

$-2.8151320$

$-2.6820250$

$-3.8187940$

$-2.0837610$

$-7.9055320$

$-8.8184660$

$-7.8441350$

$-7.9864480$

$-6.5880140$

$-5.7220620$

$-6.4921750$

$-7.4984030$

$-3.6379690$

$-4.6700880$

$-2.9852460$

$-3.3469520$

$-4.4195870$

-5.4713600
-4.1575260

\author{
1. 1710150 \\ $-0.1674560$ \\ $-0.4909760$ \\ 0.9096570 \\ 1.0345060 \\ 1. 8694810 \\ 0.1778500 \\ 5.7868000 \\ 6.8473340 \\ 5. 3192830 \\ 5.7164680 \\ 5. 2176160 \\ 4.7419330 \\ 4.7412010 \\ 6.2771670 \\ 1.2515230 \\ 0.4827690 \\ 2. 1850720 \\ 0.9385850 \\ 2. 4908090 \\ 3.4372820 \\ 2. 6910340 \\ 2. 0896840 \\ $-4.1110730$ \\ $-4.2902180$ \\ $-4.4644500$ \\ $-4.6976730$ \\ $-2.1819150$ \\ $-1.1036030$ \\ $-2.4507690$ \\ $-2.6947150$ \\ $-0.3225820$ \\ 0.1505650 \\ $-0.1192220$ \\ $-1.4080750$ \\ 1.7641640 \\ 2.1746590 \\ 2. 0255600 \\ 2. 2393640 \\ 0.3595980 \\ 0.7309010 \\ 1.0808930 \\ 0.3051280 \\ $-2.0344820$ \\ -1.7307270
-2.0642480
}

-3.5138870
-2.3491210
-4.0346860
-4.0384880
-3.7705880
-4.4093570
-4.8542290
-1.9082990
-2.0242200
-2.9018650
-1.5073520
-1.5314820
-0.8622040
-2.5175010
-1.6435020
3.0560360
2.6845870
3.1812510
4.0406630
2.6786640
2.9187830
1.9766440
3.6066220
1.3374430
1.5663350
0.3208200
2.0385610
2.9459050
3.0739270
3.3018020
3.5808010
-0.2895600
0.0958880
-1.3667100
-0.1503560
0.2656260
0.7999850
-0.7956850
0.6547910
-3.9556100
-3.9129260
-3.4574920
-5.0126230
-4.0628340
-3.9728970
-5.1290390 


$\begin{array}{rrrr}\mathrm{H} & -4.3214770 & -3.0457360 & -3.6510470 \\ \mathrm{P} & 0.2441380 & 0.5042320 & -1.0464530 \\ \mathrm{O} & -0.4966930 & 0.8859340 & -2.3035010 \\ \mathrm{O} & 0.4406800 & 1.5250510 & 0.0301130 \\ \mathrm{H} & -2.4757400 & -1.3573850 & -3.4439780 \\ \mathrm{H} & -6.7748520 & 0.0209910 & 1.5069350 \\ \mathrm{H} & -1.5464670 & -2.4756010 & 1.2618380 \\ \mathrm{H} & 4.1697130 & -0.5527850 & -2.5351490 \\ \mathrm{H} & 5.1434860 & 5.5822090 & -0.0001150 \\ \mathrm{H} & 2.8786650 & 0.5207520 & 1.9920640 \\ \mathrm{C} & -2.7560730 & 2.9253360 & -1.3579470 \\ \mathrm{C} & -1.6882730 & 3.7420750 & -0.7724820 \\ \mathrm{H} & -2.8464670 & 1.9002250 & -0.9918260 \\ \mathrm{H} & -0.8321350 & 3.2248610 & -0.3519760 \\ \mathrm{O} & -1.7705680 & 3.0225140 & -2.4497500 \\ \mathrm{C} & -1.9640390 & 2.4316680 & 2.7645570 \\ \mathrm{C} & -1.2050100 & 0.7203320 & 4.4677610 \\ \mathrm{C} & -0.5803540 & 0.6135660 & 3.2085590 \\ \mathrm{~N} & -1.0266390 & 1.5716350 & 2.3137310 \\ \mathrm{H} & -0.5873210 & 1.6358500 & 1.3692490 \\ \mathrm{~S} & -2.3562200 & 2.0423020 & 4.4394960 \\ \mathrm{~S} & -2.6772250 & 3.6997110 & 1.9256940 \\ \mathrm{H} & -1.1850100 & 2.1362780 & -2.3992060 \\ \mathrm{C} & 0.0771710 & -1.1634920 & 5.2351270 \\ \mathrm{C} & 0.6934800 & -1.2627740 & 3.9691000 \\ \mathrm{C} & 0.3648180 & -0.3698020 & 2.9413170 \\ \mathrm{C} & -0.8755720 & -0.1715430 & 5.4860560 \\ \mathrm{H} & 0.3324630 & -1.8586950 & 6.0269870 \\ \mathrm{H} & 0.8260200 & -0.4530620 & 1.9646110 \\ \mathrm{H} & -1.3490340 & -0.1083310 & 6.4618320 \\ \mathrm{C} & -3.9100160 & 5.0579960 & -2.1106990 \\ \mathrm{C} & -3.0972820 & 5.8316230 & -1.0610610 \\ \mathrm{C} & -1.6891460 & 5.2341770 & -0.8864900 \\ \mathrm{C} & -4.0741230 & 3.5873580 & -1.6919840 \\ \mathrm{H} & -4.9011230 & 5.5111630 & -2.2301280 \\ \mathrm{H} & -3.0070640 & 6.8875770 & -1.3408230 \\ \mathrm{H} & -1.0541920 & 5.4938880 & -1.7463500 \\ \mathrm{H} & -4.6909020 & 3.5250530 & -0.7872400 \\ \mathrm{H} & -4.5750150 & 2.9998630 & -2.4702470 \\ \mathrm{H} & -3.4042660 & 5.1109180 & -3.0839410 \\ \mathrm{H} & -3.6247330 & 5.7908390 & -0.0981730 \\ \mathrm{H} & -1.1823470 & 5.6380120 & -0.0026860 \\ \mathrm{O} & 1.6265180 & -2.1990490 & 3.6429790 \\ \mathrm{C} & 1.9998980 & -3.1642070 & 4.6350520 \\ \mathrm{H} & 2.4374650 & -2.6710620 & 5.5148560 \\ \mathrm{H} & 1.1299410 & -3.7604980 & 4.9452190\end{array}$

148 TS8 (R, R) G[B97-D/def2-TZVP $]=-4125.390938$

$\begin{array}{lrrr}\text { TS8 (R, R) } & \text { G }[\mathrm{B} 97-\mathrm{D} / \mathrm{def}-\mathrm{TZV}]=-4125.390938 \\ \mathrm{O} & 0.3441700 & 0.8612780 & -0.0158770 \\ \mathrm{O} & -1.7200170 & 0.2760410 & -1.3722230 \\ \mathrm{C} & 0.3871680 & 2.1560280 & -0.5229270 \\ \mathrm{C} & -0.8030080 & 2.8662740 & -0.6601280 \\ \mathrm{C} & 1.6643530 & 2.6976400 & -0.8352280 \\ \mathrm{C} & -0.7548430 & 4.1466230 & -1.3073300 \\ \mathrm{C} & 1.7012870 & 3.9806290 & -1.3477360 \\ \mathrm{C} & 0.5244910 & 4.7138200 & -1.6305950 \\ \mathrm{H} & 2.6633790 & 4.4319460 & -1.5753470 \\ \mathrm{C} & -2.4803310 & 1.0175910 & -0.4806030 \\ \mathrm{C} & -3.6984410 & 0.4356480 & -0.0361020 \\ \mathrm{C} & -2.0758400 & 2.3038500 & -0.1371550 \\ \mathrm{C} & -4.5302630 & 1.2024450 & 0.7520270 \\ \mathrm{C} & -2.9026180 & 3.0533570 & 0.7704800 \\ \mathrm{C} & -4.1619330 & 2.4997370 & 1.1858430 \\ \mathrm{H} & -5.4870970 & 0.7955650 & 1.0696220 \\ \mathrm{C} & -4.6004050 & 4.4649560 & 2.5578640 \\ \mathrm{C} & -3.3376870 & 4.9908790 & 2.1923020 \\ \mathrm{C} & -2.5129340 & 4.3047210 & 1.3238290 \\ \mathrm{C} & -4.9978680 & 3.2392670 & 2.0661460 \\ \mathrm{H} & -5.2431990 & 5.0185860 & 3.2384050 \\ \mathrm{H} & -3.0088120 & 5.9411530 & 2.6069650 \\ \mathrm{H} & -1.5442130 & 4.7200480 & 1.0694500 \\ \mathrm{H} & -5.9515200 & 2.8046100 & 2.3603930 \\ \mathrm{C} & -1.8306500 & 6.0989860 & -2.2992550 \\ \mathrm{C} & -0.5667520 & 6.6773620 & -2.5742470 \\ \mathrm{C} & 0.5857740 & 5.9925620 & -2.2500800 \\ \mathrm{C} & -1.9218230 & 4.8680470 & -1.6806510 \\ \mathrm{H} & -2.7382680 & 6.6271950 & -2.5829470 \\ \mathrm{H} & -0.5094180 & 7.6517610 & -3.0541500 \\ \mathrm{H} & 1.5643450 & 6.4120060 & -2.4770210 \\ \mathrm{H} & -2.8965540 & 4.4357300 & -1.4791260 \\ \mathrm{C} & 2.9260470 & 1.9228170 & -0.6346290 \\ \mathrm{C} & 3.7081630 & 1.5523370 & -1.7509850 \\ \mathrm{C} & 3.3657780 & 1.5937460 & 0.6768040 \\ \mathrm{C} & 4.9161330 & 0.8660090 & -1.5406150 \\ \mathrm{C} & 4.5583280 & 0.8844710 & 0.8240910 \\ \mathrm{C} & 5.3598700 & 0.5190240 & -0.2674790 \\ \mathrm{H} & 5.5178880 & 0.6046640 & -2.4053780 \\ \mathrm{H} & 4.9017850 & 0.6238250 & 1.8214040 \\ \mathrm{C} & -4.0781840 & -0.9519650 & -0.4374030 \\ \mathrm{C} & -3.9024290 & -2.0204220 & 0.4701680 \\ \mathrm{C} & -4.6180140 & -1.1854250 & -1.7203520\end{array}$




$\begin{array}{rrr}-4.3166510 & -3.3011940 & 0.0825110 \\ -5.0075910 & -2.4841940 & -2.0631560 \\ -4.8724010 & -3.5540510 & -1.1743080 \\ -4.1912780 & -4.1347610 & 0.7703380 \\ -5.4255330 & -2.6645490 & -3.0511640 \\ 2.6164280 & 2.0949840 & 1.9096220 \\ 6.6860730 & -0.1817430 & -0.0003340 \\ 3.3286680 & 1.9056700 & -3.1859520 \\ -3.2842200 & -1.8032330 & 1.8465680 \\ -5.2871000 & -4.9589940 & -1.5742050 \\ -4.8005800 & -0.0687970 & -2.7393510 \\ -6.2946220 & 0.1765040 & -3.0258670 \\ -6.7562000 & -0.7197610 & -3.4609930 \\ -6.8340940 & 0.4253690 & -2.1027730 \\ -6.4154510 & 1.0052540 & -3.7363680 \\ -4.0318700 & -0.3613060 & -4.0414880 \\ -2.9650320 & -0.5078100 & -3.8360310 \\ -4.4204210 & -1.2656960 & -4.5271970 \\ -4.1438020 & 0.4771690 & -4.7417810 \\ -4.3905030 & -5.4981910 & -2.7064480 \\ -4.6725650 & -6.5285980 & -2.9613060 \\ -4.5000870 & -4.8791020 & -3.6066810 \\ -3.3341080 & -5.4854230 & -2.4097960 \\ -6.7702810 & -5.0229250 & -1.9848590 \\ -7.4190660 & -4.6680800 & -1.1739770 \\ -6.9511450 & -4.3952560 & -2.8672830 \\ -7.0527060 & -6.0539340 & -2.2357810 \\ -4.3712400 & -1.5585670 & 2.9119700 \\ -4.9921370 & -0.6925120 & 2.6577890 \\ -5.0232480 & -2.4388320 & 2.9922120 \\ -3.9086440 & -1.3807860 & 3.8924370 \\ -2.3628190 & -2.9505770 & 2.2919290 \\ -2.9340260 & -3.8664420 & 2.4901960 \\ -1.6086930 & -3.1599650 & 1.5269340 \\ -1.8519360 & -2.6660710 & 3.2196540 \\ 2.8784750 & 3.6041600 & 2.1080040 \\ 3.9459170 & 3.7741750 & 2.3042400 \\ 2.5914410 & 4.1801630 & 1.2217960 \\ 2.3051960 & 3.9761090 & 2.9680560 \\ 2.9556580 & 1.3449600 & 3.2062550 \\ 2.8884640 & 0.2605610 & 3.0754520 \\ 3.9708320 & 1.5870470 & 3.9480350 \\ 2.2537860 & 1.6390470 & 0.7653030 \\ 7.6396020 & 0.7622600 & \\ 8.5694490 & 0.2378720 & 1.6776110 \\ 7.8917640 & 1.6281720 & \end{array}$

7.1790250

7.3915340

6.7557850

7.6754760

8.3080780

3. 3844560

4.4038170

2.7265340

3.0606010

4.2379360

5. 2813820

3.9340450

4.1935030

$-0.2627080$

0.4729790

$-0.3521290$

2. 2979440

6.4702400

1. 5439870

$-4.3932860$

$-5.1489260$

$-2.6642180$

2. 9276640

1.9685500

2. 8699080

1.0454270

1.9899000

2. 1716840

1.4730340

0.8049050

1.2163170

0.7469940

2.6218610

2.8659230

1.2923600

0.2225440

$-0.4381230$

$-0.1506920$

1.1802610

$-0.0273270$

$-0.6239460$

1.6888880

4.3854410

3.6514880

2.1704100

4.3315860
1.1306940
-0.7193170
-1.4129500
0.1033860
-1.2467580
0.6973830
0.2995840
-0.1033870
1.0044350
3.0330920
2.6923730
3.3202130
3.9209750
-0.3277480
-0.4144180
-1.5309720
2.2719540
-1.0352540
1.9665510
0.8575500
-5.6063110
-0.9017230
-2.3345240
-3.4043940
-1.4368670
-3.1301460
-2.2718330
-3.0228800
-1.8083610
-1.3908690
-2.0842080
-1.9171890
-3.0654050
-4.0384590
-1.4970030
-0.2059710
0.2173020
-0.3727400
-1.2122620
0.2792270
-0.0654200
-1.5231670
-4.0250090
-5.1478860
-4.7974780
-2.7090610

1.6893100

$-1.2521090$

$-1.8122680$

$-1.9223510$

$-0.9606580$

$-4.1372890$

$-4.2225670$

$-3.7906270$

$-5.1403880$

$-3.7167300$

$-3.7525370$

$-4.7322790$

$-3.0747620$

$-0.9624930$

$-2.2772040$

$-0.0803110$

$-3.1943240$

0.6611170

1.7355700

$-2.3208040$

$-0.6965770$

1.7886370

$-1.7338640$

$-1.4414100$

$-1.1141480$

$-0.9426000$

$-2.8658740$

2. 3050120

4.4066540

3.2438020

2. 1165020

1.2011230

4.0060120

1. 1629370

$-2.6252450$

5.6776550

4.5015720

3.2653610

5.6374690

6.6171570

2. 3419410

6.5456210

$-2.9427280$

2.1926090

$-1.9553840$

$-2.1490930$ 


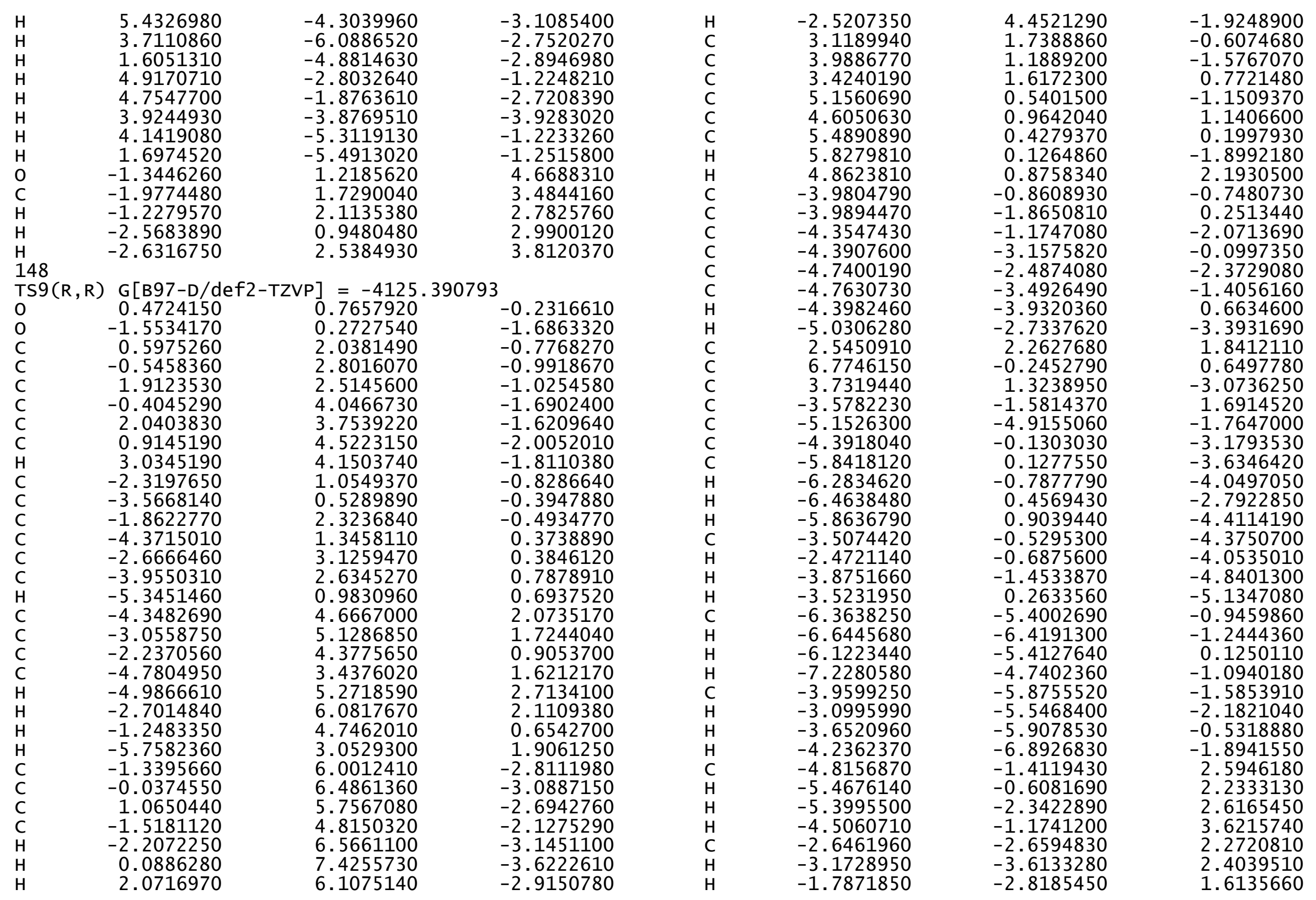




$\begin{array}{rrr}-2.2815500 & -2.3376900 & 3.2553550 \\ 2.8168810 & 3.7806750 & 1.9160640 \\ 3.8564690 & 3.9592150 & 2.2234030 \\ 2.6523820 & 4.2648740 & 0.9475930 \\ 2.1523570 & 4.2463140 & 2.6566480 \\ 2.6990450 & 1.6431470 & 3.2384220 \\ 2.6201070 & 0.5521870 & 3.2041580 \\ 3.6683130 & 1.9032730 & 3.6840610 \\ 1.9101420 & 2.0238620 & 3.8969820 \\ 8.0132920 & 0.3900160 & -0.0091920 \\ 8.9300920 & -0.0748760 & 0.3766740 \\ 7.9893820 & 0.2431290 & -1.0968720 \\ 8.0554610 & 1.4683740 & 0.1895700 \\ 6.7414660 & -1.7611640 & 0.3833190 \\ 5.8809320 & -2.2275530 & 0.8791700 \\ 6.6649400 & -1.9567230 & -0.6934690 \\ 7.6596020 & -2.2356170 & 0.7548810 \\ 3.8599920 & -0.0111290 & -3.8274430 \\ 4.8795780 & -0.4122720 & -3.7609950 \\ 3.1650110 & -0.7550160 & -3.4290820 \\ 3.6292220 & 0.1416760 & -4.8898190 \\ 4.6874400 & 2.3674630 & -3.6875250 \\ 5.7292660 & 2.0378760 & -3.5765790 \\ 4.4769560 & 2.4933410 & -4.7580520 \\ 4.5851280 & 3.3417240 & -3.1949080 \\ -0.1918630 & -0.4281670 & -1.1375630 \\ 0.6239220 & -0.7014490 & -2.3759570 \\ -0.4632110 & -1.5395130 & -0.1725750 \\ 2.7068010 & 1.6797040 & -3.2173000 \\ 6.8562150 & -0.0975120 & 1.7359440 \\ 1.4984670 & 2.1297650 & 1.5509920 \\ -4.0039880 & 0.8143250 & -2.7851730 \\ -5.4356700 & -4.9216000 & -2.8269140 \\ -3.0268060 & -0.6367700 & 1.7070300 \\ 2.8687360 & -2.7561510 & -1.4332070 \\ 1.7820910 & -3.6274100 & -0.9740840 \\ 2.9150600 & -1.7563630 & -0.9959100 \\ 0.8894540 & -3.1535240 & -0.5794490 \\ 1.9598820 & -2.7945290 & -2.5929220 \\ 1.7331650 & -2.5992100 & 2.6732390 \\ 0.7705640 & -1.0769060 & 4.4503080 \\ 0.2805560 & -0.8436030 & 3.1495250 \\ 0.8324370 & -1.7040700 & 2.2150550 \\ 0.4774230 & -1.6972720 & 1.2343200 \\ 1.9425660 & -2.3795220 & 4.4103190 \\ 2.5548800 & -3.7674000 & 1.7897110\end{array}$

\begin{tabular}{lr}
$\mathrm{H}$ & 1.3505740 \\
$\mathrm{C}$ & -0.6149900 \\
$\mathrm{C}$ & -1.0953190 \\
$\mathrm{C}$ & -0.6411920 \\
$\mathrm{C}$ & 0.3226750 \\
$\mathrm{H}$ & -0.9652530 \\
$\mathrm{H}$ & -0.9945310 \\
$\mathrm{H}$ & 0.6922660 \\
$\mathrm{C}$ & 4.1117520 \\
$\mathrm{C}$ & 3.2471350 \\
$\mathrm{C}$ & 1.8193980 \\
$\mathrm{C}$ & 4.2185450 \\
$\mathrm{H}$ & 5.1173050 \\
$\mathrm{H}$ & 3.1959410 \\
$\mathrm{H}$ & 1.2474200 \\
$\mathrm{H}$ & 4.7715960 \\
$\mathrm{H}$ & 4.7588690 \\
$\mathrm{H}$ & 3.6718280 \\
$\mathrm{H}$ & 3.7095830 \\
$\mathrm{H}$ & 1.2633760 \\
$\mathrm{O}$ & -2.0066890 \\
$\mathrm{C}$ & -2.5085270 \\
$\mathrm{H}$ & -3.0260300 \\
$\mathrm{H}$ & -1.6920840 \\
$\mathrm{H}$ & -3.2086830 \\
$\mathrm{H}$ & \\
\hline
\end{tabular}
$-1.9272930$
0.7110910
0.9391170
0.1620190
$-0.2960420$
1. 3166020
0.3457890
$-0.4566620$
$-4.8142940$
$-5.6707560$
$-5.1076920$
$-3.3736150$
$-5.2430640$
$-6.7060260$
$-5.3161630$
$-3.3645630$
$-2.7257000$
$-4.8075200$
$-5.6889860$
$-5.5789990$
1.8969700
2.7387580
2. 1429480
3. 3062240
3. 4191280

148

$$
\begin{array}{r}
-2.5189460 \\
5.2653780 \\
3.9574640 \\
2.8837750 \\
5.5131290 \\
6.0936000 \\
1.8762060 \\
6.5220650 \\
-2.2477640 \\
-1.3093420 \\
-1.1886150 \\
-1.7207700 \\
-2.3310050 \\
-1.6656700 \\
-2.1047920 \\
-0.7738890 \\
-2.4205260 \\
-3.2538080 \\
-0.3128190 \\
-0.3701370 \\
3.6334970 \\
4.6801320 \\
5.4456230 \\
5.1491530 \\
4.1919120
\end{array}
$$

$\operatorname{TS} 10(R, R)$ G $[B 97-D / d e f 2-T Z V P]=-4125.385166$

$\begin{array}{lrlr}\text { TS10 }(\mathrm{R}, \mathrm{R}) \mathrm{G}[\mathrm{B} 97-\mathrm{D} / \mathrm{de} \text { ef }-\mathrm{TZVP}]=-4125.385166 \\ \mathrm{O} & 0.3100200 & 0.8420290 & 0.0387640 \\ \mathrm{O} & -1.6901930 & 0.2377810 & -1.4107620 \\ \mathrm{C} & 0.2999080 & 2.1722890 & -0.3687550 \\ \mathrm{C} & -0.9222280 & 2.8264940 & -0.4872350 \\ \mathrm{C} & 1.5538920 & 2.7985400 & -0.6058480 \\ \mathrm{C} & -0.9279550 & 4.1530170 & -1.0367040 \\ \mathrm{C} & 1.5344340 & 4.1176440 & -1.0176780 \\ \mathrm{C} & 0.3271280 & 4.8086890 & -1.2783170 \\ \mathrm{H} & 2.4763200 & 4.6330490 & -1.1854540 \\ \mathrm{C} & -2.5223150 & 0.8944920 & -0.5192130 \\ \mathrm{C} & -3.7677890 & 0.2705690 & -0.2280860 \\ \mathrm{C} & -2.1799850 & 2.1615020 & -0.0551170 \\ \mathrm{C} & -4.6516990 & 0.9426720 & 0.5871970 \\ \mathrm{C} & -3.0720670 & 2.8095240 & 0.8690660 \\ \mathrm{C} & -4.3338480 & 2.1934010 & 1.1687230 \\ \mathrm{H} & -5.6154200 & 0.4929820 & 0.8111420 \\ \mathrm{C} & -4.8844620 & 4.0055420 & 2.7003430 \\ \mathrm{C} & -3.6220860 & 4.5935040 & 2.4430330 \\ \mathrm{C} & -2.7422320 & 4.0126400 & 1.5520400\end{array}$




-5.2271520
-5.5704500
-3.3374550
-1.7739120
-6.1813170
-2.0796570
-0.8420290
0.3364620
-2.1215080
-3.0063110
-0.8245250
1.2969710
-3.0770600
2.8486970
3.6753280
3.2749960
4.9117520
4.4955320
5.3406230
5.5445340
4.8213760
-4.1135480
-3.7707950
-4.7874570
-4.1046750
-5.0880490
-4.7565640
-3.8431850
-5.6032330
2.4782160
6.6804680
3.3125910
-3.1014780
-5.0866660
-5.2983930
-4.2978070
-4.1596810
-3.3188050
-4.6916610
-6.5991020
-7.3400010
-6.3983380
-7.0290180
-6.0375670
-6.2925090
-5.5601270

2.8247620

4.4762950

5.5072390

4.4703330

2. 3430560

6.1141190

6.7763370

6.1306000

4.8384120

6.6131810

7.7847030

6.6151450

4. 3426720

2. 0718180

1.8484580

1.6403320

1.2054110

0.9728360

0.7522510

1.0577870

0.6280100

$-1.0703860$

$-2.2291300$

$-1.1882710$

$-3.4853000$

$-2.4767540$

$-3.6323090$

$-4.3759420$

$-2.5770590$

1.9920270

0.0593530

2. 3123240

$-2.1205490$

$-5.0090690$

$-0.0190120$

1.1103800

1.7791550

0.7137680

1.7110120

0.5657960

$-0.2239130$

1.0747250

1.2981940

$-5.7775760$

$-6.7547120$

$-5.9481480$

2.0758710
3.4007380
2.9599360
1.3813960
2.2815420
-1.9201360
-2.1130820
-1.8024110
-1.3937140
-2.1950500
-2.5204530
-1.9684370
-1.2582780
-0.4417260
-1.5653480
0.8442430
-1.3866220
0.9558100
-0.1418910
-2.2562450
1.9327070
-0.7904360
-0.0492340
-2.0260430
-0.5628820
-2.4974180
-1.7908010
0.0012930
-3.4530500
2.0988910
0.0824720
-2.9730600
1.3170620
-2.3397930
-2.8727010
-3.1838210
-2.3290800
-3.4734930
-4.0144440
-2.2825670
-2.1042370
-1.3330890
-2.9794940
-1.4021760
-1.8335630
-0.4282520

$-6.9646680$

$-3.8075960$

$-3.1397710$

$-3.2626620$

$-4.0605700$

$-4.1604780$

$-4.7927470$

$-4.8055330$

$-3.6714670$

$-2.1779590$

$-2.7547770$

$-1.4541500$

$-1.6315230$

2.6547710

3.7082530

2. 3480680

2.0504810

2.8347010

2.8272590

3.8299750

2. 1045710

7.5507750

8.4880360

7.7969400

7.0322200

7.4808310

6.9129340

7.7571020

8.4047720

3.4483620

4.4870670

2.8192090

3.1377820

4.1782050

5.2377750

3.8845070

4.0751960

$-0.2000920$

0.5609920

$-0.2180400$

2.2659540

6.4621010

1.4173900

$-5.5677100$

$-5.6001860$

$-2.4817110$
$-5.2146140$
$-5.8277670$
$-5.3018010$
$-5.9943350$
$-6.8067530$
$-1.9601700$
$-1.0823450$
$-2.8486020$
$-1.8564230$
$-3.2996480$
$-4.2173240$
$-3.4785710$
$-3.0708750$
3. 4898090
3. 6994120
4.1262140
3.7527020
1.1503220
0.0797300
1.4123920
1.3380320
0.8653280
0.3306560
1.8455650
1.0296280
$-0.2065590$
$-0.8056040$
0.7393230
$-0.7438740$
1.1984480
0.8552450
0.3406410
1.5822310
3. 5229370
3.2362130
3.8847720
4.3476830
$-0.2940920$
$-0.2214610$
$-1.5732440$
2. 6295310
$-0.9116510$
1.8233490
$-0.4616330$
$-4.8657660$
$-1.2171600$

$-1.2349920$

2.6008060

$-3.2947620$

$-1.6623460$

2.4271800

2.2582200

2.4572620

3.4053060

1.6610240

1.8354130

0.8608030

2.5837520

2.4333670

2.6635520

1. 5964500

3.3122810

3. 3331940

3.1078070

3.7164280

4.1286100

1.0718090

1.2746400

0.6409890

2.0230280

$-1.1994600$

$-1.9188090$

$-1.6847380$

$-0.9535670$

$-4.0268170$

$-4.1165010$

$-3.7753850$

$-5.0074100$

$-3.3778500$

$-3.4141580$

$-4.3723180$

$-2.6626150$

$-1.0204310$

$-2.3215350$

$-0.2476870$

$-2.9772280$

0.5547220

1.8926360

$-3.8414820$

$-3.3010330$

1. 3058970 


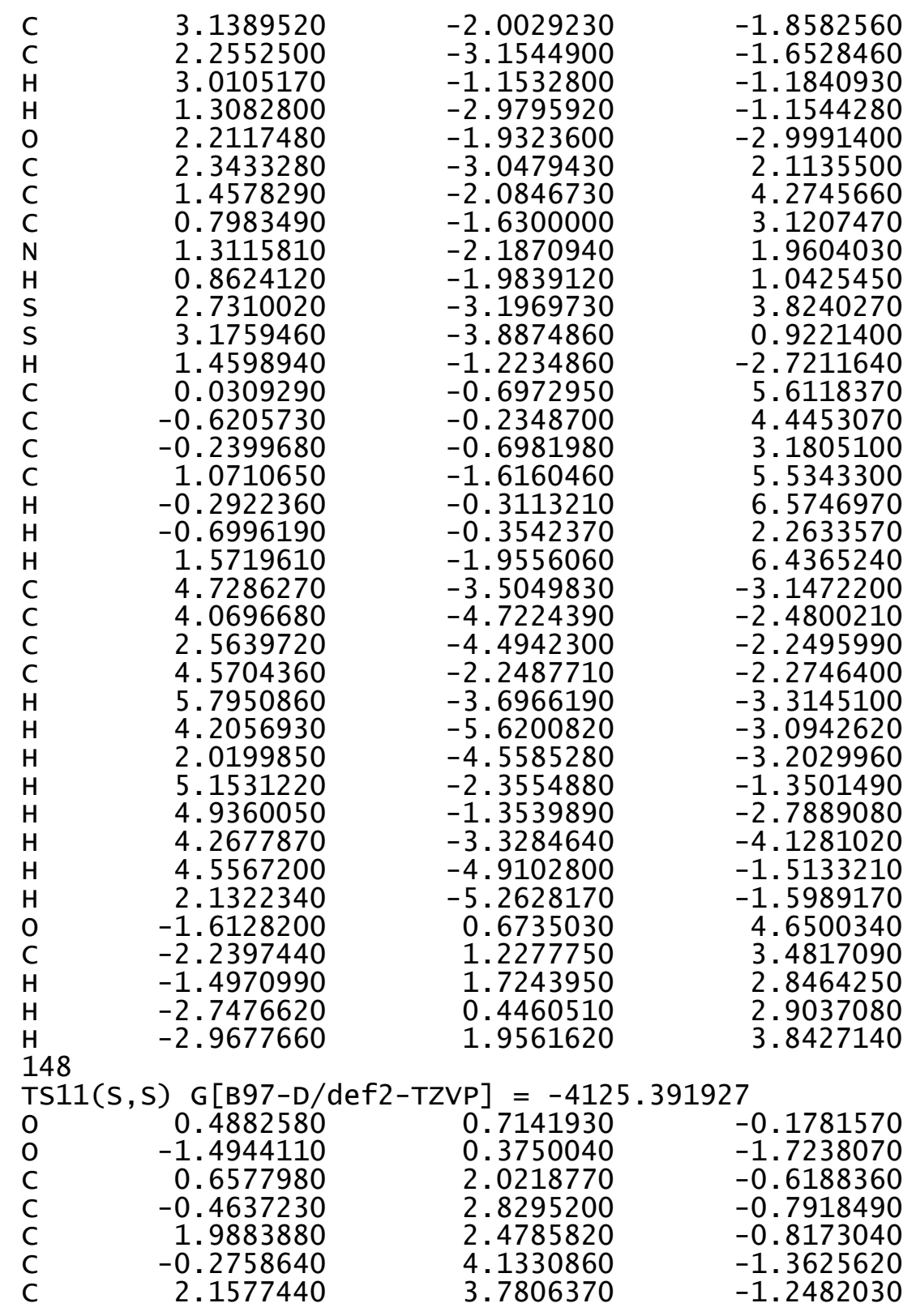

1.0613170
3.1649050
-2.2713820
-3.5395790
-1.8056810
-4.3661850
-2.6367910
-3.9510880
-5.3606820
-4.3822460
-3.0671150
-2.2159620
-4.8065440
-5.0441540
-2.7190390
-1.2079820
-5.8027110
-1.1323750
0.1868080
1.2600120
-1.3574130
-1.9763220
0.3492080
2.2804160
-2.3729950
3.1760250
3.9894680
3.5236880
5.1500150
4.6786890
5.5152490
5.7732390
4.9576320
-3.9645530
-3.9825110
-4.3499770
-4.4149900
-4.7694780
-4.8105100
-4.4289630
-5.0711830
2.7182840
6.7894460
3.6862340
-3.5464740
-5.2486890

4. 6170010

4. 1643970

1. 1055360

0.5708440

2. 3385470

1. 3505020

3. 0943170

2. 6026420

0.9880960

4.5596180

5. 0190190

4. 3013830

3. 3663830

5.1368800

5.9404760

4. 6628930

2. 9813560

6.2084630

6.7019600

5. 9167290

4.9593680

6.8175310

7. 6914080

6.2709450

4. 5936470

1.6015820

1. 2358130

1. 1837770

0.4814530

0.4195170

0.0669990

0.2134780

0.0927530

$-0.7846160$

$-1.8623430$

$-0.9890470$

$-3.1147970$

$-2.2657760$

$-3.3397620$

- 3.9471110

$-2.4277600$

1.6397820

$-0.7119050$

1.6535390

$-1.6952050$

$-4.7197010$
$-1.5655040$

$-1.3886670$

$-0.8284980$

$-0.4756500$

$-0.3827810$

0.3076060

0.5140580

0.8217780

0.5552010

2. 2074150

1. 9521370

1.1369530

1. 6624880

2. 8491900

2. 4138540

0.9677670

1.8735650

$-2.3180110$

$-2.4735890$

$-2.1075570$

$-1.7746870$

$-2.6344540$

$-2.8951130$

$-2.2433110$

$-1.6629220$

$-0.5916480$

$-1.6866310$

0.7208030

$-1.4508450$

0.8949340

$-0.1728540$

$-2.2986960$

1.8936030

$-0.9349460$

$-0.0152080$

$-2.2754050$

$-0.4607950$

$-2.6726410$

$-1.7836310$

0.2379520

$-3.7062810$

1.9347240

0.1176230

$-3.1225030$

1. 4365500

$-2.2405980$ 


$\begin{array}{rrr}-4.3563140 & 0.1359670 & -3.3023610 \\ -5.7976030 & 0.4749690 & -3.7305480 \\ -6.2709450 & -0.3924060 & -4.2092460 \\ -6.4055880 & 0.7617960 & -2.8628120 \\ -5.7957250 & 1.3063280 & -4.4482810 \\ -3.4856140 & -0.2021370 & -4.5265520 \\ -2.4563820 & -0.4197730 & -4.2196180 \\ -3.8835510 & -1.0753930 & -5.0593140 \\ -3.4731190 & 0.6457810 & -5.2243320 \\ -6.4879110 & -5.2068810 & -1.4656950 \\ -6.8076250 & -6.1909080 & -1.8339350 \\ -6.2575390 & -5.3005490 & -0.3962140 \\ -7.3226340 & -4.5028350 & -1.5755750 \\ -4.0990670 & -5.7385770 & -2.1135770 \\ -3.2194640 & -5.4104310 & -2.6816550 \\ -3.8035660 & -5.8510380 & -1.0620610 \\ -4.4149690 & -6.7213200 & -2.4885930 \\ -4.7602670 & -1.4653310 & 2.3589730 \\ -5.3342090 & -0.5828150 & 2.0569650 \\ -5.4280450 & -2.3372270 & 2.3273300 \\ -4.4256550 & -1.3235460 & 3.3958560 \\ -2.7216760 & -2.8840270 & 1.9609580 \\ -3.3460940 & -3.7786380 & 2.0810020 \\ -1.8981950 & -3.1204850 & 1.2807730 \\ -2.3070640 & -2.6345100 & 2.9453310 \\ 3.0838110 & 3.0958670 & 2.2934830 \\ 4.1406120 & 3.1563680 & 2.5874210 \\ 2.9192170 & 3.7661390 & 1.4424350 \\ 2.4691000 & 3.4427890 & 3.1352730 \\ 2.8770490 & 0.7429760 & 3.1712180 \\ 2.7197320 & -0.3121400 & 2.9218710 \\ 3.8767320 & 0.8451210 & 3.6138200 \\ 2.1402870 & 1.0326740 & 3.9286600 \\ 7.8344140 & 0.2163710 & 0.7738410 \\ 8.7334110 & -0.3514130 & 1.0486250 \\ 8.1253430 & 1.0094690 & 0.0715910 \\ 7.4279500 & 0.6888320 & 1.6761230 \\ 7.4022880 & -1.4098340 & -1.1045630 \\ 6.6808790 & -2.0676900 & -1.6047810 \\ 7.7549870 & -0.6736710 & -1.8387880 \\ 8.2635850 & -2.0135650 & -0.7937350 \\ 3.7032080 & 0.4683950 & -4.1056160 \\ 4.6957760 & 0.0023060 & -4.1532700 \\ 2.9743700 & -0.2925580 & -3.8129670 \\ 3.4504500 & 0.8252240 & -3.5879250 \\ 4.6807920 & 2.7367150 & \end{array}$

\begin{abstract}
5.7029280
4.4343740

4.6629610

$-0.1690100$

0.6848360

$-0.4811000$

2. 6807420

6.5300850

1.6573980

$-3.9356170$

$-5.5198480$

$-2.9107160$

1.8938220

2.6484920

0.8082070

2. 1535110

2. 4503510

1.1053970

0.3531340

$-0.0075700$

0.4304120

0.1360460

1. 2541330

1. 7177280

1.6997430

$-0.7248800$

$-1.0827220$

$-0.7204710$

$-0.0045990$

$-1.0042170$

$-0.9778340$

0.2687120

3. 9730380

4. 5218660

4. 0941700

2. 4344320

4.3016570

5. 6165470

4. 6585310

2. 0246300

2. 0392390

4. 3711070

4. 1463450

4. 3011130

-1.7800890
-2.1965530
\end{abstract}

0.8252240
2.7367150
2. 3350560

3. 0668930

3. 6096850

$-0.3983860$

$-0.6009030$

$-1.5742980$

2.0835310

$-1.4857120$

1. 6243250

1.0350240

$-4.6451550$

$-0.8058960$

$-3.4918950$

$-2.7491520$

$-3.4282560$

$-1.9178690$

$-2.3538190$

$-2.9391200$

$-1.3481660$

$-0.9600420$

$-1.8604690$

$-1.7707200$

$-2.8475280$

$-4.2072980$

$-1.5982470$

0.6191870

1. 0000880

0.2088850

$-0.5561030$

1. 2349400

0.5116190

$-0.8378830$

$-4.8788330$

$-4.4465920$

$-3.0132830$

$-4.8248900$

$-5.8984060$

$-4.5059400$

$-2.2803340$

$-5.5871920$

$-5.0311850$

$-4.2182320$

$-5.1311440$

$-2.7705090$

2. 1288530

2. 9740700
$-3.5937390$

4.6059560

$-2.9244240$

$-1.1878680$

$-2.4156730$

$-0.3183510$

$-3.1564360$

0.8556920

1. 6714570

$-2.8405510$

$-3.3033580$

1.4974600

$-1.5331730$

$-0.5213850$

$-1.4533850$

$-0.0329970$

$-2.2865490$

2. 6642310

4. 4741010

3. 1668700

2. 2103040

1.2068750

4.4158350

1.7508930

$-2.3370200$

5. 3348460

4. 0229710

2.9231600

5. 5621300

6.1821190

1. 9145030

6. 5750890

$-1.9843320$

$-0.6152840$

$-0.2599010$

$-2.0023090$

$-2.2172830$

$-0.6031250$

$-0.8503190$

$-1.3251010$

$-3.0046650$

$-2.7659820$

0.1574720

0.7875250

3.7235100

4.8044050 


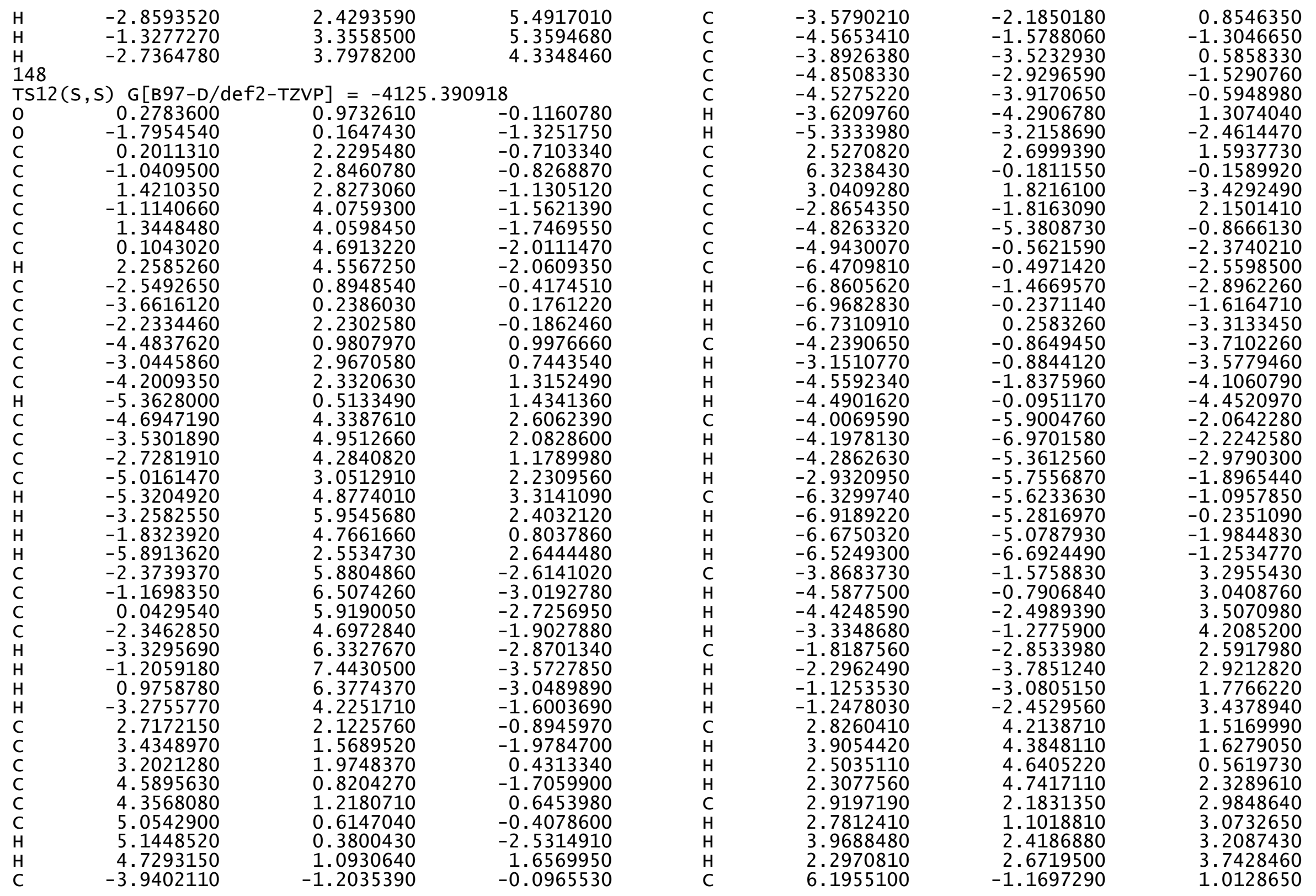




$\begin{array}{rrrr}\text { H } & 7.1065710 & -1.7768750 & \\ \text { H } & 6.0660930 & -0.6345990 & 1.0924350 \\ \text { H } & 5.3380720 & -1.8417550 & 0.8621560 \\ \mathrm{C} & 7.5139460 & 0.7723690 & 0.0744780 \\ \mathrm{H} & 7.6435400 & 1.4540570 & -0.7756430 \\ \mathrm{H} & 7.3401790 & 1.3755940 & 0.9757920 \\ \mathrm{H} & 8.4425040 & 0.2023710 & 0.2138050 \\ \mathrm{C} & 3.1726630 & 0.5898870 & -4.3400880 \\ \mathrm{H} & 4.2210000 & 0.2854000 & -4.4546430 \\ \mathrm{H} & 2.6057000 & -0.2555420 & -3.9455130 \\ \mathrm{H} & 2.7887480 & 0.8337550 & -5.3391830 \\ \mathrm{C} & 3.8929040 & 2.9754000 & -4.0014460 \\ \mathrm{H} & 4.9512560 & 2.6819620 & -4.0261060 \\ \mathrm{H} & 3.5756350 & 3.2104990 & -5.0264030 \\ \mathrm{H} & 3.8083080 & 3.8824790 & -3.3923130 \\ \mathrm{P} & -0.2743470 & -0.3073380 & -0.9755970 \\ \mathrm{O} & 0.4031480 & -0.4021730 & -2.3202200 \\ \mathrm{O} & -0.2238070 & -1.4740650 & -0.0435180 \\ \mathrm{H} & 1.9915060 & 2.1319970 & -3.4512440 \\ \mathrm{H} & 6.5321540 & -0.7576220 & -1.0717360 \\ \mathrm{H} & 1.4430690 & 2.5702480 & 1.4917490 \\ \mathrm{H} & -4.6125630 & 0.4296680 & -2.0475110 \\ \mathrm{H} & -4.5222460 & -5.9495930 & 0.0236100 \\ \mathrm{H} & -2.3301440 & -0.8770450 & 1.9709100 \\ \mathrm{C} & 1.6754950 & -3.3898660 & -1.9477480 \\ \mathrm{C} & 2.8204130 & -2.5860250 & -1.5121820 \\ \mathrm{H} & 0.7640960 & -3.2799520 & -1.3592580 \\ \mathrm{H} & 2.6205020 & -1.6863480 & -0.9397660 \\ \mathrm{O} & 1.7789590 & -2.3518740 & -2.9873790 \\ \mathrm{C} & 2.5545000 & -2.7409540 & 2.1404840 \\ \mathrm{C} & 2.0663500 & -1.3594310 & 4.1971850 \\ \mathrm{C} & 1.2542090 & -1.0632460 & 3.0893460 \\ \mathrm{~N} & 1.5588090 & -1.8405840 & 1.9849050 \\ \mathrm{H} & 0.9754230 & -1.7681740 & 1.1207840 \\ \mathrm{~S} & 3.2062610 & -2.6165180 & 3.7695700 \\ \mathrm{~S} & 3.1200780 & -3.8460070 & 1.0096750 \\ \mathrm{H} & 1.1597880 & -1.5293680 & -2.7017660 \\ \mathrm{C} & 0.8834260 & 0.2891020 & 5.4753910 \\ \mathrm{C} & 0.0766540 & 0.5878540 & 4.3533840 \\ \mathrm{C} & 0.2553130 & -0.0881990 & 3.1421870 \\ \mathrm{C} & 1.8795920 & -0.6772430 & 5.4045520 \\ \mathrm{H} & 0.7164210 & 0.8402820 & 6.3966820 \\ \mathrm{H} & -0.3300480 & 0.1226900 & 2.2571270 \\ \mathrm{H} & 2.4982130 & -0.8922840 & 6.2712590 \\ \mathrm{C} & 3.2860370 & -4.8901710 & -3.2157210 \\ \mathrm{C} & 4.4307370 & -4.3554310 & -2.3401930 \\ & & & \\ & & & \end{array}$

$\begin{array}{rr}4.2170370 & -2.8792830 \\ 1.9354950 & -4.7801860 \\ 3.4709260 & -5.9385790 \\ 5.3912830 & -4.4561880 \\ 4.4269220 & -2.2247530 \\ 1.9197340 & -5.4583590 \\ 1.1030080 & -5.0643970 \\ 3.2424220 & -4.3199710 \\ 4.4908490 & -4.9539380 \\ 4.8996930 & -2.5620580 \\ -0.8531010 & 1.5638110 \\ -1.6450410 & 1.9401680 \\ -1.0027620 & 2.3013920 \\ -2.2407120 & 1.0916370 \\ -2.3051310 & 2.7409210\end{array}$

$-1.9611450$ $-2.4855530$ $-3.4773270$ $-2.8586670$ $-2.8186810$ $-1.6212420$ $-3.1411750$ -3.1411750
-4.1531250 $-1.4210340$ $-1.1664990$ 4.5433040 3.4047740 2.5926730 3.0451890 3.7421050 148 $\operatorname{TS} 13(\mathrm{~S}, \mathrm{~S}) \mathrm{G}[\mathrm{B} 97-\mathrm{D} / \mathrm{def} 2-\mathrm{TZVP}]=-4125.390898$

$\begin{array}{lrrr}\text { O } & -0.2670800 & -1.0031100 & -0.1319350 \\ \mathrm{O} & 1.7587710 & -0.1850110 & -1.4156740 \\ \mathrm{C} & -0.2047930 & -2.2475340 & -0.7491470 \\ \mathrm{C} & 1.0325980 & -2.8604540 & -0.9225420 \\ \mathrm{C} & -1.4386060 & -2.8490240 & -1.1157630 \\ \mathrm{C} & 1.0776290 & -4.0787950 & -1.6785180 \\ \mathrm{C} & -1.3870440 & -4.0660710 & -1.7637700 \\ \mathrm{C} & -0.1564640 & -4.6868530 & -2.0942070 \\ \mathrm{H} & -2.3129320 & -4.5656920 & -2.0360570 \\ \mathrm{C} & 2.5522230 & -0.9166760 & -0.5439620 \\ \mathrm{C} & 3.6795150 & -0.2585210 & 0.0188800 \\ \mathrm{C} & 2.2474790 & -2.2539060 & -0.3158940 \\ \mathrm{C} & 4.5295630 & -1.0050250 & 0.8074610 \\ \mathrm{C} & 3.0891120 & -2.9959120 & 0.5818250 \\ \mathrm{C} & 4.2604780 & -2.3605420 & 1.1217490 \\ \mathrm{H} & 5.4195990 & -0.5388920 & 1.2220470 \\ \mathrm{C} & 4.7990190 & -4.3768380 & 2.3799410 \\ \mathrm{C} & 3.6211590 & -4.9894720 & 1.8873440 \\ \mathrm{C} & 2.7898710 & -4.3175860 & 1.0137590 \\ \mathrm{C} & 5.1052270 & -3.0851460 & 2.0059170 \\ \mathrm{H} & 5.4478750 & -4.9191100 & 3.0638750 \\ \mathrm{H} & 3.3627250 & -5.9963840 & 2.2075600 \\ \mathrm{H} & 1.8839140 & -4.7981460 & 0.6613160 \\ \mathrm{H} & 5.9912980 & -2.5883420 & 2.3969150 \\ \mathrm{C} & 2.2991550 & -5.8675620 & -2.8002990 \\ \mathrm{C} & 1.0811930 & -6.4867790 & -3.1745730 \\ \mathrm{C} & -0.1203690 & -5.9026990 & -2.8301020 \\ \mathrm{C} & 2.2969350 & -4.6957260 & -2.0698860 \\ \mathrm{H} & 3.2449730 & -6.3168250 & -3.0951610\end{array}$




$\begin{array}{lr}\mathrm{H} & 1.0971650 \\ \mathrm{H} & -1.0640430 \\ \mathrm{H} & 3.2364190 \\ \mathrm{C} & -2.7328010 \\ \mathrm{C} & -3.5144700 \\ \mathrm{C} & -3.1612650 \\ \mathrm{C} & -4.7112470 \\ \mathrm{C} & -4.3612060 \\ \mathrm{C} & -5.1464870 \\ \mathrm{H} & -5.3148260 \\ \mathrm{H} & -4.7029340 \\ \mathrm{C} & 3.9200210 \\ \mathrm{C} & 3.6080690 \\ \mathrm{C} & 4.4265500 \\ \mathrm{C} & 3.8370940 \\ \mathrm{C} & 4.6320350 \\ \mathrm{C} & 4.3417440 \\ \mathrm{H} & 3.5947930 \\ \mathrm{H} & 5.0212940 \\ \mathrm{C} & -2.3715130 \\ \mathrm{C} & -6.4440540 \\ \mathrm{C} & -3.1114610 \\ \mathrm{C} & 3.0299300 \\ \mathrm{C} & 4.5274390 \\ \mathrm{C} & 4.7687700 \\ \mathrm{C} & 6.2835820 \\ \mathrm{H} & 6.6113270 \\ \mathrm{H} & 6.8515390 \\ \mathrm{H} & 6.5235740 \\ \mathrm{C} & 3.9729470 \\ \mathrm{H} & 2.8954800 \\ \mathrm{H} & 4.2353520 \\ \mathrm{H} & 4.2046290 \\ \mathrm{C} & 5.4730770 \\ \mathrm{H} & 5.6129610 \\ \mathrm{H} & 5.0539950 \\ \mathrm{H} & 6.4542700 \\ \mathrm{C} & 3.1677630 \\ \mathrm{H} & 2.5001630 \\ \mathrm{H} & 2.6777820 \\ \mathrm{H} & 3.3057370 \\ \mathrm{C} & 4.1435740 \\ \mathrm{H} & 4.8833070 \\ \mathrm{H} & 4.6618030 \\ \mathrm{H} & 3.7126820 \\ \mathrm{C} & 1.9659630 \\ & \end{array}$

$\begin{array}{rr}-7.4136930 & -3.7434760 \\ -6.3572480 & -3.1266300 \\ -4.2287740 & -1.7914930 \\ -2.1929780 & -0.7554820 \\ -1.5579900 & -1.7469010 \\ -2.2020980 & 0.5951690 \\ -0.9367610 & -1.3667420 \\ -1.5601640 & 0.9214130 \\ -0.9182970 & -0.0402520 \\ -0.4455660 & -2.1257020 \\ -1.5503080 & 1.9533740 \\ 1.1912050 & -0.2478060 \\ 2.1530580 & 0.7441610 \\ 1.5992850 & -1.4980170 \\ 3.5031090 & 0.4633280 \\ 2.9655440 & -1.7323390 \\ 3.9318080 & -0.7694570 \\ 4.2476590 & 1.2171440 \\ 3.2854520 & -2.6978780 \\ -2.9506490 & 1.6660390 \\ -0.2339620 & 0.3519990 \\ -1.5664720 & -3.2169810 \\ 1.7511170 & 2.0967220 \\ 5.4099070 & -1.0632050 \\ 0.6077780 & -2.6021240 \\ 0.6024930 & -2.8855950 \\ 1.5880540 & -3.2416560 \\ 0.3596230 & -1.9782120 \\ -0.1399290 & -3.6585560 \\ 0.8949610 & -3.8894300 \\ 0.8751840 & -3.6898830 \\ 1.8801540 & -4.2963110 \\ 0.1389310 & -4.6514810 \\ 6.0821650 & -0.0505500 \\ 7.1416590 & -0.3029540 \\ 6.0254400 & 0.9625830 \\ 5.5906020 & -0.0426700 \\ 6.1365920 & -1.0982690 \\ 5.6844520 & -1.8428670 \\ 6.0730730 & -0.1177200 \\ 7.1975740 & -1.3463790 \\ 1.5816200 & 3.1493530 \\ 0.8364700 & 2.8377940 \\ 2.5379780 & 3.3023630 \\ 1.2651290 & 4.1091810 \\ 2.7285630 & 2.6242570\end{array}$

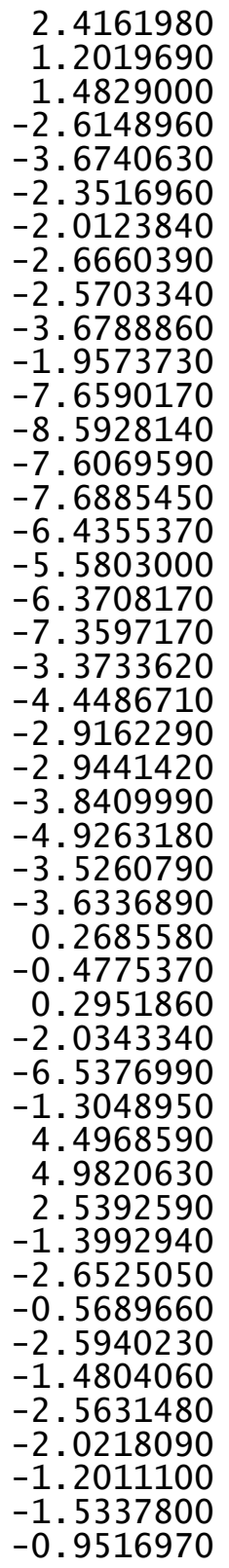

2.4161980
1.2019690
1.4829000
-2.6148960
-3.6740630
-2.3516960
-2.0123840
-2.6660390
-2.5703340
-3.6788860
-1.9573730
-7.6590170
-8.5928140
-7.6069590
-7.6885450
-6.4355370
-5.5803000
-6.3708170
-7.3597170
-3.3733620
-4.4486710
-2.9162290
-2.9441420
-3.8409990
-4.9263180
-3.5260790
-3.6336890
0.2685580
-0.4775370
0.2951860
-2.0343340
-6.5376990
-1.3048950
4.4968590
4.9820630
2.5392590
-1.3992940
-2.6525050
-0.5689660
-2.5940230
-1.4804060
-2.5631480
-2.0218090
-1.2011100
-1.5337800
-0.9516970
3.6845090

2. 9179890

2.2958030

$-4.4708780$

$-4.6948390$

$-4.8416810$

$-5.0088350$

$-2.5000810$

$-1.4161460$

$-2.7937890$

$-2.9813550$

$-0.9406590$

$-0.4634920$

$-0.8792170$

$-2.0005640$

1. 2591320

1.7761890

1.3751650

1.7455270

$-0.2292090$

$-0.0331500$

0.6010740

$-0.2626220$

$-2.7013270$

$-2.5335930$

$-2.7268650$

$-3.6792650$

0.3002550

0.4681100

1.4227240

$-1.7564170$

$-0.3089300$

$-2.7717780$

$-0.3986380$

5.4930970

0.7802740

3. 5479130

2. 8196410

3.2802660

1.8400740

2. 6494050

2. 6124070

1. 1962720

0.9585150

1.7554540

1. 7180260
2.9214690

1.8649330

3. 5087250

1.5467580

1.7329960

0.5510500

2. 2913100

3. 1037220

3. 2164130

3.4103540

3. 7875050

$-0.2800260$

0.0461800

$-1.3752710$

0.0027740

$-0.0230060$

0.4303550

$-1.1126800$

0.3161010

$-3.9305200$

$-4.0335470$

$-3.3878120$

$-4.9400800$

$-3.9654360$

$-3.9338380$

$-5.0175190$

$-3.5171050$

$-0.9681510$

$-2.2679140$

0.0176040

$-3.2706450$

1. 4445860

1.4843710

$-2.2667950$

$-2.0606320$

1.9668190

$-1.7647950$

$-1.5488480$

$-1.1121310$

$-1.0848470$

$-2.9344070$

2. 1912640

4.2128390

3. 0974340

2. 0144610

1. 1491800 


\begin{tabular}{|c|c|c|c|c|c|c|c|}
\hline $\mathrm{s}$ & -3.2092630 & 2.4204620 & 3.8173130 & C & -3.0193690 & 5.1229280 & 1.6777840 \\
\hline S & -3.1750210 & 3.7257150 & 1.0916330 & $\mathrm{C}$ & -2.2284730 & 4.3389570 & 0.8617530 \\
\hline $\mathrm{H}$ & -1.0065870 & 1.7359660 & -2.6724780 & C & -4.7409170 & 3.4268860 & 1.7076680 \\
\hline C & -0.7741770 & -0.4375800 & 5.4482040 & $\mathrm{H}$ & -4.9156130 & 5.3068810 & 2.7251520 \\
\hline C & 0.0342320 & -0.6850150 & 4.3151690 & $\mathrm{H}$ & -2.6539860 & 6.0923230 & 2.0096920 \\
\hline C & -0.1682500 & 0.0181310 & 3.1233920 & $\mathrm{H}$ & -1.2510970 & 4.6989790 & 0.5589470 \\
\hline C & -1.8040880 & 0.4946550 & 5.4039570 & $\mathrm{H}$ & -5.7059500 & 3.0522790 & 2.0443950 \\
\hline $\mathrm{H}$ & -0.5863080 & -1.0062660 & 6.3547110 & C & -1.5667480 & 5.8335860 & -2.9292340 \\
\hline $\mathrm{H}$ & 0.4242800 & -0.1461960 & 2.2333470 & $\mathrm{C}$ & -0.2901310 & 6.3337600 & -3.2861220 \\
\hline $\mathrm{H}$ & -2.4261340 & 0.6669180 & 6.2777480 & C & 0.8443910 & 5.6331230 & -2.9326280 \\
\hline C & -2.7232690 & 5.3519230 & -2.9713930 & $\mathrm{C}$ & -1.6871310 & 4.6635460 & -2.2059990 \\
\hline C & -3.9940610 & 4.8394860 & -2.2756130 & $\mathrm{H}$ & -2.4611190 & 6.3734480 & -3.2325860 \\
\hline C & -3.9599840 & 3.3128570 & -2.0832490 & $\mathrm{H}$ & -0.2090960 & 7.2606470 & -3.8495230 \\
\hline C & -1.4715170 & 5.0106150 & -2.1439260 & $\mathrm{H}$ & 1.8313890 & 5.9935750 & -3.2174080 \\
\hline $\mathrm{H}$ & -2.7804990 & 6.4380320 & -3.1088350 & $\mathrm{H}$ & -2.6710380 & 4.2890990 & -1.9421310 \\
\hline $\mathrm{H}$ & -4.8860300 & 5.1075430 & -2.8534800 & C & 3.0678050 & 1.6600070 & -0.9240770 \\
\hline $\mathrm{H}$ & -4.1358720 & 2.8004300 & -3.0401790 & C & 3.8262680 & 1.0708490 & -1.9612070 \\
\hline $\mathrm{H}$ & -1.4824650 & 5.5739250 & -1.2003260 & C & 3.4842840 & 1.5285440 & 0.4243500 \\
\hline $\mathrm{H}$ & -0.5513120 & 5.2813020 & -2.6764370 & $\mathrm{C}$ & 4.9855580 & 0.3596550 & -1.6252180 \\
\hline $\mathrm{H}$ & -2.6396560 & 4.8982850 & -3.9679310 & $\mathrm{C}$ & 4.6463330 & 0.8007870 & 0.7039510 \\
\hline $\mathrm{H}$ & -4.0839600 & 5.3204630 & -1.2919070 & $\mathrm{C}$ & 5.4117960 & 0.2081420 & -0.3051280 \\
\hline $\mathrm{H}$ & -4.7495670 & 2.9701940 & -1.4072200 & $\mathrm{H}$ & 5.5757520 & -0.0963100 & -2.4179250 \\
\hline 0 & 0.9870440 & -1.6448420 & 4.4734590 & $\mathrm{H}$ & 4.9671850 & 0.6965400 & 1.7349070 \\
\hline C & 1.7557810 & -2.0009990 & 3.3125240 & C & -4.0129350 & -0.9707590 & -0.4831690 \\
\hline $\mathrm{H}$ & 1.0970080 & -2.3434690 & 2.5053070 & $\mathrm{C}$ & -3.9995200 & -1.9226260 & 0.5620360 \\
\hline $\mathrm{H}$ & 2.3485080 & -1.1483150 & 2.9580990 & $\mathrm{C}$ & -4.4409070 & -1.3441450 & -1.7772740 \\
\hline $\mathrm{H}$ & 2.4195360 & -2.8102850 & 3.6213990 & $\mathrm{C}$ & -4.4374350 & -3.2252630 & 0.2895540 \\
\hline \multirow{2}{*}{\multicolumn{4}{|c|}{$\begin{array}{l}148 \\
\text { TS14 (S, S }) \text { G[B97-D/def2-TZVP }]=-4125.390764\end{array}$}} & C & -4.8600480 & -2.6592800 & -1.9991750 \\
\hline & & & & C & -4.8678460 & -3.6159720 & -0.9797140 \\
\hline 0 & 0.4780690 & 0.7230050 & -0.2721710 & $\mathrm{H}$ & -4.4306490 & -3.9654240 & 1.0877930 \\
\hline 0 & -1.6256220 & 0.1164510 & -1.5639200 & $\mathrm{H}$ & -5.1937590 & -2.9410540 & -2.9956500 \\
\hline C & 0.5479050 & 1.9667830 & -0.8856160 & C & 2.7372480 & 2.2368310 & 1.5510440 \\
\hline C & -0.6187990 & 2.7053000 & -1.0568170 & C & 6.7111810 & -0.5247450 & -0.0116050 \\
\hline C & 1.8372970 & 2.4439930 & -1.2448450 & C & 3.4544020 & 1.2207070 & -3.4317610 \\
\hline C & -0.5383980 & 3.9277310 & -1.8050640 & $\mathrm{C}$ & -3.5267180 & -1.5811430 & 1.9702670 \\
\hline C & 1.9114030 & 3.6701440 & -1.8749740 & C & -5.2984780 & -5.0468680 & -1.2503990 \\
\hline C & 0.7529310 & 4.4150300 & -2.2049790 & $\mathrm{C}$ & -4.5028580 & -0.3543730 & -2.9333400 \\
\hline $\mathrm{H}$ & 2.8847850 & 4.0710870 & -2.1454610 & $\mathrm{C}$ & -5.9643130 & -0.0964200 & -3.3501770 \\
\hline C & -2.3563500 & 0.9407340 & -0.7160860 & $\mathrm{H}$ & -6.4333100 & -1.0234580 & -3.7055610 \\
\hline C & -3.5839530 & 0.4323040 & -0.2105480 & $\mathrm{H}$ & -6.5513810 & 0.2812820 & -2.5030360 \\
\hline C & -1.8976080 & 2.2295650 & -0.4653080 & $\mathrm{H}$ & -6.0035070 & 0.6428490 & -4.1615360 \\
\hline C & -4.3652610 & 1.2806210 & 0.5477210 & C & -3.6651340 & -0.8233120 & -4.1369070 \\
\hline C & -2.6715850 & 3.0654630 & 0.4103560 & $\mathrm{H}$ & -2.6224350 & -0.9853910 & -3.8420840 \\
\hline C & -3.94 & 2.5887880 & 0.8839950 & $\mathrm{H}$ & -4.0622520 & -1.7604530 & -4.5487930 \\
\hline $\mathrm{H}$ & -5.3229890 & 0.9277250 & 0.9225810 & $\mathrm{H}$ & -3.6923540 & -0.0652890 & -4.9310880 \\
\hline C & -4.2978550 & 4.6754080 & 2.0904870 & C & -4.2911590 & -5.7556860 & -2.1775900 \\
\hline
\end{tabular}




$\begin{array}{rrr}-4.5874720 & -6.8009470 & -2.3388940 \\ -4.2545130 & -5.2535830 & -3.1534550 \\ -3.2821460 & -5.7380220 & -1.7464760 \\ -6.7187160 & -5.1208400 & -1.8412550 \\ -7.4484240 & -4.6438490 & -1.1746220 \\ -6.7574880 & -4.6108950 & -2.8125560 \\ -7.0139660 & -6.1673900 & -1.9940100 \\ -4.7217270 & -1.4094150 & 2.9278950 \\ -5.4069180 & -0.6303790 & 2.5727330 \\ -5.2838230 & -2.3497860 & 3.0082940 \\ -4.3677210 & -1.1317270 & 3.9301480 \\ -2.5380150 & -2.6183970 & 2.5316780 \\ -3.0293490 & -3.5850060 & 2.7018420 \\ -1.7023900 & -2.7638380 & 1.8399100 \\ -2.1424140 & -2.2655180 & 3.4922310 \\ 3.1010950 & 3.7372070 & 1.5675970 \\ 4.1710430 & 3.8590760 & 1.7853490 \\ 2.8855960 & 4.2110020 & 0.6037850 \\ 2.5281660 & 4.2563220 & 2.3480190 \\ 2.9758770 & 1.6343650 & 2.9431310 \\ 2.8394590 & 0.5490550 & 2.9386440 \\ 3.9918730 & 1.8486900 & 3.3004950 \\ 2.2678330 & 2.0700160 & 3.6574540 \\ 6.7418610 & -1.2238060 & 1.3570960 \\ 7.6524680 & -1.8293700 & 1.4446980 \\ 6.7506490 & -0.4876240 & 2.1713180 \\ 5.8710370 & -1.8754070 & 1.5035170 \\ 7.9009540 & 0.4511690 & -0.1350960 \\ 7.9209650 & 0.9213270 & -1.1260460 \\ 7.8137540 & 1.2434380 & 0.6207340 \\ 8.8506900 & -0.0774290 & 0.0236220 \\ 3.5118710 & -0.1039340 & -4.2124430 \\ 4.5381070 & -0.4881520 & -4.2737470 \\ 2.8825530 & -0.8639310 & -3.7431340 \\ 3.1566000 & 0.0605140 & -5.2380900 \\ 4.3675630 & 2.2644800 & -4.1072860 \\ 5.4118430 & 1.9248820 & -4.0800160 \\ 4.0765750 & 2.4023330 & -5.1573800 \\ 4.3125950 & 3.2349680 & -3.6002680 \\ -0.2155340 & -0.5292570 & -1.0706960 \\ 0.5174200 & -0.8494730 & -2.3492450 \\ -0.3809380 & -1.5926600 & -0.0317500 \\ 2.4240910 & 1.5860080 & -3.4887380 \\ 6.8329340 & -1.2921500 & -0.7898910 \\ 1.6638550 & 2.1610040 & -2.6003590 \\ -4.0891640 & 0.6026330 & \end{array}$

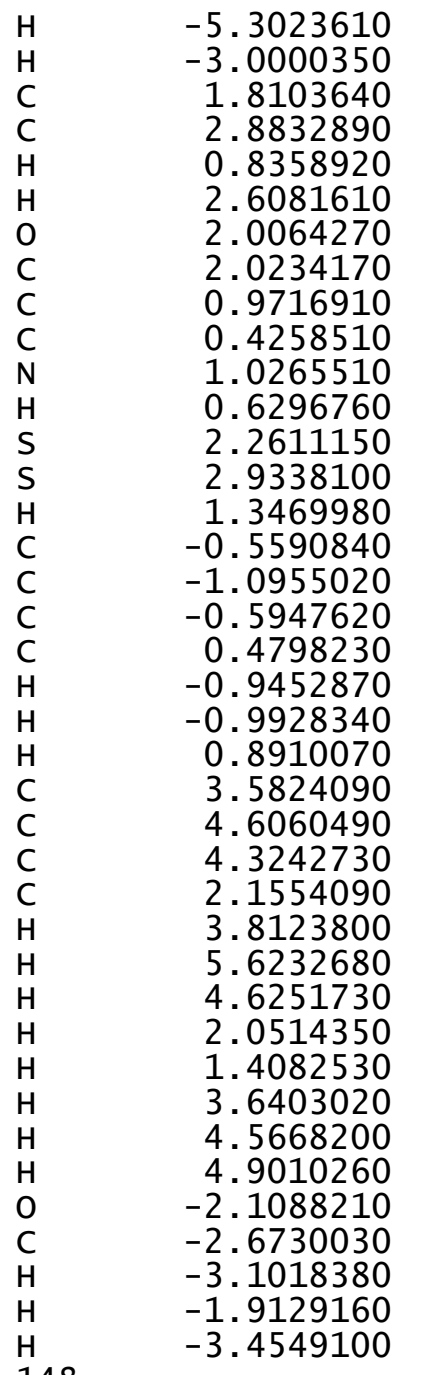
$-5.5768730$
$-3.4641330$
$-1.7389260$
$-2.8475120$
$-2.2845340$
$-0.7976290$
$-0.6573750$
$-1.4996480$
$-1.5815780$
$-1.9821290$
$-3.4017960$
$-2.0164630$
0.8728420
1.0094600
0.2467560
$-0.0285020$
1.4676560
0.3599350
$-0.1196790$
$-5.3159440$
$-4.5690600$
$-3.0572690$
$-5.1111180$
$-6.3876710$
$-4.7333430$
$-2.5788070$
$-5.5957080$
$-5.5577430$
$-4.9499270$
$-4.9659840$
$-2.5551540$
1.8589610
2. 6668190
2. 0369430
3. 3326450

$-0.2873350$

$-0.6237880 \quad 1.9299360$

$-3.6515530 \quad-1.2528840$

$-2.7305090 \quad-0.8668590$

$-0.8025110$

$-0.5229170$

$-2.4726900$

2.7606620

4.5126480

3.2205700

2. 2986270

1.3354940

4.4795850

1.8980720

$-2.4290570$

5. 3154530

4. 0162620

2.9521850

5.5645890

6.1354480

1.9508000

6.5660640

$-1.9942330$

$-1.1252030$

$-1.0867690$

$-1.4565200$

$-2.0115700$

$-1.4993120$

$-2.0290400$

$-0.4757680$

$-2.1242750$

$-3.0278750$

$-0.1014370$

$-0.3059960$

3.6951420

4.7367760

5. 5292090

5.1697380

TS15 (S , S ) G [B97-D/def2-TZVP $]=-4125.390761$

$\begin{array}{lrrr}\text { TS15 }(\mathrm{S}, \mathrm{S}) & \mathrm{G}[\mathrm{B} 97-\mathrm{D} / \mathrm{def}-\mathrm{TZVP}]=-4125.390761 \\ \mathrm{O} & -0.4501810 & -0.8058480 & -0.3560170 \\ \mathrm{O} & 1.6617830 & -0.2010500 & -1.6337620 \\ \mathrm{C} & -0.5210080 & -2.0356610 & -0.9957850 \\ \mathrm{C} & 0.6405500 & -2.7745320 & -1.1931900 \\ \mathrm{C} & -1.8158140 & -2.5128710 & -1.3338910\end{array}$




$\begin{array}{rr}\mathrm{C} & 0.5506380 \\ \mathrm{C} & -1.8998540 \\ \mathrm{C} & -0.7432050 \\ \mathrm{H} & -2.8770800 \\ \mathrm{C} & 2.3758740 \\ \mathrm{C} & 3.5708330 \\ \mathrm{C} & 1.9135770 \\ \mathrm{C} & 4.3202240 \\ \mathrm{C} & 2.6515300 \\ \mathrm{C} & 3.8891580 \\ \mathrm{H} & 5.2545130 \\ \mathrm{C} & 4.1867640 \\ \mathrm{C} & 2.9428140 \\ \mathrm{C} & 2.1956730 \\ \mathrm{C} & 4.6427150 \\ \mathrm{H} & 4.7686380 \\ \mathrm{H} & 2.5679480 \\ \mathrm{H} & 1.2413470 \\ \mathrm{H} & 5.5826170 \\ \mathrm{C} & 1.5697280 \\ \mathrm{C} & 0.2914550 \\ \mathrm{C} & -0.8395940 \\ \mathrm{C} & 1.6954540 \\ \mathrm{H} & 2.4606390 \\ \mathrm{H} & 0.2059940 \\ \mathrm{H} & -1.8274410 \\ \mathrm{H} & 2.6797370 \\ \mathrm{C} & -3.0305600 \\ \mathrm{C} & -3.8100440 \\ \mathrm{C} & -3.3899680 \\ \mathrm{C} & -4.9455430 \\ \mathrm{C} & -4.5334150 \\ \mathrm{C} & -5.3237060 \\ \mathrm{H} & -5.5509800 \\ \mathrm{H} & -4.8275260 \\ \mathrm{C} & 3.9733170 \\ \mathrm{C} & 3.8747680 \\ \mathrm{C} & 4.4228050 \\ \mathrm{C} & 4.2388680 \\ \mathrm{C} & 4.7679880 \\ \mathrm{C} & 4.6779580 \\ \mathrm{H} & 4.1562230 \\ \mathrm{H} & 5.1135330 \\ \mathrm{C} & -2.5964790 \\ \mathrm{C} & -6.5668060 \\ \mathrm{C} & -3.4740450 \\ & \end{array}$

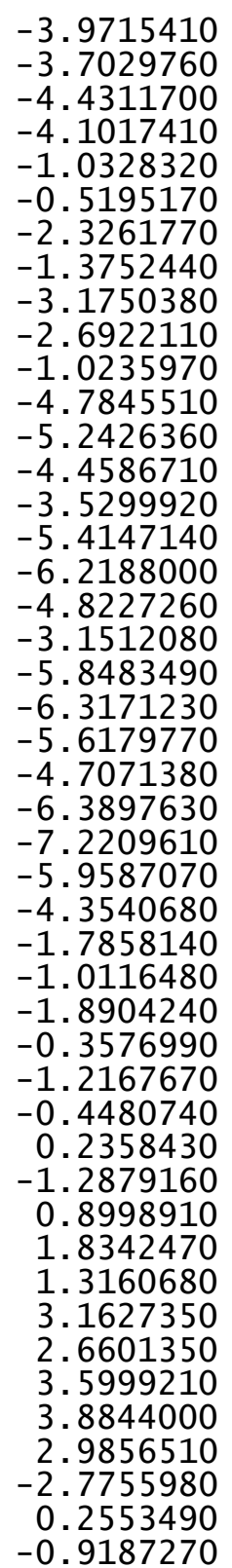
$-1.9783430$
$-2.0260420$
$-2.4034790$
$-2.2867130$
$-0.7779070$
$-0.2046300$
$-0.5700000$
0.5770840
0.3225710
0.8716940
1. 0071310
2. 0851040
1.5863560
0.7300570
1.7382090
2.7540920
1.8864350
0.3650400
2. 1359000
$-3.1568230$
$-3.5484670$
$-3.1793660$
$-2.3894550$
$-3.4675920$
$-4.1475560$
$-3.4848090$
$-2.0975730$
$-0.8542160$
$-1.7404960$
0.5123660
$-1.2449010$
0.9559100
0.0966540
$-1.9253470$
2.0001150
$-0.4286300$
0.6319260
$-1.6997280$
0.3912760
$-1.8898960$
$-0.8625220$
1.1999190
$-2.8700810$
1.4709940
0.6134720
-3.2234560

\begin{abstract}
3. 3857500
4.9995170

4. 5820750

6.0716090

6. 5100950

6.6377310

6.1832180

3.7757590

2. 7166650

4.1509180

3. 8651190

6.0662390

6. 3053820

5.7001420

6.9874940

3. 7194100

2. 9655970

3. 2877460

3. 9454230

4.5738690

5. 2671480

5.1295510

4. 2127490

2. 3956200

2.8944260

1. 5808450

1.9703020

$-2.9992880$

$-4.0582690$

$-2.8518710$

$-2.3976980$

$-2.7297950$

$-2.5130220$

$-3.7390420$

$-2.0195940$

$-7.8382100$

$-8.7300890$

$-7.8003590$

$-7.9387890$

$-6.4641190$

$-5.5738120$

$-6.4004920$

$-7.3521650$

$-3.6484960$

$-4.7031440$

$-3.0728420$
\end{abstract}

2.0214910

$-1.1122920$

$-2.8666540$

$-3.2204590$

$-3.5394930$

$-2.3533120$

$-4.0407050$

$-4.1001360$

$-3.8457790$

$-4.4904190$

$-4.8971560$

$-0.1394380$

$-0.3739780$

0.8948340

$-0.2003490$

$-1.0356020$

$-1.7485370$

$-0.0273790$

$-1.2589290$

2. 9829430

2. 6097190

3.0981390

3.9725740

2. 6225200

2.8718930

1. 9223680

3.5468380

1. 2866830

1.5475790

0.2526300

1. 9458370

2. 9507920

3. 1116310

3. 3283930

3. 5448250

$-0.0782390$

0.3339990

$-1.1557950$

0.0582180

0.4546350

0.9644700

$-0.6082640$

0.8758490

$-3.8015810$

$-3.8032580$

$-3.2275980$ 


$\begin{array}{rrr}-3.2945050 & 0.5144320 & -4.8403000 \\ -4.3293480 & -1.9224900 & -4.0236170 \\ -5.3943250 & -1.6714410 & -3.9247380 \\ -4.0627830 & -1.8880080 & -5.0886940 \\ -4.1851110 & -2.9467280 & -3.6611540 \\ 0.2691400 & 0.4600350 & -1.1118550 \\ -0.4661200 & 0.8496010 & -2.3690950 \\ 0.4793980 & 1.4814910 & -0.0398080 \\ -2.4210030 & -1.1928750 & -3.3500910 \\ -6.6474740 & 0.0326900 & 1.6868560 \\ -1.5348990 & -2.6878430 & 1.2137850 \\ 4.2015440 & -0.6311050 & -2.5651860 \\ 5.3995740 & 5.1422270 & -2.1331150 \\ 2.8588870 & 0.4853130 & 1.9381340 \\ -1.2403540 & 3.8621870 & -1.3484510 \\ -2.4975820 & 3.1568220 & -1.0790460 \\ -0.3602280 & 3.4894020 & -0.8263930 \\ -2.4398770 & 2.1240120 & -0.7514000 \\ -1.4803710 & 3.1223000 & -2.6030360 \\ -2.0257820 & 2.4347630 & 2.5803750 \\ -1.3175010 & 0.7470530 & 4.3259310 \\ -0.6541750 & 0.6264690 & 3.0877800 \\ -1.0731000 & 1.5734020 & 2.1682960 \\ -0.5921280 & 1.6346490 & 1.2414040 \\ -2.4746310 & 2.0612640 & 4.2437570 \\ -2.7098930 & 3.6976930 & 1.7080260 \\ -1.0133170 & 2.1746770 & -2.5186130 \\ -0.0422680 & -1.1124840 & 5.1612620 \\ 0.6104660 & -1.2278160 & 3.9149320 \\ 0.3054190 & -0.3539520 & 2.8642140 \\ -1.0097430 & -0.1250710 & 5.3682150 \\ 0.1939760 & -1.7935950 & 5.9710780 \\ 0.7968340 & -0.4470380 & 1.9034650 \\ -1.5119450 & -0.0502350 & 6.3285770 \\ -2.5972870 & 5.8470360 & -2.1753740 \\ -3.8068820 & 5.2981830 & -1.4030780 \\ -3.8270610 & 3.7594830 & -1.4023040 \\ -1.2819310 & 5.3626410 & -1.5412810 \\ -2.6160270 & 6.9431330 & -2.1779710 \\ -4.7434720 & 5.6682050 & -1.8358050 \\ -4.1284130 & 3.3777410 & -2.3886530 \\ -1.1613740 & 5.8083290 & -0.5439870 \\ -0.4128340 & 5.6618770 & -2.1403350 \\ -2.6451870 & 5.5143420 & -3.2207790 \\ -3.7616240 & 5.6537070 & -0.3644890 \\ -4.5572520 & 3.3622350 & -0.6911240\end{array}$

$\begin{array}{llll}\mathrm{O} & 1.5583140 & -2.1631390 & 3.6303020 \\ \mathrm{C} & 1.9073640 & -3.1120920 & 4.6465620 \\ \mathrm{H} & 2.3194840 & -2.6044540 & 5.5303730 \\ \mathrm{H} & 1.0311460 & -3.7066570 & 4.9419000 \\ \mathrm{H} & 2.6636670 & -3.7560190 & 4.1926240\end{array}$

TS16(S, S ) G[B97-D/def2-TZVP $]=-4125.390738$

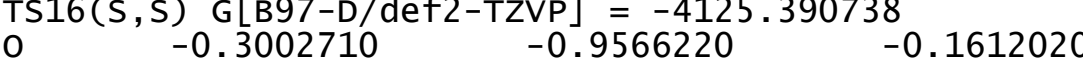

$0-1.7971840 \quad-0.1275320 \quad-1.3219150$

C $\quad-0.2560290 \quad-2.1634100 \quad-0.8511950$

C $\quad 0.9677750 \quad-2.8030510 \quad-1.0230800$

C $\quad-1.4939890 \quad-2.7042260 \quad-1.2912850$

$\mathrm{C} \quad 1.0040460 \quad-3.9712320 \quad-1.8547450$

$\begin{array}{lll}-1.4544200 & -3.8757890 & -2.0194650\end{array}$

$\mathrm{C} \quad-0.2317090 \quad-4.5104810 \quad-2.3524180$

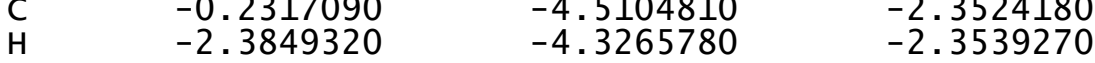

C $\quad 2.5350500 \quad-0.9389030 \quad-0.4728530$

$3.6644790 \quad-0.3563890 \quad 0.1640750$

$2.1786270 \quad-2.2759200 \quad-0.3389660$

$4.4626040 \quad-1.1794720 \quad 0.9305070$

$2.9644490 \quad-3.1008480 \quad 0.5365550$

$4.1377870 \quad-2.5413480 \quad 1.1511100$

$\begin{array}{lll}5.3544190 & -0.7729640 & 1.4004930\end{array}$

$4.5654400 \quad-4.6494850 \quad 2.2968010$

$3.3852590 \quad-5.1889050 \quad 1.7297000$

$2.6072750 \quad-4.4353580 \quad 0.8739140$

$\begin{array}{lll}4.9276450 & -3.3491460 & 2.0136730\end{array}$

$5.1724130 \quad-5.2551830 \quad 2.9657840$

$3.0831540 \quad-6.2041490 \quad 1.9770070$

$1.6997180 \quad-4.8612420 \quad 0.4601940$

$5.8168450 \quad-2.9102910 \quad 2.4626910$

$\begin{array}{lll}2.2119600 & -5.7195560 & -3.0524390\end{array}$

$0.9906280-6.2712350 \quad-3.5118540$

$-0.2048180-5.6735450 \quad-3.1694180$

$2.2178300 \quad-4.6007290 \quad-2.2432800$

$3.1540940-6.1790570 \quad-3.3432930$

$0.9997160-7.1572480 \quad-4.1426840$

$\begin{array}{lll}-1.1499610 & -6.0766790 & -3.5289610\end{array}$

$3.1597780 \quad-4.1851720 \quad-1.8996250$

$\begin{array}{lll}-2.7831240 & -2.0426950 & -0.9224020\end{array}$

$-3.5382630-1.3484610 \quad-1.8948760$

$-3.2412900 \quad-2.1169830 \quad 0.4171120$

$-4.7333200 \quad-0.7286300 \quad-1.5065000$

$\begin{array}{lll}-4.4404780 & -1.4780760 & 0.7509470\end{array}$

$\begin{array}{llllll}5.6537070 & -0.3644890 & \mathrm{C} & -5.1975490 & -0.7755080 & -0.1908100 \\ 3.3622350 & -0.6911240 & \mathrm{H} & -5.3160850 & -0.1937670 & -2.2521160\end{array}$ 


$\begin{array}{lrrr}\mathrm{H} & -4.8057290 & -1.5213180 & 1.7738460 \\ \mathrm{C} & 3.9633020 & 1.0965550 & -0.0100070 \\ \mathrm{C} & 3.6560260 & 2.0094730 & 1.0246060 \\ \mathrm{C} & 4.5243540 & 1.5562380 & -1.2213980 \\ \mathrm{C} & 3.9441700 & 3.3658790 & 0.8268990 \\ \mathrm{C} & 4.7850530 & 2.9219920 & -1.3727220 \\ \mathrm{C} & 4.5008570 & 3.8438130 & -0.3618680 \\ \mathrm{H} & 3.7081230 & 4.0816470 & 1.6115630 \\ \mathrm{H} & 5.2152780 & 3.2727940 & -2.3084890 \\ \mathrm{C} & -2.4872630 & -2.9331660 & 1.4642320 \\ \mathrm{C} & -6.4970820 & -0.1007550 & 0.2117710 \\ \mathrm{C} & -3.1172080 & -1.2985360 & -3.3588650 \\ \mathrm{C} & 3.0242840 & 1.5551370 & 2.3355210 \\ \mathrm{C} & 4.7370050 & 5.3300830 & -0.5659060 \\ \mathrm{C} & 4.8668200 & 0.6148390 & -2.3685410 \\ \mathrm{C} & 6.3878240 & 0.5776230 & -2.6135120 \\ \mathrm{H} & 6.7560930 & 1.5708100 & -2.9024320 \\ \mathrm{H} & 6.9228890 & 0.2649720 & -1.7075060 \\ \mathrm{H} & 6.6261760 & -0.1265490 & -3.4219080 \\ \mathrm{C} & 4.1135620 & 0.9926410 & -3.6577310 \\ \mathrm{H} & 3.0314090 & 0.9994830 & -3.4850780 \\ \mathrm{H} & 4.4176180 & 1.9872630 & -4.0094880 \\ \mathrm{H} & 4.3393450 & 0.2677620 & -4.4512210 \\ \mathrm{C} & 3.7520960 & 5.8963550 & -1.6090770 \\ \mathrm{H} & 3.8981310 & 6.9783950 & -1.7285720 \\ \mathrm{H} & 3.9151850 & 5.4163920 & -2.5833220 \\ \mathrm{H} & 2.7127940 & 5.7126750 & -1.3075600 \\ \mathrm{C} & 6.1894310 & 5.6387100 & -0.9721830 \\ \mathrm{H} & 6.8971730 & 5.2524600 & -0.2277390 \\ \mathrm{H} & 6.4246560 & 5.1777800 & -1.9401630 \\ \mathrm{H} & 6.3364980 & 6.7226350 & -1.0675840 \\ \mathrm{C} & 4.1010230 & 1.2937560 & 3.4077280 \\ \mathrm{H} & 4.8279830 & 0.5453460 & 3.0740140 \\ \mathrm{H} & 4.6417190 & 2.2239240 & 3.6291000 \\ \mathrm{H} & 3.6336560 & 0.9372660 & 4.3359670 \\ \mathrm{C} & 1.9743650 & 2.5362520 & 2.8832820 \\ \mathrm{H} & 2.4426420 & 3.4641310 & 3.2360170 \\ \mathrm{H} & 1.2358430 & 2.7820610 & 2.1149440 \\ \mathrm{H} & 1.4568960 & 2.0768740 & 3.7341810 \\ \mathrm{C} & -2.7362220 & -4.4422520 & 1.2507600 \\ \mathrm{H} & -3.8014000 & -4.6680960 & 1.3964950 \\ \mathrm{H} & -2.4492650 & -4.7589920 & 0.2430170 \\ \mathrm{H} & -2.1572310 & -5.0264750 & 1.9789540 \\ \mathrm{C} & -2.8211670 & -2.5653830 & 2.9169300 \\ \mathrm{H} & -2.7270170 & -1.4899430 & 3.0936950 \\ \mathrm{H} & -3.8429690 & -2.8721540 & \\ & & & \\ \mathrm{H} & & & \\ \mathrm{H} & -1770830\end{array}$

-2.1328390
-7.7028280
-8.6387300
-7.6197260
-7.7550650
-6.4575340
-5.6129830
-6.3536300
-7.3872040
-3.3132870
-4.3775830
-2.8136500
-2.8883850
-3.8872440
-4.9639900
-3.5581760
-3.7330690
0.3042090
-0.3845760
0.3234300
-2.0490820
-6.6243330
-1.4153480
4.5574030
4.5380120
2.5091570
-1.3003850
-2.5572080
-0.4782320
-2.5052430
-1.3699600
-2.5604150
-2.1242570
-1.2703580
-1.5460420
-0.9386360
-3.2680330
-3.1056950
-0.9012440
-0.9590270
-0.1164750
-0.2604520
-1.9650060
-0.8162010
0.3593950
-2.6134080
3. 5913050

$-0.5034240$

-0.1691990

$-1.5896100$

$-0.2985050$

$-0.0511580$

0.4686200

$-1.1262330$

0.2920240

$-4.0053680$

$-4.0935100$

$-3.4248770$

$-5.0173560$

$-4.1687670$

$-4.1430380$

$-5.2167470$

$-3.7622600$

$-0.9033590$

$-2.2199000$

0.1416570

$-3.4143870$

1. 2929290

1. 3243810

$-2.0944030$

0.3928280

2. 1397160

$-1.5862180$

$-1.4132750$

$-0.9349530$

$-0.9873570$

$-2.7918160$

2. 3046920

4. 2530490

3.1536700

2. 1109960

1.2623620

3.8930450

1.2539610

$-2.5641610$

5. 4300090

4. 3126080

3. 1582540

5. 4068400

6. 3065220

2. 2804870

6. 2678110 


$\begin{array}{ll}\mathrm{C} & -2.6057010 \\ \mathrm{C} & -3.8873340 \\ \mathrm{C} & -3.8573740 \\ \mathrm{C} & -1.3666780 \\ \mathrm{H} & -2.6605140 \\ \mathrm{H} & -4.7697850 \\ \mathrm{H} & -4.0238860 \\ \mathrm{H} & -1.3924490 \\ \mathrm{H} & -0.4387430 \\ \mathrm{H} & -2.5067100 \\ \mathrm{H} & -3.9935900 \\ \mathrm{H} & -4.6543380 \\ \mathrm{O} & 0.8082440 \\ \mathrm{C} & 1.6150250 \\ \mathrm{H} & 0.9825990 \\ \mathrm{H} & 2.2370780 \\ \mathrm{H} & 2.2505430 \\ \mathrm{H} & \end{array}$

$\begin{array}{rr}5.5378000 & -2.7412390 \\ 5.0017330 & -2.0842380 \\ 3.4694640 & -1.9444750 \\ 5.1655630 & -1.9078650 \\ 6.6281460 & -2.8407070 \\ 5.2906340 & -2.6667100 \\ 2.9890910 & -2.9194110 \\ 5.6921610 & -0.9436130 \\ 5.4563280 & -2.4159600 \\ 5.1195960 & -3.7516940 \\ 5.4480940 & -1.0859000 \\ 3.1062050 & -1.2885050 \\ -1.9041640 & 4.4479800 \\ -2.2083330 & 3.2983090 \\ -2.4887790 & 2.4477040 \\ -1.3492630 & 3.0174270 \\ -3.0483680 & 3.5837030\end{array}$

148

TS17 (S , S ) G[B97-D/def2-TZVP $]=-4125.390446$

$\begin{array}{lrlr}\text { OS } & 0.3743160 & 0.8719140 & 0.0739400 \\ \mathrm{O} & -1.6195300 & 0.5452440 & -1.4574460 \\ \mathrm{C} & 0.4696570 & 2.2221190 & -0.2482290 \\ \mathrm{C} & -0.6961350 & 2.9820710 & -0.3289650 \\ \mathrm{C} & 1.7705650 & 2.7664340 & -0.4289520 \\ \mathrm{C} & -0.5861910 & 4.3459440 & -0.7651780 \\ \mathrm{C} & 1.8625620 & 4.1181830 & -0.7038680 \\ \mathrm{C} & 0.7193530 & 4.9244860 & -0.9131130 \\ \mathrm{H} & 2.8456160 & 4.5670320 & -0.8209120 \\ \mathrm{C} & -2.4148840 & 1.1704450 & -0.5057290 \\ \mathrm{C} & -3.6648820 & 0.5619110 & -0.2106160 \\ \mathrm{C} & -2.0070230 & 2.3851010 & 0.0388000 \\ \mathrm{C} & -4.5245700 & 1.2305810 & 0.6351630 \\ \mathrm{C} & -2.8716440 & 3.0221570 & 0.9971410 \\ \mathrm{C} & -4.1600480 & 2.4449370 & 1.2651780 \\ \mathrm{H} & -5.5056980 & 0.8089990 & 0.8386770 \\ \mathrm{C} & -4.6520760 & 4.2180050 & 2.8624550 \\ \mathrm{C} & -3.3643700 & 4.7626030 & 2.6392650 \\ \mathrm{C} & -2.4991340 & 4.1795950 & 1.7356990 \\ \mathrm{C} & -5.0347690 & 3.0759640 & 2.1912020 \\ \mathrm{H} & -5.3258290 & 4.6894100 & 3.5742280 \\ \mathrm{H} & -3.0472530 & 5.6428020 & 3.1940640 \\ \mathrm{H} & -1.5108890 & 4.6027490 & 1.5963320 \\ \mathrm{H} & -6.0089140 & 2.6250170 & 2.3713210 \\ \mathrm{C} & -1.5633720 & 6.4594340 & -1.4946000 \\ \mathrm{C} & -0.2756940 & 7.0430630 & -1.5883690 \\ \mathrm{C} & 0.8419030 & 6.2852460 & -1.3081800\end{array}$

-1.7143550
-2.4418840
-0.1720540
1.8398780
-2.7069290
3.0102690
3.7731150
3.4594980
4.9768740
4.6568440
5.4382330
5.5573490
5.0133850
-4.0612690
-3.9475200
-4.5694530
-4.3830980
-4.9863950
-4.9062290
-4.3018700
-5.3825510
2.7165170
6.7576160
3.3756380
-3.3720520
-5.3442470
-4.6938000
-6.1712420
-6.6326340
-6.7392130
-6.2501260
-3.8833910
-2.8279210
-4.2705690
-3.9531290
-6.4459630
-6.7706380
-6.0709450
-7.3161420
-4.1438880
-3.3677040
-3.7001040
-4.4646860
-4.4804700
-5.0386100
-5.1865830

5.1474930

7. 0490850

8.0805310

6.7095640

4.7140240

1.9341130

1.7716910

1.3606340

1. 0516280

0.6423300

0.4823410

0.9350960

0.1978700

$-0.7407550$

$-1.9378020$

$-0.7652550$

$-3.1320910$

$-1.9892930$

$-3.1800830$

$-4.0566170$

$-2.0142560$

1. 6084990

$-0.2693270$

2. 3772290

$-1.9417030$

$-4.4975960$

0.4938590

0.8143100

0.0014730

0.9393470

1.7398060

0. 3808070

0.1784440

$-0.4301980$

1. 3179300

$-5.1790150$

$-6.1092680$

$-5.4289070$

$-4.5202820$

$-5.4466020$

$-4.9801500$

$-5.6950490$

$-6.3807590$

$-1.7683840$

$-0.8385400$

$-2.6082500$
$-1.0917880$

$-1.7472330$

$-1.8981490$

$-1.4030310$

$-1.0255200$

$-0.3836860$

$-1.5626240$

0.8351930

$-1.5001640$

0.8359380

$-0.3162440$

$-2.4105600$

1.7618430

$-0.8245100$

$-0.0776450$

$-2.1378500$

$-0.6590530$

$-2.6784680$

$-1.9566680$

$-0.0939100$

$-3.6927310$

2. 1444170

$-0.2114470$

-2. 9064420

1. 3348270

$-2.5713100$

$-2.9848270$

$-3.2817310$

$-3.8578400$

$-2.3507410$

$-3.8678470$

$-4.2897030$

$-4.0730720$

$-4.9203530$

$-4.8578320$

$-1.7380350$

$-2.2227280$

$-0.7368730$

$-1.6229010$

$-2.7578770$

$-3.3777310$

$-1.7847460$

$-3.2383090$

2. 3924340

2. 2414100

2. 3380240 


$\begin{array}{rrr}-4.0411400 & -1.7510490 & 3.3993360 \\ -2.5486620 & -3.2001000 & 1.6552720 \\ -3.1927520 & -4.0844170 & 1.7434510 \\ -1.7993310 & -3.3866610 & 0.8800830 \\ -2.0373690 & -3.0644300 & 2.6156180 \\ 3.0637580 & 3.0124300 & 2.6847070 \\ 4.1350640 & 3.0702300 & 2.9207440 \\ 2.8277000 & 3.7881150 & 1.9470700 \\ 2.4951850 & 3.2172060 & 3.6021800 \\ 2.9764800 & 0.5503460 & 3.2270510 \\ 2.8303840 & -0.4631700 & 2.8381600 \\ 3.9988030 & 0.6251170 & 3.6202160 \\ 2.2823360 & 0.6994610 & 4.0616190 \\ 7.8027950 & 0.5994860 & 0.5209700 \\ 8.7364380 & 0.0388280 & 0.6625680 \\ 8.0238650 & 1.4987030 & -0.0700890 \\ 7.4310420 & 0.9166270 & 1.5025970 \\ 7.3234410 & -0.7513000 & -1.5543690 \\ 6.5948540 & -1.3523570 & -2.1118050 \\ 7.6130100 & 0.0996270 & -2.1847240 \\ 8.2179770 & -1.3620940 & -1.3821760 \\ 3.3580300 & 1.3454930 & -4.0493330 \\ 4.3505610 & 0.9052100 & -4.2078980 \\ 2.6491200 & 0.5405980 & -3.8385390 \\ 3.0565600 & 1.8403540 & -4.9822190 \\ 4.3169270 & 3.5445830 & -3.2662220 \\ 5.3458250 & 3.1798160 & -3.3859470 \\ 4.0028580 & 4.0074200 & -4.2111510 \\ 4.3179510 & 4.3129780 & -2.4838040 \\ -0.2154490 & -0.1703790 & -1.0448560 \\ 0.6014590 & -0.1394430 & -2.3136660 \\ -0.4021680 & -1.4678010 & -0.3287330 \\ 2.3615940 & 2.7784200 & -2.8237790 \\ 6.5711120 & -1.1543840 & 0.4152120 \\ 1.6429070 & 1.5953840 & 1.9378500 \\ -4.2842810 & 1.3373510 & -2.4189910 \\ -5.7584640 & -4.2760030 & -3.5651940 \\ -2.6948210 & -1.0835290 & 1.4150580 \\ 2.0438590 & -3.0796460 & -2.2273120 \\ 2.7292070 & -2.5500130 & -1.0469730 \\ 0.9579810 & -3.1573850 & -2.1524010 \\ 2.1499220 & -1.9326780 & -0.3702920 \\ 2.4677870 & -1.7370910 & -2.6527260 \\ 1.5114490 & -3.6428850 & 1.9849440 \\ 1.0155340 & -2.5203820 & 4.1923650 \\ 0.4584090 & -1.8787690 & 3.0734150\end{array}$

0.7616140
0.3582660
1.9194030
1.9951820
1.6578350
0.0236830
-0.5350740
-0.3179880
0.7994190
-0.1647920
-0.7104000
1.2238090
4.2541880
4.7829730
4.1991310
2.7199140
4.6923720
5.8769700
4.6665320
2.4183580
2.3281020
4.5523940
4.5077220
4.3933100
-1.2757410
-1.8479710
-1.0611910
-2.5227480
-2.4065640
$-2.5222210$
$-2.1851660$
$-3.9262160$
$-4.6448490$
$-1.0559730$
$-0.8587250$
$-0.2200840$
$-0.7238560$
$-2.0041860$
$-0.4330800$
$-0.2523200$
$-2.4852230$
$-4.0579580$
$-3.9364680$
$-2.7080320$
$-4.1835350$
$-4.9356040$
$-3.8654410$
$-1.7849620$
$-5.1324900$
$-4.1808580$
$-3.1743080$
$-4.8384250$
$-2.7332270$
0.8905730
1. 5698640
1.9283350
0.9043780
2. 4158590

1.8872400

0.9829090

3.6730160

0.7285690

5.6082320

4.4766100

3.1892110

5.4745770

6.5899730

2. 2970580

6.3512650

$-2.9979130$

$-1.5595300$

$-0.8421450$

$-3.0115750$

$-3.4872800$

$-1.5560750$

$-1.2075740$

$-2.5462310$

$-4.0362270$

$-3.5774760$

$-0.9962780$

0.2354820

4.7372330

3.6079650

2.9327310

3.0545510

4.0102590

TS18(S,S) G[B97-D/def2-TZVP $]=-4125.390029$

$\begin{array}{lrcr}\text { TS18 }(\mathrm{S}, \mathrm{S}) \mathrm{G}[\mathrm{B} 97-\mathrm{D} / \mathrm{def2}-\mathrm{TZVP}]=-4125.390029 \\ \mathrm{O} & 0.2457990 & 1.0145290 & -0.0707120 \\ \mathrm{O} & -1.7638240 & 0.2320440 & -1.3961310 \\ \mathrm{C} & 0.1490110 & 2.3017790 & -0.5911380 \\ \mathrm{C} & -1.1057630 & 2.8925510 & -0.7023050 \\ \mathrm{C} & 1.3628680 & 2.9533690 & -0.9440940 \\ \mathrm{C} & -1.1932090 & 4.1653660 & -1.3594310 \\ \mathrm{C} & 1.2697540 & 4.2210420 & -1.4810330 \\ \mathrm{C} & 0.0196300 & 4.8382910 & -1.7351240 \\ \text { H } & 2.1768320 & 4.7598800 & -1.7399030 \\ \mathrm{C} & -2.5692800 & 0.8799620 & -0.4706310 \\ \mathrm{C} & -3.6840670 & 0.1580230 & 0.0345280 \\ \mathrm{C} & -2.2984060 & 2.2044500 & -0.1402960 \\ \mathrm{C} & -4.5532290 & 0.8218510 & 0.8747160 \\ \mathrm{C} & -3.1597640 & 2.8557450 & 0.8087090 \\ \mathrm{C} & -4.3167070 & 2.1543040 & 1.2937950\end{array}$




$\begin{array}{rrr}-5.4343450 & 0.3046780 & 1.2466550 \\ -4.9076740 & 4.0545700 & 2.6997150 \\ -3.7435560 & 4.7307360 & 2.2603830 \\ -2.8940010 & 4.1475600 & 1.3417880 \\ -5.1814710 & 2.7884370 & 2.2267220 \\ -5.5711310 & 4.5274200 & 3.4201670 \\ -3.5099500 & 5.7159540 & 2.6577750 \\ -2.0002040 & 4.6762620 & 1.0299950 \\ -6.0566330 & 2.2424430 & 2.5742790 \\ -2.4727410 & 6.0026070 & -2.3280370 \\ -1.2756490 & 6.6838480 & -2.6592950 \\ -0.0557130 & 6.1073360 & -2.3716040 \\ -2.4322890 & 4.7767840 & -1.6934690 \\ -3.4330840 & 6.4468680 & -2.5804790 \\ -1.3221430 & 7.6522040 & -3.1524350 \\ 0.8728530 & 6.6077010 & -2.6407840 \\ -3.3563930 & 4.2634200 & -1.4474350 \\ 2.6695160 & 2.2627790 & -0.7233020 \\ 3.4149420 & 1.7805590 & -1.8227130 \\ 3.1320200 & 2.0494060 & 0.6014320 \\ 4.5784030 & 1.0392020 & -1.5701080 \\ 4.2990700 & 1.3061430 & 0.7953960 \\ 5.0252150 & 0.7733120 & -0.2764360 \\ 5.1548050 & 0.6514280 & -2.4073630 \\ 4.6549240 & 1.1314560 & 1.8057170 \\ -3.9172980 & -1.2676580 & -0.3473250 \\ -3.5633090 & -2.3051710 & 0.5489880 \\ -4.4930190 & -1.5727210 & -1.5963610 \\ -3.8340740 & -3.6265980 & 0.1831150 \\ -4.7399960 & -2.9144160 & -1.9183460 \\ -4.4223880 & -3.9534410 & -1.0440030 \\ -3.5653080 & -4.4299640 & 0.8638840 \\ -5.1875740 & -3.1553530 & -2.8814500 \\ 2.4156700 & 2.6881160 & 1.7890990 \\ 6.3038740 & -0.0142250 & -0.0490090 \\ 3.0355910 & 2.1006500 & -3.2639920 \\ -2.9032810 & -2.0091640 & 1.8907690 \\ -4.6831510 & -5.4015800 & -1.4185010 \\ -4.8598520 & -0.4951850 & -2.6078220 \\ -6.3820450 & -0.4494000 & -2.8407430 \\ -6.7362760 & -1.4007460 & -3.2589910 \\ -6.9164710 & -0.2686150 & -1.8991620 \\ -6.6349540 & 0.3522850 & -3.5474870 \\ -4.1070740 & -0.6935730 & -3.9372640 \\ -3.0238050 & -0.7058880 & -3.7698940 \\ -4.3972410 & -1.6413110 & -4.4091760\end{array}$

\begin{tabular}{|c|c|}
\hline$H$ & \\
\hline c & $\begin{array}{l}-4.5474100 \\
-56498060\end{array}$ \\
\hline $\mathrm{H}$ & -5.8548270 \\
\hline $\mathrm{H}$ & -5.2100550 \\
\hline $\mathrm{H}$ & -6.6006300 \\
\hline C & -3.3664760 \\
\hline $\mathrm{H}$ & -2.6817970 \\
\hline $\mathrm{H}$ & -2.8643600 \\
\hline H & -3.5662080 \\
\hline C & -3.9525330 \\
\hline $\mathrm{H}$ & -4.6833660 \\
\hline H & -4.4915170 \\
\hline $\mathrm{H}$ & -3.4610030 \\
\hline C & -1.8443300 \\
\hline $\mathrm{H}$ & -2.3071980 \\
\hline $\mathrm{H}$ & -1.1197450 \\
\hline H & -1.3110950 \\
\hline C & 2.6990740 \\
\hline H & 3.7717510 \\
\hline $\mathrm{H}$ & 2.4055960 \\
\hline $\mathrm{H}$ & 2.1456260 \\
\hline C & 2.7715580 \\
\hline $\mathrm{H}$ & 2.6440450 \\
\hline $\mathrm{H}$ & 3.8098510 \\
\hline $\mathrm{H}$ & 2.1191760 \\
\hline C & 6.1710300 \\
\hline $\mathrm{H}$ & 7.0895950 \\
\hline $\mathrm{H}$ & 6.0164130 \\
\hline $\mathrm{H}$ & 5.3262390 \\
\hline C & 7.4735230 \\
\hline $\mathrm{H}$ & 7.6049720 \\
\hline $\mathrm{H}$ & 7.2748590 \\
\hline $\mathrm{H}$ & 8.4096060 \\
\hline C & 3.2333750 \\
\hline $\mathrm{H}$ & 4.2962260 \\
\hline $\mathrm{H}$ & 2.7035130 \\
\hline $\mathrm{H}$ & 2.8477170 \\
\hline C & 3.8506230 \\
\hline $\mathrm{H}$ & 9198240 \\
\hline $\mathrm{H}$ & 3. 5419080 \\
\hline $\mathrm{H}$ & 3.7205420 \\
\hline $\mathrm{P}$ & -0.2466450 \\
\hline 0 & 0.4789820 \\
\hline 0 & -0.2038170 \\
\hline $\mathrm{H}$ & 1.9747110 \\
\hline $\mathrm{H}$ & 10 \\
\hline
\end{tabular}

0.1218840
-6.0767250
-7.1105450
-6.1011030
-5.5315040
-6.1976230
-5.7394020
-6.2217300
-7.2324060
-1.8754500
-1.0917550
-2.8245820
-1.6307520
-3.0487930
-4.0123100
-3.2023640
-2.6943370
4.2064470
4.3800480
4.6905580
4.6761530
2.0809900
0.9946860
2.3117610
2.5093440
-1.0681210
-1.6658340
-0.5887860
-1.7437270
0.9441480
1.6740790
1.4937460
0.3827860
0.9311060
0.6836960
0.0386020
1.2149770
3.3174190
3.0659450
3.5990020
4.1839390
-0.2289240
-0.2364650
-1.4432670
2.3697430
-0.5359100

$-4.6324160$ $-0.4269360$ $-0.7354530$ 0.5789190 $-0.3715590$ $-1.5135740$ $-2.2385870$ $-0.5373600$ $-1.8225270$

3. 0117350

2.7859780

3. 1352100

3.9633770

2. 2961210

2. 5456050

1. 4908160

3. 1862010

1.8217180

1.9831410

0.8847040

2. 6461490

3.1534460

3. 1620460

3.4274250

3.9230060

1.0636180

1.1263230

2.0385420

0.8821930

0.2568290

$-0.5519700$

1. 1869270

0.3802280

$-4.2422440$

$-4.3619240$

$-3.9042780$

$-5.2299840$

$-3.7537250$

$-3.7745510$

$-4.7695900$

$-3.0959740$

$-1.0163120$

$-2.3388920$

$-0.1464720$

$-3.2874030$

$-0.9884410$ 


\begin{tabular}{|c|c|c|c|}
\hline & & & \\
\hline $\mathrm{H}$ & 1.3366900 & 2.5560890 & 1.6454940 \\
\hline $\mathrm{H}$ & -4.5621200 & 0.4787420 & -2.2050190 \\
\hline $\mathrm{H}$ & -5.1553100 & -5.4065550 & -2.4112380 \\
\hline $\mathrm{H}$ & -2.3880150 & -1.0467490 & 1.7957480 \\
\hline C & 1.7952530 & -3.2125820 & -2.0975540 \\
\hline C & 2.9269310 & -2.4228080 & -1.6063490 \\
\hline $\mathrm{H}$ & 0.8748840 & -3.1400870 & -1.5172820 \\
\hline $\mathrm{H}$ & 2.7148500 & -1.5533710 & -0.9926380 \\
\hline $\mathrm{O}$ & 1.9026340 & -2.1220630 & -3.081 \\
\hline C & 2.5651220 & -2.8124750 & 2.0194090 \\
\hline C & 1.9784350 & -1.5708830 & 4.1391010 \\
\hline $\mathrm{C}$ & 1.1927160 & -1.2283700 & 3.0258410 \\
\hline $\mathrm{N}$ & 1.5513070 & -1.9288290 & 1.8866830 \\
\hline $\mathrm{H}$ & 0.9923010 & -1.8141160 & 1.0122610 \\
\hline $\mathrm{S}$ & 3.1627810 & -2.7723000 & 3.6734190 \\
\hline $\mathrm{S}$ & 3.1960170 & -3.8295150 & 0.8416850 \\
\hline $\mathrm{H}$ & 1.2656220 & -1.3248840 & -2.7635590 \\
\hline C & 0.7199360 & -0.0244910 & 5.4723250 \\
\hline $\mathrm{c}$ & -0.0586330 & 0.3226330 & 4.3443120 \\
\hline $\mathrm{C}$ & 0.1703550 & -0.2800710 & 3.1034570 \\
\hline C & 1.7392870 & -0.9645270 & 5.3774100 \\
\hline $\mathrm{r}$ & 0.5135760 & 0.4689960 & 6.4180350 \\
\hline 1 & -0.3934070 & -0.0330100 & 2.2139830 \\
\hline $\mathrm{r}$ & 2.3360560 & -1.2165430 & 6.2494440 \\
\hline $\mathrm{C}$ & 3.4357250 & -4.6335870 & -3.4174940 \\
\hline $\mathrm{C}$ & 4.5646620 & -4.1349070 & -2.5010750 \\
\hline $\mathrm{C}$ & 4.3320330 & -2.6820280 & -2.0493850 \\
\hline $\mathrm{C}$ & 2.0751140 & -4.5719210 & -2.7006260 \\
\hline $\mathrm{r}$ & 3.6331650 & -5.6656990 & -3.7298050 \\
\hline $\mathrm{r}$ & 5.5320600 & -4.1996610 & -3.0125170 \\
\hline $\mathrm{r}$ & 4.54 & -1.9833640 & -2.86 \\
\hline 1 & 2.0547090 & -5.2924750 & -1.8714130 \\
\hline $\mathrm{r}$ & 7060 & -4.8301600 & -3.3 \\
\hline $\mathrm{r}$ & 3.3 & -4.0165010 & -4.3 \\
\hline $\mathrm{r}$ & 4.62 & -4.7803310 & -1.6 \\
\hline 1 & 5.0001510 & -2.3997990 & -1.2 \\
\hline C & -1.0116520 & 1.2709180 & 4.5 \\
\hline$c$ & -1.77 & 1.6995780 & 3. \\
\hline r & $-1.1084 \varepsilon$ & 2.1111940 & 2.6 \\
\hline r & -2.34 & 0.8645890 & 0020 \\
\hline 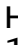 & -2.4 & 2.4731600 & 3.7742140 \\
\hline & D/ & -412 & \\
\hline $\mathrm{C}$ & 0.4783370 & 0.7544340 & -0 \\
\hline 0 & 28710 & 0.2718880 & -1.6397000 \\
\hline & & 2.0433000 & \\
\hline
\end{tabular}

-0.5816890
1.8773650
-0.4722250
1.9767450
0.8329530
2.9600870
-2.3389070
-3.5883220
-1.8808740
-4.3897630
-2.6772600
-3.9671560
-5.3628670
-4.3449380
-3.0480110
-2.2368000
-4.7871320
-4.9784320
-2.6850940
-1.2453630
-5.7675410
-1.4556500
-0.1664160
0.9529470
-1.6040980
-2.3376770
-0.0635230
1.9498650
-2.5976830
3.0925390
3.8917910
3.4531130
5.0326070
4.5988880
5.4010880
5.6546570
4.8779760
-4.0111750
-4.0442340
-4.3764810
-4.4580350
-4.7763090
-4.8223920
-4.4847070
-5.0623510
2.6647870

2.8018270

2.5378930

4.0791350

3. 8068600

4.5843350

4. 2203830

1.0309530

0.4963400

2.2899000

1.2872300

3.0571120

2. 5573950

0.9138730

4.5381950

5. 0026870

4. 2815160

3.3326750

5.1210980

5.9348630

. 6525220

2. 9437680

0784750

6.5926410

. 8549570

4.8560660

6.6497590

7. 5607150

6. 2277420

4.4726680

1.7274270

(1.2104200

1. 4989900

0.4676430

0.7440890

0.2170150

0.0664040

0.5644790

$-0.8768530$

$-1.9106650$

$-1.1452670$

$-3.1868670$

$-2.4426910$

$-3.4769330$

$-3.9827280$

$-2.6536540$

2.1332690
$-0.8912020$

$-1.0237680$

$-1.5367460$

$-1.5582790$

$-1.8632070$

$-1.7659060$

$-0.7539600$

$-0.3365960$

$-0.3813080$

0.4618780

0.5355000

0.9229640

0.7721190

2. 2904110

1. 9625990

1.1094570

1.7870620

2.9553560

2. 3900440

0.8726010

2. 0567370

$-2.5314170$

$-2.8153790$

$-2.4900760$

$-1.9067420$

$-2.8124880$

$-3.3004910$

$-2.7189490$

$-1.6982940$

$-0.7123800$

$-1.7568700$

0.6398690

$-1.4255650$

0.9135540

$-0.1035940$

$-2.2234940$

1.9462050

$-0.7390570$

0.2285790

$-2.0753560$

$-0.1657890$

$-2.4200080$

$-1.4842130$

0.5747710

$-3.4493480$

1.7827840 


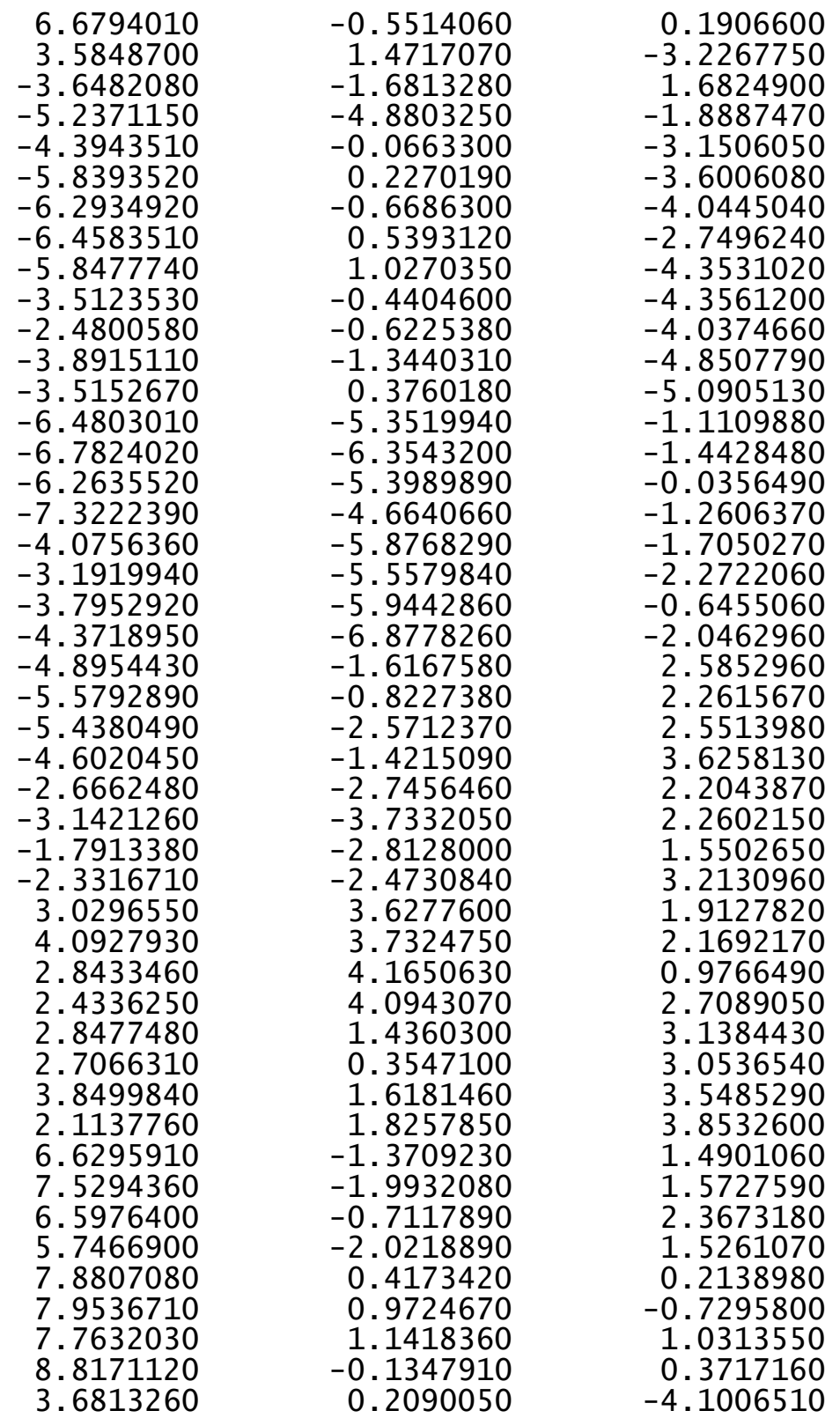

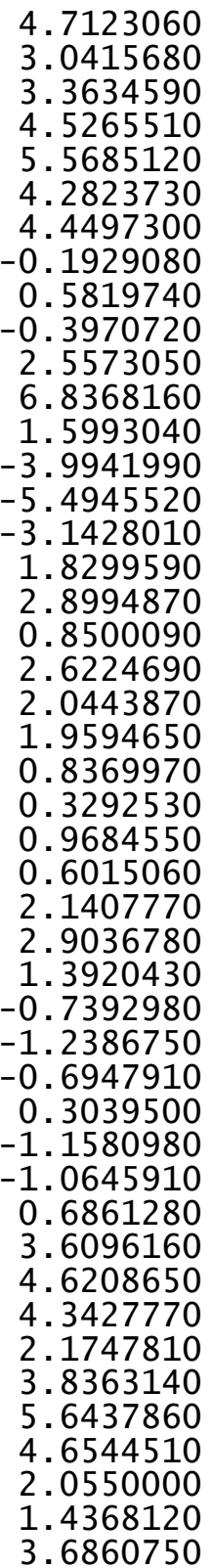
$-0.1641760$
$-0.5872240$
0.4462670
2. 5617500
2. 2172640
2.7807720
3.4902000
$-0.4227630$
$-0.6415380$
$-1.5692950$
1.8417220
$-1.2485250$
2. 0759610
0.8598550
$-4.8530140$
$-0.7142500$
$-3.5342850$
$-2.6460500$
$-3.3774610$
$-1.6785640$
$-2.6461290$
$-1.1659900$
$-0.9293640$
$-1.6903600$
$-1.6956970$
$-2.3325400$
$-3.5408220$
$-1.8128020$
0.4199130
0.6513340
$-0.0184100$
$-0.4864440$
0.9439090
0.1660460
$-0.6506780$
$-5.1498900$
$-4.4694780$
$-2.9626210$
$-4.9771920$
$-6.2188500$
$-4.6122420$
$-2.4284610$
$-5.5282560$
$-5.3733340$
$-4.7132390$

-4.1506400
-3.7121750
-5.1242320
-3.7790700
-3.7316460
-4.8272450
-3.2012940
-1.1557410
-2.4310710
-0.2171030
-3.3022820
-0.6453560
1.5389620
-2.7264200
-2.9571230
1.7528900
-1.5187530
-1.0550260
-1.0685010
-0.6478960
-2.6757390
2.5700390
4.3997500
3.1062970
2.1405580
1.1632800
4.3142790
1.6500800
-2.5809120
5.2821050
3.9815800
2.8773450
5.4923990
6.1337130
1.8759190
6.4960260
-2.3477740
-1.4122600
-1.2774080
-1.8195720
-2.4358590
-1.7796400
-2.1856310
-0.8764310
-2.5280930
-3.3524560 


\begin{tabular}{|c|c|c|c|c|c|c|c|}
\hline & & & & & & & \\
\hline $\mathrm{H}$ & 4.5627400 & -4.9352780 & -0.4188710 & C & -5.3739310 & -0.3391270 & -0.0473180 \\
\hline $\mathrm{H}$ & 4.9128160 & -2.5120400 & -0.4608270 & $\mathrm{H}$ & -5.5358330 & 0.4532260 & -2.0356320 \\
\hline 0 & -2.2563200 & 1.5090360 & 3.6964220 & $\mathrm{H}$ & -4.9378450 & -1.2792570 & 1.8236000 \\
\hline C & -2.8632610 & 2.2248700 & 4.7805440 & C & 4.0118250 & 0.8243880 & -0.1979220 \\
\hline $\mathrm{H}$ & -3.3029650 & 1.5292880 & 5.5095650 & $\mathrm{C}$ & 3.9257610 & 1.7016890 & 0.9079810 \\
\hline $\mathrm{H}$ & -2.1270830 & 2.8664040 & 5.2856230 & C & 4.5357430 & 1.2827830 & -1.4279990 \\
\hline $\mathrm{H}$ & -3.6415120 & 2.8375710 & 4.3221750 & $\mathrm{C}$ & 4.3890660 & 3.0155690 & 0.7594590 \\
\hline & & & & C & 4.9772570 & 2.6055720 & -1.5257230 \\
\hline & 5) $\mathrm{G}[\mathrm{B} 97-\mathrm{D} / \mathrm{C}$ & $Z V P]=-412$ & & C & 4.9132640 & 3.4894090 & -0.4446320 \\
\hline 0 & -0.4773840 & -0.7519340 & -0.3695470 & $\mathrm{H}$ & 4.3267180 & 3.6994170 & 1.6038470 \\
\hline 0 & 1.6953580 & -0.1275550 & -1.5342640 & $\mathrm{H}$ & 5.3851010 & 2.9527770 & -2.4726830 \\
\hline C & -0.5563190 & -1.9426180 & -1.0803290 & C & -2.7067860 & -2.7606540 & 1.2809580 \\
\hline C & 0.5942540 & -2.6964020 & -1.2825890 & C & -6.6267430 & 0.3466150 & 0.4700560 \\
\hline C & -1.8492740 & -2.3689050 & -1.4864010 & C & -3.4324500 & -0.6528500 & -3.3341590 \\
\hline C & 0.5059000 & -3.8423720 & -2.1399770 & C & 3.3511780 & 1.2672270 & 2.2516920 \\
\hline C & -1.9352150 & -3.5157380 & -2.2482130 & C & 5.3804750 & 4.9272860 & -0.5811640 \\
\hline $\mathrm{C}$ & -0.7823350 & -4.2458430 & -2.6333370 & $\mathrm{C}$ & 4.6792320 & 0.3768050 & -2.6445460 \\
\hline $\mathrm{H}$ & -2.9116690 & -3.8796610 & -2.5575590 & C & 6.1669540 & 0.1310960 & -2.9646060 \\
\hline C & 2.3548050 & -1.0278410 & -0.7040830 & $\mathrm{H}$ & 6.6661830 & 1.0751600 & -3.2199080 \\
\hline C & 3.5429360 & -0.5856150 & -0.0628610 & $\mathrm{H}$ & 6.6852490 & -0.3083330 & -2.1025240 \\
\hline C & 1.8524930 & -2.3180950 & -0.5881950 & $\mathrm{H}$ & 6.2647500 & -0.5525620 & -3.8186900 \\
\hline C & 4.2354380 & -1.5037990 & 0.7006890 & C & 3.9422950 & 0.9424000 & -3.8724560 \\
\hline C & 2.5326540 & -3.2376570 & 0.2801650 & $\mathrm{H}$ & 2.8799440 & 1.0913500 & -3.6500520 \\
\hline $\mathrm{C}$ & 3.7580670 & -2.8214380 & 0.9052630 & $\mathrm{H}$ & 4.3743060 & 1.9042580 & -4.1779260 \\
\hline $\mathrm{H}$ & 5.1607700 & -1.2016720 & 1.1846360 & $\mathrm{H}$ & 4.0307200 & 0.2463570 & -4.7173060 \\
\hline C & 3.9528590 & -4.9865890 & 2.0059740 & C & 4.5180500 & 5.6970050 & -1.6007410 \\
\hline C & 2.7206150 & -5.3822670 & 1.4305120 & $\mathrm{H}$ & 4.8353090 & 6.7469890 & -1.6563780 \\
\hline C & 2.0290150 & -4.5308330 & 0.5920750 & $\mathrm{H}$ & 4.6221570 & 5.2538310 & -2.5998890 \\
\hline C & 4.4535290 & -3.7268170 & 1.7522240 & $\mathrm{H}$ & 3.4571970 & 5.6640470 & -1.3215250 \\
\hline $\mathrm{H}$ & 4.4903200 & -5.6694850 & 2.6602370 & C & 04140 & 5.0095850 & -0.9634940 \\
\hline $\mathrm{H}$ & 2.3107930 & -6.3642 & 1.6566930 & $\mathrm{H}$ & 7.4941990 & 4.4829360 & -0.2300850 \\
\hline $\mathrm{H}$ & 1.0829300 & -4.8483020 & 0.1672350 & $\mathrm{H}$ & 7.0413440 & 4.5542550 & -1.9479700 \\
\hline H & 5.3848300 & -3.3967240 & 2.2092510 & $\mathrm{H}$ & 7.1949460 & 6.0575970 & -1.0118940 \\
\hline C & 1.52 & -5.6686440 & -3.3954220 & C & 4.4777730 & 0.9782490 & 3.2637330 \\
\hline C & 0.25 & -6.0825280 & -3.8571430 & $\mathrm{H}$ & 5.1603170 & 0.2045200 & 2.8931560 \\
\hline C & -0.877 & -5.3809030 & -3.4831890 & $\mathrm{H}$ & 5.0621310 & 1.8896290 & 3.4498560 \\
\hline C & 1.6480660 & -4.5792030 & -2.5558620 & $\mathrm{H}$ & 4.0520980 & 0.6390360 & 4.2180390 \\
\hline $\mathrm{H}$ & 2.4131970 & -6.2116790 & -3.7086670 & C & 2.3650730 & 2.2913040 & 2.8409230 \\
\hline $\mathrm{H}$ & 0.16 & -6.9462300 & -4.5129530 & $\mathrm{H}$ & 2.8800040 & 3.2129440 & 3.1410280 \\
\hline $\mathrm{H}$ & -1.8615950 & -5.6788620 & -3.8415170 & $\mathrm{H}$ & 1.5892180 & 2.5414890 & 2.1114960 \\
\hline $\mathrm{H}$ & 2.6288930 & -4.2681450 & -2.2096100 & $\mathrm{H}$ & 1.8859510 & 1.8658260 & 3.7315330 \\
\hline C & -3.0654020 & -1.6481490 & -1.0017030 & C & -3.1224700 & -4.2229110 & 1.0110050 \\
\hline C & -3.8127860 & -0.8212930 & -1.8682210 & $\mathrm{H}$ & -4.1907710 & -4.3531110 & 1.2316210 \\
\hline C & -3.4635910 & -1.8180630 & 0.3475900 & $\mathrm{H}$ & -2.9468620 & -4.5028300 & -0.0328600 \\
\hline C & -4.9560950 & -0.1822510 & -1.3709850 & $\mathrm{H}$ & -2.5501190 & -4.9034880 & 1.6560670 \\
\hline $\mathrm{C}$ & -4.6138320 & -1.1574360 & 0.7930730 & C & -2.8755420 & -2.4474580 & 2.7742360 \\
\hline
\end{tabular}




\begin{tabular}{|c|c|c|}
\hline $\begin{array}{l}-2.6428540 \\
-3.8989410 \\
-2.1943700 \\
-7.8812750 \\
-8.7810260 \\
-7.8117460 \\
-7.9932120 \\
-6.5090880 \\
-5.6297600 \\
-6.4160130 \\
-7.4045070 \\
-3.5848100 \\
-4.6378620 \\
-3.0236760 \\
-3.2016810 \\
-4.2688460 \\
-5.3352050 \\
-3.9739690 \\
-4.1384900 \\
0.2903280 \\
-0.3879920 \\
0.4689640 \\
-2.3767580 \\
-6.7408390 \\
-1.6374350 \\
4.2334230 \\
5.2558030 \\
2.7969390 \\
-1.1912280 \\
-2.4540360 \\
-0.3259760 \\
-2.4033840 \\
-1.3955520 \\
-2.1041370 \\
-1.4565150 \\
-0.7628810 \\
-1.1490400 \\
-0.6423730 \\
-2.5990920 \\
-2.7534100 \\
-0.9309560 \\
-0.2204080 \\
0.4622320 \\
0.1911670 \\
-1.1837980 \\
-0.0105410\end{array}$ & $\begin{array}{r}-1.4017630 \\
-2.6570170 \\
-3.0790910 \\
-0.1315020 \\
0.3425950 \\
0.1367830 \\
-1.2207680 \\
1.8796200 \\
2.2345030 \\
2.2023460 \\
2.3535260 \\
0.7902320 \\
1.1004260 \\
1.4891440 \\
0.8594090 \\
-1.6057260 \\
-1.3553210 \\
-1.5103240 \\
-2.6500850 \\
0.5324250 \\
0.9838620 \\
1.5092620 \\
-0.9255390 \\
0.0673190 \\
-2.6744580 \\
-0.5960250 \\
5.4088330 \\
0.3365080 \\
3.9570070 \\
3.2400730 \\
3.5640660 \\
2.1962260 \\
3.2656650 \\
2.3667300 \\
0.6005650 \\
0.5246160 \\
1.5133710 \\
1.6096220 \\
1.9289170 \\
3.6719090 \\
2.3137740 \\
-1.3053550 \\
-1.3760760 \\
-0.4570520 \\
-0.3174550 \\
-2.0211850\end{array}$ & $\begin{array}{r}2.9954500 \\
3.1130680 \\
3.3561480 \\
-0.2860190 \\
0.1283950 \\
-1.3485970 \\
-0.2143650 \\
0.3965660 \\
0.9492950 \\
-0.6485810 \\
0.8202410 \\
-3.8438610 \\
-3.8599910 \\
-3.2188250 \\
-4.8700460 \\
-4.2129290 \\
-4.1279980 \\
-5.2667800 \\
-3.9080760 \\
-1.0430990 \\
-2.3121400 \\
0.0755550 \\
-3.4425140 \\
1.5270490 \\
1.0577410 \\
-2.4150110 \\
0.3992480 \\
2.0998640 \\
-1.1765530 \\
-0.9720240 \\
-0.6445140 \\
-0.6803670 \\
-2.4646010 \\
2.6629120 \\
4.3539210 \\
3.1288960 \\
2.2394770 \\
1.3296290 \\
4.2972540 \\
1.8271720 \\
-2.4087960 \\
5.1425570 \\
3.9090580 \\
2.8880630 \\
5.3661080\end{array}$ \\
\hline
\end{tabular}
$\mathrm{H} \quad 0.7056350$
$-2.5278250$
$-3.7585920$
$-3.7748250$
$-1.2305300$
$-2.5490950$
$-4.6827030$
$-4.0440420$
$-1.1403690$
$-0.3450930$
$-2.5438170$
$-3.7455940$
$-4.5258410$
1. 4081590
1.7224930
2. 1171520
0.8335420

2. 4839450
$-0.5160360$
$-0.2771110$
5.9701180
5. 3903070
3.8527390
5.4635250
7.0654800
5.7753600
3.5086610
5.8714180
5.7863660
5.6778670
5.7059300
3. 4274170
$-2.3084420$
$-3.3029460$
$-2.8374400$
$-3.9000910$

1.9368480

6. 3160030

$-1.9674480$

$-1.2532990$

$-1.3121740$

$-1.3136280$

$-1.9283790$

$-1.6995580$

$-2.3211570$

$-0.2972540$

$-1.8754020$

$-3.0257850$

$-0.2010370$

$-0.6402370$

3. 6089080

4. 5925180

5.5069710

4.8407250

148

TS21 (S, S ) G[B97-D/def2-TZVP $]=-4125.389128$

\begin{tabular}{lrlr}
$\mathrm{O}$ & 0.2391190 & 1.0219830 & -0.1008670 \\
$\mathrm{O}$ & -1.7522990 & 0.1653760 & -1.4169620 \\
$\mathrm{C}$ & 0.1196520 & 2.2963340 & -0.6480430 \\
$\mathrm{C}$ & -1.1462700 & 2.8538360 & -0.7955460 \\
$\mathrm{C}$ & 1.3230540 & 2.9755930 & -0.9808660 \\
$\mathrm{C}$ & -1.2531620 & 4.1026850 & -1.4932060 \\
$\mathrm{C}$ & 1.2089090 & 4.2199480 & -1.5665020 \\
$\mathrm{C}$ & -0.0509740 & 4.7919180 & -1.8736190 \\
$\mathrm{H}$ & 2.1073840 & 4.7796600 & -1.8111980 \\
$\mathrm{C}$ & -2.5740020 & 0.8225810 & -0.5124930 \\
$\mathrm{C}$ & -3.6755110 & 0.0945410 & 0.0124070 \\
$\mathrm{C}$ & -2.3291290 & 2.1604120 & -0.2204310 \\
$\mathrm{C}$ & -4.5575100 & 0.7659490 & 0.8332390 \\
$\mathrm{C}$ & -3.2027620 & 2.8228230 & 0.7088740 \\
$\mathrm{C}$ & -4.3466620 & 2.1142330 & 1.2138260 \\
$\mathrm{H}$ & -5.4286090 & 0.2428640 & 1.2201570 \\
$\mathrm{C}$ & -4.9734910 & 4.0432120 & 2.5639270 \\
$\mathrm{C}$ & -3.8218400 & 4.7278800 & 2.1053530 \\
$\mathrm{C}$ & -2.9609580 & 4.1342870 & 1.2041300 \\
$\mathrm{C}$ & -5.2235190 & 2.7589670 & 2.1279740 \\
$\mathrm{H}$ & -5.6461190 & 4.5244640 & 3.2701660 \\
$\mathrm{H}$ & -3.6070350 & 5.7284810 & 2.4738800 \\
$\mathrm{H}$ & -2.0765360 & 4.6699860 & 0.8776850 \\
$\mathrm{H}$ & -6.0884790 & 2.2070040 & 2.4912930 \\
$\mathrm{C}$ & -2.5618800 & 5.8753400 & -2.5389640 \\
& & & \\
\hline
\end{tabular}




$\begin{array}{rr}-1.3763260 & 6.5722610 \\ -0.1472020 & 6.0360700 \\ -2.5016470 & 4.6734930 \\ -3.5286770 & 6.2884530 \\ -1.4386400 & 7.5213450 \\ 0.7728620 & 6.5503590 \\ -3.4165200 & 4.1470320 \\ 2.6460580 & 2.3586850 \\ 3.4715190 & 1.8562810 \\ 3.0612250 & 2.2679850 \\ 4.6794010 & 1.2270940 \\ 4.2755670 & 1.6439900 \\ 5.0942450 & 1.0976780 \\ 5.3047190 & 0.8327900 \\ 4.6061940 & 1.5687960 \\ -3.8829280 & -1.3451280 \\ -3.5086130 & -2.3514260 \\ -4.4552960 & -1.6940870 \\ -3.7576080 & -3.6868270 \\ -4.6791820 & -3.0481930 \\ -4.3425400 & -4.0571560 \\ -3.4741110 & -4.4667830 \\ -5.1244330 & -3.3228870 \\ 2.2386560 & 2.9062580 \\ 6.3812620 & 0.3928720 \\ 3.1276460 & 2.0348870 \\ -2.8489070 & -2.0067420 \\ -4.5801910 & -5.5192120 \\ -4.8414840 & -0.6516910 \\ -6.3639770 & -0.6410580 \\ -6.6996610 & -1.6099810 \\ -6.9031880 & -0.4445380 \\ -6.6308820 & 0.1360190 \\ -4.0839830 & -0.8735190 \\ -3.0007030 & -0.8548970 \\ -4.3518170 & -1.8422430 \\ -4.3434070 & -0.0861030 \\ -5.5370770 & -6.1804350 \\ -5.7249110 & -7.2262030 \\ -5.0981860 & -6.1673640 \\ -6.4966980 & -5.6493250 \\ -3.2512310 & -6.2971310 \\ -2.5736470 & -5.8499900 \\ -2.7492020 & -6.2838910 \\ -3.4344800 & -7.3437340 \\ -3.8957160 & -1.8565530\end{array}$

-2.8793600
-2.5547930
-1.8616460
-2.8182620
-3.4070120
-2.8268190
-1.6085680
-0.6607400
-1.6875490
0.6948960
-1.3418380
0.9814220
-0.0174330
-2.1363500
2.0136650
-0.3292640
0.5941910
-1.5681610
0.2653220
-1.8530140
-0.9511650
0.9672070
-2.8082180
1.8123170
0.3944100
-3.1613830
1.9245360
-1.2849650
-2.6090530
-2.8443530
-3.2367200
-1.9086820
-3.5730750
-3.9321730
-3.7653450
-4.3736270
-4.6523690
-0.2747490
-0.5523710
0.7316560
-0.2363740
-1.3566920
-2.0951810
-0.3803060
-1.6343920
3.0456700
$-4.6389170$
$-4.4199100$
$-3.4040460$
$-1.7718540$
$-2.2180760$
$-1.0470220$
$-1.2419950$
2. 3983720
3. 4454750
2. 1081480
1.7733540
2. 5657140
2. 5270280
3. 5639430
1. 8373830
7.2843540
8.2074070
6.7825830
7. 5509910
6.0668720
5. 4659400
5. 5002860
6. 9971120
3. 4375680
4.5188960
2. 9687130
3. 0557390
3. 8774560
4.9612460
3. 5920780
3. 6612630
$-0.2265070$
0.5061390
$-0.1625240$
2. 0523620
6. 9436500
1.1824030
$-4.5624490$
$-5.0515240$
$-2.3493230$
1.7964620
2. 9312460
0.8895610
2. 7211660
1.8710790
2. 6408750

-1.0905620
-2.8095490
-1.5767650
-3.0169950
-3.9803230
-3.1815440
-2.6280520
4.4420030
4.7104590
4.8599950
4.8995150
2.3876700
1.2953950
2.7160320
2.7901790
-0.0084940
-0.4632170
-0.7444920
0.8570440
-0.8403420
-0.5671430
-1.5849020
-1.3079790
0.8035660
0.6305210
-0.0952460
0.9676870
3.2593600
3.0839360
3.4297370
4.1676070
-0.2505030
-0.2728230
-1.4445430
2.2259410
1.1000160
2.6801680
0.3387330
-5.5592100
-1.0396080
-3.2644460
-2.4459290
-3.1795060
-1.5587200
-2.2090460
-2.6942390

2.8004230

3. 1991140

3.9875380

2. 3555320

2.6342280

1. 5526310

3. 2330960

1.7862660

1.9817280

0.8170920

2. 5651610

3. 2198530

3. 2698410

3. 5393020

3.9331080

$-0.7789750$

$-0.3996090$

$-1.4212780$

$-1.3984560$

1. 2675440

2. 1422500

0.6941000

1. 6162260

$-4.0290970$

$-4.1073440$

$-3.6235780$

$-5.0449710$

$-3.7281300$

$-3.6895800$

$-4.7751040$

$-3.1548790$

$-1.0218980$

$-2.3408660$

$-0.1257500$

$-3.2397000$

1.0241520

1.6235340

$-2.2338870$

$-2.2772820$

1.7995770

$-2.0401490$

$-1.6061160$

$-1.4410300$

$-1.0178850$

$-3.0657230$

2.0599270 


\begin{tabular}{|c|c|c|c|c|c|c|c|}
\hline c & 2.0222660 & -1.4221030 & 4.1534230 & C & -2.7197010 & 3.0279770 & 0.4425310 \\
\hline $\begin{array}{l}\mathrm{C} \\
\mathrm{N}\end{array}$ & $\begin{array}{l}1.2340910 \\
1.6109740\end{array}$ & $\begin{array}{l}-1.1172660 \\
-1.8328480\end{array}$ & $\begin{array}{l}3.0309620 \\
1.9070130\end{array}$ & $\begin{array}{l}\mathrm{C} \\
\mathrm{H}\end{array}$ & $\begin{array}{l}-3.9961630 \\
-5.3668170\end{array}$ & $\begin{array}{l}2.5194520 \\
0.8503430\end{array}$ & $\begin{array}{l}0.8621610 \\
0.7793480\end{array}$ \\
\hline $\mathrm{H}$ & 1.0471560 & -1.7530270 & 1.0317340 & C & -4.3872560 & 4.5335860 & 2.1763390 \\
\hline $\mathrm{S}$ & 3.2317030 & -2.6094120 & 3.7153330 & $\mathrm{C}$ & -3.1036150 & 5.0098180 & 1.8148830 \\
\hline $\mathrm{S}$ & 3.2936050 & -3.7236180 & 0.9047860 & C & -2.2923950 & 4.2768440 & 0.9717930 \\
\hline $\mathrm{H}$ & 1.2508270 & -1.3942160 & -2.7585750 & $\mathrm{C}$ & -4.8166280 & 3.3068000 & 1.7148430 \\
\hline C & 0.7146020 & 0.1100790 & 5.4560970 & $\mathrm{H}$ & -5.0206950 & 5.1253450 & 2.8334460 \\
\hline $\mathrm{C}$ & -0.0659050 & 0.4195650 & 4.3187320 & $\mathrm{H}$ & -2.7507740 & 5.9606760 & 2.2082600 \\
\hline $\mathrm{C}$ & 0.1860900 & -0.1956410 & 3.0887300 & $\mathrm{H}$ & -1.3110740 & 4.6575350 & 0.7092720 \\
\hline C & 1.7594970 & -0.8035640 & 5.3810670 & $\mathrm{H}$ & -5.7864830 & 2.9101540 & 2.0102120 \\
\hline $\mathrm{H}$ & 0.4903950 & 0.6128230 & 6.3928030 & C & -1.5671090 & 5.9477200 & -2.7616220 \\
\hline $\mathrm{H}$ & -0.3797000 & 0.0221530 & 2.1929870 & $\mathrm{C}$ & -0.2874450 & 6.4728690 & -3.0681300 \\
\hline $\mathrm{H}$ & 2.3569310 & -1.0269170 & 6.2604460 & c & 0.8457750 & 5.7721190 & -2.7102070 \\
\hline C & 3.4207150 & -4.7177170 & -3.3459670 & $\mathrm{C}$ & -1.6928350 & 4.7503410 & -2.0855110 \\
\hline C & 4.5652510 & -4.1735170 & -2.4758610 & $\mathrm{H}$ & -2.4593910 & 6.4902780 & -3.0662960 \\
\hline $\mathrm{C}$ & 4.3278740 & -2.7067440 & -2.0747360 & $\mathrm{H}$ & -0.2025550 & 7.4207830 & -3.5947710 \\
\hline $\mathrm{C}$ & 2.0760070 & -4.6417180 & -2.6007770 & $\mathrm{H}$ & 1.8355780 & 6.1549580 & -2.9528820 \\
\hline $\mathrm{H}$ & 3.6218280 & -5.7592720 & -3.6224810 & $\mathrm{H}$ & -2.6787520 & 4.3566640 & -1.8596130 \\
\hline $\mathrm{H}$ & 5.5214220 & -4.2489860 & -3.0064780 & C & 3.0655380 & 1.8165690 & -0.6621200 \\
\hline $\mathrm{H}$ & 4.5164450 & -2.0369210 & -2.9256650 & $\mathrm{C}$ & 3.9423770 & 1.2712670 & -1.6214670 \\
\hline $\mathrm{H}$ & 2.0809850 & -5.3316510 & -1.7457020 & $\mathrm{C}$ & 3.3800060 & 1.7199410 & 0.7201600 \\
\hline $\mathrm{H}$ & 1.2419140 & -4.9318780 & -3.2517180 & c & 5.1155230 & 0.6306690 & -1.1880540 \\
\hline $\mathrm{H}$ & 3.3572080 & -4.1370680 & -4.2757690 & C & 4.5645710 & 1.0844590 & 1.0942910 \\
\hline $\mathrm{H}$ & 4.6472050 & -4.7837870 & -1.5661000 & $\mathrm{C}$ & 5.4508250 & 0.5259860 & 0.1615120 \\
\hline $\mathrm{H}$ & 5.0137030 & -2.3875430 & -1.2830380 & $\mathrm{H}$ & 5.7809440 & 0.2165740 & -1.9385090 \\
\hline 0 & -1.0431950 & 1.3475650 & 4.5116450 & H & 4.8193190 & 1.0262090 & 2.1480540 \\
\hline C & -1.8036860 & 1.7431380 & 3.3579940 & C & -4.0053790 & -0.9642020 & -0.6956700 \\
\hline $\mathrm{H}$ & -1.1436590 & 2.1566840 & 2.5856840 & $\mathrm{C}$ & -4.0120550 & -1.9694340 & 0.3020410 \\
\hline $\mathrm{H}$ & -2.3560210 & 0.8904250 & 2.9433570 & $\mathrm{C}$ & -4.3813500 & -1.2775270 & -2.0191970 \\
\hline $\mathrm{H}$ & -2.5029140 & 2.5079700 & 3.6999330 & $\mathrm{C}$ & -4.4102280 & -3.2630070 & -0.0496800 \\
\hline & & & & C & -4.7640790 & -2.5905780 & -2.3211150 \\
\hline \multicolumn{4}{|c|}{$\operatorname{TS} 22(\mathrm{~S}, \mathrm{~S}) \mathrm{G}[\mathrm{B} 97-\mathrm{D} / \mathrm{def} 2-\mathrm{TZVP}]=-4125.386943$} & $\mathrm{C}$ & -4.7838250 & -3.5974950 & -1.3549460 \\
\hline 0 & 0.4635550 & 0.7493060 & -0.2730260 & $\mathrm{H}$ & -4.4160750 & -4.0366970 & 0.7146490 \\
\hline 0 & -1.6031910 & 0.1961730 & -1.6566730 & $\mathrm{H}$ & -5.0574960 & -2.8361270 & -3.3407460 \\
\hline C & 0.5377750 & 2.0286700 & -0.8100980 & C & 2.4943570 & 2.3710420 & 1.7809160 \\
\hline $\mathrm{C}$ & -0.6327090 & 2.7578760 & -0.9916390 & $\mathrm{C}$ & 6.7442040 & -0.1191590 & 0.6560200 \\
\hline $\mathrm{C}$ & 1.8321720 & 2.5502360 & -1.0789440 & $\mathrm{C}$ & 3.6952970 & 1.4067690 & -3.1199140 \\
\hline C & -0.5463170 & 4.0111800 & -1.6848790 & $\mathrm{C}$ & -3.6020350 & -1.6914590 & 1.7435520 \\
\hline C & 1.9077070 & 3.7943570 & -1.6727510 & C & -5.1756960 & $-\overline{5} .0194870$ & -1.7149000 \\
\hline C & 0.7496010 & 4.5279570 & -2.0290260 & $\mathrm{C}$ & -4.4285490 & -0.2310330 & -3.1248850 \\
\hline $\mathrm{H}$ & 2.8837010 & 4.2271560 & -1.8758180 & $\mathrm{C}$ & -5.8832790 & 0.0276870 & -3.5648140 \\
\hline C & -2.3637510 & 0.9682720 & -0.7865820 & $\mathrm{H}$ & -6.3290960 & -0.8869850 & -3.9773150 \\
\hline C & -3.5997090 & 0.4268150 & -0.3391680 & $\mathrm{H}$ & -6.4965010 & 0.3548960 & -2.7152350 \\
\hline C & -1.9217580 & 2.2442960 & -0.4583230 & H & -5.9132300 & 0.8056910 & -4.3394960 \\
\hline C & -4.4038040 & $\begin{array}{l}1.2292050 \\
\text {. }\end{array}$ & 0.4452160 & C & -3.5559130 & -0.6271020 & -4.3300600 \\
\hline
\end{tabular}




$\begin{array}{rrr}-2.5177670 & -0.7875380 & -4.0188790 \\ -3.9290740 & -1.5486500 & -4.7953730 \\ -3.5776670 & 0.1686610 & -5.0865220 \\ -6.3880100 & -5.5017490 & -0.8962780 \\ -6.6714470 & -6.5196260 & -1.1955320 \\ -6.1462350 & -5.5157250 & 0.1746390 \\ -7.2505710 & -4.8393610 & -1.0435780 \\ -3.9853830 & -5.9824060 & -1.5362890 \\ -3.1248220 & -5.6564210 & -2.1340820 \\ -3.6765070 & -6.0150850 & -0.4831200 \\ -4.2647450 & -6.9989120 & -1.8443300 \\ -4.8380690 & -1.6211280 & 2.6610920 \\ -5.5400290 & -0.8493360 & 2.3227210 \\ -5.3644690 & -2.5852520 & 2.6646410 \\ -4.5341530 & -1.3890130 & 3.6909580 \\ -2.5955580 & -2.7241750 & 2.2819640 \\ -3.0536980 & -3.7177520 & 2.3702320 \\ -1.7273420 & -2.7948690 & 1.6192450 \\ -2.2535520 & -2.4186010 & 3.2786130 \\ 2.7284000 & 3.8965690 & 1.8191720 \\ 3.7651920 & 4.1066540 & 2.1156620 \\ 2.5454600 & 4.3555930 & 0.8421230 \\ 2.0577230 & 4.3618610 & 2.5543460 \\ 2.6734430 & 1.7917640 & 3.1921080 \\ 2.6250580 & 0.6990510 & 3.1870210 \\ 3.6381420 & 2.0896830 & 3.6241340 \\ 1.8788650 & 2.1689210 & 3.8461860 \\ 7.6327090 & -0.6806750 & -0.4625900 \\ 8.5546880 & -1.0903820 & -0.0326890 \\ 7.1206410 & -1.4889930 & -1.0018280 \\ 7.9048530 & 0.0937030 & -1.1903040 \\ 6.4782390 & -1.2095010 & 1.7157540 \\ 5.9108700 & -0.8169040 & 2.5664530 \\ 5.9045100 & -2.0420740 & 1.2901240 \\ 7.4313030 & -1.6050010 & 2.0904150 \\ 3.8995390 & 0.0923770 & -3.8931050 \\ 4.9465680 & -0.2362360 & -3.8541280 \\ 3.2689250 & -0.7045760 & -3.4915240 \\ 3.6383800 & 0.2430330 & -4.9486220 \\ 4.6052010 & 2.5038080 & -3.7107360 \\ 5.6603140 & 2.2222480 & -3.5923540 \\ 4.4000680 & 2.6322630 & -4.7820610 \\ 4.4525980 & 3.4663080 & -3.2087460 \\ -0.1937650 & -0.4554200 & -1.1724400 \\ 0.5759050 & -0.6765510 & -2.4508310 \\ -0.3643370 & -1.5914370 & -0.2147150\end{array}$

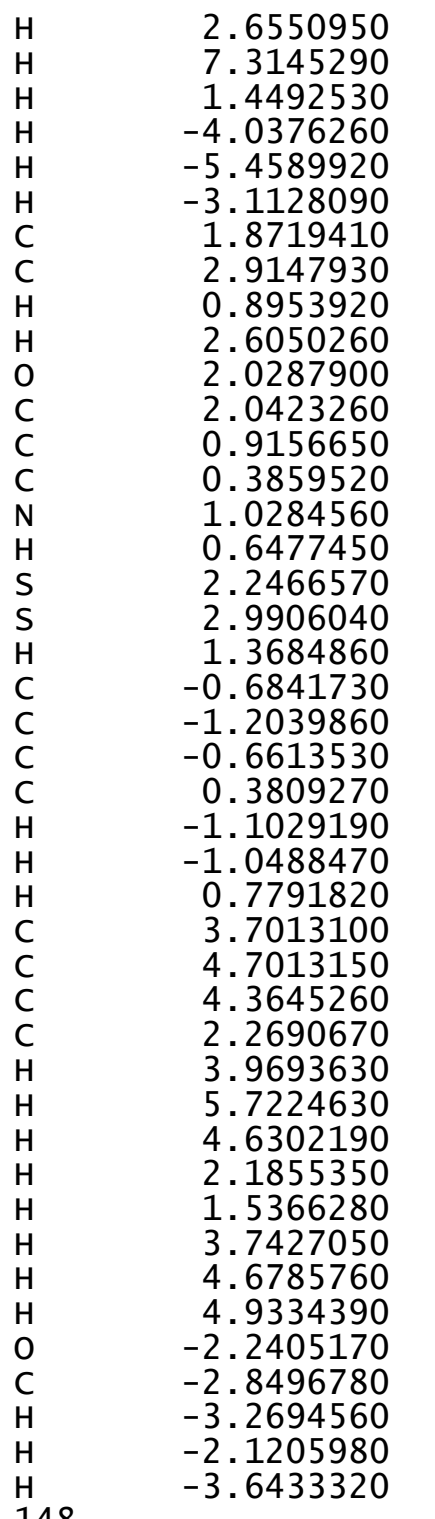

1.7137720
0.6782290
2.2046540
0.7129740
-5.0243160
-0.7141650
-3.5481890
-2.6073030
-3.4165990
-1.6424290
-2.6861730
-2.3917630
-1.0354730
-0.8424920
-1.6133640
-1.6546970
-2.1724600
-3.4371160
-1.8574490
0.5375310
0.7264810
0.0390790
-0.3411810
1.0742190
0.1893760
-0.4720860
-5.1146790
-4.3669310
-2.8693320
-4.9839210
-6.1758790
-4.4811310
-2.3555210
-5.5135740
-5.4280860
-4.7052780
-4.8025940
-2.3633710
1.5580040
2.2890350
1.6036500
2.9559290
2.8764770
-1

$-3.2701580$

1.1590090

1. 5012990

$-2.7327180$

$-2.7770500$

1.7795430

$-1.5015640$

$-1.0838710$

$-1.0362160$

$-0.6953340$

$-2.6867690$

2. 5609650

4. 3735530

3.0816470

2. 1264410

1.1553390

4. 2977980

1. 6518010

$-2.5963270$

5. 2373520

3.9380810

2.8443910

5.4561150

6.0811120

1.8441080

6.4583800

$-2.3184280$

$-1.4223950$

$-1.3304230$

$-1.7701710$

$-2.3794920$

$-1.8043340$

$-2.2647560$

$-0.8111490$

$-2.4555950$

$-3.3365170$

$-0.4140940$

$-0.5455070$

3.6432550

4.7157570

5.4658490

5.1977000

$\begin{array}{llll}14823(\mathrm{~S}, \mathrm{~S}) \mathrm{G}[\mathrm{B} 97-\mathrm{D} / \mathrm{def} 2-\mathrm{TZVP}]=-4125.384887 & \\ \mathrm{~T} & 0.3112420 & 0.8448500 & 0.0327760\end{array}$

0.0327760 


$\begin{array}{rrrr}\mathrm{O} & -1.6860370 & 0.2068710 & -1.4034150 \\ \mathrm{C} & 0.2933750 & 2.1694340 & -0.3920110 \\ \mathrm{C} & -0.9325280 & 2.8155200 & -0.5174930 \\ \mathrm{C} & 1.5441510 & 2.7975140 & -0.6415850 \\ \mathrm{C} & -0.9449850 & 4.1348600 & -1.0844160 \\ \mathrm{C} & 1.5178560 & 4.1119560 & -1.0671930 \\ \mathrm{C} & 0.3067200 & 4.7942550 & -1.3333540 \\ \mathrm{H} & 2.4567980 & 4.6296720 & -1.2436160 \\ \mathrm{C} & -2.5206020 & 0.8726410 & -0.5214890 \\ \mathrm{C} & -3.7624050 & 0.2449620 & -0.2223010 \\ \mathrm{C} & -2.1867690 & 2.1490550 & -0.0767580 \\ \mathrm{C} & -4.6531100 & 0.9239580 & 0.5794000 \\ \mathrm{C} & -3.0862050 & 2.8058800 & 0.8345680 \\ \mathrm{C} & -4.3453050 & 2.1868040 & 1.1399440 \\ \mathrm{H} & -5.6143170 & 0.4716170 & 0.8086130 \\ \mathrm{C} & -4.9141770 & 4.0214530 & 2.6378540 \\ \mathrm{C} & -3.6542990 & 4.6127050 & 2.3760970 \\ \mathrm{C} & -2.7670280 & 4.0223200 & 1.4987570 \\ \mathrm{C} & -5.2464640 & 2.8279420 & 2.0323060 \\ \mathrm{H} & -5.6062810 & 4.4997800 & 3.3270100 \\ \mathrm{H} & -3.3774430 & 5.5367980 & 2.8786070 \\ \mathrm{H} & -1.8011260 & 4.4835420 & 1.3242300 \\ \mathrm{H} & -6.1984600 & 2.3437000 & 2.2420630 \\ \mathrm{C} & -2.1065710 & 6.0785820 & -1.9932660 \\ \mathrm{C} & -0.8725200 & 6.7456790 & -2.1919110 \\ \mathrm{C} & 0.3091780 & 6.1100540 & -1.8726990 \\ \mathrm{C} & -2.1418080 & 4.8089080 & -1.4519330 \\ \mathrm{H} & -3.0356610 & 6.5688230 & -2.2757010 \\ \mathrm{H} & -0.8601080 & 7.7493580 & -2.6108860 \\ \mathrm{H} & 1.2671640 & 6.5977390 & -2.0440360 \\ \mathrm{H} & -3.0946870 & 4.3091330 & -1.3126150 \\ \mathrm{C} & 2.8415920 & 2.0741960 & -0.4809840 \\ \mathrm{C} & 3.6543260 & 1.8339650 & -1.6113460 \\ \mathrm{C} & 3.2811470 & 1.6586050 & 0.8055990 \\ \mathrm{C} & 4.8902800 & 1.1886740 & -1.4382400 \\ \mathrm{C} & 4.5025920 & 0.9916840 & 0.9130470 \\ \mathrm{C} & 5.3330190 & 0.7543640 & -0.1920340 \\ \mathrm{H} & 5.5114960 & 1.0238330 & -2.3131080 \\ \mathrm{H} & 4.8419990 & 0.6612400 & 1.8907000 \\ \mathrm{C} & -4.0946730 & -1.1063150 & -0.7673660 \\ \mathrm{C} & -3.7343420 & -2.2525330 & -0.0151120 \\ \mathrm{C} & -4.7674490 & -1.2461800 & -2.0011160 \\ \mathrm{C} & -4.0473390 & -3.5184410 & -0.5174760 \\ \mathrm{C} & -5.0481460 & -2.5435470 & -2.4604170 \\ \mathrm{C} & -4.6965120 & -3.6870880 & -1.7441640 \\ \mathrm{H} & -3.7714650 & -4.3997610 & 0.0543640\end{array}$

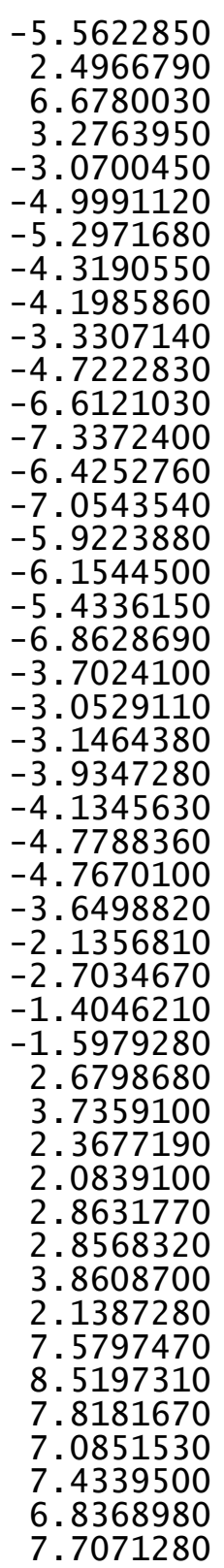

$-3.4146810$

2.0630480

0.0283670

$-3.0201990$

1. 3513890

$-2.2826760$

$-2.8584320$

$-3.1726670$

$-2.3213060$

$-3.4562040$

$-4.0085050$

$-2.2776280$

$-2.0964510$

$-1.3310870$

$-2.9811700$

$-1.3329390$

$-1.7559070$

$-0.3614650$

$-1.1625570$

$-2.5509690$

$-3.2517700$

$-1.6161760$

$-2.9745190$

2. 4556670

2. 2691130

2. 4998530

3.4330690

1.7146680

1.8961550

0.9213090

2. 6382460

2. 3781830

2. 5966760

1.5361800

3. 2590060

3. 3035110

3. 0887350

3.6765730

4.1024770

0.9347470

1. 1496870

0.4296650

1.8845820

$-1.2603140$

$-1.9283620$

$-1.8063950$ 


\begin{tabular}{|c|c|c|}
\hline $\begin{array}{l}8.3583090 \\
3.4139030 \\
4.4581810 \\
2.8110380 \\
3.0734440 \\
4.1288690 \\
5.1904130 \\
3.8258570 \\
4.0244040 \\
0.1892520 \\
0.5646140 \\
0.1823680 \\
2.2269620 \\
6.4768910 \\
1.4335050 \\
5.5529130 \\
5.5241810 \\
2.4598260 \\
2.0703350 \\
3.0572960 \\
1.0860970 \\
2.6921480 \\
2.1900720 \\
2.3623840 \\
1.4513350 \\
0.7965730 \\
1.3228310 \\
0.8678370 \\
2.7424760 \\
3.2023490 \\
1.4629470 \\
0.0019850 \\
0.6479790 \\
0.2512400 \\
1.0483250 \\
0.3380680 \\
0.7070230 \\
1.5450040 \\
3.9800560 \\
4.9438170 \\
4.5202290 \\
2.5470470 \\
4.3097470 \\
5.9649480 \\
4.7528420 \\
2.5039670\end{array}$ & $\begin{array}{r}-0.8141990 \\
1.1594760 \\
0.8391420 \\
0.2914610 \\
1.5260340 \\
3.4954030 \\
3.2164440 \\
3.8440080 \\
4.3274750 \\
-0.3070650 \\
-0.2396330 \\
-1.5793790 \\
2.5898150 \\
-0.8654530 \\
1.8583790 \\
-0.5477010 \\
-4.9486860 \\
-1.2094390 \\
-3.1336840 \\
-2.2800280 \\
-3.1759690 \\
-1.4710230 \\
-1.9869430 \\
-2.9555600 \\
-1.9915290 \\
-1.5480490 \\
-2.1070760 \\
-1.9341700 \\
-3.0880850 \\
-3.7968980 \\
-1.2546040 \\
-0.6162900 \\
-0.1638870 \\
-0.6261780 \\
-1.5239440 \\
-0.2312300 \\
-0.2893050 \\
-1.8555690 \\
-4.3255770 \\
-3.7807390 \\
-2.3855670 \\
-4.4277190 \\
-5.3164360 \\
-3.7258590 \\
-1.6217870 \\
-5.1917630\end{array}$ & $\begin{array}{l}-1.0127260 \\
-4.0650880 \\
-4.1740880 \\
-3.7883930 \\
-5.0423590 \\
-3.4434770 \\
-3.4838440 \\
-4.4399440 \\
-2.7370870 \\
-1.0147870 \\
-2.3201360 \\
-0.2316940 \\
-3.0181870 \\
0.5716320 \\
1.8678590 \\
-3.8254320 \\
-3.2401610 \\
1.3304820 \\
-2.0625550 \\
-1.3969550 \\
-1.5953000 \\
-0.7723900 \\
-2.9770570 \\
2.1707450 \\
4.3186730 \\
3.1574000 \\
2.0041230 \\
1.0805470 \\
3.8830550 \\
0.9854110 \\
-2.6999300 \\
5.6395050 \\
4.4657540 \\
3.2055200 \\
5.5738130 \\
6.5982920 \\
2.2837840 \\
6.4812430 \\
-3.2396880 \\
-2.1734520 \\
-1.6829610 \\
-2.6867430 \\
-3.5734930 \\
-2.5686860 \\
-2.4371250 \\
-1.8981010\end{array}$ \\
\hline
\end{tabular}

$\begin{array}{lr}\mathrm{H} & 1.8374210 \\ \mathrm{H} & 3.9874350 \\ \mathrm{H} & 4.9618120 \\ \mathrm{H} & 5.0630530 \\ \mathrm{O} & -1.6520310 \\ \mathrm{C} & -2.2741970 \\ \mathrm{H} & -1.5306900 \\ \mathrm{H} & -2.7686790 \\ \mathrm{H} & -3.0129260 \\ \mathrm{H} & \end{array}$
$-4.7231740$
$-3.6643440$
$-4.4697750$
$-2.0855240$
0.7334280
1. 2769880
1. 7785670
0.4879200
1.9992360
$-3.4695590$
$-4.1163220$
$-1.3178690$
$-0.7817220$
4.6594500
3.4835800
2.8532750
2. 9039580

TS24(S, S) G[B97-D/def2-TZVP $]=-4125.384660$

$\begin{array}{lrlr}\text { TS24 }(\mathrm{S}, \mathrm{S}) \mathrm{G}[\mathrm{B} 97-\mathrm{D} / \mathrm{de} \text { ef }-\mathrm{TZVP}]=-4125.384660 \\ \mathrm{O} & 0.3510130 & 0.8383840 & 0.0806910 \\ \mathrm{O} & -1.6401940 & 0.3965070 & -1.4351020 \\ \mathrm{C} & 0.3744310 & 2.1942570 & -0.2332320 \\ \mathrm{C} & -0.8309270 & 2.8873260 & -0.3086390 \\ \mathrm{C} & 1.6433880 & 2.8094660 & -0.4139570 \\ \mathrm{C} & -0.7985640 & 4.2563730 & -0.7432770 \\ \mathrm{C} & 1.6592040 & 4.1650840 & -0.6828650 \\ \mathrm{C} & 0.4727660 & 4.9068820 & -0.8908810 \\ \mathrm{H} & 2.6153940 & 4.6689800 & -0.7954090 \\ \mathrm{C} & -2.4707840 & 1.0003420 & -0.5026900 \\ \mathrm{C} & -3.7305760 & 0.3818640 & -0.2685610 \\ \mathrm{C} & -2.1097120 & 2.2236080 & 0.0560090 \\ \mathrm{C} & -4.6110080 & 1.0129450 & 0.5829280 \\ \mathrm{C} & -3.0040310 & 2.8265460 & 1.0091000 \\ \mathrm{C} & -4.2794260 & 2.2147150 & 1.2526030 \\ \mathrm{H} & -5.5856700 & 0.5669530 & 0.7630490 \\ \mathrm{C} & -4.8295390 & 3.9396010 & 2.8819800 \\ \mathrm{C} & -3.5561660 & 4.5237860 & 2.6770010 \\ \mathrm{C} & -2.6693270 & 3.9820350 & 1.7686510 \\ \mathrm{C} & -5.1773380 & 2.8020240 & 2.1846130 \\ \mathrm{H} & -5.5200210 & 4.3783680 & 3.5985260 \\ \mathrm{H} & -3.2671930 & 5.4020470 & 3.2499430 \\ \mathrm{H} & -1.6923460 & 4.4352210 & 1.6437160 \\ \mathrm{H} & -6.1410670 & 2.3227050 & 2.3464050 \\ \mathrm{C} & -1.8903560 & 6.3129310 & -1.4730710 \\ \mathrm{C} & -0.6370790 & 6.9672870 & -1.5661450 \\ \mathrm{C} & 0.5205110 & 6.2724140 & -1.2849510 \\ \mathrm{C} & -1.9690920 & 4.9947420 & -1.0699960 \\ \mathrm{H} & -2.7999800 & 6.8526470 & -1.7273300 \\ \mathrm{H} & -0.5910230 & 8.0087940 & -1.8762530 \\ \mathrm{H} & 1.4939590 & 6.7503800 & -1.3792030 \\ \mathrm{H} & -2.9365410 & 4.5078650 & -1.0066480 \\ \mathrm{C} & 2.9279510 & 2.0477180 & -0.3718650 \\ \mathrm{C} & 3.7019770 & 1.9372350 & -1.5497760 \\ \mathrm{C} & 3.4064090 & 1.4927580 & 0.8444170\end{array}$




$\begin{array}{rrr}4.9443660 & 1.2865730 & -1.4882450 \\ 4.6422890 & 0.8428530 & 0.8442490 \\ 5.4338990 & 0.7344970 & -0.3073550 \\ 5.5336000 & 1.2105450 & -2.3971310 \\ 5.0207870 & 0.4137580 & 1.7687250 \\ -4.1014840 & -0.9098640 & -0.9232780 \\ -3.8108970 & -2.1236430 & -0.2501900 \\ -4.7558570 & -0.9275880 & -2.1752590 \\ -4.1797200 & -3.3326710 & -0.8472630 \\ -5.0890550 & -2.1728230 & -2.7323310 \\ -4.8129450 & -3.3805770 & -2.0927110 \\ -3.9594880 & -4.2660390 & -0.3369800 \\ -5.5869530 & -2.1952510 & -3.7021760 \\ 2.6499630 & 1.6942850 & 2.1537770 \\ 6.7937060 & 0.0579000 & -0.2058920 \\ 3.2739520 & 2.5307050 & -2.8898060 \\ -3.1504990 & -2.1268800 & 1.1258140 \\ -5.1839060 & -4.7077800 & -2.7300340 \\ -5.2103460 & 0.3089940 & -2.9554980 \\ -4.1576700 & 1.4072150 & -3.1981810 \\ -3.9953470 & 2.0211600 & -2.3072850 \\ -3.1958270 & 0.9824080 & -3.5043160 \\ -4.5176430 & 2.0704190 & -3.9959750 \\ -6.4888970 & 0.9179610 & -2.3420230 \\ -7.2655620 & 0.1536090 & -2.2118250 \\ -6.2750740 & 1.3660230 & -1.3652150 \\ -6.8797190 & 1.7050940 & -3.0013210 \\ -6.1872940 & -5.4892080 & -1.8602380 \\ -6.4767930 & -6.4245270 & -2.3575240 \\ -5.7376070 & -5.7425860 & -0.8910380 \\ -7.0915220 & -4.8959210 & -1.6737730 \\ -3.9329450 & -5.5627150 & -3.0107310 \\ -3.2278510 & -5.0259830 & -3.6579620 \\ -3.4181110 & -5.8052850 & -2.0718930 \\ -4.2147130 & -6.5041540 & -3.5010600 \\ -4.2036930 & -1.9786590 & 2.2431580 \\ -4.7902840 & -1.0618070 & 2.1286500 \\ -4.8924560 & -2.8341730 & 2.2226350 \\ -3.7102400 & -1.9564620 & 3.2245910 \\ -2.2938810 & -3.3718650 & 1.4048360 \\ -2.9202610 & -4.2650370 & 1.5267630 \\ -1.5773460 & -3.5488470 & 0.5974830 \\ -1.7429140 & -3.2260610 & 2.3408350 \\ 2.9226740 & 3.1128110 & 2.6989640 \\ 3.9891610 & 3.2250240 & 2.9374960 \\ 2.6487070 & 3.8771750 & \\ -5 & & \\ -5 & -9627030\end{array}$

2. 3422940

2.9652700

2.8759400

3.9811100

2. 2625760

7.7833750

8.7491650

7.9498240

7.3927170

7. 3952240

6.7093820

7.6339850

8.3254030

3. 3474170

4. 3754180

2. 7136310

3.0033000

4.1282470

5.1829600

3.7899900

4.0644560

$-0.1813600$

0.6159650

$-0.2641420$

2. 2312240

6.6553430

1.5786110

$-5.4911090$

$-5.6680090$

$-2.4826020$

2.2199920

2. 9020120

1. 1419940

2. 3053600

2. 5607040

1.7866190

1.1919920

0.5929800

0.9542000

0.5332480

2.2075310

2. 3478040

1.7160180

0.0560950

$-0.5488850$

$-0.2781070$
3.2855180

0.6483580

$-0.3707950$

0.7780320

0.7579540

0.9733460

0.4675040

1.8961670

1.2470890

$-1.0044620$

0.5099980

$-0.9232960$

1.5200990

1.1732730

0.6512310

2.0021890

3.7710670

3.4864500

4.2214780

4.5275590

$-0.2174470$

$-0.1077390$

$-1.5405990$

2.8516440

$-0.8469050$

1.6245420

$-0.0660980$

$-4.4874410$

$-1.2593320$

$-2.9703730$

$-2.4098330$

$-3.1066620$

$-1.8281460$

$-1.6047870$

$-3.5960680$

$-2.5308190$

$-1.9273170$

$-2.5351020$

$-2.2215460$

$-3.8569200$

$-4.5516560$

$-0.9679970$

$-0.9671650$

$-0.3707130$

$-0.8424650$
3.6156210

3.2337260

2.8426570

3.6295260

4.0673650

0.5469680

0.6788640

$-0.0252630$

1.5342770

$-1.5528000$

$-2.1243410$

$-2.1682830$

$-1.3842360$

$-4.0492100$

$-4.2145620$

$-3.8533190$

$-4.9738970$

$-3.2231630$

$-3.3358230$

$-4.1657760$

$-2.4320660$

$-1.0513230$

$-2.3286020$

$-0.3640700$

$-2.8123330$

0.4047750

1.9469750

$-3.9491820$

$-3.6920990$

1.1712830

$-2.2603410$

$-1.0920710$

$-2.1614630$

$-0.3987410$

$-2.6860340$

1.9465120

4.1585670

3.0400160

1. 8513040

0.9467450

3.6353690

0.6864810

$-2.5163370$

5.5786370

4.4478900

3.1587180 


\begin{tabular}{|c|c|c|c|c|c|c|c|}
\hline C & 0.9258090 & -2.0430700 & 5.4425740 & $\mathrm{H}$ & -5.8250070 & 0.7408780 & 2.9033970 \\
\hline $\mathrm{H}$ & -0.1747660 & -0.5673850 & 6.5622210 & C & -2.8244750 & 5.9592600 & -1.2398060 \\
\hline $\mathrm{H}$ & -0.7046570 & -0.4008060 & 2.2672670 & C & -1.6834400 & 6.7757610 & -1.4350930 \\
\hline $\mathrm{H}$ & 1.3825790 & -2.4937470 & 6.3191350 & C & -0.4208140 & 6.2384980 & -1.2948650 \\
\hline C & 4.4624250 & -3.8251920 & -3.0856600 & $\mathrm{c}$ & -2.6866720 & 4.6308450 & -0.8907130 \\
\hline C & 5.0151710 & -3.6823990 & -1.6585320 & $\mathrm{H}$ & -3.8182270 & 6.3819970 & -1.3705800 \\
\hline C & 4.3832710 & -2.4890340 & -0.9231280 & $\mathrm{H}$ & -1.8064930 & 7.8221590 & -1.7053170 \\
\hline $\mathrm{C}$ & 2.9367050 & -4.0316930 & -3.0669410 & $\mathrm{H}$ & 0.4640730 & 6.8517160 & -1.4563660 \\
\hline $\mathrm{H}$ & 4.9354970 & -4.6759190 & -3.5898670 & $\mathrm{H}$ & -3.5666570 & 4.0115100 & -0.7459880 \\
\hline $\mathrm{H}$ & 6.1037380 & -3.5534860 & -1.6784070 & C & 2.5901530 & 2.3823400 & -0.3746420 \\
\hline $\mathrm{H}$ & 4.7912060 & -1.5415300 & -1.2972080 & $\mathrm{C}$ & 3.4660800 & 2.3110510 & -1.4835120 \\
\hline $\mathrm{H}$ & 2.6953920 & -4.9982780 & -2.6026800 & C & 3.0005600 & 1.9051210 & 0.8951730 \\
\hline $\mathrm{H}$ & 2.5229900 & -4.0430420 & -4.0829300 & $\mathrm{C}$ & 4.8078750 & 1.7925250 & -1.2993850 \\
\hline $\mathrm{H}$ & 4.7008890 & -2.9238600 & -3.6658610 & $\mathrm{C}$ & 4.3256420 & 1.3394680 & 1.0511920 \\
\hline $\mathrm{H}$ & 4.8011840 & -4.6004140 & -1.0942650 & $\mathrm{C}$ & 5.1983670 & 1.3204330 & -0.0423600 \\
\hline $\mathrm{H}$ & 4.6061420 & -2.5049500 & 0.1490590 & $\mathrm{c}$ & -3.6208360 & -1.6916280 & -0.9238850 \\
\hline 0 & -1.3920570 & 0.6642830 & 4.7095540 & C & -3.2599550 & -2.8643170 & -0.2178430 \\
\hline C & -2.0107140 & 1.2984730 & 3.5781610 & $\mathrm{C}$ & -4.0479890 & -1.7655470 & -2.2696210 \\
\hline $\mathrm{H}$ & -1.2512370 & 1.7323500 & 2.9166710 & $\mathrm{C}$ & -3.3236960 & -4.1467360 & -0.8916280 \\
\hline $\mathrm{H}$ & -2.6152420 & 0.5789090 & 3.0111390 & $\mathrm{C}$ & -4.1102590 & -3.0540730 & -2.9296010 \\
\hline $\mathrm{H}$ & -2.6488750 & 2.0869700 & 3.9793430 & $\mathrm{C}$ & -3.7472740 & -4.2066680 & -2.2237990 \\
\hline \multirow{2}{*}{\multicolumn{4}{|c|}{$\begin{array}{l}114 \\
\operatorname{TS} 25(R, R) \text { G }[B 97-D / \text { def2-TZVP }]=-3918.079799\end{array}$}} & C & 3.3771860 & -1.6009360 & -1.3526320 \\
\hline & & & & $\mathrm{C}$ & 2.5455630 & -2.8086580 & -1.3177720 \\
\hline 0 & 0.2669280 & 0.8164900 & -0.0948370 & $\mathrm{H}$ & 3.0485090 & -0.7621470 & -0.7351350 \\
\hline 0 & -1.6532970 & 0.2314940 & -1.6505630 & $\mathrm{H}$ & 1.5007910 & -2.6913640 & -1.0481790 \\
\hline C & 0.0744430 & 2.1623210 & -0.3507540 & 0 & 2.7355670 & -1.5904300 & -2.6763460 \\
\hline C & -1.2174410 & 2.6761510 & -0.3596260 & C & 1.8575990 & -2.4749830 & 2.4157420 \\
\hline C & 1.2309950 & 2.9667560 & -0.5330310 & C & 0.6733090 & -1.2338760 & 4.2773260 \\
\hline $\mathrm{C}$ & -1.4012880 & 4.0494580 & -0.7198580 & $\mathrm{C}$ & 0.1336310 & -1.0247360 & 2.9925900 \\
\hline C & 1.0494690 & 4.3064640 & -0.8180070 & $\mathrm{~N}$ & 0.8064060 & -1.7323030 & 2.0118600 \\
\hline C & -0.2437440 & 4.8714860 & -0.9473850 & $\mathrm{H}$ & 0.4801780 & -1.7119440 & 1.0152390 \\
\hline $\mathrm{H}$ & 1.9200880 & 4.9443600 & -0.9553200 & $\mathrm{~s}$ & 2.0379450 & -2.3237540 & 4.1677110 \\
\hline C & -2.5155050 & 0.5663850 & -0.6118720 & $\mathrm{~s}$ & 2.8741300 & -3.4199750 & 1.4757550 \\
\hline C & -3.5212810 & -0.3704830 & -0.2454260 & $\mathrm{P}$ & -0.1640610 & -0.2921510 & -1.2322620 \\
\hline C & -2.3448760 & 1.7888960 & 0.0290000 & 0 & 0.6830220 & -0.1595650 & -2.4675710 \\
\hline C & -4.3663580 & -0.0366740 & 0.7943670 & 0 & -0.2094180 & -1.5912500 & -0.4909250 \\
\hline C & -3.1852790 & 2.0982830 & 1.1506850 & $\mathrm{H}$ & 1.8660710 & -0.9889600 & -2.6094710 \\
\hline $\mathrm{C}$ & -4.2197610 & 1.1705830 & 1.5198680 & C & -0.9548970 & 0.2669550 & 5.1977100 \\
\hline $\mathrm{H}$ & -5.1416520 & -0.7389300 & 1.0928010 & $\mathrm{C}$ & -1.4906300 & 0.4753030 & 3.9152860 \\
\hline C & -4.8484250 & 2.5905700 & 3.3975000 & $\mathrm{C}$ & -0.9533960 & -0.1670720 & 2.8007960 \\
\hline C & -3.8068710 & 3.4879000 & 3.0585400 & $\mathrm{C}$ & 0.1324680 & -0.5895440 & 5.3925000 \\
\hline C & -2.9966850 & 3.2475220 & 1.9670150 & $\mathrm{H}$ & -1.3881600 & 0.7802000 & 6.0526940 \\
\hline C & -5.0445070 & 1.4533350 & 2.6427020 & $\mathrm{H}$ & -1.3428680 & 0.0054930 & 1.8046840 \\
\hline H & -5.4803830 & 2.7926630 & 4.2593160 & $\mathrm{H}$ & 0.5496880 & -0.7453370 & 6.3839240 \\
\hline H & -3.6356900 & 4.3710680 & 3.6699980 & C & 5.3038680 & -3.0222000 & -2.2060520 \\
\hline $\mathrm{H}$ & -2.1948820 & 3.9387210 & 1.7328330 & $\mathrm{C}$ & 4.5899910 & -4.2699770 & -1.6645610 \\
\hline
\end{tabular}




$\begin{array}{lrrr}\mathrm{C} & 3.0609710 & -4.1343380 & -1.7810200 \\ \mathrm{C} & 4.8797240 & -1.7645980 & -1.4284960 \\ \mathrm{H} & 6.3909220 & -3.1447640 & -2.1279530 \\ \mathrm{H} & 4.9144840 & -5.1676380 & -2.2041430 \\ \mathrm{H} & 2.7452720 & -4.2564530 & -2.8277160 \\ \mathrm{H} & 5.2366650 & -1.8251640 & -0.3910430 \\ \mathrm{H} & 5.3053410 & -0.8596580 & -1.8726100 \\ \mathrm{H} & 5.0639470 & -2.8910360 & -3.2697320 \\ \mathrm{H} & 4.8580210 & -4.4076190 & -0.6080400 \\ \mathrm{H} & 2.5379940 & -4.9117140 & -1.2120790 \\ \mathrm{H} & -2.3314890 & 1.1501540 & 3.7825980 \\ \mathrm{H} & -3.7881610 & -5.1727770 & -2.7259440 \\ \mathrm{H} & 6.1995200 & 0.9108180 & 0.0841280 \\ \mathrm{C} & -2.4077470 & -4.0005430 & 1.7733900 \\ \mathrm{C} & -2.7856310 & -2.8466360 & 1.1321350 \\ \mathrm{C} & -2.9298800 & -5.3258240 & -0.1851910 \\ \mathrm{C} & -2.4823330 & -5.2595010 & 1.1108920 \\ \mathrm{H} & -2.0367420 & -3.9518900 & 2.7951330 \\ \mathrm{H} & -2.7073460 & -1.8983950 & 1.6514600 \\ \mathrm{H} & -2.9856990 & -6.2802390 & -0.7064180 \\ \mathrm{H} & -2.1768310 & -6.1633830 & 1.6336600 \\ \mathrm{C} & -4.8399800 & -0.7142410 & -4.3292000 \\ \mathrm{C} & -4.4354210 & -0.6112600 & -3.0199660 \\ \mathrm{C} & -4.5377730 & -3.1182210 & -4.2918860 \\ \mathrm{C} & -4.8905930 & -1.9819660 & -4.9772830 \\ \mathrm{H} & -5.1244800 & 0.1804340 & -4.8789000 \\ \mathrm{H} & -4.4042230 & 0.3630340 & -2.5405750 \\ \mathrm{H} & -4.5755380 & -4.0935110 & -4.7745940 \\ \mathrm{H} & -5.2113560 & -2.0439260 & -6.0149740 \\ \mathrm{C} & 3.9529460 & 2.6165070 & -3.8597270 \\ \mathrm{C} & 3.0767610 & 2.7001140 & -2.8048500 \\ \mathrm{C} & 5.6935380 & 1.7403950 & -2.4200850 \\ \mathrm{C} & 5.2821700 & 2.1414130 & -3.6667210 \\ \mathrm{H} & 3.6268600 & 2.9065250 & -4.8560530 \\ \mathrm{H} & 2.0621070 & 3.0477250 & -2.9705500 \\ \mathrm{H} & 6.7020770 & 1.3616770 & -2.2619690 \\ \mathrm{H} & 5.9638180 & 2.0883050 & -4.5128470 \\ \mathrm{C} & 3.8612190 & 0.8562110 & 3.3963270 \\ \mathrm{C} & 4.7153250 & 0.8156000 & 2.3225870 \\ \mathrm{C} & 2.1604040 & 1.9556460 & 2.0518530 \\ \mathrm{C} & 2.5719340 & 1.4445770 & 3.2582430 \\ \mathrm{H} & 4.1654860 & 0.4437250 & 4.3552470 \\ \mathrm{H} & 5.7088180 & 0.3807320 & 2.4178650 \\ \mathrm{H} & 1.1710200 & 2.3940360 & 1.9701800 \\ \mathrm{H} & 1.9017140 & 1.4796710 & 4.1131480 \\ \mathrm{I} & & & \\ & & & \\ & & & \end{array}$

\begin{tabular}{|c|c|c|c|}
\hline \multicolumn{4}{|c|}{$\operatorname{TS} 26(\mathrm{~S}, \mathrm{~S}) \quad \mathrm{G}[\mathrm{B} 97-\mathrm{D} / \mathrm{def} 2-\mathrm{TZVP}]=-3918.080768$} \\
\hline 0 & -1.6916350 & 0.0843900 & -1.6618740 \\
\hline 0 & 0.2333690 & 0.8173700 & -0.1747120 \\
\hline & -2.5442490 & 0.4613770 & -0.6308740 \\
\hline & -2.4040800 & 1.7299640 & -0.0776040 \\
\hline & -3.5079580 & -0.4831820 & -0.1810960 \\
\hline & -3.2401550 & 2.0872440 & 1.0336060 \\
\hline & -4.3497440 & -0.1076300 & 0.8464550 \\
\hline & -4.2376570 & 1.1533790 & 1.4814870 \\
\hline & -5.0924610 & -0.8150780 & 1.2087820 \\
\hline & 0.0019990 & 2.1410760 & -0.5006170 \\
\hline & 1.1380460 & 2.9633180 & -0.7228860 \\
\hline & -1.3029670 & 2.6197220 & -0.5325850 \\
\hline & 0.9253670 & 4.2781690 & -1.0873200 \\
\hline & -1.5197040 & 3.9653890 & -0.9712330 \\
\hline & -0.3826440 & 4.8004680 & -1.2510000 \\
\hline & 1.7795080 & 4.9304070 & -1.2577290 \\
\hline & -1.869 & 6.6370220 & 1.8458580 \\
\hline & -2.9899180 & 5.8068560 & -1.5969170 \\
\hline & -2.8190570 & 4.5052410 & -1.1698780 \\
\hline & -0.5938920 & 6.1393350 & -1.6788620 \\
\hline & -2.0183860 & 7.6621830 & -2.1774900 \\
\hline & -3.9939830 & 6.19 & -1.7477270 \\
\hline & -3.6833370 & 3.874 & -0.9834460 \\
\hline & 0.2755840 & 6.7628990 & -1.8796790 \\
\hline & -3.8806630 & 3.5806700 & 2.8553950 \\
\hline & -4.8876820 & 2.6 & 3.2 \\
\hline & -5.0 & 1.48 & 2.5955850 \\
\hline & -3.0781830 & 3.2936640 & 1.7694220 \\
\hline & -3.729 & 4.507 & 3.40 \\
\hline & -5.5143160 & 2.914 & 4.1258080 \\
\hline & -5.808 & 0.7 & 2.9162270 \\
\hline & 3180 & 3.9 & 1.4779900 \\
\hline & -3.5554010 & -1.8 & -0.7582130 \\
\hline & -3.0999100 & -2.949 & 0.0149880 \\
\hline & -4.0198180 & -2.0503600 & -2.0785870 \\
\hline & -3.1003690 & -4.27 & -0.5657090 \\
\hline & -4.0221880 & -3.3844780 & -2.6442220 \\
\hline & -3.5617190 & -4.4587330 & -1.8742370 \\
\hline & 2.5068220 & & -0.5060990 \\
\hline & 3.3882340 & 2.2475010 & -1.5982200 \\
\hline & 2.9125060 & 2.0675150 & 0.8055040 \\
\hline & 4.7320320 & 1.7564070 & -1.3596370 \\
\hline & 4.2475510 & 1.5475070 & 1.0244180 \\
\hline & 5.1254780 & 1.4296000 & -0.058458 \\
\hline & $-0.1 / 34330$ & $-0.36108<0$ & -1.2510460 \\
\hline
\end{tabular}




$\begin{array}{rrr}-0.1384030 & -1.6199920 & -0.4457110 \\ 0.6345650 & -0.2523590 & -2.5138550 \\ 6.1312920 & 1.0482100 & 0.1124390 \\ -3.5559400 & -5.4587490 & -2.3068420 \\ 3.8770230 & 2.3073040 & -3.9920060 \\ 3.0009290 & 2.5026780 & -2.9518830 \\ 5.6155370 & 1.5789280 & -2.4688460 \\ 5.2035980 & 1.8467740 & -3.7508320 \\ 3.5522560 & 2.4941630 & -5.0131250 \\ 1.9868450 & 2.8335590 & -3.1522440 \\ 6.6234310 & 1.2168070 & -2.2717950 \\ 5.8831900 & 1.6999080 & -4.5875010 \\ 2.4644990 & 1.8050090 & 3.1953310 \\ 2.0562660 & 2.1974000 & 1.9440650 \\ 4.6354360 & 1.1530260 & 2.3421280 \\ 3.7669130 & 1.2668520 & 3.3992120 \\ 1.7806820 & 1.8923580 & 4.0356870 \\ 1.0559240 & 2.5970490 & 1.8127040 \\ 5.6371750 & 0.7512910 & 2.4855100 \\ 4.0694840 & 0.9495950 & 4.3943310 \\ -2.1181930 & -3.8853220 & 2.0499640 \\ -2.5893980 & -2.8063460 & 1.3435760 \\ -2.6074930 & -5.3760180 & 0.2059830 \\ -2.1272350 & -5.1898300 & 1.4783820 \\ -1.7220110 & -3.7400060 & 3.0528230 \\ -2.5567330 & -1.8212720 & 1.7943300 \\ -2.6150840 & -6.3664480 & -0.2463920 \\ -1.7469000 & -6.0330390 & 2.0509490 \\ -4.9363280 & -2.5099150 & -4.7303290 \\ -4.4902660 & -3.5711290 & -3.9816160 \\ -4.5003960 & -0.9772260 & -2.8928510 \\ -4.9416680 & -1.1977090 & -4.1753910 \\ -5.2874890 & -2.6656690 & -5.7481600 \\ -4.4836940 & -4.5788310 & -4.3941270 \\ -4.5120250 & 0.0290800 & -2.4834110 \\ -5.2980110 & -0.3632450 & -4.7755740 \\ 2.5379000 & -2.7670770 & -1.7688560 \\ 3.2413370 & -1.8350140 & -0.8816470 \\ 1.4812160 & -2.9199580 & -1.5514820 \\ 2.6516890 & -1.0845710 & -0.3661010 \\ 2.7521680 & -1.5905350 & -2.6304660 \\ 1.9305770 & -2.2615100 & 2.4856760 \\ 0.7411390 & -0.9568420 & 4.2967280 \\ 0.1916570 & -0.8101120 & 3.0070600 \\ 0.8679440 & -1.5515130 & 1.0604800 \\ 0.5250800 & -1.6084260 & \end{array}$

2.1225470
2.9460830
1.8676450
-0.9058770
-1.4507780
-0.9089880
0.1957550
-1.3430490
-1.3070780
0.6204640
4.7837440
5.3908940
4.7354410
3.2762370
5.2804290
6.4708050
5.0727890
3.1134500
2.8107740
4.9434670
5.2480150
5.0150880
-2.3035480
$-2.0276430$
$-3.2386810$
$-1.0079110$
0.5544770
0.7021110
0.0234400
$-0.2760090$
1.0952010
0.1503020
$-0.3848510$
$-3.7481270$
$-3.1453750$
$-1.7987820$
$-3.9945190$
$-4.6945590$
$-2.9959460$
$-1.0140810$
$-4.7783170$
$-4.3343290$
$-3.0634390$
$-3.8452310$
$-1.4601110$
1.3568080

4.2255690

1.5766280

$-2.5958740$

5. 1648260

3.8777400

2.7875370

5. 3878740

6.0006730

1. 7882820

6.3823440

$-2.4392160$

$-1.1635700$

$-0.8077590$

$-2.2577130$

$-2.6843460$

$-1.2829960$

$-1.4990710$

$-1.5044330$

$-3.1913860$

$-3.2828590$

$-0.3287330$

0.1950180

3.7217900

114

TS27(S,S) G[B97-D/def2-TZVP $]=-3918.076726$

$\begin{array}{lrcr}\text { TS27 }(\mathrm{S}, \mathrm{S}) \mathrm{G}[\mathrm{B} 97-\mathrm{D} / \mathrm{de} \text { ef }-\mathrm{TZVP}]=-3918.076726 \\ \mathrm{O} & 0.5741000 & 1.3933660 & 0.6328970 \\ \mathrm{O} & -1.4933200 & 0.6408090 & -0.6696670 \\ \mathrm{C} & 0.6517860 & 2.5025370 & -0.2054100 \\ \mathrm{C} & -0.5018970 & 3.2390200 & -0.4602790 \\ \mathrm{C} & 1.9216580 & 2.8410730 & -0.7433090 \\ \mathrm{C} & -0.4264380 & 4.3235640 & -1.3954460 \\ \mathrm{C} & 1.9977770 & 3.9257310 & -1.5938640 \\ \mathrm{C} & 0.8501260 & 4.6719840 & -1.9575910 \\ \mathrm{H} & 2.9628100 & 4.2056450 & -2.0117170 \\ \mathrm{C} & -2.2566730 & 1.5523240 & 0.0443660 \\ \mathrm{C} & -3.4992530 & 1.1088100 & 0.5716300 \\ \mathrm{C} & -1.7853490 & 2.8526180 & 0.1865020 \\ \mathrm{C} & -4.2543820 & 2.0160740 & 1.2897520 \\ \mathrm{C} & -2.5439700 & 3.7683730 & 0.9867260 \\ \mathrm{C} & -3.8038480 & 3.3374770 & 1.5305910 \\ \mathrm{H} & -5.2116110 & 1.7032440 & 1.7016570 \\ \mathrm{C} & -4.1045600 & 5.5110820 & 2.5896590 \\ \mathrm{C} & -2.8477460 & 5.9254730 & 2.0842260 \\ \mathrm{C} & -2.0881370 & 5.0773240 & 1.3037780 \\ \mathrm{C} & -4.5675940 & 4.2405800 & 2.3195230 \\ \mathrm{H} & -4.6950360 & 6.1921150 & 3.1984770\end{array}$




$\begin{array}{lr}\mathrm{H} & -2.4753320 \\ \mathrm{H} & -1.1262560 \\ \mathrm{H} & -5.5232040 \\ \mathrm{C} & -1.4554560 \\ \mathrm{C} & -0.1908680 \\ \mathrm{C} & 0.9363600 \\ \mathrm{C} & -1.5705210 \\ \mathrm{H} & -2.3466580 \\ \mathrm{H} & -0.1147520 \\ \mathrm{H} & 1.9129130 \\ \mathrm{H} & -2.5470660 \\ \mathrm{C} & 3.1617180 \\ \mathrm{C} & 3.6915070 \\ \mathrm{C} & 3.8317710 \\ \mathrm{C} & 4.9370490 \\ \mathrm{C} & 5.0928910 \\ \mathrm{C} & 5.6116990 \\ \mathrm{C} & -3.9802720 \\ \mathrm{C} & -4.0603950 \\ \mathrm{C} & -4.3762570 \\ \mathrm{C} & -4.5868240 \\ \mathrm{C} & -4.8858770 \\ \mathrm{C} & -4.9882310 \\ \mathrm{P} & -0.1404200 \\ \mathrm{O} & 0.6471860 \\ \mathrm{O} & -0.4711830 \\ \mathrm{H} & -5.3762090 \\ \mathrm{H} & 6.5459550 \\ \mathrm{C} & -3.6702200 \\ \mathrm{C} & -3.6002610 \\ \mathrm{C} & -4.6556730 \\ \mathrm{C} & -4.2112930 \\ \mathrm{H} & -3.2979870 \\ \mathrm{H} & -3.1657270 \\ \mathrm{H} & -5.0592630 \\ \mathrm{H} & -4.2608910 \\ \mathrm{C} & -4.6829770 \\ \mathrm{C} & -4.3075570 \\ \mathrm{C} & -5.2695170 \\ \mathrm{C} & -5.1660740 \\ \mathrm{H} & -4.6111960 \\ \mathrm{H} & -3.9445330 \\ \mathrm{H} & -5.6456440 \\ \mathrm{H} & -5.4578200 \\ \mathrm{C} & 3.5484100 \\ \mathrm{C} & 3.0333150\end{array}$

$\begin{array}{rr}6.9208940 & 2.3165020 \\ 5.4062990 & 0.9240120 \\ 3.9016380 & 2.7158510 \\ 6.0765790 & -2.7451440 \\ 6.4416900 & -3.2684580 \\ 5.7470530 & -2.8835930 \\ 5.0462920 & -1.8338440 \\ 6.6103120 & -3.0682670 \\ 7.2595500 & -3.9815210 \\ 6.0015620 & -3.2918320 \\ 4.7755540 & -1.4461510 \\ 2.0879920 & -0.3904870 \\ 1.1297240 & -1.2838100 \\ 2.3881350 & 0.8180210 \\ 0.4617230 & -0.9578780 \\ 1.7393500 & 1.1148950 \\ 0.7993950 & 0.2190830 \\ -0.2869380 & 0.3642170 \\ -1.1726160 & 1.4656770 \\ -0.7143300 & -0.9257330 \\ -2.5100690 & 1.2709590 \\ -2.0593110 & -1.1086820 \\ -2.9139650 & -0.0053830 \\ 0.0467180 & 0.0320950 \\ -0.5549440 & -1.0885580 \\ -0.7897490 & 1.2422740 \\ -3.9221050 & -0.1480480 \\ 0.2945140 & 0.4606870 \\ -1.7143380 & 3.8168610 \\ -0.8222430 & 2.7743440 \\ -3.4013690 & 2.3849340 \\ -3.0182320 & 3.6257620 \\ -1.4269920 & 4.7978060 \\ 0.1594940 & 2.9316540 \\ -4.3993440 & 2.2215880 \\ -3.7095000 & 4.4644510 \\ -0.3170310 & -3.3188750 \\ 0.1330490 & -2.0758820 \\ -2.4875560 & -2.4174320 \\ -1.6453320 & -3.4967630 \\ 0.3467830 & -4.1776200 \\ 1.1498550 & -1.9602320 \\ -3.5024490 & -2.5371400 \\ -1.9846910 & -4.4883030 \\ -0.2101740 & -3.3200000 \\ 0.7629840 & -2.4993330\end{array}$

5.4384260

4.7618690

3. 0155050

2. 0927370

6. 3661020

5. 1447700

5. 2280930

5.7642360

3. 3146670

3.9864470

5. 7470880

6.7094260

2. 3693160

3. 5679200

$-0.4277340$

$-0.7779700$

0.5154370

$-0.3657060$

$-1.5262680$

2. 5378620

4. 0660920

3. 2221770

2. 4341490

1.7591580

3.7810420

1. 6038630

-1. 1084080

4. 9194510

4. 0637040

3. 2106170

4. 9329170

5. 5805440

2. 5660060

5. 5890850

$-2.0654080$

$-2.0068960$

$-1.8123760$

$-0.7624310$

$-2.2212850$

$-2.9243700$

$-2.7504120$

0.0809090

$-0.8197670$

$-2.9102930$

-1.1684590
-1.5425160
-0.5446380
-0.8780040
-0.4892190
1.2410290
-1.0479970
-1.6562650
2.9465490
2.0530090
3.3176380
3.5841960
3.1712240
1.5551920
3.8121270
4.2887580
-3.9322950
-3.4532680
-3.5739180
-2.5045880
-3.0119390
-3.6884280
-3.3240390
-2.2351450
-2.4697240
-1.7439590
-4.6190290
-4.2932440
-2.0630280
-2.1244820
-1.0506270
-1.0887810
-3.2730460
-2.0664770
-0.2510820
-4.1073510
-5.8401310
-5.6181770
-4.1312490
-5.3651060
-6.9026580
-5.9808460
-3.5749880
-5.9823190
-5.4491360
-5.2821420
-6.1944840
-3.9852480

$-1.8398090$

$-2.9869280$

$-4.2259250$

$-2.7505260$

$-1.5725470$

$-3.6434740$

3. 2306960

2.3360860

1.7750390

2. 9443240

4.1600840

2. 5465360

1. 5695070

3. 6597980

0.4847800

$-0.8585830$

0.8988190

$-1.1847330$

0.7746740

$-0.9791510$

1.0073620

0.7054190

$-0.4090650$

$-0.7582840$

$-0.1370420$

$-2.2331800$

0.9949840

2.8979290

2.6037770

1. 5035720

2. 1016890

3.7592340

1. 2607910

2. 3350180

0.1758070

$-1.3443080$

$-1.6905520$

0.8430410

0.3975570

$-1.8233280$

$-1.5442810$

0.5025230

1. 9355170

0.5997680

$-1.7589110$

$-2.7430840$ 
H

TS28(R,R) G[B97-D/def2-TZVP] = -4356.205891

$\begin{array}{lrrr}\mathrm{C} 28(\mathrm{R}, \mathrm{R}) & \mathrm{G}[\mathrm{B} 97-\mathrm{D} / \mathrm{de} 2-\mathrm{TZ} \text {. } & -1.4356 .205891 \\ \mathrm{O} & -0.0349960 & -0.1834280 & -0.7437730 \\ \mathrm{O} & 0.3315440 & 1.2596370 & -0.5984280 \\ \mathrm{O} & -1.0279270 & -0.5983190 & -1.8010210 \\ \mathrm{O} & -0.5143230 & -0.6857650 & 0.7396230 \\ \mathrm{C} & 1.2538000 & -1.1365860 & -1.0283740 \\ \mathrm{C} & -0.7143680 & -2.0608470 & 0.8915650 \\ \mathrm{C} & 0.4165070 & -2.8865790 & 0.9901200 \\ \mathrm{C} & -2.0214730 & -2.5656770 & 0.8800060 \\ \mathrm{H} & -2.1539140 & -3.9535760 & 0.9861140 \\ \mathrm{C} & -3.1575510 & -4.3752380 & 0.9953900 \\ \mathrm{C} & 2.1629200 & -1.4152050 & -0.0137060 \\ \mathrm{C} & 3.4515470 & -0.8711930 & -0.1025230 \\ \mathrm{C} & 1.7708520 & -2.2649110 & 1.0326040 \\ \mathrm{H} & 4.3442100 & -1.1971970 & 0.9212880 \\ \mathrm{C} & 5.3605410 & -0.8101350 & 0.8649480 \\ \mathrm{C} & -3.2120670 & -1.7025010 & 0.6581720 \\ \mathrm{C} & 3.8519170 & 0.0018300 & -1.2377240 \\ \mathrm{C} & -3.5304900 & -0.6388350 & 1.5221840 \\ \mathrm{C} & -2.7940320 & -0.2468690 & 2.7813960 \\ \mathrm{C} & -4.8592820 & 1.2571760 & 2.3088500 \\ \mathrm{C} & -4.6658500 & 0.1619210 & 1.2899480 \\ \mathrm{H} & -2.3452320 & 1.2307640 & 2.6482170 \\ \mathrm{H} & -1.8618170 & 1.5438600 & 3.5817460 \\ \mathrm{C} & -1.6002240 & 1.2998940 & 1.8561800 \\ \mathrm{H} & -3.5767500 & 2.1328080 & 2.3348080 \\ \mathrm{H} & -3.6916850 & 2.9146010 & 3.0967670 \\ \mathrm{H} & -3.4536850 & 2.6348720 & 1.3678750 \\ \mathrm{H} & -5.7405520 & 1.8655550 & 2.0718880 \\ \mathrm{C} & -1.9295410 & -0.8914290 & 2.9614000 \\ \mathrm{H} & -5.0105120 & 0.5970910 & 3.7082780 \\ \mathrm{H} & -5.0677090 & 1.3872880 & 4.4687520 \\ \mathrm{C} & -5.9481470 & 0.0301040 & 3.7471780 \\ \mathrm{H} & -3.7882050 & -0.3365370 & 3.9730060 \\ \mathrm{H} & -3.2648390 & -0.0375560 & 4.8907450 \\ \mathrm{C} & -4.1145790 & -1.3757190 & 4.0997890 \\ \mathrm{C} & -4.0632130 & -1.9478050 & -0.4403880 \\ \mathrm{C} & -3.9100080 & -3.0232690 & -1.4944990 \\ \mathrm{C} & -6.0453620 & -1.5861640 & -1.8269640 \\ \mathrm{C} & -5.2138240 & -1.1607910 & -0.6425330 \\ \mathrm{H} & -5.1637170 & -3.9388040 & -1.4244120 \\ \mathrm{H} & -5.0868570 & -4.7030800 & -2.2090710 \\ \mathrm{C} & -5.1908840 & -4.4566370 & -0.4578860 \\ & -6.4492140 & -3.0741870 & -1.6215280\end{array}$

$-7.0134760$ $-7.1092960$ $-6.9386390$ $-2.9991550$ $-5.1713810$ $-5.7577440$ $-4.9038190$ $-3.8881410$ $-3.8320850$ $-2.9883390$ $-5.5144800$ $-6.4044200$ 4.2620570 4. 2456460 3.5742060 3.8826950 5. 2223360 5. 1602620 6. 2511010 4.8382890 4.6233430 5. 6628780 3. 2949380 4.5343890 2. 4252270 2. 2613470 1. 5031740 2. 8154010 2. 7966120 2. 1077750 4. 5885280 4. 9086180 4.1632080 4. 2029520 3. 6423430 3. 8830880 2. 8476840 3. 1793110 3. 1480290 2. 1782540 3.8439720 5. 2213430 5.5755440 5. 5318170 6.2827970 6.0322850

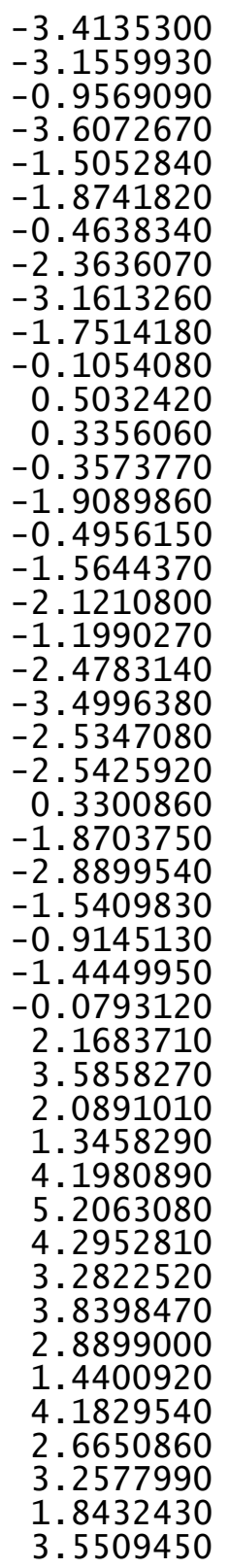

$-2.5002030$ $-0.7498540$ $-1.9261560$ $-1.3510990$ $-3.1068700$ $-3.9592070$ $-3.3120130$ $-2.9010070$ $-3.6536740$ $-2.9963860$ 0.2172520 0.0543900 $-3.6232370$ $-4.9626760$ $-2.9894680$ $-2.5529230$ $-4.8997550$ $-5.8443900$ $-4.7970590$ $-3.6943160$ $-4.0356460$ $-2.9735360$ $-2.1432680$ $-5.7670630$ $-4.0352910$ $-4.4093860$ $-3.5492340$ $-5.2049430$ $-6.1663590$ $-5.2714580$ $-2.0985880$ $-1.6931720$ 0.2925210 $-1.0220490$ $-1.0321680$ $-0.6689990$ $-1.7814130$ 0.1403080

1.0850260 $-0.0511700$ 1. 1112920 $-2.5582900$ 0.5824370

1. 5058130

0.7475610 $-0.6200090$ 


$\begin{array}{rr}\mathrm{H} & 6.2401870 \\ \mathrm{H} & 6.9513670 \\ \mathrm{C} & 4.6170500 \\ \mathrm{H} & 4.9085390 \\ \mathrm{C} & 2.6667540 \\ \mathrm{C} & 2.1857230 \\ \mathrm{C} & 3.1846290 \\ \mathrm{C} & 4.6172390 \\ \mathrm{C} & 4.9953460 \\ \mathrm{C} & 3.9736870 \\ \mathrm{H} & 2.9071620 \\ \mathrm{H} & 1.9464680 \\ \mathrm{H} & 1.2328200 \\ \mathrm{H} & 4.6812860 \\ \mathrm{H} & 5.3215270 \\ \mathrm{H} & 5.9796750 \\ \mathrm{H} & 5.0894610 \\ \mathrm{H} & 3.1289860 \\ \mathrm{C} & 0.2472380 \\ \mathrm{C} & 1.4403790 \\ \mathrm{C} & 1.0325180 \\ \mathrm{C} & -0.0665330 \\ \mathrm{C} & -1.3300560 \\ \mathrm{C} & -1.0563710 \\ \mathrm{H} & 1.9117590 \\ \mathrm{H} & 1.9843000 \\ \mathrm{H} & 2.1507310 \\ \mathrm{H} & 0.2914270 \\ \mathrm{H} & -0.2990720 \\ \mathrm{H} & -2.0343480 \\ \mathrm{H} & -1.8489730 \\ \mathrm{H} & 0.6570800 \\ \mathrm{C} & -3.1768560 \\ \mathrm{C} & -2.0806270 \\ \mathrm{H} & -3.0199040 \\ \mathrm{H} & -1.0766890 \\ \mathrm{O} & -2.6562040 \\ \mathrm{C} & -1.2534290 \\ \mathrm{C} & -0.2237510 \\ \mathrm{C} & 0.1062490 \\ \mathrm{~N} & -0.4724560 \\ \mathrm{H} & -0.2965610 \\ \mathrm{~S} & -1.2790980 \\ \mathrm{~S} & -2.0989220 \\ \mathrm{H} & -1.9347410 \\ \mathrm{C} & 1.0743000 \\ & \end{array}$

\begin{abstract}
4. 5767350
3. 1530470

1.6661610

2. 3068390

$-2.4935770$

$-3.2202580$

$-3.2215160$

$-3.4128320$

$-2.2314060$

$-1.9784990$

$-4.0147140$

$-4.2606030$

$-2.7727010$

$-4.3491260$

$-3.4901450$

$-2.3896190$

$-1.3273280$

$-2.2738880$

$-4.2913620$

$-5.2056970$

$-6.5637400$

$-7.1824130$

$-6.3155680$

$-4.8207040$

$-7.2184230$

$-5.4015740$

$-4.6923190$

$-7.2339370$

$-8.2068380$

$-6.5888260$

$-6.5242400$

$-6.4320370$

1.8033920

2. 5664790

1. 4418890

2. 3866910

0.8678590

4.4998180

4. 5093890

3. 3917040

3. 4312550

2. 6487150

5. 5702630

4.8250090

0.2289240

3. 5974010
\end{abstract}

\begin{abstract}
$-0.2878880$
$-1.0664840$

$-3.3991760$

$-4.2323870$

2. 1045560

3. 3537950

4. 5243010

4.0168540

3. 1171550

2. 0226680

5. 2297890

3. 1141810

3. 6673850

3.4422980

4.8548590

2. 6566070

3.7404380

5.0763940

0.9392320

0.6998620

0.1131940

0.9839070

0.9095560

0.9766310

0.0623360

1.6335020

0.0402530

2.0232550

0.6665340

1. 7078070

$-0.0390850$

$-0.9124850$

$-1.9050300$

$-2.5166810$

$-0.8855590$

$-2.1449510$

$-2.9004770$

0.5433330

2. 8510160

2. 0689400

0.8123280

0.1448760

1.9414200

$-0.8680530$

$-2.4105110$

4.6500770
\end{abstract}

\begin{abstract}
1. 3876440
0.9092920

0.2704300

1.4678730

1. 1161200

0.0290520

$-4.7683520$

$-3.7391810$

$-2.2958510$

$-4.5988450$

$-5.7854130$

$-3.8779370$

$-2.0396060$

$-4.8481710$

$-5.2613360$

$-4.6464210$

$-3.8952070$

$-1.5699180$

2. 1640170

2.4169310

2.9553630

1. 4784730

3.0356820
\end{abstract}

158

TS29(R,R) G[B97-D/def2-TZVP $]=-4356.205622$

$\begin{array}{lrrr}\mathrm{P} & -0.0329860 & -0.1206320 & -0.7970680 \\ \mathrm{O} & -0.9998660 & -0.4856960 & -1.8958170 \\ \mathrm{O} & 0.2787660 & 1.3189710 & -0.5359460 \\ \mathrm{O} & 1.2916500 & -1.0060460 & -1.1301220 \\ \mathrm{O} & -0.5044070 & -0.7519080 & 0.6365060 \\ \mathrm{C} & 2.1920920 & -1.3265630 & -0.1184980 \\ \mathrm{C} & 1.8204080 & -2.2897680 & 0.8408120 \\ \mathrm{C} & 3.4499210 & -0.7090950 & -0.1310470 \\ \mathrm{C} & 4.3507120 & -1.0672700 & 0.8795910 \\ \mathrm{H} & 5.3412650 & -0.6158020 & 0.8813740 \\ \mathrm{C} & -0.6687520 & -2.1379240 & 0.6861220 \\ \mathrm{C} & -1.9652360 & -2.6661980 & 0.6381430 \\ \mathrm{C} & 0.4812180 & -2.9434710 & 0.7520610 \\ \mathrm{C} & -2.0764220 & -4.0586450 & 0.6945010 \\ \mathrm{H} & -3.0726870 & -4.4972980 & 0.6828600 \\ \mathrm{C} & 3.8291590 & 0.2739710 & -1.1810970 \\ \mathrm{C} & -3.1727440 & -1.8130960 & 0.4742800 \\ \mathrm{C} & 3.9358080 & -0.1168970 & -2.5274430 \\ \mathrm{C} & 3.7210500 & -1.5063610 & -3.0812370 \\ \mathrm{C} & 4.3885540 & 0.2228180 & -4.9009380 \\ \mathrm{C} & 4.3136290 & 0.8116080 & -3.5148720\end{array}$

$\begin{array}{ll}2.4683240 & 3.8579460\end{array}$

$2.3562260 \quad 2.5493860$

$4.6185220 \quad 4.1555860$

$3.6459890 \quad 5.6616630$

$1.5076960 \quad 1.9092840$

$5.4763470 \quad 4.7773350$

$2.7995500 \quad-3.6114330$

$3.9059730 \quad-3.8936500$

$3.3869330-3.7551380$

2.2385170 -2.1897750

$3.1933720 \quad-3.7266710$

$4.3149650 \quad-4.9015680$

$2.7484330 \quad-4.6127410$

$3.0096330 \quad-1.4475900$

$1.9867760 \quad-4.3397520$

$4.7283380-3.1823390$

$4.2073820 \quad-3.7530340$

$\begin{array}{ll}1.5258920 & 4.4602210\end{array}$

$0.3217640 \quad 3.7229440$

$0.5281480 \quad 2.7905390$

3.4845530

4.3717640
$1.3856320 \quad-2.0127620$

$-0.1206320$

87660

$-1.0060460$

4. 3136290

0.8116080

$-3.5148720$ 


\begin{tabular}{|c|c|c|}
\hline 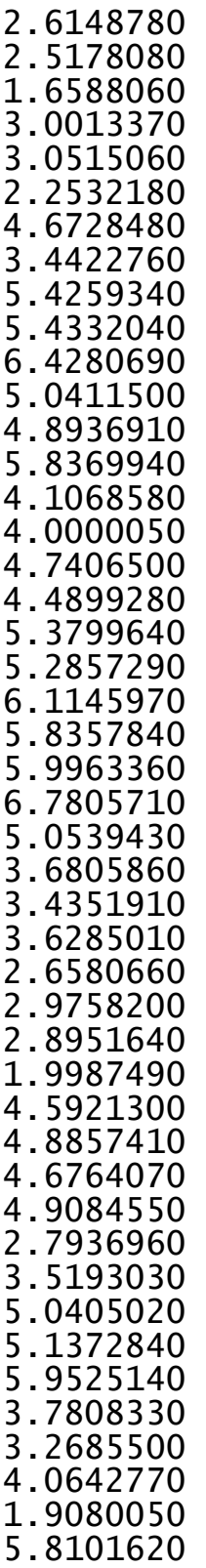 & $\begin{array}{r}-1.4456970 \\
-2.4407570 \\
-1.1980250 \\
-0.3857070 \\
-0.8429460 \\
0.4151810 \\
0.9832620 \\
-2.2178100 \\
-0.9346740 \\
-1.4196480 \\
-0.5263370 \\
-1.9569360 \\
-2.9579990 \\
-2.0286460 \\
1.6113910 \\
2.2428900 \\
3.9235540 \\
2.5323930 \\
2.8456910 \\
3.3608760 \\
2.0405650 \\
3.8416750 \\
4.8438470 \\
3.5135330 \\
4.6005830 \\
1.5183830 \\
4.4337740 \\
5.4156370 \\
4.5657100 \\
3.4072220 \\
3.8825950 \\
2.9943180 \\
2.1360080 \\
2.8526780 \\
-0.0523460 \\
0.9283000 \\
-0.5433530 \\
-0.8387780 \\
0.1235220 \\
0.8289690 \\
-0.4842000 \\
-0.7800760 \\
-0.5439260 \\
-1.8389100 \\
-1.1725370 \\
1.5266020\end{array}$ & $\begin{array}{l}-4.1702190 \\
-4.6248330 \\
-3.7014590 \\
-5.2483420 \\
-6.2456340 \\
-5.2860940 \\
-5.6384830 \\
-2.2991120 \\
-4.8844020 \\
-5.8697020 \\
-4.7074560 \\
-3.7687990 \\
-4.1953100 \\
-3.0177040 \\
-0.8479430 \\
0.5196970 \\
-1.3150390 \\
-1.8421300 \\
0.8988300 \\
1.8639930 \\
1.0216400 \\
-0.2150160 \\
0.2040590 \\
-0.6644510 \\
-2.1190210 \\
1.2729730 \\
-0.6449210 \\
-0.1923800 \\
-1.4070950 \\
0.4337640 \\
1.4193190 \\
0.1771640 \\
-3.1749920 \\
-3.9430020 \\
1.2661190 \\
2.3882040 \\
2.7184270 \\
1.4278130 \\
3.7124970 \\
4.5483230 \\
3.6817530 \\
3.8953580 \\
4.8373870 \\
3.9266110 \\
2.8377730 \\
2.2098110\end{array}$ \\
\hline
\end{tabular}

-2.3944970
-1.9010460
-1.6688900
-3.6578820
-3.7958960
-3.5570400
-5.1853720
-6.0051740
-3.8452830
-4.0192870
-5.1267260
-5.7052920
-4.8716400
-3.8320450
-3.7671160
-2.9407950
-2.9261930
-6.9078620
-5.0852880
-4.9956920
-5.1051970
-6.3850680
-6.9427060
-7.0447950
-5.5135790
-6.4192190
-0.9633460
0.3315450
-1.1948430
0.0018230
1.2936470
1.5337250
-0.1538930
-1.4116980
-2.0961420
1.2095500
2.1532110
2.4266500
1.7518920
0.0848370
4.0030630
4.9621130
4.9729090
3.5539830
2.4204750
2.7325290

1.1948670

1.1172790

1. 8412250

2. 5279460

2. 4535120

$-1.2104750$

$-1.5559310$

$-2.9791130$

$-1.9873740$

$-1.3639900$

$-1.6702360$

$-0.3063230$

$-2.2184660$

-2.9576300

$-1.5886630$

$-3.5592460$

$-0.9348060$

$-3.9169000$

$-4.6197730$

$-4.5056330$

$-3.0616700$

$-3.3434870$

$-3.2194940$

$-0.2417820$

0.3550670

$-4.9037050$

$-4.3492860$

$-6.4061900$

$-7.2313060$

$-6.7056890$

$-5.2536570$

$-8.2910850$

$-6.6881730$

$-6.6513240$

$-6.7588570$

$-7.3220660$

$-4.8572270$

$-5.2397730$

$-7.1499830$

$-1.9718390$

$-2.3724220$

-3. 9101170

$-4.4704510$

$-3.4602940$

$-2.5777330$
2. 7196310

3. 6688980

1.9233620

2. 5115510

3. 3568870

1.6073500

$-0.7797110$

$-1.9974440$

$-1.7686780$

$-0.6393910$

$-3.2633080$

$-4.1452180$

$-3.3818520$

-3. 1228530

$-3.9322450$

$-3.1732820$

$-1.6621500$

$-2.0528760$

$-1.7706530$

$-2.6094870$

$-0.8452440$

$-1.9058250$

$-2.8088360$

$-1.0443610$

0.1681820

0.0564370

0.7028230

0.6878110

0.7471210

0.2573700

0.8932110

0.4656330

0.4972510

1. 7903250

0.1697540

1.9895550

0.5996490

0.9483240

$-0.6151610$

$-0.8365700$

1.8820370

2.9756700

3. 1140230

3.4000970

3.0665810

1.8784910 


$\begin{array}{lrrr}\text { H } & 4.6484610 & -1.9325780 & \\ \mathrm{H} & 5.6695600 & -4.2141780 & 3.9364890 \\ \mathrm{H} & 3.4620700 & -4.7421860 & 4.4590120 \\ \mathrm{H} & 2.3000850 & -2.7970130 & 3.9362860 \\ \mathrm{H} & 5.9673720 & -1.9912470 & 2.7596480 \\ \mathrm{H} & 5.3500410 & -4.3266910 & 2.1710750 \\ \mathrm{H} & 3.4022240 & -5.3908470 & 2.8244600 \\ \mathrm{H} & 1.4690310 & -3.9823700 & 2.9437610 \\ \mathrm{C} & -3.1997900 & 1.8737980 & -1.8148120 \\ \mathrm{C} & -2.1263220 & 2.7088340 & -2.3709120 \\ \mathrm{H} & -3.0275210 & 1.4419780 & -0.8254650 \\ \mathrm{H} & -1.1164540 & 2.5255120 & -2.0179380 \\ \mathrm{O} & -2.6677700 & 1.0240660 & -2.8784080 \\ \mathrm{C} & -1.3627550 & 4.4191940 & 0.8390740 \\ \mathrm{C} & -0.3772830 & 4.2710760 & 3.1612330 \\ \mathrm{C} & -0.0211640 & 3.2161410 & 2.3071590 \\ \mathrm{~N} & -0.5779170 & 3.3402150 & 1.0464100 \\ \mathrm{H} & -0.3769530 & 2.6135320 & 0.3237670 \\ \mathrm{~S} & -1.4234720 & 5.3875290 & 2.3092700 \\ \mathrm{~S} & -2.1818040 & 4.8388290 & -0.5633800 \\ \mathrm{H} & -1.9310830 & 0.3666220 & -2.4421500 \\ \mathrm{C} & 0.8867310 & 3.2364830 & 4.9180990 \\ \mathrm{C} & 1.2269210 & 2.1709190 & 4.0525180 \\ \mathrm{C} & 0.7813310 & 2.1546550 & 2.7279210 \\ \mathrm{C} & 0.0867300 & 4.2866140 & 4.4814100 \\ \mathrm{H} & 1.2558750 & 3.2124600 & 5.9397140 \\ \mathrm{H} & 1.0090320 & 1.3556420 & 2.0336580 \\ \mathrm{H} & -0.1758050 & 5.0942200 & 5.1592540 \\ \mathrm{C} & -4.8254770 & 2.9539070 & -3.4352910 \\ \mathrm{C} & -3.8241620 & 4.1015440 & -3.6425710 \\ \mathrm{C} & -2.3676190 & 3.6112970 & -3.5455580 \\ \mathrm{C} & -4.6332470 & 2.2973120 & -2.0581960 \\ \mathrm{H} & -5.8519270 & 3.3315290 & -3.5165500 \\ \mathrm{H} & -3.9781940 & 4.5783010 & -4.6179770 \\ \mathrm{H} & -2.0965250 & 3.0450390 & -4.4481560 \\ \mathrm{H} & -4.8915850 & 3.0092280 & -1.2619600 \\ \mathrm{H} & -5.2773050 & 1.4208940 & -1.9375510 \\ \mathrm{H} & -4.6908330 & 2.1976640 & -4.2201490 \\ \mathrm{H} & -3.9953410 & 4.8664610 & -2.8732390 \\ \mathrm{H} & -1.6633660 & 4.4483370 & -3.4847220 \\ \mathrm{O} & 1.9915310 & 1.1872750 & 4.6028360 \\ \mathrm{C} & 2.2805480 & 0.0510990 & 3.7758760 \\ \mathrm{H} & 2.8533460 & 0.3410720 & 2.8870350 \\ \mathrm{H} & 1.3553200 & -0.4468820 & 3.4565810 \\ \mathrm{H} & 2.8764670 & -0.6216230 & 4.3951610 \\ \mathrm{I} & & & \\ & & & \end{array}$

\begin{tabular}{|c|c|c|c|}
\hline \multicolumn{4}{|c|}{$\operatorname{TS} 30(R, R) \quad G[B 97-D /$ def2-TZVP $]=-4356.205603$} \\
\hline$P$ & -0.0329560 & -0.1206380 & \\
\hline 0 & 0.2787300 & 1.3189790 & -0.5362530 \\
\hline 0 & -0.9996950 & -0.4857530 & -1.8962320 \\
\hline c & -0.5045310 & -0.7518630 & 0.6361700 \\
\hline 0 & 1.2917570 & -1.0060110 & -1.1302490 \\
\hline c & -0.6689570 & -2.1378660 & 0.6857830 \\
\hline$c$ & 0.4809600 & -2.9434770 & 0.7517610 \\
\hline & -1.9654770 & -2.6660560 & 0.6377950 \\
\hline c & -2.0767500 & -4.0584970 & 0.6940650 \\
\hline H & -3.0730390 & -4.4970930 & 0.6824090 \\
\hline$c$ & 2.1920300 & -1.3265950 & -0.1184950 \\
\hline & 3.4498810 & -0.7091660 & -0.1308120 \\
\hline$C$ & 1.8201730 & -2.2898450 & 0.8407010 \\
\hline c & 4.3505170 & -1.0674490 & 0.8799250 \\
\hline & 5.3410900 & -0.6160270 & 0.8818750 \\
\hline $\mathrm{c}$ & -3.1729060 & -1.8128090 & 0.4741210 \\
\hline$c$ & 3.8293570 & 0.2739130 & -1.1807680 \\
\hline$c$ & -3.5192290 & -0.8 & 1.4278050 \\
\hline$c$ & -2.7935130 & -0.5434730 & 2.7184360 \\
\hline$c$ & -4.9 & 0.9284660 & 2.3886730 \\
\hline$c$ & -4.6762100 & -0.0518970 & 1.2663130 \\
\hline$c$ & -2.3941580 & 0.9533810 & 2.7198920 \\
\hline $\mathrm{H}$ & -1.9003630 & 1.1944300 & 3.6690490 \\
\hline $\mathrm{H}$ & -1.6688020 & 1.1172020 & 1.9234150 \\
\hline 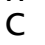 & -3.6 & 1.84 & 2.5124210 \\
\hline H & -3.7 & 2.527 & 3.3581930 \\
\hline H & -3.5 & 2.454 & 1.6086050 \\
\hline $\mathrm{H}$ & -5.80 & 1.526 & 2.2104650 \\
\hline H & -1.9 & -1.172 & 2. \\
\hline 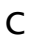 & $-\overline{5}$ & 0.1232460 & 3.7126920 \\
\hline $\mathrm{H}$ & -5.1 & 0.8284020 & 4.5487470 \\
\hline $\mathrm{H}$ & -5.9 & -0.4845180 & 3.6816270 \\
\hline C & -3 & -0.7803470 & 3.8953860 \\
\hline $\mathrm{H}$ & -3.2 & -0.5442900 & 4.8374120 \\
\hline H & -4.0640150 & -1.8391900 & 3.9265450 \\
\hline C & -4.0196100 & -1.9868640 & -0.6394580 \\
\hline $\mathrm{c}$ & -3.8459930 & -2.9786620 & -1.7687560 \\
\hline $\mathrm{c}$ & -6.0056430 & -1.5550580 & -1.9971820 \\
\hline $\mathrm{C}$ & -5.1 & -1.2097 & -0.7795930 \\
\hline $\mathrm{C}$ & -5.086 & -3.9162010 & -1.7704820 \\
\hline $\mathrm{H}$ & -4.9968 & -4.6190940 & -2.6093320 \\
\hline $\mathrm{H}$ & -5.1060270 & -4.5049290 & -0.8450680 \\
\hline C & -6.3858240 & -3.0607180 & -1.9053990 \\
\hline $\mathrm{H}$ & -6.943 & -3.3424700 & 30826 \\
\hline & -7.0453800 & -3.2183610 & -1.0437710 \\
\hline
\end{tabular}




$\begin{array}{rrr}-6.9082190 & -0.9337580 & -2.0524790 \\ -2.9270000 & -3.5589750 & -1.6623710 \\ -5.1274130 & -1.3634000 & -3.2632420 \\ -5.7062070 & -1.6696780 & -4.1449910 \\ -4.8722030 & -0.3057890 & -3.3819950 \\ -3.8328340 & -2.2180530 & -3.1229460 \\ -3.7681110 & -2.9572340 & -3.9323380 \\ -2.9415010 & -1.5883730 & -3.1735000 \\ -5.5135040 & -0.2410630 & 0.1684190 \\ -6.4190610 & 0.3559390 & 0.0568210 \\ 4.3145540 & 0.8114820 & -3.5144080 \\ 4.3899090 & 0.2226480 & -4.9004310 \\ 3.7218760 & -1.5064820 & -3.0808730 \\ 3.9364400 & -0.1169950 & -2.5270660 \\ 5.4273070 & -0.9348190 & -4.8835340 \\ 5.4349090 & -1.4198190 & -5.8688200 \\ 6.4293760 & -0.5264530 & -4.7062770 \\ 5.0421890 & -1.9570610 & -3.7680290 \\ 4.8948610 & -2.9581320 & -4.1945670 \\ 5.8378050 & -2.0287570 & -3.0166910 \\ 3.4428760 & -2.2179070 & -2.2988060 \\ 4.6744150 & 0.9830720 & -5.6379150 \\ 2.6160290 & -1.4458660 & -4.1701840 \\ 2.5190820 & -2.4409450 & -4.6247830 \\ 1.6598220 & -1.1981610 & -3.7017180 \\ 3.0028110 & -0.3859210 & -5.2482400 \\ 3.0533080 & -0.8432120 & -6.2454910 \\ 2.2546900 & 0.4149510 & -5.2862710 \\ 4.4903670 & 2.5323050 & -1.8416560 \\ 4.7410190 & 3.9234620 & -1.3145180 \\ 3.9996200 & 2.2428920 & 0.5200120 \\ 4.1069330 & 1.6113460 & -0.8475690 \\ 3.4353910 & 4.4338110 & -0.6448390 \\ 3.6286670 & 5.4156320 & -0.1921940 \\ 2.6585400 & 4.5658730 & -1.4072700 \\ 2.9755330 & 3.4072800 & 0.4336510 \\ 2.8944850 & 3.8826550 & 1.4191720 \\ 1.9985530 & 2.9944150 & 0.1766430 \\ 3.6798670 & 1.5184290 & 1.2731880 \\ 5.0546460 & 4.6004440 & -2.1184080 \\ 5.3794710 & 2.8456370 & 0.8996270 \\ 5.2848930 & 3.3609000 & 1.8647140 \\ 6.1140140 & 2.0404810 & 1.0227800 \\ 5.8357720 & 3.8415160 & -0.2141200 \\ 5.9962950 & 4.8436900 & -60632210 \\ 6.7806740 & 3.5132470 & \end{array}$

4.5929800

4.8868640

4.0026980

4.9615870

4.9722600

3.5532610

2. 4198610

2.7321340

4.6478600

5.6687970

3.4611960

2. 2993900

5.9669000

5. 3494700

3.4015160

1.4684010

0.3312070

$-0.9637190$

1.5333580

1.2931530

0.0013460

$-1.1953060$

2.1527030

1.7516470

2. 4262510

0.0844450

$-0.1544510$

$-2.0965800$

$-1.4122470$

1.2089630

$-3.1996960$

$-2.1262090$

$-3.0275030$

$-1.1163750$

$-2.6675420$

$-1.3627770$

$-0.3772610$

$-0.0211120$

$-0.5778760$

$-0.3768760$

$-1.4234950$

$-2.1819230$

$-1.9309040$

0.8867160

1.2269110
0.7813850
2.1358850

2.8525250

$-1.9720840$

$-2.3727910$

$-3.9104920$

$-4.4707430$

$-3.4605040$

$-2.5779140$

$-1.9329710$

$-4.2146430$

$-4.7425040$

$-2.7972440$

$-1.9916790$

$-4.3270450$

$-5.3911120$

$-3.9825150$

$-4.3492810$

$-4.9036230$

$-5.2537070$

$-6.7057560$

$-7.2312450$

$-6.4060990$

$-7.3221650$

$-5.2397570$

$-4.8573620$

$-7.1498400$

$-8.2910340$

$-6.6511410$

$-6.6881460$

$-6.7589990$

1.8735140

2.7086680

1.4415510

2.5253360

1.0239190

4.4190100

4.2710460

3.2160850

3.3400850

2. 6134010

5.3874240

4.8385090

0.3665180

3.2365250

. 1709210

2.1546240
$-3.1744750$ $-3.9424080$

1.8822520

2.9759810

3.1142590

3.4001380

3.0665040

1.8784950

3.9367860

3.9053130

4.4590950

3.9362150

2.7600950

2.1713320

2.8244530

2.9435300

0.6874450

0.7023520

0.4653310

0.8927820

0.2568010

0.7465320

0.5992450

$-0.6154370$

0.9481540

$-0.8371260$

0.4965850

0.1690860

1.7897010

1.9891160

$-1.8154610$

$-2.3713350$

$-0.8261660$

$-2.0182710$

$-2.8791070$

0.8388900

3.1610360

2. 3070060

1.0462560

0.3236150

2. 3090340

$-0.5635500$

$-2.4428070$

4.9179710

4.0524440

2.7278270 


\begin{tabular}{|c|c|c|c|c|c|c|c|}
\hline C & 0.0867300 & 4.2866410 & 4.4812190 & $\mathrm{H}$ & -1.7210190 & -1.0489850 & 4.0112300 \\
\hline H & 1.2558200 & 3.2125320 & 5.9396000 & C & -3.3148960 & -0.4487410 & 5.4097140 \\
\hline $\mathrm{H}$ & 1.0091090 & 1.3555800 & 2.0336060 & $\mathrm{H}$ & -3.3937760 & -0.9009290 & 6.4075260 \\
\hline H & -0.1758260 & 5.0942710 & 5.1590270 & $\mathrm{H}$ & -2.7058450 & 0.4583950 & 5.5026540 \\
\hline C & -4.8252650 & 2.9537910 & -3.4359430 & $\mathrm{H}$ & -5.2054080 & 0.6521160 & 5.5988360 \\
\hline C & -3.8239650 & 4.1014830 & -3.6429750 & $\mathrm{H}$ & -3.1817720 & -2.3680570 & 2.4849450 \\
\hline C & -2.3674160 & 3.6112700 & -3.5458840 & C & -5.5798640 & -1.3687890 & 4.8301680 \\
\hline $\mathrm{c}$ & -4.6331430 & 2.2970240 & -2.0589150 & $\mathrm{H}$ & -5.6145810 & -1.8338760 & 5.8244180 \\
\hline $\mathrm{H}$ & -5.8517190 & 3.3313930 & -3.5172490 & $\mathrm{H}$ & -6.6088130 & -1.1213410 & 4.5428930 \\
\hline $\mathrm{H}$ & -3.9779170 & 4.5783690 & -4.6183310 & C & -4.9335100 & -2.3365280 & 3.7903430 \\
\hline $\mathrm{H}$ & -2.0962130 & 3.0451370 & -4.4485280 & $\mathrm{H}$ & -4.6753550 & -3.2943700 & 4.2613660 \\
\hline $\mathrm{H}$ & -4.8915580 & 3.0088350 & -1.2626120 & $\mathrm{H}$ & -5.6312610 & -2.5468900 & 2.9708110 \\
\hline $\mathrm{H}$ & -5.2772030 & 1.4205900 & -1.9384230 & C & -4.2547060 & 1.3045460 & 0.8741610 \\
\hline $\mathrm{H}$ & -4.6905260 & 2.1976540 & -4.2208880 & $\mathrm{C}$ & -4.0852330 & 1.9381210 & -0.4876800 \\
\hline $\mathrm{H}$ & -3.9952380 & 4.8662910 & -2.8735560 & $\mathrm{c}$ & -5.2971060 & 3.4875910 & 1.2080800 \\
\hline $\mathrm{H}$ & -1.6631920 & 4.4483210 & -3.4848730 & $\mathrm{c}$ & -4.8784130 & 2.1638920 & 1.7981180 \\
\hline 0 & 1.9914370 & 1.1872540 & 4.6028440 & $\mathrm{C}$ & -5.4867210 & 2.3136530 & -1.0412830 \\
\hline $\mathrm{C}$ & 2.2802740 & 0.0509430 & 3.7760080 & $\mathrm{H}$ & -5.3576510 & 2.8494760 & -1.9912350 \\
\hline $\mathrm{H}$ & 2.8531840 & 0.3407080 & 2.8871730 & $\mathrm{H}$ & -6.0597160 & 1.4021020 & -1.2504580 \\
\hline $\mathrm{H}$ & 1.3549630 & -0.4468830 & 3.4567090 & C & -6.2333350 & 3.2076900 & -0.0013110 \\
\hline $\mathrm{H}$ & 2.8760220 & -0.6218400 & 4.3953930 & $\mathrm{H}$ & -6.5292280 & 4.1636340 & -0.4533560 \\
\hline \multirow{2}{*}{\multicolumn{4}{|c|}{$\begin{array}{l}158 \\
T S 31(R, R) \quad G[B 97-D / \text { def2-TZVP }]=-4356.205553\end{array}$}} & $\mathrm{H}$ & -7.1444350 & 2.7106780 & 0.3528960 \\
\hline & & & & $\mathrm{H}$ & -5.8086260 & 4.1082040 & 1.9539150 \\
\hline $\mathrm{P}$ & 0.0264930 & -0.0036090 & 1.1318790 & $\mathrm{H}$ & -3.5650060 & 1.2734240 & -1.1816720 \\
\hline 0 & 0.9591070 & -0.1375430 & 2.3105870 & C & -4.0307520 & 4.2076850 & 0.6699690 \\
\hline 0 & -0.5024390 & 1.3510880 & 0.7748890 & $\mathrm{H}$ & -4.3360630 & 5.1306200 & 0.1586380 \\
\hline 0 & -1.1587820 & -1.0752300 & 1.4205770 & $\mathrm{H}$ & -3.3825010 & 4.4909040 & 1.5075920 \\
\hline 0 & 0.7144570 & -0.5822480 & -0.2404140 & C & -3.2771990 & 3.2536810 & -0.3054030 \\
\hline C & -1.9693940 & -1.4413970 & 0.3435330 & $\mathrm{H}$ & -3.1406640 & 3.7256480 & -1.2872580 \\
\hline C & -1.4390210 & -2.3328710 & -0.6074570 & $\mathrm{H}$ & -2.2917560 & 3.0067780 & 0.0930090 \\
\hline C & -3.2634910 & -0.9159770 & 0.2581840 & C & -5.0579340 & 1.7696950 & 3.1239330 \\
\hline C & -4.0369950 & -1.3070960 & -0.8441360 & $\mathrm{H}$ & -5.5397590 & 2.4383120 & 3.8385340 \\
\hline $\mathrm{H}$ & -5.0530470 & -0.9259980 & -0.9276280 & C & 4.8820900 & 0.3604500 & -1.1123970 \\
\hline C & 1.0079880 & -1.9458660 & -0.3081340 & $\mathrm{C}$ & 5.0441780 & 1.2584500 & -2.3128750 \\
\hline C & 2.3462480 & -2.3526050 & -0.2543910 & $\mathrm{C}$ & 2.9766720 & -0.3084740 & -2.4633440 \\
\hline C & -0.0537080 & -2.8583030 & -0.4351640 & $\mathrm{C}$ & 3.7590210 & -0.4852310 & -1.1835450 \\
\hline C & 2.5903060 & -3.7285390 & -0.2991740 & C & 5.1686050 & 0.3587440 & -3.5746810 \\
\hline $\mathrm{H}$ & 3.6239020 & -4.0693600 & -0.2694820 & $\mathrm{H}$ & 5.2357050 & 0.9993960 & -4.4640070 \\
\hline C & -3.8272250 & 0.0214090 & 1.2638970 & $\mathrm{H}$ & 6.0939460 & -0.2264300 & -3.5151880 \\
\hline C & $3.4 \varepsilon$ & -1.3983730 & -0.1506410 & C & 3.9257040 & -0.5815220 & -3.6631920 \\
\hline C & -4.00 & -0.3640720 & 2.6042270 & $\mathrm{H}$ & 3.3723250 & -0.4048240 & -4.5951250 \\
\hline C & -3.6 & -1.6964780 & 3.2156480 & $\mathrm{H}$ & 4.2335110 & -1.6338990 & -3.6538750 \\
\hline C & -4.73 & -0.0655430 & 4.9141120 & $\mathrm{H}$ & 2.1098410 & -0.9725310 & -2.5079440 \\
\hline C & -4.6 & 0.5096990 & 3.5254270 & $\mathrm{H}$ & 5.9276700 & 1.9001520 & -2.2101560 \\
\hline C & -2.6 & -1.4440110 & 4. 3988610 & C & 2.5195530 & 1.1707000 & -2.5356660 \\
\hline H & -2.4 & 425 & 4.8865790 & $\mathrm{H}$ & 1.9538630 & 1.3310450 & -3.4609810 \\
\hline
\end{tabular}




$\begin{array}{rrrr}\mathrm{H} & 1.8446740 & 1.3605130 & -1.7008540 \\ \mathrm{C} & 3.7576640 & 2.1139430 & -2.4686910 \\ \mathrm{H} & 3.8374240 & 2.7165730 & -3.3829820 \\ \mathrm{H} & 3.6687000 & 2.8091390 & -1.6258490 \\ \mathrm{C} & 5.4706190 & -0.5900090 & 1.0183350 \\ \mathrm{C} & 6.2852020 & -0.7527410 & 2.2767230 \\ \mathrm{C} & 4.2157530 & -2.3212400 & 2.1762990 \\ \mathrm{C} & 4.3476150 & -1.4372710 & 0.9576750 \\ \mathrm{C} & 5.3599720 & -0.4657820 & 3.4921530 \\ \mathrm{H} & 5.9309730 & -0.6186550 & 4.4176770 \\ \mathrm{H} & 5.0354140 & 0.5805690 & 3.4685960 \\ \mathrm{C} & 4.1265660 & -1.4174380 & 3.4368500 \\ \mathrm{H} & 4.0954970 & -2.0624190 & 4.3249460 \\ \mathrm{H} & 3.1939620 & -0.8488460 & 3.4048840 \\ \mathrm{H} & 3.3348420 & -2.9649020 & 2.1158660 \\ \mathrm{H} & 7.1494950 & -0.0770120 & 2.2785120 \\ \mathrm{C} & 5.5080070 & -3.1749340 & 2.2967410 \\ \mathrm{H} & 5.4393640 & -3.7946050 & 3.2004650 \\ \mathrm{H} & 5.5844870 & -3.8505500 & 1.4360880 \\ \mathrm{C} & 6.7514400 & -2.2332490 & 2.3685810 \\ \mathrm{H} & 7.2931050 & -2.3774980 & 3.3127940 \\ \mathrm{H} & 7.4479870 & -2.4478760 & 1.5493990 \\ \mathrm{C} & 5.7445820 & 0.3012240 & -0.0183190 \\ \mathrm{H} & 6.6189390 & 0.9512860 & 0.0304200 \\ \mathrm{C} & 1.5632600 & -4.6751940 & -0.3395330 \\ \mathrm{C} & 0.2238500 & -4.2443010 & -0.3859310 \\ \mathrm{C} & 1.9331960 & -6.1502170 & -0.3678580 \\ \mathrm{C} & 0.7904090 & -7.0799970 & 0.0592040 \\ \mathrm{C} & -0.5023480 & -6.6784630 & -0.6600650 \\ \mathrm{C} & -0.9057520 & -5.2545860 & -0.2621860 \\ \mathrm{H} & 1.0568200 & -8.1223240 & -0.1587420 \\ \mathrm{H} & 2.2311340 & -6.4112180 & -1.3964480 \\ \mathrm{H} & 2.8200340 & -6.3134820 & 0.2589260 \\ \mathrm{H} & -0.3440100 & -6.7272830 & -1.7485030 \\ \mathrm{H} & -1.3176880 & -7.3720220 & -0.4167170 \\ \mathrm{H} & -1.7814400 & -4.9368520 & -0.8295030 \\ \mathrm{H} & -1.2234270 & -5.2602970 & 0.7935280 \\ \mathrm{H} & 0.6339910 & -6.9996140 & 1.1452550 \\ \mathrm{C} & -3.5306820 & -2.1403000 & -1.8377440 \\ \mathrm{C} & -4.3463390 & -2.5502510 & -3.0375150 \\ \mathrm{C} & -4.2224390 & -4.0722730 & -3.2648360 \\ \mathrm{C} & -2.7406500 & -4.5224070 & -3.3951240 \\ \mathrm{C} & -1.7282810 & -3.4455180 & -2.9190620 \\ \mathrm{C} & -2.2226220 & -2.6486010 & -1.7347700 \\ \mathrm{H} & -3.9773860 & -2.0200870 & -3.9274570 \\ \mathrm{H} & -4.7904280 & -4.3669680 & -4.1563310\end{array}$

\begin{tabular}{|c|c|}
\hline $\mathrm{H}$ & -2.5096850 \\
\hline $\mathrm{H}$ & -1.5801010 \\
\hline $\mathrm{H}$ & -5.3960680 \\
\hline $\mathrm{H}$ & -4.6852900 \\
\hline $\mathrm{H}$ & -2.5878290 \\
\hline $\mathrm{H}$ & -0.7554460 \\
\hline C & 2.7838870 \\
\hline C & 1.6818670 \\
\hline $\mathrm{H}$ & 2.5480220 \\
\hline $\mathrm{H}$ & 0.6731650 \\
\hline 0 & 2.5080970 \\
\hline C & 0.4968120 \\
\hline C & -0.2498420 \\
\hline C & -0.5233950 \\
\hline $\mathrm{N}$ & -0.1005610 \\
\hline $\mathrm{H}$ & -0.2252180 \\
\hline $\mathrm{S}$ & 0.5435710 \\
\hline $\mathrm{S}$ & 1.1183210 \\
\hline $\mathrm{H}$ & 1.8306430 \\
\hline C & -1.1645270 \\
\hline C & -1.4275820 \\
\hline C & -1.1121530 \\
\hline C & -0.5744340 \\
\hline $\mathrm{H}$ & -1.4139040 \\
\hline $\mathrm{H}$ & -1.2958830 \\
\hline $\mathrm{H}$ & -0.3678590 \\
\hline C & 4.4315490 \\
\hline $\mathrm{C}$ & 3.3126290 \\
\hline C & 1.9427730 \\
\hline C & 4.1660080 \\
\hline $\mathrm{H}$ & 1340 \\
\hline $\mathrm{H}$ & 77610 \\
\hline $\mathrm{H}$ & 7860 \\
\hline $\mathrm{H}$ & 07990 \\
\hline $\mathrm{H}$ & 4.9015330 \\
\hline $\mathrm{H}$ & 4.4984750 \\
\hline $\mathrm{H}$ & 33010 \\
\hline $\mathrm{H}$ & 1.12 \\
\hline 0 & -1.9858300 \\
\hline C & -2.2582680 \\
\hline $\mathrm{H}$ & -1.3413120 \\
\hline $\mathrm{H}$ & -3.0103260 \\
\hline$H_{15}$ & 1110 \\
\hline
\end{tabular}

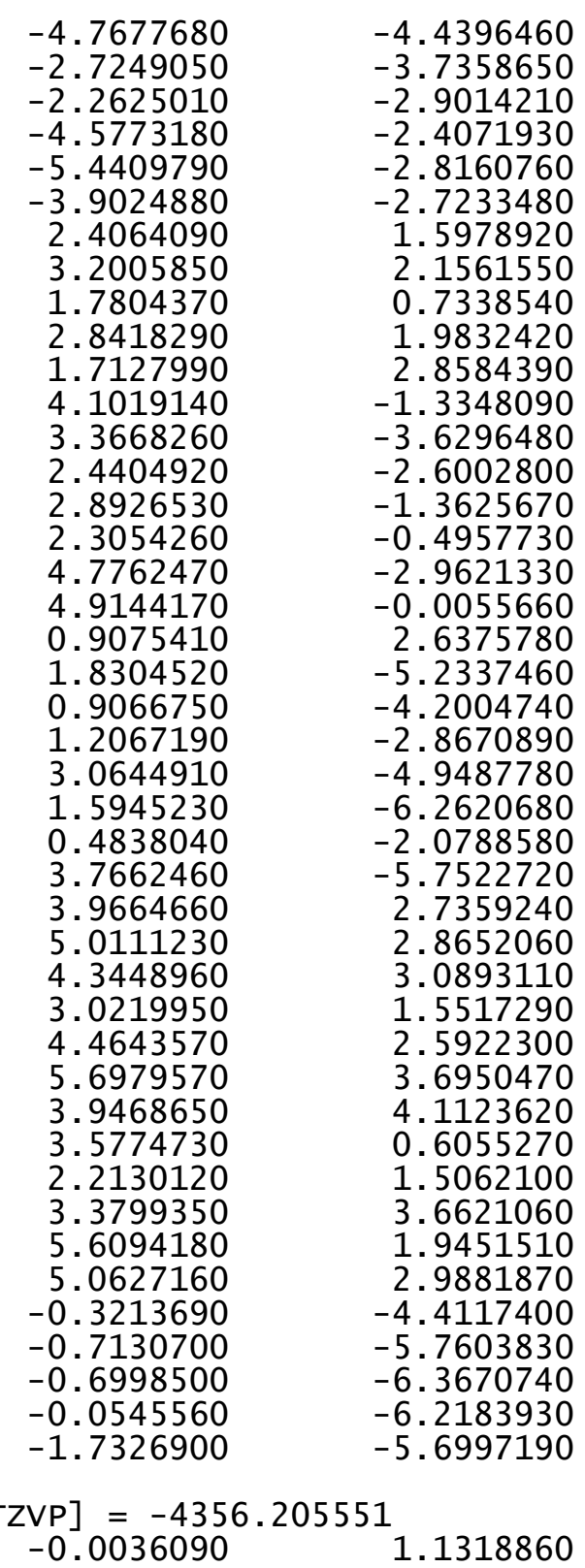

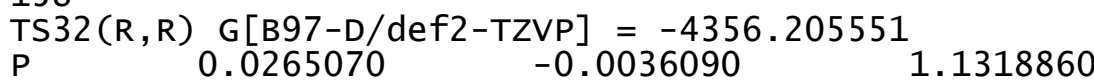




$\begin{array}{rrr}-0.5024310 & 1.3510870 & 0.7748860 \\ 0.9591210 & -0.1375390 & 2.3105880 \\ 0.7144610 & -0.5822530 & -0.2404130 \\ -1.1587780 & -1.0752230 & 1.4205840 \\ 1.0079920 & -1.9458710 & -0.3081260 \\ -0.0537050 & -2.8583080 & -0.4351480 \\ 2.3462510 & -2.3526110 & -0.2543840 \\ 2.5903090 & -3.7285450 & -0.2991610 \\ 3.6239040 & -4.0693670 & -0.2694700 \\ -1.9693890 & -1.4413950 & 0.3435420 \\ -3.2634870 & -0.9159750 & 0.2581900 \\ -1.4390190 & -2.3328780 & -0.6074420 \\ -4.0369930 & -1.3071020 & -0.8441250 \\ -5.0530440 & -0.9260050 & -0.9276190 \\ 3.4815680 & -1.3983790 & -0.1506390 \\ -3.8272200 & 0.0214190 & 1.2638970 \\ 3.7590250 & -0.4852410 & -1.1835460 \\ 2.9766780 & -0.3084910 & -2.4633460 \\ 5.0441790 & 1.2584380 & -2.3128810 \\ 4.8820920 & 0.3604430 & -1.1123990 \\ 2.5195560 & 1.1706810 & -2.5356730 \\ 1.9538620 & 1.3310220 & -3.4609860 \\ 1.8446790 & 1.3604950 & -1.7008590 \\ 3.7576640 & 2.1139280 & -2.4687050 \\ 3.8374240 & 2.7165500 & -3.3830010 \\ 3.6686980 & 2.8091310 & -1.6258690 \\ 5.9276700 & 1.9001430 & -2.2101640 \\ 2.1098480 & -0.9725500 & -2.5079440 \\ 5.1686100 & 0.3587260 & -3.5746830 \\ 5.2357130 & 0.9993730 & -4.4640120 \\ 6.0939520 & -0.2264470 & -3.5151840 \\ 3.9257110 & -0.5815410 & -3.6631930 \\ 3.3723330 & -0.4048470 & -4.5951270 \\ 4.2335190 & -1.6339180 & -3.6538720 \\ 4.3476190 & -1.4372710 & 0.9576780 \\ 4.2157570 & -2.3212360 & 2.1763070 \\ 6.2852030 & -0.7527300 & 2.2767260 \\ 5.4706210 & -0.5900060 & 1.0183370 \\ 5.5080140 & -3.1749260 & 2.2967520 \\ 5.4393710 & -3.7945950 & 3.2004770 \\ 5.5844970 & -3.8505440 & 1.4361000 \\ 6.7514430 & -2.2332370 & 2.3685930 \\ 7.2931050 & -2.3774800 & 3.3128090 \\ 7.4479950 & -2.4478660 & 2.5494150 \\ 7.1494940 & -0.0770000 & 2.1158750 \\ 3.3348490 & -2.9649000 & \end{array}$

5.3599690

5. 9309690

5.0354050

4.1265670

4.0955000

3.1939620

5.7445820

6.6189370

$-4.6134690$

$-4.7371580$

$-3.6389440$

$-4.0047370$

$-5.5798610$

$-5.6145800$

$-6.6088090$

$-4.9335090$

$-4.6753550$

$-5.6312600$

$-3.1817720$

$-5.2054010$

$-2.6644430$

$-2.4498550$

$-1.7210150$

$-3.3148910$

$-3.3937710$

$-2.7058380$

$-4.8784050$

$-5.2970950$

$-4.0852250$

$-4.2546990$

$-4.0307400$

$-4.3360480$

$-3.3824880$

$-3.2771880$

$-3.1406520$

$-2.2917470$

$-3.5649990$

$-5.8086140$

$-5.4867120$

$-5.3576420$

$-6.0597090$

$-6.2333240$

$-6.5292130$

$-7.1444260$

-5.0579260
-5.5397510
$-0.4657680$

$-0.6186310$

0.5805820

$-1.4174290$

$-2.0624070$

$-0.8488410$

0.3012250

0.9512890

0.5097250

$-0.0655070$

$-1.6964560$

$-0.3640540$

$-1.3687520$

$-1.8338330$

$-1.1213040$

$-2.3365000$

$-3.2943390$

$-2.5468670$

$-2.3680410$

0.6521570

$-1.4439820$

$-2.4042260$

$-1.0489590$

$-0.4487050$

$-0.9008860$

0.4584310

2.1639070

. 4876020

1.9381190

1.3045530

. 2076900

5.1306230

4.4909130

3.2536780

3.7256390

3.0067740

1.2734150

4.1082210

2. 3136500

2.8494640

1.4020970

3.2076970

4.1636380

2.7106900

1.7697180

2. 4383410
3.4921530

4.4176790

3.4685870

3.4368540

4.3249520

3.4048850

$-0.0183190$

0.0304190

3.5254230

4.9141120

3.2156590

2. 6042290

4.8301770

5.8244300

4.5429000

3.7903590

4.2613880

2.9708290

2.4849600

5.5988320

4.3988680

4.8865930

4.0112340

5.4097160

6.4075310

5.5026500

1.7981030

1.2080560

$-0.4876930$

0.8741520

0.6699410

0.1586040

1.5075620

$-0.3054240$

$-1.2872830$

0.0929900

$-1.1816810$

1.9538870

$-1.0412990$

$-1.9912560$

$-1.2504670$

$-0.0013340$

$-0.4533850$

0.3528750

3.1239200
3.8385170 


$\begin{array}{rrr}-3.5306830 & -2.1403170 & -1.8377260 \\ -4.3463430 & -2.5502810 & -3.0374900 \\ -4.2224440 & -4.0723060 & -3.2647950 \\ -2.7406550 & -4.5224420 & -3.3950820 \\ -1.7282840 & -3.4455470 & -2.9190350 \\ -2.2226220 & -2.6486180 & -1.7347500 \\ -3.9773920 & -2.0201270 & -3.9274390 \\ -4.7904350 & -4.3670090 & -4.1562870 \\ -2.5096930 & -4.7678150 & -4.4396010 \\ -1.5801070 & -2.7249440 & -3.7358470 \\ -5.3960720 & -2.2625290 & -2.9013970 \\ -4.6852930 & -4.5773420 & -2.4071470 \\ -2.5878330 & -5.4410070 & -2.8160240 \\ -0.7554490 & -3.9025150 & -2.7233190 \\ 0.2238530 & -4.2443060 & -0.3859070 \\ 1.5632620 & -4.6752000 & -0.3395120 \\ -0.9057500 & -5.2545890 & -0.2621460 \\ -0.5023510 & -6.6784710 & -0.6600090 \\ 0.7904120 & -7.0799980 & 0.0592530 \\ 1.9331950 & -6.1502230 & -0.3678310 \\ -1.3176900 & -7.3720260 & -0.4166460 \\ -1.2234200 & -5.2602870 & 0.7935700 \\ -1.7814410 & -4.9368600 & -0.8294620 \\ 0.6340040 & -6.9996010 & 1.1453050 \\ 1.0568210 & -8.1223280 & -0.1586820 \\ 2.8200420 & -6.3134860 & 0.2589420 \\ 2.2311170 & -6.4112330 & -1.3964230 \\ -0.3440220 & -6.7273060 & -1.7484480 \\ 2.7838780 & 2.4064430 & 1.5978740 \\ 1.6818540 & 3.2006490 & 2.1560950 \\ 2.5480130 & 1.7804630 & 0.7338400 \\ 0.6731570 & 2.8418530 & 1.9832380 \\ 2.5081560 & 1.7128220 & 2.8584190 \\ 0.4968000 & 4.1018850 & -1.3348210 \\ -0.2498700 & 3.3667930 & -3.6296480 \\ -0.5234010 & 2.4404550 & -2.6002770 \\ -0.1005570 & 2.8926200 & -1.3625680 \\ -0.2252070 & 2.3053900 & -0.4957660 \\ 0.5435380 & 4.7762210 & -2.9621430 \\ 1.1183140 & 4.9144170 & -0.0055930 \\ 1.8307160 & 0.9075870 & 2.6375850 \\ -1.1645620 & 1.8304160 & -5.2337370 \\ -1.4275940 & 0.9066330 & -4.2004640 \\ -1.1121510 & 1.2066780 & -2.8670820 \\ -0.5744770 & 3.0644590 & -4.9487740 \\ -1.4139490 & 1.5944870 & -6.2620560\end{array}$

$\begin{array}{lr}\mathrm{H} & -1.2958630 \\ \mathrm{H} & -0.3679190 \\ \mathrm{C} & 4.4315390 \\ \mathrm{C} & 3.3126140 \\ \mathrm{C} & 1.9427630 \\ \mathrm{C} & 4.1659890 \\ \mathrm{H} & 5.3981190 \\ \mathrm{H} & 3.5177460 \\ \mathrm{H} & 1.8777970 \\ \mathrm{H} & 4.2307520 \\ \mathrm{H} & 4.9015250 \\ \mathrm{H} & 4.4984780 \\ \mathrm{H} & 3.2732770 \\ \mathrm{H} & 1.1207090 \\ \mathrm{O} & -1.9858320 \\ \mathrm{C} & -2.2582870 \\ \mathrm{H} & -1.3413400 \\ \mathrm{H} & -3.0103580 \\ \mathrm{H} & -2.6471210 \\ \mathrm{H} & \end{array}$
0.4837580
$-0.3214150$
$-0.7131130$
$-0.6998840$
$-1.7327360$

$3.7662190-5.7522690$

$\begin{array}{ll}3.9665390 & 2.7358530\end{array}$

$5.0111910 \quad 2.8651380$

$4.3449550 \quad 3.0892530$

$3.0220540 \quad 1.5516710$

$4.4644360 \quad 2.5921440$

$5.6980280 \quad 3.6949770$

$3.9469090 \quad 4.1123000$

$3.5775250 \quad 0.6054640$

2. $2130820 \quad 1.5061460$

$3.3800180 \quad 3.6620400$

$5.6094850 \quad 1.9450830$

$5.0627690 \quad 2.9881570$

$-4.4117260$

$-5.7603680$

$-6.3670710$

$-6.2183620$

158

$-5.6997000$

$\operatorname{TS} 33(R, R)$ G $[B 97-D / d e f 2-T Z V P]=-4356.205092$

$\begin{array}{lrrr}\text { TS } & -0.1413300 & -0.3131240 & -0.9208760 \\ \mathrm{O} & 0.4218460 & 1.0736140 & -0.9923550 \\ \mathrm{O} & -1.1782170 & -0.7380930 & -1.9299560 \\ \mathrm{O} & -0.6893120 & -0.4928890 & 0.6111290 \\ \mathrm{O} & 1.0056240 & -1.4600700 & -1.0155650 \\ \mathrm{C} & -1.0016990 & -1.7874600 & 1.0324960 \\ \mathrm{C} & 0.0603920 & -2.6660610 & 1.3266730 \\ \mathrm{C} & -2.3471240 & -2.1603370 & 1.1295700 \\ \mathrm{C} & -2.6205290 & -3.4811030 & 1.5140880 \\ \mathrm{H} & -3.6593250 & -3.7977590 & 1.5870770 \\ \mathrm{C} & 1.9087760 & -1.5927120 & 0.0379410 \\ \mathrm{C} & 3.2267910 & -1.1595340 & -0.1492280 \\ \mathrm{C} & 1.4652030 & -2.1791370 & 1.2347750 \\ \mathrm{C} & 4.0978450 & -1.3118800 & 0.9326770 \\ \mathrm{H} & 5.1291730 & -0.9836300 & 0.8132730 \\ \mathrm{C} & -3.4584650 & -1.2283220 & 0.8019750 \\ \mathrm{C} & 3.6929990 & -0.5479390 & -1.4204150 \\ \mathrm{C} & -3.6656020 & -0.0525460 & 1.5454440 \\ \mathrm{C} & -2.8522520 & 0.4195030 & 2.7274040 \\ \mathrm{C} & -4.8407640 & 1.9966680 & 2.1692910 \\ \mathrm{C} & -4.7473320 & 0.8008390 & 1.2551280 \\ \mathrm{C} & -2.3171010 & 1.8371880 & 2.4032660 \\ \mathrm{H} & -1.7288390 & 2.2068880 & 3.2526140 \\ \mathrm{H} & -1.6476440 & 1.7614370 & 1.5465190 \\ \mathrm{C} & -3.5090850 & 2.7930670 & 2.0923620\end{array}$




$\begin{array}{lr}-3.5469420 & 3.6155320 \\ -3.3958260 & 3.2402230 \\ -5.6910280 & 2.6348210 \\ -2.0222330 & -0.2577680 \\ -4.9923460 & 1.4716840 \\ -5.0159060 & 2.3279880 \\ -5.9457470 & 0.9390900 \\ -3.7953680 & 0.5276200 \\ -3.2191470 & 0.9193900 \\ -4.1539160 & -0.4715580 \\ -4.3607330 & -1.5302570 \\ -4.3234080 & -2.7352620 \\ -6.3311790 & -1.1587640 \\ -5.4483350 & -0.6776130 \\ -5.6539360 & -3.5165210 \\ -5.6589660 & -4.3783830 \\ -5.7115910 & -3.9024340 \\ -6.8616310 & -2.5721040 \\ -7.4588580 & -2.9599560 \\ -7.5206700 & -2.4961120 \\ -7.1657950 & -0.4670970 \\ -3.4665740 & -3.3781960 \\ -5.4657200 & -1.2996570 \\ -6.0941650 & -1.6928380 \\ -5.0997750 & -0.3148540 \\ -4.2693150 & -2.2548500 \\ -4.3139990 & -3.1377130 \\ -3.3153980 & -1.7545920 \\ -5.6440620 & 0.4851550 \\ -6.4901650 & 1.1397860 \\ 4.1426550 & -0.6856370 \\ 4.0600410 & -1.5986080 \\ 3.2063250 & -2.6946490 \\ 3.6669120 & -1.2680980 \\ 4.9006480 & -2.8705410 \\ 4.7931920 & -3.5752250 \\ 5.9609440 & -2.6001440 \\ 4.4032450 & -3.5143880 \\ 4.0736920 & -4.5480860 \\ 5.2092310 & -3.5390070 \\ 2.8532660 & -3.1375310 \\ 4.4295280 & -1.0991340 \\ 2.0758910 & -2.7145620 \\ 1.7927770 & -3.7587840 \\ 1.1973070 & -2.1973140 \\ 2.5810060 & -2.0372430\end{array}$

2.8186870
1.0982920
1.8994560
2.9438910
3.6247170
4.3118890
3.7234690
3.9586020
4.8072330
4.2334210
-0.2365190
-1.1490830
-1.6322540
-0.5086260
-0.9588130
-1.6391300
0.0663640
-1.2588730
-2.0947060
-0.3858530
-1.8014170
-0.9355880
-2.9142170
-3.7245530
-3.2235630
-2.6254540
-3.2769370
-2.8069400
0.2354490
0.0240640
-3.8184800
-5.0158080
-2.8203140
-2.6285730
-4.7144720
-5.5500990
-4.6425240
-3.3819900
-3.5513480
-2.6386960
-1.8854770
-5.9197860
-3.8858650
-4.0748970
-3.4917940
-5.1975050

2.5167200

4.6821820

5.1848650

4. 2551860

4. 2016840

4. 0144460

4. 3936410

3. 2415390

3.4293450

3.4540490

2. 3909430

3. 8555510

5. 5714650

5. 7277690

5.7545250

6.3253880

6.2983230

6.6518790

7.1486360

4.6523670

5.0237720

2. 3578110

1.8727610

2. 7466070

4.2143080

4.6990520

3. 6879200

2. 4126520

1.8727900

0.8295100

4. 3016340

4.8553210

5.6342020

4.9417670

2. 6305680

$-0.2533650$

0.7652220

0.3812920

$-1.0866280$

$-1.8605250$

$-1.6004620$

0.7791310

1.0813330

-1.1042370
-1.5129470
$-2.7314310$
$-1.1598480$
1.3346040
2.7477710
1.7243270
0.7638990
3.6263750
4.6371650
3. 7096730
2. 9830460
3. 6919430
2. 6849950
1. 2788320
. 1414510
2. 1648410
2. 9194520
1.3080220
.7492220
3. 7782190
2. 1523300
0.6129720
1. 0580530
$-2.2594980$
$-2.7409840$
$-2.2374700$
$-2.5796870$
$-1.8548800$
$-1.8318490$
$-2.6953070$
$-3.8391130$
$-2.4335750$
$-3.6674940$
$-2.3117620$
$-2.3046890$
$-0.8116910$
$-1.1505810$
$-3.9995190$
$-5.1056320$
$-6.2755660$
$-6.7539180$
$-5.8532820$
$-4.3983320$
$-5.4808770$
1052640
-7.7879260
-6.0874310

$-6.0461070$

$-5.4377050$

$-2.6273190$

$-2.4650050$

$-0.2662000$

$-1.4330400$

$-1.9412950$

$-1.7386560$

$-2.7144740$

$-0.6482220$

0.1894140

$-0.8072950$

0.6470190

$-3.4129470$

$-0.0495810$

0.7480120

0.2848760

$-1.3805010$

$-1.2318720$

$-1.7316330$

$-3.8203080$

$-4.7443220$

2. 3303050

3.6863560

4.8411190

4. 5609740

3. 2972930

2.1633080

5.7812300

3.7108460

3.8271420

4.4208650

5.4115450

2. 9357720

3. 5450290

4.9373320

1.6504420

1.7753510

0.8359980

1. 0469000

2. 0448980

1.7514490

0.9947890

1. 4136560

3. 0631930 


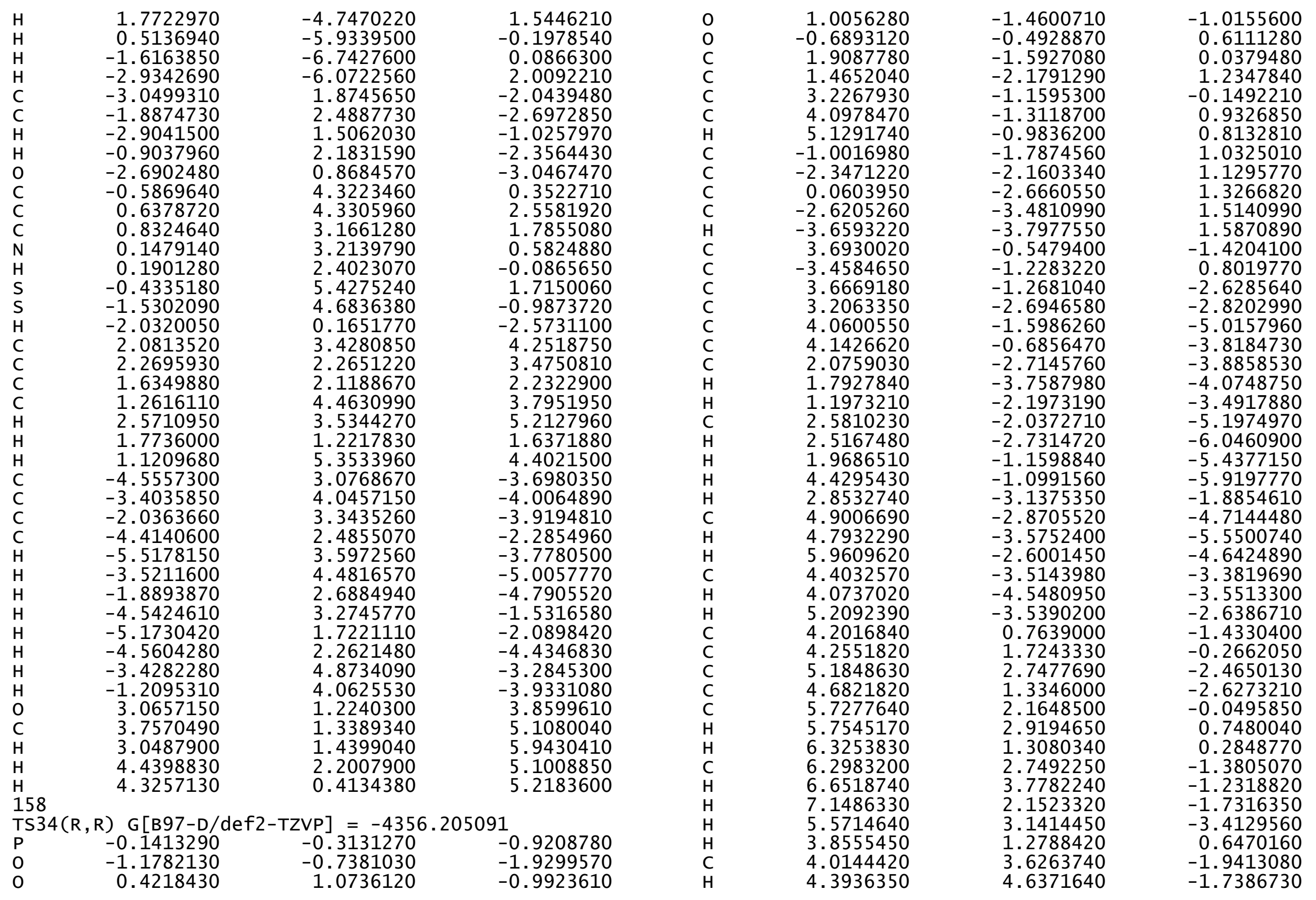




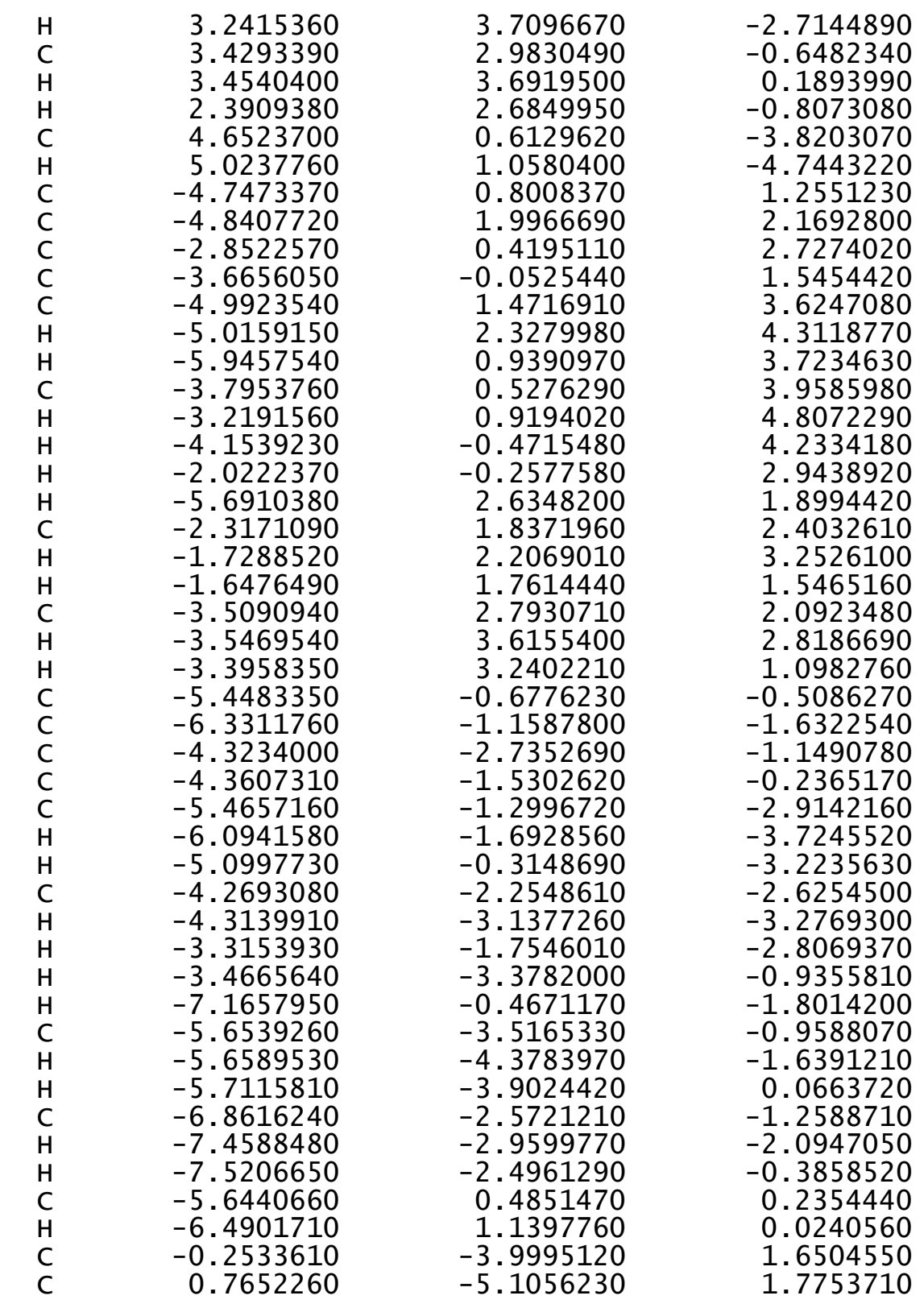
0.3813030
$-1.0866250$
$-1.8605190$
$-1.6004580$
0.7791320
1. 0813340
$-1.1042510$
$-1.5129380$
1. 7723010
0.5137300
-1.6163720
$-2.9342630$
2. 3578110
1.8727610
2. 7466050
4. 2143060
4. 6990500
3. 6879200
2. 4126490
1.8727920
0.8295090
4. 3016350
4.8553180
5.6342020
4.9417630
2. 6305640
3.0499310
-1.8874730
$-2.9041510$
$-0.9037960$
$-2.6902460$
$-0.5869700$
0.6378590
0.8324590
0.1479120
0.1901300
$-0.4335340$
$-1.5302130$
$-2.0320040$
2. 0813380
2. 2695870
1. 6349850
1. 2615930
2. 5710760
1. 7736030
1. 1209440

-6.2755650
-6.7539040
-5.8532770
-4.3983260
-5.4808620
-7.1052670
-7.7879200
-6.0874310
-4.7470130
-5.9339660
-6.7427180
-6.0722510
-2.2594850
-2.7409660
-2.2374480
-2.5796630
-1.8548590
-1.8318350
-2.6952820
-3.8390960
-2.4335590
-3.6674700
-2.3117340
-2.3046670
-0.8116680
-1.1505580
1.8745520
2.4887600
1.5061940
2.1831470
0.8684420
4.3223450
4.3306030
3.1661340
3.2139810
2.4023080
5.4275250
4.6836310
0.1651640
3.4281000
2.2651380
2.1188780
4.4631110
3.5344460
1.2217930
5.3534070

1. 0469020

2. 0449090

1.7514620

2.8104560

0.9948420

1. 4136360

3.0632010

1. 5446420

$-0.1978300$

0.0866280

2. 0092340

2. 3303150

3. 6863680

4.8411300

4. 5609870

3. 2973050

2. 1633180

5. 7812420

3. 7108610

3. 8271520

4.4208820

5.4115580

2. 9357870

3. 5450370

4.9373390

$-2.0439590$

$-2.6972960$

$-1.0258060$

$-2.3564510$

$-3.0467540$

0.3522560

2. 5581800

1.7854990

0.5824770

$-0.0865760$

1. 7149900

$-0.9873910$

$-2.5731150$

4. 2518700

3. 4750780

2. 2322850

3.7951850

5. 2127910

1.6371850

4.4021390 


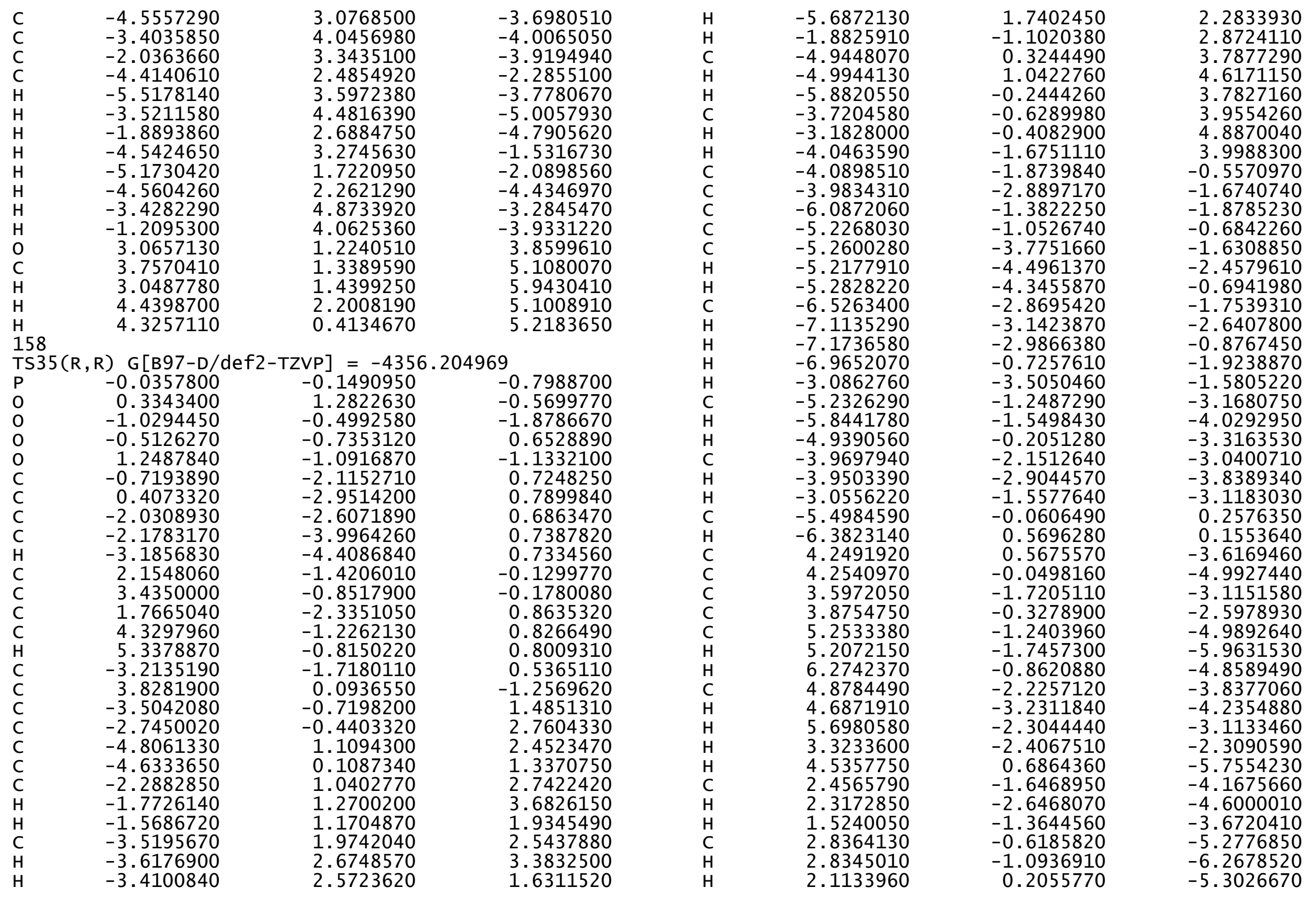




\begin{tabular}{|c|c|c|c|}
\hline 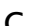 & & & \\
\hline c & $\begin{array}{l}4.5439240 \\
4.8555130\end{array}$ & $\begin{array}{l}2.3138600 \\
3.7071300\end{array}$ & $\begin{array}{l}-1.9880200 \\
-1.5000830\end{array}$ \\
\hline C & 4.1212930 & 2.0907560 & 0.3937970 \\
\hline C & 4.1640360 & 1.4266350 & -0.9619820 \\
\hline C & 3.5886470 & 4.2730900 & -0.8006900 \\
\hline $\mathrm{H}$ & 3.8267430 & 5.2578910 & -0.3767060 \\
\hline H & 2.7920860 & 4.4136170 & -1.5409970 \\
\hline C & 3.1317990 & 3.2854640 & 0.3144150 \\
\hline W & 3.0986460 & 3.7841830 & 1.2912260 \\
\hline $\mathrm{H}$ & 2.1332630 & 2.9006000 & 0.0990560 \\
\hline $\mathrm{H}$ & 3.8051910 & 1.3925620 & 1.1724450 \\
\hline $\mathrm{H}$ & 5.1636340 & 4.3563050 & -2.3286270 \\
\hline C & 5.5309640 & 2.6566660 & 0.7157190 \\
\hline $\mathrm{H}$ & 5.4847610 & 3.1945490 & 1.6719520 \\
\hline $\mathrm{H}$ & 6.2431210 & 1.8307280 & 0.8325020 \\
\hline C & 5.9823200 & 3.6134640 & -0.4335510 \\
\hline $\mathrm{H}$ & 6.1880300 & 4.6185500 & -0.042201 \\
\hline $\mathrm{H}$ & 6.9012540 & 3.2451160 & -0.9048740 \\
\hline C & 4.5845620 & 1.8877560 & -3.3150320 \\
\hline $\mathrm{H}$ & 4.8736560 & 2.5783790 & -4.1081990 \\
\hline C & 3.9733430 & -2.0917820 & 1.8672470 \\
\hline C & 2.6738470 & -2.6308670 & 1.9109240 \\
\hline C & 5.0367130 & -2.4560150 & 2.8938040 \\
\hline C & 4.5010890 & -3.1353730 & 4.1626900 \\
\hline C & 3.4142370 & -4.1560930 & 3.8087440 \\
\hline C & 2.2422410 & -3.4496960 & 3.1175690 \\
\hline H & 5.3298680 & -3.6110500 & 4.702391 \\
\hline $\mathrm{H}$ & 5.7493390 & -3.1392790 & 2.4052810 \\
\hline $\mathrm{H}$ & 5.6117000 & -1.5572320 & 3.1553400 \\
\hline $\mathrm{H}$ & 3.8305240 & -4.9204710 & 3.1352450 \\
\hline $\mathrm{H}$ & 3.0570050 & -4.6706310 & 4.7099080 \\
\hline $\mathrm{H}$ & 1.4672230 & -4.1692120 & 2.8480240 \\
\hline $\mathrm{H}$ & 1.7715060 & -2.7643820 & 3.8405070 \\
\hline $\mathrm{H}$ & 4.0688040 & -2.3842140 & 4.8390670 \\
\hline C & 0.2213950 & -4.3526290 & 0.7150190 \\
\hline C & -1.0878790 & -4.8714210 & 0.7302930 \\
\hline C & 1.4032190 & -5.2839120 & 0.4838820 \\
\hline C & 1.1189890 & -6.7416310 & 0.8643190 \\
\hline C & -0.1908890 & -7.2094880 & 0.2210200 \\
\hline $\mathrm{C}$ & -1.3588640 & -6.3676740 & 0.7491640 \\
\hline $\mathrm{H}$ & 1.9588130 & -7.3733600 & 0.5474300 \\
\hline $\mathrm{H}$ & 1.6417280 & -5.2407410 & -0.5916750 \\
\hline $\mathrm{H}$ & 2.2984930 & -4.9269620 & 0.996865 \\
\hline $\mathrm{H}$ & -0.1148840 & -7.0934320 & -0.8703650 \\
\hline $\mathrm{H}$ & -0.3740840 & 0050 & 0.426975 \\
\hline
\end{tabular}

-6.6680170
-6.8277520
1.9141970
2.7066140
1.4911040
2.4974110
1.0346860
4.4511230
4.3248750
3.2502210
3.3626460
2.6190130
5.4437540
4.8630290
0.3646140
3.3027450
2.2181720
2.1843750
4.3564760
3.2908770
1.3702050
5.1795750
3.0196630
4.1329910
3.5987080
2.3778630
3.4267740
4.6010270
3.0100890
3.1083060
1.5234430
2.2484120
4.9129840
4.4137180
1.2349780
0.0804210
0.3458020
-0.4112340
-0.5894410

1.7894080

1.9589050

$-1.8195070$

$-2.3939170$

$-0.8249200$

$-2.0435970$

$-2.8734610$

0.7727780

3.0806490

2. 2388650

0.9846720

0.2725950

2.2271800

$-0.6207740$

$-2.4311890$

4.8336940

3.9807970

2.6647610

4.3923960

5.8490340

1.9810420

5.0600470

$-3.4485540$

$-3.6767680$

$-3.5810260$

$-2.0626710$

$-3.5312970$

$-4.6577720$

$-4.4763520$

$-1.2754270$

$-1.9260580$

$-4.2227130$

$-2.9170090$

$-3.5371860$

4.5341960

3.7234480

2.8187850

3.4296680

4. 3427450

158

TS36 $(R, R)$ G [B97-D/def2-TZVP $]=-4356.204803$

$\begin{array}{lrrr}\mathrm{P} & -0.0391300 & -0.1563730 & -0.7743020 \\ \mathrm{O} & 0.3241850 & 1.2825810 & -0.5851960 \\ \mathrm{O} & -1.0276620 & -0.5419510 & -1.8464940 \\ \mathrm{O} & -0.5187210 & -0.7034280 & 0.6919180 \\ \mathrm{O} & 1.2513370 & -1.0997520 & -1.0809390\end{array}$




$\begin{array}{rr}-0.7082040 & -2.0834100 \\ 0.4277180 & -2.9009070 \\ -2.0116640 & -2.5973560 \\ -2.1361320 & -3.9874830 \\ -3.1368310 & -4.4161830 \\ 2.1635100 & -1.3960760 \\ 3.4474390 & -0.8412840 \\ 1.7795640 & -2.2726290 \\ 4.3527280 & -1.1949600 \\ 5.3654110 & -0.7979190 \\ -3.2064480 & -1.7310200 \\ 3.8321120 & 0.0691090 \\ -3.5188230 & -0.7054470 \\ -2.7726510 & -0.3682190 \\ -4.8504930 & 1.1430410 \\ -4.6593370 & 0.1001040 \\ -2.3339420 & 1.1168580 \\ -1.8401780 & 1.3879190 \\ -1.5997610 & 1.2277570 \\ -3.5738680 & 2.0255270 \\ -3.6866640 & 2.7664120 \\ -3.4631080 & 2.5777460 \\ -5.7378060 & 1.7560870 \\ -1.9033750 & -1.0150610 \\ -4.9857740 & 0.4135760 \\ -5.0408120 & 1.1653010 \\ -5.9194940 & -0.1608010 \\ -3.7554840 & -0.5239030 \\ -3.2266040 & -0.2677110 \\ -4.0738500 & -1.5704660 \\ -4.0693820 & -1.9342500 \\ -3.9307550 & -2.9744210 \\ -6.0645330 & -1.5191200 \\ -5.2206460 & -1.1388300 \\ -5.1877140 & -3.8868720 \\ -5.1226180 & -4.6252650 \\ -5.2062560 & -4.4363600 \\ -6.4721000 & -3.0119690 \\ -7.0471910 & -3.3197600 \\ -7.1229180 & -3.1206590 \\ -6.9561660 & -0.8834980 \\ -3.0209420 & -3.5667240 \\ -5.2025600 & -1.3991380 \\ -5.7994740 & -1.7366460 \\ -4.9321230 & -0.3528160 \\ -3.9207840 & -2.2688270\end{array}$

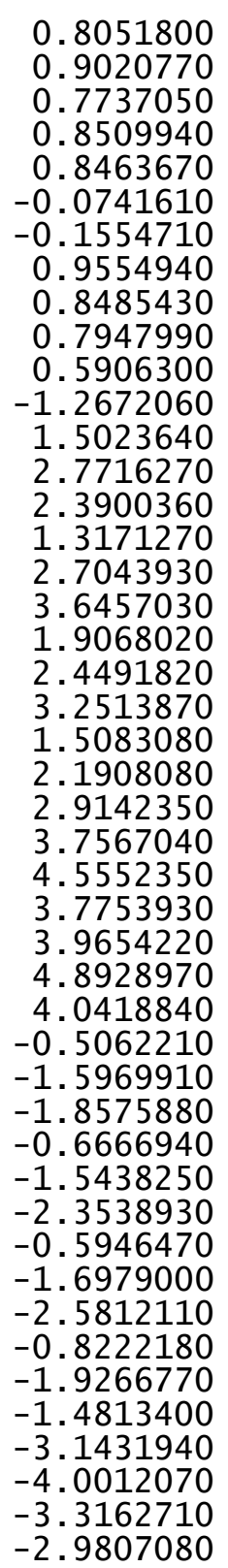

-3. 8761490

$-3.0196870$

$-5.5154310$

$-6.4082230$

4. 2213540

4. 2077310

3. 5705950

3.8601680

5.2036480

5. 1445460

6.2270600

4.8406810

4.6409950

5. 6695620

3. 3047840

4. 4817310

2. 4166690

2. 2691890

1.4910300

2. 7851840

2.7695690

2.0641800

4.5356900

4.8446660

4.1434240

4. 1711050

3. 5808420

3. 8142480

2. 7738940

3. 1471680

3. 1290760

2. 1460110

3. 8421680

5.1386950

5.5545910

5. 5201690

6.2720220

5.9840090

6.1844500

6. 9012780

4. 5596550

4.8364420

3. 9999610

2. 6926970

5.0725870

4.5463940
-3.0407960
-1.6567420
-0.1212720
0.4912740
0.4742680
-0.1825900
-1.7967630
-0.3911130
-1.3754220
-1.9079270
-0.9965620
-2.3268830
-3.3423920
-2.3880730
-2.4575470
0.5305940
-1.7492480
-2.7608550
-1.4499130
-0.7550700
-1.2587110
0.0702950
2.2678760
3.6765430
2.1129970
1.4100670
4.2541610
5.2551210
4.3613470
3.3006040
3.8300220
2.9059380
1.4359580
4.3028630
2.6934170
3.2554550
1.8735690
3.6219790
4.6393520
3.2494580
1.8029140
2.4703010
-2.0197470
-2.5367460
-2.3726170
-2.9985360

$-3.7604340$

$-3.0651880$

0.2399460

0.1109270

$-3.6452060$

$-5.0026660$

$-3.0693090$

$-2.5959450$

$-4.9773010$

$-5.9358930$

$-4.8702460$

$-3.7939720$

$-4.1609060$

$-3.0785240$

$-2.2396550$

$-5.7896570$

$-4.1091220$

$-4.5105960$

$-3.6106490$

$-5.2532900$

$-6.2291170$

$-5.2931260$

$-2.0723050$

$-1.6291170$

0.3204530

$-1.0161000$

$-0.9325700$

$-0.5454160$

$-1.6671560$

0.2210720

1. 1821650

0.0361240

1.1229780

$-2.4800450$

0.6069280

1. 5496690

0.7340600

$-0.5730680$

$-0.2117770$

$-1.0443540$

$-3.3866990$

$-4.2037970$

1.9224980

2.0030530

2.9433700

4.2443270 


\begin{tabular}{|c|c|c|}
\hline $\begin{array}{l}3.4202370 \\
2.2594090 \\
5.3737750 \\
5.7607660 \\
5.6721910 \\
3.7956420 \\
3.0680460 \\
1.4312900 \\
1.8576440 \\
4.1520500 \\
0.2684380 \\
1.4810400 \\
1.1204330 \\
0.0270460 \\
1.2878080 \\
1.0314970 \\
2.0066170 \\
1.9917090 \\
2.2078280 \\
0.2617210 \\
0.2305470 \\
2.0564140 \\
1.7186710 \\
0.8087150 \\
3.1922790 \\
2.1024330 \\
3.0287450 \\
1.0958410 \\
2.6754780 \\
1.2650690 \\
0.2310070 \\
0.1096100 \\
0.4728860 \\
0.2926850 \\
1.2981350 \\
2.1169570 \\
1.9467570 \\
1.0813510 \\
1.4080650 \\
0.9270550 \\
0.2640140 \\
1.4768590 \\
1.1441810 \\
0.0132910 \\
4.7966440 \\
3.7728820\end{array}$ & $\begin{array}{l}-3.9973100 \\
-3.2720930 \\
-3.4815210 \\
-3.0872260 \\
-1.4793820 \\
-4.7989590 \\
-4.4650390 \\
-3.9530810 \\
-2.5229920 \\
-2.2144620 \\
-4.3072290 \\
-5.2140070 \\
-6.6072850 \\
-7.1934990 \\
-6.3437490 \\
-4.8462620 \\
-7.2535090 \\
-5.3426490 \\
-4.7225630 \\
-7.1892120 \\
-8.2347350 \\
-6.6090160 \\
-6.5765930 \\
-6.5366030 \\
1.8435340 \\
2.6350820 \\
1.4433690 \\
2.4444420 \\
0.9481670 \\
4.4570250 \\
4.4000750 \\
3.3125960 \\
3.3876880 \\
2.6298710 \\
5.4802290 \\
4.8240130 \\
0.2970770 \\
3.4453580 \\
2.3477470 \\
2.2720150 \\
4.4720330 \\
3.4655910 \\
1.4472280 \\
5.3058840 \\
2.8992420 \\
4.0194050\end{array}$ & $\begin{array}{r}3.9512850 \\
3.2606800 \\
4.7796510 \\
2.4647880 \\
3.1655140 \\
3.2975350 \\
4.8795870 \\
3.0595020 \\
3.9615170 \\
4.9064210 \\
0.8493850 \\
0.6948330 \\
0.1627530 \\
0.9922520 \\
0.7921400 \\
0.8560960 \\
0.2015480 \\
1.6591170 \\
0.0359750 \\
2.0543250 \\
0.7116580 \\
1.5312730 \\
-0.1944980 \\
-0.8899660 \\
-1.8517970 \\
-2.4385140 \\
-0.8477490 \\
-2.0799620 \\
-2.8854080 \\
0.6933610 \\
2.9975570 \\
2.1780880 \\
0.9245930 \\
0.2290410 \\
2.1259840 \\
-0.7040630 \\
-2.4247080 \\
4.7634060 \\
3.9334530 \\
2.6228420 \\
4.3042700 \\
5.7751890 \\
1.9555970 \\
4.9542720 \\
-3.5096320 \\
-3.7545130\end{array}$ \\
\hline
\end{tabular}

$\begin{array}{lr}\mathrm{C} & -2.3269090 \\ \mathrm{C} & -4.6171470 \\ \mathrm{H} & -5.8156200 \\ \mathrm{H} & -3.9186850 \\ \mathrm{H} & -2.0713670 \\ \mathrm{H} & -4.8630430 \\ \mathrm{H} & -5.2771830 \\ \mathrm{H} & -4.6761670 \\ \mathrm{H} & -3.9275910 \\ \mathrm{H} & -1.6044640 \\ \mathrm{O} & 2.1994890 \\ \mathrm{C} & 2.4808280 \\ \mathrm{H} & 3.0250900 \\ \mathrm{H} & 1.5538920 \\ \mathrm{H} & 3.1000670 \\ \mathrm{H} & \end{array}$
3.5016860
3. 2936020
4.4673730
2.8983840
3. 0280270
1.4257340
2. 1150050
4.8120920
4. 3246630
1. 3973200
0.2296870
0.4852370
$-0.2918820$
$-0.4106690$

$\begin{aligned} & 158 \\ & \operatorname{TS} 37(R, R) \quad G[B 97-D / d e f 2-T Z V P]\end{aligned}=-4356.204767$

$\begin{array}{lrrr}\text { TS37 (R, R }) \text { G }[\mathrm{B} 97-\mathrm{D} / \mathrm{def}-\mathrm{TZP}] & -4356.204767 \\ \mathrm{P} & 0.0238720 & -0.0369810 & 1.1136480 \\ \mathrm{O} & -0.4794330 & 1.3390440 & 0.8033330 \\ \mathrm{O} & 0.9525020 & -0.2291420 & 2.2875500 \\ \mathrm{O} & 0.7013970 & -0.5835330 & -0.2768580 \\ \mathrm{O} & -1.1810600 & -1.0959090 & 1.3681070 \\ \mathrm{C} & 0.9749960 & -1.9502270 & -0.3731880 \\ \mathrm{C} & -0.0994490 & -2.8399750 & -0.5317930 \\ \mathrm{C} & 2.3053040 & -2.3828810 & -0.3128060 \\ \mathrm{C} & 2.5220550 & -3.7631270 & -0.3557510 \\ \mathrm{H} & 3.5482310 & -4.1245090 & -0.3134860 \\ \mathrm{C} & -1.9970040 & -1.4271790 & 0.2845000 \\ \mathrm{C} & -3.2898210 & -0.8969990 & 0.2230130 \\ \mathrm{C} & -1.4763780 & -2.2933630 & -0.6958520 \\ \mathrm{C} & -4.0718650 & -1.2566640 & -0.8838060 \\ \mathrm{H} & -5.0878730 & -0.8717500 & -0.9492910 \\ \mathrm{C} & 3.4540580 & -1.4459040 & -0.2047770 \\ \mathrm{C} & -3.8401040 & 0.0169040 & 1.2572470 \\ \mathrm{C} & 3.7323750 & -0.5224590 & -1.2287290 \\ \mathrm{C} & 2.9485600 & -0.3295680 & -2.5055240 \\ \mathrm{C} & 5.0224800 & 1.2271560 & -2.3436940 \\ \mathrm{C} & 4.8596630 & 0.3170450 & -1.1525240 \\ \mathrm{C} & 2.4972770 & 1.1519090 & -2.5635760 \\ \mathrm{H} & 1.9325240 & 1.3238560 & -3.4875130 \\ \mathrm{H} & 1.8236210 & 1.3357240 & -1.7267770 \\ \mathrm{C} & 3.7391760 & 2.0894290 & -2.4866500 \\ \mathrm{H} & 3.8201230 & 2.7037770 & -3.3929780 \\ \mathrm{H} & 3.6542690 & 2.7733340 & -1.6341820 \\ \mathrm{H} & 5.9087780 & 1.8641570 & -2.2360450 \\ \mathrm{H} & 2.0792410 & -0.9898730 & -2.5558510\end{array}$




$\begin{array}{rrr}5.1404240 & 0.3405210 & -3.6153620 \\ 5.2056700 & 0.9905240 & -4.4980080 \\ 6.0645710 & -0.2474270 & -3.5656370 \\ 3.8949270 & -0.5957050 & -3.7090780 \\ 3.3404240 & -0.4102840 & -4.6386530 \\ 4.1999220 & -1.6489420 & -3.7085030 \\ 4.3269870 & -1.5043580 & 0.8975880 \\ 4.2045990 & -2.4108220 & 2.1008140 \\ 6.2809570 & -0.8523840 & 2.2094400 \\ 5.4541740 & -0.6631630 & 0.9626890 \\ 5.4950540 & -3.2716380 & 2.1877020 \\ 5.4347130 & -3.9108710 & 3.0783210 \\ 5.5590750 & -3.9284240 & 1.3115920 \\ 6.7429280 & -2.3361830 & 2.2654200 \\ 7.2943710 & -2.5020440 & 3.2003870 \\ 7.4295030 & -2.5361100 & 1.4341990 \\ 7.1476000 & -0.1796480 & 2.2148100 \\ 3.3204070 & -3.0496100 & 2.0377260 \\ 5.3713960 & -0.5878780 & 3.4414300 \\ 5.9530620 & -0.7611440 & 4.3566180 \\ 5.0492580 & 0.4592610 & 3.4438140 \\ 4.1346820 & -1.5345490 & 3.3816230 \\ 4.1137590 & -2.1991430 & 4.2554090 \\ 3.2037210 & -0.9625110 & 3.3755510 \\ 5.7263900 & 0.2411050 & -0.0630580 \\ 6.6039820 & 0.8863940 & -0.0105090 \\ -4.6100460 & 0.4509870 & 3.5350070 \\ -4.7420040 & -0.1649120 & 4.9053230 \\ -3.6673800 & -1.7588970 & 3.1570640 \\ -4.0173560 & -0.4043510 & 2.5864570 \\ -5.6046080 & -1.4520650 & 4.7800520 \\ -5.6516290 & -1.9451440 & 5.7602520 \\ -6.6280480 & -1.1800820 & 4.4953280 \\ -4.9686280 & -2.3997980 & 3.7153540 \\ -4.7200330 & -3.3717890 & 4.1619020 \\ -5.6686890 & -2.5819420 & 2.8910880 \\ -3.2188020 & -2.4136860 & 2.4058900 \\ -5.1998750 & 0.5379500 & 5.6120350 \\ -2.6880480 & -1.5534450 & 4.3453170 \\ -2.4806440 & -2.5306620 & 4.8015080 \\ -1.7422720 & -1.1545920 & 3.9675990 \\ -3.3265190 & -0.5851750 & 5.3704760 \\ -3.4143270 & -1.0709060 & \\ -2.7049490 & 0.3093440 & \\ -4.8528480 & 2.1599310 & 3089600 \\ -5.2480910 & 3.5082790 & \end{array}$

-4.0784010
-4.2491840
-3.9696940
-4.2577570
-3.3094630
-3.2445780
-3.1111300
-2.2590720
-3.5759140
-5.7423740
-5.4765140
-5.3444200
-6.0683980
-6.1970430
-6.4749300
-7.1166210
-5.0342590
-5.5022580
-3.5737180
-4.4042430
-4.2403210
-2.7587410
-1.7515830
-2.2652000
-4.0705500
-4.8594250
-2.5694730
-1.5679040
-5.4574980
-4.6113630
-2.5652510
-0.7877850
0.1480600
-1.0120070
-0.6215070
0.6398370
1.8162290
1.4760810
-1.4570660
-1.4056490
-1.8337380
0.4606590
0.8811940
2.6770380
2.1476410
-0.4245160

1.9842900
1.3169070
4.2169760
5.1629240
4.4576250
3.2790530
3.7773590
3.0026950
1.3293220
4.1178120
2.4005440
2.9584380
1.5057000
3.2815710
4.2556280
2.7949310
1.7297660
2.3851120
-2.0591340
-2.4255700
-3.9284690
-4.2989680
-3.2855030
-2.5722790
-1.8405340
-4.2160530
-4.3485620
-2.4973790
-2.1736760
-4.4875640
-5.3032500
-3.7656120
-4.2319820
-5.2137810
-6.5993780
-7.0921780
-6.1693010
-4.6897580
-7.2957500
-5.3366360
-4.7886700
-7.0836240
-8.1244620
-6.3797870
-6.3867490
-6.5437680
$-0.4392860$

0.9062030

0.7838380

0.3055820

1.6253540

$-0.2286020$

$-1.1978490$

0.1498660

$-1.1552610$

2. 0752850

$-0.9706440$

$-1.9074070$

$-1.1984070$

0.0982510

$-0.3258520$

0.4448350

3. 1725060

3.9082900

$-1.9063060$

-3. 1108010

$-3.4147340$

$-3.6746330$

$-3.0584630$

$-1.8284580$

$-3.9806830$

$-4.2740290$

$-4.7548260$

$-3.8021510$

$-2.9360750$

$-2.5456390$

$-3.2783020$

$-2.8731280$

$-0.4894850$

$-0.4742420$

0.0560430

$-0.6622230$

$-0.3212860$

$-0.4074420$

$-0.0909200$

$-1.4912610$

0.1153330

$-1.7482630$

$-0.3780910$

$-0.9708490$

0.7066350

1.1370300 


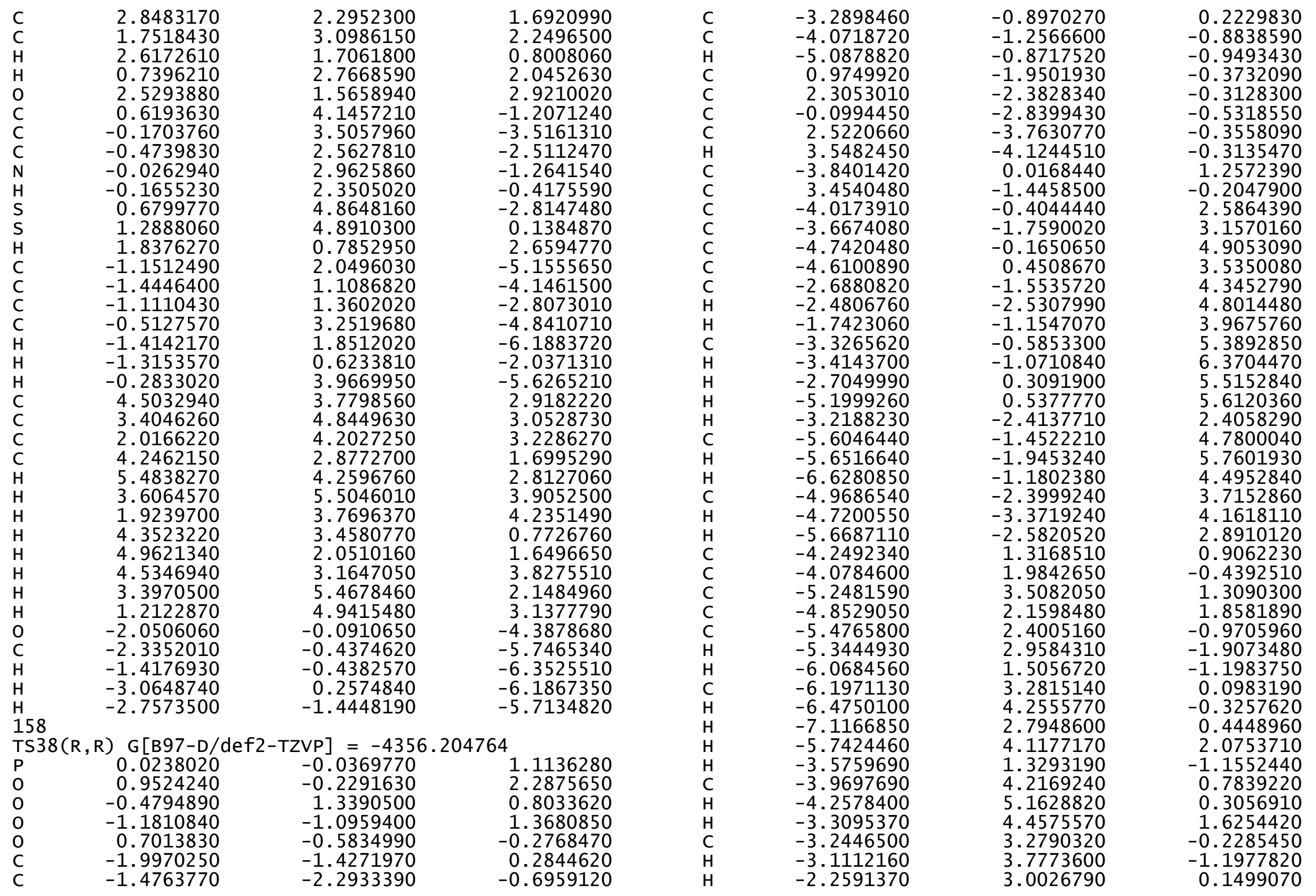




$\begin{array}{lr}\mathrm{C} & -5.0343120 \\ \mathrm{H} & -5.5023180 \\ \mathrm{C} & 4.8596550 \\ \mathrm{C} & 5.0224720 \\ \mathrm{C} & 2.9485300 \\ \mathrm{C} & 3.7323580 \\ \mathrm{C} & 5.1403930 \\ \mathrm{H} & 5.2056460 \\ \mathrm{H} & 6.0645300 \\ \mathrm{C} & 3.8948800 \\ \mathrm{H} & 3.3403680 \\ \mathrm{H} & 4.1998560 \\ \mathrm{H} & 2.0792030 \\ \mathrm{H} & 5.9087780 \\ \mathrm{C} & 2.4972640 \\ \mathrm{H} & 1.9325000 \\ \mathrm{H} & 1.8236230 \\ \mathrm{C} & 3.7391760 \\ \mathrm{H} & 3.8201220 \\ \mathrm{H} & 3.6542870 \\ \mathrm{C} & 5.4541790 \\ \mathrm{C} & 6.2809770 \\ \mathrm{C} & 4.2046100 \\ \mathrm{C} & 4.3269870 \\ \mathrm{C} & 5.3714380 \\ \mathrm{H} & 5.9531170 \\ \mathrm{H} & 5.0493160 \\ \mathrm{C} & 4.1347130 \\ \mathrm{H} & 4.1137920 \\ \mathrm{H} & 3.2037560 \\ \mathrm{H} & 3.3204140 \\ \mathrm{H} & 7.1476230 \\ \mathrm{C} & 5.4950630 \\ \mathrm{H} & 5.4347310 \\ \mathrm{H} & 5.5590670 \\ \mathrm{C} & 6.7429430 \\ \mathrm{H} & 7.2944040 \\ \mathrm{H} & 7.4295000 \\ \mathrm{C} & 5.7263940 \\ \mathrm{H} & 6.6039910 \\ \mathrm{C} & 0.1480770 \\ \mathrm{C} & -1.0119810 \\ \mathrm{C} & -0.6214800 \\ \mathrm{C} & 0.6398870 \\ \mathrm{C} & 1.8162610 \\ \mathrm{C} & 1.4761020 \\ & \end{array}$

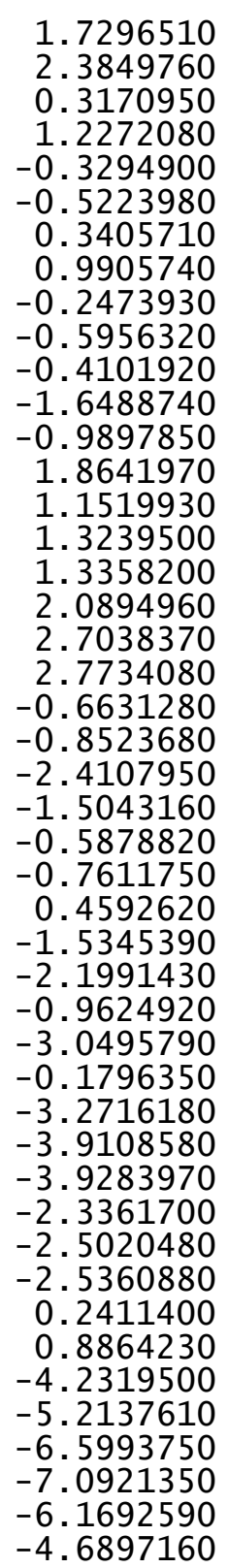

1. 7296510

2.3849760

1. 2272080

$-0.3294900$

$-0.5223980$

0.3405710

$-0.2473930$

$-0.5956320$

$-0.4101920$

$-0.9897850$

1.8641970

1.1519930

1.3358200

2.0894960

2.7038370

2.7734080

0.6631280

$-1.5043160$

$-0.5878820$

$-0.7611750$

(592620

$-2.1991430$

$-0.9624920$

$-3.2716180$

- 3.9108580

(1)

$-2.5020480$

5360880

0.2411400

$-4.2319500$

(2)

$-7.0921350$

$-4.6897160$

3.1725350
3.9083340
-1.1525410
-2.3437100
-2.5055220
-1.2287380
-3.6153790
-4.4980250
-3.5656600
-3.7090890
-4.6386550
-3.7085290
-2.5558440
-2.2360680
-2.5635620
-3.4874900
-1.7267540
-2.4866540
-3.3929870
-1.6341910
0.9626620
2.2094010
2.1007830
0.8975670
3.4414120
4.3565850
3.4438320
3.3816040
4.2553820
3.3755540
2.0376960
2.2147700
2.1876450
3.0782590
1.3115280
2.2653520
3.2003050
1.4341130
-0.0630850
-0.0105420
-0.4895910
-0.4744150
0.0558250
-0.6624280
-0.3214280
-0.4075420

-1.4570290
-1.4055950
-1.8337310
0.4607360
0.8812480
2.6770890
2.1476480
-0.4245180
-3.5737010
-4.4042010
-4.2402600
-2.7586720
-1.7515350
-2.2651790
-4.0704940
-4.8593470
-2.5693850
-1.5678560
-5.4574610
-4.6113120
-2.5651800
-0.7877330
2.8483190
1.7518650
2.6172510
0.7396270
2.5290890
0.6194180
-0.1702570
-0.4739490
-0.0262990
-0.1655490
0.6801050
1.2888410
1.8372570
-1.1510990
-1.4445750
-1.1110380
-0.5125780
-1.4140190
-1.3154150
-0.2830550
4.5033070
3.4046750
2.0166410
4.2462570
$-0.0911880$

$-1.4914500$

0.1151560

$-1.7484720$

$-0.3783300$

$-0.9709750$

0.7064950

1.1368190

$-1.9063860$

$-3.1109130$

$-3.4149120$

$-3.6748020$

$-3.0585710$

$-1.8285420$

$-3.9807610$

$-4.2742300$

$-4.7549940$

$-3.8022200$

$-2.9361960$

$-2.5458480$

$-3.2785120$

$-2.8732440$

1. 6922510

2. 2499830

0.8009490

2. 0453610

2. 9211710

$-1.2070520$

$-3.5161160$

$-2.5112450$

$-1.2641370$

$-0.4175710$

$-2.8146880$

0.1386110

2.6595100

$-5.1555950$

$-4.1461910$

$-2.8073270$

$-4.8410710$

$-6.1884150$

$-2.0371630$

$-5.6265150$

2. 9185670

3.0531860

3. 2289210

1.6998330 


\begin{tabular}{|c|c|c|c|c|c|c|c|}
\hline $\mathrm{H}$ & 5.4838650 & 4.2592680 & 2.8131110 & $\mathrm{H}$ & 5.8208080 & 0.6930300 & -3.8599740 \\
\hline $\mathrm{H}$ & 3.6065160 & 5.5042770 & 3.9055580 & C & 3.6995380 & 0.1196790 & -4.0043760 \\
\hline $\mathrm{H}$ & 1.9238990 & 3.7694640 & 4.2354730 & $\mathrm{H}$ & 3.0956180 & 0.3734230 & -4.8856770 \\
\hline $\mathrm{H}$ & 4.3524840 & 3.4577680 & 0.7730000 & $\mathrm{H}$ & 4.1202400 & -0.8797940 & -4.1667390 \\
\hline $\mathrm{H}$ & 4.9621190 & 2.0506450 & 1.6499990 & C & 4.4129650 & -1.4360160 & 0.3770110 \\
\hline $\mathrm{H}$ & 4.5346390 & 3.1643100 & 3.8278810 & C & 4.4556350 & -2.5398370 & 1.4096590 \\
\hline $\mathrm{H}$ & 3.3971390 & 5.4675030 & 2.1487990 & c & 6.3858800 & -0.8223400 & 1.6805910 \\
\hline $\mathrm{H}$ & 1.2123490 & 4.9413090 & 3.1379350 & C & 5.4567090 & -0.5042180 & 0.5357050 \\
\hline 0 & -2.0505730 & -0.0908610 & -4.3879300 & $\mathrm{C}$ & 5.8221690 & -3.2688850 & 1.2708910 \\
\hline C & -2.3351240 & -0.4372460 & -5.7466070 & $\mathrm{H}$ & 5.8868030 & -4.0464920 & 2.0433590 \\
\hline $\mathrm{H}$ & -1.4175900 & -0.4380800 & -6.3525860 & $\mathrm{H}$ & 5.8796720 & -3.7651060 & 0.2943970 \\
\hline $\mathrm{H}$ & -3.0647480 & 0.2577290 & -6.1868410 & C & 6.9837370 & -2.2363960 & 1.4319430 \\
\hline $\mathrm{H}$ & -2.7573180 & -1.4445860 & -5.7135730 & $\mathrm{H}$ & 7.6263770 & -2.5052960 & 2.2807330 \\
\hline & & & & $\mathrm{H}$ & 7.6108180 & -2.2150690 & 0.5327440 \\
\hline & 2) $G[B 97-D / c$ & ZVP] $=-4356$ & & $\mathrm{H}$ & 7.1852090 & -0.0748800 & 1.7588790 \\
\hline$P$ & 0.1394170 & -0.2800320 & 0.9820810 & $\mathrm{H}$ & 3.6307160 & -3.2450980 & 1.2859760 \\
\hline 0 & -0.4127160 & 1.1109240 & 0.9182200 & C & 5.5618850 & -0.8851740 & 2.9955730 \\
\hline 0 & 1.1647480 & -0.6166600 & 2.0369680 & $\mathrm{H}$ & 6.2300570 & -1.1795700 & 3.8159050 \\
\hline 0 & 0.7043850 & -0.5978190 & -0.5211950 & $\mathrm{H}$ & 5.1598870 & 0.1046430 & 3.2335880 \\
\hline 0 & -1.0192580 & -1.4074530 & 1.1683280 & C & 4.4019110 & -1.9118250 & 2.8288680 \\
\hline C & 1.0400440 & -1.9228010 & -0.8043980 & $\mathrm{H}$ & 4.4903880 & -2.7187970 & 3.5681470 \\
\hline $\mathrm{C}$ & -0.0004730 & -2.8416500 & -1.0059430 & $\mathrm{H}$ & 3.4307840 & -1.4325350 & 2.9733950 \\
\hline $\mathrm{C}$ & 2.3915680 & -2.2844750 & -0.8591940 & C & 5.5774010 & 0.5828280 & -0.3297310 \\
\hline C & 2.6699390 & -3.6363000 & -1.0778710 & $\mathrm{H}$ & 6.3876870 & 1.3011910 & -0.2019680 \\
\hline $\mathrm{H}$ & 3.7121300 & -3.9475800 & -1.1280000 & C & -4.3037990 & -0.4391950 & 3.7634670 \\
\hline C & -1.8879150 & -1.6423770 & 0.1012080 & c & -4.2969800 & -1.2706040 & 5.0210720 \\
\hline C & -3.206 & -1.1795550 & 0.1916350 & $\mathrm{C}$ & -3.3451280 & -2.5211940 & 2.9531940 \\
\hline $\mathrm{C}$ & -1.40 & -2.336 & -1.0198320 & $\mathrm{c}$ & -3.7732340 & -1.1054980 & 2.6430700 \\
\hline C & -4.0270590 & -1.4008630 & -0.9169930 & $\mathrm{C}$ & -5.1388890 & -2.5513160 & 4.7610370 \\
\hline $\mathrm{H}$ & -5.0557900 & -1.0465360 & -0.8738730 & $\mathrm{H}$ & -5.0837110 & -3.2002550 & 5.6454110 \\
\hline $\mathrm{C}$ & 3.4804200 & -1.2922780 & -0.6682160 & $\mathrm{H}$ & -6.1901530 & -2.2749920 & 4.6163680 \\
\hline C & -3.72 & -0.4698350 & 1.3897870 & C & -4.5831050 & -3.2869080 & 3.5008870 \\
\hline $\mathrm{C}$ & 3.61 & -0.1973610 & -1.5418050 & $\mathrm{H}$ & -4.2812070 & -4.3122620 & 3.7528090 \\
\hline C & 2.770 & 0.0967660 & -2.7580400 & $\mathrm{H}$ & -5.3489020 & -3.3477530 & 2.7182410 \\
\hline $\mathrm{C}$ & 4.6701000 & 1.8336080 & -2.4006300 & $\mathrm{H}$ & -2.9494110 & -3.0295850 & 2.0701300 \\
\hline $\mathrm{C}$ & 4.6552670 & 0.7377570 & -1.3642450 & $\mathrm{H}$ & -4.7061040 & -0.7076630 & 5.8689320 \\
\hline C & 2.159 & 1.5107450 & -2.5923710 & $\mathrm{C}$ & -2.2727580 & -2.4835920 & 4.0758620 \\
\hline $\mathrm{H}$ & 1.563 & 1.7530240 & -3.4818840 & $\mathrm{H}$ & -2.0174100 & -3.5160790 & 4.3498610 \\
\hline $\mathrm{H}$ & 1.482 & 1.4950700 & -1.7386500 & $\mathrm{H}$ & -1.3663610 & -2.0064370 & 3.6930850 \\
\hline C & 3.295 & 2.5572070 & -2.3813090 & C & -2.8350960 & -1.7113950 & 5.3096530 \\
\hline $\mathrm{H}$ & 3.28 & 3.3137960 & -3.1767030 & $\mathrm{H}$ & -2.8239590 & -2.3456830 & 6.2061460 \\
\hline $\mathrm{H}$ & 3.16 & 3.0824630 & -1.4281190 & $\mathrm{H}$ & -2.2240970 & -0.8256270 & 5.5207140 \\
\hline $\mathrm{H}$ & 5.48 & 2.5440520 & -2.2134420 & C & -4.7500870 & 1.5002700 & 2.4110180 \\
\hline $\mathrm{H}$ & 1.97 & -0.6446520 & -2.8913620 & $\mathrm{C}$ & -5.2261510 & 2.9034250 & 2.1271820 \\
\hline C & 4.8 & 1.1695050 & -3.7963760 & c & -4.1974730 & 1.7221640 & 0.0549610 \\
\hline $\mathrm{H}$ & 4.791 & 1.9485330 & -4.5690980 & $\mathrm{C}$ & -4.2129770 & 0.8455400 & 1.2859470 \\
\hline
\end{tabular}




-4.0178120
-4.3681290
-3.2820000
-3.3818700
-3.3681320
-2.3530920
-3.7560190
-5.6561710
-5.6529120
-5.6323090
-6.2423940
-6.2817050
-6.6092080
-7.1596070
-4.7951060
-5.2101520
-2.2382420
-1.6881910
-2.4568800
-3.9488250
-4.5137310
-3.5647970
-2.0677530
-1.7554710
-0.6218330
-4.0680450
-4.5211340
-5.4533400
-4.7738390
-2.3036500
0.3138040
-0.7876150
-0.2851570
0.9301090
2.0866430
1.6660690
-1.0935780
-1.2244250
-1.6001060
0.6504710
1.2487060
2.8767220
2.5460980
0.0016910
3.0111500
1.8491470

3.7316100

3.8505060

3.0011460

3.6524960

2.7118860

1.2105130

3. 3640170

2. 1559950

2.8494430

1.2806500

2.8418640

3.8624050

2.2836990

0.8617500

1.3727050

$-2.4724650$

$-3.0120120$

-2. 4821470

$-2.7901800$

$-2.0701480$

$-2.0076400$

$-2.9512270$

$-4.1083000$

$-2.7686020$

$-3.8773320$

$-2.5001580$

$-2.5451080$

$-1.0358240$

$-1.3992570$

$-4.2152760$

$-5.2621810$

$-6.6483760$

$-7.0313380$

$-6.0651040$

$-4.6054020$

$-7.3817820$

$-5.3630730$

$-4.9209900$

$-6.9743930$

$-8.0623720$

$-6.1707340$

$-6.3414100$

$-6.6344240$

2. 0348620

2.6708240

1.6089970
1.3271470
2.4131920
0.3880710
-0.4950600
0.6105840
-0.8025130
3.0249290
-0.2665610
-1.1180390
-0.5654530
0.9876040
0.7483560
1.3344420
3.6498560
4.5196320
-2.1548260
-3.4635080
-4.6805870
-4.5086330
-3.2736770
-2.0880720
-5.5936060
-3.4839390
-3.5369730
-4.3903670
-5.4001160
-2.9582600
-3.5378770
-4.7666150
-1.1308810
-1.1186190
-0.6936250
-1.5458780
-1.2584970
-1.1759290
-0.8081330
-2.1209190
-0.4656010
-2.6087720
-1.3458160
-2.0149300
-0.2963080
0.3684420
1.9605050
2.5923300

2.8598670

0.8653040

2.6683700

0.5781240

$-0.6554540$

$-0.7845670$

$-0.0932680$

$-0.1208410$

0.3599960

1.4990570

2. 0123160

$-2.0632990$

$-2.1817230$

$-1.5330140$

$-1.2954570$

$-2.5677080$

$-1.6185710$

$-1.2077310$

4.5210850

3. 3636840

2. 0001490

4.3690610

5.4793040

3.4858460

1.8616990

4.4691970

5.1396040

4.5405690

3. 3774350

1.1695070

$-2.9190380$

$-3.5985190$

$-2.8875300$

$-4.3445370$

$-4.0930890$
1.6114700

2. 3413780

1.0802820

4.3458170

4.2340160

3.0813930

3.1926800

2. 4098120

5.4071630

4.7893970

0.3403690

3.2149850

2.0628870

1.9827410

4. 3027680

3.2732090

1.0955290

5.1842380

3. 3244010

4.2958590

3.5820790

2.6760330

3.8556660

4.7702740

2.9663290

3.4389110

1.9211120

. 5403820

5.0955050

4.2966980

0.9685010

0.9971810

1.1300620

1.8045320

0.0283010
0.9647310

2. 2746120

3.0174420

$-0.5348210$

$-2.7332980$

$-1.9310040$

$-0.7360110$

$-0.0352660$

$-1.9239750$

0.7946430

2.5924790

$-4.3908930$

$-3.5846880$

$-2.3439400$

$-3.9673860$

$-5.3484820$

$-1.7248150$

$-4.5966960$

3.5416210

3.8223510

3.7738200

2.1555250

3.5903470

4.8034140

4.6743280

1.3711100

1.9749060

4.3105450

3.0692400

3.7601870

$-3.9367740$

$-5.1961790$

$-6.0243630$

$-5.2208030$

$-5.2883620$ 158

TS40 (R, R) G [B97-D/def2-TZVP $]=-4356.204684$

$\begin{array}{lrrr}\mathrm{P} & -0.0553490 & -0.2222090 & -0.6828130 \\ \mathrm{O} & 0.3438290 & 1.2167630 & -0.5945850 \\ \mathrm{O} & -1.0818070 & -0.6487980 & -1.7017610 \\ \mathrm{O} & -0.5007180 & -0.6703290 & 0.8261780 \\ \mathrm{O} & 1.2012770 & -1.2160300 & -0.9610020 \\ \mathrm{C} & -0.7157520 & -2.0362160 & 1.0299820 \\ \mathrm{C} & 0.4121670 & -2.8753440 & 1.1572400 \\ \mathrm{C} & -2.0318260 & -2.5148050 & 1.0464790 \\ \mathrm{C} & -2.2053850 & -3.8951970 & 1.2261690\end{array}$




$\begin{array}{rrr}-3.2173470 & -4.2948460 & 1.2440770 \\ 2.1391120 & -1.4628050 & 0.0349790 \\ 3.4219060 & -0.9201660 & -0.1162340 \\ 1.7826600 & -2.2864750 & 1.1180030 \\ 4.3527190 & -1.2158370 & 0.8820260 \\ 5.3611660 & -0.8170420 & 0.7836560 \\ -3.2055740 & -1.6432500 & 0.7678870 \\ 3.7811870 & -0.0698130 & -1.2823920 \\ -3.5254360 & -0.5401090 & 1.5789320 \\ -2.8031480 & -0.1014190 & 2.8307550 \\ -4.8414390 & 1.4056050 & 2.2573500 \\ -4.6454910 & 0.2640280 & 1.2910690 \\ -2.3318190 & 1.3615190 & 2.6335440 \\ -1.8496560 & 1.7111030 & 3.5546790 \\ -1.5810810 & 1.3816940 & 1.8444660 \\ -3.5479650 & 2.2652760 & 2.2688070 \\ -3.6634890 & 3.0768120 & 2.9988930 \\ -3.4056540 & 2.7289710 & 1.2856640 \\ -5.7101210 & 2.0144870 & 1.9785950 \\ -1.9495220 & -0.7476170 & 3.0529930 \\ -5.0264060 & 0.8080520 & 3.6800300 \\ -5.0960160 & 1.6303910 & 4.4046270 \\ -5.9676870 & 0.2473440 & 3.7222170 \\ -3.8154270 & -0.1190450 & 4.0109050 \\ -3.3018360 & 0.2257820 & 4.9180280 \\ -4.1522720 & -1.1470920 & 4.1899200 \\ -4.0416140 & -1.9277350 & -0.3329670 \\ -3.8853970 & -3.0524730 & -1.3338430 \\ -5.9981790 & -1.6080560 & -1.7652860 \\ -5.1800790 & -1.1387030 & -0.5881130 \\ -5.1513310 & -3.9493730 & -1.2396350 \\ -5.0707520 & -4.7520310 & -1.9846110 \\ -5.2010070 & -4.4183160 & -0.2493710 \\ -6.4229020 & -3.0807110 & -1.5013740 \\ -6.9732390 & -3.4534660 & -2.3753270 \\ -7.1011880 & -3.1166480 & -0.6406170 \\ -6.8823730 & -0.9741730 & -1.9071120 \\ -2.9842640 & -3.6401040 & -1.1491920 \\ -5.1026660 & -1.5931670 & -3.0321790 \\ -5.6804930 & -1.9874690 & -3.8789430 \\ -4.8161210 & -0.5643210 & -3.2731990 \\ -3.8365720 & -2.4617370 & -2.7701190 \\ -3.7871160 & -3.2965950 & -3.4819800 \\ -2.9249200 & -1.8713430 & -2.8849870 \\ -5.4805190 & -0.0416630 & 0.2178760 \\ -6.3605150 & 0.5690900 & 0.0142330\end{array}$

4. 1034410 4.0332630 3.4210480 3.7569140 5.0019600 4.9012690 6. 0365930 4. 6541700 4. 4185350 5. 5038890 3.1676020 4. 2990120 2. 2357440 2. 0490580 1. 3345110 2. 5914640 2. 5342160 1. 8885960 4.5054740 4.8607580 4.1842900 4.1556920 3. 6328410 3. 9007120 2. 8066210 3. 2120350 3. 2311010 2. 1982560 3. 8926950 5.1433330 5. 6163440 5. 6250830 6. 3179140 6.0304120 6. 2660360 6.9236430 4.4783750 4.7440310 2.7360590 2. 3515750 3. 3526110 4. 7867070 5.0986480 4.0326750 3. 1241470 2. 2691930

0.2242560
-0.4884400
-2.0041000
-0.5870860
-1.7025000
-2.2726810
-1.3442860
-2.5949070
-3.6192050
-2.6480160
-2.6228920
0.1846710
-1.9704200
-2.9940890
-1.6244580
-1.0367250
-1.5820310
-0.1966110
2.0761260
3.4948810
2.0355270
1.2734860
4.1331200
5.1438850
4.2282440
3.2408280
3.8122470
2.8604130
1.4037120
4.0752750
2.5933080
3.1963640
1.7622110
3.4581290
4.4849960
3.0426630
1.5545630
2.1797850
-2.5185300
-3.3088040
-3.2023340
-3.3453780
-2.1897100
-1.9871020
-3.9795650
-4.3689070

$-3.6868180$ $-5.0141750$ $-2.9939040$ $-2.5902640$ $-5.9009610$ $-4.9077330$ $-3.7354430$ $-4.0533790$ -3.0442050 $-2.1290170$ $-5.8383680$ $-3.9990020$ $-4.3505620$ $-3.4865480$ $-5.1968530$ $-6.1484240$ $-5.2518140$ $-2.2046230$ $-1.8347790$ 0.2032100 $-1.1013330$ $-1.1278220$ $-0.7917290$ $-1.8423860$ 0.0780550 1. 0147500 $-0.0618950$ 1.0454240 $-2.7213810$

0.4209830

1. 3386260 0.5627470 $-0.8113860$ $-0.5020030$ $-1.2929440$ $-3.4973650$ $-4.3507360$

2. 1416850

3. 3835910

4. 5446050

4.0245650

3. 0672270

2. 0044880

5. 2848910

3. 1209560 


$\begin{array}{lrrr}\text { H } & 1.3478350 & -3.0040720 & \\ \text { H } & 4.8844770 & -4.3032110 & 3.7070860 \\ \text { H } & 5.5070680 & -3.3533800 & 4.8517460 \\ \text { H } & 6.0694180 & -2.3411390 & 2.5766830 \\ \text { H } & 5.1935870 & -1.2610460 & 3.6532110 \\ \text { H } & 3.2497080 & -2.2360150 & 5.0559090 \\ \mathrm{C} & 0.1934600 & -4.2654270 & 1.2282280 \\ \mathrm{C} & 1.2754160 & -5.3065630 & 1.0732070 \\ \mathrm{C} & 0.8951230 & -6.2698230 & -0.0784670 \\ \mathrm{C} & -0.5119290 & -6.9056710 & 0.1239380 \\ \mathrm{C} & -1.2809550 & -6.2638400 & 1.3104620 \\ \mathrm{C} & -1.1215700 & -4.7657720 & 1.2908660 \\ \mathrm{H} & 1.3676590 & -5.8975700 & 1.9977870 \\ \mathrm{H} & 1.6605810 & -7.0513710 & -0.1607630 \\ \mathrm{H} & -0.4230050 & -7.9846960 & 0.3022200 \\ \mathrm{H} & -0.8648150 & -6.6630680 & 2.2481540 \\ \mathrm{H} & 2.2471920 & -4.8440710 & 0.8785240 \\ \mathrm{H} & 0.9128010 & -5.6980570 & -1.0144290 \\ \mathrm{H} & -1.1063580 & -6.7767940 & -0.7886630 \\ \mathrm{H} & -2.3404130 & -6.5439500 & 1.2786640 \\ \mathrm{C} & -3.1543600 & 1.8076690 & -1.9132110 \\ \mathrm{C} & -2.0341850 & 2.5278620 & -2.5327510 \\ \mathrm{H} & -3.0165970 & 1.4733340 & -0.8820520 \\ \mathrm{H} & -1.0389180 & 2.3344170 & -2.1452440 \\ \mathrm{O} & -2.6445960 & 0.8321110 & -2.8756190 \\ \mathrm{C} & -1.1619070 & 4.5137230 & 0.4814940 \\ \mathrm{C} & -0.1115780 & 4.5498420 & 2.7801960 \\ \mathrm{C} & 0.1881730 & 3.4097130 & 2.0188850 \\ \mathrm{~N} & -0.4001530 & 3.4358150 & 0.7665710 \\ \mathrm{H} & -0.2472970 & 2.6345280 & 0.1157930 \\ \mathrm{~S} & -1.1538890 & 5.6129230 & 1.8576080 \\ \mathrm{~S} & -2.0128830 & 4.8247590 & -0.9300620 \\ \mathrm{H} & -1.9504650 & 0.1876610 & -2.3557770 \\ \mathrm{C} & 1.1873880 & 3.6492980 & 4.5846000 \\ \mathrm{C} & 1.4710390 & 2.4982840 & 3.8129450 \\ \mathrm{C} & 0.9747310 & 2.3678690 & 2.5128630 \\ \mathrm{C} & 0.3977540 & 4.6754200 & 4.0774990 \\ \mathrm{H} & 1.5935980 & 3.7117840 & 5.5904200 \\ \mathrm{H} & 1.1577670 & 1.5021890 & 1.8805320 \\ \mathrm{H} & 0.1800070 & 5.5504570 & 4.6838090 \\ \mathrm{C} & -4.7035180 & 2.7940470 & -3.6637240 \\ \mathrm{C} & -3.6468760 & 3.8707550 & -3.9585820 \\ \mathrm{C} & -2.2175030 & 3.3218110 & -3.7929370 \\ \mathrm{C} & -4.5636930 & 2.2629550 & -2.2271930 \\ \mathrm{H} & -5.7103080 & 3.2065350 & -3.8014570 \\ \mathrm{H} & -3.7657300 & 4.2601780 & -4.9767410 \\ & & & \\ & & & \end{array}$

$\begin{array}{lr}\mathrm{H} & -1.9686160 \\ \mathrm{H} & -4.8094570 \\ \mathrm{H} & -5.2436000 \\ \mathrm{H} & -4.5895290 \\ \mathrm{H} & -3.7910690 \\ \mathrm{H} & -1.4721630 \\ \mathrm{O} & 2.2399920 \\ \mathrm{C} & 2.4796450 \\ \mathrm{H} & 3.0039620 \\ \mathrm{H} & 1.5375200 \\ \mathrm{H} & 3.1086150 \\ \mathrm{H} & \end{array}$
2.6561520
3. 0548560
1.4255670
1.9619350
4. 7123720
4. 1246070
1.5560590
0.3390860
0.5295700
$-0.1824640$
$-0.2690920$

TS41 $(R, R)$ G $[B 97-D / d e f 2-T Z V P]=-4356.204670$

$\begin{array}{lrrr}\text { TS41(R, R) G }[\mathrm{B} 97-\mathrm{D} / \mathrm{de} \text { ef }- \text { TZVP }]=-4356.204670 \\ \mathrm{P} & -0.0553420 & -0.2221940 & -0.6827600 \\ \mathrm{O} & -1.0818540 & -0.6487110 & -1.7016820 \\ \mathrm{O} & 0.3438630 & 1.2167650 & -0.5944710 \\ \mathrm{O} & 1.2012440 & -1.2160300 & -0.9610630 \\ \mathrm{O} & -0.5006440 & -0.6703950 & 0.8262260 \\ \mathrm{C} & 2.1391340 & -1.4628700 & 0.0348490 \\ \mathrm{C} & 1.7827290 & -2.2865890 & 1.1178520 \\ \mathrm{C} & 3.4219220 & -0.9202230 & -0.1164030 \\ \mathrm{C} & 4.3527850 & -1.2159570 & 0.8817900 \\ \mathrm{H} & 5.3612280 & -0.8171580 & 0.7833950 \\ \mathrm{C} & -0.7156890 & -2.0362910 & 1.0299540 \\ \mathrm{C} & -2.0317690 & -2.5148640 & 1.0464450 \\ \mathrm{C} & 0.4122260 & -2.8754390 & 1.1571320 \\ \mathrm{C} & -2.2053390 & -3.8952620 & 1.2260830 \\ \mathrm{H} & -3.2173060 & -4.2949010 & 1.2439960 \\ \mathrm{C} & 3.7810970 & -0.0697460 & -1.2825050 \\ \mathrm{C} & -3.2055110 & -1.6432880 & 0.7678880 \\ \mathrm{C} & 3.7566700 & -0.5868670 & -2.5904350 \\ \mathrm{C} & 3.4208870 & -2.0038660 & -2.9941900 \\ \mathrm{C} & 4.0327150 & -0.4879330 & -5.0143690 \\ \mathrm{C} & 4.1029850 & 0.2246260 & -3.6869440 \\ \mathrm{C} & 2.2354710 & -1.9702100 & -3.9991550 \\ \mathrm{H} & 2.0488600 & -2.9938620 & -4.3508020 \\ \mathrm{H} & 1.3342530 & -1.6244010 & -3.4865720 \\ \mathrm{C} & 2.5909590 & -1.0363550 & -5.1969470 \\ \mathrm{H} & 2.5336730 & -1.5815700 & -6.1485670 \\ \mathrm{H} & 1.8879920 & -0.1963130 & -5.2517530 \\ \mathrm{H} & 4.2983000 & 0.1852940 & -5.8385200 \\ \mathrm{H} & 3.1676060 & -2.6227780 & -2.1293400 \\ \mathrm{C} & 5.0015520 & -1.7018910 & -4.9682170 \\ \mathrm{H} & 4.9008260 & -2.2719830 & -5.9014430 \\ \mathrm{H} & 6.0361500 & -1.3435670 & -4.9082320 \\ \mathrm{C} & 4.6539900 & -2.5944720 & -3.7359300\end{array}$

$-4.6319650$

1.5060010

$-2.0405770$

.

4889440

4. 3534920

(1)

2. 2481540

- 7886630

1. 2786640

2.5327510

$-0.8820520$

$-2.8756190$

4814940

7801960

0.7665710

.1157930

-.9300620
-2.3557770

4.5846000

8129450

4. 07128630
5.5904200

5.5904200

(2)

4.6838090
-3.6637240

(

$-4.9767410$

4.6539900

$-2.5944720$

. 5944710

10630

.1178520

(1)

0.7833950

1.0299540

(2)

1. 2260830

1. 2439960

0.7678880

2.5904350

9941900

-

$-3.4865720$

(1)

5.8385200

-4.9082320
-3.7359300 


\begin{tabular}{|c|c|c|c|}
\hline $\begin{array}{l}\mathrm{H} \\
\mathrm{H} \\
\mathrm{C} \\
\mathrm{C} \\
\mathrm{C} \\
\mathrm{C} \\
\mathrm{C} \\
\mathrm{H} \\
\mathrm{H} \\
\mathrm{C} \\
\mathrm{H} \\
\mathrm{H} \\
\mathrm{H} \\
\mathrm{H} \\
\mathrm{C} \\
\mathrm{H} \\
\mathrm{H} \\
\mathrm{C} \\
\mathrm{H} \\
\mathrm{H} \\
\mathrm{C} \\
\mathrm{H} \\
\mathrm{C} \\
\mathrm{C} \\
\mathrm{C} \\
\mathrm{C} \\
\mathrm{C} \\
\mathrm{H} \\
\mathrm{H} \\
\mathrm{C} \\
\mathrm{H} \\
\mathrm{H} \\
\mathrm{H} \\
\mathrm{H} \\
\mathrm{C} \\
\mathrm{H} \\
\mathrm{H} \\
\mathrm{C} \\
\mathrm{H} \\
\mathrm{H} \\
\mathrm{C} \\
\mathrm{C} \\
\mathrm{C} \\
\mathrm{C} \\
\mathrm{C} \\
\mathrm{H}\end{array}$ & $\begin{array}{r}4.4184190 \\
5.5037910 \\
4.1555880 \\
4.1844330 \\
4.8604090 \\
4.5051260 \\
5.6165130 \\
5.6254330 \\
6.3181400 \\
6.0302850 \\
6.2659360 \\
6.9234280 \\
5.1427740 \\
3.8930390 \\
3.6326140 \\
3.9005240 \\
2.8062360 \\
3.2121080 \\
3.2313450 \\
2.1983140 \\
4.4778470 \\
4.7433230 \\
-4.6454080 \\
-4.8413530 \\
-2.8030860 \\
-3.5253700 \\
-5.0263440 \\
-5.0959720 \\
-5.9676230 \\
-3.8153670 \\
-3.3017760 \\
-4.1522150 \\
-1.9494700 \\
-5.7100250 \\
-2.3317350 \\
-1.8495460 \\
-1.5810140 \\
-3.5478710 \\
-3.6633900 \\
-3.4055530 \\
-5.1799950 \\
-5.9980950 \\
-3.8853450 \\
-4.0415460 \\
-5.1025740 \\
-5.6804060\end{array}$ & $\begin{array}{r}-3.6187570 \\
-2.6475720 \\
1.2735440 \\
2.0354140 \\
3.4950720 \\
2.0763460 \\
2.5932140 \\
3.1961340 \\
1.7621180 \\
3.4582250 \\
4.4850570 \\
3.0428590 \\
4.0755980 \\
1.4034800 \\
4.1331640 \\
5.1438910 \\
4.2283630 \\
3.2406890 \\
3.8119770 \\
2.8602540 \\
1.5549390 \\
2.1802820 \\
0.2639900 \\
1.4055240 \\
-0.1015500 \\
-0.5401790 \\
0.8079060 \\
1.6302100 \\
0.2471910 \\
-0.1192020 \\
0.2256030 \\
-1.1472520 \\
-0.7477690 \\
2.0144300 \\
1.3613880 \\
1.7109280 \\
1.3815750 \\
2.2651810 \\
3.0766690 \\
2.7289430 \\
-1.1386480 \\
-1.6079370 \\
-3.0524120 \\
-1.9277100 \\
-1.5930150 \\
-1.9872650\end{array}$ & $\begin{array}{r}-4.0539540 \\
-3.0447940 \\
-1.1013390 \\
0.2033020 \\
-1.8346320 \\
-2.2045910 \\
0.4208420 \\
1.3385710 \\
0.5623310 \\
-0.8114920 \\
-0.5020150 \\
-1.2932980 \\
-2.7212160 \\
1.0454970 \\
-1.1273200 \\
-0.7911420 \\
-1.8416890 \\
0.0785230 \\
1.0152930 \\
-0.0612600 \\
-3.4973920 \\
-4.3507310 \\
1.2911380 \\
2.2574680 \\
2.8308240 \\
1.5789790 \\
3.6801170 \\
4.4047520 \\
3.7222590 \\
4.0109710 \\
4.9181020 \\
4.1899630 \\
3.0530390 \\
1.9787320 \\
2.6336650 \\
3.5548020 \\
1.8445720 \\
2.2689840 \\
2.9991250 \\
1.2858730 \\
-0.5881110 \\
-1.7653100 \\
-1.3339080 \\
-0.3329880 \\
-3.0321970 \\
-3.8789820\end{array}$ \\
\hline
\end{tabular}

-4.8160020
-3.8365040
-3.7870740
-2.9248350
-2.9842240
-6.8822750
-5.1512980
-5.0707290
-5.2009910
-6.4228500
-6.9731820
-7.1011480
-5.4804250
-6.3604070
0.1935020
1.2754380
0.8949940
-0.5119080
-1.2809450
-1.1215320
1.3677590
1.6605350
-0.4227460
-0.8648350
2.2471980
0.9123240
-1.1064430
-2.3404080
2.7361820
2.3517620
3.3528770
4.7869400
5.0988190
4.0327950
3.1244550
2.2693320
1.3480520
4.8846760
5.5073520
6.0695730
5.1937600
3.2500190
-3.1543300
-2.0341340
-3.0166100
-1.0388810
$-0.5641660$
$-2.4616290$
$-3.2964670$
$-1.8712570$
$-3.6400660$
$-0.9740310$
$-3.9492900$
$-4.7519280$
$-4.4182610$
$-3.0805940$
$-3.4533050$
$-3.1165510$
$-0.0416360$
0.5691420
$-4.2655220$
$-5.3066600$
$-6.2697980$
$-6.9058660$
$-6.2639180$
$-4.7658530$
$-5.8977400$
$-7.9848280$
$-6.6631100$
$-4.8441580$
$-5.6978640$
$-6.7773500$
$-6.5440040$
$-2.5187080$
$-3.3090470$
$-3.2026810$
$-3.3456910$
$-2.1899460$
$-1.9872810$
$-3.9799720$
$-4.3691330$
$-3.0043070$
$-4.3034820$
$-3.3537590$
$-2.3413160$
$-1.2613250$
$-2.2364060$
1.8077820
2. 5280300
1.4733750
2. 3345600

$-3.2731700$

$-2.7701650$

$-3.4820520$

$-2.8850170$

$-1.9071160$

$-1.2397360$

$-1.9847360$

$-1.5014600$

$-2.3754350$

$-0.6407140$

0.2179180

0.0142890

1. 2280660

1.0729000

$-0.0788190$

0.1238790

1. 3103430

1. 2907240

1. 9974260

$-0.1614690$

0.3024310

2. 2480650

0.8781580

$-1.0146860$

$-0.7887030$

1. 2785470

2. 1414670

3. 3833520

4.5443080

4. 0241670

3. 0669030

2. 0042270

5. 2845440

3.1206590

3. 7069310

3.4912660

4.8519900

2. 5763070

3. 6529540

5. 0557020

$-2.5325640$

$-0.8819840$

$-2.1450340$ 


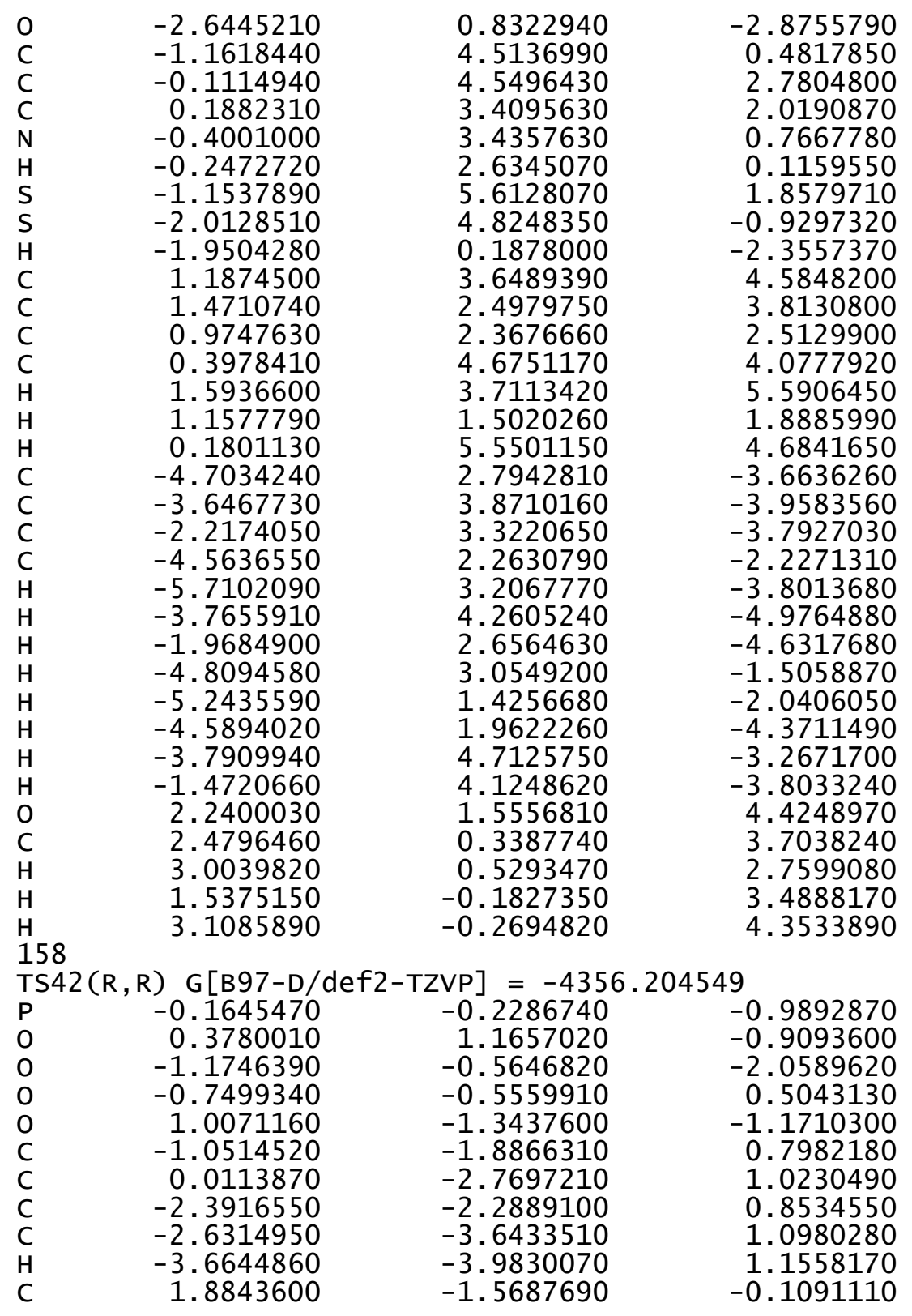

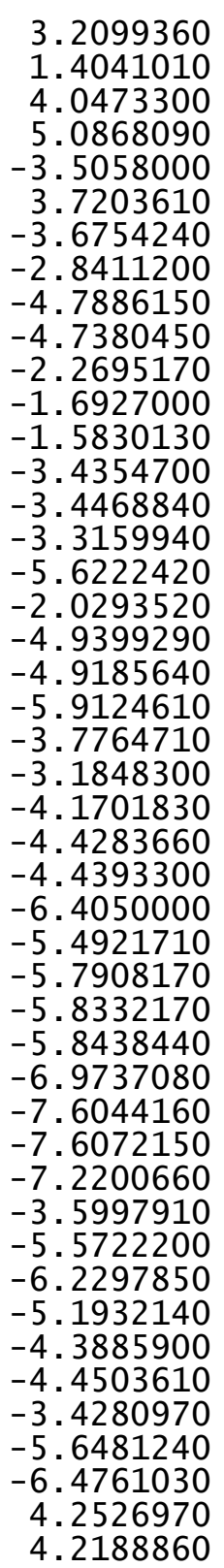
$-1.1318370$
$-2.2389170$
$-1.3749480$
$-1.0581350$
$-1.3260360$
$-0.4421190$
$-0.2451950$
0.0541130
1. 7403550
0.6622010
1. 4881150
1.7325620
1.5079090
2. 5030740
3. 2509960
3.0409210
2. 4301350
$-0.6667610$
1. 0502630
1. 8189080
0.5466330
0.0284740
0.2824420
$-0.9851020$
$-1.4828070$
$-2.5811220$
$-0.9040680$
$-0.5738080$
$-3.3405010$
$-4.1144340$
$-3.8435930$
$-2.3324890$
$-2.6099110$
$-2.3304670$
$-0.1739610$
$-3.2685000$
$-0.9443100$
$-1.2539210$
0.0545030
$-1.9433340$
$-2.7468220$
$-1.4391900$
0.4974040
1. 1958780
$-0.4422570$
$-1.2891800$

$-0.2215300$

1. 0279860

0.8719000

0.8041280

0.6615720

$-1.4348650$

1.5465740

2. 7706590

2.4308040

1. 3769820

2. 6331080

3. 5345290

1.7867860

2. 4273300

3. 2309520

1. 4806120

2. 2512210

2. 8955700

3.8155730

4. 5996030

3. 8697500

4. 0117850

4. 9013310

4.1531770

$-0.3892290$

$-1.4274750$

$-1.7013970$

$-0.5465830$

$-1.3025670$

$-2.0801670$

$-0.3294140$

$-1.4653910$

$-2.3203580$

$-0.5704630$

$-1.7814580$

$-1.2995180$

- 3.0122550

$-3.8355260$

$-3.2496440$

$-2.8421990$

$-3.5879480$

$-2.9747670$

0.3332300

0.2089670

-3.8198670
-5.0667160 


$\begin{array}{lrr}3.2970350 & -2.5088050 & -2.9668850 \\ 3.7404110 & -1.0924140 & -2.6816000 \\ 5.0584490 & -2.5712350 & -4.8053160 \\ 4.9854240 & -3.2302740 & -5.6808950 \\ 6.1133620 & -2.2987470 & -4.6813430 \\ 4.5201170 & -3.2891980 & -3.5274160 \\ 4.2066410 & -4.3149970 & -3.7628060 \\ 5.2993340 & -3.3471070 & -2.7578640 \\ 2.9140350 & -3.0036330 & -2.0704890 \\ 4.6160820 & -0.7392920 & -5.9286890 \\ 2.2052350 & -2.4788670 & -4.0710440 \\ 1.9398020 & -3.5133120 & -4.3274370 \\ 1.3080940 & -1.9919300 & -3.6793930 \\ 2.7499220 & -1.7254440 & -5.3241340 \\ 2.7197010 & -2.3707440 & -6.2123020 \\ 2.1399010 & -0.8390680 & -5.5353910 \\ 4.7328970 & 1.5116520 & -2.5000810 \\ 5.2088850 & 2.9199660 & -2.2432810 \\ 4.2426050 & 1.7562160 & -0.1320290 \\ 4.2229200 & 0.8696410 & -1.3553250 \\ 4.0081230 & 3.7459710 & -1.7023380 \\ 4.3573140 & 4.7556860 & -1.4470020 \\ 3.2488280 & 3.8447120 & -2.4871820 \\ 3.4133460 & 3.0306490 & -0.4510510 \\ 3.4305180 & 3.6927050 & 0.4241450 \\ 2.3758830 & 2.7405410 & -0.6333500 \\ 3.8336630 & 1.2492120 & 0.7431600 \\ 5.6112630 & 3.3741010 & -3.1570280 \\ 5.7058470 & 2.1958460 & 0.1426550 \\ 5.7091820 & 2.8924180 & 0.9918700 \\ 6.3059350 & 1.3227760 & 0.4271080 \\ 6.2949540 & 2.8777940 & -1.1324590 \\ 6.6185270 & 3.9034080 & -0.9100900 \\ 7.1688960 & 2.3254190 & -1.4980540 \\ 4.7483800 & 0.8591390 & -3.7323280 \\ 5.1435150 & 1.3596130 & -4.6173380 \\ 3.5908030 & -1.9636190 & 2.0539170 \\ 2.2472800 & -2.3706480 & 2.1530210 \\ 4.5765110 & -2.1782150 & 3.1907180 \\ 3.9150480 & -2.3857890 & 4.5594780 \\ 2.7505050 & -3.3755030 & 4.4406070 \\ 1.6858220 & -2.8049200 & 3.4971700 \\ 4.6616720 & -2.7374980 & 5.2836860 \\ 5.1812130 & -3.0692790 & 2.9566420 \\ 5.2793830 & -1.3352590 & 3.2207230 \\ 3.1197010 & -4.3349900 & 4.0470570\end{array}$

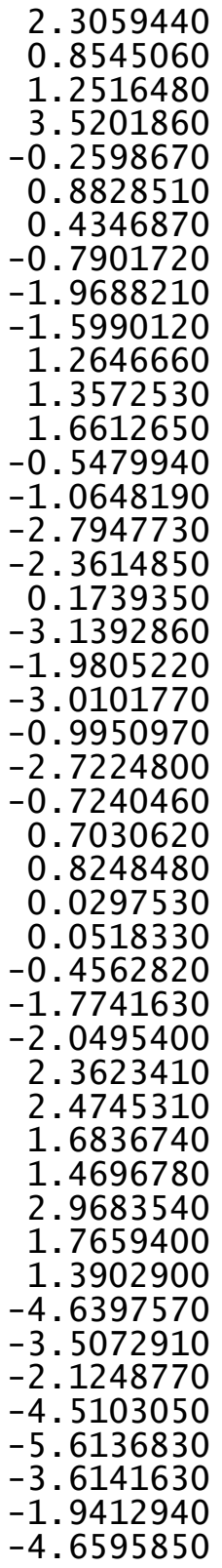

\author{
$-3.5722780$ \\ $-3.5026180$ \\ $-1.9067660$ \\ $-1.4296720$ \\ $-4.1498940$ \\ $-5.1527710$ \\ $-6.5908750$ \\ $-6.9382770$ \\ $-6.0470730$ \\ $-4.5796080$ \\ $-7.2802110$ \\ $-5.1367470$ \\ $-4.8377440$ \\ $-6.7712130$ \\ $-7.9950350$ \\ $-6.1394520$ \\ $-6.4039410$ \\ $-6.6909950$ \\ 1.9959090 \\ 2. 7027240 \\ 1. 5171750 \\ 2. 3903240 \\ 1. 1185450 \\ 4. 2985430 \\ 4.1947810 \\ 3. 0769510 \\ 3. 1838980 \\ 2. 4269520 \\ 5.3219240 \\ 4.7275190 \\ 0.3832320 \\ 3. 2400630 \\ 2. 1241850 \\ 2.0224100 \\ 4.2749130 \\ 3. 3114400 \\ 1. 1658530 \\ 5. 1287990 \\ 3. 3334790 \\ 4.3562680 \\ 3. 6806010 \\ 2. 6051710 \\ 3.8353200 \\ 4.8871900 \\ 3. 1260260 \\ 3. 3133950
}

5.4248100

3. 3768790

3.9674620

4.9284970

1. 1783210

1. 2260570

0.9330310

1.7863970

1.3756720

1. 2288190

1.1342630

2. 2174170

0.5199130

2. 8470180

1.6749280

2. 0943710

0.4101700

$-0.1311310$

$-1.9038220$

$-2.4614550$

$-0.9303200$

$-2.1310980$

$-3.0032730$

0.7146360

2.7960190

1. 9449520

0.8162120

0.0880510

2. 1242490

$-0.5203870$

$-2.5980170$

4. 2482140

3. 3916790

2. 2387700

3.9554330

5.1444370

1. 5785310

4. 6227910

$-3.4571040$

$-3.6383500$

$-3.5897270$

$-2.1090420$

$-3.5059990$

$-4.5918620$

-4.5213710
-1.2822240 


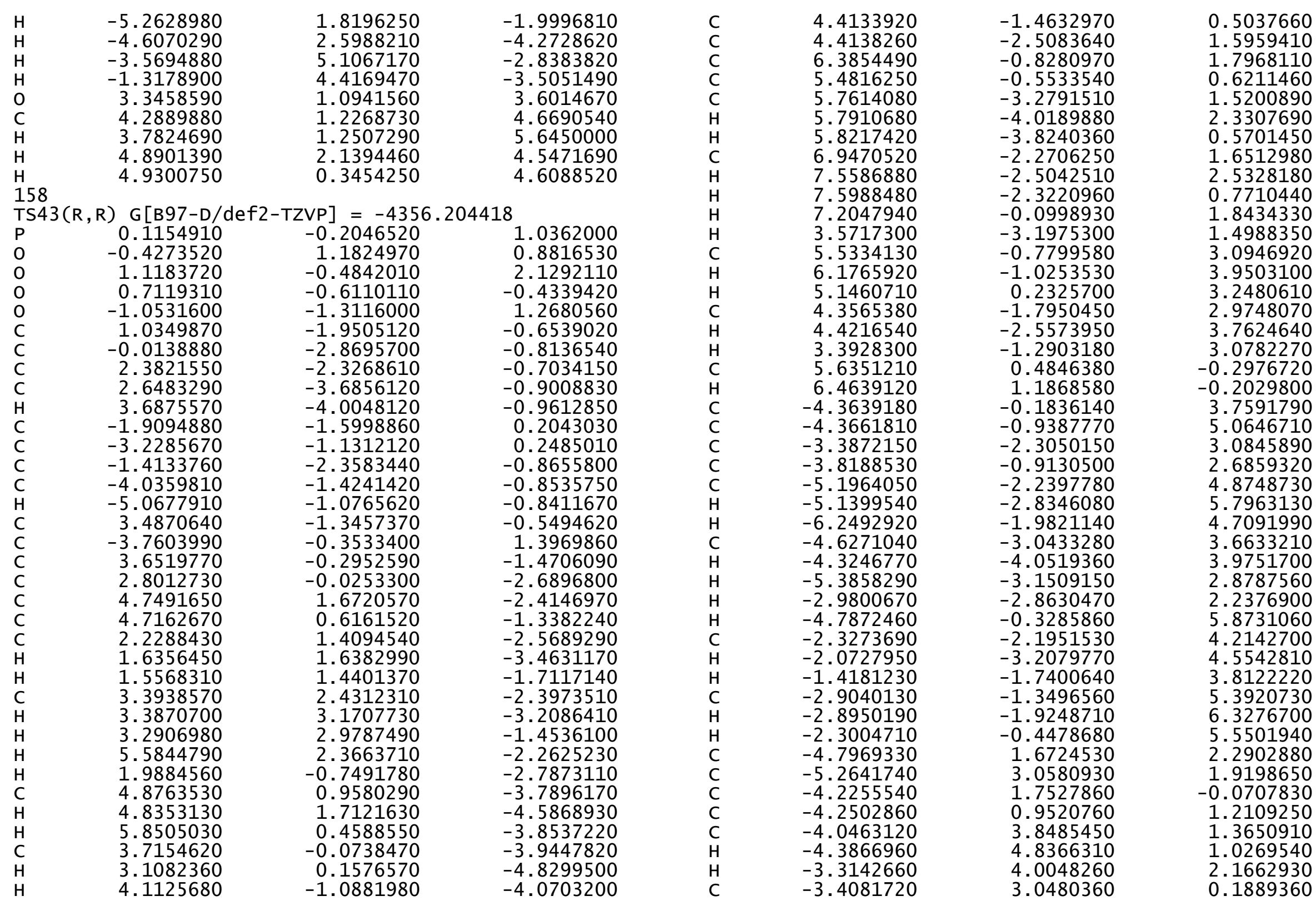




$\begin{array}{rrr}-3.3918620 & 3.6473710 & -0.7303710 \\ -2.3797900 & 2.7715460 & 0.4296930 \\ -3.7809680 & 1.1902330 & -0.8944090 \\ -5.6988360 & 3.5730370 & 2.7852660 \\ -5.6780080 & 2.1683910 & -0.4263900 \\ -5.6525790 & 2.8047880 & -1.3211400 \\ -6.2680540 & 1.2765970 & -0.6708340 \\ -6.3107040 & 2.9350610 & 0.7775110 \\ -6.6273270 & 3.9416060 & 0.4732600 \\ -7.1961060 & 2.4066900 & 1.1508730 \\ -4.8549470 & 1.1076120 & 3.5641160 \\ -5.2782700 & 1.6688040 & 4.3981910 \\ -2.2297850 & -2.5605810 & -2.0034830 \\ -1.6539290 & -3.1998760 & -3.2569720 \\ -2.4573900 & -2.8612010 & -4.5188590 \\ -3.9424170 & -3.1597790 & -4.2841670 \\ -4.4963850 & -2.2680940 & -3.1635770 \\ -3.5590710 & -2.1025740 & -1.9797340 \\ -2.0723250 & -3.4435410 & -5.3658990 \\ -1.6272280 & -4.2938990 & -3.1488040 \\ -0.6092910 & -2.8854060 & -3.3703060 \\ -4.0497220 & -4.2182720 & -4.0034560 \\ -4.5271320 & -3.0090880 & -5.2015630 \\ -5.4588640 & -2.6603870 & -2.8066560 \\ -4.7084360 & -1.2666460 & -3.5637400 \\ -2.3265250 & -1.7981640 & -4.7580050 \\ 0.2887260 & -4.2464080 & -0.9015840 \\ 1.6367240 & -4.6474620 & -0.9772190 \\ -0.8076590 & -5.2911030 & -0.7655460 \\ -0.4188710 & -6.6591380 & -1.3379110 \\ 0.9338190 & -7.0986370 & -0.7647210 \\ 2.0272460 & -6.1038630 & -1.1760600 \\ -1.1998150 & -7.3933610 & -1.1013700 \\ -1.0041200 & -5.4090540 & 0.3130810 \\ -1.7480140 & -4.9394580 & -1.1954250 \\ 0.8603180 & -7.1352120 & 0.3323670 \\ 1.1995110 & -8.1063360 & -1.1097170 \\ 2.9595100 & -6.3077600 & -0.6326820 \\ 2.2537000 & -6.2558640 & -2.2440500 \\ -0.3473750 & -6.5954510 & -2.4346670 \\ 2.9749980 & 2.1399870 & 1.8561390 \\ 1.8350370 & 2.8300260 & 2.4718650 \\ 2.7937090 & 1.6584760 & 0.8917320 \\ 0.8406330 & 2.4928270 & 2.1979560 \\ 2.6461900 & 1.2574440 & 2.9771430 \\ 0.5888530 & 4.3110930 & -0.7650340\end{array}$

$\begin{array}{lr}\mathrm{C} & -0.5521300 \\ \mathrm{C} & -0.7410740 \\ \mathrm{~N} & -0.0992450 \\ \mathrm{H} & -0.1489770 \\ \mathrm{~S} & 0.4515900 \\ \mathrm{~S} & 1.4593260 \\ \mathrm{H} & 1.9768160 \\ \mathrm{C} & -1.8988040 \\ \mathrm{C} & -2.0819610 \\ \mathrm{C} & -1.4939380 \\ \mathrm{C} & -1.1306760 \\ \mathrm{H} & -2.3519240 \\ \mathrm{H} & -1.6253480 \\ \mathrm{H} & -0.9936640 \\ \mathrm{C} & 4.5354640 \\ \mathrm{C} & 3.3957570 \\ \mathrm{C} & 2.0251510 \\ \mathrm{C} & 4.3461420 \\ \mathrm{H} & 5.5008640 \\ \mathrm{H} & 3.5461550 \\ \mathrm{H} & 1.9077420 \\ \mathrm{H} & 4.4452870 \\ \mathrm{H} & 5.0997540 \\ \mathrm{H} & 4.5623840 \\ \mathrm{H} & 3.3992440 \\ \mathrm{H} & 1.1999720 \\ \mathrm{O} & -2.8274430 \\ \mathrm{C} & -3.4555910 \\ \mathrm{H} & -2.7075740 \\ \mathrm{H} & -4.1665170 \\ \mathrm{H} & -3.9862850 \\ \mathrm{H} & \end{array}$
4.0380170
2.9022090
1.8269660
1.8434440
4. 0101090
2. 8847860
1.0132970
4.8316420
3.5105850
4.5136670
3.8122130
2.7703600
4.0309510
5.0527070
3.2555340
3.4747280
1.9896840
2. 7811630
5.2593090
4.5323890
0.7205350
0.6605000
0.6908800
1.4893260
$-0.2932410$

$-2.1097900$

$3.1581160 \quad-0.8989900$

$2.4257060 \quad-0.1471540$

$5.2573430 \quad-2.2447300$

$4.8462310 \quad 0.5644700$

2. 6099240

$-3.7363970$

$-2.4632860$

$-4.2645410$

$-5.6163160$

$-1.7770410$

$-4.9624170$

3. 3166980

3. 5554070

3.5890060

1. 9816840

3. 3085310

4.4984530

4.5300730

1.1443540

1.8418710

4. 1372070

2. 7486070

3.5538710

$-4.0285340$

$-5.3132140$

$-6.1185920$

$-5.4418770$

158

TS44 $(R, R)$ G $[B 97-D /$ def2-TZVP $]=-4356.204205$

$\begin{array}{lrrr}\text { TS44 (R, R ) G }[\mathrm{B} 97-\mathrm{D} / \mathrm{def} 2-\mathrm{TZVP}]=-4356.204205 \\ \mathrm{P} & -0.0319010 & -0.1567050 & -0.8592070 \\ \mathrm{O} & 0.3180360 & 1.2811070 & -0.6399610 \\ \mathrm{O} & -0.9876670 & -0.5297730 & -1.9660620 \\ \mathrm{O} & -0.5611320 & -0.7210310 & 0.5843740 \\ \mathrm{O} & 1.2761620 & -1.0867750 & -1.1374390 \\ \mathrm{C} & -0.7805720 & -2.0970450 & 0.6787230 \\ \mathrm{C} & 0.3381210 & -2.9430020 & 0.7544100 \\ \mathrm{C} & -2.0950890 & -2.5802190 & 0.6644630 \\ \mathrm{C} & -2.2522210 & -3.9681990 & 0.7483430 \\ \mathrm{H} & -3.2628350 & -4.3719690 & 0.7718980 \\ \mathrm{C} & 2.1341870 & -1.4240470 & -0.0945300 \\ \mathrm{C} & 3.4228020 & -0.8736000 & -0.0805650 \\ \mathrm{C} & 1.6964370 & -2.3399530 & 0.8738180\end{array}$




$\begin{array}{rrr}4.2603660 & -1.2529110 & 0.9711020 \\ 5.2734120 & -0.8540510 & 0.9943990 \\ -3.2721600 & -1.6841530 & 0.5189570 \\ 3.9020560 & 0.0419670 & -1.1503400 \\ -3.5390570 & -0.6682140 & 1.4557580 \\ -2.7575340 & -0.3753030 & 2.7145270 \\ -4.8043970 & 1.1937310 & 2.4084990 \\ -4.6618940 & 0.1692410 & 1.3104120 \\ -2.2839730 & 1.0993180 & 2.6656580 \\ -1.7604350 & 1.3395060 & 3.5989360 \\ -1.5675180 & 1.2080160 & 1.8519060 \\ -3.5058190 & 2.0435200 & 2.4588960 \\ -3.5831740 & 2.7680650 & 3.2799680 \\ -3.4017240 & 2.6142300 & 1.5282840 \\ -5.6804510 & 1.8319510 & 2.2414520 \\ -1.9011910 & -1.0452350 & 2.8253110 \\ -4.9293170 & 0.4386600 & 3.7623120 \\ -4.9508920 & 1.1743010 & 4.5772310 \\ -5.8755880 & -0.1144910 & 3.7879460 \\ -3.7173470 & -0.5310560 & 3.9266660 \\ -3.1625100 & -0.3043420 & 4.8466710 \\ -4.0585810 & -1.5711720 & 3.9912750 \\ -4.1652620 & -1.8485980 & -0.5591990 \\ -4.0813670 & -2.8806390 & -1.6625930 \\ -6.1694360 & -1.3512630 & -1.8672850 \\ -5.2943990 & -1.0165860 & -0.6850440 \\ -5.3678060 & -3.7502650 & -1.5974740 \\ -5.3410630 & -4.4814500 & -2.4161270 \\ -5.3886340 & -4.3090150 & -0.6537930 \\ -6.6246970 & -2.8315530 & -1.7203580 \\ -7.2229460 & -3.1084660 & -2.5984730 \\ -7.2652410 & -2.9304220 & -0.8359970 \\ -7.0399370 & -0.6849750 & -1.9137250 \\ -3.1910440 & -3.5058960 & -1.5688160 \\ -5.3248910 & -1.2450010 & -3.1663690 \\ -5.9475190 & -1.5529080 & -4.0171320 \\ -5.0223300 & -0.2069820 & -3.3339440 \\ -4.0698200 & -2.1581210 & -3.0369070 \\ -4.0620120 & -2.9196460 & -3.8280070 \\ -3.1508720 & -1.5732140 & -3.1276570 \\ -5.5432480 & -0.0078060 & 0.2453740 \\ -6.4204900 & 0.6317620 & 0.1436580 \\ 4.5760280 & 0.4250820 & -3.4675300 \\ 4.7191370 & -0.2447760 & -4.8110840 \\ 3.7903660 & -1.8222030 & -2.9661020 \\ 4.0688310 & -0.4238500 & -2.4660110\end{array}$

5.6702920

.7397880

6.6756410

5.1149230

4.9135580

5.8423840

3. 4009580

5.1134850

2.7719710

2. 6126830

1.8135250

3.3266270

3.4321510

2.6439750

4.7376120

5.0254830

4.0594760

4.2312070

3.7023440

3.9132060

2. 9921440

3.1097900

2.9884020

2.1300460

3.6457590

5.4348540

5.4376160

5. 3007920

6.1162320

6.0273020

6.2067960

6.9854900

4.9135860

5. 3082120

2. 5411460

2.0230880

2. 9232710

4.3954930

4.8057570

3.8422480

2.6221300

1.9074000

1. 0102620

4.5284540

5.0398210
5.8152660
-1.4638290
-1.9947220
-1.1099370
-2.4063370
-3.4068540
-2.5170350
-2.4684460
0.4521190
-1.7341890
-2.7435150
-1.3729170
-0.7834520
-1.3157140
0.0591760
2.2211570
3.6242510
2.0879000
1.3782840
4.2356180
5.2316320
4.3597680
3.2976220
3.8323850
2.9223720
1.4209930
4.2395420
2.6391760
3.2090700
1.8057580
3.5494350
4.5643640
3.1528170
1.7467480
2.4018630
-2.6427420
-3.5010280
-3.5048440
-3.6337960
-2.4100780
-2.1085500
-4.3303550
-4.5394040
-3.1662420
-4.5468650
-3.7230630
-2.5388220

$-5.6037320$

$-4.3871700$

$-3.5316280$

$-3.9369620$

$-2.7184750$

$-2.1748420$

$-5.5606150$

$-4.1354960$

$-4.5380440$

$-3.7518110$

$-5.2419530$

$-6.1968580$

$-5.4035830$

$-1.8737100$

$-1.4004030$

0.4563750

$-0.8655400$

$-0.8626370$

$-0.4506920$

$-1.6886650$

0.2327230

1.1829750

$-0.0701920$

1. 2164590

$-2.2108050$

0.9094440

1.8381430

1.1279310

$-0.2142640$

0.1645640

$-0.5704820$

$-3.1731340$

$-3.9508040$

1.9711690

3.1184790

4.3637520

3.9596270

3.1330940

1.9977780

5.0209860

2.7842210

3.3784030

3.3599430

4.8434250

2.7203040 


$\begin{array}{lrrr}\text { H } & 4.8567950 & -1.5337960 & \\ \text { H } & 2.7877610 & -2.5768320 & 4.7992920 \\ \mathrm{C} & 0.1426200 & -4.3403480 & 0.68458500 \\ \mathrm{C} & -1.1687580 & -4.8512030 & 0.7345950 \\ \mathrm{C} & 1.3048760 & -5.2785400 & 0.3983020 \\ \mathrm{C} & 1.0471160 & -6.7178870 & 0.8587400 \\ \mathrm{C} & -0.2863460 & -7.2134230 & 0.2855930 \\ \mathrm{C} & -1.4423730 & -6.3459290 & 0.8021290 \\ \mathrm{H} & 1.8735370 & -7.3637880 & 0.5352310 \\ \mathrm{H} & 1.4426820 & -5.2859500 & -0.6957650 \\ \mathrm{H} & 2.2427390 & -4.8940950 & 0.8058260 \\ \mathrm{H} & -0.2426270 & -7.1552900 & -0.8120470 \\ \mathrm{H} & -0.4634160 & -8.2636210 & 0.5514450 \\ \mathrm{H} & -2.3678430 & -6.5725560 & 0.2563200 \\ \mathrm{H} & -1.6344980 & -6.6087410 & 1.8551450 \\ \mathrm{H} & 1.0107920 & -6.7545720 & 1.9585520 \\ \mathrm{C} & -3.1489070 & 1.8850010 & -1.8970000 \\ \mathrm{C} & -2.0668850 & 2.6628850 & -2.5157830 \\ \mathrm{H} & -2.9656310 & 1.4892030 & -0.8945470 \\ \mathrm{H} & -1.0540600 & 2.4665270 & -2.1785240 \\ \mathrm{O} & -2.6662930 & 0.9779610 & -2.9369540 \\ \mathrm{C} & -1.2046240 & 4.4990650 & 0.6029310 \\ \mathrm{C} & -0.1986850 & 4.4318230 & 2.9195200 \\ \mathrm{C} & 0.1104060 & 3.3232170 & 2.1162950 \\ \mathrm{~N} & -0.4553660 & 3.4041540 & 0.8560220 \\ \mathrm{H} & -0.2861130 & 2.6383580 & 0.1670520 \\ \mathrm{~S} & -1.2160450 & 5.5399320 & 2.0235160 \\ \mathrm{~S} & -2.0265310 & 4.8773710 & -0.8091190 \\ \mathrm{H} & -1.9276420 & 0.3207670 & -2.5001720 \\ \mathrm{C} & 1.0510990 & 3.4454600 & 4.7132320 \\ \mathrm{C} & 1.3413260 & 2.3250340 & 3.9005390 \\ \mathrm{C} & 0.8813710 & 2.2574100 & 2.5825300 \\ \mathrm{C} & 0.2831350 & 4.4996740 & 4.2314430 \\ \mathrm{H} & 1.4310680 & 3.4597150 & 5.7310450 \\ \mathrm{H} & 1.0764600 & 1.4180430 & 1.9267320 \\ \mathrm{H} & 0.0577350 & 5.3503060 & 4.8688480 \\ \mathrm{C} & -4.7834150 & 2.9417960 & -3.5242760 \\ \mathrm{C} & -3.7559960 & 4.0493380 & -3.8078240 \\ \mathrm{C} & -2.3123540 & 3.5199620 & -3.7235040 \\ \mathrm{C} & -4.5744710 & 2.3433660 & -2.1236900 \\ \mathrm{H} & -5.8008510 & 3.3445390 & -3.5980460 \\ \mathrm{H} & -3.9207340 & 4.4856390 & -4.8002530 \\ \mathrm{H} & -2.0827510 & 2.9050350 & -4.6055300 \\ \mathrm{H} & -4.7878370 & 3.0991270 & -1.3549690 \\ \mathrm{H} & -5.2430870 & 1.4965850 & -1.9450340 \\ \mathrm{H} & -4.6893350 & 2.1473860 & -4.2766110 \\ & & & \\ & & & \end{array}$

$\begin{array}{lrrr}\mathrm{H} & -3.8865770 & 4.8525750 & -3.0703950 \\ \mathrm{H} & -1.5820590 & 4.3366370 & -3.7187540 \\ \mathrm{O} & 2.0743110 & 1.3431470 & 4.4945320 \\ \mathrm{C} & 2.2744510 & 0.1359050 & 3.7459470 \\ \mathrm{H} & 2.8387080 & 0.3231130 & 2.8249950 \\ \mathrm{H} & 1.3130530 & -0.3280120 & 3.4870670 \\ \mathrm{H} & 2.8488110 & -0.5229420 & 4.3970070\end{array}$

158

TS45 $(R, R)$ G $[B 97-D /$ def2-TZVP $]=-4356.204146$

$\begin{array}{lrrr}\text { P } & -0.1834120 & -0.2973200 & -0.8969630 \\ \text { O } & 0.4003980 & 1.0800410 & -0.9894770 \\ \text { O } & -1.2305150 & -0.7201620 & -1.8964300 \\ \text { O } & -0.7303940 & -0.4481130 & 0.6391640 \\ \text { O } & 0.9489730 & -1.4602870 & -0.9803840 \\ \text { C } & -1.0474760 & -1.7368010 & 1.0770500 \\ \text { C } & 0.0122320 & -2.6104900 & 1.3857240 \\ \text { C } & -2.3931140 & -2.1104420 & 1.1712520 \\ \text { C } & -2.6669410 & -3.4293420 & 1.5608580 \\ \text { H } & -3.7057370 & -3.7458670 & 1.6340460 \\ \mathrm{C} & 1.8628570 & -1.5892010 & 0.0642270 \\ \mathrm{C} & 3.1902370 & -1.1996120 & -0.1518360 \\ \mathrm{C} & 1.4199020 & -2.1324650 & 1.2821790 \\ \mathrm{C} & 4.0742450 & -1.3614380 & 0.9190180 \\ \mathrm{H} & 5.1144380 & -1.0738940 & 0.7754450 \\ \mathrm{C} & -3.5038740 & -1.1773250 & 0.8475410 \\ \mathrm{C} & 3.6549720 & -0.6311490 & -1.4436300 \\ \mathrm{C} & -3.7032930 & -0.0020070 & 1.5941410 \\ \mathrm{C} & -2.8787880 & 0.4678770 & 2.7695190 \\ \mathrm{C} & -4.8787880 & 2.0401690 & 2.2395920 \\ \mathrm{C} & -4.7904600 & 0.8493350 & 1.3182680 \\ \mathrm{C} & -2.3515560 & 1.8892230 & 2.4470030 \\ \mathrm{H} & -1.7577520 & 2.2566910 & 3.2939120 \\ \mathrm{H} & -1.6887960 & 1.8218520 & 1.5837280 \\ \mathrm{C} & -3.5510020 & 2.8416640 & 2.1530350 \\ \mathrm{H} & -3.5839900 & 3.6610760 & 2.8831160 \\ \mathrm{H} & -3.4498080 & 3.2924290 & 1.1598690 \\ \mathrm{H} & -5.7338210 & 2.6767620 & 1.9813370 \\ \mathrm{H} & -2.0449020 & -0.2077180 & 2.9752910 \\ \mathrm{C} & -5.0145220 & 1.5069340 & 3.6934390 \\ \mathrm{H} & -5.0352520 & 2.3596530 & 4.3851150 \\ \mathrm{H} & -5.9645380 & 0.9694750 & 3.7986870 \\ \mathrm{C} & -3.8100490 & 0.5667620 & 4.0106320 \\ \mathrm{H} & -3.2268810 & 0.9570290 & 4.8551930 \\ \mathrm{H} & -4.1613050 & -0.4352890 & 4.2844300 \\ \mathrm{C} & -4.4164000 & -1.4791130 & -0.1816890 \\ \mathrm{C} & -4.3898540 & -2.6859020 & -1.0920830\end{array}$




-6.4008510
-5.5067080
-5.7193280
-5.7324920
-5.7667770
-6.9290330
-7.5341480
-7.5799880
-7.2364330
-3.5314630
-5.5490020
-6.1868470
-5.1846190
-4.3510580
-4.4046600
-3.3982040
-5.6971530
-6.5469450
4.0511170
3.9080210
3.0697740
3.5807730
4.7071130
4.5436560
5.7793880
4.2270780
3.8640170
5.0514970
2.7239240
4.2732230
1.9155130
1.5996600
1.0607500
2.4098290
2.2975660
1.8236380
4.6824150
5.2272280
4.3233660
4.2097340
4.0912860
4.4968490
3.2993410
3.5286240
3.6097880
2.4734710

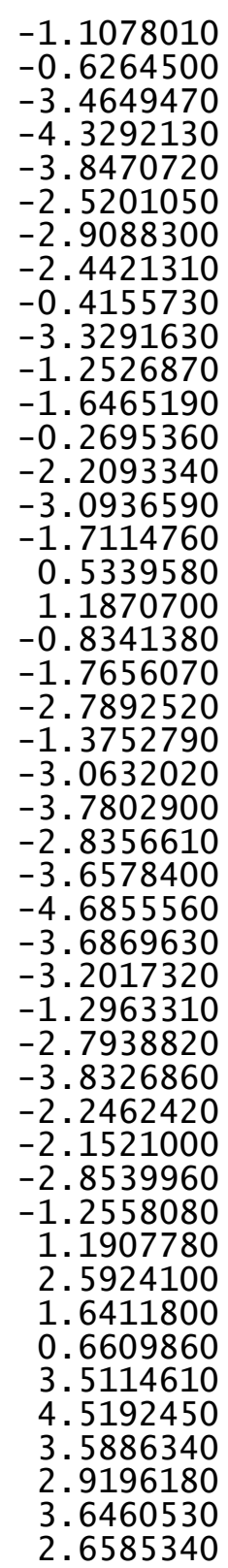

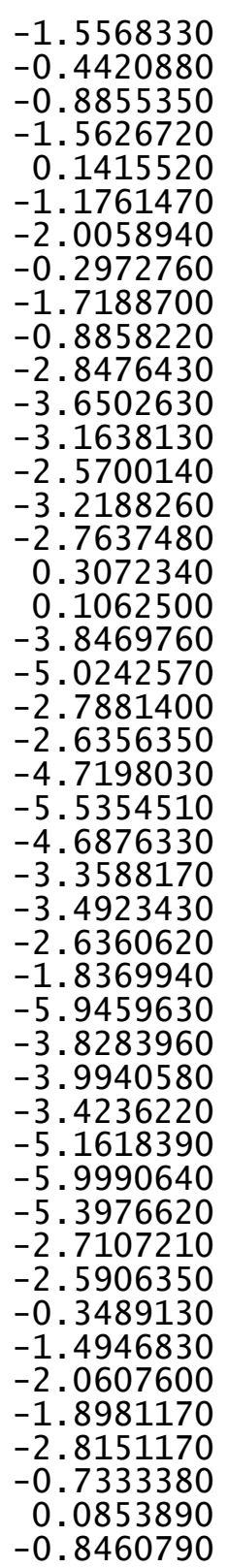

\begin{abstract}
3.9389100
5. 6011150

5.8145330

5.8882910

6. 3934560

6. 3655090

6.7422310

7.1959300

4.6030890

4. 9668620

3.6668050

2. 3231900

4.7075570

4.1115380

2. 9485070

1.8332900

4.8908780

5. 2948070

5. 4154790

3. 3040690

2. 5518600

1. 0253400

1. 3899120

3. 7289080

$-0.3006290$

0.7242630

0.3653250

$-1.1111320$

$-1.9067190$

$-1.6467270$

0.7314910

1. 0560070

$-1.1455410$

$-1.5788090$

1.7315890

0.5358120

$-1.6143430$

$-2.9789100$

$-3.1270310$

$-1.9635700$

$-2.9891500$

$-0.9799410$

$-2.7501460$

$-0.5907660$

0.8592720

0.9618940
\end{abstract}

1.2254410
2.9553950
2.0375510
2.7993490
1.1651720
2.5888290
3.6129720
1.9700000
0.4463560
0.8605020
-1.8376570
-2.2070530
-1.9687190
-2.0530970
-3.0499220
-2.5623240
-2.3396270
-2.8822590
-1.1324970
-4.0366290
-3.1699530
-3.2943190
-1.6490730
-1.0680650
-3.9435690
-5.0391140
-6.2443690
-6.7109950
-5.7952230
-4.3443120
-5.3759430
-7.0688980
-7.7410570
-6.0091330
-4.6804980
-5.9461120
-6.7066870
-6.0186740
1.8830720
2.5140770
1.5009910
2.2087410
0.8949740
4.3357050
4.3780340
3.1972130

0.5837450

$-3.5557470$

$-0.1795820$

0.6082420

0.1477010

$-1.5324850$

$-1.4091540$

$-1.8931040$

$-3.8873550$

$-4.8286230$

2. 1673150

2. 3674380

3. 2669470

4.6772770

4.6937770

3. 7619660

5.3957300

3. 0800480

3. 1916580

4. 3601200

5. 7102920

3.7231910

4.1923400

4.9759430

1.7142490

1.8861480

0.9824990

1.1583220

2. 1234350

1.8090710

2.9348660

1.1988220

1.5347750

3. 1528320

1. 6569240

$-0.0591950$

0.1837540

2. 0709590

$-1.9818370$

$-2.6163090$

$-0.9677370$

$-2.2749770$

$-2.9974600$

0.4013690

2. 4690760

1.7041890 


\begin{tabular}{|c|c|c|c|c|c|c|c|}
\hline & & & & & & & \\
\hline$\underset{\mathrm{H}}{\mathrm{N}}$ & 0.1553130 & $\begin{array}{l}3.2257370 \\
2.4112840\end{array}$ & $\begin{array}{r}0.5792390 \\
-0.0870170\end{array}$ & $\mathrm{C}$ & $\begin{array}{r}3.6550170 \\
-3.5038700\end{array}$ & -0.6311050 & -1.4435510 \\
\hline $\mathrm{S}$ & -0.3011510 & $\begin{array}{l}2.4112840 \\
5.4597810\end{array}$ & $\begin{array}{r}-0.0876230 \\
1.7276230\end{array}$ & c & $\begin{array}{r}-3.5038120 \\
3.5808700\end{array}$ & $\begin{array}{l}-1.1 / 73 / 40 \\
-1.3752700\end{array}$ & $\begin{array}{r}0.8475570 \\
-2.6355370\end{array}$ \\
\hline $\mathrm{s}$ & -1.6492140 & 4.6831850 & -0.8530870 & $\mathrm{C}$ & 3.0699590 & -2.7892790 & -2.7880050 \\
\hline $\mathrm{H}$ & -2.0897980 & 0.1895540 & -2.5311690 & $\mathrm{C}$ & 3.9082000 & -1.7656610 & -5.0241350 \\
\hline C & 2.5336200 & 3.5263170 & 3.9672730 & $\mathrm{C}$ & 4.0512070 & -0.8341390 & -3.8468860 \\
\hline $\mathrm{C}$ & 2.6265060 & 2.3471050 & 3.1975680 & $\mathrm{C}$ & 1.9157250 & -2.7940170 & -3.8282880 \\
\hline C & 1.8189070 & 2.1620380 & 2.0666250 & $\mathrm{H}$ & 1.5999360 & -3.8328460 & -3.9939180 \\
\hline C & 1.6431560 & 4.5419820 & 3.6080660 & $\mathrm{H}$ & 1.0609200 & -2.2464100 & -3.4235510 \\
\hline $\mathrm{H}$ & 3.1535730 & 3.6605690 & 4.8465230 & $\mathrm{C}$ & 2.4100370 & -2.1522560 & -5.1617430 \\
\hline $\mathrm{H}$ & 1.8880270 & 1.2562810 & 1.4736680 & $\mathrm{H}$ & 2.2978400 & -2.8541920 & -5.9989430 \\
\hline $\mathrm{H}$ & 1.5786240 & 5.4452940 & 4.2085860 & $\mathrm{H}$ & 1.8237950 & -1.2560110 & -5.3976150 \\
\hline C & -4.6250180 & 3.1032520 & -3.6311350 & $\mathrm{H}$ & 4.2733960 & -1.2963980 & -5.9458500 \\
\hline $\mathrm{C}$ & -3.4767810 & 4.0850570 & -3.9118540 & $\mathrm{H}$ & 2.7241080 & -3.2017450 & -1.8368530 \\
\hline C & -2.1065230 & 3.3893310 & -3.8245480 & C & 4.7073750 & -3.0631880 & -4.7196080 \\
\hline C & -4.4925150 & 2.4895730 & -2.2273420 & $\mathrm{H}$ & 4.5440040 & -3.7803170 & -5.5352380 \\
\hline $\mathrm{H}$ & -5.5901690 & 3.6177960 & -3.7118990 & $\mathrm{H}$ & 5.7796310 & -2.8355660 & -4.6874060 \\
\hline $\mathrm{H}$ & -3.5879740 & 4.5378500 & -4.9043460 & C & 4.2273360 & -3.6578160 & -3.3586180 \\
\hline $\mathrm{H}$ & -1.9478650 & 2.7505800 & -4.7056840 & $\mathrm{H}$ & 3.8643430 & -4.6855580 & -3.4921240 \\
\hline $\mathrm{H}$ & -4.6325390 & 3.2649930 & -1.4615420 & $\mathrm{H}$ & 5.0517340 & -3.6868630 & -2.6358370 \\
\hline $\mathrm{H}$ & -5.2489240 & 1.7193490 & -2.0506750 & C & 4.2097190 & 0.6610530 & -1.4946280 \\
\hline $\mathrm{H}$ & -4.6161680 & 2.3011350 & -4.3814110 & C & 4.3232980 & 1.6412750 & -0.3488750 \\
\hline $\mathrm{H}$ & -3.5130830 & 4.8997540 & -3.1757390 & $\mathrm{C}$ & 5.2271490 & 2.5924930 & -2.5906080 \\
\hline $\mathrm{H}$ & -1.2837990 & 4.1130760 & -3.8173400 & $\mathrm{C}$ & 4.6823930 & 1.1908360 & -2.7106720 \\
\hline 0 & 3.4953680 & 1.3306810 & 3.4742350 & C & 5.8144480 & 2.0377080 & -0.1795360 \\
\hline C & 4.4654410 & 1.5453030 & 4.5039470 & $\mathrm{H}$ & 5.8881660 & 2.7995280 & 0.6082710 \\
\hline $\mathrm{H}$ & 3.9840460 & 1.6472890 & 5.4875730 & $\mathrm{H}$ & 6.3934040 & 1.1653610 & 0.1477740 \\
\hline $\mathrm{H}$ & 5.0633870 & 2.4441630 & 4.2952110 & C & 6.3654180 & 2.5889780 & -1.5324450 \\
\hline $\mathrm{H}$ & 5.1045700 & 0.6604040 & 4.4974070 & $\mathrm{H}$ & 6.7420980 & 3.6131390 & -1.4091320 \\
\hline & & & & $\mathrm{H}$ & 7.1958680 & 1.9701740 & -1.8930410 \\
\hline & 2) $\mathrm{G}[\mathrm{B} 97-\mathrm{D}$ & {$[\mathrm{VP}]=-4356$} & & $\mathrm{H}$ & 5.6010300 & 2.9554760 & -3.5557230 \\
\hline $\mathrm{P}$ & -0.1833690 & -0.2973360 & -0.8969870 & $\mathrm{H}$ & 3.9388470 & 1.2255350 & 0.5837860 \\
\hline 0 & -1.2304230 & -0.7201960 & -1.8965010 & C & 4.0911630 & 3.5115080 & -2.0607620 \\
\hline 0 & 0.4003990 & 1.0800440 & -0.9894660 & $\mathrm{H}$ & 4.4966840 & 4.5193130 & -1.8981380 \\
\hline 0 & 0.9490500 & -1.4602690 & -0.9803920 & $\mathrm{H}$ & 3.2992230 & 3.5886330 & -2.8151300 \\
\hline 0 & -0.7303930 & -0.4481580 & 0.6391230 & C & 3.5285110 & 2.9196730 & -0.7333350 \\
\hline C & 1.8628960 & -1.5891870 & 0.0642530 & $\mathrm{H}$ & 3.6096390 & 3.6461270 & 0.0853780 \\
\hline c & 1.4199120 & -2.1324860 & 1.2821760 & $\mathrm{H}$ & 2.4733690 & 2.6585440 & -0.8460770 \\
\hline C & 3.1902740 & -1.1995630 & -0.1517600 & C & 4.6031220 & 0.4463790 & -3.8872870 \\
\hline c & 4.0742510 & -1.3613790 & 0.9191180 & $\mathrm{H}$ & 4.9668940 & 0.8605160 & -4.8285600 \\
\hline $\mathrm{H}$ & 5.1144410 & -1.0738030 & 0.7755860 & C & -4.7904300 & 0.8492930 & 1.3183380 \\
\hline C & -1.0474660 & -1.7368500 & 1.0770000 & $\mathrm{C}$ & -4.8787090 & 2.0401330 & 2.2396570 \\
\hline C & -2.3931020 & -2.1104980 & 1.1712100 & $\mathrm{C}$ & -2.8786960 & 0.4678330 & 2.7695060 \\
\hline $\mathrm{C}$ & 0.0122470 & -2.6105350 & 1.3856690 & C & -3.7032570 & -0.0020570 & 1.5941690 \\
\hline c & -2.6669190 & -3.4294120 & 1.5607820 & $\mathrm{C}$ & -5.0143870 & 1.5069030 & 3.6935110 \\
\hline $\mathrm{H}$ & -3.7057130 & 15013 & 1.6339650 & $\mathrm{H}$ & 5.0351090 & 2.3596230 & 4.3851 \\
\hline
\end{tabular}




\begin{abstract}
$-5.9643920$
$-3.8098920$

$-3.2266770$

$-4.1611250$

$-2.0448050$

$-5.7337490$

$-2.3514690$

$-1.7576030$

$-1.6887680$

$-3.5509220$

$-3.5838700$

$-3.4497730$

$-5.5067500$

$-6.4009100$

$-4.3899130$

$-4.4164400$

$-5.5490740$

$-6.1869300$

$-5.1846950$

$-4.3511300$

$-4.4047380$

$-3.3982770$

$-3.5315230$

$-7.2364910$

$-5.7193880$

$-5.7325610$

$-5.7668270$

$-6.9290930$

$-7.5342010$

$-7.5800530$

$-5.6971620$

$-6.5469510$

$-0.3006050$

0.7242960

0.3654540

$-1.1110920$

$-1.9066740$

$-1.6466980$

0.7314780

1.0560050

$-1.1456980$

$-1.5787350$

1.7316280

$-1.6141830$

$-2.9788610$
\end{abstract}

3.7987870
4.0106650
4.8551800
4.2845180
2.9752500
1.9814350
2.4469430
3.2938080
1.5836250
2.1530410
2.8831300
1.1598730
-0.4419990
-1.5567470
-1.0920700
-0.1816410
-2.8475710
-3.6501960
-3.1637160
-2.5699860
-3.2188270
-2.7637110
-0.8858400
-1.7187540
-0.8855270
-1.5626780
0.1415540
-1.1761090
-2.0058740
-0.2972390
0.3073360
0.1063780
1.7141600
1.8861030
0.9824800
1.1579740
2.1232210
1.8089630
2.9348280
1.1990870
1.5341150
3.1525860
1.6569280
-0.0592070
0.1833450
2.0707370

2.3231660
3.6667790
1.8332260
2.9484230
4.1114250
4.7074940
2.5517430
1.3898030
1.0252990
3.7287560
4.8907460
5.4153900
5.2947840
3.3040280
-3.1270550
-1.9636240
-2.9891360
-0.9799810
-2.7501820
-0.5908210
0.8592260
0.9618230
0.1552440
0.1539410
-0.3011790
-1.6492760
-2.0897910
2.5335350
2.6263940
1.8188040
1.6431020
3.1534830
1.8878980
1.5785880
-4.6251110
-3.4768950
-2.1066200
-4.4925460
-5.5902700
-3.5881220
-1.9479600
-4.6325180
-5.2489590
-4.6162830
-3.5131920
-1.2839120

-2.2070660
-1.8376290
-2.5623640
-3.0499330
-2.0530740
-1.9686670
-3.1699840
-1.6491300
-3.2943820
-1.0680550
-2.3395880
-1.1324200
-2.8821840
-4.0366280
1.8830130
2.5139990
1.5009730
2.2086940
0.8948920
4.3357840
4.3781480
3.1973020
3.2258110
2.4113420
5.4598950
4.6832330
0.1894850
3.5264280
2.3471900
2.1621130
4.5421070
3.6606850
1.2563360
5.4454370
3.1030880
4.0848890
3.3891950
2.4895050
3.6176180
4.5376000
2.7504070
3.2649840
1.7193060
2.3009250
4.8996430
4.1129590
2. 3674660

2.1673930

3.7619730

4.6938220

4.6773700

3.2670640

5.7103220

4.1923370

3.7231630

4.9760310

5.3958490

3.1918070

3.0801800

4. 3601710

$-1.9818040$

$-2.6163450$

$-0.9676930$

$-2.2750270$

$-2.9974050$

0.4012400

2.4689420

1.7040900

0.5791390

$-0.0870940$

1.7274600

$-0.8532180$

$-2.5311350$

3.9671790

3.1975080

2. 0665610

3.6079350

4.8464310

1.4736290

4.2084300

$-3.6311140$

$-3.9119410$

$-3.8246200$

$-2.2272860$

$-3.7118710$

$-4.9044650$

$-4.7057300$

$-1.4615360$

$-2.0505230$

$-4.3813400$

$-3.1758890$

$-3.8174580$ 


\begin{tabular}{|c|c|c|c|c|c|c|c|}
\hline & & & & & & & \\
\hline 0 & $\begin{array}{l}3.4952240 \\
4.4652860\end{array}$ & $\begin{array}{l}1.3307500 \\
1.5453740\end{array}$ & $\begin{array}{l}3.4742190 \\
4.5039400\end{array}$ & $\mathrm{C}$ & $\begin{array}{l}-5.6371130 \\
-5.6212310\end{array}$ & 2.2217870 & $\begin{array}{l}-0.1399350 \\
-0.9586990\end{array}$ \\
\hline $\mathrm{H}$ & 3.9838780 & $\begin{array}{l}1.3453740 \\
1.6473990\end{array}$ & $\begin{array}{l}4.3039400 \\
5.4875560\end{array}$ & $\mathrm{H}$ & -6.2353720 & $\begin{array}{l}2.95371<0 \\
1.3648180\end{array}$ & $\begin{array}{l}-0.9586990 \\
-0.4727260\end{array}$ \\
\hline $\mathrm{H}$ & 5.0632580 & 2.4442120 & 4.2951870 & C & -6.2481480 & 2.8523650 & 1.1516630 \\
\hline $\mathrm{H}$ & 5.1043920 & 0.6604570 & 4.4974340 & $\mathrm{H}$ & -6.5752030 & 3.8834680 & 0.9623080 \\
\hline & & & & $\mathrm{H}$ & -7.1233650 & 2.2812880 & 1.4839710 \\
\hline & 2) $\mathrm{G}[\mathrm{B} 97-\mathrm{D} / \mathrm{C}$ & {$[\mathrm{VP}]=-4356$} & 9 & $\mathrm{H}$ & -5.5950180 & 3.2840930 & 3.2015120 \\
\hline$P$ & 0.1426420 & -0.3162920 & 0.9570510 & $\mathrm{H}$ & -3.7522190 & 1.2885880 & -0.7385590 \\
\hline 0 & 1.1618910 & -0.7149790 & 1.9951920 & C & -3.9732940 & 3.7052670 & 1.7813760 \\
\hline 0 & -0.4010440 & 1.0796270 & 0.9669240 & $\mathrm{H}$ & -4.3248950 & 4.7181560 & 1.5425650 \\
\hline 0 & -1.0227610 & -1.4436850 & 1.0777210 & $\mathrm{H}$ & -3.2284780 & 3.7918650 & 2.5813320 \\
\hline 0 & 0.7085140 & -0.5587820 & -0.5590600 & C & -3.3524290 & 3.0220480 & 0.5262380 \\
\hline $\mathrm{C}$ & -1.9017260 & -1.6087040 & 0.0068470 & $\mathrm{H}$ & -3.3384250 & 3.7099640 & -0.3287870 \\
\hline C & -1.4381230 & -2.2509790 & -1.1535720 & $\mathrm{H}$ & -2.3251600 & 2.7140230 & 0.7293400 \\
\hline $\mathrm{C}$ & -3.2161260 & -1.1457120 & 0.1393470 & C & -4.7444870 & 0.7493180 & 3.7055320 \\
\hline $\mathrm{C}$ & -4.0589890 & -1.3201920 & -0.9611000 & $\mathrm{H}$ & -5.1464010 & 1.2232390 & 4.6020350 \\
\hline $\mathrm{H}$ & -5.0859710 & -0.9665640 & -0.8839540 & C & 4.6808910 & 0.7670490 & -1.3284900 \\
\hline C & 1.0235180 & -1.8689720 & -0.9237100 & $\mathrm{C}$ & 4.7211520 & 1.9111960 & -2.3102910 \\
\hline C & 2.3699320 & -2.2437640 & -1.0001090 & $\mathrm{C}$ & 2.7950810 & 0.2274790 & -2.7630920 \\
\hline $\mathrm{C}$ & -0.0368530 & -2.7549570 & -1.1948000 & $\mathrm{C}$ & 3.6299370 & -0.1410140 & -1.5586110 \\
\hline $\mathrm{C}$ & 2.6455330 & -3.5807250 & -1.3204780 & $\mathrm{C}$ & 4.8850360 & 1.3124860 & -3.7354820 \\
\hline $\mathrm{H}$ & 3.6844750 & -3.9001460 & -1.3781770 & $\mathrm{H}$ & 4.8610960 & 2.1286890 & -4.4697370 \\
\hline C & -3.7120190 & -0.4875880 & 1.3755290 & $\mathrm{H}$ & 5.8622060 & 0.8214660 & -3.8153060 \\
\hline $\mathrm{C}$ & 3.4689690 & -1.2765820 & -0.7430390 & C & 3.7321530 & 0.2951410 & -4.0016800 \\
\hline C & -3.7544980 & -1.1801270 & 2.5983550 & $\mathrm{H}$ & 3.1387880 & 0.6023680 & -4.8730770 \\
\hline $\mathrm{C}$ & -3.3407200 & -2.6139550 & 2.8374460 & $\mathrm{H}$ & 4.1358700 & -0.7028660 & -4.2099840 \\
\hline C & -4.2637060 & -1.4520640 & 4.9700610 & $\mathrm{H}$ & 1.9914310 & -0.4926080 & -2.9354960 \\
\hline C & -4.2658590 & -0.5604240 & 3.7540470 & $\mathrm{H}$ & 5.5462700 & 2.5972840 & -2.0836970 \\
\hline $\mathrm{C}$ & -2.2598150 & -2.6406560 & 3.9524250 & C & 2.2060680 & 1.6413270 & -2.5287850 \\
\hline $\mathrm{H}$ & -2.0083710 & -3.6873620 & 4.1704730 & $\mathrm{H}$ & 1.6156160 & 1.9366500 & -3.4053400 \\
\hline $\mathrm{H}$ & -1.3532490 & -2.1489180 & 3.5889710 & $\mathrm{H}$ & 1.5287970 & 1.5937500 & -1.6767860 \\
\hline C & -2.8084490 & -1.9307640 & 5.2292180 & C & 3.3588090 & 2.6571680 & -2.2653650 \\
\hline $\mathrm{H}$ & -2.8071820 & -2.6155590 & 6.0878610 & $\mathrm{H}$ & 3.3619370 & 3.4481350 & -3.0267010 \\
\hline $\mathrm{H}$ & -2.1829350 & -1.0686080 & 5.4895130 & $\mathrm{H}$ & 3.2318320 & 3.1417510 & -1.2904300 \\
\hline $\mathrm{H}$ & -4.6598460 & -0.9254810 & 5.8469430 & C & 5.4503210 & -0.5828740 & 0.5089260 \\
\hline $\mathrm{H}$ & -2.9577070 & -3.0846550 & 1.9281990 & $\mathrm{C}$ & 6.3661450 & -0.9725290 & 1.6419900 \\
\hline C & -5.1274170 & -2.7041820 & 4.6511050 & C & 4.4134750 & -2.6460370 & 1.2700760 \\
\hline $\mathrm{H}$ & -5.0857340 & -3.3938660 & 5.5049150 & $\mathrm{C}$ & 4.3932240 & -1.4889870 & 0.2971270 \\
\hline $\mathrm{H}$ & -6.1732720 & -2.4024490 & 4.5184160 & $\mathrm{C}$ & 5.5292190 & -1.0892460 & 2.9455000 \\
\hline C & -4.5831760 & -3.3911320 & 3.3591100 & $\mathrm{H}$ & 6.1864810 & -1.4295450 & 3.7569120 \\
\hline $\mathrm{H}$ & -4.2883580 & -4.4282900 & 3.5671300 & $\mathrm{H}$ & 5.1362850 & -0.1066990 & 3.2259540 \\
\hline $\mathrm{H}$ & -5.3528790 & -3.4137480 & 2.5782950 & C & 4.3594880 & -2.0936110 & 2.7206360 \\
\hline C & -4.1892690 & 0.8352990 & 1.3370140 & $\mathrm{H}$ & 4.4377700 & -2.9399140 & 3.4158150 \\
\hline $\mathrm{C}$ & -4.1810180 & 1.7651390 & 0.1453740 & $\mathrm{H}$ & 3.3926820 & -1.6130110 & 2.8880560 \\
\hline C & -5.1781950 & 2.8602170 & 2.2797500 & $\mathrm{H}$ & 3.5792150 & -3.3310750 & 1.1011670 \\
\hline C & 72750 & 1.4434000 & 2.4966560 & $\mathrm{H}$ & 7.1752580 & -0.2416520 & 1.7643860 \\
\hline
\end{tabular}




\begin{tabular}{|c|c|c|}
\hline $\begin{array}{l}5.7707660 \\
5.8191180 \\
5.8284230 \\
6.9457860 \\
7.5765060 \\
7.5814940 \\
5.5941550 \\
6.4153430 \\
0.2776500 \\
0.7445860 \\
0.3849870 \\
1.0847100 \\
1.8842510 \\
1.6250090 \\
0.7420720 \\
1.0853270 \\
1.1045250 \\
1.5587960 \\
1.7534560 \\
0.5364410 \\
1.5946280 \\
2.9559330 \\
2.3014890 \\
1.7897670 \\
2.6121770 \\
4.0966600 \\
4.6029140 \\
3.6256620 \\
1.8227910 \\
2.2605800 \\
4.2171720 \\
5.5575940 \\
0.7338890 \\
2.4632250 \\
4.7025390 \\
4.8166250 \\
3.0195600 \\
1.8621710 \\
2.8648470 \\
0.8763320 \\
2.6702250 \\
0.5956820 \\
0.6180370 \\
0.7689560 \\
0.0881330 \\
0.1248890\end{array}$ & $\begin{array}{l}-3.3859080 \\
-4.2051740 \\
-3.8292080 \\
-2.3807980 \\
-2.7026560 \\
-2.3224450 \\
0.5448810 \\
1.2420800 \\
-4.1002420 \\
-5.2041000 \\
-6.3474780 \\
-6.8378900 \\
-5.9710990 \\
-4.5059700 \\
-5.6094480 \\
-7.1788360 \\
-7.8822130 \\
-6.2359240 \\
-4.8323190 \\
-5.9751410 \\
-6.8012980 \\
-6.1933020 \\
-2.3569250 \\
-2.8986960 \\
-2.4080010 \\
-2.7038170 \\
-1.9322330 \\
-1.8940070 \\
-3.9967330 \\
-2.9025220 \\
-3.7847440 \\
-2.3524790 \\
-2.6275720 \\
-1.3277950 \\
-2.4430140 \\
-0.8922210 \\
1.9255070 \\
2.5461600 \\
1.5428980 \\
2.2369090 \\
0.9327670 \\
4.3384780 \\
4.3095420 \\
3.1342300 \\
3.2007730 \\
2.3945670\end{array}$ & $\begin{array}{l}1.1013470 \\
1.8307440 \\
0.0996390 \\
1.3257140 \\
2.1649910 \\
0.4342620 \\
-0.2987000 \\
-0.1295860 \\
-1.4629760 \\
-1.5728340 \\
-0.5922100 \\
-0.7590130 \\
-1.7664950 \\
-1.5272330 \\
-2.5966230 \\
-0.7406860 \\
-1.0950650 \\
-2.7847080 \\
-1.3719820 \\
0.4282880 \\
0.2113000 \\
-1.7004150 \\
-2.2703110 \\
-3.5932420 \\
-4.7905580 \\
-4.5494580 \\
-3.3221630 \\
-2.1591820 \\
-3.5814250 \\
-5.7053330 \\
-4.3834940 \\
-2.9763810 \\
-3.7118850 \\
-4.9150170 \\
-5.4275750 \\
-3.6071950 \\
2.0351920 \\
2.6906070 \\
1.0235650 \\
2.3594200 \\
3.0544870 \\
-0.3848570 \\
-2.5968870 \\
-1.8318560 \\
-0.6275380 \\
0.0475620\end{array}$ \\
\hline
\end{tabular}

0.4025300
1.5110210
2.0125280
-2.0126720
-2.1552980
-1.5247240
-1.2399100
-2.5012120
-1.6272160
-1.1334570
4.5384090
3.3888510
2.0192890
4.3830880
5.5007900
3.5139840
1.8730020
4.4935010
5.1458550
4.5508170
3.4100770
1.1948680
-2.8993730
-3.5733240
-2.8563770
-4.3004900
-4.0906690

$\begin{array}{ll}\mathrm{S} & 0.4025300 \\ \mathrm{~S} & 1.5110210\end{array}$

$\mathrm{H} \quad 2.0125280$

$\begin{array}{lrrr}\text { TS48 }(\mathrm{R}, \mathrm{R}) \mathrm{G}[\mathrm{B} 97-\mathrm{D} / \mathrm{def}-\mathrm{TZVP}]=-4356.203741 \\ \mathrm{P} & 0.0443200 & -0.0902760 & 0.9871510 \\ \mathrm{O} & -0.4300070 & 1.3228200 & 0.8346610 \\ \mathrm{O} & 0.9929540 & -0.4268570 & 2.1098630 \\ \mathrm{O} & 0.6632900 & -0.5073550 & -0.4709310 \\ \mathrm{O} & -1.1780000 & -1.1439020 & 1.1590010 \\ \mathrm{C} & 0.8728160 & -1.8634930 & -0.7301090 \\ \mathrm{C} & -0.2472800 & -2.6798760 & -0.9935650 \\ \mathrm{C} & 2.1819610 & -2.3561910 & -0.7115450 \\ \mathrm{C} & 2.3591030 & -3.7247880 & -0.9612260 \\ \mathrm{H} & 3.3693720 & -4.1296860 & -0.9505500 \\ \mathrm{C} & -2.0525360 & -1.3261050 & 0.0873700 \\ \mathrm{C} & -3.3369000 & -0.7792600 & 0.1734160 \\ \mathrm{C} & -1.6133880 & -2.0872640 & -1.0143870 \\ \mathrm{C} & -4.2059970 & -1.0148960 & -0.9005390 \\ \mathrm{H} & -5.2180110 & -0.6174990 & -0.8486010 \\ \mathrm{C} & 3.3654760 & -1.5086870 & -0.4068720 \\ \mathrm{C} & -3.7889040 & 0.0162850 & 1.3437520\end{array}$

$\begin{array}{lr}5.4452750 & -1.7418020 \\ 4.7299630 & 0.9653900 \\ 0.2142230 & 2.5998820 \\ 3.3562590 & -4.3038110 \\ 2.1817150 & -3.5345610 \\ 2.0570480 & -2.2876090 \\ 4.4224780 & -3.8368680 \\ 3.4475840 & -5.2668920 \\ 1.1518790 & -1.6973630 \\ 5.3213070 & -4.4381780 \\ 3.1412700 & 3.6650050 \\ 4.1110570 & 3.9809570 \\ 3.4117880 & 3.9043970 \\ 2.5459250 & 2.2557340 \\ 3.6626210 & 3.7343340 \\ 4.5477390 & 4.9790160 \\ 2.7648990 & 4.7815970 \\ 3.3353230 & 1.4993850 \\ 1.7892300 & 2.0504160 \\ 2.3286920 & 4.4039050 \\ 4.9380190 & 3.2581480 \\ 4.1337170 & 3.9151790 \\ 1.1072490 & -3.9319230 \\ 1.1908140 & -5.1917540 \\ 1.3323610 & -6.0134250 \\ 2.0156500 & -5.1941660 \\ 0.2371960 & -5.3151000\end{array}$

5.4452750

-4. 3038110

- 3.8368680

$-5.2668920$

1.6973630

3. 6650050

3.9809570

(1)

3.7343340

4.7815970

1.4993850

4.4039050

3. 2581480

$-5.1917540$

5.1941660

$-5.3151000$ 


\begin{tabular}{|c|c|c|}
\hline $\begin{array}{l}3.7616160 \\
3.0576530 \\
5.2361390 \\
4.9421520 \\
2.7244790 \\
2.2082520 \\
2.0357400 \\
4.0319900 \\
4.2035690 \\
3.9584560 \\
6.1623720 \\
2.1433530 \\
5.3496210 \\
5.5210650 \\
6.2153650 \\
4.0359860 \\
3.5457000 \\
4.2515070 \\
4.1629810 \\
3.9015620 \\
6.0893650 \\
5.3473550 \\
5.1099890 \\
4.9489950 \\
5.1694280 \\
6.4226260 \\
6.8999130 \\
7.1411080 \\
7.0079360 \\
2.9713050 \\
5.1416260 \\
5.6585930 \\
4.9009790 \\
3.8411220 \\
3.7317180 \\
2.9570620 \\
5.7427820 \\
6.6652930 \\
4.3573390 \\
4.4105600 \\
3.5468440 \\
3.8780440 \\
5.3383250 \\
5.3291980 \\
6.3680720 \\
4.8318570\end{array}$ & $\begin{array}{r}-0.4664760 \\
-0.0190050 \\
1.3169100 \\
0.2589490 \\
1.4863260 \\
1.8436340 \\
1.5943660 \\
2.2958020 \\
3.0176830 \\
2.8648570 \\
1.8544860 \\
-0.5901200 \\
0.6174830 \\
1.3796580 \\
-0.0553650 \\
-0.1751880 \\
0.2056240 \\
-1.2380890 \\
-1.7894760 \\
-2.8407400 \\
-1.5037140 \\
-1.0655820 \\
-3.8174940 \\
-4.5734490 \\
-4.3408070 \\
-3.0166640 \\
-3.3595360 \\
-3.1596450 \\
-0.9196770 \\
-3.3810120 \\
-1.3303260 \\
-1.6720160 \\
-0.2691030 \\
-2.1547790 \\
-2.9366520 \\
-1.5152950 \\
-0.0486500 \\
0.5039490 \\
0.1914590 \\
-0.5857260 \\
-1.9851150 \\
-0.5588450 \\
-1.8163110 \\
-2.4285080 \\
-1.4754830 \\
-2.6420150\end{array}$ & $\begin{array}{l}-1.2623230 \\
-2.5213630 \\
-2.0450680 \\
-1.0118870 \\
-2.3615570 \\
-3.2608740 \\
-1.5230430 \\
-2.1104460 \\
-2.9197040 \\
-1.1767300 \\
-1.8080930 \\
-2.7017690 \\
-3.4293910 \\
-4.2010950 \\
-3.4272800 \\
-3.7180860 \\
-4.6238920 \\
-3.8794940 \\
0.7188220 \\
1.7727330 \\
2.1923270 \\
0.9552820 \\
1.7826870 \\
2.5626520 \\
0.8205100 \\
2.0551780 \\
2.9826850 \\
1.2392460 \\
2.3290910 \\
1.5830970 \\
3.4100610 \\
4.3167290 \\
3.5412570 \\
3.1666310 \\
3.9299130 \\
3.2145790 \\
0.0874810 \\
0.2684770 \\
3.7133340 \\
5.0048030 \\
2.9939070 \\
2.6232940 \\
4.7945300 \\
5.7063450 \\
4.6335980 \\
3.5704130\end{array}$ \\
\hline
\end{tabular}

-4.5987170
-5.5999320
-3.1879320
-4.7796220
-2.4734760
-2.2874010
-1.5375090
-2.9826180
-3.0171110
-2.3116750
-4.6571300
-5.0197240
-4.0932030
-4.1744160
-3.7492580
-4.0161750
-3.0034010
-3.1787860
-3.1254410
-2.1728430
-3.6949800
-5.4093230
-5.5125950
-5.4445520
-6.1694800
-6.0784110
-6.3167380
-7.0007270
-4.7496150
-5.1233030
-3.8002540
-4.7256000
-4.6466570
-3.1905190
-2.1258730
-2.4967650
-4.4282870
-5.3019670
-3.0616700
-2.0563290
-5.7536950
-5.0312770
-2.9971670
-1.1413670
-0.0330730
-1.1365980
3.8685270

2.7888930

2.1331490

5.8275510

4.1162500

4.4332340

3. 7168340

5. 3164240

6.2372900

5.4941910

2. 2784310

1.9327850

$-0.0857320$

1. 1833470

1. 3833150

1.0648810

2. 1822620

0.1892240

$-0.7205450$

0.4186690

$-0.9208150$

2.8092250

$-0.4252610$

$-1.3097890$

$-0.6792510$

0.7951680

0.5090580

1.1649080

3.5434790

4. 3900810

$-2.0264860$

$-3.1870970$

$-3.5903870$

$-3.9112780$

$-3.4111980$

$-2.0994020$

$-4.0438390$

$-4.4491300$

$-4.9944470$

$-4.1474290$

$-2.9259600$

$-2.7510800$

$-3.4563190$

$-3.3720870$

$-1.1769420$ 


-0.8919930
0.5312610
1.4318740
1.2777950
-1.1487830
-1.6590490
0.4679600
1.1281920
-2.1177520
-1.0281330
1.0139730
2.4804100
3.0275010
1.9151880
2.8436420
0.9092170
2.6158820
0.8426440
-0.0390330
-0.4275330
0.0673730
-0.0991630
0.9610200
1.6163580
1.9116510
-1.2114620
-1.5911750
-1.2016000
-0.4338770
-1.5174760
-1.4795990
-0.1418880
4.6325180
3.5463370
2.1405630
4.4322670
5.6258750
3.7090170
1.9839550
4.6044680
5.1352980
4.5997670
3.6026100
1.3539530
-2.3436850
-2.7069370

$-6.2196690$

$-6.8402150$

$-6.0609780$

$-4.5737000$

$-5.5187550$

$-6.9928810$

$-7.8863680$

$-6.3278160$

$-4.6334480$

$-5.7985070$

$-6.8374650$

$-6.3606240$

2. 0345850

2. 8209130

1.5102140

2. 5319100

1.2308030

4.1754990

3.7494880

2.7888690

3.0759720

2.4202450

4.9692360

4.7655340

0.5058730

2. 5111820

1.5516900

1.6831140

3.6136170

2.4063080

0.9360070

4.3436310

3.4014810

4.4783580

3.8524700

2. 5831080

3.8659860

5.0745560

3.3562980

3.2185270

1.7465310

2.7288960

5.1628720

4.6101780

0.4503850

0.2339140

-0.2533100
-0.3712110
-1.3642880
-1.1772090
-2.2894770
-0.3854270
-0.6960990
-2.3886950
-1.1119360
0.7502700
0.6136600
-1.2495580
1.8205020
2.3709430
0.8793510
2.0846760
2.9751410
-1.0082630
-3.3331960
-2.3745640
-1.1141190
-0.3063290
-2.5762860
0.3579990
2.6170320
-5.0239360
-4.0617410
-2.7200840
-4.6606490
-6.0584150
-1.9830950
-5.4107100
3.2395070
3.3816730
3.4348400
1.9522640
3.2218240
4.2875090
4.4036790
1.0723440
1.8900250
4.1072420
2.5241160
3.3469760
-4.3553580
-5.7221750

$\begin{array}{llrl}\mathrm{H} & -1.8138650 & 0.1514700 & -6.3584910 \\ \mathrm{H} & -3.3480570 & 1.0461700 & -6.0939820 \\ \mathrm{H} & -3.2585230 & -0.7091080 & -5.7377590\end{array}$

158

$-0.7091080$

$-5.7377590$

TS49 (R, R) G[B97-D/def2-TZVP $]=-4356.203726$

$\begin{array}{lrrr}\mathrm{P} & 0.0443290 & -0.0902690 & 0.9871730 \\ \mathrm{O} & 0.9930260 & -0.4268800 & 2.1098190 \\ \mathrm{O} & -0.4300530 & 1.3228180 & 0.8347560 \\ \mathrm{O} & -1.1779640 & -1.1439300 & 1.1590380 \\ \mathrm{O} & 0.6632320 & -0.5072770 & -0.4709610 \\ \mathrm{C} & -2.0525300 & -1.3261370 & 0.0874350 \\ \mathrm{C} & -1.6134040 & -2.0872700 & -1.0143520 \\ \mathrm{C} & -3.3369040 & -0.7793210 & 0.1735260 \\ \mathrm{C} & -4.2060440 & -1.0149880 & -0.9003870 \\ \mathrm{H} & -5.2180650 & -0.6176160 & -0.8484070 \\ \mathrm{C} & 0.8728050 & -1.8633990 & -0.7301790 \\ \mathrm{C} & 2.1819700 & -2.3560470 & -0.7116700 \\ \mathrm{C} & -0.2472690 & -2.6798230 & -0.9936090 \\ \mathrm{C} & 2.3591610 & -3.7246260 & -0.9614110 \\ \mathrm{H} & 3.3694460 & -4.1294850 & -0.9507790 \\ \mathrm{C} & -3.7888890 & 0.0162520 & 1.3438510 \\ \mathrm{C} & 3.3654660 & -1.5085260 & -0.4069700 \\ \mathrm{C} & -3.8780100 & -0.5588370 & 2.6234100 \\ \mathrm{C} & -3.5467690 & -1.9850860 & 2.9940660 \\ \mathrm{C} & -4.4105190 & -0.5856540 & 5.0049190 \\ \mathrm{C} & -4.3573170 & 0.1914910 & 3.7134280 \\ \mathrm{C} & -2.4733980 & -1.9724400 & 4.1164050 \\ \mathrm{H} & -2.2872840 & -3.0074920 & 4.4334190 \\ \mathrm{H} & -1.5374470 & -1.5721530 & 3.7169710 \\ \mathrm{C} & -2.9825640 & -1.1144510 & 5.3165570 \\ \mathrm{H} & -3.0170430 & -1.7120160 & 6.2374380 \\ \mathrm{H} & -2.3116430 & -0.2656390 & 5.4943030 \\ \mathrm{H} & -4.7795970 & 0.0389360 & 5.8276480 \\ \mathrm{H} & -3.1878430 & -2.5548060 & 2.1333250 \\ \mathrm{C} & -5.3382540 & -1.8162690 & 4.7946860 \\ \mathrm{H} & -5.3291180 & -2.4284350 & 5.7065210 \\ \mathrm{H} & -6.3680070 & -1.4754700 & 4.6337350 \\ \mathrm{C} & -4.8317610 & -2.6420040 & 3.5705990 \\ \mathrm{H} & -4.5985880 & -3.6729160 & 3.8687520 \\ \mathrm{H} & -5.5998350 & -2.6866880 & 2.7890810 \\ \mathrm{C} & -4.1744230 & 1.3589310 & 1.1834050 \\ \mathrm{C} & -4.0932330 & 2.1765440 & -0.0857010 \\ \mathrm{C} & -5.0197710 & 3.5228040 & 1.9327760 \\ \mathrm{C} & -4.6571440 & 2.1003480 & 2.2784670 \\ \mathrm{C} & -5.5126370 & 2.7063590 & -0.4252420 \\ \mathrm{H} & -5.4446070 & 3.3537060 & -1.3097870\end{array}$




-6.1695050
-6.0784670
-6.3168260
-7.0007660
-5.4093760
-3.6950050
-3.7493260
-4.0162720
-3.0034680
-3.1788390
-3.1255030
-2.1728890
-4.7496180
-5.1233160
4.9421460
5.2361280
3.0575940
3.7615920
5.3495510
5.5210110
6.2152670
4.0358770
3.5455700
4.2513550
2.1432740
6.1623830
2.7244610
2.2081770
2.0357850
4.0320020
4.2035640
3.9585160
5.3473790
6.0894430
3.9016190
4.1629940
5.1417680
5.6587830
4.9011300
3.8412500
3.7318820
2.9571910
2.9713540
7.0080210
5.1100480
4.9490910

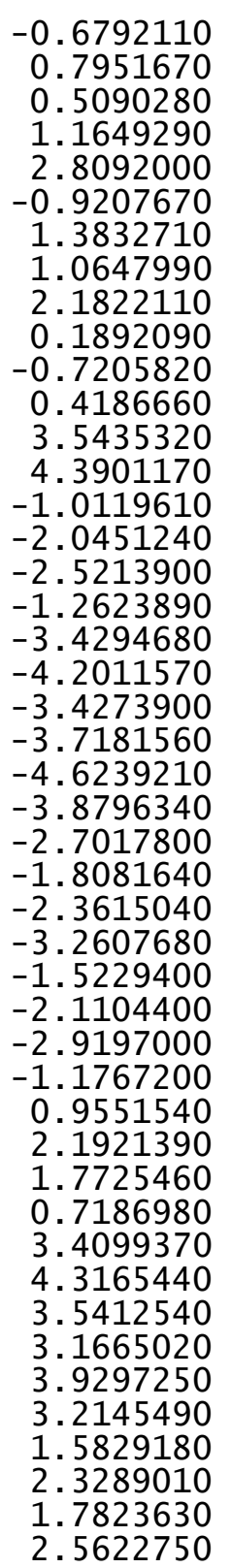

\begin{abstract}
5.1694410
6.4226980

6.9000260

7.1411430

5.7428010

6.6653300

$-0.0330090$

$-1.1364890$

$-0.8917850$

0.5315030

1.4320120

1.2778810

-1.1486930

$-1.6588010$

0.4682530

1.1282640

$-2.1176590$

$-1.0279200$

1.0142660

2.4805690

$-3.8003310$

$-4.7257150$

$-4.6467540$

$-3.1906030$

$-2.1259850$

$-2.4968250$

$-4.4284570$

$-5.3020320$

$-3.0617510$

$-2.0565640$

$-5.7538080$

$-5.0314150$

$-2.9972250$

$-1.1414430$

3.0277550

1.9153990

2. 8439950

0.9094500

2.6160280

0.8426370

$-0.0392210$

$-0.4277060$

0.0672820

$-0.0992340$

0.9609370

1.6165320
\end{abstract}

-4.3406820
-3.0166410
-3.3595850
-3.1595590
-0.0485340
0.5040380
-4.0598710
-5.0853280
-6.2196630
-6.8401230
-6.0608440
-4.5735750
-5.5187240
-6.9929170
-7.8862860
-6.3276830
-4.6334820
-5.7985340
-6.8373190
-6.3604470
-1.7251960
-1.9897680
-3.4780920
-3.9148670
-2.9017680
-2.2527320
-1.3672170
-3.6712100
-4.0368140
-2.0886110
-1.7109830
-4.0719100
-4.8925940
-3.3719180
2.0343370
2.8206720
1.5099020
2.5316580
1.2306200
4.1753710
3.7494340
2.7888220
3.0758980
2.4201860
4.9691260
4.7653130

0.8201400

2.0548570

2.9823170

1. 2388810

0.0873740

0.2683600

$-1.1770340$

$-1.2779080$

$-0.2534020$

$-0.3713610$

$-1.3644970$

$-1.1773820$

$-2.2895440$

$-0.3855160$

$-0.6962230$

$-2.3888830$

$-1.1119510$

0.7501920

0.6134860

$-1.2498440$

$-2.0263510$

$-3.1869280$

$-3.5902690$

$-3.9111190$

$-3.4111510$

$-2.0993290$

$-4.0436630$

$-4.4490450$

$-4.9942720$

$-4.1473970$

$-2.9257440$

$-2.7510030$

$-3.4560400$

$-3.3721180$

1.8206270

2.3709560

0.8794940

2.0846220

2.9752780

$-1.0083110$

$-3.3331890$

$-2.3745430$

$-1.1141240$

$-0.3063200$

$-2.5763300$

0.3578840 


\begin{tabular}{|c|c|c|c|c|c|c|c|}
\hline $\mathrm{H}$ & 19117890 & 1 55740 & 26171120 & & 17500270 & & \\
\hline c & $\begin{array}{r}1.911 / 890 \\
-1.2117850\end{array}$ & $\begin{array}{l}0.5057460 \\
2.5111860\end{array}$ & $\begin{array}{r}2.0171430 \\
-5.0238760\end{array}$ & C & $\begin{array}{l}-4.75003 / 0 \\
-4.7119080\end{array}$ & $\begin{array}{l}1.7563550 \\
0.6886540\end{array}$ & 2.4600730 \\
\hline C & -1.5914790 & 1.5516970 & -4.0616710 & $\mathrm{C}$ & -2.2328290 & $\begin{array}{l}1.4781650 \\
1.470\end{array}$ & 2.6509380 \\
\hline C & -1.2018290 & 1.6830960 & -2.7200330 & $\mathrm{H}$ & -1.6480360 & 1.7084720 & 3.5508680 \\
\hline C & -0.4341430 & 3.6135910 & -4.6606210 & $\mathrm{H}$ & -1.5514910 & 1.4988000 & 1.8005190 \\
\hline $\mathrm{H}$ & -1.5178510 & 2.4063290 & -6.0583410 & C & -3.3898010 & 2.5065460 & 2.4617590 \\
\hline $\mathrm{H}$ & -1.4798060 & 0.9359910 & -1.9830350 & $\mathrm{H}$ & -3.3917160 & 3.2442270 & 3.2749060 \\
\hline $\mathrm{H}$ & -0.1421670 & 4.3435990 & -5.4106940 & $\mathrm{H}$ & -3.2679790 & 3.0556790 & 1.5217920 \\
\hline C & 4.6326560 & 3.4013360 & 3.2396720 & $\mathrm{H}$ & -5.5777470 & 2.4555870 & 2.2898080 \\
\hline C & 3.5464510 & 4.4782070 & 3.3816870 & $\mathrm{H}$ & -2.0119220 & -0.6809170 & 2.8928940 \\
\hline C & 2.1406860 & 3.8522940 & 3.4348060 & C & -4.9044650 & 1.0528080 & 3.8377740 \\
\hline C & 4.4325060 & 2.5828720 & 1.9524720 & $\mathrm{H}$ & -4.8756020 & 1.8128300 & 4.6299360 \\
\hline H & 5.6260080 & 3.8658520 & 3.2220260 & $\mathrm{H}$ & -5.8808950 & 0.5563860 & 3.8881750 \\
\hline $\mathrm{H}$ & 3.7090590 & 5.0744720 & 4.2874910 & $\mathrm{C}$ & -3.7488950 & 0.0197000 & 4.0210820 \\
\hline $\mathrm{H}$ & 1.9840260 & 3.3561820 & 4.4036680 & $\mathrm{H}$ & -3.1523700 & 0.2609990 & 4.9108910 \\
\hline $\mathrm{H}$ & 4.6047710 & 3.2182300 & 1.0725190 & $\mathrm{H}$ & -4.1505580 & -0.9917870 & 4.1548720 \\
\hline $\mathrm{H}$ & 5.1355480 & 1.7462960 & 1.8903380 & C & -4.4270180 & -1.4405290 & -0.3938670 \\
\hline $\mathrm{H}$ & 4.5998540 & 2.7288130 & 4.1074530 & C & -4.4506140 & -2.5272880 & -1.4441910 \\
\hline $\mathrm{H}$ & 3.6027670 & 5.1626610 & 2.5240850 & $\mathrm{C}$ & -6.4041910 & -0.8323110 & -1.6920310 \\
\hline $\mathrm{H}$ & 1.3540620 & 4.6099760 & 3.3468400 & $\mathrm{C}$ & -5.4838740 & -0.5214100 & -0.5378130 \\
\hline 0 & -2.3440370 & 0.4504170 & -4.3552670 & $\mathrm{C}$ & -5.8073290 & -3.2775760 & -1.3217590 \\
\hline C & -2.7073500 & 0.2339820 & -5.7220750 & $\mathrm{H}$ & -5.8587130 & -4.0433540 & -2.1068890 \\
\hline $\mathrm{H}$ & -1.8143030 & 0.1514950 & -6.3584210 & $\mathrm{H}$ & -5.8605530 & -3.7900560 & -0.3535080 \\
\hline $\mathrm{H}$ & -3.3484340 & 1.0462800 & -6.0938520 & C & -6.9828680 & -2.2588510 & -1.4696090 \\
\hline $\mathrm{H}$ & -3.2589920 & -0.7090070 & -5.7376560 & $\mathrm{H}$ & -7.6184760 & -2.5221740 & -2.3254010 \\
\hline 15 & & & & $\mathrm{H}$ & -7.6134470 & -2.2617480 & -0.5726110 \\
\hline & 2) $\mathrm{G}[\mathrm{B} 9$ & {$[V P]=-435$} & & $\mathrm{H}$ & -7.2140070 & -0.0952110 & -1.7603890 \\
\hline $\mathrm{P}$ & -0.1663910 & -0.2262590 & -0.9821060 & $\mathrm{H}$ & -3.6160590 & -3.2225860 & -1.3281030 \\
\hline 0 & 0.3899330 & 1.1615170 & -0.8865600 & C & -5.5768000 & -0.8633840 & -3.0062320 \\
\hline 0 & -1.1939220 & -0.5360280 & -2.0433030 & $\mathrm{H}$ & -6.2394970 & -1.1572780 & $-3.831144 \mathrm{C}$ \\
\hline 0 & -0.7322220 & -0.5749900 & 0.5141500 & $\mathrm{H}$ & -5.1902690 & 0.1353200 & -3.2318490 \\
\hline 0 & 0.9901940 & -1.3508400 & -1.1966010 & C & -4.4010650 & -1.8742320 & -2.8520150 \\
\hline C & -1.0528800 & -1.9066320 & 0.7821630 & $\mathrm{H}$ & -4.4727710 & -2.6689810 & -3.6062110 \\
\hline C & -0.0023870 & -2.8154090 & 0.9720390 & $\mathrm{H}$ & -3.4370450 & -1.3767780 & -2.9828350 \\
\hline C & -2.3999790 & -2.2842740 & 0.8374250 & C & -5.6273730 & 0.5421070 & 0.3533340 \\
\hline C & -2.6645420 & -3.6370700 & 1.0659720 & $\mathrm{H}$ & -6.4500530 & 1.2485980 & 0.2398580 \\
\hline $\mathrm{H}$ & -3.7031250 & -3.9584000 & 1.1256510 & C & 4.2232170 & -0.3999310 & -3.8397700 \\
\hline C & 1.8725990 & -1.6000910 & -0.1440900 & $\mathrm{C}$ & 4.1761150 & -1.2231780 & -5.1019830 \\
\hline $\mathrm{C}$ & 3.1944600 & -1.1483270 & -0.2493270 & $\mathrm{C}$ & 3.2601870 & -2.4758040 & -3.0194730 \\
\hline $\mathrm{C}$ & 1.3990910 & -2.3016120 & 0.9759520 & $\mathrm{C}$ & 3.7116240 & -1.0667030 & -2.7108120 \\
\hline C & 4.0316850 & -1.3974420 & 0.8415740 & $\mathrm{C}$ & 5.0078770 & -2.5163130 & -4.8712960 \\
\hline $\mathrm{H}$ & 5.0658220 & -1.0619490 & 0.7840410 & $\mathrm{H}$ & 4.9199530 & -3.1592200 & -5.7573960 \\
\hline C & -3.5002570 & -1.3034490 & 0.6563590 & $\mathrm{H}$ & 6.0663030 & -2.2548950 & -4.7537120 \\
\hline C & 3.7002110 & -0.4378940 & -1.4525890 & C & 4.4763310 & -3.2515530 & -3.6005360 \\
\hline $\mathrm{C}$ & 5574800 & -0.2303370 & 1.5525960 & $\mathrm{H}$ & 4.1577820 & -4.2 & .8488990 \\
\hline C & -2.8173110 & 0.0486190 & 2.7773280 & $\mathrm{H}$ & 5.2608710 & -3.3235940 & 2.8375970 \\
\hline
\end{tabular}




\begin{tabular}{|c|c|c|c|}
\hline $\begin{array}{l}\mathrm{H} \\
\mathrm{H} \\
\mathrm{C} \\
\mathrm{H} \\
\mathrm{H} \\
\mathrm{C} \\
\mathrm{H} \\
\mathrm{H} \\
\mathrm{C} \\
\mathrm{C} \\
\mathrm{C} \\
\mathrm{C} \\
\mathrm{C} \\
\mathrm{H} \\
\mathrm{H} \\
\mathrm{C} \\
\mathrm{H} \\
\mathrm{H} \\
\mathrm{H} \\
\mathrm{H} \\
\mathrm{C} \\
\mathrm{H} \\
\mathrm{H} \\
\mathrm{C} \\
\mathrm{H} \\
\mathrm{H} \\
\mathrm{C} \\
\mathrm{H} \\
\mathrm{C} \\
\mathrm{C} \\
\mathrm{C} \\
\mathrm{C} \\
\mathrm{C} \\
\mathrm{C} \\
\mathrm{H} \\
\mathrm{H} \\
\mathrm{H} \\
\mathrm{H} \\
\mathrm{H} \\
\mathrm{H} \\
\mathrm{H} \\
\mathrm{H} \\
\mathrm{C} \\
\mathrm{C} \\
\mathrm{C} \\
\mathrm{C}\end{array}$ & $\begin{array}{r}2.8803560 \\
4.5714120 \\
2.1625170 \\
1.8938580 \\
1.2685250 \\
2.7019950 \\
2.6591800 \\
2.0968220 \\
4.7216880 \\
5.2103450 \\
4.2425680 \\
4.2108720 \\
4.0186700 \\
4.3766950 \\
3.2569900 \\
3.4233850 \\
3.4498700 \\
2.3829090 \\
3.8317700 \\
5.6131360 \\
5.7108270 \\
5.7246800 \\
6.3043420 \\
6.3001910 \\
6.6321820 \\
7.1685880 \\
4.7281660 \\
5.1234570 \\
3.5820290 \\
2.2486450 \\
4.5674960 \\
3.8993590 \\
2.7893740 \\
1.7018450 \\
4.6507610 \\
5.2013400 \\
5.2422560 \\
3.2144360 \\
2.3447040 \\
0.9421030 \\
1.1781590 \\
3.4533800 \\
-0.3026050 \\
-1.6500310 \\
0.8065130 \\
0.3938860\end{array}$ & $\begin{array}{l}-2.9859670 \\
-0.6595880 \\
-2.4210840 \\
-3.4495750 \\
-1.9408950 \\
-1.6431760 \\
-2.2675320 \\
-0.7477050 \\
1.5273530 \\
2.9271200 \\
1.7355740 \\
0.8696440 \\
3.7538980 \\
4.7565900 \\
3.8714740 \\
3.0225610 \\
3.6694170 \\
2.7442300 \\
1.2181310 \\
3.3929580 \\
2.1571630 \\
2.8374940 \\
1.2735350 \\
2.8573040 \\
3.8764020 \\
2.3051120 \\
0.8962670 \\
1.4093050 \\
-2.0182170 \\
-2.4588860 \\
-2.2204570 \\
-2.4656770 \\
-3.5109980 \\
-2.9863810 \\
-2.7868680 \\
-3.0896290 \\
-1.3556630 \\
-4.4418370 \\
-3.7506390 \\
-3.7533070 \\
-2.1518070 \\
-1.5317980 \\
-4.1909130 \\
-4.5935950 \\
-5.2315370 \\
-6.5946410\end{array}$ & $\begin{array}{r}-2.1304960 \\
-5.9559520 \\
-4.1167900 \\
-4.3929660 \\
-3.7099880 \\
-5.3570710 \\
-6.2596100 \\
-5.5424730 \\
-2.4884240 \\
-2.2098210 \\
-0.1153490 \\
-1.3530260 \\
-1.6508630 \\
-1.3805040 \\
-2.4308290 \\
-0.4091290 \\
0.4770880 \\
-0.5919410 \\
0.7527530 \\
-3.1174650 \\
0.1608430 \\
1.0229810 \\
0.4258700 \\
-1.1042130 \\
-0.8650240 \\
-1.4830240 \\
-3.7317770 \\
-4.6094840 \\
2.0103780 \\
2.0956830 \\
3.1491830 \\
4.5072980 \\
4.3589360 \\
3.4139360 \\
5.2407120 \\
2.9100210 \\
3.1944100 \\
3.9531480 \\
5.3334850 \\
3.2466480 \\
3.9087930 \\
4.8753840 \\
1.1076790 \\
1.1763450 \\
1.0596470 \\
1.6275480\end{array}$ \\
\hline
\end{tabular}

-0.9384570
-2.0418980
1.1841540
1.0879910
1.7071800
-0.8353300
-1.2110600
-2.9645490
-2.2853810
0.2835640
-3.1185230
-1.9529650
-2.9908980
-0.9703390
-2.7187160
-0.6718540
0.7442470
0.8552910
0.0664780
0.0800500
-0.3959660
-1.7119760
-2.0543270
2.3811020
2.4832910
1.6980790
1.5049430
2.9821180
1.7713200
1.4332220
-4.6085380
-3.4635310
-2.0900090
-4.4827810
-5.5760270
-3.5678960
-1.9192770
-4.6210170
-5.2439750
-4.5890180
-3.5120150
-1.2727180
3.3387820
4.2689110
3.7489870
4.8848200
$-7.0420030$
$-6.0465630$
$-7.3296100$
$-5.3592610$
$-4.8682260$
$-7.0822740$
$-8.0488070$
$-6.2599930$
$-6.1839870$
$-6.5206060$
2. 0494430
2.7441630
1.5644090
2. 4160720
1.1724070
4. 3011250
4.1636800
3. 0502530
3.1757040
2.4234640
5. 3114240
4.7525020
0.4260310
3. 1757200
2. 0644820
1. 9820700
4.2249820
3. 2325100
1. 1285740
5.0750570
3.4150790
4.4240970
3.7296600
2.6766500
3. 9298880
4.9623610
3. 1773660
3. 3811860
1.8988900
2.6852320
5. 1702520
4.4546920
1.0208460
1.1287140
1.1496760
2.0336560

1. 0158250

1. 3946940

1.4273360

0.0015310

1.5608190

$-0.0788160$

1. 3579480

0.8386830

2. 4609230

2.7204340

$-1.8681430$

$-2.4269720$

$-0.8975390$

$-2.1034720$

$-2.9735430$

0.7564900

2.8429050

1.9847140

0.8533070

0.1206060

2. 1737410

$-0.4788850$

$-2.5732540$

4. 2980890

3. 4342520

2. 2758680

4.0072320

5. 1986390

1.6107190

4. 6802870

$-3.4069210$

$-3.5871180$

$-3.5496210$

$-2.0640220$

$-3.4484260$

$-4.5367830$

$-4.4850410$

$-1.2321590$

$-1.9574280$

$-4.2273870$

$-2.7821450$

$-3.4660880$

3. 6425340

4.7244610

5.6933750

4.6207480 
H

TS51 (R, R) G[B97-D/def2-TZVP $]=-4356.202330$

\begin{tabular}{|c|c|c|c|}
\hline & & & \\
\hline$P$ & -0.0385250 & -0.1621010 & -0.7051450 \\
\hline 0 & -1.0316390 & -0.5820190 & -1.7588130 \\
\hline 0 & 0.3060450 & 1.2853050 & -0.5475890 \\
\hline 0 & 1.2585070 & -1.0966650 & -0.9998690 \\
\hline 0 & -0.4898880 & -0.6901110 & 0.7752940 \\
\hline C & 2.1955190 & -1.3373470 & -0.0011950 \\
\hline C & 1.8613700 & -2.2218610 & 1.0469230 \\
\hline C & 3.4525610 & -0.7310800 & -0.1191390 \\
\hline C & 4.4024370 & -1.0244690 & 0.8677200 \\
\hline $\mathrm{H}$ & 5.3915040 & -0.5764950 & 0.7912290 \\
\hline C & -0.6447830 & -2.0696820 & 0.9322880 \\
\hline C & -1.9388980 & -2.6044770 & 0.9214590 \\
\hline C & 0.5153890 & -2.8627860 & 1.0649230 \\
\hline C & -2.0589840 & -3.9913760 & 1.0890950 \\
\hline $\mathrm{H}$ & -3.0538270 & -4.4323240 & 1.0912970 \\
\hline C & 3.7776510 & 0.1887230 & -1.2420030 \\
\hline C & -3.1468870 & -1.7787260 & 0.6515250 \\
\hline C & 3.7883190 & -0.2653570 & -2.5729750 \\
\hline $\mathrm{C}$ & 3.5201050 & -1.6745840 & -3.0479550 \\
\hline C & 4.0924390 & -0.0402620 & -4.9844730 \\
\hline C & 4.1137040 & 0.6115510 & -3.6246740 \\
\hline C & 2.3489980 & -1.6460910 & -4.0692460 \\
\hline $\mathrm{H}$ & 2.2152320 & -2.6590140 & -4.4722200 \\
\hline $\mathrm{H}$ & 1.4252750 & -1.3676760 & -3.5553330 \\
\hline C & 2.6785270 & -0.6408840 & -5.21 \\
\hline $\mathrm{H}$ & 2.6580600 & -1.1414090 & -6.19 \\
\hline $\mathrm{H}$ & 1.9400790 & 0.1695090 & -5.2 \\
\hline $\mathrm{H}$ & 4.33 & 0.6817000 & -5.77 \\
\hline $\mathrm{H}$ & 3.2 & -2.3 & -2.2 \\
\hline C & 5.1136110 & -1.2120730 & -4.9797730 \\
\hline $\mathrm{H}$ & 5.0510480 & -1.7415800 & -5.9 \\
\hline $\mathrm{H}$ & 6.13 & -0.8118680 & -4.8 \\
\hline C & 5650 & -2.1760160 & -3.7 \\
\hline $\mathrm{H}$ & 4.6029010 & -3.1935250 & -4.16 \\
\hline $\mathrm{H}$ & 5.6298740 & -2.2240550 & -3.0934050 \\
\hline C & 4.0930910 & 1.5360700 & -0.9914790 \\
\hline C & 4.0757180 & 2.2336460 & 0.3488380 \\
\hline C & 4.7088950 & 3.8181800 & -1.6073300 \\
\hline C & 4.4199140 & 2.4053540 & -2.0503620 \\
\hline C & 5.4792190 & 2.8418750 & 0.6120750 \\
\hline $\mathrm{H}$ & 5.4501090 & 3.4003330 & 1.5571100 \\
\hline $\mathrm{H}$ & 55570 & 2.0367190 & 0.7244320 \\
\hline C & 5. & 3.7 & -0 . \\
\hline
\end{tabular}

6.0554110

6.7836570

4.9751260

3.8015770

3.4459430

3. 6647760

2. 6239580

3.0536000

3. 0405970

2. 0590400

4. 4301810

4.6802540

$-4.6955380$

$-4.9663430$

$-2.8480630$

$-3.5341610$

$-5.1268240$

$-5.2495440$

$-6.0339100$

$-3.8663900$

$-3.3823890$

$-4.1436920$

$-1.9596520$

$-5.8674810$

$-2.4616770$

$-1.9997880$

$-1.7131450$

$-3.7261260$

-3.8942260

$-3.6021580$

$-5.1326370$

$-5.9092310$

$-3.7201980$

$-3.9536160$

$-5.0011710$

$-5.5451860$

$-4.7707190$

$-3.6905170$

$-3.5877210$

$-2.8127620$

$-2.7882100$

$-6.8277190$

$-4.9326620$

$-4.7980110$

-4.9661290
-6.2493850
4.8020810

3.4293730

4.4533090

1.5500010

4. 3671770

5. 3716220

4. 4589910

3. 4002050

3.9257770

2.9834270

1.9456070

. 6211780

0.0227770

1.1079760

$-0.3008860$

$-0.7273480$

0.4393300

1.2244780

$-0.1764590$

$-0.4295860$

$-0.0963350$

$-1.4826000$

$-0.9048930$

1. 6760280

1. 1931840

1.5326470

1. 2849340

2. 0413580

2.8075050

2. 5619540

$-1.3327120$

$-1.8020520$

$-3.1383260$

$-2.0651190$

$-1.6826690$

$-2.0721220$

$-0.6296990$

$-2.4891170$

$-3.2909340$

$-1.8442220$

$-3.6791680$

$-1.2158410$

$-4.1097170$

$-4.8787630$

$-4.6171160$

$-3.3068620$
$-0.2109450$

$-1.0617930$

$-2.4608900$

1.1559920

$-0.8864750$

$-0.4998610$

$-1.6062310$

0.2712640

1. 2344840

0.1016450

$-3.3668140$

$-4.1858210$

1. 2320200

2. 2435360

2.7759050

1.4999330

3. 6380790

4. 3959320

3. 6467730

3. 9429540

4.8703890

4.0724340

2. 9792480

1.9822090

2. 6414350

3. 5765280

1.8549400

2.3083330

3. 0763970

1. 3514390

$-0.7069640$

$-1.9119010$

$-1.5092480$

$-0.4690650$

$-3.1645140$

$-4.0355930$

$-3.3573860$

$-2.9211520$

$-3.6645080$

$-3.0022720$

$-1.3340030$

$-2.0407170$

$-1.4663180$

$-2.2385140$

$-0.4944970$

$-1.7124970$ 


\begin{tabular}{|c|c|c|}
\hline $\begin{array}{r}-6.7654380 \\
-6.9355400 \\
-5.5023710 \\
-6.4147670 \\
0.3521620 \\
1.4850880 \\
1.2029670 \\
-0.2017430 \\
-1.0403270 \\
-0.9415020 \\
1.5772900 \\
1.9891130 \\
-0.1047380 \\
-0.6464460 \\
2.4413140 \\
1.2794830 \\
-0.7513060 \\
-2.0860910 \\
4.1040440 \\
5.1110860 \\
5.1524170 \\
3.7430830 \\
2.6012890 \\
2.8316060 \\
4.8241960 \\
5.8542470 \\
3.6855170 \\
2.5787210 \\
6.1016580 \\
5.5428820 \\
3.5706550 \\
1.6335060 \\
-3.2223450 \\
-2.1305800 \\
-3.0800350 \\
-1.1304490 \\
-2.6615730 \\
-1.3581300 \\
-0.3436240 \\
0.0208510 \\
-0.5486100 \\
-0.3506160 \\
-1.4182830 \\
-2.2082630 \\
-1.9391460 \\
0.9541390\end{array}$ & $\begin{array}{r}-3.6752010 \\
-3.4169030 \\
-0.2872530 \\
0.2792060 \\
-4.2603350 \\
-5.2563100 \\
-6.3067420 \\
-6.9644460 \\
-6.3171450 \\
-4.8151030 \\
-5.7750640 \\
-7.0713730 \\
-8.0392210 \\
-6.6675100 \\
-4.7622010 \\
-5.8030610 \\
-6.8583820 \\
-6.6407030 \\
-1.8612070 \\
-2.1889010 \\
-3.7155530 \\
-4.2900990 \\
-3.2736610 \\
-2.4576530 \\
-1.6977100 \\
-3.9513300 \\
-4.5938910 \\
-2.5720810 \\
-1.8072480 \\
-4.1823120 \\
-5.1886160 \\
-3.7780790 \\
1.7674520 \\
2.5728600 \\
1.3795220 \\
2.4027280 \\
0.8704760 \\
4.4400480 \\
4.4000810 \\
3.3181920 \\
3.3863100 \\
2.6284100 \\
5.4645710 \\
4.7828810 \\
0.2377990 \\
3.4542690\end{array}$ & $\begin{array}{r}-2.6089150 \\
-0.8643500 \\
0.1382230 \\
-0.0503240 \\
1.1447370 \\
1.0797700 \\
-0.0222340 \\
0.1179660 \\
1.2498190 \\
1.1863340 \\
2.0459150 \\
0.0072570 \\
0.3161890 \\
2.2165670 \\
0.8908400 \\
-0.9935920 \\
-0.8252830 \\
1.1880240 \\
1.9407470 \\
3.0141890 \\
3.2458900 \\
3.5640120 \\
3.2949260 \\
2.0437050 \\
3.9583380 \\
4.0553510 \\
4.6166840 \\
4.1426110 \\
2.7396890 \\
2.3326790 \\
2.9623780 \\
3.2847180 \\
-1.8677720 \\
-2.4323280 \\
-0.8558530 \\
-2.0463630 \\
-2.8770500 \\
0.6882860 \\
3.0026540 \\
2.1865200 \\
0.9269810 \\
0.2365260 \\
2.1197700 \\
-0.7165360 \\
-2.3852460 \\
4.7846940\end{array}$ \\
\hline
\end{tabular}

$\begin{array}{lr}\mathrm{C} & 1.3031910 \\ \mathrm{C} & 0.8415300 \\ \mathrm{C} & 0.1326880 \\ \mathrm{H} & 1.3349470 \\ \mathrm{H} & 1.0735540 \\ \mathrm{H} & -0.1360970 \\ \mathrm{C} & -4.7996830 \\ \mathrm{C} & -3.7896270 \\ \mathrm{C} & -2.3384870 \\ \mathrm{C} & -4.6478320 \\ \mathrm{H} & -5.8225620 \\ \mathrm{H} & -3.9168420 \\ \mathrm{H} & -2.0504480 \\ \mathrm{H} & -4.9341480 \\ \mathrm{H} & -5.2919390 \\ \mathrm{H} & -4.6446110 \\ \mathrm{H} & -3.9777050 \\ \mathrm{H} & -1.6309880 \\ \mathrm{O} & 2.0953900 \\ \mathrm{C} & 2.4036740 \\ \mathrm{H} & 2.9524980 \\ \mathrm{H} & 1.4887900 \\ \mathrm{H} & 3.0300680 \\ \mathrm{H} & \end{array}$

2.3611190
2.2841750
4.4737940
3.4764040
1.4625030
5.3028340
2.7811870
3.9182160
3.4258420
2.1772780
3.1567380
4.3584470
2.8205270
2.9171880
1.3022550
1.9958000
4.7125210
4.2610490
1.4166790
0.2565800
0.5264730
-0.2807250
-0.3731710

3.9575290

3.9575290
2.6402020

4.3163950

5.8020780

1. 9741350

4.9653480

$-3.5780480$

$-3.8012050$

$-3.6494430$

$-2.1718640$

$-3.7024440$

$-4.7974120$

$-4.5209490$

$-1.4115770$

$-2.0380040$

$-4.3297820$

$-3.0660010$

$-3.6065370$

4. 5363980

3.7508900

2. 8407880

3.4688560

4.3849770

158

TS52 $(R, R) \quad G[B 97-D /$ def2-TZVP $]=-4356.202329$

$\begin{array}{lrrr}\text { TS52 (R, R) G[B97-D/def2-TZVP] }=-4356.202329 \\ \mathrm{P} & -0.0385320 & -0.1621070 & -0.7051440 \\ \mathrm{O} & 0.3060350 & 1.2852990 & -0.5476020 \\ \mathrm{O} & -1.0316500 & -0.5820340 & -1.7588100 \\ \mathrm{O} & -0.4898950 & -0.6901070 & 0.7752970 \\ \mathrm{O} & 1.2584920 & -1.0966780 & -0.9998670 \\ \mathrm{C} & -0.6447950 & -2.0696760 & 0.9323020 \\ \mathrm{C} & 0.5153740 & -2.8627850 & 1.0649390 \\ \mathrm{C} & -1.9389130 & -2.6044650 & 0.9214810 \\ \mathrm{C} & -2.0590050 & -3.9913620 & 1.0891280 \\ \mathrm{H} & -3.0538490 & -4.4323060 & 1.0913370 \\ \mathrm{C} & 2.1955080 & -1.3373580 & -0.0011950 \\ \mathrm{C} & 3.4525520 & -0.7310960 & -0.1191470 \\ \mathrm{C} & 1.8613590 & -2.2218650 & 1.0469290 \\ \mathrm{C} & 4.4024300 & -1.0244850 & 0.8677090 \\ \mathrm{H} & 5.3914990 & -0.5765170 & 0.7912110 \\ \mathrm{C} & -3.1468970 & -1.7787080 & 0.6515420 \\ \mathrm{C} & 3.7776390 & 0.1887010 & -1.2420170 \\ \mathrm{C} & -3.5341600 & -0.7273180 & 1.4999400 \\ \mathrm{C} & -2.8480610 & -0.3008530 & 2.7759100 \\ \mathrm{C} & -4.9663210 & 1.1080330 & 2.2435220 \\ \mathrm{C} & -4.6955290 & 0.0228190 & 1.2320170\end{array}$




$\begin{array}{lrr}-2.4616550 & 1.1932110 & 2.6414280 \\ -1.9997680 & 1.5326770 & 3.5765210 \\ -1.7131180 & 1.2849470 & 1.8549370 \\ -3.7260920 & 2.0413980 & 2.3083100 \\ -3.8941840 & 2.8075570 & 3.0763630 \\ -3.6021150 & 2.5619800 & 1.3514100 \\ -5.8674510 & 1.6760940 & 1.9821860 \\ -1.9596580 & -0.9048690 & 2.9792610 \\ -5.1268140 & 0.4394050 & 3.6380720 \\ -5.2495220 & 1.2245630 & 4.3959160 \\ -6.0339090 & -0.1763710 & 3.6467730 \\ -3.8663930 & -0.4295280 & 3.9429570 \\ -3.3823900 & -0.0962760 & 4.8703900 \\ -4.1437110 & -1.4825350 & 4.0724450 \\ -3.9536270 & -2.0651030 & -0.4690460 \\ -3.7202220 & -3.1383240 & -1.5092190 \\ -5.9092380 & -1.8020260 & -1.9118880 \\ -5.1326400 & -1.3326840 & -0.7069540 \\ -4.9326970 & -4.1096980 & -1.4662790 \\ -4.7980540 & -4.8787560 & -2.2384650 \\ -4.9661720 & -4.6170850 & -0.4944510 \\ -6.2494100 & -3.3068310 & -1.7124710 \\ -6.7654630 & -3.6751720 & -2.6088880 \\ -6.9355700 & -3.4168560 & -0.8643260 \\ -6.8277190 & -1.2158050 & -2.0407110 \\ -2.7882400 & -3.6791750 & -1.3339680 \\ -5.0011740 & -1.6826650 & -3.1645000 \\ -5.5451940 & -2.0721180 & -4.0355770 \\ -4.7707080 & -0.6297000 & -3.3573820 \\ -3.6905330 & -2.4891300 & -2.9211300 \\ -3.5877480 & -3.2909580 & -3.6644780 \\ -2.8127680 & -1.8442490 & -3.0022590 \\ -5.5023640 & -0.2872130 & 0.1382230 \\ -6.4147530 & 0.2792550 & -0.0503320 \\ 4.1136810 & 0.6115160 & -3.6246910 \\ 4.0924090 & -0.0403030 & -4.9844870 \\ 3.5200820 & -1.6746150 & -3.0479580 \\ 3.7882990 & -0.2653850 & -2.5729870 \\ 5.1135790 & -1.2121160 & -4.9797860 \\ 5.0510120 & -1.7416280 & -5.9398130 \\ 6.1301560 & -0.8119110 & -4.8863710 \\ 4.7885380 & -2.1760520 & -3.7952040 \\ 4.6028720 & -3.1935620 & -4.1639250 \\ 5.6298500 & -2.2240880 & -3.0934150 \\ 3.2816520 & -2.3445290 & -2.2175960 \\ 4.3393100 & 0.6816560 & -5.7724790\end{array}$

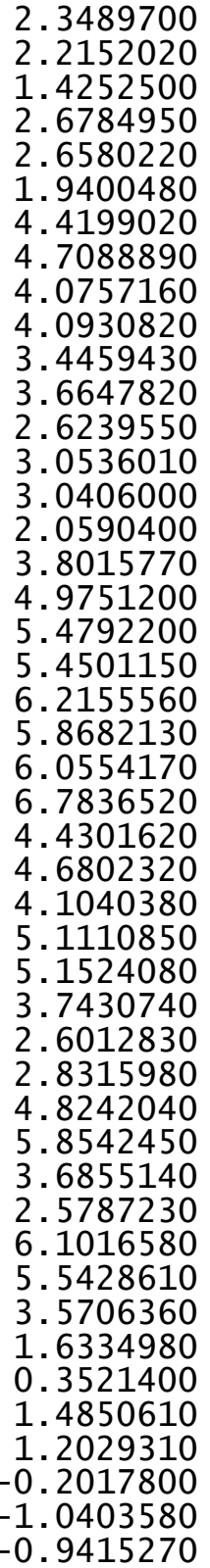

-1.6461250
-2.6590500
-1.3677080
-0.6409240
-1.1414520
0.1694700
2.4053260
3.8181540
2.2336310
1.5360480
4.3671590
5.3716040
4.4589740
3.4001920
3.9257680
2.9834150
1.5499920
4.4532770
2.8418580
3.4003210
2.0367000
3.7821100
4.8020570
3.4293420
1.9455740
2.6211400
-1.8612170
-2.1889130
-3.7155640
-4.2900980
-3.2736560
-2.4576570
-1.6977150
-3.9513430
-4.5938790
-2.5720700
-1.8072670
-4.1823290
-5.1886210
-3.7780690
-4.2603320
-5.2563120
-6.3067510
-6.9644500
-6.3171350
-4.8150940
$-4.0692450$ $-4.4722140$ $-3.5553290$ $-5.2153840$ $-6.1927600$ $-5.2411240$ $-2.0503890$ $-1.6073660$ 0.3488130 $-0.9915010$ $-0.8865070$ $-0.4998980$ $-1.6062600$

0.2712360

1. 2344540

0.1016200

1.1559710

$-2.4609310$

0.6120410

1.5570730

0.7244000

$-0.5721840$

$-0.2109930$

$-1.0618360$

$-3.3668400$

$-4.1858510$

1. 9407410

3. 0141790

3. 2458850

3. 5640230

3. 2949350

2.0437080

3. 9583270

4.0553400

4. 6166990

4. 1426160

2. 7396710

2. 3326720

2. 9624000

3. 2847350

1. 1447650

1. 0798020

$-0.0221940$

0.1180190

1. 2498680

1.1863700 


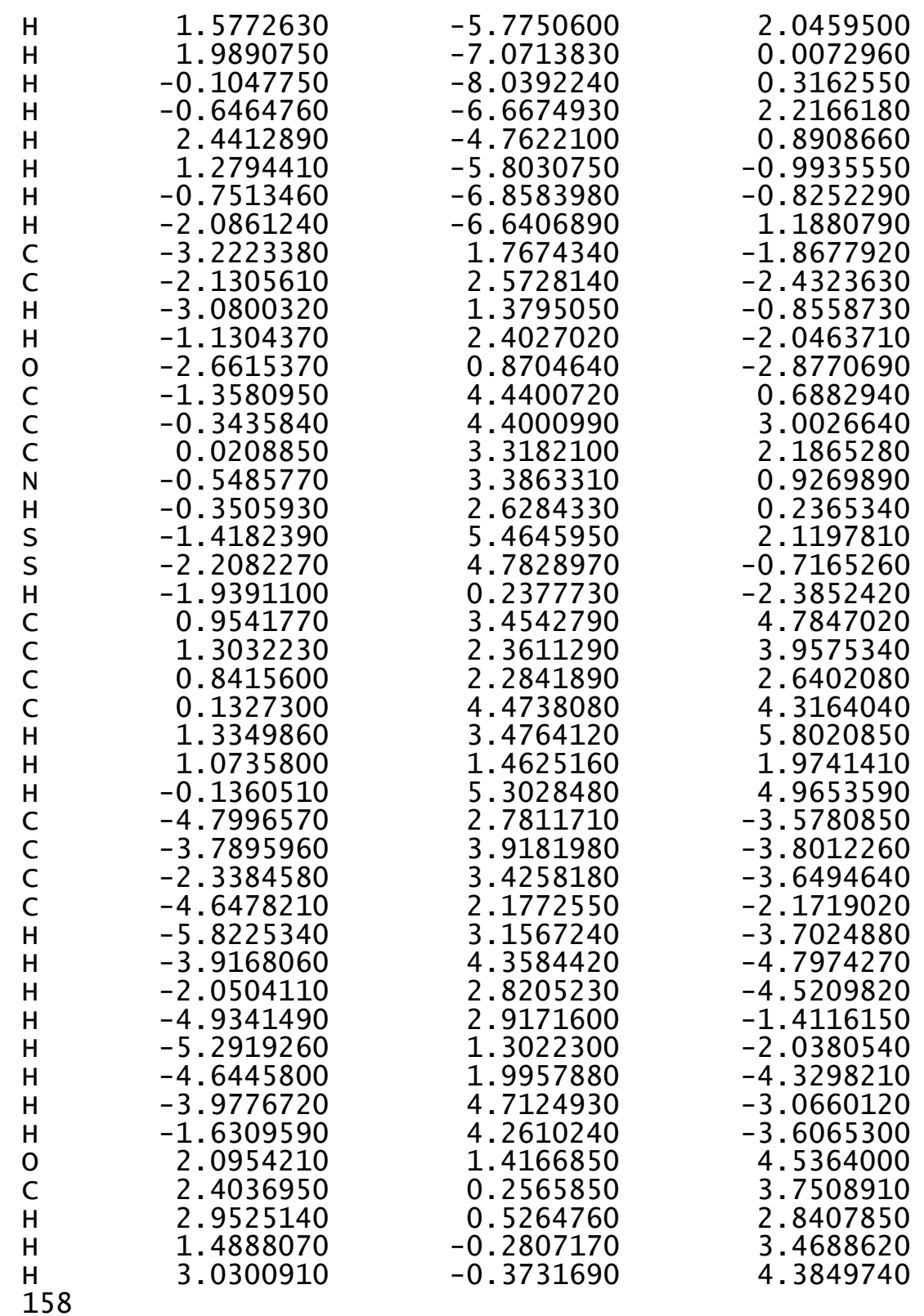

$\begin{array}{lrrr}\text { TS53(R, R) G[B97-D/def2-TZVP] }=-4356.202253 \\ \text { P } & -0.0549960 & -0.1978740 & -0.6967490 \\ \text { O } & 0.3347990 & 1.2421730 & -0.5851950 \\ \text { O } & -1.0752270 & -0.6163320 & -1.7248780 \\ \text { O } & -0.5007080 & -0.6709070 & 0.8040680 \\ \text { O } & 1.2082740 & -1.1796330 & -0.9865250 \\ \mathrm{C} & -0.6914910 & -2.0424270 & 0.9929700 \\ \mathrm{C} & 0.4503520 & -2.8595360 & 1.1258420 \\ \mathrm{C} & -1.9982700 & -2.5467260 & 0.9970830 \\ \mathrm{C} & -2.1468640 & -3.9306220 & 1.1693080 \\ \mathrm{H} & -3.1510760 & -4.3495790 & 1.1813000 \\ \mathrm{C} & 2.1548640 & -1.4229980 & 0.0016690 \\ \mathrm{C} & 3.4322320 & -0.8705050 & -0.1588220 \\ \mathrm{C} & 1.8123890 & -2.2509590 & 1.0868280 \\ \mathrm{C} & 4.3812530 & -1.1808980 & 0.8176570 \\ \mathrm{H} & 5.3878350 & -0.7806840 & 0.7063350 \\ \mathrm{C} & -3.1866220 & -1.6922360 & 0.7286580 \\ \mathrm{C} & 3.7676450 & 0.0037960 & -1.3147150 \\ \mathrm{C} & -3.5266280 & -0.6086480 & 1.5577790 \\ \mathrm{C} & -2.8133720 & -0.1787980 & 2.8179660 \\ \mathrm{C} & -4.8774770 & 1.3014980 & 2.2677340 \\ \mathrm{C} & -4.6611090 & 0.1795450 & 1.2828670 \\ \mathrm{C} & -2.3678950 & 1.2953910 & 2.6465760 \\ \mathrm{H} & -1.8927520 & 1.6377000 & 3.5741090 \\ \mathrm{H} & -1.6169670 & 1.3414210 & 1.8586800 \\ \mathrm{C} & -3.5994050 & 2.1837580 & 2.2952480 \\ \mathrm{H} & -3.7298370 & 2.9805470 & 3.0390050 \\ \mathrm{H} & -3.4636020 & 2.6673000 & 1.3208100 \\ \mathrm{H} & -5.7567260 & 1.8994280 & 1.9985300 \\ \mathrm{H} & -1.9487900 & -0.8135540 & 3.0303670 \\ \mathrm{C} & -5.0521210 & 0.6776880 & 3.6806270 \\ \mathrm{H} & -5.1334520 & 1.4870660 & 4.4184510 \\ \mathrm{H} & -5.9846570 & 0.1020100 & 3.7145760 \\ \mathrm{C} & -3.8266040 & -0.2358380 & 3.9957430 \\ \mathrm{H} & -3.3202360 & 0.0991790 & 4.9105590 \\ \mathrm{H} & -4.1468340 & -1.2726220 & 4.1537920 \\ \mathrm{C} & -4.0168780 & -1.9737410 & -0.3766390 \\ \mathrm{C} & -3.8379180 & -3.0781720 & -1.3956410 \\ \mathrm{C} & -5.9792880 & -1.6689400 & -1.8033880 \\ \mathrm{C} & -5.1705910 & -1.2028710 & -0.6184440 \\ \mathrm{C} & -5.0854170 & -4.0015560 & -1.3163690 \\ \mathrm{H} & -4.9888360 & -4.7912210 & -2.0732550 \\ \mathrm{H} & -5.1259530 & -4.4863460 & -0.3333400 \\ \mathrm{C} & -6.3737790 & -3.1545330 & -1.5652990 \\ \mathrm{H} & -6.9151420 & -3.5231870 & -2.4465570 \\ \mathrm{H} & -7.0525010 & -3.2190420 & -0.7065620\end{array}$




$\begin{array}{rrr}-6.8763680 & -1.0510400 & -1.9346510 \\ -2.9248650 & -3.6500270 & -1.2199270 \\ -5.0838760 & -1.6132650 & -3.0692790 \\ -5.6535020 & -2.0027570 & -3.9238270 \\ -4.8169260 & -0.5746960 & -3.2909790 \\ -3.8013760 & -2.4624020 & -2.8220900 \\ -3.7370650 & -3.2840940 & -3.5479320 \\ -2.9010930 & -1.8530520 & -2.9276590 \\ -5.4910240 & -0.1245850 & 0.2050270 \\ -6.3824210 & 0.4727310 & 0.0113300 \\ 4.0549990 & 0.3429540 & -3.7174280 \\ 3.9827540 & -0.3500120 & -5.0550220 \\ 3.4150000 & -1.9068010 & -3.0535960 \\ 3.7335060 & -0.4915580 & -2.6306780 \\ 4.9719970 & -1.5483770 & -5.0369250 \\ 4.8722030 & -2.1053710 & -5.9780900 \\ 6.0008820 & -1.1735300 & -4.9802140 \\ 4.6505580 & -2.4661400 & -3.8154620 \\ 4.4283620 & -3.4889040 & -4.1476430 \\ 5.5076250 & -2.5161270 & -3.1331220 \\ 3.1794430 & -2.5426720 & -2.1959910 \\ 4.2296350 & 0.3400200 & -5.8710710 \\ 2.2202430 & -1.8775660 & -4.0474050 \\ 2.0489760 & -2.8985640 & -4.4141930 \\ 1.3173530 & -1.5567700 & -3.5217040 \\ 2.5484330 & -0.9186180 & -5.2328360 \\ 2.4907390 & -1.4490470 & -6.1927700 \\ 1.8315070 & -0.0893900 & -5.2672600 \\ 4.4462760 & 2.1762200 & -2.2093380 \\ 4.7809580 & 3.5946110 & -1.8192290 \\ 4.1692400 & 2.0860530 & 0.2029250 \\ 4.1270240 & 1.3485720 & -1.1152960 \\ 3.5527800 & 4.1953290 & -1.0793370 \\ 3.8040690 & 5.2073790 & -0.7345380 \\ 2.7107330 & 4.2803640 & -1.7764120 \\ 3.1760740 & 3.2771790 & 0.1222660 \\ 3.2109500 & 3.8335690 & 1.0675650 \\ 2.1649100 & 2.8838930 & 0.0013770 \\ 3.9045810 & 1.4332090 & 1.0379370 \\ 5.0366240 & 4.1967050 & -2.6995180 \\ 5.5960500 & 2.6626100 & 0.4032230 \\ 5.6148940 & 3.2447840 & 1.3341150 \\ 6.3137510 & 1.8401570 & 0.5111370 \\ 5.9692960 & 3.5617400 & -0.8172680 \\ 6.1882360 & 4.5868690 & -0.4902610 \\ 6.8624970 & 3.1761800 & -1.3230300\end{array}$
4.4101380
4.6540380
4.0813520
2. 7816200
5.2042140
4.7415320
3. 6278750
2.4209380
5.5970980
5.8742790
5.8062540
3. 9967370
3. 3208470
1. 6260300
2. 0042980
4. 3561250
0.2583020
1. 3702700
1. 0569100
$-0.3598240$
$-1.1767670$
$-1.0466330$
1. 4549670
1.8271420
$-0.2821790$
$-0.7834640$
2. 3360390
1. 1330570
$-0.9152130$
$-2.2293850$
$-3.1860750$
$-2.0753490$
$-3.0456170$
$-1.0779780$
$-2.6599930$
$-1.2211660$
$-0.1749470$
0.1572360
$-0.4296050$
$-0.2616660$
$-1.2435240$
$-2.0821820$
$-1.9562400$
1. 1402230
1. 4578730
1.4578730
0.9698350

1. 6760790

2. 3197630

$-1.9690280$

$-2.4879810$

$-2.2707220$

$-2.8376790$

$-3.8694280$

$-3.2050320$

$-3.2788930$

- 3.0041620

$-1.3644530$

$-4.6896320$

$-4.3062060$

$-3.9333070$

$-2.4574760$

$-2.0279460$

$-4.2539960$

$-5.2738300$

$-6.3047860$

$-6.9352290$

$-6.2801220$

$-4.7798640$

$-5.8087680$

$-7.0858630$

$-8.0130100$

$-6.6473050$

$-4.7965150$

$-5.7910120$

$-6.8121680$

$-6.5815990$

1.8007590

2. 5566880

1.4366630

2. 3660080

0.8622150

4.4745170

4.4694050

3. 3614870

3. 4100510

2.6294810

5. 5318230

4.8051680

0.2171610

3. 5485200

2. 4300560

2. 3262660
$-3.5103960$

4.3565140

1.9333510

2.0931900

2. 9156060

4. 2654100

4. 0581330

3. 3844190

4.7924900

2. 4397620

3.0662730

3. 4241050

5. 0171000

3. 2278620

4.0784680

4. 9015070

1. 2008020

1. 1212500

0.0091980

0.1578540

1. 3018140

1. 2504170

2. 0802150

0.0211240

0.3481640

2. 2625050

0.9341310

$-0.9569180$

$-0.7799220$

1. 2446090

$-1.8823660$

$-2.4764700$

$-0.8616350$

$-2.0931570$

$-2.8724040$

0.5929810

2.8933510

2. 0984050

0.8457950

0.1730020

2. 0011090

$-0.8080310$

$-2.3678520$

4.6754800

3. 8699910

2.5639330 


\begin{tabular}{|c|c|c|c|c|c|c|c|}
\hline C & 0.3258070 & 4.5685100 & 4.1962080 & $\mathrm{H}$ & 1.3173530 & -1.5567720 & -3.5217040 \\
\hline H & 1.5405690 & 3.5905160 & 5.6847050 & C & 2.5484370 & -0.9186280 & -5.2328370 \\
\hline $\mathrm{H}$ & 1.1780430 & 1.4848350 & 1.9148760 & $\mathrm{H}$ & 2.4907460 & -1.4490620 & -6.1927690 \\
\hline $\mathrm{H}$ & 0.0820470 & 5.4182000 & 4.8281170 & H & 1.8315110 & -0.0894010 & -5.2672670 \\
\hline C & -4.7464320 & 2.8196810 & -3.6047240 & $\mathrm{H}$ & 4.2296390 & 0.3400090 & -5.8710710 \\
\hline $\mathrm{C}$ & -3.7046790 & 3.9203570 & -3.8616930 & $\mathrm{H}$ & 3.1794410 & -2.5426770 & -2.1959880 \\
\hline C & -2.2681060 & 3.3862540 & -3.7119850 & C & 4.9720000 & -1.5483870 & -5.0369200 \\
\hline $\mathrm{C}$ & -4.6014870 & 2.2441790 & -2.1858610 & $\mathrm{H}$ & 4.8722110 & -2.1053810 & -5.9780860 \\
\hline H & -5.7587270 & 3.2222940 & -3.7308890 & H & 6.0008850 & -1.1735390 & -4.9802050 \\
\hline $\mathrm{H}$ & -3.8278980 & 4.3424290 & -4.8662160 & C & 4.6505570 & -2.4661500 & -3.8154580 \\
\hline $\mathrm{H}$ & -2.0092770 & 2.7505560 & -4.5710020 & $\mathrm{H}$ & 4.4283590 & -3.4889120 & -4.1476400 \\
\hline $\mathrm{H}$ & -4.8616110 & 3.0084150 & -1.4402880 & $\mathrm{H}$ & 5.5076230 & -2.5161390 & -3.1331170 \\
\hline $\mathrm{H}$ & -5.2686710 & 1.3907240 & -2.0279590 & C & 4.1270220 & 1.3485680 & -1.1152980 \\
\hline $\mathrm{H}$ & -4.6197240 & 2.0129580 & -4.3389320 & $\mathrm{C}$ & 4.1692390 & 2.0860510 & 0.2029220 \\
\hline $\mathrm{H}$ & -3.8613250 & 4.7357370 & -3.1424260 & $\mathrm{C}$ & 4.7809580 & 3.5946050 & -1.8192330 \\
\hline $\mathrm{H}$ & -1.5338620 & 4.1990650 & -3.6965180 & $\mathrm{C}$ & 4.4462770 & 2.1762140 & -2.2093410 \\
\hline 0 & 2.2487280 & 1.4898630 & 4.4572060 & $\mathrm{C}$ & 5.5960510 & 2.6626050 & 0.4032200 \\
\hline C & 2.5317270 & 0.3090180 & 3.6938570 & $\mathrm{H}$ & 5.6148960 & 3.2447790 & 1.3341110 \\
\hline $\mathrm{H}$ & 3.0713520 & 0.5512250 & 2.7705940 & $\mathrm{H}$ & 6.3137490 & 1.8401520 & 0.5111320 \\
\hline $\mathrm{H}$ & 1.6062790 & -0.2216340 & 3.4346960 & C & 5.9692960 & 3.5617360 & -0.8172710 \\
\hline $\mathrm{H}$ & 3.1560720 & -0.3176150 & 4.3317960 & $\mathrm{H}$ & 6.1882340 & 4.5868650 & -0.4902650 \\
\hline & & & & $\mathrm{H}$ & 6.8624980 & 3.1761770 & -1.3230320 \\
\hline & 2) $G[B 97-D /$ & $\mathrm{VP}]=-43$ & & H & 5.0366250 & 4.1966980 & -2.6995230 \\
\hline $\mathrm{P}$ & -0.0549960 & -0.1978760 & -0.6967460 & $\mathrm{H}$ & 3.9045800 & 1.4332070 & 1.0379350 \\
\hline 0 & -1.0752290 & -0.6163330 & -1.7248750 & C & 3.5527810 & 4.1953240 & -1.0793430 \\
\hline 0 & 0.3347990 & 1.2421710 & -0.5851910 & $\mathrm{H}$ & 3.8040690 & 5.2073760 & -0.7345480 \\
\hline 0 & 1.2082720 & -1.1796350 & -0.9865240 & H & 2.7107340 & 4.2803570 & -1.7764190 \\
\hline 0 & -0.5007090 & -0.6709090 & 0.8040700 & C & 3.1760750 & 3.2771780 & 0.1222630 \\
\hline C & 2.1548620 & -1.4230010 & 0.0016700 & $\mathrm{H}$ & 3.2109540 & 3.8335700 & 1.0675610 \\
\hline C & 1.8123860 & -2.2509610 & 1.0868290 & $\mathrm{H}$ & 2.1649100 & 2.8838940 & 0.0013770 \\
\hline $\mathrm{C}$ & 3.4322300 & -0.8705080 & -0.1588210 & C & 4.4101390 & 1.6760710 & -3.5103980 \\
\hline C & 4.3812510 & -1.1809000 & 0.8176590 & $\mathrm{H}$ & 4.6540400 & 2.3197530 & -4.3565160 \\
\hline $\mathrm{H}$ & 5.3878320 & -0.7806860 & 0.7063370 & C & -4.6611080 & 0.1795470 & 1.2828730 \\
\hline C & -0.6914940 & -2.0424280 & 0.9929700 & $\mathrm{C}$ & -4.8774730 & 1.3014990 & 2.2677420 \\
\hline C & -1.9982720 & -2.5467270 & 0.9970820 & $\mathrm{C}$ & -2.8133670 & -0.1787980 & 2.8179680 \\
\hline $\mathrm{C}$ & 0.4503490 & -2.8595380 & 1.1258420 & $\mathrm{C}$ & -3.5266260 & -0.6086470 & 1.5577820 \\
\hline C & -2.1468670 & -3.9306230 & 1.1693040 & C & -5.0521140 & 0.6776880 & 3.6806340 \\
\hline $\mathrm{H}$ & -3.1510790 & -4.3495800 & 1.1812950 & $\mathrm{H}$ & -5.1334450 & 1.4870640 & 4.4184600 \\
\hline C & 3.7676440 & 0.0037920 & -1.3147150 & $\mathrm{H}$ & -5.9846490 & 0.1020090 & 3.7145840 \\
\hline C & -3.1866230 & -1.6922350 & 0.7286600 & C & -3.8265960 & -0.2358370 & 3.9957470 \\
\hline C & 3.7335060 & -0.4915640 & -2.6306770 & $\mathrm{H}$ & -3.3202250 & 0.0991800 & 4.9105610 \\
\hline C & 3.4149990 & -1.9068070 & -3.0535940 & $\mathrm{H}$ & -4.1468250 & -1.2726220 & 4.1537980 \\
\hline $\mathrm{C}$ & 3.9827570 & -0.3500220 & -5.0550210 & $\mathrm{H}$ & -1.9487860 & -0.8135550 & 3.0303660 \\
\hline C & 0000 & 0.3429 & -3.7174280 & $\mathrm{H}$ & -5.7567220 & 1.8994300 & 1.9985400 \\
\hline C & 2.22 & -1.877 & -4.0474040 & C & -2.3678900 & 1.2953900 & 2.6465770 \\
\hline $\mathrm{H}$ & 2.04 & -2 . & -4.4141880 & $\mathrm{H}$ & -1.8927430 & 1.6376990 & 3.5741080 \\
\hline
\end{tabular}




$\begin{array}{rr}-1.6169660 & 1.3414210 \\ -3.5994000 & 2.1837590 \\ -3.7298310 & 2.9805460 \\ -3.4636000 & 2.6673040 \\ -5.1705950 & -1.2028660 \\ -5.9792940 & -1.6689310 \\ -3.8379260 & -3.0781680 \\ -4.0168820 & -1.9737380 \\ -5.0838830 & -1.6132550 \\ -5.6535120 & -2.0027430 \\ -4.8169310 & -0.5746860 \\ -3.8013850 & -2.4623960 \\ -3.7370780 & -3.2840880 \\ -2.9011010 & -1.8530490 \\ -2.9248740 & -3.6500250 \\ -6.8763730 & -1.0510280 \\ -5.0854280 & -4.0015490 \\ -4.9888500 & -4.7912150 \\ -5.1259640 & -4.4863400 \\ -6.3737880 & -3.1545230 \\ -6.9151520 & -3.5231750 \\ -7.0525100 & -3.2190320 \\ -5.4910250 & -0.1245800 \\ -6.3824210 & 0.4727380 \\ 0.2582990 & -4.2539980 \\ 1.3702670 & -5.2738320 \\ 1.0569090 & -6.3047880 \\ -0.3598260 & -6.9352310 \\ -1.1767710 & -6.2801240 \\ -1.0466370 & -4.7798650 \\ 1.4549630 & -5.8087710 \\ 1.8271400 & -7.0858650 \\ -0.2821810 & -8.0130120 \\ -0.7834700 & -6.6473060 \\ 2.3360360 & -4.7965170 \\ 1.1330570 & -5.7910140 \\ -0.9152130 & -6.8121700 \\ -2.2293890 & -6.5816000 \\ 2.7816160 & -2.4879830 \\ 4.0813490 & -1.9690290 \\ 2.4209330 & -3.2050330 \\ 3.6278700 & -3.8694280 \\ 4.7415260 & -2.8376780 \\ 5.2042090 & -2.2707230 \\ 3.3208410 & -4.3062050 \\ 2.0042910 & -2.4574770\end{array}$

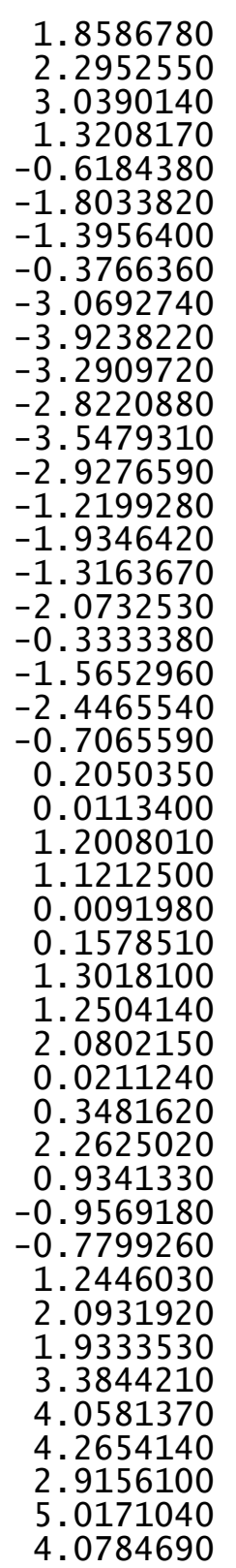

1.6260260
4.3561180
5.5970910
5.8062500
5.8742750
3.9967330
-3.1860760
-2.0753500
-3.0456190
-1.0779800
-2.6599950
-1.2211600
-0.1749330
0.1572400
-0.4296070
-0.2616760
-1.2435070
-2.0821800
-1.9562400
1.1402400
1.4578800
0.9698360
0.3258270
1.5405900
1.1780370
0.0820750
-4.7464340
-3.7046810
-2.2681080
-4.6014890
-5.7587290
-3.8279000
-2.0092800
-4.8616120
-5.2686730
-4.6197260
-3.8613270
-1.5338630
2.2487330
2.5317240
3.0713470
1.6062720
3.1560670

3.2278630

4.9015090

4.7924950

3.0662760

2.4397680

3.4241100

$-1.8823630$

$-2.4764670$

$-0.8616310$

$-2.0931550$

$-2.8723980$

0.5929770

2.8933410

2.0983990

0.8457920

0.1730020

2.0011000

$-0.8080340$

$-2.3678450$

4.6754690

3.8699830

2.5639270

4.1961970

5.6846930

1.9148730

4.8281030

$-3.6047220$

$-3.8616940$

$-3.7119850$

$-2.1858580$

$-3.7308880$

$-4.8662190$

$-4.5710000$

$-1.4402870$

$-2.0279540$

$-4.3389290$

$-3.1424300$

$-3.6965220$

4.4571980

3.6938520

2.7705860

3.4346950

4.3317900

158

$\operatorname{TS} 55(R, R)$ G $[B 97-D /$ def2-TZVP $]=-4356.201895$

$P \quad-0.0314120 \quad-0.1390760 .201895-0.7608080$ 


$\begin{array}{rrr}-1.0082190 & -0.5303070 & -1.8414600 \\ 0.2913630 & 1.3053890 & -0.5427370 \\ 1.2836350 & -1.0431850 & -1.0806520 \\ -0.4942330 & -0.7286720 & 0.6934460 \\ 2.1981950 & -1.3338530 & -0.0736690 \\ 1.8365820 & -2.2590860 & 0.9273280 \\ 3.4584340 & -0.7254030 & -0.1302630 \\ 4.3730130 & -1.0529680 & 0.8785510 \\ 5.3655830 & -0.6068750 & 0.8491730 \\ -0.6545840 & -2.1141190 & 0.7778970 \\ -1.9486660 & -2.6497910 & 0.7314760 \\ 0.4968570 & -2.9134000 & 0.8672690 \\ -2.0514550 & -4.0421470 & 0.7923180 \\ -3.0450370 & -4.4866840 & 0.7717010 \\ 3.8196560 & 0.2253790 & -1.2156290 \\ -3.1596350 & -1.8063190 & 0.5449540 \\ 3.8819060 & -0.1972820 & -2.5553240 \\ 3.6374680 & -1.5964830 & -3.0713700 \\ 4.2662240 & 0.0849600 & -4.9488510 \\ 4.2377580 & 0.7053250 & -3.5746750 \\ 2.5002440 & -1.5489090 & -4.1291490 \\ 2.3821790 & -2.5528890 & -4.5584550 \\ 1.5601330 & -1.2831890 & -3.6386890 \\ 2.8639220 & -0.5170960 & -5.2413400 \\ 2.8799060 & -0.9964390 & -6.2293610 \\ 2.1224160 & 0.2901520 & -5.2748650 \\ 4.5354960 & 0.8259770 & -5.7114030 \\ 3.3742020 & -2.2869400 & -2.2654520 \\ 5.2926770 & -1.0821990 & -4.9368350 \\ 5.2654960 & -1.5892540 & -5.9105630 \\ 6.3035050 & -0.6801110 & -4.7994090 \\ 4.9323980 & -2.0752710 & -3.7870720 \\ 4.7636600 & -3.0843510 & -4.1858820 \\ 5.7498130 & -2.1371900 & -3.0586410 \\ 4.1155340 & 1.5685050 & -0.9227660 \\ 4.0476270 & 2.2339650 & 0.4314260 \\ 4.7414100 & 3.8667870 & -1.4631730 \\ 4.4745250 & 2.4636700 & -1.9491200 \\ 5.4386250 & 2.8414640 & 0.7580620 \\ 5.3730850 & 3.3782530 & 1.7137720 \\ 6.1745990 & 2.0370920 & 0.8778870 \\ 5.8647680 & 3.8106080 & -0.3902520 \\ 6.0355310 & 4.8224130 & 0.0007160 \\ 6.7977960 & 3.4719250 & -0.8560790 \\ 5.0338770 & 4.5231290 & -2.2917620 \\ 3.7483040 & 1.5290570 & 1.2107290\end{array}$

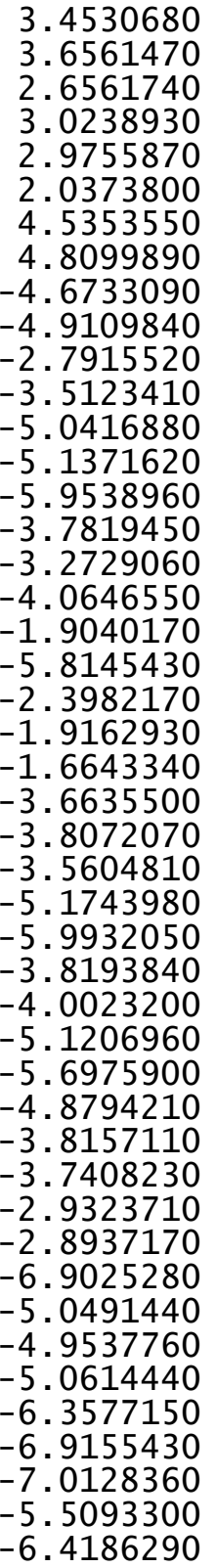

4. 3947940

5. 3895020

4.5036810

3. 3987450

3.9004310

2.9833160

2.0350770

2.7313050

$-0.0310510$

0.9824930

$-0.4709940$

$-0.8065280$

0.2187960

0.9499800

$-0.3892960$

$-0.6788020$

$-0.4186320$

$-1.7367770$

$-1.0932870$

1.5717220

1.0273570

1. 2965180

1.1733700

1.9031080

2. 6189710

2.4834610

$-1.2504870$

$-1.6366860$

$-3.0313290$

$-2.0144380$

$-1.4701430$

$-1.8098060$

$-0.4132840$

$-2.3042880$

$-3.0627450$

$-1.6650870$

$-3.5982670$

$-1.0267190$

$-3.9819970$

$-4.7035140$

$-4.5489410$

$-3.1432850$

3.4540610

$-3.2848640$

$-0.2578030$

0.3300200
$-0.7730080$

$-0.3540290$

$-1.5182170$

0.3461550

1. 3208480

0.1319490

$-3.2745760$

$-4.0679870$

1.2835830

2.3749160

2.7533430

1.4695390

3.7234220

4.5370040

3.7130060

3.9329660

4.8703780

3.9898850

2.8933400

2.1766760

2.7161960

3.6640550

1.9239780

2.4712400

3.2911180

1.5465000

$-0.7282970$

$-1.9345930$

$-1.6724430$

$-0.5666750$

$-3.2077610$

$-4.0784850$

$-3.3588750$

$-3.0450530$

$-3.8355790$

$-3.1111680$

$-1.5549640$

$-2.0040690$

$-1.6494630$

$-2.4716920$

$-0.7104750$

$-1.8020720$

$-2.6953610$

$-0.9343110$

0.1918480

0.0633470 


\begin{tabular}{|c|c|c|}
\hline $\begin{array}{l}0.3575190 \\
1.5817670 \\
1.2531550 \\
0.1030470 \\
1.1671010 \\
0.9335270 \\
2.1487750 \\
2.0495450 \\
2.3302900 \\
0.3790280 \\
0.0830880 \\
1.9370620 \\
1.5884110 \\
0.9574340 \\
4.0360480 \\
5.0125530 \\
4.9890910 \\
3.5759160 \\
2.4392580 \\
2.7622840 \\
4.7343770 \\
5.7202050 \\
3.5133310 \\
2.2845630 \\
6.0196240 \\
5.2945120 \\
3.4082110 \\
1.4944500 \\
3.2170600 \\
2.1428950 \\
3.0501410 \\
1.1342770 \\
2.6732000 \\
1.3820210 \\
0.3751150 \\
0.0112680 \\
0.5795580 \\
0.3763060 \\
1.4447980 \\
2.2198350 \\
1.9359690 \\
0.9233680 \\
1.2719080 \\
0.8111840 \\
0.1019060 \\
1.3042740\end{array}$ & $\begin{array}{l}-4.3215680 \\
-5.2128280 \\
-6.6010510 \\
-7.2189010 \\
-6.3826470 \\
-4.8820960 \\
-7.2340180 \\
-5.3545480 \\
-4.7046840 \\
-7.2323400 \\
-8.2569640 \\
-6.6675290 \\
-6.6088150 \\
-6.5164630 \\
-1.9151320 \\
-2.2653730 \\
-3.7883280 \\
-4.2736360 \\
-3.3129240 \\
-2.5151050 \\
-1.7549020 \\
-4.0663840 \\
-4.3702860 \\
-2.5817840 \\
-1.9224070 \\
-4.2794240 \\
-5.2754700 \\
-3.8515230 \\
1.8129930 \\
2.6387270 \\
1.4036690 \\
2.4676210 \\
0.9425490 \\
4.4315880 \\
4.3550450 \\
3.2838930 \\
3.3692730 \\
2.6259810 \\
5.4345590 \\
4.8056940 \\
0.3005170 \\
3.3869350 \\
2.3049470 \\
2.2455360 \\
4.4123510 \\
3.3956440\end{array}$ & $\begin{array}{r}0.8122030 \\
0.6809140 \\
0.1165730 \\
0.9189590 \\
0.7207210 \\
0.8025670 \\
0.1590950 \\
1.6627020 \\
0.0605470 \\
1.9843200 \\
0.6153940 \\
1.4510670 \\
-0.2715440 \\
-0.9397460 \\
1.9210760 \\
3.0173570 \\
3.2576510 \\
3.6628880 \\
3.2046320 \\
1.9602200 \\
3.9540040 \\
4.0269500 \\
4.7540810 \\
4.0116840 \\
2.7511140 \\
2.3244160 \\
3.2502720 \\
3.1039100 \\
-1.8368530 \\
-2.4053740 \\
-0.8370250 \\
-2.0425810 \\
-2.8775640 \\
0.7559410 \\
3.0720950 \\
2.2415930 \\
0.9824730 \\
0.2780090 \\
2.2022720 \\
-0.6480730 \\
-2.4185080 \\
4.8412830 \\
3.9997110 \\
2.6813900 \\
4.3863460 \\
5.8588180\end{array}$ \\
\hline
\end{tabular}

$\begin{array}{lr}\mathrm{H} & 1.0446850 \\ \mathrm{H} & -0.1662420 \\ \mathrm{C} & -4.8361580 \\ \mathrm{C} & -3.8377890 \\ \mathrm{C} & -2.3802010 \\ \mathrm{C} & -4.6503960 \\ \mathrm{H} & -5.8635060 \\ \mathrm{H} & -3.9872130 \\ \mathrm{H} & -2.1022120 \\ \mathrm{H} & -4.9175280 \\ \mathrm{H} & -5.2912730 \\ \mathrm{H} & -4.6935870 \\ \mathrm{H} & -4.0172040 \\ \mathrm{H} & -1.6791490 \\ \mathrm{O} & 2.0618610 \\ \mathrm{C} & 2.3595110 \\ \mathrm{H} & 2.9164940 \\ \mathrm{H} & 1.4387940 \\ \mathrm{H} & 2.9737160 \\ \mathrm{H} & \end{array}$
1.4332270
5.2334350
2.8496710
3. 9963380
3. 5133630
2. 2246420
3. 2213180
4.4498180
2.9273760
2. 9526210
1. 3480350
2.0768210
4.7784080
4. 3539660
1. 3511570
0.1968710
0.4692540
$-0.3246440$
$-0.4479940$

158

2.0046200

5. 0455640

$-3.4922000$

$-3.7190450$

$-3.6019610$

$-2.0996790$

$-3.5884040$

$-4.7061960$

$-4.4896990$

$-1.3210910$

$-1.9640660$

$-4.2592560$

$-2.9689530$

$-3.5560680$

4.5664860

3. 7691210

2.8647870

3.4759600

4. 3998950

TS56 (R, R) G $[B 97-D / \operatorname{def} 2-T Z V P]=-4356.201895$

$\begin{array}{lrrr}\text { TS56(R, R) G }[\mathrm{B} 97-\mathrm{D} / \mathrm{de} f 2-\mathrm{TZVP}]=-4356.201895 \\ \mathrm{P} & -0.0314120 & -0.1390770 & -0.7608110 \\ \mathrm{O} & 0.2913620 & 1.3053870 & -0.5427380 \\ \mathrm{O} & -1.0082180 & -0.5303080 & -1.8414640 \\ \mathrm{O} & -0.4942340 & -0.7286750 & 0.6934420 \\ \mathrm{O} & 1.2836350 & -1.0431860 & -1.0806550 \\ \mathrm{C} & -0.6545860 & -2.1141220 & 0.7778910 \\ \mathrm{C} & 0.4968550 & -2.9134040 & 0.8672630 \\ \mathrm{C} & -1.9486680 & -2.6497930 & 0.7314690 \\ \mathrm{C} & -2.0514580 & -4.0421500 & 0.7923070 \\ \mathrm{H} & -3.0450400 & -4.4866860 & 0.7716890 \\ \mathrm{C} & 2.1981950 & -1.3338540 & -0.0736700 \\ \mathrm{C} & 3.4584330 & -0.7254040 & -0.1302610 \\ \mathrm{C} & 1.8365800 & -2.2590890 & 0.9273240 \\ \mathrm{C} & 4.3730100 & -1.0529700 & 0.8785550 \\ \mathrm{H} & 5.3655800 & -0.6068760 & 0.8491790 \\ \mathrm{C} & -3.1596370 & -1.8063190 & 0.5449520 \\ \mathrm{C} & 3.8196570 & 0.2253790 & -1.2156250 \\ \mathrm{C} & -3.5123380 & -0.8065310 & 1.4695420 \\ \mathrm{C} & -2.7915450 & -0.4710030 & 2.7533460 \\ \mathrm{C} & -4.9109740 & 0.9824910 & 2.3749290 \\ \mathrm{C} & -4.6733040 & -0.0310490 & 1.2835920 \\ \mathrm{C} & -2.3982060 & 1.0273470 & 2.7162030 \\ \mathrm{H} & -1.9162780 & 1.2965040 & 3.6640610 \\ \mathrm{H} & -1.6643260 & 1.1733610 & 1.9239820 \\ \mathrm{C} & -3.6635380 & 1.9031020 & 2.4712540\end{array}$




$\begin{array}{lrr}-3.8071910 & 2.6189610 & 3.2911360 \\ -3.5604690 & 2.4834610 & 1.5465170 \\ -5.8145320 & 1.5717230 & 2.1766930 \\ -1.9040120 & -1.0932990 & 2.8933390 \\ -5.0416770 & 0.2187890 & 3.7234320 \\ -5.1371480 & 0.9499700 & 4.5370170 \\ -5.9538870 & -0.3893000 & 3.7130150 \\ -3.7819360 & -0.6788120 & 3.9329700 \\ -3.2728940 & -0.4186470 & 4.8703820 \\ -4.0646480 & -1.7367880 & 3.9898860 \\ -4.0023250 & -2.0144330 & -0.5666760 \\ -3.8193960 & -3.0313210 & -1.6724460 \\ -5.9932130 & -1.6366700 & -1.9345860 \\ -5.1744010 & -1.2504770 & -0.7282920 \\ -5.0491590 & -3.9819850 & -1.6494650 \\ -4.9537960 & -4.7035000 & -2.4716970 \\ -5.0614590 & -4.5489320 & -0.7104790 \\ -6.3577280 & -3.1432680 & -1.8020660 \\ -6.9155610 & -3.4540400 & -2.6953530 \\ -7.0128460 & -3.2848460 & -0.9343030 \\ -6.9025340 & -1.0267000 & -2.0040580 \\ -2.8937300 & -3.5982630 & -1.5549710 \\ -5.1207070 & -1.4701280 & -3.2077570 \\ -5.6976050 & -1.8097890 & -4.0784800 \\ -4.8794300 & -0.4132700 & -3.3588710 \\ -3.8157240 & -2.3042780 & -3.0450540 \\ -3.7408400 & -3.0627330 & -3.8355830 \\ -2.9323830 & -1.6650780 & -3.1111690 \\ -5.5093280 & -0.2577950 & 0.1918570 \\ -6.4186250 & 0.3300320 & 0.0633600 \\ 4.2377630 & 0.7053260 & -3.5746710 \\ 4.2662330 & 0.0849610 & -4.9488470 \\ 3.6374760 & -1.5964830 & -3.0713670 \\ 3.8819110 & -0.1972820 & -2.5553210 \\ 5.2926870 & -1.0821960 & -4.9368290 \\ 5.2655080 & -1.5892510 & -5.9105570 \\ 6.3035150 & -0.6801070 & -4.7994020 \\ 4.9324080 & -2.0752680 & -3.7870670 \\ 4.7636730 & -3.0843490 & -4.1858770 \\ 5.7498230 & -2.1371850 & -3.0586340 \\ 3.3742100 & -2.2869410 & -2.2654490 \\ 4.5355050 & 0.8259790 & -5.7113980 \\ 2.5002540 & -1.5489110 & -4.1291490 \\ 2.3821920 & -2.5528910 & -4.5584550 \\ 1.5601420 & -1.2831940 & -3.6386900 \\ 2.8639320 & -0.5170970 & -5.2413380\end{array}$

2.8799180
2.1224240
4.4745250
4.7414080
4.0476220
4.1155330
3.4530640
3.6561400
2.6561710
3.0238870
2.9755800
2.0373760
3.7482980
5.0338760
5.4386190
5.3730770
6.1745940
5.8647640
6.0355230
6.7977930
4.5353580
4.8099930
4.0360440
5.0125460
4.9890850
3.5759100
2.4392520
2.7622800
4.7343670
5.7201970
3.5133210
2.2845560
6.0196170
5.2945110
3.4082070
1.4944450
0.3575170
1.5817650
1.2531540
0.1030440
-1.1671030
0.9335290
2.1487740
2.0495410
2.3302890
0.3790230

$-0.9964380$

0.2901500

2. 4636710

3. 8667880

2.2339650

1. 5685050

4. 3947940

5. 3895030

4. 5036790

3.3987450

3.9004310

2. 9833160

1.5290570

4.5231300

2.8414660

3. 3782530

2.0370930

3. 8106100

4.8224160

. 4719300

2. 0350780

2.7313060

$-1.9151360$

$-2.2653770$

$-3.7883320$

$-4.2736430$

$-3.3129310$

$-2.5151090$

$-1.7549070$

$-4.0663880$

$-4.3702960$

$-2.5817910$

$-1.9224100$

$-4.2794270$

$-5.2754760$

$-3.8515300$

$-4.3215710$

$-5.2128310$

$-6.6010530$

$-7.2189040$

$-6.3826500$

$-4.8820990$

$-7.2340210$

$-5.3545520$

$-4.7046870$

$-7.2323440$
$-6.2293590$

5.2748630

$-1.9491150$

$-1.4631670$

0.4314300

$-0.9227620$

$-0.7730060$

$-0.3540260$

$-1.5182160$

0.3461580

1. 3208500

0.1319500

1. 2107320

$-2.2917560$

0.7580690

1. 7137790

0.8778950

$-0.3902430$

0.0007260

$-0.8560680$

$-3.2745700$

$-4.0679810$

1. 9210770

3. 0173600

3. 2576530

3. 6628840

3. 2046280

1.9602170

3. 9540070

4.0269550

4.7540770

4.0116810

2. 7511200

2. 3244190

3. 2502650

3. 1039030

0.8121940

0.6809050

0.1165620

0.9189440

0.7207050

0.8025540

0.1590850

1. 6626940

0.0605400

1. 9843060 


\begin{tabular}{|c|c|c|c|c|c|c|c|}
\hline & & & & & & & \\
\hline $\mathrm{H}$ & -0.0830900 & -8.2569670 & 0.6153780 & 0 & 1.1905550 & -1.2288490 & -0.9559890 \\
\hline & -1.9370670 & -6.6675330 & 1.4510470 & 0 & -0.5351680 & -0.6918520 & 0.8042520 \\
\hline $\mathrm{H}$ & -1.5884110 & -6.6088150 & -0.2715630 & C & 2.0982690 & -1.5093080 & 0.0601990 \\
\hline $\mathrm{H}$ & 0.9574350 & -6.5164650 & -0.9397580 & C & 1.7063170 & -2.3675050 & 1.1106280 \\
\hline C & -3.2170590 & 1.8129940 & -1.8368540 & $\mathrm{C}$ & 3.3928810 & -0.9900880 & -0.0560730 \\
\hline c & -2.1428940 & 2.6387300 & -2.4053720 & $\mathrm{c}$ & 4.3169230 & -1.3366530 & 0.9386160 \\
\hline $\mathrm{H}$ & -3.0501420 & 1.4036700 & -0.8370270 & $\mathrm{H}$ & 5.3351740 & -0.9594050 & 0.8594250 \\
\hline $\mathrm{H}$ & -1.1342760 & 2.4676240 & -2.0425790 & C & -0.7822100 & -2.0518940 & 1.0032630 \\
\hline 0 & -2.6731960 & 0.9425510 & -2.8775650 & C & -2.1103770 & -2.4937650 & 1.0351380 \\
\hline C & -1.3820190 & 4.4315890 & 0.7559390 & $\mathrm{C}$ & 0.3229640 & -2.9201670 & 1.1326820 \\
\hline c & -0.3751090 & 4.3550460 & 3.0720920 & $\mathrm{C}$ & -2.3214380 & -3.8667910 & 1.2316410 \\
\hline C & -0.0112670 & 3.2838920 & 2.2415910 & $\mathrm{H}$ & -3.3440990 & -4.2371690 & 1.2673040 \\
\hline $\mathrm{N}$ & -0.5795590 & 3.3692720 & 0.9824720 & C & 3.8068710 & -0.1666840 & -1.2250410 \\
\hline $\mathrm{H}$ & -0.3763110 & 2.6259770 & 0.2780090 & C & -3.2601010 & -1.5853080 & 0.7764230 \\
\hline $\mathrm{S}$ & -1.4447910 & 5.4345630 & 2.2022690 & C & 3.8456670 & -0.7321440 & -2.5124330 \\
\hline $\mathrm{s}$ & -2.2198350 & 4.8056960 & -0.6480730 & $\mathrm{C}$ & 3.4787220 & -2.1496520 & -2.8852030 \\
\hline $\mathrm{H}$ & -1.9359650 & 0.3005180 & -2.4185080 & C & 4.2722170 & -0.7370060 & -4.9158540 \\
\hline c & 0.9233730 & 3.3869350 & 4.8412800 & C & 4.2867240 & 0.0245570 & -3.6140680 \\
\hline C & 1.2719090 & 2.3049440 & 3.9997080 & C & 2.3548520 & -2.1045570 & -3.9568170 \\
\hline C & 0.8111820 & 2.2455340 & 2.6813880 & $\mathrm{H}$ & 2.1369770 & -3.1319310 & -4.2782030 \\
\hline c & 0.1019140 & 4.4123530 & 4.3863420 & $\mathrm{H}$ & 1.4453750 & -1.6952470 & -3.5081130 \\
\hline $\mathrm{H}$ & 1.3042810 & 3.3956430 & 5.8588130 & C & 2.8224400 & -1.2382680 & -5.1679040 \\
\hline H & 1.0446800 & 1.4332220 & 2.0046190 & $\mathrm{H}$ & 2.8027150 & -1.8224640 & -6.0976640 \\
\hline $\mathrm{H}$ & -0.1662300 & 5.2334380 & 5.0455600 & $\mathrm{H}$ & 2.1590070 & -0.3755000 & -5.3020910 \\
\hline C & -4.8361560 & 2.8496710 & -3.4922030 & $\mathrm{H}$ & 4.6134910 & -0.1069170 & -5.7462680 \\
\hline C & -3.8377880 & 3.9963400 & -3.7190460 & $\mathrm{H}$ & 3.1496680 & -2.7262570 & -2.0170950 \\
\hline C & -2.3802000 & 3.5133660 & -3.6019600 & C & 5.1882950 & -1.9841870 & -4.7636510 \\
\hline C & -4.6503950 & 2.2246410 & -2.0996830 & $\mathrm{H}$ & 5.1279190 & -2.5836370 & -5.6818810 \\
\hline $\mathrm{H}$ & -5.8635040 & 3.2213180 & -3.5884080 & $\mathrm{H}$ & 6.2297020 & -1.6615850 & -4.6459190 \\
\hline $\mathrm{H}$ & -3.9872110 & 4.4498210 & -4.7061970 & C & 4.7252910 & -2.8176410 & -3.5283360 \\
\hline $\mathrm{H}$ & -2.1022100 & 2.9273780 & -4.4896980 & $\mathrm{H}$ & 4.4632940 & -3.8411930 & -3.8277520 \\
\hline H & -4.9175300 & 2.9526200 & -1.3210940 & $\mathrm{H}$ & 5.5276310 & -2.8840130 & -2.7835990 \\
\hline $\mathrm{H}$ & -5.2912710 & 1.3480340 & -1.9640700 & C & 4.2021880 & 1.1724610 & -1.0716390 \\
\hline $\mathrm{H}$ & -4.6935830 & 2.0768230 & -4.2592590 & $\mathrm{C}$ & 4.1560380 & 1.9849740 & 0.2000870 \\
\hline $\mathrm{H}$ & -4.0172050 & 4.7784090 & -2.9689540 & $\mathrm{C}$ & 5.0162990 & 3.3428050 & -1.8376440 \\
\hline $\mathrm{H}$ & -1.6791490 & 4.3539700 & -3.5560670 & C & 4.6492490 & 1.9203470 & -2.1780590 \\
\hline 0 & 2.0618590 & 1.3511520 & 4.5664830 & C & 5.5803640 & 2.5225410 & 0.5034420 \\
\hline C & 2.3595020 & 0.1968640 & 3.7691190 & $\mathrm{H}$ & 5.5324920 & 3.1656460 & 1.3922300 \\
\hline $\mathrm{H}$ & 2.9164850 & 0.4692440 & 2.8647840 & $\mathrm{H}$ & 6.2499720 & 1.6852200 & 0.7345160 \\
\hline $\mathrm{H}$ & 1.4387830 & -0.3246480 & 3.4759590 & C & 6.1055200 & 3.3241480 & -0.7296240 \\
\hline & 2.9737060 & -0.4480030 & 4.3998930 & $\mathrm{H}$ & 6.3430900 & 4.3581420 & -0.4463940 \\
\hline & & & & $\mathrm{H}$ & 7.0212040 & 2.8691770 & -1.1258360 \\
\hline & 口 & $/ P]=-43$ & & $\mathrm{H}$ & 5.3778040 & 3.8799410 & -2.7228870 \\
\hline $\mathrm{P}$ & -0.0746730 & -0.2415310 & -0.6977930 & $\mathrm{H}$ & 3.7873010 & 1.3917140 & 1.0401910 \\
\hline 0 & -1.0877330 & -0.6711000 & -1.7301120 & C & 3.7560520 & 4.0374940 & -1.2520380 \\
\hline 0 & 0.316217 & 1.1993740 & -0.6048330 & $\mathrm{H}$ & 4.0231650 & 5.0554920 & -0.9378510 \\
\hline
\end{tabular}




$\begin{array}{rrrr}\mathrm{H} & 2.9881610 & 4.1191240 & -2.0303860 \\ \mathrm{C} & 3.2245390 & 3.2057840 & -0.0446850 \\ \mathrm{H} & 3.1912430 & 3.8154810 & 0.8668360 \\ \mathrm{H} & 2.2139940 & 2.8423060 & -0.2433390 \\ \mathrm{C} & 4.6929740 & 1.3491960 & -3.4493590 \\ \mathrm{H} & 5.0347060 & 1.9324170 & -4.3053850 \\ \mathrm{C} & -4.6231850 & 0.3713200 & 1.3242520 \\ \mathrm{C} & -4.7622100 & 1.5194760 & 2.2922840 \\ \mathrm{C} & -2.7612770 & -0.0502780 & 2.8253120 \\ \mathrm{C} & -3.5240690 & -0.4686170 & 1.5902500 \\ \mathrm{C} & -4.9362840 & 0.9300070 & 3.7196320 \\ \mathrm{H} & -4.9646550 & 1.7546290 & 4.4444030 \\ \mathrm{H} & -5.8939970 & 0.3998270 & 3.7820790 \\ \mathrm{C} & -3.7493110 & -0.0353710 & 4.0260780 \\ \mathrm{H} & -3.2063130 & 0.2923520 & 4.9223840 \\ \mathrm{H} & -4.1150830 & -1.0522360 & 4.2120840 \\ \mathrm{H} & -1.9250770 & -0.7236060 & 3.0313090 \\ \mathrm{H} & -5.6166900 & 2.1552160 & 2.0303620 \\ \mathrm{C} & -2.2480500 & 1.3979630 & 2.6184610 \\ \mathrm{H} & -1.7424140 & 1.7328130 & 3.5325780 \\ \mathrm{H} & -1.5082830 & 1.3976890 & 1.8184800 \\ \mathrm{C} & -3.4421160 & 2.3377210 & 2.2724950 \\ \mathrm{H} & -3.5168040 & 3.1576900 & 2.9984850 \\ \mathrm{H} & -3.3061470 & 2.7889040 & 1.2825730 \\ \mathrm{C} & -5.2426270 & -1.0144780 & -0.5410160 \\ \mathrm{C} & -6.0970460 & -1.4543100 & -1.7035860 \\ \mathrm{C} & -4.0330000 & -2.9765810 & -1.3037470 \\ \mathrm{C} & -4.1297040 & -1.8446530 & -0.3039770 \\ \mathrm{C} & -5.2231030 & -1.4790160 & -2.9859130 \\ \mathrm{H} & -5.8292280 & -1.8599430 & -3.8188530 \\ \mathrm{H} & -4.9061010 & -0.4629130 & -3.2410050 \\ \mathrm{C} & -3.9820150 & -2.3873830 & -2.7401540 \\ \mathrm{H} & -3.9658690 & -3.2218710 & -3.4538760 \\ \mathrm{H} & -3.0539030 & -1.8246690 & -2.8636730 \\ \mathrm{H} & -3.1520920 & -3.5979840 & -1.1315950 \\ \mathrm{H} & -6.9582160 & -0.7869880 & -1.8331990 \\ \mathrm{C} & -5.3306800 & -3.8251690 & -1.1907090 \\ \mathrm{H} & -5.2930510 & -4.6267480 & -1.9401550 \\ \mathrm{H} & -5.3809370 & -4.2965700 & -0.2015940 \\ \mathrm{C} & -6.5734460 & -2.9085740 & -1.4274300 \\ \mathrm{H} & -7.1568060 & -3.2612410 & -2.2882350 \\ \mathrm{H} & -7.2335670 & -2.9165630 & -0.5519430 \\ \mathrm{C} & -5.4888450 & 0.0936810 & 0.2681030 \\ \mathrm{H} & -6.3502520 & 0.7349660 & 1.2288660 \\ \mathrm{C} & 0.0660960 & -4.3030170 & -1262640 \\ \mathrm{C} & 1.1261970 & -5.3725070 & \\ & & & \end{array}$
0.7268170
$-0.7007520$
$-1.4627430$
$-1.2617000$
1. 2263270
1.4701250
$-0.6469530$
$-1.0683020$
2. 1020870
0.7746500
$-1.2804640$
$-2.5295570$
2. 6635180
2. 3840520
2. 9575950
4.4509500
4. 9815110
3. 9725270
2. 8515690
2. 8322730
5.0750660
5. 2279300
1. 3114170
2. 3488740
4. 5862700
5. 9133400
$-3.1428760$
-2.0230420
$-2.9946210$
$-1.0250970$
$-2.6611100$
$-1.1031390$
0.0221240
0.2488800
$-0.3805220$
$-0.2623370$
$-1.0092670$
$-1.9761960$
$-1.9638620$
1. 3669650
1. 5757530
1. 0188540
0.5914770
1. 8195580
1.1541450
0.4314830
$-6.3991820$
$-6.9813850$
$-6.2566510$
$-4.7657440$
$-5.8951050$
$-7.2058880$
$-8.0519140$
$-6.6185720$
$-4.9403310$
$-5.8919800$
$-6.8828650$
$-6.5079400$
$-2.6861460$
$-3.5640050$
$-2.9620040$
$-2.5491320$
$-2.6309510$
$-4.5465590$
$-3.6923510$
$-3.1910460$
$-3.6837520$
$-3.7421210$
$-2.0917100$
$-1.5254580$
$-2.0618130$
1. 8198260
2. 5068090
1. 5143360
2. 3117050
0.8106590
4.5771800
4.7107480
3. 5131070
3. 4854840
2.6501470
5.7564620
4.8440430
0.1680890
3.8821750
2. 6737200
2.4769290
4.9015910
3.9951490
1. 5695220
5.8223250

0.0378920

0.2548930

1.3953490

1. 3084100

2. 0894760

0.0174350

0.4895030

2. 3575220

0.8902780

$-0.9336170$

$-0.6709700$

1. 3662270

2.0992950

3. 2980240

4.6000750

4. 4772860

3. 0234960

1.9972590

3.1336580

5.4086770

5. 1109650

2. 8117970

3.4128220

4.8681030

4.8471280

2. 9233320

$-1.9466010$

$-2.6021580$

$-0.9079210$

$-2.2220460$

$-2.8883250$

0.3385530

2. 5975410

1. 9017090

0.6697030

0.0566530

1.6433970

$-1.0682650$

$-2.3677820$

4.4025320

3. 6984590

2. 4320630

3.8613730

5. 3838270

1.8564070

4.4157790 


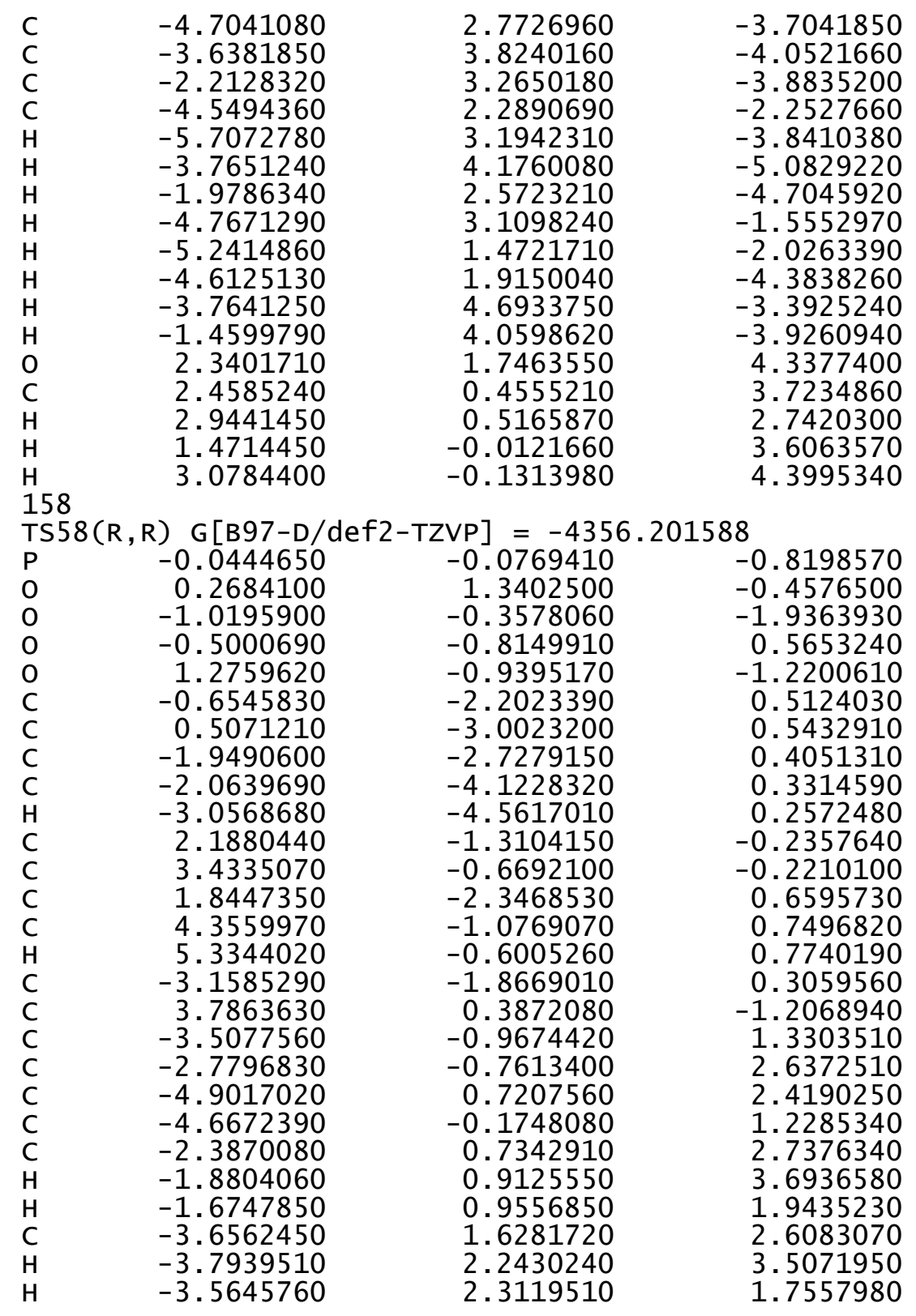

-5.8065890
-1.8900790
-5.0290490
-5.1353870
-5.9350890
-3.7609610
-3.2461580
-4.0350990
-4.0076490
-3.8283900
-5.9951370
-5.1770870
-5.0639060
-4.9690490
-5.0826110
-6.3681000
-6.9257040
-7.0254270
-6.9001390
-2.9069180
-5.1139300
-5.6880590
-4.8641740
-3.8153400
-3.7443380
-2.9268030
-5.5062350
-6.4142710
4.2532350
4.3357470
3.6847620
3.8873830
5.3863850
5.4043500
6.3827210
5.0081780
4.8678800
5.8040200
3.4128710
4.6130270
2.5781820
2.4883560
1.6205010
2.9555730
3.0123750
2.1992120

1. 3259210

-1.3919790

$-0.1789780$

0.4618890

$-0.7914770$

$-1.0810650$

$-0.9004300$

$-2.1422370$

$-1.9613080$

$-2.8566310$

$-1.4293190$

$-1.1810970$

$-3.7960820$

$-4.4213100$

$-4.4641960$

$-2.9387620$

$-3.1479290$

$-3.1709970$

$-0.8091330$

$-3.4396680$

$-1.1284600$

$-0.0630400$

$-1.9850650$

$-2.6534660$

$-1.3499100$

$-0.2860300$

0.3140730

1.0788160

0.5780570

$-1.2678880$

0.0855650

$-0.5670920$

$-0.9876470$

$-0.1594640$

$-1.6631380$

$-2.6348820$

$-1.7788830$

$-2.0283530$

1.3855510

$-1.1498460$

$-2.1160150$

$-0.9389310$

$-0.0227240$

$-0.4185120$

0.7711870
2.2856280

2.7105670

3.6804830

4.5658550

3. 6020950

3. 7997480

4.7526580

3.7654960

$-0.8163650$

$-2.0229380$

$-2.1378740$

$-0.8955390$

$-2.1090790$

$-3.0066290$

$-1.2392750$

$-2.1716960$

$-3.0942860$

$-1.3253750$

$-2.1433760$

$-1.9648250$

$-3.3804660$

$-4.2872030$

$-3.4070540$

$-3.3084710$

$-4.1767780$

$-3.3025700$

0.1214190

0.0548610

$-3.5036440$

$-4.9237580$

$-3.2160210$

$-2.5765050$

$-4.9751270$

$-5.9894320$

$-4.7665830$

$-3.9297730$

$-4.4214920$

$-3.1841470$

$-2.4792370$

$-5.6123080$

$-4.2990680$

$-4.8134810$

$-3.8158620$

$-5.3105190$

-6.3334100
-5.3019670 


\begin{tabular}{|c|c|c|c|}
\hline C & 4.4181810 & 26902960 & -1.7239700 \\
\hline$c$ & 4.6555490 & 4.0472990 & -1.1098190 \\
\hline c & 3.9653920 & 2.2391680 & 0.6205850 \\
\hline C & 4.0557720 & 1.7018940 & -0.7887530 \\
\hline $\mathrm{C}$ & 3.3512150 & 4.4921080 & -0.3925220 \\
\hline $\mathrm{H}$ & 3.5315000 & 5.4503780 & 0.1128300 \\
\hline $\mathrm{H}$ & 2.5596480 & 4.6504240 & -1.1344770 \\
\hline C & 2.9283770 & 3.3955930 & 0.6317220 \\
\hline $\mathrm{H}$ & 2.8675970 & 3.8080670 & 1.6466750 \\
\hline H & 1.9495170 & 2.9892530 & 0.3732010 \\
\hline $\mathrm{H}$ & 3.6682580 & 1.4626840 & 1.3307440 \\
\hline $\mathrm{H}$ & 4.9477340 & 4.7802630 & -1.8716110 \\
\hline C & 5.3466990 & 2.8298540 & 1.0137770 \\
\hline $\mathrm{H}$ & 5.2702190 & 3.2689650 & 2.0173620 \\
\hline $\mathrm{H}$ & 6.0922730 & 2.0265990 & 1.0586840 \\
\hline C & 5.7662120 & 3.9124750 & -0.0311530 \\
\hline H & 5.9094610 & 4.8859220 & 0.4562690 \\
\hline $\mathrm{H}$ & 6.7122440 & 3.6391940 & -0.513419 \\
\hline C & 4.5173500 & 2.3818960 & -3.0804520 \\
\hline $\mathrm{H}$ & 4.7986370 & 3.1503560 & -3.8016090 \\
\hline $\mathrm{C}$ & 4.0441810 & -2.0631710 & 1.6825050 \\
\hline C & 5.0229520 & -2.5030740 & 2.7422070 \\
\hline C & 5.1064600 & -4.0440410 & 2.7784410 \\
\hline C & 3.7057450 & -4.6987560 & 2.9367060 \\
\hline C & 2.5364860 & -3.6941940 & 2.7578560 \\
\hline $\mathrm{C}$ & 2.7881670 & -2.7004510 & 1.6488010 \\
\hline H & 4.6916370 & -2.1432550 & 3.7298350 \\
\hline $\mathrm{H}$ & 5.7721200 & -4.3620780 & 3.5902850 \\
\hline $\mathrm{H}$ & 3.6091210 & -5.1602440 & 3.9272760 \\
\hline $\mathrm{H}$ & 2.4382400 & -3.1256430 & 3.6952030 \\
\hline $\mathrm{H}$ & 6.0103070 & -2.0637810 & 2.556229 \\
\hline $\mathrm{H}$ & 5.5622770 & -4.3768850 & 1.8 \\
\hline $\mathrm{H}$ & 3.6004360 & -5.5024930 & 2.2015120 \\
\hline $\mathrm{H}$ & 1.5939330 & -4.2274650 & 2.6205660 \\
\hline$C$ & -0.9435220 & -4.9479480 & 0.2941720 \\
\hline C & -1.0509380 & -6.4440720 & 0.1371290 \\
\hline C & -0.1445510 & -7.1559110 & 1.1640070 \\
\hline$C$ & $1 . \overline{3} 208700$ & -6.6383670 & 1.1034820 \\
\hline C & 1.4846590 & -5.3754080 & 0.2174000 \\
\hline$c$ & 0.3488760 & -4.3953810 & 0.3890190 \\
\hline $\mathrm{H}$ & -0.7289540 & -6.7291990 & -0.8777830 \\
\hline $\mathrm{H}$ & -0.1750510 & -8.2400520 & 0.9991160 \\
\hline $\mathrm{H}$ & 1.9862910 & -7.4184200 & 0.713083 \\
\hline $\mathrm{H}$ & 1.4755110 & -5.6995840 & -0.8352330 \\
\hline $\mathrm{H}$ & -2.0933000 & -6.7668080 & 0.247497 \\
\hline $\mathrm{H}$ & -0.5576050 & -6.9695860 & 2.163517 \\
\hline
\end{tabular}

$\begin{array}{lr}\text { H } & 1.6629460 \\ \text { H } & 2.4576980 \\ \mathrm{C} & -3.1831040 \\ \mathrm{C} & -2.1054810 \\ \mathrm{H} & -3.0010480 \\ \mathrm{H} & -1.0933610 \\ \mathrm{O} & -2.6837710 \\ \mathrm{C} & -1.3360990 \\ \mathrm{C} & -0.3928550 \\ \mathrm{C} & -0.0271340 \\ \mathrm{~N} & -0.5652290 \\ \mathrm{H} & -0.3641080 \\ \mathrm{~S} & -1.4207090 \\ \mathrm{~S} & -2.1133460 \\ \mathrm{H} & -1.9498210 \\ \mathrm{C} & 0.8429940 \\ \mathrm{C} & 1.1956760 \\ \mathrm{C} & 0.7694470 \\ \mathrm{C} & 0.0510490 \\ \mathrm{H} & 1.1971050 \\ \mathrm{H} & 1.0068440 \\ \mathrm{H} & -0.2197530 \\ \mathrm{C} & -4.8171930 \\ \mathrm{C} & -3.8011800 \\ \mathrm{C} & -2.3506400 \\ \mathrm{C} & -4.6135080 \\ \mathrm{H} & -5.8386150 \\ \mathrm{H} & -3.9629490 \\ \mathrm{H} & -2.1002560 \\ \mathrm{H} & -4.8469260 \\ \mathrm{H} & -5.2687430 \\ \mathrm{H} & -4.7067190 \\ \mathrm{H} & -3.9483470 \\ \mathrm{H} & -1.6338320 \\ \mathrm{O} & 1.9517340 \\ \mathrm{C} & 2.2657510 \\ \mathrm{H} & 2.8542570 \\ \mathrm{H} & 1.3512260 \\ \mathrm{H} & 2.8531160 \\ \mathrm{H} & \\ \mathrm{H} & \\ \mathrm{H} & \end{array}$
$-6.4092700$
-4.9110500
2.0354200
2.8858580
1.5429280
2. 6663570
1.2443640
4.3473390
3. 9893420
3.0117370
3. 2461200
2. 5786490
5.1813440
4.9007860
0.5472010
2.7996360
1.8146490
1. 9169140
3. 8866050
2.6848430
1. 1819440
4.6326190
3. 2358400
4. 3784160
3.8632370
2. 4940680
3. 6330180
4.9138760
3. 3512440
3. 1609770
1.6209380
2. 5259670
5.0996280
4.6854520
0.7842540
$-0.2638190$
0.1171880
$-0.7327770$
$-0.9918250$

2.1181200

0.3889980

$-1.6476820$

$-2.1701930$

$-0.6891330$

$-1.8447560$

$-2.7702490$

1.1499810

3.4674670

2. 5297170

1.2769920

0.4998080

2.6991660

$-0.2295030$

$-2.3937540$

5.1450330

4.1933440

2. 8664100

4.7907710

6.1658060

2.1078000

5.5328990

$-3.1713830$

$-3.3262860$

$-3.2820890$

$-1.8393500$

$-3.2128500$

$-4.2694870$

$-4.2223280$

$-0.9976380$

$-1.7599690$

$-4.0020720$

$-2.5110720$

$-3.1795390$

4.6636470

3.7353560

2.8920360

3.3485980

158

4.2975680

$\operatorname{TS} 59(R, R) G[B 97-D / d e f 2-T Z V P]=-4356.201422$

$\begin{array}{lrrr}\text { TS59(R, R) G }[B 97-D / d e f 2-T Z V P] & =-4356.201422 \\ P & 0.0242550 & 0.1452300 & 1.0794880 \\ O & -0.5142160 & 1.4393540 & 0.5527370 \\ 0 & 0.9706100 & 0.1738370 & 2.2542550 \\ 0 & 0.6936780 & -0.6149430 & -0.2086710 \\ O & -1.1470770 & -0.8876330 & 1.5185680 \\ 0 & & \end{array}$




\begin{tabular}{lrrr} 
C & 0.9698330 & -1.9786490 & -0.0846100 \\
C & -0.1060500 & -2.8910010 & -0.1255780 \\
C & 2.3006510 & -2.3781090 & 0.0784170 \\
C & 2.5435970 & -3.7489950 & 0.2425320 \\
H & 3.5710430 & -4.0849580 & 0.3698270 \\
C & -1.9872470 & -1.3719200 & 0.5143170 \\
C & -3.2798930 & -0.8468050 & 0.4110130 \\
C & -1.4921440 & -2.3807560 & -0.3368220 \\
C & -4.0935460 & -1.3572700 & -0.6100760 \\
H & -5.1068420 & -0.9729300 & -0.7098340 \\
C & 3.4402130 & -1.4227390 & 0.0970160 \\
C & -3.7928950 & 0.2143460 & 1.3162230 \\
C & 3.7690010 & -0.6574740 & -1.0365920 \\
C & 3.0204980 & -0.6217830 & -2.3483650 \\
C & 5.1377190 & 0.8863690 & -2.3452840 \\
C & 4.9177790 & 0.1563960 & -1.0441020 \\
C & 2.6151690 & 0.8501460 & -2.6211900 \\
H & 2.0780890 & 0.9060760 & -3.5755460 \\
H & 1.9250000 & 1.1688710 & -1.8386670 \\
C & 3.8841050 & 1.7547390 & -2.6416700 \\
H & 4.0048260 & 2.2328040 & -3.6226590 \\
H & 3.8013060 & 2.5538420 & -1.8966440 \\
H & 6.0400420 & 1.5084060 & -2.3018390 \\
H & 2.1336340 & -1.2596350 & -2.3285160 \\
C & 5.2568290 & -0.1705630 & -3.4784940 \\
H & 5.3674950 & 0.3491180 & -4.4395740 \\
H & 6.1586460 & -0.7738350 & -3.3203340 \\
C & 3.9830600 & -1.0714840 & -3.4817990 \\
H & 3.4552190 & -0.9909780 & -4.4414200 \\
H & 4.2523060 & -2.1246390 & -3.3383450 \\
C & 4.2689680 & -1.3283400 & 1.2304120 \\
C & 4.0719890 & -2.0300590 & 2.5546170 \\
C & 6.2051990 & -0.5459330 & 2.4974810 \\
C & 5.4213700 & -0.5186440 & 1.2097660 \\
C & 5.3232070 & -2.9119310 & 2.8220300 \\
H & 5.2100040 & -3.3983520 & 3.7998300 \\
H & 5.3881680 & -3.7020550 & 2.0640350 \\
C & 6.6044330 & -2.0192600 & 2.7966510 \\
H & 7.1220050 & -2.0566420 & 3.76434100 \\
H & 7.3063730 & -2.3699040 & 2.0306470 \\
H & 7.0978510 & 0.0880300 & 2.4295640 \\
H & 3.1640620 & -2.6378480 & 2.5593540 \\
C & 5.2735710 & -0.0632310 & 3.6423930 \\
H & 5.8205830 & -0.1188400 & 4.5931300 \\
H & 4.9967040 & 0.9842440 & 3.4764660 \\
C & 4.0008010 & -0.9613840 & 3.6807000 \\
& & & \\
\hline
\end{tabular}

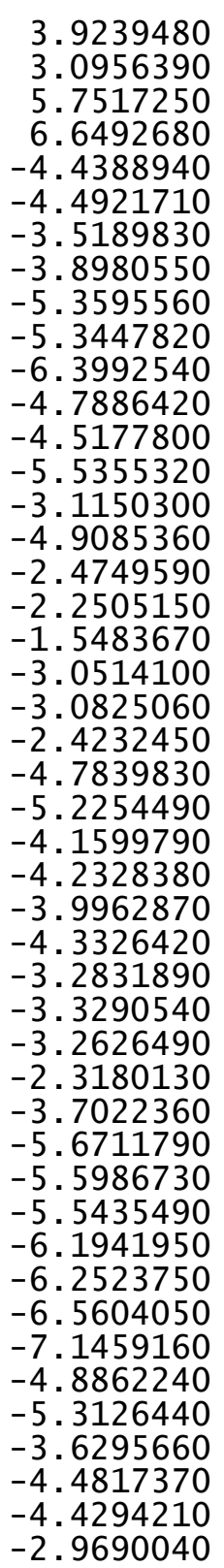
$-1.4812140$
$-0.3639430$
0.2165880
0.8359560
1. 0020380
0.6113520
$-1.2462440$
0.0055910
$-0.6716320$
$-1.0058160$
$-0.4393590$
$-1.7777660$
$-2.6731800$
$-2.0753140$
$-2.0161390$
1.4215810
$-0.8695500$
$-1.7659380$
$-0.5487050$
0.2601540
$-0.0603240$
1.1571450
2. 4278120
3.6733750
1.8881910
1.4431370
4. 2882740
5.1396030
4.6686470
3. 1994470
3.5344470
2.9883880
1.1291640
4.3981270
2. 2104770
2. 6020210
1.2898970
3.2591400
4.1531920
2. 8417140
2. 2108050
2. 9762470
$-2.3171430$
$-2.8465440$
$-4.3894380$
$-4.9284290$

4.6449500

3.5510110

0.0721580

0.0563150

3.5379610

4.9937350

3.4601270

2.7033370

5.1243960

6.1705880

4.8644590

4.1834800

4.7586290

3.4375460

2.7982940

5.6047530

4.5481050

5.1416190

4.0649830

5.4569390

6.5070340

5.4006380

1.6307980

0.9028000

$-0.6549920$

0.7886720

0.1799580

$-0.4271110$

0.9207430

$-0.7125730$

$-1.7561340$

$-0.3603890$

$-1.2938120$

1.5949400

$-1.1440510$

$-2.1687120$

$-1.1727080$

$-0.1884210$

$-0.7469140$

0.2911010

3.0044750

3.6540760

$-1.5042830$

$-2.6286400$

$-2.6661830$

$-2.6656370$ 


$\begin{array}{rrr}-1.9083890 & -3.8204730 & -2.4410380 \\ -2.3261680 & -2.8352290 & -1.3767540 \\ -4.1026080 & -2.4505780 & -3.5820850 \\ -4.9733650 & -4.7566730 & -3.5455910 \\ -2.7463740 & -5.4304130 & -3.6159360 \\ -1.7953430 & -3.2542370 & -3.3757600 \\ -5.5160250 & -2.4974050 & -2.5220970 \\ -4.9577270 & -4.7666320 & -1.7815130 \\ -2.8634060 & -5.6831790 & -1.8791700 \\ -0.9351100 & -4.2659700 & -2.2242350 \\ 1.5053760 & -4.6749710 & 0.2824270 \\ 1.7405230 & -6.1456860 & 0.5184920 \\ 0.9729890 & -6.9881910 & -0.5243810 \\ -0.5299540 & -6.5909000 & -0.6046650 \\ -0.8720480 & -5.3335670 & 0.2354860 \\ 0.1742230 & -4.2536680 & 0.0976070 \\ 1.3772480 & -6.4149330 & 1.5236180 \\ 1.0755660 & -8.0539470 & -0.2849940 \\ -1.1630570 & -7.4179170 & -0.2597710 \\ -0.8993610 & -5.6305400 & 1.2959610 \\ 2.8129280 & -6.3734460 & 0.4899970 \\ 1.4468330 & -6.8307040 & -1.5016490 \\ -0.7991420 & -6.4006540 & -1.6492050 \\ -1.8709700 & -4.9690910 & -0.0122480 \\ 2.7415100 & 2.6429440 & 1.1793710 \\ 1.6475920 & 3.4888170 & 1.6691640 \\ 2.4854290 & 1.9250340 & 0.3966420 \\ 0.6376020 & 3.1095200 & 1.5570550 \\ 2.5022350 & 2.0970180 & 2.5174150 \\ 0.5139340 & 3.8899770 & -1.9029090 \\ -0.1675020 & 2.8000000 & -4.0747530 \\ -0.4867510 & 2.0544930 & -2.9194970 \\ -0.0937110 & 2.6942770 & -1.7569840 \\ -0.2394420 & 2.2430600 & -0.8154240 \\ 0.6264220 & 4.2902890 & -3.6157550 \\ 1.0940650 & 4.9042330 & -0.6989070 \\ 1.8291580 & 1.2636700 & 2.4142020 \\ -1.0867590 & 1.0483110 & -5.4371000 \\ -1.4029390 & 0.3096260 & -4.2775540 \\ -1.1050970 & 0.8090850 & -3.0009150 \\ -0.4681580 & 2.2972300 & -5.3372180 \\ -1.3182940 & 0.6564670 & -6.4209760 \\ -1.3309810 & 0.2298290 & -2.1112490 \\ -0.2251770 & 2.8567220 & -6.2364790 \\ 4.4040360 & 4.3191340 & 2.1029070 \\ 3.2842620 & 5.3716390 & 2.1481690\end{array}$

1.9167450
4.1151500
5.3651780
3.5021370
1.8586560
4.1424770
4.8576160
4.4931510
3.2289570
1.0929480
-2.0030420
-2.2815850
-1.3585910
-2.9916610
-2.7256410

$\begin{array}{ll}C & 1.9167450 \\ \text { C } & 4.1151500\end{array}$

$\mathrm{H} \quad 5.3651780$

$\mathrm{H}$ 158

TS60 (S, S ) G[B97-D/def2-TZVP $]=-4356.206312$

$\begin{array}{lrrr}\text { TS60 (S , S }) \text { G }[\mathrm{B} 97-\mathrm{D} / \mathrm{def}-\mathrm{TZVP}] & =-4356.206312 \\ \mathrm{P} & -0.1596660 & -0.3453770 & -0.9700060 \\ \mathrm{O} & 0.3489020 & 1.0568430 & -1.0975940 \\ \mathrm{O} & -1.1577300 & -0.8536150 & -1.9831360 \\ \mathrm{O} & -0.7468660 & -0.4662160 & 0.5555660 \\ \mathrm{O} & 1.0206950 & -1.4611350 & -0.9883250 \\ \mathrm{C} & -1.0595840 & -1.7493230 & 1.0128890 \\ \mathrm{C} & 0.0021700 & -2.6079480 & 1.3512180 \\ \mathrm{C} & -2.4044030 & -2.1285480 & 1.1049750 \\ \mathrm{C} & -2.6723130 & -3.4492300 & 1.4920600 \\ \mathrm{H} & -3.7094340 & -3.7716670 & 1.5630940 \\ \mathrm{C} & 1.8882360 & -1.5608070 & 0.0973540 \\ \mathrm{C} & 3.2155120 & -1.1463140 & -0.0642500 \\ \mathrm{C} & 1.4045490 & -2.1063280 & 1.2982830 \\ \mathrm{C} & 4.0550380 & -1.2795650 & 1.0454890 \\ \mathrm{H} & 5.0944360 & -0.9715570 & 0.9448420 \\ \mathrm{C} & -3.5148490 & -1.1841720 & 0.8146150 \\ \mathrm{C} & 3.7295530 & -0.5930810 & -1.3438540 \\ \mathrm{C} & -3.6788830 & -0.0157250 & 1.5832580 \\ \mathrm{C} & -2.8278800 & 0.4180670 & 2.7546590 \\ \mathrm{C} & -4.7981420 & 2.0419210 & 2.2763440 \\ \mathrm{C} & -4.7560270 & 0.8578390 & 1.3429740 \\ \mathrm{C} & -2.2728630 & 1.8335530 & 2.4555560 \\ \mathrm{H} & -1.6776430 & 2.1761840 & 3.3117110 \\ \mathrm{H} & -1.6042780 & 1.7679610 & 1.5971520 \\ \mathrm{C} & -3.4527390 & 2.8125960 & 2.1714800 \\ \mathrm{H} & -3.4576610 & 3.6363020 & 2.8972030 \\ \mathrm{H} & -3.3526800 & 3.2587490 & 1.1751960 \\ \mathrm{H} & -5.6420160 & 2.7014960 & 2.0400120 \\ \mathrm{H} & -2.0065300 & -0.2780730 & 2.9401920\end{array}$
2.4593960
1.0277450
1.8879210
2. 9027100
3. 5225180
0.0314520
1.0380040
3. 0823140
1.1743000
2. 2813050
$-4.3061530$
$-5.5824850$
$-6.1660490$
$-6.1520770$
$-5.3711590$

$\begin{array}{rr}\mathrm{H} & 1.3772480 \\ \mathrm{H} & 1.0755660 \\ \mathrm{H} & -1.1630570 \\ \mathrm{H} & -0.8993610 \\ \mathrm{H} & 2.8129280 \\ \mathrm{H} & 1.4468330 \\ \mathrm{H} & -0.7991420 \\ \mathrm{H} & -1.8709700 \\ \mathrm{C} & 2.7415100 \\ \mathrm{C} & 1.6475920 \\ \mathrm{H} & 2.4854290 \\ \mathrm{H} & 0.6376020 \\ \mathrm{O} & 2.5022350 \\ \mathrm{C} & 0.5139340 \\ \mathrm{C} & -0.1675020 \\ \mathrm{C} & -0.4867510 \\ \mathrm{~N} & -0.0937110 \\ \mathrm{H} & -0.2394420 \\ \mathrm{~S} & 0.6264220 \\ \mathrm{~S} & 1.0940650 \\ \mathrm{H} & 1.8291580 \\ \mathrm{C} & -1.0867590 \\ \mathrm{C} & -1.4029390 \\ \mathrm{C} & -1.1050970 \\ \mathrm{C} & -0.4681580 \\ \mathrm{H} & -1.3182940 \\ \mathrm{H} & -1.3309810 \\ \mathrm{H} & -0.2251770 \\ \mathrm{C} & 4.4040360 \\ \mathrm{C} & 3.2842620 \\ & \\ & \end{array}$

5. 3716390

2. 1481690 


-4.9193390
-4.8934720
-5.8852730
-3.7423000
-3.1384310
-4.1225220
-4.4593240
-4.4832060
-6.4742220
-5.5435420
-5.8217310
-5.8683080
-5.8510100
-7.0193730
-7.6396820
-7.6593950
-7.3003530
-3.6320400
-5.6520280
-6.3153280
-5.2578220
-4.4823040
-4.5968750
-3.5195310
-5.6943060
-6.5368210
4.2641000
4.2193690
3.2646610
3.7364400
5.0321860
4.9536700
6.0912630
4.4711550
4.1340180
5.2454710
2.8699220
4.6305640
2.1767940
1.8875020
1.2899700
2.7436020
2.7065380
2.1519420
4.7886420
5.3039550

1.5000220

2. 3461140

0.9958640

0.5145600

0.8642140

$-0.4826700$

$-1.4595220$

$-2.6615740$

$-1.0399070$

$-0.5871600$

$-3.4133510$

$-4.2826010$

$-3.7857780$

$-2.4472280$

$-2.8194320$

$-2.3672240$

$-0.3305350$

$-3.3227130$

$-1.1800080$

$-1.5302720$

$-0.2009520$

$-2.1832050$

$-3.0642070$

$-1.7242760$

0.5687890

1.2388310

$-0.8472350$

$-1.8172110$

$-2.7962610$

$-1.3676990$

$-3.0806430$

$-3.8231890$

$-2.8163460$

$-3.6554240$

$-4.6916990$

$-3.6561700$

$-3.1898010$

$-1.3659590$

$-2.8549580$

$-3.9039960$

$-2.3088510$

$-2.2502770$

$-2.9869140$

$-1.3808170$

1.2187450

2.6315250

3.7284820
4.4279930
3.8508820
4.0084370
4.8562400
4.2601580
-0.1918090
-1.1088080
-1.5078410
-0.4117210
-0.8636820
-1.5328220
0.1676750
-1.1324250
-1.9585510
-0.2457950
-1.6416580
-0.9299060
-2.8179710
-3.6201790
-3.1125840
-2.5867410
-3.2322420
-2.8217240
0.3526230
0.1774750
-3.7148060
-4.8693200
-2.6597570
-2.5181490
-4.4709410
-5.2764390
-4.3658040
-3.1335310
-3.2690420
-2.3568850
-1.7197370
-5.7805870
-3.7673780
-3.9162160
-3.4347800
-5.0896290
-5.9034180
-5.4000120
-2.5980350
-2.4802310

\begin{abstract}
4.2875350
4.2580850

4.1281680

4. 5122010

3. 3818990

3. 4929970

3. 5069800

2. 4525640

3.8573270

5.7266090

5.7585290

5.7701770

6.3321300

6.3806750

6.7397900

7.2360860

4.7914690

5.2021300

3.6032940

2. 2608760

4. 5958160

3.9365700

2.7999710

1.7166890

4.6877850

5.2050000

5.2932320

3. 1967620

2. 3594140

0.9348070

1. 2244510

3.5162190

$-0.3035900$

0.7304130

0.4078110

$-1.0665950$

$-1.8973900$

$-1.6469560$

0.7164470

1.1032580

$-1.0966120$

$-1.5937690$

1.7382430

0.5985160

$-1.5462070$

$-2.9651400$
\end{abstract}

$-0.2715960$

$-1.3973890$

$-2.0363850$

$-1.8685280$

$-2.8375760$

$-0.7339960$

0.0716410

$-0.9080820$

0.6467260

$-3.4307980$

$-0.0255960$

0.7413600

0.3635590

$-1.3595530$

$-1.2433000$

$-1.6554550$

$-3.7573840$

$-4.6863880$

2.2787340

2.4227700

3.4253980

4.8076680

4.7880840

3.7975060

5.5674020

3.2810480

3.3682770

4.4879790

5.7869420

3.7299700

4.1949370

5.0681090

1.6818180

1.8889760

0.9885170

1.1349650

2.0703370

1.7513600

2.9407260

1. 2282280

1.5227390

3.1098560

1.6811040

$-0.0517300$

0.1486840

1.9927040 


$\begin{array}{rrr}-2.0415800 & 2.0116480 & -3.1401010 \\ -2.7880160 & 2.1604630 & -1.8871660 \\ -0.9612680 & 1.9029220 & -3.0328490 \\ -2.3435080 & 1.7562200 & -0.9842350 \\ -2.7304160 & 0.7234580 & -3.0323910 \\ -0.6529040 & 4.3281280 & 0.1990350 \\ 0.7512350 & 4.4497820 & 2.2928510 \\ 0.8592760 & 3.2341490 & 1.5845410 \\ 0.0788440 & 3.2199920 & 0.4411440 \\ 0.0995860 & 2.3904540 & -0.2083270 \\ -0.3820390 & 5.5068110 & 1.4794190 \\ -1.6776130 & 4.6209070 & -1.0963310 \\ -2.0460880 & 0.0255970 & -2.5686060 \\ 2.3769750 & 3.6520330 & 3.8710560 \\ 2.4756620 & 2.4376370 & 3.1587390 \\ 1.6963150 & 2.2088240 & 2.0159100 \\ 1.5084840 & 4.6590320 & 3.4422980 \\ 2.9757580 & 3.8190640 & 4.7591350 \\ 1.7736870 & 1.2759590 & 1.4671900 \\ 1.4383550 & 5.5897760 & 3.9987050 \\ -4.0175600 & 3.0118590 & -4.3782610 \\ -4.5268650 & 3.5877810 & -3.0471910 \\ -4.1941560 & 2.6663220 & -1.8611540 \\ -2.4912940 & 2.8167710 & -4.3417610 \\ -4.2795450 & 3.6837500 & -5.2044080 \\ -5.6114710 & 3.7456840 & -3.0853100 \\ -4.8490540 & 1.7846200 & -1.8486500 \\ -1.9890110 & 3.7922260 & -4.2782610 \\ -2.1323280 & 2.3189480 & -5.2513610 \\ -4.5020210 & 2.0458070 & -4.5724640 \\ -4.0600680 & 4.5679430 & -2.8779000 \\ -4.3566870 & 3.1648070 & -0.8996000 \\ 3.3242830 & 1.4264320 & 3.5069420 \\ 4.2685210 & 1.6793380 & 4.5522320 \\ 3.7618570 & 1.8320440 & 5.5163420 \\ 4.8820940 & 2.5612340 & 4.3182790 \\ 4.8973670 & 0.7887960 & 4.6044420 \\ -2.030 & \end{array}$

158

0.7887960

1.0039150

2. 0320300

1.0959500

1.0399400

$-0.5164560$

$-0.0554320$

$-1.2312070$
-3.2261070
-4.0474400
-5.0765660
1.0381470
2.3859200
-0.0186220
2.6656270
3.7055530
-3.7459300
3.4852530
-3.8066540
-3.3883020
-4.3585170
-4.3425080
-2.3280140
-2.0744800
-1.4175310
-2.9054290
-2.9160440
-2.2905220
-4.7743560
-2.9855420
-5.2074010
-5.1772600
-6.2526840
-4.6340470
-4.3351520
-5.3891940
-4.2277880
-4.1984780
-5.2442870
-4.7709540
-5.6500180
-5.6202880
-6.2370270
-6.2915700
-6.6211030
-7.1695500
-5.6815240
-3.7504210
-4.0352440
-4.3892770
-3.3086380
-3.3811220
-3.3463880
-2.3584330

$\begin{array}{lr}\mathrm{C} & -3.2261070 \\ \mathrm{C} & -4.0474400 \\ \mathrm{H} & -5.0765660 \\ \mathrm{C} & 1.0381470 \\ \mathrm{C} & 2.3859200 \\ \mathrm{C} & -0.0186220 \\ \mathrm{C} & 2.6656270 \\ \mathrm{H} & 3.7055530 \\ \mathrm{C} & -3.7459300 \\ \mathrm{C} & 3.4852530 \\ \mathrm{C} & -3.8066540 \\ \mathrm{C} & -3.3883020 \\ \mathrm{C} & -4.3585170 \\ \mathrm{C} & -4.3425080 \\ \mathrm{C} & -2.3280140 \\ \mathrm{H} & -2.0744800 \\ \mathrm{H} & -1.4175310 \\ \mathrm{C} & -2.9054290 \\ \mathrm{H} & -2.9160440 \\ \mathrm{H} & -2.2905220 \\ \mathrm{H} & -4.7743560 \\ \mathrm{H} & -2.9855420 \\ \mathrm{C} & -5.2074010 \\ \mathrm{H} & -5.1772600 \\ \mathrm{H} & -6.2526840 \\ \mathrm{C} & -4.6340470 \\ \mathrm{H} & -4.3351520 \\ \mathrm{H} & -5.3891940 \\ \mathrm{C} & -4.2277880 \\ \mathrm{C} & -4.1984780 \\ \mathrm{C} & -5.2442870 \\ \mathrm{C} & -4.7709540 \\ \mathrm{C} & -5.6500180 \\ \mathrm{H} & -5.6202880 \\ \mathrm{H} & -6.2370270 \\ \mathrm{C} & -6.2915700 \\ \mathrm{H} & -6.6211030 \\ \mathrm{H} & -7.1695500 \\ \mathrm{H} & -5.6815240 \\ \mathrm{H} & -3.7504210 \\ \mathrm{C} & -4.0352440 \\ \mathrm{H} & -4.3892770 \\ \mathrm{H} & -3.3086380 \\ \mathrm{C} & -3.3811220 \\ \mathrm{H} & -3.3463880 \\ & -2.3584330\end{array}$

-1.1350670
-1.2659420
-0.9174810
-1.8124240
-2.1807710
-2.6862600
-3.5039320
-3.8185740
-0.5299630
-1.2198180
-1.2713740
-2.7111510
-1.6402080
-0.7006980
-2.7755560
-3.8287890
-2.2647560
-2.1206150
-2.8393960
-1.2661120
-1.1508800
-3.1429520
-2.8836360
-3.6057200
-2.5835900
-3.5159940
-4.5583180
-3.5144470
0.7914920
1.7699160
2.7723180
1.3499750
2.2336600
3.0000580
1.3896290
2.8056860
3.8420550
2.2161130
3.1566790
1.3319920
3.6427380
4.6612500
3.7048330
3.0112480
3.7330130
2.6958690
0.0713670

$-1.0513940$

$-0.9797270$

$-0.9454940$

$-1.0338570$

$-1.2665710$

$-1.4037840$

$-1.4691520$

1. 3248240

$-0.7535560$

2. 5182690

2. 7080960

4.8679320

3. 6880560

3. 8419740

4. 0229670

3. 5170490

5. 1354820

5.9659010

5. 4420960

5.7571780

1.7882130

4.4829070

5. 3099930

4. 3421380

3. 1766590

3. 3502250

2. 3814130

1. 3310410

0.1790670

2. 3355000

2. 5040730

$-0.1174170$

$-0.9037710$

$-0.4997160$

1.1865250

1.0337780

1. 4766140

3.2650690

$-0.7152550$

1.8961170

1.6866610

2.7146220

0.6309480

$-0.1951150$

0.8484060 


$\begin{array}{lrrr}\text { C } & -4.8274530 & 0.6074910 & \\ \text { H } & -5.2481900 & 1.0433610 & 4.5829760 \\ \text { C } & 4.6973640 & 0.8346310 & -1.3010220 \\ \text { C } & 4.7299840 & 2.0035070 & -2.2538550 \\ \text { C } & 2.7952930 & 0.3366420 & -2.7284810 \\ \text { C } & 3.6402480 & -0.0628630 & -1.5408420 \\ \text { C } & 4.8773880 & 1.4408970 & -3.6955980 \\ \text { H } & 4.8452910 & 2.2751650 & -4.4089130 \\ \text { H } & 5.8533690 & 0.9520020 & -3.7984750 \\ \mathrm{C} & 3.7212640 & 0.4306090 & -3.9736760 \\ \text { H } & 3.1207290 & 0.7569560 & -4.8330990 \\ \text { H } & 4.1219480 & -0.5631610 & -4.2065320 \\ \text { H } & 1.9881220 & -0.3767380 & -2.9112660 \\ \text { H } & 5.5592180 & 2.6819690 & -2.0192600 \\ \mathrm{C} & 2.2119520 & 1.7456140 & -2.4559160 \\ \mathrm{H} & 1.6161730 & 2.0643200 & -3.3204870 \\ \mathrm{H} & 1.5392240 & 1.6788710 & -1.6014120 \\ \mathrm{C} & 3.3698050 & 2.7511400 & -2.1767350 \\ \mathrm{H} & 3.3670520 & 3.5629560 & -2.9157150 \\ \mathrm{H} & 3.2523120 & 3.2110200 & -1.1885500 \\ \mathrm{C} & 5.4868620 & -0.5663360 & 0.4881920 \\ \mathrm{C} & 6.4150390 & -0.9878390 & 1.5988770 \\ \mathrm{C} & 4.4568980 & -2.6500110 & 1.2032880 \\ \mathrm{C} & 4.4219320 & -1.4621850 & 0.2682210 \\ \mathrm{C} & 5.5850570 & -1.1281500 & 2.9040990 \\ \mathrm{H} & 6.2477610 & -1.4622910 & 3.7136840 \\ \mathrm{H} & 5.1750870 & -0.1524450 & 3.1874350 \\ \mathrm{C} & 4.4314620 & -2.1500700 & 2.6738080 \\ \mathrm{H} & 4.5496730 & -3.0199270 & 3.3336000 \\ \mathrm{H} & 3.4597940 & -1.7006100 & 2.8898560 \\ \mathrm{H} & 3.6194810 & -3.3288460 & 1.0248860 \\ \mathrm{H} & 7.2271020 & -0.2620320 & 1.7310420 \\ \mathrm{C} & 5.8109960 & -3.3818990 & 0.9842290 \\ \mathrm{H} & 5.8670820 & -4.2363930 & 1.6714170 \\ \mathrm{H} & 5.8563220 & -3.7748720 & -0.0389450 \\ \mathrm{C} & 6.9888560 & -2.3892680 & 1.2438580 \\ \mathrm{H} & 7.6137080 & -2.7384450 & 2.0765980 \\ \mathrm{H} & 7.6298940 & -2.3082360 & 0.3580440 \\ \mathrm{C} & 5.6257470 & 0.5807630 & -0.2917730 \\ \mathrm{H} & 6.4534310 & 1.2690320 & -0.1163860 \\ \mathrm{C} & 0.2998490 & -4.0195710 & -1.5852810 \\ \mathrm{C} & -0.7202410 & -5.1188180 & -1.7482460 \\ \mathrm{C} & -0.3713710 & -6.2973140 & -0.8061150 \\ \mathrm{C} & 1.1010540 & -6.7796430 & -0.9723400 \\ \mathrm{C} & 1.9104260 & -5.8778230 & -1.9404900 \\ \mathrm{C} & 1.6479550 & -4.4222630 & -1.6512940 \\ & & & \end{array}$
$-0.7049470$
$-1.0685530$
1. 1266150
1. 5950300
$-1.7316940$
$-0.5365890$
1. 5996110
2.9815030
$-2.2632730$
$-1.7272370$
$-2.5278050$
$-4.0159450$
$-4.5466390$
$-3.5904060$
$-1.7613710$
$-2.1585880$
$-4.1367670$
$-5.5052190$
$-0.6689660$
$-2.3784050$
$-4.6063380$
$-4.7600140$
1.9486970
2.6736820
0.8700180
2.2251070
2.6679000
0.5629490
$-0.6375360$
$-0.7820640$
$-0.1104390$
$-0.1552310$
0.3710810
1. 4612160
1.9969750
$-2.0091720$
$-2.1456450$
$-1.5234350$
$-1.2516020$
$-2.4903580$
$-1.6207110$
$-1.1494580$
3.9162890
4.3968920
4.0665640
2. 3943150

\begin{tabular}{rr}
-5.4862700 & -2.7861260 \\
-7.1232800 & -0.9943390 \\
-7.8118960 & -1.3434930 \\
-6.1064130 & -2.9705480 \\
-4.7549170 & -1.5459990 \\
-5.9635860 & 0.2255860 \\
-6.7754770 & 0.0045350 \\
-6.1020060 & -1.8715960 \\
-2.2477940 & -2.3672970 \\
-2.7391490 & -3.7003680 \\
-2.2026670 & -4.8929050 \\
-2.5096870 & -4.6906500 \\
-1.7880810 & -3.4434770 \\
-1.7916180 & -2.2626010 \\
-3.8368480 & -3.7312460 \\
-2.6610810 & -5.8194900 \\
-3.5965060 & -4.5695930 \\
-2.2254950 & -3.1314300 \\
-2.4656510 & -3.7884770 \\
-1.1183340 & -4.9721900 \\
-2.2155130 & -5.5688040 \\
-0.7386280 & -3.6910760 \\
2.0263170 & 3.1958200 \\
2.2027080 & 1.9342520 \\
1.8925630 & 3.1006450 \\
1.7926120 & 1.0357280 \\
0.7565470 & 3.0730960 \\
4.3140930 & -0.1751320 \\
4.3922640 & -2.3921650 \\
3.1754730 & -1.6919760 \\
3.1844640 & -0.4811550 \\
2.3569080 & 0.1699950 \\
5.4876750 & -1.4736660 \\
4.6319000 & 1.2049390 \\
0.0449990 & 2.6128340 \\
3.5193860 & -4.1595950 \\
2.3035920 & -3.4562730 \\
2.1176600 & -2.2127830 \\
4.5661090 & -3.6290540 \\
3.6572820 & -5.1208570 \\
1.1787930 & -1.6769290 \\
5.4970660 & -4.1801230 \\
3.0646690 & 4.4145350 \\
3.6599030 & 3.0812590 \\
2.7425450 & 1.8912020 \\
2.8361460 & 4.3958910 \\
& \\
\hline
\end{tabular}




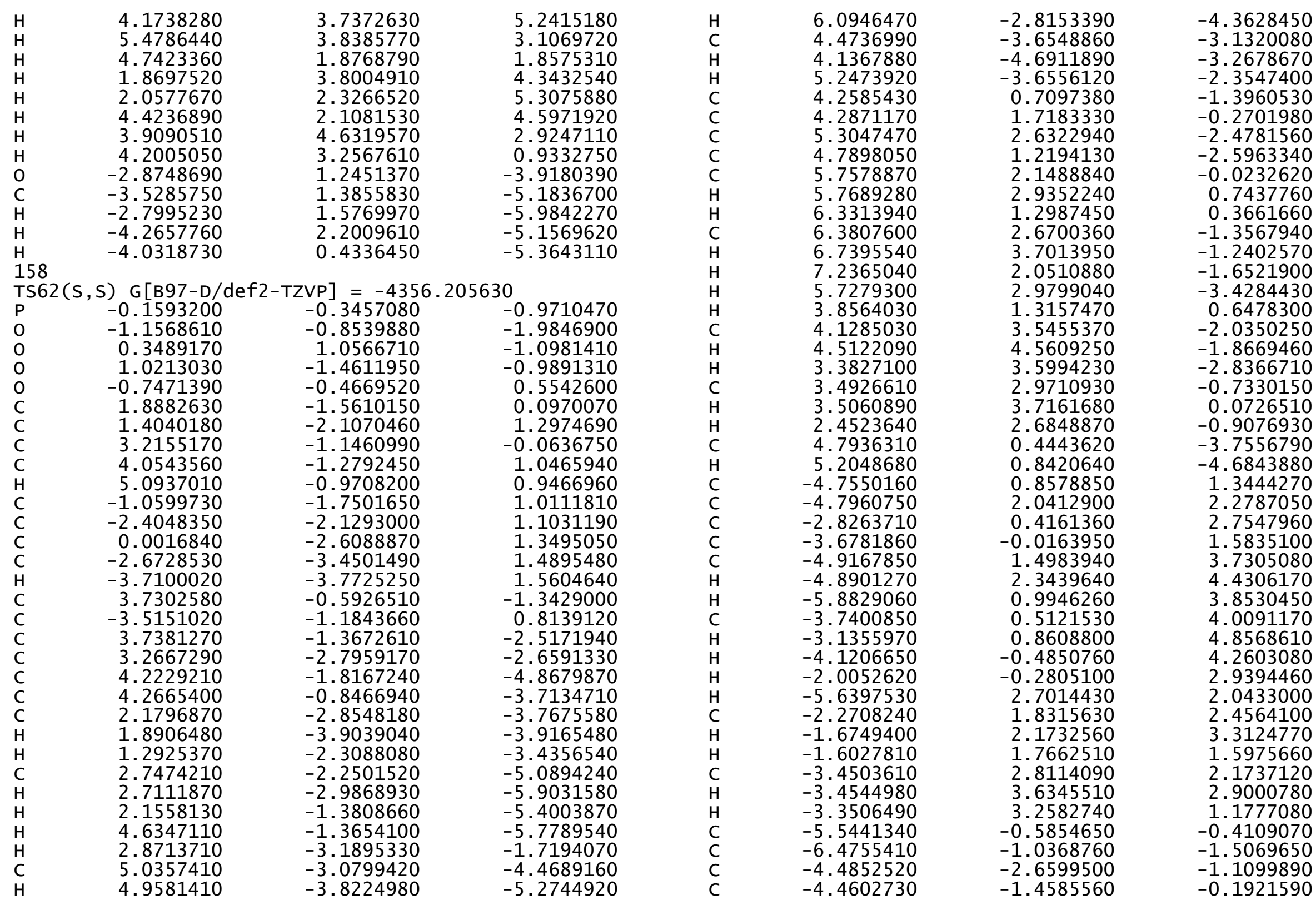




\begin{tabular}{|c|c|c|}
\hline $\begin{array}{l}-5.6539860 \\
-6.3178180 \\
-5.2593710 \\
-4.4846940 \\
-4.5999630 \\
-3.5217940 \\
-3.6343760 \\
-7.3013310 \\
-5.8240900 \\
-5.8714340 \\
-5.8531360 \\
-7.0213130 \\
-7.6421940 \\
-7.6608940 \\
-5.6939130 \\
-6.5361440 \\
-0.3041640 \\
0.7298140 \\
0.4075090 \\
-1.0668900 \\
-1.8980450 \\
-1.6475490 \\
0.7155340 \\
1.1029670 \\
-1.0969030 \\
-1.5947960 \\
1.7376910 \\
0.5983990 \\
-1.5462310 \\
-2.9657600 \\
2.2596380 \\
3.6019710 \\
1.7146470 \\
2.7974630 \\
3.9334200 \\
4.5937030 \\
2.3562110 \\
1.2213290 \\
0.9334420 \\
3.5122840 \\
4.6841800 \\
5.2907600 \\
5.2033970 \\
3.1951040 \\
-2.0411670 \\
-2.7872390\end{array}$ & $\begin{array}{l}-1.1764920 \\
-1.5258170 \\
-0.1974440 \\
-2.1804790 \\
-3.0609210 \\
-1.7218380 \\
-3.3216970 \\
-0.3269400 \\
-3.4111560 \\
-4.2798670 \\
-3.7843640 \\
-2.4441690 \\
-2.8154170 \\
-2.3644590 \\
0.5700320 \\
1.2406410 \\
-3.9430110 \\
-5.0233520 \\
-6.2408160 \\
-6.7244030 \\
-5.8091220 \\
-4.3572550 \\
-5.3508820 \\
-7.0548370 \\
-7.7504350 \\
-6.0101760 \\
-4.6528780 \\
-5.9494820 \\
-6.7382730 \\
-6.0461920 \\
-2.1543760 \\
-1.7549000 \\
-2.5102440 \\
-2.9513760 \\
-1.9239830 \\
-1.8515760 \\
-3.0663190 \\
-1.6076230 \\
-3.2686990 \\
-0.9434450 \\
-2.1766690 \\
-1.0049580 \\
-2.7581960 \\
-3.9329000 \\
2.0115700 \\
2.1599190\end{array}$ & $\begin{array}{r}-2.8175520 \\
-3.6197270 \\
-3.1116690 \\
-2.5875570 \\
-3.2336950 \\
-2.8225630 \\
-0.9319180 \\
-1.6399190 \\
-0.8648510 \\
-1.5346360 \\
0.1662310 \\
-1.1323490 \\
-1.9584750 \\
-0.2453760 \\
0.3543140 \\
0.1799790 \\
1.6795580 \\
1.8867030 \\
0.9857650 \\
1.1317570 \\
2.0670440 \\
1.7485740 \\
2.9383430 \\
1.2253380 \\
1.5193880 \\
3.1066240 \\
1.6792620 \\
-0.0543420 \\
0.1453440 \\
1.9889820 \\
2.4224980 \\
2.2794540 \\
3.7967680 \\
4.7881880 \\
4.8085870 \\
3.4268300 \\
5.7867110 \\
4.1939150 \\
3.7285100 \\
5.0685700 \\
5.5689460 \\
3.3702000 \\
3.2829780 \\
4.4884250 \\
-3.1413160 \\
-1.8881090\end{array}$ \\
\hline
\end{tabular}

$\begin{array}{lr}\mathrm{H} & -0.9607820 \\ \mathrm{H} & -2.3422550 \\ \mathrm{O} & -2.7295900 \\ \mathrm{C} & -0.6530920 \\ \mathrm{C} & 0.7503540 \\ \mathrm{C} & 0.8584810 \\ \mathrm{~N} & 0.0784260 \\ \mathrm{H} & 0.0993510 \\ \mathrm{~S} & -0.3824550 \\ \mathrm{~S} & -1.6773590 \\ \mathrm{H} & -2.0450520 \\ \mathrm{C} & 2.3752510 \\ \mathrm{C} & 2.4740300 \\ \mathrm{C} & 1.6951620 \\ \mathrm{C} & 1.5071320 \\ \mathrm{H} & 2.9736620 \\ \mathrm{H} & 1.7725810 \\ \mathrm{H} & 1.4369110 \\ \mathrm{C} & -4.0179350 \\ \mathrm{C} & -4.5270230 \\ \mathrm{C} & -4.1935230 \\ \mathrm{C} & -2.4915730 \\ \mathrm{H} & -4.2804940 \\ \mathrm{H} & -5.6117090 \\ \mathrm{H} & -4.8481580 \\ \mathrm{H} & -1.9896440 \\ \mathrm{H} & -2.1327370 \\ \mathrm{H} & -4.5020540 \\ \mathrm{H} & -4.0605810 \\ \mathrm{H} & -4.3557720 \\ \mathrm{O} & 3.3222770 \\ \mathrm{C} & 4.2660150 \\ \mathrm{H} & 3.7588700 \\ \mathrm{H} & 4.8799120 \\ \mathrm{H} & 4.8946240 \\ \mathrm{H} & \end{array}$
1. 9031660
1.7556540
0.7231800
4.3279950
4.4498250
3. 2340500
3. 2197920
2. 3902250
5.5068880
4.6206760
0.0253140
3.6520770
2.4375320
2.2086610
4.6591520
3. 8191610
1. 2756610
5. 5900070
3. 0112320
3.5867770
2. 6653240
2. 8167570
3.6831140
3.7442280
1.7834270
3.7923940
2. 3192340
2. 0449980
4. 5671150
3.1636760
1.4262230
1.6791580
1.8322080
2. 5608570

$-3.0344420$

$-0.9854200$

$-3.0336250$

0.1982130

2. 2924690

1. 5844190

0.4407620

$-0.2086490$

1.4784450

$-1.0975090$

$-2.5700450$

3. 8715290

3.1594780

2. 0163300

3.4422070

4.7598460

1.4678400

3.9984170

$-4.3786050$

$-3.0472840$

$-1.8614620$

$-4.3426780$

$-5.2045780$

$-3.0850220$

$-1.8487300$

$-4.2791750$

$-5.2524940$

$-4.5727550$

$-2.8780410$

$-0.8997900$

3. 5082960

4.5540360

5. 5178370

4. 3201960

158

TS63 (S , S ) G [B97-D/def2-TZVP $]=-4356.205478$

\begin{tabular}{lrrr} 
TS63 $(\mathrm{S}, \mathrm{S})$ & \multicolumn{4}{c}{$\mathrm{G}[\mathrm{B} 97-\mathrm{D} / \mathrm{def}-\mathrm{TZVP}]=-4356.205478$} \\
$\mathrm{P}$ & -0.1418890 & -0.3567570 & -0.9741720 \\
$\mathrm{O}$ & 0.3700060 & 1.0465310 & -1.0712810 \\
$\mathrm{O}$ & -1.1487180 & -0.8374870 & -1.9913600 \\
$\mathrm{O}$ & -0.7150010 & -0.5128480 & 0.5528190 \\
$\mathrm{O}$ & 1.0342330 & -1.4758910 & -1.0232830 \\
$\mathrm{C}$ & -1.0300600 & -1.8030460 & 0.9856950 \\
$\mathrm{C}$ & 0.0293790 & -2.6760410 & 1.3010870 \\
$\mathrm{C}$ & -2.3767390 & -2.1737040 & 1.0779270 \\
$\mathrm{C}$ & -2.6534990 & -3.4949250 & 1.4569840
\end{tabular}




$\begin{array}{lrrr}\mathrm{H} & -3.6927380 & -3.8112100 & 1.5252090 \\ \mathrm{C} & 1.9041270 & -1.5894820 & 0.0597480 \\ \mathrm{C} & 3.2208540 & -1.1390480 & -0.0890090 \\ \mathrm{C} & 1.4320620 & -2.1772720 & 1.2449510 \\ \mathrm{C} & 4.0590460 & -1.2691880 & 1.0212360 \\ \mathrm{H} & 5.0883730 & -0.9252420 & 0.9325010 \\ \mathrm{C} & -3.4782670 & -1.2219250 & 0.7763350 \\ \mathrm{C} & 3.7210030 & -0.5402580 & -1.3533950 \\ \mathrm{C} & -3.6474780 & -0.0536430 & 1.5430460 \\ \mathrm{C} & -2.8186460 & 0.3678240 & 2.7342970 \\ \mathrm{C} & -4.7535630 & 2.0194690 & 2.2088400 \\ \mathrm{C} & -4.7053140 & 0.8352380 & 1.2758460 \\ \mathrm{C} & -2.2369560 & 1.7749820 & 2.4482230 \\ \mathrm{H} & -1.6551570 & 2.1094070 & 3.3164490 \\ \mathrm{H} & -1.5513740 & 1.6982950 & 1.6046310 \\ \mathrm{C} & -3.3950570 & 2.7709830 & 2.1378640 \\ \mathrm{H} & -3.4055750 & 3.5942430 & 2.8640160 \\ \mathrm{H} & -3.2649060 & 3.2166040 & 1.1447800 \\ \mathrm{H} & -5.5821010 & 2.6911280 & 1.9530300 \\ \mathrm{H} & -2.0112380 & -0.3400630 & 2.9373740 \\ \mathrm{C} & -4.9169990 & 1.4789390 & 3.6574730 \\ \mathrm{H} & -4.8967700 & 2.3243720 & 4.3579970 \\ \mathrm{H} & -5.8924620 & 0.9881040 & 3.7558300 \\ \mathrm{C} & -3.7610110 & 0.4771710 & 3.9660910 \\ \mathrm{H} & -3.1730910 & 0.8187210 & 4.8282930 \\ \mathrm{H} & -4.1612790 & -0.5144700 & 4.2085120 \\ \mathrm{C} & -4.4009380 & -1.4845030 & -0.2533780 \\ \mathrm{C} & -4.4164640 & -2.6847410 & -1.1730540 \\ \mathrm{C} & -6.3824530 & -1.0432710 & -1.6123540 \\ \mathrm{C} & -5.4682360 & -0.5982820 & -0.4992940 \\ \mathrm{C} & -5.7671660 & -3.4242440 & -0.9592240 \\ \mathrm{H} & -5.8100970 & -4.2869140 & -1.6371160 \\ \mathrm{H} & -5.8198240 & -3.8059110 & 0.0678760 \\ \mathrm{C} & -6.9496060 & -2.4434180 & -1.2417860 \\ \mathrm{H} & -7.5658030 & -2.8085380 & -2.0741250 \\ \mathrm{H} & -7.5978710 & -2.3541460 & -0.3620120 \\ \mathrm{H} & -7.1985570 & -0.3256580 & -1.7628930 \\ \mathrm{H} & -3.5759480 & -3.3545610 & -0.9769430 \\ \mathrm{C} & -5.5391600 & -1.1968320 & -2.9075250 \\ \mathrm{H} & -6.1914560 & -1.5511790 & -3.7170250 \\ \mathrm{H} & -5.1365730 & -0.2218580 & -3.2027920 \\ \mathrm{C} & -4.3780060 & -2.2035360 & -2.6497280 \\ \mathrm{H} & -4.4794360 & -3.0832000 & -3.2992840 \\ \mathrm{H} & -3.4077590 & -1.7473870 & -2.8591820 \\ \mathrm{C} & -5.6213410 & 0.5604310 & 0.2606610 \\ \mathrm{H} & -6.4509450 & 1.2413560 & 0.0664100\end{array}$

4.2700590
4.2593020
3.3302570
3.7561910
5.1126000
5.0636770
6.1613470
4.5647310
4.2601630
5.3358880
2.9451900
4.6583080
2.2474040
1.9882510
1.3445950
2.7998420
2.7914810
2.1809550
4.7280710
5.2086330
4.1990340
4.2059860
4.0102950
4.3720370
3.2697760
3.3761090
3.3556730
2.3496890
3.7657790
5.6302350
5.6563920
5.6415940
6.2483710
6.2765170
6.6137490
7.1462570
4.7589740
5.1630080
2.2915970
1.7741070
2.5976640
4.0807200
4.5933060
3.6187310
2.2420170
1.8003790

-0.7196270
-1.6622030
-2.7246850
-1.2855570
-2.9068060
-3.6313090
-2.6091360
-3.5339060
-4.5761040
-3.5312420
-3.1529280
-1.1763460
-2.7897720
-3.8429480
-2.2764880
-2.1391740
-2.8596840
-1.2837080
1.3332190
2.7546920
1.7625270
0.7797980
3.6304710
4.6478670
3.6940940
3.0036360
3.7280260
2.6889250
1.3288640
3.1347830
2.2245700
2.9953810
1.3816050
2.7881590
3.8235240
2.1931100
0.5870620
1.0188640
-2.2400580
-2.7261750
-2.1949850
-2.5086710
-1.7873250
-1.7894510
-2.6525000
-3.8239800
$-3.7275320$ $-4.9050210$ $-2.7231460$ $-2.5456240$ $-4.5340390$ $-5.3581280$ $-4.4157040$ $-3.2141470$ $-3.3782860$ $-2.4344360$ $-1.7940960$ $-5.8038330$ $-3.8352620$ $-4.0085400$ $-3.4933030$ $-5.1416890$ $-5.9706070$ $-5.4375110$ $-2.5582660$ $-2.4028250$ $-0.2244740$ $-1.3733050$ $-1.9441490$ $-1.7426490$ $-2.7499920$ $-0.6666100$

0.1576960 $-0.8665370$ 0.6792050 $-3.3413310$ 0.0446850 0.8271320 0.4217020 $-1.2731620$ $-1.1308040$ $-1.5766400$ $-3.7358740$ $-4.6524420$

2. 3679640

3. 7102890

4.8893530

4. 6616010

3.4068590

2. 2411140

5.8216570

3. 7414410 


$\begin{array}{lr}\mathrm{H} & 0.7195390 \\ \mathrm{H} & 4.1947810 \\ \mathrm{H} & 4.6871410 \\ \mathrm{H} & 5.5463080 \\ \mathrm{H} & 4.8118460 \\ \mathrm{H} & 2.4549420 \\ \mathrm{C} & -0.2864350 \\ \mathrm{C} & 0.7343770 \\ \mathrm{C} & 0.3757900 \\ \mathrm{C} & -1.0952890 \\ \mathrm{C} & -1.8942290 \\ \mathrm{C} & -1.6339940 \\ \mathrm{H} & 0.7287180 \\ \mathrm{H} & 1.0742800 \\ \mathrm{H} & -1.1181400 \\ \mathrm{H} & -1.5680850 \\ \mathrm{H} & 1.7441550 \\ \mathrm{H} & 0.5314130 \\ \mathrm{H} & -1.6033900 \\ \mathrm{H} & -2.9660550 \\ \mathrm{C} & -1.9535100 \\ \mathrm{C} & -2.6846950 \\ \mathrm{H} & -0.8768690 \\ \mathrm{H} & -2.2462230 \\ \mathrm{O} & -2.6856750 \\ \mathrm{C} & -0.5516550 \\ \mathrm{C} & 0.6649030 \\ \mathrm{C} & 0.8042530 \\ \mathrm{~N} & 0.1231920 \\ \mathrm{H} & 0.1611020 \\ \mathrm{~S} & -0.3517330 \\ \mathrm{~S} & -1.4572500 \\ \mathrm{H} & -2.0224940 \\ \mathrm{C} & 2.0564250 \\ \mathrm{C} & 2.1884700 \\ \mathrm{C} & 1.5532110 \\ \mathrm{C} & 1.2911440 \\ \mathrm{H} & 2.5474720 \\ \mathrm{H} & 1.6481390 \\ \mathrm{H} & 1.1931950 \\ \mathrm{C} & -3.9015370 \\ \mathrm{C} & -4.3840210 \\ \mathrm{C} & -4.0725000 \\ \mathrm{C} & -2.3825830 \\ \mathrm{H} & -4.1452520 \\ \mathrm{H} & -5.4634190 \\ & \end{array}$

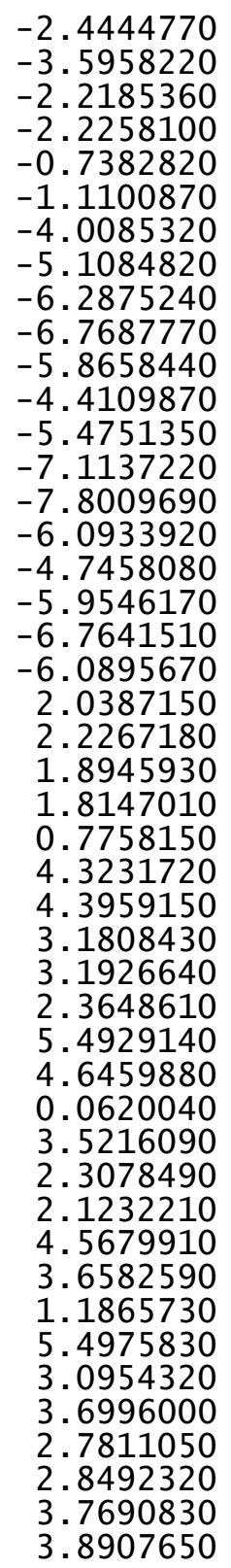

3.8156760
4.5368010
5.5301220
3.0796420
3.6518300
4.9721580
1.6261640
1.7804840
0.8426180
1.0231790
1.9989700
1.7045410
2.8187440
1.0248570
1.3946910
3.0258700
1.5681410
-0.1908370
0.0512310
1.9413090
-3.1641360
-1.9078350
-3.0615820
-1.0052120
-3.0415090
0.2012470
2.4097020
1.7053540
0.4998750
-0.1517110
1.5019460
-1.1731420
-2.5761940
4.1608250
3.4530410
2.2159020
3.6407530
5.1172560
1.6757540
4.1948750
-4.3995150
-3.0710570
-1.8767980
-4.3696290
-5.2298240
-3.1042680

\begin{tabular}{lr}
$\mathrm{H}$ & -4.7575500 \\
$\mathrm{H}$ & -1.8475930 \\
$\mathrm{H}$ & -2.0454210 \\
$\mathrm{H}$ & -4.4192510 \\
$\mathrm{H}$ & -3.8860670 \\
$\mathrm{H}$ & -4.2093900 \\
$\mathrm{O}$ & 2.9263200 \\
$\mathrm{C}$ & 3.5984580 \\
$\mathrm{H}$ & 2.8807630 \\
$\mathrm{H}$ & 4.3316600 \\
$\mathrm{H}$ & 4.1086190 \\
\hline
\end{tabular}
1.9227230
3.8077990
2.3333510
1.2506580
1. 3922530
1.5793040
2. 2108490

$2.1446770 \quad-4.5835210$

$4.6663410 \quad-2.9134510$

$3.2985590 \quad-0.9210850$

3.9038920

5.1597260

5.9714980

5.1230500

158

5.3313800

TS64 (S, S) G[B97-D/def2-TZVP $]=-4356.205478$

$\begin{array}{lrcr}\text { TS64 (S , S }) \text { G }[\mathrm{B} 97-\mathrm{D} / \mathrm{def}-\mathrm{TZVP}]=-4356.205478 \\ \mathrm{P} & -0.1418850 & -0.3567590 & -0.9741780 \\ \mathrm{O} & -1.1487140 & -0.8374830 & -1.9913680 \\ \mathrm{O} & 0.3700150 & 1.0465270 & -1.0712820 \\ \mathrm{O} & 1.0342320 & -1.4758970 & -1.0232920 \\ \mathrm{O} & -0.7149990 & -0.5128510 & 0.5528130 \\ \mathrm{C} & 1.9041260 & -1.5894950 & 0.0597370 \\ \mathrm{C} & 1.4320590 & -2.1772890 & 1.2449380 \\ \mathrm{C} & 3.2208540 & -1.1390640 & -0.0890170 \\ \mathrm{C} & 4.0590460 & -1.2692150 & 1.0212270 \\ \mathrm{H} & 5.0883750 & -0.9252720 & 0.9324940 \\ \mathrm{C} & -1.0300630 & -1.8030500 & 0.9856870 \\ \mathrm{C} & -2.3767430 & -2.1737020 & 1.0779230 \\ \mathrm{C} & 0.0293730 & -2.6760510 & 1.3010720 \\ \mathrm{C} & -2.6535090 & -3.4949220 & 1.4569750 \\ \mathrm{H} & -3.6927490 & -3.8112030 & 1.5252020 \\ \mathrm{C} & 3.7210030 & -0.5402610 & -1.3533960 \\ \mathrm{C} & -3.4782670 & -1.2219160 & 0.7763380 \\ \mathrm{C} & 3.7561820 & -1.2855410 & -2.5456370 \\ \mathrm{C} & 3.3302410 & -2.7246650 & -2.7231810 \\ \mathrm{C} & 4.2592810 & -1.6621510 & -4.9050430 \\ \mathrm{C} & 4.2700490 & -0.7195950 & -3.7275390 \\ \mathrm{C} & 2.2473830 & -2.7897290 & -3.8352930 \\ \mathrm{H} & 1.9882260 & -3.8429020 & -4.0085870 \\ \mathrm{H} & 1.3445760 & -2.2764500 & -3.4933210 \\ \mathrm{C} & 2.7998160 & -2.1391100 & -5.1417110 \\ \mathrm{H} & 2.7914450 & -2.8596050 & -5.9706420 \\ \mathrm{H} & 2.1809320 & -1.2836350 & -5.4375120 \\ \mathrm{H} & 4.6582840 & -1.1762820 & -5.8038490 \\ \mathrm{H} & 2.9451770 & -3.1529200 & -1.7941360 \\ \mathrm{C} & 5.1125730 & -2.9067660 & -4.5340870 \\ \mathrm{H} & 5.0636380 & -3.6312560 & -5.3581870 \\ \mathrm{H} & 6.1613230 & -2.6091060 & -4.4157560 \\ \mathrm{C} & 4.5647090 & -3.5338830 & -3.2142000\end{array}$




\begin{tabular}{|c|c|c|c|}
\hline $\begin{array}{l}\mathrm{H} \\
\mathrm{H} \\
\mathrm{C} \\
\mathrm{C} \\
\mathrm{C} \\
\mathrm{C} \\
\mathrm{C} \\
\mathrm{H} \\
\mathrm{H} \\
\mathrm{C} \\
\mathrm{H} \\
\mathrm{H} \\
\mathrm{H} \\
\mathrm{H} \\
\mathrm{C} \\
\mathrm{H} \\
\mathrm{H} \\
\mathrm{C} \\
\mathrm{H} \\
\mathrm{H} \\
\mathrm{C} \\
\mathrm{H} \\
\mathrm{C} \\
\mathrm{C} \\
\mathrm{C} \\
\mathrm{C} \\
\mathrm{C} \\
\mathrm{H} \\
\mathrm{H} \\
\mathrm{C} \\
\mathrm{H} \\
\mathrm{H} \\
\mathrm{H} \\
\mathrm{H} \\
\mathrm{C} \\
\mathrm{H} \\
\mathrm{H} \\
\mathrm{C} \\
\mathrm{H} \\
\mathrm{H} \\
\mathrm{C} \\
\mathrm{C} \\
\mathrm{C} \\
\mathrm{C} \\
\mathrm{C} \\
\mathrm{H}\end{array}$ & $\begin{array}{r}4.2601380 \\
5.3358720 \\
4.2059930 \\
4.1990480 \\
5.2086440 \\
4.7280760 \\
5.6564080 \\
5.6416130 \\
6.2483850 \\
6.2765330 \\
6.6137730 \\
7.1462670 \\
5.6302450 \\
3.7657940 \\
4.0103120 \\
4.3720600 \\
3.2697920 \\
3.3761260 \\
3.3556900 \\
2.3497050 \\
4.7589700 \\
5.1630020 \\
-4.7053040 \\
-4.7535470 \\
-2.8186420 \\
-3.6474730 \\
-4.9169890 \\
-4.8967530 \\
-5.8924560 \\
-3.7610090 \\
-3.1730900 \\
-4.1612850 \\
-2.0112370 \\
-5.5820810 \\
-2.2369420 \\
-1.6551500 \\
-1.5513540 \\
-3.3950370 \\
-3.4055520 \\
-3.2648810 \\
-5.4682340 \\
-6.3824540 \\
-4.4164740 \\
-4.4009400 \\
-5.5391620 \\
-6.1914620\end{array}$ & $\begin{array}{r}-4.5760780 \\
-3.5312310 \\
0.7797940 \\
1.7625070 \\
2.7546990 \\
1.3332300 \\
2.2245420 \\
2.9953440 \\
1.3815710 \\
2.7881440 \\
3.8235050 \\
2.1930920 \\
3.1348010 \\
1.3288330 \\
3.6304770 \\
4.6478670 \\
3.6941180 \\
3.0036240 \\
3.7280010 \\
2.6889160 \\
0.5870910 \\
1.0189050 \\
0.8352500 \\
2.0194740 \\
0.3678140 \\
-0.0536380 \\
1.4789330 \\
2.3243610 \\
0.9881060 \\
0.4771540 \\
0.8186900 \\
-0.5144870 \\
-0.3400810 \\
2.6911400 \\
1.7749710 \\
2.1093860 \\
1.6982880 \\
2.7709800 \\
3.5942380 \\
3.2166040 \\
-0.5982540 \\
-1.0432310 \\
-2.6847150 \\
-1.4844820 \\
-1.1967900 \\
-1.5511270\end{array}$ & $\begin{array}{r}-3.3783510 \\
-2.4344940 \\
-1.3732880 \\
-0.2244440 \\
-2.4027840 \\
-2.5582430 \\
0.0447180 \\
0.8271730 \\
0.4217250 \\
-1.2731240 \\
-1.1307540 \\
-1.5766130 \\
-3.3412850 \\
0.6792310 \\
-1.9440910 \\
-1.7425740 \\
-2.7499310 \\
-0.6665610 \\
0.1577560 \\
-0.8664930 \\
-3.7358630 \\
-4.6524260 \\
1.2758620 \\
2.2088660 \\
2.7343140 \\
1.5430570 \\
3.6574950 \\
4.3580250 \\
3.7558470 \\
3.9661060 \\
4.8283130 \\
4.2085140 \\
2.9373860 \\
1.9530600 \\
2.4482560 \\
3.3164910 \\
1.6046680 \\
2.1378970 \\
2.8640510 \\
1.1448140 \\
-0.4992870 \\
-1.6123490 \\
-1.1730600 \\
-0.2533770 \\
-2.9075220 \\
-3.7170230\end{array}$ \\
\hline
\end{tabular}

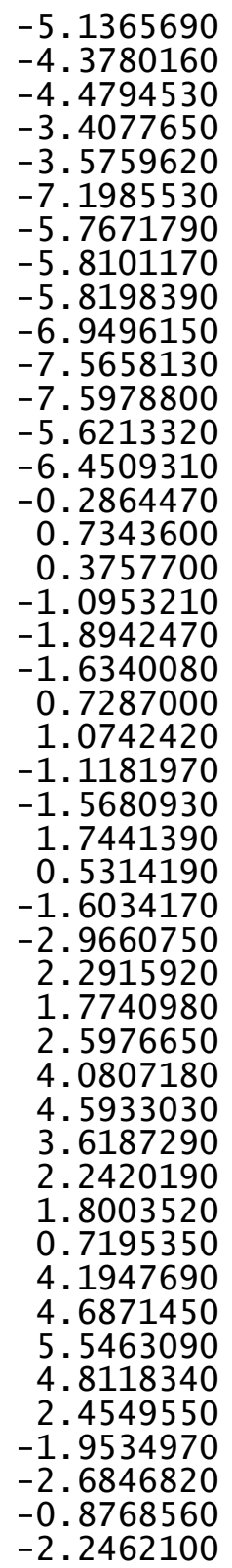

-0.2218160
-2.2035030
-3.0831630
-1.7473600
-3.3545410
-0.3256130
-3.4242110
-4.2868790
-3.8058810
-2.4433770
-2.8084890
-2.3541060
0.5604550
1.2413870
-4.0085430
-5.1084990
-6.2875380
-6.7687670
-5.8658510
-4.4109910
-5.4751560
-7.1137460
-7.8009720
-6.0934170
-4.7458280
-5.9546400
-6.7640940
-6.0895680
-2.2400810
-2.7262000
-2.1950330
-2.5087280
-1.7873630
-1.7894810
-2.6525570
-3.8240050
-2.4444840
-3.5958780
-2.2186150
-2.2258360
-0.7383210
-1.1101350
2.0387250
2.2267220
1.8946040
1.8147010
$-3.2027830$ $-2.6497320$ $-3.2992910$ $-2.8591850$ $-0.9769530$ $-1.7628830$ $-0.9592310$ $-1.6371270$ 0.0678680 $-1.2417880$ $-2.0741300$ $-0.3620140$ 0.2606750 0.0664290

1.6261430 1.7804560 0.8425870 1.0231210 1.9989370

1.7045230 2. 8187140 1.0248440

1. 3945940

3. 0258290

1. 5681150 $-0.1908680$ 0.0511700

1. 9412820

2. 3679510

3. 7102750

4.8893420

4.6615790

3. 4068490

2. 2411030

5.8216420

3.7414180

3. 8156660

4.5367570

5.5301030

3. 0796270

3. 6518350 4. 9721620 $-3.1641400$ $-1.9078390$ $-3.0615870$ $-1.0052180$ 


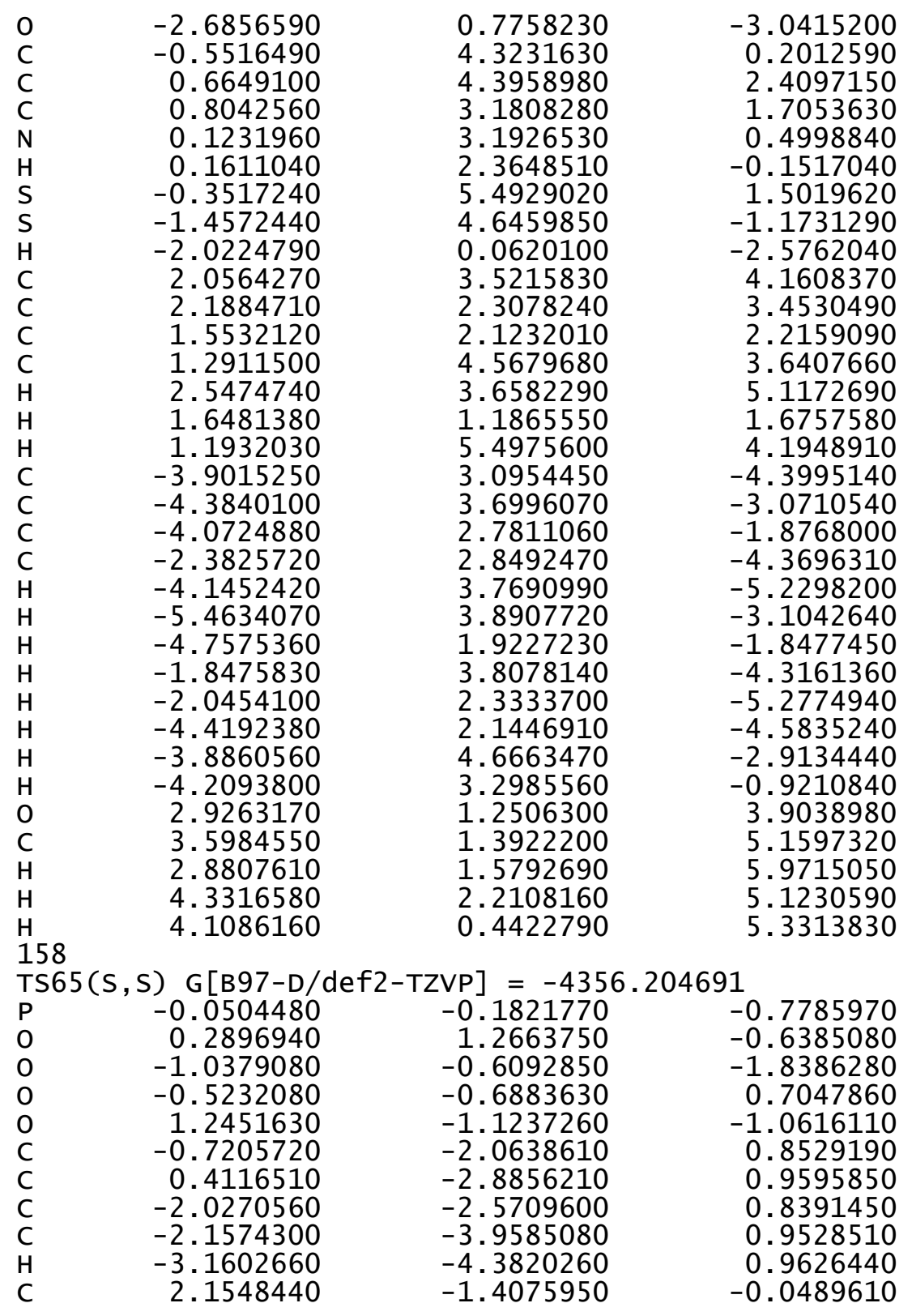

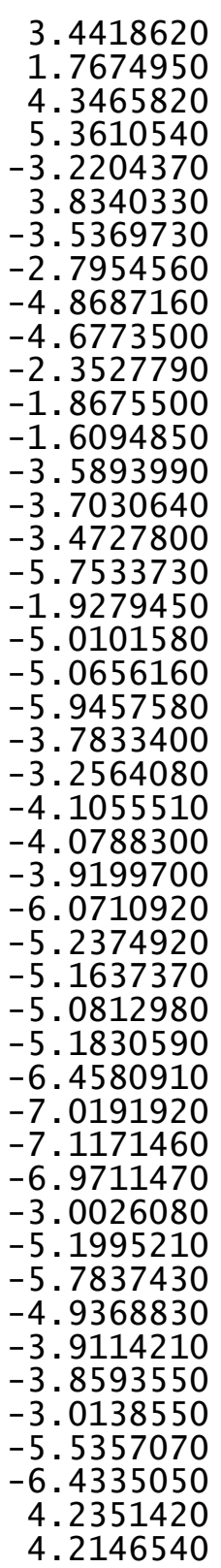

-0.8618010
-2.2650220
-1.2042430
-0.8130790
-1.7106600
0.0305500
-0.6430620
-0.2437470
1.2503900
0.1508720
1.2352280
1.5531270
1.3065250
2.1307000
2.9161090
2.6302660
1.8547990
-0.8841280
0.5957740
1.3885340
0.0260950
-0.3330300
-0.0299140
-1.3729100
-1.9646680
-3.0335610
-1.6159040
-1.1870020
-3.9619490
-4.7222640
-4.4841510
-3.1095150
-3.4481520
-3.2055390
-0.9952960
-3.6092100
-1.5099140
-1.8670740
-0.4624040
-2.3652080
-3.1595650
-1.7504310
-0.1283420
0.4711680
-0.2649630
-0
$-0.1409020$

0.9957670

0.8674900

0.8058360

0.6192890

$-1.2643640$

1.4805340

2.7348630

2. 2680140

1.2530390

2. 5963240

3. 5271090

1.8022830

2. 2861240

3. 0444150

1. 3176000

2. 0331360

2. 9139430

3.6711430

4.4291080

3.7169300

3.9319490

4.8462740

4.0629100

$-0.4711250$

$-1.5311320$

$-1.8462810$

$-0.6654010$

$-1.4618870$

$-2.2498350$

$-0.4974820$

$-1.6518930$

$-2.5328900$

$-0.7808350$

$-1.9373200$

$-1.3947850$

$-3.1259920$

$-3.9848960$

$-3.3071310$

$-2.9346120$

$-3.6912330$

$-3.0356080$

0.1903890

0.0348780

$-3.6452300$

$-4.9960640$ 


\begin{tabular}{|c|c|c|}
\hline $\begin{array}{l}0 \\
0 \\
0 \\
0 \\
0 \\
0 \\
0 \\
0 \\
0 \\
0 \\
0 \\
0 \\
0 \\
0 \\
0 \\
0 \\
0 \\
0 \\
0 \\
0 \\
0 \\
0 \\
0 \\
0 \\
0 \\
0 \\
0 \\
0 \\
0\end{array}$ & $\begin{array}{r}-1.8507450 \\
-0.4444550 \\
-1.4717980 \\
-2.0130120 \\
-1.1065220 \\
-2.4058190 \\
-3.4221190 \\
-2.4715120 \\
-2.4988840 \\
0.4360170 \\
-1.7972640 \\
-2.8106750 \\
-1.4793400 \\
-0.8203440 \\
-1.3338690 \\
0.0148210 \\
2.2107510 \\
3.6200380 \\
2.0907030 \\
1.3698890 \\
4.2255290 \\
5.2223980 \\
4.3476930 \\
3.2850160 \\
3.8225850 \\
2.8941860 \\
1.4283670 \\
4.2313940 \\
2.6615410 \\
3.2427840 \\
1.8370460 \\
3.5616870 \\
4.5808880 \\
3.1634120 \\
1.7310470 \\
2.3854490 \\
-2.0097530 \\
-2.5183990 \\
-2.3507300 \\
-2.9519930 \\
-3.9516420 \\
-3.2334130 \\
-3.4283900 \\
-3.0767560 \\
-1.4567870 \\
-4.7642460\end{array}$ & $\begin{array}{l}-3.0472010 \\
-2.5879010 \\
-4.9567970 \\
-5.9101200 \\
-4.8520640 \\
-3.7647410 \\
-4.1216790 \\
-3.0474120 \\
-2.2110850 \\
-5.7896770 \\
-4.0892640 \\
-4.4804920 \\
-3.5954300 \\
-5.2431660 \\
-6.2137010 \\
-5.2929500 \\
-2.0908450 \\
-1.6618620 \\
0.2997440 \\
-1.0271710 \\
-0.9835710 \\
-0.5960760 \\
-1.7275330 \\
0.1672070 \\
1.1230850 \\
-0.0401490 \\
1.1079960 \\
-2.5174870 \\
0.5967750 \\
1.5274670 \\
0.7518680 \\
-0.5945430 \\
-0.2483590 \\
-1.0508780 \\
-3.3999390 \\
-4.2233090 \\
1.9548390 \\
2.0455470 \\
2.9792370 \\
4.2910580 \\
4.0148970 \\
3.3151330 \\
4.8345370 \\
2.5114660 \\
3.1850180 \\
3.3727120\end{array}$ \\
\hline
\end{tabular}

3.0551470
1.4181050
1.8487810
4.1455500
0.2446740
1.4503520
1.0749290
-0.0641460
-1.3232240
-1.0578220
1.9579220
1.9708700
2.1727970
0.2400910
-0.2775170
-2.0804800
-1.7726910
0.7491210
-2.1701120
-2.9002250
-1.0821360
-2.4089610
-2.7730770
-1.1537560
-0.1679570
0.1497560
-0.4063560
-0.2310880
-1.1836420
-1.9488080
-2.0158320
1.0915890
1.3973700
0.9296490
0.3117830
1.4748220
1.1313390
0.0792690
-4.2282330
-4.7602100
-4.3410520
-2.6914090
-4.5510470
-5.8541850
-4.9274890
-2.2582260
-4.4042880
-3.9155940
-2.4733640
-2.1548580
-4.2917520
-5.2079690
-6.6063010
-7.1742830
-6.3202960
-4.8232840
-7.2570400
-5.3258610
-4.7309070
-7.1570880
-8.2180770
-6.5678360
-6.5677400
-6.5482690
2.2059630
2.3362530
2.1698960
1.9877240
0.8837640
4.5228490
4.4790020
3.3626060
3.4304510
2.6590900
5.5735160
4.8754800
0.2208720
3.5238730
2.3991150
2.3080630
4.5642180
3.5549170
1.4632310
5.4198220
3.0391370
3.6027570
2.7359000
2.9551870
3.6739880
3.6757610
1.8076260
3.9645020
4.9505300

3. 1260520

4.0055190

4. 9391320

0.9359200

0.7830280

0.2752700

1. 1288360

0.9352500

0.9675330

0.3108750

1. 7434840

0.1089930

2. 1864340

0.8656810

1.6920510

$-0.0394280$

$-0.7739550$

$-2.8498910$

$-1.5858450$

$-2.7617870$

$-0.6835190$

$-2.6981080$

0.5466900

2.8735690

2.0836830

0.8190370

0.1357410

1.9594160

$-0.8868670$

$-2.2834130$

4.6778580

3. 8761240

2. 5619550

4.1855690

5. 6940900

1. 9151990

4. 8137120

$-4.0727100$

$-2.7448640$

$-1.5442400$

$-4.0593040$

$-4.9065860$

$-2.7694480$

$-1.5048590$

$-4.0239870$ 


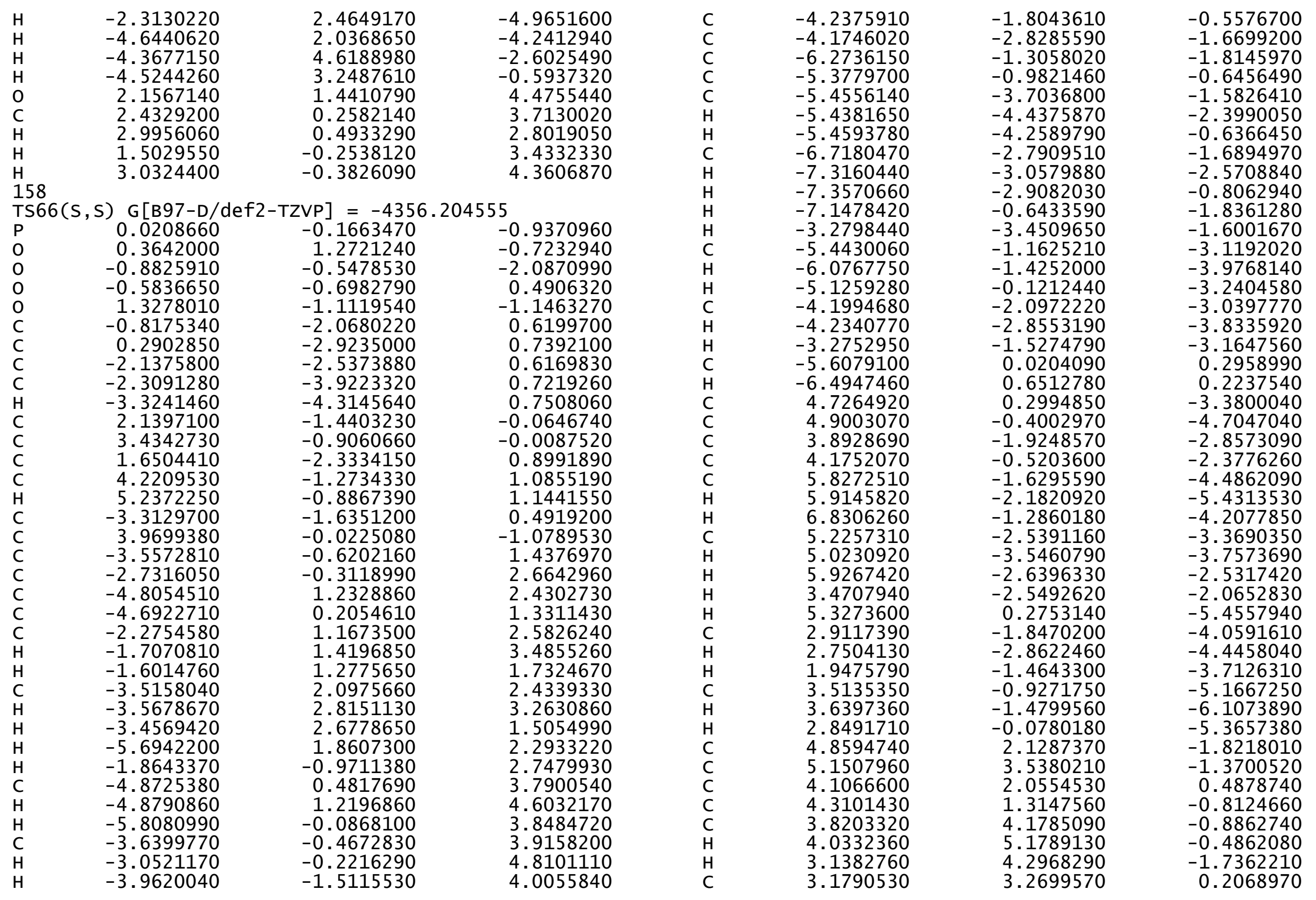




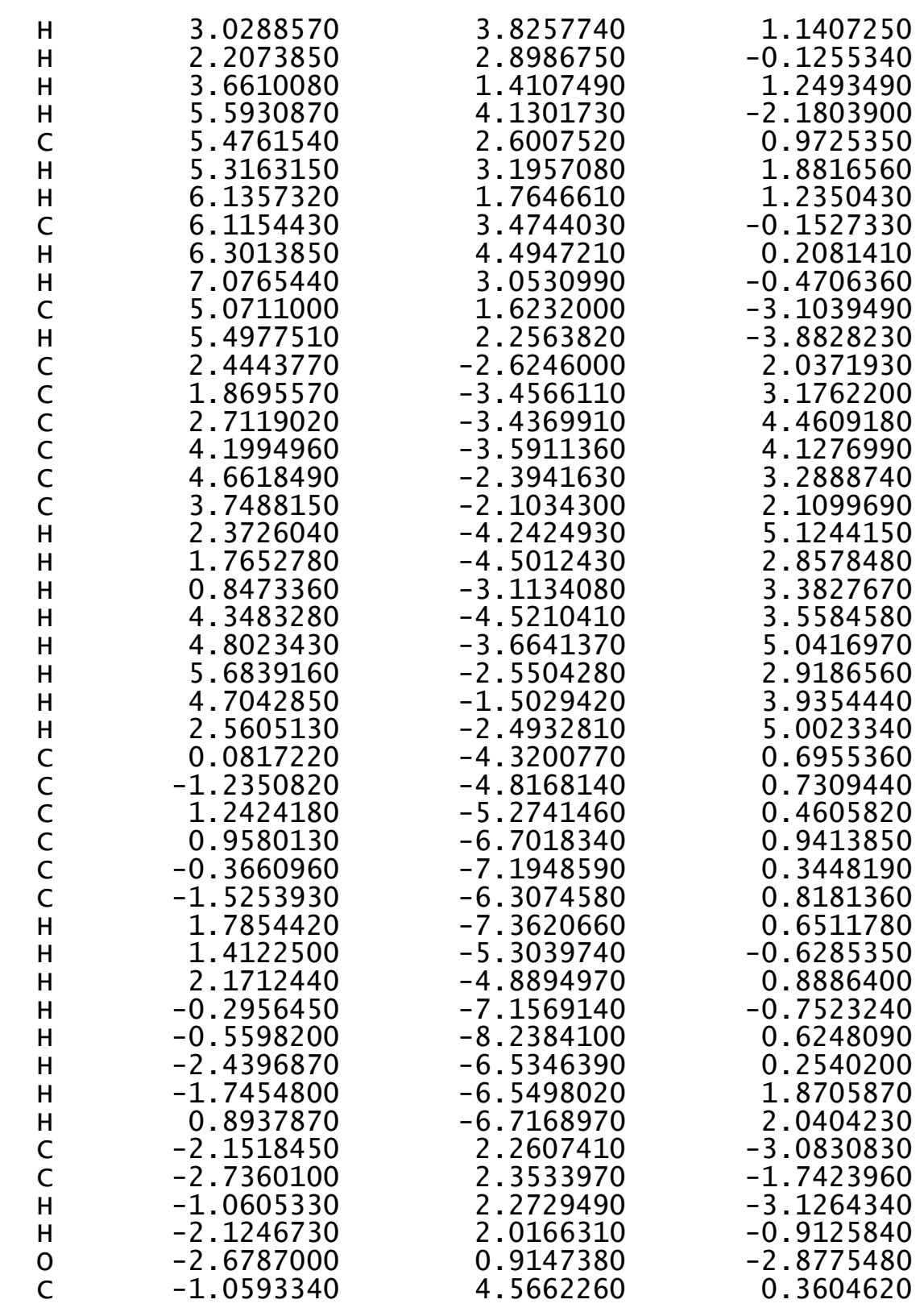

$\begin{array}{lr}\mathrm{C} & -0.1860960 \\ \mathrm{C} & 0.1329630 \\ \mathrm{~N} & -0.3628440 \\ \mathrm{H} & -0.1667270 \\ \mathrm{~S} & -1.1234440 \\ \mathrm{~S} & -1.7695450 \\ \mathrm{H} & -1.8770070 \\ \mathrm{C} & 0.9559610 \\ \mathrm{C} & 1.2598660 \\ \mathrm{C} & 0.8575130 \\ \mathrm{C} & 0.2354890 \\ \mathrm{H} & 1.2891140 \\ \mathrm{H} & 1.0616110 \\ \mathrm{H} & 0.0022200 \\ \mathrm{C} & -4.3737240 \\ \mathrm{C} & -4.7706710 \\ \mathrm{C} & -4.1755650 \\ \mathrm{C} & -2.8435330 \\ \mathrm{H} & -4.8170570 \\ \mathrm{H} & -5.8621900 \\ \mathrm{H} & -4.7167390 \\ \mathrm{H} & -2.4529090 \\ \mathrm{H} & -2.5541280 \\ \mathrm{H} & -4.7636250 \\ \mathrm{H} & -4.4083280 \\ \mathrm{H} & -4.2665520 \\ \mathrm{O} & 1.9468790 \\ \mathrm{C} & 2.1672020 \\ \mathrm{H} & 2.7716820 \\ \mathrm{H} & 1.2133840 \\ \mathrm{H} & 2.7071800 \\ \mathrm{H} & \end{array}$

4.5279300

2.7333460
0.9746060

$3.4544150 \quad 0.6856750$

$2.6748220 \quad 0.0179570$

$5.6388980 \quad 1.7566610$

$4.9170340 \quad-1.1170160$

$0.2749670 \quad-2.5089210$

$3.5587650 \quad 4.6074980$

$2.4123430 \quad 3.8364540$

$\begin{array}{ll}2.3210440 & 2.5017160\end{array}$

$\begin{array}{ll}4.6171730 & 4.0649540\end{array}$

$3.5910770 \quad 5.6411780$

$\begin{array}{ll}1.4617990 & 1.8752240\end{array}$

$5.4888810 \quad 4.6702540$

$3.0117280 \quad-4.0479690$

$3.5461260 \quad-2.6623150$

$2.6944760 \quad-1.5274690$

$2.9936200 \quad-4.2146830$

$3.6359440 \quad-4.8330980$

$3.5696510 \quad-2.5581590$

$1.7436660 \quad-1.4229270$

$4.0208040 \quad-4.2188200$

$2.5268190 \quad-5.1646600$

$1.9934980 \quad-4.1777990$

$4.5778550 \quad-2.5562760$

$3.1954980 \quad-0.5574230$

$1.4324180 \quad 4.4859210$

$0.2070590 \quad 3.7730850$

$0.3685820 \quad 2.8728110$

$-0.2547650 \quad 3.4840270$

$-0.4417330 \quad 4.4626920$ 158

$\begin{array}{lrrr}\text { TS67 (S , S ) G }[\mathrm{B} 97-\mathrm{D} / \mathrm{def} 2-\mathrm{TZVP}]=-4356.204096 \\ \mathrm{P} & 0.1007420 & -0.2308300 & 1.0943110 \\ \mathrm{O} & -0.4013510 & 1.1749760 & 0.9919070 \\ \mathrm{O} & 1.0817040 & -0.5759300 & 2.1904090 \\ \mathrm{O} & 0.7154150 & -0.5871010 & -0.3836790 \\ \mathrm{O} & -1.0906010 & -1.3225910 & 1.2677710 \\ \mathrm{C} & 1.0430820 & -1.9204420 & -0.6376210 \\ \mathrm{C} & -0.0018440 & -2.8389390 & -0.8228960 \\ \mathrm{C} & 2.3915370 & -2.2919190 & -0.6942120 \\ \mathrm{C} & 2.6621540 & -3.6468290 & -0.9102640 \\ \mathrm{H} & 3.7025360 & -3.9613620 & -0.9751480 \\ \mathrm{C} & -1.9216980 & -1.5899970 & 0.1794680 \\ \mathrm{C} & -3.2424780 & -1.1242260 & 0.2028310 \\ \mathrm{C} & -1.4013830 & -2.3301110 & -0.8914860\end{array}$




$\begin{array}{lrrr}\mathrm{C} & -4.0261760 & -1.4041040 & -0.9196330 \\ \mathrm{H} & -5.0591910 & -1.0599570 & -0.9232720 \\ \mathrm{C} & 3.4944280 & -1.3084390 & -0.5422430 \\ \mathrm{C} & -3.8015560 & -0.3620460 & 1.3489750 \\ \mathrm{C} & 3.6484850 & -0.2560400 & -1.4640430 \\ \mathrm{C} & 2.7922640 & 0.0056120 & -2.6812470 \\ \mathrm{C} & 4.7407210 & 1.7049250 & -2.4264880 \\ \mathrm{C} & 4.7125680 & 0.6559530 & -1.3423870 \\ \mathrm{C} & 2.2198070 & 1.4407390 & -2.5719370 \\ \mathrm{H} & 1.6191090 & 1.6597350 & -3.4634720 \\ \mathrm{H} & 1.5528980 & 1.4797740 & -1.7108180 \\ \mathrm{C} & 3.3854380 & 2.4648800 & -2.4222920 \\ \mathrm{H} & 3.3788880 & 3.1865840 & -3.2495230 \\ \mathrm{H} & 3.2817930 & 3.0367940 & -1.4931950 \\ \mathrm{H} & 5.5763690 & 2.4006810 & -2.2824130 \\ \mathrm{H} & 1.9787790 & -0.7185700 & -2.7706340 \\ \mathrm{C} & 4.8664930 & 0.9764730 & -3.7945880 \\ \mathrm{H} & 4.8272950 & 1.7223080 & -4.5997010 \\ \mathrm{H} & 5.8393320 & 0.4741980 & -3.8527770 \\ \mathrm{C} & 3.7027660 & -0.0541440 & -3.9389440 \\ \mathrm{H} & 3.0937000 & 0.1721950 & -4.8241560 \\ \mathrm{H} & 4.0972470 & -1.0702400 & -4.0577640 \\ \mathrm{C} & 4.4344580 & -1.4302940 & 0.4974120 \\ \mathrm{C} & 4.4557520 & -2.4856570 & 1.5798050 \\ \mathrm{C} & 6.4241490 & -0.7991680 & 1.7646640 \\ \mathrm{C} & 5.5041660 & -0.5199960 & 0.6030210 \\ \mathrm{C} & 5.8047390 & -3.2503280 & 1.4709990 \\ \mathrm{H} & 5.8479150 & -4.0056180 & 2.2666710 \\ \mathrm{H} & 5.8545470 & -3.7762750 & 0.5097680 \\ \mathrm{C} & 6.9880030 & -2.2396250 & 1.6066320 \\ \mathrm{H} & 7.6022890 & -2.4783600 & 2.4849450 \\ \mathrm{H} & 7.6381530 & -2.2834670 & 0.7247710 \\ \mathrm{H} & 7.2418270 & -0.0687520 & 1.8049000 \\ \mathrm{H} & 3.6143880 & -3.1767370 & 1.4898530 \\ \mathrm{C} & 5.5841540 & -0.7546860 & 3.0699810 \\ \mathrm{H} & 6.2375030 & -0.9791750 & 3.9236240 \\ \mathrm{H} & 5.1781040 & 0.2526510 & 3.2122190 \\ \mathrm{C} & 4.4245620 & -1.7916880 & 2.9696360 \\ \mathrm{H} & 4.5299350 & -2.5646560 & 3.7424760 \\ \mathrm{H} & 3.4548890 & -1.3098140 & 3.1131150 \\ \mathrm{C} & 5.6452140 & 0.5213260 & -0.3136650 \\ \mathrm{H} & 6.4767520 & 1.2218030 & -0.2279480 \\ \mathrm{C} & -4.4583760 & -0.2200020 & 3.6983780 \\ \mathrm{C} & -4.4857820 & -0.9880320 & 4.9958400 \\ \mathrm{C} & -3.4645610 & -2.3329670 & 3.0220840 \\ \mathrm{C} & -3.8884150 & -0.9368920 & 2.6296270 \\ & & & \end{array}$

-5.3101690
-5.2705660
-6.3600670
-4.7165980
-4.4209350
-5.4590940
-3.0394320
-4.9239390
-2.4290220
-2.1835080
-1.5101930
-3.0301650
-3.0392930
-2.4310020
-4.8658510
-5.3274510
-4.2277710
-4.2915170
-4.0986230
-4.4375330
-3.3942460
-3.4152670
-3.3551160
-2.3997200
-3.7582210
-5.7881720
-5.6662540
-5.6068420
-6.2482890
-6.3412250
-6.6627280
-7.2293860
-4.9501000
-5.3931110
-2.1937830
-1.5902540
-2.3742730
-3.8611150
-4.4403450
-3.5253790
-1.9708010
-1.5542060
-0.5469360
-3.9665170
-4.4307480
-5.4064760
4.7762280

5.6918590

4.5932430

3. 5670850

3. 8731880

2. 7658850

2.1778060

5.8016250

4.1755860

4. 5112870

3. 7986710

5. 3487030

6.2792130

5. 5268440

2. 2404720

1.8751050

$-0.1022480$

1. 1662800

1.3627780

1.0154090

2. 1871290

0.2066320

$-0.7039990$

0.4894480

$-0.9200650$

2.7343680

$-0.4978170$

$-1.3731650$

$-0.7881350$

0.7023630

0.4079850

1. 0413940

3. 5063190

4.3371330

$-2.0485900$

$-3.2989450$

$-4.5712980$

$-4.3651810$

$-3.2465020$

$-2.0445850$

$-5.4166890$

$-3.2018460$

$-3.3905470$

$-4.0955620$

$-5.2913010$

$-2.9105870$ 


$\begin{array}{rrr}-4.6515420 & -1.2214820 & -3.6404730 \\ -2.2461340 & -1.7275210 & -4.7986240 \\ 0.3045530 & -4.2137360 & -0.9302290 \\ 1.6537210 & -4.6107760 & -1.0015930 \\ -0.7917380 & -5.2620790 & -0.8225400 \\ -0.3933530 & -6.6217390 & -1.4080510 \\ 0.9543240 & -7.0654150 & -0.8263960 \\ 2.0494970 & -6.0636240 & -1.2151900 \\ -1.1750220 & -7.3606380 & -1.1891930 \\ -1.0024540 & -5.3945940 & 0.2516410 \\ -1.7268730 & -4.9062420 & -1.2606010 \\ 0.8699950 & -7.1148830 & 0.2693910 \\ 1.2255670 & -8.0684530 & -1.1804890 \\ 2.9769520 & -6.2721880 & -0.6653880 \\ 2.2864550 & -6.2019710 & -2.2827540 \\ -0.3104890 & -6.5440440 & -2.5031270 \\ 1.9618210 & 2.4128170 & 2.9833030 \\ 2.6437410 & 2.4539060 & 1.6869190 \\ 0.8802110 & 2.2754290 & 2.9391630 \\ 2.1597820 & 1.9582690 & 0.8526010 \\ 2.6745570 & 1.1338790 & 2.9613350 \\ 0.5617920 & 4.3010870 & -0.6181360 \\ -0.5663190 & 4.0862510 & -2.8643850 \\ -0.7370600 & 2.9722670 & -2.0154430 \\ -0.1045210 & 3.1402450 & -0.7956930 \\ -0.1643610 & 2.3987140 & -0.0502640 \\ 0.4143970 & 5.2943940 & -2.0649180 \\ 1.4121680 & 4.7944020 & 0.7399190 \\ 1.9813880 & 0.3788360 & 2.6109890 \\ -1.8856700 & 2.9858800 & -4.5425640 \\ -2.0482840 & 1.8735910 & -3.6891830 \\ -1.4657400 & 1.8546050 & -2.4135890 \\ -1.1415450 & 4.0944190 & -4.1318330 \\ -2.3359990 & 2.9962380 & -5.5283690 \\ -1.5830050 & 0.9965040 & -1.7594680 \\ -1.0198040 & 4.9443470 & -4.7978320 \\ 3.9721720 & 3.5611930 & 4.0208010 \\ 4.4043600 & 4.0177920 & 2.6179300 \\ 4.0357090 & 2.9806370 & 1.5425870 \\ 2.4506730 & 3.3366430 & 4.0795250 \\ 4.2581740 & 4.3125740 & 4.7666390 \\ 5.4859580 & 4.1965640 & 2.5872730 \\ 4.7117300 & 2.1156680 & 1.5836030 \\ 1.9241930 & 4.2925360 & 3.9499560 \\ 2.1473300 & 2.9218090 & 4.2802130 \\ 4.4874620 & 2.6269770 & \end{array}$

$\begin{array}{lr}\mathrm{H} & 3.9084560 \\ \mathrm{H} & 4.1406920 \\ \mathrm{O} & -2.7673650 \\ \mathrm{C} & -3.3851710 \\ \mathrm{H} & -2.6333130 \\ \mathrm{H} & -4.1191440 \\ \mathrm{H} & -3.8875700\end{array}$

TS68 (S, S) G[B97-D/def2-TZVP $]=-4356.204001$

$\begin{array}{lrrr}\text { TS68 }(\mathrm{S}, \mathrm{S}) \mathrm{G}[\mathrm{B} 97-\mathrm{D} / \mathrm{de} \text { ef }-\mathrm{TZVP}]=-4356.204001 \\ \mathrm{P} & -0.0500680 & -0.2108060 & -0.7782310 \\ \mathrm{O} & 0.2634250 & 1.2481900 & -0.6954710 \\ \mathrm{O} & -1.0117830 & -0.6997830 & -1.8353350 \\ \mathrm{O} & -0.5443850 & -0.6574320 & 0.7183960 \\ \mathrm{O} & 1.2695870 & -1.1371330 & -1.0002510 \\ \mathrm{C} & -0.7425110 & -2.0262870 & 0.9183240 \\ \mathrm{C} & 0.3898480 & -2.8444600 & 1.0488760 \\ \mathrm{C} & -2.0482150 & -2.5349720 & 0.9194960 \\ \mathrm{C} & -2.1752290 & -3.9198600 & 1.0678550 \\ \mathrm{H} & -3.1773510 & -4.3446350 & 1.0883110 \\ \mathrm{C} & 2.1512850 & -1.3858890 & 0.0462910 \\ \mathrm{C} & 3.4406340 & -0.8420630 & -0.0240070 \\ \mathrm{C} & 1.7399560 & -2.2161050 & 1.1007540 \\ \mathrm{C} & 4.3108630 & -1.1418970 & 1.0265730 \\ \mathrm{H} & 5.3275120 & -0.7540630 & 0.9825620 \\ \mathrm{C} & -3.2416340 & -1.6854350 & 0.6638470 \\ \mathrm{C} & 3.8832670 & -0.0157290 & -1.1781400 \\ \mathrm{C} & -3.5621570 & -0.5833210 & 1.4788520 \\ \mathrm{C} & -2.8262800 & -0.1326890 & 2.7192580 \\ \mathrm{C} & -4.9002460 & 1.3378390 & 2.1841620 \\ \mathrm{C} & -4.7018090 & 0.1996420 & 1.2139600 \\ \mathrm{C} & -2.3847630 & 1.3398500 & 2.5219090 \\ \mathrm{H} & -1.8989570 & 1.6948340 & 3.4388760 \\ \mathrm{H} & -1.6421870 & 1.3803280 & 1.7250720 \\ \mathrm{C} & -3.6226960 & 2.2211890 & 2.1778120 \\ \mathrm{H} & -3.7414490 & 3.0300650 & 2.9102110 \\ \mathrm{H} & -3.5030690 & 2.6902270 & 1.1945800 \\ \mathrm{H} & -5.7846280 & 1.9310380 & 1.9212530 \\ \mathrm{H} & -1.9582020 & -0.7634160 & 2.9279960 \\ \mathrm{C} & -5.0497460 & 0.7359260 & 3.6095120 \\ \mathrm{H} & -5.1187700 & 1.5559530 & 4.3367500 \\ \mathrm{H} & -5.9811540 & 0.1605490 & 3.6677220 \\ \mathrm{C} & -3.8183860 & -0.1724460 & 3.9159920 \\ \mathrm{H} & -3.2957880 & 0.1765280 & 4.8163780 \\ \mathrm{H} & -4.1349240 & -1.2068570 & 4.0950240 \\ \mathrm{C} & -4.0943240 & -1.9838170 & -0.4202730 \\ \mathrm{C} & -3.9285520 & -3.0933370 & -1.4365990\end{array}$

$\begin{array}{rrr}3.9084560 & 4.9689510 & 2.3801650 \\ 4.1406920 & 3.3900970 & 0.5321420 \\ -2.7673650 & 0.7630770 & -4.0280690 \\ -3.3851710 & 0.7374070 & -5.3190090 \\ -2.6333130 & 0.8223310 & -6.1168590 \\ -4.1191440 & 1.5499090 & -5.4191210 \\ -3.8875700 & -0.2293530 & -5.3889110\end{array}$

4. 9689510 0.5321420 $-5.3190090$ 5.3889110

16410

0.2693910 ( . 5031270 . 6869190 2.9391630 8526010 $-2.8643850$ 2.0154430 0502640 $-2.0649180$ 7399190 6891830 $-4.1318330$ . 4.7978320 .0208010 5425870 4. 0795250 2. 5872730 4.2802130

\begin{abstract}
$-3.9285520$
\end{abstract}
$-1.8353350$

(2)

0.0240070

0.9825620

0.6638470

400

2. 7192580

1.2139600

5219090

1.9212530

(2)

3. 9159920

$-1.4365990$ 


-6.0812080
-5.2532970
-5.1702700
-5.0824670
-5.1925040
-6.4657750
-7.0230340
-7.1274730
-6.9822200
-3.0105640
-5.2054520
-5.7852800
-4.9458600
-3.9150680
-3.8551820
-3.0194620
-5.5562050
-6.4541990
4.4539680
4.5282560
3.6886330
3.9927010
5.4799180
5.4888200
6.5013430
4.9846720
4.7684990
5.7520450
3.3382450
4.8862760
2.6147690
2.4424440
1.6736510
3.1118780
3.1624870
2.4252590
4.6819070
4.9773100
4.0957830
4.2217970
3.6698990
3.8874520
2.9282810
3.1258070
3.0318090
2.1398190

-1.8168810
-0.6505660
-1.3364400
-2.0945710
-0.3528910
-1.5644110
-2.4330710
-0.6923340
-1.9362150
-1.2743370
-3.0966490
-3.9415650
-3.3220370
-2.8648750
-3.5895800
-2.9847790
0.1607950
-0.0220850
-3.5446020
-4.8412740
-2.8429730
-2.4609400
-4.6372130
-5.5550550
-4.4670880
-3.4245900
-3.7358220
-2.6422870
-1.9868690
-5.6586330
-3.9653310
-4.2884850
-3.5625310
-5.1605610
-6.0829650
-5.3390280
-2.1000670
-1.7474970
0.2587970
-1.0103630
-1.2083810
-0.8832080
-2.0138150
-0.0222780
0.8876530
-0.2611930

\begin{abstract}
3.7195240
5.3493570

5.4861230

5. 3816490

6.1785030

6.0246800

6.2091630

6.9721540

4.8017290

5.1568430

2.6127060

2.1064030

3.0759530

4.5202090

4.9182760

3.9191910

2.7835740

1.8839370

1.1416110

4.5974300

5.2055050

5.9110230

5.0026720

3.0045000

0.2267940

1.4248360

1.0230890

$-0.0744150$

$-1.3419200$

$-1.0746670$

1.9050650

1. 9742720

2. 1290100

0.2821630

$-0.3016820$

$-2.0469920$

$-1.8574460$

0.6478880

$-2.1635020$

$-2.9025340$

$-1.0758230$

$-2.4113740$

$-2.7542560$

$-1.1946640$

$-0.2105130$

0.1063310
\end{abstract}

1.5404290

4.0899300

2.7386720

3. 3781440

1. 9273150

3.5615740

4.6041380

3.1440740

1.5294930

2.1239650

$-2.4207070$

$-3.1253230$

$-3.0897790$

$-3.2914150$

$-2.1316660$

$-1.9023210$

$-3.8634780$

$-4.1734880$

$-2.6809360$

$-4.2410740$

$-3.3481620$

$-2.3024920$

$-1.2135600$

$-2.1265410$

$-4.2503960$

$-5.1636300$

$-6.5385090$

$-7.1392320$

$-6.2804360$

$-4.7833810$

$-7.1906330$

$-5.3304040$

$-4.6634190$

$-7.1619650$

$-8.1726150$

$-6.5371020$

$-6.5147750$

$-6.4352580$

2.0621240

2.2349000

2.0399430

1.9269370

0.7403010

.5473010

4.6318970

3.4730550
1.0865650

$-2.6188890$

0.6135220

1.5001790

0.8691140

$-0.5995310$

$-0.3082880$

$-0.9605230$

$-3.3666000$

$-4.2092980$

2.1967800

3.4489820

4.6429910

4.1742280

3.2551930

2.1348830

5.3639350

3.2269200

3.7286290

3.6236950

5.0294240

2.8177210

3.8589990

5.1655910

1.0323370

0.8146060

0.2641990

1. 1494500

1.0507690

1.0813130

0.2307390

1.7506220

0.1382960

2.1903040

0.8584560

1.8538040

0.1062550

$-0.7648400$

$-2.9756130$

$-1.7220120$

$-2.8796940$

$-0.8049420$

$-2.7752120$

0.3240050

2.6501750

1.9234780
1. 


\begin{tabular}{|c|c|c|c|c|c|c|c|}
\hline & & & & & & & \\
\hline $\mathrm{N}$ & -0.4481540 & 3.4715150 & 0.6566490 & C & -3.5159090 & -1.3272260 & 0.6182010 \\
\hline $\begin{array}{c}\mathrm{H} \\
\mathrm{S}\end{array}$ & -0.2682150 & 2.6670290 & 0.0138960 & C & 3.7861320 & -0.3981170 & -1.3575170 \\
\hline $\mathrm{s}$ & $\begin{array}{l}-1.2269070 \\
-1.9869780\end{array}$ & $\begin{array}{l}5.67395 / 0 \\
4.8189540\end{array}$ & $\begin{array}{r}1.6768430 \\
-1\end{array}$ & c & -3.6850940 & -0.2647790 & 1.5263990 \\
\hline $\mathrm{H}$ & -1.9952950 & 0.1028400 & -2.3279780 & c & -4.8060350 & 1.6918840 & 2.4630770 \\
\hline C & 1.0482110 & 3.7766510 & 4.5045530 & $\mathrm{C}$ & -4.7646820 & 0.6277340 & 1.3947750 \\
\hline $\mathrm{C}$ & 1.3496750 & 2.6072470 & 3.7679430 & $\mathrm{C}$ & -2.2819650 & 1.4660440 & 2.6256500 \\
\hline C & 0.8835850 & 2.4450790 & 2.4600760 & $\mathrm{H}$ & -1.7070280 & 1.7037800 & 3.5299640 \\
\hline C & 0.2705310 & 4.7895460 & 3.9549050 & $\mathrm{H}$ & -1.5947790 & 1.5080550 & 1.7806910 \\
\hline $\mathrm{H}$ & 1.4312540 & 3.8634800 & 5.5176160 & C & -3.4611580 & 2.4691070 & 2.4386490 \\
\hline $\mathrm{H}$ & 1.0841440 & 1.5655700 & 1.8609460 & $\mathrm{H}$ & -3.4664820 & 3.2186230 & 3.2406610 \\
\hline $\mathrm{H}$ & 0.0401820 & 5.6794570 & 4.5342610 & $\mathrm{H}$ & -3.3638630 & 3.0081010 & 1.4895930 \\
\hline C & -4.2223470 & 2.8271880 & -4.2417360 & $\mathrm{H}$ & -5.6507060 & 2.3745040 & 2.3090440 \\
\hline C & -4.7672790 & 3.4375820 & -2.9402910 & $\mathrm{H}$ & -2.0126150 & -0.6892320 & 2.8432190 \\
\hline C & -4.3470230 & 2.6228600 & -1.7041630 & C & -4.9203520 & 0.9847510 & 3.8430630 \\
\hline C & -2.6849700 & 2.7573490 & -4.2168850 & $\mathrm{H}$ & -4.8800390 & 1.7437940 & 4.6356850 \\
\hline $\mathrm{H}$ & -4.5455560 & 3.4261420 & -5.1016230 & $\mathrm{H}$ & -5.8902910 & 0.4787310 & 3.9158130 \\
\hline $\mathrm{H}$ & -5.8617740 & 3.4984240 & -2.9736150 & C & -3.7506200 & -0.0374490 & 3.9968610 \\
\hline $\mathrm{H}$ & -4.9261910 & 1.6921290 & -1.6324930 & $\mathrm{H}$ & -3.1470290 & 0.1962070 & 4.8838890 \\
\hline $\mathrm{H}$ & -2.2611350 & 3.7712160 & -4.2202110 & $\mathrm{H}$ & -4.1388150 & -1.0557660 & 4.1176030 \\
\hline $\mathrm{H}$ & -2.2973600 & 2.2347240 & -5.1004510 & C & -4.4566890 & -1.4798290 & -0.4161410 \\
\hline $\mathrm{H}$ & -4.6288990 & 1.8156170 & -4.3737620 & $\mathrm{C}$ & -4.4682020 & -2.5558960 & -1.4777630 \\
\hline $\mathrm{H}$ & -4.3860110 & 4.4624200 & -2.8355080 & $\mathrm{C}$ & -6.4582030 & -0.8976140 & -1.6882200 \\
\hline $\mathrm{H}$ & -4.5410330 & 3.1709010 & -0.7756520 & $\mathrm{C}$ & -5.5397960 & -0.5868210 & -0.5332680 \\
\hline 0 & 2.1033740 & 1.6812100 & 4.4214220 & $\mathrm{C}$ & -5.8075130 & -3.3348090 & -1.3485410 \\
\hline C & 2.3530620 & 0.4437150 & 3.7404000 & $\mathrm{H}$ & -5.8439850 & -4.1066940 & -2.1284320 \\
\hline $\mathrm{H}$ & 2.9042680 & 0.6041900 & 2.8065000 & $\mathrm{H}$ & -5.8479400 & -3.8413850 & -0.3765310 \\
\hline $\mathrm{H}$ & 1.4123000 & -0.0758550 & 3.5148710 & C & -7.0039310 & -2.3417720 & -1.5011850 \\
\hline $\mathrm{H}$ & 2.9588900 & -0.1517690 & 4.4230280 & $\mathrm{H}$ & -7.6169890 & -2.6051990 & -2.3732900 \\
\hline & & & & $\mathrm{H}$ & -7.6515750 & -2.3764000 & -0.6171230 \\
\hline & 5) $G[B 97-D / d$ & $\mathrm{ZVP}]=-435$ & & $\mathrm{H}$ & -7.2845930 & -0.1777960 & -1.7401210 \\
\hline $\mathrm{P}$ & -0.1342200 & -0.2409160 & -1.0629180 & $\mathrm{H}$ & -3.6174600 & -3.2340660 & -1.3768360 \\
\hline 0 & 0.3449300 & 1.1762750 & -1.0112040 & C & -5.6235470 & -0.8702960 & -2.9976590 \\
\hline 0 & -1.0981070 & -0.6451560 & -2.1532400 & $\mathrm{H}$ & -6.2779130 & -1.1204670 & -3.8433120 \\
\hline 0 & -0.7588550 & -0.5432680 & 0.4229040 & $\mathrm{H}$ & -5.2307990 & 0.1383990 & -3.1665300 \\
\hline 0 & 1.0754350 & -1.3212710 & -1.1832690 & C & -4.4507130 & -1.8902310 & -2.8812850 \\
\hline C & -1.0532070 & -1.8722990 & 0.7338060 & $\mathrm{H}$ & -4.5495080 & -2.6795710 & -3.6381770 \\
\hline c & 0.0125360 & -2.7456100 & 0.9834600 & $\mathrm{H}$ & -3.4879560 & -1.3991900 & -3.0383420 \\
\hline C & -2.3914350 & -2.2829990 & 0.7855500 & C & -5.6958280 & 0.4647080 & 0.3691140 \\
\hline c & -2.6237430 & -3.6392380 & 1.0276230 & $\mathrm{H}$ & -6.5363460 & 1.1529210 & 0.2729480 \\
\hline $\mathrm{H}$ & -3.6549950 & -3.9848410 & 1.0809100 & C & 4.4058820 & -0.4021440 & -3.7210740 \\
\hline C & 1.9147860 & -1.5326220 & -0.0900630 & $\mathrm{C}$ & 4.4394330 & -1.2593260 & -4.9610430 \\
\hline C & 3.2414670 & -1.0900320 & -0.1601080 & $\mathrm{C}$ & 3.4599060 & -2.4817880 & -2.8885640 \\
\hline C & 1.4008760 & -2.2049400 & 1.0303790 & C & 3.8649420 & -1.0552950 & -2.5979540 \\
\hline c & 4.0462240 & -1.3344870 & 0.9570270 & $\mathrm{C}$ & 5.2954500 & -2.5198850 & -4.6542510 \\
\hline $\mathrm{H}$ & 5.0870090 & -1.0166170 & 0.9211690 & $\mathrm{H}$ & 5.2755360 & -3.1858370 & -5.5274040 \\
\hline
\end{tabular}




\begin{tabular}{|c|c|c|}
\hline $\begin{array}{l}850 \\
300 \\
900 \\
520 \\
220 \\
910 \\
260 \\
670 \\
190 \\
480 \\
160 \\
800 \\
580 \\
400 \\
980 \\
530 \\
260 \\
730 \\
860 \\
160 \\
970 \\
080 \\
900 \\
350 \\
490 \\
930 \\
340 \\
070 \\
460 \\
590 \\
790 \\
680 \\
820 \\
370\end{array}$ & $\begin{array}{r}-2.2225490 \\
-3.2422730 \\
-4.2744040 \\
-3.2833920 \\
-2.9769450 \\
-0.7078440 \\
-2.4795510 \\
-3.5203640 \\
-2.0007480 \\
-1.7318620 \\
-2.3911890 \\
-0.8630860 \\
1.5689400 \\
2.9897550 \\
1.8155710 \\
0.9226060 \\
3.7892720 \\
4.8084260 \\
3.8651890 \\
3.0673890 \\
3.7348360 \\
2.7512490 \\
1.3030460 \\
3.4480280 \\
2.2920610 \\
2.9942020 \\
1.4356970 \\
2.9804600 \\
4.0151770 \\
2.4477430 \\
0.9098420 \\
1.4134510 \\
-1.9307960 \\
-2.3375310 \\
-2.1603720 \\
-2.3794200 \\
-3.3621270 \\
-2.7774900 \\
-2.7429140 \\
-3.0522430 \\
-1.3223260 \\
-4.3191700 \\
-3.5668850 \\
-3.4692700 \\
-1.8799510 \\
-1.4256990\end{array}$ & $\begin{array}{l}-4.4865670 \\
-3.3961420 \\
-3.6393920 \\
-2.5954090 \\
-2.0033490 \\
-5.8114050 \\
-4.0341380 \\
-4.2888880 \\
-3.6830030 \\
-5.2738950 \\
-6.1519220 \\
-5.5258580 \\
-2.3978980 \\
-2.1339500 \\
-0.0500420 \\
-1.2677770 \\
-1.6408390 \\
-1.3796190 \\
-2.4522150 \\
-0.4069310 \\
0.4637460 \\
-0.6243600 \\
0.8129270 \\
-3.0359890 \\
0.2730810 \\
1.1167000 \\
0.5854120 \\
-0.9848110 \\
-0.7579490 \\
-1.3150040 \\
-3.6245340 \\
-4.4982890 \\
2.1216310 \\
2.1800930 \\
3.2818850 \\
4.6314200 \\
4.4716910 \\
3.5062540 \\
5.3699400 \\
3.0536790 \\
3.3380390 \\
4.0778540 \\
5.4418190 \\
3.3603390 \\
3.9722230 \\
5.0015060\end{array}$ \\
\hline
\end{tabular}

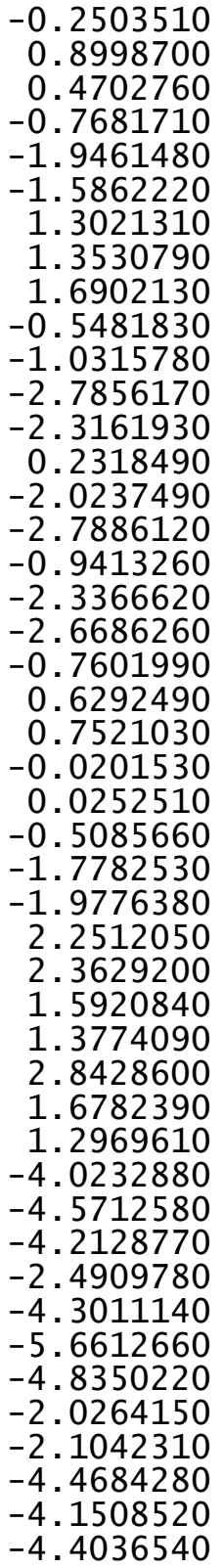

-4.1278290
-5.1206630
-6.5628790
-6.9203300
-6.0392510
-4.5686920
-7.2445970
-5.0992770
-4.8001120
-6.7505910
-7.9794310
-6.1392730
-6.3987890
-6.6665970
2.3426980
2.3060780
2.2600630
1.8071320
1.0288040
4.2832950
4.2035900
3.0668150
3.1623340
2.4108210
5.3278840
4.6984500
0.2952950
3.2621640
2.1259780
2.0083010
4.3009350
3.3450200
1.1360450
5.1704580
3.4202220
3.8035450
2.7539690
3.2774450
4.1816140
3.9188530
1.8557930
4.2540390
2.9134000
2.4701470
4.7740210
3.1187570
1.1372700

1.2102590

0.9100520

1.7393530

1. 3054420

1. 1664720

1.1287910

2. 2113780

0.5200850

2.8043750

1.6231520

2.0072650

0.3320160

$-0.1589810$

$-2.9845700$

$-1.7352970$

$-2.8738550$

$-0.8846490$

$-3.0507220$

0.6109700

2.7163180

1.8899940

0.7443580

0.0087870

2.0063750

$-0.6551150$

$-2.6589630$

4.2172900

3.3877580

2. 2227270

3.8864050

5.1220430

1.5835020

4.5330980

$-4.1156820$

$-2.7312640$

$-1.6647400$

$-4.0812180$

$-4.8544430$

$-2.7679740$

$-1.7746610$

$-3.8864910$

$-5.0413870$

$-4.4392210$

$-2.4337960$

$-0.6500200$ 


\begin{tabular}{|c|c|c|c|c|c|c|c|}
\hline & & & & & & & \\
\hline 0 & $\begin{array}{l}3.2163760 \\
4.1506670\end{array}$ & $\begin{array}{l}1.0902710 \\
1.2431720\end{array}$ & $\begin{array}{l}3.6380380 \\
4.7109470\end{array}$ & $\mathrm{C}$ & $\begin{array}{l}-5.8210280 \\
-5.8651370\end{array}$ & -3.2762880 & $\begin{array}{l}-1.3640250 \\
-2 \\
-2500330\end{array}$ \\
\hline $\mathrm{H}$ & 3.6353610 & $\begin{array}{l}1.2431 / 20 \\
1.2973960\end{array}$ & $\begin{array}{l}4.7109470 \\
5.6810730\end{array}$ & $\mathrm{H}$ & -5.8620230 & $\begin{array}{l}-4.0415630 \\
-3.7905110\end{array}$ & -2.1500330 \\
\hline $\mathrm{H}$ & 4.7606520 & 2.1471050 & 4.5703830 & C & -7.0110120 & -2.2739340 & -1.5047490 \\
\hline $\mathrm{H}$ & 4.7846930 & 0.3553880 & 4.6802840 & $\mathrm{H}$ & -7.6282130 & -2.5257430 & -2.3773590 \\
\hline & & & & $\mathrm{H}$ & -7.6564640 & -2.3117590 & -0.6192080 \\
\hline & 5) $\mathrm{G}[\mathrm{B} 97-\mathrm{D} / \mathrm{C}$ & {$[\mathrm{VP}]=-4356$} & & $\mathrm{H}$ & -7.2777950 & -0.1062660 & -1.7238880 \\
\hline$P$ & -0.1378380 & -0.2389020 & -1.0553000 & $\mathrm{H}$ & -3.6307940 & -3.1904220 & -1.3994140 \\
\hline 0 & 0.3545530 & 1.1730360 & -0.9912170 & C & -5.6251520 & -0.7982520 & -2.9925550 \\
\hline 0 & -1.1209450 & -0.6196340 & -2.1371240 & $\mathrm{H}$ & -6.2835500 & -1.0352680 & -3.8388550 \\
\hline 0 & -0.7411420 & -0.5585510 & 0.4356880 & $\mathrm{H}$ & -5.2252110 & 0.2091290 & -3.1519420 \\
\hline 0 & 1.0588300 & -1.3295010 & -1.2061620 & C & -4.4597630 & -1.8279540 & -2.8889260 \\
\hline C & -1.0542940 & -1.8888270 & 0.7224700 & $\mathrm{H}$ & -4.5673440 & -2.6103330 & -3.6518610 \\
\hline C & -0.0002880 & -2.7879220 & 0.9386620 & $\mathrm{H}$ & -3.4938710 & -1.3431140 & -3.0453870 \\
\hline $\mathrm{C}$ & -2.3995900 & -2.2750110 & 0.7734310 & C & -5.6778270 & 0.5076050 & 0.3853270 \\
\hline $\mathrm{C}$ & -2.6565570 & -3.6294900 & 0.9998010 & $\mathrm{H}$ & -6.5139390 & 1.2023150 & 0.2980130 \\
\hline $\mathrm{H}$ & -3.6935440 & -3.9569350 & 1.0536340 & C & 4.3760810 & -0.3682390 & -3.7408150 \\
\hline C & 1.9037450 & -1.5629860 & -0.1212710 & $\mathrm{C}$ & 4.3952280 & -1.2045220 & -4.9951170 \\
\hline C & 3.2266030 & -1.1061540 & -0.1853440 & C & 3.4226880 & -2.4550670 & -2.9365680 \\
\hline $\mathrm{C}$ & 1.3970980 & -2.2641620 & 0.9839680 & $\mathrm{C}$ & 3.8359420 & -1.0351140 & -2.6254090 \\
\hline $\mathrm{C}$ & 4.0315870 & -1.3549980 & 0.9293890 & $\mathrm{C}$ & 5.2447350 & -2.4761570 & -4.7164850 \\
\hline $\mathrm{H}$ & 5.0667940 & -1.0188150 & 0.9026760 & $\mathrm{H}$ & 5.2103000 & -3.1287980 & -5.5991850 \\
\hline C & -3.5106740 & -1.3024320 & 0.6141400 & $\mathrm{H}$ & 6.2908220 & -2.1894160 & 5150 \\
\hline C & 3.7668220 & -0.3964410 & -1.3742920 & C & 4.6750600 & -3.2125870 & -3.4630470 \\
\hline $\mathrm{C}$ & -3.6697050 & -0.2466030 & 1.5314070 & $\mathrm{H}$ & 4.3849220 & -4.2408920 & -3.7168350 \\
\hline $\mathrm{C}$ & -2.8155660 & 0.0238400 & 2.7487250 & $\mathrm{H}$ & 5.4290380 & -3.2654620 & -2.6684450 \\
\hline $\mathrm{C}$ & -4.7745950 & 1.7097960 & 2.4877190 & $\mathrm{H}$ & 3.0182200 & -2.9633550 & -2.0572590 \\
\hline C & -4.7426860 & 0.6552000 & 1.4096560 & $\mathrm{H}$ & 4.8121710 & -0.6412730 & -5.8389390 \\
\hline $\mathrm{C}$ & -2.2520370 & 1.4632110 & 2.6431540 & C & 2.3672520 & -2.4309340 & -4.0758510 \\
\hline $\mathrm{H}$ & -1.6722070 & 1.6886020 & 3.5474450 & $\mathrm{H}$ & 2.1242020 & -3.4667740 & -4.3485860 \\
\hline $\mathrm{H}$ & -1.5673580 & 1.5070870 & 1.7962820 & $\mathrm{H}$ & 1.4512770 & -1.9587250 & -3.7104970 \\
\hline C & -3.4238950 & 2.4771260 & 2.4689470 & C & 2.9425800 & -1.6606740 & -5.3047280 \\
\hline $\mathrm{H}$ & -3.4219800 & 3.2181760 & 3.2788290 & $\mathrm{H}$ & 2.9526580 & -2.3005470 & -6.1972590 \\
\hline $\mathrm{H}$ & -3.3240970 & 3.0256290 & 1.5255730 & $\mathrm{H}$ & 2.3267890 & -0.7821760 & -5.5314660 \\
\hline $\mathrm{H}$ & -5.6144310 & 2.4000530 & 2.3413250 & C & 4.7900210 & 1.5785500 & -2.3889880 \\
\hline $\mathrm{H}$ & -1.9982730 & -0.6955690 & 2.8414490 & $\mathrm{C}$ & 5.2365430 & 2.9914040 & -2.1058190 \\
\hline C & -4.8923060 & 0.9903390 & 3.8611110 & C & 4.2192050 & 1.7958460 & -0.0367170 \\
\hline $\mathrm{H}$ & -4.8461970 & 1.7415080 & 4.6608940 & $\mathrm{C}$ & 4.2519750 & 0.9196180 & -1.2668250 \\
\hline $\mathrm{H}$ & -5.8655330 & 0.4901250 & 3.9299020 & $\mathrm{C}$ & 4.0062800 & 3.7945780 & -1.5983510 \\
\hline C & -3.7293130 & -0.0411430 & 4.0040820 & $\mathrm{H}$ & 4.3314400 & 4.8069500 & -1.3226490 \\
\hline $\mathrm{H}$ & -3.1225740 & 0.1809510 & 4.8919240 & $\mathrm{H}$ & 3.2718250 & 3.8890550 & -2.4067880 \\
\hline $\mathrm{H}$ & -4.1243070 & -1.0577490 & 4.1169710 & C & 3.3811000 & 3.0594490 & -0.3740060 \\
\hline C & -4.4547410 & -1.4386720 & -0.4199620 & $\mathrm{H}$ & 3.3575030 & 3.7144990 & 0.5062030 \\
\hline $\mathrm{C}$ & -4.4768900 & -2.5053800 & -1.4910440 & $\mathrm{H}$ & 2.3556140 & 2.7541560 & -0.5946150 \\
\hline C & -6.4560530 & -0.8319760 & -1.6810290 & $\mathrm{H}$ & 3.7887340 & 1.2753800 & 0.8199260 \\
\hline C & 317370 & -0.5371290 & -0.5265790 & $\mathrm{H}$ & 5.6617520 & 3.4586100 & -3.0024300 \\
\hline
\end{tabular}




\begin{tabular}{|c|c|c|}
\hline $\begin{array}{l}5.6650170 \\
5.6282170 \\
6.2689860 \\
6.2860160 \\
6.5865640 \\
7.1798250 \\
4.8525050 \\
5.2684440 \\
3.5493670 \\
2.2135770 \\
4.5052590 \\
3.8043800 \\
2.6908750 \\
1.6308610 \\
4.5366320 \\
5.1381040 \\
5.1860400 \\
3.1192320 \\
2.2206070 \\
0.8686460 \\
1.1036490 \\
3.3562810 \\
-0.2921270 \\
-1.6368360 \\
0.8268660 \\
0.4122530 \\
-0.9022800 \\
-2.0217480 \\
1.2129490 \\
1.1373090 \\
1.7105730 \\
-0.7748860 \\
-1.1738530 \\
-2.9301130 \\
-2.2877350 \\
0.2764090 \\
-2.0084970 \\
-2.7643380 \\
-0.9264110 \\
-2.3122160 \\
-2.6719630 \\
-0.7061310 \\
0.6745210 \\
0.7845600 \\
0.0171440 \\
0.0536180\end{array}$ & $\begin{array}{r}2.2560660 \\
2.9470670 \\
1.3907220 \\
2.9563080 \\
3.9848690 \\
2.4197750 \\
0.9381730 \\
1.4520000 \\
-1.9818200 \\
-2.4206980 \\
-2.1998330 \\
-2.4540420 \\
-3.4899310 \\
-2.9499770 \\
-2.7883780 \\
-3.0714710 \\
-1.3413490 \\
-4.4203570 \\
-3.7346040 \\
-3.7091310 \\
-2.1129680 \\
-1.5212300 \\
-4.1658440 \\
-4.5792640 \\
-5.1969900 \\
-6.5629330 \\
-7.0216180 \\
-6.0354910 \\
-7.2914950 \\
-5.3232270 \\
-4.8252400 \\
-7.0606550 \\
-8.0307830 \\
-6.2557540 \\
-6.1756110 \\
-6.4887770 \\
2.3832970 \\
2.3527850 \\
2.2854520 \\
1.8447990 \\
1.0790070 \\
4.2885690 \\
4.1768370 \\
3.0432690 \\
3.1560940 \\
2.4083610\end{array}$ & $\begin{array}{r}0.2898810 \\
1.1429450 \\
0.5893110 \\
-0.9599160 \\
-0.7193580 \\
-1.3002310 \\
-3.6259840 \\
-4.4936220 \\
2.0818060 \\
2.1284440 \\
3.2428590 \\
4.5827800 \\
4.3975440 \\
3.4303580 \\
5.3295290 \\
3.0100980 \\
3.3115110 \\
3.9943390 \\
5.3587390 \\
3.2412060 \\
3.9173200 \\
4.9512110 \\
1.0737770 \\
1.1193940 \\
1.0552910 \\
1.6147450 \\
0.9736480 \\
1.3286680 \\
1.4334900 \\
0.0051670 \\
1.5798930 \\
-0.1184860 \\
1.3095520 \\
0.7521850 \\
2.3891470 \\
2.7047560 \\
-2.9522380 \\
-1.6973100 \\
-2.8501440 \\
-0.8520930 \\
-3.0169950 \\
0.6476930 \\
2.7570630 \\
1.9246360 \\
0.7771910 \\
0.0376950\end{array}$ \\
\hline
\end{tabular}

$\begin{array}{lr}\mathrm{S} & -0.4443320 \\ \mathrm{~S} & -1.7134370 \\ \mathrm{H} & -1.9884080 \\ \mathrm{C} & 2.2772300 \\ \mathrm{C} & 2.3769810 \\ \mathrm{C} & 1.6085670 \\ \mathrm{C} & 1.4192490 \\ \mathrm{H} & 2.8659740 \\ \mathrm{H} & 1.6836110 \\ \mathrm{H} & 1.3481840 \\ \mathrm{C} & -4.0017710 \\ \mathrm{C} & -4.5344010 \\ \mathrm{C} & -4.1824560 \\ \mathrm{C} & -2.4712950 \\ \mathrm{H} & -4.2745660 \\ \mathrm{H} & -5.6230070 \\ \mathrm{H} & -4.8162520 \\ \mathrm{H} & -1.9918220 \\ \mathrm{H} & -2.0970640 \\ \mathrm{H} & -4.4624260 \\ \mathrm{H} & -4.0988430 \\ \mathrm{H} & -4.3624200 \\ \mathrm{O} & 3.2158670 \\ \mathrm{C} & 4.1374160 \\ \mathrm{H} & 3.6092160 \\ \mathrm{H} & 4.7623340 \\ \mathrm{H} & 4.7589430 \\ \mathrm{H} & \end{array}$
5. 3214250
4. 7257360
0.3345340
3.2040590
2. 0714190
1. 9712320
4. 2567010
3. 2738540
1. 1009740
5.1232500
3.4916780
3.8769990
2. 8184130
3. 3277940
4.2596870
4.0070500
1. 9284220
4.2973320
2. 9613490
2. 5491340
4.8405180
3. 1814040
1.0230890
1.1520380
1.2043310
.2555750

2.0498430

$-0.6193540$

$-2.6313350$

4.2585620

3. 4226070

2. 2542400

3.9305380

5. 1662600

1.6109430

4. 5822810

$-4.0647800$

$-2.6748920$

$-1.6151590$

$-4.0424400$

$-4.7985600$

$-2.7032240$

$-1.7252730$

$-3.8486840$

$-5.0066580$

$-4.3885920$

$-2.3767740$

$-0.5978470$

3.6699220

4.7570780

5.7203200

4.6340300

158

TS71(S, S ) G [B97-D/def2-TZVP $]=-4356.203746$

\begin{tabular}{|c|c|c|c|}
\hline & & & \\
\hline $\mathrm{P}$ & -0.0329710 & -0.1322650 & -0.7999380 \\
\hline 0 & 0.2397080 & 1.3222010 & -0.5878440 \\
\hline 0 & -0.9849670 & -0.5526960 & -1.8932950 \\
\hline 0 & -0.4996370 & -0.7217330 & 0.653392 \\
\hline 0 & 1.3036230 & -1.0079840 & -1.101892 \\
\hline C & -0.6521640 & -2.1061750 & 0.750392 \\
\hline C & 0.5039440 & -2.9017680 & 0.8379170 \\
\hline C & -1.9450040 & -2.6447210 & 0.7243620 \\
\hline C & -2.0464520 & -4.0348920 & 0.833835 \\
\hline $\mathrm{H}$ & -3.0398620 & -4.4801510 & 0.8411730 \\
\hline C & 2.2075150 & -1.3046200 & -0.0878100 \\
\hline C & 3.4680690 & -0.6941910 & -0.131093 \\
\hline C & 1.8414260 & -2.2409560 & 0.900011 \\
\hline C & 4.37 & -1.02 & 338 \\
\hline $\mathrm{H}$ & & -0.5 & 1 \\
\hline C & -3 & - & 51 \\
\hline C & & & \\
\hline
\end{tabular}




$\begin{array}{rrr}-3.5296920 & -0.7991650 & 1.4185520 \\ -2.8170580 & -0.4282300 & 2.6972050 \\ -4.9493260 & 0.9953400 & 2.2776240 \\ -4.6983480 & -0.0417230 & 1.2114340 \\ -2.4378490 & 1.0722450 & 2.6273570 \\ -1.9608840 & 1.3682400 & 3.5694810 \\ -1.7018290 & 1.2061620 & 1.8346320 \\ -3.7109640 & 1.9298750 & 2.3600810 \\ -3.8646020 & 2.6602870 & 3.1650560 \\ -3.6092150 & 2.4963430 & 1.4267150 \\ -5.8576050 & 1.5716260 & 2.0629420 \\ -1.9233780 & -1.0379490 & 2.8536370 \\ -5.0772850 & 0.2624310 & 3.6435230 \\ -5.1829420 & 1.0113430 & 4.4395760 \\ -5.9833350 & -0.3547370 & 3.6439690 \\ -3.8093630 & -0.6175030 & 3.8783820 \\ -3.3063480 & -0.3299180 & 4.8109870 \\ -4.0820400 & -1.6764420 & 3.9598210 \\ -3.9960810 & -2.0544050 & -0.5938820 \\ -3.7839820 & -3.0767160 & -1.6887360 \\ -5.9783520 & -1.7210040 & -1.9851740 \\ -5.1799640 & -1.3122830 & -0.7732790 \\ -4.9972860 & -4.0479010 & -1.6729550 \\ -4.8770550 & -4.7783730 & -2.4839490 \\ -5.0145430 & -4.6025260 & -0.7266760 \\ -6.3168940 & -3.2334540 & -1.8569130 \\ -6.8485400 & -3.5560940 & -2.7618790 \\ -6.9892980 & -3.3859750 & -1.0042820 \\ -6.8977340 & -1.1281710 & -2.0684010 \\ -2.8503400 & -3.6269900 & -1.5558540 \\ -5.0879140 & -1.5334440 & -3.2428430 \\ -5.6462180 & -1.8675150 & -4.1277660 \\ -4.8531910 & -0.4715180 & -3.3726370 \\ -3.7778860 & -2.3582540 & -3.0670420 \\ -3.6951060 & -3.1228380 & -3.8509680 \\ -2.8977450 & -1.7149140 & -3.1352080 \\ -5.5306850 & -0.3051470 & 0.1243210 \\ -6.4479760 & 0.2670950 & -0.0187320 \\ 4.2751460 & 0.7479700 & -3.5640090 \\ 4.3175190 & 0.1312760 & -4.9394490 \\ 3.6798430 & -1.5576760 & -3.0716310 \\ 3.9140360 & -0.1586840 & -2.5501850 \\ 5.3478590 & -1.0323020 & -4.9225860 \\ 5.3298400 & -1.5369380 & -5.8978040 \\ 6.3562400 & -0.6271440 & -4.7765110 \\ 4.9822810 & -2.0295810 & -3.7781870\end{array}$

5.7941030

3.4129510

4.5900520

2. 5510410

2. 4407890

1.6057220

2. 9195580

2. 9448090

2.1755280

4.4849100

4.7359390

4.0279950

4.1197880

3.4349260

3.6273290

2.6478490

2. 9951670

2. 9239920

2.0167630

3.7230300

5.0348870

5.4087480

5.3236220

6.1492780

5.8450840

6.0054860

6.7859810

4.5624580

4.8406130

4.0367830

5.0063920

5.0107850

3.5932790

2.4607380

2.7635690

4.7055720

5.7179990

3. 5143190

2. 3525310

6.0107060

5.3703780

3.4288020

1. 5059060

0.3626870

$-0.9282080$

\begin{tabular}{rr}
-3.0382830 & -4.1808590 \\
-2.0902870 & -3.0434250 \\
-2.2514500 & -2.2696650 \\
0.8753290 & -5.6978530 \\
-1.5117560 & -4.1385740 \\
-2.5148990 & -4.5719510 \\
-1.2515640 & -3.6551530 \\
-0.4749580 & -5.2446740 \\
-0.9510460 & -6.2340740 \\
0.3298390 & -5.2812130 \\
2.5043830 & -1.9326190 \\
3.9083870 & -1.4410020 \\
2.2677280 & 0.4419590 \\
1.6051780 & -0.9120120 \\
4.4264480 & -0.7671530 \\
5.4200350 & -0.3405600 \\
4.5347500 & -1.5226500 \\
3.4232250 & 0.3414470 \\
3.9217520 & 1.3162690 \\
2.9989510 & 0.1071720 \\
1.5591260 & 1.2159560 \\
4.5681900 & -2.2644920 \\
2.8868180 & 0.7895360 \\
3.4242730 & 1.7433420 \\
2.0886750 & 0.9221800 \\
3.8581040 & -0.3532780 \\
4.8707900 & 0.0397940 \\
3.5241100 & -0.8065640 \\
2.0787650 & -3.2580780 \\
2.7783760 & -4.0472470 \\
-1.9029540 & 1.9099440 \\
-2.2801150 & 3.0026490 \\
-3.8139740 & 3.1798140 \\
-4.3567070 & 3.5026180 \\
-3.3509110 & 3.1516070 \\
-2.5025850 & 1.9362640 \\
-1.8150740 & 3.9556000 \\
-4.1024680 & 3.9672660 \\
-4.5928930 & 4.5711100 \\
-2.6630040 & 4.0034110 \\
-1.9092140 & 2.7655040 \\
-4.2568620 & 2.2419980 \\
-5.2945750 & 2.9596350 \\
-3.8720630 & 3.0539270 \\
-4.3101840 & 0.8200630 \\
-4.8719740 & 0.8655160 \\
& \\
\hline
\end{tabular}




$\begin{array}{rrr}1.5672290 & -5.2149530 & 0.6124260 \\ 1.3422470 & -6.6531290 & 1.0917960 \\ 0.0462620 & -7.2082610 & 0.4904280 \\ -1.1495520 & -6.3732920 & 0.9653620 \\ 2.2022420 & -7.2735940 & 0.8082780 \\ 1.7695090 & -5.2362540 & -0.4714060 \\ 2.4648710 & -4.7976850 & 1.0677650 \\ 0.1159310 & -7.1647050 & -0.6065930 \\ -0.1000740 & -8.2598680 & 0.7690390 \\ -2.0562740 & -6.6438920 & 0.4082260 \\ -1.3519280 & -6.6191400 & 2.0205150 \\ 1.2723490 & -6.6697050 & 2.1902670 \\ -2.1878490 & 2.2950260 & -2.8051580 \\ -2.9815870 & 2.2541800 & -1.5743380 \\ -1.1055270 & 2.3248270 & -2.6635700 \\ -2.5108690 & 1.8431570 & -0.6870290 \\ -2.7014660 & 0.9269310 & -2.8214130 \\ -1.3774350 & 4.4312830 & 0.7596000 \\ -0.3556030 & 4.3537960 & 3.0692990 \\ -0.0028440 & 3.2787580 & 2.2382290 \\ -0.5731110 & 3.3688320 & 0.9812900 \\ -0.3674720 & 2.6340040 & 0.2666390 \\ -1.4279230 & 5.4349420 & 2.2053500 \\ -2.2244220 & 4.7893100 & -0.6425760 \\ -1.9456740 & 0.2769130 & -2.3942220 \\ 0.9412560 & 3.3763760 & 4.8347080 \\ 1.2756210 & 2.2888280 & 3.9940070 \\ 0.8103470 & 2.2324560 & 2.6770680 \\ 0.1280850 & 4.4088560 & 4.3814430 \\ 1.3259660 & 3.3827680 & 5.8508310 \\ 1.0348220 & 1.4167310 & 2.0012060 \\ -0.1301130 & 5.2330800 & 5.0407090 \\ -4.2438870 & 3.1087290 & -4.0455290 \\ -4.8777130 & 3.4993490 & -2.7003750 \\ -4.4465610 & 2.5521490 & -1.5665810 \\ -2.7072170 & 3.1286850 & -3.9587720 \\ -4.5728990 & 3.8003450 & -4.8303980 \\ -5.9718230 & 3.4931500 & -2.7755530 \\ -4.9644220 & 1.5857430 & -1.6409190 \\ -2.3520160 & 4.1555810 & -3.7960090 \\ -2.2526390 & 2.7644190 & -4.8886350 \\ -4.5768840 & 2.1023890 & -4.3322070 \\ -4.5744840 & 4.5237140 & -2.4433800 \\ -4.7073650 & 2.9538960 & -0.5811100 \\ 2.0564860 & 1.3279990 & 4.5606250 \\ 2.3453370 & 0.1716030 & 3.7624680\end{array}$

$\begin{array}{llrl}\mathrm{H} & 2.9016920 & 0.4415540 & 2.8570860 \\ \mathrm{H} & 1.4208860 & -0.3447530 & 3.4718840 \\ \mathrm{H} & 2.9573880 & -0.4769790 & 4.3915780\end{array}$

158

3. 4718840

TS72 (S, S) G[B97-D/def2-TZVP $]=-4356.203745$

\begin{tabular}{|c|c|c|c|}
\hline $\mathrm{P}$ & -0.0329690 & -0.1322630 & -0.7999370 \\
\hline 0 & -0.9849640 & -0.5526960 & -1.8932950 \\
\hline 0 & 0.2397070 & 1. 3222030 & -0.5878420 \\
\hline 0 & 1. 3036260 & -1.0079800 & -1.1018900 \\
\hline 0 & -0.4996370 & -0.7217310 & 0.6533930 \\
\hline C & 2.2075180 & -1.3046150 & -0.0878080 \\
\hline C & 1.8414300 & -2.2409520 & 0.9000130 \\
\hline C & 3.4680720 & -0.6941860 & -0.1310920 \\
\hline $\mathrm{C}$ & 4.3784350 & -1.0286290 & 0.8788380 \\
\hline $\mathrm{H}$ & 5.3712980 & -0.5828170 & 0.8571300 \\
\hline $\mathrm{C}$ & -0.6521600 & -2.1061740 & 0.7503940 \\
\hline C & -1.9449990 & -2.6447220 & 0.7243630 \\
\hline C & 0.5039490 & -2.9017660 & 0.8379200 \\
\hline C & -2.0464450 & -4.0348940 & 0.8338380 \\
\hline $\mathrm{H}$ & -3.0398540 & -4.4801540 & 0.8411750 \\
\hline $\mathrm{C}$ & 3.8352330 & 0.2611930 & -1.2105030 \\
\hline $\mathrm{C}$ & -3.1612590 & -1.8135770 & 0.5163620 \\
\hline $\mathrm{C}$ & 3.9140360 & -0.1586810 & -2.5501850 \\
\hline $\mathrm{C}$ & 3.6798460 & -1.5576730 & -3.0716300 \\
\hline C & 4.3175160 & 0.1312780 & -4.9 \\
\hline C & 4.2751440 & 0.7479730 & -3.5 \\
\hline C & 2.5510420 & -1.5117560 & -4.1 \\
\hline $\mathrm{H}$ & 2.44 & -2.5149000 & -4.5 \\
\hline $\mathrm{H}$ & 1.6057220 & -1.2515680 & -3.6 \\
\hline C & 2.9195540 & -0.4749580 & 6720 \\
\hline $\mathrm{H}$ & 2.9448040 & -0.9510450 & -6 \\
\hline $\mathrm{H}$ & 5230 & 0.3298380 & -5 \\
\hline $\mathrm{H}$ & 4.59 & 0.8753 & -5 \\
\hline $\mathrm{H}$ & 3.4 & -2.2514470 & 6620 \\
\hline C & 5.3478570 & -1.0322980 & -4.92 \\
\hline $\mathrm{H}$ & 5.3298350 & -1.5369360 & -5.8 \\
\hline $\mathrm{H}$ & 6.3562380 & -0.6271400 & -4.7765170 \\
\hline C & 4.9822840 & -2.0295770 & -3.7781860 \\
\hline $\mathrm{H}$ & 4.8205310 & -3.0382800 & -4.1808560 \\
\hline $\mathrm{H}$ & 5.7941070 & -2.0902780 & -3.0434260 \\
\hline C & 4.1197880 & 1.6051840 & -0.9120140 \\
\hline C & 4.0279960 & 2.2677340 & 0.4419570 \\
\hline C & 4.7359330 & 3.9083930 & -1.4410060 \\
\hline C & 49070 & 2.5043880 & -1.9326220 \\
\hline$c_{-1}$ & 7480 & 2.8868280 & 0.7895310 \\
\hline & 5.3236220 & 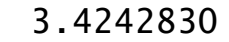 & 1.1433300 \\
\hline
\end{tabular}




$\begin{array}{lrrr}\text { H } & 6.1492800 & 2.0886850 & \\ \mathrm{C} & 5.8450800 & 3.8581130 & -0.9221740 \\ \mathrm{H} & 6.0054790 & 4.8708010 & 0.0397830 \\ \mathrm{H} & 6.7859770 & 3.5241220 & -0.8065710 \\ \mathrm{H} & 5.0348780 & 4.5681960 & -2.2644970 \\ \mathrm{H} & 3.7230340 & 1.5591320 & 1.2159550 \\ \mathrm{C} & 3.4349200 & 4.4264520 & -0.7671560 \\ \mathrm{H} & 3.6273200 & 5.4200400 & -0.3405660 \\ \mathrm{H} & 2.6478410 & 4.5347500 & -1.5226520 \\ \mathrm{C} & 2.9951650 & 3.4232290 & 0.3414460 \\ \mathrm{H} & 2.9239910 & 3.9217570 & 1.3162670 \\ \mathrm{H} & 2.0167610 & 2.9989530 & 0.1071740 \\ \mathrm{C} & 4.5624530 & 2.0787690 & -3.2580810 \\ \mathrm{H} & 4.8406060 & 2.7783800 & -4.0472510 \\ \mathrm{C} & -4.6983500 & -0.0417300 & 1.2114350 \\ \mathrm{C} & -4.9493300 & 0.9953310 & 2.2776250 \\ \mathrm{C} & -2.8170600 & -0.4282350 & 2.6972070 \\ \mathrm{C} & -3.5296920 & -0.7991700 & 1.4185530 \\ \mathrm{C} & -5.0772880 & 0.2624220 & 3.6435240 \\ \mathrm{H} & -5.1829440 & 1.0113340 & 4.4395770 \\ \mathrm{H} & -5.9833380 & -0.3547460 & 3.6439710 \\ \mathrm{C} & -3.8093650 & -0.6175110 & 3.8783820 \\ \mathrm{H} & -3.3063520 & -0.3299280 & 4.8109890 \\ \mathrm{H} & -4.0820420 & -1.6764510 & 3.9598180 \\ \mathrm{H} & -1.9233770 & -1.0379510 & 2.8536390 \\ \mathrm{H} & -5.8576100 & 1.5716150 & 2.0629430 \\ \mathrm{C} & -2.4378540 & 1.0722420 & 2.6273600 \\ \mathrm{H} & -1.9608910 & 1.3682370 & 3.5694860 \\ \mathrm{H} & -1.7018320 & 1.2061610 & 1.8346370 \\ \mathrm{C} & -3.7109700 & 1.9298690 & 2.3600820 \\ \mathrm{H} & -3.8646110 & 2.6602810 & 3.1650580 \\ \mathrm{H} & -3.6092210 & 2.4963370 & 1.4267170 \\ \mathrm{C} & -5.1799600 & -1.3122900 & -0.7732800 \\ \mathrm{C} & -5.9783460 & -1.7210130 & -1.9851750 \\ \mathrm{C} & -3.7839740 & -3.0767210 & -1.6887360 \\ \mathrm{C} & -3.9960760 & -2.0544100 & -0.5938820 \\ \mathrm{C} & -5.0879080 & -1.5334510 & -3.2428440 \\ \mathrm{H} & -5.6462100 & -1.8675230 & -4.1277680 \\ \mathrm{H} & -4.8531860 & -0.4715240 & -3.3726370 \\ \mathrm{C} & -3.7778780 & -2.3582590 & -3.0670420 \\ \mathrm{H} & -3.6950950 & -3.1228430 & -3.8509680 \\ \mathrm{H} & -2.8977380 & -1.7149170 & -3.1352080 \\ \mathrm{H} & -2.8503310 & -3.6269930 & -1.5558530 \\ \mathrm{H} & -6.8977300 & -1.1281820 & -2.0684030 \\ \mathrm{C} & -4.9972750 & -4.0479080 & -1.6729560 \\ \mathrm{H} & -4.8770420 & -4.7783800 & -2.4839500 \\ & & & \end{array}$

-5.0145320
-6.3168850
-6.8485290
-6.9892900
-5.5306840
-6.4479760
-0.9281990
0.3626950
-1.1495410
0.0462740
1.3422580
1.5672370
-0.1000600
-1.3519150
-2.0562630
1.2723610
2.2022540
2.4648790
1.7695180
0.1159430
4.0367870
5.0063960
5.0107920
3.5932860
2.4607440
2.7635740
4.7055760
5.7180040
3.5143250
2.3525380
6.0107110
5.3703890
3.4288110
1.5059120
-2.1878520
-2.9815940
-1.1055300
-2.5108770
-2.7014640
-1.3774480
-0.3556130
-0.0028510
-0.5731200
-0.3674790
-1.4279380
-2.2244380
-4.6025330
-3.2334630
-3.5561050
-3.3859870
-0.3051550
0.2670850
-4.8719740
-4.3101820
-6.3732920
-7.2082590
-6.6531260
-5.2149490
-8.2598670
-6.6191410
-6.6438930
-6.6697030
-7.2735880
-4.7976800
-5.2362480
-7.1647010
-1.9029470
-2.2801060
-3.8139640
-4.3567000
-3.3509040
-2.5025790
-1.8150630
-4.1024550
-4.5928880
-2.6629960
-1.9092030
-4.2568530
-5.2945670
-3.8720570
2.2950230
2.2541720
2.3248290
1.8431480
0.9269270
4.4312790
4.3537960
3.2787590
3.3688320
2.6340040
5.4349380
4.7893030
$-0.7266770$

$-1.8569150$

$-2.7618820$

$-1.0042860$

0.1243200

$-0.0187340$

0.8655190

0.8200660

0.9653650

0.4904290

1.0917970

0.6124280

0.7690380

2.0205180

0.4082300

2.1902680

0.8082780

1.0677680

$-0.4714040$

$-0.6065920$

1.9099460

3.0026500

3.1798190

3.5026200

3.1516100

1.9362660

3.9556010

3.9672730

4.5711110

4.0034140

2.7655050

2. 2420040

2.9596350

3.0539310

$-2.8051600$

$-1.5743420$

$-2.6635690$

$-0.6870330$

$-2.8214200$

0.7596020

3.0693000

2.2382300

0.9812910

0.2666400

2.2053520
-0.6425730 


\begin{tabular}{|c|c|c|c|c|c|c|c|}
\hline $\mathrm{H}$ & & & & & & & \\
\hline C & -1.9456710 & 0.2769100 & -2.3942280 & $\mathrm{C}$ & 4.6905400 & 1. 8795510 & $\begin{array}{l}-2.3691190 \\
-1\end{array}$ \\
\hline C & 1.2756190 & 2.2888330 & 3.9940060 & c & 2.1731050 & 1.5879670 & -2.5199740 \\
\hline C & 0.8103440 & 2.2324590 & 2.6770680 & $\mathrm{H}$ & 1.5681900 & 1.8496360 & -3.3972240 \\
\hline C & 0.1280770 & 4.4088580 & 4.3814440 & $\mathrm{H}$ & 1.5074110 & 1.5689600 & -1.6578570 \\
\hline $\mathrm{H}$ & 1.3259620 & 3.3827730 & 5.8508300 & C & 3.3244230 & 2.6178980 & -2.3112980 \\
\hline $\mathrm{H}$ & 1.0348200 & 1.4167350 & 2.0012060 & $\mathrm{H}$ & 3.3019780 & 3.3908300 & -3.0904800 \\
\hline $\mathrm{H}$ & -0.1301240 & 5.2330810 & 5.0407100 & $\mathrm{H}$ & 3.2183700 & 3.1259240 & -1.3456590 \\
\hline C & -4.2438890 & 3.1087230 & -4.0455350 & $\mathrm{H}$ & 5.5167240 & 2.5780350 & -2.1884670 \\
\hline C & -4.8777200 & 3.4993370 & -2.7003810 & $\mathrm{H}$ & 1.9628490 & -0.5601730 & -2.8422800 \\
\hline C & -4.4465690 & 2.5521360 & -1.5665890 & C & 4.8204050 & 1.2343650 & -3.7774200 \\
\hline C & -2.7072200 & 3.1286830 & -3.9587740 & $\mathrm{H}$ & 4.7662790 & 2.0244710 & -4.5381400 \\
\hline $\mathrm{H}$ & -4.5729010 & 3.8003400 & -4.8304020 & $\mathrm{H}$ & 5.8003630 & 0.7511290 & -3.8683080 \\
\hline H & -5.9718300 & 3.4931360 & -2.7755620 & C & 3.6719730 & 0.1964420 & -3.9752030 \\
\hline $\mathrm{H}$ & -4.9644260 & 1.5857280 & -1.6409330 & $\mathrm{H}$ & 3.0550110 & 0.4634740 & -4.8434210 \\
\hline $\mathrm{H}$ & -2.3520210 & 4.1555800 & -3.7960080 & $\mathrm{H}$ & 4.0808550 & -0.8051450 & -4.1537620 \\
\hline $\mathrm{H}$ & -2.2526380 & 2.7644210 & -4.8886360 & C & 4.4471670 & -1.4217690 & 0.3716900 \\
\hline $\mathrm{H}$ & -4.5768820 & 2.1023830 & -4.3322170 & $\mathrm{C}$ & 4.5004050 & -2.5420630 & 1.3860960 \\
\hline $\mathrm{H}$ & -4.5744950 & 4.5237030 & -2.4433820 & c & 6.4465650 & -0.8408200 & 1.6498280 \\
\hline $\mathrm{H}$ & -4.7073780 & 2.9538770 & -0.5811170 & $\mathrm{C}$ & 5.5055660 & -0.5040420 & 0.5207080 \\
\hline 0 & 2.0564870 & 1.3280070 & 4.5606240 & $\mathrm{C}$ & 5.8588760 & -3.2770230 & 1.2075730 \\
\hline C & 2.3453390 & 0.1716100 & 3.7624680 & $\mathrm{H}$ & 5.9270390 & -4.0799920 & 1.9533060 \\
\hline $\mathrm{H}$ & 2.9016930 & 0.4415620 & 2.8570850 & $\mathrm{H}$ & 5.9005990 & -3.7411070 & 0.2145260 \\
\hline $\mathrm{H}$ & 1.4208900 & -0.3447480 & 3.4718850 & C & 7.0296390 & -2.2586020 & 1.3879820 \\
\hline $\mathrm{H}$ & 2.9573920 & -0.4769690 & 4.3915780 & $\mathrm{H}$ & 7.6639930 & -2.5441960 & 2.2375860 \\
\hline & & & & $\mathrm{H}$ & 7.6632460 & 2339700 & 0.4934980 \\
\hline & $\mathrm{G}[\mathrm{BS}$ & $=-43$ & & $\mathrm{H}$ & 7.2533720 & -0.1011230 & 1.7248970 \\
\hline $\mathrm{P}$ & 0.1088430 & -0.3248430 & 1.0534910 & $\mathrm{H}$ & 3.6679370 & -3.2391720 & 1.2655210 \\
\hline 0 & -0.3900180 & 1.0859830 & 1.0644090 & C & 5.6289810 & -0.8971080 & 2.9686280 \\
\hline 0 & 1.0932330 & -0.7597220 & 2.1139780 & $\mathrm{H}$ & 6.3007940 & -1.1687630 & 3.7937860 \\
\hline 0 & 0.7154780 & -0.5640730 & -0.4509760 & $\mathrm{H}$ & 5.2121710 & 0.0921520 & 3.1880140 \\
\hline 0 & -1.0810490 & -1.4292710 & 1.1435500 & C & 4.4825510 & -1.9419920 & 2.8187860 \\
\hline $\mathrm{c}$ & 1.0505400 & -1.8763330 & -0.7923560 & $\mathrm{H}$ & 4.6132850 & -2.7632650 & 3.5358430 \\
\hline c & 0.0108230 & -2.7829450 & -1.0474020 & $\mathrm{H}$ & 3.5089960 & -1.4863570 & 3.0111240 \\
\hline $\mathrm{c}$ & 2.4020180 & -2.2390850 & -0.8527660 & C & 5.6244580 & 0.5905750 & -0.3345430 \\
\hline c & 2.6788230 & -3.5840790 & -1.1119640 & $\mathrm{H}$ & 6.4459910 & 1.2975290 & -0.2135170 \\
\hline $\mathrm{H}$ & 3.7208520 & -3.8951240 & -1.1666700 & C & -4.4531550 & -0.5495720 & 3.6567140 \\
\hline C & -1.9107640 & -1.6153830 & 0.0379700 & C & -4.5063260 & -1.4350110 & 4.8762410 \\
\hline C & -3.2301190 & -1.1505970 & 0.0995610 & C & -3.4891580 & -2.6077540 & 2.7927620 \\
\hline c & -1.3900760 & -2.2717800 & -1.0885660 & $\mathrm{C}$ & -3.8895510 & -1.1745510 & 2.5286090 \\
\hline c & -4.0115180 & -1.3269540 & -1.0450630 & $\mathrm{C}$ & -5.3543220 & -2.6891540 & 4.5261420 \\
\hline $\mathrm{H}$ & -5.0395740 & -0.9685750 & -1.0260540 & $\mathrm{H}$ & -5.3479540 & -3.3746410 & 5.3842730 \\
\hline c & 3.4964150 & -1.2542560 & -0.6527130 & $\mathrm{H}$ & -6.3940410 & -2.3899090 & 4.3476280 \\
\hline c & -3.7877280 & -0.4868510 & 1.3062790 & C & -4.7541910 & -3.3811900 & 3.2625950 \\
\hline c & 3.6306120 & -0.1489030 & -1.5149330 & $\mathrm{H}$ & -4.4702640 & -4.4179660 & 3.4871860 \\
\hline & 2.7655970 & 0.1695870 & -2.7128900 & $\mathrm{H}$ & -5.4886980 & -3.4061810 & 2.4486450 \\
\hline
\end{tabular}




$\begin{array}{ll}-3.0662610 & -3.0833150 \\ -4.9409120 & -0.9035460 \\ -2.4590340 & -2.6294740 \\ -2.2186660 & -3.6752310 \\ -1.5368820 & -2.1398440 \\ -3.0642690 & -1.9126100 \\ -3.1019560 & -2.5931390 \\ -2.4508970 & -1.0491730 \\ -4.8330510 & 1.4491620 \\ -5.2879910 & 2.8670690 \\ -4.1978260 & 1.7617860 \\ -4.2623230 & 0.8359520 \\ -4.0584010 & 3.7054940 \\ -4.3938420 & 4.7195800 \\ -3.3502030 & 3.7900480 \\ -3.3835470 & 3.0176390 \\ -3.3303440 & 3.7031870 \\ -2.3665200 & 2.7086070 \\ -3.7319590 & 1.2813790 \\ -5.7446270 & 3.2947030 \\ -5.6381450 & 2.2211590 \\ -5.5834890 & 2.9484250 \\ -6.2229970 & 1.3636050 \\ -6.3050730 & 2.8607350 \\ -6.6160160 & 3.8931850 \\ -7.1985320 & 2.2966820 \\ -4.9271940 & 0.7604540 \\ -5.3677960 & 1.2393850 \\ -2.1855250 & -2.3669950 \\ -1.5958850 & -2.8697860 \\ -2.3129060 & -2.2845350 \\ -3.8124700 & -2.5866490 \\ -4.4198250 & -1.9159430 \\ -3.5114250 & -1.8969680 \\ -1.8936060 & -2.7211970 \\ -1.6814240 & -3.9635880 \\ -0.5237410 & -2.6426120 \\ -3.9422180 & -3.6768710 \\ -4.3490560 & -2.2543160 \\ -5.3675420 & -2.4052830 \\ -4.6747820 & -0.8727600 \\ -2.1481790 & -1.2006450 \\ 0.3223660 & -4.1527980 \\ -0.7836190 & -5.1941220 \\ -0.2934210 & -6.5964270 \\ 0.9346120 & -6.9548590\end{array}$

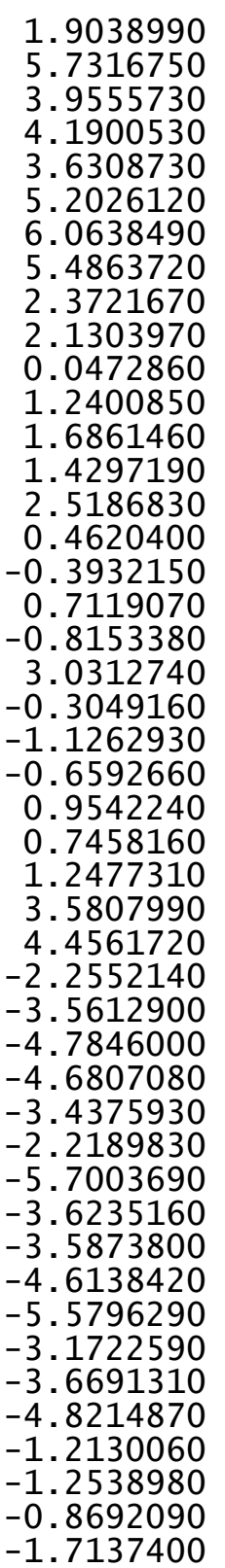

2.0898070

1.6735710

$-1.1026530$

$-1.2056100$

$-1.6042720$

0.6739440

1.2452330

2.8940710

2. 5289240

$-0.0241250$

1.9455080

2.6557070

0.8646290

2.1906260

2.6548070

0.5746150

$-0.6171910$

$-0.7551300$

$-0.0875630$

$-0.1373770$

0.3794590

1.4611390

1. 9722040

$-1.9832390$

$-2.1112820$

$-1.4884310$

$-1.2318720$

$-2.4668340$

$-1.5807240$

$-1.1357680$

3.9384010

4.4066380

4.0524300

2.4140260

4.2145830

5.4902610

4.7215090

1.8976550

2.0853490

4.4383430

3.9255980

4.1768180

$-2.8339610$

$-3.4690430$

$-2.7301770$

$-4.2209530$
$-6.0048820$
$-4.5469400$
$-7.3218380$
$-5.2581770$
$-4.8716560$
$-6.8584810$
$-7.9938940$
$-6.0893710$
$-6.3153900$
$-6.6205280$
2.1678140
2.2889380
2.0384270
1.8490770
0.8902760
4. 3289490
4. 3199600
3. 1274240
3.1830810
2. 3815370
5.4544250
.7025780
0.1613540
3. 3798540
2.1879220
2.0476430
4.4484180
3. 4824840
1.1273940
5. 3613260
3.2303430
3.7726790
2.8151610
3.0174880
3.9286130
3.9415720
1.9439120
3.9842020
2. 5451370
2.2753160
. 7430120
3.2931700
. 1096790
1.1919920
1.3684540
1.9940690

$-1.3719610$

$-1.2494060$

$-1.0223760$

$-2.2652900$

$-0.6015320$

$-2.7786040$

$-1.5450200$

$-2.1159640$

$-0.4107180$

0.1972240

3.1416090

1.8652230

3.0657930

0.9892250

3.0558180

$-0.3059590$

$-2.5287980$

$-1.7873860$

$-0.5754940$

0.1053920

$-1.6458230$

1.0668110

2.6358310

$-4.2654050$

$-3.5209020$

$-2.2721750$

$-3.7709040$

$-5.2297820$

$-1.7041110$

$-4.3524080$

4.2942240

2.9341120

1.7830500

4.3046610

5.0934360

2.9394770

1.7726370

4.2231990

5.2389600

4.5031210

2.7488630

0.8053290

$-3.9450840$

$-5.2254350$

-6.0203130
-5.2397720 
H

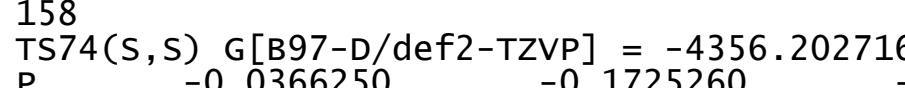

$\begin{array}{lrrr}\text { TS74(S , S }) \text { G }[B 97-D / d e f 2-T Z V P] & -4356.202716 \\ \text { P } & -0.0366250 & -0.1725260 & -0.8465630 \\ \text { O } & 0.2667890 & 1.2826060 & -0.6884170 \\ \text { O } & -0.9812720 & -0.6144360 & -1.9391400 \\ \text { O } & -0.5445990 & -0.6905920 & 0.6214560 \\ \text { O } & 1.2855470 & -1.0877520 & -1.0929060 \\ \mathrm{C} & -0.7365990 & -2.0666550 & 0.7634080 \\ \mathrm{C} & 0.3977360 & -2.8861060 & 0.8816680 \\ \mathrm{C} & -2.0426720 & -2.5742890 & 0.7437770 \\ \mathrm{C} & -2.1742070 & -3.9619930 & 0.8537480 \\ \mathrm{H} & -3.1767870 & -4.3857940 & 0.8619450 \\ \mathrm{C} & 2.1592770 & -1.3733470 & -0.0492060 \\ \mathrm{C} & 3.4410520 & -0.8084530 & -0.0864940 \\ \mathrm{C} & 1.7486290 & -2.2539400 & 0.9656040 \\ \mathrm{C} & 4.3112370 & -1.1425670 & 0.9527730 \\ \mathrm{H} & 5.3200020 & -0.7326450 & 0.9341360 \\ \mathrm{C} & -3.2385750 & -1.7094230 & 0.5595600 \\ \mathrm{C} & 3.8790820 & 0.0744550 & -1.2002630 \\ \mathrm{C} & -3.5381320 & -0.6678120 & 1.4587930 \\ \mathrm{C} & -2.7686050 & -0.3020850 & 2.7063600 \\ \mathrm{C} & -4.8580370 & 1.1948240 & 2.3346530 \\ \mathrm{C} & -4.6864150 & 0.1277680 & 1.2821490 \\ \mathrm{C} & -2.3360500 & 1.1823310 & 2.5993610 \\ \mathrm{H} & -1.8274370 & 1.4758210 & 3.5256640 \\ \mathrm{H} & -1.6142910 & 1.2779970 & 1.7882870 \\ \mathrm{C} & -3.5830790 & 2.0812370 & 2.3461200 \\ \mathrm{H} & -3.6815010 & 2.8429660 & 3.1303060 \\ \mathrm{H} & -3.4929190 & 2.6102510 & 1.3899260 \\ \mathrm{H} & -5.7512560 & 1.8009200 & 2.1402280 \\ \mathrm{H} & -1.8943440 & -0.9425990 & 2.8460110 \\ \mathrm{C} & -4.9616640 & 0.4981670 & 3.7210400 \\ \mathrm{H} & -4.9990390 & 1.2682340 & 4.5030400 \\ \mathrm{H} & -5.8944200 & -0.0755130 & 3.7742220 \\ \mathrm{C} & -3.7264060 & -0.4341440 & 3.9231150 \\ \mathrm{H} & -3.1787420 & -0.1587930 & 4.8340760 \\ \mathrm{H} & -4.0420680 & -1.4790440 & 4.0274860 \\ \mathrm{C} & -4.1229520 & -1.9369680 & -0.5147270 \\ \mathrm{C} & -3.9938720 & -2.9864550 & -1.5977050 \\ \mathrm{C} & -6.1391710 & -1.5447460 & -1.8417570 \\ \mathrm{C} & -5.2835820 & -1.1523320 & -0.6640890 \\ \mathrm{C} & -5.2447270 & -3.9049970 & -1.5211890 \\ \mathrm{H} & -5.1820010 & -4.6545180 & -2.3210810 \\ \mathrm{H} & -5.2522540 & -4.4406850 & -0.5639730 \\ \mathrm{C} & -6.5351410 & -3.0396570 & -1.6770090 \\ & & & \end{array}$

-7.1087940
-7.1847830
-7.0350950
-3.0792910
-5.2860890
-5.8890650
-5.0108960
-4.0068790
-3.9836840
-3.1032810
-5.5661450
-6.4661060
4.4652760
4.5638340
3.7376100
4.0085990
5.5384300
5.5664950
6.5510520
5.0520280
4.8602610
5.8140020
3.3924610
4.9152310
2.6739780
2.5284240
1.7206240
3.1618310
3.2317970
2.4590140
4.6581910
4.9374520
4.0561890
4.1989020
3.6215040
3.8288440
2.8842830
3.0777120
2.9717450
2.0977580
3.6787310
5.3100650
5.4384220
5.3213540
6.1376340
5.9771780
$-2.5588610$ $-0.8007530$ $-1.9035480$ $-1.4891330$ $-3.1326730$ $-3.9914550$ $-3.2915320$ $-2.9877800$ $-3.7605440$ $-3.0986160$ 0.2298780 0.1089500 $-3.5512660$ $-4.8779200$ $-2.9472180$ $-2.5044900$ $-4.7155100$ $-5.6569970$ $-4.5226930$ $-3.5426750$ $-3.8967800$ $-2.7560560$ $-2.1193570$ $-5.6652220$ $-4.0788540$ $-4.4463840$ $-3.6702350$ $-5.2319230$ $-6.1780900$ $-5.3805360$ $-2.0244490$ $-1.6081810$ 0.3299380 $-0.9719070$ $-1.0496340$ $-0.6771860$ $-1.8565070$ 0.0931290 1.0279650 $-0.1705790$ 1.1267080 $-2.4520970$ 0.7198140 1.6322080 0.9450360 $-0.4531360$ 


$\begin{array}{rrrr}\mathrm{H} & 6.1505790 & 4.6749730 & -0.1158180 \\ \mathrm{H} & 6.9306110 & 3.2510650 & -0.8253330 \\ \mathrm{C} & 4.7934430 & 1.7233890 & -3.3133530 \\ \mathrm{H} & 5.1474390 & 2.3577810 & -4.1268860 \\ \mathrm{C} & 3.9299490 & -1.9691840 & 2.0155990 \\ \mathrm{C} & 2.6307170 & -2.5087130 & 2.0456480 \\ \mathrm{C} & 4.9692310 & -2.2931690 & 3.0798400 \\ \mathrm{C} & 4.4078570 & -2.9398760 & 4.3546040 \\ \mathrm{C} & 3.3310800 & -3.9724920 & 4.0052510 \\ \mathrm{C} & 2.1737120 & -3.2877260 & 3.2693250 \\ \mathrm{H} & 5.2259850 & -3.3983690 & 4.9245820 \\ \mathrm{H} & 5.7000590 & -2.9831210 & 2.6292520 \\ \mathrm{H} & 5.5293800 & -1.3815050 & 3.3287360 \\ \mathrm{H} & 3.7627810 & -4.7542160 & 3.3620240 \\ \mathrm{H} & 2.9549940 & -4.4627850 & 4.9122360 \\ \mathrm{H} & 1.4035060 & -4.0153090 & 3.0085410 \\ \mathrm{H} & 1.6905750 & -2.5783470 & 3.9601360 \\ \mathrm{H} & 3.9591620 & -2.1722570 & 5.0012900 \\ \mathrm{C} & 0.2287340 & -4.2914270 & 0.8609860 \\ \mathrm{C} & -1.0742670 & -4.8244840 & 0.8838220 \\ \mathrm{C} & 1.4247730 & -5.2156740 & 0.6812660 \\ \mathrm{C} & 1.1516320 & -6.6642290 & 1.1034310 \\ \mathrm{C} & -0.1464810 & -7.1667100 & 0.4623340 \\ \mathrm{C} & -1.3284510 & -6.3220270 & 0.9532850 \\ \mathrm{H} & 2.0015730 & -7.2958920 & 0.8146520 \\ \mathrm{H} & 1.6821430 & -5.2036710 & -0.3907120 \\ \mathrm{H} & 2.3065080 & -4.8316390 & 1.1983150 \\ \mathrm{H} & -0.0615110 & -7.0839000 & -0.6314150 \\ \mathrm{H} & -0.3197460 & -8.2243150 & 0.6997520 \\ \mathrm{H} & -2.2379510 & -6.5615360 & 0.3865820 \\ \mathrm{H} & -1.5389320 & -6.5889560 & 2.0017430 \\ \mathrm{H} & 1.0601440 & -6.7179610 & 2.1992410 \\ \mathrm{C} & -2.1694460 & 2.2130330 & -2.9212080 \\ \mathrm{C} & -2.9251100 & 2.2436050 & -1.6663020 \\ \mathrm{H} & -1.0826890 & 2.2264550 & -2.8145960 \\ \mathrm{H} & -2.4337920 & 1.8669090 & -0.7749130 \\ \mathrm{O} & -2.7096230 & 0.8570990 & -2.8542150 \\ \mathrm{C} & -1.2505970 & 4.4848830 & 0.5380540 \\ \mathrm{C} & -0.2271220 & 4.4865160 & 2.8477940 \\ \mathrm{C} & 0.1033630 & 3.3694290 & 2.0638830 \\ \mathrm{~N} & -0.4694590 & 3.4150690 & 0.8055700 \\ \mathrm{H} & -0.2843290 & 2.6455960 & 0.1225710 \\ \mathrm{~S} & -1.2801870 & 5.5496190 & 1.9395290 \\ \mathrm{~S} & -2.0886670 & 4.8030330 & -0.8787410 \\ \mathrm{H} & -1.9464450 & 0.2093910 & 4.6513520 \\ \mathrm{C} & 1.0585000 & 3.5665590 & \\ & & & \end{array}$

1.3708030
0.9015810
0.2622870
1.4458790
1.1122150
0.0211160
-4.2484860
-4.8324410
-4.3829670
-2.7097110
-4.5911370
-5.9283590
-4.9228350
-2.3337560
-2.2894130
-4.6063840
-4.5026630
-4.6032160
2.1341440
2.3851820
2.9452300
1.4441240
2.9793000

2. 4368500

2. 3304410

4. 5919820

3. 6107070

1.4826460

5.4496820

3.0045220

3. 4727520

2. 5729290

3. 0020250

3.6615270

3.4839740

1. 6159500

4.0292160

2. 5880450

1. 9909910

4.5020480

3. 0279110

1. 4886720

0.2818670

0.4820800

$-0.2219370$

$-0.3499230$
3.8593190

2. 5465780

4. 1545400

5.6655120

1. 9064700

4.7765380

$-4.1359180$

$-2.7927850$

$-1.6279510$

$-4.0964590$

$-4.9443160$

$-2.8337930$

$-1.6388450$

$-3.9913380$

$-5.0216200$

$-4.3601410$

$-2.5960960$

$-0.6558910$

4.4688470

3.7354090

2. 8146590

3.4769020

4. 3967220
158

TS75 (S, S) G[B97-D/def2-TZVP $]=-4356.202611$

$\begin{array}{lrrr}\text { TS75 (S, S }) \text { G }[\mathrm{B} 97-\mathrm{D} / \mathrm{de} \text { ef }-\mathrm{TZVP}]=-4356.202611 \\ \mathrm{P} & -0.0691080 & -0.2528300 & -0.7295000 \\ \mathrm{O} & 0.2725770 & 1.2021490 & -0.7040330 \\ \mathrm{O} & -1.0560070 & -0.7589240 & -1.7542750 \\ \mathrm{O} & -0.5389400 & -0.6409440 & 0.7891030 \\ \mathrm{O} & 1.2240640 & -1.2175030 & -0.9327840 \\ \mathrm{C} & -0.7508660 & -1.9984740 & 1.0432070 \\ \mathrm{C} & 0.3787280 & -2.8255920 & 1.2160550 \\ \mathrm{C} & -2.0651370 & -2.4817350 & 1.0644720 \\ \mathrm{C} & -2.2334620 & -3.8575110 & 1.2817670 \\ \mathrm{H} & -3.2434230 & -4.2618800 & 1.3025690 \\ \mathrm{C} & 2.1272830 & -1.4281750 & 0.1035040 \\ \mathrm{C} & 3.4130690 & -0.8874980 & -0.0231930 \\ \mathrm{C} & 1.7443380 & -2.2263310 & 1.1966350 \\ \mathrm{C} & 4.3147720 & -1.1495330 & 1.0102340 \\ \mathrm{H} & 5.3252000 & -0.7519910 & 0.9276840 \\ \mathrm{C} & -3.2403650 & -1.6207310 & 0.7632870 \\ \mathrm{C} & 3.8258050 & -0.0978190 & -1.2136090 \\ \mathrm{C} & -3.5520970 & -0.4881890 & 1.5382620 \\ \mathrm{C} & -2.8192000 & -0.0076000 & 2.7689500 \\ \mathrm{C} & -4.8675640 & 1.4737450 & 2.1679570 \\ \mathrm{C} & -4.6779750 & 0.3015330 & 1.2372470\end{array}$




$\begin{array}{lrr}-2.3540840 & 1.4501140 & 2.5244290 \\ -1.8658250 & 1.8287890 & 3.4306600 \\ -1.6083390 & 1.4510710 & 1.7297800 \\ -3.5769670 & 2.3379790 & 2.1448690 \\ -3.6891040 & 3.1701370 & 2.8517720 \\ -3.4433420 & 2.7758200 & 1.1490250 \\ -5.7408870 & 2.0710640 & 1.8786450 \\ -1.9620040 & -0.6434010 & 3.0053920 \\ -5.0388000 & 0.9194100 & 3.6099200 \\ -5.1071510 & 1.7626440 & 4.3102010 \\ -5.9768320 & 0.3556870 & 3.6754080 \\ -3.8208150 & 0.0083640 & 3.9586090 \\ -3.3004600 & 0.3842430 & 4.8494050 \\ -4.1510530 & -1.0149740 & 4.1729290 \\ -4.0888360 & -1.9450990 & -0.3161320 \\ -3.9337320 & -3.0957930 & -1.2874280 \\ -6.0644360 & -1.6819870 & -1.7333330 \\ -5.2353370 & -1.1710640 & -0.5817540 \\ -5.1898490 & -4.0013580 & -1.1571670 \\ -5.1104950 & -4.8206960 & -1.8839150 \\ -5.2237570 & -4.4475000 & -0.1557570 \\ -6.4720930 & -3.1504340 & -1.4239780 \\ -7.0316560 & -3.5513700 & -2.2794040 \\ -7.1377420 & -3.1688640 & -0.5528150 \\ -6.9557440 & -1.0598400 & -1.8815880 \\ -3.0251870 & -3.6703470 & -1.0976850 \\ -5.1825560 & -1.6911570 & -3.0098720 \\ -5.7647360 & -2.1124050 & -3.8405500 \\ -4.9073660 & -0.6653300 & -3.2762280 \\ -3.9054380 & -2.5409320 & -2.7386320 \\ -3.8542870 & -3.3929890 & -3.4296560 \\ -3.0007420 & -1.9451390 & -2.8778760 \\ -5.5288310 & -0.0475380 & 0.1890520 \\ -6.4169850 & 0.5495560 & -0.0210550 \\ 4.3305000 & 0.0397690 & -3.5999000 \\ 4.3523610 & -0.7581510 & -4.8793020 \\ 3.5574160 & -2.1304700 & -2.8211970 \\ 3.8912950 & -0.6964320 & -2.4838680 \\ 5.2928420 & -1.9801210 & -4.6770920 \\ 5.2632790 & -2.6052250 & -5.5795930 \\ 6.3242580 & -1.6305340 & -4.5491090 \\ 4.8257910 & -2.7913670 & -3.4280770 \\ 4.5863860 & -3.8264010 & -3.7060860 \\ 5.6174640 & -2.8248310 & -2.6698960 \\ 3.2272430 & -2.6888860 & -1.9415220 \\ 4.6929410 & -0.1448570 & -5.7224310\end{array}$

2.4486540
2.2539480
1.5253510
2.9180780
2.9278860
2.2385680
4.6291500
4.9565820
4.1068410
4.1877370
3.6728460
3.9121150
2.9117670
3.1482490
3.0881690
2.1504580
3.7426300
5.3146480
5.5142800
5.4417670
6.2010870
6.0333510
6.2414930
6.9646510
4.7033020
5.0425660
2.6674700
2.2496240
3.2099350
4.6604950
5.0021020
3.9652750
2.9600340
2.1888070
1.2320910
4.7767450
5.3541950
5.9839880
5.0889000
3.0855780
0.1672480
1.2576790
0.9057350
-0.5053510
-1.2964490
-1.1457700
$-2.1372050$
$-3.1774130$
$-1.7318430$
$-1.2987130$
$-1.9124560$
$-0.4556840$
1. 9829620
3.4229270
2. 0992850
1.2543930
4. 1013530
5.1331870
4.1436990
3. 2884460
3.9205560
2.8928170
1.5218670
3.9455900
2. 6789070
3.3437640
1.8643060
3.4604600
4.5072730
3. 0186490
1. 3778360
1. 9453960
$-2.4250920$
$-3.1835770$
$-3.0288190$
$-3.1849480$
$-2.0633320$
$-1.8912500$
$-3.7793410$
$-4.2519250$
$-2.8823200$
$-4.1608450$
$-3.1616860$
$-2.2364030$
$-1.1159680$
-2. 0443380
$-4.2141440$
$-5.2527480$
$-6.2512980$
$-6.8832170$
$-6.2181130$
$-4.7201080$

$-3.9092430$

$-4.2031790$

$-3.4854900$

$-5.1391200$

$-6.0498070$

$-5.3121390$

$-2.2120410$

$-1.9060280$

0.1586320

$-1.0908170$

$-1.3524960$

$-1.0620640$

$-2.1403130$

$-0.1293430$

0.7654850

$-0.3306790$

1. 0118980

$-2.8012410$

0.4621000

1.3331410

0.7224020

$-0.7861640$

$-0.5279130$

$-1.1601470$

$-3.4663490$

$-4.3338000$

2. 2550070

3. 5061150

4.6952180

4. 2274710

3. 2400110

2.1434630

5.4558490

3. 2726210

3.7864900

3.7325430

5.0775010

2. 7795740

3.7963370

5. 1656110

1. 3251470

1. 2212930

0.0905860

0.2789660

1.4377060

1. 3817330 


$\begin{array}{lrrr}\text { H } & 1.3330430 & -5.8171900 & \\ \mathrm{H} & 1.6738610 & -7.0335820 & 0.1637020 \\ \mathrm{H} & -0.4201460 & -7.9585920 & 0.4796050 \\ \mathrm{H} & -0.8921150 & -6.5913090 & 2.3911550 \\ \mathrm{H} & 2.2314830 & -4.7910200 & 1.0344020 \\ \mathrm{H} & 0.9456940 & -5.7092870 & -0.8622170 \\ \mathrm{H} & -1.0827940 & -6.7717690 & -0.6467440 \\ \mathrm{H} & -2.3535190 & -6.5058790 & -1.3965190 \\ \mathrm{C} & -2.1299510 & 2.0018380 & -2.9896160 \\ \mathrm{C} & -2.8805120 & 2.2117370 & -1.7487800 \\ \mathrm{H} & -1.0439640 & 1.9641580 & -2.8802480 \\ \mathrm{H} & -2.4055510 & 1.9145290 & -0.8196960 \\ \mathrm{O} & -2.7446940 & 0.6935410 & -2.7719050 \\ \mathrm{C} & -1.1190760 & 4.553340 & 0.2408520 \\ \mathrm{C} & -0.0841160 & 4.6950700 & 2.5417620 \\ \mathrm{C} & 0.1883050 & 3.5034160 & 1.8510800 \\ \mathrm{~N} & -0.3920770 & 3.4716660 & 0.5961560 \\ \mathrm{H} & -0.2384350 & 2.6444720 & -0.0242930 \\ \mathrm{~S} & -1.0954370 & 5.7273570 & 1.5527590 \\ \mathrm{~S} & -1.9347010 & 4.7947810 & -1.2039940 \\ \mathrm{H} & -2.0082680 & 0.0537920 & -2.2938650 \\ \mathrm{C} & 1.1952680 & 3.8759470 & 4.3981580 \\ \mathrm{C} & 1.4524470 & 2.6739680 & 3.6981350 \\ \mathrm{C} & 0.9516820 & 2.4758270 & 2.4080890 \\ \mathrm{C} & 0.4293950 & 4.8871510 & 3.8293280 \\ \mathrm{H} & 1.6042730 & 3.9902960 & 5.3982520 \\ \mathrm{H} & 1.1178070 & 1.5702920 & 1.8377290 \\ \mathrm{H} & 0.2337430 & 5.8028250 & 4.3806120 \\ \mathrm{C} & -4.1622120 & 2.7734340 & -4.2944350 \\ \mathrm{C} & -4.7142010 & 3.4169090 & -3.0119790 \\ \mathrm{C} & -4.3190610 & 2.6216870 & -1.7550590 \\ \mathrm{C} & -2.6264070 & 2.6818720 & -4.2493960 \\ \mathrm{H} & -4.4663100 & 3.3598330 & -5.1697980 \\ \mathrm{H} & -5.8073660 & 3.4908790 & -3.0595910 \\ \mathrm{H} & -4.9127330 & 1.7012270 & -1.6705430 \\ \mathrm{H} & -2.1878490 & 3.6893150 & -4.2647000 \\ \mathrm{H} & -2.2359110 & 2.1383900 & -5.1189970 \\ \mathrm{H} & -4.5817040 & 1.7653080 & -4.4115000 \\ \mathrm{H} & -4.3214940 & 4.4389670 & -2.9233130 \\ \mathrm{H} & -4.5148680 & 3.1911840 & -0.8399350 \\ \mathrm{O} & 2.2020240 & 1.7556750 & 4.3668800 \\ \mathrm{C} & 2.4236510 & 0.4948940 & 3.7193740 \\ \mathrm{H} & 2.9489180 & 0.6217090 & 2.7656030 \\ \mathrm{H} & 1.4740290 & -0.0260610 & 3.5378400 \\ \mathrm{H} & 3.0460210 & -0.0818400 & 4.4031520 \\ \mathrm{I} & & & \\ & & & \end{array}$

$\begin{array}{lrrr}\text { TS76(S, S }) \text { G }[\mathrm{B} 97-\mathrm{D} / \mathrm{def} 2-\mathrm{TZVP}]=-4356.202611 \\ \mathrm{P} & -0.0691060 & -0.2528290 & -0.7295130 \\ \mathrm{O} & -1.0559970 & -0.7589230 & -1.7542900 \\ \mathrm{O} & 0.2725760 & 1.2021520 & -0.7040380 \\ \mathrm{O} & 1.2240760 & -1.2174920 & -0.9327900 \\ \mathrm{O} & -0.5389380 & -0.6409480 & 0.7890910 \\ \mathrm{C} & 2.1272910 & -1.4281640 & 0.1035000 \\ \mathrm{C} & 1.7443450 & -2.2263260 & 1.1966260 \\ \mathrm{C} & 3.4130760 & -0.8874830 & -0.0231900 \\ \mathrm{C} & 4.3147760 & -1.1495200 & 1.0102390 \\ \mathrm{H} & 5.3252030 & -0.7519740 & 0.9276940 \\ \mathrm{C} & -0.7508590 & -1.9984780 & 1.0431930 \\ \mathrm{C} & -2.0651290 & -2.4817450 & 1.0644580 \\ \mathrm{C} & 0.3787370 & -2.8255930 & 1.2160390 \\ \mathrm{C} & -2.2334480 & -3.8575220 & 1.2817480 \\ \mathrm{H} & -3.2434080 & -4.2618940 & 1.3025500 \\ \mathrm{C} & 3.8258150 & -0.0977980 & -1.2136000 \\ \mathrm{C} & -3.2403610 & -1.6207440 & 0.7632800 \\ \mathrm{C} & 3.8913140 & -0.6964060 & -2.4838610 \\ \mathrm{C} & 3.5574450 & -2.1304440 & -2.8211960 \\ \mathrm{C} & 4.3523980 & -0.7581170 & -4.8792920 \\ \mathrm{C} & 4.3305250 & 0.0397990 & -3.5998880 \\ \mathrm{C} & 2.4486910 & -2.1371820 & -3.9092500 \\ \mathrm{H} & 2.2539900 & -3.1773910 & -4.2031890 \\ \mathrm{H} & 1.5253820 & -1.7318250 & -3.4855030 \\ \mathrm{C} & 2.9181200 & -1.2986870 & -5.1391220 \\ \mathrm{H} & 2.9279400 & -1.9124280 & -6.0498090 \\ \mathrm{H} & 2.2386080 & -0.4556610 & -5.3121450 \\ \mathrm{H} & 4.6929820 & -0.1448200 & -5.7224170 \\ \mathrm{H} & 3.2272670 & -2.6888640 & -1.9415240 \\ \mathrm{C} & 5.2928840 & -1.9800840 & -4.6770770 \\ \mathrm{H} & 5.2633310 & -2.6051860 & -5.5795800 \\ \mathrm{H} & 6.3242970 & -1.6304920 & -4.5490850 \\ \mathrm{C} & 4.8258270 & -2.7913350 & -3.4280670 \\ \mathrm{H} & 4.5864290 & -3.8263690 & -3.7060810 \\ \mathrm{H} & 5.6174940 & -2.8247970 & -2.6698800 \\ \mathrm{C} & 4.1877410 & 1.2544150 & -1.0908020 \\ \mathrm{C} & 4.1068340 & 2.0993030 & 0.1586490 \\ \mathrm{C} & 4.9565840 & 3.4229540 & -1.9060010 \\ \mathrm{C} & 4.6291580 & 1.9829900 & -2.2120200 \\ \mathrm{C} & 5.5142680 & 2.6789290 & 0.4621290 \\ \mathrm{H} & 5.4417480 & 3.3437830 & 1.3331710 \\ \mathrm{H} & 6.2010760 & 1.8643290 & 0.7224340 \\ \mathrm{C} & 6.0333460 & 3.4604860 & -0.7861290 \\ \mathrm{H} & 6.2414850 & 4.5072990 & -0.5278740 \\ \mathrm{H} & 6.9646490 & 3.0186780 & -1.1601080 \\ & & & \\ & & & \\ & & & \end{array}$




$\begin{array}{rrr}5.3146540 & 3.9456220 & -2.8012090 \\ 3.7426190 & 1.5218810 & 1.0119110 \\ 3.6728440 & 4.1013750 & -1.3524740 \\ 3.9121090 & 5.1332080 & -1.0620350 \\ 2.9117700 & 4.1437240 & -2.1402960 \\ 3.1482390 & 3.2884610 & -0.1293280 \\ 3.0881490 & 3.9205680 & 0.7655010 \\ 2.1504520 & 2.8928290 & -0.3306750 \\ 4.7033210 & 1.3778670 & -3.4663300 \\ 5.0425890 & 1.9454310 & -4.3337770 \\ -4.6779780 & 0.3015120 & 1.2372520 \\ -4.8675690 & 1.4737190 & 2.1679680 \\ -2.8191990 & -0.0076230 & 2.7689500 \\ -3.5520960 & -0.4882080 & 1.5382610 \\ -5.0388000 & 0.9193770 & 3.6099290 \\ -5.1071520 & 1.7626070 & 4.3102130 \\ -5.9768310 & 0.3556510 & 3.6754160 \\ -3.8208120 & 0.0083320 & 3.9586120 \\ -3.3004570 & 0.3842080 & 4.8494080 \\ -4.1510480 & -1.0150080 & 4.1729270 \\ -1.9620000 & -0.6434220 & 3.0053880 \\ -5.7408940 & 2.0710370 & 1.8786600 \\ -2.3540880 & 1.4500940 & 2.5244360 \\ -1.8658290 & 1.8287660 & 3.4306680 \\ -1.6083450 & 1.4510560 & 1.7297850 \\ -3.5769740 & 2.3379560 & 2.1448820 \\ -3.6891130 & 3.1701120 & 2.8517880 \\ -3.4433530 & 2.7758020 & 1.1490390 \\ -5.2353370 & -1.1710780 & -0.5817560 \\ -6.0644360 & -1.6820000 & -1.7333340 \\ -3.9337260 & -3.0957990 & -1.2874410 \\ -4.0888330 & -1.9451110 & -0.3161400 \\ -5.1825600 & -1.6911610 & -3.0098750 \\ -5.7647410 & -2.1124090 & -3.8405540 \\ -4.9073740 & -0.6653320 & -3.2762280 \\ -3.9054370 & -2.5409320 & -2.7386420 \\ -3.8542850 & -3.3929850 & -3.4296700 \\ -3.0007440 & -1.9451340 & -2.8778840 \\ -3.0251790 & -3.6703500 & -1.0977020 \\ -6.9557480 & -1.0598560 & -1.8815850 \\ -5.1898390 & -4.0013690 & -1.1571800 \\ -5.1104840 & -4.8207050 & -1.8839310 \\ -5.2237430 & -4.4475160 & -0.1557720 \\ -6.4720870 & -3.1504500 & -1.4239850 \\ -7.0316500 & -3.5513850 & -2.2794120 \\ -7.1377340 & -3.1688860 & -0.5528210\end{array}$
$-5.5288330$
$-6.4169900$
0.1672630
1. 2576980
0.9057570
$-0.5053270$
$-1.2964260$
$-1.1457530$
1. 3330640
1.6738860
$-0.4201180$
$-0.8920890$
2. 2315000
0.9457150
$-1.0827710$
$-2.3534950$
2. 6674740
2. 2496260
3.2099330
4. 6604950
5. 0021030
3. 9652780
2. 9600310
2. 1888130
1.2320910
4.7767480
5. 3541920
5. 9839900
5.0888960
3.0855720
$-2.1299790$
$-2.8805210$
$-1.0439900$
$-2.4055620$
$-2.7447090$
$-1.1191010$
$-0.0841430$
0.1882850
$-0.3920970$
$-0.2384430$
$-1.0954720$
$-1.9347290$
$-2.0082930$
1. 1952460
1. 4524320
0.9516690

-0.0475560
0.5495350
-4.2141460
-5.2527450
-6.2512910
-6.8832160
-6.2181210
-4.7201150
-5.8171930
-7.0335720
-7.9585920
-6.5913210
-4.7910130
-5.7092750
-6.7717620
-6.5058910
-2.4250900
-3.1835830
-3.0288280
-3.1849510
-2.0633290
-1.8912440
-3.7793530
-4.2519300
-2.8823300
-4.1608450
-3.1616910
-2.2363950
-1.1159680
-2.0443480
2.0018460
2.2117690
1.9641710
1.9145330
0.6935470
4.5533150
4.6950490
3.5033970
3.4716410
2.6444480
5.7273280
4.7947530
0.0538100
3.8759370
2.6739580
2.4758120

0.1890560

$-0.0210460$

1. 3251250

1. 2212660

0.0905530

0.2789300

1. 4376750

1. 3817090

2. 1636710

0.0514350

0.4795610

2. 3911210

1.0343770

$-0.8622470$

$-0.6467790$

1. 3964870

2. 2550000

3. 5061030

4.6952090

4. 2274660

3. 2400130

2. 1434630

5.4558370

3. 2726040

3. 7864760

3.7325340

5.0774990

2.7795790

3. 7963460

5.1656070

$-2.9896110$

$-1.7487670$

$-2.8802530$

$-0.8196900$

$-2.7719210$

0.2408550

2. 5417610

1.8510810

0.5961560

$-0.0242960$

1. 5527590

$-1.2039920$

$-2.2938940$

4.3981570

3. 6981360

2. 4080910 


\begin{tabular}{|c|c|c|c|c|c|c|c|}
\hline C & 0.4293680 & 4.8871360 & 3.8293270 & $\mathrm{H}$ & -1.6160640 & 14012650 & 1.7824590 \\
\hline $\mathrm{H}$ & 1.6042520 & 3.9902900 & 5.3982510 & C & -3.5937500 & 2.2592060 & 2.2153630 \\
\hline $\mathrm{H}$ & 1.1177990 & 1.5702780 & 1.8377310 & $\mathrm{H}$ & -3.7188520 & 3.0686360 & 2.9461050 \\
\hline $\mathrm{H}$ & 0.2337110 & 5.8028090 & 4.3806100 & $\mathrm{H}$ & -3.4603170 & 2.7283440 & 1.2340590 \\
\hline C & -4.1622470 & 2.7734500 & -4.2944150 & $\mathrm{H}$ & -5.7525860 & 1.9759510 & 1.9268810 \\
\hline C & -4.7142310 & 3.4169180 & -3.0119540 & $\mathrm{H}$ & -1.9507570 & -0.7265980 & 3.0057560 \\
\hline C & -4.3190760 & 2.6216950 & -1.7550390 & C & -5.0479720 & 0.7876000 & 3.6325150 \\
\hline C & -2.6264410 & 2.6818900 & -4.2493850 & $\mathrm{H}$ & -5.1289960 & 1.6120400 & 4.3535290 \\
\hline $\mathrm{H}$ & -4.4663500 & 3.3598520 & -5.1697750 & $\mathrm{H}$ & -5.9802850 & 0.2125580 & 3.6784700 \\
\hline $\mathrm{H}$ & -5.8073970 & 3.4908800 & -3.0595590 & C & -3.8219420 & -0.1189630 & 3.9650200 \\
\hline $\mathrm{H}$ & -4.9127300 & 1.7012220 & -1.6705340 & $\mathrm{H}$ & -3.3107640 & 0.2394780 & 4.8682290 \\
\hline $\mathrm{H}$ & -2.1878840 & 3.6893340 & -4.2646860 & $\mathrm{H}$ & -4.1424710 & -1.1507820 & 4.1516180 \\
\hline H & -2.2359500 & 2.1384130 & -5.1189920 & C & -4.0457540 & -1.9658600 & -0.3584740 \\
\hline H & -4.5817370 & 1.7653240 & -4.4114830 & $\mathrm{C}$ & -3.8745080 & -3.0917800 & -1.3554320 \\
\hline $\mathrm{H}$ & -4.3215310 & 4.4389780 & -2.9232880 & C & -6.0173310 & -1.6894000 & -1.7785060 \\
\hline H & -4.5148960 & 3.1911780 & -0.8399100 & $\mathrm{C}$ & -5.1989760 & -1.1973890 & -0.6111700 \\
\hline 0 & 2.2020120 & 1.7556710 & 4.3668830 & $\mathrm{C}$ & -5.1218310 & -4.0125290 & -1.2509520 \\
\hline C & 2.4236440 & 0.4948880 & 3.7193800 & $\mathrm{H}$ & -5.0304960 & -4.8158130 & -1.9940470 \\
\hline $\mathrm{H}$ & 2.9489140 & 0.6217020 & 2.7656100 & $\mathrm{H}$ & -5.1563020 & -4.4795590 & -0.2591340 \\
\hline $\mathrm{H}$ & 1.4740240 & -0.0260700 & 3.5378450 & C & -6.4111080 & -3.1691440 & -1.5068400 \\
\hline 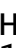 & 3.0460140 & -0.0818430 & 4.4031620 & $\mathrm{H}$ & -6.9600020 & -3.5553230 & -2.3758690 \\
\hline & & & & $\mathrm{H}$ & -7.0829710 & -3.2155630 & -0.6415140 \\
\hline & $\mathrm{G}[\mathrm{B} 97-\mathrm{D} / \mathrm{c}$ & $Z \mathrm{VP}]=-4356$ & & $\mathrm{H}$ & -6.9143940 & -1.0731040 & -1.9162670 \\
\hline $\mathrm{P}$ & -0.0618630 & -0.2239130 & -0.7213650 & $\mathrm{H}$ & -2.9608070 & -3.6609820 & -1.1743580 \\
\hline 0 & 0.3006040 & 1.2247760 & -0.6574310 & C & -5.1293910 & -1.6581350 & -3.0503300 \\
\hline 0 & -1.0713990 & -0.6872730 & -1.7434910 & $\mathrm{H}$ & -5.7037280 & -2.0626510 & -3.8947470 \\
\hline 0 & -0.5105770 & -0.6558400 & 0.7915490 & $\mathrm{H}$ & -4.8621350 & -0.6231840 & -3.2879210 \\
\hline 0 & 1.2127860 & -1.1995770 & -0.9759940 & C & -3.8463960 & -2.5041980 & -2.7942140 \\
\hline $\mathrm{C}$ & -0.7128250 & -2.0218360 & 1.0083870 & $\mathrm{H}$ & -3.7877010 & -3.3406970 & -3.5034950 \\
\hline C & 0.4227570 & -2.8444230 & 1.1578670 & $\mathrm{H}$ & -2.9456450 & -1.8989990 & -2.9184270 \\
\hline c & -2.0235 & -2.5 & 1.0189360 & C & -5.5075950 & -0.0954460 & 0.1844920 \\
\hline C & -2.1816260 & -3.8961910 & 1.2137560 & $\mathrm{H}$ & -6.4016850 & 0.4964820 & -0.0150340 \\
\hline $\mathrm{H}$ & -3.1886970 & -4.3079240 & 1.2311400 & C & 4.1096950 & 0.2676820 & -3.6938730 \\
\hline c & 2.1459610 & -1.4330290 & 0.0272430 & $\mathrm{C}$ & 4.0494980 & -0.4426870 & -5.0228690 \\
\hline C & 3.4281520 & -0.8901790 & -0.1275800 & $\mathrm{C}$ & 3.4457790 & -1.9686120 & -3.0076900 \\
\hline c & 1.7887720 & -2.2449110 & 1.1199910 & $\mathrm{C}$ & 3.7670480 & -0.5494190 & -2.6003900 \\
\hline C & 4.3654730 & -1.1914880 & 0.8627700 & $\mathrm{C}$ & 5.0295330 & -1.6476310 & -4.9762930 \\
\hline $\mathrm{H}$ & 5.3749200 & -0.7972740 & 0.7560900 & $\mathrm{H}$ & 4.9368620 & -2.2170280 & -5.9107700 \\
\hline c & -3.2063870 & -1.6596370 & 0.7332660 & $\mathrm{H}$ & 6.0605000 & -1.2795840 & -4.9127570 \\
\hline C & 3.7833610 & -0.0350400 & -1.2915690 & $\mathrm{C}$ & 4.6871460 & -2.5457650 & -3.7464040 \\
\hline C & -3.5343840 & -0.5504390 & 1.5342920 & $\mathrm{H}$ & 4.4628920 & -3.5718280 & -4.0668180 \\
\hline C & -2.8137550 & -0.0941330 & 2.7812750 & $\mathrm{H}$ & 5.5352940 & -2.5912510 & -3.0526730 \\
\hline $\mathrm{C}$ & -4.8745 & 1.3807350 & 2.2062810 & $\mathrm{H}$ & 3.1955540 & -2.5912140 & -2.1444960 \\
\hline c & -4. & 0.2353060 & 1.2465580 & $\mathrm{H}$ & 4.3111490 & 0.2345890 & -5.8449370 \\
\hline C & -2.3630 & 1.3738740 & 2.575453 & C & 2.2636990 & -1.9449540 & -4.0167440 \\
\hline H & -1.8800190 & 1.7344560 & 3.491699 & $\mathrm{H}$ & 2.0900010 & -2.9695390 & -4.3722590 \\
\hline
\end{tabular}




$\begin{array}{rrrr}\mathrm{H} & 1.3566520 & -1.6112440 & -3.5064390 \\ \mathrm{C} & 2.6132650 & -1.0037490 & -5.2105130 \\ \mathrm{H} & 2.5630200 & -1.5462050 & -6.1641010 \\ \mathrm{H} & 1.9029630 & -0.1698190 & -5.2641390 \\ \mathrm{C} & 4.4924570 & 2.1191550 & -2.2060640 \\ \mathrm{C} & 4.8324800 & 3.5403690 & -1.8311030 \\ \mathrm{C} & 4.1634610 & 2.0688200 & 0.2007630 \\ \mathrm{C} & 4.1467870 & 1.3103980 & -1.1059390 \\ \mathrm{C} & 3.5956000 & 4.1642860 & -1.1266920 \\ \mathrm{H} & 3.8531760 & 5.1757390 & -0.7846920 \\ \mathrm{H} & 2.7711600 & 4.2550280 & -1.8435640 \\ \mathrm{C} & 3.1782170 & 3.2627710 & 0.0733380 \\ \mathrm{H} & 3.1827380 & 3.8311070 & 1.0120510 \\ \mathrm{H} & 2.1706540 & 2.8700550 & -0.0764700 \\ \mathrm{H} & 3.8748020 & 1.4316690 & 1.0401370 \\ \mathrm{H} & 5.1123990 & 4.1260530 & -2.7150270 \\ \mathrm{C} & 5.5881270 & 2.6420010 & 0.4260140 \\ \mathrm{H} & 5.5853190 & 3.2445460 & 1.3440740 \\ \mathrm{H} & 6.2982570 & 1.8187200 & 0.5708920 \\ \mathrm{C} & 5.9986990 & 3.5124800 & -0.8035400 \\ \mathrm{H} & 6.2237910 & 4.5409440 & -0.4915740 \\ \mathrm{H} & 6.8974550 & 3.1059280 & -1.2824140 \\ \mathrm{C} & 4.4728260 & 1.6006560 & -3.5001890 \\ \mathrm{H} & 4.7347240 & 2.2303520 & -4.3513950 \\ \mathrm{C} & 4.0508030 & -1.9630760 & 1.9857640 \\ \mathrm{C} & 2.7472850 & -2.4744160 & 2.1387250 \\ \mathrm{C} & 5.1625020 & -2.2548670 & 2.9834580 \\ \mathrm{C} & 4.6844630 & -2.8046200 & 4.3349030 \\ \mathrm{C} & 3.5701560 & -3.8355670 & 4.1277990 \\ \mathrm{C} & 2.3718130 & -3.1753780 & 3.4349170 \\ \mathrm{H} & 5.5332510 & -3.2419250 & 4.8760060 \\ \mathrm{H} & 5.8364310 & -2.9948620 & 2.5235330 \\ \mathrm{H} & 5.7642640 & -1.3476780 & 3.1295940 \\ \mathrm{H} & 3.9428930 & -4.6634050 & 3.5061230 \\ \mathrm{H} & 3.2522650 & -4.2612620 & 5.0881990 \\ \mathrm{H} & 1.5767060 & -3.9034950 & 3.2793340 \\ \mathrm{H} & 1.9503390 & -2.4192370 & 4.1165540 \\ \mathrm{H} & 4.2948110 & -1.9863090 & 4.9573810 \\ \mathrm{C} & 0.2210000 & -4.2362160 & 1.2523680 \\ \mathrm{C} & 1.3260650 & -5.2643030 & 1.1865210 \\ \mathrm{C} & 1.0050790 & -6.3056920 & 0.0864770 \\ \mathrm{C} & -0.4142980 & -6.9274730 & 0.2458070 \\ \mathrm{C} & -1.2281750 & -6.2505580 & 1.3795070 \\ \mathrm{C} & -1.0875600 & -4.7520300 & 1.3077220 \\ \mathrm{H} & 1.4069270 & -5.7885770 & 0.1045400 \\ \mathrm{H} & 1.7714480 & -7.0904350 & \\ & & & \end{array}$

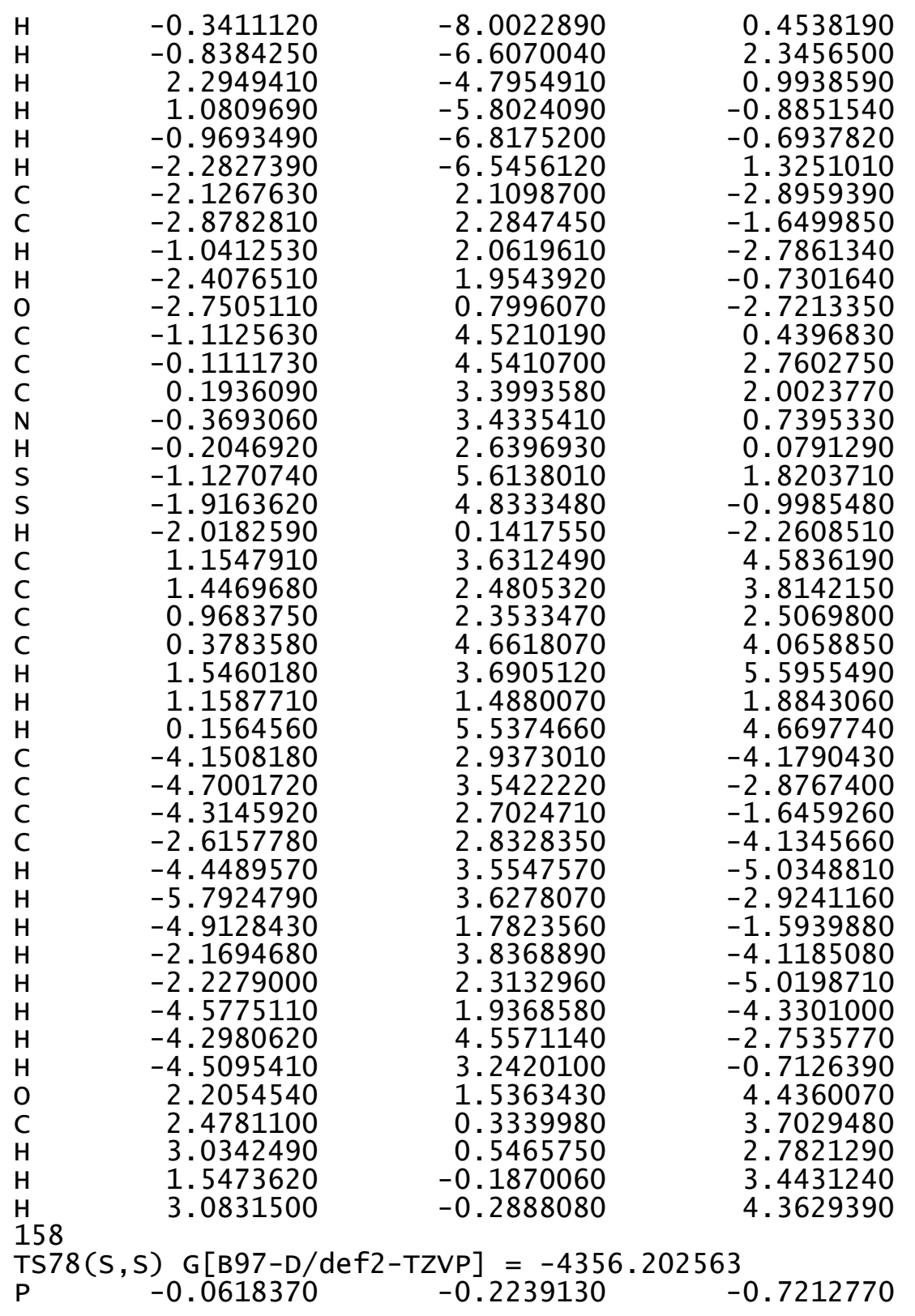




$\begin{array}{rrr}-1.0714390 & -0.6871910 & -1.7433690 \\ 0.3006720 & 1.2247610 & -0.6572810 \\ 1.2127640 & -1.1996090 & -0.9760220 \\ -0.5104740 & -0.6559060 & 0.7916390 \\ 2.1460020 & -1.4331050 & 0.0271440 \\ 1.7888650 & -2.2450050 & 1.1198970 \\ 3.4281830 & -0.8902460 & -0.1277380 \\ 4.3655580 & -1.1915870 & 0.8625530 \\ 5.3749990 & -0.7973700 & 0.7558340 \\ -0.7127330 & -2.0219110 & 1.0084080 \\ -2.0234700 & -2.5161760 & 1.0189450 \\ 0.4228470 & -2.8445110 & 1.1578250 \\ -2.1815430 & -3.8962650 & 1.2137040 \\ -3.1886160 & -4.3079950 & 1.2310720 \\ 3.7832750 & -0.0350090 & -1.2916940 \\ -3.2063000 & -1.6596890 & 0.7332960 \\ 3.7667650 & -0.5492500 & -2.6005690 \\ 3.4454900 & -1.9684120 & -3.0079710 \\ 4.0489150 & -0.4422800 & -5.0230750 \\ 4.1092310 & 0.2679710 & -3.6940200 \\ 2.2633020 & -1.9447180 & -4.0168980 \\ 2.0896170 & -2.9692810 & -4.3724830 \\ 1.3562900 & -1.6110980 & -3.5064740 \\ 2.6126940 & -1.0033950 & -5.2106210 \\ 2.5623820 & -1.5457710 & -6.1642500 \\ 1.9023430 & -0.1694970 & -5.2641070 \\ 4.3104470 & 0.2350840 & -5.8451090 \\ 3.1953820 & -2.5911040 & -2.1448080 \\ 5.0290160 & -1.6471820 & -4.9767170 \\ 4.9362670 & -2.2164950 & -5.9112370 \\ 6.0599700 & -1.2790890 & -4.9132580 \\ 4.6868030 & -2.5454490 & -3.7468700 \\ 4.4625600 & -3.5714920 & -4.0673550 \\ 5.5350300 & -2.5909600 & -3.0532390 \\ 4.1467380 & 1.3104110 & -1.1059820 \\ 4.1637480 & 2.0686670 & 0.2008160 \\ 4.8321600 & 3.5405050 & -1.8310400 \\ 4.4921410 & 2.1193120 & -2.2060870 \\ 5.5884670 & 2.6418390 & 0.4257350 \\ 5.5859430 & 3.2442000 & 1.3439160 \\ 6.2986510 & 1.8185360 & 0.5702290 \\ 5.9986470 & 3.5125560 & -0.8037770 \\ 6.2237240 & 4.5409900 & -0.4917030 \\ 6.8973180 & 3.1061640 & -1.2829440 \\ 5.1118200 & 4.1263140 & -2.7149640 \\ 3.8753410 & 1.4314010 & 1.0401890\end{array}$

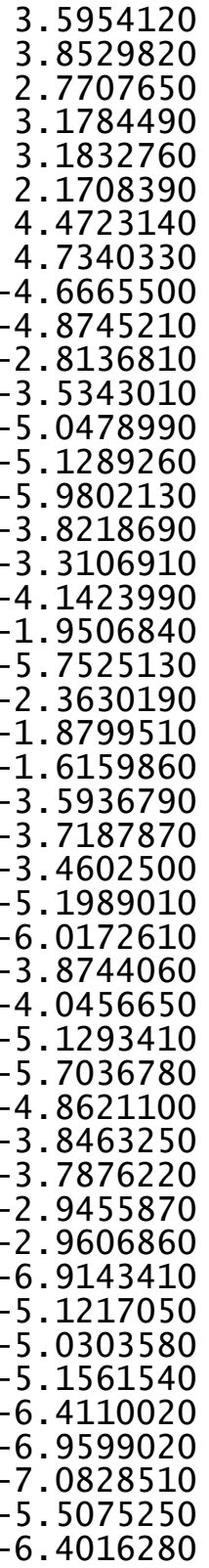

4.1642530

. 1757060

4.2549650

3. 2626090

3.8308460

2.8699040

1.6009460

2. 2307430

0.2352460

1. 3806710

$-0.0941970$

$-0.5504990$

0.7875310

1. 6119680

0.2124890

$-0.1190330$

0.2394000

$-1.1508530$

$-0.7266640$

1.9758860

1.3738130

1.7343880

1. 4012140

2. 2591460

3. 0685670

2.7282980

$-1.1974330$

$-1.6894330$

$-3.0917760$

$-1.9658940$

$-1.6581030$

$-2.0626010$

$-0.6231360$

$-2.5041480$

$-3.3406270$

$-1.8989270$

$-3.6609550$

$-1.0731560$

$-4.0125590$

$-4.8158150$

$-4.4796250$

$-3.1691950$

$-3.5553590$

$-3.2156510$

$-0.0955020$

0.4964130
$-1.1262360$ $-0.7842340$

$-1.8428740$

0.0738410

1. 0126130

$-0.0756630$

$-3.5002620$

$-4.3514480$

1. 2466060

2. 2063330

2.7813210

1.5343320

3. 6325660

4. 3535830

3. 6785170

3. 9650640

4.8682760

4.1516570

3. 0057990

1. 9269320

2. 5755090

3. 4917600

1.7825190

2. 2154230

2.9461740

1. 2341250

$-0.6111270$

$-1.7784620$

$-1.3554510$

$-0.3584530$

$-3.0502960$

$-3.8947220$

- 3.2878490

$-2.7942190$

$-3.5035230$

$-2.9184260$

$-1.1744050$

$-1.9161900$

$-1.2509890$

$-1.9941130$

$-0.2591870$

$-1.5068340$

$-2.3758650$

$-0.6414990$

0.1845460

$-0.0149620$ 


\begin{tabular}{|c|c|c|}
\hline $\begin{array}{l}0.2210820 \\
1.3261420 \\
1.0051880 \\
-0.4142160 \\
-1.2281040 \\
-1.0874810 \\
1.4069750 \\
1.7715340 \\
-0.3410740 \\
-0.8383690 \\
2.2950270 \\
1.0811480 \\
-0.9692380 \\
-2.2826700 \\
2.7474280 \\
4.0509430 \\
2.3720050 \\
3.5703700 \\
4.6846970 \\
5.1626920 \\
3.2525110 \\
1.9505370 \\
1.5769010 \\
4.2950770 \\
5.5334990 \\
5.7645230 \\
5.8365410 \\
3.9430800 \\
-2.1267730 \\
-2.8782590 \\
-1.0412620 \\
-2.4076130 \\
-2.7505530 \\
-1.1124860 \\
-0.1111760 \\
0.1936360 \\
-0.3692320 \\
-0.2046180 \\
-1.1270340 \\
-1.9162510 \\
-2.0183170 \\
1.1546980 \\
1.4469130 \\
0.9683770 \\
0.3782910 \\
1.5458790\end{array}$ & $\begin{array}{l}-4.2363060 \\
-5.2643950 \\
-6.3057780 \\
-6.9275190 \\
-6.2506440 \\
-4.7521140 \\
-5.7886780 \\
-7.0905430 \\
-8.0023500 \\
-6.6071300 \\
-4.7955860 \\
-5.8025040 \\
-6.8174860 \\
-6.5456910 \\
-2.4745260 \\
-1.9631970 \\
-3.1754890 \\
-3.8356610 \\
-2.8047170 \\
-2.2550310 \\
-4.2613170 \\
-2.4193490 \\
-3.9036140 \\
-1.9863730 \\
-3.2420100 \\
-1.3478780 \\
-2.9950860 \\
-4.6635270 \\
2.1099480 \\
2.2848900 \\
2.0620170 \\
1.9545720 \\
0.7997080 \\
4.5210730 \\
4.5409630 \\
3.3993060 \\
3.4335770 \\
2.6397540 \\
5.6137640 \\
4.8334810 \\
0.1418560 \\
3.6309970 \\
2.4803430 \\
2.3532570 \\
4.6615980 \\
3.6901810\end{array}$ & $\begin{array}{r}1.2522880 \\
1.1864500 \\
0.0863900 \\
0.2456420 \\
1.3793580 \\
1.3076310 \\
2.1516000 \\
0.1045010 \\
0.4535860 \\
2.3454930 \\
0.9938230 \\
-0.8852410 \\
-0.6939560 \\
1.3249270 \\
2.1385760 \\
1.9855490 \\
3.4347810 \\
4.1276390 \\
4.3346630 \\
2.9831760 \\
5.0880680 \\
4.1164220 \\
3.2792220 \\
4.9571180 \\
4.8757540 \\
3.1292490 \\
2.5232320 \\
3.5059840 \\
-2.8957700 \\
-1.6498130 \\
-2.7859880 \\
-0.7299890 \\
-2.7210960 \\
0.4398140 \\
2.7604490 \\
2.0024810 \\
0.7396180 \\
0.0791920 \\
1.8205770 \\
-0.9984170 \\
-2.2606430 \\
4.5837840 \\
3.8143010 \\
2.5070370 \\
4.0660930 \\
5.5957370\end{array}$ \\
\hline
\end{tabular}

1.1587900
0.1563600
-4.1508270
-4.7001330
-4.3145500
-2.6157900
-4.4489670
-5.7924380
-4.9128340
-2.1694420
-2.2279490
-4.5775540
-4.2979890
-4.5094480
2.2053770
2.4781020
3.0342680
1.5473820
3.0831330

1. 4879590

5. 5372100

2. 9373900

3.5423790

2.7026650

2.8328750

3.5548210

3. 6279950

1.7825740

3.8369130

2. 3132830

1.9369530

4. 5572630

3. 2422490

1. 5361020

0.3338480

0.5465500

$-0.1871550$

$-0.2890150$
1.8843120

4.6700390

$-4.1788620$

$-2.8765700$

$-1.6457310$

$-4.1344180$

$-5.0347170$

$-2.9239260$

$-1.5937300$

$-4.1184120$

$-5.0197080$

$-4.3298700$

$-2.7534550$

$-0.7124590$

4.4360420

3. 7028610

2. 7820860

3. 4429350

4. 3628050
158

TS79(S, S) G[B97-D/def2-TZVP $]=-4356.202523$

$\begin{array}{lrrr}\mathrm{P} & 0.0178270 & -0.1083290 & 1.0520840 \\ \mathrm{O} & -0.3846080 & 1.3243460 & 0.8891160 \\ \mathrm{O} & 0.9108580 & -0.4845270 & 2.2092020 \\ \mathrm{O} & 0.6743990 & -0.5441260 & -0.3862660 \\ \mathrm{O} & -1.2434870 & -1.1198680 & 1.1808210 \\ \mathrm{C} & 0.8654000 & -1.9065390 & -0.6269020 \\ \mathrm{C} & -0.2616300 & -2.7037000 & -0.9113230 \\ \mathrm{C} & 2.1666770 & -2.4196030 & -0.5809460 \\ \mathrm{C} & 2.3243480 & -3.7971450 & -0.7858830 \\ \mathrm{H} & 3.3271920 & -4.2189690 & -0.7518000 \\ \mathrm{C} & -2.0768190 & -1.2942530 & 0.0767620 \\ \mathrm{C} & -3.3510850 & -0.7191750 & 0.1066790 \\ \mathrm{C} & -1.6111860 & -2.0790960 & -0.9963680 \\ \mathrm{C} & -4.1789680 & -0.9411070 & -1.0019450 \\ \mathrm{H} & -5.1826880 & -0.5201360 & -0.9962030 \\ \mathrm{C} & 3.3513690 & -1.5529810 & -0.3408410 \\ \mathrm{C} & -3.8310090 & 0.0828860 & 1.2615630 \\ \mathrm{C} & 3.7145370 & -0.5667370 & -1.2762990 \\ \mathrm{C} & 3.0121920 & -0.2633870 & -2.5785420 \\ \mathrm{C} & 5.1428800 & 1.1776650 & -2.2153750 \\ \mathrm{C} & 4.8680770 & 0.2160450 & -1.0851970 \\ \mathrm{C} & 2.6327580 & 1.2383100 & -2.5801070 \\ \mathrm{H} & 2.1152340 & 1.4835570 & -3.5156430 \\ \mathrm{H} & 1.9327650 & 1.4131130 & -1.7628270 \\ \mathrm{C} & 3.9135660 & 2.1074890 & -2.4069670\end{array}$




\begin{tabular}{|c|c|c|c|}
\hline $\begin{array}{l}\mathrm{H} \\
\mathrm{H} \\
\mathrm{H} \\
\mathrm{H} \\
\mathrm{C} \\
\mathrm{H} \\
\mathrm{H} \\
\mathrm{C} \\
\mathrm{H} \\
\mathrm{H} \\
\mathrm{C} \\
\mathrm{C} \\
\mathrm{C} \\
\mathrm{C} \\
\mathrm{C} \\
\mathrm{H} \\
\mathrm{H} \\
\mathrm{C} \\
\mathrm{H} \\
\mathrm{H} \\
\mathrm{H} \\
\mathrm{H} \\
\mathrm{C} \\
\mathrm{H} \\
\mathrm{H} \\
\mathrm{C} \\
\mathrm{H} \\
\mathrm{H} \\
\mathrm{C} \\
\mathrm{H} \\
\mathrm{C} \\
\mathrm{C} \\
\mathrm{C} \\
\mathrm{C} \\
\mathrm{C} \\
\mathrm{H} \\
\mathrm{H} \\
\mathrm{C} \\
\mathrm{H} \\
\mathrm{H} \\
\mathrm{H} \\
\mathrm{H} \\
\mathrm{C} \\
\mathrm{H} \\
\mathrm{H} \\
\mathrm{C}\end{array}$ & $\begin{array}{l}4.0798620 \\
3.8100660 \\
6.0490750 \\
2.1184520 \\
5.3006300 \\
5.4647460 \\
6.1839190 \\
4.0132340 \\
3.5271420 \\
4.2593730 \\
4.1695580 \\
3.9766440 \\
6.1015990 \\
5.3250440 \\
5.2341520 \\
5.1234680 \\
5.3062050 \\
6.5081920 \\
7.0097280 \\
7.2258930 \\
6.9914960 \\
3.0716010 \\
5.1633970 \\
5.7125350 \\
4.8624480 \\
3.9089150 \\
3.8671510 \\
2.9886930 \\
5.6758770 \\
6.5771870 \\
-4.4852200 \\
-4.6110710 \\
-3.7139900 \\
-3.9882110 \\
-5.5661550 \\
-5.6089910 \\
-6.5785740 \\
-5.0383100 \\
-4.8461800 \\
-5.7776170 \\
-3.3398570 \\
-4.9921960 \\
-2.6837310 \\
-2.5413790 \\
-1.7214340 \\
-3.2127490\end{array}$ & $\begin{array}{r}2.7365530 \\
2.7787630 \\
1.7652990 \\
-0.8783550 \\
0.3416490 \\
1.0247140 \\
-0.3023340 \\
-0.5132210 \\
-0.2412310 \\
-1.5805970 \\
-1.7375760 \\
-2.7568110 \\
-1.2968290 \\
-0.9515150 \\
-3.6705420 \\
-4.4093830 \\
-4.2191880 \\
-2.7961660 \\
-3.0777560 \\
-2.9368530 \\
-0.6628180 \\
-3.3494240 \\
-1.1207280 \\
-1.3887750 \\
-0.0708540 \\
-2.0285290 \\
-2.7896680 \\
-1.4429390 \\
0.0234920 \\
0.6217280 \\
0.2561990 \\
-0.5293240 \\
-1.9384800 \\
-0.5000480 \\
-1.7297860 \\
-2.3484760 \\
-1.3572470 \\
-2.5615610 \\
-3.6007750 \\
-2.5788920 \\
-2.5122150 \\
0.0994960 \\
-1.9669150 \\
-3.0096980 \\
-1.5921760 \\
-1.1038890\end{array}$ & $\begin{array}{l}-3.2912030 \\
-1.5472450 \\
-2.0241030 \\
-2.7112410 \\
-3.5181960 \\
-4.3622330 \\
-3.4331790 \\
-3.7413040 \\
-4.6877260 \\
-3.7886700 \\
0.7875600 \\
1.8869050 \\
2.2142400 \\
0.9691000 \\
1.9062590 \\
2.7109530 \\
0.9591580 \\
2.1331730 \\
3.0685790 \\
1.3161340 \\
2.3125980 \\
1.7316170 \\
3.4386340 \\
4.3511960 \\
3.5231330 \\
3.2581290 \\
4.0486270 \\
3.3120860 \\
0.0359100 \\
0.1756010 \\
3.6089770 \\
4.8906170 \\
2.9014910 \\
2.5313310 \\
4.6351570 \\
5.5415650 \\
4.4387600 \\
3.4243180 \\
3.7228800 \\
2.6144590 \\
2.0497940 \\
5.7045770 \\
4.0637570 \\
4.3779210 \\
3.7043820 \\
5.2515290\end{array}$ \\
\hline
\end{tabular}

-3.3015380
-2.5238280
-4.6675270
-4.9718700
-4.0197970
-4.1694690
-3.6630320
-3.8897800
-2.9417290
-3.0768790
-2.9705620
-2.0926940
-3.6119050
-5.3737090
-5.4101420
-5.2932180
-6.0837780
-5.9924140
-6.1887300
-6.9404430
-4.8266510
-5.2123730
-3.7423400
-4.6241200
-4.5658670
-3.1095620
-2.0397010
-2.4515720
-4.2745100
-5.1921190
-2.9400450
-1.9091880
-5.6548660
-4.9961990
-2.9601500
-1.0734740
-0.0686600
-1.1896230
-1.0229740
0.3975610
1.3572140
1.2304460
-1.1640770
-1.7897080
0.3387740
1.0913280

-1.7082870
-0.2778400
2.1858340
3.6215420
2.2595610
1.4372470
4.2909520
5.3160710
4.3515640
3.4537610
4.0692880
3.0627520
1.6703310
4.1606620
2.8350560
3.4851150
2.0165360
3.6378220
4.6796870
3.2003100
1.5981180
2.1809000
-1.6702480
-1.9223010
-3.4160690
-3.8900820
-2.9046220
-2.2326840
-1.3163300
-3.6029310
-4.0153010
-2.1010500
-1.6149570
-3.9918940
-4.8727690
-3.4047400
-4.0903840
-5.0912700
-6.2228620
-6.8610920
-6.1246960
-4.6306260
-5.5331530
-6.9872830
-7.9170170
-6.4068700
6.1643450

5.4639700

2. 1816890

1.8346690

$-0.1617370$

1. 0982150

1. 3314890

1.0087850

2. 1547680

0.1534400

$-0.7496440$

0.4176220

$-0.9870990$

2. 7012380

$-0.5443490$

$-1.4218390$

$-0.8260270$

0.6620270

0.3754770

0.9971380

3. 4368540

4. 2743500

$-2.1045420$

$-3.3012740$

$-3.6872160$

$-3.9479820$

$-3.4044680$

$-2.1164990$

$-4.1498300$

$-4.5687630$

$-5.0251560$

$-4.1433130$

$-3.0867360$

$-2.8576370$

$-3.4869360$

$-3.3129910$

$-1.0651070$

$-1.2061170$

$-0.1627840$

$-0.1876130$

$-1.1571660$

$-1.0068570$

$-2.2140010$

$-0.3403100$

$-0.4800310$

$-2.1882010$ 


\begin{tabular}{|c|c|c|c|c|c|c|c|}
\hline $\mathrm{H}$ & -2.1667340 & -4.6153530 & -1.0906330 & 0 & 0.6885670 & -0.6778610 & -0.2447080 \\
\hline $\mathrm{H}$ & -1.2171190 & -5.7957380 & 0.8286260 & 0 & -1.1039930 & -0.8367000 & 1.5414190 \\
\hline $\mathrm{H}$ & 0.8300370 & -6.8295490 & 0.8198420 & C & 0.9339650 & -2.0440770 & -0.0895970 \\
\hline $\mathrm{H}$ & 2.3926350 & -6.4455070 & -0.9915980 & C & -0.1678010 & -2.9235610 & -0.0784560 \\
\hline c & 1.9516440 & 2.4918690 & 2.8862450 & C & 2.2585680 & -2.4723350 & 0.0592990 \\
\hline C & 2.7111940 & 2.3783910 & 1.6386910 & C & 2.4697490 & -3.8430010 & 0.2564900 \\
\hline $\mathrm{H}$ & 0.8674570 & 2.4344580 & 2.7784960 & $\mathrm{H}$ & 3.4899100 & -4.2043670 & 0.3727460 \\
\hline $\mathrm{H}$ & 2.2410740 & 1.8566010 & 0.8119690 & C & -1.9821080 & -1.3250430 & 0.5734730 \\
\hline 0 & 2.5602850 & 1.1648670 & 3.0160460 & C & -3.2616560 & -0.7655940 & 0.4940350 \\
\hline c & 0.9310350 & 4.1501710 & -0.9415420 & c & -1.5438230 & -2.3741340 & -0.2619850 \\
\hline C & 0.0583740 & 3.7678350 & -3.2762310 & C & -4.1257110 & -1.2854440 & -0.4790870 \\
\hline c & -0.3312220 & 2.7863980 & -2.3391220 & $\mathrm{H}$ & -5.1315750 & -0.8767950 & -0.5546080 \\
\hline $\mathrm{N}$ & 0.1626690 & 3.0473390 & -1.0721570 & C & 3.4004340 & -1.5197590 & 0.0291150 \\
\hline $\mathrm{H}$ & -0.0285200 & 2.3947720 & -0.2650330 & C & -3.7143170 & 0.3386730 & 1.3797540 \\
\hline $\mathrm{s}$ & 1.0526080 & 4.9738080 & -2.4921740 & $\mathrm{C}$ & 3.6905830 & -0.7817480 & -1.1330490 \\
\hline $\mathrm{S}$ & 1.6872250 & 4.7117210 & 0.4469360 & C & 2.9751800 & -0.8800500 & -2.4599180 \\
\hline $\mathrm{H}$ & 1.8456020 & 0.4396880 & 2.6643140 & $\mathrm{C}$ & 4.9596020 & 0.7973100 & -2.4972030 \\
\hline C & -1.0878460 & 2.5527210 & -5.0007960 & C & 4.7750230 & 0.1141280 & -1.1643980 \\
\hline C & -1.4661410 & 1.5703970 & -4.0609290 & $\mathrm{C}$ & 2.4578990 & 0.5296250 & -2.8371960 \\
\hline C & -1.0936990 & 1.6816190 & -2.7128180 & $\mathrm{H}$ & 1.9522270 & 0.4837800 & -3.8097510 \\
\hline C & -0.3244110 & 3.6547840 & -4.6094650 & $\mathrm{H}$ & 1.7176090 & 0.8309020 & -2.0971750 \\
\hline $\mathrm{H}$ & -1.3827910 & 2.4644470 & -6.0400100 & C & 3.6467530 & 1.5362310 & -2.8745440 \\
\hline $\mathrm{H}$ & -1.3706630 & 0.9168540 & -1.9938380 & $\mathrm{H}$ & 3.7579760 & 1.9713650 & -3.8762450 \\
\hline $\mathrm{H}$ & -0.0315950 & 4.4012360 & -5.3428080 & $\mathrm{H}$ & 3.4710310 & 2.3648890 & -2.1798490 \\
\hline C & 3.9801920 & 3.5704550 & 3.9644200 & $\mathrm{H}$ & 5.8054030 & 1.4952590 & -2.4708120 \\
\hline C & 4.5362310 & 3.8701960 & 2.5632950 & $\mathrm{H}$ & 2.1436740 & -1.5888210 & -2.4193430 \\
\hline C & 4.1469910 & 2.7817550 & 1.5477030 & C & 5.1973820 & -0.3063790 & -3.5665670 \\
\hline C & 2.4448190 & 3.4670540 & 3.9350800 & $\mathrm{H}$ & 5.2806670 & 0.1668280 & -4.5541120 \\
\hline $\mathrm{H}$ & 4.2782630 & 4.3600840 & 4.6646510 & $\mathrm{H}$ & 6.1457570 & -0.8165960 & -3.3607110 \\
\hline $\mathrm{H}$ & 5.6289850 & 3.9580510 & 2.5934780 & C & 4.0081000 & -1.3176410 & -3.5371360 \\
\hline $\mathrm{H}$ & 4.7428790 & 1.8700540 & 1.6952660 & $\mathrm{H}$ & 3.5049940 & -1.3512900 & -4.5127110 \\
\hline $\mathrm{H}$ & 2.0071420 & 4.4449680 & 3.6898660 & $\mathrm{H}$ & 4.3653380 & -2.3297380 & -3.3128010 \\
\hline $\mathrm{H}$ & 2.0476830 & 3.1639140 & 4.9119980 & C & 4.2359460 & -1.3574440 & 1.1494380 \\
\hline $\mathrm{H}$ & 4.4001200 & 2.6260720 & 4.3352070 & C & 4.1194270 & -2.0721910 & 2.4767840 \\
\hline $\mathrm{H}$ & 4.1400330 & 4.8349020 & 2.2169280 & $\mathrm{C}$ & 6.1578380 & -0.4639660 & 2.3637350 \\
\hline $\mathrm{H}$ & 4.3475990 & 3.0987480 & 0.5190440 & C & 5.3372500 & -0.4797130 & 1.0990110 \\
\hline 0 & -2.1978280 & 0.4639350 & -4.3853990 & C & 5.4295300 & -2.8826650 & 2.6890620 \\
\hline $\mathrm{C}$ & -2.5227280 & 0.2588130 & -5.7637870 & $\mathrm{H}$ & 5.3789900 & -3.3868180 & 3.6631510 \\
\hline $\mathrm{H}$ & -1.6127410 & 0.2076570 & -6.3790260 & $\mathrm{H}$ & 5.5153740 & -3.6573390 & 1.9172330 \\
\hline $\mathrm{H}$ & -3.1746400 & 1.0604880 & -6.1397500 & C & 6.6540680 & -1.9135820 & 2.6343300 \\
\hline $\mathrm{H}$ & -3.0500730 & -0.6970720 & -5.8065050 & $\mathrm{H}$ & 7.2027260 & -1.9289680 & 3.5854520 \\
\hline & & & & $\mathrm{H}$ & 7.3513900 & -2.2148680 & 1.8435340 \\
\hline & {$[\mathrm{B} 97$} & ZVP] $=-43$ & & $\mathrm{H}$ & 7.0090020 & 0.2220600 & 2.2719660 \\
\hline $\mathrm{P}$ & 0.1068660 & 0.1288150 & 1.0563500 & $\mathrm{H}$ & 3.2490200 & -2.7321100 & 2.5056980 \\
\hline 0 & -0.3642680 & 1.4502000 & 0.5333930 & C & 5.2384980 & -0.0461320 & 3.5424620 \\
\hline 0 & 1.0861190 & 0.1104950 & 2.2033740 & $\mathrm{H}$ & 5.8218230 & -0.0668620 & 4.4729110 \\
\hline
\end{tabular}




$\begin{array}{rrrr}\mathrm{H} & 4.8859950 & 0.9795840 & 3.3888360 \\ \mathrm{C} & 4.0307190 & -1.0263860 & 3.6225790 \\ \mathrm{H} & 4.0355030 & -1.5661600 & 4.5790120 \\ \mathrm{H} & 3.0827680 & -0.4894770 & 3.5493980 \\ \mathrm{C} & 5.6020790 & 0.2637590 & -0.0505830 \\ \mathrm{H} & 6.4559020 & 0.9416370 & -0.0853200 \\ \mathrm{C} & -4.2885170 & 1.2085330 & 3.5899600 \\ \mathrm{C} & -4.3280930 & 0.8579260 & 5.0561920 \\ \mathrm{C} & -3.4503730 & -1.0730800 & 3.5561190 \\ \mathrm{C} & -3.7988830 & 0.1714840 & 2.7735240 \\ \mathrm{C} & -5.2380220 & -0.3896810 & 5.2357390 \\ \mathrm{H} & -5.2145110 & -0.6975950 & 6.2897890 \\ \mathrm{H} & -6.2736230 & -0.1264720 & 4.9893640 \\ \mathrm{C} & -4.7257910 & -1.5399230 & 4.3134060 \\ \mathrm{H} & -4.4784770 & -2.4306140 & 4.9062340 \\ \mathrm{H} & -5.4962860 & -1.8268070 & 3.5876220 \\ \mathrm{H} & -3.0858200 & -1.8733140 & 2.9072790 \\ \mathrm{H} & -4.7044370 & 1.6978120 & 5.6529490 \\ \mathrm{C} & -2.3748250 & -0.7070660 & 4.6165060 \\ \mathrm{H} & -2.1739580 & -1.5950650 & 5.2307610 \\ \mathrm{H} & -1.4450760 & -0.4357990 & 4.1094230 \\ \mathrm{C} & -2.8926070 & 0.4674650 & 5.5033290 \\ \mathrm{H} & -2.9154980 & 0.1770780 & 6.5623410 \\ \mathrm{H} & -2.2338200 & 1.3392620 & 5.4114080 \\ \mathrm{C} & -4.6207020 & 2.5935050 & 1.6503860 \\ \mathrm{C} & -5.0309150 & 3.8331540 & 0.8946950 \\ \mathrm{C} & -4.0538980 & 1.9701390 & -0.6296000 \\ \mathrm{C} & -4.1199300 & 1.5674280 & 0.8266260 \\ \mathrm{C} & -3.7919020 & 4.3846830 & 0.1371210 \\ \mathrm{H} & -4.1078250 & 5.2300990 & -0.4890010 \\ \mathrm{H} & -3.0543100 & 4.7601630 & 0.8560710 \\ \mathrm{C} & -3.1767150 & 3.2482450 & -0.7334520 \\ \mathrm{H} & -3.1122420 & 3.5521120 & -1.7865460 \\ \mathrm{H} & -2.1696610 & 3.0094950 & -0.3874190 \\ \mathrm{H} & -3.6333230 & 1.1778360 & -1.2535170 \\ \mathrm{H} & -5.4402650 & 4.5919310 & 1.5727200 \\ \mathrm{C} & -5.4864390 & 2.3312970 & -1.1073640 \\ \mathrm{H} & -5.4319470 & 2.6956300 & -2.1420800 \\ \mathrm{H} & -6.1143280 & 1.4319780 & -1.1054650 \\ \mathrm{C} & -6.0889860 & 3.4249310 & -0.1695030 \\ \mathrm{H} & -6.3769060 & 4.3139760 & -0.7463450 \\ \mathrm{H} & -6.9875860 & 3.0502200 & 0.3351700 \\ \mathrm{C} & -4.7044790 & 2.4173620 & 3.0313480 \\ \mathrm{H} & -5.0915630 & 3.2145630 & -1.3561340 \\ \mathrm{C} & -3.7202500 & -2.2863100 & \\ \mathrm{C} & -4.6338840 & -2.8357320 & \\ & & & \\ \mathrm{H} & -3211430\end{array}$

-4.6031780
-3.1535860
-2.0653840
-2.4253940
-4.2999540
-5.2152260
-2.9923480
-1.9724500
-5.6565220
-5.0673410
-3.0151930
-1.0940090
1.4063000
1.6064030
0.7952330
-0.6934770
-0.9880420
0.0823830
1.2575740
0.8618060
-1.3547210
-1.0025090
2.6713200
1.2628310
-0.9581780
-1.9819170
1.8861660
2.6472080
0.8126030
2.2258440
2.6186400
0.6474360
-0.3182310
-0.5406410
-0.0001820
-0.1019760
0.5876130
1.4143220
1.9644560
-1.4349570
-1.6411060
-1.1999980
-0.7719410
-1.7839390
-1.3437790
-0.6100600
$-2.4072580$ $-2.4955040$ $-2.3203340$ $-1.2608460$ $-3.4052570$ $-3.2290130$ $-3.4633880$ $-3.2673760$ $-2.2734170$ $-1.4715540$ $-1.7263340$ $-2.1289030$ 0.3404040 0.6159560 $-0.3816840$ $-0.4647100$ 0.3569000 0.1726340

1. 6365510 $-0.0952710$ $-0.1079950$

1. 4235810

0.5733420

$-1.3698850$ $-1.5111990$

0.1199700

2. 0619850

0.8569120

1.9999230

0.1979950

2. 4918900

$-2.0180680$

$-4.1152910$

$-2.9430270$

$-1.8211680$

$-0.8646570$

$-3.7257580$

$-0.8653270$

2. 3612770

$-5.3966930$

$-4.2218340$

$-2.9767110$

$-5.3449160$

$-6.3557210$

$-2.0774330$ 


\begin{tabular}{|c|c|c|c|c|c|c|c|}
\hline & & & & & & & \\
\hline c & $\begin{array}{l}3.8022010 \\
4.3133540\end{array}$ & $\begin{array}{l}4.7183090 \\
4.7228230\end{array}$ & $\begin{array}{l}2.7907640 \\
1.3407580\end{array}$ & $\begin{array}{l}\mathrm{H} \\
\mathrm{H}\end{array}$ & $\begin{array}{l}4.3678720 \\
3.2852820\end{array}$ & $\begin{array}{r}0.6094320 \\
-2\end{array}$ & $\begin{array}{l}-5.7637290 \\
-2 \\
-2\end{array}$ \\
\hline C & 4.0361510 & 3.3853690 & 0.6326390 & C & 5.1319090 & -1.2798280 & -4.9509700 \\
\hline C & 2.2853540 & 4.4617530 & 2.8343040 & $\mathrm{H}$ & 5.0709640 & -1.8180100 & -5.9062830 \\
\hline H & 4.0236200 & 5.6787060 & 3.2715800 & $\mathrm{H}$ & 6.1496600 & -0.8825800 & -4.8576100 \\
\hline $\mathrm{H}$ & 5.3904210 & 4.9271750 & 1.3119830 & C & 4.7990830 & -2.2314860 & -3.7587600 \\
\hline $\mathrm{H}$ & 4.7211520 & 2.6012030 & 0.9849370 & $\mathrm{H}$ & 4.6113050 & -3.2517590 & -4.1186660 \\
\hline $\mathrm{H}$ & 1.7483360 & 5.3060040 & 2.3798450 & $\mathrm{H}$ & 5.6376090 & -2.2758290 & -3.0533940 \\
\hline $\mathrm{H}$ & 1.9282000 & 4. 3616010 & 3.8669760 & C & 4.1019770 & 1.5099190 & -0.9928390 \\
\hline $\mathrm{H}$ & 4.3198240 & 3.9361030 & 3.3619510 & $\mathrm{C}$ & 4.0714800 & 2.2223810 & 0.3393640 \\
\hline $\mathrm{H}$ & 3.8124000 & 5.5274510 & 0.7848400 & C & 4.7299780 & 3.7838420 & -1.6274740 \\
\hline $\mathrm{H}$ & 4.2014910 & 3.4595360 & -0.4476260 & $\mathrm{C}$ & 4.4392150 & 2.3674660 & -2.0580750 \\
\hline 0 & -2.2602730 & -0.8922580 & -4.2075650 & $\mathrm{C}$ & 5.4719430 & 2.8331430 & 0.6126030 \\
\hline $\mathrm{C}$ & -2.6573440 & -1.4560220 & -5.4610730 & $\mathrm{H}$ & 5.4294980 & 3.4071910 & 1.5477770 \\
\hline $\mathrm{H}$ & -1.7948000 & -1.5652200 & -6.1342680 & $\mathrm{H}$ & 6.2057930 & 2.0294550 & 0.7489870 \\
\hline $\mathrm{H}$ & -3.4254990 & -0.8370000 & -5.9468580 & C & 5.8791980 & 3.7537620 & -0.5810540 \\
\hline $\mathrm{H}$ & -3.0697360 & -2.4404230 & -5.2263060 & $\mathrm{H}$ & 6.0709330 & 4.7771960 & -0.2323140 \\
\hline & & & & $\mathrm{H}$ & 6.7964320 & 3.3870430 & -1.0569810 \\
\hline & 5) $\mathrm{G}[\mathrm{B}$ & $\mathrm{ZVP}]=-43$ & & $\mathrm{H}$ & 5.0069940 & 4.4086280 & -2.4851760 \\
\hline $\mathrm{P}$ & -0.0451760 & -0.1876730 & -0.7206260 & $\mathrm{H}$ & 3.7863410 & 1.5489400 & 1.1514780 \\
\hline 0 & -1.0311420 & -0.6452330 & -1.7681540 & C & 3.4633190 & 4.3461880 & -0.9236380 \\
\hline 0 & 0.2729280 & 1.2679880 & -0.6031070 & $\mathrm{H}$ & 3.6869340 & 5.3500580 & -0.5382220 \\
\hline 0 & 1.2605870 & -1.1155810 & -0.9910190 & $\mathrm{H}$ & 2.6497360 & 4.4426270 & -1.6520420 \\
\hline 0 & -0.4950060 & -0.6842940 & 0.7713980 & C & 3.0504550 & 3.3874080 & 0.2331100 \\
\hline C & 2.1900380 & -1.3469500 & 0.0170730 & $\mathrm{H}$ & 3.0153690 & 3.9198680 & 1.1919630 \\
\hline c & 1.8482100 & -2.2192150 & 1.0732070 & $\mathrm{H}$ & 2.0609170 & 2.9667120 & 0.0452080 \\
\hline C & 3.4493060 & -0.7452710 & -0.1008130 & C & 4.4547650 & 1.8947750 & -3.3698140 \\
\hline c & 4.3926480 & -1.0287800 & 0.8951950 & $\mathrm{H}$ & 4.7112080 & 2.5614310 & -4.1941030 \\
\hline $\mathrm{H}$ & 5.3828690 & -0.5832890 & 0.8193920 & C & -4.6957230 & 0.0600610 & 1.1861140 \\
\hline c & -0.6567320 & -2.0605340 & 0.9503230 & $\mathrm{C}$ & -4.9637140 & 1.1709520 & 2.1708640 \\
\hline c & -1.9532350 & -2.5899980 & 0.9447680 & $\mathrm{C}$ & -2.8499100 & -0.2307610 & 2.7382270 \\
\hline C & 0.5002820 & -2.8558150 & 1.0949490 & $\mathrm{C}$ & -3.5382240 & -0.6877180 & 1.4735750 \\
\hline c & -2.0781790 & -3.9738890 & 1.1339320 & $\mathrm{C}$ & -5.1285670 & 0.5358610 & 3.5803470 \\
\hline H & -3.0745290 & -4.4112520 & 1.1416050 & $\mathrm{H}$ & -5.2551020 & 1.3382530 & 4.3193170 \\
\hline c & 3.7837550 & 0.1612120 & -1.2316650 & $\mathrm{H}$ & -6.0348450 & -0.0807490 & 3.5996610 \\
\hline c & -3.1573790 & -1.7655130 & 0.6546150 & C & -3.8679480 & -0.3237620 & 3.9093810 \\
\hline C & 3.8004340 & -0.3061530 & -2.5579830 & $\mathrm{H}$ & -3.3830540 & 0.0373040 & 4.8259210 \\
\hline c & 3.5294730 & -1.7188630 & -3.0208400 & $\mathrm{H}$ & -4.1454040 & -1.3721570 & 4.0710320 \\
\hline C & 4.1153610 & -0.1044320 & -4.9701950 & $\mathrm{H}$ & -1.9635550 & -0.8317330 & 2.9580200 \\
\hline C & 4.1339100 & 0.5596510 & -3.6163420 & $\mathrm{H}$ & -5.8627910 & 1.7355850 & 1.8950830 \\
\hline c & 2.3624480 & -1.6955490 & -4.0470570 & C & -2.4582090 & 1.2581270 & 2.5658640 \\
\hline $\mathrm{H}$ & 2.2268720 & -2.7115700 & -4.4415680 & $\mathrm{H}$ & -1.9905510 & 1.6191580 & 3.4896940 \\
\hline $\mathrm{H}$ & 1.4376010 & -1.4095320 & -3.5394220 & $\mathrm{H}$ & -1.7118450 & 1.3297970 & 1.7744930 \\
\hline c & 2.6999740 & -0.7016840 & -5.2007730 & $\mathrm{C}$ & -3.7214280 & 2.1019360 & 2.2197020 \\
\hline H & 2.6808250 & -1.2106100 & -6.1738180 & $\mathrm{H}$ & -3.8867040 & 2.8832910 & 2.9727670 \\
\hline $\mathrm{H}$ & 1.9650300 & 0.1115750 & -5.2361040 & $\mathrm{H}$ & -3.5978090 & 2.6055060 & 1.2546130 \\
\hline
\end{tabular}




$\begin{array}{rrr}-5.1433790 & -1.3478820 & -0.7130100 \\ -5.9231840 & -1.8449350 & -1.9043530 \\ -3.7366530 & -3.1760230 & -1.4725840 \\ -3.9668590 & -2.0784600 & -0.4569830 \\ -5.0157640 & -1.7544360 & -3.1596540 \\ -5.5605150 & -2.1621830 & -4.0219730 \\ -4.7836560 & -0.7055150 & -3.3710890 \\ -3.7064400 & -2.5581180 & -2.8988560 \\ -3.6060900 & -3.3772080 & -3.6236030 \\ -2.8265820 & -1.9178880 & -2.9958560 \\ -2.8057780 & -3.7150850 & -1.2863520 \\ -6.8401690 & -1.2594210 & -2.0454880 \\ -4.9506900 & -4.1438490 & -1.4079020 \\ -4.8178400 & -4.9291880 & -2.1638510 \\ -4.9842550 & -4.6305910 & -0.4255820 \\ -6.2660060 & -3.3438650 & -1.6699810 \\ -6.7843810 & -3.7315330 & -2.5568800 \\ -6.9509230 & -3.4335360 & -0.8183950 \\ -5.5057570 & -0.2764560 & 0.1018980 \\ -6.4188930 & 0.2853020 & -0.0986820 \\ 0.3319360 & -4.2515020 & 1.1933970 \\ 1.4610270 & -5.2524100 & 1.1395070 \\ 1.1724570 & -6.3141750 & 0.0501690 \\ -0.2327440 & -6.9676400 & 0.2038360 \\ -1.0683940 & -6.3011330 & 1.3269720 \\ -0.9638580 & -4.8004350 & 1.2429720 \\ 1.5526320 & -5.7603130 & 2.1114340 \\ 1.9572970 & -7.0799510 & 0.0841490 \\ -0.1366140 & -8.0391190 & 0.4195710 \\ -0.6752260 & -6.6394850 & 2.2982520 \\ 2.4187940 & -4.7640520 & 0.9433290 \\ 1.2453590 & -5.8208380 & -0.9267510 \\ -0.7838980 & -6.8765070 & -0.7400270 \\ -2.1153720 & -6.6215680 & 1.2700810 \\ 4.0864010 & -1.8523310 & 1.9760870 \\ 5.0856790 & -2.1675400 & 3.0604280 \\ 5.1255010 & -3.6913820 & 3.3104350 \\ 3.7136050 & -4.2629610 & 3.6228230 \\ 2.5744350 & -3.2484330 & 3.3365240 \\ 2.8126890 & -2.4461850 & 2.0778170 \\ 4.7915970 & -1.6651150 & 3.9964720 \\ 5.8202960 & -3.9171130 & 4.6775380 \\ 3.6479030 & -4.5578670 & 2.7887740 \\ 2.5481350 & -2.5382720 & \\ 6.0782300 & -1.7890330 & \\ 5.5242550 & -4.1687000 & \\ -6 & & \\ -6 & -13063370\end{array}$

3. 5452520

1.6062130

$-2.1751980$

$-2.9311780$

$-1.0891320$

$-2.4565070$

$-2.7746920$

$-1.2401470$

$-0.2960480$

0.0507910

$-0.4827890$

$-0.2849030$

$-1.3084380$

$-2.0148850$

$-2.0191140$

0.9381200

1.2739540

0.8330090

0.1558670

1. 3005630

1.0547070

$-0.0996040$

$-4.2111920$

$-4.7727400$

$-4.3752330$

$-2.6747220$

$-4.5191590$

$-5.8662190$

$-4.9567420$

$-2.2468280$

$-2.2754640$

$-4.6196600$

$-4.3871480$

$-4.5823930$

2. 0341540

2. 3516940

2. 9207480

1.4400640

2. 9615080
$-5.1664170$

$-3.7518350$

2.1398380

2.2910470

2.1104580

1.9643160

0.8170260

4.5036940

4.4622790

3. 3543910

3.4212070

2.6534200

5.5484300

4.8471310

0.1669190

3. 5100400

2. 3940240

2. 3057740

5443120

3.5394430

1.4664960

5.3927460

2.9394190

. 5246400

2.6820880

2. 8626360

3.5578380

3.5926690

1.7507220

3.8745560

2.3563040

1.9326460

4.5447970

3.2118910

1.4420690

0.2739190

0.5343330

$-0.2610250$

$-0.3541720$
3.0274890

3. 3269600

$-2.8328890$

$-1.5863310$

$-2.7219760$

$-0.6672190$

$-2.6675920$

0.5497860

2. 8945200

2.1054080

0.8313160

0.1498130

1.9660780

$-0.8974700$

$-2.2337700$

4.7181840

3.9158610

2. 5924700

4.2167110

5.7420680

1.9451220

4.8456640

$-4.1140970$

$-2.8080870$

$-1.5829940$

$-4.0673620$

$-4.9657560$

$-2.8565360$

$-1.5399450$

$-4.0435170$

$-4.9552620$

$-4.2732910$

$-2.6759660$

$-0.6469430$

4.5238040

3.7538930

2.8533380

3.4573700

4.4055040

TS82 (S, S) G[B97-D/def2-TZVP] $=-4356.201999$

$\begin{array}{lrrr}\text { TS82(S, S }) \text { G G }[\mathrm{B} 97-\mathrm{D} / \mathrm{def} 2-\mathrm{TZVP}]=-4356.201999 \\ \mathrm{P} & -0.0452720 & -0.1876440 & -0.7204670 \\ 0 & 0.2728380 & 1.2680070 & -0.6028550 \\ 0 & -1.0313710 & -0.6451080 & -1.7679080 \\ 0 & -0.4948920 & -0.6844210 & 0.7715680 \\ 0 & 1.2604620 & -1.1155200 & -0.9911160\end{array}$




$\begin{array}{rr}-0.6565210 & -2.0606840 \\ 0.5005580 & -2.8559050 \\ -1.9529920 & -2.5902250 \\ -2.0778390 & -3.9741360 \\ -3.0741620 & -4.4115600 \\ 2.1900720 & -1.3468820 \\ 3.4492740 & -0.7450910 \\ 1.8484480 & -2.2192260 \\ 4.3927760 & -1.0285870 \\ 5.3829510 & -0.5830110 \\ -3.1571910 & -1.7657990 \\ 3.7834830 & 0.1615030 \\ -3.5380870 & -0.6880310 \\ -2.8498260 & -0.2310880 \\ -4.9636110 & 1.1706290 \\ -4.6955850 & 0.0597400 \\ -2.4581160 & 1.2578030 \\ -1.9905090 & 1.6188160 \\ -1.7117110 & 1.3295000 \\ -3.7213250 & 2.1016090 \\ -3.8866330 & 2.8829610 \\ -3.5976730 & 2.6051840 \\ -5.8626760 & 1.7352670 \\ -1.9634770 & -0.8320660 \\ -5.1285200 & 0.5355280 \\ -5.2550820 & 1.3379140 \\ -6.0347990 & -0.0810820 \\ -3.8679120 & -0.3240970 \\ -3.3830510 & 0.0369600 \\ -4.1453750 & -1.3724930 \\ -3.9666350 & -2.0787440 \\ -3.7363790 & -3.1762820 \\ -5.9229330 & -1.8452420 \\ -5.1431650 & -1.3481870 \\ -4.9503890 & -4.1441420 \\ -4.8175010 & -4.9294580 \\ -4.9839600 & -4.6309120 \\ -6.2657190 & -3.3441850 \\ -6.7840710 & -3.7318510 \\ -6.9506470 & -3.4338870 \\ -6.8399310 & -1.2597520 \\ -2.8054920 & -3.7153200 \\ -5.0154950 & -1.7547060 \\ -5.5602210 & -2.1624700 \\ -4.7834220 & -0.7057770 \\ -3.7061500 & -2.5583480\end{array}$

0.9503980
1.0948550
0.9449110
1.1340030
1.1417120
0.0168330
-0.1011890
1.0729680
0.8946710
0.8187620
0.6548050
-1.2320240
1.4737750
2.7384600
2.1710330
1.1862860
2.5661380
3.4900010
1.7748090
2.2199310
2.9729930
1.2548500
1.8952220
2.9582730
3.5805040
4.3194760
3.5997800
3.9095740
4.8261350
4.0712050
-0.4568220
-1.4724430
-1.9042340
-0.7128680
-1.4078040
-2.1637720
-0.4254980
-1.6698910
-2.5568030
-0.8183170
-2.0453780
-1.2862010
-3.1595200
-4.0218470
-3.3709500
-2.8987020

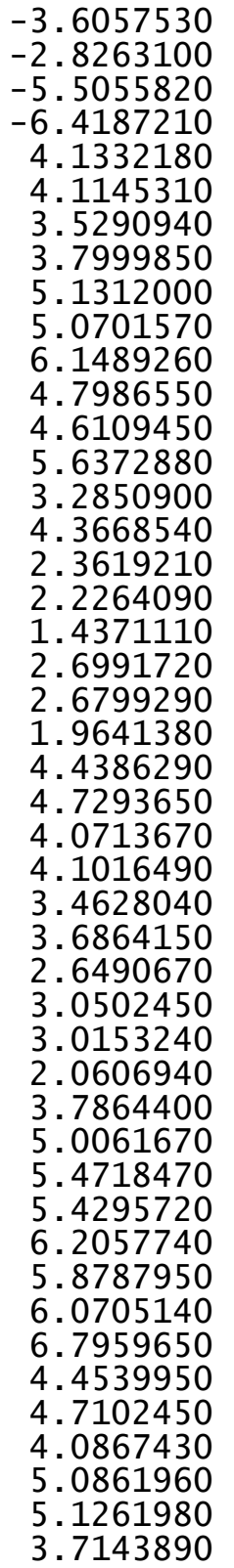
$-3.3774210$
19180860
$-0.2767740$
0.2849730
0.5601550
$-0.1038310$
$-1.7184610$
$-0.3057580$
$-1.2791290$
$-1.8172540$
$-0.8817860$
$-2.2308970$
$-3.2511710$
$-2.3797070$
0.6101140
$-1.6952010$
$-2.7112070$
$-1.4093280$
$-0.7012100$
$-1.2100610$
0.1119750
2. 3678750
3. 7842370
2. 2225630
1.5102140
4. 3464310
5. 3502900
4.4428620
3. 3875320
3. 9199110
2. 9667950
1. 5490310
4.4091140
2. 8333880
3.4073320
2. 0297270
3.7541540
4.7775710
3. 3875550
1.8952870
2. 5620280
$-1.8522370$
$-2.1674440$
$-3.6913060$
$-4.2630610$

-3.6234630
-2.9956620
0.1020400
-0.0985570
-3.6167290
-4.9706280
-3.0213010
-2.5583830
-4.9516370
-5.9069750
-4.8584100
-3.7594350
-4.1193740
-3.0541930
-2.1855710
-5.7641490
-4.0473530
-4.4419210
-3.5395710
-5.2010400
-6.1741220
-5.2361980
-2.0583730
-1.6277110
0.3391250
-0.9931470
-0.9235790
-0.5381320
-1.6518120
0.2331780
1.1920820
0.0454470
1.1512390
-2.4854150
0.6121290
1.5473750
0.7482690
-0.5815190
-0.2327200
-1.0576600
-3.3701530
-4.1944330
1.9755480
3.0597280
3.3096000
3.6220700
-3 


\begin{tabular}{|c|c|c|c|}
\hline C & 2.5751030 & -3.2485730 & 3.3360970 \\
\hline C & 2.8130890 & -2.4461920 & 2.0774230 \\
\hline H & 4.7921880 & -1.6651270 & 3.9958530 \\
\hline $\mathrm{H}$ & 5.8210930 & -3.9170310 & 4.1278550 \\
\hline $\mathrm{H}$ & 3.6488470 & -4.5581620 & 4.6767410 \\
\hline $\mathrm{H}$ & 2.5489220 & -2.5385050 & 4.1764750 \\
\hline $\mathrm{H}$ & 6.0786750 & -1.7888210 & 2.7879750 \\
\hline $\mathrm{H}$ & 5.5249200 & -4.1685050 & 2.4054260 \\
\hline $\mathrm{H}$ & 3.5460310 & -5.1664230 & 3.0265940 \\
\hline $\mathrm{H}$ & 1.6069080 & -3.7520310 & 3.3266590 \\
\hline C & 0.3322990 & -4.2516070 & 1.1932350 \\
\hline C & 1.4614410 & -5.2524440 & 1.1391790 \\
\hline C & 1.1728430 & -6.3141420 & 0.0497820 \\
\hline $\mathrm{c}$ & -0.2323030 & -6.9677080 & 0.2035340 \\
\hline C & -1.0678910 & -6.3013330 & 1.3267960 \\
\hline $\mathrm{C}$ & -0.9634570 & -4.8006210 & 1.2428960 \\
\hline $\mathrm{H}$ & 1.5531500 & -5.7604200 & 2.1110580 \\
\hline $\mathrm{H}$ & 1.9577340 & -7.0798710 & 0.0836300 \\
\hline $\mathrm{H}$ & -0.1360840 & -8.0391950 & 0.4191860 \\
\hline H & -0.6746070 & -6.6397290 & 2.2980140 \\
\hline $\mathrm{H}$ & 2.4191640 & -4.7640170 & 0.9429660 \\
\hline H & 1.2456220 & -5.8207250 & -0.9271060 \\
\hline $\mathrm{H}$ & -0.7835520 & -6.8765440 & -0.7402710 \\
\hline $\mathrm{H}$ & -2.1148530 & -6.6218340 & 1.2699810 \\
\hline C & -2.1753990 & 2.1400720 & -2.8325170 \\
\hline c & -2.9313220 & 2.2912200 & -1.5859180 \\
\hline $\mathrm{H}$ & -1.0893280 & 2.1106960 & -2.7216560 \\
\hline $\mathrm{H}$ & -2.4566030 & 1.9644520 & -0.6668460 \\
\hline 0 & -2.7748810 & 0.8172500 & -2.6672600 \\
\hline C & -1.2402630 & 4.5036450 & 0.5503160 \\
\hline C & -0.2960840 & 4.4619840 & 2.8950150 \\
\hline C & 0.0507650 & 3.3542020 & 2.1057610 \\
\hline $\mathrm{N}$ & -0.4828740 & 3.4211410 & 0.8317000 \\
\hline $\mathrm{H}$ & -0.2850030 & 2.6534230 & 0.1501200 \\
\hline $\mathrm{s}$ & -1.3085410 & 5.5482100 & 1.9667340 \\
\hline $\mathrm{S}$ & -2.0150330 & 4.8472410 & -0.8968870 \\
\hline $\mathrm{H}$ & -2.0193060 & 0.1671110 & -2.2334800 \\
\hline C & 0.9382180 & 3.5095910 & 4.7185070 \\
\hline $\mathrm{C}$ & 1.2740760 & 2.3936900 & 3.9160340 \\
\hline $\mathrm{C}$ & 0.8330590 & 2.3055660 & 2.5926590 \\
\hline $\mathrm{C}$ & 0.1558870 & 4.5438820 & 4.2171960 \\
\hline $\mathrm{H}$ & 1.3007140 & 3.5388940 & 5.742376 \\
\hline $\mathrm{H}$ & 1.0547630 & 1.4663710 & 1.945205 \\
\hline $\mathrm{H}$ & -0.0995940 & 5.3922290 & 4.8462620 \\
\hline C & -4.2114530 & 2.9397240 & 112 \\
\hline C & -4.7729490 & 3.5248640 & .80752 \\
\hline
\end{tabular}

$\begin{array}{lr}\mathrm{C} & -4.3753820 \\ \mathrm{C} & -2.6749800 \\ \mathrm{H} & -4.5194560 \\ \mathrm{H} & -5.8664300 \\ \mathrm{H} & -4.9568790 \\ \mathrm{H} & -2.2470830 \\ \mathrm{H} & -2.2757620 \\ \mathrm{H} & -4.6199230 \\ \mathrm{H} & -4.3873570 \\ \mathrm{H} & -4.5825050 \\ \mathrm{O} & 2.0343820 \\ \mathrm{C} & 2.3519920 \\ \mathrm{H} & 2.9209050 \\ \mathrm{H} & 1.4404000 \\ \mathrm{H} & 2.9619650 \\ \mathrm{I} & \end{array}$

2.6822440
2.8629390
3.5581950
3.5928900
1.7508710
3.8748570
2.3566610
1.9329600
4.5450170
3.2119930
1.4417160
0.2737040
0.5343070
-0.2613260
-0.3543840

$-1.5824930$ $-4.0669260$ $-4.9651970$ $-2.8559190$ $-1.5394680$ $-4.0430370$ $-4.9548750$ $-4.2728250$ $-2.6753570$ $-0.6464030$

4.5238130 3.7537210 2.8531300

3.4572340

4.4051840 158 $\operatorname{TS} 83(\mathrm{~S}, \mathrm{~S}) \mathrm{G}[\mathrm{B} 97-\mathrm{D} / \mathrm{def2}-\mathrm{TZVP}]=-4356.201934$

$\begin{array}{lrrr}\text { TS } & \text { P }(\mathrm{S}, \mathrm{S}) \mathrm{G}[\mathrm{B} 97-\mathrm{D} / \mathrm{def}-\mathrm{TZVP}] & -4356.201934 \\ \mathrm{O} & 0.0271080 & -0.0408480 & 1.1270000 \\ \mathrm{O} & 0.9422040 & -0.2871790 & 2.3025340 \\ \mathrm{O} & -0.4088310 & 1.3614000 & 0.8358710 \\ \mathrm{O} & -1.2124390 & -1.0613880 & 1.3652170 \\ \mathrm{C} & 0.6883200 & -0.5909380 & -0.2711300 \\ \mathrm{C} & -2.0292730 & -1.3790580 & 0.2793830 \\ \mathrm{C} & -1.5233980 & -2.2552900 & -0.7000560 \\ \mathrm{C} & -3.3136990 & -0.8291150 & 0.2197180 \\ \mathrm{H} & -4.1056870 & -1.1808990 & -0.8819670 \\ \mathrm{C} & -5.1168630 & -0.7827530 & -0.9442000 \\ \mathrm{C} & 0.9346720 & -1.9624070 & -0.3713510 \\ \mathrm{C} & 2.2567010 & -2.4202320 & -0.3068620 \\ \mathrm{C} & -0.1570330 & -2.8293320 & -0.5349810 \\ \mathrm{H} & 2.4479060 & -3.8035600 & -0.3498920 \\ \mathrm{C} & 3.4666720 & -4.1851380 & -0.3054920 \\ \mathrm{C} & -3.8430330 & 0.0965790 & 1.2545710 \\ \mathrm{C} & 3.4144590 & -1.4935610 & -0.2010350 \\ \mathrm{C} & -4.0377120 & -0.3241040 & 2.5811930 \\ \mathrm{C} & -3.7284870 & -1.6905070 & 3.1468440 \\ \mathrm{C} & -4.7680940 & -0.0745380 & 4.8967810 \\ \mathrm{C} & -4.6090750 & 0.5443920 & 3.5307190 \\ \mathrm{H} & -2.7504090 & -1.5175290 & 4.3411870 \\ \mathrm{H} & -2.5694750 & -2.5026340 & 4.7917730 \\ \mathrm{C} & -1.7931490 & -1.1397760 & 3.9707290 \\ \mathrm{H} & -3.3700940 & -0.5409220 & 5.3887720 \\ \mathrm{H} & -3.4822400 & -1.0339040 & 6.3638460 \\ \mathrm{H} & -2.7237040 & 0.3334480 & 5.5299390 \\ \mathrm{H} & -5.2098330 & 0.6376600 & 5.6043770 \\ & -3.2936180 & -2.3537110 & 2.3947330\end{array}$




\begin{tabular}{lr}
$\mathrm{C}$ & -5.6682300 \\
$\mathrm{H}$ & -5.7419370 \\
$\mathrm{H}$ & -6.6793720 \\
$\mathrm{C}$ & -5.0494400 \\
$\mathrm{H}$ & -4.8280490 \\
$\mathrm{H}$ & -5.7484760 \\
$\mathrm{C}$ & -4.2112840 \\
$\mathrm{C}$ & -4.0179380 \\
$\mathrm{C}$ & -5.1389070 \\
$\mathrm{C}$ & -4.7914780 \\
$\mathrm{C}$ & -5.4017360 \\
$\mathrm{H}$ & -5.2527940 \\
$\mathrm{H}$ & -6.0227880 \\
$\mathrm{C}$ & -6.0911720 \\
$\mathrm{H}$ & -6.3279980 \\
$\mathrm{H}$ & -7.0310510 \\
$\mathrm{H}$ & -5.6136860 \\
$\mathrm{H}$ & -3.5371210 \\
$\mathrm{C}$ & -3.8350230 \\
$\mathrm{H}$ & -4.0881820 \\
$\mathrm{H}$ & -3.1675370 \\
$\mathrm{C}$ & -3.1427390 \\
$\mathrm{H}$ & -2.9969270 \\
$\mathrm{H}$ & -2.1652610 \\
$\mathrm{C}$ & -4.9920210 \\
$\mathrm{H}$ & -5.4425390 \\
$\mathrm{C}$ & 4.8208280 \\
$\mathrm{C}$ & 5.0077830 \\
$\mathrm{C}$ & 2.9553380 \\
$\mathrm{C}$ & 3.7051130 \\
$\mathrm{C}$ & 5.1748240 \\
$\mathrm{H}$ & 5.2629180 \\
$\mathrm{H}$ & 6.1019860 \\
$\mathrm{C}$ & 3.9408750 \\
$\mathrm{H}$ & 3.4142550 \\
$\mathrm{H}$ & 4.2548210 \\
$\mathrm{H}$ & 2.0946370 \\
$\mathrm{H}$ & 5.8836760 \\
$\mathrm{C}$ & 2.4907340 \\
$\mathrm{H}$ & 1.9481270 \\
$\mathrm{H}$ & 1.7928650 \\
$\mathrm{C}$ & 3.7212930 \\
$\mathrm{H}$ & 3.8271440 \\
$\mathrm{H}$ & 3.6018050 \\
$\mathrm{C}$ & 5.3930750 \\
$\mathrm{C}$ & 6.2204820 \\
\hline &
\end{tabular}

\begin{tabular}{|c|c|}
\hline $\begin{array}{l}-1.3344170 \\
-1.8293480 \\
-1.0300340 \\
-2.2978380 \\
-3.2756070 \\
-2.4624570 \\
1.4091980 \\
2.0737990 \\
3.6303730 \\
2.2671010 \\
2.5353340 \\
3.0860610 \\
1.6595860 \\
3.4423590 \\
4.4274840 \\
2.9924070 \\
4.2538890 \\
1.4038830 \\
4.2958870 \\
5.2551610 \\
4.5055630 \\
3.3402510 \\
3.8391020 \\
3.0319570 \\
1.8373060 \\
2.5039910 \\
0.2634090 \\
1.1328590 \\
-0.4507860 \\
-0.5897740 \\
0.1982960 \\
0.8141080 \\
-0.3778980 \\
-0.7533850 \\
-0.6083220 \\
-1.8027080 \\
-1.1228920 \\
1.7840250 \\
1.0207700 \\
1.1505600 \\
1.2281920 \\
1.9731860 \\
2.5433330 \\
2.6992870 \\
-0.6788830 \\
-0.8640180\end{array}$ & $\begin{array}{r}4.7570460 \\
5.7347550 \\
4.4614020 \\
3.6962360 \\
4.1445090 \\
2.8674510 \\
0.9067050 \\
-0.4368440 \\
1.3152880 \\
1.8600910 \\
-0.9671050 \\
-1.9056320 \\
-1.1913200 \\
0.1006210 \\
-0.3230260 \\
0.4422630 \\
2.0826870 \\
-1.1539390 \\
0.7967110 \\
0.3252760 \\
1.6405670 \\
-0.2225110 \\
-1.1896970 \\
0.1523040 \\
3.1717280 \\
3.9082350 \\
-1.1599400 \\
-2.3785280 \\
-2.5435160 \\
-1.2393530 \\
-3.6101350 \\
-4.5151020 \\
-3.5075710 \\
-3.7069160 \\
-4.6595880 \\
-3.6566850 \\
-2.5922380 \\
-2.2697940 \\
-2.6739700 \\
-3.6182160 \\
-1.8635160 \\
-2.6025230 \\
-3.5347800 \\
-1.7908590 \\
0.9797460 \\
2.2267050\end{array}$ \\
\hline
\end{tabular}

\begin{abstract}
4. 1643290
4. 2747400

5.3089060

5.8955380

4.9638110

4. 0923280

4.0991660

3. 1488380

3.2879010

7.0789420

5.4668080

5.4166440

5. 5387920

6.7016200

7.2482560

7.3969670

5.6670350

6. 5388510

0.0644830

$-1.1138550$

$-0.7518450$

0.5029660

1. 6949520

1. 3833500

$-1.5997830$

$-1.5056420$

$-1.9298310$

0.3288690

0.7235110

2.5545820

2. 0174910

$-0.5586020$

$-3.6227410$

$-4.4668770$

$-4.3137000$

$-2.8378610$

$-1.8177150$

$-2.3199600$

$-4.1403070$

$-4.9458020$

$-2.6605970$

$-1.6255840$

$-5.5169160$

$-4.6742220$

-2.6470260
-0.8595250
\end{abstract}

-2.4485140
-1.5328000
-0.6194590
-0.7817330
0.4202360
-1.5919850
-2.2710470
-1.0419890
-3.0976320
-0.1808530
-3.2939590
-3.9516500
-3.9317040
-2.3425160
-2.5092380
-2.5262150
0.2187760
0.8719770
-4.2262090
-5.1860150
-6.5807270
-7.0946070
-6.1954260
-4.7094110
-7.2606190
-5.2985250
-4.7475000
-7.0785260
-8.1323440
-6.4200730
-6.4223880
-6.5326300
-1.9941110
-2.3576150
-3.8614860
-4.2376760
-3.2388840
-2.5225810
-1.7754920
-4.1484020
-4.2729970
-2.4499160
-2.1001190
-4.4177320
-5.2488480
-3.7311290

0.9109900

3.4604200

4.3745280

3. 4626930

3. 4019100

4.2648490

3. 4194620

2.0408480

2. 2363230

2. 1770260

3. 0547610

1. 2875390

2. 2719560

3. 2095850

1.4442810

$-0.0515850$

0.0034220

$-0.4949640$

$-0.4872480$

0.0399210

$-0.6746800$

$-0.3250340$

$-0.4079670$

$-0.1132020$

$-1.5061450$

0.1004560

$-1.7614730$

$-0.3935140$

$-0.9714190$

0.7036360

1. 1219380

$-1.9033240$

$-3.0993730$

$-3.4029080$

$-3.6831560$

$-3.0634520$

$-1.8304280$

$-3.9735700$

$-4.2529170$

$-4.7659500$

$-3.8043670$

$-2.9137190$

$-2.5275090$

$-3.3034240$

$-2.8806930$ 


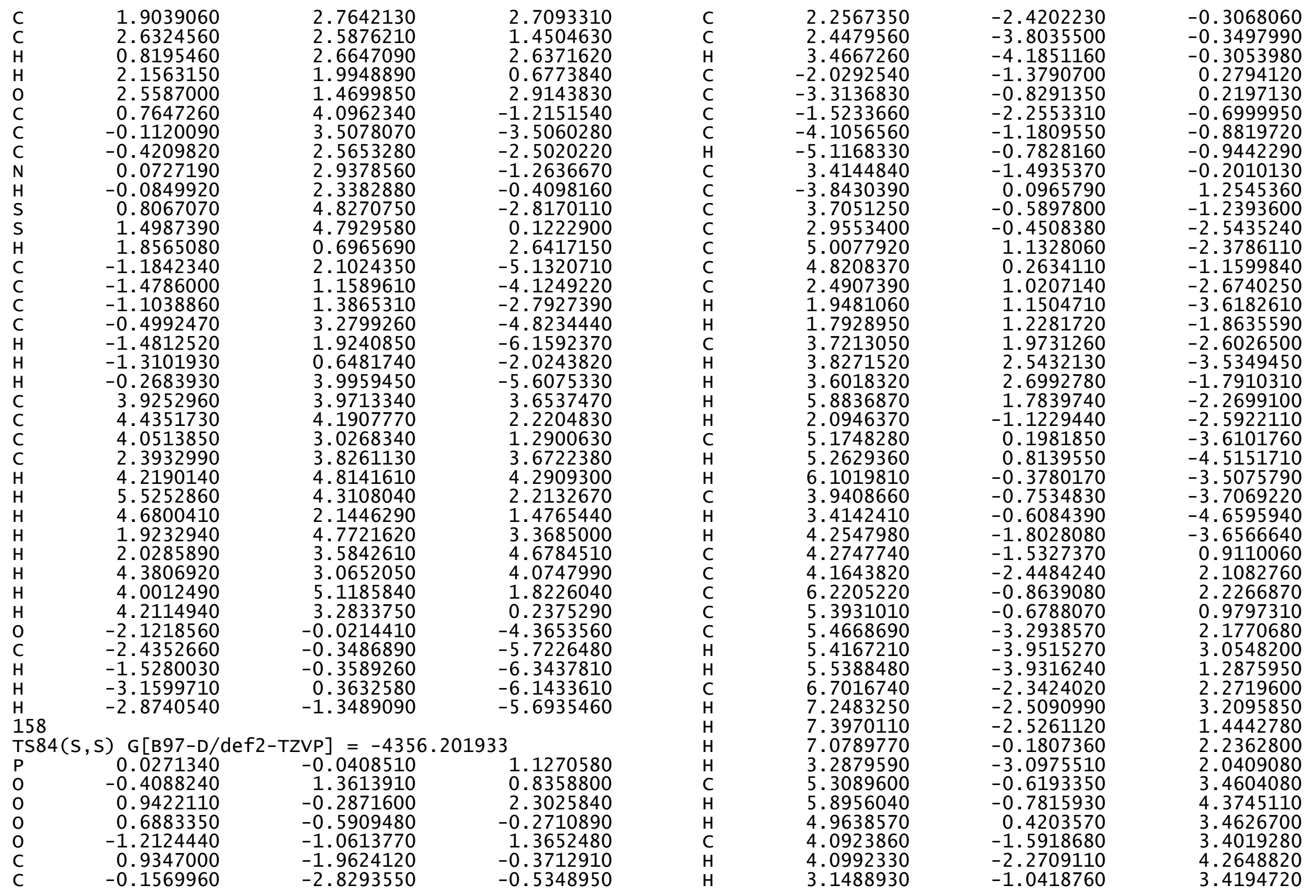




$\begin{array}{lrrr}\text { C } & 5.6670470 & 0.2188240 & -0.0516280 \\ \text { H } & 6.5388620 & 0.8720290 & 0.0033510 \\ \text { C } & -4.6091180 & 0.5444380 & 3.5306630 \\ \text { C } & -4.7681420 & -0.0744580 & 4.8967400 \\ \text { C } & -3.7285010 & -1.6904600 & 3.1468540 \\ \text { C } & -4.0377350 & -0.3240750 & 2.5811650 \\ \text { C } & -5.6682620 & -1.3343510 & 4.7570320 \\ \text { H } & -5.7419660 & -1.8292600 & 5.7347530 \\ \text { H } & -6.6794060 & -1.0299900 & 4.4613760 \\ \text { C } & -5.0494540 & -2.2977910 & 3.6962490 \\ \text { H } & -4.8280580 & -3.2755480 & 4.1445460 \\ \text { H } & -5.7484810 & -2.4624350 & 2.8674610 \\ \text { H } & -3.2936180 & -2.3536790 & 2.3947640 \\ \text { H } & -5.2098960 & 0.6377540 & 5.6043130 \\ \text { C } & -2.7504360 & -1.5174410 & 4.3412020 \\ \text { H } & -2.5695000 & -2.5025330 & 4.7918170 \\ \text { H } & -1.7931750 & -1.1396910 & 3.9707440 \\ \text { C } & -3.3701400 & -0.5408110 & 5.3887540 \\ \text { H } & -3.4822870 & -1.0337660 & 6.3638420 \\ \text { H } & -2.7237610 & 0.3335700 & 5.5298990 \\ \text { C } & -4.7915150 & 2.2671060 & 1.8599930 \\ \text { C } & -5.1389480 & 3.6303620 & 1.3151530 \\ \text { C } & -4.0179250 & 2.0737610 & -0.4369220 \\ \text { C } & -4.2112960 & 1.4091870 & 0.9066360 \\ \text { C } & -3.8350610 & 4.2958790 & 0.7965890 \\ \text { H } & -4.0882220 & 5.2551370 & 0.3251210 \\ \text { H } & -3.1675970 & 4.5055870 & 1.6404550 \\ \mathrm{C} & -3.1427400 & 3.3402240 & -0.2225910 \\ \text { H } & -2.9969020 & 3.8390550 & -1.1897840 \\ \text { H } & -2.1652730 & 3.0319430 & 0.1522630 \\ \text { H } & -3.5370860 & 1.4038340 & -1.1539900 \\ \text { H } & -5.6137490 & 4.2538880 & 2.0825300 \\ \text { C } & -5.4017140 & 2.5352750 & -0.9672240 \\ \text { H } & -5.2527570 & 3.0859870 & -1.9057580 \\ \text { H } & -6.0227520 & 1.6595170 & -1.1914380 \\ \text { C } & -6.0911850 & 3.4423130 & 0.1004700 \\ \text { H } & -6.3280130 & 4.4274270 & -0.3232020 \\ \text { H } & -7.0310660 & 2.9923560 & 0.4421000 \\ \text { C } & -4.9920740 & 1.8373390 & 3.1716370 \\ \text { H } & -5.4426080 & 2.5040370 & 3.9081220 \\ \text { C } & -3.6226980 & -1.9942040 & -1.9032950 \\ \text { C } & -4.4668230 & -2.3577570 & -3.0993350 \\ \text { C } & -4.3136450 & -3.8616420 & -3.4028040 \\ \text { C } & -2.8378030 & -4.2378460 & -3.6830200 \\ \text { C } & -1.8176630 & -3.2390240 & -3.0633560 \\ \text { C } & -2.3199160 & -2.5226680 & -1.8303650 \\ & & & \end{array}$

-4.1402460
-4.9457380
-2.6605300
-1.6255300
-5.5168640
-4.6741780
-2.6469720
-0.8594730
0.0645360
-1.1137930
-0.7517630
0.5030450
1.6950260
1.3834090
-1.5996960
-1.5055890
-1.9297660
0.3289340
0.7236030
2.5546500
2.0175790
-0.5585050
1.9040120
2.6323820
0.8196450
2.1562600
2.5588120
0.7645690
-0.1121710
-0.4210250
0.0726840
-0.0850070
0.8064610
1.4985270
1.8567090
-1.1843470
-1.4785970
-1.1038460
-0.4994450
-1.4813920
-1.3100650
-0.2686830
3.9254110
4.4351710
4.0513070
2.3934220
$-1.7756740$
$-4.1485940$
$-4.2732200$
$-2.4500880$
$-2.1002520$
$-4.4178490$
$-5.2490000$
$-3.7312580$
$-4.2262280$
$-5.1860460$
$-6.5807330$
$-7.0946290$
$-6.1954230$
$-4.7094140$
$-7.2606400$
$-5.2986000$
$-4.7475150$
$-7.0785920$
$-8.1323530$
$-6.4200860$
$-6.4223420$
$-6.5325890$
2.7642790
2. 5878970
2.6647560
1.9950040
1.4701130
4.0961850
3.5077420
2.5652080
2.9377470
2. 3381570
4.8270650
4.7930020
0.6967650
2. 1023350
1.1588060
1.3863650
3.2798730
1.9239890
0.6479700
3.9959350
3.9715450
4.1910040
3.0270690
3.8262580

$-3.9735570$

$-4.2528080$

$-4.7658110$

$-3.8043060$

$-2.9137020$

$-2.5273850$

$-3.3032360$

$-2.8805710$

$-0.4948340$

$-0.4870700$

0.0401520

$-0.6744440$

$-0.3248480$

$-0.4078330$

$-0.1129320$

$-1.5059580$

0.1006260

$-1.7612360$

$-0.3932400$

$-0.9712340$

0.7038280

1.1221640

2.7092380

1.4502210

2.6371720

0.6772530

2.9144100

$-1.2151230$

$-3.5059690$

$-2.5019780$

$-1.2636290$

$-0.4097600$

$-2.8169500$

0.1223270

2. 6418150

$-5.1320080$

$-4.1248740$

$-2.7927000$

$-4.8233780$

$-6.1591670$

$-2.0243550$

$-5.6074540$

3.6534580

2.2201540

1.2897580

3.6720650 


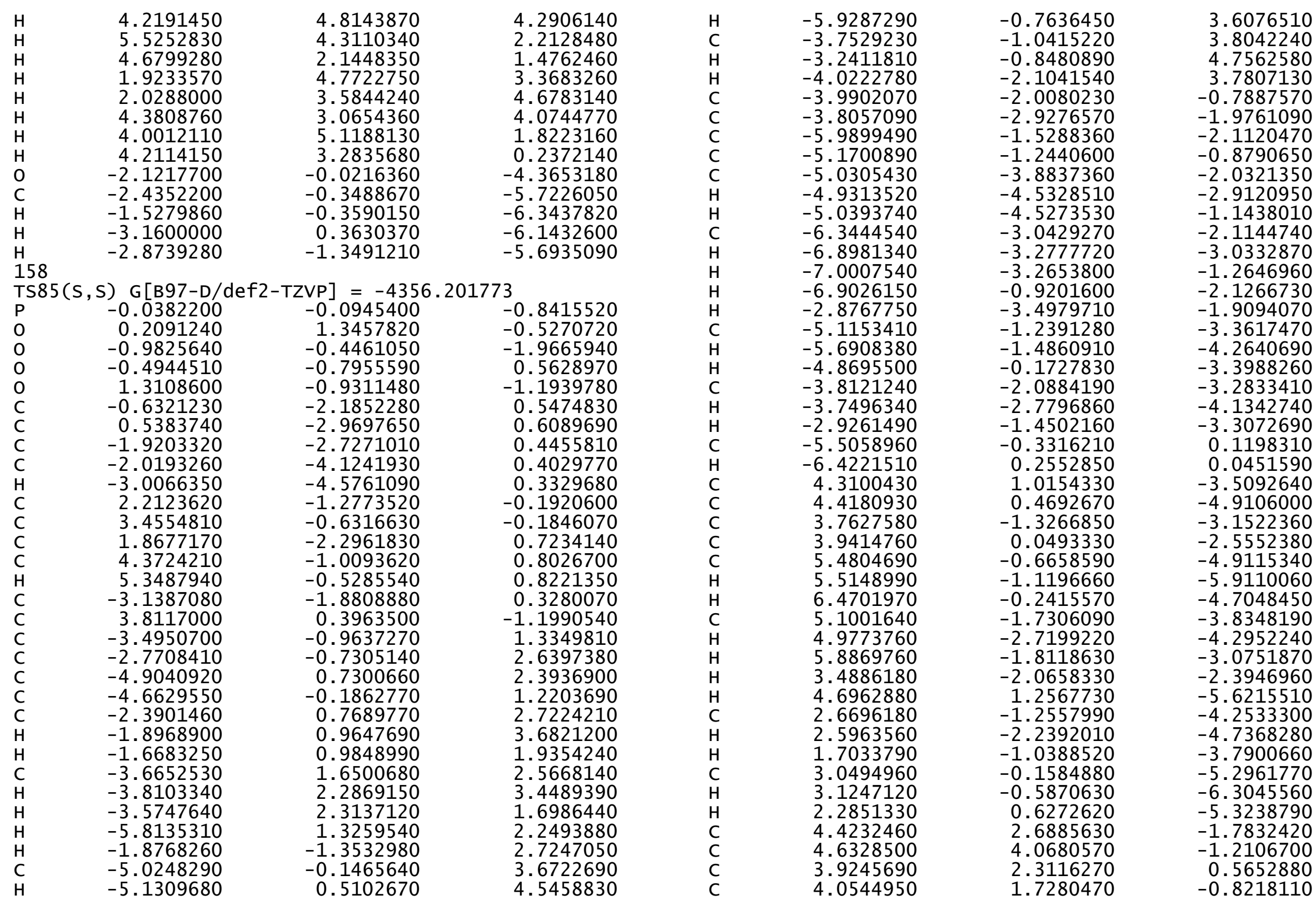




$\begin{array}{rrrr}\text { C } & 3.3089040 & 4.5219550 & -0.5358430 \\ \text { H } & 3.4708300 & 5.4960760 & -0.0551770 \\ \text { H } & 2.5327280 & 4.6515190 & -1.2991600 \\ \text { C } & 2.8724700 & 3.4528620 & 0.5113000 \\ \text { H } & 2.7784680 & 3.8967140 & 1.5103160 \\ \text { H } & 1.9062610 & 3.0246980 & 0.2392450 \\ \text { H } & 3.6194620 & 1.5557430 & 1.2940440 \\ \text { H } & 4.9335190 & 4.7783200 & -1.9904230 \\ \mathrm{C} & 5.2876620 & 2.9352410 & 0.9710310 \\ \text { H } & 5.1778070 & 3.4132910 & 1.9534920 \\ \text { H } & 6.0416530 & 2.1446060 & 1.0683240 \\ \mathrm{C} & 5.7219090 & 3.9810540 & -0.1050930 \\ \mathrm{H} & 5.8496020 & 4.9728610 & 0.3485490 \\ \mathrm{H} & 6.6792500 & 3.6961860 & -0.5574820 \\ \mathrm{C} & 4.5510860 & 2.3354320 & -3.1262440 \\ \mathrm{H} & 4.8354730 & 3.0823660 & -3.8684330 \\ \mathrm{C} & 4.0572480 & -1.9726270 & 1.7581590 \\ \mathrm{C} & 5.0287920 & -2.3770070 & 2.8384510 \\ \mathrm{C} & 5.1224530 & -3.9155440 & 2.9188220 \\ \mathrm{C} & 3.7244730 & -4.5757700 & 3.0779410 \\ \mathrm{C} & 2.5506460 & -3.5841350 & 2.8627080 \\ \mathrm{C} & 2.8051100 & -2.6179670 & 1.7296440 \\ \mathrm{H} & 4.6861250 & -1.9919210 & 3.8125720 \\ \mathrm{H} & 5.7800050 & -4.2057520 & 3.7475130 \\ \mathrm{H} & 3.6201960 & -5.0141960 & 4.0781810 \\ \mathrm{H} & 2.4413870 & -2.9910670 & 3.7837850 \\ \mathrm{H} & 6.0148450 & -1.9361840 & 2.6492720 \\ \mathrm{H} & 5.5924320 & -4.2714490 & 1.9933620 \\ \mathrm{H} & 3.6322660 & -5.3974860 & 2.3612180 \\ \mathrm{H} & 1.6124140 & -4.1268900 & 2.7327730 \\ \mathrm{C} & -0.8890890 & -4.9368110 & 0.3927020 \\ \mathrm{C} & -0.9790170 & -6.4369180 & 0.2665340 \\ \mathrm{C} & -0.0761850 & -7.1182020 & 1.3169160 \\ \mathrm{C} & 1.3832470 & -6.5826360 & 1.2649520 \\ \mathrm{C} & 1.5438330 & -5.3396770 & 0.3505570 \\ \mathrm{C} & 0.3963810 & -4.3680800 & 0.4872230 \\ \mathrm{H} & -0.6435320 & -6.7395000 & -0.7388860 \\ \mathrm{H} & -0.0908400 & -8.2053770 & 1.1709850 \\ \mathrm{H} & 2.0646870 & -7.3630640 & 0.9041250 \\ \mathrm{H} & 1.5491460 & -5.6889680 & -0.6940900 \\ \mathrm{H} & -2.0190860 & -6.7682950 & 0.3728330 \\ \mathrm{H} & -0.5039220 & -6.9198860 & 2.3079060 \\ \mathrm{H} & 1.7066320 & -6.3238910 & 2.2785090 \\ \mathrm{H} & 2.5100210 & -4.8607520 & 0.5210630 \\ \mathrm{C} & -2.2048950 & 2.4635600 & -2.6767100 \\ \mathrm{C} & -2.9706680 & 2.3618690 & -1.4318450 \\ & & & \\ & & & \end{array}$

$\begin{array}{lr}\mathrm{H} & -1.1195930 \\ \mathrm{H} & -2.4757660 \\ \mathrm{O} & -2.7170890 \\ \mathrm{C} & -1.3989760 \\ \mathrm{C} & -0.4125610 \\ \mathrm{C} & -0.0548700 \\ \mathrm{~N} & -0.6112230 \\ \mathrm{H} & -0.3999000 \\ \mathrm{~S} & -1.4638940 \\ \mathrm{~S} & -2.2108090 \\ \mathrm{H} & -1.9500590 \\ \mathrm{C} & 0.8533570 \\ \mathrm{C} & 1.1945130 \\ \mathrm{C} & 0.7510080 \\ \mathrm{C} & 0.0528500 \\ \mathrm{H} & 1.2221110 \\ \mathrm{H} & 0.9826120 \\ \mathrm{H} & -0.2100190 \\ \mathrm{C} & -4.2910790 \\ \mathrm{C} & -4.8950370 \\ \mathrm{C} & -4.4370710 \\ \mathrm{C} & -2.7527630 \\ \mathrm{H} & -4.6406710 \\ \mathrm{H} & -5.9905490 \\ \mathrm{H} & -4.9537270 \\ \mathrm{H} & -2.3975210 \\ \mathrm{H} & -2.3187010 \\ \mathrm{H} & -4.6256670 \\ \mathrm{H} & -4.5859100 \\ \mathrm{H} & -4.6779550 \\ \mathrm{O} & 1.9564300 \\ \mathrm{C} & 2.2573850 \\ \mathrm{H} & 2.8377510 \\ \mathrm{H} & 1.3374440 \\ \mathrm{H} & 2.8487980 \\ \mathrm{H} & \end{array}$

$\begin{array}{rr}2.4885820 & -2.5591270 \\ 1.9147820 & -0.5761010 \\ 1.0973630 & -2.7424940 \\ 4.3703680 & 1.0159160 \\ 4.1182210 & 3.3291360 \\ 3.1033930 & 2.4276480 \\ 3.2840540 & 1.1744860 \\ 2.6020150 & 0.4116610 \\ 5.2676360 & 2.5295640 \\ 4.8399450 & -0.3738970 \\ 0.4209760 & -2.3742870 \\ 3.0060100 & 5.0369940 \\ 1.9811470 & 4.1237580 \\ 2.0264030 & 2.7992630 \\ 4.0744330 & 4.6484450 \\ 2.9355780 & 6.0566030 \\ 1.2607980 & 2.0696090 \\ 4.8503870 & 5.3621700 \\ 3.3228730 & -3.8324790 \\ 3.6490920 & -2.4568090 \\ 2.6514070 & -1.3780030 \\ 3.3464120 & -3.7798010 \\ 4.0474490 & -4.5777110 \\ 3.6443910 & -2.5076170 \\ 1.6878610 & -1.4875800 \\ 4.3671970 & -3.5815910 \\ 3.0251520 & -4.7350400 \\ 2.3285900 & -4.1570910 \\ 4.6607060 & -2.1593320 \\ 3.0059180 & -0.3694850 \\ 0.9718380 & 4.6285050 \\ -0.1166150 & 3.7430730 \\ 0.2261370 & 2.8782360 \\ -0.5994710 & 3.3871590 \\ -0.8215280 & 4.3299060\end{array}$
158

TS86(S, S ) G[B97-D/def2-TZVP $]=-4356.201735$

$\begin{array}{lrrr}\mathrm{P} & -0.0322400 & -0.1505160 & -0.7676510 \\ \mathrm{O} & -0.9880640 & -0.6029230 & -1.8447710 \\ \mathrm{O} & 0.2452680 & 1.3091500 & -0.6018110 \\ \mathrm{O} & 1.3004880 & -1.0386270 & -1.0483170 \\ \mathrm{O} & -0.4947250 & -0.6958230 & 0.7044510 \\ \mathrm{C} & 2.2136130 & -1.3033740 & -0.0345910 \\ \mathrm{C} & 1.8547260 & -2.2027040 & 0.9907600 \\ \mathrm{C} & 3.4759410 & -0.7019510 & -0.1150530 \\ \mathrm{C} & 4.3962250 & -1.0072400 & 0.8950980\end{array}$




$\begin{array}{rrrr}\mathrm{H} & 5.3910600 & -0.5679160 & 0.8465820 \\ \mathrm{C} & -0.6407630 & -2.0788350 & 0.8372810 \\ \mathrm{C} & -1.9301120 & -2.6273400 & 0.8105910 \\ \mathrm{C} & 0.5180320 & -2.8651280 & 0.9506040 \\ \mathrm{C} & -2.0204440 & -4.0175410 & 0.9218900 \\ \mathrm{H} & -3.0101650 & -4.4711830 & 0.9181760 \\ \mathrm{C} & 3.8306620 & 0.2185140 & -1.2283790 \\ \mathrm{C} & -3.1508960 & -1.8088710 & 0.5809510 \\ \mathrm{C} & 3.8815600 & -0.2391810 & -2.5572050 \\ \mathrm{C} & 3.6327030 & -1.6510200 & -3.0351950 \\ \mathrm{C} & 4.2387840 & -0.0164920 & -4.9612910 \\ \mathrm{C} & 4.2250260 & 0.6380190 & -3.6027320 \\ \mathrm{C} & 2.4838770 & -1.6306810 & -4.0817800 \\ \mathrm{H} & 2.3640910 & -2.6450330 & -4.4855830 \\ \mathrm{H} & 1.5480160 & -1.3559840 & -3.5882550 \\ \mathrm{C} & 2.8334380 & -0.6257350 & -5.2224080 \\ \mathrm{H} & 2.8376370 & -1.1283610 & -6.1989100 \\ \mathrm{H} & 2.0913280 & 0.1805150 & -5.2661620 \\ \mathrm{H} & 4.4987490 & 0.7054570 & -5.7450430 \\ \mathrm{H} & 3.3791940 & -2.3206450 & -2.2088230 \\ \mathrm{C} & 5.2657150 & -1.1825860 & -4.9321330 \\ \mathrm{H} & 5.2274290 & -1.7138290 & -5.8925100 \\ \mathrm{H} & 6.2778970 & -0.7769540 & -4.8168470 \\ \mathrm{C} & 4.9197900 & -2.1468050 & -3.7537790 \\ \mathrm{H} & 4.7471710 & -3.1656060 & -4.1252770 \\ \mathrm{H} & 5.7458480 & -2.1899570 & -3.0337940 \\ \mathrm{C} & 4.1233740 & 1.5697960 & -0.9738770 \\ \mathrm{C} & 4.0555160 & 2.2712220 & 0.3621390 \\ \mathrm{C} & 4.7272200 & 3.8573470 & -1.5798180 \\ \mathrm{C} & 4.4700490 & 2.4394350 & -2.0262410 \\ \mathrm{C} & 5.4413790 & 2.9006080 & 0.6667270 \\ \mathrm{H} & 5.3735850 & 3.4638160 & 1.6069680 \\ \mathrm{H} & 6.1851860 & 2.1068410 & 0.8071940 \\ \mathrm{C} & 5.8547230 & 3.8403970 & -0.5099670 \\ \mathrm{H} & 6.0183560 & 4.8641990 & -0.1481810 \\ \mathrm{H} & 6.7889460 & 3.4966590 & -0.9697020 \\ \mathrm{H} & 5.0104860 & 4.4933570 & -2.4271990 \\ \mathrm{H} & 3.7660470 & 1.5854230 & 1.1619260 \\ \mathrm{C} & 3.4356800 & 4.3918590 & -0.8995040 \\ \mathrm{H} & 3.6308590 & 5.3994480 & -0.5084680 \\ \mathrm{H} & 2.6350740 & 4.4723390 & -1.6441690 \\ \mathrm{C} & 3.0196440 & 3.4227060 & 0.2480240 \\ \mathrm{H} & 2.9682640 & 3.9506410 & 1.2086470 \\ \mathrm{H} & 2.0365090 & 2.9915030 & 0.0478160 \\ \mathrm{C} & 4.5208750 & 1.9761290 & -3.3403790 \\ \mathrm{H} & 4.7838840 & 2.6525060 & -4.1546220\end{array}$

-4.7011370
-4.9613600
-2.8207810
-3.5278320
-5.0878740
-5.1998580
-5.9901540
-3.8150060
-3.3159690
-4.0811380
-1.9236060
-5.8727540
-2.4510060
-1.9798340
-1.7127420
-3.7292770
-3.8899940
-3.6288240
-5.1703370
-5.9649780
-3.7578510
-3.9805210
-5.0777310
-5.6325360
-4.8554560
-3.7586620
-3.6659410
-2.8857810
-2.8183960
-6.8903680
-4.9608370
-4.8329960
-4.9728330
-6.2885150
-6.8172270
-6.9588550
-5.5301800
-6.4520000
0.3901830
1.6202480
1.2976020
0.1610550
-1.1179520
-0.8957230
2.1989970
2.0974740
1. 2246460

2.2607750

2.7246030

1.4545040

3. 6463510

4. 4208400

3. 6625550

3. 9082970

4.8334890

4. 0191660

2. 9009660

2. 0275900

2. 6148050

3. 5504200

1. 8214610

2. 3198330

3. 1032240

1. 3710190

$-0.7261710$

$-1.9273020$

$-1.5974630$

$-0.5263490$

$-3.1900600$

$-4.0651620$

-3. 3491400

$-2.9925720$

$-3.7575700$

$-3.0755070$

$-1.4534210$

$-2.0243310$

$-1.5587330$

$-2.3524560$

$-0.5998120$

$-1.7615670$

$-2.6580010$

$-0.9048990$

0.1442300

$-0.0146300$

0.9384250

0.8219430

0.2977300

1.1282700

0.9216700

0.9547320

0.3477650

1.8028200 


$\begin{array}{rrrr}\text { H } & 2.3595360 & -4.6648810 & 0.1813010 \\ \text { H } & 0.4486120 & -7.1555430 & 2.1905180 \\ \text { H } & -0.0198790 & -8.2193430 & 0.8541670 \\ \text { H } & -1.8761590 & -6.6197000 & 1.6704280 \\ \text { H } & -1.5506110 & -6.6106500 & -0.0574980 \\ \text { H } & 0.9904520 & -6.5124660 & -0.7573260 \\ \mathrm{C} & 4.0622870 & -1.8405800 & 1.9618960 \\ \mathrm{C} & 5.0467490 & -2.1685950 & 3.0580250 \\ \mathrm{C} & 5.0151010 & -3.6844240 & 3.3380520 \\ \mathrm{C} & 3.6042300 & -4.1465990 & 3.7748440 \\ \mathrm{C} & 2.4674250 & -3.1973110 & 3.2924160 \\ \mathrm{C} & 2.7862360 & -2.4336160 & 2.0251490 \\ \mathrm{H} & 4.7822340 & -1.6315790 & 3.9837380 \\ \mathrm{H} & 5.7542890 & -3.9489250 & 4.1044150 \\ \mathrm{H} & 3.5526940 & -4.2015830 & 4.8695200 \\ \mathrm{H} & 2.3161130 & -2.4442030 & 4.0796110 \\ \mathrm{H} & 6.0533480 & -1.8400260 & 2.7725490 \\ \mathrm{H} & 5.3035060 & -4.2021260 & 2.4137600 \\ \mathrm{H} & 3.4279820 & -5.1627510 & 3.4028620 \\ \mathrm{H} & 1.5216090 & -3.7371290 & 3.2093040 \\ \mathrm{C} & -2.1919170 & 2.2137990 & -2.8421760 \\ \mathrm{C} & -2.9905270 & 2.2016670 & -1.6137910 \\ \mathrm{H} & -1.1101990 & 2.2509160 & -2.6968430 \\ \mathrm{H} & -2.5213550 & 1.8159990 & -0.7142490 \\ \mathrm{O} & -2.7013230 & 0.8441980 & -2.8247870 \\ \mathrm{C} & -1.3984010 & 4.4433440 & 0.6634880 \\ \mathrm{C} & -0.3694100 & 4.4338790 & 2.9710570 \\ \mathrm{C} & -0.0052540 & 3.3437470 & 2.1647170 \\ \mathrm{~N} & -0.5804570 & 3.3962640 & 0.9076880 \\ \mathrm{H} & -0.3697340 & 2.6449830 & 0.2117950 \\ \mathrm{~S} & -1.4594640 & 5.4790810 & 2.0856490 \\ \mathrm{~S} & -2.2515970 & 4.7607530 & -0.7447000 \\ \mathrm{H} & -1.9455900 & 0.2083540 & -2.3755220 \\ \mathrm{C} & 0.9519930 & 3.5205290 & 4.7524150 \\ \mathrm{C} & 1.2985100 & 2.4178670 & 3.9367350 \\ \mathrm{C} & 0.8258030 & 2.3208370 & 2.6247700 \\ \mathrm{C} & 0.1205440 & 4.5288970 & 4.2785430 \\ \mathrm{H} & 1.3427040 & 3.5581780 & 5.7655670 \\ \mathrm{H} & 1.0600670 & 1.4933780 & 1.9667950 \\ \mathrm{H} & -0.1462030 & 5.3656070 & 4.9184160 \\ \mathrm{C} & -4.2455150 & 2.9862590 & -4.1119590 \\ \mathrm{C} & -4.8873100 & 3.4084850 & -2.7801240 \\ \mathrm{C} & -4.4568820 & 2.4934590 & -1.6198720 \\ \mathrm{C} & -2.7093810 & 3.0156970 & -4.0188250 \\ \mathrm{H} & -4.5742220 & 3.6557950 & -4.9158700 \\ \mathrm{H} & -5.9810260 & 3.3946140 & -2.8599870 \\ & & & \\ & & & \end{array}$

$\begin{array}{lr}\mathrm{H} & -4.9704200 \\ \mathrm{H} & -2.3597860 \\ \mathrm{H} & -2.2487220 \\ \mathrm{H} & -4.5728120 \\ \mathrm{H} & -4.5907510 \\ \mathrm{H} & -4.7233640 \\ \mathrm{O} & 2.0995940 \\ \mathrm{C} & 2.3980600 \\ \mathrm{H} & 2.9460970 \\ \mathrm{H} & 1.4783240 \\ \mathrm{H} & 3.0212530 \\ \mathrm{H} & \end{array}$

1.5232100
4.0480240
2.6304610
1.9713400
4.4407960
2.9207960
1.4850370
0.3117880
0.5633660
-0.2228380
-0.3129390

$-1.6707950$ $-3.8798710$ $-4.9371720$ $-4.3736330$ $-2.5486150$ $-0.6468210$ 4.5215530 3.7528280 2.8372360 3. 4813980 4.3947910 TS87(S, S ) G[B97-D/def2-TZVP $]=-4356.201732$

$\begin{array}{lrrr}\text { TS87 }(\mathrm{S}, \mathrm{S}) \mathrm{G}[\mathrm{B} 97-\mathrm{D} / \mathrm{de} 2-\mathrm{TZVP}]=-4356.201732 \\ \mathrm{P} & -0.0322140 & -0.1505020 & -0.7676610 \\ \mathrm{O} & 0.2453000 & 1.3091670 & -0.6018530 \\ \mathrm{O} & -0.9880430 & -0.6029280 & -1.8447670 \\ \mathrm{O} & -0.4946950 & -0.6957910 & 0.7044510 \\ \mathrm{O} & 1.3005150 & -1.0386140 & -1.0483180 \\ \mathrm{C} & -0.6407360 & -2.0788050 & 0.8372800 \\ \mathrm{C} & 0.5180580 & -2.8651020 & 0.9506010 \\ \mathrm{C} & -1.9300850 & -2.6273100 & 0.8105800 \\ \mathrm{C} & -2.0204180 & -4.0175110 & 0.9218760 \\ \mathrm{H} & -3.0101400 & -4.4711520 & 0.9181530 \\ \mathrm{C} & 2.2136400 & -1.3033520 & -0.0345900 \\ \mathrm{C} & 3.4759640 & -0.7019190 & -0.1150470 \\ \mathrm{C} & 1.8547540 & -2.2026840 & 0.9907590 \\ \mathrm{C} & 4.3962500 & -1.0072130 & 0.8951010 \\ \mathrm{H} & 5.3910830 & -0.5678830 & 0.8465890 \\ \mathrm{C} & -3.1508700 & -1.8088480 & 0.5809190 \\ \mathrm{C} & 3.8306760 & 0.2185670 & -1.2283590 \\ \mathrm{C} & -3.5278400 & -0.7725980 & 1.4544740 \\ \mathrm{C} & -2.8208210 & -0.3618750 & 2.7245890 \\ \mathrm{C} & -4.9614440 & 1.0354050 & 2.2607630 \\ \mathrm{C} & -4.7011570 & -0.0291880 & 1.2246030 \\ \mathrm{C} & -2.4510950 & 1.1388700 & 2.6148130 \\ \mathrm{H} & -1.9798890 & 1.4636700 & 3.5504130 \\ \mathrm{H} & -1.7128710 & 1.2565680 & 1.8214380 \\ \mathrm{C} & -3.7294040 & 1.9798510 & 2.3199170 \\ \mathrm{H} & -3.8901640 & 2.7317700 & 3.1033900 \\ \mathrm{H} & -3.6289690 & 2.5198590 & 1.3711660 \\ \mathrm{H} & -5.8728540 & 1.5994470 & 2.0275770 \\ \mathrm{H} & -1.9236250 & -0.9608580 & 2.9009510 \\ \mathrm{C} & -5.0879770 & 0.3404940 & 3.6462990 \\ \mathrm{H} & -5.2000800 & 1.1107950 & 4.4208240 \\ \mathrm{H} & -5.9902000 & -0.2820660 & 3.6624090 \\ \mathrm{C} & -3.8150480 & -0.5243060 & 3.9082760\end{array}$




$\begin{array}{lrr}-3.3160400 & -0.2073330 & 4.8334630 \\ -4.0811020 & -1.5822510 & 4.0191730 \\ -3.9804700 & -2.0830460 & -0.5263990 \\ -3.7577790 & -3.1283150 & -1.5975060 \\ -5.9649150 & -1.8027380 & -1.9273770 \\ -5.1702970 & -1.3558590 & -0.7262340 \\ -4.9607540 & -4.1113360 & -1.5587680 \\ -4.8328900 & -4.8593180 & -2.3524610 \\ -4.9727640 & -4.6436870 & -0.5998270 \\ -6.2884360 & -3.3148130 & -1.7616550 \\ -6.8171040 & -3.6647990 & -2.6581130 \\ -6.9588110 & -3.4529850 & -0.9050170 \\ -6.8903120 & -1.2214610 & -2.0244180 \\ -2.8183170 & -3.6652080 & -1.4534520 \\ -5.0776510 & -1.6379890 & -3.1901200 \\ -5.6324460 & -2.0023990 & -4.0652380 \\ -4.8553660 & -0.5773080 & -3.3491750 \\ -3.7585930 & -2.4429280 & -2.9926270 \\ -3.6658940 & -3.2254100 & -3.7576090 \\ -2.8857000 & -1.7916930 & -3.0755890 \\ -5.5301750 & -0.3284620 & 0.1441700 \\ -6.4520060 & 0.2321870 & -0.0146980 \\ 4.2250220 & 0.6381230 & -3.6027050 \\ 4.2387790 & -0.0163600 & -4.9612780 \\ 3.6327150 & -1.6509300 & -3.0352120 \\ 3.8815670 & -0.2391000 & -2.5571950 \\ 5.2657200 & -1.1824480 & -4.9321450 \\ 5.2274380 & -1.7136700 & -5.8925330 \\ 6.2778980 & -0.7768100 & -4.8168490 \\ 4.9198010 & -2.1466950 & -3.7538110 \\ 4.7471850 & -3.1654880 & -4.1253320 \\ 5.7458620 & -2.1898590 & -3.0338290 \\ 3.3792130 & -2.3205730 & -2.2088520 \\ 4.4987370 & 0.7056060 & -5.7450170 \\ 2.4838840 & -1.6305750 & -4.0817920 \\ 2.3640980 & -2.6449200 & -4.4856120 \\ 1.5480250 & -1.3558880 & -3.5882570 \\ 2.8334370 & -0.6256080 & -5.2224040 \\ 2.8376390 & -1.1282180 & -6.1989130 \\ 2.0913220 & 0.1806380 & -5.2661450 \\ 4.4700450 & 2.4395090 & -2.0261800 \\ 4.7272100 & 3.8574130 & -1.5797280 \\ 4.0555240 & 2.2712440 & 0.3621990 \\ 4.1233820 & 1.5698450 & -0.9738320 \\ 3.4356720 & 4.3919060 & -0.8993940 \\ 3.6308510 & 5.3994830 & -0.5083300\end{array}$

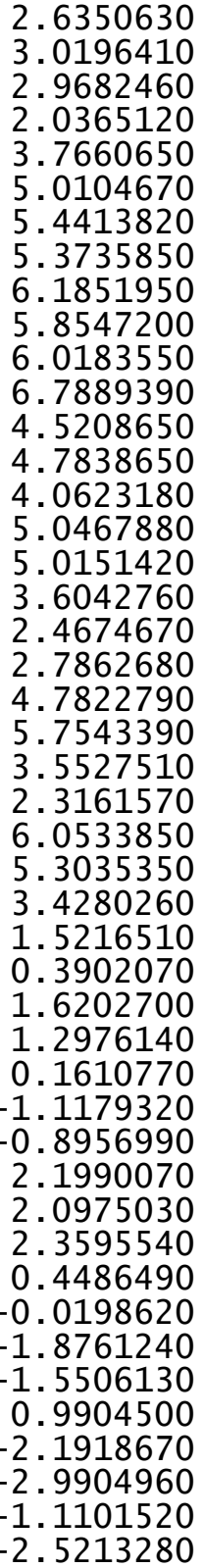

4.4724070
3.4227190
3.9506290
2.9915110
1.5854260
4.4934430
2.9006360
3.4638300
2.1068720
3.8404460
4.8642410
3.4967150
1.9762290
2.6526240
-1.8405680
-2.1685990
-3.6844330
-4.1466140
-3.1973220
-2.4336070
-1.6315980
-3.9489490
-4.2016100
-2.4442260
-1.8400240
-4.2021170
-5.1627620
-3.7371410
-4.2758130
-5.1612040
-6.5665650
-7.1721840
-6.3514790
-4.8473050
-7.1906910
-5.2734020
-4.6648430
-7.1555480
-8.2193150
-6.6196810
-6.6106060
-6.5123960
2.2137980
2.2016190
2.2509300
1.8159210

$-1.6440530$

0.2481080

1.2087450

0.0478810

1.1619730

$-2.4270970$

0.6667950

1.6070450

0.8072510

$-0.5098840$

$-0.1480820$

$-0.9696320$

$-3.3403270$

$-4.1545590$

1. 9618900

3. 0580070

3. 3380060

3. 7748060

3. 2923990

2. 0251420

3. 9837310

4.1043550

4.8694820

4.0796070

2. 7725300

2. 4137010

3. 4028150

3. 2092860

0.9384090

0.8219020

0.2976560

1. 1281960

0.9216350

0.9547130

0.3476630

1.8027730

0.1812660

2. 1904400

0.8540700

1. 6704060

$-0.0575270$

$-0.7573940$

$-2.8422160$

$-1.6138440$

$-2.6968650$

$-0.7143130$ 


\begin{tabular}{|c|c|c|c|c|c|c|c|}
\hline & & & & & & & \\
\hline 0 & -2.7012460 & 0.8441880 & -2.8248880 & $\mathrm{C}$ & -2.3262250 & -2.3774810 & 0.6785560 \\
\hline $\begin{array}{l}\mathrm{C} \\
\mathrm{C}\end{array}$ & $\begin{array}{l}-1.3984570 \\
-0.3695540\end{array}$ & $\begin{array}{l}4.4432620 \\
4.4337410\end{array}$ & $\begin{array}{l}0.6635420 \\
2.9711490\end{array}$ & $\begin{array}{l}\mathrm{C} \\
\mathrm{C}\end{array}$ & $\begin{array}{r}0.0906760 \\
-2.5344990\end{array}$ & $\begin{array}{l}-2.8042810 \\
-3.7450460\end{array}$ & $\begin{array}{l}0.8369570 \\
0.8738320\end{array}$ \\
\hline C & -0.0053000 & 3.3436720 & 2.1647670 & $\mathrm{H}$ & -3.5591290 & -4.1107330 & 0.9176870 \\
\hline $\mathrm{N}$ & -0.5804550 & 3.3962210 & 0.9077180 & C & 3.7849750 & -0.1676930 & -1.3335070 \\
\hline $\mathrm{H}$ & -0.3696630 & 2.6449860 & 0.2117940 & $\mathrm{C}$ & -3.4708990 & -1.4400720 & 0.5448170 \\
\hline $\mathrm{s}$ & -1.4596340 & 5.4789220 & 2.0857520 & $\mathrm{C}$ & 3.8998380 & -0.7772330 & -2.5961560 \\
\hline $\mathrm{s}$ & -2.2516210 & 4.7606870 & -0.7446620 & $\mathrm{C}$ & 3.5554910 & -2.2073210 & -2.9406900 \\
\hline $\mathrm{H}$ & -1.9455350 & 0.2083480 & -2.3755910 & C & 4.5029870 & -0.8760060 & -4.9591560 \\
\hline C & 0.9518160 & 3.5203660 & 4.7525190 & $\mathrm{c}$ & 4.4251550 & -0.0650020 & -3.6902580 \\
\hline C & 1.2984310 & 2.4177670 & 3.9367970 & $\mathrm{c}$ & 2.5179880 & -2.2090720 & -4.0965040 \\
\hline $\mathrm{C}$ & 0.8257900 & 2.3207810 & 2.6248040 & $\mathrm{H}$ & 2.3258640 & -3.2496030 & -4.3911640 \\
\hline C & 0.1203330 & 4.5287140 & 4.2786630 & $\mathrm{H}$ & 1.5762960 & -1.7842870 & -3.7382300 \\
\hline $\mathrm{H}$ & 1.3424760 & 3.5579810 & 5.7656920 & C & 3.0782790 & -1.3923550 & -5.3021100 \\
\hline $\mathrm{H}$ & 1.0601220 & 1.4933650 & 1.9667990 & $\mathrm{H}$ & 3.1340140 & -2.0162120 & -6.2043100 \\
\hline $\mathrm{H}$ & -0.1464910 & 5.3653730 & 4.9185700 & $\mathrm{H}$ & 2.4266480 & -0.5394840 & -5.5260760 \\
\hline C & -4.2454590 & 2.9862670 & -4.1120040 & $\mathrm{H}$ & 4.9070760 & -0.2786800 & 7856560 \\
\hline $\mathrm{C}$ & -4.8872820 & 3.4084420 & -2.7801650 & $\mathrm{H}$ & 3.1611380 & -2.7498900 & -2.0777230 \\
\hline C & -4.4568540 & 2.4933880 & -1.6199340 & C & 5.4051640 & -2.1132160 & -4.6888470 \\
\hline C & -2.7093270 & 3.0157250 & -4.0188470 & $\mathrm{H}$ & 5.4147090 & -2.7494900 & -5.5839700 \\
\hline $\mathrm{H}$ & -4.5741660 & 3.6558210 & -4.9158990 & $\mathrm{H}$ & 6.4343610 & -1.7828150 & -4.5045260 \\
\hline $\mathrm{H}$ & -5.9809960 & 3.3945530 & -2.8600460 & $\mathrm{C}$ & 4.8481600 & -2.8985840 & -3.4595690 \\
\hline $\mathrm{H}$ & -4.9703770 & 1.5231320 & -1.6708890 & $\mathrm{H}$ & 4.6080040 & -3.9334010 & -3.7378710 \\
\hline $\mathrm{H}$ & -2.3597490 & 4.0480530 & -3.8798580 & $\mathrm{H}$ & 5.5906710 & -2.9337400 & -2.6532880 \\
\hline $\mathrm{H}$ & -2.2486500 & 2.6305230 & -4.9372000 & C & 4.2080870 & 1.1659340 & -1.1940930 \\
\hline $\mathrm{H}$ & -4.5727360 & 1.9713510 & -4.3737130 & $\mathrm{C}$ & 4.1247240 & 2.0119730 & 0.0545210 \\
\hline H & -4.5907450 & 4.4407500 & -2.5486200 & $\mathrm{C}$ & 5.1009020 & 3.2986660 & -1.9784220 \\
\hline $\mathrm{H}$ & -4.7233520 & 2.9206930 & -0.6468740 & $\mathrm{C}$ & 4.7280950 & 1.8720940 & -2.2957910 \\
\hline 0 & 2.0995380 & 1.4849480 & 4.5216010 & C & 5.5449060 & 2.5311350 & 0.4038120 \\
\hline C & 2.3980750 & 0.3117430 & 3.7528370 & $\mathrm{H}$ & 5.4702410 & 3.1962920 & 1.2747710 \\
\hline $\mathrm{H}$ & 2.9461270 & 0.5633800 & 2.8372710 & $\mathrm{H}$ & 6.1872070 & 1.6874750 & 0.6847340 \\
\hline $\mathrm{H}$ & 1.4783670 & -0.2229070 & 3.4813570 & C & 6.1394110 & 3.2933940 & -0.8221590 \\
\hline $\mathrm{H}$ & 3.0212740 & -0.3129870 & 4.3947910 & $\mathrm{H}$ & 6.3799670 & 4.3311200 & 546450 \\
\hline & & & & $\mathrm{H}$ & 7.0649080 & 2.8151360 & -1.1645320 \\
\hline & $\mathrm{G}[\mathrm{B} 97-\mathrm{D}$ & VP] & & $\mathrm{H}$ & 5.5106840 & 3.8052220 & -2.8607390 \\
\hline $\mathrm{P}$ & -0.1236910 & -0.1700230 & -1.0531910 & H & 3.7131930 & 1.4528690 & 0.8960660 \\
\hline 0 & -1.0690800 & -0.5365830 & -2.1725970 & C & 3.8268990 & 4.0289490 & -1.4687260 \\
\hline 0 & 0.3087950 & 1.2556750 & -0.9104580 & $\mathrm{H}$ & 4.0965980 & 5.0523640 & -1.1747070 \\
\hline 0 & 1.1189620 & -1.2018920 & -1.2316190 & $\mathrm{H}$ & 3.0948810 & 4.0967380 & -2.2819700 \\
\hline 0 & -0.7384360 & -0.5876090 & 0.4081610 & C & 3.2308880 & 3.2429540 & -0.2605590 \\
\hline C & 1.9578470 & -1.4445130 & -0.1438380 & $\mathrm{H}$ & 3.1767180 & 3.8788320 & 0.6323200 \\
\hline C & 1.4647650 & -2.2293770 & 0.9146440 & $\mathrm{H}$ & 2.2209000 & 2.8964100 & -0.4906230 \\
\hline C & 3.2605070 & -0.9297720 & -0.1693350 & $\mathrm{C}$ & 4.8372530 & 1.2599610 & -3.5440270 \\
\hline C & 4.0716560 & -1.1890090 & 0.9439350 & $\mathrm{H}$ & 5.2395820 & 1.8098210 & -4.3958500 \\
\hline $\mathrm{H}$ & 5.0935370 & -0.8143020 & 0.9434880 & C & -4.7698660 & 0.4503440 & 1.3948290 \\
\hline C & 66380 & -1.9392900 & 0.6405550 & $\mathrm{C}$ & -4.8418310 & 1.4654980 & 2.5084230 \\
\hline
\end{tabular}




$\begin{array}{lr}-2.8425070 & -0.1769240 \\ -3.6729890 & -0.4246780 \\ -4.9557250 & 0.6963160 \\ -4.9313020 & 1.4208900 \\ -5.9187190 & 0.1739780 \\ -3.7722110 & -0.3150070 \\ -3.1840960 & -0.1176860 \\ -4.1461770 & -1.3438190 \\ -2.0069250 & -0.8771370 \\ -5.6979570 & 2.1382730 \\ -2.3163750 & 1.2795890 \\ -1.7597100 & 1.4867680 \\ -1.6174470 & 1.3712320 \\ -3.5118660 & 2.2677490 \\ -3.5410790 & 2.9821840 \\ -3.4134540 & 2.8498260 \\ -5.5002450 & -0.6937030 \\ -6.3985410 & -0.9710110 \\ -4.3769510 & -2.5959650 \\ -4.3987970 & -1.5671540 \\ -5.5479540 & -0.8657200 \\ -6.1863880 & -1.0879920 \\ -5.1728640 & 0.1570530 \\ -4.3570000 & -1.8676190 \\ -4.4318400 & -2.6236010 \\ -3.4021280 & -1.3514330 \\ -3.5137020 & -3.2608150 \\ -7.2396840 & -0.2671500 \\ -5.7008260 & -3.4064330 \\ -5.7115480 & -4.1467280 \\ -5.7417880 & -3.9525430 \\ -6.9157700 & -2.4330630 \\ -7.5096630 & -2.6692520 \\ -7.5759060 & -2.5212860 \\ -5.6881600 & 0.3126860 \\ -6.5428650 & 0.9860020 \\ -1.4807260 & -4.6581500 \\ -0.1519820 & -4.1941000 \\ -1.8119650 & -6.1308160 \\ -0.6550950 & -7.0717380 \\ 0.6409840 & -6.5687030 \\ 1.0009870 & -5.1845180 \\ -0.8895280 & -8.0924670 \\ -2.0801520 & -6.2929670 \\ -2.7076250 & -6.3778410 \\ 0.5037520 & -6.5074520 \\ -5 & \end{array}$

2.7360420
1.4980320
3.8552620
4.6801080
3.8991630
3.9738110
4.8798120
4.0370720
2.8127130
2.3756970
2.6930550
3.6160090
1.8617300
2.5332810
3.3662920
1.6100330
-0.5930660
-1.7722510
-1.6116310
-0.5043330
-3.0680260
-3.9334310
-3.1829160
-2.9836580
-3.7766500
-3.1067960
-1.5294890
-1.8021090
-1.5316940
-2.3423660
-0.5813900
-1.6588050
-2.5517400
-0.7877970
0.3538550
0.2797630
0.9799430
0.9454770
1.1679780
0.8098020
1.4551550
0.9036670
1.1386210
2.2247390
0.5825530
2.5457460

\begin{abstract}
1.4687110
1.8779780

1. 2997160

$-0.5238110$

3.5948920

4.4442880

4.3352840

2. 8622930

1.8346470

2. 2857830

4. 0791790

4.9614160

2. 6914680

1.7238090

5.4865020

4.7301000

2.6688160

0.8522920

$-2.0723170$

$-2.8490300$

$-0.9891180$

$-2.3929080$

$-2.6764710$

$-0.9090750$

0.4512200

0.6286790

$-0.1310720$

$-0.0558100$

$-0.7147590$

$-1.9237920$

$-1.9697410$

2. 0789760

2. 2490150

1. 5030830

1.1738670

2. 6492610

1.6317540

1.0490560

$-4.0896740$

$-4.6676130$

$-4.2869180$

$-2.5543530$

$-4.3815610$

$-4.8794370$

$-2.1235290$
\end{abstract}

-7.2649370
-4.7873010
-5.2927900
-7.0993130
-1.8943660
-2.1865590
-3.6851560
-4.0936280
-3.0684960
-2.4090130
-1.5993190
-3.9149250
-4.1917470
-2.2647390
-1.8963630
-4.2628790
-5.0786690
-3.5347760
2.4604290
2.3396530
2.4042300
1.8097750
1.1339160
4.2601970
4.1158260
3.0318580
3.1606920
2.4453870
5.2367760
4.7059520
0.4017360
3.1485590
2.0673060
1.9884020
4.1728020
3.1997340
1.1573260
5.0002130
3.5361990
3.8299500
2.7408220
3.4388440
4.3260750
3.9096090
1.8303570
4.4171440

1.2677330

1.4164690

$-0.1520650$

$-0.2822020$

2. 0465740

3. 2567440

3. 6133240

3. 8850090

3. 3280330

2. 0447040

4. 1126770

4.4846850

4.9645060

4.0728430

3.0758600

2.7675340

3. 4445270

3. 2215080

$-2.8613500$

$-1.6250890$

$-2.7411620$

$-0.7955260$

$-3.0049290$

0.8337750

2.9547840

2. 0697500

0.9190700

0.1510280

2. 2865360

$-0.4246340$

$-2.6397060$

4.4330140

3. 5421270

2. 3577970

4.1434420

5.3537600

1.6726520

4.8366730

$-3.9623260$

$-2.5685660$

$-1.5503870$

$-3.9126760$

$-2.6150110$

$-1.7107510$

$-3.6575820$ 


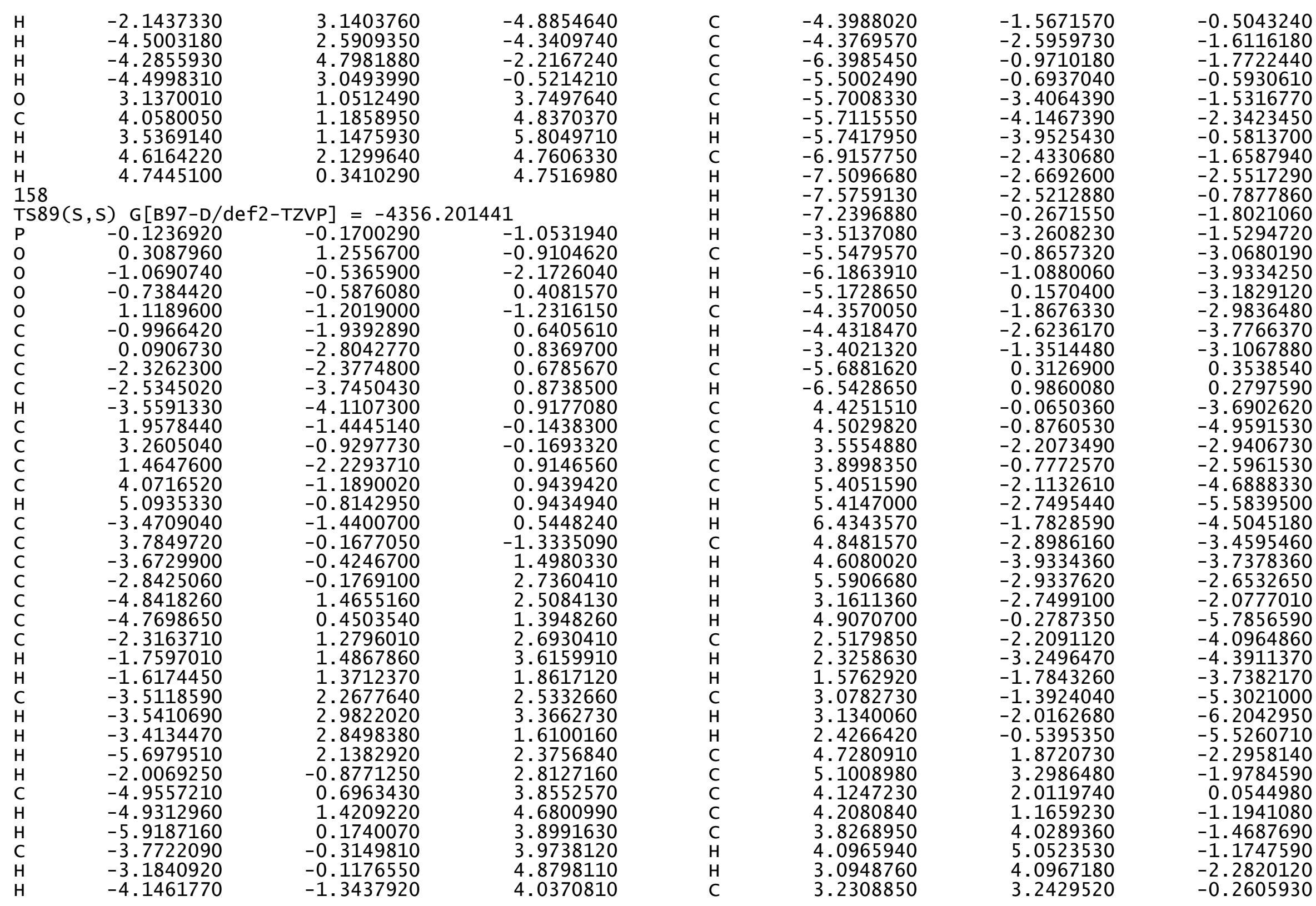




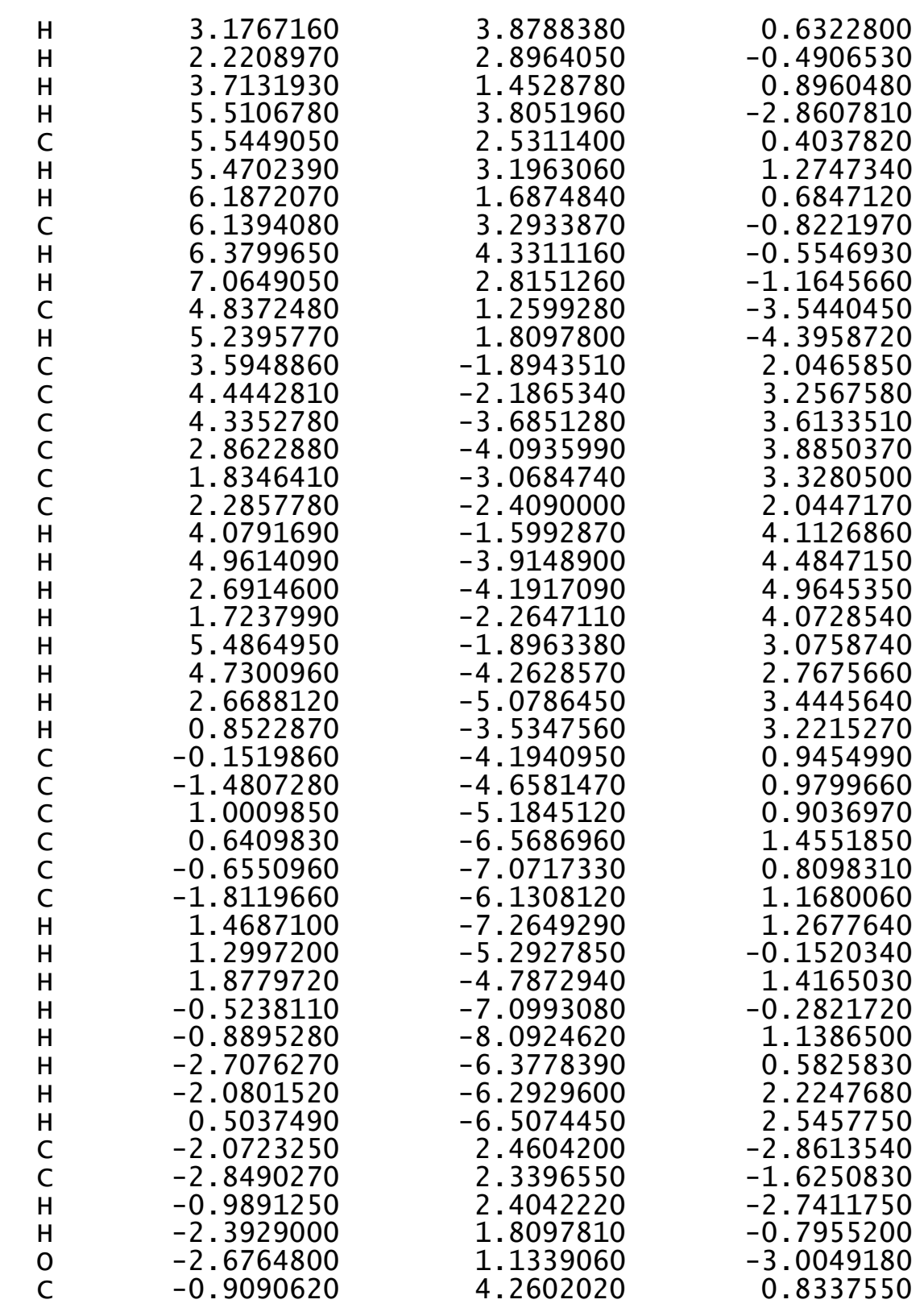

\begin{tabular}{lr}
$\mathrm{C}$ & 0.4512390 \\
$\mathrm{C}$ & 0.6286930 \\
$\mathrm{~N}$ & -0.1310620 \\
$\mathrm{H}$ & -0.0558060 \\
$\mathrm{~S}$ & -0.7147400 \\
$\mathrm{~S}$ & -1.9237810 \\
$\mathrm{H}$ & -1.9697410 \\
$\mathrm{C}$ & 2.0789960 \\
$\mathrm{C}$ & 2.2490300 \\
$\mathrm{C}$ & 1.5030950 \\
$\mathrm{C}$ & 1.1738880 \\
$\mathrm{H}$ & 2.6492830 \\
$\mathrm{H}$ & 1.6317630 \\
$\mathrm{H}$ & 1.0490800 \\
$\mathrm{C}$ & -4.0896900 \\
$\mathrm{C}$ & -4.6676180 \\
$\mathrm{C}$ & -4.2869160 \\
$\mathrm{C}$ & -2.5543700 \\
$\mathrm{H}$ & -4.3815820 \\
$\mathrm{H}$ & -5.7604520 \\
$\mathrm{H}$ & -4.8794340 \\
$\mathrm{H}$ & -2.1235440 \\
$\mathrm{H}$ & -2.1437570 \\
$\mathrm{H}$ & -4.5003380 \\
$\mathrm{H}$ & -4.2855930 \\
$\mathrm{H}$ & -4.4998250 \\
$\mathrm{O}$ & 3.1370140 \\
$\mathrm{C}$ & 4.0580210 \\
$\mathrm{H}$ & 3.5369320 \\
$\mathrm{H}$ & 4.6164390 \\
$\mathrm{H}$ & 4.7445230 \\
$\mathrm{H}$ & \\
\hline & \\
\hline &
\end{tabular}

4.1158480
3.0318700
3.1606960
2.4453790
5.2367930
4.7059500
0.4017250
3.1485900
2.0673290
1.9884150
4.1728330
3.1997730
1.1573320
5.0002500
3.5361840
3.8299450
2.7408230
3.4388280
4.3260550
3.9096060
1.8303570
4.4171300
3.1403550
2.5909180
4.7981850
3.0494050
1.0512710
1.1859250
1.1476340
2.1299920
0.3410580

2. 9547620 2.0697370

0.9190570

0.1510230

2. 2865070

$-0.4246560$

$-2.6397000$

4.4329970

3. 5421200

2. 3577920

4.1434170

5. 3537400

1.6726540

4.8366410

$-3.9623200$

$-2.5685570$

$-1.5503750$

$-3.9126810$

$-4.6648590$

$-2.6149940$

$-1.7107350$

$-3.6575970$

$-4.8854710$

$-4.3409580$

$-2.2167260$

$-0.5214100$

3.7497650

4.8370340

5.8049700

4.7606200

158

4.7517020

$\operatorname{TS} 90(\mathrm{~S}, \mathrm{~S})$ G[B97-D/def2-TZVP $]=-4356.200535$

$\begin{array}{lrrr}\mathrm{P} & -0.1411060 & -0.2391000 & -0.9777210 \\ \mathrm{O} & -1.0946590 & -0.6823750 & -2.0615860 \\ \mathrm{O} & 0.2800180 & 1.1964370 & -0.9287890 \\ \mathrm{O} & 1.1080460 & -1.2694960 & -1.1051340 \\ \mathrm{O} & -0.7300360 & -0.5758230 & 0.5121520 \\ \mathrm{C} & 1.9671200 & -1.4438980 & -0.0208860 \\ \mathrm{C} & 1.5061570 & -2.1760810 & 1.0918620 \\ \mathrm{C} & 3.2655310 & -0.9280040 & -0.1089130 \\ \mathrm{C} & 4.1148250 & -1.1379860 & 0.9862330 \\ \mathrm{H} & 5.1333120 & -0.7571670 & 0.9369100 \\ \mathrm{C} & -0.9720230 & -1.9150080 & 0.8237140 \\ \mathrm{C} & -2.2971300 & -2.3642160 & 0.8777910 \\ \mathrm{C} & 0.1315710 & -2.7501390 & 1.0814830\end{array}$




$\begin{array}{lr}\mathrm{C} & -2.5044840 \\ \mathrm{H} & -3.5255490 \\ \mathrm{C} & 3.7544310 \\ \mathrm{C} & -3.4443150 \\ \mathrm{C} & 3.8587890 \\ \mathrm{C} & 3.5186810 \\ \mathrm{C} & 4.4341710 \\ \mathrm{C} & 4.3643530 \\ \mathrm{C} & 2.4659310 \\ \mathrm{H} & 2.2703180 \\ \mathrm{H} & 1.5287630 \\ \mathrm{C} & 3.0092610 \\ \mathrm{H} & 3.0602280 \\ \mathrm{H} & 2.3502160 \\ \mathrm{H} & 4.8249440 \\ \mathrm{H} & 3.1370630 \\ \mathrm{C} & 5.3478410 \\ \mathrm{H} & 5.3554900 \\ \mathrm{H} & 6.3756760 \\ \mathrm{C} & 4.8073510 \\ \mathrm{H} & 4.5668330 \\ \mathrm{H} & 5.5593340 \\ \mathrm{C} & 4.1622770 \\ \mathrm{C} & 4.0827410 \\ \mathrm{C} & 5.0353510 \\ \mathrm{C} & 4.6653460 \\ \mathrm{C} & 5.5031050 \\ \mathrm{H} & 5.4328350 \\ \mathrm{H} & 6.1512520 \\ \mathrm{C} & 6.0845810 \\ \mathrm{H} & 6.3259780 \\ \mathrm{H} & 7.0075720 \\ \mathrm{H} & 5.4358450 \\ \mathrm{H} & 3.6797830 \\ \mathrm{C} & 3.7644160 \\ \mathrm{H} & 4.0349980 \\ \mathrm{H} & 3.0256000 \\ \mathrm{C} & 3.1800210 \\ \mathrm{H} & 3.1248450 \\ \mathrm{H} & 2.1717250 \\ \mathrm{C} & 4.7673010 \\ \mathrm{H} & 5.1576710 \\ \mathrm{C} & -4.7454840 \\ \mathrm{C} & -4.8231880 \\ \mathrm{C} & -2.8347380 \\ \mathrm{C} & -3.6530380 \\ & \end{array}$

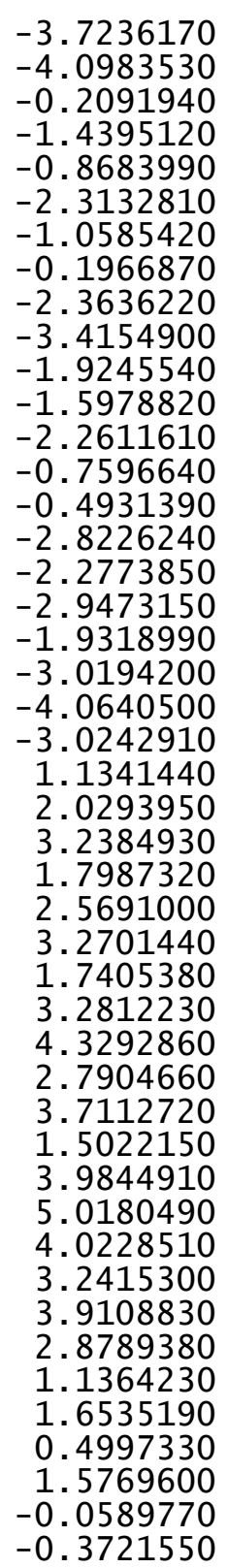

1.1476250
1.1922870
-1.3153680
0.6828960
-2.5528090
-2.8349560
-4.9167830
-3.6814780
-3.9758600
-4.2242290
-3.6225920
-5.2222010
-6.0961580
-5.4776580
-5.7716240
-1.9462660
-4.6066300
-5.4768460
-4.4436070
-3.3435550
-3.5818700
-2.5453060
-1.2362050
-0.0222220
-2.1143390
-2.3711350
0.2940860
1.1368920
0.6049670
-0.9680940
-0.7455230
-1.2993440
-3.0194010
0.8441560
-1.6210250
-1.3658600
-2.4300880
-0.3810790
0.4867320
-0.5927850
-3.5940560
-4.4715950
1.4134450
2.4667050
2.8083450
1.5767250

-4.9554410
-4.9369920
-5.9215030
-3.7783840
-3.1981740
-4.1581840
-2.0044810
-5.6739170
-2.3003910
-1.7551180
-1.5902990
-3.4888310
-3.5232830
-3.3775480
-5.4640920
-6.3634260
-4.3401560
-4.3648830
-5.5196190
-6.1615100
-5.1538960
-4.3207990
-4.3869920
-3.3690540
-3.4751050
-7.2048250
-5.6632190
-5.6752150
-5.7018980
-6.8797150
-7.4821670
-7.5312640
-5.6541720
-6.5062200
-0.1140780
0.9668040
0.6882690
-0.7513570
-1.6225880
-1.4373930
0.9774590
1.4344560
-0.7143220
-1.3127910
1.9558480
0.8320170

0.8866330

1. 6564130

0.3718130

$-0.1211770$

0.1253440

$-1.14$

-0.7585300
2.2455540

1. 3904380

1. 6469720

1. 4327350

2. 3727410

3. 1359890

2. 8965820

$-0.7557220$

$-1.1067610$

$-2.7165190$

$-1.6246120$

$-1.0884060$

$-1.3779870$

$-0.0741440$

$-2.0732370$

$-2.8769420$

$-1.5589570$

$-3.3729750$

$-0.4063160$

$-3.5232750$

$-4.3056070$

$-4.0181240$

$-2.5587870$

$-2.8508070$

$-2.5912590$

0.3069100

0.9770120

$-4.1193710$

$-5.1652370$

$-6.3230380$

$-6.9008210$

$-6.0785300$

$-4.5991770$

$-5.5701310$

$-7.1124750$

$-7.9418560$

$-6.3291900$

$-4.7381740$

$-5.9370890$
3.8535470

4.6365990

3.9160390

4.0416840

4.9407670

4. 1608980

2. 9327320

2.2867540

2. 6905410

3. 6079820

1. 8651800

2. 4586310

3. 2468640

1. 5025420

$-0.5099320$

$-1.6684730$

$-1.4086690$

$-0.3639120$

$-2.9720440$

$-3.8148390$

$-3.1653000$

$-2.8226370$

$-3.5679290$

$-2.9730700$

$-1.2864440$

$-1.7411390$

$-1.2814550$

$-2.0516750$

$-0.3033190$

$-1.4582310$

$-2.3286410$

$-0.5769500$

0.3728880

0.2529700

1. 3018960

1. 4213740

0.4324580

0.5736700

1. 5580090

1. 3367480

2. 4447840

0.5865920

2. 5848350

1. 2359400

$-0.5840910$ 


$\begin{array}{lr}\mathrm{H} & -1.2417030 \\ \mathrm{H} & -2.6788270 \\ \mathrm{C} & 3.6808770 \\ \mathrm{C} & 4.5728220 \\ \mathrm{C} & 4.4946920 \\ \mathrm{C} & 3.0304300 \\ \mathrm{C} & 1.9836670 \\ \mathrm{C} & 2.3724480 \\ \mathrm{H} & 4.2293460 \\ \mathrm{H} & 5.1291830 \\ \mathrm{H} & 2.8814230 \\ \mathrm{H} & 1.9323150 \\ \mathrm{H} & 5.6052800 \\ \mathrm{H} & 4.9009220 \\ \mathrm{H} & 2.8368600 \\ \mathrm{H} & 0.9904360 \\ \mathrm{C} & -2.0730660 \\ \mathrm{C} & -2.8894480 \\ \mathrm{H} & -0.9945040 \\ \mathrm{H} & -2.4608350 \\ \mathrm{O} & -2.6705320 \\ \mathrm{C} & -0.9654990 \\ \mathrm{C} & 0.4293780 \\ \mathrm{C} & 0.5845530 \\ \mathrm{~N} & -0.1917540 \\ \mathrm{H} & -0.1200980 \\ \mathrm{~S} & -0.7406540 \\ \mathrm{~S} & -2.0015180 \\ \mathrm{H} & -1.9751410 \\ \mathrm{C} & 2.0699910 \\ \mathrm{C} & 2.2134930 \\ \mathrm{C} & 1.4521370 \\ \mathrm{C} & 1.1717370 \\ \mathrm{H} & 2.6552390 \\ \mathrm{H} & 1.5630080 \\ \mathrm{H} & 1.0669070 \\ \mathrm{C} & -4.0526060 \\ \mathrm{C} & -4.6731850 \\ \mathrm{C} & -4.3293090 \\ \mathrm{C} & -2.5198700 \\ \mathrm{H} & -4.3214790 \\ \mathrm{H} & -5.7635930 \\ \mathrm{H} & -4.9162230 \\ \mathrm{H} & -2.0958970 \\ \mathrm{H} & -2.0790980 \\ \mathrm{H} & -4.4526930 \\ & \end{array}$

$\begin{array}{rr}-6.9034540 & -0.4073530 \\ -6.3570520 & 1.4622830 \\ -1.8048960 & 2.1282830 \\ -2.0462570 & 3.3190300 \\ -3.5311990 & 3.7364500 \\ -3.9627550 & 4.0295790 \\ -2.9374240 & 3.5157960 \\ -2.3205300 & 2.1931740 \\ -1.4316630 & 4.1650860 \\ -3.7089940 & 4.6138590 \\ -4.0896900 & 5.1093250 \\ -2.1142730 & 4.2461060 \\ -1.7523080 & 3.0942290 \\ -4.1346700 & 2.9145930 \\ -4.9350100 & 3.5636560 \\ -3.3895750 & 3.4835640 \\ 2.2732230 & -2.9628040 \\ 2.2327810 & -1.7461380 \\ 2.2286680 & -2.8041420 \\ 1.7587330 & -0.8699030 \\ 0.9385850 & -3.0390230 \\ 4.3008510 & 0.6182100 \\ 4.2920740 & 2.7227250 \\ 3.1490020 & 1.9114070 \\ 3.2056630 & 0.7664090 \\ 2.4431200 & 0.0450690 \\ 5.3736700 & 1.9971260 \\ 4.6632050 & -0.6504540 \\ 0.2302770 & -2.6092220 \\ 3.4097330 & 4.2395940 \\ 2.2669610 & 3.4236580 \\ 2.1176250 & 2.2565280 \\ 4.4227850 & 3.8935220 \\ 3.5163950 & 5.1460110 \\ 1.2419760 & 1.6257240 \\ 5.2972490 & 4.5300090 \\ 3.2695520 & -4.1979690 \\ 3.6545240 & -2.8451220 \\ 2.6342770 & -1.7457820 \\ 3.1770670 & -4.0934300 \\ 4.0113820 & -4.9595940 \\ 3.7341230 & -2.9311760 \\ 1.7139690 & -1.8660540 \\ 4.1709090 & -3.8934440 \\ 2.8119000 & -5.0295950 \\ 2.3011660 & -4.5264000 \\ -3\end{array}$

$\begin{array}{lrrr}\mathrm{H} & -4.2973650 & 4.6424150 & -2.5448700 \\ \mathrm{H} & -4.5775830 & 3.0103210 & -0.7476830 \\ \mathrm{O} & 3.0890160 & 1.2537180 & 3.6897370 \\ \mathrm{C} & 4.0141920 & 1.4406390 & 4.7656260 \\ \mathrm{H} & 3.4932590 & 1.4803360 & 5.7336120 \\ \mathrm{H} & 4.5941480 & 2.3642840 & 4.6254320 \\ \mathrm{H} & 4.6814550 & 0.5767290 & 4.7381360\end{array}$

TS91(S, S) G[B97-D/def2-TZVP $]=-4356.198812$

\begin{tabular}{|c|c|c|c|}
\hline & 5) $\mathrm{G}[\mathrm{B} 97-\mathrm{D} /$ & & \\
\hline $\mathrm{P}$ & -0.0847320 & -0.2617880 & -0.7152560 \\
\hline 0 & -1.0871230 & -0.7418550 & -1.7380610 \\
\hline 0 & 0.2760000 & 1.1881390 & -0.6753870 \\
\hline 0 & 1.1923430 & -1.2407740 & -0.9383450 \\
\hline 0 & -0.5438010 & -0.6655610 & 0.8005930 \\
\hline C & 2.0908510 & -1.5089720 & 0.0887840 \\
\hline $\mathrm{C}$ & 1.6892300 & -2.3460530 & 1.1528570 \\
\hline C & 3.3907110 & -1.0045160 & -0.0339640 \\
\hline C & 4.3097860 & -1.3415030 & 0.9683790 \\
\hline $\mathrm{H}$ & 5.3317180 & -0.9755290 & 0.8841060 \\
\hline C & -0.7981340 & -2.0187220 & 1.0346720 \\
\hline C & -2.1289760 & -2.4517810 & 1.0765570 \\
\hline C & 0.3023650 & -2.8898080 & 1. 1828160 \\
\hline C & -2.3472410 & -3.8187970 & 1.3054580 \\
\hline $\mathrm{H}$ & -3.3718970 & -4.1825460 & 1.3496290 \\
\hline C & 3.8153770 & -0.2073580 & -1.2172180 \\
\hline C & -3.2755250 & -1.5472330 & 0.7906800 \\
\hline C & 3.8566710 & -0.7980630 & -2.4929890 \\
\hline C & 3.4795310 & -2.2192770 & -2.8404670 \\
\hline C & 4.2950420 & -0.8524400 & -4.8933630 \\
\hline C & 4.3087430 & -0.0660010 & -3.6066700 \\
\hline C & 2.3614080 & -2.1850210 & -3.91 \\
\hline $\mathrm{H}$ & 2.1357080 & -3.2164760 & -4.2207570 \\
\hline $\mathrm{H}$ & 1.4534180 & -1.759 & -3.4822370 \\
\hline C & 2.8426190 & -1.3468430 & -5.1437800 \\
\hline $\mathrm{H}$ & 2.8234010 & -1.9492780 & -6.0618390 \\
\hline $\mathrm{H}$ & 2.1871810 & -0.4815260 & -5.2990390 \\
\hline $\mathrm{H}$ & 4.6458200 & -0.2413020 & -5.7338990 \\
\hline $\mathrm{H}$ & 3.1409880 & -2.7766760 & -1.9634250 \\
\hline C & 5.2005870 & -2.1036480 & -4.7121980 \\
\hline $\mathrm{H}$ & 5.1415720 & -2.7199130 & -5.6193240 \\
\hline $\mathrm{H}$ & 6.2435360 & -1.7864960 & -4.5935160 \\
\hline C & 4.7233150 & -2.9104130 & -3.4641520 \\
\hline $\mathrm{H}$ & 4.4532490 & -3.9367060 & -3.7465140 \\
\hline $\mathrm{H}$ & 5.5210040 & -2.9706140 & -2.7139290 \\
\hline C & 4.2186320 & 1.1318930 & -1.0876790 \\
\hline C & 4.1708310 & 1.9687350 & 0.1679080 \\
\hline
\end{tabular}




\begin{tabular}{|c|c|c|}
\hline $\begin{array}{l}5.0459010 \\
4.6746060 \\
5.5955050 \\
5.5455200 \\
6.2606140 \\
6.1298810 \\
6.3707640 \\
7.0453750 \\
5.4136770 \\
3.7958440 \\
3.7850230 \\
4.0540970 \\
3.0210560 \\
3.2450700 \\
3.2093330 \\
2.2340710 \\
4.7217910 \\
5.0707270 \\
4.6385900 \\
4.7753950 \\
2.7698490 \\
3.5364140 \\
4.9447630 \\
4.9757410 \\
5.8999830 \\
3.7532950 \\
3.2080390 \\
4.1144690 \\
1.9320020 \\
5.6320290 \\
2.2589260 \\
1.7473960 \\
1.5229700 \\
3.4567050 \\
3.5305510 \\
3.3234350 \\
5.2690630 \\
6.1264360 \\
4.0454580 \\
4.1488830 \\
5.2564710 \\
5.8608930 \\
4.9474810 \\
4.0073970 \\
3.9898600 \\
3.0838500\end{array}$ & $\begin{array}{r}3.2830680 \\
1.8557490 \\
2.5068960 \\
3.1679400 \\
1.6718560 \\
3.2815110 \\
4.3198790 \\
2.8148040 \\
3.8015230 \\
1.3933940 \\
3.9934340 \\
5.0164510 \\
4.0617730 \\
3.1874810 \\
3.8151710 \\
2.8240180 \\
1.2594010 \\
1.8239570 \\
0.4250400 \\
1.6038560 \\
0.0555610 \\
-0.4028290 \\
1.0579010 \\
1.9037550 \\
0.5256650 \\
0.1071250 \\
0.4662050 \\
-0.9044310 \\
-0.6093170 \\
2.2298540 \\
1.4967910 \\
1.8606360 \\
1.4724590 \\
2.4241700 \\
3.2623810 \\
2.8533920 \\
-1.0264200 \\
-1.5041910 \\
-2.9961440 \\
-1.8418990 \\
-1.5449180 \\
-1.9456380 \\
-0.5297060 \\
-2.4389160 \\
-3.2900340 \\
-1.8735790\end{array}$ & $\begin{array}{r}-1.8919650 \\
-2.2063070 \\
0.4675190 \\
1.3429560 \\
0.7190700 \\
-0.7786430 \\
-0.5148510 \\
-1.1614110 \\
-2.7857050 \\
1.0177900 \\
-1.3264280 \\
-1.0308440 \\
-2.1096490 \\
-0.1052640 \\
0.7937570 \\
-0.3021320 \\
-3.4657530 \\
-4.3313150 \\
1.2799110 \\
2.2109300 \\
2.7857260 \\
1.5671190 \\
3.6562800 \\
4.3560440 \\
3.7354160 \\
3.9894440 \\
4.8722170 \\
4.2098240 \\
3.0107600 \\
1.9326870 \\
2.5315340 \\
3.4310350 \\
1.7277640 \\
2.1659720 \\
2.8708180 \\
1.1660770 \\
-0.5312930 \\
-1.6760780 \\
-1.2509310 \\
-0.2774020 \\
-2.9605600 \\
-3.7854960 \\
-3.2302550 \\
-2.7009500 \\
-3.3947720 \\
-2.8441900\end{array}$ \\
\hline
\end{tabular}
$-3.1573550$
$-6.9950270$
$-5.3334590$
$-5.2908800$
$-5.3749220$
$-6.5860630$
$-7.1697950$
$-7.2422690$
$-5.5143320$
-6.3827920
0.0375550
1.0907020
0.6807910
$-0.7496840$
$-1.5022390$
$-1.2926970$
1. 1914310
1.4188960
$-0.7019630$
$-1.1054000$
2. 0682640
0.7284170
$-1.3326650$
$-2.5706030$
2. 6420830
2. 3540570
2. 9311860
4. 4270170
4.9592620
3.9557260
2. 8136300
2. 8012310
5. 0464150
5. 1977120
1. 2800010
2. 3275110
4. 5677580
5. 8958440
$-2.1029390$
$-2.8504060$
$-1.0176860$
$-2.3822820$
$-2.7401460$
$-1.0089140$
0.1140370
0.3063980
$-3.6043580$
$-0.8488430$
$-3.8552140$
$-4.6732080$
$-2.9564750$
$-3.3344770$
$-2.9514170$
0.1060920
0.7347440
$-4.2690410$
$-5.3468430$
$-6.3915970$
$-6.9603840$
$-6.2095810$
$-4.7219960$
$-5.8517150$
$-7.2032730$
$-8.0267450$
$-6.5549310$
$-4.9250270$
$-5.9036010$
$-6.8748960$
$-6.4550690$
$-2.6551590$
$-3.5116190$
$-2.8959260$
$-2.4939680$
$-2.6043140$
$-2.1585450$
$-4.5003000$
$-3.6152320$
$-3.1281750$
$-3.6623990$
$-3.6793950$
$-2.0188740$
$-1.4647440$
$-2.0444820$
. 0411300
2.2685000
1.9856840
1.9662950
0.7447620
4. 6068840
4. 7805600
3. 5538680

$-1.0688690$

$-1.8159480$

$-1.1132620$

$-1.8445580$

$-0.1137030$

$-1.3648710$

$-2.2145940$

$-0.4863590$

0.2429970

0.0427870

1. 3069480

1. 2210700

0.1539330

0.3872580

1. 5169980

1. 3995120

2. 1935880

0.1465800

0.6413350

2. 4842430

0.9732900

$-0.8273530$

$-0.5378370$

1.4974370

2. 1492600

3. 3615030

4.6553960

4. 5277710

3. 0766560

2. 0411370

3. 2126430

5.4731220

5. 1737120

2. 8829160

3.4779950

4.9126090

4.8798320

2. 9686750

$-2.9801480$

$-1.7407100$

$-2.8701010$

$-0.8096930$

$-2.7595990$

0.2041430

2.4610530

1.8055890 


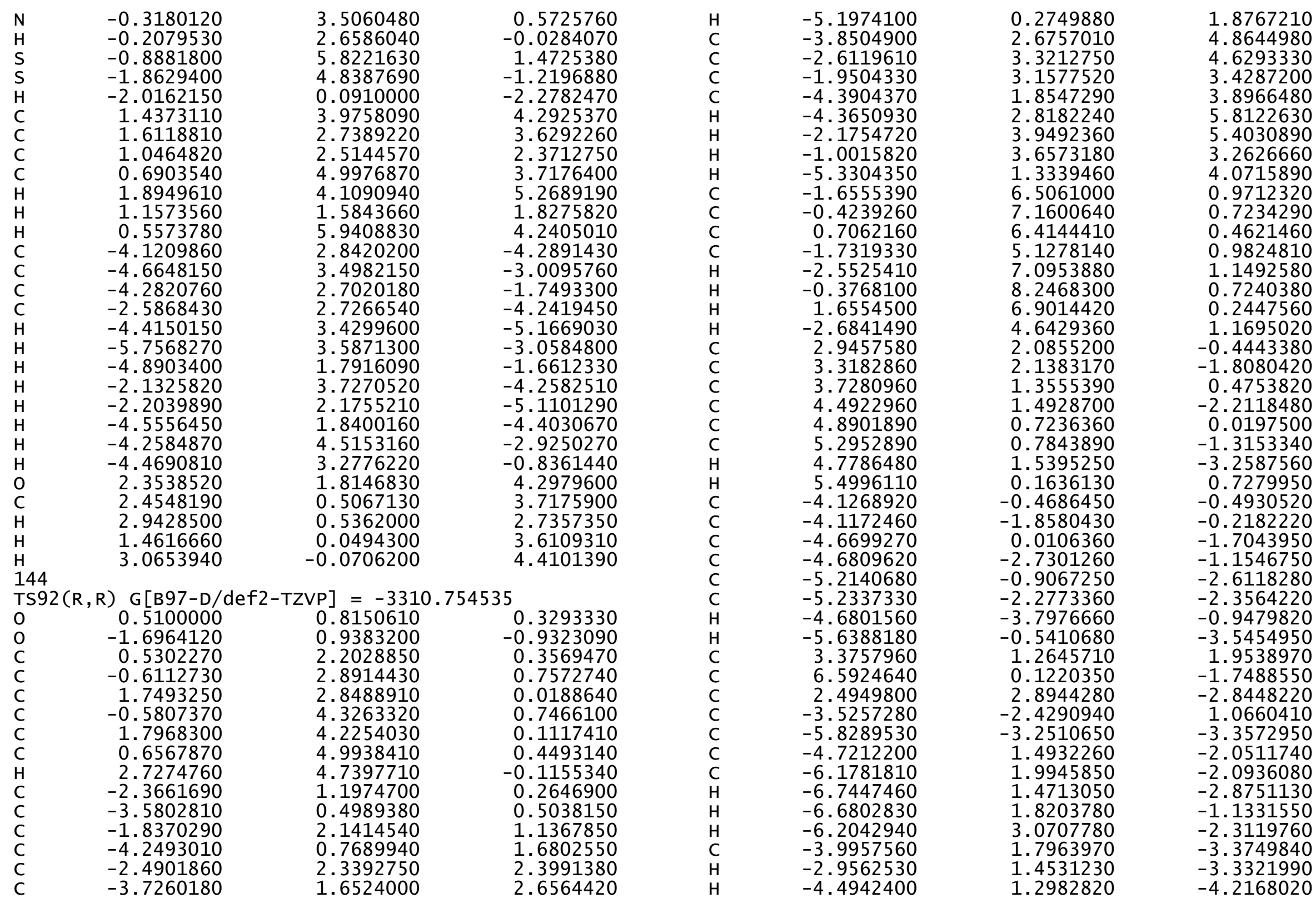




$\begin{array}{rrr}-4.0024440 & 2.8775410 & -3.5679030 \\ -7.0209440 & -4.0197290 & -2.7556160 \\ -7.4645090 & -4.6893130 & -3.5049030 \\ -6.6912070 & -4.6303610 & -1.9045660 \\ -7.7949910 & -3.3285360 & -2.3990350 \\ -4.7654990 & -4.2335900 & -3.8855300 \\ -3.9252290 & -3.6944330 & -4.3405570 \\ -4.3719650 & -4.8473210 & -3.0645460 \\ -5.2027500 & -4.9054940 & -4.6364080 \\ -4.6357840 & -2.7424310 & 2.0891840 \\ -5.2207720 & -1.8487290 & 2.3334820 \\ -5.3221690 & -3.4983680 & 1.6836390 \\ -4.1986700 & -3.1356510 & 3.0172410 \\ -2.6726670 & -3.6887620 & 0.8262850 \\ -3.2947370 & -4.5398570 & 0.5199460 \\ -1.9193920 & -3.5059570 & 0.0545290 \\ -2.1618170 & -3.9677560 & 1.7565380 \\ 4.4281170 & 1.9944130 & 2.8111110 \\ 5.4136380 & 1.5247160 & 2.6933820 \\ 4.5142010 & 3.0470890 & 2.5129060 \\ 4.1509700 & 1.9522290 & 3.8729910 \\ 3.2112070 & -0.1952080 & 2.4127740 \\ 2.4181270 & -0.6918450 & 1.8458710 \\ 4.1449340 & -0.7573560 & 2.2815360 \\ 2.9415260 & -0.2309650 & 3.4761800 \\ 7.7389650 & 1.1537570 & -1.7612770 \\ 8.6894100 & 0.6723520 & -2.0293260 \\ 7.5291080 & 1.9399680 & -2.4989920 \\ 7.8492050 & 1.6286750 & -0.7784560 \\ 6.4919790 & -0.5861380 & -3.1104860 \\ 5.6597230 & -1.2991420 & -3.1287760 \\ 6.3353690 & 0.1389160 & -3.9189540 \\ 7.4235090 & -1.1266300 & -3.3222360 \\ 2.2694170 & 2.0885650 & -4.1368700 \\ 3.2116470 & 1.9226330 & -4.6754940 \\ 1.8107710 & 1.1207320 & -3.9148230 \\ 1.5992940 & 2.6479970 & -4.8026230 \\ 3.1543580 & 4.2488280 & -3.1751940 \\ 4.1531150 & 4.0889410 & -3.6037110 \\ 2.5482220 & 4.7992140 & -3.9074260 \\ 3.2632280 & 4.8689690 & -2.2779290 \\ -0.3599140 & 0.0199220 & -0.8124250 \\ 0.3176270 & 0.0625770 & -2.1502660 \\ -0.6703050 & -1.3181470 & -0.2001260 \\ 1.5080480 & 3.0959380 & -2.4150800 \\ 6.8371150 & -0.6367970 & -0.9904030\end{array}$

2. 4164940

$-4.2112410$

$-6.1994800$

$-2.8654490$

2. 5348300

2. 2697870

0.4709750

2.2000320

1.4491550

4. 2885440

3. 2748070

1. 8388720

3. 9622160

5. 3042150

3. 5131050

1. 6800480

4. 0765280

4.6321210

4. 2696070

3. 3221790

1. 0908500

1. 1113290

0.3628200

1.7124630

2. 5400480

1. 4841280

3.1309560

2. 7091230

2. 0792230

0.8472280

2. 9030880

3. 7698710

1. 9012660

3. 3658130

$-0.0418910$

1. 3379930
1.7665580

2. 0578020

$-2.6629910$

$-1.6713520$

$-2.0601170$

$-3.0687930$

$-1.2942730$

$-2.7535440$

$-1.6389100$

$-0.9277700$

$-3.6972670$

$-4.8410590$

$-4.3597090$

$-2.5472560$

$-4.0608230$

$-5.6688220$

$-4.2083420$

$-2.8881260$

$-1.6966620$

$-3.3225710$

$-5.2256680$

$-5.0961680$

$-3.3931600$

$-2.3234340$

$-3.8912030$

$-5.0260530$

$-3.2358950$

$-5.4986120$

$-5.5271380$

$-3.7098180$

$-2.3575220$

$-4.8400300$

$-6.3782150$

$-3.1967690$

$-5.2073040$

$-1.9579400$

$-3.9726190$
2. 1159370

$-1.2647470$

$-4.2092020$

1. 4998210

$-1.1390910$

$-1.4233510$

$-0.4066640$

$-1.3255000$

$-2.4996940$

$-2.4105370$

$-1.9680460$

$-1.8110770$

$-2.0860380$

$-1.0024230$

$-1.7708250$

$-2.4894100$

$-3.1642650$

0.0359930

$-1.1448970$

$-3.0001960$

$-0.7843810$

$-1.7700030$

1.7253150

1.8625500

3. 0014340

2. 9697720

4. 2238150

4.1410490

2. 0209730

5. 3934260

4.2484350

5. 3548660

4. 1093090

6.3357310

6.2684250

0.9766120

0.6506840

$\operatorname{TS} 93(R, R)$ G [B97-D/def2-TZVP] $=-3310.754388$

$\begin{array}{lrlr}\text { O } & 0.5104390 & 0.6607280 & 0.1186220 \\ \mathrm{O} & -1.7392970 & 0.9255790 & -1.0492780 \\ \mathrm{C} & 0.6556300 & 2.0393320 & 0.1175120 \\ \mathrm{C} & -0.4135730 & 2.8407180 & 0.5085820 \\ \mathrm{C} & 1.9319630 & 2.5612870 & -0.2234110 \\ \mathrm{C} & -0.2598380 & 4.2654840 & 0.4378520 \\ \mathrm{C} & 2.0945650 & 3.9326910 & -0.1981050\end{array}$




$\begin{array}{lrrr}\text { C } & 1.0248190 & 4.8110620 & \\ \text { H } & 3.0667660 & 4.3545330 & -0.0966230 \\ \text { C } & -2.3319850 & 1.2902490 & 0.1597460 \\ \text { C } & -3.5863510 & 0.7059930 & 0.4831220 \\ \text { C } & -1.6857290 & 2.2209680 & 0.9632190 \\ \text { C } & -4.1763720 & 1.0850690 & 1.6724400 \\ \text { C } & -2.2608120 & 2.5285830 & 2.2417390 \\ \text { C } & -3.5374610 & 1.9630650 & 2.5821440 \\ \text { H } & -5.1488330 & 0.6764760 & 1.9349790 \\ \mathrm{C} & -3.4724270 & 3.0815740 & 4.7461380 \\ \mathrm{C} & -2.1953120 & 3.6058940 & 4.4299420 \\ \mathrm{C} & -1.6061940 & 3.3377270 & 3.2104000 \\ \mathrm{C} & -4.1247970 & 2.2729680 & 3.8391240 \\ \mathrm{H} & -3.9283800 & 3.3069750 & 5.7077440 \\ \mathrm{H} & -1.6709020 & 4.2230670 & 5.1563930 \\ \mathrm{H} & -0.6259330 & 3.7438050 & 2.9835130 \\ \mathrm{H} & -5.0972870 & 1.8443990 & 4.0756940 \\ \mathrm{C} & -1.1443670 & 6.5356330 & 0.5757590 \\ \mathrm{C} & 0.1325660 & 7.0708410 & 0.2766160 \\ \mathrm{C} & 1.1923430 & 6.2215360 & 0.0378570 \\ \mathrm{C} & -1.3351320 & 5.1708280 & 0.6549980 \\ \mathrm{H} & -1.9850310 & 7.2064250 & 0.7396190 \\ \mathrm{H} & 0.2699300 & 8.1485910 & 0.2228450 \\ \mathrm{H} & 2.1751070 & 6.6165330 & -0.2144130 \\ \mathrm{H} & -2.3202620 & 4.7762730 & 0.8813440 \\ \mathrm{C} & 3.0803130 & 1.6880350 & -0.6078530 \\ \mathrm{C} & 3.5342710 & 1.6855950 & -1.9432070 \\ \mathrm{C} & 3.7611630 & 0.9282930 & 0.3754770 \\ \mathrm{C} & 4.6976680 & 0.9699270 & -2.2638060 \\ \mathrm{C} & 4.9116760 & 0.2299910 & 0.0029330 \\ \mathrm{C} & 5.4088420 & 0.2508110 & -1.3060030 \\ \mathrm{H} & 5.0436890 & 0.9784040 & -3.2934690 \\ \mathrm{H} & 5.4520850 & -0.3485920 & 0.7489630 \\ \mathrm{C} & -4.2450840 & -0.2788560 & -0.4242530 \\ \mathrm{C} & -4.3607840 & -1.6351270 & -0.0306020 \\ \mathrm{C} & -4.7651070 & 0.1433900 & -1.6668500 \\ \mathrm{C} & -5.0203200 & -2.5271560 & -0.8814270 \\ \mathrm{C} & -5.4079750 & -0.7935870 & -2.4853080 \\ \mathrm{C} & -5.5491960 & -2.1299250 & -2.1128420 \\ \mathrm{H} & -5.1123100 & -3.5684280 & -0.5818810 \\ \mathrm{H} & -5.8134480 & -0.4697670 & -3.4426210 \\ \mathrm{C} & 3.3081020 & 0.9356680 & -1.8305720 \\ \mathrm{C} & 6.7056570 & -0.4818970 & -1.6139920 \\ \mathrm{C} & 2.8184310 & 2.4449010 & -3.0536910 \\ \mathrm{C} & -3.7970830 & -2.1556400 & 1.2872800 \\ \mathrm{C} & -6.2576830 & -3.1237940 & -3.0160100 \\ & & & \end{array}$

-4.6892100
-6.0966620
-6.7094070
-6.6094540
-6.0280350
-3.9491530
-2.9450090
-4.4985190
-3.8598550
-7.5510840
-8.0732980
-7.3179390
-8.2276790
-5.3356420
-4.4208810
-5.0449750
-5.8514780
-4.9195950
-5.4595370
-5.6432430
-4.5014650
-3.0202900
-3.6946200
-2.2637140
-2.5208910
3.9287810
5.0236280
3.6629590
3.5711320
3.6116900
3.2584770
4.6878930
3.1101500
7.9093070
8.8499030
7.9487550
7.8248680
6.8760260
6.0178620
6.9854790
7.7811760
2.5092160
3.4314740
1.9048410
1.9488300
3.6400700

1.5882100

2.2099600

1.6747370

2.1618200

3.2617960

1.7043970

1.2731540

1.1795170

2.7600000

$-3.6473710$

$-4.3368150$

$-4.1881820$

$-2.8203290$

$-4.2950540$

$-3.9301090$

$-4.8665070$

$-4.9767500$

$-2.3406710$

$-1.4042250$

$-3.0869690$

$-2.6918700$

$-3.4759250$

$-4.3045070$

$-3.3835010$

$-3.7279030$

2.1396830

2.0499530

. 0809600

2.1773680

$-0.3699230$

$-1.2419010$

$-0.4911390$

$-0.3629300$

0.3673630

$-0.1774530$

1. 3028890

0.6220010

$-0.8783840$

$-1.4540420$

0.0110990

$-1.4865710$

1. 5487220

1.1882820

0.6875020

2.1244220

3.6738900
$-2.1438490$ $-2.2350310$ $-2.9724180$ $-1.2657440$ $-2.5440980$ $-3.4894320$ $-3.4162870$ $-4.2820860$ $-3.7795790$ $-2.3622520$ $-3.0394390$ $-1.4354630$ $-2.1125450$ $-3.4042800$ $-3.8878070$ $-2.5129320$ $-4.0938080$

2. 3281720

2.5089740

1.9732040

3.2815170

1.1229990

0.8699110

0.3381720

2.0673500

2.5683490

2. 5810270

2.0729110

3.6063700

2. 5812400

2. 0199630

2.7611780

3.5560310

$-1.1517140$

$-1.3103580$

$-1.7257750$

$-0.0882710$

$-3.0876410$

$-3.4562520$

$-3.7216790$

$-3.2074730$

$-4.2675030$

$-3.9673430$

$-5.0159120$

$-3.4894850$ 


$\begin{array}{lrrr}\mathrm{H} & 4.6120530 & 3.3593200 & -3.8931590 \\ \mathrm{H} & 3.1064390 & 4.2308500 & -4.2712010 \\ \mathrm{H} & 3.8242180 & 4.3484070 & -2.6443530 \\ \mathrm{P} & -0.4796200 & -0.0963340 & -0.9500670 \\ \mathrm{O} & 0.1284160 & -0.1623690 & -2.3190910 \\ \mathrm{O} & -0.8618320 & -1.3769730 & -0.2570140 \\ \mathrm{H} & 1.8600320 & 2.8033820 & -2.6648730 \\ \mathrm{H} & 6.7021590 & -1.4039150 & -1.0131390 \\ \mathrm{H} & 2.2220470 & 1.0637670 & 1.8494580 \\ \mathrm{H} & -4.1267010 & 2.1736240 & -1.4102100 \\ \mathrm{H} & -6.5353370 & -2.5917720 & -3.9372890 \\ \mathrm{H} & -3.0925180 & -1.4125040 & 1.6735680 \\ \mathrm{C} & 2.4070500 & -2.2275550 & -1.4403000 \\ \mathrm{C} & 1.3292240 & -3.2116360 & -1.2574240 \\ \mathrm{H} & 2.3255420 & -1.3038400 & -0.8617630 \\ \mathrm{H} & 0.3408690 & -2.8250930 & -1.0344270 \\ \mathrm{O} & 1.7738160 & -2.0909870 & -2.7557760 \\ \mathrm{H} & 1.0918670 & -1.3214290 & -2.6548660 \\ \mathrm{C} & 3.9141230 & -4.1180550 & -2.2312370 \\ \mathrm{C} & 2.9591380 & -5.1278510 & -1.5770230 \\ \mathrm{C} & 1.5020120 & -4.6379090 & -1.6461380 \\ \mathrm{C} & 3.8244610 & -2.7517260 & -1.5329130 \\ \mathrm{H} & 4.9467470 & -4.4842160 & -2.1803760 \\ \mathrm{H} & 3.0335250 & -6.1072270 & -2.0642710 \\ \mathrm{H} & 1.1103070 & -4.7310110 & -2.6706550 \\ \mathrm{H} & 4.2008530 & -2.8282830 & -0.5039080 \\ \mathrm{H} & 4.4299880 & -1.9993250 & -2.0430970 \\ \mathrm{H} & 3.6596750 & -4.0020840 & -3.2935070 \\ \mathrm{H} & 3.2354440 & -5.2589850 & -0.5227380 \\ \mathrm{H} & 0.8342740 & -5.2345900 & -1.0134900 \\ \mathrm{C} & 1.0875390 & -2.9927520 & 1.9797720 \\ \mathrm{O} & 0.1250480 & -2.1004400 & 1.9600620 \\ \mathrm{C} & 1.6157930 & -3.2906960 & 3.3488610 \\ \mathrm{C} & 2.6828090 & -4.1929750 & 3.4885410 \\ \mathrm{C} & 1.0841320 & -2.6672570 & 4.4905520 \\ \mathrm{C} & 3.2132430 & -4.4656680 & 4.7488880 \\ \mathrm{H} & 3.0896450 & -4.6680840 & 2.6004940 \\ \mathrm{C} & 1.6160820 & -2.9433360 & 5.7505310 \\ \mathrm{H} & 0.2630380 & -1.9654700 & 4.3841460 \\ \mathrm{C} & 2.6813910 & -3.8406500 & 5.8825650 \\ \mathrm{H} & 4.0418630 & -5.1630880 & 4.8488850 \\ \mathrm{H} & 1.2012540 & -2.4560950 & 6.6299630 \\ \mathrm{H} & 3.0966170 & -4.0517090 & 6.8657390 \\ \mathrm{H} & -0.2316600 & -1.8539210 & 1.0186010 \\ \mathrm{O} & 1.5609300 & -3.5652850 & 0.9849800 \\ \mathrm{I} & & & \\ & & & \end{array}$

\begin{tabular}{|c|c|c|c|}
\hline \multicolumn{4}{|c|}{ TS94 (R, R) G[B97-D/def2-TZVP] $=-3310.754246$} \\
\hline 0 & 0.4774720 & 0.7375520 & 0.1564840 \\
\hline $\mathrm{O}$ & -1.7579070 & 0.9434810 & -1.0452690 \\
\hline $\mathrm{C}$ & 0.6013120 & 2.1183530 & 0.1390720 \\
\hline & -0.4877590 & 2.9065460 & 0.5013830 \\
\hline & 1.8752960 & 2.6554000 & -0.1876540 \\
\hline & -0.3555530 & 4.3324410 & 0.4123610 \\
\hline & 2.0156120 & 4.0294040 & -0.1774910 \\
\hline & 0.9262310 & 4.8938170 & 0.0860590 \\
\hline & 2.9848840 & 4.4641930 & -0.4106760 \\
\hline & -2.3676780 & 1.3075090 & 0.1548870 \\
\hline & -3.6061770 & 0.6908310 & 0.4786450 \\
\hline & -1.7543500 & 2.2691240 & 0.9483760 \\
\hline & -4.2219210 & 1.0786280 & 1.6519230 \\
\hline & -2.3538610 & 2.5863270 & 2.2136190 \\
\hline & -3.6201760 & 1.9950710 & 2.5488290 \\
\hline & -5.1854860 & 0.6482530 & 1.9124850 \\
\hline & -3.6164040 & 3.1620550 & 4.6881240 \\
\hline & -2.3480720 & 3.7112670 & 4.3791120 \\
\hline & -1.7340600 & 3.4315510 & 3.1745270 \\
\hline & -4.2339600 & 2.3171000 & 3.7900010 \\
\hline & -4.0924920 & 3.3967070 & 5.6376750 \\
\hline & -1.8500370 & 4.3569040 & 430 \\
\hline & -0.7610050 & 3.857 & 2.9533090 \\
\hline & -5.198 & 1.86 & 4.0220590 \\
\hline & -1.2788010 & 6.589 & 0.5026120 \\
\hline & -0.0054130 & 7.141 & 0.2187130 \\
\hline & 1.071 & 6.3( & 0.0106090 \\
\hline & -1.4489980 & 5.22 & 0.5976430 \\
\hline & -2.1328650 & 7.22 & 0.62 \\
\hline & 0.1157010 & 8.220 & 0.1524160 \\
\hline & 2.0 & 6.71 & -0.2296370 \\
\hline & -2.431 & 4.8150 & 0.8126730 \\
\hline & 3.041 & 1.791 & -0.5390870 \\
\hline & 3.523 & 1.777 & -1.8648860 \\
\hline & 3.7057590 & 1.0453500 & 0.4656890 \\
\hline & 4.6956990 & 1.06 & -2.15 \\
\hline & 4.8657150 & 0.346 & 0.1236340 \\
\hline & 5.3887180 & 0.355 & -1.1751970 \\
\hline & 5.0639520 & 1.0643060 & -3.1760210 \\
\hline & 5.3938120 & -0.2211380 & 0.8869080 \\
\hline & -4.2207010 & -0.3384640 & -0.4107020 \\
\hline & -4.2787990 & -1.6903530 & 0.0100320 \\
\hline & -4.7539920 & 0.0359070 & -1.6625280 \\
\hline & -4.8958600 & -2.6268200 & -0.8244100 \\
\hline & & & -2.4628200 \\
\hline
\end{tabular}




\begin{tabular}{|c|c|c|}
\hline $\begin{array}{l}-5.4398420 \\
-4.9449310 \\
-5.7725410 \\
3.2306990 \\
6.6946080 \\
2.8292000 \\
-3.6994100 \\
-6.0791450 \\
-4.7352630 \\
-6.1665170 \\
-6.7542070 \\
-6.6798210 \\
-6.1400070 \\
-3.9957940 \\
-2.9736330 \\
-4.5184450 \\
-3.9525590 \\
-7.2382260 \\
-7.7087490 \\
-6.8686710 \\
-8.0006850 \\
-5.0303720 \\
-4.2188700 \\
-4.5914570 \\
-5.4940740 \\
-4.8157540 \\
-5.3806370 \\
-5.5189350 \\
-4.3873450 \\
-2.8838640 \\
-3.5343910 \\
-2.1358730 \\
-2.3703040 \\
3.8652080 \\
4.9580370 \\
3.6224650 \\
3.4956100 \\
3.4954930 \\
3.1171410 \\
4.5668420 \\
2.9905600 \\
7.8861500 \\
8.8329020 \\
7.9244100 \\
7.7874560 \\
6.8825930\end{array}$ & $\begin{array}{r}-2.2770370 \\
-3.6649400 \\
-0.6558300 \\
1.0731640 \\
-0.3743700 \\
2.5256640 \\
-2.1583460 \\
-3.3238710 \\
1.4725660 \\
2.0340400 \\
1.4553330 \\
1.9890790 \\
3.0796570 \\
1.5909320 \\
1.2067640 \\
1.0241810 \\
2.6426210 \\
-4.0507950 \\
-4.7734740 \\
-4.5986390 \\
-3.3385970 \\
-4.3380700 \\
-3.8307760 \\
-4.8788230 \\
-5.0722980 \\
-2.3494940 \\
-1.4243290 \\
-3.1232770 \\
-2.6669130 \\
-3.4592580 \\
-4.3158510 \\
-3.3716310 \\
-3.6649330 \\
2.2719780 \\
2.1629720 \\
3.2128270 \\
2.3268500 \\
-0.2315860 \\
-1.0993670 \\
-0.3811300 \\
-0.1984120 \\
0.4965360 \\
-0.0442930 \\
1.4169750 \\
0.7782800 \\
-0.8060820\end{array}$ & $\begin{array}{r}-2.0637990 \\
-0.5045880 \\
-3.4267010 \\
1.9134120 \\
-1.4509300 \\
-2.9969140 \\
1.3408450 \\
-2.9587410 \\
-2.1688390 \\
-2.2778270 \\
-3.0025390 \\
-1.3086910 \\
-2.6131150 \\
-3.5146560 \\
-3.4292810 \\
-4.2964860 \\
-3.8286250 \\
-2.2515750 \\
-2.9315280 \\
-1.3748450 \\
-1.9118840 \\
-3.4570240 \\
-3.9936220 \\
-2.6080910 \\
-4.1298930 \\
2.3873340 \\
2.5474420 \\
2.0505700 \\
3.3479480 \\
1.2132730 \\
0.9930950 \\
0.4201060 \\
2.1611260 \\
2.6479530 \\
2.6747640 \\
2.1396050 \\
3.6810110 \\
2.6799650 \\
2.1286820 \\
2.8665660 \\
3.6527290 \\
-0.9979730 \\
-1.1318520 \\
-1.5960300 \\
0.0574010 \\
-2.9126950\end{array}$ \\
\hline
\end{tabular}

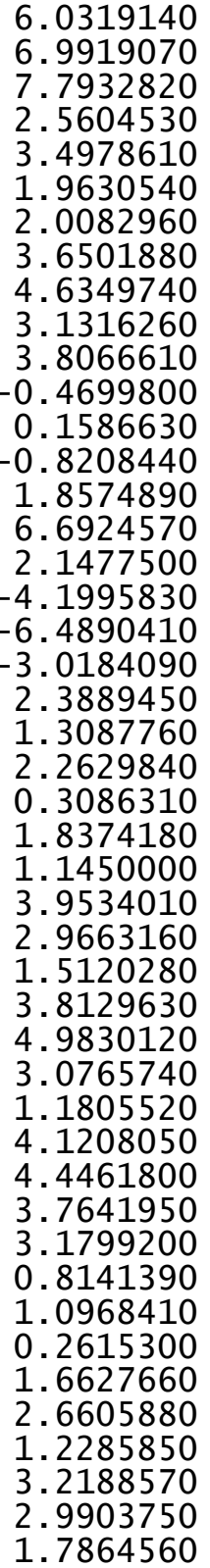

-1.3967830
0.0682590
-1.4102800
1.6237740
1.2765110
0.7536640
2.1905380
3.7608740
3.4536960
4.3099750
4.4400130
-0.0402990
-0.0725300
-1.3389120
2.8755490
-1.2812290
1.2243870
2.0951030
-2.8029280
-1.3831690
-2.1926710
-3.1898390
-1.3184990
-2.8223540
-1.9572850
-1.1941480
-4.0064330
-5.0700060
-4.5788190
-2.7052590
-4.3752720
-6.0032000
-4.5880080
-2.8717730
-1.9122240
-3.7984450
-5.2911010
-5.2270710
-3.1192950
-2.1072350
-3.4852550
-4.4710940
-2.8534700
-4.8183990
-4.9533760
-3.2060130
$-3.2745830$

$-3.5674300$

$-3.0086730$

$-4.2160200$

$-4.6694640$

$-3.9285810$

$-4.9772280$

$-3.4159930$

$-3.7935740$

$-4.2132490$

$-2.5691030$

$-0.9338670$

$-2.2954690$

$-0.2593580$

$-2.6344700$

$-0.8273170$

1.9167840

$-1.4456040$

$-3.8359350$

1.7062680

$-1.3954720$

$-1.3571360$

$-0.7513350$

$-1.1555670$

$-2.7319860$

$-2.6217040$

$-2.2438860$

$-1.7395050$

$-1.8528860$

$-1.4375560$

$-2.1612180$

$-2.3044070$

$-2.9023130$

$-0.3960760$

$-1.8409650$

$-3.3057100$

$-0.6858810$

$-1.3100600$

1.8655610

1.8957200

3. 2021450

3. 2744180

4. 3800900

4. 5041260

2. 3586860

5.6093200 


\begin{tabular}{|c|c|c|c|c|c|c|c|}
\hline $\mathrm{H}$ & 0.4606540 & -2.0883440 & 4.3253780 & $c$ & 4.7380040 & 1.2825220 & -0.7266050 \\
\hline C & 2.7824710 & -4.1866020 & 5.6742160 & $\mathrm{H}$ & 4.5206250 & 1.1702150 & -2.8540030 \\
\hline $\mathrm{H}$ & 3.9936210 & -5.5802580 & 4.5518820 & $\mathrm{H}$ & 4.6672090 & 1.5933540 & 1.3989120 \\
\hline $\mathrm{H}$ & 1.4458210 & -2.7140450 & 6.5175090 & $\mathrm{C}$ & -3.9506880 & -1.5108100 & 0.3379990 \\
\hline $\mathrm{H}$ & 3.2178370 & -4.4572640 & 6.6337920 & $\mathrm{C}$ & -3.5119690 & -2.5962750 & 1.1299960 \\
\hline $\mathrm{H}$ & -0.1438980 & -1.8426810 & 0.9783540 & $\mathrm{C}$ & -4.5393690 & -1.7468570 & -0.9250110 \\
\hline 0 & 1.4275250 & -3.7486130 & 0.8477030 & C & -3.6909280 & -3.8972780 & 0.6432110 \\
\hline \multirow{2}{*}{\multicolumn{4}{|c|}{$\begin{array}{l}144 \\
\text { TS95 (R, R) G[B97-D/def2-TZVP }]=-3310.754223\end{array}$}} & $\mathrm{C}$ & -4.6855130 & -3.0639880 & -1.3687990 \\
\hline & & & & $\mathrm{C}$ & -4.2668740 & -4.1542870 & -0.6016820 \\
\hline 0 & 0.0573460 & 1.0796600 & 0.1977590 & $\mathrm{H}$ & -3.3554160 & -4.7399810 & 1.2447980 \\
\hline 0 & -2.0850130 & 0.2229330 & -0.8531350 & $\mathrm{H}$ & -5.1367510 & -3.2409590 & -2.3426580 \\
\hline C & -0.2330790 & 2.3708060 & -0.2226710 & C & 2.3502440 & 2.8908860 & 1.7831640 \\
\hline $\mathrm{C}$ & -1.5221830 & 2.8634780 & -0.0563940 & $\mathrm{C}$ & 6.1083160 & 0.6261510 & -0.7371360 \\
\hline C & 0.8445810 & 3.1420480 & -0.7403030 & C & 2.1450390 & 2.4019000 & -3.3218450 \\
\hline $\mathrm{C}$ & -1.8149680 & 4.1686970 & -0.5755880 & $\mathrm{C}$ & -2.8655920 & -2.3998660 & 2.4964640 \\
\hline $\mathrm{C}$ & 0.5715950 & 4.4304110 & -1.1482630 & $\mathrm{c}$ & -4.3955830 & -5.5746440 & -1.1224770 \\
\hline C & -0.7432480 & 4.9608070 & -1.1152810 & C & -5.0558060 & -0.6187240 & -1.8089470 \\
\hline $\mathrm{H}$ & 1.3786650 & 5.0590060 & -1.5151540 & $\mathrm{C}$ & -6.5920340 & -0.6802750 & -1.9229310 \\
\hline C & -2.7882670 & 0.7324460 & 0.2307810 & $\mathrm{H}$ & -6.9049750 & -1.6184190 & -2.4002970 \\
\hline C & -3.7634970 & -0.1127780 & 0.8254280 & $\mathrm{H}$ & -7.0618400 & -0.6288170 & -0.9322540 \\
\hline $\mathrm{C}$ & -2.5436810 & 2.0376220 & 0.6423690 & $\mathrm{H}$ & -6.9630670 & 0.1559900 & -2.5309190 \\
\hline C & -4.5049570 & 0.4012910 & 1.8689820 & C & -4.4035110 & -0.6330140 & -3.2033970 \\
\hline $\mathrm{C}$ & -3.2527540 & 2.5263900 & 1.7920480 & $\mathrm{H}$ & -3.3134390 & -0.5636060 & -3.1222380 \\
\hline $\mathrm{C}$ & -4.2654480 & 1.6976090 & 2.3889640 & $\mathrm{H}$ & -4.6564960 & -1.5549030 & -3.7437220 \\
\hline $\mathrm{H}$ & -5.2729590 & -0.2151320 & 2.3297230 & $\mathrm{H}$ & -4.7668840 & 0.2171850 & -3.7961240 \\
\hline C & -4.7043740 & 3.4112360 & 4.0655570 & C & -3.4491280 & -5.8053360 & -2.3175190 \\
\hline C & -3.6758430 & 4.2095070 & 3.5088980 & $\mathrm{H}$ & -3.5193530 & -6.8431010 & -2.6712180 \\
\hline C & -2.9699730 & 3.7790440 & 2.4030800 & $\mathrm{H}$ & -3.7174530 & -5.1392190 & -3.1481090 \\
\hline $\mathrm{C}$ & -4.9857270 & 2.1778550 & 3.5163990 & $\mathrm{H}$ & -2.4084000 & -5.5972660 & -2.0382550 \\
\hline $\mathrm{H}$ & -5.2577170 & 3.7656400 & 4.9324630 & C & -5.8451080 & -5.9236360 & -1.5072800 \\
\hline $\mathrm{H}$ & -3.4349950 & 5.1699570 & 3.9596590 & $\mathrm{H}$ & -6.5238530 & -5.7782020 & -0.6575450 \\
\hline $\mathrm{H}$ & -2.1816810 & 4.4012550 & 1.9931170 & $\mathrm{H}$ & -6.1897620 & -5.2861490 & -2.3319990 \\
\hline $\mathrm{H}$ & -5.7560690 & 1.5403990 & 3.9472590 & $\mathrm{H}$ & -5.9116960 & -6.9691860 & -1.8366010 \\
\hline C & -3.3721240 & 5.9662680 & -1.1187750 & C & -3.8890010 & -2.6595770 & 3.6204190 \\
\hline C & -2.3093480 & 6.7597680 & -1.6151080 & $\mathrm{H}$ & -4.7595090 & -1.9996410 & 3.5283470 \\
\hline C & -1.0229830 & 6.2614570 & -1.6149010 & $\mathrm{H}$ & -4.2431110 & -3.6985040 & 3.5750500 \\
\hline C & -3.1306640 & 4.7051470 & -0.6114250 & $\mathrm{H}$ & -3.4278350 & -2.4939820 & 4.6037170 \\
\hline $\mathrm{H}$ & -4.3891470 & 6.3518510 & -1.1417180 & C & -1.6202770 & -3.2816560 & 2.7076230 \\
\hline $\mathrm{H}$ & -2.5120400 & 7.7545150 & -2.0059320 & $\mathrm{H}$ & -1.8911030 & -4.3431130 & 2.7818050 \\
\hline $\mathrm{H}$ & -0.1986090 & 6.8523560 & -2.0107280 & $\mathrm{H}$ & -0.9125320 & -3.1552090 & 1.8833770 \\
\hline H & -3.9537060 & 4.1053300 & -0.2362110 & $\mathrm{H}$ & -1.1249550 & -2.9988030 & 3.6457880 \\
\hline C & 2.2170970 & 2.5535390 & -0.7615980 & C & 3.1598920 & 4.1231850 & 2.2338990 \\
\hline C & 2.8084130 & 2.1351970 & -1.9761180 & $\mathrm{H}$ & 4.2092710 & 3.8489760 & 2.4066280 \\
\hline C & 2.8990410 & 2.3660670 & 0.4616650 & $\mathrm{H}$ & 3.1339490 & 4.9094330 & 1.4686180 \\
\hline , & 4.0585670 & 1.5062830 & -1.9278780 & $\mathrm{H}$ & 2.7499310 & 4.5297270 & 3.1684870 \\
\hline$C$ & 4.1473510 & 1.73487 & 0.4548400 & $\mathrm{C}$ & 2.3232460 & 1.8196100 & 2.8850540 \\
\hline
\end{tabular}




$\begin{array}{rrr}1.7575100 & 0.9415140 & 2.5582240 \\ 3.3397860 & 1.4987130 & 3.1456710 \\ 1.8559200 & 2.2278330 & 3.7910880 \\ 6.3441270 & -0.3214650 & 0.4502590 \\ 7.2979580 & -0.8500700 & 0.3261370 \\ 6.3928000 & 0.2344210 & 1.3948110 \\ 5.5429580 & -1.0629240 & 0.5425230 \\ 7.2124840 & 1.7024060 & -0.7891100 \\ 7.0836320 & 2.3547220 & -1.6617270 \\ 7.1712450 & 2.3267680 & 0.1136910 \\ 8.2056450 & 1.2354510 & -0.8418240 \\ 2.3192840 & 1.2663910 & -4.3430130 \\ 3.3659550 & 1.1715420 & -4.6617510 \\ 1.9912330 & 0.3085930 & -3.9323200 \\ 1.7212050 & 1.4855600 & -5.2367210 \\ 2.6889240 & 3.7163380 & -3.9219110 \\ 3.7639210 & 3.6173690 & -4.1260390 \\ 2.1771540 & 3.9443960 & -4.8668030 \\ 2.5505980 & 4.5611750 & -3.2385320 \\ -0.5188660 & -0.1754620 & -0.6786050 \\ 0.0307150 & -0.1921410 & -2.0769240 \\ -0.3291750 & -1.3776680 & 0.2055590 \\ 1.0700440 & 2.5240610 & -3.1494870 \\ 6.1770220 & 0.0353010 & -1.6619230 \\ 1.3189360 & 3.2223630 & 1.6297100 \\ -4.8044880 & 0.3384620 & -1.3408040 \\ -4.0876520 & -6.2509340 & -0.3119280 \\ -2.5389310 & -1.3573880 & 2.5725480 \\ 2.6074680 & -1.9110530 & -1.6832130 \\ 1.7623130 & -3.0624050 & -1.3239160 \\ 2.4826070 & -1.0147590 & -1.0693990 \\ 0.7883390 & -2.8421930 & -0.9007740 \\ 1.7201480 & -1.8865420 & -2.8408400 \\ 0.9674350 & -1.2061550 & -2.5821580 \\ 4.2270730 & -3.5095270 & -2.8103760 \\ 3.5867990 & -4.6689900 & -2.0306250 \\ 2.0811150 & -4.4345980 & -1.8134310 \\ 4.0513410 & -2.1835220 & -2.0520800 \\ 5.2957840 & -3.7021350 & -2.9641040 \\ 3.7311520 & -5.6184060 & -2.5596580 \\ 1.5298320 & -4.5685150 & -2.7559750 \\ 4.6198090 & -2.2134270 & -1.1134170 \\ 4.4250320 & -1.3319340 & -2.6314370 \\ 3.7617620 & -3.4279620 & -3.8017250 \\ 4.0704460 & -4.7565530 & -1.0494460 \\ 1.6517690 & -5.1497390 & -1.1026160\end{array}$

\begin{abstract}
2. 5746520
1. 6928590

3. 7422060

4.8113770

3. 8110560

5. 9380510

4.7490540

4.9391670

2. 9877810

6.0051330

6.7652770

4.9885030

6.8858940

0.8927670

2. 4964000
\end{abstract}

144

TS96 $(R, R)$ G $[B 97-D /$ def2-TZVP $]=-3310.753983$

$\begin{array}{lrrr}\mathrm{O} & 0.4651770 & 0.8645350 & 0.3854080 \\ \mathrm{O} & -1.7563260 & 0.8423690 & -0.8564470 \\ \mathrm{C} & 0.4078130 & 2.2518630 & 0.3969860 \\ \mathrm{C} & -0.7696820 & 2.8777030 & 0.7948640 \\ \mathrm{C} & 1.5856270 & 2.9625410 & 0.0412580 \\ \mathrm{C} & -0.8262030 & 4.3108870 & 0.7580770 \\ \mathrm{C} & 1.5490910 & 4.3411240 & 0.1061450 \\ \mathrm{C} & 0.3663170 & 5.0459820 & 0.4362520 \\ \mathrm{H} & 2.4447370 & 4.9065480 & -0.1392390 \\ \mathrm{C} & -2.4230650 & 1.0724070 & 0.3474900 \\ \mathrm{C} & -3.5820520 & 0.2957210 & 0.6170000 \\ \mathrm{C} & -1.9440550 & 2.0610670 & 1.1987870 \\ \mathrm{C} & -4.2503690 & 0.5391400 & 1.7998760 \\ \mathrm{C} & -2.5919320 & 2.2367350 & 2.4668150 \\ \mathrm{C} & -3.7754460 & 1.4731520 & 2.7533710 \\ \mathrm{H} & -5.1577910 & -0.0174310 & 2.0194500 \\ \mathrm{C} & -3.9386380 & 2.5231220 & 4.9462890 \\ \mathrm{C} & -2.7494720 & 3.2458300 & 4.6826210 \\ \mathrm{C} & -2.0944580 & 3.1066210 & 3.4753980 \\ \mathrm{C} & -4.4352810 & 1.6515120 & 3.9997550 \\ \mathrm{H} & -4.4487970 & 2.6472180 & 5.8990290 \\ \mathrm{H} & -2.3456480 & 3.9145010 & 5.4398360 \\ \mathrm{H} & -1.1831650 & 3.6651300 & 3.2885470 \\ \mathrm{H} & -5.3359150 & 1.0721500 & 4.1962460 \\ \mathrm{C} & -2.0311440 & 6.4247930 & 0.9466470 \\ \mathrm{C} & -0.8439200 & 7.1473360 & 0.6734630 \\ \mathrm{C} & 0.3280760 & 6.4669790 & 0.4183130 \\ \mathrm{C} & -2.0224790 & 5.0450380 & 0.9879960 \\ \mathrm{H} & -2.9611280 & 6.9618670 & 1.1205120\end{array}$

1. 5928740

1. 7494980

2. 5210180

2. 3421860

3. 5378500

3. 1586880

1. 5537340

4.3547150

3. 6735860

4.1651100

3. 0102910

5.1377740

4.8003710

1.0931500

0.7456700
$-2.9611280$

6.9618670

1. 1205120

$-4.4345980$

$-4.5685150$

$-1.3319340$

$-5.1497390$

$-1.1026160$ 


$\begin{array}{lr}\mathrm{H} & -0.8636480 \\ \mathrm{H} & 1.2439420 \\ \mathrm{H} & -2.9418420 \\ \mathrm{C} & 2.8210200 \\ \mathrm{C} & 3.2031230 \\ \mathrm{C} & 3.6253810 \\ \mathrm{C} & 4.4033850 \\ \mathrm{C} & 4.8109840 \\ \mathrm{C} & 5.2234850 \\ \mathrm{H} & 4.6961040 \\ \mathrm{H} & 5.4359850 \\ \mathrm{C} & -4.0696940 \\ \mathrm{C} & -3.9749000 \\ \mathrm{C} & -4.6443400 \\ \mathrm{C} & -4.4846310 \\ \mathrm{C} & -5.1305800 \\ \mathrm{C} & -5.0646540 \\ \mathrm{H} & -4.4205330 \\ \mathrm{H} & -5.5788550 \\ \mathrm{C} & 3.2767920 \\ \mathrm{C} & 6.5472520 \\ \mathrm{C} & 2.3644980 \\ \mathrm{C} & -3.3518340 \\ \mathrm{C} & -5.5974730 \\ \mathrm{C} & -4.7925790 \\ \mathrm{C} & -6.2799910 \\ \mathrm{H} & -6.7955130 \\ \mathrm{H} & -6.7853360 \\ \mathrm{H} & -6.3773840 \\ \mathrm{C} & -4.0695340 \\ \mathrm{H} & -3.0096930 \\ \mathrm{H} & -4.5196000 \\ \mathrm{H} & -4.1485710 \\ \mathrm{C} & -4.8149670 \\ \mathrm{H} & -5.1839150 \\ \mathrm{H} & -4.9360120 \\ \mathrm{H} & -3.7436020 \\ \mathrm{C} & -7.1047650 \\ \mathrm{H} & -7.6711440 \\ \mathrm{H} & -7.2955320 \\ \mathrm{H} & -7.4795000 \\ \mathrm{C} & -4.4442910 \\ \mathrm{H} & -5.0998890 \\ \mathrm{H} & -5.0657120 \\ \mathrm{H} & -3.9870440 \\ \mathrm{C} & -2.4092030 \\ & \end{array}$

$\begin{array}{rr}8.2347060 & 0.6505370 \\ 7.0064640 & 0.1826080 \\ 4.5069340 & 1.1935270 \\ 2.2589110 & -0.4144140 \\ 2.3325370 & -1.7747780 \\ 1.5556840 & 0.5084710 \\ 1.7327490 & -2.1722180 \\ 0.9657800 & 0.0576580 \\ 1.0470650 & -1.2741700 \\ 1.7967030 & -3.2164110 \\ 0.4261370 & 0.7680130 \\ -0.7305350 & -0.3509950 \\ -2.1062070 & -0.0377070 \\ -0.3207050 & -1.5761670 \\ -3.0376880 & -0.9514860 \\ -1.2917540 & -2.4560070 \\ -2.6558320 & -2.1620350 \\ -4.0989850 & -0.7188170 \\ -0.9705790 & -3.3939910 \\ 1.4592120 & 1.9874310 \\ 0.4346000 & -1.7004370 \\ 3.0616820 & -2.8185550 \\ -2.6091160 & 1.2598960 \\ -3.6922390 & -3.1349320 \\ 1.1454500 & -1.9642030 \\ 1.5423680 & -2.0426500 \\ 0.9586230 & -2.8165920 \\ 1.3605290 & -1.0853510 \\ 2.6074550 & -2.2931750 \\ 1.4593970 & -3.2869240 \\ 1.1911290 & -3.2198860 \\ 0.9013900 & -4.1185700 \\ 2.5309230 & -3.5142880 \\ -3.6673300 & -4.4620870 \\ -4.4481380 & -5.1409660 \\ -2.6959460 & -4.9595480 \\ -3.8284770 & -4.2887000 \\ -3.5044620 & -3.3915130 \\ -3.5521940 & -2.4529180 \\ -2.5275750 & -3.8548130 \\ -4.2831770 & -4.0692910 \\ -2.9856400 & 2.2814410 \\ -2.1355590 & 2.5020090 \\ -3.8010660 & 1.8867290 \\ -3.3245390 & 3.2212150 \\ -3.8084880 & 1.0465410\end{array}$
$-2.9669480$
$-1.6639700$
$-1.8888910$
4. 2904430
5. 2977530
4. 3261010
4. 0128230
3. 1875080
2. 4223290
4. 1493020
2. 9190600
7.6620060
8.6316040
7.4417360
7.7395900
6.4923450
5. 6753960
6. 3387830
7. 4395130
2. 1592870
3. 1071340
1. 7160490
1.4833790
2. 9952090
3. 9954740
2. 3757230
3. 0956600
$-0.3570990$
0.3019770
$-0.5688340$
1. 3727110
6. 8016640
2. 2932810
$-4.3360890$
$-5.4524670$
$-2.7527290$
2.6209870
1. 6337230
2. 3198660
0.5885750
2.2779230
1.4930560
4.4450500
3. 4769990
2. 0249840
4.0657010

-4.7054240
-3.5848370
-4.0371770
2.2525620
1.8291210
3.3035130
2.2129890
-0.0010310
-0.5459800
-0.5165740
-0.0360370
1.4999840
1.0544100
2.2980430
1.9526540
-0.2409560
-0.9694550
0.5009720
-0.7566560
2.2397130
2.0842220
1.2662940
2.7812830
4.4270240
4.2846900
4.9606300
5.0550780
0.0199030
0.1237610
-1.3410910
3.2451170
-0.3356400
1.9129460
1.7660360
-4.6805160
-1.7978830
-1.9292770
-2.9622150
-1.2125120
-2.6950640
-1.4531570
-0.7837190
-3.4479380
-4.6376800
-4.2010570
-2.3662600

0.2780010

1.9852160

2.8353530

2.7265410

2. 5212750

3. 8972480

2.4635410

1. 9029560

2. 3412900

3. 5275020

$-1.6652260$

$-1.9265900$

$-2.3870140$

$-0.6688830$

$-3.0810120$

$-3.1359280$

$-3.8746710$

$-3.2847850$

$-4.1041500$

$-4.6358950$

$-3.8752110$

$-4.7788870$

$-3.1596130$

$-3.5907210$

$-3.8930800$

$-2.2666400$

$-0.7553370$

$-2.0992140$

$-0.1528850$

$-2.3927530$

$-0.9567550$

2. 1456990

$-1.1869670$

$-2.6750470$

1. 6858310

$-1.1693710$

$-1.5155050$

$-0.4020080$

$-1.4087000$

$-2.5095940$

$-2.3924070$

$-2.0673800$

$-1.9809300$

$-2.2447380$

$-1.0435220$ 


$\begin{array}{llll}\mathrm{H} & 5.4730610 & -3.7810340 & -1.8805310 \\ \mathrm{H} & 3.7545510 & -5.4184360 & -2.6989020 \\ \mathrm{H} & 1.8680340 & -3.9989520 & -3.3147750 \\ \mathrm{H} & 4.1829460 & -2.7615820 & -0.0246860 \\ \mathrm{H} & 4.7033810 & -1.4838380 & -1.1306500 \\ \mathrm{H} & 4.4187780 & -3.0186480 & -3.0777380 \\ \mathrm{H} & 3.5316230 & -5.0729870 & -0.9751560 \\ \mathrm{H} & 1.3057410 & -4.9829010 & -1.9745270 \\ \mathrm{C} & 1.3320630 & -3.4554810 & 1.6004700 \\ \mathrm{O} & 0.6104880 & -2.3791470 & 1.8088210 \\ \mathrm{C} & 1.9821960 & -4.0083140 & 2.8285540 \\ \mathrm{C} & 2.7999790 & -5.1452240 & 2.7159190 \\ \mathrm{C} & 1.8070150 & -3.4045160 & 4.0858410 \\ \mathrm{C} & 3.4329700 & -5.6707570 & 3.8417360 \\ \mathrm{H} & 2.9278220 & -5.6063150 & 1.7408140 \\ \mathrm{C} & 2.4435390 & -3.9318900 & 5.2098920 \\ \mathrm{H} & 1.1778070 & -2.5246250 & 4.1733220 \\ \mathrm{C} & 3.2568840 & -5.0640770 & 5.0907630 \\ \mathrm{H} & 4.0636450 & -6.5518180 & 3.7473500 \\ \mathrm{H} & 2.3057760 & -3.4591400 & 6.1795900 \\ \mathrm{H} & 3.7517000 & -5.4732750 & 5.9689960 \\ \mathrm{H} & 0.1463940 & -1.9949470 & 0.9596850 \\ \mathrm{O} & 1.4976190 & -3.9986380 & 0.4959870 \\ 14 & & & \end{array}$

144

$\begin{array}{lrcr}\text { TS97 (R, R) G }[\mathrm{B} 97-\mathrm{D} / \mathrm{def}-\mathrm{TZV}]=-3310.753199 \\ \mathrm{O} & 0.4945160 & 0.6282610 & 0.0752060 \\ \mathrm{O} & -1.8200120 & 0.8115650 & -0.9831250 \\ \mathrm{C} & 0.5964240 & 2.0103050 & 0.0704380 \\ \mathrm{C} & -0.4826750 & 2.7780040 & 0.4997100 \\ \mathrm{C} & 1.8445740 & 2.5724020 & -0.3081440 \\ \mathrm{C} & -0.3807870 & 4.2061480 & 0.4155300 \\ \mathrm{C} & 1.9595210 & 3.9490510 & -0.3005380 \\ \mathrm{C} & 0.8702780 & 4.7927510 & 0.0221710 \\ \mathrm{H} & 2.9096240 & 4.4015420 & -0.5757030 \\ \mathrm{C} & -2.3609550 & 1.1593840 & 0.2556270 \\ \mathrm{C} & -3.5724700 & 0.5298200 & 0.6498540 \\ \mathrm{C} & -1.7080710 & 2.1174430 & 1.0197770 \\ \mathrm{C} & -4.1080040 & 0.8884260 & 1.8705610 \\ \mathrm{C} & -2.2223140 & 2.4089070 & 2.3277280 \\ \mathrm{C} & -3.4535980 & 1.7939920 & 2.7416290 \\ \mathrm{H} & -5.0488110 & 0.4453270 & 2.1870200 \\ \mathrm{C} & -3.3109210 & 2.9232760 & 4.8962300 \\ \mathrm{C} & -2.0760640 & 3.4969950 & 4.5067620 \\ \mathrm{C} & -1.5467010 & 3.2474300 & 3.2562650 \\ \mathrm{C} & -3.9805650 & 2.0851700 & 4.0294150 \\ \mathrm{H} & -3.7204320 & 3.1344750 & 5.8816610\end{array}$

-1.5364780
-0.5972250
-4.9199540
-1.3367430
-0.0914930
0.9868410
-1.4774400
-2.1928120
0.0065000
1.9452000
-2.4389620
3.0189820
3.4622790
3.7380600
4.6507940
4.9099150
5.3957720
4.9862250
5.4761220
-4.2492680
-4.2776890
-4.8766190
-4.9549220
-5.5335630
-5.5863700
-4.9837420
-6.0184080
3.2972230
6.7108130
2.7058200
-3.6118710
-6.3097230
-4.8992120
-6.3350860
-6.9804660
-6.7668900
-6.3372250
-4.2750120
-3.2473610
-4.8568610
-4.2629740
-5.7077760
-6.2132260
-5.8298610
-4.6357940
-7.8200580

4.1377920

3. 6904930

1.6187420

6.4451850

7.0216460

6. 2076220

5. 0751820

7.0881610

8. 1030920

6.6341710

4. 6479360

1.7366680

1.7369770

1.0156990

1. 0643770

0.3568970

0.3822160

1.0742890

$-0.1943370$

$-0.4712210$

$-1.8355600$

$-0.0475360$

$-2.7406860$

$-0.9928910$

$-2.3443520$

$-3.7932840$

$-0.6620910$

1. 0219300

$-0.3160290$

2. 4508190

$-2.3519200$

$-3.3512530$

1. 4101080

1.9697840

1.4238870

1.8769810

3. 0303850

1. 5914570

1.2126660

1.0536270

2. 6556500

$-3.4047280$

$-4.1710970$

$-2.4376570$

-3.6352150
-3.0520530
5.2009220

2.9747900

4.3216330

0.5767870

0.2249610

$-0.0508030$

0.6704360

0.7698080

0.1604930

$-0.3423740$

0.9359820

$-0.6969770$

$-2.0353140$

0.2895410

$-2.3586240$

$-0.0865020$

$-1.3999140$

$-3.3918160$

0.6606800

$-0.2260960$

0.1444610

$-1.4202460$

$-0.6830510$

$-2.2126360$

$-1.8627430$

$-0.4074010$

$-3.1287290$

1.7490230

$-1.7103420$

$-3.1483490$

1. 4151910

$-2.7391420$

$-1.8637570$

$-1.8272540$

$-2.5280840$

$-0.8223900$

-2. 1128150

$-3.2593600$

$-3.2744460$

$-4.0194790$

$-3.5306280$

$-4.1558120$

$-4.7587810$

$-4.6612200$

$-4.1185430$

$-2.8041190$ 


$\begin{array}{rrr}-8.2630090 & -3.0480220 & -1.8001430 \\ -7.9952400 & -2.0674210 & -3.2575430 \\ -8.3360880 & -3.8063890 & -3.4134470 \\ -4.6584180 & -2.5771930 & 2.5254050 \\ -5.2096440 & -1.6570400 & 2.7502280 \\ -5.3831880 & -3.3402040 & 2.2104440 \\ -4.1696670 & -2.9243100 & 3.4460860 \\ -2.8117760 & -3.6486500 & 1.1886410 \\ -3.4785990 & -4.4950150 & 0.9783830 \\ -2.1149250 & -3.5314890 & 0.3535290 \\ -2.2407660 & -3.8900800 & 2.0942750 \\ 3.8240660 & 2.2893220 & 2.4538950 \\ 4.9226330 & 2.2884430 & 2.4595190 \\ 3.4797140 & 3.1945950 & 1.9404520 \\ 3.4715730 & 2.3213040 & 3.4938620 \\ 3.7107470 & -0.2353920 & 2.5294820 \\ 3.4305990 & -1.1484270 & 1.9926250 \\ 4.7939400 & -0.2616020 & 2.7067500 \\ 3.2129400 & -0.2455990 & 3.5060050 \\ 7.8934080 & 0.5515940 & -1.2283810 \\ 8.8460990 & 0.0288970 & -1.3890530 \\ 7.9167970 & 1.4961890 & -1.7883320 \\ 7.7976700 & 0.7887310 & -0.1619190 \\ 6.8988370 & -0.6885940 & -3.1877070 \\ 6.0570280 & -1.2802280 & -3.5683860 \\ 6.9900830 & 0.2115770 & -3.8094910 \\ 7.8190690 & -1.2731430 & -3.3099950 \\ 2.4028690 & 1.5159670 & -4.3344000 \\ 3.3280140 & 1.1732980 & -4.8160160 \\ 1.8329250 & 0.6428930 & -4.0018770 \\ 1.8099540 & 2.0535330 & -5.0859790 \\ 3.4825230 & 3.6932340 & -3.6260670 \\ 4.4571620 & 3.3991460 & -4.0387290 \\ 2.9200280 & 4.2163420 & -4.4109550 \\ 3.6598890 & 4.3922010 & -2.7993890 \\ -0.5210580 & -0.1643160 & -0.9441400 \\ 0.0236580 & -0.2163630 & -2.3402910 \\ -0.8226490 & -1.4546590 & -0.2298830 \\ 1.7443330 & 2.7900180 & -2.7502330 \\ 6.7231980 & -1.2463400 & -1.1223840 \\ 2.2044800 & 1.0628480 & 1.7747900 \\ -4.3061380 & 2.0037540 & -1.1613440 \\ -6.1807420 & -4.3411910 & -2.2780230 \\ -2.9050500 & -1.5918060 & 1.7623600 \\ 2.4242920 & -2.1657480 & -1.5215330 \\ 1.4108830 & -3.2050190 & -1.2824180\end{array}$

2.3134750
0.4123770
1.7301670
1.0177060
3.9996040
3.1338340
1.6495930
3.8639980
5.0525790
3.2454980
1.2232420
4.2895570
4.4026670
3.6910040
3.4607770
1.0429850
1.2732500
0.2649420
1.8508080
2.9646270
1.3185780
3.5412150
3.3715530
1.8964630
0.4608860
3.0086960
4.4060940
1.4807710
3.4597080
-0.1291310
1.7501670
1.9
$-1.2403830$
$-2.8696840$
$-2.0847120$
$-1.3452680$
$-3.9824940$
$-5.0304580$
$-4.6268380$
$-2.6112120$
$-4.2892290$
$-6.0151880$
$-4.7630120$
$-2.6484200$
$-1.8351270$
$-3.9010630$
$-5.1200180$
$-5.2474140$
$-2.9478610$
$-2.1072280$
$-3.2050770$
$-4.0517910$
$-2.5961360$
$-4.2826570$
$-4.5162680$
$-2.8306490$
$-1.9373550$
$-3.6717800$
$-4.9366930$
$-2.3545260$
$-3.8504410$
$-3.5033660$

$-0.9505300$

$-1.0249570$

$-2.8111970$

$-2.6949620$

$-2.3500580$

$-1.6353180$

$-1.6533470$

$-1.6696280$

$-2.3407280$

$-2.1043510$

$-2.6590890$

$-0.6579910$

$-2.2179790$

$-3.4011230$

$-0.5912650$

$-0.9831360$

1. 9619050

1. 9609670

3. 3192820

3. 4397230

4.4684960

4.6880810

2. 5460760

5. 7165740

4.3772100

5.8291740

4.7730050

6.6016710

6.8029450

1.0278370

0.9592940

144 TS98 $(R, R)$ G $[B 97-D /$ def2-TZVP $]=-3310.753158$

\begin{tabular}{|c|c|c|c|}
\hline & R) $\mathrm{G}[\mathrm{B} 97-\mathrm{D} / \mathrm{C}$ & {$[\mathrm{VP}]=-33$} & \\
\hline 0 & -0.0146480 & 1.1540100 & 0.2115820 \\
\hline 0 & -2.0799880 & 0.1259690 & -0.839494 \\
\hline & -0.3375560 & 2.3984830 & -0.3128970 \\
\hline & -1.6532390 & 2.8428960 & -0.2464320 \\
\hline & 0.73 & 3.1782700 & -0.8290700 \\
\hline & -1.9740710 & 4.0858820 & -0.8868690 \\
\hline & 0.4253390 & 4.4113490 & -1.3654170 \\
\hline & -0.9114390 & 4.8780200 & -1.4444430 \\
\hline & 1.2240450 & 5.0417440 & -1.7 \\
\hline & -2.83 & 0.6857690 & 0.1 \\
\hline & 70 & -0.1589020 & \\
\hline & & 2.033 & \\
\hline & & 0 & \\
\hline
\end{tabular}




$\begin{array}{rrr}-3.4385070 & 2.5850910 & 1.5620050 \\ -4.4261420 & 1.7590140 & 2.2036170 \\ -5.3224180 & -0.2060060 & 2.3021560 \\ -5.0181750 & 3.5934990 & 3.6951660 \\ -4.0139130 & 4.3933610 & 3.0981520 \\ -3.2454210 & 3.9027620 & 2.0612620 \\ -5.2122290 & 2.3003090 & 3.2570070 \\ -5.6212120 & 3.9949910 & 4.5066220 \\ -3.8413840 & 5.4037200 & 3.4629250 \\ -2.4774940 & 4.5290620 & 1.6202870 \\ -5.9620250 & 1.6625490 & 3.7222600 \\ -3.5785200 & 5.7563610 & -1.6521100 \\ -2.5275120 & 6.5527910 & -2.1684290 \\ -1.2225730 & 6.1167510 & -2.0680460 \\ -3.3080860 & 4.5555630 & -1.0266310 \\ -4.6086860 & 6.0911720 & -1.7529970 \\ -2.7530970 & 7.5000660 & -2.6533530 \\ -0.4064400 & 6.7105610 & -2.4764900 \\ -4.1210720 & 3.9518520 & -0.6357390 \\ 2.1370640 & 2.6822200 & -0.7173430 \\ 2.8520040 & 2.2625680 & -1.8628420 \\ 2.7485120 & 2.6304830 & 0.5550250 \\ 4.1677400 & 1.8130700 & -1.7036580 \\ 4.0641240 & 2.1631400 & 0.6611990 \\ 4.7942070 & 1.7509290 & -0.4548010 \\ 4.7305970 & 1.4999770 & -2.5814690 \\ 4.5279710 & 2.1351150 & 1.6430860 \\ -3.8514980 & -1.6077350 & 0.4867270 \\ -3.3284350 & -2.5774630 & 1.3727150 \\ -4.4197760 & -2.0034880 & -0.7443460 \\ -3.4074030 & -3.9275950 & 1.0089820 \\ -4.4649830 & -3.3635000 & -1.0645530 \\ -3.9620530 & -4.3416910 & -0.2032990 \\ -3.0058890 & -4.6842510 & 1.6803700 \\ -4.9016490 & -3.6638170 & -2.0146810 \\ 2.0548050 & 3.1399200 & 1.8131170 \\ 6.2106130 & 1.2039680 & -0.3576710 \\ 2.2486970 & 2.3289280 & -3.2602650 \\ -2.6945590 & -2.1998130 & 2.7065450 \\ -3.9653010 & -5.8090670 & -0.5941240 \\ -5.0091040 & -1.0024180 & -1.7291830 \\ -6.5338730 & -1.1919500 & -1.8506140 \\ -6.7673030 & -2.1906510 & -2.2431460 \\ -7.0210400 & -1.0877650 & -0.8725350 \\ -6.9579940 & -0.4452010 & -2.5354380 \\ -4.3336210 & -1.0921840 & -3.1098860\end{array}$

-3.2524920
-4.5131220
-4.7429790
-2.9531620
-2.9224820
-3.2401570
-1.9458160
-5.3660430
-6.0887560
-5.7230050
-5.3396590
-3.7104910
-4.6057790
-4.0217540
-3.2587590
-1.4181070
-1.6500990
-0.7152110
-0.9340130
2.6689030
3.7320590
2.5892540
2.1518300
2.0937130
1.6818180
3.1225840
1.5057100
6.1848340
7.2066030
5.6562510
5.6716780
6.9945210
6.9940990
6.5611700
8.0335430
2.5206800
3.5894720
2.1802810
1.9828150
2.7649010
3.8542850
2.3029140
2.5392230
-0.5001370
0.0957240
-0.2795330
-0.9407930
-2.0731020
-0.3241360
-6.0666930
-7.1345000
-5.5036230
-5.7454880
-6.3050590
-6.1384520
-5.7732570
-7.3778390
-2.3668500
-1.7572450
-3.4176870
-2.0701330
-3.0017180
-4.0539140
-2.9565120
-2.5833830
4.4860890
4.3603700
5.2284640
4.8744970
2.1240310
1.1601210
1.9686920
2.4995220
-0.3387800
-0.7423970
-0.7167400
-0.7096680
1.7059480
2.8019250
1.3135140
1.3568960
1.0674510
0.9609140
0.1698130
1.1359820
3.5755030
3.5144980
3.6428540
4.4953990
-0.1753820
-0.2423380
-1.3137060
$-3.0205540$

$-3.5695510$

$-3.7797070$

$-1.7291010$

$-1.9856970$

$-2.6271630$

$-1.4344800$

$-0.9962950$

$-0.1877510$

$-1.8876970$

$-1.2295450$

3.8547710

3. 6888200

3. 9305550

4. 8113100

3. 0211300

3.2323240

2. 1846860

3. 9132080

2. 2464900

2. 4916600

1. 4420940

3.1345040

2.9671760

2. 6504970

3. 3182060

3. 8148910

$-0.3737660$

$-0.3628110$

0.5094510

$-1.2680650$

0.8637400

0.9171030

1.7924600

0.8091230

$-4.0975030$

$-4.3253140$

$-3.5744800$

$-5.0520150$

$-4.0078590$

$-4.1360550$

$-5.0021480$

$-3.4558300$

$-0.6045500$

$-1.9820940$

0.3544200 


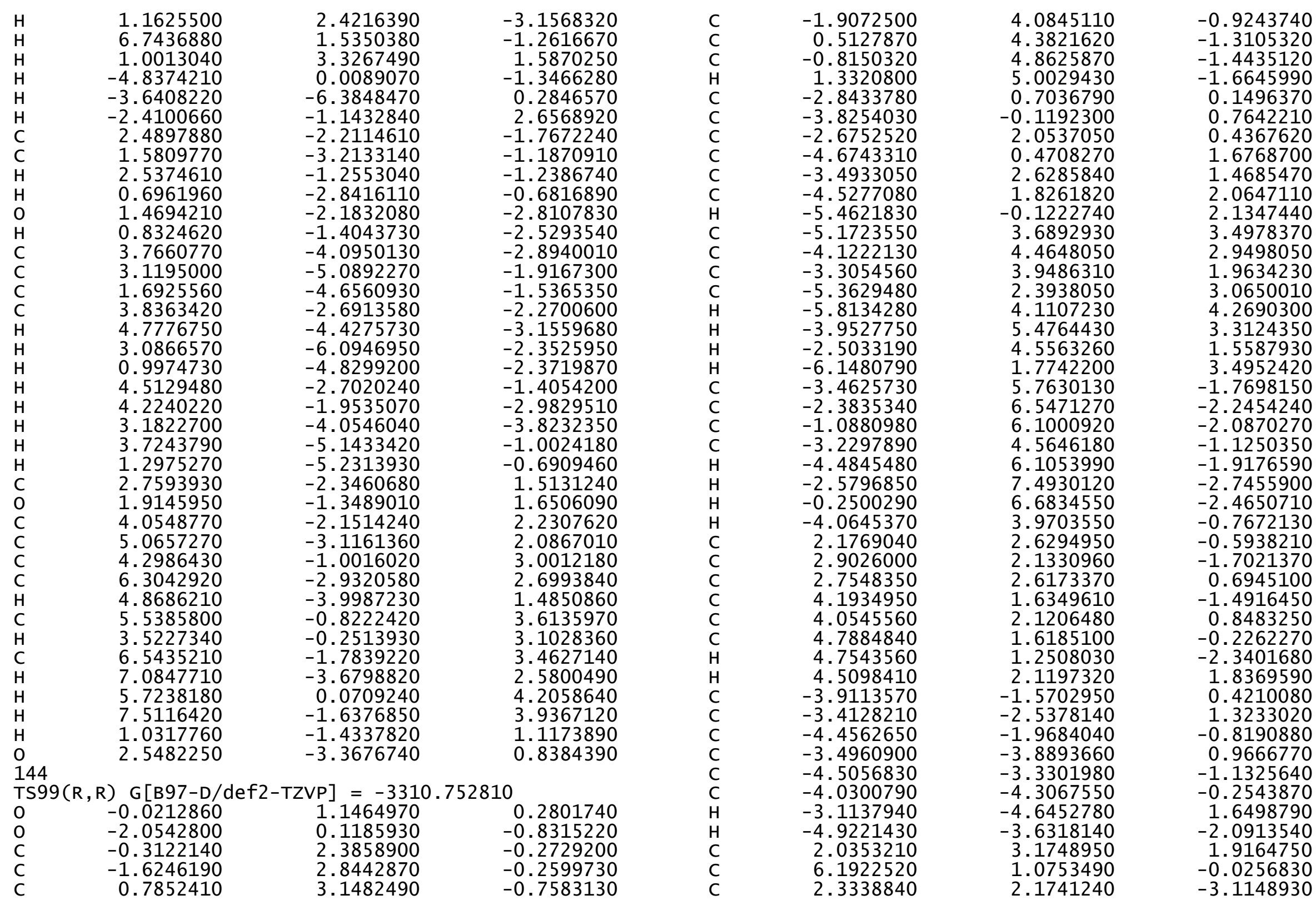




$\begin{array}{lrr}-2.7999560 & -2.1488120 & 2.6639600 \\ -4.0326280 & -5.7767710 & -0.6358110 \\ -5.0050000 & -0.9669170 & -1.8266850 \\ -6.5224280 & -1.1561770 & -2.0168570 \\ -6.7394480 & -2.1551920 & -2.4176510 \\ -7.0529960 & -1.0497910 & -1.0618970 \\ -6.9144730 & -0.4103580 & -2.7214050 \\ -4.2669810 & -1.0554670 & -3.1753940 \\ -3.1913960 & -0.9011750 & -3.0372650 \\ -4.4226510 & -2.0377990 & -3.6409980 \\ -4.6473010 & -0.2892340 & -3.8642800 \\ -2.9688870 & -6.0526460 & -1.7183570 \\ -2.9366280 & -7.1225320 & -1.9660370 \\ -3.2062430 & -5.4931000 & -2.6330240 \\ -1.9738600 & -5.7394430 & -1.3770920 \\ -5.4165480 & -6.2617200 & -1.1035370 \\ -6.1780080 & -6.0771390 & -0.3355000 \\ -5.7210100 & -5.7378830 & -2.0187370 \\ -5.3907470 & -7.3374860 & -1.3223680 \\ -3.8537640 & -2.2338610 & 3.7870960 \\ -4.7126780 & -1.5857850 & 3.5812460 \\ -4.2181150 & -3.2660050 & 3.8818570 \\ -3.4133720 & -1.9333240 & 4.7477640 \\ -1.5699270 & -2.9931570 & 3.0427670 \\ -1.8533750 & -4.0259870 & 3.2850660 \\ -0.8421180 & -3.0078920 & 2.2267640 \\ -1.0928350 & -2.5635010 & 3.9331770 \\ 2.6470600 & 4.5302850 & 2.3223800 \\ 3.7042320 & 4.4072500 & 2.5934710 \\ 2.5878420 & 5.2488310 & 1.4949350 \\ 2.1140300 & 4.9476640 & 3.1875170 \\ 2.0382060 & 2.1944590 & 3.1021950 \\ 1.6372800 & 1.2198540 & 2.8035040 \\ 3.0547510 & 2.0509900 & 3.4924850 \\ 1.4229140 & 2.5943600 & 3.9187140 \\ 7.2290740 & 1.8812490 & -0.8309420 \\ 8.2417710 & 1.5011220 & -0.6401710 \\ 7.0295180 & 1.7948680 & -1.9071760 \\ 7.1957510 & 2.9447750 & -0.5629100 \\ 6.2750300 & -0.4201390 & -0.3815910 \\ 5.5830140 & -1.0057510 & -3.2330760 \\ 6.0203860 & -0.5759880 & -3.1591090 \\ 7.2924040 & -0.7979670 & -4093250 \\ 2.6386620 & 0.9113400 & \end{array}$

2.1055830
2.8595110
3.9527480
2.4260760
2.6097160
-0.4874650
0.1577980
-0.3109240
1.2446170
6.4338760
0.9888940
-4.8507020
-3.7571810
-2.4668800
2.4965880
1.5658330
2.5449940
0.6719100
1.5023180
0.8739920
3.7786790
3.0994650
1.6687120
3.8490630
4.7924040
3.0658320
0.9931300
4.5107680
4.2559050
3.2164180
3.6822700
1.2445030
2.7199880
1.8889900
4.0514530
5.0175770
4.3750820
6.2898960
4.7588590
5.6505080
3.6350010
6.6095500
7.0348110
5.8991730
7.6050930
0.9945620
0.9601900

3. 4182950

3.3648630

3. 4702610

4. 3418720

$-0.1920160$

$-0.2878870$

$-1.3140780$

2. 2579080

1.1783580

3. 3592990

0.0440720

$-6.3500220$

$-2.2947030$

$-3.2727390$

$-1.3271400$

$-2.2749630$

$-1.4820150$

$-4.2194740$

$-5.1809980$

$-4.7237220$

$-2.7996130$

$-4.5690020$

$-6.1971710$

-4.9065080

$-2.7891560$

$-2.0853630$

$-4.1990850$

$-5.2170420$

$-5.2764800$

$-2.3221680$

$-1.3102200$

$-2.0931440$

$-3.1121690$

$-0.8593900$

$-2.8990430$

$-4.0607120$

$-0.6491650$

$-0.0679650$

$-1.6657870$

$-3.6901310$

0.3109920

$-1.4973900$

-1. 4140960
$-4.8953550$

-3.8609810

$-3.9559910$

$-4.8689990$

$-0.5318520$

$-1.8847320$

0.4558440

$-3.0388640$

1. 0412080

1.6565770

$-1.4352400$

0.2612260

2. 5877630

$-1.5929000$

$-1.0034980$

$-1.0854130$

$-0.5320110$

$-2.6606350$

$-2.4087820$

$-2.6406280$

$-1.6527710$

$-1.3178040$

$-2.0544570$

$-2.8703350$

$-2.0629820$

$-2.1674340$

$-1.1784850$

$-2.7802340$

$-3.5837340$

$-0.7234150$

$-0.4713500$

1.6362850

1.7454990

2.2710140

2. 2199520

2.8607190

2.7478500

1. 7583200

3. 3832700

2. 8849250

3. 3277710

2. 7038570

3.8295720

3. 7326880

1. 2338140 
0

2.4677500

$-3.3838430$

1.0405720

144

\begin{tabular}{|c|c|c|c|}
\hline \multicolumn{4}{|c|}{$\operatorname{TS} 100(R, R) \quad G[B 97-D /$ def2-TZVP $]=-3310.752495$} \\
\hline & 0.4553380 & 0.7162010 & 0.0647430 \\
\hline 0 & -1.8987200 & 0.6897190 & -0.9197830 \\
\hline $\mathrm{C}$ & 0.4616410 & 2.0985120 & -0.0495920 \\
\hline $\mathrm{C}$ & -0.6446290 & 2.8267910 & 0.3781210 \\
\hline $\mathrm{C}$ & 1.6511150 & 2.7081250 & -0.5313960 \\
\hline $\mathrm{C}$ & -0.6355510 & 4.2499510 & 0.1940210 \\
\hline $\mathrm{C}$ & 1.6847360 & 4.0850380 & 6139540 \\
\hline $\mathrm{C}$ & 0.5588610 & 4.8824990 & -0.2938070 \\
\hline $\mathrm{H}$ & 2.5970950 & 4.5750960 & -0.9 \\
\hline $\mathrm{C}$ & -2.4261520 & 1.0906370 & 0790 \\
\hline $\mathrm{C}$ & -3.5946510 & 0.4277170 & 0.7687680 \\
\hline $\mathrm{C}$ & -1.8090060 & 2.1318020 & 0.9865130 \\
\hline $\mathrm{C}$ & -4.1183560 & 0.8339260 & 1.9 \\
\hline C & -2.3076910 & 2.4805510 & 00 \\
\hline $\mathrm{C}$ & -3.4924260 & 1.8276010 & 70 \\
\hline $\mathrm{H}$ & -5.0251520 & 0.36 & \\
\hline C & -3.3603540 & 3.1021520 & 4.8 \\
\hline $\mathrm{C}$ & -2.1702160 & 3.7166120 & $4.3 \xi$ \\
\hline C & -1.6587750 & 3.4150170 & 3.13 \\
\hline $\mathrm{C}$ & -4.0028380 & 2.1735870 & 4.05 \\
\hline $\mathrm{H}$ & -3.7566260 & 3.3542480 & 617 \\
\hline $\mathrm{H}$ & -1.6513260 & 4.4305240 & 5.0 \\
\hline $\mathrm{H}$ & -0.7448500 & 3.8921100 & 2.80 \\
\hline $\mathrm{H}$ & -4.9065870 & 1.6762000 & 4.40 \\
\hline C & -1.7203390 & 6.4348570 & 0.25778 \\
\hline C & -0.5288680 & 7.0596670 & -0.18 \\
\hline $\mathrm{C}$ & 0.5850570 & 6.2939440 & -0.45 \\
\hline C & -1.7721020 & 5.0678930 & 0.42 \\
\hline $\mathrm{H}$ & -2.6057840 & 7.0372640 & 0.4 \\
\hline $\mathrm{H}$ & -0.5001800 & 8.1385800 & -0.3 \\
\hline $\mathrm{H}$ & 1.5023670 & 6.7561270 & -0.8 \\
\hline $\mathrm{H}$ & -2.69 & 4.6032490 & \\
\hline C & 2.8617820 & 1.9025840 & -0 \\
\hline C & 3.1910990 & 1.6484830 & -2.2 \\
\hline $\mathrm{C}$ & 3. & 1.4436220 & \\
\hline $\mathrm{C}$ & 4.3881680 & 0.9837100 & -2 \\
\hline $\mathrm{C}$ & 4.8892600 & 0.7730170 & 578 \\
\hline $\mathrm{C}$ & 5.2584950 & 0.5480010 & 1090 \\
\hline $\mathrm{H}$ & 030 & 0.7875660 & \\
\hline $\mathrm{H}$ & 50 & 0.4282980 & $U<y$ \\
\hline $\mathrm{C}$ & -4 & -0.6547880 & \\
\hline $\mathrm{C}$ & -4.1 & -1.9953310 & 0004 \\
\hline & -4.8912540 & -0.3337340 & \\
\hline
\end{tabular}

$-4.8225420$

$-5.4996970$

$-5.4783960$

$-4.7922110$

$-6.0051880$

3. 3850800

6.5700310

2. 2907670

$-3.4823030$

$-6.1242890$

$-4.9951500$

$-6.4594790$

$-7.0774980$

$-6.8792190$

$-6.5229880$

$-4.3913910$

$-3.3469420$

$-4.9530970$

$-4.4331170$

$-5.4024550$

$-5.8507070$

$-5.4854260$

$-4.3364140$

$-7.6266510$

$-8.1546630$

$-7.7766980$

$-8.0773590$

$-4.5045520$

$-5.1040300$

$-5.1891650$

$-3.9898380$

$-2.6111950$

$-3.2296360$

$-1.9236350$

$-2.0247390$

4.4524540

5.4339240

4. 5407510

4.1874350

3. 2259630

2. 4242820

4.1545580

2.9734920

7.1679140

8.0536280
7.4826980

$\begin{array}{rr}-2.9805120 & -0.3569460 \\ -1.3563040 & -1.9788360 \\ -2.6865970 & -1.5530780 \\ -4.0155860 & -0.0219020 \\ -1.1040170 & -2.9086360 \\ 1.6636730 & 1.6300400 \\ -0.1144110 & -1.8947530 \\ 2.1105850 & -3.3666640 \\ -2.4051150 & 1.6985020 \\ -3.7830940 & -2.3812210 \\ 1.0927340 & -1.7723820 \\ 1.5742870 & -1.7550330 \\ 0.9513750 & -2.4150730 \\ 1.5189000 & -0.7423230 \\ 2.6138370 & -2.1040480 \\ 1.2270230 & -3.1824660 \\ 0.8973960 & -3.1887530 \\ 0.6217150 & -3.9060310 \\ 2.2742140 & -3.5109020 \\ -3.9499420 & -3.7331830 \\ -4.7701230 & -4.3102490 \\ -3.0283140 & -4.3240260 \\ -4.1629790 & -3.5853020 \\ -3.5253920 & -2.6014690 \\ -3.4288190 & -1.6444340 \\ -2.5978380 & -3.1692940 \\ -4.3494800 & -3.1706970 \\ -2.6287460 & 2.8312570 \\ -1.7293920 & 3.0135310 \\ -3.4453530 & 2.5645700 \\ -2.8996790 & 3.7632850 \\ -3.6645400 & 1.5295520 \\ -4.5561560 & 1.3619190 \\ -3.5473050 & 0.6866260 \\ -3.8297520 & 2.4423100 \\ 2.5465820 & 2.3036020 \\ 2.0553260 & 2.2783230 \\ 3.5132110 & 1.7913000 \\ 2.7286600 & 3.3536630 \\ 0.3236450 & 2.3699690 \\ -0.2662720 & 1.9166140 \\ -0.2606590 & 2.3334980 \\ 0.4957140 & 3.4242090 \\ -1.0266300 & -0.8144470 \\ -1.5401820 & -1.2085610 \\ -0.4431320 & 0.0605690\end{array}$




$\begin{array}{lrrr}\text { H } & 6.4456610 & -1.7807780 & -0.4798360 \\ \mathrm{C} & 7.5970900 & 0.9610910 & -2.3086520 \\ \mathrm{H} & 7.2017940 & 1.5922860 & -3.1137200 \\ \mathrm{H} & 7.8295400 & 1.6064960 & -1.4507300 \\ \mathrm{H} & 8.5289180 & 0.4925010 & -2.6533380 \\ \mathrm{C} & 2.1909700 & 1.0935710 & -4.5159880 \\ \mathrm{H} & 3.1414460 & 1.0100200 & -5.0597730 \\ \mathrm{H} & 1.9118600 & 0.1031660 & -4.1456230 \\ \mathrm{H} & 1.4273560 & 1.4257450 & -5.2304350 \\ \mathrm{C} & 2.7683050 & 3.4704930 & -3.9155240 \\ \mathrm{H} & 3.7875570 & 3.3776550 & -4.3150930 \\ \mathrm{H} & 2.1079080 & 3.8060010 & -4.7265780 \\ \mathrm{H} & 2.7739470 & 4.2371450 & -3.1334290 \\ \mathrm{P} & -0.5388320 & -0.1989360 & -0.8696380 \\ \mathrm{O} & -0.0503480 & -0.2863990 & -2.2854160 \\ \mathrm{O} & -0.7316990 & -1.4663930 & -0.0816720 \\ \mathrm{H} & 1.2808390 & 2.2411560 & -2.9619550 \\ \mathrm{H} & 6.3648450 & -0.7321180 & -2.7823720 \\ \mathrm{H} & 2.4292560 & 2.1918570 & 1.7072630 \\ \mathrm{H} & -4.4306670 & 1.7571890 & -1.1109790 \\ \mathrm{H} & -6.0190070 & -4.7239630 & -1.8220230 \\ \mathrm{H} & -2.8158870 & -1.5892290 & 1.9958920 \\ \mathrm{C} & 2.4373050 & -2.1178500 & -1.6444710 \\ \mathrm{C} & 1.4911090 & -3.1775880 & -1.2633980 \\ \mathrm{H} & 2.3432910 & -1.1708400 & -1.1064580 \\ \mathrm{H} & 0.5084250 & -2.8643500 & -0.9292980 \\ \mathrm{O} & 1.6215850 & -2.1232020 & -2.8621490 \\ \mathrm{H} & 0.8980480 & -1.3966960 & -2.7006120 \\ \mathrm{C} & 3.9995220 & -3.9185900 & -2.5319450 \\ \mathrm{C} & 3.2409080 & -4.9583800 & -1.6941850 \\ \mathrm{C} & 1.7467760 & -4.6067900 & -1.5968040 \\ \mathrm{C} & 3.8734710 & -2.5211960 & -1.9051750 \\ \mathrm{H} & 5.0600710 & -4.1893180 & -2.6029050 \\ \mathrm{H} & 3.3484530 & -5.9608030 & -2.1251920 \\ \mathrm{H} & 1.2407250 & -4.7995060 & -2.5551120 \\ \mathrm{H} & 4.3854570 & -2.4977160 & -0.9346990 \\ \mathrm{H} & 4.3357410 & -1.7544050 & -2.5325270 \\ \mathrm{H} & 3.5965000 & -3.8995600 & -3.5535120 \\ \mathrm{H} & 3.6621380 & -4.9839160 & -0.6808950 \\ \mathrm{H} & 1.2250780 & -5.2185450 & -0.8514090 \\ \mathrm{C} & 1.5980160 & -2.8480780 & 1.9605800 \\ \mathrm{O} & 0.5062030 & -2.1205360 & 2.0310230 \\ \mathrm{C} & 2.2929540 & -3.0348880 & 3.2729370 \\ \mathrm{C} & 3.4698950 & -3.8001250 & 3.3209270 \\ \mathrm{C} & 1.8065760 & -2.4406520 & 4.4497580 \\ \mathrm{C} & 4.1510870 & -3.9675990 & 4.5259800 \\ & & & \\ & & & \end{array}$

$\begin{array}{ll}\mathrm{H} & 3.8409730 \\ \mathrm{C} & 2.4903200 \\ \mathrm{H} & 0.9016670 \\ \mathrm{C} & 3.6627830 \\ \mathrm{H} & 5.0625270 \\ \mathrm{H} & 2.1100970 \\ \mathrm{H} & 4.1952660 \\ \mathrm{H} & 0.0533680 \\ \mathrm{O} & 2.0609670\end{array}$
$-4.2538850$
$-2.6104520$
$-1.8428450$
$-3.3725340$
$-4.5603730$
$-3.5018850$
$-1.9013820$

2.4064130

5.6539550

4.4122960

5.6949070

4.5554990

6.5606230

6.6347740

6.6347740
1.1263930

144

0.9270820

TS101(R, R) G[B97-D/def2-TZVP] = -3310.752355

$\begin{array}{lrrr}\text { TS101(R, R) G[B97-D/def2-TZVP] }=-3310.752355 \\ \text { O } & 0.0689040 & -1.1611000 & 0.2773170 \\ \text { O } & 2.0531620 & -0.1390880 & -0.9218960 \\ \text { C } & 0.3870380 & -2.4142180 & -0.2275180 \\ \text { C } & 1.7117930 & -2.8374120 & -0.2127960 \\ \text { C } & -0.6952250 & -3.2241760 & -0.6682470 \\ \text { C } & 2.0184730 & -4.0977590 & -0.8263830 \\ \text { C } & -0.3970970 & -4.4733980 & -1.1702460 \\ \text { C } & 0.9412130 & -4.9255880 & -1.2977050 \\ \text { H } & -1.2038550 & -5.1302630 & -1.4858750 \\ \text { C } & 2.8780700 & -0.6501110 & 0.0708940 \\ \text { C } & 3.8431210 & 0.2321650 & 0.6268230 \\ \text { C } & 2.7524020 & -1.9876170 & 0.4278260 \\ \text { C } & 4.7199960 & -0.2860280 & 1.5569590 \\ \text { C } & 3.6035950 & -2.4871860 & 1.4719130 \\ \text { C } & 4.6203090 & -1.6247040 & 2.0119830 \\ \text { H } & 5.4899000 & 0.3541630 & 1.9806390 \\ \text { C } & 5.3392380 & -3.3941370 & 3.5265770 \\ \text { C } & 4.3077000 & -4.2282210 & 3.0317460 \\ \text { C } & 3.4633070 & -3.7864400 & 2.0325340 \\ \text { C } & 5.4844070 & -2.1165530 & 3.0278820 \\ \text { H } & 6.0024810 & -3.7567280 & 4.3088680 \\ \text { H } & 4.1747230 & -5.2253980 & 3.4459170 \\ \text { H } & 2.6753690 & -4.4367030 & 1.6679340 \\ \text { H } & 6.2550920 & -1.4531320 & 3.4169730 \\ \text { C } & 3.6074760 & -5.7714030 & -1.6173520 \\ \text { C } & 2.5439820 & -6.6030340 & -2.0449700 \\ \text { C } & 1.2391740 & -6.1827620 & -1.8900780 \\ \text { C } & 3.3507890 & -4.5522560 & -1.0226030 \\ \text { H } & 4.6363060 & -6.0938440 & -1.7625160 \\ \text { H } & 2.7591490 & -7.5646660 & -2.5057500 \\ \text { H } & 0.4123600 & -6.8032960 & -2.2319240 \\ \text { H } & 4.1736590 & -3.9220590 & -0.7005690 \\ \text { C } & -2.0978350 & -2.7339460 & -0.5055450 \\ \text { C } & -2.8396570 & -2.2693320 & -1.6172200 \\ \text { C } & -2.6663800 & -2.7086870 & 0.7861370\end{array}$




-4.1361920
-3.9720750
-4.7210520
-4.7092410
-4.4182030
3.8675760
3.3948020
4.3195780
3.4016810
4.2983720
3.8401020
3.0348570
4.6426120
-1.9268810
-6.1284290
-2.2778190
2.8885710
3.7734610
4.8551800
6.3619570
6.5440690
6.9232520
6.7489390
4.0763930
3.0092290
4.1953990
4.4556930
4.5452580
4.5089670
4.1022780
5.5955290
2.3078000
1.7602750
1.8026080
2.2604440
3.9922300
4.8922530
4.2700830
3.6354280
1.6162330
1.8283210
0.8476810
1.2236250
-2.5465950
-3.5949390
-2.5172490

-1.4053370
0.9420880
-0.1351220
-2.2552800
1.9349700
0.2196080
1.1124370
-1.0667030
0.6959940
-1.4374370
-0.5757070
1.3764890
-2.4312060
2.0142010
0.0669460
-3.0318760
2.5099200
-1.0239280
-2.0611970
-2.3040500
-2.7413630
-1.3631430
-2.9974050
-3.3892860
-3.2156790
-3.8936020
-4.0620750
-0.0766790
-0.4459500
0.9276500
0.0088420
-1.1635370
-1.8597680
-0.1894290
-1.5312550
3.5583120
3.3391960
3.5658920
4.5613970
2.9009150
3.0504100
2.1298090
3.8471980
2.4949180
2.7858250
1.6994600

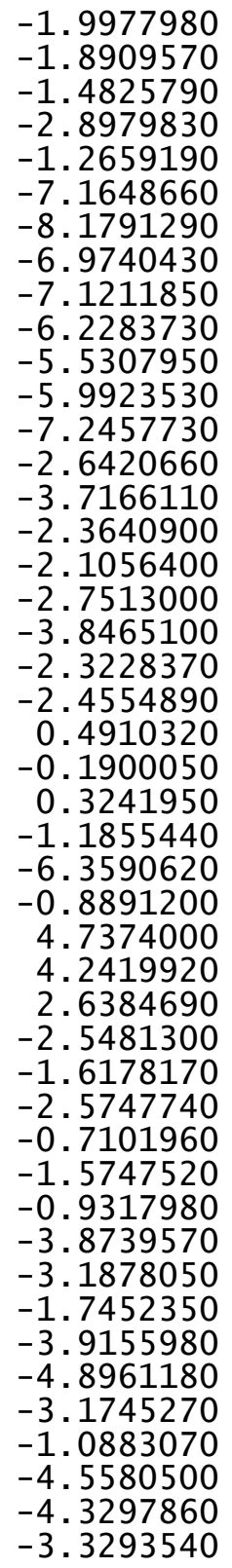
$-4.9371580$
$-2.1914070$
$-1.2375650$
$-2.0149170$
$-2.5631450$
$-2.0544920$
$-1.6779510$
$-1.9917660$
$-3.1111910$
0.2646910
0.8686140
0.3991320
0.6386770
$-1.1096730$
$-1.0846750$
$-0.1776100$
$-1.1631950$
$-3.6190550$
$-3.6182230$
$-3.6817660$
$-4.5136630$
0.1581470
0.2020160
1. 3080860
$-2.3628060$
$-1.3039070$
$-3.4353660$
0.0071020
5.8848590
1. 2545880
2. 1879370
3.1928760
1.2340930
2. 8236260
2.1501940
1.3719280
4.0682950
5.0646640
4.6334540
2.6647080
4. 3991920
6.0695390
4.8022190
2.6721150
1.9258900
4.0281980

3. 3649310

3.1537130

2. 8026360

3.5550840

3.9762160

$-0.7111400$

$-0.5215340$

$-1.7906190$

$-0.4186840$

$-0.3201710$

0.2700750

$-1.3833760$

$-0.1448750$

$-3.8935880$

$-4.1184730$

$-3.3952090$

$-4.8495420$

$-3.7354370$

$-3.8231750$

$-4.7450020$

$-3.1762400$

$-0.5888080$

$-1.9266790$

0.3679350

$-2.9587430$

1.1380710

1.7385780

$-1.6335270$

$-2.0168680$

2. 5287590

$-1.6444660$

$-1.1021810$

$-1.1097500$

$-0.6368340$

$-2.7311630$

$-2.4701850$

$-2.7183130$

$-1.7706430$

$-1.4519420$

$-2.0916270$

$-2.9379810$

$-2.2089230$

$-2.3188580$

$-1.2013910$

$-2.7880980$

$-3.6711170$ 


\begin{tabular}{|c|c|c|c|c|c|c|c|}
\hline & & & & & & & \\
\hline $\mathrm{H}$ & -3.7527570 & 5.1194180 & -0.8312030 & C & 3.3509080 & -4.5522280 & -1.0226360 \\
\hline $\mathrm{H}$ & -1.3101330 & 5.2130970 & -0.6294640 & $\mathrm{H}$ & 4.6364540 & -6.0938310 & -1.7624700 \\
\hline C & -2.6999620 & 2.2951900 & 1.5900150 & $\mathrm{H}$ & 2.7593220 & -7.5647400 & -2.5055950 \\
\hline 0 & -1.8380130 & 1.3117540 & 1.7185270 & $\mathrm{H}$ & 0.4125180 & -6.8034130 & -2.2317840 \\
\hline C & -4.0146770 & 2.0468940 & 2.2520710 & $\mathrm{H}$ & 4.1737640 & -3.9219880 & -0.7006450 \\
\hline C & -5.0073780 & 3.0396620 & 2.1960520 & C & -2.0977410 & -2.7340070 & -0.5055690 \\
\hline $\mathrm{C}$ & -4.2972500 & 0.8170040 & 2.8704720 & $\mathrm{c}$ & -2.8395890 & -2.2693370 & -1.6172040 \\
\hline $\mathrm{C}$ & -6.2652110 & 2.8050280 & 2.7490560 & C & -2.6662450 & -2.7087940 & 0.7861310 \\
\hline $\mathrm{H}$ & -4.7802830 & 3.9846670 & 1.7110240 & C & -4.1361020 & -1.7873190 & -1.4052580 \\
\hline C & -5.5585400 & 0.5848570 & 3.4175060 & $\mathrm{C}$ & -3.9719340 & -2.2278280 & 0.9421400 \\
\hline $\mathrm{H}$ & -3.5369440 & 0.0449190 & 2.8961050 & C & -4.7209230 & -1.7551750 & -0.1350230 \\
\hline C & -6.5437590 & 1.5759170 & 3.3582910 & $\mathrm{H}$ & -4.7091710 & -1.4252570 & -2.2551620 \\
\hline $\mathrm{H}$ & -7.0308680 & 3.5758650 & 2.7018190 & $\mathrm{H}$ & -4.4180320 & -2.2147380 & 1.9350360 \\
\hline $\mathrm{H}$ & -5.7759280 & -0.3725410 & 3.8855440 & C & 3.8674490 & 1.6685190 & 0.2196150 \\
\hline $\mathrm{H}$ & -7.5281180 & 1.3905920 & 3.7827310 & $\mathrm{C}$ & 3.3946170 & 2.6608610 & 1.1124850 \\
\hline $\mathrm{H}$ & -0.9604880 & 1.4167550 & 1.1795580 & C & 4.3194370 & 2.0299660 & -1.0666790 \\
\hline 0 & -2.4883040 & 3.3439500 & 0.9571360 & $\mathrm{c}$ & 3.4014200 & 3.9953820 & 0.6960990 \\
\hline & & & & C & 4.2981440 & 3.3802120 & -1.4373570 \\
\hline & R) $G[B$ & {$[Z \mathrm{VP}]=-33$} & 355 & C & 3.8398130 & 4.3763920 & -0.5755860 \\
\hline 0 & 0.0689610 & -1.1610560 & 0.2770750 & $\mathrm{H}$ & 3.0345420 & 4.7598180 & 1.3766270 \\
\hline 0 & 2.0532660 & -0.1390820 & -0.9220890 & $\mathrm{H}$ & 4.6423690 & 3.6632410 & -2.4311120 \\
\hline C & 0.3871250 & -2.4141980 & -0.2276740 & $\mathrm{C}$ & -1.9267000 & -3.2251310 & 2.0141580 \\
\hline C & 1.7118870 & -2.8373740 & -0.2129180 & $\mathrm{c}$ & -6.1282790 & -1.2219150 & 0.0671130 \\
\hline C & -0.6951280 & -3.2242170 & -0.6683180 & $\mathrm{C}$ & -2.2777900 & -2.3296960 & -3.0318770 \\
\hline C & 2.0185840 & -4.0977510 & -0.8264340 & $\mathrm{c}$ & 2.8883860 & 2.3205660 & 2.5099510 \\
\hline C & -0.3969840 & -4.4734700 & -1.1702250 & c & 3.7730440 & 5.8255410 & -1.0237690 \\
\hline C & 0.9413370 & -4.9256340 & -1.2976860 & $\mathrm{C}$ & 4.8551430 & 1.0083530 & -2.0611950 \\
\hline $\mathrm{H}$ & -1.20 & -5.1303860 & -1.4857720 & $\mathrm{C}$ & 6.3619280 & 1.2245090 & -2.3039350 \\
\hline C & 2.87 & -0.6500530 & 0.0707740 & $\mathrm{H}$ & 6.5440290 & 2.2150920 & -2.7411770 \\
\hline C & 3.8430760 & 0.2322780 & 0.6267830 & $\mathrm{H}$ & 6.9231630 & 1.1585780 & -1.3629950 \\
\hline $\mathrm{C}$ & 2.75 & -1.9875550 & 0.4277190 & $\mathrm{H}$ & 6.7489870 & 0.4656650 & -2.9973090 \\
\hline C & 4.71 & -0.2858690 & 1.5569960 & C & 4.0764480 & 1.0343890 & -3.3893380 \\
\hline C & 3.60 & -2.4870830 & 1.4718710 & $\mathrm{H}$ & 3.0092770 & 0.8590640 & -3.2158130 \\
\hline C & 4.62 & -1.6245500 & 2.0120120 & $\mathrm{H}$ & 4.1954690 & 2.0025050 & -3.8936110 \\
\hline $\mathrm{H}$ & 5.48 & 0.3543710 & 1.9807580 & $\mathrm{H}$ & 4.4558130 & 0.2535550 & -4.0621280 \\
\hline C & 5.33 & -3.3939350 & 3.5266790 & C & 4.5445440 & 6.7622580 & -0.0763770 \\
\hline C & 4.3 & -4.228 & 3.0317780 & $\mathrm{H}$ & 4.5081980 & 7.7958990 & -0.4456680 \\
\hline C & 3.4 & -3.786 & 2.0324970 & $\mathrm{H}$ & 4.1013720 & 6.7445270 & 0.9278670 \\
\hline C & $5.4 \varepsilon$ & -2.116 & 3.0279840 & $\mathrm{H}$ & 5.5948350 & 6.4565070 & 0.0093530 \\
\hline $\mathrm{H}$ & 6.00 & -3.7564880 & 4.3090250 & C & 2.3073330 & 6.2849580 & -1.1636020 \\
\hline H & 4.17 & -5.2252490 & 3.4459490 & $\mathrm{H}$ & 1.7600230 & 5.6370100 & -1.8599560 \\
\hline $\mathrm{H}$ & 2.67 & -4.4366400 & 1.6678500 & $\mathrm{H}$ & 1.8019870 & 6.2421680 & -0.1895780 \\
\hline $\mathrm{H}$ & 6.2 & -1.4528890 & 3.4171280 & $\mathrm{H}$ & 2.2598730 & 7.3190620 & -1.5312740 \\
\hline C & 3.60 & -5.7714040 & -1.6173140 & C & 3.9919790 & 2.5690220 & 3.5583810 \\
\hline C & 2.5 & -6.6030860 & -2.0448700 & $\mathrm{H}$ & 4.8920900 & 1.9835660 & 392800 \\
\hline C & 1.2 (2) & 10 & -1.8899850 & $\mathrm{H}$ & 4.2696870 & 3.6319520 & 3.5659960 \\
\hline
\end{tabular}




$\begin{array}{rrr}3.6351840 & 2.2977070 & 4.5614490 \\ 1.6159370 & 3.0943420 & 2.9009110 \\ 1.8278790 & 4.1613360 & 3.0503880 \\ 0.8474110 & 2.9914650 & 2.1297940 \\ 1.2233680 & 2.6995700 & 3.8471970 \\ -2.5463560 & -4.5519810 & 2.4948410 \\ -3.5947060 & -4.4022910 & 2.7857510 \\ -2.5169810 & -5.3075120 & 1.6993610 \\ -1.9975420 & -4.9373250 & 3.3648420 \\ -1.8907870 & -2.1915720 & 3.1537090 \\ -1.4825080 & -1.2376820 & 2.8026450 \\ -2.8978020 & -2.0151770 & 3.5551530 \\ -1.2656660 & -2.5632910 & 3.9761570 \\ -7.1647780 & -2.0545650 & -0.7108110 \\ -8.1790220 & -1.6779690 & -0.5212160 \\ -6.9739930 & -1.9919990 & -1.7903060 \\ -7.1211160 & -3.1112240 & -0.4182080 \\ -6.2282450 & 0.2646570 & -0.3201310 \\ -5.5306340 & 0.8686390 & 0.2700200 \\ -5.9922800 & 0.3990040 & -1.3833590 \\ -7.2456380 & 0.6386590 & -0.1448200 \\ -2.6421370 & -1.1097470 & -3.8936340 \\ -3.7167130 & -1.0847400 & -4.1183710 \\ -2.3640630 & -0.1776480 & -3.3953760 \\ -2.1058480 & -1.1633640 & -4.8496610 \\ -2.7511990 & -3.6191300 & -3.7353680 \\ -3.8464110 & -3.6183800 & -3.8230800 \\ -2.3227550 & -3.6818570 & -4.7449390 \\ -2.4553110 & -4.5136910 & -3.1761360 \\ 0.4911240 & 0.1581660 & -0.5890760 \\ -0.1898530 & 0.2020060 & -1.9269780 \\ 0.3242480 & 1.3081280 & 0.3676310 \\ -1.1855110 & -2.3627330 & -2.9587690 \\ -6.3588210 & -1.3038080 & 1.1382640 \\ -0.8889370 & -3.4354450 & 1.7385040 \\ 4.7373800 & 0.0073630 & -1.6335870 \\ 4.2417220 & 5.8851230 & -2.0166330 \\ 2.6384190 & 1.2545440 & 2.5287710 \\ -2.5481250 & 2.1877880 & -1.6445220 \\ -1.6178910 & 3.1928360 & -1.1023040 \\ -2.5746340 & 1.2339530 & -1.1097860 \\ -0.7102040 & 2.8236850 & -0.6370090 \\ -1.5748160 & 2.1501230 & -2.7312810 \\ -0.9317900 & 1.3718960 & -2.4703640 \\ -3.8742090 & 4.0679760 & -2.7183410 \\ -3.1881130 & 5.0644400 & -1.7707280\end{array}$

\begin{tabular}{ll}
$\mathrm{C}$ & -1.7454760 \\
$\mathrm{C}$ & -3.9156730 \\
$\mathrm{H}$ & -4.8964140 \\
$\mathrm{H}$ & -3.1749720 \\
$\mathrm{H}$ & -1.0886190 \\
$\mathrm{H}$ & -4.5580650 \\
$\mathrm{H}$ & -4.3298390 \\
$\mathrm{H}$ & -3.3296430 \\
$\mathrm{H}$ & -3.7530190 \\
$\mathrm{H}$ & -1.3103840 \\
$\mathrm{C}$ & -2.6998270 \\
$\mathrm{O}$ & -1.8378240 \\
$\mathrm{C}$ & -4.0144800 \\
$\mathrm{C}$ & -5.0072210 \\
$\mathrm{C}$ & -4.2969550 \\
$\mathrm{C}$ & -6.2650000 \\
$\mathrm{H}$ & -4.7802020 \\
$\mathrm{C}$ & -5.5581890 \\
$\mathrm{H}$ & -3.5366200 \\
$\mathrm{C}$ & -6.5434490 \\
$\mathrm{H}$ & -7.0306890 \\
$\mathrm{H}$ & -5.7755000 \\
$\mathrm{H}$ & -7.5277640 \\
$\mathrm{H}$ & -0.9603560 \\
$\mathrm{O}$ & -2.4882690 \\
$\mathrm{H}$ & \\
\hline
\end{tabular}

$\begin{array}{lr}4.6333910 & -1.4521010 \\ 2.6644060 & -2.0916040 \\ 4.3987610 & -2.9379710 \\ 6.0693120 & -2.2090200 \\ 4.8021950 & -2.3190630 \\ 2.6717810 & -1.2013230 \\ 1.9255200 & -2.7880150 \\ 4.0279010 & -3.6711660 \\ 5.1191390 & -0.8312570 \\ 5.2131070 & -0.6296690 \\ 2.2951230 & 1.5899620 \\ 1.3117280 & 1.7184280 \\ 2.0467900 & 2.2521260 \\ 3.0395210 & 2.1961800 \\ 0.8168950 & 2.8705610 \\ 2.8048470 & 2.7492920 \\ 3.9845290 & 1.7111210 \\ 0.5847090 & 3.4177080 \\ 0.0448380 & 2.8961330 \\ 1.5757330 & 3.3585680 \\ 3.5756550 & 2.7021100 \\ -0.3726930 & 3.8857720 \\ 1.3903780 & 3.7830960 \\ 1.4167480 & 1.1793690 \\ 3.3438780 & 0.9570390\end{array}$

TS103(R,R) G[B97-D/def2-TZVP $]=-3310.752107$

$\begin{array}{lrrr}\text { TS103(R, R) G }[\mathrm{B} 97-\mathrm{D} / \mathrm{def}-\mathrm{TZVP}]=-3310.752107 \\ \mathrm{O} & -0.0060840 & 1.1304790 & 0.2140760 \\ \mathrm{O} & -2.0699080 & 0.1487910 & -0.8836400 \\ \mathrm{C} & -0.3345250 & 2.3972200 & -0.2514680 \\ \mathrm{C} & -1.6513590 & 2.8346750 & -0.1605780 \\ \mathrm{C} & 0.7316680 & 3.2039370 & -0.7353910 \\ \mathrm{C} & -1.9773400 & 4.1062610 & -0.7397170 \\ \mathrm{C} & 0.4197650 & 4.4623140 & -1.2055380 \\ \mathrm{C} & -0.9182580 & 4.9289850 & -1.2579620 \\ \mathrm{H} & 1.2155200 & 5.1147980 & -1.5559700 \\ \mathrm{C} & -2.8274050 & 0.6579990 & 0.1630800 \\ \mathrm{C} & -3.7689700 & -0.2175050 & 0.7673750 \\ \mathrm{C} & -2.6634430 & 1.9891630 & 0.5285870 \\ \mathrm{C} & -4.5765550 & 0.2989060 & 1.7595220 \\ \mathrm{C} & -3.4401210 & 2.4862440 & 1.6303390 \\ \mathrm{C} & -4.4302390 & 1.6291410 & 2.2253610 \\ \mathrm{H} & -5.3239140 & -0.3398220 & 2.2238820 \\ \mathrm{C} & -5.0258830 & 3.3853540 & 3.8069260 \\ \mathrm{C} & -4.0193820 & 4.2141740 & 3.2548850 \\ \mathrm{C} & -3.2479850 & 3.7766270 & 2.1965520\end{array}$




$\begin{array}{rrr}-5.2193200 & 2.1164520 & 3.3026400 \\ -5.6312110 & 3.7451330 & 4.6360600 \\ -3.8474610 & 5.2043350 & 3.6716100 \\ -2.4782430 & 4.4236970 & 1.7900660 \\ -5.9706910 & 1.4562890 & 3.7327920 \\ -3.5879740 & 5.8068360 & -1.4213560 \\ -2.5401040 & 6.6320970 & -1.8972280 \\ -1.2336090 & 6.1961980 & -1.8188770 \\ -3.3131190 & 4.5775120 & -0.8562030 \\ -4.6194340 & 6.1421890 & -1.5058810 \\ -2.7693360 & 7.6015070 & -2.3343460 \\ -0.4195270 & 6.8118950 & -2.1979300 \\ -4.1243170 & 3.9522090 & -0.4970510 \\ 2.1361610 & 2.7021340 & -0.6555490 \\ 2.8322840 & 2.3012700 & -1.8155980 \\ 2.7619970 & 2.6145790 & 0.6106620 \\ 4.1413660 & 1.8119110 & -1.6848720 \\ 4.0682240 & 2.1281040 & 0.6858400 \\ 4.7786030 & 1.7139740 & -0.4460800 \\ 4.6720980 & 1.5083280 & -2.5817740 \\ 4.5539810 & 2.0705960 & 1.6584640 \\ -3.8476670 & -1.6489580 & 0.3524480 \\ -3.3613230 & -2.6569030 & 1.2163000 \\ -4.3827470 & -1.9932490 & -0.9090230 \\ -3.4435990 & -3.9919850 & 0.8010670 \\ -4.4324090 & -3.3400420 & -1.2798500 \\ -3.9672380 & -4.3555240 & -0.4405250 \\ -3.0710060 & -4.7767180 & 1.4569430 \\ -4.8451020 & -3.6001190 & -2.2524500 \\ 2.0856280 & 3.0975700 & 1.8883030 \\ 6.1946930 & 1.1798380 & -0.2696930 \\ 2.2235540 & 2.4434180 & -3.2051960 \\ -2.7625140 & -2.3414630 & 2.5824860 \\ -3.9811900 & -5.8081900 & -0.8823280 \\ -4.9393500 & -0.9521320 & -1.8714690 \\ -6.4631410 & -1.1208340 & -2.0314600 \\ -6.6987260 & -2.1032290 & -2.4619560 \\ -6.9698150 & -1.0438570 & -1.0608020 \\ -6.8646240 & -0.3468310 & -2.6994750 \\ -4.2362540 & -1.0005380 & -3.2402700 \\ -3.1559150 & -0.8616770 & -3.1255460 \\ -4.4147340 & -1.9629920 & -3.7380120 \\ -4.6258240 & -0.2060540 & -3.8908360 \\ -2.9501610 & -6.0412050 & -2.0053790 \\ -2.9291160 & -7.0998650 & -2.2981220 \\ -3.2117220 & -5.4436300 & -2.8887790\end{array}$

-1.9446180
-5.3799320
-6.1183280
-5.7082740
-5.3644390
-3.7953060
-4.7002090
-4.0866270
-3.3678510
-1.4720810
-1.6796060
-0.7535190
-1.0191400
2.7336030
3.7929760
2.6733100
2.2255880
2.1045900
1.6492000
3.1329130
1.5481870
6.9787350
8.0062700
6.5182330
7.0155340
6.1805820
5.6503240
5.6753960
7.2054060
2.5081970
3.5747330
2.1971670
1.9530230
2.7240580
3.8134860
2.2574520
2.4930590
-0.4915720
0.1022290
-0.2696710
1.1364210
6.7310850
1.0363800
-4.7665770
-3.6828030
-2.5022540

-5.7446550
-6.2697380
-6.1162410
-5.7060340
-7.3353000
-2.5976260
-1.9969120
-3.6567970
-2.3491460
-3.1306380
-4.2007570
-3.0074790
-2.7637590
4.4113090
4.2506610
5.1836150
4.7805080
2.0367650
1.1034350
1.8236390
2.4017960
1.0228660
0.7053280
0.2588710
1.9633080
-0.1545990
-0.0632720
-0.9268250
-0.4933310
1.2484950
1.1817350
0.3085880
1.3721860
3.7430490
3.7002990
3.8718750
4.6222610
-0.1642290
-0.1725790
-1.3434720
2.5152670
1.9106680
3.3181330
0.0438390
-6.4179480
-1.2779290

$-1.6804580$

$-1.3299110$

$-0.5330250$

$-2.2127070$

$-1.5950820$

3.6985360

3.5508630

3.7065850

4.6796330

2.8718530

3.0023900

2.0568470

3.8022160

2. 3688100

2.6099580

1. 5913100

3.2700740

3.0016510

2. 6547240

3.3213640

3.8751950

$-1.5790310$

$-1.3627300$

$-2.2200320$

$-2.1429850$

0.5036920

1.4572290

$-0.0889180$

0.7052430

$-4.1304860$

$-4.3833580$

$-3.6679700$

$-5.0691500$

$-3.8702030$

$-4.0060450$

$-4.8563480$

$-3.2581700$

$-0.6579750$

$-2.0384800$

0.2492660

$-3.0933900$

0.3556440

1. 6714200

$-1.4510870$

$-0.0172650$

2.6021090 
144

$\begin{array}{lll}2.6174610 & -1.9783680 & -1.7532610 \\ 1.7686100 & -3.0768510 & -1.2672160 \\ 2.5640420 & -1.0488540 & -1.1797770 \\ 0.8365580 & -2.8015690 & -0.7858030 \\ 1.6420190 & -1.9863100 & -2.8395630 \\ 0.9379180 & -1.2612360 & -2.5638570 \\ 4.0845840 & -3.6897200 & -2.9208090 \\ 3.4803600 & -4.7846880 & -2.0268160 \\ 2.0093750 & -4.4860970 & -1.6856900 \\ 4.0173510 & -2.3225950 & -2.2199190 \\ 5.1292400 & -3.9278050 & -3.1544320 \\ 3.5449190 & -5.7635100 & -2.5164410 \\ 1.3633110 & -4.6687520 & -2.5574530 \\ 4.6555080 & -2.3364450 & -1.3267380 \\ 4.3759920 & -1.5157740 & -2.8701480 \\ 3.5376590 & -3.6413270 & -3.8718050 \\ 4.0517750 & -4.8430520 & -1.0914330 \\ 1.6317420 & -5.1359690 & -0.8876020 \\ 2.7119690 & -2.3523120 & 1.6021980 \\ 1.8045770 & -1.4101900 & 1.7323220 \\ 3.8780350 & -2.2066030 & 2.5248990 \\ 4.9816060 & -3.0610480 & 2.3692980 \\ 3.9074440 & -1.2099040 & 3.5149310 \\ 6.1030550 & -2.9144710 & 3.1841750 \\ 4.9500020 & -3.8271830 & 1.6000170 \\ 5.0299620 & -1.0683910 & 4.3308050 \\ 3.0586980 & -0.5449980 & 3.6299020 \\ 6.1299890 & -1.9171170 & 4.1654940 \\ 6.9578950 & -3.5739700 & 3.0536480 \\ 5.0483110 & -0.2931070 & 5.0933940 \\ 7.0066250 & -1.8012990 & 4.7992040 \\ 0.9917760 & -1.4740630 & 1.0977160 \\ 2.6556900 & -3.2892140 & 0.7889790\end{array}$

2.6556900

$-3.2892140$

2.7790670

4.6530870

3.6047400

4.5759400

5. 3906570

5. 2905210

4. 3020240

3.4819110

5.4166840

5.9352240

4. 1830170

2.7272580

6.1535380

3.7992560

2.7716520

1.4520690

3.4924990

4.8401900

3. 0262240

0.6527060

4. 2883210

$-2.0179790$

$-2.7561950$

$-2.6218300$

$-4.0858960$

$-3.9535110$

$-4.7052730$

$-4.6663100$

$-4.4115690$

3.8019230

3. 2191260

4. 3502920

3. 2168060

4.3191240

3.7531940

2.7626380

4.7398740

$-1.8996820$

$0.0865600 \quad-1.1609030$

$\begin{array}{ll}2.0834640 & -0.0960110 \\ 0.4500970 & -2.4115570\end{array}$

$1.7843460 \quad-2.7995630$

$-0.5970730 \quad-3.2519800$

2. 1432770

$-4.0535280$

$-4.4941510$

1.1031270

$-4.9108330$

$-1.0287340$

$-5.1711550$

2.8800000
3.7961790

$-0.5798830$

0.3304330

$-0.2471230$

$-0.1897560$

$-0.7138000$

$-0.7874920$

$-1.2025340$

$-1.2882230$

$-1.5445860$

0.1242740

0.7157270
$-6.1381560$

$-2.1609350$

2.6062170

3.6778990

4.9888970

6.5030210

6.9938710
6.6914070
-1.9195150
-0.1604420
-2.3925760
-1.5005870
0.5022650
-3.2460170
-4.1101770
-3.6946080
-1.9655300
-3.5882970
-5.1102520
-4.3685850
-1.2795940
-5.6859100
-6.5462450
-6.1605990
-4.4727410
-5.9811560
-7.5025140
-6.8031330
-3.8198490
-2.8037730
-2.4507530
-2.7313310
-2.0432360
-2.3105680
-1.9619470
-1.7792040
-2.2666510
1.7658180
2.7425640
2.1376430
4.0750310
3.4861410
4.4673630
4.8293470
3.7779930
-3.1714450
-1.4587120
-2.5470900
2.3791820
5.9135720
1.1272950
1.3829650
2.3774580
1.3332820

1.6781550

1.5583640

2.1329640

2.1236120

3. 6768800

3. 1471010

2. 1169320

3.1805620

4.4834400

3. 5585490

1. 7260880

3.5952520

$-1.5249460$

$-1.9823880$

$-1.8682030$

$-0.9415350$

$-1.6381610$

$-2.4341830$

$-2.2337680$

$-0.5973870$

$-0.6063630$

$-1.7598100$

0.6682370

$-1.6043740$

0.7700650

$-0.3528870$

$-2.4868330$

1.7541180

0.3033110

1.1471620

$-0.9408290$

0.7251530

$-1.3185930$

$-0.5054050$

1. 3624720

$-2.2799520$

1.9364410

$-0.2626130$

$-3.1590190$

2. 4950760

$-0.9617600$

$-1.8840270$

$-2.0145360$

$-2.4403580$

$-1.0339660$ 


\begin{tabular}{|c|c|c|c|}
\hline & & & \\
\hline $\mathrm{H}$ & 6.9609800 & 0.6336160 & $\begin{array}{l}-2.6743340 \\
-3.2658250\end{array}$ \\
\hline $\mathrm{H}$ & $\begin{array}{l}4.3098470 \\
3.2364690\end{array}$ & $\begin{array}{l}1.1 \angle 6 \perp 170 \\
0.9302580\end{array}$ & $\begin{array}{l}-3.2658<50 \\
-3.1679440\end{array}$ \\
\hline $\mathrm{H}$ & 4.4444320 & 2.0931300 & -3.7684050 \\
\hline $\mathrm{H}$ & 4.7535420 & 0.3487470 & -3.9021870 \\
\hline C & 4.3602500 & 6.8702230 & 0.0332830 \\
\hline $\mathrm{H}$ & 4.3233760 & 7.9023480 & -0.3403130 \\
\hline $\mathrm{H}$ & 3.8506840 & 6.8421120 & 1.0054100 \\
\hline $\mathrm{H}$ & 5.4095670 & 6.5906170 & 0.1905050 \\
\hline C & 2.2130040 & 6.3340760 & -1.1987320 \\
\hline $\mathrm{H}$ & 1.7326480 & 5.6764090 & -1.9343290 \\
\hline $\mathrm{H}$ & 1.6445230 & 6.2696900 & -0.2614790 \\
\hline $\mathrm{H}$ & 2.1617780 & 7.3691510 & -1.5630480 \\
\hline C & 3.6300750 & 2.5859040 & 3.6301570 \\
\hline $\mathrm{H}$ & 4.5339010 & 1.9880460 & 3.4706100 \\
\hline $\mathrm{H}$ & 3.9235960 & 3.6433930 & 3.681365 \\
\hline $\mathrm{H}$ & 3.1910480 & 2.3019030 & 4.5964440 \\
\hline C & 1.3198870 & 3.1627460 & 2.8140130 \\
\hline $\mathrm{H}$ & 1.5376870 & 4.2186220 & 3.0227910 \\
\hline $\mathrm{H}$ & 0.6122410 & 3.1062740 & 1.9824920 \\
\hline $\mathrm{H}$ & 0.8472780 & 2.7395240 & 3.7097700 \\
\hline C & -2.4613190 & -4.5227630 & 2.4213170 \\
\hline $\mathrm{H}$ & -3.5262860 & -4.4264890 & 2.6718480 \\
\hline $\mathrm{H}$ & -2.3617560 & -5.2887740 & 1.6416850 \\
\hline $\mathrm{H}$ & -1.9230880 & -4.8612060 & 3.3170770 \\
\hline C & -1.9652530 & -2.1175540 & 3.0548050 \\
\hline $\mathrm{H}$ & -1.5851770 & -1.1529890 & 2.7019690 \\
\hline $\mathrm{H}$ & -2.9968640 & -1.9813790 & 3.4056680 \\
\hline $\mathrm{H}$ & -1.3621950 & -2.4448370 & 3.9118940 \\
\hline C & -6.1611020 & 0.0824300 & -0.3335730 \\
\hline $\mathrm{H}$ & -7.1949170 & 0.4541580 & -0.3259480 \\
\hline $\mathrm{H}$ & -5.6357970 & 0.5073300 & 0.5300630 \\
\hline $\mathrm{H}$ & -5.6681990 & 0.4365510 & -1.2457310 \\
\hline C & -6.8962960 & -1.9407640 & 0.9828040 \\
\hline $\mathrm{H}$ & -6.8603250 & -3.0334710 & 1.0759750 \\
\hline $\mathrm{H}$ & -6.4691350 & -1.5007280 & 1.8929140 \\
\hline $\mathrm{H}$ & -7.9463900 & -1.6272990 & 0.9243210 \\
\hline C & -2.5025650 & -1.3401170 & -4.0494550 \\
\hline $\mathrm{H}$ & -3.5760090 & -1.3015370 & -4.2768700 \\
\hline $\mathrm{H}$ & -2.2105490 & -0.4028460 & -3.5685380 \\
\hline $\mathrm{H}$ & -1.9640880 & -1.4220620 & -5.0024710 \\
\hline$C$ & -2.6199770 & -3.8495350 & -3.8466110 \\
\hline $\mathrm{H}$ & -3.7118690 & -3.8469860 & -3.9682350 \\
\hline $\mathrm{H}$ & -2.1614040 & -3.9387680 & -4.8407920 \\
\hline $\mathrm{H}$ & -2.3465840 & -4.7318960 & -3.2565270 \\
\hline & 0.4983510 & 0.1517410 & 64637 \\
\hline
\end{tabular}
$-0.1276520$
0.2524870
$-1.0706490$
$-6.6672680$
$-0.8420010$
4.8652370
4.2095150
2. 3402560
$-2.5788440$
$-1.6929290$
$-2.5846550$
$-0.7869190$
$-1.5787330$
$-0.9120920$
$-3.9395030$
$-3.3070390$
$-1.8594650$
$-3.9501610$
$-4.9668730$
$-3.3156180$
$-1.1865990$
$-4.6086360$
$-4.3284600$
$-3.3739330$
$-3.8957220$
$-1.4661990$
$-2.7967860$
$-1.9132170$
$-4.0773650$
$-5.1287550$
$-4.2671690$
$-6.3549410$
$-4.9729560$
$-5.4946310$
$-3.4598160$
$-6.5406830$
$-7.1674240$
$-5.6383780$
$-7.4992520$
$-1.0434430$
$-2.6322640$

144

$\operatorname{TS} 105(R, R) \quad G[B 97-D /$ def2-TZVP $]=-3310.751801$

$\begin{array}{lrrr} & & \\ 0 & 0.4106500 & 0.6947810 & 0.0671120 \\ O & -1.9350270 & 0.6492560 & -0.9327960 \\ C & 0.4336710 & 2.0739010 & -0.0711850\end{array}$

$-2.0121000$

0.2697500

$-3.0620120$

$-1.1495920$

1.7060880

$-1.4588000$

$-1.9214560$

2.4651930

$-1.8254020$

$-1.3069390$

$-1.2557690$

$-0.8046040$

$-2.8880160$

$-2.5890730$

$-3.0014160$

$-2.0793350$

$-1.7113670$

$-3.2545510$

$-2.5565460$

$-2.5670280$

$-1.4406380$

$-2.9915460$

$-3.9408730$

$-1.1557520$

$-0.8984610$

1.4445350

1.6082190

2. 1820570

2.0099620

2.9972010

2.6379750

1. 3741200

3.6252740

3.1195490

3.4451240

2. 4962400

4.2514050

3.9306940

$\begin{array}{ll}1.4281300 & 1.0550400 \\ 3.2961140 & 0.7301290\end{array}$

$\begin{array}{ll}1.4790760 & 3.9306940 \\ 1.4281300 & 1.0550400\end{array}$

$-0.0711850$ 


-0.6758000
1.6405110
-0.6557990
1.6789680
0.5500500
2.5982030
-2.4637640
-3.6341750
-1.8498660
-4.1665790
-2.3570590
-3.5466480
-5.0749740
-3.4286660
-2.2332170
-1.7125480
-4.0665580
-3.8323300
-1.7173070
-0.7940560
-4.9738450
-1.7313750
-0.5301290
0.5848820
-1.7922790
-2.6168850
-0.4944760
1.5102530
-2.7201770
2.8512040
3.2561270
3.6236540
4.4544990
4.8128010
5.2543290
4.7773400
5.4103470
-4.2643370
-4.2133270
-4.9165880
-4.8405750
-5.5167730
-5.4926500
-4.8076880
-6.0190070
3.2300110

2.8190530

2.6631860

4.2359810

4.0368420

4.8477580

4.5123430

1.0846200

0.4356440

2.1460340

0.8811810

2.5360950

1.9007800

0.4209800

3.2407680

3.8373820

3.4954050

2.2884240

3.5248240

4.5695730

3.9591060

1. 8036500

6.4258780

7.0313210

6.2530770

5.0657180

7.0377910

8.1052320

6.7002080

4.6148680

1.8439720

1.6556500

1. 3121240

0.9781230

0.6364570

0.4726970

0.8347680

0.2350110

$-0.6770570$

$-2.0004660$

$-0.4025300$

$-3.0153830$

$-1.4535300$

$-2.7673590$

$-4.0374560$

-1.2373970
1.5447060
0.3168710

$-0.5344790$

0.0927470

$-0.6637700$

$-0.3937070$

$-0.9976000$

0.2799810

0.7575750

0.9317530

1.9503670

2.2165670

2.7146780

2.3309940

4.7467400

4.2769600

3.0448310

3.9796250

5.7161520

4.8944910

2.7003400

4.3363670

0.0704490

$-0.3732390$

$-0.6038260$

0.2979530

0.2284940

$-0.542$

-0.9632830
0.6344270

$-0.8412140$

$-2.1817290$

0.2162530

$-2.4301930$

$-0.0873190$

$-1.4022510$

$-3.4600170$

0.7245930

$-0.0123070$

0.4817880

$-1.2366530$

$-0.2523550$

$-1.9351940$

$-1.4609040$

0.1203520

-2.8757910
1.6704350
6.5759290

2. 4488300

$-3.5092200$

$-6.1290680$

$-5.0251170$

$-6.4920030$

$-7.1045070$

$-6.9146200$

$-6.5600920$

$-4.4165570$

$-3.3712000$

$-4.9737730$

$-4.4599850$

$-5.3820500$

$-5.8227010$

$-5.4486110$

$-4.3201890$

$-7.6259250$

$-8.1715500$

$-7.7627860$

$-8.0685430$

$-4.5340070$

$-5.1413400$

$-5.2107930$

$-4.0207110$

$-2.6231640$

$-3.2308290$

$-1.9314950$

$-2.0409210$

3.7720520

4.8699990

3.4013420

3.4594900

3.6777810

3. 3815230

4.7667100

3.2159110

7.1444760

8.0424290

7.4316570

6.4161600

7.6162250

7.2443590

7.8278820

8.5554810

2.2540180
-0.1932500
2.1971520
-2.3605210
-3.8966070
1.0042070
1.4783570
0.8284430
1.4569390
2.5042660
1.0934190
0.7667980
0.4629830
2.1290340
-4.1265680
-4.9703900
-3.2323650
-4.3371440
-3.6445950
-3.5039270
-2.7431510
-4.4922140
-2.5577480
-1.6578600
-3.3880030
-2.7950280
-3.6145540
-4.5175870
-3.5146770
-3.7465840
2.9083190
2.9100480
3.7206420
3.1028950
0.4283210
-0.5576290
0.4295930
0.5760940
-1.1033450
-1.6143450
-0.5183390
-1.8601210
0.8801890
1.5129840
1.5247200
0.4091670
1.1596340

$-1.7547590$

$-3.3553970$

1.7850150

$-2.2517390$

$-1.8120530$

$-1.8162740$

$-2.4550870$

$-0.8034540$

$-2.2026860$

$-3.2236060$

$-3.2147050$

$-3.9289500$

$-3.5868260$

$-3.5803710$

$-4.1285670$

$-4.2141670$

$-3.4018440$

$-2.5118130$

$-1.5702500$

$-3.1234050$

$-3.0520280$

2. 9202910

3.0717830

2.6768360

3.8622350

1.6594180

1.5146160

0.8177410

2.5805170

2. 1469760

2.1092170

1. 5113540

3. 1819760

2. 6258150

2.2535950

2.7662590

3.6093270

$-0.6574960$

$-1.0260560$

0.2260660

$-0.3421460$

$-2.1411050$

$-2.9558700$

$-1.2771580$

$-2.4613540$

$-4.4743280$ 


\begin{tabular}{lrr}
3.2096180 & 0.8951790 & -4.9454580 \\
1.7904710 & 0.2491140 & -4.0842520 \\
1.6013760 & 1.5764060 & -5.2522270 \\
3.1093850 & 3.4709460 & -3.9197880 \\
4.1146790 & 3.2408420 & -4.2982360 \\
2.5119070 & 3.8741830 & -4.7483840 \\
3.2036630 & 4.2456230 & -3.1496840 \\
-0.5694620 & -0.2292020 & -0.8711330 \\
-0.0765510 & -0.3174710 & -2.2852300 \\
-0.7490200 & -1.4936150 & -0.0742370 \\
1.4535770 & 2.4688060 & -2.9883110 \\
6.3961890 & -0.8125020 & -2.6467970 \\
2.1378170 & 1.5882950 & 1.7257430 \\
-4.4661850 & 1.6935860 & -1.1717200 \\
-6.0386740 & -4.8121780 & -1.6494940 \\
-2.8538010 & -1.5274440 & 2.0584800 \\
2.4530420 & -2.1054660 & -1.7025430 \\
1.5192190 & -3.1583260 & -1.2728600 \\
2.3771820 & -1.1471320 & -1.1815740 \\
0.5427760 & -2.8423770 & -0.9230940 \\
1.6042460 & -2.1352090 & -2.8969760 \\
0.8770570 & -1.4169950 & -2.7226470 \\
3.9917320 & -3.9252470 & -2.5962740 \\
3.2595990 & -4.9460990 & -1.7130930 \\
1.7681470 & -4.5939910 & -1.5838700 \\
3.8809880 & -2.5141070 & -1.9984450 \\
5.0503630 & -4.1960390 & -2.6918260 \\
3.3573740 & -5.9583280 & -2.1230300 \\
1.2383850 & -4.8058180 & -2.5251570 \\
4.4234270 & -2.4657220 & -1.0460020 \\
4.3215410 & -1.7605590 & -2.6571380 \\
3.5588820 & -3.9307610 & -3.6057320 \\
3.7084990 & -4.9473780 & -0.7112990 \\
1.2664130 & -5.1911070 & -0.8134040 \\
1.6752040 & -2.7760480 & 1.9391150 \\
0.5904350 & -2.0398290 & 2.0151650 \\
2.3817780 & -2.9583730 & 3.2466430 \\
3.5878840 & -3.6776830 & 3.2767960 \\
1.8761900 & -2.4062280 & 4.4358810 \\
4.2803090 & -3.8391510 & 4.4761830 \\
3.9736780 & -4.0981510 & 2.3526140 \\
2.5700810 & -2.5720170 & 5.6346920 \\
0.9482610 & -1.8439090 & 4.4121910 \\
3.7727710 & -3.2863970 & 5.6575420 \\
5.2153970 & -4.3944170 & 6.5514090 \\
2.1743060 & -2.1409600 & \\
\hline & &
\end{tabular}

$\begin{array}{llll}\mathrm{H} & 4.3137550 & -3.4113780 & 6.5931200 \\ \mathrm{H} & 0.1035240 & -1.8615570 & 1.1197360 \\ \mathrm{O} & 2.1257230 & -3.2956740 & 0.9058250\end{array}$

TS106 $(R, R)$ G [B97-D/def2-TZVP] $=-3310.751710$

$\begin{array}{lrlr}\mathrm{O} & 0.1945290 & 0.9533290 & 0.1577320 \\ \mathrm{O} & -2.0286350 & 0.4188570 & -0.9530420 \\ \mathrm{C} & 0.0588350 & 2.3004690 & -0.1494280 \\ \mathrm{C} & -1.1713760 & 2.9170960 & 0.0425160 \\ \mathrm{C} & 1.2255060 & 2.9934310 & -0.5764780 \\ \mathrm{C} & -1.3127670 & 4.2828450 & -0.3739530 \\ \mathrm{C} & 1.0990970 & 4.3309090 & -0.8893380 \\ \mathrm{C} & -0.1526220 & 4.9945200 & -0.8368560 \\ \mathrm{H} & 1.9774290 & 4.8923460 & -1.1979420 \\ \mathrm{C} & -2.6803140 & 0.9296760 & 0.1611000 \\ \mathrm{C} & -3.7704760 & 0.1781260 & 0.6772670 \\ \mathrm{C} & -2.2865320 & 2.1611910 & 0.6727870 \\ \mathrm{C} & -4.4515810 & 0.6964750 & 1.7595370 \\ \mathrm{C} & -2.9428520 & 2.6449290 & 1.8556100 \\ \mathrm{C} & -4.0549180 & 1.9033770 & 2.3862650 \\ \mathrm{H} & -5.3033700 & 0.1525820 & 2.1609370 \\ \mathrm{C} & -4.2992290 & 3.5245700 & 4.1895090 \\ \mathrm{C} & -3.1774530 & 4.2331590 & 3.6947970 \\ \mathrm{C} & -2.5178760 & 3.8052230 & 2.5598790 \\ \mathrm{C} & -4.7220640 & 2.3795150 & 3.5477330 \\ \mathrm{H} & -4.8140780 & 3.8767330 & 5.0807180 \\ \mathrm{H} & -2.8274370 & 5.1212820 & 4.2166700 \\ \mathrm{H} & -1.6566630 & 4.3567740 & 2.1988640 \\ \mathrm{H} & -5.5687310 & 1.8099680 & 3.9275090 \\ \mathrm{C} & -2.6598290 & 6.2730350 & -0.7923560 \\ \mathrm{C} & -1.5095810 & 6.9832920 & -1.2141250 \\ \mathrm{C} & -0.2834020 & 6.3518050 & -1.2378330 \\ \mathrm{C} & -2.5631550 & 4.9584540 & -0.3815790 \\ \mathrm{H} & -3.6295980 & 6.7660430 & -0.7961480 \\ \mathrm{H} & -1.5983180 & 8.0206180 & -1.5294450 \\ \mathrm{H} & 0.6063460 & 6.8800830 & -1.5765440 \\ \mathrm{H} & -3.4518930 & 4.4235260 & -0.0620820 \\ \mathrm{C} & 2.5348660 & 2.2790270 & -0.6150210 \\ \mathrm{C} & 3.1598020 & 1.9759800 & -1.8459830 \\ \mathrm{C} & 3.1338820 & 1.8780380 & 0.6021740 \\ \mathrm{C} & 4.3594900 & 1.2527600 & -1.8274660 \\ \mathrm{C} & 4.3213260 & 1.1415980 & 0.5635560 \\ \mathrm{C} & 4.9444120 & 0.8063050 & -0.6407030 \\ \mathrm{H} & 4.8499780 & 1.0132610 & -2.7687020 \\ \mathrm{H} & 4.7734900 & 0.8292240 & 1.5014680 \\ \mathrm{C} & -4.1692090 & -1.1232230 & 0.0647720\end{array}$




$\begin{array}{rrr}-3.9256220 & -2.3332370 & 0.7573300 \\ -4.7991490 & -1.1450010 & -1.1977030 \\ -4.3484690 & -3.5348470 & 0.1812560 \\ -5.1955720 & -2.3761780 & -1.7343460 \\ -4.9844340 & -3.5802130 & -1.0628720 \\ -4.1639300 & -4.4657160 & 0.7124810 \\ -5.6861350 & -2.3960490 & -2.7065070 \\ 2.5796440 & 2.2954880 & 1.9594800 \\ 6.2523840 & 0.0349490 & -0.6678890 \\ 2.5953220 & 2.4543920 & -3.1777160 \\ -3.2254760 & -2.3692810 & 2.1110270 \\ -5.4143430 & -4.9022900 & -1.6726160 \\ -5.0922730 & 0.1216010 & -1.9919130 \\ -6.6114030 & 0.3477150 & -2.1221580 \\ -7.0785770 & -0.4790350 & -2.6733450 \\ -7.0849620 & 0.4094540 & -1.1338590 \\ -6.8124430 & 1.2805610 & -2.6660010 \\ -4.4210530 & 0.0897900 & -3.3774060 \\ -3.3391120 & -0.0489120 & -3.2783380 \\ -4.8252410 & -0.7292980 & -3.9868620 \\ -4.6075400 & 1.0330290 & -3.9083640 \\ -6.4342460 & -5.6334940 & -0.7792390 \\ -6.7636830 & -6.5662410 & -1.2564660 \\ -5.9825350 & -5.8867160 & 0.1890170 \\ -7.3135700 & -5.0046960 & -0.5910290 \\ -4.1985680 & -5.8059790 & -1.9564700 \\ -3.4842760 & -5.3038240 & -2.6210210 \\ -3.6789410 & -6.0501990 & -1.0205510 \\ -4.5187460 & -6.7454410 & -2.4271740 \\ -4.2471190 & -2.5800860 & 3.2458330 \\ -5.0053170 & -1.7881670 & 3.2510320 \\ -4.7603720 & -3.5427760 & 3.1170400 \\ -3.7411530 & -2.5854780 & 4.2209820 \\ -2.1259310 & -3.4454950 & 2.1827680 \\ -2.5549720 & -4.4557090 & 2.1498740 \\ -1.4226120 & -3.3321900 & 1.3524870 \\ -1.5742700 & -3.3470910 & 3.1267670 \\ 3.5004950 & 3.3589100 & 2.5924060 \\ 4.4990280 & 2.9407100 & 2.7774030 \\ 3.6107310 & 4.2268060 & 1.9295940 \\ 3.0854420 & 3.6991190 & 3.5507580 \\ 2.3792040 & 1.1087000 & 2.9149630 \\ 1.7033060 & 0.3670540 & 3.1400630 \\ 3.3324080 & 0.6156140 & 3.8627290 \\ 1.9510120 & 1.4619860 & \\ 6.2726610 & -1.1445710 & \\ -4 & & \\ -4 & -3192540\end{array}$

7.1893560

6.2524130

5.4061930

7.4379510

7.4568120

7. 3482020

8.3906740

2. 6161070

3.6428270

2.0774820

2. 1330900

3. 3616990

4.4163300

2. 9310520

3.3272770

$-0.5087560$

0.0375170

$-0.4438320$

1. 5485930

6.3723310

1. 6021170

$-4.6832440$

$-5.9021210$

$-2.7389570$

2.6244530

1.8321790

2. 5836070

0.9081560

1. 5849120

0.8741330

4.0875290

3. 5698760

2. 1027180

4.0022490

5.1278170

3.6554920

1.4358220

4.6973490

4.2778290

3.4925190

4.1794700

1.7722620

2.4020990

1.2353950

3.2782830

4.5403040
$-1.7337500$
$-0.7882680$
$-1.8008560$
0.9835700
1.8056980
1.4174090
0.4395980
1.3813540
1.1009480
0.4839260
1.7751160
3.7068640
3.4576490
4.0878490
4.5054600
$-0.1487900$
$-0.0900120$
$-1.4422210$
2.7358470
$-0.3674350$
2.7646450
0.9805810
$-4.6797650$
$-1.4010640$
$-1.7795560$
$-2.8880090$
$-0.8376940$
$-2.6310120$
$-1.8407170$
$-1.1528890$
$-3.4860980$
-4. 5610010
$-4.3020110$
$-2.0924610$
$-3.6959480$
$-5.5592600$
$-4.5294330$
$-2.0285920$
$-1.3033200$
$-3.5018270$
$-4.5499200$
$-4.9416460$
$-2.5905190$
$-2.0334550$
-2.7091410
-3.3128090

1.3566580

0.1790600

$-0.3960080$

$-1.1223090$

0.6089280

$-0.4545250$

$-4.2786440$

$-4.5478080$

$-3.9642160$

$-5.1821470$

$-3.6518100$

$-3.8324490$

$-4.5877180$

$-2.9014320$

$-0.8280420$

$-2.2259030$

$-0.0624890$

$-3.0226860$

$-1.6839810$

1. 8141270

$-1.4506620$

$-2.6326150$

2.2696330

$-2.1580720$

$-1.6075840$

$-1.6070020$

$-1.1018710$

$-3.1897110$

$-2.8651230$

$-3.3464100$

$-2.3776280$

$-1.9934940$

$-2.7025270$

$-3.6232550$

$-2.8228250$

$-2.8388130$

$-1.8570000$

3.4108990

$-4.2694370$

$-1.4647980$

$-1.1667820$

1.5291850

1.7691640

2.7361660

2.6088090 


$\begin{array}{llll}\mathrm{C} & 2.8739510 & -2.2119820 & 3.9870270 \\ \mathrm{C} & 5.3869710 & -3.4129040 & 3.7116460 \\ \mathrm{H} & 4.8478780 & -3.6917850 & 1.6393070 \\ \mathrm{C} & 3.7233290 & -2.3139050 & 5.0888660 \\ \mathrm{H} & 1.9024800 & -1.7401380 & 4.0867470 \\ \mathrm{C} & 4.9806980 & -2.9125370 & 4.9539950 \\ \mathrm{H} & 6.3640830 & -3.8781060 & 3.6036360 \\ \mathrm{H} & 3.4056550 & -1.9227140 & 6.0526010 \\ \mathrm{H} & 5.6424470 & -2.9885880 & 5.8141770 \\ \mathrm{H} & 0.6331480 & -1.8416440 & 0.9560240 \\ \mathrm{O} & 2.7938010 & -3.0008200 & 0.4258600\end{array}$

TS107 (R, R) G[B97-D/def2-TZVP $]=-3310.751651$

\begin{tabular}{|c|c|c|c|}
\hline 0 & -0.0210570 & 1.0726210 & 0.2704510 \\
\hline 0 & -2.0932730 & 0.2305080 & -0.9225230 \\
\hline C & -0.3044740 & 2.3770090 & -0.1153300 \\
\hline C & -1.6070240 & 2.8475340 & 0.0014280 \\
\hline C & 0.7879490 & 3.1773800 & -0.5514530 \\
\hline c & -1.8913840 & 4.1661430 & -0.4875660 \\
\hline C & 0.5165800 & 4.4747850 & -0.9331490 \\
\hline C & -0.8051620 & 4.9880690 & -0.9481530 \\
\hline $\mathrm{H}$ & 1.3314990 & 5.1215160 & -1.2483170 \\
\hline C & -2 & 0.6909560 & 0.1437670 \\
\hline C & -3.8487480 & -0.1867610 & 0.6550060 \\
\hline C & -2.6518160 & 1.9834770 & 0.6141580 \\
\hline C & -4.6560780 & 0.2822580 & 1.6703400 \\
\hline C & -3.4299800 & 2.4221930 & 1.7391160 \\
\hline C & -4.4645280 & 1.5622930 & 2.2469500 \\
\hline $\mathrm{H}$ & -5.4417050 & -0.3580290 & 2.0637170 \\
\hline C & -5.0201240 & 3.2108390 & 3.9538500 \\
\hline C & -3.9711430 & 4.0389870 & 3.4861640 \\
\hline C & -3.1975890 & 3.6551110 & 2.4088660 \\
\hline C & -5.2543980 & 1.9949810 & 3.3466970 \\
\hline $\mathrm{H}$ & -5.6265760 & 3.5283860 & 4.7992520 \\
\hline $\mathrm{H}$ & -3.7686410 & 4.9853700 & 3.9831350 \\
\hline $\mathrm{H}$ & -2.3950110 & 4.2999530 & 2.0666140 \\
\hline $\mathrm{H}$ & -6.0397800 & 1.3347000 & 3.7108320 \\
\hline C & -3.4442390 & 5.9604610 & -1.0530410 \\
\hline C & -2.3692870 & 6.7820580 & -1.4714150 \\
\hline C & -1.0777130 & 6.3000060 & -1.4218080 \\
\hline C & -3.2108660 & 4.6873500 & -0.5725950 \\
\hline $\mathrm{H}$ & -4.4641280 & 6.3339530 & -1.1150410 \\
\hline $\mathrm{H}$ & -2.5662530 & 7.7856860 & -1.8419100 \\
\hline $\mathrm{H}$ & -0.2428770 & 6.9128490 & -1.7578100 \\
\hline $\mathrm{H}$ & -4.0430860 & 4.0661890 & -0.2573690 \\
\hline$\Omega$ & 2.1 & & 40313 \\
\hline
\end{tabular}

\begin{abstract}
2.8462900
2.7846790

4.1140120

4.0501680

4.7279710

4.6444700

4.5167860

$-3.9885240$

$-3.5390530$

$-4.5407870$

$-3.6753510$

$-4.6506330$

$-4.2232710$

$-3.3272140$

$-5.0778800$

2. 1554830

6.1254660

2. 2665920

-2.9217440

$-4.3208470$

$-5.0427570$

$-6.5695700$

$-6.8412870$

$-7.0871690$

$-6.9273220$

$-4.3209110$

$-3.2385360$

$-4.5206890$

$-4.6771750$

$-5.1837670$

$-5.2723620$

$-4.7272100$

$-6.1902120$

$-2.9199030$

$-2.3079290$

-2. 4025730

$-2.9962360$

$-3.9697060$

$-4.8326740$

$-4.3304590$

$-3.5263910$

$-1.6877090$

$-1.9695020$

$-0.9668830$

-1.2037960
2.8575250
\end{abstract}

$-1.7494950$

0.6997930

$-1.6833450$

0.7101620

$-0.4704380$

$-2.6078300$

1.6668710

0.1148690

0.8831150

$-1.1661310$

0.3543380

$-1.6519230$

$-0.9113340$

0.9377440

$-2.6400070$

2.0257490

$-0.4642650$

$-3.1076800$

2.2659790

$-1.4714850$

$-2.0339590$

$-2.2257220$

$-2.7467050$

$-1.2578340$

$-2.8246910$

$-3.3937580$

$-3.2544480$

$-3.9699670$

$-3.9806800$

$-0.5783690$

$-1.0221500$

0.4144160

$-0.4475160$

$-1.6727120$

$-2.3287800$

$-0.7082950$

$-2.1171030$

3.3656210

3.2673340

3.2976540

4. 3600040

2.4953100

2.5667990

1. 6813640

3.4403980

2.5579810 


$\begin{array}{rrr}3.9189650 & 3.7935020 & 2.7522210 \\ 2.7951770 & 4.8161120 & 1.8281300 \\ 2.3903570 & 4.3288150 & 3.4957940 \\ 2.1707780 & 1.6143160 & 3.0788830 \\ 1.6908020 & 0.7101910 & 2.6912810 \\ 3.1974690 & 1.3639120 & 3.3751450 \\ 1.6347490 & 1.9451390 & 3.9782310 \\ 6.3691020 & -0.1795070 & 0.6794310 \\ 7.3492920 & -0.6576260 & 0.5578340 \\ 6.3663590 & 0.3278270 & 1.6519520 \\ 5.6015060 & -0.9603750 & 0.7107280 \\ 7.1793690 & 1.9439850 & -0.4220120 \\ 7.0495650 & 2.6361700 & -1.2631830 \\ 7.0784360 & 2.5156110 & 0.5104140 \\ 8.1943460 & 1.5253580 & -0.4636340 \\ 2.4439420 & 1.6174040 & -4.1813910 \\ 3.5038680 & 1.4533130 & -4.4164960 \\ 2.0073830 & 0.6690000 & -3.8590840 \\ 1.9451380 & 1.9341770 & -5.1065290 \\ 2.8971480 & 4.0244770 & -3.5977940 \\ 3.9754240 & 3.8897650 & -3.7590050 \\ 2.4414040 & 4.3348130 & -4.5477300 \\ 2.7633090 & 4.8300160 & -2.8665610 \\ -0.5287800 & -0.1441100 & -0.6960240 \\ 0.0773040 & -0.0590400 & -2.0730840 \\ -0.3431440 & -1.3937540 & 0.1148820 \\ 1.1908550 & 2.8672180 & -2.9859170 \\ 6.2526450 & 0.2810040 & -1.4159370 \\ 1.1080860 & 2.9960470 & 1.8546310 \\ -4.8334420 & 0.3130530 & -1.5214690 \\ -4.8055030 & -5.5283460 & -2.4560970 \\ -2.5898840 & -1.4574610 & 2.3594130 \\ 2.7144440 & -1.6765520 & -1.6700970 \\ 1.8631670 & -2.8632040 & -1.5279910 \\ 2.5239610 & -0.8457800 & -0.9864420 \\ 0.8724590 & -2.7191230 & -1.1146340 \\ 1.8576610 & -1.6002000 & -2.8566870 \\ 1.0574180 & -0.9563630 & -2.5700610 \\ 4.4284430 & -3.1028180 & -2.8842730 \\ 3.7746860 & -4.3513680 & -2.2719600 \\ 2.2519160 & -4.1772400 & -2.1312570 \\ 4.1778870 & -1.8711140 & -1.9991790 \\ 5.5087930 & -3.2560740 & -2.9950500 \\ 3.9817510 & -5.2370530 & -2.8842460 \\ 1.7630570 & -4.2406490 & -3.1133580 \\ 4.7042250 & -1.9850180 & -1.0423360\end{array}$

\begin{abstract}
4.5505240
4. 0173350

4. 1990460

1.8107490

2. 5527710

1. 6598580

3.7573250

4.8173420

3. 8727440

5. 9791120

4. 7182570

5.0364560

3.0583960

6. 0918810

6.7982730

5. 1220290

7.0004130

0.8667870

2. 4476580
\end{abstract}

144

TS108(R, R) G[B97-D/def2-TZVP $]=-3310.751477$

$\begin{array}{lrrr}\text { O } & 0.0509120 & 1.0539480 & 0.1946080 \\ \mathrm{O} & -2.0863340 & 0.2883850 & -0.9411470 \\ \mathrm{C} & -0.2020950 & 2.3744920 & -0.1523500 \\ \mathrm{C} & -1.4877460 & 2.8780260 & 0.0028630 \\ \mathrm{C} & 0.9047160 & 3.1573290 & -0.5846610 \\ \mathrm{C} & -1.7470800 & 4.2111390 & -0.4587680 \\ \mathrm{C} & 0.6601000 & 4.4666400 & -0.9425660 \\ \mathrm{C} & -0.6494320 & 5.0115140 & -0.9304300 \\ \mathrm{H} & 1.4867710 & 5.0995650 & -1.2553600 \\ \mathrm{C} & -2.7910990 & 0.7534930 & 0.1620790 \\ \mathrm{C} & -3.7857770 & -0.1035540 & 0.7040610 \\ \mathrm{C} & -2.5353630 & 2.0345240 & 0.6390760 \\ \mathrm{C} & -4.5378120 & 0.3723110 & 1.7577650 \\ \mathrm{C} & -3.2567740 & 2.4801780 & 1.7987540 \\ \mathrm{C} & -4.2904670 & 1.6399690 & 2.3404160 \\ \mathrm{H} & -5.3279380 & -0.2500230 & 2.1707250 \\ \mathrm{C} & -4.7398970 & 3.2839600 & 4.0824180 \\ \mathrm{C} & -3.6908940 & 4.0918360 & 3.5805710 \\ \mathrm{C} & -2.9694160 & 3.7007810 & 2.4702170 \\ \mathrm{C} & -5.0260440 & 2.0797490 & 3.4743500 \\ \mathrm{H} & -5.3052720 & 3.6076190 & 4.9535660 \\ \mathrm{H} & -3.4468800 & 5.0286530 & 4.0770550 \\ \mathrm{H} & -2.1662170 & 4.3305290 & 2.1027860 \\ \mathrm{H} & -5.8123500 & 1.4344150 & 3.8625740 \\ \mathrm{C} & -3.2646740 & 6.0524500 & -0.9641360\end{array}$

$-2.4646420$

-3.8864650

$-1.2753620$

1.4401640

1. 7019100

2. 3224110

2.0583220

3. 3749520

2.8270190

1. 2418770

4. 1424510

3. 5766390

3.8692450

2. 6126960

4.9529280

4.4662900

1.0479780

0.5310810
$-1.5119850$

$\begin{array}{lr}-2.9225480 & -3.8864650 \\ -4.5278290 & -1.2753620 \\ -4.9658340 & -1.5119850 \\ -2.6175810 & 1.4401640 \\ -1.6826820 & 1.7019100 \\ -2.5788950 & 2.3224110 \\ -3.4622700 & 2.0583220 \\ -1.6554350 & 3.3749520 \\ -3.4166970 & 2.8270190 \\ -4.1717580 & 1.2418770 \\ -1.6128830 & 4.1424510 \\ -0.9688560 & 3.5766390 \\ -2.4895060 & 3.8692450 \\ -4.0991510 & 2.6126960 \\ -0.8925490 & 4.9529280 \\ -2.4500480 & 4.4662900 \\ -1.6564860 & 1.0479780 \\ -3.4522620 & 0.5310810\end{array}$

$6.0524500 \quad-0.9641360$ 


-2.1781580
-0.8984240
-3.0539030
-4.2754660
-2.3571780
-0.0555700
-3.8942250
2.2715500
2.9435290
2.8746820
4.1895850
4.1176090
4.7852460
4.7146480
4.5740950
-4.0116770
-3.5376070
-4.6915020
-3.7856120
-4.9083710
-4.4655910
-3.4247990
-5.4346750
2.2572350
6.1518940
2.3792580
-2.7779420
-4.6859010
-5.2129750
-6.7523430
-7.1056740
-7.1898120
-7.1187050
-4.6002330
-3.5067680
-4.8876570
-4.9598200
-5.4871930
-5.6561110
-4.9380500
-6.4597710
-3.3444090
-2.7806240
-2.7291570
-3.5144780
-3.7297560

6.8511960

6.3360640

4.7673960

6.4533190

7.8642390

6.9324260

4.1630000

2. 5567220

2.2653160

2. 2408940

1.6288990

1.6002170

1.2696770

1. 3966150

1.3559710

$-1.4669830$

$-2.6070210$

$-1.6119070$

$-3.8707650$

$-2.9005300$

$-4.0404080$

$-4.7513540$

$-3.0175260$

2.6601100

0.6056500

2. 6734610

$-2.4904230$

$-5.4261610$

$-0.4189610$

$-0.4353420$

$-1.3346130$

$-0.4308710$

0.4447640

$-0.3622320$

$-0.3212330$

$-1.2460170$

0.5293250

$-6.3272640$

$-7.3139740$

$-6.4716250$

$-5.8793340$

$-6.0894610$

$-5.4653700$

$-6.2294950$

$-7.0736990$

$-2.6804890$

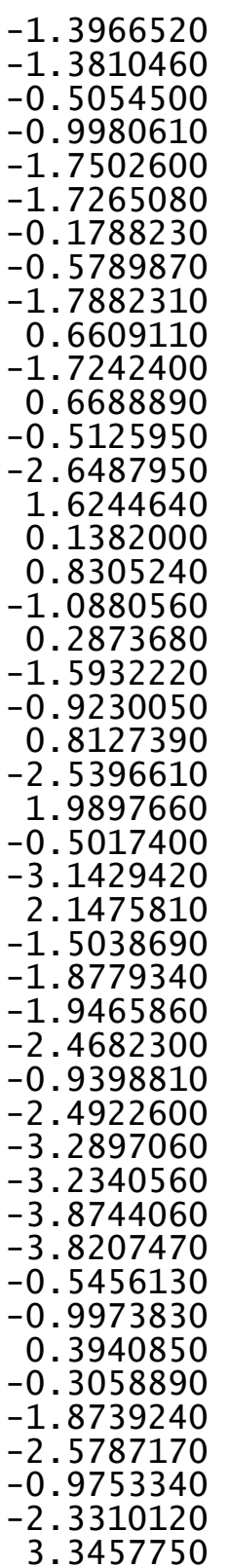

\begin{abstract}
$-4.5372940$
$-4.1815660$

$-3.1780650$

$-1.6018570$

$-1.9572190$

$-0.9617150$

$-1.0015650$

3.0140850

4.0632960

2.9957740

2.5563600

2.2148330

1.6889910

3.2263830

1.6961400

6. 3119120

7.2675920

6.3082380

5.5016070

7.2586810

7.1848510

7.1606580

8.2527630

2. 5319830

3.5878640

2. 0819220

2.0336240

3.0461610

4.1222550

2.6069850

2.9255410

$-0.5213140$

0.0400950

$-0.3412430$

1. 3076130

6.2754960

1.2241100

$-4.9210780$

$-5.2707020$

$-2.3582800$

2. 6442280

1. 8194280

2.4834170

0.8317860

1.7816040

1.0095610
\end{abstract}

-1.9402030
-3.6813110
-2.5821560
-3.4778560
-4.5108540
-3.4189090
-3.2356890
3.8835930
3.6257240
4.7145990
4.2201390
1.5256770
0.6541360
1.2157810
1.8694490
-0.4503690
-0.9769670
0.0140320
-1.1871040
1.6745080
2.4062610
2.2118490
1.2078740
1.5968540
1.4182900
0.6518610
1.9321510
3.9838050
3.8241050
4.3165400
4.7838590
-0.1239210
-0.0287000
-1.3947960
2.8637080
0.1030180
2.9744890
0.4998730
-5.3074440
-1.4799060
-1.7229150
-2.9163410
-0.8794310
-2.7504560
-1.6311840
-0.9864740

3.3370180

3. 3118020

4.2908060

2. 2574090

2. 3695650

1. 3726470

3.1438380

2. 5462860

2.7440820

1.8296360

3.4864260

3.0251330

2.6234110

3. 3164170

3.9299930

0.6038810

0.4864100

1. 5978310

0.5798780

$-0.3849860$

$-1.1990120$

0.5678840

$-0.4192950$

$-4.2298430$

$-4.4726570$

$-3.9156400$

$-5.1486000$

$-3.6124620$

$-3.7655870$

$-4.5626870$

$-2.8728520$

$-0.7870030$

$-2.1780660$

$-0.0034580$

$-3.0225120$

$-1.4718820$

1. 8169970

$-1.3587110$

$-2.4273230$

2.2041970

$-1.8491740$

$-1.6018100$

$-1.1721970$

$-1.1862830$

$-3.0232150$

$-2.7330560$ 


$\begin{array}{lll}4.3245390 & -3.1901620 & -3.0616430 \\ 3.6983190 & -4.4213660 & -2.3882560 \\ 2.1831990 & -4.2398250 & -2.1855920 \\ 4.1014940 & -1.9339670 & -2.2042440 \\ 5.4004570 & -3.3454340 & -3.2073590 \\ 3.8763470 & -5.3234590 & -2.9854590 \\ 1.6530270 & -4.3171300 & -3.1463100 \\ 4.6489960 & -2.0280970 & -1.2576050 \\ 4.4667590 & -1.0300430 & -2.7031600 \\ 3.8763400 & -3.0409520 & -4.0529740 \\ 4.1650740 & -4.5730230 & -1.4066470 \\ 1.7591470 & -5.0163400 & -1.5387910 \\ 2.5107060 & -2.6273660 & 1.4115990 \\ 1.5951450 & -1.7096750 & 1.6240580 \\ 3.6047570 & -2.6553660 & 2.4287480 \\ 4.6952040 & -3.5183330 & 2.2284240 \\ 3.5824160 & -1.8195260 & 3.5580890 \\ 5.7513730 & -3.5399090 & 3.1375110 \\ 4.7045640 & -4.1601460 & 1.3523510 \\ 4.6408800 & -1.8444490 & 4.4665540 \\ 2.7439400 & -1.1496150 & 3.7119420 \\ 5.7270790 & -2.7012790 & 4.2577200 \\ 6.5946990 & -4.2066170 & 2.9732600 \\ 4.6195750 & -1.1928750 & 5.3371230 \\ 6.5523300 & -2.7158960 & 4.9664440 \\ 0.8370270 & -1.6596920 & 0.9217150 \\ 2.5192980 & -3.4174070 & 0.4532230\end{array}$

144

$\begin{array}{lrrr}\text { TS109(R, R) } \text { G }[\mathrm{B} 97-\mathrm{D} / \mathrm{def} 2-\mathrm{TZVP}]=-3310.751448 \\ \mathrm{O} & 0.0670000 & -1.1283130 & 0.2764640 \\ \mathrm{O} & 2.0802260 & -0.0798760 & -0.8409790 \\ \mathrm{C} & 0.3260080 & -2.3568190 & -0.3182140 \\ \mathrm{C} & 1.6326270 & -2.8274400 & -0.3563180 \\ \mathrm{C} & -0.7949640 & -3.0877930 & -0.8014170 \\ \mathrm{C} & 1.8831910 & -4.0463670 & -1.0713050 \\ \mathrm{C} & -0.5508020 & -4.3025570 & -1.4065390 \\ \mathrm{C} & 0.7682140 & -4.7917400 & -1.5907510 \\ \mathrm{H} & -1.3844740 & -4.8986280 & -1.7693530 \\ \mathrm{C} & 2.8811430 & -0.7106960 & 0.0995410 \\ \mathrm{C} & 3.8810320 & 0.0811750 & 0.7274230 \\ \mathrm{C} & 2.7084050 & -2.0699350 & 0.3398700 \\ \mathrm{C} & 4.7380090 & -0.5469540 & 1.6070470 \\ \mathrm{C} & 3.5421620 & -2.6887750 & 1.3332670 \\ \mathrm{C} & 4.5905330 & -1.9154090 & 1.9438740 \\ \mathrm{H} & 5.5250020 & 0.0298040 & 2.0873870 \\ \mathrm{C} & 5.2457560 & -3.8348110 & 3.2959070\end{array}$

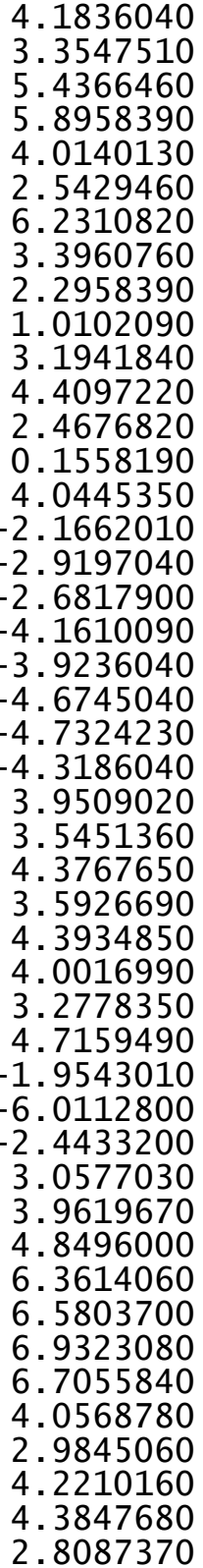
$-4.5835680$ $-4.0258020$ $-2.5246310$ $-4.2885540$ $-5.6076240$ $-4.6139950$ $-1.9253560$ $-5.7069490$ $-6.4595680$ $-6.0054820$ $-4.5326080$ $-6.0543710$ $-7.3866770$ $-6.5642650$ $-3.9615460$ $-2.5338940$ $-2.0230240$ $-2.4657590$ $-1.4226650$ $-1.8654590$ $-1.3180290$
$-1.0203470$ $-1.8021720$
1.5485300 2. 4570240 2. 0281880 3. 8289570 3.4065650 4. 3248710 4.5360300
3.7710050 $-3.0769710$ $-0.6688520$ $-2.1508140$ 1.9985490 5.8147470 1.0976040 1. 2733240 2. 2966170
1. 0841300
0.5770060 1. 3042210 1.1673020 2. 3127660 0.5804680 6.1414200

2.7340270
1.7809240
2.9101180
4.0407850
3.0599010
1.3669640
3.3519200
-2.0222230
-2.4998660
-2.2893840
-1.3247800
-2.2102370
-3.0423620
-2.6680180
-0.9656730
-0.5901000
-1.6693970
0.7266360
-1.4051890
0.9342750
-0.1110590
-2.2364160
1.9457290
0.4649240
1.4691960
-0.7943730
1.1919750
-1.0243990
-0.0465250
1.9573430
-1.9971740
1.9183880
0.2152370
-3.1106250
2.8386600
-0.3370170
-1.9035590
-2.1485380
-2.4816030
-1.2304090
-2.9252680
-3.2070980
-3.0322890
-3.6089180
-3.9651670
-1.3080270 


$\begin{array}{lr}\mathrm{H} & 2.7477600 \\ \mathrm{H} & 2.9715600 \\ \mathrm{H} & 1.8490320 \\ \mathrm{C} & 5.2989800 \\ \mathrm{H} & 6.1215950 \\ \mathrm{H} & 5.5332430 \\ \mathrm{H} & 5.2463700 \\ \mathrm{C} & 4.1586530 \\ \mathrm{H} & 5.0748130 \\ \mathrm{H} & 4.4052280 \\ \mathrm{H} & 3.8175050 \\ \mathrm{C} & 1.7607190 \\ \mathrm{H} & 1.9318530 \\ \mathrm{H} & 0.9911930 \\ \mathrm{H} & 1.3930630 \\ \mathrm{C} & -2.6513560 \\ \mathrm{H} & -3.6824500 \\ \mathrm{H} & -2.6860520 \\ \mathrm{H} & -2.1147690 \\ \mathrm{C} & -1.8350640 \\ \mathrm{H} & -1.3806180 \\ \mathrm{H} & -2.8215870 \\ \mathrm{H} & -1.2156100 \\ \mathrm{C} & -7.0476030 \\ \mathrm{H} & -7.9890720 \\ \mathrm{H} & -7.2555480 \\ \mathrm{H} & -6.6779480 \\ \mathrm{C} & -6.5732020 \\ \mathrm{H} & -5.8501180 \\ \mathrm{H} & -6.8325960 \\ \mathrm{H} & -7.4850860 \\ \mathrm{C} & -2.6851380 \\ \mathrm{H} & -3.7568550 \\ \mathrm{H} & -2.2328690 \\ \mathrm{H} & -2.2342670 \\ \mathrm{C} & -3.1173450 \\ \mathrm{H} & -4.2043200 \\ \mathrm{H} & -2.7402980 \\ \mathrm{H} & -2.9299690 \\ \mathrm{P} & 0.5094320 \\ \mathrm{O} & -0.1323240 \\ \mathrm{O} & 0.3102890 \\ \mathrm{H} & -1.3627470 \\ \mathrm{H} & -5.8467930 \\ \mathrm{H} & -0.9366310 \\ \mathrm{H} & 4.6906430 \\ & \end{array}$

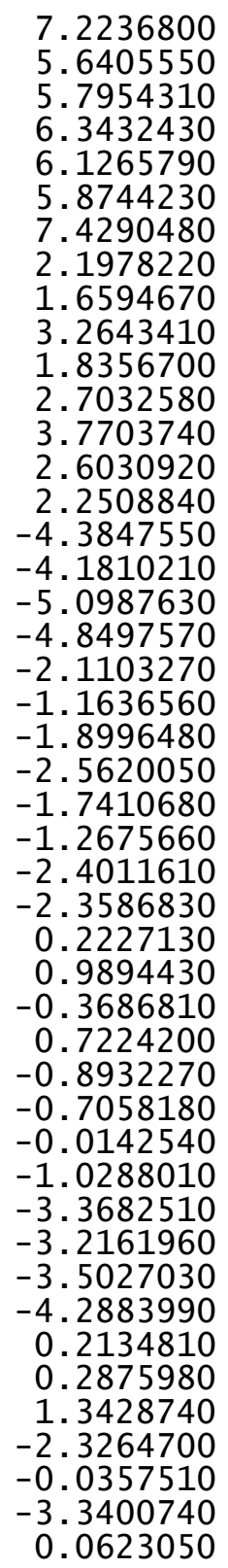
$-1.4868250$
$-2.2716360$
$-0.9032000$
$-0.8866330$
$-0.1936890$
$-1.8510010$
$-1.0419510$
3.8993370
3. 6281670
3.9919460
4.8788210
3. 2783900
3.4716270
2. 5070550
4. 2090540
2. 3443120
2. 6633460
1. 5116100
3. 1826510
3. 1086720
2. 7988820
3.5422070
3.8945480
0.6109740
0.9215360
$-0.2424010$
1.4383720
$-0.8982360$
$-1.1989260$
$-1.7868050$
$-0.5484160$
$-3.9608640$
$-4.1080760$
$-3.4957540$
$-4.9523500$
$-3.7772950$
$-3.8258110$
$-4.8002970$
$-3.2118390$
$-0.5412050$
$-1.8981150$
0.4335770
$-3.0959470$
1. 0970370
1. 6157230
$-1.5853260$

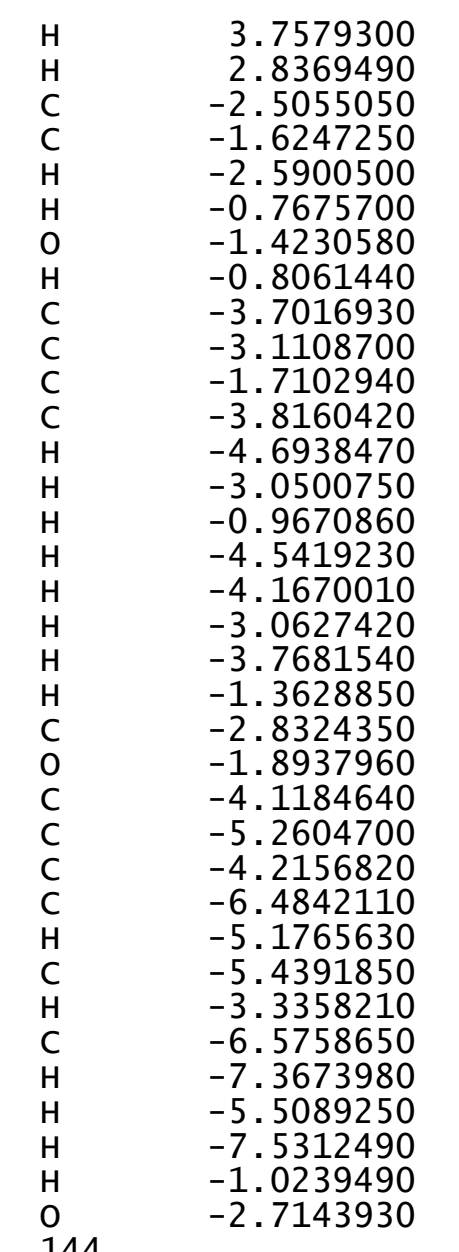
6.3293520
$-0.203$
1. 1199840
1. 4437040

$\begin{array}{ll}.2001260 & -1.1204940\end{array}$

$-1.3264870$

$2.2559270 \quad-2.7813350$

$1.4667010 \quad-2.4848050$

$4.1891960 \quad-2.9006790$

$5.1240200 \quad-1.8334330$

$4.6611000 \quad-1.3961820$

$2.7551210 \quad-2.3574880$

$4.5431690-3.2055320$

$6.1520710 \quad-2.2094190$

$4.8759860 \quad-2.1788460$

$2.7308940 \quad-1.5335560$

$2.0581790-3.1278610$

$4.1931660 \quad-3.7938170$

$5.1301490 \quad-0.9542530$

$5.1865560 \quad-0.4988000$

2. $2149400 \quad 1.5502370$

$1.3155660 \quad 1.7485890$

$1.9164910 \quad 2.2505740$

$2.6671580 \quad 1.9272180$

$0.8805750 \quad 3.1944110$

$\begin{array}{ll}2.3793630 & 2.5301500\end{array}$

$3.4657100 \quad 1.1957980$

$0.6015920 \quad 3.8026310$

$0.2949290 \quad 3.4354680$

$1.3463910 \quad 3.4694980$

$2.9561520 \quad 2.2659050$

4.5305150

3.9376950

1.2107290

144

$\begin{array}{lrcr}\text { TS110 (R, R) G[B97-D/def2-TZVP] }=-3310.751117 \\ \text { O } & 0.4150550 & 0.7320700 & 0.0432360 \\ \text { O } & -1.8985920 & 0.7873190 & -1.0281770 \\ \text { C } & 0.4951270 & 2.1109910 & -0.0736040 \\ \text { C } & -0.5981010 & 2.8926890 & 0.2881060 \\ \text { C } & 1.7395720 & 2.6591360 & -0.4843840 \\ \text { C } & -0.5155590 & 4.3109460 & 0.0886230 \\ \text { C } & 1.8364910 & 4.0323610 & -0.5843990 \\ \text { C } & 0.7305010 & 4.8825330 & -0.3414620 \\ \text { H } & 2.7860100 & 4.4774220 & -0.8717320\end{array}$




$\begin{array}{lr}\mathrm{C} & -2.4438550 \\ \mathrm{C} & -3.6428120 \\ \mathrm{C} & -1.8140570 \\ \mathrm{C} & -4.1937690 \\ \mathrm{C} & -2.3423310 \\ \mathrm{C} & -3.5647380 \\ \mathrm{H} & -5.1247170 \\ \mathrm{C} & -3.4591810 \\ \mathrm{C} & -2.2321450 \\ \mathrm{C} & -1.6893390 \\ \mathrm{C} & -4.1064490 \\ \mathrm{H} & -3.8796980 \\ \mathrm{H} & -1.7091080 \\ \mathrm{H} & -0.7463880 \\ \mathrm{H} & -5.0384220 \\ \mathrm{C} & -1.5035650 \\ \mathrm{C} & -0.2637700 \\ \mathrm{C} & 0.8280820 \\ \mathrm{C} & -1.6259350 \\ \mathrm{H} & -2.3699150 \\ \mathrm{H} & -0.1800510 \\ \mathrm{H} & 1.7830370 \\ \mathrm{H} & -2.5828240 \\ \mathrm{C} & 2.9316350 \\ \mathrm{C} & 3.3671190 \\ \mathrm{C} & 3.6599120 \\ \mathrm{C} & 4.5608750 \\ \mathrm{C} & 4.8431200 \\ \mathrm{C} & 5.3202130 \\ \mathrm{H} & 4.9108990 \\ \mathrm{H} & 5.4083380 \\ \mathrm{C} & -4.2798060 \\ \mathrm{C} & -4.2638550 \\ \mathrm{C} & -4.8993330 \\ \mathrm{C} & -4.8897430 \\ \mathrm{C} & -5.5049390 \\ \mathrm{C} & -5.5130390 \\ \mathrm{H} & -4.8776120 \\ \mathrm{H} & -5.9866610 \\ \mathrm{C} & 3.2237940 \\ \mathrm{C} & 6.6468950 \\ \mathrm{C} & 2.5914870 \\ \mathrm{C} & -3.5984070 \\ \mathrm{C} & -6.1569550 \\ \mathrm{C} & -4.9685930 \\ \mathrm{C} & -6.4258950 \\ & \end{array}$

\begin{abstract}
1. 2220320
0.6008830

2. 2558650

1.0525690

2. 6479720

2. 0463330

0.6157190

3. 3597990

3. 9211040

3.5755530

2.4373830

3. 6465960

4. 6285130

4.0114580

1.9787380

6. 5416280

7.1064070

6.2886740

5. 1812540

7. 1853870

8.1807660

6.7047160

4.7619060

1.8011000

1. 5990450

1.2504130

0.9011360

0.5509500

0.3813120

0.7496300

0.1362240

$-0.4944120$

$-1.8231740$

$-0.2033160$

$-2.8229770$

$-1.2434700$

$-2.5575920$

$-3.8439770$

$-1.0208260$

1. 4834360

$-0.2947560$

2. 1437090

$-2.1991360$

$-3.6704110$

1. 2068410

1.7082200
\end{abstract}

0.1780390
0.6212210
0.8585400
1.8032580
2.1343380
2.5934690
2.1558520
4.6434300
4.2132070
2.9917110
3.8481910
5.6048260
4.8532280
2.6788050
4.1744850
0.0664530
-0.3211610
-0.5242940
0.2666660
0.2026570
-0.4695620
-0.8411650
0.5602270
-0.7591020
-2.0876250
0.3201210
-2.3018450
0.0510500
-1.2508600
-3.3214860
0.8796740
-0.1679610
0.3219200
-1.4022270
-0.4283780
-2.1179730
-1.6512870
-0.0546470
-3.0690020
1.7620660
-1.5580760
-3.2811030
1.6412350
-2.4584930
-1.9745830
-2.0140910

$-7.0343220$

$-6.8740920$

$-6.4643430$

$-4.3225970$

$-3.2815640$

$-4.3446740$

$-7.2760680$

$-7.7570720$

$-8.0393670$

$-5.1036170$

$-4.3205720$

$-4.6261280$

$-5.5726050$

$-4.6543280$

$-5.2423600$

$-5.3457730$

$-4.1693090$

$-2.7423870$

$-3.3698770$

$-2.0315790$

$-2.1827360$

3.8095380

4.9071590

3. 4987970

3.4686480

3. 5814520

3. 2375300

4.6640850

3. 1020440

7.0820910

7.9951980

7. 3041950

6.3049790

7. 7482850

7.4712420

7.8927250

8.7012090

2. 4664450

3.4414330

2.0451950

1.8059830

3.2375500

4.2632030

2. 6632790
1.0723350

1.6916060

2. 7363950

1. 2899090

0.9514170

0.6663090

2. 3263180

$-4.3762130$

$-5.1429610$

$-4.8693150$

$-3.6591330$

$-4.6911570$

$-4.2001550$

$-5.1735350$

$-5.4719100$

$-2.3695590$

$-1.4550640$

$-3.1824850$

$-2.6199070$

$-3.4759280$

$-4.3642280$

$-3.4000150$

$-3.6177030$

2.8141590

2.7675120

3. 6506410

3. 0095780

0.3335400

$-0.6300450$

0.2701030

0.4992000

$-1.3438370$

$-1.8466550$

$-0.8742560$

$-2.1006070$

0.7743390

1. 5052260

1. 3116820

0.

1. 1268670

0.9243090

0.1821440

1.5319190

3.4440760

3. 2460120

3. 8422650
$-2.6706060$

$-1.0122510$

$-2.3984410$

$-3.3698610$

$-3.3341020$

$-4.0897210$

$-3.7329270$

$-1.6702100$

$-2.2922020$

$-0.7789040$

$-1.3428950$

$-2.9323570$

$-3.5236620$

$-2.0692510$

$-3.5465870$

2.7522610

2.8902640

2. 4920100

3. 7057340

1.5396360

1. 3880670

0.7116940

2. 4731690

2. 2778400

2. 2791850

1. 6411700

3. 3037210

2. 7162240

2.3263230

2.8866580

3.6885380

$-0.5237710$

$-0.8662160$

0.4433420

$-0.3650560$

$-1.7235800$

$-2.4929910$

$-0.7765510$

$-2.0068560$

$-4.4290430$

$-4.8914910$

$-4.0738580$

$-5.2062720$

$-3.8000570$

-4.1400760
-4.6473760 


\begin{tabular}{|c|c|c|c|c|c|c|c|}
\hline & & 2101870 & 30176660 & & & 0150510 & \\
\hline $\begin{array}{l}H \\
P\end{array}$ & $\begin{array}{r}3.2778710 \\
-0.5652530\end{array}$ & $\begin{array}{r}4.2101870 \\
-0.1370410\end{array}$ & -3.0176660 & 0 & 2.0807840 & -0.0159540 & -0.8732580 \\
\hline 0 & -0.0276030 & -0.2110760 & -2.3443760 & c & $\begin{array}{l}. .47857850 \\
1.785780\end{array}$ & -2.7738240 & -0.3263310 \\
\hline 0 & -0.8129800 & -1.4113140 & -0.1837830 & c & -0.6352280 & -3.1665640 & -0.7090360 \\
\hline $\mathrm{H}$ & 1.5758170 & 2.3791090 & -2.9448850 & C & 2.0816570 & -3.9931010 & -1.0233450 \\
\hline $\mathrm{H}$ & 6.5332470 & -0.8029530 & -2.5275620 & C & -0.3427500 & -4.3819830 & -1.2913900 \\
\hline $\mathrm{H}$ & 2.1334210 & 1.5780660 & 1.7768260 & C & 0.9949670 & -4.8072810 & -1.4969690 \\
\hline $\mathrm{H}$ & -4.4134820 & 1.8830460 & -1.3168900 & $\mathrm{H}$ & -1.1529780 & -5.0263030 & -1.6233290 \\
\hline $\mathrm{H}$ & -6.6083170 & -3.2117070 & -3.3501040 & C & 2.9255140 & -0.5847510 & 0.0674450 \\
\hline H & -2.9293070 & -1.3820220 & 1.9291800 & C & 3.8811790 & 0.2748250 & 0.6740570 \\
\hline C & 2.3443560 & -2.1748760 & -1.6253140 & C & 2.8357930 & -1.9480420 & 0.3301770 \\
\hline C & 1.3396040 & -3.2136580 & -1.3490660 & C & 4.7978750 & -0.2911660 & 1.5353140 \\
\hline $\mathrm{H}$ & 2.2520510 & -1.2499150 & -1.0498970 & C & 3.7279250 & -2.5042400 & 1.3105980 \\
\hline $\mathrm{H}$ & 0.3523770 & -2.8766200 & -1.0524680 & C & 4.7459090 & -1.6638890 & 1.8827010 \\
\hline 0 & 1.6039930 & -2.0936150 & -2.8863450 & $\mathrm{H}$ & 5.5539650 & 0.3386020 & 1.9986150 \\
\hline $\mathrm{H}$ & 0.9014990 & -1.3433590 & -2.7384280 & C & 5.5497470 & -3.5254220 & 3.2356380 \\
\hline C & 3.8894410 & -3.9871250 & -2.5143070 & C & 4.5156810 & -4.3404190 & 2.7153680 \\
\hline c & 3.0449260 & -5.0388450 & -1.7793310 & $\mathrm{c}$ & 3.6290700 & -3.8430630 & 1.7808610 \\
\hline C & 1.5612270 & -4.6332810 & -1.7411710 & C & 5.6533430 & -2.2115050 & 2.8300160 \\
\hline C & 3.7782610 & -2.6213000 & -1.8188360 & $\mathrm{H}$ & 6.2466270 & -3.9315560 & 3.9655340 \\
\hline $\mathrm{H}$ & 4.9414830 & -4.2963960 & -2.5399090 & $\mathrm{H}$ & 4.4137710 & -5.3677720 & 3.0585690 \\
\hline $\mathrm{H}$ & 3.1382000 & -6.0197230 & -2.2602840 & $\mathrm{H}$ & 2.8399850 & -4.4818470 & 1.3994330 \\
\hline $\mathrm{H}$ & 1.0991290 & -4.7600420 & -2.7320080 & $\mathrm{H}$ & 6.4238570 & -1.5622560 & 3.2426540 \\
\hline $\mathrm{H}$ & 4.2247890 & -2.6748120 & -0.8174770 & C & 3.6557160 & -5.5905790 & -1.9828370 \\
\hline $\mathrm{H}$ & 4.3100210 & -1.8403320 & -2.3696960 & $\mathrm{C}$ & 2.5851760 & -6.4111440 & -2.4138650 \\
\hline H & 3.5479540 & -3.8970730 & -3.5543230 & C & 1.2830420 & -6.0217040 & -2.1769130 \\
\hline $\mathrm{H}$ & 3.4076950 & -5.1374590 & -0.7480760 & C & 3.4096170 & -4.4140220 & -1.3036160 \\
\hline $\mathrm{H}$ & 0.9795810 & -5.2594760 & -1.0545200 & $\mathrm{H}$ & 4.6811440 & -5.8872700 & -2.1925530 \\
\hline c & 1.4409620 & -2.9000910 & 1.8655080 & $\mathrm{H}$ & 2.7920600 & -7.3392760 & -2.9421950 \\
\hline 0 & 0.4873440 & -1.9989050 & 1.9167770 & $\mathrm{H}$ & 0.4501830 & -6.6327780 & -2.5211940 \\
\hline c & 2.1459170 & -3.1167000 & 3.1682250 & $\mathrm{H}$ & 4.2369570 & -3.7907660 & -0.9791440 \\
\hline C & 3.2642720 & -3.9656140 & 3.2078930 & C & -2.0283280 & -2.6727490 & -0.4931340 \\
\hline C & 1.7241530 & -2.4736460 & 4.3444320 & C & -2.8278180 & -2.2533690 & -1.5830950 \\
\hline C & 3.9548720 & -4.1633390 & 4.4030510 & $\mathrm{C}$ & -2.5237570 & -2.5706560 & 0.8271150 \\
\hline $\mathrm{H}$ & 3.5838890 & -4.4582650 & 2.2940450 & C & -4.0934660 & -1.7140880 & -1.3216610 \\
\hline C & 2.4143780 & -2.6772310 & 5.5392870 & C & -3.7943650 & -2.0240130 & 1.0330850 \\
\hline $\mathrm{H}$ & 0.8636910 & -1.8129790 & 4.3136510 & C & -4.5891610 & -1.5745110 & -0.0219310 \\
\hline c & 3.5309100 & -3.5197360 & 5.5712850 & $\mathrm{H}$ & -4.7101170 & -1.3912840 & -2.1567880 \\
\hline $\mathrm{H}$ & 4.8232740 & -4.8177920 & 4.4252540 & $\mathrm{H}$ & -4.1778510 & -1.9348290 & 2.0470710 \\
\hline H & 2.0832130 & -2.1761380 & 6.4459660 & C & 3.8292620 & 1.7453030 & 0.4253080 \\
\hline $\mathrm{H}$ & 4.0697840 & -3.6738460 & 6.5037220 & C & 3.3572660 & 2.6037590 & 1.4449980 \\
\hline $\mathrm{H}$ & -0.0006180 & -1.8158990 & 1.0221070 & C & 4.1978340 & 2.2731540 & -0.8317260 \\
\hline & 1.7744470 & -3.5403410 & 0.8557860 & C & 3.2821170 & 3.9772210 & 1.1833300 \\
\hline & & & & & $\begin{array}{l}4.09 \\
2.62\end{array}$ & 3.6504260 & -1.0456120 \\
\hline & 0.1400050 & -1.1409920 & 0.3043940 & H & $\begin{array}{l}3.634 / 980 \\
2.9172780\end{array}$ & $\begin{array}{l}4.5203130 \\
4.6466060\end{array}$ & $\begin{array}{r}-0.0530490 \\
1.9603240\end{array}$ \\
\hline
\end{tabular}




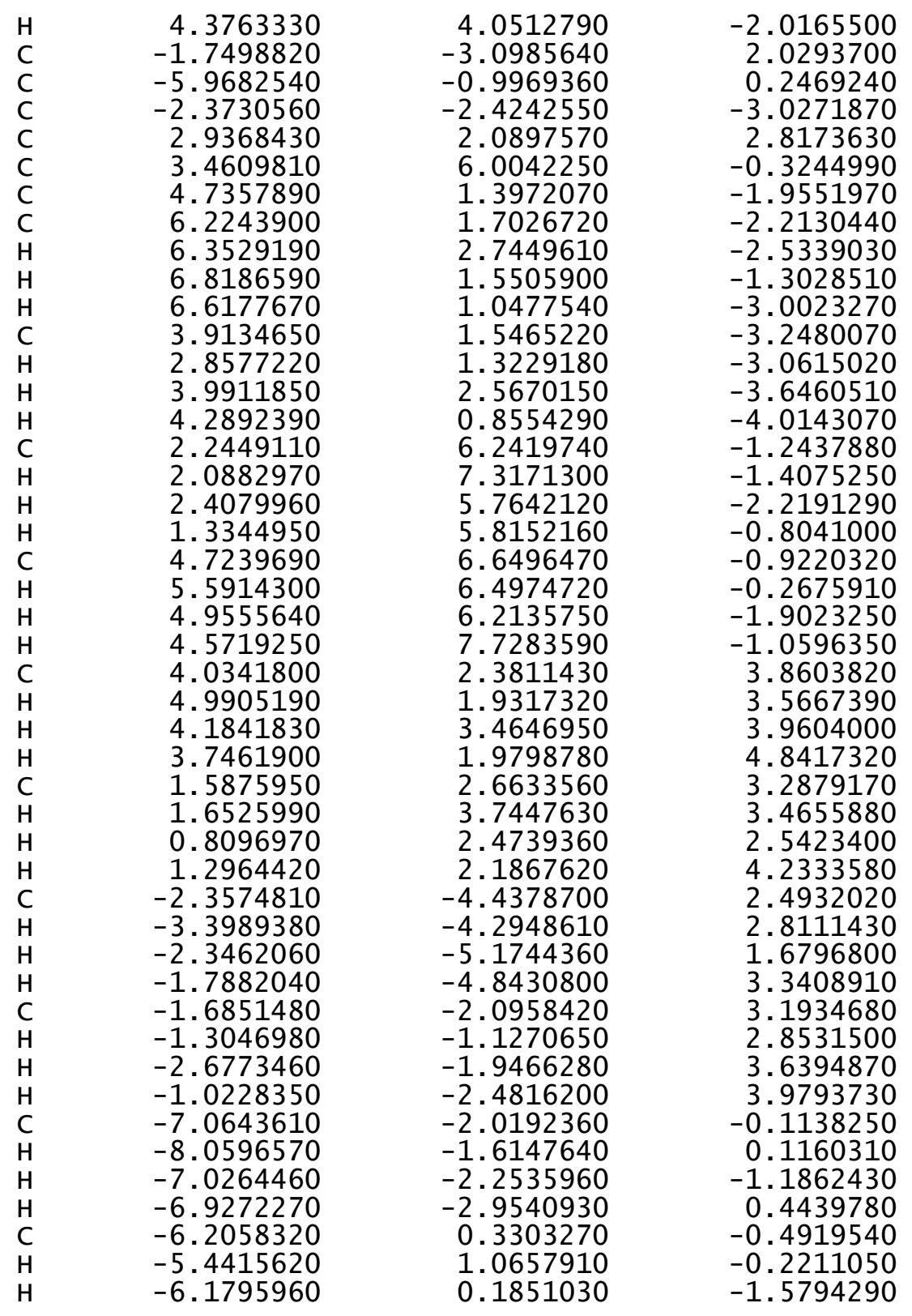

-7.1884380
-2.6827540
-3.7640000
-2.2457750
-2.2578880
-3.0103160
-4.1029490
-2.6449360
-2.7794720
0.4964740
-0.1574340
0.2340390
-1.2862090
-6.0336850
-0.7194030
4.6684260
3.2539590
2.8173250
-2.6318780
-1.8113820
-2.7060040
-0.9555700
-1.5118370
-0.8729710
-3.8378340
-3.3324740
-1.9375930
-3.9312860
-4.8248780
-3.2912970
-1.1674820
-4.6970570
-4.2175790
-3.1553640
-4.0301180
-1.6488990
-2.9415730
-1.9313530
-4.1477780
-5.3070810
-4.1544970
-6.4585140
-5.2939670
-5.3079670
-3.2623210
-6.4618710
0.7416250

$-1.2144850$

$-1.0682040$

$-0.3015940$

$-1.3801570$

$-3.6947190$

$-3.5860570$

$-3.8600030$

$-4.5815830$

0.1912910

0.1631390

1.3498730

$-2.5547660$

$-0.7938040$

$-3.3036160$

0.3496380

6.4925580

1.0033590

2. 0089450

. 0155140

1.0358690

2. 6581410

2. 0416680

. 2903200

3.8943850

4.8704300

4.4669530

2.4689820

4.2077760

5.8931760

4.6949350

2. 4331960

1.7443230

3.9025000

4.8681360

5.0203440

2. 1193130

1.3097890

1.8384990

2. 6081080

0.7979470

2. 3368530

3.4081210

0.5293090

0.1986980

1.2953560
$-0.2270860$

$-3.9237700$

$-4.0448000$

$-3.5126290$

$-4.9221360$

$-3.6277350$

$-3.6634840$

$-4.6504140$

$-3.0261720$

$-0.5725810$

$-1.9252940$

0.3509500

$-3.0288370$

1. 3245680

1.7260390

$-1.6443960$

0.6387110

2.7549640

$-1.9723150$

$-1.2808260$

$-1.4790560$

$-0.7182810$

$-2.9115530$

$-2.5697850$

$-3.1714400$

$-2.0974460$

$-1.5883690$

$-2.6020470$

$-3.5322200$

$-2.4902700$

$-2.3408350$

$-1.8169040$

$-3.3738510$

$-4.0317840$

$-1.2496130$

$-0.6868850$

1. 4960580

1.7276100

2. 3334520

2. 1442640

3. 2773290

2. 8815770

1. 4099270

4. 0138620

3. 4167810

3. 8165130 


\begin{tabular}{|c|c|c|c|c|c|c|c|}
\hline $\mathrm{H}$ & 72511200 & 20231710 & 27261510 & & 18191270 & 0816170 & \\
\hline $\begin{array}{r}\mathrm{H} \\
\mathrm{H}\end{array}$ & $\begin{array}{l}-7.3544300 \\
-5.3087020\end{array}$ & $\begin{array}{r}2.9334 / 100 \\
-0.2811500\end{array}$ & $\begin{array}{l}2.7264540 \\
4.7392680\end{array}$ & $\mathrm{H}$ & $\begin{array}{r}4.8191270 \\
-4.1511370\end{array}$ & $\begin{array}{r}0.8916470 \\
-1\end{array}$ & $\begin{array}{l}1.3884220 \\
0.2792390\end{array}$ \\
\hline $\mathrm{H}$ & -7.3616510 & 1.0816220 & 4.3894340 & $\mathrm{C}$ & -3.8871500 & -2.3278810 & 1.0157750 \\
\hline $\mathrm{H}$ & -1.0996680 & 1.4313100 & 1.1334890 & $\mathrm{c}$ & -4.8160670 & -1.2286340 & -0.9660210 \\
\hline 0 & -2.9461810 & 3.0405400 & 0.6637390 & $\mathrm{C}$ & -4.3245160 & -3.5552600 & 0.5008680 \\
\hline & & & & $\mathrm{C}$ & -5.2242230 & -2.4786710 & -1.4388830 \\
\hline & ,R) $G[B 97-D$ & $\mathrm{ZVP}]=-33$ & 922 & $\mathrm{C}$ & -4.9918620 & -3.6546400 & -0.7205950 \\
\hline 0 & 0.2110370 & 0.9269510 & 0.1347070 & $\mathrm{H}$ & -4.1282840 & -4.4667330 & 1.0627030 \\
\hline 0 & -2.0486960 & 0.3439230 & -0.8771810 & $\mathrm{H}$ & -5.7412520 & -2.5333370 & -2.3946980 \\
\hline C & 0.0627560 & 2.2601240 & -0.2239290 & C & 2.6235540 & 2.3642150 & 1.8244150 \\
\hline c & -1.1627740 & 2.8826310 & -0.0225820 & $\mathrm{c}$ & 6.2620190 & 0.0136180 & -0.7724950 \\
\hline C & 1.2150270 & 2.9385080 & -0.7098370 & $\mathrm{C}$ & 2.5404510 & 2.2989230 & -3.3127660 \\
\hline $\mathrm{C}$ & -1.3215250 & 4.2277610 & -0.4956040 & $\mathrm{C}$ & -3.1509340 & -2.3062040 & 2.3501760 \\
\hline C & 1.0743780 & 4.2600260 & -1.0793850 & C & -5.4284630 & -5.0027850 & -1.2661250 \\
\hline $\mathrm{C}$ & -0.1785900 & 4.9210780 & -1.0242760 & $\mathrm{C}$ & -5.1371680 & 0.0039160 & -1.8022940 \\
\hline $\mathrm{H}$ & 1.9423020 & 4.8092450 & -1.4355190 & $\mathrm{C}$ & -6.6602420 & 0.2291510 & -1.8778900 \\
\hline C & -2.6641560 & 0.9031930 & 0.2342510 & $\mathrm{H}$ & -7.1518030 & -0.6209920 & -2.3690370 \\
\hline C & -3.7361800 & 0.1762520 & 0.8197540 & $\mathrm{H}$ & -7.0904700 & 0.3380920 & -0.8739750 \\
\hline C & -2.2551130 & 2.1559120 & 0.6769100 & $\mathrm{H}$ & -6.8836370 & 1.1364410 & -2.4553000 \\
\hline C & -4.3817290 & 0.7440610 & 1.8992520 & C & -4.5245190 & -0.0883940 & -3.2117520 \\
\hline C & -2.8721030 & 2.6930030 & 1.8573290 & $\mathrm{H}$ & -3.4396360 & -0.2270790 & -3.1523270 \\
\hline C & -3.9660110 & 1.9781010 & 2.4571570 & $\mathrm{H}$ & -4.9551100 & -0.9300710 & -3.7702330 \\
\hline $\mathrm{H}$ & -5.2187340 & 0.2190680 & 2.3534680 & $\mathrm{H}$ & -4.7310890 & 0.8328900 & -3.7728130 \\
\hline C & -4.1499170 & 3.6804790 & 4.1915860 & C & -4.6601010 & -5.3524350 & -2.5556830 \\
\hline C & -3.0442470 & 4.3633710 & 3.6291920 & $\mathrm{H}$ & -4.9542260 & -6.3455440 & -2.9215800 \\
\hline C & -2.4229450 & 3.8829920 & 2.4937610 & $\mathrm{H}$ & -4.8809230 & -4.6177750 & -3.3411000 \\
\hline C & -4.5945690 & 2.5082140 & 3.6169310 & $\mathrm{H}$ & -3.5769630 & -5.3469080 & -2.3805280 \\
\hline $\mathrm{H}$ & -4.6353110 & 4.0738620 & 5.0821060 & C & -6.9479510 & -5.0545510 & -1.5125440 \\
\hline $\mathrm{H}$ & -2.6765940 & 5.2732310 & 4.0988740 & $\mathrm{H}$ & -7.5033820 & -4.8383420 & -0.5912530 \\
\hline $\mathrm{H}$ & -1.5735700 & 4.4162100 & 2.0803850 & $\mathrm{H}$ & -7.2393090 & -4.3131760 & -2.2680970 \\
\hline $\mathrm{H}$ & -5.4286840 & 1.9585250 & 4.0500400 & $\mathrm{H}$ & -7.2435070 & -6.0473490 & -1.8776090 \\
\hline C & -2.6891740 & 6.1923370 & -0.9645160 & C & -4.1397600 & -2.4774700 & 3.5202140 \\
\hline C & -1.5552450 & 6.8856250 & -1.4534830 & $\mathrm{H}$ & -4.9038980 & -1.6911570 & 3.5133280 \\
\hline $\mathrm{C}$ & -0.3273460 & 6.2576880 & -1.4842020 & $\mathrm{H}$ & -4.6486510 & -3.4480820 & 3.4459430 \\
\hline C & -2.5744320 & 4.8985070 & -0.4966300 & $\mathrm{H}$ & -3.6073620 & -2.4387410 & 4.4804080 \\
\hline $\mathrm{H}$ & -3.6607810 & 6.6817260 & -0.9619650 & C & -2.0415640 & -3.3712870 & 2.4336630 \\
\hline $\mathrm{H}$ & -1.6580880 & 7.9068300 & -1.8138960 & $\mathrm{H}$ & -2.4634000 & -4.3849610 & 2.4434450 \\
\hline $\mathrm{H}$ & 0.5496610 & 6.7725800 & -1.8730280 & $\mathrm{H}$ & -1.3573400 & -3.2805290 & 1.5848910 \\
\hline $\mathrm{H}$ & -3.4507410 & 4.3756780 & -0.1269500 & $\mathrm{H}$ & -1.4702850 & -3.2370350 & 3.3614680 \\
\hline C & 2.5275520 & 2.2300550 & -0.7447480 & C & 3.5381470 & 3.4777790 & 2.3752560 \\
\hline C & 3.1316720 & 1.8789450 & -1.9731440 & $\mathrm{H}$ & 4.5493440 & 3.0884420 & 2.5543240 \\
\hline C & 3.1532570 & 1.8874350 & 0.4768580 & $\mathrm{H}$ & 3.6138760 & 4.3113050 & 1.6651600 \\
\hline C & 4.3369550 & 1.1653090 & -1.9467450 & $\mathrm{H}$ & 3.1415910 & 3.8610980 & 3.3252010 \\
\hline C & 4.3458150 & 1.1589810 & 0.4470350 & C & 2.4717130 & 1.2269810 & 2.8462470 \\
\hline C & 4.9484820 & 757830 & -0.7534400 & $\mathrm{H}$ & 1.8091030 & 0.4456490 & 2.4635600 \\
\hline H & 4.8106 & 83330 & -2.8863400 & $\mathrm{H}$ & 3.4412830 & 0.7718560 & 3.0832010 \\
\hline
\end{tabular}




$\begin{array}{rrrr}\mathrm{H} & 2.0494490 & 1.6201550 & 3.7807860 \\ \mathrm{C} & 6.3097410 & -1.1240420 & 0.2620790 \\ \mathrm{H} & 7.2311700 & -1.7082220 & 0.1395690 \\ \mathrm{H} & 6.3001120 & -0.7258480 & 1.2843120 \\ \mathrm{H} & 5.4496760 & -1.7960200 & 0.1628970 \\ \mathrm{C} & 7.4450060 & 0.9801910 & -0.5601930 \\ \mathrm{H} & 7.4445170 & 1.7728680 & -1.3186770 \\ \mathrm{H} & 7.3703620 & 1.4526830 & 0.4283570 \\ \mathrm{H} & 8.4003180 & 0.4404450 & -0.6145950 \\ \mathrm{C} & 2.5425490 & 1.1805700 & -4.3678730 \\ \mathrm{H} & 3.5644240 & 0.8863770 & -4.6407120 \\ \mathrm{H} & 2.0073040 & 0.2978970 & -4.0084350 \\ \mathrm{H} & 2.0467230 & 1.5376680 & -5.2797000 \\ \mathrm{C} & 3.2941360 & 3.5317510 & -3.8546400 \\ \mathrm{H} & 4.3454840 & 3.2769590 & -4.0463350 \\ \mathrm{H} & 2.8431460 & 3.8709460 & -4.7969270 \\ \mathrm{H} & 3.2733710 & 4.3619640 & -3.1387210 \\ \mathrm{P} & -0.5242560 & -0.2158230 & -0.7790640 \\ \mathrm{O} & -0.0240780 & -0.2150830 & -2.1952550 \\ \mathrm{O} & -0.4327390 & -1.4759860 & 0.0373320 \\ \mathrm{H} & 1.4963280 & 2.5852460 & -3.1495580 \\ \mathrm{H} & 6.3670750 & -0.4298280 & -1.7731100 \\ \mathrm{H} & 1.6331350 & 2.8056650 & 1.6798530 \\ \mathrm{H} & -4.7048620 & 0.8840060 & -1.3162280 \\ \mathrm{H} & -5.1817100 & -5.7607360 & -0.5086610 \\ \mathrm{H} & -2.6681840 & -1.3291070 & 2.4573020 \\ \mathrm{C} & 2.5787750 & -1.8845480 & -2.1023160 \\ \mathrm{C} & 1.8040560 & -2.9814350 & -1.5052150 \\ \mathrm{H} & 2.5383170 & -0.9258710 & -1.5807380 \\ \mathrm{H} & 0.8868180 & -2.7157900 & -0.9914900 \\ \mathrm{O} & 1.5257480 & -1.9848950 & -3.1164990 \\ \mathrm{H} & 0.8142180 & -1.2910200 & -2.8052480 \\ \mathrm{C} & 4.0354030 & -3.6148930 & -3.2625590 \\ \mathrm{C} & 3.5409180 & -4.6652230 & -2.2555610 \\ \mathrm{C} & 2.0786230 & -4.4043490 & -1.8542650 \\ \mathrm{C} & 3.9514860 & -2.2048960 & -2.6552840 \\ \mathrm{H} & 5.0723550 & -3.8259480 & -3.5507760 \\ \mathrm{H} & 3.6255580 & -5.6752150 & -2.6736460 \\ \mathrm{H} & 1.3998150 & -4.6601210 & -2.6817990 \\ \mathrm{H} & 4.6562040 & -2.1168100 & -1.8199540 \\ \mathrm{H} & 4.2151430 & -1.4328060 & -3.3862820 \\ \mathrm{H} & 3.4251360 & -3.6593280 & -4.1745970 \\ \mathrm{H} & 4.1654960 & -4.6243480 & -1.3538300 \\ \mathrm{H} & 1.7662690 & -5.0219710 & -1.0041140 \\ \mathrm{C} & 2.4553030 & -2.5498650 & 1.6011170 \\ \mathrm{O} & 1.3027270 & -1.9667780 & 1.8476800 \\ & & & \end{array}$

$\begin{array}{ll}3.3740770 & -2.6040190 \\ 4.6277290 & -3.2227250 \\ 3.0188010 & -2.0306720 \\ 5.5153220 & -3.2607040 \\ 4.8969780 & -3.6618330 \\ 3.9089490 & -2.0708270 \\ 2.0536790 & -1.5477170 \\ 5.1583230 & -2.6834450 \\ 6.4858640 & -3.7375380 \\ 3.6293180 & -1.6206930 \\ 5.8520800 & -2.7109250 \\ 0.6735700 & -1.8211940 \\ 2.8034680 & -3.0298990\end{array}$
2.7806470
2. 6432020
4. 0137120
3. 7173210
1. 6876810
5. 0869490
4. 1211280
4.9412680
3. 6011960
6.0366490
5. 7788650
5.
1.0454340
0.5113710

144

$\operatorname{TS} 113(\mathrm{R}, \mathrm{R}) \mathrm{G}[\mathrm{B} 97-\mathrm{D} / \mathrm{def} 2-\mathrm{TZVP}]=-3310.750727$

$\begin{array}{lrrr}\text { TS113(R, R) G }[\mathrm{B} 97-\mathrm{D} / \mathrm{def}-\mathrm{TZVP}]=-3310.750727 \\ \mathrm{O} & 0.1293600 & -1.0921550 & 0.2877930 \\ \mathrm{O} & 2.1032520 & -0.0840640 & -0.9309330 \\ \mathrm{C} & 0.3874420 & -2.3533380 & -0.2353980 \\ \mathrm{C} & 1.6987450 & -2.8093730 & -0.2822950 \\ \mathrm{C} & -0.7367630 & -3.1255710 & -0.6414560 \\ \mathrm{C} & 1.9465970 & -4.0631890 & -0.9353120 \\ \mathrm{C} & -0.4918310 & -4.3687500 & -1.1860840 \\ \mathrm{C} & 0.8284420 & -4.8497340 & -1.3824160 \\ \mathrm{H} & -1.3273510 & -4.9930800 & -1.4927790 \\ \mathrm{C} & 2.9284010 & -0.6542330 & 0.0265090 \\ \mathrm{C} & 3.9299410 & 0.1826670 & 0.5889880 \\ \mathrm{C} & 2.7809990 & -2.0010630 & 0.3427960 \\ \mathrm{C} & 4.8220490 & -0.3873200 & 1.4727100 \\ \mathrm{C} & 3.6500750 & -2.5556900 & 1.3444730 \\ \mathrm{C} & 4.7057580 & -1.7391120 & 1.8811880 \\ \mathrm{H} & 5.6136070 & 0.2234540 & 1.9006840 \\ \mathrm{C} & 5.4270690 & -3.5773420 & 3.3102490 \\ \mathrm{C} & 4.3570030 & -4.3661680 & 2.8233630 \\ \mathrm{C} & 3.4925360 & -3.8686530 & 1.8684780 \\ \mathrm{C} & 5.5892360 & -2.2873890 & 2.8504070 \\ \mathrm{H} & 6.1059480 & -3.9837730 & 4.0567550 \\ \mathrm{H} & 4.2097140 & -5.3728540 & 3.2087120 \\ \mathrm{H} & 2.6750640 & -4.4860220 & 1.5123220 \\ \mathrm{H} & 6.3891070 & -1.6570730 & 3.2356480 \\ \mathrm{C} & 3.4560620 & -5.7531080 & -1.8386530 \\ \mathrm{C} & 2.3539900 & -6.5437360 & -2.2455170 \\ \mathrm{C} & 1.0682550 & -6.0955830 & -2.0229880 \\ \mathrm{C} & 3.2567440 & -4.5460280 & -1.1987630 \\ \mathrm{H} & 4.4690070 & -6.0972960 & -2.0361800 \\ \mathrm{H} & 2.5241620 & -7.4958960 & -2.7432900 \\ \mathrm{H} & 0.2116690 & -6.6842830 & -2.3474110\end{array}$




$\begin{array}{lrrr}\mathrm{H} & 4.1083880 & -3.9463610 & -0.8935760 \\ \mathrm{C} & -2.1096510 & -2.5804560 & -0.4230100 \\ \mathrm{C} & -2.9218410 & -2.1903900 & -1.5097410 \\ \mathrm{C} & -2.5747470 & -2.4040140 & 0.9037270 \\ \mathrm{C} & -4.1696700 & -1.6014260 & -1.2479770 \\ \mathrm{C} & -3.8215230 & -1.8146520 & 1.1087090 \\ \mathrm{C} & -4.6338680 & -1.3898870 & 0.0513410 \\ \mathrm{H} & -4.7873270 & -1.2985070 & -2.0881500 \\ \mathrm{H} & -4.1768600 & -1.6717830 & 2.1260990 \\ \mathrm{C} & 3.9653430 & 1.6368440 & 0.2549500 \\ \mathrm{C} & 3.5174170 & 2.5806520 & 1.2099710 \\ \mathrm{C} & 4.3981790 & 2.0651260 & -1.0168440 \\ \mathrm{C} & 3.5308590 & 3.9365440 & 0.8702450 \\ \mathrm{C} & 4.3866020 & 3.4342640 & -1.3096600 \\ \mathrm{C} & 3.9537820 & 4.3847380 & -0.3847240 \\ \mathrm{H} & 3.1801120 & 4.6635830 & 1.5986420 \\ \mathrm{H} & 4.7164480 & 3.7696250 & -2.2918130 \\ \mathrm{C} & -1.7958980 & -2.9131700 & 2.1116160 \\ \mathrm{C} & -5.9755410 & -0.7463810 & 0.3735610 \\ \mathrm{C} & -2.5101160 & -2.4398930 & -2.9551630 \\ \mathrm{C} & 3.0305670 & 2.1663570 & 2.5939460 \\ \mathrm{C} & 3.8923830 & 5.8569090 & -0.7505720 \\ \mathrm{C} & 4.8986230 & 1.0925710 & -2.0761280 \\ \mathrm{C} & 6.4042880 & 1.2967010 & -2.3350320 \\ \mathrm{H} & 6.5958440 & 2.3073780 & -2.7192500 \\ \mathrm{H} & 6.9815880 & 1.1683390 & -1.4103930 \\ \mathrm{H} & 6.7654040 & 0.5720480 & -3.0773030 \\ \mathrm{C} & 4.0961250 & 1.2065980 & -3.3851490 \\ \mathrm{H} & 3.0293880 & 1.0419120 & -3.1991390 \\ \mathrm{H} & 4.2240430 & 2.1991290 & -3.8371300 \\ \mathrm{H} & 4.4465440 & 0.4573580 & -4.1076290 \\ \mathrm{C} & 4.6567280 & 6.7406760 & 0.2517490 \\ \mathrm{H} & 4.6261150 & 7.7920310 & -0.0640490 \\ \mathrm{H} & 4.2039920 & 6.6731730 & 1.2495500 \\ \mathrm{H} & 5.7055360 & 6.4282820 & 0.3315260 \\ \mathrm{C} & 2.4274140 & 6.3233080 & -0.8750840 \\ \mathrm{H} & 1.8841940 & 5.7126130 & -1.6075230 \\ \mathrm{H} & 1.9161580 & 6.2292270 & 0.0922600 \\ \mathrm{H} & 2.3820020 & 7.3753530 & -1.1882390 \\ \mathrm{C} & 4.1361140 & 2.3955660 & 3.6440140 \\ \mathrm{H} & 5.0489100 & 1.8450240 & 3.3874960 \\ \mathrm{H} & 4.3862590 & 3.4638360 & 3.7001410 \\ \mathrm{H} & 3.7971230 & 2.0668350 & 4.6360070 \\ \mathrm{C} & 1.7379440 & 2.8864650 & 3.0195210 \\ \mathrm{H} & 1.9152860 & 3.9558170 & 2.2506030 \\ \mathrm{H} & 0.9674350 & 2.7774590 & \\ & & & \end{array}$

1.3676890
-2.4176790
-3.4532850
-2.4268700
-1.8448130
-1.7058110
-1.3084100
-2.6915520
-1.0465750
-6.9210360
-7.8607200
-7.1546270
-6.4610940
-6.6707480
-6.0205410
-6.9586360
-7.5826820
-2.7757220
-3.8500910
-2.2813850
-2.3846950
-3.2259500
-4.3123020
-2.8945960
-3.0249890
0.5293700
-0.1265470
0.3116010
-1.4324320
-5.7797460
-0.7708400
4.7692090
4.3678790
2.8092340
-2.5909120
-1.7401290
-2.5834060
-0.8311540
-1.5711370
-0.8951080
-3.9797940
-3.3858430
-1.9352150
-3.9640290
-5.0105980
-3.4109240

2.4535980 $-4.2347620$

$-4.0697010$

$-4.9860650$

$-4.6329980$

$-1.8897270$

$-0.9356140$

$-1.7113160$

$-2.2740160$

$-1.7702240$

$-1.2854300$

$-2.5806030$

$-2.2135540$

$-0.0916980$

0.6417780

$-0.8442270$

0.4191550

$-1.2489220$

- 1.0481640

$-0.3459530$

$-1.4744510$

$-3.6972880$

$-3.5347690$

$-3.9201010$

$-4.5707110$

0.1949250

0.1500030

1. 3907500

$-2.6300900$

0.0389910

$-3.1386560$

0.0716730

5.9718320

1.0940580

1.9865590

3. 0649880

1.0542090

2. 7712210

1. 9707520

1. 2411350

3. 7357210

4.8127260

4.4806640

2. 3622760

3. 9961480

5. 7948700
3.9583030

2.6069480

2.9334240

1.8070220

3.4555620

3.2553420

2.8941030

3.7049990

4.0445430

1. 0358690

1. 3333660

0.3317950

1.9267600

$-0.8259290$

$-1.3160660$

$-1.5727300$

$-0.4926640$

$-3.8904010$

$-3.9928980$

$-3.5240900$

$-4.8910240$

$-3.4910000$

$-3.5063350$

$-4.5143350$

$-2.8592430$

$-0.6339380$

$-1.9857860$

0.2532280

$-2.9755520$

1.1159570

1.8050680

$-1.7017730$

$-1.7353670$

2. 5706710

$-1.8886430$

$-1.3633270$

$-1.3172980$

$-0.8486910$

$-2.9338540$

$-2.6100400$

-3.0980360

$-2.1760870$

1.7830810

$-2.4066960$

- 3.3668060

$-2.6627130$ 


\begin{tabular}{|c|c|c|c|c|c|c|c|}
\hline & & & & & & & \\
\hline $\mathrm{H}$ & -1.2542750 & 4.6520650 & -2.6303440 & H & -6.1939060 & 1.9690460 & 3.3763790 \\
\hline $\mathrm{H}$ & -4.6355950 & 2.3780460 & -1.5377660 & C & -3.3563750 & 5.7290210 & -2.0092530 \\
\hline $\mathrm{H}$ & -4.3109070 & 1.5684030 & -3.0786060 & C & -2.2525130 & 6.4732650 & -2.4915920 \\
\hline $\mathrm{H}$ & -3.4009370 & 3.6830580 & -4.0297950 & C & -0.9696180 & 6.0079910 & -2.2889550 \\
\hline $\mathrm{H}$ & -3.9900690 & 4.8803570 & -1.2622280 & $\mathrm{C}$ & -3.1605300 & 4.5517870 & -1.3149620 \\
\hline H & -1.5712070 & 5.1192980 & -0.9697200 & $\mathrm{H}$ & -4.3680890 & 6.0852600 & -2.1910630 \\
\hline C & -2.8111950 & 2.2834020 & 1.4075220 & $\mathrm{H}$ & -2.4195160 & 7.4027980 & -3.0314420 \\
\hline 0 & -1.8586320 & 1.4032990 & 1.6237490 & $\mathrm{H}$ & -0.1126230 & 6.5603260 & -2.6711340 \\
\hline C & -4.0721990 & 2.0150590 & 2.1625050 & H & -4.0137370 & 3.9873650 & -0.9521900 \\
\hline C & -5.2315480 & 2.7336410 & 1.8272440 & C & 2.1868510 & 2.5090590 & -0.6097670 \\
\hline C & -4.1329960 & 1.0313740 & 3.1633420 & C & 2.9279130 & 1.9846120 & -1.6905400 \\
\hline C & -6.4368140 & 2.4641550 & 2.4743590 & $\mathrm{c}$ & 2.7114370 & 2.4475860 & 0.7039410 \\
\hline $\mathrm{H}$ & -5.1757080 & 3.4910930 & 1.0508540 & $\mathrm{C}$ & 4.1672990 & 1.3775860 & -1.4312580 \\
\hline C & -5.3381280 & 0.7707170 & 3.8147250 & C & 3.9518140 & 1.8429550 & 0.9062180 \\
\hline $\mathrm{H}$ & -3.2403360 & 0.4700410 & 3.4145110 & $\mathrm{C}$ & 4.6903910 & 1.2809500 & -0.1406340 \\
\hline C & -6.4923230 & 1.4820270 & 3.4694890 & $\mathrm{H}$ & 4.7283910 & 0.9631390 & -2.2634720 \\
\hline $\mathrm{H}$ & -7.3337200 & 3.0148930 & 2.2009200 & $\mathrm{H}$ & 4.3562380 & 1.7863320 & 1.9139950 \\
\hline $\mathrm{H}$ & -5.3800500 & 0.0049220 & 4.5858340 & C & -3.9665490 & -1.5212950 & 0.4671030 \\
\hline $\mathrm{H}$ & -7.4334810 & 1.2685270 & 3.9712700 & $\mathrm{C}$ & -3.5700740 & -2.4394320 & 1.4662990 \\
\hline H & -1.0051640 & 1.5130260 & 1.0556660 & C & -4.4005830 & -1.9899510 & -0.7936600 \\
\hline 0 & -2.7243600 & 3.2537190 & 0.6377160 & C & -3.6331470 & -3.8092990 & 1.1820140 \\
\hline & & & & C & -4.4335350 & -3.3668970 & -1.0305170 \\
\hline & , R) $G[B 97-L$ & {$[\mathrm{ZVP}]=-3$} & 423 & C & -4.0497130 & -4.2944230 & -0.0582460 \\
\hline 0 & -0.0519550 & 1.1217380 & 0.2691900 & $\mathrm{H}$ & -3.3250620 & -4.5237550 & 1.9432510 \\
\hline 0 & -2.0800500 & 0.0890550 & -0.8376250 & $\mathrm{H}$ & -4.7622660 & -3.7227040 & -2.0043900 \\
\hline C & -0.3052980 & 2.3507680 & -0.3263490 & C & 1.9956100 & 3.0804520 & 1.8913310 \\
\hline C & -1.6081240 & 2.8324130 & -0.3568280 & C & 6.0230280 & 0.6214020 & 0.1825060 \\
\hline C & 0.8188740 & 3.0732 & -0.8148740 & $\mathrm{C}$ & 2.4395950 & 2.1033570 & -3.1285540 \\
\hline C & -1.8523120 & 4.0541260 & -1.0693900 & C & -3.0771120 & -1.9935490 & 2.8380210 \\
\hline C & 0.5818850 & 4.2908630 & -1.4168630 & c & -4.0264640 & -5.7831400 & -0.3563590 \\
\hline C & -0.7340200 & 4.7910960 & -1.5936930 & c & -4.8654960 & -1.0489110 & -1.8974930 \\
\hline $\mathrm{H}$ & 1.4186870 & 4.8805960 & -1.7827970 & $\mathrm{C}$ & -6.3794100 & -1.2079690 & -2.1410930 \\
\hline C & -2.8729480 & 0.7267980 & 0.1051080 & $\mathrm{H}$ & -6.6091890 & -2.2271320 & -2.4795140 \\
\hline C & -3.8785990 & -0.0559400 & 0.7353180 & $\mathrm{H}$ & -6.9471080 & -1.0180160 & -1.2211380 \\
\hline C & -2.6867340 & 2.0842180 & 0.3451130 & $\mathrm{H}$ & -6.7175230 & -0.5039480 & -2.9135280 \\
\hline C & -4.7247990 & 0.5793100 & 1.6202320 & C & -4.0771080 & -1.2573390 & -3.2034230 \\
\hline C & -3.5096220 & 2.7 & 1.3432500 & $\mathrm{H}$ & -3.0032410 & -1.1307260 & -3.0301940 \\
\hline c & -4.5617440 & 1.9455930 & 1.9588410 & $\mathrm{H}$ & -4.2511730 & -2.2627760 & -3.6087830 \\
\hline H & -5.5156030 & 0.0098900 & 2.1029880 & $\mathrm{H}$ & -4.4000170 & -0.5276400 & -3.9579380 \\
\hline C & -5.1909010 & 3.8692270 & 3.3172460 & C & -2.8786330 & -6.1172710 & -1.3312110 \\
\hline c & -4.1252670 & 4.6088590 & 2.7498670 & $\mathrm{H}$ & -2.8299470 & -7.1991690 & -1.5158850 \\
\hline C & -3.3073620 & 146500 & 1.7911400 & $\mathrm{H}$ & -3.0374040 & -5.6094810 & -2.2918520 \\
\hline C & -5.3 & 2.5614280 & 2.9308470 & $\mathrm{H}$ & -1.9144670 & -5.7841610 & -0.9262120 \\
\hline $\mathrm{H}$ & -5.8 & 279940 & 4.0666060 & C & -5.3699850 & -6.2948430 & -0.9058590 \\
\hline H & 42950 & 5.6309040 & 3.0759310 & $\mathrm{H}$ & -6.1889850 & -6.0729580 & -0.2102960 \\
\hline $\mathrm{H}$ & -2. & 57690 & 1.3729430 & $\mathrm{H}$ & -5.6012 & 5.8191310 & 675880 \\
\hline
\end{tabular}




$\begin{array}{rrr}-5.3290050 & -7.3803840 & -1.0664550 \\ -4.1807170 & -2.1849000 & 3.8974610 \\ -5.0903460 & -1.6343040 & 3.6289260 \\ -4.4401110 & -3.2488390 & 3.9845620 \\ -3.8354640 & -1.8319180 & 4.8788500 \\ -1.7890440 & -2.7161290 & 3.2746680 \\ -1.9730380 & -3.7821310 & 3.4621430 \\ -1.0179410 & -2.6210320 & 2.5043270 \\ -1.4164580 & -2.2732360 & 4.2079330 \\ 2.6524810 & 4.4331650 & 2.2334590 \\ 3.7016960 & 4.2846760 & 2.5226850 \\ 2.6287720 & 5.1088010 & 1.3689430 \\ 2.1264920 & 4.9146430 & 3.0692400 \\ 1.9466110 & 2.1687960 & 3.1284440 \\ 1.5152470 & 1.1942520 & 2.8760800 \\ 2.9504590 & 2.0113140 & 3.5444720 \\ 1.3332740 & 2.6366490 & 3.9094980 \\ 7.0665740 & 1.6834260 & 0.5864020 \\ 8.0037030 & 1.2008240 & 0.8960030 \\ 7.2812810 & 2.3470510 & -0.2625440 \\ 6.7002080 & 2.2987200 & 1.4169220 \\ 6.5802440 & -0.2673210 & -0.9355880 \\ 5.8543860 & -1.0304450 & -1.2389100 \\ 6.8405670 & 0.3271320 & -1.8218750 \\ 7.4908520 & -0.7716810 & -0.5890690 \\ 2.6704090 & 0.8388340 & -3.9715180 \\ 3.7402680 & 0.6485120 & -4.1283780 \\ 2.2216330 & -0.0357280 & -3.4949200 \\ 2.2094840 & 0.9675700 & -4.9592730 \\ 3.1110460 & 3.3138510 & -3.8103430 \\ 4.1975260 & 3.1600560 & -3.8643080 \\ 2.7277560 & 3.4393780 & -4.8321580 \\ 2.9279720 & 4.2393880 & -3.2523850 \\ -0.5124540 & -0.2206370 & -0.5377370 \\ 0.1257170 & -0.3137920 & -1.8950470 \\ -0.3244550 & -1.3437480 & 0.4466940 \\ 1.3596950 & 2.2825490 & -3.1055240 \\ 5.8533670 & -0.0158550 & 1.0604490 \\ 0.9596060 & 3.2897440 & 1.6100040 \\ -4.6955680 & -0.0168000 & -1.5745800 \\ -3.8260310 & -6.3046110 & 0.5907460 \\ -2.8435590 & -0.9251280 & 2.7869990 \\ 2.4873750 & -2.2753880 & -1.7725250 \\ 1.6006540 & -3.2258350 & -1.0844980 \\ 2.5756020 & -1.2884100 & -0.5606410 \\ 0.7439600 & -2.8153440 & \end{array}$
1.4076420
0.7941410
3.6788870
3.0800450
1.6800120
3.7970540
4.6707960
3.0160750
0.9392010
4.5212830
4.1522300
3.0434400
3.7340080
1. 3260750
2.8109220
1.8779520
4.1030240
5.2360570
4.2149170
6.4650680
5.1410880
5.4438070
3. 3429040
6.5712300
7. 3411410
5.5248750
7.5308540
1.0065490
2.6827160

144

TS115(R,R) G[B97-D/def2-TZVP] $=-3310.750304$

$\begin{array}{lrrr}\text { TS115(R, R) G[B97-D/def2-TZVP }=-3310.750304 \\ \text { O } & 0.1142520 & -1.0820150 & 0.2751880 \\ \mathrm{O} & 2.1081460 & -0.0741600 & -0.9143840 \\ \mathrm{C} & 0.3722150 & -2.3433240 & -0.2470530 \\ \mathrm{C} & 1.6823000 & -2.8035430 & -0.2834880 \\ \mathrm{C} & -0.7512040 & -3.1125690 & -0.6609020 \\ \mathrm{C} & 1.9318420 & -4.0561330 & -0.9381570 \\ \mathrm{C} & -0.5054920 & -4.3549450 & -1.2070550 \\ \mathrm{C} & 0.8150240 & -4.8382460 & -1.3961880 \\ \mathrm{H} & -1.3404220 & -4.9770290 & -1.5199170 \\ \mathrm{C} & 2.9160690 & -0.6538120 & 0.0528560 \\ \mathrm{C} & 3.9140980 & 0.1748140 & 0.6326780 \\ \mathrm{C} & 2.7599010 & -2.0022870 & 0.3580940 \\ \mathrm{C} & 4.7992210 & -0.4063060 & 1.5153640 \\ \mathrm{C} & 3.6164200 & -2.5670940 & 1.3649720 \\ \mathrm{C} & 4.6730160 & -1.7602880 & 1.9141410\end{array}$

$-2.7570500$ $-2.4700340$ $-2.8470540$ $-1.7707550$ $-1.3444000$ $-2.3211850$ $-2.1346160$ $-2.1281760$ $-1.4960570$ $-3.0994440$ $-3.7426150$ $-0.8891240$ $-0.4432010$

1.5738350

1.7658940

2.2620690

1.9499990

3.1829540

2. 5422390

1.2360730

3.7800320

3.4137410

3.4588940

2.2870670

4.4893460

3.9183330

1.2305060
0.8796690 $-3.1435710$
0.2379870
$-1.1198830$
$-1.4395210$
$-1.5036790$
4.6899720
4.9091500
$-5.1626850$
$-2.2166900$
$-1.9166390$
$-0.8617880$
$-3.4987230$
$-0.5824890$
. 2606480

$-1.7602880$

1. 9141410

3.4393780

. 2825490

.0158550

.8321580

$-0.5377370$

$-3.1055240$

5745800

0.5907460
2.7869990

$-0.5606410$ 


$\begin{array}{rrrr}\mathrm{H} & 5.5985850 & 0.1939510 & 1.9433030 \\ \mathrm{C} & 5.3755750 & -3.6120750 & 3.3347020 \\ \mathrm{C} & 4.3033280 & -4.3909570 & 2.8368270 \\ \mathrm{C} & 3.4476270 & -3.8822870 & 1.8799510 \\ \mathrm{C} & 5.5480740 & -2.3201450 & 2.8842090 \\ \mathrm{H} & 6.0476800 & -4.0275100 & 4.0823720 \\ \mathrm{H} & 4.1474760 & -5.3990640 & 3.2150380 \\ \mathrm{H} & 2.6287980 & -4.4926880 & 1.5151300 \\ \mathrm{H} & 6.3494910 & -1.6971500 & 3.2780270 \\ \mathrm{C} & 3.4437850 & -5.7474680 & -1.8344190 \\ \mathrm{C} & 2.3427630 & -6.5337850 & -2.2523340 \\ \mathrm{C} & 1.0565110 & -6.0828100 & -2.0385890 \\ \mathrm{C} & 3.2427050 & -4.5417640 & -1.1924730 \\ \mathrm{H} & 4.4572940 & -6.0938770 & -2.0250320 \\ \mathrm{H} & 2.5142320 & -7.4849260 & -2.7516080 \\ \mathrm{H} & 0.2009600 & -6.6683370 & -2.3713930 \\ \mathrm{H} & 4.0934220 & -3.9451450 & -0.8787350 \\ \mathrm{C} & -2.1241320 & -2.5665480 & -0.4452750 \\ \mathrm{C} & -2.9337600 & -2.1742270 & -1.5329790 \\ \mathrm{C} & -2.5921310 & -2.3921290 & 0.8808560 \\ \mathrm{C} & -4.1814170 & -1.5839710 & -1.2729860 \\ \mathrm{C} & -3.8384870 & -1.8014320 & 1.0840200 \\ \mathrm{C} & -4.6479570 & -1.3734350 & 0.0256390 \\ \mathrm{H} & -4.7969870 & -1.2793830 & -2.1140800 \\ \mathrm{H} & -4.1958980 & -1.6601460 & 2.1008500 \\ \mathrm{C} & 3.9735820 & 1.6275550 & 0.2909350 \\ \mathrm{C} & 3.4287030 & 2.5787420 & 1.1866650 \\ \mathrm{C} & 4.5324860 & 2.0415090 & -0.9340100 \\ \mathrm{C} & 3.4816080 & 3.9308820 & 0.8382960 \\ \mathrm{C} & 4.5545780 & 3.4086760 & -1.2399910 \\ \mathrm{C} & 4.0331030 & 4.3666740 & -0.3713270 \\ \mathrm{H} & 3.0608670 & 4.6677860 & 1.5172800 \\ \mathrm{H} & 4.9812150 & 3.7336540 & -2.1880630 \\ \mathrm{C} & -1.8172940 & -2.9057830 & 2.0895020 \\ \mathrm{C} & -5.9883610 & -0.7262770 & 0.3462670 \\ \mathrm{C} & -2.5197560 & -2.4231950 & -2.9778220 \\ \mathrm{C} & 2.8024850 & 2.1595030 & 2.5123810 \\ \mathrm{C} & 4.0194640 & 5.8384290 & -0.7455050 \\ \mathrm{C} & 5.1117190 & 1.0536020 & -1.9369530 \\ \mathrm{C} & 6.6253640 & 1.2752550 & -2.1196290 \\ \mathrm{H} & 6.8236800 & 2.2791960 & -2.5177030 \\ \mathrm{H} & 7.1533960 & 1.1779420 & -1.1622820 \\ \mathrm{H} & 7.0364250 & 0.5391940 & -2.8236030 \\ \mathrm{C} & 4.3776850 & 1.1251190 & -3.2890320 \\ \mathrm{H} & 3.3051920 & 0.9458740 & -3.1554540 \\ \mathrm{H} & 4.5119230 & 2.1112730 & -3.7531790 \\ & & & \end{array}$

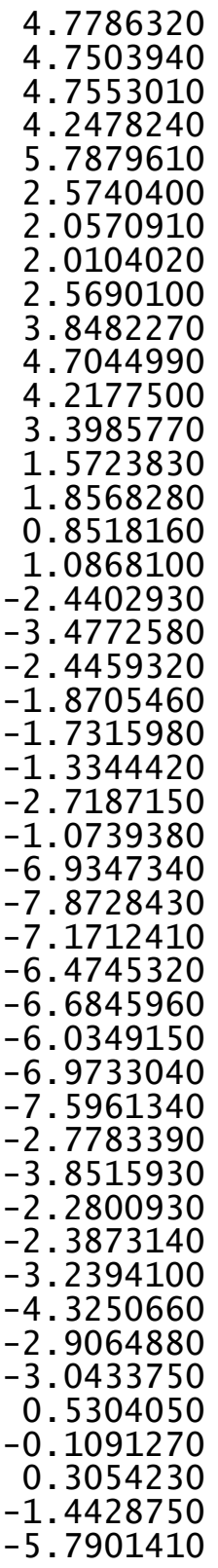

4.7786320

4.7553010

4.2478240

2. 0570910

2. 0104020

2. 5690100

3.8482270

4. 2177500

3. 3985770

1. 5723830

0.8518160

1.0868100

$-2.4402930$

-3.4772580
-2.4459320

$-1.8705460$

$-1.7315980$

$-1.3344420$

$-2.7187150$

1.0739380

-7.8728430

$-7.1712410$

$-6.4745320$

$-6.6845960$

$-6.0349150$

-6.9733040
-7.5961340

$-2.7783390$

$-3.8515930$

$-2.2800930$

- 3.2394100

$-4.3250660$

$-2.9064880$

0.5304050

-1.4428750
-5.7901410
$-3.9765480$

0.2958830

$-0.0171100$

1. 2699870

0.4258430

$-0.9453980$

$-1.7092200$

$-0.0070570$

$-1.2546290$

3. 6451950

3.4304120

3. 7671290

4.5949640

2. 9041120

3. 1776140

2. 0824040

3.7783720

2. 5768210

2.8999040

1.7736440

3. 4259420

3. 2381020

2. 8823590

3. 6861100

4.0272430

1. 0161270

1. 3122930

0.3171860

1.9089750

$-0.8558390$

$-1.3503400$

$-1.5985530$

$-0.5242010$

$-3.9116990$

$-4.0149990$

$-3.5436440$

$-4.9122220$

$-3.5168080$

$-3.5345420$

$-4.5395710$

$-2.8853850$

$-0.6389330$

$-1.9986320$

0.2500900

$-2.9961450$

1.0838630 


\begin{tabular}{|c|c|c|c|}
\hline $\mathrm{H}$ & -0.7910640 & -3.1296410 & 1.7858640 \\
\hline $\mathrm{H}$ & 4.9759210 & 0.0399950 & -1.5444410 \\
\hline $\mathrm{H}$ & 4.5485040 & 5.9402840 & -1.7039310 \\
\hline $\mathrm{H}$ & 2.4667250 & 1.1214060 & 2.4074340 \\
\hline C & -2.5856210 & 1.9935820 & -1.8929130 \\
\hline C & -1.7373600 & 3.0744910 & -1.3683770 \\
\hline $\mathrm{H}$ & -2.5749830 & 1.0612300 & -1.3216520 \\
\hline $\mathrm{H}$ & -0.8271170 & 2.7824230 & -0.8550560 \\
\hline 0 & -1.5677570 & 1.9806230 & -2.9398070 \\
\hline $\mathrm{H}$ & -0.8878880 & 1.2538490 & -2.6180230 \\
\hline C & -3.9817800 & 3.7391820 & -3.0993620 \\
\hline C & -3.3889850 & 4.8177740 & -2.1785350 \\
\hline C & -1.9367170 & 4.4894690 & -1.7884220 \\
\hline C & -3.9607230 & 2.3656120 & -2.4084170 \\
\hline $\mathrm{H}$ & -5.0138850 & 3.9966890 & -3.3659600 \\
\hline $\mathrm{H}$ & -3.4175630 & 5.7998250 & -2.6651440 \\
\hline $\mathrm{H}$ & -1.2581710 & 4.6618590 & -2.6373900 \\
\hline $\mathrm{H}$ & -4.6307750 & 2.3790680 & -1.5382880 \\
\hline $\mathrm{H}$ & -4.3065680 & 1.5711290 & -3.0800460 \\
\hline $\mathrm{H}$ & -3.4047220 & 3.6883380 & -4.0323310 \\
\hline $\mathrm{H}$ & -3.9915920 & 4.8838280 & -1.2634980 \\
\hline $\mathrm{H}$ & -1.5725020 & 5.1296030 & -0.9763370 \\
\hline C & -2.8108600 & 2.2919450 & 1.4006060 \\
\hline 0 & -1.8625370 & 1.4081430 & 1.6185750 \\
\hline C & -4.0766100 & 2.0266380 & 2.1486900 \\
\hline C & -5.2305440 & 2.7533800 & 1.8122780 \\
\hline C & -4.1473610 & 1.0377540 & 3.1 \\
\hline C & -6.4401380 & 2.48 & 560 \\
\hline $\mathrm{H}$ & -5.1670420 & 3.51 & 1.0 \\
\hline C & -5.3568130 & 0.7800020 & 2020 \\
\hline $\mathrm{H}$ & -3.2589360 & 0.4700950 & 60020 \\
\hline C & -6.5055060 & 1.4996190 & 3.4418970 \\
\hline $\mathrm{H}$ & -7.3327510 & 3.0442660 & 2.1783320 \\
\hline $\mathrm{H}$ & -5.4064270 & 0.0099930 & 4.5546460 \\
\hline $\mathrm{H}$ & -7.4500570 & 1.2885320 & 3.9382900 \\
\hline $\mathrm{H}$ & -1.0063040 & 1.5169020 & 1.0529270 \\
\hline $\begin{array}{l}0 \\
144\end{array}$ & -2.7168840 & 3.2641440 & 0.6336900 \\
\hline
\end{tabular}

$\operatorname{TS} 116(R, R)$ G [B97-D/def2-TZVP $]=-3310.749685$

$\begin{array}{lrlr}\text { O } & 0.4351390 & 0.7656340 & 0.0590930 \\ \mathrm{O} & -1.8826730 & 0.7857240 & -1.0071090 \\ \mathrm{C} & 0.4833820 & 2.1473320 & -0.0584810 \\ \mathrm{C} & -0.6218590 & 2.9042730 & 0.3182480 \\ \mathrm{C} & 1.7087070 & 2.7254410 & -0.4874070 \\ \mathrm{C} & -0.5718980 & 4.3251510 & 0.1243250 \\ \mathrm{C} & 1.7775220 & 4.1004470 & -0.5792040\end{array}$

\begin{abstract}
0.6565680
2.7151840

$-2.4360170$

$-3.6269700$

$-1.8216710$

$-4.1808550$

$-2.3534080$

$-3.5639190$

$-5.1058020$

$-3.4742360$

$-2.2594520$

$-1.7147720$

$-4.1079040$

$-3.8963030$

$-1.7476620$

$-0.7818940$

$-5.0306730$

$-1.6079060$

$-0.3850020$

0.7217970

$-1.6986610$

$-2.4864060$

$-0.3260350$

1.6638530

$-2.6431100$

2. 9136350

3. 3195420

3. 6741820

4.5055490

4.8492230

5. 2904670

4.8325510

5.4319730

$-4.2578550$

$-4.2371720$

$-4.8810140$

$-4.8635500$

$-5.4862680$

$-5.4913910$

$-4.8488200$

$-5.9707020$

3. 2934760

6.6026100

2. 5209400

$-3.5655450$

$-6.1392470$
\end{abstract}

$\begin{array}{rr}4.9258060 & -0.3172410 \\ 4.5666730 & -0.8721540 \\ 1.2027700 & 0.2015570 \\ 0.5619110 & 0.6375940 \\ 2.2397820 & 0.8910500 \\ 0.9906800 & 1.8268910 \\ 2.6107470 & 2.1713310 \\ 1.9836600 & 2.6276640 \\ 0.5379940 & 2.1752200 \\ 3.2780380 & 4.6904500 \\ 3.8663090 & 4.2614160 \\ 3.5425080 & 3.0347800 \\ 2.3522370 & 3.8881710 \\ 3.5476420 & 5.6561310 \\ 4.5775370 & 4.9062800 \\ 3.9994560 & 2.7215280 \\ 1.8742300 & 4.2128310 \\ 6.5342510 & 0.1251090 \\ 7.1276900 & -0.2729680 \\ 6.3346850 & -0.4928780 \\ 5.1705070 & 0.3192290 \\ 7.1583550 & 0.2743700 \\ 8.2043650 & -0.4164330 \\ 6.7728650 & -0.8183770 \\ 4.7293000 & 0.6206740 \\ 1.8871680 & -0.7653610 \\ 1.6356820 & -2.0968120 \\ 1.3903480 & 0.3128740 \\ 0.9271880 & -2.3105860 \\ 0.6743280 & 0.0451510 \\ 0.4440230 & -1.2575640 \\ 0.7366880 & -3.3312330 \\ 0.3039580 & 0.8843020 \\ -0.5241680 & -0.1689700 \\ -1.8606350 & 0.2989580 \\ -0.2148570 & -1.3972110 \\ -2.8496070 & -0.4652720 \\ -1.2448280 & -2.1277960 \\ -2.5660270 & -1.6817050 \\ -3.8764970 & -0.1080540 \\ -1.0078930 & -3.0739370 \\ 1.6420220 & 1.7659200 \\ -0.2559240 & -1.5733970 \\ 2.1520280 & -3.2877850 \\ -2.2569810 & 1.6090520 \\ -3.6667770 & -2.5022000 \\ & \end{array}$




$\begin{array}{rrr}-4.9549970 & 1.2036430 & -1.9483440 \\ -6.4138140 & 1.7011600 & -1.9807250 \\ -7.0201330 & 1.0742460 & -2.6477330 \\ -6.8625360 & 1.6673610 & -0.9795430 \\ -6.4550960 & 2.7352860 & -2.3484680 \\ -4.3090180 & 1.3089920 & -3.3421220 \\ -3.2666960 & 0.9740210 & -3.3107120 \\ -4.8526440 & 0.6934240 & -4.0707810 \\ -4.3349190 & 2.3503420 & -3.6904370 \\ -7.2616640 & -4.3758970 & -1.7214700 \\ -7.7455590 & -5.1345360 & -2.3511190 \\ -6.8538100 & -4.8791390 & -0.8349210 \\ -8.0221390 & -3.6589850 & -1.3872870 \\ -5.0907660 & -4.6872870 & -2.9870170 \\ -4.3054020 & -4.1937840 & -3.5731380 \\ -4.6156150 & -5.1813460 & -2.1292450 \\ -5.5635290 & -5.4591100 & -3.6095520 \\ -4.6158370 & -2.4513540 & 2.7213020 \\ -5.2062980 & -1.5414650 & 2.8782790 \\ -5.3057300 & -3.2618480 & 2.4496500 \\ -4.1257670 & -2.7169750 & 3.6680140 \\ -2.7054450 & -3.5285730 & 1.4799450 \\ -3.3305520 & -4.4161880 & 1.3153970 \\ -1.9996370 & -3.4348890 & 0.6493880 \\ -2.1398190 & -3.6851610 & 2.4072730 \\ 4.3385940 & 2.5414570 & 2.4538840 \\ 5.3188620 & 2.0472940 & 2.4752920 \\ 4.4474740 & 3.4938380 & 1.9192870 \\ 4.0370840 & 2.7520820 & 3.4887290 \\ 3.0987370 & 0.3270190 & 2.5394110 \\ 2.3068730 & -0.2722460 & 2.0814250 \\ 4.0226440 & -0.2658970 & 2.5565710 \\ 2.8129590 & 0.5365380 & 3.5784190 \\ 7.0789100 & -1.2317150 & -0.4876890 \\ 7.9785190 & -1.7588130 & -0.8291150 \\ 7.3366090 & -0.6968150 & 0.4356580 \\ 6.3078970 & -1.9739570 & -0.2488340 \\ 7.6964500 & 0.7946310 & -1.8620750 \\ 7.3870710 & 1.4701180 & -2.6688290 \\ 7.8821490 & 1.3980670 & -0.9632100 \\ 8.6358900 & 0.3048090 & -2.1524520 \\ 2.4270470 & 1.1397890 & -4.4421240 \\ 3.4035100 & 0.9881260 & -4.9211080 \\ 2.0559240 & 0.1728400 & -4.0914290 \\ 1.7376480 & 1.5210430 & -5.2059960 \\ 3.1199640 & 3.4761590 & -3.8041850\end{array}$

\author{
4.1520490 \\ 2. 5318300 \\ 3. 1324450 \\ $-0.5356890$ \\ $-0.0005770$ \\ $-0.7697920$ \\ 1. 4991130 \\ 6.4468380 \\ 2. 3383300 \\ $-4.4022890$ \\ $-6.5883560$ \\ $-2.8979680$ \\ 2. 3753040 \\ 1. 3779200 \\ 2. 2803130 \\ 0.3915890 \\ 1.6242840 \\ 0.9213680 \\ 3. 9250790 \\ 3.0905360 \\ 1. 6046900 \\ 3. 8105350 \\ 4.9786100 \\ 3. 1874420 \\ 1.1396710 \\ 4. 2669290 \\ 4. 3315490 \\ 3. 5770300 \\ 3.4585440 \\ 1.0294560 \\ 1.4683580 \\ 0.4704590 \\ 2. 1733200 \\ 3.3044660 \\ 1. 7417860 \\ 3. 9962560 \\ 3. 6324310 \\ 2. 4342620 \\ 0.8720970 \\ 3. 5623680 \\ 4.8735650 \\ 2. 0958180 \\ 4. 1025400 \\ 0.0058360 \\ 1. 8410040
}

3. 3141640

3. 8543910

4.2420270

$-0.1196410$

$-0.1867120$

$-1.3990600$

2. 3508250

$-0.8315720$

2. 1762670

1. 8721440

$-3.1961640$

$-1.4429320$

$-2.1485230$

$-3.1939460$

$-1.2280330$

$-2.8635480$

$-2.0647300$

$-1.3157400$

- 3.9474500

$-5.0069300$

$-4.6102760$

$-2.5845340$

$-4.2508440$

$-5.9853480$

$-4.7337040$

$-2.6364930$

$-1.7988880$

$-3.8553870$

$-5.1069870$

$-5.2438880$

$-2.9337360$

$-2.0823890$

$-3.1537000$

$-3.9858510$

$-2.5267850$

$-4.1849290$

$-4.4654960$

$-2.7303940$

$-1.8783530$

$-3.5574110$

$-4.8275630$

$-2.2409750$

$-3.7120570$

$-1.8603200$

$-3.5257760$
$-4.1440210$

$-4.6511930$

$-3.0210870$

$-0.9261270$

$-2.3264960$

$-0.1692940$

$-2.9462320$

$-2.4985290$

1.7915580

$-1.2809440$

$-3.3887100$

1. 9089280

$-1.6369880$

$-1.3610830$

$-1.0552010$

$-1.0548250$

$-2.8917270$

$-2.7340110$

$-2.5443080$

$-1.8091060$

$-1.7627350$

$-1.8434760$

$-2.5772540$

$-2.2943260$

$-2.7525980$

$-0.8464310$

$-2.3976700$

$-3.5819860$

$-0.7799320$

$-1.0775140$

1.8608440

1.9294530

3. 1626680

3.1959690

4. 3439340

4. 3901830

2. 2781640

5. 5376760

4.3183920

5. 5635940

4.4076040

6.4480420

6.4952060

1.0303310

0.8358490 
$\operatorname{TS} 117(\mathrm{~S}, \mathrm{~S}) \mathrm{G}[\mathrm{B} 97-\mathrm{D} / \mathrm{def} 2-\mathrm{TZVP}]=-3310.755010$

\begin{tabular}{|c|c|c|c|}
\hline & & & \\
\hline 0 & 0.5605340 & $0 . \overline{7270830}$ & 0.3228130 \\
\hline 0 & -1.6677540 & 0.9741200 & -0.8924820 \\
\hline C & 0.6159400 & 2.1082320 & 0.4507000 \\
\hline $\mathrm{C}$ & -0.5097570 & 2.7898060 & 0.9024050 \\
\hline $\mathrm{C}$ & 1.8501410 & 2.7496460 & 0.1618530 \\
\hline $\mathrm{C}$ & -0.4537080 & 4.2211270 & 0.9819130 \\
\hline $\mathrm{C}$ & 1.9202730 & 4.1177260 & 0.3324610 \\
\hline C & 0.7929020 & 4.8844510 & 0.7146200 \\
\hline $\mathrm{H}$ & 2.8610670 & 4.6278900 & 0.1403210 \\
\hline C & -2.3049950 & 1.1603450 & 0.3339710 \\
\hline $\mathrm{C}$ & -3.5257300 & 0.4670120 & 0.5540540 \\
\hline $\mathrm{C}$ & -1.7417880 & 2.0362210 & 1.2543470 \\
\hline C & -4.1610210 & 0.6639310 & 1.7635250 \\
\hline $\mathrm{C}$ & -2.3617380 & 2.1578740 & 2.5424310 \\
\hline C & -3.6011220 & 1.4712470 & 2.7841350 \\
\hline $\mathrm{H}$ & -5.1097190 & 0.1670220 & 1.9500250 \\
\hline C & -3.6548600 & 2.3413040 & 5.0597020 \\
\hline C & -2.4133220 & 2.9856260 & 4.8369660 \\
\hline C & -1.7844740 & 2.8967400 & 3.6112120 \\
\hline C & -4.2311120 & 1.5961720 & 4.0522580 \\
\hline $\mathrm{H}$ & -4.1433060 & 2.4250130 & 6.0281010 \\
\hline $\mathrm{H}$ & -1.9482790 & 3.5528060 & 5.6404480 \\
\hline $\mathrm{H}$ & -0.8319100 & 3.3929140 & 3.4559800 \\
\hline $\mathrm{H}$ & -5.1739950 & 1.0767310 & 4.2151810 \\
\hline C & -1.4899430 & 6.3993700 & 1.3498960 \\
\hline C & -0.2499500 & 7.0470000 & 1.1284550 \\
\hline C & 0.8656960 & 6.3007290 & 0.8121720 \\
\hline $\mathrm{C}$ & -1.5891090 & 5.0243570 & 1.2791270 \\
\hline $\mathrm{H}$ & -2.3750480 & 6.9914790 & 1.5724330 \\
\hline $\mathrm{H}$ & -0.1848990 & 8.1308830 & 1.1 \\
\hline $\mathrm{H}$ & 1.8209460 & 6.7851450 & 0.6165690 \\
\hline $\mathrm{H}$ & 70770 & 4.5432640 & 1.4463890 \\
\hline C & 3.0445890 & 1.9853130 & -0.3054090 \\
\hline C & 090 & 2.0954720 & -1 \\
\hline C & 4910 & 1. 1948440 & 0.6052100 \\
\hline C & 4.63 & 1.4340050 & -430 \\
\hline C & 4.9 & 0.5567660 & 0.1 \\
\hline C & $5.38-1$ & 0.6621100 & -1.1 \\
\hline $\mathrm{H}$ & 4.95 & 1.519 & -3.0 \\
\hline $\mathrm{H}$ & $5.5]$ & -0.0464730 & 15040 \\
\hline C & -4.11 & -0.4241590 & $-0.4 \varepsilon$ \\
\hline C & -4.14 & -1.8238660 & -0.2 \\
\hline $\mathrm{C}$ & -4.6624510 & 0.1398680 & -1.66 \\
\hline C & -4.7 & -2.6232410 & -1.2664060 \\
\hline C & -5.2502340 & -0.7044670 & -2.6096150 \\
\hline
\end{tabular}

-5.3152300
-4.7941500
-5.6735040
-0.3266130
0.3287620
-0.5982390
3.3763500
2.4190490
6.6760490
6.9205700
2.7104250
1.7164440
-4.6740770
-4.1390230
-5.9482540
-5.9409640
-3.5481760
-2.8810340
-2.7041650
-2.2079430
-3.3307610
-1.9403880
-4.6538910
-5.3456650
-4.2140050
-5.2340740
3.1762680
4.1078530
2.8614720
2.4008510
4.4089640
4.0970650
5.3923840
4.5158290
-3.9564490
-4.4809970
-3.9320820
-2.9276930
-6.1165180
-6.1139730
-6.7058340
-6.6134980
2.5062880
1.8836390
3.4622190
2.0039260

-2.0881320
-3.7015240
-0.2667030
0.0557220
0.2528420
-1.3444840
1.0463010
1.5536970
-0.0219150
-0.7687400
2.9342310
3.1509250
1.6408300
2.1464650
-2.9859000
-4.0112890
-2.4927480
-1.7748500
-3.7337210
-4.1039020
-4.5444920
-3.4844830
-2.8767370
-3.5977140
-3.3395110
-2.0007670
-0.4285170
-0.9977300
-0.5038880
-0.8893880
1.7251520
1.6423990
1.2484270
2.7880720
1.9959640
1.5607980
3.0858570
1.6196770
2.1844900
3.2718550
1.7236020
1.9682980
2.2066500
2.8249420
2.0321160
1.2471830

$-2.4286340$ $-1.1231520$ $-3.5109520$ $-0.8880770$ $-2.2231860$ $-0.4144250$ 2. 0668700 2. 2208350 $-1.5892910$ $-0.8191710$ $-2.6737790$ $-2.2689180$ $-1.9262280$ $-1.1167340$ $-3.4768500$ $-3.0798120$ 0.9408370 1. 4290120

0.5948290

1.5007670 0.2009100 $-0.1478630$

1. 9448210

1.4882940 2.8390190 2. 2575000 2. 4588900 2.3433670 3.5076410 1.8397260

2.9872610 4.0370040 2.8809590 2.7359850 $-3.2412880$ $-4.1020190$ $-3.3741710$ $-3.2331370$ $-1.9218550$ $-2.0773560$ $-2.7255660$ $-0.9673060$ $-4.0146150$ $-4.6741600$ -4.5256260
-3.8637220 


$\begin{array}{lrr}3.4313490 & 4.2780300 & -2.9054770 \\ 4.4379950 & 4.1034180 & -3.3089410 \\ 2.8721070 & 4.8911610 & -3.6249320 \\ 3.5322080 & 4.8436790 & -1.9717730 \\ -7.4114710 & -2.5960890 & -3.7582870 \\ -8.0082440 & -2.6163890 & -2.8377220 \\ -7.4653720 & -1.5828540 & -4.1776030 \\ -7.8593200 & -3.2882860 & -4.4838410 \\ -5.1261650 & -2.9757970 & -4.7806470 \\ -4.0899380 & -3.2835730 & -4.5926890 \\ -5.5684900 & -3.6565830 & -5.5206460 \\ -5.1077080 & -1.9657230 & -5.2105710 \\ 7.8331410 & 0.9964650 & -1.6383160 \\ 7.9597460 & 1.4922210 & -0.6678640 \\ 8.7750630 & 0.4988790 & -1.9064520 \\ 7.6232500 & 1.7680240 & -2.3911940 \\ 6.5512790 & -0.7543680 & -2.9370000 \\ 5.7276850 & -1.4782550 & -2.9277460 \\ 6.3645230 & -0.0443630 & -3.7521740 \\ 7.4831780 & -1.2884570 & -3.1630500 \\ 1.6790240 & -2.6688390 & -2.1459540 \\ 2.4355230 & -2.2084060 & -0.9749040 \\ 0.5953370 & -2.6296120 & -2.0386030 \\ 1.9910380 & -1.4351860 & -0.3603770 \\ 2.2100670 & -1.4227470 & -2.7105140 \\ 1.4838400 & -0.7011230 & -2.5481640 \\ 3.7297960 & -3.9720830 & -2.8838750 \\ 4.2877310 & -3.8668070 & -1.4558930 \\ 3.8673010 & -2.5489270 & -0.7844290 \\ 2.1936350 & -3.8961080 & -2.8706720 \\ 4.0459520 & -4.9159570 & -3.3436590 \\ 5.3825440 & -3.9305350 & -1.4625650 \\ 4.4347230 & -1.6998570 & -1.1881020 \\ 1.7821490 & -4.7687150 & -2.3443420 \\ 1.7838550 & -3.8974290 & -3.8882870 \\ 4.1298340 & -3.1561420 & -3.5002720 \\ 3.9087010 & -4.7050930 & -0.8571180 \\ 4.0697990 & -2.5438050 & 0.2922740 \\ 1.0830710 & -3.3741230 & 1.6339190 \\ 0.1979490 & -2.4083780 & 1.7212580 \\ 1.3369700 & -4.0931650 & 2.9206220 \\ 2.3520120 & -5.0630720 & 2.9733800 \\ 0.5851130 & -3.8152760 & 4.0755400 \\ 2.6134780 & -5.7435930 & 4.1621210 \\ 2.9266790 & -5.2714940 & 2.0754180 \\ 0.8485590 & -4.4990260 & 5.2629990\end{array}$

$\begin{array}{lr}\mathrm{H} & -0.1996230 \\ \mathrm{C} & 1.8619760 \\ \mathrm{H} & 3.4018150 \\ \mathrm{H} & 0.2633870 \\ \mathrm{H} & 2.0655690 \\ \mathrm{H} & -0.0706140 \\ \mathrm{O} & 1.6872550 \\ 144 & \end{array}$
$-3.0662560$
$-5.4628100$
$-6.4921420$
$-4.2803120$
$-5.9939980$
$-1.9838100$

4.0353840

5. 3091210

4. 1963660

6.1532740

6.2364750

144

0.8084730

$\operatorname{TS} 118(\mathrm{~S}, \mathrm{~S})$ G[B97-D/def2-TZVP $]=-3310.753855$

$\begin{array}{lrrr}\text { TS118(S }, \mathrm{S}) \mathrm{G}[\mathrm{B} 97-\mathrm{D} / \mathrm{def}-\mathrm{TZVP}]=-3310.753855 \\ \mathrm{O} & 0.0307970 & -1.1493470 & 0.2387950 \\ \mathrm{O} & 2.0581160 & -0.0917860 & -0.8559270 \\ \mathrm{C} & 0.3387830 & -2.3890640 & -0.3022520 \\ \mathrm{C} & 1.6574330 & -2.8281370 & -0.2873770 \\ \mathrm{C} & -0.7496450 & -3.1690660 & -0.7791140 \\ \mathrm{C} & 1.9558610 & -4.0652950 & -0.9507550 \\ \mathrm{C} & -0.4616460 & -4.3985720 & -1.3325090 \\ \mathrm{C} & 0.8737280 & -4.8582890 & -1.4692330 \\ \mathrm{H} & -1.2724690 & -5.0338920 & -1.6807940 \\ \mathrm{C} & 2.8450590 & -0.6693830 & 0.1295400 \\ \mathrm{C} & 3.8146820 & 0.1655390 & 0.7479210 \\ \mathrm{C} & 2.6948380 & -2.0224960 & 0.4129670 \\ \mathrm{C} & 4.6695640 & -0.4126380 & 1.6616230 \\ \mathrm{C} & 3.5186790 & -2.5868490 & 1.4464120 \\ \mathrm{C} & 4.5404080 & -1.7709170 & 2.0465980 \\ \mathrm{H} & 5.4490850 & 0.1903120 & 2.1208180 \\ \mathrm{C} & 5.2087860 & -3.6277740 & 3.4772140 \\ \mathrm{C} & 4.1709550 & -4.4169860 & 2.9255420 \\ \mathrm{C} & 3.3488300 & -3.9103690 & 1.9385940 \\ \mathrm{C} & 5.3814670 & -2.3287240 & 3.0474150 \\ \mathrm{H} & 5.8544420 & -4.0415550 & 4.2487280 \\ \mathrm{H} & 4.0152210 & -5.4317350 & 3.2856400 \\ \mathrm{H} & 2.5562960 & -4.5287700 & 1.5312730 \\ \mathrm{H} & 6.1568310 & -1.6989120 & 3.4805320 \\ \mathrm{C} & 3.5337950 & -5.7218230 & -1.7975140 \\ \mathrm{C} & 2.4654330 & -6.5203550 & -2.2729630 \\ \mathrm{C} & 1.1639220 & -6.0914640 & -2.1134200 \\ \mathrm{C} & 3.2846880 & -4.5271190 & -1.1518830 \\ \mathrm{H} & 4.5603230 & -6.0499850 & -1.9461100 \\ \mathrm{H} & 2.6742900 & -7.4631040 & -2.7739170 \\ \mathrm{H} & 0.3339730 & -6.6864890 & -2.4912110 \\ \mathrm{H} & 4.1110600 & -3.9211610 & -0.7940960 \\ \mathrm{C} & -2.1460170 & -2.6704100 & -0.5883390 \\ \mathrm{C} & -2.8879480 & -2.1473480 & -1.6731670 \\ \mathrm{C} & -2.7052180 & -2.6897740 & 0.7079490 \\ \mathrm{C} & -4.1791170 & -1.6637890 & -1.4336170 \\ \mathrm{C} & -4.0024930 & -2.1969550 & 0.8938110\end{array}$




\begin{tabular}{|c|c|c|}
\hline $\begin{array}{l}-4.7547420 \\
-4.7541870 \\
-4.4398660 \\
3.8768840 \\
3.3004170 \\
4.4558370 \\
3.3288230 \\
4.4580020 \\
3.8926390 \\
2.8754850 \\
4.8997830 \\
-1.9634860 \\
-6.1615020 \\
-2.3287700 \\
2.6662060 \\
3.8334920 \\
5.0789170 \\
6.5947430 \\
6.7920660 \\
7.0924420 \\
7.0388220 \\
4.3899880 \\
3.3158290 \\
4.5281730 \\
4.8232410 \\
4.4370280 \\
4.4154020 \\
3.8630430 \\
5.4759900 \\
2.3805910 \\
1.9598750 \\
1.7527190 \\
2.3359590 \\
3.7018870 \\
4.5705220 \\
4.0535110 \\
3.2493820 \\
1.4237120 \\
1.6965520 \\
0.7124470 \\
0.9318290 \\
-2.6087060 \\
-3.6488120 \\
-2.6090170 \\
-2.0574130 \\
-1.8903930\end{array}$ & $\begin{array}{r}-1.6766270 \\
-1.2635910 \\
-2.2153100 \\
1.6152460 \\
2.5773140 \\
2.0122640 \\
3.9213530 \\
3.3717410 \\
4.3386920 \\
4.6650720 \\
3.6830400 \\
-3.2584480 \\
-1.1529330 \\
-2.1379210 \\
2.1790850 \\
5.7948080 \\
1.0150270 \\
1.2552380 \\
2.2551100 \\
1.1816050 \\
0.5133020 \\
1.0518860 \\
0.8618430 \\
2.0302190 \\
0.2870750 \\
6.7407470 \\
7.7779150 \\
6.6948990 \\
6.4682410 \\
6.2000290 \\
5.5523890 \\
6.1046070 \\
7.2421810 \\
2.2680900 \\
1.6300690 \\
3.3036440 \\
1.9577070 \\
3.0101770 \\
4.0410350 \\
3.0320980 \\
2.5653360 \\
-4.5821680 \\
-4.4151570 \\
-5.3172980 \\
-5.0035030 \\
-2.2554400\end{array}$ & $\begin{array}{r}-0.1587020 \\
-2.2651110 \\
1.8906530 \\
0.3930070 \\
1.2568400 \\
-0.8290190 \\
0.8741570 \\
-1.1667000 \\
-0.3361270 \\
1.5238970 \\
-2.1124600 \\
1.9113360 \\
0.0725580 \\
-3.0899680 \\
2.5847080 \\
-0.7629770 \\
-1.7962760 \\
-1.9331310 \\
-2.3419310 \\
-0.9575770 \\
-2.6104810 \\
-3.1731000 \\
-3.0722290 \\
-3.6523070 \\
-3.8316130 \\
0.2909700 \\
-0.0694020 \\
1.2255610 \\
0.5150130 \\
-1.0862450 \\
-1.8657100 \\
-0.1903450 \\
-1.4310570 \\
3.7247500 \\
3.5292310 \\
3.8304380 \\
4.6766530 \\
2.9496540 \\
3.2126350 \\
2.1193320 \\
3.8242230 \\
2.3661300 \\
2.6764570 \\
1.5512350 \\
3.2177160 \\
3.0762630\end{array}$ \\
\hline
\end{tabular}

-1.4672500
-2.8890860
-1.2630280
-7.2039810
-8.2191330
-7.0334250
-7.1409240
-6.2933590
-5.5866450
-6.0931860
-7.3091970
-2.6781760
-3.7521820
-2.3878650
-2.1404020
-2.8174720
-3.9126690
-2.3899650
-2.5311540
0.4764590
-0.1411940
0.2399040
-1.2371180
-6.3650690
-0.9344790
4.9448680
4.4242740
2.3446800
-2.8235480
-1.8885970
-4.0704720
-5.0903330
-4.2689910
-6.2922450
-4.9283470
-5.4784210
-3.4852700
-6.4902680
-7.0775150
-5.6340140
-7.4322570
-1.0315350
-2.7281820
-1.1722860
-2.3697610
-0.3148450
2.7433000

3.4935260

3.8810510

$-0.6689610$

$-0.4661550$

$-1.7528290$

$-0.3577630$

$-0.3328870$

0.2287820

$-1.4052820$

$-0.1307120$

$-3.8784900$

$-4.1021980$

$-3.3233420$

$-4.8350690$

- 3.8619870

$-3.9482970$

$-4.8738430$

$-3.3509070$

$-0.5962520$

$-1.9637220$

0.3595600

$-3.0198870$

1.1501440

1. 6172350

$-1.3909370$

$-1.6850950$

2.4988310

1.5033490

1.7296120

2. 3002340

2. 2110820

3.0784040

2. 8914840

1.5992750

3.7461570

3.1354810

3.6555070

2. 8222890

4.3363310

4.1784120

1.1428840

0.6743160

$-1.7437520$

$-1.4143050$

$-1.0951410$ 


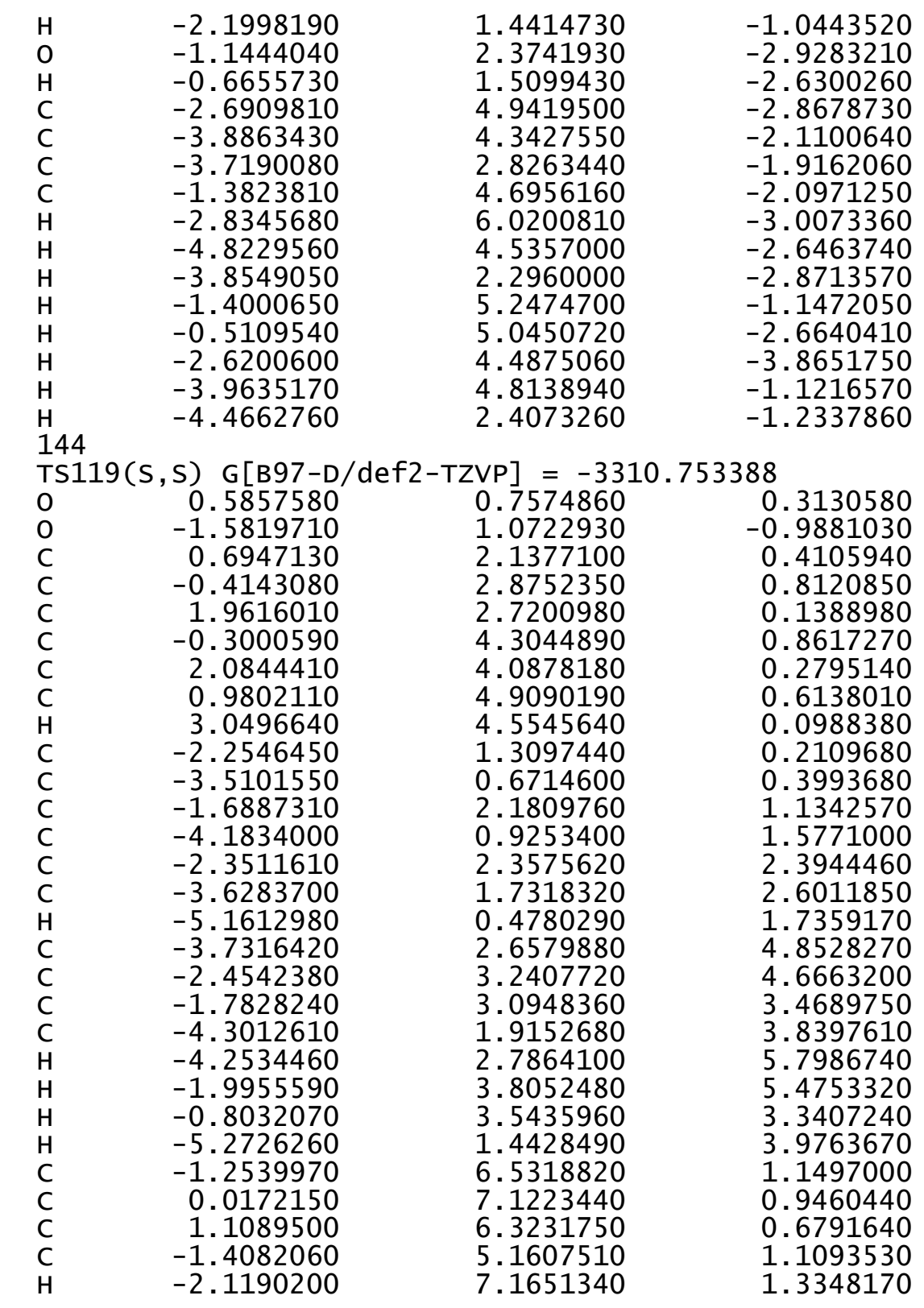
0.1256590
2. 0884100
$-2.3888800$
3. 1256060
3.5767610
3. 7963670
4.7071750
4.9244240
5. 3966730
5. 0521300
5.4530890
$-4.0919280$
$-4.1509080$
$-4.5974900$
$-4.7436680$
$-5.1725250$
$-5.2592730$
$-4.7923350$
$-5.5660030$
3. 3687850
6.6455660
2. 8911970
$-3.6032360$
$-5.8795140$
$-4.5720310$
$-6.0023440$
$-6.5731190$
$-6.5360930$
$-5.9718860$
$-3.8025230$
$-2.7796430$
$-4.3009500$
$-3.7597010$
$-7.1129410$
$-7.5716000$
$-6.8242790$
$-7.8631010$
$-4.8492190$
$-3.9799920$
$-4.4948730$
$-5.3019280$
$-4.7394830$
$-5.2786490$
$-5.4602460$
$-4.3338130$
$-2.8177860$

8.2039550

6.7630350

4.7232710

1.8907920

1.9557280

1. 0722050

1. 2172560

0.3572110

0.4132370

1. 2657620

$-0.2663330$

$-0.2195160$

$-1.6194330$

0.3425310

$-2.4172580$

$-0.5034560$

$-1.8823240$

$-3.4921270$

$-0.0734980$

0.9773270

$-0.3551050$

2.8253340

$-2.2796590$

$-2.7791570$

1.8405110

2.4128160

1.9565110

2. 2162290

3.4983190

2. 1691930

1.7803160

1.7288390

3. 2569700

$-3.5250990$

$-4.1348970$

$-4.1936400$

$-2.8206230$

$-3.7786480$

$-3.2539860$

$-4.4475760$

$-4.3938740$

$-2.5765380$

$-1.6640530$

$-3.2766810$

$-3.0324310$

$-3.5723400$
0.9865450

0.4987020

1.2643090

$-0.2918220$

$-1.6312170$

0.6422700

$-1.9976410$

0.2253440

$-1.0873730$

$-3.0267900$

0.9447520

$-0.6480640$

$-0.4409920$

$-1.8397210$

$-1.4242850$

$-2.7964430$

$-2.6085650$

$-1.2697540$

$-3.7163570$

2. 1014630

$-1.4870360$

$-2.6789260$

0.8201610

$-3.6647810$

$-2.1161500$

$-2.1664740$

$-2.9859490$

$-1.2276410$

$-2.3314560$

$-3.4088110$

$-3.3586210$

$-4.2822890$

$-3.5542920$

$-3.1213390$

$-3.9114730$

$-2.2995020$

$-2.7405470$

$-4.2257730$

$-4.6415290$

$-3.4305530$

$-5.0150620$

1.8193120

2. 0972750

1. 3750770

2.7329490

0.5310530 


$\begin{array}{rr}\mathrm{H} & -3.4786480 \\ \mathrm{H} & -2.0325170 \\ \mathrm{H} & -2.3518350 \\ \mathrm{C} & 4.4222850 \\ \mathrm{H} & 5.3845360 \\ \mathrm{H} & 4.5777730 \\ \mathrm{H} & 4.0981570 \\ \mathrm{C} & 3.0960880 \\ \mathrm{H} & 2.3035480 \\ \mathrm{H} & 3.9999810 \\ \mathrm{H} & 2.7715090 \\ \mathrm{C} & 7.8681140 \\ \mathrm{H} & 8.7810210 \\ \mathrm{H} & 7.7256030 \\ \mathrm{H} & 8.0056450 \\ \mathrm{C} & 6.5042380 \\ \mathrm{H} & 5.6273880 \\ \mathrm{H} & 6.3950980 \\ \mathrm{H} & 7.3990500 \\ \mathrm{C} & 2.6706630 \\ \mathrm{H} & 3.6240990 \\ \mathrm{H} & 2.1109310 \\ \mathrm{H} & 2.0973150 \\ \mathrm{C} & 3.6965030 \\ \mathrm{H} & 4.6977910 \\ \mathrm{H} & 3.1894030 \\ \mathrm{H} & 3.8152170 \\ \mathrm{P} & -0.2882510 \\ \mathrm{O} & 0.4236460 \\ \mathrm{O} & -0.6486380 \\ \mathrm{H} & 1.9037070 \\ \mathrm{H} & 6.8223590 \\ \mathrm{H} & 2.4351510 \\ \mathrm{H} & -4.0545680 \\ \mathrm{H} & -6.2101690 \\ \mathrm{H} & -2.9070130 \\ \mathrm{C} & 1.5829660 \\ \mathrm{C} & 2.3529670 \\ \mathrm{H} & 0.5029080 \\ \mathrm{H} & 1.9525870 \\ \mathrm{O} & 2.2007910 \\ \mathrm{H} & 1.5198250 \\ \mathrm{C} & 3.5542380 \\ \mathrm{C} & 3.1021170 \\ \mathrm{C} & 2.0261980 \\ \mathrm{C} & \\ & \end{array}$

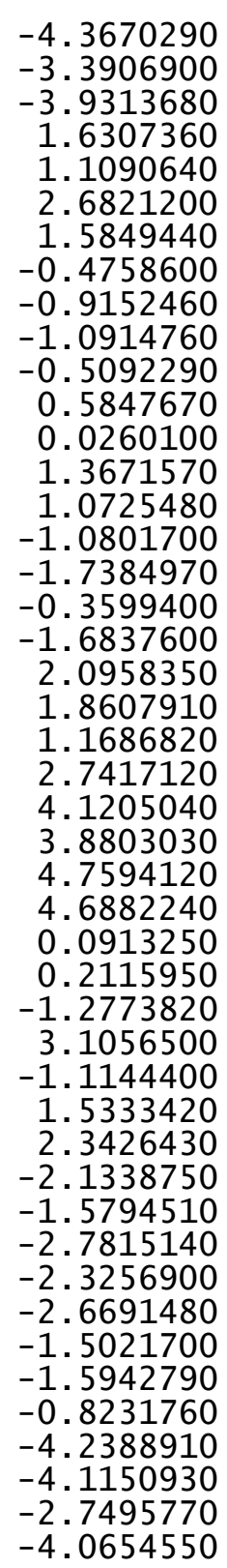

0.1610310
-0.2088860
1.4570930
3.0169740
2.9298400
2.7436050
4.0653020
2.5287120
1.9157350
2.4341290
3.5766260
-1.5097690
-1.7568990
-2.2671440
-0.5367920
-2.8371200
-2.8511640
-3.6575620
-3.0363760
-4.0161110
-4.5071730
-3.8639850
-4.6937860
-2.9087000
-3.2910540
-3.6442200
-1.9784120
-0.9089400
-2.2240460
-0.4029220
-2.2976290
-0.7105580
2.2311820
-1.2928380
-4.4913690
1.2930900
-2.0213750
-0.8573910
-1.9318500
-0.2786270
-2.6236840
-2.4986930
-2.6827600
-1.2524320
-0.6333070
-2.6927210

\begin{abstract}
3. 8146410
5. 1907820

4. 3843620

1. 5539610

1. 6283930

4. 0123720

3. 6650090

3.9438900

0.8685230

0.0315980

1. 0387310

2. 0097320

0.2512210

2. 1934410

2. 6121740

0.4363010

$-0.4998150$

1. 4067000

2. 9484330

$-0.1764850$

1.5494080

$-0.1871800$
\end{abstract}

144

TS120(S, S ) G[B97-D/def2-TZVP $]=-3310.752991$

$\begin{array}{lrcr}\text { TS120(S S) G }[\mathrm{B} 97-\mathrm{D} / \mathrm{def}-\mathrm{TZVP}]=-3310.752991 \\ \mathrm{O} & 0.5722270 & 0.6788790 & 0.2826640 \\ \mathrm{O} & -1.6687110 & 0.9974220 & -0.8957150 \\ \mathrm{C} & 0.6571750 & 2.0553990 & 0.4367120 \\ \mathrm{C} & -0.4482580 & 2.7506610 & 0.9169740 \\ \mathrm{C} & 1.9011520 & 2.6772930 & 0.1487850 \\ \mathrm{C} & -0.3622770 & 4.1784770 & 1.0246420 \\ \mathrm{C} & 2.0016900 & 4.0397060 & 0.3479650 \\ \mathrm{C} & 0.8943400 & 4.8218620 & 0.7566220 \\ \mathrm{H} & 2.9527960 & 4.5332080 & 0.1633990 \\ \mathrm{C} & -2.2867580 & 1.1731950 & 0.3425360 \\ \mathrm{C} & -3.5191440 & 0.5024620 & 0.5660600 \\ \mathrm{C} & -1.6906980 & 2.0164200 & 1.2721580 \\ \mathrm{C} & -4.1327130 & 0.6885530 & 1.7884660 \\ \mathrm{C} & -2.2887290 & 2.1245380 & 2.5717030 \\ \mathrm{C} & -3.5400280 & 1.4613360 & 2.8172640 \\ \mathrm{H} & -5.0892890 & 0.2085270 & 1.9790580 \\ \mathrm{C} & -3.5394300 & 2.2818240 & 5.1117560 \\ \mathrm{C} & -2.2863510 & 2.9016650 & 4.8842690 \\ \mathrm{C} & -1.6782060 & 2.8257540 & 3.6472590 \\ \mathrm{C} & -4.1482290 & 1.5731250 & 4.0972140 \\ \mathrm{H} & -4.0113760 & 2.3553400 & 6.0891250\end{array}$

$-3.1026050$ $-1.2422940$ $-1.0557510$ $-2.1402480$ $-3.7149850$ $-3.3237650$ $-0.6286300$ 0.4461740

1.7597130

1.7862580

3.0753030

3. 1977340

4.1888480

4.4145970

2.3311010

5. 4044290

4.0947210

5. 5202120

4.5027800

6.2623550

6.4694640

0.8515910

0.7559920 


$\begin{array}{rrr}-1.7959800 & 3.4393210 & 5.6929630 \\ -0.7161510 & 3.3020720 & 3.4882730 \\ -5.1007430 & 1.0726060 & 4.2632780 \\ -1.3499430 & 6.3686970 & 1.4495230 \\ -0.0996510 & 6.9957900 & 1.2265620 \\ 0.9971510 & 6.2340220 & 0.8825100 \\ -1.4777220 & 4.9977590 & 1.3519970 \\ -2.2201680 & 6.9737910 & 1.6946310 \\ -0.0119400 & 8.0765900 & 1.3128470 \\ 1.9597980 & 6.7030620 & 0.6857240 \\ -2.4431830 & 4.5324710 & 1.5211070 \\ 3.0863640 & 1.9034370 & -0.3295510 \\ 3.4860480 & 1.9925380 & -1.6842430 \\ 3.8453070 & 1.1429150 & 0.5844210 \\ 4.6676970 & 1.3580580 & -2.0798260 \\ 5.0226550 & 0.5299640 & 0.1385860 \\ 5.4572420 & 0.6311250 & -1.1840320 \\ 4.9791640 & 1.4319020 & -3.1182540 \\ 5.6182880 & -0.0471420 & 0.8445780 \\ -4.1396830 & -0.3567670 & -0.4844780 \\ -4.1999300 & -1.7587760 & -0.3117600 \\ -4.6907200 & 0.2393720 & -1.6421760 \\ -4.8410240 & -2.5275770 & -1.2919350 \\ -5.3088640 & -0.5752270 & -2.5947480 \\ -5.4006220 & -1.9603040 & -2.4374490 \\ -4.8972920 & -3.6071980 & -1.1666410 \\ -5.7352880 & -0.1115390 & -3.4815980 \\ -0.3524380 & 0.0448940 & -0.9223650 \\ 0.2911350 & 0.2399600 & -2.2635900 \\ -0.6516980 & -1.3529080 & -0.4583230 \\ 3.4470950 & 0.9896550 & 2.0465770 \\ 2.4931810 & 1.5023530 & 2.2051370 \\ 6.7637750 & -0.0082600 & -1.6232230 \\ 7.1040920 & -0.6560900 & -0.8019700 \\ 2.6805470 & 2.7841990 & -2.7082750 \\ 1.6697910 & 2.9203660 & -2.3088440 \\ -4.6730510 & 1.7445610 & -1.8793490 \\ -4.1224630 & 2.2256420 & -1.0651660 \\ -6.0651130 & -2.8256990 & -3.4933290 \\ -6.0765770 & -3.8576130 & -3.1136840 \\ -3.6028870 & -2.4617650 & 0.9028440 \\ -2.9182610 & -1.7655390 & 1.3980090 \\ -2.7850340 & -3.7111260 & 0.5254870 \\ -2.2893000 & -4.1085560 & 1.4202260 \\ -3.4295790 & -4.5018620 & 0.1198250 \\ -2.0220250 & -3.4609550 & -0.2175800\end{array}$

-4.7065100
-5.4152230
-4.2672710
-5.2682210
3.2355190
4.1617980
2.9266120
2.4526530
4.4842690
4.1696750
5.4638660
4.6008210
-3.9572400
-4.4973210
-3.9107080
-2.9368350
-6.1039030
-6.0796630
-6.7076330
-6.5993030
2.5393350
1.8625940
3.5056860
2.1257480
3.3035400
4.3238810
2.7080460
3.3531590
-7.5221210
-8.1093810
-7.5579090
-7.9927700
-5.2568090
-4.2264930
-5.7235650
-5.2181210
7.8405920
7.9862970
8.7984850
7.5352210
6.6098310
5.8664960
6.2912700
7.5696600
1.6435290
2.3844870

-2.8439710
-3.5441790
-3.3303870
-1.9634890
-0.4883330
-1.0630960
-0.5745910
-0.9349650
1.6504830
1.5607790
1.1664520
2.7146870
2.1060610
1.6959160
3.1972270
1.7078190
2.3177860
3.4070560
1.8812880
2.0973640
2.0596750
2.6299200
1.9792430
1.0564450
4.1778890
4.0768690
4.7501800
4.7473450
-2.3989400
-2.4216870
-1.3778610
-3.0687440
-2.8115080
-3.1478550
-3.4675090
-1.7943280
1.0716540
1.6762620
0.6096630
1.7423380
-0.8792400
-1.6719160
-0.2719350
-1.3468990
-2.6958300
-2.2194660

1.9097510

1.4468960

2.7916420

2.2429270

2.4221900

2. 2920210

3.4718680

1.8014020

2.9742190

4.0225260

2.8663390

2.7329760

$-3.1937300$

$-4.0571760$

$-3.3092590$

$-3.1997060$

$-1.8569030$

$-1.9961400$

$-2.6635110$

$-0.9025060$

$-4.0588490$

$-4.7078800$

$-4.5736970$

$-3.9256200$

$-2.9281510$

$-3.3231310$

$-3.6521740$

$-1.9934590$

$-3.7522390$

$-2.8256300$

$-4.1540060$

$-4.4843430$

$-4.8057180$

$-4.6350420$

$-5.5530670$

$-5.2171650$

$-1.8507310$

$-0.9469590$

$-2.1251860$

$-2.6648020$

$-2.8837680$

$-2.7394610$

$-3.7401210$

$-3.1393280$

-2.1551180
-0.9806790 


\begin{tabular}{|c|c|c|c|c|c|c|c|}
\hline$H$ & & 25050250 & & & & & \\
\hline $\mathrm{H}$ & $\begin{array}{l}0.5584440 \\
1.9277730\end{array}$ & $\begin{array}{l}-2.6650260 \\
-1.4476630\end{array}$ & $\begin{array}{l}-2.0597450 \\
-0.3739930\end{array}$ & C & -2.3252960 & $\begin{array}{l}2.1299630 \\
1.4579900\end{array}$ & $\begin{array}{l}2.5694050 \\
2.8037310\end{array}$ \\
\hline 0 & 2.1709960 & -1.4488130 & -2.7220410 & $\mathrm{H}$ & -5.1050830 & 0.1913730 & 1.9525230 \\
\hline $\mathrm{H}$ & 1.4411740 & -0.7277260 & -2.5717310 & C & -3.6047070 & 2.2863210 & 5.0952260 \\
\hline C & 3.7155440 & -3.9789200 & -2.8656480 & $\mathrm{C}$ & -2.3541480 & 2.9152840 & 4.8790490 \\
\hline C & 4.2588730 & -3.8604060 & -1.4330980 & $\mathrm{C}$ & -1.7320140 & 2.8397300 & 3.6489970 \\
\hline $\mathrm{C}$ & 3.8154730 & -2.5460970 & -0.7698450 & $\mathrm{C}$ & -4.1968860 & 1.5692780 & 4.0766800 \\
\hline $\mathrm{C}$ & 2.1785480 & -3.9221930 & -2.8661980 & $\mathrm{H}$ & -4.0877360 & 2.3594960 & 6.0671900 \\
\hline $\mathrm{H}$ & 4.0473750 & -4.9210520 & -3.3178800 & $\mathrm{H}$ & -1.8768680 & 3.4597500 & 5.6910170 \\
\hline $\mathrm{H}$ & 5.3543740 & -3.9114820 & -1.4286960 & $\mathrm{H}$ & -0.7721720 & 3.3232590 & 3.4986100 \\
\hline $\mathrm{H}$ & 4.3792530 & -1.6888780 & -1.1629930 & $\mathrm{H}$ & -5.1471530 & 1.0617620 & 4.2341600 \\
\hline $\mathrm{H}$ & 1.7731040 & -4.7959300 & -2.3370440 & C & -1.4058010 & 6.3785990 & 1.4420260 \\
\hline $\mathrm{H}$ & 1.7778500 & -3.9359580 & -3.8873330 & $\mathrm{C}$ & -0.1590480 & 7.0146180 & 1.2246980 \\
\hline $\mathrm{H}$ & 4.1107190 & -3.1608850 & -3.4822740 & $\mathrm{C}$ & 0.9460650 & 6.2600300 & 0.8916800 \\
\hline $\mathrm{H}$ & 3.8845420 & -4.7011370 & -0.8346460 & $\mathrm{C}$ & -1.5219940 & 5.0062100 & 1.3502610 \\
\hline $\mathrm{H}$ & 4.0013700 & -2.5384160 & 0.3097470 & $\mathrm{H}$ & -2.2825550 & 6.9778360 & 1.6781070 \\
\hline C & 1.0125510 & -3.3643300 & 1.6297920 & $\mathrm{H}$ & -0.0805550 & 8.0964840 & 1.3064110 \\
\hline 0 & 0.1272270 & -2.3965300 & 1.6977470 & $\mathrm{H}$ & 1.9062720 & 6.7358670 & 0.6993660 \\
\hline C & 1.2572040 & -4.0643380 & 2.9288280 & $\mathrm{H}$ & -2.4849610 & 4.5341820 & 1.5147270 \\
\hline $\mathrm{C}$ & 2.2697630 & -5.0354590 & 3.0020200 & C & 3.0727710 & 1.9399100 & -0.2963210 \\
\hline $\mathrm{C}$ & 0.4988960 & -3.7680940 & 4.0749160 & $\mathrm{C}$ & 3.4945380 & 2.0629050 & -1.6416260 \\
\hline $\mathrm{C}$ & 2.5223800 & -5.6992950 & 4.2020560 & $\mathrm{C}$ & 3.8108270 & 1.1456240 & 0.6069040 \\
\hline $\mathrm{H}$ & 2.8493380 & -5.2580600 & 2.1106410 & $\mathrm{C}$ & 4.6680360 & 1.4155980 & -2.0412070 \\
\hline C & 0.7535100 & -4.4351210 & 5.2737510 & C & 4.9818380 & 0.5236100 & 0.1578470 \\
\hline $\mathrm{H}$ & -0.2839880 & -3.0181000 & 4.0191940 & $\mathrm{C}$ & 5.4314670 & 0.6477440 & -1.1579720 \\
\hline C & 1.7644110 & -5.4003730 & 5.3401700 & $\mathrm{H}$ & 4.9941980 & 1.5148340 & -3.0731190 \\
\hline $\mathrm{H}$ & 3.3088300 & -6.4489540 & 4.2519270 & $\mathrm{H}$ & 5.5624670 & -0.0777550 & 0.8559610 \\
\hline $\mathrm{H}$ & 0.1633150 & -4.2022640 & 6.1571010 & C & -4.1255390 & -0.3720210 & -0.5001190 \\
\hline $\mathrm{H}$ & 1.9610020 & -5.9185690 & 6.2763460 & $\mathrm{C}$ & -4.1777860 & -1.7742080 & -0.3266400 \\
\hline $\mathrm{H}$ & -0.1341910 & -1.9845770 & 0.7779940 & $\mathrm{C}$ & -4.6677090 & 0.2189710 & -1.6647160 \\
\hline 0 & 1.6223470 & -3.6992410 & 0.6012560 & $\mathrm{C}$ & -4.8023980 & -2.5486040 & -1.3131060 \\
\hline \multirow{2}{*}{\multicolumn{4}{|c|}{$\begin{array}{l}144 \\
\mathrm{TS} 121(\mathrm{~S}, \mathrm{~S}) \mathrm{G}[\mathrm{B} 97-\mathrm{D} / \mathrm{def} 2-\mathrm{TZVP}]=-3310.752790\end{array}$}} & C & -5.2689470 & -0.6010670 & -2.6234140 \\
\hline & & & & C & -5.3527100 & -1.9865880 & -2.4655900 \\
\hline 0 & 0.5693350 & 0.6986880 & 0.3156390 & $\mathrm{H}$ & -4.8525980 & -3.6284560 & -1.1873240 \\
\hline 0 & -1.6602030 & 0.9968940 & -0.8877720 & $\mathrm{H}$ & -5.6879670 & -0.1416640 & -3.5160120 \\
\hline $\mathrm{C}$ & 0.6433330 & 2.0766430 & 0.4651930 & $\mathrm{P}$ & -0.3347200 & 0.0565300 & -0.8995520 \\
\hline C & -0.4717010 & 2.7652210 & 0.9326300 & 0 & 0.3201390 & 0.2578830 & -2.2342770 \\
\hline $\mathrm{C}$ & 1.8843030 & 2.7072680 & 0.1822570 & 0 & -0.6273850 & -1.3442030 & -0.4402510 \\
\hline $\mathrm{C}$ & -0.3977870 & 4.1941750 & 1.0349150 & $\mathrm{C}$ & 3.4061270 & 0.9776160 & 2.0657980 \\
\hline C & 1.9716430 & 4.0717370 & 0.3736550 & $\mathrm{H}$ & 2.4483890 & 1.4826700 & 2.2246870 \\
\hline C & 0.8555350 & 4.8464300 & 0.7722330 & C & 6.7301070 & -0.0059880 & -1.5993120 \\
\hline $\mathrm{H}$ & 2.9183040 & 4.5728170 & 0.1864340 & $\mathrm{H}$ & 7.0424910 & -0.6898410 & -0.7963720 \\
\hline C & -2.2919040 & 1.1717740 & 0.3434300 & C & 2.7306360 & 2.9058460 & -2.6564450 \\
\hline $\mathrm{C}$ & -3.5220420 & 0.4930070 & 0.5556760 & $\mathrm{H}$ & 1.7352130 & 3.1114360 & -2.2493320 \\
\hline C & -1.7123350 & 2.0224490 & 1.2768770 & C & -4.6582230 & 1.7240030 & -1.9033190 \\
\hline C & -4 & 0.6777990 & 1.7709420 & $\mathrm{H}$ & -4.1182060 & 2.2095980 & -1.0847980 \\
\hline
\end{tabular}




-5.9998500
-6.0049770
-3.5894750
-2.9146790
-2.7599600
-2.2709000
-3.3955090
-1.9910150
-4.7016570
-5.4006460
-4.2690450
-5.2725330
3.2046400
4.1350130
2.8926750
2.4266580
4.4361300
4.1201640
5.4195730
4.5447020
-3.9331790
-4.4617610
-3.8943320
-2.9095370
-6.0931810
-6.0753410
-6.6868020
-6.5953560
2.5321130
1.9065150
3.4897420
2.0353230
3.4418020
4.4497530
2.8780730
3.5381280
-7.4585460
-8.0531160
-7.5002090
-7.9171650
-5.1809440
-4.1487680
-5.6348490
-5.1490650
7.8339980
7.9787640

-3.5275760
-3.1484400
0.8951980
1.3970210
0.5282220
1.4284650
0.1173850
-0.2076060
1.8904480
1.4205060
2.7776610
2.2163980
2.4356380
2.3079480
3.4839710
1.8116850
2.9988550
4.0461800
2.8901270
2.7622190
-3.2115000
-4.0794860
-3.3279860
-3.2073930
-1.8937320
-2.0329940
-2.7054900
-0.9436270
-4.0040510
-4.6586040
-4.5153370
-3.8633650
-2.8762970
-3.2811670
-3.5905120
-1.9377880
-3.7980760
-2.8763220
-4.1989120
-4.5347030
-4.8331920
-4.6538290
-5.5842090
-5.2446810
-1.7719260
-0.8453070

\subsection{0 \\ 7.5545920 \\ 6.5779960 \\ 5.8055260 \\ 6. 3000750 \\ 7.5281040 \\ 1. 6620630 \\ 2.4035420 \\ 0.5770560 \\ 1. 9519210 \\ 2.2046400 \\ 1.4777550 \\ 3.7208820 \\ 4.2611760 \\ 3.8311280 \\ 2. 1847320 \\ 4.0428780 \\ 5.3560150 \\ 4.4047360 \\ 1.7663590 \\ 1.7878830 \\ 4.1282220 \\ 3.8755450 \\ 4.0157200 \\ 1.0224420 \\ 0.1488620 \\ 1. 2624230 \\ 2. 2651210 \\ 0.5099320 \\ 2. 5139130 \\ 2.8402380 \\ 0.7606800 \\ $-0.2652320$ \\ 1.7619070 \\ 3. 2927360 \\ 0.1750790 \\ 1.9555940 \\ $-0.1120830$ \\ 1.6260440}

144

TS122 (S, S)

$\begin{array}{lr}\text { TS122(S, S) } & \text { G }[\mathrm{B} 97-\mathrm{D} / \mathrm{def} 2-\mathrm{TZ} \\ \mathrm{O} & 0.5361920 \\ \mathrm{O} & -1.7975980 \\ \mathrm{C} & 0.6124540 \\ \mathrm{C} & -0.4711510 \\ \mathrm{C} & 1.8387670\end{array}$

0.5840900

1.7620090

$-0.8271500$

$-1.5986730$

$-0.1792770$

$-1.3167870$

$-2.6697040$

$-2.2206180$

$-2.6282520$

$-1.4533140$

$-1.4196080$

$-0.6972950$

$-3.9673330$

$-3.8753340$

$-2.5651700$

$-3.8913650$

$-4.9069820$

$-3.9388100$

$-1.7098520$

$-4.7679100$

$-3.8843310$

$-3.1456920$

$-4.7199420$

$-2.5740540$

$-3.3956440$

$-2.4190180$

$-4.1221490$

$-5.1047950$

$-3.8386800$

$-5.7922940$

$-5.3176230$

$-4.5294300$

$-3.0797480$

$-5.5058610$

$-6.5507280$

$-4.3063140$

$-6.0425000$

$-1.9909860$

$-3.7187200$
$-2.0490740$

$-2.5661350$

$-2.8929190$

$-2.7943510$

$-3.7335510$

$-3.1434800$

$-2.1605490$

$-0.9758780$

$-2.0682640$

$-0.3595170$

$-2.7059620$

$-2.5490520$

$-2.8833560$

$-1.4474910$

$-0.7671250$

$-2.8887100$

$-3.3477130$

$-1.4409910$

$-1.1492710$

$-2.3744970$

$-3.9114030$

$-3.4871530$

$-0.8618930$

0.3127510

1.6115300

1.6990270

2.8967510

2.9499620

4.0499110

4.1374090

2.0533280

5.2360700

4.0095400

5.2826750

4.1719440

6.1249710

6.2090140

0.7859560

0.5753970

$-3.1171970$

1.0570950

1. 6262800

0.6783170

$\begin{array}{rr}0.9103260 & -0.9173600 \\ 2.0595650 & 0.1927930\end{array}$

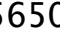

2.7674290

0.1927930

0.7047550
-0.1726820 


-0.3956050
1.9356830
0.8386160
2.8769600
-2.3233330
-3.5179390
-1.674530
-4.0379900
-2.1741850
-3.3857200
-4.9659690
-3.2325180
-2.0177050
-1.5028500
-3.8974290
-3.6309060
-1.4824030
-0.5691290
-4.8216900
-1.3865690
-0.1565440
0.9309500
-1.5026560
-2.2503710
-0.0775620
1.8770350
-2.4524370
3.0192490
3.3570830
3.8328700
4.5306350
5.0014210
5.3761340
4.8003880
5.6231970
-4.2008660
-4.2079800
-4.8624310
-4.9046200
-5.5372580
-5.5742220
-4.9182200
-6.0503370
3.4958400
6.6730190
2.4967460

4.1993030

4.0474410

4.8401120

4.5380280

1.1400030

0.4574960

2.0411420

0.6963710

2. 2108940

1.5360160

0.2105010

2.4839810

3.1180830

2.9853440

1.7057460

2.6026110

3.7126260

3.4738730

1.1953130

6.3979460

7.0249280

6.2575030

5.0226770

7.0062620

8.1094540

6.7254790

4.5562110

1.8848220

1.8485560

1. 2280510

1. 1916420

0.5883970

0.5683910

1.1584300

0.0911430

$-0.4644710$

$-1.8579640$

0.0650500

$-2.6866150$

$-0.8068080$

$-2.1853870$

$-3.7607620$

$-0.3953550$

1.1974890

$-0.0626270$

2. 5413420
0.7374200 $-0.0397370$

0.3769180

$-0.2756230$

0.3543110

0.7081410

1.1883730

1. 9642420

2.5231150

2.9005640

2.2548450

5.1401900

4.7826830

3.5084840

4.2158760

6.1454760

5.5199380

.2503040

4.4816670

1.1063260

0.7897590

0.4287370

1.0823920

1. 3657770

0.8185120

0.1615390

1.3232150

$-0.6349170$

$-2.0082690$

0.3111340

$-2.3907080$

$-0.1221970$

$-1.4659110$

$-3.4443910$

0.6173050

$-0.2466670$

$-0.0079650$

$-1.3786080$

$-0.8971330$

$-2.2374560$

$-2.0141370$

$-0.7226230$

$-3.1043920$

1.7965360

-1.9519310
-3.0582940
$-3.4951450$

$-6.3139840$

$-4.8963380$

$-6.3334520$

$-6.9817930$

$-6.7571800$

$-6.3417290$

$-4.2826140$

$-3.2573610$

$-4.2665980$

$-5.7189710$

$-6.2415060$

$-5.8239040$

$-4.6515120$

$-7.8216520$

$-8.2607850$

$-8.3464470$

$-4.4977320$

$-5.0467530$

$-5.2286780$

$-3.9716010$

$-2.6968150$

$-3.3653480$

$-2.0224220$

$-2.1011690$

4.5887590

5. 5487750

4.7289620

4.3119130

3.2578100

2.4346970

4.1594750

2.9960610

7. 2170040

8.0978070

7.5256460

6.4675570

7.7430410

7.3837860

7.9778160

8.6662710

2. 3547660

3.3091800
2.0000070
-2.4862400
-3.1107370
1.5564400
2.1048690
1.6177240
1.9244100
3.1858260
1.8619570
1.4810090
1.3981260
2.9463120
-3.0509970
-3.7514040
-2.0404620
-3.3042330
-2.7946710
-2.8673880
-1.7756570
-3.4934630
-2.8235870
-1.9343100
-3.5624020
-3.2487970
-3.7475700
-4.5723820
-3.5399210
-4.0760870
1.8870470
1.3650330
2.9272080
1.8820300
-0.2447820
-0.7028400
-0.8565450
-0.2539000
-1.1742870
-1.6367040
-0.7697810
-1.9540280
1.0326950
1.8128880
1.5020640
0.6022930
1.7380740
1.6793000
0.7226000
1. 1842650

$-2.9638910$

$-1.6884180$

$-1.5939910$

$-2.3340900$

$-0.5976920$

$-1.7886780$

$-3.0677010$

$-3.1276980$

$-3.8676500$

$-3.2413150$

$-4.3837370$

$-5.0492010$

$-4.7999170$

$-4.3730360$

$-2.9966070$

$-1.9936060$

$-3.3693170$

$-3.6620110$

2. 3064840

2.6366690

1.9505170

3.1723600

0.8055980

0.5264310

$-0.0303340$

1.6665980

2.6345800

2.5284300

2. 3139580

3.6971690

2.2805570

1.7242050

2.1459280

3.3463950

$-1.0426890$

$-1.5052850$

$-0.0698710$

$-0.8654130$

$-2.1480770$

$-2.8298670$

$-1.1830760$

$-2.5590860$

$-4.3623910$

$-4.9021920$

$-4.1659960$ 


$\begin{array}{rrrr}\mathrm{H} & 1.6313110 & 2.2374990 & -5.0190350 \\ \mathrm{C} & 3.0558760 & 3.9447490 & -3.3710690 \\ \mathrm{H} & 4.0729840 & 3.8629250 & -3.7784780 \\ \mathrm{H} & 2.4241220 & 4.4475490 & -4.1157450 \\ \mathrm{H} & 3.0956390 & 4.5693770 & -2.4721730 \\ \mathrm{P} & -0.4771830 & -0.0329670 & -0.9901540 \\ \mathrm{O} & 0.0541530 & 0.0814830 & -2.3882260 \\ \mathrm{O} & -0.7225760 & -1.4005530 & -0.4166160 \\ \mathrm{H} & 1.4910200 & 2.6614220 & -2.6406050 \\ \mathrm{H} & 6.4667640 & -0.5028670 & -2.9393580 \\ \mathrm{H} & 2.5652850 & 1.7519140 & 1.9551150 \\ \mathrm{H} & -4.2997330 & 2.0878560 & -0.9405640 \\ \mathrm{H} & -6.1911190 & -4.1365480 & -2.5873570 \\ \mathrm{H} & -2.7797580 & -1.7559340 & 1.5756290 \\ \mathrm{C} & 1.4562950 & -2.8255310 & -2.1804360 \\ \mathrm{C} & 2.3003700 & -2.2264810 & -1.1380770 \\ \mathrm{H} & 0.3861460 & -2.8019240 & -1.9766320 \\ \mathrm{H} & 1.8938650 & -1.4002500 & -0.5678880 \\ \mathrm{O} & 1.8887700 & -1.6359130 & -2.9226830 \\ \mathrm{H} & 1.1674900 & -0.9114830 & -2.7555840 \\ \mathrm{C} & 3.4746950 & -4.1655720 & -2.9519130 \\ \mathrm{C} & 4.1436940 & -3.8954150 & -1.5960040 \\ \mathrm{C} & 3.7534180 & -2.5129420 & -1.0491060 \\ \mathrm{C} & 1.9431060 & -4.1126010 & -2.8160640 \\ \mathrm{H} & 3.7737940 & -5.1493170 & -3.3328790 \\ \mathrm{H} & 5.2353950 & -3.9505580 & -1.6840410 \\ \mathrm{H} & 4.2561430 & -1.7099190 & -1.6062910 \\ \mathrm{H} & 1.5989620 & -4.9321360 & -2.1698520 \\ \mathrm{H} & 1.4503500 & -4.2280820 & -3.7894400 \\ \mathrm{H} & 3.8033510 & -3.4147870 & -3.6829600 \\ \mathrm{H} & 3.8278750 & -4.6614170 & -0.8759820 \\ \mathrm{H} & 4.0573870 & -2.3703060 & -0.0066290 \\ \mathrm{C} & 1.2115880 & -3.1494420 & 1.6833500 \\ \mathrm{O} & 0.2430900 & -2.2655040 & 1.7416330 \\ \mathrm{C} & 1.6552280 & -3.6641730 & 3.0158640 \\ \mathrm{C} & 2.7282610 & -4.5688760 & 3.0755700 \\ \mathrm{C} & 1.0351790 & -3.2472870 & 4.2065660 \\ \mathrm{C} & 3.1762380 & -5.0486970 & 4.3057960 \\ \mathrm{H} & 3.2013960 & -4.8845950 & 2.1501340 \\ \mathrm{C} & 1.4866790 & -3.7283890 & 5.4359430 \\ \mathrm{H} & 0.2078490 & -2.5461060 & 4.1615680 \\ \mathrm{C} & 2.5564300 & -4.6288560 & 5.4884110 \\ \mathrm{H} & 4.0080980 & -5.7483160 & 4.3447050 \\ \mathrm{H} & 1.0043090 & -3.4007610 & 6.3540110 \\ \mathrm{H} & 2.9064490 & -5.0024480 & 6.4483750 \\ \mathrm{H} & -0.1038690 & -1.9370600 & 0.8155620\end{array}$

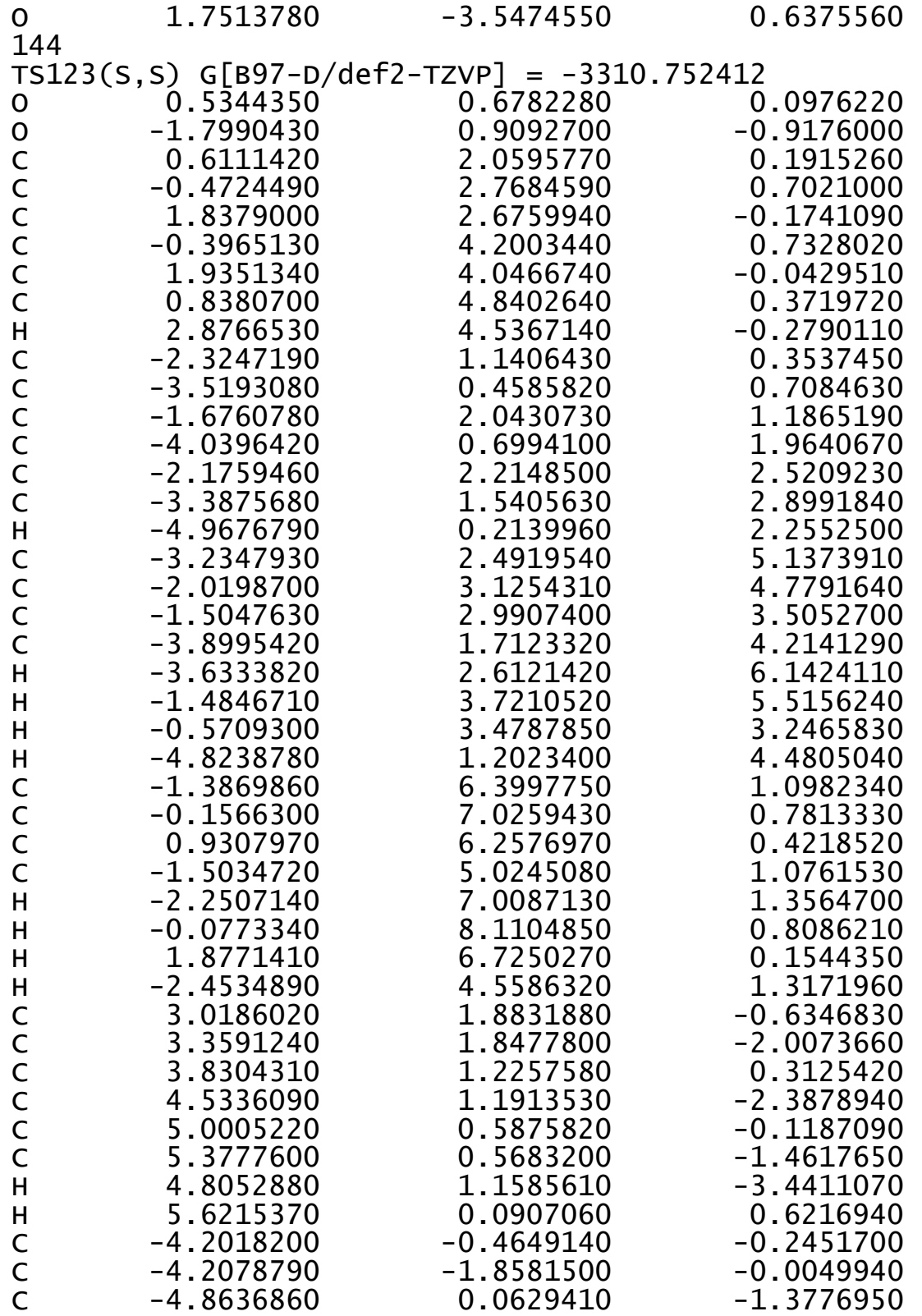




-4.9038390
-5.5377970
-5.5737270
-4.9166600
-6.0510330
-0.4782460
0.0532650
-0.7230920
3.4910770
2.5509110
6.6759230
6.4723200
2.5007630
1.4948370
-4.8985240
-4.3021330
-6.3126330
-6.1892920
-3.4947130
-2.7793600
-2.6962410
-2.1002440
-3.3646920
-2.0221630
-4.4971110
-5.2279090
-3.9708250
-5.0462890
3.2774340
4.1921850
3.0057760
2.4690880
4.5712980
4.2955090
5.5406020
4.6919760
-4.2851340
-4.8769710
-4.2699250
-3.2596030
-6.3359530
-6.3449110
-6.9840910
-6.7594500
2.3587760
1.6373170

$-2.6882570$

$-0.8103140$

$-2.1886850$

$-3.7622360$

$-0.4001830$

$-0.0335270$

0.0810690

$-1.4011320$

1.1959110

1.7349880

$-0.0618070$

$-0.5000570$

2. 5407100

2.6628290

1.5539770

2.0865190

$-3.1156130$

$-4.1408940$

$-2.4844570$

$-1.7534040$

$-3.7462840$

$-4.0731010$

$-4.5717570$

$-3.5400420$

$-2.8201500$

$-3.5597030$

$-3.2438300$

$-1.9304480$

$-0.2471910$

$-0.8419130$

$-0.2533770$

$-0.7263300$

1.9086460

1.9021290

1.4038770

2.9501240

1.8584430

1. 3933160

2.9426200

1.4781860

2.1016380

3.1823900

1.6133390

1.9219590

1.7360090

2.2361360

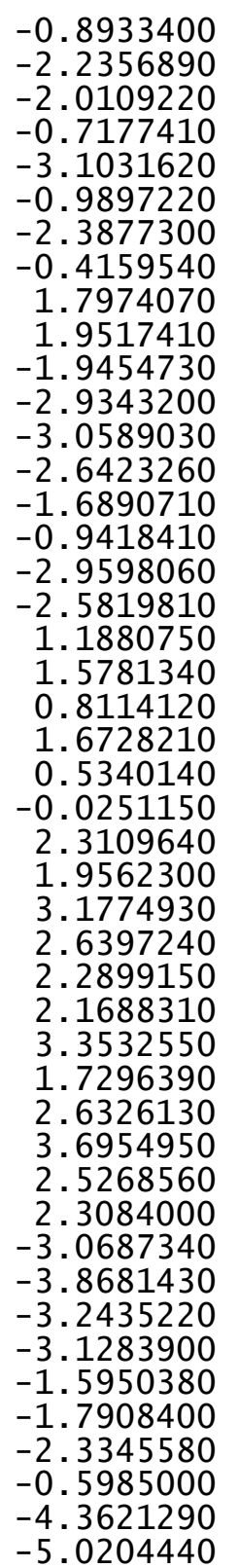

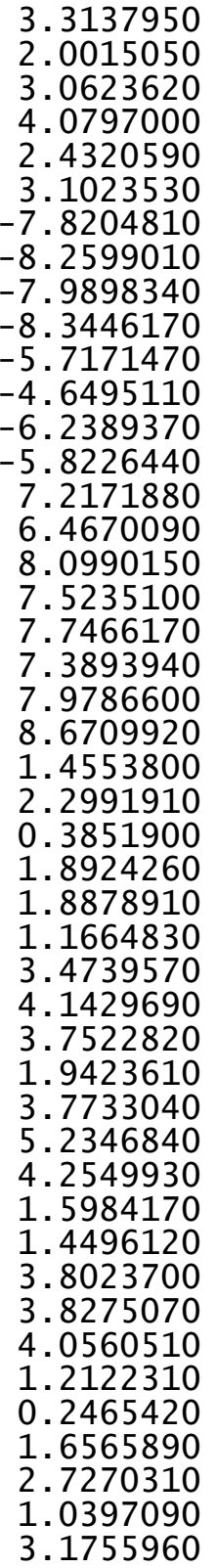

0.7215970

3.9428700

3.8590020

4.4458160

4. 5684720

$-2.8005160$

$-2.8722480$

$-1.7820810$

$-3.5004720$

$-3.0572720$

$-3.3097350$

$-3.7589090$

$-2.0473520$

$-1.1753660$

$-1.9551610$

$-1.6372440$

$-0.7727790$

1.0337430

1.8151660

1.5013880

0.6039720

$-2.8256810$

$-2.2276950$

$-2.8023020$

$-1.4018790$

$-1.6355460$

$-0.9112880$

$-4.1646460$

$-3.8957110$

$-2.5139970$

$-4.1121880$

$-5.1479290$

$-3.9503800$

$-1.7100640$

$-4.9323400$

$-4.2269440$

$-3.4130350$

$-4.6626520$

$-2.3727640$

$-3.1505360$

$-2.2636420$

$-3.6662390$

$-4.5741190$

-3.2473380
-5.0550620
$-4.9005300$

4.1648090

$-3.3728250$

$-3.7792360$

$-4.1186270$

$-2.4746030$

$-2.9934320$

$-1.9904840$

$-3.3674790$

$-3.6581310$

$-4.3795070$

$-4.3680840$

$-5.0442610$

$-4.7970440$

$-1.0369000$

$-0.8629620$

$-1.4980900$

$-0.0625570$

$-2.1365110$

$-2.8179700$

$-1.1700160$

$-2.5456020$

$-2.1816850$

$-1.1384770$

$-1.9779950$

$-0.5678360$

$-2.9229450$

$-2.7553960$

$-2.9543090$

$-1.5981720$

$-1.0495760$

$-2.8183400$

$-3.3362710$

$-1.6863480$

$-1.6055130$

$-2.1728090$

$-3.7918050$

$-3.6846100$

$-0.8789870$

$-0.0068730$

1.6820070

1.7415830

3. 0138860

3.0719820

4.2055330

4.3015530 


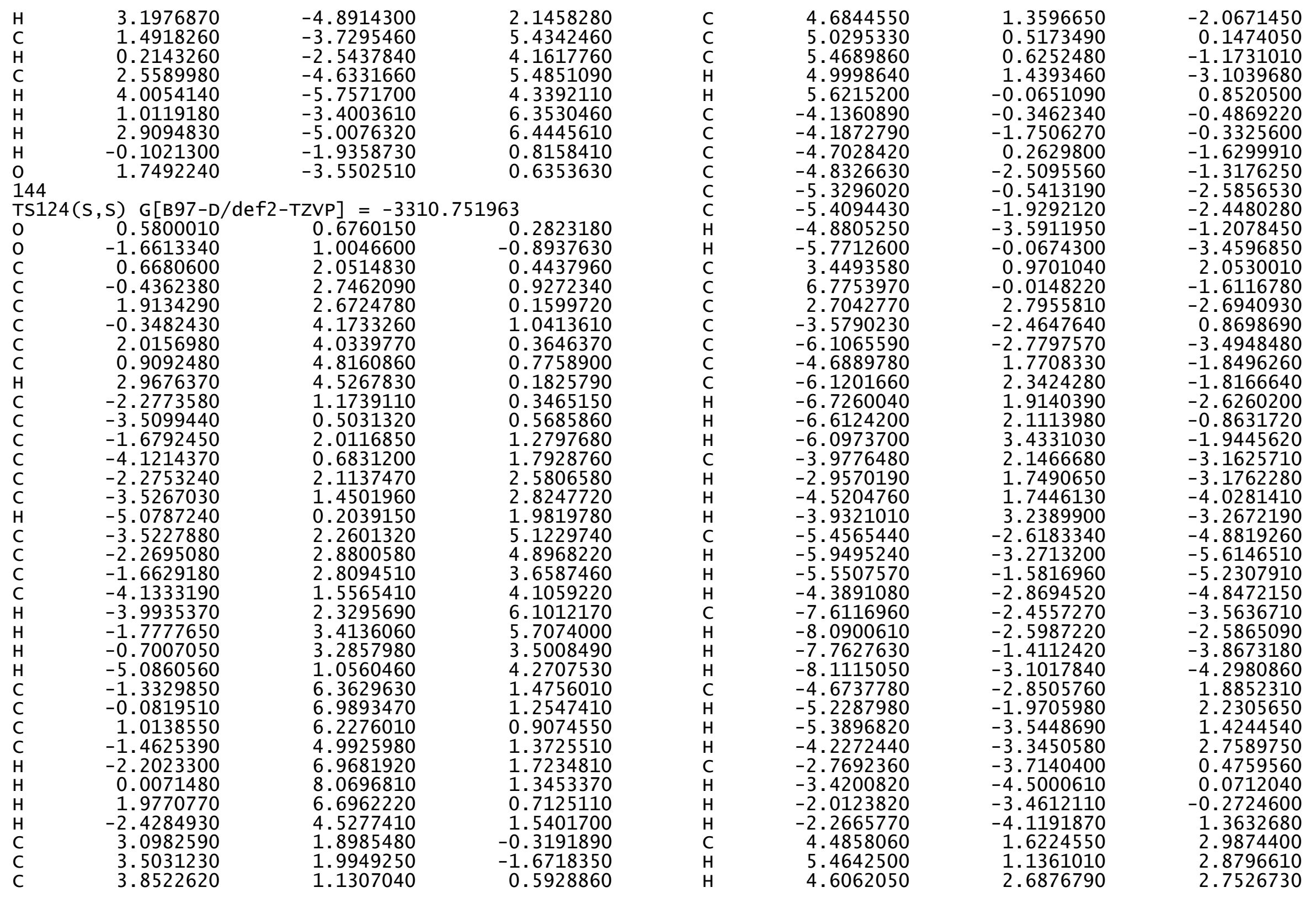




$\begin{array}{rrr}4.1678090 & 1.5276180 & 4.0342690 \\ 3.2323420 & -0.5094350 & 2.4191710 \\ 2.4491760 & -0.9496230 & 1.7941910 \\ 4.1570390 & -1.0863630 & 2.2872580 \\ 2.9210950 & -0.6011120 & 3.4677100 \\ 7.8551760 & 1.0639630 & -1.8306080 \\ 8.8128980 & 0.6013400 & -2.1045950 \\ 7.5534370 & 1.7391910 & -2.6422580 \\ 7.9994490 & 1.6638110 & -0.9234450 \\ 6.6232880 & -0.8788230 & -2.8772590 \\ 5.8773100 & -1.6702050 & -2.7396940 \\ 6.3093040 & -0.2660510 & -3.7314050 \\ 7.5827010 & -1.3477430 & -3.1320790 \\ 2.5618220 & 2.0772520 & -4.0478710 \\ 3.5290430 & 1.9921190 & -4.5603110 \\ 2.1411440 & 1.0763430 & -3.9196320 \\ 1.8907600 & 2.6544760 & -4.6966350 \\ 3.3361930 & 4.1863520 & -2.9067820 \\ 4.3565700 & 4.0808550 & -3.3004890 \\ 2.7454720 & 4.7655710 & -3.6291930 \\ 3.3877970 & 4.7513040 & -1.9694310 \\ -0.3453080 & 0.0517390 & -0.9272650 \\ 0.2988130 & 0.2556580 & -2.2669300 \\ -0.6455770 & -1.3490380 & -0.4732100 \\ 1.6935220 & 2.9362510 & -2.2962710 \\ 7.1118410 & -0.6676260 & -0.7927930 \\ 2.4965820 & 1.4847110 & 2.2120320 \\ -4.1360450 & 2.2433260 & -1.0318710 \\ -5.9998130 & -3.8308190 & -3.1900270 \\ -2.8874730 & -1.7738800 & 1.3629050 \\ 1.6459580 & -2.6841400 & -2.1778880 \\ 2.3858280 & -2.2181800 & -0.9987770 \\ 0.5607770 & -2.6518320 & -2.0843480 \\ 1.9287090 & -1.4513700 & -0.3861650 \\ 2.1769990 & -1.4331900 & -2.7331740 \\ 1.4480180 & -0.7119370 & -2.5790310 \\ 3.7169110 & -3.9655620 & -2.8948620 \\ 4.2580820 & -3.8588070 & -1.4604900 \\ 3.8158420 & -2.5488050 & -0.7880260 \\ 2.1799890 & -3.9056380 & -2.8979680 \\ 4.0476460 & -4.9049580 & -3.3535380 \\ 5.3534960 & -3.9117280 & -1.4547890 \\ 4.3815890 & -1.6895290 & -1.1737600 \\ 1.7717340 & -4.7827160 & -2.3765510 \\ 1.7812740 & -3.9104130 & -3.9199540 \\ 4.1149720 & -3.1437600 & -3.5045700\end{array}$

3.8814630
4.0000050
1.0080500
0.1281330
1.2455280
2.2518750
0.4864880
2.4977470
2.8320060
0.7343710
-0.2916250
1.7391760
3.2793910
0.1436500
1.9305290
-0.1307810
1.6180290
$-4.7033900$
$-2.5496640$
$-3.3821760$
$-2.4098550$
$-4.0956300$
$-5.0736640$
$-3.8051120$
$-5.7498320$
$-5.2919110$
- 4.4844570
$-5.4564750$
$-6.5048200$
$-4.2559410$
$-5.9842920$
$-1.9902730$
$-3.7108180$

$-0.8689440$

0.2918970

1.6010910

1. 6761580

2.8941670

2. 9607340

4.0412620

4. 1552820

2. 0686400

5. 2345840

3.9906760

5. 2944480

4.2000640

6.1187170

6.2263250

0.7592260

0.5707420

TS125(S, S) G[B97-D/def2-TZVP $]=-3310.751127$

$\begin{array}{lrrr}\text { TS125(S }, \mathrm{S}) \text { G }[\mathrm{B} 97-\mathrm{D} / \mathrm{def}-\mathrm{TZVP}]=-3310.751127 \\ \mathrm{O} & 0.5673550 & 0.6512850 & 0.0717240 \\ \mathrm{O} & -1.7126980 & 1.0482960 & -1.0059000 \\ \mathrm{C} & 0.7235510 & 2.0242850 & 0.1812960 \\ \mathrm{C} & -0.3243460 & 2.7901420 & 0.6850640 \\ \mathrm{C} & 1.9863450 & 2.5733340 & -0.1673870 \\ \mathrm{C} & -0.1643570 & 4.2144410 & 0.7408880 \\ \mathrm{C} & 2.1616410 & 3.9343570 & -0.0150190 \\ \mathrm{C} & 1.1104310 & 4.7850890 & 0.4043160 \\ \mathrm{H} & 3.1308350 & 4.3726790 & -0.2413180 \\ \mathrm{C} & -2.2654700 & 1.2935110 & 0.2525640 \\ \mathrm{C} & -3.5132530 & 0.6862140 & 0.5570620 \\ \mathrm{C} & -1.5842980 & 2.1342770 & 1.1231590 \\ \mathrm{C} & -4.0575760 & 0.9421010 & 1.7995410 \\ \mathrm{C} & -2.1132320 & 2.3143900 & 2.4448140 \\ \mathrm{C} & -3.3802550 & 1.7194840 & 2.7710250 \\ \mathrm{H} & -5.0247260 & 0.5155530 & 2.0527470 \\ \mathrm{C} & -3.2317830 & 2.6118180 & 5.0336460 \\ \mathrm{C} & -1.9643380 & 3.1643690 & 4.7268100 \\ \mathrm{C} & -1.4204970 & 3.0207380 & 3.4658910 \\ \mathrm{C} & -3.9205410 & 1.9009160 & 4.0732930 \\ \mathrm{H} & -3.6524150 & 2.7392980 & 6.0287350 \\ \mathrm{H} & -1.4117940 & 3.7043690 & 5.4927550 \\ \mathrm{H} & -0.4467400 & 3.4462770 & 3.2461030 \\ \mathrm{H} & -4.8865150 & 1.4528150 & 4.3002640 \\ \mathrm{C} & -1.0266730 & 6.4636740 & 1.1287190 \\ \mathrm{C} & 0.2426030 & 7.0201070 & 0.8358500 \\ \mathrm{C} & 1.2867040 & 6.1936520 & 0.4773370\end{array}$




$\begin{array}{rrr}-1.2244550 & 5.0983700 & 1.0836730 \\ -1.8558500 & 7.1190790 & 1.3864620 \\ 0.3860570 & 8.0974380 & 0.8809910 \\ 2.2625400 & 6.6074370 & 0.2282890 \\ -2.2033450 & 4.6868560 & 1.3063510 \\ 3.1279930 & 1.7339770 & -0.6458130 \\ 3.4748390 & 1.7333730 & -2.0150700 \\ 3.9207460 & 1.0294290 & 0.2870700 \\ 4.6541630 & 1.0875910 & -2.4079540 \\ 5.0868940 & 0.3964500 & -0.1562650 \\ 5.4869140 & 0.4360810 & -1.4955280 \\ 4.9431600 & 1.1038790 & -3.4568160 \\ 5.7070450 & -0.1253140 & 0.5701330 \\ -4.2143050 & -0.1856000 & -0.4307000 \\ -4.3147250 & -1.5801430 & -0.2024330 \\ -4.7882770 & 0.3859830 & -1.5866490 \\ -5.0072300 & -2.3625450 & -1.1311400 \\ -5.4656070 & -0.4446760 & -2.4879130 \\ -5.5870750 & -1.8182900 & -2.2809200 \\ -5.0845850 & -3.4333970 & -0.9607040 \\ -5.9134280 & -0.0060910 & -3.3783980 \\ 3.5703270 & 0.9737540 & 1.7689920 \\ 6.8026130 & -0.1794630 & -1.9437530 \\ 2.6300970 & 2.4595140 & -3.0549730 \\ -3.7048180 & -2.2551780 & 1.0217590 \\ -6.3047240 & -2.7014710 & -3.2860020 \\ -4.7342510 & 1.8803880 & -1.8782410 \\ -6.1449350 & 2.4991980 & -1.8195490 \\ -6.7937100 & 2.0526530 & -2.5845370 \\ -6.6072480 & 2.3297010 & -0.8385320 \\ -6.0934710 & 3.5812940 & -2.0008290 \\ -4.0629010 & 2.1748110 & -3.2323260 \\ -3.0575810 & 1.7412930 & -3.2669070 \\ -4.6525730 & 1.7568740 & -4.0588230 \\ -3.9850370 & 3.2596090 & -3.3851730 \\ -7.4985810 & -3.4379210 & -2.6498360 \\ -8.0265390 & -4.0356170 & -3.4049990 \\ -7.1543160 & -4.1169500 & -1.8583500 \\ -8.2073990 & -2.7280290 & -2.2051890 \\ -5.3326130 & -3.7067890 & -3.9334350 \\ -4.4949440 & -3.1872510 & -4.4151550 \\ -4.9211540 & -4.3824830 & -3.1720330 \\ -5.8524660 & -4.3139950 & -4.6869370 \\ -4.7859790 & -2.5340390 & 2.0854380 \\ -5.2952850 & -1.6136190 & 2.3929740 \\ -5.5416090 & -3.2225430 & 1.6832220\end{array}$

-4.3363520
-2.9665640
-3.6689120
-2.2360860
-2.4402540
4.6486640
5.6163140
4.7751780
4.3673970
3.3444230
2.5360510
4.2558410
3.0666810
6.8305150
7.8010440
6.6770080
6.0462380
7.9932960
7.9947680
7.9378680
8.9416160
2.4603970
3.4122030
2.0708090
1.7532660
3.2266890
4.2404900
2.6074070
3.2844580
-0.4663290
0.1185590
-0.8301230
1.6296680
6.9106600
2.6318260
-4.1359700
-6.6942060
-2.9675860
0.8695410
-0.0224660
1.2211950
2.2477160
0.5616390
2.6120100
2.7512250
0.9274000

-2.9965590
-3.5631140
-4.3449460
-3.3976470
-3.9291510
1.6680270
1.1609710
2.7138520
1.6458160
-0.4734680
-0.9419400
-1.0728960
-0.4904250
-1.7069040
-2.1129440
-1.9687920
-2.1929470
0.4726060
1.5598010
0.2742980
0.0649160
1.6627150
1.5720700
0.6600510
2.1861870
3.8479330
3.7407780
4.3707120
4.4669650
0.0077600
0.0693550
-1.3334160
2.6051740
0.0268110
1.5148930
2.3707040
-2.0477960
-1.5728500
-3.1347100
-2.1709590
-3.6315290
-4.5807670
-3.1562280
-5.0464320
-4.9408560
-3.6256320

2.9748270

0.6798300

0.3619360

$-0.1174480$

1. 5704860

2. 6220490

2. 5122240

2. 3148330

3. 6832920

2. 2419460

1. 6722210

2. 1184340

3. 3034460

$-1.7549490$

$-2.0693710$

$-0.6996890$

$-2.3470060$

$-1.2144030$

$-1.3608060$

$-0.1359870$

$-1.5888250$

$-4.3602060$

$-4.9000860$

$-4.1626970$

$-5.0160120$

$-3.3640680$

$-3.7737320$

$-4.1054160$

$-2.4620800$

$-1.0406700$

$-2.4204050$

$-0.4677070$

$-2.6328510$

$-3.0179850$

1.9256040

$-1.1039910$

$-4.0798210$

1.4560440

1.7795750

1. 7687090

3. 1455360

3. 2819430

4. 2922990

4. 5447990

2. 3892730

5.5543150 


\begin{tabular}{|c|c|c|c|c|c|c|c|}
\hline & & & & & & & \\
\hline H & -0.2296200 & -2.4204880 & 4.1877380 & $\mathrm{C}$ & -3.9409750 & 1. 9635450 & 4.0445760 \\
\hline H & $\frac{1}{3.9521230}$ & $\begin{array}{l}-4.5698690 \\
-5.7798230\end{array}$ & $\begin{array}{l}5.6834080 \\
4.6433380\end{array}$ & $\stackrel{\mathrm{H}}{\mathrm{H}}$ & $\begin{array}{l}-3.6856150 \\
-1.4673830\end{array}$ & $\begin{array}{l}2.8612050 \\
3.8581430\end{array}$ & $\begin{array}{l}5.9753250 \\
5.4055700\end{array}$ \\
\hline $\mathrm{H}$ & 0.4136970 & -3.2544570 & 6.4381860 & $\mathrm{H}$ & -0.5026670 & 3.5578140 & 3.1644700 \\
\hline $\mathrm{H}$ & 2.2356490 & -4.9335580 & 6.6687890 & $\mathrm{H}$ & -4.8969370 & 1.5020220 & 4.2865510 \\
\hline $\mathrm{H}$ & -0.3104520 & -1.8610460 & 0.8165270 & C & -1.1214140 & 6.4984470 & 0.9741190 \\
\hline 0 & 1.4107880 & -3.6133000 & 0.7692530 & $\mathrm{C}$ & 0.1436860 & 7.0644840 & 0.6818020 \\
\hline C & 1.3965530 & -2.8946260 & -2.0757230 & $\mathrm{C}$ & 1.2026550 & 6.2438030 & 0.3547360 \\
\hline C & 2.1731140 & -2.3256720 & -0.9665720 & $\mathrm{C}$ & -1.3006040 & 5.1298720 & 0.9598310 \\
\hline H & 0.3146210 & -2.8322730 & -1.9603770 & $\mathrm{H}$ & -1.9618810 & 7.1486890 & 1.2071850 \\
\hline $\mathrm{H}$ & 1.7499000 & -1.4873590 & -0.4287720 & $\mathrm{H}$ & 0.2725070 & 8.1444100 & 0.7026260 \\
\hline 0 & 1.9333060 & -1.7211950 & -2.7756510 & $\mathrm{H}$ & 2.1757200 & 6.6647890 & 0.1069690 \\
\hline $\mathrm{H}$ & 1.2269300 & -0.9708330 & -2.6756600 & $\mathrm{H}$ & -2.2762940 & 4.7103070 & 1.1819770 \\
\hline C & 3.4253430 & -4.2988800 & -2.6839100 & C & 3.1087780 & 1.7691830 & -0.6244930 \\
\hline $\mathrm{C}$ & 3.9915600 & -4.0539000 & -1.2769490 & c & 3.4587110 & 1.6887480 & -1.9929100 \\
\hline $\mathrm{C}$ & 3.5986920 & -2.6649110 & -0.7512580 & $\mathrm{C}$ & 3.8830410 & 1.0989240 & 0.3446380 \\
\hline C & 1.8903380 & -4.1976250 & -2.6718500 & C & 4.6062020 & 0.9728240 & -2.3477050 \\
\hline H & 3.7230310 & -5.2907170 & -3.0445070 & $\mathrm{C}$ & 5.0270510 & 0.3997480 & -0.0615140 \\
\hline $\mathrm{H}$ & 5.0842650 & -4.1390140 & -1.2780690 & C & 5.4144140 & 0.3345360 & -1.4000840 \\
\hline H & 4.1791020 & -1.8722650 & -1.2439910 & $\mathrm{H}$ & 4.8864150 & 0.9046090 & -3.3968830 \\
\hline H & 1.4695100 & -5.0046920 & -2.0559620 & $\mathrm{H}$ & 5.6189270 & -0.1076790 & 0.6952800 \\
\hline $\mathrm{H}$ & 1.4740080 & -4.2992010 & -3.6817470 & $\mathrm{C}$ & -4.2113510 & -0.2404390 & -0.4045570 \\
\hline $\mathrm{H}$ & 3.8348690 & -3.5576190 & -3.3832360 & $\mathrm{C}$ & -4.2851610 & -1.6317790 & -0.1494150 \\
\hline H & 3.5969400 & -4.8146110 & -0.5908420 & C & -4.8061140 & 0.2996040 & -1.5651830 \\
\hline H & 3.8095780 & -2.5453180 & 0.3166470 & C & -4.9735100 & -2.4432590 & -1.0560030 \\
\hline & & & & C & -5.4771710 & -0.5594420 & -2.4443590 \\
\hline & , S) $\mathrm{G}[\mathrm{B} 97-\mathrm{D} /$ & {$[Z V P]=-331$} & L07 & $\mathrm{C}$ & -5.5736570 & -1.9305530 & -2.2098310 \\
\hline 0 & 0.5578210 & $0 . \overrightarrow{6} 852050$ & 0.0665300 & $\mathrm{H}$ & -5.0326950 & -3.5117950 & -0.8648850 \\
\hline 0 & -1.7302130 & 1.0177270 & -1.0167910 & H & -5.9401510 & -0.1459130 & -3.3391670 \\
\hline C & 0.6953040 & 2.0623800 & 0.1496400 & $\mathrm{P}$ & -0.4621970 & 0.0030540 & -1.0337080 \\
\hline $\mathrm{C}$ & -0.3666900 & 2.8253720 & 0.6269460 & 0 & 0.1162040 & 0.0533930 & -2.4166920 \\
\hline C & 1.9567240 & 2.6190170 & -0.1931800 & 0 & -0.7989820 & -1.3357190 & -0.4389850 \\
\hline C & -0.2252960 & 4.2525620 & 0.6492870 & $\mathrm{C}$ & 3.5228640 & 1.1050730 & 1.8250370 \\
\hline $\mathrm{C}$ & 2.1139530 & 3.9848390 & -0.0703950 & $\mathrm{H}$ & 2.6369470 & 1.7329410 & 1.9666620 \\
\hline $\mathrm{C}$ & 1.0458710 & 4.8317110 & 0.3134670 & C & 6.6901060 & -0.3578490 & -1.8571540 \\
\hline $\mathrm{H}$ & 3.0820240 & 4.4291700 & -0.2892580 & $\mathrm{H}$ & 6.4815610 & -0.7999640 & -2.8431660 \\
\hline C & -2.2835590 & 1.2851190 & 0.2365210 & C & 2.6407380 & 2.3969020 & -3.0661680 \\
\hline $\mathrm{C}$ & -3.5194270 & 0.6635820 & 0.5606950 & $\mathrm{H}$ & 1.6318400 & 2.5539200 & -2.6688040 \\
\hline $\mathrm{C}$ & -1.6166560 & 2.1620330 & 1.0820060 & C & -4.7796620 & 1.7887430 & -1.8855140 \\
\hline C & -4.0649420 & 0.9413610 & 1.7978730 & $\mathrm{H}$ & -4.1847350 & 2.3037950 & -1.1249390 \\
\hline C & -2.1463730 & 2.3686800 & 2.3997610 & C & -6.2873680 & -2.8455780 & -3.1891910 \\
\hline C & -3.4002800 & 1.7575620 & 2.7461290 & $\mathrm{H}$ & -6.6982190 & -2.2146850 & -3.9905340 \\
\hline $\mathrm{H}$ & -5.0238360 & 0.5045000 & 2.0647880 & $\mathrm{C}$ & -3.6515600 & -2.2719480 & 1.0811850 \\
\hline C & -3.2 & 2.7149620 & 4.9828490 & $\mathrm{H}$ & -2.9190970 & -1.5701050 & 1.4919680 \\
\hline $\mathrm{C}$ & -2.0 & 3.2851840 & 4.6568050 & C & -2.8993540 & -3.5764350 & 0.7574410 \\
\hline C & -1 & 3.1176390 & 3.3988410 & $\mathrm{H}$ & -2.3547700 & -3.9153980 & 1.6476680 \\
\hline
\end{tabular}




$\begin{array}{rrr}-3.5948320 & -4.3747840 & 0.4668500 \\ -2.1831570 & -3.4179600 & -0.0541780 \\ -4.7158450 & -2.5431000 & 2.1637680 \\ -5.4663220 & -3.2506310 & 1.7856680 \\ -4.2490070 & -2.9799930 & 3.0572570 \\ -5.2339890 & -1.6232230 & 2.4577610 \\ 3.1620580 & -0.3129360 & 2.3053210 \\ 4.0087750 & -0.9983510 & 2.1661450 \\ 2.9006570 & -0.3042720 & 3.3712660 \\ 2.3030150 & -0.6957440 & 1.7464940 \\ 4.6526280 & 1.7073960 & 2.6810980 \\ 4.3522000 & 1.7383150 & 3.7367580 \\ 5.5662530 & 1.1037590 & 2.6039630 \\ 4.8892730 & 2.7277610 & 2.3537840 \\ -4.1216620 & 2.0677380 & -3.2493900 \\ -4.7089230 & 1.6233330 & -4.0637120 \\ -4.0632770 & 3.1504290 & -3.4242220 \\ -3.1092410 & 1.6508190 & -3.2814320 \\ -6.1998640 & 2.3854110 & -1.8289920 \\ -6.1670330 & 3.4648000 & -2.0296650 \\ -6.8461000 & 1.9151930 & -2.5818530 \\ -6.6532410 & 2.2260160 & -0.8421370 \\ 2.4967550 & 1.5850040 & -4.3645100 \\ 1.7971950 & 2.0972880 & -5.0368510 \\ 3.4574070 & 1.4953590 & -4.8889200 \\ 2.1113780 & 0.5815700 & -4.1634960 \\ 3.2508400 & 3.7782430 & -3.3827550 \\ 4.2734390 & 3.6593010 & -3.7666630 \\ 2.6516220 & 4.2913930 & -4.1470230 \\ 3.2904170 & 4.4122350 & -2.4905080 \\ -5.3053650 & -3.8463390 & -3.8286770 \\ -4.4822840 & -3.3220710 & -4.3300430 \\ -4.8738620 & -4.5002650 & -3.0593640 \\ -5.8231960 & -4.4766450 & -4.5643970 \\ -7.4592360 & -3.5906590 & -2.5225980 \\ -8.1794450 & -2.8847280 & -2.0901000 \\ -7.9806430 & -4.2199300 & -3.2563310 \\ -7.0922470 & -4.2398110 & -1.7163910 \\ 7.1711100 & -1.4812660 & -0.9268300 \\ 6.3879840 & -2.2283450 & -0.7545120 \\ 8.0400030 & -1.9838420 & -1.3695760 \\ 7.4800130 & -1.0794360 & 0.0469720 \\ 7.8099720 & 0.6878290 & -2.0454690 \\ 7.4984450 & 1.4734200 & -2.7444180 \\ 8.0454630 & 1.1596590 & -1.0818510 \\ 8.7217390 & 0.2124090 & -2.4318180\end{array}$
1. 3669940
2. 2035760
0.2930100
1.8146580
1.8697720
1.1792000
3. 3521260
3.9896160
3.6402860
1.8205370
3.6199990
5.0802710
4.1922060
1.4212760
1. 3545520
3.7357810
3. 6197350
3.9190010
0.9779740
0.0420400
1. 3482390
2.4031100
0.6776110
2.7844210
2.9153360
1.0609190
$-0.1356740$
2. 1138800
3.6034850
0.5389210
2.4111670
$-0.2542120$
1. 5440010

144

TS127(S,S) G[B97-D/def2-TZVP $]=-3310.750339$

$\begin{array}{lllr}\mathrm{O} & 1.5113250 & -0.3155120 & -0.6727820 \\ \mathrm{O} & 0.1406860 & -1.6551710 & 0.9715340 \\ \mathrm{C} & 2.6752710 & -0.9167120 & -0.2155030 \\ \mathrm{C} & 2.6960330 & -2.2962840 & -0.0369870 \\ \mathrm{C} & 3.7985370 & -0.0735510 & 0.0050020 \\ \mathrm{C} & 3.8899440 & -2.8880550 & 0.4962970 \\ \mathrm{C} & 4.9738060 & -0.6736110 & 0.4128650 \\ \mathrm{C} & 5.0496820 & -2.0606590 & 0.6908290 \\ \mathrm{H} & 5.8565020 & -0.0591850 & 0.5728960 \\ \mathrm{C} & 0.2437800 & -2.7470440 & 0.1167410 \\ \mathrm{C} & -0.9506400 & -3.4639210 & -0.1696220\end{array}$

$-2.0698210$

$-2.9228700$

$-1.0245380$

$-1.9006480$

$-0.4940870$

$-2.8388180$

$-2.7168170$

$-2.7253330$

$-1.3594610$

$-2.6427730$

$-3.0573210$

$-1.4097430$

$-1.4513890$

$-1.9791140$

$-3.6271740$

$-3.4745010$

$-0.6210970$

0.1651660

1.7900030

1.7808370

3.1552860

3.2902310

4.3033310

4.5526640

2. 3971210

5.5649680

4.2005070

5.6925830

4.6498030

6.4497080

6.6776590

0.8319120

$-4.8486040$

$-1.8641250$

$-3.5483030$
0.7807490

$-3.4639210$

$-0.1696220$

. 0901000

1.7163910

$-2.4318180$ 


\begin{tabular}{|c|c|c|c|}
\hline$r$ & & & \\
\hline c & $\begin{array}{r}1.4940320 \\
-0.8427710\end{array}$ & -3.1032170 & $\begin{array}{l}-0.3813300 \\
-0.9429940\end{array}$ \\
\hline C & 1.5692430 & -4.2203670 & -1.2817500 \\
\hline c & 0.3855080 & -4.9998010 & -1.5247010 \\
\hline $\mathrm{H}$ & -1.7374100 & -5.1811040 & -1.1614210 \\
\hline C & 1.6286400 & -6.4550340 & -3.0331890 \\
\hline C & 2.7827730 & -5.6581720 & -2.8378580 \\
\hline c & 2.7535870 & -4.5715900 & -1.9865570 \\
\hline$c$ & 0.4535030 & -6.1241060 & -2.3921420 \\
\hline $\mathrm{H}$ & 1.6677420 & -7.3131770 & -3.7005610 \\
\hline H & 3.7014110 & -5.9003650 & -3.3680090 \\
\hline $\mathrm{H}$ & 3.6460770 & -3.9692780 & -1.8556860 \\
\hline H & -0.4513950 & -6.7076870 & -2.5536630 \\
\hline C & 5.1395120 & -4.7861540 & 1.3848240 \\
\hline C & 6.2936470 & -3.9789490 & 1.5345880 \\
\hline C & 6.2437910 & -2.6425920 & 1.1975970 \\
\hline C & 3.9701030 & -4.2548440 & 0.8788780 \\
\hline H & 5.1727970 & -5.8337740 & 1.6762130 \\
\hline $\mathrm{H}$ & 7.2107040 & -4.4115920 & 1.9285260 \\
\hline H & 7.1153790 & -2.0035750 & 1.3296740 \\
\hline $\mathrm{H}$ & 3.0924440 & -4.8836890 & 0.7708330 \\
\hline C & 3.6897240 & 1.4070730 & -0.151628 \\
\hline C & 3.8325790 & 2.2455800 & 0.9811110 \\
\hline C & 3.4376430 & 1.9796340 & -1.4208010 \\
\hline $\mathrm{C}$ & 3.7401050 & 3.6330220 & 0.8136980 \\
\hline $\mathrm{C}$ & 3.3442040 & 3.3703450 & -1.5289200 \\
\hline C & 3.4944500 & 4.2177880 & -0.4286530 \\
\hline H & 3.8514500 & 4.2687320 & 1.6872820 \\
\hline H & 3.1583180 & 3.8119520 & -2.5073860 \\
\hline C & -2.2799780 & -2.9603790 & 0.2837910 \\
\hline C & $-\overline{3} .2043990$ & -2.4831010 & -0.6755500 \\
\hline $\mathrm{C}$ & -2.6164710 & -2.9439330 & 1.6572790 \\
\hline C & -4.4468910 & -2.0115300 & -0.2336190 \\
\hline C & -3.8647650 & -2.4494940 & 2.0446130 \\
\hline $\mathrm{C}$ & -4.7938730 & -1.9728960 & 1.1158390 \\
\hline $\mathrm{H}$ & -5.1622430 & -1.6455130 & -0.9679920 \\
\hline $\mathrm{H}$ & -4.1191080 & -2.4407300 & 3.102346 \\
\hline $\mathrm{P}$ & 0.2673240 & -0.1500810 & 0.368959 \\
\hline 0 & 0.6219410 & 0.7179780 & 1.5435770 \\
\hline 0 & -0.8899460 & 0.2256830 & -0.5107960 \\
\hline C & 3.3159560 & 1.1522290 & -2.6947540 \\
\hline $\mathrm{H}$ & 3.3544630 & 0.0907040 & -2.4344830 \\
\hline C & 3.4493050 & 5.7240040 & 319350 \\
\hline H & 2.7792380 & 5.9143700 & -1.4844100 \\
\hline C & 4.1032490 & 1.7073160 & 2.383173 \\
\hline $\mathrm{H}$ & 3.9596320 & 0.6232130 & 2.3725500 \\
\hline
\end{tabular}

-1.6898860
-0.7342320
-6.1335210
-6.6675230
-2.9180310
-1.8600910
-1.0953420
-0.6083040
-1.5972900
-0.3317090
0.3401100
0.5510590
-1.0789820
-1.0097600
-0.2415560
-1.7428060
-1.6458460
-0.5300970
0.8429680
-2.7984840
-1.7152720
-0.0687380
-2.0298170
-0.3918650
-3.1717100
-2.9304450
-4.2224490
-2.5440280
-3.7437180
-4.8177090
-3.5060070
-3.5392850
1.9799380
1.9047620
1.9094940
1.1340460
4.5065600
4.4309950
4.5181360
5.4588600
-1.3971070
-2.3164020
-0.6920210
-0.9593360
-2.2787600
-1.5942750
-3.5014460
-3.7660220
-1.4175030
-1.0814530
-2.4691850
-2.6999120
3.3039930
3.3533360
2.3797540
2.4152950
3.2314860
2.2118640
5.8417880
5.8521490
4.6368580
4.5535220
6.7107060
6.7715810
4.7515060
4.5284890
4.5012500
5.9183460
5.8321090
4.5110490
-1.0935910
-1.1407240
-0.7915650
-0.3313370
-3.5580900
-3.3571730
-3.5768220
-4.5505730
1.4025420
2.4472710
0.7621010
1.1774790
1.4314680
0.8120430
2.4861910
1.2098180
-2.4723760
-2.2031860
-2.8947450
-1.5623010
-4.7954510
-5.2152030
2.7301500

2.2676180

1. 5641640

0.6633320

$-2.1739930$

$-2.3292160$

1.1778370

$-0.2068270$

1.4682300

$-0.6706170$

1.4426010

1.4889090

1.2248530

$-0.3107610$

$-0.8607340$

1.7392170

1. 5799620

$-0.6668810$

$-0.7206480$

1.4343610

2.8346570

1.6459420

$-0.7159960$

$-1.9394000$

$-2.8185220$

$-3.8889090$

$-2.7232330$

$-2.3492910$

$-2.8876440$

$-2.7759070$

$-3.9598910$

$-2.4670050$

$-3.4183010$

$-3.7480950$

$-4.3074450$

$-2.7605120$

$-3.6339840$

$-4.5377680$

$-3.9390760$

$-3.1353820$

3.8366170

4.3733410

4.5649490

3.4126820

3.3271240

4.0766660 


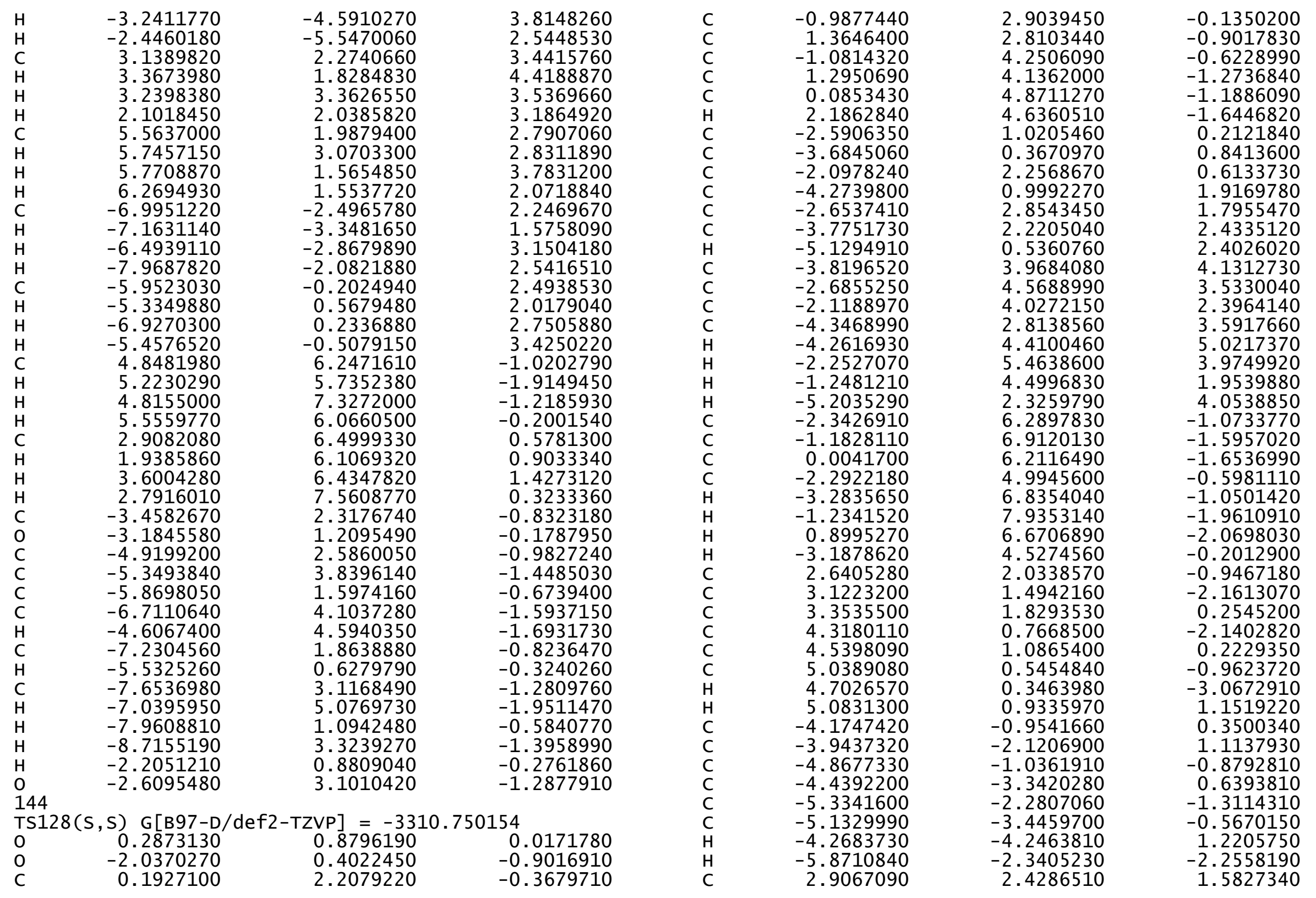




6.3566420
2.3905910
-3.1831510
-5.6315380
-5.1585560
-6.6723050
-7.2135670
-7.0672490
-6.8738620
-4.5912940
-3.5121180
-5.0738620
-4.7748870
-4.8998750
-5.2353090
-5.1079920
-3.8142220
-7.1561090
-7.6855260
-7.4313430
-7.4981660
-4.1603640
-4.8767160
-4.7275420
-3.6098110
-2.1395420
-2.6214200
-1.4662080
-1.5451840
3.8864220
4.8877920
3.9663920
3.5444230
2.7515470
2.0292940
3.7082310
2.4007600
6.6135280
7.5192220
6.7650850
5.7724320
7.5238550
7.3741410
7.5883000
8.4772210
2.4503470

$-0.2104810$

1.7256890

$-2.0918760$

$-4.7897180$

0.1879240

0.4766080

$-0.3647920$

0.6313950

1. 3765970

0.0373300

$-0.1471910$

$-0.7964410$

0.9542210

$-5.2034940$

$-6.1952210$

$-4.4836370$

$-5.2323540$

$-4.7883540$

$-4.5187340$

$-4.0615470$

$-5.7799480$

$-2.1530620$

$-1.3237590$

$-3.0935230$

$-2.1060220$

$-3.2182400$

$-4.2025640$

$-3.2088810$

$-3.0753910$

3.5340500

3.1150880

4. 3166880

3. 9932120

1.3618600

0.5970540

0.8683310

1.8296520

$-1.0840270$

$-1.6862940$

$-0.4642500$

$-1.7572210$

0.7769130

1.3736870

1.4639510

0.2376880

0.5281320

-1.0110160
-3.4775650
2.4343620
-1.0677650
-1.7385690
-1.7775760
-2.2300870
-0.7652280
-2.3741480
-3.1618100
-3.1303420
-3.6885190
-3.7378400
-2.3597910
-2.6925980
-3.1623670
-2.2030520
-1.2868840
-0.3643470
-2.0623830
-1.6123040
3.6254960
3.5971650
3.6011140
4.5750920
2.5505310
2.6163210
1.6884600
3.4624260
2.0242680
2.1907830
1.2589900
2.9615350
2.6796720
2.3788000
2.8920630
3.6094030
0.2282540
0.0820790
1.1210660
0.4314730
-1.2189970
-2.1271380
-0.3646690
-1.3040750
-4.4393330

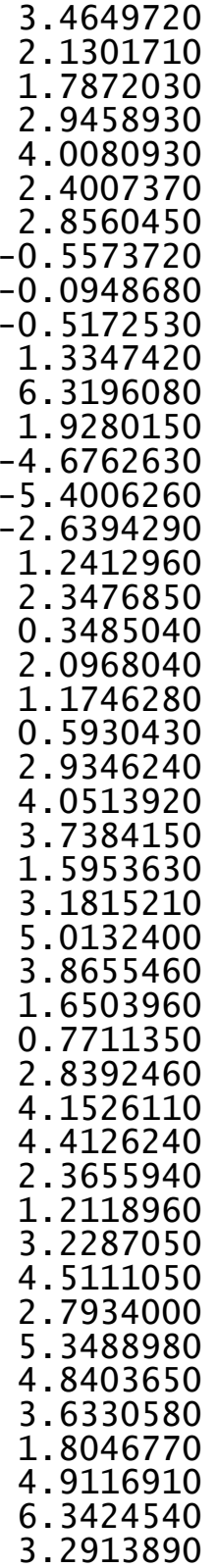

0.3838450

$-0.3942620$

0.7134140

2.9804080

2.8357030

3.1642230

3.8688550

$-0.2622480$

$-0.4001450$

$-1.4622090$

1.9030830

$-0.8704220$

2.8988810

1.0599760

$-5.5360250$

$-1.1431700$

$-3.2839120$

$-2.3664460$

$-3.1601040$

$-1.3683070$

$-2.4689270$

$-1.6421710$

$-4.8767850$

$-4.1397410$

$-2.6414400$

$-4.7284720$

$-5.9407490$

$-4.2606370$

$-2.1197930$

$-5.2417720$

$-5.1793270$

$-4.4664710$

$-4.5693140$

$-2.1374930$

$-2.3819930$

$-1.7836440$

$-2.4196280$

$-2.9856780$

$-1.8810250$

$-3.0063550$

$-3.4009600$

$-1.9044100$

$-1.4397320$

$-2.4645740$

-3.4419790
-1.4823010
$-4.8344460$

$-3.9486630$

$-5.2935220$

$-4.1829580$

$-4.4238120$

$-5.1187550$

$-3.5494220$

$-0.8007710$

$-2.2230950$

0.1034350

$-3.2443710$

$-1.8905140$

1.4454340

$-1.2860620$

$-0.2939900$

2.4920060

$-1.7620500$

$-1.4541960$

$-1.1498670$

$-1.1118740$

$-2.9784390$

$-2.7400980$

$-2.7975550$

$-2.0424070$

$-1.9041290$

$-2.0555740$

$-2.8951410$

$-2.5548620$

$-2.8636330$

$-1.0853260$

$-2.6221620$

$-3.8116800$

$-1.0371910$

$-1.2067440$

1.7494670

1.9282450

2.9713650

2. 8844100

4.1943310

3.9985950

1.9368940

5.3079360

4.2628770

5.2126580

3.9213960

6.2501790 


\begin{tabular}{|c|c|c|c|}
\hline $\begin{array}{l}\mathrm{H} \\
\mathrm{H} \\
\mathrm{O} \\
1\end{array}$ & $\begin{array}{l}5.5658520 \\
0.5851780 \\
2.7611590\end{array}$ & $\begin{array}{l}-2.4786240 \\
-1.7097660 \\
-2.8896210\end{array}$ & $\begin{array}{l}6.0818410 \\
1.1059690 \\
0.6873110\end{array}$ \\
\hline \multicolumn{4}{|c|}{$\begin{array}{l}144 \\
\operatorname{TS} 129(\mathrm{~S}, \mathrm{~S}) \quad \mathrm{G}[\mathrm{B} 97-\mathrm{D} / \mathrm{def} 2-\mathrm{TZVP}]=-3310.750087\end{array}$} \\
\hline 0 & 0.1419070 & 0.9684980 & 0.0974910 \\
\hline 0 & -2.0694520 & 0.3239450 & -0.9854020 \\
\hline C & -0.0159710 & 2.3019390 & -0.2511320 \\
\hline $\mathrm{C}$ & -1.2651770 & 2.8947950 & -0.1187090 \\
\hline C & 1.1510890 & 3.0107700 & -0.6469980 \\
\hline C & -1.4262150 & 4.2348500 & -0.6041210 \\
\hline C & 1.0020140 & 4.3270590 & -1.0311630 \\
\hline C & -0.2700730 & 4.9535230 & -1.0666180 \\
\hline $\mathrm{H}$ & 1.8791100 & 4.9026530 & -1.3162020 \\
\hline C & -2.7241840 & 0.8756730 & 0.1068380 \\
\hline C & -3.7820730 & 0.1179570 & 0.6777590 \\
\hline C & -2.3683730 & 2.1471330 & 0.5423470 \\
\hline C & -4.4810560 & 0.6784350 & 1.7263670 \\
\hline C & -3.0399670 & 2.6799010 & 1.6957750 \\
\hline C & -4.1278040 & 1.9368930 & 2.2730330 \\
\hline $\mathrm{H}$ & -5.3121060 & 0.1304960 & 2.1641240 \\
\hline C & -4.4266420 & 3.6569120 & 3.9736270 \\
\hline C & -3.3265710 & 4.3682790 & 3.4364180 \\
\hline C & -2.6522600 & 3.8930520 & 2.3293380 \\
\hline C & -4.8116980 & 2.4618660 & 3.4031850 \\
\hline $\mathrm{H}$ & -4.9537530 & 4.0463050 & 4.8418770 \\
\hline $\mathrm{H}$ & -3.0046560 & 5.2966400 & 3.9034940 \\
\hline $\mathrm{H}$ & -1.8082580 & 4.4492790 & 9366500 \\
\hline $\mathrm{H}$ & -5.6398960 & 1.8894230 & 8176830 \\
\hline $\mathrm{C}$ & 98310 & 6.1658190 & 1557670 \\
\hline $\mathrm{C}$ & -1.6660610 & 6.8834470 & 5826880 \\
\hline $\mathrm{C}$ & -0.4237610 & 6.2848610 & -1.5396390 \\
\hline $\mathrm{C}$ & $-2.6 s$ & 3762820 & -0.6768380 \\
\hline $\mathrm{H}$ & -3.79 & 5331650 & 340 \\
\hline $\mathrm{H}$ & -1.7 & 0310 & -1.9524110 \\
\hline $\mathrm{H}$ & 0.46 & 2200 & -1.8779100 \\
\hline $\mathrm{H}$ & -3.5 & 50 & 120 \\
\hline C & 2.4 & 50 & -0 \\
\hline C & 3.2 & 1.9860260 & 670 \\
\hline C & 3.0 & 2.1108990 & 56710 \\
\hline C & 4.4 & 1.3705450 & 2540 \\
\hline C & 4.2 & 1.5000710 & 660860 \\
\hline C & 5.0 & 1.1136390 & 13950 \\
\hline $\mathrm{H}$ & 5.0 & 1.0984210 & 00070 \\
\hline $\mathrm{H}$ & 4.68 & 1.3339110 & 91280 \\
\hline C & -4 & -1.2362850 & 15190 \\
\hline
\end{tabular}

-3.7640610
-4.8080170
-4.1295930
-5.1449720
-4.8188640
-3.8563470
-5.6776760
2.3196620
6.3925570
2.7329920
-3.0020880
-5.1800970
-5.2147830
-6.7491720
-7.1887600
-7.1794200
-7.0332020
-4.6061120
-3.5137550
-4.9827130
-4.8771530
-6.0944830
-6.3732380
-5.5775550
-7.0099440
-3.9159740
-3.2761750
-3.3324080
-4.1879520
-3.9660050
-4.7414440
-4.4610020
-3.4156700
-1.8708280
-2.2711820
-1.2071650
-1.2824540
3.0665230
4.0862000
3.1353680
2.5414550
2.1687480
1.6206730
3.1475690
1.6214700
7.1504850
0.8871760 $-1.0757840$

0.3891680

$-1.5346350$

$-0.8185120$

0.9503400

$-2.4793790$

2. 0299980

$-0.0400980$

$-3.0863790$

2. 2049150

$-1.3445930$

$-1.9108500$

$-1.9769400$

$-2.4655710$

$-0.9704230$

$-2.5527660$

$-3.3242300$

$-3.2711470$

$-3.8764110$

$-3.8874400$

$-0.3652100$

$-0.7803230$

0.5885960

$-0.1601080$

$-1.6548560$

$-2.3784060$

$-0.7389230$

$-2.0665370$

3. 4000020

3. 3783560

3. 3731300

4. 3471650

2. 3144920

2. 3859400

1.4471170

3. 2211690

2. 6356210

2. 9255910

1. 9116410

3. 5296770

3.0648560

2. 6402820

3.4070820

3. 9414890

$-1.3372090$ 


$\begin{array}{rr}\mathrm{H} & 8.1439820 \\ \mathrm{H} & 6.6167630 \\ \mathrm{H} & 7.2770110 \\ \mathrm{C} & 6.3053120 \\ \mathrm{H} & 5.8029770 \\ \mathrm{H} & 5.7434470 \\ \mathrm{H} & 7.3124700 \\ \mathrm{C} & 2.9253470 \\ \mathrm{H} & 3.9898780 \\ \mathrm{H} & 2.4348680 \\ \mathrm{H} & 2.4872010 \\ \mathrm{C} & 3.4378560 \\ \mathrm{H} & 4.5190020 \\ \mathrm{H} & 3.0543440 \\ \mathrm{H} & 3.2843570 \\ \mathrm{P} & -0.5243070 \\ \mathrm{O} & 0.0054600 \\ \mathrm{O} & -0.3685410 \\ \mathrm{H} & 1.6587790 \\ \mathrm{H} & 6.9904370 \\ \mathrm{H} & 1.3118070 \\ \mathrm{H} & -4.8334800 \\ \mathrm{H} & -5.7323560 \\ \mathrm{H} & -2.5367460 \\ \mathrm{C} & 2.5594370 \\ \mathrm{O} & 1.4215610 \\ \mathrm{C} & 3.4189980 \\ \mathrm{C} & 4.6187120 \\ \mathrm{C} & 3.0647990 \\ \mathrm{C} & 5.4536660 \\ \mathrm{H} & 4.8869010 \\ \mathrm{C} & 3.9067750 \\ \mathrm{H} & 2.1414500 \\ \mathrm{C} & 5.1015510 \\ \mathrm{H} & 6.3809680 \\ \mathrm{H} & 3.6313700 \\ \mathrm{H} & 5.7561580 \\ \mathrm{H} & 0.7783350 \\ \mathrm{O} & 2.9380520 \\ \mathrm{C} & 1.5110410 \\ \mathrm{C} & 2.5213340 \\ \mathrm{H} & 0.6022940 \\ \mathrm{H} & 2.1686660 \\ \mathrm{O} & 1.3932260 \\ \mathrm{H} & 0.7525620 \\ \mathrm{C} & 3.3651030 \\ & \end{array}$

$\begin{array}{rr}-0.2335620 & -1.0989360 \\ -0.5894100 & -1.9301700 \\ 1.0599980 & -1.9593760 \\ -0.7850900 & 0.8391550 \\ -0.5851840 & 1.7909230 \\ -1.5775510 & 0.3304440 \\ -1.1663160 & 1.0538550 \\ 1.1486040 & -4.0833540 \\ 0.9365970 & -4.2515720 \\ 0.2375470 & -3.7328170 \\ 1.4238110 & -5.0513000 \\ 3.5698850 & -3.6158740 \\ 3.3927010 & -3.6969870 \\ 3.8318840 & -4.6112120 \\ 4.4246420 & -2.9469860 \\ -0.1682870 & -0.8768280 \\ -0.0887310 & -2.2815380 \\ -1.4516370 & -0.1112420 \\ 2.5094970 & -3.0326650 \\ 1.2163320 & 0.5199840 \\ 2.9122640 & 1.7783640 \\ 0.7507920 & -1.4277510 \\ -5.0299860 & -2.2848520 \\ -1.3106340 & 2.2613040 \\ -2.4083800 & 1.4382890 \\ -1.7939050 & 1.6655940 \\ -2.5710730 & 2.6515070 \\ -3.2939290 & 2.5478760 \\ -1.9932130 & 3.8826810 \\ -3.4343770 & 3.6548710 \\ -3.7337770 & 1.5922710 \\ -2.1283260 & 4.9868930 \\ -1.4302590 & 3.9634400 \\ -2.8479590 & 4.8759240 \\ -3.9956270 & 3.5661800 \\ -1.6706340 & 5.9342630 \\ -2.9522040 & 5.7386130 \\ -1.6936770 & 0.8619650 \\ -2.8366290 & 0.3359890 \\ -2.9462550 & -2.1751240 \\ -1.9911040 & -1.7041210 \\ -2.9950690 & -1.5752800 \\ -1.0919490 & -1.2118500 \\ -1.9649260 & -3.2567820 \\ -1.2160880 & -2.9178970 \\ -4.2032400 & -3.3842240\end{array}$

$\begin{array}{ll}\mathrm{C} & 4.3969230 \\ \mathrm{C} & 3.9362630 \\ \mathrm{C} & 2.0052750 \\ \mathrm{H} & 3.7149120 \\ \mathrm{H} & 5.3705780 \\ \mathrm{H} & 4.0136210 \\ \mathrm{H} & 2.0930480 \\ \mathrm{H} & 1.2380970 \\ \mathrm{H} & 3.2459270 \\ \mathrm{H} & 4.5305710 \\ \mathrm{H} & 4.5515730 \\ \mathrm{I} & \end{array}$
$-3.4831450$
$-2.0582850$
$-4.2918040$
$-5.2138490$
$-3.4354430$
$-1.3971200$
$-4.9368950$
$-4.7274210$
$-3.6600330$
$-4.0439010$
$-1.5893500$

$-2.5014110$

$-2.1566350$

$-2.6688880$

$-3.6267630$

$-3.0036340$

$-3.0314460$

$-1.7836400$

$-3.3210790$

$-4.3311650$

$-1.5673300$

TS130(S, S ) G[B97-D/def2-TZVP $]=-3310.750078$

$\begin{array}{lrcr}\text { TS130 (S , S }) \text { G }[\mathrm{B} 97-\mathrm{D} / \mathrm{def}-\mathrm{TZVP}]=-3310.750078 \\ \mathrm{O} & 0.2051680 & 0.9434220 & 0.1049410 \\ \mathrm{O} & -2.0484530 & 0.4383790 & -0.9632580 \\ \mathrm{C} & 0.0940150 & 2.2905520 & -0.2082040 \\ \mathrm{C} & -1.1234870 & 2.9323490 & -0.0187560 \\ \mathrm{C} & 1.2744290 & 2.9593980 & -0.6337250 \\ \mathrm{C} & -1.2408820 & 4.2946030 & -0.4528510 \\ \mathrm{C} & 1.1714560 & 4.2936850 & -0.9677440 \\ \mathrm{C} & -0.0695690 & 4.9784800 & -0.9293990 \\ \mathrm{H} & 2.0611430 & 4.8369200 & -1.2761480 \\ \mathrm{C} & -2.6689360 & 0.9768500 & 0.1557210 \\ \mathrm{C} & -3.7590920 & 0.2506460 & 0.7073340 \\ \mathrm{C} & -2.2447450 & 2.2095220 & 0.6382740 \\ \mathrm{C} & -4.4119320 & 0.7998620 & 1.7918230 \\ \mathrm{C} & -2.8710610 & 2.7253010 & 1.8236870 \\ \mathrm{C} & -3.9856710 & 2.0125440 & 2.3875380 \\ \mathrm{H} & -5.2624920 & 0.2756330 & 2.2210530 \\ \mathrm{C} & -4.1687680 & 3.6698710 & 4.1652690 \\ \mathrm{C} & -3.0437110 & 4.3492580 & 3.6383560 \\ \mathrm{C} & -2.4126370 & 3.8897190 & 2.4996620 \\ \mathrm{C} & -4.6233300 & 2.5212700 & 3.5519370 \\ \mathrm{H} & -4.6612730 & 4.0467720 & 5.0590010 \\ \mathrm{H} & -2.6685800 & 5.2398190 & 4.1381490 \\ \mathrm{H} & -1.5485910 & 4.4200340 & 2.1143040 \\ \mathrm{H} & -5.4726450 & 1.9736290 & 3.9572570 \\ \mathrm{C} & -2.5535580 & 6.3029440 & -0.8916040 \\ \mathrm{C} & -1.3927080 & 6.9854990 & -1.3298950 \\ \mathrm{C} & -0.1780730 & 6.3318180 & -1.3498590 \\ \mathrm{C} & -2.4786150 & 4.9928190 & -0.4626130 \\ \mathrm{H} & -3.5139860 & 6.8138750 & -0.8968560 \\ \mathrm{H} & -1.4641330 & 8.0195930 & -1.6598630 \\ \mathrm{H} & 0.7194120 & 6.8396560 & -1.6992900 \\ \mathrm{H} & -3.3744550 & 4.4781400 & -0.1296900 \\ \mathrm{C} & 2.5783260 & 2.2326020 & -0.6301970\end{array}$




$\begin{array}{rrr}3.2178260 & 1.8807340 & -1.8404720 \\ 3.1626050 & 1.8803800 & 0.6086860 \\ 4.4263320 & 1.1754810 & -1.7794480 \\ 4.3627580 & 1.1625640 & 0.6132280 \\ 5.0101590 & 0.7966470 & -0.5682710 \\ 4.9295470 & 0.9021420 & -2.7049160 \\ 4.8035370 & 0.8905180 & 1.5690390 \\ -4.1773030 & -1.0597740 & 0.1284280 \\ -3.9251440 & -2.2576810 & 0.8387070 \\ -4.8225610 & -1.1029000 & -1.1257610 \\ -4.3507640 & -3.4692700 & 0.2859120 \\ -5.2220940 & -2.3431450 & -1.6383820 \\ -4.9984060 & -3.5360620 & -0.9511820 \\ -4.1571770 & -4.3910220 & 0.8294680 \\ -5.7237730 & -2.3796840 & -2.6043590 \\ 2.5705600 & 2.3055100 & 1.9473830 \\ 6.3421300 & 0.0657920 & -0.5530260 \\ 2.6573580 & 2.2896290 & -3.1964220 \\ -3.2135730 & -2.2699520 & 2.1868900 \\ -5.4234660 & -4.8693470 & -1.5396030 \\ -5.1313850 & 0.1511480 & -1.9337620 \\ -6.6532110 & 0.3785410 & -2.0270130 \\ -7.1362370 & -0.4569820 & -2.5506010 \\ -7.0996680 & 0.4584540 & -1.0274530 \\ -6.8676360 & 1.3022150 & -2.5813880 \\ -4.4972690 & 0.0966940 & -3.3357520 \\ -3.4132040 & -0.0422910 & -3.2633050 \\ -4.9183280 & -0.7303680 & -3.9225800 \\ -4.6963720 & 1.0322790 & -3.8755140 \\ -6.4208820 & -5.6036140 & -0.6238020 \\ -6.7478140 & -6.5437450 & -1.0880880 \\ -5.9512510 & -5.8444800 & 0.3390490 \\ -7.3038890 & -4.9827660 & -0.4266060 \\ -4.2010230 & -5.7613140 & -1.8326430 \\ -3.5028210 & -5.2572300 & -2.5127250 \\ -3.6645900 & -5.9893810 & -0.9021670 \\ -4.5164550 & -6.7093390 & -2.2891990 \\ -4.2273250 & -2.4593220 & 3.3326320 \\ -4.9857690 & -1.6675220 & 3.3280210 \\ -4.7413170 & -3.4242700 & 3.2256860 \\ -3.7145380 & -2.4462270 & 4.3041490 \\ -2.1132640 & -3.3442380 & 2.2700770 \\ -2.5417570 & -4.3551300 & 2.2546600 \\ -1.4129120 & -3.2428590 & 1.4360280 \\ -1.5577910 & -3.2311780 & 3.2101970 \\ 3.5006060 & 3.3180690 & 2.6454280\end{array}$

4.4693870

3. 6821690

3. 0489470

2. 2826000

1. 5898780

3. 2059520

1.8375270

6.4088830

7.3449830

6.3830820

5. 5659930

7.4960500

7.4837580

7.3943770

8.4659520

2.7155290

3. 7522960

2. 2118660

2. 2168460

3. 3995690

4.4620610

2. 9719520

3. 3359040

$-0.5354700$

$-0.0085300$

$-0.4650530$

1.6027980

6. 4720480

1.6192350

$-4.7066300$

$-5.9281640$

$-2.7263530$

2.4652680

1.2590840

3. 2619120

4. 5261690

2. 7821850

5.3007460

4.8908600

3. 5618800

1.8080110

4.8213370

6.2786390

3. 1875430

5.4275790

0.6647690
2.8562950

4. 1906720

3. 6607690

1.1039840

0.4075010

0.5624260

1. 4497520

$-1.0487590$

$-1.6125380$

$-0.6290410$

$-1.7429010$

1.0685840

1.8431420

1.5611190

0.5536410

1. 1683760

0.9157960

0.2672710

1.5015510

3. 5368160

3. 3046980

3. 8608500

4. 3700550

$-0.1498400$

$-0.0996420$

$-1.4404040$

2. 5526580

$-0.3954860$

2. 8155740

1. 0176310

$-4.6624750$

$-1.2988830$

$-2.5182670$

$-2.0502760$

$-2.7393950$

$-3.3445620$

$-2.3289450$

$-3.5332600$

$-3.6584680$

$-2.5142860$

$-1.8572770$

$-3.1157900$

$-4.0025000$

$-2.1875310$

-3.2589770
-1.8358570
2.8777760

2. 0049050

3.5860500

2.8633430

2. 3822250

3. 1045660

3. 8061090

0.5052380

0.4010250

1. 5186950

0.4145510

$-0.3493700$

$-1.1261300$

0.6268590

$-0.3788890$

$-4.2472160$

$-4.5067160$

$-3.8886020$

$-5.1663710$

$-3.7203060$

$-3.8761640$

$-4.6787090$

$-3.0107440$

$-0.8658870$

$-2.2722120$

$-0.0999020$

$-3.0630070$

$-1.5435260$

1.7692320

$-1.4171460$

$-2.4943350$

2. 3247160

1.4413290

1.6586440

2. 6890230

2. 5974420

3. 9448110

3. 7410840

1.6244700

5. 0866410

4.0175680

4. 9879280

3.6613030

6. 0540390

5.8798790

0.8404570 


\begin{tabular}{|c|c|c|c|c|c|c|c|}
\hline & & & & & & & \\
\hline 0 & $\begin{array}{l}2.9585020 \\
1.4298790\end{array}$ & $\begin{array}{l}-2.7732190 \\
-2.9559860\end{array}$ & $\begin{array}{r}0.3306780 \\
-2.1320300\end{array}$ & $\begin{array}{l}\mathrm{C} \\
\mathrm{C}\end{array}$ & $\begin{array}{l}4.7241410 \\
5.0785480\end{array}$ & $\begin{array}{l}-5.6604290 \\
-4.3473600\end{array}$ & $\begin{array}{l}1.3633770 \\
1.1355510\end{array}$ \\
\hline C & 2.5055330 & -2.0364500 & -1.7366630 & $\mathrm{C}$ & 2.4513630 & -5.1958900 & 0.6380870 \\
\hline $\mathrm{H}$ & 0.5397390 & -2.9309930 & -1.5040890 & $\mathrm{H}$ & 3.1068790 & -7.1095880 & 1.3313820 \\
\hline $\mathrm{H}$ & 2.2236010 & -1.0913780 & -1.2839370 & $\mathrm{H}$ & 5.4577310 & -6.3697070 & 1.7402590 \\
\hline 0 & 1. 3203440 & -2.0201670 & -3.2540150 & $\mathrm{H}$ & 6.0907230 & -4.0014260 & 1.3386820 \\
\hline $\mathrm{H}$ & 0.7109640 & -1.2458470 & -2.9222650 & $\mathrm{H}$ & 1.4351280 & -5.5335040 & 0.4639440 \\
\hline C & 3.1650080 & -4.3717980 & -3.3425890 & $\mathrm{C}$ & 3.9065480 & 0.3484200 & 0.0659240 \\
\hline$c$ & 4.2650420 & -3.6717320 & -2.5297580 & C & 4.2194420 & 1.0174770 & 1.2773640 \\
\hline $\mathrm{C}$ & 3.8976600 & -2.2092210 & -2.2321400 & $\mathrm{C}$ & 4.0002190 & 1.0399150 & -1.1596700 \\
\hline $\mathrm{C}$ & 1.8313220 & -4.3485840 & -2.5766910 & $\mathrm{C}$ & 4.6506520 & 2.3441190 & 1.2183870 \\
\hline $\mathrm{H}$ & 3.4492810 & -5.4107400 & -3.5483200 & C & 4.4437850 & 2.3703350 & -1.1622070 \\
\hline $\mathrm{H}$ & 5.2212420 & -3.6997820 & -3.0658550 & $\mathrm{C}$ & 4.7801280 & 3.0412710 & 0.0136030 \\
\hline $\mathrm{H}$ & 3.9817920 & -1.5898070 & -3.1364830 & $\mathrm{H}$ & 4.8876990 & 2.8612630 & 2.1465170 \\
\hline $\mathrm{H}$ & 1.9175060 & -4.9561560 & -1.6647850 & $\mathrm{H}$ & 4.5319960 & 2.8809090 & -2.1171830 \\
\hline $\mathrm{H}$ & 1.0160980 & -4.7695240 & -3.1782400 & C & -3.1425910 & -2.3343540 & 0.2853260 \\
\hline $\mathrm{H}$ & 3.0412430 & -3.8668090 & -4.3099310 & $\mathrm{C}$ & -4.0233460 & -1.6028840 & -0.5501140 \\
\hline $\mathrm{H}$ & 4.4030200 & -4.1984390 & -1.5763540 & $\mathrm{C}$ & -3.3924300 & -2.4163060 & 1.6730560 \\
\hline$H$ & 4.5674800 & -1.7516640 & -1.4998130 & $\mathrm{C}$ & -5.1430660 & -0.9923270 & 0.0232970 \\
\hline & & & & $\mathrm{C}$ & -4.5227870 & -1.7771220 & 2.1965530 \\
\hline & S) G[B97- & $T Z V P]=-331$ & 047 & $\mathrm{C}$ & -5.4134310 & -1.0665730 & 1.3921990 \\
\hline 0 & 1.3093780 & -0.6139330 & -0.6648800 & $\mathrm{H}$ & -5.8255010 & -0.4412180 & -0.6198980 \\
\hline 0 & -0.4488570 & -1.6143690 & 0.8728000 & $\mathrm{H}$ & -4.7232090 & -1.8485400 & 3.2644490 \\
\hline C & 2.2282340 & -1.5535520 & -0.2180510 & $\mathrm{P}$ & 0.0816760 & -0.1782470 & 0.3241460 \\
\hline C & 1.8448680 & -2.8892210 & -0.1484110 & 0 & 0.5865070 & 0.5555200 & 1.5360880 \\
\hline C & 3.5358020 & -1.0974390 & 0.1020280 & 0 & -0.9051220 & 0.4926830 & -0.5897960 \\
\hline C & 2.7881250 & -3.8416650 & 0.3642590 & C & 3.6879980 & 0.3872200 & -2.5003900 \\
\hline C & 4.4660130 & -2.0430160 & 0.4879870 & $\mathrm{H}$ & 3.3390090 & -0.6340750 & -2.3237370 \\
\hline $\mathrm{C}$ & 4.1274380 & -3.4074740 & 0.6524970 & C & 5.3083810 & 4.4686280 & 0.0408840 \\
\hline $\mathrm{H}$ & 5.4797240 & -1.7237860 & 0.7173190 & $\mathrm{H}$ & 4.7815100 & 4.9887260 & 0.8558280 \\
\hline C & -0.6369800 & -2.6554930 & -0.0309500 & C & 4.1191650 & 0.3422710 & 2.6419040 \\
\hline C & -1.9748820 & -3.0457370 & -0.3117560 & $\mathrm{H}$ & 3.6014860 & -0.6137580 & 2.5180880 \\
\hline C & 0.4768960 & -3.2945220 & -0.5665840 & C & -2.5179340 & -3.2260300 & 2.6224450 \\
\hline C & -2.1616580 & -4.1096910 & -1.1725590 & $\mathrm{H}$ & -1.6614500 & -3.6197990 & 2.0675540 \\
\hline C & 0.2640480 & -4.3252800 & -1.5431980 & C & -6.6549160 & -0.4221080 & 1.9835270 \\
\hline C & -1.0798460 & -4.7501370 & -1.8232110 & $\mathrm{H}$ & -6.6084520 & -0.5571420 & 3.0736620 \\
\hline $\mathrm{H}$ & -3.1734550 & -4.4439600 & -1.3883790 & C & -3.8176700 & -1.4759860 & -2.0562790 \\
\hline C & -0.2492670 & -6.3640420 & -3.4490770 & $\mathrm{H}$ & -2.8164100 & -1.8429970 & -2.3014340 \\
\hline C & 1.0726090 & -5.9163920 & -3.2079070 & C & 0.1807670 & 3.6130430 & 1.0265240 \\
\hline C & 1.32 & -4.9250070 & -2.2801680 & $\mathrm{C}$ & 0.5070820 & 3.3440570 & -0.3804830 \\
\hline C & -1.3019500 & -5.7858460 & -2.7715160 & $\mathrm{H}$ & -0.6520280 & 3.0391900 & 1.4377890 \\
\hline $\mathrm{H}$ & -0.43 & -7.1511760 & -4.1774900 & $\mathrm{H}$ & 0.3024410 & 2.3523940 & -0.7623270 \\
\hline $\mathrm{H}$ & $1.8 \mathrm{~s}$ & -6.3539370 & -3.7627020 & 0 & 1.4513490 & 2.9171570 & 1.2144780 \\
\hline $\mathrm{H}$ & 2.340 & -4.5903700 & -2.1100740 & $\mathrm{H}$ & 1.1965320 & 1.9168330 & 1.3531810 \\
\hline $\mathrm{H}$ & -2.3262180 & -6.1020610 & -2.9619760 & C & 1.3507860 & 5.8533810 & 0.8087420 \\
\hline C & 3.3927990 & -6.0805170 & 1.1244500 & $\mathrm{C}$ & 1.2266780 & 5.7460340 & -0.7187690 \\
\hline
\end{tabular}




$\begin{array}{rrr}1.3219790 & 4.2843430 & -1.1889410 \\ 0.2326040 & 5.0525800 & 1.4987340 \\ 1.2934300 & 6.9030810 & 1.1203530 \\ 2.0088870 & 6.3350370 & -1.2117490 \\ 2.3568030 & 3.9139790 & -1.1288000 \\ -0.7437220 & 5.5023610 & 1.2689740 \\ 0.3510410 & 5.0577100 & 2.5894100 \\ 2.3267580 & 5.4660470 & 1.1286300 \\ 0.2574120 & 6.1551030 & -1.0328590 \\ 1.0220090 & 4.1639970 & -2.2364210 \\ -3.8929140 & -0.0186290 & -2.5469700 \\ -3.6908800 & 0.0170050 & -3.6258480 \\ -4.8888370 & 0.4106160 & -2.3776120 \\ -3.1544300 & 0.5992130 & -2.0299520 \\ -4.8404300 & -2.3451260 & -2.8152050 \\ -5.8614750 & -1.9976640 & -2.6077480 \\ -4.6675720 & -2.2813470 & -3.8981350 \\ -4.7739980 & -3.3972550 & -2.5126120 \\ 2.5670240 & 1.1352250 & -3.2456740 \\ 2.8713440 & 2.1648120 & -3.4761870 \\ 2.3400940 & 0.6284630 & -4.1929060 \\ 1.6532710 & 1.1641310 & -2.6427240 \\ 4.9554970 & 0.2897870 & -3.3718250 \\ 4.7261530 & -0.2126560 & -4.3209960 \\ 5.3484410 & 1.2896530 & -3.5986980 \\ 5.7413310 & -0.2768080 & -2.8562540 \\ -1.9664680 & -2.3630450 & 3.7719920 \\ -2.7831160 & -1.9861890 & 4.4017790 \\ -1.2989870 & -2.9629550 & 4.4049290 \\ -1.4041660 & -1.5089940 & 3.3798680 \\ -3.2949100 & -4.4393010 & 3.1725250 \\ -2.6520310 & -5.0333420 & 3.8362190 \\ -4.1706570 & -4.1086940 & 3.7463350 \\ -3.6452950 & -5.0834680 & 2.3557450 \\ 3.3058270 & 1.1658270 & 3.6576280 \\ 3.2501210 & 0.6204700 & 4.6090960 \\ 3.7774520 & 2.1372750 & 3.8535400 \\ 2.2878140 & 1.3350730 & 3.2953200 \\ 5.5250210 & 0.0487940 & 3.2036010 \\ 6.0764230 & 0.9857840 & 3.3605790 \\ 5.4490260 & -0.4720750 & 4.1675330 \\ 6.1075150 & -0.5756230 & 2.5154750 \\ -6.7134990 & 1.0887600 & 1.6953860 \\ -5.8137080 & 1.5953240 & 0.6152310 \\ -6.7896130 & 1.2688030 & 2.1766030 \\ -7.5927990 & 1.5376330 & \end{array}$

-7.9326040
-7.9127040
-8.8243730
-8.0191920
5.0753250
4.0160700
5.4164960
5.6402480
6.8122280
6.9967650
7.3802810
7.1872420
-2.5499640
-2.6453430
-3.8026040
-3.7722770
-5.0081340
-4.9296730
-2.8367830
-6.1643050
-5.0323240
-6.1276420
-4.9002110
-7.0937180
-7.0302860
-1.8653200
-1.5261970

TS132(S,S) G[B97-D/def2-TZVP $]=-3310.749235$

$\begin{array}{llcr}\text { O } & -0.0235130 & 1.1375480 & 0.2313100 \\ \mathrm{O} & -2.0725600 & 0.1008740 & -0.8350290 \\ \mathrm{C} & -0.2840390 & 2.3663540 & -0.3587700 \\ \mathrm{C} & -1.5877480 & 2.8469330 & -0.3816710 \\ \mathrm{C} & 0.8364340 & 3.0916590 & -0.8481790 \\ \mathrm{C} & -1.8364090 & 4.0647780 & -1.0989660 \\ \mathrm{C} & 0.5964250 & 4.3066060 & -1.4538790 \\ \mathrm{C} & -0.7216590 & 4.8014660 & -1.6315070 \\ \mathrm{H} & 1.4319270 & 4.9015270 & -1.8146100 \\ \mathrm{C} & -2.8462350 & 0.7454230 & 0.1175200 \\ \mathrm{C} & -3.8346620 & -0.0348780 & 0.7774430 \\ \mathrm{C} & -2.6587000 & 2.1055810 & 0.3396650 \\ \mathrm{C} & -4.6672040 & 0.6072400 & 1.6701870 \\ \mathrm{C} & -3.4670170 & 2.7402600 & 1.3442740 \\ \mathrm{C} & -4.5052500 & 1.9789610 & 1.9872660 \\ \mathrm{H} & -5.4436430 & 0.0397080 & 2.1780450 \\ \mathrm{C} & -5.1191450 & 3.9161090 & 3.3336790\end{array}$

1.4729400

1.6993640

1.9380550

0.3843980

$-1.2520320$

$-1.5324500$

$-1.1216180$

$-2.0858530$

0.3909150

1. 3347190

$-0.4006690$

0.4828830

$-0.7485970$

$-0.1295880$

$-0.7140720$

$-1.1725240$

$-0.2356600$

$-1.1467200$

$-1.5460600$

$-0.2128580$

0.1123370

$-0.6666800$

$-1.5004700$

0.1586210

$-0.6477120$ $\begin{array}{ll}1.6255200 & -0.3039490 \\ 3.8645900 & -1.3083280\end{array}$

$\begin{array}{ll}1.6255200 & -0.3039490 \\ 3.8645900 & -1.3083280\end{array}$

3. 9161090

3. 3336790

3.3798680

.1725250

.8362190

2.3557450

(2)

090960

2953200

036010

2. 5154750

2. 1766030 


$\begin{array}{rrr}-4.0666170 & 4.6531610 & 2.7392530 \\ -3.2628410 & 4.0808580 & 1.7733990 \\ -5.3254330 & 2.6029470 & 2.9663540 \\ -5.7495300 & 4.3810550 & 4.0885110 \\ -3.8843540 & 5.6797710 & 3.0499090 \\ -2.4580410 & 4.6606950 & 1.3349010 \\ -6.1125180 & 2.0125460 & 3.4325830 \\ -3.3479500 & 5.7328730 & -2.0385920 \\ -2.2476280 & 6.4766450 & -2.5297600 \\ -0.9628680 & 6.0148330 & -2.3308970 \\ -3.1466880 & 4.5593310 & -1.3396960 \\ -4.3611010 & 6.0866880 & -2.2170890 \\ -2.4188540 & 7.4033190 & -3.0731840 \\ -0.1088090 & 6.5673600 & -2.7193280 \\ -3.9968020 & 3.9949120 & -0.9695070 \\ 2.2111630 & 2.5496860 & -0.6206000 \\ 2.9547050 & 1.9814060 & -1.6811530 \\ 2.7463530 & 2.5725980 & 0.6863860 \\ 4.2208330 & 1.4511990 & -1.4066920 \\ 4.0189980 & 2.0334860 & 0.9068210 \\ 4.7702430 & 1.4631290 & -0.1206210 \\ 4.7968670 & 1.0163330 & -2.2198400 \\ 4.4381160 & 2.0526740 & 1.9111330 \\ -3.9079070 & -1.5054490 & 0.5348580 \\ -3.4787110 & -2.4004260 & 1.5417870 \\ -4.3499350 & -2.0019460 & -0.7124560 \\ -3.5168470 & -3.7753250 & 1.2783920 \\ -4.3562990 & -3.3825490 & -0.9288860 \\ -3.9392440 & -4.2873840 & 0.0510470 \\ -3.1834610 & -4.4716160 & 2.0457930 \\ -4.6907880 & -3.7594380 & -1.8927330 \\ 2.0073870 & 3.2015250 & 1.8611360 \\ 6.1595840 & 0.9119010 & 0.1497530 \\ 2.4286110 & 1.9733800 & -3.1106160 \\ -2.9778840 & -1.9255930 & 2.9008500 \\ -3.8828430 & -5.7792210 & -0.2270210 \\ -4.8501110 & -1.0877800 & -1.8233310 \\ -6.3641680 & -1.2801870 & -2.0403780 \\ -6.5786250 & -2.3091350 & -2.3586040 \\ -6.9212390 & -1.0865420 & -1.1147270 \\ -6.7281660 & -0.5957790 & -2.8186680 \\ -4.0787650 & -1.3018310 & -3.1385920 \\ -3.0041270 & -1.1591580 & -2.9836980 \\ -4.2458890 & -2.3141610 & -3.5294090 \\ -4.4226290 & -0.5857510 & -3.8968780 \\ -2.7144750 & -6.1004570 & -1.1819090\end{array}$

-2.6432700
-2.8683280
-1.7628610
-5.2070890
-6.0412510
-5.4332250
-5.1412480
-4.0585180
-4.9906770
-4.2795860
-3.7122010
-1.6585520
-1.7979040
-0.8976780
-1.2967170
2.6808690
3.7142280
2.7055130
2.1335030
1.8990200
1.4567330
2.8882960
1.2724110
7.2371180
8.2420500
7.1222630
7.1528250
6.3262970
5.5872280
6.2044330
7.3274700
2.7328790
3.8089190
2.3762640
2.2261350
2.9967080
4.0907430
2.5888120
2.7521440
-0.4963070
0.1109160
-0.2718820
1.3393670
6.3023700
0.9868820
-4.6953420

-7.1827590
-5.6033290
-5.7472450
-6.3276230
-6.1105170
-5.8745500
-7.4146980
-2.1432610
-1.6280360
-3.2143690
-1.7665150
-2.5988790
-3.6725770
-2.4679970
-2.1466420
4.5265020
4.3464930
5.2287700
4.9940720
2.2487350
1.2922140
2.0570410
2.7007290
1.8263620
1.4448300
1.8673320
2.8487400
-0.5314050
-1.1930920
-0.5777740
-0.9084520
0.6715210
0.5584730
-0.1979430
0.6874710
3.1769440
3.0998270
3.1991450
4.1241820
-0.2017400
-0.2817640
-1.3298700
2.0754170
0.9027900
3.4420470
-0.0477330

$-1.3568820$ $-2.1490510$ $-0.7654250$ $-0.7874810$ $-0.1086120$ $-1.7612590$ $-0.9278210$ 3.9786410 3.7164610 4.0818830

4.9507410

3. 3225000

3.5044670

2. 5472360

4.2555740

2. 2697060

2. 5950930

1.4267210

3.0994540

3.0644170

2.7659680

3.5012780

3.8443850

$-0.4660340$

$-0.2393620$

$-1.5575230$

$-0.0766870$

$-0.3553330$

0.1088490

$-1.4452960$

$-0.1090000$

$-3.8697910$

$-4.0582600$

$-3.3120660$

$-4.8430780$

$-3.8904870$

$-3.9561280$

$-4.9102280$

$-3.3969590$

$-0.5768640$

$-1.9483560$

0.3926440

$-3.0684800$

1. 2391910

1.5490840

1.5187950
-1.5 


\begin{tabular}{|c|c|c|c|c|c|c|c|}
\hline $\mathrm{H}$ & -3.6836160 & -6.2841100 & 0.7292450 & C & -0.0924010 & -2.7267290 & 0.0223690 \\
\hline H & -2.7809860 & -0.8506110 & 2.8377320 & $\mathrm{C}$ & -1.3516010 & -3.2973280 & -0.3098180 \\
\hline C & 2.8462820 & -2.1477130 & 1.5010050 & C & 1.1207830 & -3.1920610 & -0.4748870 \\
\hline 0 & 1.8916330 & -1.2767920 & 1.7231690 & C & -1.3516600 & -4.4002470 & -1.1404340 \\
\hline C & 4.0633000 & -1.9622730 & 2.3452490 & $\mathrm{C}$ & 1.0934320 & -4.2659520 & -1.4281590 \\
\hline C & 5.0963910 & -2.9118030 & 2.2748090 & C & -0.1630920 & -4.8969610 & -1.7284100 \\
\hline C & 4.2185100 & -0.8261420 & 3.1570590 & $\mathrm{H}$ & -2.2982030 & -4.8716740 & -1.3937200 \\
\hline C & 6.2683980 & -2.7285160 & 3.0066540 & C & 0.9467630 & -6.4069470 & -3.2863050 \\
\hline H & 4.9687780 & -3.7817940 & 1.6371530 & C & 2.1777010 & -5.7555760 & -3.0295390 \\
\hline C & 5.3983590 & -0.6395060 & 3.8768220 & $\mathrm{C}$ & 2.2487070 & -4.7142960 & -2.1256780 \\
\hline H & 3.4245180 & -0.0895110 & 3.2008470 & C & -0.1994930 & -5.9782590 & -2.6507520 \\
\hline C & 6.4234860 & -1.5889920 & 3.8047770 & $\mathrm{H}$ & 0.9062470 & -7.2310200 & -3.9952560 \\
\hline $\mathrm{H}$ & 7.0638340 & -3.4679900 & 2.9512900 & $\mathrm{H}$ & 3.0764680 & -6.0751720 & -3.5526670 \\
\hline $\mathrm{H}$ & 5.5199330 & 0.2483680 & 4.4932530 & $\mathrm{H}$ & 3.1994000 & -4.2247780 & -1.9436390 \\
\hline $\mathrm{H}$ & 7.3421690 & -1.4412190 & 4.3684590 & H & -1.1589450 & -6.4495310 & -2.8574050 \\
\hline $\mathrm{H}$ & 1.0388860 & -1.3880610 & 1.1365800 & C & 4.4849180 & -5.3646100 & 1.3008840 \\
\hline 0 & 2.7931560 & -3.0553290 & 0.6495360 & $\mathrm{C}$ & 5.7191520 & -4.7054890 & 1.5215760 \\
\hline C & 1.1292360 & -3.2793050 & -1.6983100 & C & 5.8329970 & -3.3575370 & 1.2540170 \\
\hline $\mathrm{C}$ & 2.3414070 & -2.4861110 & -1.4434650 & $\mathrm{C}$ & 3.4011140 & -4.6779960 & 0.7914960 \\
\hline H & 0.2999660 & -3.0802300 & -1.0199330 & $\mathrm{H}$ & 4.3876810 & -6.4212620 & 1.5408350 \\
\hline $\mathrm{H}$ & 2.1926670 & -1.4700000 & -1.0920770 & $\mathrm{H}$ & 6.5673330 & -5.2602490 & 1.9167460 \\
\hline 0 & 1.0557570 & -2.4470610 & -2.9024700 & $\mathrm{H}$ & 6.7664070 & -2.8301100 & 1.4435850 \\
\hline H & 0.5944630 & -1.5716080 & -2.6045050 & $\mathrm{H}$ & 2.4606180 & -5.1952790 & 0.6322540 \\
\hline C & 2.5888840 & -5.0163500 & -2.8500270 & $\mathrm{C}$ & 3.8365280 & 1.0240300 & 0.0709980 \\
\hline C & 3.8196020 & -4.4052020 & -2.1616780 & c & 4.0691900 & 1.7583390 & 1.2598250 \\
\hline C & 3.6666880 & -2.8843080 & -1.9915070 & C & 3.7666370 & 1.7023670 & -1.1687390 \\
\hline C & 1.3187120 & -4.7474850 & -2.0248540 & $\mathrm{C}$ & 4.2964710 & 3.1373150 & 1.1680910 \\
\hline $\mathrm{H}$ & 2.7228940 & -6.0978470 & -2.9718880 & C & 3.9795520 & 3.0849930 & -1.2004300 \\
\hline $\mathrm{H}$ & 4.7298120 & -4.6130200 & -2.7365070 & $\mathrm{C}$ & 4.2746950 & 3.8188340 & -0.0481760 \\
\hline $\mathrm{H}$ & 3.7690160 & -2.3715350 & -2.9601910 & $\mathrm{H}$ & 4.5003130 & 3.7006220 & 2.0770320 \\
\hline $\mathrm{H}$ & 1.3807880 & -5.2763510 & -1.0635450 & $\mathrm{H}$ & 3.9515920 & 3.5939800 & -2.1620360 \\
\hline H & 0.4205530 & -5.1068090 & -2.5418320 & C & -2.6262230 & -2.6887940 & 0.1713960 \\
\hline $\mathrm{H}$ & 2.4727720 & -4.5839140 & -3.8528050 & $\mathrm{C}$ & -3.5012240 & -2.0753290 & -0.7567940 \\
\hline H & 3.9413580 & -4.8565030 & -1.1684300 & $\mathrm{C}$ & -2.9606820 & -2.7154410 & 1.5453830 \\
\hline $\mathrm{H}$ & 4.4414660 & -2.4572820 & -1.3458440 & C & -4.6936340 & -1.5133220 & -0.2838950 \\
\hline \multirow{2}{*}{\multicolumn{4}{|c|}{$\operatorname{Ts} 133(\mathrm{~s}, \mathrm{~s}) \mathrm{G}[$}} & C & -4.1581770 & -2.1301890 & 1.9641360 \\
\hline & & & & C & -5.0382250 & -1.5196020 & 1.0670650 \\
\hline 0 & 1.4633820 & -0.4120050 & -0.6361290 & $\mathrm{H}$ & -5.3706650 & -1.0412460 & -0.9931220 \\
\hline 0 & -0.0874100 & -1.6659640 & 0.9234410 & $\mathrm{H}$ & -4.4124800 & -2.1583400 & 3.0213500 \\
\hline C & 2.5336910 & -1.1609330 & -0.1675360 & $\mathrm{P}$ & 0.1913770 & -0.1681800 & 0.3583510 \\
\hline C & 2.3939310 & -2.5423110 & -0.0616090 & 0 & 0.5711040 & 0.6540340 & 1.5582160 \\
\hline C & 3.7344070 & -0.4651840 & 0.1386830 & 0 & -0.8978130 & 0.3149000 & -0.5573620 \\
\hline $\mathrm{C}$ & 3.4910810 & -3.2943770 & 0.4758900 & $\mathrm{C}$ & 3.5351450 & 0.9833800 & -2.4916140 \\
\hline C & 4.8186140 & -1.2192160 & 0.5458570 & $\mathrm{H}$ & 3.3909360 & -0.0828520 & -2.2965470 \\
\hline C & 4.7296940 & -2.6177290 & 0.7471020 & C & 4.6217610 & 5.2962520 & -0.1195100 \\
\hline H & 5.7586740 & -0.7190080 & 0.7651350 & $\mathrm{H}$ & 4.7655870 & 5.6497230 & 0.9115980 \\
\hline
\end{tabular}




$\begin{array}{rrr}4.1008310 & 1.1126710 & 2.6417850 \\ 3.7469880 & 0.0815010 & 2.5504110 \\ -2.0945150 & -3.4157130 & 2.5847440 \\ -1.1631620 & -3.7382630 & 2.1100490 \\ -6.3322620 & -0.8888160 & 1.5489920 \\ -6.8135470 & -0.4186620 & 0.6790930 \\ -3.2169140 & -2.0194280 & -2.2546420 \\ -2.1779200 & -2.3183470 & -2.4221490 \\ -0.6497510 & 3.4978590 & 1.0831580 \\ -0.2567860 & 3.3569240 & -0.3246090 \\ -1.2979240 & 2.7105350 & 1.4726150 \\ -0.1976160 & 2.3540560 & -0.7275330 \\ 0.7622230 & 3.1648640 & 1.2689280 \\ 0.7820770 & 2.1302600 & 1.3939220 \\ -0.1309660 & 5.9738630 & 0.9226840 \\ -0.1984280 & 5.8701100 & -0.6095900 \\ 0.2825260 & 4.4964110 & -1.1078720 \\ -0.9964310 & 4.8860560 & 1.5814840 \\ -0.4750720 & 6.9625110 & 1.2489510 \\ 0.4093710 & 6.6541290 & -1.0759120 \\ 1.3783610 & 4.4165170 & -1.0546420 \\ -2.0558280 & 5.0562520 & 1.3420500 \\ -0.8967160 & 4.9024990 & 2.6738350 \\ 0.9078430 & 5.8603850 & 1.2591050 \\ -1.2368940 & 6.0168310 & -0.9340330 \\ 0.0205730 & 4.3241370 & -2.1584620 \\ -3.3748480 & -0.6072480 & -2.8487260 \\ -3.1376110 & -0.6340970 & -3.9206560 \\ -4.4028290 & -0.2378880 & -2.7420320 \\ -2.6961610 & 0.0943450 & -2.3571510 \\ -4.1215130 & -3.0155900 & -3.0080660 \\ -5.1771050 & -2.7359550 & -2.8893640 \\ -3.8824780 & -3.0130930 & -4.0802120 \\ -3.9970370 & -4.0358960 & -2.6252410 \\ 2.2679810 & 1.4992460 & -3.1984060 \\ 2.3629950 & 2.5675500 & -3.4343450 \\ 2.1111650 & 0.9557280 & -4.1395480 \\ 1.3872240 & 1.3538310 & -2.5643160 \\ 4.7663890 & 1.1100590 & -3.4108090 \\ 4.6054160 & 0.5452100 & -4.3387150 \\ 4.9498670 & 2.1595820 & -3.6757130 \\ 5.6652400 & 0.7213270 & -2.9154200 \\ -1.7170440 & -2.4849920 & 3.7512160 \\ -2.6092640 & -2.1775300 & 4.3125660 \\ -1.0454710 & -3.0079750 & 4.4450150 \\ -1.2091400 & -1.5873600 & 3.3824650\end{array}$

$\begin{array}{lr}\mathrm{C} & -2.8023360 \\ \mathrm{H} & -2.1651530 \\ \mathrm{H} & -3.7464660 \\ \mathrm{H} & -3.0302510 \\ \mathrm{C} & 3.1665110 \\ \mathrm{H} & 3.1930190 \\ \mathrm{H} & 3.4809190 \\ \mathrm{H} & 2.1358970 \\ \mathrm{C} & 5.5382820 \\ \mathrm{H} & 5.9252560 \\ \mathrm{H} & 5.5551090 \\ \mathrm{H} & 6.2141930 \\ \mathrm{C} & -7.2996500 \\ \mathrm{H} & -7.5131060 \\ \mathrm{H} & -6.8576230 \\ \mathrm{H} & -8.2463440 \\ \mathrm{C} & -6.0727200 \\ \mathrm{H} & -5.3893220 \\ \mathrm{H} & -7.0151670 \\ \mathrm{H} & -5.6207450 \\ \mathrm{C} & 3.5014620 \\ \mathrm{H} & 2.5765560 \\ \mathrm{H} & 3.7945020 \\ \mathrm{H} & 3.2950590 \\ \mathrm{C} & 5.9464040 \\ \mathrm{H} & 6.7588080 \\ \mathrm{H} & 5.8442460 \\ \mathrm{H} & 6.2237950 \\ \mathrm{C} & -3.2602720 \\ \mathrm{O} & -3.0624480 \\ \mathrm{C} & -4.6918410 \\ \mathrm{C} & -5.0147090 \\ \mathrm{C} & -5.7191860 \\ \mathrm{C} & -6.3466330 \\ \mathrm{H} & -4.2132750 \\ \mathrm{C} & -7.0502070 \\ \mathrm{H} & -5.4655230 \\ \mathrm{C} & -7.3665210 \\ \mathrm{H} & -6.5918520 \\ \mathrm{H} & -7.8409110 \\ \mathrm{H} & -8.4051430 \\ \mathrm{H} & -2.1329850 \\ \mathrm{O} & -2.3671480 \\ \mathrm{H} & -\end{array}$
$-4.6840340$
$-5.2077820$
$-4.4217110$
$-5.3706210$
1. 8204540
1. 2928960
2.8575100
1.8230700
1. 0771870
2. 0980840
0.5857700
0.5351250
$-1.9531530$
$-2.7191920$
$-2.4498520$
$-1.4905410$
0.2045790
0.9699270
0.6875980
$-0.2327820$
6.1389500
6.0590350
7.1963480
5.8043190
5. 5045000
4.9313700
5. 1689090
6. 5672070
2. 6803060
1. 5461220
3. 1045880
4.4002010
2. 2221740
4.8108760
5.0715930
2.6348900
1. 2192750
3. 9291520
5.8165970
1. 9468440
4. 2503920
1.1168690
3. 3679790

TS134(S, S) G[B97-D/def2-TZVP $]=-3310.748890$

$\begin{array}{lll}0 & 0.0953370 & -1.2158490\end{array}$
3.1027200

3. 8282670

3.5984260

2. 2771810

3.6413670

4. 6040860

3. 8160610

3. 2751680

3. 1974380

3. 3160040

4.1794970

2. 5253560

2. 1017800

1. 3458470

2. 9754200

2. 4124380

2.6025670

2. 2169670

2.8935550

3. 5021460

$-0.7523790$

$-0.1719420$

$-0.7939380$

$-1.7775470$

$-0.8805750$

$-0.4167940$

$-1.9211080$

$-0.8871270$

$-0.7393380$

$-0.1003900$

$-0.7863950$

$-1.2218460$

$-0.4116940$

$-1.2714450$

$-0.4659830$

$-0.0871980$

$-0.8933860$

$-1.6050780$

$-0.1750320$

$-0.9339170$

$-0.2561820$

$-1.2575920$

0.2671640 


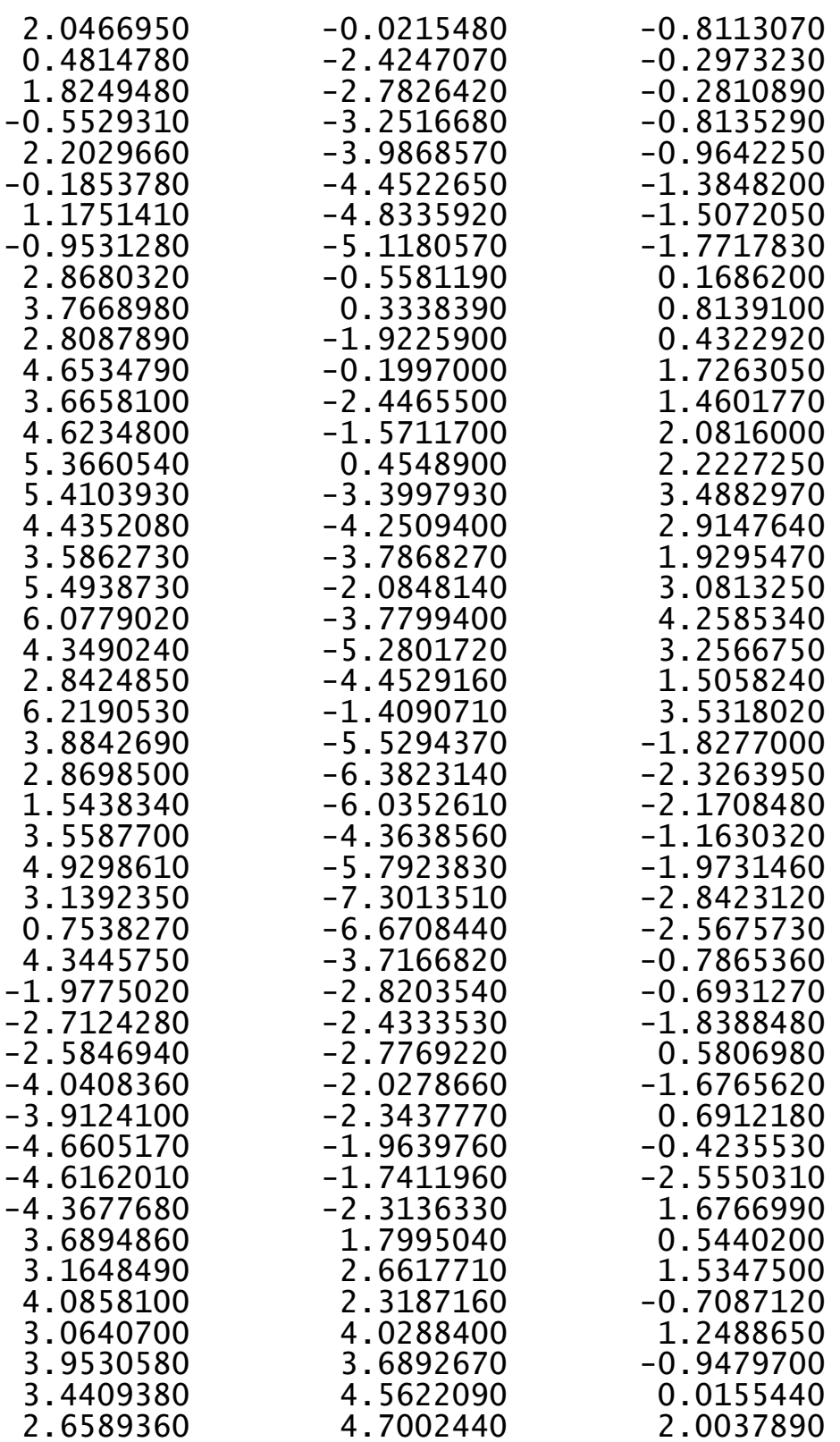

4. 2560740 $-1.8717910$ $-6.0933380$ $-2.1118960$ 2.7135690 3.2364200 4.6892830 6.1769560 6.2870430 6.7415590 6.6173390 3. 9119460 2.8575530 3.9726390 4.3384750 2. 0308710 1.8475200 2. 2238610 1.1252510 4.4924140 5.3523100 4.7521330 4.3143850 3.7734220 4.7467120 3.8997360 3.4640360 1. 3402640 1. 3825330 0.5910880 1.0245940 $-2.4543270$ $-3.5179330$ $-2.3655460$ $-1.9224500$ $-1.9273630$ $-1.5399410$ $-2.9580420$ $-1.3276390$ $-6.1392810$ $-7.1752930$ $-5.5771810$ $-5.6993100$ $-6.7822480$ $-6.7566560$ $-6.2947160$
4.0824610 $-3.2542040$ $-1.4561530$ $-2.4698390$ 2.1600920

6.0364950

1.4438460

1.7900090

2.8317930

1.6659230

1.1376720

1.5520660

1.2962190

2.5699170

0.8648810

6.2248810

7.2919460

5.7334310

5.7821170

6.7043770

6.5862670

. 2588210

7.7762150

2.4888920

2.0539810

3.5765390

.0970990

2.7151240

3.7985260

2. 5091670

. 2400870

$-4.6073930$

$-4.5004260$

$-5.3584930$

$-4.9729450$

$-2.2242700$

$-1.2552010$

$-2.0865770$

$-2.5768560$

0.0557910

0.4208640

0.6098040

0.2681730

$-1.7422310$

$-2.8119410$

$-1.1908570$
$-1.9156600$

1.8404450

$-0.3323830$

$-3.2388990$

2. 9017150

$-0.2849890$

$-1.7997010$

$-2.0075420$

$-2.3365670$

$-1.0745370$

$-2.7737540$

$-3.1242040$

$-2.9754160$

$-3.5318970$

$-3.8670780$

$-1.2287180$

$-1.4159400$

$-2.1917820$

$-0.7966840$

$-0.8722370$

$-0.2009770$

$-1.8411580$

$-1.0306870$

3.9722040

3.7145580

4.0593020

4.9508410

3. 3229660

3.4956000

2.5528640

4.2612920

2. 2953540

2. 5472980

1. 5000290

3.1842790

2. 9818610

2. 6512790

3. 3345220

3.8313110

$-0.6379200$

$-0.6327210$

0.1240990

$-1.6193050$

1.0085600

1.2518050

1.8227610 


$\begin{array}{rrr}-7.8295880 & -1.4181610 & 0.9652370 \\ -2.4256820 & -1.2065710 & -4.0599270 \\ -3.4971100 & -1.1345050 & -4.2893530 \\ -2.1159160 & -0.3079860 & -3.5192720 \\ -1.8835000 & -1.2430690 & -5.0138390 \\ -2.5898830 & -3.7242130 & -3.9978690 \\ -3.6810370 & -3.6976010 & -4.1229880 \\ -2.1287370 & -3.7668810 & -4.9939580 \\ -2.3331540 & -4.6420590 & -3.4557510 \\ 0.4491260 & 0.1472450 & -0.5582310 \\ -0.1648030 & 0.1541810 & -1.9290500 \\ 0.1372260 & 1.2708570 & 0.3922480 \\ -1.0233830 & -2.5275560 & -3.1388710 \\ -6.6647180 & -1.9665580 & -1.1229770 \\ -0.8159970 & -3.4215550 & 1.6089870 \\ 4.6421540 & 0.3987650 & -1.4773820 \\ 2.9995050 & 6.5363430 & 0.6653360 \\ 2.6169950 & 1.0708020 & 2.8529030 \\ -3.0231140 & 2.0296930 & 1.4199820 \\ -2.0392730 & 1.2027980 & 1.6771860 \\ -4.2356200 & 1.8281570 & 2.2686160 \\ -5.3614020 & 2.6417360 & 2.0575600 \\ -4.2873510 & 0.8107750 & 3.2371320 \\ -6.5234540 & 2.4386870 & 2.8004390 \\ -5.3136130 & 3.4213390 & 1.3026930 \\ -5.4528560 & 0.6088940 & 3.9758940 \\ -3.4218690 & 0.1757290 & 3.3908650 \\ -6.5719600 & 1.4206500 & 3.7592370 \\ -7.3926390 & 3.0698280 & 2.6307200 \\ -5.4910220 & -0.1854020 & 4.7178200 \\ -7.4808830 & 1.2582670 & 4.3344480 \\ -1.1804640 & 1.3194870 & 1.0968010 \\ -2.9993840 & 2.9095220 & 0.5388680 \\ -1.2623050 & 3.1318240 & -1.7779330 \\ -2.4644380 & 2.3207670 & -1.5313210 \\ -0.4438230 & 2.9674510 & -1.0773320 \\ -2.3037440 & 1.3182800 & -1.1487520 \\ -1.1489590 & 2.2676290 & -2.9573090 \\ -0.6722710 & 1.4119210 & -2.6266730 \\ -2.7258440 & 4.8120130 & -3.0072880 \\ -3.9594930 & 4.1958100 & -2.3294150 \\ -3.7839890 & 2.6821270 & -2.1194230 \\ -1.4698620 & 4.5868090 & -2.1486520 \\ -2.8759370 & 5.8875730 & -3.1592690 \\ -4.8613340 & 4.3734790 & -2.9271270 \\ -3.8559260 & 2.1459260 & -3.0779970\end{array}$

$\begin{array}{ll}\mathrm{H} & -1.5617420 \\ \mathrm{H} & -0.5665270 \\ \mathrm{H} & -2.5805120 \\ \mathrm{H} & -4.1092640 \\ \mathrm{H} & -4.5654340 \\ \mathrm{H} 44 & \end{array}$
5.1403930
4.9483170
4. 3567980
4.6683690
2. 2587340

$\operatorname{TS} 135(\mathrm{~S}, \mathrm{~S}) \mathrm{G}[\mathrm{B} 97-\mathrm{D} / \mathrm{def} 2-\mathrm{TZVP}]=-3310.748883$

$\begin{array}{lrcr}\text { TS135 }(\mathrm{S}, \mathrm{S}) \mathrm{G}[\mathrm{B} 97-\mathrm{D} / \mathrm{def}-\mathrm{TZV}] \mathrm{r}-3310.748883 \\ \mathrm{O} & 0.0279830 & 1.0737850 & 0.1582970 \\ \mathrm{O} & -2.0887350 & 0.1713160 & -0.9022130 \\ \mathrm{C} & -0.1603530 & 2.3254040 & -0.4081480 \\ \mathrm{C} & -1.4335370 & 2.8808470 & -0.4240480 \\ \mathrm{C} & 1.0028420 & 2.9971410 & -0.8735120 \\ \mathrm{C} & -1.6094350 & 4.1208100 & -1.1245680 \\ \mathrm{C} & 0.8354060 & 4.2299550 & -1.4665770 \\ \mathrm{C} & -0.4529570 & 4.7980130 & -1.6477090 \\ \mathrm{H} & 1.7057070 & 4.7850280 & -1.8080810 \\ \mathrm{C} & -2.8163080 & 0.8537810 & 0.0603950 \\ \mathrm{C} & -3.8517930 & 0.1318470 & 0.7142920 \\ \mathrm{C} & -2.5433430 & 2.1971230 & 0.2954890 \\ \mathrm{C} & -4.6320970 & 0.8126730 & 1.6248250 \\ \mathrm{C} & -3.3012200 & 2.8689730 & 1.3153620 \\ \mathrm{C} & -4.3779370 & 2.1657950 & 1.9609120 \\ \mathrm{H} & -5.4432720 & 0.2911480 & 2.1274300 \\ \mathrm{C} & -4.8534390 & 4.1197420 & 3.3387430 \\ \mathrm{C} & -3.7642170 & 4.7977420 & 2.7401570 \\ \mathrm{C} & -3.0092630 & 4.1889120 & 1.7574860 \\ \mathrm{C} & -5.1460010 & 2.8271040 & 2.9575770 \\ \mathrm{H} & -5.4448500 & 4.6130680 & 4.1069900 \\ \mathrm{H} & -3.5148740 & 5.8070300 & 3.0607960 \\ \mathrm{H} & -2.1748720 & 4.7229240 & 1.3160920 \\ \mathrm{H} & -5.9637000 & 2.2815130 & 3.4257450 \\ \mathrm{C} & -3.0202120 & 5.8860520 & -2.0426960 \\ \mathrm{C} & -1.8783600 & 6.5685950 & -2.5279340 \\ \mathrm{C} & -0.6227030 & 6.0304780 & -2.3342090 \\ \mathrm{C} & -2.8882300 & 4.6951330 & -1.3567570 \\ \mathrm{H} & -4.0107370 & 6.3014410 & -2.2154510 \\ \mathrm{H} & -1.9951010 & 7.5092960 & -3.0615940 \\ \mathrm{H} & 0.2625210 & 6.5365640 & -2.7159280 \\ \mathrm{H} & -3.7696250 & 4.1782890 & -0.9901040 \\ \mathrm{C} & 2.3400100 & 2.3816920 & -0.6117960 \\ \mathrm{C} & 3.0717660 & 1.7522250 & -1.6424150 \\ \mathrm{C} & 2.8383540 & 2.3840440 & 0.7114400 \\ \mathrm{C} & 4.2896560 & 1.1323800 & -1.3254440 \\ \mathrm{C} & 4.0553180 & 1.7508440 & 0.9754410 \\ \mathrm{C} & 4.7947030 & 1.1111920 & -0.0220170 \\ \mathrm{H} & 4.8523430 & 0.6487910 & -2.1187660\end{array}$

$-1.2037470$ $-2.6541440$ $-3.9960660$ $-1.4799190$ $-1.3498830$ 


$\begin{array}{lr}\mathrm{H} & 4.4381540 \\ \mathrm{C} & -4.0403840 \\ \mathrm{C} & -3.6452990 \\ \mathrm{C} & -4.5630300 \\ \mathrm{C} & -3.7936620 \\ \mathrm{C} & -4.6873020 \\ \mathrm{C} & -4.3014500 \\ \mathrm{H} & -3.4815440 \\ \mathrm{H} & -5.0873300 \\ \mathrm{C} & 2.1131250 \\ \mathrm{C} & 6.1347410 \\ \mathrm{C} & 2.5847420 \\ \mathrm{C} & -3.0662020 \\ \mathrm{C} & -4.3782710 \\ \mathrm{C} & -5.0173700 \\ \mathrm{C} & -6.5465960 \\ \mathrm{H} & -6.8511780 \\ \mathrm{H} & -7.0635420 \\ \mathrm{H} & -6.8721890 \\ \mathrm{C} & -4.2957650 \\ \mathrm{H} & -3.2098910 \\ \mathrm{H} & -4.5491570 \\ \mathrm{H} & -4.6012450 \\ \mathrm{C} & -5.1939400 \\ \mathrm{H} & -5.2582520 \\ \mathrm{H} & -4.7170830 \\ \mathrm{H} & -6.2108050 \\ \mathrm{C} & -2.9625010 \\ \mathrm{H} & -2.3905310 \\ \mathrm{H} & -2.4182690 \\ \mathrm{H} & -3.0149630 \\ \mathrm{C} & -4.1403340 \\ \mathrm{H} & -5.0125640 \\ \mathrm{H} & -4.4804030 \\ \mathrm{H} & -3.7305270 \\ \mathrm{C} & -1.8195560 \\ \mathrm{H} & -2.0732870 \\ \mathrm{H} & -1.0722280 \\ \mathrm{H} & -1.3815750 \\ \mathrm{C} & 2.9085910 \\ \mathrm{H} & 3.8936640 \\ \mathrm{H} & 3.0632580 \\ \mathrm{H} & 2.3705890 \\ \mathrm{C} & 1.8361510 \\ \mathrm{H} & 1.3085000 \\ \mathrm{H} & 2.7755100 \\ & \end{array}$

$\begin{array}{rr}1.7422210 & 1.9944090 \\ -1.3216860 & 0.4323700 \\ -2.2798080 & 1.3966480 \\ -1.7401480 & -0.8094720 \\ -3.6366920 & 1.0942600 \\ -3.1111850 & -1.0642970 \\ -4.0746760 & -0.1322780 \\ -4.3756660 & 1.8281950 \\ -3.4381780 & -2.0230330 \\ 3.0784550 & 1.8575580 \\ 0.4888560 & 0.3376220 \\ 1.7762530 & -3.0860210 \\ -1.8805540 & 2.7496030 \\ -5.5550500 & -0.4619400 \\ -0.7548180 & -1.8780880 \\ -0.8188200 & -2.0610280 \\ -1.8165010 & -2.4041450 \\ -0.6101080 & -1.1155650 \\ -0.0832650 & -2.8090540 \\ -0.9892720 & -3.2182290 \\ -0.9335330 & -3.0862370 \\ -1.9750680 & -3.6295600 \\ -0.2280580 & -3.9485320 \\ -6.3427560 & 0.5792860 \\ -7.4012880 & 0.2939440 \\ -6.2856300 & 1.5664300 \\ -5.9403100 & 0.6683720 \\ -6.1501410 & -0.6043410 \\ -5.6165060 & -1.3739200 \\ -6.0627680 & 0.3453030 \\ -7.2131070 & -0.8768490 \\ -1.9953400 & 3.8497830 \\ -1.3710910 & 3.6218120 \\ -3.0361520 & 3.9385100 \\ -1.6816450 & 4.8196500 \\ -2.6972590 & 3.1353540 \\ -3.7479380 & 3.3285320 \\ -2.6542860 & 2.3376540 \\ -2.2867950 & 4.0548300 \\ 4.3115930 & 2.3289080 \\ 4.0096990 & 2.7094780 \\ 5.0177520 & 1.5031470 \\ 4.8276240 & 3.1357210 \\ 2.1260600 & 3.0341610 \\ 1.2292290 & 2.6926340 \\ 1.8183080 & 3.5113030\end{array}$

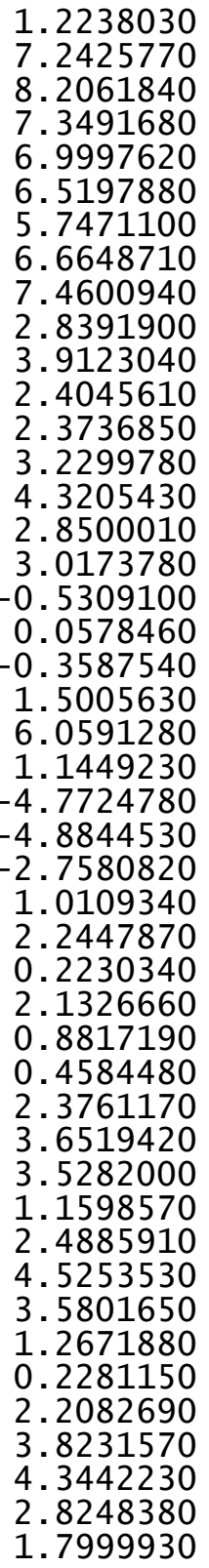

2.6334190

1. 5616870

1.1388920

1.9394550

2. 4097050

$-0.7196310$

$-1.4942850$

$-0.4328930$

$-1.1527940$

0.4676300

0.2875060

$-0.3831990$

0.5265290

2. 9560220

2.8274600

3. 0037950

3. 9106880

$-0.2261630$

$-0.3313660$

$-1.3719050$

1. 9303980

0.1428650

3.4379710

0.2597490

$-5.6507390$

$-0.8312990$

$-2.5631070$

$-3.1159750$

$-1.5333650$

$-2.5553890$

$-1.6585260$

$-5.1485320$

$-4.5313670$

$-3.0033180$

$-4.8309900$

$-6.2356030$

$-4.7755940$

$-2.5303750$

$-5.3311170$

$-5.1906420$

$-4.7502610$

$-2.5642020$

$-2.1581230$
3.7912180

0.2800200

0.5962270

$-0.7461130$

0.9320510

$-0.5249300$

$-0.4768030$

$-1.5746370$

$-0.1616400$

$-3.8501180$

$-3.9976520$

$-3.3203690$

$-4.8421810$

$-3.8421740$

$-3.8809660$

$-4.8717810$

$-3.3481130$

$-0.6588690$

$-2.0374400$

0.3003450

$-3.0702200$

1. 3773920

1.4970220

$-1.5473730$

$-1.4333260$

2.6920390

$-1.7450820$

$-1.5965550$

$-1.0301000$

$-1.2741220$

$-2.9713020$

$-2.6779450$

$-2.9206480$

$-2.3264510$

$-2.2097290$

$-2.0340960$

$-3.0103930$

$-2.9425690$

$-3.2014320$

$-1.0614280$

$-2.4878120$

$-3.9301790$

$-1.3247430$

$-1.6272670$

1. 3937570

1.6577970 


\begin{tabular}{|c|c|c|c|c|c|c|c|}
\hline$C$ & 1 & & 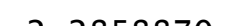 & & 2 & & 15127180 \\
\hline c & $\begin{array}{l}4.0038980 \\
5.1750920\end{array}$ & $\begin{array}{l}-1.9398300 \\
-2.6827690\end{array}$ & $\begin{array}{l}2.2858870 \\
2.0665390\end{array}$ & $\begin{array}{l}\mathrm{H} \\
\mathrm{C}\end{array}$ & $\begin{array}{r}-2.3 / 89 / 40 \\
3.0425320\end{array}$ & $\begin{array}{l}4.5074170 \\
1.8647200\end{array}$ & $\begin{array}{r}1.5127180 \\
-0.6275040\end{array}$ \\
\hline C & 3.9768110 & -0.9781180 & 3.3098590 & $\mathrm{C}$ & 3.3631220 & 1.9249060 & -2.0018640 \\
\hline C & 6.3058870 & -2.4602610 & 2.8507490 & $\mathrm{C}$ & 3.8866270 & 1.1709550 & 0.2680760 \\
\hline $\mathrm{H}$ & 5.1884610 & -3.4231460 & 1.2723480 & $\mathrm{C}$ & 4.5646420 & 1.3508930 & -2.4367840 \\
\hline C & 5.1102670 & -0.7557310 & 4.0909320 & $\mathrm{c}$ & 5.0740450 & 0.6120280 & -0.2167090 \\
\hline $\mathrm{H}$ & 3.0743470 & -0.4012290 & 3.4757120 & $\mathrm{C}$ & 5.4459510 & 0.7135200 & -1.5609530 \\
\hline C & 6.2772640 & -1.4933410 & 3.8616350 & $\mathrm{H}$ & 4.8323750 & 1.4150570 & -3.4895040 \\
\hline $\mathrm{H}$ & 7.2117600 & -3.0342210 & 2.6704620 & $\mathrm{H}$ & 5.7349930 & 0.1016890 & 0.4812570 \\
\hline $\mathrm{H}$ & 5.0856640 & -0.0036180 & 4.8762290 & C & -4.2064220 & -0.4064560 & -0.2896700 \\
\hline $\mathrm{H}$ & 7.1620430 & -1.3147780 & 4.4688680 & $\mathrm{c}$ & -4.2439640 & -1.8069920 & -0.0982960 \\
\hline $\mathrm{H}$ & 0.9688890 & -1.4854880 & 1.0470370 & $\mathrm{C}$ & -4.8466930 & 0.1740820 & -1.4088240 \\
\hline 0 & 2.8648220 & -3.0086220 & 0.4861390 & $\mathrm{C}$ & -4.9478760 & -2.5908300 & -1.0216450 \\
\hline & & & & C & -5.5310080 & -0.6544170 & -2.3022480 \\
\hline & S) G[B97-D/ & {$[\mathrm{ZVP}]=-331$} & 28 & $\mathrm{C}$ & -5.5967210 & -2.0388170 & -2.1269320 \\
\hline 0 & 0.5507550 & 0.6408270 & 0.1149150 & $\mathrm{H}$ & -4.9834250 & -3.6698050 & -0.8839880 \\
\hline 0 & -1.7755320 & 0.9518260 & -0.8949960 & $\mathrm{H}$ & -6.0289200 & -0.2031420 & -3.1579660 \\
\hline C & 0.6453160 & 2.0165900 & 0.2585150 & $\mathrm{P}$ & -0.4753030 & -0.0162490 & -0.9963760 \\
\hline C & -0.4257920 & 2.7183400 & 0.8049480 & 0 & 0.0659650 & 0.1314450 & -2.3874690 \\
\hline C & 1.8724140 & 2.6339000 & -0.1025780 & 0 & -0.7488630 & -1.3959770 & -0.4665380 \\
\hline C & -0.3324990 & 4.1469460 & 0.8927080 & $\mathrm{C}$ & 3.5673910 & 1.0488820 & 1.7526210 \\
\hline C & 1.9861920 & 3.9978290 & 0.0799640 & $\mathrm{H}$ & 2.6116900 & 1.5466990 & 1.9453300 \\
\hline C & 0.9055050 & 4.7864300 & 0.5432740 & $\mathrm{C}$ & 6.7815010 & 0.1799570 & -2.0527480 \\
\hline $\mathrm{H}$ & 2.9276260 & 4.4873180 & -0.1581420 & $\mathrm{H}$ & 6.8594500 & 0.4297590 & -3.1203140 \\
\hline C & -2.3038390 & 1.1394970 & 0.3827500 & C & 2.4641650 & 2.6403840 & -3.0030870 \\
\hline C & -3.51 & 0.4666460 & 0.7027750 & H & 1.4683790 & 2.7302940 & -2.5553210 \\
\hline C & -1.6410510 & 1.9913530 & 1.2569250 & C & -4.8482130 & 1.6755310 & -1.6685960 \\
\hline c & -4.0346730 & 0.6634950 & 1.9656630 & $\mathrm{H}$ & -4.2504790 & 2.1697940 & -0.8965230 \\
\hline C & -2.1435150 & 2.1146470 & 2.5953970 & C & -6.3453010 & -2.9158190 & -3.1147920 \\
\hline C & -3.37 & 1.4496910 & 2.9390070 & $\mathrm{H}$ & -6.2390640 & -3.9571220 & -2.7779760 \\
\hline $\mathrm{H}$ & -4.9 & 0.1833300 & 2.2323830 & c & -3.5555650 & -2.4910440 & 1.0777050 \\
\hline C & -3.208 & 2.2997920 & 5.2169620 & $\mathrm{H}$ & -2.8338010 & -1.7879580 & 1.5052730 \\
\hline C & -1.979 & 2.9235380 & 4.8913220 & C & -2.7717050 & -3.7494240 & 0.6604350 \\
\hline C & -1.46 & 2.8338420 & 3.6146910 & $\mathrm{H}$ & -2.1956340 & -4.1221690 & 1.5168540 \\
\hline C & -3.8 & 1.5749570 & 4.2581870 & $\mathrm{H}$ & -3.4485480 & -4.5513140 & 0.3377680 \\
\hline $\mathrm{H}$ & -3.6 & 2.3842800 & 6.2247470 & H & -2.0806980 & -3.5194890 & -0.1558060 \\
\hline $\mathrm{H}$ & -1.436 & 3.4758610 & 5.6550300 & C & -4.5769800 & -2.8544020 & 2.1745380 \\
\hline H & -0.517 & 3.3151960 & 3.3794810 & $\mathrm{H}$ & -5.3178770 & -3.5640050 & 1.7815690 \\
\hline H & -4.8207290 & 1.0730550 & 4.4988160 & $\mathrm{H}$ & -4.0688340 & -3.3242030 & 3.0280330 \\
\hline C & -1.291 & 6.3420790 & 1.3555440 & $\mathrm{H}$ & -5.1131540 & -1.9688580 & 2.5346730 \\
\hline C & -0.057 & 6.9658640 & 1.0503640 & C & 3.4057410 & -0.4221230 & 2.1762710 \\
\hline C & 1.016 & 6.1996260 & 0.6483190 & $\mathrm{H}$ & 4.3370220 & -0.9815730 & 2.0168910 \\
\hline C & -1.425 & 4.9703250 & 1.2799410 & $\mathrm{H}$ & 3.1485000 & -0.4869450 & 3.2411850 \\
\hline H & -2.145 & 6.9503830 & 1.6472020 & $\mathrm{H}$ & 2.6048490 & -0.9013340 & 1.6049670 \\
\hline H & 0.035 & 8.0474080 & 1.1200500 & C & 4.6335080 & 1.7534340 & 2.6125770 \\
\hline $\mathrm{H}$ & 1.9655780 & 6.6657170 & 0.3887400 & $\mathrm{H}$ & 4.3681710 & 1.6883270 & 3.6761280 \\
\hline
\end{tabular}




$\begin{array}{lrrr}\text { H } & 5.6163070 & 1.2838110 & 2.4746660 \\ \text { H } & 4.7199930 & 2.8120660 & 2.3370570 \\ \mathrm{C} & -4.2129930 & 2.0146730 & -3.0301670 \\ \mathrm{H} & -4.8076330 & 1.5935520 & -3.8516020 \\ \mathrm{H} & -4.1697990 & 3.1037920 & -3.1651790 \\ \mathrm{H} & -3.1965420 & 1.6116490 & -3.0940510 \\ \mathrm{C} & -6.2755720 & 2.2487680 & -1.5722190 \\ \mathrm{H} & -6.2605250 & 3.3354450 & -1.7313080 \\ \mathrm{H} & -6.9241530 & 1.7989200 & -2.3353380 \\ \mathrm{H} & -6.7147790 & 2.0441240 & -0.5873670 \\ \mathrm{C} & 2.2998720 & 1.8709640 & -4.3254950 \\ \mathrm{H} & 1.5560970 & 2.3797700 & -4.9516440 \\ \mathrm{H} & 3.2422620 & 1.8361940 & -4.8877050 \\ \mathrm{H} & 1.9591830 & 0.8472220 & -4.1478670 \\ \mathrm{C} & 2.9922070 & 4.0609930 & -3.2890380 \\ \mathrm{H} & 3.9985900 & 4.0084680 & -3.7266100 \\ \mathrm{H} & 2.3321430 & 4.5748220 & -4.0008490 \\ \mathrm{H} & 3.0469280 & 4.6594080 & -2.3730430 \\ \mathrm{C} & -7.8479130 & -2.5755830 & -3.1411300 \\ \mathrm{H} & -8.2931780 & -2.6812790 & -2.1438000 \\ \mathrm{H} & -7.9988390 & -1.5399140 & -3.4735410 \\ \mathrm{H} & -8.3802030 & -3.2389380 & -3.8361980 \\ \mathrm{C} & -5.7436080 & -2.8092370 & -4.5291740 \\ \mathrm{H} & -4.6798310 & -3.0776040 & -4.5246950 \\ \mathrm{H} & -6.2732960 & -3.4756590 & -5.2232370 \\ \mathrm{H} & -5.8329310 & -1.7818830 & -4.9057880 \\ \mathrm{C} & 6.8877850 & -1.3505350 & -1.9224080 \\ \mathrm{H} & 6.1154290 & -1.8523840 & -2.5169020 \\ \mathrm{H} & 7.8699960 & -1.6957400 & -2.2713140 \\ \mathrm{H} & 6.7704940 & -1.6598440 & -0.8753400 \\ \mathrm{C} & 7.9524330 & 0.8630300 & -1.3198720 \\ \mathrm{H} & 7.9017180 & 1.9532580 & -1.4309320 \\ \mathrm{H} & 7.9233000 & 0.6284160 & -0.2477780 \\ \mathrm{H} & 8.9122930 & 0.5118120 & -1.7211410 \\ \mathrm{C} & 1.5059690 & -2.7632610 & -2.2161990 \\ \mathrm{C} & 2.2777180 & -2.2169660 & -1.0921520 \\ \mathrm{H} & 0.4249780 & -2.7594340 & -2.0767840 \\ \mathrm{H} & 1.8265450 & -1.4274430 & -0.5051660 \\ \mathrm{O} & 1.9706420 & -1.5309260 & -2.8633600 \\ \mathrm{H} & 1.2282100 & -0.8249920 & -2.7134550 \\ \mathrm{C} & 3.5863190 & -4.0271140 & -2.9407940 \\ \mathrm{C} & 4.1704710 & -3.8333510 & -1.5336020 \\ \mathrm{C} & 3.7216530 & -2.4980500 & -0.9203790 \\ \mathrm{C} & 2.0486920 & -4.0059940 & -2.8930430 \\ \mathrm{H} & 3.9259970 & -4.9801450 & -3.3634360 \\ \mathrm{H} & 5.2657030 & -3.8631930 & -1.5605390 \\ & & & \\ & & & \end{array}$

\begin{abstract}
4.2531730
1. 6822570

1.6156340

3.9417660

3.8294430

3.9465810

1.0937630

0.1688020

1. 5122800

2.5469280

0.9072230

2. 9719070

3.0080190

1. 3355680

0.1091550

2. 3671220

3.7737430

0.8646680

2.6988070

$-0.1643930$

1. 6137420
\end{abstract}

144

TS137(S,S) G[B97-D/def2-TZVP] $=-3310.748734$

$\begin{array}{lrrr}\text { TS137 (S, S }) \text { G }[\mathrm{B} 97-\mathrm{D} / \mathrm{def} 2-\mathrm{TZVP}]=-3310.748734 \\ \mathrm{O} & 0.0104660 & -1.1904270 & 0.2284350 \\ \mathrm{O} & 2.0452690 & -0.1006250 & -0.8195750 \\ \mathrm{C} & 0.3667260 & -2.4235180 & -0.2989950 \\ \mathrm{C} & 1.6930390 & -2.8332900 & -0.2286860 \\ \mathrm{C} & -0.6824380 & -3.2244800 & -0.8256030 \\ \mathrm{C} & 2.0462730 & -4.0663970 & -0.8718820 \\ \mathrm{C} & -0.3434970 & -4.4472800 & -1.3659780 \\ \mathrm{C} & 1.0050570 & -4.8815530 & -1.4376890 \\ \mathrm{H} & -1.1242200 & -5.0943020 & -1.7590760 \\ \mathrm{C} & 2.8155430 & -0.6443500 & 0.1988470 \\ \mathrm{C} & 3.7462050 & 0.2209640 & 0.8344940 \\ \mathrm{C} & 2.6843600 & -1.9954560 & 0.5001180 \\ \mathrm{C} & 4.5714740 & -0.3212490 & 1.7966170 \\ \mathrm{C} & 3.4788410 & -2.5244930 & 1.5737640 \\ \mathrm{C} & 4.4535660 & -1.6741560 & 2.2035150 \\ \mathrm{H} & 5.3187700 & 0.3068790 & 2.2750030 \\ \mathrm{C} & 5.1006280 & -3.4905760 & 3.6945110 \\ \mathrm{C} & 4.1099310 & -4.3151630 & 3.1087330 \\ \mathrm{C} & 3.3210310 & -3.8450470 & 2.0776220 \\ \mathrm{C} & 5.2611310 & -2.1945220 & 3.2510400 \\ \mathrm{H} & 5.7201260 & -3.8757310 & 4.5014800 \\ \text { H } & 3.9642790 & -5.3283220 & 3.4774480 \\ \mathrm{H} & 2.5640940 & -4.4898330 & 1.6440990\end{array}$

$-1.3735910$ $-2.3162380$ $-3.8988810$ $-3.6040270$ $-0.8849880$

0.1494010

1.6347220

1.7032910

2.9605120

3.0084020

4.1562910

4.2320000

2.0787850

5.3790340

4.1202800

5.4197090

4.2617710

6.3011010

6.3745180

0.7778730

0.5828390
2. 5640940

$-4.4898330$

1.6440990

8877850

.8699960

.9233000

0.4249780

3.5863190

3.9259970

$-3.8631930$

$-1.5605390$ 


\begin{tabular}{|c|c|c|c|}
\hline $\begin{array}{l}\mathrm{H} \\
\mathrm{C} \\
\mathrm{C} \\
\mathrm{C} \\
\mathrm{C} \\
\mathrm{H} \\
\mathrm{H} \\
\mathrm{H} \\
\mathrm{H} \\
\mathrm{C} \\
\mathrm{C} \\
\mathrm{C} \\
\mathrm{C} \\
\mathrm{C} \\
\mathrm{C} \\
\mathrm{H} \\
\mathrm{H} \\
\mathrm{C} \\
\mathrm{C} \\
\mathrm{C} \\
\mathrm{C} \\
\mathrm{C} \\
\mathrm{C} \\
\mathrm{H} \\
\mathrm{H} \\
\mathrm{C} \\
\mathrm{C} \\
\mathrm{C} \\
\mathrm{C} \\
\mathrm{C} \\
\mathrm{C} \\
\mathrm{C} \\
\mathrm{H} \\
\mathrm{H} \\
\mathrm{H} \\
\mathrm{C} \\
\mathrm{H} \\
\mathrm{H} \\
\mathrm{H} \\
\mathrm{C} \\
\mathrm{H} \\
\mathrm{H} \\
\mathrm{H} \\
\mathrm{C} \\
\mathrm{H} \\
\mathrm{H}\end{array}$ & $\begin{array}{r}6.0008360 \\
3.6934850 \\
2.6641850 \\
1.3487320 \\
3.3916930 \\
4.7318700 \\
2.9144900 \\
0.5487840 \\
4.1884400 \\
-2.0959200 \\
-2.8043810 \\
-2.7182820 \\
-4.1265800 \\
-4.0391190 \\
-4.7637650 \\
-4.6841950 \\
-4.5094220 \\
3.8088000 \\
3.2128720 \\
4.4194440 \\
3.2560310 \\
4.4366300 \\
3.8556330 \\
2.7893530 \\
4.9063820 \\
-2.0286230 \\
-6.1870190 \\
-2.1826440 \\
2.5452160 \\
3.8195480 \\
5.0664740 \\
6.5864870 \\
6.7987000 \\
7.0558960 \\
7.0473540 \\
4.4175690 \\
3.3403790 \\
4.5726200 \\
4.8669410 \\
4.3665260 \\
4.3697030 \\
3.7411770 \\
5.3900880 \\
2.3863590 \\
2.0086670 \\
1.7111110\end{array}$ & $\begin{array}{l}-1.5388940 \\
-5.6944070 \\
-6.5137500 \\
-6.1096330 \\
-4.5032310 \\
-6.0036760 \\
-7.4530700 \\
-6.7207550 \\
-3.8819680 \\
-2.7532900 \\
-2.3258820 \\
-2.7207240 \\
-1.8947820 \\
-2.2655180 \\
-1.8494150 \\
-1.5767470 \\
-2.2488850 \\
1.6617120 \\
2.6452410 \\
2.0296680 \\
3.9805400 \\
3.3812080 \\
4.3692560 \\
4.7396300 \\
3.6700400 \\
-3.2322540 \\
-1.3165160 \\
-2.3545640 \\
2.2829450 \\
5.8158360 \\
1.0110920 \\
1.2445470 \\
2.2343060 \\
1.1916570 \\
0.4862160 \\
1.0220300 \\
0.8369150 \\
1.9900460 \\
0.2427950 \\
6.7871990 \\
7.8143280 \\
6.7688120 \\
6.5178330 \\
6.2113410 \\
5.5448930 \\
6.1363790\end{array}$ & $\begin{array}{r}3.7075610 \\
-1.6384050 \\
-2.1622180 \\
-2.0651700 \\
-1.0087580 \\
-1.7366880 \\
-2.6504850 \\
-2.4800110 \\
-0.6120170 \\
-0.7217420 \\
-1.8686120 \\
0.5454450 \\
-1.7163230 \\
0.6460350 \\
-0.4717030 \\
-2.5956220 \\
1.6249530 \\
0.4446260 \\
1.2702880 \\
-0.7716250 \\
0.8584570 \\
-1.1383470 \\
-0.3440140 \\
1.4804900 \\
-2.0777100 \\
1.8048250 \\
-0.3868210 \\
-3.2593180 \\
2.5915960 \\
-0.8046970 \\
-1.7004630 \\
-1.7988120 \\
-2.2245110 \\
-0.8080120 \\
-2.4461670 \\
-3.0968940 \\
-3.0231280 \\
-3.5916530 \\
-3.7270400 \\
0.2566820 \\
-0.1317710 \\
1.1586630 \\
0.5459060 \\
-1.2147950 \\
-2.0003000 \\
-0.3520730\end{array}$ \\
\hline
\end{tabular}

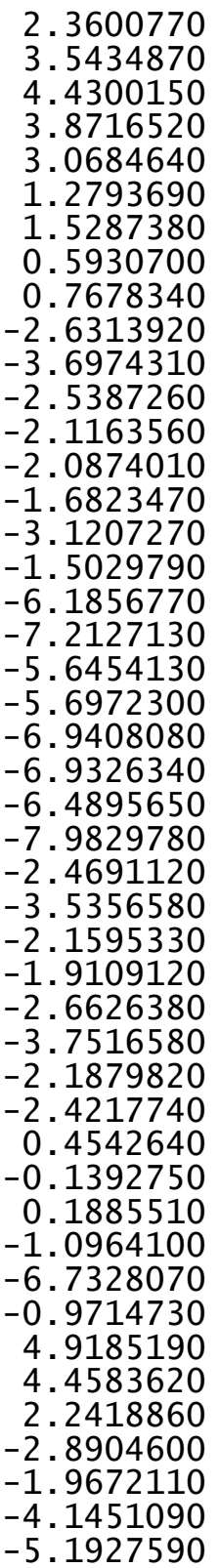

7.2449170

2. 4306510

1.8046950

3.4759890

2. 1421450

3. 1072420

4. 1512520

3. 0877280

2. 6869460

$-4.5881130$

$-4.4752560$

$-5.3235370$

$-4.9779350$

$-2.2255700$

$-1.2551470$

$-2.0826340$

$-2.6020980$

0.2207600

0.6106620

0.6738990

0.5260300

$-1.7300030$

$-2.8190760$

$-1.2685180$

$-1.3914470$

$-1.0804850$

$-0.9953590$

$-0.1894150$

$-1.1137110$

$-3.5968900$

$-3.5581450$

$-3.6349800$

$-4.5217290$

0.1509940

0.1984930

1. 2819970

$-2.4266850$

$-1.7234520$

$-3.4058250$

0.0110080

5. 8934790

1.2317110

2.1521390

1.2543420

1.9817480

2. 9016920
$-1.5863810$

3.7582980

3.6094590

3.8399300

4.7060750

2.8880540

3. 1204740

2.0368670

3.7635190

2. 2236780

2. 4626530

1.4143070

3. 1122590

2. 9663870

2.6609450

3. 3093290

3. 8161460

$-0.5252270$

$-0.5238320$

0.3148090

$-1.4583790$

0.8848130

1. 0185010

1.7725110

0.8268520

$-4.0732910$

$-4.3203990$

$-3.5198600$

$-5.0179570$

$-4.0367990$

$-4.1766610$

$-5.0267130$

$-3.4994060$

$-0.5910190$

$-1.9689900$

0.3647020

$-3.1427250$

$-1.2516590$

1.5847270

$-1.2794760$

$-1.6962830$

2. 5343960

1.4397550

1.6812490

2. 2296990

2.0554830 


\begin{tabular}{|c|c|c|c|c|c|c|c|}
\hline C & -4.3203670 & 0.8858160 & 3.0922250 & $\mathrm{H}$ & -5.2794880 & 0.6673290 & 2.2586390 \\
\hline C & -6.4002550 & 2.7259060 & 2.7294050 & $\mathrm{C}$ & -4.0715240 & 4.2423970 & 3.7567980 \\
\hline $\mathrm{H}$ & -5.0487570 & 3.7422980 & 1.3828090 & $\mathrm{C}$ & -2.9175010 & 4.8049560 & 3.1599510 \\
\hline C & -5.5324780 & 0.7099240 & 3.7588340 & $\mathrm{C}$ & -2.3013190 & 4.1821950 & 2.0927480 \\
\hline $\mathrm{H}$ & -3.5149760 & 0.1705140 & 3.2168010 & $\mathrm{C}$ & -4.5683190 & 3.0445040 & 3.2877230 \\
\hline C & -6.5738890 & 1.6271970 & 3.5784600 & $\mathrm{H}$ & -4.5524110 & 4.7472390 & 4.5917840 \\
\hline $\mathrm{H}$ & -7.2081960 & 3.4402890 & 2.5898440 & $\mathrm{H}$ & -2.5079230 & 5.7350470 & 3.5482850 \\
\hline $\mathrm{H}$ & -5.6679680 & -0.1461890 & 4.4158030 & $\mathrm{H}$ & -1.4154550 & 4.6267280 & 1.6517440 \\
\hline $\mathrm{H}$ & -7.5193000 & 1.4851240 & 4.0974810 & $\mathrm{H}$ & -5.4395940 & 2.5852510 & 3.7518200 \\
\hline $\mathrm{H}$ & -1.0972310 & 1.3490460 & 1.1095610 & $\mathrm{C}$ & -2.3261640 & 6.1676900 & -1.5673170 \\
\hline 0 & -2.7793660 & 3.0706170 & 0.6046350 & $\mathrm{C}$ & -1.1355990 & 6.7436940 & -2.0734390 \\
\hline C & -1.1380910 & 3.2132930 & -1.7715880 & $\mathrm{C}$ & 0.0512000 & 6.0441770 & -1.9985480 \\
\hline $\mathrm{C}$ & -2.3598490 & 2.4485170 & -1.4756000 & $\mathrm{C}$ & -2.3084480 & 4.9182470 & -0.9798870 \\
\hline $\mathrm{H}$ & -0.3012160 & 3.0166850 & -1.1019330 & $\mathrm{H}$ & -3.2645710 & 6.7123560 & -1.6463400 \\
\hline $\mathrm{H}$ & -2.2205040 & 1.4388440 & -1.1029190 & $\mathrm{H}$ & -1.1622800 & 7.7310770 & -2.5291480 \\
\hline 0 & -1.0988060 & 2.3507020 & -2.9563510 & $\mathrm{H}$ & 0.9713380 & 6.4675470 & -2.3979940 \\
\hline $\mathrm{H}$ & -0.6385750 & 1.4800100 & -2.6474870 & $\mathrm{H}$ & -3.2268830 & 4.4846300 & -0.5966650 \\
\hline C & -2.5878850 & 4.9522650 & -2.9343730 & $\mathrm{C}$ & 2.6270910 & 1.9439390 & -0.7874360 \\
\hline C & -3.8154830 & 4.3787490 & -2.2094400 & $\mathrm{C}$ & 3.2661740 & 1.4669620 & -1.9490960 \\
\hline C & -3.6870020 & 2.8589100 & -2.0118050 & $\mathrm{C}$ & 3.1846280 & 1.6751980 & 0.4892410 \\
\hline C & -1.3073050 & 4.6768690 & -2.1281730 & $\mathrm{C}$ & 4.4590910 & 0.7376710 & -1.8174920 \\
\hline $\mathrm{H}$ & -2.7042560 & 6.0333800 & -3.0761960 & $\mathrm{C}$ & 4.3497970 & 0.9119560 & 0.5671320 \\
\hline $\mathrm{H}$ & -4.7333090 & 4.5920460 & -2.7699830 & C & 5.0139610 & 0.4380740 & -0.5735790 \\
\hline $\mathrm{H}$ & -3.8128410 & 2.3309820 & -2.9696050 & $\mathrm{H}$ & 4.9500690 & 0.3899830 & -2.7206580 \\
\hline $\mathrm{H}$ & -1.3400020 & 5.2276710 & -1.1779280 & $\mathrm{H}$ & 4.7711650 & 0.6916580 & 1.5440020 \\
\hline $\mathrm{H}$ & -0.4128810 & 5.0085240 & -2.6690720 & C & -4.2279310 & -0.9707750 & 0.3773590 \\
\hline $\mathrm{H}$ & -2.4995990 & 4.4972140 & -3.9300030 & $\mathrm{C}$ & -3.9663560 & -2.0708610 & 1.2242370 \\
\hline $\mathrm{H}$ & -3.9081570 & 4.8515980 & -1.2231190 & $\mathrm{C}$ & -4.9202240 & -1.1585830 & -0.8395130 \\
\hline $\mathrm{H}$ & -4.4594530 & 2.4600310 & -1.3457320 & C & -4.4353820 & -3.3350990 & 0.8449630 \\
\hline & & & & $\mathrm{C}$ & -5.3618370 & -2.4415970 & -1.1750270 \\
\hline & s) $G[B 97-D$ & $Z V P]=-3$ & 718 & $\mathrm{C}$ & -5.1332000 & -3.5429270 & -0.3458240 \\
\hline 0 & 0.2021100 & 0.8576790 & 0.0825830 & $\mathrm{H}$ & -4.2424470 & -4.1900860 & 1.4903460 \\
\hline 0 & -2.0726700 & 0.3096050 & -0.9106450 & $\mathrm{H}$ & -5.8990580 & -2.5832980 & -2.1104600 \\
\hline C & 0.1465480 & 2.1619270 & -0.3848410 & C & 2.6118260 & 2.3172320 & 1.7490570 \\
\hline C & -1.0432640 & 2.8710100 & -0.2782720 & C & 6.2986450 & -0.3689530 & -0.4049570 \\
\hline C & 1.3577050 & 2.7253580 & -0.8729850 & $\mathrm{C}$ & 2.7283440 & 1.7532940 & -3.3460060 \\
\hline C & -1.1011380 & 4.1764410 & -0.8720410 & C & -3.2034710 & -1.9199380 & 2.5354970 \\
\hline C & 1.3128370 & 4.0150110 & -1.3596900 & C & -5.6088950 & -4.9297650 & -0.7401080 \\
\hline C & 0.1006680 & 4.7494760 & -1.4149740 & C & -5.2244870 & -0.0089880 & -1.7911100 \\
\hline $\mathrm{H}$ & 2.2251900 & 4.4817520 & -1.7231350 & C & -6.7427010 & 0.2386530 & -1.8838940 \\
\hline C & -2.6628210 & 1.0087180 & 0.1327050 & $\mathrm{H}$ & -7.2546030 & -0.6461420 & -2.2848460 \\
\hline C & -3.7726640 & 0.3948770 & 0.7729720 & $\mathrm{H}$ & -7.1646390 & 0.4573280 & -0.8945290 \\
\hline C & -2.1935620 & 2.2776570 & 0.4551920 & $\mathrm{H}$ & -6.9504540 & 1.0872310 & -2.5496420 \\
\hline C & -4.4113110 & 1.1027370 & 1.7697500 & $\mathrm{C}$ & -4.6202220 & -0.2521400 & -3.1863560 \\
\hline C & -2.8028990 & 2.9610250 & 1.5631320 & $\mathrm{H}$ & -3.5373420 & -0.4020950 & -3.1166640 \\
\hline C & -3 & 3681100 & 2.2039300 & $\mathrm{H}$ & -5.0666960 & -1.1391250 & -3.6548800 \\
\hline
\end{tabular}




$\begin{array}{rrr}-4.8152450 & 0.6109280 & -3.8369490 \\ -4.8912810 & -5.4207700 & -2.0126700 \\ -5.2109010 & -6.4405930 & -2.2667270 \\ -5.1287110 & -4.7651830 & -2.8609350 \\ -3.8027020 & -5.4164570 & -1.8746080 \\ -7.1369390 & -4.9766990 & -0.9290990 \\ -7.6556730 & -4.6483330 & -0.0196040 \\ -7.4424350 & -4.3175240 & -1.7522850 \\ -7.4627920 & -5.9973100 & -1.1708070 \\ -4.1793250 & -1.8794500 & 3.7287610 \\ -4.8961810 & -1.0560430 & 3.6306050 \\ -4.7450730 & -2.8195300 & 3.7852690 \\ -3.6277370 & -1.7507720 & 4.6702370 \\ -2.1533800 & -3.0248030 & 2.7497230 \\ -2.6290780 & -4.0004270 & 2.9145930 \\ -1.4880750 & -3.0956370 & 1.8842280 \\ -1.5520950 & -2.7923120 & 3.6384580 \\ 3.2856170 & 3.6897500 & 1.9586560 \\ 4.3638340 & 3.5611360 & 2.1253460 \\ 3.1463220 & 4.3320040 & 1.0802730 \\ 2.8554310 & 4.1952770 & 2.8339310 \\ 2.7246010 & 1.4554200 & 3.0134880 \\ 2.2383730 & 0.4869980 & 2.8686190 \\ 3.7685900 & 1.2802680 & 3.3028720 \\ 2.2331560 & 1.9713070 & 3.8486150 \\ 7.3560730 & 0.4309060 & 0.3856790 \\ 8.2500350 & -0.1841490 & 0.5540080 \\ 7.6512540 & 1.3238100 & -0.1815970 \\ 6.9707070 & 0.7553340 & 1.3583930 \\ 6.9134120 & -0.8668760 & -1.7201040 \\ 6.2095520 & -1.4748580 & -2.2990530 \\ 7.2229380 & -0.0191050 & -2.3457630 \\ 7.8014910 & -1.4745690 & -1.5081480 \\ 2.7218230 & 0.5145860 & -4.2584050 \\ 3.7421470 & 0.1683320 & -4.4717540 \\ 2.1598330 & -0.3052920 & -3.8038250 \\ 2.2509530 & 0.7670980 & -5.2172000 \\ 3.5378550 & 2.8882750 & -4.0051830 \\ 4.5850500 & 2.5814260 & -4.1321430 \\ 3.1252730 & 3.1269940 & -4.9946750 \\ 3.5233680 & 3.7971770 & -3.3922830 \\ -0.5784920 & -0.3127340 & -0.7548240 \\ -0.0681170 & -0.4369890 & -2.1627840 \\ -0.5282410 & -1.5072580 & 0.1565220 \\ 1.6904370 & 2.0879360 & -3.2489370 \\ 6.0418820 & -1.2513320 & \\ -1.2038740\end{array}$
1. 5455350 $-4.7711570$ $-5.3478870$ $-2.6669850$ 2. 4222620 1. 2807960 3.3825690 4.7381980 2.9678400 5.6699540 5.0490300 3.8990970 1. 9209180 5.2513350 6.7207010 3. 5718400 5.9771260 0.6093850 2.7358120 1.2448100 2. 3465720 0.3446540 2. 0897660 1.1957790 0.6131630 2.9509400 4.0592060 3.7435090 1.6035280 3.1998770 5.0264680 3.8843930 1.6489620 0.7863120 2.8659270
4.1501780
4.4064050

2. 5042700

0.9048700

$-5.6132910$

$-0.9653680$

$-2.3261430$

$-1.7104660$

$-2.2284080$

$-2.5132790$

-1.8282880

$-2.3796910$

$-2.8263790$

$-1.7126710$

$-1.6035830$

$-1.9803380$

$-2.5860500$

$-1.4055730$

$-1.8785080$

$-1.7078080$

$-2.9438730$

$-3.3164490$

$-2.4028400$

$-3.1990630$

$-1.4083940$

$-2.4861520$

$-1.6622690$

$-4.8944250$

$-4.1672040$

$-2.6714390$

$-4.7571570$

$-5.9567680$

$-4.2798670$

$-2.1341410$

$-5.2817850$

$-5.2025140$

$-4.4704940$

$-4.6110510$

$-2.1799990$
1.5950230

$-1.3937330$

0.0806760

2.5059970

1.7400950

1.9446500

2. 8812440

2.6506930

4.1619530

3.6790460

1.6583660

5.1939540

4.3389830

4.9535330

3.4887430

6.1845050

5.7574720

1.1564170

0.7101710

$-1.7488130$

$-1.4168340$

$-1.1458340$

$-1.0687210$

$-2.9559250$

$-2.7069790$

$-2.7846480$

$-2.0076310$

$-1.8504710$

$-2.0550660$

$-2.8938680$

$-2.5118080$

$-2.7990980$

$-1.0904320$

$-2.6359850$

$-3.7940300$

$-1.0077170$

$-1.1332430$

$\operatorname{TS} 139(\mathrm{~S}, \mathrm{~S})$ G[B97-D/def2-TZVP $]=-3310.748390$

$\begin{array}{lrrr}\mathrm{O} & 1.3645550 & -0.4681520 & -0.6512970 \\ \mathrm{O} & -0.1597280 & -1.7007860 & 0.9554640 \\ \mathrm{C} & 2.4373030 & -1.2551950 & -0.2586860 \\ \mathrm{C} & 2.2636670 & -2.6320800 & -0.1533200 \\ \mathrm{C} & 3.6808490 & -0.6018740 & -0.0383420 \\ \mathrm{C} & 3.3692770 & -3.4198040 & 0.3140320 \\ \mathrm{C} & 4.7650620 & -1.3890790 & 0.2971550\end{array}$




$\begin{array}{lr}\mathrm{C} & 4.6434490 \\ \mathrm{H} & 5.7337100 \\ \mathrm{C} & -0.2218410 \\ \mathrm{C} & -1.5003340 \\ \mathrm{C} & 0.9550200 \\ \mathrm{C} & -1.5603900 \\ \mathrm{C} & 0.8587260 \\ \mathrm{C} & -0.4172600 \\ \mathrm{H} & -2.5228220 \\ \mathrm{C} & 0.5810100 \\ \mathrm{C} & 1.8288870 \\ \mathrm{C} & 1.9635340 \\ \mathrm{C} & -0.5191680 \\ \mathrm{H} & 0.4903530 \\ \mathrm{H} & 2.6897810 \\ \mathrm{H} & 2.9261470 \\ \mathrm{H} & -1.4923150 \\ \mathrm{C} & 4.3472600 \\ \mathrm{C} & 5.6129980 \\ \mathrm{C} & 5.7522590 \\ \mathrm{C} & 3.2562240 \\ \mathrm{H} & 4.2314870 \\ \mathrm{H} & 6.4662370 \\ \mathrm{H} & 6.7119630 \\ \mathrm{H} & 2.2921250 \\ \mathrm{C} & 3.8180520 \\ \mathrm{C} & 4.1340810 \\ \mathrm{C} & 3.6704590 \\ \mathrm{C} & 4.3264140 \\ \mathrm{C} & 3.8633760 \\ \mathrm{C} & 4.2006880 \\ \mathrm{H} & 4.5769320 \\ \mathrm{H} & 3.7614680 \\ \mathrm{C} & -2.7396510 \\ \mathrm{C} & -3.6935480 \\ \mathrm{C} & -2.9711110 \\ \mathrm{C} & -4.8616570 \\ \mathrm{C} & -4.1552160 \\ \mathrm{C} & -5.1137000 \\ \mathrm{H} & -5.5964250 \\ \mathrm{H} & -4.3421360 \\ \mathrm{P} & 0.1515140 \\ \mathrm{O} & 0.6257420 \\ \mathrm{O} & -0.9549200 \\ \mathrm{C} & 3.3759170 \\ \mathrm{H} & 3.2160200 \\ & \end{array}$

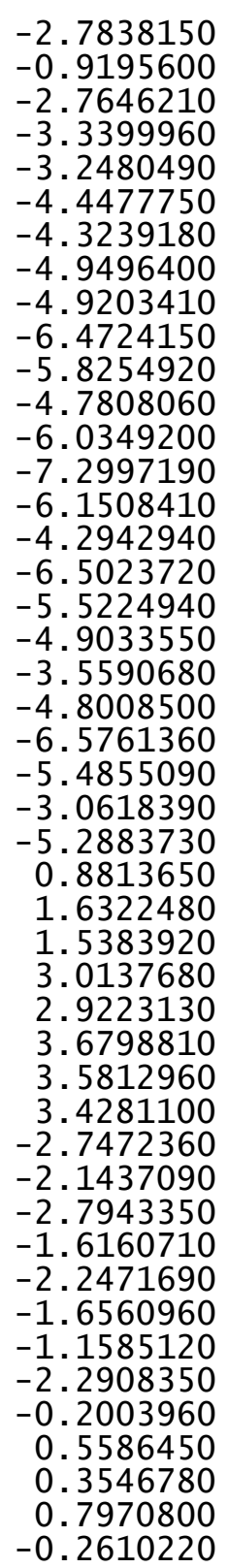

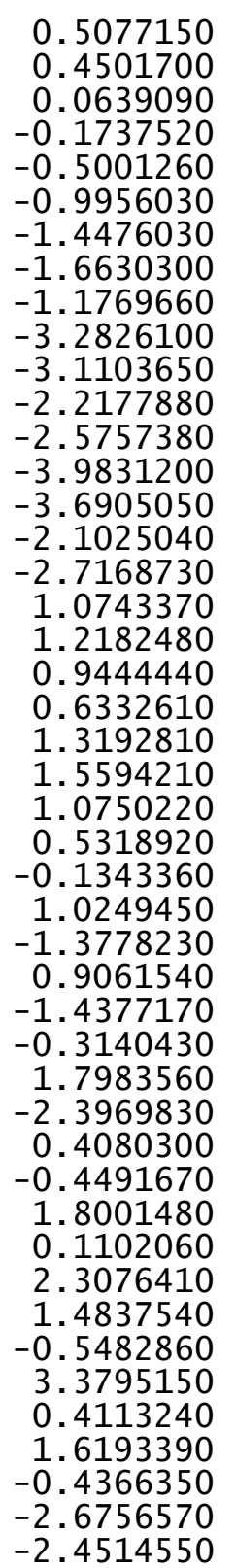

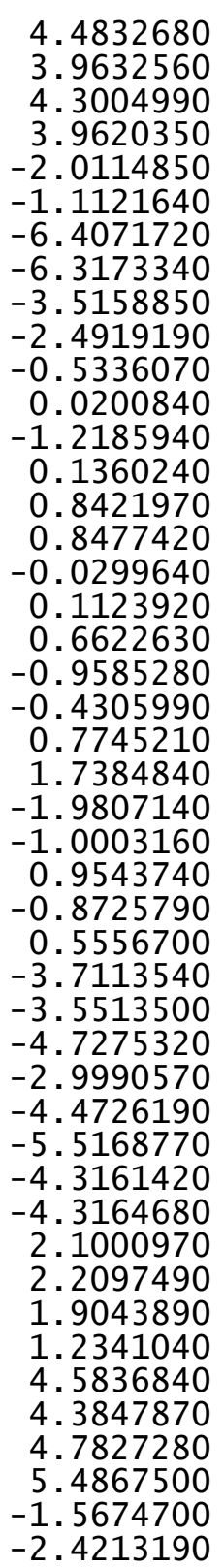

\author{
5. 1661470 \\ 5. 5072850 \\ 0.9970770 \\ $-0.0416580$ \\ $-3.4726900$ \\ $-3.7734020$ \\ $-1.1126370$ \\ $-1.1512500$ \\ $-2.0734110$ \\ 3. 4253730 \\ 3. 3968400 \\ 2. 6127040 \\ 2. 4286390 \\ 3.0747470 \\ 2. 0325470 \\ 5. 9093940 \\ 5.9251490 \\ 4.5924360 \\ 4.7699100 \\ 6.8682010 \\ 6.7399720 \\ 4.4920840 \\ 4.9561990 \\ 4.6988930 \\ 5. 7761240 \\ 6. 1040860 \\ 4.5119130 \\ $-0.6539240$ \\ $-0.6647240$ \\ $-0.2842800$ \\ 0.0394020 \\ $-3.0609940$ \\ $-2.7877920$ \\ $-3.0413990$ \\ $-4.0866120$ \\ 1. 3216000 \\ 2. 3797010 \\ 0.7541660 \\ 1. 2153900 \\ 0.8788910 \\ 0.3032230 \\ 1. 9201870 \\ 0.4774490 \\ -2.5281380
-2.2377070
}

$-0.4606010$

1.3685090

2. 4022970

2.3502050

2.7695180

2. 2242190

2. 0644260

3. 1595600

$-1.9632390$

$-2.2074220$

1. 2274590

$-0.1338890$

1.4741130

$-0.6042940$

1. 5650360

1.6027990

1. 3315240

$-0.1989810$

$-0.7373160$

1.7832730

1.6814840

$-0.5151370$

$-0.5351050$

1.4245240

2.8771540

1.7974580

$-0.6491010$

$-1.8252630$

$-2.5256400$

$-2.3389320$

$-2.0716430$

$-2.6608810$

$-2.4575230$

3.7479330

$-2.3044360$

$-3.3593300$

(

4.5445800

9164180

3. 9005630

4.5270370 


\begin{tabular}{|c|c|c|c|}
\hline $\mathrm{H}$ & -0.8329830 & -3.0321970 & 4.5427530 \\
\hline $\mathrm{H}$ & -1.1093750 & -1.6218830 & 3.4901480 \\
\hline C & -2.6417640 & -4.7583080 & 3.3416590 \\
\hline $\mathrm{H}$ & -1.9333520 & -5.2632000 & 4.0123430 \\
\hline $\mathrm{H}$ & -3.5493850 & -4.5234970 & 3.9131880 \\
\hline $\mathrm{H}$ & -2.9155400 & -5.4517060 & 2.5359710 \\
\hline C & 3.4524490 & 1.6880960 & 3.4860120 \\
\hline H & 3.6070090 & 1.1824650 & 4.4484920 \\
\hline H & 3.7367830 & 2.7409320 & 3.6086370 \\
\hline $\mathrm{H}$ & 2.3886690 & 1.6392650 & 3.2370090 \\
\hline C & 5.7861970 & 0.9894170 & 2.8151770 \\
\hline $\mathrm{H}$ & 6.1631040 & 2.0172900 & 2.9041780 \\
\hline $\mathrm{H}$ & 5.9094980 & 0.4919530 & 3.7864860 \\
\hline $\mathrm{H}$ & 6.4032250 & 0.4661640 & 2.0744940 \\
\hline C & -6.6578030 & 0.3512240 & 1.6613430 \\
\hline H & -5.8083140 & 0.9877490 & 1.9366710 \\
\hline $\mathrm{H}$ & -6.8066650 & 0.4293110 & 0.5764790 \\
\hline $\mathrm{H}$ & -7.5611040 & 0.7355150 & 2.1536080 \\
\hline C & -7.6041680 & -1.9939670 & 1.6555300 \\
\hline H & -7.4523100 & -3.0334260 & 1.9721010 \\
\hline H & -8.5321340 & -1.6195180 & 2.1090300 \\
\hline $\mathrm{H}$ & -7.7260210 & -1.9843620 & 0.5640640 \\
\hline C & 5.9939360 & 5.3961790 & -0.6748520 \\
\hline H & 6.3562720 & 4.8343070 & -1.5444940 \\
\hline $\mathrm{H}$ & 6.2044330 & 6.4630420 & -0.8309030 \\
\hline H & 6.5523980 & 5.0579460 & 0.2083080 \\
\hline C & 3.9786660 & 6.0114830 & 0.7194210 \\
\hline H & 2.9108090 & 5.8474320 & 0.8998370 \\
\hline $\mathrm{H}$ & 4.5201220 & 5.7625310 & 1.6407600 \\
\hline $\mathrm{H}$ & 4.1398910 & 7.0775440 & 0.5142360 \\
\hline C & -2.9424100 & 3.0242270 & -0.8705820 \\
\hline 0 & -2.9678740 & 1.8747420 & -0.2290410 \\
\hline C & -4.2777010 & 3.6765230 & -1.0156190 \\
\hline C & -4.3524210 & 4.9733330 & -1.5513940 \\
\hline C & -5.4568390 & 3.0183980 & -0.6265300 \\
\hline C & -5.5880170 & 5.6041900 & -1.6918320 \\
\hline $\mathrm{H}$ & -3.4357480 & 5.4714810 & -1.8541100 \\
\hline C & -6.6910960 & 3.6525080 & -0.7686180 \\
\hline H & -5.3991990 & 2.0176920 & -0.2136280 \\
\hline C & -6.7595200 & 4.9450460 & -1.3005220 \\
\hline H & -5.6399090 & 6.6085500 & -2.1058280 \\
\hline H & -7.5995920 & 3.1379400 & -0.4639150 \\
\hline $\mathrm{H}$ & -7.7231460 & 5.4378620 & -1.4105750 \\
\hline $\mathrm{H}$ & -2.0876190 & 1.3326780 & -0.2844000 \\
\hline 0 & -1.9153220 & 3.5599770 & -1.3168050 \\
\hline
\end{tabular}

\begin{tabular}{|c|c|c|c|}
\hline \multicolumn{4}{|c|}{, s) G[B97-D/def2-TZVP] $=-331$} \\
\hline 0 & 0.1883630 & 0.8625130 & 0.0794490 \\
\hline$V$ & -2.0703510 & 0.3669250 & -0.9789470 \\
\hline C & 0.1442410 & 2.1856960 & -0.3316300 \\
\hline C & -1.0457110 & 2.8928890 & -0.2162480 \\
\hline 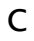 & 1.3659920 & 2.7682220 & -0.7683050 \\
\hline C & -1.0898970 & 4.2237720 & -0.7514230 \\
\hline & 1.3325840 & 4.0782400 & -1.1982090 \\
\hline C & 0.1231060 & 4.8175140 & -1.2446210 \\
\hline & 2.2526500 & 4.5581570 & -1.5227640 \\
\hline C & -2.6799080 & 1.0225570 & 0.0814770 \\
\hline & -3.8082400 & 0.3896330 & 0.6695840 \\
\hline & -2.2103450 & 2.2724230 & 0.4698510 \\
\hline C & -4.4594780 & 1.0547380 & 1.6875200 \\
\hline C & -2.8348870 & 2.9080070 & 1.5970330 \\
\hline C & -3.9917500 & 2.2940140 & 2.1904880 \\
\hline $\mathrm{H}$ & -5.3414260 & 0.6054190 & 2.1376600 \\
\hline$c$ & -4.1319220 & 4.0956290 & 3.8260670 \\
\hline C & -2.9649470 & 4.6774340 & 3.2745280 \\
\hline C & -2.334 & 4.1001860 & 2.1901730 \\
\hline C & -4.6283590 & 2.9238980 & 3.2942940 \\
\hline $\mathrm{H}$ & -4.6235430 & 4.5646760 & 4.6755680 \\
\hline H & -2.5564520 & 5.5858850 & 3.7121250 \\
\hline $\mathrm{H}$ & -1.4 & 4.5584620 & 58680 \\
\hline $\mathrm{H}$ & -5.5102130 & 2.4498340 & 3.7221410 \\
\hline C & -2.2979760 & 6.2479510 & -1.3778330 \\
\hline C & -1.0968130 & 6.8431330 & -1.8342760 \\
\hline C & 0.0870050 & 6.1375970 & -1.7693570 \\
\hline C & -2.29 & 4.9729070 & -0.8480200 \\
\hline $\mathrm{H}$ & -3.2336500 & 6.7983170 & -1.4493050 \\
\hline $\mathrm{H}$ & -1.112 & 7.8505550 & -2.2443370 \\
\hline H & 1.0152360 & 6.5767000 & -2.1314000 \\
\hline $\mathrm{H}$ & -3.2199240 & 4.5243520 & -0.5026740 \\
\hline C & 2.632 & 1.9822320 & -0.6880570 \\
\hline C & 3.29 & 1.5501830 & -1.8544050 \\
\hline C & 3.1637160 & 1.6622810 & 0.5882950 \\
\hline C & 4.4832860 & 0.8131660 & -1.7274740 \\
\hline C & 4.3265300 & 0.8948130 & 0.6596840 \\
\hline C & 5.0125440 & 0.4642180 & -0.4853810 \\
\hline $\mathrm{H}$ & 4.9915320 & 0.4987410 & -2.6332490 \\
\hline $\mathrm{H}$ & 4.7271910 & 0.6342550 & 1.6353630 \\
\hline 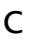 & -4.2660680 & -0.9485720 & 0.1917500 \\
\hline C & -4.0282960 & -2.0984400 & 0.9817340 \\
\hline C & -4.9256080 & -1.0641090 & -1.0495560 \\
\hline C & -4.4825880 & -3.3364740 & 0.5178630 \\
\hline 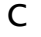 & -5.3569810 & -2.3282050 & -1.4707810 \\
\hline
\end{tabular}




$\begin{array}{rr}-5.1468280 & -3.4747350 \\ -4.3006420 & -4.2229680 \\ -5.8698240 & -2.4215770 \\ 2.5621670 & 2.2524080 \\ 6.2924990 & -0.3517590 \\ 2.7853380 & 1.8931760 \\ -3.3033000 & -2.0220050 \\ -5.5942450 & -4.8386840 \\ -5.2057310 & 0.1381970 \\ -6.7211520 & 0.3947360 \\ -7.2248050 & -0.4655790 \\ -7.1654700 & 0.5608720 \\ -6.9118650 & 1.2787410 \\ -4.5698420 & -0.0258120 \\ -3.4894540 & -0.1850330 \\ -5.0090460 & -0.8807230 \\ -4.7463950 & 0.8755910 \\ -6.5542790 & -5.5231130 \\ -6.8964540 & -6.4864670 \\ -6.0485500 & -5.7135890 \\ -7.4308630 & -4.8933180 \\ -4.3801980 & -5.7439640 \\ -3.7098940 & -5.2769300 \\ -3.8085220 & -5.9180140 \\ -4.7084720 & -6.7165940 \\ -4.3145970 & -2.0363500 \\ -5.0244080 & -1.2046690 \\ -4.8866830 & -2.9742270 \\ -3.7915270 & -1.9592410 \\ -2.2651880 & -3.1426210 \\ -2.7492210 & -4.1236840 \\ -1.5707210 & -3.1678330 \\ -1.6944480 & -2.9632950 \\ 3.2146390 & 3.6250720 \\ 4.2916710 & 3.5036980 \\ 3.0812680 & 4.2968240 \\ 2.7643380 & 4.0936580 \\ 2.6646550 & 1.3486300 \\ 2.1961150 & 0.3790470 \\ 3.7056240 & 1.1789900 \\ 2.1493130 & 1.8273680 \\ 7.3334100 & 0.4127080 \\ 8.2233340 & -0.2100540 \\ 7.6407350 & 1.3280620 \\ 6.9276900 & 0.6968450 \\ 6.9347850 & -0.7954470\end{array}$

-0.7048980
1.1202250
-2.4271790
1.8598870
-0.3228130
-3.2489350
2.3204130
-1.1995260
-1.9416160
-2.0556280
-2.5157860
-1.0658510
-2.6790220
-3.3345510
-3.2491570
-3.8653640
-3.9366690
-0.2085390
-0.6099150
0.7471830
-0.0109580
-1.4884710
-2.2210140
-0.5672530
-1.8795000
3.4843560
3.4068460
3.4754110
4.4474780
2.5084660
2.6023520
1.6635850
3.4290640
2.1290530
2.3087620
1.2720520
3.0147450
3.0953570
2.9070550
3.3988220
3.9381350
0.5228590
0.6840640
-0.0004350
1.4999190
-1.6442380

6. 2427930

(2585360

7.8173930

2.7988580

3. 8234600

2.2304630

2. 3448260

3. 6063400

4.6575040

3.2171500

3. 5735060

$-0.5829940$

$-0.0446740$

$-0.5577910$

1. 7448880

6.0219230

1. 4962270

$-4.7609970$

$-6.1347550$

$-2.7611430$

2. 3559990

1.2061160

3. 2826430

4.6470480

2. 8278020

5. 5481600

4. 9887660

3.7285010

1.7739800

5.0897240

6. 6060490

3. 3703760

5.7915800

0.5563760

2. 7031580

1.2625460

2. 3574230

0.3500970

2. 0939890

1.2388630

0.6500220

2. 9893910

4.0809960

3. 7623290

1.6267660
3.2399020
-1.3772050
0.0773930
-1.4133060
0.6928630
0.3565380
-0.1452370
0.9830150
3.0552290
2.7564880
3.3302850
3.9400140
-0.2703030
-0.3462600
-1.4977600
2.2225410
-1.2585780
2.4314330
1.0271180
-4.6855140
-1.0709660
-2.3714240
-1.7696510
-2.3198780
-2.5807840
-1.9845590
-2.4869620
-2.8429040
-1.9088680
-1.7777330
-2.1522820
-2.6737490
-1.6519000
-2.0815290
-1.7348620
-2.9418260
-3.2355620
-2.3342130
-3.1365300
-1.3515370
-2.3674540
-1.5524840
-4.7835440
-4.0813930
-2.5905860
-4.6665350
-5.8423990

$-2.2631540$ $-2.2267200$

$-1.4389590$

$-4.2110280$

$-4.4193150$

$-3.7996340$

$-5.1672970$

$-3.8440150$

$-3.9568070$

$-4.8335300$

$-3.1973820$

$-0.8164840$

$-2.2173310$

0.0512320

$-3.1600240$

0.2425950

1.6944480

$-1.4826620$

$-2.1446610$

2. 3564920

1.6775650

1.8764180

2. 8491930

2.6442200

4.1349820

3.7038540

1. 6472830

5. 1973250

4.2922260

4.9832630

3. 5339700

6. 1914570

5.8114160

1. 0711690

0.6313020

$-1.8691770$

$-1.4849540$

$-1.2817990$

$-1.1090840$

$-3.0503260$

$-2.7879620$

$-2.9167790$

$-2.0944760$

$-1.9001240$

$-2.2124360$ 


\begin{tabular}{|c|c|c|c|c|c|c|c|}
\hline & & & & & & & \\
\hline $\mathrm{H}$ & 5.0588710 & -4.1798500 & -2.5808320 & C & 5.1269940 & 0.8172240 & -0.7036560 \\
\hline $\mathrm{H}$ & 3.9189030 & -2.0277270 & -2.8312630 & $\mathrm{H}$ & 4.8955530 & 0.5370340 & -2.8215220 \\
\hline $\mathrm{H}$ & 1.6511800 & -5.2205910 & -1.2636170 & $\mathrm{H}$ & 5.0282090 & 1.2871330 & 1.3924160 \\
\hline $\mathrm{H}$ & 0.8216690 & -5.0927450 & -2.8238550 & C & -4.1717210 & -1.0432680 & 0.3547290 \\
\hline $\mathrm{H}$ & 2.9264920 & -4.3288930 & -3.9143460 & $\mathrm{C}$ & -3.9211770 & -2.1841680 & 1.1498880 \\
\hline $\mathrm{H}$ & 4.1502280 & -4.5546350 & -1.1063610 & $\mathrm{C}$ & -4.8604130 & -1.1705620 & -0.8732120 \\
\hline $\mathrm{H}$ & 4.4128320 & -2.1193920 & -1.1586520 & $\mathrm{C}$ & -4.3939070 & -3.4268160 & 0.7083020 \\
\hline & & & & $\mathrm{C}$ & -5.3048370 & -2.4342840 & -1.2713290 \\
\hline & , S) $\mathrm{G}[\mathrm{B} 97-\mathrm{D}$ & $Z \mathrm{ZVP}]=-33$ & 852 & $\mathrm{C}$ & -5.0850880 & -3.5751430 & -0.4948910 \\
\hline 0 & 0.2560280 & 0.8869000 & 0.0083680 & $\mathrm{H}$ & -4.2086460 & -4.3118680 & 1.3144230 \\
\hline 0 & -2.0524550 & 0.3271750 & -0.9157660 & $\mathrm{H}$ & -5.8400830 & -2.5290520 & -2.2137500 \\
\hline C & 0.1364490 & 2.2066720 & -0.3983690 & C & 2.7214160 & 2.6001490 & 1.6729590 \\
\hline $\mathrm{C}$ & -1.0678350 & 2.8741510 & -0.2082060 & $\mathrm{C}$ & 6.5054370 & 0.1708440 & -0.5748620 \\
\hline $\mathrm{C}$ & 1.3060790 & 2.8354030 & -0.9056610 & $\mathrm{C}$ & 2.5385100 & 1.7327370 & -3.3848930 \\
\hline C & -1.1908050 & 4.2027800 & -0.7366440 & $\mathrm{C}$ & -3.1634930 & -2.1059610 & 2.4701620 \\
\hline $\mathrm{C}$ & 1.2036930 & 4.1457040 & -1.3231970 & C & -5.5634020 & -4.9394510 & -0.9582700 \\
\hline $\mathrm{c}$ & -0.0316720 & 4.8415020 & -1.2976910 & $\mathrm{C}$ & -5.1681970 & 0.0239200 & -1.7672660 \\
\hline $\mathrm{H}$ & 2.0900160 & 4.6640280 & -1.6806810 & $\mathrm{C}$ & -6.6865750 & 0.2827310 & -1.8265500 \\
\hline C & -2.6288870 & 0.9616760 & 0.1763580 & $\mathrm{H}$ & -7.2084370 & -0.5804230 & -2.2604680 \\
\hline C & -3.7125100 & 0.3002910 & 0.8146500 & $\mathrm{H}$ & -7.0930160 & 0.4573200 & -0.8220250 \\
\hline $\mathrm{C}$ & -2.1704000 & 2.2205400 & 0.5455090 & $\mathrm{H}$ & -6.8994680 & 1.1622830 & -2.4490870 \\
\hline C & -4.3246090 & 0.9466130 & 1.8689730 & $\mathrm{C}$ & -4.5853340 & -0.1549530 & -3.1810160 \\
\hline $\mathrm{C}$ & -2.7503500 & 2.8378290 & 1.7059100 & $\mathrm{H}$ & -3.5032430 & -0.3181520 & -3.1346320 \\
\hline C & -3.8 & 2.1936460 & 2.3547220 & $\mathrm{H}$ & -5.0472830 & -1.0121290 & 882810 \\
\hline $\mathrm{H}$ & -5.17 & 0.4753730 & 2.3619270 & $\mathrm{H}$ & -4.7805400 & 0.7420060 & -3.7839380 \\
\hline C & -3.958 & 3.9860020 & 4.0031440 & C & -4.8289600 & -5.3765450 & -2.2410060 \\
\hline C & -2.83 & 4.5985670 & 3.3947000 & $\mathrm{H}$ & -5.1498560 & -6.3819950 & -2.5457680 \\
\hline C & -2.2 & 4.0405290 & 2.2765890 & $\mathrm{H}$ & -5.0505290 & -4.6820080 & -3.0621670 \\
\hline C & -4.4 & 2.8041280 & 3.4927150 & $\mathrm{H}$ & -3.7425720 & -5.3844500 & -2.0869770 \\
\hline $\mathrm{H}$ & -4.4174620 & 4.4404400 & 4.8786930 & C & -7.0885070 & -4.9677970 & -1.1729350 \\
\hline $\mathrm{H}$ & -2.4 & 5.5159210 & 3.8143380 & $\mathrm{H}$ & -7.6198160 & -4.6807680 & -0.2567570 \\
\hline $\mathrm{H}$ & -1.3 & 4.5224790 & 1.8263240 & $\mathrm{H}$ & -7.3771920 & -4.2674440 & -1.9676870 \\
\hline H & -5.3 & 2.3077480 & 3.9632600 & $\mathrm{H}$ & -7.4157340 & -5.9733710 & -1.4694580 \\
\hline C & -2.5( & 6.1861130 & -1.2766900 & C & -4.1394590 & -2.1753790 & 3.6616050 \\
\hline c & -1.3 & 6.8263300 & -1.7986380 & $\mathrm{H}$ & -4.8802400 & -1.3683130 & 3.6171580 \\
\hline C & -0.1 & 6.1625640 & -1.8094530 & $\mathrm{H}$ & -4.6782120 & -3.1326570 & 3.6545640 \\
\hline C & -2.42 & 4.9093480 & -0.7576500 & $\mathrm{H}$ & -3.5915130 & -2.0942510 & 4.6103780 \\
\hline $\mathrm{H}$ & -3.4 & 6.7029330 & -1.2889220 & C & -2.0845140 & -3.1962040 & 2.6041910 \\
\hline $\mathrm{H}$ & -1.42 & 7.8347230 & -2.1995260 & $\mathrm{H}$ & -2.5350200 & -4.1946220 & 2.6791600 \\
\hline $\mathrm{H}$ & 0.74 & 6.6360740 & -2.2223050 & $\mathrm{H}$ & -1.4085220 & -3.1746740 & 1.7443330 \\
\hline $\mathrm{H}$ & -3.3 & 4.4273230 & -0.3622480 & $\mathrm{H}$ & -1.4985490 & -3.0238970 & 3.5165430 \\
\hline C & 2.61 & 2.1204070 & -0.8575840 & C & 3.6163150 & 3.7534440 & 2.1676790 \\
\hline C & 3.20 & 1.5778960 & -2.0212130 & $\mathrm{H}$ & 4.6215780 & 3.3845120 & 2.4109640 \\
\hline C & 3.2 & 2.0031600 & 0.3861320 & $\mathrm{H}$ & 3.7145190 & 4.5318030 & 1.4001210 \\
\hline C & 4.4 & 0.939529 & -1.9194180 & $\mathrm{H}$ & 3.1878660 & 4.2058250 & 3.0722090 \\
\hline C & & 35667 & 0.4343460 & C & 2.5418210 & 1.5347600 & 2.7681670 \\
\hline
\end{tabular}




\begin{tabular}{|c|c|c|c|}
\hline & & & \\
\hline $\mathrm{H}$ & 1.8771890 & 0.7349910 & 2.4272300 \\
\hline $\mathrm{H}$ & $\begin{array}{l}3.5051860 \\
2.1096470\end{array}$ & 1.0877170 & $\begin{array}{l}3.0447720 \\
3.6688230\end{array}$ \\
\hline C & 7.0870200 & $\begin{array}{r}1.9912900 \\
-0.3457150\end{array}$ & $\begin{array}{r}3.6688230 \\
-1.8985280\end{array}$ \\
\hline $\mathrm{H}$ & 8.0938260 & -0.7464930 & -1.7294390 \\
\hline H & 6.4662540 & -1.1525600 & -2.3104040 \\
\hline $\mathrm{H}$ & 7.1530210 & 0.4504170 & -2.6500320 \\
\hline C & 6.5175360 & -0.9567030 & 0.4787020 \\
\hline H & 6.1400730 & -0.6131670 & 1.4470880 \\
\hline H & 5.8905090 & -1.7977780 & 0.1592870 \\
\hline $\mathrm{H}$ & 7.5410740 & -1.3289080 & 0.6192530 \\
\hline C & 2.6811100 & 0.4990410 & -4.2914180 \\
\hline H & 3.7250740 & 0.3400260 & -4.5940540 \\
\hline H & 2.3164390 & -0.4033140 & -3.7950970 \\
\hline H & 2.0921810 & 0.6488530 & -5.2049600 \\
\hline C & 3.1013960 & 2.9728850 & -4.1095730 \\
\hline H & 4.1784070 & 2.8473420 & -4.2869840 \\
\hline H & 2.6036510 & 3.1066670 & -5.079579 \\
\hline $\mathrm{H}$ & 2.9562130 & 3.8819960 & -3.5164760 \\
\hline $\mathrm{P}$ & -0.5513790 & -0.2846180 & -0.8064180 \\
\hline 0 & -0.0817320 & -0.4113830 & -2.2280640 \\
\hline 0 & -0.4697580 & -1.4785320 & 0.1022080 \\
\hline $\mathrm{H}$ & 1.4664640 & 1.8895160 & -3.220661 \\
\hline $\mathrm{H}$ & 7.1817830 & 0.9576250 & -0.2046170 \\
\hline $\mathrm{H}$ & 1.7339390 & 3.0238800 & 1.465635 \\
\hline H & -4.7060930 & 0.9168570 & -1.3349260 \\
\hline H & -5.3191190 & -5.6611710 & -0.1655500 \\
\hline H & -2.6511320 & -1.1391130 & 2.510847 \\
\hline C & 2.4908520 & -2.2670970 & 1.7157810 \\
\hline 0 & 3990 & -1.7377320 & 1.905372 \\
\hline C & 3.3464800 & -2.2941310 & 2.9435580 \\
\hline $\mathrm{C}$ & 4.6348980 & -2.8473640 & 2.8660220 \\
\hline C & 2.8965720 & -1.7610110 & 4.163796 \\
\hline C & 5.4631300 & -2.8635280 & 3.987358 \\
\hline H & 4.9772700 & -3.2533300 & 1.9196320 \\
\hline C & 3.7282280 & -1.7766760 & 5.2836470 \\
\hline H & 1.9031230 & -1.3297010 & 4.225756 \\
\hline C & 5.0119820 & -2.3266290 & 5.1984090 \\
\hline H & 6.4609280 & -3.2905900 & 3.917352 \\
\hline H & 3.3756580 & -1.3574940 & 6.2231960 \\
\hline H & 5.6591680 & -2.3362050 & 6.0728780 \\
\hline $\mathrm{H}$ & 0.6744730 & -1.6815490 & 1.087710 \\
\hline 0 & 2.9182850 & -2.7217920 & 0.642928 \\
\hline C & 1.3533430 & -3.2561590 & -1.751747 \\
\hline C & 2.4081050 & -2.2647590 & -1.502872 \\
\hline $\mathrm{H}$ & 0.4741920 & -3.1739880 & -1.1134 \\
\hline
\end{tabular}

\begin{abstract}
2. 1057930
1.1992130

0.5973750

3. 1204800

4.2071440

3.7962580

1.7908950

3.4327820

5.1599230

3.8491210

1.8985770

0.9845420

2. 9748580

4.3652130

4.4553920
\end{abstract}

144

TS142(S, S) G[B97-D/def2-TZVP] $=-3310.747831$

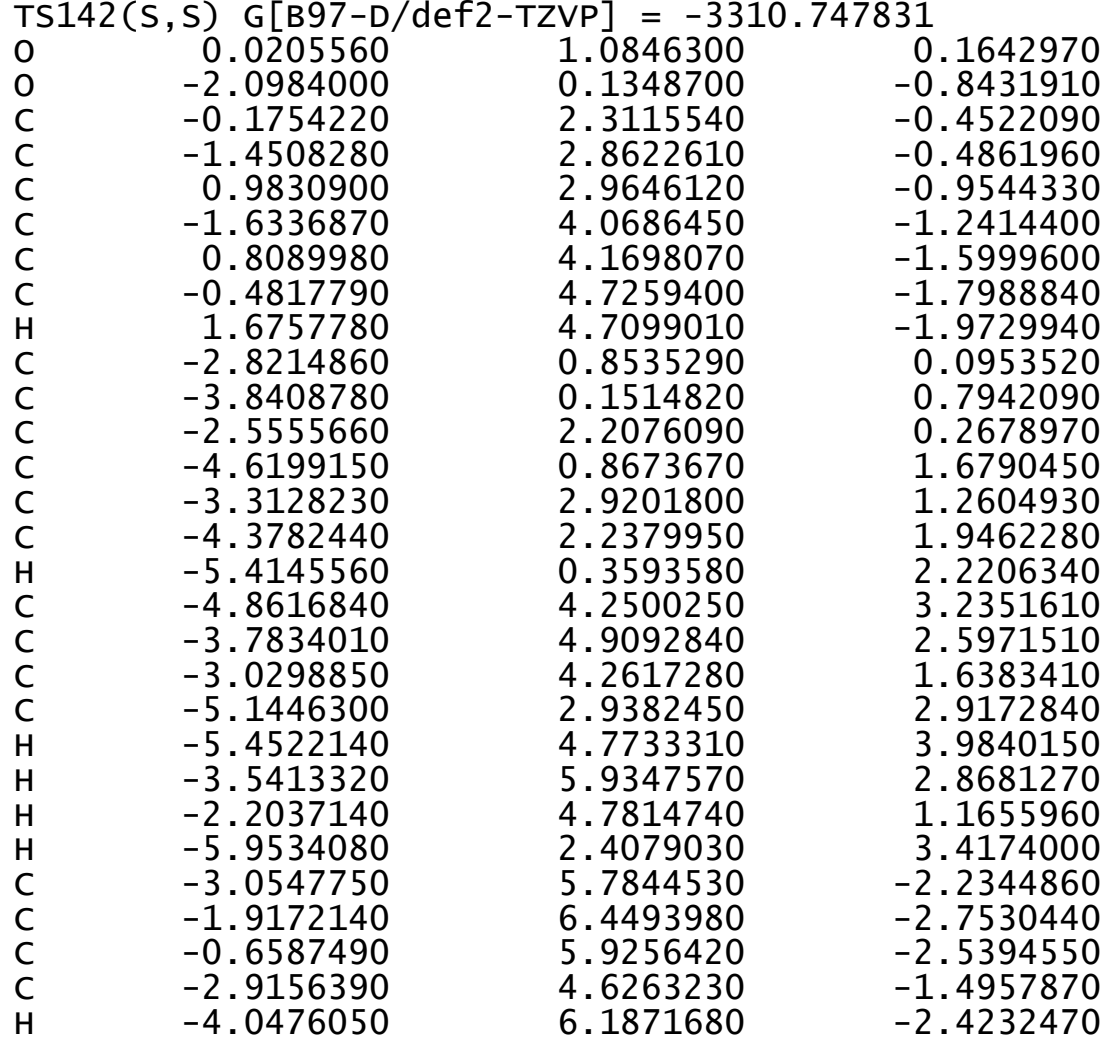

$-1.1655820$

$-2.9814730$

$-2.7952420$

$-2.0940480$

$-1.9958590$

$-2.0251500$

$-2.8680010$

$-2.9792270$

$-1.0455510$

$-2.5623690$

$-3.8182710$

$-1.0795740$

$-1.3491100$
$-2.7404990$
$-5.1680300$

$-4.3941370$

$-4.3238350$

.1698070

8535290

2.2076090

0.3593580

.9382450

.4079030

6.1871680
$-2.4232470$

2. 9728850

3.1066670

3.8819960

$-0.4113830$

$-1.4785320$

. 8895160

3.0238800

0.9168570

. 6611710

$-1.1391130$

$-1.7377320$

$-2.2941310$

2.8473640

$-1.3297010$

. 3266290

$-2.3362050$

- . 6815490

$-3.1739880$

$-1.1134840$ 


$\begin{array}{lr}\mathrm{H} & -2.0394970 \\ \mathrm{H} & 0.2230440 \\ \mathrm{H} & -3.7938780 \\ \mathrm{C} & 2.3233330 \\ \mathrm{C} & 3.0504890 \\ \mathrm{C} & 2.8318060 \\ \mathrm{C} & 4.2719360 \\ \mathrm{C} & 4.0528100 \\ \mathrm{C} & 4.7868170 \\ \mathrm{H} & 4.8303770 \\ \mathrm{H} & 4.4433570 \\ \mathrm{C} & -3.9991800 \\ \mathrm{C} & -3.6140460 \\ \mathrm{C} & -4.4779770 \\ \mathrm{C} & -3.7300600 \\ \mathrm{C} & -4.5620690 \\ \mathrm{C} & -4.1870750 \\ \mathrm{H} & -3.4305010 \\ \mathrm{H} & -4.9231980 \\ \mathrm{C} & 2.1145650 \\ \mathrm{C} & 6.1326310 \\ \mathrm{C} & 2.5578080 \\ \mathrm{C} & -3.0749680 \\ \mathrm{C} & -4.2105700 \\ \mathrm{C} & -4.9378360 \\ \mathrm{C} & -6.4624370 \\ \mathrm{H} & -6.7362520 \\ \mathrm{H} & -6.9987480 \\ \mathrm{H} & -6.7958120 \\ \mathrm{C} & -4.1931480 \\ \mathrm{H} & -3.1107860 \\ \mathrm{H} & -4.4173810 \\ \mathrm{H} & -4.5083190 \\ \mathrm{C} & -3.0770960 \\ \mathrm{H} & -3.0634380 \\ \mathrm{H} & -3.2236390 \\ \mathrm{H} & -2.1015440 \\ \mathrm{C} & -5.5703040 \\ \mathrm{H} & -6.3802980 \\ \mathrm{H} & -5.7919990 \\ \mathrm{H} & -5.5598460 \\ \mathrm{C} & -4.1511690 \\ \mathrm{H} & -5.0597780 \\ \mathrm{H} & -4.4244510 \\ \mathrm{H} & -3.7741010 \\ \mathrm{C} & -1.7835800 \\ & \end{array}$

$\begin{array}{rr}7.3645720 & -3.3282580 \\ 6.4175330 & -2.9468650 \\ 4.1223320 & -1.1044750 \\ 2.3599930 & -0.6842070 \\ 1.7071280 & -1.7036950 \\ 2.3979630 & 0.6347530 \\ 1.0983170 & -1.3796830 \\ 1.7754730 & 0.9058350 \\ 1.1121610 & -0.0800950 \\ 0.5970060 & -2.1649380 \\ 1.7949860 & 1.9217720 \\ -1.3195100 & 0.6016630 \\ -2.2034750 & 1.6354960 \\ -1.8314230 & -0.6260820 \\ -3.5822670 & 1.4180610 \\ -3.2157440 & -0.7964610 \\ -4.1098740 & 0.2101370 \\ -4.2701200 & 2.2069110 \\ -3.6055710 & -1.7455300 \\ 3.1200620 & 1.7687800 \\ 0.5047570 & 0.2837360 \\ 1.6942870 & -3.1456580 \\ -1.7146110 & 2.9752900 \\ -5.6103550 & -0.0214830 \\ -0.9281220 & -1.7630380 \\ -1.0438570 & -1.9612600 \\ -2.0689020 & -2.2444550 \\ -0.7901780 & -1.0378420 \\ -0.3655570 & -2.7584090 \\ -1.2271870 & -3.0771180 \\ -1.1355040 & -2.9382870 \\ -2.2420220 & -3.4310190 \\ -0.5204520 & -3.8563940 \\ -6.0183680 & -0.9852170 \\ -7.1075840 & -1.1284780 \\ -5.5424320 & -1.9640290 \\ -5.7012450 & -0.5956440 \\ -6.1076160 & -0.5437220 \\ -5.8320840 & 0.1431800 \\ -5.6699350 & -1.5255050 \\ -7.1999850 & -0.6552900 \\ -1.8492800 & 4.0708470 \\ -1.2949070 & 3.8053130 \\ -2.9046040 & 4.2063000 \\ -1.4643940 & 5.0281880 \\ -2.4390090 & 3.3988420\end{array}$
$-1.9705650$
$-1.0261040$
$-1.3904820$
2. 9169230
3.9017340
3. 0721370
2. 3833310
1.8398150
1. 3064660
2. 7808090
1. 2344330
7.2354790
8. 2037490
7. 3308010
6.9953090
6.5141560
5.7446710
6.6487770
7.4591970
2.7910130
3. 8610630
2. 3399460
2. 3287380
3. 2176470
4.3059390
2. 8340810
3. 0223580
$-0.5370910$
0.0363080
$-0.3491680$
1. 4760930
6.0690210
1.1461480
$-4.7241470$
$-4.0202200$
$-2.8279970$
2.8546700
1.8328260
4.0451550
5. 2166270
4.0283600
6. 3581120
5. 2216440
5. 1723250
3. 1252560
6. 3397040

-3.5010080
-2.3605520
-1.9843150
4.3595060
4.0616350
5.0470200
4.8947580
2.1938000
1.2922140
1.8923760
2.7204730
1.5798870
1.1697650
1.9291710
2.4447940
-0.7247800
-1.5004770
-0.4651560
-1.1452530
0.3588230
0.1554030
-0.4674570
0.3959860
2.8411320
2.6948130
2.8645380
3.8133260
-0.2465030
-0.3928380
-1.3607440
1.8644440
0.1868090
3.4760070
0.1114160
-6.0954850
-0.6524850
-2.1244220
-1.3433480
-1.8802230
-2.6228630
-0.8940450
-2.3755510
-3.3824950
-0.6472030
-0.3179700
-1.3841860

3. 6051760

2. 6128590

4. 3180600

2. 2107930

2. 5951300

1. 3695470

3. 0078840

2. 9669050

2. 6479040

3. 4449300

3. 7164020

0.1859340

0.5045640

$-0.8513170$

0.8164040

$-0.5501940$

$-0.4751500$

$-1.6083070$

$-0.1842570$

$-3.8695070$

$-4.0087250$

$-3.3151730$

$-4.8641830$

$-3.9389270$

$-3.9779740$

$-4.9680490$

$-3.4723470$

$-0.6026000$

$-1.9841760$

0.3899840

$-3.1316900$

1. 3332300

1.4051660

$-1.4947720$

0.9467170

2. 8802840

1.4530980

1. 7097040

2. 3228700

2. 1040910

3. 3235680

2. 8651080

1.3281940

4. 0816000

3.4893890

3. 8522480 


\begin{tabular}{|c|c|c|c|c|c|c|c|}
\hline $\mathrm{H}$ & 7.2641540 & -2.9492530 & 2.6849120 & $\mathrm{H}$ & -1.3226390 & 3.8603040 & 5.2903680 \\
\hline $\mathrm{H}$ & 5.1557390 & 0.1236020 & 4.8487630 & $\mathrm{H}$ & -0.4020910 & 3.5074180 & 3.0398390 \\
\hline $\mathrm{H}$ & 7.2326920 & -1.1861980 & 4.4411620 & $\mathrm{H}$ & -4.7792250 & 1.4900110 & 4.2899390 \\
\hline $\mathrm{H}$ & 0.9932890 & -1.4612910 & 1.1142550 & C & -1.0608480 & 6.3925680 & 0.7751180 \\
\hline 0 & 2.8831750 & -2.9994460 & 0.5688890 & $\mathrm{C}$ & 0.2041910 & 6.9492800 & 0.4634580 \\
\hline C & 0.9962210 & -3.3994920 & -1.6222280 & $\mathrm{C}$ & 1.2623920 & 6.1189910 & 0.1601320 \\
\hline C & 2.2314900 & -2.6074050 & -1.5160510 & $\mathrm{C}$ & -1.2411790 & 5.0245370 & 0.8017240 \\
\hline $\mathrm{H}$ & 0.2199050 & -3.1450300 & -0.9012850 & $\mathrm{H}$ & -1.9003450 & 7.0500800 & 0.9906190 \\
\hline $\mathrm{H}$ & 2.1241790 & -1.5691340 & -1.2198900 & $\mathrm{H}$ & 0.3332290 & 8.0293080 & 0.4521490 \\
\hline 0 & 0.8474350 & -2.6389010 & -2.8675700 & $\mathrm{H}$ & 2.2359730 & 6.5315890 & -0.0993820 \\
\hline $\mathrm{H}$ & 0.4271730 & -1.7344400 & -2.5920720 & $\mathrm{H}$ & -2.2168640 & 4.6122290 & 1.0375270 \\
\hline C & 2.3432800 & -5.2282700 & -2.7701210 & $\mathrm{C}$ & 3.2026880 & 1.6327160 & -0.6191520 \\
\hline C & 3.6284620 & -4.5943840 & -2.2146580 & $\mathrm{C}$ & 3.5035330 & 1.4569710 & -1.9930360 \\
\hline C & 3.5055630 & -3.0638020 & -2.1369970 & $\mathrm{C}$ & 4.0664300 & 1.1060740 & 0.3705610 \\
\hline C & 1.1414840 & -4.8873550 & -1.8724520 & $\mathrm{C}$ & 4.6789830 & 0.7900390 & -2.3518100 \\
\hline $\mathrm{H}$ & 2.4554100 & -6.3173350 & -2.8317960 & $\mathrm{C}$ & 5.2329850 & 0.4482230 & -0.0514450 \\
\hline $\mathrm{H}$ & 4.4914120 & -4.8547860 & -2.8388510 & $\mathrm{C}$ & 5.5651650 & 0.2868600 & -1.3967020 \\
\hline $\mathrm{H}$ & 3.5420260 & -2.6174780 & -3.1416460 & $\mathrm{H}$ & 4.9135180 & 0.6652700 & -3.4052050 \\
\hline $\mathrm{H}$ & 1.2662400 & -5.3602370 & -0.8882950 & $\mathrm{H}$ & 5.9081430 & 0.0464800 & 0.7046630 \\
\hline $\mathrm{H}$ & 0.2025060 & -5.2596970 & -2.3001570 & $\mathrm{C}$ & -4.1867720 & -0.3633590 & -0.3686680 \\
\hline $\mathrm{H}$ & 2.1579390 & -4.8581250 & -3.7872780 & C & -4.2687240 & -1.7433950 & -0.0713830 \\
\hline $\mathrm{H}$ & 3.8172550 & -4.9830160 & -1.2053700 & $\mathrm{C}$ & -4.7959450 & 0.1483100 & -1.5376890 \\
\hline $\mathrm{H}$ & 4.3302640 & -2.6091260 & -1.5790380 & $\mathrm{C}$ & -4.9788280 & -2.5757470 & -0.9463310 \\
\hline & & & & $\mathrm{C}$ & -5.4882900 & -0.7267470 & -2.3789240 \\
\hline \multicolumn{4}{|c|}{$\operatorname{TS} 143(\mathrm{~S}, \mathrm{~S}) \mathrm{G}[\mathrm{B} 97-\mathrm{D} / \mathrm{def} 2-\mathrm{TZVP}]=-3310.746529$} & $\mathrm{C}$ & -5.5935440 & -2.0921260 & -2.1019580 \\
\hline 0 & 0.5996400 & 0.5596380 & -0.0066300 & $\mathrm{H}$ & -5.0486110 & -3.6396080 & -0.7275030 \\
\hline 0 & -1.7087560 & 0.8709840 & -1.0576740 & $\mathrm{H}$ & -5.9616280 & -0.3286010 & -3.2740810 \\
\hline $\mathrm{C}$ & 0.7509590 & 1.9330260 & 0.0696290 & $\mathrm{C}$ & 3.8333810 & 1.1517290 & 1.8851520 \\
\hline C & -0.3099780 & 2.7118220 & 0.5264390 & $\mathrm{C}$ & 6.8653840 & -0.3843280 & -1.8063850 \\
\hline C & 2.0238600 & 2.4759920 & -0.2460350 & $\mathrm{C}$ & 2.6050380 & 2.0340040 & -3.0825190 \\
\hline C & -0.1667320 & 4.1380100 & 0.5139510 & C & -3.6267270 & -2.3533120 & 1.1698280 \\
\hline C & 2.1736140 & 3.8465160 & -0.1798480 & C & -6.3535500 & -3.0216200 & -3.0313990 \\
\hline $\mathrm{C}$ & 1.1044950 & 4.7062630 & 0.1622030 & $\mathrm{C}$ & -4.7642020 & 1.6278820 & -1.9003970 \\
\hline $\mathrm{H}$ & 3.1465520 & 4.2816760 & -0.3943300 & $\mathrm{C}$ & -6.1792310 & 2.2358760 & -1.8355300 \\
\hline C & -2.2434400 & 1.1734390 & 0.1975980 & $\mathrm{H}$ & -6.8414300 & 1.7501500 & -2.5642960 \\
\hline C & -3.4747050 & 0.5633020 & 0.5591770 & $\mathrm{H}$ & -6.6167100 & 2.1058120 & -0.8372850 \\
\hline C & -1.5581580 & 2.0660420 & 1.0101530 & $\mathrm{H}$ & -6.1430850 & 3.3092960 & -2.0655010 \\
\hline C & -3.9957550 & 0.8713590 & 1.7997930 & $\mathrm{C}$ & -4.1293280 & 1.8632260 & -3.2832010 \\
\hline C & -2.0625360 & 2.3055030 & 2.3325590 & $\mathrm{H}$ & -3.1191890 & 1.4412940 & -3.3205520 \\
\hline C & -3.3111820 & 1.7066020 & 2.7165840 & $\mathrm{H}$ & -4.7322590 & 1.3969360 & -4.0735640 \\
\hline $\mathrm{H}$ & -4.9496430 & 0.4419340 & 2.0950900 & $\mathrm{H}$ & -4.0702880 & 2.9399300 & -3.4916620 \\
\hline C & -3.1309040 & 2.7132250 & 4.9282900 & $\mathrm{C}$ & -5.7390370 & -3.0340430 & -4.4437610 \\
\hline C & -1.8813150 & 3.2721880 & 4.5653910 & $\mathrm{H}$ & -6.2757700 & -3.7419210 & -5.0897100 \\
\hline C & -1.3619390 & 3.0748820 & 3.3014540 & $\mathrm{H}$ & -5.8060610 & -2.0373110 & -4.8993820 \\
\hline C & -3.8266770 & 1.9430120 & 4.0201090 & $\mathrm{H}$ & -4.6806750 & -3.3208980 & -4.4090090 \\
\hline $\mathrm{H}$ & -3.5322290 & 2.8832070 & 5.9250010 & $\mathrm{C}$ & -7.8479350 & -2.6507930 & -3.0947080 \\
\hline
\end{tabular}




$\begin{array}{rrr}-8.3020490 & -2.6776960 & -2.0960830 \\ -7.9734640 & -1.6374340 & -3.4984270 \\ -8.3903540 & -3.3488800 & -3.7466380 \\ -4.6843600 & -2.6009900 & 2.2646970 \\ -5.2059190 & -1.6760930 & 2.5365700 \\ -5.4329970 & -3.3224120 & 1.9100600 \\ -4.2101970 & -3.0113200 & 3.1668460 \\ -2.8735590 & -3.6634030 & 0.8722530 \\ -3.5691280 & -4.4687670 & 0.6022340 \\ -2.1606620 & -3.5215390 & 0.0547880 \\ -2.3247160 & -3.9814110 & 1.7678430 \\ 2.8787930 & 0.0320810 & 2.3477460 \\ 1.8622160 & 0.2020220 & 1.9846840 \\ 3.2151050 & -0.9472720 & 1.9857560 \\ 2.8486290 & -0.0044670 & 3.4447490 \\ 3.3982620 & 2.5003040 & 2.4907050 \\ 3.9897430 & 3.3318320 & 2.0896260 \\ 2.3410480 & 2.7119410 & 2.3063280 \\ 3.5483220 & 2.4591370 & 3.5778600 \\ 7.8426320 & 0.6511810 & -2.3976840 \\ 8.8005060 & 0.1770760 & -2.6504830 \\ 7.4223430 & 1.0886360 & -3.3128590 \\ 8.0287910 & 1.4644930 & -1.6853320 \\ 6.6475950 & -1.5409690 & -2.7999070 \\ 6.0109560 & -2.3238180 & -2.3717620 \\ 6.1683140 & -1.1795290 & -3.7184780 \\ 7.6107060 & -1.9914710 & -3.0734440 \\ 2.5224060 & 1.1566070 & -4.3437620 \\ 3.4658670 & 1.1771300 & -4.9056740 \\ 2.2871200 & 0.1181520 & -4.0967650 \\ 1.7348210 & 1.5431050 & -5.0019400 \\ 3.0754820 & 3.4497260 & -3.4770620 \\ 4.0994270 & 3.4079160 & -3.8733530 \\ 2.4198760 & 3.8611210 & -4.2563600 \\ 3.0647700 & 4.1321790 & -2.6212240 \\ -0.4550730 & -0.1604990 & -1.0498660 \\ 0.1063870 & -0.1917670 & -2.4400610 \\ -0.7912980 & -1.4611540 & -0.3770110 \\ 1.5908650 & 2.1103680 & -2.6762270 \\ 7.3226560 & -0.7993980 & -0.8964460 \\ 4.8133140 & 0.9270390 & 2.3290680 \\ -4.1524850 & 2.1604420 & -1.1656900 \\ -6.2746030 & -4.0375310 & -2.6180510 \\ -2.8937750 & -1.6407570 & 1.5602420 \\ 1.4941070 & -3.0837880 & -1.7942640 \\ 2.2811260 & -2.3646150 & -0.7838210\end{array}$

\begin{tabular}{|c|c|}
\hline & \\
\hline $\mathrm{H}$ & 0.4147280 \\
\hline $\mathrm{H}$ & 1.8450100 \\
\hline 0 & 1.9607540 \\
\hline $\mathrm{H}$ & 1.2293980 \\
\hline C & 3.5598110 \\
\hline C & 4.1577920 \\
\hline C & 3.7256490 \\
\hline C & 2.0227310 \\
\hline $\mathrm{H}$ & 3.8861530 \\
\hline $\mathrm{H}$ & 5.2531810 \\
\hline $\mathrm{H}$ & 4.2527990 \\
\hline $\mathrm{H}$ & 1.6529920 \\
\hline $\mathrm{H}$ & 1.5808410 \\
\hline $\mathrm{H}$ & 3.9189640 \\
\hline $\mathrm{H}$ & 3.8164290 \\
\hline $\mathrm{H}$ & 3.9703130 \\
\hline C & 1.0012900 \\
\hline 0 & 0.0129870 \\
\hline C & 1.3606550 \\
\hline C & 2.4102770 \\
\hline C & 0.6857360 \\
\hline C & 2.7798080 \\
\hline $\mathrm{H}$ & 2.9262050 \\
\hline C & 1.0596780 \\
\hline $\mathrm{H}$ & -0.12 \\
\hline C & 2.1 \\
\hline $\mathrm{H}$ & 20 \\
\hline $\mathrm{H}$ & 0.5 \\
\hline H & 190 \\
\hline $\mathrm{H}$ & -0.2 \\
\hline 0 & \\
\hline
\end{tabular}
$-3.0503500$
$-1.4899970$
$-1.9738790$
$-1.2411150$
$-4.4696140$
$-4.0488320$
$-2.6260590$
$-4.4234370$
$-5.4839910$
$-4.0954830$
$-1.8703860$
$-5.1763070$
$-4.6456590$
$-3.7963790$
$-4.7429730$
$-2.3800160$
$-2.9280780$
$-2.0735150$
$-3.2319240$
$-4.1274810$
$-2.6230110$
$-4.4112820$
$-4.5923970$
$-2.9070510$
$-1.9256460$
$-3.8006360$
$-5.1064550$
$-2.4303270$
$-4.0202650$
$-1.8686630$

$-1.6502570$

$-0.3200640$

$-2.6353240$

$-2.6020280$

$-2.3205790$

$-0.9690620$

$-0.5856020$

$-2.2668980$

$-2.5790460$

$-0.9986950$

$-1.1855220$

$-1.5566510$

$-3.2460300$

$-3.1100960$

$-0.1900150$

0.4534090

2.0689860

1.9376380

3. 4895520

3.7528220

4. 5618350

5.0672540

2. 9175020

5.8756440

4. 3588160

6.1311200

5.2640990

6.7010340

7. 1565880

0.9564760

144

1. 1289300

TS144 (S, S) G[B97-D/def2-TZVP $]=-3310.746104$

\begin{tabular}{lrrr}
\multicolumn{4}{c}{ TS144(S, S) G $[\mathrm{B} 97-\mathrm{D} / \mathrm{def} 2-\mathrm{TZVP}]=-3310.746104$} \\
$\mathrm{O}$ & 1.5150120 & -0.5229460 & -0.6602170 \\
$\mathrm{O}$ & -0.2067080 & -1.5560970 & 0.8865490 \\
$\mathrm{C}$ & 2.5057530 & -1.2971350 & -0.0700180 \\
$\mathrm{C}$ & 2.2693160 & -2.6546010 & 0.1255800 \\
$\mathrm{C}$ & 3.7284230 & -0.6545950 & 0.2653840 \\
$\mathrm{C}$ & 3.2809050 & -3.4311180 & 0.7835740 \\
$\mathrm{C}$ & 4.7335690 & -1.4401930 & 0.7967020 \\
$\mathrm{C}$ & 4.5414710 & -2.8119040 & 1.0893050 \\
H & 5.6891100 & -0.9831350 & 1.0420310 \\
$\mathrm{C}$ & -0.2262780 & -2.6828980 & 0.0708170 \\
$\mathrm{C}$ & -1.4958700 & -3.2122230 & -0.2870420 \\
$\mathrm{C}$ & 0.9823510 & -3.2538130 & -0.3187720 \\
$\mathrm{C}$ & -1.5131680 & -4.3758570 & -1.0320520
\end{tabular}




$\begin{array}{rrr}0.9449680 & -4.3932810 & -1.1913910 \\ -0.3271460 & -4.9788650 & -1.5144920 \\ -2.4704590 & -4.8149760 & -1.3026990 \\ 0.7752050 & -6.6576620 & -2.8941780 \\ 2.0256630 & -6.0522230 & -2.6188400 \\ 2.1080640 & -4.9510820 & -1.7902940 \\ -0.3765480 & -6.1241350 & -2.3555680 \\ 0.7255200 & -7.5297670 & -3.5424970 \\ 2.9305660 & -6.4553030 & -3.0685890 \\ 3.0732910 & -4.4964970 & -1.5941290 \\ -1.3488860 & -6.5596560 & -2.5798380 \\ 4.0879860 & -5.4931690 & 1.8088110 \\ 5.3449520 & -4.8936710 & 2.0679690 \\ 5.5610990 & -3.5774320 & 1.7184890 \\ 3.0838480 & -4.7813220 & 1.1837810 \\ 3.9097800 & -6.5233520 & 2.1096550 \\ 6.1296280 & -5.4681320 & 2.5554480 \\ 6.5128180 & -3.0944310 & 1.9337230 \\ 2.1252510 & -5.2530030 & 0.9947560 \\ 3.9266780 & 0.8160760 & 0.0965930 \\ 4.1305440 & 1.6272100 & 1.2399880 \\ 3.9657630 & 1.3981180 & -1.1924120 \\ 4.4181290 & 2.9860930 & 1.0599730 \\ 4.2452970 & 2.7633470 & -1.3135840 \\ 4.4946960 & 3.5728030 & -0.2025200 \\ 4.5917640 & 3.6104660 & 1.9348410 \\ 4.3026840 & 3.1973030 & -2.3100200 \\ -2.7742790 & -2.5258870 & 0.0628520 \\ -3.5811790 & -1.9979930 & -0.9714340 \\ -3.2078740 & -2.4344210 & 1.4062700 \\ -4.8253650 & -1.4411530 & -0.6453330 \\ -4.4449020 & -1.8453230 & 1.6784450 \\ -5.2839190 & -1.3596190 & 0.6690300 \\ -5.4409650 & -1.0532370 & -1.4511340 \\ -4.7780690 & -1.7841020 & 2.7131280 \\ 0.2130100 & -0.1238810 & 0.2389460 \\ 0.5874080 & 0.7547740 & 1.3990130 \\ -0.7957680 & 0.3663640 & -0.7646810 \\ 3.7851730 & 0.5893090 & -2.4707800 \\ 3.5624090 & -0.4480950 & -2.2074830 \\ 4.8982410 & 5.0284770 & -0.3646720 \\ 4.9637860 & 5.4660910 & 2.6417660 \\ 4.0727390 & 1.0856610 & 2.5654770 \\ 3.6942580 & 0.0600770 & \\ -2.4097950 & -3.0126810 & -1875530 \\ -1.4591910 & -3.3970470 & \end{array}$

-6.6504760
-6.4808610
-3.1679000
-2.1265130
-0.3950130
-0.1253970
-1.0582660
-0.1366710
1.0025170
0.9605050
0.2403690
0.0627570
0.4227240
-0.6322960
-0.0300800
0.6835120
1.5132590
-1.6953580
-0.4556150
1.2923760
-0.9843980
0.0814670
-3.2386850
-2.8982230
-4.2658760
-2.5977830
-4.0281120
-5.0850760
-3.7078420
-3.9483750
2.6076870
2.7890600
2.4856320
1.6753540
5.0857010
4.9557380
5.3547780
5.9191960
-2.0851510
-3.0060010
-1.4724390
-1.5336310
-3.1572090
-2.5594880
-4.1197470
-3.3544820
-0.7937750
-0.0073040
-2.0085840
-2.3366050
3.6465270
3.4078530
2.9225520
2.3811450
3.2389750
2.2171420
6.0787680
5.8961310
4.4695730
5.0782340
7.1012400
6.6131290
4.3224220
5.2935900
5.1492450
5.9284660
6.0921110
4.2591720
-0.6073710
-0.6598750
-0.2198080
0.0935530
-3.0043950
-2.7077810
-3.0261620
-4.0187490
1.1152680
2.1511280
0.5009780
1.0790860
0.5754610
-0.0424770
1.5913820
0.1692530
-1.9447570
-1.5544260
-2.3821580
-1.1108450
-4.2035140
-4.6388280
-3.8788550
-4.9831110
1.0402170

1.7931390

$-2.4404720$

$-2.5081970$

0.8830160

$-0.5422020$

1. 3611570

$-0.8869410$

1. 0050640

1.1693420

0.5432520

$-0.9726550$

$-1.4218090$

1. 3198590

0.8325900

$-1.5225420$

$-1.4344600$

1.1421300

2. 4002220

0.8188850

$-1.2376950$

$-2.4424800$

$-3.0770030$

-4. 1199170

$-3.0772730$

$-2.5346470$

$-3.2428990$

$-3.2108410$

$-4.2933540$

$-2.8328400$

3. 3125430

$-3.6293640$

$-4.2144220$

$-2.7392870$

$-3.2985620$

$-4.1971680$

$-3.6161970$

$-2.7113330$

3. 6278210

4. 0813420

4.4275120

3. 1809890

3. 2008080

4. 0129810

3. 6175020

2. 4537710 


\begin{tabular}{|c|c|c|c|c|c|c|c|}
\hline C & 3.1147490 & 18895260 & 3.5648770 & $c$ & 5.5661260 & 26530210 & -3.6425660 \\
\hline $\mathrm{H}$ & 3.1008570 & $\begin{array}{l}1.0091860 \\
1.4491\end{array}$ & 4.5708280 & $\mathrm{H}$ & 5.1776780 & 2.8849800 & -1.5295960 \\
\hline $\mathrm{H}$ & 3.4376350 & 2.9343510 & 3.6577720 & C & 3.6486660 & 2.0594540 & -4.9994850 \\
\hline $\mathrm{H}$ & 2.0972750 & 1.8703900 & 3.1637300 & $\mathrm{H}$ & 1.7841980 & 1.8228040 & -3.9400370 \\
\hline C & 5.4815680 & 1.0513590 & 3.2907330 & $\mathrm{C}$ & 5.0127470 & 2.3510100 & -4.8920970 \\
\hline $\mathrm{H}$ & 5.8909420 & 2.0677550 & 3.3631740 & $\mathrm{H}$ & 6.6265030 & 2.8790560 & -3.5559700 \\
\hline $\mathrm{H}$ & 5.4399280 & 0.6233860 & 4.3013270 & $\mathrm{H}$ & 3.2163800 & 1.8238640 & -5.9693510 \\
\hline $\mathrm{H}$ & 6.1723110 & 0.4513060 & 2.6860890 & $\mathrm{H}$ & 5.6426750 & 2.3424620 & -5.7790920 \\
\hline C & -7.4148130 & -0.1694840 & -0.1344700 & $\mathrm{H}$ & 0.7399020 & 1.8606400 & -0.7078760 \\
\hline $\mathrm{H}$ & -6.8277170 & 0.6090370 & -0.6335890 & 0 & 3.0560530 & 2.6437090 & -0.2669340 \\
\hline $\mathrm{H}$ & -7.6697090 & -0.9365780 & -0.8780910 & $\mathrm{C}$ & 3.0099750 & 0.9587550 & 2.0446250 \\
\hline $\mathrm{H}$ & -8.3499440 & 0.2780540 & 0.2238510 & $\mathrm{C}$ & 2.3624530 & 2.2664330 & 1.8626340 \\
\hline c & -7.5225280 & -1.8809490 & 1.7052450 & $\mathrm{H}$ & 2.7175810 & 0.1798670 & 1.3363090 \\
\hline $\mathrm{H}$ & -7.0231620 & -2.3153860 & 2.5788540 & $\mathrm{H}$ & 1.3485090 & 2.2715010 & 1.4774560 \\
\hline $\mathrm{H}$ & -8.4840220 & -1.4579610 & 2.0263290 & 0 & 2.1667890 & 0.9405700 & 3.2407140 \\
\hline $\mathrm{H}$ & -7.7210780 & -2.6890060 & 0.9882890 & $\mathrm{H}$ & 1.2797430 & 0.4919910 & 2.9441970 \\
\hline C & 3.8697160 & 5.8437520 & -1.1677530 & C & 4.9129590 & 2.1437810 & 3.2464460 \\
\hline $\mathrm{H}$ & 2.8953830 & 5.8479610 & -0.6679550 & $\mathrm{C}$ & 4.4632560 & 3.4682710 & 2.6123250 \\
\hline $\mathrm{H}$ & 4.2069080 & 6.8831080 & -1.2764950 & $\mathrm{C}$ & 2.9347400 & 3.5105290 & 2.4464650 \\
\hline $\mathrm{H}$ & 3.7400810 & 5.4233660 & -2.1737850 & $\mathrm{C}$ & 4.4889650 & 0.9496790 & 2.3753740 \\
\hline C & 6.2908440 & 5.1318380 & -1.0192710 & $\mathrm{H}$ & 6.0028100 & 2.1337350 & 3.3700050 \\
\hline $\mathrm{H}$ & 7.0399930 & 4.5818120 & -0.4363270 & $\mathrm{H}$ & 4.7840890 & 4.3210780 & 3.2223200 \\
\hline $\mathrm{H}$ & 6.2662170 & 4.7055100 & -2.0308690 & $\mathrm{H}$ & 2.4428780 & 3.6529420 & 3.4208640 \\
\hline $\mathrm{H}$ & 6.6040660 & 6.1819100 & -1.0952460 & $\mathrm{H}$ & 5.0235890 & 0.9749880 & 1.4162940 \\
\hline C & -3.1095130 & 2.8367130 & -0.5896630 & $\mathrm{H}$ & 4.7266630 & -0.0026150 & 2.8596500 \\
\hline 0 & -2.9059150 & 1.6299470 & -0.1093760 & $\mathrm{H}$ & 4.4691110 & 2.0420010 & 4.2459890 \\
\hline $\mathrm{C}$ & -4.4388800 & 3.4164650 & -0.2307740 & $\mathrm{H}$ & 4.9268750 & 3.5737020 & 1.6228260 \\
\hline c & -4.7430840 & 4.7303670 & -0.6255540 & $\mathrm{H}$ & 2.6079850 & 4.3482050 & 1.8189910 \\
\hline c & -5.3793780 & 2.6796560 & 0.5098710 & 0 & -2.0974870 & -0.1357460 & 1.0546710 \\
\hline $\mathrm{C}$ & -5.9683450 & 5.3005750 & -0.2824960 & 0 & 0.0147570 & -0.8012940 & -0.1922380 \\
\hline $\mathrm{H}$ & -4.0102900 & 5.2895270 & -1.2005170 & $\mathrm{C}$ & -2.7953630 & -0.2868400 & -0.1373910 \\
\hline C & -6.6033710 & 3.2543750 & 0.8517540 & C & -2.6213150 & -1.4491840 & -0.8809520 \\
\hline $\mathrm{H}$ & -5.1467530 & 1.6630090 & 0.8090050 & $\mathrm{C}$ & -3.6733570 & 0.7604290 & -0.5236200 \\
\hline c & -6.9002100 & 4.5636390 & 0.4582480 & $\mathrm{C}$ & -3.3121990 & -1.5660080 & -2.1344440 \\
\hline $\mathrm{H}$ & -6.1986130 & 6.3176680 & -0.5912850 & $\mathrm{C}$ & -4.3885760 & 0.6093350 & -1.6942570 \\
\hline $\mathrm{H}$ & -7.3288680 & 2.6782690 & 1.4209040 & $\mathrm{C}$ & -4.2269210 & -0.5266520 & -2.5248940 \\
\hline $\mathrm{H}$ & -7.8562470 & 5.0085170 & 0.7259250 & $\mathrm{H}$ & -5.0700290 & 1.3979630 & -2.0067150 \\
\hline $\mathrm{H}$ & -2.0209040 & 1.1795710 & -0.4041200 & C & -0.3536550 & $-\overline{2} .1343910$ & -0.1297380 \\
\hline 0 & -2.2863540 & 3.4796200 & -1.2627900 & $\mathrm{C}$ & 0.6586130 & -3.0806100 & 0.1866540 \\
\hline & & & & $\mathrm{C}$ & -1.6674340 & -2.4919070 & -0.4167290 \\
\hline & , R) $\mathrm{G}[\mathrm{B} 97-\mathrm{D} /$ & $\mathrm{ZVP}]=-3$ & 980 & C & 0.2947150 & -4.4105100 & 0.2802830 \\
\hline C & 2.5591440 & 2.3356800 & -1.3610260 & C & -2.0511390 & -3.8621610 & -0.2448130 \\
\hline 0 & 1.3139700 & 1.9648730 & -1.5580970 & $\mathrm{C}$ & -1.0459430 & -4.8306560 & 0.1007300 \\
\hline $\mathrm{C}$ & 3.3903290 & 2.3633520 & -2.6063060 & $\mathrm{H}$ & 1.0531160 & -5.1539980 & 0.5161970 \\
\hline $\mathrm{C}$ & 4.7586560 & 2.6605790 & -2.5060520 & C & -2.7310220 & -6.5902380 & 0.1249070 \\
\hline C & 839433 & 2.0634540 & 3.8629430 & C & -3.7281380 & -5.6322240 & -0.1826310 \\
\hline
\end{tabular}




$\begin{array}{rr}-3.3969640 & -4.3043780 \\ -1.4180700 & -6.1928330 \\ -3.0056030 & -7.6341300 \\ -4.7661790 & -5.9450480 \\ -4.1708810 & -3.5801060 \\ -0.6433550 & -6.9146980 \\ -3.7800930 & -2.7106000 \\ -4.7135010 & -1.7083240 \\ -4.9245570 & -0.6362390 \\ -3.0981210 & -2.6409650 \\ -3.5939010 & -3.5421950 \\ -5.2493660 & -1.7795280 \\ -5.6208280 & 0.1551590 \\ -2.3820310 & -3.4141860 \\ -3.7885890 & 2.0017140 \\ -3.2050480 & 3.1994740 \\ -4.4743560 & 1.9750510 \\ -3.3249600 & 4.4099130 \\ -4.5840690 & 3.1901980 \\ -4.0102410 & 4.3724370 \\ 2.0719400 & -2.6597150 \\ 2.6780390 & -2.8253480 \\ 2.8120840 & -2.1163860 \\ 4.0807790 & -2.4996760 \\ 4.1981240 & -1.7419960 \\ 4.7994620 & -1.9686070 \\ -0.5072060 & 0.2183470 \\ -0.2597750 & 1.5901280 \\ 0.0487730 & -0.1475030 \\ 5.8466420 & -1.7037880 \\ -4.0952130 & 5.2839530 \\ 2.5752250 & -3.4203500 \\ 1.9551350 & -3.2708410 \\ 4.6922210 & -2.6909580 \\ 3.9640210 & -3.1410920 \\ 1.9978030 & -3.7451230 \\ 0.8921020 & -3.4681550 \\ 5.7504570 & -2.4566610 \\ 4.4392540 & -3.2731640 \\ 2.9877530 & -1.3483530 \\ 2.2549220 & -1.9203800 \\ 4.9239910 & -1.1461190 \\ 4.3350410 & -0.9422670 \\ 2.5322510 & -1.1927370 \\ 1.2283500 & -2.2191300 \\ 5.9569680 & -0.8502720\end{array}$

-0.3632160
0.2665000
0.2594100
-0.2726020
-0.5963760
0.5193570
-4.2394310
-4.5997380
-3.7587560
-3.0411210
-4.9157110
-5.5436550
-4.0310940
-2.7848400
0.2906610
-0.1832660
1.5259790
0.6063750
2.3080570
1.8260550
0.3913030
1.6609080
-0.6881990
1.8294460
-0.4880190
0.7543270
0.9832340
0.4192400
2.3269470
0.8941340
2.4169500
4.0303830
2.8134090
3.1070930
4.1799830
4.8930540
2.7211220
3.2119040
5.1495800
-3.0025300
-1.9915280
-1.5658260
-2.7878350
-3.9774830
-2.1783290
-1.3897010

$\begin{array}{ll}\mathrm{H} & 4.8866270 \\ \mathrm{C} & -1.8968130 \\ \mathrm{C} & -2.4627830 \\ \mathrm{C} & -2.7272090 \\ \mathrm{C} & -2.0341100 \\ \mathrm{H} & -1.3258790 \\ \mathrm{H} & -2.3306670 \\ \mathrm{H} & -2.8313430 \\ \mathrm{H} & -1.5808580 \\ \mathrm{C} & -5.8395860 \\ \mathrm{C} & -5.2788570 \\ \mathrm{C} & -5.0808320 \\ \mathrm{C} & -5.7385430 \\ \mathrm{H} & -6.3634120 \\ \mathrm{H} & -5.3520560 \\ \mathrm{H} & -5.0121480 \\ \mathrm{H} & -6.1864330 \\ \mathrm{H} & \end{array}$
$-0.4630620$
4.4479070
3.2702560
5.6163400
5.6397460
4.4693790
2. 3676240
6.5178800
6.5640290
1. 9864500
3. 1515960
0.7876930
0.7911580
1. 9706450
4. 0714310
$-0.1308950$
$-0.1268040$

114

$-3.5925150$

$-1.8281990$

$-1.4048680$

0.1255450

$-1.0594460$

$-2.7540310$

$-1.9928060$

0.7273500

$-1.4118690$

4. 0190910

3. 5565660

2. 0435960

3. 2500960

4.9725070

4.1347220

1. 4675120

TS146(S,S) G[B97-D/def2-TZVP $]=-3217.918128$

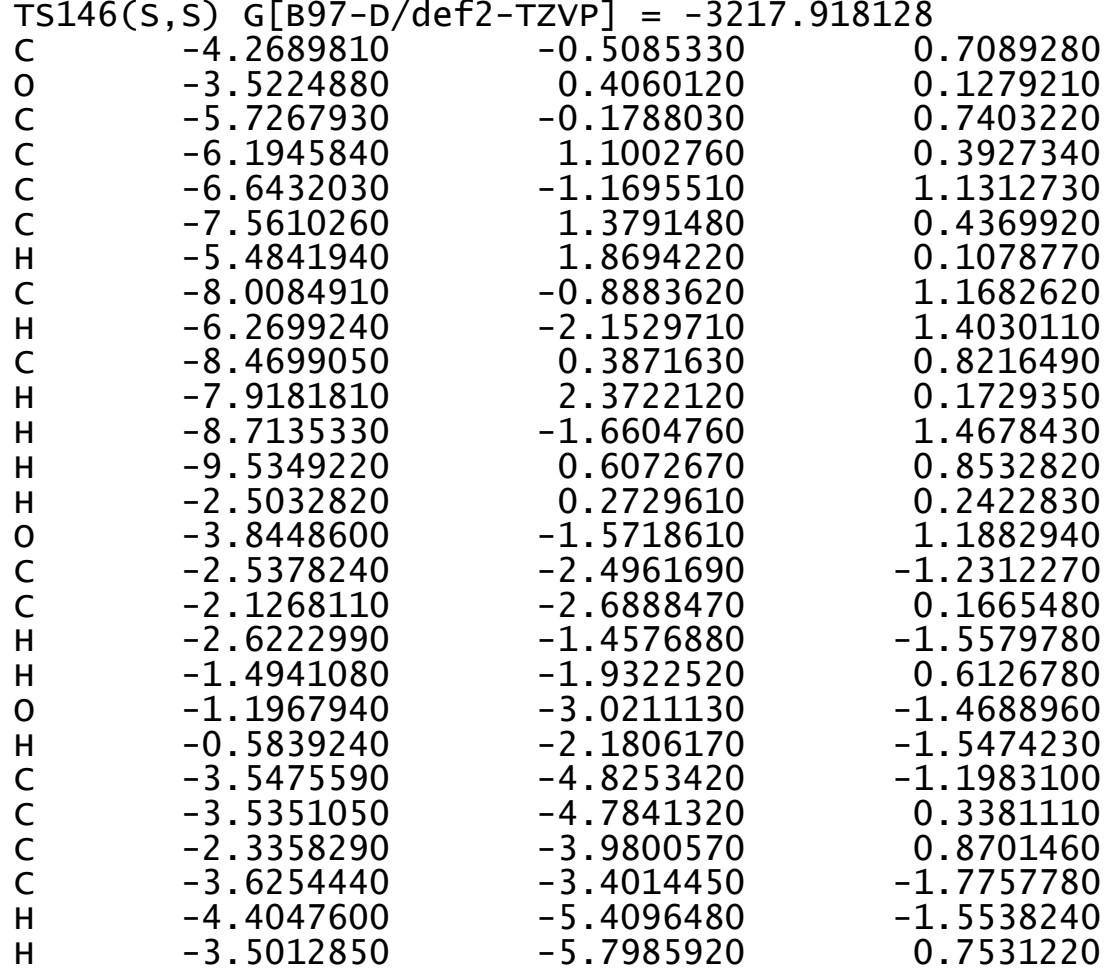




$\begin{array}{lrrr}\text { H } & -1.3999170 & -4.5471950 & \\ \text { H } & -4.5886220 & -2.9444760 & -1.5086270 \\ \text { H } & -3.5558240 & -3.4092690 & -2.8705890 \\ \text { H } & -2.6372680 & -5.3176780 & -1.5651830 \\ \text { H } & -4.4603810 & -4.3112350 & 0.6924060 \\ \text { H } & -2.4238190 & -3.7623430 & 1.9408700 \\ \text { O } & 0.9189010 & 1.3937930 & -0.9325520 \\ \text { O } & 1.2991160 & -0.5461800 & 0.6686410 \\ \mathrm{C} & 1.4862140 & 2.2416080 & 0.0101290 \\ \mathrm{C} & 2.6878290 & 1.8802020 & 0.6106020 \\ \mathrm{C} & 0.8099950 & 3.4592940 & 0.2900370 \\ \mathrm{C} & 3.2214640 & 2.7342370 & 1.6320650 \\ \mathrm{C} & 1.3640610 & 4.3062860 & 1.2293100 \\ \mathrm{C} & 2.5500190 & 3.9713010 & 1.9277040 \\ \mathrm{H} & 0.8667080 & 5.2471810 & 1.4555150 \\ \mathrm{C} & 2.6283510 & -0.5835650 & 0.2788780 \\ \mathrm{C} & 3.1934600 & -1.8519580 & -0.0223960 \\ \mathrm{C} & 3.3492170 & 0.6051090 & 0.2226140 \\ \mathrm{C} & 4.5158390 & -1.8932110 & -0.4197120 \\ \mathrm{C} & 4.6982880 & 0.5612630 & -0.2601170 \\ \mathrm{C} & 5.2874850 & -0.7143770 & -0.5673370 \\ \mathrm{H} & 4.9717960 & -2.8535100 & -0.6517090 \\ \mathrm{C} & 7.3562800 & 0.3852260 & -1.2358050 \\ \mathrm{C} & 6.7650410 & 1.6450070 & -0.9713790 \\ \mathrm{C} & 5.4718860 & 1.7307950 & -0.4962340 \\ \mathrm{C} & 6.6268260 & -0.7687620 & -1.0408400 \\ \mathrm{H} & 8.3784490 & 0.3326690 & -1.6040440 \\ \mathrm{H} & 7.3340600 & 2.5549960 & -1.1494740 \\ \mathrm{H} & 5.0304300 & 2.7031900 & -0.3039120 \\ \mathrm{H} & 7.0596540 & -1.7435250 & -1.2591970 \\ \mathrm{C} & 4.8434230 & 3.2410860 & 3.3836170 \\ \mathrm{C} & 4.2029610 & 4.4769690 & 3.6450660 \\ \mathrm{C} & 3.0759190 & 4.8283890 & 2.9325010 \\ \mathrm{C} & 4.3655290 & 2.3930150 & 2.4047510 \\ \mathrm{H} & 5.7184460 & 2.9542440 & 3.9629350 \\ \mathrm{H} & 4.5941900 & 5.1385780 & 4.4147540 \\ \mathrm{H} & 2.5604410 & 5.7656200 & 3.1350010 \\ \mathrm{H} & 4.8646030 & 1.4472340 & 2.2206440 \\ \mathrm{C} & -0.4534440 & 3.8174110 & -0.4125760 \\ \mathrm{C} & -1.6748920 & 3.8367430 & 0.3005840 \\ \mathrm{C} & -0.4189740 & 4.1568600 & -1.7857690 \\ \mathrm{C} & -2.8921200 & 4.2345070 & -0.3810820 \\ \mathrm{C} & -1.6467900 & 4.5266780 & -2.4613730 \\ \mathrm{C} & -2.8457310 & 4.5621530 & -1.7402880 \\ \mathrm{C} & 2.3906120 & -3.1037310 & 0.0770840 \\ \mathrm{C} & 2.0548290 & -3.8159050 & -1.0994280 \\ & & & \\ & & & \end{array}$

$\begin{array}{lr}\text { C } & 1.9890790 \\ \mathrm{C} & 1.3321310 \\ \mathrm{C} & 1.2550780 \\ \mathrm{C} & 0.9612530 \\ \mathrm{P} & 0.1972520 \\ \mathrm{O} & -1.0108830 \\ \mathrm{O} & 0.0953270 \\ \mathrm{H} & 0.4203050 \\ \mathrm{H} & -3.7641180 \\ \mathrm{C} & 2.0185730 \\ \mathrm{C} & 2.3692550 \\ \mathrm{C} & 0.9959470 \\ \mathrm{C} & 1.3302590 \\ \mathrm{H} & 2.2576600 \\ \mathrm{H} & 2.8738660 \\ \mathrm{H} & 0.4568870 \\ \mathrm{H} & 1.0620520 \\ \mathrm{C} & 1.8680800 \\ \mathrm{C} & 2.2873240 \\ \mathrm{C} & 0.8424080 \\ \mathrm{C} & 1.1323510 \\ \mathrm{H} & 2.1000210 \\ \mathrm{H} & 2.8508280 \\ \mathrm{H} & 0.2879470 \\ \mathrm{H} & 0.8081970 \\ \mathrm{C} & -2.9771110 \\ \mathrm{C} & -1.7758140 \\ \mathrm{C} & -4.1181800 \\ \mathrm{C} & -4.1631520 \\ \mathrm{H} & -3.0272550 \\ \mathrm{H} & -0.8847490 \\ \mathrm{H} & -5.0201540 \\ \mathrm{H} & -5.1048690 \\ \mathrm{C} & -0.4204990 \\ \mathrm{C} & -1.6036850 \\ \mathrm{C} & 0.7909250 \\ \mathrm{C} & 0.7910120 \\ \mathrm{H} & -0.4018910 \\ \mathrm{H} & -2.5355540 \\ \mathrm{H} & 1.7243450 \\ \mathrm{H} & 1.7250520 \\ \mathrm{H} & -\end{array}$

1. 3449280 $-0.9951120$

1.4341700

0.2653310

$-0.4030650$

0.4391610

$-1.6121070$

0.3392010

$-2.2499210$

$-3.5322160$

$-2.4101280$

$-2.1867730$

$-3.4231960$

$-4.5168970$

$-2.5114880$

$-2.0889330$

$-4.3238420$

3.7785140

2.5635930

2.7175010

3.8597320

4.6889800

2. 5202580

2.7672130

4.8300630

2. 3411280

1.6758720

0.3520290

1.6775640

3.3836330

2. 1899850

$-0.1719380$

2. 2215160

$-4.5474210$

$-3.8510690$

$-2.5486360$

$-3.8835790$

$-5.6053910$

$-4.3451620$

$-2.0587900$

$-4.4412790$

TS147(S, S) G[B97-D/def2-TZVP $]=-3217.917606$

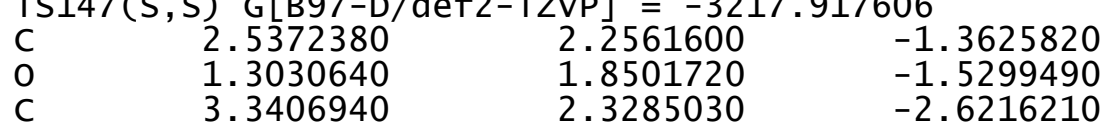




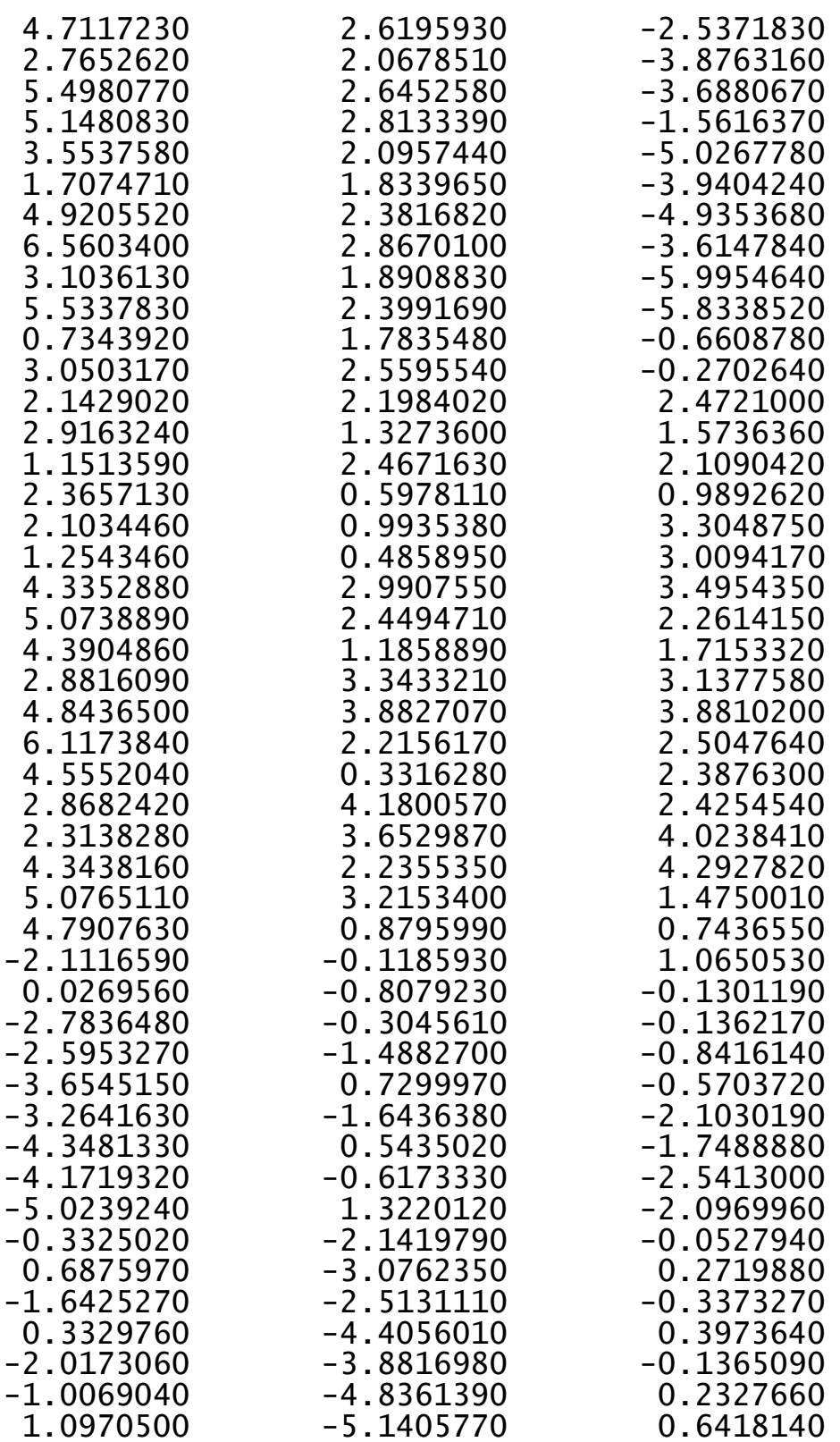

-2.6828910
-3.6844140
-3.3599160
-1.3724270
-2.9519750
-4.7201990
-4.1360090
-0.5946600
-3.6943890
-4.6216770
-4.8476980
-3.0335550
-3.4961250
-5.1410140
-5.5392890
-2.3217650
-3.7842850
-3.1836770
-4.4989570
-3.3148070
-4.6202140
-4.0282590
2.1010110
2.7266820
2.8170730
4.1280290
4.2059090
4.8296460
-0.5168120
-0.2301370
0.0017770
5.8782530
-4.1212120
2.6567260
2.0230280
4.7532390
4.0407530
2.0935940
0.9628440
5.8086090
4.5255360
2.9389970
2.2318210
4.9070860
4.2895510
2.4598930
0.2929530 $-0.0406480$ $-0.2506970$ 0.4291480

0.4509190

$-0.1275680$

$-0.5044670$

0.7001730

$-4.1803820$

$-4.5874430$

$-3.7834760$

$-2.9729970$

$-4.8274330$

$-5.5379270$

$-4.0921710$

$-2.6814530$

0.2056510

$-0.2861050$

1. 4246380

0.4688490

2.1718330

1. 6727550

0.4372920

1.7034150

$-0.6778740$

1.8352470

$-0.5210590$

0.7194800

1.0320150

0.4675760

2. 3888250

0.8281110

2. 2375370

4.1034960

2. 8861880

3. 1167300

4. 2225290

4.9897820

2. 8150490

3. 1968410

5. 1945180

$-3.0188200$

$-1.9749160$

$-1.6339170$

-2.8476820
-3.9868680 


\begin{tabular}{|c|c|c|c|c|c|c|}
\hline & & & & & & \\
\hline 1.2023990 & -2.2907980 & -2.1305420 & $\mathrm{H}$ & -4.7924960 & -2.4243340 & $\begin{array}{l}-2.3412940 \\
-0\end{array}$ \\
\hline 4.8217690 & -0.5669210 & -3.6785690 & $\mathrm{H}$ & -6.3731600 & -1.0081150 & -0.9795820 \\
\hline-1.8311390 & 4.3745400 & -1.9323990 & $\mathrm{H}$ & -2.3142890 & 0.9488290 & -2.5964880 \\
\hline-2.4135590 & 3.2112380 & -1.4918240 & $\mathrm{C}$ & -5.7144540 & 0.1194390 & -2.7442310 \\
\hline-2.6986390 & 5.5975640 & -0.0289780 & $\mathrm{H}$ & -6.0680410 & -0.6298210 & -3.4650650 \\
\hline-1.9781250 & 5.5863290 & -1.1975460 & $\mathrm{H}$ & -6.5086210 & 0.8654830 & -2.6224550 \\
\hline-1.2392100 & 4.3691300 & -2.8451960 & $\mathrm{C}$ & -4.3980140 & 0.7905500 & -3.2477410 \\
\hline-2.2741520 & 2.2929360 & -2.0531800 & $\mathrm{H}$ & -4.1210960 & 0.4036880 & -4.2373890 \\
\hline-2.8102450 & 6.5148020 & 0.5471780 & $\mathrm{H}$ & -4.5259980 & 1.8758110 & -3.3382190 \\
\hline-1.5102550 & 6.4983830 & -1.5624190 & $\mathrm{C}$ & -3.5449230 & 2.3612820 & 1.1006770 \\
\hline-5.9225090 & 2.0874910 & 3.8835070 & $\mathrm{C}$ & -2.9806600 & 3.3723280 & 2.0721650 \\
\hline-5.3443490 & 3.2372130 & 3.4040930 & $\mathrm{C}$ & -5.2299210 & 2.3413320 & 2.8674280 \\
\hline-5.1235660 & 0.8332100 & 1.9589610 & $\mathrm{C}$ & -4.7626520 & 1.8047290 & 1.5385040 \\
\hline-5.8097620 & 0.8718060 & 3.1488510 & $\mathrm{C}$ & -4.0334390 & 4.5016810 & 2.2498590 \\
\hline-6.4689880 & 2.0994700 & 4.8241750 & $\mathrm{H}$ & -3.6505510 & 5.2314810 & 2.9753080 \\
\hline-5.4259760 & 4.1723920 & 3.9558950 & $\mathrm{H}$ & -4.1777130 & 5.0245420 & 1.2965450 \\
\hline-5.0461460 & -0.1006670 & 1.4091300 & C & -5.3792420 & 3.8847410 & 2.7480960 \\
\hline-6.2714290 & -0.0337490 & 3.5366750 & $\mathrm{H}$ & -5.6503780 & 4.2907940 & 3.7316250 \\
\hline & & & $\mathrm{H}$ & -6.1949050 & 4.1210710 & 2.0544260 \\
\hline R) G[B97-D & {$[Z \mathrm{VP}]=-354$} & 722 & $\mathrm{H}$ & -6.1824870 & 1.8850430 & 3.1641400 \\
\hline 0.3271270 & 0.0951640 & 0.8083320 & $\mathrm{H}$ & -2.0309030 & 3.7833230 & 1.7214180 \\
\hline 0.5774050 & -1.3801920 & 0.6355170 & C & -4.1280980 & 2.0492590 & 3.9226530 \\
\hline-0.3309160 & 0.5735300 & 2.0712390 & $\mathrm{H}$ & -4.4433480 & 2.4631430 & 4.8898820 \\
\hline-0.5112700 & 0.5414170 & -0.5269970 & $\mathrm{H}$ & -4.0109920 & 0.9665690 & 4.0444170 \\
\hline 1.6994450 & 0.9572930 & 0.6873050 & C & -2.7865150 & 2.6897490 & 3.4545630 \\
\hline-0.5549440 & 1.9009970 & -0.8426130 & $\mathrm{H}$ & -2.4521900 & 3.4517500 & 4.1710830 \\
\hline 0.6029040 & 2.5029290 & -1.3550980 & $\mathrm{H}$ & -1.9980400 & 1.9376760 & 3.3766690 \\
\hline-1.7404590 & 2.6144740 & -0.6309890 & $\mathrm{C}$ & -5.4218380 & 0.8465420 & 0.7696840 \\
\hline-1.7107650 & 3.9841690 & -0.9066970 & $\mathrm{H}$ & -6.3641800 & 0.4173550 & 1.1114600 \\
\hline-2.6215650 & 4.5612370 & -0.7535420 & $\mathrm{C}$ & 5.1527090 & -0.5511350 & 2.7254770 \\
\hline 2.3701690 & 0.9509190 & -0.5371610 & C & 5.5386290 & 0.3175360 & 3.8960670 \\
\hline 3.5680050 & 0.2366730 & -0.6490630 & $\mathrm{C}$ & 4.4664270 & 1.6428720 & 1.9361000 \\
\hline 1.8121250 & 1.6682920 & -1.6061730 & C & 4.5588870 & 0.1588500 & 1.6652120 \\
\hline 4.1877330 & 0.2449150 & -1.9032370 & $\mathrm{C}$ & 6.5421010 & 1.3918800 & 3.3919720 \\
\hline 5.1299730 & -0.2891040 & -2.0152670 & $\mathrm{H}$ & 6.7827490 & 2.0718470 & 4.2202770 \\
\hline-2.9822150 & 1.9713310 & -0.1280930 & $\mathrm{H}$ & 7.4738060 & 0.9050410 & 3.0799410 \\
\hline 4.1599240 & -0.5078530 & 0.4922370 & C & 5.9031290 & 2.1752780 & 2.2024650 \\
\hline-3.6507 & 77290 & -0.8914650 & $\mathrm{H}$ & 5.8439290 & 3.2474260 & 2.4325340 \\
\hline-3.2432 & 3350 & -2.2525860 & $\mathrm{H}$ & 6.5078830 & 2.0623740 & 1.2946340 \\
\hline-5.4459 & 5940 & -1.3828490 & $\mathrm{H}$ & 4.0157390 & 2.1827680 & 1.0994690 \\
\hline-4.8578 & 2090 & -0.4370020 & $\mathrm{H}$ & 5.9862060 & -0.2792760 & 4.7002460 \\
\hline-3.0781 & 33800 & -2.1717300 & C & 3.6318810 & 1.8613100 & 3.2283700 \\
\hline-2.8467 & -1 & -3.1719340 & $\mathrm{H}$ & 3.6210900 & 2.9351670 & 3.4590500 \\
\hline-2.2268 & 4930 & -1.5280960 & $\mathrm{H}$ & 2.5995410 & 1.5481380 & 3.0504650 \\
\hline-4.3902 & 92 & -1.6258710 & $\mathrm{C}$ & 4.2679640 & 1.0547320 & 4.4028540 \\
\hline
\end{tabular}




$\begin{array}{lrrr}\text { H } & 4.5507260 & 1.7228590 & \\ \text { H } & 3.5545380 & 0.3212930 & 4.7974960 \\ \mathrm{C} & 4.9631350 & -2.5975190 & 1.4737870 \\ \mathrm{C} & 5.1034490 & -4.0785330 & 1.2239220 \\ \mathrm{C} & 3.9524630 & -2.8062440 & -0.7275060 \\ \mathrm{C} & 4.3596320 & -1.8984780 & 0.4106650 \\ \mathrm{C} & 3.6859220 & -4.6690500 & 0.9902050 \\ \mathrm{H} & 3.7813520 & -5.7347840 & 0.7412590 \\ \mathrm{H} & 3.1025680 & -4.5939840 & 1.9158250 \\ \mathrm{C} & 2.9882420 & -3.8881110 & -0.1651630 \\ \mathrm{H} & 2.7101230 & -4.5685850 & -0.9809410 \\ \mathrm{H} & 2.0782670 & -3.3991770 & 0.1900690 \\ \mathrm{H} & 3.4606690 & -2.2526270 & -1.5311250 \\ \mathrm{H} & 5.5965610 & -4.5763450 & 2.0678350 \\ \mathrm{C} & 5.2206390 & -3.5295650 & -1.2575850 \\ \mathrm{H} & 4.9210020 & -4.2364670 & -2.0428760 \\ \mathrm{H} & 5.9012090 & -2.7984950 & -1.7109040 \\ \mathrm{C} & 5.9231380 & -4.2799340 & -0.0815900 \\ \mathrm{H} & 5.9946380 & -5.3547940 & -0.2956390 \\ \mathrm{H} & 6.9418780 & -3.9018800 & 0.0657190 \\ \mathrm{C} & 5.3588110 & -1.9277720 & 2.6311510 \\ \mathrm{H} & 5.8242580 & -2.4734960 & 3.4530070 \\ \mathrm{C} & 3.6235180 & 0.8717540 & -3.0184830 \\ \mathrm{C} & 2.4067410 & 1.5679180 & -2.8829090 \\ \mathrm{C} & 4.3692140 & 0.8152290 & -4.3429650 \\ \mathrm{C} & 3.4856790 & 1.1048380 & -5.5635360 \\ \mathrm{C} & 2.6160030 & 2.3410260 & -5.3040130 \\ \mathrm{C} & 1.6809220 & 2.0779890 & -4.1177110 \\ \mathrm{H} & 4.1141600 & 1.2440960 & -6.4527480 \\ \mathrm{H} & 5.1793060 & 1.5619300 & -4.3116520 \\ \mathrm{H} & 4.8581020 & -0.1629290 & -4.4437960 \\ \mathrm{H} & 3.2629880 & 3.2031590 & -5.0807560 \\ \mathrm{H} & 2.0237550 & 2.5951660 & -6.1926350 \\ \mathrm{H} & 1.0793480 & 2.9605210 & -3.8887050 \\ \mathrm{H} & 0.9587700 & 1.2982880 & -4.4123350 \\ \mathrm{H} & 2.8285280 & 0.2437270 & -5.7568990 \\ \mathrm{C} & 0.6272600 & 3.9054350 & -1.5425080 \\ \mathrm{C} & 1.9283630 & 4.6062940 & -1.9037560 \\ \mathrm{C} & 1.9057570 & 6.1108920 & -1.6024110 \\ \mathrm{C} & 0.6351740 & 6.7390190 & -2.1856990 \\ \mathrm{C} & -0.5943530 & 6.1566320 & -1.4778960 \\ \mathrm{C} & -0.5503990 & 4.6442560 & -1.3253840 \\ \mathrm{H} & 2.8059020 & 6.5803410 & -2.0193720 \\ \mathrm{H} & 2.1440700 & 4.4743160 & -2.9736520 \\ \mathrm{H} & 2.7546650 & 4.1179590 & -1.3723400 \\ \mathrm{H} & 0.5866650 & 6.5162540 & -3.2624420 \\ & & & \\ & & & \end{array}$

0.6456830
-1.5163500
-0.6668640
1.9231270
-1.5506740
-0.4592440
-2.1490740
-3.2622280
-1.6451400
-3.8628730
-3.6494200
-2.2514320
-0.7910350
-3.3600160
-4.7245940
-1.8607090
-3.8315420
-0.0961370
-2.0933660
-2.7711940
-1.7422460
-2.6810760
-0.7481610
-2.0754350
-1.3437090
-4.3091440
-3.4250360
-1.9490130
-4.2028300
-5.3555200
-3.5132670
-1.5147180
-4.6208880
-4.7651380
-3.9996110
-3.7576690
-1.3362790

7.8310750

6.4387760

6.6032410

6.2732720

$-3.6912010$

$-3.0342470$

$-4.4626510$

$-5.2839100$

$-4.3477980$

$-5.9804760$

$-5.3614490$

$-5.0418310$

$-3.7054530$

$-5.8588150$

$-6.6149370$

$-4.9437380$

$-6.3983290$

$-2.3757480$

$-3.7022060$

$-1.4559170$

$-2.4903640$

$-0.8521460$

$-2.2773610$

$-0.8364150$

$-0.2318710$

$-2.7716540$

$-4.0111080$

$-3.6171060$

$-1.8241000$

$-3.0714920$

$-4.6983990$

$-3.2971640$

$-2.3075320$

$-0.8997920$

$-2.2416890$

$-4.5482990$

$-4.4585390$
$-2.0765680$

$-2.0047830$

$-0.4733920$

$-0.5143840$

$-0.6451650$

$-0.9587350$

$-1.7789160$

$-1.5375200$

$-3.0852940$

$-2.5852600$

$-0.5258170$

$-4.1327800$

$-3.2736890$

$-3.8857340$

$-2.3908750$

$-5.1430450$

$-4.7044020$

$-0.2410780$

0.4719580

2.1293540

2. 3297880

1.2221220

1.9528530

3.2563740

2. 8290490

3.6686980

3.4635740

3.2834420

2.4637370

3.8036190

4.3134850

4.2425580

1.5704480

2.6249580

4.5796730

2. 5658210

2.9394620

$\operatorname{TS} 149(\mathrm{R}, \mathrm{R}) \quad \mathrm{G}[\mathrm{B} 97-\mathrm{D} / \mathrm{def2}-\mathrm{TZVP}]=-3541.575978$

$\begin{array}{lrrr}\mathrm{P} & -0.3717250 & -0.1218050 & 0.9053420 \\ \mathrm{O} & -0.6414380 & 1.3428440 & 0.6805890 \\ \mathrm{O} & 0.2638540 & -0.5490090 & 2.1987850 \\ \mathrm{O} & 0.5130120 & -0.5915770 & -0.3938080 \\ \mathrm{O} & -1.7294340 & -1.0063090 & 0.7757650 \\ \mathrm{C} & 0.5996640 & -1.9542720 & -0.6825810 \\ \mathrm{C} & -0.5419750 & -2.6086680 & -1.1710220\end{array}$




\begin{tabular}{|c|c|c|}
\hline $\begin{array}{r}1.8176840 \\
1.8396560 \\
2.7789590 \\
-2.3496380 \\
-3.5267440 \\
-1.7533240 \\
-4.0616880 \\
-4.9799070 \\
3.0505370 \\
-4.1883200 \\
3.6487290 \\
3.1665730 \\
5.3509630 \\
4.8482520 \\
2.9368010 \\
2.6632390 \\
2.0937910 \\
4.2349790 \\
4.5746600 \\
4.0513600 \\
6.2729260 \\
2.2466020 \\
5.5957080 \\
5.8796750 \\
6.4314680 \\
4.2951720 \\
3.9624370 \\
4.4719140 \\
3.6768270 \\
3.1919820 \\
5.4141050 \\
4.8820200 \\
4.3016010 \\
3.9757820 \\
4.4390710 \\
5.6332590 \\
5.9592750 \\
6.4335240 \\
6.3517390 \\
2.2531440 \\
4.3308040 \\
4.6930130 \\
4.1694460 \\
3.0042400 \\
2.7179890 \\
2.1849930\end{array}$ & $\begin{array}{r}-2.6168650 \\
-3.9896140 \\
-4.5253850 \\
-1.0375480 \\
-0.3065130 \\
-1.8006840 \\
-0.3238940 \\
0.2317730 \\
-1.9091040 \\
0.4565870 \\
-0.9454110 \\
-0.5145280 \\
0.6857590 \\
-0.3140110 \\
1.0185410 \\
1.3493650 \\
1.2412250 \\
1.7395040 \\
2.4643430 \\
2.2925660 \\
1.1689040 \\
-1.0309490 \\
-0.0596080 \\
0.6746680 \\
-0.7598000 \\
-0.8155540 \\
-0.4932600 \\
-1.8970020 \\
-2.2220730 \\
-3.2101670 \\
-2.0387510 \\
-1.5923570 \\
-4.2786990 \\
-4.9826760 \\
-4.8475790 \\
-3.5770000 \\
-3.9217100 \\
-3.8091440 \\
-1.5241220 \\
-3.6810270 \\
-1.7475660 \\
-2.1047160 \\
-0.6668950 \\
-2.4640530 \\
-3.1979370 \\
-1.7471440\end{array}$ & $\begin{array}{l}-0.4920450 \\
-0.7564570 \\
-0.6269430 \\
-0.4770290 \\
-0.6768250 \\
-1.4880400 \\
-1.9683990 \\
-2.1520100 \\
-0.0587520 \\
0.4126080 \\
-0.8904490 \\
-2.2554720 \\
-1.5220690 \\
-0.5110260 \\
-2.2369860 \\
-3.2468730 \\
-1.5814300 \\
-1.7600510 \\
-2.5104300 \\
-0.8321650 \\
-1.1760100 \\
-2.5417650 \\
-2.8644980 \\
-3.6301920 \\
-2.7487720 \\
-3.2807140 \\
-4.2762940 \\
-3.3222590 \\
1.1602180 \\
2.1951760 \\
2.8655040 \\
1.5272640 \\
2.3978830 \\
3.1748070 \\
1.4703250 \\
2.8149580 \\
3.8052910 \\
2.1022040 \\
3.1096100 \\
1.8925930 \\
3.9414020 \\
4.9147410 \\
4.0218910 \\
3.5451810 \\
4.3101610 \\
3.4486810\end{array}$ \\
\hline
\end{tabular}

5.4725260
6.4059270
-5.3506460
-5.8243730
-4.6032090
-4.6753160
-6.7835000
-7.0825540
-7.6911680
-6.0553550
-6.0141340
-6.5884530
-4.0887410
-6.3338150
-3.8701450
-3.8755810
-2.8275550
-4.5945410
-4.9369040
-3.9143020
-5.0715100
-5.2015420
-3.8939360
-4.3822610
-3.7739220
-3.8548230
-3.2624400
-2.9865160
-2.6589980
-2.0978930
-3.3367000
-5.7629550
-5.1249850
-4.7762410
-5.7656950
-5.9154960
-5.9667140
-6.9437660
-5.5541610
-6.0858470
-2.2686090
-1.5231570
-1.9356790
-3.4639660
-4.0428900
-3.4428370
-0.6453820
-0.1607680
0.5209400
-0.3373800
-1.6798300
-0.1983220
-1.4203060
-2.0938120
-0.9402560
-2.2118300
-3.2823070
-2.1061990
-2.2264440
0.2657410
-1.8839430
-2.9552230
-1.5703810
-1.0658790
-1.7258880
-0.3261860
2.5538440
4.0306740
2.7420760
1.8459450
4.6280530
5.6918390
4.5609780
3.8437140
4.5178240
3.3722150
2.1814000
4.5351290
3.4430840
4.1353310
2.6955280
4.2119010
5.2835230
3.8380080
1.8953600
2.4480390
-1.7274650
-2.3873510
-1.8464680
-1.8293490
-0.8428800
-0.9869620
0.6911100

0.9798520

2.5623380

3. 7082020

1.8489300

1. 5584440

3. 1388190

3.9531970

2.7538950

2.0078150

2. 2493500

1.0552550

1.0546010

4.4696710

3. 2029910

3. 4451010

3. 1001570

4. 3172500

5. 1256750

4.7557760

1. 3054990

1.0261960

$-0.8132050$

0.3019080

0.8977260

0.6355250

1.8655170

$-0.1953240$

$-0.9978680$

0.2296490

$-1.5678690$

1.8220880

$-1.4491820$

$-2.2272880$

$-1.9325680$

$-0.3428680$

$-0.5776570$

$-0.2706360$

2. 4361310

3. 2118460

$-2.8032250$

$-3.9535500$

5.4450090

$-4.4235560$

$-3.0342320$ 


$\begin{array}{rrr}-1.4866820 & -2.4670970 & -6.1154890 \\ -1.7003360 & -3.4723270 & -3.9440340 \\ -0.4442770 & -2.2623370 & -3.7969910 \\ -3.8496290 & -2.8408330 & -5.2460240 \\ -3.7828310 & -1.5494830 & -6.4572780 \\ -5.1344750 & -0.9477560 & -4.3569430 \\ -3.8542510 & 0.1831710 & -4.7778840 \\ -1.5521890 & -0.8231530 & -5.4586590 \\ -0.5135130 & -4.0108830 & -1.3367750 \\ 0.6995550 & -4.6994240 & -1.1454610 \\ -1.7988580 & -4.7852720 & -1.5794530 \\ -1.5674920 & -6.1754710 & -2.1826330 \\ -0.5254900 & -6.9362710 & -1.3537660 \\ 0.8180940 & -6.1958560 & -1.3911150 \\ -2.5168470 & -6.7253730 & -2.2169070 \\ -2.2909900 & -4.9085650 & -0.6003670 \\ -2.5000220 & -4.2081140 & -2.1863930 \\ -0.8802500 & -7.0063560 & -0.3146980 \\ -0.3958360 & -7.9606140 & -1.7265090 \\ 1.5177080 & -6.6290640 & -0.6638940 \\ 1.2734070 & -6.3465090 & -2.3835040 \\ -1.2072710 & -6.0746090 & -3.2181450 \\ 1.4097260 & 3.6942950 & -0.6271540 \\ 0.3740850 & 2.9608760 & -0.9584380 \\ 2.0303280 & 4.4335040 & -1.7705590 \\ 3.1174590 & 5.2872490 & -1.5234870 \\ 1.5792090 & 4.2527850 & -3.0885580 \\ 3.7465840 & 5.9485900 & -2.5770300 \\ 3.4638700 & 5.4152960 & -0.5021430 \\ 2.2127630 & 4.9133470 & -4.1416160 \\ 0.7451520 & 3.5858660 & -3.2810320 \\ 3.2971520 & 5.7605390 & -3.8891010 \\ 4.5894310 & 6.6065600 & -2.3780650 \\ 1.8630180 & 4.7645910 & -5.1606730 \\ 3.7912940 & 6.2720850 & -4.7123520 \\ 0.0084490 & 2.3238320 & -0.2216020 \\ 1.8880300 & 3.7959740 & 0.5145430 \\ 2.6296810 & 1.5612810 & 2.1394980 \\ 1.5970640 & 2.5815550 & 2.3806900 \\ 2.5021870 & 0.9522660 & 1.2407300 \\ 0.5934510 & 2.3555730 & 2.0380300 \\ 1.9921460 & 0.9410570 & 3.3000960 \\ 1.2630740 & 0.3069570 & 2.9081890 \\ 4.2191330 & 2.9012310 & 3.5996040 \\ 3.3075820 & 4.1271200 & 3.4360390 \\ 1.8299500 & 3.7135500 & \\ -1.3222160\end{array}$

$\begin{array}{lll}4.0701960 & 1.9498110 & 2.4022670 \\ 5.2659620 & 3.2172560 & 3.6847730 \\ 3.4245690 & 4.8155280 & 4.2815520 \\ 1.4421800 & 3.3911130 & 4.3001090 \\ 4.4372180 & 2.4357850 & 1.4880430 \\ 4.6525980 & 1.0341900 & 2.5374440 \\ 3.9613250 & 2.3672610 & 4.5243260 \\ 3.5904080 & 4.6687380 & 2.5241340 \\ 1.1929070 & 4.5465160 & 3.0026960\end{array}$

TS150(R,R) G[B97-D/def2-TZVP] = -3541.575907

$\begin{array}{lrrr}\text { TS150(R, R) G[B97-D/def2-TZVP] }=-3541.575907 \\ \text { P } & 0.3231570 & 0.1328700 & 0.8088130 \\ \text { O } & 0.6203190 & -1.3347170 & 0.6414870 \\ \text { O } & -0.3458730 & 0.5957170 & 2.0708980 \\ \text { O } & -0.5335430 & 0.5440500 & -0.5267070 \\ \text { O } & 1.6681070 & 1.0354470 & 0.6717330 \\ \text { C } & -0.5982710 & 1.8948720 & -0.8727970 \\ \text { C } & 0.5485950 & 2.5021610 & -1.4072810 \\ \text { C } & -1.7909720 & 2.5956390 & -0.6606430 \\ \text { C } & -1.7881690 & 3.9571510 & -0.9761330 \\ \text { H } & -2.7044670 & 4.5247960 & -0.8211060 \\ \text { C } & 2.3312210 & 0.9858210 & -0.5577420 \\ \text { C } & 3.5142270 & 0.2453020 & -0.6599010 \\ \text { C } & 1.7646080 & 1.6692760 & -1.6427720 \\ \text { C } & 4.1022670 & 0.1803820 & -1.9267940 \\ \text { H } & 5.0270100 & -0.3835580 & -2.0373500 \\ \text { C } & -3.0094500 & 1.9466040 & -0.1103740 \\ \text { C } & 4.1230960 & -0.4491570 & 0.5040490 \\ \text { C } & -3.6813770 & 0.9452270 & -0.8332730 \\ \text { C } & -3.3075910 & 0.4192750 & -2.1986150 \\ \text { C } & -5.4579110 & -0.6790810 & -1.2392030 \\ \text { C } & -4.8595080 & 0.3638960 & -0.3290080 \\ \text { C } & -3.1069930 & -1.1147210 & -2.0973870 \\ \text { H } & -2.8928950 & -1.5142730 & -3.0965840 \\ \text { H } & -2.2346830 & -1.3112770 & -1.4719300 \\ \text { C } & -4.3894710 & -1.7748250 & -1.5049660 \\ \text { H } & -4.8009720 & -2.5169570 & -2.2006750 \\ \text { H } & -4.1544050 & -2.2964800 & -0.57132000 \\ \text { H } & -6.3619340 & -1.1170480 & -0.7983440 \\ \text { H } & -2.4003150 & 0.8952900 & -2.5801870 \\ \text { C } & -5.7875530 & -0.0011400 & -2.5992210 \\ \text { H } & -6.1564490 & -0.7655060 & -3.2961260 \\ \text { H } & -6.5874940 & 0.7352340 & -2.4579660 \\ \text { C } & -4.5004710 & 0.6815350 & -3.1603740 \\ \text { H } & -4.2495100 & 0.2779080 & -4.1502320 \\ \text { H } & -4.6511640 & 1.7621340 & -3.2695380 \\ & & & \end{array}$




\begin{tabular}{|c|c|c|}
\hline $\begin{array}{l}-3.5426610 \\
-2.9751410 \\
-5.1684130 \\
-4.7312770 \\
-4.0531900 \\
-3.6666400 \\
-4.2444170 \\
-5.3632880 \\
-5.6093810 \\
-6.2093670 \\
-6.0978320 \\
-2.0497370 \\
-4.0245620 \\
-4.3194530 \\
-3.8733330 \\
-2.7173360 \\
-2.3840450 \\
-1.9095880 \\
-5.3914110 \\
-6.3114650 \\
5.1739640 \\
5.5877000 \\
4.4589220 \\
4.5507530 \\
6.5724350 \\
6.8339560 \\
7.4965080 \\
5.8977680 \\
5.8368470 \\
6.4798120 \\
3.9841780 \\
6.0593710 \\
3.6549330 \\
3.6389610 \\
2.6220220 \\
4.3274630 \\
4.6287730 \\
3.6291430 \\
4.9582390 \\
5.1106910 \\
3.8956180 \\
4.3218210 \\
3.6976540 \\
3.8022180 \\
3.1352060 \\
2.9619110\end{array}$ & $\begin{array}{r}2.3518880 \\
3.4009070 \\
2.3292140 \\
1.7755690 \\
4.5049290 \\
5.2643320 \\
4.9974780 \\
3.8650560 \\
4.2860320 \\
4.0647480 \\
1.8548950 \\
3.8299920 \\
2.0901830 \\
2.5154630 \\
1.0136430 \\
2.7579730 \\
3.5466160 \\
2.0276250 \\
0.7856880 \\
0.3420820 \\
-0.3844180 \\
0.5395810 \\
1.7683950 \\
0.2734030 \\
1.5922300 \\
2.3089890 \\
1.0937300 \\
2.3200050 \\
3.4006310 \\
2.1726830 \\
2.2675400 \\
-0.0174830 \\
2.0405140 \\
3.1237980 \\
1.7095920 \\
1.2950560 \\
2.0039730 \\
0.5790540 \\
-2.4881010 \\
-3.9770910 \\
-2.8043460 \\
-1.8423030 \\
-4.5961620 \\
-5.6704050 \\
-4.4889050 \\
-3.8749080\end{array}$ & $\begin{array}{l}1.1264780 \\
2.0550030 \\
2.9478500 \\
1.6156620 \\
2.2400280 \\
2.9324210 \\
1.2787820 \\
2.8004300 \\
3.7842840 \\
2.1321460 \\
3.2867450 \\
1.6631170 \\
3.9711640 \\
4.9398630 \\
4.1100800 \\
3.4461170 \\
4.1338390 \\
3.3600530 \\
0.8890420 \\
1.2706690 \\
2.7108920 \\
3.8286980 \\
1.8379380 \\
1.6336710 \\
3.2477810 \\
4.0377920 \\
2.9315820 \\
2.0435720 \\
2.2292950 \\
1.1258750 \\
0.9895430 \\
4.6474730 \\
3.1394940 \\
3.3200110 \\
3.0020790 \\
4.3341490 \\
5.1171470 \\
4.7834740 \\
1.5633830 \\
1.3751490 \\
-0.5997680 \\
0.4858730 \\
1.2020320 \\
0.9970010 \\
2.1374540 \\
0.0325330\end{array}$ \\
\hline
\end{tabular}

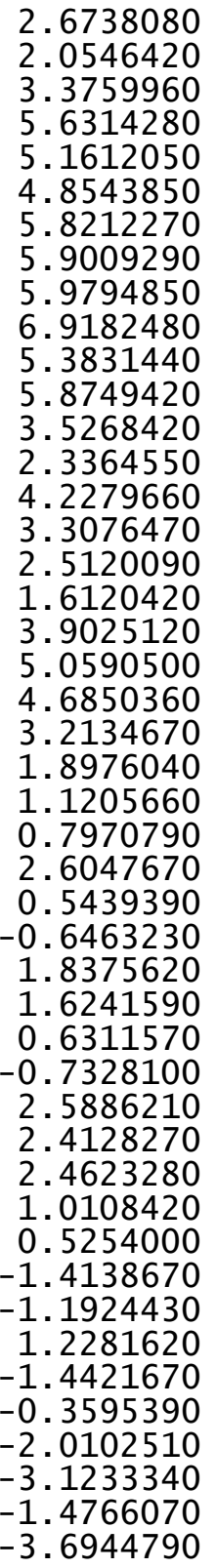

-4.5922730
-3.3836450
-2.2921940
-4.4312610
-3.5327120
-4.2748940
-2.8120760
-4.2247180
-5.3068920
-3.8295390
-1.7631810
-2.2678740
0.7714700
1.5116170
0.6159520
0.8571230
2.1512930
2.0299760
0.9030710
1.3385100
-0.3810100
2.9889340
2.3785510
2.9842300
1.3215540
0.0167160
3.8971370
4.6214850
4.6248730
6.0261370
6.8151170
6.1142120
6.5462440
4.7155050
4.0274040
6.8663140
7.8453160
6.5775480
6.2601930
5.9424400
-3.6808700
-3.0055370
-4.4723590
-5.2974550
-4.3743060
-6.0145260

$-0.7472350$

0.3899350

$-1.4132930$

2. 2270750

$-1.1300560$

$-1.8792650$

$-1.6283480$

0.0591240

$-0.1108030$

0.1649890

2.6762670

3. 5088960

$-3.0566170$

$-2.9255280$

$-4.3968560$

$-5.5999360$

$-5.3939890$

$-4.1586040$

$-6.5213670$

$-4.4404760$

$-4.4528290$

$-5.2585890$

$-6.2747640$

$-3.9511460$

$-4.3815840$

$-5.7027040$

$-1.6382430$

$-1.4348400$

$-1.9739570$

$-2.5588640$

$-1.6986440$

$-1.7115220$

$-2.6228130$

$-1.0378690$

$-2.6426400$

$-0.6672270$

$-2.0626480$

$-0.9853130$

$-2.7026460$

$-3.5827190$

$-0.6651570$

$-0.9699580$

$-1.5731200$

$-3.0971290$

$-2.6236540$ 


\begin{tabular}{|c|c|c|c|c|c|c|c|}
\hline & & & & & & & \\
\hline $\mathrm{H}$ & -3.5341890 & -5.3612110 & -0.5698700 & C & -3.6455510 & 0.9694350 & -0.9532090 \\
\hline C & -2.0523870 & -5.0900750 & -4.1472490 & $\mathrm{C}$ & -3.2540330 & 0.3737200 & -2.2848360 \\
\hline $\mathrm{H}$ & -0.6226580 & -3.7288210 & -3.2756640 & C & -5.4539230 & -0.6265970 & -1.3313590 \\
\hline C & -3.1610890 & -5.9108550 & -3.9134680 & C & -4.8499710 & 0.4404780 & -0.4532330 \\
\hline $\mathrm{H}$ & -4.5567510 & -6.6513660 & -2.4396910 & $\mathrm{C}$ & -3.0961020 & -1.1588830 & -2.1102080 \\
\hline $\mathrm{H}$ & -1.6380820 & -5.0056370 & -5.1493230 & $\mathrm{H}$ & -2.8691080 & -1.6079750 & -3.0852450 \\
\hline $\mathrm{H}$ & -3.6091400 & -6.4669740 & -4.7342160 & $\mathrm{H}$ & -2.2437090 & -1.3484440 & -1.4560770 \\
\hline $\mathrm{H}$ & -0.0155320 & -2.3393150 & -0.2491470 & $\mathrm{C}$ & -4.4092090 & -1.7602860 & -1.5217900 \\
\hline 0 & -1.9994220 & -3.6944280 & 0.4448840 & $\mathrm{H}$ & -4.8232180 & -2.5214230 & -2.1952690 \\
\hline C & -2.7390700 & -1.4972700 & 2.1579450 & $\mathrm{H}$ & -4.2122670 & -2.2471210 & -0.5606760 \\
\hline C & -1.6722820 & -2.5000510 & 2.3216030 & $\mathrm{H}$ & -6.3793370 & -1.0210670 & -0.8938880 \\
\hline $\mathrm{H}$ & -2.6920220 & -0.8816090 & 1.2554040 & $\mathrm{H}$ & -2.3258550 & 0.8074560 & -2.6659690 \\
\hline $\mathrm{H}$ & -0.6930820 & -2.2479610 & 1.9303380 & C & -5.7335010 & -0.0015930 & -2.7273030 \\
\hline 0 & -2.0390640 & -0.8670800 & 3.2766380 & $\mathrm{H}$ & -6.1074590 & -0.7857350 & -3.3990870 \\
\hline $\mathrm{H}$ & -1.3325570 & -0.2402480 & 2.8425350 & H & -6.5164520 & 0.7606970 & -2.6373740 \\
\hline C & -4.1968710 & -2.8919080 & 3.7094940 & C & -4.4163760 & 0.6222100 & -3.2864950 \\
\hline $\mathrm{C}$ & -3.2785920 & -4.0969860 & 3.4556800 & $\mathrm{H}$ & -4.1527010 & 0.1676660 & -4.2506080 \\
\hline C & -1.8209380 & -3.6489010 & 3.2584890 & $\mathrm{H}$ & -4.5357470 & 1.7001400 & -3.4481290 \\
\hline C & -4.1501870 & -1.9141650 & 2.5242190 & C & -3.5162510 & 2.4558750 & 0.9477280 \\
\hline $\mathrm{H}$ & -5.2297600 & -3.2280780 & 3.8616660 & C & -2.9397200 & 3.5240120 & 1.8483790 \\
\hline $\mathrm{H}$ & -3.3283960 & -4.8076330 & 4.2892790 & C & -5.1832410 & 2.5519280 & 2.7292930 \\
\hline $\mathrm{H}$ & -1.3817260 & -3.3297450 & 4.2157580 & C & -4.7307660 & 1.9320190 & 1.4315720 \\
\hline $\mathrm{H}$ & -4.5815350 & -2.3890100 & 1.6328040 & $\mathrm{C}$ & -3.9878600 & 4.6654370 & 1.9656980 \\
\hline $\mathrm{H}$ & -4.7320800 & -1.0091190 & 2.7226860 & $\mathrm{H}$ & -3.5953430 & 5.4386440 & 2.6391890 \\
\hline $\mathrm{H}$ & -3.8826570 & -2.3729420 & 4.6252060 & $\mathrm{H}$ & -4.1413660 & 5.1271940 & 0.9826880 \\
\hline $\mathrm{H}$ & -3.6107070 & -4.6220020 & 2.5505200 & C & -5.3296220 & 4.0854220 & 2.5159950 \\
\hline $\mathrm{H}$ & -1.1841900 & -4.4614170 & 2.8895250 & $\mathrm{H}$ & -5.5886780 & 4.5527650 & 3.4752620 \\
\hline 15 & & & & $\mathrm{H}$ & -6.1522590 & 4.2811070 & 1.8179090 \\
\hline & , R) G[B97-D/ & {$[Z \mathrm{VP}]=-354$} & 547 & $\mathrm{H}$ & -6.1341940 & 2.1188750 & 3.0638020 \\
\hline$P$ & 0.3100460 & 0.1464070 & 0.7495790 & $\mathrm{H}$ & -1.9927930 & 3.9102990 & 1.4634630 \\
\hline 0 & 0.5619100 & -1.3356390 & 0.6554770 & C & -4.0710430 & 2.3216130 & 3.7887810 \\
\hline 0 & -0.3523380 & 0.6911080 & 1.9828170 & $\mathrm{H}$ & -4.3742120 & 2.7951650 & 4.7320940 \\
\hline 0 & -0.5168200 & 0.5244620 & -0.6120600 & $\mathrm{H}$ & -3.9562560 & 1.2477700 & 3.9751970 \\
\hline 0 & 1.6798270 & 1.0035210 & 0.5869830 & C & -2.7325160 & 2.9271990 & 3.2679300 \\
\hline C & -0.5500020 & 1.8656310 & -1.0011450 & $\mathrm{H}$ & -2.3874180 & 3.7299990 & 3.9330190 \\
\hline C & 0.6157890 & 2.4276350 & -1.5569780 & $\mathrm{H}$ & -1.9473710 & 2.1687280 & 3.2266900 \\
\hline C & -1.7293240 & 2.5951210 & -0.8142380 & C & -5.4000100 & 0.9294680 & 0.7311310 \\
\hline C & -1.7076890 & 3.9531800 & -1.1621860 & $\mathrm{H}$ & -6.3404320 & 0.5265280 & 1.1082970 \\
\hline $\mathrm{H}$ & -2.6104500 & 4.5435520 & -1.0159780 & $\mathrm{C}$ & 5.0961030 & -0.4261190 & 2.7498400 \\
\hline C & 2.3613850 & 0.9150860 & -0.6278820 & C & 5.4815360 & 0.5074930 & 3.8699230 \\
\hline C & 3.5476220 & 0.1755600 & -0.6791260 & C & 4.4207050 & 1.7216370 & 1.8333420 \\
\hline C & 1.8304050 & 1.5827170 & -1.7431610 & C & 4.5109000 & 0.2244020 & 1.6472190 \\
\hline C & 4.1853340 & 0.0999090 & -1.9209610 & $\mathrm{C}$ & 6.4893860 & 1.5487260 & 3.3086180 \\
\hline $\mathrm{H}$ & 5.1145500 & -0.4633960 & -1.9898960 & $\mathrm{H}$ & 6.7311130 & 2.2720760 & 4.0989730 \\
\hline C & -2.9659210 & 1.9858990 & -0.2586250 & $\mathrm{H}$ & 7.4198730 & 1.0420450 & 3.0259160 \\
\hline $\mathrm{C}$ & 4.1144320 & -0 . & 0.5126080 & C & 5.8558290 & 2.2679700 & 2.0770830 \\
\hline
\end{tabular}




$\begin{array}{rrrr}\mathrm{H} & 5.7951030 & 3.3507020 & 2.2501190 \\ \mathrm{H} & 6.4647810 & 2.1079560 & 1.1792070 \\ \mathrm{H} & 3.9742980 & 2.2138250 & 0.9657140 \\ \mathrm{H} & 5.9252350 & -0.0434660 & 4.7082650 \\ \mathrm{C} & 3.5791960 & 2.0110540 & 3.1072900 \\ \mathrm{H} & 3.5637590 & 3.0962400 & 3.2762500 \\ \mathrm{H} & 2.5489290 & 1.6844200 & 2.9422160 \\ \mathrm{C} & 4.2110680 & 1.2747310 & 4.3294650 \\ \mathrm{H} & 4.4930280 & 1.9902500 & 5.1136780 \\ \mathrm{H} & 3.4954740 & 0.5674460 & 4.7653110 \\ \mathrm{C} & 4.8985090 & -2.5411090 & 1.6195340 \\ \mathrm{C} & 5.0379670 & -4.0340000 & 1.4534950 \\ \mathrm{C} & 3.8977710 & -2.8718370 & -0.5713640 \\ \mathrm{C} & 4.3028350 & -1.9017490 & 0.5152780 \\ \mathrm{C} & 3.6223990 & -4.6374830 & 1.2448170 \\ \mathrm{H} & 3.7199860 & -5.7146790 & 1.0524670 \\ \mathrm{H} & 3.0343870 & -4.5149880 & 2.1624160 \\ \mathrm{C} & 2.9296850 & -3.9189320 & 0.0474350 \\ \mathrm{H} & 2.6508470 & -4.6417620 & -0.7306710 \\ \mathrm{H} & 2.0208910 & -3.4082460 & 0.3735360 \\ \mathrm{H} & 3.4097300 & -2.3634820 & -1.4067120 \\ \mathrm{H} & 5.5268220 & -4.4843950 & 2.3260720 \\ \mathrm{C} & 5.1677420 & -3.6241940 & -1.0546850 \\ \mathrm{H} & 4.8719620 & -4.3738890 & -1.8008540 \\ \mathrm{H} & 5.8508020 & -2.9201210 & -1.5454840 \\ \mathrm{C} & 5.8646100 & -4.3069350 & 0.1651390 \\ \mathrm{H} & 5.9379300 & -5.3919350 & 0.0117520 \\ \mathrm{H} & 6.8819270 & -3.9195090 & 0.2966270 \\ \mathrm{C} & 5.2950460 & -1.8068850 & 2.7368890 \\ \mathrm{H} & 5.7562360 & -2.3059080 & 3.5902200 \\ \mathrm{C} & 3.6588570 & 0.6866360 & -3.0755060 \\ \mathrm{C} & 2.4609470 & 1.4238730 & -2.9991080 \\ \mathrm{C} & 4.4226090 & 0.5313330 & -4.3813910 \\ \mathrm{C} & 3.5613520 & 0.7703220 & -5.6276140 \\ \mathrm{C} & 2.7519090 & 2.0607170 & -5.4580710 \\ \mathrm{C} & 1.7944700 & 1.9334450 & -4.2673710 \\ \mathrm{H} & 4.2003780 & 0.8193560 & -6.5188530 \\ \mathrm{H} & 5.2537740 & 1.2551540 & -4.3851020 \\ \mathrm{H} & 4.8836660 & -0.4648580 & -4.4142310 \\ \mathrm{H} & 3.4416470 & 2.9015470 & -5.2877630 \\ \mathrm{H} & 2.1786700 & 2.2863330 & -6.3666440 \\ \mathrm{H} & 1.2878180 & 2.8834360 & -4.0891830 \\ \mathrm{H} & 0.9983080 & 1.2168400 & -4.5292050 \\ \mathrm{H} & 2.8672260 & -0.0723720 & -5.7652330 \\ \mathrm{C} & 0.6146540 & 3.8054240 & -1.8483270 \\ \mathrm{C} & 1.8348930 & 4.5819950 & -2.2811070 \\ & & & \end{array}$

\begin{abstract}
2.0308720
0.7427900

$-0.4740280$

$-0.5554550$

1.6979010

2.8637290

0.9132390

$-0.3524710$

2. 7303310

2. 3257450

0.4920460

$-1.4020390$

$-1.5563950$

$-0.4663160$

$-2.1473180$

$-3.2637860$

$-1.6308100$

$-3.8545440$

$-3.6610100$

$-2.2265560$

$-0.7739280$

$-3.3379790$

$-4.7188710$

$-1.8253140$

$-3.8012630$

$-0.1066580$

$-2.1024840$

$-2.7879070$

$-1.7518400$

$-2.7074230$

$-0.7610750$

$-2.0888160$

$-1.3607730$

$-4.3113570$

$-3.4199270$

$-1.9473140$

$-4.2156340$

$-5.3553430$

$-3.5009620$

$-1.5142880$

$-4.6384190$

$-4.7802740$

$-4.0026330$

$-3.7513610$

$-1.3285660$
\end{abstract}

$\begin{array}{ll}5.8105110 & -1.3584500 \\ 6.6782230 & -1.2320520 \\ 6.0382620 & -1.9495390 \\ 4.5628130 & -1.6524660 \\ 4.9384720 & -3.3142580 \\ 6.4144620 & -1.7395330 \\ 7.6794830 & -1.6472670 \\ 6.1832470 & -3.0346250 \\ 3.9542490 & -2.2739350 \\ 5.4426570 & -0.3679570 \\ 6.8078140 & -0.1721680 \\ 6.5443770 & -1.6579580 \\ -3.7211270 & -0.4938440 \\ -3.0811100 & -0.8452720 \\ -4.5659550 & -1.5782680 \\ -5.3678160 & -1.2912290 \\ -4.5422890 & -2.8847360 \\ -6.1358790 & -2.2937220 \\ -5.3747460 & -0.2804550 \\ -5.3086680 & -3.8869310 \\ -3.9152800 & -3.1088230 \\ -6.1065440 & -3.5942290 \\ -6.7548780 & -2.0641810 \\ -5.2819920 & -4.8974920 \\ -6.7027130 & -4.3775580 \\ -2.3806930 & -0.1670720 \\ -3.6633840 & 0.6200170 \\ -1.3352830 & 2.1636170 \\ -2.3522990 & 2.4112750 \\ -0.7800600 & 1.2249460 \\ -2.1539540 & 2.0175560 \\ -0.6534210 & 3.2519030 \\ -0.0711740 & 2.7889650 \\ -2.5822170 & 3.7749690 \\ -3.8242830 & 3.6270390 \\ -3.4292930 & 3.4230120 \\ -1.6923220 & 2.5258200 \\ -2.8818190 & 3.9277910 \\ -4.4698190 & 4.5097440 \\ -3.0557380 & 4.3630990 \\ -2.2180970 & 1.6591330 \\ -0.7632670 & 2.6475990 \\ -2.0073130 & 4.6585590 \\ -4.4068990 & 2.7575920 \\ -4.2828920 & 3.1226450 \\ -5 & \end{array}$




$\begin{array}{lrrr}\text { TS152(R, R) G[B97-D/def2-TZVP] }=-3541.575322 \\ \text { P } & 0.3405770 & 0.1573210 & 0.7964800 \\ \text { O } & 0.6247340 & -1.3164270 & 0.6664040 \\ \text { O } & -0.3154720 & 0.6598910 & 2.0506460 \\ \text { O } & -0.5217630 & 0.5417710 & -0.5434480 \\ \text { O } & 1.6913100 & 1.0457200 & 0.6278080 \\ \text { C } & -0.5852530 & 1.8875450 & -0.9103800 \\ \mathrm{C} & 0.5604550 & 2.4846230 & -1.4598830 \\ \mathrm{C} & -1.7753970 & 2.5941530 & -0.7039880 \\ \mathrm{C} & -1.7629870 & 3.9562470 & -1.0165080 \\ \mathrm{H} & -2.6749750 & 4.5297460 & -0.8569370 \\ \mathrm{C} & 2.3399880 & 0.9709370 & -0.6078450 \\ \mathrm{C} & 3.5187300 & 0.2240760 & -0.7093090 \\ \mathrm{C} & 1.7666790 & 1.6404180 & -1.6987620 \\ \mathrm{C} & 4.0814840 & 0.1175270 & -1.9846610 \\ \mathrm{H} & 4.9966850 & -0.4616990 & -2.0966080 \\ \mathrm{C} & -2.9987750 & 1.9533320 & -0.1555740 \\ \mathrm{C} & 4.1423770 & -0.4454450 & 0.4612650 \\ \mathrm{C} & -3.6648870 & 0.9409900 & -0.8687680 \\ \mathrm{C} & -3.2821300 & 0.3962620 & -2.2243150 \\ \mathrm{C} & -5.4362350 & -0.6922530 & -1.2624380 \\ \mathrm{C} & -4.8466450 & 0.3666680 & -0.3647850 \\ \mathrm{C} & -3.0804100 & -1.1359260 & -2.1023710 \\ \mathrm{H} & -2.8665910 & -1.5482480 & -3.0964190 \\ \mathrm{H} & -2.2078370 & -1.3252390 & -1.4754300 \\ \mathrm{C} & -4.3631030 & -1.7893760 & -1.5027810 \\ \mathrm{H} & -4.7684540 & -2.5463090 & -2.1860360 \\ \mathrm{H} & -4.1315090 & -2.2927010 & -0.5579520 \\ \mathrm{H} & -6.3422090 & -1.1254060 & -0.8208340 \\ \mathrm{H} & -2.3733450 & 0.8680640 & -2.6076340 \\ \mathrm{C} & -5.7581130 & -0.0366520 & -2.6351860 \\ \mathrm{H} & -6.1181500 & -0.8130260 & -3.3234160 \\ \mathrm{H} & -6.5626320 & 0.6979020 & -2.5114630 \\ \mathrm{C} & -4.4698680 & 0.6436850 & -3.1964310 \\ \mathrm{H} & -4.2114540 & 0.2292530 & -4.1798740 \\ \mathrm{H} & -4.6233950 & 1.7224360 & -3.3191330 \\ \mathrm{C} & -3.5420900 & 2.3771920 & 1.0709760 \\ \mathrm{C} & -2.9798020 & 3.4363110 & 1.9914730 \\ \mathrm{C} & -5.1832730 & 2.3819190 & 2.8790070 \\ \mathrm{C} & -4.7356420 & 1.8095140 & 1.5581480 \\ \mathrm{C} & -4.0559450 & 4.5456180 & 2.1539030 \\ \mathrm{H} & -3.6731640 & 5.3119250 & 2.8407570 \\ \mathrm{H} & -4.2372700 & 5.0275190 & 1.1853870 \\ \mathrm{C} & -5.3728150 & 3.9161600 & 2.7101610 \\ \mathrm{H} & -5.6269640 & 4.3503390 & 3.6861900 \\ \mathrm{H} & -6.2121430 & 4.1092920 & 2.0315210\end{array}$
$-6.1169730$ $-2.0495790$ $-4.0491180$ $-4.3506910$ $-3.9024180$ $-2.7354310$ $-2.4031760$ $-1.9304170$ $-5.3895290$
-6. 3123640 5.2197360 5.6468990 4.4973280
4.5854320
6.6254800 6.8952450 7.5461230 5.9383410 5. 8797540 6. 5107010 4.0136830 6.1272180 3.7075870 3.6948050 2. 6729250 4.3919510 4.7006920 3.6980890 4.9828730 5.1265120 3.8965760 4.3369840 3.7087240 3.8059340 3.1557710 2. 9650360 2. 6690170 2. 0617200 3. 3705420 5.6537590 5.1549000 4.8397220 5.8132850 5.9019860 5.9719980 6.9227430
3.2158170
1.6031280
3.9153440
4.8755530
4.0704610
3.3920930
4.0729280
3. 3207440
0.8413520
1.2223220
2.6534950
3.7478610
1.7455110
1.5702480
3.1355680
3.9086050
2. 8196730
1.9245760
2.0894280
1.0038510
0.8925540
4.5723410
3.0500150
3.2095080
2. 9299850
4.2517300
5.0185840
4.7211890
1. 5538860
1.3963790
$-0.5907130$
0.4700290
1.2513940
1.0704970
2. 1893660
0.0723780
$-0.6891780$
0.4256550
$-1.4102520$
2.2525910
$-1.1177590$
$-1.8507180$
$-1.6347900$
0.0781500
$-0.0699530$
0.1658470 


$\begin{array}{rrr}5.4236600 & -1.7176710 & 2.6458630 \\ 5.9230150 & -2.2064610 & 3.4834500 \\ 2.3148320 & 1.4452170 & -2.9872990 \\ 1.5998160 & 1.9744240 & -4.2189770 \\ 1.9633510 & 1.1974710 & -5.4913350 \\ 3.4884010 & 1.1528490 & -5.6404570 \\ 4.1010830 & 0.3617910 & -4.4768180 \\ 3.4863720 & 0.6754560 & -3.1207780 \\ 1.4967940 & 1.6778850 & -6.3609460 \\ 1.8657730 & 3.0268580 & -4.3887320 \\ 0.5173600 & 1.9573540 & -4.0428180 \\ 3.8750870 & 2.1831290 & -5.6375600 \\ 3.7822090 & 0.6966520 & -6.5945380 \\ 5.1866970 & 0.5246680 & -4.4282450 \\ 3.9627820 & -0.7138530 & -4.6695400 \\ 1.5708850 & 0.1713720 & -5.4281860 \\ 0.5652980 & 3.8802360 & -1.6899600 \\ 1.8505120 & 4.5951760 & -2.0714520 \\ 1.8337440 & 6.0833210 & -1.6972670 \\ 0.5747610 & 6.7373180 & -2.2775990 \\ -0.6739340 & 6.1272310 & -1.6273150 \\ -0.6152230 & 4.6151360 & -1.4676650 \\ 2.7409740 & 6.5676550 & -2.0805020 \\ 2.0104210 & 4.5310400 & -3.1561970 \\ 2.7007320 & 4.0820170 & -1.6061930 \\ 0.5539490 & 6.5622480 & -3.3638340 \\ 0.5811850 & 7.8236770 & -2.1214290 \\ -1.5754340 & 6.3959900 & -2.1953700 \\ -0.8025040 & 6.5681210 & -0.6261900 \\ 1.8349580 & 6.1948660 & -0.6027220 \\ -1.4640350 & -3.6967370 & -0.5346800 \\ -0.3948420 & -3.0215010 & -0.8836190 \\ -2.0729570 & -4.4963720 & -1.6431340 \\ -3.1617570 & -5.3376630 & -1.3633310 \\ -1.6056100 & -4.3867100 & -2.9635290 \\ -3.7749660 & -6.0587060 & -2.3869700 \\ -3.5207570 & -5.4110770 & -0.3409760 \\ -2.2247800 & -5.1049390 & -3.9869020 \\ -0.7702120 & -3.7293220 & -3.1817080 \\ -3.3094280 & -5.9413570 & -3.7016490 \\ -4.6177320 & -6.7086350 & -2.1628300 \\ -1.8630420 & -5.0103220 & -5.0082450 \\ -3.7913260 & -6.4993800 & -4.5016620 \\ -0.0316750 & -2.3433590 & -0.1838060 \\ -1.9801020 & -3.7025550 & 0.5950970 \\ -2.7014130 & -1.4315010 & 2.1991180\end{array}$

-1.6418500
-2.6390810
-0.6585470
-2.0106200
-1.3002480
-4.1866250
-3.2686970
-1.8067210
-4.1186910
-5.2226680
-3.3294380
-1.3737980
-4.5347940
-4.7030750
-3.8850870
-3.5930000
-1.1722190

$-2.4306950$

2.4174080

$-0.8538110 \quad 1.2728030$

$-2.2015160 \quad 2.0218960$

$\begin{array}{ll}-0.7592110 & 3.2988530\end{array}$

$-0.1486980$

$-2.7481040$

2.8459520

3.7903160

$-3.9666510$

$-3.5350400$

$-1.8286450$

$-3.0743280$

$-4.6333550$

$-3.1752480$

$-2.3445090$

$-0.9155140$

$-2.1862350$

$-4.5369890$

3.6091080

3.4042530

2. 5610620

3.9426370

4.4775930

4. 3497760

1.6851390

2.7069200

4.6847340

$\begin{array}{ll}\mathrm{H} & -3.5930000 \\ \mathrm{H} & -1.1722190\end{array}$

$-4.3675520$

2. 7289020

154

$\operatorname{TS} 153(R, R)$ G $[B 97-D /$ def2-TZVP $]=-3541.575201$

$\begin{array}{llll}P & 0.3156920 & 0.1527830 & 0.7439560\end{array}$

$0 \quad 0.5591710$

$-1.3298880$

0.6409140

$-0.3497870$

0.6925450

1. 9777950

$-0.5008310$

0.5464650

$-0.6189400$

1.6911400

1.0026800

1.8911450

0.6309810

2. 4705390

0.5946480

$-1.7270810$

2. 6071360

3. 9662730

$-1.7170890$

$-2.6279750$

2. 3700020

4. 5460980

0.9320340

0.1806140

3.5480450

1.6326950

4.1729520

5.0909200

$-2.9596960$

4.1175640

$-3.6336260$

$-3.2447950$

$-5.4280390$

$-4.8303660$

$-3.0709860$

$-2.8526520$

$-2.2089510$

$-4.3717530$

$-4.7820040$

0.1096310

$-0.4699180$

1.9868860

$-0.5219970$

0.9679730

0.3846670

$-0.6440320$

0.4253940

$-1.1474450$

$-1.5871460$

$-1.3353490$

$-1.7649190$

-2.5308570
-2.2498660

$-0.9937440$

$-1.5277240$

$-0.8178300$

$-1.1627820$

$-1.0247320$

$-0.6229700$

$-0.6923280$

$-1.7215950$

$-1.9407170$

$-2.0251410$

$-0.2650010$

0.4866560

$-0.9607280$

$-2.2983720$

$-1.3362970$

$-0.4567540$

$-2.1368160$

$-3.1181340$

$-1.4952590$

$-1.5373450$

$-2.2076110$

$\mathrm{H}$

$-4.1608010$

$-0.5781280$ 


-6.3465020
-2.3234090
-5.7227010
-6.0893570
-6.5153250
-4.4166710
-4.1543330
-4.5499160
-3.5093790
-2.9410690
-5.1712750
-4.7169570
-4.0010790
-3.6146490
-4.1627640
-5.3346440
-5.5948440
-6.1619430
-6.1165150
-1.9995230
-4.0534700
-4.3590490
-3.9261510
-2.7232990
-2.3857410
-1.9296390
-5.3793340
-6.3141050
5.1112360
5.5076080
4.4482550
4.5252420
6.5211210
6.7695280
7.4475140
5.8890940
5.8385060
6.4930750
4.0029460
5.9501160
3.6148000
3.6113220
2.5804760
4.2443540
4.5338770
3.5245800

$-1.0507320$

0.8309390

$-0.0156710$

$-0.8014040$

0.7356190

0.6285560

0.1856290

1.7064190

2.4484450

3.5218820

2.5257300

1.9119390

4.6520080

5.4296940

5.1115750

4.0577510

4.5218900

4.2452000

2.0824520

3.9186890

2.3067770

2.7761680

1.2341620

2.9274880

3.7343510

2.1780170

0.9047550

0.4914950

$-0.4768680$

0.4386950

1.6875470

0.1921000

1.4799840

2.1919210

0.9707440

2. 2185410

3.2997330

2.0633410

2. 1949420

$-0.1267090$

1. 9681520

3.0510580

1.6545390

1.2090060

1.9107160

0.5007130

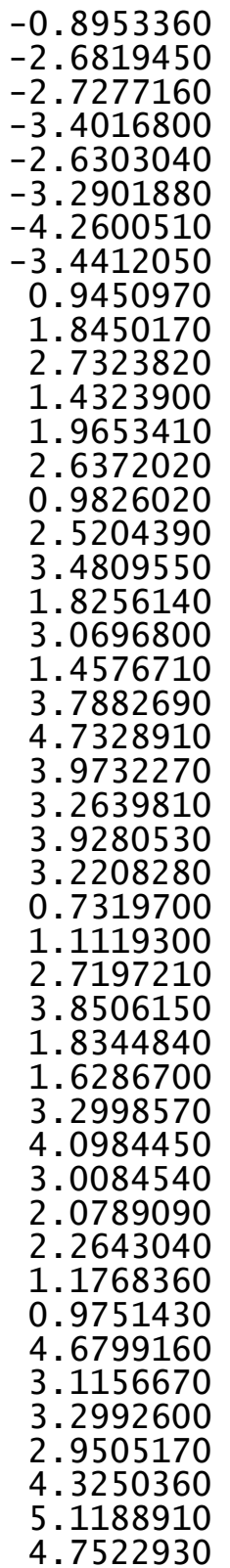

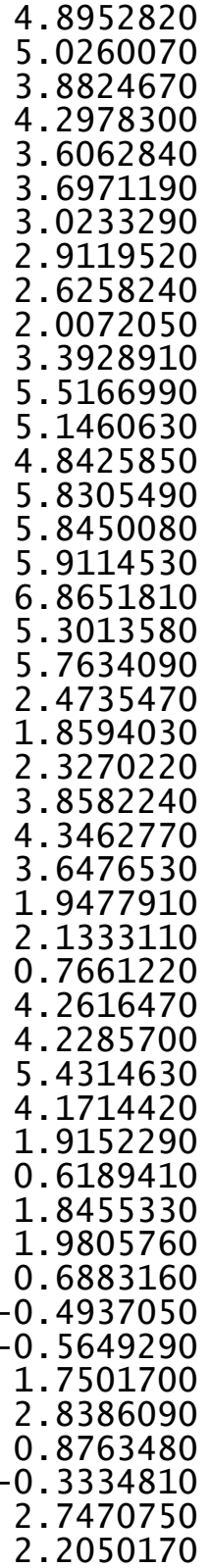
$-2.5751330$
$-4.0663920$
$-2.8709090$
$-1.9175270$
$-4.6595260$
$-5.7345650$
$-4.5461370$
$-3.9213300$
$-4.6321740$
$-3.4107790$
$-2.3491500$
$-4.5309390$
$-3.6233950$
$-4.3613360$
$-2.9164170$
$-4.3264300$
$-5.4096690$
$-3.9466420$
$-1.8585390$
$-2.3718810$
1. 4927350
2. 1220760
1.4611210
1. 3924600
0.4797520
0.7214620
2.0328470
3.1851370
2. 0943620
2. 4069480
1.0209930
0.5807060
$-0.5679730$
0.4433100
3.8497000
4.6512100
5.8527290
6.7197910
6.0710660
4.5915540
5.0385720
6.4648290
7.7170460
6.2296540
4.0328590
5.4549800

1. 5619530

1.3752550

$-0.6280560$

0.4693840

1. 1654340

0.9583070

2.0874290

$-0.0190120$

$-0.8054310$

0.3180350

$-1.4541630$

2. 2393330

$-1.1277000$

$-1.8824210$

$-1.6122770$

0.0793550

$-0.0889660$

0.2110360

2. 6872220

3.5315510

$-2.9833620$

$-4.2222600$

$-5.5254070$

$-5.5445220$

$-4.4125840$

$-3.0829130$

$-6.3822290$

$-4.2676210$

$-4.1383450$

$-5.4058420$

$-6.5086540$

$-4.2736650$

$-4.7051550$

$-5.5987920$

$-1.8085100$

$-2.1685620$

$-1.2004460$

$-1.1405570$

$-1.9086910$

$-1.6313200$

$-3.1948440$

$-1.5050330$

$-2.9865460$

$-2.1345960$

$-0.2030550$ 


\begin{tabular}{|c|c|c|c|c|c|c|c|}
\hline $\mathrm{H}$ & 0.3903950 & 6.8597610 & -0.0943230 & $\mathrm{H}$ & 26110280 & -45912020 & $-0,6007190$ \\
\hline $\mathrm{H}$ & -1.4385020 & 6.5615670 & -1.6460490 & C & -2.3693350 & -0.9136450 & -0.4741960 \\
\hline C & -1.5623220 & -3.7238580 & -0.4821850 & $\mathrm{C}$ & -3.5335360 & -0.1525660 & -0.6312020 \\
\hline 0 & -0.4813560 & -3.0768280 & -0.8483800 & $\mathrm{c}$ & -1.8314630 & -1.7151990 & -1.4965920 \\
\hline C & -2.1722270 & -4.5596270 & -1.5628420 & $\mathrm{c}$ & -4.1422730 & -0.1743390 & -1.8940460 \\
\hline $\mathrm{C}$ & -3.2801280 & -5.3679100 & -1.2609490 & $\mathrm{H}$ & -5.0566260 & 0.3974150 & -2.0415590 \\
\hline C & -1.6842940 & -4.5194320 & -2.8797770 & C & 2.9667350 & -1.9891790 & -0.0330660 \\
\hline $\mathrm{C}$ & -3.8911160 & -6.1253800 & -2.2592550 & C & -4.1157610 & 0.6448010 & 0.4797400 \\
\hline $\mathrm{H}$ & -3.6550520 & -5.3877090 & -0.2418480 & $\mathrm{C}$ & 3.6484920 & -1.0660170 & -0.8452780 \\
\hline C & -2.3007430 & -5.2746860 & -3.8778740 & $\mathrm{C}$ & 3.2470450 & -0.6213090 & -2.2311330 \\
\hline $\mathrm{H}$ & -0.8338790 & -3.8878630 & -3.1152950 & $\mathrm{c}$ & 5.4692010 & 0.4549520 & -1.4216180 \\
\hline C & -3.4038430 & -6.0785250 & -3.5705310 & C & 4.8642490 & -0.4973560 & -0.4208220 \\
\hline $\mathrm{H}$ & -4.7487300 & -6.7493080 & -2.0181700 & $\mathrm{C}$ & 3.1073660 & 0.9229190 & -2.2295180 \\
\hline $\mathrm{H}$ & -1.9221570 & -5.2346180 & -4.8966940 & $\mathrm{H}$ & 2.8725160 & 1.2630080 & -3.2458800 \\
\hline $\mathrm{H}$ & -3.8833720 & -6.6658620 & -4.3507490 & $\mathrm{H}$ & 2.2654370 & 1.1942290 & -1.5906300 \\
\hline $\mathrm{H}$ & -0.1145350 & -2.3773170 & -0.1731250 & C & 4.4335950 & 1.5716130 & -1.7265780 \\
\hline 0 & -2.0868320 & -3.6782710 & 0.6425430 & $\mathrm{H}$ & 4.8464900 & 2.2482940 & -2.4853900 \\
\hline C & -2.7738080 & -1.3466740 & 2.1708190 & $\mathrm{H}$ & 4.2521940 & 2.1648820 & -0.8236780 \\
\hline $\mathrm{C}$ & -1.7311970 & -2.3554810 & 2.4226930 & $\mathrm{H}$ & 6.4044190 & 0.8857630 & -1.0431020 \\
\hline $\mathrm{H}$ & -2.6980390 & -0.7969360 & 1.2285710 & $\mathrm{H}$ & 2.3097740 & -1.0859440 & -2.5487230 \\
\hline $\mathrm{H}$ & -0.7431410 & -2.1541120 & 2.0237040 & C & 5.7241690 & -0.3275990 & -2.7412780 \\
\hline 0 & -2.0761000 & -0.6549400 & 3.2536520 & $\mathrm{H}$ & 6.0944840 & 0.3723070 & -3.5021520 \\
\hline H & -1.3519490 & -0.0706830 & 2.7858990 & $\mathrm{H}$ & 6.5018760 & -1.0824830 & -2.5757500 \\
\hline C & -4.2870840 & -2.5939530 & 3.7914140 & C & 4.3945710 & -0.9980550 & -3.2095230 \\
\hline C & -3.3874370 & -3.8308830 & 3.6498950 & $\mathrm{H}$ & 4.1241420 & -0.6581750 & -4.2179530 \\
\hline $\mathrm{C}$ & -1.9177620 & -3.4273560 & 3.4416390 & $\mathrm{H}$ & 4.5014460 & -2.0888590 & -3.2433760 \\
\hline C & -4.1987670 & -1.7104480 & 2.5371250 & C & 3.5238750 & -2.3226010 & 1.2140950 \\
\hline $\mathrm{H}$ & -5.3288610 & -2.8996470 & 3.9472130 & $\mathrm{c}$ & 2.9510910 & -3.2838420 & 2.2293770 \\
\hline $\mathrm{H}$ & -3.4631590 & -4.4715050 & 4.5366420 & $\mathrm{C}$ & 5.2095870 & -2.2367690 & 2.9774100 \\
\hline $\mathrm{H}$ & -1.48 & -3.0450200 & 4.3787830 & $\mathrm{C}$ & 4.7476170 & -1.7588960 & 1.6246100 \\
\hline $\mathrm{H}$ & -4.61 & -2.2435580 & 1.6737440 & $\mathrm{C}$ & 3.9949370 & -4.4126630 & 2.4572620 \\
\hline H & -4.76 & -0.7844030 & 2.6550900 & $\mathrm{H}$ & 3.6070380 & -5.1062210 & 3.2148530 \\
\hline $\mathrm{H}$ & -3.98 & -2.0118010 & 4.6712730 & $\mathrm{H}$ & 4.1340750 & -4.9788570 & 1.5282650 \\
\hline $\mathrm{H}$ & -3.71 & -4.4208490 & 2.7843200 & C & 5.3464240 & -3.7852390 & 2.9266810 \\
\hline H & -1.2 & -4.2787320 & 3.1457130 & $\mathrm{H}$ & 5.6158280 & -4.1499410 & 3.9267520 \\
\hline 15 & & & & $\mathrm{H}$ & 6.1590720 & -4.0583930 & 2.2430750 \\
\hline & R) & TZVP. & 03 & $\mathrm{H}$ & 6.1656150 & -1.7748120 & 3.2534070 \\
\hline $\mathrm{P}$ & -0.3226480 & -0.0387720 & 0.8371960 & $\mathrm{H}$ & 1.9981540 & -3.7016030 & 1.8950980 \\
\hline 0 & -0.5462530 & 1.4291680 & 0.5828880 & $\mathrm{C}$ & 4.1102510 & -1.8890940 & 4.0183570 \\
\hline 0 & 0.3269580 & -0.4560920 & 2.1257970 & $\mathrm{H}$ & 4.4232720 & -2.2599080 & 5.0036110 \\
\hline 0 & 0.5070660 & -0.5692220 & -0.4719760 & $\mathrm{H}$ & 4.0009730 & -0.8011700 & 4.0904200 \\
\hline 0 & -1.7074050 & -0.8843120 & 0.7562800 & C & 2.7633160 & -2.5404560 & 3.5812350 \\
\hline C & 0.5339520 & -1.9397200 & -0.7311360 & $\mathrm{H}$ & 2.4265490 & -3.2690810 & 4.3305600 \\
\hline $\mathrm{C}$ & -0.6318480 & -2.5560980 & -1.2148900 & $\mathrm{H}$ & 1.9796140 & -1.7872700 & 3.4730220 \\
\hline C & 1.7210750 & -2.6464000 & -0.5091500 & C & 5.4208430 & -0.8520260 & 0.8073320 \\
\hline $\mathrm{C}$ & 1.6969540 & -4.0209010 & -0.7592930 & $\mathrm{H}$ & 6.3695250 & -0.4200140 & $\begin{array}{l}1.1272830 \\
\text { 1. }\end{array}$ \\
\hline
\end{tabular}




-5.1460290
-5.5763940
-4.5150170
-4.5552890
-6.6040840
-6.8740190
-7.5176050
-5.9720490
-5.9510770
-6.5585730
-4.0694720
-6.0175150
-3.7088670
-3.7375340
-2.6641590
-4.3364080
-4.6510750
-3.6067530
-4.8806170
-4.9838500
-3.8287180
-4.2761620
-3.5509170
-3.6198940
-2.9845590
-2.8509670
-2.5427110
-1.9591180
-3.3349540
-5.4802350
-5.0713280
-4.7429270
-5.7599860
-5.7771370
-5.8215450
-6.8063050
-5.3133480
-5.7787600
-3.5917230
-4.2160220
-4.3108590
-2.9235660
-1.8564790
-2.4291180
-3.5951300
-4.7586590

0.7774080

$-0.0467890$

$-1.4562880$

0.0221920

$-1.1045930$

$-1.7560490$

$-0.5999700$

$-1.9357510$

$-3.0034560$

$-1.8257070$

$-2.0330450$

0.5853120

$-1.6662520$

$-2.7330960$

$-1.3918920$

$-0.8072880$

$-1.4427450$

$-0.0845940$

2.7815710

4.2571580

2.9022630

2.0375710

4.8107830

5.8699810

4.7524400

3.9800210

4.6283460

3.4824070

2. 3148190

4.7899880

3.6370500

4. 3140430

2.9078220

4.4368180

5.5061490

4.0853540

2.1556810

2.7362790

$-0.8836180$

$-0.8999970$

$-2.3554220$

$-3.0536950$

$-2.3094300$

$-1.6539660$

$-0.3174820$

$-2.3771130$

2.6930860
3.8805050
1.9743360
1.6628420
3.3904190
4.2324670
3.0538410
2.2301230
2.4860910
1.3100270
1.1600440
4.6609530
3.2857330
3.5455480
3.1164490
4.4271390
5.2659600
4.8113450
1.3887720
1.0934550
-0.7992500
0.3571200
0.8664210
0.5831480
1.8037290
-0.2523760
-1.0833370
0.1343860
-1.5772410
1.9137180
-1.3724560
-2.1724490
-1.8163460
-0.2314360
-0.4781890
-0.0907740
2.5572160
3.3548190
-2.9603250
-4.3339480
-4.8426270
-4.8571400
-4.0063920
-2.7704610
-5.0343600
-5.8440850

$-2.5474200$

$-1.4332530$

$-5.2065210$

$-4.9897030$

$-3.0288610$

$-0.6448660$

0.5402120

$-1.9470210$

$-0.7587720$

0.6105710

$-2.7188540$

$-2.5015270$

$-2.5834450$

$-1.1357630$

$-0.6614690$

1. 2860750

1.0714220

$-1.3592970$

1. 5959670

0.4785370

2.1783760

3. 3258140

1. 6225230

3.9085950

3.7534120

2. 2103450

0.7414640

3.3529580

4.7973180

1.7784920

3.8099670

0.1204980

2.1737730

2.8060030

1.8024310

2.7064700

0.8047050

2.0865360

1. 3460900

4. 3710900

3. 5217100

2. 0364790

4.2453190

5.4247790

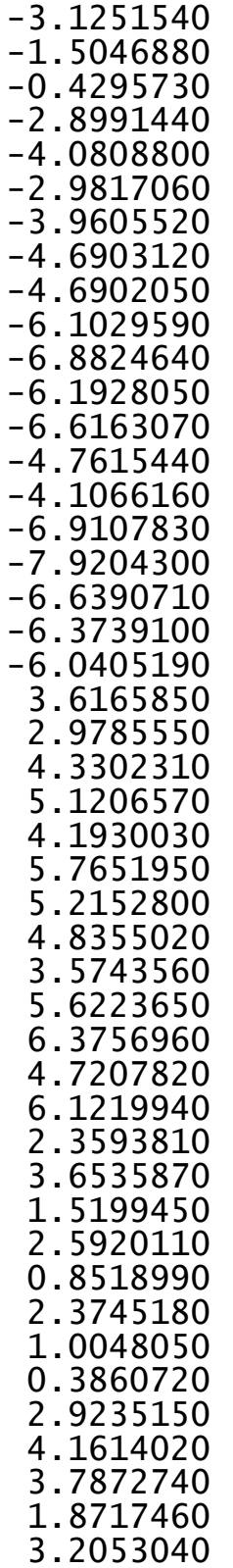

$-5.8853930$

$-4.6276840$

$-4.3097870$

$-4.1727420$

$-4.4881960$

$-3.7602180$

$-1.3846450$

$-1.1694740$

$-1.6751730$

$-2.2358970$

$-1.3649480$

$-1.3960660$

$-2.2843290$

$-0.7248570$

$-2.3413030$

$-0.3317080$

$-1.7086650$

$-0.6541320$

$-2.3808240$

$-3.2633360$

$-0.8634150$

$-1.1194130$

$-2.0424170$

$-1.8677780$

$-3.3253690$

$-2.9580590$

$-0.8739810$

$-4.4153660$

$-3.4622000$

$-4.2346810$

$-2.8152470$

$-5.4069470$

$-5.0862450$

$-0.3646320$

0.2353810

2.0663890

2.1803250

1.2063780

1.8162810

3.2312560

2. 8424090

3.5011380

3.1768880

3.0398520

2. 3871670

3.6166480 


\begin{tabular}{|c|c|c|c|c|c|c|c|}
\hline & & & & & & & \\
\hline $\mathrm{H}$ & 3.6315410 & $\begin{array}{l}4.9253330 \\
3.5526600\end{array}$ & 3.9558080 & $\mathrm{C}$ & 5.1905480 & -2.3275740 & 2.9491300 \\
\hline $\mathrm{H}$ & 4.6925390 & $\begin{array}{l}3.25200060 \\
2.2520060\end{array}$ & $\begin{array}{l}4.0201130 \\
1.4590670\end{array}$ & C & 3.9395070 & $\begin{array}{l}-1.0100400 \\
-4.4745380\end{array}$ & $\begin{array}{l}1.0040020 \\
2.3947200\end{array}$ \\
\hline $\mathrm{H}$ & 4.7721490 & 0.9479880 & 2.6431590 & $\mathrm{H}$ & 3.5400630 & -5.1720170 & 3.1426680 \\
\hline $\mathrm{H}$ & 4.0410690 & 2.4888650 & 4.4544080 & $\mathrm{H}$ & 4.0683070 & -5.0299270 & 1.4577610 \\
\hline $\mathrm{H}$ & 3.8632680 & 4.5983470 & 2.2296380 & C & 5.3016810 & -3.8771760 & 2.8720780 \\
\hline $\mathrm{H}$ & 1.4367470 & 4.6104950 & 2.6346620 & $\mathrm{H}$ & 5.5666770 & -4.2629290 & 3.8653790 \\
\hline 15 & & & & $\mathrm{H}$ & 6.1087570 & -4.1518610 & 2.1825150 \\
\hline & , R) $\mathrm{G}[\mathrm{B} 97-\mathrm{D}$ & {$[Z V P]=-35$} & 394 & $\mathrm{H}$ & 6.1542780 & -1.8866010 & 3.2326100 \\
\hline$P$ & -0.3206450 & -0.0364950 & 0.8183440 & $\mathrm{H}$ & 1.9554280 & $-\overline{3} .7207370$ & 1.8441640 \\
\hline 0 & -0.5393200 & 1.4367830 & 0.5919830 & C & 4.0974710 & -1.9798390 & 3.9965350 \\
\hline 0 & 0.3234750 & -0.4806390 & 2.1006990 & $\mathrm{H}$ & 4.4034330 & -2.3748640 & 4.9745590 \\
\hline 0 & 0.5103030 & -0.5449300 & -0.4986900 & $\mathrm{H}$ & 4.0077390 & -0.8916770 & 4.0894340 \\
\hline 0 & -1.7068710 & -0.8773830 & 0.7188650 & C & 2.7395250 & -2.5988030 & 3.5469450 \\
\hline $\mathrm{C}$ & 0.5284210 & -1.9123860 & -0.7768830 & $\mathrm{H}$ & 2.3874320 & -3.3323200 & 4.2843220 \\
\hline C & -0.6371060 & -2.5108120 & -1.2805730 & $\mathrm{H}$ & 1.9703640 & -1.8294710 & 3.4489700 \\
\hline C & 1.7048300 & -2.6352950 & -0.5488280 & $\mathrm{C}$ & 5.4227360 & -0.9075460 & 0.8037170 \\
\hline $\mathrm{C}$ & 1.6627030 & -4.0092880 & -0.7986320 & $\mathrm{H}$ & 6.3769360 & -0.4940990 & 1.1316230 \\
\hline $\mathrm{H}$ & 2.5668760 & -4.5928030 & -0.6313030 & C & -5.1337930 & 0.7302390 & 2.7104020 \\
\hline C & -2.3728070 & -0.8876610 & -0.5089290 & $\mathrm{C}$ & -5.5466700 & -0.1145270 & 3.8894000 \\
\hline $\mathrm{C}$ & -3.5455960 & -0.1367140 & -0.6429180 & $\mathrm{C}$ & -4.4932700 & -1.4880910 & 1.9528970 \\
\hline C & -1.8345990 & -1.6647800 & -1.5510990 & $\mathrm{C}$ & -4.5482390 & -0.0056400 & 1.6633300 \\
\hline C & -4.1627630 & -0.1418290 & -1.9018820 & $\mathrm{c}$ & -6.5710220 & -1.1728590 & 3.3935430 \\
\hline $\mathrm{H}$ & -5.0833420 & 0.4240690 & -2.0327400 & $\mathrm{H}$ & -6.8287020 & -1.8381110 & 4.2286190 \\
\hline C & 2.9554960 & -1.9978390 & -0.0598420 & $\mathrm{H}$ & -7.4911480 & -0.6701770 & 3.0724130 \\
\hline $\mathrm{C}$ & -4.1247140 & 0.6377230 & 0.4855430 & C & -5.9438920 & -1.9829320 & 2.2157870 \\
\hline C & 3.6506490 & -1.0699730 & -0.8552420 & $\mathrm{H}$ & -5.9118580 & -3.0538470 & 2.4567240 \\
\hline $\mathrm{C}$ & 3.2583300 & -0.5965320 & -2.2342730 & $\mathrm{H}$ & -6.5402950 & -1.8648290 & 1.3030700 \\
\hline C & 2510 & 0.4383940 & -1.4010600 & $\mathrm{H}$ & -4.0509070 & -2.0494010 & 1.1261430 \\
\hline $\mathrm{C}$ & 4.8725150 & -0.5240380 & -0.4186100 & $\mathrm{H}$ & -5.9851580 & 0.5026290 & 4.6831780 \\
\hline $\mathrm{c}$ & 3.1374710 & 0.9491340 & -2.2061250 & $\mathrm{C}$ & -3.6727760 & -1.7107930 & 3.2534210 \\
\hline $\mathrm{H}$ & 2.9091580 & 1.3096500 & -3.2168750 & $\mathrm{H}$ & -3.6913040 & -2.7814800 & 3.4978700 \\
\hline $\mathrm{H}$ & 2.2973870 & 1.2193400 & -1.5642590 & $\mathrm{H}$ & -2.6316710 & -1.4265080 & 3.0782730 \\
\hline C & 4.4699690 & 1.5726120 & -1.6886850 & C & -4.2956110 & -0.8731440 & 4.4131680 \\
\hline $\mathrm{H}$ & 4.8931760 & 2.2575510 & -2.4343040 & $\mathrm{H}$ & -4.5972250 & -1.5228570 & 5.2458380 \\
\hline $\mathrm{H}$ & 4.2928020 & 2.1519030 & -0.7760360 & $\mathrm{H}$ & -3.5676530 & -0.1503750 & 4.8004450 \\
\hline $\mathrm{H}$ & 6.4306460 & 0.8510430 & -1.0127740 & C & -4.8914140 & 2.7560420 & 1.4350390 \\
\hline $\mathrm{H}$ & 2.3163940 & -1.0440450 & -2.5623220 & $\mathrm{C}$ & -5.0026630 & 4.2357940 & 1.1644110 \\
\hline C & 5.7400740 & -0.3235600 & -2.7338920 & $\mathrm{C}$ & -3.8618370 & 2.9159670 & -0.7611730 \\
\hline $\mathrm{H}$ & 6.1202830 & 0.3854450 & -3.4813560 & $\mathrm{C}$ & -4.2940700 & 2.0312460 & 0.3857750 \\
\hline $\mathrm{H}$ & 6.5084420 & -1.0904140 & -2.5802360 & $\mathrm{C}$ & -3.5735620 & 4.7977970 & 0.9322680 \\
\hline C & 4.4036660 & -0.9697130 & -3.2167190 & $\mathrm{H}$ & -3.6486430 & 5.8612720 & 0.6671880 \\
\hline $\mathrm{H}$ & 4.1396040 & -0.6086900 & -4.2194850 & $\mathrm{H}$ & -2.9978880 & 4.7260560 & 1.8629370 \\
\hline $\mathrm{H}$ & 4.4975760 & -2.0609270 & -3.2698840 & C & -2.8820060 & 3.9879530 & -0.2069110 \\
\hline C & 3.5048610 & -2.3576540 & 1.1836990 & $\mathrm{H}$ & -2.5851750 & 4.6512710 & -1.0301750 \\
\hline $\mathrm{C}$ & 2.9153880 & -3.3244740 & 2.1841310 & $\mathrm{H}$ & -1.9841780 & 3.4871450 & 0.1621470 \\
\hline
\end{tabular}




$\begin{array}{rrr}-3.3741830 & 2.3428770 & -1.5535730 \\ -5.4924540 & 4.7538070 & 1.9980260 \\ -5.1128260 & 3.6550810 & -1.3101260 \\ -4.7949590 & 4.3458190 & -2.1025840 \\ -5.8033370 & 2.9304310 & -1.7586580 \\ -5.8098540 & 4.4338930 & -0.1494340 \\ -5.8604910 & 5.5068640 & -0.3784910 \\ -6.8362710 & 4.0763500 & -0.0041340 \\ -5.3097150 & 2.1095970 & 2.5975660 \\ -5.7699340 & 2.6752560 & 3.4088090 \\ -3.6114250 & -0.8219930 & -2.9861780 \\ -4.2436630 & -0.8021570 & -4.3566870 \\ -4.2911930 & -2.2369920 & -4.9220980 \\ -2.8752930 & -2.8598010 & -5.0147110 \\ -1.8397290 & -2.1750140 & -4.0760680 \\ -2.4402120 & -1.5860400 & -2.8197400 \\ -3.6480090 & -0.1702890 & -5.0365100 \\ -4.7734830 & -2.2443090 & -5.9076810 \\ -2.4994970 & -2.7848730 & -6.0429730 \\ -1.3969260 & -1.3325260 & -4.6303950 \\ -5.2489100 & -0.3661880 & -4.3059550 \\ -4.9156650 & -2.8401330 & -4.2497830 \\ -2.9372330 & -3.9295340 & -4.7815500 \\ -1.0166520 & -2.8563530 & -3.8468090 \\ -0.6689850 & -3.9147850 & -1.4536440 \\ -1.9662600 & -4.6085670 & -1.8347110 \\ -1.9678930 & -6.1082700 & -1.5113930 \\ -0.6951690 & -6.7585100 & -2.0647930 \\ 0.5291460 & -6.1779410 & -1.3467000 \\ 0.4991480 & -4.6629920 & -1.2162190 \\ -2.8662160 & -6.5727550 & -1.9379620 \\ -2.1415390 & -4.4914160 & -2.9115140 \\ -2.8039280 & -4.1028400 & -1.3387000 \\ -0.6277080 & -6.5522930 & -3.1438620 \\ -0.7186630 & -7.8486440 & -1.9396450 \\ 1.4562170 & -6.4775040 & -1.8546680 \\ 0.5809950 & -6.6102910 & -0.3346990 \\ -2.0061830 & -6.2552980 & -0.4217360 \\ 1.6282690 & 3.6302020 & -0.8138350 \\ 0.5035450 & 3.0088130 & -1.0790220 \\ 2.2170360 & 4.3568840 & -1.9817380 \\ 3.3708830 & 5.1351510 & -1.7949320 \\ 1.6604380 & 4.2435790 & -3.2667410 \\ 3.9590950 & 5.7913960 & -2.8753060 \\ 3.7989680 & 5.2112250 & -0.7997500 \\ 2.2537460 & 4.8976350 & -4.3468290\end{array}$

$\begin{array}{ll}\mathrm{H} & 0.7745720 \\ \mathrm{C} & 3.4026390 \\ \mathrm{H} & 4.8526420 \\ \mathrm{H} & 1.8212990 \\ \mathrm{H} & 3.8638970 \\ \mathrm{H} & 0.1396110 \\ \mathrm{O} & 2.2074390 \\ \mathrm{C} & 2.8169030 \\ \mathrm{C} & 1.8213860 \\ \mathrm{H} & 2.7146960 \\ \mathrm{H} & 0.8228630 \\ \mathrm{O} & 2.0899480 \\ \mathrm{H} & 1.3459750 \\ \mathrm{C} & 4.3872710 \\ \mathrm{C} & 3.5497680 \\ \mathrm{C} & 2.0619460 \\ \mathrm{C} & 4.2577290 \\ \mathrm{H} & 5.4426740 \\ \mathrm{H} & 3.6619980 \\ \mathrm{H} & 1.6238970 \\ \mathrm{H} & 4.7090950 \\ \mathrm{H} & 4.7784730 \\ \mathrm{H} & 4.0489510 \\ \mathrm{H} & 3.8999630 \\ \mathrm{H} & 1.4718300 \\ \mathrm{H} & \\ \mathrm{H} & \end{array}$

$\begin{array}{lr}3.6340480 & -3.4129550 \\ 5.6723950 & -4.1540210 \\ 6.3925340 & -2.7232210 \\ 4.8013780 & -5.3401190 \\ 6.1811590 & -4.9978520 \\ 2.3799180 & -0.3353730 \\ 3.6427910 & 0.2847830 \\ 1.4750940 & 2.0839070 \\ 2.5531240 & 2.2120340 \\ 0.8215700 & 1.2131740 \\ 2.3496070 & 1.8419900 \\ 0.9465320 & 3.2379690 \\ 0.3404490 & 2.8360950 \\ 2.8399550 & 3.5488550 \\ 4.0907610 & 3.2435100 \\ 3.7319800 & 3.0923120 \\ 1.8109140 & 2.4144910 \\ 3.1107840 & 3.6744400 \\ 4.8395080 & 4.0367060 \\ 3.4853730 & 4.0713040 \\ 2.2074930 & 1.4952570 \\ 0.8789130 & 2.6527480 \\ 2.3903970 & 4.4922150 \\ 4.5418610 & 2.3060980 \\ 4.5671550 & 2.6975530\end{array}$

TS156(R,R) G[B97-D/def2-TZVP] $=-3541.574156$

$\begin{array}{lrrr}\text { TS156(R, R) } \mathrm{G}[\mathrm{B} 97-\mathrm{D} / \mathrm{def} 2-\mathrm{TZVP}]=-3541.574156 \\ \mathrm{P} & 0.3422070 & 0.0770330 & 0.8957390 \\ \mathrm{O} & 0.6476320 & -1.3701080 & 0.6084430 \\ \mathrm{O} & -0.3145330 & 0.4316910 & 2.1994580 \\ \mathrm{O} & -0.5352920 & 0.5860280 & -0.3911870 \\ \mathrm{O} & 1.6782820 & 0.9983700 & 0.8136730 \\ \mathrm{C} & -0.6223310 & 1.9588150 & -0.6278290 \\ \mathrm{C} & 0.5133630 & 2.6215490 & -1.1286850 \\ \mathrm{C} & -1.8316540 & 2.6149250 & -0.3721000 \\ \mathrm{C} & -1.8685230 & 4.0000640 & -0.5821230 \\ \mathrm{H} & -2.8010750 & 4.5318590 & -0.4016430 \\ \mathrm{C} & 2.3271360 & 1.0671000 & -0.4225630 \\ \mathrm{C} & 3.5259650 & 0.3660740 & -0.5961610 \\ \mathrm{C} & 1.7439350 & 1.8385380 & -1.4390010 \\ \mathrm{C} & 4.1148460 & 0.4349170 & -1.8619360 \\ \mathrm{H} & 5.0522900 & -0.0948990 & -2.0232820 \\ \mathrm{C} & -3.0466260 & 1.8868200 & 0.0789180 \\ \mathrm{C} & 4.1614450 & -0.4139800 & 0.4973380 \\ \mathrm{C} & -3.6724570 & 0.9507640 & -0.7641150 \\ \mathrm{C} & -3.2369260 & 0.5662950 & -2.1583040\end{array}$




\begin{tabular}{|c|c|c|}
\hline $\begin{array}{l}-5.3993890 \\
-4.8563790 \\
-3.0082830 \\
-2.7440810 \\
-2.1584810 \\
-4.2997870 \\
-4.6673500 \\
-4.0932990 \\
-6.3110650 \\
-2.3257380 \\
-5.6893100 \\
-6.0144410 \\
-6.5097760 \\
-4.3968480 \\
-4.1004540 \\
-4.5652070 \\
-3.6295500 \\
-3.1177730 \\
-5.3123510 \\
-4.8199920 \\
-4.2275690 \\
-3.8824350 \\
-4.3988230 \\
-5.5404280 \\
-5.8326420 \\
-6.3664430 \\
-6.2398150 \\
-2.1906800 \\
-4.1953170 \\
-4.5306690 \\
-4.0217760 \\
-2.8881850 \\
-2.5953600 \\
-2.0610580 \\
-5.4371580 \\
-6.3599670 \\
5.2582500 \\
5.6840940 \\
4.5027680 \\
4.6041530 \\
6.6464740 \\
6.9140360 \\
7.5703550 \\
5.9416890 \\
5.8775330 \\
6.5051700\end{array}$ & $\begin{array}{r}-0.6551580 \\
0.3023820 \\
-0.9668570 \\
-1.2676140 \\
-1.2035230 \\
-1.7032450 \\
-2.3800610 \\
-2.3109780 \\
-1.1481800 \\
1.0907690 \\
0.1455930 \\
-0.5541710 \\
0.8506890 \\
0.9016800 \\
0.6030480 \\
1.9851960 \\
2.1550380 \\
3.1163700 \\
1.9137410 \\
1.5089660 \\
4.1724900 \\
4.8626370 \\
4.7595780 \\
3.4517990 \\
3.7670780 \\
3.6990730 \\
1.3872380 \\
3.6004910 \\
1.5974590 \\
1.9168580 \\
0.5161600 \\
2.3408960 \\
3.0623830 \\
1.6392670 \\
0.5891430 \\
0.0939730 \\
-0.5112530 \\
0.3270160 \\
1.6947780 \\
0.2213430 \\
1.4333140 \\
2.0915610 \\
0.9722110 \\
2.2420550 \\
3.3056830 \\
2.1666880\end{array}$ & $\begin{array}{l}-1.3956090 \\
-0.3648420 \\
-2.1925800 \\
-3.2141880 \\
-1.5504400 \\
-1.7205120 \\
-2.5021920 \\
-0.8327690 \\
-1.0362300 \\
-2.4574940 \\
-2.6964940 \\
-3.4778660 \\
-2.5181150 \\
-3.1364790 \\
-4.1506730 \\
-3.1446230 \\
1.3299470 \\
2.3770600 \\
3.0825070 \\
1.7164310 \\
2.6380780 \\
3.4190580 \\
1.7276440 \\
3.0821590 \\
4.0926030 \\
2.4047550 \\
3.3399150 \\
2.0597170 \\
4.1149360 \\
5.1107610 \\
4.1519860 \\
3.7041860 \\
4.4785010 \\
3.5729540 \\
0.8699440 \\
1.1740160 \\
2.6799790 \\
3.8590080 \\
1.9925160 \\
1.6717600 \\
3.3434620 \\
4.1810090 \\
2.9742330 \\
2.2094220 \\
2.4751100 \\
1.2716260\end{array}$ \\
\hline
\end{tabular}

6.1773560

3.7242640

3.7032480

2.6911090

4.4274440

4.7385750

3.7448060

5.0499270

5.2159050

3.9343180

4. 3782250

3. 8052440

3.9153620

3.2675720

3.0305290

2.7298990

2.1271210

3.3865130

5.7652700

5.1927140

4.8738830

5.8307360

5.9715350

6.0564810

6.9876710

5.4877600

6.0055380

3.5311170

2. 3253320

4.2442420

3. 3346120

2. 5156310

1.6019670

3.9396670

5.0691700

4.7107990

3.1996970

1.9088820

1.0807560

0.8127090

2.6460000

$-0.7395520$

$-0.7465840$

$-0.1067240$

1. 3304920
2. 2524730

$-0.2865980$

1.8577540

2.9235290

1.5288370

1.0278470

1.6767960

0.2716410

$-2.5178050$

$-3.9845740$

$-2.6749190$

$-1.7974730$

$-4.6060150$

$-5.6596350$

$-4.5783810$

$-3.8051300$

$-4.4632100$

$-3.3563260$

$-2.1056960$

$-4.4978480$

$-3.3417990$

$-4.0262450$

$-2.5746310$

$-4.1167070$

$-5.1809180$

$-3.7193700$

$-1.8789770$

$-2.4413740$

1.1256890

1.8249750

1.1235230

1.4894370

2.7381130

2.4549850

1.6506120

1.8530870

0.1423520

3.5644960

3.0591830

3.3648520

1.7526180

0.6571770

4.7066190

6.2006500

6.5369250

5.9595960
1.1948740

4.6227720

3.3266630

3.5915980

3.1841920

4.4456130

5.2753440

4.8510430

1. 3684660

1.0574140

$-0.7861490$

0.3617020

0.8737530

0.5826790

1.8292430

$-0.2162910$

$-1.0423520$

0.2016840

$-1.5415020$

1.8560750

$-1.4057320$

$-2.2033300$

$-1.8610520$

$-0.2950920$

$-0.5530030$

$-0.1854210$

2.5281950

3.3063380

$-2.9285470$

$-2.7278760$

$-4.2718100$

$-5.4511830$

$-5.1060210$

$-3.9078070$

$-6.3528130$

$-4.2266460$

$-4.4319500$

$-4.8594200$

$-5.9626560$

$-3.6067810$

$-4.2232760$

$-5.6610660$

$-0.9929670$

$-1.2028950$

-2.5685780
-2.6914560 


$\begin{array}{lrrr}\mathrm{C} & 1.6498530 & 4.8857190 & -1.6146340 \\ \mathrm{C} & 0.4596300 & 4.0221480 & -1.2657970 \\ \mathrm{H} & -0.1645200 & 6.6926460 & -0.4064300 \\ \mathrm{H} & -0.0961120 & 7.6231870 & -2.7225030 \\ \mathrm{H} & 2.0710280 & 6.7636760 & -2.5985200 \\ \mathrm{H} & 1.9432150 & 5.4112650 & -0.6924320 \\ \mathrm{H} & -1.7700790 & 6.5905350 & -1.1448410 \\ \mathrm{H} & -0.7445730 & 6.1047450 & -3.3505950 \\ \mathrm{H} & 1.4631680 & 5.5221490 & -3.6882090 \\ \mathrm{H} & 2.5106730 & 4.2850150 & -1.9186300 \\ \mathrm{C} & -1.3808490 & -3.6223960 & -0.8927280 \\ \mathrm{O} & -0.2917030 & -2.9313830 & -1.1328690 \\ \mathrm{C} & -1.9288290 & -4.3389810 & -2.0862380 \\ \mathrm{C} & -3.0437260 & -5.1782790 & -1.9299710 \\ \mathrm{C} & -1.3744500 & -4.1576900 & -3.3643110 \\ \mathrm{C} & -3.5963220 & -5.8270230 & -3.0332960 \\ \mathrm{H} & -3.4709100 & -5.3069960 & -0.9398660 \\ \mathrm{C} & -1.9316070 & -4.8054210 & -4.4673700 \\ \mathrm{H} & -0.5186180 & -3.5017160 & -3.4870370 \\ \mathrm{C} & -3.0423490 & -5.6404870 & -4.3049350 \\ \mathrm{H} & -4.4602640 & -6.4750460 & -2.9043310 \\ \mathrm{H} & -1.5010970 & -4.6567380 & -5.4550120 \\ \mathrm{H} & -3.4758230 & -6.1435270 & -5.1667260 \\ \mathrm{H} & 0.0358830 & -2.3120850 & -0.3642390 \\ \mathrm{O} & -1.9596360 & -3.7051930 & 0.2030350 \\ \mathrm{C} & -2.6981310 & -1.6599240 & 2.0892610 \\ \mathrm{C} & -1.6406470 & -2.6815670 & 2.1737270 \\ \mathrm{H} & -2.6352200 & -0.9650670 & 1.2472730 \\ \mathrm{H} & -0.6563440 & -2.4036980 & 1.8142890 \\ \mathrm{O} & -2.0027310 & -1.1404610 & 3.2669890 \\ \mathrm{H} & -1.2939190 & -0.4765350 & 2.8953530 \\ \mathrm{C} & -4.1840120 & -3.1797750 & 3.4891100 \\ \mathrm{C} & -3.2723860 & -4.3631440 & 3.1320820 \\ \mathrm{C} & -1.8091640 & -3.9095430 & 3.0017200 \\ \mathrm{C} & -4.1168540 & -2.0936620 & 2.4029000 \\ \mathrm{H} & -5.2212570 & -3.5191510 & 3.5986190 \\ \mathrm{H} & -3.3405800 & -5.1507400 & 3.8919320 \\ \mathrm{H} & -1.3875110 & -3.6786700 & 3.9916270 \\ \mathrm{H} & -4.5467640 & -2.4717620 & 1.4658000 \\ \mathrm{H} & -4.6895070 & -1.2051760 & 2.6842050 \\ \mathrm{H} & -3.8750130 & -2.7531590 & 4.4530340 \\ \mathrm{H} & -3.5941460 & -4.7937670 & 2.1750110 \\ \mathrm{H} & -1.1691690 & -4.6895840 & 2.5734150 \\ \mathrm{H} & & & \\ \mathrm{H} & & & \end{array}$

TS157(R, R) G[B97-D/def2-TZVP $]=-3541.574094$

$\mathrm{P}$

0.3519230

0.1906940

0.7867000

0.6676450
-0.3084430
-0.5201050
1.6793890
-0.6156730
0.5070380
-1.8241270
-1.8620810
-2.7903780
2.3295470
3.5229620
1.7436380
4.1158370
5.0402330
-3.0198900
4.1450900
-3.6738070
-3.3022420
-5.4143770
-4.8313970
-3.0632940
-2.8508300
-2.1794000
-4.3228450
-4.7235600
-4.0659580
-6.3024210
-2.4110770
-5.7762280
-6.1374230
-6.5902980
-4.5118890
-4.2624780
-4.6891340
-3.5501690
-3.0002250
-5.1536040
-4.7175970
-4.1052440
-3.7310980
-4.3200280
-5.3914790
-5.6386200
-6.2496700
-6.0656880
-2.0916610
0.6525130

2.0451640

$-0.5487850$

0.6103100

$-0.9223310$

$-1.4958520$

$-0.7171250$

$-1.0683420$

$-0.9116740$

$-0.6250390$

$-0.7214820$

$-1.7156090$

$-1.9901230$

$-2.0973210$

$-0.1368670$

0.4511740

$-0.8254960$

$-2.1818950$

$-1.1712990$

$-0.2931290$

$-2.0465480$

$-3.0383870$

$-1.4274870$

$-1.4241820$

$-2.0978490$

$-0.4813450$

$-0.7096820$

$-2.5853690$

$-2.5421040$

$-3.2165250$

$-2.4069130$

$-3.1358980$

$-4.1178620$

$-3.2709610$

1.0944250

1.9880190

2.9354330
1.6126770

2.1580760

2.8262800

1.1870600

2.7500560

3.7250390

2.0862980

3.2986830

1. 5732820 


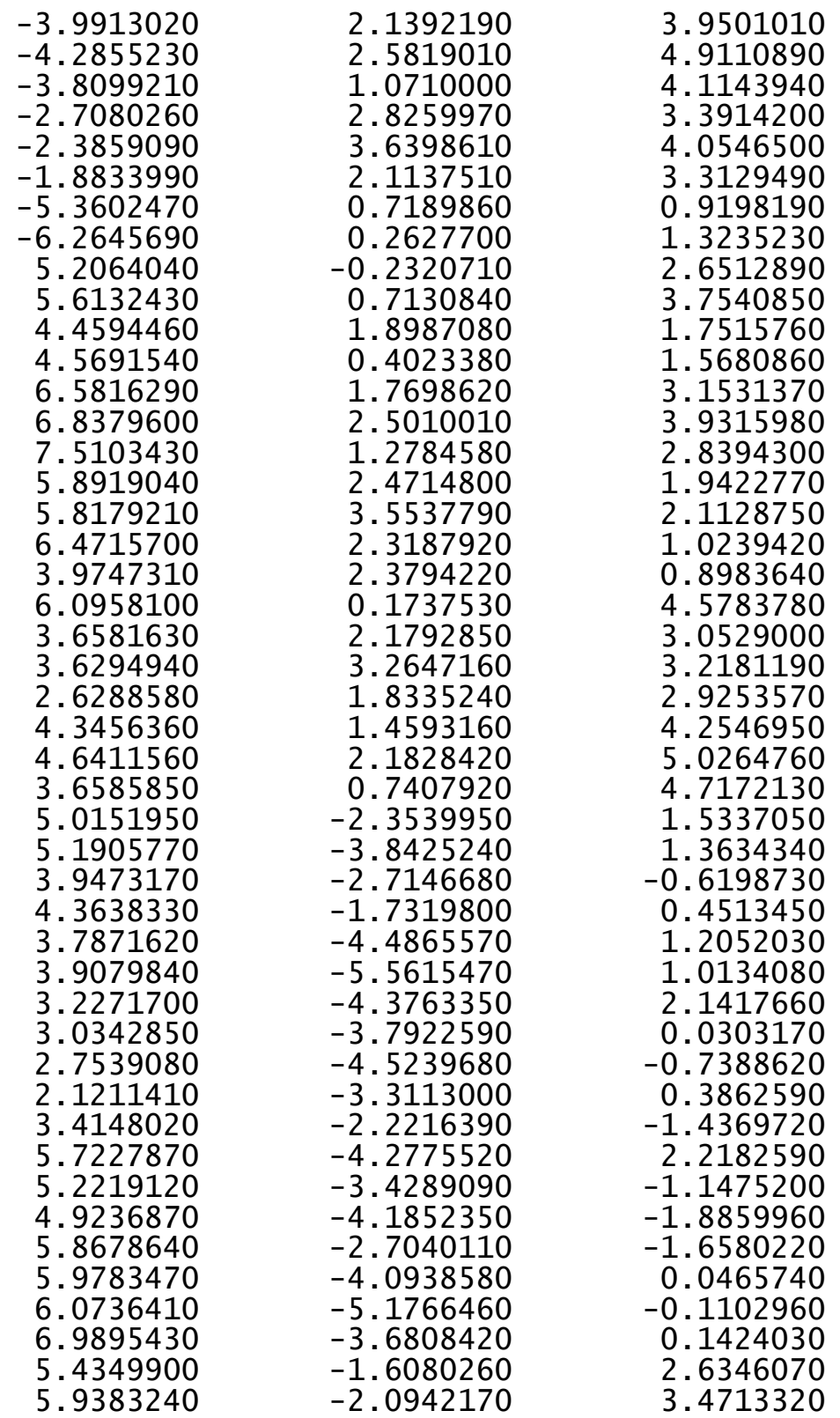

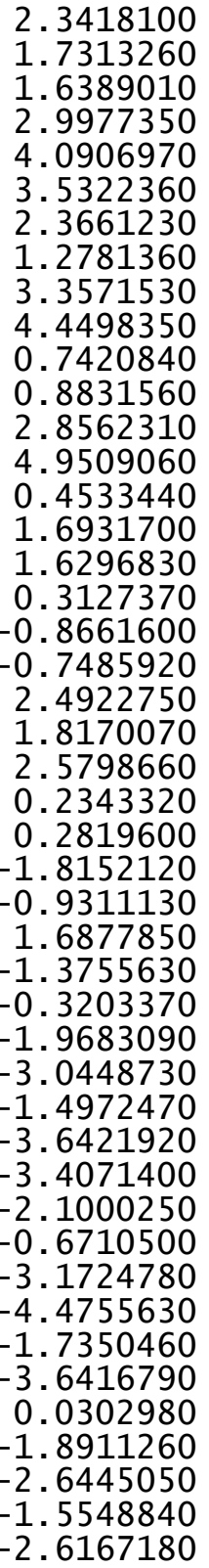

1.5750800
2.0683840
0.9032870
0.1645570
0.6749960
0.8334050
2.8579170
1.2943150
0.2828760
1.6514580
2.5032380
0.1974230
-0.9098350
-0.0049230
3.9067300
4.6404470
6.1536670
6.7082840
6.1064210
4.6081300
6.6374700
4.4767860
4.2045620
6.4419720
7.8033570
6.3183290
6.6010930
6.3618160
-3.7118480
-3.0201030
-4.5389310
-5.3922460
-4.4454040
-6.1411560
-5.4530350
-5.1922620
-3.7792540
-6.0406170
-6.8002470
-5.1106400
-6.6209690
-2.3258140
-3.7130030
-1.4667990
-2.4377970
-0.8884670
$-2.9804330$ $-4.2682830$ $-5.2847540$ $-5.4749230$ $-4.5000870$ $-3.1093130$ $-4.6998960$ $-6.2442830$ $-6.5049240$ $-4.8613980$ $-4.0973020$ $-4.9187410$ $-5.3049650$ $-4.4978650$ $-1.7879570$ $-2.2704230$ $-2.0263550$ $-2.5808590$ $-1.8060650$ $-1.5717650$ $-2.5023040$ $-3.3496390$ $-1.7943110$ $-3.6457300$ $-2.5134120$ $-2.3175410$ $-0.8238570$ $-0.9474760$ $-0.5151890$ $-0.8739410$ $-1.6122450$ $-1.3217930$ $-2.9326340$ $-2.3347910$ $-0.2997810$ $-3.9453180$ $-3.1593380$ $-3.6493790$ $-2.1023890$ $-4.9666230$ $-4.4410130$ $-0.1836130$ 0.6148200 2. 2383470 2. 4342550 1. 3108170 


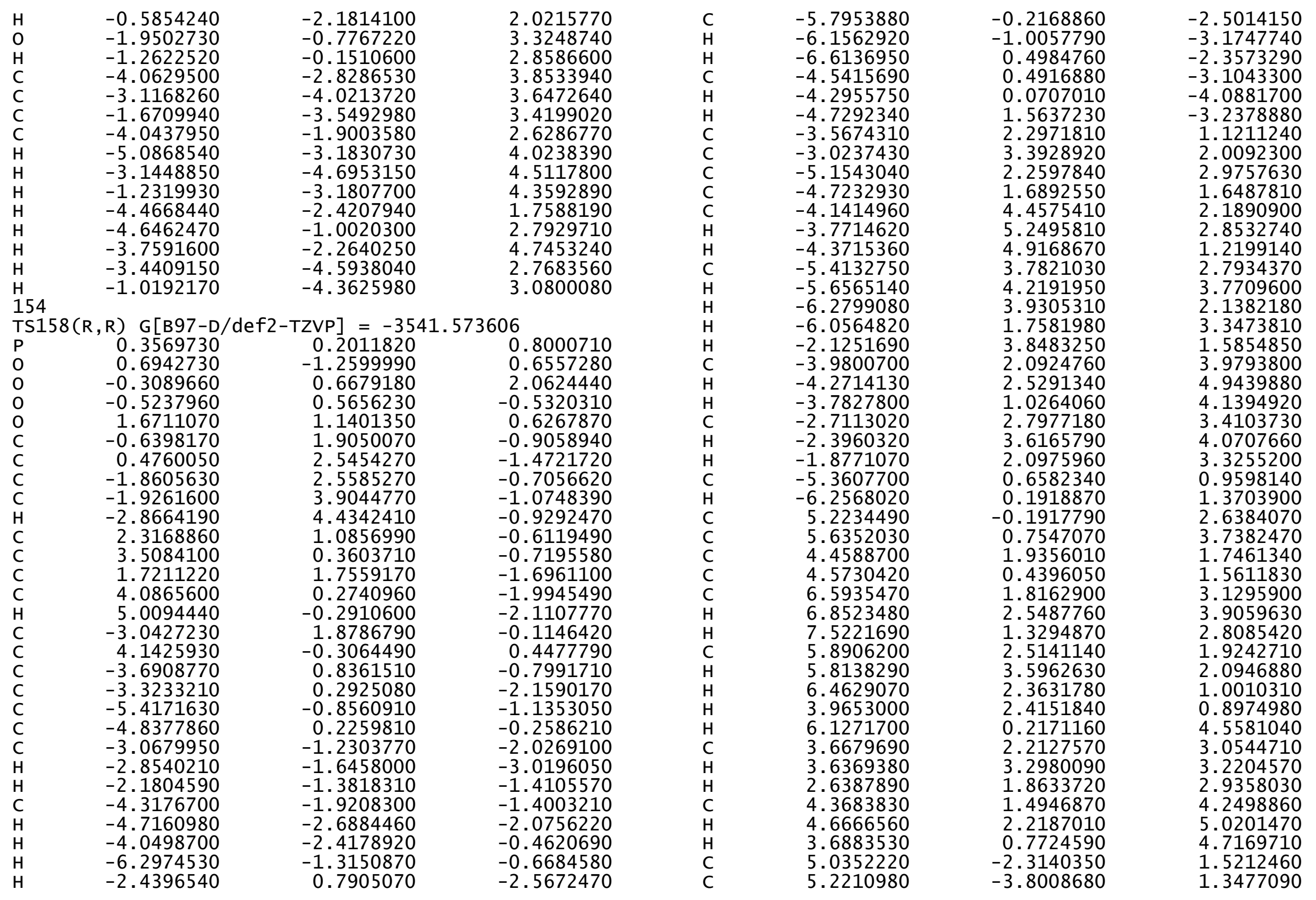




$\begin{array}{rrr}3.9485820 & -2.6809920 & -0.6215590 \\ 4.3689300 & -1.6957110 & 0.4459050 \\ 3.8216660 & -4.4563850 & 1.2032030 \\ 3.9492350 & -5.5302480 & 1.0093830 \\ 3.2701710 & -4.3513130 & 2.1454000 \\ 3.0514640 & -3.7672340 & 0.0365920 \\ 2.7695810 & -4.5002880 & -0.7307260 \\ 2.1380240 & -3.2947440 & 0.4024350 \\ 3.4033860 & -2.1916240 & -1.4324260 \\ 5.7657030 & -4.2321870 & 2.1965820 \\ 5.2233310 & -3.3844650 & -1.1630790 \\ 4.9236400 & -4.1424650 & -1.8992390 \\ 5.8582240 & -2.6539000 & -1.6793620 \\ 5.9972670 & -4.0444870 & 0.0225080 \\ 6.0996890 & -5.1263170 & -0.1364800 \\ 7.0060490 & -3.6234560 & 0.1083660 \\ 5.4605900 & -1.5661680 & 2.6186490 \\ 5.9752120 & -2.0496380 & 3.4500600 \\ 2.3035050 & 1.6041060 & -2.9682530 \\ 1.6877150 & 2.1150660 & -4.2481230 \\ 1.5838080 & 0.9649140 & -5.2801140 \\ 2.9306510 & 0.2043490 & -5.4719560 \\ 4.0357040 & 0.7023990 & -4.5053390 \\ 3.4905420 & 0.8605990 & -3.1090600 \\ 2.3224790 & 2.9094280 & -4.6718890 \\ 1.2360960 & 1.3748920 & -6.2365340 \\ 3.2866700 & 0.3105760 & -6.5044390 \\ 4.4006330 & 1.6768830 & -4.8665720 \\ 0.7022500 & 2.5540440 & -4.0662820 \\ 0.8136040 & 0.2671730 & -4.9290320 \\ 2.7735990 & -0.8667110 & -5.2945560 \\ 4.8900110 & 0.0150580 & -4.5125660 \\ 0.3972220 & 3.9260930 & -1.7705910 \\ -0.8255720 & 4.6000200 & -1.5827600 \\ 1.6462020 & 4.7043110 & -2.1575200 \\ 1.3552520 & 6.0679680 & -2.7965920 \\ 0.3310200 & 6.8427600 & -1.9609830 \\ -0.9934030 & 6.0706760 & -1.9290320 \\ 2.2917950 & 6.6324860 & -2.8925850 \\ 2.2295980 & 4.8619170 & -1.2354970 \\ 2.2887230 & 4.1148980 & -2.8136090 \\ 0.7160270 & 6.9602160 & -0.9369440 \\ 0.1680620 & 7.8484520 & -2.3696710 \\ -1.6945980 & 6.5308250 & -1.2201890 \\ -1.4654060 & 6.1443980 & -2.9223410 \\ 0.9554360 & 5.9213220 & -3.8116870\end{array}$
C $\quad-1.3144120$
$-0.2660860$
$-1.8913210$
$-2.9635710$
$-1.4098620$
$-3.5464980$
$-3.3341650$
$-1.9978370$
$-0.5871330$
$-3.0661900$
$-4.3767910$
$-1.6247050$
$-3.5239750$
0.0743270
$-1.8358850$
$-2.6144360$
$-1.5121950$
$-2.5989610$
$-0.5478780$
$-1.9223940$
$-1.2456910$
$-4.0059850$
$-3.0486790$
$-1.6094840$
$-4.0063120$
$-5.0246570$
$-3.0645510$
$-1.1692690$
$-4.4306670$
$-4.6166180$
$-3.7013740$
$-3.3718340$
$-0.9502480$

154

$\operatorname{TS} 159(R, R) G[B 97-D / d e f 2-T Z V P]=-3541.573541$

$\begin{array}{lrcr}\text { TS159(R, R) } \quad \text { G }[\mathrm{B} 97-\mathrm{D} / \mathrm{def}-\mathrm{TZVP}]=-3541.573541 \\ \mathrm{P} & 0.3539190 & 0.0935990 & 0.9015480 \\ \mathrm{O} & 0.6639000 & -1.3561520 & 0.6321960 \\ \mathrm{O} & -0.2952000 & 0.4627050 & 2.2049590 \\ \mathrm{O} & -0.5364680 & 0.5796270 & -0.3857870 \\ \mathrm{O} & 1.6854150 & 1.0193700 & 0.7952990 \\ \mathrm{C} & -0.6307960 & 1.9471520 & -0.6475840 \\ \mathrm{C} & 0.4995400 & 2.6070030 & -1.1662430 \\ \mathrm{C} & -1.8421080 & 2.6015270 & -0.3997390 \\ \mathrm{C} & -1.8852260 & 3.9826180 & -0.6333770 \\ \mathrm{H} & -2.8183380 & 4.5142300 & -0.4550480 \\ \mathrm{C} & 2.3209720 & 1.0648720 & -0.4494050\end{array}$

$\begin{array}{ll}-3.7065100 & -0.5434450 \\ -2.9997260 & -0.8929670 \\ -4.5345550 & -1.6483210 \\ -5.3971520 & -1.3697740 \\ -4.4330570 & -2.9643840 \\ -6.1474280 & -2.3901470 \\ -5.4636590 & -0.3511400 \\ -5.1817890 & -3.9843880 \\ -3.7595580 & -3.1819880 \\ -6.0393570 & -3.7002650 \\ -6.8135330 & -2.1668970 \\ -5.0942810 & -5.0022560 \\ -6.6209320 & -4.4976630 \\ -2.3074330 & -0.1952620 \\ -3.7206650 & 0.5838070 \\ -1.5075830 & 2.2453870 \\ -2.4680680 & 2.4218920 \\ -0.9175640 & 1.3250390 \\ -2.1937400 & 2.0088010 \\ -0.8228550 & 3.3368310 \\ -0.1827550 & 2.8745100 \\ -2.9085430 & 3.8502700 \\ -4.0873940 & 3.6187300 \\ -3.5956790 & 3.3909350 \\ -1.9616430 & 2.6393080 \\ -3.2766400 & 4.0230630 \\ -4.7759270 & 4.4719540 \\ -3.2366810 & 4.3335070 \\ -2.4739750 & 1.7653380 \\ -1.0717590 & 2.8206690 \\ -2.3541180 & 4.7482940 \\ -4.6483210 & 2.7320810 \\ -4.3958990 & 3.0347920 \\ -5 & \end{array}$

1.0648720
$-0.4494050$

0679680

5.9213220

$-3.8116870$ 


\begin{tabular}{|c|c|c|}
\hline $\begin{array}{l}3.5155610 \\
1.7268520 \\
4.0757260 \\
5.0069300 \\
-3.0519850 \\
4.1710780 \\
-3.6828260 \\
-3.2585280 \\
5.4107440 \\
4.8618830 \\
-3.0261070 \\
-2.7719830 \\
-2.1690390 \\
-4.3105770 \\
4.6833690 \\
-4.0930040 \\
6.3178110 \\
-2.3513200 \\
-5.7139730 \\
6.0417570 \\
6.5361920 \\
4.4279920 \\
4.1394550 \\
-4.6002420 \\
-3.6247100 \\
-3.1064230 \\
5.2924710 \\
-4.8109410 \\
-4.2166930 \\
-3.8664890 \\
-4.3977110 \\
-5.5234880 \\
-5.8056140 \\
6.3570520 \\
6.2166280 \\
-2.1830030 \\
4.1659930 \\
-4.4940780 \\
-3.9886550 \\
-2.8647500 \\
-2.5699100 \\
-2.0354030 \\
-5.4330950 \\
6.3521220 \\
5.2968070 \\
5.7347420\end{array}$ & $\begin{array}{r}0.3573250 \\
1.8181850 \\
0.3851430 \\
-0.1542140 \\
1.8772100 \\
-0.3906260 \\
0.9291550 \\
0.5276950 \\
-0.6893920 \\
0.2836580 \\
-1.0051060 \\
-1.3179580 \\
-1.2320140 \\
-1.7384740 \\
-2.4279220 \\
-2.3320050 \\
-1.1801330 \\
1.0507680 \\
0.0922230 \\
-0.6197510 \\
0.7961980 \\
0.8475730 \\
0.5380780 \\
1.9303500 \\
2.1606130 \\
3.1378170 \\
1.9405120 \\
1.5180130 \\
4.1951710 \\
4.8985300 \\
4.7671250 \\
3.4780640 \\
3.8062690 \\
3.7148970 \\
1.4157580 \\
3.6193150 \\
1.6400510 \\
1.9700120 \\
0.5597890 \\
2.3827860 \\
3.1167870 \\
1.6829780 \\
0.5856430 \\
0.0923370 \\
-0.4258850 \\
0.4448820\end{array}$ & $\begin{array}{l}-0.6270690 \\
-1.4715770 \\
-1.9074230 \\
-2.0735950 \\
0.0705790 \\
0.4768620 \\
-0.7547930 \\
-2.1475170 \\
-1.3498120 \\
-0.3368750 \\
-2.1648190 \\
-3.1853070 \\
-1.5287390 \\
-1.6696710 \\
-2.4376760 \\
-0.7750630 \\
-0.9759730 \\
-2.4611070 \\
-2.6593270 \\
-3.4284740 \\
-2.4846440 \\
-3.1194760 \\
-4.1326790 \\
-3.1387440 \\
1.3228640 \\
2.3520130 \\
3.0921950 \\
1.7277160 \\
2.6061500 \\
3.3729680 \\
1.6880140 \\
3.0734040 \\
4.0825970 \\
2.4015440 \\
3.3645520 \\
2.0202410 \\
4.1189340 \\
5.1137420 \\
4.1671000 \\
3.6886280 \\
4.4503280 \\
3.5621840 \\
0.8987530 \\
1.2169630 \\
2.6468850 \\
3.7975590\end{array}$ \\
\hline
\end{tabular}

4.6267760

6.6845570

6.9598680

7.6057420

5.9612420

5.8952250

6. 5129120

4. 0123860

6.2411950

3. 7609960

3.7394770

2.7273770

4.4821030

4.7999320

3. 8082440

5.0757850

5. 2403100

3. 9374240

4. 3904230

3.8282440

3. 9360000

3. 2997240

3. 0424710

2. 7403250

2. 1394610

3. 3810700

5.7985430

5. 1922110

4.8689710

5. 8249590

5. 9812600

6.0606600

6. 9994490

5. 5272520

6. 0556470

2. 2780160

1. 5598280

2. 0037400

3. 5342490

4.0996200

3. 4684780

1. 5671960

1.7451900

0.4769350

3. 9077620
1.7583670

0.2773990

1. 5420030

2. 2228080

1. 0757720

2. 3179220

3. 3877410

2. 2212980

2. 2928670

$-0.1463510$

1.9526510

3. 0248270

1.6163710

1.1543830

1.8252630

0.4052450

$-2.4681010$

$-3.9427290$

$-2.6860180$

$-1.7768380$

$-4.5701820$

$-5.6323890$

$-4.5127690$

$-3.8037920$

$-4.4859660$

$-3.3479770$

$-2.1383930$

$-4.4332480$

$-3.3649980$

$-4.0682520$

$-2.6071590$

$-4.1122860$

$-5.1832250$

$-3.7149220$

$-1.7968310$

$-2.3369710$

1.7644090

2.4336860

1.9084880

1.9035390

0.9153630

1.0431940

2. 5335920

3. 5148000

2. 3127470

2. 9167830
1.9118370

1.6281620

3.2401970

4.0569210

2. 8707340

2. 0954610

2. 3345180

1.1525880

1.1065960

4. 5702680

3. 2517040

3.4892030

3. 1315840

4. 3823390

5. 1918520

4. 8145960

1. 3937240

1. 1215310

$-0.7434690$

0.3759110

0.9704780

0.7114110

1.9297360

$-0.1362240$

$-0.9418920$

0.2749320

$-1.5084520$

1. 9283180

$-1.3571250$

$-2.1363680$

$-1.8349550$

$-0.2348680$

$-0.4651210$

$-0.1467690$

2. 5296570

3. 3163670

$-2.7724100$

$-3.9320860$

5.3038160

$-5.3870250$

$-4.3595730$

$-2.9823950$

6.0934100

3. 9022640

$-3.8025470$

$-5.1740800$ 


$\begin{array}{rrr}3.8766960 & 1.6323640 & -6.3939710 \\ 5.1885490 & 1.0292820 & -4.2689990 \\ 3.9276940 & -0.1097620 & -4.7250160 \\ 1.6301130 & 0.8838840 & -5.4503300 \\ -0.7615300 & 4.6874590 & -1.0601850 \\ -0.7785030 & 6.1809980 & -1.2746680 \\ -0.1203780 & 6.5177590 & -2.6291460 \\ 1.3337720 & 5.9833070 & -2.7063970 \\ 1.6348170 & 4.8666520 & -1.6658470 \\ 0.4392880 & 4.0039920 & -1.3313970 \\ -0.2137050 & 6.6805430 & -0.4702010 \\ -0.1366590 & 7.6012590 & -2.8013370 \\ 2.0445060 & 6.8017490 & -2.5365670 \\ 1.9338260 & 5.3598220 & -0.7275820 \\ -1.8067160 & 6.5605760 & -1.2327670 \\ -0.7256170 & 6.0537430 & -3.4190280 \\ 1.5254480 & 5.6069530 & -3.7186150 \\ 2.4906520 & 4.2653320 & -1.9828140 \\ -1.3621490 & -3.6257210 & -0.8440670 \\ -0.2753770 & -2.9341280 & -1.0934170 \\ -1.9127970 & -4.3521640 & -2.0304610 \\ -3.0234800 & -5.1950230 & -1.8639140 \\ -1.3657150 & -4.1756140 & -3.3123580 \\ -3.5791360 & -5.8519070 & -2.9609030 \\ -3.4451400 & -5.3198950 & -0.8709490 \\ -1.9260030 & -4.8313360 & -4.4090510 \\ -0.5134580 & -3.5165540 & -3.4431170 \\ -3.0325880 & -5.6698890 & -4.2363940 \\ -4.4397800 & -6.5026880 & -2.8240130 \\ -1.5013500 & -4.6860400 & -5.3997330 \\ -3.4686230 & -6.1790830 & -5.0932650 \\ 0.0533770 & -2.3077000 & -0.3308530 \\ -1.9369200 & -3.7014190 & 0.2543200 \\ -2.6755710 & -1.6391610 & 2.1254800 \\ -1.6145440 & -2.6567240 & 2.2156180 \\ -2.6188520 & -0.9530310 & 1.2759730 \\ -0.6327740 & -2.3796440 & 1.8487520 \\ -1.9758420 & -1.1055430 & 3.2944790 \\ -1.2708160 & -0.4432630 & 2.9136110 \\ -4.1500230 & -3.1495190 & 3.5478690 \\ -3.2368010 & -4.3336290 & 3.1974390 \\ -1.7755380 & -3.8769760 & 3.0561680 \\ -4.0914260 & -2.0736940 & 2.4509180 \\ -5.1857550 & -3.4907060 & 3.6657460 \\ -3.2992690 & -5.1144540 & 4.0419110 \\ -1.3501510 & -3.6353080 & \end{array}$

$\begin{array}{llll}H & -4.5251910 & -2.4622270 & 1.5198960 \\ H & -4.6651770 & -1.1840500 & 2.7262960 \\ H & -3.8374420 & -2.7129030 & 4.5061520 \\ -3.5617570 & -4.7737530 & 2.2457810 \\ H & -1.1350280 & -4.6591200 & 2.6324800\end{array}$

154

$\operatorname{TS} 160(R, R)$ G $[B 97-D / d e f 2-T Z V P]=-3541.572906$

\begin{tabular}{|c|c|c|c|}
\hline & , R) G[B97-D & rZVF & \\
\hline $\mathrm{P}$ & 0.2947690 & 0.07572040 & 0.7527790 \\
\hline 0 & 0.4739640 & -1.4283070 & 0.5801590 \\
\hline 0 & -0.3523270 & 0.5648770 & 2.0096480 \\
\hline 0 & -0.4972250 & 0.5476670 & 0.5921680 \\
\hline 0 & 1.7033180 & 0.8563680 & 0.6459530 \\
\hline C & -0.4822770 & 1.9080710 & -0.9050520 \\
\hline C & 0.7051960 & 2.4661650 & -1.4208490 \\
\hline $\mathrm{C}$ & -1.6459150 & 2.6569240 & -0.7010770 \\
\hline $\mathrm{C}$ & -1.5929260 & 4.0255760 & -0.9999970 \\
\hline $\mathrm{H}$ & -2.4855680 & 4.6290470 & .8451990 \\
\hline $\mathrm{C}$ & 2.3905620 & 0.8258870 & -( \\
\hline $\mathrm{C}$ & 3.5522280 & 0.0517200 & 20 \\
\hline $\mathrm{C}$ & 1.8992860 & 1.6015950 & -1.6 \\
\hline $\mathrm{C}$ & 4.2141170 & 0.0311120 & -1.8 \\
\hline $\mathrm{H}$ & 5.1255390 & -0.5565450 & -1.9 \\
\hline $\mathrm{C}$ & -2.9061000 & 2.0585740 & -0 \\
\hline $\mathrm{C}$ & 4.0832590 & -0.7192970 & 0 \\
\hline $\mathrm{C}$ & -3.6190700 & 1.1129600 & - \\
\hline $\mathrm{C}$ & -3.2317220 & 0.5695380 & - \\
\hline $\mathrm{C}$ & -5.4906250 & -0.3771810 & -1 \\
\hline $\mathrm{C}$ & -4.8538390 & 0.6129240 & -0.4892900 \\
\hline $\mathrm{C}$ & -3.1447800 & -0.9754030 & -2.1978120 \\
\hline $\mathrm{H}$ & -2.9154250 & -1.3873320 & -3.1884220 \\
\hline $\mathrm{H}$ & -2.3164180 & -1.2327240 & -1.53584 \\
\hline C & -4.4946110 & -1.5460310 & -1.664909 \\
\hline $\mathrm{H}$ & -4.9294940 & -2.2505650 & -2.3852640 \\
\hline $\mathrm{H}$ & -4.3375510 & -2.0918080 & -0.7284920 \\
\hline $\mathrm{H}$ & -6.4404960 & -0.7508550 & -1.02962 \\
\hline $\mathrm{H}$ & -2.2786430 & 0.9798650 & -2.642417 \\
\hline $\mathrm{C}$ & -5.7171140 & 0.3304080 & -2.79760 \\
\hline $\mathrm{H}$ & -6.1134670 & -0.4015090 & 39660 \\
\hline $\mathrm{H}$ & -6.4663830 & 1.1222310 & -2.6805520 \\
\hline $\mathrm{C}$ & -4.3638130 & 0.9206390 & -3.3042620 \\
\hline $\mathrm{H}$ & -4.1040480 & 0.5036860 & -4.2862720 \\
\hline $\mathrm{H}$ & -4.4317240 & 2.0096830 & -3.4126400 \\
\hline $\mathrm{C}$ & -3.4503530 & 2.4823470 & 1.0394150 \\
\hline $\mathrm{C}$ & -2.84 & 3.4751650 & 80 \\
\hline $\mathrm{C}$ & -5.7 & 2.5616460 & 2. \\
\hline$c$ & -4 . & & \\
\hline
\end{tabular}




\begin{tabular}{|c|c|c|}
\hline $\begin{array}{l}-3.8395480 \\
-3.4252390 \\
-3.9550570 \\
-5.2150610 \\
-5.4711240 \\
-6.0155380 \\
-6.1147100 \\
-1.8718500 \\
-4.0572280 \\
-4.3554380 \\
-3.9922300 \\
-2.6849230 \\
-2.3177750 \\
-1.9328650 \\
-5.3988940 \\
-6.3632060 \\
5.0445910 \\
5.4478470 \\
4.4588300 \\
4.4940270 \\
6.4994690 \\
6.7493880 \\
7.4182550 \\
5.9116930 \\
5.8928600 \\
6.5253720 \\
4.0432030 \\
5.8595860 \\
3.6158610 \\
3.6497970 \\
2.5733900 \\
4.1979830 \\
4.4907000 \\
3.4495990 \\
4.7881940 \\
4.8769390 \\
3.8001970 \\
4.2267090 \\
3.4423850 \\
3.5035820 \\
2.8495490 \\
2.7881760 \\
2.4926210 \\
1.8940820 \\
3.3402420 \\
5.3398360\end{array}$ & $\begin{array}{r}4.6556970 \\
5.3743090 \\
5.1747080 \\
4.1092120 \\
4.5393530 \\
4.3754430 \\
2.1554420 \\
3.8360620 \\
2.2302610 \\
2.6706500 \\
1.1446800 \\
2.8006150 \\
3.5546630 \\
2.0113450 \\
1.0603170 \\
0.6825170 \\
-0.8053340 \\
0.0449230 \\
1.4158580 \\
-0.0702840 \\
1.0818550 \\
1.7517230 \\
0.5611900 \\
1.8890910 \\
2.9630250 \\
1.7508600 \\
1.9759440 \\
-0.5710510 \\
1.6658700 \\
2.7382540 \\
1.3998820 \\
0.8278650 \\
1.4793490 \\
0.1200780 \\
-2.8411660 \\
-4.3253290 \\
-3.0048720 \\
-2.1167020 \\
-4.8632680 \\
-5.9299850 \\
-4.7739240 \\
-4.0507290 \\
-4.7161000 \\
-3.5277490 \\
-2.4306420 \\
-4.8444290\end{array}$ & $\begin{array}{r}2.1631620 \\
2.8825000 \\
1.2038740 \\
2.6619780 \\
3.6392550 \\
1.9614880 \\
3.0980870 \\
1.6497740 \\
3.8630590 \\
4.8239240 \\
3.9987420 \\
3.3928620 \\
4.1016830 \\
3.3273460 \\
0.7134480 \\
1.0545740 \\
2.7443530 \\
3.9227390 \\
1.9506020 \\
1.6777160 \\
3.4383040 \\
4.2720430 \\
3.1430180 \\
2.2386700 \\
2.4672980 \\
1.3403280 \\
1.1093290 \\
4.7316440 \\
3.2317340 \\
3.4668110 \\
3.0369410 \\
4.4123990 \\
5.2468710 \\
4.7883060 \\
1.4875970 \\
1.2336020 \\
-0.7276500 \\
0.4187550 \\
0.9771850 \\
0.7218590 \\
1.8954630 \\
-0.1821380 \\
-1.0041270 \\
0.1649900 \\
-1.5357220 \\
2.0817890\end{array}$ \\
\hline
\end{tabular}

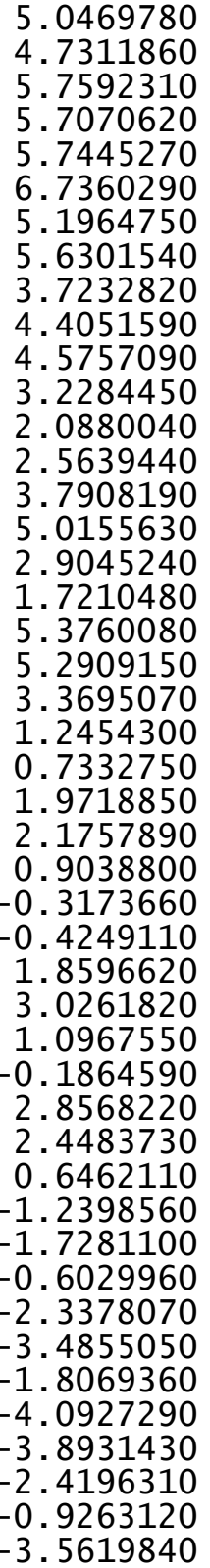
$-3.7735550$
$-4.4676100$
$-3.0673860$
$-4.5509810$
$-5.6270420$
$-4.2086180$
$-2.1890660$
$-2.7536720$
0.7249850
0.6956640
2. 1339170
2. 9130200
2.1704290
1.5160160
0.1215460
2. 1051890
3. 0874190
1. 3680130
0.1905860
2. 6532170
3.8966510
2.8427940
3.8527900
4. 6175520
5.8240660
6.7144720
6.1067130
4. 6266530
4.9913350
6.4189930
7.7173840
6.2713470
3. 9763480
5.4307450
6.8360150
6.6208740
$-3.6638720$
$-3.0624890$
$-4.4346980$
$-5.2080690$
$-4.3677330$
$-5.9055840$
$-5.2490050$
$-5.0626950$
-3.7615830
-5.8326610

-1.2444370
-2.0348140
-1.6884030
-0.0616250
-0.2783090
0.1010860
2.6506070
3.4770780
-2.9944990
-4.3393350
-4.8778650
-4.8969840
-4.1510840
-2.8759000
-5.0521320
-5.8825900
-5.9306970
-4.8107490
-4.2673350
-4.2271400
-4.4359630
-3.9761570
-1.6664380
-2.0631650
-1.1143070
-0.9884720
-1.7254070
-1.4615850
-3.0922250
-1.4701660
-1.3896560
-2.8066420
-2.0509650
-0.1272100
0.0706970
-1.4303540
-0.7007960
-1.0067360
-1.8289440
-1.5904480
-3.1280400
-2.6338340
-0.5846250
-4.1711680
-3.3140930
-3.9268710 


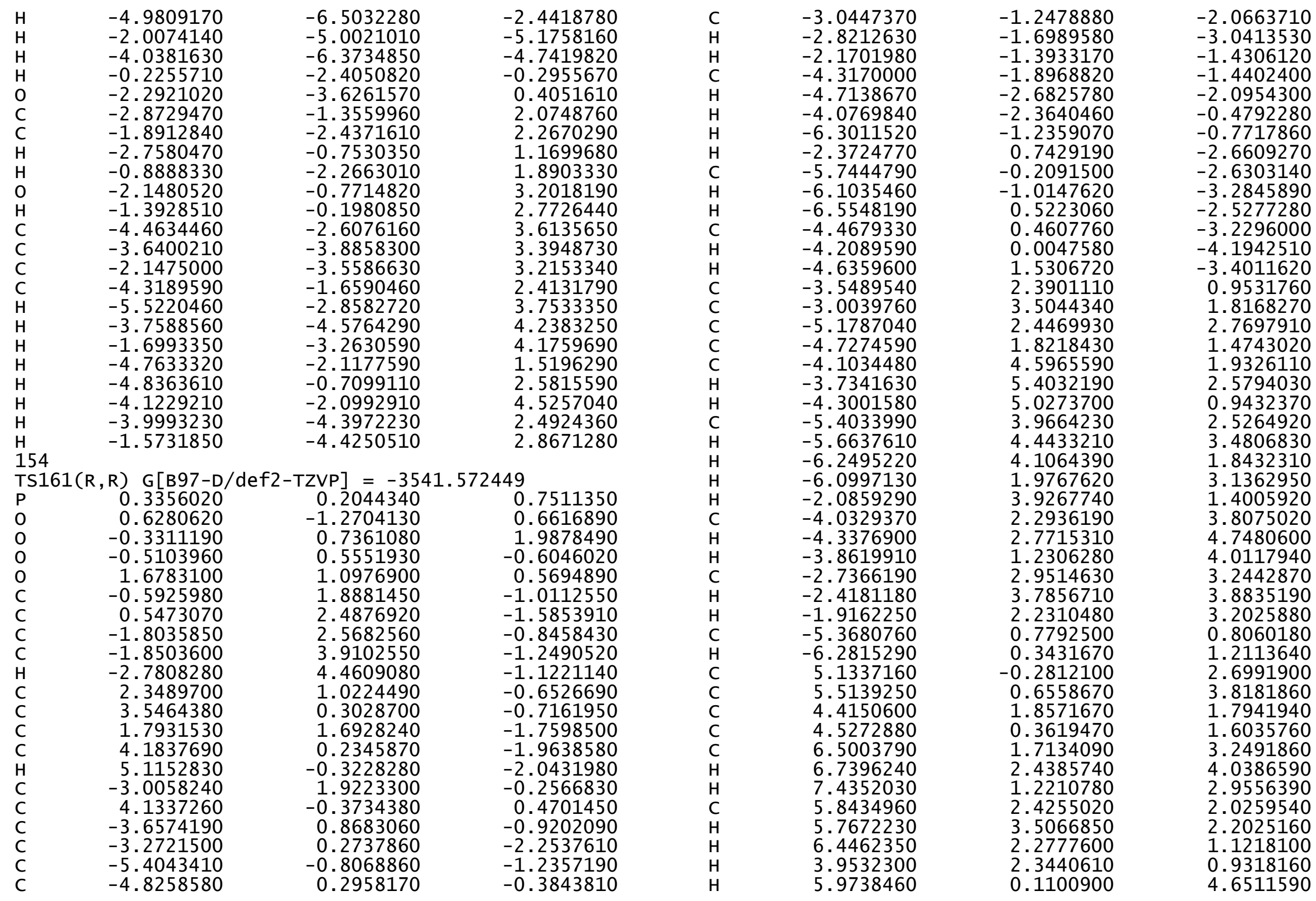




$\begin{array}{lrrr}\mathrm{C} & 3.5806990 & 2.1304690 & \\ \mathrm{H} & 3.5486600 & 3.2149430 & 3.0762300 \\ \mathrm{H} & 2.5545700 & 1.7867890 & 2.9201960 \\ \mathrm{C} & 4.2362060 & 1.4027040 & 4.2910540 \\ \mathrm{H} & 4.5148060 & 2.1216300 & 5.0733520 \\ \mathrm{H} & 3.5360660 & 0.6838720 & 4.7330740 \\ \mathrm{C} & 4.9690430 & -2.3951670 & 1.5622190 \\ \mathrm{C} & 5.1407530 & -3.8837560 & -1.3881360 \\ \mathrm{C} & 3.9563170 & -2.7383900 & -0.6208930 \\ \mathrm{C} & 4.3502000 & -1.7639020 & 0.4661740 \\ \mathrm{C} & 3.7378800 & -4.5184820 & 1.1896340 \\ \mathrm{H} & 3.8578970 & -5.5923930 & 0.9915650 \\ \mathrm{H} & 3.1554030 & -4.4132050 & 2.1129130 \\ \mathrm{C} & 3.0182880 & -3.8102310 & 0.0022240 \\ \mathrm{H} & 2.7476450 & -4.5352770 & -0.7766920 \\ \mathrm{H} & 2.1019630 & -3.3220530 & 0.3401450 \\ \mathrm{H} & 3.4487030 & -2.2376710 & -1.4492040 \\ \mathrm{H} & 5.6478620 & -4.3265620 & 2.2541620 \\ \mathrm{C} & 5.2379880 & -3.4605170 & -1.1193620 \\ \mathrm{H} & 4.9514280 & -4.2138520 & -1.8654770 \\ \mathrm{H} & 5.9007900 & -2.7399020 & -1.6139730 \\ \mathrm{C} & 5.9614280 & -4.1323350 & 0.0910860 \\ \mathrm{H} & 6.0579530 & -5.2147310 & -0.0678290 \\ \mathrm{H} & 6.9708640 & -3.7223940 & 0.2148280 \\ \mathrm{C} & 5.3597800 & -1.6576080 & 2.6793840 \\ \mathrm{H} & 5.8385360 & -2.1505370 & 3.5265530 \\ \mathrm{C} & 2.4417200 & 1.5689630 & -3.0034710 \\ \mathrm{C} & 1.8926310 & 2.1041020 & -4.3029350 \\ \mathrm{C} & 1.8682390 & 0.9829610 & -5.3711050 \\ \mathrm{C} & 3.2338820 & 0.2457830 & -5.5081670 \\ \mathrm{C} & 4.2715360 & 0.7243980 & -4.4608940 \\ \mathrm{C} & 3.6426510 & 0.8391110 & -3.0958280 \\ \mathrm{H} & 2.5331480 & 2.9246840 & -4.6614530 \\ \mathrm{H} & 1.5682500 & 1.4150360 & -6.3339910 \\ \mathrm{H} & 3.6494230 & 0.3918040 & -6.5131580 \\ \mathrm{H} & 4.6461660 & 1.7128540 & -4.7706830 \\ \mathrm{H} & 0.8890840 & 2.5169750 & -4.1689240 \\ \mathrm{H} & 1.0903240 & 0.2645730 & -5.0843430 \\ \mathrm{H} & 3.0813950 & -0.8325290 & -5.3769120 \\ \mathrm{H} & 5.1329480 & 0.0463850 & -4.4348570 \\ \mathrm{C} & 0.4734890 & 3.8477790 & -1.9438510 \\ \mathrm{C} & 1.6484550 & 4.6635200 & -2.4244970 \\ \mathrm{C} & 1.7820290 & 5.9544870 & -1.5782380 \\ \mathrm{C} & 0.4477440 & 6.7517960 & -1.4740970 \\ \mathrm{C} & -0.7338910 & 6.0128340 & -2.1521420 \\ \mathrm{C} & -0.7345030 & 4.5524830 & -1.7787320 \\ & & & \end{array}$

\begin{abstract}
1. 4930450
2. 5707670

0.5566300

$-0.6212070$

2. 5775440

2. 1159010

0.1974300

$-1.6873060$

$-1.4281540$

$-0.3711100$

$-2.0155580$

$-3.1060790$

$-1.5233430$

$-3.6958620$

$-3.4849360$

$-2.1178630$

$-0.6859080$

$-3.2040080$

$-4.5403730$

$-1.7355310$

$-3.6667640$

$-0.0204920$

$-1.9478380$

$-2.6904220$

$-1.6120000$

$-2.6466160$

$-0.6365110$

$-2.0004040$

$-1.3007910$

$-4.1414100$

$-3.2026760$

$-1.7502400$

$-4.0979530$

$-5.1705350$

$-3.2474910$

$-1.3233890$

$-4.5158680$

$-4.6939530$

$-3.8443780$

$-3.5196400$

$-1.0995710$
\end{abstract}

$\mathrm{H}$
154
$-3.4777360$

$-2.0108520$

$-1.9303520$

$-3.2437120$

$-2.3797130$

$-0.5745070$

$-0.4177950$

$-1.8823980$

$-0.4118920$

$-0.7962030$

$-1.4736570$

$-1.1550040$

$-2.7895590$

$-2.1356970$

$-0.1371960$

$-3.7698970$

$-3.0382690$

$-3.4458550$

$-1.8817190$

$-4.7878740$

$-4.2122200$

$-0.1335980$

0.7149000

2.2343830

2.4810260

1.2854960

2.0666210

3. 3009040

2.8181320

3.8828650

3.7354140

3.5100700

2.6243430

4.0527450

4.6247420

4.4399490

1.7690850

2.7481590

4.7567360

2.8746760

3.2145900

$\operatorname{TS} 162(R, R)$ G $[B 97-D /$ def2-TZVP $]=-3541.571383$

$\begin{array}{lrrr} & & \\ \text { TS162 (R, R }) & \text { G }[\mathrm{B} 97-\mathrm{D} / \mathrm{def2}-\mathrm{TZVP}] & = & -3541.571383 \\ \mathrm{P} & 0.3654610 & 0.1520800 & 0.8641430 \\ \mathrm{O} & 0.7240030 & -1.2900650 & 0.6150970 \\ \mathrm{O} & -0.2858790 & 0.5189170 & 2.1666700\end{array}$




$\begin{array}{rrr}-0.5452090 & 0.5898700 & -0.4239280 \\ 1.6597290 & 1.1228320 & 0.7322640 \\ -0.6789300 & 1.9456550 & -0.7251110 \\ 0.4232170 & 2.6206630 & -1.2874300 \\ -1.9072920 & 2.5702540 & -0.4846220 \\ -2.0109670 & 3.9324330 & -0.7972300 \\ -2.9605160 & 4.4366010 & -0.6262000 \\ 2.2993100 & 1.1578640 & -0.5099300 \\ 3.5160520 & 0.4835200 & -0.6576980 \\ 1.6846130 & 1.8753030 & -1.5534620 \\ 4.1075790 & 0.5053580 & -1.9292150 \\ 5.0528300 & -0.0147800 & -2.0732090 \\ -3.0757130 & 1.8349040 & 0.0682660 \\ 4.1676290 & -0.2342700 & 0.4692960 \\ -3.7161820 & 0.8354970 & -0.6847800 \\ -3.3453910 & 0.3866210 & -2.0780640 \\ -5.4220160 & -0.8527810 & -1.1279340 \\ -4.8541740 & 0.1766330 & -0.1834560 \\ -3.0697340 & -1.1385700 & -2.0435850 \\ -2.8519130 & -1.4870000 & -3.0609170 \\ -2.1795480 & -1.3157030 & -1.4377880 \\ -4.3085750 & -1.8842970 & -1.4598250 \\ -4.6979970 & -2.6135540 & -2.1814640 \\ -4.0309910 & -2.4350530 & -0.5550660 \\ -6.2954040 & -1.3519330 & -0.6904340 \\ -2.4688170 & 0.9212630 & -2.4538840 \\ -5.8098570 & -0.1352640 & -2.4515880 \\ -6.1560970 & -0.8870220 & -3.1733650 \\ -6.6407110 & 0.5553450 & -2.2649130 \\ -4.5679180 & 0.6308470 & -3.0064680 \\ -4.3183540 & 0.2818650 & -4.0172280 \\ -4.7714260 & 1.7066540 & -3.0667150 \\ -3.5975750 & 2.1577480 & 1.3329390 \\ -3.0640080 & 3.1990360 & 2.2891590 \\ -5.1698190 & 1.9692540 & 3.1904390 \\ -4.7422580 & 1.4994530 & 1.8234180 \\ -4.1972330 & 4.2310480 & 2.5477670 \\ -3.8340290 & 4.9835940 & 3.2599610 \\ -4.4435150 & 4.7491830 & 1.6129930 \\ -5.4528670 & 3.4964570 & 3.1160980 \\ -5.6919850 & 3.8613650 & 4.1238230 \\ -6.3283140 & 3.6766580 & 2.4809190 \\ -6.0610730 & 1.4288580 & 3.5327500 \\ -2.1759660 & 3.6953560 & 1.8891870 \\ -3.9846180 & 1.7519990 & 4.1713770 \\ -4.2757280 & 2.1148350 & \\ -4 & & \\ -4 & & \\ -4 & 1662740\end{array}$

$\begin{array}{lr}\mathrm{H} & -3.7682610 \\ \mathrm{C} & -2.7321690 \\ \mathrm{H} & -2.4300040 \\ \mathrm{H} & -1.8844930 \\ \mathrm{C} & -5.3739290 \\ \mathrm{H} & -6.2611950 \\ \mathrm{C} & 5.2481270 \\ \mathrm{C} & 5.6409080 \\ \mathrm{C} & 4.4368920 \\ \mathrm{C} & 4.5821580 \\ \mathrm{C} & 6.5759100 \\ \mathrm{H} & 6.8194060 \\ \mathrm{H} & 7.5146990 \\ \mathrm{C} & 5.8579870 \\ \mathrm{H} & 5.7618510 \\ \mathrm{H} & 6.4310750 \\ \mathrm{H} & 3.9308840 \\ \mathrm{H} & 6.1450980 \\ \mathrm{C} & 3.6443380 \\ \mathrm{H} & 3.5947800 \\ \mathrm{H} & 2.6208440 \\ \mathrm{C} & 4.3600230 \\ \mathrm{H} & 4.6448570 \\ \mathrm{H} & 3.6947590 \\ \mathrm{C} & 5.1068180 \\ \mathrm{C} & 5.3202930 \\ \mathrm{C} & 4.0189720 \\ \mathrm{C} & 4.4241700 \\ \mathrm{C} & 3.9316680 \\ \mathrm{H} & 4.0770280 \\ \mathrm{H} & 3.3822810 \\ \mathrm{C} & 3.1452930 \\ \mathrm{H} & 2.8772890 \\ \mathrm{H} & 2.2228810 \\ \mathrm{H} & 3.4615420 \\ \mathrm{H} & 5.8763050 \\ \mathrm{C} & 5.3046670 \\ \mathrm{H} & 5.0165100 \\ \mathrm{H} & 5.9241180 \\ \mathrm{C} & 6.0943390 \\ \mathrm{H} & 6.2140890 \\ \mathrm{H} & 7.0966570 \\ \mathrm{C} & 5.5166060 \\ \mathrm{H} & 2.0430200 \\ \mathrm{C} & 1.6640380 \\ \mathrm{C} & \end{array}$

0.6809630
2.5179380
3.2974250
1.8421750
0.5139830
0.0083250
-0.2197010
0.6726740
1.9374510
0.4566200
1.7860500
2.4814830
1.3374660
2.5327240
3.6030490
2.4395990
2.4516010
0.1011670
2.1280260
3.2018620
1.7680270
1.3576620
2.0430300
0.5968080
-2.2785860
-3.7497290
-2.5477830
-1.6155270
-4.4212360
-5.4812490
-4.3740340
-3.6859750
-4.3827170
-3.2529530
-2.0236480
-4.2166360
-3.1958880
-3.9170880
-2.4255540
-3.9070000
-4.9765900
-3.4749630
-1.5850600
-2.1035110
1.8347050
2.4168950

4. 2560950

3. 6457730

4. 3579520

3. 5115620

1.0660010

1.4483670

2. 6626780

3.8131830

1.8893470

1.6229820

3.2632620

4.0778190

2. 9170120

2. 0961340

2. 3223880

1.1657810

1.0684890

4. 6020700

3. 2119060

3. 4375270

3. 0759170

4. 3649510

5. 1745940

4.7901090

1.4258460

1.1702300

$-0.7314470$

0.3878500

0.9956210

0.7462390

1.9437060

$-0.1314510$

$-0.9367340$

0.2601320

$-1.5120140$

1. 9924320

$-1.3143320$

$-2.0909030$

$-1.7896510$

$-0.1691660$

$-0.3879440$

$-0.0639500$

2. 5641290

3. 3665940

$-2.8256970$

$-4.0717350$ 


$\begin{array}{rrr}1.6228750 & 1.3470220 & -5.1903710 \\ 3.0044520 & 0.6653810 & -5.4243390 \\ 4.0716490 & 1.1290140 & -4.4002660 \\ 3.5015970 & 1.1496950 & -3.0054370 \\ 2.2667820 & 3.2700680 & -4.4188430 \\ 1.2639280 & 1.8126190 & -6.1167820 \\ 3.3687830 & 0.8761440 & -6.4376390 \\ 4.3934430 & 2.1463880 & -4.6734910 \\ 0.6578380 & 2.7965450 & -3.8745020 \\ 0.8830330 & 0.5899350 & -4.9023300 \\ 2.8938060 & -0.4228230 & -5.3417450 \\ 4.9579990 & 0.4853620 & -4.4492680 \\ -0.9283570 & 4.6499160 & -1.3002690 \\ -1.0073360 & 6.1198890 & -1.6286640 \\ -0.4269150 & 6.3780570 & -3.0379010 \\ 1.0120430 & 5.8062700 & -3.1881680 \\ 1.4259160 & 4.8920260 & -2.0053010 \\ 0.2973900 & 3.9998890 & -1.5442220 \\ -0.4203810 & 6.6951090 & -0.8940750 \\ -0.4360200 & 7.4540790 & -3.2519610 \\ 1.7404220 & 6.6237670 & -3.2596910 \\ 1.6918620 & 5.5405800 & -1.1555540 \\ -2.0437070 & 6.4727360 & -1.5635050 \\ -1.0915130 & 5.8979460 & -3.7676830 \\ 1.0822900 & 5.2338900 & -4.1198340 \\ 2.3226120 & 4.3222960 & -2.2571800 \\ -1.2441670 & -3.6631140 & -0.7786090 \\ -0.1944490 & -2.9293440 & -1.0634250 \\ -1.7905910 & -4.4278770 & -1.9430200 \\ -2.8680080 & -5.3057770 & -1.7428650 \\ -1.2728390 & -4.2534790 & -3.2375380 \\ -3.4201460 & -5.9990500 & -2.8192060 \\ -3.2669100 & -5.4291230 & -0.7403480 \\ -1.8291010 & -4.9462950 & -4.3132400 \\ -0.4464500 & -3.5676550 & -3.3943490 \\ -2.9029050 & -5.8193270 & -4.1071690 \\ -4.2550100 & -6.6768640 & -2.6563600 \\ -1.4272070 & -4.8028850 & -5.3136690 \\ -3.3361420 & -6.3570490 & -4.9478790 \\ 0.1276620 & -2.2798960 & -0.3175120 \\ -1.7887450 & -3.7480240 & 0.3344200 \\ -2.5687720 & -1.6820460 & 2.1901260 \\ -1.4650700 & -2.6538380 & 2.2716520 \\ -2.5605610 & -1.0135550 & 1.3250680 \\ -0.5043250 & -2.3437040 & 1.8761160 \\ -1.8651300 & -1.0952330 & 3.3299860\end{array}$

$\begin{array}{ll}\mathrm{H} & -1.1984140 \\ \mathrm{C} & -3.9440770 \\ \mathrm{C} & -2.9928580 \\ \mathrm{C} & -1.5547760 \\ \mathrm{C} & -3.9572910 \\ \mathrm{H} & -4.9615990 \\ \mathrm{H} & -3.0040730 \\ \mathrm{H} & -1.1132340 \\ \mathrm{H} & -4.3979010 \\ \mathrm{H} & -4.5599650 \\ \mathrm{H} & -3.6249490 \\ \mathrm{H} & -3.3245020 \\ \mathrm{H} & -0.8949710 \\ \mathrm{H} & \end{array}$

-0.4116000
-3.2204230
-4.3739000
-3.8626620
-2.1658510
-3.6007140
-5.1410830
-3.5854470
-2.5929530
-1.2937160
-2.7520260
-4.8454370
-4.6270740

2. 9181850

3. 6793270

3. 3276190

3. 1383560

2. 5600680

3.8311100

4. 1109040

4.1075420

1.6494600

2.8312560

4. 6202100

4.6202100
2.3934220

2. 7118650

$\operatorname{TS} 163(R, R) \quad G[B 97-D / d e f 2-T Z V P]=-3541.570669$

$\begin{array}{lrrr}\text { TS163(R, R) G }[\mathrm{B} 97-\mathrm{D} / \mathrm{def}-\mathrm{TZVP}]=-3541.570669 \\ \mathrm{P} & -0.3237630 & -0.0053550 & 0.8622010 \\ \mathrm{O} & -0.5571120 & 1.4357640 & 0.4910070 \\ \mathrm{O} & 0.3325730 & -0.3148120 & 2.1777330 \\ \mathrm{O} & 0.4976980 & -0.6371510 & -0.4046460 \\ \mathrm{O} & -1.7026280 & -0.8632890 & 0.8527800 \\ \mathrm{C} & 0.5304250 & -2.0249600 & -0.5445160 \\ \mathrm{C} & -0.6347690 & -2.6831990 & -0.9874100 \\ \mathrm{C} & 1.7232380 & -2.6980630 & -0.2588320 \\ \mathrm{C} & 1.7184380 & -4.0940590 & -0.3748720 \\ \mathrm{H} & 2.6352390 & -4.6401030 & -0.1598610 \\ \mathrm{C} & -2.3662190 & -0.9843090 & -0.3714500 \\ \mathrm{C} & -3.5212200 & -0.2229290 & -0.5833770 \\ \mathrm{C} & -1.8430130 & -1.8763780 & -1.3276870 \\ \mathrm{C} & -4.1341250 & -0.3305040 & -1.8392100 \\ \mathrm{H} & -5.0342910 & 0.2501550 & -2.0316740 \\ \mathrm{C} & 2.9626970 & -1.9770090 & 0.1345260 \\ \mathrm{C} & -4.0981860 & 0.6566390 & 0.4673190 \\ \mathrm{C} & 3.6082860 & -1.1177830 & -0.7727240 \\ \mathrm{C} & 3.1810520 & -0.8267020 & -2.1920610 \\ \mathrm{C} & 5.3817390 & 0.3816510 & -1.5246560 \\ \mathrm{C} & 4.8107560 & -0.4751310 & -0.4229090 \\ \mathrm{C} & 3.0008440 & 0.7051720 & -2.3469610 \\ \mathrm{H} & 2.7459180 & 0.9309370 & -3.3900940 \\ \mathrm{H} & 2.1595420 & 1.0202250 & -1.7280720 \\ \mathrm{C} & 4.3160610 & 1.4353870 & -1.9338300 \\ \mathrm{H} & 4.7045080 & 2.0348810 & -2.7668360 \\ \mathrm{H} & 4.1316100 & 2.1189710 & -1.0982720 \\ \mathrm{H} & 6.3088080 & 0.8726630 & -1.2039630 \\ \mathrm{H} & 2.2539080 & -1.3444700 & -2.4512940 \\ \mathrm{C} & 5.6459910 & -0.5297660 & -2.7560000 \\ \mathrm{H} & 5.9995450 & 0.0922800 & -3.5890310\end{array}$




$\begin{array}{lrrr}\text { H } & 6.4395750 & -1.2473230 & -2.5161500 \\ \mathrm{C} & 4.3286120 & -1.2723270 & -3.1414440 \\ \mathrm{H} & 4.0409850 & -1.0387050 & -4.1749560 \\ \mathrm{H} & 4.4607200 & -2.3585050 & -3.0698730 \\ \mathrm{C} & 3.5486430 & -2.1806770 & 1.3964350 \\ \mathrm{C} & 3.0284210 & -3.0704300 & 2.5007310 \\ \mathrm{C} & 5.2524770 & -1.8747140 & 3.1183450 \\ \mathrm{C} & 4.7546310 & -1.5372610 & 1.7355880 \\ \mathrm{C} & 4.1204850 & -4.1323980 & 2.8106880 \\ \mathrm{H} & 3.7715370 & -4.7689250 & 3.6343800 \\ \mathrm{H} & 4.2685050 & -4.7749310 & 1.9341690 \\ \mathrm{C} & 5.4531730 & -3.4148000 & 3.1966230 \\ \mathrm{H} & 5.7558960 & -3.6820020 & 4.2177570 \\ \mathrm{H} & 6.2634550 & -3.7125690 & 2.5205190 \\ \mathrm{H} & 6.1923270 & -1.3531350 & 3.3384650 \\ \mathrm{H} & 2.0898390 & -3.5543560 & 2.2188690 \\ \mathrm{C} & 4.1537470 & -1.4854620 & 4.1454490 \\ \mathrm{H} & 4.4934650 & -1.7657870 & 5.1514330 \\ \mathrm{H} & 4.0041350 & -0.4002390 & 4.1343560 \\ \mathrm{C} & 2.8259410 & -2.2173150 & 3.7835960 \\ \mathrm{H} & 2.5193670 & -2.8861890 & 4.5986570 \\ \mathrm{H} & 2.0167750 & -1.5028330 & 3.6159840 \\ \mathrm{C} & 5.3879030 & -0.6891800 & 0.8281220 \\ \mathrm{H} & 6.3247260 & -0.1988580 & 1.0946740 \\ \mathrm{C} & -5.1531990 & 0.9442890 & 2.6541950 \\ \mathrm{C} & -5.6042630 & 0.2042600 & 3.8885900 \\ \mathrm{C} & -4.5335620 & -1.3373330 & 2.0927980 \\ \mathrm{C} & -4.5569970 & 0.1174390 & 1.6835900 \\ \mathrm{C} & -6.6363590 & -0.8744440 & 3.4560580 \\ \mathrm{H} & -6.9245550 & -1.4652350 & 4.3359950 \\ \mathrm{H} & -7.5399790 & -0.3848230 & 3.0736100 \\ \mathrm{C} & -5.9973610 & -1.7874950 & 2.3631560 \\ \mathrm{H} & -5.9874910 & -2.8351820 & 2.6920800 \\ \mathrm{H} & -6.5722870 & -1.7365330 & 1.4307150 \\ \mathrm{H} & -4.0843700 & -1.9704740 & 1.3238350 \\ \mathrm{H} & -6.0481570 & 0.8904840 & 4.6201940 \\ \mathrm{C} & -3.7431860 & -1.4649270 & 3.4236100 \\ \mathrm{H} & -3.7788530 & -2.5122450 & 3.7527620 \\ \mathrm{H} & -2.6956750 & -1.2053670 & 3.2478420 \\ \mathrm{C} & -4.3785760 & -0.5300360 & 4.4989950 \\ \mathrm{H} & -4.7103530 & -1.1082260 & 5.37193800 \\ \mathrm{H} & -3.6475920 & 0.2092900 & 4.8473260 \\ \mathrm{C} & -4.8610720 & 2.8540590 & 1.2206720 \\ \mathrm{C} & -4.9589270 & 4.3052560 & 0.8213890 \\ \mathrm{C} & -3.7764760 & 2.8215450 & -0.9546660 \\ \mathrm{C} & -4.2456360 & 2.0390750 & 0.2508590 \\ & & & \end{array}$

-3.5232020
-3.5882470
-2.9707940
-2.8059000
-2.4795800
-1.9238140
-3.2706750
-5.4679900
-5.0068170
-4.6617210
-5.6883860
-5.7324670
-5.7784040
-6.7618780
-5.3115800
-5.7849640
-3.6073290
-4.2217070
-4.4000360
-3.0886010
-1.9559330
-2.4644710
-3.5579540
-4.7488610
-2.7220050
-1.5428720
-5.1819270
-5.1894140
-3.2992260
-1.1382810
0.5674210
0.5259280
-0.1129410
-1.4943780
-1.8156840
-0.6142330
-0.0801820
-0.2110620
-2.2968080
-2.1244830
1.5340680
0.5748130
-1.5166470
-2.6664180
1.5661400
0.4579530

4.8432770

5.8789330

4.8539960

3.9340710

4.5209170

3.4638340

2. 1811900

4.8937880

3. 5175340

.1414900

2. 7619870

4. 3898120

5.4393260

4.0428490

2. 3107230

2. 9458710

$-1.1419750$

$-1.2362710$

$-2.7146340$

$-3.5356170$

$-2.7320130$

$-1.9264370$

$-0.7451040$

$-2.7675690$

$-3.8853040$

$-2.0248220$

$-0.7073070$

$-3.1474540$

$-4.4285240$

$-3.3964900$

$-4.7906310$

$-6.2950980$

$-6.7319910$

$-6.0572550$

$-4.9482460$

$-4.0904770$

$-6.6961270$

$-7.8244520$

$-6.8044650$

$-5.4391000$

$-6.7141340$

$-6.4527200$

$-5.6211100$

$-4.3499420$

3.5413480
2.8747310
0.5770950

0.2163590

1. 5244940

$-0.4672400$

$-1.3361210$

$-0.0284300$

$-1.6817970$

1. 5944840

$-1.5978830$

$-2.4331990$

$-2.0072390$

$-0.5245190$

$-0.8450180$

$-0.3747270$

2. 4232620

3.1731290

$-2.8415870$

$-4.2153280$

$-4.6271120$

$-4.4509370$

$-3.7623540$

$-2.5909120$

$-4.9454230$

$-5.6659740$

$-5.4241580$

$-4.4993420$

$-4.2447560$

$-3.9997330$

$-3.8525200$

$-3.4744570$

$-0.7358540$

$-0.8272370$

$-2.1647920$

$-2.4014010$

$-1.3658250$

$-1.0494030$

0.0014440

$-2.1885000$

$-2.3630830$

$-0.4293120$

$-0.7228200$

$-2.9731690$

$-3.4058200$

$-1.6993720$

$-1.0913960$

$-1.3124610$ 


$\begin{array}{lll}2.1526570 & 4.1797070 & -2.3105460 \\ 3.2883910 & 4.9946830 & -2.1774280 \\ 1.6141880 & 3.9480910 & -3.5871990 \\ 3.8778840 & 5.5693910 & -3.3023110 \\ 3.7024120 & 5.1629420 & -1.1876310 \\ 2.2088230 & 4.5210790 & -4.7119040 \\ 0.7414360 & 3.3114730 & -3.6911050 \\ 3.3403780 & 5.3319190 & -4.5725660 \\ 4.7578500 & 6.1988890 & -3.1912420 \\ 1.7908400 & 4.3333140 & -5.6982730 \\ 3.8027860 & 5.7768080 & -5.4511350 \\ 0.1005860 & 2.3056020 & -0.5191020 \\ 2.1335070 & 3.6596880 & 0.0070200 \\ 2.7779090 & 1.6912080 & 1.9945160 \\ 1.7530960 & 2.7479340 & 2.0192350 \\ 2.7005070 & 0.9585070 & 1.1865180 \\ 0.7640840 & 2.4845830 & 1.6615040 \\ 2.0550550 & 1.2511350 & 3.1882050 \\ 1.3290160 & 0.5923220 & 2.8388520 \\ 4.2981240 & 3.2247020 & 3.3398810 \\ 3.4280340 & 4.4196690 & 2.9227730 \\ 1.9525300 & 4.0076850 & 2.7912510 \\ 4.2066990 & 2.0960580 & 2.3001150 \\ 5.3444150 & 3.5361530 & 3.4462510 \\ 3.5118280 & 5.2355560 & 3.6505850 \\ 1.5099220 & 3.8378320 & 3.7843160 \\ 4.6527090 & 2.4226140 & 1.3510840 \\ 4.7519360 & 1.2047320 & 2.6235010 \\ 3.9666920 & 2.8492690 & 4.3175280 \\ 3.7750510 & 4.8008190 & 1.9535420 \\ 1.3449020 & 4.7874030 & 2.3175360\end{array}$

1. 3449020

4.7874030

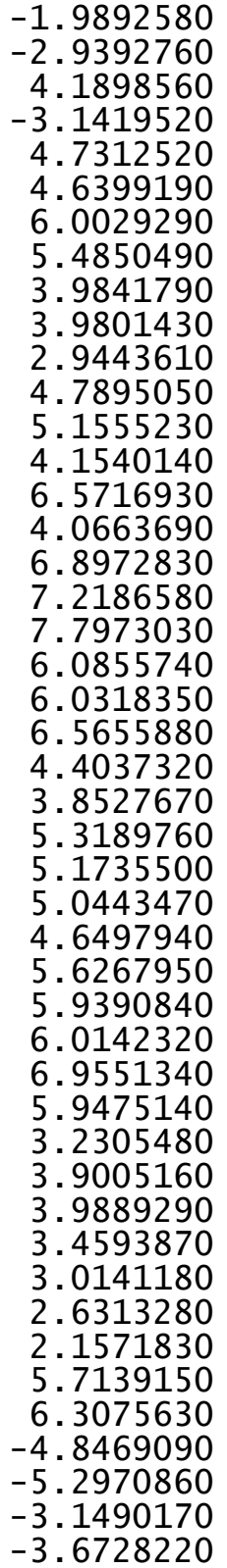

3.9045000

4.4216520

$-0.3014520$

1.7895720

0.4562450

1.9545610

0.7934400

$-0.1646150$

2.2529300

3.3397740

1. 9141260

1.5377060

2. 2633050

0.8169360

0.2645960

2. 4250620

1.8502000

2. 5956710

1. 3637020

2. 5267440

3. 6129640

2. 3508440

$-1.6921970$

$-2.6880120$

$-3.7933050$

$-2.3010760$

$-3.4291380$

$-4.1999660$

$-2.7243530$

$-4.0732360$

$-5.1590230$

$-3.6622760$

$-4.2195340$

$-2.2021970$

$-4.4257380$

$-5.5042080$

$-4.2983900$

$-3.7410780$

$-4.4822480$

$-3.2420250$

$-1.5405730$

$-2.0160430$

0.1058860

$-0.8898210$

0.4057650

0.7964650
$-0.8321040$

$-0.7042390$

0.3782860

$-0.1154380$

1.4324620

1.6071010

3. 4895250

2.4463570

2.9830320

3.1404090

2. 9681620

4. 1122560

4.8510510

4.6403800

4.2641030

0.8046080

2. 7819020

3. 5214610

2. 3872700

1.6334260

1.7855610

0.6631400

0.3739150

$-0.6210200$

1. 2182130

1. 3836360

$-1.2860710$

$-1.9615380$

$-1.8922520$

$-0.1797660$

$-0.3257970$

$-0.2156350$

2.0096570

$-1.3770610$

1. 2425280

1.0528900

2. 2384690

0.1573840

$-0.5564770$

0.6149500

2. 4202330

3. 2020790

$-0.6085610$

$-1.6494190$

$-2.3235810$

$-0.9604570$ 


$\begin{array}{rrr}-5.5559700 & -0.1130710 & -2.9717340 \\ -5.8226270 & -0.8315060 & -3.7582760 \\ -6.4067140 & 0.5653500 & -2.8374170 \\ -4.2712890 & 0.6820860 & -3.3632910 \\ -3.9144330 & 0.3761740 & -4.3556460 \\ -4.4774070 & 1.7583450 & -3.4006390 \\ -2.2456530 & 0.9616470 & -2.5869640 \\ -6.2050440 & -1.4150750 & -1.3284400 \\ -2.8592270 & -1.1171610 & -2.3277240 \\ -2.5340540 & -1.4150920 & -3.3328340 \\ -2.0323760 & -1.3197450 & -1.6447510 \\ -4.1447940 & -1.8968030 & -1.9146960 \\ -4.4510590 & -2.5893080 & -2.7090090 \\ -3.9599760 & -2.4976610 & -1.0184860 \\ -4.9892610 & 1.3815370 & 1.4285050 \\ -5.5779450 & 1.8104820 & 2.7491120 \\ -3.4174080 & 3.1109870 & 2.1119640 \\ -3.8134240 & 2.0775410 & 1.0838570 \\ -4.5023160 & 1.6010260 & 3.8510490 \\ -4.9099750 & 1.9351870 & 4.8143330 \\ -4.2671030 & 0.5342350 & 3.9431890 \\ -3.2199820 & 2.4064060 & 3.4815820 \\ -3.0139800 & 3.1732260 & 4.2398750 \\ -2.3470920 & 1.7528310 & 3.4261820 \\ -2.5022040 & 3.6344530 & 1.8234140 \\ -6.4871880 & 1.2419950 & 2.9808130 \\ -4.5981490 & 4.1096410 & 2.2644350 \\ -4.3359330 & 4.8516150 & 3.0298680 \\ -4.7522830 & 4.6457180 & 1.3201360 \\ -5.8900420 & 3.3321000 & 2.6721400 \\ -6.2525890 & 3.6696580 & 3.6521350 \\ -6.6916000 & 3.5043020 & 1.9440790 \\ -5.5085160 & 0.4005040 & 0.5844650 \\ -6.4218830 & -0.1310030 & 0.8548920 \\ 0.3441640 & 3.9561620 & -1.4913350 \\ 1.5897050 & 4.7257140 & -1.8972510 \\ 1.5001040 & 6.2210940 & -1.5645170 \\ 0.1914250 & 6.7918300 & -2.1232960 \\ -1.0027420 & 6.1355150 & -1.4181050 \\ -0.8707630 & 4.6291000 & -1.2599560 \\ 2.3688220 & 6.7428510 & -1.9859640 \\ 1.7522140 & 4.6343950 & -2.9797220 \\ 2.4652270 & 4.2698340 & -1.4189090 \\ 0.1459840 & 6.5850290 & -3.2032430 \\ 0.1457810 & 7.8809800 & -1.9957440 \\ -1.9382530 & 6.3629620 & -1.9478280\end{array}$
$-1.1055700$
1. 5251740
2. 0796870
1. 2649910
1. 5454040
3. 0560500
3.7569590
3. 2440300
1. 0130780
1.4942450
0.1988330
3.4337420
3. 2842340
4.8426160
3. 6078920
1.1677610
$-1.3210070$
$-0.2612380$
$-1.7504150$
$-2.8807640$
$-1.0675480$
$-3.3243360$
$-3.4052560$
$-1.5158470$
$-0.1964020$
$-2.6437270$
$-4.2014380$
$-0.9861790$
$-2.9916970$
0.0501920
$-1.9630970$
$-1.4217520$
$-2.3640570$
$-0.3734770$
$-2.0271620$
$-1.8052990$
$-1.1487450$
$-3.3338550$
$-4.1359540$
$-3.8168330$
$-1.8240500$
$-3.5795480$
$-5.2130810$
$-4.2771670$
$-1.5309310$
$-1.2383290$

6. 5766460

6. 3638700

1. 5806780

2. 1071430

1. 3498150

1. 3213580

0.5221540

0.8218540

1.8354930

3. 1673430

2.0647320

2. 3548820

0.8791180

0.6887230

$-0.5522390$

0.3191120

$-3.6125930$

$-2.8971100$

$-4.5341260$

$-5.3454150$

$-4.5763650$

$-6.1847130$

$-5.3013180$

$-5.4146360$

$-3.9461900$

$-6.2192840$

$-6.8091380$

$-5.4400350$

$-6.8712990$

$-2.2175030$

$-3.5656570$

$-2.1234420$

$-1.8325380$

$-2.1639640$

$-1.1832530$

$-0.7827450$

$-0.1203090$

$-3.1924250$

$-3.2847050$

$-2.1120990$

$-3.1931810$

$-4.0375830$

$-3.2958670$

$-1.1784880$

$-4.1584350$

$-3.0512360$
$-0.4139370$

$-0.4739330$

$-2.9088390$

$-4.0789470$

$-5.3837310$

$-5.6423260$

$-4.5356740$

$-3.1352330$

$-6.2116110$

$-4.2540880$

$-3.8249310$

$-5.6544050$

$-6.6206500$

$-4.5672480$

$-4.7276160$

$-5.3058310$

$-0.4574080$

$-0.7393930$

$-1.5555200$

$-1.3655510$

$-2.7828930$

$-2.3864220$

$-0.4156950$

$-3.8042020$

$-2.9311840$

$-3.6089590$

$-2.2324360$

$-4.7538100$

$-4.4073430$

$-0.0076000$

0.6063460

3.0656530

1.9750040

2. 7698830

1.1747820

3. 5055570

3. 0431150

4. 3558270

3. 0490060

2. 1079570

4.0623530

5.0100520

3.2547980

2. 4622990

3. 6261960

4.9790990 


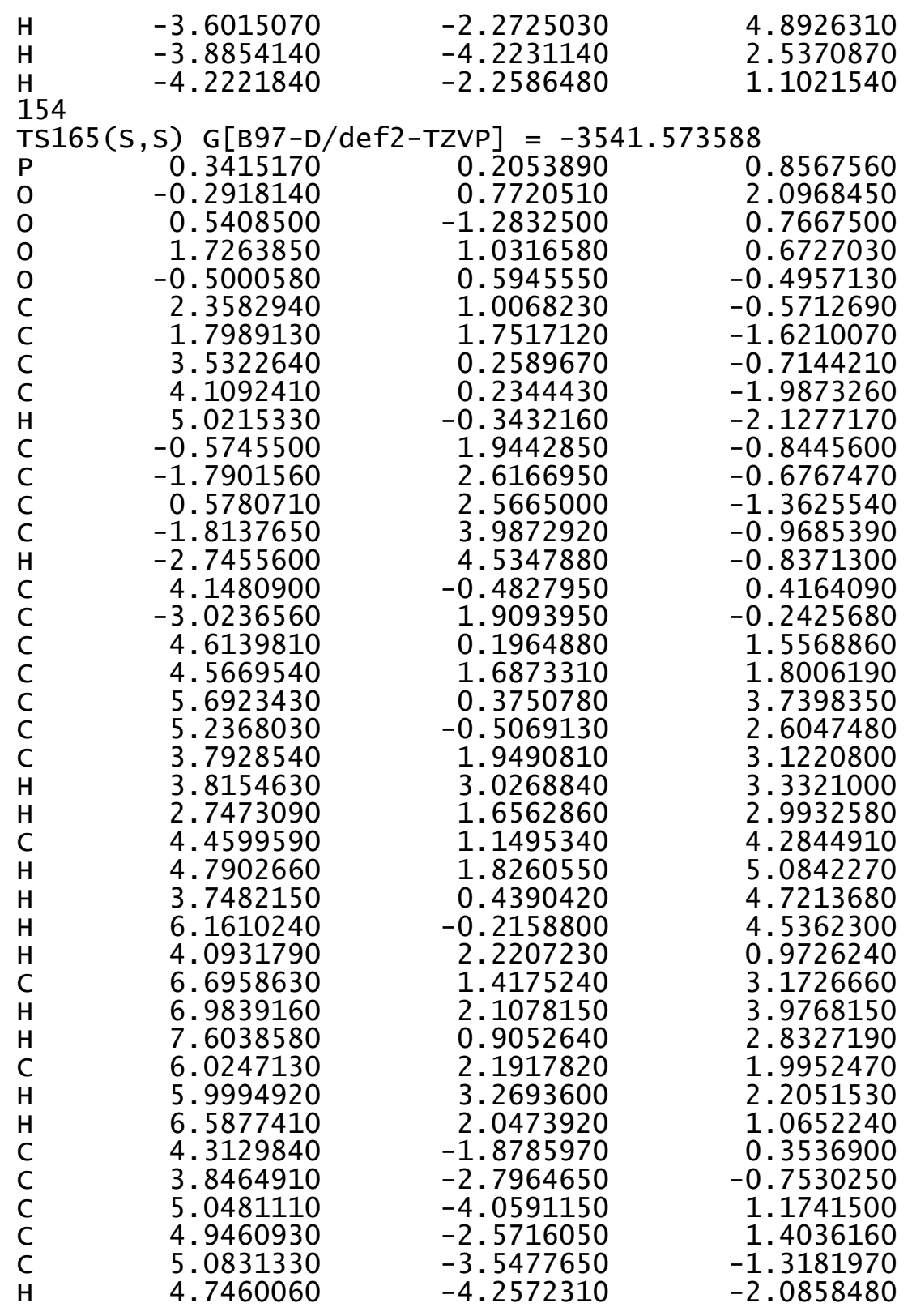

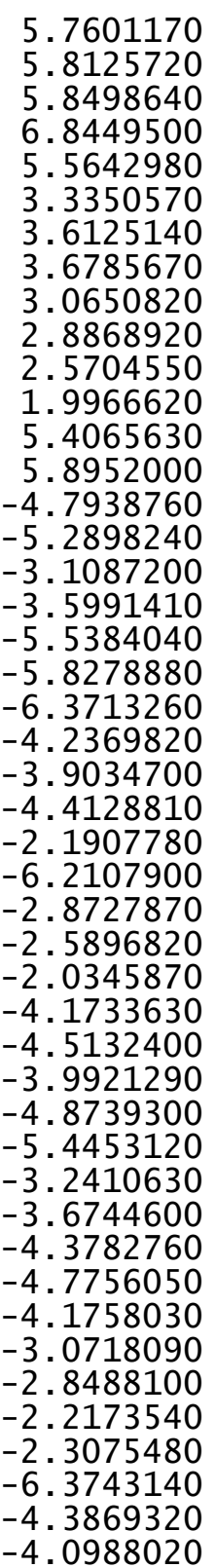
$-2.8329020$
$-4.2980470$
$-5.3772850$
$-3.9429830$
$-4.5525600$
$-4.6264350$
$-5.6982800$
$-4.5227510$
$-3.8540330$
$-4.5432590$
$-3.3470610$
$-1.8896370$
$-2.4305320$
0.2819770
$-0.7133150$
0.5006070
0.9245730
0.0495100
$-0.6743720$
0.7507550
0.8063400
0.4876970
1.8879900
1.0229140
$-1.2031340$
$-1.0316300$
$-1.3489220$
$-1.2608980$
$-1.7613220$
$-2.4679210$
$-2.3396880$
1. 5961130
2. 0772590
3. 2833660
2.2403680
1.8564990
2. 2230580
0.7853100
2.6136030
3. 3974750
1. 9347740
3.7664170
1. 5472410
4.3268410
5. 0840380

$-1.8017790$

$-0.1584450$

$-0.3584550$

$-0.0559450$

2.0067360

$-1.5470320$

1.0045720

0.7730470

1.9490950

$-0.1388230$

$-0.9330120$

0.2395040

2. 5294550

3. 3409540

$-0.6936310$

$-1.7134740$

$-2.4328100$

$-1.0676050$

$-3.0460160$

-3.8194260

$-2.9173390$

$-3.4580810$

$-4.4545270$

$-3.4955130$

$-2.7142290$

$-1.3739060$

$-2.4284560$

$-3.4405020$

$-1.7684560$

$-1.9717030$

$-2.7395420$

$-1.0597900$

1. 3215760

2. 6313860

1.9624950

0.9582590

3. 7386120

4.6945010

3.8507650

3. 3532450

4.0891380

3. 3237840

1.6627800

2.8757100

2.0828640

2.8237490 


$\begin{array}{rr}-4.5242470 & 4.8365560 \\ -5.7038480 & 3.6066940 \\ -6.0486140 & 3.9819880 \\ -6.5028220 & 3.7888830 \\ -5.4349290 & 0.6201460 \\ -6.3663540 & 0.1287770 \\ 0.5303180 & 3.9527680 \\ 1.7342470 & 4.7926780 \\ 1.8516980 & 5.9661510 \\ 0.5389780 & 6.7988610 \\ -0.6333320 & 6.1481010 \\ -0.6708580 & 4.6592660 \\ 1.6164650 & 5.2109640 \\ 2.6911390 & 6.6081670 \\ 0.6997220 & 7.8137010 \\ -0.4821950 & 6.3423540 \\ 2.6495820 & 4.1935720 \\ 2.0963080 & 5.5441960 \\ 0.2461420 & 6.8964080 \\ -1.5870200 & 6.6100800 \\ 2.3775240 & 1.6613770 \\ 1.7192840 & 2.3401820 \\ 2.1373730 & 1.7345120 \\ 3.6664320 & 1.6616200 \\ 4.1899570 & 0.7002300 \\ 3.5437610 & 0.8914540 \\ 1.7304730 & 2.3440170 \\ 1.9922890 & 3.4044160 \\ 0.6296880 & 2.3088850 \\ 4.0793340 & 2.6679110 \\ 4.0004350 & 1.3303720 \\ 5.2803220 & 0.7885900 \\ 3.9959160 & -0.3334920 \\ 1.7180650 & 0.7222440 \\ -1.5620030 & -3.6472570 \\ -0.4970250 & -2.9618820 \\ -2.0481510 & -4.5828410 \\ -3.1869000 & -5.3665330 \\ -1.4109190 & -4.6653320 \\ -3.6830300 & -6.2185490 \\ -3.6761160 & -5.2921870 \\ -1.9119980 & -5.5162210 \\ -0.5340740 & -4.0557690 \\ -3.0475360 & -6.2934740 \\ -4.5660160 & -6.8216130 \\ -1.4175860 & -5.5725210\end{array}$

1.1214460
2.5161290
3.4888450
1.7876590
0.4986780
0.7831630
-1.5998610
-1.9478030
-0.9442690
-0.8459330
-1.6271340
-1.3965850
-2.9595550
-1.2388980
-1.2310540
-2.7005920
-1.9454760
0.0382490
0.2064640
-1.3456020
-2.9098060
-4.0992280
-5.4454980
-5.5222950
-4.4482090
-3.0843400
-6.2626820
-4.1096610
-3.9771470
-5.3549550
-6.5139920
-4.3466240
-4.7763370
-5.5483850
-0.4433340
-0.7755000
-1.5046010
-1.2563750
-2.7541290
-2.2418790
-0.2896140
-3.7399110
-2.9472390
-3.4867000
-2.0429930
-4.7070060

$\begin{array}{ll}\mathrm{H} & -3.4367660 \\ \mathrm{H} & -0.1443330 \\ \mathrm{O} & -2.1661230 \\ \mathrm{C} & -1.5651160 \\ \mathrm{C} & -2.5047410 \\ \mathrm{H} & -0.5191720 \\ \mathrm{H} & -2.1520980 \\ \mathrm{O} & -1.9159020 \\ \mathrm{H} & -1.2434800 \\ \mathrm{C} & -3.4960490 \\ \mathrm{C} & -4.3082140 \\ \mathrm{C} & -3.9648900 \\ \mathrm{C} & -1.9882350 \\ \mathrm{H} & -3.7606570 \\ \mathrm{H} & -5.3839690 \\ \mathrm{H} & -4.3970260 \\ \mathrm{H} & -1.7225080 \\ \mathrm{H} & -1.3942920 \\ \mathrm{H} & -3.7357710 \\ \mathrm{H} & -4.0850170 \\ \mathrm{H} & -4.3797450 \\ \mathrm{H} & \end{array}$

-6.9553230
-2.2739550
-3.5621120
-2.0304410
-1.7628060
-2.1075000
-1.1536710
-0.6649350
-0.0406420
-2.9944280
-3.1202600
-1.9965740
-3.0478170
-3.8038780
-3.0947590
-1.0365730
-4.0378350
-2.8825820
-2.0452150
-4.0854000
-2.1776280

$-4.2574560$ $-0.0707510$

0.6402270

3. 0183780

1. 9198410

2. 7198030

1.0949380

3. 4025770

2. 9075910

4. 3601220

3. 0621670

2. 0709050

4. 0604230

5.0511000

3. 2739080

2. 3877440

3. 6641670

4. 9679570

4.8577370

2. 5888560

TS166(S, S) G[B97-D/def2-TZVP $]=-3541.573490$

$\begin{array}{lrrr}\text { TS166(S S) G }[\text { B } 97-D / d e f 2-T Z V P] & =-3541.573490 \\ \mathrm{P} & 0.3822970 & 0.1719870 & 0.9786390 \\ \mathrm{O} & -0.2216670 & 0.6585780 & 2.2662090 \\ \mathrm{O} & 0.6374420 & -1.3024880 & 0.8166910 \\ \mathrm{O} & 1.7273470 & 1.0583950 & 0.7699630 \\ \mathrm{O} & -0.5460150 & 0.5689960 & -0.3191540 \\ \mathrm{C} & 2.3145590 & 1.0574950 & -0.4980500 \\ \mathrm{C} & 1.6736240 & 1.7672560 & -1.5229900 \\ \mathrm{C} & 3.5042370 & 0.3494720 & -0.6984430 \\ \mathrm{C} & 4.0215410 & 0.3501410 & -1.9981720 \\ \mathrm{H} & 4.9515070 & -0.1856680 & -2.1815550 \\ \mathrm{C} & -0.6587870 & 1.9186030 & -0.6569590 \\ \mathrm{C} & -1.8822860 & 2.5726760 & -0.4670350 \\ \mathrm{C} & 0.4588460 & 2.5665960 & -1.1979400 \\ \mathrm{C} & -1.9153750 & 3.9431680 & -0.7348860 \\ \mathrm{H} & -2.8560160 & 4.4749210 & -0.5982610 \\ \mathrm{C} & 4.1962160 & -0.3782200 & 0.3963230 \\ \mathrm{C} & -3.1080060 & 1.8352180 & -0.0646710 \\ \mathrm{C} & 4.7010910 & 0.3098240 & 1.5140730 \\ \mathrm{C} & 4.6193340 & 1.7972960 & 1.7683970 \\ \mathrm{C} & 5.8961560 & 0.5144960 & 3.6326560 \\ \mathrm{C} & 5.4052440 & -0.3777700 & 2.5204590 \\ \mathrm{C} & 3.9161340 & 2.0292930 & 3.1335910 \\ \mathrm{H} & 3.9186390 & 3.1061470 & 3.3491440\end{array}$




$\begin{array}{rrrr}\mathrm{H} & 2.8739490 & 1.7057610 & 3.0624410 \\ \mathrm{C} & 4.6716170 & 1.2440970 & 4.2508420 \\ \mathrm{H} & 5.0224740 & 1.9253080 & 5.0377460 \\ \mathrm{H} & 4.0082240 & 0.5072630 & 4.7189230 \\ \mathrm{H} & 6.4294330 & -0.0636070 & 4.3971840 \\ \mathrm{H} & 4.0803680 & 2.3172220 & 0.9722210 \\ \mathrm{C} & 6.8299480 & 1.5934280 & 3.0143830 \\ \mathrm{H} & 7.1380550 & 2.2908400 & 3.8048240 \\ \mathrm{H} & 7.7349460 & 1.1138870 & 2.6226440 \\ \mathrm{C} & 6.0691510 & 2.3475950 & 1.8796300 \\ \mathrm{H} & 6.0227860 & 3.4237280 & 2.0935540 \\ \mathrm{H} & 6.5822050 & 2.2217300 & 0.9185000 \\ \mathrm{C} & 4.3981970 & -1.7683020 & 0.3173300 \\ \mathrm{C} & 3.8955050 & -2.6947740 & -0.7661080 \\ \mathrm{C} & 5.2430820 & -3.9285730 & 1.0817820 \\ \mathrm{C} & 5.1122690 & -2.4454590 & 1.3246020 \\ \mathrm{C} & 5.1200580 & -3.4038080 & -1.4057480 \\ \mathrm{H} & 4.7634090 & -4.1145000 & -2.1633850 \\ \mathrm{H} & 5.7493500 & -2.6637150 & -1.9151900 \\ \mathrm{C} & 5.9313920 & -4.1441330 & -0.2953010 \\ \mathrm{H} & 5.9790050 & -5.2213160 & -0.5035990 \\ \mathrm{H} & 6.9605950 & -3.7683320 & -0.2518190 \\ \mathrm{H} & 5.8210310 & -4.4102400 & 1.8799620 \\ \mathrm{H} & 3.3231880 & -2.1557510 & -1.5253740 \\ \mathrm{C} & 3.8147100 & -4.5332950 & 0.9967500 \\ \mathrm{H} & 3.8941810 & -5.6046220 & 0.7668380 \\ \mathrm{H} & 3.3195290 & -4.4372530 & 1.9705050 \\ \mathrm{C} & 3.0052800 & -3.7860470 & -0.1064490 \\ \mathrm{H} & 2.6744270 & -4.4849530 & -0.8861350 \\ \mathrm{H} & 2.1173760 & -3.3116520 & 0.3170820 \\ \mathrm{C} & 5.6153740 & -1.7538250 & 2.4262900 \\ \mathrm{H} & 6.1669880 & -2.2824830 & 3.2047850 \\ \mathrm{C} & -4.8534600 & 0.2060190 & -0.5970410 \\ \mathrm{C} & -5.3341230 & -0.7443120 & -1.6660200 \\ \mathrm{C} & -3.1693410 & 0.5338130 & -2.3209910 \\ \mathrm{C} & -3.6697320 & 0.8853110 & -0.9385560 \\ \mathrm{C} & -5.5922360 & 0.0808580 & -2.9589030 \\ \mathrm{H} & -5.8777270 & -0.6063380 & -3.7664290 \\ \mathrm{H} & -6.4304920 & 0.7679450 & -2.7938220 \\ \mathrm{C} & -4.2984790 & 0.8680060 & -3.3361330 \\ \mathrm{H} & -3.9588470 & 0.5938730 & -4.3436580 \\ \mathrm{H} & -4.4861510 & 1.9482600 & -3.3305360 \\ \mathrm{H} & -2.2589500 & 1.0826060 & -2.5756680 \\ \mathrm{H} & -6.2482800 & -1.2635890 & -1.3528970 \\ \mathrm{C} & -2.9088950 & -0.9932030 & -2.3824950 \\ \mathrm{H} & -2.6049710 & -1.2622100 & -3.4022330\end{array}$

-2.0761550
-4.2030440
-4.5302550
-4.0181030
-4.9454670
-5.5115480
-3.3336600
-3.7596460
-4.4285210
-4.8203150
-4.2105320
-3.1362440
-2.9119330
-2.2743530
-2.4118390
-6.4290530
-4.4942630
-4.2099470
-4.6488360
-5.7955750
-6.1389470
-6.6029430
-5.4947160
-6.4157400
0.4197130
1.6814020
1.6059250
0.2888860
-0.8885890
-0.7835530
2.4692030
1.8743580
2.5399680
0.2092430
0.2583160
-1.8417580
-0.9321080
1.6570300
2.1658980
3.3679760
1.3344610
2.1466450
2.9896230
3.9925670
1.4670370
0.5619230

\author{
$-1.2352200$ \\ $-1.7627620$ \\ $-2.4265740$ \\ $-2.3931620$ \\ 1. 4102390 \\ 1.8056370 \\ 3. 0860540 \\ 2. 0944020 \\ 1. 5391800 \\ 1.8467950 \\ 0.4660900 \\ 2. 3349470 \\ 3. 0746990 \\ 1. 6698610 \\ 3. 6015900 \\ 1. 2469370 \\ 4.1005130 \\ 4.8139870 \\ 4.6676870 \\ 3. 3344340 \\ 3. 6456550 \\ 3. 5459540 \\ 0.4708710 \\ $-0.0513550$ \\ 3. 9674330 \\ 4.7142610 \\ 6.2216830 \\ 6.7877050 \\ 6.1652240 \\ 4.6563410 \\ 6.7213570 \\ 4.5711270 \\ 4.2681410 \\ 6.5473840 \\ 7.8807180 \\ 6.4141760 \\ 6.6113280 \\ 6.4032170 \\ 1. 6637140 \\ 0.9657430 \\ 2.1572090 \\ 2.3568180 \\ 1.1092480 \\ 0.8819260 \\ 2.5730280 \\ 1.3931280
}

$-1.7199970$

$-1.9767720$

$-2.7871010$

$-1.1010170$

1.4860230

2.8267430

2. 2088150

1.1519070

3. 9090870

4.8877540

3. 9611450

3. 5537080

4.3335070

3. 4706270

1. 9268400

3. 0496360

2. 4051000

3.1894830

1. 4792440

2.8040040

3. 7994960

2.0929430

0.6138930

0.8763420

$-1.3884940$

$-1.7934560$

$-1.5154430$

$-2.0587460$

$-1.2981150$

$-1.1490520$

$-1.9732160$

$-2.8665690$

$-1.2760350$

$-3.1298700$

$-1.9639470$

$-1.7846480$

$-0.2915850$

$-0.4315410$

$-2.8424530$

$-3.0700260$

$-4.0166850$

$-5.3018220$

$-5.5919710$

$-4.4537220$

$-6.1363530$

$-4.2059050$ 


\begin{tabular}{|c|c|c|c|c|c|c|c|}
\hline $\mathrm{H}$ & 0.7856390 & 3.0662940 & -3.7604050 & C & 2.3148570 & 1.0922530 & -0.4565110 \\
\hline H & 2.3227150 & 0.2381420 & -5.6773720 & $\mathrm{C}$ & 1.6808380 & 1.8451650 & -1.4518490 \\
\hline $\mathrm{H}$ & 3.5242720 & 1.2087240 & -6.5455560 & $\mathrm{C}$ & 3.4948170 & 0.3755610 & -0.6873200 \\
\hline H & 4.4944410 & -0.0881030 & -4.5674670 & C & 3.9932420 & 0.3954740 & -1.9933170 \\
\hline $\mathrm{H}$ & 4.7814610 & 1.6481960 & -4.5257980 & $\mathrm{H}$ & 4.9120660 & -0.1506150 & -2.2011840 \\
\hline $\mathrm{H}$ & 2.8131960 & 3.2254440 & -5.1868380 & C & -0.6605540 & 1.9445820 & -0.6113580 \\
\hline C & -1.4231530 & -3.5929840 & -0.5866010 & $\mathrm{C}$ & -1.9016910 & 2.5687640 & -0.4411160 \\
\hline 0 & -0.3578370 & -2.8802290 & -0.8533310 & C & 0.4588990 & 2.6271320 & -1.1111280 \\
\hline C & -1.8903470 & -4.4488690 & -1.7217990 & $\mathrm{c}$ & -1.9649560 & 3.9384960 & -0.7102660 \\
\hline $\mathrm{C}$ & -3.0135900 & -5.2722040 & -1.5416350 & $\mathrm{H}$ & -2.9224770 & 4.4446790 & -0.5954170 \\
\hline $\mathrm{C}$ & -1.2544750 & -4.4116590 & -2.9742670 & C & 4.1988380 & -0.3738350 & 0.3849610 \\
\hline $\mathrm{C}$ & -3.4957500 & -6.0457050 & -2.5964500 & $\mathrm{C}$ & -3.1184040 & 1.8079300 & -0.0552300 \\
\hline $\mathrm{H}$ & -3.5024790 & -5.2891720 & -0.5720420 & $\mathrm{C}$ & 4.7193370 & 0.2942050 & 1.5079250 \\
\hline C & -1.7420680 & -5.1832160 & -4.0295670 & $\mathrm{C}$ & 4.6393640 & 1.7766140 & 1.7915190 \\
\hline $\mathrm{H}$ & -0.3898290 & -3.7711720 & -3.1159790 & $\mathrm{C}$ & 5.9466290 & 0.4615730 & 3.6116080 \\
\hline C & -2.8622730 & -6.0006230 & -3.8437440 & $\mathrm{C}$ & 5.4407260 & -0.4104630 & 2.4900270 \\
\hline $\mathrm{H}$ & -4.3668270 & -6.6801990 & -2.4495540 & $\mathrm{C}$ & 3.9568310 & 1.9810350 & 3.1713820 \\
\hline $\mathrm{H}$ & -1.2492430 & -5.1459770 & -4.9984230 & $\mathrm{H}$ & 3.9607580 & 3.0535510 & 3.4075620 \\
\hline $\mathrm{H}$ & -3.2411760 & -6.6003410 & -4.6686070 & $\mathrm{H}$ & 2.9142050 & 1.6568200 & 3.1090190 \\
\hline $\mathrm{H}$ & -0.0204010 & -2.2397430 & -0.0972690 & C & 4.7301150 & 1.1760390 & 4.2620060 \\
\hline 0 & -2.0427600 & -3.5907480 & 0.4917300 & $\mathrm{H}$ & 5.0913510 & 1.8426930 & 5.0566590 \\
\hline C & -1.4729930 & -2.2311620 & 2.9886810 & H & 4.0752150 & 0.4288100 & 4.7255430 \\
\hline $\mathrm{C}$ & -2.4086950 & -1.8925810 & 1.9057710 & $\mathrm{H}$ & 6.4925640 & -0.1299770 & 4.3566690 \\
\hline $\mathrm{H}$ & -0.4254010 & -2.2851920 & 2.6920530 & $\mathrm{H}$ & 4.0878200 & 2.3112830 & 1.0140760 \\
\hline $\mathrm{H}$ & -2.0585840 & -1.2267200 & 1.1248890 & C & 6.8688570 & 1.5544150 & 3.0002640 \\
\hline 0 & -1.8303680 & -0.8964690 & 3.4656370 & $\mathrm{H}$ & 7.1863560 & 2.2379650 & 3.7991090 \\
\hline H & -1.1617620 & -0.2335950 & 3.0203760 & H & 7.7695710 & 1.0848170 & 2.5871400 \\
\hline C & $-\overline{3} .4071180$ & -3.2923880 & 4.2508930 & C & 6.0900590 & 2.3270780 & 1.8905600 \\
\hline $\mathrm{C}$ & -4.2108510 & -3.3350370 & 2.9425500 & $\mathrm{H}$ & 6.0455260 & 3.3992640 & 2.1238600 \\
\hline C & -3.8672410 & -2.1457440 & 2.0316730 & $\mathrm{H}$ & 6.5885290 & 2.2186670 & 0.9197110 \\
\hline $\mathrm{C}$ & -1.8978960 & -3.3189290 & 3.9560740 & C & 4.4019570 & -1.7618350 & 0.2774440 \\
\hline $\mathrm{H}$ & -3.6710850 & -4.1476510 & 4.8845360 & $\mathrm{c}$ & 3.8801830 & -2.6701560 & -0.8123100 \\
\hline $\mathrm{H}$ & -5.2880350 & -3.3291380 & 3.1482380 & C & 5.2668750 & -3.9333890 & 0.9860830 \\
\hline H & -4.3086620 & -1.2120830 & 2.4091350 & $\mathrm{C}$ & 5.1352470 & -2.4556370 & 1.2593340 \\
\hline $\mathrm{H}$ & -1.6262020 & -4.2785370 & 3.4944490 & $\mathrm{C}$ & 5.0927590 & -3.3647950 & -1.4888720 \\
\hline $\mathrm{H}$ & -1.3091020 & -3.2141700 & 4.8758610 & $\mathrm{H}$ & 4.7221700 & -4.0643480 & -2.2502020 \\
\hline H & -3.6544380 & -2.3799110 & 4.8096230 & $\mathrm{H}$ & 5.7091160 & -2.6147670 & -1.9994460 \\
\hline $\mathrm{H}$ & -3.9795130 & -4.2651500 & 2.4070100 & c & 5.9292550 & -4.1201810 & -0.4077460 \\
\hline $\mathrm{H}$ & -4.2750610 & -2.2597670 & 1.0226490 & $\mathrm{H}$ & 5.9787780 & -5.1931310 & -0.6363900 \\
\hline & & & & $\mathrm{H}$ & 6.9569520 & -3.7390420 & -0.3771550 \\
\hline & , S) G[B97-1 & {$[Z \mathrm{VP}]=-3$} & & $\mathrm{H}$ & 5.8617970 & -4.4276500 & 1.7638250 \\
\hline $\mathrm{P}$ & 0.3856980 & 0.1712660 & 0.9983680 & $\mathrm{H}$ & 3.2915210 & -2.1188520 & -1.5500130 \\
\hline 0 & -0.2278850 & 0.6360300 & 2.2896510 & C & 3.8392780 & -4.5418840 & 0.9168960 \\
\hline 0 & 0.6417360 & -1.3002860 & 0.8140270 & $\mathrm{H}$ & 3.9178870 & -5.6077910 & 0.6627900 \\
\hline 0 & 1.7327530 & 1.0596790 & 0.8140000 & H & 3.3632660 & -4.4681940 & 1.9020670 \\
\hline 0 & -0.5325470 & 0.5906440 & -0.2993010 & C & 3.0053570 & -3.7746380 & -0.1538140 \\
\hline
\end{tabular}




$\begin{array}{lrrr}\text { H } & 2.6569750 & -4.4588480 & -0.9388560 \\ \text { H } & 2.1268820 & -3.3091260 & 0.2982670 \\ \mathrm{C} & 5.6542120 & -1.7835950 & 2.3656960 \\ \mathrm{H} & 6.2205380 & -2.3250710 & 3.1245890 \\ \mathrm{C} & -4.8434690 & 0.1672310 & -0.6152040 \\ \mathrm{C} & -5.3063450 & -0.7781530 & -1.6964010 \\ \mathrm{C} & -3.1468790 & 0.5238590 & -2.3213340 \\ \mathrm{C} & -3.6626680 & 0.8593660 & -0.9407980 \\ \mathrm{C} & -5.5594940 & 0.0554590 & -2.9849090 \\ \mathrm{H} & -5.8310730 & -0.6276060 & -3.8007030 \\ \mathrm{H} & -6.4055560 & 0.7334860 & -2.8222390 \\ \mathrm{C} & -4.2695840 & 0.8575250 & -3.3437430 \\ \mathrm{H} & -3.9186710 & 0.5954460 & -4.3505970 \\ \mathrm{H} & -4.4671120 & 1.9359700 & -3.3301370 \\ \mathrm{H} & -2.2383620 & 1.0821860 & -2.5619910 \\ \mathrm{H} & -6.2189260 & -1.3076630 & -1.3960220 \\ \mathrm{C} & -2.8733560 & -1.0002630 & -2.3943570 \\ \mathrm{H} & -2.5602100 & -1.2574800 & -3.4143190 \\ \mathrm{H} & -2.0428930 & -1.2410350 & -1.7287450 \\ \mathrm{C} & -4.1636430 & -1.7842030 & -2.0043030 \\ \mathrm{H} & -4.4780210 & -2.4458680 & -2.8214300 \\ \mathrm{H} & -3.9806200 & -2.4182880 & -1.1308150 \\ \mathrm{C} & -4.9646670 & 1.3535790 & 1.4766240 \\ \mathrm{C} & -5.5450660 & 1.7339330 & 2.8156500 \\ \mathrm{C} & -3.3735350 & 3.0380110 & 2.2256630 \\ \mathrm{C} & -3.7824390 & 2.0509250 & 1.1578470 \\ \mathrm{C} & -4.4684850 & 1.4684540 & 3.9047890 \\ \mathrm{H} & -4.8705360 & 1.7657860 & 4.8824540 \\ \mathrm{H} & -4.2422960 & 0.3967790 & 3.9509220 \\ \mathrm{C} & -3.1798290 & 2.2771040 & 3.5655410 \\ \mathrm{H} & -2.9667720 & 3.0117970 & 4.3532340 \\ \mathrm{H} & -2.3122740 & 1.6192610 & 3.4829360 \\ \mathrm{H} & -2.4542730 & 3.5640430 & 1.9550160 \\ \mathrm{H} & -6.4594850 & 1.1657740 & 3.0270290 \\ \mathrm{C} & -4.5442440 & 4.0408520 & 2.4212500 \\ \mathrm{H} & -4.2724710 & 4.7497500 & 3.2141810 \\ \mathrm{H} & -4.6959040 & 4.6149210 & 1.4991630 \\ \mathrm{C} & -5.8421650 & 3.2603880 & 2.8023880 \\ \mathrm{H} & -6.1972040 & 3.5606940 & 3.7971270 \\ \mathrm{H} & -6.6449750 & 3.4704560 & 2.08575100 \\ \mathrm{C} & -5.4980370 & 0.4168430 & 0.59183800 \\ \mathrm{H} & -6.4168600 & -0.1154350 & 0.8413570 \\ \mathrm{C} & -0.8438320 & 4.6807240 & -1.0961220 \\ \mathrm{C} & 0.3878840 & 4.0265910 & -1.2878410 \\ \mathrm{C} & -1.0078550 & 6.1713590 & -1.3499090 \\ \mathrm{C} & 0.3144960 & 6.9501070 & -1.3409510 \\ & & & \end{array}$

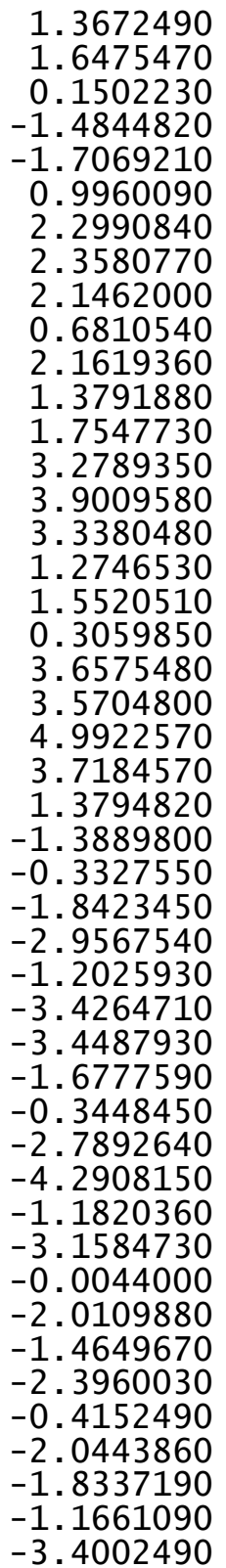

6.2083960

4.8346390

7.9649850

6.3013890

6.5902980

6.0815120

6.7858560

4.2684100

4.9863400

7.0451130

1.7769910

2.4261650

1.8793280

1.8796010

0.9078970

1.0497040

2. 4888690

3.5119890

2. 2976570

2. 8971890

1. 5961950

1.0296180

$-0.1229300$

0.8506930

$-3.6048020$

$-2.8765580$

$-4.4605770$

$-5.2974960$

$-4.4102230$

$-6.0713520$

$-5.3246280$

$-5.1822260$

$-3.7592580$

$-6.0131380$

$-6.7163380$

$-5.1347440$

$-6.6131510$

$-2.2366270$

$-3.6162100$

$-2.2694600$

$-1.9284550$

$-2.3148920$

$-1.2551850$

$-0.9409140$

-0.2697630
-3.3501990
$-2.1736580$

$-1.5538230$

$-1.7256700$

$-2.3351490$

$-0.6138070$

$-3.2023530$

$-2.2291620$

$-2.1604950$

$-0.5821700$

$-0.3079980$

$-2.7804760$

$-3.9120220$

$-5.2958830$

$-5.4580030$

$-4.4477740$

$-3.0426000$

$-6.0721420$

$-3.9146860$

$-3.7231850$

$-5.2777730$

$-6.4774690$

$-4.4123960$

$-4.7911860$

$-5.4055820$

$-0.6078890$

$-0.8684920$

$-1.7486930$

$-1.5763980$

$-2.9987260$

$-2.6365500$

$-0.6086040$

$-4.0593440$

$-3.1345480$

$-3.8813880$

$-2.4956750$

$-5.0262700$

$-4.7104160$

$-0.1080280$

0.4690070

2.9758130

1.8897450

2.6851740

1.1159200

3.4606300

3.0253550

4.2188570 


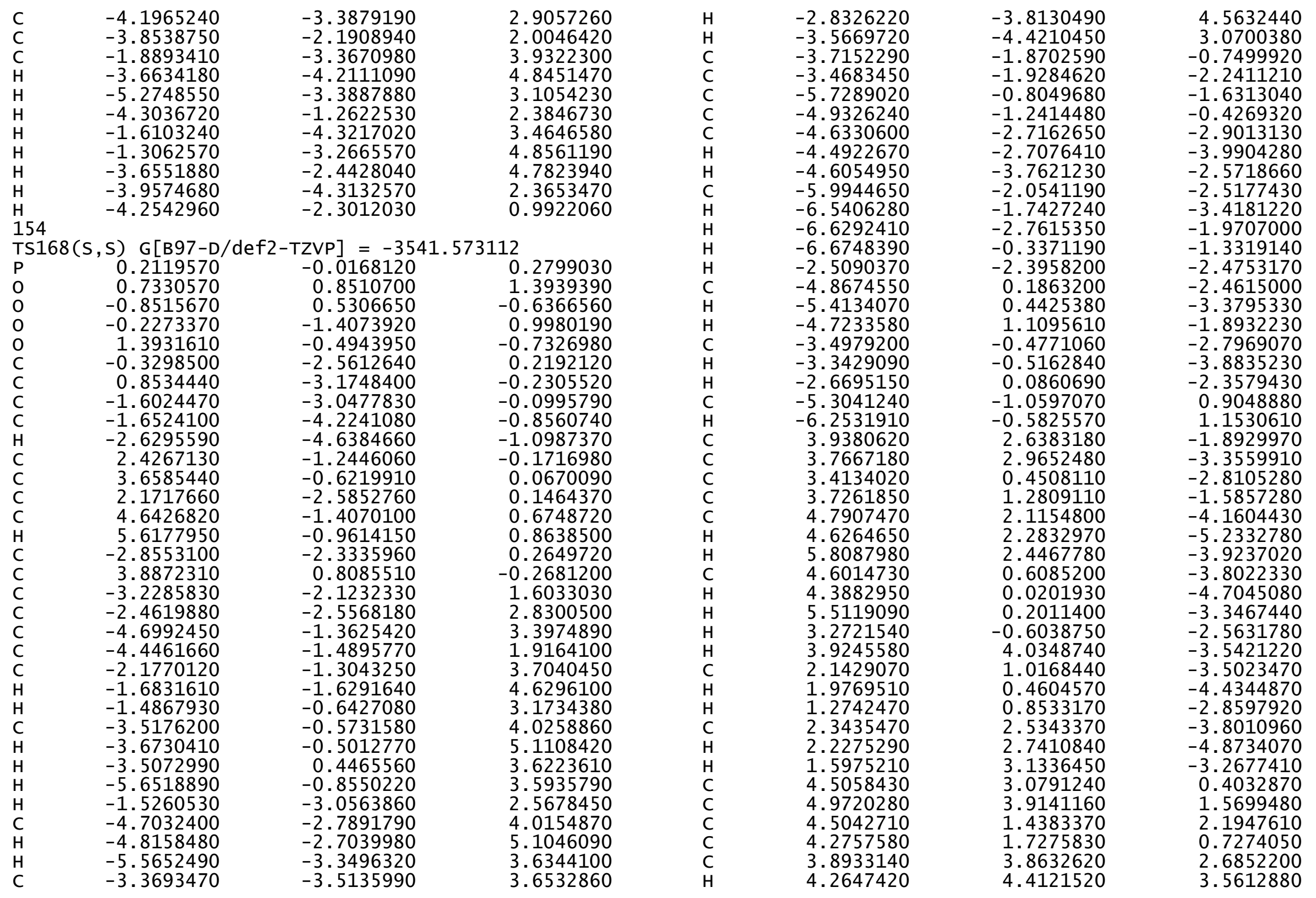




$\begin{array}{rrrr}\text { H } & 2.9812350 & 4.3571910 & \\ \mathrm{C} & 3.6030800 & 2.3752270 & 3.3360700 \\ \mathrm{H} & 3.8138910 & 2.1858460 & 4.1067560 \\ \mathrm{H} & 2.5542590 & 2.1244260 & 2.8654350 \\ \mathrm{H} & 4.2893840 & 0.3967740 & 2.4431770 \\ \mathrm{H} & 5.1618960 & 4.9496790 & 1.2614140 \\ \mathrm{C} & 5.9833690 & 1.7825780 & 2.5237190 \\ \mathrm{H} & 6.1528540 & 1.6237010 & 3.5969170 \\ \mathrm{H} & 6.6509400 & 1.1047050 & 1.9779570 \\ \mathrm{C} & 6.2693320 & 3.2684020 & 2.1350590 \\ \mathrm{H} & 6.5978660 & 3.8413980 & 3.0122960 \\ \mathrm{H} & 7.0644930 & 3.3259480 & 1.3822270 \\ \mathrm{C} & 4.3269050 & 3.5386160 & -0.9005810 \\ \mathrm{H} & 4.5094390 & 4.5858990 & -1.1457350 \\ \mathrm{C} & 3.1532410 & -3.3180970 & 0.8541480 \\ \mathrm{C} & 2.8293080 & -4.7016870 & 1.3967050 \\ \mathrm{C} & 3.7897940 & -5.1511940 & 2.5059330 \\ \mathrm{C} & 5.2393670 & -4.9372390 & 2.0558600 \\ \mathrm{C} & 5.5007630 & -3.4375500 & 1.8700900 \\ \mathrm{C} & 4.4041720 & -2.7200090 & 1.0986550 \\ \mathrm{H} & 3.6023190 & -6.2053410 & 2.7469700 \\ \mathrm{H} & 2.8691560 & -5.4454150 & 0.5880770 \\ \mathrm{H} & 1.7921060 & -4.7078250 & 1.7547290 \\ \mathrm{H} & 5.4012530 & -5.4648480 & 1.1035010 \\ \mathrm{H} & 5.9468590 & -5.3517210 & 2.7854100 \\ \mathrm{H} & 6.4636980 & -3.2695800 & 1.3683230 \\ \mathrm{H} & 5.5865380 & -2.9693580 & 2.8637440 \\ \mathrm{H} & 3.6046630 & -4.5660500 & 3.4190900 \\ \mathrm{C} & 0.7593120 & -4.2879930 & -1.0952520 \\ \mathrm{C} & -0.5077030 & -4.8441220 & -1.3625990 \\ \mathrm{C} & 1.9743460 & -4.7809830 & -1.8660610 \\ \mathrm{C} & 1.8395340 & -6.2266200 & -2.3577020 \\ \mathrm{C} & 0.5184340 & -6.3999290 & -3.1161190 \\ \mathrm{C} & -0.6663100 & -6.1142110 & -2.1842670 \\ \mathrm{H} & 2.6931220 & -6.4781070 & -3.0005140 \\ \mathrm{H} & 2.0789030 & -4.1241890 & -2.7459390 \\ \mathrm{H} & 2.8945570 & -4.6478140 & -1.2942980 \\ \mathrm{H} & 0.5001780 & -5.6978500 & -3.9632280 \\ \mathrm{H} & 0.4308160 & -7.4135200 & -3.5285760 \\ \mathrm{H} & -1.6034350 & -6.0641050 & -2.7545010 \\ \mathrm{H} & -0.7752780 & -6.9608740 & -1.4869640 \\ \mathrm{H} & 1.8612450 & -6.9139030 & -1.4978750 \\ \mathrm{C} & -3.1623040 & 2.9709760 & -0.4224200 \\ \mathrm{O} & -2.9093170 & 1.7850110 & 0.0802000 \\ \mathrm{C} & -4.5618600 & 3.4404430 & -0.1887810 \\ \mathrm{C} & -5.5286980 & 2.5805100 & 0.3610700 \\ & & & \\ & & & \end{array}$

$\begin{array}{lr}\text { C } & -4.9171360 \\ \text { C } & -6.8320620 \\ \text { H } & -5.2544860 \\ \text { C } & -6.2208270 \\ \text { H } & -4.1638270 \\ \text { C } & -7.1806050 \\ \text { H } & -7.5778030 \\ \text { H } & -6.4908770 \\ \text { H } & -8.1979740 \\ \text { H } & -2.0136340 \\ \text { O } & -2.3426570 \\ \text { C } & -0.3444360 \\ \text { C } & -0.1644370 \\ \text { H } & -0.9739290 \\ \text { H } & -0.1806740 \\ \text { O } & 1.0561610 \\ \text { H } & 1.0312970 \\ \text { C } & 0.2690550 \\ \text { C } & -0.0211160 \\ \text { C } & 0.3292530 \\ \text { C } & -0.5515380 \\ \text { H } & 0.0246100 \\ \text { H } & 0.5443480 \\ \text { H } & 1.4200560 \\ \text { H } & -1.6232980 \\ \text { H } & -0.3005750 \\ \text { H } & 1.3386250 \\ \text { H } & -1.0885990 \\ \text { H } & -0.0633310 \\ \text { H } & \end{array}$
4.7548580
4.5766870

$-0.5339120$

$\begin{array}{ll} & -0.5339120 \\ 1.5342730 & 0.5618630\end{array}$

$1.5613040 \quad 0.6147340$

$5.2053620-0.3278000$

$5.4089570 \quad-0.9641410$

$4.3458930 \quad 0.2198580$

$2.3636800 \quad 0.9832760$

$6.2245010-0.5949960$

$4.6977060 \quad 0.3776250$

$1.3413600 \quad-0.2198900$

$3.6775010-1.0352630$

$3.7165560 \quad 1.0279260$

$3.5800130 \quad-0.4247850$

$2.9564810 \quad 1.4954900$

$2.5775420 \quad-0.8347950$

$3.3149610 \quad 1.0279180$

$2.2765230 \quad 1.1571210$

$6.1691220 \quad 0.8287450$

$6.0971520 \quad-0.6786100$

$4.7130340-1.2536440$

$5.1112090 \quad 1.5860520$

$7.1663900 \quad 1.2136620$

$6.8651100 \quad-1.2195890$

$4.5837810 \quad-1.3293130$

$5.3390060 \quad 1.4988970$

$5.0992050 \quad 2.6540550$

$5.9980630 \quad 1.0092750$

$6.2889620 \quad-0.8500240$

154

$-2.2681200$

\section{TS169(S,S) G[B97-D/def2-TZVP $]=-3541.573073$}

$\begin{array}{lrrr}\text { P } & 0.4047070 & 0.2717980 & 0.9680130 \\ \mathrm{O} & -0.2079970 & 0.7813140 & 2.2426740 \\ \mathrm{O} & 0.7074490 & -1.1980280 & 0.8534680 \\ \mathrm{O} & 1.7156420 & 1.1949130 & 0.7166710 \\ \mathrm{O} & -0.5374030 & 0.5984540 & -0.3373550 \\ \mathrm{C} & 2.2878190 & 1.1653480 & -0.5582740 \\ \mathrm{C} & 1.6251260 & 1.8494250 & -1.5939190 \\ \mathrm{C} & 3.4809050 & 0.4609050 & -0.7490460 \\ \mathrm{C} & 3.9889570 & 0.4102400 & -2.0560320 \\ \mathrm{H} & 4.9101020 & -0.1413420 & -2.2346150 \\ \mathrm{C} & -0.7044950 & 1.9310770 & -0.7153290 \\ \mathrm{C} & -1.9557190 & 2.5330700 & -0.5400030 \\ \mathrm{C} & 0.3790220 & 2.6084980 & -1.2954640 \\ \mathrm{C} & -2.0598670 & 3.8871890 & -0.8661080 \\ \mathrm{H} & -3.0230340 & 4.3783250 & -0.7345290\end{array}$




$\begin{array}{lr}\mathrm{C} & 4.1949680 \\ \mathrm{C} & -3.1434300 \\ \mathrm{C} & 4.6997990 \\ \mathrm{C} & 4.5911320 \\ \mathrm{C} & 5.9164610 \\ \mathrm{C} & 5.4332880 \\ \mathrm{C} & 3.8998600 \\ \mathrm{H} & 3.8847940 \\ \mathrm{H} & 2.8631690 \\ \mathrm{C} & 4.6825680 \\ \mathrm{H} & 5.0255050 \\ \mathrm{H} & 4.0397740 \\ \mathrm{H} & 6.4703030 \\ \mathrm{H} & 4.0336920 \\ \mathrm{C} & 6.8196570 \\ \mathrm{H} & 7.1177930 \\ \mathrm{H} & 7.7322750 \\ \mathrm{C} & 6.0309200 \\ \mathrm{H} & 5.9647790 \\ \mathrm{H} & 6.5359680 \\ \mathrm{C} & 4.4292180 \\ \mathrm{C} & 3.9341190 \\ \mathrm{C} & 5.3502820 \\ \mathrm{C} & 5.1770370 \\ \mathrm{C} & 5.1663610 \\ \mathrm{H} & 4.8160100 \\ \mathrm{H} & 5.7623090 \\ \mathrm{C} & 6.0227460 \\ \mathrm{H} & 6.1068750 \\ \mathrm{H} & 7.0377850 \\ \mathrm{H} & 5.9565390 \\ \mathrm{H} & 3.3315850 \\ \mathrm{C} & 3.9411170 \\ \mathrm{H} & 4.0509750 \\ \mathrm{H} & 3.4598430 \\ \mathrm{C} & 3.0885880 \\ \mathrm{H} & 2.7606750 \\ \mathrm{H} & 2.1974720 \\ \mathrm{C} & 5.6779020 \\ \mathrm{H} & 6.2552860 \\ \mathrm{C} & -4.8250940 \\ \mathrm{C} & -5.2769800 \\ \mathrm{C} & -3.1664350 \\ \mathrm{C} & -3.6708930 \\ \mathrm{C} & -5.5752620 \\ \mathrm{H} & -5.8422720 \\ & \end{array}$

$\begin{array}{rr}-0.2185560 & 0.3633440 \\ 1.7571740 & -0.0972350 \\ 0.5154480 & 1.4520800 \\ 2.0081660 & 1.6624710 \\ 0.8102170 & 3.5487400 \\ -0.1250650 & 2.4688070 \\ 2.2678090 & 3.0287600 \\ 3.3504310 & 3.2123540 \\ 1.9231550 & 2.9802660 \\ 1.5300610 & 4.1593390 \\ 2.2396280 & 4.9243740 \\ 0.7919820 & 4.6535550 \\ 0.2662360 & 4.3236590 \\ 2.4940190 & 0.8578760 \\ 1.8906000 & 2.8892150 \\ 2.6193200 & 3.6548220 \\ 1.4201520 & 2.5042380 \\ 2.5903850 & 1.7389510 \\ 3.6719490 & 1.9173910 \\ 2.4431540 & 0.7766130 \\ -1.6055940 & 0.3246860 \\ -2.5774610 & -0.7222560 \\ -3.7181770 & 1.1339180 \\ -2.2339080 & 1.3388840 \\ -3.2700290 & -1.3649550 \\ -4.0159970 & -2.0909260 \\ -2.5292900 & -1.9122530 \\ -3.9483350 & -0.2487750 \\ -5.0285390 & -0.4277630 \\ -3.5338120 & -0.2338670 \\ -4.1595820 & 1.9343860 \\ -2.0778680 & -1.4852640 \\ -4.3700200 & 1.0892060 \\ -5.4433480 & 0.8820420 \\ -4.2678540 & 2.0693150 \\ -3.6746090 & -0.0157380 \\ -4.4028010 & -0.7692910 \\ -3.2134660 & 0.4148880 \\ -1.4972810 & 2.4118750 \\ -1.9880370 & 3.1964440 \\ 0.0409880 & -0.5543280 \\ -0.9639870 & -1.5853660 \\ 0.3701650 & -2.3024720 \\ 0.7523400 & -0.9298070 \\ -0.1947660 & -2.9036470 \\ -0.9196350 & -3.6841440\end{array}$

-6.4366260
-4.3138150
-3.9706970
-4.5412440
-2.2784910
-6.1688030
-2.8494360
-2.5410960
-2.0049210
-4.1116250
-4.4206530
-3.8984310
-4.9527130
-5.5254400
-3.4064320
-3.7978310
-4.4246180
-4.8223880
-4.1612220
-3.1691280
-2.9713620
-2.2805900
-2.5090510
-6.4173240
-4.6068960
-4.3475260
-4.7910890
-5.8727800
-6.2221770
-6.6928040
-5.4689280
-6.3671380
0.2580390
1.4569000
1.3244240
-0.0315290
-1.1594470
-0.9742150
2.1502390
1.5774660
2.3680110
-0.1256920
-0.1121170
-2.1334310
-1.2071500
1.3990130
0.4669600
0.6248240
0.3256290
1.6969380
0.9423920
$-1.5053980$
$-1.1473430$
$-1.4401530$
$-1.3350610$
$-1.9500760$
$-2.6507740$
$-2.5443190$
1. 3183190
1. 7415410
3.0857240
2. 0363190
1. 5626970
1.8905450
0.5024810
2. 3975080
3.1755950
1.7663330
3.6278910
1.1544290
4.0586590
4.8132590
4.5825350
3.2555050
3. 5912210
3. 4056740
0.3252780
$-0.2222380$
3.9904700
4.7783820
6.2893980
6.7748040
6.1250990
4.6317890
6.8095600
4.6080150
4. 3896000
6.4942620
7.8678330
6.3034080
6.6114470
6. 5123240




$\begin{array}{rrr}2.1374700 & 1.7348380 & -2.8989200 \\ 1.4275260 & 2.2299320 & -4.1348280 \\ 1.2773490 & 1.0679430 & -5.1480080 \\ 2.6317930 & 0.3585770 & -5.4485150 \\ 3.7839060 & 0.8835700 & -4.5519560 \\ 3.3257150 & 1.0122620 & -3.1224390 \\ 2.0182920 & 3.0303260 & -4.6070790 \\ 0.8340180 & 1.4553220 & -6.0738370 \\ 2.9103470 & 0.4944020 & -6.5011480 \\ 4.0894140 & 1.8733970 & -4.9255810 \\ 0.4471490 & 2.6491210 & -3.8907730 \\ 0.5671750 & 0.3455060 & -4.7268360 \\ 2.5249880 & -0.7200350 & -5.2796970 \\ 4.6578720 & 0.2255030 & -4.6240330 \\ -1.2677660 & -3.6274550 & -0.4354260 \\ -0.2374520 & -2.8805570 & -0.7431870 \\ -1.7007100 & -4.5564950 & -1.5255110 \\ -2.8078450 & -5.3934510 & -1.3118920 \\ -1.0456750 & -4.5802230 & -2.7684790 \\ -3.2562780 & -6.2397100 & -2.3248460 \\ -3.3110070 & -5.3634810 & -0.3499830 \\ -1.4985850 & -5.4259290 & -3.7815870 \\ -0.1923670 & -3.9304580 & -2.9349610 \\ -2.6035410 & -6.2559490 & -3.5628730 \\ -4.1154920 & -6.8839050 & -2.1525630 \\ -0.9904830 & -5.4371660 & -4.7431790 \\ -2.9552860 & -6.9134820 & -4.3550440 \\ 0.0787250 & -2.1967230 & -0.0169340 \\ -1.8811230 & -3.6021030 & 0.6459800 \\ -1.3382170 & -2.1217090 & 3.0800610 \\ -2.3005090 & -1.8642140 & 1.9989050 \\ -0.2930600 & -2.1394560 & 2.7709430 \\ -1.9867970 & -1.2153430 & 1.1889080 \\ -1.7487260 & -0.7867330 & 3.5117950 \\ -1.1108510 & -0.1143320 & 3.0363490 \\ -3.2073400 & -3.2244660 & 4.4045610 \\ -4.0242270 & -3.3459430 & 3.1092540 \\ -3.7450690 & -2.1744710 & 2.1540550 \\ -1.7014340 & -3.1913730 & 4.0920040 \\ -3.4244930 & -4.0699800 & 5.0683560 \\ -5.0979470 & -3.3798480 & 3.3299300 \\ -4.2214100 & -1.2480770 & 2.5064120 \\ -1.3899070 & -4.1532990 & 3.6613610 \\ -1.1081250 & -3.0259550 & 4.9998840 \\ -3.4901540 & -2.3069720 & 2.6014260 \\ -3.7595100 & -4.2826520 & \end{array}$

154

TS170(S,S) G[B97-D/def2-TZVP $]=-3541.572826$

\begin{tabular}{|c|c|c|c|}
\hline & S) $\mathrm{G}[\mathrm{B} 97-\mathrm{D}$ & $=-3$ & \\
\hline $\mathrm{P}$ & 0.3443620 & 0.2017860 & 0.857655 \\
\hline 0 & -0.2819470 & 0.7692170 & 2.100631 \\
\hline 0 & 0.5593100 & -1.2853150 & 0.774323 \\
\hline 0 & 1.7179170 & 1.0425820 & 0.653213 \\
\hline 0 & -0.5170680 & 0.5709610 & 488852 \\
\hline C & 2.3485630 & 0.9928290 & 590647 \\
\hline $\mathrm{C}$ & 1.7733160 & 1.6968680 & -1.660063 \\
\hline $\mathrm{C}$ & 3.5303900 & 0.2546050 & -0.716285 \\
\hline $\mathrm{C}$ & 4.1111240 & 0.2129660 & -1.987 \\
\hline $\mathrm{H}$ & 5.0326390 & -0.3528540 & -2.114193 \\
\hline C & -0.5905350 & 1.9158850 & -0.8574200 \\
\hline C & -1.7964060 & 2.6019910 & $-0.672943 c$ \\
\hline C & 0.5553530 & 2.5196030 & -1.4074800 \\
\hline C & -1.8109070 & 3.9704910 & -0.97 \\
\hline $\mathrm{H}$ & -2.7344130 & 4.5288070 & -0.83 \\
\hline $\mathrm{C}$ & 4.1481590 & -0.4624040 & 0.42 \\
\hline $\mathrm{C}$ & -3.0299310 & 1.9060390 & -0.22 \\
\hline $\mathrm{C}$ & 4.6038390 & 0.2393110 & 1.56 \\
\hline $\mathrm{C}$ & 4.5425010 & 1.7334030 & 1.77 \\
\hline $\mathrm{C}$ & 5.6725550 & 0.4624830 & 3.74 \\
\hline $\mathrm{C}$ & 5.2281410 & -0.4419040 & 2.62 \\
\hline $\mathrm{C}$ & 3.7613790 & 2.0092140 & 3.09 \\
\hline $\mathrm{H}$ & 3.7733760 & 3.0904550 & 3.28 \\
\hline $\mathrm{H}$ & 2.7190210 & 1.7047950 & 2.96 \\
\hline C & 4.4317980 & 1.2349000 & 4.27 \\
\hline $\mathrm{H}$ & 4.7536940 & 1.9273190 & 5.06 \\
\hline $\mathrm{H}$ & 3.7248050 & 0.5255980 & 4.71 \\
\hline $\mathrm{H}$ & 6.1438060 & -0.1113240 & 4.5511 \\
\hline $\mathrm{H}$ & 4.0665090 & 2.2487900 & 0.94 \\
\hline C & 6.6689660 & 1.5039730 & 3.16 \\
\hline $\mathrm{H}$ & 6.9499120 & 2.2088420 & 3.9 \\
\hline $\mathrm{H}$ & 7.5816060 & 0.9935080 & 2 \\
\hline C & 5.9946600 & 2.2545780 & 1 \\
\hline $\mathrm{H}$ & 9583360 & 3.3347920 & \\
\hline $\mathrm{H}$ & & 2020 & \\
\hline C & 0 & -1.8578990 & $\partial($ \\
\hline C & 6857 & -2.79 & -1 \\
\hline C & 0 & -4.0190660 & 1 \\
\hline C & & -2.5286430 & 1 \\
\hline C & & 5640 & \\
\hline $\mathrm{H}$ & & -4 & \\
\hline $\mathrm{H}$ & 5.7 & 6090 & -1 \\
\hline & & $-4.2 / 4 / 630$ & \\
\hline
\end{tabular}




$\begin{array}{lr}\mathrm{H} & 5.8878040 \\ \mathrm{H} & 6.8716030 \\ \mathrm{H} & 5.5847400 \\ \mathrm{H} & 3.3570930 \\ \mathrm{C} & 3.6385900 \\ \mathrm{H} & 3.7131250 \\ \mathrm{H} & 3.0855790 \\ \mathrm{C} & 2.9135880 \\ \mathrm{H} & 2.6078640 \\ \mathrm{H} & 2.0170850 \\ \mathrm{C} & 5.4078720 \\ \mathrm{H} & 5.8970880 \\ \mathrm{C} & -4.8158390 \\ \mathrm{C} & -5.3313290 \\ \mathrm{C} & -3.1497060 \\ \mathrm{C} & -3.6214510 \\ \mathrm{C} & -5.5903130 \\ \mathrm{H} & -5.8966740 \\ \mathrm{H} & -6.4146670 \\ \mathrm{C} & -4.2870930 \\ \mathrm{H} & -3.9676890 \\ \mathrm{H} & -4.4538180 \\ \mathrm{H} & -2.2316920 \\ \mathrm{H} & -6.2518620 \\ \mathrm{C} & -2.9242230 \\ \mathrm{H} & -2.6494570 \\ \mathrm{H} & -2.0833220 \\ \mathrm{C} & -4.2266600 \\ \mathrm{H} & -4.5806720 \\ \mathrm{H} & -4.0401490 \\ \mathrm{C} & -4.8639080 \\ \mathrm{C} & -5.4167320 \\ \mathrm{C} & -3.2143650 \\ \mathrm{C} & -3.6648200 \\ \mathrm{C} & -4.3373940 \\ \mathrm{H} & -4.7217980 \\ \mathrm{H} & -4.1378450 \\ \mathrm{C} & -3.0327530 \\ \mathrm{H} & -2.8001760 \\ \mathrm{H} & -2.1804130 \\ \mathrm{H} & -2.2820490 \\ \mathrm{H} & -6.3454620 \\ \mathrm{C} & -4.3538690 \\ \mathrm{H} & -4.0529490 \\ \mathrm{H} & -4.5011500 \\ \mathrm{C} & -5.6684200 \\ & \end{array}$

\begin{tabular}{rr}
-5.3568470 & -0.2558050 \\
-3.9109050 & 0.0281040 \\
-4.4950110 & 2.0941530 \\
-2.2643590 & -1.5083450 \\
-4.5991650 & 1.0839070 \\
-5.6744560 & 0.8717040 \\
-4.4827460 & 2.0237120 \\
-3.8520670 & -0.0764450 \\
-4.5571760 & -0.8608140 \\
-3.3458560 & 0.2879450 \\
-1.8244550 & 2.5706540 \\
-2.3481730 & 3.3929930 \\
0.2893720 & -0.6496130 \\
-0.7024140 & -1.6633710 \\
0.4947920 & -2.4083390 \\
0.9238950 & -1.0381470 \\
0.0629500 & -2.9925570 \\
-0.6580380 & -3.7621440 \\
0.7721720 & -2.8529390 \\
0.8071990 & -3.4214460 \\
0.4829970 & -4.4207340 \\
1.8902420 & -3.4600380 \\
1.0105510 & -2.7012430 \\
-1.1852840 & -1.3128370 \\
-1.0390570 & -2.4037260 \\
-1.3599480 & -3.4168990 \\
-1.2725100 & -1.7483600 \\
-1.7591830 & -1.9373820 \\
-2.4583890 & -2.7056420 \\
-2.3445930 & -1.0312040 \\
1.6062000 & 1.3649280 \\
2.0926550 & 2.6805810 \\
3.2860890 & 1.9828910 \\
2.2427830 & 0.9866180 \\
1.8677560 & 3.7748540 \\
2.2356140 & 4.7355350 \\
0.7957150 & 3.8841900 \\
2.6199420 & 3.3739210 \\
3.4054910 & 4.1050580 \\
1.9387970 & 3.3380790 \\
3.7635220 & 1.6705070 \\
1.5678990 & 2.9369270 \\
4.3354290 & 2.1129640 \\
5.0948160 & 2.8464810 \\
4.8410980 & 1.1508800 \\
3.6233130 & 2.5663660 \\
-4 & \\
\hline &
\end{tabular}

$-5.9966300$ $-6.4769980$ $-5.4404930$ $-6.3714440$ 0.5170370 1. 7130140 1. 8928890 1.8928890
0.5853830 $-0.6243340$ $-0.6700630$ 1.5526230 1.5526230
2.7049620 0.7265870 $-0.5163290$ 2. 6220820 2. 2107180 0.3444780 $-1.5603920$ 2. 3471740 3. 5352630 1. 6223380 2. 5071650 3. 2961240 4.2296650 1.8809370 0.7889010 1.1575560 2. 5916040 3. 8796680 4.6976050 5. 0513720 3. 2139950 $-1.5541860$ $-0.4786690$ $-2.0415120$ $-3.1877890$ $-1.3972760$ $-3.6842890$ $-3.6825170$ $-1.8987150$ $-0.5146280$ $-3.0417380$ $-4.5730770$ $-1.3987610$ $-3.4312410$ $-0.1256430$

$\begin{array}{rr}4.0018890 & 3.5435230 \\ 3.8091040 & 1.8494870 \\ 0.6326330 & 0.5500180 \\ 0.1472440 & 0.8460880 \\ 3.9042940 & -1.6591520 \\ 4.7197400 & -2.0868970 \\ 5.9275390 & -1.1343950 \\ 6.7591240 & -0.9680170 \\ 6.1109620 & -1.6902120 \\ 4.6262810 & -1.4336920 \\ 5.1017670 & -3.1076470 \\ 6.5620310 & -1.5107090 \\ 7.7758230 & -1.3557410 \\ 6.2866690 & -2.7723310 \\ 4.1118460 & -2.1096090 \\ 5.5414020 & -0.1581580 \\ 6.8516530 & 0.0979560 \\ 6.5886290 & -1.3771810 \\ 1.5748850 & -2.9472300 \\ 0.8336140 & -3.0998140 \\ 2.1214060 & -4.1678560 \\ 2.2400190 & -5.4148720 \\ 0.9452320 & -5.6376540 \\ 0.7016990 & -4.4459810 \\ 2.4736740 & -6.2856560 \\ 1.4335780 & -4.3871810 \\ 3.0842060 & -3.9484670 \\ 0.1054370 & -5.7345390 \\ 0.9945030 & -6.5661480 \\ -0.2892960 & -4.5151770 \\ 1.4349350 & -4.4896530 \\ -3.0740150 & -5.2862520 \\ -3.6278380 & -0.4580030 \\ -2.9535040 & -0.7782470 \\ -4.5538460 & -1.5274180 \\ -5.3300240 & -1.2907660 \\ -4.6342380 & -2.7735240 \\ -6.1726910 & -2.2841290 \\ -5.2572790 & -0.3267220 \\ -5.4756220 & -3.7672320 \\ -4.0303440 & -2.9578520 \\ -6.2455210 & -3.5254280 \\ -6.7700620 & -2.0941000 \\ -5.5302200 & -4.7315800 \\ -6.9000130 & -4.3023130 \\ -2.2697560 & -0.0689940\end{array}$




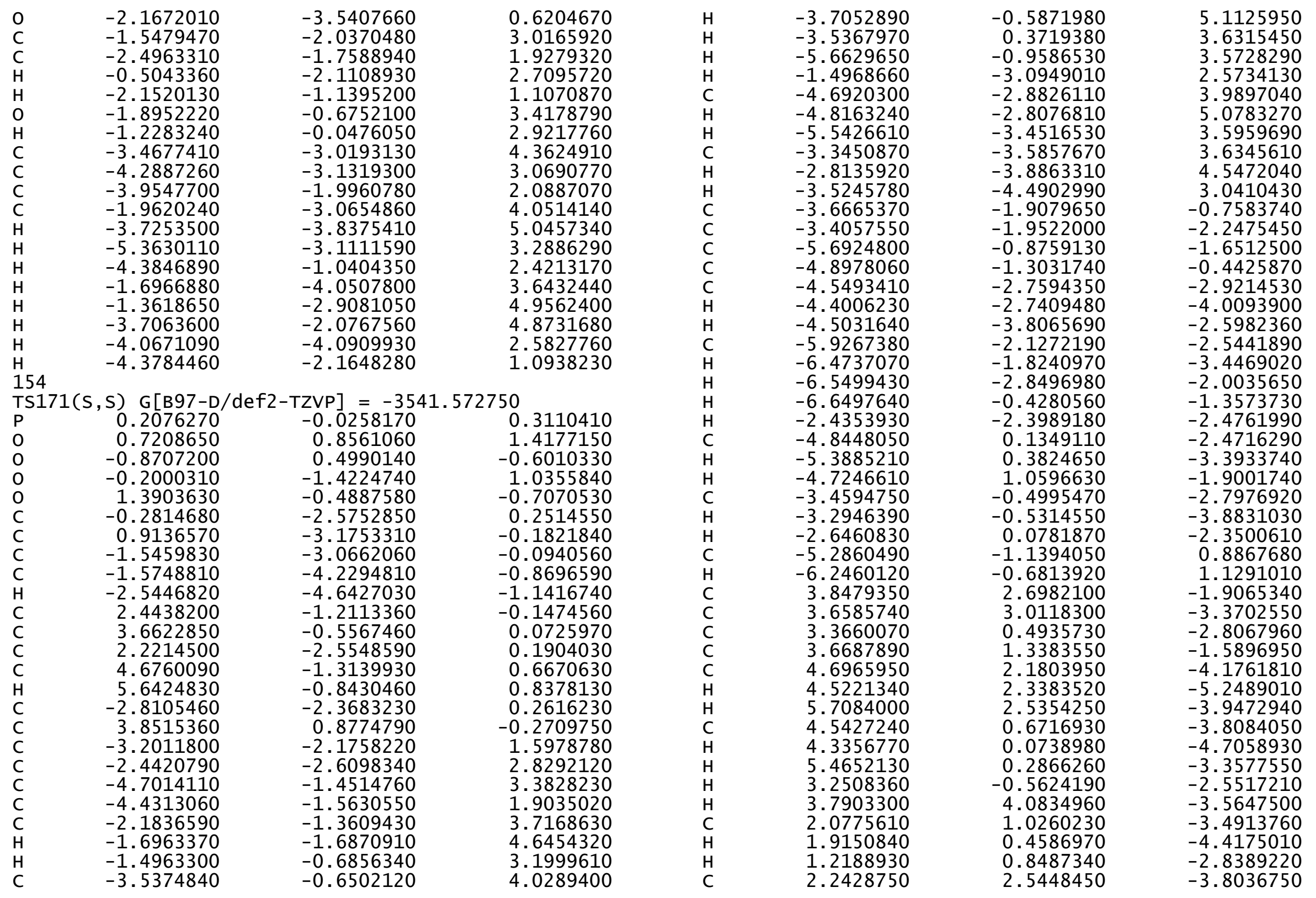




\begin{tabular}{|c|c|c|}
\hline $\begin{array}{l}2.1168180 \\
1.4863990 \\
4.4261770 \\
4.8841540 \\
4.4779630 \\
4.2288340 \\
3.8178840 \\
4.1857040 \\
2.8914960 \\
3.5649010 \\
3.7914180 \\
2.5200750 \\
4.2895310 \\
5.0473510 \\
5.9521120 \\
6.1351660 \\
6.6299110 \\
6.2012850 \\
6.5250040 \\
6.9879090 \\
4.2259100 \\
4.3837070 \\
4.4782410 \\
3.2337560 \\
5.6367360 \\
5.2039550 \\
4.2151730 \\
2.9485610 \\
6.0859140 \\
6.3249450 \\
6.2063440 \\
4.6880220 \\
3.9466280 \\
2.2723870 \\
2.3990610 \\
4.7148480 \\
0.8431310 \\
0.4168980 \\
2.0893880 \\
1.9412040 \\
0.6453770 \\
0.5597970 \\
2.8143120 \\
2.2930660 \\
2.9669130 \\
0.6506050\end{array}$ & $\begin{array}{r}2.7397510 \\
3.1318440 \\
3.1646800 \\
4.0162440 \\
1.5337520 \\
1.8104230 \\
3.9470780 \\
4.5089610 \\
4.4183800 \\
2.4548570 \\
2.2762850 \\
2.1796730 \\
0.4889070 \\
5.0541950 \\
1.9134200 \\
1.7643980 \\
1.2479760 \\
3.4032610 \\
3.9881570 \\
3.4746270 \\
3.6124520 \\
4.6619630 \\
-2.6310120 \\
-3.2564740 \\
-3.3574240 \\
-4.5660270 \\
-5.4261230 \\
-4.6202880 \\
-5.1494770 \\
-3.7057900 \\
-2.6455480 \\
-5.7483110 \\
-6.3320100 \\
-5.1998920 \\
-4.4486980 \\
-4.2203750 \\
-4.2898330 \\
-4.8394410 \\
-4.8011280 \\
-6.2194770 \\
-6.3376540 \\
-6.0851890 \\
-6.4584410 \\
-4.1089220 \\
-4.7431210 \\
-5.5917130\end{array}$ & $\begin{array}{r}-4.8770610 \\
-3.2717470 \\
0.3823210 \\
1.5400450 \\
2.1821220 \\
0.7158000 \\
2.6662200 \\
3.5355420 \\
2.3237390 \\
3.0377230 \\
4.0973200 \\
2.8702090 \\
2.4381990 \\
1.2243500 \\
2.4951630 \\
3.5675450 \\
1.9467170 \\
2.0960680 \\
2.9672310 \\
1.3354860 \\
-0.9225720 \\
-1.1753360 \\
1.0958150 \\
0.8845990 \\
1.7620000 \\
2.6015830 \\
1.8063600 \\
1.4949860 \\
2.8962240 \\
0.9747560 \\
2.3742270 \\
0.8658170 \\
2.3650730 \\
0.8611740 \\
2.4350500 \\
3.5245210 \\
-1.0511780 \\
-1.3593750 \\
-1.7602900 \\
-2.3239450 \\
-3.1332460 \\
-2.2195190 \\
-2.9448970 \\
-2.5941390 \\
-1.1129190 \\
-3.9419870\end{array}$ \\
\hline
\end{tabular}
0.5601760
$-1.4846860$
$-0.6829170$
1. 9196380
$-3.2147290$
$-2.9444020$
$-4.6195160$
$-5.5760740$
$-4.9900480$
$-6.8848610$
$-5.2891980$
$-6.2989350$
$-4.2443850$
$-7.2486540$
$-7.6227940$
$-6.5809550$
$-8.2701430$
$-2.0438610$
$-2.4068810$
$-0.4055270$
$-0.2266530$
$-1.0224250$
$-0.2284520$
1.0009430
0.9931730
0.1717350
$-0.1206970$
0.2475670
$-0.6326880$
$-0.0859060$
0.4326160
1. 3397840
$-1.7077410$
$-0.3810940$
1. 2439560
$-1.1911790$
$-0.1469630$

154
$-7.3278540$
$-6.0148790$
$-6.9548790$
$-6.9449820$
2.9065860
1.7295480
3. 3603230
2.4904170
4. 6697040
2.9289800
1. 4753140
5. 1053190
5.3316010
4.2357010
2. 2504770
6. 1206940
4.5758590
1. 2947740
3. 6174340
3. 7019180
3. 5589530
2. 9355540
2.5537030
3. 3219040
2. 2841130
6.1616490
6.0766580
4.6942990
5.0968270
7.1576480
6.8492200
4. 5802350
5. 3081820
5.0954740
.0066520
6.2525730
4. 5464210

$\operatorname{TS} 172(\mathrm{~S}, \mathrm{~S})$ G[B97-D/def2-TZVP $]=-3541.572570$

\begin{tabular}{lrrr} 
TS172(S, S & \multicolumn{3}{c}{ G $[\mathrm{B} 97-\mathrm{D} / \mathrm{def}-\mathrm{TZP}=-3541.572570$} \\
$\mathrm{P}$ & 0.3834850 & 0.1817690 & 0.9818450 \\
$\mathrm{O}$ & -0.2353760 & 0.6537380 & 2.2678250 \\
$\mathrm{O}$ & 0.6535990 & -1.2892120 & 0.8125310 \\
$\mathrm{O}$ & 1.7205340 & 1.0834850 & 0.7881250 \\
$\mathrm{O}$ & -0.5356160 & 0.5793540 & -0.3222310 \\
$\mathrm{C}$ & 2.3090740 & 1.0935900 & -0.4798220 \\
$\mathrm{C}$ & 1.6736570 & 1.8195590 & -1.4959300
\end{tabular}

$-3.5993710$

$-2.8073030$

$-1.5536210$

$-1.4961520$

$-0.4033110$

0.1113130

$-0.5187670$

0.5805100

0.6371870

$-0.3135350$

$-0.9504060$

0.2356430

1. 0029840

$-0.5826990$

0.3923820

$-0.1872390$

$-1.0266970$

1.0322080

$-0.4200520$

1. 5061360

$-0.8233760$

1.0309390

1.1683730

0.8169360

$-0.6891980$

$-1.2570460$

1.5817740

1.1965420

$-1.2361590$

$-1.3366040$

1.4935770

2. 6496910

0.9964000

$-0.8590210$

$-2.2691270$
$-0.1704810$

0.3812990 


\begin{tabular}{|c|c|c|}
\hline $\begin{array}{l}3.4904050 \\
3.9982940 \\
4.9166490 \\
-0.6650840 \\
-1.9001630 \\
0.4482040 \\
-1.9579830 \\
-2.9079990 \\
4.1838990 \\
-3.1150290 \\
4.6927490 \\
4.6095660 \\
5.9002270 \\
5.4056600 \\
3.9140650 \\
3.9158950 \\
2.8720110 \\
4.6773310 \\
5.0306860 \\
4.0183350 \\
6.4392810 \\
4.0653710 \\
6.8278050 \\
7.1376340 \\
7.7323670 \\
6.0590210 \\
6.0116900 \\
6.5667000 \\
4.3912500 \\
3.8844040 \\
5.2567900 \\
5.1166410 \\
5.1072940 \\
4.7480110 \\
5.7262560 \\
5.9349320 \\
5.9906630 \\
6.9609130 \\
5.8454390 \\
3.3009290 \\
3.8327380 \\
3.9185150 \\
3.3456320 \\
3.0076690 \\
2.6721370 \\
2.1218420\end{array}$ & $\begin{array}{r}0.3726340 \\
0.3681480 \\
-0.1841520 \\
1.9311090 \\
2.5618710 \\
2.6047210 \\
3.9329710 \\
4.4467780 \\
-0.3620510 \\
1.8046950 \\
0.3206840 \\
1.8065700 \\
0.5154970 \\
-0.3708050 \\
2.0284760 \\
3.1039370 \\
1.7035650 \\
1.2375730 \\
1.9142410 \\
0.4958130 \\
-0.0663570 \\
2.3312410 \\
1.6008690 \\
2.2945970 \\
1.1264110 \\
2.3590560 \\
3.4340300 \\
2.2390520 \\
-1.7510030 \\
-2.6737090 \\
-3.9113470 \\
-2.4311650 \\
-3.3702950 \\
-4.0793430 \\
-2.6230050 \\
-4.1108450 \\
-5.1860400 \\
-3.7257160 \\
-4.3943770 \\
-2.1335880 \\
-4.5267350 \\
-5.5952000 \\
-4.4437540 \\
-3.7754170 \\
-4.4698230 \\
-3.3095480\end{array}$ & $\begin{array}{r}-0.6880310 \\
-1.9905970 \\
-2.1828970 \\
-0.6449700 \\
-0.4522210 \\
-1.1675740 \\
-0.7110390 \\
-0.5709930 \\
0.4010860 \\
-0.0540090 \\
1.5207070 \\
1.7849470 \\
3.6336980 \\
2.5183330 \\
3.1556650 \\
3.3781240 \\
3.0882390 \\
4.2635030 \\
5.0532130 \\
4.7300230 \\
4.3913040 \\
0.9956770 \\
3.0173390 \\
3.8103750 \\
2.6184500 \\
1.8908360 \\
2.1103710 \\
0.9261280 \\
0.3122290 \\
-0.7726120 \\
1.0541330 \\
1.3093370 \\
-1.4286040 \\
-2.1865520 \\
-1.9401460 \\
-0.3304330 \\
-0.5468320 \\
-0.2930660 \\
1.8436230 \\
-1.5224720 \\
0.9764820 \\
0.7357000 \\
1.9554910 \\
-0.1121010 \\
-0.8937710 \\
0.3247710\end{array}$ \\
\hline
\end{tabular}

\begin{abstract}
5.6230530
6.1833510

$-4.8450210$

$-5.3162850$

$-3.1606860$

$-3.6659660$

$-5.5786840$

$-5.8567570$

$-6.4230930$

$-4.2909880$

$-3.9477220$

$-4.4875930$

$-2.2541320$

$-6.2269000$

$-2.8885210$

$-2.5821120$

$-2.0540660$

$-4.1766860$

$-4.4975250$

$-3.9876590$

$-4.9530690$

$-5.5270230$

$-3.3577480$

$-3.7718800$

$-4.4455250$

$-4.8433400$

$-4.2180410$

$-3.1594010$

$-2.9458420$

$-2.2906960$

$-2.4391780$

$-6.4407770$

$-4.5274110$

$-4.2517840$

$-4.6837380$

$-5.8234410$

$-6.1723080$

$-6.6304410$

$-5.4920990$

$-6.4100810$

$-0.8395270$

0.3802160

$-0.9940600$

0.3390020

1.3461710
1.6352080
\end{abstract}

-1.7448210
-2.2758660
0.1637550
-0.7831460
0.5166490
0.8544410
0.0488100
-0.6351450
0.7275600
0.8495350
0.5860680
1.9281350
1.0743660
-1.3116590
-1.0077600
-1.2669150
-1.2479030
-1.7904030
-2.4520020
-2.4245850
1.3564620
1.7419180
3.0428040
2.0517870
1.4796300
1.7784630
0.4082560
2.2888720
3.0281910
1.6326390
3.5669380
1.1749940
4.0469500
4.7602100
4.6158480
3.2685190
3.5729050
3.4762260
0.4172460
-0.1132120
4.6683530
4.0052140
6.1644620
6.9230480
6.1955880
4.7959880

3. 1833460

$-0.5994310$

$-1.6756440$

$-2.3182410$

$-0.9341550$

$-2.9633150$

$-3.7761700$

$-2.7951630$

$-3.3325500$

$-4.3416900$

$-3.3188520$

$-2.5674160$

$-1.3676640$

$-2.3907950$

$-3.4123250$

$-1.7299120$

$-1.9912110$

$-2.8058910$

$-1.1190750$

1.4894640

2.8297610

2. 2245950

1. 1623080

3. 9145720

4.8935270

3.9614760

3. 5677140

4.3509440

3. 4863650

1.9482240

3. 0472480

2. 4202340

3. 2079060

1.4957010

2. 8121930

3.8078830

2. 0995940

0.6108240

0.8672740

$-1.1192570$

$-1.3501380$

$-1.3426700$

$-1.3456980$

$-2.2434160$

$-1.6878640$ 


$\begin{array}{lrrr}\mathrm{H} & 0.1803370 & 7.9546270 & -1.6856830 \\ \mathrm{H} & -1.4893270 & 6.3219180 & -2.3146970 \\ \mathrm{H} & -1.6716660 & 6.5755030 & -0.5824570 \\ \mathrm{H} & 0.9298290 & 6.1102670 & -3.2590400 \\ \mathrm{H} & 2.2839080 & 6.7608070 & -2.3203370 \\ \mathrm{H} & 2.2738530 & 4.2366940 & -2.3768600 \\ \mathrm{H} & 2.2205460 & 4.8969690 & -0.7592360 \\ \mathrm{H} & 0.7402050 & 6.9707180 & -0.3223430 \\ \mathrm{C} & 2.1667260 & 1.7265880 & -2.8178730 \\ \mathrm{C} & 3.3500290 & 1.0015620 & -3.0558120 \\ \mathrm{C} & 1.3604920 & 2.2888970 & -3.9798100 \\ \mathrm{C} & 2.1638660 & 2.4326570 & -5.2784000 \\ \mathrm{C} & 2.9452900 & 1.1467270 & -5.5693280 \\ \mathrm{C} & 3.9579370 & 0.8967920 & -4.4453050 \\ \mathrm{H} & 1.4826380 & 2.6749280 & -6.1043290 \\ \mathrm{H} & 0.5184840 & 1.5993660 & -4.1561900 \\ \mathrm{H} & 0.9026630 & 3.2450910 & -3.7136450 \\ \mathrm{H} & 2.2419950 & 0.3024560 & -5.6291440 \\ \mathrm{H} & 3.4651980 & 1.2118620 & -6.5339280 \\ \mathrm{H} & 4.4308940 & -0.0876200 & -4.5595220 \\ \mathrm{H} & 4.7665740 & 1.6406220 & -4.5323550 \\ \mathrm{H} & 2.8727190 & 3.2694070 & -5.1820120 \\ \mathrm{C} & -1.3773710 & -3.5977510 & -0.6055750 \\ \mathrm{O} & -0.3222630 & -2.8683920 & -0.8674650 \\ \mathrm{C} & -1.8330600 & -4.4515670 & -1.7469220 \\ \mathrm{C} & -2.9472710 & -5.2886020 & -1.5739390 \\ \mathrm{C} & -1.1960000 & -4.3988270 & -2.9982410 \\ \mathrm{C} & -3.4195400 & -6.0600840 & -2.6346940 \\ \mathrm{H} & -3.4372260 & -5.3176220 & -0.6051500 \\ \mathrm{C} & -1.6736620 & -5.1684910 & -4.0594200 \\ \mathrm{H} & -0.3383660 & -3.7478170 & -3.1345960 \\ \mathrm{C} & -2.7850710 & -5.9994000 & -3.8808000 \\ \mathrm{H} & -4.2837770 & -6.7050960 & -2.4932960 \\ \mathrm{H} & -1.1800090 & -5.1191340 & -5.0273110 \\ \mathrm{H} & -3.1563120 & -6.5975020 & -4.7103040 \\ \mathrm{H} & 0.0074190 & -2.2287320 & -0.1072260 \\ \mathrm{O} & -1.9967480 & -3.6116750 & 0.4728880 \\ \mathrm{C} & -1.4495250 & -2.2563360 & 2.9757770 \\ \mathrm{C} & -2.3861480 & -1.9244830 & 1.8916170 \\ \mathrm{H} & -0.4006010 & -2.2970420 & 2.6817870 \\ \mathrm{H} & -2.0411970 & -1.2511530 & 1.1148400 \\ \mathrm{O} & -1.8236890 & -0.9281140 & 3.4574290 \\ \mathrm{H} & -1.1630480 & -0.2548990 & 3.0154620 \\ \mathrm{C} & -3.3742660 & -3.3446210 & 4.2290080 \\ \mathrm{C} & -4.1745260 & -3.3909020 & 2.9186500 \\ \mathrm{C} & -3.8421470 & -2.1944150 & 2.0129550\end{array}$

$\begin{array}{lll}\mathrm{C} & -1.8641860 & -3.3530120 \\ \mathrm{H} & -3.6301010 & -4.2054390 \\ \mathrm{H} & -5.2521700 & -3.3977110 \\ \mathrm{H} & -4.2951670 & -1.2670970 \\ \mathrm{H} & -1.5806600 & -4.3075500 \\ \mathrm{H} & -1.2787070 & -3.2454950 \\ \mathrm{H} & -3.6330660 & -2.4373430 \\ \mathrm{H} & -3.9317470 & -4.3163110 \\ \mathrm{H} & -4.2461060 & -2.3096300 \\ \mathrm{H} 54 & & \end{array}$
3.9375700
4. 8584550
3. 1219130
2.3925310
3.4725490
4.8591540
4.7909960
2. 3800220
1.0025060

$\operatorname{TS} 173(\mathrm{~S}, \mathrm{~S}) \mathrm{G}[\mathrm{B} 97-\mathrm{D} / \mathrm{def} 2-\mathrm{TZVP}]=-3541.572233$

$\begin{array}{lrcr}\text { TS173(S, S }(\mathrm{G}[\mathrm{B} 97-\mathrm{D} / \mathrm{def}-\mathrm{TZVP}]=-3541.572233 \\ \mathrm{P} & 0.3986560 & 0.2719530 & 0.9718750 \\ \mathrm{O} & -0.2275250 & 0.7675700 & 2.2455430 \\ \mathrm{O} & 0.7066780 & -1.1957600 & 0.8464070 \\ \mathrm{O} & 1.7096530 & 1.2008880 & 0.7418580 \\ \mathrm{O} & -0.5319150 & 0.6085690 & -0.3394110 \\ \mathrm{C} & 2.2853310 & 1.1895230 & -0.5322300 \\ \mathrm{C} & 1.6277820 & 1.8937610 & -1.5563180 \\ \mathrm{C} & 3.4737440 & 0.4791800 & -0.7335910 \\ \mathrm{C} & 3.9791070 & 0.4413110 & -2.0417740 \\ \mathrm{H} & 4.8966550 & -0.1132950 & -2.2291080 \\ \mathrm{C} & -0.7086260 & 1.9446880 & -0.7001360 \\ \mathrm{C} & -1.9684590 & 2.5297980 & -0.5290180 \\ \mathrm{C} & 0.3760680 & 2.6438560 & -1.2529900 \\ \mathrm{C} & -2.0871920 & 3.8842690 & -0.8474360 \\ \mathrm{H} & -3.0577030 & 4.3621930 & -0.7216490 \\ \mathrm{C} & 4.1884400 & -0.2143080 & 0.3697360 \\ \mathrm{C} & -3.1501050 & 1.7397140 & -0.0955000 \\ \mathrm{C} & 4.6961720 & 0.5074000 & 1.4655830 \\ \mathrm{C} & 4.5872780 & 1.9975940 & 1.6941880 \\ \mathrm{C} & 5.9220470 & 0.7789190 & 3.5602750 \\ \mathrm{C} & 5.4352760 & -0.1439710 & 2.4713720 \\ \mathrm{C} & 3.9021300 & 2.2405570 & 3.0665760 \\ \mathrm{H} & 3.8873100 & 3.3209430 & 3.2630120 \\ \mathrm{H} & 2.8653480 & 1.8959800 & 3.0184540 \\ \mathrm{C} & 4.6902670 & 1.4900370 & 4.1848890 \\ \mathrm{H} & 5.0359600 & 2.1907510 & 4.9568230 \\ \mathrm{H} & 4.0503640 & 0.7454160 & 4.6729960 \\ \mathrm{H} & 6.4798810 & 0.2262190 & 4.3261100 \\ \mathrm{H} & 4.0260230 & 2.4928920 & 0.8981760 \\ \mathrm{C} & 6.8212990 & 1.8680000 & 2.9096890 \\ \mathrm{H} & 7.1215420 & 2.5882330 & 3.6824980 \\ \mathrm{H} & 7.7329990 & 1.4032610 & 2.5156990 \\ \mathrm{C} & 6.0270520 & 2.5799110 & 1.7708310 \\ \mathrm{H} & 5.9610080 & 3.6594140 & 1.9614950 \\ \mathrm{H} & 6.5277990 & 2.4436120 & 0.8046600\end{array}$




$\begin{array}{rrr}4.4252760 & -1.6003670 & 0.3136320 \\ 3.9259610 & -2.5611560 & -0.7414180 \\ 5.3570140 & -3.7201110 & 1.0918370 \\ 5.1798480 & -2.2391050 & 1.3163000 \\ 5.1553580 & -3.2431980 & -1.4003900 \\ 4.8017630 & -3.9826290 & -2.1314720 \\ 5.7450270 & -2.4950120 & -1.9442670 \\ 6.0219030 & -3.9304990 & -0.2976680 \\ 6.1101030 & -5.0080830 & -0.4900400 \\ 7.0349790 & -3.5111660 & -0.2840020 \\ 5.9697140 & -4.1691930 & 1.8830770 \\ 3.3171020 & -2.0540020 & -1.4943320 \\ 3.9500110 & -4.3764940 & 1.0472610 \\ 4.0623340 & -5.4463230 & 0.8239190 \\ 3.4749600 & -4.2901220 & 2.0318980 \\ 3.0877080 & -3.6683170 & -0.0417550 \\ 2.7540950 & -4.3872920 & -0.8016050 \\ 2.1997170 & -3.2137220 & 0.4020660 \\ 5.6835780 & -1.5146100 & 2.3961120 \\ 6.2662690 & -2.0134310 & 3.1716230 \\ -4.8180420 & 0.0152220 & -0.5698070 \\ -5.2596970 & -0.9857770 & -1.6091190 \\ -3.1531900 & 0.3644230 & -2.3081610 \\ -3.6663820 & 0.7355850 & -0.9356960 \\ -5.5561500 & -0.2095470 & -2.9237300 \\ -5.8163460 & -0.9306550 & -3.7099940 \\ -6.4213830 & 0.4469460 & -2.7739060 \\ -4.2971300 & 0.6189670 & -3.3296130 \\ -3.9478220 & 0.3271630 & -4.3288980 \\ -4.5300650 & 1.6900420 & -3.3579640 \\ -2.2669340 & 0.9431210 & -2.5810750 \\ -6.1502080 & -1.5340250 & -1.2778070 \\ -2.8279610 & -1.1512410 & -2.3254530 \\ -2.5126930 & -1.4360300 & -3.3375860 \\ -1.9858140 & -1.3383390 & -1.6572790 \\ -4.0878730 & -1.9637700 & -1.8959910 \\ -4.3896410 & -2.6604500 & -2.6884060 \\ -3.8758380 & -2.5632450 & -1.0048700 \\ -4.9642620 & 1.2809320 & 1.4730310 \\ -5.5461210 & 1.6936010 & 2.8017470 \\ -3.4318270 & 3.0540240 & 2.1347080 \\ -3.8121640 & 2.0080800 & 1.1133510 \\ -4.4496560 & 1.5151210 & 3.8883850 \\ -4.8539750 & 1.8355360 & 4.8577450 \\ -4.1808330 & 0.4558740 & 3.9748850 \\ -3.1970760 & 2.3590820 & \\ -3.5032660\end{array}$

-3.0070880
-2.3047510
-2.5361850
-6.4358370
-4.6386640
-4.3874390
-4.8211860
-5.9020770
-6.2591130
-6.7189490
-5.4700050
-6.3664680
-1.0065360
0.2410260
-1.2309080
0.0658090
1.1004680
1.4545930
-0.1392010
-1.7278990
-1.9305310
0.6843170
2.0127810
2.1013050
2.0521320
0.4659830
2.1390390
1.4505710
1.2889000
2.6242670
3.7818590
3.3196860
2.0615230
0.8868220
2.9178270
4.1106010
0.4769960
0.5407060
2.4827670
4.6421920
-1.2446250
-0.2212200
-1.6603310
-2.7668450
-0.9882720
-3.1981300

1.7333050

3.6032840

1. 0999920

4. 0190640

4.7702730

4. 5479550

3. 2058690

3. 5340230

3. 3549990

0.2892870

$-0.2651290$

4.6468040

4. 0277820

6. 1218240

6.9393080

6.2235130

4.8576210

7. 9493000

. 2123710

6. 5338140

0842390

6. 8252810

. 3037250

5.0149220

7.0430070

1.7941280

2. 3463040

1.2305760

0.4820640

0.9611930

1. 0642380

3. 1548770

1. 6709290

. 6179860

1.9531660

2. 7778370

0.5191120

$-0.5942070$

0.2866790

$-3.6374420$

$-2.8788480$

$-4.5712280$

$-5.4118830$

$-4.5974100$

$-6.2639540$
4.2575820

3.4392600

1.8328790

3. 0460380

2. 3012810

3. 0613810

1. 3579310

2.7285440

3.7137500

2. 0124930

0.6333280

0.9139990

$-1.3027040$

$-1.5123080$

$-1.5950830$

$-1.6293240$

$-2.5040810$

$-1.9026960$

$-2.0072570$

$-2.5746930$

$-0.8556800$

$-2.6061510$

$-2.5861220$

$-0.9899140$

$-0.6096820$

$-2.8636680$

$-4.0889170$

$-5.1504520$

$-5.4432350$

$-4.5297120$

$-3.0990310$

$-4.5205770$

$-6.0714250$

$-6.4917440$

$-4.8776690$

$-3.8396070$

$-4.7796150$

$-5.2851610$

$-4.6136190$

$-0.4573820$

$-0.7592840$

$-1.5500600$

-1.3479980

$-2.7838810$

$-2.3635360$ 


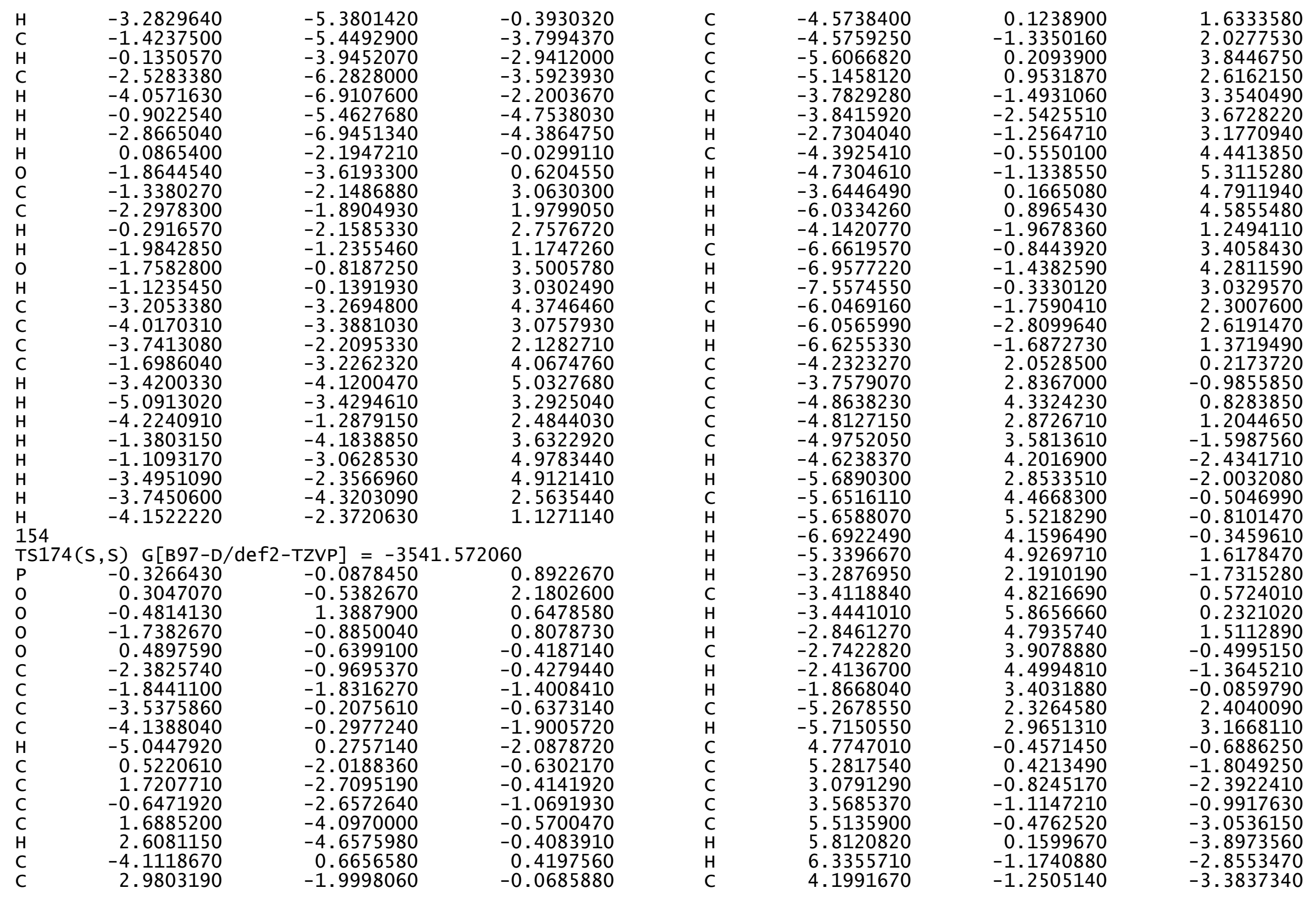




\begin{tabular}{lr}
$\mathrm{H}$ & 3.8668290 \\
$\mathrm{H}$ & 4.3586180 \\
$\mathrm{H}$ & 2.1524720 \\
$\mathrm{H}$ & 6.2108930 \\
$\mathrm{C}$ & 2.8671800 \\
$\mathrm{H}$ & 2.5884300 \\
$\mathrm{H}$ & 2.0340030 \\
$\mathrm{C}$ & 4.1796340 \\
$\mathrm{H}$ & 4.5272530 \\
$\mathrm{H}$ & 4.0096040 \\
$\mathrm{C}$ & 4.8443420 \\
$\mathrm{C}$ & 5.4148130 \\
$\mathrm{C}$ & 3.1907150 \\
$\mathrm{C}$ & 3.6334810 \\
$\mathrm{C}$ & 4.3557890 \\
$\mathrm{H}$ & 4.7514390 \\
$\mathrm{H}$ & 4.1704710 \\
$\mathrm{C}$ & 3.0357950 \\
$\mathrm{H}$ & 2.8053250 \\
$\mathrm{H}$ & 2.1912390 \\
$\mathrm{H}$ & 2.2504670 \\
$\mathrm{H}$ & 6.3524430 \\
$\mathrm{C}$ & 4.3224860 \\
$\mathrm{H}$ & 4.0268120 \\
$\mathrm{H}$ & 4.4472850 \\
$\mathrm{C}$ & 5.6517740 \\
$\mathrm{H}$ & 5.9968750 \\
$\mathrm{H}$ & 6.4438080 \\
$\mathrm{C}$ & 5.4157890 \\
$\mathrm{H}$ & 6.3555180 \\
$\mathrm{C}$ & -0.6655120 \\
$\mathrm{C}$ & -1.9606280 \\
$\mathrm{C}$ & -1.9438030 \\
$\mathrm{C}$ & -0.6712110 \\
$\mathrm{C}$ & 0.5570760 \\
$\mathrm{C}$ & 0.5169670 \\
$\mathrm{H}$ & -2.8420950 \\
$\mathrm{H}$ & -2.1488630 \\
$\mathrm{H}$ & -2.7975280 \\
$\mathrm{H}$ & -0.6201700 \\
$\mathrm{H}$ & -0.6817900 \\
$\mathrm{H}$ & 1.4803310 \\
$\mathrm{H}$ & 0.6234240 \\
$\mathrm{H}$ & -1.9683250 \\
$\mathrm{C}$ & -4.5883740 \\
$\mathrm{C}$ & \\
& \\
\hline
\end{tabular}

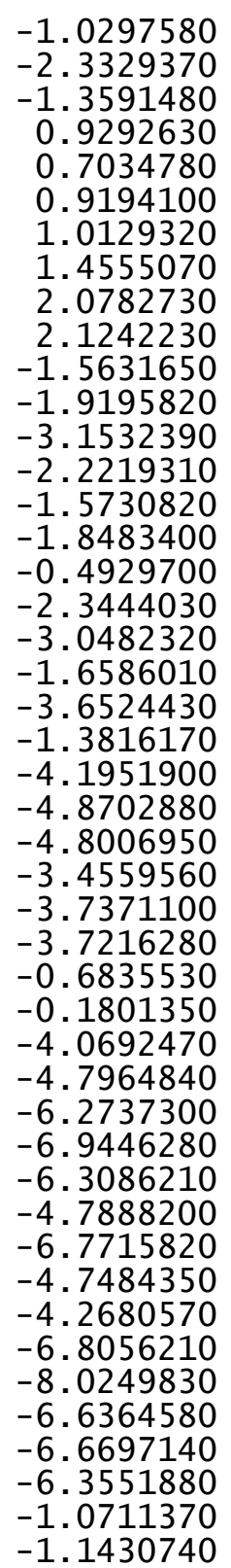
$-4.4065630$
$-3.3115000$
$-2.6173270$
$-1.5181010$
$-2.5452660$
$-3.5848700$
$-1.9128740$
$-2.1650800$
$-2.9992260$
$-1.3146500$
1. 4485500
2. 7981570
2. 2601460
1. 1557530
3.8810060
4.8677680
3.8844520
3.5773640
4. 3881960
3.4836590
2. 0125710
2. 9860490
2.4815040
3. 2951760
1.5755700
2.8352930
3.8391120
2. 1251470
0.5298570
0.7606830
$-1.1541380$
$-1.4740000$
$-1.0597620$
$-1.5885210$
$-0.9260460$
$-0.8984220$
$-1.4469530$
$-2.5541490$
$-1.0009240$
$-2.6792430$
$-1.3952980$
$-1.4233240$
0.1127240
0.0371190
$-2.9210050$
$-4.2991280$

-4.2624150
-2.8565540
-1.8235520
-2.4323720
-3.5838440
-4.7267540
-2.4641760
-1.3690910
-5.1981200
-4.9101980
-2.9411590
-1.0087040
1.6580920
0.6005280
2.1551040
3.2886520
1.5332790
3.7940440
3.7659030
2.0434860
0.6608990
3.1732050
4.6728650
1.5607150
3.5695740
0.2287690
2.2463840
1.6575810
2.5707070
0.6086740
2.1891720
1.9828550
1.2863640
3.6364870
4.4273240
4.0386160
2.1256740
3.9338660
5.5059860
4.4540020
1.8785810
1.5444670
3.8611270
4.2173180
4.4394840
$-4.7600060$

$-4.7787480$

$-3.8834690$

$-2.6803000$

$-5.0131780$

$-5.7517260$

$-5.8034010$

$-4.4941970$

$-4.2966640$

$-4.0585420$

$-4.4584340$

$-3.5969080$

$-0.8000030$

$-1.0702700$

$-1.9504110$

$-1.7751350$

$-3.2091970$

$-2.8412300$

$-0.8000440$

$-4.2751760$

$-3.3467630$

$-2.6984140$

$-5.2484410$

$-4.9272650$

$-0.2946440$

0.2952050

2.8092060

1.7244800

2. 5217640

0.9624670

3. 3077790

2.8833960

4.0288340

2.7114540

1.8301690

3.7491950

4.6439850

2. 9064880

2. 2251650

3. 2708570

4.6772560

4.6009770

2. 1593640

0.8146590 


$\begin{array}{lrrr}\text { TS175 (S , S }) \text { G[B97-D/def2-TZVP] }=-3541.571915 \\ \text { P } & -0.3180520 & -0.0825470 & 0.8935350 \\ \text { O } & 0.3219350 & -0.5213780 & 2.1812550 \\ \text { O } & -0.4644320 & 1.3919400 & 0.6320350 \\ \text { O } & -1.7362590 & -0.8695660 & 0.8323330 \\ \text { O } & 0.4811060 & -0.6578840 & -0.4179090 \\ \mathrm{C} & -2.3857190 & -0.9706560 & -0.3997760 \\ \mathrm{C} & -1.8628780 & -1.8622480 & -1.3532160 \\ \mathrm{C} & -3.5303320 & -0.1961040 & -0.6227500 \\ \mathrm{C} & -4.1346440 & -0.3007110 & -1.8832290 \\ \mathrm{H} & -5.0318890 & 0.2828950 & -2.0807130 \\ \mathrm{C} & 0.5110860 & -2.0395440 & -0.6099630 \\ \mathrm{C} & 1.7154010 & -2.7234250 & -0.4047030 \\ \mathrm{C} & -0.6656440 & -2.6872820 & -1.0180950 \\ \mathrm{C} & 1.6912280 & -4.1107380 & -0.5647250 \\ \mathrm{H} & 2.6172280 & -4.6650330 & -0.4189920 \\ \mathrm{C} & -4.0957410 & 0.6982850 & 0.4212630 \\ \mathrm{C} & 2.9739190 & -2.0084280 & -0.0653450 \\ \mathrm{C} & -4.5627250 & 0.1771110 & 1.6422110 \\ \mathrm{C} & -4.5755850 & -1.2760160 & 2.0578150 \\ \mathrm{C} & -5.5984210 & 0.3016950 & 3.8504620 \\ \mathrm{C} & -5.1304550 & 1.0244870 & 2.6121260 \\ \mathrm{C} & -3.7855130 & -1.4200470 & 3.3874090 \\ \mathrm{H} & -3.8509720 & -2.4644160 & 3.7212030 \\ \mathrm{H} & -2.7312590 & -1.1924180 & 3.2086290 \\ \mathrm{C} & -4.3907690 & -0.4626200 & 4.4602300 \\ \mathrm{H} & -4.7343510 & -1.0267900 & 5.3377600 \\ \mathrm{H} & -3.6385300 & 0.2584840 & 4.8014350 \\ \mathrm{H} & -6.0215330 & 1.0024940 & 4.5805410 \\ \mathrm{H} & -4.1454760 & -1.9234850 & 1.2896120 \\ \mathrm{C} & -6.6604500 & -0.7507550 & 3.4246600 \\ \mathrm{H} & -6.9627680 & -1.3293000 & 4.3079950 \\ \mathrm{H} & -7.5512730 & -0.2383980 & 3.0420440 \\ \mathrm{C} & -6.0497350 & -1.6861460 & 2.3349040 \\ \mathrm{H} & -6.0665780 & -2.7319580 & 2.6694490 \\ \mathrm{H} & -6.6263980 & -1.6249890 & 1.4041450 \\ \mathrm{C} & -4.2072570 & 2.0829310 & 0.1986310 \\ \mathrm{C} & -3.7262480 & 2.8456230 & -1.0151800 \\ \mathrm{C} & -4.8265800 & 4.3749530 & 0.7742430 \\ \mathrm{C} & -4.7837540 & 2.9208850 & 1.1727090 \\ \mathrm{C} & -4.9378150 & 3.5883940 & -1.6417160 \\ \mathrm{H} & -4.5808440 & 4.1951170 & -2.4847060 \\ \mathrm{H} & -5.6547340 & 2.8589760 & -2.0380870 \\ \mathrm{C} & -5.6120740 & 4.4926200 & -0.5617720 \\ \mathrm{H} & -5.6149670 & 5.5429540 & -0.8829450 \\ \mathrm{H} & -6.6540000 & 4.1915650 & -0.3997870\end{array}$

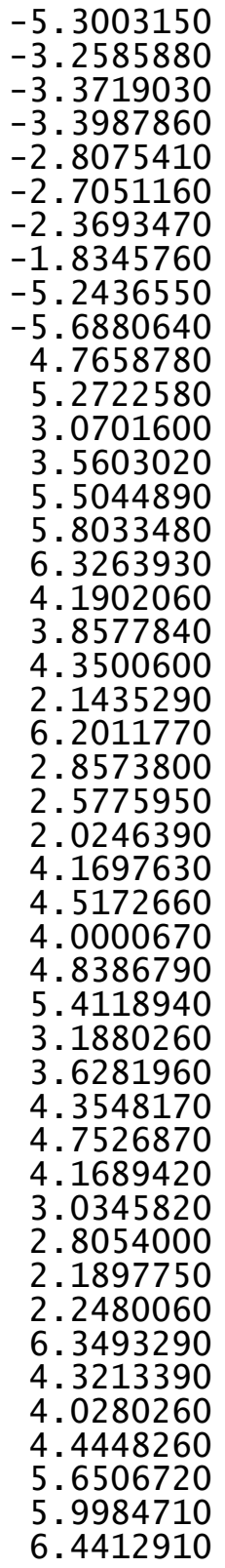

4.9841320

2. 1855850

4.8528480

5.8910140

4.8381080

3. 9177560

4.4931990

3. 4138950

2. 3952280

3.0477650

$-0.4676680$

0.4025010

$-0.8490940$

$-1.1285590$

$-0.5049670$

0.1244600

$-1.2011560$

$-1.2820030$

$-1.0686780$

$-2.3638330$

$-1.3859380$

0.9131070

0.6775190

0.8852840

0.9913610

1.4332690

2. 0482480

2. 1100820

$-1.5607300$

$-1.9107850$

-3. 1489940

$-2.2223410$

$-1.5614740$

$-1.8336360$

$-0.4815280$

$-2.3344930$

$-3.0348850$

$-1.6492290$

$-3.6504200$

$-1.3710880$

$-4.1888270$

$-4.8605030$

$-4.7982930$

$-3.4467780$

-3.7234360
-3.7144810
1.5537680

$-1.7501870$

0.5122270

0.1540850

1.4522600

$-0.5428250$

$-1.4159260$

$-0.1180980$

2. 3796150

3. 1322420

$-0.6960150$

$-1.8190910$

$-2.3965110$

$-0.9942680$

$-3.0605840$

-3. 9092660

$-2.8563190$

$-3.3850790$

$-4.4094550$

$-3.3050880$

$-2.6167000$

$-1.5363200$

$-2.5611750$

$-3.6021680$

$-1.9304510$

$-2.1880020$

$-3.0279810$

$-1.3438840$

1.4476910

2. 7978160

2. 2691160

1.1598010

3. 8816000

4.8683390

3. 8821240

3. 5830460

4. 3972280

3. 4874270

2.0250540

2. 9817450

2. 4930100

3. 3103720

1. 5895450

2. 8405900

3.8447260

2. 1296230 


5.4082210
6.3479640
0.5203150
-0.6761320
0.5904780
-0.7740850
-1.7963730
-1.9835160
-0.6805590
1.0270080
1.2869520
-1.4381860
-2.7630220
-2.6492040
-2.4992470
-1.1209030
-3.6014590
-4.2178520
-4.3637650
-3.0074460
-1.9126550
-2.4601940
-3.5707010
-4.7888840
-2.6311920
-1.4798200
-5.1901630
-5.0813900
-3.1577570
-1.0994360
1.6653480
0.6123430
2.1570540
3.2852470
1.5351620
3.7852060
3.7625400
2.0399380
0.6670330
3.1642640
4.6598600
1.5571240
3.5563530
0.2427230
2.2533070
1.6737360

$-0.6858400$

$-0.1805160$

$-4.8098060$

$-4.0997580$

$-6.3232700$

$-7.0153170$

$-6.2881330$

$-4.8487930$

$-8.0695960$

$-6.5613810$

$-6.7224130$

$-6.2781040$

$-6.8081070$

$-4.3017490$

$-4.8739670$

$-6.9912720$

$-1.1096080$

$-1.2160690$

$-2.7026870$

$-3.4584380$

$-2.6666060$

$-1.8927720$

$-0.7151710$

$-2.7844560$

$-3.6672650$

$-1.9305010$

$-0.7092900$

$-3.1622510$

$-4.4290950$

$-3.3340100$

3.5736980

2.8429300

4.3867510

5.2057980

4.3186700

5.9455110

5.2472340

5.0574240

3.6812080

5.8713980

6.5769210

4.9974020

6.4457500

2.2521160

3.6193880

2.3352870

0.5233070
0.7498240
-0.8769940
-1.0941730
-1.0134950
-0.9059880
-1.7867920
-1.2941870
-1.1970820
-1.9971770
-0.2640710
-2.8277100
-1.7774390
-1.9632610
-0.3200270
0.1378650
-2.8854550
-4.2581290
-4.6515000
-4.5734200
-3.8067290
-2.6288630
-4.9967550
-5.6596960
-5.5827530
-4.5023820
-4.2820520
-3.9596160
-4.0861670
-3.5111290
-0.8341130
-1.0996780
-1.9897570
-1.8198660
-3.2479520
-2.8906270
-0.8451250
-4.3185490
-3.3814820
-4.1428990
-2.7518990
-5.2912730
-4.9795610
-0.3191700
0.2609130
2.7832710

\begin{abstract}
2.5843070
0.6237780

2. 2013950

2.0037410

1. 3066150

3.6541570

4.4402060

4.0521450

2. 1420960

3.9511920

5.5196800

4.4708980

1.8901480

1. 5646030

3.8838040

4.2248120

4.4497880
\end{abstract}

154

TS176(S,S) G[B97-D/def2-TZVP] $=-3541.571777$

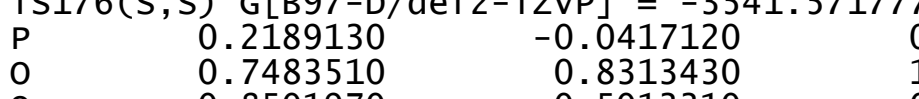

$-0.8501970$

0.5013310

$-0.2150560$

1.3931800

$-0.3223620$

0.8592620

$-1.5975570$

$-1.6521030$

$-2.6313680$

2.4293020

3.6605270

2. 1772150

4.6484010

5.6206570

$-2.8474210$

3.8836480

$-3.2330720$

$-2.4782440$

$-4.7211970$

$-4.4540530$

$-2.2016340$

$-1.7121150$

$-1.5102050$

$-3.5460490$

$-3.7106960$

$-3.5340420$

$-1.4307180$

$-0.5271130$

$-2.5751830$

$-3.2009130$

$-3.0299940$

$-4.1697730$

$-4.5474690$

$-1.2732720$

$-0.6451370$

$-2.6125250$

$-1.4185540$

$-0.9652900$

$-2.3125770$

0.7835190

$-2.1316250$

$-2.5915490$

$-1.4110640$

$-1.5056430$

$-1.3568310$

$-1.6989660$

$-0.6830690$

$-0.6356250$

$-0.5909100$

0.3938150

0.3053350

1.4112960

6072110

1.0294740

$-0.7121370$

0.2355410

$-0.1998160$

$-0.1193960$

$-0.9268550$

$-1.2165570$

$-0.1508600$

0.0737660

0.1847360

0.6902650

0.8756840

0.2489580

$-0.2733270$

1. 5881940

2.8126770

3. 3850670

1.9039200

3.7138770

4.6354810

3.2002730

4.0423370

5.1273850

3.6644380 


$\begin{array}{rrr}-5.6758740 & -0.9080790 & 3.5829700 \\ -1.5399780 & -3.0858260 & 2.5495490 \\ -4.7309270 & -2.8509180 & 3.9710210 \\ -4.8561300 & -2.7905500 & 5.0604320 \\ -5.5879220 & -3.4032760 & 3.5674440 \\ -3.3923630 & -3.5656950 & 3.6075830 \\ -2.8639240 & -3.8823380 & 4.5165460 \\ -3.5823420 & -4.4616360 & 3.0043620 \\ -3.6977640 & -1.8251770 & -0.7636000 \\ -3.4416900 & -1.8550520 & -2.2541080 \\ -5.7156460 & -0.7628470 & -1.6396720 \\ -4.9211950 & -1.2098940 & -0.4378370 \\ -4.5949810 & -2.6442700 & -2.9329370 \\ -4.4500800 & -2.6145040 & -4.0211450 \\ -4.5573660 & -3.6954520 & -2.6220280 \\ -5.9649690 & -2.0036010 & -2.5431870 \\ -6.5133970 & -1.6871210 & -3.4404130 \\ -6.5919930 & -2.7256050 & -2.0063830 \\ -6.6676000 & -0.3089350 & -1.3379630 \\ -2.4760940 & -2.3074940 & -2.4912980 \\ -4.8620190 & 0.2473250 & -2.4542490 \\ -5.4064590 & 0.5068440 & -3.3722490 \\ -4.7323000 & 1.1663880 & -1.8759980 \\ -3.4836120 & -0.3966650 & -2.7898500 \\ -3.3218670 & -0.4197850 & -3.8759320 \\ -2.6634450 & 0.1690320 & -2.3394750 \\ -5.3052410 & -1.0586470 & 0.8942390 \\ -6.2596270 & -0.5931130 & 1.1441650 \\ 3.9212740 & 2.6028060 & -1.9101870 \\ 3.7385730 & 2.9201310 & -3.3739340 \\ 3.3912260 & 0.4090450 & -2.8096420 \\ 3.7131710 & 1.2471640 & -1.5927600 \\ 4.7569060 & 2.0658840 & -4.1808140 \\ 4.5846090 & 2.2272730 & -5.2533800 \\ 5.7765650 & 2.3988360 & -3.9535160 \\ 4.5707200 & 0.5609800 & -3.8123290 \\ 4.3498760 & -0.0324920 & -4.7093850 \\ 5.4850900 & 0.1562480 & -3.3623730 \\ 3.2524070 & -0.6440950 & -2.5543450 \\ 3.8940650 & 3.9886230 & -3.5684300 \\ 2.1144730 & 0.9699400 & -3.4940080 \\ 1.9398900 & 0.4067410 & -4.4204720 \\ 1.2518230 & 0.8108530 & -2.8422820 \\ 2.3123280 & 2.4852470 & -3.8053920 \\ 2.1885480 & 2.6839810 & -4.8783290 \\ 1.5699570 & 3.0882290 & -3.2711610\end{array}$

4.5035530
4.9760460
4.5135690
4.2765730
3.9041710
4.2806430
2.9896000
3.6173380
3.8354040
2.5675980
4.3005570
5.1634470
5.9944930
6.1706230
6.6592130
6.2771100
6.6098840
7.0681240
4.3160600
4.4960080
3.1636590
2.8461610
3.7527770
5.2187000
5.5067580
4.4125560
3.5480020
2.9760420
1.7886310
5.4038750
5.8989760
6.4632390
5.6257960
3.5366560
0.7646230
2.0045960
1.6875450
0.6011760
-0.7017280
-0.5084710
2.6017640
2.4973230
2.7286310
0.9412110
0.4240370
-1.4034030

3.0593990

3. 9029070

1.4315160

1.7099220

3.8595140

4.4149150

4. 3503280

2. 3739790

2.1922080

. 1211230

0.3919880

4.9363420

1.7786910

1.6272400

1.0975760

3. 2621160

3. 8412980

3. 3152570

3. 5099100

4.5557020

$-3.3306400$

$-4.6924910$

$-5.0444600$

$-4.8820630$

$-3.4092770$

$-2.7233030$

$-6.0723320$

$-5.4779360$

$-4.7195510$

$-5.5020880$

$-5.2302680$

- 3.3098170

$-2.8589660$

$-4.3779800$

$-4.2934560$

$-4.8533870$

$-5.5669210$

$-6.6201300$

$-5.9308070$

$-4.7908690$

$-6.0280970$

$-5.5843900$

$-4.0443490$

$-7.3024670$

$-7.2258740$

$-6.6622070$
0.3794410

1. 5374180

2. 1822900

0.7140000

2.6594920

3. 5292880

2. 3125990

3. 0323840

4.0932140

2. 8608010

2. 4404340

1.2203630

2. 5000220

3.5732330

1.9547630

2. 0996320

2. 9711890

1. 3421250

$-0.9263730$

$-1.1796670$

0.8995610

1. 4934310

2. 6802530

2. 2622750

1.9420410

1.1371510

3. 0059420

0.7370950

1.7832500

1. 3720950

3. 0500750

1. 4100910

2.8887040

3. 5285290

$-1.0951380$

$-1.7727720$

$-3.0939620$

$-2.8511300$

$-2.4247600$

$-1.4355940$

$-3.4891080$

$-1.1179090$

1.9268450

$-2.0575330$

$-3.7490690$

$-2.0000120$ 


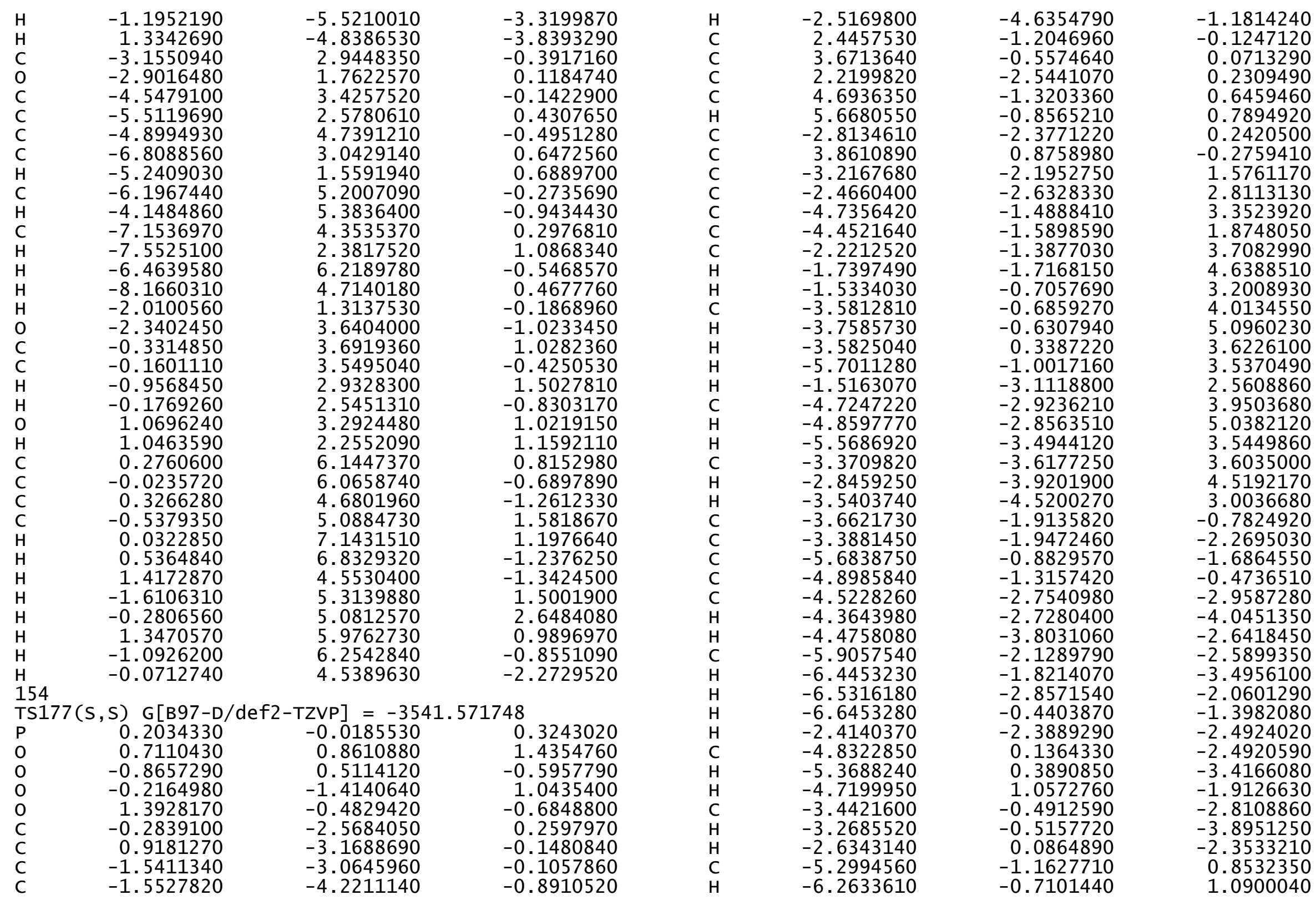




\begin{tabular}{|c|c|c|}
\hline $\begin{array}{l}0 \\
0 \\
0 \\
0 \\
0 \\
0 \\
0 \\
0 \\
0 \\
0 \\
0 \\
0 \\
0 \\
0 \\
0 \\
0 \\
0 \\
0 \\
0 \\
0 \\
0 \\
0 \\
0 \\
0 \\
0 \\
0 \\
0 \\
0 \\
0 \\
0 \\
0 \\
0 \\
0 \\
0 \\
0 \\
0\end{array}$ & $\begin{array}{r}2.6977780 \\
3.0124920 \\
0.4942050 \\
1.3377800 \\
2.1792480 \\
2.3388300 \\
2.5315400 \\
0.6703340 \\
0.0743330 \\
0.2824660 \\
-0.5615160 \\
4.0839700 \\
1.0299250 \\
0.4635360 \\
0.8540470 \\
2.5486430 \\
2.7444200 \\
3.1370050 \\
3.1627530 \\
4.0139910 \\
1.5291230 \\
1.8077190 \\
3.9393100 \\
4.5013760 \\
4.4076960 \\
2.4456730 \\
2.2666700 \\
2.1670870 \\
0.4829110 \\
5.0530350 \\
1.9135550 \\
1.7635190 \\
1.2511940 \\
3.4047610 \\
3.9894930 \\
3.4799520 \\
3.6115130 \\
4.6615690 \\
-2.6318300 \\
-3.2429760 \\
-3.3763320 \\
-4.5766970 \\
-5.4162030 \\
-4.5722500 \\
-5.1799620 \\
-3.7383080\end{array}$ & $\begin{array}{l}-1.9095420 \\
-3.3711080 \\
-2.8063970 \\
-1.5924250 \\
-4.1884020 \\
-5.2591140 \\
-3.9694860 \\
-3.8208930 \\
-4.7168290 \\
-3.3812510 \\
-2.5496390 \\
-3.5665560 \\
-3.4766610 \\
-4.4013340 \\
-2.8152860 \\
-3.7898300 \\
-4.8617310 \\
-3.2496310 \\
0.3758880 \\
1.5330080 \\
2.1732210 \\
0.7088030 \\
2.6626260 \\
3.5315430 \\
2.3237290 \\
3.0332350 \\
4.0919360 \\
2.8684360 \\
2.4278310 \\
1.2184640 \\
2.4812580 \\
3.5527170 \\
1.9293060 \\
2.0835640 \\
2.9544870 \\
1.3204650 \\
-0.9273580 \\
-1.1800920 \\
1.0885750 \\
0.9189610 \\
1.7122130 \\
2.5739640 \\
1.8405600 \\
1.5937780 \\
2.8187360 \\
0.8967490\end{array}$ \\
\hline
\end{tabular}

6.2734850

4.6068070

3.9348970

2. 1863800

2.4813050

4.8229630

0.8685470

2. 1445540

1.8884210

0.8044920

$-0.5214970$

$-0.3842770$

2. 8244340

2. 6841480

2. 8162550

1. 1089970

0.6786560

$-1.2575880$

$-0.9442280$

1. 5560760

$-3.2265760$

$-2.9525350$

$-4.6342140$

$-5.5877800$

$-5.0103060$

$-6.8992020$

$-5.2965040$

$-6.3218170$

$-4.2668650$

$-7.2685900$

$-7.6346920$

$-6.6081960$

$-8.2921080$

$-2.0478050$

$-2.4202910$

$-0.4181290$

$-0.2383760$

$-1.0337960$

$-0.2385710$

0.9891480

0.9824710

0.1539860

$-0.1388510$

0.2329510

$-0.6476170$

$-0.1058530$
-2.6738050
-5.7592070
-6.3085200
-5.1386130
-4.3410380
-4.2211770
-4.2821470
-4.8305420
-5.6806080
-6.7223540
-6.0102630
-4.8242280
-6.1612090
-5.4537210
-3.9943130
-7.3175200
-7.4160850
-6.7139970
-5.6480380
-5.0366070
2.9038790
1.7225590
3.3483960
2.4683400
4.6594620
2.8983840
1.4522520
5.0865360
5.3294420
4.2067100
2.2120160
6.1032420
4.5402870
1.2963830
3.6250160
3.7063680
3.5685650
2.9372580
2.5646200
3.3282970
2.2901350
6.1680990
6.0873970
4.7078530
5.0988750
7.1622120

2. 2971700

0.8781940

2.4238050

1.0435880

2. 5688010

3. 5232490

$-1.0222380$

$-1.6446020$

$-2.8963070$

$-2.6004200$

$-2.3091680$

$-1.3667380$

$-3.2084660$

$-0.9177120$

$-1.8750160$

$-1.7260670$

$-3.4415950$

$-1.8965800$

$-3.2597830$

$-3.7243420$

$-0.3895990$

0.1132170

$-0.1559310$

0.3847900

$-0.4917360$

0.5852200

0.6315520

$-0.2852000$

$-0.9146420$

0.2526790

0.9991540

$-0.5445590$

0.4103490

$-0.1841730$

$-1.0029840$

1. 0557910

$-0.3967930$

1. 5269270

$-0.8035160$

1. 0532850

1.1885960

0.8486520

$-0.6576810$

$-1.2300830$

1.6103300

1.2316820 


\begin{tabular}{|c|c|c|c|c|c|c|c|}
\hline $\mathrm{H}$ & 0.4121750 & 6.8632440 & -1.2022780 & $c$ & -5.7413510 & -0.6409570 & -1.6241270 \\
\hline $\mathrm{H}$ & 1.3254370 & 4.5972680 & -1.3100880 & $\mathrm{C}$ & -4.9458880 & -1.1042050 & -0.4289970 \\
\hline $\mathrm{H}$ & -1.7231790 & 5.3082180 & 1.5236080 & c & -4.6805660 & -2.5528830 & -2.9229850 \\
\hline$H$ & -0.3952540 & 5.0942760 & 2.6780560 & $\mathrm{H}$ & -4.5454640 & -2.5317580 & -4.0126420 \\
\hline$H$ & 1.2266590 & 6.0150790 & 1.0271790 & $\mathrm{H}$ & -4.6668790 & -3.6033090 & -2.6076310 \\
\hline H & -1.2098710 & 6.2609680 & -0.8264760 & C & -6.0299140 & -1.8758840 & -2.5231070 \\
\hline $\mathrm{H}$ & -0.1611400 & 4.5621210 & -2.2426600 & $\mathrm{H}$ & -6.5771510 & -1.5462660 & -3.4163270 \\
\hline & & & & $\mathrm{H}$ & -6.6713080 & -2.5804440 & -1.9801330 \\
\hline & S) $\mathrm{G}[\mathrm{B} 97-\mathrm{D}$ & {$[Z \mathrm{VP}]=-354$} & 724 & $\mathrm{H}$ & -6.6786560 & -0.1628740 & -1.3140520 \\
\hline $\mathrm{P}$ & 0.2342970 & -0.0484900 & 0.2624810 & $\mathrm{H}$ & -2.5493750 & -2.2684020 & -2.5036470 \\
\hline 0 & 0.7913740 & 0.8029120 & 1.3715380 & C & -4.8703660 & 0.3467180 & -2.4482280 \\
\hline 0 & -0.8257340 & 0.5280270 & -0.6400620 & $\mathrm{H}$ & -5.4173030 & 0.6198680 & -3.3607520 \\
\hline 0 & -0.2280830 & -1.4314160 & 0.9784420 & $\mathrm{H}$ & -4.7107690 & 1.2621790 & -1.8716900 \\
\hline 0 & 1.3888440 & -0.5602380 & -0.7623370 & C & -3.5123930 & -0.3328000 & -2.7977380 \\
\hline c & -0.3716430 & -2.5734870 & 0.1876440 & $\mathrm{H}$ & -3.3634730 & -0.3621800 & -3.8854880 \\
\hline c & 0.7871440 & -3.2394470 & -0.2523920 & $\mathrm{H}$ & -2.6733780 & 0.2131910 & -2.3575840 \\
\hline C & -1.6628340 & -3.0012000 & -0.1411990 & C & -5.3108830 & -0.9390940 & 0.9067800 \\
\hline c & -1.7611990 & -4.1558330 & -0.9230450 & $\mathrm{H}$ & -6.2516830 & -0.4514980 & 1.1658540 \\
\hline $\mathrm{H}$ & -2.7547070 & -4.5114060 & -1.1914190 & C & 3.9913000 & 2.5427250 & -1.9154740 \\
\hline c & 2.4100420 & -1.3374810 & -0.2164750 & $\mathrm{C}$ & 3.8139440 & 2.8951620 & -3.3719200 \\
\hline c & 3.6556740 & -0.7387930 & 0.0037830 & $\mathrm{c}$ & 3.3742630 & 0.3886250 & -2.8542320 \\
\hline C & 2.1267470 & -2.6824180 & 0.0951930 & $\mathrm{C}$ & 3.7402350 & 1.1883170 & -1.6237270 \\
\hline c & 4.6502980 & -1.5405790 & 0.5834270 & $\mathrm{C}$ & 4.7965120 & 2.0193800 & -4.1993390 \\
\hline $\mathrm{H}$ & 5.6309600 & -1.1071970 & 0.7695700 & $\mathrm{H}$ & 4.6350790 & 2.2159460 & -5.2677620 \\
\hline c & -2.8891760 & -2.2495440 & 0.2383730 & $\mathrm{H}$ & 5.8286490 & 2.3027420 & -3.9611960 \\
\hline c & 3.9044450 & 0.6928830 & -0.3151970 & c & 4.5455410 & 0.5147120 & -3.8700540 \\
\hline $\mathrm{C}$ & -3.2538650 & -2.0532610 & 1.5811310 & $\mathrm{H}$ & 4.2837030 & -0.0417440 & -4.7797800 \\
\hline c & -2.4935350 & -2.5228930 & 2.7986020 & $\mathrm{H}$ & 5.4465230 & 0.0550570 & -3.4466860 \\
\hline $\mathrm{c}$ & -4.7046470 & -1.2934210 & 3.3922130 & $\mathrm{H}$ & 3.1958240 & -0.6629820 & -2.6187070 \\
\hline C & -4.4575030 & -1.4000790 & 1.9083690 & $\mathrm{H}$ & 4.0094980 & 3.9606490 & -3.5456930 \\
\hline $\mathrm{c}$ & -2.1803640 & -1.2898540 & 3.6894050 & C & 2.1120530 & 1.0146280 & -3.5078070 \\
\hline H & -1.6851880 & -1.6369240 & 4.6061450 & $\mathrm{H}$ & 1.8929640 & 0.4703180 & -4.4360640 \\
\hline H & -1.4823210 & -0.6330440 & 3.1627770 & $\mathrm{H}$ & 1.2560010 & 0.8870610 & -2.8404900 \\
\hline c & -3.5056130 & -0.5401780 & 4.0320950 & C & 2.3710750 & 2.5231930 & -3.8064290 \\
\hline H & -3.6558300 & -0.4890770 & 5.1189610 & $\mathrm{H}$ & 2.2563410 & 2.7359650 & -4.8777090 \\
\hline$H$ & -3.4773260 & 0.4878180 & 3.6510940 & $\mathrm{H}$ & 1.6533910 & 3.1514860 & -3.2679610 \\
\hline $\mathrm{H}$ & -5.6461990 & -0.7698290 & 3.5993610 & C & 4.5965820 & 2.9353170 & 0.3796150 \\
\hline$H$ & -1.5690060 & -3.0374370 & 2.5260460 & C & 5.0957030 & 3.7410890 & 1.5535110 \\
\hline c & -4.7369960 & -2.7302480 & 3.9850230 & C & 4.5604630 & 1.2721040 & 2.1500350 \\
\hline H & -4.8480230 & -2.6623070 & 5.0755180 & $\mathrm{C}$ & 4.3294960 & 1.5869890 & 0.6881490 \\
\hline $\mathrm{H}$ & -5.6097960 & -3.2666760 & 3.5940550 & $\mathrm{c}$ & 4.0231950 & 3.7071060 & 2.6754320 \\
\hline c & -3.4176500 & -3.4741410 & 3.6095370 & $\mathrm{H}$ & 4.4159630 & 4.2331790 & 3.5561700 \\
\hline $\mathrm{H}$ & -2.8848270 & -3.7970550 & 4.5137550 & $\mathrm{H}$ & 3.1238190 & 4.2323040 & 2.3389870 \\
\hline $\mathrm{H}$ & -3.6331360 & -4.3691230 & 3.0134850 & C & 3.6913400 & 2.2236560 & 3.0181480 \\
\hline C & -3.7406060 & -1.7487320 & -0.7659180 & $\mathrm{H}$ & 3.9010840 & 2.0141320 & 4.0755610 \\
\hline C & -3.5009810 & -1.79080 & -2.2587200 & $\mathrm{H}$ & 2.6350190 & 2.0058080 & 2.8394260 \\
\hline
\end{tabular}




$\begin{array}{lrrr}\text { H } & 4.3167900 & 0.2341180 & \\ \text { H } & 5.3137630 & 4.7746240 & 1.3861230 \\ \mathrm{C} & 6.0509380 & 1.5695490 & 2.4735550 \\ \mathrm{H} & 6.2224090 & 1.3924720 & 3.5436340 \\ \mathrm{H} & 6.6955490 & 0.8796030 & 1.9154260 \\ \mathrm{C} & 6.3771150 & 3.0508230 & 2.1012460 \\ \mathrm{H} & 6.7283430 & 3.6029830 & 2.9830400 \\ \mathrm{H} & 7.1684040 & 3.0947100 & 1.3434140 \\ \mathrm{C} & 4.4201550 & 3.4170880 & -0.9165780 \\ \mathrm{H} & 4.6331320 & 4.4611670 & -1.1505500 \\ \mathrm{C} & 3.1303630 & -3.4317870 & 0.7360590 \\ \mathrm{C} & 2.9182550 & -4.8055690 & 1.3205870 \\ \mathrm{C} & 3.3569570 & -4.8118920 & 2.8057070 \\ \mathrm{C} & 4.8077200 & -4.2794940 & 3.0012370 \\ \mathrm{C} & 5.4081780 & -3.7040420 & 1.6915390 \\ \mathrm{C} & 4.3952780 & -2.8552760 & 0.9666560 \\ \mathrm{H} & 3.5288310 & -5.5409730 & 0.7740330 \\ \mathrm{H} & 3.2660790 & -5.8308910 & 3.2019870 \\ \mathrm{H} & 5.4628790 & -5.0791100 & 3.3693490 \\ \mathrm{H} & 5.7030830 & -4.5469210 & 1.0468450 \\ \mathrm{H} & 1.8729540 & -5.1152100 & 1.2288160 \\ \mathrm{H} & 2.6545180 & -4.1828130 & 3.3659300 \\ \mathrm{H} & 4.8071170 & -3.4886530 & 3.7612370 \\ \mathrm{H} & 6.3160930 & -3.1277640 & -1.9057890 \\ \mathrm{C} & 0.6483450 & -4.3641190 & -1.1022800 \\ \mathrm{C} & 1.8680560 & -5.0167690 & -1.7309450 \\ \mathrm{C} & 1.5363240 & -5.8103540 & -3.0011280 \\ \mathrm{C} & 0.3933140 & -6.7884600 & -2.7085210 \\ \mathrm{C} & -0.8772670 & -6.0036150 & -2.3586090 \\ \mathrm{C} & -0.6426100 & -4.8307640 & -1.4179870 \\ \mathrm{H} & 2.4327400 & -6.3429310 & -3.3439640 \\ \mathrm{H} & 2.3262060 & -5.7098340 & -1.0125980 \\ \mathrm{H} & 2.6240190 & -4.2491620 & -1.9365710 \\ \mathrm{H} & 0.6822130 & -7.4300040 & -1.8623080 \\ \mathrm{H} & 0.2001010 & -7.4452570 & -3.5664030 \\ \mathrm{H} & -1.6351170 & -6.6692740 & -1.9226860 \\ \mathrm{H} & -1.3163530 & -5.6098100 & -3.2888840 \\ \mathrm{H} & 1.2321270 & -5.1229100 & -3.8046070 \\ \mathrm{C} & -3.0803080 & 3.0131020 & -0.3923990 \\ \mathrm{O} & -2.8514480 & 1.8199680 & 0.1046770 \\ \mathrm{C} & -4.4609770 & 3.5230240 & -0.1336420 \\ \mathrm{C} & -5.4383400 & 2.6966810 & 0.4479510 \\ \mathrm{C} & -4.7874740 & 4.8432550 & -0.4850260 \\ \mathrm{C} & -6.7230340 & 3.1896090 & 0.6748690 \\ \mathrm{H} & -5.1874560 & 1.6724380 & 0.7050150 \\ \mathrm{C} & -6.0728300 & 5.3326310 & -0.2539810 \\ & & & \end{array}$

$\begin{array}{lr}\mathrm{H} & -4.0265600 \\ \mathrm{C} & -7.0428900 \\ \mathrm{H} & -7.4768580 \\ \mathrm{H} & -6.3204290 \\ \mathrm{H} & -8.0458750 \\ \mathrm{H} & -1.9693210 \\ \mathrm{O} & -2.2514670 \\ \mathrm{C} & -0.2456440 \\ \mathrm{C} & -0.0755890 \\ \mathrm{H} & -0.8797140 \\ \mathrm{H} & -0.1049410 \\ \mathrm{O} & 1.1507870 \\ \mathrm{H} & 1.1135710 \\ \mathrm{C} & 0.3872290 \\ \mathrm{C} & 0.0857420 \\ \mathrm{C} & 0.4237990 \\ \mathrm{C} & -0.4354170 \\ \mathrm{H} & 0.1530210 \\ \mathrm{H} & 0.6523170 \\ \mathrm{H} & 1.5132520 \\ \mathrm{H} & -1.5059130 \\ \mathrm{H} & -0.1748130 \\ \mathrm{H} & 1.4568220 \\ \mathrm{H} & -0.9817120 \\ \mathrm{H} & 0.0262860 \\ \mathrm{H} & \end{array}$
5.4712290
4.5068350
2. 5450790
6.3559720
4.8889550
1. 3577210
3.6963900
3.6820790
3. 5625980
2. 9219530
2. 5656350
3.2656870
2. 2268070
6.1323390
6.0811460
4.7020980
5.0709350
7. 1264020
6.8521940
4.5663520
5. 3092620
5.0419790
5.9511060
6.2813700
4.5816920

154

$-0.9400520$

0.3264160

1.1217670

$-0.5267890$

0.5044350

$-0.2074990$

$-1.0191500$

1.0381260

$-0.4173100$

1.4992940

$-0.8399450$

1.0241680

1.1425600

0.8662270

$-0.6398820$

$-1.2339570$

1.6164240

1.2654650

$-1.1752470$

$-1.3152720$

1. 5428120

2.6817910

$-0.8014610$

TS179(S,S) G[B97-D/def2-TZVP $]=-3541.571716$

$\begin{array}{lrrr}\text { TS179(S, S) } \mathrm{G}[\mathrm{B} 97-\mathrm{D} / \mathrm{def} 2-\mathrm{TZVP}]=-3541.571716 \\ \mathrm{P} & 0.3680760 & 0.2476830 & 0.8594610 \\ \mathrm{O} & -0.2580830 & 0.8153450 & 2.1025710 \\ \mathrm{O} & 0.6059910 & -1.2365820 & 0.7868270 \\ \mathrm{O} & 1.7251990 & 1.1075690 & 0.6374020 \\ \mathrm{O} & -0.5065660 & 0.5945680 & -0.4825510 \\ \mathrm{C} & 2.3455880 & 1.0735340 & -0.6119840 \\ \mathrm{C} & 1.7497230 & 1.7843040 & -1.6715330 \\ \mathrm{C} & 3.5408370 & 0.3600450 & -0.7478090 \\ \mathrm{C} & 4.1308710 & 0.3379030 & -2.0201440 \\ \mathrm{H} & 5.0594330 & -0.2141510 & -2.1538380 \\ \mathrm{C} & -0.6182650 & 1.9319760 & -0.8659800 \\ \mathrm{C} & -1.8487190 & 2.5773140 & -0.7029680 \\ \mathrm{C} & 0.5061180 & 2.5648710 & -1.4311420 \\ \mathrm{C} & -1.9204530 & 3.9306140 & -1.0598040 \\ \mathrm{H} & -2.8664880 & 4.4550180 & -0.9361020 \\ \mathrm{C} & 4.1728890 & -0.3534150 & 0.3928730 \\ \mathrm{C} & -3.0551510 & 1.8531910 & -0.2216140 \\ \mathrm{C} & 4.6224340 & 0.3493240 & 1.5257110 \\ \mathrm{C} & 4.5415430 & 1.8414780 & 1.7521690\end{array}$




\begin{tabular}{|c|c|c|c|}
\hline$c$ & 56973010 & & \\
\hline c & $\begin{array}{l}5.6973040 \\
5.2606520\end{array}$ & $\begin{array}{r}0.5775870 \\
-0.3779070\end{array}$ & $\begin{array}{l}3.7058010 \\
2.5817580\end{array}$ \\
\hline $\mathrm{C}$ & 3.7630970 & 2.1017480 & 3.0714280 \\
\hline $\mathrm{H}$ & 3.7621060 & 3.1822940 & 3.2682360 \\
\hline $\mathrm{H}$ & 2.7240810 & 1.7845590 & 2.9481120 \\
\hline C & 4.4490060 & 1.3315590 & 4.2426210 \\
\hline H & 4.7655930 & 2.0249620 & 5.0334210 \\
\hline $\mathrm{H}$ & 3.7533530 & 0.6113210 & 4.6893580 \\
\hline H & 6.1797220 & 0.0067200 & 4.5086370 \\
\hline H & 4.0545070 & 2.3535700 & 0.9184070 \\
\hline C & 6.6771960 & 1.6345690 & 3.1239410 \\
\hline $\mathrm{H}$ & 6.9528660 & 2.3398280 & 3.9194400 \\
\hline H & 7.5947450 & 1.1373340 & 2.7873810 \\
\hline C & 5.9875030 & 2.3810870 & 1.9395650 \\
\hline H & 5.9377810 & 3.4599110 & 2.1385190 \\
\hline $\mathrm{H}$ & 6.5529280 & 2.2401050 & 1.0104950 \\
\hline C & 4.3691250 & -1.7457280 & 0.3465690 \\
\hline C & 3.9250030 & -2.6861370 & -0.7506310 \\
\hline C & 5.1511170 & -3.8998810 & 1.1926580 \\
\hline C & 5.0162420 & -2.4124220 & 1.4048430 \\
\hline c & 5.1792530 & -3.4157430 & -1.3053220 \\
\hline H & 4.8594870 & -4.1406080 & -2.0660020 \\
\hline $\mathrm{H}$ & 5.8415050 & -2.6913330 & -1.7951070 \\
\hline C & 5.9225520 & -4.1373550 & -0.1361680 \\
\hline $\mathrm{H}$ & 5.9830400 & -5.2177230 & -0.3240870 \\
\hline $\mathrm{H}$ & 6.9469420 & -3.7591780 & -0.0363670 \\
\hline $\mathrm{H}$ & 5.6766440 & -4.3723560 & 2.0315660 \\
\hline H & 3.4027410 & -2.1561920 & -1.5514130 \\
\hline C & 3.7286270 & -4.5003710 & 1.0278360 \\
\hline $\mathrm{H}$ & 3.8181700 & -5.5734780 & 0.8103500 \\
\hline $\mathrm{H}$ & 3.1771340 & -4.3963340 & 1.9699640 \\
\hline C & 2.9884940 & -3.7585370 & -0.1262250 \\
\hline H & 2.6903090 & -4.4641550 & -0.9130870 \\
\hline H & 2.0861110 & -3.2680840 & 0.2445580 \\
\hline C & 5.4608800 & -1.7073360 & 2.5228220 \\
\hline $\mathrm{H}$ & 5.9608140 & -2.2278730 & 3.3407210 \\
\hline C & -4.8022470 & 0.1830560 & -0.5976950 \\
\hline $\mathrm{C}$ & -5.3053890 & -0.8374490 & -1.5887750 \\
\hline $\mathrm{C}$ & -3.1687880 & 0.4073470 & -2.3853360 \\
\hline $\mathrm{C}$ & -3.6315120 & 0.8432570 & -1.0144290 \\
\hline $\mathrm{C}$ & -5.6051200 & -0.1000090 & -2.9250680 \\
\hline $\mathrm{H}$ & -5.9025440 & -0.8407310 & -3.679285 \\
\hline $\mathrm{H}$ & -6.4470640 & 0.5878400 & 7834400 \\
\hline C & -4.3296860 & 0.6739720 & 3846570 \\
\hline $\mathrm{H}$ & -4.0168810 & 0.3445280 & 384320 \\
\hline П & -4.5272980 & 1.7513220 & 3.435515 \\
\hline
\end{tabular}

-2.2691240
-6.2067440
-2.9020210
-2.6376990
-2.0435060
-4.1760850
-4.5229020
-3.9595430
-4.8566120
-5.4040400
-3.2457540
-3.6806180
-4.3030920
-4.6846090
-4.0703880
-3.0264220
-2.8096760
-2.1541670
-2.3323650
-6.3138340
-4.4129790
-4.1237590
-4.5882160
-5.7000170
-6.0233420
-6.5244560
-5.4180670
-6.3312810
0.4072970
1.5652540
1.6840550
0.3376490
-0.8381600
-0.8119450
1.3980190
2.4589050
0.4257930
-0.7371500
2.5036740
2.0304060
0.0954100
-1.7967720
2.3489170
1.7443800
1.6812560
3.0466440
$-2.6985990$ $-1.2168870$ $-2.3638180$ $-3.3777470$ $-1.7211480$ $-1.8636780$ $-2.6137590$ $-0.9506740$

1. 3962300

2.7105300

1.9578840

0.9896210

3.7925860

4.7521500

3.9174000

3. 3600970

4.0717190

3. 3281420

1.6210110

2.9907960

2.0826780

2.7950970

1.1122310

2.5705800

3.5453980

1.8633030

0.6060260

0.9234990

$-1.7484810$

$-2.2201800$

$-1.3489620$

$-1.2187460$

$-1.8987040$

$-1.5579960$

$-3.2666080$

$-1.7743680$

$-1.6594130$

$-2.9893420$

$-2.1925240$

$-0.3538410$

$-0.1576440$

$-1.6119340$

$-2.9432440$

$-4.2023020$

-5.3003650
-5.5223350 


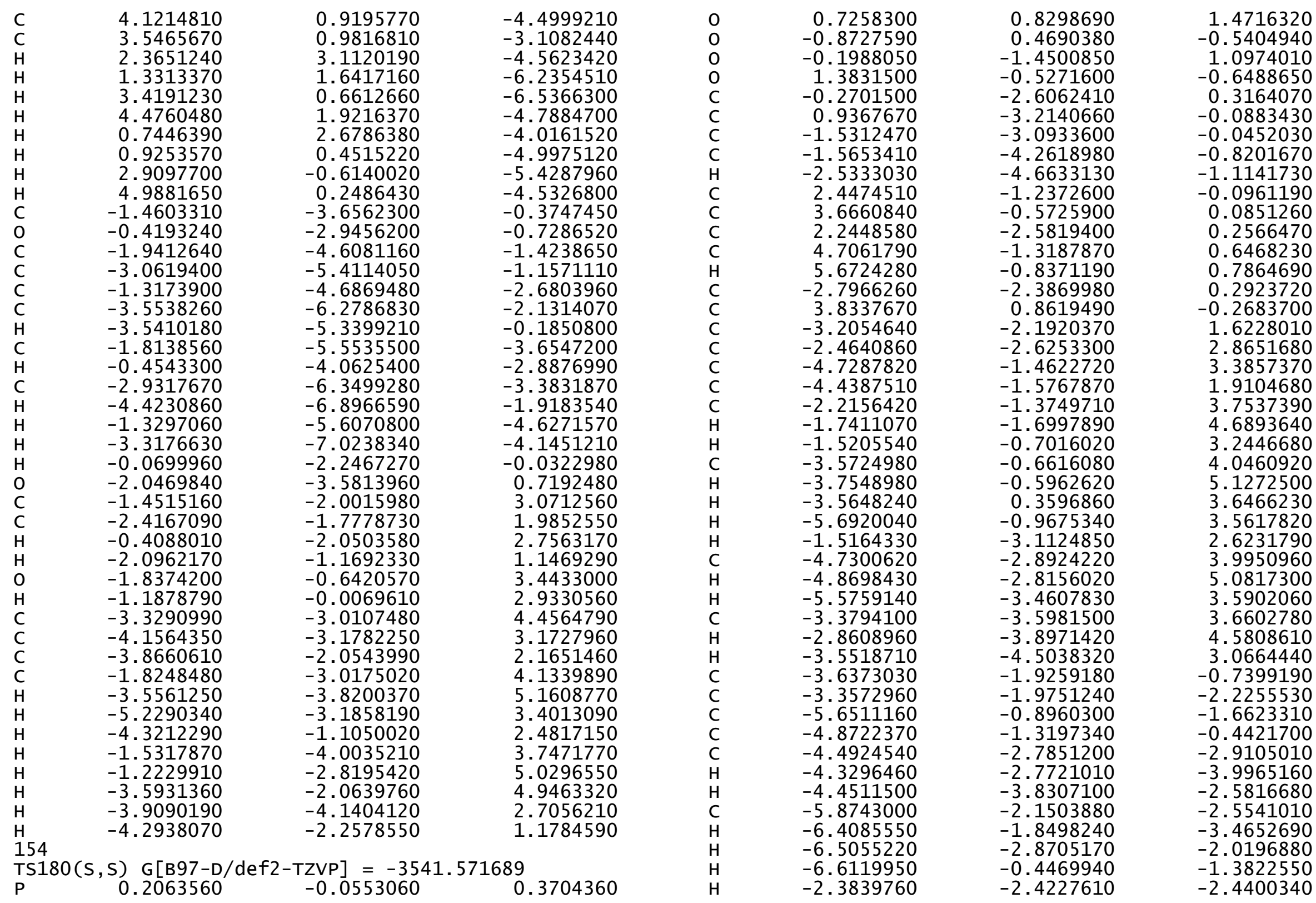




\begin{tabular}{|c|c|c|}
\hline $\begin{array}{l}-4.7929280 \\
-5.3253760 \\
-4.6789520 \\
-3.4044660 \\
-3.2276980 \\
-2.5959680 \\
-5.2788900 \\
-6.2418920 \\
3.7845910 \\
3.5695120 \\
3.3057260 \\
3.6230470 \\
4.6021740 \\
4.4127440 \\
5.6145650 \\
4.4627280 \\
4.2421230 \\
5.3954610 \\
3.2031350 \\
3.6891810 \\
2.0010160 \\
1.8222530 \\
1.1570680 \\
2.1515990 \\
2.0111570 \\
1.3972320 \\
4.3990780 \\
4.8693450 \\
4.4990450 \\
4.2206780 \\
3.8248490 \\
4.2040020 \\
2.8880900 \\
3.5918510 \\
3.8373400 \\
2.5470470 \\
4.3263740 \\
5.0162710 \\
5.9747100 \\
6.1776130 \\
6.6497930 \\
6.2024310 \\
6.5363480 \\
6.9742930 \\
4.1717630 \\
4.3162070\end{array}$ & $\begin{array}{r}0.1115690 \\
0.3570780 \\
1.0377880 \\
-0.5249520 \\
-0.5622970 \\
0.0547480 \\
-1.1538120 \\
-0.6949500 \\
2.6748330 \\
2.9786440 \\
0.4610130 \\
1.3150210 \\
2.1509850 \\
2.3043500 \\
2.5130800 \\
0.6424300 \\
0.0411920 \\
0.2632570 \\
-0.5943610 \\
4.0501420 \\
0.9799390 \\
0.4033360 \\
0.8039890 \\
2.4967120 \\
2.6802540 \\
3.0830890 \\
3.1564240 \\
4.0160160 \\
1.5314480 \\
1.8017630 \\
3.9395280 \\
4.5065530 \\
4.4019450 \\
2.4459930 \\
2.2725060 \\
2.1607880 \\
0.4851710 \\
5.0546790 \\
1.9273720 \\
1.7847250 \\
1.2664330 \\
3.4178760 \\
4.0092020 \\
3.4937840 \\
3.5966930 \\
4.6463500\end{array}$ & $\begin{array}{l}-2.4754350 \\
-3.4042770 \\
-1.9051080 \\
-2.7829000 \\
-3.8662810 \\
-2.3293090 \\
0.8814150 \\
1.1096170 \\
-1.9109380 \\
-3.3731840 \\
-2.7918780 \\
-1.5851540 \\
-4.1899890 \\
-5.2608310 \\
-3.9752420 \\
-3.8154930 \\
-4.7074640 \\
-3.3812230 \\
-2.5285210 \\
-3.5762930 \\
-3.4550010 \\
-4.3723870 \\
-2.7831410 \\
-3.7838060 \\
-4.8573710 \\
-3.2487220 \\
0.3658190 \\
1.5126970 \\
2.1696120 \\
0.7075610 \\
2.6586680 \\
3.5197430 \\
2.3329760 \\
3.0382710 \\
4.0944420 \\
2.8884390 \\
2.4314910 \\
1.1914330 \\
2.4556770 \\
3.5253630 \\
1.8985180 \\
2.0464970 \\
2.9094230 \\
1.2713520 \\
-0.9374320 \\
-1.1975780\end{array}$ \\
\hline
\end{tabular}

4.5376330
3.2916600
5.7286010
5.3412540
4.3305110
3.0459670
6.2394940
6.3834910
6.3201710
4.7711140
4.0893500
2.3577670
2.5278770
4.8857760
0.8584180
2.0469610
1.7921080
0.4429480
-0.4106800
-0.3996380
2.1972700
2.6286290
0.6238310
0.0200290
2.9664200
1.7905620
-0.1378080
-1.4372720
-3.2125130
-2.9491550
-4.6190770
-5.5835100
-4.9833710
-6.8939140
-5.3012510
-6.2938530
-4.2315160
-7.2514880
-7.6380520
-6.5708650
-8.2742470
-2.0467880
-2.3972940
-0.4104240
-0.2214560
-1.0303860

-2.6393360
-3.2764570
-3.3573960
-4.5670740
-5.4368540
-4.6434610
-5.1418610
-3.7035920
-2.6406750
-5.7576850
-6.3431630
-5.2324410
-4.4771590
-4.2237770
-4.3490550
-5.0009390
-5.1205940
-5.8253550
-6.0846710
-4.8823240
-6.0108700
-5.6595770
-6.7814840
-6.9454410
-4.4393020
-4.1067230
-5.2002020
-6.3539790
2.8813610
1.7103000
3.3396100
2.4769230
4.6457620
2.9195450
1.4641540
5.0855930
5.3018250
4.2231680
2.2465830
6.0985710
4.5663750
1.2704720
3.5835240
3.6724220
3.5266830
2.9067020
1.0747180

0.9066920

1.6904450

2.5494720

1.7935740

1.5257380

2.8100110

0.8742790

2.2755160

0.8372150

2.3640720

0.9171960

2.4844750

3.4903850

$-0.9198260$

$-1.5867010$

$-3.1090860$

$-3.4382870$

$-2.1692220$

$-1.2610390$

$-1.1732130$

$-3.5710720$

$-3.9453840$

$-1.6342650$

$-1.4006440$

$-3.5281510$

$-4.1275400$

$-2.4444210$

$-0.3900730$

0.1414930

$-0.1778670$

0.3715360

$-0.5442130$

0.5502970

0.6415300

$-0.3591380$

$-0.9740210$

0.1876370

0.9707570

$-0.6421690$

0.3286370

$-0.1436440$

$-1.0136760$

1.0596070

$-0.3911600$

1. 5305180 


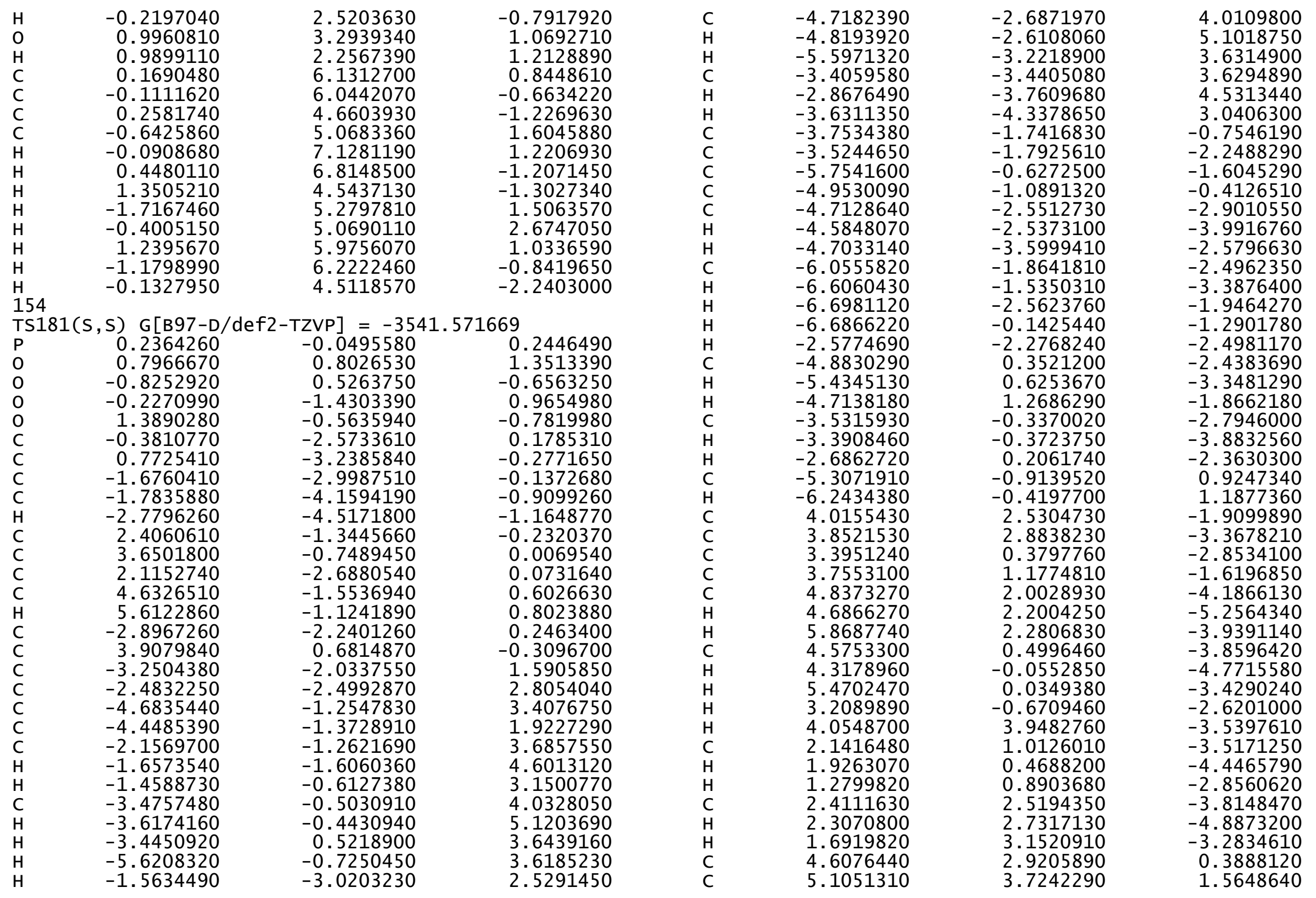




\begin{tabular}{|c|c|c|}
\hline $\begin{array}{l}4.5505390 \\
4.3309290 \\
4.0267220 \\
4.4191040 \\
3.1331430 \\
3.6818720 \\
3.8822890 \\
2.6253860 \\
4.2984830 \\
5.3315550 \\
6.0408000 \\
6.2038890 \\
6.6846940 \\
6.3790820 \\
6.7301470 \\
7.1740600 \\
4.4424930 \\
4.6629910 \\
3.1049480 \\
2.8829200 \\
3.2991420 \\
4.7416840 \\
5.3663030 \\
4.3667920 \\
3.4998600 \\
3.2119990 \\
5.3948930 \\
5.6757040 \\
1.8396500 \\
2.5821440 \\
4.7185780 \\
6.2689610 \\
0.6238580 \\
0.6711080 \\
1.8284400 \\
1.5810240 \\
0.2655190 \\
0.9003590 \\
2.4271150 \\
2.0897420 \\
2.7027980 \\
0.3034340 \\
0.1138500 \\
1.8375220 \\
1.0442100 \\
1.5296820\end{array}$ & $\begin{array}{r}1.2593080 \\
1.5739690 \\
3.6982370 \\
4.2210420 \\
4.2305440 \\
2.2173290 \\
2.0064850 \\
2.0070150 \\
0.2233500 \\
4.7560290 \\
1.5477410 \\
1.3717070 \\
0.8525090 \\
3.0260220 \\
3.5771000 \\
3.0631520 \\
3.4027670 \\
4.4455070 \\
-3.4398220 \\
-4.8214880 \\
-4.8478770 \\
-4.3047880 \\
-3.7198510 \\
-2.8671610 \\
-5.5521260 \\
-5.8741430 \\
-5.1003730 \\
-4.5576840 \\
-5.1306450 \\
-4.2350450 \\
-3.5162890 \\
-3.1436710 \\
-4.3600810 \\
-4.8362000 \\
-4.9492700 \\
-6.3399850 \\
-6.3610130 \\
-6.0656360 \\
-6.6145150 \\
-4.2535600 \\
-4.9777640 \\
-5.5946950 \\
-7.3317820 \\
-5.9433050 \\
-6.9376820 \\
-7.0846840\end{array}$ & $\begin{array}{r}2.1600780 \\
0.6962890 \\
2.6812170 \\
3.5640670 \\
2.3404680 \\
3.0216520 \\
4.0806210 \\
2.8351930 \\
2.3958880 \\
1.2686460 \\
2.4928300 \\
3.5643720 \\
1.9404260 \\
2.1198170 \\
3.0023500 \\
1.3654940 \\
-0.9086060 \\
-1.1415670 \\
0.7335540 \\
1.2989080 \\
2.7899340 \\
3.0175510 \\
1.7238360 \\
0.9846780 \\
0.7519920 \\
3.1678060 \\
3.3975880 \\
1.0790840 \\
1.1845300 \\
3.3496860 \\
3.7796470 \\
1.9597200 \\
-1.1273830 \\
-1.4151140 \\
-1.8472000 \\
-2.4450990 \\
-3.2299270 \\
-2.2797750 \\
-3.0883670 \\
-2.6617140 \\
-1.1962150 \\
-4.0185800 \\
-3.7195030 \\
-2.8387650 \\
-1.6210240 \\
-1.6361270\end{array}$ \\
\hline
\end{tabular}

\begin{tabular}{lr}
$\mathrm{C}$ & -3.0668750 \\
$\mathrm{O}$ & -2.8407740 \\
$\mathrm{C}$ & -4.4439710 \\
$\mathrm{C}$ & -5.4218760 \\
$\mathrm{C}$ & -4.7665340 \\
$\mathrm{C}$ & -6.7030980 \\
$\mathrm{H}$ & -5.1742460 \\
$\mathrm{C}$ & -6.0484580 \\
$\mathrm{H}$ & -4.0053030 \\
$\mathrm{C}$ & -7.0190040 \\
$\mathrm{H}$ & -7.4573430 \\
$\mathrm{H}$ & -6.2929770 \\
$\mathrm{H}$ & -8.0193110 \\
$\mathrm{H}$ & -1.9625120 \\
$\mathrm{O}$ & -2.2379310 \\
$\mathrm{C}$ & -0.2266620 \\
$\mathrm{C}$ & -0.0614190 \\
$\mathrm{H}$ & -0.8610950 \\
$\mathrm{H}$ & -0.0945620 \\
$\mathrm{O}$ & 1.1687860 \\
$\mathrm{H}$ & 1.1282920 \\
$\mathrm{C}$ & 0.4111530 \\
$\mathrm{C}$ & 0.1045130 \\
$\mathrm{C}$ & 0.4385120 \\
$\mathrm{C}$ & -0.4111310 \\
$\mathrm{H}$ & 0.1803130 \\
$\mathrm{H}$ & 0.6706040 \\
$\mathrm{H}$ & 1.5275550 \\
$\mathrm{H}$ & -1.4813540 \\
$\mathrm{H}$ & -0.1467750 \\
$\mathrm{H}$ & 1.4809520 \\
$\mathrm{H}$ & -0.9631390 \\
$\mathrm{H}$ & 0.0388190 \\
$\mathrm{H}$ & \\
\hline & \\
$\mathrm{H}$ & -8
\end{tabular}
3.0227100
3.7004930
3.6834200
3. 5617570
2.9253810
2. 5644260
3. 2634000
2. 2251560
6.1321310
6.0795800
4.6991850
5.0733340
7.1271650
6.8489700
4.5613540
5. 3140220
5.0448660
5.9489160
6.2812800
4.5783820

$-0.4027030$

-0.40967980
0.1389040

$3.5395750 \quad-0.1389040$

$2.7198430 \quad 0.4510910$

$4.8598490 \quad-0.4937550$

$3.2194410 \quad 0.6830140$

$\begin{array}{ll}1.6954880 & 0.7108260\end{array}$

$5.3558600 \quad-0.2578310$

$5.4826670 \quad-0.9552910$

$4.5366980 \quad 0.3310950$

$2.5800760 \quad 1.1365710$

$6.3791920 \quad-0.5334230$

$4.9239560 \quad 0.5130270$

$1.3629310 \quad-0.2192230$

$-1.0353110$

-1.0353110
1.0168730

$-0.4389320$

1.4809160

$-0.8603720$

0.9989520

1.1186720

0.8400300

$-0.6649760$

$-1.2582400$

1.5942780

1.2388030

$-1.2032320$

$-1.3412190$

1.5243360

2. 6587320

1.0067080

$-0.8233360$

154

$-2.2718220$

TS182(S, S ) G[B97-D/def2-TZVP $]=-3541.571573$

$\begin{array}{lrcr}\mathrm{P} & 0.4041190 & 0.1752560 & 1.0033090 \\ \mathrm{O} & -0.2258680 & 0.5627210 & 2.3120940 \\ \mathrm{O} & 0.7161940 & -1.2772220 & 0.7610650 \\ \mathrm{O} & 1.7100590 & 1.1259820 & 0.8566550 \\ \mathrm{O} & -0.5206120 & 0.6166880 & -0.2790110 \\ \mathrm{C} & 2.3024720 & 1.2473200 & -0.4025780 \\ \mathrm{C} & 1.6491280 & 2.0319150 & -1.3710200 \\ \mathrm{C} & 3.5203390 & 0.6009900 & -0.6392270 \\ \mathrm{C} & 4.0694370 & 0.7225880 & -1.9241360 \\ \mathrm{H} & 5.0132600 & 0.2237720 & -2.1363880 \\ \mathrm{C} & -0.7065950 & 1.9774740 & -0.5282310\end{array}$




$\begin{array}{rrr}-1.9710160 & 2.5351070 & -0.3111400 \\ 0.3768370 & 2.7255220 & -1.0293460 \\ -2.1164290 & 3.9119760 & -0.5263760 \\ -3.0935500 & 4.3649570 & -0.3681240 \\ 4.2206860 & -0.1935380 & 0.4033110 \\ -3.1457280 & 1.7075310 & 0.0686660 \\ 4.6847730 & 0.4098300 & 1.5860620 \\ 4.5317740 & 1.8610790 & 1.9785180 \\ 5.8522380 & 0.4680990 & 3.7296590 \\ 5.4105670 & -0.3369250 & 2.5333810 \\ 3.8046510 & 1.9258100 & 3.3502100 \\ 3.7468390 & 2.9760720 & 3.6664470 \\ 2.7820510 & 1.5557970 & 3.2343590 \\ 4.5909880 & 1.0797680 & 4.4001190 \\ 4.9056740 & 1.7035390 & 5.2476320 \\ 3.9614450 & 0.2735240 & 4.7950550 \\ 6.4061760 & -0.1548190 & 4.4426230 \\ 3.9751340 & 2.4294280 & 1.2293660 \\ 6.7398850 & 1.6406000 & 3.2256450 \\ 7.0148500 & 2.2718570 & 4.0813480 \\ 7.6661460 & 1.2395800 & 2.7969630 \\ 5.9512780 & 2.4660210 & 2.1620300 \\ 5.8497040 & 3.5119060 & 2.4812460 \\ 6.4769500 & 2.4615810 & 1.1995610 \\ 4.4829780 & -1.5609130 & 0.2014590 \\ 4.0229280 & -2.4064630 & -0.9640220 \\ 5.4263570 & -3.7409790 & 0.7685670 \\ 5.2207330 & -2.2959780 & 1.1491400 \\ 5.2769910 & -2.9994160 & -1.6624920 \\ 4.9497330 & -3.6638850 & -2.4735440 \\ 5.8658870 & -2.1909360 & -2.1126470 \\ 6.1308010 & -3.7859970 & -0.6172500 \\ 6.2480780 & -4.8340160 & -0.9239690 \\ 7.1335360 & -3.3507980 & -0.5302160 \\ 6.0263450 & -4.2666870 & 1.5213960 \\ 3.4249910 & -1.8276390 & -1.6726160 \\ 4.0326610 & -4.4077070 & 0.6133350 \\ 4.1693960 & -5.4417500 & 0.2686010 \\ 3.5348960 & -4.4448460 & 1.5897880 \\ 3.1827560 & -3.5892220 & -0.4051360 \\ 2.8660610 & -4.2226130 & -1.2445130 \\ 2.2847210 & -3.1890870 & 0.0705200 \\ 5.6846720 & -1.6872820 & 2.3150570 \\ 6.2565280 & -2.2595250 & -0.5261560 \\ -4.8306890 & 0.0388020 & -6367160 \\ -5.2871460 & -0.8753800 & \end{array}$

-3.1717010
-3.6732390
-5.5820050
-5.8489650
-6.4426510
-4.3194670
-3.9788540
-4.5442990
-2.2815240
-6.1813210
-2.8625000
-2.5627700
-2.0134830
-4.1250980
-4.4371960
-3.9098200
-4.9572210
-5.5342360
-3.4050400
-3.7981410
-4.4404850
-4.8416420
-4.1824130
-3.1803300
-2.9872090
-2.2921710
-2.5023050
-6.4314250
-4.6008410
-4.3411140
-4.7778600
-5.8723050
-6.2232890
-6.6891820
-5.4768180
-6.3794410
-1.0436910
-1.1670550
-0.5752550
0.8820830
1.3127310
0.2075370
-0.6105810
-0.6147950
1.5858640
1.5588920

0.5058510

0.7735900

0.0016440

$-0.6553770$

0.6498700

0.8514480

0.6401150

1. 9228920

1.0965750

$-1.4393210$

$-1.0068230$

$-1.2134620$

$-1.2538180$

$-1.8391660$

$-2.4760790$

$-2.5023670$

1.1475620

1.4719830

2.8524260

1.8869540

1.2027670

. 4547360

0.1373020

2. 0609160

2.7808840

1.4345580

3.4104900

0.8737760

3. 8171560

4. 5116870

4. 4115360

2.9898930

3. 2503960

3.1989620

0.2287890

$-0.3347540$

4. 7044960

6.1910070

. 5705550

6.0542410

5. 0681860

4. 1143050

6.7280810

7.6587310

6.8959440

5.6593810
$-2.2425270$ 0.8422270 $-2.8870260$ $-3.7253810$ $-2.6844290$ $-3.2329350$ $-4.2551310$ $-3.1719930$ $-2.4743200$

$-1.3436030$ $-2.3831770$ $-3.4186110$ $-1.7438250$ $-2.0026600$ $-2.8401490$ $-1.1586380$

1. 6075520

2. 9625350

2. 3926050

1. 2989540

4.0329000

5.0236210

4.0402270

3.7097090

4.5159780

3. 6063920

2. 1306360

3.1646170

2. 6261830

3.4356530

1.7216420

2. 9979260

4.0052230

2. 2970780

0.6961700

0.9357020

$-0.9287110$

$-1.1487550$

$-2.5257690$

$-2.6969180$

$-1.5791820$

$-1.1924280$

$-0.3635700$

$-2.6601070$

$-2.7009560$

$-0.6829140$ 


$\begin{array}{lrrr}\mathrm{H} & -2.2154240 & 6.5049770 & -1.0761370 \\ \mathrm{H} & -1.2156470 & 6.1262100 & -3.2985900 \\ \mathrm{H} & 0.9787420 & 5.5523680 & -3.6657750 \\ \mathrm{H} & 2.2248950 & 4.5408610 & -1.8668920 \\ \mathrm{C} & 2.2086000 & 2.0947610 & -2.6613130 \\ \mathrm{C} & 1.5485610 & 2.7630150 & -3.8420930 \\ \mathrm{C} & 1.4722630 & 1.7801260 & -5.0361990 \\ \mathrm{C} & 2.8415760 & 1.1067740 & -5.3520150 \\ \mathrm{C} & 3.9444710 & 1.5151110 & -4.3425240 \\ \mathrm{C} & 3.4244290 & 1.4374940 & -2.9300750 \\ \mathrm{H} & 2.1424950 & 3.6390740 & -4.1444950 \\ \mathrm{H} & 1.0979970 & 2.3185790 & -5.9159230 \\ \mathrm{H} & 3.1716150 & 1.3660360 & -6.3658220 \\ \mathrm{H} & 4.2487410 & 2.5507660 & -4.5620790 \\ \mathrm{H} & 0.5494350 & 3.1269040 & -3.5894150 \\ \mathrm{H} & 0.7309190 & 1.0094780 & -4.7908970 \\ \mathrm{H} & 2.7296550 & 0.0160590 & -5.3184960 \\ \mathrm{H} & 4.8319450 & 0.8830720 & -4.4653130 \\ \mathrm{C} & -1.2480690 & -3.6089090 & -0.7182840 \\ \mathrm{O} & -0.2237860 & -2.8305600 & -0.9599680 \\ \mathrm{C} & -1.6700120 & -4.4478990 & -1.8833250 \\ \mathrm{C} & -2.7562330 & -5.3259930 & -1.7378860 \\ \mathrm{C} & -1.0271160 & -4.3446370 & -3.1285500 \\ \mathrm{C} & -3.1946410 & -6.0885300 & -2.8195050 \\ \mathrm{H} & -3.2512140 & -5.3947340 & -0.7735910 \\ \mathrm{C} & -1.4706990 & -5.1058380 & -4.2103690 \\ \mathrm{H} & -0.1909420 & -3.6623420 & -3.2436450 \\ \mathrm{C} & -2.5540480 & -5.9782250 & -4.0589930 \\ \mathrm{H} & -4.0370960 & -6.7658320 & -2.6991590 \\ \mathrm{H} & -0.9721860 & -5.0177140 & -5.1730020 \\ \mathrm{H} & -2.8985930 & -6.5699440 & -4.9044260 \\ \mathrm{H} & 0.0881540 & -2.2036580 & -0.1814240 \\ \mathrm{O} & -1.8651560 & -3.6786730 & 0.3593340 \\ \mathrm{C} & -1.3354940 & -2.4052590 & 2.9059000 \\ \mathrm{C} & -2.2932040 & -2.0633490 & 1.8434660 \\ \mathrm{H} & -0.2884710 & -2.3966260 & 2.6019770 \\ \mathrm{H} & -1.9783920 & -1.3469770 & 1.0925510 \\ \mathrm{O} & -1.7516260 & -1.1120540 & 3.4441440 \\ \mathrm{H} & -1.1168350 & -0.3995370 & 3.0241560 \\ \mathrm{C} & -3.2083510 & -3.6091390 & 4.1306180 \\ \mathrm{C} & -4.0187650 & -3.6316130 & 2.8260030 \\ \mathrm{C} & -3.7382790 & -2.3878630 & 1.9673260 \\ \mathrm{C} & -1.7017410 & -3.5536040 & 3.8263600 \\ \mathrm{H} & -3.4285050 & -4.5021070 & 4.7279530 \\ \mathrm{H} & -5.0935350 & -3.6858630 & 3.0373570 \\ \mathrm{H} & -4.2190330 & -1.4929670 & 2.3886790 \\ & & & \end{array}$

$\begin{array}{llll}\mathrm{H} & -1.3895570 & -4.4788320 & 3.3221260 \\ \mathrm{H} & -1.1114340 & -3.4613760 & 4.7465510 \\ \mathrm{H} & -3.4926720 & -2.7335580 & 4.7292660 \\ \mathrm{H} & -3.7477960 & -4.5248770 & 2.2479790 \\ \mathrm{H} & -4.1489650 & -2.4764920 & 0.9569610\end{array}$

154

TS183(S, S) G[B97-D/def2-TZVP $]=-3541.571564$

$\begin{array}{lrrr}\text { TS183(S, S G }[\text { B } 97-D / d e f 2-T Z V P] & =-3541.571564 \\ \text { P } & 0.2315050 & -0.0607180 & 0.2945300 \\ \text { O } & 0.7868670 & 0.7947920 & 1.4012700 \\ \text { O } & -0.8284840 & 0.5117520 & -0.6103920 \\ \text { O } & -0.2272410 & -1.4419980 & 1.0138710 \\ \text { O } & 1.3856200 & -0.5744800 & -0.7280010 \\ \mathrm{C} & -0.3618870 & -2.5950320 & 0.2383820 \\ \mathrm{C} & 0.8078020 & -3.2594160 & -0.1914880 \\ \mathrm{C} & -1.6506540 & -3.0398310 & -0.0742630 \\ \mathrm{C} & -1.7557650 & -4.2261850 & -0.8151760 \\ \mathrm{H} & -2.7478820 & -4.5958940 & -1.0673500 \\ \mathrm{C} & 2.4155710 & -1.3427270 & -0.1873640 \\ \mathrm{C} & 3.6608490 & -0.7365060 & 0.0121880 \\ \mathrm{C} & 2.1481270 & -2.6907550 & 0.1241540 \\ \mathrm{C} & 4.6750710 & -1.5349190 & 0.5611630 \\ \mathrm{H} & 5.6565150 & -1.0950780 & 0.7276840 \\ \mathrm{C} & -2.8779130 & -2.2796130 & 0.2867040 \\ \mathrm{C} & 3.8922680 & 0.6977810 & -0.3082270 \\ \mathrm{C} & -3.2475350 & -2.0572790 & 1.6238660 \\ \mathrm{C} & -2.4912240 & -2.5035050 & 2.8525250 \\ \mathrm{C} & -4.7031940 & -1.2604860 & 3.4144610 \\ \mathrm{C} & -4.4519340 & -1.3972690 & 1.9339710 \\ \mathrm{C} & -2.1799260 & -1.2535120 & 3.7205260 \\ \mathrm{H} & -1.6900920 & -1.5835170 & 4.6464070 \\ \mathrm{H} & -1.4777760 & -0.6082200 & 3.1851260 \\ \mathrm{C} & -3.5054420 & -0.4948530 & 4.0419490 \\ \mathrm{H} & -3.6593500 & -0.4195480 & 5.1268920 \\ \mathrm{H} & -3.4745330 & 0.5243730 & 3.6382160 \\ \mathrm{H} & -5.6449820 & -0.7322930 & 3.6084530 \\ \mathrm{H} & -1.5660170 & -3.0233750 & 2.5924810 \\ \mathrm{C} & -4.7380070 & -2.6849430 & 4.0365690 \\ \mathrm{H} & -4.8512290 & -2.5944150 & 5.1252100 \\ \mathrm{H} & -5.6105730 & -3.2284980 & 3.6550370 \\ \mathrm{C} & -3.4185740 & -3.4377200 & 3.6793730 \\ \mathrm{H} & -2.8887370 & -3.7434890 & 4.5912690 \\ \mathrm{H} & -3.6337540 & -4.3438740 & 3.1003440 \\ \mathrm{C} & -3.7248500 & -1.7980310 & -0.7301400 \\ \mathrm{C} & -3.4764680 & -1.8654700 & -2.2205460 \\ \mathrm{C} & -5.7170960 & -0.6984390 & -1.6174590 \\ \mathrm{C} & -4.9303090 & -1.1452100 & -0.4105070\end{array}$




$\begin{array}{rrr}-4.6545520 & -2.6336670 & -2.8802360 \\ -4.5121980 & -2.6310090 & -3.9691930 \\ -4.6469650 & -3.6788960 & -2.5478290 \\ -6.0039380 & -1.9453640 & -2.5002730 \\ -6.5431560 & -1.6267560 & -3.4023270 \\ -6.6522380 & -2.6393360 & -1.9518810 \\ -6.6549090 & -0.2127590 & -1.3209310 \\ -2.5251020 & -2.3509600 & -2.4512710 \\ -4.8371620 & 0.2740800 & -2.4502710 \\ -5.3772100 & 0.5361650 & -3.3701290 \\ -4.6776380 & 1.1969980 & -1.8855870 \\ -3.4794400 & -0.4153860 & -2.7811750 \\ -3.3236960 & -0.4611600 & -3.8674320 \\ -2.6416260 & 0.1342530 & -2.3431510 \\ -5.3007280 & -0.9544310 & 0.9203610 \\ -6.2415750 & -0.4600370 & 1.1659040 \\ 3.9439090 & 2.5474950 & -1.9094850 \\ 3.7522640 & 2.8955710 & -3.3652270 \\ 3.3507440 & 0.3834830 & -2.8425610 \\ 3.7120540 & 1.1902700 & -1.6154170 \\ 4.7421510 & 2.0326280 & -4.1976280 \\ 4.5701860 & 2.2244130 & -5.2652580 \\ 5.7716380 & 2.3317150 & -3.9673830 \\ 4.5157240 & 0.5250740 & -3.8636250 \\ 4.2588820 & -0.0376380 & -4.7709240 \\ 5.4253460 & 0.0804570 & -3.4426810 \\ 3.1892510 & -0.6703640 & -2.6043310 \\ 3.9313670 & 3.9634570 & -3.5419420 \\ 2.0768610 & 0.9898050 & -3.4917560 \\ 1.8634790 & 0.4426070 & -4.4196770 \\ 1.2247690 & 0.8490460 & -2.8219840 \\ 2.3124290 & 2.5021670 & -3.7908150 \\ 2.1878250 & 2.7140060 & -4.8611540 \\ 1.5891710 & 3.1193470 & -3.2473500 \\ 4.5644440 & 2.9476880 & 0.3806050 \\ 5.0659400 & 3.7577230 & 1.5505730 \\ 4.5658650 & 1.2815780 & 2.1493650 \\ 4.3162010 & 1.5962080 & 0.6909310 \\ 4.0056730 & 3.7093450 & 2.6836180 \\ 4.4016130 & 4.2387270 & 3.5609580 \\ 3.0968980 & 4.2245310 & 2.3574070 \\ 3.6947820 & 2.2215260 & 3.0279060 \\ 3.9179790 & 2.0133600 & 4.0828000 \\ 2.6392780 & 1.9914830 & 2.8596910 \\ 4.3375810 & 0.2399010 & 2.3854090 \\ 5.2686940 & 4.7942530 & 1.2536980\end{array}$

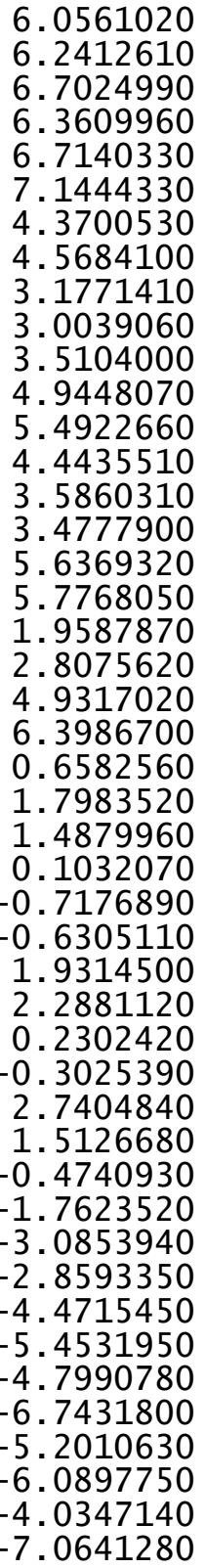

1. 5960280

1.4192770

0.9145060

3.0818310

3.6368220

3.1363390

3.4273390

4.4738900

$-3.4428120$

$-4.8441370$

$-4.9264050$

$-4.3452120$

$-3.7169390$

$-2.8592960$

$-5.5449140$

$-5.9726670$

$-5.1274200$

$-4.5323700$

$-5.1609650$

$-4.3687260$

$-3.5714150$

$-3.1342850$

$-4.4166070$

$-5.1382380$

$-5.3166930$

$-5.9803650$

$-6.1483030$

$-49099720$

$-6.1309410$

$-5.9088070$

$-6.9643070$

$-7.0004530$

$-4.5987440$

$-4.3237430$

$-5.3626690$

-6. 3886890

2. 9969570

1.8085830

3.5036280

2.6791580

4.8183490

3.1686470

1.6591950

5.3042330

5.4447460

4.4803640
2.4576440

3.5254640

1.8912830

2.0843760

2.9636700

1. 3191020

$-0.9139210$

$-1.1497760$

0.7230210

1.2545250

2.7157880

2.8933950

1.5869230

0.9261260

0.6365220

3.0443240

3.2298380

0.9031070

1.1994870

3.3466160

3.6707340

1.7903110

$-0.9813610$

$-1.6593140$

$-3.1662910$

$-3.4235640$

$-2.1191800$

$-1.2655930$

$-1.2024890$

$-3.6278180$

$-3.8923660$

$-1.5585230$

$-1.5347290$

$-3.6321680$

$-4.1224040$

$-2.3494960$

$-0.4018360$

0.1077500

$-0.1669660$

0.4100960

$-0.5374350$

0.6135780

0.6826270

$-0.3299940$

$-0.9888420$

0.2459380 


\begin{tabular}{|c|c|c|c|c|c|c|c|}
\hline & & & & & & & \\
\hline $\mathrm{H}$ & -7.5003110 & 2.5256740 & 1.0571510 & C & -2.2079370 & -1.3292390 & 3.7436110 \\
\hline $\mathrm{H}$ & -6.3381270 & 6.3233000 & -0.6177090 & $\mathrm{H}$ & -1.7260680 & -1.6571580 & 4.6743770 \\
\hline $\mathrm{H}$ & -8.0712510 & 4.8596740 & 0.4056500 & $\mathrm{H}$ & -1.5116950 & -0.6641810 & 3.2251580 \\
\hline $\mathrm{H}$ & -1.9737280 & 1.3448100 & -0.1921800 & C & -3.5540210 & -0.6014180 & 4.0500140 \\
\hline 0 & -2.2503240 & 3.6784130 & -1.0223110 & $\mathrm{H}$ & -3.7255220 & -0.5362710 & 5.1329710 \\
\hline C & -0.2596260 & 3.6711510 & 1.0511710 & $\mathrm{H}$ & -3.5389180 & 0.4206640 & 3.6527360 \\
\hline $\mathrm{C}$ & -0.0793830 & 3.5488420 & -0.4029150 & $\mathrm{H}$ & -5.6812020 & -0.8823380 & 3.5838390 \\
\hline $\mathrm{H}$ & -0.8961880 & 2.9114340 & 1.5095790 & $\mathrm{H}$ & -1.5391840 & -3.0761270 & 2.6099920 \\
\hline $\mathrm{H}$ & -0.1038000 & 2.5507840 & -0.8232080 & C & -4.7380290 & -2.8181760 & 4.0097450 \\
\hline 0 & 1.1372150 & 3.2570920 & 1.0484820 & $\mathrm{H}$ & -4.8685780 & -2.7392540 & 5.0973590 \\
\hline $\mathrm{H}$ & 1.1018680 & 2.2182800 & 1.1698370 & $\mathrm{H}$ & -5.5932300 & -3.3771620 & 3.6115120 \\
\hline C & 0.3739880 & 6.1208630 & 0.8795980 & C & -3.3979080 & -3.5392990 & 3.6651610 \\
\hline $\mathrm{C}$ & 0.0844470 & 6.0673080 & -0.6287190 & $\mathrm{H}$ & -2.8747970 & -3.8417900 & 4.5819980 \\
\hline C & 0.4247150 & 4.6869540 & -1.2184970 & $\mathrm{H}$ & -3.5851730 & -4.4445030 & 3.0750740 \\
\hline C & -0.4552280 & 5.0611600 & 1.6248660 & C & -3.6763690 & -1.8698150 & -0.7349450 \\
\hline $\mathrm{H}$ & 0.1370620 & 7.1157660 & 1.2751460 & $\mathrm{C}$ & -3.4090020 & -1.9232000 & -2.2227680 \\
\hline $\mathrm{H}$ & 0.6562120 & 6.8367160 & -1.1609430 & $\mathrm{C}$ & -5.6852090 & -0.8165350 & -1.6413000 \\
\hline $\mathrm{H}$ & 1.5143960 & 4.5498150 & -1.2955850 & $\mathrm{C}$ & -4.9011920 & -1.2482730 & -0.4272110 \\
\hline $\mathrm{H}$ & -1.5250540 & 5.2990950 & 1.5411680 & $\mathrm{C}$ & -4.5592530 & -2.7195500 & -2.8983960 \\
\hline $\mathrm{H}$ & -0.2040340 & 5.0346280 & 2.6925550 & $\mathrm{H}$ & -4.4053600 & -2.7081410 & -3.9857300 \\
\hline $\mathrm{H}$ & 1.4420410 & 5.9393440 & 1.0587680 & $\mathrm{H}$ & -4.5276470 & -3.7656760 & -2.5702120 \\
\hline $\mathrm{H}$ & -0.9814260 & 6.2689940 & -0.7988280 & C & -5.9304120 & -2.0685920 & -2.5302860 \\
\hline $\mathrm{H}$ & 0.0313720 & 4.5652910 & -2.2344700 & $\mathrm{H}$ & -6.4690660 & -1.7622910 & -3.4369270 \\
\hline 15 & & & & $\mathrm{H}$ & -6.5652380 & -2.7810670 & -1.9899200 \\
\hline & S) $G[B 97-D$ & ZVP] & 17 & $\mathrm{H}$ & -6.6384270 & -0.3560830 & -1.3537200 \\
\hline $\mathrm{P}$ & 0.2143230 & -0.0541970 & 0.3380260 & $\mathrm{H}$ & -2.4427810 & -2.3824510 & -2.4448290 \\
\hline 0 & 0.7394760 & 0.8249200 & 1.4412500 & C & -4.8219990 & 0.1803380 & -2.4623080 \\
\hline 0 & -0.8543770 & 0.4828410 & -0.5782830 & $\mathrm{H}$ & -5.3588110 & 0.4305850 & 873590 \\
\hline 0 & -0.2132900 & -1.4422840 & 1.0650400 & $\mathrm{H}$ & -4.6930850 & 1.1058870 & -1.8941750 \\
\hline 0 & 1.3895430 & -0.5424350 & -0.6748790 & C & -3.4433730 & -0.4724640 & -2.7797540 \\
\hline $\mathrm{C}$ & -0.3094890 & -2.5968910 & 0.2851880 & $\mathrm{H}$ & -3.2747590 & -0.5116290 & -3.8643570 \\
\hline C & 0.8838800 & -3.2243170 & -0.1336380 & $\mathrm{H}$ & -2.6247660 & 0.0974330 & -2.3318640 \\
\hline $\mathrm{C}$ & -1.5816290 & -3.0664250 & -0.0586650 & C & -5.2940890 & -1.0758830 & 0.8997300 \\
\hline C & -1.6430280 & -4.2375940 & -0.8284670 & $\mathrm{H}$ & -6.2495670 & -0.6054170 & 1.1357370 \\
\hline $\mathrm{H}$ & -2.6205600 & -4.6253730 & -1.1087910 & C & 3.8754980 & 2.6074550 & -1.9011030 \\
\hline C & 2.4341920 & -1.2767330 & -0.1153130 & $\mathrm{C}$ & 3.6838140 & 2.9162810 & -3.3655790 \\
\hline C & 3.6615220 & -0.6362040 & 0.0936450 & $\mathrm{C}$ & 3.3649320 & 0.4039850 & -2.7893040 \\
\hline $\mathrm{C}$ & 2.2029040 & -2.6212990 & 0.2162890 & $\mathrm{C}$ & 3.6827500 & 1.2510710 & -1.5776190 \\
\hline $\mathrm{C}$ & 4.6703330 & -1.4010430 & 0.6862700 & $\mathrm{C}$ & 4.7089300 & 2.0693180 & -4.1718080 \\
\hline H & 5.6396240 & -0.9361030 & 0.8579490 & $\mathrm{H}$ & 4.5322140 & 2.2247880 & -5.2445220 \\
\hline C & -2.8326970 & -2.3403800 & 0.2904190 & $\mathrm{H}$ & 5.7256870 & 2.4134830 & -3.9483050 \\
\hline $\mathrm{C}$ & 3.8655630 & 0.7942510 & -0.2576810 & C & 4.5389680 & 0.5640040 & -3.7971030 \\
\hline $\mathrm{C}$ & -3.2267250 & -2.1379820 & 1.6240010 & $\mathrm{H}$ & 4.3206130 & -0.0349590 & -4.6911330 \\
\hline $\mathrm{C}$ & -2.47 & -2.5781180 & 2.8600760 & $\mathrm{H}$ & 5.4590020 & 0.1699260 & -3.3492370 \\
\hline $\mathrm{C}$ & -4.72 & -1.3 & 3.3996100 & $\mathrm{H}$ & 3.2386820 & -0.6494480 & -2.5286170 \\
\hline $\mathrm{C}$ & -4.4 & 5731 & 1.9218950 & $\mathrm{H}$ & 3.8268650 & 3.9854380 & -3.5658870 \\
\hline
\end{tabular}




$\begin{array}{lrrr}\mathrm{C} & 2.0796770 & 0.9479860 & -3.4707740 \\ \mathrm{H} & 1.9063610 & 0.3780370 & -4.3933600 \\ \mathrm{H} & 1.2217910 & 0.7839220 & -2.8139110 \\ \mathrm{C} & 2.2611900 & 2.4633710 & -3.7911060 \\ \mathrm{H} & 2.1330250 & 2.6546510 & -4.8648640 \\ \mathrm{H} & 1.5136820 & 3.0613960 & -3.2588880 \\ \mathrm{C} & 4.4678140 & 3.0776820 & 0.3835560 \\ \mathrm{C} & 4.9404020 & 3.9285850 & 1.5360120 \\ \mathrm{C} & 4.5077950 & 1.4538300 & 2.1901750 \\ \mathrm{C} & 4.2565500 & 1.7271420 & 0.7236070 \\ \mathrm{C} & 3.8782770 & 3.8766220 & 2.6670440 \\ \mathrm{H} & 4.2570590 & 4.4368150 & 3.5327490 \\ \mathrm{H} & 2.9563290 & 4.3584700 & 2.3273620 \\ \mathrm{C} & 3.6083690 & 2.3890050 & 3.0448900 \\ \mathrm{H} & 3.8354220 & 2.2116140 & 4.1045500 \\ \mathrm{H} & 2.5599640 & 2.1257530 & 2.8807920 \\ \mathrm{H} & 4.3080560 & 0.4122990 & 2.4515090 \\ \mathrm{H} & 5.1146500 & 4.9632490 & 1.2154850 \\ \mathrm{C} & 5.9874720 & 1.8172630 & 2.4961190 \\ \mathrm{H} & 6.1728400 & 1.6708990 & 3.5684700 \\ \mathrm{H} & 6.6552440 & 1.1413170 & 1.9482040 \\ \mathrm{C} & 6.2524030 & 3.3023170 & 2.0893530 \\ \mathrm{H} & 6.5864030 & 3.8871760 & 2.9566390 \\ \mathrm{H} & 7.0367720 & 3.3611020 & 1.3253660 \\ \mathrm{C} & 4.2674380 & 3.5218080 & -0.9226280 \\ \mathrm{H} & 4.4360780 & 4.5683200 & -1.1807790 \\ \mathrm{C} & 3.2215130 & -3.3409420 & 0.8848750 \\ \mathrm{C} & 2.9562580 & -4.7432260 & 1.4053520 \\ \mathrm{C} & 3.9221040 & -5.1607140 & 2.5219310 \\ \mathrm{C} & 5.3657790 & -4.9143690 & 2.0696160 \\ \mathrm{C} & 5.5906110 & -3.4112860 & 1.8633330 \\ \mathrm{C} & 4.4656120 & -2.7186400 & 1.1089750 \\ \mathrm{H} & 3.7614480 & -6.2181580 & 2.7682110 \\ \mathrm{H} & 3.0578210 & -5.4656220 & 0.5843390 \\ \mathrm{H} & 1.9143730 & -4.8108420 & 1.7421160 \\ \mathrm{H} & 5.5403130 & -5.4509690 & 1.1246760 \\ \mathrm{H} & 6.0843680 & -5.3007880 & 2.8037700 \\ \mathrm{H} & 6.5407270 & -3.2286750 & 1.3425790 \\ \mathrm{H} & 5.6852490 & -2.9311340 & 2.8503810 \\ \mathrm{H} & 3.7199590 & -4.5749620 & 3.4311410 \\ \mathrm{C} & 0.7777660 & -4.3577820 & -0.9620010 \\ \mathrm{C} & 1.9428630 & -5.0044930 & -1.6714610 \\ \mathrm{C} & 1.6387190 & -5.0951260 & -3.1871650 \\ \mathrm{C} & 0.2932070 & -5.8205550 & -3.4820860 \\ \mathrm{C} & -0.5322900 & -6.0756830 & -2.1925720 \\ \mathrm{C} & -0.4927770 & -4.8751680 & -1.2834990 \\ & & & \end{array}$

\begin{abstract}
2.0983590
2.4682910

0.4771570

$-0.0928340$

2. 8687160

1.6015000

$-0.3077510$

$-1.5663110$

$-3.1628630$

$-2.9112200$

$-4.5601940$

$-5.5288860$

$-4.9113740$

$-6.8300580$

$-5.2576780$

$-6.2128290$

$-4.1566010$

$-7.1744920$

$-7.5773720$

$-6.4796040$

$-8.1901190$

$-2.0165890$

$-2.3433830$

$-0.3492170$

$-0.1669720$

$-0.9773160$

$-0.1795780$

1.0522790

1.0307330

0.2592440

$-0.0284470$

0.3243070

$-0.5612550$

0.0126120

0.5365850

1.4152290

$-1.6332490$

$-0.3135440$

1. 3287240

$-1.0959810$

$-0.0693610$
\end{abstract}

154
$-6.0234330$
$-5.6065530$
$-6.7811070$
$-6.9384920$
$-4.4471330$
$-4.0720920$
$-5.2119070$
$-6.3399630$
2. 9250320
1.7479830
3.4031080
2.5558820
4.7130240
3.0178340
1.5398050
5.1718510
5.3570700
4. 3251040
2. 3570170
6.1875630
4.6832890
1. 2978520
3.6178110
3. 6823740
3.5334110
2. 9249000
2.5270480
3. 2849340
2. 2481790
6.1341250
0489190
4.6606290
5.0813560
7.1342460
. 8131120
4. 5322680
5. 3060160
5.0793930
5.9661870
6. 2378730
4.5153650

TS185(S, S) G[B97-D/def2-TZVP $]=-3541.571005$

$\begin{array}{llrr} & \\ \mathrm{P} & -0.3616000 & -0.0912900 & 0.9779750 \\ \mathrm{O} & 0.2768630 & -0.4548280 & 2.2894220 \\ \mathrm{O} & -0.6277880 & 1.3614740 & 0.6873020\end{array}$

$-1.2836600$

$-3.6910700$

$-3.9797230$

$-1.6683350$

$-1.5039330$

$-3.5816590$

$-4.1687170$

$-2.4434810$

$-0.4025920$

0.1211840

$-0.1736890$

0.3921720

$-0.5394310$

0.5885540

0.6610960

$-0.3376510$

$-0.9820810$

0.2262940

1.0224460

$-0.6207160$

0.3809070

$-0.1727670$

$-1.0312180$

1.0353160

$-0.4160310$

1.5088200

$-0.8163990$

1.0408870

1.1834740

0.8162700

$-0.6907320$

$-1.2541010$

1.5806370

1.1923190

$-1.2374820$

$-1.3306210$

1. 4881590

2.6494640

1.0002560

$-0.8650960$

$-2.2669010$

-2.1925720
-1.2834990

0

1. 3614740

0.6873020 


$\begin{array}{rrr}-1.7043810 & -0.9992680 & 0.8902230 \\ 0.5245560 & -0.6104120 & -0.3033690 \\ -2.3255870 & -1.1389760 & -0.3529360 \\ -1.7189680 & -1.9624590 & -1.3130600 \\ -3.5275700 & -0.4617730 & -0.5864110 \\ -4.0978850 & -0.6145050 & -1.8533170 \\ -5.0375310 & -0.1046930 & -2.0601740 \\ 0.6484360 & -1.9873190 & -0.4989420 \\ 1.8847660 & -2.5970920 & -0.2574140 \\ -0.4755850 & -2.7019770 & -0.9516170 \\ 1.9558090 & -3.9888320 & -0.4007800 \\ 2.9093830 & -4.4842380 & -0.2268210 \\ -4.1845040 & 0.3854800 & 0.4423170 \\ 3.0963060 & -1.8081320 & 0.0867630 \\ -4.6503840 & -0.1698730 & 1.6475450 \\ -4.5393870 & -1.6139270 & 2.0779490 \\ -5.7812150 & -0.1294820 & 3.8110630 \\ -5.3340190 & 0.6266990 & 2.5852110 \\ -3.7897400 & -1.6645250 & 3.4380570 \\ -3.7579350 & -2.7070970 & 3.7821790 \\ -2.7588050 & -1.3291290 & 3.2942540 \\ -4.5305190 & -0.7662850 & 4.4775150 \\ -4.8527660 & -1.3577630 & 5.3450880 \\ -3.8681320 & 0.0275110 & 4.8429080 \\ -6.3008850 & 0.5314180 & 4.5155930 \\ -4.0154200 & -2.2212820 & 1.3357090 \\ -6.7168950 & -1.2841270 & 3.3563490 \\ -6.9974760 & -1.8815600 & 4.2341810 \\ -7.6367840 & -0.8639470 & 2.9322460 \\ -5.9750150 & -2.1653580 & 2.3029090 \\ -5.9023360 & -3.2046000 & 2.6504380 \\ -6.5175280 & -2.1703170 & 1.3498610 \\ -4.4004680 & 1.7556010 & 0.2080720 \\ -3.9243510 & 2.5542740 & -0.9837420 \\ -5.2592410 & 3.9814910 & 0.7295950 \\ -5.0990560 & 2.5405360 & 1.1455810 \\ -5.1642170 & 3.1747540 & -1.6834880 \\ -4.8221730 & 3.8084250 & -2.5128580 \\ -5.7861020 & 2.3776660 & -2.1088420 \\ -5.9785750 & 4.0154550 & -0.6488070 \\ -6.0649750 & 5.0587960 & -0.9807060 \\ -6.9940130 & 3.6162660 & -0.5391520 \\ -5.8316810 & 4.5467820 & 1.4751710 \\ -3.3554440 & 1.9371490 & -1.6837880 \\ -3.8453040 & 4.5943910 & 0.1706460 \\ -3.9499490 & 5.6230970 & \end{array}$

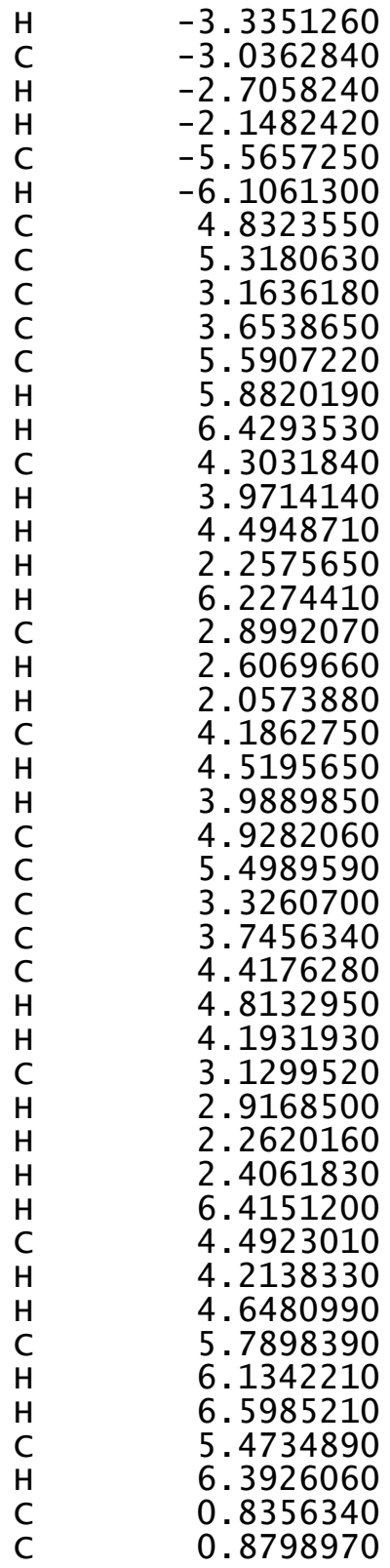

4.6403140
3.7198370
4.3198630
3.2994690
1.9792710
2.5901370
-0.2128510
0.6481980
-0.6891350
-0.9231610
-0.2809010
0.3367680
-0.9483810
-1.1024590
-0.9150410
-2.1776060
-1.2618830
1.1956820
0.8261610
1.0050310
1.1207140
1.6329310
2.2283630
2.3333440
-1.2569320
-1.5560660
-2.8856000
-1.9688830
-1.2179760
-1.4499520
-0.1454070
-2.0457250
-2.7335530
-1.3945300
-3.4243000
-0.9801580
-3.8791870
-4.5423370
-4.5042110
-3.0817170
-3.3211880
-3.3383140
-0.3826780
-6.24612070
-123820
-1

1. 5114110 -0.4637310 $-1.3221310$ 0.0133850 2. 3339780 3. 0583490 $-0.5619550$ $-1.7016160$ $-2.2654010$ $-0.8550630$ $-2.9186820$ $-3.7785100$ $-2.6882540$ $-3.2398910$ $-4.2695770$ $-3.1424100$ $-2.4800110$ $-1.4243440$ $-2.4597560$ $-3.5025430$ $-1.8309150$ $-2.1065450$ $-2.9661380$ $-1.2886660$ 1.6054350 2. 9686970 2. 4471060

1. 3217280

4. 0320680

5.0298470

4.0037910

3.7390430

4. 5680810

3. 6175140

2. 2054320

3. 1495020

2. 7070550

3.5364170

1. 8193650

3. 0517650

4. 0665310

2. 3572230

0.6658840

0.8874550

$-0.7417340$

$-0.8810650$ 


0.2227790
-1.2348560
-1.5609070
-0.3863960
0.3272320
0.2453270
-1.9445740
-1.8329030
1.9148860
0.8288300
-1.4105400
-2.4370360
-2.2824450
-3.4934220
-1.5351560
-2.4277770
-3.2573850
-4.1882360
-1.8043720
-0.7509990
-1.0064450
-2.5763260
-3.8472850
-4.6661130
-5.0035190
-3.1043850
1.4059140
0.3497900
1.8470190
2.9620480
1.1923360
3.4166450
3.4661690
1.6519080
0.3342370
2.7634480
4.2813790
1.1438900
3.1203920
0.0228500
2.0365340
1.4834860
2.4237810
0.4348140
2.0830250
1.8654310

-2.2165670
-2.3298270
-1.3090720
-1.0218460
-0.0509530
-2.3250990
-2.1666360
-0.3556640
-0.8267360
-3.0303710
-3.3466500
-1.6349290
-2.6085670
-2.8671120
-3.7403740
-4.9317150
-5.3610300
-4.2172400
-5.7595130
-4.0858560
-3.3808770
-5.6089120
-6.2598860
-4.4410810
-4.1392260
-4.6466980
-0.8856810
-1.0937950
-2.0786110
-1.9659470
-3.3162340
-3.0721190
-1.0073630
-4.4226060
-3.4061660
-4.3036130
-2.9770230
-5.3790820
-5.1680290
-0.2929260
0.1834500
2.7774750
1.7240360
2.4795640
1.0031540
3.3616920

1.2077750
3.3989390
4.1982750
3.8784780
1.8885880
3.6467860
5.2758770
4.3344570
1.5949470
1.3049080
3.6667310
3.9439760
4.2876030

0.4968050

3.6878780

3.6346610

2. 3628220

3. 6586480

4.5988510

3.6705530

1.4743120

4.5718970

3. 6166590

2.8308260

4. 5088090

2. 3946780

154

TS186(S, S) G[B97-D/def2-TZVP] $=-3541.570512$

$-1.3636290$

$-2.7225340$

$-1.8727540$

-3. 4499460

$-2.0285730$

$-3.6061360$

$-1.0448040$

$-2.0942080$

$-0.4881880$

$-2.1888010$

$-3.9078760$

3. 5662380

2.8216510

4. 3548270

4.2359330

5.9175400

5. 2818940

4.9511820

3.5775080

5.7924960

6.5703400

6.3483630

2. 2314230

3.6450740

2.4867350

2.0757940

2.4969390

1.2026770
-0.3767280
0.2510990

$-0.6499990$

$-1.7125440$

0.5288150

$-2.3140990$

$-1.6913670$

$-3.5108280$

$-4.0453190$

$-4.9780440$

0.6644670

1.9061210

$-0.4512720$

1.9885080

2.9454450

$-4.1964020$

3.1121400

$-4.6858010$

$-4.5746890$

$-5.8712880$

$-5.3980330$

$-3.8602050$

$-3.8306090$

$-2.8280780$

$-4.6336830$

$-4.9739400$

$-3.9865410$

$-6.4139820$

$-4.0273710$

$-6.7865880$

$-7.0868430$
$-0.1129800$

$-0.4887250$

1. 3416320

$-1.0282340$

$-1.1452340$

$-1.9512150$

$-0.4621520$

$-0.0566130$

$-1.9780880$

$-2.5806490$

$-2.6943890$

$-3.9689320$

$-4.4597880$

0.3520050

$-1.7877820$

$-1.6952590$

$-0.2699350$

0.5247230

$-1.7807780$

$-2.8328290$

$-1.4353340$

$-0.9178380$

$-1.5364800$

$-0.1307260$

0.3666100

$-2.2775040$

-1.4167680
-2.0412290
$-0.6067830$

$-0.5748710$

$-0.2400210$
2.9688470

3.9401320

2.6288250

1.8281570

3.6506860

4.4982070

2.8292930

2.2884420

3.1145900

4.5787470

4.5723070

2.0152280

0.8138400
0.9989020

2. 3120430

0.7245060

0.8830420

$-0.2795160$

$-0.3727430$

$-1.3356460$

$-0.6175270$

$-1.9041010$

$-2.1212780$

$-0.5021120$

$-0.2731570$

$-0.9744860$

$-0.4412600$

$-0.2727820$

0.4191890

0.0807630

1.5974390

1.9889370

3.7317160

2. 5404550

3. 3659170

3. 6801180

3. 2586120

4.4115240

5. 2529220

4.8163330

4.4412660

1. 2435170

3. 2180300

4. 0702640 


$\begin{array}{lrrr}\mathrm{H} & -7.6974340 & -0.9897330 & 2.7815040 \\ \mathrm{C} & -6.0113100 & -2.2625830 & 2.1602350 \\ \mathrm{H} & -5.9400560 & -3.3108840 & 2.4797710 \\ \mathrm{H} & -6.5285950 & -2.2436990 & 1.1934430 \\ \mathrm{C} & -4.4164470 & 1.7270580 & 0.2189570 \\ \mathrm{C} & -3.9195460 & 2.5627250 & -0.9386090 \\ \mathrm{C} & -5.3015540 & 3.9321300 & 0.7844580 \\ \mathrm{C} & -5.1420220 & 2.4804090 & 1.1619050 \\ \mathrm{C} & -5.1483660 & 3.1941680 & -1.6474900 \\ \mathrm{H} & -4.7936760 & 3.8502870 & -2.4538220 \\ \mathrm{H} & -5.7566430 & 2.4043970 & -2.1048470 \\ \mathrm{C} & -5.9884640 & 4.0038450 & -0.6087920 \\ \mathrm{H} & -6.0687170 & 5.0562300 & -0.9125740 \\ \mathrm{H} & -7.0054440 & 3.6005220 & -0.5346800 \\ \mathrm{H} & -5.8940330 & 4.4722780 & 1.5330040 \\ \mathrm{H} & -3.3310810 & 1.9691280 & -1.6428480 \\ \mathrm{C} & -3.8870330 & 4.5575550 & 0.6474790 \\ \mathrm{H} & -3.9890340 & 5.5971540 & 0.3072410 \\ \mathrm{H} & -3.3988090 & 4.5742380 & 1.6292890 \\ \mathrm{C} & -3.0513510 & 3.7194100 & -0.3669770 \\ \mathrm{H} & -2.7097780 & 4.3467170 & -1.2011640 \\ \mathrm{H} & -2.1687940 & 3.2929630 & 0.1148830 \\ \mathrm{C} & -5.6332180 & 1.8824480 & 2.3222010 \\ \mathrm{H} & -6.1952250 & 2.4689540 & 3.0502710 \\ \mathrm{C} & 4.8395340 & -0.1769260 & -0.5520200 \\ \mathrm{C} & 5.3184720 & 0.7004710 & -1.6822210 \\ \mathrm{C} & 3.1687350 & -0.6395770 & -2.2574830 \\ \mathrm{C} & 3.6639160 & -0.8893520 & -0.8515610 \\ \mathrm{C} & 5.5917050 & -0.2116830 & -2.9119460 \\ \mathrm{H} & 5.8776560 & 0.4185300 & -3.7644350 \\ \mathrm{H} & 6.4340650 & -0.8782450 & -2.6927290 \\ \mathrm{C} & 4.3071000 & -1.0348270 & -3.2405310 \\ \mathrm{H} & 3.9715950 & -0.8354030 & -4.2667400 \\ \mathrm{H} & 4.5039490 & -2.1102850 & -3.1578010 \\ \mathrm{H} & 2.2644240 & -1.2132570 & -2.4770140 \\ \mathrm{H} & 6.2262380 & 1.2484730 & -1.4007860 \\ \mathrm{C} & 2.8965950 & 0.8768940 & -2.4306100 \\ \mathrm{H} & 2.5991690 & 1.0685800 & -3.4695770 \\ \mathrm{H} & 2.0558660 & 1.1586140 & -1.7944850 \\ \mathrm{C} & 4.1810080 & 1.6849880 & -2.0711860 \\ \mathrm{H} & 4.5087270 & 2.2928620 & -2.9241660 \\ \mathrm{H} & 3.9830610 & 2.3741040 & -1.2439260 \\ \mathrm{C} & 4.9413750 & -1.2430850 & 1.6045070 \\ \mathrm{C} & 5.5125570 & -1.5523150 & 2.9654030 \\ \mathrm{C} & 3.3470660 & -2.8884760 & 2.4292670 \\ \mathrm{C} & 3.7626210 & -1.9583370 & 1.3135540 \\ & & & \end{array}$

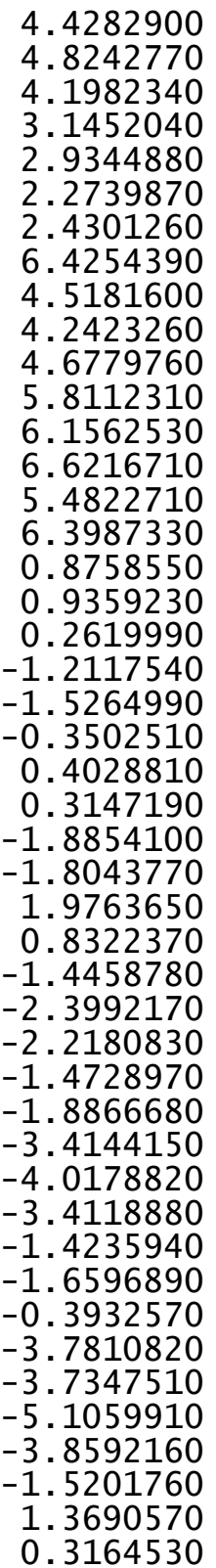
$-1.2302810$
$-1.4700820$
$-0.1586280$
$-2.0618090$
$-2.7582290$
$-1.4141160$
$-3.4293950$
$-0.9733260$
$-3.8783030$
$-4.5500220$
$-4.4947460$
$-3.0771670$
$-3.3243580$
$-3.3229980$
$-0.3568500$
0.1892960
$-4.7289700$
$-6.2294250$
$-6.6466490$
$-6.1699340$
$-5.0042130$
$-4.0951170$
$-6.7088250$
$-7.7351090$
$-7.0021600$
$-5.4516520$
$-6.5746980$
$-6.1944130$
$-5.8641740$
$-4.4400380$
$-1.9844700$
$-2.7142740$
$-2.2638280$
$-2.2824250$
$-1.2525270$
$-1.2918530$
$-2.9226730$
$-3.7924030$
$-2.5856830$
$-3.2889770$
$-2.0653810$
$-1.3834270$
$-0.2449920$
$-1.2435050$
3.5717900
2.8243590

4.0309510
5.0267470
4.0135150
3.7286740
4.5511020
3.6120160
2.1812240
3.1527570
2.6812860
3.5045890
1.7883430
3.0343070
4.0469970
2.3379320
0.6735810
0.9005520
-0.7975870
-0.9437560
-2.2679680
-2.3327170
-1.3511610
-1.0755510
-0.1061490
-2.3962170
-2.0930920
-0.3839630
-0.9067060
-3.0901630
-3.3595580
-1.6898900
-2.6479790
-3.7531960
-5.1602100
-5.2810020
-4.3182320
-2.9243650
-5.9060570
-3.6701400
-3.6064800
-5.0281740
-6.3081540
-4.2416210
-4.7348000
-5.3483550
-0.8277780
-1.0435850 


\begin{tabular}{|c|c|c|c|c|c|c|c|}
\hline & & & & & & & \\
\hline C & 1.8091240 & 4.3711450 & -2.0139470 & $\mathrm{C}$ & -1.6078990 & 4.1338420 & -0.7902650 \\
\hline C & 2.9188350 & 5.2231440 & -1.8922560 & $\mathrm{H}$ & -2.5178100 & 4.7145690 & -0.6491430 \\
\hline C & 1.1596330 & 4.2558080 & -3.2546230 & $\mathrm{C}$ & 4.0627970 & -0.7583350 & 0.4355930 \\
\hline C & 3.3734870 & 5.9484400 & -2.9925970 & $\mathrm{C}$ & -2.9251140 & 2.0710710 & -0.2175720 \\
\hline $\mathrm{H}$ & 3.4190060 & 5.3010470 & -0.9313670 & $\mathrm{C}$ & 4.5109780 & -0.2141180 & 1.6529700 \\
\hline C & 1.6194090 & 4.9797380 & -4.3552220 & $\mathrm{C}$ & 4.5363680 & 1.2483940 & 2.0327730 \\
\hline $\mathrm{H}$ & 0.3057410 & 3.5929230 & -3.3515840 & $\mathrm{C}$ & 5.4982100 & -0.2989270 & 3.8846140 \\
\hline C & 2.7257900 & 5.8265350 & -4.2272990 & $\mathrm{C}$ & 5.0446650 & -1.0457280 & 2.6553150 \\
\hline $\mathrm{H}$ & 4.2341150 & 6.6055990 & -2.8905260 & $\mathrm{C}$ & 3.7213040 & 1.4361730 & 3.3419340 \\
\hline $\mathrm{H}$ & 1.1156680 & 4.8821750 & -5.3142120 & $\mathrm{H}$ & 3.7971630 & 2.4870360 & 3.6522430 \\
\hline $\mathrm{H}$ & 3.0829640 & 6.3891050 & -5.0872720 & $\mathrm{H}$ & 2.6672470 & 1.2215670 & 3.1462100 \\
\hline $\mathrm{H}$ & -0.0073720 & 2.2246840 & -0.2483570 & $\mathrm{C}$ & 4.2882550 & 0.4953840 & 4.4501470 \\
\hline 0 & 1.9976740 & 3.6446190 & 0.2429810 & $\mathrm{H}$ & 4.6195990 & 1.0748880 & 5.3223750 \\
\hline C & 1.4514510 & 2.4559230 & 2.8265130 & $\mathrm{H}$ & 3.5185330 & -0.2077290 & 4.7899530 \\
\hline C & 2.3925260 & 2.0596940 & 1.7680390 & $\mathrm{H}$ & 5.8964650 & -0.9870330 & 4.6403320 \\
\hline $\mathrm{H}$ & 0.4026600 & 2.4671180 & 2.5292590 & $\mathrm{H}$ & 4.1310280 & 1.8823160 & 1.2402020 \\
\hline $\mathrm{H}$ & 2.0538760 & 1.3323130 & 1.0383810 & C & 6.5834590 & 0.7287770 & 3.4560040 \\
\hline 0 & 1.8367340 & 1.1661590 & 3.3959280 & $\mathrm{H}$ & 6.8741260 & 1.3252110 & 4.3312590 \\
\hline $\mathrm{H}$ & 1.1802400 & 0.4623250 & 2.9975470 & $\mathrm{H}$ & 7.4752980 & 0.1950620 & 3.1064010 \\
\hline C & 3.3642620 & 3.6468860 & 4.0032840 & C & 6.0101750 & 1.6444620 & 2.3301980 \\
\hline C & 4.1632300 & 3.6124880 & 2.6913280 & $\mathrm{H}$ & 6.0362080 & 2.6983340 & 2.6378390 \\
\hline C & 3.8462770 & 2.3501500 & 1.8744500 & $\mathrm{H}$ & 6.6052440 & 1.5509440 & 1.4137600 \\
\hline C & 1.8540590 & 3.6183860 & 3.7133840 & $\mathrm{C}$ & 4.1538080 & -2.1498320 & 0.2506750 \\
\hline $\mathrm{H}$ & 3.6103840 & 4.5509710 & 4.5731700 & C & 3.6838210 & -2.9340030 & -0.9536320 \\
\hline $\mathrm{H}$ & 5.2408380 & 3.6484350 & 2.8917580 & $\mathrm{C}$ & 4.7186660 & -4.4366350 & 0.8962400 \\
\hline $\mathrm{H}$ & 4.3061110 & 1.4573840 & 2.3225850 & $\mathrm{C}$ & 4.6945940 & -2.9724740 & 1.2574360 \\
\hline $\mathrm{H}$ & 1.5589130 & 4.5373160 & 3.1878820 & $\mathrm{C}$ & 4.8946400 & -3.7134600 & -1.5359660 \\
\hline $\mathrm{H}$ & 1.2702680 & 3.5644570 & 4.6407540 & $\mathrm{H}$ & 4.5452470 & -4.3338740 & 721600 \\
\hline $\mathrm{H}$ & 3.6337970 & 2.7820950 & 4.6240620 & $\mathrm{H}$ & 5.6329590 & -3.0064270 & -1.9336170 \\
\hline $\mathrm{H}$ & 3.9066330 & 4.4938220 & 2.0890340 & $\mathrm{C}$ & 5.5286100 & -4.6030240 & -0.4200660 \\
\hline $\mathrm{H}$ & 4.2540210 & 2.3960560 & 0.8601500 & $\mathrm{H}$ & 5.5164850 & -5.6609830 & -0.7148230 \\
\hline & & & & $\mathrm{H}$ & 6.5729850 & -4.3188020 & -0.2440980 \\
\hline & S) G[B97-D, & $\mathrm{ZVP}]=-35$ & 124 & $\mathrm{H}$ & 5.1646350 & -5.0345790 & 1.7004280 \\
\hline $\mathrm{P}$ & 0.2966980 & 0.1225320 & 0.8234820 & $\mathrm{H}$ & 3.2444500 & -2.2842170 & -1.7148880 \\
\hline 0 & -0.3379110 & 0.6435670 & 2.0830910 & $\mathrm{C}$ & 3.2606910 & -4.8942010 & 0.6162940 \\
\hline 0 & 0.4026860 & -1.3666160 & 0.6378220 & $\mathrm{H}$ & 3.2748160 & -5.9417370 & 0.2858770 \\
\hline 0 & 1.7341070 & 0.8685580 & 0.7359390 & $\mathrm{H}$ & 2.6778490 & -4.8443440 & 1.5437340 \\
\hline 0 & -0.4700270 & 0.6574970 & -0.5212900 & C & 2.6331480 & -3.9750960 & -0.4759920 \\
\hline C & 2.4043660 & 0.9112450 & -0.4868760 & $\mathrm{H}$ & 2.3054240 & -4.5672750 & -1.3409100 \\
\hline C & 1.9227100 & 1.7798240 & -1.4858970 & $\mathrm{H}$ & 1.7627830 & -3.4450790 & -0.0833860 \\
\hline C & 3.5441220 & 0.1163970 & -0.6484310 & $\mathrm{C}$ & 5.1396040 & -2.4237490 & 2.4597360 \\
\hline C & 4.1960740 & 0.1779410 & -1.8879130 & $\mathrm{H}$ & 5.5574760 & -3.0640980 & 3.2376160 \\
\hline $\mathrm{H}$ & 5.0897640 & -0.4250910 & -2.0373530 & C & -4.7425810 & 0.5359560 & -0.7875010 \\
\hline C & -0.4660940 & 2.0310450 & -0.7689210 & $\mathrm{C}$ & -5.2570040 & -0.3794850 & -1.8700920 \\
\hline C & -1.6534640 & 2.7486960 & -0.5846340 & $\mathrm{C}$ & -3.0366330 & 0.8143860 & -2.4977350 \\
\hline C & 0.7294570 & 2.6297310 & -1.2122590 & C & 3.5241900 & 1.1614690 & -1.1094920 \\
\hline
\end{tabular}




$\begin{array}{rrr}-5.4726350 & 0.4699280 & -3.1553280 \\ -5.7670380 & -0.1979840 & -3.9757750 \\ -6.2922600 & 1.1797370 & -2.9930420 \\ -4.1503810 & 1.2225290 & -3.5039160 \\ -3.8179880 & 0.9699450 & -4.5193390 \\ -4.3000490 & 2.3079830 & -3.4622400 \\ -2.1024160 & 1.3278860 & -2.7397470 \\ -6.1936020 & -0.8639120 & -1.5673510 \\ -2.8473430 & -0.7207210 & -2.6005910 \\ -2.5851400 & -0.9744410 & -3.6360670 \\ -2.0118880 & -1.0240290 & -1.9683350 \\ -4.1660570 & -1.4401580 & -2.1813450 \\ -4.5215210 & -2.1018050 & -2.9815510 \\ -4.0015570 & -2.0644300 & -1.2964320 \\ -4.8067600 & 1.7287620 & 1.3022770 \\ -5.3834600 & 2.1535850 & 2.6290610 \\ -3.1313840 & 3.3213380 & 2.0617150 \\ -3.5819780 & 2.3527410 & 0.9927810 \\ -4.3427080 & 1.8362860 & 3.7379900 \\ -4.7433650 & 2.1629340 & 4.7069410 \\ -4.1774590 & 0.7544410 & 3.7912410 \\ -3.0055650 & 2.5687490 & 3.4145720 \\ -2.7691080 & 3.3030130 & 4.1961640 \\ -2.1735050 & 1.8638560 & 3.3597620 \\ -2.1787160 & 3.7907330 & 1.8035560 \\ -6.3328790 & 1.6415430 & 2.8297390 \\ -4.2450930 & 4.3934960 & 2.2258600 \\ -3.9451320 & 5.0961230 & 3.0142870 \\ -4.3489160 & 4.9629860 & 1.2942480 \\ -5.5922030 & 3.6942760 & 2.5953410 \\ -5.9436620 & 4.0261710 & 3.5813530 \\ -6.3708950 & 3.9422640 & 1.8643470 \\ -5.3872980 & 0.8220400 & 0.4162640 \\ -6.3376690 & 0.3454250 & 0.6602070 \\ 0.7483000 & 4.0293010 & -1.3665760 \\ 1.9905410 & 4.8282260 & -1.6743010 \\ 2.1532620 & 5.9704240 & -0.6420620 \\ 0.8656500 & 6.8345600 & -0.4912620 \\ -0.3227940 & 6.2719030 & -1.3125260 \\ -0.4258680 & 4.7766550 & -1.1531640 \\ 1.9053700 & 5.2693310 & -2.6790520 \\ 3.0036770 & 6.5982920 & -0.9358280 \\ 1.0579860 & 7.8675350 & -0.8073510 \\ -0.1536330 & 6.5105250 & -2.3746000 \\ 2.8799540 & 4.1926840 & -1.6786740 \\ 2.4064820 & 5.5161160 & 0.3236250\end{array}$

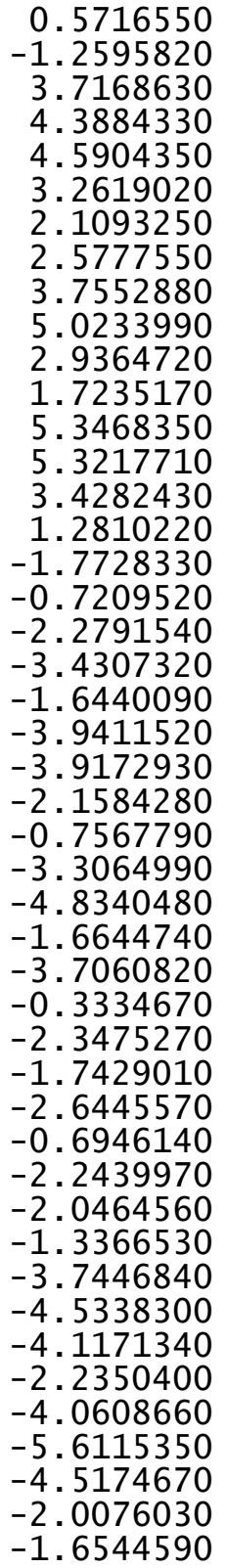

6.8705800

6.7594960

0.9728660

1. 0365120

2. 5091700

3.3179830

2. 5415950

1.7790660

0.5373070

2.5518290

3.5852700

1.8013540

0.5037870

2.9565820

4.2563340

3. 2146400

$-3.6053340$

$-2.8966390$

$-4.4925450$

$-5.2647180$

$-4.5454460$

$-6.0768220$

$-5.2137380$

$-5.3576760$

$-3.9452720$

$-6.1238220$

$-6.6710920$

$-5.3928380$

$-6.7555530$

$-2.2682000$

$-3.5763400$

-2.1414040

$-1.7965460$

$-2.2538660$

$-1.1795290$

$-0.7757350$

$-0.1604570$

$-3.0587070$

$-3.1066470$

$-1.9712540$

$-3.1733800$

$-3.8762810$

$-3.0367280$

$-1.0029300$

$-4.1600750$

$-3.0689320$
0.5647670

$-1.0174810$

$-2.9261880$

$-4.2750980$

$-4.6961910$

$-4.6410030$

$-3.9510800$

$-2.7336630$

$-5.0267280$

$-5.7034200$

$-5.6542330$

$-4.6701700$

$-4.2530470$

$-4.0110030$

$-4.1004960$

$-3.7215700$

$-0.6704270$

$-0.9957340$

$-1.7631480$

$-1.5386200$

$-3.0154760$

$-2.5503170$

$-0.5689260$

$-4.0266470$

$-3.1897300$

$-3.7969970$

$-2.3700420$

$-4.9949740$

$-4.5874790$

$-0.2551110$

0.4319420

2. 8617750

1.7533760

2.5827890

0.9569450

3. 2827020

2. 8254310

4.1285080

2. 8110910

1.8620530

3. 8575360

4.7875420

3. 0018210

2. 1948590

3.4306440

4.7825870 


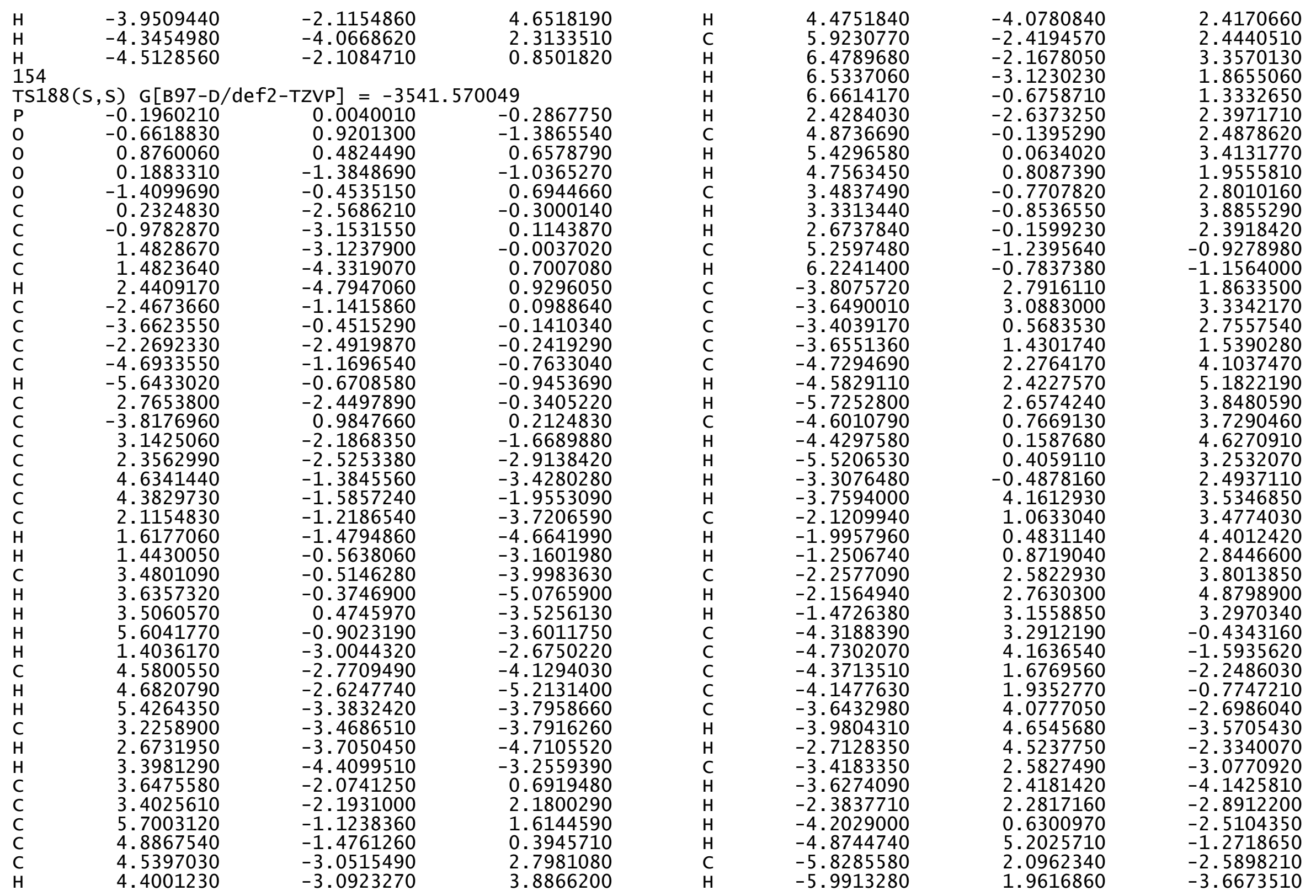




\begin{tabular}{|c|c|c|}
\hline $\begin{array}{l}-6.5345380 \\
-6.0504070 \\
-6.3433780 \\
-6.8498080 \\
-4.1389100 \\
-4.2778770 \\
-4.5143850 \\
-5.5996830 \\
-5.7330150 \\
-4.3924150 \\
-3.1611240 \\
-3.2977130 \\
-5.3439690 \\
-6.5242670 \\
-4.4233510 \\
-3.0705930 \\
-6.5509560 \\
-6.0431990 \\
-4.2542720 \\
-2.2436540 \\
-0.9334040 \\
0.3103340 \\
-2.1860530 \\
-2.0852790 \\
-0.7927340 \\
0.4200720 \\
-2.9646000 \\
-2.3463820 \\
-3.0701250 \\
-0.7840180 \\
-0.7366280 \\
1.3443980 \\
0.5261530 \\
-2.0882820 \\
3.2760700 \\
2.9936740 \\
4.6944770 \\
5.6456420 \\
5.0822330 \\
6.9663010 \\
5.3446810 \\
6.4029150 \\
4.3399300 \\
7.3472630 \\
7.6997810 \\
6.6981840\end{array}$ & $\begin{array}{l}1.4425940 \\
3.5879020 \\
4.1880450 \\
3.6710070 \\
3.7230240 \\
4.7738720 \\
-2.4855370 \\
-3.2644900 \\
-4.6613100 \\
-5.4437060 \\
-4.5300100 \\
-3.1507650 \\
-3.3865750 \\
-5.2352670 \\
-6.2035660 \\
-4.3920570 \\
-2.7206220 \\
-4.5184510 \\
-5.9794020 \\
-5.0236950 \\
-4.3216960 \\
-4.9320700 \\
-4.8424680 \\
-6.3088480 \\
-6.5382340 \\
-6.2416360 \\
-6.5740740 \\
-4.2203370 \\
-4.6940340 \\
-5.8689260 \\
-7.5684070 \\
-6.2382990 \\
-7.0598270 \\
-6.9574220 \\
2.8538320 \\
1.6826940 \\
3.2783080 \\
2.3871390 \\
4.5828030 \\
2.7999200 \\
1.3769430 \\
4.9928360 \\
5.2614460 \\
4.1019130 \\
2.1052750 \\
6.0047760\end{array}$ & $\begin{array}{r}-2.0632040 \\
-2.1816430 \\
-3.0533450 \\
-1.4357170 \\
0.8788530 \\
1.1371610 \\
-1.1878280 \\
-1.8899540 \\
-1.2454430 \\
-1.2864400 \\
-1.5470890 \\
-0.9442070 \\
-2.9553680 \\
-1.7437600 \\
-2.0772540 \\
-2.6358460 \\
-1.8445040 \\
-0.2020340 \\
-0.3397490 \\
-1.2172930 \\
0.9116400 \\
1.1674680 \\
1.5996530 \\
2.0334990 \\
2.8237640 \\
1.9334190 \\
2.6348120 \\
2.4959170 \\
0.9797230 \\
3.6971930 \\
3.1988510 \\
2.5262960 \\
1.2025180 \\
1.1439390 \\
0.5526450 \\
0.0320510 \\
0.3493420 \\
-0.1776790 \\
0.6970690 \\
-0.3520750 \\
-0.4367810 \\
0.5157890 \\
1.1080720 \\
-0.0078590 \\
-0.7555210 \\
0.7836390\end{array}$ \\
\hline
\end{tabular}

8.3779150
2.0744660
2.4689510
0.5413140
0.3106360
1.1553620
0.2724630
-0.8730930
-0.8912210
0.0219420
0.2552430
-0.1665170
0.8255660
0.3207180
-0.2991150
-1.2633100
1.9018290
0.6145850
-1.0466210
1.3225770
0.1876920

1.2662590

3.5820700

3.7329830

3. 5761610

2.9575720

2. 5659180

3. 3911480

2. 3529480

6. 2044460

6.0913000

4.7113480

5.1287310

7. 1980500

6.8700080

4.6224680

5. 3091000

.1495190

6.0821880

6. 2382140

4. 5411070
$-0.1458030$

0.2986520

1.1567680

$-0.9602330$

0.4835200

$-1.4232270$

0.8726460

$-1.0128390$

$-1.1511250$

$-0.7301810$

0.7843950

1.3189900

$-1.4804510$

$-1.0852870$

1. 3214890

1. 3580450

$-1.3487940$

$-2.5569270$

$-0.9527450$

0.9963120

2. 3423320

TS189(S, S) G[B97-D/def2-TZVP $]=-3541.570038$

\begin{tabular}{lrrr} 
TS189(S, S & \multicolumn{3}{c}{ G $[\mathrm{B} 97-\mathrm{D} / \mathrm{de} 2-\mathrm{TZVP}=-3541.570038$} \\
$\mathrm{P}$ & 0.2029460 & -0.0103220 & 0.2809280 \\
$\mathrm{O}$ & 0.6990200 & 0.8529780 & 1.4099190 \\
$\mathrm{O}$ & -0.8852730 & 0.5173640 & -0.6175910 \\
$\mathrm{O}$ & -0.1763090 & -1.4318490 & 0.9689190 \\
$\mathrm{O}$ & 1.3932660 & -0.4281380 & -0.7466350 \\
$\mathrm{C}$ & -0.2275730 & -2.5701950 & 0.1613340 \\
$\mathrm{C}$ & 0.9886900 & -3.1408780 & -0.2659950 \\
$\mathrm{C}$ & -1.4805720 & -3.0830870 & -0.1940060 \\
$\mathrm{C}$ & -1.4934080 & -4.2392270 & -0.9872730 \\
$\mathrm{H}$ & -2.4537090 & -4.6706510 & -1.2638100 \\
$\mathrm{C}$ & 2.4667680 & -1.1373230 & -0.2099690 \\
$\mathrm{C}$ & 3.6728970 & -0.4581510 & 0.0014960 \\
$\mathrm{C}$ & 2.2843670 & -2.4946450 & 0.1012470 \\
$\mathrm{C}$ & 4.7220570 & -1.2042960 & 0.5462380 \\
$\mathrm{H}$ & 5.6798640 & -0.7117640 & 0.7049440 \\
$\mathrm{C}$ & -2.7583620 & -2.4186160 & 0.1795740 \\
$\mathrm{C}$ & 3.8179020 & 0.9905860 & -0.3018850 \\
$\mathrm{C}$ & -3.1501040 & -2.2650260 & 1.5205820 \\
$\mathrm{C}$ & -2.3811420 & -2.7133810 & 2.7405550 \\
$\mathrm{C}$ & -4.6590310 & -1.6074520 & 3.3240780 \\
$\mathrm{C}$ & -4.3907360 & -1.6824380 & 1.8421420 \\
$\mathrm{C}$ & -2.1425280 & -1.4810460 & 3.6566890 \\
$\mathrm{H}$ & -1.6507050 & -1.8212280 & 4.5778530
\end{tabular}




-1.4655590
-3.5076060
-3.6745760
-3.5249880
-5.6284880
-1.4282700
-4.6232890
-4.7438440
-5.4658560
-3.2660240
-2.7269320
-3.4328300
-3.6256840
-3.3684850
-5.6729700
-4.8676060
-4.4988920
-4.3535940
-4.4321880
-5.8867410
-6.4420480
-6.4948120
-6.6375280
-2.3906490
-4.8460460
-5.3968500
-4.7406420
-3.4501600
-3.2888490
-2.6460360
-5.2557820
-6.2236520
3.7452370
3.5288800
3.3087620
3.6054780
4.5779030
4.3865130
5.5830300
4.4669990
4.2626320
5.4050540
3.2233380
3.6304760
1.9983240
1.8377760

$-0.7830930$

$-0.7995220$

$-0.7603700$

0.2299370

$-1.1355070$

$-3.1761750$

$-3.0503080$

$-3.0005800$

$-3.6255760$

$-3.7227060$

$-4.0365940$

$-4.6153260$

$-1.9525640$

$-1.9616020$

$-0.9403890$

$-1.3786540$

$-2.7754530$

$-2.7308660$

$-3.8281810$

$-2.1770770$

$-1.8655650$

$-2.9220530$

$-0.5164560$

$-2.3853650$

0.1029180

0.3601590

1.0173450

$-0.4992610$

$-0.5059100$

0.0844290

$-1.2523730$

$-0.8184070$

2.8603910

3.2132490

0.6721220

1.4871910

2.4341290

2.6209020

2.8077720

0.9109970

0.3365170

0.5359590

$-0.3933810$

4.2934440

1.1913060

0.6477250

3.1566520
3.9842860
5.0691820
3.6066320
3.5266200
2.4718750
3.9006000
4.9910920
3.4986470
3.5247680
4.4284040
2.9098740
-0.8280060
-2.3180070
-1.6940860
-0.4964830
-3.0060130
-4.0936300
-2.7054390
-2.6122070
-3.5069600
-2.0850280
-1.3887700
-2.5583480
-2.4948140
-3.4096580
-1.9039770
-2.8383850
-3.9247840
-2.3817140
0.8369160
1.0918920
-1.8790850
-3.3300120
-2.8409190
-1.6027360
-4.1734510
-5.2386350
-3.9447870
-3.8514610
-4.7646060
-3.4251560
-2.6152400
-3.4928560
-3.4928550
-4.4334020

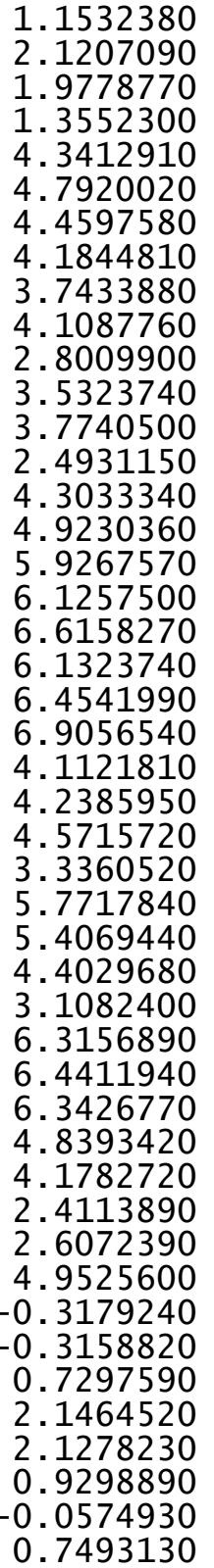

2.7222250

2.9452650

3.2733330

3.2706750

4.0971150

1.5850160

1.9017570

3.9628240

. 5045720

4.4216700

2.4532120

2.2461980

. 1566940

0.5273160

5.1486880

1.9952340

1. 8192580

1. 3647520

3. 5021560

4.0687740

3.6166010

3.7534930

4.8142520

$-2.5392340$

$-3.1885510$

$-3.2595840$

$-4.5169050$

$-5.3654740$

$-4.5752170$

$-5.0893880$

$-3.5515680$

$-2.5582650$

$-5.6369550$

$-6.2998280$

$-5.1476710$

$-4.4442840$

$-4.2279120$

$-6.0375350$

$-7.0510680$

$-6.4204280$

$-4.8692490$

$-4.2550930$

$-5.7523970$

$-7.9349420$
$-2.8349380$

$-3.7617850$

$-4.8276140$

$-3.2054210$

0.4159740

1.5950050

2. 1618820

0.7086280

2.7317150

3.6147460

2. 4170250

3.0563260

4.1073580

2.8899600

2.3850000

1. 3110590

2.4706140

3.5360540

1.8955640

2.1138760

2.9977360

1.3449020

$-0.8716760$

$-1.0932600$

0.9354440

0.7434100

1.5309720

2.3305890

1.5418610

1. 3225480

2.5574720

0.7054750

2.1541310

0.5683610

2.0724640

0.7087670

2.2957730

3.2900520

$-1.4645280$

$-2.3468120$

$-1.8320360$

$-1.7343400$

$-1.8160980$

$-1.1276110$

$-3.3799470$

$-2.4818020$ 


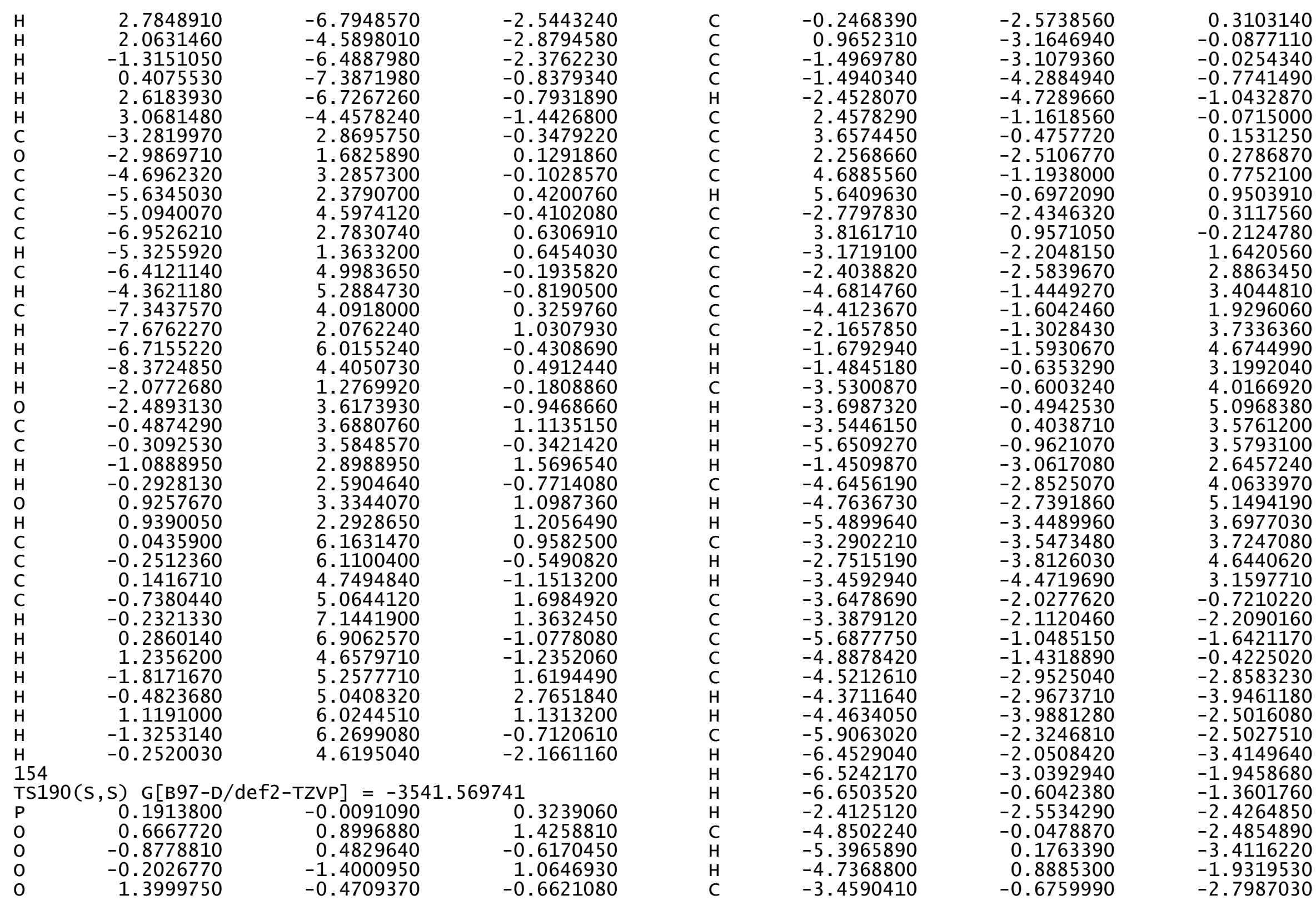




$\begin{array}{lrrr}\mathrm{H} & -3.2964490 & -0.7356500 & -3.8832800 \\ \mathrm{H} & -2.6510590 & -0.0773120 & -2.3686890 \\ \mathrm{C} & -5.2751200 & -1.2271430 & 0.9012920 \\ \mathrm{H} & -6.2403130 & -0.7731380 & 1.1300890 \\ \mathrm{C} & 3.7971750 & 2.7515810 & -1.8766790 \\ \mathrm{C} & 3.6266550 & 3.0376400 & -3.3483730 \\ \mathrm{C} & 3.3791260 & 0.5229040 & -2.7488590 \\ \mathrm{C} & 3.6434980 & 1.3931410 & -1.5408830 \\ \mathrm{C} & 4.6982070 & 2.2168300 & -4.1208780 \\ \mathrm{H} & 4.5429350 & 2.3557280 & -5.1991250 \\ \mathrm{H} & 5.6973310 & 2.5964740 & -3.8763470 \\ \mathrm{C} & 4.5681750 & 0.7105590 & -3.7340810 \\ \mathrm{H} & 4.3870580 & 0.0964090 & -4.6261090 \\ \mathrm{H} & 5.4907790 & 0.3500180 & -3.2637710 \\ \mathrm{H} & 3.2823690 & -0.5310080 & -2.4781000 \\ \mathrm{H} & 3.7382180 & 4.1087220 & -3.5582420 \\ \mathrm{C} & 2.0911250 & 1.0160430 & -3.4624040 \\ \mathrm{H} & 1.9547230 & 0.4283770 & -4.3799330 \\ \mathrm{H} & 1.2264340 & 0.8331520 & -2.8194960 \\ \mathrm{C} & 2.2301730 & 2.5317290 & -3.8003570 \\ \mathrm{H} & 2.1214520 & 2.7035550 & -4.8795650 \\ \mathrm{H} & 1.4506480 & 3.1118560 & -3.2950880 \\ \mathrm{C} & 4.3313210 & 3.2664310 & 0.4125670 \\ \mathrm{C} & 4.7567530 & 4.1457990 & 1.5614380 \\ \mathrm{C} & 4.3946510 & 1.6655860 & 2.2383670 \\ \mathrm{C} & 4.1583640 & 1.9137790 & 0.7646720 \\ \mathrm{C} & 3.6803970 & 4.0723060 & 2.6776550 \\ \mathrm{H} & 4.0284990 & 4.6542510 & 3.5418500 \\ \mathrm{H} & 2.7481260 & 4.5193420 & 2.3191400 \\ \mathrm{C} & 3.4532760 & 2.5811410 & 3.0693650 \\ \mathrm{H} & 3.6720950 & 2.4235090 & 4.1339270 \\ \mathrm{H} & 2.4157620 & 2.2829760 & 2.8957560 \\ \mathrm{H} & 4.2247520 & 0.6214740 & 2.5099780 \\ \mathrm{H} & 4.9018130 & 5.1817780 & 1.2307600 \\ \mathrm{C} & 5.8567750 & 2.0817490 & 2.5626930 \\ \mathrm{H} & 6.0291890 & 1.9547090 & 3.6396350 \\ \mathrm{H} & 6.5553650 & 1.4215370 & 2.0344070 \\ \mathrm{C} & 6.0803400 & 3.5695010 & 2.1412730 \\ \mathrm{H} & 6.3835080 & 4.1750190 & 3.0057300 \\ \mathrm{H} & 6.8730190 & 3.6440850 & 1.3873150 \\ \mathrm{C} & 4.1407780 & 3.6890910 & -0.9021110 \\ \mathrm{H} & 4.2810770 & 4.7374730 & -1.1695930 \\ \mathrm{C} & 4.5057250 & -2.5041430 & 1.2137950 \\ \mathrm{C} & 5.5881210 & -3.2680050 & 1.9369940 \\ \mathrm{C} & 5.7036420 & -4.6868170 & 1.3438810 \\ \mathrm{C} & 4.3640790 & -5.4595850 & \\ & & & \end{array}$

\begin{abstract}
3. 1298380
3.2866630

5.3373660

6.5057460

4. 3931600

3. 0043810

6.5432940

5. 9842840

4. 2368770

2. 2161420

0.9264430

2. 2067490

1. 9647850

0.8920600

$-0.4419260$

$-0.3186180$

2. 9067190

2. 7192370

2.8923010

1. 2060990

0.7714650

$-1.1641100$

$-0.8775980$

1. 6286840

$-3.2581420$

$-2.9785830$

$-4.6739070$

$-5.6282600$

$-5.0565500$

$-6.9469810$

$-5.3314890$

$-6.3752410$

$-4.3120370$

$-7.3227690$

$-7.6831090$

$-6.6665160$

$-8.3519140$

$-2.0659360$

$-2.4506350$

$-0.4983120$

$-0.2828870$

$-1.1191420$

$-0.2628090$

0.9114670

0.9154220

0.0571060
\end{abstract}

-4.5263950
-3.1695080
-3.3498100
-5.2458760
-6.1405260
-4.3401520
-2.7335010
-4.5857310
-6.0850770
-5.0252920
-4.3115260
-4.8766980
-5.7505270
-6.7985410
-6.0982940
-4.8850190
-6.2275620
-5.4922180
-4.0523570
-7.3767980
-7.5068500
-6.8027320
-5.7663290
-5.1252800
2.8682440
1.7011050
3.3009360
2.4166100
4.6051140
2.8358640
1.4060300
5.0217770
5.2782280
4.1377010
2.1462960
6.0335000
4.4628370
1.2749510
3.5861960
3.7278490
3.5607660
2.9636710
2.5482520
3.3651500
2.3286320
6.1897260

1.6248360

0.9777450

3. 0076350

1.8419160

2. 3131420

2.7030770

1.8671660

0.2871190

0.5618370

1. 2924250

$-0.9174500$

$-1.5099960$

$-2.7473640$

$-2.4330560$

$-2.1484750$

$-1.2387790$

$-3.0472870$

$-0.7603160$

$-1.7417190$

$-1.5508150$

$-3.2628010$

$-1.7131430$

$-3.1042590$

$-3.5881700$

$-0.5016400$

0.0297910

$-0.2968520$

0.2358470

$-0.6512190$

0.4094570

0.4983860

$-0.4704950$

$-1.0672840$

0.0589790

0.8168150

$-0.7434980$

0.1963070

$-0.2444300$

$-1.1173150$

0.9797300

$-0.4651450$

1.4521340

$-0.8495030$

1. 0208060

1.1703630

0.7333060 


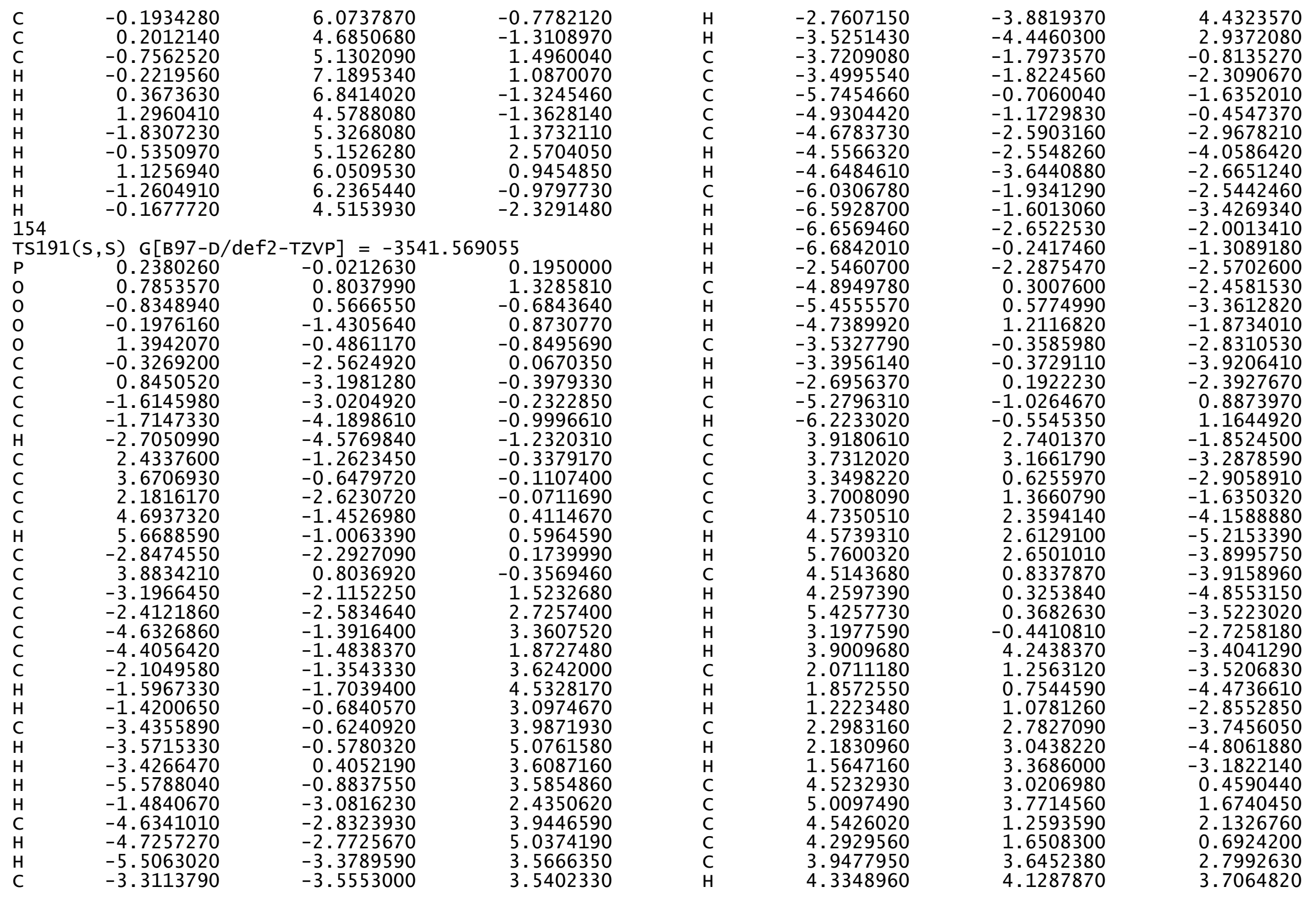




\begin{tabular}{|c|c|c|c|}
\hline & & & \\
\hline${ }_{\mathrm{C}}^{\mathrm{H}}$ & $\begin{array}{l}3.0334850 \\
3.6545250\end{array}$ & $\begin{array}{l}4.1668150 \\
2.1366050\end{array}$ & $\begin{array}{r}2.4997730 \\
3.0571010\end{array}$ \\
\hline $\mathrm{H}$ & 3.8740520 & 1.8718610 & 4.1001210 \\
\hline $\mathrm{H}$ & 2.6032940 & 1.9020680 & 2.8690490 \\
\hline $\mathrm{H}$ & 4.3280680 & 0.2033640 & 2.3096310 \\
\hline $\mathrm{H}$ & 5.1985550 & 4.8255070 & 1.4356440 \\
\hline C & 6.0270280 & 1.5772600 & 2.4640910 \\
\hline $\mathrm{H}$ & 6.2104070 & 1.3452150 & 3.5216470 \\
\hline $\mathrm{H}$ & 6.6851100 & 0.9361940 & 1.8648850 \\
\hline C & 6.3130880 & 3.0848710 & 2.1729130 \\
\hline $\mathrm{H}$ & 6.6587860 & 3.5958740 & 3.0813470 \\
\hline $\mathrm{H}$ & 7.0960880 & 3.1906390 & 1.4125720 \\
\hline C & 4.3283740 & 3.5689770 & -0.8078640 \\
\hline $\mathrm{H}$ & 4.5139990 & 4.6294510 & -0.9846870 \\
\hline C & 3.2155570 & -3.3786230 & 0.5146490 \\
\hline C & 3.0529440 & -4.7921300 & 1.0166600 \\
\hline $\mathrm{C}$ & 3.5494440 & -4.8960810 & 2.4794320 \\
\hline $\mathrm{C}$ & 4.9811530 & -4.3135260 & 2.6734200 \\
\hline C & 5.5285400 & -3.6533180 & 1.3823330 \\
\hline $\mathrm{C}$ & 4.4753850 & -2.7892420 & 0.7372640 \\
\hline $\mathrm{H}$ & 3.6479690 & -5.4756130 & 0.3920510 \\
\hline $\mathrm{H}$ & 3.5180170 & -5.9473510 & 2.7917750 \\
\hline $\mathrm{H}$ & 5.6756890 & -5.1006510 & 2.9929480 \\
\hline $\mathrm{H}$ & 5.8209120 & -4.4520500 & 0.6823630 \\
\hline $\mathrm{H}$ & 2.0123810 & -5.1197930 & 0.9447070 \\
\hline $\mathrm{H}$ & 2.8409660 & -4.3499620 & 3.1140310 \\
\hline $\mathrm{H}$ & 4.9633160 & -3.5579760 & 3.4683760 \\
\hline $\mathrm{H}$ & 6.4301600 & -3.0688860 & 1.6011500 \\
\hline C & -0.5880660 & -4.8353850 & -1.5003740 \\
\hline C & -0.6776210 & -6.0796320 & 3487900 \\
\hline $\mathrm{C}$ & 0.3291100 & -7.1362590 & -1.8417770 \\
\hline $\mathrm{C}$ & 1.7771660 & -6.5721540 & -1.7747510 \\
\hline $\mathrm{C}$ & 1.8310020 & -5.0303150 & -1.9375980 \\
\hline C & 0.6980210 & -4.3311310 & -1.2234570 \\
\hline $\mathrm{H}$ & -0.4359310 & -5.8332240 & -3.3957930 \\
\hline $\mathrm{H}$ & 0.2935930 & -8.0251000 & -2.4840200 \\
\hline $\mathrm{H}$ & 2.3966740 & -7.0203000 & -2.5617280 \\
\hline $\mathrm{H}$ & 1.7216570 & -4.8033670 & -3.0100930 \\
\hline $\mathrm{H}$ & -1.6995690 & -6.4775700 & -2.3375300 \\
\hline $\mathrm{H}$ & 0.0099710 & -7.4488980 & -0.8391930 \\
\hline $\mathrm{H}$ & 2.2310580 & -6.8451460 & -0.8157830 \\
\hline $\mathrm{H}$ & 2.8076950 & -4.6478890 & -1.6365000 \\
\hline C & 3.1389140 & 2.9993120 & -0.3484850 \\
\hline 0 & -2.8873090 & 1.7929290 & 0.1040800 \\
\hline $\mathrm{C}$ & -4.5340200 & 3.4664350 & -0.0864200 \\
\hline $\mathrm{C}$ & -5.4974980 & 2.5953590 & 0.4511210 \\
\hline
\end{tabular}

$\begin{array}{lr}\text { C } & -4.8885460 \\ \text { C } & -6.7969190 \\ \text { H } & -5.2238490 \\ \text { C } & -6.1884930 \\ \text { H } & -4.1380120 \\ \text { C } & -7.1450050 \\ \text { H } & -7.5398470 \\ \text { H } & -6.4583520 \\ \text { H } & -8.1594490 \\ \text { H } & -1.9949290 \\ \text { O } & -2.3199400 \\ \text { C } & -0.3215030 \\ \text { C } & -0.1452270 \\ \text { H } & -0.9441410 \\ \text { H } & -0.1565000 \\ \text { O } & 1.0818840 \\ \text { H } & 1.0658050 \\ \text { C } & 0.2688360 \\ \text { C } & -0.0285950 \\ \text { C } & 0.3362790 \\ \text { C } & -0.5355470 \\ \text { H } & 0.0154870 \\ \text { H } & 0.5248900 \\ \text { H } & 1.4281160 \\ \text { H } & -1.6099850 \\ \text { H } & -0.2752520 \\ \text { H } & 1.3413020 \\ \text { H } & -1.0992200 \\ \text { H } & -0.0558880\end{array}$

154 TS192(S, S) G[B97-D/def2-TZVP $]=-3541.568937$

$\begin{array}{lrrr}\text { TS192(S, S) } \text { G }[\mathrm{B} 97-\mathrm{D} / \mathrm{def2}-\mathrm{TZVP}]=-3541.568937 \\ \mathrm{P} & 0.2011300 & -0.0207440 & 0.3476100 \\ \mathrm{O} & 0.6766130 & 0.9044330 & 1.4356300 \\ \mathrm{O} & -0.8704380 & 0.4542250 & -0.5988800 \\ \mathrm{O} & -0.1851070 & -1.4009880 & 1.1100240 \\ \mathrm{O} & 1.4073590 & -0.4977380 & -0.6320310 \\ \mathrm{C} & -0.2271950 & -2.5927830 & 0.3857440 \\ \mathrm{C} & 0.9912940 & -3.1908480 & -0.0021550 \\ \mathrm{C} & -1.4782250 & -3.1421880 & 0.0830620 \\ \mathrm{C} & -1.4903600 & -4.3605210 & -0.6119370 \\ \mathrm{H} & -2.4506100 & -4.8107620 & -0.8565230 \\ \mathrm{C} & 2.4723890 & -1.1759660 & -0.0408120 \\ \mathrm{C} & 3.6711630 & -0.4814450 & 0.1596420 \\ \mathrm{C} & 2.2869890 & -2.5253640 & 0.3179330 \\ \mathrm{C} & 4.7248360 & -1.1918240 & 0.7518560 \\ \mathrm{H} & 5.6765390 & -0.6863640 & 0.9042830\end{array}$

$\begin{array}{lr}4.7909700 & -0.3919090 \\ 3.0474340 & 0.6791040 \\ 1.5689780 & 0.6736560 \\ 5.2394460 & -0.1596420 \\ 5.4541700 & -0.8128830 \\ 4.3685780 & 0.3756550 \\ 2.3680510 & 1.0912840 \\ 6.2660150 & -0.3969150 \\ 4.7191520 & 0.5537440 \\ 1.3611260 & -0.2202870 \\ 3.7259570 & -0.9380440 \\ 3.6792070 & 1.1285150 \\ 3.6079160 & -0.3292530 \\ 2.8945670 & 1.5637960 \\ 2.6246120 & -0.7837160 \\ 3.2876620 & 1.1044770 \\ 2.2432900 & 1.1761770 \\ 6.1450430 & 1.0322600 \\ 6.1341480 & -0.4756390 \\ 4.7803670 & -1.1092610 \\ 5.0465170 & 1.7484030 \\ 7.1219210 & 1.4611400 \\ 6.9313410 & -0.9858090 \\ 4.6665300 & -1.1905840 \\ 5.2682760 & 1.6808820 \\ 4.9897000 & 2.8127380 \\ 5.9785510 & 1.1992620 \\ 6.3195150 & -0.6339270 \\ 4.6837450 & -2.1282840\end{array}$

4.7909700

0.6736560

$-0.8128830$

0.3756550

1.0912840

0.5537440

0.2202870

1.1285150

$-0.3292530$

5637960

1.1044770

1761770

.4756390

1.1092610

1.4611400

1.1905840

1.6808820

(

$-2.1282840$
5.6765390

$-0.6863640$

0.9042830

. 5721540

0.4511210 


-2.7586200
3.8117000
-3.1489670
-2.3763240
-4.6569830
-4.3911670
-2.1410060
-1.6522740
-1.4621030
-3.5070990
-3.6730850
-3.5266830
-5.6277520
-1.4224410
-4.6126040
-4.7257250
-5.4565800
-3.2564370
-2.7134140
-3.4248990
-3.6285460
-3.3668930
-5.6704260
-4.8700280
-4.4972150
-4.3459030
-4.4369710
-5.8842980
-6.4311090
-6.5003910
-6.6344300
-2.3903580
-4.8343880
-5.3798930
-4.7241110
-3.4407660
-3.2748440
-2.6359980
-5.2568680
-6.2230530
3.7665930
3.5850250
3.3611020
3.6265190
4.6586470
4.4968790

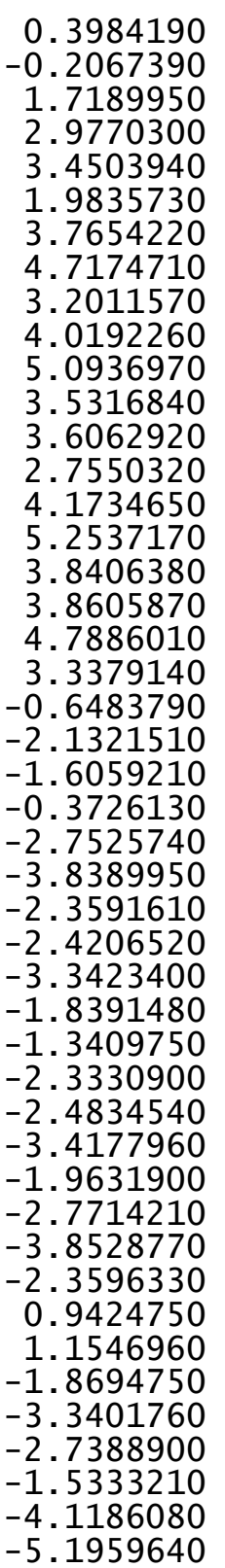

5.6561340
4.5419110
4.3591400
5.4703070
3.2741110
3.6865990
2.0644350
1.9246890
1.2061620
2.1901780
2.0749840
1.4085570
4.3091330
4.7321970
4.3976800
4.1514200
3.6628080
4.0115220
2.7249700
3.4497930
3.6718080
2.4148900
4.2400020
4.8653340
5.8567520
6.0337150
6.5603060
6.0644270
6.3674500
6.8517970
4.1074240
4.2368880
4.5687980
5.6813950
5.8139150
4.4738180
3.2541650
3.3472600
5.4557250
6.6082230
4.5293310
3.2355290
6.6253730
6.1223140
4.3021460
2.3217050
2.6061290

0.7116710

0.0969220

0.3574130

$-0.5394170$

4. 1036550

0.9979590

0.4080440

0.8100460

2. 5140810

2. 6832110

3. 0889110

3. 2662040

4.1479970

1.6630430

1.9116570

4.0621060

4.6439800

4. 5032200

2. 5678900

2. 4098890

2.2604960

0.6164220

5. 1859120

2. 0941470

1.9694220

1. 4405440

3. 5836860

4. 1919250

3.6652850

3.6878050

4.7375380

$-2.5098970$

$-3.2839150$

$-4.6742350$

$-5.4612840$

$-4.5671650$

$-3.1820180$

$-3.4177920$

$-5.2495490$

$-6.2692630$

$-4.4434750$

$-2.7295740$

$-4.5212920$

$-5.9318540$

$-5.0651040$
$-3.8788200$

$-3.7321390$

$-4.6234340$

$-3.2684950$

$-2.4663260$

$-3.5512800$

$-3.4432230$

$-4.3588200$

$-2.7930730$

$-3.7843120$

$-4.8632640$

$-3.2765400$

0.4172600

1. 5652030

2. 2406190

0.7688680

2. 6873300

3. 5513280

2. 3360160

3. 0760330

4.1398730

2. 9031380

2. 5106010

1.2356070

2. 5598080

3. 6362870

2. 0299280

2.1370190

2. 9996140

1. 3782240

$-0.8962800$

$-1.1639890$

1.1761750

1.8388210

1. 1796980

1. 2154110

1. 5689490

0.9738360

2.9095410

1. 6714800

1.9556560

2. 6633220

1.7728210

0.1373570

0.2411400

1.2955860 


\begin{tabular}{|c|c|c|}
\hline $\begin{array}{l}0.9320670 \\
2.1281850 \\
1.8567080 \\
0.5176570 \\
0.3042870 \\
0.3144300 \\
2.3166670 \\
2.6985380 \\
0.7099730 \\
0.1618390 \\
3.0324670 \\
1.8313970 \\
0.0906530 \\
-1.3268210 \\
-3.2612050 \\
2.9903020 \\
4.6815290 \\
-5.6441140 \\
-5.0597680 \\
6.9667200 \\
5.3501660 \\
6.3824480 \\
4.3085370 \\
7.3382730 \\
-7.7092460 \\
6.6702470 \\
-8.3705300 \\
2.0691180 \\
-2.4423700 \\
0.5388280 \\
0.2920990 \\
-1.1556010 \\
0.2474180 \\
0.8759970 \\
0.8970370 \\
0.0237760 \\
0.2396670 \\
0.1901780 \\
0.8332250 \\
0.3290200 \\
0.3191130 \\
1.2873990 \\
-1.9083970 \\
0.6348910 \\
1.0424880 \\
-1.3048660\end{array}$ & $\begin{array}{l}-4.3804190 \\
-5.0445520 \\
-5.2893260 \\
-6.0427380 \\
-6.2373930 \\
-4.9737340 \\
-6.0133570 \\
-5.8479590 \\
-7.0248260 \\
-7.0449310 \\
-4.4435890 \\
-4.3126550 \\
-5.4745370 \\
-6.5555780 \\
2.8302290 \\
1.6696460 \\
3.2624560 \\
2.3849880 \\
4.5597000 \\
2.8042680 \\
1.3799810 \\
4.9764870 \\
5.2272820 \\
4.0992030 \\
2.1200990 \\
5.9828780 \\
4.4241360 \\
1.2454730 \\
3.5432970 \\
3.7095690 \\
3.5456370 \\
2.9346940 \\
2.5332140 \\
3.3728690 \\
2.3358850 \\
6.1810560 \\
6.0594020 \\
4.6777110 \\
5.1071610 \\
7.1757330 \\
6.8364570 \\
4.5901660 \\
5.2839140 \\
5.1341950 \\
6.0626210 \\
6.2032030\end{array}$ & $\begin{array}{r}-0.7553140 \\
-1.3935030 \\
-2.8982900 \\
-3.1544180 \\
-1.8541210 \\
-1.0339940 \\
-0.9063030 \\
-3.3261700 \\
-3.6044250 \\
-1.2680030 \\
-1.2695740 \\
-3.3971820 \\
-3.8687490 \\
-2.0895190 \\
-0.5648080 \\
-0.0151160 \\
-0.3943940 \\
0.1348220 \\
-0.7776360 \\
0.2759660 \\
0.4209090 \\
-0.6293190 \\
-1.1905540 \\
-0.1034440 \\
0.6807700 \\
-0.9247730 \\
0.0086380 \\
-0.2622480 \\
-1.1711540 \\
0.9691670 \\
-0.4711220 \\
1.4293060 \\
-0.8537120 \\
1.0386900 \\
1.1863250 \\
0.7321200 \\
-0.7843350 \\
-1.3076100 \\
1.4786860 \\
1.0786170 \\
-1.3192550 \\
-1.3378570 \\
1.3335940 \\
2.5574200 \\
0.9675550 \\
-1.0088520\end{array}$ \\
\hline
\end{tabular}

$\mathrm{H}$

TS193 (S, S) G[B97-D/def2-TZVP $]=-3541.568765$

$\begin{array}{lrrr}\text { TS193 (S , S }) \text { G [B97-D/def2-TZVP] }=-3541.568765 \\ \mathrm{P} & 0.2099620 & -0.0133730 & 0.2610490 \\ \mathrm{O} & 0.7173840 & 0.8431870 & 1.3901260 \\ \mathrm{O} & -0.8672390 & 0.5310190 & -0.6411320 \\ \mathrm{O} & -0.1958470 & -1.4269690 & 0.9509080 \\ \mathrm{O} & 1.3958710 & -0.4525420 & -0.7628890 \\ \mathrm{C} & -0.2773330 & -2.5624830 & 0.1421360 \\ \mathrm{C} & 0.9234120 & -3.1574750 & -0.2991070 \\ \mathrm{C} & -1.5435740 & -3.0478660 & -0.2025030 \\ \mathrm{C} & -1.5868570 & -4.1995320 & -1.0013450 \\ \mathrm{H} & -2.5583070 & -4.6088700 & -1.2723910 \\ \mathrm{C} & 2.4482240 & -1.1888230 & -0.2193640 \\ \mathrm{C} & 3.6651600 & -0.5377500 & 0.0190500 \\ \mathrm{C} & 2.2319280 & -2.5436390 & 0.0759400 \\ \mathrm{C} & 4.6778500 & -1.3047870 & 0.6019100 \\ \mathrm{H} & 5.6401750 & -0.8329170 & 0.7926370 \\ \mathrm{C} & -2.8050920 & -2.3590670 & 0.1821980 \\ \mathrm{C} & 3.8536980 & 0.9047750 & -0.2899090 \\ \mathrm{C} & -3.1847290 & -2.2000400 & 1.5259510 \\ \mathrm{C} & -2.4141740 & -2.6607110 & 2.7402670 \\ \mathrm{C} & -4.6711360 & -1.5212910 & 3.3404470 \\ \mathrm{C} & -4.4136240 & -1.5978220 & 1.8566110 \\ \mathrm{C} & -2.1506440 & -1.4322030 & 3.6541460 \\ \mathrm{H} & -1.6537540 & -1.7786070 & 4.5702290 \\ \mathrm{H} & -1.4698330 & -0.7433060 & 3.1467240 \\ \mathrm{C} & -3.5033290 & -0.7328710 & 3.9954320 \\ \mathrm{H} & -3.6615990 & -0.6969510 & 5.0817500 \\ \mathrm{H} & -3.5088370 & 0.2987690 & 3.6234080 \\ \mathrm{H} & -5.6320440 & -1.0351670 & 3.5500590 \\ \mathrm{H} & -1.4701360 & -3.1372450 & 2.4647710 \\ \mathrm{C} & -4.6536140 & -2.9659370 & 3.9138080 \\ \mathrm{H} & -4.7682000 & -2.9167760 & 5.0049490 \\ \mathrm{H} & -5.5063760 & -3.5278700 & 3.5145050 \\ \mathrm{C} & -3.3081600 & -3.6573700 & 3.5302410 \\ \mathrm{H} & -2.7680070 & -3.9783540 & 4.4307550 \\ \mathrm{H} & -3.4909530 & -4.5479880 & 2.9169720 \\ \mathrm{C} & -3.6715060 & -1.8772920 & -0.8188850 \\ \mathrm{C} & -3.4245950 & -1.8884060 & -2.3107120 \\ \mathrm{C} & -5.7095940 & -0.8344190 & -1.6703250 \\ \mathrm{C} & -4.9021180 & -1.2845860 & -0.4784200 \\ \mathrm{C} & -4.5713530 & -2.6849090 & -2.9919730 \\ \mathrm{H} & -4.4333580 & -2.6401360 & -4.0805330 \\ \mathrm{H} & -4.5174990 & -3.7391090 & -2.6940140 \\ \mathrm{C} & -5.9476030 & -2.0677150 & -2.5868510 \\ & & & \end{array}$




-6.5057990
-6.5615180
-6.6657300
-2.4547120
-4.8738210
-5.4269260
-4.7527080
-3.4884770
-3.3328730
-2.6736270
-5.2789900
-6.2383790
3.8526410
3.6636060
3.3693720
3.6725390
4.7007480
4.5261880
5.7128500
4.5460660
4.3383530
5.4684220
3.2531090
3.7964650
2.0812720
1.9186920
1.2222490
2.2475030
2.1212330
1.4917210
4.4282890
4.8870880
4.4765880
4.2296550
3.8197710
4.1875710
2.8947940
3.5633840
3.7868800
2.5184680
4.2860390
5.0523080
5.9507090
6.1320280
6.6280340
6.2028070

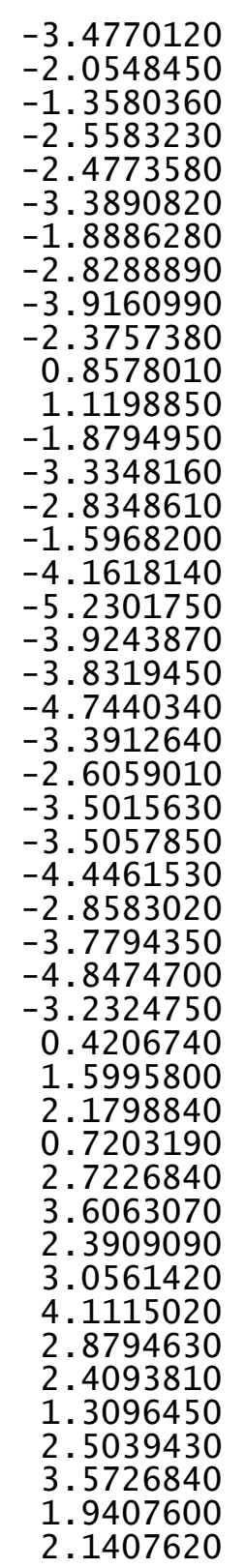

6.5278330

6.9895600

4.2298600

4.3883550

3.2483990

2.9861620

3.9517000

5. 3951190

5.6081000

4.4833530

3.7988310

3.0874360

1. 9440530

5.5780990

6.1136020

6. 5608660

5.6897760

3.7412430

$-0.4288980$

$-0.4682100$

0.5877000

2.0128030

2.0091400

0.8342510

$-0.2466600$

0.5620590

2. 5725920

1.9223730

$-1.4718880$

0.3173730

2. 5575010

2.9620970

$-3.2226020$

$-2.9447790$

$-4.6275010$

$-5.5751980$

$-5.0071560$

$-6.8843400$

$-5.2812650$

$-6.3164680$

$-4.2684540$

$-7.2573610$

$-7.6151940$

$-6.6058130$

$-8.2791670$

$-2.0439470$
3.9313960

3. 4561330

3.6554810

4.7108810

$-3.2630310$

$-4.6749450$

$-5.1105480$

$-4.8413870$

$-3.3317630$

$-2.6333170$

$-6.1747550$

$-5.3837670$

$-4.7503650$

$-5.3527350$

$-5.2402820$

$-3.1280890$

$-2.8744350$

$-4.5481590$

$-4.7921350$

$-5.9984320$

$-7.0264750$

$-6.4138330$

$-4.8588800$

$-4.2621220$

$-5.6947660$

$-7.9117920$

$-6.7378440$

$-4.5159330$

$-6.4408360$

$-7.3562840$

$-6.7945910$

$-4.4735420$

2.9241820

1.7325070

3.3649490

2. 4766870

4.6810210

2.9033370

1.4569740

5.1044630

5.3576520

4.2163870

2.2106370

6.1248490

4.5473430

1. 3113540
3.0256190

1.3820570

$-0.8727980$

$-1.0992120$

0.7469690

1.2425180

2. 3522250

1.9123180

1.7445330

0.9956000

2.5731190

0.4108800

1.5771080

0.9549800

2.6398270

1.2365870

2.7435340

3.2742020

$-1.4973330$

$-2.4033870$

$-1.9473420$

$-1.9305580$

$-1.9105010$

$-1.1687320$

$-3.4401120$

$-2.5950330$

$-2.8169480$

$-2.9536440$

$-2.4040460$

$-0.9356530$

$-1.0583320$

$-1.5409920$

$-0.3592610$

0.1162920

$-0.1034340$

0.4340510

$-0.4150000$

0.6554070

0.6616100

$-0.1881110$

$-0.8355550$

0.3465030

1.0669650

$-0.4290750$

0.5200270
-0.1992300 


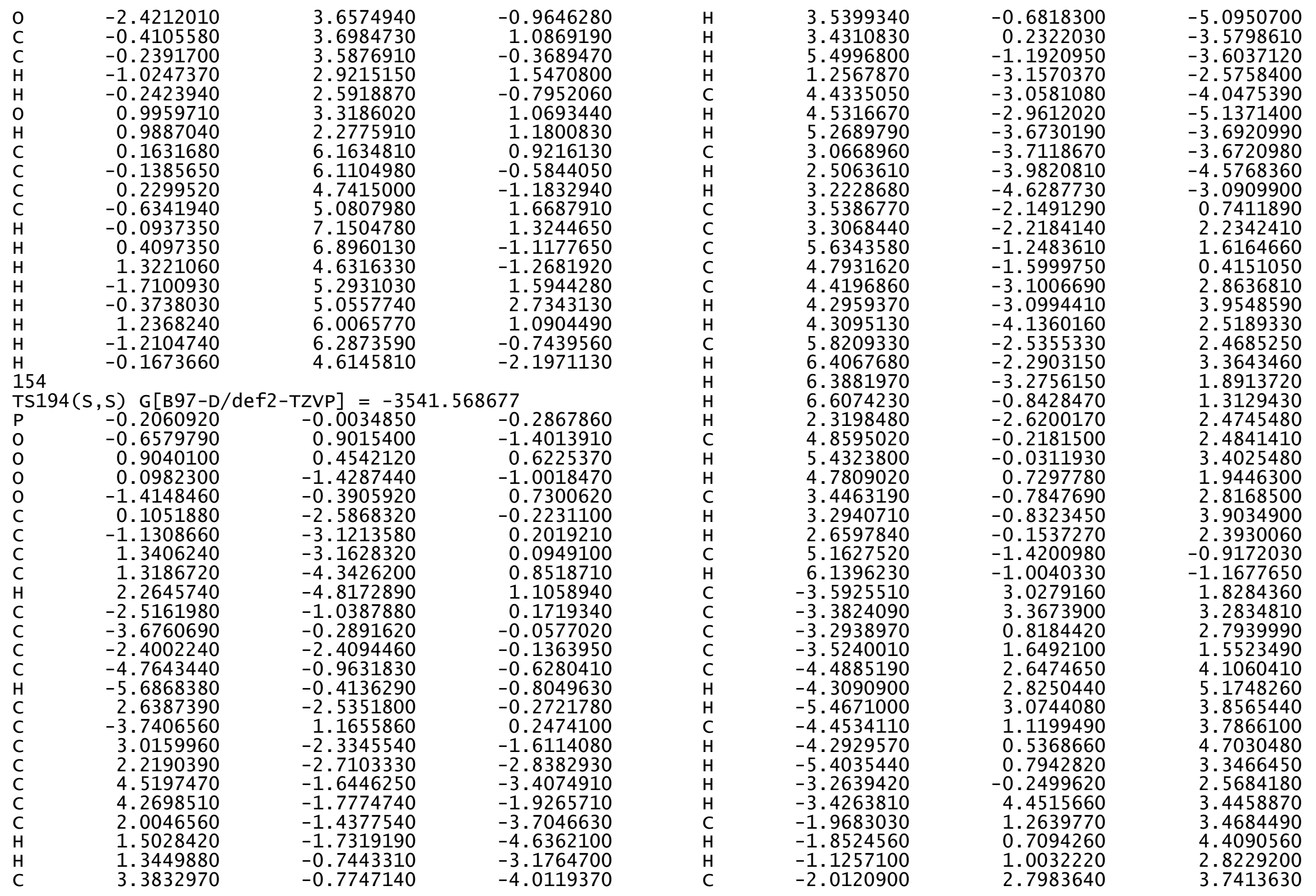




$\begin{array}{rrr}-1.8789970 & 3.0097940 & 4.8108020 \\ -1.2061170 & 3.3074690 & 3.2032520 \\ -4.1306150 & 3.4711790 & -0.4746430 \\ -4.5232590 & 4.3219770 & -1.6568070 \\ -4.3097810 & 1.7972830 & -2.2260830 \\ -4.0403180 & 2.0965320 & -0.7676770 \\ -3.4702920 & 4.1382050 & -2.7821280 \\ -3.7994780 & 4.6983160 & -3.6679380 \\ -2.5097590 & 4.5496280 & -2.4570470 \\ -3.3297270 & 2.6208180 & -3.1070930 \\ -3.5694640 & 2.4276910 & -4.1612490 \\ -2.3082600 & 2.2736050 & -2.9301820 \\ -4.2023430 & 0.7339810 & -2.4513760 \\ -4.6053070 & 5.3779840 & -1.3710250 \\ -5.7506350 & 2.2818570 & -2.5534310 \\ -5.9425090 & 2.1203810 & -3.6224650 \\ -6.4790940 & 1.6845420 & -1.9916840 \\ -5.8856240 & 3.7955770 & -2.1925900 \\ -6.1712010 & 4.3805520 & -3.0769610 \\ -6.6598490 & 3.9445170 & -1.4304760 \\ -3.8967640 & 3.9399450 & 0.8174370 \\ -3.9717450 & 5.0056060 & 1.0390130 \\ -4.6758910 & -2.3007670 & -1.0075340 \\ -5.8236780 & -3.0302270 & -1.6611450 \\ -6.0417640 & -4.3927370 & -0.9690180 \\ -4.7444620 & -5.2476130 & -0.9495220 \\ -3.4727270 & -4.4382920 & -1.3196480 \\ -3.4928300 & -3.0298760 & -0.7748400 \\ -5.5951870 & -3.2035900 & -2.7256850 \\ -6.8527880 & -4.9403000 & -1.4651430 \\ -4.8309840 & -6.0853160 & -1.6528330 \\ -3.4320480 & -4.3584840 & -2.4174330 \\ -6.7353850 & -2.4218450 & -1.6208730 \\ -6.3672500 & -4.1959930 & 0.0606390 \\ -4.6148400 & -5.6837430 & 0.0464950 \\ -2.5738780 & -4.9780490 & -1.0133190 \\ 0.1274010 & -4.8858890 & 1.3233700 \\ 0.0917120 & -6.1172650 & 2.1934980 \\ -0.9737980 & -7.1131610 & 1.6841230 \\ -2.3566600 & -6.4312690 & 1.4682660 \\ -2.3127340 & -4.8951630 & 1.6712080 \\ -1.1033100 & -4.2735930 & 1.0145470 \\ -0.1642140 & -5.8251280 & 3.2250960 \\ -1.0640560 & -7.9479860 & 2.3901820 \\ -3.1022020 & -6.8494910 & 2.7527510 \\ -2.2391360 & -4.6986200 & \end{array}$

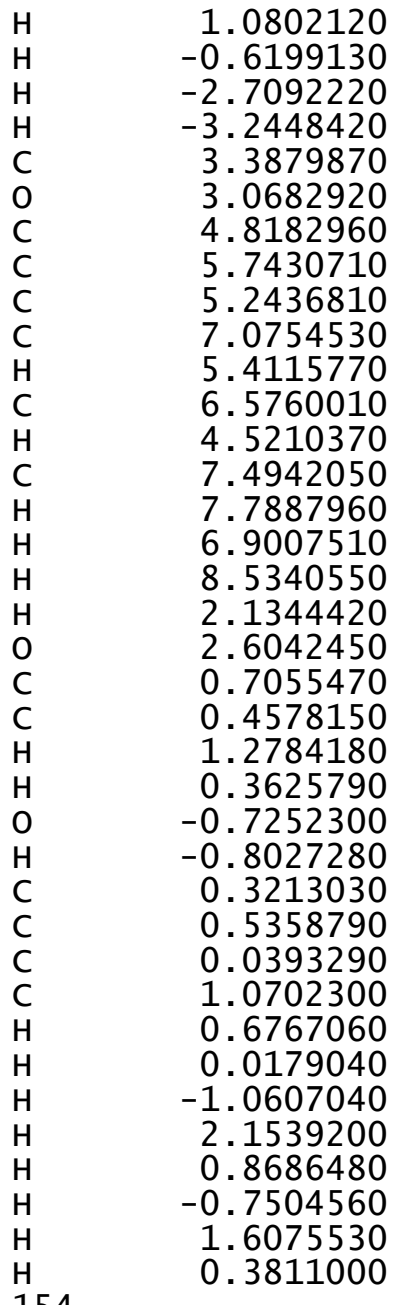
$-6.5909810$
$-7.5319050$
$-6.6419190$
$-4.4384820$
2.7200950
1.5582890
3. 1018290
2. 1838920
4.3941950
2.5578210
1.1835220
4.7654840
5. 0939230
3.8476150
1.8426480
5.7680240
4.1371550
1.1769930
3. 4730690
3. 6561580
3. 5595040
2. 8332470
2. 5660840
3. 3919720
2.3533120
6.1582960
6.0836370
4. 7462750
5.0162010
7. 1217910
6.9072990
4.7163230
5. 1412940
5.0129330
6. 0869150
6. 1832280
4.5919480

2. 2289690

0.7336550

0.4530010

1. 3334520

0.4967780

$-0.0233380$

0.2937410

$-0.2337340$

0.6427070

$-0.4067630$

$-0.4932660$

0.4630430

1.0533340

$-0.0605400$

$-0.8104520$

0.7321240

$-0.1968730$

0.2456860

1. 1012560

$-1.0435230$

0.4029110

$-1.4763830$

0.8246190

$-1.0949660$

$-1.2034190$

$-0.8988330$

0.6208760

1.1970790

$-1.6060190$

$-1.2834860$

1. 1263460

1. 2341340

$-1.4709440$

$-2.6844660$

$-1.1279990$

0.8384880

154

2. 2270110

TS195 (S, S) G[в97-D/def2-TZVP $]=-3541.568529$

\begin{tabular}{lrrr} 
TS195(S, S & \multicolumn{2}{c}{ G $[\mathrm{B} 97-\mathrm{D} / \mathrm{def}-\mathrm{TZV}]=-3541.568529$} \\
$\mathrm{P}$ & -0.3291820 & -0.0403900 & 0.9254010 \\
$\mathrm{O}$ & 0.3181460 & -0.3847770 & 2.2375420 \\
$\mathrm{O}$ & -0.5318870 & 1.4115010 & 0.5849940 \\
$\mathrm{O}$ & -1.7116620 & -0.8890300 & 0.8945750 \\
$\mathrm{O}$ & 0.4973390 & -0.6509470 & -0.3526460 \\
$\mathrm{C}$ & -2.3579190 & -1.0471050 & -0.3334380 \\
$\mathrm{C}$ & -1.8157390 & -1.9591810 & -1.2597110
\end{tabular}




\begin{tabular}{|c|c|c|}
\hline $\begin{array}{l}-3.5211720 \\
-4.1261780 \\
-5.0318770 \\
0.5633770 \\
1.7794750 \\
-0.5919240 \\
1.8047230 \\
2.7393110 \\
-4.1082420 \\
3.0140250 \\
-4.5668090 \\
-4.5421600 \\
-5.6163980 \\
-5.1641720 \\
-3.7550420 \\
-3.7938900 \\
-2.7065090 \\
-4.3904930 \\
-4.7211610 \\
-3.6595000 \\
-6.0621350 \\
-4.0904660 \\
-6.6466450 \\
-6.9351740 \\
-7.5504490 \\
-6.0060730 \\
-5.9958350 \\
-6.5800410 \\
-4.2576810 \\
-3.7900860 \\
-4.9596170 \\
-4.8693330 \\
-5.0199780 \\
-4.6755740 \\
-5.7072250 \\
-5.7361080 \\
-5.7752550 \\
-6.7675410 \\
-5.4628640 \\
-3.2917750 \\
-3.5202730 \\
-3.5788380 \\
-2.9645710 \\
-2.8121540 \\
-2.4899890 \\
-1.9281480\end{array}$ & $\begin{array}{r}-0.3076990 \\
-0.4633450 \\
0.1004570 \\
-2.0391180 \\
-2.6782610 \\
-2.7325920 \\
-4.0760410 \\
-4.5956950 \\
0.6052730 \\
-1.9155080 \\
0.1109530 \\
-1.3278190 \\
0.2779630 \\
0.9728530 \\
-1.4071500 \\
-2.4413280 \\
-1.1568630 \\
-0.4313920 \\
-0.9749080 \\
0.3213120 \\
0.9901020 \\
-1.9874430 \\
-0.8171830 \\
-1.3755910 \\
-0.3429080 \\
-1.7690530 \\
-2.8039840 \\
-1.7522820 \\
1.9782920 \\
2.7147940 \\
4.2660570 \\
2.8294210 \\
3.3920890 \\
3.9821110 \\
2.6251700 \\
4.3080030 \\
5.3455440 \\
3.9740710 \\
4.8848990 \\
2.0460520 \\
4.7855890 \\
5.8097510 \\
4.8222170 \\
3.8387370 \\
4.3948680 \\
3.3811860\end{array}$ & $\begin{array}{r}-0.5757950 \\
-1.8302920 \\
-2.0457520 \\
-0.4832490 \\
-0.2172510 \\
-0.8971280 \\
-0.2997720 \\
-0.0962980 \\
0.4401510 \\
0.1062560 \\
1.6751910 \\
2.1372130 \\
3.8740150 \\
2.6141190 \\
3.4738170 \\
3.8418140 \\
3.2913880 \\
4.5123490 \\
5.4076900 \\
4.8307960 \\
4.5792340 \\
1.3921690 \\
3.4795510 \\
4.3802800 \\
3.0786350 \\
2.4213180 \\
2.7884400 \\
1.4870620 \\
0.1719450 \\
-1.0629890 \\
0.6624220 \\
1.1125520 \\
-1.7265780 \\
-2.5864910 \\
-2.1042960 \\
-0.6837360 \\
-1.0417780 \\
-0.5189950 \\
1.4154050 \\
-1.7695310 \\
0.3965700 \\
0.0034280 \\
1.3412770 \\
-0.6202600 \\
-1.5107810 \\
-0.1719870\end{array}$ \\
\hline
\end{tabular}

-5.3199010
-5.7907360
4.7958010
5.3141380
3.1317770
3.6023250
5.5761740
5.8944350
6.3934100
4.2700780
3.9532900
4.4323380
2.2133650
6.2339890
2.9076970
2.6279850
2.0696720
4.2126880
4.5706600
4.0279920
4.8517870
5.4120290
3.2095860
3.6529490
4.3378700
4.7268150
4.1394470
3.0311880
2.8012350
2.1790780
2.2783560
6.3411610
4.3523370
4.0573490
4.4949470
5.6678130
6.0046000
6.4714690
5.4237700
6.3549180
0.6612390
0.6508870
-0.0008940
-1.3984940
-1.7280530
-0.5403060
.

$-5.3199010$

2. 3305080

2.9933980

$-0.3867260$

0.4220480

$-0.8791300$

$-1.0768800$

$-0.5563870$

0.0219310

$-1.2374830$

$-1.3541390$

$-1.1918740$

$-2.4301610$

$-1.4358390$

0.9561020

0.6345030

0.7839140

0.9724400

1.4200530

1. 9716210

. 1573130

$-1.3663000$

$-1.6435570$

$-2.9334170$

$-2.0564450$

$-1.2549870$

$-1.4729220$

$-0.1784370$

$-2.0587480$

$-2.7219220$

$-1.3904170$

$-3.4574320$

$-1.0848050$

$-3.9476050$

$-4.5850640$

$-4.5963030$

$-3.1730450$

$-3.3962280$

$-3.4648950$

$-0.5334140$

$-0.0061880$

$-4.8068370$

$-6.3139190$

$-6.8038990$

$-6.1627750$

$-5.0302300$

$-4.1404730$
2.3343020

3.0614190

$-0.5787320$

$-1.7416570$

$-2.2812940$

$-0.8589390$

$-2.9217500$

$-3.7992640$

$-2.6567540$

$-3.2292940$

$-4.2679450$

$-3.0948140$

$-2.4854020$

$-1.4728110$

$-2.5302980$

$-3.5811770$

$-1.9187160$

$-2.1951390$

$-3.0738870$

$-1.4071660$

1. 6194850

2.9916560

2.4974520

1. 3496660

4.0445440

5.0481190

3.9902790

3.7688410

4.6133240

3.6300070

2. 2676210

3.1599350

2.7832910

3.6270440

1. 9104230

3.1120540

4.1330830

2.4256160

0.6586880

0.8700430

$-0.6148430$

$-0.6624460$

$-1.9749110$

$-2.2102260$

$-1.2033310$ 


$\begin{array}{lrrr}\text { H } & 0.0682660 & -6.7027870 & \\ \mathrm{H} & -0.0784190 & -7.8982020 & -1.9635370 \\ \mathrm{H} & -2.1853430 & -6.9239570 & -2.1387710 \\ \mathrm{H} & -2.0175230 & -5.4985420 & -0.2492600 \\ \mathrm{H} & 1.6696040 & -6.7081170 & -0.5640210 \\ \mathrm{H} & 0.6686520 & -6.5372200 & -2.8026030 \\ \mathrm{H} & -1.4431800 & -5.7570560 & -3.2263750 \\ \mathrm{H} & -2.5932340 & -4.4574670 & -1.5450610 \\ \mathrm{C} & -3.5836480 & -1.2988970 & -2.8038450 \\ \mathrm{C} & -4.1871990 & -1.4414880 & -4.1782140 \\ \mathrm{C} & -4.3420320 & -2.9326610 & -4.5529030 \\ \mathrm{C} & -3.0262620 & -3.7346930 & -4.3287550 \\ \mathrm{C} & -1.9062450 & -2.8920170 & -3.6677670 \\ \mathrm{C} & -2.4301920 & -2.0576560 & -2.5238000 \\ \mathrm{H} & -3.5248430 & -0.9609590 & -4.9166200 \\ \mathrm{H} & -4.6691560 & -3.0162110 & -5.5967820 \\ \mathrm{H} & -2.6488180 & -4.1264290 & -5.2815750 \\ \mathrm{H} & -1.5038030 & -2.2031360 & -4.4277500 \\ \mathrm{H} & -5.1542590 & -0.9268600 & -4.2290150 \\ \mathrm{H} & -5.1395060 & -3.3576050 & -3.9304970 \\ \mathrm{H} & -3.2352590 & -4.6004420 & -3.6912360 \\ \mathrm{H} & -1.0786590 & -3.5337040 & -3.3577890 \\ \mathrm{C} & 1.5559500 & 3.5148350 & -1.0484240 \\ \mathrm{O} & 0.4894440 & 2.7814550 & -1.2426200 \\ \mathrm{C} & 2.0177610 & 4.2612650 & -2.2603600 \\ \mathrm{C} & 3.1482380 & 5.0891250 & -2.1655370 \\ \mathrm{C} & 1.3651400 & 4.1223190 & -3.4970090 \\ \mathrm{C} & 3.6197790 & 5.7678800 & -3.2882650 \\ \mathrm{H} & 3.6503620 & 5.1860440 & -1.2073860 \\ \mathrm{C} & 1.8413370 & 4.8002780 & -4.6197620 \\ \mathrm{H} & 0.4951880 & 3.4778970 & -3.5729400 \\ \mathrm{C} & 2.9679760 & 5.6235790 & -4.5184190 \\ \mathrm{H} & 4.4964060 & 6.4065230 & -3.2069770 \\ \mathrm{H} & 1.3345120 & 4.6850910 & -5.5751630 \\ \mathrm{H} & 3.3378160 & 6.1504000 & -5.3955200 \\ \mathrm{H} & 0.1442260 & 2.2269500 & -0.4244300 \\ \mathrm{O} & 2.1792150 & 3.6147300 & 0.0231950 \\ \mathrm{C} & 1.5881190 & 2.5414980 & 2.6462770 \\ \mathrm{C} & 2.5206750 & 2.0771240 & 1.6088180 \\ \mathrm{H} & 0.5408000 & 2.5662680 & 2.34477300 \\ \mathrm{H} & 2.1625320 & 1.3306840 & 0.9083310 \\ \mathrm{O} & 1.9395940 & 1.2666100 & 3.2687160 \\ \mathrm{H} & 1.2676540 & 0.5644930 & 2.8941530 \\ \mathrm{C} & 3.5291190 & 3.7364470 & 3.7748190 \\ \mathrm{C} & 4.3299610 & 3.6204420 & 2.4679360 \\ \mathrm{C} & 3.9816410 & 2.3307900 & 1.7090440 \\ & & & \end{array}$

2.0183780
3.7958580
5.4075710
4.4117490
1.7444700
1.4334580
3.7777110
4.0978300
4.3949640

3.7299510

4.6608780

3.6375080

1.4467180

4.6321060

3.7292190

2.8968720

4.4793510

2. 3183470

3.4843880

4.3012090

2.6707400

2.2013730

2.9199350

4.4125930

4.4376930

1.8248840

$\mathrm{H}$

TS196 (R, R) G[B97-D/def2-TZVP $]=-3633.751531$

$\begin{array}{lrrr}\text { O } & 0.6424080 & 0.6596360 & 0.1675820 \\ \mathrm{O} & -1.5506810 & 1.0628250 & -1.0658150 \\ \mathrm{C} & 0.8197000 & 2.0357620 & 0.2486990 \\ \mathrm{C} & -0.2424410 & 2.8344960 & 0.6598800 \\ \mathrm{C} & 2.1136130 & 2.5469470 & -0.0379520 \\ \mathrm{C} & -0.0580610 & 4.2571290 & 0.6788070 \\ \mathrm{C} & 2.3033390 & 3.9110420 & 0.0690460 \\ \mathrm{C} & 1.2448610 & 4.7932800 & 0.3948060 \\ \mathrm{H} & 3.2885630 & 4.3257580 & -0.1309430 \\ \mathrm{C} & -2.1801960 & 1.3589250 & 0.1470910 \\ \mathrm{C} & -3.4560630 & 0.7827860 & 0.3878420 \\ \mathrm{C} & -1.5406390 & 2.2150740 & 1.0338370 \\ \mathrm{C} & -4.0758660 & 1.0906700 & 1.5829430 \\ \mathrm{C} & -2.1496360 & 2.4458970 & 2.3124490 \\ \mathrm{C} & -3.4476020 & 1.8864690 & 2.5723260 \\ \mathrm{H} & -5.0644200 & 0.6879480 & 1.7872260 \\ \mathrm{C} & -3.4247260 & 2.8432940 & 4.8134090 \\ \mathrm{C} & -2.1276290 & 3.3602810 & 4.5740010 \\ \mathrm{C} & -1.5074260 & 3.1680850 & 3.3552660 \\ \mathrm{C} & -4.0668450 & 2.1180870 & 3.8312400 \\ \mathrm{H} & -3.9047170 & 3.0085110 & 5.7754140 \\ \mathrm{H} & -1.6131170 & 3.9099360 & 5.3591710 \\ \mathrm{H} & -0.5115200 & 3.5650070 & 3.1866670 \\ \mathrm{H} & -5.0547870 & 1.6965440 & 4.0083860 \\ \mathrm{C} & -0.8988920 & 6.5337920 & 0.9330940 \\ \mathrm{C} & 0.3951460 & 7.0576930 & 0.6913290 \\ \mathrm{C} & 1.4426110 & 6.2011050 & 0.4236690 \\ \mathrm{C} & -1.1192070 & 5.1708070 & 0.9280780 \\ \mathrm{H} & -1.7285870 & 7.2122420 & 1.1194190 \\ \mathrm{H} & 0.5555850 & 8.1334120 & 0.7044900 \\ \mathrm{H} & 2.4385040 & 6.5888570 & 0.2163540 \\ \mathrm{H} & -2.1170940 & 4.7853120 & 1.1097200 \\ \mathrm{C} & 3.2480400 & 1.6642200 & -0.4399470 \\ \mathrm{C} & 3.7544830 & 1.7295340 & -1.7571860 \\ \mathrm{C} & 3.8613290 & 0.8164860 & 0.5124520\end{array}$




$\begin{array}{rrr}4.8970740 & 0.9852820 & -2.0821080 \\ 4.9960850 & 0.0924660 & 0.1363280 \\ 5.5400790 & 0.1710070 & -1.1495750 \\ 5.2858750 & 1.0452930 & -3.0947260 \\ 5.4827190 & -0.5502620 & 0.8680220 \\ -4.1064650 & -0.1176890 & -0.6083120 \\ -4.2771610 & -1.4918960 & -0.3173300 \\ -4.5703230 & 0.4093160 & -1.8364910 \\ -4.9287620 & -2.3008890 & -1.2582050 \\ -5.2052050 & -0.4438570 & -2.7436360 \\ -5.3974640 & -1.8016740 & -2.4744310 \\ -5.0683520 & -3.3585110 & -1.0425740 \\ -5.5646330 & -0.0338420 & -3.6849500 \\ 3.3754000 & 0.7238140 & 1.9535120 \\ 6.8168310 & -0.5913390 & -1.4673050 \\ 3.1161570 & 2.5907680 & -2.8409400 \\ -3.7922260 & -2.1230260 & 0.9836700 \\ -6.0881210 & -2.7069250 & -3.4788790 \\ -4.4485180 & 1.8849820 & -2.1969510 \\ -5.8404070 & 2.5430320 & -2.2756560 \\ -6.4434320 & 2.0786990 & -3.0670620 \\ -6.3792660 & 2.4325450 & -1.3257370 \\ -5.7421960 & 3.6135420 & -2.5007230 \\ -3.6706330 & 2.0903470 & -3.5099350 \\ -2.6742570 & 1.6398230 & -3.4426400 \\ -4.2042120 & 1.6348740 & -4.3543650 \\ -3.5594620 & 3.1632650 & -3.7165420 \\ -5.2840620 & -2.8013220 & -4.7900800 \\ -5.7787460 & -3.4832940 & -5.4945960 \\ -5.2094990 & -1.8135020 & -5.2635110 \\ -4.2668410 & -3.1671550 & -4.6014100 \\ -7.5286230 & -2.2405040 & -3.7636530 \\ -8.1169560 & -2.1916920 & -2.8385480 \\ -7.5241170 & -1.2416510 & -4.2197560 \\ -8.0235480 & -2.9313510 & -4.4592330 \\ -4.9704290 & -2.3490400 & 1.9527550 \\ -5.4965900 & -1.4128840 & 2.1722990 \\ -5.6919310 & -3.0503550 & 1.5119670 \\ -4.6083530 & -2.7760300 & 2.8980590 \\ -3.0410850 & -3.4495320 & 0.7635040 \\ -3.7165700 & -4.2318050 & 0.3940840 \\ -2.2240360 & -3.3213600 & 0.0472160 \\ -2.6188760 & -3.7941240 & 1.7159000 \\ 4.3381830 & 1.4813780 & 2.8892080 \\ 5.3385130 & 1.0294800 & 2.5888600 \\ 4.4258210 & 2.5334660 & \end{array}$

3.9741700

(

2. 5152850

4.1486020

2.7608570

8.0434650

8.9704040

8.0900310

7.9824500

6.9506540

6.0723420

7.0683380

7.8390860

2.8178210

3.7421060

2.1689350

2. 3105750

4.0057580

4. 9749790

3. 5237340

4.1913870

$-0.3192900$

0.3394010

$-0.7584030$

2. 1592460

6.8055390

2.4006460

$-3.8949590$

$-6.1377760$

$-3.0914210$

2.4332110

1.2974540

2. 2982200

0.3109130

1.9388560

1. 2757750

3.9581580

2.8803530

1.4662830

3.8304150

4.9571860

2.9894410

1.2236750

4.0681460

4.5238830

3.8596720
1.4416400

$-0.7305170$

$-1.2733210$

$-1.2633450$

$-0.7487950$

0.2412380

$-0.3233630$

1.1686340

0.5088200

-1.0089160

$-1.5693840$

$-0.1304550$

$-1.6396600$

1.4179410

0.9344970

2.4325160

. 8067450

3.4745220

4.4412390

4.4112120

0.0058910

0.0194130

$-1.3160930$

2. 9672580

$-1.5052000$

1.2132200

2.3993620

$-3.7125670$

$-1.4298690$

$-2.2838240$

$-3.1884390$

$-1.4744240$

$-2.8106330$

$-1.9006720$

$-1.1061300$

$-4.0759320$

$-5.1221550$

$-4.5393350$

$-2.8589850$

$-4.5126920$

$-6.0028660$

$-4.4423880$

$-3.1483040$

$-2.0628130$

$-3.7503660$
3.9247410

2.4193440

1.7494350

2.4497680

3.4289490

$-1.0367830$

$-1.2055440$

$-1.6236320$

0.0253760

$-2.9399280$

$-3.2832230$

$-3.5875590$

$-3.0681320$

$-4.1213500$

$-4.5833540$

$-3.8998000$

$-4.8513590$

$-3.1648720$

$-3.5605260$

$-3.9206210$

$-2.2686710$

$-0.9842960$

$-2.3354800$

$-0.4167620$

$-2.4650210$

$-0.8550310$

2.0297680

$-1.4056340$

$-3.0376110$

1.4592610

$-1.4802660$

$-1.6846020$

$-0.7606400$

$-1.4439160$

$-2.8207780$

$-2.6704120$

$-2.4396820$

$-2.1200020$

$-2.2990490$

$-1.5091780$

$-2.3257250$

$-2.7630670$

$-3.3678150$

$-0.4760790$

$-1.7884240$

$-3.4840000$ 


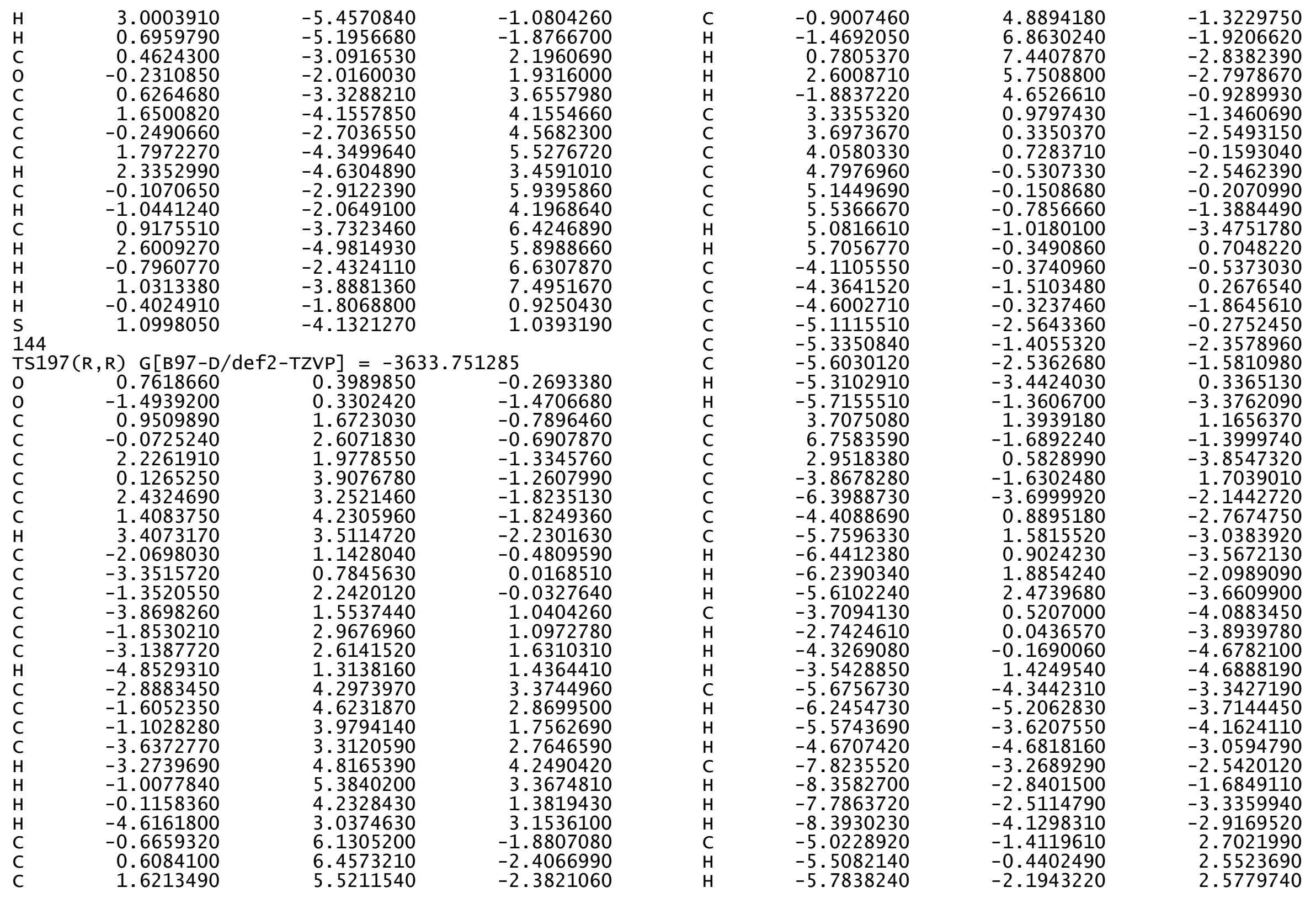




\begin{tabular}{|c|c|}
\hline $\mathrm{H}$ & -4.6467310 \\
\hline C & -3.1844390 \\
\hline $\mathrm{H}$ & -3.9076460 \\
\hline $\mathrm{H}$ & -2.3927590 \\
\hline $\mathrm{H}$ & -2.7387170 \\
\hline C & 4.8803520 \\
\hline $\mathrm{H}$ & 5.7560610 \\
\hline $\mathrm{H}$ & 5.1707360 \\
\hline $\mathrm{H}$ & 4.5939740 \\
\hline C & 3.2659640 \\
\hline $\mathrm{H}$ & 2.3956170 \\
\hline $\mathrm{H}$ & 4.0751760 \\
\hline $\mathrm{H}$ & 2.9950290 \\
\hline C & 7.9894650 \\
\hline $\mathrm{H}$ & 8.8872170 \\
\hline $\mathrm{H}$ & 7.8315930 \\
\hline $\mathrm{H}$ & 8.1640030 \\
\hline C & 6.5635750 \\
\hline $\mathrm{H}$ & 5.6851060 \\
\hline $\mathrm{H}$ & 6.4321240 \\
\hline $\mathrm{H}$ & 7.4480270 \\
\hline C & 2.5911650 \\
\hline $\mathrm{H}$ & 3.4926570 \\
\hline $\mathrm{H}$ & 2.0096260 \\
\hline $\mathrm{H}$ & 1.9916790 \\
\hline C & 3.7751560 \\
\hline $\mathrm{H}$ & 4.7314390 \\
\hline $\mathrm{H}$ & 3.2273390 \\
\hline $\mathrm{H}$ & 3.9899420 \\
\hline $\mathrm{P}$ & -0.3322310 \\
\hline 0 & 0.2488680 \\
\hline 0 & -0.8341470 \\
\hline $\mathrm{H}$ & 2.0128090 \\
\hline $\mathrm{H}$ & 6.9546320 \\
\hline $\mathrm{H}$ & 2.8642490 \\
\hline $\mathrm{H}$ & -3.7742430 \\
\hline $\mathrm{H}$ & -6.4821580 \\
\hline $\mathrm{H}$ & -3.1224510 \\
\hline C & 2. 3190140 \\
\hline C & 1.1897200 \\
\hline $\mathrm{H}$ & 2.1751860 \\
\hline $\mathrm{H}$ & 0.1999760 \\
\hline 0 & 1.8418000 \\
\hline $\mathrm{H}$ & 1.1990290 \\
\hline C & 3.8603750 \\
\hline C & 2.7896200 \\
\hline
\end{tabular}

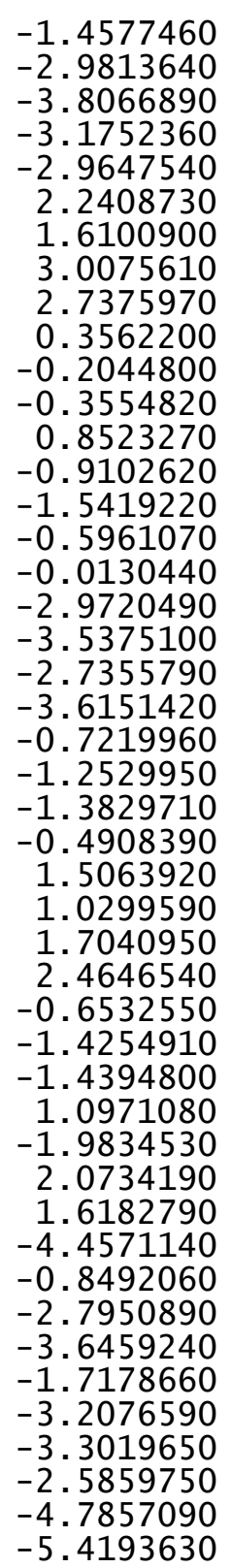

3.7332780
1.9876000
1.9545820
1.2577130
2.9905600
1.6951780
1.8963160
0.9653900
2.6316410
2.2114880
1.8579410
2.4200380
3.1520590
-1.9078190
-1.8695160
-2.9484970
-1.3007700
-2.2272360
-1.8936050
-3.2907420
-2.1339580
-4.5878870
-4.9197450
-3.9369210
-5.4782390
-4.7749740
-5.0304070
-5.7062000
-4.2861690
-0.8941480
-2.0417810
0.2891980
-3.6214570
-0.3586470
1.0032060
-2.2548490
-1.3516340
1.8744480
0.1103990
0.5056550
0.1944730
0.4543550
-1.1965550
-1.5756980
0.4539040
1.3542560

\begin{abstract}
1. 3725910
3. 7189450

4.8624360

2. 9068860

1. 1320270

3. 9446320

4.4126180

3. 7608140

2. 9072250

0.6017110

0.2255490

$-0.5551290$

0.4272710

0.9819750

0.0732160

1.1777020

1. 2521690

0.2823470

$-0.3499010$

0.8327980

1. 6001300

0.0143510

0.9921910

$-0.6371950$

0.9581580
\end{abstract}

$$
\begin{array}{r}
-5.0825030 \\
-3.2553400 \\
-5.0527100 \\
-6.5080490 \\
-5.6680090 \\
-2.8532220 \\
-2.7843290 \\
-5.1721170 \\
-5.0344460 \\
-5.3439630 \\
-1.0999470 \\
-0.5317600 \\
-0.2639560 \\
-0.7943450 \\
1.1023090 \\
0.0152940 \\
-1.8459950 \\
1.9109320 \\
1.5302640 \\
1.3715490 \\
-0.4112790 \\
2.9640830 \\
2.0031580 \\
-1.0032560 \\
-2.6028530
\end{array}
$$

0.8548730

0.8091030

1. 3962110

$-0.0460080$

1. 4415720

$-0.2559530$

$-0.5693750$

2. 3766560

1. 5901910

3. 4611130

2. 5835850

4.6769960

5.8566750

4.6461520

6.9741050

5.8899270

5.7624660

3.7430080

6.9303230

7.8808650

5.7189490

7.8015060

1. 6497110

144

3.2555520

$\begin{array}{lrcr}\text { TS198(R, R) G }[\mathrm{B} 97-\mathrm{D} / \mathrm{def}-\mathrm{TZVP}]=-3633.750983 \\ \mathrm{O} & 0.6240310 & 0.2348870 & -0.2838440 \\ \mathrm{O} & -1.6420410 & 0.1475950 & -1.4670820 \\ \mathrm{C} & 0.9012090 & 1.4106560 & -0.9683880 \\ \mathrm{C} & -0.0640150 & 2.4099320 & -1.0316040 \\ \mathrm{C} & 2.2036910 & 1.5610610 & -1.5126700 \\ \mathrm{C} & 0.2165140 & 3.5888900 & -1.7974530 \\ \mathrm{C} & 2.4868310 & 2.7277020 & -2.1945600 \\ \mathrm{C} & 1.5197000 & 3.7442810 & -2.3813380 \\ \mathrm{H} & 3.4824920 & 2.8710330 & -2.6071150 \\ \mathrm{C} & -2.1465900 & 1.1238580 & -0.5963960 \\ \mathrm{C} & -3.4343020 & 0.9173010 & -0.0321250 \\ \mathrm{C} & -1.3611290 & 2.2362040 & -0.3308590 \\ \mathrm{C} & -3.8945970 & 1.8683510 & 0.8578120 \\ \mathrm{C} & -1.8096650 & 3.1664790 & 0.6630700 \\ \mathrm{C} & -3.1034920 & 2.9739550 & 1.2561090 \\ \mathrm{H} & -4.8826010 & 1.7498620 & 1.2947000 \\ \mathrm{C} & -2.7531650 & 4.9199750 & 2.6795690 \\ \mathrm{C} & -1.4644820 & 5.0932510 & 2.1174890 \\ \mathrm{C} & -1.0061100 & 4.2414970 & 1.1321770\end{array}$




$\begin{array}{rrr}-3.5537500 & 3.8798000 & 2.2549280 \\ -3.1028360 & 5.6008630 & 3.4522820 \\ -0.8272710 & 5.9015950 & 2.4695020 \\ -0.0141260 & 4.3819490 & 0.7154570 \\ -4.5388600 & 3.7232700 & 2.6906480 \\ -0.4392670 & 5.7212440 & -2.7849950 \\ 0.8566920 & 5.8857370 & -3.3338790 \\ 1.8136090 & 4.9120370 & -3.1366350 \\ -0.7512390 & 4.6037540 & -2.0364650 \\ -1.1982170 & 6.4807700 & -2.9597340 \\ 1.0892280 & 6.7736730 & -3.9174370 \\ 2.8094990 & 5.0161010 & -3.5637850 \\ -1.7494750 & 4.4904840 & -1.6260040 \\ 3.2716800 & 0.5332440 & -1.3304620 \\ 3.6201480 & -0.3170250 & -2.3979460 \\ 3.9862270 & 0.4795130 & -0.1081940 \\ 4.7194460 & -1.1761750 & -2.2498430 \\ 5.0645340 & -0.4023600 & -0.0072430 \\ 5.4592210 & -1.2259130 & -1.0692250 \\ 4.9895670 & -1.8250240 & -3.0775270 \\ 5.6236340 & -0.4602440 & 0.9238100 \\ -4.2641800 & -0.2728270 & -0.3800300 \\ -4.5469530 & -1.2532030 & 0.6042480 \\ -4.7950640 & -0.4054590 & -1.6826290 \\ -5.3689900 & -2.3312990 & 0.2617630 \\ -5.6091950 & -1.5081420 & -1.9730970 \\ -5.9104750 & -2.4797950 & -1.0186980 \\ -5.5890050 & -3.0836250 & 1.0158370 \\ -6.0259400 & -1.6100050 & -2.9739240 \\ 3.6383500 & 1.4134420 & 1.0468290 \\ 6.6749830 & -2.1231460 & -0.8896970 \\ 2.8571880 & -0.3027140 & -3.7163830 \\ -3.9999010 & -1.1756150 & 2.0251940 \\ -6.7850470 & -3.6723750 & -1.3629480 \\ -4.5587920 & 0.6256300 & -2.7793920 \\ -5.8730840 & 1.3442510 & -3.1443930 \\ -6.6065470 & 0.6293180 & -3.5394640 \\ -6.3098420 & 1.8318610 & -2.2631910 \\ -5.6881020 & 2.1079040 & -3.9117280 \\ -3.9149810 & -0.0043620 & -4.0284040 \\ -2.9757510 & -0.5054000 & -3.7686590 \\ -4.5889150 & -0.7405120 & -4.4854470 \\ -3.7042790 & 0.7730830 & -4.7749340 \\ -8.0340020 & -3.7412550 & -0.4641690 \\ -8.6681980 & -4.5887210 & -0.7564560 \\ -7.7437990 & -3.8785730 & 0.5857490\end{array}$

$\begin{array}{lr}\mathrm{H} & -8.6237370 \\ \mathrm{C} & -5.9860740 \\ \mathrm{H} & -5.1115310 \\ \mathrm{H} & -5.6322900 \\ \mathrm{H} & -6.6178650 \\ \mathrm{C} & -5.0999780 \\ \mathrm{H} & -5.5265550 \\ \mathrm{H} & -5.9134540 \\ \mathrm{H} & -4.6896860 \\ \mathrm{C} & -3.3811870 \\ \mathrm{H} & -4.1499890 \\ \mathrm{H} & -2.6340700 \\ \mathrm{H} & -2.8946670 \\ \mathrm{C} & 4.3457340 \\ \mathrm{H} & 5.4348680 \\ \mathrm{H} & 4.0817510 \\ \mathrm{H} & 4.0606980 \\ \mathrm{C} & 3.9628020 \\ \mathrm{H} & 3.5083900 \\ \mathrm{H} & 5.0467790 \\ \mathrm{H} & 3.5710530 \\ \mathrm{C} & 7.9685000 \\ \mathrm{H} & 8.8493630 \\ \mathrm{H} & 8.0511360 \\ \mathrm{H} & 7.9698040 \\ \mathrm{C} & 6.7276740 \\ \mathrm{H} & 5.8130590 \\ \mathrm{H} & 6.8551850 \\ \mathrm{H} & 7.5819100 \\ \mathrm{C} & 2.5433980 \\ \mathrm{H} & 3.4587440 \\ \mathrm{H} & 2.0223370 \\ \mathrm{H} & 1.9002700 \\ \mathrm{C} & 3.6324850 \\ \mathrm{H} & 4.6056040 \\ \mathrm{H} & 3.0688660 \\ \mathrm{H} & 3.8095890 \\ \mathrm{P} & -0.5230060 \\ \mathrm{O} & 0.0058860 \\ \mathrm{O} & -1.0492490 \\ \mathrm{H} & 1.8985980 \\ \mathrm{H} & 6.6321940 \\ \mathrm{H} & 2.5578910 \\ \mathrm{H} & -3.8699330 \\ \mathrm{H} & -7.1203230 \\ \mathrm{H} & -3.2063190 \\ & \end{array}$
$-2.8187680$
$-4.9877840$
$-4.9600300$
$-5.1515000$
$-5.8395130$
$-0.7349870$
0.2352370
$-1.4730050$
$-0.6546190$
$-2.5049200$
$-3.2782340$
$-2.8620890$
$-2.3557250$
2. 7720430
2.6322720
3. 2302900
3.4598530
0.8435280
$-0.1431560$
0.7563670
1. 5174860
$-1.2894670$
$-1.9177190$
$-0.8720610$
$-0.4583590$
$-3.3205000$
$-3.9242280$
$-2.9863890$
$-3.9601320$
$-1.7150720$
$-2.2448500$
$-2.3055420$
$-1.6423950$
0.4996940
0.0280270
0.5282760
1. 5292770
$-0.8233450$
$-1.7276410$
$-1.4579900$
0.2005600
$-2.5149200$
1.5978230
1. 3895260
$-3.5468220$
$-0.4241570$

-0.5384340
-1.2767880
-1.9390120
-0.2501780
-1.5625200
3.0116000
2.7315910
3.0252590
4.0274020
2.4986100
2.6237630
1.7835240
3.4714000
0.8616970
0.8931410
-0.0977200
1.6693650
2.4356400
2.5810780
2.5868180
3.2053920
-1.0121340
-0.8230810
-2.0249400
-0.2964150
-1.8489730
-1.7962440
-2.8870540
-1.5954530
-4.2410030
-4.5344830
-3.4808870
-5.1275550
-4.7801300
-4.9732750
-5.7224280
-4.4481990
-0.7953060
-1.8693210
0.4667670
-3.5475830
0.1377390
1.0192930
-2.4071160
-2.4023170
2.0467040 


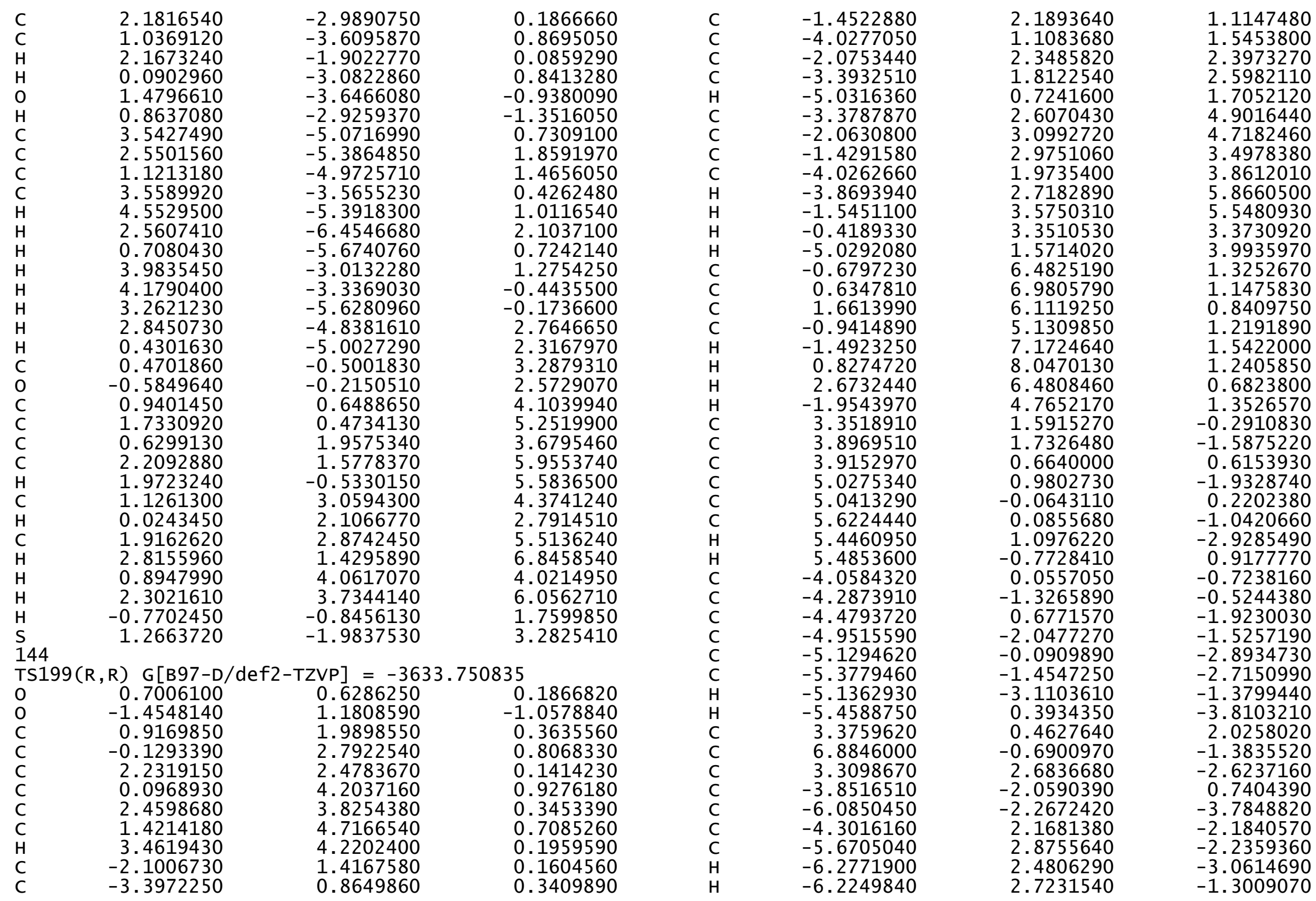




$\begin{array}{rrr}-5.5357990 & 3.9543620 & -2.3919440 \\ -3.4999480 & 2.4334450 & -3.4716350 \\ -2.5185680 & 1.9489020 & -3.4228120 \\ -4.0354980 & 2.0505160 & -4.3501400 \\ -3.3536320 & 3.5134950 & -3.6065640 \\ -5.2701150 & -2.3004210 & -5.0923190 \\ -5.7793910 & -2.9165870 & -5.8453550 \\ -5.1577720 & -1.2862610 & -5.4976650 \\ -4.2676750 & -2.7122400 & -4.9199780 \\ -7.5066250 & -1.7354870 & -4.0500590 \\ -8.1029370 & -1.7309260 & -3.1288060 \\ -7.4647510 & -0.7083240 & -4.4360070 \\ -8.0157720 & -2.3603340 & -4.7959380 \\ -5.0562720 & -2.3104510 & 1.6701030 \\ -5.5579100 & -1.3747000 & 1.9425210 \\ -5.7899380 & -2.9579550 & 1.1709250 \\ -4.7266610 & -2.8102040 & 2.5913690 \\ -3.1409270 & -3.3927810 & 0.4435530 \\ -3.8329830 & -4.1225670 & 0.0041540 \\ -2.3028940 & -3.2437480 & -0.2437550 \\ -2.7546020 & -3.8188100 & 1.3779010 \\ 4.3721020 & 1.0030240 & 3.0702700 \\ 5.3231120 & 0.4576540 & 3.0094570 \\ 4.5762830 & 2.0683520 & 2.9026410 \\ 3.9661600 & 0.8789250 & 4.0831540 \\ 3.0401370 & -1.0132130 & 2.3028780 \\ 2.3312120 & -1.3979620 & 1.5632560 \\ 3.9436630 & -1.6352520 & 2.2706260 \\ 2.5898190 & -1.1184990 & 3.2980440 \\ 8.1274040 & 0.1094920 & -0.9377920 \\ 9.0438670 & -0.4649840 & -1.1290150 \\ 8.1855600 & 1.0525670 & -1.4979610 \\ 8.0774800 & 0.3471220 & 0.1321160 \\ 7.0034150 & -1.0712310 & -2.8678070 \\ 6.1146640 & -1.6093740 & -3.2197200 \\ 7.1310070 & -0.1785780 & -3.4935780 \\ 7.8812530 & -1.7120470 & -3.0179550 \\ 3.0422510 & 1.9862670 & -3.9709480 \\ 3.9764050 & 1.6442410 & -4.4348460 \\ 2.3809060 & 1.1240750 & -3.8352870 \\ 2.5616670 & 2.6915050 & -4.6617900 \\ 4.2278220 & 3.9054830 & -2.8249860 \\ 5.2085250 & 3.5863190 & -3.2028010 \\ 3.7860430 & 4.5984560 & -3.5536220 \\ 4.3840710 & 4.4438640 & -1.8822760 \\ -0.2636900 & 0.0763420 & -1.0158420\end{array}$

0.4223920
-0.7610830
2.3465140
6.8596320
2.4493130
-3.7427940
-6.1720670
-3.1389630
2.4173400
1.2697980
2.2777070
0.2848420
1.9690870
1.3269970
3.9344590
2.8254090
1.4310450
3.8034060
4.9201280
2.9369150
1.2285020
4.0058010
4.5199790
3.8750740
2.9041020
0.6325940
0.2102530
-0.4109220
0.2103370
1.1612050
-0.7570720
1.1481110
1.9150520
-0.7764200
-1.4974730
0.1773390
1.8971140
-1.5360180
0.1652460
-0.5032960
0.9262450
1
0.1313960
$-1.2560460$
3.0479030
$-1.6194360$
1.0333630
2. 6112530
$-3.2979130$
$-1.4239640$
$-2.2669610$
$-3.1404800$
$-1.4769240$
$-2.7495270$
$-1.8329290$
$-1.0240620$
$-4.0654030$
$-5.0948570$
$-4.4772480$
$-2.8688580$
$-4.5263340$
$-5.9634950$
$-4.3464200$
$-3.1868040$
$-2.0797760$
$-3.7130880$
$-5.4546690$
$-5.1273950$
$-3.1886920$
$-2.0660200$
$-3.5426740$
$-4.4283180$
$-2.9763050$
$-4.7366530$
$-4.8597090$
$-3.3003790$
$-2.2942110$
$-4.1778020$
$-5.4124660$
$-2.8663630$
$-4.4241060$
$-1.7967980$
$-4.1699840$

$-2.3520250$

$-0.5253920$

$-2.2518720$

$-0.7956270$

$-1.3541830$

$-3.4125600$

1.2753540

$-1.5232370$

$-1.7886320$

$-0.7835890$

$-1.5637980$

$-2.8652470$

$-2.7085810$

$-2.4843470$

$-2.2230980$

$-2.4347890$

$-1.5283290$

$-2.3509320$

$-2.8819560$

$-3.5083150$

$-0.4960720$

$-1.7668860$

$-3.5229190$

$-1.1878690$

$-2.0575300$

2.0632200

1.8162880

3. 5083150

4.0492550

4.3643450

5.4083500

3.3969240

5.7204880

3.9584640

6.2481250

5.8143610

6.3665420

7. 3075220

0.8117710

144

0.9010760

TS200(R,R) G[B97-D/def2-TZVP $]=-3633.750757$

$\begin{array}{lccc}\text { TS } & 0.5969730 & 0.1706250 & -0.2828110 \\ \mathrm{O} & -1.6616180 & 0.1459590 & -1.4941520 \\ \mathrm{O} & 0.9115350 & 1.3463770 & -0.9504590\end{array}$




$\begin{array}{rrr}-0.0362090 & 2.3623120 & -1.0243510 \\ 2.2286390 & 1.4847150 & -1.4618130 \\ 0.2704620 & 3.5379730 & -1.7835310 \\ 2.5337810 & 2.6472600 & -2.1437510 \\ 1.5820140 & 3.6734890 & -2.3525290 \\ 3.5408960 & 2.7819660 & -2.5308740 \\ -2.1508610 & 1.1216910 & -0.6145150 \\ -3.4455250 & 0.9369620 & -0.0589150 \\ -1.3418860 & 2.2122440 & -0.3339110 \\ -3.8870390 & 1.8872760 & 0.8413680 \\ -1.7731660 & 3.1422040 & 0.6674760 \\ -3.0727430 & 2.9706130 & 1.2546640 \\ -4.8787820 & 1.7860670 & 1.2742010 \\ -2.6844080 & 4.8934430 & 2.6997100 \\ -1.3909270 & 5.0455670 & 2.1426010 \\ -0.9475700 & 4.1942650 & 1.1500540 \\ -3.5058430 & 3.8749140 & 2.2624030 \\ -3.0216320 & 5.5733520 & 3.4787850 \\ -0.7380560 & 5.8368220 & 2.5044980 \\ 0.0494280 & 4.3158510 & 0.7391140 \\ -4.4950080 & 3.7347460 & 2.6944930 \\ -0.3416280 & 5.6811910 & -2.7743250 \\ 0.9618760 & 5.8245550 & -3.3115480 \\ 1.9017220 & 4.8362720 & -3.1051540 \\ -0.6786890 & 4.5686580 & -2.0294280 \\ -1.0861950 & 6.4533840 & -2.9552720 \\ 1.2139030 & 6.7087810 & -3.8926270 \\ 2.9035390 & 4.9255550 & -3.5216210 \\ -1.6822380 & 4.4711210 & -1.6277510 \\ 3.3206980 & 0.4870080 & -1.2380310 \\ 3.7567470 & -0.3322580 & -2.3016600 \\ 4.0202110 & 0.4912580 & -0.0084410 \\ 4.9399820 & -1.0649390 & -2.1487760 \\ 5.1947640 & -0.2642340 & 0.0951210 \\ 5.6887980 & -1.0219310 & -0.9701100 \\ 5.2890040 & -1.6769850 & -2.9771450 \\ 5.7565140 & -0.2521310 & 1.0261060 \\ -4.2990020 & -0.2295020 & -0.4289620 \\ -4.6111110 & -1.2179270 & 0.5381430 \\ -4.8194020 & -0.3335100 & -1.7385180 \\ -5.4482930 & -2.2764400 & 0.1714820 \\ -5.6502400 & -1.4168120 & -2.0531000 \\ -5.9782360 & -2.3972750 & -1.1166010 \\ -5.6904880 & -3.0343410 & 0.9129250 \\ -6.0585580 & -1.4961100 & -3.0594460 \\ 3.5664750 & 1.3688030 & 1.1539570\end{array}$

7. 0142180 2. 9940130 $-4.0830350$ $-6.8684210$ $-4.5552370$ $-5.8520270$ $-6.5985880$ $-6.2828250$ $-5.6465770$ $-3.9200610$ $-2.9945110$ $-4.6087830$ $-3.6867460$ $-8.1255160$ $-8.7707850$ $-7.8461990$ $-8.6997010$ $-6.0903860$ $-5.2092620$ $-5.7490980$ $-6.7325760$ $-5.1911510$ $-5.5996660$ $-6.0152560$ $-4.7949120$ $-3.4928110$ $-4.2750470$ $-2.7377730$ $-3.0224970$ 4. 1887740 5. 2828660 3. 9310500 3.8265160 3.8689630 3. 4604750 4. 9491000 3. 4129970 8.0485090 9.0215840 7.7206370 8.1744540 6.8753370 6.2255020 6.4500390 7.8604810 2.7764070

-1.7571810
-0.4082500
-1.1681230
-3.5695060
0.7099520
1.4633080
0.7719780
1.9434660
2.2372230
0.0876570
-0.4406150
-0.6234370
0.8735130
-3.6360880
-4.4671330
-3.7994980
-2.7028250
-4.8985510
-4.8718610
-5.0874270
-5.7346610
-0.7218590
0.2571610
-1.4481200
-0.6577610
-2.5128710
-3.2763830
-2.8711210
-2.3848570
2.7758760
2.7086500
3.2467270
3.4156070
0.7734580
-0.2391610
0.7363500
1.4016020
-1.1623670
-1.6553380
-1.3101630
-0.0854610
-3.2734960
-3.7329940
-3.4778290
-3.7553040
-1.8598130

$-0.8538410$ $-3.6178430$ 1.9674390 $-1.4889260$ $-2.8170550$ $-3.1746670$ $-3.5870240$ $-2.2864660$ $-3.9263670$ $-4.0742140$ $-3.8193280$ $-4.5485850$ $-4.8050640$ $-0.6014460$ $-0.9158010$

0.4476920 $-0.6615770$ $-1.4208650$ $-2.0743230$ $-0.3943220$ $-1.7287470$

2. 9424010

2.6658390

2.9362100

3. 9649060

2. 4330810

2.5327890

1.7269180

3. 4167820

1. 0396470

1.1113260

0.0842990

1.8558780

2. 5377740

2.6357090

2. 7297410

3. 3108400

$-1.8308110$

1.7044090

$-2.8683420$

$-1.6616690$

$-1.0835710$

$-0.3308670$

$-2.0748650$

$-1.0298590$

$-4.0817870$ 


$\begin{array}{rrrr}\mathrm{H} & 3.7288930 & -2.3410200 & -4.3395450 \\ \mathrm{H} & 2.2876840 & -2.4510520 & -3.3004510 \\ \mathrm{H} & 2.1406080 & -1.8707390 & -4.9764330 \\ \mathrm{C} & 3.7104410 & 0.4015730 & -4.7160810 \\ \mathrm{H} & 4.7129520 & -0.0094820 & -4.8965160 \\ \mathrm{H} & 3.1432410 & 0.3537720 & -5.6553660 \\ \mathrm{H} & 3.8169470 & 1.4536420 & -4.4267050 \\ \mathrm{P} & -0.5667720 & -0.8555900 & -0.8303020 \\ \mathrm{O} & -0.0377580 & -1.7407320 & -1.9206990 \\ \mathrm{O} & -1.1136010 & -1.5100100 & 0.4118890 \\ \mathrm{H} & 2.0044150 & 0.0359030 & -3.4625020 \\ \mathrm{H} & 7.3876210 & -1.6026900 & 0.1683860 \\ \mathrm{H} & 2.4789240 & 1.4848550 & 1.0864270 \\ \mathrm{H} & -3.8517370 & 1.4527410 & -2.4297220 \\ \mathrm{H} & -7.1933000 & -3.4197360 & -2.5283950 \\ \mathrm{H} & -3.2781870 & -0.4299070 & 2.0113810 \\ \mathrm{C} & 2.1178800 & -2.9189900 & 0.1741980 \\ \mathrm{C} & 1.0133240 & -3.6409310 & 0.8192260 \\ \mathrm{H} & 2.0231190 & -1.8359210 & 0.0973270 \\ \mathrm{H} & 0.0314010 & -3.1833340 & 0.7897510 \\ \mathrm{O} & 1.4898340 & -3.6007130 & -0.9798270 \\ \mathrm{H} & 0.8453780 & -2.9073460 & -1.4001170 \\ \mathrm{C} & 3.6300000 & -4.8978080 & 0.6761570 \\ \mathrm{C} & 2.6488420 & -5.3261740 & 1.7773370 \\ \mathrm{C} & 1.1954230 & -5.0093750 & 1.3797360 \\ \mathrm{C} & 3.5313160 & -3.3855750 & 0.4237400 \\ \mathrm{H} & 4.6570840 & -5.1499870 & 0.9647420 \\ \mathrm{H} & 2.7381870 & -6.3983380 & 1.9856730 \\ \mathrm{H} & 0.8425090 & -5.7194270 & 0.6156600 \\ \mathrm{H} & 3.8922120 & -2.8327560 & 1.3013820 \\ \mathrm{H} & 4.1436660 & -3.0690050 & -0.4238390 \\ \mathrm{H} & 3.4073700 & -5.4427600 & -0.2511270 \\ \mathrm{H} & 2.8916010 & -4.7891750 & 2.7048590 \\ \mathrm{H} & 0.5019680 & -5.1160660 & 2.2228910 \\ \mathrm{C} & 0.3520860 & -0.5943930 & 3.2840980 \\ \mathrm{O} & -0.6817860 & -0.2927800 & 2.5447360 \\ \mathrm{C} & 0.8063780 & 0.5407160 & 4.1280050 \\ \mathrm{C} & 1.5732510 & 0.3447710 & 5.2904420 \\ \mathrm{C} & 0.5089540 & 1.8568530 & 3.7175640 \\ \mathrm{C} & 2.0367540 & 1.4364760 & 6.0215390 \\ \mathrm{H} & 1.8026650 & -0.6674180 & 5.6112570 \\ \mathrm{C} & 0.9929320 & 2.9460290 & 4.4402510 \\ \mathrm{H} & -0.0763940 & 2.0224020 & 2.8188010 \\ \mathrm{C} & 1.7571900 & 2.7405240 & 6.9226800 \\ \mathrm{H} & 2.6228670 & 1.2723600 & 4.0981760 \\ \mathrm{H} & 0.7724490 & 3.9542820 & \\ & & & \end{array}$

$\begin{array}{lrrr}\mathrm{H} & 2.1335730 & 3.5908930 & 6.1581270 \\ \mathrm{H} & -0.8550910 & -0.9148230 & 1.7243790 \\ \mathrm{~S} & 1.1376090 & -2.0833510 & 3.2800230 \\ 144 & & \end{array}$

TS201(R, R) G[B97-D/def2-TZVP $]=-3633.750507$

$\begin{array}{lrrr}\text { TS201(R, R) G }[\mathrm{B} 97-\mathrm{D} / \mathrm{de} \text { ef }-\mathrm{TZVP}]=-3633.750507 \\ \mathrm{O} & 0.5305030 & 0.6738590 & -0.0733060 \\ \mathrm{O} & -1.7626450 & 0.8672900 & -1.1751240 \\ \mathrm{C} & 0.7022400 & 2.0480200 & -0.1732770 \\ \mathrm{C} & -0.3528480 & 2.8927860 & 0.1562140 \\ \mathrm{C} & 1.9936610 & 2.5187830 & -0.5299970 \\ \mathrm{C} & -0.1818590 & 4.3024190 & -0.0438370 \\ \mathrm{C} & 2.1709050 & 3.8840580 & -0.6392740 \\ \mathrm{C} & 1.1069900 & 4.7975100 & -0.4422140 \\ \mathrm{H} & 3.1532260 & 4.2715720 & -0.8984120 \\ \mathrm{C} & -2.2904750 & 1.3275910 & 0.0333630 \\ \mathrm{C} & -3.5151800 & 0.7620390 & 0.4808050 \\ \mathrm{C} & -1.6107050 & 2.3295680 & 0.7125080 \\ \mathrm{C} & -4.0460380 & 1.2491550 & 1.6584470 \\ \mathrm{C} & -2.1196400 & 2.7529200 & 1.9863620 \\ \mathrm{C} & -3.3721110 & 2.2160990 & 2.4443670 \\ \mathrm{H} & -4.9963480 & 0.8590120 & 2.0131400 \\ \mathrm{C} & -3.2025090 & 3.5265850 & 4.4919580 \\ \mathrm{C} & -1.9457790 & 4.0209000 & 4.0640560 \\ \mathrm{C} & -1.4194100 & 3.6454410 & 2.8439640 \\ \mathrm{C} & -3.8963100 & 2.6380500 & 3.6969970 \\ \mathrm{H} & -3.6089810 & 3.8367370 & 5.4520260 \\ \mathrm{H} & -1.3865610 & 4.6987080 & 4.7053250 \\ \mathrm{H} & -0.4523640 & 4.0281060 & 2.5355120 \\ \mathrm{H} & -4.8515460 & 2.2297560 & 4.0224480 \\ \mathrm{C} & -1.0396870 & 6.5854040 & -0.1041820 \\ \mathrm{C} & 0.2403010 & 7.0746200 & -0.4648650 \\ \mathrm{C} & 1.2892910 & 6.1946550 & -0.6330130 \\ \mathrm{C} & -1.2448400 & 5.2356930 & 0.1024960 \\ \mathrm{H} & -1.8701200 & 7.2793400 & 0.0062260 \\ \mathrm{H} & 0.3890200 & 8.1410860 & -0.6185250 \\ \mathrm{H} & 2.2743980 & 6.5536290 & -0.9258980 \\ \mathrm{H} & -2.2311400 & 4.8736750 & 0.3757040 \\ \mathrm{C} & 3.1436870 & 1.5902920 & -0.7430890 \\ \mathrm{C} & 3.6836590 & 1.4165840 & -2.0373890 \\ \mathrm{C} & 3.7372240 & 0.9421060 & 0.3661960 \\ \mathrm{C} & 4.8318610 & 0.6310750 & -2.1865770 \\ \mathrm{C} & 4.8818710 & 0.1620810 & 0.1608380 \\ \mathrm{C} & 5.4529450 & 0.0033100 & -1.1043610 \\ \mathrm{H} & 5.2557280 & 0.4983460 & -3.1806100 \\ \mathrm{H} & 5.3395720 & -0.3289990 & 1.0128710 \\ \mathrm{C} & -4.1998600 & -0.3189020 & -0.2879040\end{array}$




-4.2482070
-4.8084130
-4.9259350
-5.4625000
-5.5353880
-4.9716430
-5.9333250
3.2086650
6.7286100
3.0759540
-3.6033170
-6.2402690
-4.8202590
-6.2548310
-6.8958930
-6.6930740
-6.2515920
-4.1879040
-3.1613190
-4.7678120
-4.1706270
-5.5275910
-6.0189030
-5.5614470
-4.4748800
-7.7243320
-8.2474950
-7.8178980
-8.2211630
-4.6650090
-5.2088060
-5.3940320
-4.1892900
-2.8181760
-3.4920220
-2.0946150
-2.2770250
3.8019250
4.8935620
3.5716320
3.3900770
3.4589890
3.1285650
4.5230020
2.9051200
7.0439030

$-1.6340430$ $-0.0256760$ $-2.6189860$ $-1.0482100$ $-2.3518470$ $-3.6331500$ $-0.8170370$ 1.1544660 $-0.7909080$ 2.0761780 $-2.0150640$ $-3.4493180$ 1. 3768740 1.9405070 1. 3226750 1.9567960 2.9640570 1.4091590

1.0274680 0.7969240 2.4391730 $-3.7283540$ $-4.5548150$ $-2.8391700$ $-3.9914220$ $-3.1139590$ $-2.9416020$ $-2.2072110$ $-3.9368950$ $-2.1036570$ $-1.1592970$ $-2.8903700$ $-2.3523350$ $-3.3384980$ $-4.1933330$ $-3.3213940$ $-3.4914610$ 2.4501610 2. 3607870 3.3114850 2.6352820 $-0.0312680$ $-0.9742240$ $-0.1267480$ 0.1202360
-1.8263590

0.2320680
-1.5301520
-0.4984320
-2.2240220
-1.7256260
-0.1064120
-3.1772620
1.7815620
-1.3408090
-3.2697020
1.5604320
-2.5027760
-2.1256490
-2.1592000
-2.8017330
-1.1529450
-2.5573510
-3.5291020
-3.4990680
-4.2319490
-3.9098680
-3.8403650
-4.3712460
-4.4838360
-3.6768400
-2.7428630
-1.7936800
-3.3549530
-3.2737190
2.6761480
2.7921300
2.4387570
3.6347530
1.4920800
1.3507870
0.6717840
2.4342200
2.3727310
2.4569900
1.7346740
3.3741470
2.7272400
2.2775290
2.9811480
3.6613800
-0.2523300

7.9176060
7.2792900
6.1999520
7.9216970
7.7298640
8.0899780
8.8362920
2.8469940
3.7975600
2.2157590
2.3501950
3.9584380
4.9527190
3.5045780
4.0870390
-0.4800500
0.0699010
-0.8048120
2.0981850
6.5996730
2.1246960
-4.2295130
-6.1925560
-2.8914450
2.3744050
1.2902240
2.3164080
0.3347570
1.6170160
0.9486540
3.8242690
2.9061740
1.4442580
3.7797310
4.8572780
2.9605360
1.0045720
4.2254030
4.3503960
3.5091180
3.2408910
0.8139430
0.8962300
0.0476700
1.1645610
2.2859610

-2.4206980
-1.3345650
-2.5060740
0.1735720
0.8932650
0.7339530
-0.3858720
1.0790020
0.6736380
0.2462180
1.5880690
3.2471020
2.8800300
3.7356670
3.9958250
-0.1240310
-0.2126510
-1.3974120
2.4854610
-1.3286660
1.2887160
2.0386830
-4.3647840
-1.2285560
-2.2895540
-3.2643350
-1.3783460
-2.8923400
-2.1539350
-1.3657750
-4.1548150
-5.1740580
-4.6908080
-2.8022840
-4.5218440
-6.1488370
-4.7866130
-2.9001320
-2.0352930
-4.0214040
-5.3153000
-5.2984910
-2.6606530
-1.7244550
-2.7010150
-3.3703420

-0.5467800
0.7007040
-0.0844380
-1.5157410
-2.3208390
-0.5859460
-1.7539900
-4.4201550
-4.7900180
-4.0944860
-5.2563150
-3.7464340
-4.0341140
-4.6190890
-2.9553920
-1.0836420
-2.4801290
-0.3514760
-2.9948450
-2.2922130
1.7265680
-1.4847820
-1.8959530
1.8300890
-1.7480250
-1.5799690
-1.1492490
-1.2282570
-3.0098720
-2.8492770
-2.6900820
-1.9995730
-1.9937770
-1.9615610
-2.7033280
-2.4974740
-2.9979930
-0.9632090
-2.4926110
-3.7337530
-0.9624410
-1.3335070
2.3288690
1.9961120
3.7920950
4.3171020
- 


\begin{tabular}{|c|c|c|c|c|c|c|c|}
\hline & & & & & & & \\
\hline $\begin{array}{c}C \\
C\end{array}$ & $\begin{array}{l}0.2888750 \\
2.5286530\end{array}$ & $\begin{array}{l}-2.0431430 \\
-3.3785770\end{array}$ & $\begin{array}{l}4.6812260 \\
5.6891530\end{array}$ & C & $\begin{array}{l}-2.2337060 \\
-2.1498210\end{array}$ & $\begin{array}{l}-2.9615820 \\
-3.0125690\end{array}$ & $\begin{array}{r}-1.7799600 \\
0.6688830\end{array}$ \\
\hline $\mathrm{H}$ & 2.9711510 & -3.8697840 & 3.6385450 & $\mathrm{C}$ & -3.5895610 & -2.6151960 & -1.6914800 \\
\hline C & 0.5291780 & -2.0632940 & 6.0544800 & $\mathrm{C}$ & -3.5023830 & -2.6650430 & 0.7014940 \\
\hline $\mathrm{H}$ & -0.5821980 & -1.5257890 & 4.2922350 & $\mathrm{c}$ & -4.2386860 & -2.4480900 & -0.4672520 \\
\hline C & 1.6504390 & -2.7273020 & 6.5635560 & $\mathrm{H}$ & -4.1600590 & -2.4648900 & -2.6058450 \\
\hline $\mathrm{H}$ & 3.4059740 & -3.8904140 & 6.0775080 & $\mathrm{H}$ & -3.9962520 & -2.5595140 & 1.6644450 \\
\hline $\mathrm{H}$ & -0.1594980 & -1.5588790 & 6.7282740 & C & 3.4957850 & 2.2531050 & 0.2415650 \\
\hline $\mathrm{H}$ & 1.8398140 & -2.7366820 & 7.6346240 & C & 2.7508900 & 3.1283300 & 1.0697150 \\
\hline $\mathrm{H}$ & -0.2301580 & -1.6756970 & 0.9963190 & $\mathrm{C}$ & 3.9324260 & 2.6798260 & -1.0295680 \\
\hline S & 1.6161550 & -3.7320230 & 1.2498280 & C & 2.4637600 & 4.4135820 & 0.6007820 \\
\hline & & & & C & 3.6183300 & 3.9775530 & -1.4531040 \\
\hline & R) $G[B 97-D$ & TZVP] $=-36$ & +51 & $\mathrm{C}$ & 2.8765240 & 4.8545070 & -0.6607920 \\
\hline 0 & 0.3088630 & -1.2154190 & 0.2639360 & $\mathrm{H}$ & 1.8791730 & 5.0832620 & 1.2256570 \\
\hline 0 & 2.1208390 & 0.1064020 & -0.9052920 & H & 3.9486850 & 4.3097290 & -2.4361700 \\
\hline C & 0.8661600 & -2.4160080 & -0.1661410 & C & -1.4398300 & -3.3191050 & 1.9830180 \\
\hline C & 2.242 & -2.5898070 & -0.0808690 & C & -5.7058560 & -2.0628060 & -0.4051990 \\
\hline $\mathrm{C}$ & -0.0356970 & -3.4183160 & -0.6190190 & $\mathrm{C}$ & -1.6092260 & -3.1783530 & -3.1528850 \\
\hline C & 2.7971050 & -3.7991710 & -0.6199450 & C & 2.2655320 & 2.7112220 & 2.4540290 \\
\hline $\mathrm{C}$ & 0.5037600 & -4.6133540 & -1.0473790 & $\mathrm{C}$ & 2.4691630 & 6.2204920 & -1.1839930 \\
\hline C & 1.9058870 & -4.8241130 & -1.0936420 & $\mathrm{C}$ & 4.7437870 & 1.7821940 & -1.9538590 \\
\hline $\mathrm{H}$ & -0.1557330 & -5.4111430 & -1.3791120 & C & 6.1719500 & 2.3330950 & -2.1331000 \\
\hline C & 2.9782660 & -0.2124600 & 0.1409950 & $\mathrm{H}$ & 6.1442120 & 3.3244370 & -2.6047420 \\
\hline C & 3.7438240 & 0.8508510 & 0.6921870 & $\mathrm{H}$ & 6.6795790 & 2.4286100 & -1.1645940 \\
\hline $\mathrm{C}$ & 3.0816930 & -1.5365540 & 0.5529360 & $\mathrm{H}$ & 6.7613600 & 1.6633680 & -2.7736140 \\
\hline C & 4.6651890 & 0.5360450 & 1.6687470 & C & 4.0571590 & 1.5927060 & -3.3195070 \\
\hline C & 3.9631620 & -1.8312370 & 1.6486730 & $\mathrm{H}$ & 3.0477850 & 1.1859390 & -3.1918880 \\
\hline C & 4.7899580 & -0.7804320 & 2.1795600 & $\mathrm{H}$ & 3.9844040 & 2.5480540 & -3.8556410 \\
\hline $\mathrm{H}$ & 5.2882580 & 1.3210080 & 2.0899200 & $\mathrm{H}$ & 4.6404230 & 0.8991550 & -3.9399420 \\
\hline C & 5.74 & -2.3292440 & 3.7997230 & C & 2.8133860 & 7.3573610 & -0.2053300 \\
\hline C & $4.8 \mathrm{~s}$ & -3.3523580 & 3.3145030 & $\mathrm{H}$ & 2.5426650 & 8.3281720 & -0.6409910 \\
\hline C & 4.0 & -3.1096940 & 2.2684480 & $\mathrm{H}$ & 2.2582930 & 7.2421920 & 0.7346360 \\
\hline C & 5.6871210 & -1.0679940 & 3.2443990 & $\mathrm{H}$ & 3.8854750 & 7.3644850 & 0.0288980 \\
\hline $\mathrm{H}$ & 6.4 & -2.5360080 & 4.6188050 & C & 0.9636690 & 6.2333220 & -1.5217100 \\
\hline $\mathrm{H}$ & 4.9 & -4.3381960 & 3.7737750 & $\mathrm{H}$ & 0.7204390 & 5.4490030 & -2.2494720 \\
\hline $\mathrm{H}$ & 4460 & -3.9034380 & 1.9139720 & $\mathrm{H}$ & 0.3703060 & 6.0534330 & -0.6153210 \\
\hline $\mathrm{H}$ & 6.3 & -0.2626680 & 3.6241200 & $\mathrm{H}$ & 0.6705600 & 7.2057100 & -1.9404220 \\
\hline c & 4.6916330 & -5.1910810 & -1.2729970 & $\mathrm{C}$ & 3.2578830 & 3.1857940 & 3.5357240 \\
\hline $\mathrm{C}$ & 3.8 & -6.2154790 & -1.7022350 & $\mathrm{H}$ & 4.2661940 & 2.7973230 & 3.3539610 \\
\hline C & 2.4 & -6.0298650 & -1.6163220 & $\mathrm{H}$ & 3.3084750 & 4.2833160 & 3.5399210 \\
\hline C & 4.1 & -4.0151600 & -0.7446570 & $\mathrm{H}$ & 2.9289550 & 2.8513100 & 4.5291290 \\
\hline $\mathrm{H}$ & 5.7 & -5.3297470 & -1.3643880 & C & 0.8514550 & 3.2191830 & 2.7895220 \\
\hline H & 7700 & -7.1396180 & -2.1104290 & $\mathrm{H}$ & 0.8426560 & 4.3073120 & 2.9350630 \\
\hline $\mathrm{H}$ & 1.7 & -6.7999530 & -1.9612860 & $\mathrm{H}$ & 0.1461240 & 2.9674260 & 1.9927910 \\
\hline $\mathrm{H}$ & 4.8 & -3.2363530 & -0.4230330 & $\mathrm{H}$ & 0.5097460 & 2.7577800 & 3.7253330 \\
\hline C & -1 & 1461350 & -0.5839260 & C & -1.9107500 & -4.6851250 & 2.5226320 \\
\hline
\end{tabular}




$\begin{array}{rrr}-2.9876310 & -4.6595430 & 2.7367390 \\ -1.7261160 & -5.4806330 & 1.7892350 \\ -1.3780530 & -4.9330280 & 3.4507820 \\ -1.6299280 & -2.2218080 & 3.0426680 \\ -1.3209170 & -1.2465530 & 2.6522360 \\ -2.6793810 & -2.1542780 & 3.3557980 \\ -1.0298420 & -2.4556120 & 3.9318580 \\ -5.9128230 & -0.7409470 & 0.3560690 \\ -6.9741660 & -0.4597400 & 0.3537990 \\ -5.5864760 & -0.8360180 & 1.3984420 \\ -5.3368340 & 0.0699390 & -0.1022080 \\ -6.5510560 & -3.1852760 & 0.2279150 \\ -6.4286850 & -4.1276470 & -0.3210050 \\ -6.2436810 & -3.3529500 & 1.2686240 \\ -7.6147480 & -2.9119420 & 0.2252000 \\ -2.0099400 & -2.1163490 & -4.1912710 \\ -3.0786570 & -2.1776930 & -4.4346530 \\ -1.7920800 & -1.1084540 & -3.8278100 \\ -1.4484520 & -2.2831260 & -5.1197760 \\ -1.9759050 & -4.5822070 & -3.6786910 \\ -3.0630940 & -4.6581160 & -3.8166710 \\ -1.4902620 & -4.7671230 & -4.6465200 \\ -1.6674140 & -5.3664830 & -2.9777080 \\ 0.5121040 & 0.0948840 & -0.6842300 \\ -0.0505310 & -0.1116180 & -2.0666790 \\ 0.0341010 & 1.2596020 & 0.1351110 \\ -0.5206770 & -3.1332100 & -3.0440540 \\ -6.0526740 & -1.9196570 & -1.4387440 \\ -0.3654870 & -3.4029190 & 1.7949990 \\ 4.8309810 & 0.7933760 & -1.4908460 \\ 3.0244790 & 6.3958780 & -2.1166110 \\ 2.2330070 & 1.6166040 & 2.4847670 \\ -2.8080510 & 1.4385790 & -1.9357910 \\ -2.0057100 & 2.6630110 & -1.8145570 \\ -2.6485740 & 0.6701390 & -1.1774870 \\ -1.0672600 & 2.5943990 & -1.2769610 \\ -1.8404230 & 1.3112700 & -3.0346120 \\ -1.0446620 & 0.7192060 & -2.6466830 \\ -4.4444840 & 2.7080290 & -3.4043070 \\ -3.8921750 & 4.0218270 & -2.8288280 \\ -2.3843440 & 3.9193430 & -2.5336630 \\ -4.2402720 & 1.5523500 & -2.4100150 \\ -5.5137270 & 2.8114450 & -3.6227930 \\ -4.0655400 & 4.8513900 & -3.5239140 \\ -1.8091160 & 3.9400970 & -3.4707730 \\ -4.8534230 & 1.7233750 & -1.5147240\end{array}$

$\begin{array}{ll}\mathrm{H} & -4.5458900 \\ \mathrm{H} & -3.9361630 \\ \mathrm{H} & -4.4228700 \\ \mathrm{H} & -2.0245870 \\ \mathrm{C} & -3.0327850 \\ \mathrm{O} & -2.1462800 \\ \mathrm{C} & -4.1618860 \\ \mathrm{C} & -5.3966670 \\ \mathrm{C} & -4.0161940 \\ \mathrm{C} & -6.4579780 \\ \mathrm{H} & -5.5190640 \\ \mathrm{C} & -5.0766290 \\ \mathrm{H} & -3.0708890 \\ \mathrm{C} & -6.3018630 \\ \mathrm{H} & -7.4107040 \\ \mathrm{H} & -4.9480840 \\ \mathrm{H} & -7.1313050 \\ \mathrm{H} & -1.2968070 \\ \mathrm{~S} & -2.9156120 \\ \mathrm{I} & \end{array}$
0.5895750
1.2534230

$-4.3491950$

$4.2557560 \quad-1.8959200$

$4.7693920 \quad-1.9430710$

$2.0087850 \quad 1.5239360$

$1.0463010 \quad 1.4251020$

$\begin{array}{ll}1.6666450 & 2.4230680\end{array}$

$\begin{array}{ll}2.3364290 & 2.3315830\end{array}$

$0.6327170 \quad 3.3705750$

$1.9783610 \quad 3.1598970$

$\begin{array}{ll}1.97230110 & 1.5927150\end{array}$

$0.2860030 \quad 4.2053840$

$0.1081920 \quad 3.4485080$

$0.9537160 \quad 4.1009450$

$2.4942910 \quad 3.0681510$

$-0.5087260 \quad 4.9365680$

$0.6761910 \quad 4.7475110$

$\begin{array}{ll}3.4809980 & 0.7256920\end{array}$

TS203(R,R) G[B97-D/def2-TZVP $]=-3633.750421$

$\begin{array}{lrcr}\text { TS203(R, R) G[B97-D/def2-TZVP] }=-3633.750421 \\ \text { O } & 0.5927520 & 0.6325470 & -0.0839180 \\ \text { O } & -1.6160680 & 0.8992300 & -1.3319740 \\ \mathrm{C} & 0.8309900 & 1.9921300 & -0.2284710 \\ \mathrm{C} & -0.1950420 & 2.8965760 & 0.0258530 \\ \mathrm{C} & 2.1542230 & 2.3886860 & -0.5567940 \\ \mathrm{C} & 0.0541190 & 4.2899400 & -0.2043210 \\ \mathrm{C} & 2.4044110 & 3.7399300 & -0.6941730 \\ \mathrm{C} & 1.3820140 & 4.7101630 & -0.5587460 \\ \mathrm{H} & 3.4124220 & 4.0699480 & -0.9337510 \\ \mathrm{C} & -2.1998350 & 1.4296050 & -0.1768590 \\ \mathrm{C} & -3.4788430 & 0.9416240 & 0.2046010 \\ \mathrm{C} & -1.5094530 & 2.4111460 & 0.5210260 \\ \mathrm{C} & -4.0522910 & 1.4837920 & 1.3374560 \\ \mathrm{C} & -2.0703450 & 2.8906580 & 1.7522150 \\ \mathrm{C} & -3.3737930 & 2.4294530 & 2.1451850 \\ \mathrm{H} & -5.0426880 & 1.1543660 & 1.6401820 \\ \mathrm{C} & -3.2523360 & 3.7715880 & 4.1755240 \\ \mathrm{C} & -1.9495540 & 4.1929100 & 3.8119730 \\ \mathrm{C} & -1.3749060 & 3.7655150 & 2.6313690 \\ \mathrm{C} & -3.9457640 & 2.9038690 & 3.3574640 \\ \mathrm{H} & -3.6957580 & 4.1216330 & 5.1050280 \\ \mathrm{H} & -1.3936120 & 4.8552700 & 4.4719550 \\ \mathrm{H} & -0.3737830 & 4.0921710 & 2.3706530 \\ \mathrm{H} & -4.9382890 & 2.5524830 & 3.6340680 \\ \mathrm{C} & -0.6846100 & 6.6097480 & -0.3644200\end{array}$




$\begin{array}{rrr}0.6328900 & 7.0256570 & -0.6788710 \\ 1.6424300 & 6.0908040 & -0.7771250 \\ -0.9661540 & 5.2781810 & -0.1319950 \\ -1.4835530 & 7.3460690 & -0.3106630 \\ 0.8415120 & 8.0787950 & -0.8533160 \\ 2.6559450 & 6.3923020 & -1.0357710 \\ -1.9811520 & 4.9737200 & 0.1025960 \\ 3.2559610 & 1.3971840 & -0.7351760 \\ 3.7977190 & 1.1721040 & -2.0167610 \\ 3.8002790 & 0.7335920 & 0.3940630 \\ 4.9095240 & 0.3248080 & -2.1460120 \\ 4.8992550 & -0.1068700 & 0.2096910 \\ 5.4813660 & -0.3144820 & -1.0481900 \\ 5.3244620 & 0.1617940 & -3.1362510 \\ 5.3293640 & -0.6180200 & 1.0676990 \\ -4.1743480 & -0.1188730 & -0.5826260 \\ -4.3339160 & -1.4168220 & -0.0345760 \\ -4.6818980 & 0.1741380 & -1.8670020 \\ -5.0146500 & -2.3814360 & -0.7837210 \\ -5.3488630 & -0.8317630 & -2.5783980 \\ -5.5261660 & -2.1134840 & -2.0573980 \\ -5.1423900 & -3.3772670 & -0.3669920 \\ -5.7455700 & -0.6074370 & -3.5674120 \\ 3.2639060 & 0.9972530 & 1.7975600 \\ 6.7087000 & -1.2087390 & -1.1508710 \\ 3.2344380 & 1.8317040 & -3.2701340 \\ -3.8041120 & -1.7959120 & 1.3447370 \\ -6.2429650 & -3.1903400 & -2.8521040 \\ -4.5720030 & 1.5568380 & -2.4972780 \\ -5.9650670 & 2.1991710 & -2.6482810 \\ -6.5956450 & 1.5984340 & -3.3167560 \\ -6.4695390 & 2.2728710 & -1.6761770 \\ -5.8745270 & 3.2076630 & -3.0738310 \\ -3.8413130 & 1.5129620 & -3.8522790 \\ -2.8446710 & 1.0717260 & -3.7406910 \\ -4.4072320 & 0.9183970 & -4.5812110 \\ -3.7331980 & 2.5293760 & -4.2536790 \\ -7.5026620 & -3.6897440 & -2.1188820 \\ -8.0278310 & -4.4389450 & -2.7263580 \\ -7.2291670 & -4.1556170 & -1.1628270 \\ -8.1913600 & -2.8610350 & -1.9107210 \\ -5.3024160 & -4.3688620 & -3.1708540 \\ -4.4127130 & -4.0249570 & -3.7133750 \\ -4.9710570 & -4.8542450 & -2.2433750 \\ -5.8211550 & -5.1180220 & -3.7838580 \\ -4.9433430 & -1.7964020 & 2.3846590\end{array}$
$-5.4404550$
$-5.6972670$
$-4.5496070$
$-3.0926860$
$-3.8028580$
$-2.3174020$
$-2.6241510$
3. 9175440
5. 0012490
3.7441730
3. 5009030
3.4385790
3. 0517150
4. 4942380
2. 8921520
7.9404080
8.8149470
8.1744870
7.7539400
7.0168130
6. 1527420
7. 3032220
7.8546350
2.9546400
3.8822930
2. 2746450
2. 4904270
4. 1823690
5. 1587130
3.7631620
4. 3426870
$-0.4182700$
0.2074140
$-0.8820240$
2. 2787600
6. 5137270
2. 1893040
$-3.9904040$
$-6.5595810$
$-3.0684980$
2. 3025020
1. 1420920
2. 2447570
0.1835070
1. 6969670
1. 0682340

-0.8212010
-2.5489760
-2.0427360
-3.1619840
-3.9825820
-3.2080150
-3.3180900
2.2695040
2.1225510
3.1307550
2.4940450
-0.1827510
-1.1127220
-0.3366260
0.0196200
-0.4867370
-1.1509150
0.4047890
-0.1686180
-1.7048690
-2.2079130
-0.8701380
-2.4121470
0.8152470
0.3468690
0.0308700
1.3272130
2.9388370
2.5118040
3.4245710
3.7025910
-0.1705770
-0.4200800
-1.3532250
2.3010320
-2.0890020
1.1883070
2.2028800
-2.7421050
-1.0440530
-2.5700680
-3.4618660
-1.6161740
-3.0075000
-2.4804230
-1.6469410

2.4395320

2. 1162890

3. 3803030

1. 3617430

1. 1971600

0.5916230

2. 3415920

2. 3759860

2.4793560

1. 7200870

3.3672760

2. 7661350

2. 3340530

3. 0259670

3. 6947130

$-0.5635830$

$-0.5742950$

$-1.1613250$

0.4691240

$-2.5702810$

$-3.0219030$

$-3.2235770$

$-2.5419220$

$-4.3927080$

$-4.7459650$

$-4.0443960$

$-5.2458240$

$-3.7720870$

$-4.0379580$

$-4.6634320$

$-3.0013780$

$-1.0893280$

$-2.4328450$

$-0.2823570$

$-3.0139070$

$-0.5199870$

1. 7240690

$-1.8323720$

$-3.8045910$

1. 6472460

$-1.2603840$

$-1.1526190$

$-0.7329060$

$-0.9312520$

$-2.6064090$

$-2.5850440$ 


\begin{tabular}{|c|c|c|c|c|c|c|c|}
\hline & & & & & & & \\
\hline C & 3.7137070 & -4.5826370 & -1.9106560 & $\mathrm{C}$ & -1.2138920 & 4.9599250 & 2.3633300 \\
\hline C & 2.6593650 & -5.4911710 & -1.2616850 & $\mathrm{C}$ & -0.8291810 & 4.1477030 & 1.3151640 \\
\hline c & 1.2416870 & -4.9209630 & -1.4514130 & $\mathrm{C}$ & -3.3426680 & 3.8187800 & 2.5233450 \\
\hline C & 3.6846680 & -3.1805440 & -1.2812230 & $\mathrm{H}$ & -2.7758950 & 5.4521400 & 3.7918800 \\
\hline $\mathrm{H}$ & 4.7146220 & -5.0122440 & -1.7862100 & $\mathrm{H}$ & -0.5320730 & 5.7234160 & 2.7314670 \\
\hline $\mathrm{H}$ & 2.7000840 & -6.5012350 & -1.6849900 & $\mathrm{H}$ & 0.1511280 & 4.2706430 & 0.8659140 \\
\hline $\mathrm{H}$ & 0.9074410 & -5.0625970 & -2.4902010 & $\mathrm{H}$ & -4.3142540 & 3.6748700 & 2.9925030 \\
\hline $\mathrm{H}$ & 4.0187270 & -3.2300800 & -0.2357950 & C & -0.3698240 & 5.7954110 & -2.5740380 \\
\hline $\mathrm{H}$ & 4.3540130 & -2.4906280 & -1.8004210 & $\mathrm{C}$ & 0.9133860 & 5.9441450 & -3.1565810 \\
\hline $\mathrm{H}$ & 3.5214900 & -4.5049510 & -2.9892350 & $\mathrm{C}$ & 1.8457970 & 4.9355400 & -3.0290460 \\
\hline $\mathrm{H}$ & 2.8699700 & -5.5756410 & -0.1866490 & C & -0.6934670 & 4.6572450 & -1.8624630 \\
\hline $\mathrm{H}$ & 0.5033450 & -5.4396180 & -0.8281820 & $\mathrm{H}$ & -1.1097940 & 6.5838190 & -2.6933550 \\
\hline C & 0.5141210 & -2.4303690 & 2.6275040 & $\mathrm{H}$ & 1.1554370 & 6.8484580 & -3.7103360 \\
\hline 0 & -0.2397740 & -1.4824120 & 2.1389210 & $\mathrm{H}$ & 2.8317500 & 5.0289280 & -3.4809660 \\
\hline C & 0.7711190 & -2.2705760 & 4.0853570 & $\mathrm{H}$ & -1.6827860 & 4.5552320 & -1.4277970 \\
\hline C & 1.7583000 & -3.0258590 & 4.7460870 & C & 3.2716030 & 0.5000870 & -1.3650510 \\
\hline C & 0.0315800 & -1.3256000 & 4.8278030 & $\mathrm{C}$ & 3.6578630 & -0.3098590 & -2.4519410 \\
\hline C & 2.0017220 & -2.8418310 & 6.1055840 & $\mathrm{c}$ & 3.9973210 & 0.4600900 & -0.1488250 \\
\hline $\mathrm{H}$ & 2.3415300 & -3.7460790 & 4.1797750 & $\mathrm{C}$ & 4.8133230 & -1.0942180 & -2.3296970 \\
\hline C & 0.2730020 & -1.1496900 & 6.1896310 & $\mathrm{C}$ & 5.1362720 & -0.3477380 & -0.0755450 \\
\hline $\mathrm{H}$ & -0.7326150 & -0.7328350 & 4.3357560 & $\mathrm{C}$ & 5.5761630 & -1.1116630 & -1.1619350 \\
\hline C & 1.2595870 & -1.9041130 & 6.8335120 & $\mathrm{H}$ & 5.1312840 & -1.7073150 & -3.1708400 \\
\hline $\mathrm{H}$ & 2.7744900 & -3.4274620 & 6.5978980 & $\mathrm{H}$ & 5.7102350 & -0.3692450 & 0.8461930 \\
\hline $\mathrm{H}$ & -0.3090720 & -0.4206060 & 6.7485080 & C & -4.3118640 & -0.1594160 & -0.2980680 \\
\hline $\mathrm{H}$ & 1.4504250 & -1.7617580 & 7.8948520 & $\mathrm{C}$ & -4.5967290 & -1.1773940 & 0.6469390 \\
\hline $\mathrm{H}$ & -0.4593080 & -1.5297440 & 1.1213260 & $\mathrm{C}$ & -4.8820110 & -0.2158830 & -1.5898600 \\
\hline $\mathrm{s}$ & 1.1335840 & -3.7146010 & 1.7323780 & C & -5.4582080 & -2.2153110 & 0.2776170 \\
\hline & & & & C & -5 & 19170 & 39610 \\
\hline & R) $\mathrm{G}[\mathrm{B} 97-\mathrm{D} /$ & {$[Z \mathrm{VP}]=-36$} & 236 & C & -6.0362670 & -2.2895150 & -0.9931620 \\
\hline 0 & 0.5834980 & 0.1696810 & -0.3580950 & $\mathrm{H}$ & -5.6814530 & -2.9942840 & 1.0029540 \\
\hline 0 & -1.7248420 & 0.2371320 & -1.4723350 & $\mathrm{H}$ & -6.1799240 & -1.3237180 & -2.9011120 \\
\hline C & 0.8894410 & 1.3723030 & -0.9818030 & C & 3.6093960 & 1.3463070 & 1.0313700 \\
\hline $\mathrm{C}$ & -0.0450060 & 2.4027820 & -0.9756150 & $\mathrm{c}$ & 6.8751620 & -1.8976540 & -1.0981930 \\
\hline C & 2.1899570 & 1.5183830 & -1.5322850 & C & 2.8789180 & -0.3200510 & -3.7606310 \\
\hline C & 0.2494510 & 3.6050870 & -1.6970670 & $\mathrm{c}$ & -4.0140160 & -1.1824570 & 2.0559140 \\
\hline C & 2.4845300 & 2.7019440 & -2.1809620 & $\mathrm{c}$ & -6.9595980 & -3.4357340 & -1.3657360 \\
\hline C & 1.5391620 & 3.7469570 & -2.3123810 & $\mathrm{C}$ & -4.6529690 & 0.8633570 & -2.6407140 \\
\hline $\mathrm{H}$ & 3.4789250 & 2.8414000 & -2.5979840 & $\mathrm{c}$ & -5.9590350 & 1.6315730 & -2.9254310 \\
\hline C & -2.1601140 & 1.1773550 & -0.5288660 & $\mathrm{H}$ & -6.7200450 & 0.9578790 & -3.3406650 \\
\hline C & -3.4306810 & 0.9841640 & 0.0770470 & $\mathrm{H}$ & -6.3600210 & 2.0765520 & -2.0055770 \\
\hline C & -1.3225880 & 2.2425980 & -0.2369940 & $\mathrm{H}$ & -5.7765840 & 2.4345970 & -3.6522580 \\
\hline C & -3.8178660 & 1.9006280 & 1.0358430 & C & -4.0664190 & 0.2828640 & -3.9409160 \\
\hline $\mathrm{C}$ & -1.6940650 & 3.1325010 & 0.8227850 & $\mathrm{H}$ & -3.1355540 & -0.2584830 & -3.7380690 \\
\hline C & -2.9 & 2.9539790 & 1.4584630 & $\mathrm{H}$ & -4.7762950 & -0.4078260 & -4.4143600 \\
\hline H & -4.7 & 1.79230 & 1.5093260 & $\mathrm{H}$ & -3.8544940 & 1.0924020 & -4.6521060 \\
\hline C & -2 . & 324 & 2.9689530 & c & -8.2032750 & -3.4784230 & -0.4576840 \\
\hline
\end{tabular}




\begin{tabular}{|c|c|c|}
\hline $\begin{array}{l}-8.8733390 \\
-7.9096160 \\
-8.7558990 \\
-6.2175720 \\
-5.3456950 \\
-5.8670650 \\
-6.8876140 \\
-5.0803370 \\
-5.4930500 \\
-5.9090640 \\
-4.6439760 \\
-3.4162020 \\
-4.1988110 \\
-2.6908760 \\
-2.9083810 \\
4.2654870 \\
5.3591180 \\
3.9840520 \\
3.9551430 \\
3.9506030 \\
3.5364120 \\
5.0363220 \\
3.5266080 \\
6.9921240 \\
7.9464400 \\
6.9626190 \\
6.1777750 \\
8.0763910 \\
8.0254760 \\
8.0784850 \\
9.0207360 \\
2.6202450 \\
3.5565780 \\
2.1288050 \\
1.9713620 \\
3.6047810 \\
4.5943490 \\
3.0269850 \\
3.7419070 \\
-0.6175610 \\
-0.1540880 \\
-1.1207800 \\
1.9021630 \\
6.9073020 \\
2.5231560 \\
-3.9332560\end{array}$ & $\begin{array}{r}-4.2919780 \\
-3.6541970 \\
-2.5313760 \\
-4.7860820 \\
-4.7751280 \\
-4.9995970 \\
-5.5994050 \\
-0.7644600 \\
0.2258810 \\
-1.4854410 \\
-0.7379500 \\
-2.5467350 \\
-3.3097170 \\
-2.8821520 \\
-2.4576010 \\
2.7362650 \\
2.6365830 \\
3.2197850 \\
3.3822640 \\
0.7446490 \\
-0.2640760 \\
0.6974620 \\
1.3758900 \\
-2.7854500 \\
-3.3276040 \\
-2.1789230 \\
-3.5160480 \\
-0.9325350 \\
-0.3181910 \\
-0.2609130 \\
-1.4933530 \\
-1.7444750 \\
-2.2360380 \\
-2.3591820 \\
-1.7003370 \\
0.5160930 \\
0.0870140 \\
0.5216750 \\
1.5521150 \\
-0.8103520 \\
-1.6444390 \\
-1.5227970 \\
0.1417030 \\
-2.5512290 \\
1.4911880 \\
1.5904630\end{array}$ & $\begin{array}{r}-0.7659230 \\
0.5855520 \\
-0.5027990 \\
-1.3263790 \\
-1.9925450 \\
-0.3078470 \\
-1.6358200 \\
3.0884180 \\
2.8625830 \\
3.0900730 \\
4.0961930 \\
2.4492490 \\
2.5498330 \\
1.7018720 \\
3.4184740 \\
0.8952070 \\
0.9226570 \\
-0.0466260 \\
1.7277450 \\
2.4032220 \\
2.5122800 \\
2.5594220 \\
3.1918650 \\
0.1534760 \\
0.1395380 \\
1.0677100 \\
0.2009520 \\
-1.1798850 \\
-2.0876700 \\
-0.3108400 \\
-1.1861060 \\
-4.2833230 \\
-4.5778220 \\
-3.5222750 \\
-5.1674280 \\
-4.8331790 \\
-5.0413150 \\
-5.7672300 \\
-4.5020340 \\
-0.9090730 \\
-2.0674980 \\
0.3202260 \\
-3.5772220 \\
-1.9816380 \\
1.0053900 \\
-2.2533920\end{array}$ \\
\hline
\end{tabular}

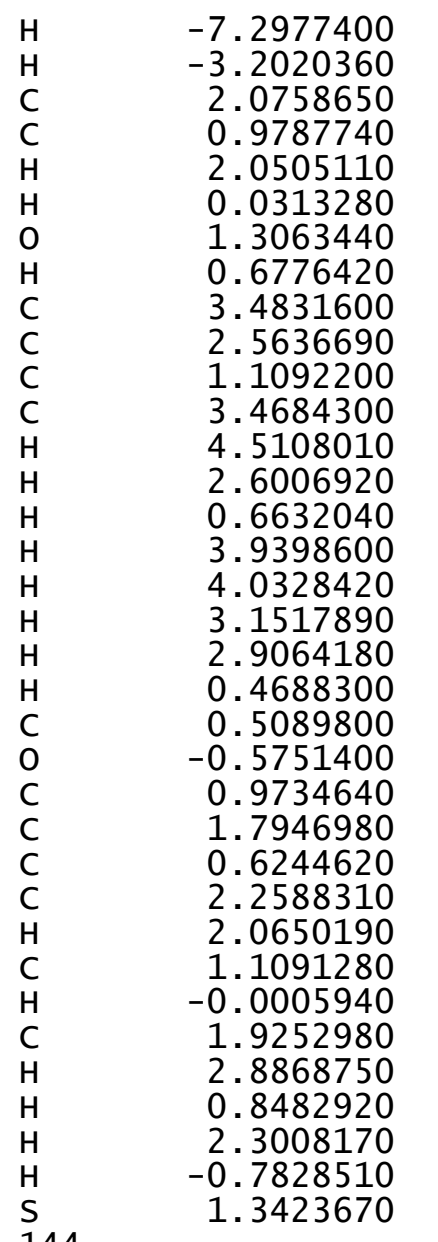
$-3.2647860$
$-0.4518770$
$-3.0356350$
$-3.6954320$
$-1.9459970$
$-3.1717560$
$-3.6444210$
$-2.8959920$
$-5.1243400$
$-5.4946320$
$-5.0840860$
$-3.6067930$
$-5.4463700$
$-6.5709590$
$-5.7559530$
$-3.0872280$
$-3.3292930$
$-5.6463440$
$-4.9757040$
$-5.1639400$
$-0.7538060$
$-0.4322850$
0.3262630
0.0628750
1. 6609380
1.1070480
$-0.9642630$
2.7039010
1.8792830
2.4314810
0.8902840
3.7289070
3.2458620
$-1.0022880$
$-2.2062040$

$-2.3976750$

2.0965950

$-0.2266960$

0.4956430

$-0.2766330$

0.5532470

$-1.3345820$

$-1.6778710$

0.1387300

1. 3111690

1. 0199430

$-0.1021510$

0.3446720

1. 5136950

0.2701430

0.7420930

$-0.9969910$

$-0.7690590$

2.2170480

1.9066820

3. 1517370

2. 4972370

4.0599920

5. 1704910

3.7669740

5.9676240

5.3984540

4. 5543930

2.9069430

5.6576470

6.8284940

4. 3023210

6.2733240

1.6471830

2. 9838530

$\operatorname{TS} 205(R, R) \quad G[B 97-D / d e f 2-T Z V P]=-3633.750232$

\begin{tabular}{lrcr} 
TS205(R, R) & \multicolumn{4}{c}{ G $[\mathrm{B} 97-\mathrm{D} / \mathrm{def}-\mathrm{TZVP}]=-3633.750232$} \\
$\mathrm{O}$ & 0.3365260 & 0.9144510 & -0.0903640 \\
$\mathrm{O}$ & -1.9907050 & 0.4499060 & -0.9970120 \\
$\mathrm{C}$ & 0.2626310 & 2.2295180 & -0.5356980 \\
$\mathrm{C}$ & -0.9039500 & 2.9538620 & -0.3218950 \\
$\mathrm{C}$ & 1.4374960 & 2.7846130 & -1.1149520 \\
$\mathrm{C}$ & -0.9818510 & 4.2791630 & -0.8676980 \\
$\mathrm{C}$ & 1.3826710 & 4.0917630 & -1.5502060 \\
$\mathrm{C}$ & 0.1867260 & 4.8512860 & -1.4794920 \\
$\mathrm{H}$ & 2.2737340 & 4.5586420 & -1.9616220
\end{tabular}




$\begin{array}{lr}\mathrm{C} & -2.5294950 \\ \mathrm{C} & -3.6160200 \\ \mathrm{C} & -2.0184420 \\ \mathrm{C} & -4.1888970 \\ \mathrm{C} & -2.5568100 \\ \mathrm{C} & -3.6762970 \\ \mathrm{H} & -5.0426330 \\ \mathrm{C} & -3.6904360 \\ \mathrm{C} & -2.5577400 \\ \mathrm{C} & -2.0068180 \\ \mathrm{C} & -4.2323850 \\ \mathrm{H} & -4.1193310 \\ \mathrm{H} & -2.1137280 \\ \mathrm{H} & -1.1372320 \\ \mathrm{H} & -5.0876710 \\ \mathrm{C} & -2.2150450 \\ \mathrm{C} & -1.0525660 \\ \mathrm{C} & 0.1214480 \\ \mathrm{C} & -2.1795360 \\ \mathrm{H} & -3.1456380 \\ \mathrm{H} & -1.0920300 \\ \mathrm{H} & 1.0179090 \\ \mathrm{H} & -3.0772950 \\ \mathrm{C} & 2.6881980 \\ \mathrm{C} & 3.1303120 \\ \mathrm{C} & 3.3940440 \\ \mathrm{C} & 4.2502260 \\ \mathrm{C} & 4.5042900 \\ \mathrm{C} & 4.9360940 \\ \mathrm{H} & 4.5979660 \\ \mathrm{H} & 5.0395550 \\ \mathrm{C} & -4.1226260 \\ \mathrm{C} & -3.8770950 \\ \mathrm{C} & -4.8508270 \\ \mathrm{C} & -4.3945950 \\ \mathrm{C} & -5.3375630 \\ \mathrm{C} & -5.1239130 \\ \mathrm{H} & -4.2160190 \\ \mathrm{H} & -5.9021460 \\ \mathrm{C} & 3.0221720 \\ \mathrm{C} & 6.1411830 \\ \mathrm{C} & 2.4472690 \\ \mathrm{C} & -3.0838510 \\ \mathrm{C} & -5.6503810 \\ \mathrm{C} & -5.1604600 \\ \mathrm{C} & -6.6724110 \\ & \end{array}$

$\begin{array}{rr}1.1095930 & 0.1048050 \\ 0.4836200 & 0.7721750 \\ 2.3527200 & 0.4580470 \\ 1.1593640 & 1.8302590 \\ 2.9981120 & 1.6230880 \\ 2.3959660 & 2.2946930 \\ 0.7228210 & 2.3422770 \\ 4.2037490 & 3.9290250 \\ 4.7739380 & 3.2981790 \\ 4.1878610 & 2.1755120 \\ 3.0348810 & 3.4364630 \\ 4.6803330 & 4.8076800 \\ 5.6802890 & 3.7041900 \\ 4.6366660 & 1.7068710 \\ 2.5705820 & 3.9242650 \\ 6.3175960 & -1.3943770 \\ 6.8938920 & -1.9626280 \\ 6.1702550 & -2.0057890 \\ 5.0448100 & -0.8597920 \\ 6.8808120 & -1.3827580 \\ 7.8999080 & -2.3742840 \\ 6.5929790 & -2.4561970 \\ 4.6130690 & -0.4289240 \\ 1.9677120 & -1.1370770 \\ 1.3388000 & -2.3230760 \\ 1.7841310 & 0.0728470 \\ 0.5000240 & -2.2557310 \\ 0.9337910 & 0.0873420 \\ 0.2650850 & -1.0600890 \\ -0.0009840 & -3.1563420 \\ 0.7886530 & 1.0225230 \\ -0.8515920 & 0.3366860 \\ -1.9951580 & 1.1314370 \\ -0.9675130 & -0.8693310 \\ -3.2273680 & 0.7104560 \\ -2.2220430 & -1.2487610 \\ -3.3643170 & -0.4722940 \\ -4.1141550 & 1.3156160 \\ -2.3083320 & -2.1745120 \\ 2.5237610 & 1.3524750 \\ -0.6589730 & -1.0307390 \\ 1.6045860 & -3.6589050 \\ -1.9270920 & 2.4316040 \\ -4.7186800 & -0.9124830 \\ 0.2323410 & -1.7555650 \\ 0.5325690 & -1.7536770\end{array}$

-7.2336660
-7.0320740
-6.8861470
-4.6427080
-3.5643370
-5.1492530
-4.8371930
-4.9576090
-5.3135430
-5.1788800
-3.8685960
-7.1801010
-7.6826430
-7.4670000
-7.5408900
-4.0345330
-4.7249250
-4.6297010
-3.4594420
-2.0808100
-2.5984260
-1.4350720
-1.4522960
4.0974240
5.0656270
4.2213220
3.8151100
2.8040230
2.0115160
3.7176090
2.5187000
6.1282450
6.9527680
6.2563240
5.1826710
7.4497460
7.4901770
7.5156530
8.3205270
2.4224900
3.4313300
1.9879530
1.8193050
3.1271550
4.1728090
2.6115180
$-0.3170990$
0.7198120
1.4170200
0.0345620
$-0.1597050$
$-0.8095260$
0.9361070
$-5.1864600$
$-6.1850380$
$-4.4930700$
$-5.2251820$
$-4.7063050$
$-4.3942240$
$-4.0085440$
$-5.7062120$
$-1.8945080$
$-1.0451850$
$-2.8172770$
$-1.8189480$
$-3.0850470$
$-4.0416760$
$-3.1660470$
$-2.9062110$
3. 5792920
3. 0953200
4.2854490
4.1423580
1. 5749320
0.8543270
1.0163490
2. 1497620
$-1.6128430$
$-2.3332560$
$-1.0560440$
$-2.1648630$
0.1567310
0.8078930
0.7878660
$-0.5126130$
0.3968540
0.1415600
$-0.4821320$
0.6409510
2. 7965670
2. 5485550

$-2.1644740$

$-0.7335920$

$-2.3687640$

$-3.1919130$

$-3.1902630$

$-3.6777350$

$-3.7880700$

$-2.2076510$

$-2.4950080$

$-3.0299860$

$-2.0774930$

$-1.0929650$

$-0.1685990$

$-1.8906460$

$-1.3682930$

3. 6458610

3. 5933860

3. 6806170

4.5788950

2. 5906060

2. 7382850

1. 7111610

3.4722380

1.6808360

1.8662410

0.8495120

2. 5806470

2. 5416550

2. 3222660

2. 7802020

3.4326680

0.1768630

0.0983720

1. 1136940

0.2429330

$-1.0544310$

$-1.9366800$

$-0.1579260$

$-1.0708850$

$-4.6081040$

$-4.9574730$

$-4.1258720$

$-5.4917530$

$-4.3659910$

-4.5945870
-5.3087080 


\begin{tabular}{lrrr} 
H & 3.1187200 & 3.6939350 & -3.7385120 \\
$\mathrm{P}$ & -0.5286290 & -0.2450870 & -0.8494770 \\
$\mathrm{O}$ & -0.0689660 & -0.4697470 & -2.2650390 \\
$\mathrm{O}$ & -0.5387710 & -1.4069710 & 0.1050070 \\
$\mathrm{H}$ & 1.4067100 & 1.8800510 & -3.4517590 \\
$\mathrm{H}$ & 6.1084230 & -1.2666040 & -1.9458360 \\
$\mathrm{H}$ & 2.0822640 & 3.0605090 & 1.1881750 \\
$\mathrm{H}$ & -4.6565510 & 1.1132510 & -1.3458640 \\
$\mathrm{H}$ & -5.4078130 & -5.4401880 & -0.1194360 \\
$\mathrm{H}$ & -2.5071360 & -0.9959960 & 2.4263180 \\
$\mathrm{C}$ & 2.3254330 & -2.4734450 & -1.9367020 \\
$\mathrm{C}$ & 1.3451810 & -3.3978870 & -1.3556280 \\
$\mathrm{H}$ & 2.4154880 & -1.4893950 & -1.4727790 \\
$\mathrm{H}$ & 0.5135010 & -2.9687420 & -0.8095570 \\
$\mathrm{O}$ & 1.2421370 & -2.4956810 & -2.9397050 \\
$\mathrm{H}$ & 0.6358190 & -1.6674710 & -2.6937240 \\
$\mathrm{C}$ & 3.4982740 & -4.4644380 & -2.9963010 \\
$\mathrm{C}$ & 2.8208000 & -5.3703500 & -1.9567020 \\
$\mathrm{C}$ & 1.4059610 & -4.8694400 & -1.6147900 \\
$\mathrm{C}$ & 3.6300200 & -3.0284710 & -2.4618300 \\
$\mathrm{H}$ & 4.4945280 & -4.8501000 & -3.2423180 \\
$\mathrm{H}$ & 2.7582580 & -6.4013660 & -2.3231880 \\
$\mathrm{H}$ & 0.7141500 & -5.0821040 & -2.4432300 \\
$\mathrm{H}$ & 4.3366330 & -3.0073120 & -1.6222140 \\
$\mathrm{H}$ & 4.0163420 & -2.3459720 & -3.2270250 \\
$\mathrm{H}$ & 2.9089430 & -4.4612330 & -3.9230210 \\
$\mathrm{H}$ & 3.4283040 & -5.3840810 & -1.0412010 \\
$\mathrm{H}$ & 0.9916870 & -5.3813540 & -0.7384120 \\
$\mathrm{C}$ & 2.0197840 & -2.2000200 & 2.2833820 \\
$\mathrm{O}$ & 0.9699690 & -1.4314210 & 2.1408540 \\
$\mathrm{C}$ & 2.8201770 & -1.8626690 & 3.4895500 \\
$\mathrm{C}$ & 4.1915460 & -2.1701020 & 3.5604130 \\
$\mathrm{C}$ & 2.2153940 & -1.1877380 & 4.5688700 \\
$\mathrm{C}$ & 4.9411560 & -1.8032450 & 4.6765670 \\
$\mathrm{H}$ & 4.6653560 & -2.6737970 & 2.7237070 \\
$\mathrm{C}$ & 2.9640530 & -0.8416350 & 5.6923020 \\
$\mathrm{H}$ & 1.1597930 & -0.9401930 & 4.5216720 \\
$\mathrm{C}$ & 4.3297280 & -1.1419540 & 5.7480730 \\
$\mathrm{H}$ & 6.0042600 & -2.0297560 & 4.7091020 \\
$\mathrm{H}$ & 2.4832760 & -0.3304100 & 6.5230080 \\
$\mathrm{H}$ & 4.9147540 & -0.8600400 & 6.6206520 \\
$\mathrm{H}$ & 0.4056820 & -1.5489330 & 1.2829310 \\
$\mathrm{~S}$ & 2.4444240 & -3.4632040 & 1.2613000 \\
$\mathrm{I}$ & & & \\
$\mathrm{F}$ & & & \\
\hline & & &
\end{tabular}

TS206 $(R, R)$ G $[B 97-D /$ def2-TZVP $]=-3633.749920$

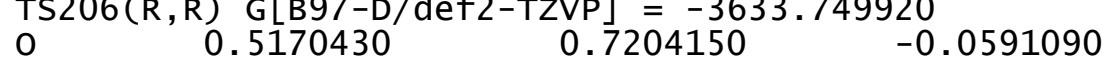

$\begin{array}{rll}-0.0591090 & \text { C } & -5.4835250 \\ \text { H } & -4.9535250\end{array}$
$-1.7284460$
0.7120400
$-0.3421530$
2.0226720
$-0.1417260$
2.2260170
1.1692070
3.2227280
$-2.2919060$
$-3.5361230$
$-1.6274460$
$-4.1056680$
$-2.1797910$
$-3.4527830$
$-5.0723560$
$-3.3499110$
$-2.0744210$
$-1.5054620$
$-4.0201080$
$-3.7896220$
$-1.5345810$
$-0.5251230$
$-4.9901030$
$-0.9617910$
0.3386540
1. 3798520
$-1.1952510$
$-1.7854150$
0.5095850
2. 3807810
$-2.1972860$
3.1566910
3.7252550
3.6958920
4.8472790
4.8163920
5.4149280
5.2939170
5.2337950
$-4.2003360$
$-4.2571050$
$-4.7738770$
$-4.9060980$
$-5.4055310$

0.9342480
2.0909150
2.9530110
2.5381890
4.3580450
3.8992720
4.8301340
4.2702490
1.4079760
0.8557740
2.4100970
1.3581270
2.8504660
2.3282030
0.9804230
3.6613470
4.1415630
3.7477870
2.7684200
3.9858840
4.8232740
4.1204830
2.3709360
6.6528970
7.1197420
6.2227050
5.3083200
7.3599540
8.1824300
6.5644830
4.9636590
1.5814630
1.3740230
0.9261410
0.5452520
0.1026400
-0.0923200
0.3857750
-0.3936320
-0.2249720
-1.5441960
0.0692780
-2.5298440
-0.9570870
-2.2607800
-3.5427780

$-1.2471380$

$-0.1623890$

0.1209340

$-0.4765490$

$-0.0841040$

$-0.5883180$

$-0.4368800$

$-0.8143130$

$-0.0607540$

0.3479920

0.6336440

1.5006060

1.8835340

2. 3006780

1.8230640

4.3381250

3.9517470

2.7568030

3.5279220

5.2785770

4.6053900

2.4797960

3.8215820

$-0.1979140$

$-0.5117740$

$-0.6315970$

0.0122180

$-0.1267520$

$-0.6684720$

$-0.8892700$

0.2476530

$-0.6450720$

$-1.9220980$

0.4871680

$-2.0307770$

0.3221780

$-0.9250800$

$-3.0107130$

1.1916570

$-0.4395000$

0.0758480

$-1.6949390$

$-0.6741480$

$-2.4093260$

$-1.9193360$

$-0.2821410$ 


\begin{tabular}{|c|c|c|}
\hline $\begin{array}{l}-5.8525180 \\
3.1353490 \\
6.6685660 \\
3.1775370 \\
-3.6506970 \\
-6.1507300 \\
-4.7679570 \\
-6.2008080 \\
-6.8166070 \\
-6.6763360 \\
-6.1836180 \\
-4.0811570 \\
-3.0558350 \\
-4.6327590 \\
-4.0504830 \\
-7.3003750 \\
-7.7936910 \\
-6.9172640 \\
-8.0481070 \\
-5.1204220 \\
-4.3136620 \\
-4.6723680 \\
-5.6029770 \\
-4.7425570 \\
-5.2767890 \\
-5.4751550 \\
-4.2960090 \\
-2.8815040 \\
-3.5623030 \\
-2.1390080 \\
-2.3647980 \\
3.7465280 \\
4.8325670 \\
3.5571280 \\
3.3122430 \\
3.3290130 \\
2.9923700 \\
4.3821750 \\
2.7494560 \\
6.9275200 \\
7.7834410 \\
7.1645190 \\
6.0564490 \\
7.8963320 \\
7.7470270 \\
8.0626200\end{array}$ & $\begin{array}{r}-0.7318720 \\
1.1731820 \\
-0.9328930 \\
2.0451330 \\
-1.9205630 \\
-3.3607600 \\
1.4712590 \\
2.0323290 \\
1.4108690 \\
2.0526060 \\
3.0539860 \\
1.5036430 \\
1.1236060 \\
0.8903460 \\
2.5335700 \\
-4.0270900 \\
-4.7850320 \\
-4.5237320 \\
-3.2856650 \\
-4.4159440 \\
-3.9531000 \\
-4.9064530 \\
-5.1859240 \\
-1.9843060 \\
-1.0321020 \\
-2.7638040 \\
-2.2300810 \\
-3.2544370 \\
-4.1030910 \\
-3.2572770 \\
-3.4013720 \\
2.4614310 \\
2.3441930 \\
3.3191970 \\
2.6718970 \\
-0.0041810 \\
-0.9457110 \\
-0.1187450 \\
0.1718180 \\
-1.9505240 \\
-2.5835060 \\
-1.4428750 \\
-2.5944430 \\
-0.0123500 \\
0.6913120 \\
0.5669690\end{array}$ & $\begin{array}{r}-3.3765190 \\
1.8845230 \\
-1.1157690 \\
-3.1759450 \\
1.4239170 \\
-2.7255390 \\
-2.2912020 \\
-2.3813660 \\
-3.0446750 \\
-1.3922720 \\
-2.7840810 \\
-3.6693490 \\
-3.5997500 \\
-4.3937430 \\
-4.0495530 \\
-1.9470550 \\
-2.5698830 \\
-1.0460150 \\
-1.6380290 \\
-3.1748900 \\
-3.7572620 \\
-2.3005850 \\
-3.7918890 \\
2.5120240 \\
2.6053430 \\
2.2620040 \\
3.4853630 \\
1.3910630 \\
1.2457430 \\
0.5878540 \\
2.3476620 \\
2.4738380 \\
2.5900440 \\
1.8178820 \\
3.4606700 \\
2.8531990 \\
2.4058590 \\
3.1419400 \\
3.7672310 \\
0.0035120 \\
-0.2606650 \\
0.9478090 \\
0.1745620 \\
-1.2878900 \\
-2.1157900 \\
-0.3694020\end{array}$ \\
\hline
\end{tabular}

8.7969060

2. 9750500

3.9311690

2. 3176970

2. 5191490

4.0998310

5.0962080

3.6877480

4.2147000

$-0.4567880$

0.1441040

$-0.8161140$

2. 1968390

6.5420100

2.0570700

$-4.2033640$

$-6.5753350$

$-2.9357010$

2. 4020260

1.2892960

2. 3811690

0.3466840

1.6388760

0.9911500

3.7863380

2. 8427440

1. 3966600

3.7892180

4.8076000

2.8659530

0.9555480

4.2488990

4. 3724850

3.4672330

3.1778840

0.7474400

0.8326560

0.0009260

1.0652430

2.1758020

0.1671030

2. 3860760

2.8789710

0.3747880

$-0.6957250$

1.4855840
$-0.6076290$
1.0572960
0.6369840
0.2345550
1.5798220
. 2002350
2. 8134490
3.6999690
3.9437600
$-0.0664810$
$-0.1483630$
$-1.3414190$
2.4704180
$-1.4890520$
1.3352940
2.1342920
$-2.8971250$
$-1.1402610$
$-2.2807600$
$-3.2220560$
$-1.3667510$
$-2.8205520$
$-2.1225090$
$-1.3181940$
$-4.1954710$
$-5.1803420$
$-4.6528650$
$-2.8363340$
$-4.5938870$
$-6.1613010$
$-4.7332250$
$-2.9374130$
$-2.0899360$
$-4.0614530$
$-5.3205510$
$-5.2410340$
$-2.6553450$
$-1.7061290$
$-2.7197740$
$-3.3949100$
$-2.0782150$
$-3.4246060$
$-3.8806850$
$-2.1202760$
-1.5563980
-2.7897090

$-1.4901260$

$-4.3389160$

$-4.6765690$

$-4.0422170$

$-5.1899690$

$-3.6148550$

$-3.8675410$

$-4.5017380$

$-2.8170050$

$-1.1118010$

$-2.4875230$

$-0.3987230$

$-2.9379730$

$-2.0564780$

1.7978980

$-1.6281510$

$-3.6274000$

1.7040090

$-1.7762760$

$-1.5959500$

$-1.1786500$

$-1.2423060$

$-3.0312090$

$-2.8635580$

$-2.7229120$

$-2.0173050$

$-2.0109700$

$-2.0051470$

$-2.7409760$

$-2.5054480$

$-3.0158960$

$-1.0137350$

$-2.5521100$

$-3.7653240$

$-0.9800750$

$-1.3516280$

2.2991500

1.9601230

3.7675210

4. 3079420

4.6459800

5.6849960

3.6378870

6.0240570

4.2447730

6.5487310 


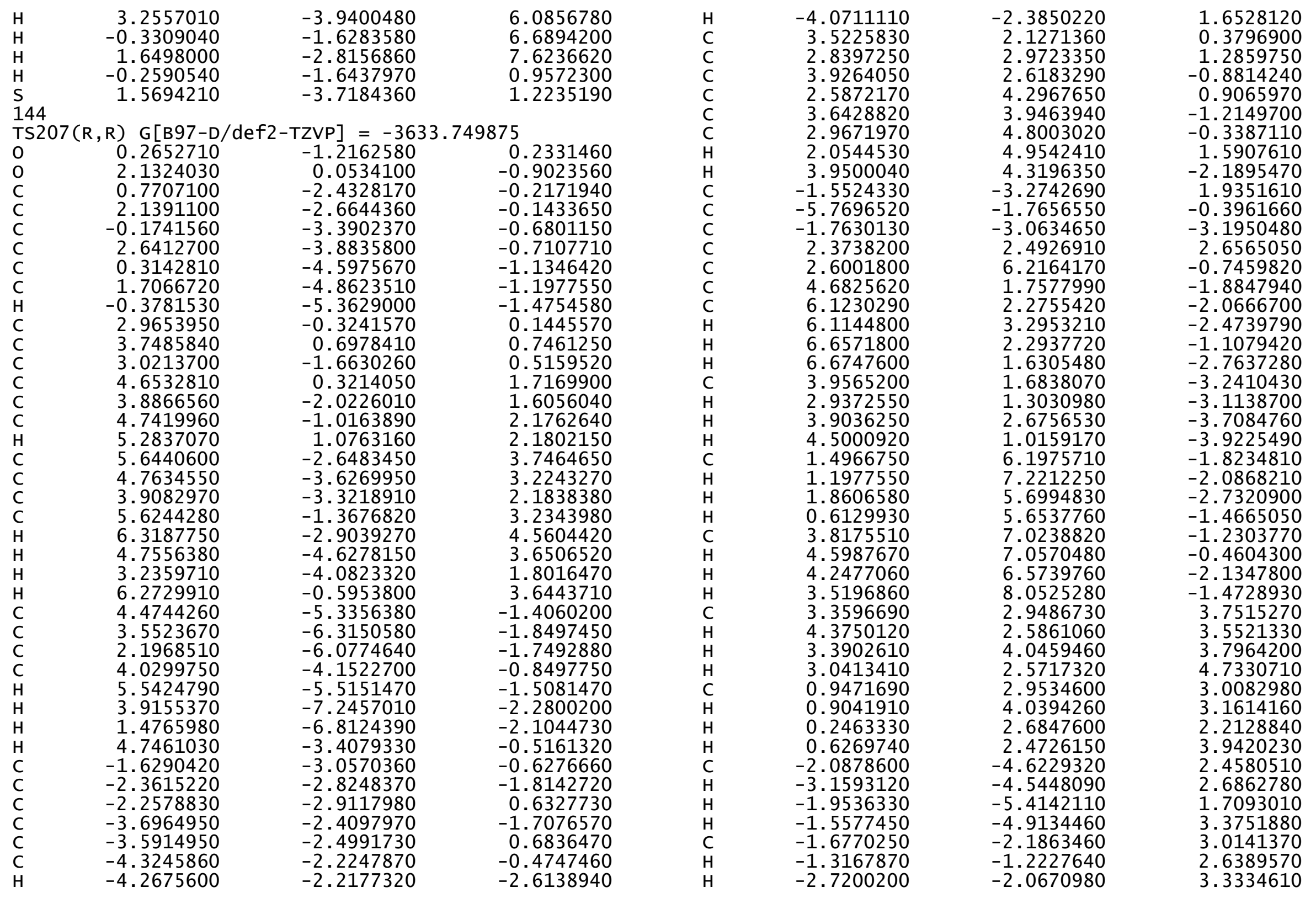




$\begin{array}{rrr}-1.0870630 & -2.4684960 & 3.8960840 \\ -5.9191300 & -0.4857290 & 0.4449100 \\ -6.9630570 & -0.1458580 & 0.4414710 \\ -5.6221210 & -0.6644140 & 1.4850430 \\ -5.2894860 & 0.3198590 & 0.0530560 \\ -6.6760870 & -2.8810770 & 0.1608990 \\ -6.6057540 & -3.7891610 & -0.4512620 \\ -6.3775340 & -3.1349360 & 1.1866980 \\ -7.7231720 & -2.5500830 & 0.1806240 \\ -2.1290180 & -1.9843080 & -4.2279080 \\ -3.2026040 & -1.9956030 & -4.4567750 \\ -1.8601070 & -0.9878820 & -3.8676650 \\ -1.5884310 & -2.1764280 & -5.1638530 \\ -2.1961320 & -4.4495460 & -3.7177240 \\ -3.2863600 & -4.4760670 & -3.8498870 \\ -1.7244460 & -4.6569040 & -4.6878440 \\ -1.9206610 & -5.2464360 & -3.0173990 \\ 0.5217380 & 0.0932910 & -0.7038820 \\ -0.0308420 & -0.0863490 & -2.0945250 \\ 0.0714500 & 1.2703280 & 0.1139000 \\ -0.6723840 & -3.0643430 & -3.0985870 \\ -6.0984260 & -1.5414360 & -1.4210020 \\ -0.4856140 & -3.4102010 & 1.7350820 \\ 4.7498390 & 0.7381630 & -1.4907640 \\ 2.1922680 & 6.7181830 & 0.1430780 \\ 2.3649350 & 1.3975470 & 2.6499340 \\ -2.7599290 & 1.5147350 & -1.9314840 \\ -1.9409660 & 2.7283290 & -1.8133670 \\ -2.6049300 & 0.7428510 & -1.1758450 \\ -0.9989360 & 2.6447680 & -1.2844990 \\ -1.8025200 & 1.3772390 & -3.0379920 \\ -1.0148250 & 0.7683930 & -2.6599430 \\ -4.3894170 & 2.8065550 & -3.3875220 \\ -3.8171220 & 4.1125510 & -2.8135950 \\ -2.3087600 & 3.9905400 & -2.5280950 \\ -4.1938690 & 1.6475100 & -2.3955350 \\ -5.4585310 & 2.9238930 & -3.5995520 \\ -3.9840980 & 4.9452580 & -3.5064820 \\ -1.7391920 & 4.0058800 & -3.4687610 \\ -4.7991240 & 1.8257640 & -1.4965830 \\ -4.5148850 & 0.6888850 & -2.8202680 \\ -3.8895770 & 2.5679950 & -4.3356290 \\ -4.3393750 & 4.3518560 & -1.8772700 \\ -1.9355400 & 4.8356830 & -1.9384510 \\ -2.9510350 & 2.0959660 & 1.5453240 \\ -2.1022140 & 1.1029410 & 1.4212890\end{array}$

$\begin{array}{ll}\mathrm{C} & -4.0615540 \\ \mathrm{C} & -5.2782030 \\ \mathrm{C} & -3.9163450 \\ \mathrm{C} & -6.3233960 \\ \mathrm{H} & -5.4002650 \\ \mathrm{C} & -4.9598720 \\ \mathrm{H} & -2.9844220 \\ \mathrm{C} & -6.1679790 \\ \mathrm{H} & -7.2632650 \\ \mathrm{H} & -4.8316790 \\ \mathrm{H} & -6.9848640 \\ \mathrm{H} & -1.2552010 \\ \mathrm{~S} & -2.8091830 \\ \mathrm{I} & \end{array}$

1.7953000
2.5004630
0.7658130
2.1805650
3.2837520
0.4573130
0.2150010
1.1595740
2.7231160
-0.3344580
0.9116500
1.2871020
3.5611860

2.4812490 2.4216510 3.4334330 3.2851970 1.6792490 4. 3037220 3. 4866290 4. 2303870 3. 2177170 5. 0381750 4.9043940

$\operatorname{TS} 208(R, R) \quad G[B 97-D / d e f 2-T Z V P]=-3633.749830$

$\begin{array}{lrrr}\text { TS208(R, R) } G[\mathrm{~B} 97-\mathrm{D} / \mathrm{def} 2-\mathrm{TZVP}]=-3633.749830 \\ \mathrm{O} & 0.1977700 & -1.2197570 & 0.2247420 \\ \mathrm{O} & 2.1235470 & -0.0259820 & -0.8985410 \\ \mathrm{C} & 0.6613510 & -2.4550850 & -0.2198330 \\ \mathrm{C} & 2.0189970 & -2.7378880 & -0.1293390 \\ \mathrm{C} & -0.3127350 & -3.3777900 & -0.6928790 \\ \mathrm{C} & 2.4823390 & -3.9760640 & -0.6883490 \\ \mathrm{C} & 0.1358360 & -4.6030000 & -1.1402260 \\ \mathrm{C} & 1.5180210 & -4.9200300 & -1.1856580 \\ \mathrm{H} & -0.5805390 & -5.3428940 & -1.4875040 \\ \mathrm{C} & 2.9319100 & -0.4306050 & 0.1580370 \\ \mathrm{C} & 3.7514620 & 0.5619480 & 0.7596250 \\ \mathrm{C} & 2.9305060 & -1.7682920 & 0.5375050 \\ \mathrm{C} & 4.6267930 & 0.1560240 & 1.7456000 \\ \mathrm{C} & 3.7675250 & -2.1556560 & 1.6395260 \\ \mathrm{C} & 4.6539000 & -1.1804030 & 2.2162480 \\ \mathrm{H} & 5.2838030 & 0.8876480 & 2.2091570 \\ \mathrm{C} & 5.4695050 & -2.8371700 & 3.8075790 \\ \mathrm{C} & 4.5596040 & -3.7842380 & 3.2775020 \\ \mathrm{C} & 3.7316410 & -3.4520910 & 2.2233530 \\ \mathrm{C} & 5.5070290 & -1.5596560 & 3.2886540 \\ \mathrm{H} & 6.1220700 & -3.1139910 & 4.6325970 \\ \mathrm{H} & 4.5074380 & -4.7817760 & 3.7083560 \\ \mathrm{H} & 3.0367580 & -4.1885770 & 1.8345720 \\ \mathrm{H} & 6.1797120 & -0.8110870 & 3.7037490 \\ \mathrm{C} & 4.2685500 & -5.4971090 & -1.3574770 \\ \mathrm{C} & 3.3162070 & -6.4420480 & -1.8118070 \\ \mathrm{C} & 1.9693910 & -6.1536600 & -1.7291710 \\ \mathrm{C} & 3.8617010 & -4.2968630 & -0.8086340 \\ \mathrm{H} & 5.3303380 & -5.7168540 & -1.4453080 \\ \mathrm{H} & 3.6497160 & -7.3864190 & -2.2361400 \\ \mathrm{H} & 1.2268430 & -6.8618000 & -2.0928640\end{array}$




$\begin{array}{rrrr}\mathrm{H} & 4.6006080 & -3.5793880 & -0.4658300 \\ \mathrm{C} & -1.7547900 & -2.9906850 & -0.6533040 \\ \mathrm{C} & -2.4646510 & -2.7178010 & -1.8448190 \\ \mathrm{C} & -2.3893630 & -2.8306500 & 0.6021950 \\ \mathrm{C} & -3.7803440 & -2.2435360 & -1.7470410 \\ \mathrm{C} & -3.7022900 & -2.3555860 & 0.6446960 \\ \mathrm{C} & -4.4099990 & -2.0371420 & -0.5179980 \\ \mathrm{H} & -4.3339940 & -2.0195680 & -2.6566380 \\ \mathrm{H} & -4.1854160 & -2.2284190 & 1.6104620 \\ \mathrm{C} & 3.6060070 & 1.9959580 & 0.3721730 \\ \mathrm{C} & 2.9717400 & 2.8931220 & 1.2631790 \\ \mathrm{C} & 4.0516070 & 2.4463010 & -0.8905900 \\ \mathrm{C} & 2.8160140 & 4.2288170 & 0.8711410 \\ \mathrm{C} & 3.8633020 & 3.7875340 & -1.2377090 \\ \mathrm{C} & 3.2426880 & 4.6946100 & -0.3738020 \\ \mathrm{H} & 2.3246500 & 4.9270900 & 1.5459190 \\ \mathrm{H} & 4.2042840 & 4.1307440 & -2.2119560 \\ \mathrm{C} & -1.7130130 & -3.2395470 & 1.9058800 \\ \mathrm{C} & -5.8341450 & -1.5150490 & -0.4481350 \\ \mathrm{C} & -1.8625720 & -2.9757050 & -3.2205760 \\ \mathrm{C} & 2.4530730 & 2.4580370 & 2.6298790 \\ \mathrm{C} & 2.9910190 & 6.1320360 & -0.7932270 \\ \mathrm{C} & 4.7588970 & 1.5270600 & -1.8775260 \\ \mathrm{C} & 6.2408840 & 1.9259210 & -2.0218480 \\ \mathrm{H} & 6.3272030 & 2.9478690 & -2.4138950 \\ \mathrm{H} & 6.7521380 & 1.8877320 & -1.0512000 \\ \mathrm{H} & 6.7535480 & 1.2453310 & -2.7149820 \\ \mathrm{C} & 4.0603350 & 1.5083930 & -3.2494590 \\ \mathrm{H} & 3.0083010 & 1.2211860 & -3.1427540 \\ \mathrm{H} & 4.1065960 & 2.4966770 & -3.7253170 \\ \mathrm{H} & 4.5567360 & 0.7888330 & -3.9140510 \\ \mathrm{C} & 1.9309320 & 6.1929080 & -1.9118040 \\ \mathrm{H} & 1.7193390 & 7.2358800 & -2.1840370 \\ \mathrm{H} & 2.2923370 & 5.6695130 & -2.8070460 \\ \mathrm{H} & 0.9962220 & 5.7158690 & -1.5909570 \\ \mathrm{C} & 4.2811540 & 6.8483490 & -1.2311760 \\ \mathrm{H} & 5.0314010 & 6.8318980 & -0.4304390 \\ \mathrm{H} & 4.7140790 & 6.3621040 & -2.1152900 \\ \mathrm{H} & 4.0667620 & 7.8937120 & -1.4897510 \\ \mathrm{C} & 3.4426550 & 2.8652840 & 3.7404370 \\ \mathrm{H} & 4.4360750 & 2.4339970 & 3.5696540 \\ \mathrm{H} & 3.5446530 & 3.9586570 & 3.7709650 \\ \mathrm{H} & 3.0784370 & 2.5251600 & 4.7194450 \\ \mathrm{C} & 1.0503400 & 3.0097620 & 2.9471380 \\ \mathrm{H} & 1.0727480 & 4.0972440 & 3.0941000 \\ \mathrm{H} & 0.3507260 & 2.7807710 & 2.1381510\end{array}$

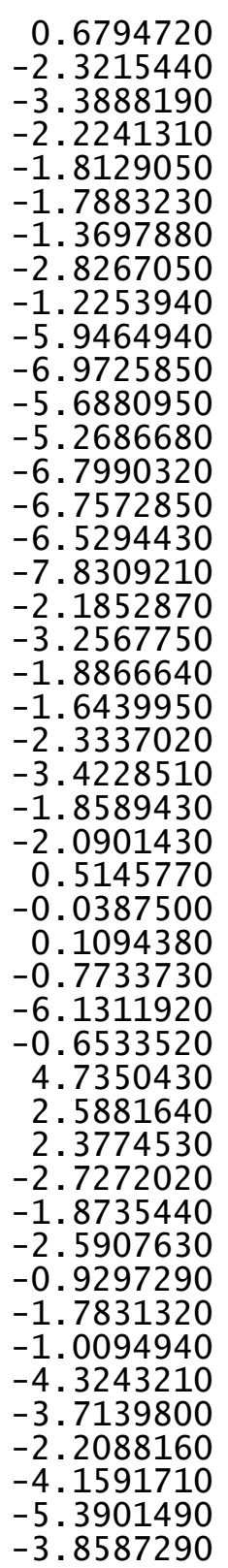

2.5564450
-4.5629040
-4.4310430
-5.3533260
-4.8887050
-2.1558110
-1.2113120
-1.9807320
-2.4783440
-0.2669950
0.1229280
-0.5041400
0.5229590
-2.6127490
-3.4949570
-2.9245090
-2.2368230
-1.8877460
-1.8638590
-0.8999740
-2.0998480
-4.3485380
-4.3415450
-4.5713310
-5.1530600
0.0801860
-0.0731190
1.2728870
-3.0100760
-1.2333640
-3.4291160
0.5055950
6.6634010
1.3655320
1.6030910
2.7929300
0.8279330
2.6808350
1.4356460
0.8056720
2.9394480
4.2297350
4.0652880
1.7776100
3.0865580
5.0657080

2. 4134910

2.6366120

1.6581760

3. 3308980

2.9931390

2. 6303990

3. 3013080

3. 8789270

0.4447270

0.4292600

1.4834640

0.1058470

0.0428750

$-0.6084670$

1.0609170

0.0604290

$-4.2582960$

$-4.4958200$

$-3.8980530$

$-5.1894820$

$-3.7459120$

$-3.8892290$

$-4.7110870$

$-3.0425730$

$-0.7078110$

$-2.1013530$

0.1110460

$-3.1139390$

$-1.4684850$

1. 7110690

$-1.4839190$

0.0807290

2.6337190

$-1.9124360$

$-1.7989600$

$-1.1563750$

$-1.2788590$

$-3.0256960$

$-2.6545490$

$-3.3634270$

$-2.7932500$

$-2.5113460$

$-2.3691960$

$-3.5733760$

- 3.4872050 


\begin{tabular}{|c|c|c|c|c|c|c|c|}
\hline $\mathrm{H}$ & -1.6412200 & 4.0660500 & -3.4534120 & H & -5.1130350 & 2.1173680 & 3.6775850 \\
\hline $\mathrm{H}$ & -4.7544850 & 1.9761700 & -1.4681460 & C & -0.3920840 & 6.6437900 & 0.9490420 \\
\hline H & -4.5104070 & 0.8279480 & -2.7900780 & $\mathrm{C}$ & 0.9558390 & 7.0600450 & 0.8179220 \\
\hline H & -3.8332270 & 2.6849670 & -4.3118960 & C & 1.9456960 & 6.1226770 & 0.6083300 \\
\hline $\mathrm{H}$ & -4.2272810 & 4.4854690 & -1.8563220 & C & -0.7237030 & 5.3044600 & 0.8971520 \\
\hline $\mathrm{H}$ & -1.8106690 & 4.8989280 & -1.9215490 & $\mathrm{H}$ & -1.1745170 & 7.3869100 & 1.0866190 \\
\hline C & -2.8721660 & 2.1948550 & 1.5734200 & $\mathrm{H}$ & 1.2036330 & 8.1179730 & 0.8676900 \\
\hline 0 & -2.0487090 & 1.1806080 & 1.4518700 & $\mathrm{H}$ & 2.9828620 & 6.4286080 & 0.4825050 \\
\hline C & -3.9867480 & 1.9269950 & 2.5147860 & $\mathrm{H}$ & -1.7612810 & 5.0013390 & 0.9937810 \\
\hline C & -5.1776670 & 2.6760580 & 2.4693130 & C & 3.4188970 & 1.4718670 & -0.2693030 \\
\hline C & -3.8735580 & 0.8844650 & 3.4571860 & $\mathrm{c}$ & 3.9843520 & 1.5296170 & -1.5641570 \\
\hline C & -6.2288530 & 2.3861400 & 3.3361190 & $\mathrm{C}$ & 3.9202100 & 0.5536470 & 0.6809180 \\
\hline $\mathrm{H}$ & -5.2758560 & 3.4700480 & 1.7348120 & $\mathrm{C}$ & 5.0725440 & 0.7013680 & -1.8651710 \\
\hline c & -4.9230960 & 0.6049360 & 4.3300430 & $\mathrm{C}$ & 5.0084110 & -0.2517610 & 0.3292180 \\
\hline $\mathrm{H}$ & -2.9622830 & 0.2993570 & 3.5002100 & $\mathrm{C}$ & 5.6066360 & -0.1890530 & -0.9322980 \\
\hline c & -6.1054710 & 1.3508370 & 4.2703990 & $\mathrm{H}$ & 5.5085590 & 0.7554190 & -2.8587830 \\
\hline $\mathrm{H}$ & -7.1486580 & 2.9632060 & 3.2793730 & $\mathrm{H}$ & 5.4066110 & -0.9530620 & 1.0609930 \\
\hline $\mathrm{H}$ & -4.8198830 & -0.1984690 & 5.0557330 & C & -4.0097180 & 0.2574310 & -0.8837580 \\
\hline $\mathrm{H}$ & -6.9272980 & 1.1256650 & 4.9463790 & C & -4.2989970 & -1.1036470 & -0.6117720 \\
\hline H & -1.2041280 & 1.3365580 & 0.8751520 & $\mathrm{C}$ & -4.3603670 & 0.8197600 & -2.1307110 \\
\hline s & -2.6967250 & 3.6510150 & 0.7561910 & $\mathrm{c}$ & -4.9504240 & -1.8593620 & -1.5914120 \\
\hline \multirow{2}{*}{\multicolumn{4}{|c|}{ TS209(R,R) G[B97-D/def2-TZVP] $=-3633.749806$}} & C & -5.0014310 & 0.0144110 & -3.0811840 \\
\hline & & & & $\mathrm{C}$ & -5.3070980 & -1.3236910 & -2.8328970 \\
\hline 0 & 0.7149570 & 0.6695200 & 0.2013870 & $\mathrm{H}$ & -5.1796740 & -2.9020880 & -1.3858140 \\
\hline 0 & -1.3501550 & 1.2497960 & -1.1730680 & $\mathrm{H}$ & -5.2750970 & 0.4459280 & -4.0428090 \\
\hline C & 0.9958250 & 2.0265320 & 0.3049800 & c & 3.3582520 & 0.4487290 & 2.0929500 \\
\hline C & -0.0236170 & 2.9061760 & 0.6552420 & $\mathrm{C}$ & 6.8233320 & -1.0469390 & -1.2382070 \\
\hline $\mathrm{C}$ & 2.3412420 & 2.4341910 & 0.1049520 & $\mathrm{C}$ & 3.4664860 & 2.4725450 & -2.6442200 \\
\hline C & 0.2743920 & 4.3085240 & 0.7098730 & C & -3.9414160 & -1.7708290 & 0.7126200 \\
\hline C & 2.6357370 & 3.7751010 & 0.2571220 & $\mathrm{C}$ & -5.9942800 & -2.1800900 & -3.8818840 \\
\hline C & 1.63 & 4.7373180 & 0.5327170 & $\mathrm{C}$ & -4.1139460 & 2.2839520 & -2.4727590 \\
\hline $\mathrm{H}$ & 3.6624570 & 4.1093230 & 0.1282520 & C & -5.4495800 & 3.0384330 & -2.6276750 \\
\hline c & -2.0319610 & 1.5883030 & 0.0006390 & $\mathrm{H}$ & -6.0367670 & 2.6170210 & -3.4541150 \\
\hline C & -3.3584510 & 1.1046560 & 0.1574980 & $\mathrm{H}$ & -6.0481530 & 2.9659950 & -1.7104320 \\
\hline C & -1.3883360 & 2.3894410 & 0.9352310 & $\mathrm{H}$ & -5.2635370 & 4.0992770 & -2.8425700 \\
\hline C & -4.0280750 & 20930 & 1.3141230 & C & -3.2491360 & 2.4394010 & -3.7374400 \\
\hline C & -2.0586230 & 2.6584380 & 2.1754670 & $\mathrm{H}$ & -2.2926680 & 1.9182570 & -3.6188450 \\
\hline C & -3.4079100 & 2.1954800 & 2.3481170 & $\mathrm{H}$ & -3.7652350 & 2.0285450 & -4.6150000 \\
\hline $\mathrm{H}$ & -5.0540830 & 1.1219770 & 1.4524840 & $\mathrm{H}$ & -3.0491320 & 3.5023900 & -3.9274680 \\
\hline C & -3.4575330 & 62810 & 4.5954000 & C & -7.3458850 & -2.7182910 & -3.3747240 \\
\hline C & -2.1131860 & 3680 & 4.4432210 & $\mathrm{H}$ & -7.8394010 & -3.3113960 & -4.1560810 \\
\hline C & -1.4325290 & 6390 & 3.2643370 & $\mathrm{H}$ & -7.1960540 & -3.3632980 & -2.4987220 \\
\hline C & -4.0875280 & 7570 & 3.5675950 & $\mathrm{H}$ & -8.0122900 & -1.8962390 & -3.0839760 \\
\hline $\mathrm{H}$ & -3.9841770 & 17370 & 5.5268550 & C & -5.0875350 & -3.3408810 & -4.3343910 \\
\hline $\mathrm{H}$ & -1.6102010 & 17790 & 5.2647400 & $\mathrm{H}$ & -4.1317810 & -2.9646380 & -4.7204590 \\
\hline $\mathrm{H}$ & -0.4013870 & 9560 & 3.1641360 & $\mathrm{H}$ & -4.8758470 & -4.0108200 & -3.4905320 \\
\hline
\end{tabular}




$\begin{array}{rrr}-5.5796380 & -3.9265020 & -5.1224510 \\ -5.1867760 & -1.9003160 & 1.6134510 \\ -5.6534640 & -0.9269310 & 1.8026450 \\ -5.9323640 & -2.5481530 & 1.1326660 \\ -4.9127900 & -2.3482100 & 2.5785370 \\ -3.2943850 & -3.1561930 & 0.5309450 \\ -4.0118350 & -3.8792070 & 0.1220560 \\ -2.4300130 & -3.1004620 & -0.1371000 \\ -2.9596530 & -3.5343830 & 1.5049390 \\ 4.3699820 & 0.9943490 & 3.1196480 \\ 5.2920070 & 0.3984670 & 3.1016640 \\ 4.6308490 & 2.0368820 & 2.8966450 \\ 3.9474630 & 0.9475420 & 4.1322910 \\ 2.9476620 & -0.9922210 & 2.4443490 \\ 2.2127740 & -1.3732650 & 1.7286170 \\ 3.8168100 & -1.6625380 & 2.4410420 \\ 2.4975830 & -1.0228600 & 3.4448620 \\ 8.1146380 & -0.2581490 & -0.9360780 \\ 8.9987330 & -0.8882960 & -1.1028920 \\ 8.1826270 & 0.6164610 & -1.5972480 \\ 8.1247020 & 0.0945030 & 0.1028230 \\ 6.8538990 & -1.5857080 & -2.6784550 \\ 5.9313760 & -2.1242470 & -2.9264420 \\ 6.9754230 & -0.7681000 & -3.4003500 \\ 7.7026200 & -2.2700740 & -2.8025270 \\ 3.1756960 & 1.7403230 & -3.9680260 \\ 4.0939250 & 1.3253270 & -4.4031410 \\ 2.4620610 & 0.9255160 & -3.8090990 \\ 2.7483070 & 2.4457000 & -4.6928890 \\ 4.4577080 & 3.6293630 & -2.8788080 \\ 5.4210100 & 3.2379860 & -3.2324780 \\ 4.0659420 & 4.3193720 & -3.6382550 \\ 4.6358950 & 4.1925930 & -1.9547550 \\ -0.2160430 & 0.0959260 & -1.0169720 \\ 0.5357880 & 0.0513130 & -2.3178020 \\ -0.7992160 & -1.1847460 & -0.4867870 \\ 2.5220880 & 2.9067930 & -2.3006680 \\ 6.7918170 & -1.9083950 & -0.5548920 \\ 2.4589320 & 1.0678860 & 2.1611020 \\ -3.5737880 & 2.7557440 & -1.6463670 \\ -6.1886120 & -1.5410440 & -4.7549100 \\ -3.2125510 & -1.1385340 & 1.2289220 \\ 2.3108860 & -2.4509490 & -1.2674610 \\ 1.1374310 & -3.2461300 & -1.6399300 \\ 2.1530810 & -1.6507930 & -0.5433450 \\ 0.1651540 & -2.7864390 & -1.5074310\end{array}$

2.0257120
1.4199490
3.7874520
2.5931870
1.2665420
3.6432330
4.7230800
2.7070000
1.1647790
3.7052650
4.4375220
3.8512850
2.5540590
0.3973000
-0.0166000
-0.4932430
-0.0393380
0.7118440
-0.8251960
0.6804000
1.3262320
-0.8611500
-1.4123930
-0.1077750
1.2735500
-1.4781070
-0.1333430
-0.5696910
0.5523430
-1
$-1.9935260$
$-6.1715890$
$-4.4523580$
$-3.4937080$
$-2.4157600$
$-3.9865520$
$-5.6720120$
$-5.1879970$
$-3.1323020$
$-1.9500290$
$-3.4264670$
$-4.4822550$
$-2.6312120$
$-4.7352450$
$-5.0934430$
$-2.8938500$
$-1.8158100$
$-3.9442200$
$-5.5487170$
$-2.2774360$
$-4.1448310$
$-1.7127930$
$-4.2343120$

$-2.0882860$

$\begin{array}{ll}-4.5928050 & -1.9435900 \\ -3.1508290 & -2.2723300\end{array}$

$-3.1508290-1.1342270$

$-1.8671200$

$-2.6003230$

$-3.3588450$

$-0.0919030$

$-1.2793800$

$-3.1236340$

$-0.9101120$

$-1.9672730$

2.1628980

1.8753710

3. 6219310

4.1729310

4.4829230

5.5429660

3. 5182230

5.8518240

4.0730800

6. 3873650

5. 9540380

6.5015040

7.4561490

0.8627980

S 144

1.0269580

TS210(R, R) G[B97-D/def2-TZVP] $=-3633.749491$

$\begin{array}{llrr}\text { TS210 }(\mathrm{R}, \mathrm{R}) & \mathrm{G}[\mathrm{B} 97-\mathrm{D} / \mathrm{def}-\mathrm{TZVP}]=-3633.749491 \\ \mathrm{O} & 0.2834830 & -1.2319480 & 0.2856640 \\ \mathrm{O} & 2.1081600 & 0.0514590 & -0.9064510 \\ \mathrm{C} & 0.7294260 & -2.4355710 & -0.2514440 \\ \mathrm{C} & 2.0945200 & -2.6978940 & -0.2873440 \\ \mathrm{C} & -0.2666720 & -3.3563290 & -0.6770680 \\ \mathrm{C} & 2.5258400 & -3.8978390 & -0.9455700 \\ \mathrm{C} & 0.1598940 & -4.5509470 & -1.2190600 \\ \mathrm{C} & 1.5371880 & -4.8369210 & -1.4022610 \\ \mathrm{H} & -0.5731160 & -5.2906030 & -1.5305080 \\ \mathrm{C} & 2.9959650 & -0.3951080 & 0.0653870 \\ \mathrm{C} & 3.8349790 & 0.5789360 & 0.6710620 \\ \mathrm{C} & 3.0439160 & -1.7525020 & 0.3618390 \\ \mathrm{C} & 4.7842110 & 0.1342240 & 1.5681250 \\ \mathrm{C} & 3.9667580 & -2.1876620 & 1.3744680 \\ \mathrm{C} & 4.8723610 & -1.2281620 & 1.9481900\end{array}$




$\begin{array}{rrrr}\mathrm{H} & 5.4531940 & 0.8521400 & 2.0364740 \\ \mathrm{C} & 5.8337610 & -2.9590960 & 3.3704270 \\ \mathrm{C} & 4.9081100 & -3.8940470 & 2.8462510 \\ \mathrm{C} & 3.9995500 & -3.5180490 & 1.8765190 \\ \mathrm{C} & 5.8073680 & -1.6514390 & 2.9323340 \\ \mathrm{H} & 6.5496680 & -3.2699920 & 4.1280240 \\ \mathrm{H} & 4.9082340 & -4.9176640 & 3.2145420 \\ \mathrm{H} & 3.2946200 & -4.2469160 & 1.4916710 \\ \mathrm{H} & 6.4923800 & -0.9132300 & 3.3458390 \\ \mathrm{C} & 4.2714080 & -5.3505570 & -1.8370060 \\ \mathrm{C} & 3.2983200 & -6.2933230 & -2.2503220 \\ \mathrm{C} & 1.9592860 & -6.0358100 & -2.0390870 \\ \mathrm{C} & 3.8945620 & -4.1848020 & -1.1996870 \\ \mathrm{H} & 5.3248020 & -5.5443590 & -2.0272570 \\ \mathrm{H} & 3.6088720 & -7.2111810 & -2.7445080 \\ \mathrm{H} & 1.1994930 & -6.7422160 & -2.3690060 \\ \mathrm{H} & 4.6485190 & -3.4680320 & -0.8897120 \\ \mathrm{C} & -1.7100800 & -3.0240650 & -0.4809180 \\ \mathrm{C} & -2.5358080 & -2.7065810 & -1.5862040 \\ \mathrm{C} & -2.2385660 & -2.9902010 & 0.8292100 \\ \mathrm{C} & -3.8667420 & -2.3450100 & -1.3495950 \\ \mathrm{C} & -3.5794190 & -2.6311730 & 1.0098750 \\ \mathrm{C} & -4.4060430 & -2.2894860 & -0.0604630 \\ \mathrm{H} & -4.5030490 & -2.0932400 & -2.1942650 \\ \mathrm{H} & -3.9920410 & -2.6109530 & 2.0166690 \\ \mathrm{C} & 3.6235170 & 2.0293740 & 0.3906210 \\ \mathrm{C} & 3.0432800 & 2.8510910 & 1.3868070 \\ \mathrm{C} & 3.9458830 & 2.5703540 & -0.8727900 \\ \mathrm{C} & 2.8095270 & 4.1983520 & 1.0897090 \\ \mathrm{C} & 3.6812490 & 3.9216110 & -1.1233530 \\ \mathrm{C} & 3.1061110 & 4.7519290 & -0.1582260 \\ \mathrm{H} & 2.3583630 & 4.8381760 & 1.8459330 \\ \mathrm{H} & 3.9277300 & 4.3283900 & -2.1008900 \\ \mathrm{C} & -1.4192620 & -3.3849820 & 2.0518280 \\ \mathrm{C} & -5.8455850 & -1.8646000 & 0.1685580 \\ \mathrm{C} & -2.0279370 & -2.7824980 & -3.0210330 \\ \mathrm{C} & 2.6699870 & 2.3249350 & 2.7688210 \\ \mathrm{C} & 2.7182410 & 6.1900110 & -0.4573140 \\ \mathrm{C} & 4.6065170 & 1.7427800 & -1.9674870 \\ \mathrm{C} & 6.0536080 & 2.2192150 & -2.2038050 \\ \mathrm{H} & 6.0613970 & 3.2610700 & -2.5506030 \\ \mathrm{H} & 6.6403960 & 2.1618000 & -1.2779790 \\ \mathrm{H} & 6.5401030 & 1.5975230 & -2.9674850 \\ \mathrm{C} & 3.8030980 & 1.7716470 & -3.2807980 \\ \mathrm{H} & 2.7782640 & 1.4216940 & -3.1150660 \\ \mathrm{H} & 3.7632390 & 2.7883860 & -3.6928020 \\ & & & \end{array}$

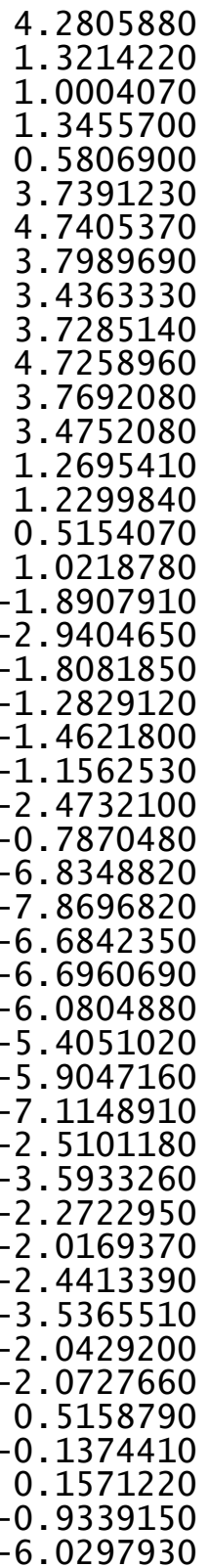

1.1227400
6.2207570
7.2556350
5.6900950
5.7294160
6.9402390
6.9105770
6.5005970
7.9892300
2.7459400
2.3857650
3.8411370
2.3411520
2.7718510
3.8560380
2.5042640
2.2797080
-4.7513250
-4.6948990
-5.5244230
-5.0530880
-2.3197860
-1.3407550
-2.2309660
-2.6053720
-2.8261180
-2.5200550
-2.8195930
-3.8543670
-0.4177310
0.2741530
-0.3341380
-0.1113610
-1.6196370
-1.6690150
-0.6537500
-1.6753100
-4.1245460
-4.1943130
-4.1994480
-4.9779880
0.1190390
0.0260810
1.2587240
-2.7410500
-1.8985370

$-4.0270180$

$-1.1138430$

$-1.2954560$

$-2.0754040$

$-0.4709000$

$-1.3277670$

$-0.8799830$

$-2.3316970$

$-1.4403510$

3.8079240

3. 5284700

3. 8827670

4.7972840

3. 2272020

3.3934020

2. 4810960

4.1771020

2. 5880680

2.9058520

1.8133320

3. 4517360

3.1619720

2.7768660

3. 5807350

3. 9792530

$-0.5165450$

$-0.3125470$

$-1.6041490$

$-0.1587720$

$-0.3028960$

0.2117130

$-1.3830600$

$-0.0984340$

$-3.9054850$

$-4.0769200$

$-3.4516610$

$-4.8846000$

$-3.6610100$

$-3.7111380$

$-4.6819740$

$-3.0805230$

$-0.5972780$

$-1.9514110$

0.3149310

$-2.9963550$

1. 2509570 


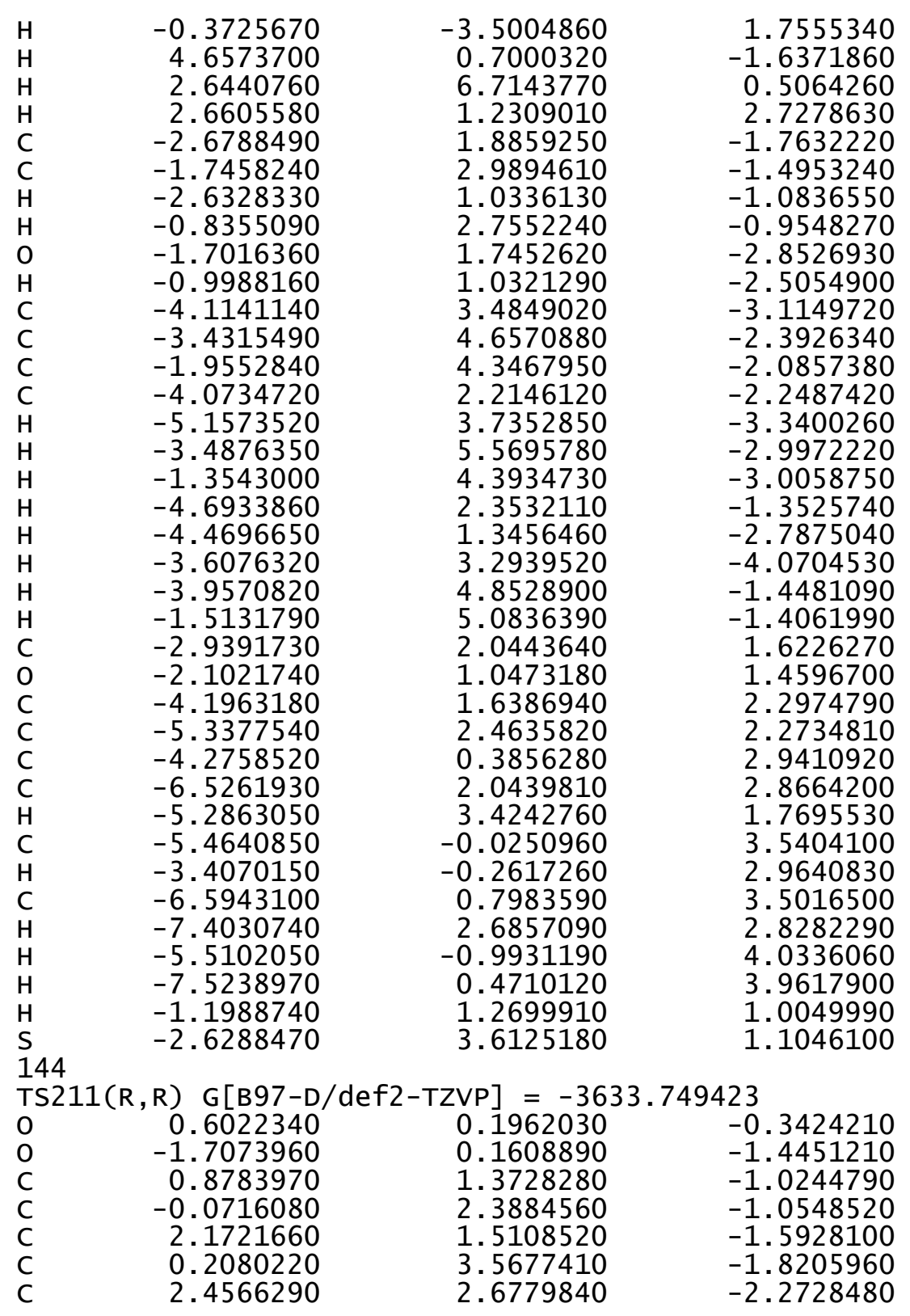

1.4990160
3.4468010
-2.1637670
-3.4351780
-1.3483930
-3.8488720
-1.7458970
-3.0240410
-4.8241260
-2.5899800
-1.3162390
-0.9058820
-3.4242000
-2.9015980
-0.6522050
0.0763560
-4.3976950
-0.4390410
0.8457790
1.7923930
-0.7497970
-1.1906190
1.0777030
2.7793890
-1.7400480
3.2410940
3.5079550
4.0339730
4.6104390
5.1231680
5.4371370
4.8316930
5.7368010
-4.2926740
-4.5493360
-4.8692810
-5.3932510
-5.7028600
-5.9800730
-5.5939680
-6.1547530
3.7561180
6.6527240
2.6427150
-3.9493630
-6.8804870

3. 7082590

2. 8128110

1. 1417940

0.9554940

2. 2371580

1. 9124000

3. 1718860

3. 0018590

1. 8113680

4.9342970

5. 0841330

4. 2276280

3. 9120830

5. 6185860

5.8779740

4. 3480300

3.7728890

5.7166860

5.8659820

4.8766540

4.5985690

6.4884170

6.7544970

4.9690600

4.4967780

0.4819060

$-0.4548750$

0.5125640

$-1.3037820$

$-0.3634630$

$-1.2663040$

$-2.0289250$

$-0.3388210$

$-0.2222870$

$-1.2061780$

$-0.3426060$

$-2.2742480$

$-1.4362880$

$-2.4102390$

$-3.0298110$

$-1.5290450$

1. 5178550

$-2.1776430$

$-0.5267180$

$-1.1409880$

$-3.5909740$
$-2.4340450$ $-2.7012820$

$-0.5545620$

0.0514790

$-0.3125370$

0.9574600

0.6992810

1. 3324810

1.4260290

2. 7514760

2. 1501530

1.1478680

2. 3483790

3. 5373530

2. 4851220

0.7024420

2. 8149500

$-2.7775180$

$-3.3559860$

$-3.1882510$

$-2.0293300$

$-2.9290960$

$-3.9388910$

$-3.6381260$

$-1.5969780$

$-1.4129890$

$-2.4346660$

$-0.2439710$

$-2.2907400$

$-0.1454160$

$-1.1638400$

$-3.0714200$

0.7496650

$-0.2718730$

0.7161250

$-1.5560410$

0.3965070

$-1.8246460$

$-0.8653650$

1. 1526770

$-2.8110270$

0.8689260

$-1.1038490$

$-3.6862970$

2. 1162380

$-1.1832380$ 


-4.6604320
-5.9795430
-6.7314330
-6.3833480
-5.8132890
-4.0647830
-3.1235730
-4.7619140
-3.8693790
-8.1083070
-8.7612860
-7.7946210
-8.6864190
-6.1018200
-5.2441320
-5.7250360
-6.7553350
-5.0008260
-5.4159280
-5.8296630
-4.5502520
-3.3445000
-4.1258530
-2.6363370
-2.8146380
4.5217540
5.6038550
4.2569770
4.2882200
4.0810520
3.5964840
5.1634120
3.7226480
7.0610060
7.8788850
7.4181020
6.2223910
7.8433800
7.5822900
8.1262530
8.7121310
2.4342980
3.3637890
2.0796390
1.6879850
3.2347480

0.6933040

1.4258790

0.7198570

1. 9105850

2.1936450

0.0661660

$-0.4479680$

$-0.6584650$

0.8469260

$-3.6349760$

$-4.4753070$

$-3.7684990$

$-2.7046210$

$-4.9191550$

$-4.9100540$

$-5.0840070$

$-5.7611730$

$-0.6735190$

0.3056140

$-1.3928370$

$-0.6007610$

$-2.4833630$

$-3.2371650$

$-2.8612770$

$-2.3419250$

2. 8310940

2.6420410

3.2562530

3.5687720

0.9947270

0.0305880

0.8765100

1.7120190

$-2.5946200$

$-3.3243470$

$-1.7309110$

$-3.0454240$

$-1.5083000$

$-1.2508630$

$-0.5853090$

$-2.1803090$

$-1.9599690$

$-2.3744320$

$-2.6224840$

$-1.9524190$

0.3520050

-2.6536720
-2.9701390
-3.3462320
-2.0718060
-3.7377100
-3.9277200
-3.7030360
-4.3679860
-4.6749230
-0.2540690
-0.5247630
0.7895800
-0.3213340
-1.1098330
-1.7942480
-0.0916340
-1.3747190
3.1424230
2.8770370
3.1894940
4.1416280
2.5699280
2.7311480
1.8263890
3.5210320
0.6065580
0.6213220
-0.3678630
1.3863380
2.2769250
2.4668480
2.4184010
3.0241060
0.3170860
0.2691340
0.8924880
0.8601590
-1.8235810
-2.8574800
-1.2993770
-1.8364060
-4.2034910
-4.6153170
-3.4074190
-5.0076570
-4.8069800

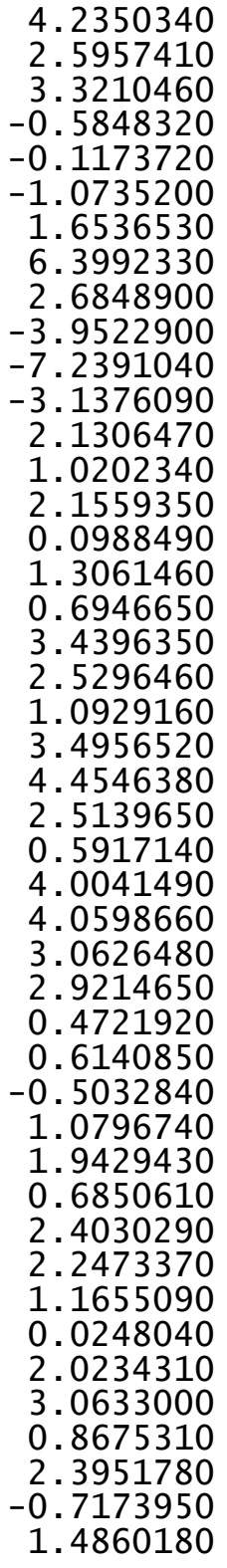

-0.0110030
0.3102850
1.3969140
-0.8343970
-1.7383940
-1.4675560
-0.1296870
-3.0895300
1.7520670
1.4479850
-3.4647170
-0.4089330
-3.0877090
-3.6376520
-2.0043970
-3.0678060
-3.7348600
-2.9867520
-5.2171910
-5.4582900
-4.9891370
-3.7214250
-5.5754490
-6.5193460
-5.6881790
-3.1691810
-3.5384570
-5.7826730
-4.9013240
-4.9685580
-0.5124690
-0.2574270
0.6267210
0.4443340
1.9345380
1.5412190
-0.5617700
3.0302730
2.0904920
2.8382160
1.3868510
4.0332250
3.6935790
-0.8711090
-1.9461560

$-5.0802100$

$-5.6994390$

$-4.4899520$

$-0.8185950$

$-1.9209310$

0.4591960

$-3.4301300$

$-1.6646130$

0.8522260

$-2.2989600$

$-2.2146970$

2.1059120

$-0.0603580$

0.7307970

$-0.1907620$

0.7675130

$-1.1048050$

$-1.4775990$

0.4299570

1. 6429010

1. 3542520

0.0833170

0.6381480

1.9163740

0.6666870

0.8843700

$-0.8350530$

$-0.4329990$

2.5052130

2. 2583030

3.1939580

2. 5659540

4.0262270

5.1212210

3.6755950

5.8467500

5.3954180

4.3908700

2.8283480

5.4791670

6.6969100

4.0949120

1.7488990

3.0700740 
$\operatorname{TS} 212(R, R) G[B 97-D / d e f 2-T Z V P]=-3633.749324$

\begin{tabular}{|c|c|c|c|}
\hline & & & \\
\hline 0 & 0.5705110 & 0.1428100 & -0.3062350 \\
\hline 0 & -1.7646340 & 0.0352080 & -1.3503090 \\
\hline $\mathrm{C}$ & 0.8328160 & 1.2676540 & -1.0766250 \\
\hline C & -0.1203360 & 2.2782650 & -1.1597060 \\
\hline C & 2.1107220 & 1.3641900 & -1.6862090 \\
\hline $\mathrm{C}$ & 0.1345390 & 3.3956820 & -2.0199980 \\
\hline C & 2.3731200 & 2.4788430 & -2.4587410 \\
\hline $\mathrm{C}$ & 1.4090000 & 3.4920180 & -2.6743170 \\
\hline $\mathrm{H}$ & 3.3525730 & 2.5826950 & -2.9193300 \\
\hline C & -2.1960140 & 1.0726820 & -0.5129850 \\
\hline C & -3.4471660 & 0.9291650 & 0.1446120 \\
\hline C & -1.3769560 & 2.1832520 & -0.3754290 \\
\hline C & -3.8386760 & 1.9530050 & 0.9856900 \\
\hline C & -1.7493050 & 3.1933120 & 0.5715380 \\
\hline C & -3.0099380 & 3.0711980 & 1.2496000 \\
\hline $\mathrm{H}$ & -4.7980260 & 1.8848980 & 1.4916570 \\
\hline C & -2.5482010 & 5.1126410 & 2.4975250 \\
\hline C & -1.2902990 & 5.2158910 & 1.8544860 \\
\hline C & -0.9023670 & 4.2828570 & 0.9136020 \\
\hline C & -3.3878400 & 4.0595230 & 2.1992190 \\
\hline $\mathrm{H}$ & -2.8429670 & 5.8572180 & 3.2334970 \\
\hline $\mathrm{H}$ & -0.6210160 & 6.0351950 & 2.1077420 \\
\hline $\mathrm{H}$ & 0.0672400 & 4.3694170 & 0.4343180 \\
\hline $\mathrm{H}$ & -4.3487640 & 3.9563850 & 2.7000500 \\
\hline C & -0.5468610 & 5.4618030 & -3.1234490 \\
\hline C & 0.7228520 & 5.5698000 & -3.7435160 \\
\hline C & 1.6783160 & 4.5995060 & -3.5238050 \\
\hline C & -0.8335380 & 4.4041830 & -2.2835160 \\
\hline $\mathrm{H}$ & -1.3056450 & 6.2172730 & -3.3156530 \\
\hline $\mathrm{H}$ & 0.9356950 & 6.4113550 & -4.3989110 \\
\hline $\mathrm{H}$ & 2.6530160 & 4.6594820 & -4.0048650 \\
\hline $\mathrm{H}$ & -1.8124620 & 4.3327440 & -1.8203380 \\
\hline C & 3.1979100 & 0.3586030 & -1.4803830 \\
\hline C & 3.4818840 & -0. & $-2.4 \varepsilon$ \\
\hline C & 4.0202380 & 0.4 & 24050 \\
\hline C & 4.63 & -1.3 & 2690 \\
\hline C & 5.15867 & -0.3 & -0 \\
\hline C & 5.49640 & -1.2 & -1.2 \\
\hline $\mathrm{H}$ & 4.8 & -2.05 & -3. \\
\hline $\mathrm{H}$ & 5.8 & -0.27 & 0.6 \\
\hline C & -4.3 & -0.2 & -0.0 \\
\hline C & -4.5 & -1.16 & 00 \\
\hline C & -4.9 & -0.5128980 & 00750 \\
\hline C & $-5 \cdot 3$ & -2.2667340 & $\lfloor 35600$ \\
\hline C & -5.7370880 & -1.6284780 & -1 \\
\hline
\end{tabular}

-5.9873060
-5.5617440
-6.2061060
3.7177450
6.7895800
2.5918310
-3.9169240
-6.8882900
-4.7400190
-6.0758830
-6.8303790
-6.4596630
-5.9399250
-4.1709200
-3.2137730
-4.8661170
-4.0135670
-6.3131070
-6.9550360
-6.2573340
-5.3025220
-8.3209700
-8.7499800
-8.3222900
-8.9655410
-4.9626630
-5.4052590
-5.7731310
-4.4970620
-3.2768530
-4.0396410
-2.5664380
-2.7425600
4.3531070
5.4465130
3.9998210
4.1015920
4.1603620
3.7558280
5.2547810
3.7960820
7.0650390
8.0009900
7.1715680
6.2558910
7.9732240

-2.5185370
-2.9564680
-1.8013500
1.4679830
-2.0560990
-0.7383550
-0.9743290
-3.7246800
0.4278050
1.1177530
0.3746530
1.6766980
1.8154790
-0.2997520
-0.7755930
-1.0727570
0.4150350
-4.6862270
-5.5715330
-4.1897690
-5.0138350
-3.3021130
-2.6405850
-2.7655910
-4.1853800
-0.4444440
0.4986640
-1.1753130
-0.2756810
-2.2626970
-3.0191450
-2.6820470
-2.0359000
2.8339630
2.7366840
3.2138270
3.5673560
1.0217660
0.0348570
0.9849690
1.7412440
-2.7162030
-3.2877940
-1.9602700
-3.3969530
-1.1509560

-0.4170620
1.6353850
-2.4312880
0.7662900
-1.2235270
-3.7131370
2.4021990
-0.6148290
-2.4973380
-2.8386750
-3.1283190
-1.9754110
-3.6759000
-3.7291050
-3.4888600
-4.0820650
-4.5478520
-1.6725130
-1.7736250
-2.6502080
-1.3977240
-0.9922100
-0.2288750
-1.9502910
-1.0943040
3.4040380
3.0628810
3.5286050
4.3845040
2.9540200
3.1804200
2.2353600
3.8859160
0.4311520
0.3872320
-0.5335710
1.2094150
2.1678220
2.4196290
2.2466590
2.9092910
0.1386620
0.0934070
0.9269440
0.4239810
-1.6248610




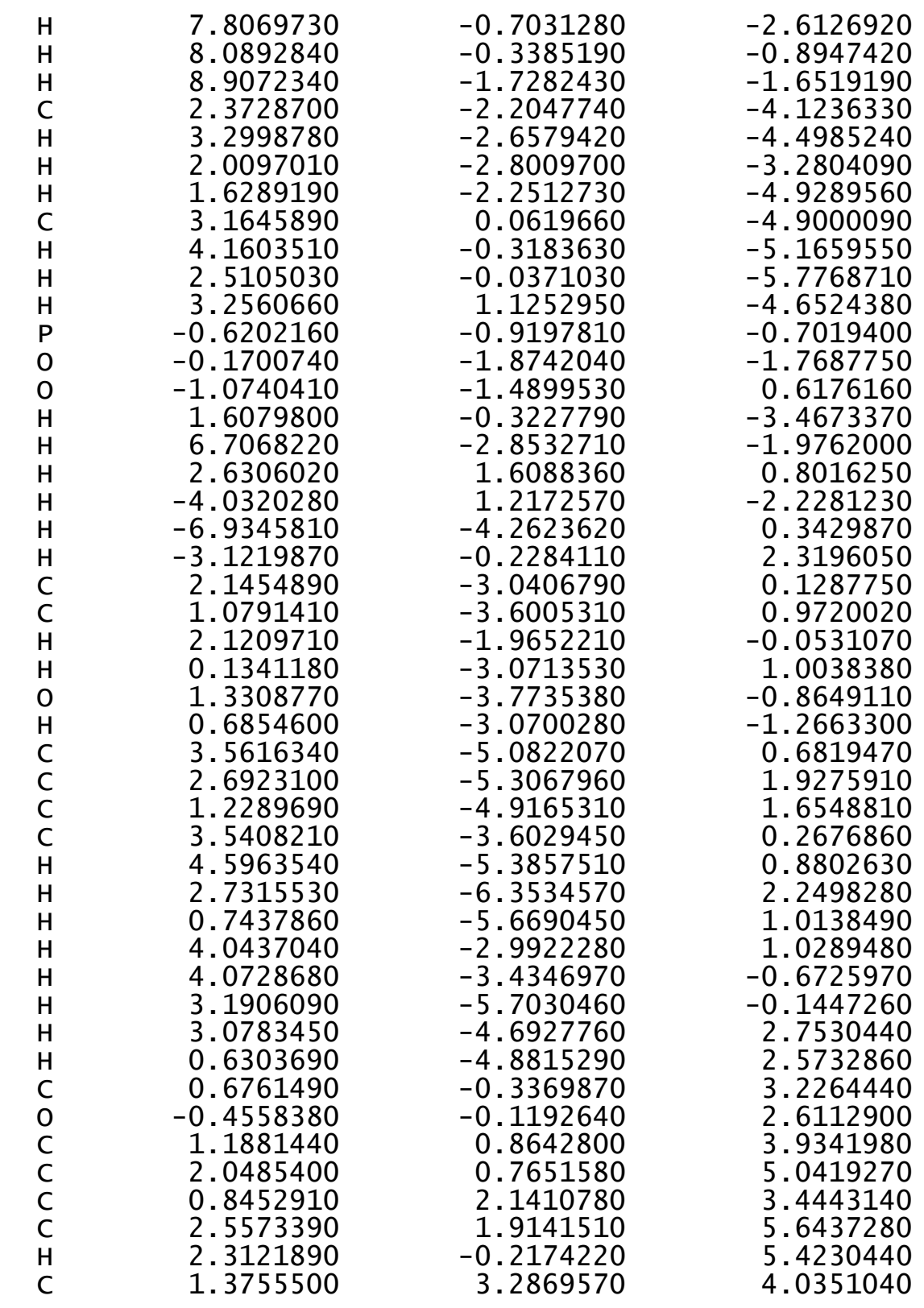

$\begin{array}{lrrr}\mathrm{H} & 0.1869150 & 2.2304790 & 2.5862940 \\ \mathrm{C} & 2.2312020 & 3.1782050 & 5.1365340 \\ \mathrm{H} & 3.2148760 & 1.8256810 & 6.5053170 \\ \mathrm{H} & 1.1180140 & 4.2634890 & 3.6320970 \\ \mathrm{H} & 2.6423980 & 4.0728810 & 5.5988250 \\ \mathrm{H} & -0.6994150 & -0.7967670 & 1.8554500 \\ \mathrm{~S} & 1.5220890 & -1.7914510 & 3.2152280\end{array}$

TS213(R,R) G[B97-D/def2-TZVP $]=-3633.749230$

\begin{tabular}{|c|c|c|c|}
\hline & & & \\
\hline 0 & 0.6086990 & 0.1779320 & -0.2666190 \\
\hline 0 & -1.6435580 & 0.1507590 & -1.4862210 \\
\hline C & 0.9201160 & 1.3573360 & -0.9297590 \\
\hline C & -0.0291240 & 2.3721630 & -0.9981150 \\
\hline C & 2.2352320 & 1.4983210 & -1.4449070 \\
\hline C & 0.2770580 & 3.5536450 & -1.7487930 \\
\hline C & 2.5408770 & 2.6668470 & -2.1160340 \\
\hline C & 1.5894450 & 3.6953250 & -2.3144560 \\
\hline $\mathrm{H}$ & 3.5467350 & 2.8029250 & -2.5058960 \\
\hline C & -2.1408630 & 1.1216530 & -0.6056840 \\
\hline C & -3.4387400 & 0.9311840 & -0.0598210 \\
\hline C & -1.3372370 & 2.2137220 & -0.3146150 \\
\hline C & -3.8889330 & 1.8759930 & 0.8422320 \\
\hline C & -1.7778860 & 3.1375040 & 0.6883610 \\
\hline C & -3.0806430 & 2.9594250 & 1.26630 \\
\hline $\mathrm{H}$ & -4.8831520 & 1.7695010 & 1.2( \\
\hline C & -2.7077090 & 4.8769960 & 2.72 \\
\hline C & -1.4111510 & 6080 & 2.17 \\
\hline C & -0.9588770 & 4.1901200 & $1.1 \varepsilon$ \\
\hline C & -3.52 & 3.8579020 & 2.2 \\
\hline $\mathrm{H}$ & -3.05 & 4030 & 3.5 \\
\hline $\mathrm{H}$ & -0.7631130 & 5.8272950 & 2.5 \\
\hline $\mathrm{H}$ & 0.0400580 & 4.3170640 & 0.7763290 \\
\hline $\mathrm{H}$ & -4.5146690 & 3.7128610 & 2.7002320 \\
\hline C & -0.3363400 & 5.7029670 & -2.7258480 \\
\hline C & 0.9683410 & 5.8528240 & -3.2583810 \\
\hline C & 1.9091440 & 4.8643970 & -3.0573230 \\
\hline C & -0.6733290 & 4.5843400 & -1.9901090 \\
\hline $\mathrm{H}$ & -1.0819550 & 6.4749870 & -2.9031740 \\
\hline $\mathrm{H}$ & 1.2203580 & 6.7418560 & -3.8320850 \\
\hline $\mathrm{H}$ & 2.9116780 & 4.9580670 & -3.4710880 \\
\hline $\mathrm{H}$ & -1.6778680 & 4.4821610 & -1.5922030 \\
\hline C & 3.3194580 & 0.4882920 & -1.2442290 \\
\hline C & 3.73 & -0.3182750 & -2.3242140 \\
\hline C & 4.024 & 0.4599540 & -0.0177220 \\
\hline C & 4.9080630 & -1.0745120 & -2.1895960 \\
\hline C & 5.18 & 32 & 0.068671 \\
\hline
\end{tabular}




$\begin{array}{rrr}5.6580710 & -1.0703750 & -1.0114820 \\ 5.2437080 & -1.6752870 & -3.0311950 \\ 5.7460560 & -0.3398620 & 0.9986370 \\ -4.2879880 & -0.2348900 & -0.4396140 \\ -4.6158750 & -1.2198350 & 0.5258270 \\ -4.7924430 & -0.3406310 & -1.7553910 \\ -5.4560480 & -2.2739630 & 0.1529630 \\ -5.6232050 & -1.4217370 & -2.0771430 \\ -5.9699070 & -2.3965250 & -1.1413560 \\ -5.7135440 & -3.0260010 & 0.8952640 \\ -6.0190710 & -1.5020400 & -3.0883930 \\ 3.5915130 & 1.3289520 & 1.1589480 \\ 6.9753030 & -1.8221820 & -0.9103770 \\ 2.9691280 & -0.3587010 & -3.6389380 \\ -4.1010230 & -1.1729290 & 1.9602300 \\ -6.8920950 & -3.5442360 & -1.5127620 \\ -4.5131670 & 0.6990010 & -2.8339770 \\ -5.8049970 & 1.4506420 & -3.2128030 \\ -6.5460510 & 0.7577710 & -3.6323880 \\ -6.2479220 & 1.9346900 & -2.3327160 \\ -5.5889040 & 2.2212450 & -3.9648870 \\ -3.8600580 & 0.0718230 & -4.0794480 \\ -2.9364150 & -0.4519830 & -3.8092650 \\ -4.5405540 & -0.6439200 & -4.5587050 \\ -3.6197220 & 0.8545280 & -4.8114190 \\ -8.2345210 & -3.4380690 & -0.7625220 \\ -8.9072140 & -4.2527310 & -1.0627150 \\ -8.0716420 & -3.5085950 & 0.3212180 \\ -8.7274020 & -2.4802580 & -0.9725940 \\ -6.2370370 & -4.9138600 & -1.2543930 \\ -5.2908320 & -5.0066820 & -1.8021810 \\ -6.0275250 & -5.0447810 & -0.1846930 \\ -6.9084860 & -5.7227860 & -1.5714720 \\ -5.2206480 & -0.7389340 & 2.9274610 \\ -5.6327920 & 0.2389780 & 2.6520140 \\ -6.0400060 & -1.4703750 & 2.9099380 \\ -4.8342340 & -0.6776840 & 3.9538710 \\ -3.5066690 & -2.5170650 & 2.4227760 \\ -4.2846550 & -3.2869250 & 2.5058420 \\ -2.7402380 & -2.8640400 & 1.7232530 \\ -3.0496710 & -2.3939460 & 3.4133890 \\ 4.2328940 & 2.7283980 & 1.0546110 \\ 5.3265150 & 2.6447550 & 1.1154300 \\ 3.9735340 & 3.2137200 & 1.8811420 \\ 3.8872240 & 3.3640480 & 2.5340210 \\ 3.8962270 & 0.7152140 & \end{array}$

3.4725880

4. 9772430

3. 4565490

8.0597230

9.0296600

7.7917190

8.1635490

6.8711410

6.1526000

6. 5526610

7.8500990

2. 7255890

3. 6685640

2. 2295420

2. 0860620

3. 6953900

4. 6899990

3. 1241540

3. 8211880

$-0.5496550$

$-0.0137380$

$-1.1022340$

1. 9871690

7. 2850960

2. 5052120

$-3.8157140$

$-7.0991050$

$-3.3009660$

2. 1347890

1.0178190

2. 0474420

0.0392310

1. 5172270

0.8767760

3. 6270320

2. 6283030

1. 1828400

3. 5416650

4.6484130

2. 7070910

0.8341690

3. 8924270

4. 1687430

3. 4136860

2.8627080

0.4777070
-0.2916740
0.6594970
1.3430620
-1.0978280
-1.6000530
-1.1041960
-0.0534880
-3.2943140
-3.8459160
-3.3689180
-3.7827690
-1.7978240
-2.2905990
-2.3936180
-1.7815730
0.4591280
0.0343500
0.4388650
1.5035140
-0.8504500
-1.7334670
-1.5079940
0.0982180
-1.8025020
1.4613160
1.4438780
-3.4648180
-0.4300980
-2.9263680
-3.6452130
-1.8420840
-3.1810260
-3.5963380
-2.9001330
-4.9194990
-5.3490830
-5.0184840
-3.4048540
-5.1801470
-6.4234510
-5.7207840
-2.8607090
-3.0864120
-5.4565800
-4.8211570
-5.1251540

2.6264070

2. 7163770

3. 3167610

$-1.7348520$

$-1.6200000$

$-2.7999460$

$-1.4143050$

$-1.3473690$

$-0.7308360$

$-2.3950000$

$-1.2567820$

$-4.1282230$

$-4.3988670$

$-3.3551590$

$-5.0201680$

$-4.7246780$

$-4.9166900$

$-5.6625220$

$-4.4158610$

$-0.8197320$

$-1.9082740$

0.4185630

$-3.4728270$

0.1442190

1.1013220

$-2.4399270$

$-2.5894800$

2. 0154640

0.1972630

0.8244300

0.1257530

0.7861030

$-0.9692710$

$-1.3892750$

0.7076880

1. 7924610

1. 3782510

0.4643940

1. 0089250

1.9935630

0.6050990

1. 3515320

$-0.3715950$

0.2263910

2. 7272870 


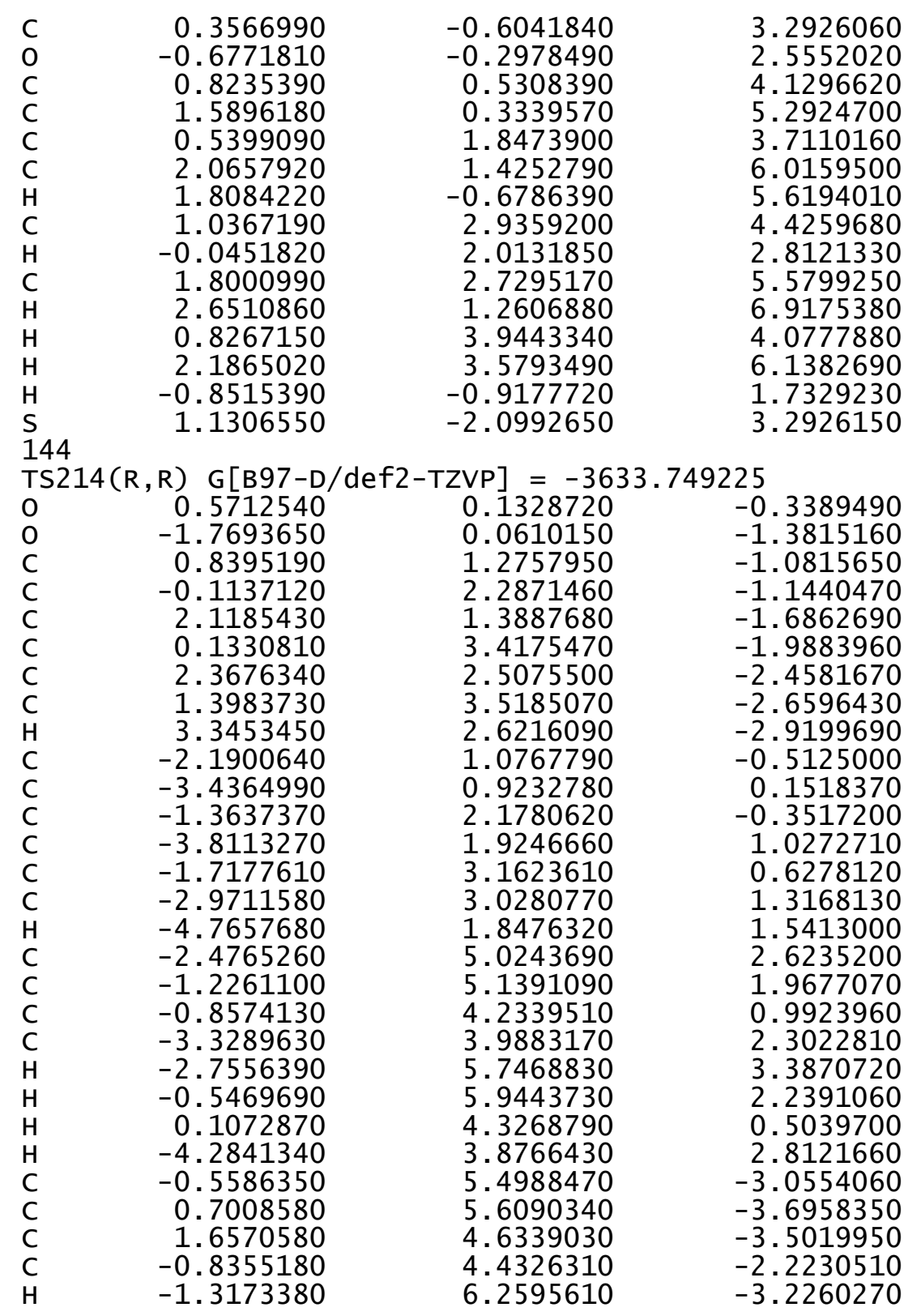
0.9055520
2.6245400
$-1.8070060$
3. 2270010
3. 6106320
3. 9824580
4.7901630
5. 1462690
5.5818090
5.1039460
5.7435610
$-4.3069540$
$-4.5495180$
$-4.9146540$
$-5.4046190$
$-5.7548210$
$-6.0152620$
$-5.5961350$
-6.2259790
3.5883460
6.9033520
2. 8029680
$-3.9281120$
$-6.9302780$
$-4.7372200$
$-6.0657020$
$-6.8302750$
$-6.4390610$
$-5.9245740$
$-4.1833340$
$-3.2327110$
$-4.8915810$
$-4.0179200$
$-6.3655440$
$-7.0186570$
$-6.3018750$
$-5.3597130$
$-8.3575310$
$-8.7794990$
$-8.3515730$
$-9.0122030$
$-4.9720700$
$-5.4132710$
$-5.7838990$
$-4.5050980$
$-3.2864870$

6.4570010

4.6969810

4. 3587950

0.4100700

$-0.4647530$

0.4750010

$-1.2082890$

$-0.2932870$

$-1.1212380$

$-1.8702050$

$-0.2321770$

$-0.2669330$

$-1.1809150$

$-0.4664270$

$-2.2657580$

$-1.5684490$

$-2.4807130$

$-2.9732000$

$-1.7126630$

1.4260190

$-1.8659100$

$-0.5906100$

$-1.0308620$

$-3.6710260$

0.5017960

1. 2170380

0.4905630

1.7587390

1.9339710

$-0.2012380$

$-0.6980240$

$-0.9537330$

0.5326040

$-4.6148480$

$-5.4891980$

$-4.0965260$

$-4.9617830$

$-3.2230690$

$-2.5738880$

$-2.6653160$

$-4.0962760$

$-0.5324840$

0.4220750

$-1.2652370$

-0.3961140
-2.3360440
$-4.3455370$

$-3.9970160$

$-1.7445460$

$-1.4670950$

$-2.5033570$

$-0.2692250$

$-2.3557950$

$-0.1699700$

$-1.2110730$

$-3.1608960$

0.7349030

$-0.0744930$

0.9791480

$-1.3372600$

0.7435200

$-1.5190070$

$-0.4926930$

1. 5482920

$-2.4887650$

0.8586140

$-1.1224360$

$-3.7884880$

2. 3632440

$-0.7191660$

$-2.5011010$

$-2.8188820$

$-3.1237740$

$-1.9401810$

$-3.6388480$

$-3.7538520$

$-3.5306340$

$-4.1245070$

$-4.5539140$

$-1.7982150$

$-1.9206760$

$-2.7640070$

$-1.5297610$

$-1.0879940$

$-0.3102700$

$-2.0338400$

$-1.2103090$

3. 3826940

3. 0725450

3.4848260

4.3675360

2.8719630 


\begin{tabular}{|c|c|c|}
\hline $\begin{array}{l}-4.0477050 \\
-2.5707830 \\
-2.7579520 \\
4.1233720 \\
5.2214220 \\
3.7535950 \\
3.8081980 \\
4.0444130 \\
3.7211460 \\
5.1366970 \\
3.6081960 \\
7.0434470 \\
8.0176820 \\
6.9823660 \\
6.2571030 \\
8.0778710 \\
8.0101400 \\
8.0631430 \\
9.0367250 \\
2.5436560 \\
3.4790550 \\
2.0601650 \\
1.8867250 \\
3.4980350 \\
4.4845780 \\
2.8976630 \\
3.6372040 \\
-0.6211660 \\
-0.1710980 \\
-1.0714780 \\
1.8277590 \\
6.9527890 \\
2.4937670 \\
-4.0181290 \\
-6.9840400 \\
-3.1338340 \\
2.1456760 \\
1.0883980 \\
2.0973010 \\
0.1332460 \\
1.3511300 \\
0.6961860 \\
3.6061760 \\
2.7370440 \\
1.2655130 \\
3.5529280\end{array}$ & $\begin{array}{r}-3.1027720 \\
-2.7268870 \\
-2.1409970 \\
2.8474090 \\
2.8339040 \\
3.2366840 \\
3.5293250 \\
0.9673020 \\
-0.0590560 \\
1.0201660 \\
1.6254750 \\
-2.7040960 \\
-3.2097780 \\
-2.0661760 \\
-3.4629690 \\
-0.8724870 \\
-0.2920610 \\
-0.1710060 \\
-1.4079090 \\
-2.0572360 \\
-2.5716870 \\
-2.5995080 \\
-2.0961470 \\
0.1526410 \\
-0.2889170 \\
0.0760590 \\
1.2138570 \\
-0.9149500 \\
-1.8278990 \\
-1.5358950 \\
-0.1197380 \\
-2.5502090 \\
1.4846770 \\
1.2749210 \\
-4.2301310 \\
-0.2822720 \\
-3.0258340 \\
-3.6403240 \\
-1.9451770 \\
-3.1306830 \\
-3.7389140 \\
-3.0344210 \\
-5.0520920 \\
-5.3397850 \\
-4.9766600 \\
-3.5601180\end{array}$ & $\begin{array}{r}3.0663030 \\
2.1423750 \\
3.8142080 \\
0.5821220 \\
0.5510170 \\
-0.3721870 \\
1.3836560 \\
2.2514140 \\
2.4593550 \\
2.3496930 \\
3.0103000 \\
0.1611120 \\
0.1736330 \\
1.0522120 \\
0.2291240 \\
-1.2392870 \\
-2.1680820 \\
-0.3944000 \\
-1.2271640 \\
-4.1767640 \\
-4.4326550 \\
-3.3579010 \\
-5.0552500 \\
-4.9462510 \\
-5.1416030 \\
-5.8627170 \\
-4.7085010 \\
-0.7727890 \\
-1.8756850 \\
0.5246060 \\
-3.6229480 \\
-1.9813550 \\
0.8818650 \\
-2.2150180 \\
0.2258880 \\
2.3043020 \\
0.0005830 \\
0.8161850 \\
-0.1389680 \\
0.8637930 \\
-1.0241260 \\
-1.4062000 \\
0.4850690 \\
1.7178120 \\
1.4509480 \\
0.1217650\end{array}$ \\
\hline
\end{tabular}

4.6466240
2.8011740
0.8025880
4.0427980
4.0807530
3.2530230
3.1036830
0.6616330
0.6197870
-0.4935300
1.0868250
1.9458230
0.7002040
2.4118760
2.2437380
1.1878140
0.0424820
2.0432240
3.0695230
0.8980100
2.4213180
-0.7146690
1.4841290

4.6466240

$\mathrm{H}$

$\mathrm{H}$

$\mathrm{H}$

$\mathrm{H}$

$\mathrm{H}$
$-5.3388330$
$-6.3954600$
$-5.7168640$
$-2.9645110$
$-3.3471170$
$-5.6526450$
$-4.7444960$
$-4.9901810$
$-0.4757100$
$-0.2357100$
0.6878800
0.5373360
1. 9854140
1. 6553120
3. 1019000
2. 1166040
2. 9415400
1.5257670
4.0958860
3. 8131240
$-0.8830450$
$-1.9176590$

0.6783370

2. 0046910

0.7798860

0.9029530

$-0.8122540$

$-0.3640750$

2. 5654900

2. 3663940

3. 2161630

2. 5762100

4. 0131890

5. 1163290

3. 6185240

5.8049280

5.4249950

4.2958240

2. 7653330

5.3911280

6.6612230

3.9636770

5. 9209050

1. 7881670

3.1440780

TS215 (R, R) G[B97-D/def2-TZVP] $=-3633.749157$

\begin{tabular}{lrrr}
\multicolumn{1}{c}{ O } & 0.2712100 & -1.2181730 & 0.2607340 \\
$\mathrm{O}$ & 2.1094280 & 0.0072590 & -0.9743460 \\
$\mathrm{C}$ & 0.7116780 & -2.4442830 & -0.2271080 \\
$\mathrm{C}$ & 2.0750110 & -2.7160300 & -0.2487760 \\
$\mathrm{C}$ & -0.2885630 & -3.3780620 & -0.6126980 \\
$\mathrm{C}$ & 2.5008800 & -3.9445740 & -0.8557230 \\
$\mathrm{C}$ & 0.1318440 & -4.5964900 & -1.1036130 \\
$\mathrm{C}$ & 1.5078660 & -4.8962800 & -1.2750830 \\
$\mathrm{H}$ & -0.6041780 & -5.3474200 & -1.3788040 \\
$\mathrm{C}$ & 2.9924720 & -0.4078520 & 0.0162550 \\
$\mathrm{C}$ & 3.8400860 & 0.5818850 & 0.5828040 \\
$\mathrm{C}$ & 3.0284350 & -1.7525750 & 0.3661870 \\
$\mathrm{C}$ & 4.7849390 & 0.1652730 & 1.4972510 \\
$\mathrm{C}$ & 3.9437960 & -2.1542610 & 1.3989840 \\
$\mathrm{C}$ & 4.8582670 & -1.1809960 & 1.9342720 \\
$\mathrm{H}$ & 5.4655200 & 0.8934900 & 1.9311570 \\
$\mathrm{C}$ & 5.7987290 & -2.8607460 & 3.4298400 \\
$\mathrm{C}$ & 4.8632880 & -3.8066560 & 2.9440820 \\
$\mathrm{C}$ & 3.9608430 & -3.4623960 & 1.9570690 \\
$\mathrm{C}$ & 5.7875020 & -1.5725630 & 2.9367700 \\
$\mathrm{H}$ & 6.5099320 & -3.1471980 & 4.2013650
\end{tabular}




$\begin{array}{lrrr}\text { H } & 4.8508110 & -4.8133770 & \\ \text { H } & 3.2476870 & -4.1987700 & -3561630 \\ \text { H } & 6.4798440 & -0.8249230 & 3.3199750 \\ \mathrm{C} & 4.2403960 & -5.4429200 & -1.6806010 \\ \mathrm{C} & 3.2627780 & -6.3960310 & -2.0578990 \\ \mathrm{C} & 1.9246810 & -6.1227070 & -1.8607970 \\ \mathrm{C} & 3.8686390 & -4.2496870 & -1.0931420 \\ \mathrm{H} & 5.2931650 & -5.6508430 & -1.8589820 \\ \mathrm{H} & 3.5691760 & -7.3352370 & -2.5130410 \\ \mathrm{H} & 1.1618860 & -6.8381620 & -2.1631130 \\ \mathrm{H} & 4.6257020 & -3.5250380 & -0.8099950 \\ \mathrm{C} & -1.7295430 & -3.0340930 & -0.4167700 \\ \mathrm{C} & -2.5582830 & -2.7272010 & -1.5224220 \\ \mathrm{C} & -2.2493750 & -2.9769370 & 0.8955120 \\ \mathrm{C} & -3.8856160 & -2.3538150 & -1.2840720 \\ \mathrm{C} & -3.5872450 & -2.6075950 & 1.0785520 \\ \mathrm{C} & -4.4172000 & -2.2765510 & 0.0074500 \\ \mathrm{H} & -4.5254120 & -2.1089490 & -2.1281200 \\ \mathrm{H} & -3.9940350 & -2.5681900 & 2.0871670 \\ \mathrm{C} & 3.6482670 & 2.0206750 & 0.2332740 \\ \mathrm{C} & 3.0345160 & 2.8868190 & 1.1718900 \\ \mathrm{C} & 4.0201450 & 2.5022070 & -1.0387920 \\ \mathrm{C} & 2.8158630 & 4.2200500 & 0.8106880 \\ \mathrm{C} & 3.7796270 & 3.8462500 & -1.3508670 \\ \mathrm{C} & 3.1727750 & 4.7184570 & -0.4463670 \\ \mathrm{H} & 2.3345540 & 4.8859970 & 1.5221790 \\ \mathrm{H} & 4.0651370 & 4.2231120 & -2.3319870 \\ \mathrm{C} & -1.4213070 & -3.3499920 & 2.1190220 \\ \mathrm{C} & -5.8525500 & -1.8382690 & 0.2378790 \\ \mathrm{C} & -2.0532110 & -2.8294740 & -2.9563990 \\ \mathrm{C} & 2.6206840 & 2.4151440 & 2.5618880 \\ \mathrm{C} & 2.8572720 & 6.1507700 & -0.8399580 \\ \mathrm{C} & 4.6955190 & 1.6188460 & -2.0789820 \\ \mathrm{C} & 6.1465380 & 2.0778080 & -2.3235520 \\ \mathrm{H} & 6.1623200 & 3.0997840 & -2.7249180 \\ \mathrm{H} & 6.7235800 & 2.0672100 & -1.3899100 \\ \mathrm{H} & 6.6386350 & 1.4153650 & -3.0483620 \\ \mathrm{C} & 3.9055530 & 1.5833330 & -3.4006620 \\ \mathrm{H} & 2.8786320 & 1.2429050 & -3.2275480 \\ \mathrm{H} & 3.8708340 & 2.5795850 & -3.8603000 \\ \mathrm{H} & 4.3895760 & 0.8987020 & -4.1098760 \\ \mathrm{C} & 3.3927820 & 7.1741070 & 0.1775360 \\ \mathrm{H} & 3.1839580 & 8.1958820 & -0.1658650 \\ \mathrm{H} & 2.9097070 & 7.0397820 & 1.1539680 \\ \mathrm{H} & 4.4761780 & 7.0643250 & 0.3135050 \\ \mathrm{C} & 1.3383630 & 6.3292300 & -1.0399160 \\ & & & \\ & & & \end{array}$

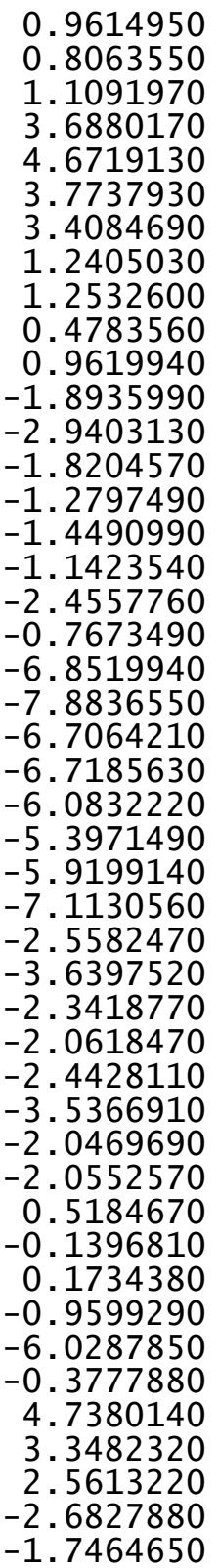

5.6299780

6.1348280

7.3540350

2. 8146160

2.4065690

3.9087070

2. 4448310

2.9365530

4.0230140

2.6984250

2.4682220

$-4.7019320$

$-4.6344060$

$-5.4925620$

$-4.9869860$

$-2.2598220$

$-1.2912060$

$-2.1568390$

$-2.5292630$

$-2.8085390$

$-2.4905010$

$-2.8284810$

$-3.8290790$

$-0.4005020$

0.2976810

$-0.3413700$

$-0.0826280$

$-1.6997430$

$-1.7767310$

$-0.7177200$

$-1.7693940$

$-4.1949630$

$-4.2869170$

$-4.2884750$

$-5.0253670$

0.1016920

$-0.0222460$

1.2721850

$-2.7663230$

$-1.8481390$

$-3.4762190$

0.5946080

6.3428130

1. 3216010

1.8465970

2.9550660
$-1.7963430$ $-0.0992010$

$-1.3622820$

3.6015030

3. 3422640

3.6503040

4.5975200

3.0016160

3.1576340

2.2540940

3.9548810

2.6897940

3.0151520

1. 9320040

3.5549420

3.2050210

2.7945920

3.6309160

4.0222940

$-0.4196740$

$-0.2180220$

$-1.5078630$

$-0.0385200$

$-0.2623780$

0.2289730

$-1.3461060$

$-0.0524580$

$-3.8700610$

$-4.0421170$

$-3.4407740$

$-4.8465290$

$-3.5604560$

$-3.6038350$

$-4.5809290$

$-2.9598120$

$-0.6647960$

$-2.0144500$

0.2128590

$-2.9345320$

1. 3220790

1.8156830

$-1.6934400$

$-1.8047610$

2. 5470920

$-1.8274140$

$-1.5938140$ 


$\begin{array}{lrrr}\mathrm{H} & -2.6298100 & 1.0095860 & -1.1293260 \\ \mathrm{H} & -0.8319440 & 2.7320000 & -1.0556410 \\ \mathrm{O} & -1.7145240 & 1.6845780 & -2.9223200 \\ \mathrm{H} & -1.0083890 & 0.9757180 & -2.5706330 \\ \mathrm{C} & -4.1295220 & 3.4167680 & -3.2003100 \\ \mathrm{C} & -3.4421860 & 4.6037150 & -2.5072730 \\ \mathrm{C} & -1.9626040 & 4.3007090 & -2.2088080 \\ \mathrm{C} & -4.0814550 & 2.1645470 & -2.3083840 \\ \mathrm{H} & -5.1746220 & 3.6618320 & -3.4225010 \\ \mathrm{H} & -3.5053160 & 5.5042760 & -3.1288160 \\ \mathrm{H} & -1.3716170 & 4.3297560 & -3.1361690 \\ \mathrm{H} & -4.6944710 & 2.3215480 & -1.4106280 \\ \mathrm{H} & -4.4816270 & 1.2847600 & -2.8262630 \\ \mathrm{H} & -3.6299470 & 3.2066800 & -4.1553880 \\ \mathrm{H} & -3.9588790 & 4.8169650 & -1.5616670 \\ \mathrm{H} & -1.5154660 & 5.0504560 & -1.5467190 \\ \mathrm{C} & -2.8996020 & 2.0805300 & 1.5636940 \\ \mathrm{O} & -2.0595190 & 1.0837450 & 1.4139740 \\ \mathrm{C} & -4.1438310 & 1.6869200 & 2.2694350 \\ \mathrm{C} & -5.2847290 & 2.5128970 & 2.2556360 \\ \mathrm{C} & -4.2123420 & 0.4441850 & 2.9336530 \\ \mathrm{C} & -6.4615860 & 2.1040190 & 2.8783060 \\ \mathrm{H} & -5.2421930 & 3.4658460 & 1.7364230 \\ \mathrm{C} & -5.3889720 & 0.0438220 & 3.5621310 \\ \mathrm{H} & -3.3442570 & -0.2039780 & 2.9498040 \\ \mathrm{C} & -6.5187730 & 0.8681720 & 3.5335010 \\ \mathrm{H} & -7.3381540 & 2.7466100 & 2.8480920 \\ \mathrm{H} & -5.4259240 & -0.9167790 & 4.0705270 \\ \mathrm{H} & -7.4394970 & 0.5492920 & 4.0168320 \\ \mathrm{H} & -1.1663540 & 1.2969820 & 0.9358520 \\ \mathrm{~S} & -2.6065200 & 3.6360610 & 1.0002350 \\ \mathrm{H} & - & & \end{array}$

144

$\begin{array}{lrcr}\text { TS216(R, R) } \text { G }[\mathrm{B} 97-\mathrm{D} / \mathrm{def} 2-\mathrm{TZVP}]=-3633.749148 \\ \text { O } & 0.2536180 & 0.9170690 & 0.0047490 \\ \mathrm{O} & -2.0179090 & 0.5396770 & -1.0765780 \\ \mathrm{C} & 0.2141020 & 2.2748840 & -0.2910500 \\ \mathrm{C} & -0.9716670 & 2.9732820 & -0.1014660 \\ \mathrm{C} & 1.4321910 & 2.8894360 & -0.6922870 \\ \mathrm{C} & -1.0182240 & 4.3458350 & -0.5163890 \\ \mathrm{C} & 1.3955380 & 4.2314340 & -1.0113020 \\ \mathrm{C} & 0.1897690 & 4.9760620 & -0.9749010 \\ \text { H } & 2.3123440 & 4.7340470 & -1.3087960 \\ \mathrm{C} & -2.6136670 & 1.0951030 & 0.0520830 \\ \mathrm{C} & -3.7333460 & 0.4137610 & 0.6006480 \\ \mathrm{C} & -2.1294770 & 2.3009070 & 0.5448080 \\ \mathrm{C} & -4.3670870 & 0.9915350 & 1.6818070\end{array}$

-2.7309070
-3.8853090
-5.2463830
-3.9911970
-2.8246090
-2.2117690
-4.5043410
-4.4688330
-2.4033600
-1.3154070
-5.3849780
-2.2253290
-1.0278230
0.1529420
-2.2195740
-3.1585500
-1.0446390
1.0775730
-3.1430000
2.6954990
3.3319520
3.2393110
4.4805300
4.3775270
5.0044120
4.9790050
4.7911310
-4.2001980
-3.9547950
-4.8760160
-4.4176760
-5.3136650
-5.0967150
-4.2301240
-5.8380550
2.6613280
6.2606710
2.8472970
-3.2152910
-5.5616290
-5.1735920
-6.6908370
-7.2084250
-7.1086230
-6.8947420
-4.5791760

8362610

2.1795400

0.5109330

3. 8303700

4.4496770

3. 9662000

2.7135780

4. 2266440

5.3120640

4504890

2.2098670

6.4233140

7. 0524200

6.3388410

5. 1052470

6.9822470

8.0932010

.8055050

4.6320840

2. 0946770

1.7579630

1.6590980

0.9549970

0.8490620

0.4711810

0.6888730

0.5030510

$-0.8802890$

$-2.0922640$

$-0.8938690$

- 3.2879410

$-2.1193690$

$-3.3253150$

$-4.2215410$

$-2.1339990$

2.1494370

$-0.3823840$

2. 2870820

$-2.1276550$

$-4.6423940$

0.3781730

0.6459030

$-0.1697250$

0.7245650

1.5823540

0.3268050
1.7343670

2. 2853590

2.1031210

4.0754450

3. 5640270

2. 4250460

3.4489940

4.9687470

4.0761320

2. 0529150

3. 8440680

$-0.9365370$

$-1.3572830$

$-1.3779340$

$-0.5247700$

$-0.9415120$

$-1.6728550$

$-1.7133020$

$-0.2056430$

$-0.6990270$

$-1.9130730$

0.5369250

$-1.8677970$

0.5236790

$-0.6690770$

$-2.7976560$

1.4654920

0.0205040

0.7110260

$-1.2175120$

0.1530140

$-1.7363380$

$-1.0695980$

0.6777280

$-2.6905670$

1.8613690

$-0.6721170$

$-3.2576910$

2. 0439870

$-1.6642490$

$-2.0011010$

$-2.0516380$

$-2.5734900$

$-1.0396050$

$-2.5880890$

$-3.4205570$ 


$\begin{array}{rrr}-3.4971780 & 0.1583320 & -3.3798600 \\ -5.0366640 & -0.4811800 & -4.0064380 \\ -4.7682670 & 1.2745480 & -3.9422230 \\ -6.5607790 & -5.3624800 & -0.7388540 \\ -6.9143080 & -6.2909810 & -1.2067050 \\ -6.0813220 & -5.6216940 & 0.2143470 \\ -7.4279290 & -4.7250620 & -0.5239870 \\ -4.3655140 & -5.5603450 & -1.9847670 \\ -3.6649800 & -5.0666480 & -2.6702150 \\ -3.8219950 & -5.8136430 & -1.0649520 \\ -4.7122390 & -6.4940660 & -2.4475520 \\ -4.2149590 & -2.2109250 & 3.2156360 \\ -4.9154730 & -1.3682710 & 3.2045430 \\ -4.7967330 & -3.1403710 & 3.1480240 \\ -3.6798120 & -2.2073790 & 4.1750830 \\ -2.1974900 & -3.2789630 & 2.1440290 \\ -2.7011150 & -4.2533540 & 2.1885190 \\ -1.5171100 & -3.2726760 & 1.2873250 \\ -1.6071090 & -3.1667920 & 3.0624260 \\ 3.1627700 & 3.5839910 & 2.1355700 \\ 4.2555910 & 3.5829660 & 2.2482220 \\ 2.8986610 & 4.2578320 & 1.3125200 \\ 2.7184520 & 3.9718890 & 3.0623250 \\ 2.9632080 & 1.2471790 & 3.0656150 \\ 2.6673920 & 0.2118400 & 2.8743090 \\ 4.0310210 & 1.2617980 & 3.3214950 \\ 2.4081490 & 1.6103450 & 3.9395070 \\ 6.2337180 & -1.5142080 & 0.3696960 \\ 7.1133220 & -2.1584410 & 0.2450950 \\ 6.2566290 & -1.1097230 & 1.3895790 \\ 5.3305630 & -2.1293390 & 0.2734300 \\ 7.5060990 & 0.5043750 & -0.4632000 \\ 7.5603910 & 1.2902940 & -1.2269510 \\ 7.4627360 & 0.9857410 & 0.5231960 \\ 8.4222290 & -0.0996410 & -0.5138530 \\ 2.8000700 & 1.2174640 & -4.3613010 \\ 3.8011400 & 0.8307320 & -4.5906960 \\ 2.1624460 & 0.3787660 & -4.0691860 \\ 2.3951430 & 1.6584710 & -5.2813180 \\ 3.7364430 & 3.4662910 & -3.7042000 \\ 4.7680330 & 3.1228760 & -3.8600320 \\ 3.3654600 & 3.8879550 & -4.6480550 \\ 3.7536890 & 4.2615250 & -2.9493210 \\ -0.5418540 & -0.1312410 & -0.9626230 \\ -0.0163950 & -0.1175190 & -2.3740140 \\ -0.5465140 & -1.4256290 & -0.1983220\end{array}$
1.8281130
6. 3401030
1. 5716350
$-4.7129720$
$-6.0764910$
$-2.6553500$
2. 4994820
1. 5380740
2. 4704840
0.6294060
1. 5258730
0.8320410
3.9100980
3. 2016890
1.7281360
3.8859890
4.9506890
3. 2476990
1.1371730
4. 5032340
4. 2952770
3.4146010
3. 7178580
1. 2694650
1.9235330
0.9470040
2. 6143520
3. 9699410
1. 9141440
4.6121230
4. 5158630
2. 5545570
0.8693680
3. 9060880
5.6653520
2. 0004740
4.4073980
0.4048520

144

TS217(R,R) G[B97-D/def2-TZVP $]=-3633.749084$

\begin{tabular}{lllr} 
TS217(R,R) & \multicolumn{1}{c}{ G $[\mathrm{B} 97-\mathrm{D} / \mathrm{def}-\mathrm{TZVP}]=-3633.749084$} \\
$\mathrm{O}$ & 0.1889880 & -1.2346600 & 0.2902820 \\
$\mathrm{O}$ & 2.0921370 & -0.0479650 & -0.8838730 \\
$\mathrm{C}$ & 0.5719180 & -2.4585120 & -0.2501620 \\
$\mathrm{C}$ & 1.9206730 & -2.7952800 & -0.2752290 \\
$\mathrm{C}$ & -0.4685560 & -3.3216550 & -0.6904610
\end{tabular}

$-3.1302530$

$-1.6683830$

1.7695750

$-1.4840220$

$-2.6085720$

2. 1447980

$-2.1417950$

$-1.8638640$

$-1.4873230$

$-1.3361450$

$-3.2437250$

$-2.9155320$

$-3.4412180$

$-2.6863370$

$-2.4058930$

$-2.6179750$

$-3.6492340$

$-3.2582390$

$-3.3308690$

$-1.7183100$

$-3.1810900$

$-4.4069710$

$-1.7320410$

$-1.7020800$

1.9489820

1.8465960

3. 2627380

3. 3907830

4. 3991610

4.6232700

2.5133980

5.6346030

4.3069450

5. 7497610

4.7053180

6. 5075220

6.7121390

0.9678000

0.7741860
1.9206730
-0.4685560
$-3.3216550$

$-0.6904610$

3. 4662910

3.8879550

$-1.4256290$ 


$\begin{array}{lrrr} & & & \\ \mathrm{C} & 2.2923640 & -4.0137360 & -0.9360830 \\ \mathrm{C} & -0.1025580 & -4.5350140 & -1.2351760 \\ \mathrm{C} & 1.2585870 & -4.8953560 & -1.4067310 \\ \mathrm{H} & -0.8724580 & -5.2310160 & -1.5582750 \\ \mathrm{C} & 2.9504050 & -0.5483900 & 0.0882130 \\ \mathrm{C} & 3.8514690 & 0.3712370 & 0.6893820 \\ \mathrm{C} & 2.9161180 & -1.9063960 & 0.3841430 \\ \mathrm{C} & 4.7712490 & -0.1304300 & 1.5871220 \\ \mathrm{C} & 3.8086830 & -2.3963460 & 1.3988940 \\ \mathrm{C} & 4.7718560 & -1.4944100 & 1.9718050 \\ \mathrm{H} & 5.4879930 & 0.5436880 & 2.0498550 \\ \mathrm{C} & 5.6233380 & -3.2798710 & 3.3964840 \\ \mathrm{C} & 4.6418500 & -4.1562280 & 2.8727960 \\ \mathrm{C} & 3.7588800 & -3.7258490 & -1.9019650 \\ \mathrm{C} & 5.6783730 & -1.9736280 & 2.9569310 \\ \mathrm{H} & 6.3180240 & -3.6336990 & 4.1549950 \\ \mathrm{H} & 4.5781820 & -5.1774130 & 3.2423440 \\ \mathrm{H} & 3.0103260 & -4.4101910 & 1.5175920 \\ \mathrm{H} & 6.4076070 & -1.2789410 & 3.3701830 \\ \mathrm{C} & 3.9649960 & -5.5537880 & -1.8206700 \\ \mathrm{C} & 2.9461680 & -6.4405310 & -2.2474000 \\ \mathrm{C} & 1.6211280 & -6.1121850 & -2.0463320 \\ \mathrm{C} & 3.6458550 & -4.3728210 & -1.1798890 \\ \mathrm{H} & 5.0080350 & -5.8032070 & -2.0029730 \\ \mathrm{H} & 3.2109790 & -7.3712940 & -2.7440880 \\ \mathrm{H} & 0.8275210 & -6.7747230 & -2.3874300 \\ \mathrm{H} & 4.4345850 & -3.6994420 & -0.8593220 \\ \mathrm{C} & -1.8941450 & -2.9149700 & -0.5060970 \\ \mathrm{C} & -2.6908400 & -2.5440890 & -1.6158680 \\ \mathrm{C} & -2.4335390 & -2.8692850 & 0.7989390 \\ \mathrm{C} & -4.0040200 & -2.1175360 & -1.3880490 \\ \mathrm{C} & -3.7557150 & -2.4431130 & 0.9710400 \\ \mathrm{C} & -4.5530540 & -2.0491050 & -0.1036680 \\ \mathrm{H} & -4.6178670 & -1.8242460 & -2.2358810 \\ \mathrm{H} & -4.1785510 & -2.4138790 & 1.9733830 \\ \mathrm{C} & 3.7440440 & 1.8302540 & 0.3937610 \\ \mathrm{C} & 3.2194670 & 2.7018760 & 1.3767020 \\ \mathrm{C} & 4.1121070 & 2.3316580 & -0.8750650 \\ \mathrm{C} & 3.0820300 & 4.0605320 & 1.0635480 \\ \mathrm{C} & 3.9502500 & 3.6946620 & -1.1388470 \\ \mathrm{C} & 3.4291580 & 4.5755020 & -0.1865030 \\ \mathrm{H} & 2.6725540 & 4.7390290 & -1.8095070 \\ \mathrm{H} & 4.2325380 & 4.0768060 & -2.1173740 \\ \mathrm{C} & -1.6498950 & -3.3284970 & 2.0227340 \\ \mathrm{C} & -5.9698960 & -1.5499240 & 0.1169400 \\ \mathrm{C} & -2.1723930 & -2.6338030 & -3.0459650 \\ & & & \end{array}$

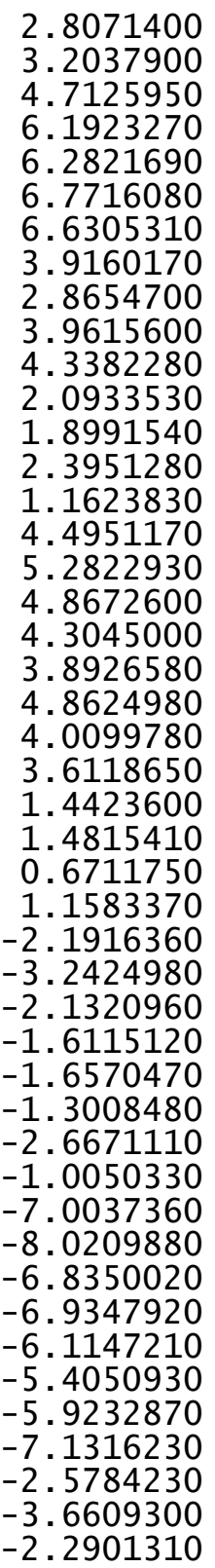

2. 2182480

6.0401160

1.4445550

1.8065960

2.8430320

1.7079260

1.1453470

1.5186260

1.2583740

2. 5282500

0.8197450

6.1923790

7.2539850

5.7096930

5.7226410

6.7369220

6.6561780

6.2815520

7. 8003830

2.5717060

2. 1411000

3. 6617440

2. 1920580

2.7692200

3.8544400

2. 5523110

2. 3026070

$-4.6837870$

$-4.5864400$

$-5.4416500$

$-5.0329750$

$-2.2896210$

$-1.3175080$

$-2.1631630$

$-2.6259680$

$-2.4353420$

$-2.0696920$

$-2.4166950$

$-3.4770730$

$-0.0809040$

0.5569520

0.0181110

0.2801750

$-1.4362660$

-1.4217110
-0.4911230
2.7624910 $-0.5162000$ $-1.9577180$ $-2.1934390$ $-2.5446210$

$-1.2663310$ $-2.9531200$ $-3.2732690$ $-3.1035440$ $-3.7021870$ $-4.0075790$ $-1.5752860$ $-1.7797000$ $-2.5144880$ $-1.2344050$ $-0.9825110$ $-0.2221020$ $-1.9097010$

$-1.1786630$

3.7992110

3.5227270

3.8668190

4.7911220

3.2155330

3.3750810

2. 4702900

4.1680780

2. 5192850

2.8227890

1.7275710

3. 3841290

3.1578630

2.7996290

3.5691610

3.9745330

$-0.6037000$

$-0.4097260$

$-1.6885020$

$-0.2651820$

$-0.3210480$

0.2166920

$-1.3971180$

$-0.1182920$

$-3.9218470$

-4.1033310
-3.4541160 


$\begin{array}{rrr}-2.0796490 & -1.5091620 & -4.8969970 \\ -2.6533160 & -3.9438870 & -3.7048130 \\ -3.7502460 & -3.9529270 & -3.7652630 \\ -2.2502570 & -4.0289770 & -4.7231650 \\ -2.3370840 & -4.8227160 & -3.1312050 \\ 0.5044160 & 0.1077890 & -0.5804330 \\ -0.1468280 & 0.0639680 & -1.9380120 \\ 0.2060480 & 1.2579630 & 0.3404950 \\ -1.0780470 & -2.6549260 & -3.0092380 \\ -6.1706020 & -1.5985080 & 1.1957930 \\ -0.6056350 & -3.4854870 & 1.7368110 \\ 4.6834470 & 0.4050980 & -1.6152220 \\ 2.8633700 & 6.5369490 & 0.4034150 \\ 2.7193940 & 1.1273070 & 2.7321290 \\ -2.5990400 & 2.0276230 & -1.7375240 \\ -1.6257920 & 3.0919560 & -1.4547130 \\ -2.5875450 & 1.1654330 & -1.0689930 \\ -0.7269600 & 2.8170640 & -0.9148730 \\ -1.6241590 & 1.8636770 & -2.8257920 \\ -0.9538130 & 1.1167140 & -2.4849980 \\ -3.9684030 & 3.6934690 & -3.0769390 \\ -3.2443000 & 4.8318590 & -2.3412530 \\ -1.7819520 & 4.4628680 & -2.0318400 \\ -3.9786580 & 2.4149440 & -2.2222160 \\ -5.0006890 & 3.9857020 & -3.3020750 \\ -3.2631440 & 5.7516360 & -2.9370090 \\ -1.1754270 & 4.4969480 & -2.9488640 \\ -4.5930550 & 2.5692940 & -1.3249190 \\ -4.4078660 & 1.5670130 & -2.7688900 \\ -3.4670100 & 3.4922520 & -4.0330000 \\ -3.7658250 & 5.0381290 & -1.3968190 \\ -1.3185050 & 5.1760920 & -1.3409190 \\ -2.8575560 & 2.1768670 & 1.6489440 \\ -2.0681610 & 1.1432090 & 1.4760150 \\ -4.1325980 & 1.8246660 & 2.3199410 \\ -5.2311000 & 2.7062020 & 2.3119560 \\ -4.2740260 & 0.5670040 & 2.9436350 \\ -6.4380220 & 2.3373740 & 2.9012540 \\ -5.1319800 & 3.6713770 & 1.8240570 \\ -5.4806460 & 0.2063840 & 3.5384350 \\ -3.4387270 & -0.1232570 & 2.9552800 \\ -6.5677550 & 1.0865330 & 3.5164490 \\ -7.2813390 & 3.0232350 & 2.8761300 \\ -5.5746730 & -0.7660970 & 4.0158140 \\ -7.5118610 & 0.7992130 & 1.0269450 \\ -1.1541330 & 1.3279970 & \end{array}$

14

$-2.4740430$

3.7335280

1.1452580

TS218(R,R) G[B97-D/def2-TZVP $]=-3633.749067$

\begin{tabular}{|c|c|c|c|}
\hline & & & \\
\hline 0 & 0.7115180 & 0.2505250 & -0.4343040 \\
\hline 0 & -1.5869300 & 0.2624710 & -1.5626370 \\
\hline$C$ & 0.9752830 & 1.4724880 & -1.0375930 \\
\hline & 0.0143500 & 2.4767340 & -0.9988630 \\
\hline & 2.2681840 & 1.6669000 & -1.5924430 \\
\hline & 0.2832890 & 3.7137710 & -1.6709680 \\
\hline & 2.5431120 & 2.8849870 & -2.1811230 \\
\hline & 1.5746180 & 3.9144490 & -2.2673750 \\
\hline & 3.5364040 & 3.0646660 & -2.5863170 \\
\hline & -2.0698120 & 1.1783860 & -0.6149840 \\
\hline & -3.3460570 & 0.9378120 & -0.0372350 \\
\hline & -1.2648600 & 2.2560450 & -0.2788120 \\
\hline C & -3.7667090 & 1.8094960 & 0.9489450 \\
\hline$C$ & -1.6693530 & 3.0949170 & 0.8104080 \\
\hline C & -2.9461570 & 2.8617300 & 1.4251050 \\
\hline H & -4.7417760 & 1. 6612610 & 1.4052210 \\
\hline & -2.5129230 & 4.6500860 & 3.02 \\
\hline & -1.2402180 & 4.8596930 & 2.4 \\
\hline & -0.8307730 & 4.1046270 & 1.3 \\
\hline 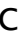 & -3.3466850 & 3.6703790 & 2.5 \\
\hline & -2.824 & 5.2536900 & 3.8 \\
\hline & -0.57 & 5.6167210 & 0 \\
\hline & 0.150 & 4.2670380 & 0.9 \\
\hline & $-4.3]$ & 3.4864930 & 50 \\
\hline & -0.38 & 5.9247910 & -2 \\
\hline & 0.9005310 & 6.1315250 & -3 \\
\hline & 1.8569 & 5. 1416070 & -2 \\
\hline & -0.68 & 4.7480870 & -1 \\
\hline & -1.1426750 & 6.6988720 & 50 \\
\hline & 1. 1248110 & 7.0654780 & -3 \\
\hline & 2.8439430 & 5.2794910 & 10 \\
\hline & -1.6748820 & 4.6017810 & 90 \\
\hline & 3.3524550 & 0.6478780 & -1.454 \\
\hline & 3.5917100 & -0.2945940 & 880 \\
\hline & 4. 1756710 & 0.6823670 & 7510 \\
\hline & 4.6813170 & -1.1610700 & -2.3 \\
\hline & 5.246 & -0.2169640 & -0.2 \\
\hline & 5.525 & -1.1352040 & -1.2311280 \\
\hline & 4.88 & -1.8902870 & -3.1298450 \\
\hline & 5.87 & -0.1949060 & 0.66979 \\
\hline & -4.19 & -0.2111470 & -0.4664500 \\
\hline & -4.49 & -1.2586950 & 0.4428680 \\
\hline & -4 & 30 & \\
\hline
\end{tabular}




$\begin{array}{rrr}-5.3158120 & -2.3049260 & 0.0188380 \\ -5.5408490 & -1.3199360 & -2.1501630 \\ -5.8495130 & -2.3590190 & -1.2720540 \\ -5.5446650 & -3.1064490 & 0.7172640 \\ -5.9554550 & -1.3438710 & -3.1567240 \\ 3.9004740 & 1.6317370 & 0.8505150 \\ 6.7193760 & -2.0750970 & -1.1784370 \\ 2.7150210 & -0.3352410 & -3.7236270 \\ -3.9567430 & -1.2892850 & 1.8709010 \\ -6.7294520 & -3.5165450 & -1.7077990 \\ -4.4921080 & 0.8682310 & -2.7908730 \\ -5.8065710 & 1.6201290 & -3.0805440 \\ -6.5456310 & 0.9450640 & -3.5316290 \\ -6.2349210 & 2.0296060 & -2.1565570 \\ -5.6244880 & 2.4479220 & -3.7791880 \\ -3.8675460 & 0.3376910 & -4.0947760 \\ -2.9298690 & -0.1902400 & -3.8878590 \\ -4.5526600 & -0.3535880 & -4.6027910 \\ -3.6583860 & 1.1717880 & -4.7777450 \\ -8.0044540 & -3.6133800 & -0.8484510 \\ -8.6442280 & -4.4320860 & -1.2039820 \\ -7.7451220 & -3.8130850 & 0.1996220 \\ -8.5766900 & -2.6777730 & -0.8872480 \\ -5.9556030 & -4.8490730 & -1.6750830 \\ -5.0568910 & -4.7970010 & -2.3025440 \\ -5.6432050 & -5.0836540 & -0.6488390 \\ -6.5904420 & -5.6693320 & -2.0360850 \\ -5.0740880 & -0.9419130 & 2.8754870 \\ -5.5165880 & 0.0387670 & 2.6632820 \\ -5.8735300 & -1.6934780 & 2.8250400 \\ -4.6753690 & -0.9298350 & 3.8990020 \\ -3.3267880 & -2.6449000 & 2.2436750 \\ -4.0852470 & -3.4373900 & 2.2866080 \\ -2.5601320 & -2.9287650 & 1.5166590 \\ -2.8608130 & -2.5719460 & 3.2350180 \\ 5.1579170 & 2.3829760 & 1.3219060 \\ 5.9033900 & 1.6933860 & 1.7376170 \\ 5.6229360 & 2.9320720 & 0.4931280 \\ 4.8876230 & 3.0991840 & 2.1088090 \\ 3.2478710 & 0.8653300 & 2.0162070 \\ 2.3263480 & 0.3743730 & 1.6880950 \\ 3.9288590 & 0.0929090 & 2.3977570 \\ 3.0047380 & 1.5490750 & 0.2417300 \\ 7.1280340 & -2.4953980 & 0.1912160 \\ 7.9282990 & -3.2442350 & \\ 7.5095450 & -1.6375580 & \\ -5 & & \\ -5 & & \\ -5 & -103480\end{array}$

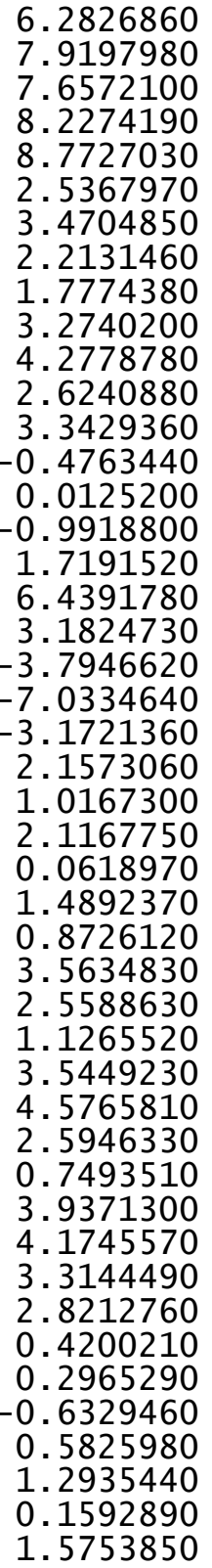
$-2.9234720$
$-1.4374640$
$-1.1793030$
$-0.5186330$
$-2.1294270$
$-1.7450900$
$-2.1117240$
$-2.4558890$
$-1.7177980$
0.6130240
0.2854230
0.6034750
1. 6421100
$-0.7590390$
$-1.6394650$
$-1.4300000$
0.0243400
2. 3870590
1.5971970
$-3.3263840$
$-0.5346590$
$-2.9558290$
$-3.6386590$
$-1.8666480$
$-3.1262600$
$-3.5726040$
$-2.8448310$
$-5.0271830$
$-5.4222530$
$-5.0285030$
$-3.5079340$
$-5.3343660$
$-6.5001710$
$-5.7018670$
-2.9899740
$-3.2186220$
$-5.5449340$
$-4.9094430$
$-5.1223860$
$-0.7214330$
$-0.3267490$
0.2832460
$-0.0513350$
1. 6174470
0.9197230

0.7925480

$-1.9103410$

$-2.9436870$

$-1.3929010$

$-1.9258400$

$-4.3089160$

$-4.7551710$

$-3.5417890$

$-5.1001690$

$-4.8048810$

$-5.1085560$

$-5.6904210$

$-4.4354570$

$-0.9548140$

$-2.0663350$

0.2917740

$-3.4388770$

$-1.7329600$

0.5132750

$-2.3681710$

$-2.7469500$

1.9632500

0.0101310

0.6373090

$-0.0366320$

0.6245430

$-1.1584450$

$-1.5600840$

0.4741850

1. 5657410

1.1633520

0.2441650

0.7590400

1.7598990

0.3779710

1. 1298090

$-0.6007980$

$-0.4619330$

2. 5014680

1. 9970260

3. 3174750

2. 4901350

4.3777360

5.5449300

4.1964990

6. 5041860 


$\begin{array}{lrrr}\mathrm{H} & 1.6182610 & -1.0774080 & 5.6929560 \\ \mathrm{C} & 0.4548080 & 2.5876510 & 5.1524010 \\ \mathrm{H} & -0.3796890 & 1.8970300 & 3.2972430 \\ \mathrm{C} & 1.1620210 & 2.2434440 & 6.3096100 \\ \mathrm{H} & 2.1195790 & 0.6451660 & 7.4048110 \\ \mathrm{H} & 0.1329430 & 3.6133130 & 4.9882070 \\ \mathrm{H} & 1.3905840 & 3.0009610 & 7.0561520 \\ \mathrm{H} & -0.7494740 & -0.8876540 & 1.6144830 \\ \mathrm{~S} & 1.1164750 & -2.1881020 & 3.2047000 \\ \mathrm{I} 44 & & & \\ \mathrm{TS} 219(\mathrm{R}) \mathrm{R}) & \mathrm{G}[\mathrm{B} 97-\mathrm{D} / \mathrm{def} 2-\mathrm{TZVP}] & -3633.748892 \\ \mathrm{O} & 0.2580090 & 0.9156400 & 0.0133230 \\ \mathrm{O} & -2.0138030 & 0.5419380 & -1.0692470 \\ \mathrm{C} & 0.2213670 & 2.2734020 & -0.2835260 \\ \mathrm{C} & -0.9629710 & 2.9742050 & -0.0944120 \\ \mathrm{C} & 1.4405100 & 2.8851120 & -0.6859590 \\ \mathrm{C} & -1.0068630 & 4.3467060 & -0.5099420 \\ \mathrm{C} & 1.4064500 & 4.2270280 & -1.0056240 \\ \mathrm{C} & 0.2023130 & 4.9742300 & -0.9690340 \\ \mathrm{H} & 2.3241170 & 4.7274770 & -1.3040930 \\ \mathrm{C} & -2.6120010 & 1.1016380 & 0.0557400 \\ \mathrm{C} & -3.7385410 & 0.4271680 & 0.5994040 \\ \mathrm{C} & -2.1233060 & 2.3048270 & 0.5506090 \\ \mathrm{C} & -4.3686070 & 1.0048310 & 1.6831050 \\ \mathrm{C} & -2.7237860 & 2.8415110 & 1.7400150 \\ \mathrm{C} & -3.8807070 & 2.1884240 & 2.2900810 \\ \mathrm{H} & -5.2482500 & 0.5250120 & 2.1050530 \\ \mathrm{C} & -3.9792500 & 3.8360650 & 4.0835460 \\ \mathrm{C} & -2.8110030 & 4.4523830 & 3.5722580 \\ \mathrm{C} & -2.2005150 & 3.9686750 & 2.4321080 \\ \mathrm{C} & -4.4968250 & 2.7223070 & 3.4553700 \\ \mathrm{H} & -4.4548470 & 4.2323830 & 4.9779160 \\ \mathrm{H} & -2.3865710 & 5.3125720 & 4.0854300 \\ \mathrm{H} & -1.3027150 & 4.4505150 & 2.0602200 \\ \mathrm{H} & -5.3791370 & 2.2211420 & 3.8499560 \\ \mathrm{C} & -2.2097010 & 6.4265080 & -0.9310250 \\ \mathrm{C} & -1.0110000 & 7.0529030 & -1.3523930 \\ \mathrm{C} & 0.1682610 & 6.3368520 & -1.3728420 \\ \mathrm{C} & -2.2066200 & 5.1086720 & -0.5184920 \\ \mathrm{H} & -3.1417710 & 6.9873570 & -0.9361310 \\ \mathrm{H} & -1.0257280 & 8.0935250 & -1.6685920 \\ \mathrm{H} & 1.0938040 & 6.8013590 & -1.7086910 \\ \mathrm{H} & -3.1310460 & 4.6377370 & -0.1990090 \\ \mathrm{C} & 2.7021830 & 2.0877900 & -0.6944780 \\ \mathrm{C} & 3.3367190 & 1.7509520 & -1.9095360 \\ \mathrm{C} & 3.2467270 & 1.6503730 & 0.5405100\end{array}$

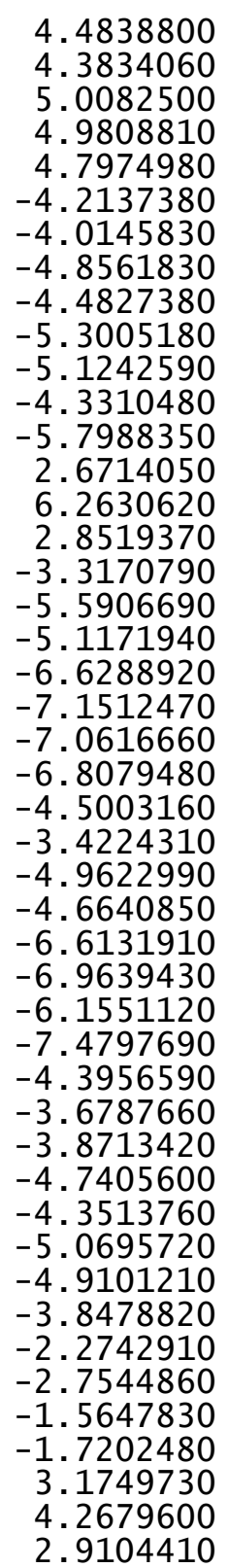

$\begin{array}{ll}\text { C } & 4.4838800 \\ \text { C } & 4.3834060\end{array}$

\author{
0.9458600 \\ 0.8382070 \\ 0.4601460 \\ 0.6796650 \\ 0.4907330 \\ $-0.8622260$ \\ $-2.0720100$ \\ $-0.8756160$ \\ $-3.2646270$ \\ $-2.0978780$ \\ - 3.3018190 \\ $-4.1954910$ \\ $-2.1118450$ \\ 2. 1411410 \\ $-0.3955160$ \\ 2. 2821370 \\ $-2.1161800$ \\ $-4.6162300$ \\ 0.3940630 \\ 0.6862130 \\ $-0.1271770$ \\ 0.7839600 \\ 1. 6192840 \\ 0.3201770 \\ 0.1333560 \\ $-0.4841110$ \\ 1. 2669300 \\ $-5.3255440$ \\ $-6.2532170$ \\ $-5.5845400$ \\ $-4.6810120$ \\ $-5.5442730$ \\ $-5.0579210$ \\ $-5.7980990$ \\ $-6.4769780$ \\ $-2.2496390$ \\ $-1.4215010$ \\ $-3.1892490$ \\ $-2.2571800$ \\ $-3.2451380$ \\ $-4.2322100$ \\ -3. 1942340 \\ $-3.1488340$ \\ 3. 5750520 \\ 3. 5726620 \\ 4.2493910
}

-1.8661680
0.5253260
-0.6684510
-2.7967940
1.4663880
0.0167790
0.7255810
-1.2396670
0.1650680
-1.7597570
-1.0771330
0.7064160
-2.7278670
1.8659450
-0.6735820
-3.2533190
2.0807410
-1.6763860
-2.0404600
-2.1191840
-2.6397620
-1.1151810
-2.6701560
-3.4491080
-3.3886670
-4.0365190
-3.9810300
-0.7687000
-1.2401760
0.1949560
-0.5737430
-1.9717120
-2.6454120
-1.0409370
-2.4379490
3.2166470
3.2010950
3.1084980
4.1929900
2.1832080
2.1775930
1.3518860
3.1259560
2.1396440
2.2506480
1.3171450 


$\begin{array}{rrr}2.7325340 & 3.9632980 & 3.0671500 \\ 2.9742960 & 1.2383320 & 3.0695310 \\ 2.6775220 & 0.2032370 & 2.8783570 \\ 4.0424970 & 1.2521770 & 3.3238550 \\ 2.4207630 & 1.6016650 & 3.9443210 \\ 6.2347410 & -1.5288190 & 0.3665560 \\ 7.1131300 & -2.1744350 & 0.2405350 \\ 6.2588790 & -1.1258960 & 1.3870190 \\ 5.3304350 & -2.1421710 & 0.2697890 \\ 7.5101390 & 0.4888130 & -0.4641570 \\ 7.5653280 & 1.2757290 & -1.2268190 \\ 7.4682500 & 0.9688460 & 0.5229530 \\ 8.4251900 & -0.1167160 & -0.5162550 \\ 2.8010270 & 1.2133430 & -4.3575620 \\ 3.8008940 & 0.8241140 & -4.5879860 \\ 2.1614650 & 0.3761460 & -4.0654390 \\ 2.3965320 & 1.6559870 & -5.2769880 \\ 3.7436030 & 3.4593670 & -3.7000530 \\ 4.7740580 & 3.1133220 & -3.8575460 \\ 3.3724710 & 3.8827130 & -4.6430930 \\ 3.7640010 & 4.2539890 & -2.9446090 \\ -0.5388730 & -0.1313310 & -0.9540900 \\ -0.0136110 & -0.1180160 & -2.3655850 \\ -0.5446010 & -1.4262120 & -0.1901810 \\ 1.8338410 & 2.6638920 & -3.1246370 \\ 6.3411180 & -0.8514490 & -1.6705730 \\ 1.5816210 & 2.1920860 & 1.7758740 \\ -4.6519560 & 1.2393380 & -1.5235000 \\ -6.0852300 & -4.3870000 & -2.6310820 \\ -2.7845100 & -1.1696450 & 2.2220020 \\ 2.4993850 & -2.0041340 & -2.1407870 \\ 1.5361260 & -3.0756670 & -1.8628930 \\ 2.4739060 & -1.1316510 & -1.4845720 \\ 0.6289160 & -2.8100650 & -1.3332280 \\ 1.5240110 & -1.8793060 & -3.2405010 \\ 0.8321520 & -1.1507380 & -2.9095550 \\ 3.9032000 & -3.6714190 & -3.4469880 \\ 3.1937300 & -4.8068860 & -2.6932620 \\ 1.7217060 & -4.4566590 & -2.4085500 \\ 3.8840830 & -2.3751580 & -2.6204440 \\ 4.9426490 & -3.9488200 & -3.6580010 \\ 3.2361330 & -5.7394910 & -3.2675150 \\ 1.1284450 & -4.5222030 & -3.3323190 \\ 4.5026600 & -2.4959010 & -1.7222170 \\ 4.2945270 & -1.5294740 & -3.1822280 \\ 3.4059290 & -3.5008590 & -4.4111880\end{array}$

3.7116830
1.2630030
1.9348280
0.9624190
2.6294440
3.9839800
1.9339870
4.6299410
4.5261320
2.5781070
0.8899410
3.9286800
5.6824010
2.0276940
4.4329480
0.4169570
2.3749940
$-4.9846380$
$-5.1614130$
$-2.5655120$
$-1.6938350$
$-2.5001300$
$-2.8596980$
$-2.0452960$
$-2.7570670$
$-3.1952630$
$-1.9666710$
$-1.7617500$
$-2.3159710$
$-3.0189440$
$-1.6276720$
$-2.2430870$
$-1.6920150$
$-3.6778870$

$-1.7405970$

$-1.7054310$

1.9467040

1.8466070

3. 2584950

3. 3828320

4. 3968320

4. 6135290

2. 5040010

5.6304810

4. 3074810

5. 7418900

4.6927130

6.5049210

6.7028560

0.9705110

0.7711220

TS220(R,R) G[B97-D/def2-TZVP $]=-3633.748808$

$\begin{array}{lrrr}\text { TS220 (R, R) G }[\mathrm{B} 97-\mathrm{D} / \mathrm{def}-\mathrm{TZVP}]=-3633.748808 \\ \mathrm{O} & 0.1990120 & -1.2369680 & 0.2956450 \\ \mathrm{O} & 2.0914090 & -0.0350960 & -0.8798860 \\ \mathrm{C} & 0.5909730 & -2.4568240 & -0.2472710 \\ \mathrm{C} & 1.9421550 & -2.7836690 & -0.2724430 \\ \mathrm{C} & -0.4430780 & -3.3263000 & -0.6901120 \\ \mathrm{C} & 2.3228210 & -3.9989380 & -0.9340620 \\ \mathrm{C} & -0.0682400 & -4.5368020 & -1.2351550 \\ \mathrm{C} & 1.2955300 & -4.8876590 & -1.4056000 \\ \mathrm{H} & -0.8329340 & -5.2378590 & -1.5596490 \\ \mathrm{C} & 2.9551330 & -0.5291890 & 0.0904930 \\ \mathrm{C} & 3.8506670 & 0.3970440 & 0.6898840 \\ \mathrm{C} & 2.9311880 & -1.8873770 & 0.3866250 \\ \mathrm{C} & 4.7743900 & -0.0977350 & 1.5874730 \\ \mathrm{C} & 3.8279850 & -2.3705400 & 1.4008260 \\ \mathrm{C} & 4.7847250 & -1.4613950 & 1.9731150 \\ \mathrm{H} & 5.4865680 & 0.5817460 & 2.0494110 \\ \mathrm{C} & 5.6493090 & -3.2401600 & 3.3983050 \\ \mathrm{C} & 4.6742580 & -4.1239070 & 2.8749500 \\ \mathrm{C} & 3.7880630 & -3.7002610 & 1.9041170 \\ \mathrm{C} & 5.6948180 & -1.9336910 & 2.9582950 \\ \mathrm{H} & 6.3466570 & -3.5886410 & 4.1568510 \\ \mathrm{H} & 4.6182000 & -5.1454430 & 3.2447580 \\ \mathrm{H} & 3.0445010 & -4.3900790 & 1.5197970 \\ \mathrm{H} & 6.4191090 & -1.2336220 & 3.3711590 \\ \mathrm{C} & 4.0066300 & -5.5267300 & -1.8188240 \\ \mathrm{C} & 2.9942940 & -6.4205570 & -2.2462080 \\ \mathrm{C} & 1.6669020 & -6.1016410 & -2.0455320\end{array}$




$\begin{array}{rrr}3.6789060 & -4.3482830 & -1.1777230 \\ 5.0514820 & -5.7686120 & -2.0009100 \\ 3.2659070 & -7.3492240 & -2.7431440 \\ 0.8780960 & -6.7695850 & -2.3872170 \\ 4.4628170 & -3.6694790 & -0.8568310 \\ -1.8715700 & -2.9283370 & -0.5090730 \\ -2.6665600 & -2.5592490 & -1.6206510 \\ -2.4153410 & -2.8882160 & 0.7942610 \\ -3.9828380 & -2.1405730 & -1.3961390 \\ -3.7404390 & -2.4699170 & 0.9630300 \\ -4.5364640 & -2.0780760 & -0.1134000 \\ -4.5955010 & -1.8485860 & -2.2452550 \\ -4.1666890 & -2.4453690 & 1.9640480 \\ 3.7337300 & 1.8549520 & 0.3928010 \\ 3.2123040 & 2.7260450 & 1.3781030 \\ 4.0906570 & 2.3563990 & -0.8790950 \\ 3.0688970 & 4.0838360 & 1.0645960 \\ 3.9202910 & 3.7183400 & -1.1441050 \\ 3.4048160 & 4.5989850 & -0.1886580 \\ 2.6630650 & 4.7617450 & 1.8131430 \\ 4.1926580 & 4.0988490 & -2.1259010 \\ -1.6327500 & -3.3458220 & 2.0193010 \\ -5.9568020 & -1.5872710 & 0.1036590 \\ -2.1426640 & -2.6423400 & -3.0492050 \\ 2.8079890 & 2.2428650 & 2.7664910 \\ 3.1582090 & 6.0605460 & -0.5181540 \\ 4.6908790 & 1.4716520 & -1.9638890 \\ 6.1683990 & 1.8401880 & -2.2038680 \\ 6.2523900 & 2.8768350 & -2.5558740 \\ 6.7506240 & 1.7447320 & -1.2782690 \\ 6.6075260 & 1.1804690 & -2.9643560 \\ 3.8905190 & 1.5422550 & -3.2772860 \\ 2.8413080 & 1.2785760 & -3.1047440 \\ 3.9315730 & 2.5516370 & -3.7071850 \\ 4.3132010 & 0.8443240 & -4.0122120 \\ 1.9602910 & 6.1963820 & -1.4804120 \\ 1.7455410 & 7.2545030 & -1.6826820 \\ 2.1822300 & 5.6998590 & -2.4345650 \\ 1.0635690 & 5.7300390 & -1.0540520 \\ 4.4021990 & 6.7527000 & -1.1037700 \\ 5.2554540 & 6.6762430 & -0.4178920 \\ 4.6879310 & 6.2929790 & -2.0587120 \\ 4.1939870 & 7.8148380 & -1.2881890 \\ 3.8932600 & 2.6071330 & 3.7995890 \\ 4.8655630 & 2.1827650 & 3.5219650 \\ 4.0029150 & 3.6981560 & 3.8640190\end{array}$

3.6175640
1.4399700
1.4707260
0.6685100
1.1624460
-2.1667750
-3.2193440
-2.0993620
-1.5872720
-1.6512110
-1.3002290
-2.6636680
-1.0000590
-6.9834160
-8.0034130
-6.8123960
-6.9086170
-6.1086680
-5.4046950
-5.9138380
-7.1283530
-2.5564800
-3.6383770
-2.2788170
-2.0533600
-2.6098840
-3.7064840
-2.2026890
-2.2879880
0.5032920
-0.1503430
0.1990400
-1.0482790
-6.1603720
-0.5864250
4.6673970
2.8956600
2.7281470
-2.6088990
-1.6396910
-2.5949370
-0.7404020
-1.6325000
-0.9599460
-3.9828350
-3.2631280

2.2282220

2. 7848420

3. 8711280

2. 5578900

2.3206000

$-4.7064930$

$-4.6173040$

$-5.4611920$

$-5.0547080$

$-2.3108240$

$-1.3351710$

$-2.1925040$

$-2.6454100$

$-2.4778460$

$-2.1190740$

$-2.4560510$

$-3.5197660$

$-0.1186570$

0.5226540

$-0.0173290$

0.2363920

$-1.4473640$

$-1.4426740$

$-0.5001730$

$-1.5143860$

$-3.9555690$

$-3.9739940$

$-4.0354730$

$-4.8325470$

0.1093040

0.0631240

1. 2556070

$-2.6536250$

$-1.6382030$

$-3.4944890$

0.4320750

6.5703450

1.1512420

.0170870

3. 0841040

1.1533810

2. 8112660

1.8587450

1. 1136760

3.6813350

4.8205150
4.7932070

3. 2208380

3.3748090

2. 4787880

4.1764700

2. 5093870

2. 8095260

1.7152810

3. 3750430

3. 1578110

2.8040850

3.5655840

3.9758870

$-0.6209640$

$-0.4285830$

$-1.7053480$

$-0.2842760$

$-0.3331650$

0.2078670

$-1.4084010$

$-0.1337440$

$-3.9249460$

$-4.1106490$

$-3.4549420$

$-4.8982890$

$-3.7116620$

$-3.7758140$

$-4.7287870$

$-3.1384580$

$-0.5729090$

$-1.9292150$

0.3509560

$-3.0088510$

1. 1818780

1.7364300

$-1.6214900$

0.4197220

2.7391750

$-1.7293220$

$-1.4429140$

$-1.0628320$

$-0.9026320$

$-2.8170580$

$-2.4767130$

$-3.0660380$

$-2.3272720$ 


\begin{tabular}{|c|c|c|c|c|c|c|c|}
\hline c & $\begin{array}{l}-1.1999310 \\
-3.9894490\end{array}$ & $\begin{array}{l}4.4556800 \\
2.4008600\end{array}$ & $\begin{array}{l}-2.0170320 \\
-2.2142120\end{array}$ & $\mathrm{C}$ & $\begin{array}{l}-3.4646010 \\
-2.9611490\end{array}$ & $\begin{array}{l}4.0722070 \\
5.9000110\end{array}$ & $\begin{array}{l}2.1324040 \\
3.1345130\end{array}$ \\
\hline $\mathrm{H}$ & -5.0159240 & 3.9705820 & -3.2913590 & $\mathrm{H}$ & -0.7374360 & 6.1008670 & 2.0159140 \\
\hline$H$ & -3.2843370 & 5.7414450 & -2.9211720 & $\mathrm{H}$ & -0.0080930 & 4.4176800 & 0.3775390 \\
\hline$H$ & -1.1922620 & 4.4940900 & -2.9331180 & $\mathrm{H}$ & -4.4258990 & 3.9593690 & 2.6304270 \\
\hline$H$ & -4.6050680 & 2.5511590 & -1.3170340 & $\mathrm{C}$ & -0.6154670 & 5.4405400 & -3.1919700 \\
\hline $\mathrm{H}$ & -4.4154840 & 1.5527730 & -2.7631160 & $\mathrm{C}$ & 0.6544630 & 5.5594870 & -3.8094070 \\
\hline$H$ & -3.4800220 & 3.4839090 & -4.0221470 & $\mathrm{C}$ & 1.6238500 & 4.6069840 & -3.5732750 \\
\hline $\mathrm{H}$ & -3.7863310 & 5.0232140 & -1.3829820 & $\mathrm{C}$ & -0.8883360 & 4. 3906180 & 3378150 \\
\hline$H$ & -1.3393770 & 5.1687020 & -1.3240880 & $\mathrm{H}$ & -1.3853500 & 6.1811600 & -3.3973910 \\
\hline c & -2.8722720 & 2.1546960 & 1.6556060 & $\mathrm{H}$ & 0.8565720 & 6.3949010 & -4.4759730 \\
\hline 0 & -2.0765030 & 1.1258520 & 1.4829530 & $\mathrm{H}$ & 2.5987510 & 4.6747180 & -4.0528980 \\
\hline c & -4.1477190 & 1.7934420 & 2.3209420 & $\mathrm{H}$ & -1.8677140 & 4.3102640 & -1.8771740 \\
\hline c & -5.2525590 & 2.6669550 & 2.3074790 & C & 3.1807860 & 0.3949080 & -1.4816270 \\
\hline c & -4.2828270 & 0.5350530 & 2.9445620 & $\mathrm{C}$ & 3.4382280 & -0.5862310 & -2.4642980 \\
\hline c & -6.4595610 & 2.2895130 & 2.8911620 & $\mathrm{C}$ & 4.0002700 & 0.4908340 & -0.3346100 \\
\hline $\mathrm{H}$ & -5.1582410 & 3.6325940 & 1.8195350 & $\mathrm{C}$ & 4.5521500 & -1.4161370 & -2.3039900 \\
\hline c & -5.4895360 & 0.1659350 & 3.5339300 & $\mathrm{C}$ & 5.1012480 & -0.3687060 & -0.2187910 \\
\hline H & -3.4423900 & -0.1488800 & 2.9608100 & C & 5.4034810 & -1.3162520 & -1.1994950 \\
\hline c & -6.5830330 & 1.0379820 & 3.5062350 & $\mathrm{H}$ & 4.7631470 & -2.1748900 & -3.0549030 \\
\hline $\mathrm{H}$ & -7.3077930 & 2.9691060 & 2.8616460 & $\mathrm{H}$ & 5.7328190 & -0.2949750 & 0.6603890 \\
\hline$H$ & -5.5785350 & -0.8069150 & 4.0115180 & $\mathrm{C}$ & -4.2841520 & -0.3215120 & -0.0427090 \\
\hline$H$ & -7.5271660 & 0.7438670 & 3.9591590 & C & -4.5015600 & -1.1986290 & 1.0469990 \\
\hline $\mathrm{H}$ & -1.1626110 & 1.3168680 & 1.0363710 & C & -4.8759690 & -0.5951130 & -1.2987550 \\
\hline S & -2.4958010 & 3.7147840 & 1.1572990 & C & -5.3162660 & -2.3220080 & 0.8529510 \\
\hline & R) & 20 & 26 & $\begin{array}{l}\mathrm{C} \\
\mathrm{C}\end{array}$ & $\begin{array}{l}-5.6744060 \\
-5.9089740\end{array}$ & $\begin{array}{l}-1.7337180 \\
-2\end{array}$ & $\begin{array}{l}-1.4389670 \\
-0.3768960\end{array}$ \\
\hline 0 & 0.5783490 & 0.1830600 & -0.2908860 & $\mathrm{H}$ & -5.4877540 & -3.0007680 & 1.6861020 \\
\hline 0 & -1.7495160 & 0.0157770 & -1.3361740 & $\mathrm{H}$ & -6.1327580 & -1.9354590 & -2.4047240 \\
\hline $\mathrm{C}$ & 0.8217550 & 1.2983480 & -1.0815520 & $\mathrm{C}$ & 3.7326530 & 1.5485610 & 0.7316550 \\
\hline $\mathrm{c}$ & -0.1461630 & 2.2932520 & -1.1795030 & $\mathrm{C}$ & 6.6199990 & -2.2256780 & -1.1228620 \\
\hline C & 2.0971590 & 1.4014600 & -1.6963780 & C & 2.5553530 & -0.7266770 & -3.6984220 \\
\hline C & 0.0943500 & 3.4014080 & -2.0563430 & $\mathrm{C}$ & -3.8964810 & -0.9659770 & 2.4270220 \\
\hline C & 2.3464150 & 2.5094040 & -2.4817220 & C & -6.7740020 & -3.8456840 & -0.5593640 \\
\hline C & 1.3689620 & 3.5074330 & -2.7091800 & C & -4.7253850 & 0.3305510 & -2.5002590 \\
\hline H & 3.32 & 2.6188500 & -2.9461080 & $\mathrm{C}$ & -6.0771100 & 0.9808900 & -2.8565540 \\
\hline c & -2.2014310 & 1.0599200 & -0.5187380 & $\mathrm{H}$ & -6.8116340 & 0.2150560 & -3.1385710 \\
\hline $\mathrm{C}$ & -3.4524900 & 0.9041910 & 0.1364790 & $\mathrm{H}$ & -6.4784190 & 1.5419670 & -2.0026840 \\
\hline C & -1.40 & 2.1885630 & -0.3979560 & $\mathrm{H}$ & -5.9554050 & 1.6701110 & -3.7029830 \\
\hline c & -3.86 & 1.9350380 & 0.9571170 & C & -4.1326900 & -0.4007320 & -3.7185470 \\
\hline C & -1.80 & 3.2081120 & 0.5289440 & $\mathrm{H}$ & -3.1651960 & -0.8488760 & -3.4669040 \\
\hline c & -3.0 & 3.0739020 & 1.2035110 & $\mathrm{H}$ & -4.8068600 & -1.1957310 & -4.0633610 \\
\hline $\mathrm{H}$ & -4.8 & 1.8574080 & 1.4605890 & $\mathrm{H}$ & -3.9891260 & 0.3056830 & -4.5470460 \\
\hline C & -2.6 & 5.1475580 & 2.4143680 & C & -6.1562510 & -4.8124740 & -1.5880200 \\
\hline c & -1.3 & 5.2635840 & 1.7754560 & $\mathrm{H}$ & -6.7696410 & -5.7189880 & -1.6780680 \\
\hline C & -0 & 06 & 0.8545680 & $\mathrm{H}$ & -6.1020870 & -4.3353910 & -2.5754330 \\
\hline
\end{tabular}




$\begin{array}{rrr}-5.1399870 & -5.1028210 & -1.2930540 \\ -8.2125900 & -3.4728430 & -0.9651920 \\ -8.6710600 & -2.8076740 & -0.2223810 \\ -8.2159340 & -2.9572730 & -1.9347110 \\ -8.8305640 & -4.3760570 & -1.0565420 \\ -4.9610140 & -0.4399170 & 3.4109830 \\ -5.4243140 & 0.4842550 & 3.0462980 \\ -5.7544140 & -1.1871540 & 3.5477410 \\ -4.5055260 & -0.2380080 & 4.3899530 \\ -3.2322850 & -2.2289040 & 3.0083090 \\ -3.9802730 & -2.9968160 & 3.2452280 \\ -2.5089020 & -2.6472360 & 2.3021340 \\ -2.7087900 & -1.9724860 & 3.9386530 \\ 4.3997230 & 2.8858860 & 0.3450790 \\ 5.4901790 & 2.7598120 & 0.2992560 \\ 4.0507170 & 3.2417550 & -0.6301980 \\ 4.1715830 & 3.6517180 & 1.0989820 \\ 4.1761930 & 1.1439340 & 2.1453330 \\ 3.7575920 & 0.1737110 & 2.4352820 \\ 5.2704900 & 1.0915040 & 2.2184900 \\ 3.8286620 & 1.8951310 & 2.8630850 \\ 7.1537840 & -2.4555240 & 0.2978780 \\ 7.9586150 & -3.2006290 & 0.2755200 \\ 7.5679820 & -1.5289350 & 0.7164510 \\ 6.3658820 & -2.8129510 & 0.9713360 \\ 7.7432400 & -1.6794420 & -2.0302000 \\ 7.3910390 & -1.5587960 & -3.0619120 \\ 8.0780480 & -0.6995150 & -1.6633990 \\ 8.6033600 & -2.3624840 & -2.0295720 \\ 2.3173000 & -2.1887780 & -4.1129520 \\ 3.2379240 & -2.6525920 & -4.4906520 \\ 1.9461120 & -2.7826640 & -3.2718310 \\ 1.5727960 & -2.2224260 & -4.9183590 \\ 3.1515160 & 0.0632430 & -4.8811210 \\ 4.1427740 & -0.3352520 & -5.1371970 \\ 2.5036040 & -0.0261360 & -5.7636080 \\ 3.2593890 & 1.1252160 & -4.6348720 \\ -0.5900300 & -0.9079780 & -0.6686870 \\ -0.1237410 & -1.8751880 & -1.7164100 \\ -1.0375120 & -1.4625720 & 0.6601610 \\ 1.5761800 & -0.2952540 & -3.4615270 \\ 6.3151160 & -3.2014440 & -1.5303550 \\ 2.6495960 & 1.7182730 & 0.7696050 \\ -4.0393120 & 1.1424890 & -2.2409920 \\ -6.8181560 & -4.3635840 & 2.3360380 \\ -3.1180060 & -0.2037600 & \end{array}$

\begin{tabular}{lr}
$\mathrm{C}$ & 2.2015400 \\
$\mathrm{C}$ & 1.1258450 \\
$\mathrm{H}$ & 2.2073690 \\
$\mathrm{H}$ & 0.1944140 \\
$\mathrm{O}$ & 1.3597490 \\
$\mathrm{H}$ & 0.7253660 \\
$\mathrm{C}$ & 3.5591850 \\
$\mathrm{C}$ & 2.6933480 \\
$\mathrm{C}$ & 1.2409510 \\
$\mathrm{C}$ & 3.5801610 \\
$\mathrm{H}$ & 4.5862140 \\
$\mathrm{H}$ & 2.7030660 \\
$\mathrm{H}$ & 0.7308370 \\
$\mathrm{H}$ & 4.1118860 \\
$\mathrm{H}$ & 4.1055240 \\
$\mathrm{H}$ & 3.1633850 \\
$\mathrm{H}$ & 3.1041300 \\
$\mathrm{H}$ & 0.6475900 \\
$\mathrm{C}$ & 0.7125210 \\
$\mathrm{O}$ & -0.4347670 \\
$\mathrm{C}$ & 1.2161110 \\
$\mathrm{C}$ & 2.0908360 \\
$\mathrm{C}$ & 0.8500170 \\
$\mathrm{C}$ & 2.5909390 \\
$\mathrm{H}$ & 2.3725310 \\
$\mathrm{C}$ & 1.3722020 \\
$\mathrm{H}$ & 0.1803160 \\
$\mathrm{C}$ & 2.2420690 \\
$\mathrm{H}$ & 3.2598630 \\
$\mathrm{H}$ & 1.0975870 \\
$\mathrm{H}$ & 2.6469240 \\
$\mathrm{H}$ & -0.6729680 \\
$\mathrm{~S}$ & 1.5870830 \\
$\mathrm{H}$ & \\
\hline
\end{tabular}
$-3.0498990$
$-3.5565370$
$-1.9780230$
$-3.0035490$
$-3.7798270$
$-3.0702620$
$-5.1217560$
$-5.2849820$
$-4.8580660$
$-3.6550920$
$-5.4494360$
$-6.3230790$
$-5.6115930$
$-3.0394670$
$-3.5353600$
$-5.7549210$
$-4.6607370$
$-4.7826350$
$-0.2475510$
$-0.0630270$
0.9767880
0.9165510
2. 2360280
2. 0869250
$-0.0528180$
3.4032370
2. 2943930
3. 3333250
2. 0289310
4. 3659870
4.2446670
$-0.7525820$
$-1.6855910$

144

0.1795800

1. 0456840

$-0.0265320$

1.0663200

$-0.7922290$

$-1.1996460$

0.7788720

2. 0361870

1. 7610380

0.3215130

0.9783360

2. 3871650

1.1406630

1.0585550

$-0.6295370$

$-0.0268200$

2. 8418880

2. 6804340

3. 2310040

2. 6340490

3. 9044100

5.0035270

3.3868460

5.5703990

5.4050190

3. 9419650

2.5348210

5.0354470

6.4257870

3. 5176300

5.4699430

1.8860330

3. 2325170

\section{TS222(R, R) G[B97-D/def2-TZVP $]=-3633.748079$}

\begin{tabular}{lrrr} 
TS22L(R, R $)$ & \multicolumn{2}{c}{ G $[\mathrm{B} 97-\mathrm{D} / \mathrm{def}-\mathrm{TZVP}]=-3633.748079$} \\
$\mathrm{O}$ & 0.5956980 & 0.2141940 & -0.2700150 \\
$\mathrm{O}$ & -1.7045920 & -0.0088630 & -1.3630100 \\
$\mathrm{C}$ & 0.8373850 & 1.3187850 & -1.0772950 \\
$\mathrm{C}$ & -0.1431440 & 2.2969390 & -1.2119480 \\
$\mathrm{C}$ & 2.1191380 & 1.4260970 & -1.6781270 \\
$\mathrm{C}$ & 0.0937610 & 3.3887950 & -2.1098440 \\
$\mathrm{C}$ & 2.3604400 & 2.5150160 & -2.4928820 \\
$\mathrm{C}$ & 1.3732780 & 3.4945620 & -2.7536050 \\
$\mathrm{H}$ & 3.3389200 & 2.6218850 & -2.9549820 \\
$\mathrm{C}$ & -2.1933620 & 1.0433970 & -0.5772250 \\
$\mathrm{C}$ & -3.4581570 & 0.8797930 & 0.0494230
\end{tabular}




-1.4161040
-3.9093680
-1.8501290
-3.1261230
-4.8801210
-2.7693780
-1.4966060
-1.0501350
-3.5645960
-3.1104630
-0.8629610
-0.0711830
-4.5365330
-0.6292900
0.6446840
1.6227680
-0.8978240
-1.4057390
0.8431600
2.6011340
-1.8806830
3.2080620
3.5574960
3.9438010
4.6667360
5.0308090
5.4186780
4.9337480
5.6039360
-4.2603510
-4.4915520
-4.8022130
-5.2686790
-5.5615570
-5.8070460
-5.4512200
-5.9785330
3.6032690
6.6408800
2.7890700
-3.9400400
-6.6191620
-4.6427060
-6.0009310
-6.7099650
-6.4355110

2.1856190

1. 9185580

3.2136050

3.0726890

1.8350210

5.1712950

5.2947040

4.3426030

4.0803630

5.9309220

6.1450900

4.4450710

3.9628830

5.3920740

5.5113000

4.5761460

4.3591910

6.1186960

6.3332310

4.6440360

4.2778830

0.4339520

$-0.4837790$

0.4797680

$-1.3206380$

$-0.3835100$

$-1.2817330$

$-2.0214870$

$-0.3661490$

$-0.3655940$

$-1.2233860$

$-0.6833980$

$-2.3729100$

$-1.8484250$

$-2.7086970$

$-3.0370190$

$-2.0871130$

1.4967550

$-2.1583700$

$-0.5751530$

$-0.9432720$

$-3.9778710$

0.2231250

0.8340040

0.0456310

1.3995270

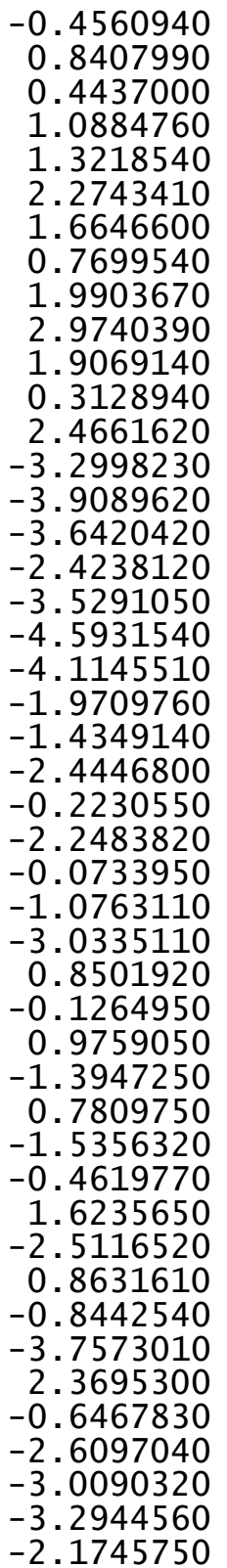

-5.8757450
-4.0034870
-3.0329530
-4.6511300
-3.8548570
-5.9092010
-6.4827740
-5.8161100
-4.9014410
-8.0451390
-8.5686520
-8.0133870
-8.6237420
-5.0503720
-5.5264890
-5.8277950
-4.6328730
-3.2672930
-4.0035370
-2.5041640
-2.7896850
4.1957230
5.2919300
3.8369960
3.9161900
4.0605480
3.6874620
5.1560930
3.6772490
7.9289200
8.8127970
8.0182330
7.9170240
6.7101020
5.7984360
6.8442480
7.5657880
2.4221670
3.3186110
1.8523990
1.8071540
3.5882530
4.5426930
3.0205820
3.8055880
-0.5527180

\author{
1. 5115190 \\ $-0.5138940$ \\ $-0.9361350$ \\ $-1.3287850$ \\ 0.1827230 \\ $-4.9536540$ \\ $-5.8856170$ \\ $-4.5061710$ \\ $-5.1945650$ \\ $-3.6746430$ \\ $-3.0020680$ \\ $-3.1942190$ \\ $-4.6035490$ \\ $-0.4178420$ \\ 0.4816200 \\ $-1.1821130$ \\ $-0.1742290$ \\ $-2.1740750$ \\ $-2.9566470$ \\ $-2.5885530$ \\ $-1.8820950$ \\ 2.8788420 \\ 2.8156520 \\ 3. 2376310 \\ 3. 6119640 \\ 1.0948000 \\ 0.1026730 \\ 1.0931190 \\ 1.8206030 \\ $-1.3210630$ \\ $-1.9315370$ \\ $-0.9545070$ \\ $-0.4548160$ \\ $-3.4008010$ \\ $-4.0066570$ \\ $-3.1168940$ \\ $-4.0220540$ \\ $-2.0232360$ \\ $-2.6240790$ \\ -2. 4973520 \\ $-2.0243140$ \\ 0.0862960 \\ $-0.4368620$ \\ 0.0415120 \\ 1.1371210 \\ $-0.9002800$
}

$-3.8643860$

$-3.8005710$

$-3.5178590$

$-4.1494880$

$-4.6364220$

$-1.6055510$

$-1.6986540$

$-2.6039500$

$-1.2443470$

$-1.1442820$

$-0.4528790$

$-2.1311920$

$-1.2356760$

3. 3022130

2.8947420

3.4367660

4.2886280

3.0068490

3.2317260

2. 3412830

3.9512480

0.5119200

0.4763880

$-0.4582200$

1.2806570

2. 2725150

2. 5504990

2. 3473600

2.9974410

$-0.9970910$

$-0.7682800$

$-2.0288560$

$-0.3245180$

$-1.7426580$

$-1.6677080$

$-2.7948000$

$-1.4511810$

$-4.1297220$

$-4.3306970$

$-3.3242950$

$-5.0391600$

$-4.8976270$

$-5.0456170$

$-5.8367800$

$-4.6712220$

$-0.6409320$ 


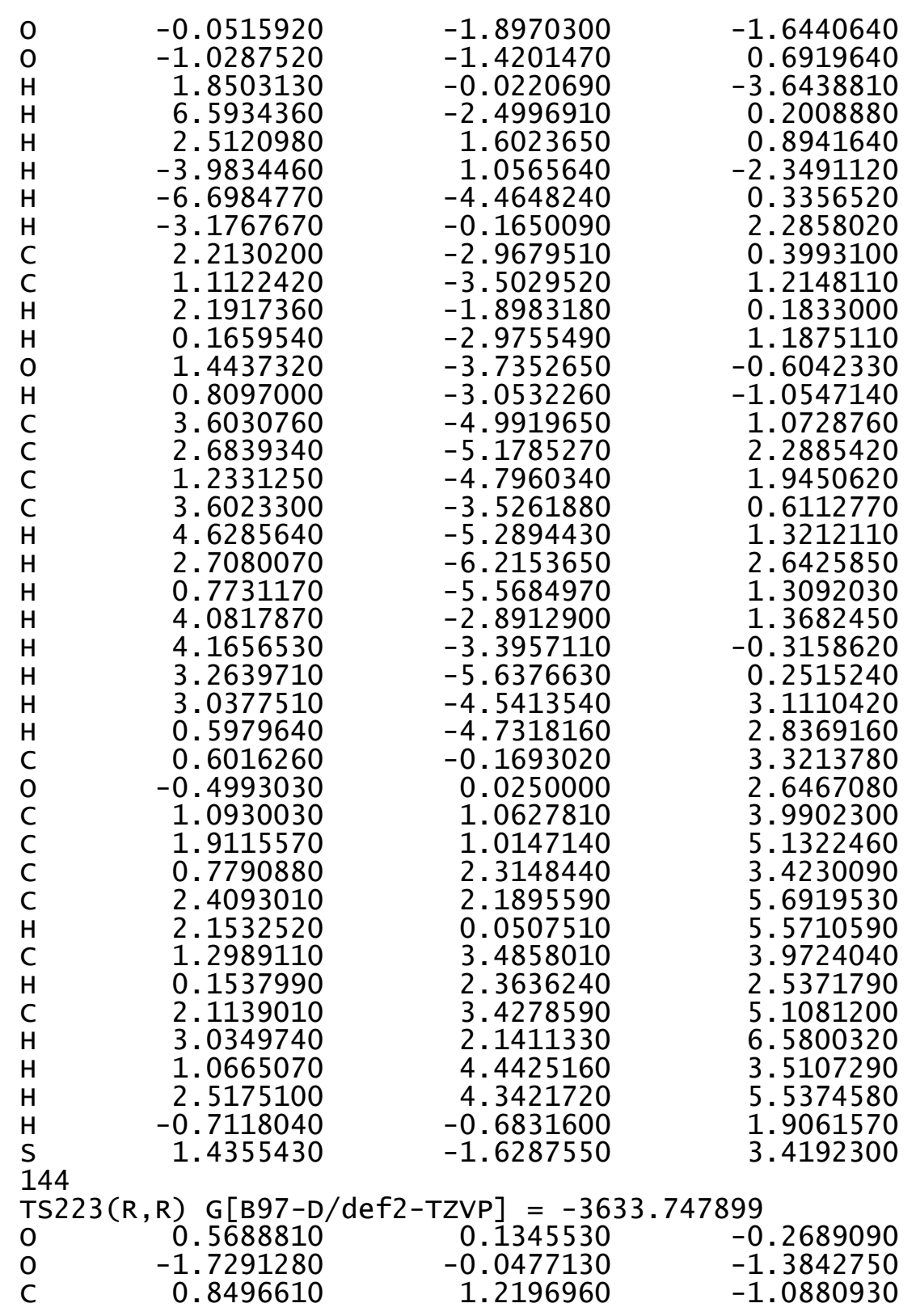

$\begin{array}{lr}\mathrm{C} & -0.1091260 \\ \mathrm{C} & 2.1474730 \\ \mathrm{C} & 0.1610010 \\ \mathrm{C} & 2.4196650 \\ \mathrm{C} & 1.4524050 \\ \mathrm{H} & 3.4126450 \\ \mathrm{C} & -2.1959290 \\ \mathrm{C} & -3.4668480 \\ \mathrm{C} & -1.3902530 \\ \mathrm{C} & -3.8927950 \\ \mathrm{C} & -1.8014900 \\ \mathrm{C} & -3.0815400 \\ \mathrm{H} & -4.8661490 \\ \mathrm{C} & -2.6763870 \\ \mathrm{C} & -1.4009780 \\ \mathrm{C} & -0.9758090 \\ \mathrm{C} & -3.4966950 \\ \mathrm{H} & -3.0003520 \\ \mathrm{H} & -0.7487620 \\ \mathrm{H} & 0.0058300 \\ \mathrm{H} & -4.4715700 \\ \mathrm{C} & -0.5062960 \\ \mathrm{C} & 0.7789910 \\ \mathrm{C} & 1.7357510 \\ \mathrm{C} & -0.8073950 \\ \mathrm{H} & -1.2651300 \\ \mathrm{H} & 1.0033780 \\ \mathrm{H} & 2.7230280 \\ \mathrm{H} & -1.7976100 \\ \mathrm{C} & 3.2549770 \\ \mathrm{C} & 3.6487600 \\ \mathrm{C} & 4.0050840 \\ \mathrm{C} & 4.8435290 \\ \mathrm{C} & 5.1870840 \\ \mathrm{C} & 5.6409240 \\ \mathrm{H} & 5.1629750 \\ \mathrm{H} & 5.7873470 \\ \mathrm{C} & -4.3002750 \\ \mathrm{C} & -4.5574980 \\ \mathrm{C} & -4.8435910 \\ \mathrm{C} & -5.3588930 \\ \mathrm{C} & -5.6287270 \\ \mathrm{C} & -5.8980470 \\ \mathrm{H} & -5.5601050 \\ \mathrm{H} & -6.0468130 \\ \mathrm{C} & 3.5906020 \\ & \end{array}$

2.2143840
1.2985950
3.2836950
2.3675780
3.3542610
2.4578480
1.0265220
0.9039720
2.1496970
1.9649700
3.2036190
3.1032850
1.9130280
5.2126110
5.2966600
4.3192180
4.1358650
5.9915440
6.1370280
4.3908240
4.0494890
5.2773350
5.3613480
4.4142610
4.2662880
6.0141300
6.1664790
4.4569870
4.2118340
0.3409160
-0.6158570
0.5112630
-1.3187320
-0.2242140
-1.1162130
-2.0357680
-0.0891920
-0.3222870
-1.1635830
-0.6392130
-2.2973650
-1.7877010
-2.6328270
-2.9491540
-2.0255070
1.5323820

$-1.2567630$

$-1.6575170$

$-2.1713380$

$-2.4904010$

$-2.7948950$

$-2.9242200$

$-0.6154190$

0.0079800

$-0.5093490$

0.7838280

0.3708320

1.0153650

1. 2640780

2. 1665710

1.5559160

0.6783850

1.9001120

2. 8530480

1.7833560

0.2214420

2. 3766310

$-3.4082970$

$-3.9994540$

-3. 6991650

$-2.5176010$

$-3.6626380$

$-4.6954860$

$-4.1558150$

$-2.0765460$

$-1.3485320$

$-2.3096130$

$-0.1619320$

$-2.1115540$

$-0.0074700$

$-0.9825180$

$-2.8636370$

0.8891210

$-0.1582170$

0.9509170

$-1.4261390$

0.7625760

$-1.5601270$

$-0.4800880$

1.6105260

-2.5358310
0.8940800 


$\begin{array}{rrr}6.9798390 & -1.8193540 & -0.8284000 \\ 2.8251150 & -0.8668800 & -3.5661590 \\ -4.0111050 & -0.8797240 & 2.3456130 \\ -6.7361550 & -3.8860730 & -0.6584190 \\ -4.6587640 & 0.2531650 & -2.6480440 \\ -6.0004980 & 0.8953080 & -3.0539930 \\ -6.7287380 & 0.1227240 & -3.3340160 \\ -6.4215960 & 1.4779910 & -2.2244640 \\ -5.8572730 & 1.5626720 & -3.9144760 \\ -4.0364280 & -0.5083770 & -3.8322820 \\ -3.0758310 & -0.9500200 & -3.5453060 \\ -4.7019370 & -1.3114430 & -4.1751440 \\ -3.8717550 & 0.1777740 & -4.6737640 \\ -6.0403810 & -4.8856980 & -1.6029540 \\ -6.6328970 & -5.8062000 & -1.6913090 \\ -5.9310220 & -4.4497710 & -2.6048090 \\ -5.0404390 & -5.1443580 & -1.2324120 \\ -8.1518420 & -3.5573360 & -1.1686800 \\ -8.6661850 & -2.8684470 & -0.4865210 \\ -8.1030570 & -3.0857830 & -2.1591350 \\ -8.7489980 & -4.4747540 & -1.2567480 \\ -5.1178220 & -0.3165230 & 3.2606650 \\ -5.5710960 & 0.5869750 & 2.8361250 \\ -5.9126860 & -1.0620330 & 3.3986750 \\ -4.7039560 & -0.0678170 & 4.2473570 \\ -3.3731130 & -2.1173800 & 3.0044990 \\ -4.1291240 & -2.8795200 & 3.2339650 \\ -2.6150510 & -2.5583890 & 2.3502450 \\ -2.8957380 & -1.8241830 & 3.9486220 \\ 4.1452490 & 2.9298500 & 0.5455110 \\ 5.2435370 & 2.9058160 & 0.5423010 \\ 3.8023500 & 3.2628480 & -0.4399880 \\ 3.8173210 & 3.6619260 & 1.2959440 \\ 4.0064800 & 1.1553300 & 2.3241530 \\ 3.6561160 & 0.1520370 & 2.5926670 \\ 5.0971660 & 1.1912920 & 2.4441910 \\ 3.5686800 & 1.8702750 & 3.0289020 \\ 7.9756190 & -1.3107940 & -1.8908650 \\ 8.9584880 & -1.7802770 & -1.7513560 \\ 7.6150890 & -1.5588930 & -2.8980100 \\ 8.0935910 & -0.2215510 & -1.8286260 \\ 6.8643880 & -3.3532870 & -0.8981750 \\ 6.2378360 & -3.7433270 & -0.0887550 \\ 6.4251260 & -3.6670230 & -1.8540790 \\ 7.8591740 & -3.8103700 & -0.8157160 \\ 2.6489190 & -2.3664250 & -3.8667210\end{array}$

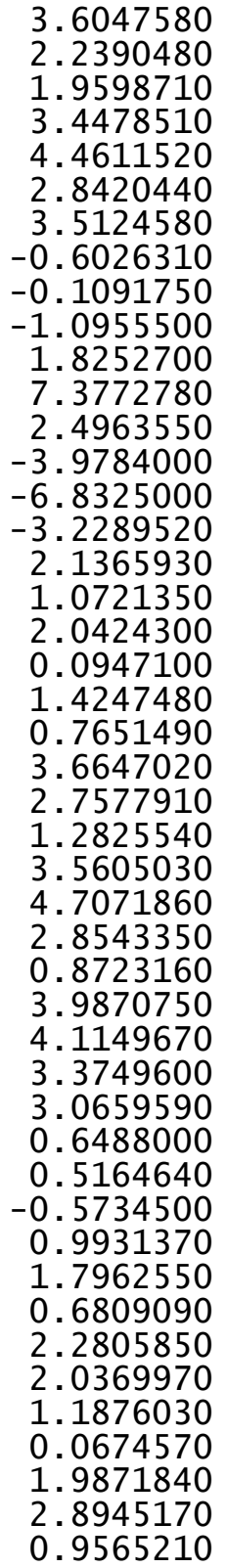

-2.8315760
-2.8968650
-2.4935010
-0.1516640
-0.5338250
-0.3293170
0.9293780
-0.9573800
-1.9630340
-1.4623580
-0.4493980
-1.5544840
1.5994620
1.0711330
-4.3618030
-0.1208910
-2.9068830
-3.5166770
-1.8428760
-3.0491680
-3.7285730
-3.0821710
-4.8235430
-5.0810730
-4.7988830
-3.3585640
-5.0512150
-6.1155380
-5.6014970
-2.6970170
-3.1711080
-5.4859490
-4.4249490
-4.7766520
-0.1685230
0.0139580
1.0740460
1.0430640
2.3180290
2.2265710
0.0852330
3.4975520
2.3545280
3.4566920
2.1911680
4.4472920
$-4.1401840$ $-3.0007560$ $-4.7114560$ $-4.7812240$ $-4.9655160$ $-5.6800040$ $-4.6127430$ $-0.6470690$ $-1.6460410$ 0.6845960 $-3.4014860$ 0.1614850 0.8846330 $-2.3927730$ 0.3279910 2. 2594010 0.4477790

1. 2555000

0.2315130

1. 2304650 $-0.5570550$ $-1.0273060$

1.1122320

2. 3249970

1.9853650

0.6620280

1. 3636280

2. 6734990

1. 3530500

1.4279020

$-0.2607110$

0.2854130

3. 1508790

2. 8804110

3. 3231280

2. 6266330

3. 9831400

5.1367120

3.3976240

5.6898350

5.5892310

3.9406910

2. 5030400

5.0879930

6.5866670

3. 4644360 


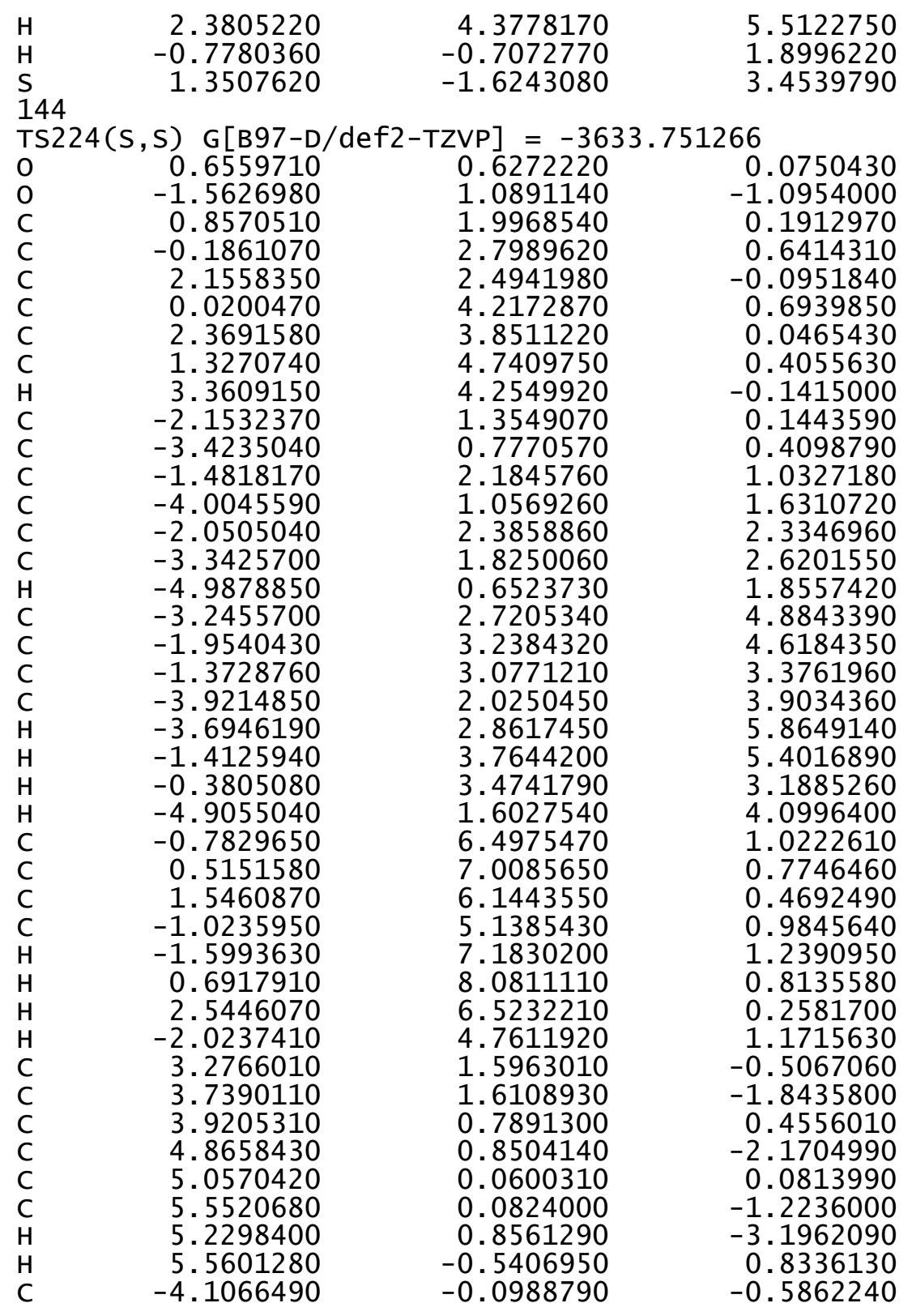

-4.2728590
-4.6053050
-4.9524390
-5.2672110
-5.4535960
-5.0883600
-5.6549650
3.4585950
6.8140390
3.0684200
-3.7525910
-6.1678650
-4.5021030
-5.8991230
-6.5404000
-6.3867750
-5.8180370
-3.7960890
-2.7967390
-4.3724870
-3.6976410
-5.3790120
-5.8888320
-5.2983430
-4.3640750
-7.6064830
-8.1835960
-7.5978080
-8.1160690
-4.9020160
-5.4196370
-5.6376230
-4.5126560
-3.0130000
-3.7016750
-2.2159430
-2.5665490
4.4315180
5.4335770
4.5089170
4.0841380
3.2837150
2.6016180
4.2442620
2.8684510
7.2194970
$-0.3208260$

$-1.7878910$

$-1.2608960$

$-2.6959510$

$-2.4522600$

$-1.0648960$

$-3.6157160$

1.9063860

$-1.6468980$

$-2.9221150$

0.9510690

$-3.4591280$

$-2.1103400$

$-2.0950340$

$-2.8668250$

$-1.1217370$

$-2.2935110$

$-3.4548060$

$-3.4561730$

$-4.2858080$

$-3.6294250$

$-4.7803710$

$-5.4884980$

$-5.2409200$

$-4.6075220$

$-3.7218550$

$-2.7894770$

$-4.1673330$

$-4.4191380$

1.9493830

2.2047490

1. 5155550

2.8740280

0.6782210

0.3092880

$-0.0577100$

1.6101540

2. 8118930

2.7717880

2. 4890700

3.8536350

2.4068560

1.7599620

2. 4360870

3. 4225380

3.4225380
-0.7195290 


$\begin{array}{rrrr}\mathrm{H} & 8.0878220 & -2.3327700 & -1.1392300 \\ \mathrm{H} & 7.5021720 & -1.4347280 & 0.2726760 \\ \mathrm{H} & 6.4066310 & -2.5344930 & -0.5916230 \\ \mathrm{C} & 7.9826390 & 0.3436760 & -1.7861890 \\ \mathrm{H} & 7.7286000 & 1.1502310 & -2.4845620 \\ \mathrm{H} & 8.2097980 & 0.7926970 & -0.8095800 \\ \mathrm{H} & 8.8835770 & -0.1675780 & -2.1512210 \\ \mathrm{C} & 2.8491630 & 1.6839370 & -4.2365720 \\ \mathrm{H} & 3.8038960 & 1.4221110 & -4.7105830 \\ \mathrm{H} & 2.2797440 & 0.7662250 & -4.0616890 \\ \mathrm{H} & 2.2880880 & 2.3129740 & -4.9398140 \\ \mathrm{C} & 3.8856090 & 3.7339230 & -3.1901340 \\ \mathrm{H} & 4.8863160 & 3.4722300 & -3.5596820 \\ \mathrm{H} & 3.3871370 & 4.3522710 & -3.9487490 \\ \mathrm{H} & 4.0031380 & 4.3299150 & -2.2778190 \\ \mathrm{P} & -0.3336730 & 0.0287990 & -1.0878960 \\ \mathrm{O} & 0.3016400 & 0.1042670 & -2.4486070 \\ \mathrm{O} & -0.7431590 & -1.3215430 & -0.5679600 \\ \mathrm{H} & 2.0817490 & 2.7551820 & -2.5535410 \\ \mathrm{H} & 6.6206000 & -1.0756360 & -2.6457650 \\ \mathrm{H} & 2.4826780 & 1.2187090 & 1.9841080 \\ \mathrm{H} & -3.9092280 & 2.4346690 & -1.3335350 \\ \mathrm{H} & -6.2228590 & -3.6331700 & -3.0303500 \\ \mathrm{H} & -3.0355770 & -1.4606390 & 1.4215320 \\ \mathrm{C} & 1.3692850 & -2.9407100 & -2.2528190 \\ \mathrm{C} & 2.2360880 & -2.4302540 & -1.1869420 \\ \mathrm{H} & 0.3006610 & -2.7968730 & -2.1039630 \\ \mathrm{H} & 1.8612350 & -1.6313180 & -0.5597200 \\ \mathrm{O} & 1.9940630 & -1.7491780 & -2.8712040 \\ \mathrm{H} & 1.3241180 & -0.9577730 & -2.7269880 \\ \mathrm{C} & 3.2796350 & -4.4395340 & -3.0044770 \\ \mathrm{C} & 3.8987950 & -4.2926850 & -1.6067320 \\ \mathrm{C} & 3.6421850 & -2.8930860 & -1.0227460 \\ \mathrm{C} & 1.7565700 & -4.2279670 & -2.9458310 \\ \mathrm{H} & 3.4916060 & -5.4355770 & -3.4097800 \\ \mathrm{H} & 4.9794810 & -4.4726760 & -1.6408750 \\ \mathrm{H} & 4.2819320 & -2.1410740 & -1.5039200 \\ \mathrm{H} & 1.2900900 & -5.0415110 & -2.3723790 \\ \mathrm{H} & 1.3093390 & -4.2349300 & -3.9474180 \\ \mathrm{H} & 3.7275710 & -3.7040470 & -3.6859190 \\ \mathrm{H} & 3.4612500 & -5.0456120 & -0.9365790 \\ \mathrm{H} & 3.8875320 & -2.8380430 & 0.0437920 \\ \mathrm{C} & 0.3978540 & -2.9936180 & 2.1334600 \\ \mathrm{O} & -0.2963480 & -1.9326120 & 1.8187470 \\ \mathrm{C} & 0.4142450 & -3.2631860 & 3.5956640 \\ \mathrm{C} & 1.3942810 & -4.0877210 & 4.1800870\end{array}$

$\begin{array}{lr}\mathrm{C} & -0.5642720 \\ \mathrm{C} & 1.3986070 \\ \mathrm{H} & 2.1559530 \\ \mathrm{C} & -0.5637260 \\ \mathrm{H} & -1.3272800 \\ \mathrm{C} & 0.4184830 \\ \mathrm{H} & 2.1687420 \\ \mathrm{H} & -1.3302250 \\ \mathrm{H} & 0.4209980 \\ \mathrm{H} & -0.4264510 \\ \mathrm{~S} & 1.1850850 \\ \mathrm{1} 44 & \end{array}$
$-2.6759740$
$-4.3169290$
$-4.5345040$
$-2.9185090$
$-2.0406310$
$-3.7363110$
$-4.9470520$
$-2.4679580$
$-3.9194340$
$-1.7498100$
$-3.9811150$

TS225(S, S) G[B97-D/def2-TZVP $]=-3633.751150$

$\begin{array}{lrcr}\text { TS225(S, S }(\mathrm{G}[\mathrm{B} 97-\mathrm{D} / \mathrm{de} 2-\mathrm{TZVP}]=-3633.751150 \\ \mathrm{O} & 0.7298680 & 0.6853570 & 0.1893360 \\ \mathrm{O} & -1.3114130 & 1.2239450 & -1.2389710 \\ \mathrm{C} & 1.0126250 & 2.0437220 & 0.2666980 \\ \mathrm{C} & -0.0151190 & 2.9303330 & 0.5688320 \\ \mathrm{C} & 2.3644590 & 2.4426020 & 0.0910830 \\ \mathrm{C} & 0.2823650 & 4.3332240 & 0.5893400 \\ \mathrm{C} & 2.6554930 & 3.7877610 & 0.2049700 \\ \mathrm{C} & 1.6461580 & 4.7569500 & 0.4257100 \\ \mathrm{H} & 3.6849320 & 4.1200080 & 0.0933280 \\ \mathrm{C} & -2.0067060 & 1.5804290 & -0.0791810 \\ \mathrm{C} & -3.3284470 & 1.0832160 & 0.0752820 \\ \mathrm{C} & -1.3823980 & 2.4134960 & 0.8408940 \\ \mathrm{C} & -4.0130510 & 1.4460130 & 1.2182030 \\ \mathrm{C} & -2.0704380 & 2.7062120 & 2.0660130 \\ \mathrm{C} & -3.4138000 & 2.2250790 & 2.2382720 \\ \mathrm{H} & -5.0354230 & 1.1041580 & 1.3555150 \\ \mathrm{C} & -3.5023000 & 3.2258720 & 4.4584210 \\ \mathrm{C} & -2.1640040 & 3.6651600 & 4.3076320 \\ \mathrm{C} & -1.4672420 & 3.4126630 & 3.1425170 \\ \mathrm{C} & -4.1099240 & 2.5163180 & 3.4434920 \\ \mathrm{H} & -4.0417710 & 3.4376070 & 5.3789050 \\ \mathrm{H} & -1.6782340 & 4.2018830 & 5.1195890 \\ \mathrm{H} & -0.4407180 & 3.7499060 & 3.0443910 \\ \mathrm{H} & -5.1305810 & 2.1537790 & 3.5528570 \\ \mathrm{C} & -0.3898380 & 6.6732060 & 0.7476760 \\ \mathrm{C} & 0.9600160 & 7.0864670 & 0.6278090 \\ \mathrm{C} & 1.9544370 & 6.1444100 & 0.4649770 \\ \mathrm{C} & -0.7195750 & 5.3324970 & 0.7297480 \\ \mathrm{H} & -1.1747940 & 7.4193990 & 0.8496190 \\ \mathrm{H} & 1.2063060 & 8.1456750 & 0.6501570 \\ \mathrm{H} & 2.9935070 & 6.4480020 & 0.3497490 \\ \mathrm{H} & -1.7581490 & 5.0297540 & 0.8172930 \\ \mathrm{C} & 3.4391860 & 1.4495680 & -0.2038180\end{array}$




$\begin{array}{rrr}4.0514860 & 1.4308550 & -1.4786760 \\ 3.8736030 & 0.5561650 & 0.8017870 \\ 5.1025590 & 0.5350830 & -1.7112580 \\ 4.9278690 & -0.3176110 & 0.5180660 \\ 5.5571860 & -0.3470380 & -0.7292310 \\ 5.5738720 & 0.5288930 & -2.6904190 \\ 5.2725860 & -1.0004580 & 1.2925950 \\ -3.9602550 & 0.2118910 & -0.9587040 \\ -4.2339680 & -1.1492310 & -0.6717330 \\ -4.3083390 & 0.7534920 & -2.2156220 \\ -4.8695510 & -1.9253290 & -1.6459410 \\ -4.9314410 & -0.0721870 & -3.1604410 \\ -5.2233060 & -1.4104460 & -2.8969030 \\ -5.0878050 & -2.9680280 & -1.4287720 \\ -5.2021340 & 0.3430700 & -4.1300150 \\ 3.2811280 & 0.5561290 & 2.2057240 \\ 6.7279470 & -1.2864280 & -0.9686910 \\ 3.6290230 & 2.3685540 & -2.6039470 \\ -3.8765140 & -1.7949320 & 0.6632620 \\ -5.8929790 & -2.2879570 & -3.9396680 \\ -4.0793230 & 2.2165100 & -2.5746470 \\ -5.4242230 & 2.9512100 & -2.7444550 \\ -6.0012830 & 2.5132440 & -3.5693900 \\ -6.0265880 & 2.8803930 & -1.8295680 \\ -5.2514110 & 4.0121650 & -2.9696660 \\ -3.2109400 & 2.3683000 & -3.8372430 \\ -2.2477680 & 1.8621870 & -3.7079010 \\ -3.7171210 & 1.9394080 & -4.7119180 \\ -3.0248570 & 3.4315170 & -4.0396070 \\ -7.2435510 & -2.8322940 & -3.4363240 \\ -7.7247820 & -3.4406810 & -4.2136050 \\ -7.0944590 & -3.4641090 & -2.5506260 \\ -7.9203830 & -2.0130780 & -3.1621210 \\ -4.9719340 & -3.4463720 & -4.3690840 \\ -4.0159740 & -3.0667930 & -4.7513150 \\ -4.7623140 & -4.1041250 & -3.5152170 \\ -5.4519640 & -4.0458780 & -5.1541270 \\ -5.1252480 & -1.9273630 & 1.5588350 \\ -5.6040630 & -0.9568920 & 1.7323310 \\ -5.8606710 & -2.5902230 & 1.0828970 \\ -4.8511660 & -2.3590900 & 2.5312240 \\ -3.2117130 & -3.1745380 & 0.5030700 \\ -3.9142790 & -3.9081960 & 0.0876890 \\ -2.3361410 & -3.1144850 & -0.1502400 \\ -2.8919790 & -3.5424770 & 1.4857860 \\ 4.2824480 & 1.1632770 & 3.2082730\end{array}$

5.1970250

4.5599400

3.8423610

2. 8424950

2.1282180

3.7021990

2. 3578910

8.0599530

8.9105940

8.1549580

8.1068530

6.7051720

5.7607490

6.8330170

7.5276980

3. 3691760

4.2846860

2. 6036400

3.0215580

4.6865140

5.6446960

4.3653360

4.8492870

$-0.1537320$

0.6314920

$-0.6985250$

2.6912100

6.6684030

2. 3901970

$-3.5492460$

$-6.0859900$

$-3.1586930$

1. 3710200

2. 1661380

0.3128360

1.8056790

2.1763230

1. 5750180

3.1891320

3.6760440

3.4927650

1.6962230

3.3443980

4.7336810

4.2515620

1.0969720
0.5569790

2. 1836020

1.1954580

$-0.8479720$

$-1.2800170$

$-1.5249510$

$-0.7944340$

$-0.5365980$

$-1.2204220$

0.2716680

$-0.0937450$

$-1.9567020$

$-2.4853490$

$-1.2140190$

$-2.6787660$

1.6259920

1.1533400

0.8552780

2. 3394650

3.4713090

3.0259730

4.1643360

4.0427710

0.0991430

0.0872070

$-1.2058890$

2.8539820

$-2.0779860$

1.1907780

2.7055410

$-1.6628050$

$-1.1472950$

$-3.0479520$

$-2.6390080$

$-2.7952980$

$-1.8113040$

$-1.9241050$

$-1.0706820$

$-4.7227990$

$-4.6538320$

$-3.2458770$

$-4.3628610$

$-5.7301340$

$-4.9334280$

$-2.5537390$

$-5.1303390$
3.2495680

2. 9142960

4. 2140170

2.6553490

1.9478940

2.7391900

3.6385950

$-0.7603440$

$-0.8838110$

$-1.4980220$

0.2423730

$-2.3533450$

$-2.5321920$

3.1506940

$-2.4323520$

$-3.9277110$

$-4.3057370$

$-3.7951870$

$-4.6865310$

$-2.8102040$

$-3.1102110$

$-3.5993650$

$-1.8883900$

$-1.0584220$

$-2.3404530$

$-0.5479400$

$-2.3152630$

$-0.2075120$

2.2135470

$-1.7518350$

$-4.8229830$

1.1758210

$-2.0360910$

$-0.8743720$

$-1.9991990$

$-0.2769570$

$-2.5639480$

$-2.4910660$

2.6206750

$-1.1649660$

$-0.5730660$

$-2.7084420$

$-3.0237150$

$-1.0960760$

$-0.9599580$

$-2.1982550$ 


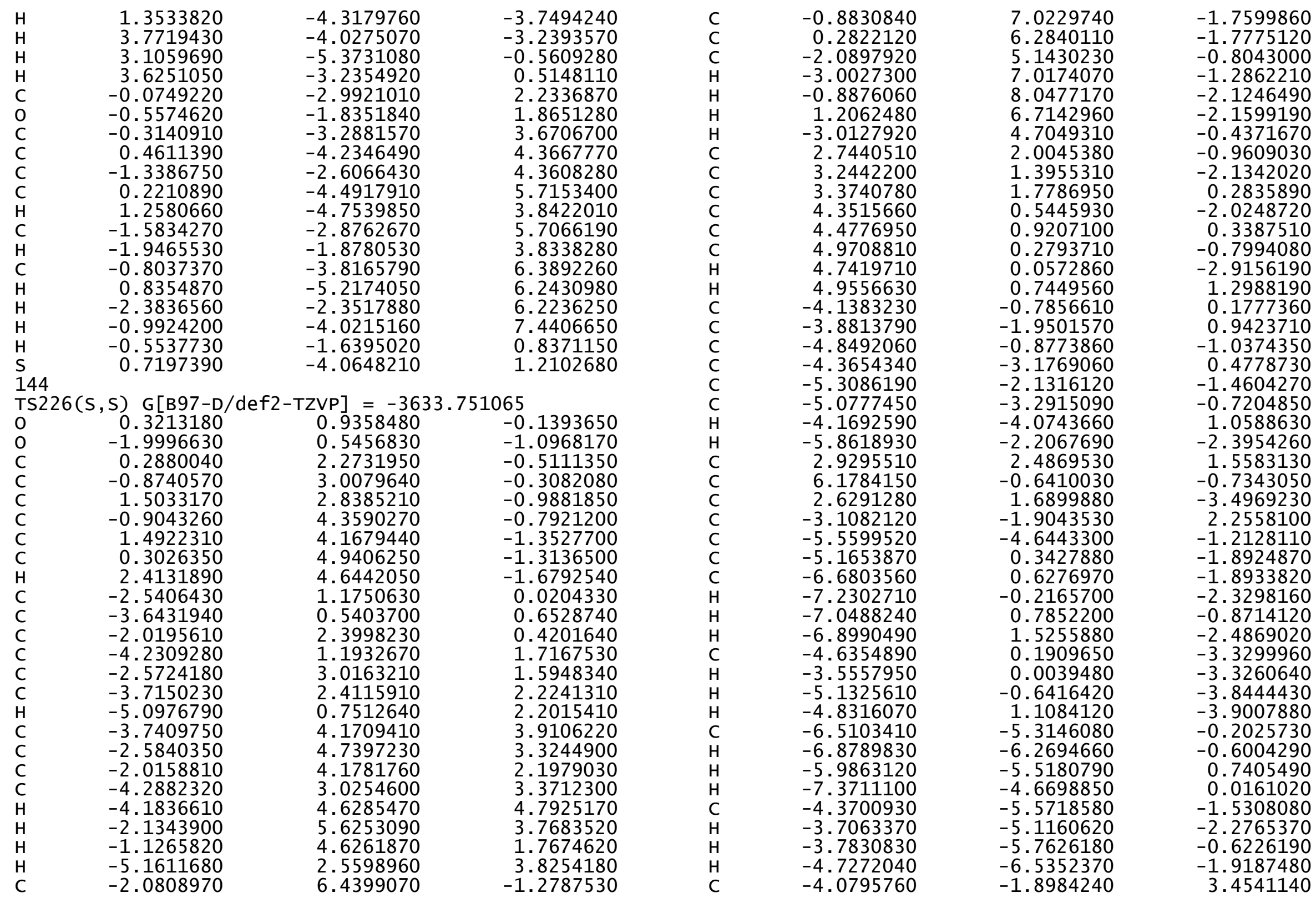




$\begin{array}{rr}-4.7715990 & -1.0502280 \\ -4.6724280 & -2.8233280 \\ -3.5210040 & -1.8397300 \\ -2.1024610 & -3.0603730 \\ -2.6177870 & -4.0209160 \\ -1.4473240 & -3.1276980 \\ -1.4843320 & -2.8909440 \\ 3.9353130 & 3.6016520 \\ 4.9248320 & 3.1703810 \\ 4.0356610 & 4.3167110 \\ 3.6036350 & 4.1440870 \\ 2.7388270 & 1.5296090 \\ 2.0102310 & 0.7495260 \\ 3.6818990 & 1.0437800 \\ 2.3792160 & 2.0899530 \\ 6.1661170 & -1.5657580 \\ 6.9944870 & -2.2832080 \\ 6.2881840 & -0.9875010 \\ 5.2236980 & -2.1221490 \\ 7.4830540 & 0.1812550 \\ 7.5251600 & 0.8101950 \\ 7.5393000 & 0.8349490 \\ 8.3574270 & -0.4835630 \\ 2.6977160 & 0.5150650 \\ 3.7299930 & 0.3280720 \\ 2.3014700 & -0.4021520 \\ 2.1086960 & 0.7548420 \\ 3.3056600 & 2.9254380 \\ 4.3713160 & 2.7208620 \\ 2.8384950 & 3.1621650 \\ 3.2249600 & 3.8026310 \\ -0.5435880 & -0.1644770 \\ -0.0765660 & -0.2977130 \\ -0.5481440 & -1.3866290 \\ 1.5701930 & 1.9259670 \\ 6.1567470 & -1.2702460 \\ 1.9638360 & 2.9686930 \\ -4.6729970 & 1.2165700 \\ -6.1180390 & -4.4770070 \\ -2.5364150 & -0.9703840 \\ 0.9838810 & -3.3421860 \\ 2.1583330 & -2.5612050 \\ 0.0758700 & -3.1686690 \\ 1.9903860 & -1.6169110 \\ 1.1238380 & -2.3653240 \\ 0.5797830 & -1.5051300\end{array}$

3.4062980
3.4598640
4.3983390
2.4099980
2.5398280
1.5364310
3.3007080
1.9115110
2.1147050
1.0848260
2.8071670
2.7463810
2.5083330
3.0269370
3.6196300
0.4950470
0.4353500
1.4196380
0.5715120
-0.7711150
-1.6691510
0.1097530
-0.7654240
-4.4844600
-4.8088790
-4.0425640
-5.3786070
-4.1276430
-4.2998030
-5.0932480
-3.4782110
-0.9823580
-2.4076690
-0.1081270
-3.3392150
-1.6357550
1.3746600
-1.4539420
-2.1449500
2.2796470
-2.0663190
-1.6654960
-1.4912960
-1.1611090
-3.1650180
-2.8854480

$\begin{array}{ll}\mathrm{C} & 2.5475440 \\ \mathrm{C} & 3.6930500 \\ \mathrm{C} & 3.5438600 \\ \mathrm{C} & 1.1901770 \\ \mathrm{H} & 2.6859940 \\ \mathrm{H} & 4.6631930 \\ \mathrm{H} & 3.7995240 \\ \mathrm{H} & 1.1270150 \\ \mathrm{H} & 0.3559130 \\ \mathrm{H} & 2.5530950 \\ \mathrm{H} & 3.6900430 \\ \mathrm{H} & 4.2284380 \\ \mathrm{C} & 1.9368220 \\ \mathrm{O} & 0.9360470 \\ \mathrm{C} & 2.6353870 \\ \mathrm{C} & 3.9754210 \\ \mathrm{C} & 1.9625710 \\ \mathrm{C} & 4.6284270 \\ \mathrm{H} & 4.5016130 \\ \mathrm{C} & 2.6137700 \\ \mathrm{H} & 0.9297460 \\ \mathrm{C} & 3.9493720 \\ \mathrm{H} & 5.6692960 \\ \mathrm{H} & 2.0806110 \\ \mathrm{H} & 4.4590680 \\ \mathrm{H} & 0.3907120 \\ \mathrm{~S} & 2.4010750 \\ \mathrm{H} & \end{array}$
$-4.9847640$
$-5.3962660$
$-5.0509890$
$-4.4652660$
$-4.9937360$
$-2.6054900$
$-2.2955580$
$-1.4709890$
$-2.1030660$
$-2.4976170$
$-1.4882410$
$-2.2762980$
$-2.9568980$
$-1.2850480$
$-1.1783660$
$-1.6729320$
$-2.5703620$
$-0.8194930$
$-1.5043100$
$-1.5499160$

$-4.7689660-2.5250480$

$-6.0536640 \quad-3.4168120$

$-2.8156070$

$-2.9609790$

$-1.6248780$

$-3.1792340$

$-4.1846300$

$-1.3813910$

$-1.2851880$

2. 0993840

1.9335610

3. 3961380

3. 5666430

4.4718060

4.7777930

2.7357590

5.6870770

4.3496680

5.8428960

4.8902010

6.5126220

6.7889060

1.0540860

144

1. 0004510

TS227(S, S) G[B97-D/def2-TZVP $]=-3633.750985$

$\begin{array}{lrcr}\text { O } & 0.6712580 & 0.5893400 & 0.0479980 \\ \mathrm{O} & -1.5463990 & 1.1267750 & -1.0969210 \\ \mathrm{C} & 0.8970030 & 1.9517840 & 0.1979110 \\ \mathrm{C} & -0.1289490 & 2.7596710 & 0.6780240 \\ \mathrm{C} & 2.2006460 & 2.4361130 & -0.0875060 \\ \mathrm{C} & 0.1008790 & 4.1722790 & 0.7688150 \\ \mathrm{C} & 2.4368230 & 3.7854240 & 0.0909510 \\ \mathrm{C} & 1.4140220 & 4.6821070 & 0.4847510 \\ \mathrm{H} & 3.4334940 & 4.1774080 & -0.0963030 \\ \mathrm{C} & -2.1273700 & 1.3700150 & 0.1527920 \\ \mathrm{C} & -3.4068480 & 0.8087180 & 0.4097770 \\ \mathrm{C} & -1.4336770 & 2.1589920 & 1.0606660 \\ \mathrm{C} & -3.9740340 & 1.0640570 & 1.6429780 \\ \mathrm{C} & -1.9894510 & 2.3321340 & 2.3721300 \\ \mathrm{C} & -3.2903000 & 1.7882560 & 2.6499270 \\ \mathrm{H} & -4.9629060 & 0.6709180 & 1.8631930 \\ \mathrm{C} & -3.1595030 & 2.6101880 & 4.9400870\end{array}$




-1.8601550
-1.2912020
-3.8561200
-3.5986200
-1.3028880
-0.2926360
-4.8469710
-0.6600190
0.6448420
1.6581300
-0.9246360
-1.4627450
0.8402130
2.6614110
-1.9297630
3.3166960
3.7875250
3.9767000
4.9573770
5.1412280
5.6649610
5.3428010
5.6684590
-4.1104740
-4.3105530
-4.5941950
-5.0051520
-5.2729200
-5.4901350
-5.1659530
-5.6498120
-0.3448480
0.2888390
-0.7834440
3.5087840
2.5233180
6.9822740
7.2058850
3.0913640
2.1020330
-4.4594330
-3.8556160
-6.2197500
-6.2950220
-3.8102390
-3.0851290

3. 1113190

1.9592780

2. 7291910

3. 6019230

3. 3613930

1.5505190

6.4552960

6.9513060

6.0790450

5. 1023790

7.1479470

8.0190690

6.4465640

4.7369460

1. 5484210

1.6318850

0.7178330

0.9406870

0.0496700

0.1691440

1.0217010

$-0.5600250$

$-0.0312270$

$-1.4114110$

0.5561410

$-2.1653290$

$-0.2438050$

$-1.6070370$

$-3.2269520$

0.2140090

0.0365440

0.1167350

$-1.3113870$

0.5991250

1.0649340

$-0.4873030$

$-0.1950350$

2. 4830860

2.7576140

2.0450780

2. 5109550

$-2.4576180$

$-3.4749980$

$-2.1090160$

$-1.4525650$

4.6802570
3.4290870
3.9432000
5.9280740
5.4752790
3.2463160
4.1336310
1.1699910
0.9267650
0.5874590
1.0943220
1.4134170
0.9957570
0.3789530
1.2782150
-0.5356590
-1.8644650
0.3997520
-2.2081220
0.0073850
-1.2847000
-3.2228330
0.7377640
-0.6027290
-0.3613820
-1.7954020
-1.3167980
-2.7192840
-2.5004070
-1.1392940
-3.6313210
-1.1168520
-2.4782070
-0.6149250
1.8451970
1.9350260
-1.6651420
-2.7007390
-2.9199160
-2.5378380
-2.0910190
-1.3063980
-3.5246870
-3.1151050
0.8992840
1.3895850

\begin{abstract}
$-3.0913440$
$-2.6579240$

$-3.7901990$

$-2.2867150$

$-4.9703880$

$-5.7132220$

$-4.5943520$

$-5.4761410$

3. 3581820

4. 3240100

2. 9650380

2. 6655860

4.4624940

4. 1069370

5. 4716320

4.5261290

$-3.7506940$

$-4.3375220$

$-3.6302400$

$-2.7609520$

$-5.8428380$

$-5.7404170$

$-6.4936870$

$-6.3321500$

2. 8764210

2. 3026840

3.8334280

2. 3216260

3. 8776970

4.8828620

3. 3615360

3. 9840450

$-7.6482220$

$-8.2259190$

$-7.6191290$

$-8.1696450$

$-5.4306600$

$-4.4233110$

$-5.9519380$

$-5.3306220$

6.9090070

6.1653770

7.8842790

6.6343980

8.1296070

8.2056560
\end{abstract}

-3.4381890
-3.8357770
-4.1886840
-3.2925970
-2.3569640
-3.0275570
-2.8294350
-1.4230820
-0.8635340
-1.3843330
-0.9002710
-1.4090060
1.3669500
1.3106700
0.9362910
2.4229560
2.3066430
1.9065230
3.3870080
1.8369500
2.7259320
3.8044670
2.3057660
2.5781380
1.7243720
2.3518720
1.4810880
0.7961820
3.7841530
3.5511620
4.3981450
4.3735140
-1.9405120
-1.9037840
-0.9285580
-2.5946400
-2.5339890
-2.9359060
-3.1780840
-1.5339710
-2.0244980
-2.4078950
-2.4591040
-2.3673420
0.0212850
1.1150500

1. 5279230

0.2103840

$-0.1252850$

1.8844170

1.4315100

2. 8021760

2. 1557190

2. 2972930

2. 2871020

3. 3210970

1. 6473350

2.7812490

3.8188500

2.7363940

2. 4892640

$-3.4322310$

$-4.2692700$

$-3.5878180$

$-3.4442000$

$-2.0620730$

$-2.8402230$

$-1.0906030$

$-4.2421080$

$-4.9364670$

$-4.7213910$

$-4.0739760$

$-3.1775750$

$-3.5536870$

$-3.9277830$

$-2.2594960$

$-3.7798160$

$-2.8474770$

$-4.2049870$

$-4.4912770$

$-4.8463850$

$-4.6794260$

$-5.5672030$

$-5.2885190$

$-1.6167670$

$-2.3254200$

$-1.8722220$

$-0.6107510$

$-0.7702440$

$-0.8130930$ 


\begin{tabular}{|c|c|c|c|c|c|c|c|}
\hline $\mathrm{H}$ & 79583810 & $-0,2691540$ & 0.2746800 & $c$ & -21331080 & 13809250 & 0.1258330 \\
\hline $\mathrm{H}$ & 9.0855350 & -0.4115530 & -1.0934050 & c & -3.4179880 & 0.8006610 & 0.3006960 \\
\hline C & 1.3702570 & -2.9271200 & -2.2637020 & $\mathrm{C}$ & -1.5292210 & 2.2104850 & 1.0628480 \\
\hline C & 2.1829000 & -2.4374880 & -1.1474600 & $\mathrm{C}$ & -4.0831350 & 1.0728030 & 1.4799400 \\
\hline $\mathrm{H}$ & 0.2949500 & -2.7884960 & -2.1649770 & $\mathrm{C}$ & -2.1875940 & 2.4034960 & 2.3230350 \\
\hline $\mathrm{H}$ & 1.7714300 & -1.6602340 & -0.5171080 & $\mathrm{c}$ & -3.4946440 & 1.8382140 & 2.5162310 \\
\hline 0 & 2.0230250 & -1.7179530 & -2.8191430 & $\mathrm{H}$ & -5.0791700 & 0.6655890 & 1.6326710 \\
\hline $\mathrm{H}$ & 1.3399010 & -0.9328420 & -2.7042500 & C & -3.5598180 & 2.7310020 & 4.7826530 \\
\hline C & 3.3258700 & -4.3849230 & -2.9693160 & C & -2.2538440 & 3.2521600 & 4.6093790 \\
\hline $\mathrm{C}$ & 3.8842300 & -4.2714960 & -1.5427090 & $\mathrm{C}$ & -1.5863170 & 3.0934720 & 3.4111630 \\
\hline $\mathrm{C}$ & 3.5789050 & -2.8979830 & -0.9221270 & $\mathrm{C}$ & -4.1628760 & 2.0346020 & 3.7558930 \\
\hline $\mathrm{C}$ & 1.7992460 & -4.1938100 & -2.9693260 & $\mathrm{H}$ & -4.0773520 & 2.8695750 & 5.7292850 \\
\hline $\mathrm{H}$ & 3.5684990 & -5.3660220 & -3.3934320 & $\mathrm{H}$ & -1.7699720 & 3.7776640 & 5.4297400 \\
\hline $\mathrm{H}$ & 4.9684020 & -4.4304810 & -1.5366350 & $\mathrm{H}$ & -0.5837200 & 3.4917570 & 3.2948810 \\
\hline $\mathrm{H}$ & 4.2384380 & -2.1211650 & -1.3303240 & $\mathrm{H}$ & -5.1570850 & 1.6094190 & 3.8819200 \\
\hline $\mathrm{H}$ & 1.3193360 & -5.0259040 & -2.4347930 & C & -0.8528080 & 6.5313280 & 1.1278830 \\
\hline $\mathrm{H}$ & 1.3943540 & -4.1819200 & -3.9886990 & $\mathrm{C}$ & 0.4547330 & 7.0501270 & 0.9587920 \\
\hline $\mathrm{H}$ & 3.7906600 & -3.6245560 & -3.6109410 & $\mathrm{C}$ & 1.5058430 & 6.1926800 & 0.7083840 \\
\hline $\mathrm{H}$ & 3.4332580 & -5.0540300 & -0.9166300 & $\mathrm{C}$ & -1.0840170 & 5.1713780 & 1.0692090 \\
\hline $\mathrm{H}$ & 3.7608900 & -2.8866110 & 0.1583130 & $\mathrm{H}$ & -1.6839740 & 7.2115660 & 1.3006620 \\
\hline C & 0.2771380 & -3.0092040 & 2.1038290 & $\mathrm{H}$ & 0.6232090 & 8.1232800 & 1.0138310 \\
\hline 0 & -0.3767100 & -1.9239750 & 1.7819870 & $\mathrm{H}$ & 2.5128460 & 6.5773270 & 0.5565860 \\
\hline $\mathrm{C}$ & 0.2713170 & -3.2756170 & 3.5665020 & $\mathrm{H}$ & -2.0916230 & 4.7889050 & 1.1960030 \\
\hline C & 1.2288730 & -4.1175170 & 4.1630740 & C & 3.2955280 & 1.6602090 & -0.2475630 \\
\hline C & -0.705 & -2.6687590 & 4.3835470 & $\mathrm{C}$ & 3.8372050 & 1.7552400 & -1.5505780 \\
\hline C & 1.212 & -4.3449650 & 5.5379530 & $\mathrm{C}$ & 3.8507830 & 0.7551780 & 0.6861840 \\
\hline $\mathrm{H}$ & 1.9893860 & -4.5784880 & 3.5394050 & $\mathrm{C}$ & 4.9449980 & 0.9647090 & -1.8812720 \\
\hline C & -0.7268570 & -2.9104150 & 5.7565620 & $\mathrm{C}$ & 4.9591420 & -0.0079130 & 0.3059700 \\
\hline $\mathrm{H}$ & -1.4511250 & -2.0196200 & 3.9349700 & $\mathrm{C}$ & 5.5226600 & 0.0791790 & -0.9698710 \\
\hline C & 0.23 & -3.7456710 & 6.3389680 & $\mathrm{H}$ & 5.3600800 & 1.0409910 & -2.8823570 \\
\hline $\mathrm{H}$ & 1.9 & -4.9884190 & 5.9866370 & $\mathrm{H}$ & 5.4017490 & -0.6972870 & 1.0227030 \\
\hline $\mathrm{H}$ & -1.492 & -2.4454890 & 6.3731380 & C & -4.0361030 & -0.0644540 & -0.7463260 \\
\hline $\mathrm{H}$ & 0.2190970 & -3.9279420 & 7.4112320 & $\mathrm{C}$ & -4.2225530 & -1.4459750 & -0.5047600 \\
\hline $\mathrm{H}$ & -0.4947200 & -1.7425820 & 0.7596510 & $\mathrm{C}$ & -4.4623450 & 0.5046840 & -1.9693900 \\
\hline & 1.0 & -4.0258480 & 0.9996720 & C & -4.8554730 & -2.2194480 & -1.4872290 \\
\hline & & & & $\mathrm{C}$ & -5.0780600 & -0.3145460 & -2.9202140 \\
\hline & S) $\mathrm{G}[\mathrm{B} 97-\mathrm{D} /$ & TZVP] $=-36$ & 36 & C & -5.2885260 & -1.6783790 & -2.6985900 \\
\hline 0 & 0.6689960 & 0.6727260 & 0.2491060 & $\mathrm{H}$ & -5.0087160 & -3.2820650 & -1.3089000 \\
\hline 0 & -1.4614970 & 1.1224330 & -1.0729140 & $\mathrm{H}$ & -5.4100470 & 0.1278090 & -3.8571210 \\
\hline C & 0.8585430 & 2.0438950 & 0.3756700 & C & 3.3325580 & 0.6393350 & 2.1142710 \\
\hline C & -0.2148560 & 2.8371730 & 0.7641670 & C & 6.7504760 & -0.7473280 & -1.3151670 \\
\hline C & 2.1664050 & 2.5503680 & 0.1525630 & C & 3.2762210 & 2.7042050 & -2.6035990 \\
\hline C & -0.0206210 & 4.2564840 & 0.8356300 & C & -3.7719420 & -2.1237630 & 0.7851940 \\
\hline C & 2.3629670 & 3.9079220 & 0.3130380 & $\mathrm{C}$ & -5.9635090 & -2.5453480 & -3.7464530 \\
\hline C & 1.2981740 & 4.7884670 & 0.6247390 & $\mathrm{C}$ & -4.3229330 & 1.9905150 & -2.2782920 \\
\hline H & 3.3 & 4.3200440 & 0.1654610 & C & -5.7091290 & 2.6581630 & -2.3748260 \\
\hline
\end{tabular}




$\begin{array}{rrr}-6.2910950 & 2.2222810 & -3.1974690 \\ -6.2751500 & 2.5197480 & -1.4445850 \\ -5.6001770 & 3.7348300 & -2.5623300 \\ -3.5079720 & 2.2341410 & -3.5620060 \\ -2.5137390 & 1.7810230 & -3.4810420 \\ -4.0177900 & 1.8050470 & -4.4344620 \\ -3.3906510 & 3.3126550 & -3.7329360 \\ -5.1375100 & -2.5977920 & -5.0462140 \\ -5.6234740 & -3.2516630 & -5.7827010 \\ -5.0497300 & -1.5944500 & -5.4832530 \\ -4.1256460 & -2.9760820 & -4.8533170 \\ -7.3972110 & -2.0627220 & -4.0386590 \\ -8.0007760 & -2.0450170 & -3.1222950 \\ -7.3807150 & -1.0478130 & -4.4574770 \\ -7.8829270 & -2.7254890 & -4.7672480 \\ -4.9734520 & -2.3850930 & 1.7158370 \\ -5.5032510 & -1.4568160 & 1.9592770 \\ -5.6852210 & -3.0674050 & 1.2315600 \\ -4.6343750 & -2.8490070 & 2.6522800 \\ -3.0141450 & -3.4404790 & 0.5325550 \\ -3.6765490 & -4.2050200 & 0.1069110 \\ -2.1745960 & -3.2818160 & -0.1509540 \\ -2.6239990 & -3.8276420 & 1.4819630 \\ 4.2850210 & 1.3668450 & 3.0839460 \\ 5.2806190 & 0.9038240 & 3.0603430 \\ 4.3904140 & 2.4235480 & 2.8066880 \\ 3.9002340 & 1.3106200 & 4.1111710 \\ 3.1179430 & -0.8199420 & 2.5515370 \\ 2.4580640 & -1.3466790 & 1.8545130 \\ 4.0686410 & -1.3648160 & 2.6053240 \\ 2.6567610 & -0.8481420 & 3.5467660 \\ 8.0314540 & 0.0810100 & -1.0857360 \\ 8.9240970 & -0.5283740 & -1.2814730 \\ 8.0472840 & 0.9458390 & -1.7628210 \\ 8.0779670 & 0.4518050 & -0.0541420 \\ 6.7285850 & -1.3124050 & -2.7456080 \\ 5.8150710 & -1.8887330 & -2.9370440 \\ 6.7806680 & -0.5056110 & -3.4875980 \\ 7.5942190 & -1.9684020 & -2.9017410 \\ 3.0373970 & 2.0161120 & -3.9606550 \\ 3.9797640 & 1.6748640 & -4.4079710 \\ 2.3690170 & 1.1566880 & -3.8482590 \\ 2.5758750 & 2.7295490 & -4.6560160 \\ 4.2066370 & 3.9208790 & -2.7828580 \\ 5.1931430 & 3.5942100 & -3.1387020 \\ 3.7860620 & 4.6166520 & -3.5213600\end{array}$

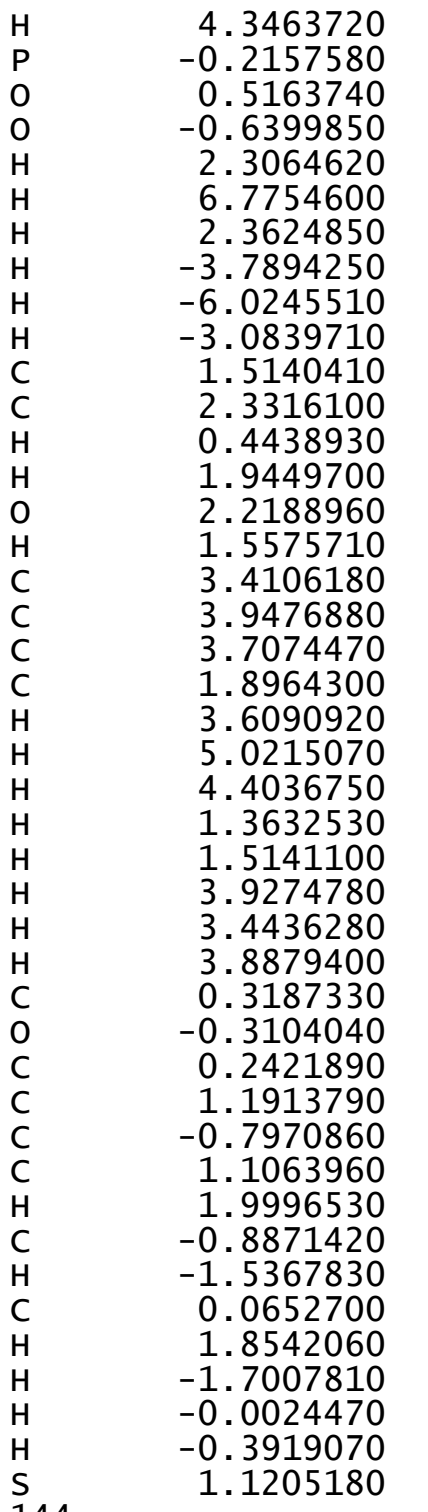
4.4587260
0.0821530
0.2021240
$-1.2868000$
3. 0731920
$-1.5968190$
1.1396220
2.4761960
$-3.5654530$
$-1.4491800$
$-2.8480640$
$-2.4432350$
$-2.6714850$
$-1.6747310$
$-1.6407870$
$-0.8430200$
$-4.3704980$
$-4.3417170$
$-2.9756320$
$-4.0985700$
$-5.3458010$
$-4.5612120$
$-2.2230850$
$-4.9300210$
$-4.0161110$
$-3.6121420$
$-5.1206480$
$-3.0053570$
$-3.1669860$
$-2.0525880$
$-3.5479160$
$-4.4083710$
$-3.0343470$
$-4.7440560$
$-4.7971650$
$-3.3854740$
$-2.3731760$
$-4.2377500$
$-5.3993880$
$-2.9929340$
$-4.5050660$
$-1.7977010$

$-1.8375090$

$-0.9955000$

$-2.3036500$

$-0.5404890$

$-2.2537700$

$-0.6166930$

2. 1789080

$-1.4556990$

$-3.3412940$

1. 3043840

$-2.2301040$

$-1.0833920$

$-2.1365180$

$-0.4259160$

$-2.7203430$

$-2.5687550$

$-2.9717770$

$-1.5324420$

$-0.8690410$

$-2.9894200$

$-3.4308330$

$-1.5153880$

$-1.2594330$

$-2.5071610$

$-4.0142530$

$-3.5749850$

$-0.9437850$

0.2114470

2. 0870010

1.8215470

3. 5216340

4. 1046130

4.3253480

5.4545830

3.4919210

5.6716820

3. 8849810

6.2417970

5.8948700

6.2771740

7. 2939790

0.8107870

0.9467420

$\begin{array}{lrrr}\text { TS229(S, S) } & \text { G }[\mathrm{B} 97-\mathrm{D} / \mathrm{def2}-\mathrm{TZVP}]=-3633.750215 \\ 0 & 0.5809860 & 0.1635920 & -0.3039300\end{array}$ 


\begin{tabular}{|c|c|c|c|}
\hline 0 & & & \\
\hline c & $\begin{array}{r}-1.7214800 \\
0.8401870\end{array}$ & $\begin{array}{r}-0.1050500 \\
1.2184840\end{array}$ & $\begin{array}{l}-1.3870790 \\
-1.1672260\end{array}$ \\
\hline $\mathrm{C}$ & -0.1337860 & 2.1923160 & -1.3611890 \\
\hline C & 2.1340030 & 1.2943560 & -1.7465050 \\
\hline C & 0.1205250 & 3.2383810 & -2.3069520 \\
\hline$c$ & 2.3944570 & 2.3469150 & -2.6021920 \\
\hline C & 1.4123490 & 3. 3124010 & -2.9301200 \\
\hline H & 3.3849640 & 2.4392080 & -3.0409080 \\
\hline C & -2.1942560 & 0.9905710 & -0.6540320 \\
\hline C & -3.4560750 & 0.8706890 & -0.0120230 \\
\hline C & -1.4074910 & 2.1316530 & -0.6006330 \\
\hline C & -3.8940230 & 1.9532020 & 0.7262680 \\
\hline C & -1.8269920 & 3. 2088210 & 0.2475560 \\
\hline$c$ & -3.0995860 & 3.1122030 & 0.9073290 \\
\hline $\mathrm{H}$ & -4.8616500 & 1.9031320 & 1.2181450 \\
\hline C & -2.7159650 & 5.2651920 & 1.9825470 \\
\hline C & -1.4458050 & 5.3449660 & 1.360498 \\
\hline C & -1.0136680 & 4.3443200 & 0.5129700 \\
\hline C & -3.5228100 & 4.1686530 & 1.7595440 \\
\hline $\mathrm{H}$ & -3.0456750 & 6.0625460 & 2.6447260 \\
\hline $\mathrm{H}$ & -0.8023660 & 6.1999720 & 1.5562420 \\
\hline H & -0.0348130 & 4.4126240 & 0.049769 \\
\hline $\mathrm{H}$ & -4.4923200 & 4.0845590 & 2.2473060 \\
\hline C & -0.5760940 & 5.1874940 & -3.5972710 \\
\hline C & 0.7092210 & 5.2768560 & -4.1873540 \\
\hline C & 1.6804450 & 4.3533320 & -3.8606600 \\
\hline C & -0.8628080 & 4.1952300 & 580990 \\
\hline $\mathrm{H}$ & -1.3460610 & 5.9049400 & -3.8725500 \\
\hline $\mathrm{H}$ & 0.9225760 & 6.0670970 & -4.9036190 \\
\hline $\mathrm{H}$ & 2.6677620 & 4.4000850 & -4.3168640 \\
\hline $\mathrm{H}$ & -1.8528750 & 4.1352210 & -2.2401420 \\
\hline C & 3.2349090 & 0.3400320 & -1.4075240 \\
\hline C & 3.5859260 & -0.6917650 & -2.3048440 \\
\hline C & 3.9947860 & 0.5588700 & -0.2351900 \\
\hline C & 4.7372530 & -1.4452840 & -2.0457180 \\
\hline C & 5.1435650 & -0.2138040 & -0.0283320 \\
\hline C & 5.5407350 & -1.2075400 & -0.9271580 \\
\hline $\mathrm{H}$ & 5.0188840 & -2.2318920 & -2.7407400 \\
\hline $\mathrm{H}$ & 5.7498150 & -0.0454570 & 0.8588470 \\
\hline C & -4.2663090 & -0.3772110 & -0.1199650 \\
\hline C & -4.4874580 & -1.1818310 & 1.0233890 \\
\hline C & -4.8205680 & -0.7526460 & -1.3669420 \\
\hline $\mathrm{C}$ & -5.2642140 & -2.3404450 & 0.889036 \\
\hline C & -5.5800110 & -1.9233090 & -1.446586 \\
\hline C & -5.8125720 & .7341870 & $-0 \quad 332336$ \\
\hline & -5.43917 & & \\
\hline
\end{tabular}

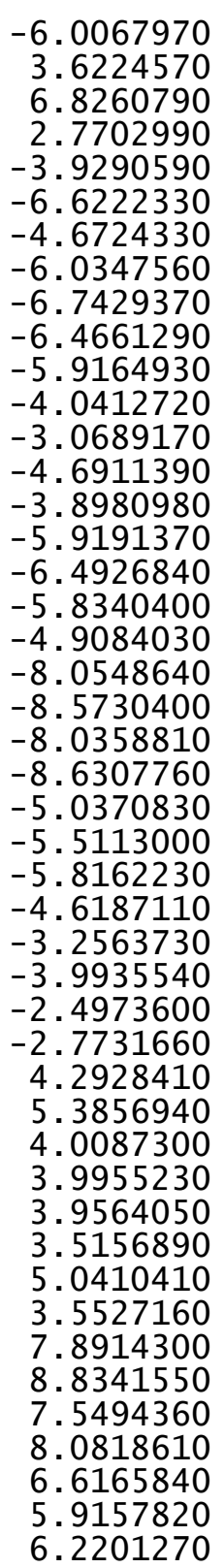

-2.2065830
1.6616260
-1.9871730
-0.9588300
-0.8316620
-4.0127800
0.0970600
0.6871410
-0.1145280
1.2858750
1.3285530
-0.6924470
-1.1020410
-1.5219010
-0.0335850
-5.0276830
-5.9629850
-4.6223240
-5.2527350
-3.7365070
-3.0357070
-3.3001740
-4.6705080
-0.2559770
0.6229180
-1.0108560
0.0358810
-2.0282990
-2.7955660
-2.4791320
-1.6876450
2.9911610
2.8868240
3.2854500
3.7904190
1.3274320
0.3709280
1.2810270
2.1087300
-1.5695330
-2.1021040
-1.8127650
-0.4899450
-3.5123380
-3.8412610
-3.8266740
$-2.4062980$

0.7512720

$-0.7039460$

$-3.5636720$

2. 3981570

$-0.4514810$

$-2.6235220$

$-3.0397180$

$-3.2879330$

$-2.2270640$

$-3.9234400$

$-3.7846070$

$-3.4898760$

$-4.0932770$

$-4.6514910$

$-1.3738630$

$-1.4232740$

$-2.3907850$

$-1.0107330$

$-0.9458840$

$-0.2789560$

$-1.9533250$

$-0.9895960$

3. 3038990

2.8514300

3.4764400

4.2767880

3.0965560

3.3662230

2. 4500930

4.0214800

0.3476370

0.3871110

$-0.6689690$

1.0401020

2.2133650

2. 5175460

2. 3758940

2.8671040

$-1.7379320$

$-1.5543060$

$-2.7526920$

$-1.6904310$

$-0.7427120$

0.0334960
-1.7163120 


\begin{tabular}{|c|c|c|}
\hline $\begin{array}{l}7.5726190 \\
2.6043350 \\
3.5589960 \\
2.2175930 \\
1.9017410 \\
3.4007220 \\
4.4150430 \\
2.8012090 \\
3.4647650 \\
0.5619300 \\
0.0505090 \\
1.0026610 \\
1.7674320 \\
7.2017160 \\
2.5378580 \\
4.0124280 \\
6.6889070 \\
3.1644360 \\
0.5146250 \\
0.5258850 \\
0.9363570 \\
1.7611380 \\
0.5489680 \\
2.1910280 \\
2.0635540 \\
0.9982250 \\
0.0805420 \\
1.8186070 \\
2.8236380 \\
0.7064660 \\
2.1677490 \\
0.7105640 \\
1.3360050 \\
1.1313900 \\
2.0737250 \\
0.0771300 \\
1.7461550 \\
1.6147100 \\
0.9585190 \\
3.0288470 \\
3.7779670 \\
3.5005420 \\
1.5116660 \\
3.2471920 \\
4.8585020 \\
4.0537660\end{array}$ & $\begin{array}{r}-4.0275710 \\
-2.4571890 \\
-2.9102070 \\
-3.0012160 \\
-2.5825940 \\
-0.2441190 \\
-0.6281960 \\
-0.4253550 \\
0.8371230 \\
-0.9713790 \\
-1.9753930 \\
-1.4665280 \\
-0.5453590 \\
-1.7217290 \\
1.8107490 \\
0.9425690 \\
-4.4555240 \\
-0.0605310 \\
-0.0253050 \\
0.1223920 \\
1.2417260 \\
1.2521270 \\
2.4704480 \\
2.4578980 \\
0.3078820 \\
3.6737310 \\
2.4795830 \\
3.6726430 \\
2.4523290 \\
4.6111050 \\
4.6123730 \\
-0.6465360 \\
-1.4815520 \\
-3.7483680 \\
-2.6306160 \\
-3.4906890 \\
-1.6376110 \\
-3.6146340 \\
-2.9842440 \\
-5.3333860 \\
-4.1542040 \\
-2.8418580 \\
-5.0791090 \\
-6.2597430 \\
-4.3389330 \\
-2.7990850\end{array}$ & $\begin{array}{r}-0.5831790 \\
-3.8723240 \\
-4.1696580 \\
-3.0053640 \\
-4.7057910 \\
-4.7762510 \\
-4.9513020 \\
-5.6785820 \\
-4.6122650 \\
-0.6486720 \\
-1.6395310 \\
0.7039830 \\
-3.4065480 \\
0.2943970 \\
0.6970000 \\
-2.4068510 \\
0.5524950 \\
2.2720780 \\
3.3298670 \\
2.5581090 \\
3.9818880 \\
5.1212810 \\
3.4068420 \\
5.6707110 \\
5.5649500 \\
3.9481290 \\
2.5229060 \\
5.0814050 \\
6.5551570 \\
3.4806670 \\
5.5036340 \\
1.8674130 \\
3.5569070 \\
0.8750100 \\
1.0189040 \\
0.9559700 \\
0.7402010 \\
-0.5162160 \\
-1.0067320 \\
1.4725240 \\
2.1106700 \\
1.3605500 \\
1.4884820 \\
2.0161540 \\
2.1195790 \\
0.4109090\end{array}$ \\
\hline
\end{tabular}

$\begin{array}{llll}\mathrm{H} & 1.1497040 & -5.0579200 & 2.5262070 \\ \mathrm{H} & 0.9644590 & -5.8733710 & 0.9662280 \\ \mathrm{H} & 3.3684120 & -5.4710010 & 0.4372340 \\ \mathrm{H} & 3.4565500 & -4.0446080 & 3.1557680 \\ \mathrm{H} & 3.8441520 & -1.9607060 & 1.9145740\end{array}$

$\begin{array}{llll}144 & & \\ \text { TS230 } & (\mathrm{S}, \mathrm{S}) & \mathrm{G}[\mathrm{B} 97-\mathrm{D} / \mathrm{def} 2-\mathrm{TZVP}]= & -3633.749986 \\ 0 & 0.7324860 & 0.3377610 & -0.3499190\end{array}$

$0 \quad-1.4779180 \quad 0.2202340 \quad-1.6315290$

C $\quad 0.9964850 \quad 1.5493650 \quad-0.9725770$

C $\quad-0.0019730 \quad-2.5148210 \quad-1.0207760$

C $\quad 2.3130390 \quad 1.7716190 \quad-1.4559720$

C $\quad 0.2655660 \quad 3.7445330 \quad-1.7064160$

$\begin{array}{lll}2.5829370 & 2.9861370 & -2.0538480\end{array}$

$\begin{array}{lll}1.5857030 & 3.9779370 & -2.2237250\end{array}$

$3.5914820 \quad 3.1925780 \quad-2.4041710$

$\begin{array}{lll}-2.0502060 & 1.1321080 & -0.7327600\end{array}$

$\begin{array}{lll}-3.3491820 & 0.8494500 & -0.2299930\end{array}$

$\begin{array}{lll}-1.3153840 & 2.2544410 & -0.3783270\end{array}$

$\begin{array}{lll}-3.8710410 & 1.7273230 & 0.7004090\end{array}$

$\begin{array}{lll}-1.8265660 & 3.1026730 & 0.6581090\end{array}$

$\begin{array}{lll}-3.1303270 & 2.8285390 & 1.1958090\end{array}$

$\begin{array}{lll}-4.8660320 & 1.5464040 & 1.0982960\end{array}$

$\begin{array}{lll}-2.8838980 & 4.6775950 & 2.7637040\end{array}$

$\begin{array}{lll}-1.5855800 & 4.9292050 & 2.2560770\end{array}$

$\begin{array}{lll}-1.0722580 & 4.1649900 & 1.2268880\end{array}$

$\begin{array}{lll}-3.6381050 & 3.6472050 & 2.2415210\end{array}$

$\begin{array}{lll}-3.2774970 & 5.2895550 & 3.5721490\end{array}$

$\begin{array}{lll}-0.9844220 & 5.7277990 & 2.6851640\end{array}$

$\begin{array}{lll}-0.0734800 & 4.3639230 & 0.8519540\end{array}$

$\begin{array}{lll}-4.6297610 & 3.4304960 & 2.6346560\end{array}$

$\begin{array}{lll}-0.4272690 & 5.9088430 & -2.5931480\end{array}$

$0.8830390 \quad 6.1495130 \quad-3.0756080$

$\begin{array}{lll}1.8664640 & 5.1989590 & -2.8952020\end{array}$

$\begin{array}{lll}-0.7280290 & 4.7384200 & -1.9251450\end{array}$

$\begin{array}{lll}-1.2053640 & 6.6517680 & -2.7543490\end{array}$

$1.1067280 \quad 7.0783070 \quad-3.5955720$

$\begin{array}{lll}2.8742420 & 5.3629990 & -3.2727450\end{array}$

$\begin{array}{lll}-1.7369450 & 4.5660080 & -1.5638630\end{array}$

$3.4101350 \quad 0.7785700 \quad-1.2436690$

$3.7906080-0.1051370 \quad-2.2790200$

$4.1060540 \quad 0.7809460 \quad-0.0163980$

$4.8884470-0.9472440 \quad-2.0746420$

$5.1943330 \quad-0.0867670 \quad 0.1419990$

$5.6079780 \quad-0.9496410 \quad-0.8746040$

$5.1930980-1.6200830 \quad-2.8716720$ 


$\begin{array}{lr}\mathrm{H} & 5.7346720 \\ \mathrm{C} & -4.1140270 \\ \mathrm{C} & -4.4125500 \\ \mathrm{C} & -4.5558680 \\ \mathrm{C} & -5.1504460 \\ \mathrm{C} & -5.2863500 \\ \mathrm{C} & -5.5925030 \\ \mathrm{H} & -5.3796310 \\ \mathrm{H} & -5.6325610 \\ \mathrm{C} & 3.7025810 \\ \mathrm{C} & 6.8246510 \\ \mathrm{C} & 3.0616740 \\ \mathrm{C} & -3.9757370 \\ \mathrm{C} & -6.3700880 \\ \mathrm{C} & -4.3198600 \\ \mathrm{C} & -5.6524050 \\ \mathrm{H} & -6.3231470 \\ \mathrm{H} & -6.1606320 \\ \mathrm{H} & -5.4718890 \\ \mathrm{C} & -3.5823050 \\ \mathrm{H} & -2.6296790 \\ \mathrm{H} & -4.1903180 \\ \mathrm{H} & -3.3803480 \\ \mathrm{C} & -7.6810180 \\ \mathrm{H} & -8.2469510 \\ \mathrm{H} & -7.4670770 \\ \mathrm{H} & -8.3092030 \\ \mathrm{C} & -5.5107140 \\ \mathrm{H} & -4.5881990 \\ \mathrm{H} & -5.2329850 \\ \mathrm{H} & -6.0695220 \\ \mathrm{C} & -5.1737060 \\ \mathrm{H} & -5.6539270 \\ \mathrm{H} & -5.9264280 \\ \mathrm{H} & -4.8434580 \\ \mathrm{C} & -3.2982350 \\ \mathrm{H} & -4.0114600 \\ \mathrm{H} & -2.4638760 \\ \mathrm{H} & -2.9124100 \\ \mathrm{C} & 4.8788180 \\ \mathrm{H} & 5.6752090 \\ \mathrm{H} & 5.3062650 \\ \mathrm{H} & 4.5357840 \\ \mathrm{C} & 3.0846980 \\ \mathrm{H} & 2.2279760 \\ \mathrm{H} & 3.8235580 \\ & \end{array}$

$\begin{array}{rr}-0.0938070 & 1.0871250 \\ -0.3506870 & -0.6765490 \\ -1.3872240 & 0.2439000 \\ -0.4477670 & -2.0156690 \\ -2.4892110 & -0.2000880 \\ -1.5764440 & -2.4080220 \\ -2.6085980 & -1.5208210 \\ -3.2840870 & 0.5057750 \\ -1.6507610 & -3.4376840 \\ 1.6851310 & 1.1415530 \\ -1.8412770 & -0.6903110 \\ -0.1097040 & -3.6164050 \\ -1.3462100 & 1.7043360 \\ -3.8292740 & -1.9785190 \\ 0.6505350 & -3.0453370 \\ 1.3184080 & -3.4399150 \\ 0.5899190 & -3.9139060 \\ 1.7288690 & -2.5577170 \\ 2.1351310 & -4.1517790 \\ 0.1249520 & -4.2907960 \\ -0.3376390 & -4.0096240 \\ -0.6205020 & -4.8200300 \\ 0.9521340 & -4.9843650 \\ -3.9979340 & -1.1870480 \\ -4.8604390 & -1.5635700 \\ -4.1679570 & -0.1236620 \\ -3.1019960 & -1.2709710 \\ -5.1045450 & -1.8766030 \\ -5.0036810 & -2.4621640 \\ -5.2941820 & -0.8313240 \\ -5.9744180 & -2.2470250 \\ -1.0324300 & 2.6228600 \\ -0.0850860 & 2.3497580 \\ -1.8288160 & 2.5486850 \\ -0.9666840 & 3.6684670 \\ -2.6523190 & 2.1604950 \\ -3.4868230 & 2.1705900 \\ -2.9081190 & 1.5005730 \\ -2.5282260 & 3.1806610 \\ 2.5374730 & 1.6503090 \\ 1.9056460 & 2.0643590 \\ 3.1390080 & 0.8379200 \\ 3.2140140 & 2.4440970 \\ 0.8613850 & 2.2860250 \\ 0.2828690 & 1.9265850 \\ 0.1658540 & 2.7052950\end{array}$

2. 7392880 7.9933860

8.8876240

7.7246660

8. 2367190

6.5277670

5.7315830

6.2133060

7.4289860

2.9185550

3.8906780

2. 4724070

2. 2729770

3. 7680120

4.7908790

3. 2259810

3.8238780

$-0.3445760$

0.2774430

$-0.8661350$

2. 0495510

7. 1324900

2.9318030

$-3.6929940$

$-6.6287040$

$-3.2417670$

1. 2853360

2. 0717680

0.2097510

1.6710370

1. 9641690

1. 3135000

3. 2342910

3. 7496650

3.4448840

1. 7125440

3.4708220

4.8310020

4.1324400

1. 2007430

1.3392800

3.7337430

3. 2698690

3. 5899440

0.0360610
-0.7121810
1. 5204730

$-1.3440270$

$-1.9589150$

$-1.4099720$

$-0.2986560$

$-3.3219650$

$-3.7113000$

$-3.4513720$

$-3.9276500$

$-1.5109600$

$-1.9074940$

$-2.2122750$

$-1.4548450$

0.8329290

0.4794190

0.8533610

1.8552030

$-0.7393040$

$-1.5498800$

$-1.4762570$

0.2745760

$-1.7630400$

2.3776330

1.4286260

$-3.6779320$

$-0.5454210$

$-3.7446490$

$-2.6388060$

$-3.5861020$

$-1.6361730$

$-3.2145250$

$-2.5516550$

$-5.2961830$

$-4.2742260$

$-2.8329900$

$-5.1600160$

$-6.3143130$

$-4.3816190$

$-2.5046560$

$-5.4352130$

$-5.8259990$

$-5.1380540$

$-4.4628550$

$-2.1098390$

$-0.7483320$

$-0.3186970$
3. 0926520

$-1.5647840$

$-1.3966020$

$-2.6275590$

$-1.3369430$

$-0.9908950$

$-0.3459160$

$-2.0342840$

$-0.8296170$

$-4.2338410$

$-4.5550190$

$-3.5221510$

$-5.1193480$

$-4.6124990$

$-4.8018200$

$-5.5676970$

$-4.2222630$

$-0.9702030$

$-2.0690860$

0.2345710

$-3.4446760$

0.3622190

0.7868450

$-2.5999880$

$-3.0361790$

1.8235760

0.2000780

0.7617220

0.1509770

0.6973480

$-1.0042060$

$-1.4622050$

0.7033870

1. 7278860

1. 2879790

0.5229040

1. 0329950

1.8724530

0.4957420

1. 4560290

$-0.2645960$

$-0.2615090$

2.6982140

2. 0989260

3. 4068780

2. 4286670 


\begin{tabular}{|c|c|c|c|}
\hline & & & \\
\hline c & $\begin{array}{l}0.1189340 \\
0.6930640\end{array}$ & $\begin{array}{r}0.2212540 \\
-0.1289970\end{array}$ & $\begin{array}{l}4.5336540 \\
5.7700740\end{array}$ \\
\hline C & -0.3693820 & 1.5347320 & 4.3584100 \\
\hline$C$ & 0.7731040 & 0.8024120 & 6.8036580 \\
\hline $\mathrm{H}$ & 1.0725010 & -1.1370330 & 5.9112380 \\
\hline C & -0.2767090 & 2.4661020 & 5.3912980 \\
\hline $\mathrm{H}$ & -0.8053870 & 1.8278670 & 3.4091430 \\
\hline C & 0.2914550 & 2.1039300 & 6.6176790 \\
\hline $\mathrm{H}$ & 1.2131180 & 0.5144070 & 7.7555180 \\
\hline $\mathrm{H}$ & -0.6483080 & 3.4762160 & 5.2355590 \\
\hline $\mathrm{H}$ & 0.3602890 & 2.8311640 & 7.4237610 \\
\hline $\mathrm{H}$ & -0.7426620 & -0.9041550 & 1.5579800 \\
\hline & 0.8413110 & -2.2296940 & 3.3986450 \\
\hline & G[B97-L & {$[Z V P]=-363$} & 58 \\
\hline 0 & 0.3502990 & 0.8806910 & -0. 1637840 \\
\hline 0 & -2.0059970 & 0.4652100 & -1.0173070 \\
\hline C & 0.3160380 & 2.1889950 & -0.6290550 \\
\hline $\mathrm{C}$ & -0.8279770 & 2.9527360 & -0.4295780 \\
\hline C & 1.5118630 & 2.6990660 & 2056290 \\
\hline C & -0.8660250 & 4.2660750 & 5072220 \\
\hline C & 1.4968010 & 3.9970250 & -1.6698340 \\
\hline C & 0.3205170 & 4.7893990 & -1.6282410 \\
\hline $\mathrm{H}$ & 2.4054120 & 4.4333500 & -2.0764340 \\
\hline C & -2.5002620 & 1.1691700 & 0.0771720 \\
\hline C & -3.5838730 & 4870 & 78680 \\
\hline C & -1.9504370 & 2.4084480 & 0.3810770 \\
\hline $\mathrm{C}$ & -4.1174290 & 1.3069440 & 1.8372160 \\
\hline $\mathrm{C}$ & -2.4497070 & 3.1023690 & 1.5363980 \\
\hline C & -3.5680290 & 2.5463760 & 090 \\
\hline $\mathrm{H}$ & -4.9675540 & & \\
\hline C & -3.5096960 & 4.4041150 & 0 \\
\hline $\mathrm{C}$ & -2.3 & 3050 & 300 \\
\hline $\mathrm{C}$ & -1.8623890 & 4.2952740 & 2.0417010 \\
\hline $\mathrm{C}$ & -4.0862390 & & \\
\hline $\mathrm{H}$ & -3.9101480 & 4. & 79190 \\
\hline $\mathrm{H}$ & -1.90 & 5.8 & 244060 \\
\hline $\mathrm{H}$ & -0.9916180 & 4.7 & 43880 \\
\hline $\mathrm{H}$ & -4.9409660 & 2.8021570 & 3.9010210 \\
\hline C & -2.0387840 & 6.3254080 & 65720 \\
\hline $\mathrm{C}$ & -0.8590490 & 6.8534210 & 1660990 \\
\hline C & 0.2942750 & 6.0961070 & -2.1869570 \\
\hline$c$ & -2.0410060 & & 0198930 \\
\hline $\mathrm{H}$ & -2.9526030 & 55430 & 5915550 \\
\hline H & -0.8688640 & 194310 & 30990 \\
\hline$\Pi$ & 1.2036930 & 6.4820820 & -2.6442870 \\
\hline
\end{tabular}

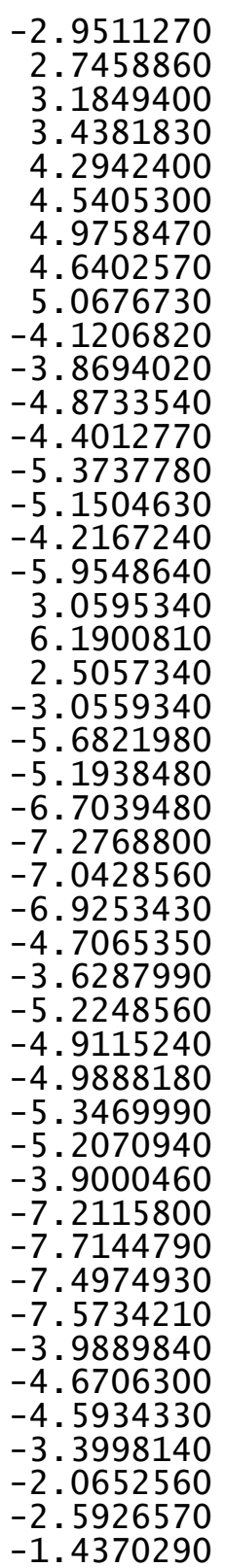
.9511270
3.4381830
4.2942400
4.5405300
4.6402570
5.0676730
$-4.1206820$
$-3.8694020$
$-4.4012770$
$-5.3737780$
$-5.1504630$
2. 5057340
.0559340
$-6.7039480$
$-7.2768800$
$-7.0428560$
$-6.9253430$
$-4.7065350$
$-4.9888180$
$-5.3469990$
. 2070940
$-7.7144790$
.
$-4.6706300$
.
$-1.4370290$

4.6708770

1.8560330

1.1553350

1.7247330

0.3086600

0.8655380

0.1369200

$-0.2452520$

0.7644970

$-0.7511610$

$-1.8772240$

$-0.8896440$

$-3.1156010$

$-2.1494050$

$-3.2754870$

$-3.9895730$

$-2.2529840$

2.5258350

$-0.7740820$

1.3530910

$-1.7833920$

$-4.6385590$

0.2920740

0.6003870

$-0.2545610$

0.8096480

1.4733720

0.0598110

$-0.1369430$

$-0.7941060$

0.9477300

$-5.1388550$

$-6.1429910$

$-4.4639690$

$-5.1773070$

$-4.6282860$

$-4.2916930$

$-3.9515880$

$-5.6345920$

$-1.7046610$

$-0.8493130$

$-2.6193390$

$-1.6084560$

$-2.9491730$

-3.8934840
-3.0666340
$-0.5794480$

$-1.1743560$

$-2.3203620$

0.0499170
-2.2014330

$-0.9919240$

$-3.0714760$

1.0621170

0.4015420

1.2189790

$-0.7868350$

0.8355810

$-1.1288840$

$-0.3317030$

1.4572700

$-2.0425310$

1.2904150

$-0.9192910$

$-3.6692290$

2.5049530

$-0.7375230$

$-1.6932630$

$-1.6679640$

$-2.0507550$

$-0.6450540$

$-2.2965660$

$-3.1351350$

$-3.1511150$

$-3.5905300$

$-3.7480100$

$-2.0203490$

$-2.2846030$

$-2.8587150$

$-1.8875880$

$-0.9196000$

$-0.0040740$

$-1.7355310$

$-1.1689590$

3.7308430

3.6628910

3.8008970

4.6531600

2.6824530

2.8695750

1.7944840 


$\begin{array}{rr}-1.4187040 & -2.7513230 \\ 4.1489630 & 3.5734380 \\ 5.1038030 & 3.0799520 \\ 4.3026470 & 4.2403550 \\ 3.8608320 & 4.1802900 \\ 2.8005440 & 1.6320760 \\ 1.9992130 & 0.9160120 \\ 3.6997890 & 1.0693120 \\ 2.5091150 & 2.2502160 \\ 6.2381130 & -1.6172320 \\ 7.0777280 & -2.3227500 \\ 6.3810720 & -0.9788540 \\ 5.3097020 & -2.1839840 \\ 7.4862930 & 0.0488320 \\ 7.4826520 & 0.6185890 \\ 7.5793090 & 0.7582470 \\ 8.3640950 & -0.6113920 \\ 2.5021320 & 0.1028240 \\ 3.5136980 & -0.1376870 \\ 2.1026330 & -0.7649930 \\ 1.8795500 & 0.2885640 \\ 3.1726080 & 2.5198900 \\ 4.2228450 & 2.2754050 \\ 2.6594290 & 2.6940530 \\ 3.1482190 & 3.4474100 \\ -0.5590820 & -0.2634270 \\ -0.1375840 & -0.5108840 \\ -0.5593180 & -1.4168870 \\ 1.4605080 & 1.6225820 \\ 6.1331870 & -1.4613480 \\ 2.1335740 & 3.0727100 \\ -4.6770270 & 1.1787370 \\ -5.4414260 & -5.3409930 \\ -2.4688570 & -0.8594100 \\ 0.9303000 & -3.5137930 \\ 2.1321060 & -2.7035060 \\ 0.0729580 & -3.2697980 \\ 2.0094880 & -1.7036810 \\ 0.9772730 & -2.6685760 \\ 0.4620960 & -1.7789790 \\ 2.3995230 & -5.2898040 \\ 3.6098650 & -4.6788130 \\ 3.4821750 & -3.1500770 \\ 1.1003230 & -4.9847460 \\ 2.5206160 & -6.3754870 \\ 4.5400250 & -4.9218830\end{array}$

3.5467030
1.5951350
1.8203240
0.7368540
2.4640860
2.5143160
2.3113070
2.7938380
3.3739080
0.3665010
0.3225720
1.2476410
0.5106650
-1.0690790
-2.0067520
-0.2357340
-1.0613390
-4.5619640
-4.9145760
-4.0321020
-5.4462260
-4.4291350
-4.6402550
-5.3847210
-3.8476610
-0.8956120
-2.3194440
0.0681250
-3.4772190
-1.7757720
1.0858390
-1.3129410
0.0730130
2.4669880
-1.7514600
-1.5355410
-1.1272050
-1.1353310
-2.9626740
-2.7330410
-2.8274610
-2.1044690
-1.9895380
-2.0617940
-2.9175940
-2.6307430

3.6714290
1.1061310
0.2174650
2.3316030
3.6819480
4.2227060
2.0138180
0.9519680
2.7344920
4.1044170
2.0520870
4.7767720
4.6375470
2.7240090
0.9963900
4.0888000
5.8394160
2.1840140
4.6137240
0.3984350
2.5345910
$-2.6707510$
$-5.5066940$
$-5.3301830$
$-4.8798260$
$-5.1085100$
$-2.7243920$
$-2.1156220$
$-1.3734410$
$-1.8091210$
$-2.0970850$
$-1.1856820$
$-1.7626170$
$-2.5611250$
$-0.8700090$
$-0.9559590$
$-1.1517000$
$-1.9748470$
$-0.3987880$
$-0.8947140$
$-1.5088580$
$-3.3192840$

$-2.9605730$

$-1.0946710$

$-2.6138930$

$-3.8439590$

$-1.0956480$

$-1.3050880$

2. 2571230

2. 0864850

3. 5200170

3.6645720

4. 5843900

4.8386040

2.8408150

5.7639140

4.4817920

5.8935060

4. 9297010

6.5818020

6.8108790

$\begin{array}{ll}\mathrm{H} & 0.3984350 \\ \mathrm{~S} & 2.5345910\end{array}$

1. 2181700

TS232(S,S) G[B97-D/def2-TZVP $]=-3633.749658$

$\begin{array}{lrrr}\text { TS232(S, S } r \text { G } \\ \text { O } & 0.7072950 & 0.6062410 & 0.0160230 \\ \mathrm{O} & -1.4279860 & 1.2059960 & -1.2451010 \\ \mathrm{C} & 1.0068900 & 1.9586200 & 0.1136340 \\ \mathrm{C} & 0.0113580 & 2.8480530 & 0.5065550 \\ \mathrm{C} & 2.3499910 & 2.3493950 & -0.1289850 \\ \mathrm{C} & 0.3245810 & 4.2472360 & 0.5435070 \\ \mathrm{C} & 2.6619720 & 3.6885810 & 0.0032700 \\ \mathrm{C} & 1.6796090 & 4.6625060 & 0.3063010 \\ \mathrm{H} & 3.6886840 & 4.0119750 & -0.1507010 \\ \mathrm{C} & -2.0413830 & 1.5449550 & -0.0346590 \\ \mathrm{C} & -3.3589380 & 1.0665170 & 0.1950400 \\ \mathrm{C} & -1.3419320 & 2.3443720 & 0.8596480 \\ \mathrm{C} & -3.9640340 & 1.4238120 & 1.3839780 \\ \mathrm{C} & -1.9433770 & 2.6222880 & 2.1327380 \\ \mathrm{C} & -3.2849500 & 2.1692420 & 2.3787160 \\ \mathrm{H} & -4.9816660 & 1.0970240 & 1.5802040 \\ \mathrm{C} & -3.2090680 & 3.1173550 & 4.6221800 \\ \mathrm{C} & -1.8719840 & 3.5275550 & 4.3971210 \\ \mathrm{C} & -1.2562460 & 3.2878690 & 3.1845260 \\ \mathrm{C} & -3.8972230 & 2.4482990 & 3.6314680 \\ \mathrm{H} & -3.6839730 & 3.3193790 & 5.5796550 \\ \mathrm{H} & -1.3227130 & 4.0322040 & 5.1889350 \\ \mathrm{H} & -0.2299600 & 3.6036540 & 3.0288090\end{array}$




$\begin{array}{rrr}-4.9175410 & 2.1066390 & 3.7967090 \\ -0.3096510 & 6.5887620 & 0.8040820 \\ 1.0347070 & 6.9922490 & 0.6103680 \\ 2.0061930 & 6.0451960 & 0.3616300 \\ -0.6553120 & 5.2524470 & 0.7724650 \\ -1.0781600 & 7.3395940 & 0.9743810 \\ 1.2937320 & 8.0480980 & 0.6443120 \\ 3.0396050 & 6.3410420 & 0.1895020 \\ -1.6898860 & 4.9583720 & 0.9183460 \\ 3.4249450 & 1.3738440 & -0.4858830 \\ 3.9585220 & 1.3673720 & -1.7935070 \\ 3.9862530 & 0.5443160 & 0.5127340 \\ 5.0941820 & 0.5877890 & -2.0538120 \\ 5.1211390 & -0.2119760 & 0.2029500 \\ 5.7084230 & -0.1845680 & -1.0667010 \\ 5.5285190 & 0.5992650 & -3.0518300 \\ 5.5737560 & -0.8214310 & 0.9822330 \\ -4.0608720 & 0.2046880 & -0.8002290 \\ -4.3379900 & -1.1512060 & -0.4908140 \\ -4.4631930 & 0.7429120 & -2.0422030 \\ -5.0265500 & -1.9259220 & -1.4294550 \\ -5.1387230 & -0.0814080 & -2.9513960 \\ -5.4310830 & -1.4151810 & -2.6666520 \\ -5.2438650 & -2.9652930 & -1.1953610 \\ -5.4499190 & 0.3314430 & -3.9098090 \\ 3.4488020 & 0.5167840 & 1.9385600 \\ 6.9987480 & -0.9350990 & -1.3534390 \\ 3.3671030 & 2.2119580 & -2.9159910 \\ -3.9214350 & -1.7957930 & 0.8276750 \\ -6.1545260 & -2.2924380 & -3.6727930 \\ -4.2342020 & 2.2006120 & -2.4222910 \\ -5.5768950 & 2.9484460 & -2.5443720 \\ -6.1920340 & 2.5097410 & -3.3409540 \\ -6.1421420 & 2.8927210 & -1.6050950 \\ -5.4017480 & 4.0052830 & -2.7865190 \\ -3.4161730 & 2.3320480 & -3.7205580 \\ -2.4517190 & 1.8216260 & -3.6234490 \\ -3.9596200 & 1.8954820 & -4.5687040 \\ -3.2323800 & 3.3916420 & -3.9432900 \\ -7.4952050 & -2.8048810 & -3.1130360 \\ -8.0175600 & -3.4110620 & -3.8651250 \\ -7.3251380 & -3.4308540 & -2.2269770 \\ -8.1447770 & -1.9695400 & -2.8220890 \\ -5.2706050 & -3.4724480 & -4.1205930 \\ -4.3234540 & -3.1146740 & -4.5437030 \\ -5.0394790 & -4.1221490 & -3.2660990\end{array}$

-5.7899810
-5.1277280
-5.5944880
-5.8867980
-4.8111930
-3.2709110
-3.9985950
-2.4353540
-2.8938920
4.4003520
5.3851950
4.5355290
3.9954710
3.1993910
2.5065300
4.1344040
2.7606690
6.8404640
7.8013840
6.5023470
6.1100830
8.1398140
8.2800340
7.9132210
9.0805010
3.1361840
4.0844380
2.4893920
2.6524960
4.2621900
5.2536230
3.8150710
4.3944590
-0.2894630
0.3988310
-0.8197130
2.3904910
7.2742180
2.4865970
-3.6661440
-6.3687390
-3.1765830
-0.0006990
-0.5387930
-0.1376730
0.6879080

$\begin{array}{rr}-4.0741100 & -4.8784800 \\ -1.9194280 & 1.7807230 \\ -0.9462370 & 1.9714320 \\ -2.5809840 & 1.3416570 \\ -2.3484220 & 2.7414020 \\ -3.1790830 & 0.6402460 \\ -3.9137280 & 0.2721990 \\ -3.1269870 & -0.0641060 \\ -3.5392620 & 1.6055990 \\ 1.2756950 & 2.8841650 \\ 0.7903320 & 2.9067120 \\ 2.3121410 & 2.5495700 \\ 1.2869780 & 3.9050640 \\ -0.9140240 & 2.4451480 \\ -1.4503540 & 1.7880670 \\ -1.4860760 & 2.5013360 \\ -0.8838190 & 3.4505500 \\ -2.4594870 & -1.2053670 \\ -2.9598110 & -1.3828540 \\ -2.7165530 & -0.1933020 \\ -2.8532210 & -1.9218870 \\ -0.4265840 & -0.4502970 \\ 0.6554910 & -0.5662210 \\ -0.6312980 & 0.6042860 \\ -0.9333430 & -0.7032140 \\ 1.4001240 & -4.2037530 \\ 1.0422480 & -4.6244910 \\ 0.5388500 & -4.0109150 \\ 2.0356960 & -4.9570480 \\ 3.4321010 & -3.2096150 \\ 3.1028460 & -3.5489840 \\ 4.0513540 & -3.9987710 \\ 4.0506720 & -2.3142410 \\ 0.0533520 & -1.1643250 \\ 0.0318470 & -2.5006420 \\ -1.2433500 & -0.6182750 \\ 2.5825620 & -2.5860840 \\ -0.7259570 & -2.3965290 \\ 1.0362710 & 1.9619610 \\ 2.6905800 & -1.6255270 \\ -1.6741870 & -4.5560950 \\ -1.1518210 & 1.3048300 \\ -2.8809710 & 2.2198610 \\ -1.7614670 & 1.8141560 \\ -3.0896130 & 3.6855460 \\ -3.9929190 & 4.3811760\end{array}$




$\begin{array}{rrr}-1.1127250 & -2.3661130 & 4.4045680 \\ 0.5442680 & -4.1681870 & 5.7563630 \\ 1.4475490 & -4.5438220 & 3.8340820 \\ -1.2606020 & -2.5532910 & 5.7781670 \\ -1.7577600 & -1.6683220 & 3.8802080 \\ -0.4321560 & -3.4520600 & 6.4592570 \\ 1.1962370 & -4.8618330 & 6.2820110 \\ -2.0233710 & -1.9967740 & 6.3178190 \\ -0.5455060 & -3.5930590 & 7.5318370 \\ -0.5951140 & -1.6235570 & 0.7795570 \\ 0.7547730 & -3.9936810 & 1.2092370 \\ 1.2716360 & -3.0772240 & -2.1053750 \\ 2.0706860 & -2.5788680 & -0.9822520 \\ 0.2026390 & -2.8771490 & -2.0551440 \\ 1.6806550 & -1.7499950 & -0.4062650 \\ 2.0079240 & -1.9352870 & -2.6953690 \\ 1.3714680 & -1.1063290 & -2.6420440 \\ 3.1677240 & -4.6682790 & -2.6697960 \\ 3.6844900 & -4.5154600 & -1.2316050 \\ 3.4323500 & -3.0995340 & -0.6870180 \\ 1.6545700 & -4.3997880 & -2.7306040 \\ 3.3715450 & -5.6798420 & -3.0393010 \\ 4.7578430 & -4.7297650 & -1.1791420 \\ 4.1438600 & -2.3779510 & -1.1097410 \\ 1.1161190 & -5.1766430 & -2.1693010 \\ 1.2821430 & -4.4220810 & -3.7620880 \\ 3.6928880 & -3.9645430 & -3.3291340 \\ 3.1719320 & -5.2424780 & -0.5864430 \\ 3.5826480 & -3.0420990 & 0.3966640 \\ & & \end{array}$

5. 4067110

5. 3699230

4. 3750610

3. 5325770

5. 4789880

6.0320850

4. 2686020

2. 7727970

6. 2191480

3. 7706420

2.7332880

1.4153150

3.4756050

4.8088570

2. 9786860

0.6087010

4. 2781130

$-2.0250860$

$-2.7962360$

$-2.5685910$

$-4.0925920$

$-3.8732880$

$-4.6471780$

$-4.6869940$

$-4.2976120$

3.7649410

3. 2043600

4.2537830

3. 1633280

4.1851680

3. 6409850

2. 7282340

4.5615370

$-1.8034230$

$-6.0451350$

$-2.2620800$

2. 6607840

3. 5282040

4.8775660

6.3834000

6.5435250

6.9037060

6.8320150

4.1600790

3. 0934450

4.2625810
0.3725200
-3.4355980
-4.2852290
-3.8366560
-2.1362990
-3.8039480
-5.3000990
-4.5005230
-1.4612510
-5.7059290
-6.5613720
-6.1883660
-4.5104350
-5.9911740
-7.5039350
-6.8275740
-3.8607310
-2.8768280
-2.4218730
-2.8634440
-1.9532220
-2.3898400
-1.9186250
-1.5954330
-2.3801610
1.6956770
2.6130150
2.1481160
3.9676480
3.5152370
4.4397720
4.6745620
3.8691900
-3.3870660
-1.3731540
-2.4638870
2.1717860
5.9081020
1.2052630
1.4930550
2.5141850
1.3926030
0.7917940
1.2741130
1.0504390
2.2719770

2.0946710

3. 4924000

2.9503100

1.9520450

3. 0423700

4. 2727690

3. 3273570

1.5540810

3. 4685460

$-1.7573720$

$-2.2028270$

$-2.0352420$

$-1.1321490$

$-1.9127180$

$-2.6870960$

$-2.3901280$

$-0.7970670$

$-0.5512770$

$-1.6468510$

0.7522230

$-1.4072110$

0.9374630

$-0.1235240$

$-2.2439640$

1. 9397160

0.3650470

1. 2870260

$-0.8779840$

0.9432460

$-1.1760530$

$-0.2836520$

1. 6448430

$-2.1346550$

1. 9620400

0.1083870

$-3.0727250$

2. 6415720

$-0.6530260$

$-1.8981210$

$-2.0567080$

$-2.4273060$

$-1.0953710$

$-2.7731610$

$-3.2588340$

- 3.1444540

$-3.7051930$ 


$\begin{array}{lrrr}\text { H } & 4.6000830 & 0.5453890 & -3.9526300 \\ \mathrm{C} & 4.1956770 & 6.8235670 & 0.3896280 \\ \mathrm{H} & 4.1313460 & 7.8727520 & 0.0721010 \\ \mathrm{H} & 3.6941700 & 6.7316480 & 1.3618230 \\ \mathrm{H} & 5.2529340 & 6.5616820 & 0.5239410 \\ \mathrm{C} & 2.0518650 & 6.3051340 & -0.8559750 \\ \mathrm{H} & 1.5806910 & 5.6793160 & -1.6236610 \\ \mathrm{H} & 1.4910940 & 6.1771460 & 0.0793180 \\ \mathrm{H} & 1.9751310 & 7.3569810 & -1.1630950 \\ \mathrm{C} & 3.7097570 & 2.4148120 & 3.7459210 \\ \mathrm{H} & 4.6459690 & 1.8866600 & 3.5318220 \\ \mathrm{H} & 3.9313790 & 3.4881410 & 3.8218240 \\ \mathrm{H} & 3.3290480 & 2.0705750 & 4.7172230 \\ \mathrm{C} & 1.3353700 & 2.8585550 & 3.0190340 \\ \mathrm{H} & 1.4852230 & 3.9250190 & 3.2323120 \\ \mathrm{H} & 0.6025310 & 2.7631220 & 2.2125820 \\ \mathrm{H} & 0.9281820 & 2.3938350 & 3.9267810 \\ \mathrm{C} & -2.4129280 & -4.7185440 & 2.4438320 \\ \mathrm{H} & -3.4544730 & -4.5706610 & 2.7586840 \\ \mathrm{H} & -2.4001900 & -5.4670100 & 1.6411240 \\ \mathrm{H} & -1.8452500 & -5.1110490 & 3.2983020 \\ \mathrm{C} & -1.7469780 & -2.3658780 & 3.1119720 \\ \mathrm{H} & -1.3413560 & -1.4085640 & 2.7663710 \\ \mathrm{H} & -2.7464610 & -2.1912760 & 3.5312420 \\ \mathrm{H} & -1.1092780 & -2.7486370 & 3.9195440 \\ \mathrm{C} & -7.1065800 & -2.1938160 & -0.6483730 \\ \mathrm{H} & -8.1113570 & -1.8018840 & -0.4410830 \\ \mathrm{H} & -6.9345910 & -2.1370060 & -1.7314020 \\ \mathrm{H} & -7.0732770 & -3.2499750 & -0.3518820 \\ \mathrm{C} & -6.1350800 & 0.1151230 & -0.2797100 \\ \mathrm{H} & -5.4163620 & 0.7109210 & 0.2932860 \\ \mathrm{H} & -5.9215670 & 0.2452550 & -1.3486830 \\ \mathrm{H} & -7.1437210 & 0.5008830 & -0.0811730 \\ \mathrm{C} & -2.6564300 & -1.2364280 & -3.9115800 \\ \mathrm{H} & -3.7332410 & -1.2278340 & -4.1252640 \\ \mathrm{H} & -2.3937640 & -0.3085370 & -3.3965780 \\ \mathrm{H} & -2.1280740 & -1.2643200 & -4.8732840 \\ \mathrm{C} & -2.7347300 & -3.7506780 & -3.7810960 \\ \mathrm{H} & -3.8313050 & -3.7604210 & -3.8486150 \\ \mathrm{H} & -2.3249470 & -3.7976900 & -4.7992860 \\ \mathrm{H} & -2.4184480 & -4.6480410 & -3.2372060 \\ \mathrm{P} & 0.4735150 & 0.0751360 & -0.6530310 \\ \mathrm{O} & -0.1457530 & 0.0445260 & -2.0246050 \\ \mathrm{O} & 0.1889930 & 1.2423000 & 0.2500940 \\ \mathrm{H} & -1.1683940 & -2.4868830 & -3.0205740 \\ \mathrm{H} & -6.2545890 & -1.4520270 & 1.1838390 \\ & & & \\ & & & \end{array}$
$-0.7710060$
4.7809550
4.0495620
2. 4665500
$-1.0678400$
$-2.2977870$
$-0.1833520$
$-2.1872370$
$-1.2431520$
$-0.7497940$
$-2.5094800$
$-3.6990530$
$-3.6504240$
$-1.1777170$
$-2.5758980$
$-4.6484270$
$-3.9265320$
$-1.0875310$
$-0.3189500$
$-2.5360190$
$-3.6736030$
$-4.3674590$
$-2.7927970$
$-1.9813860$
$-4.0449260$
$-5.1046600$
$-4.2119150$
$-6.2972670$
$-4.9868330$
$-5.4065180$
$-3.4105890$
$-6.4540940$
$-7.1089790$
$-5.5237100$
$-7.3886210$
$-1.0940880$
$-2.4572620$

144

TS234(S, S) G[B97-D/def2-TZVP $]=-3633.749504$

$\begin{array}{lrrr}\mathrm{O} & 0.7136150 & 0.2628520 & -0.4247970 \\ \mathrm{O} & -1.5581550 & 0.2530260 & -1.6023970 \\ \mathrm{C} & 0.9874950 & 1.4852560 & -1.0199830 \\ \mathrm{C} & 0.0178650 & 2.4809480 & -0.9918640 \\ \mathrm{C} & 2.2876690 & 1.6865400 & -1.5538770 \\ \mathrm{C} & 0.2913650 & 3.7255350 & -1.6466620 \\ \mathrm{C} & 2.5661600 & 2.9132420 & -2.1234560\end{array}$

1.6649410

$-3.5949780$

0.1788230

6.0479610

1.0946940

3.1236910

2.4744410

.9575090

1.5670570

2.0557110

1.2087560

4.7385160

4.3763940

2.9000430

.5097800

5.7882110

4.5699230

2. 2427090

.2174470

4.6756400

4.1251230

5.0058740

. 6828400

2.1569510

1.1325290

1.8508460

2.7774130

0.5887740

.4494760

3.7464370

0.2657880

$-0.1389000$

1.1920780

3.1722760

$-0.7132420$

0.9352360

1.2963970

3.6569680
1.5291970

$-1.6107370$

2. 5951170

$-2.0489150$

$-1.5804120$

$-1.4344570$

$-0.9980950$

$-3.0501490$

$-2.6682020$

$-3.3823790$

$-2.4795830$

$-2.0483530$

$-2.6460900$

$-3.6911120$

$-2.9917800$

$-2.8864090$

$-1.8103360$

$-3.3080490$

$-4.2927950$

$-1.5794150$

1.6335780

1.5415730

2. 3680850

2.4266620

2.9776520

3.0668060

1.9508090

3.6165980

2.9333760

3.6619290

3.0963640

4.0753680

4.1556580

1. 0196720

0.9442050
2. 5661600

2. 9132420

$-1.6466620$

$-6.2545890$

$-1.4520270$

3. 0205740
1.1838390 


$\begin{array}{lrrr}\mathrm{C} & 1.5934380 & 3.9387120 & -2.2153490 \\ \mathrm{H} & 3.5646980 & 3.1031640 & -2.5102780 \\ \mathrm{C} & -2.0584450 & 1.1535920 & -0.6491170 \\ \mathrm{C} & -3.3389890 & 0.8936500 & -0.0897150 \\ \mathrm{C} & -1.2709420 & 2.2392440 & -0.2949180 \\ \mathrm{C} & -3.7832630 & 1.7519490 & 0.8976160 \\ \mathrm{C} & -1.7005740 & 3.0645630 & 0.7954740 \\ \mathrm{C} & -2.9825250 & 2.8109370 & 1.3916840 \\ \mathrm{H} & -4.7617530 & 1.5872840 & 1.3407800 \\ \mathrm{C} & -2.5935630 & 4.5919150 & -30085320 \\ \mathrm{C} & -1.3153030 & 4.8207310 & 2.4428300 \\ \mathrm{C} & -0.8819510 & 4.0795500 & 1.3613330 \\ \mathrm{C} & -3.4080330 & 3.6059420 & 2.4910270 \\ \mathrm{H} & -2.9237620 & 5.1850480 & 3.8583100 \\ \mathrm{H} & -0.6653180 & 5.5814560 & 2.8694370 \\ \mathrm{H} & 0.1036320 & 4.2574290 & 0.9434080 \\ \mathrm{H} & -4.3845210 & 3.4064880 & 2.9286320 \\ \mathrm{C} & -0.3724330 & 5.9411460 & -2.4214060 \\ \mathrm{C} & 0.9216090 & 6.1603470 & -2.9555270 \\ \mathrm{C} & 1.8816150 & 5.1746310 & -2.8558790 \\ \mathrm{C} & -0.6795200 & 4.7558620 & -1.7832060 \\ \mathrm{H} & -1.1330620 & 6.7126940 & -2.5188710 \\ \mathrm{H} & 1.1507260 & 7.1010010 & -3.4512380 \\ \mathrm{H} & 2.8760010 & 5.3226700 & -3.2734300 \\ \mathrm{H} & -1.6762120 & 4.5995820 & -1.3827230 \\ \mathrm{C} & 3.3743840 & 0.6672480 & -1.4222010 \\ \mathrm{C} & 3.6661950 & -0.2128910 & -2.4856190 \\ \mathrm{C} & 4.1653520 & 0.6609780 & -0.2506440 \\ \mathrm{C} & 4.7857890 & -1.0484200 & -2.3763500 \\ \mathrm{C} & 5.2652520 & -0.2013270 & -0.1849030 \\ \mathrm{C} & 5.6082780 & -1.0444300 & -1.2470050 \\ \mathrm{H} & 5.0384920 & -1.7145970 & -3.1984840 \\ \mathrm{H} & 5.8796000 & -0.2047980 & 0.7127490 \\ \mathrm{C} & -4.1662750 & -0.2624500 & -0.5409750 \\ \mathrm{C} & -4.4554890 & -1.3247750 & 0.3527710 \\ \mathrm{C} & -4.6774300 & -0.2901020 & -1.8584950 \\ \mathrm{C} & -5.2529260 & -2.3826630 & -0.0957640 \\ \mathrm{C} & -5.4663190 & -1.3766360 & -2.2566440 \\ \mathrm{C} & -5.7648320 & -2.4329610 & -1.3955860 \\ \mathrm{H} & -5.4755870 & -3.1970630 & 0.5895540 \\ \mathrm{H} & -5.8665670 & -1.3968820 & -3.2690570 \\ \mathrm{C} & 3.8458810 & 1.5599320 & 0.9385610 \\ \mathrm{C} & 6.8559220 & -1.9109420 & -1.1902130 \\ \mathrm{C} & 2.8267740 & -0.2149600 & -3.7564430 \\ \mathrm{C} & -3.9476260 & -1.3565950 & 1.7899460 \\ \mathrm{C} & -6.6124250 & -3.6039840 & -1.8584040 \\ & & & \end{array}$

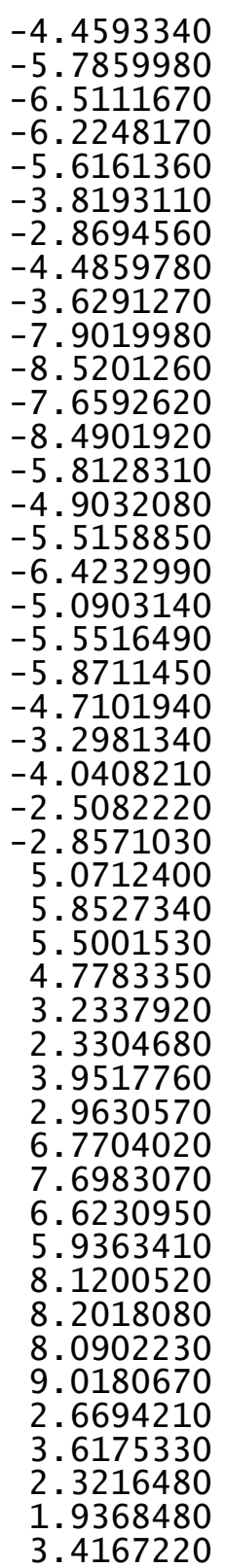

0.8443590

1.5777260

0.8972320

1.9634840

2. 4204210

0.3504370

$-0.1577290$

$-0.3484850$

1.2009940

$-3.7330280$

$-4.5590610$

$-3.9392900$

$-2.8074200$

$-4.9209300$

$-4.8452980$

$-5.1578090$

$-5.7508500$

$-1.0374540$

$-0.0666480$

$-1.8070940$

$-1.0191860$

$-3.5102910$

$-2.9627160$

$-2.6285400$

2. 3559060

1.6887020

2.9489060

3.0361990

0.7427390

0.2200160

$-0.0006930$

1.4015900

$-2.9853000$

$-3.5711730$

$-2.5213580$

$-3.6723260$

$-1.0495310$

$-0.2942610$

$-0.5294580$

$-1.6808590$

$-1.6079680$

$-1.9619090$

$-2.3409160$

$-1.5576310$

0.7641850
$-2.8523980$

$-3.1342350$

$-3.5994960$

$-2.2049850$

$-3.8180140$

$-4.1629530$

$-3.9622480$

$-4.6848280$

$-4.8309250$

$-1.0251650$

$-1.4015270$

0.0256210

$-1.0653800$

$-1.8211000$

$-2.4301820$

$-0.7908400$

$-2.2014900$

2.7752260

2.5573780

2.7089160

3.8057160

2. 1651080

2.1834140

1.4545040

3.1676830

1.4213000

1.8067110

0.6035270

2.2316090

2.0913990

1.7608650

2.4620420

2.9263400

$-0.0906060$

$-0.0575080$

0.8933980

$-0.2744500$

$-1.0017070$

$-1.7934660$

$-0.0352910$

$-1.0230740$

$-4.3879150$

$-4.8131690$

$-3.6534040$

$-5.2029650$

$-4.7926530$ 


$\begin{array}{lrrr}\text { H } & 4.4293270 & 0.4457360 & -5.0761910 \\ \mathrm{H} & 2.7932690 & 0.7809560 & -5.6969170 \\ \mathrm{H} & 3.4742580 & 1.7819390 & -4.3914510 \\ \mathrm{P} & -0.4324470 & -0.7616520 & -1.0143690 \\ \mathrm{O} & 0.1136120 & -1.5741600 & -2.1515520 \\ \mathrm{O} & -0.9250590 & -1.5035220 & 0.1995020 \\ \mathrm{H} & 1.8222920 & 0.1343770 & -3.4905690 \\ \mathrm{H} & 6.9402950 & -2.4269210 & -2.1569170 \\ \mathrm{H} & 3.0912940 & 2.2886020 & 0.6238400 \\ \mathrm{H} & -3.7767530 & 1.5776730 & -2.4131700 \\ \mathrm{H} & -6.8999100 & -3.4108720 & -2.9017060 \\ \mathrm{H} & -3.1795500 & -0.5878510 & 1.9031080 \\ \mathrm{C} & 1.2027700 & -3.7947310 & 0.0692200 \\ \mathrm{C} & 2.0252230 & -2.6952870 & 0.5901850 \\ \mathrm{H} & 0.1273820 & -3.6273660 & 0.0718670 \\ \mathrm{H} & 1.6302630 & -1.6900990 & 0.5468500 \\ \mathrm{O} & 1.8276160 & -3.2672820 & -1.1659540 \\ \mathrm{H} & 1.1640870 & -2.5960620 & -1.5902930 \\ \mathrm{C} & 3.1594250 & -5.3624090 & 0.4947830 \\ \mathrm{C} & 3.7192430 & -4.3404150 & 1.4952700 \\ \mathrm{C} & 3.4201490 & -2.9005510 & 1.0494340 \\ \mathrm{C} & 1.6327980 & -5.2140190 & 0.3697200 \\ \mathrm{H} & 3.3986030 & -6.3816170 & 0.8191600 \\ \mathrm{H} & 4.8020640 & -4.4604370 & 1.6091800 \\ \mathrm{H} & 4.0746690 & -2.5953740 & 0.2201770 \\ \mathrm{H} & 1.1543150 & -5.4888600 & 1.3204500 \\ \mathrm{H} & 1.2250630 & -5.8745170 & -0.4052390 \\ \mathrm{H} & 3.6254480 & -5.2115660 & -0.4882300 \\ \mathrm{H} & 3.2640030 & -4.5123540 & 2.4805290 \\ \mathrm{H} & 3.6162030 & -2.1696690 & 1.8420520 \\ \mathrm{C} & 0.1321960 & -0.7939110 & 3.3324610 \\ \mathrm{O} & -0.6690630 & -0.3674390 & 2.3957980 \\ \mathrm{C} & 0.2763880 & 0.1790230 & 4.4500210 \\ \mathrm{C} & 0.9042520 & -0.1717930 & 5.6600090 \\ \mathrm{C} & -0.2060090 & 1.4966980 & 4.2911720 \\ \mathrm{C} & 1.0434960 & 0.7639140 & 6.6833510 \\ \mathrm{H} & 1.2780970 & -1.1834850 & 5.7895300 \\ \mathrm{C} & -0.0534250 & 2.4322510 & 5.3130210 \\ \mathrm{H} & -0.6837640 & 1.7911940 & 3.3626870 \\ \mathrm{C} & 0.5685860 & 2.0701560 & 6.5128940 \\ \mathrm{H} & 1.5243230 & 0.4754790 & 7.6151430 \\ \mathrm{H} & -0.4213000 & 3.4450610 & 5.1672390 \\ \mathrm{H} & 0.6841970 & 2.8006780 & 7.3106290 \\ \mathrm{H} & -0.7391540 & -0.9478770 & 1.5243860 \\ \mathrm{~S} & 0.9363930 & -2.2746420 & 3.2849260 \\ \mathrm{I} & & & \\ & & & \end{array}$

\begin{tabular}{|c|c|c|c|}
\hline \multicolumn{4}{|c|}{$\operatorname{TS} 235(\mathrm{~S}, \mathrm{~S}) \mathrm{G}[\mathrm{B} 97-\mathrm{D} / \mathrm{def} 2-\mathrm{TZVP}]=-3633.749249$} \\
\hline 0 & 0.5798360 & 0.1456140 & -0.3583470 \\
\hline 0 & -1.7578040 & 0.0025120 & -1.3886600 \\
\hline & 0.8410470 & 1.2553650 & -1.1487460 \\
\hline & -0.1153140 & 2.2602060 & -1.2491830 \\
\hline & 2.1245020 & 1.3496400 & -1.7477960 \\
\hline & 0.1439930 & 3.3698230 & -2.1182270 \\
\hline & 2.3950740 & 2.4625260 & -2.5193320 \\
\hline & 1.4274660 & 3.4689060 & -2.7547600 \\
\hline & 3.3803670 & 2.5692850 & -2.9665750 \\
\hline & -2.1848180 & 1.0557090 & -0.5688150 \\
\hline & -3.4300770 & 0.9204430 & 0.1018820 \\
\hline & -1.3697300 & 2.1731940 & -0.4602900 \\
\hline & -3.8195000 & 1.9590480 & 0.9257780 \\
\hline & -1.7374190 & 3.1977270 & 0.4735200 \\
\hline & -2.9924660 & 3.0842060 & 1.1631800 \\
\hline & -4.7739060 & 1.8961540 & 1.4416630 \\
\hline & -2.5239220 & 5.1438730 & 2.3782890 \\
\hline & -1.2703040 & 5.2380420 & 1.7256830 \\
\hline & -0.8883700 & 4.2918820 & 0.7954400 \\
\hline & -3.3646890 & 4.0860100 & 2.1010020 \\
\hline & -2.8140560 & 5.8986230 & 3.1056940 \\
\hline & -0.5988160 & 6.060 & 1.9635670 \\
\hline & 0.0788420 & 4.3713720 & 0.3103110 \\
\hline & -4.3216470 & 3.9889010 & 2.6105790 \\
\hline & -0.5344530 & 5.4240550 & -3.2447220 \\
\hline & 0.7430250 & 5.5349600 & -3 \\
\hline & 1.7 & 4.5 & -3 \\
\hline & -0.8261050 & 4.3706200 & -2.4012050 \\
\hline & -1.2 & 6.17 & -3.4 \\
\hline & 0.9 & 6.37 & -4.5065490 \\
\hline & 2.682 & 4.63 & -4.0760960 \\
\hline & -1.810 & 4.2962 & -1.9489130 \\
\hline & 3.204 & 0.3426060 & -1.5117370 \\
\hline & 3.4728380 & -0.6536270 & -2.4740390 \\
\hline & 4.0199790 & 0.4673570 & -0.3635710 \\
\hline & 4.5965130 & -1.4702370 & -2.2951550 \\
\hline & 5.1417690 & -0.3598690 & -0.2404850 \\
\hline & 5.4554620 & -1.3254490 & -1.2020290 \\
\hline & 4.8187270 & -2.2394100 & -3.0319740 \\
\hline & 5.7811760 & -0.2487530 & 0.6301080 \\
\hline & -4.2817180 & -0.2937240 & -0.0603560 \\
\hline & -4.5027800 & -1.1578070 & 1.0390610 \\
\hline & -4.8905500 & -0.5683890 & -1.3081740 \\
\hline & -5.3363780 & -2.2701340 & 0.8623260 \\
\hline & & & \\
\hline
\end{tabular}




$\begin{array}{rr}-5.9460900 & -2.5600950 \\ -5.5102420 & -2.9388400 \\ -6.1808850 & -1.8966250 \\ 3.7310590 & 1.5291380 \\ 6.7136330 & -2.1743440 \\ 2.5973970 & -0.8146780 \\ -3.8829850 & -0.9235200 \\ -6.8372720 & -3.7788060 \\ -4.7388030 & 0.3453190 \\ -6.0836610 & 1.0157310 \\ -6.8335620 & 0.2601750 \\ -6.4661040 & 1.5886570 \\ -5.9602990 & 1.6974350 \\ -4.1739270 & -0.4050600 \\ -3.2101330 & -0.8661390 \\ -4.8647980 & -1.1923280 \\ -4.0306230 & 0.2923680 \\ -6.2649990 & -4.7571900 \\ -6.9001680 & -5.6498350 \\ -6.2237510 & -4.2806400 \\ -5.2487460 & -5.0702880 \\ -8.2780820 & -3.3772000 \\ -8.7044940 & -2.7041310 \\ -8.2950640 & -2.8601230 \\ -8.9156820 & -4.2680570 \\ -4.9352370 & -0.3854970 \\ -5.3953140 & 0.5402180 \\ -5.7325740 & -1.1266030 \\ -4.4700370 & -0.1823510 \\ -3.2223440 & -2.1887690 \\ -3.9726680 & -2.9536740 \\ -2.5023760 & -2.6097590 \\ -2.6950640 & -1.9342460 \\ 4.4879620 & 2.8315050 \\ 5.5714240 & 2.6518030 \\ 4.2220190 & 3.1997410 \\ 4.2470720 & 3.6094660 \\ 4.0530270 & 1.0822100 \\ 3.5510850 & 0.1402860 \\ 5.1331330 & 0.9523810 \\ 3.7091210 & 1.8471830 \\ 7.0700220 & -2.6196440 \\ 7.9428830 & -3.2840910 \\ 7.3249630 & -1.7583110 \\ 6.2395560 & -3.1555540 \\ 7.8996360 & -1.4168670\end{array}$

-0.3588640
1.7030710
-2.3896060
0.6926510
-1.1144600
-3.7107910
2.4122480
-0.5205510
-2.5186400
-2.8637390
-3.1325620
-2.0091030
-3.7159960
-3.7385650
-3.4962710
-4.0679750
-4.5746860
-1.5641600
-1.6401540
-2.5524270
-1.2934130
-0.8900340
-0.1353780
-1.8585940
-0.9669040
3.4029070
3.0376990
3.5496290
4.3770380
2.9918780
3.2311980
2.2838100
3.9205280
0.3608610
0.3875130
-0.6366200
1.0980420
2.1276570
2.3774340
2.2741490
2.8334090
0.3129860
0.2879390
0.9431150
0.7857830
-1.7479480

7.6758580

8. 1067640

8.8033930

2.4002460

3. 3331400

2. 0509240

1. 6550230

3. 1745860

4. 1768710

2. 5310140

3. 2522980

$-0.6000400$

$-0.1376600$

$-1.0193650$

1. 6068940

6.5365970

2. 6585130

$-4.0357620$

$-6.8675230$

$-3.0994430$

0.6143040

$-0.4456120$

1. 0682950

1. 9280550

0.6738980

2. 3861420

2. 2352710

1.1510280

0.0166130

2. 0072190

3. 0456440

0.8527430

2. 3783660

$-0.6646500$

1. 4272370

1.0464130

2. 0425100

0.0076760

1.7539030

1. 5001160

0.8587060

2. 8812150

3. 6894480

3. 4671160

1. 3774810
3. 0656130
-1.1358720
-0.4995300
-2.0410570
-2.2800120
-2.7138130
-2.8923990
-2.3292610
-0.0047420
-0.3752580
-0.1100820
1.0590860
-0.9372990
-1.8879920
-1.5075660
-0.4103070
-3.0774150
1.7544690
1.1473120
-4.2960400
-0.1678750
-0.2827770
-0.0669140
0.9255170
0.8395030
2.1992030
1.9945640
-0.1386940
3.3524000
2.2836300
3.2551840
1.9137910
4.3257570
4.1556910
-0.7811790
-1.7587300
-3.8467740
-2.7805970
-3.5479470
-1.7607490
-3.6540350
-2.9686050
-5.5445480
-4.4308660
-3.0769750
-5.2258650
-6.5048490

$-1.1799710$

$-1.7392070$

$-4.1343740$

$-4.5178600$

$-3.2975660$

$-4.9381230$

$-4.8897320$

$-5.1449470$

$-5.7736330$

$-4.6412330$

$-0.7423700$

$-1.8071200$

0.5872290

$-3.4729240$

$-1.7162860$

0.6608720

$-2.2744450$

0.4490650

2. 3118500

3. 2582690

2. 5310690

3. 9946880

5.1050360

3.5334370

5.7348160

5.4638200

4.1542750

2. 6742400

5. 2563440

6.5956480

3. 7722460

5.7407370

1.7928210

3.3499200

0.5596370

0.7342430

0.6836870

0.5146920

$-0.8358030$

$-1.2669960$

1.0367690

1.7185730

1. 0260110

1.0885740

1.5319410 


\begin{tabular}{|c|c|c|c|c|c|c|c|}
\hline & & & & & & & \\
\hline $\mathrm{H}$ & 4.7597870 & -4.6664580 & 1.7089170 & C & -4.5969940 & -1.5050240 & -0.0908920 \\
\hline $\mathrm{H}$ & 3.9992950 & -3.0304430 & 0.0643710 & $\mathrm{H}$ & -4.6492100 & -1.0069600 & -2.1784230 \\
\hline $\mathrm{H}$ & 1.0297930 & -5.2490390 & 2.1310640 & $\mathrm{H}$ & -4.2590230 & -2.1904260 & 1.9117590 \\
\hline $\mathrm{H}$ & 0.7886070 & -5.9638550 & 0.5302960 & C & 3.8472490 & 1.5783920 & 0.5079650 \\
\hline $\mathrm{H}$ & 3.2019390 & -5.6461540 & -0.0086660 & C & 3.2658060 & 2.4596050 & 1.4518930 \\
\hline $\mathrm{H}$ & 3.3817780 & -4.3508540 & 2.7704700 & $\mathrm{C}$ & 4.3997360 & 2.0823260 & -0.6869250 \\
\hline $\mathrm{H}$ & 3.8713310 & -2.2375150 & 1.6029450 & c & 3.2653190 & 3.8304310 & 1.1777730 \\
\hline 14 & & & & C & 4.3713300 & 3.4641400 & -0.9162280 \\
\hline & S) G[B97-D, & {$[\mathrm{ZVP}]=-36$} & 93 & $\mathrm{C}$ & 3.8040520 & 4.3531840 & -0.0025740 \\
\hline 0 & 0.0683210 & -1.1862760 & 0.1227260 & $\mathrm{H}$ & 2.8139200 & 4.5106110 & 1.8952530 \\
\hline 0 & 2.1078660 & -0.0932880 & -0.9059130 & $\mathrm{H}$ & 4.7945450 & 3.8582820 & -1.8389740 \\
\hline C & 0.3753220 & -2.4205280 & -0.4397160 & C & -1.8897200 & -3.4571590 & 1.7703430 \\
\hline $\mathrm{C}$ & 1.6947780 & -2.8545630 & -0.4381860 & $\mathrm{c}$ & -5.9301290 & -0.8662450 & 0.2618810 \\
\hline C & -0.7156900 & -3.1958960 & -0.9203390 & $\mathrm{C}$ & -2.3471820 & -2.0690650 & -3.1464450 \\
\hline C & 1.9939570 & -4.0737190 & -1.1338270 & $\mathrm{C}$ & 2.6571630 & 1.9547040 & 2.7559410 \\
\hline C & -0.4265520 & -4.4113460 & -1.5026570 & C & 3.7305020 & 5.8396560 & -0.3028430 \\
\hline C & 0.9112770 & -4.8583370 & -1.6639750 & $\mathrm{C}$ & 5.0393610 & 1.1775610 & -1.7311790 \\
\hline $\mathrm{H}$ & -1.2354750 & -5.0481240 & -1.8517230 & $\mathrm{C}$ & 6.5557430 & 1.4375200 & -1.8230140 \\
\hline C & 2.8741490 & -0.7085590 & 0.0759850 & $\mathrm{H}$ & 6.7490060 & 2.4708440 & -2.1404170 \\
\hline C & 3.8164990 & 0.1067140 & 0.7594000 & $\mathrm{H}$ & 7.0397800 & 1.2818970 & -0.8502050 \\
\hline C & 2.7250210 & -2.0730150 & 0.2993780 & $\mathrm{H}$ & 7.0151430 & 0.7602920 & -2.5555710 \\
\hline C & 4.6538420 & -0.5050510 & 1.6681570 & C & 4.3730800 & 1.3347500 & -3.1107200 \\
\hline $\mathrm{C}$ & 3.5287640 & -2.6766650 & 1.3270770 & $\mathrm{H}$ & 3.2980980 & 1.1319890 & -3.0446920 \\
\hline $\mathrm{C}$ & 4.5303110 & -1.8809550 & 1.9861710 & $\mathrm{H}$ & 4.5142680 & 2.3520100 & -3.4984710 \\
\hline $\mathrm{H}$ & 5.4071000 & 0.0856300 & 2.1836700 & $\mathrm{H}$ & 4.8209760 & 0.6328290 & -3.8267150 \\
\hline C & 5.1763400 & -3.7914600 & 3.3554860 & C & 4.3757420 & 6.6895280 & 0.8070950 \\
\hline C & 4.1558980 & -4.5622750 & 2.7472600 & H & 4.3375490 & 7.7546060 & 0.5427100 \\
\hline $\mathrm{C}$ & 3.3543350 & -4.0198360 & 1.7621280 & $\mathrm{H}$ & 3.8405760 & 6.5554830 & 1.7561700 \\
\hline C & 5.3511150 & -2.4746320 & 2.9838290 & $\mathrm{H}$ & 5.4239540 & 6.4038760 & 0.9621570 \\
\hline $\mathrm{H}$ & 5.8056390 & -4.2335550 & 4.1246630 & C & 2.2694740 & 6.2743950 & -0.5376480 \\
\hline $\mathrm{H}$ & 3.9966490 & -5.5911560 & 3.0627660 & $\mathrm{H}$ & 1.8153970 & 5.6958450 & -1.3514930 \\
\hline $\mathrm{H}$ & 2.5740780 & -4.6256250 & 1.3145330 & $\mathrm{H}$ & 1.6731200 & 6.1116890 & 0.3699100 \\
\hline $\mathrm{H}$ & 6.1111840 & -1.8585600 & 3.4611710 & $\mathrm{H}$ & 2.2223610 & 7.3412430 & -0.7948590 \\
\hline C & 3.5743040 & -5.7011740 & -2.0304140 & C & 3.6789910 & 2.0733860 & 3.9053710 \\
\hline $\mathrm{C}$ & 2.5058990 & -6.4921210 & -2.5193210 & $\mathrm{H}$ & 4.6003560 & 1.5245960 & 3.6799270 \\
\hline C & 1.2029060 & -6.0740350 & -2.3404640 & $\mathrm{H}$ & 3.9388000 & 3.1285650 & 4.0673400 \\
\hline $\mathrm{C}$ & 3.3237870 & -4.5242010 & -1.3527330 & $\mathrm{H}$ & 3.2543710 & 1.6751040 & 4.8370330 \\
\hline $\mathrm{H}$ & 10430 & -6.0214370 & -2.1935630 & C & 1.3511580 & 2.6719620 & 3.1424600 \\
\hline H & 2.7158520 & -7.4209010 & -3.0450950 & $\mathrm{H}$ & 1.5379360 & 3.7158270 & 3.4263800 \\
\hline $\mathrm{H}$ & 0.3734540 & -6.6638110 & -2.7270730 & $\mathrm{H}$ & 0.6375810 & 2.6545000 & 2.3135620 \\
\hline $\mathrm{H}$ & 4.1492720 & -3.9233260 & -0.9837270 & $\mathrm{H}$ & 0.9009220 & 2.1692020 & 4.0085420 \\
\hline C & -2.1031290 & -2.6932040 & -0.6777880 & C & -2.6725550 & -4.7025510 & 2.2304330 \\
\hline $\mathrm{C}$ & -2.8477260 & -2.0764150 & -1.7081540 & $\mathrm{H}$ & -3.6570970 & -4.4131890 & 2.6217080 \\
\hline $\mathrm{C}$ & 39710 & -2.7550740 & 0.6344090 & $\mathrm{H}$ & -2.8265460 & -5.3992640 & 1.3964340 \\
\hline $\mathrm{C}$ & 0480 & -1.4962340 & -1.3915750 & $\mathrm{H}$ & -2.1250000 & -5.2240620 & 3.0271210 \\
\hline $\mathrm{C}$ & -3 & -2.1631680 & 0.8982580 & C & -1.6187710 & -2.5187030 & 2.9600710 \\
\hline
\end{tabular}




$\begin{array}{rrr}-1.0896760 & -1.6169220 & 2.6333500 \\ -2.5603060 & -2.2180250 & 3.4366070 \\ -1.0092210 & -3.0348300 & 3.7135330 \\ -7.0753460 & -1.8839830 & 0.0828480 \\ -8.0341460 & -1.4422680 & 0.3873260 \\ -7.1518520 & -2.1855260 & -0.9709190 \\ -6.8962900 & -2.7834920 & 0.6851880 \\ -6.2257280 & 0.4158510 & -0.5310010 \\ -5.4067620 & 1.1368440 & -0.4300470 \\ -6.3676850 & 0.2003060 & -1.5979280 \\ -7.1449250 & 0.8824840 & -0.1560100 \\ -2.7252680 & -0.8047790 & -3.9341660 \\ -3.8048560 & -0.7625580 & -4.1294900 \\ -2.4308920 & 0.0989090 & -3.3949800 \\ -2.2133360 & -0.8120400 & -4.9048440 \\ -2.8669810 & -3.3159720 & -3.8930340 \\ -3.9642020 & -3.2908510 & -3.9462670 \\ -2.4715520 & -3.3391750 & -4.9177790 \\ -2.5713600 & -4.2402640 & -3.3849090 \\ 0.5149940 & 0.1514480 & -0.6953420 \\ -0.0517630 & 0.1785310 & -2.0906040 \\ 0.2172370 & 1.2958210 & 0.2324030 \\ -1.2534420 & -2.1204320 & -3.1169000 \\ -5.8853510 & -0.5972220 & 1.3254700 \\ -0.9193970 & -3.8031570 & 1.4005230 \\ 4.9050750 & 0.1363350 & -1.4193700 \\ 4.2887200 & 6.0160330 & -1.2333730 \\ 2.4179180 & 0.8930980 & 2.6281330 \\ -0.9933230 & 3.2497370 & -2.0034920 \\ -2.2519330 & 2.5433640 & -1.7455390 \\ -0.1873540 & 3.0494360 & -1.2991880 \\ -2.1941550 & 1.5995760 & -1.2146610 \\ -1.0302080 & 2.2522940 & -3.0911960 \\ -0.5720810 & 1.3794540 & -2.7132550 \\ -2.2999030 & 4.9403820 & -3.3835670 \\ -3.5798370 & 4.4910470 & -2.6614460 \\ -3.5485210 & 2.9860350 & -2.3422200 \\ -1.0601550 & 4.6728390 & -2.5121870 \\ -2.3539460 & 6.0102680 & -3.6159960 \\ -4.4652220 & 4.7104610 & -3.2689660 \\ -3.7079670 & 2.3936560 & -3.2552430 \\ -1.0814350 & 5.3193430 & -1.6239620 \\ -0.1326290 & 4.8948140 & -3.0539760 \\ -2.2040810 & 4.4014660 & -4.3355920 \\ -3.6780350 & 5.0501130 & -1.7206000 \\ -4.3518090 & 2.6980940 & -1.6547140\end{array}$

-2.8901530
-1.9725590
-4.0553910
-5.2858770
-3.9499200
-6.3816350
-5.3778770
-5.0446800
-3.0093100
-6.2653800
-7.3302010
-4.9481530
-7.1222950
-1.1101320
-2.7714440

144

$\operatorname{TS} 237(\mathrm{~S}, \mathrm{~S}) \mathrm{G}[\mathrm{B} 97-\mathrm{D} / \mathrm{def2}-\mathrm{TZVP}]=-3633.748852$

$\begin{array}{lrrr}\text { O } & -1.4676500 & 0.5853190 & -0.6455560 \\ \mathrm{O} & 0.3283520 & 1.5912470 & 0.8408630 \\ \mathrm{C} & -2.3983170 & 1.4761440 & -0.1220510 \\ \mathrm{C} & -2.0553360 & 2.8202200 & -0.0151980 \\ \mathrm{C} & -3.6754330 & 0.9600140 & 0.2290810 \\ \mathrm{C} & -3.0051810 & 3.7166980 & 0.5802880 \\ \mathrm{C} & -4.6158720 & 1.8549410 & 0.7018950 \\ \mathrm{C} & -4.3127350 & 3.2217500 & 0.9138060 \\ \mathrm{H} & -5.6085480 & 1.4943480 & 0.9598110 \\ \mathrm{C} & 0.4362350 & 2.6686510 & -0.0364720 \\ \mathrm{C} & 1.7450600 & 3.1015130 & -0.3827220 \\ \mathrm{C} & -0.7245530 & 3.2899530 & -0.4851530 \\ \mathrm{C} & 1.8543210 & 4.2021600 & -1.2100320 \\ \mathrm{C} & -0.5953200 & 4.3624040 & -1.4312720 \\ \mathrm{C} & 0.7186320 & 4.8375790 & -1.7682700 \\ \mathrm{H} & 2.8421790 & 4.5709750 & -1.4747370 \\ \mathrm{C} & -0.2471390 & 6.4870610 & -3.2799740 \\ \mathrm{C} & -1.5405860 & 5.9899760 & -2.9853340 \\ \mathrm{C} & -1.7096850 & 4.9563100 & -2.0854650 \\ \mathrm{C} & 0.8583230 & 5.9153400 & -2.6854210 \\ \mathrm{H} & -0.1288220 & 7.3068860 & -3.9849630 \\ \mathrm{H} & -2.4092640 & 6.4227440 & -3.4767370 \\ \mathrm{H} & -2.7066290 & 4.5836840 & -1.8747670 \\ \mathrm{H} & 1.8609180 & 6.2688650 & -2.9197820 \\ \mathrm{C} & -3.6478990 & 5.8989510 & 1.4641280 \\ \mathrm{C} & -4.9499030 & 5.4199660 & 1.7508510 \\ \mathrm{C} & -5.2702980 & 4.1051160 & 1.4840410 \\ \mathrm{C} & -2.7020070 & 5.0704830 & 0.8936450 \\ \mathrm{H} & -3.3885660 & 6.9286910 & 1.7003650\end{array}$

$\begin{array}{rr}2.0003800 & 1.5786970 \\ 1.0704990 & 1.4944200 \\ 1.5940350 & 2.4033980 \\ 2.2721050 & 2.3154200 \\ 0.4866380 & 3.2701820 \\ 1.8504130 & 3.0645910 \\ 3.1158390 & 1.6382270 \\ 0.0755910 & 4.0271590 \\ -0.0463550 & 3.3438640 \\ 0.7519840 & 3.9242790 \\ 2.3737160 & 2.9726540 \\ -0.7788020 & 4.6930030 \\ 0.4231400 & 4.5078360 \\ 1.3024170 & 0.9657250 \\ 3.5039340 & 0.8321730\end{array}$

2.0003800

1.5940350

2.2721050

1.8504130

$-0.7788020$

0.9657250

0.8321730

4944200

2. 3154200

1.6382270

3.3438640

3.9242790

2.9726540
0.0755910

$-0.8120400$

1514480

.

0.5972220

2. 2522940

1.3794540

4.9403820

4.4910470

4104610

2. 6980940

$-1.6547140$ 


$\begin{array}{rrrr}\mathrm{H} & -5.6872480 & 6.0857130 & 2.1937880 \\ \mathrm{H} & -6.2586170 & 3.7145300 & 1.7202550 \\ \mathrm{H} & -1.7086200 & 5.4527600 & 0.6833500 \\ \mathrm{C} & -3.9940590 & -0.4947690 & 0.1224620 \\ \mathrm{C} & -4.2342830 & -1.2489710 & 1.3000660 \\ \mathrm{C} & -4.0912600 & -1.1182970 & -1.1397100 \\ \mathrm{C} & -4.5833590 & -2.5955300 & 1.1748550 \\ \mathrm{C} & -4.4537780 & -2.4710830 & -1.2084580 \\ \mathrm{C} & -4.7078340 & -3.2300030 & -0.0655070 \\ \mathrm{H} & -4.7618120 & -3.1779270 & 2.0767040 \\ \mathrm{H} & -4.5396790 & -2.9324680 & -2.1881740 \\ \mathrm{C} & 2.9595680 & 2.3865520 & 0.1077100 \\ \mathrm{C} & 3.7878420 & 1.6953210 & -0.8082790 \\ \mathrm{C} & 3.2972010 & 2.4155870 & 1.4814060 \\ \mathrm{C} & 4.9398300 & 1.0610010 & -0.3257670 \\ \mathrm{C} & 4.4553670 & 1.7608810 & 1.9104600 \\ \mathrm{C} & 5.2915930 & 1.0763570 & 1.0243780 \\ \mathrm{H} & 5.5838690 & 0.5334360 & -1.0267370 \\ \mathrm{H} & 4.7160920 & 1.7998560 & 2.9654950 \\ \mathrm{P} & -0.1830450 & 0.1605380 & 0.2645470 \\ \mathrm{O} & -0.5927060 & -0.6256610 & 1.4825560 \\ \mathrm{O} & 0.7776280 & -0.4516970 & -0.7164390 \\ \mathrm{C} & -3.8721180 & -0.3720420 & -2.4494430 \\ \mathrm{H} & -3.5726400 & 0.6560570 & -2.2266980 \\ \mathrm{C} & -5.1233010 & -4.6952190 & -0.1085820 \\ \mathrm{H} & -4.4630980 & -5.2345450 & 0.5883400 \\ \mathrm{C} & -4.1516720 & -0.6462310 & 2.6990980 \\ \mathrm{H} & -3.7002520 & 0.3477810 & 2.6236510 \\ \mathrm{C} & 2.4808050 & 3.1852570 & 2.5123760 \\ \mathrm{H} & 1.5842080 & 3.5849420 & 2.0295410 \\ \mathrm{C} & 6.5673020 & 0.4061480 & 1.5025770 \\ \mathrm{H} & 6.9546860 & -0.1918480 & 0.6658140 \\ \mathrm{C} & 3.4980670 & 1.6326470 & -2.3041530 \\ \mathrm{H} & 2.4861660 & 2.0112140 & -2.4785310 \\ \mathrm{C} & 3.5435350 & 0.1991430 & -2.8645310 \\ \mathrm{H} & 3.3003490 & 0.2172010 & -3.9351480 \\ \mathrm{H} & 4.5414260 & -0.2447290 & -2.7545120 \\ \mathrm{H} & 2.8186000 & -0.4396170 & -2.3530970 \\ \mathrm{C} & 4.4794940 & 2.5360240 & -3.0781220 \\ \mathrm{H} & 5.5088000 & 2.1753470 & -2.9488380 \\ \mathrm{H} & 4.2422140 & 2.5255690 & -4.1504630 \\ \mathrm{H} & 4.4351850 & 3.5720170 & -2.7206020 \\ \mathrm{C} & -2.7479660 & -1.0111260 & -3.2865330 \\ \mathrm{H} & -3.0093970 & -2.0393160 & -3.5689720 \\ \mathrm{H} & -2.5919420 & -0.4350240 & -4.2082080 \\ \mathrm{H} & -1.8083610 & -1.0301500 & -2.7240570\end{array}$

-5.1804500
-5.0203580
-5.5238470
-5.9744240
2.0178810
2.8780770
1.3861190
1.4417300
3.2829830
2.6810470
4.1968390
3.5736390
-3.2724210
-3.2276920
-3.6848390
-2.2543410
-5.5651210
-6.0457590
-5.5095900
-6.2002920
7.6352270
7.8426600
7.2864760
8.5707390
6.3264150
5.5750910
7.2602540
5.9753420
-4.9933660
-3.9765560
-5.2501100
-5.6802920
-6.5682260
-6.6782800
-7.2674620
-6.8448500
0.0696090
-0.4986370
0.9001210
-0.3637830
-1.2280750
-1.0299510
-0.9980990
-1.0921000
-1.3438410
0.1521590
$-3.2614470$ $-4.1848360$ $-3.5355810$ $-2.6804630$ 3. 6710010 4. 2358410 4. 3625420 3. 2922810 3.0449840 3.7663750 3. 5499090 2. 2258120 3. 6573830

4.6342250

3. 8120910

3. 2687170

3. 2931210

3. 4083660

4. 2813380

2.6443570

1.8569100

0.9988590

2. 6879620

2. 1623890

2.6948430

2. 4529080

2.9718990

3. 5681350

$-1.4880780$

$-1.8894070$

$-1.4154640$

$-2.2070610$

0.4113510

1. 4163750

$-0.2589700$

0.4472180

0.9322330

$-0.3933070$

1. 2428300

$-0.8159360$

1. 2352320

1. 3487720

0.8711190

$-0.6583290$

$-1.1042900$

1.4118540 


\begin{tabular}{|c|c|c|c|c|c|c|c|}
\hline & & & & & & & \\
\hline $\mathrm{H}$ & -0.8318230 & -7.0155040 & 1.1634490 & $\mathrm{H}$ & -0.6216450 & 5.7839080 & 2.6222080 \\
\hline $\mathrm{H}$ & -1.8966590 & -6.4967020 & -1.0432140 & $\mathrm{H}$ & 0.0944490 & 4. 3196980 & 0.7805410 \\
\hline $\mathrm{H}$ & -2.3876060 & -4.1162160 & -0.9223800 & $\mathrm{H}$ & -4.3619470 & 3.6632930 & 2.9064750 \\
\hline $\mathrm{H}$ & 1.1162640 & -5.4992500 & 1.0616880 & C & -0.4095030 & 5.7972870 & -2.6558570 \\
\hline $\mathrm{H}$ & 0.1746360 & -5.1066440 & 2.5086090 & $\mathrm{C}$ & 0.8790990 & 5.9616470 & -3.2217770 \\
\hline $\mathrm{H}$ & -1.9442060 & -5.6496510 & 1.3257950 & $\mathrm{C}$ & 1.8237500 & 4.9665920 & -3.0780090 \\
\hline $\mathrm{H}$ & -0.1511040 & -6.2122290 & -1.1022300 & $\mathrm{C}$ & -0.7263070 & 4.6574450 & -1.9438510 \\
\hline $\mathrm{H}$ & -1.1857140 & -4.2769820 & -2.1810570 & $\mathrm{H}$ & -1.1587420 & 6.5748040 & -2.7880740 \\
\hline C & 3.0111700 & -3.0214310 & -0.8243540 & $\mathrm{H}$ & 1.1159120 & 6.8670530 & -3.7760130 \\
\hline 0 & 2.7446240 & -1.9347680 & -0.1375710 & $\mathrm{H}$ & 2.8138960 & 5.0720740 & -3.5179470 \\
\hline C & 4.3870450 & -3.5277110 & -0.5943770 & $\mathrm{H}$ & -1.7190830 & 4.5432550 & -1.5201610 \\
\hline $\mathrm{C}$ & 4.7328700 & -4.8665640 & -0.8617720 & C & 3.2594170 & 0.5239760 & -1.4045450 \\
\hline C & 5.3762960 & -2.6518520 & -0.1010260 & C & 3.5948650 & -0.3359780 & -2.4716700 \\
\hline $\mathrm{C}$ & 6.0324800 & -5.3174140 & -0.6399600 & $\mathrm{C}$ & 3.9862400 & 0.4774660 & -0.1920230 \\
\hline $\mathrm{H}$ & 3.9709870 & -5.5461950 & -1.2328610 & C & 4.6868480 & -1.1986000 & -2.3189680 \\
\hline C & 6.6774840 & -3.1051490 & 0.1071750 & C & 5.0805470 & -0.3903280 & -0.0962000 \\
\hline $\mathrm{H}$ & 5.1202540 & -1.6169220 & 0.0993260 & $\mathrm{C}$ & 5.4527440 & -1.2298400 & -1.1503280 \\
\hline C & 7.0102400 & -4.4377990 & -0.1587660 & $\mathrm{H}$ & 4.9564520 & -1.8668970 & -3.1345780 \\
\hline $\mathrm{H}$ & 6.2842230 & -6.3562140 & -0.8398690 & $\mathrm{H}$ & 5.6473220 & -0.4207250 & 0.8288780 \\
\hline $\mathrm{H}$ & 7.4323980 & -2.4176600 & 0.4801450 & C & -4.2709980 & -0.2243600 & -0.3160960 \\
\hline $\mathrm{H}$ & 8.0252760 & -4.7907350 & 0.0094520 & $\mathrm{C}$ & -4.5303290 & -1.2328910 & 0.6460760 \\
\hline $\mathrm{H}$ & 1.8843230 & -1.4207090 & -0.4074020 & C & -4.8420950 & -0.3144400 & -1.6055410 \\
\hline $\mathrm{s}$ & 1.9283050 & -3.7662150 & -1.8688480 & $\mathrm{C}$ & -5.3678490 & -2.2963670 & 0.2951760 \\
\hline & & & & C & -5.6692350 & -1.4046000 & -1.9055810 \\
\hline & S) G[B97-I & TZVP] $=-36$ & & C & -5.9464180 & -2.4042410 & -0.9729690 \\
\hline 0 & 0.6071270 & 0.2105290 & -0.3777540 & $\mathrm{H}$ & -5.5713430 & -3.0692210 & 1.0328490 \\
\hline 0 & -1.6963700 & 0.2031360 & -1.4933060 & $\mathrm{H}$ & -6.1173160 & -1.4730940 & -2.8956430 \\
\hline C & 0.8935080 & 1.4039820 & -1.0239550 & C & 3.6385210 & 033230 & 0.9700900 \\
\hline C & -0.0569510 & 2.4188090 & -1.0332080 & C & 6.6682630 & -2.1425450 & -1.0825020 \\
\hline c & 2.1934040 & 1.5575210 & -1.5739940 & $\mathrm{C}$ & 2.8286440 & -0.3081050 & -3.7878970 \\
\hline c & 0.2289020 & 3.6195470 & -1.7613400 & $\mathrm{C}$ & -3.9462810 & -1.1997750 & 2.0540990 \\
\hline C & 2.4827390 & 2.7428880 & -2.2198100 & $\mathrm{C}$ & -6.8418780 & -3.5788490 & -1.3249240 \\
\hline C & 1.5248500 & 3.7763220 & -2.3609640 & $\mathrm{C}$ & -4.6398810 & 0.7533850 & -2.6734110 \\
\hline $\mathrm{H}$ & 3.4772840 & 2.8923560 & -2.6326060 & C & -5.9628960 & 1.4903080 & -2.9625100 \\
\hline C & -2.1492600 & 1.1547340 & -0.5695930 & $\mathrm{H}$ & -6.7125290 & 0.7939700 & -3.3605640 \\
\hline C & -3.4161100 & 0.9455290 & 0.0389900 & $\mathrm{H}$ & -6.3668360 & 1.9428440 & -2.0476320 \\
\hline C & -1.3349900 & 2.2442020 & -0.2974750 & $\mathrm{H}$ & -5.8016740 & 2.2841690 & -3.7042640 \\
\hline $\mathrm{C}$ & -3.824 & 1.8701500 & 0.9803020 & C & -4.0474400 & 0.1661230 & -3.9678400 \\
\hline C & -1.72 & 3.1429620 & 0.7482390 & $\mathrm{H}$ & -3.1045960 & -0.3528490 & -3.7617000 \\
\hline C & -2.999 & 2.9481120 & 1.3856510 & $\mathrm{H}$ & -4.7447370 & -0.5461230 & -4.4277390 \\
\hline $\mathrm{H}$ & -4.7953640 & 1.7492970 & 1.4542960 & $\mathrm{H}$ & -3.8557770 & 0.9693730 & -4.6917610 \\
\hline C & -2.5564740 & 4.8291380 & 2.8695040 & C & -8.0820160 & -3.6385130 & -0.4129540 \\
\hline $\mathrm{C}$ & -1.2877910 & 5.00219 & 2.2637590 & $\mathrm{H}$ & -8.7324460 & -4.4729420 & -0.7071620 \\
\hline C & -0.8845700 & 4.1826670 & 1.2281910 & $\mathrm{H}$ & -7.7817750 & -3.7913330 & 0.6320320 \\
\hline C & -3.3 & 3.82080 & 2.4368140 & $\mathrm{H}$ & -8.6581270 & -2.7062700 & 706960 \\
\hline $\mathrm{H}$ & -2.8 & 5.48387 & 6823910 & C & -6.0654950 & -4.9089830 & -1.2657280 \\
\hline
\end{tabular}




$\begin{array}{rrr}-5.1960580 & -4.8865180 & -1.9348190 \\ -5.7065790 & -5.0960220 & -0.2449180 \\ -6.7150460 & -5.7444620 & -1.5594250 \\ -5.0206780 & -0.7860140 & 3.0799030 \\ -5.4546310 & 0.1911760 & 2.8372740 \\ -5.8337000 & -1.5245080 & 3.0947060 \\ -4.5845160 & -0.7328020 & 4.0867150 \\ -3.3192950 & -2.5434040 & 2.4717210 \\ -4.0854130 & -3.3209150 & 2.5867520 \\ -2.5863740 & -2.8761510 & 1.7307680 \\ -2.8134120 & -2.4258860 & 3.4388760 \\ 4.3861760 & 2.7445180 & 0.8231330 \\ 5.4706640 & 2.5761190 & 0.8709260 \\ 4.1511680 & 3.2245470 & -0.1337010 \\ 4.1044990 & 3.4270360 & 1.6364950 \\ 3.9097080 & 0.7975860 & 2.3560220 \\ 3.4210100 & -0.1768500 & 2.4715780 \\ 4.9853660 & 0.6727560 & 2.5354970 \\ 3.5170480 & 1.4672200 & 3.1296520 \\ 7.0318920 & -2.6070580 & 0.3357590 \\ 7.8477890 & -3.3388240 & 0.2886590 \\ 7.3748150 & -1.7640840 & 0.9494610 \\ 6.1767970 & -3.0712760 & 0.8397940 \\ 7.8803720 & -1.4497910 & -1.7411980 \\ 7.6519640 & -1.1582790 & -2.7736920 \\ 8.1461360 & -0.5447080 & -1.1779940 \\ 8.7495900 & -2.1212290 & -1.7488590 \\ 2.5486260 & -1.7088530 & -4.3588400 \\ 3.4759560 & -2.2038090 & -4.6746760 \\ 2.0467570 & -2.3422140 & -3.6211840 \\ 1.9007390 & -1.6206800 & -5.2402860 \\ 3.5864820 & 0.5421490 & -4.8278050 \\ 4.5693470 & 0.0960150 & -5.0323710 \\ 3.0225460 & 0.5848850 & -5.7693930 \\ 3.7418670 & 1.5655750 & -4.4677290 \\ -0.5677680 & -0.8112410 & -0.9117260 \\ -0.0781890 & -1.6518830 & -2.0538990 \\ -1.0360390 & -1.5142150 & 0.3352070 \\ 1.8588740 & 0.1681660 & -3.6038970 \\ 6.4328680 & -3.0348200 & -1.6814850 \\ 2.5652150 & 1.6201910 & 0.9217990 \\ -3.9330450 & 1.5001290 & -2.3000240 \\ -7.1866020 & -3.4331030 & -2.3585560 \\ -3.1498820 & -0.4513950 & 2.0799550 \\ -0.5364770 & -0.6510810 & 3.2141930 \\ -0.3215460 & 2.4543370\end{array}$
0.8974460
1.7013590
0.5348920
2.1343520
1.9848970
0.9861480
$-0.0767020$
1.7855590
2.7501900
0.7124500
2.1365010
$-0.7278740$
1.2425170
0.9924330
1.9758710
$-0.0527440$
1. 6998070
1. 5214380
0.8981270
2.7999670
3.5688130
3. 3834740
1. 2955000
2.9578670
4.6385390
3.9622040
0.8914410
0.7387490
3.1765080
3.2030700
3.7599470

144

TS239(S, S) G[B97-D/def2-TZVP] $=-3633.748651$

$\begin{array}{lrrr}\text { TS239(S, S }) \text { G }[B 97-\mathrm{D} / \mathrm{def}-\mathrm{TZVP}]=-3633.748651 \\ \mathrm{O} & -1.4884530 & 0.5435950 & -0.6770610 \\ \mathrm{O} & 0.2220720 & 1.6009750 & 0.8692310 \\ \mathrm{C} & -2.4758230 & 1.3797380 & -0.1672530 \\ \mathrm{C} & -2.1992160 & 2.7353780 & -0.0242370 \\ \mathrm{C} & -3.7370150 & 0.7963170 & 0.1327480 \\ \mathrm{C} & -3.2069010 & 3.5734880 & 0.5618770 \\ \mathrm{C} & -4.7322980 & 1.6366930 & 0.5933620 \\ \mathrm{C} & -4.4988710 & 3.0105760 & 0.8449460 \\ \mathrm{H} & -5.7154320 & 1.2266590 & 0.8101380 \\ \mathrm{C} & 0.2978160 & 2.6980710 & 0.0149660 \\ \mathrm{C} & 1.5927000 & 3.1990060 & -0.2910180 \\ \mathrm{C} & -0.8799400 & 3.2760760 & -0.4484110 \\ \mathrm{C} & 1.6697260 & 4.3256790 & -1.0861000\end{array}$

4.1165950

5.2469200

3.7997360

6.0383690

5.4946010

4.5837520

2.9257670

5.7054930

6.9129630

4. 3141160

6.3175420

1.6268130

3.1750280

0.1151010

0.4817370

0.2169180

$-1.2166830$

$-1.5954960$

0.4681040

1. 3312120

0.8059470

0.4799140

1. 3493390

$-0.1157330$

1.4900540

$-0.2006990$

$-0.5630450$

2. 3660290

1.5030210

$-5.6037260$

$-4.6798510$

$-2.4370660$

$-1.0861000$
0.3807870

0.8433790 


\begin{tabular}{|c|c|c|}
\hline $\begin{array}{l}-0.7782630 \\
0.5191970 \\
2.6456750 \\
-0.4861840 \\
-1.7608810 \\
-1.9027120 \\
0.6300240 \\
-0.3898000 \\
-2.6366110 \\
-2.8855240 \\
1.6200590 \\
-3.9750410 \\
-5.2607780 \\
-5.5122260 \\
-2.9759730 \\
-3.7709460 \\
-6.0404740 \\
-6.4876380 \\
-1.9956100 \\
-3.9782440 \\
-4.2468970 \\
-3.9659180 \\
-4.5208510 \\
-4.2344940 \\
-4.5224590 \\
-4.7312960 \\
-4.2311300 \\
2.8278890 \\
3.7027930 \\
3.1377830 \\
4.8713380 \\
4.3099800 \\
5.1908780 \\
5.5513720 \\
4.5463700 \\
-0.2257560 \\
-0.6546690 \\
0.7828090 \\
-3.7361900 \\
-3.5086750 \\
-4.8768740 \\
-4.3251640 \\
-4.2762940 \\
-3.8709970 \\
2.2768770 \\
1.3745290\end{array}$ & $\begin{array}{r}4.3759630 \\
4.9220500 \\
4.7451020 \\
6.5619320 \\
5.9952280 \\
4.9321120 \\
6.0286820 \\
7.4045270 \\
6.3979490 \\
4.5070050 \\
6.4354370 \\
5.7015670 \\
5.1559410 \\
3.8348900 \\
4.9316980 \\
6.7359510 \\
5.7758840 \\
3.3940460 \\
5.3632550 \\
-0.6693410 \\
-1.4576930 \\
-1.2722820 \\
-2.8217050 \\
-2.6400950 \\
-3.4342290 \\
-3.4188440 \\
-3.1044670 \\
2.5214760 \\
1.9013320 \\
2.5127640 \\
1.2979230 \\
1.8842430 \\
1.2677780 \\
0.8269220 \\
1.8888200 \\
0.1546060 \\
-0.6374280 \\
-0.4300220 \\
-0.4847590 \\
0.5539470 \\
-4.8994180 \\
-5.2463520 \\
-0.8837480 \\
0.1320580 \\
3.2188880 \\
3.6021820\end{array}$ & $\begin{array}{r}-1.3663110 \\
-1.6564850 \\
-1.3178310 \\
-3.1526580 \\
-2.9060660 \\
-2.0362750 \\
-2.5426110 \\
-3.8337350 \\
-3.4103530 \\
-1.8620140 \\
-2.7413220 \\
1.4767520 \\
1.7140220 \\
1.4072080 \\
0.9147490 \\
1.7446800 \\
2.1509400 \\
1.6056110 \\
0.7423320 \\
-0.0160270 \\
1.1295530 \\
-1.2962280 \\
0.9650590 \\
-1.4019360 \\
-0.2888030 \\
1.8476440 \\
-2.3871570 \\
0.2002780 \\
-0.7231560 \\
1.5810910 \\
-0.2402840 \\
2.0102100 \\
1.1172250 \\
-0.9472900 \\
3.0718390 \\
0.2771700 \\
1.4843310 \\
-0.6705240 \\
-2.5799850 \\
-2.3247780 \\
-0.4884170 \\
-1.3749830 \\
2.5431810 \\
2.5140920 \\
2.6215250 \\
2.1360700\end{array}$ \\
\hline
\end{tabular}

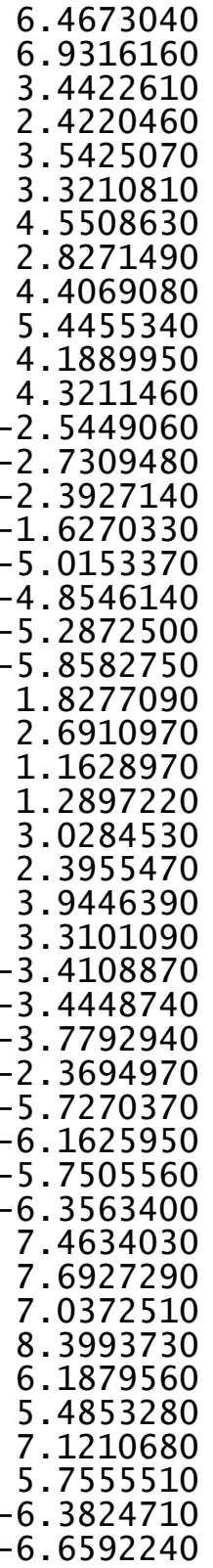

0.6065880

0.1106730

1.8738280

2.2245800

0.4574550

0.4968400

0.0411510

$-0.2161520$

2.8287370

2.4994380

2.8392300

3.8528470

$-1.0352680$

$-2.0715660$

$-0.4315630$

$-1.0066680$

$-0.4680260$

0.1339490

$-1.4862740$

$-0.0420930$

2. 2659330

1.8979650

2.7943330

1.4062130

4.4329270

4.9595180

4.1081530

5.1370000

$-1.6852640$

$-1.2049480$

$-2.7126560$

$-1.7235410$

$-0.8013190$

$-1.8072850$

$-0.3488170$

$-0.2023050$

1. 6561130

2. 4038730

2.1763770

1. 1740450

$-0.4613130$

$-1.2177330$

$-0.9617270$

0.0011110

$-5.0384250$

$-4.4385410$
1.6054260

0.7414060

$-2.2258160$

$-2.4089560$

$-2.8225640$

$-3.8973140$

$-2.7019010$

$-2.3428160$

$-2.9572720$

$-2.8189140$

$-4.0336860$

$-2.5738610$

$-3.3856280$

$-3.6968060$

$-4.2901050$

$-2.7889610$

$-3.4409060$

$-4.3452780$

$-3.7483890$

$-2.8815540$

3. 7441100

4.3138370

4.4402760

3. 3302270

3. 2043290

3. 9313160

3. 7148640

2. 4106620

3.5334890

4.5203160

3. 6463970

3. 2004850

3. 0594750

3. 1249910

4.0599040

2. 3903090

2. 1365590

1. 3669530

3.0044730

2. 4494200

2.6789920

2. 3108250

2.9694350

3.5755650

$-0.7991320$

$-1.6748880$ 


\begin{tabular}{|c|c|c|c|c|c|c|c|}
\hline $\mathrm{H}$ & -6.6391930 & -6.0881020 & $-0 \quad 9959410$ & C & 0.2029710 & 3.5355090 & -1.8824390 \\
\hline $\mathrm{H}$ & -6.9752750 & -4.6908560 & 0.0579160 & $\mathrm{C}$ & 2.4849630 & 2.6860680 & -2.2357270 \\
\hline $\mathrm{c}$ & -4.4921810 & -5.8039230 & 0.6915180 & C & 1.5117300 & 3.6909600 & -2.4534340 \\
\hline $\mathrm{H}$ & -3.4324410 & -5.6960560 & 0.9485770 & $\mathrm{H}$ & 3.4890850 & 2.8390630 & -2.6233760 \\
\hline $\mathrm{H}$ & -5.0845840 & -5.5618600 & 1.5832900 & C & -2.1637510 & 1.0844340 & -0.6424680 \\
\hline $\mathrm{H}$ & -4.6849660 & -6.8532040 & 0.4357600 & $\mathrm{C}$ & -3.4445150 & 0.8849600 & -0.0606650 \\
\hline C & 0.3152190 & -3.5825990 & 0.9827740 & $\mathrm{C}$ & -1.3735550 & 2.1983080 & -0.3988530 \\
\hline $\mathrm{C}$ & -0.3451680 & -3.4521310 & -0.3198140 & C & -3.8926970 & 1.8450400 & 0.8256230 \\
\hline $\mathrm{H}$ & 1.0652730 & -2.8307540 & 1.2266400 & $\mathrm{C}$ & -1.8081240 & 3.1382350 & 0.5927840 \\
\hline $\mathrm{H}$ & -0.3552060 & -2.4749920 & -0.7862390 & $\mathrm{C}$ & -3.0948210 & 2.9527750 & 1.2038880 \\
\hline 0 & -1.0379310 & -3.1146670 & 1.3333690 & $\mathrm{H}$ & -4.8746880 & 1.7311090 & 1.2771160 \\
\hline $\mathrm{H}$ & -0.9566570 & -2.0672370 & 1.4038360 & $\mathrm{C}$ & -2.7216580 & 4.9064850 & 2.6108870 \\
\hline C & -0.4105440 & -6.0137430 & 1.0555150 & $\mathrm{C}$ & -1.4399980 & 5.0730700 & 2.0311380 \\
\hline C & -0.6111620 & -5.9692240 & -0.4676690 & $\mathrm{C}$ & -0.9959130 & 4.2139570 & 1.0455400 \\
\hline C & -1.0921100 & -4.5851830 & -0.9401720 & C & -3.5300350 & 3.8658100 & 2.2027810 \\
\hline C & 0.6236050 & -4.9678920 & 1.5050540 & $\mathrm{H}$ & -3.0595510 & 5.5926830 & 3.3841280 \\
\hline $\mathrm{H}$ & -0.0734750 & -7.0107970 & 1.3617260 & $\mathrm{H}$ & -0.7962080 & 5.8817060 & 2.3702250 \\
\hline $\mathrm{H}$ & -1.3387820 & -6.7264690 & -0.7818920 & $\mathrm{H}$ & -0.0080970 & 4.3485230 & 0.6171140 \\
\hline $\mathrm{H}$ & -2.1522540 & -4.4323340 & -0.6958270 & $\mathrm{H}$ & -4.5095380 & 3.7138080 & 2.6524460 \\
\hline $\mathrm{H}$ & 1.6172490 & -5.2334310 & 1.1181580 & C & -0.4515950 & 5.6565600 & -2.8931110 \\
\hline $\mathrm{H}$ & 0.6985100 & -4.9229790 & 2.5985170 & $\mathrm{C}$ & 0.8485290 & 5.8201260 & -3.4325730 \\
\hline $\mathrm{H}$ & -1.3641610 & -5.8214290 & 1.5630470 & C & 1.8079040 & 4.8526390 & -3.2170970 \\
\hline $\mathrm{H}$ & 0.3424170 & -6.2016660 & -0.9607990 & $\mathrm{C}$ & -0.7662280 & 4.5448820 & -2.1371380 \\
\hline $\mathrm{H}$ & -1.0236970 & -4.4788600 & -2.0290180 & $\mathrm{H}$ & -1.2111220 & 6.4123820 & -3.0808770 \\
\hline C & 3.1289540 & -2.8539540 & -0.9189730 & $\mathrm{H}$ & 1.0828790 & 6.7034340 & -4.0223840 \\
\hline 0 & 2.8495200 & -1.7958850 & -0.1943970 & $\mathrm{H}$ & 2.8075470 & 4.9577880 & -3.6351010 \\
\hline C & 4.5394830 & -3.2938680 & -0.7816520 & $\mathrm{H}$ & -1.7670400 & 4.4313490 & -1.7325970 \\
\hline C & 4.9391570 & -4.6033210 & -1.1115370 & C & 3.3020520 & 0.5401390 & -1.3027620 \\
\hline $\mathrm{C}$ & 5.5078590 & -2.3820810 & -0.3129320 & $\mathrm{C}$ & 3.7241120 & -0.3243160 & -2.3326040 \\
\hline C & 6.2705750 & -4.9905190 & -0.9732900 & $\mathrm{C}$ & 4.0140750 & 0.5941090 & -0.0797360 \\
\hline $\mathrm{H}$ & 4.1950580 & -5.3110930 & -1.4655570 & $\mathrm{C}$ & 4.8987630 & -1.0695390 & -2.1520340 \\
\hline C & 6.8401090 & -2.7706810 & -0.1880140 & $\mathrm{C}$ & 5.1884400 & -0.1537330 & 0.0432660 \\
\hline $\mathrm{H}$ & 5.2089000 & -1.3699620 & -0.0637100 & $\mathrm{C}$ & 5.6650940 & -0.9710490 & -0.9889510 \\
\hline C & 7.2262500 & -4.0752170 & -0.5147020 & $\mathrm{H}$ & 5.2338450 & -1.7153760 & -2.9582670 \\
\hline $\mathrm{H}$ & 6.5645220 & -6.0077230 & -1.2209020 & $\mathrm{H}$ & 5.7656760 & -0.0911820 & 0.9633800 \\
\hline $\mathrm{H}$ & 7.5773450 & -2.0547660 & 0.1673240 & C & -4.2774270 & -0.3095140 & -0.3853250 \\
\hline $\mathrm{H}$ & 8.2657120 & -4.3786130 & -0.4116520 & $\mathrm{C}$ & -4.5486500 & -1.2773080 & 0.6144580 \\
\hline H & 1.9483710 & -1.3245510 & -0.4075510 & $\mathrm{C}$ & -4.8214530 & -0.4592320 & -1.6806550 \\
\hline & 2.0220160 & -3.6324060 & -1.9129330 & $\mathrm{C}$ & -5.3705010 & -2.3620390 & 0.2934370 \\
\hline & & & & C & -5.6353360 & -1.5675280 & -1.9492210 \\
\hline & S) G[B97- & $=-3$ & 37 & $\mathrm{C}$ & -5.9239870 & -2.5280730 & -0.9796870 \\
\hline 0 & 0.6010100 & 0.1981460 & -0.3414400 & $\mathrm{H}$ & -5.5810500 & -3.1053240 & 1.0590910 \\
\hline 0 & -1.6742450 & 0.1011360 & -1.5126620 & $\mathrm{H}$ & -6.0628150 & -1.6826820 & -2.9440590 \\
\hline C & 0.8881940 & 1.3675700 & -1.0289950 & C & 3.5722970 & 1.5214980 & 1.0485200 \\
\hline C & -0.0796170 & 2.3631890 & -1.1086210 & $\mathrm{C}$ & 6.9724260 & -1.7286370 & -0.7993810 \\
\hline & 2.2011870 & 1.5237930 & -1.5453250 & C & 2.9762140 & -0.4098140 & -3.6572250 \\
\hline
\end{tabular}




-3.9921230
-6.7995520
-4.6020190
-5.9233200
-6.6599830
-6.3493350
-5.7512600
-3.9727870
-3.0279040
-4.6498160
-3.7757660
-8.0388600
-8.6735010
-7.7375180
-8.6320240
-5.9968150
-5.1308300
-5.6295880
-6.6304380
-5.0901840
-5.5297990
-5.8952140
-4.6733500
-3.3550770
-4.1129970
-2.6018070
-2.8716510
4.2405970
5.3309010
4.0108720
3.8877580
3.8350970
3.3808830
4.9102460
3.3994870
7.7246060
8.7067540
7.1749980
7.8702550
6.7437720
6.2772950
6.0860400
7.6984910
2.7901190
3.7532390
2.3002250

$-1.1775930$ $-3.7267940$ 0.5611470 1. 2721580 0.5506580 1.7683700 2.0276550 $-0.0787950$ $-0.5732830$ $-0.8222280$ 0.6919180 $-3.7865570$ $-4.6397060$ $-3.9084620$ $-2.8668230$ $-5.0393010$ $-5.0191050$ $-5.1879480$ $-5.8961030$ $-0.7370190$ 0.2247400 $-1.4837560$ $-0.6389580$ $-2.4943700$ $-3.2777150$ $-2.8453270$ $-2.3317380$ 2.9032100 2.8043100 3.3468780 3.5833460 0.9586460 $-0.0307000$ 0.8793290 1.6295020 $-2.0013710$ $-2.4385070$ $-2.7139500$ $-1.0784120$ $-3.0464080$ $-2.8569160$ $-3.7128580$ $-3.5632990$ $-1.8552690$ -2.3155930
-2.4721400

2.0303060
-1.2995760
-2.7909020
-3.1456170
-3.5223550
-2.2639580
-3.9239670
-4.0421400
-3.7905810
-4.4823680
-4.7992820
-0.3869150
-0.6610570
0.6617380
-0.4678510
-1.2052090
-1.8788650
-0.1810620
-1.4710300
3.0189720
2.7295720
3.0483580
4.0305430
2.5134590
2.6428190
1.8018270
3.4855760
0.8974480
0.9871840
-0.0780300
1.6845670
2.4542670
2.5829070
2.6600830
3.2034700
-2.1110610
-1.8921840
-2.7399010
-2.6861380
-0.0322470
0.9407040
-0.6067180
0.1327750
-4.1505960
-4.4067920
-3.3913800

2.1675910
3.6936440
4.7038710
3.1379690
3.7821260
-0.5386170
-0.0006350
-1.0187850
1.9776610
7.6139530
2.4901380
-3.9107750
-7.1461480
-3.2071540
0.3869180
-0.6054410
0.7720510
1.5570520
0.3900640
1.9537440
1.8557570
0.8055190
-0.2078490
1.5872120
2.5556640
0.5179580
1.9104830
-0.7620810
1.1869420
1.1336730
2.0417120
0.0706630
1.7021850
1.6724690
1.0187340
3.0404810
3.7258390
3.4582260
1.5180750
3.2566080
4.8075650
4.0518520
1.1040690
1.0187240
3.4340930
3.3445040

-1.8567580
0.4255390
0.0302430
0.3805340
1.4753670
-0.8673280
-1.7320320
-1.5397250
0.0164280
-1.0947640
1.6694110
1.3305780
-3.6151780
-0.4167640
-0.5221970
-0.2444030
0.6230280
0.4511090
1.9279870
1.5525910
-0.5507870
3.0283520
2.0794060
2.8455900
1.4049580
4.0278620
3.7048450
-0.9021400
-2.0075950
-3.8267900
-2.7264840
-3.6034140
-1.7092280
-3.4737770
-2.7926470
-5.4503690
-4.3662260
-2.9635330
-5.2303060
-6.4436840
-4.5326930
-2.7826670
-5.3817530
-5.9411970
-5.4235660
-4.4150920

$-5.0544850$ $-4.7370270$ $-4.9100850$ $-5.6833630$ $-4.4349190$ $-0.8699270$

$-1.9717890$ 0.3891350 $-3.5087280$ $-0.1697000$ 0.9609060 $-2.4347790$ $-2.3368180$

2.0389450

3.2678540

2. 4697840

4.1328490

5.2875440

3.7549640

6.0426030

5.5822540

4.5028000

2.8620820

5.6485510

6.9361370

4.1857210

6.2320510

1.6655440

3. 3108680

0.3046220

0.6590750

0.3737670

0.5177210

$-1.0259590$

$-1.4455150$

0.7475840

1. 5922320

1.0252650

0.7221560

1.1574190

1.6340910

0.1170800

1.7290270

0.0525000

$-0.2773630$

2. 6217180 


$\begin{array}{lrrr}\text { TS241(S, S }(\mathrm{G}[\mathrm{B} 97-\mathrm{D} / \mathrm{def}-\mathrm{TZVP}]=-3633.748389 \\ \mathrm{O} & 0.5794810 & 0.7086740 & -0.0038970 \\ \mathrm{O} & -1.6294110 & 1.0399160 & -1.2334890 \\ \mathrm{C} & 0.8029740 & 2.0784260 & -0.0329580 \\ \mathrm{C} & -0.2379770 & 2.9428410 & 0.2891530 \\ \mathrm{C} & 2.1231750 & 2.5164030 & -0.3198660 \\ \mathrm{C} & -0.0064350 & 4.3535490 & 0.1759390 \\ \mathrm{C} & 2.3571640 & 3.8772010 & -0.3348050 \\ \mathrm{C} & 1.3190350 & 4.8189300 & -0.1285710 \\ \mathrm{H} & 3.3624390 & 4.2416290 & -0.5316350 \\ \mathrm{C} & -2.2154850 & 1.4572230 & -0.0361060 \\ \mathrm{C} & -3.4849000 & 0.9153530 & 0.3022270 \\ \mathrm{C} & -1.5461940 & 2.3968570 & 0.7377580 \\ \mathrm{C} & -4.0741500 & 1.3588490 & 1.4692420 \\ \mathrm{C} & -2.1230510 & 2.7713010 & 1.9979090 \\ \mathrm{C} & -3.4197100 & 2.2579020 & 2.3469820 \\ \mathrm{H} & -5.0584180 & 0.9868290 & 1.7410580 \\ \mathrm{C} & -3.3418890 & 3.4568250 & 4.4670870 \\ \mathrm{C} & -2.0449360 & 3.9273560 & 4.1456760 \\ \mathrm{C} & -1.4521650 & 3.5946670 & 2.9436880 \\ \mathrm{C} & -4.0104890 & 2.6343190 & 3.5843960 \\ \mathrm{H} & -3.7995680 & 3.7329640 & 5.4143940 \\ \mathrm{H} & -1.5076630 & 4.5530300 & 4.8551130 \\ \mathrm{H} & -0.4562050 & 3.9593900 & 2.7158180 \\ \mathrm{H} & -4.9977720 & 2.2446380 & 3.8261860 \\ \mathrm{C} & -0.7771260 & 6.6681840 & 0.1974880 \\ \mathrm{C} & 0.5373350 & 7.1261120 & -0.0672510 \\ \mathrm{C} & 1.5613910 & 6.2162250 & -0.2301790 \\ \mathrm{C} & -1.0414200 & 5.3183240 & 0.3175730 \\ \mathrm{H} & -1.5871150 & 7.3867470 & 0.3021350 \\ \mathrm{H} & 0.7323900 & 8.1927130 & -0.1533000 \\ \mathrm{H} & 2.5726510 & 6.5518100 & -0.4532340 \\ \mathrm{H} & -2.0540130 & 4.9814850 & 0.5155180 \\ \mathrm{C} & 3.2346190 & 1.5502720 & -0.5715270 \\ \mathrm{C} & 3.7742600 & 1.4157960 & -1.8715140 \\ \mathrm{C} & 3.7842920 & 0.8147850 & 0.5031580 \\ \mathrm{C} & 4.8804450 & 0.5793160 & -2.0554950 \\ \mathrm{C} & 4.8999970 & 0.0015260 & 0.2681860 \\ \mathrm{C} & 5.4701220 & -0.1235180 & -1.0008400 \\ \mathrm{H} & 5.3018760 & 0.4715330 & -3.0532650 \\ \mathrm{H} & 5.3284540 & -0.5481520 & 1.0997940 \\ \mathrm{C} & -4.1556640 & -0.0969140 & -0.5664630 \\ \mathrm{C} & -4.2715500 & -1.4393790 & -1.8199510 \\ \mathrm{C} & -4.6805930 & 0.2844800 & \end{array}$

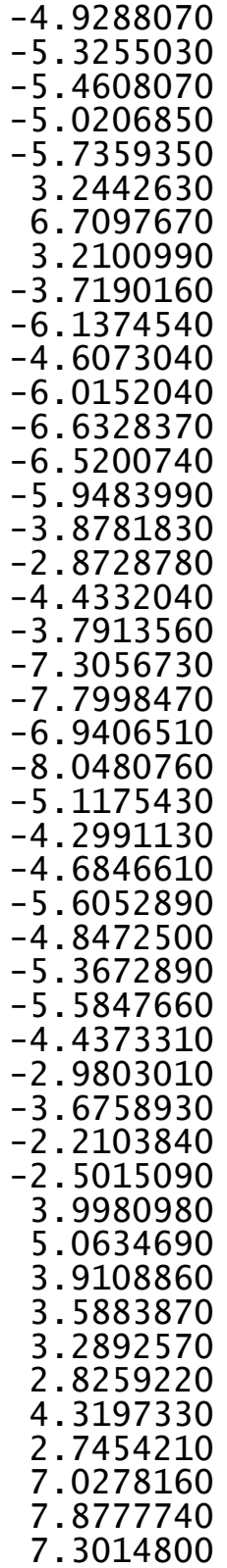

$-0.9482950$

$-2.6066050$

$-2.1915420$

$-0.6160940$

$-3.5717980$

1. 9218910

$-1.2754870$

$-3.0660030$

1. 2152860

$-3.0757690$

$-2.3396950$

$-2.4394890$

$-3.1550750$

$-1.4649650$

$-2.7820620$

$-3.6941570$

$-3.6166510$

$-4.4669650$

$-4.0152500$

$-2.3553970$

$-3.0312520$

$-1.4830530$

$-2.0089380$

$-3.5822200$

$-4.1275290$

$-2.7366840$

$-4.2506200$

2. 2624090

2. 3934900

1. 9444510

3. 2334750

1. 1244000

0.9042750

0.3478040

2. 0867160

2. 6608400

2. 7485960

2. 1182980

3. 6710680

2. 7312720

2. 1772660

2. 9767650

3. 6746890

$-0.1926630$

$-0.5089700$

0.7513380 


$\begin{array}{lrrr}\text { H } & 6.1732220 & -2.6624260 & 0.0006010 \\ \mathrm{C} & 7.9299350 & -0.0445030 & -1.4977030 \\ \mathrm{H} & 7.7370810 & 0.6780810 & -2.2997240 \\ \mathrm{H} & 8.1501550 & 0.5132910 & -0.5772050 \\ \mathrm{H} & 8.8147630 & -0.6379610 & -1.7640390 \\ \mathrm{C} & 2.9876670 & 1.2807790 & -4.2980450 \\ \mathrm{H} & 3.9363200 & 0.8835110 & -4.6806440 \\ \mathrm{H} & 2.3275440 & 0.4422740 & -4.0584100 \\ \mathrm{H} & 2.5242280 & 1.8706090 & -5.0996910 \\ \mathrm{C} & 4.1278450 & 3.3596880 & -3.4331620 \\ \mathrm{H} & 5.1177900 & 2.9909420 & -3.7339590 \\ \mathrm{H} & 3.7006710 & 3.9261770 & -4.2714110 \\ \mathrm{H} & 4.2601560 & 4.0396530 & -2.5833770 \\ \mathrm{P} & -0.3876050 & 0.0005330 & -1.1190890 \\ \mathrm{O} & 0.2540170 & -0.0236610 & -2.4795350 \\ \mathrm{O} & -0.7823930 & -1.3042570 & -0.4865360 \\ \mathrm{H} & 2.2337820 & 2.5824460 & -2.7811320 \\ \mathrm{H} & 6.5298890 & -1.5018530 & -2.2193190 \\ \mathrm{H} & 2.1938580 & 1.2542210 & 1.8644320 \\ \mathrm{H} & -4.0388150 & 2.3200440 & -1.6269390 \\ \mathrm{H} & -6.5436450 & -2.5076080 & -3.9496940 \\ \mathrm{H} & -2.9958480 & -1.1663800 & 1.5653650 \\ \mathrm{C} & 1.2391620 & -3.0737790 & -2.1552890 \\ \mathrm{C} & 2.1721900 & -2.5064230 & -1.1777440 \\ \mathrm{H} & 0.1829700 & -2.8946210 & -1.9621490 \\ \mathrm{H} & 1.8496620 & -1.6519950 & -0.5956530 \\ \mathrm{O} & 1.8530360 & -1.9455090 & -2.8911610 \\ \mathrm{H} & 1.2108330 & -1.1271330 & -2.7546730 \\ \mathrm{C} & 3.0741100 & -4.6755970 & -2.8857040 \\ \mathrm{C} & 3.7680770 & -4.4379730 & -1.5366050 \\ \mathrm{C} & 3.5781410 & -2.9887490 & -1.0586550 \\ \mathrm{C} & 1.5615120 & -4.4178240 & -2.7692480 \\ \mathrm{H} & 3.2407930 & -5.7054420 & -3.2220210 \\ \mathrm{H} & 4.8405940 & -4.6524050 & -1.6085520 \\ \mathrm{H} & 4.2026310 & -2.2993530 & -1.6423640 \\ \mathrm{H} & 1.1041780 & -5.1752800 & -2.1171230 \\ \mathrm{H} & 1.0652170 & -4.4846970 & -3.7452680 \\ \mathrm{H} & 3.5041560 & -4.0078230 & -3.6441130 \\ \mathrm{H} & 3.3454050 & -5.1212720 & -0.7867990 \\ \mathrm{H} & 3.8894070 & -2.8506150 & -0.0178450 \\ \mathrm{C} & 0.5606940 & -2.7992150 & 2.2501890 \\ \mathrm{O} & -0.1461830 & -1.7553650 & 1.9056180 \\ \mathrm{C} & 0.6902520 & -2.9518010 & 3.7229640 \\ \mathrm{C} & 1.7239830 & -3.7188190 & 4.2927620 \\ \mathrm{C} & -0.2286380 & -2.3063150 & 4.5765950 \\ \mathrm{C} & 1.8381990 & -3.8356920 & 5.6765890 \\ & & & \\ & & & \end{array}$

$\begin{array}{lrl}\mathrm{H} & 2.4422710 & -4.2068950 \\ \mathrm{C} & -0.1187500 & -2.4372680 \\ \mathrm{H} & -1.0314790 & -1.7137400 \\ \mathrm{C} & 0.9158440 & -3.1987380 \\ \mathrm{H} & 2.6494570 & -4.4212900 \\ \mathrm{H} & -0.8404490 & -1.9432600 \\ \mathrm{H} & 1.0042690 & -3.2940060 \\ \mathrm{H} & -0.3537150 & -1.6525250 \\ \mathrm{~S} & 1.2483160 & -3.8861590 \\ 144 & & \end{array}$
3.6403850
5.9603150
4.1497200
6.5153280
6.1025360
6.6066830
7.5952420

1.1662140

TS242(S,S) G[B97-D/def2-TZVP $]=-3633.748319$

$\begin{array}{lrrr}\text { TS242(S, S) G[B97-D/def2-TZVP] }=-3633.748319 \\ \text { O } & 0.5921390 & 0.1889930 & -0.3748560 \\ \text { O } & -1.7115980 & 0.2104860 & -1.4905780 \\ \mathrm{C} & 0.8905500 & 1.3834790 & -1.0129470 \\ \mathrm{C} & -0.0512570 & 2.4069230 & -1.0155640 \\ \mathrm{C} & 2.1903070 & 1.5287170 & -1.5645290 \\ \mathrm{C} & 0.2427610 & 3.6091040 & -1.7375430 \\ \mathrm{C} & 2.4875960 & 2.7161980 & -2.2041680 \\ \mathrm{C} & 1.5389280 & 3.7584860 & -2.3381710 \\ \mathrm{H} & 3.4826160 & 2.8590500 & -2.6182140 \\ \mathrm{C} & -2.1549390 & 1.1591340 & -0.5592820 \\ \mathrm{C} & -3.4231830 & 0.9574550 & 0.0487600 \\ \mathrm{C} & -1.3305100 & 2.2391250 & -0.2804690 \\ \mathrm{C} & -3.8239480 & 1.8808700 & 0.9948420 \\ \mathrm{C} & -1.7148480 & 3.1357850 & 0.7698880 \\ \mathrm{C} & -2.9893970 & 2.9494690 & 1.4056320 \\ \mathrm{H} & -4.7951150 & 1.7658220 & 1.4686430 \\ \mathrm{C} & -2.5298720 & 4.8196730 & 2.8982040 \\ \mathrm{C} & -1.2592140 & 4.9838570 & 2.2941240 \\ \mathrm{C} & -0.8627490 & 4.1652950 & 1.2552510 \\ \mathrm{C} & -3.3750010 & 3.8209060 & 2.4605790 \\ \mathrm{H} & -2.8302180 & 5.4735980 & 3.7137550 \\ \mathrm{H} & -0.5863530 & 5.7580340 & 2.6563600 \\ \mathrm{H} & 0.1178530 & 4.2949960 & 0.8088610 \\ \mathrm{H} & -4.3458770 & 3.6701080 & 2.9290080 \\ \mathrm{C} & -0.3793590 & 5.7960930 & -2.6207040 \\ \mathrm{C} & 0.9099290 & 5.9530580 & -3.1873930 \\ \mathrm{C} & 1.8467360 & 4.9498670 & -3.0497650 \\ \mathrm{C} & -0.7044740 & 4.6554640 & -1.9138330 \\ \mathrm{H} & -1.1225740 & 6.5801180 & -2.7483990 \\ \mathrm{H} & 1.1532040 & 6.8592560 & -3.7375130 \\ \mathrm{H} & 2.8370750 & 5.0493750 & -3.4906420 \\ \mathrm{H} & -1.6977960 & 4.5471330 & -1.4899340 \\ \mathrm{C} & 3.2598520 & 0.4945260 & -1.4151470 \\ \mathrm{C} & 3.6134650 & -0.3211900 & -2.5084480 \\ \mathrm{C} & 4.0005310 & 0.4368120 & -0.2084530 \\ & & & \end{array}$




$\begin{array}{rrr}4.7539400 & -1.1309500 & -2.4027430 \\ 5.1335250 & -0.3797010 & -0.1574170 \\ 5.5424050 & -1.1512080 & -1.2522250 \\ 5.0503500 & -1.7471830 & -3.2492730 \\ 5.7254920 & -0.4105030 & 0.7534760 \\ -4.2868320 & -0.2045160 & -0.3105010 \\ -4.5542820 & -1.2138030 & 0.6486960 \\ -4.8569170 & -0.2872600 & -1.6008960 \\ -5.3982740 & -2.2708520 & 0.2937510 \\ -5.6907780 & -1.3711370 & -1.9049460 \\ -5.9757250 & -2.3715420 & -0.9754870 \\ -5.6079600 & -3.0441990 & 1.0291540 \\ -6.1379640 & -1.4339460 & -2.8957980 \\ 3.6336570 & 1.3175530 & 0.9824000 \\ 6.8249810 & -1.9643930 & -1.1990330 \\ 2.8273270 & -0.2959380 & -3.8130610 \\ -3.9725680 & -1.1883480 & 2.0578930 \\ -6.8782360 & -3.5392680 & -1.3322110 \\ -4.6463940 & 0.7821850 & -2.6655300 \\ -5.9639560 & 1.5293380 & -2.9533590 \\ -6.7180120 & 0.8397430 & -3.3548400 \\ -6.3657720 & 1.9814330 & -2.0373320 \\ -5.7963250 & 2.3247050 & -3.6920800 \\ -4.0574020 & 0.1947060 & -3.9614260 \\ -3.1188640 & -0.3323680 & -3.7563160 \\ -4.7599210 & -0.5103390 & -4.4244700 \\ -3.8587420 & 0.9989300 & -4.6823880 \\ -8.1194530 & -3.5945460 & -0.4214710 \\ -8.7747550 & -4.4238770 & -0.7192490 \\ -7.8210590 & -3.7530070 & 0.6231990 \\ -8.6897260 & -2.6585430 & -0.4763460 \\ -6.1102260 & -4.8744620 & -1.2773300 \\ -5.2400740 & -4.8550070 & -1.9455750 \\ -5.7534080 & -5.0676580 & -0.2569300 \\ -6.7648280 & -5.7046780 & -1.5747360 \\ -5.0469430 & -0.7725600 & 3.0828980 \\ -5.4759230 & 0.2072970 & 2.8421010 \\ -5.8634420 & -1.5072720 & 3.0941570 \\ -4.6124250 & -0.7241390 & 4.0906580 \\ -3.3526800 & -2.5362390 & 2.4722190 \\ -4.1222830 & -3.3112490 & 2.5806640 \\ -2.6180380 & -2.8686320 & 1.7328370 \\ -2.8503600 & -2.4249620 & 3.4419760 \\ 4.3443330 & 2.6824560 & 0.8721390 \\ 5.4331920 & 2.5416550 & -0.0675500 \\ 4.0897290 & 3.1858530 & \end{array}$

4.0503070
3.9312400
3.4543680
5.0105060
3.5417050
6.7749760
7.7310740
6.5870290
5.9806100
8.0443660
8.1017960
7.9740250
8.9717330
2.5566840
3.4877140
2.0620280
1.9058090
3.5556090
4.5417300
2.9752070
3.7007050
-0.5914830
-0.1050240
-1.0634040
1.8545760
6.9465750
2.5548040
-3.9346510
-7.2212220
-3.1725310
0.4463830
-0.5549190
0.8890950
1.6857850
0.5487370
2.1332400
1.9519660
1.0150070
-0.0577370
1.8068950
2.7429560
0.7585120
2.1692880
-0.7528880
1.1966990
1.0104920

3. 3309400

0.6797050

$-0.3023540$

0.5626170

1. 3254410

-3.0675710

- 3.6058850

$-2.6346980$

$-3.7918270$

-1.0495040

$-0.2713830$

$-0.5564270$

$-1.6369340$

$-2.1909350$

$-2.3354270$

$-1.6237490$

0.5642840

0.1340310

0.5977820

1. 5903590

$-0.8188250$

$-1.6489030$

$-1.5318320$

0.1672670

$-2.4507920$

1. 5066560

1.5229440

$-3.3876430$

$-0.4439990$

$-0.6873480$

$-0.3440340$

0.4074760

0.1608890

1.7393120

1. 2160540

$-0.8623060$

2. 7929030

1.9477560

2. 5361150

1. 0111850

3. 8140790

3. 3589990

$-0.9620640$

-2.1980460
-3.8761940
1.7086180

2. 3483700

2. 4477720

2. 5100940

3. 1435790

$-0.1262190$

$-0.0895820$

0.8648810

$-0.3406340$

$-0.9695500$

$-1.7411190$

0.0089300

$-0.9916310$

$-4.3774010$

$-4.6913900$

$-3.6351030$

$-5.2576550$

$-4.8654710$

$-5.0872890$

$-5.7973270$

$-4.5083490$

$-0.9199090$

$-2.0713410$

0.3197140

$-3.6127850$

$-2.1770050$

0.9466620

$-2.2895470$

$-2.3655810$

2. 0880180

3.2062230

2. 4450310

4.1081490

5. 2411520

3.7890430

6.0331180

5.4902600

4.5732540

2. 9135850

5.6977090

6. 9099200

4. 3020370

6. 3099580

1. 6196420

3. 1675910

0.0801250 


\begin{tabular}{|c|c|c|c|c|c|c|c|}
\hline 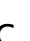 & 19641960 & 28257670 & 01616130 & & -08328520 & 2850010 & 23667000 \\
\hline $\mathrm{H}$ & -0.0425180 & -3.6195650 & 0.1793020 & $\mathrm{H}$ & -1.3009510 & $\begin{array}{l}4.3850910 \\
6.1954370\end{array}$ & $\begin{array}{l}-2.366 / 990 \\
-3.4058990\end{array}$ \\
\hline $\mathrm{H}$ & 1.6589030 & -1.7913560 & 0.3782360 & $\mathrm{H}$ & 0.9551730 & 6.4037130 & -4.4549440 \\
\hline 0 & 1.5369270 & -3.4795260 & -1.2437180 & $\mathrm{H}$ & 2.6780090 & 4.6639210 & -4.0345300 \\
\hline H & 0.8960580 & -2.7604800 & -1.6178490 & $\mathrm{H}$ & -1.8174280 & 4.3069350 & -1.9166580 \\
\hline c & 2.8647300 & -5.5856320 & 0.4190200 & C & 3.2027560 & 0.3502470 & -1.5244560 \\
\hline C & 3.6004420 & -4.5645780 & 1.2989190 & $\mathrm{C}$ & 3.4900820 & -0.5986380 & -2.5271890 \\
\hline C & 3.3781690 & -3.1318910 & 0.7915180 & $\mathrm{C}$ & 4.0264520 & 0.4525440 & -0.3783360 \\
\hline C & 1.3512310 & -5.3100120 & 0.4273390 & C & 4.6513650 & -1.3740280 & -2.4033320 \\
\hline $\mathrm{H}$ & 3.0497610 & -6.6032930 & 0.7816340 & $\mathrm{c}$ & 5.1772800 & -0.3380270 & -0.3055510 \\
\hline $\mathrm{H}$ & 4.6759490 & -4.7717790 & 1.3215540 & $\mathrm{C}$ & 5.5228570 & -1.2374950 & -1.3210080 \\
\hline $\mathrm{H}$ & 3.9580020 & -2.9399490 & -0.1233200 & $\mathrm{H}$ & 4.8969910 & -2.0929140 & -3.1822660 \\
\hline $\mathrm{H}$ & 0.9469000 & -5.4918650 & 1.4331400 & $\mathrm{H}$ & 5.8290940 & -0.2432370 & 0.5589810 \\
\hline $\mathrm{H}$ & 0.8166210 & -5.9718800 & -0.2649350 & C & -4.2858740 & -0.3059060 & -0.0682500 \\
\hline $\mathrm{H}$ & 3.2446040 & -5.5278800 & -0.6098540 & $\mathrm{C}$ & -4.5074080 & -1.1748300 & 1.0273860 \\
\hline $\mathrm{H}$ & 3.2293200 & -4.6409230 & 2.3304590 & $\mathrm{C}$ & -4.8914530 & -0.5769300 & -1.3183730 \\
\hline $\mathrm{H}$ & 3.7286980 & -2.3752590 & 1.5021020 & $\mathrm{C}$ & -5.3386020 & -2.2879150 & 0.8445850 \\
\hline & & & & $\mathrm{C}$ & -5.7069860 & -1.7047270 & -1.4468890 \\
\hline & , S) $\mathrm{G}[\mathrm{B} 97-\mathrm{D}$ & {$[\mathrm{ZVP}]=-36$} & 229 & C & -5.9452540 & -2.5742680 & -0.3789790 \\
\hline 0 & 0.5722440 & 0.1478450 & -0.3509630 & $\mathrm{H}$ & -5.5129890 & -2.9602440 & 1.6823260 \\
\hline 0 & -1.7604450 & 0.0068650 & -1.3920280 & $\mathrm{H}$ & -6.1767710 & -1.9035860 & -2.4077430 \\
\hline C & 0.8355940 & 1.2618560 & -1.1340690 & C & 3.7211780 & 1.4663620 & 0.7195760 \\
\hline $\mathrm{C}$ & -0.1216720 & 2.2666620 & -1.2291970 & $\mathrm{C}$ & 6.8100750 & -2.0426350 & -1.2529110 \\
\hline C & 2.1178730 & 1.3584660 & -1.7344740 & C & 2.6005410 & -0.7512230 & -3.7547500 \\
\hline C & 0.1375350 & 3.3828880 & -2.0895040 & $\mathrm{C}$ & -3.8902650 & -0.9452990 & 2.4026250 \\
\hline C & 2.3884340 & 2.4783440 & -2.4967280 & $\mathrm{C}$ & -6.8337190 & -3.7940910 & -0.5470310 \\
\hline C & 1.421 & 3.4873110 & -2.7235770 & $\mathrm{C}$ & -4.7393290 & 0.3421290 & -2.5247130 \\
\hline $\mathrm{H}$ & 3.3728160 & 2.5869660 & -2.9456210 & $\mathrm{C}$ & -6.0852100 & 1.0103510 & -2.8701150 \\
\hline c & -2.1913040 & 1.0527420 & -0.5646850 & $\mathrm{H}$ & -6.8326290 & 0.2538670 & -3.1432100 \\
\hline C & -3.4380460 & 0.9099140 & 0.1016600 & $\mathrm{H}$ & -6.4708700 & 1.5791300 & -2.0141490 \\
\hline C & -1.3785750 & 2.1710090 & -0.4450130 & $\mathrm{H}$ & -5.9619030 & 1.6955190 & -3.7195960 \\
\hline C & -3.83 & 23390 & 0.9308910 & C & -4.1696930 & -0.4017360 & -3.7463880 \\
\hline C & -1.7514360 & 3.1879590 & 0.4949820 & $\mathrm{H}$ & -3.2050460 & -0.8609640 & -3.5039860 \\
\hline C & -3.0089470 & 3.0675330 & 1.1789020 & $\mathrm{H}$ & -4.8575990 & -1.1896850 & -4.0802800 \\
\hline $\mathrm{H}$ & -4.7884970 & 1.8736580 & 1.4433100 & $\mathrm{H}$ & -4.0266970 & 0.2993730 & -4.5794770 \\
\hline c & -2.5487230 & 5.1191560 & 2.4106180 & C & -6.2557550 & -4.7687850 & -1.5909510 \\
\hline C & -1.2925770 & 5.2199090 & 1.7638200 & $\mathrm{H}$ & -6.8886650 & -5.6626750 & -1.6711220 \\
\hline c & -0.9055220 & 4.2812310 & 0.8281370 & $\mathrm{H}$ & -6.2122250 & -4.2898510 & -2.5779660 \\
\hline $\mathrm{C}$ & -3.3866840 & 520630 & 2.1222100 & $\mathrm{H}$ & -5.2397410 & -5.0802420 & -1.3174430 \\
\hline $\mathrm{H}$ & -2.8430070 & 5.8682400 & 3.1421970 & C & -8.2739340 & -3.3943050 & -0.9206740 \\
\hline $\mathrm{H}$ & -0.6233070 & 6.0412990 & 2.0103380 & $\mathrm{H}$ & -8.7042900 & -2.7238260 & -0.1659400 \\
\hline $\mathrm{H}$ & 0.0633300 & 4.3660180 & 0.3472100 & $\mathrm{H}$ & -8.2885370 & -2.8750230 & -1.8880960 \\
\hline $\mathrm{H}$ & -4.3455880 & 3.9597730 & 2.6270710 & $\mathrm{H}$ & -8.9095850 & -4.2861740 & -1.0018180 \\
\hline C & -0.5404810 & 5.4446980 & -3.2022290 & C & -4.9448460 & -0.4144300 & 3.3945980 \\
\hline C & 0.7379180 & 5.5608590 & -3.8026790 & $\mathrm{H}$ & -5.4071960 & 0.5116080 & 3.0331390 \\
\hline C & 1.6966320 & 71980 & -3.5681760 & $\mathrm{H}$ & -5.7403020 & -1.1582630 & 3.5376690 \\
\hline
\end{tabular}




\begin{tabular}{|c|c|c|c|}
\hline & & & \\
\hline $\mathrm{H}$ & $\begin{array}{l}-4.4809290 \\
-3.2269360\end{array}$ & $\begin{array}{l}-0.2142350 \\
-2\end{array}$ & $\begin{array}{l}4.3699470 \\
2.9773910\end{array}$ \\
\hline $\mathrm{H}$ & -3.9750070 & -2.9808630 & 3.2087680 \\
\hline $\mathrm{H}$ & -2.5018560 & -2.6250860 & 2.2702710 \\
\hline $\mathrm{H}$ & -2.7049020 & -1.9602440 & 3.9099050 \\
\hline C & 4.4746600 & 2.7841280 & 0.4485690 \\
\hline $\mathrm{H}$ & 5.5587520 & 2.6094320 & 0.4785790 \\
\hline $\mathrm{H}$ & 4.2155720 & 3.1876930 & -0.5371990 \\
\hline $\mathrm{H}$ & 4.2216490 & 3.5319380 & 2123130 \\
\hline C & 4.0310230 & 0.9581260 & 2.1366090 \\
\hline $\mathrm{H}$ & 3.5095260 & 0.0178340 & 2.3500710 \\
\hline $\mathrm{H}$ & 5.1075850 & 0.8009200 & 2.2806880 \\
\hline $\mathrm{H}$ & 3.6988590 & 1.7004740 & 2.8715640 \\
\hline C & 6.7741890 & -3.0924880 & -0.1261340 \\
\hline $\mathrm{H}$ & 7.7234370 & -3.6429560 & -0.0880650 \\
\hline $\mathrm{H}$ & 6.6184120 & -2.6090380 & 0.8470850 \\
\hline $\mathrm{H}$ & 5.9641260 & -3.8143570 & -0.2840470 \\
\hline $\mathrm{C}$ & 8.0395780 & -1.1282270 & -1.0936540 \\
\hline $\mathrm{H}$ & 8.0852810 & -0.3873010 & -1.9016810 \\
\hline $\mathrm{H}$ & 7.9995570 & -0.5897450 & -0.1378860 \\
\hline $\mathrm{H}$ & 8.9610580 & -1.7247150 & -1.1103270 \\
\hline C & 2.4099350 & -2.2162150 & -4.1839820 \\
\hline $\mathrm{H}$ & 3.3428960 & -2.6418450 & -4.5760570 \\
\hline $\mathrm{H}$ & 2.0717410 & -2.8339100 & -3.3463300 \\
\hline $\mathrm{H}$ & 1.6584190 & -2.2675990 & -4.9818110 \\
\hline C & 3.1538770 & 0.0727780 & -4.9348640 \\
\hline $\mathrm{H}$ & 4.1556500 & -0.2858600 & -5.2082680 \\
\hline $\mathrm{H}$ & 2.4985440 & -0.0307470 & 8102700 \\
\hline $\mathrm{H}$ & 3.2255510 & 1.1351780 & -4.6779310 \\
\hline $\mathrm{P}$ & -0.6017990 & -0.9361450 & -0.7525500 \\
\hline 0 & -0.1265720 & -1.8695650 & -1.8270170 \\
\hline 0 & -1.0230280 & -1.5255770 & 0.5679080 \\
\hline $\mathrm{H}$ & 1.6098760 & -0.3578180 & -3.500229 \\
\hline $\mathrm{H}$ & 6.9097630 & -2.5796600 & -2.2066160 \\
\hline $\mathrm{H}$ & 2.6484220 & 1.6889680 & 0.6878550 \\
\hline $\mathrm{H}$ & -4.0390440 & 1.1449700 & -2.2754040 \\
\hline $\mathrm{H}$ & -6.8665130 & -4.3138800 & 0.4211420 \\
\hline $\mathrm{H}$ & -3.1083020 & -0.1874280 & 2.3067370 \\
\hline C & 0.5668300 & -0.3236450 & 3.2767710 \\
\hline 0 & -0.4682360 & -0.0848710 & 2.5207130 \\
\hline C & 1.0356250 & 0.8784230 & 4.0137650 \\
\hline C & 1.8843330 & 0.7794240 & 5.1316020 \\
\hline C & 0.6688240 & 2.1583000 & 3.5464020 \\
\hline$C$ & 2.3583930 & 1.9273660 & 5.762565 \\
\hline $\mathrm{H}$ & 2.1708000 & -0.2035200 & 5.494574 \\
\hline & 1.16 & & \\
\hline
\end{tabular}

$\begin{array}{lr}\mathrm{H} & 0.0201470 \\ \mathrm{C} & 2.0061150 \\ \mathrm{H} & 3.0094980 \\ \mathrm{H} & 0.8828460 \\ \mathrm{H} & 2.3894180 \\ \mathrm{H} & -0.6861100 \\ \mathrm{~S} & 1.3309260 \\ \mathrm{C} & 1.1150430 \\ \mathrm{C} & 2.0546400 \\ \mathrm{H} & 0.0604160 \\ \mathrm{H} & 1.7195440 \\ \mathrm{O} & 1.5873730 \\ \mathrm{H} & 0.9185250 \\ \mathrm{C} & 3.0238610 \\ \mathrm{C} & 3.7675380 \\ \mathrm{C} & 3.4845440 \\ \mathrm{C} & 1.5052590 \\ \mathrm{H} & 3.2497820 \\ \mathrm{H} & 4.8483570 \\ \mathrm{H} & 4.0357220 \\ \mathrm{H} & 1.1475810 \\ \mathrm{H} & 0.9597220 \\ \mathrm{H} & 3.3599440 \\ \mathrm{H} & 3.4443670 \\ \mathrm{H} & 3.8286510 \\ \mathrm{H} & \\ \mathrm{H} & \end{array}$

2.2530020
3.1936450
1.8365800
4.2818220
4.0882980
-0.7996480
-1.8230880
-3.8217480
-2.7161180
-3.5769850
-1.7044170
-3.5604270
-2.9005160
-5.4428920
-4.3195790
-2.9506100
-5.2002670
-6.4126130
-4.4977240
-2.8298040
-5.2750330
-5.9468830
-5.4848790
-4.3007180
-2.1182230

2.6818210 5.2786760

6.6287760

3.7834800

5.7644460

1.7839430

3.3971840

0.5236260

0.7558070

0.6351580

0.5693210

$-0.8538510$

$-1.2836170$

0.9683890

1.7058060

1.0683040

1.0120700

1.4265390

1.6987500

0.1245110

2.0488020

0.4221260

$-0.0762860$

144

2.7559050
1.6924200

TS244(S,S) G[B97-D/def2-TZVP $]=-3633.748122$

$\begin{array}{lrrr}\text { TS244(S, S) G[B97-D/def2-TZVP }]=-3633.748122 \\ \text { O } & 0.6131540 & 0.2086030 & -0.3198750 \\ \mathrm{O} & -1.6462420 & 0.0901310 & -1.5153050 \\ \mathrm{C} & 0.9073270 & 1.3654610 & -1.0264300 \\ \mathrm{C} & -0.0576140 & 2.3612680 & -1.1325330 \\ \mathrm{C} & 2.2246500 & 1.5087600 & -1.5364430 \\ \mathrm{C} & 0.2366130 & 3.5203770 & -1.9224150 \\ \mathrm{C} & 2.5201200 & 2.6598170 & -2.2398080 \\ \mathrm{C} & 1.5523260 & 3.6648490 & -2.4804270 \\ \mathrm{H} & 3.5274160 & 2.8022710 & -2.6231550 \\ \mathrm{C} & -2.1472880 & 1.0895790 & -0.6708270 \\ \mathrm{C} & -3.4363960 & 0.9005550 & -0.1040610 \\ \mathrm{C} & -1.3612870 & 2.2089650 & -0.4378560 \\ \mathrm{C} & -3.8992380 & 1.8788270 & 0.7544660 \\ \mathrm{C} & -1.8116450 & 3.1681960 & 0.5280760 \\ \mathrm{C} & -3.1080300 & 2.9948140 & 1.1219640 \\ \mathrm{H} & -4.8876770 & 1.7728980 & 1.1937400 \\ \mathrm{C} & -2.7579520 & 4.9768560 & 2.4948570 \\ \mathrm{C} & -1.4667140 & 5.1315380 & 1.9333340 \\ \mathrm{C} & -1.0069290 & 4.2530030 & 0.9724060\end{array}$




$\begin{array}{rrr}-3.5596790 & 3.9280780 & 2.0945360 \\ -3.1085250 & 5.6787420 & 3.2481130 \\ -0.8282680 & 5.9467200 & 2.2667970 \\ -0.0125600 & 4.3793400 & 0.5569850 \\ -4.5467520 & 3.7853450 & 2.5305150 \\ -0.4024680 & 5.6258870 & -2.9750920 \\ 0.9044780 & 5.7795510 & -3.5005840 \\ 1.8595690 & 4.8142130 & -3.2583400 \\ -0.7278740 & 4.5265940 & -2.2056860 \\ -1.1587070 & 6.3793720 & -3.1842970 \\ 1.1474410 & 6.6532410 & -4.1011400 \\ 2.8642590 & 4.9113110 & -3.6660770 \\ -1.7338050 & 4.4208270 & -1.8120840 \\ 3.3063110 & 0.5090400 & -1.2791540 \\ 3.7073990 & -0.3820170 & -2.2978650 \\ 3.9980770 & 0.5438040 & -0.0457920 \\ 4.8315620 & -1.1884850 & -2.0831940 \\ 5.1236410 & -0.2724270 & 0.1133170 \\ 5.5641270 & -1.1352340 & -0.8944730 \\ 5.1507850 & -1.8663340 & -2.8707390 \\ 5.6766560 & -0.2436870 & 1.0492690 \\ -4.2606270 & -0.3039980 & -0.4127630 \\ -4.5477460 & -1.2454620 & 0.6076910 \\ -4.7792440 & -0.4911380 & -1.7136300 \\ -5.3581170 & -2.3426700 & 0.3002240 \\ -5.5828020 & -1.6102750 & -1.9676890 \\ -5.8856120 & -2.5461030 & -0.9783910 \\ -5.5795130 & -3.0660780 & 1.0816470 \\ -5.9907280 & -1.7541710 & -2.9669930 \\ 3.5838500 & 1.5075270 & 1.0624470 \\ 6.8212310 & -1.9688120 & -0.7096640 \\ 2.9795510 & -0.4440820 & -3.6346540 \\ -4.0200860 & -1.1054670 & 2.0310820 \\ -6.7449500 & -3.7601130 & -1.2845320 \\ -4.5450900 & 0.5010340 & -2.8464880 \\ -5.8633380 & 1.1969270 & -3.2407060 \\ -6.5919280 & 0.4624040 & -3.6078120 \\ -6.3040880 & 1.7160150 & -2.3797310 \\ -5.6819320 & 1.9307690 & -4.0374130 \\ -3.8931420 & -0.1676830 & -4.0706990 \\ -2.9487420 & -0.6481450 & -3.7920570 \\ -4.5584690 & -0.9273270 & -4.5008590 \\ -3.6906270 & 0.5843450 & -4.8449720 \\ -7.9888370 & -3.8237220 & -0.3787320 \\ -8.6103910 & -4.6888990 & -0.6449420 \\ -7.6929320 & -3.9274050 & 0.6733720\end{array}$

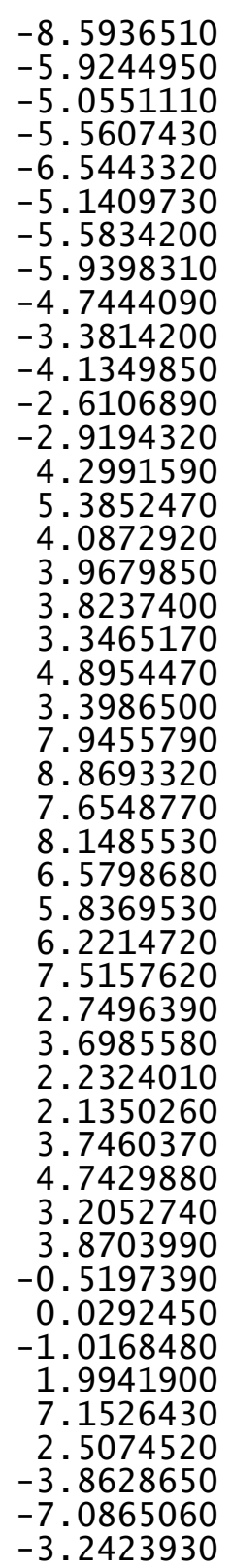

-2.9132140
-5.0598630
-5.0372510
-5.1878970
-5.9295130
-0.6500510
0.2996850
-1.4032020
-0.5217890
-2.4043010
-3.1914110
-2.7649880
-2.2146300
2.8621980
2.7280080
3.2915950
3.5716050
0.9685640
-0.0072940
0.8691190
1.6648640
-1.4586020
-2.0275800
-1.5800640
-0.3952640
-3.4711740
-3.8751390
-3.6501860
-4.0288610
-1.8825990
-2.3756850
-2.4808240
-1.8632040
0.3636230
-0.0705350
0.3407100
1.4089300
-0.8670380
-1.7541820
-1.5152160
0.0173560
-1.8384250
1.6898060
1.2824940
-3.6687330
-0.3371770
$-0.4760330$

$-1.1663350$

$-1.8355370$

$-0.1381590$

$-1.4228500$

2. 9869540

2.6639630

3.0207350

4.0032620

2. 5586680

2.6908330

1.8710550

3.5362870

0.8829310

0.9783290

$-0.1028480$

1.6534430

2. 4814720

2.6279880

2. 6965640

3. 2132670

$-1.6336560$

$-1.4636540$

$-2.6856100$

$-1.4546630$

$-0.9475250$

$-0.2497140$

$-1.9692380$

$-0.8128420$

$-4.1297390$

$-4.3782090$

$-3.3736700$

$-5.0388240$

$-4.7015890$

$-4.8575210$

$-5.6572980$

$-4.3966200$

$-0.8400460$

$-1.9183430$

0.4252890

$-3.5045460$

0.3303550

0.9675110

$-2.4993610$

$-2.3254250$

2. 0361170 


\begin{tabular}{|c|c|c|c|c|c|c|c|}
\hline C & 0.3493080 & -0.4509730 & 3.3008520 & C & 0.4958090 & -3.2873960 & -0.5276390 \\
\hline 0 & -0.6292530 & -0.1792480 & 2.4838290 & $\mathrm{C}$ & -2.1477840 & -4.0896210 & -1.1233240 \\
\hline C & 0.7429040 & 0.7113510 & 4.1390030 & c & 0.2752250 & -4.3271280 & -1.4934910 \\
\hline C & 1.5150670 & 0.5594140 & 5.3050350 & C & -1.0718640 & -4.7428580 & -1.7705130 \\
\hline$c$ & 0.3834600 & 2.0109810 & 3.7227410 & $\mathrm{H}$ & -3.1615950 & -4.4230630 & -1.3304960 \\
\hline C & 1.9216280 & 1.6750310 & 6.0336670 & C & -0.2550730 & -6.3814370 & -3.3784000 \\
\hline $\mathrm{H}$ & 1.7963630 & -0.4384640 & 5.6290480 & $\mathrm{C}$ & 1.0709710 & -5.9431550 & -3.1404780 \\
\hline c & 0.8086990 & 3.1250560 & 4.4442850 & C & 1.3290290 & -4.9444900 & -2.2224730 \\
\hline $\mathrm{H}$ & -0.2053440 & 2.1466710 & 2.8213610 & C & -1.3034590 & -5.7866760 & -2.7080760 \\
\hline c & 1.5779260 & 2.9621050 & 5.6014200 & $\mathrm{H}$ & -0.4432670 & -7.1745200 & -4.0986030 \\
\hline $\mathrm{H}$ & 2.5136230 & 1.5428770 & 6.9362040 & $\mathrm{H}$ & 1.8943710 & -6.3937560 & -3.6903390 \\
\hline $\mathrm{H}$ & 0.5385860 & 4.1197280 & 4.0978150 & $\mathrm{H}$ & 2.3500620 & -4.6172260 & -2.0562930 \\
\hline $\mathrm{H}$ & 1.9090820 & 3.8321170 & 6.1641870 & $\mathrm{H}$ & -2.3303050 & -6.0957350 & -2.8954960 \\
\hline $\mathrm{H}$ & -0.7814060 & -0.8525990 & 1.6913290 & C & 3.3450370 & -6.1085160 & 1.2134180 \\
\hline s & 1.1241490 & -1.9474590 & 3.3933690 & c & 4.6881660 & -5.7171100 & 1.4370680 \\
\hline C & 1.1028630 & -3.8273090 & 0.4036510 & $\mathrm{C}$ & 5.0744040 & -4.4179930 & 1.1810750 \\
\hline C & 2.0179190 & -2.7360270 & 0.7679270 & C & 2.4227100 & -5.2098210 & 0.7153620 \\
\hline $\mathrm{H}$ & 0.0417360 & -3.5891820 & 0.4492950 & $\mathrm{H}$ & 3.0352800 & -7.1261310 & 1.4412980 \\
\hline $\mathrm{H}$ & 1.6942820 & -1.7158210 & 0.6098150 & $\mathrm{H}$ & 5.4058820 & -6.4373630 & 1.8232680 \\
\hline 0 & 1.6736360 & -3.4930210 & -0.9183830 & $\mathrm{H}$ & 6.0964270 & -4.0940960 & 1.3698250 \\
\hline $\mathrm{H}$ & 1.0347370 & -2.8110410 & -1.3592630 & $\mathrm{H}$ & 1.3974000 & -5.5256510 & 0.5531660 \\
\hline c & 2.9790260 & -5.4733860 & 0.8926830 & C & 4.0288340 & 0.2760980 & -0.0272950 \\
\hline $\mathrm{C}$ & 3.6664610 & -4.3933760 & 1.7411570 & $\mathrm{C}$ & 4.4324900 & 0.9787120 & 1.1352850 \\
\hline C & 3.4225230 & -2.9892270 & 1.1668580 & C & 4.0776090 & 0.9162590 & -1.2873460 \\
\hline C & 1.4606240 & -5.2320110 & 0.8409530 & $\mathrm{C}$ & 4.9081420 & 2.2890940 & 1.0044150 \\
\hline $\mathrm{H}$ & 3.1745710 & -6.4666430 & 1.3127590 & $\mathrm{C}$ & 4.5558590 & 2.2287650 & -1.3597970 \\
\hline H & 4.7463820 & -4.5728920 & 1.7983640 & $\mathrm{C}$ & 4.9847770 & 2.9322690 & -0.2321910 \\
\hline $\mathrm{H}$ & 4.0324950 & -2.8134690 & 0.2687420 & $\mathrm{H}$ & 5.2257010 & 2.8182730 & 1.8989720 \\
\hline H & 1.0279490 & -5.3680970 & 1.8422400 & $\mathrm{H}$ & 4.6075390 & 2.7189220 & -2.3306560 \\
\hline $\mathrm{H}$ & 0.9626400 & -5.9423730 & 0.1697820 & C & -3.1210960 & -2.3165180 & 0.3353220 \\
\hline $\mathrm{H}$ & 3.3889960 & -5.4597590 & -0.1260080 & $\mathrm{C}$ & -4.0354780 & -1.6158330 & -0.4910230 \\
\hline $\mathrm{H}$ & 3.2732 & -4.4344050 & 2.7664890 & C & -3.3515200 & -2.4010070 & 1.7272430 \\
\hline $\mathrm{H}$ & 3.7206 & -2.1946680 & 1.8603170 & C & -5.1676640 & -1.0376730 & 0.0940960 \\
\hline \multirow{2}{*}{\multicolumn{8}{|c|}{ TS245(S, S) G[B97-D/def2-TZVP $]=-3633.747940$}} \\
\hline & & & & & & & \\
\hline 0 & 1.3815690 & -0.6216500 & -0.6493120 & $\mathrm{H}$ & -5.8790610 & $-0 . \overline{5} 184090$ & -0.5444680 \\
\hline 0 & -0.4143390 & -1.5728590 & 0.8829450 & $\mathrm{H}$ & -4.6852900 & -1.8766800 & 3.3308950 \\
\hline C & 2.2847530 & -1.5788360 & -0.2014390 & $\mathrm{P}$ & 0.1595980 & -0.1566190 & 0.3265030 \\
\hline $\mathrm{C}$ & 1.8691220 & -2.9025460 & -0.1085660 & 0 & 0.6656670 & 0.5629980 & 1.5498830 \\
\hline C & 3.6109860 & -1.1523530 & 0.0810820 & 0 & -0.7976930 & 0.5388630 & -0.5991430 \\
\hline C & 2.7916390 & -3.8695350 & 0.4153090 & $\mathrm{C}$ & 3.6867700 & 0.2235240 & -2.5870580 \\
\hline C & 4.5187910 & -2.1148460 & 0.4799910 & $\mathrm{H}$ & 3.3039270 & -0.7748440 & -2.3585210 \\
\hline C & 4.1436620 & -3.4642550 & 0.6849020 & C & 5.5723570 & 4.3252000 & -0.3858110 \\
\hline $\mathrm{H}$ & 5.5458330 & -1.8216560 & 0.6826130 & $\mathrm{H}$ & 5.2050560 & 4.7289540 & -1.3407500 \\
\hline C & -0.6121830 & -2.6285110 & -0.0047560 & C & 4.3921860 & 0.3640480 & 2.5312910 \\
\hline$c$ & -1.9530220 & -3.017526 & -0.2736270 & $\mathrm{H}$ & 3.8625960 & -0.5920730 & 2.4770550 \\
\hline
\end{tabular}




$\begin{array}{rrr}-2.4482580 & -3.1872460 & 2.6700920 \\ -1.5851770 & -3.5588060 & 2.1107820 \\ -6.6923860 & -0.5514600 & 2.0716720 \\ -6.6048490 & -0.6401830 & 3.1637620 \\ -3.8613340 & -1.4981380 & -2.0023180 \\ -2.8684240 & -1.8731300 & -2.2685460 \\ 0.1095210 & 3.6228390 & 1.0330200 \\ 0.7764890 & 3.3922300 & -0.2525700 \\ -0.7436620 & 2.9861010 & 1.2639020 \\ 0.6624720 & 2.4199370 & -0.7148820 \\ 1.3765350 & 2.9709440 & 1.4129200 \\ 1.1494350 & 1.9448690 & 1.4808100 \\ 1.1753700 & 5.9227840 & 1.1202800 \\ 1.3965390 & 5.8473940 & -0.3986740 \\ 1.6812530 & 4.4083040 & -0.8646380 \\ -0.0092850 & 5.0401650 & 1.5464600 \\ 0.9824660 & 6.9588710 & 1.4214710 \\ 2.2313750 & 6.4913000 & -0.6985840 \\ 2.7077310 & 4.1074010 & -0.6133350 \\ -0.9453640 & 5.4453060 & 1.1371110 \\ -0.1149870 & 5.0104770 & 2.6378830 \\ 2.0797500 & 5.5923740 & 1.6468470 \\ 0.4960550 & 6.2145580 & -0.9090620 \\ 1.6073660 & 4.3096020 & -1.9538070 \\ -3.9399900 & -0.0427430 & -2.4983480 \\ -3.7901460 & -0.0155730 & -3.5857780 \\ -4.9196840 & 0.4013030 & -2.2816510 \\ -3.1682310 & 0.5691420 & -2.0240300 \\ -4.9049930 & -2.3661170 & -2.7337700 \\ -5.9193360 & -2.0102670 & -2.5089610 \\ -4.7526750 & -2.3106030 & -3.8200380 \\ -4.8380690 & -3.4165170 & -2.4248750 \\ 2.5716090 & 0.9873260 & -3.3248550 \\ 2.9122950 & 1.9883260 & -3.6201280 \\ 2.2828030 & 0.4445060 & -4.2345140 \\ 1.6870860 & 1.0917750 & -2.6876180 \\ 4.9175970 & 0.0366750 & -3.4965510 \\ 4.6321730 & -0.4903770 & -4.4167450 \\ 5.3448800 & 1.0089320 & -3.7746670 \\ 5.6954490 & -0.5458120 & -2.9861880 \\ -1.9135050 & -2.3100160 & 3.8165130 \\ -2.7355560 & -1.9574560 & 4.4531020 \\ -1.2235640 & -2.8907140 & 4.4430920 \\ -1.3796370 & -1.4390680 & 3.4206620 \\ -3.1879330 & -4.4216060 & 3.2248810 \\ -2.5228650 & -4.9982140 & 3.8818460\end{array}$
H $\quad-4.0674660$
3.6327660
3. 6044630
4. 1270310
2. 6047010
5.8196800
6.3851430
5.7835890
6.3640810
$-6.8911090$
$-6.0319430$
$-7.0123450$
$-7.7939240$
$-7.9191200$
$-7.8034170$
$-8.8324980$
$-8.0412110$
7. 1103760
7.4183280
7.5435880
7. 5177370
5.1561380
4.0659780
5.5579000
5.5465740
$-2.7082860$
$-2.6206070$
$-4.0093700$
$-4.1479540$
$-5.1352320$
$-5.3787580$
$-3.2800960$
$-6.3668320$
$-5.0405200$
$-6.4930740$
$-5.4699520$
$-7.2280180$
$-7.4542570$
$-1.8091090$
$-1.4884110$
$-4.1161290$
$-5.0742500$
1.2429560
0.7386710
2. 2132080
1.4195460
0.0827460
1.0203520
$-0.3903790$
$-0.5803000$
0.9345020
1.5341890
1.0660950
1. 3218490
$-1.3730160$
$-2.4297890$
$-0.9861370$
$-1.3114540$
4. 2389160
3. 5777510
5.2342550
3. 8369940
5.2950840
5.3567770
4.9737490
6.2989640
3. 3076150
2. 2267360
4.0063030
5. 3685490
3.2998460
6. 0068590
5. 9186040
3. 9400440
. 2493250
5. 2942370
7. 0616490
3. 3808470
5. 7929610
1.6069770
3.8532170

3.8065860

2.4095850

3.5431100

4. 5181300

3. 6791860

3. 2127050

3. 0413730

3. 1272880

4. 0318000

2. 3580090

1.7299490

2. 0528140

0.6474010

2. 2200310

1. 6264340

1.8984620

2. 0981560

0.5367430

$-0.4748080$

$-1.2944430$

$-0.6425750$

0.4628290

0.7313990

0.8188680

1.7007500

0.5218670

$-0.9075390$

$-0.1668170$

$-0.7681230$

$-1.0979830$

$-0.2967230$

$-0.9600770$

$-1.4504130$

$-0.1713410$

$-0.0441550$

$-0.5004350$

$-1.2081600$

0.1850510

$-0.3976220$

$-0.3653390$

$-1.9233830$

$\operatorname{TS} 246(\mathrm{~S}, \mathrm{~S}) \mathrm{G}[\mathrm{B} 97-\mathrm{D} / \mathrm{def} 2-\mathrm{TZVP}]=-3633.747324$

$\begin{array}{rrrr}0 & 1.3525080 & -0.6565590 & -0.6479210 \\ \mathrm{O} & -0.5183130 & -1.5527110 & 0.8294200 \\ \mathrm{C} & 2.1915930 & -1.6608520 & -0.1762900\end{array}$




\begin{tabular}{|c|c|c|c|}
\hline & & & \\
\hline C & 1.7156320 & -2.9672060 & $\begin{array}{r}-0.1369690 \\
0.1911330\end{array}$ \\
\hline $\begin{array}{l}\mathrm{C} \\
\mathrm{C}\end{array}$ & $\begin{array}{l}3.5156 / 00 \\
2.5713850\end{array}$ & $\begin{array}{l}-1.2992680 \\
-3.9892260\end{array}$ & $\begin{array}{l}0.1911330 \\
0.3962170\end{array}$ \\
\hline $\mathrm{C}$ & 4.3612130 & -2.3097710 & 0.6063860 \\
\hline C & 3.9238950 & -3.6488700 & 0.7432170 \\
\hline $\mathrm{H}$ & 5.3859140 & -2.0649690 & 0.8745600 \\
\hline$c$ & -0.7490330 & -2.5836570 & -0.0808720 \\
\hline C & -2.1007140 & -2.9097580 & -0.3765180 \\
\hline C & 0.3382380 & -3.2759860 & -0.6033280 \\
\hline C & -2.3268310 & -3.9430220 & -1.2654540 \\
\hline 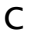 & 0.0901480 & -4.2764520 & -1.6022040 \\
\hline C & -1.2687390 & -4.6204500 & -1.9178640 \\
\hline H & -3.3505880 & -4.2266050 & -1.4964630 \\
\hline C & -0.4931130 & -6.2434330 & -3.5614730 \\
\hline C & 0.8466220 & -5.8774130 & -3.2811100 \\
\hline$c$ & 1.1304520 & -4.9211950 & -2.3262920 \\
\hline $\mathrm{C}$ & -1.5276120 & -5.6216690 & -2.8938130 \\
\hline H & -0.7020420 & -7.0031640 & -4.3113050 \\
\hline $\mathrm{H}$ & 1.6599040 & -6.3503470 & -3.8272410 \\
\hline $\mathrm{H}$ & 2.1613200 & -4.6483080 & -2.1253560 \\
\hline $\mathrm{H}$ & -2.5631960 & -5.8762630 & -3.1124580 \\
\hline C & 2.9963390 & -6.2734140 & 1.1442380 \\
\hline C & 4.3417900 & -5.9450200 & 1.4433680 \\
\hline C & 4.7909410 & -4.6555220 & 1.2501180 \\
\hline C & 2.1353290 & -5.3220990 & 0.634144 \\
\hline H & 2.6358270 & -7.2841510 & 1.3227000 \\
\hline H & 5.0110430 & -6.7062740 & 1.8379070 \\
\hline H & 5.8148230 & -4.3807170 & 1.4975990 \\
\hline $\mathrm{H}$ & 1.1076480 & -5.5902930 & 0.4128030 \\
\hline C & 4.0026010 & 0.1118900 & 0.1586870 \\
\hline C & 4.3159420 & 0.7710800 & $1 . \overline{3} 746680$ \\
\hline C & 4.2219360 & 0.7667190 & -1.0710710 \\
\hline C & 4.863 & 2.0547790 & 1.3196620 \\
\hline C & 4.7829050 & 2.0519590 & -1.0702280 \\
\hline C & 5.1145620 & 2.7146300 & 0.1123730 \\
\hline H & 5.1025850 & 2.5653480 & 2.2507070 \\
\hline H & 4.9586190 & 2.5376900 & -2.0260470 \\
\hline C & -3.2459420 & -2.1895000 & 0.2530020 \\
\hline C & -4.1307020 & -1.4254340 & -0.5485730 \\
\hline C & -3.4843050 & -2.3169870 & 1.6405840 \\
\hline C & -5.2444200 & -0.8302850 & 0.0547520 \\
\hline C & -4.6081620 & -1.6920670 & 2.1944900 \\
\hline C & -5.5062260 & -0.9548040 & 1.4221650 \\
\hline $\mathrm{H}$ & -5.9332530 & -0.2601580 & -0.5645160 \\
\hline $\mathrm{H}$ & -4.8030660 & -1.8030750 & 3.259995 \\
\hline & 0.1061440 & -0.1548270 & 0.2805 \\
\hline
\end{tabular}
0.5904510
$-0.8113880$
3.9179480
3.4747680
5.7580620
5.2278380
4.1074030
3.5258420
$-2.6121240$
$-1.7648620$
$-6.7551580$
$-6.6860450$
$-3.9417090$
$-2.9578020$
0.4117670
0.9202030
$-0.4973970$
0.6349840
1.6067270
1.2561110
1.7875050
1.8263060
1.8906160
0.5451670
1.7684500
2.6916880
2.8876290
$-0.3641570$
0.5521290
2.6940340
0.9233970
1.7236500
$-3.9744520$
$-3.8114560$
$-4.9439650$
$-3.1915890$
$-5.0051510$
$-6.0100050$
$-4.8414600$
$-4.9737190$
2. 8998100
3.2979480
2.6785460
1.9641670
5.2102650
4.9807950

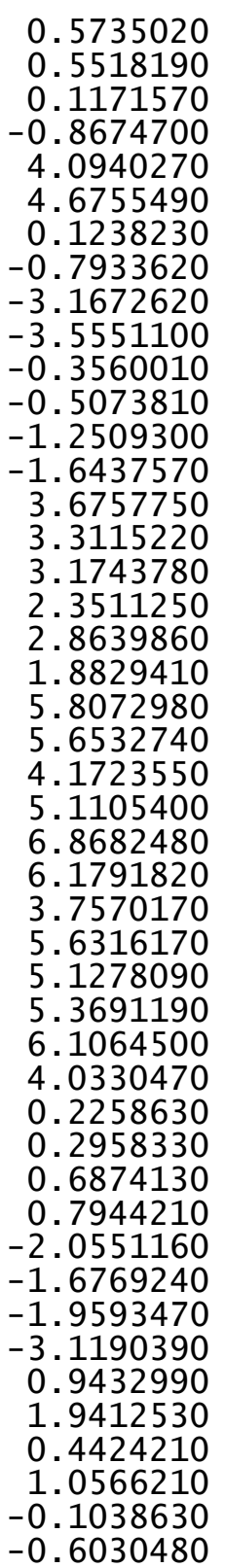

1.5074560

$-0.6788230$

$-2.4152700$

$-2.2399460$

0.1456040

0.9154010

2.7405170

2.6046680

2.5563620

1.9840920

2.0447980

3.1313210
-2.0526290

$-2.3273890$

0.9152670

$-0.4120290$

1. 2465430

$-0.8202850$

1.2098160

1.3457230

0.7922400

$-0.7358370$

$-1.1458980$

1. 3753640

1.0668270

$-1.1550100$

$-0.9430710$

1.0440890

2. 4722170

1.2304130

$-1.1673020$

$-2.2197460$

$-2.4863480$

$-3.5699570$

$-2.2604930$

$-1.9781210$

$-2.8273120$

$-2.5960840$

$-3.9091070$

$-2.5622330$

$-3.2228360$

$-3.4477400$

$-4.1745630$

$-2.6648680$

$-3.2253530$

$-4.1761590$ 


\begin{tabular}{|c|c|c|}
\hline $\begin{array}{l}5.6965760 \\
5.9206330 \\
-2.0414920 \\
-2.8485410 \\
-1.3743860 \\
-1.4746910 \\
-3.3985380 \\
-2.7541540 \\
-4.2619100 \\
-3.7673100 \\
3.3190380 \\
3.1825530 \\
3.8564100 \\
2.3326610 \\
5.4633620 \\
6.0776610 \\
5.3096190 \\
6.0211000 \\
-6.8726700 \\
-5.9895480 \\
-6.9693730 \\
-7.7624840 \\
-8.0165430 \\
-7.9566140 \\
-8.9142430 \\
-8.1246180 \\
5.6712290 \\
4.6367370 \\
6.0854080 \\
6.2510230 \\
7.2320210 \\
7.3112000 \\
7.8015230 \\
7.6862930 \\
-2.5703520 \\
-2.4814070 \\
-3.8209170 \\
-3.9143560 \\
-4.9454770 \\
-5.0989490 \\
-3.0474740 \\
-6.1321980 \\
-4.8871690 \\
-6.2128750 \\
-5.1543000 \\
-6.9940320\end{array}$ & $\begin{array}{r}0.8546110 \\
-0.7257420 \\
-2.3469120 \\
-1.9819760 \\
-2.9732910 \\
-1.4860480 \\
-4.3880400 \\
-5.0118960 \\
-4.0661820 \\
-4.9996150 \\
1.0207620 \\
0.4941700 \\
1.9551760 \\
1.2653960 \\
-0.2661530 \\
0.6297010 \\
-0.7595250 \\
-0.9474950 \\
1.1548750 \\
1.6907550 \\
1.3502270 \\
1.5611300 \\
-1.0868690 \\
-2.1624310 \\
-0.6832340 \\
-0.9541290 \\
4.8704810 \\
4.9658350 \\
5.8772160 \\
4.3695290 \\
3.9771830 \\
3.4668810 \\
3.3995920 \\
4.9730840 \\
3.4578150 \\
2.3679560 \\
4.2187340 \\
5.5900670 \\
3.5623750 \\
6.2865310 \\
6.1005810 \\
4.2605310 \\
2.5046690 \\
5.6236930 \\
7.3475680 \\
3.7387490\end{array}$ & $\begin{array}{r}-3.4490150 \\
-2.6656050 \\
3.7277240 \\
4.3763600 \\
4.3347780 \\
3.3564250 \\
3.0758570 \\
3.7098240 \\
3.6727260 \\
2.2422350 \\
3.7132420 \\
4.6671140 \\
3.9190210 \\
3.3074880 \\
3.3631660 \\
3.5244700 \\
4.3321750 \\
2.7094320 \\
1.7818890 \\
2.1494600 \\
0.7064130 \\
2.2805760 \\
1.5426910 \\
1.7516970 \\
2.0300820 \\
0.4578290 \\
-1.1752710 \\
-1.5241700 \\
-1.0409620 \\
-1.9618640 \\
0.5903730 \\
1.5575170 \\
-0.1505780 \\
0.6787870 \\
-0.8353860 \\
-0.1068250 \\
-0.5947340 \\
-0.9022750 \\
-0.0530360 \\
-0.6720110 \\
-1.3119530 \\
0.1633520 \\
0.1796920 \\
-0.1422750 \\
-0.9034650 \\
0.5710150\end{array}$ \\
\hline
\end{tabular}

$\begin{array}{lllr}\mathrm{H} & -7.1385380 & 6.1676710 & 0.0321730 \\ \mathrm{H} & -1.7315700 & 1.6996630 & -0.3734060 \\ \mathrm{~S} & -1.4073590 & 3.9495590 & -1.9402580\end{array}$

TS247(S, S) G[B97-D/def2-TZVP $]=-3633.747145$

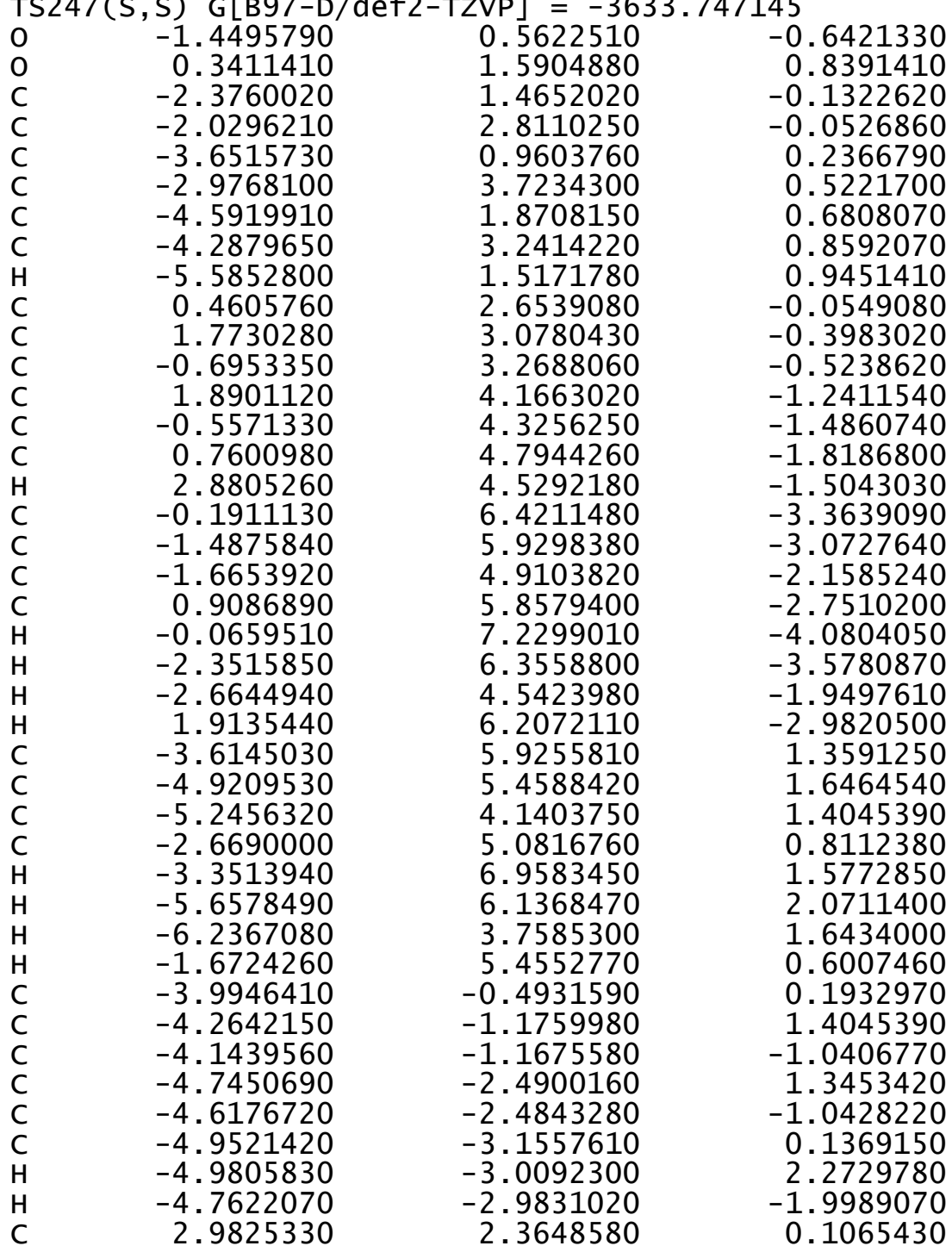




\begin{tabular}{rrr}
3.8161820 & 1.6660120 & -0.7991040 \\
3.3101090 & 2.4014300 & 1.4825550 \\
4.9617880 & 1.0303560 & -0.3035640 \\
4.4619990 & 1.7443330 & 1.9247740 \\
5.3023470 & 1.0511880 & 1.0493350 \\
5.6098640 & 0.4968870 & -0.9963190 \\
4.7147840 & 1.7888150 & 2.9815260 \\
-0.1600970 & 0.1541490 & 0.2695980 \\
-0.5687440 & -0.6352020 & 1.4857460 \\
0.8069710 & -0.4508290 & -0.7097720 \\
-3.8778500 & -0.4974080 & -2.3827700 \\
-3.5156840 & 0.5191380 & -2.2060110 \\
-5.5838560 & -4.5372150 & 0.1060820 \\
-5.7070010 & -4.8646170 & 1.1480780 \\
-4.0801490 & -0.5314520 & 2.7750410 \\
-3.5586890 & 0.4214640 & 2.6413790 \\
2.4910610 & 3.1841450 & 2.5016370 \\
1.5987560 & 3.5826780 & 2.0100200 \\
6.5707820 & 0.3771500 & 1.5413810 \\
6.9642180 & -0.2238690 & 0.7095890 \\
3.5404300 & 1.5967270 & -2.2973740 \\
2.5314330 & 1.9770870 & -2.4836310 \\
0.0324210 & -3.6510130 & 0.8676520 \\
-0.5204940 & -3.3707580 & -0.4600930 \\
0.8526170 & -3.0137380 & 1.1981520 \\
-0.3756390 & -2.3796650 & -0.8699000 \\
-1.2744440 & -3.0306700 & 1.1608720 \\
-1.0656370 & -2.0103420 & 1.3071400 \\
-0.9990170 & -5.9658110 & 0.7477980 \\
-1.0715430 & -5.8212420 & -0.7804130 \\
-1.3467070 & -4.3665400 & -1.1994780 \\
0.1245900 & -5.0888170 & 1.3268990 \\
-0.8190140 & -7.0121490 & 1.0201220 \\
-1.8559840 & -6.4658660 & -1.1921340 \\
-2.3990390 & -4.0988690 & -1.0240300 \\
1.1020760 & -5.4621270 & 0.9904100 \\
0.1247090 & -5.1092260 & 2.4236440 \\
-1.9569940 & -5.6705680 & 1.1961210 \\
-0.1165470 & -6.1444050 & -1.2162720 \\
-1.1783020 & -4.2107140 & -2.2713320 \\
3.5872860 & 0.1607470 & -2.8511300 \\
3.3559150 & 0.1752000 & -3.9244200 \\
4.5822970 & -0.2861670 & -2.7284370 \\
2.8546160 & -0.4735430 & -2.3453630 \\
4.5322890 & 2.4936050 & -3.0656910 \\
5.5589660 & 2.1296600 & \\
-5 & & \\
\hline & &
\end{tabular}

4.3050400
4.4887210
-2.7910000
-3.1064210
-2.6032230
-1.8528760
-5.1755850
-4.9765050
-5.5833220
-5.9380010
2.0187160
2.8745360
1.3864290
1.4405590
3.2955320
2.6929560
4.2065030
3.5913080
-3.2179090
-3.0947260
-3.6927660
-2.2269750
-5.4437130
-5.9962690
-5.2987730
-6.0589080
7.6391510
7.8576590
7.2845390
8.5696840
6.3140020
5.5619810
7.2429180
5.9561640
-4.7078230
-3.7427320
-5.2099430
-4.5203460
-6.9825690
-7.6259080
-6.9030640
-7.4609580
3.0236830
2.7579800
4.3939580
4.7332970
2.4798130

3.5311470

$-1.2439090$

$-2.2720530$

$-0.7329630$

$-1.2789640$

$-0.3783540$

0.1389000

$-1.3715870$

0.1849800

2965060

1.9160530

2.8797670

1.4437770

4.3910770

4. 9687050

4.0529550

5. 0528510

$-1.3922560$

$-0.8736830$

$-2.3615010$

$-1.5691610$

$-0.2372020$

$-1.1719290$

0.2528540

0.4156490

1.4276860

2. 0772520

2. 0579910

0.9368410

$-0.5626120$

$-1.3204100$

$-1.0721830$

0.0045750

$-5.5762340$

$-5.6916560$

$-6.5523920$

$-5.2754530$

$-4.4744440$

$-3.7579500$

$-4.1571930$

$-5.4626650$

$-3.0203560$

$-1.9447160$

$-3.5401610$

$-4.8767830$
$-4.1401640$

-2.7126120

$-3.1781970$

$-3.3984610$

$-4.1317770$

$-2.6138340$

$-3.2058780$

$-4.1538240$

$-3.4348340$

$-2.6527000$

3.6673620

4. 2402950

4. 3499050

3. 2950210

3.0258260

3.7397750

3. 5369910

2. 2014690

3. 7178410

4.6779010

3. 9160640

3. 2889740

3.4320280

3.5966290

4.4040620

2.8015550

1.9046930

1.0477090

2.7311910

2. 2203500

2.7326080

2. 4840950

3. 0200560

3. 6012520

$-0.6139780$

$-0.1105980$

$-0.6284460$

$-1.6529280$

$-0.5397970$

$-0.0136430$

$-1.5880160$

$-0.5141020$

$-0.8231620$

$-0.1193770$

$-0.5896140$

$-0.8751810$ 


\begin{tabular}{|c|c|c|c|}
\hline & & & \\
\hline $\begin{array}{l}C \\
C\end{array}$ & $\begin{array}{l}5.3845260 \\
6.0279150\end{array}$ & $\begin{array}{l}-2.6787570 \\
-5.3397910\end{array}$ & $\begin{array}{l}-0.0740870 \\
-0.6488400\end{array}$ \\
\hline $\mathrm{H}$ & 3.9702510 & -5.5452090 & -1.2639020 \\
\hline C & 6.6807780 & -3.1438850 & 0.1387050 \\
\hline $\mathrm{H}$ & 5.1336900 & -1.6451950 & 0.1392360 \\
\hline C & 7.0070760 & -4.4745310 & -0.1449310 \\
\hline $\mathrm{H}$ & 6.2746480 & -6.3769680 & -0.8629530 \\
\hline $\mathrm{H}$ & 7.4367560 & -2.4670820 & 0.5288590 \\
\hline $\mathrm{H}$ & 8.0181480 & -4.8369660 & 0.0269240 \\
\hline $\mathrm{H}$ & 1.9042660 & -1.4198860 & -0.3930530 \\
\hline $\mathrm{s}$ & 1.9457410 & -3.7371980 & -1.8919610 \\
\hline & {$[\mathrm{B} 97$} & & 6 \\
\hline 0 & ) G[BG/-D & $-P P J=-36=$ & $56 \quad 6762200$ \\
\hline 0 & -0.1813450 & 1.5171140 & -0.8963890 \\
\hline $\mathrm{C}$ & 2.5513960 & 1.3471000 & 0.0502980 \\
\hline C & 2.2848450 & 2.6871910 & -0.2131410 \\
\hline C & 3.7896300 & 0.7144550 & -0.2431670 \\
\hline $\mathrm{C}$ & 3.2786370 & 3.4475300 & -0.9158420 \\
\hline $\mathrm{C}$ & 4.7760400 & 1.4905350 & -0.8213210 \\
\hline C & 4.5512380 & 2.8385140 & -1.1919960 \\
\hline $\mathrm{H}$ & 5.7429620 & 1.0439760 & -1.0386600 \\
\hline C & -0.2117370 & 2.6790710 & -0.1282150 \\
\hline C & -1.4865740 & 3.1984890 & 0.2248420 \\
\hline C & 0.9923070 & 62020 & 0.2165530 \\
\hline C & -1.5140050 & & 0.9161830 \\
\hline C & 0.9467940 & 4.4647550 & 1.0358880 \\
\hline C & -0.3318530 & 5.041 & 1.35 \\
\hline $\mathrm{H}$ & -2.4741640 & 4.8297310 & 1.1826380 \\
\hline C & 0.7579510 & 6.804 & 2.63 \\
\hline C & 2.0159220 & & 50 \\
\hline C & 2.1076250 & 5.0707440 & 1.5912520 \\
\hline C & -0.3912930 & 6.2 & 2.1363490 \\
\hline $\mathrm{H}$ & 0.7010230 & 7.7 & 3.2382690 \\
\hline $\mathrm{H}$ & 2.9188660 & 6.6 & \\
\hline $\mathrm{H}$ & 3.078 & 4.6247 & 1.4041320 \\
\hline H & -1.3682880 & 6.6531560 & 2.3536610 \\
\hline C & 4.0391870 & 5.4676350 & -2.0538680 \\
\hline C & 5.3081620 & 4.8809420 & -2.2846160 \\
\hline C & 5.5533040 & 0450 & -1.8653120 \\
\hline $\mathrm{C}$ & 3.0516900 & 4.7697790 & -1.3871940 \\
\hline $\mathrm{H}$ & 3.8390740 & 6.4760180 & -2.4095360 \\
\hline $\mathrm{H}$ & 6.0793030 & 5.4447010 & -2.8048550 \\
\hline $\mathrm{H}$ & 6.5145990 & 3.1164850 & -2.0569010 \\
\hline $\mathrm{H}$ & 2.0835060 & 5.2304070 & -1.2201710 \\
\hline$\pi$ & 4.0206680 & -0.7358840 & 0.0296110 \\
\hline
\end{tabular}

\begin{abstract}
4. 2421880
4.0661600

4.5424560

4.3596410

4.6185510

4.7305060

4.4149620

$-2.7577740$

$-3.5280650$

$-3.2207870$

$-4.7629690$

$-4.4514240$

$-5.2489680$

$-5.3503280$

$-4.8118650$

0.2756430

0.6441990

$-0.7114540$

3.8770780

3.6375910

5.0314350

4.9573820

4.1980990

3.7973280

$-2.4560830$

$-1.5062070$

$-6.6134960$

$-6.4903530$

$-3.0793740$

$-2.0397680$

$-3.1256440$

$-2.7374000$

$-4.1535660$

$-2.5168140$

$-3.9284360$

$-4.9838370$

$-3.5878080$

$-3.8614140$

2.7133960

2. 9181860

2. 5807170

1. 7795050

5.1816900

5.0450990

5.4690670

6.0047610
\end{abstract}
$-1.6264840$
$-1.2207070$
$-2.9640770$
$-2.5733880$
$-3.4582110$
$-3.6398170$
$-2.9418530$
2. 4726290
1.9614010
2. 3263690
1. 3599140
1.7017270
1.2230490
0.9788600
1. 5966610
0.1250700
$-0.7887960$
$-0.3500980$
$-0.3241630$
0.6880740
$-4.8943230$
$-5.0568690$
$-1.1830580$
$-0.1659120$
2.8717900
3. 2891620
0.6286450
$-0.0015940$
2.0339400
2.3742140
0.6600160
0.7531700
0.2810400
$-0.0713410$
3.0545060
2.7507710
3.1136580
4.0544280
$-0.8060160$
$-1.8081610$
$-0.1226540$
$-0.8394160$
$-0.2291500$
0.4497020
$-1.2151060$
0.1483730

$-1.0528480$

1.3562920

$-0.7769950$

1.5751500

0.5280610

$-1.6079780$

2.5983580

$-0.0718760$

0.9996670

$-1.4004690$

0.7219480

$-1.6229150$

$-0.5779160$

1.5522450

$-2.6453050$

$-0.1929940$

$-1.3306540$

0.8401250

2.5738630

2.2366790

0.8025720

1.8874030

$-2.5127300$

$-2.5548590$

$-2.6000300$

$-2.2528360$

$-0.8937240$

$-1.7862720$

2.4563750

2.4843460

3.1520480

4.1752160

3.2167800

2.6123470

3.2398860

3.2385200

4.2824740

2.7940020

3.4598530

3.8590280

4.3090780

2. 8886810

3.3900240

4.2424660

3.7779670

2.7696340 


$\begin{array}{rrr}-2.1289200 & 1.7654510 & -3.6193940 \\ -3.0495550 & 1.3329070 & -4.0328890 \\ -1.5453430 & 2.1814270 & -4.4516670 \\ -1.5471390 & 0.9653200 & -3.1487720 \\ -3.2365360 & 4.0195060 & -3.2711290 \\ -2.6585280 & 4.4312570 & -4.1093310 \\ -4.1974080 & 3.6581610 & -3.6603750 \\ -3.4387390 & 4.8262170 & -2.5547290 \\ 3.2782660 & -2.0669520 & -3.3759660 \\ 3.2578520 & -1.6798760 & -4.4034020 \\ 3.6403380 & -3.1022670 & -3.4138340 \\ 2.2567920 & -2.0684990 & -2.9840630 \\ 5.6175470 & -1.1576600 & -3.1143510 \\ 6.0475700 & -2.1681110 & -3.1134290 \\ 5.5853760 & -0.7973760 & -4.1513180 \\ 6.2849020 & -0.5048790 & -2.5389870 \\ -7.1990140 & -0.2464420 & 0.2225020 \\ -6.5069280 & -1.0448240 & 0.5113280 \\ -7.4264760 & 0.3563430 & 1.1119710 \\ -8.1341360 & -0.7059770 & -0.1199650 \\ -7.6047260 & 1.7556620 & -1.2568800 \\ -7.2231600 & 2.3653540 & -2.0850820 \\ -8.5757040 & 1.3331520 & -1.5493700 \\ -7.7598180 & 2.4123460 & -0.3897450 \\ 6.4995140 & -5.1206140 & 0.3882760 \\ 7.1655980 & -4.4175180 & 0.9040890 \\ 6.8132500 & -6.1449750 & 0.6299110 \\ 6.6152800 & -4.9709990 & -0.6933270 \\ 4.1154860 & -5.9157340 & 0.1060070 \\ 3.0827940 & -5.8241510 & 0.4579550 \\ 4.1189460 & -5.7635310 & -0.9808360 \\ 4.4601790 & -6.9380070 & 0.3098440 \\ -0.4081790 & -3.6866320 & -0.7046720 \\ 0.0503760 & -3.4315770 & 0.6662230 \\ -1.1108030 & -2.9638280 & -1.1197290 \\ 0.0273670 & -2.4093360 & 1.0234230 \\ 0.9813090 & -3.2604620 & -0.9317720 \\ 0.9287970 & -2.2295010 & -1.1004020 \\ 0.2876010 & -6.1119170 & -0.4381350 \\ 0.2788130 & -5.9220900 & 1.0876360 \\ 0.6901490 & -4.4940630 & 1.4917940 \\ -0.6669150 & -5.1180670 & -1.1209510 \\ -0.0138240 & -7.1353240 & -0.6895300 \\ 0.9544380 & -6.6391110 & 1.5683950 \\ 1.7774040 & -4.3512090 & -0.8414430 \\ -1.7058520 & -5.3418830 & \end{array}$
$-0.5982880$
1.3028600
$-0.7330690$
0.4626230
$-3.2895580$
$-2.8106840$
$-4.5663780$
$-5.4078380$
$-4.9539430$
$-6.6072050$
$-5.1161020$
$-6.1421000$
$-4.3221930$
$-6.9757500$
$-7.2553940$
$-6.4239190$
$-7.9090440$
$-1.9327700$
$-2.5243010$

144

TS249(S,S) G[B97-D/def2-TZVP] $=-3633.746756$

\begin{tabular}{|c|c|c|c|}
\hline & & & \\
\hline 0 & -1.5606770 & 0.5694390 & -0.6329770 \\
\hline 0 & 0.2264320 & 1.5434420 & 0.8820690 \\
\hline C & -2.5137500 & 1.3729470 & -0.0148720 \\
\hline C & -2.2276230 & 2.7207070 & 0.1824000 \\
\hline C & -3.7484760 & 0.7672320 & 0.3431890 \\
\hline & -3.1975690 & 3.5253000 & 0.8687460 \\
\hline & -4.7131770 & 1.5814860 & 0.9063950 \\
\hline C & -4.4689450 & 2.9440300 & 1.2032480 \\
\hline $\mathrm{H}$ & -5.6772300 & 1.1547060 & 1.1716440 \\
\hline C & 0.2660220 & 2.6759310 & 0.0698960 \\
\hline C & 1.5442940 & 3.1631240 & -0.3151850 \\
\hline & -0.9334190 & 3.2828390 & -0.2901540 \\
\hline C & 1.5801830 & 4.3282550 & -1.0575300 \\
\hline C & -0.8806410 & 4.4234710 & -1.1604300 \\
\hline C & 0.4021050 & 4.9690300 & -1.5111980 \\
\hline $\mathrm{H}$ & 2.5432340 & 4.7393320 & -1.3498000 \\
\hline & -0.6779540 & 6.6852240 & -2.8626110 \\
\hline & -1.9408590 & 6.1191170 & -2.5597520 \\
\hline & -2.0393030 & 89180 & -1.7311750 \\
\hline & 0.4688080 & 6.1144460 & -2.3514030 \\
\hline & -0.6 & 7.5567610 & 5104370 \\
\hline & -2.8421580 & 6.5518460 & 884970 \\
\hline & -3 & 40640 & 144510 \\
\hline & 1.448910 & 200 & -2.5970290 \\
\hline & -3. & & 2214 \\
\hline
\end{tabular}

$-2.2137180$

$-5.1843360$

$-5.9641360$

$-6.1179620$

$-4.2845190$

$-1.4818710$

$-3.0128990$

$-3.9300690$

$-2.5185380$

$-4.3331930$

-4. 3118910

$-2.9450350$

$-1.8009620$

$-3.8473830$

$-5.0286240$

$-2.5628350$

$-4.1689830$

$-1.0860680$

1.4667370

2.5438100

0.6006800

0.0407170

$-0.0090290$

$-1.2722560$

0.0666910

1.6233830

$-1.8618680$

$-1.7845980$

$-1.1937600$

0.5944600

$-2.8400190$

$-1.6503980$

0.4452540

1.8537140
0.6491690

5.6068300

1.9221490

1.7556620

. 3653540

1.3331520

9709990

$-3.6866320$

3.2604620

(2)

$-4.4940630$

$-4.3512090$

$-0.8414430$ 


-5.1820710
-5.4485130
-2.9477160
-3.6963610
-5.9358900
-6.4094610
-1.9797180
-4.0055980
-4.2118130
-4.1056980
-4.5631630
-4.4485430
-4.7040620
-4.7423270
-4.5532430
2.8068550
3.5667110
3.2704990
4.7903800
4.4884470
5.2745570
5.3694390
4.8486160
-0.2421120
-0.5873150
0.7267490
-3.9258810
-3.6516370
-5.1915290
-5.2337840
-4.0914130
-3.6753610
2.5245610
1.5785440
6.6256130
6.4848630
3.1213840
2.0854900
3.1541150
2.7677860
4.1778240
2.5369670
3.9837440
5.0350030
3.6438930
3.9305080

5.0452480

4.8669540

6.6295210

5.6419260

3.2839370

5.3094950

$-0.6934910$

$-1.5096690$

$-1.2578160$

$-2.8527490$

$-2.6082960$

$-3.4196640$

$-3.4785530$

$-3.0274270$

2.4312850

1.8718080

2. 3224380

1.2563120

1.6805520

1. 1511110

0.8368920

1.6031070

0.1366450

$-0.7583130$

$-0.3580730$

$-0.4425040$

0.5813980

$-4.8491830$

$-5.2998430$

$-0.9896140$

0.0217200

2. 9340960

3.3504710

0.5368770

$-0.0738960$

1. 9094590

2.2596490

0.5181890

0.5899820

0.1265480

$-0.1929750$

2.8993770

2. 5815930

2.9371250

3.9108940

2.2102710
1.8613610
1.2693770
2.2226760
2.7190870
2.0975180
1.0587940
0.1634400
1.3030260
-1.1304520
1.1164270
-1.2587500
-0.1500040
1.9890780
-2.2573820
0.0022020
-1.0527400
1.3343190
-0.7560030
1.5765430
0.5474590
-1.5734330
2.6015230
0.2185250
1.3782920
-0.8233010
-2.4047650
-2.1369290
-0.3146350
0.6870050
2.7323420
2.7014170
2.5134430
2.1551560
0.8828410
1.7862760
-2.5120230
-2.5514970
-3.1726590
-4.1982690
-3.2271560
-2.6159020
-3.3202210
-3.3110870
-4.3640640
-2.8994930

\begin{abstract}
$-2.7971030$
$-3.0341170$

$-2.6714340$

$-1.8491780$

$-5.2470990$

$-5.1149710$

$-5.5677040$

$-6.0456650$

2. 1895090

3.1069910

1.6120640

1. 5987590

3. 3298660

2. 7691740

4. 2902770

3.5351710

$-3.1406720$

$-3.0578140$

$-3.5191440$

$-2.1421050$

$-5.4778520$

$-5.9247020$

$-5.3871260$

$-6.1612520$

7.1996360

6.4920500

7.4443390

8.1228000

7.6367490

7.2629730

8.5975580

7.8096160

$-4.2505740$

$-3.2566100$

$-4.6532080$

$-4.1424590$

$-6.6178040$

$-7.3048410$

$-6.6157880$

$-6.9944480$

0.2813170

$-0.1269240$

1.0280840

$-0.0193100$

$-1.0777740$

$-0.9575570$
\end{abstract}

-1.0100150
-2.0317010
-0.3885520
-1.0274730
-0.3624110
0.2614240
-1.3617040
0.0717920
1.8841450
1.4640750
2.3465460
1.0671950
4.1005820
4.5618100
3.7433120
4.8688140
-1.8452630
-1.4063740
-2.8695390
-1.8869210
-0.9088180
-1.9094530
-0.5002620
-0.2704800
-0.3705370
-1.1591130
0.2109360
-0.8431390
1.6509910
2.2838290
1.2151610
2.2882190
-5.7049720
-5.7763350
-6.7204570
-5.2732430
-4.8617520
-4.2783730
-4.4231410
-5.8908530
-3.7248350
-3.3994750
-3.0690170
-2.3734650
-3.1996980
-2.1796950

$-3.2854620$

$-3.6103060$

$-4.1819480$

$-2.7371810$

$-3.1953640$

$-4.0895030$

$-3.5175090$

$-2.5800690$

3.5879280

4.0207610

4.3996220

3.1592110

3.1201790

3.9444000

3.5142870

2. 3634580

3. 5914120

4.5946130

3.7014860

3.1456360

3.4022200

3.4716520

4.4175980

2.8293860

$-0.2129930$

$-0.4908870$

$-1.1120270$

0.1436880

1.2308370

2.0450860

1.5370150

0.3525860

$-1.1794910$

$-0.7263720$

$-1.2888920$

$-2.1830160$

$-0.9018660$

$-0.2759910$

$-1.9085370$

$-0.9743430$

0.7227360

$-0.6494190$

1.1718460

$-0.9782220$

0.9286120
1.1241880 


$\begin{array}{lrrr}\mathrm{C} & -0.5958930 & -6.0785450 & 0.3671860 \\ \mathrm{C} & -0.5183990 & -5.8509260 & -1.1511990 \\ \mathrm{C} & -0.8176370 & -4.3881770 & -1.5219390 \\ \mathrm{C} & 0.4121770 & -5.1824940 & 1.1074130 \\ \mathrm{H} & -0.3878850 & -7.1288260 & 0.6014790 \\ \mathrm{H} & -1.2247880 & -6.5045680 & -1.6753430 \\ \mathrm{H} & -1.8949400 & -4.1761500 & -1.4444530 \\ \mathrm{H} & 1.4380730 & -5.4847240 & 0.8544000 \\ \mathrm{H} & 0.3017390 & -5.2698040 & 2.1952410 \\ \mathrm{H} & -1.6095710 & -5.8570570 & 0.7261210 \\ \mathrm{H} & 0.4906700 & -6.1075830 & -1.5006650 \\ \mathrm{H} & -0.5489120 & -4.1635990 & -2.5609800 \\ \mathrm{C} & 3.2492160 & -2.6365490 & -0.5277130 \\ \mathrm{O} & 2.7791250 & -1.5488260 & 0.0242710 \\ \mathrm{C} & 4.5176310 & -3.0991530 & 0.0924840 \\ \mathrm{C} & 5.3379350 & -4.0515450 & -0.5422010 \\ \mathrm{C} & 4.9208450 & -2.5776100 & 1.3399090 \\ \mathrm{C} & 6.5311080 & -4.4628150 & 0.0472540 \\ \mathrm{H} & 5.0345400 & -4.4548000 & -1.5041560 \\ \mathrm{C} & 6.1031340 & -3.0105650 & 1.9365740 \\ \mathrm{H} & 4.3062800 & -1.8331790 & 1.8344400 \\ \mathrm{C} & 6.9153520 & -3.9486160 & 1.2916150 \\ \mathrm{H} & 7.1622670 & -5.1866990 & -0.4626130 \\ \mathrm{H} & 6.3972790 & -2.6056390 & 2.9018850 \\ \mathrm{H} & 7.8440310 & -4.2758730 & 1.7536350 \\ \mathrm{H} & 1.9213870 & -1.1333310 & -0.3997250 \\ \mathrm{~S} & 2.4840670 & -3.4557020 & -1.7840640 \\ \mathrm{I} 44 & & & -1.20 \\ \mathrm{TS} 250(\mathrm{~S}, \mathrm{~S}) \mathrm{G}[\mathrm{B} 97-\mathrm{D} / \mathrm{def} 2-\mathrm{TZVP}] & -1.36033 .742975 \\ \mathrm{O} & -1.6831850 & 0.7136920 & -0.7701550 \\ \mathrm{O} & 0.2922300 & 1.3111940 & 0.7106880 \\ \mathrm{C} & -2.5184360 & 1.4601260 & 0.0581220 \\ \mathrm{C} & -2.1140910 & 2.7355940 & 0.4367710 \\ \mathrm{C} & -3.7588040 & 0.8801780 & 0.4385940 \\ \mathrm{C} & -2.9563110 & 3.4747540 & 1.3329380 \\ \mathrm{C} & -4.6072440 & 1.6472150 & 1.2132830 \\ \mathrm{C} & -4.2352960 & 2.9267530 & 1.6937990 \\ \mathrm{H} & -5.5736480 & 1.2419250 & 1.5020930 \\ \mathrm{C} & 0.3485400 & 2.5490870 & 0.0747580 \\ \mathrm{C} & 1.6188620 & 3.0117250 & -0.3621520 \\ \mathrm{C} & -0.8269940 & 3.2822890 & -0.0689990 \\ \mathrm{C} & 1.6755440 & 4.2722480 & -0.9267460 \\ \mathrm{C} & -0.7664270 & 4.5396700 & -0.7582610 \\ \mathrm{C} & 0.5162110 & 5.0503890 & -1.1562220 \\ \mathrm{H} & 2.6379380 & 4.6649040 & -1.2446400 \\ \mathrm{C} & -0.5450250 & 7.0279990 & -2.1054820\end{array}$

-1.8149240
-1.9225040
0.5951770
-0.4744440
-2.7151000
-2.9029330
1.5769070
-3.4140100
-4.6928170
-5.0898550
-2.5692970
-3.0929270
-5.3515610
-6.0596810
-1.5926450
-4.1134700
-4.2185500
-4.3559360
-4.5779320
-4.7047500
-4.8238800
-4.6657060
-4.9052710
2.8686160
3.4775580
3.4986960
4.7327860
4.7537690
5.4018400
5.2142270
5.2409880
-0.3550140
-0.7037350
0.5131740
-4.3231150
-3.9780120
-5.2254020
-5.3473940
-3.9901030
-3.5243010
2.8811760
1.9114220
6.8243810
6.9789580
2.8258390
1.7834190

6.5039420

5.2933770

6. 3090550

7.9864590

7.0578840

4. 9049050

6.6845560

5. 3957770

4.8753520

3.6623950

4.7143390

6.3395540

5.4266130

3.2378870

5.1247000

$-0.5170350$

$-1.5178500$

$-0.8444110$

$-2.8141590$

$-2.1611130$

$-3.1597260$

$-3.5813430$

$-2.4119950$

2. 1960160

1.7385530

1.9631270

1.1259950

1. 3427840

0.9393680

0.7888390

1. 1918760

0.0521230

$-0.9601300$

$-0.3668510$

0.1927330

1.1455830

$-4.5742930$

$-4.6005060$

$-1.2330030$

$-0.2475200$

2.3957650

2.8621350

0.4037850

$-0.1180510$

1. 8947570

2. 1960030
$-1.7587730$

$-1.1035500$

$-1.8140080$

$-2.6149060$

$-2.0160610$

$-0.8491680$

$-2.0975540$

2.7660690

3.0840820

2. 5604950

1. 9124930

3. 2014750

3.7514270

2.8145380

1.6775210

0.0513090

1. 0512220

$-1.3010150$

0.6696410

$-1.6289630$

$-0.6622120$

1.4350780

$-2.6695400$

$-0.2735920$

$-1.4672250$

0.9685060

$-1.3890330$

0.9917680

$-0.1775740$

$-2.3053370$

1. 9522240

$-0.0999660$

0.9569240

$-1.2582300$

$-2.4164660$

$-2.0045960$

$-1.0415680$

$-2.1337560$

2. 5329460

2. 6291410

2. 2913180

2. 0931760

$-0.1734510$

$-1.1291410$

$-2.8371180$ 


$\begin{array}{rrr}2.8103080 & 0.5746960 & -3.6317680 \\ 2.2546700 & 0.7170450 & -4.5683120 \\ 3.8283150 & 0.2562750 & -3.8909970 \\ 2.3297180 & -0.2236010 & -3.0582940 \\ 3.5278200 & 2.9920460 & -3.6622390 \\ 4.5766180 & 2.7180190 & -3.8395860 \\ 3.0336930 & 3.1106350 & -4.6361220 \\ 3.5104330 & 3.9577780 & -3.1437690 \\ -3.3529940 & -0.2007820 & -3.5457340 \\ -3.6740560 & -1.1330680 & -4.0283310 \\ -3.3299710 & 0.5866480 & -4.3106780 \\ -2.3392330 & -0.3396190 & -3.1547940 \\ -5.7412450 & 0.4272310 & -2.9747470 \\ -5.7194690 & 1.2013460 & -3.7536220 \\ -6.1376440 & -0.4963340 & -3.4167440 \\ -6.4269050 & 0.7486700 & -2.1801460 \\ 2.6313790 & 1.1833430 & 3.2081800 \\ 3.5788070 & 0.6913210 & 3.4643700 \\ 2.1500640 & 1.5060610 & 4.1411080 \\ 1.9811940 & 0.4534890 & 2.7128380 \\ 3.7549850 & 3.4483710 & 3.0003870 \\ 3.2751460 & 3.7744210 & 3.9329260 \\ 4.7397740 & 3.0314990 & 3.2485450 \\ 3.9066550 & 4.3259670 & 2.3587870 \\ -3.0440450 & -2.2456880 & 3.2044020 \\ -2.8860330 & -1.9581980 & 4.2524120 \\ -3.4678260 & -3.2580080 & 3.1953170 \\ -2.0744710 & -2.2666000 & 2.6982330 \\ -5.3372320 & -1.1959070 & 3.2832470 \\ -5.8303430 & -2.1752630 & 3.2208520 \\ -5.1775630 & -0.9557860 & 4.3430790 \\ -6.0140240 & -0.4474430 & 2.8539190 \\ 7.8180610 & 1.5841130 & -0.1187200 \\ 7.6485400 & 2.2802640 & -0.9498560 \\ 7.6915510 & 2.1360170 & 0.8227070 \\ 8.8528560 & 1.2187140 & -0.1698260 \\ 7.1176290 & -0.5893430 & 0.9604770 \\ 6.4430630 & -1.4494430 & 0.9230400 \\ 8.1514710 & -0.9505250 & 0.8810910 \\ 7.0065610 & -0.1098150 & 1.9415730 \\ -6.5737000 & -4.9610680 & -0.4032220 \\ -7.3629500 & -4.2570940 & -0.6959560 \\ -6.8697620 & -5.9715070 & -0.7153040 \\ -6.4941420 & -4.9501020 & 0.6918830 \\ -4.1383550 & -5.5960590 & -1.1458080 \\ -3.1859850 & -5.3547420 & \end{array}$

\begin{tabular}{lr}
$\mathrm{H}$ & -3.9730680 \\
$\mathrm{H}$ & -4.4387680 \\
$\mathrm{C}$ & 0.3568740 \\
$\mathrm{C}$ & 0.1319210 \\
$\mathrm{H}$ & 0.9854360 \\
$\mathrm{H}$ & 0.1728610 \\
$\mathrm{O}$ & -1.0489700 \\
$\mathrm{H}$ & -1.0156880 \\
$\mathrm{C}$ & -0.2644010 \\
$\mathrm{C}$ & 0.0005480 \\
$\mathrm{C}$ & -0.3576860 \\
$\mathrm{C}$ & 0.5561710 \\
$\mathrm{H}$ & -0.0009200 \\
$\mathrm{H}$ & -0.5746560 \\
$\mathrm{H}$ & -1.4488350 \\
$\mathrm{H}$ & 1.6287280 \\
$\mathrm{H}$ & 0.3096770 \\
$\mathrm{H}$ & -1.3324940 \\
$\mathrm{H}$ & 1.0648050 \\
$\mathrm{H}$ & 0.0261250 \\
$\mathrm{C}$ & 3.1828610 \\
$\mathrm{O}$ & 2.6196740 \\
$\mathrm{C}$ & 4.1300070 \\
$\mathrm{C}$ & 5.0768680 \\
$\mathrm{C}$ & 4.0641580 \\
$\mathrm{C}$ & 5.9388080 \\
$\mathrm{H}$ & 5.1314440 \\
$\mathrm{C}$ & 4.9108430 \\
$\mathrm{H}$ & 3.3472960 \\
$\mathrm{C}$ & 5.8533560 \\
$\mathrm{H}$ & 6.6763570 \\
$\mathrm{H}$ & 4.8417290 \\
$\mathrm{H}$ & 6.5211000 \\
$\mathrm{H}$ & 1.8476300 \\
$\mathrm{~S}$ & 2.8170870 \\
$\mathrm{H}$ & \\
\hline
\end{tabular}
$-5.5961700$
$-6.6078410$
$-3.8310660$
$-3.5128340$
$-3.1328340$
$-2.4708710$
$-3.4121870$
$-2.3912040$
$-6.2428360$
$-5.9821700$
$-4.5391500$
$-5.2811260$
$-7.2764090$
$-6.6779580$
$-4.4114950$
$-5.4912850$
$-5.3978320$
$-6.1136680$
$-6.1527390$
$-4.2774060$
$-2.4504360$
$-1.3056080$
$-2.9006230$
$-3.9116620$
$-2.3298290$
$-4.3365340$
$-4.3521150$
$-2.7772800$
$-1.5421860$
$-3.7769950$
$-5.1071010$
$-2.3374540$
$-4.1169960$
$-0.9972640$
$-3.3546960$

0.4243230

$-0.9636210$

0.3853910

$-1.0327570$

0.9396250

$-1.3311450$

0.4273730

0.6376170

$-0.1063450$

$-1.5981740$

$-1.9950820$

0.7704550

0.1463220

$-2.2196790$

$-2.0617120$

0.6547280

1.8329080

0.1095400

$-1.8093660$

$-2.9883560$

$-0.4322930$

$-0.1488510$

0.6153690

0.3650240

1.9044520

1. 3734680

$-0.6263880$

2. 9161180

2. 1081580

2.6543470

1.1629340

3.9081750

3.4426350

$-0.7583060$

144

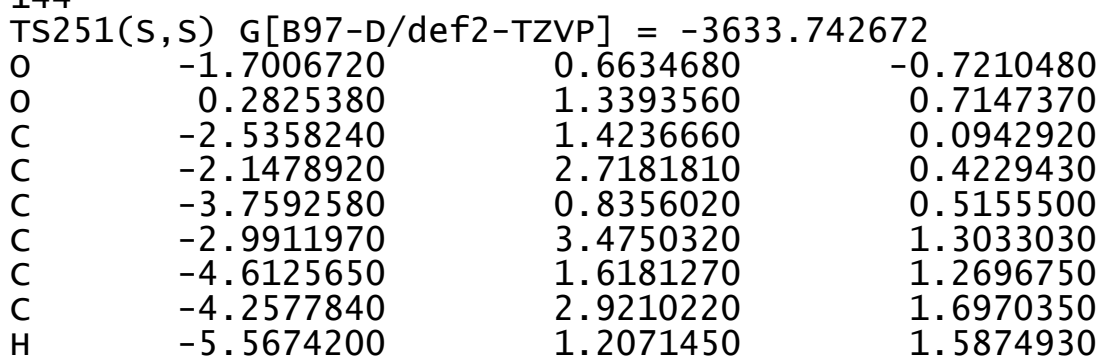




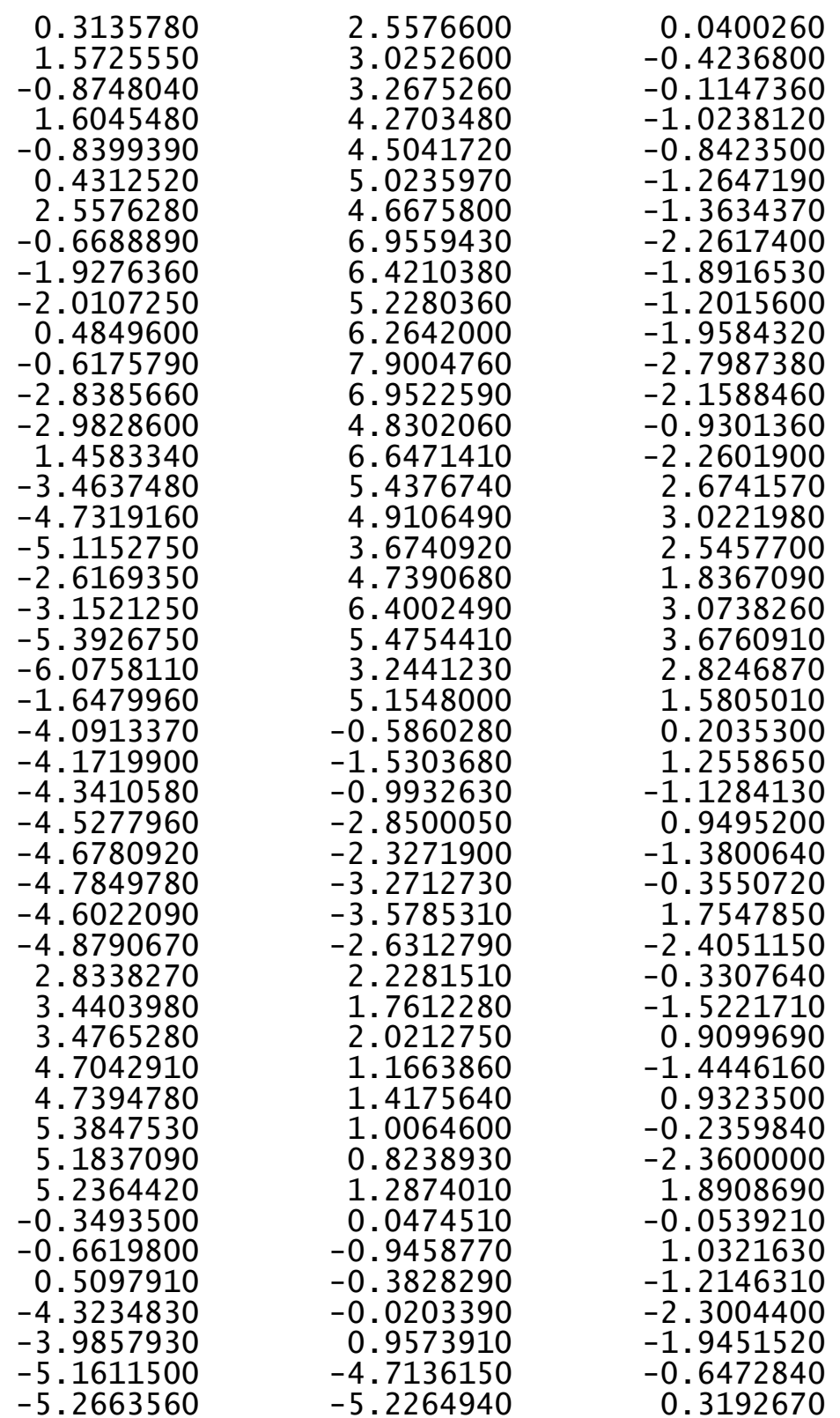

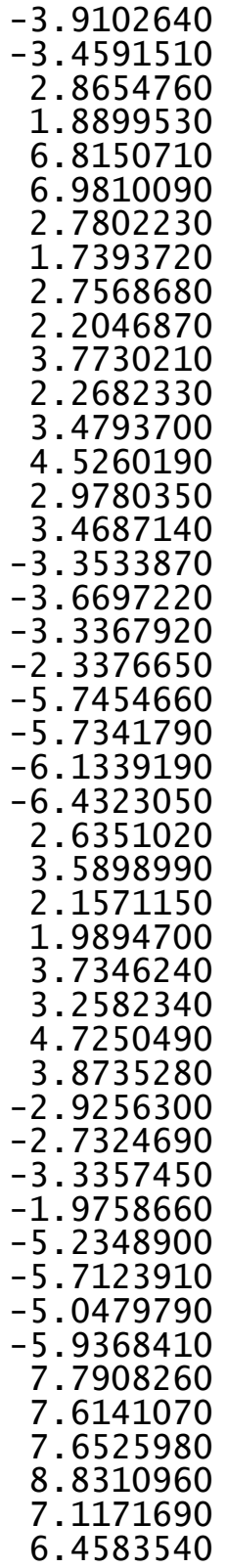

-1.1724360
-0.1763410
2.4669440
2.9215160
0.4925340
-0.0169240
1.8888750
2.1959360
0.5505020
0.6744180
0.2177900
-0.2294550
2.9662640
2.6858810
3.0663370
3.9423860
-0.4694910
-1.4289820
0.2742460
-0.5800790
0.1693620
0.9011670
-0.7802290
0.5256210
1.2653960
0.7878530
1.5966400
0.5212830
3.5377540
3.8727530
3.1339190
4.4073530
-2.1385010
-1.7967450
-3.1548840
-2.1727260
-1.1240180
-2.1130670
-0.8341700
-0.4062660
1.6867100
2.3895010
2.2264000
1.3372270
-0.5082040
-1.3796940

2.7157670

2.7541620

2. 2314560

2. 0346700

$-0.2323510$

$-1.1927390$

$-2.8910980$

$-2.7458760$

$-3.6546130$

$-4.5959090$

$-3.9028700$

$-3.0628540$

$-3.7441810$

$-3.9240150$

$-4.7164140$

$-3.2451090$

$-3.4086870$

$-3.8384110$

$-4.2164040$

$-3.0135070$

$-2.8659400$

$-3.6849010$

$-3.2570290$

$-2.0873740$

3. 1675720

3.4240050

4.0992430

2.6879230

2.9188490

3. 8499740

3.1660870

2. 2636110

3. 4012080

4.4268130

3.4580770

2.8593800

3. 5041180

3. 5005380

4.5469270

3. 0628190

$-0.1605460$

$-0.9846090$

0.7863200

$-0.2114980$

0.8927420 


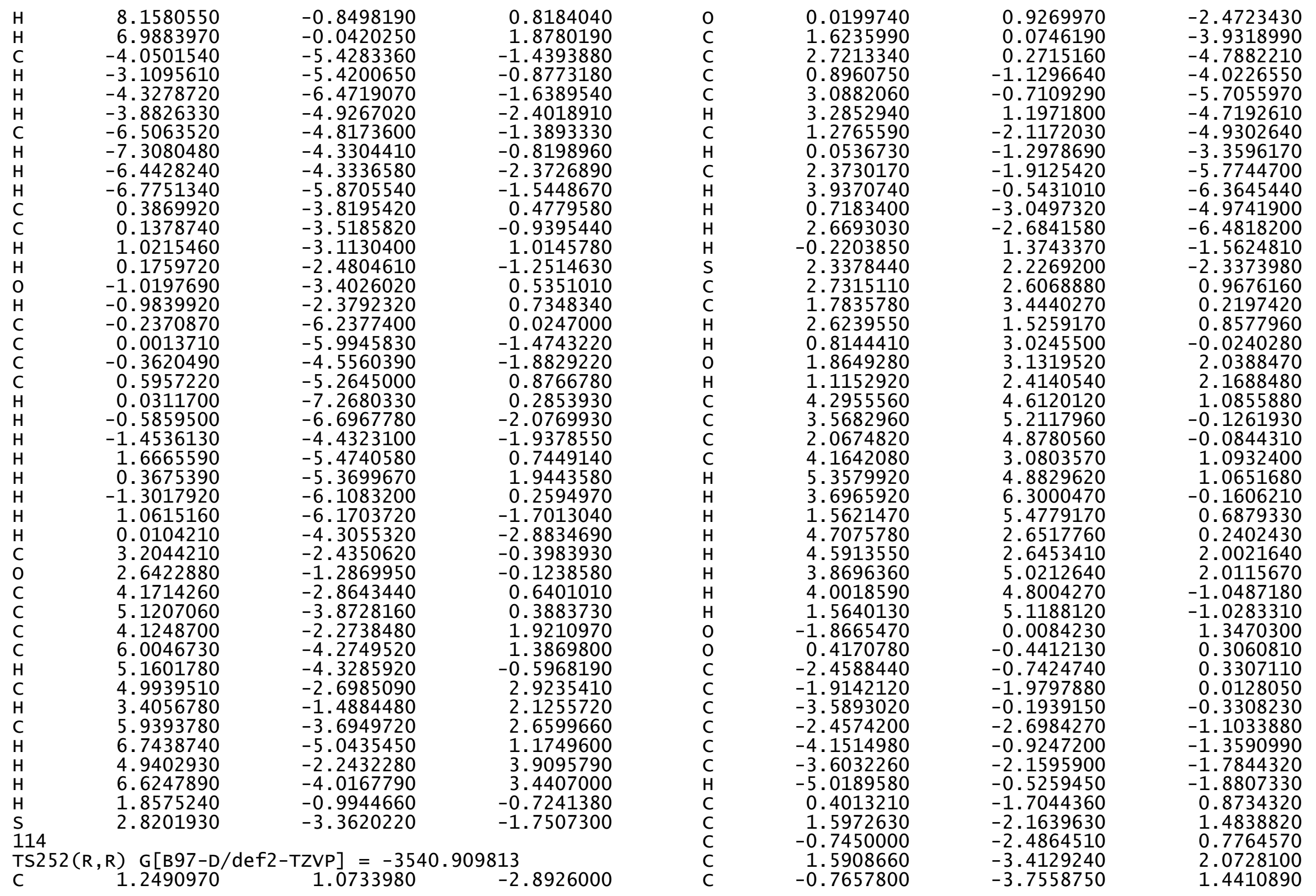




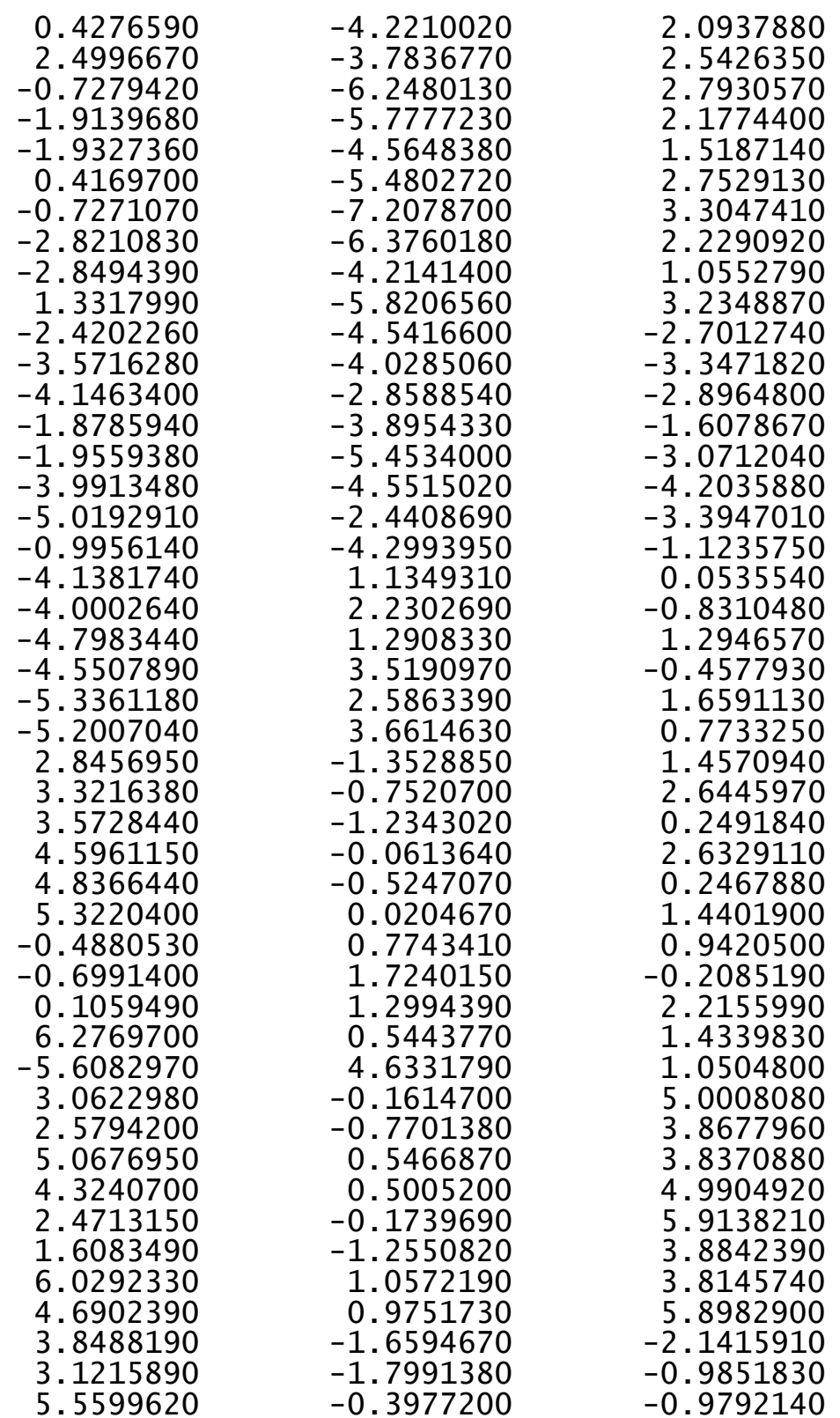
5. 0802850
3. 4725280
2. 1786010
6.5022860
5. 6335910
$-3.1823710$
$-3.3055720$
$-4.4063150$
$-3.7446820$
$-2.6378150$
$-2.8502580$
$-4.8308740$
$-3.6389280$
$-6.1366350$
$-5.9992230$
$-4.9757730$
$-5.6197890$
$-6.6418450$
$-6.3941210$
$-4.5892880$
$-5.7365940$

114

$\operatorname{TS} 253(\mathrm{R}, \mathrm{R}) \mathrm{G}[\mathrm{B} 97-\mathrm{D} / \mathrm{def} 2-\mathrm{TZVP}]=-3540.909775$

$\begin{array}{lllr}\mathrm{C} & 1.2504880 & 1.0757930 & -2.8919640 \\ \mathrm{O} & 0.0213770 & 0.9284830 & -2.4719750 \\ \mathrm{C} & 1.6255890 & 0.0779410 & -3.9319310 \\ \mathrm{C} & 2.7235820 & 0.2757990 & -4.7877080 \\ \mathrm{C} & 0.8983880 & -1.1264450 & -4.0239270 \\ \mathrm{C} & 3.0910410 & -0.7058280 & -5.7057250 \\ \mathrm{H} & 3.2872750 & 1.2015580 & -4.7178340 \\ \mathrm{C} & 1.2794370 & -2.1131530 & -4.9322040 \\ \mathrm{H} & 0.0558070 & -1.2953690 & -3.3613050 \\ \mathrm{C} & 2.3761680 & -1.9075550 & -5.7758240 \\ \mathrm{H} & 3.9401110 & -0.5372680 & -6.3642240 \\ \mathrm{H} & 0.7214620 & -3.0457820 & -4.9771000 \\ \mathrm{H} & 2.6729040 & -2.6785340 & -6.4836800 \\ \mathrm{H} & -0.2194490 & 1.3752330 & -1.5619720 \\ \mathrm{~S} & 2.3385820 & 2.2294640 & -2.3357790 \\ \mathrm{C} & 2.7309720 & 2.6057080 & 0.9694610 \\ \mathrm{C} & 1.7834930 & 3.4438510 & 0.2221510 \\ \mathrm{H} & 2.6228290 & 1.5248720 & 0.8588990 \\ \mathrm{H} & 0.8142350 & 3.0249420 & -0.0221180 \\ \mathrm{O} & 1.8644830 & 3.1304500 & 2.0409590 \\ \mathrm{H} & 1.1146710 & 2.4125980 & 2.1703970 \\ \mathrm{C} & 4.2961290 & 4.6099030 & 1.0887220 \\ \mathrm{C} & 3.5691670 & 5.2108130 & -0.1226790\end{array}$

$-2.1427870$ 3.0703350 $-1.0058820$ $-0.9675360$ $-3.0723630$ $-2.9087830$ $-2.0786350$ $-2.5489300$ $-3.8456100$ $-2.3576560$ $-1.0614800$ $-3.2203990$ 3.7751350

2. 9166050

2. 2155710

3.4155170

4.7301260

3. 1776110

1.9566860

4.0997500 $-1.3540690$
16726050
0.2109510
0.3946960
3.7159740
-0.7706330
-0.4429920

1.2504880
0.0213770
1.6255890
2.7235820
0.8983880
3.0910410
3.2872750
1.2794370
0.0558070
2.3761680
3.9401110
0.7214620
2.6729040
0.2194490
2.3385820
2.7309720
1.7834930
2.6228290
0.8142350
1.8644830
1.1146710
4.2961290
3.5691670
$-0.1226790$ 


2.0681520
4.1638930
5.3587230
3.6981190
1.5632670
4.7071550
4.5906710
3.8704920
4.0024000
1.5647620
-1.8669210
0.4168770
-2.4591430
-1.9146170
-3.5894740
-2.4577720
-4.1516330
-3.6034310
-5.0189990
0.4007550
1.5964280
-0.7456390
1.5897040
-0.7667700
0.4264210
2.4982980
-0.7295870
-1.9153760
-1.9338270
0.4154060
-0.7290000
-2.8225660
-2.8503550
1.3300530
-2.4205710
-3.5718360
-4.1464820
-1.8790130
-1.9563280
-3.9915070
-5.0193290
-0.9961340
-4.1382440
-3.9998440
-4.7987200
-4.5500510

4.8779580

3.0783140

4.8802410

6.2990060

5.4775820

2.6498630

2.6425860

5.0189010

4.7997040

5.1196780

0.0076440

$-0.4416590$

$-0.7425030$

$-1.9796620$

$-0.1933600$

$-2.6974320$

$-0.9233600$

$-2.1579810$

$-0.5241260$

$-1.7052600$

$-2.1653250$

$-2.4870580$

$-3.4147240$

$-3.7569830$

$-4.2227140$

$-3.7858960$

$-6.2501330$

$-5.7792700$

$-4.5658960$

$-5.4824690$

$-7.2103690$

$-6.3775120$

$-4.2147760$

$-5.8232850$

$-4.5395140$

$-4.0257730$

$-2.8563870$

$-3.8941370$

$-5.4510330$

$-4.5481060$

$-2.4379560$

$-4.2985480$

1.1352540

2. 2313460

1.2901930

3. 5200010

-0.0810070
1.0955500
1.0684060
-0.1564750
0.6918320
0.2424240
2.0043060
2.0149460
-1.0454810
-1.0246990
1.3464030
0.3057260
0.3294890
0.0107880
-0.3317820
-1.1059960
-1.3606420
-1.7867990
-1.8820790
0.8722200
1.4827830
0.7743200
2.0707740
1.4380030
2.0907350
2.5406670
2.7881520
2.1725200
1.5146850
2.7489270
3.2991260
2.2234470
1.0512240
3.2309430
-2.7052120
-3.3508910
-2.8994120
-1.6112660
-3.0757460
-4.2077260
-3.3974400
-1.1271580
0.0535520
-0.8300290
1.2946130
-0.4557060

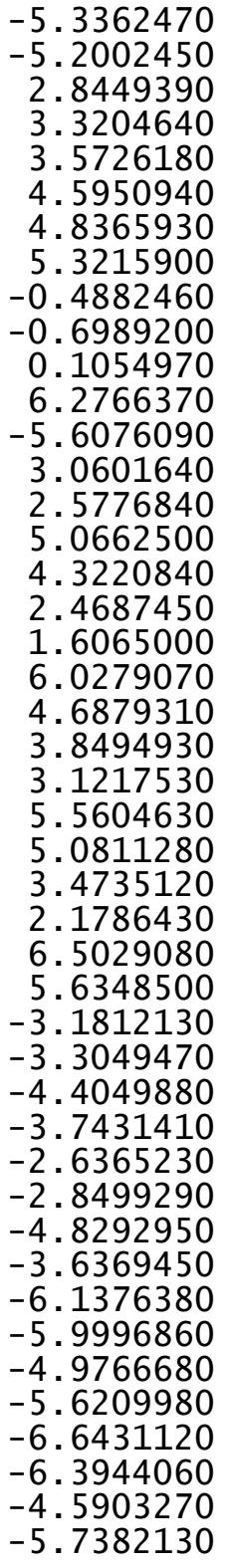

2. 5855050

3.6614210

$-1.3543240$

$-0.7545940$

$-1.2347990$

$-0.0641560$

$-0.5255010$

0.0184960

0.7736380

1.7239850

1.2979200

0.5422020

4.6330030

$-0.1658500$

$-0.7734930$

0.5428100

0.4958700

$-0.1789650$

$-1.2582370$

1.0531550

0.9697090

$-1.6577890$

$-1.7983800$

$-0.3975540$

$-0.9441830$

$-2.0761990$

$-2.3362600$

0.1478140

$-0.8309200$

3.2275240

2. 1396060

4.6253000

4.4863080

3.1310910

1.1957030

5.5842510

5. 3365260

1.6700170

2.7335440

0.2095100

0.3923110

1.7969260

3.7140080

$-0.7719230$

$-0.4459750$
1.6601110

0.7753740

1.4571710

2.6453950

0.2496770

2.6348740

0.2484630

1.4425590

0.9421740

$-0.2079050$

2.2161850

1.4372330

1.0533330

5.0019680

3.8682380

3.8397640

4.9927880

5.9146900

3.8838570

3.8181080

5.9011390

$-2.1413770$

$-0.9854020$

$-0.9771170$

$-2.1414050$

$-3.0706560$

$-1.0070020$

$-0.9645320$

$-3.0706440$

$-2.9066790$

$-2.0775710$

$-1.3509030$

$-2.5457490$

$-3.8435020$

$-2.3574080$

$-1.0575090$

$-3.2163970$

3.7750430

2.9175430

2. 2144880

3.4144090

4.7299950

3.1793450

1.9548140

4.0978390 


$\begin{array}{lrcr}\text { TS2 } 54(\mathrm{~S}, \mathrm{~S}) \mathrm{G}[\mathrm{B} 97-\mathrm{D} / \mathrm{def} 2-\mathrm{TZVP}]=-3540.909917 \\ \mathrm{C} & 2.4179800 & 2.1756630 & -1.6715610 \\ \mathrm{O} & 1.2623910 & 1.5768570 & -1.5728840 \\ \mathrm{C} & 3.0134010 & 2.0687370 & -3.0316960 \\ \mathrm{C} & 4.3598470 & 2.4013860 & -3.2701920 \\ \mathrm{C} & 2.2330460 & 1.5930340 & -4.1056510 \\ \mathrm{C} & 4.9120470 & 2.2594840 & -4.5414210 \\ \mathrm{H} & 4.9681940 & 2.7586270 & -2.4444980 \\ \mathrm{C} & 2.7869240 & 1.4581100 & -5.3774200 \\ \mathrm{H} & 1.1957480 & 1.3257660 & -3.9346980 \\ \mathrm{C} & 4.1280960 & 1.7870870 & -5.6002730 \\ \mathrm{H} & 5.9566710 & 2.5130470 & -4.7066450 \\ \mathrm{H} & 2.1715540 & 1.0923300 & -6.1963460 \\ \mathrm{H} & 4.5600030 & 1.6757650 & -6.5925680 \\ \mathrm{H} & 0.7764120 & 1.6377190 & -0.6523740 \\ \mathrm{~S} & 3.1700500 & 2.9960440 & -0.4086460 \\ \mathrm{C} & 2.2340310 & 1.9698850 & 2.6969570 \\ \mathrm{C} & 2.9979280 & 1.1724550 & 1.7320530 \\ \mathrm{H} & 1.2458630 & 2.2876510 & 2.3670540 \\ \mathrm{H} & 2.4443530 & 0.6011980 & 0.9961230 \\ \mathrm{O} & 2.1990920 & 0.6243540 & 3.2986940 \\ \mathrm{H} & 1.2971900 & 0.1894120 & 2.9636140 \\ \mathrm{C} & 4.4201680 & 2.5509130 & 3.8622450 \\ \mathrm{C} & 5.1651090 & 2.1723640 & 2.5731380 \\ \mathrm{C} & 4.4764670 & 0.9985380 & 1.8569440 \\ \mathrm{C} & 2.9766420 & 2.9790600 & 3.5462870 \\ \mathrm{H} & 4.9384820 & 3.3698580 & 4.3751210 \\ \mathrm{H} & 6.2040600 & 1.8982170 & 2.7922820 \\ \mathrm{H} & 4.6406840 & 0.0634400 & 2.4093380 \\ \mathrm{H} & 2.9820800 & 3.9202290 & 2.9784680 \\ \mathrm{H} & 2.4009250 & 3.1541710 & 4.4637280 \\ \mathrm{H} & 4.4058190 & 1.6903780 & 4.5444250 \\ \mathrm{H} & 5.1892310 & 3.0397650 & 1.8989350 \\ \mathrm{H} & 4.8831770 & 0.8331910 & 0.8535500 \\ \mathrm{O} & -2.0920230 & -0.0431870 & 1.1510720 \\ \mathrm{O} & -0.0553420 & -0.8821840 & -0.1103270 \\ \mathrm{C} & -2.8101780 & -0.2130090 & -0.0264560 \\ \mathrm{C} & -2.7336150 & -1.4265380 & -0.7030540 \\ \mathrm{C} & -3.6104370 & 0.8744520 & -0.4652050 \\ \mathrm{C} & -3.4657640 & -1.5669620 & -1.9311250 \\ \mathrm{C} & -4.3692520 & 0.7055650 & -1.6048770 \\ \mathrm{C} & -4.3185630 & -0.4911020 & -2.3614990 \\ \mathrm{H} & -4.9942620 & 1.5247690 & -1.9546540 \\ \mathrm{C} & -0.4826860 & -2.1957080 & 0.0030080 \\ \mathrm{C} & 0.5010600 & -3.1743680 & 0.3060700 \\ \mathrm{C} & -1.8221330 & -2.4972180 & -0.2164810 \\ & & & \end{array}$

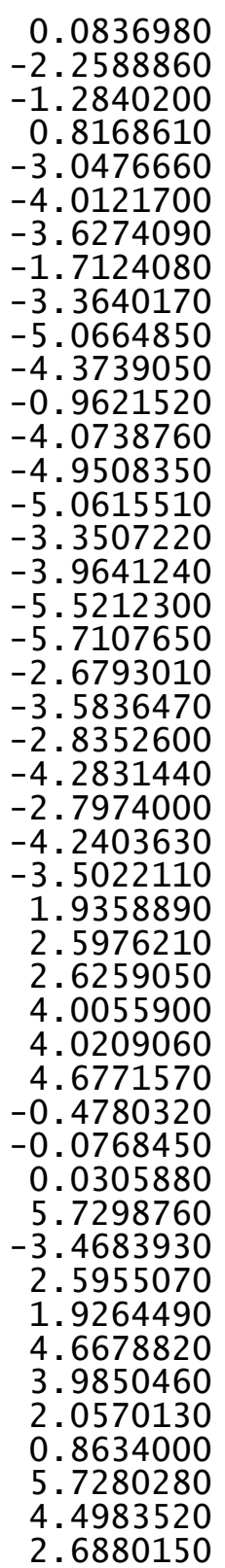
$-4.4800850$
$-3.8397840$
$-4.8399160$
$-5.2507840$
$-6.5125900$
$-5.5218200$
$-4.2203280$
$-6.1737900$
$-7.5355020$
$-5.7878150$
$-3.4673910$
$-6.9216700$
$-2.7975690$
$-1.7580480$
$-0.6257360$
$-2.7039840$
$-3.6782910$
$-1.8486200$
0. 1947180
$-3.5080350$
2. 1776790
3. 2482650
2. 3307370
4. 5157650
3.6032130
4.6593790
$-2.7896750$
$-2.8537460$
$-2.3428190$
$-2.5152910$
$-1.9630190$
$-2.0871390$
0.1592630
1. 5086160
$-0.2905390$
$-1.8160820$
5. 6132500
$-3.2375950$
$-3.2029400$
$-2.5818400$
$-2.9318530$
$-3.4899770$
$-2.3358790$
$-2.9677820$
$-1.7511160$

0.4729670
0.0299520
0.3739970
0.7017040
0.5459730
0.2385210
-0.0138180
0.6147290
0.7376380
0.2058230
-0.2467050
0.8655860
-3.9507270
-4.3448450
-3.5659830
-2.7785270
-4.5799030
-5.2665840
-3.8669550
-2.4980170
0.2564110
-0.2846060
1.4734150
0.4185470
2.1661310
1.6190460
0.4100370
1.6589910
-0.7437820
1.7379140
-0.6405840
0.5884570
1.0827640
0.5602250
2.4242470
0.6581800
2.1446100
4.0729410
2.8734980
3.0027390
4.1411760
4.9836210
2.8406430
3.0429280
5.0996430
-3.1151060 


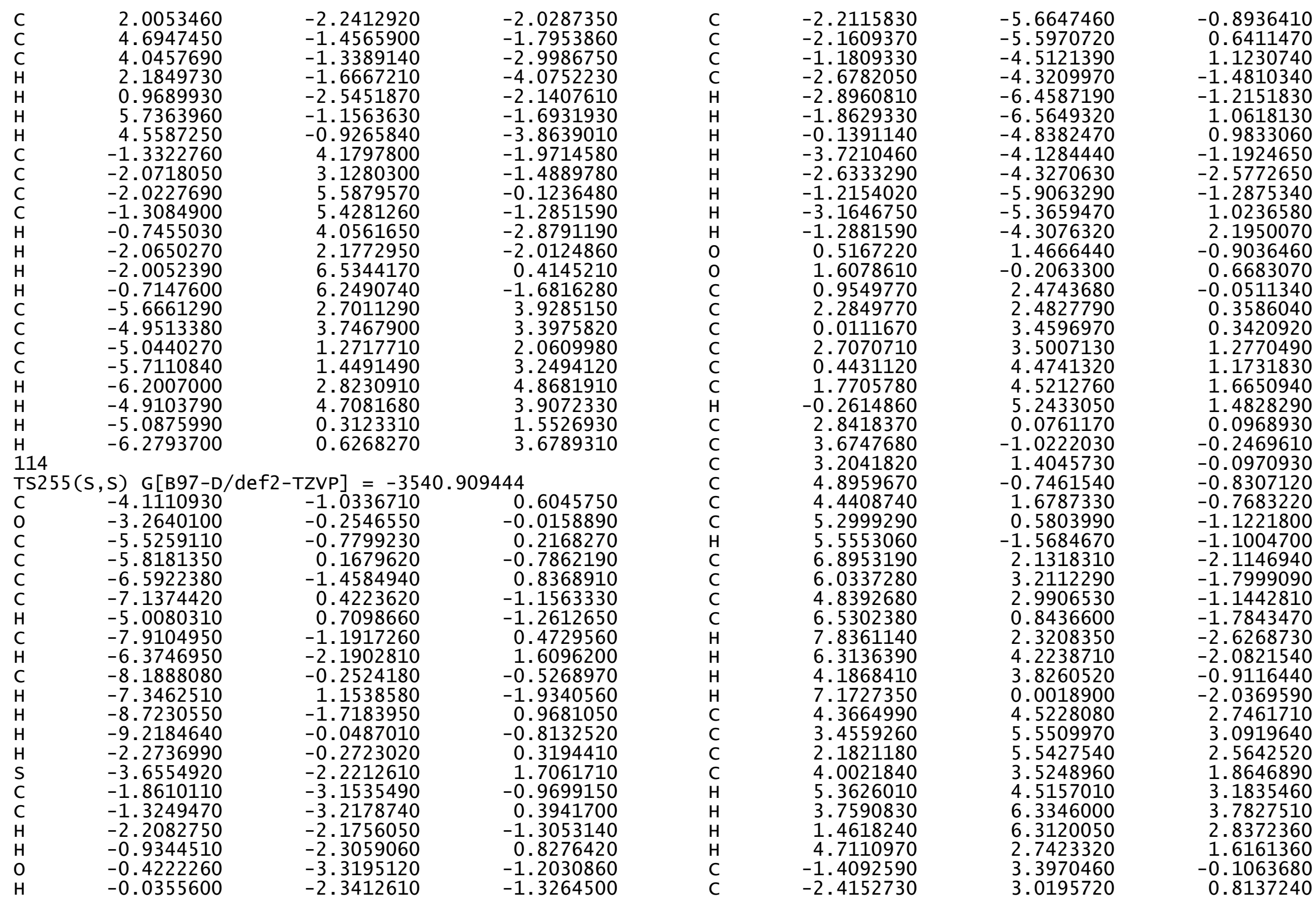




$\begin{array}{rrr}-1.7440060 & 3.7287480 & -1.4390710 \\ -3.8016950 & 3.0075600 & 0.3884320 \\ -3.1323760 & 3.7013870 & -1.8554030 \\ -4.1216910 & 3.3539050 & -0.9282860 \\ 3.2363920 & -2.4247550 & 0.0019730 \\ 2.9504680 & -3.2768830 & -1.0920340 \\ 3.1145640 & -2.9012670 & 1.3298090 \\ 2.5614700 & -4.6522090 & -0.8443840 \\ 2.7051460 & -4.2719190 & 1.5633620 \\ 2.4531370 & -5.1113410 & 0.4720170 \\ 0.2920290 & -0.0233160 & -0.2836520 \\ -0.8633100 & -0.0812350 & 0.6744540 \\ 0.3290160 & -0.9500200 & -1.4685920 \\ 2.1597980 & -6.1452080 & 0.6529340 \\ -5.1650210 & 3.3430320 & -1.2414350 \\ 2.6975560 & -3.6839290 & -3.4890740 \\ 2.9912820 & -2.8325430 & -2.4517020 \\ 2.2734530 & -5.5069960 & -1.9521810 \\ 2.3417010 & -5.0408460 & -3.2411540 \\ 2.7242640 & -3.3153390 & -4.5121960 \\ 3.2372620 & -1.7962220 & -2.6573300 \\ 1.9878550 & -6.5374400 & -1.7463560 \\ 2.1134970 & -5.6996420 & -4.0763470 \\ 3.2483770 & -2.5711550 & 3.7487520 \\ 3.3908040 & -2.0851210 & 2.4713950 \\ 2.5694020 & -4.7391640 & 2.9070890 \\ 2.8274510 & -3.9133640 & 3.9732930 \\ 3.4585890 & -1.9256960 & 4.5987270 \\ 3.7153280 & -1.0601130 & 2.3196820 \\ 2.2571130 & -5.7703630 & 3.0653050 \\ 2.7177270 & -4.2806100 & 4.9914220 \\ -3.1244290 & 2.2224270 & 3.0117810 \\ -2.1244010 & 2.6079660 & 2.1527200 \\ -4.8122350 & 2.6147310 & 1.3185200 \\ -4.4865400 & 2.2292030 & 2.5944660 \\ -2.8739560 & 1.8922600 & 4.0174620 \\ -1.0899600 & 2.5775510 & 2.4790220 \\ -5.8463080 & 2.6030360 & 0.9809490 \\ -5.2640140 & 1.9136430 & 3.2869210 \\ -2.4821400 & 4.3805040 & -4.1069820 \\ -3.4594270 & 4.0352290 & -3.2059630 \\ -0.7622570 & 4.1049830 & -2.4088470 \\ -1.1173270 & 4.4165580 & -3.6994400 \\ -2.7445140 & 4.6300060 & -5.1329120 \\ -4.5065490 & 4.0092190 & -3.5038940 \\ 0.2826560 & 4.1377830 & -2.1134140\end{array}$

$\begin{array}{lrrr}\mathrm{H} & -0.3500690 & 4.6929600 & -4.4195070 \\ \mathrm{C} 14 & & & \\ \mathrm{TS} 256(\mathrm{~S}, \mathrm{~S}) \mathrm{G}[\mathrm{B} 97-\mathrm{D} / \mathrm{def} 2-\mathrm{TZVP}]=-3540.908136 \\ \mathrm{C} & 0.9182600 & 0.9958100 & -2.9588820 \\ \mathrm{O} & -0.2216050 & 0.7681350 & -2.3712470 \\ \mathrm{C} & 1.2834060 & -0.0598220 & -3.9440860 \\ \mathrm{C} & 2.2830070 & 0.1405470 & -4.9128630 \\ \mathrm{C} & 0.6563550 & -1.3206440 & -3.8626780 \\ \mathrm{C} & 2.6490230 & -0.8908260 & -5.7751050 \\ \mathrm{H} & 2.7735550 & 1.1077760 & -4.9729870 \\ \mathrm{C} & 1.0384840 & -2.3553450 & -4.7150800 \\ \mathrm{H} & -0.1098110 & -1.4934540 & -3.1141500 \\ \mathrm{C} & 2.0345890 & -2.1451250 & -5.6745780 \\ \mathrm{H} & 3.4201940 & -0.7200030 & -6.5227980 \\ \mathrm{H} & 0.5576580 & -3.3268150 & -4.6255880 \\ \mathrm{H} & 2.3319840 & -2.9529780 & -6.3397710 \\ \mathrm{H} & -0.4163020 & 1.2990140 & -1.4814310 \\ \mathrm{~S} & 1.9173550 & 2.3166310 & -2.6454690 \\ \mathrm{O} & -1.8208000 & -0.0306950 & 1.4333170 \\ \mathrm{O} & 0.4927390 & -0.3399010 & 0.3911440 \\ \mathrm{C} & -2.3677310 & -0.8160790 & 0.4163910 \\ \mathrm{C} & -1.7423720 & -2.0131260 & 0.0954340 \\ \mathrm{C} & -3.5310290 & -0.3407750 & -0.2461210 \\ \mathrm{C} & -2.2270370 & -2.7579420 & -1.0300120 \\ \mathrm{C} & -4.0380610 & -1.1035140 & -1.2803980 \\ \mathrm{C} & -3.4026800 & -2.2927530 & -1.7143550 \\ \mathrm{H} & -4.9285550 & -0.7616670 & -1.8034630 \\ \mathrm{C} & 0.5541420 & -1.6028420 & 0.9531030 \\ \mathrm{C} & 1.7812130 & -2.0013950 & 1.5452690 \\ \mathrm{C} & -0.5458670 & -2.4487470 & 0.8599350 \\ \mathrm{C} & 1.8481700 & -3.2510750 & 2.1296120 \\ \mathrm{C} & -0.4902480 & -3.7211300 & 1.5156030 \\ \mathrm{C} & 0.7314030 & -4.1216280 & 2.1583600 \\ \mathrm{H} & 2.7821610 & -3.5758290 & 2.5837820 \\ \mathrm{C} & -0.3013000 & -6.2186020 & 2.8434170 \\ \mathrm{C} & -1.5150030 & -5.8137620 & 2.2351180 \\ \mathrm{C} & -1.6073450 & -4.5979180 & 1.5881980 \\ \mathrm{C} & 0.7965550 & -5.3847740 & 2.8070480 \\ \mathrm{H} & -0.2421940 & -7.1814930 & 3.3458910 \\ \mathrm{H} & -2.3848030 & -6.4653840 & 2.2829460 \\ \mathrm{H} & -2.5441820 & -4.2973610 & 1.1297500 \\ \mathrm{H} & 1.7317830 & -5.6762230 & -2.2818960 \\ \mathrm{C} & -2.0426770 & -4.5702860 & -3.3025230 \\ \mathrm{C} & -3.2218870 & -4.1315400 & -1.5438850\end{array}$




$\begin{array}{lrrr}\mathrm{H} & -1.5103320 & -5.4403510 & -3.0306880 \\ \mathrm{H} & -3.5944030 & -4.6698680 & -4.1711450 \\ \mathrm{H} & -4.7806180 & -2.6520980 & -3.3421260 \\ \mathrm{H} & -0.6531060 & -4.2490510 & -1.0576500 \\ \mathrm{C} & -4.1666900 & 0.9486370 & 0.1386220 \\ \mathrm{C} & -4.1201650 & 2.0444800 & -0.7558000 \\ \mathrm{C} & -4.8146600 & 1.0697010 & 1.3904810 \\ \mathrm{C} & -4.7495060 & 3.2962170 & -0.3806510 \\ \mathrm{C} & -5.4307020 & 2.3294850 & 1.7566140 \\ \mathrm{C} & -5.3843720 & 3.4043590 & 0.8615860 \\ \mathrm{C} & 2.9933870 & -1.1379800 & 1.4887960 \\ \mathrm{C} & 3.4802340 & -0.5218190 & 2.6633620 \\ \mathrm{C} & 3.6848910 & -0.9949150 & 0.2615140 \\ \mathrm{C} & 4.7281610 & 0.2159750 & 2.6181900 \\ \mathrm{C} & 4.9312090 & -0.2565820 & 0.2299250 \\ \mathrm{C} & 5.4267000 & 0.3107310 & 1.4096510 \\ \mathrm{P} & -0.4983620 & 0.8216610 & 1.0146810 \\ \mathrm{O} & -0.7675110 & 1.7329130 & -0.1538280 \\ \mathrm{O} & 0.0731600 & 1.4054280 & 2.2707220 \\ \mathrm{H} & 6.3676840 & 0.8593020 & 1.3801020 \\ \mathrm{H} & -5.8496820 & 4.3491560 & 1.1408510 \\ \mathrm{C} & 3.2712340 & 0.0440400 & 5.0302990 \\ \mathrm{C} & 2.7769110 & -0.5755270 & 3.9082620 \\ \mathrm{C} & 5.2105390 & 0.8385220 & 3.8108270 \\ \mathrm{C} & 4.5051470 & 0.7554930 & 4.9858100 \\ \mathrm{H} & 2.7108720 & 0.0014120 & 5.9615550 \\ \mathrm{H} & 1.8270780 & -1.0995080 & 3.9507330 \\ \mathrm{H} & 6.1504020 & 1.3862280 & 3.7628090 \\ \mathrm{H} & 4.8801660 & 1.2386290 & 5.8854770 \\ \mathrm{C} & 3.8929350 & -1.3823650 & -2.1437750 \\ \mathrm{C} & 3.2102290 & -1.5584240 & -0.9650270 \\ \mathrm{C} & 5.6167660 & -0.1059480 & -1.0146140 \\ \mathrm{C} & 5.1098160 & -0.6439420 & -2.1721760 \\ \mathrm{H} & 3.4939070 & -1.7960260 & -3.0657850 \\ \mathrm{H} & 2.2811360 & -2.1201790 & -0.9639520 \\ \mathrm{H} & 6.5487710 & 0.4571680 & -1.0246640 \\ \mathrm{H} & 5.6340250 & -0.5086960 & -3.1156180 \\ \mathrm{C} & -3.4053560 & 3.0793870 & -2.8521310 \\ \mathrm{C} & -3.4420020 & 1.9912730 & -2.0149180 \\ \mathrm{C} & -4.6949300 & 4.4022800 & -1.2849300 \\ \mathrm{C} & -4.0450120 & 4.2999570 & -2.4898810 \\ \mathrm{H} & -2.8696280 & 3.0135650 & -3.7967220 \\ \mathrm{H} & -2.9285440 & 1.0793310 & -2.2988700 \\ \mathrm{H} & -5.1772530 & 5.3327010 & -0.9893600 \\ \mathrm{H} & -4.0062960 & 5.1508460 & -3.1668830 \\ \mathrm{C} & -6.1267440 & 1.3835320 & 3.8951020\end{array}$

$\begin{array}{lr}\mathrm{C} & -6.0771560 \\ \mathrm{C} & -4.9040370 \\ \mathrm{C} & -5.5349780 \\ \mathrm{H} & -6.6193490 \\ \mathrm{H} & -6.5302440 \\ \mathrm{H} & -4.4611650 \\ \mathrm{H} & -5.5838750 \\ \mathrm{C} & 1.4784310 \\ \mathrm{C} & 2.4078450 \\ \mathrm{H} & 0.4260700 \\ \mathrm{H} & 2.0459840 \\ \mathrm{O} & 1.8824860 \\ \mathrm{H} & 1.1736560 \\ \mathrm{C} & 3.4215570 \\ \mathrm{C} & 4.1729770 \\ \mathrm{C} & 3.8592100 \\ \mathrm{C} & 1.9032860 \\ \mathrm{H} & 3.6749410 \\ \mathrm{H} & 5.2558690 \\ \mathrm{H} & 4.3611950 \\ \mathrm{H} & 1.5781080 \\ \mathrm{H} & 1.3484330 \\ \mathrm{H} & 3.7246920 \\ \mathrm{H} & 3.8748040 \\ \mathrm{H} & 4.2305370 \\ \mathrm{H} & \end{array}$
2. 4454510 $-0.0119070$
0.1391860
1.4858410
3.4003050
$-0.9690060$
$-0.6989800$
3.7422090
2. 6843660
3.5549230
1.6639170
3. 1902650
2. 4697180
5. 3870600
4.4148670
2. 9520600
5.1839620
6.4236340
4. 5745520
2. 6565510
5.4785130
5.8019370
5. 2177840
4.6002830
2. 2529110

TS257 $(R, R) \quad G[B 97-D /$ def2-TZVP $]=-3864.567330$

$\begin{array}{lrrr}\text { TS257(R, R) } \text { G }[\mathrm{B} 97-\mathrm{D} / \mathrm{def}-\mathrm{TZVP}]=-3864.567330 \\ \mathrm{P} & 0.2630360 & 0.5264130 & 0.9225220 \\ \mathrm{O} & 0.8196580 & -0.8648110 & 1.0836970 \\ \mathrm{O} & -0.4293370 & 1.1481130 & 2.1044290 \\ \mathrm{O} & -0.7116050 & 0.4321370 & -0.3863810 \\ \mathrm{O} & 1.4021390 & 1.5813450 & 0.4590010 \\ \mathrm{C} & -1.0258340 & 1.5902170 & -1.0985200 \\ \mathrm{C} & -0.0181190 & 2.2037420 & -1.8680230 \\ \mathrm{C} & -2.3379970 & 2.0686110 & -1.0526880 \\ \mathrm{C} & -2.6205100 & 3.2482520 & -1.7552890 \\ \mathrm{H} & -3.6349310 & 3.6424350 & -1.7276140 \\ \mathrm{C} & 2.0301160 & 1.2910780 & -0.7605390 \\ \mathrm{C} & 3.3041410 & 0.7161270 & -0.7452180 \\ \mathrm{C} & 1.3277910 & 1.5765740 & -1.9455310 \\ \mathrm{C} & 3.8692530 & 0.3913170 & -1.9879160 \\ \mathrm{H} & 4.8564710 & -0.0665780 & -2.0055900 \\ \mathrm{C} & -3.4191120 & 1.3413630 & -0.3365660 \\ \mathrm{C} & 4.0471800 & 0.4340530 & 0.5104940 \\ \mathrm{C} & -3.8767200 & 0.1029940 & -0.8200180 \\ \mathrm{C} & -3.3606650 & -0.6323200 & -2.0344040\end{array}$

3.0261320

2.3219400

3.5333870

.2879910

2.0620520

4.2252180

0.5552640

0.3528090

0.0981470

1.8635020

0.5092470

$-0.4122780$

0.3741060

0.2571160

0.8754280

$-0.6340200$

1.0908920

1. 5513290

$-1.4538150$

-1.4538150
-0.8157270

.9507330

3.7628090
5.8854770

$-2.1437750$

0.9650270

-1.0146140
-2.1721760

$-3.0657850$

0.9639520

0246640

$-2.8521310$

-

3.8951020

$-3.3606650$

$-0.6323200$

$-2.0344040$ 


\begin{tabular}{|c|c|c|}
\hline $\begin{array}{l}-5.3407930 \\
-4.9532050 \\
-2.9109920 \\
-2.5484790 \\
-2.0757720 \\
-4.1026090 \\
-4.3632870 \\
-3.8313330 \\
-6.1781060 \\
-2.5287880 \\
-5.7264380 \\
-5.9670180 \\
-6.6251880 \\
-4.5352780 \\
-4.1803960 \\
-4.8445850 \\
-4.0504790 \\
-3.7050330 \\
-5.6902450 \\
-5.1288760 \\
-4.9694430 \\
-4.7443210 \\
-5.2228630 \\
-6.1558880 \\
-6.5020870 \\
-7.0051080 \\
-6.5258090 \\
-2.8634150 \\
-4.5453400 \\
-4.9343940 \\
-4.2081190 \\
-3.3663410 \\
-3.1919580 \\
-2.4399440 \\
-5.5862620 \\
-6.4260430 \\
5.2040510 \\
5.5337970 \\
4.1141320 \\
4.4223990 \\
6.3046930 \\
6.4839580 \\
7.2812690 \\
5.4615760 \\
5.2485490 \\
6.0070030\end{array}$ & $\begin{array}{r}-1.8496620 \\
-0.5559820 \\
-2.0460280 \\
-2.6049690 \\
-1.9427090 \\
-2.7874030 \\
-3.6959160 \\
-3.0955550 \\
-2.3308440 \\
-0.1034100 \\
-1.5246730 \\
-2.4613720 \\
-0.8966810 \\
-0.7962890 \\
-1.3748020 \\
0.1902460 \\
1.8976370 \\
3.2080740 \\
1.9714350 \\
1.2331140 \\
4.1105010 \\
5.0575110 \\
4.3435850 \\
3.3773370 \\
3.9486150 \\
3.2692170 \\
1.4210950 \\
3.7009660 \\
2.1719780 \\
2.7364410 \\
1.1966130 \\
2.9345990 \\
3.8972090 \\
2.3581710 \\
0.0126570 \\
-0.4960110 \\
1.2068080 \\
2.4349660 \\
2.9446600 \\
1.4744860 \\
3.4310980 \\
4.3584870 \\
3.0072580 \\
3.7156100 \\
4.7889600 \\
3.4065960\end{array}$ & $\begin{array}{l}-0.8703810 \\
-0.1978630 \\
-1.5857010 \\
-2.4568970 \\
-0.8905780 \\
-0.9087720 \\
-1.4675710 \\
0.1072750 \\
-0.3502050 \\
-2.5072800 \\
-2.3413650 \\
-2.8615700 \\
-2.3530890 \\
-3.0391350 \\
-3.9022430 \\
-3.4043200 \\
0.7874350 \\
1.4543290 \\
2.5926550 \\
1.4036030 \\
1.4152220 \\
1.9229040 \\
0.3739450 \\
2.1185620 \\
2.9899810 \\
1.4334170 \\
3.0424560 \\
0.9599240 \\
3.6236770 \\
4.4814050 \\
3.9929900 \\
2.9458800 \\
3.4444930 \\
3.0041500 \\
0.9077810 \\
1.3825480 \\
2.5197650 \\
3.3307970 \\
1.2160940 \\
1.3798100 \\
2.4191940 \\
2.9794720 \\
2.1564150 \\
1.1371740 \\
1.0444280 \\
0.2373420\end{array}$ \\
\hline
\end{tabular}

3.5196410 6. 1333870 3. 3576020 3.1933030 2. 3774700 4.2008650 4.4279540 3.6480810 5.2489050 5.6226500 4.0995140 4.4494720 4.3167920 4.5739840 3. 8106210 3. 3960870 3. 1749020 2. 4476980 3.4477860 6.2692380 5.4210140 5.1808510 5.9215500 6. 3447190 6.5829090 7.2911070 5.6258310 6.2445610 1.9104590 1.2105860 1.2528340 2.6837930 3.7259360 3.1875520 1.7191830 0.8516170 3.0078870 3.9354200 0.1742920 0.5792090 2.6708210 4.6710120 $-0.3296780$ 0.6799610 0.6140180 $-0.8332880$

\begin{tabular}{rr}
3.1402620 & 0.3204890 \\
2.1772410 & 4.2124160 \\
3.4354840 & 2.4810330 \\
4.5177420 & 2.3918420 \\
2.9521040 & 2.5265100 \\
3.1118010 & 3.7539520 \\
4.0280960 & 4.3152190 \\
2.4401880 & 4.4214440 \\
-1.1315530 & 1.9533540 \\
-2.5833620 & 2.1201600 \\
-2.1380400 & 0.0625370 \\
-0.8783240 & 0.8225400 \\
-3.4102330 & 2.2619680 \\
-4.4765460 & 2.3206540 \\
-3.1382360 & 3.1960900 \\
-3.1261710 & 1.0364520 \\
-4.0536590 & 0.4924610 \\
-2.6942050 & 1.3610780 \\
-1.9302850 & -0.7895740 \\
-2.7264310 & 2.9945690 \\
-2.8050220 & -0.4081650 \\
-3.7520320 & -0.9098120 \\
-2.1590820 & -1.1398120 \\
-3.0554240 & 0.8264340 \\
-4.1232250 & 0.9211810 \\
-2.5121140 & 0.7187040 \\
-0.0921080 & 2.8035110 \\
-0.2922220 & 3.6792620 \\
1.2020170 & -3.1702490 \\
1.2910140 & -4.5034650 \\
-0.0850340 & -5.2160750 \\
-0.7005760 & -5.2629070 \\
0.1751860 & -4.5217400 \\
0.6086450 & -3.1825380 \\
2.0350910 & -5.1355030 \\
0.0284850 & -6.2309010 \\
-0.8413430 & -6.3016990 \\
1.0639220 & -5.1375470 \\
1.6203270 & -4.3899000 \\
-0.7626660 & -4.6770290 \\
-1.6914740 & -4.7918010 \\
-0.3679200 & -4.4032190 \\
3.3991970 & -2.5417880 \\
4.2344590 & -3.2899820 \\
5.7052880 & -2.8089310 \\
6.2822030 & -2.8174520 \\
& \\
\hline
\end{tabular}




\begin{tabular}{|c|c|c|c|}
\hline$c$ & 1001070 & ᄃ & -32010350 \\
\hline c & $\begin{array}{l}-1.8918740 \\
-1.6366510\end{array}$ & $\begin{array}{l}5.2172340 \\
3.9202040\end{array}$ & $\begin{array}{l}-3.2010350 \\
-2.4772440\end{array}$ \\
\hline $\mathrm{H}$ & 0.4556230 & 4.2016310 & -4.3674700 \\
\hline $\mathrm{H}$ & 1.2731170 & 6.3154020 & -3.4389300 \\
\hline $\mathrm{H}$ & -0.9066060 & 7.1238230 & -3.5175870 \\
\hline $\mathrm{H}$ & -1.8282100 & 5.0418110 & -4.2867080 \\
\hline $\mathrm{H}$ & 1.6928540 & 3.8426970 & -3.1618260 \\
\hline $\mathrm{H}$ & 1.0180530 & 5.7426980 & -1.7898200 \\
\hline $\mathrm{H}$ & -1.0775110 & 6.6708510 & -1.8212790 \\
\hline $\mathrm{H}$ & -2.9029830 & 5.5865100 & -2.9921290 \\
\hline C & -0.2136290 & -3.7110060 & -0.3666550 \\
\hline 0 & 0.5040340 & -2.6519110 & -0.6133130 \\
\hline C & -0.3603050 & -4.5989970 & -1.5547140 \\
\hline C & -1.3326680 & -5.6148040 & -1.5996710 \\
\hline C & 0.4682380 & -4.4023550 & -2.6792200 \\
\hline C & -1.4757210 & -6.4094910 & -2.7355380 \\
\hline $\mathrm{H}$ & -1.9822700 & -5.7615670 & -0.7419120 \\
\hline C & 0.3284330 & -5.2057480 & -3.8098230 \\
\hline $\mathrm{H}$ & 1.2201910 & -3.6201490 & 588230 \\
\hline C & -0.6451380 & -6.2098410 & -3.8441390 \\
\hline $\mathrm{H}$ & -2.2390480 & -7.1839160 & -2.7580490 \\
\hline $\mathrm{H}$ & 0.9791710 & -5.0465430 & -4.6668630 \\
\hline $\mathrm{H}$ & -0.7568830 & -6.8321730 & -4.7294540 \\
\hline $\mathrm{H}$ & 0.6037320 & -1.9513690 & 0.1648280 \\
\hline S & -0.9125800 & -4.0806780 & 1.1205290 \\
\hline C & -2.3444080 & -1.3484470 & 2.6113710 \\
\hline C & -1.1293200 & -2.0219270 & 3.0908520 \\
\hline $\mathrm{H}$ & -2.3248760 & -0.9621750 & \\
\hline $\mathrm{H}$ & -0.1897180 & -1.7439850 & 2.6278060 \\
\hline 0 & -1.8027680 & -0.3718520 & 3.5701910 \\
\hline $\mathrm{H}$ & -1.2114730 & 0.2926980 & 3.0012280 \\
\hline C & -3.6581760 & -2.5582670 & 4.4218080 \\
\hline C & -2.5283550 & -3.5957150 & 4.5021940 \\
\hline C & -1.1564450 & -2.9369200 & 4.2730990 \\
\hline C & -3.6903630 & -1.8962480 & 3.0349350 \\
\hline H & -4.6263940 & -3.0345970 & 4.6172990 \\
\hline $\mathrm{H}$ & -2.5293560 & -4.0998050 & 5.4758030 \\
\hline $\mathrm{H}$ & -0.8685870 & -2.3399540 & 5.1514250 \\
\hline $\mathrm{H}$ & -3.9822600 & -2.6342120 & 2.2753980 \\
\hline $\mathrm{H}$ & -4.4216170 & -1.0835330 & 2.9877280 \\
\hline H & -3.5088410 & -1.7894120 & 5.1919850 \\
\hline $\mathrm{H}$ & -2.6884860 & -4.3648070 & 3.7335440 \\
\hline $\mathrm{H}$ & -0.3617720 & -3.6803130 & 4.1387740 \\
\hline
\end{tabular}

TS258(R, R) G[B97-D/def2-TZVP $]=-3864.566972$$$
\mathrm{P}
$$

0.2580070

0.3932170

1.0214870

0.7640060

$-0.4512370$

$-0.6871310$

1.4485140

$-0.9701970$

0.0634760

$-2.2735370$

$-2.4888560$

$-3.4942340$

2.0694640

3. 3169280

1. 3785340

3.8389230

4.8074840

$-3.3923990$

4.0655280

$-3.7992930$

-3. 1996850

$-5.2012680$

$-4.8921880$

$-2.7326650$

$-2.3173600$

$-1.9315190$

$-3.9332800$

$-4.1302360$

$-3.7095920$

$-6.0512010$

$-2.3601060$

$-5.5112810$

$-5.6770660$

$-6.4345600$

$-4.3120260$

$-3.8904790$

$-4.6365490$

$-4.0970250$

$-3.8144230$

$-5.8259510$

$-5.1906800$

$-5.0939920$

$-4.9138700$

$-5.2993000$

$-6.2996240$

$-6.7070640$

$-7.1067750$

$-6.6696750$

$-2.9610010$

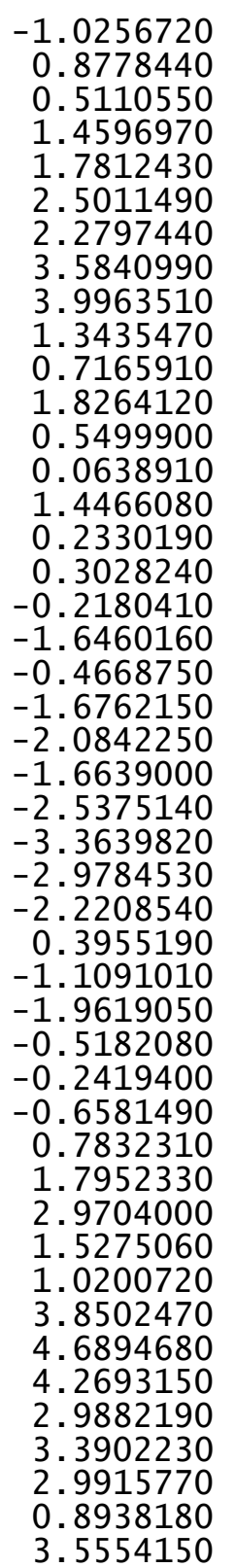

1.0083370

2.2576120

$-0.3105460$

0.7392300

$-0.8164950$

$-1.4308210$

$-0.7088860$

$-1.1606120$

$-1.0883410$

$-0.5132750$

$-0.6023560$

$-1.6321380$

$-1.8891800$

$-1.9918450$

$-0.1962540$

0.5859470

$-0.9089990$

$-2.1941180$

$-1.3573220$

$-0.4687510$

$-1.9496790$

$-2.8794080$

$-1.2099430$

$-1.4559460$

$-2.1509020$

$-0.4775480$

$-0.9696250$

$-2.5299080$

$-2.7833190$

$-3.4548480$

$-2.7596970$

$-3.2792290$

$-4.2037870$

$-3.4937080$

0.9677770

1.8742380

2.6693660

1. 3999650

1.9342390

2.6187410

0.9417000

2. 4265210

3.3636310

1.6844730

2.9696810

1. 5207780 


$\begin{array}{ll}\mathrm{C} & -4.7384570 \\ \mathrm{H} & -5.1816020 \\ \mathrm{H} & -4.3996820 \\ \mathrm{C} & -3.5418620 \\ \mathrm{H} & -3.4110740 \\ \mathrm{H} & -2.6082840 \\ \mathrm{C} & -5.5954920 \\ \mathrm{H} & -6.4457390 \\ \mathrm{C} & 5.2562260 \\ \mathrm{C} & 5.6234040 \\ \mathrm{C} & 4.2239670 \\ \mathrm{C} & 4.4810400 \\ \mathrm{C} & 6.4291120 \\ \mathrm{H} & 6.6395570 \\ \mathrm{H} & 7.3912150 \\ \mathrm{C} & 5.5981570 \\ \mathrm{H} & 5.4231920 \\ \mathrm{H} & 6.1340460 \\ \mathrm{H} & 3.6377850 \\ \mathrm{H} & 6.2126190 \\ \mathrm{C} & 3.4819440 \\ \mathrm{H} & 3.3506230 \\ \mathrm{H} & 2.4879660 \\ \mathrm{C} & 4.3136220 \\ \mathrm{H} & 4.5724600 \\ \mathrm{H} & 3.7382120 \\ \mathrm{C} & 5.2295530 \\ \mathrm{C} & 5.5633820 \\ \mathrm{C} & 4.0316630 \\ \mathrm{C} & 4.4284310 \\ \mathrm{C} & 4.2360580 \\ \mathrm{H} & 4.4637790 \\ \mathrm{H} & 3.7513670 \\ \mathrm{C} & 3.3066710 \\ \mathrm{H} & 3.0359510 \\ \mathrm{H} & 2.3828710 \\ \mathrm{H} & 3.3775920 \\ \mathrm{H} & 6.2150660 \\ \mathrm{C} & 5.3257730 \\ \mathrm{H} & 5.0498970 \\ \mathrm{H} & 5.8366200 \\ \mathrm{C} & 6.2572860 \\ \mathrm{H} & 6.4695440 \\ \mathrm{H} & 7.2153100 \\ \mathrm{C} & 5.6408800 \\ \mathrm{H} & 6.2579940 \\ & \end{array}$

$\begin{array}{rr}1.5565870 & 3.7796350 \\ 1.9588670 & 4.7003590 \\ 0.5362160 & 3.9917390 \\ 2.4390570 & 3.3090940 \\ 3.2996950 & 3.9782350 \\ 1.8704590 & 3.3128500 \\ -0.1028940 & 0.6791230 \\ -0.6962680 & 1.0171410 \\ 0.6764630 & 2.6743350 \\ 1.7655890 & 3.6510070 \\ 2.6158110 & 1.6346410 \\ 1.1272210 & 1.5892360 \\ 2.8534750 & 2.8877030 \\ 3.6859480 & 3.5725440 \\ 2.4386160 & 2.5641770 \\ 3.3439640 & 1.6610380 \\ 4.4265040 & 1.7198220 \\ 3.1442340 & 0.7252710 \\ 2.9554740 & 0.7772210 \\ 1.3655670 & 4.4851700 \\ 2.9472000 & 2.9581970 \\ 4.0356220 & 3.0231170 \\ 2.4911960 & 2.9375360 \\ 2.4210990 & 4.1690430 \\ 3.2419190 & 4.8513550 \\ 1.6824130 & 4.7394840 \\ -1.5561950 & 1.7775900 \\ -3.0268850 & 1.7305320 \\ -2.2505140 & -0.2206350 \\ -1.1219550 & 0.7039280 \\ -3.8314500 & 1.7704680 \\ -4.9006050 & 1.6620900 \\ -3.6916280 & 2.7442100 \\ -3.3410010 & 0.6197040 \\ -4.1723980 & -0.0441380 \\ -2.9240820 & 1.0252870 \\ -1.9038320 & -1.0243310 \\ -3.3105270 & 2.5659600 \\ -2.8845440 & -0.7996660 \\ -3.7461750 & -1.4224560 \\ -2.1593040 & -1.4448990 \\ -3.3283750 & 0.3725970 \\ -4.4040530 & 0.3123770 \\ -2.7967860 & 0.3279450 \\ -0.6612940 & 2.7650300 \\ -1.0026840 & 3.5971710 \\ -4 & \end{array}$

3.1475200
1.8972280
3.7790550
2.7624950
1.8603470
1.0553480
3.2873620
4.5226250
4.3364390
2.4847220
1.1716300
0.5001670
0.2901060
2.1400930
-0.1673980
-1.4604650
0.9918090
0.5506430
-0.5473850
-1.7842660
1.4178940
1.5578050
1.6921050
-0.1711410
-0.8176740
-2.5212360
-2.2725710
0.1621330
-0.3145030
0.4263320
-0.5126020
-1.5368430
0.3188180
-1.7285400
-2.1886130
0.1316330
1.1111800
-0.8941350
-2.5326930
0.7864430
-1.0433060
0.5406130
-0.9876920
-2.4124900
-1.2324190
-2.3313640

0.9370980

1. 5735350

0.6629550

0.6387010

1.8746740

1.8591480

0.5981680

1. 4498870

$-0.2821800$

2.7803830

1.9163940

2. 7941670

1.0680660

$-0.2659330$

3. 8402740

4.3801940

4. 7140360

5.9853650

6.7024520

5.8014800

6.6407580

5. 0037160

4.1413320

6.9313800

7.6546280

6.2313070

5.7636990

5.7198830

$-3.6899810$

$-2.6265490$

$-4.4279380$

$-5.3809690$

$-4.1488200$

$-6.0329290$

$-5.5910840$

$-4.8121090$

$-3.4139600$

$-5.7535560$

$-6.7582720$

$-4.5924890$

$-6.2652810$

$-2.0111840$

$-1.6332470$

$-2.3648290$

$-1.1477930$
$-3.0414300$

$-2.9223290$

$-4.3970850$

$-5.5455870$

$-5.4618280$

$-4.1567980$

$-6.5088280$

$-4.6033160$

$-4.3508740$

$-5.5000360$

$-6.3154690$

$-4.0446220$

$-4.2208060$

$-5.4702040$

$-1.8164080$

$-1.6781760$

$-2.2703320$

$-3.0064040$

$-2.2132070$

$-2.1108660$

$-3.1577980$

$-1.3698370$

$-2.8847430$

$-1.2049320$

$-2.6877100$

$-1.4195220$

$-3.0982880$

$-4.0017140$

$-0.7267940$

$-0.8648140$

$-2.0058660$

$-2.1539520$

$-3.1102450$

$-3.3705200$

$-1.3114000$

$-4.3219970$

$-3.0107650$

$-4.4586170$

$-3.4713120$

$-5.1623470$

$-5.4069910$

$-0.0205090$

0.7217530

2. 3858310

2.8639470

1.4109550 


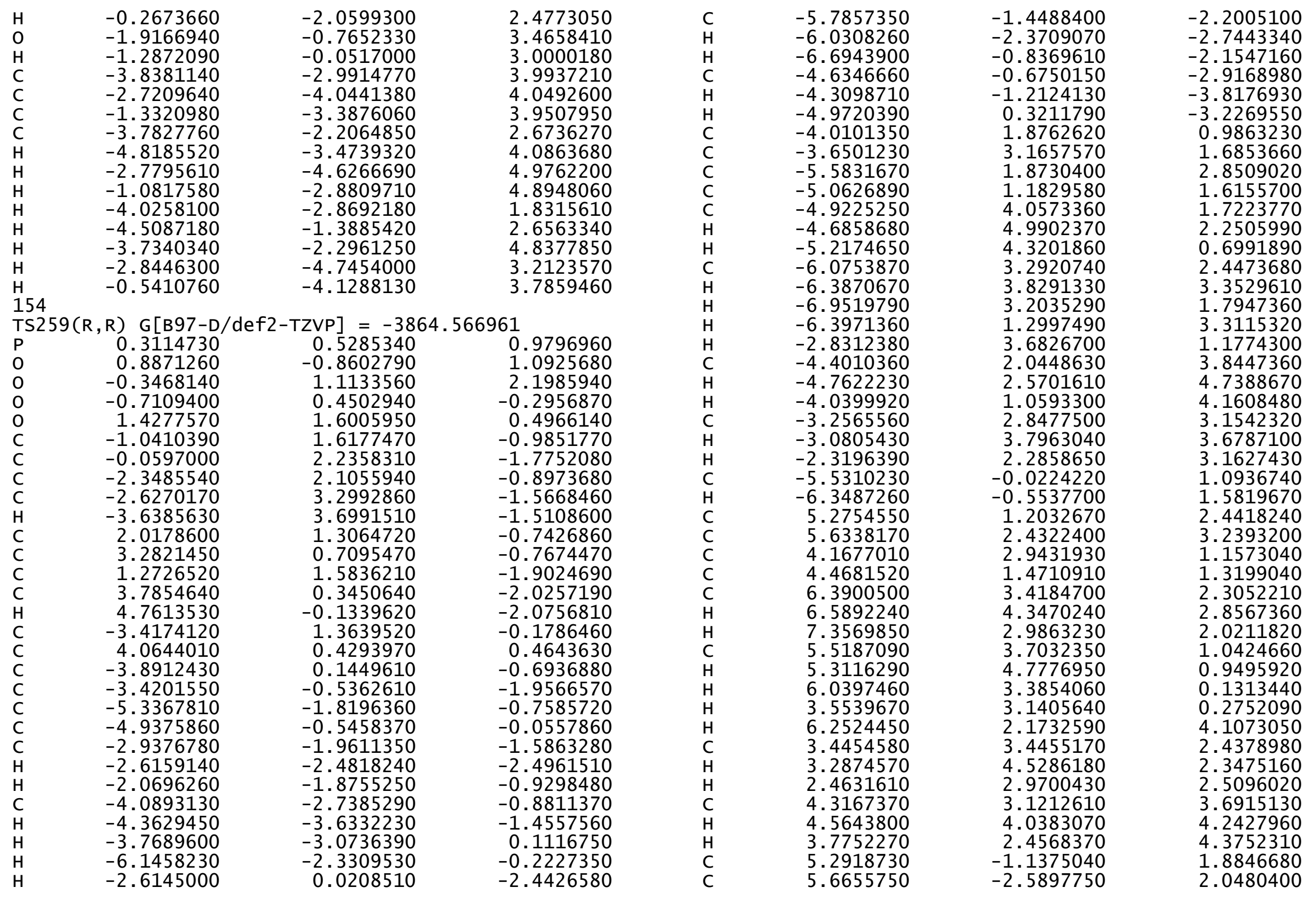




$\begin{array}{rrr}4.0971300 & -2.1467220 & 0.0244540 \\ 4.4675610 & -0.8846510 & 0.7715580 \\ 4.3626110 & -3.4150260 & 2.2202990 \\ 4.6202730 & -4.4814210 & 2.2750080 \\ 3.8781560 & -3.1406410 & 3.1652190 \\ 3.4149760 & -3.1319960 & 1.0156790 \\ 3.1806100 & -4.0598020 & 0.4778270 \\ 2.4744370 & -2.6993690 & 1.3613460 \\ 3.4257500 & -1.9424570 & -0.8125470 \\ 6.3313330 & -2.7312030 & 2.9082330 \\ 5.4067200 & -2.8175610 & -0.4741180 \\ 5.1532570 & -3.7659650 & -0.9664230 \\ 5.8912160 & -2.1752770 & -1.2197110 \\ 6.3586490 & -3.0648170 & 0.7394670 \\ 6.5999250 & -4.1322470 & 0.8310160 \\ 7.3018660 & -2.5209830 & 0.6089090 \\ 5.6948340 & -0.0971400 & 2.7214680 \\ 6.3326060 & -0.2978030 & 3.5832960 \\ 1.7857360 & 1.1545900 & -3.1390400 \\ 1.0081690 & 1.1772570 & -4.4308790 \\ 0.9780590 & -0.2459470 & -5.0446690 \\ 2.3971210 & -0.8807110 & -5.1574710 \\ 3.4969570 & 0.0200250 & -4.5359590 \\ 3.0485210 & 0.5336890 & -3.1923120 \\ 1.4963050 & 1.8575400 & -5.1458460 \\ 0.5007960 & -0.1984680 & -6.0314010 \\ 2.6475210 & -1.0771480 & -6.2075570 \\ 3.6753310 & 0.8684330 & -5.2146460 \\ -0.0110470 & 1.5429380 & -4.2761310 \\ 0.3420980 & -0.8731150 & -4.4081140 \\ 2.4089820 & -1.8467460 & -4.6373890 \\ 4.4406270 & -0.5308910 & -4.4473220 \\ -0.3588840 & 3.4635470 & -2.4093630 \\ 0.7207230 & 4.2112130 & -3.1739290 \\ 0.3992610 & 5.6982860 & -3.3735390 \\ -1.0194770 & 5.8504660 & -3.9338480 \\ -2.0348960 & 5.3373120 & -2.9057370 \\ -1.6565750 & 3.9985030 & -2.2923850 \\ 1.1400170 & 6.1451460 & -4.0489090 \\ 0.8467420 & 3.7525820 & -4.1645970 \\ 1.6812210 & 4.0865290 & -2.6589720 \\ -1.1022670 & 5.2658080 & -4.8625060 \\ -1.2377800 & 6.8967070 & -4.1834510 \\ -3.0354780 & 5.2594850 & -3.3525900 \\ -2.1182050 & 6.0771290 & -2.0934930 \\ 0.4678580 & 6.2278690 & -2.4115910\end{array}$
$-0.1814030$
0.5607070
$-0.4083220$
$-1.3537780$
0.3046060
$-1.5835410$
$-1.9159590$
0.0762510
1.0321270
$-0.8699170$
$-2.3242560$
0.6347990
$-1.0517200$
0.6758310
$-0.8360730$
$-2.2348880$
$-1.0065240$
$-2.2397170$
$-0.0783820$
$-1.6790360$
$-1.1075330$
$-3.5012540$
$-2.3663730$
$-1.0024390$
$-3.5677670$
$-4.4629170$
$-2.3416970$
$-0.6901700$
$-3.8702980$
$-4.3063470$
$-3.3380590$
$-2.5429120$
$-0.2107420$

154

TS260 (R,R) G[B97-D/def2-TZVP] $=-3864.566755$

\begin{tabular}{lrrr} 
PS & \multicolumn{3}{c}{ P R, $\mathrm{R}) \mathrm{G}[\mathrm{B} 97-\mathrm{D} / \mathrm{def2}-\mathrm{TZVP}]=-3864.566755$} \\
$\mathrm{O}$ & 0.2617780 & 0.3967740 & 1.0504150 \\
$\mathrm{O}$ & 0.7643970 & -1.0232040 & 1.0257030 \\
$\mathrm{O}$ & -0.4422570 & 0.8734790 & 2.2925960 \\
$\mathrm{O}$ & -0.6917520 & 0.5244830 & -0.2747940 \\
$\mathrm{C}$ & 1.4552050 & 1.4606850 & 0.7696310 \\
$\mathrm{C}$ & -0.9807690 & 1.7944220 & -0.7765790 \\
$\mathrm{C}$ & 0.0515140 & 2.5256070 & -1.3815270 \\
$\mathrm{C}$ & -2.2901690 & 2.2787070 & -0.6866100 \\
$\mathrm{H}$ & -2.5172820 & 3.5759520 & -1.1547420 \\
$\mathrm{C}$ & -3.5299340 & 3.9733860 & -1.1029830 \\
$\mathrm{C}$ & 2.0633890 & 1.3546600 & -0.4906290
\end{tabular}

$-0.4960540$

$-3.6150260$

$-2.5608720$

$-4.3870600$

$-5.4265980$

$-6.1012770$

$-5.6856760$

$-4.7250170$

$-3.2400690$

$-5.7541740$

$-6.8968930$

$-4.4481830$

$-1.9037560$

$-4.0861740$

$-1.4387950$

$-1.1239320$

$-1.8235570$

$-0.4944480$

0.1991190

$-2.7199120$

$-3.7546010$

$-2.0115870$

$-3.2072740$

$-4.2907720$

$-2.5207820$

$-2.7265020$

$-1.2044800$

$-1.9769930$

$-4.4984420$

$-3.8196430$

$-0.6804$

$-1.7514920$

$-1.8179470$

$-2.9196410$

$-3.0154810$

$-0.9256790$

$-4.1141780$

$-2.8868400$

$-4.1682470$

$-3.0513110$

$-5.0056530$

$-5.1026530$

0.1351910

0.9835410

2. 6388300

3.0662950

1.6323560

2. 5943240

3.6221780

3.0713970

4.4338850

4.4528330

4.2117190

3.0715670

4.6345490

5.4088200

5.1041360

2. 2942720

3.0679380

5.2263920

3.6631830

4.0266020
$-3.0838190$

1. 3546600

$-0.4906290$

8504660

(5)

5.2658080

6.2278690

$-2.4115910$ 


\begin{tabular}{|c|c|c|}
\hline $\begin{array}{l}3.3084490 \\
1.3639730 \\
3.8099130 \\
4.7764260 \\
3.4062530 \\
4.0786670 \\
3.8040340 \\
3.1943060 \\
5.1933550 \\
4.8950490 \\
2.7210630 \\
2.2976410 \\
1.9246920 \\
3.9201690 \\
4.1087270 \\
3.6999490 \\
6.0425070 \\
2.3555620 \\
5.4979930 \\
5.6564290 \\
6.4239710 \\
4.2999640 \\
3.8710740 \\
4.6281720 \\
4.1169480 \\
3.8428060 \\
5.8500450 \\
5.2082550 \\
5.1268340 \\
4.9530250 \\
5.3306490 \\
6.3300890 \\
6.7434420 \\
7.1341450 \\
6.6915340 \\
2.9913480 \\
4.7662400 \\
5.2143040 \\
4.4239630 \\
3.5715840 \\
3.4454160 \\
2.6359660 \\
5.6048470 \\
6.4533210 \\
5.3258820 \\
5.7257260\end{array}$ & $\begin{array}{r}0.7265010 \\
1.8532060 \\
0.5648720 \\
0.0781760 \\
1.4370910 \\
0.2383230 \\
0.2943090 \\
-0.2189710 \\
-1.6598580 \\
-0.4823500 \\
-1.6752560 \\
-2.0781070 \\
-1.6608730 \\
-2.5444320 \\
-3.3692090 \\
-2.9879440 \\
-2.2404930 \\
0.3999540 \\
-1.1194040 \\
-1.9706350 \\
-0.5326350 \\
-0.2453190 \\
-0.6572340 \\
0.7788450 \\
1.7778160 \\
2.9512870 \\
1.4955430 \\
0.9956820 \\
3.8246550 \\
4.6614690 \\
4.2474150 \\
2.9547190 \\
3.3511880 \\
2.9567390 \\
0.8567300 \\
3.5418090 \\
1.5265620 \\
1.9246220 \\
0.5071300 \\
2.4153200 \\
3.2735310 \\
1.8498390 \\
-0.1262650 \\
-0.7253520 \\
0.6758510 \\
1.7637810\end{array}$ & $\begin{array}{r}-0.6023430 \\
-1.5960440 \\
-1.8978830 \\
-2.0165400 \\
-0.1819440 \\
0.5702200 \\
-0.9005590 \\
-2.1836980 \\
-1.3626640 \\
-0.4681620 \\
-1.9408150 \\
-2.8690750 \\
-1.1959510 \\
-1.4573030 \\
-2.1565690 \\
-0.4792960 \\
-0.9819400 \\
-2.5120020 \\
-2.7885390 \\
-3.4638220 \\
-2.7678720 \\
-3.2754320 \\
-4.1985180 \\
-3.4891980 \\
0.9803610 \\
1.8914200 \\
2.6746900 \\
1.4055260 \\
1.9512330 \\
2.6402900 \\
0.9599940 \\
2.4352720 \\
3.3721450 \\
1.6898670 \\
2.9703530 \\
1.5420950 \\
3.7886960 \\
4.7088500 \\
3.9995420 \\
3.3247910 \\
3.9979140 \\
3.3288450 \\
0.6783430 \\
1.0106970 \\
2.6263650 \\
3.5915190\end{array}$ \\
\hline
\end{tabular}

4. 5208030

6.5085390

6.7473480

7.4565790

5.6370690

5. 4623940

6.1422000

3. 6506270

6. 3407850

3. 5625030

3. 4268510

2. 5709230

4.4344400

4.7162820

3.8782150

5. 2663510

5. 5913780

4.0167990

4. 4403720

4. 2611110

4.4802520

3. 8003710

3. 3072550

3. 0187390

2. 3943190

3. 3452230

6. 2615740

5. 2937230

4.9988750

5.7928150

6.2505200

6.4544030

7.2104970

5. 7086110

6. 3474670

1.8595730

1. 0217280

1.4463430

2.9631100

3. 6786140

3. 1014330

0.9091980

1.0967260

$-0.0351760$

3. 2430930
2. 6177760

1296790

2. 8504160

3.6802350

2. 4319370

3. 3478610

4.4296360

3. 1556540

2.9586770

1. 3626530

2. 9443060

4.0318180

2. 4825270

2. 4213830

3. 2441390

1.6849240

$-1.5563950$

$-3.0286450$

$-2.2432700$

$-1.1178090$

$-3.8259690$

$-4.8965200$

$-3.6823880$

$-3.3316980$

$-4.1617420$

$-2.9117180$

$-1.8925830$

$-3.3164090$

$-2.8823180$

$-3.7414480$

$-2.1587200$

$-3.3332260$

$-4.4101610$

$-2.8075880$

$-0.6632430$

-1.0071080

1.6043350

1.9576490

1. 1938810

1. 3051630

0.6109210

0.9574930

1.5956830

3. 0346980

1. 7710880

2. 3690800
1. 6211980

1.5645020

2.8022240

3.4810040

2.4437140

1. 6068450

1.6790030

0.6525970

0.7833940

4.4062570

2. 9676160

3. 0378930

2. 9775930

4.1511640

4.8219160

4.7430850

1.7297760

1.6737340

$-0.2392630$

0.6770670

1.7458140

1.6335960

2. 7305640

0.6168380

$-0.0410610$

1. 0435410

$-1.0266760$

2. 4929360

$-0.8499240$

$-1.4674790$

$-1.5060970$

0.2992650

0.2332010

0.2319130

2.7054620

3.5200080

$-2.8970530$

$-4.1158170$

$-5.3774250$

$-5.5674290$

$-4.4019300$

$-3.0384860$

$-6.2460890$

$-4.3257500$

$-3.8884430$

$-5.5966500$ 


$\begin{array}{rrr}3.2780020 & 0.8567910 & -6.5184380 \\ 4.7521170 & 0.8443170 & -4.4100660 \\ 3.6011870 & -0.4795050 & -4.5402200 \\ 1.1693560 & 0.1331880 & -5.2807600 \\ -0.1894650 & 3.8597360 & -1.7729240 \\ -1.4930880 & 4.3815190 & -1.6643610 \\ 0.9653230 & 4.7585090 & -2.1826360 \\ 0.5237250 & 5.9912920 & -2.9802800 \\ -0.6122440 & 6.7108870 & -2.2434760 \\ -1.8331040 & 5.7880510 & -2.1326620 \\ 1.3804240 & 6.6614720 & -3.1273930 \\ 1.4565380 & 5.0993980 & -1.2562170 \\ 1.7276440 & 4.1943350 & -2.7252640 \\ -0.2649210 & 6.9878540 & -1.2369550 \\ -0.8924400 & 7.6375720 & -2.7608090 \\ -2.5862380 & 6.2222080 & -1.4617100 \\ -2.3089140 & 5.7162480 & -3.1242470 \\ 0.1728590 & 5.6814450 & -3.9767210 \\ -0.2981440 & -3.6797420 & -0.7263750 \\ 0.4382780 & -2.6125390 & -0.8576850 \\ -0.4888010 & -4.4130480 & -2.0092510 \\ -1.5132250 & -5.3641900 & -2.1673150 \\ 0.3496550 & -4.1304770 & -3.1073590 \\ -1.6983920 & -6.0108430 & -3.3877490 \\ -2.1704130 & -5.5766070 & -1.3295590 \\ 0.1691860 & -4.7887270 & -4.3228770 \\ 1.1422090 & -3.3969310 & -2.9999250 \\ -0.8570020 & -5.7281980 & -4.4695840 \\ -2.5029890 & -6.7345490 & -3.4964940 \\ 0.8293860 & -4.5666070 & -5.1583390 \\ -1.0011240 & -6.2357920 & -5.4209500 \\ 0.5483060 & -2.0006820 & -0.0097500 \\ -0.9742910 & -4.2205190 & 0.7173620 \\ -2.4051790 & -1.6415450 & 2.3933350 \\ -1.2271620 & -2.3760670 & 2.8716860 \\ -2.3201700 & -1.1499000 & 1.4219110 \\ -0.2608180 & -2.0688490 & 2.4902940 \\ -1.9139250 & -0.7804780 & 3.4813020 \\ -1.2825520 & -0.0632800 & 3.0240620 \\ -3.8374370 & -3.0119860 & 3.9852510 \\ -2.7200240 & -4.0645320 & 4.0381130 \\ -1.3311830 & -3.4062990 & 3.9509400 \\ -3.7763470 & -2.2174840 & 4.6711770 \\ -4.8180840 & -3.4955280 & 4.9603390 \\ -2.7825020 & -4.6541170 & -8996510 \\ -1.0858990 & -2.9059940 & \end{array}$

$\begin{array}{ll}\mathrm{H} & -4.0146620 \\ \mathrm{H} & -4.5030950 \\ \mathrm{H} & -3.7376380 \\ \mathrm{H} & -2.8392480 \\ \mathrm{H} & -0.5387780 \\ \mathrm{H} & \end{array}$
$-2.8745610$
$-1.4003030$
$-2.3228690$
$-4.7594070$
$-4.1457350$
2.6558100
4.8349330
3.1952260

1.8233890

TS261 $(R, R)$ G [B97-D/def2-TZVP $]=-3864.566740$

\begin{tabular}{|c|c|c|c|}
\hline Q & & ט & \\
\hline U & 0.7328770 & -1.1090790 & 0.9330400 \\
\hline 0 & -0.4576710 & 0.8355190 & 1462470 \\
\hline 0 & -0.6624170 & 0.4467500 & -0.4238310 \\
\hline 0 & 1.4651560 & 1.3655930 & 0.6571290 \\
\hline C & -0.8991230 & 1.7164230 & -0.9545820 \\
\hline C & 0.1611530 & 2.3932280 & -1.5736260 \\
\hline C & -2.1815300 & 2.2659890 & -0.8513090 \\
\hline C & -2.3536990 & 3.5638780 & -1.3387920 \\
\hline $\mathrm{H}$ & -3.3434850 & 4.0131390 & -1.2714760 \\
\hline C & 2.1304240 & 1.2479660 & -0.5689370 \\
\hline C & 3.4003590 & 0.6632350 & -0.5980290 \\
\hline C & 1.4768140 & 1.7087600 & -1.7209870 \\
\hline C & 4.0033710 & 0.5445030 & -1.8549710 \\
\hline H & 4.9995090 & 0.1086090 & -1.9086950 \\
\hline C & -3.3202260 & 1.5232380 & -0.2502840 \\
\hline C & 4.0794430 & 0.1650210 & 0.6258490 \\
\hline C & -3.8406450 & 0.3751090 & -0.87 \\
\hline C & -3.3448510 & -0.2549260 & -2.1 \\
\hline C & -5.4071580 & -1.4769470 & -1.1 \\
\hline C & -4.9631880 & -0.2847120 & -0.3 \\
\hline C & -2.979 & -1.7307740 & -1.8 \\
\hline $\mathrm{H}$ & -2.63 & -2.2 & -2.7 \\
\hline $\mathrm{H}$ & -2.15 & -1.7 & -1.1 \\
\hline C & -4.22 & -2.4 & -1.2 \\
\hline $\mathrm{H}$ & -4.52 & -3.2 & -1 \\
\hline $\mathrm{H}$ & -3.9 & 46700 & -0 \\
\hline $\mathrm{H}$ & -6.27 & -1.9638 & -0 . \\
\hline $\mathrm{H}$ & -2.47 & 0.2748350 & -2 \\
\hline C & -5.74 & -0.9785090 & -2 . \\
\hline $\mathrm{H}$ & -6.0289770 & -1.8413930 & -3.2 \\
\hline $\mathrm{H}$ & -6.6129940 & -0.3048070 & -2.5 \\
\hline C & -4.5081370 & -0.2469210 & -3.1840670 \\
\hline $\mathrm{H}$ & -4.1689850 & -0.7500530 & -4.0991440 \\
\hline $\mathrm{H}$ & -4.7575940 & 0.7879840 & -3.44672 \\
\hline C & -3.9365050 & 1.9859470 & 0.9 \\
\hline C & -3.52 & 3. 1821480 & 1. \\
\hline C & -5.60 & 1.9628260 & 2.70 \\
\hline$\sigma$ & -5 & 1.3 & 1 \\
\hline
\end{tabular}




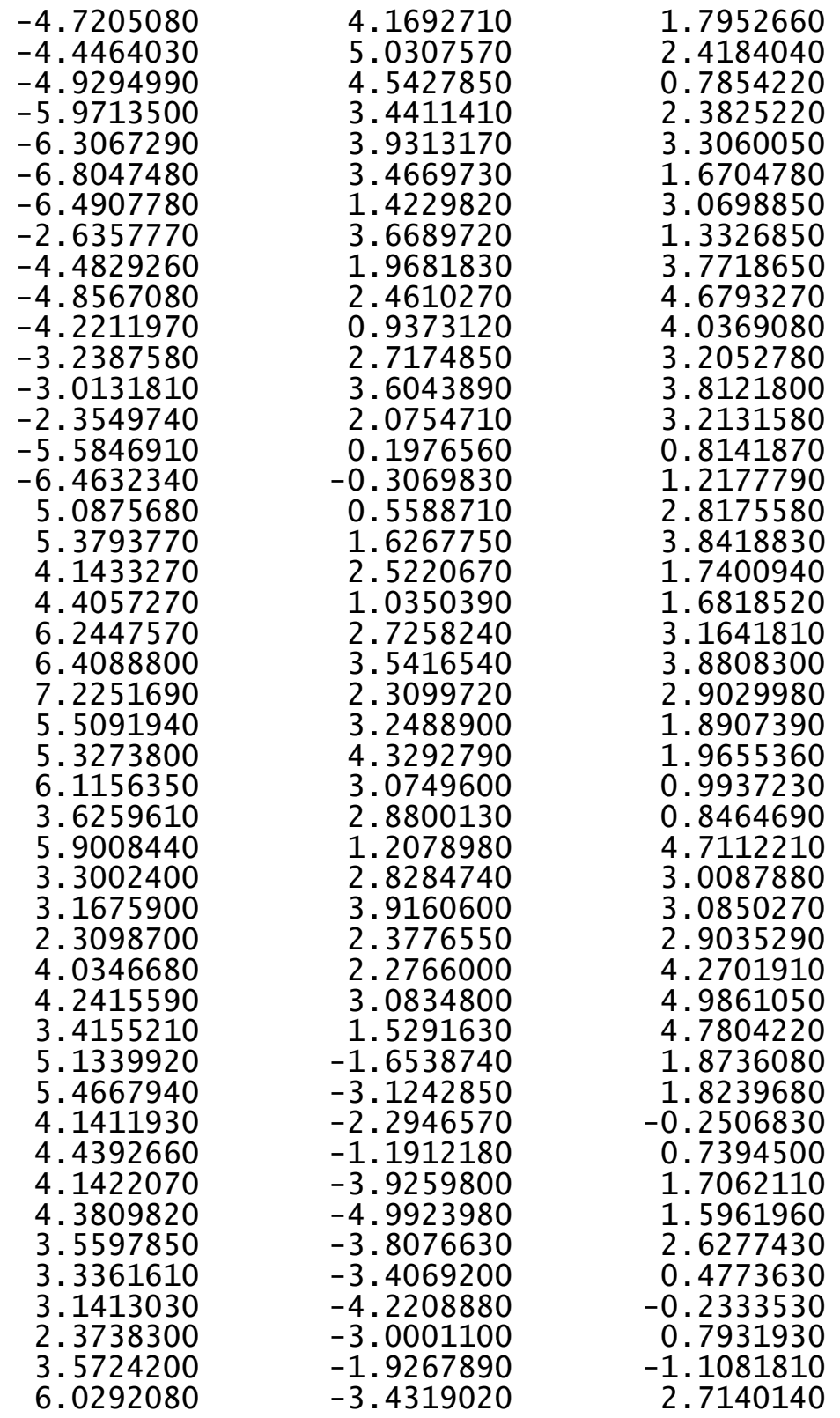

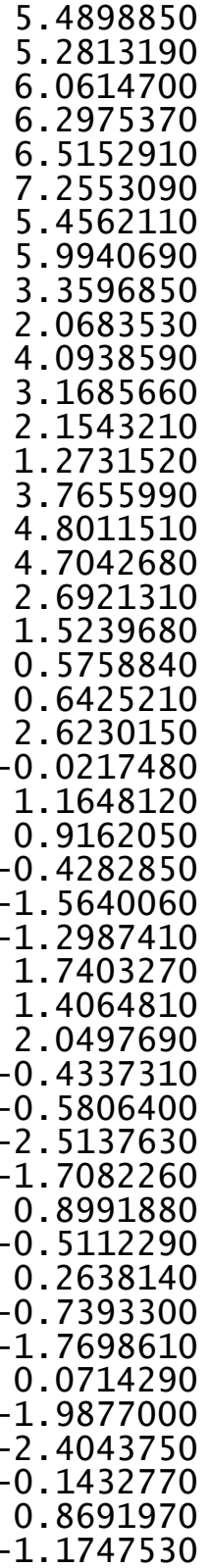

-2.9118440
-3.7549700
-2.1675380
-3.3905100
-4.4647400
-2.8609080
-0.7829470
-1.1456930
0.9178370
1.4777890
0.7354260
0.7039230
1.8517140
1.6868420
0.7667480
1.5725830
-0.1763030
2.8107840
1.8767830
2.5218920
0.7925290
-0.2514130
3.7354630
4.5542720
6.0675630
6.3999450
5.7627000
4.3164490
6.5931680
4.2882430
4.2840160
6.0035980
7.4848180
5.8208110
6.3453740
6.4009810
-3.7550140
-2.7196020
-4.5109370
-5.4623550
-4.2523850
-6.1318280
-5.6598040
-4.9317000
-3.5203780
-5.8711100

$-0.7123530$

$-1.3845810$

$-1.2802070$

0.5361630

0.4676870

0.6044200

2.9140000

3. 7908870

$-3.0385000$

$-2.9819540$

$-4.3578640$

$-5.5814900$

$-5.5041270$

$-4.2605140$

$-6.5005210$

$-4.4749860$

$-4.3112590$

$-5.4518860$

$-6.4024090$

$-4.1621780$

$-4.3969090$

$-5.6085670$

$-1.9801110$

$-2.4670010$

$-2.4065840$

$-3.0630520$

$-2.2533540$

$-1.8671130$

$-2.9055340$

$-3.5062330$

$-1.8773810$

$-4.0898100$

$-3.1291000$

$-2.8027130$

$-1.3295160$

$-1.3582500$

$-0.7463680$

$-0.9080640$

$-2.0103480$

$-2.1240550$

$-3.1351990$

$-3.3266310$

$-1.2652470$

$-4.3334210$

$-3.0625160$

$-4.4355370$ 


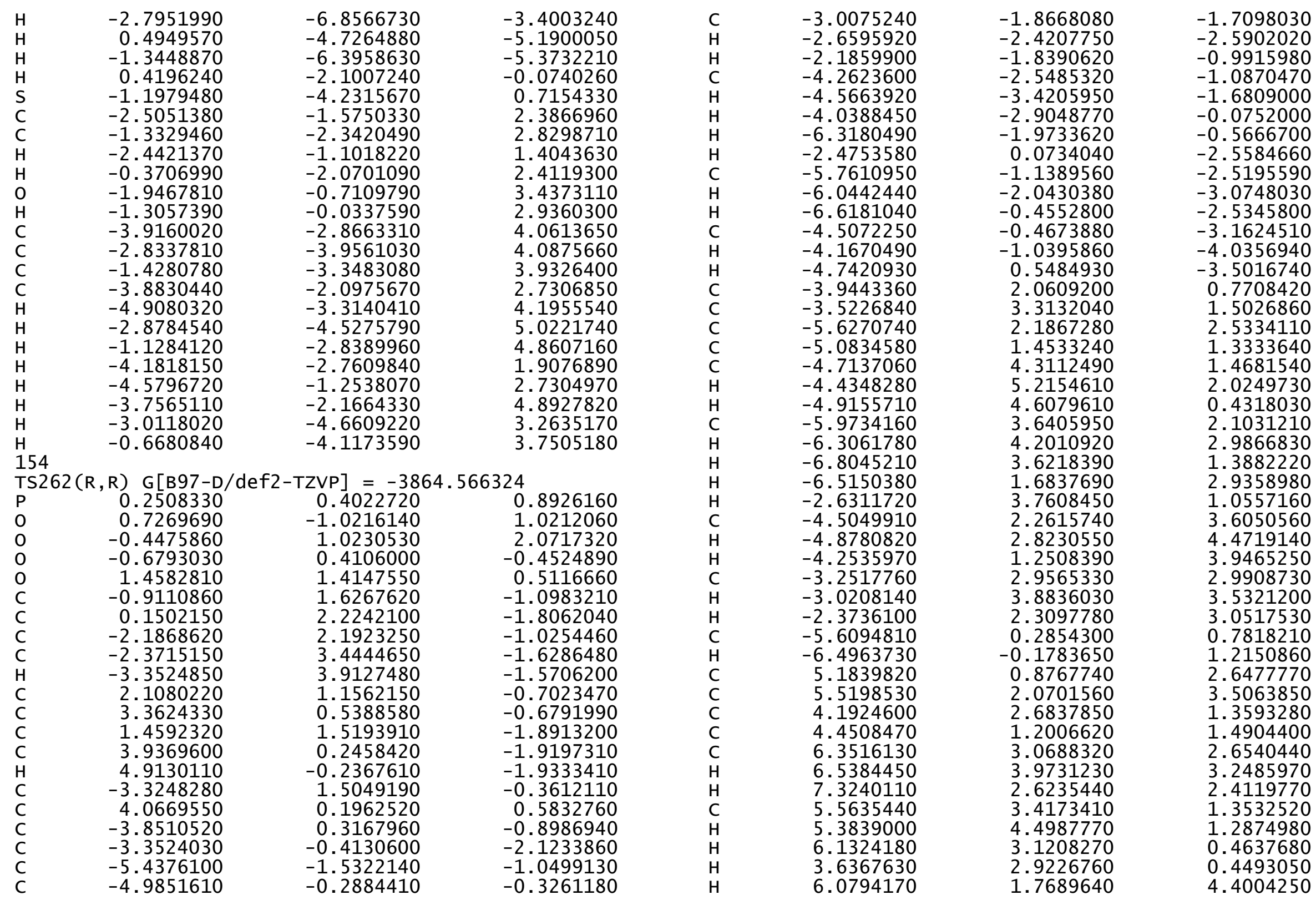




$\begin{array}{lrrr}\mathrm{C} & 3.4038110 & 3.1579940 & \\ \mathrm{H} & 3.2699360 & 4.2463630 & 2.6111970 \\ \mathrm{H} & 2.4112430 & 2.6986590 & 2.5492250 \\ \mathrm{C} & 4.1925170 & 2.7743530 & 3.9024130 \\ \mathrm{H} & 4.4261780 & 3.6676930 & 4.4969670 \\ \mathrm{H} & 3.5975760 & 2.1014480 & 4.5312620 \\ \mathrm{C} & 5.1675610 & -1.4463370 & 2.0202690 \\ \mathrm{C} & 5.4783630 & -2.9151370 & 2.1639540 \\ \mathrm{C} & 4.0405230 & -2.3633340 & 0.0709480 \\ \mathrm{C} & 4.4134190 & -1.1366630 & 0.8721280 \\ \mathrm{C} & 4.1353450 & -3.6899860 & 2.2466410 \\ \mathrm{H} & 4.3468670 & -4.7670240 & 2.2905380 \\ \mathrm{H} & 3.6108110 & -3.4161800 & 3.1700590 \\ \mathrm{C} & 3.2669810 & -3.3436140 & 0.9990190 \\ \mathrm{H} & 3.0279650 & -4.2499590 & 0.4274520 \\ \mathrm{H} & 2.3259100 & -2.8802730 & 1.3020630 \\ \mathrm{H} & 3.4266100 & -2.1087560 & -0.7966010 \\ \mathrm{H} & 6.0901670 & -3.1038240 & 3.0545800 \\ \mathrm{C} & 5.3498560 & -3.0706390 & -0.3742280 \\ \mathrm{H} & 5.0898020 & -3.9941290 & -0.9086880 \\ \mathrm{H} & 5.8993670 & -2.4267420 & -1.0717020 \\ \mathrm{C} & 6.2207890 & -3.3891530 & 0.8827670 \\ \mathrm{H} & 6.4087330 & -4.4683510 & 0.9595780 \\ \mathrm{H} & 7.1931860 & -2.8864570 & 0.8181160 \\ \mathrm{C} & 5.5519830 & -0.4430470 & 2.9094870 \\ \mathrm{H} & 6.1328740 & -0.6876300 & 3.7997110 \\ \mathrm{C} & 2.0402120 & 1.1555700 & -3.1282410 \\ \mathrm{C} & 1.2948760 & 1.4022410 & -4.4286900 \\ \mathrm{C} & 1.7858510 & 0.5153600 & -5.5806180 \\ \mathrm{C} & 3.3119440 & 0.6087260 & -5.6888580 \\ \mathrm{C} & 3.9540480 & 0.0401310 & -4.4173590 \\ \mathrm{C} & 3.2929820 & 0.5124300 & -3.1318980 \\ \mathrm{H} & 1.3045410 & 0.8299940 & -6.5154850 \\ \mathrm{H} & 1.4212910 & 2.4519060 & -4.7280640 \\ \mathrm{H} & 0.2197000 & 1.2631110 & -4.2605050 \\ \mathrm{H} & 3.5970490 & 1.6646830 & -5.8107380 \\ \mathrm{H} & 3.6824150 & 0.0657590 & -6.5678570 \\ \mathrm{H} & 5.0253990 & 0.2806910 & -4.3822150 \\ \mathrm{H} & 3.8884730 & -1.0592230 & -4.4511120 \\ \mathrm{H} & 1.4969660 & -0.5304530 & -5.3961720 \\ \mathrm{C} & -0.0618840 & 3.4941490 & -2.3727670 \\ \mathrm{C} & 1.0219400 & 4.3323300 & -3.00381100 \\ \mathrm{C} & 1.0613710 & 5.7329280 & -2.3428380 \\ \mathrm{C} & -0.3402710 & 6.4126440 & -2.2917790 \\ \mathrm{C} & -1.4622240 & 5.4933880 & -2.8400590 \\ \mathrm{C} & -1.3276100 & 4.1040140 & -2.2727080 \\ & & & \end{array}$
0.8066510
1.7731920
$-0.3339510$
$-1.3727470$
1. 9988090
1.4512090
$-0.5835450$
$-2.4492450$
$-0.4895860$
0.3098140
$-0.6954620$
$-1.7167470$
0.1273780
$-1.9120650$
$-2.3611460$
$-0.0659650$
0.9169230
$-1.0868950$
$-2.7114260$
0.5807400
$-1.2393610$
0.4460930
$-1.2273770$
$-2.5177120$
$-1.3468300$
$-2.4588360$
$-0.3861790$
$-1.9449410$
$-1.3021760$
$-3.9249450$
$-2.8469440$
$-1.4400530$
$-3.8963000$
$-4.9182230$
$-2.8906920$
$-1.1377440$
$-4.2047020$
$-4.5880280$
$-3.7574480$
$-3.0298600$

$-0.6820430$

154

TS263(R,R) G[B97-D/def2-TZVP] $=-3864.565740$

$\begin{array}{lrrr}\mathrm{P} & 0.2819090 & 0.4121920 & 0.9560310 \\ \mathrm{O} & 0.7819320 & -1.0057840 & 1.0608780 \\ \mathrm{O} & -0.3987770 & 1.0102340 & 2.1570420\end{array}$

$-4.0760750$

4.4607700

$5.4509540-3.9369580$

$3.8468730-2.9237480$

$5.6155080-1.3243740$

$6.6712080-1.2540650$

$5.9123590-2.6110280$

$-3.7846130$

$\begin{aligned} & -0.78761300 \\ & -4.6364500\end{aligned}-0.6927600$

$-4.6364840$

$-5.6031950$

$-4.4570410$

$-6.3666460$

$-5.7385000$

$-5.2286400$

$-6.1840950$

$-7.1036410$

$-5.0831730$

$-6.7820150$

$-2.0940880$

$-4.1277580$

$-2.0906210$

$-0.9441240$

$-1.8546290$

$-0.4202690$

0.2157130

$-3.6123850$

$-3.0199310$

$-1.8391200$

$-2.9555310$

$-4.1244850$

$-2.5510940$

$-0.9928440$

$-1.7716940$

$-4.3665960$

$-1.6782990$

$-1.7313080$

$-2.8098270$

$-2.8805780$

$-0.8678130$

$-2.7838510$

$-3.9960870$

$-2.9078990$

$-4.8171360$

$-4.8921040$

0.0916770

1.0017140

2.4934030

2.9821060

1.4809950

2.5391610

3.4799420

2.9315960

4.2575190

4.3474040

4.1505130

2.8803310

4.4252080

5.3158670

5.0420920

2. 1026510

2.8303270

5.0419790

3. 5692750

4.0181020 $\begin{array}{ll}6.3632310 & -2.8901920 \\ 7.3485830 & -2.8643790\end{array}$

$-1.3496240$

$-3.9546910$

$-0.3987770$

1. 0102340

2.1570420

5.4933880

$-2.2727080$ 


$\begin{array}{rrr}-0.6867670 & 0.4108530 & -0.3648350 \\ 1.4675360 & 1.4451330 & 0.5561530 \\ -0.9460670 & 1.6243550 & -1.0048560 \\ 0.0926280 & 2.2447880 & -1.7147300 \\ -2.2287790 & 2.1740510 & -0.9204670 \\ -2.4176510 & 3.4303250 & -1.5023110 \\ -3.4064250 & 3.8830700 & -1.4433050 \\ 2.0898630 & 1.1928670 & -0.6748350 \\ 3.3481720 & 0.5826770 & -0.6893360 \\ 1.3959270 & 1.5337300 & -1.8431180 \\ 3.8716220 & 0.2667740 & -1.9469040 \\ 4.8483450 & -0.2124440 & -1.9910730 \\ -3.3587250 & 1.4661800 & -0.2652130 \\ 4.0994160 & 0.2575700 & 0.5499630 \\ -3.8779980 & 0.2839810 & -0.8213960 \\ -3.3779520 & -0.4198250 & -2.0605620 \\ -5.4501230 & -1.5744550 & -0.9993470 \\ -5.0047100 & -0.3403600 & -0.2547950 \\ -3.0192860 & -1.8771690 & -1.6736390 \\ -2.6671820 & -2.4123020 & -2.5637850 \\ -2.1976130 & -1.8552820 & -0.9551150 \\ -4.2671550 & -2.5808950 & -1.0618320 \\ -4.5671950 & -3.4417220 & -1.6738310 \\ -4.0377620 & -2.9583430 & -0.0590060 \\ -6.3249030 & -2.0316490 & -0.5208190 \\ -2.5066350 & 0.0820100 & -2.4898590 \\ -5.7828380 & -1.1565240 & -2.4602820 \\ -6.0632930 & -2.0521150 & -3.0304620 \\ -6.6438100 & -0.4777000 & -2.4590080 \\ -4.5358520 & -0.4660230 & -3.0967270 \\ -4.1944800 & -1.0221680 & -3.9798110 \\ -4.7790370 & 0.5532930 & -3.4193470 \\ -3.9758070 & 1.9959840 & 0.8805300 \\ -3.5566780 & 3.2352500 & 1.6350720 \\ -5.6488430 & 2.0760700 & 2.6545880 \\ -5.1078230 & 1.3697060 & 1.4372240 \\ -4.7529380 & 4.2272450 & 1.6285030 \\ -4.4756390 & 5.1207500 & 2.2031570 \\ -4.9620360 & 4.5450700 & 0.5999130 \\ -6.0054240 & 3.5363410 & 2.2559030 \\ -6.3368790 & 4.0766260 & 3.1524420 \\ -6.8400640 & 3.5272550 & 1.5449480 \\ -6.5317090 & 1.5603360 & 3.0519740 \\ -2.6700220 & 3.6960090 & 1.1919510 \\ -4.5208740 & 2.1354350 & 4.6010430 \\ -4.8916870 & 2.6778390 & \end{array}$

$\begin{array}{lr}\mathrm{H} & -4.2627340 \\ \mathrm{C} & -3.2746000 \\ \mathrm{H} & -3.0435740 \\ \mathrm{H} & -2.3935260 \\ \mathrm{C} & -5.6283100 \\ \mathrm{H} & -6.5092690 \\ \mathrm{C} & 5.2728120 \\ \mathrm{C} & 5.6241520 \\ \mathrm{C} & 4.2139410 \\ \mathrm{C} & 4.4969600 \\ \mathrm{C} & 6.4120910 \\ \mathrm{H} & 6.6074770 \\ \mathrm{H} & 7.3811910 \\ \mathrm{C} & 5.5741570 \\ \mathrm{H} & 5.3790100 \\ \mathrm{H} & 6.1145480 \\ \mathrm{H} & 3.6227690 \\ \mathrm{H} & 6.2191720 \\ \mathrm{C} & 3.4645470 \\ \mathrm{H} & 3.3148290 \\ \mathrm{H} & 2.4781810 \\ \mathrm{C} & 4.3031070 \\ \mathrm{H} & 4.5463880 \\ \mathrm{H} & 3.7395210 \\ \mathrm{C} & 5.2646190 \\ \mathrm{C} & 5.5966200 \\ \mathrm{C} & 4.0906690 \\ \mathrm{C} & 4.4734200 \\ \mathrm{C} & 4.2665800 \\ \mathrm{H} & 4.4931510 \\ \mathrm{H} & 3.7679460 \\ \mathrm{C} & 3.3550200 \\ \mathrm{H} & 3.1034070 \\ \mathrm{H} & 2.4214350 \\ \mathrm{H} & 3.4478710 \\ \mathrm{H} & 6.2365020 \\ \mathrm{C} & 5.3934140 \\ \mathrm{H} & 5.1269470 \\ \mathrm{H} & 5.9134160 \\ \mathrm{C} & 6.3072960 \\ \mathrm{H} & 6.5147450 \\ \mathrm{H} & 7.2690670 \\ \mathrm{C} & 5.6646970 \\ \mathrm{H} & 6.2759810 \\ \mathrm{C} & 1.9126710 \\ \mathrm{C} & \\ & \end{array}$

1.1194190
2.8484310
3.7647370
2.2040330
0.2086940
-0.2700860
0.9629950
2.1657720
2.7499070
1.2711550
3.1699880
4.0802670
2.7358340
3.5001610
4.5788740
3.2044690
2.9771530
1.8767280
3.2213240
4.3075150
2.7498930
2.8556510
3.7554010
2.1790550
-1.3637670
-2.8280600
-2.3056590
-1.0694070
-3.6190460
-4.6931870
-3.3500080
-3.2862050
-4.1974540
-2.8263290
-2.0646520
-3.0044140
-3.0026890
-3.9340770
-2.3596550
-3.2993210
-4.3748930
-2.7816990
-0.3508850
-0.5837360
1.1237990
1.2685910

4. 0413500

3. 1136770

3. 6728820

3.1550950

0.8662660

1.2953650

2. 5746650

3. 4140810

1. 3124400

1. 4410520

2. 5264950

3. 1089640

2. 2530440

1. 2522860

1. 1861930

0.3449740

0.4219940

4. 2890280

2. 5890480

2.5257150

2. 6226790

3.8536310

4.4344990

4.5068910

1. 9601810

2. 1029060

0.0509090

0.8332210

2. 2288340

2. 2676080

3.1678270

1. 0085600

0.4503080

1.3392340

$-0.7991280$

2. 9761580

$-0.4284820$

$-0.9458040$

$-1.1490360$

0.8029340

0.8804940

0.7053780

2. 8314980

3.7043290

$-3.0930880$

$-4.3557080$ 


$\begin{array}{rrr}1.4977720 & 0.2810820 & -5.4536270 \\ 3.0079990 & 0.3947880 & -5.6917910 \\ 3.7654380 & -0.0414070 & -4.4304820 \\ 3.1680340 & 0.4874880 & -3.1352790 \\ 0.9364920 & 0.4945090 & -6.3722570 \\ 1.1831610 & 2.2830480 & -4.7648730 \\ 0.0199620 & 1.1475470 & -4.1033340 \\ 3.2511090 & 1.4409810 & -5.9310250 \\ 3.3247980 & -0.2177500 & -6.5456270 \\ 4.8211360 & 0.2574390 & -4.4904940 \\ 3.7632370 & -1.1418440 & -4.3798240 \\ 1.2513170 & -0.7461880 & -5.1449880 \\ -0.1078440 & 3.5450380 & -2.2313530 \\ 1.0557820 & 4.3257240 & -2.8198690 \\ 0.8243380 & 5.8426700 & -2.7999310 \\ -0.5321670 & 6.1610860 & -3.4387500 \\ -1.6572840 & 5.5575990 & -2.5883060 \\ -1.3798920 & 4.1384710 & -2.1169980 \\ 1.6400200 & 6.3446180 & -3.3357650 \\ 1.2185980 & 4.0279790 & -3.8647440 \\ 1.9749080 & 4.0630570 & -2.2821610 \\ -0.5579210 & 5.7322570 & -4.4519230 \\ -0.6813290 & 7.2439160 & -3.5376310 \\ -2.6100440 & 5.5773650 & -3.1353460 \\ -1.8032540 & 6.1890860 & -1.6976530 \\ 0.8345680 & 6.2088810 & -1.7625270 \\ -0.4134240 & -3.7298720 & -0.5148970 \\ 0.3755190 & -2.7101570 & -0.7049280 \\ -0.6259500 & -4.5358490 & -1.7508660 \\ -1.6374380 & -5.5108020 & -1.8300560 \\ 0.1787820 & -4.3006720 & -2.8853230 \\ -1.8419060 & -6.2270460 & -3.0078040 \\ -2.2676900 & -5.6894940 & -0.9639670 \\ -0.0238900 & -5.0247250 & -4.0592160 \\ 0.9611710 & -3.5501400 & -2.8396570 \\ -1.0357290 & -5.9883540 & -4.1266290 \\ -2.6338800 & -6.9710040 & -3.0546760 \\ 0.6083750 & -4.8355710 & -4.9239340 \\ -1.1959020 & -6.5489760 & -5.0451230 \\ 0.5108410 & -2.0508930 & 0.1023080 \\ -1.1340060 & -4.1334410 & 0.9531070 \\ -2.4413840 & -1.4024820 & 2.5219960 \\ -1.2680550 & -2.1501110 & 2.9946150 \\ -2.3829730 & -0.9730350 & 1.5194230 \\ -0.3078260 & -1.8995760 & 2.5589000 \\ -1.8744400 & -0.4935040 & 3.5313550\end{array}$

$\begin{array}{ll}\mathrm{H} & -1.2404660 \\ \mathrm{C} & -3.8457220 \\ \mathrm{C} & -2.7650500 \\ \mathrm{C} & -1.3594810 \\ \mathrm{C} & -3.8183440 \\ \mathrm{H} & -4.8378530 \\ \mathrm{H} & -2.8068470 \\ \mathrm{H} & -1.0560560 \\ \mathrm{H} & -4.1226860 \\ \mathrm{H} & -4.5137010 \\ \mathrm{H} & -3.6805410 \\ \mathrm{H} & -2.9467800 \\ \mathrm{H} & -0.6005030 \\ \mathrm{I} & \end{array}$

0.1636720
-2.6181550
-3.7075080
-3.1080540
-1.9060420
-3.0577970
-4.2404970
-2.5604640
-2.6031280
-1.0620020
-1.8843760
-4.4451480
-3.8844610
2.9997810
4. 2583360
4.3244380
4. 1394890
2.8961880
4. 4162240
5.2816440
5.0442780
2. 1037300
2.8624760
5.0588980
3.5303250

TS264 (R, R) G[B97-D/def2-TZVP $]=-3864.565635$

$\begin{array}{lrrr}\text { TS264(R, R) G[B97-D/def2-TZVP] }=-3864.565635 \\ \text { P } & 0.3051420 & 0.5155280 & 0.9756860 \\ \text { O } & 0.8861770 & -0.8729140 & 1.0626510 \\ \text { O } & -0.3641930 & 1.0709190 & 2.2021700 \\ \text { O } & -0.7073350 & 0.4605950 & -0.3086050 \\ \text { O } & 1.4204470 & 1.6024560 & 0.5232790 \\ \mathrm{C} & -1.0440120 & 1.6414530 & -0.9712690 \\ \mathrm{C} & -0.0622270 & 2.2897630 & -1.7371870 \\ \mathrm{C} & -2.3557670 & 2.1165610 & -0.8740870 \\ \mathrm{C} & -2.6446220 & 3.3181180 & -1.5241740 \\ \mathrm{H} & -3.6593780 & 3.7087000 & -1.4617540 \\ \mathrm{C} & 2.0138210 & 1.3338970 & -0.7208360 \\ \mathrm{C} & 3.2723540 & 0.7239230 & -0.7555360 \\ \mathrm{C} & 1.2734370 & 1.6427350 & -1.8746180 \\ \mathrm{C} & 3.7712890 & 0.3748810 & -2.0195950 \\ \mathrm{H} & 4.7420240 & -0.1134020 & -2.0783520 \\ \mathrm{C} & -3.4146540 & 1.3585880 & -0.1574190 \\ \mathrm{C} & 4.0532540 & 0.4198990 & 0.4716580 \\ \mathrm{C} & -3.8829970 & 0.1417030 & -0.6820490 \\ \mathrm{C} & -3.4219830 & -0.5179850 & -1.9600910 \\ \mathrm{C} & -5.3084770 & -1.8371110 & -0.7534820 \\ \mathrm{C} & -4.9142460 & -0.5677780 & -0.0401170 \\ \mathrm{C} & -2.9201280 & -1.9428800 & -1.6166800 \\ \mathrm{H} & -2.6088610 & -2.4483880 & -2.5386840 \\ \mathrm{H} & -2.0419880 & -1.8573140 & -0.9740400 \\ \mathrm{C} & -4.0526020 & -2.7404120 & -0.9030640 \\ \mathrm{H} & -4.3239960 & -3.6326770 & -1.4824880 \\ \mathrm{H} & -3.7140710 & -3.0814360 & 0.0816110 \\ \mathrm{H} & -6.1047640 & -2.3639760 & -0.2136140 \\ \mathrm{H} & -2.6285520 & 0.0541050 & -2.4489540 \\ \mathrm{C} & -5.7802880 & -1.4539380 & -2.1849230 \\ \mathrm{H} & -6.0203340 & -2.3722790 & -2.7372400\end{array}$




\begin{tabular}{|c|c|c|c|}
\hline $\mathrm{H}$ & -6.6960420 & -0.8545060 & -2.1201260 \\
\hline C & -4.6481220 & -0.6565110 & -2.9055760 \\
\hline $\mathrm{H}$ & -4.3299620 & -1.1769060 & -3.8186320 \\
\hline $\mathrm{H}$ & -5.0010860 & 0.3401670 & -3.1961870 \\
\hline C & -4.0004170 & 1.8520290 & 1.0195290 \\
\hline C & -3.6489500 & 3.1387290 & 1.7281580 \\
\hline C & -5.5539180 & 1.8111450 & 2.9002040 \\
\hline C & -5.0386120 & 1.1403800 & 1.6522390 \\
\hline C & -4.9320730 & 4.0139910 & 1.7863900 \\
\hline $\mathrm{H}$ & -4.7013490 & 4.9451270 & 2.3203500 \\
\hline $\mathrm{H}$ & -5.2412100 & 4.2820020 & 0.7687230 \\
\hline C & -6.0676710 & 3. 2281860 & 2.5166730 \\
\hline $\mathrm{H}$ & -6.3750390 & 3.7525010 & 3.4311750 \\
\hline $\mathrm{H}$ & -6.9506310 & 3.1358320 & 1.8732200 \\
\hline $\mathrm{H}$ & -6.3558770 & 1.2230380 & 3.3632790 \\
\hline $\mathrm{H}$ & -2.8420280 & 3.6710340 & 1.2170820 \\
\hline C & -4.3636200 & 1.9871470 & 3.8834980 \\
\hline $\mathrm{H}$ & -4.7221190 & 2.4977300 & 4.7872230 \\
\hline $\mathrm{H}$ & -3.9866780 & 1.0028000 & 4.1845020 \\
\hline C & -3.2370010 & 2.8126120 & 3.1901950 \\
\hline $\mathrm{H}$ & -3.0702470 & 3.7589480 & 3.7217380 \\
\hline $\mathrm{H}$ & -2.2921080 & 2.26 & 48960 \\
\hline $\mathrm{C}$ & -5.4996700 & -0.0641710 & 2140 \\
\hline $\mathrm{H}$ & -6.3064260 & -0.6094400 & 1.6 \\
\hline C & 5.2653350 & 1.1602290 & 0090 \\
\hline C & 5.6251460 & 2.375 & 8050 \\
\hline C & 4.1623780 & 2.9225750 & 1.20 \\
\hline C & 4.4591750 & 1.4472200 & 1.3438950 \\
\hline C & 6.3846920 & 3.3747940 & 2.3624920 \\
\hline $\mathrm{H}$ & 6.5851640 & 4.2940450 & 2.9289320 \\
\hline $\mathrm{H}$ & 7.3510630 & 2.9450980 & 2.0728320 \\
\hline C & 5.5153660 & 3.6812080 & 1.1036000 \\
\hline $\mathrm{H}$ & 5.3108090 & 4.7574780 & 1.0273300 \\
\hline $\mathrm{H}$ & 6.0366590 & 3.3766590 & 0.1881030 \\
\hline $\mathrm{H}$ & 3.5503940 & 3.1360220 & 0.3254600 \\
\hline $\mathrm{H}$ & 6.2423260 & 2.1008560 & 4.1440320 \\
\hline C & 3.4396000 & 3.4051340 & 2.4925230 \\
\hline $\mathrm{H}$ & 3. 2821890 & 4.4896350 & 2.4194100 \\
\hline $\mathrm{H}$ & 2.4570460 & 2.9288850 & 2.5552590 \\
\hline C & 4.3092400 & 3.0601610 & 3.7416210 \\
\hline $\mathrm{H}$ & 4.5584470 & 3.9681530 & 4.3069840 \\
\hline $\mathrm{H}$ & 3.7658970 & 2.3865940 & 4.4148750 \\
\hline C & 5260 & -1.1708600 & 1.8664900 \\
\hline C & 5.6528780 & -2.6256090 & 2.0064860 \\
\hline C & 4.08 & -2.1495430 & -0.0084890 \\
\hline C & 4.4548750 & -0.8994630 & 0.7578290 \\
\hline
\end{tabular}

4.3497460
4.6068100
3.8663380
3.4010250
3.1650800
2.4613090
3.4109000
6.3193480
5.3915070
5.1366630
5.8758320
6.3443180
6.5851880
7.2876610
5.6833000
6.3207720
1.7833430
1.0186750
0.9650710
2.3692640
3.4836690
3.0368440
1.5274500
0.5106410
2.6288060
3.6894510
0.0066370
0.2998630
2.3490640
4.4130920
-0.3712650
-1.6789740
0.7216490
0.1895590
-0.9019580
-2.0881080
1.0198160
1.3242330
1.4082380
-0.4920210
-1.2377590
-2.8153750
-2.6152780
-0.2317790
-0.1684090
0.5693700
$-3.4528960$
$-4.5202110$
$-3.1937350$
$-3.1498900$
$-4.0687890$
$-2.7222220$
$-1.9316750$
$-2.7807640$
$-2.8137880$
$-3.7543220$
$-2.1606370$
$-3.0803370$
$-4.1491680$
$-2.5349350$
$-0.1449050$
$-0.3605040$
1. 2305310
1. 3158370
$-0.0809770$
$-0.7494850$
0.1066160
0.5934740
2. 0141110
0.0173740
$-0.9293640$
0.9694390
1.6994850
$-0.7153060$
$-1.7267540$
$-0.4692440$
3. 5282980
4.0399830
4. 3500390
5.4599690
6.2647650
5.3475880
6.1091060
4.8075550
3. 7062520
6.6810290
7.1077920
5. 8626220
5. 1155520
5. 0130220
$-3.6092960$
$-2.5495190$

2. 1669560 2. 2041340

3.1167250

0.9680460

0.4158640

1. 3219080

$-0.8414090$

2.8637610

$-0.5185170$

$-1.0249700$

$-1.2547380$

0.6900860

0.7647110

0.5672090

2. 7203940

3. 5788110

$-3.1190420$

$-4.4166610$

$-5.0859840$

$-5.1939910$

$-4.5375650$

$-3.1830090$

$-5.0997940$

$-6.0796760$

$-6.2448070$

$-5.1900900$

$-4.2585480$

$-4.4875590$

$-4.6955660$

$-4.4567180$

$-2.3448810$

$-2.2333000$

$-3.0106500$

$-3.9260650$

$-3.2124060$

$-2.8918800$

$-4.2328770$

$-2.2090240$

$-3.5645850$

$-2.2800250$

$-3.8299690$

$-2.2501570$

$-4.8395850$

-0.5653220
-0.7350460 


\begin{tabular}{|c|c|c|c|c|c|c|c|}
\hline c & -0.4011600 & -4.3593300 & -1.8331300 & $c$ & 3.7625510 & 0.5715470 & -19903190 \\
\hline c & -1.3357270 & -5.4080690 & -1.9091960 & $\mathrm{H}$ & 4.7456320 & 0.1120740 & -2.0705980 \\
\hline C & 0.2925390 & -3.9842920 & -3.0029130 & $\mathrm{C}$ & -3.4408350 & 1.3227650 & -0.0258440 \\
\hline C & -1.5731330 & -6.0607770 & -3.1173440 & $\mathrm{C}$ & 4.0434500 & 0.5019230 & 0.4998460 \\
\hline $\mathrm{H}$ & -1.8840730 & -5.6911760 & -1.0156180 & $\mathrm{C}$ & -3.8927970 & 0.1187860 & -0.5932240 \\
\hline $\mathrm{C}$ & 0.0565650 & -4.6430490 & -4.2082410 & $\mathrm{C}$ & -3.4396740 & -0.4786700 & -1.9046130 \\
\hline $\mathrm{H}$ & 1.0105980 & -3.1720140 & -2.9628410 & C & -5.2795960 & -1.8841890 & -0.7244240 \\
\hline C & -0.8783710 & -5.6818950 & -4.2716050 & $\mathrm{C}$ & -4.8982620 & -0.6382290 & 0.0354920 \\
\hline $\mathrm{H}$ & -2.3052420 & -6.8639710 & -3.1602370 & $\mathrm{C}$ & -2.9045790 & -1.9064880 & -1.6290980 \\
\hline $\mathrm{H}$ & 0.5999540 & -4.3411670 & -5.1009520 & $\mathrm{H}$ & -2.5990160 & -2.3668360 & -2.5764700 \\
\hline $\mathrm{H}$ & -1.0663340 & -6.1912020 & -5.2143900 & $\mathrm{H}$ & -2.0177060 & -1.8316490 & -0.9971760 \\
\hline $\mathrm{H}$ & 0.6820640 & -1.9033150 & 0.0895770 & $\mathrm{C}$ & -4.0093260 & -2.7557050 & -0.9319760 \\
\hline $\mathrm{s}$ & -0.8137250 & -4.1083830 & 0.9090180 & $\mathrm{H}$ & -4.2739380 & -3.6279420 & -1.5441640 \\
\hline C & -2.2297500 & -1.5072390 & 2.6173580 & $\mathrm{H}$ & -3.6478580 & -3.1315640 & 0.0314580 \\
\hline $\mathrm{C}$ & -0.9881370 & -2.1817080 & 3.0230590 & $\mathrm{H}$ & -6.0565170 & -2.4492020 & -0.1949990 \\
\hline $\mathrm{H}$ & -2.2505990 & -1.0697070 & 1.6164490 & $\mathrm{H}$ & -2.6654070 & 0.1289930 & -2.3812110 \\
\hline $\mathrm{H}$ & -0.0689230 & -1.8620650 & 2.5462740 & C & -5.7826620 & -1.4515030 & -2.1311160 \\
\hline 0 & -1.6771340 & -0.5683330 & 3.6074000 & $\mathrm{H}$ & -6.0138870 & -2.3509210 & -2.7171700 \\
\hline $\mathrm{H}$ & -1.1161440 & 0.1376870 & 3.0618940 & $\mathrm{H}$ & -6.7087890 & -0.8739720 & -2.0267330 \\
\hline C & -3.4588240 & -2.8282520 & 4.4097850 & C & -4.6783180 & -0.6025650 & -2.8357620 \\
\hline C & -2.3105640 & -3.8481490 & 4.4034510 & $\mathrm{H}$ & -4.3655770 & -1.0782320 & -3.7746850 \\
\hline C & -0.9582020 & -3.1570900 & 4.1546280 & $\mathrm{H}$ & -5.0551930 & 0.3980090 & -3.0791950 \\
\hline $\mathrm{C}$ & -3.5498830 & -2.1042410 & 3.0569660 & C & -4.0170990 & 1.7551820 & 1.1792050 \\
\hline $\mathrm{H}$ & -4.4118020 & -3.3305760 & 4.6149530 & $\mathrm{C}$ & -3.6808220 & 3.0193880 & 1.9334920 \\
\hline $\mathrm{H}$ & -2.2679560 & -4.3952490 & 5.3525960 & $\mathrm{C}$ & -5.5353240 & 1.6032750 & 3.0827630 \\
\hline $\mathrm{H}$ & -0.6413580 & -2.6026260 & 5.0508240 & $\mathrm{C}$ & -5.0287660 & 0.9956040 & 1.7993700 \\
\hline $\mathrm{H}$ & -3.8507390 & -2.8141280 & 2.2743860 & $\mathrm{C}$ & -4.9812790 & 3.8630950 & 2.0500910 \\
\hline $\mathrm{H}$ & -4.2995800 & -1.3076400 & 3.0719260 & $\mathrm{H}$ & -4.7604520 & 4.7766590 & 2.6176220 \\
\hline $\mathrm{H}$ & -3.2963890 & -2.0929420 & 5.2094780 & $\mathrm{H}$ & -5.3151660 & 4.1651100 & 1.0500520 \\
\hline $\mathrm{H}$ & -2.4869970 & -4.5849200 & 3.6072200 & $\mathrm{C}$ & -6.0859620 & 3.0233540 & 2.7677010 \\
\hline $\mathrm{H}$ & -0.1599240 & -3.8803220 & 3.9492130 & $\mathrm{H}$ & -6.3864550 & 3.5027620 & 3.7087600 \\
\hline 15 & & & & $\mathrm{H}$ & -6.9790020 & 2.9384120 & 2.1372790 \\
\hline TS & , R) $G[B 97-D$ & [ZVP] & 358 & $\mathrm{H}$ & -6.3161250 & 0.9795200 & 3.5353800 \\
\hline $\mathrm{P}$ & 0.3105620 & 0.5036860 & 1.0360840 & $\mathrm{H}$ & -2.8949960 & 3.5893380 & 1.4299010 \\
\hline 0 & 0.9246920 & -0.8741390 & 1.0436960 & C & -4.3307920 & 1.7645480 & 4.0512850 \\
\hline 0 & -0.3436270 & 0.9812060 & 2.3022200 & $\mathrm{H}$ & -4.6830340 & 2.2279480 & 4.9825280 \\
\hline 0 & -0.7280980 & 0.4868020 & -0.2270650 & $\mathrm{H}$ & -3.9270400 & 0.7768880 & 4.3030590 \\
\hline 0 & 1.3918090 & 1.6330550 & 0.6086180 & $\mathrm{C}$ & -3.2355030 & 2.6441580 & 3.3740310 \\
\hline C & -1.0879180 & 1.6823490 & -0.8500910 & $\mathrm{H}$ & -3.0822360 & 3.5721350 & 3.9408700 \\
\hline C & -0.1230480 & 2.3593170 & -1.6209480 & $\mathrm{H}$ & -2.2784260 & 2.1191890 & 3.3311950 \\
\hline C & -2.4070010 & 2.1295500 & -0.7272760 & $\mathrm{C}$ & -5.4730840 & -0.1960590 & 1.2279000 \\
\hline C & -2.7390370 & 3.3409020 & -1.3473090 & $\mathrm{H}$ & -6.2593280 & -0.7784550 & 1.7096790 \\
\hline $\mathrm{H}$ & -3.7618560 & 3.7055660 & -1.2684210 & $\mathrm{C}$ & 5.2366350 & 1.1711740 & 2.5264830 \\
\hline C & 1.9807370 & 1.4210660 & -0.6485940 & $\mathrm{c}$ & 5.5657550 & 2.3520720 & 3.4050060 \\
\hline C & 3.2542640 & 0.8461890 & -0.7115210 & $\mathrm{C}$ & 4.0964280 & 2.9677130 & 1.3534330 \\
\hline & 1.2269730 & 1.7547970 & -1.7876320 & $\mathrm{C}$ & 4.4254930 & 1.4941680 & 1.4216610 \\
\hline
\end{tabular}




$\begin{array}{lrr}6.3053400 & 3.4119280 & 2.5404380 \\ 6.4826270 & 4.3071550 & 3.1513880 \\ 7.2823170 & 3.0187810 & 2.2348490 \\ 5.4335130 & 3.7587640 & 1.2938650 \\ 5.2068660 & 4.8329860 & 1.2669570 \\ 5.9644230 & 3.5086780 & 0.3674170 \\ 3.4818980 & 3.2099600 & 0.4833680 \\ 6.1866940 & 2.0490570 & 4.2569300 \\ 3.3600890 & 3.3703820 & 2.6602700 \\ 3.1780290 & 4.4532440 & 2.6396840 \\ 2.3888080 & 2.8688270 & 2.6950210 \\ 4.2335310 & 2.9839170 & 3.8941730 \\ 4.4606740 & 3.8680510 & 4.5048260 \\ 3.7033400 & 2.2660570 & 4.5311960 \\ 5.3093620 & -1.1257480 & 1.8141820 \\ 5.7198740 & -2.5755900 & 1.8810200 \\ 4.1386890 & -2.0386140 & -0.1096670 \\ 4.4775650 & -0.8199400 & 0.7198460 \\ 4.4374520 & -3.4420240 & 1.9989460 \\ 4.7206290 & -4.5033190 & 1.9847380 \\ 3.9468890 & -3.2413830 & 2.9591430 \\ 3.4825710 & -3.1048250 & 0.8138240 \\ 3.2726760 & -4.0011240 & 0.2158100 \\ 2.5310660 & -2.7209560 & 1.1858630 \\ 3.4612950 & -1.7958920 & -0.9313270 \\ 6.3903530 & -2.7568130 & 2.7300170 \\ 5.4644210 & -2.6411320 & -0.6507500 \\ 5.2346150 & -3.5592720 & -1.2077120 \\ 5.9326650 & -1.9371420 & -1.3497030 \\ 6.4215810 & -2.9473050 & 0.5445690 \\ 6.6857110 & -4.0129760 & 0.5656960 \\ 7.3526990 & -2.3757150 & 0.4514830 \\ 5.6864760 & -0.1345110 & 2.7196470 \\ 6.3284110 & -0.3775020 & 3.5673480 \\ 1.7514390 & 1.4233650 & -3.0500670 \\ 0.9884120 & 1.5555950 & -4.3439040 \\ 0.9833050 & 0.2001950 & -5.0955850 \\ 2.4054030 & -0.4247190 & -5.2238570 \\ 3.4903910 & 0.4297890 & -4.5194240 \\ 3.0244790 & 0.8291540 & -3.1428610 \\ 1.4724010 & 2.3118400 & -4.9805810 \\ 0.5381910 & 0.3462700 & -6.0876530 \\ 2.6764220 & -0.5495400 & -6.2797400 \\ 3.6697830 & 1.3327010 & -5.1240200 \\ -0.0369400 & 1.8920430 & -4.5456350 \\ 0.3280290 & -0.4862800 & \end{array}$
2.4090990
4.4376780
$-1.7930640$
$-2.1166240$
$-1.6262150$
$-0.1278770$
0.4716060
$-0.4815240$
$-1.6087140$
$-1.7913130$
0.4713240
0.6614490
$-3.1937760$
$-2.2448470$
$-0.0224830$
1.4377930
$-0.0720840$
0.6303430
$-0.2868940$
$-1.1699490$
0.3725700
$-1.3899760$
$-1.6920280$
0.1534230
1. 0512400
$-0.7297530$
$-2.0816040$
0.6697710
$-0.9046020$
0.7352640
$-0.6953050$
$-2.1633500$
$-0.8991440$
$-2.2115500$
0.0025710
$-1.6175570$
$-1.0786560$
$-3.3267090$
$-2.1553660$
$-0.8247690$
$-3.4607260$
$-4.2637030$
$-2.0826530$
$-0.5063270$
$-3.7610350$
$-4.2269630$
$-1.4237030$
$-0.1194810$
4. 0800600
5. 3926850
5. 3801210
4.9731390
4.4828210
3. 5883970
6. 2117920
6.3660840
5.8210300
5.3667930
5.5932850
4.6634830
4. 1744680
4.0011300
$-3.5289190$
$-2.4391880$
$-4.2210350$
$-5.3092530$
$-3.7506780$
$-5.9080310$
$-5.6673970$
$-4.3545740$
$-2.9079180$
$-5.4340190$
$-6.7437540$
$-3.9788750$
$-5.9012130$
$-1.8346310$
$-4.1226960$
$-1.6555050$
$-2.3211850$
$-1.1642670$
$-1.9504360$
$-0.7587500$
$-0.0144780$
$-3.1103310$
$-4.0993280$
$-3.3608400$
$-2.3079630$
$-3.6470280$
$-2.8529160$
$-2.9764300$
$-1.5308580$

$-4.7701320$

$-4.4653950$

$-2.0552950$

$-2.7226230$

$-4.1893270$

$-4.3040730$

$-2.9603040$

$-2.2049420$

$-2.1880150$

$-4.6413140$

$-4.6584630$

$-2.3311620$

$-2.6756400$

$-4.7445240$

$-5.0466330$

$-3.1263450$

$-0.7317060$

$-0.8497080$

$-2.0357000$

$-2.1586630$

$-3.1906770$

$-3.3976920$

$-1.2763360$

$-4.4274880$

$-3.1128910$

$-4.5370380$

$-3.4762490$

$-5.3081650$

$-5.5038570$

0.0104720

0.7172470

2. 6002410

2.9456280

1. 6256130

2. 4725190

3. 6329150

3. 1221510

4. 3332200

4. 2435190

4.0155350

3. 0282640

4.5243240

5.1559000

4. 9385290

2. 2101210

3. 1047610 


$\begin{array}{lrrr}\mathrm{H} & -3.1651160 & -2.4215220 & 5.1734250 \\ \mathrm{H} & -2.3295390 & -4.7894680 & 3.4063010 \\ \mathrm{H} & -0.0119360 & -4.0489310 & 3.7544070 \\ \mathrm{I} 54 & & & \\ \mathrm{TS} 266(\mathrm{R}, \mathrm{R}) & \mathrm{G}[\mathrm{B} 97-\mathrm{D} / \mathrm{de} \text { f2 } & -\mathrm{TZVP}]=-3864.564747 \\ \mathrm{P} & -0.2427790 & -0.2619750 & 0.9942360 \\ \mathrm{O} & -0.6896920 & 1.1723500 & 0.8830400 \\ \mathrm{O} & 0.4708480 & -0.6862490 & 2.2498970 \\ \mathrm{O} & 0.6620410 & -0.5256740 & -0.3429190 \\ \mathrm{O} & -1.4799840 & -1.2944680 & 0.8172080 \\ \mathrm{C} & 0.8851770 & -1.8434600 & -0.7472030 \\ \mathrm{C} & -0.1835810 & -2.5687600 & -1.2949360 \\ \mathrm{C} & 2.1687290 & -2.3838870 & -0.6112150 \\ \mathrm{C} & 2.3299420 & -3.7258430 & -0.9649330 \\ \mathrm{H} & 3.3192610 & -4.1701880 & -0.8662370 \\ \mathrm{C} & -2.1276890 & -1.2622300 & -0.4252920 \\ \mathrm{C} & -3.3610530 & -0.6116420 & -0.5299120 \\ \mathrm{C} & -1.4850860 & -1.8764850 & -1.5149910 \\ \mathrm{C} & -3.9422890 & -0.5584130 & -1.8053580 \\ \mathrm{H} & -4.9063150 & -0.0657310 & -1.9171160 \\ \mathrm{C} & 3.3240440 & -1.5694500 & -0.1515600 \\ \mathrm{C} & -4.0443480 & 0.0043460 & 0.6372430 \\ \mathrm{C} & 3.7866750 & -0.4927690 & -0.9307220 \\ \mathrm{C} & 3.2195920 & -0.0288650 & -2.2520680 \\ \mathrm{C} & 5.2928300 & 1.3442490 & -1.4980430 \\ \mathrm{C} & 4.9163190 & 0.2469020 & -0.5340570 \\ \mathrm{C} & 2.8320990 & 1.4659840 & -2.1126410 \\ \mathrm{H} & 2.4385810 & 1.8281030 & -3.0704570 \\ \mathrm{H} & 2.0317150 & 1.5504190 & -1.3765450 \\ \mathrm{C} & 4.0776310 & 2.2952060 & -1.6774370 \\ \mathrm{H} & 4.3265940 & 3.0494360 & -2.4351480 \\ \mathrm{H} & 3.8734530 & 2.8274710 & -0.7410710 \\ \mathrm{H} & 6.1707840 & 1.8978050 & -1.1432470 \\ \mathrm{H} & 2.3490540 & -0.6178350 & -2.5515560 \\ \mathrm{C} & 5.5827320 & 0.6887650 & -2.8779410 \\ \mathrm{H} & 5.8084140 & 1.4779540 & -3.6073350 \\ \mathrm{H} & 6.4674590 & 0.0459290 & -2.7983440 \\ \mathrm{C} & 4.3376410 & -0.1388330 & -3.3264190 \\ \mathrm{H} & 3.9455500 & 0.2385950 & -4.2799540 \\ \mathrm{H} & 4.6041570 & -1.1927200 & -3.4702120 \\ \mathrm{C} & 4.0110030 & -1.8819150 & 1.0339750 \\ \mathrm{C} & 3.6680110 & -2.9821660 & 2.0106150 \\ \mathrm{C} & 5.7542080 & -1.6006420 & 2.7195750 \\ \mathrm{C} & 5.1434450 & -1.1391790 & 1.4204940 \\ \mathrm{C} & 4.8988790 & -3.9233580 & 2.1293570 \\ \mathrm{H} & 4.6756390 & -4.7058720 & 2.8663710\end{array}$

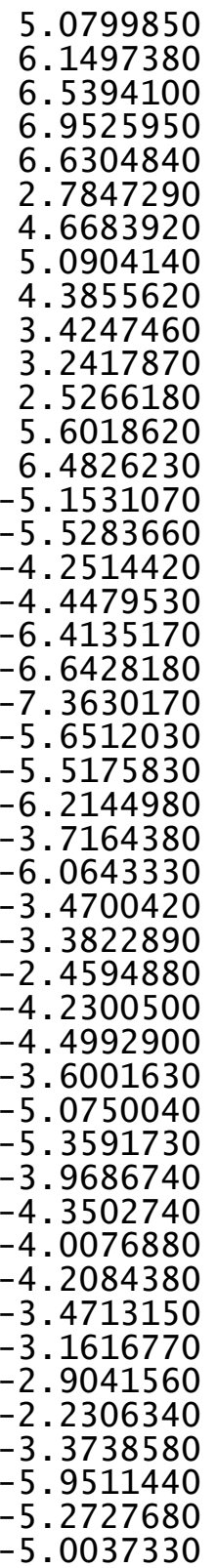

5. 0799850

6.5394100

5950

(1)

5. 0904140

4.3855620

4247460

2. 5266180

. 6018620

$-5.1531070$

$-5.5283660$

14420

$-6.6428180$

$-7.3630170$

$-6.2144980$

$-3.7164380$

$-6.0643330$

$-3.4700420$

$-3.3822890$

$-3.6001630$

$-5.0750040$

. 3591730

$-4.0076880$

2084380

$-2.9041560$

. 2306340

$-5.0037330$
-4.4160610
-3.0964690
-3.4610850
-3.1878620
-0.9949160
-3.5423950
-1.5055120
-1.8758760
-0.4575560
-2.3470590
-3.1534050
-1.7258710
-0.0823480
0.4855060
-0.2006900
-1.1847900
-2.2683950
-0.7792400
-2.2909100
-3.0487210
-1.8532430
-2.9288100
-4.0077590
-2.7944150
-2.7066320
-0.6881940
-2.5201050
-3.6041950
-2.1129440
-1.8582040
-2.6078220
-1.1046530
1.9421770
3.4138570
2.3995860
1.3781930
4.1687690
5.2302190
4.1043580
3.5370750
4.2904160
3.1267900
1.9542110
3.8001840
3.0247500
3.8152870

1. 1663260

2. 5670160

3. 5266880

1.8258490

2. 9818510

1. 6925860

3.8273100

4.7709800

3.9779700

3. 4076050

4. 1299620

3. 3677040

0.6348900

0.9367720

2. 8061050

3.8862720

1.9003940

1.7338290

3. 2459250

4.0071030

2. 9154530

2. 0430860

2. 1981350

1.1117660

1.0543370

4. 7043350

3. 2190940

3. 3715380

3. 1245840

4.4104810

5. 1667440

4. 8978810

1.7142410

1.5453400

$-0.4015020$

0.6469660

1.4327320

1. 2338040

2. 3870140

0.2860260

$-0.4698720$

0.6805780

$-1.2029360$

2. 3840520

$-0.9684490$

$-1.6819030$ 


-5.8414850
-6.1229800
-6.3088390
-7.0962740
-5.4739960
-6.0350400
-3.3032840
-3.8933100
-3.7971140
-2.3316070
-1.3870920
-2.0666540
-3.3336750
-4.2310700
-1.9299020
-1.0412610
-4.9339220
-4.4025960
-2.3134290
-0.4931200
-0.0038600
-1.1834390
-0.9602780
0.3949720
1.5210650
1.2681370
-1.7788110
-1.3705110
-2.0884960
0.4285000
0.5352160
2.4821080
1.6351010
-0.9758480
0.5228230
-0.2489210
0.7324860
1.7915140
-0.1256100
1.9909160
2.4630880
0.0694550
-0.9455330
1.1299260
2.8218730
-0.6063200
-

2.2632230

3.6088710

4.6804300

3.1072830

1.1567460

1.5975650

$-1.0855960$

$-0.9932020$

$-2.3669570$

$-2.8717820$

$-2.1538070$

$-1.7459370$

$-0.2523200$

$-2.3137790$

$-2.7180700$

$-1.2231520$

$-0.6502870$

$-3.0773630$

$-3.9526910$

$-2.7539440$

$-3.9395440$

$-4.7831100$

$-6.2883170$

$-6.6913430$

$-5.9966790$

$-4.5181870$

$-6.8436610$

$-4.6040680$

$-4.4506580$

$-6.3873930$

$-7.7793640$

$-6.1167840$

$-6.4941920$

$-6.5328200$

3.6876050

2. 6407450

4.3589180

5.2626370

4.0655680

5.8522760

5.4840420

4.6675930

3.3689690

5.5595210

6.5392060

4.4384350

-1.5159980
0.2044380
0.0518070
0.2638070
2.7955060
3.6205440
-2.9256140
-4.3113720
-5.0085620
-5.0810510
-4.0760790
-2.7896390
-4.9064810
-6.0148900
-6.0904560
-4.5531300
-4.2616110
-4.4307660
-4.8967770
-3.8891090
-1.5885900
-2.0423440
-1.8466380
-2.4382740
-1.6628180
-1.4150980
-2.3223110
-3.1090840
-1.5191410
-3.4956610
-2.4047680
-2.1814290
-0.6864520
-0.7740260
-0.9940830
-1.0777390
-2.3075540
-2.5109920
-3.3874650
-3.7577590
-1.6869070
-4.6295920
-3.2449390
-4.8213640
-3.9012840
-5.4505540

$-5.4505540$

\begin{abstract}
1.2853700
$-0.3957750$

1.2214390

2. 5428140

1. 3916720

2. 4393070

0.4141450

2.0239300

1.3584560

4.0231380

2. 9409780

1. 5315360

3.9332810

5.0194790

3.0252800

1.2764150

4.1952260

4.6304540

3. 9024260

3.0804510

0.7613200
\end{abstract}

154

TS267 (R,R) G[B97-D/def2-TZVP $]=-3864.564630$

$\begin{array}{lrrr}\mathrm{P} & 0.2455940 & 0.4001130 & 0.8788860 \\ \mathrm{O} & 0.7228810 & -1.0223680 & 1.0184250 \\ \mathrm{O} & -0.4577510 & 1.0286440 & 2.0508770 \\ \mathrm{O} & -0.6792680 & 0.3985800 & -0.4700220 \\ \mathrm{O} & 1.4536300 & 1.4121810 & 0.4972040 \\ \mathrm{C} & -0.9066570 & 1.6140580 & -1.1195990 \\ \mathrm{C} & 0.1571350 & 2.2018360 & -1.8295520 \\ \mathrm{C} & -2.1767880 & 2.1923410 & -1.0394560 \\ \mathrm{C} & -2.3502470 & 3.4486980 & -1.6366500 \\ \mathrm{H} & -3.3261920 & 3.9266950 & -1.5739660 \\ \mathrm{C} & 2.1125050 & 1.1485860 & -0.7104300 \\ \mathrm{C} & 3.3696770 & 0.5378320 & -0.6733840 \\ \mathrm{C} & 1.4669960 & 1.4947120 & -1.9069210 \\ \mathrm{C} & 3.9595580 & 0.2489960 & -1.9077210 \\ \mathrm{H} & 4.9390180 & -0.2265270 & -1.9114050 \\ \mathrm{C} & -3.3186290 & 1.5130630 & -0.3738440 \\ \mathrm{C} & 4.0614710 & 0.1958980 & 0.5962940 \\ \mathrm{C} & -3.8489980 & 0.3248790 & -0.9077570 \\ \mathrm{C} & -3.3530820 & -0.4108200 & -2.1302150 \\ \mathrm{C} & -5.4426990 & -1.5186650 & -1.0537660 \\ \mathrm{C} & -4.9858560 & -0.2741050 & -0.3340870 \\ \mathrm{C} & -3.0140080 & -1.8648080 & -1.7123890 \\ \mathrm{H} & -2.6692590 & -2.4228620 & -2.5915030\end{array}$

$-5.7934100$

$-0.2005580$

0.4227680

2.2189150

2.6570600

1.2734720

2. 2930910

3.3494370

2.9279620

3.7358290

3.7326660

3.6833510

2.4673780

3.7933030

4.6216020

4.6567210

1.5847240

2. 5007830

4.6220850

2.8531860

$\begin{array}{ll}1.4297620 & 2.50220850 \\ 2.4972480 & 4.6220850 \\ 4.8693460 & 2.8531860\end{array}$

$-2.6692590$

-1.8648080
-2.4228620

$-2.5915030$ 


-2.1917010
-4.2712940
-4.5785450
-4.0491790
-6.3248970
-2.4743710
-5.7644110
-6.0500620
-6.6193460
-4.5082020
-4.1700260
-4.7394100
-3.9376140
-3.5128610
-5.6221580
-5.0794910
-4.7006450
-4.4194980
-4.9003900
-5.9632730
-6.2950470
-6.7937040
-6.5122520
-2.6193300
-4.5012090
-4.8736350
-4.2532350
-3.2452280
-3.0127220
-2.3688110
-5.6090690
-6.4980720
5.1587660
5.4869030
4.1800350
4.4367430
6.3273460
6.5100120
7.3012980
5.5513640
5.3725430
6.1284460
3.6330090
6.0377990
3.3798050
3.2469240

$-1.8384720$ $-2.5394580$ $-3.4128330$ $-2.8923990$ $-1.9548420$ 0.0706530 $-1.1292430$ $-2.0342550$ $-0.4430360$ $-0.4635950$ $-1.0389790$ 0.5523300 2.0756220 3.3292590 2. 2135670 1.4741130 4. 3310180

5.2365270

4.6242880

3.6669820

4.2318780

3.6483790

1.7148970

3.7725680

2.2886310

2.8541770

1.2783110

2.9777540

3.9064330

2. 3290670

0.3060140

$-0.1528930$

0.8773920

2.0712750

2.6838040

1.2007220

3.0685060

3.9729800

2.6218860

3.4170910

4.4985620

3.1204790

2.9219090

1.7706630

3.1594550

4.2478150

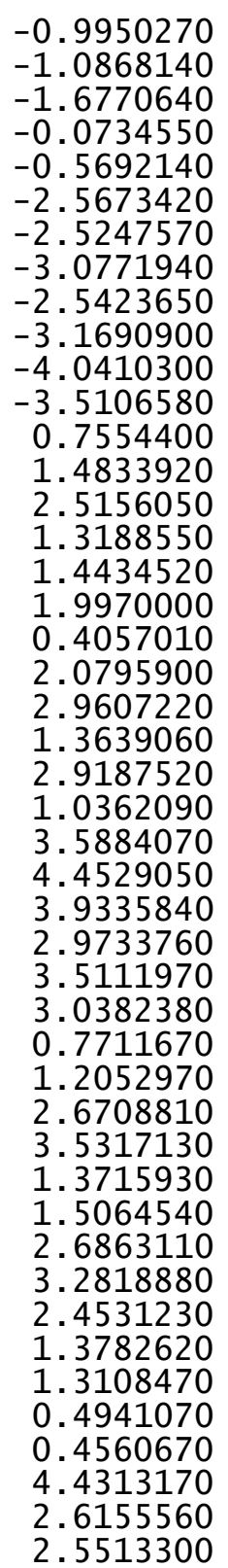

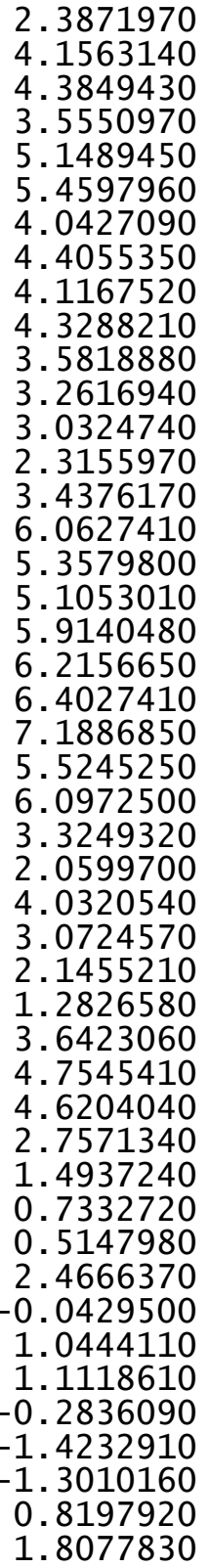

2.7005240

2.7766900

3.6705440

2.1048870

-1. 4459270

$-2.9144560$

$-2.3635750$

$-1.1368540$

$-3.6906820$

$-4.7674750$

$-3.4164610$

$-3.3465250$

$-4.2534400$

$-2.8867530$

$-2.1095560$

$-3.1027140$

$-3.0680000$

$-2.4218700$

$-3.3875050$

$-4.4667680$

$-2.8847750$

$-0.4422400$

$-0.6862600$

0.5072790

1.1272740

0.1154490

$-0.0746220$

1.1413680

1.2724190

$-0.2155250$

0.9058730

$-0.7951110$

2.0481350

1.0653820

2. 2157960

0.4816320

$-0.9799400$

3.4779870

4.2981430

5.7056970

6.3945500

5.4935420

4.1006930

4.4163520

6.3253900
2.6029360

3.9144310

4.5101470

4.5384510

2. 0440400

2.1905210

0.0830800

0.8887800

2.2593520

2. 3067840

3.1767130

1.0019860

0.4272960

1.2944620

$-0.7908130$

3.0872560

$-0.3490760$

$-0.8880170$

$-1.0392780$

0.9168030

0.9948180

0.8627560

2.9366110

3.8322710

$-3.1262250$

$-3.1363050$

$-4.4128940$

$-5.5933590$

$-5.6978230$

$-4.4357610$

$-6.5209180$

$-4.6733750$

$-4.2383940$

$-5.8215060$

$-6.5777770$

$-4.4579750$

$-4.4448350$

$-5.4361470$

$-2.3881020$

$-3.0381460$

$-2.3945180$

$-2.3029620$

$-2.8430860$

$-2.2808160$

$-4.1101640$

$-2.9734790$ 


\begin{tabular}{|c|c|c|c|c|c|c|c|}
\hline $\mathrm{H}$ & -0.2813420 & 7.3408760 & -2.8581810 & $\mathrm{C}$ & -0.8403520 & 1.7621020 & -0.9998250 \\
\hline $\mathrm{H}$ & -1.3462280 & 5.4542660 & -3.9411610 & $\mathrm{C}$ & 0.2565850 & 2.4239260 & -1.5877120 \\
\hline$H$ & 2.0154450 & 3.7998810 & -2.9661260 & $\mathrm{C}$ & -2.1091370 & 2.3401480 & -0.8969480 \\
\hline $\mathrm{H}$ & 1.5358490 & 5.5989180 & -1.3886570 & $\mathrm{C}$ & -2.2599490 & 3.6522490 & -1.3665430 \\
\hline $\mathrm{H}$ & -0.5042040 & 6.6352960 & -1.2559000 & $\mathrm{H}$ & -3.2366320 & 4.1261530 & -1.2869610 \\
\hline$H$ & -2.4020650 & 5.9247830 & -2.6016550 & C & 2.1513940 & 1.1685650 & -0.5470170 \\
\hline $\mathrm{C}$ & -0.5053020 & -3.7902410 & -0.4600880 & $\mathrm{C}$ & 3.3921480 & 0.5239120 & -0.5490310 \\
\hline 0 & 0.2989790 & -2.7892400 & -0.6849040 & $\mathrm{C}$ & 1.5664350 & 1.7240270 & -1.7008000 \\
\hline $\mathrm{C}$ & -0.7122480 & -4.6489360 & -1.6606800 & $\mathrm{C}$ & 4.0538210 & 0.4241440 & -1.7814710 \\
\hline $\mathrm{c}$ & -1.7400400 & -5.6089010 & -1.7108870 & $\mathrm{H}$ & 5.0260150 & -0.0641920 & -1.8130010 \\
\hline c & 0.1163530 & -4.4832480 & -2.7900760 & $\mathrm{C}$ & -3.2661380 & 1.6185040 & -0.3035710 \\
\hline c & -1.9360670 & -6.3792110 & -2.8554430 & $\mathrm{C}$ & 4.0028530 & -0.0428800 & 0.6819830 \\
\hline $\mathrm{H}$ & -2.3888240 & -5.7333870 & -0.8490680 & $\mathrm{C}$ & -3.8178020 & 0.4911590 & -0.9368430 \\
\hline c & -0.0776460 & -5.2618600 & -3.9300930 & C & -3.3400480 & -0.1387280 & -2.2234380 \\
\hline H & 0.9111000 & -3.7448000 & -2.7660980 & $\mathrm{C}$ & -5.4395620 & -1.3095360 & -1.2355020 \\
\hline c & -1.1050460 & -6.2104870 & -3.9687720 & $\mathrm{C}$ & -4.9591590 & -0.1417650 & -0.4094440 \\
\hline $\mathrm{H}$ & -2.7404720 & -7.1107690 & -2.8807970 & $\mathrm{C}$ & -3.0199290 & -1.6283210 & -1.9395420 \\
\hline $\mathrm{H}$ & 0.5737050 & -5.1272960 & -4.7908120 & $\mathrm{H}$ & -2.6814120 & -2.1095690 & -2.8653090 \\
\hline $\mathrm{H}$ & -1.2579540 & -6.8138910 & -4.8610320 & $\mathrm{H}$ & -2.1970870 & -1.6764160 & -1.2245760 \\
\hline $\mathrm{H}$ & 0.4374850 & -2.1004270 & 0.0953920 & $\mathrm{C}$ & -4.2853130 & -2.3400000 & -1.3755230 \\
\hline $\mathrm{s}$ & -1.2475710 & -4.1212500 & 1.0146840 & $\mathrm{H}$ & -4.6105370 & -3.1453330 & -2.0471500 \\
\hline $\mathrm{c}$ & -2.5322000 & -1.3333220 & 2.4900570 & $\mathrm{H}$ & -4.0639590 & -2.7963120 & -0.4044480 \\
\hline c & -1.3618570 & -2.0730840 & 2.9819170 & $\mathrm{H}$ & -6.3250070 & -1.7752320 & -0.7854800 \\
\hline $\mathrm{H}$ & -2.4736810 & -0.9332940 & 1.4754640 & $\mathrm{H}$ & -2.4564450 & 0.3689060 & -2.6194810 \\
\hline $\mathrm{H}$ & -0.4011050 & -1.8409630 & 2.5371640 & C & -5.7663920 & -0.7805570 & -2.6615400 \\
\hline 0 & -1.9571880 & -0.3994250 & 3.4708790 & $\mathrm{H}$ & -6.0764070 & -1.6246910 & -3.2917750 \\
\hline H & -1.3131840 & 0.2313340 & 2.9177230 & $\mathrm{H}$ & -6.6068940 & -0.0785150 & -2.6071870 \\
\hline c & -3.9395450 & -2.4952570 & 4.2612310 & $\mathrm{C}$ & -4.5018700 & -0.0834960 & -3.2554720 \\
\hline C & -2.8625220 & -3.5860410 & 4.3559320 & $\mathrm{H}$ & -4.1784300 & -0.5881730 & -4.1753170 \\
\hline $\mathrm{C}$ & -1.4552440 & -2.9956700 & 4.1556890 & $\mathrm{H}$ & -4.7178540 & 0.9613690 & -3.5080810 \\
\hline $\mathrm{c}$ & -3.9110820 & -1.8190150 & 2.8806580 & $\mathrm{C}$ & -3.8713150 & 2.0876920 & 0.8755180 \\
\hline $\mathrm{H}$ & -4.9331630 & -2.9267670 & 4.4317060 & $\mathrm{C}$ & -3.4279610 & 3.2684060 & 1.7071750 \\
\hline H & -2.9063980 & -4.0934590 & 5.3268520 & $\mathrm{C}$ & -5.5496520 & 2.0981590 & 2.6474120 \\
\hline $\mathrm{H}$ & -1.1526390 & -2.4214300 & 5.0440010 & $\mathrm{C}$ & -5.0195270 & 1.4552980 & 1.3907530 \\
\hline H & -4.2210830 & -2.5342790 & 2.1066170 & $\mathrm{C}$ & -4.6021180 & 4.2853440 & 1.7532740 \\
\hline$H$ & -4.6016450 & -0.9720070 & 2.8271410 & $\mathrm{H}$ & -4.3081860 & 5.1379600 & 2.3795730 \\
\hline $\mathrm{H}$ & -3.7707760 & -1.7410970 & 5.0418640 & $\mathrm{H}$ & -4.7990590 & 4.6667730 & 0.7439310 \\
\hline & -3.0463480 & -4.3437890 & 3.5814530 & C & -5.8723210 & 3.5871340 & 2.3350920 \\
\hline & -0.6977310 & -3.7777540 & 4.0276350 & $\mathrm{H}$ & -6.1960060 & 4.0812350 & 3.2606540 \\
\hline & & & & $\mathrm{H}$ & -6.7038220 & 3.6385690 & 1.6222130 \\
\hline & , R) G[B97-D, & {$[Z \mathrm{VP}]=-3$} & 98 & $\mathrm{H}$ & -6.4458610 & 1.5793700 & 3.0093410 \\
\hline & 0.2049720 & 0.22628070 & 0.8648060 & $\mathrm{H}$ & -2.5293750 & 3.7355160 & 1.2964300 \\
\hline & 0.6368970 & -1.1808560 & 0.8408690 & $\mathrm{C}$ & -4.4263590 & 2.0674690 & 3.7197910 \\
\hline 0 & -0.5295060 & 0.7622330 & 2.0789930 & $\mathrm{H}$ & -4.7898910 & 2.5614350 & 4.6307910 \\
\hline 0 & -0.6557170 & 0.4682680 & -0.5084720 & $\mathrm{H}$ & -4.1908400 & 1.0278780 & 3.9752600 \\
\hline c & 1.4530890 & 1.274945 & 0.6618320 & $\mathrm{C}$ & -3.1625860 & 2.7904400 & 3.1622470 \\
\hline
\end{tabular}




$\begin{array}{lr}\mathrm{H} & -2.9196890 \\ \mathrm{H} & -2.2943080 \\ \mathrm{C} & -5.5681690 \\ \mathrm{H} & -6.4619340 \\ \mathrm{C} & 4.9756400 \\ \mathrm{C} & 5.2913460 \\ \mathrm{C} & 4.1356450 \\ \mathrm{C} & 4.3390800 \\ \mathrm{C} & 6.2157710 \\ \mathrm{H} & 6.4044510 \\ \mathrm{H} & 7.1808880 \\ \mathrm{C} & 5.5255450 \\ \mathrm{H} & 5.3845740 \\ \mathrm{H} & 6.1418710 \\ \mathrm{H} & 3.6515790 \\ \mathrm{H} & 5.7775670 \\ \mathrm{C} & 3.2785420 \\ \mathrm{H} & 3.1815910 \\ \mathrm{H} & 2.2755380 \\ \mathrm{C} & 3.9674300 \\ \mathrm{H} & 4.1956400 \\ \mathrm{H} & 3.3088600 \\ \mathrm{C} & 4.9556570 \\ \mathrm{C} & 5.2365430 \\ \mathrm{C} & 3.9807080 \\ \mathrm{C} & 4.3029150 \\ \mathrm{C} & 3.8874820 \\ \mathrm{H} & 4.0897470 \\ \mathrm{H} & 3.2925360 \\ \mathrm{C} & 3.1224410 \\ \mathrm{H} & 2.9100590 \\ \mathrm{H} & 2.1703930 \\ \mathrm{H} & 3.4417900 \\ \mathrm{H} & 5.7711280 \\ \mathrm{C} & 5.3134150 \\ \mathrm{H} & 5.0848520 \\ \mathrm{H} & 5.9223840 \\ \mathrm{C} & 6.0806030 \\ \mathrm{H} & 6.2614880 \\ \mathrm{H} & 7.0548950 \\ \mathrm{C} & 5.2903780 \\ \mathrm{H} & 5.7965370 \\ \mathrm{C} & 3.4877210 \\ \mathrm{C} & 4.1730780 \\ \mathrm{C} & 4.1566000 \\ \mathrm{C} & 2.7179890 \\ & \end{array}$

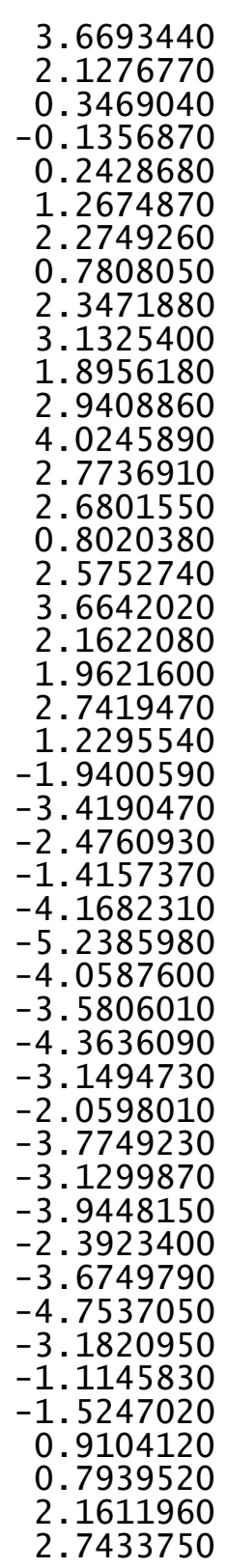

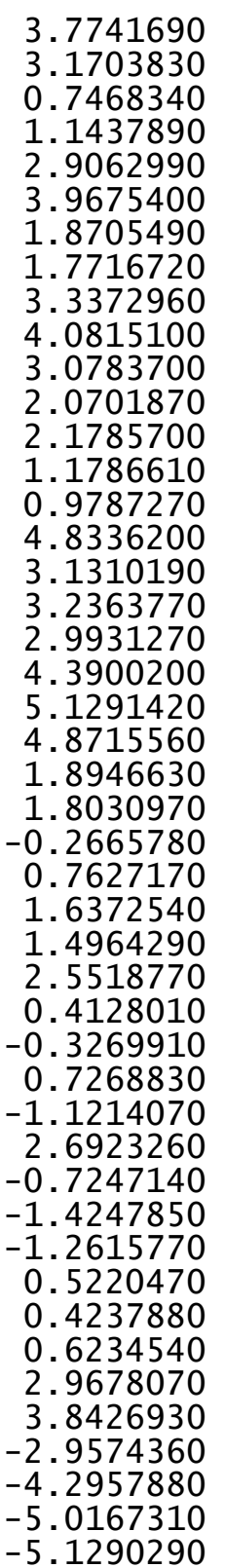

\begin{abstract}
1. 6825210
2. 2384630

3.6413010

4. 6087940

2. 3801230

1.4204790

5. 2008050

4.7875450

2.7236630

0.7603810

0.0771610

1. 1892600

1. 2147590

$-0.1874860$

$-1.2925790$

$-1.1879980$

1. 0225830

1. 9318450

$-0.1661960$

$-1.1605550$

2. 1595180

1. 5943930

$-0.4587680$

$-2.2851850$

$-0.6282050$

0.1591170

$-0.8375940$

$-1.8820770$

0.0080100

$-2.0793640$

$-2.5431730$

$-0.1860350$

0.8169900

$-1.2314490$

$-2.8981070$

0.4793170

$-1.3854060$

0.3154660

$-1.3482440$

$-2.6468660$

$-1.4826260$

$-2.5834880$

$-0.5174190$

$-2.0783130$

$-1.4186950$

$-4.0675260$
\end{abstract}

$\begin{array}{ll}1.9697590 & -4.2705190 \\ 1.5601920 & -2.9274930 \\ 0.0580450 & -4.9209770 \\ 2.0637360 & -6.0115370 \\ 2.7239400 & -6.1727410 \\ 1.0451700 & -4.8090820 \\ 0.4320470 & -4.1712730 \\ 2.8503010 & -4.4409130 \\ 3.7929160 & -4.8147100 \\ 2.5463730 & -4.1734010 \\ 3.7541610 & -2.0115260 \\ 4.6372750 & -2.5206030 \\ 5.9765500 & -1.7423750 \\ 6.6498510 & -1.6474380 \\ 5.8041010 & -2.3285740 \\ 4.3627920 & -1.9009330 \\ 4.8527950 & -3.5871330 \\ 6.6514120 & -2.2261160 \\ 7.6472720 & -2.1040250 \\ 5.8732230 & -3.4199400 \\ 4.1400810 & -2.4433200 \\ 5.7740640 & -0.7335020 \\ 6.7832700 & -0.5930360 \\ 6.2071390 & -2.0949100 \\ -3.7700530 & -0.9020640 \\ -2.7407700 & -1.0396200 \\ -4.5135710 & -2.1767060 \\ -5.4450450 & -2.3238800 \\ -4.2641860 & -3.2776870 \\ -6.1039340 & -3.5358550 \\ -5.6356720 & -1.4837430 \\ -4.9335850 & -4.4850230 \\ -3.5476750 & -3.1790360 \\ -5.8530280 & -4.6205950 \\ -6.8128580 & -3.6357900 \\ -4.7360210 & -5.3225460 \\ -6.3698060 & -5.5654770 \\ -2.1382110 & -0.1931100 \\ -4.2546090 & 0.5411450 \\ -1.6107900 & 2.2575400 \\ -2.3988560 & 2.6858520 \\ -1.1257040 & 1.2808690 \\ -2.1293650 & 2.2729170 \\ -0.7683530 & 3.3195810 \\ -0.0981850 & 2.8344680 \\ -2.9137410 & 3.9166050 \\ & \end{array}$




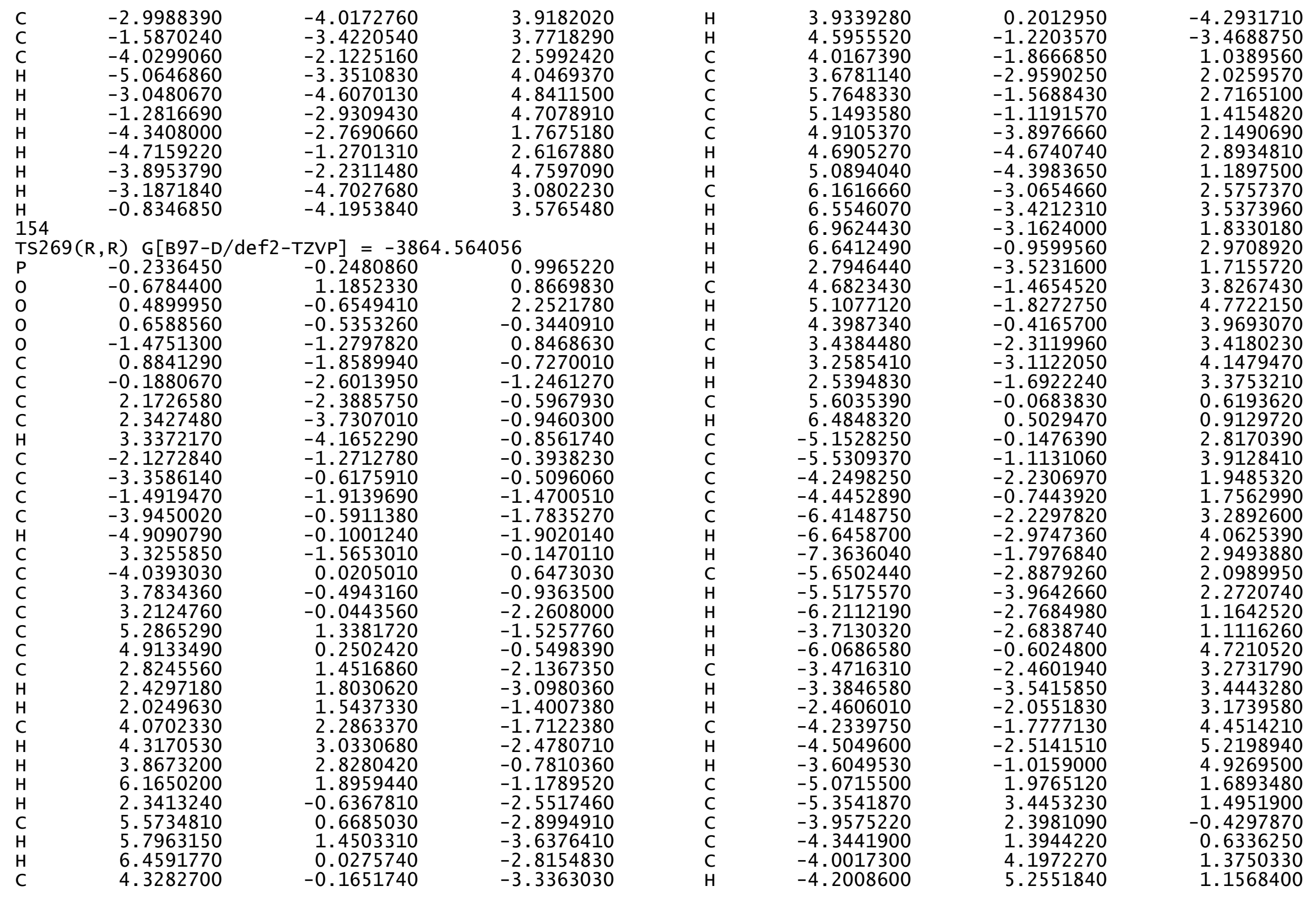




-3.4693290
-3.1513620
-2.8880770
-2.2234780
-3.3604200
-5.9488260
-5.2583090
-4.9851260
-5.8254350
-6.1130720
-6.2988940
-7.0863500
-5.4736550
-6.0367800
-3.3137630
-3.9130820
-3.8584170
-2.4133180
-1.4337690
-2.0793600
-3.3397670
-4.2714340
-2.0142550
-1.1061210
-4.9446080
-4.5060730
-2.4320080
-0.5366060
0.0006580
1.2839420
-1.1882060
-0.7961660
0.3090420
1.5656850
-1.6813200
-1.7223520
-1.9026010
-0.0438600
0.5476330
2.3104870
2.0306560
-0.4324930
0.5179690
-0.2502670
0.7133870
1.7738270

4.1491230

3.5450190

4.2854030

3.1389270

1.9385690

3.8458630

3.0162340

3.7958560

2.2473660

3.6186560

4.6875350

3.1175650

1.2094660

1.6642690

$-1.1505170$

$-1.1110750$

$-2.5201760$

$-3.0919710$

$-2.2995010$

$-1.8111370$

$-0.4149270$

$-2.4916780$

$-3.0798500$

$-1.4075430$

$-0.7406340$

$-3.1775610$

$-4.1411420$

$-2.8906030$

$-3.9738950$

$-4.5353740$

$-4.8561610$

$-6.1828860$

$-6.8704390$

$-5.9915280$

$-6.8267720$

$-5.0720520$

$-4.3191150$

$-7.0250960$

$-7.8571610$

$-6.3872330$

$-6.0340630$

$-5.9926110$

3.6885360

2.6382870

4.3550950

5.2533190

2.3324890
0.2431760
-0.5234050
0.6494510
-1.2215700
2.3253050
-1.0117960
-1.7356000
-1.5505940
0.1485140
-0.0219160
0.2123000
2.7825580
3.5985300
-2.8929490
-4.2770810
-4.9092790
-4.9288170
-4.0206590
-2.7450470
-4.9113050
-5.9253280
-5.9508090
-4.5774650
-4.2381430
-4.3150680
-4.6105650
-3.8205520
-1.5268630
-1.3797310
-1.8734910
-2.5344060
-1.7254390
-1.7154900
-2.6166260
-0.9336770
-2.4995380
-0.6948590
-2.1429550
-1.0122290
-2.7138570
-3.5558580
-1.0355030
-1.1080760
-2.3534150
-2.5731220

$$
\begin{array}{r}
-0.1608060 \\
1.9586460 \\
2.4576110 \\
0.0194930 \\
-0.9818310 \\
1.0813250 \\
2.7908280 \\
-0.6688350 \\
1.2252100 \\
-0.3910220 \\
1.2264670 \\
2.5613680 \\
1.4089890 \\
2.4575020 \\
0.4314780 \\
2.0471580 \\
1.3809450 \\
4.0408750 \\
2.9554160 \\
1.5479930 \\
3.9508940 \\
5.0360200 \\
3.0401760 \\
1.2982030 \\
4.2102930 \\
4.6500580 \\
3.9239380 \\
3.0902020 \\
0.7742510
\end{array}
$$

154

TS270(R,R) G[B97-D/def2-TZVP $]=-3864.563858$

$\begin{array}{lrrr}\mathrm{P} & 0.2413230 & 0.3034170 & 1.0211080 \\ \mathrm{O} & 0.7459710 & -1.1102960 & 0.8950010 \\ \mathrm{O} & -0.4900300 & 0.6837660 & 2.2805120 \\ \mathrm{O} & -0.6698420 & 0.5455930 & -0.3147900 \\ \mathrm{O} & 1.4358130 & 1.3881200 & 0.8588480 \\ \mathrm{C} & -0.9544870 & 1.8579410 & -0.6981100 \\ \mathrm{C} & 0.0863470 & 2.6382650 & -1.2365740 \\ \mathrm{C} & -2.2617020 & 2.3325680 & -0.5514280 \\ \mathrm{C} & -2.4992640 & 3.6705160 & -0.8940020 \\ \mathrm{H} & -3.5092940 & 4.0635770 & -0.7927550 \\ \mathrm{C} & 2.0806350 & 1.4058430 & -0.38501400 \\ \mathrm{C} & 3.3419340 & 0.8120550 & -0.5039480 \\ \mathrm{C} & 1.4178440 & 2.0072700 & -1.4644160 \\ \mathrm{C} & 3.9103130 & 0.8034000 & -1.7812160 \\ \mathrm{H} & 4.8924820 & 0.3498380 & -1.9040600\end{array}$

$\begin{array}{lr}4.0633780 & -3.4206760 \\ 5.8395890 & -3.8237110 \\ 5.4730750 & -1.7586690 \\ 4.6625440 & -4.6664340 \\ 3.3708570 & -3.2651150 \\ 5.5491860 & -4.8745530 \\ 6.5221890 & -3.9802280 \\ 4.4352110 & -5.4774070 \\ 6.0100250 & -5.8494510 \\ 2.0844850 & -0.2261780 \\ 4.2798890 & 0.3721970 \\ 1.7610110 & 2.1896420 \\ 2.5617410 & 2.6218020 \\ 1.2139330 & 1.2499370 \\ 2.2620170 & 2.2632750 \\ 0.9858490 & 3.3292520 \\ 0.2715190 & 2.9168360 \\ 3.1876950 & 3.6877400 \\ 4.2747520 & 3.6743330 \\ 3.6517600 & 3.6366270 \\ 2.3095040 & 2.4296870 \\ 3.6458410 & 3.7377230 \\ 4.9222130 & 4.5550700 \\ 3.2159340 & 4.6154590 \\ 2.8991360 & 1.5396720 \\ 1.4695520 & 2.4721320 \\ 2.5600860 & 4.5816900 \\ 4.9081610 & 2.7864180 \\ 4.4012390 & 3.4325800\end{array}$

$4.0633780 \quad-3.4206760$

$-3.8237110$

$-4.6664340$

$-3.2651150$

$-4.8745530$

$-5.4774070$

$-5.8494510$

$-0.2261780$

$\begin{array}{ll}1.7610110 & 2.1896420\end{array}$

2.6218020

. 2499370

2.2632750
3.3292520

2.9168360

6877400

3.6366270

.

4.5550700

1.5396720

4721320

.4325800

0.2413230

0.7459710

0.9544870

4.8924820

0.3498380

$-1.9040600$ 


\begin{tabular}{|c|c|c|}
\hline $\begin{array}{l}-3.3714270 \\
4.0618440 \\
-3.7894990 \\
-3.2163450 \\
-5.2118070 \\
-4.8774620 \\
-2.7555570 \\
-2.3585970 \\
-1.9417010 \\
-3.9540070 \\
-4.1737140 \\
-3.7141110 \\
-6.0581190 \\
-2.3781530 \\
-5.5480100 \\
-5.7403340 \\
-6.4632110 \\
-4.3503950 \\
-3.9516380 \\
-4.6699010 \\
-4.0610400 \\
-3.7672520 \\
-5.7690080 \\
-5.1495770 \\
-5.0460470 \\
-4.8582410 \\
-5.2631960 \\
-6.2457230 \\
-6.6416420 \\
-7.0618900 \\
-6.6093220 \\
-2.9185580 \\
-4.6678210 \\
-5.0987820 \\
-4.3265720 \\
-3.4770190 \\
-3.3363500 \\
-2.5445420 \\
-5.5643000 \\
-6.4121820 \\
5.1895900 \\
5.5270410 \\
4.1749180 \\
4.4426640 \\
6.3475010 \\
6.5438930\end{array}$ & $\begin{array}{r}1.4609070 \\
0.2091230 \\
0.3633820 \\
-0.0712500 \\
-1.5431520 \\
-0.4303200 \\
-1.5458980 \\
-1.8875180 \\
-1.5914790 \\
-2.4343150 \\
-3.2012690 \\
-2.9542710 \\
-2.1388570 \\
0.5593220 \\
-0.9018660 \\
-1.7011050 \\
-0.3045430 \\
-0.0125980 \\
-0.3670540 \\
1.0274480 \\
1.7401290 \\
2.8607930 \\
1.3736380 \\
0.9413400 \\
3.7350800 \\
4.5346130 \\
4.2093350 \\
2.8455060 \\
3.1928880 \\
2.8908440 \\
0.7233770 \\
3.4666850 \\
1.3381950 \\
1.6844260 \\
0.3070410 \\
2.2469390 \\
3.0667150 \\
1.6786340 \\
-0.1364760 \\
-0.7476050 \\
0.4305420 \\
1.4114130 \\
2.4666960 \\
0.9926820 \\
2.5737850 \\
3.3282820\end{array}$ & $\begin{array}{r}-0.0844100 \\
0.6477250 \\
-0.8591030 \\
-2.1873620 \\
-1.4126390 \\
-0.4508930 \\
-2.0555480 \\
-3.0195000 \\
-1.3312160 \\
-1.6052740 \\
-2.3592170 \\
-0.6704450 \\
-1.0491080 \\
-2.4948320 \\
-2.7889240 \\
-3.5168540 \\
-2.7001950 \\
-3.2488820 \\
-4.2084380 \\
-3.3852860 \\
1.1080880 \\
2.0779280 \\
2.8127110 \\
1.5080700 \\
2.2034480 \\
2.9320060 \\
1.2386200 \\
2.6600550 \\
3.6235530 \\
1.9292920 \\
3.0858980 \\
1.7498390 \\
3.9087070 \\
4.8574860 \\
4.0544620 \\
3.4757110 \\
4.1924700 \\
3.4322470 \\
0.7267310 \\
1.0379080 \\
2.8047870 \\
3.9000150 \\
1.9490120 \\
1.7522580 \\
3.2743300 \\
4.0479120\end{array}$ \\
\hline
\end{tabular}

7.3155440
5.5419380
5.3564800
6.1010400
3.6092310
6.0963860
3.3970000
3.2582500
2.4062690
4.2012670
4.4387740
3.6155860
5.2010400
5.5514800
4.0711190
4.4311380
4.2353830
4.4821280
3.7218190
3.3307420
3.0784280
2.3977700
3.4379220
6.1801470
5.3866350
5.1349900
5.9053320
6.2936580
6.5338000
7.2394680
5.5780830
6.1731350
1.9906200
1.2251380
1.7300210
3.2528070
3.9130780
3.2536320
1.2357410
1.3141870
0.1565250
3.5152470
3.6296930
4.9806420
3.8647540
1.4662560

2. 1927000

3.1933790

4. 2615380

3.1018550

2.8938600

0.9268150

2.6538930

3. 7291330

2. 2000980

0059730

2.7515430

1. 2104620

$-1.6943870$

- 3.1482620

$-2.1726390$

$-1.1488440$

$-3.9653050$

5.0109100

-3. 9488620

$-3.3505750$

$-4.1028560$

$-2.9806570$

$-1.7443330$

$-3.5179740$

$-2.7336600$

$-3.5313870$

$-1.9437760$

$-3.2855370$

$-4.3433320$

$-2.7324240$

$-0.9085790$

$-1.3347940$

1. 9218620

2. 4233340

1.8285380

1.9782710

1.1516360

1. 3178210

2. 3324060

3. 5155090

2. 2161310

3.0403560

1. 6560880

1. 3968590

0.0864140

0.7615230
2.9279130

2.0898600

2. 2646590

1.1508780

1.1174530

4.7026080

3.2806380

3.4563320

3. 1877840

4.4505100

5. 2212060

4.9263470

1.6756920

1. 4772680

$-0.4228220$

0.6305390

1. 3815770

1. 1529240

2. 3503340

0.2713300

$-0.4872010$

0.7003680

$-1.2039010$

2. 2965220

$-1.0287780$

$-1.7406290$

$-1.5854860$

0.1170580

$-0.0545050$

0.1619020

2. 7645020

3. 5732520

$-2.7545200$

$-3.9671660$

$-5.2880140$

$-5.3739030$

$-4.2638030$

$-2.9036100$

6.1285330

$-4.0283170$

$-3.8309690$

$-5.2539130$

$-6.3531090$

4.1791600

$-4.5413860$

$-5.3414850$ 


\begin{tabular}{|c|c|c|}
\hline $\begin{array}{l}-1.4741780 \\
-1.7030300 \\
-1.0538800 \\
0.4643020 \\
0.8907180 \\
-0.1747880 \\
-1.2469340 \\
-1.2201720 \\
1.0437420 \\
1.0804630 \\
-2.7766480 \\
-1.5589950 \\
0.7392220 \\
1.8363870 \\
-0.3374610 \\
0.3933400 \\
-0.5143860 \\
-1.5544610 \\
0.3547450 \\
-1.7246080 \\
-2.2345560 \\
0.1899330 \\
1.1595440 \\
-0.8519800 \\
-2.5416560 \\
0.8743930 \\
-0.9841790 \\
0.5084620 \\
-1.0214930 \\
-2.4499060 \\
-1.2543640 \\
-2.3868910 \\
-0.2987980 \\
-1.9526220 \\
-1.3274230 \\
-3.8341100 \\
-2.7001080 \\
-1.3244530 \\
-3.8084820 \\
-4.8059920 \\
-2.7399360 \\
-1.0799370 \\
-4.0585580 \\
-4.5438190 \\
-3.7298910 \\
-2.8202880\end{array}$ & $\begin{array}{r}4.5052850 \\
5.9585480 \\
6.2855500 \\
5.9651540 \\
4.9952370 \\
3.9927040 \\
6.5960810 \\
7.3390030 \\
6.8902060 \\
5.6028640 \\
6.1823810 \\
5.6803360 \\
5.5384300 \\
4.5039430 \\
-3.6712900 \\
-2.5951670 \\
-4.3503930 \\
-5.2728920 \\
-4.0451990 \\
-5.8698890 \\
-5.5019780 \\
-4.6556190 \\
-3.3333150 \\
-5.5664990 \\
-6.5710700 \\
-4.4180040 \\
-6.0358830 \\
-2.0287050 \\
-4.2799900 \\
-1.8239140 \\
-2.5704900 \\
-1.2749580 \\
-2.2255380 \\
-1.0226410 \\
-0.2804230 \\
-3.3196000 \\
-4.3551560 \\
-3.6667220 \\
-2.4351550 \\
-3.8233120 \\
-5.0106790 \\
-3.2212350 \\
-3.0339380 \\
-1.6277520 \\
-2.6895160 \\
-4.9888960\end{array}$ & $\begin{array}{l}-1.3351640 \\
-1.6685800 \\
-3.0301680 \\
-3.0334550 \\
-1.8949190 \\
-1.5156340 \\
-0.8932520 \\
-3.2878100 \\
-2.9221910 \\
-0.9960640 \\
-1.6826710 \\
-3.7944400 \\
-4.0053700 \\
-2.1376660 \\
-0.9903940 \\
-1.0705930 \\
-2.3043720 \\
-2.5201730 \\
-3.3718660 \\
-3.7677790 \\
-1.7052680 \\
-4.6143410 \\
-3.2188340 \\
-4.8188320 \\
-3.9218840 \\
-5.4256490 \\
-5.7913020 \\
-0.1928400 \\
0.4217240 \\
2.2638150 \\
2.6754790 \\
1.3217750 \\
2.2994630 \\
3.3927570 \\
2.9670790 \\
3.7857470 \\
3.7482120 \\
3.6904760 \\
2.5291060 \\
3.8525510 \\
4.6261260 \\
4.6663770 \\
1.6426040 \\
2.5877190 \\
4.6795540 \\
2.8584010\end{array}$ \\
\hline
\end{tabular}

$\begin{array}{lrrr}\text { H } & -0.5193750 & -4.3780420 & 3.4717590 \\ \text { I54 } & & & \\ \text { TS271(R, R) G[B97-D/def2-TZVP] }=-3864.563721 \\ \text { P } & 0.2408890 & 0.2920200 & 1.0209860 \\ \text { O } & 0.7541540 & -1.1177980 & 0.8851670 \\ \text { O } & -0.4914040 & 0.6603730 & 2.2832970 \\ \text { O } & -0.6728820 & 0.5364920 & -0.3125360 \\ \text { O } & 1.4285780 & 1.3853000 & 0.8649870 \\ \mathrm{C} & -0.9583300 & 1.8501590 & -0.6912410 \\ \mathrm{C} & 0.0808530 & 2.6285010 & -1.2330990 \\ \mathrm{C} & -2.2640140 & 2.3274590 & -0.5361910 \\ \mathrm{C} & -2.5017040 & 3.6659780 & -0.8761420 \\ \mathrm{H} & -3.5109590 & 4.0599970 & -0.7710710 \\ \mathrm{C} & 2.0807810 & 1.4125120 & -0.3744830 \\ \mathrm{C} & 3.3498280 & 0.8343180 & -0.4847620 \\ \mathrm{C} & 1.4159550 & 2.0039600 & -1.4592320 \\ \mathrm{C} & 3.9346680 & 0.8491570 & -1.7549190 \\ \mathrm{H} & 4.9250080 & 0.4119320 & -1.8703390 \\ \mathrm{C} & -3.3726760 & 1.4550550 & -0.0679040 \\ \mathrm{C} & 4.0615860 & 0.2206970 & 0.6663830 \\ \mathrm{C} & -3.7956460 & 0.3625250 & -0.8471040 \\ \mathrm{C} & -3.2311430 & -0.0629400 & -2.1820740 \\ \mathrm{C} & -5.2252880 & -1.5364930 & -1.4072970 \\ \mathrm{C} & -4.8827660 & -0.4321180 & -0.4386110 \\ \mathrm{C} & -2.7733340 & -1.5397490 & -2.0660160 \\ \mathrm{H} & -2.3833610 & -1.8737880 & -3.0354920 \\ \mathrm{H} & -1.9549920 & -1.5939250 & -1.3472930 \\ \mathrm{C} & -3.9710390 & -2.4291780 & -1.6159710 \\ \mathrm{H} & -4.1978630 & -3.1884300 & -2.3756030 \\ \mathrm{H} & -3.7262320 & -2.9585890 & -0.6877140 \\ \mathrm{H} & -6.0707920 & -2.1332250 & -1.0435660 \\ \mathrm{H} & -2.3933810 & 0.5680720 & -2.4895270 \\ \mathrm{C} & -5.5687120 & -0.8824980 & -2.7758460 \\ \mathrm{H} & -5.7680890 & -1.6748630 & -3.5093830 \\ \mathrm{H} & -6.4816710 & -0.2835040 & -2.6759110 \\ \mathrm{C} & -4.3717130 & 0.0075240 & -3.2359560 \\ \mathrm{H} & -3.9799450 & -0.3398630 & -4.2009730 \\ \mathrm{H} & -4.6892170 & 1.0495480 & -3.3614860 \\ \mathrm{C} & -4.0567680 & 1.7285460 & 1.1289690 \\ \mathrm{C} & -3.7580900 & 2.8442640 & 2.1028910 \\ \mathrm{C} & -5.7583510 & 1.3553800 & 2.8384540 \\ \mathrm{C} & -5.1444400 & 0.9286960 & 1.5293700 \\ \mathrm{C} & -5.0356810 & 3.7191000 & 2.2372560 \\ \mathrm{H} & -4.8444270 & 4.5152240 & 2.9686380 \\ \mathrm{H} & -5.2561640 & 4.1978080 & 1.2753830 \\ \mathrm{C} & -6.2343050 & 2.8284150 & 2.6945190\end{array}$




\begin{tabular}{|c|c|c|}
\hline $\begin{array}{l}-6.6260200 \\
-7.0534080 \\
-6.5982660 \\
-2.9101360 \\
-4.6529810 \\
-5.0800060 \\
-4.3121170 \\
-3.4631520 \\
-3.3194000 \\
-2.5311540 \\
-5.5636220 \\
-6.4110060 \\
5.1644050 \\
5.4800040 \\
4.1389610 \\
4.4237140 \\
6.2948340 \\
6.4737880 \\
7.2711430 \\
5.4966310 \\
5.2982800 \\
6.0672600 \\
3.5780450 \\
6.0455080 \\
3.3441950 \\
3.1917540 \\
2.3595030 \\
4.1416430 \\
4.3616070 \\
3.5588600 \\
5.2042070 \\
5.5645160 \\
4.0955820 \\
4.4414060 \\
4.2534750 \\
4.5074720 \\
3.7321610 \\
3.3544010 \\
3.1099790 \\
2.4173070 \\
3.4669440 \\
6.1879740 \\
5.4184060 \\
5.1759820 \\
5.9376720 \\
6.3192270\end{array}$ & $\begin{array}{r}3.1716320 \\
2.8778960 \\
0.7046940 \\
3.4508710 \\
1.3138030 \\
1.6556720 \\
0.2816260 \\
2.2238000 \\
3.0401760 \\
1.6552550 \\
-0.1444840 \\
-0.7562500 \\
0.4168890 \\
1.3823070 \\
2.4572310 \\
0.9895080 \\
2.5641000 \\
3.3074330 \\
2.1996290 \\
3.1950690 \\
4.2582800 \\
3.1244050 \\
2.8927730 \\
0.8897890 \\
2.6131690 \\
3.6835730 \\
2.1507060 \\
1.9530180 \\
2.6868920 \\
1.1429080 \\
-1.6900700 \\
-3.1391180 \\
-2.1456740 \\
-1.1341530 \\
-3.9615230 \\
-5.0024180 \\
-3.9618340 \\
-3.3353710 \\
-4.0785610 \\
-2.9738750 \\
-1.7103010 \\
-3.5167290 \\
-2.6934830 \\
-3.4843350 \\
-1.8950990 \\
-3.2539710\end{array}$ & $\begin{array}{r}3.6612230 \\
1.9673110 \\
3.1118640 \\
1.7741330 \\
3.9299410 \\
4.8820710 \\
4.0692990 \\
3.4967760 \\
4.2167870 \\
3.4472600 \\
0.7440580 \\
1.0553420 \\
2.8386560 \\
3.9537650 \\
2.0057630 \\
1.7872670 \\
3.3574080 \\
4.1458710 \\
3.0162580 \\
2.1740720 \\
2.3640450 \\
1.2402260 \\
1.1753270 \\
4.7542530 \\
3.3314710 \\
3.5244150 \\
3.2189850 \\
4.4992500 \\
5.2861960 \\
4.9535970 \\
1.6771440 \\
1.4619110 \\
-0.4375890 \\
0.6323460 \\
1.3439140 \\
1.1021280 \\
2.3086670 \\
0.2354170 \\
-0.5346100 \\
0.6627350 \\
-1.2184310 \\
2.2815440 \\
-1.0398270 \\
-1.7624380 \\
-1.5837610 \\
0.1066290\end{array}$ \\
\hline
\end{tabular}

6.5681750

608060

5.5641730

6.1535370

3.2833260

2. 0021350

3.9942570

3.0417030

2.0775980

1. 2088120

3.6179290

4.7102400

4.5910500

2.6573750

1.4327790

0.5929770

0.4991770

2.4609560

$-1.4776370$

$-1.7034090$

$-1.0898740$

0.4173790

0.8733480

$-0.1803110$

$-1.2196830$

$-1.2304400$

1.0330960

1.0689680

$-2.7746400$

$-1.6445750$

0.6266380

1. 8192310

$-0.3341550$

0.3992470

$-0.5081340$

$-1.5513850$

0.3669750

$-1.7188260$

$-2.2359970$

0.2048380

1. 1742570

$-0.8402940$

$-2.5383420$

0.8939420

$-0.9703520$

0.5144340
-4.3077790
-2.6951170
-0.9182850
-1.3526620
1.3628330
1.9319610
1.3027630
1.4017900
2.5749870
2.3374420
1.5215370
2.1389520
0.3824690
3.5019180
2.7115870
3.2148590
1.5212070
0.4713830
4.4983500
5.9481590
6.2519660
5.8781260
4.9790560
3.9837600
6.5922940
7.3111660
6.7860840
5.6327600
6.1829570
5.6678020
5.3646880
4.4863600
-3.6578420
-2.5825050
-4.3237230
-5.2394780
-4.0124320
-5.8240380
-5.4730030
-4.6105870
-3.3056290
-5.5147850
-6.5200270
-4.3685760
-5.9744810
-2.0260670

$-0.0764190$

0.1668780

2.7816560

3.5902190

$-2.8801080$

$-2.7435760$

$-4.2229610$

$-5.4209530$

$-5.2163690$

$-3.9758570$

$-6.3475390$

$-4.2764290$

$-4.2753450$

$-5.0878130$

$-6.0941900$

$-3.7743110$

$-4.1901560$

$-5.5107220$

$-1.3254200$

$-1.6744790$

$-3.0599030$

$-3.1211080$

$-1.9390790$

$-1.5105890$

$-0.9221000$

$-3.3090110$

$-3.1130710$

$-1.0746550$

$-1.6627630$

$-3.8056790$

4.0671200

$-2.1789900$

$-1.0287070$

$-1.0959800$

$-2.3498060$

$-2.5786140$

$-3.4106110$

$-3.8324440$

$-1.7687840$

$-4.6594130$

$-3.2473660$

$-4.8768510$

$-3.9966460$

$-5.4654820$

$-5.8542270$

$-0.2119590$ 


\begin{tabular}{|c|c|c|c|c|c|c|c|}
\hline $\mathrm{S}$ & -1.0249450 & -4.2783890 & 0.3748490 & $\mathrm{H}$ & 4.2717730 & 30305120 & -25549700 \\
\hline c & -2.4513610 & -1.8468850 & 2.2512770 & $\mathrm{H}$ & 3.8036910 & 2.8886950 & -0.8565810 \\
\hline C & -1.2515690 & -2.5944540 & 2.6485190 & $\mathrm{H}$ & 6.1266770 & 1.9895660 & -1.1867210 \\
\hline $\mathrm{H}$ & -2.3948690 & -1.2874760 & 1.3149790 & $\mathrm{H}$ & 2.3760620 & -0.6803230 & -2.4996930 \\
\hline $\mathrm{H}$ & -0.2987210 & -2.2427170 & 2.2718510 & C & 5.5836030 & 0.6803930 & -2.8621270 \\
\hline 0 & -1.9502130 & -1.0564360 & 3.3863440 & $\mathrm{H}$ & 5.7994890 & 1.4365560 & -3.6285540 \\
\hline $\mathrm{H}$ & -1.3277580 & -0.3098570 & 2.9653960 & $\mathrm{H}$ & 6.4814350 & 0.0626860 & -2.7411330 \\
\hline C & -3.8227110 & -3.3646740 & 3.7633550 & C & 4.3619980 & -0.1965730 & -3.2801870 \\
\hline C & -2.6858650 & -4.3961400 & 3.7071040 & $\mathrm{H}$ & 3.9719700 & 0.1233890 & -4.2553430 \\
\hline C & -1.3128070 & -3.7025270 & 3.6510840 & $\mathrm{H}$ & 4.6528870 & -1.2502290 & -3.3676400 \\
\hline C & -3.8067520 & -2.4650070 & 2.5172250 & C & 4.0428650 & -1.7373440 & 1.1491690 \\
\hline $\mathrm{H}$ & -4.7927400 & -3.8720410 & 3.8294510 & $\mathrm{C}$ & 3.7208890 & -2.8016670 & 2.1722130 \\
\hline $\mathrm{H}$ & -2.7193020 & -5.0626770 & 4.5769550 & $\mathrm{C}$ & 5.7705410 & -1.3422760 & 2.8279110 \\
\hline $\mathrm{H}$ & -1.0651780 & -3.2676910 & 4.6310080 & $\mathrm{C}$ & 5.1540300 & -0.9503630 & 1.5088720 \\
\hline $\mathrm{H}$ & -4.0601590 & -3.0538200 & 1.6250080 & C & 4.9728510 & -3.7079960 & 2.3362390 \\
\hline $\mathrm{H}$ & -4.5440010 & -1.6604510 & 2.5896750 & $\mathrm{H}$ & 4.7648330 & -4.4613640 & 3.1072720 \\
\hline $\mathrm{H}$ & -3.7152820 & -2.7451230 & 4.6641040 & $\mathrm{H}$ & 5.1690350 & -4.2393270 & 1.3970210 \\
\hline $\mathrm{H}$ & -2.8088140 & -5.0191290 & 2.8101290 & C & 6.2024320 & -2.8331170 & 2.7393770 \\
\hline $\mathrm{H}$ & -0.5062820 & -4.4082770 & 3.4201260 & $\mathrm{H}$ & 6.5988230 & -3.1470260 & 3.7141030 \\
\hline 15 & & & & $\mathrm{H}$ & 7.0089000 & -2.9362820 & 2.0036680 \\
\hline TS & R) $\mathrm{G}[\mathrm{B} 97-\mathrm{D} /$ & {$[Z \mathrm{VP}]=-386$} & 479 & $\mathrm{H}$ & 6.6317630 & -0.7060210 & 3.0666810 \\
\hline $\mathrm{P}$ & -0.2022780 & -0.2081880 & 1.0038560 & $\mathrm{H}$ & 2.8522280 & -3.3961060 & 1.8775590 \\
\hline 0 & -0.6945580 & 1.2008080 & 0.8004890 & C & 4.6791900 & -1.2260760 & 3.9277570 \\
\hline 0 & 0.5502470 & -0.5201920 & 2.2691120 & $\mathrm{H}$ & 5.1061040 & -1.5478880 & 4.8868950 \\
\hline 0 & 0.6731740 & -0.5453640 & -0.3340170 & $\mathrm{H}$ & 4.3732590 & -0.1791040 & 4.0342650 \\
\hline 0 & -1.4089920 & -1.2874220 & 0.9298190 & C & 3.4567780 & -2.1121750 & 3.5391410 \\
\hline $\mathrm{C}$ & 0.9298560 & -1.8824690 & -0.6439780 & $\mathrm{H}$ & 3.2884810 & -2.8909930 & 4.2945580 \\
\hline C & -0.1281920 & -2.6747770 & -1.1324210 & $\mathrm{H}$ & 2.5447000 & -1.5147280 & 3.4682290 \\
\hline $\mathrm{C}$ & 2.2307420 & -2.3679820 & -0.4790450 & C & 5.5894100 & 0.0828710 & 0.6801140 \\
\hline C & 2.4506060 & -3.7240670 & -0.7552460 & $\mathrm{H}$ & 6.4542130 & 0.6856680 & 0.9592530 \\
\hline H & 3.4550430 & -4.1255250 & -0.6338810 & C & -5.0813850 & -0.1501410 & 2.9314010 \\
\hline C & -2.0878580 & -1.3649010 & -0.2932470 & C & -5.3944960 & -1.0562710 & 4.0958110 \\
\hline $\mathrm{C}$ & -3.3435720 & -0.7591240 & -0.4042780 & C & -4.1049180 & -2.2455390 & 2.1793130 \\
\hline $\mathrm{C}$ & -1.4619270 & -2.0458710 & -1.3548060 & $\mathrm{C}$ & -4.3679820 & -0.7844820 & 1.8963730 \\
\hline C & -3.9700670 & -0.8193950 & -1.6575400 & $\mathrm{C}$ & -6.2379940 & -2.2513980 & 3.5691080 \\
\hline $\mathrm{H}$ & -4.9506290 & -0.3611360 & -1.7710930 & $\mathrm{H}$ & -6.4132770 & -2.9544390 & 4.3944930 \\
\hline C & 3.3555320 & -1.4946600 & -0.0528780 & $\mathrm{H}$ & -7.2151200 & -1.8879240 & 3.2291640 \\
\hline $\mathrm{C}$ & -4.0139120 & -0.0764450 & 0.7334980 & C & -5.4724650 & -2.9493410 & 2.4026060 \\
\hline $\mathrm{C}$ & 3.7950720 & -0.4419880 & -0.8759430 & $\mathrm{H}$ & -5.2904630 & -4.0067400 & 2.6364460 \\
\hline C & 3.2305650 & -0.0574290 & -2.2233540 & $\mathrm{H}$ & -6.0580100 & -2.9095560 & 1.4761630 \\
\hline C & 5.2646920 & 1.3989340 & -1.5205640 & $\mathrm{H}$ & -3.5652270 & -2.7294220 & 1.3618450 \\
\hline C & 4.9031360 & 0.3426230 & -0.5060040 & $\mathrm{H}$ & -5.9384730 & -0.5167140 & 4.8807100 \\
\hline C & 2.8086610 & 1.4331770 & -2.1647760 & C & -3.2917740 & -2.3540020 & 3.4983400 \\
\hline H & 2.4180390 & 1.7358620 & -3.1443730 & $\mathrm{H}$ & -3.1568830 & -3.4168050 & 3.7400740 \\
\hline $\mathrm{H}$ & 1.9980810 & 74400 & -1.4424220 & $\mathrm{H}$ & -2.3000270 & -1.9179290 & 3.3480860 \\
\hline c & 4.0307130 & 18970 & -1.7607470 & C & -4.0563100 & -1.6228640 & 4.6458460 \\
\hline
\end{tabular}




\begin{tabular}{|c|c|c|c|}
\hline & & & \\
\hline $\mathrm{H}$ & $\begin{array}{l}-4.2732460 \\
-3.4516120\end{array}$ & -2.3126990 & $\begin{array}{l}5.4724620 \\
5.0481200\end{array}$ \\
\hline C & -5.1045290 & 1.9002730 & 1.6725030 \\
\hline c & -5.4425050 & 3.3440590 & 1.3956260 \\
\hline C & -4.0229330 & 2.2370000 & -0.4778550 \\
\hline C & -4.3694990 & 1.2825730 & 0.6427960 \\
\hline C & -4.1186050 & 4.1353340 & 1.2204990 \\
\hline $\mathrm{H}$ & -4.3570330 & 5.1687850 & 0.9348820 \\
\hline $\mathrm{H}$ & -3.5819780 & 4.1698970 & 2.1762230 \\
\hline C & -3.2487870 & 3.4421020 & 0.1285450 \\
\hline $\mathrm{H}$ & -3.0025890 & 4.1447740 & -0.6780990 \\
\hline $\mathrm{H}$ & -2.3122300 & 3.0820220 & 0.5581810 \\
\hline $\mathrm{H}$ & -3.4155100 & 1.7532240 & -1.2470530 \\
\hline $\mathrm{H}$ & -6.0456700 & 3.7708920 & 2.2062390 \\
\hline C & -5.3444640 & 2.7826660 & -1.0849880 \\
\hline $\mathrm{H}$ & -5.0986390 & 3.5355850 & -1.8458960 \\
\hline $\mathrm{H}$ & -5.8869060 & 1.9712930 & -1.5853720 \\
\hline C & -6.2163450 & 3.4110580 & 0.0482940 \\
\hline $\mathrm{H}$ & -6.4495600 & 4.4598280 & -0.1787010 \\
\hline $\mathrm{H}$ & -7.1665640 & 2.8729820 & 0.1486760 \\
\hline C & -5.4596630 & 1.1876660 & 2.8175760 \\
\hline $\mathrm{H}$ & -6.0283230 & 1.6700300 & 3.6136530 \\
\hline C & -3.3584770 & -1.4239430 & -2.7527610 \\
\hline c & -3.9941330 & -1.4623800 & -4.1200110 \\
\hline C & -3.9436650 & -2.8960520 & -4.6943650 \\
\hline c & -2.5071710 & -3.4934040 & -4.6528840 \\
\hline C & -1.5021420 & -2.6059730 & -3.8746360 \\
\hline C & -2.1011810 & -2.0430430 & -2.6092060 \\
\hline $\mathrm{H}$ & -3.4418380 & -0.7915500 & -4.7983950 \\
\hline $\mathrm{H}$ & -4.3291060 & -2.8954390 & -5.7215150 \\
\hline $\mathrm{H}$ & -2.1232080 & -3.6379380 & -5.670433 \\
\hline $\mathrm{H}$ & -1.2298070 & -1.7544870 & -4.5184830 \\
\hline H & -5.0274350 & -1.0977630 & -4.0742890 \\
\hline $\mathrm{H}$ & -4.6183910 & -3.5224730 & -4.0973280 \\
\hline $\mathrm{H}$ & -2.5400670 & -4.4824330 & -4.1834240 \\
\hline $\mathrm{H}$ & -0.5794330 & -3.1562880 & -3.678131 \\
\hline C & 1.4147350 & -4.5624810 & -1.1606540 \\
\hline C & 1.6162450 & -6.0336030 & -1.4210870 \\
\hline C & 1.0118680 & -6.4268440 & -2.7887410 \\
\hline C & -0.4585360 & -5.9403210 & -2.943536 \\
\hline C & -0.9307050 & -5.0447310 & -1.7699450 \\
\hline C & 0.1214050 & -4.0415570 & -1.362509 \\
\hline $\mathrm{H}$ & 1.1115820 & -6.6148220 & -0.632304 \\
\hline $\mathrm{H}$ & 1.0715560 & -7.5146880 & -2.91747 \\
\hline $\mathrm{H}$ & -1.1395800 & -6.7972000 & -3.018544 \\
\hline $\mathrm{H}$ & -1.1250280 & -5.6945720 & -0.9019480 \\
\hline
\end{tabular}
2.6817710
1. 6313440
$-0.5567890$
$-1.8784860$
0.3863810
$-0.3584560$
0.4942050
1. 5413370
$-0.4561290$
1.6379760
2.2829930
$-0.3646350$
$-1.2664290$
0.6838690
2.4603820
$-1.1115670$
0.7580040
$-0.4535750$
1.1547560
2. 5698060
1. 3789950
2. 5113500
0.4215170
2.0531370
1. 4150660
3.9540630
2.8332380
1.4502000
3.9320780
4.9312270
2.8716030
1.1877730
4.1961510
4.6591160
3.8313190
2. 9716910
0.6568040

154

$\operatorname{TS} 273(\mathrm{~S}, \mathrm{~S}) \mathrm{G}[\mathrm{B} 97-\mathrm{D} / \mathrm{def} 2-\mathrm{TZVP}]=-3864.565187$

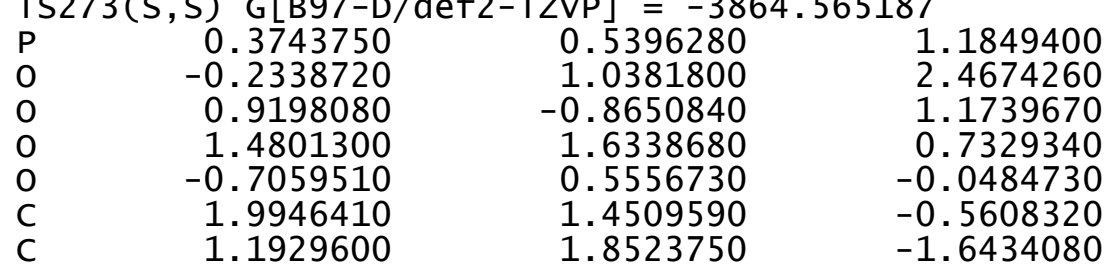

$-1.3801260$

$-3.5738410$

$-3.8772570$

$-1.2546780$

$-1.2537750$

$-2.6004490$

$-2.9161490$

$-4.1899570$

$-2.1580860$

$-4.8628270$

$-3.3628750$

$-5.1668180$

$-4.4223090$

$-5.6163820$

$-6.1592800$

$-0.3434300$

0.0921420

2.0994330

2. 4491070

1.1963190

2.0926350

3.2754450

2.8954730

3.5355070

3.4174250

3.3882920

2. 3384750

3.5814930

4.2517940

4.3888510

1.4172460

2.4569740

4.4666680

2.4907750

3.1104970
$-2.0179000$

$-5.6945720$

$-0.9019480$ 


\begin{tabular}{|c|c|c|}
\hline $\begin{array}{l}3.2447470 \\
3.6725300 \\
4.6343610 \\
1.0831600 \\
2.4117350 \\
0.1354930 \\
2.7579280 \\
3.7885580 \\
4.0925700 \\
3.4509390 \\
4.5672080 \\
4.2886820 \\
5.8636460 \\
5.4316470 \\
3.6522730 \\
3.5022910 \\
2.6705730 \\
4.5937360 \\
4.8979590 \\
4.0810940 \\
6.5259250 \\
3.6283620 \\
6.5886370 \\
6.8566280 \\
7.5189760 \\
5.6455840 \\
5.4544760 \\
6.1024520 \\
4.4905820 \\
4.0614040 \\
5.7352530 \\
5.3729220 \\
5.3313430 \\
5.0368840 \\
5.7807380 \\
6.3480910 \\
6.5832780 \\
7.2875080 \\
6.4465200 \\
3.3461170 \\
4.4340830 \\
4.6816250 \\
4.0075970 \\
3.4221860 \\
3.1421540 \\
2.5103950\end{array}$ & $\begin{array}{r}0.8433930 \\
0.5997170 \\
0.1140000 \\
1.7658110 \\
2.1830710 \\
2.4737980 \\
3.4184070 \\
3.7600230 \\
0.4388860 \\
1.2811700 \\
1.3927800 \\
2.8781990 \\
2.1606660 \\
1.0128190 \\
3.2742240 \\
4.3620830 \\
2.8009600 \\
2.8356490 \\
3.7013440 \\
2.1305660 \\
1.8152890 \\
3.1623360 \\
3.2064510 \\
4.0761370 \\
2.7742020 \\
3.6261980 \\
4.7072450 \\
3.3926260 \\
-0.9023570 \\
-2.0839580 \\
-2.7312100 \\
-1.2667130 \\
-2.7144180 \\
-3.6106360 \\
-2.0098810 \\
-3.0817730 \\
-4.1541130 \\
-2.5316780 \\
-2.9603580 \\
-1.7934060 \\
-3.5609100 \\
-4.6290620 \\
-3.3802820 \\
-3.1531820 \\
-4.0223650 \\
-2.7469220\end{array}$ & $\begin{array}{r}-0.7159940 \\
-2.0299000 \\
-2.1813440 \\
-0.6290580 \\
-0.5023280 \\
-1.3910050 \\
-1.0636980 \\
-0.9825500 \\
0.4353980 \\
0.0630020 \\
1.3544070 \\
1.3396410 \\
3.2767950 \\
2.3988120 \\
2.6999960 \\
2.7131130 \\
2.7910400 \\
3.8644270 \\
4.4680120 \\
4.5294660 \\
4.0799980 \\
0.5168030 \\
2.3830000 \\
2.9978900 \\
1.9956200 \\
1.2131170 \\
1.2390040 \\
0.2438990 \\
0.5955410 \\
-0.2454380 \\
1.6330500 \\
1.6306310 \\
-0.8802080 \\
-1.4426240 \\
-1.5910030 \\
0.2472820 \\
0.2222660 \\
0.1154510 \\
2.4359860 \\
-1.0179220 \\
1.7980500 \\
1.7313410 \\
2.7920840 \\
0.6851740 \\
0.0758880 \\
1.1267080\end{array}$ \\
\hline
\end{tabular}

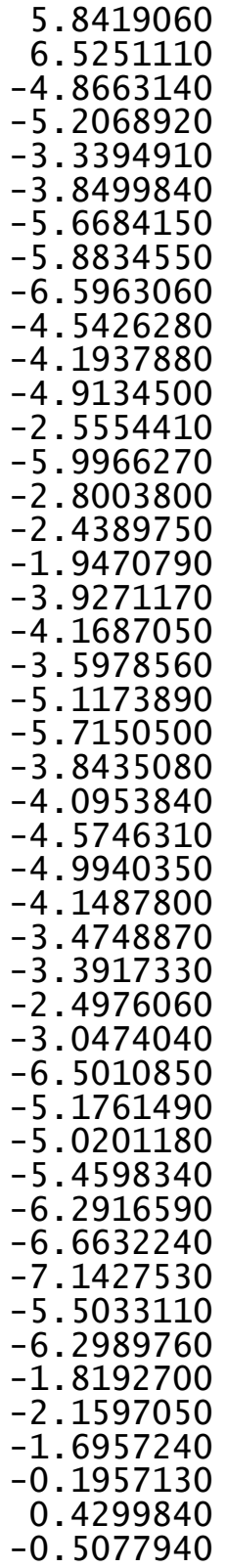

-0.3133250
-0.6007060
-0.6936620
-1.8091170
-0.2314220
0.1578120
-1.1551590
-1.9476200
-0.5959730
-0.2109380
-0.5387760
0.8158320
0.4453480
-2.4534930
-1.6813610
-1.9856990
-1.7000320
-2.6355940
-3.3820380
-3.1834610
0.6864940
1.1239470
2.7140810
1.5402970
1.1987670
1.5329510
0.2004300
2.1859130
3.0447310
1.7005280
3.3585280
0.4323740
3.5043310
4.3503990
3.9123910
2.5569980
2.9146790
2.5226340
-0.4283470
-1.0819150
4.2008280
5.5328800
5.5633660
5.1769050
4.6391600
3.7223050
2.5341610 3.3344510 $-0.2164280$ $-1.1750040$ $-2.0530010$ $-0.6851500$ $-2.5098260$ $-3.2387120$ $-2.3409000$ $-3.0378970$ $-4.0260600$ $-3.1399770$ $-2.4021570$ $-0.7695860$ $-1.9799680$ $-2.9699460$ $-1.2998710$ $-1.4850740$ $-2.2527890$ $-0.5952120$ 1. 7419800 3.0550640 2. 1958340 1. 2801730 4. 1074050 5.0656880 4. 2608220 3. 6091230 4. 2881850 3.5704830 1.8113080 3. 3827070 2. 3264160 3. 0083290 1. 3488200 2.8723550 3.8417120 2.1820430 0.9973830 1. 3573540 $-1.7355480$ $-2.3545640$ $-3.8298330$ $-3.9827660$ $-2.6689700$ $-1.9218800$ 


$\begin{array}{lr}\mathrm{H} & -1.6452520 \\ \mathrm{H} & -1.8805000 \\ \mathrm{H} & 0.3894750 \\ \mathrm{H} & 0.6384380 \\ \mathrm{H} & -3.2367260 \\ \mathrm{H} & -2.3166010 \\ \mathrm{H} & -0.0943020 \\ \mathrm{H} & 1.3898460 \\ \mathrm{C} & 1.6342430 \\ \mathrm{C} & 0.7991150 \\ \mathrm{C} & 0.7072020 \\ \mathrm{C} & 2.1027400 \\ \mathrm{C} & 3.2510020 \\ \mathrm{C} & 2.8799330 \\ \mathrm{H} & 1.2679000 \\ \mathrm{H} & 0.1896810 \\ \mathrm{H} & 2.3009780 \\ \mathrm{H} & 3.4161780 \\ \mathrm{H} & -0.2026640 \\ \mathrm{H} & 0.0833960 \\ \mathrm{H} & 2.1137640 \\ \mathrm{H} & 4.1852710 \\ \mathrm{C} & -0.1159120 \\ \mathrm{O} & 0.5789810 \\ \mathrm{C} & -0.2462150 \\ \mathrm{C} & -1.0813460 \\ \mathrm{C} & 0.4482530 \\ \mathrm{C} & -1.2231100 \\ \mathrm{H} & -1.6258620 \\ \mathrm{C} & 0.3042270 \\ \mathrm{H} & 1.0925910 \\ \mathrm{C} & -0.5332350 \\ \mathrm{H} & -1.8771670 \\ \mathrm{H} & 0.8436590 \\ \mathrm{H} & -0.6485260 \\ \mathrm{H} & 0.6908310 \\ \mathrm{~S} & -0.8164230 \\ \mathrm{C} & -0.8764530 \\ \mathrm{C} & -1.8616380 \\ \mathrm{H} & 0.1599430 \\ \mathrm{H} & -1.6215450 \\ \mathrm{O} & -1.5633780 \\ \mathrm{H} & -1.0131500 \\ \mathrm{C} & -2.5686200 \\ \mathrm{C} & -3.3031020 \\ \mathrm{C} & -3.2270020 \\ & \end{array}$

$\begin{array}{rr}6.3379480 & -1.8053340 \\ 6.5580330 & -4.2543190 \\ 6.0447360 & -4.3112550 \\ 5.5005440 & -2.0150130 \\ 5.7268640 & -2.2829080 \\ 4.8531200 & -4.3908740 \\ 4.4107830 & -4.7593120 \\ 4.1581650 & -2.8722300 \\ 1.5491970 & -2.9443930 \\ 1.7332370 & -4.1873880 \\ 0.3910640 & -4.9565320 \\ -0.2566340 & -5.2029690 \\ 0.5492390 & -4.5418000 \\ 0.9196480 & -3.1291580 \\ 2.4852010 & -4.8404380 \\ 0.5609580 & -5.9089070 \\ -0.3456020 & -6.2784730 \\ 1.4650390 & -5.1306290 \\ 2.0972040 & -3.9457010 \\ -0.2917000 & -4.3667910 \\ -1.2722130 & -4.7884540 \\ -0.0242260 & -4.5618990 \\ -3.4191510 & -0.7359460 \\ -2.3206590 & -0.7952570 \\ -4.0917750 & -2.0603890 \\ -5.2091230 & -2.2429230 \\ -3.5766720 & -3.1755260 \\ -5.7915140 & -3.5010390 \\ -5.6065110 & -1.3915420 \\ -4.1616030 & -4.4320490 \\ -2.7126410 & -3.0513820 \\ -5.2696300 & -4.6009440 \\ -6.6515660 & -3.6253500 \\ -3.7495770 & -5.2820890 \\ -5.7228230 & -5.5832430 \\ -1.7616500 & 0.0984690 \\ -4.0335740 & 0.6696360 \\ -2.0065740 & 3.4251380 \\ -1.9486670 & 2.3398910 \\ -1.8484610 & 3.1326610 \\ -1.3704590 & 1.4568970 \\ -0.7303390 & 3.6958450 \\ 0.0281180 & 3.2110140 \\ -3.3067940 & 4.8086990 \\ -3.6704060 & 3.5096900 \\ -2.5283270 & 2.4830670\end{array}$

$\begin{array}{llll}\mathrm{C} & -1.0829710 & -3.0254250 & 4.5254720 \\ \mathrm{H} & -2.6521370 & -4.1246450 & 5.5342460 \\ \mathrm{H} & -4.3555200 & -3.9036640 & 3.7102630 \\ \mathrm{H} & -3.8995470 & -1.7026070 & 2.7560820 \\ \mathrm{H} & -0.5871440 & -3.9481650 & 4.1916440 \\ \mathrm{H} & -0.5611290 & -2.6791070 & 5.4262750 \\ \mathrm{H} & -3.0305200 & -2.4180160 & 5.2590400 \\ \mathrm{H} & -2.8445270 & -4.5703970 & 3.0761400 \\ \mathrm{H} & -3.5614450 & -2.8440310 & 1.4897350\end{array}$

TS274(S, S) G[B97-D/def2-TZVP $]=-3864.564992$

$\begin{array}{lrrr}\text { TS274(S, S }) \text { G }[\mathrm{B} 97-\mathrm{D} / \mathrm{def} 2-\mathrm{TZVP}]=-3864.564992 \\ \mathrm{P} & 0.2095300 & -0.0700830 & 0.3396760 \\ \mathrm{O} & 0.7367880 & 0.7712220 & 1.4761800 \\ \mathrm{O} & -0.8012110 & 0.5323630 & -0.5999620 \\ \mathrm{O} & -0.3008960 & -1.4399980 & 1.0419220 \\ \mathrm{O} & 1.3974040 & -0.5913190 & -0.6387350 \\ \mathrm{C} & -0.4248140 & -2.5858600 & 0.2501750 \\ \mathrm{C} & 0.7518190 & -3.2546560 & -0.1489000 \\ \mathrm{C} & -1.7061140 & -3.0061390 & -0.1202740 \\ \mathrm{C} & -1.7942330 & -4.1570900 & -0.9178850 \\ \mathrm{H} & -2.7794790 & -4.5025400 & -1.2249170 \\ \mathrm{C} & 2.3909830 & -1.3834380 & -0.0611770 \\ \mathrm{C} & 3.6461340 & -0.8112910 & 0.1762300 \\ \mathrm{C} & 2.0851600 & -2.7180170 & 0.2480250 \\ \mathrm{C} & 4.5996840 & -1.6323330 & 0.7852910 \\ \mathrm{H} & 5.5895500 & -1.2224160 & 0.9782320 \\ \mathrm{C} & -2.9411820 & -2.2576110 & 0.2367890 \\ \mathrm{C} & 3.9453610 & 0.6008960 & -0.1802290 \\ \mathrm{C} & -3.3408800 & -2.0826910 & 1.5727250 \\ \mathrm{C} & -2.6049470 & -2.5568530 & 2.8032990 \\ \mathrm{C} & -4.8403710 & -1.3530220 & 3.3565880 \\ \mathrm{C} & -4.5586260 & -1.4446510 & 1.8780240 \\ \mathrm{C} & -2.3236270 & -1.3274850 & 3.7102850 \\ \mathrm{H} & -1.8459770 & -1.6766500 & 4.6354240 \\ \mathrm{H} & -1.6205940 & -0.6599180 & 3.2043070 \\ \mathrm{C} & -3.6632730 & -0.5925400 & 4.0279580 \\ \mathrm{H} & -3.8380740 & -0.5493580 & 5.1114390 \\ \mathrm{H} & -3.6357900 & 0.4379100 & 3.6535440 \\ \mathrm{H} & -5.7914070 & -0.8400460 & 3.5464220 \\ \mathrm{H} & -1.6695780 & -3.0609060 & 2.5488200 \\ \mathrm{C} & -4.8721900 & -2.7944850 & 3.9374160 \\ \mathrm{H} & -5.0085430 & -2.7365300 & 5.0256140 \\ \mathrm{H} & -5.7308350 & -3.3358940 & 3.5226910 \\ \mathrm{C} & -3.5378900 & -3.5228400 & 3.5859690 \\ \mathrm{H} & -3.0223220 & -3.8473890 & 4.4994990 \\ \mathrm{H} & -3.7312410 & -4.4152120 & 2.9785570\end{array}$




$\begin{array}{rrr}-3.7705450 & -1.7490140 & -0.7819080 \\ -3.4924730 & -1.7679260 & -2.2682610 \\ -5.7616410 & -0.6524630 & -1.6751140 \\ -4.9911490 & -1.1229190 & -0.4670290 \\ -4.6460210 & -2.5347740 & -2.9720070 \\ -4.4838220 & -2.4972590 & -4.0574550 \\ -4.6275690 & -3.5891490 & -2.6702020 \\ -6.0128880 & -1.8789160 & -2.5972290 \\ -6.5414600 & -1.5436820 & -3.4995190 \\ -6.6593280 & -2.5981590 & -2.0800390 \\ -6.7118220 & -0.1892720 & -1.3823930 \\ -2.5293590 & -2.2314180 & -2.4948490 \\ -4.8814430 & 0.3559500 & -2.4630140 \\ -5.4082670 & 0.6374920 & -3.3845840 \\ -4.7467790 & 1.2658800 & -1.8713810 \\ -3.5080690 & -0.3032540 & -2.7891850 \\ -3.3345420 & -0.3172130 & -3.8734430 \\ -2.6851210 & 0.2493480 & -2.3273680 \\ -5.3917980 & -0.9805820 & 0.8611860 \\ -6.3432850 & -0.5048390 & 1.1012950 \\ 4.1294570 & 2.3835440 & -1.8465670 \\ 4.0124530 & 2.6805750 & -3.3212200 \\ 3.4749580 & 0.2122520 & -2.7180910 \\ 3.8275170 & 1.0507780 & -1.5102430 \\ 4.9980040 & 1.7414960 & -4.0732930 \\ 4.8873250 & 1.9019440 & -5.1540200 \\ 6.0286310 & 1.9995730 & -3.8021930 \\ 4.6818630 & 0.2597460 & -3.6988130 \\ 4.4290630 & -0.3206240 & -4.5959320 \\ 5.5517950 & -0.2150580 & -3.2297720 \\ 3.2554570 & -0.8229500 & -2.4470070 \\ 4.2469570 & 3.7314620 & -3.5316270 \\ 2.2547830 & 0.8492700 & -3.4362770 \\ 2.0383820 & 0.2685150 & -4.3427270 \\ 1.3780140 & 0.7859530 & -2.7866170 \\ 2.5770740 & 2.3310210 & -3.7989120 \\ 2.5164520 & 2.4908500 & -4.8835650 \\ 1.8572250 & 3.0075620 & -3.3272390 \\ 4.6905380 & 2.8442920 & 0.4487500 \\ 5.1921040 & 3.6760500 & 1.6030920 \\ 4.5863280 & 1.2412940 & 2.2728100 \\ 4.3779670 & 1.5160470 & 0.7995210 \\ 4.1043820 & 3.7042220 & 2.7101920 \\ 4.4943480 & 4.2575770 & 3.5751290 \\ 3.2187020 & 4.2311600 & 2.3416490 \\ 3.7393260 & 2.2423440 & \\ -4 & & \\ -4 & & \\ -4 & -1063810\end{array}$

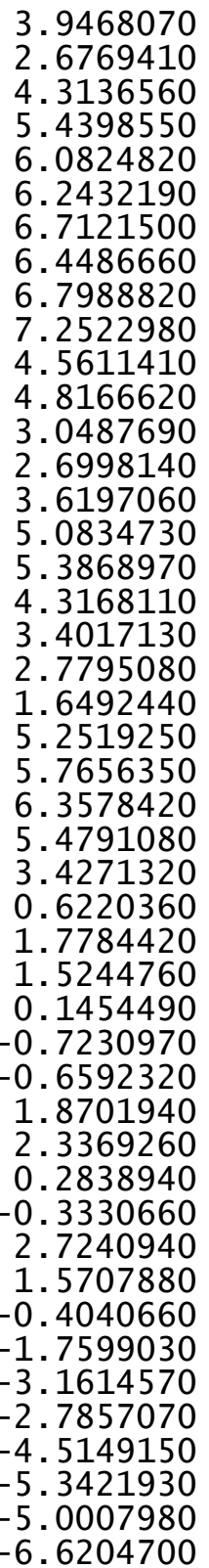

2.0663500

2. 0447830

0.2175920

4.6937490

1.5097700

1.3559600

0.7895760

2.9720920

3.5406300

2.9768490

3.2813760

4.3077310

$-3.4960320$

$-4.8894130$

$-5.3723640$

$-5.1910670$

$-3.6980090$

$-2.9403740$

$-6.4243000$

$-5.6049440$

$-4.9064810$

$-5.6249860$

$-3.5542850$

$-3.2380840$

$-4.7923240$

$-4.3680380$

$-5.0531510$

$-5.0972730$

$-5.7260990$

$-5.9946070$

$-4.8257850$

$-6.0865400$

$-5.6548970$

$-6.6693620$

$-6.8928020$

$-4.5463540$

$-4.0680680$

$-5.0489150$

$-6.2070530$

2.9470410

1.8554010

3.4090360

2.5533880

4.6812030

2. 9596390
4.1702250

2. 9392850

2.5384150

1.2767880

2. 5979880

3.6732050

2.0614950

2. 1902480

3.0618880

1.4443270

$-0.8688980$

$-1.1364120$

0.9324590

1.4270080

2.5560600

2. 1386880

1.9634730

1.1925380

2.7807090

0.5975370

1.7422960

1.1882300

2.8809600

1.4697400

2.9603070

3.4709850

$-1.0006750$

$-1.6874180$

$-3.2140780$

$-3.5726180$

$-2.3148540$

$-1.3661730$

$-1.3182040$

$-3.6963810$

$-4.1158910$

$-1.8114600$

$-1.4761910$

$-3.5909900$

$-4.2378030$

$-2.6011240$

$-0.4722580$

0.1424470

$-0.0626040$

0.6946250

$-0.4204510$

1.0725810 


$\begin{array}{lrrr}\mathrm{H} & -4.9853260 & 1.5649320 & 0.9618400 \\ \mathrm{C} & -6.2776710 & 5.0845800 & -0.0356390 \\ \mathrm{H} & -4.3669420 & 5.3462590 & -0.9998590 \\ \mathrm{C} & -7.0931640 & 4.2255180 & 0.7106800 \\ \mathrm{H} & -7.2503330 & 2.2844640 & 1.6477500 \\ \mathrm{H} & -6.6385980 & 6.0712340 & -0.3169070 \\ \mathrm{H} & -8.0911500 & 4.5417270 & 1.0068590 \\ \mathrm{H} & -1.9201020 & 1.3778830 & -0.2142220 \\ \mathrm{~S} & -2.2286910 & 3.7553950 & -1.6157930 \\ \mathrm{C} & -0.1416490 & 3.7097590 & 0.9748630 \\ \mathrm{C} & 0.3075620 & 3.5319160 & -0.4102600 \\ \mathrm{H} & -0.8933410 & 3.0053660 & 1.3325970 \\ \mathrm{H} & 0.2160660 & 2.5467100 & -0.8497550 \\ \mathrm{O} & 1.2118830 & 3.1761220 & 1.1429050 \\ \mathrm{H} & 1.0818410 & 2.1204810 & 1.2717440 \\ \mathrm{C} & 0.7414330 & 6.0850140 & 0.9149370 \\ \mathrm{C} & 0.7073610 & 6.0304750 & -0.6201010 \\ \mathrm{C} & 1.0211620 & 4.6183730 & -1.1464980 \\ \mathrm{C} & -0.2939520 & 5.1171290 & 1.5124400 \\ \mathrm{H} & 0.5304830 & 7.1036360 & 1.2617770 \\ \mathrm{H} & 1.4240020 & 6.7410780 & -1.0487630 \\ \mathrm{H} & 2.0963910 & 4.3965340 & -1.0687710 \\ \mathrm{H} & -1.3093910 & 5.4488590 & 1.2528520 \\ \mathrm{H} & -0.2258300 & 5.0838150 & 2.6070640 \\ \mathrm{H} & 1.7431160 & 5.8147180 & 1.2752020 \\ \mathrm{H} & -0.2938760 & 6.3236380 & -0.9649680 \\ \mathrm{H} & 0.7720400 & 4.5223580 & -2.2094850 \\ \mathrm{H} & & & \end{array}$

TS275(S, S) G[B97-D/def2-TZVP] $=-3864.564947$

$\begin{array}{lrrr}\mathrm{P} & 0.2124710 & -0.0406210 & 0.2911750 \\ \mathrm{O} & 0.7330680 & 0.8083060 & 1.4247920 \\ \mathrm{O} & -0.8062290 & 0.5493810 & -0.6483090 \\ \mathrm{O} & -0.2874020 & -1.4127280 & 0.9996860 \\ \mathrm{O} & 1.4063030 & -0.5502740 & -0.6883660 \\ \mathrm{C} & -0.4130400 & -2.5564790 & 0.2050060 \\ \mathrm{C} & 0.7575400 & -3.2103260 & -0.2147310 \\ \mathrm{C} & -1.6940500 & -2.9842810 & -0.1610600 \\ \mathrm{C} & -1.7652720 & -4.1253240 & -0.9665210 \\ \mathrm{H} & -2.7492080 & -4.4862230 & -1.2608120 \\ \mathrm{C} & 2.3983510 & -1.3411610 & -0.1044650 \\ \mathrm{C} & 3.6468380 & -0.7652380 & 0.1596790 \\ \mathrm{C} & 2.0865740 & -2.6730080 & 0.2054710 \\ \mathrm{C} & 4.5929120 & -1.5889150 & 0.7774310 \\ \mathrm{H} & 5.5809190 & -1.1808200 & 0.9830400 \\ \mathrm{C} & -2.9343380 & -2.2537060 & 0.2121370 \\ \mathrm{C} & 3.9431830 & 0.6520740 & -0.1778940\end{array}$

-3.3188730
-2.5649860
-4.8030180
-4.5384240
-2.2827730
-1.7943370
-1.5887400
-3.6239700
-3.7866770
-3.6081600
-5.7557410
-1.6289910
-4.8163000
-4.9372710
-5.6767780
-3.4817750
-2.9525600
-3.6769590
-3.7818110
-3.5223500
-5.7940150
-5.0041880
-4.6775600
-4.5294000
-4.6454840
-6.0456780
-6.5891650
-6.6784450
-6.7445090
-2.5580890
-4.9328350
-5.4735070
-4.7992510
-3.5574530
-3.3971190
-2.7338510
-5.3888900
-6.3414430
4.1410940
4.0529750
3.5232200
3.8437560
5.0633800
4.9771130
6.0850920
4.7506880
1. 5537770

2. 7739710

3. 3584500

1.8762770

3. 6837660

4. 6027170

3.1746430

4. 0178430

5. 1033150

3. 6448740

3. 5613140

2.5069680

3.9353470

5.0255520

3. 5314340

3. 5625210

4.4674020

2.9520020

$-0.7945480$

$-2.2841940$

$-1.6588690$

$-0.4621490$

$-2.9765320$

$-4.0637390$

$-2.6795340$

$-2.5819220$

$-3.4760790$

$-2.0588220$

$-1.3524890$

$-2.5248110$

$-2.4538840$

$-3.3678960$

$-1.8607010$

$-2.7994380$

$-3.8857560$

$-2.3457730$

0.8714760

1.1253510

$-1.8239000$

$-3.2980280$

$-2.7275060$

$-1.5052540$

$-4.0351660$

$-5.1167620$

$-3.7372300$

$-3.6811970$ 


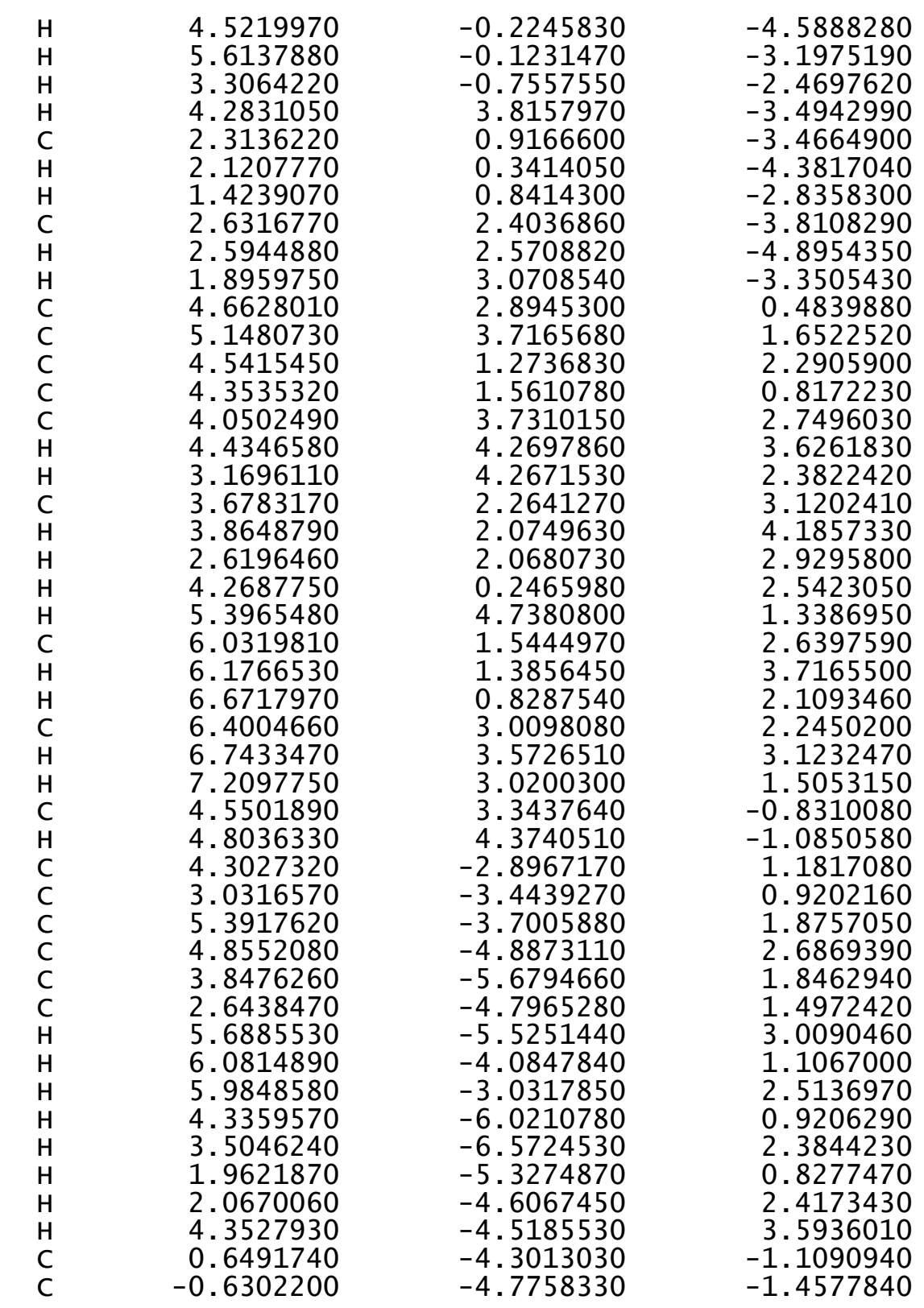

\begin{abstract}
1.8859410
1.6719180

0.3913730

$-0.8184120$

2. 5456670

2. 1657940

2.7394630

0.4624350

0.2648320

$-1.7271560$

$-0.9957910$

1. 5883820

$-3.1893730$

$-2.7944570$

$-4.5408430$

$-5.3470410$

$-5.0459100$

$-6.6234270$

$-4.9760480$

$-6.3207780$

$-4.4287260$

$-7.1152850$

$-7.2369440$

$-6.6964840$

$-8.1118040$

$-1.9285450$

$-2.2807870$

$-0.1595290$

0.2712930

$-0.9004130$

0.1819810

1. 2006880

1.0784840

0.7016620

0.6443880

0.9654170

$-0.3157930$

0.4866970

1. 3462680

2.0435730

$-1.3378910$

$-0.2308640$

1.7111120

$-0.3654300$
\end{abstract}

154

$\begin{array}{rr}-4.8629630 & -1.7961620 \\ -6.2459720 & -2.4233950 \\ -6.2581170 & -3.2651100 \\ -5.9814480 & -2.3645750 \\ -6.5086520 & -3.0336610 \\ -4.1516770 & -2.5907450 \\ -4.8879650 & -1.1150920 \\ -5.4795090 & -4.0392810 \\ -7.2212250 & -3.7763180 \\ -5.8432560 & -2.9651810 \\ -6.8683950 & -1.7346290 \\ -7.0026720 & -1.6282780 \\ 2.9400430 & -0.4827730 \\ 1.8502430 & 0.1226100 \\ 3.3882680 & -0.0516770 \\ 2.5248640 & 0.7194210 \\ 4.6548050 & -0.4030200 \\ 2.9181740 & 1.1170290 \\ 1.5403150 & 0.9817800 \\ 5.0452160 & 0.0014010 \\ 5.3259240 & -0.9933640 \\ 4.1785030 & 0.7613620 \\ 2.2370280 & 1.7027330 \\ 6.0276130 & -0.2753810 \\ 4.4843480 & 1.0729520 \\ 1.3818860 & -0.2469980 \\ 3.7608060 & -1.6370810 \\ 3.7373420 & 0.9273920 \\ 3.5601280 & -0.4636680 \\ 3.0274310 & 1.2967040 \\ 2.5731160 & -0.8994650 \\ 3.2146430 & 1.0776420 \\ 2.1599150 & 1.2112830 \\ 6.1201940 & 0.8492410 \\ 6.0612240 & -0.6849240 \\ 4.6510510 & -1.2120420 \\ 5.1444330 & 1.4645060 \\ 7.1377230 & 1.1967960 \\ 6.7784420 & -1.1267490 \\ 4.4402740 & -1.1463650 \\ 5.4667260 & 1.2195850 \\ 5.1139920 & 2.5580370 \\ 5.8597840 & 1.1947380 \\ 6.3418720 & -1.0148020 \\ 4.5495870 & -2.2715460 \\ & \end{array}$




$\begin{array}{lrrr}\text { TS276(S , S }) \text { G [B97-D/def2-TZVP] }=-3864.564802 \\ \text { P } & 0.2152690 & -0.0576800 & 0.2936710 \\ \text { O } & 0.7559910 & 0.7736910 & 1.4310060 \\ \text { O } & -0.7960950 & 0.5573140 & -0.6374090 \\ \text { O } & -0.3064920 & -1.4254570 & 0.9943410 \\ \text { O } & 1.3959440 & -0.5828620 & -0.6937050 \\ \mathrm{C} & -0.4498490 & -2.5619150 & 0.1920590 \\ \mathrm{C} & 0.7097870 & -3.2335490 & -0.2317460 \\ \mathrm{C} & -1.7377290 & -2.9666460 & -0.1751950 \\ \mathrm{C} & -1.8279250 & -4.0929400 & -0.9986000 \\ \mathrm{H} & -2.8182150 & -4.4303710 & -1.2999990 \\ \mathrm{C} & 2.3771630 & -1.3916690 & -0.1160560 \\ \mathrm{C} & 3.6363060 & -0.8375110 & 0.1427810 \\ \mathrm{C} & 2.0455160 & -2.7196630 & 0.1923660 \\ \mathrm{C} & 4.5647270 & -1.6723860 & 0.7715550 \\ \mathrm{H} & 5.5570440 & -1.2772950 & 0.9821800 \\ \mathrm{C} & -2.9661790 & -2.2197730 & 0.2047240 \\ \mathrm{C} & 3.9593080 & 0.5738970 & -0.1950110 \\ \mathrm{C} & -3.3496620 & -2.0576830 & 1.5470380 \\ \mathrm{C} & -2.6028690 & -2.5496710 & 2.7640360 \\ \mathrm{C} & -4.8251110 & -1.3396690 & 3.3557920 \\ \mathrm{C} & -4.5598560 & -1.4156800 & 1.8732290 \\ \mathrm{C} & -2.3043550 & -1.3321780 & 3.6810150 \\ \mathrm{H} & -1.8170620 & -1.6940310 & 4.5961980 \\ \mathrm{H} & -1.6042440 & -0.6627260 & 3.1735110 \\ \mathrm{C} & -3.6362440 & -0.5942760 & 4.0234120 \\ \mathrm{H} & -3.7985670 & -0.5644490 & 5.1092750 \\ \mathrm{H} & -3.6072830 & 0.4408820 & 3.6623700 \\ \mathrm{H} & -5.7709680 & -0.8234080 & 3.5618790 \\ \mathrm{H} & -1.6733690 & -3.0563620 & 2.4935810 \\ \mathrm{C} & -4.8591510 & -2.7875880 & 3.9201460 \\ \mathrm{H} & -4.9835180 & -2.7416220 & 5.0103270 \\ \mathrm{H} & -5.7251450 & -3.3193880 & 3.5082940 \\ \mathrm{C} & -3.5327440 & -3.5192580 & 3.5459910 \\ \mathrm{H} & -3.0090550 & -3.8570520 & 4.4500630 \\ \mathrm{H} & -3.7376430 & -4.4036820 & 2.9307760 \\ \mathrm{C} & -3.8037840 & -1.6941230 & -0.7989180 \\ \mathrm{C} & -3.5436180 & -1.6980830 & -2.2886900 \\ \mathrm{C} & -5.7988640 & -0.5762460 & -1.6569130 \\ \mathrm{C} & -5.0167280 & -1.0638080 & -0.4629850 \\ \mathrm{C} & -4.7098460 & -2.4508090 & -2.9867290 \\ \mathrm{H} & -4.5605800 & -2.4018230 & -4.0735600 \\ \mathrm{H} & -4.6936430 & -3.5085790 & -2.6969700 \\ \mathrm{C} & -6.0682980 & -1.7916260 & -2.5881530 \\ \mathrm{H} & -6.6064040 & -1.4442560 & -3.4801580 \\ \mathrm{H} & -6.7120780 & -2.5126580 & -2.0701540 \\ & & & \end{array}$

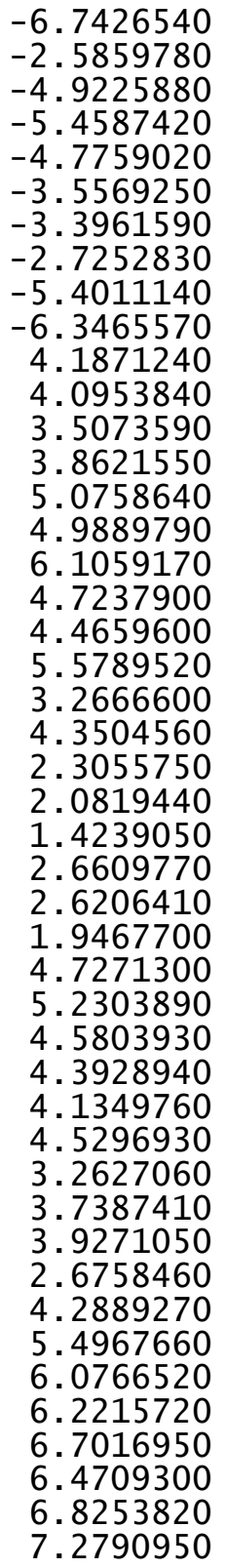

$-6.7426540$

$-4.9225880$

$-5.4587420$

$-4.7759020$

$-3.5569250$

$-3.3961590$

. 7252830

4. 1871240

4.0953840

3.5073590

4.9889790

. 1059170

5.5789520

. 2666600

2. 0819440

1.4239050

.6609770

.6206410

4.7271300

.2303890

(1)

4.1349760

. 5296930

(27060

3. 9271050

2. 6758460

2889270

.4967660

6.2215720

6.7016950

6.8253820

7.2790950
-1.3476380
-2.5325210
-2.4449620
-3.3573470
-1.8461160
-2.7940880
-3.8803690
-2.3364530
0.8712920
1.1279810
-1.8358040
-3.3078640
-2.7425440
-1.5200720
-4.0569260
-5.1368660
-3.7621890
-3.7117230
-4.6212220
-3.2443730
-2.4872520
-3.5018870
-3.4625430
-4.3751440
-2.8183040
-3.8100360
-4.8944470
-3.3450450
0.4713510
1.6405630
2.2741220
0.8016620
2.7398650
3.6163670
2.3743670
3.1095270
4.1737040
2.9240590
2.5251260
1.3289230
2.6193970
3.6951800
2.0846710
2.2288450
3.1083380
1.4878290

$\begin{array}{rr}-0.1108430 & -1.3476380 \\ -2.1638080 & -2.5325210 \\ 0.4354850 & -2.4449620 \\ 0.7290360 & -3.3573470 \\ 1.3388190 & -1.8461160 \\ -0.2280530 & -2.7940880 \\ -0.2316080 & -3.8803690 \\ 0.3148830 & -2.3364530 \\ -0.9340360 & 0.8712920 \\ -0.4549160 & 1.1279810 \\ 2.3751630 & -1.8358040 \\ 2.6921720 & -3.3078640 \\ 0.2262650 & -2.7425440 \\ 1.0426680 & -1.5200720 \\ 1.7455040 & -4.0569260 \\ 1.9247830 & -5.1368660 \\ 1.9786830 & -3.7621890 \\ 0.2647240 & -3.7117230 \\ -0.2937580 & -4.6212220 \\ -0.2377560 & -3.2443730 \\ -0.8082620 & -2.4872520 \\ 3.7414220 & -3.5018870 \\ 0.8950450 & -3.4625430 \\ 0.3268890 & -4.3751440 \\ 0.8445290 & -2.8183040 \\ 2.3726180 & -3.8100360 \\ 2.5404510 & -4.8944470 \\ 3.0596250 & -3.3450450 \\ 2.7992170 & 0.4713510 \\ 3.6092630 & 1.6405630 \\ 1.1766280 & 2.2741220 \\ 1.4710840 & 0.8016620 \\ 3.6411640 & 2.7398650 \\ 4.1725240 & 3.6163670 \\ 4.1920710 & 2.3743670 \\ 2.1806190 & 3.1095270 \\ 1.9859030 & 4.1737040 \\ 2.0037880 & 2.9240590 \\ 0.1546260 & 2.5251260 \\ 4.6268310 & 1.3289230 \\ 1.4188810 & 2.6193970 \\ 1.2535240 & 3.6951800 \\ 0.6933420 & 2.0846710 \\ 2.8784070 & 2.2288450 \\ 3.4320190 & 3.1083380 \\ 2.8765500 & 1.4878290\end{array}$




\begin{tabular}{|c|c|c|c|}
\hline & & & \\
\hline$c$ & 4.6191750 & 3.2546440 & -0.8419870 \\
\hline $\mathrm{C}$ & 2.9732850 & -3.5015690 & 0.9189380 \\
\hline $\mathrm{C}$ & 2.5696760 & -4.8541210 & 1.4820330 \\
\hline $\mathrm{C}$ & 3.4240660 & -5.2680400 & 2.6872590 \\
\hline $\mathrm{C}$ & 4.9069210 & -5.1811630 & 2.3083370 \\
\hline C & 5.2831380 & -3.7228290 & 2.0143240 \\
\hline $\mathrm{C}$ & 4.2470410 & -2.9674120 & 1.1952630 \\
\hline $\mathrm{H}$ & 3.1557310 & -6.2869010 & 2.9945370 \\
\hline $\mathrm{H}$ & 2.6818340 & -5.6345730 & 0.7175810 \\
\hline $\mathrm{H}$ & 1.5043480 & -4.8312040 & 1.7416460 \\
\hline H & 5.0803200 & -5.7995410 & 1.4147260 \\
\hline $\mathrm{H}$ & 5.5470910 & -5.5753440 & 3.1079090 \\
\hline H & 6.2553140 & -3.6702720 & 1.5046140 \\
\hline $\mathrm{H}$ & 5.4111940 & -3.1908150 & 2.9701380 \\
\hline $\mathrm{H}$ & 3.2219870 & -4.6004840 & 3.5381400 \\
\hline C & 0.5832020 & -4.3094380 & -1.1430480 \\
\hline C & 1.8095460 & -4.9114010 & -1.8094890 \\
\hline C & 1.4832610 & -5.5979300 & -3.1427720 \\
\hline $\mathrm{C}$ & 0.3492740 & -6.6063730 & -2.9275420 \\
\hline C & -0.9297150 & -5.8654750 & -2.5158900 \\
\hline C & -0.7041560 & -4.7497360 & -1.5066070 \\
\hline $\mathrm{H}$ & 2.3833140 & -6.0936380 & -3.5284520 \\
\hline H & 2.2637620 & -5.6676730 & -1.1555610 \\
\hline $\mathrm{H}$ & 2.5669330 & -4.1300840 & -1.9451000 \\
\hline H & 0.6470430 & -7.3088870 & -2.1347060 \\
\hline H & 0.1615400 & -7.1961210 & -3.8338830 \\
\hline $\mathrm{H}$ & -1.6718400 & -6.5700880 & -2.1154440 \\
\hline $\mathrm{H}$ & -1.3861640 & -5.4208350 & -3.4141200 \\
\hline $\mathrm{H}$ & 1.1729010 & -4.8483770 & -3.8860480 \\
\hline C & -3.1352570 & 2.9897360 & -0.4635990 \\
\hline 0 & -2.7613940 & 1.8909300 & 0.1392130 \\
\hline C & -4.4801010 & 3.4600080 & -0.0355070 \\
\hline C & -5.3071900 & 2.6050820 & 0.7228370 \\
\hline C & -4.9582620 & 4.7394490 & -0.3774980 \\
\hline C & -6.5779720 & 3.0193130 & 1.1170630 \\
\hline $\mathrm{H}$ & -4.9568620 & 1.6110270 & 0.9777470 \\
\hline C & -6.2275750 & 5.1507050 & 0.0236350 \\
\hline H & -4.3243900 & 5.4038550 & -0.9576550 \\
\hline C & -7.0430690 & 4.2923670 & 0.7707540 \\
\hline H & -7.2081020 & 2.3447610 & 1.6926660 \\
\hline $\mathrm{H}$ & -6.5825660 & 6.1428670 & -0.2455420 \\
\hline H & -8.0352320 & 4.6146630 & 1.0796570 \\
\hline H & -1.9037850 & 1.4092430 & -0.2311750 \\
\hline $\mathrm{S}$ & -2.2088800 & 3.7981850 & -1.6123230 \\
\hline & -0.0 & 3.7190940 & 0.952 \\
\hline
\end{tabular}

0.3391420
-0.8423090
0.2351840
1.2613160
1.1217450
0.8099440
0.7554750
1.0522730
-0.2250610
0.6108990
1.4714690
2.1266490
-1.2409930
-0.1443520
1.8141660
-0.2481130
0.7898670

3.5455960

3. 0184980

2. 5634140

3. 1738520

2. 1198410

6.0881350

6.0421540

4.6306150

5. 1246620

7.1063760

6.7499410

4.4008940

5.4646300

5.0852880

5.8085530

6.3441080

4. 5421540

154

TS277(S, S) G[B97-D/def2-TZVP $]=-3864.564640$

$\begin{array}{lrcr}\mathrm{P} & 0.2175370 & 0.4859170 & 1.0409170 \\ \mathrm{O} & -0.4993640 & 1.0533480 & 2.2373280 \\ \mathrm{O} & 0.7086510 & -0.9345310 & 1.1266790 \\ \mathrm{O} & 1.4043530 & 1.5362120 & 0.6939360 \\ \mathrm{O} & -0.7237310 & 0.5061340 & -0.3013700 \\ \mathrm{C} & 2.0095660 & 1.3752900 & -0.5608570 \\ \mathrm{C} & 1.3002000 & 1.8052840 & -1.6899290 \\ \mathrm{C} & 3.2661990 & 0.7666380 & -0.6396570 \\ \mathrm{C} & 3.7670110 & 0.5373220 & -1.9248920 \\ \mathrm{H} & 4.7398170 & 0.0574330 & -2.0184610 \\ \mathrm{C} & -1.0392220 & 1.7440440 & -0.8638460 \\ \mathrm{C} & -2.3587650 & 2.2037200 & -0.7956000 \\ \mathrm{C} & -0.0217620 & 2.4679850 & -1.4990350 \\ \mathrm{C} & -2.6030040 & 3.4916070 & -1.2781420 \\ \mathrm{H} & -3.6206610 & 3.8769360 & -1.2327480 \\ \mathrm{C} & 4.0469940 & 0.3682030 & 0.5596860 \\ \mathrm{C} & -3.4561660 & 1.3321950 & -0.3011060 \\ \mathrm{C} & 4.4725340 & 1.3320050 & 1.4919100 \\ \mathrm{C} & 4.1885520 & 2.8156930 & 1.4494860 \\ \mathrm{C} & 5.6585910 & 2.1207490 & 3.4752720 \\ \mathrm{C} & 5.2813540 & 0.9640530 & 2.5839290 \\ \mathrm{C} & 3.4767910 & 3.2175310 & 2.7698810 \\ \mathrm{H} & 3.3221100 & 4.3048100 & 2.7680800 \\ \mathrm{H} & 2.4931430 & 2.7404560 & 2.8069830 \\ \mathrm{C} & 4.3529550 & 2.7892310 & 3.9879490 \\ \mathrm{H} & 4.6164080 & 3.6588520 & 4.6047830 \\ \mathrm{H} & 3.8081270 & 2.0820430 & 4.6245120\end{array}$

$-0.4392040$

1.3165950

$-0.8826330$

1.1010860

1.2270030

0.8942820

1.4995190

1.2491610

$-1.1140440$

1.2530980

2.5930720

1.2400300

$-0.9705570$

$-2.2387500$
$-0.6404530$

$-1.1782830$

1.0533480
10.9345310
1.5362120
0.5061340
1.3752900
1.8052840
0.7666380
0.5373220
0.0574330
1.7440440
2.2037200
2.4679850
3.4916070
3.8769360
0.3682030
1.3321950
1.3320050
2.8156930
2.1207490
0.9640530
3.2175310
4.3048100
2.7404560
2.7892310
3.6588520
2.0820430

2. 2373280

(1)

0.3013700

1.6899290

. 6396570

1060

1. 4494860

.

2.7698810

4.6245120 


$\begin{array}{rrrr}\mathrm{H} & 6.2783020 & 1.7855780 & 4.3158760 \\ \mathrm{H} & 3.5719590 & 3.0894080 & 0.5898750 \\ \mathrm{C} & 6.4233240 & 3.1682860 & 2.6175260 \\ \mathrm{H} & 6.6417290 & 4.0459400 & 3.2405600 \\ \mathrm{H} & 7.3812840 & 2.7460500 & 2.2912180 \\ \mathrm{C} & 5.5479630 & 3.5678110 & 1.3892580 \\ \mathrm{H} & 5.3534050 & 4.6486090 & 1.3877490 \\ \mathrm{H} & 6.0601380 & 3.3213490 & 0.4513780 \\ \mathrm{C} & 4.4300120 & -0.9713290 & 0.7577240 \\ \mathrm{C} & 4.0201170 & -2.1639110 & -0.0768610 \\ \mathrm{C} & 5.5968580 & -2.7937600 & 1.8910530 \\ \mathrm{C} & 5.2563640 & -1.3250610 & 1.8418720 \\ \mathrm{C} & 5.3033990 & -2.8363090 & -0.6368700 \\ \mathrm{H} & 5.0164670 & -3.7444320 & -1.1840860 \\ \mathrm{H} & 5.7951380 & -2.1617110 & -1.3484240 \\ \mathrm{C} & 6.2643850 & -3.1864590 & 0.5432270 \\ \mathrm{H} & 6.4863440 & -4.2618120 & 0.5554470 \\ \mathrm{H} & 7.2159360 & -2.6515220 & 0.4388940 \\ \mathrm{H} & 6.2668330 & -3.0160630 & 2.7305630 \\ \mathrm{H} & 3.3474530 & -1.8758810 & -0.8884880 \\ \mathrm{C} & 4.2748460 & -3.5982700 & 2.0151790 \\ \mathrm{H} & 4.5061330 & -4.6713670 & 1.9753290 \\ \mathrm{H} & 3.8105820 & -3.3940000 & 2.9873990 \\ \mathrm{C} & 3.3165990 & -3.1923900 & 0.8548180 \\ \mathrm{H} & 3.0334610 & -4.0695670 & 0.2586690 \\ \mathrm{H} & 2.4017460 & -2.7468860 & 1.2503850 \\ \mathrm{C} & 5.6820690 & -0.3610360 & 2.7554850 \\ \mathrm{H} & 6.3203890 & -0.6404890 & 3.5946330 \\ \mathrm{C} & -4.8642880 & -0.6454390 & -0.6015590 \\ \mathrm{C} & -5.1328580 & -1.8110290 & -1.5223280 \\ \mathrm{C} & -3.1774010 & -0.2977720 & -2.3165980 \\ \mathrm{C} & -3.8020160 & 0.1740530 & -1.0238750 \\ \mathrm{C} & -5.4648700 & -1.2357090 & -2.9293210 \\ \mathrm{H} & -5.6181110 & -2.0696920 & -3.6268740 \\ \mathrm{H} & -6.3996840 & -0.6649510 & -2.8798970 \\ \mathrm{C} & -4.2870650 & -0.3282420 & -3.4053420 \\ \mathrm{H} & -3.8534550 & -0.7144130 & -4.3372950 \\ \mathrm{H} & -4.6372830 & 0.6924380 & -3.5993600 \\ \mathrm{H} & -2.3579510 & 0.3519370 & -2.6340840 \\ \mathrm{H} & -5.9636070 & -2.4246760 & -1.1526110 \\ \mathrm{C} & -2.6626090 & -1.7461240 & -2.1126080 \\ \mathrm{H} & -2.2256690 & -2.1080910 & -3.0520850 \\ \mathrm{H} & -1.8676810 & -1.7338800 & -1.3651220 \\ \mathrm{C} & -3.8404410 & -2.6615020 & -1.6628530 \\ \mathrm{H} & -4.0196050 & -3.4552290 & -2.3999070 \\ \mathrm{H} & -3.6000520 & -3.1507480 & -0.7125580 \\ & & & \end{array}$

-5.2563590
-5.9441260
-3.9969000
-4.1951730
-4.8839150
-5.3682370
-4.4860040
-3.7347900
-3.6805900
-2.7669910
-3.1680020
-6.7592020
-5.3246850
-5.2059750
-5.5318670
-6.4899640
-6.9316130
-7.2854460
-5.5949810
-6.4225290
-0.2810410
0.8525650
0.5434470
-0.8192040
-1.9247270
-1.5860880
1.3391570
1.0524370
1.7718420
-0.8090850
-1.0216520
-2.8713960
-2.1094480
0.5216150
1.7992380
0.9562650
1.3465450
2.8553680
3.6203580
3.0479510
0.7789630
1.0734480
-0.1037890
3.1122100
3.1525680
4.6804120

0.8224410

1. 3106570

2. 8511080

1.6497650

1. 4115060

1. 7896390

0.4149980

2. 3629520

3. 2417920

1.8592700

3.4760460

0.6367340

3.6594770

4.5217430

4. 0446640

2. 7412480

3.1340130

2. 6916790

$-0.3200680$

$-0.9524000$

3.7916850

4.6684220

6.1667780

. 4612760

5.7432970

4.3062750

6.7451040

4.4509090

4.4138440

6.1046530

7.5396720

5.7624560

6.2968340

6.4609870

1.4923780

1. 7466740

0.8421890

0.9517220

.4104250

0.8499380

1.1355760

2. 7888680

1.6234930

2. 0092760

0.4006550

0.6948750
1. 2670620

2. 5166560

1.7439410

0.8493140

3. 6481090

4. 5581670

3. 8716110

3. 1951340

3. 8511780

3. 2382670

1. 4006240

2.8083240

1.7567140

2. 4257120

0.7510190

2. 2446060

3.1700630

1. 4917200

0.5424300

0.8666000

$-1.9174860$

$-2.4214540$

$-2.3021940$

$-2.9401440$

$-2.1549510$

$-1.7909950$

$-2.7888770$

$-3.4796410$

$-1.8796340$

$-3.9812060$

$-2.9650360$

$-2.7125010$

$-1.2204670$

$-1.2422710$

$-2.9753250$

$-4.2133250$

$-5.3895510$

$-5.6369410$

4.4220890

$-3.0826870$

-6. 2819200

$-4.5415490$

$-3.9609090$

$-5.7999940$

$-6.5384100$

$-4.4740920$ 


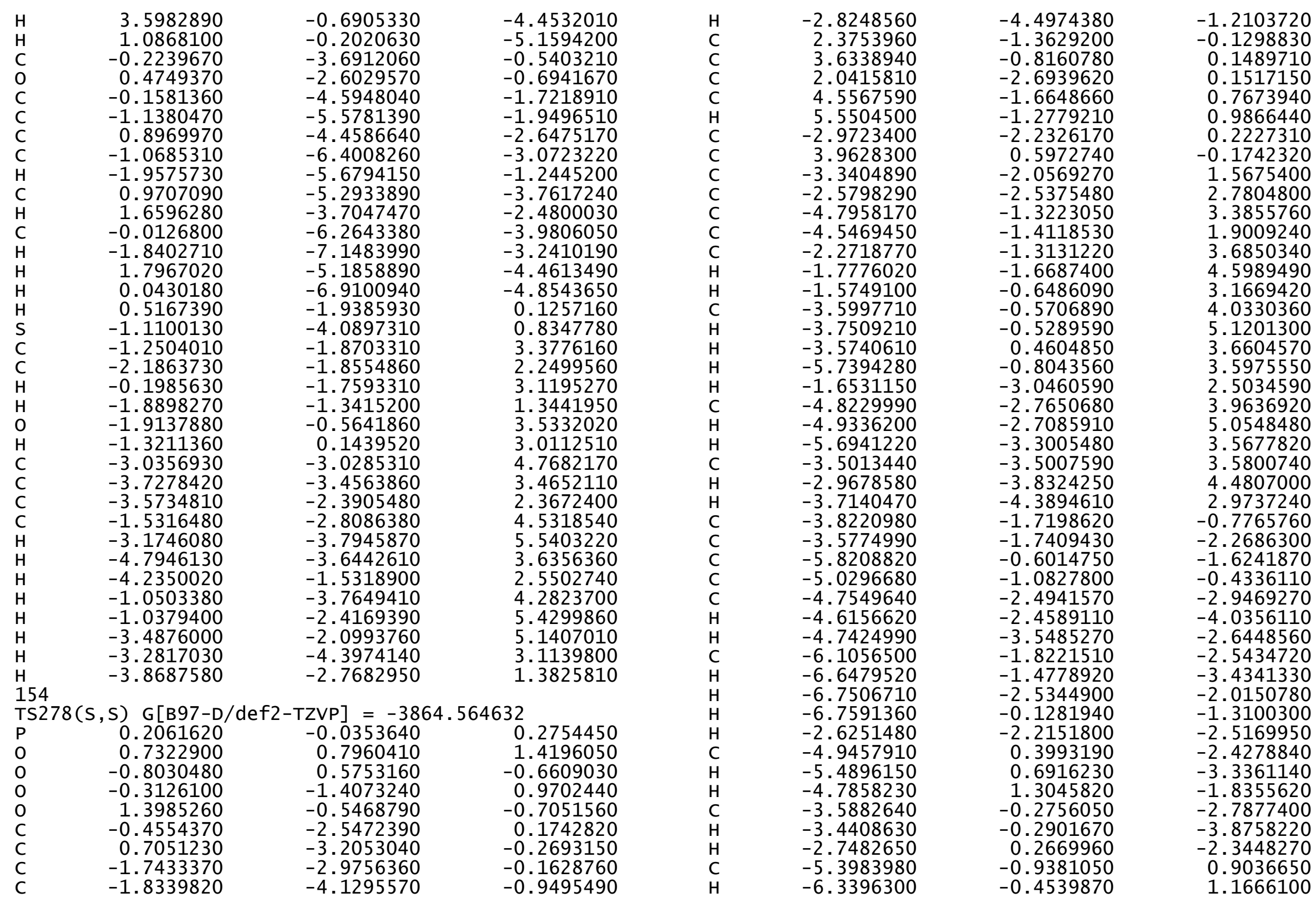




$\begin{array}{lrr}4.2103240 & 2.4071440 & -1.8026110 \\ 4.1406170 & 2.7307070 & -3.2744690 \\ 3.5445560 & 0.2621520 & -2.7291160 \\ 3.8815700 & 1.0731250 & -1.4979160 \\ 5.1335430 & 1.7875260 & -4.0115660 \\ 5.0650110 & 1.9719720 & -5.0919400 \\ 6.1584200 & 2.0190600 & -3.6981070 \\ 4.7755210 & 0.3051330 & -3.6794990 \\ 4.5312720 & -0.2484990 & -4.5956850 \\ 5.6231390 & -0.2003490 & -3.2018040 \\ 3.3002260 & -0.7734570 & -2.4818490 \\ 4.3980310 & 3.7808930 & -3.4601350 \\ 2.3535520 & 0.9338510 & -3.4638690 \\ 2.1421590 & 0.3687710 & -4.3813030 \\ 1.4629370 & 0.8817330 & -2.8320320 \\ 2.7147520 & 2.4122120 & -3.8016470 \\ 2.6933960 & 2.5823110 & -4.8862380 \\ 1.9923440 & 3.0979610 & -3.3477790 \\ 4.7230590 & 2.8184160 & 0.5131360 \\ 5.2141640 & 3.6207710 & 1.6926740 \\ 4.5579370 & 1.1837100 & 2.3036640 \\ 4.3858710 & 1.4882170 & 0.8318260 \\ 4.1082270 & 3.6450590 & 2.7817900 \\ 4.4955260 & 4.1685670 & 3.6662920 \\ 3.2403100 & 4.2005820 & 2.4127700 \\ 3.7063020 & 2.1819930 & 3.1358650 \\ 3.8806870 & 1.9796000 & 4.2009970 \\ 2.6457580 & 2.0074820 & 2.9350920 \\ 4.2646700 & 0.1594340 & 2.5435100 \\ 5.4833670 & 4.6404910 & 1.3906940 \\ 6.0503200 & 1.4245090 & 2.6667330 \\ 6.1832370 & 1.2537660 & 3.7432240 \\ 6.6814060 & 0.7016920 & 2.1354690 \\ 6.4489830 & 2.8859570 & 2.2879230 \\ 6.7960080 & 3.4344430 & 3.1735610 \\ 7.2634840 & 2.8876000 & 1.5538760 \\ 4.6299170 & 3.2813510 & -0.7987220 \\ 4.9057730 & 4.3085530 & -1.0417390 \\ 2.9635450 & -3.4950570 & 0.8654340 \\ 2.5547400 & -4.8742700 & 1.3595840 \\ 3.4612040 & -5.4059470 & 2.4782700 \\ 4.9315150 & -5.2530540 & 1.9379200 \\ 5.2728350 & -3.7641630 & 1571550 \\ 4.2369100 & -2.9711800 & \\ 3.2144220 & -6.4560480 & -6802470 \\ 2.5768250 & -5.5959780 & \\ 2.530170 & \end{array}$

\begin{abstract}
1.5096830
5. 0929030

5. 5971010

6.2572200

5. 3527810

3. 2809870

0.5751730

$-0.7109520$

1. 7806180

1. 5859370

0.2733750

$-0.9146200$

2. 4390770

1. 9337710

2. 6944560

0.3064630

0.1454090

$-1.8366170$

-1. 0784200

1. 5562730

$-3.1397130$

$-2.7678980$

$-4.4904480$

$-5.3260410$

$-4.9658970$

$-6.6023480$

$-4.9778130$

$-6.2408040$

$-4.3254340$

$-7.0646040$

$-7.2389600$

$-6.5936600$

$-8.0610640$

$-1.9090430$

$-2.2052630$

$-0.0991930$

0.3372680

$-0.8559710$

0.2319790

1. 2496010

1. 1055320

0.8098440

0.7634470

1. 0578240

$-0.2318700$

0.6125750
\end{abstract}

$\begin{array}{rr}-4.8365190 & 1.6917000 \\ -5.7610920 & 1.1095580 \\ -5.7234370 & 2.8076110 \\ -3.6315680 & 1.4683120 \\ -3.3281920 & 2.9466030 \\ -4.8409540 & 3.4050290 \\ -4.2927350 & -1.1614410 \\ -4.7834230 & -1.4618980 \\ -4.8239360 & -1.9217740 \\ -6.2483660 & -2.4539570 \\ -6.3352380 & -3.2419600 \\ -6.0197810 & -2.3240040 \\ -6.5248470 & -3.0869500 \\ -4.1514200 & -2.7824350 \\ -4.7492150 & -1.3293110 \\ -5.6078930 & -4.0668150 \\ -7.3305820 & -3.6868500 \\ -5.9066300 & -2.9095680 \\ -6.8804090 & -1.6550880 \\ -6.9577320 & -1.6125970 \\ 3.0136130 & -0.5012080 \\ 1.9161900 & 0.1055360 \\ 3.4787650 & -0.0863800 \\ 2.6163130 & 0.6539830 \\ 4.7603120 & -0.4240150 \\ 3.0256610 & 1.0352280 \\ 1.6204910 & 0.9053340 \\ 5.1666470 & -0.0357020 \\ 5.4302520 & -0.9904170 \\ 4.3010570 & 0.6936990 \\ 2.3455250 & 1.5969540 \\ 6.1606060 & -0.3010290 \\ 4.6195580 & 0.9925410 \\ 1.4332920 & -0.2600140 \\ 3.8250700 & -1.6409570 \\ 3.7440720 & 0.9370090 \\ 3.5668410 & -0.4522320 \\ 3.0466400 & 1.2977670 \\ 2.5846790 & -0.8953840 \\ 3.1942400 & 1.0929300 \\ 2.1408990 & 1.2186310 \\ 6.1097650 & 0.8795780 \\ 6.0617320 & -0.6553490 \\ 4.6482590 & -1.1893850 \\ 5.1509710 & 1.4807880 \\ 7.1292420 & 1.2318950 \\ & \end{array}$




\begin{tabular}{|c|c|c|c|c|c|c|c|}
\hline $\mathrm{H}$ & 1.4845320 & 6.7660760 & -1.0868510 & C & -5.7680160 & -0.6640410 & -1.6923130 \\
\hline $\mathrm{H}$ & 2.1310590 & 4.4150100 & -1.1194670 & $\mathrm{C}$ & -5.0003860 & -1.1471660 & -0.4872980 \\
\hline $\mathrm{H}$ & -1.2452700 & 5.4943770 & 1.2287460 & C & -4.6388120 & -2.5236660 & -3.0093600 \\
\hline $\mathrm{H}$ & -0.1569320 & 5.1129540 & 2.5748020 & $\mathrm{H}$ & -4.4730800 & -2.4697370 & -4.0935530 \\
\hline $\mathrm{H}$ & 1.8112090 & 5.8271080 & 1.2310690 & $\mathrm{H}$ & -4.6158320 & -3.5820820 & -2.7225240 \\
\hline $\mathrm{H}$ & -0.2371230 & 6.3671250 & -0.9914310 & C & -6.0103290 & -1.8801130 & -2.6303930 \\
\hline $\mathrm{H}$ & 0.8001330 & 4.5589460 & -2.2509180 & $\mathrm{H}$ & -6.5382990 & -1.5364190 & -3.5298510 \\
\hline \multirow{2}{*}{\multicolumn{4}{|c|}{$\begin{array}{l}154 \\
\text { TS279(S,S) G[B97-D/def2-TZVP }]=-3864.564358\end{array}$}} & $\mathrm{H}$ & -6.6542650 & -2.6092370 & -2.1240170 \\
\hline & & & & $\mathrm{H}$ & -6.7213770 & -0.2087370 & -1.3976300 \\
\hline$P$ & 0.1932770 & -0.0393430 & 0.3536540 & $\mathrm{H}$ & -2.5255730 & -2.2173630 & -2.5202980 \\
\hline 0 & 0.7144160 & 0.7866610 & 1.5045690 & C & -4.8894000 & 0.3581880 & -2.4645950 \\
\hline 0 & -0.8178450 & 0.5726520 & -0.5790600 & $\mathrm{H}$ & -5.4141640 & 0.6474340 & -3.3849160 \\
\hline 0 & -0.3158800 & -1.4201310 & 1.0366210 & $\mathrm{H}$ & -4.7616080 & 1.2619150 & -1.8621530 \\
\hline 0 & 1.3878000 & -0.5345710 & -0.6320370 & C & -3.5110680 & -0.2896600 & -2.7928810 \\
\hline C & -0.4232250 & -2.5592220 & 0.2326780 & $\mathrm{H}$ & -3.3320100 & -0.2879030 & -3.8763180 \\
\hline C & 0.7557060 & -3.2040970 & -0.1717080 & $\mathrm{H}$ & -2.6933910 & 0.2607980 & -2.3190360 \\
\hline C & -1.6957750 & -2.9975010 & -0.1512950 & C & -5.4078540 & -1.0274580 & 0.8412540 \\
\hline C & -1.7471700 & -4.1348510 & -0.9625650 & $\mathrm{H}$ & -6.3635450 & -0.5617690 & 1.0848940 \\
\hline $\mathrm{H}$ & -2.7247990 & -4.5043480 & -1.2677570 & C & 4.1118130 & 2.4376370 & -1.8706630 \\
\hline C & 2.3885700 & -1.3291290 & -0.0712070 & $\mathrm{c}$ & 3.9706180 & 2.7424920 & -3.3416390 \\
\hline C & 3.6506310 & -0.7646470 & 0.1460870 & C & 3.4324420 & 0.2738090 & -2.7411950 \\
\hline C & 2.0792240 & -2.6597780 & 0.2497110 & $\mathrm{C}$ & 3.8118390 & 1.1040320 & -1.5357050 \\
\hline C & 4.6129520 & -1.5968870 & 0.7284250 & C & 4.9383380 & 1.8027090 & -4.1156410 \\
\hline $\mathrm{H}$ & 5.6127960 & -1.1983540 & 0.8917870 & $\mathrm{H}$ & 4.8109470 & 1.9707190 & -5.1933790 \\
\hline C & -2.9453870 & -2.2768300 & 0.2095330 & $\mathrm{H}$ & 5.9746740 & 2.0532540 & -3.8596960 \\
\hline C & 3.9495400 & 0.6478160 & -0.2095570 & C & 4.6198800 & 0.3203450 & -3.7455660 \\
\hline $\mathrm{C}$ & -3.3521470 & -2.1245180 & 1.5460780 & $\mathrm{H}$ & 4.3446760 & -0.2514030 & -4.6416520 \\
\hline C & -2.6154670 & -2.6073690 & 2.7727030 & $\mathrm{H}$ & 5.4959780 & -0.1638790 & -3.2980840 \\
\hline C & -4.8652520 & -1.4339990 & 3.3336130 & $\mathrm{H}$ & 3.2127220 & -0.7617950 & -2.4716920 \\
\hline C & -4.5767040 & -1.5020530 & 1.8551030 & $\mathrm{H}$ & 4.2065570 & 3.7932650 & -3.5509320 \\
\hline C & -2.3500580 & -1.3873480 & 3.6972660 & C & 2.2017320 & 0.9210840 & -3.4318120 \\
\hline H & -1.8724050 & -1.7443960 & 4.6193990 & $\mathrm{H}$ & 1.9634140 & 0.3450100 & -4.3356750 \\
\hline $\mathrm{H}$ & -1.6520660 & -0.7057400 & 3.2032270 & $\mathrm{H}$ & 1.3381710 & 0.8605140 & -2.7646030 \\
\hline C & -3.6984330 & -0.6708140 & 4.0198340 & C & 2.5255530 & 2.4020930 & -3.7957340 \\
\hline $\mathrm{H}$ & -3.8776290 & -0.6443390 & 5.1031220 & $\mathrm{H}$ & 2.4476260 & 2.5653340 & -4.8787730 \\
\hline $\mathrm{H}$ & -3.6806110 & 0.3647640 & 3.6594360 & $\mathrm{H}$ & 1.8170950 & 3.0812070 & -3.3105260 \\
\hline $\mathrm{H}$ & -5.8220080 & -0.9331690 & 3.5268300 & C & 4.7063610 & 2.8876990 & 0.4182730 \\
\hline $\mathrm{H}$ & -1.6739760 & -3.0983620 & 2.5145900 & C & 5.2141970 & 3.7164950 & 1.5718280 \\
\hline C & -4.8846060 & -2.8835550 & 3.8943590 & C & 4.6249830 & 1.2761460 & 2.2364060 \\
\hline $\mathrm{H}$ & -5.0265860 & -2.8421980 & 4.9825790 & C & 4.3989880 & 1.5576480 & 0.7671870 \\
\hline $\mathrm{H}$ & -5.7356010 & -3.4282150 & 3.4682570 & C & 4.1319380 & 3.7348040 & 2.6848660 \\
\hline C & -3.5411350 & -3.5931370 & 3.5391870 & $\mathrm{H}$ & 4.5202550 & 4.2923990 & 3.5478070 \\
\hline $\mathrm{H}$ & -3.0257670 & -3.9236850 & 4.4506610 & $\mathrm{H}$ & 3.2391190 & 4.2524260 & 2.3199760 \\
\hline $\mathrm{H}$ & -3.7224580 & -4.4797580 & 2.9197640 & C & 3.7823660 & 2.2696390 & 3.0833250 \\
\hline C & -3.7748550 & -1.7620870 & -0.8059070 & $\mathrm{H}$ & 4.0052490 & 2.0931850 & 4.1439500 \\
\hline C & -3.4913970 & -1.7608750 & -2.2912420 & $\mathrm{H}$ & 2.7191450 & 2.0654710 & 2.9299160 \\
\hline
\end{tabular}




\begin{tabular}{|c|c|c|c|}
\hline $\mathrm{H}$ & 43596380 & 02407760 & 21001300 \\
\hline $\mathrm{H}$ & 5.4546600 & 4.7367690 & 1.2481060 \\
\hline C & 6.1231490 & 1.5491240 & 2.5474740 \\
\hline $\mathrm{H}$ & 6.2967740 & 1.3868910 & 3.6194310 \\
\hline $\mathrm{H}$ & 6.7499890 & 0.8365860 & 1.9975950 \\
\hline C & 6.4777700 & 3.0166600 & 2.1482940 \\
\hline $\mathrm{H}$ & 6.8279340 & 3. 5814930 & 3.0223640 \\
\hline $\mathrm{H}$ & 7.2782360 & 3.0320210 & 1.3991320 \\
\hline C & 4.5581570 & 3. 3308790 & -0.8953420 \\
\hline $\mathrm{H}$ & 4.8085570 & 4.3588220 & -1.1615490 \\
\hline C & 4.3253800 & -2.8997330 & 1.1485330 \\
\hline C & 3.0378640 & -3.4325360 & 0.9405930 \\
\hline C & 5.4350700 & -3.7221110 & 1.7868890 \\
\hline C & 4.9272350 & -4.9115240 & 2.6139190 \\
\hline C & 3.8512410 & -5.6768850 & 1.8340700 \\
\hline C & 2.6515780 & -4.7600120 & 1.5708040 \\
\hline $\mathrm{H}$ & 5.7678090 & -5.5678360 & 2.8742510 \\
\hline H & 6.0817540 & -4.1064600 & 0.9814520 \\
\hline $\mathrm{H}$ & 6.0670430 & -3.0665850 & 2.4009030 \\
\hline $\mathrm{H}$ & 4.2697320 & -6.0234340 & 0.8766060 \\
\hline $\mathrm{H}$ & 3.5238480 & -6.5643420 & 2.3908120 \\
\hline $\mathrm{H}$ & 1.8868290 & -5.2710170 & 0.9812060 \\
\hline H & 2.1723020 & -4.5298680 & 2.5368120 \\
\hline H & 4.4908490 & -4.5472480 & 3.5558680 \\
\hline C & 0.6698620 & -4.2904410 & -1.0759400 \\
\hline C & 1.9298750 & -4.8709170 & -1.7015880 \\
\hline C & 1.6523240 & -5.6881690 & -2.9705840 \\
\hline C & 0.5241500 & -6.6907830 & -2.7051170 \\
\hline C & -0.7749700 & -5.9295950 & -2.4159240 \\
\hline C & -0.5999430 & -4.7723200 & -1.4450940 \\
\hline $\mathrm{H}$ & 2.5715890 & -6.1997750 & -3.2831040 \\
\hline $\mathrm{H}$ & 2.4456760 & -5.5227720 & -0.9828210 \\
\hline $\mathrm{H}$ & 2.6292240 & -4.0527790 & -1.9148880 \\
\hline $\mathrm{H}$ & 0.7939950 & -7.3130650 & -1.8383340 \\
\hline H & 0.3802880 & -7.3635320 & -3.5602540 \\
\hline H & -1.5460790 & -6.6099370 & -2.0288990 \\
\hline H & -1.1679450 & -5.5287890 & -3.3637620 \\
\hline $\mathrm{H}$ & 36930 & -5.0168570 & -3.7895770 \\
\hline C & 1839780 & 2.9828860 & -0.4606560 \\
\hline 0 & 14560 & 1.8890990 & 0.1528350 \\
\hline C & -4.5529840 & 3.4241490 & -0.0817560 \\
\hline C & .1530 & 2.5274600 & 0.5884610 \\
\hline C & 240 & 5580 & -0.3868040 \\
\hline C & -6 . & 2.9 & 0.9327240 \\
\hline $\mathrm{H}$ & -5.066 & 1.5 & 0.8123590 \\
\hline C & -6.3158830 & 5.0987140 & -0.0337040 \\
\hline
\end{tabular}

$\begin{array}{lr}\mathrm{H} & -4.3654920 \\ \mathrm{C} & -7.1623260 \\ \mathrm{H} & -7.3594020 \\ \mathrm{H} & -6.6648280 \\ \mathrm{H} & -8.1724240 \\ \mathrm{H} & -1.9400670 \\ \mathrm{~S} & -2.2357490 \\ \mathrm{C} & -0.1675010 \\ \mathrm{C} & 0.2953800 \\ \mathrm{H} & -0.9247780 \\ \mathrm{H} & 0.2031130 \\ \mathrm{O} & 1.1830240 \\ \mathrm{H} & 1.0520730 \\ \mathrm{C} & 0.7237060 \\ \mathrm{C} & 0.7042200 \\ \mathrm{C} & 1.0185340 \\ \mathrm{C} & -0.3201190 \\ \mathrm{H} & 0.5125870 \\ \mathrm{H} & 1.4271930 \\ \mathrm{H} & 2.0927150 \\ \mathrm{H} & -1.3321700 \\ \mathrm{H} & -0.2618020 \\ \mathrm{H} & 1.7211930 \\ \mathrm{H} & -0.2926540 \\ \mathrm{H} & 0.7771920 \\ \mathrm{H} & \end{array}$
5.4112570
4.5771640

$-0.8995280$

$4.1991180 \quad 0.6252330$

$2.2066680 \quad 1.4388830$

$6.1009270 \quad-0.2717640$

$4.4994630 \quad 0.8957650$

$1.4171190 \quad-0.1952950$

$3.8131400 \quad-1.5744870$

$3.7353650 \quad 1.0328280$

$3.5702070 \quad-0.3493410$

$3.0296060 \quad 1.3760360$

$2.5904860 \quad-0.8008820$

$3.1962090 \quad 1.2073570$

2.1377710 1.3215380

$6.1081760 \quad 1.0052170$

$6.0693160 \quad-0.5306200$

$4.6617520 \quad-1.0685390$

$5.1376310 \quad 1.5835200$

$7.1239120 \quad 1.3603190$

$6.7820260 \quad-0.9449180$

$4.4362830 \quad-0.9856950$

$5.4752760 \quad 1.3184390$

$5.0928990 \quad 2.6783000$

$5.8312790 \quad 1.3720850$

$6.3693930 \quad-0.8821810$

154

$-2.1343060$

TS280(S,S) G[B97-D/def2-TZVP $]=-3864.564153$

$\begin{array}{lrrr}\text { TS280 }(\mathrm{S}, \mathrm{S}) \mathrm{G}[\mathrm{B} 97-\mathrm{D} / \mathrm{def}-\mathrm{TZVP}]=-3864.564153 \\ \mathrm{P} & 0.2014720 & 0.4030680 & 1.0195850 \\ \mathrm{O} & -0.5407500 & 0.9104760 & 2.2278280 \\ \mathrm{O} & 0.6789240 & -1.0243750 & 1.0338000 \\ \mathrm{O} & 1.4043200 & 1.4632230 & 0.7768630 \\ \mathrm{O} & -0.6986450 & 0.5188130 & -0.3446010 \\ \mathrm{C} & 2.0393090 & 1.4163250 & -0.4704260 \\ \mathrm{C} & 1.3579410 & 1.9426230 & -1.5745480 \\ \mathrm{C} & 3.3085930 & 0.8360160 & -0.5680850 \\ \mathrm{C} & 3.8597000 & 0.7546010 & -1.8504220 \\ \mathrm{H} & 4.8479500 & 0.3105800 & -1.9579720 \\ \mathrm{C} & -1.0094590 & 1.7918060 & -0.8264070 \\ \mathrm{C} & -2.3343210 & 2.2359600 & -0.7599800 \\ \mathrm{C} & 0.0199640 & 2.5694620 & -1.3765860 \\ \mathrm{C} & -2.5871030 & 3.5372710 & -1.2016270 \\ \mathrm{H} & -3.6118360 & 3.9044510 & -1.1690900 \\ \mathrm{C} & 4.0507310 & 0.3213490 & 0.6116490 \\ \mathrm{C} & -3.4324820 & 1.3520720 & -0.2893760 \\ \mathrm{C} & 4.4294450 & 1.1816480 & 1.6582770 \\ \mathrm{C} & 4.1404020 & 2.6614280 & 1.7601010\end{array}$




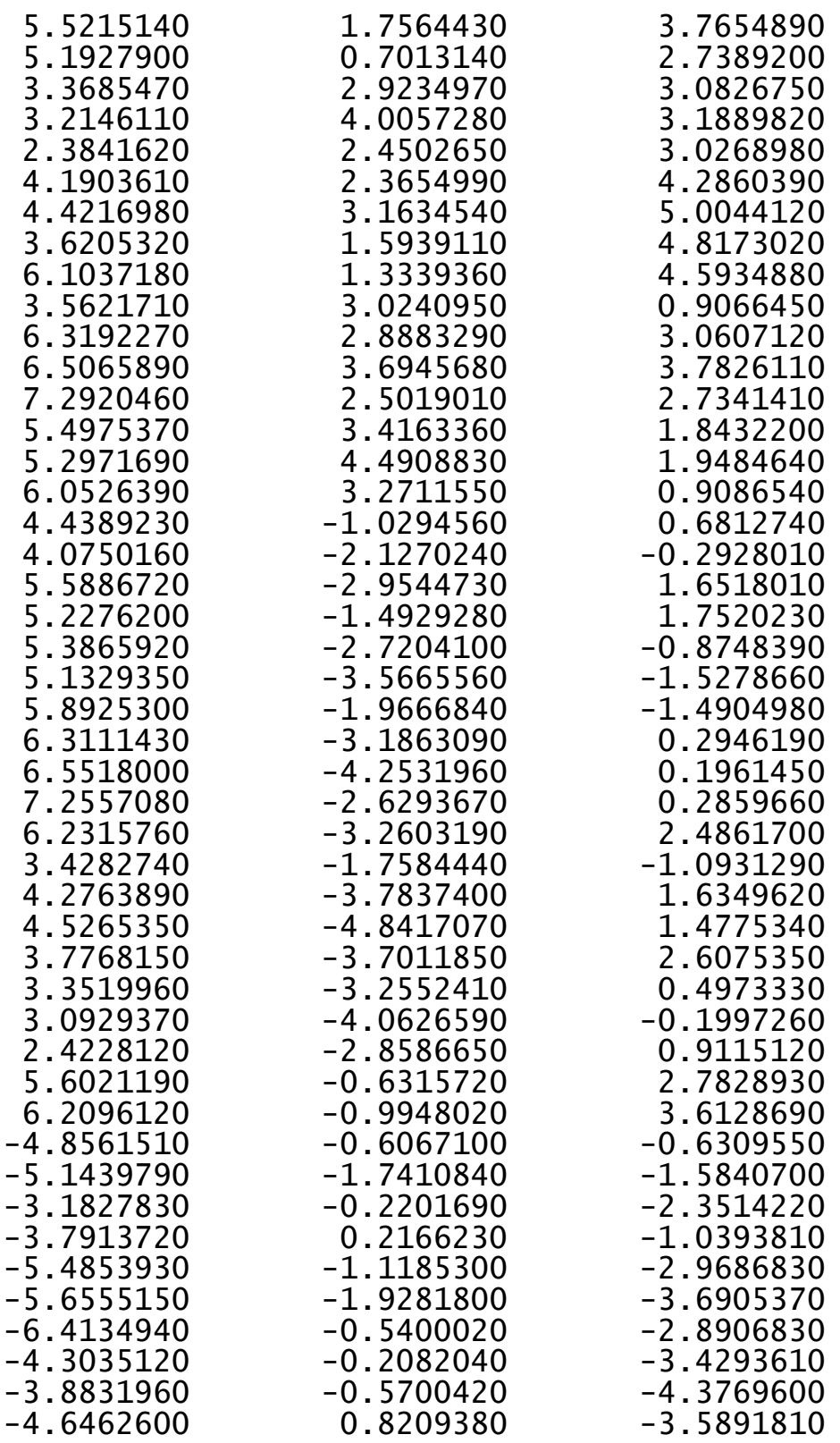

-2.3603480
-5.9764920
-2.6789560
-2.2506010
-1.8796480
-3.8609140
-4.0541410
-3.6154930
-5.2271420
-5.9066820
-3.9553510
-4.1630890
-4.8402570
-5.3182100
-4.4436110
-3.6910450
-3.6358530
-2.7230840
-3.1254730
-6.7239760
-5.2796700
-5.1546750
-5.4892060
-6.4468180
-6.8807780
-7.2470040
-5.5770260
-6.4077140
-1.5715060
-0.2489470
-1.9387400
-0.7469810
0.4432380
0.8911810
-1.0361880
-2.3648210
-2.7369800
0.1467550
1.2828460
1.6959110
1.3288270
-0.4531780
1.9066920
1.0992240
1.6068520
3.1239030

0.4329560

$-2.3588290$

$-1.6780740$

$-2.0149680$

$-1.6938080$

-2. 5979370

$-3.3648070$

$-3.1212050$

0.8153530

1.2783560

2.8261460

1.6458480

1. 3471010

1. 7022290

0.3439440

2. 3069190

3. 1722630

1.8023270

3. 4554000

0.6021160

3. 6396690

4.4885260

4.0448110

2.7171720

3. 0918890

2. 6880880

$-0.3066160$

$-0.9408300$

4.3871920

3. 9095620

.7967860

6.7629310

6.1094170

4.8602740

7.6990730

5. 7434090

6.1817970

5.8289530

6.8115480

4. 3434580

5. 1800870

7. 0122760

1.7735310

2.1580870

1.4921070

1.6742130
$-2.6550070$

$-1.2251480$

$-2.1960170$

$-3.1486390$

$-1.4538040$

$-1.7658800$

$-2.5274730$

$-0.8353180$

1. 2769360

2.5404780

1. 7939490

0.8734250

3.6683960

4. 5910420

3.8613890

3. 2340110

3. 9078350

3. 2655350

1. 4613930

2.8204320

1.8280600

2. 5129210

0.8306980

2. 3029770

3. 2394800

1. 5540190

0.5261060

0.8383110

$-1.6530180$

$-1.7301600$

$-2.0903600$

$-2.1126630$

$-2.8247770$

$-2.0561610$

$-2.6075210$

$-3.1055100$

$-1.4419270$

$-3.8472820$

$-2.9073970$

$-2.5848840$

$-1.0960030$

$-1.0821820$

$-2.8675080$

$-4.0977590$

$-5.3839130$

$-5.5016220$ 


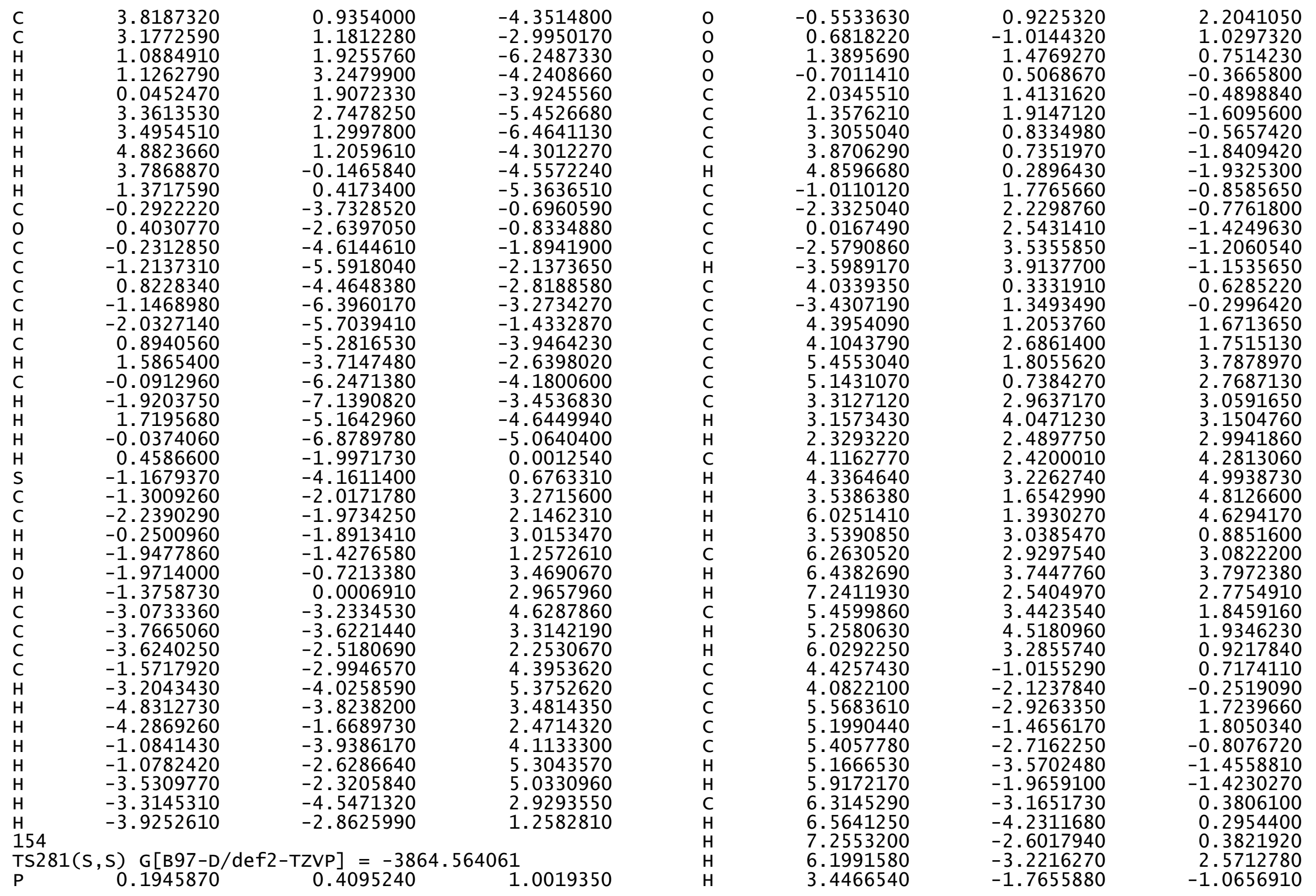




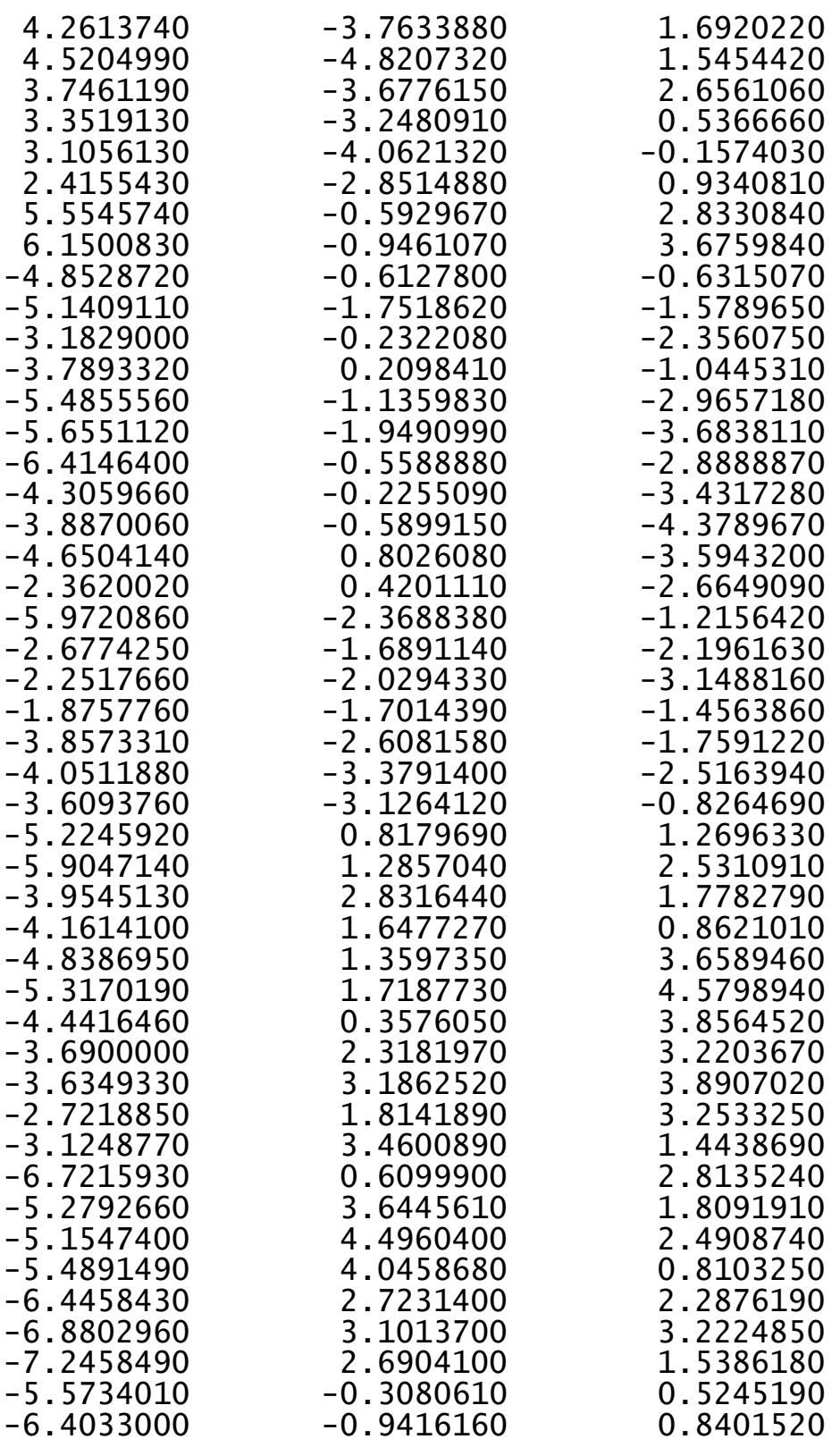

$-1.5620910$

$-0.2475710$

$-1.9198840$

$-0.7132000$

0.4377680

0.8957460

$-0.9992150$

$-2.3658010$

$-2.6994070$

0.0956280

1.2869420

1.6354810

1.4206830

$-0.3834600$

1. 9230230

3. 1997060

1.1091390

1.9526640

2.9051880

3.8834100

1. 2895140

0.3770100

0.5150550

2. 3180440

3. 4611060

4.4780610

4. 5994550

2. 5423000

$-0.2805190$

0.4130430

$-0.2166930$

$-1.1973770$

0.8385040

$-1.1277950$

$-2.0171380$

0.9124040

1.6009540

$-0.0712110$

$-1.8998930$

1.7386780

$-0.0151960$

0.4660560

$-1.1571010$

$-1.2956640$

$-2.2354320$

$-0.2456640$

$\begin{array}{ll}4.3774520 & -1.6711980 \\ 3.8851850 & -1.7805340 \\ 5.7983090 & -2.0778050 \\ 6.7445940 & -2.1040240 \\ 6.0912830 & -2.8770240 \\ 4.8110180 & -2.1668930 \\ 7.7018250 & -2.5587320 \\ 5.7719530 & -3.0854820 \\ 6.1813730 & -1.4058890 \\ 5.8469140 & -3.8944540 \\ 6.7798300 & -2.9740690 \\ 4.2824650 & -2.7747630 \\ 5.0885640 & -1.2382040 \\ 6.9533060 & -1.0753020 \\ 1.7298030 & -2.8920670 \\ 1.1449770 & -2.9975200 \\ 2.0345070 & -4.1408280 \\ 2.1355630 & -5.4173840 \\ 0.9398410 & -5.5235210 \\ 0.9546670 & -4.3422730 \\ 2.1884240 & -6.2904190 \\ 1.2188280 & -4.2605890 \\ 2.9425220 & -4.0090610 \\ 0.0092710 & -5.5064330 \\ 0.9617490 & -6.4697980 \\ 0.0317320 & -4.3176110 \\ 1.7793630 & -4.4908270 \\ 3.0648870 & -5.3967510 \\ -3.7334110 & -0.6889830 \\ -2.6396630 & -0.8299250 \\ -4.6197010 & -1.8835010 \\ -5.5994520 & -2.1240670 \\ -4.4722230 & -2.8072840 \\ -6.4081220 & -3.2568000 \\ -5.7099490 & -1.4206290 \\ -5.2933710 & -3.9315350 \\ -3.7203900 & -2.6301310 \\ -6.2612420 & -4.1626240 \\ -7.1530900 & -3.4351050 \\ -5.1775470 & -4.6294530 \\ -6.8965180 & -5.0440070 \\ -1.9932990 & 0.0022600 \\ -4.1579880 & 0.6841130 \\ -2.0031610 & 3.2694120 \\ -1.9682500 & 2.1451630 \\ -1.8748240 & 3.0111190\end{array}$




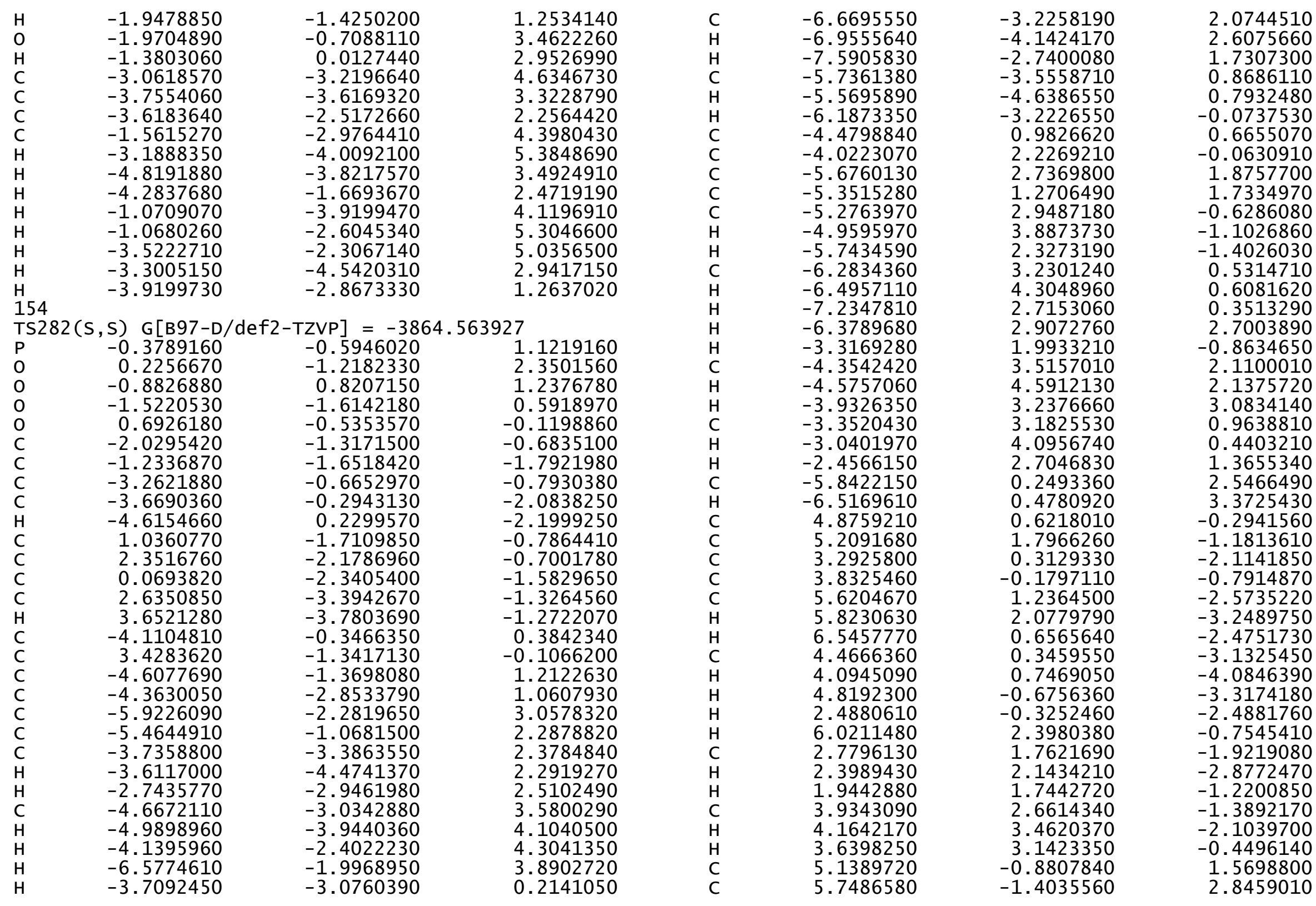




$\begin{array}{rrr}3.8386500 & -2.9112660 & 1.9257220 \\ 4.0941080 & -1.6885820 & 1.0769510 \\ 4.6229180 & -1.5237520 & 3.9102620 \\ 5.0522160 & -1.9214630 & 4.8394610 \\ 4.2150790 & -0.5306850 & 4.1314200 \\ 3.4995910 & -2.4623290 & 3.3727760 \\ 3.4118720 & -3.3578350 & 4.0019780 \\ 2.5302390 & -1.9599300 & 3.3780910 \\ 3.0264890 & -3.5193170 & 1.5166380 \\ 6.5501940 & -0.7441850 & 3.2013060 \\ 5.1600230 & -3.7281610 & 1.9888180 \\ 4.9994910 & -4.6102390 & 2.6222790 \\ 5.4230160 & -4.0821950 & 0.9848290 \\ 6.2992070 & -2.8325030 & 2.5720060 \\ 6.6781830 & -3.2517790 & 3.5134530 \\ 7.1409490 & -2.7720350 & 1.8721060 \\ 5.5319840 & 0.2703290 & 0.8868020 \\ 6.3448810 & 0.8875720 & 1.2715970 \\ 0.3736010 & -3.5832230 & -2.1819070 \\ -0.6918640 & -4.3306780 & -2.9655430 \\ -0.3827160 & -5.8244960 & -3.1306400 \\ 1.0520010 & -6.0052010 & -3.6398310 \\ 2.0412080 & -5.4825770 & -2.5906150 \\ 1.6655650 & -4.1221560 & -2.0266730 \\ -1.1057260 & -6.2735370 & -3.8235870 \\ -0.7805200 & -3.8854460 & -3.9667810 \\ -1.6660810 & -4.1850190 & -2.4827810 \\ 1.1732060 & -5.4423650 & -4.5778030 \\ 1.2642440 & -7.0593640 & -3.8596380 \\ 3.0578660 & -5.4344420 & -3.0041420 \\ 2.0823420 & -6.2022120 & -1.7571740 \\ -0.4911520 & -6.3369170 & -2.1630920 \\ -1.6492160 & -1.2185730 & -3.0635990 \\ -0.7961060 & -1.2972260 & -4.3049300 \\ -0.6528570 & 0.1152520 & -4.9271130 \\ -2.0267070 & 0.8213950 & -5.1327440 \\ -3.2089090 & -0.0141190 & -4.5740940 \\ -2.8725220 & -0.5349940 & -3.2006150 \\ -1.2740950 & -1.9607470 & -5.0423660 \\ -0.1183860 & 0.0320970 & -5.8816490 \\ -2.2011120 & 1.0194650 & -6.1977930 \\ -3.3875240 & -0.8596550 & -5.2560940 \\ 0.1907350 & -1.7130690 & -4.0833730 \\ -0.0255940 & 0.7158040 & -4.2571420 \\ -2.0196890 & 1.7922850 & -4.6222010 \\ -4.1263240 & 0.5856340 & -4.5494990\end{array}$

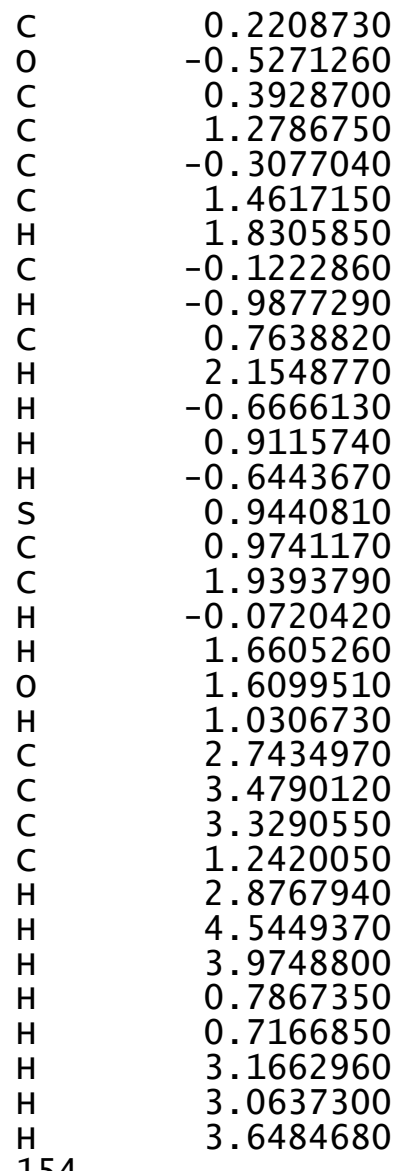
3.4910010
2.4349120
4.2420320
5.3299130
3.8280710
5.9834220
5.6451630
4.4834250
2.9850700
5.5625410
6.8191620
4.1479340
6.0707140
1.8084320
3.9753920
1.7293460
1.7268890
1.6413410
1.2335490
0.4087560
$-0.2879290$
2.8212510
3.2675530
2.2371390
2.6379570
3. 5648260
3.4255920
1.3627160
3. 6090900
2.2371570
1.8741740
4.2281350
2. 6394010

154

$-0.4668710$

$-0.5986950$

$-1.7432610$

$-1.8463470$

$-2.8957430$

$-3.0635350$

$-0.9658580$

$-4.1111370$

$-2.8347340$

$-4.2010930$

$-3.1267390$

$-4.9911340$

$-5.1516480$

0.2483920

0.9780500

3. 5562190

2. 4520690

3.2687330

1. 5299780

3. 7088080

3. 1693590

5.0182440

3.7455030

2.6136640

4.7373680

5.8129770

3.9482050

2.7797320

4.4962930

5. 6132410

5.3793900

3.4097080

1.6461480

\section{TS283(S, S) G[B97-D/def2-TZVP $]=-3864.563812$}

\begin{tabular}{lrrr} 
TS283(S, S & \multicolumn{4}{c}{ G $[\mathrm{B} 97-\mathrm{D} / \mathrm{def}-\mathrm{TZV}]=-3864.563812$} \\
$\mathrm{P}$ & 0.2704240 & 0.6050580 & 1.0283940 \\
$\mathrm{O}$ & -0.4052130 & 1.2531450 & 2.2072320 \\
$\mathrm{O}$ & 0.7796660 & -0.8012880 & 1.2020760 \\
$\mathrm{O}$ & 1.4270170 & 1.6316350 & 0.5478370 \\
$\mathrm{O}$ & -0.7214990 & 0.5137100 & -0.2718980 \\
$\mathrm{C}$ & 2.0049740 & 1.3586730 & -0.7005590 \\
$\mathrm{C}$ & 1.2692980 & 1.6973290 & -1.8500910 \\
$\mathrm{C}$ & 3.2636530 & 0.7522790 & -0.7480030 \\
$\mathrm{C}$ & 3.7696390 & 0.4385870 & -2.0187010 \\
$\mathrm{H}$ & 4.7412520 & -0.0472450 & -2.0853200 \\
$\mathrm{C}$ & -1.0614060 & 1.6816810 & -0.9551800
\end{tabular}




-2.3889640
-0.0682970
-2.6904660
-3.7158490
4.0555210
-3.4642680
4.4959400
4.2185420
5.7193350
5.3200560
3.5309940
3.3819680
2.5456540
4.4267240
4.7059920
3.8897470
6.3514180
3.5893110
6.4753210
6.7128210
7.4230550
5.5793800
5.3888730
6.0730700
4.4403770
4.0244740
5.6272970
5.2802970
5.3040680
5.0148440
5.7826920
6.2828270
6.5153370
7.2279130
6.3081800
3.3404910
4.3126290
4.5509530
3.8588600
3.3374050
3.0480110
2.4278520
5.7199350
6.3708120
-4.8719140
-5.1516090

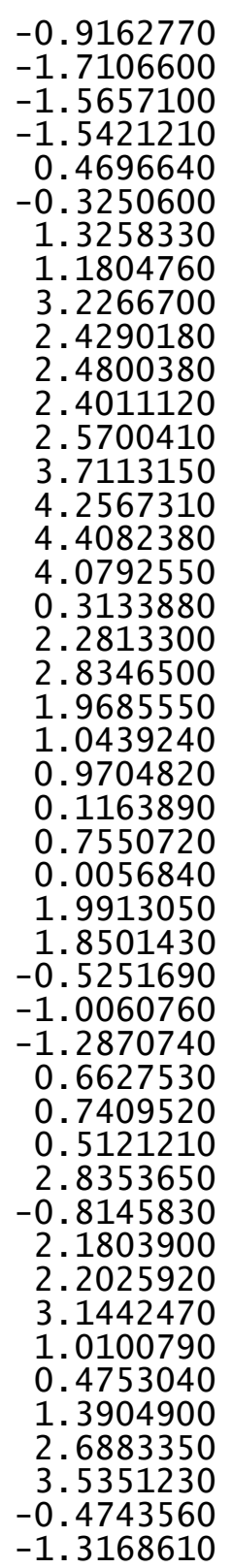

$\begin{array}{rr}2.1191420 & -0.9162770 \\ 2.3323920 & -1.7106600 \\ 3.3238510 & -1.5657100 \\ 3.6891880 & -1.5421210 \\ 0.4377500 & 0.4696640 \\ 1.2779240 & -0.3250600 \\ 1.4632060 & 1.3258330 \\ 2.9413540 & 1.1804760 \\ 2.3866230 & 3.2266700 \\ 1.1701830 & 2.4290180 \\ 3.4427760 & 2.4800380 \\ 4.5280270 & 2.4011120 \\ 2.9764980 & 2.5700410 \\ 3.1000650 & 3.7113150 \\ 4.0115220 & 4.2567310 \\ 2.4457610 & 4.4082380 \\ 2.1097530 & 4.0792550 \\ 3.1558780 & 0.3133880 \\ 3.3623710 & 2.2813300 \\ 4.2807670 & 2.8346500 \\ 2.9080410 & 1.9685550 \\ 3.6795900 & 1.0439240 \\ 4.7585830 & 0.9704820 \\ 3.3655780 & 0.1163890 \\ -0.8854050 & 0.7550720 \\ -2.1315750 & 0.0056840 \\ -2.6264450 & 1.9913050 \\ -1.1652290 & 1.8501430 \\ -2.8344610 & -0.5251690 \\ -3.7786870 & -1.0060760 \\ -2.2071100 & -1.2870740 \\ -3.0998640 & 0.6627530 \\ -4.1702930 & 0.7409520 \\ -2.5644580 & 0.5121210 \\ -2.7909650 & 2.8353650 \\ -1.9006680 & -0.8145830 \\ -3.4291400 & 2.1803900 \\ -4.5011650 & 2.2025920 \\ -3.1692560 & 3.1442470 \\ -3.0999510 & 1.0100790 \\ -4.0138500 & 0.4753040 \\ -2.6315280 & 1.3904900 \\ -0.1408110 & 2.6883350 \\ -0.3621500 & 3.5351230 \\ -0.7181850 & -0.4743560 \\ -1.9395100 & -1.3168610\end{array}$

-3.2112250
-3.8181780
-5.5055480
-5.6698470
-6.4395960
-4.3358710
-3.9150260
-4.6896790
-2.3972750
-5.9756960
-2.6906090
-2.2595210
-1.8903850
-3.8600670
-4.0485640
-3.6053750
-5.2394150
-5.9101290
-3.9814650
-4.1865130
-4.8330900
-5.3058640
-4.4267750
-3.6957380
-3.6369160
-2.7248450
-3.1601050
-6.7178730
-5.3129620
-5.1889600
-5.5373520
-6.4657580
-6.8918850
-7.2749460
-5.5858580
-6.4069360
-0.4004270
0.5900960
0.5227750
-0.9313840
-1.9863630
-1.7131750
0.3444380
1.1532970
-1.0293870
-1.9331950

\author{
$-0.4771580$ \\ 0.0743930 \\ $-1.4526180$ \\ $-2.3286510$ \\ $-0.8791800$ \\ $-0.5777250$ \\ $-1.0235460$ \\ 0.4282950 \\ 0.1519550 \\ $-2.5296390$ \\ $-1.9082200$ \\ $-2.3290560$ \\ $-1.8451830$ \\ $-2.7961900$ \\ $-3.6319200$ \\ $-3.2288950$ \\ 0.8693050 \\ 1.4395830 \\ 2.9309040 \\ 1.6695010 \\ 1.6223920 \\ 2. 0592190 \\ 0.6452520 \\ 2.5462960 \\ 3.4680690 \\ 2.0505660 \\ 3.5318770 \\ 0.7849850 \\ 3.7328800 \\ 4.6422230 \\ 4.0423860 \\ 2. 8472400 \\ 3.3016390 \\ 2.7443980 \\ $-0.3195830$ \\ $-0.9299190$ \\ 3. 5456640 \\ 4.4103440 \\ 5.8665450 \\ 6.4251240 \\ 5.3696150 \\ 4.0471380 \\ 4.4081920 \\ 6. 5014530 \\ 7. 3032440 \\ 5.2389530
}

$-2.2342280$

$-0.9650100$

$-2.7514980$

$-3.3927520$

$-2.7227200$

$-3.3024710$

$-4.2135190$

$-3.5572270$

$-2.6026060$

$-0.8975730$

$-1.9443200$

$-2.8615950$

$-1.2052150$

$-1.4246360$

$-2.1111990$

$-0.4507540$

1. 2987020

2. 5225610

1.6159790

0.8115370

3.6274320

4.5170250

3.9135630

3.0936920

3.6872480

3.1571430

1.2154800

2. 8726160

1.5909480

2.1932780

0.5630490

2.1624880

3.0667880

1.4297900

0.6568750

1.0352510

$-2.3399830$

$-3.0789870$

$-2.5547770$

$-2.5003100$

$-2.9172760$

$-2.2478600$

$-4.1522770$

$-3.1895320$

$-3.1507770$

$-4.0100390$ 


$\begin{array}{rr}\mathrm{H} & 1.6074340 \\ \mathrm{H} & 0.9595870 \\ \mathrm{H} & -1.1580730 \\ \mathrm{H} & -2.9977740 \\ \mathrm{C} & 1.7954140 \\ \mathrm{C} & 1.0524970 \\ \mathrm{C} & 1.0146650 \\ \mathrm{C} & 2.4160730 \\ \mathrm{C} & 3.5193910 \\ \mathrm{C} & 3.0499060 \\ \mathrm{H} & 1.5672400 \\ \mathrm{H} & 0.5886070 \\ \mathrm{H} & 2.6968380 \\ \mathrm{H} & 3.7391120 \\ \mathrm{H} & 0.0347860 \\ \mathrm{H} & 0.3292560 \\ \mathrm{H} & 2.3779010 \\ \mathrm{H} & 4.4465120 \\ \mathrm{C} & -0.1857440 \\ \mathrm{O} & 0.5033280 \\ \mathrm{C} & -0.1645810 \\ \mathrm{C} & -1.1409170 \\ \mathrm{C} & 0.8418010 \\ \mathrm{C} & -1.1148910 \\ \mathrm{H} & -1.9237330 \\ \mathrm{C} & 0.8722420 \\ \mathrm{H} & 1.6004090 \\ \mathrm{C} & -0.1070110 \\ \mathrm{H} & -1.8834760 \\ \mathrm{H} & 1.6606480 \\ \mathrm{H} & -0.0858650 \\ \mathrm{H} & 0.5664160 \\ \mathrm{~S} & -1.0171790 \\ \mathrm{C} & -1.1273970 \\ \mathrm{C} & -2.0755660 \\ \mathrm{H} & -0.0781470 \\ \mathrm{H} & -1.7865360 \\ \mathrm{O} & -1.7843430 \\ \mathrm{H} & -1.2025660 \\ \mathrm{C} & -2.9055760 \\ \mathrm{C} & -3.6149810 \\ \mathrm{C} & -3.4627770 \\ \mathrm{C} & -1.4021830 \\ \mathrm{H} & -3.0424940 \\ \mathrm{H} & -4.6814790 \\ \mathrm{H} & -4.1203920 \\ & \end{array}$

$\begin{array}{rr}4.0204090 & -2.9822700 \\ 5.8824720 & -1.5488300 \\ 6.7543920 & -1.4789440 \\ 5.7224540 & -2.6828360 \\ 1.3410180 & -3.1057380 \\ 1.4891640 & -4.4100960 \\ 0.1317700 & -5.1570090 \\ -0.5415810 & -5.2627230 \\ 0.2799770 & -4.5483750 \\ 0.7050120 & -3.1805680 \\ 2.2294430 & -5.0417450 \\ 0.2888710 & -6.1557580 \\ -0.6813410 & -6.3141870 \\ 1.1720330 & -5.1557280 \\ 1.8545580 & -4.2513670 \\ -0.5307660 & -4.6143090 \\ -1.5373390 & -4.8035250 \\ -0.3010450 & -4.4772390 \\ -3.6369800 & -0.3144980 \\ -2.5531300 & -0.5277380 \\ -4.5732520 & -1.4719840 \\ -5.5736650 & -1.6275920 \\ -4.4461270 & -2.4515450 \\ -6.4223760 & -2.7328690 \\ -5.6662820 & -0.8807160 \\ -5.3057860 & -3.5483980 \\ -3.6777930 & -2.3413470 \\ -6.2942730 & -3.6953020 \\ -7.1836360 & -2.8454840 \\ -5.2040690 & -4.2909320 \\ -6.9601360 & -4.5553850 \\ -1.8565470 & 0.2650730 \\ -3.9911210 & 1.1066300 \\ -1.6244850 & 3.5315130 \\ -1.6724510 & 2.4154090 \\ -1.5346720 & 3.2551100 \\ -1.2141090 & 1.4780770 \\ -0.3080520 & 3.6193200 \\ 0.3712320 & 3.0527150 \\ -2.6827850 & 5.0068850 \\ -3.1866070 & 3.7405320 \\ -2.1937510 & 2.5756010 \\ -2.4907000 & 4.7423680 \\ -3.3966810 & 5.8278120 \\ -3.3532340 & 3.9332570 \\ -1.3225690 & 2.7058540\end{array}$

$\begin{array}{ll}\mathrm{H} & -0.9314050 \\ \mathrm{H} & -0.8956570 \\ \mathrm{H} & -3.3462160 \\ \mathrm{H} & -3.1807180 \\ \mathrm{H} & -3.7656850 \\ 154 & \end{array}$
$-3.4642910$
$-2.0504700$
$-1.7279140$
$-4.1523000$

4.5449620

5.6103730

5.3232160

3. 4460590

TS284(S, S) G[B97-D/def2-TZVP $]=-3864.563745$

$\begin{array}{lrrr}\text { TS284(S S }) \text { G }[\mathrm{B} 97-\mathrm{D} / \mathrm{def}-\mathrm{TZVP}]=-3864.563745 \\ \mathrm{P} & 0.2003550 & -0.0238260 & 0.2729460 \\ \mathrm{O} & 0.7116790 & 0.7953400 & 1.4326290 \\ \mathrm{O} & -0.8189080 & 0.5880180 & -0.6518740 \\ \mathrm{O} & -0.2910620 & -1.4184750 & 0.9388600 \\ \mathrm{O} & 1.3989080 & -0.4994430 & -0.7165130 \\ \mathrm{C} & -0.3987900 & -2.5461470 & 0.1190800 \\ \mathrm{C} & 0.7863830 & -3.1840050 & -0.2979670 \\ \mathrm{C} & -1.6746230 & -2.9830940 & -0.2536460 \\ \mathrm{C} & -1.7440080 & -4.1196780 & -1.0722430 \\ \mathrm{H} & -2.7243480 & -4.4911300 & -1.3646530 \\ \mathrm{C} & 2.4023130 & -1.2939820 & -0.1586550 \\ \mathrm{C} & 3.6487270 & -0.7110650 & 0.0986570 \\ \mathrm{C} & 2.1121720 & -2.6390460 & 0.1189320 \\ \mathrm{C} & 4.6193720 & -1.5399990 & 0.6690920 \\ \mathrm{H} & 5.6059570 & -1.1240160 & 0.8659670 \\ \mathrm{C} & -2.9211150 & -2.2712460 & 0.1366140 \\ \mathrm{C} & 3.9255200 & 0.7180660 & -0.2048380 \\ \mathrm{C} & -3.3082250 & -2.1396680 & 1.4813910 \\ \mathrm{C} & -2.5503280 & -2.6330060 & 2.6909560 \\ \mathrm{C} & -4.7978960 & -1.4803820 & 3.3008310 \\ \mathrm{C} & -4.5326900 & -1.5309920 & 1.8172730 \\ \mathrm{C} & -2.2778050 & -1.4230850 & 3.6264890 \\ \mathrm{H} & -1.7861460 & -1.7892910 & 4.5376470 \\ \mathrm{H} & -1.5896010 & -0.7321250 & 3.1318490 \\ \mathrm{C} & -3.6248870 & -0.7170140 & 3.9762610 \\ \mathrm{H} & -3.7874560 & -0.7013690 & 5.0623820 \\ \mathrm{H} & -3.6179910 & 0.3219580 & 3.6252770 \\ \mathrm{H} & -5.7545710 & -0.9876710 & 3.5145300 \\ \mathrm{H} & -1.6103770 & -3.1156470 & 2.4125320 \\ \mathrm{C} & -4.7992570 & -2.9353840 & 3.8472610 \\ \mathrm{H} & -4.9201790 & -2.9056310 & 4.9384040 \\ \mathrm{H} & -5.6552850 & -3.4806850 & 3.4322340 \\ \mathrm{C} & -3.4588300 & -3.6336060 & 3.4589070 \\ \mathrm{H} & -2.9265860 & -3.9752600 & 4.3565030 \\ \mathrm{H} & -3.6469790 & -4.5118660 & 2.8296590 \\ \mathrm{C} & -3.7712240 & -1.7504420 & -0.8584090 \\ \mathrm{C} & -3.5106660 & -1.7269220 & -2.3474420 \\ \mathrm{C} & -5.7881640 & -0.6601570 & -1.6994520 \\ \mathrm{C} & -4.9975440 & -1.1518580 & -0.5129260\end{array}$




$\begin{array}{rrr}-4.6617130 & -2.4905320 & -3.0587650 \\ -4.5130600 & -2.4198380 & -4.1444440 \\ -4.6242960 & -3.5525840 & -2.7873230 \\ -6.0332910 & -1.8656380 & -2.6501340 \\ -6.5785860 & -1.5150980 & -3.5365480 \\ -6.6622900 & -2.6077500 & -2.1438140 \\ -6.7410180 & -0.2180700 & -1.3838780 \\ -2.5442340 & -2.1708690 & -2.5974830 \\ -4.9308830 & 0.3811350 & -2.4701220 \\ -5.4720290 & 0.6784840 & -3.3783140 \\ -4.8024650 & 1.2776290 & -1.8567520 \\ -3.5515760 & -0.2492630 & -2.8286220 \\ -3.3896260 & -0.2309270 & -3.9145940 \\ -2.7315370 & 0.3022250 & -2.3601400 \\ -5.3849300 & -1.0526630 & 0.8230390 \\ -6.3405420 & -0.5986200 & 1.0876300 \\ 4.1006150 & 2.5596350 & -1.8066000 \\ 4.0036510 & 2.9035070 & -3.2724560 \\ 3.4898420 & 0.4096550 & -2.7594690 \\ 3.8137340 & 1.2120010 & -1.5193510 \\ 5.0170430 & 2.0030670 & -4.0345070 \\ 4.9295640 & 2.2005570 & -5.1112210 \\ 6.0377920 & 2.2629190 & -3.7298210 \\ 4.7102850 & 0.5055310 & -3.7197650 \\ 4.4759480 & -0.0434920 & -4.6412900 \\ 5.5780430 & 0.0215690 & -3.2561280 \\ 3.2807980 & -0.6366470 & -2.5255990 \\ 4.2267580 & 3.9638380 & -3.4442810 \\ 2.2718360 & 1.0545360 & -3.4733500 \\ 2.0709160 & 0.4979340 & -4.3982660 \\ 1.3886890 & 0.9636840 & -2.8353880 \\ 2.5830110 & 2.5492150 & -3.7898470 \\ 2.5444960 & 2.7362650 & -4.8711520 \\ 1.8442870 & 3.2044130 & -3.3175150 \\ 4.6355050 & 2.9476310 & 0.5081000 \\ 5.1243170 & 3.7421870 & 1.6937570 \\ 4.5407250 & 1.2790230 & 2.2729720 \\ 4.3375030 & 1.6041420 & 0.8096430 \\ 4.0343950 & 3.7197140 & 2.7990190 \\ 4.4201900 & 4.2398780 & 3.6861350 \\ 3.1467690 & 4.2570370 & 2.4507300 \\ 3.6774460 & 2.2406960 & 3.1354190 \\ 3.8749530 & 2.0258970 & 4.1940470 \\ 2.6187510 & 2.0410500 & 2.9489180 \\ 4.2778780 & 0.2431020 & 2.4981340 \\ 5.3630240 & 4.7734450 & 1.4055550\end{array}$

6.0320900

6.1873270

6.6725160

6.3863440

6.7313900

7.1900510

4.5112160

4.7572270

4.3580700

3.0878800

5.4809250

4.9962140

3.9692730

2.7412420

5.8524370

6.1604920

6.0739050

4.4242500

3.6612950

2.0330890

2. 2104000

4.5228640

$-0.5981530$

$-0.6549360$

0.3234200

1.7736280

1. 8468020

0.6749880

$-0.3658040$

0.2977640

2. 3938110

1.8187980

$-1.6781410$

$-0.0283270$

2.2211140

2.8023630

$-3.2153010$

$-2.8107780$

$-4.5772930$

$-5.3843030$

$-5.0920490$

$-6.6709410$

$-5.0062290$

$-6.3771370$

$-4.4743080$

$-7.1723680$
1. 5515430

1.3647950

0.8551120

3.0296430

3. 5716960

3.0657750

3.4288720

4.4665520

$-2.8659280$

$-3.4213450$

$-3.6817010$

$-4.9272460$

$-5.6848750$

$-4.8021880$

$-5.5672180$

$-4.0020210$

$-3.0358950$

$-5.9608590$

$-6.6146260$

$-5.3089240$

$-4.6621100$

$-4.6268110$

$-4.7485860$

$-5.9503570$

$-7.0337770$

$-6.4900640$

$-4.2728620$

$-5.6576980$

$-7.9049090$

$-6.8769230$

$-4.6251060$

$-6.3417260$

$-7.3687470$

$-6.8522320$

$-4.5942080$

2.9627680

1.8644300

3. 3809450

2. 4809370

4.6536700

2.8452000

1.4913060

5.0149280

5.3524910

4.1121290
2.6167670

3.6875860

2.0619910

2. 2584150

3.1487930

1.5134370

$-0.7945620$

$-1.0247950$

1.0293600

0.7794110

1.6528310

2.4064260

1. 5575220

1. 3110710

2.6560070

0.8464690

2. 3142650

0.5938510

2.0531380

0.6540580

2. 2671970

3.3530730

$-1.5526430$

$-2.4631840$

$-1.9584400$

$-1.8354530$

$-1.8705980$

$-1.1857180$

$-3.4859500$

$-2.6249360$

$-2.6536960$

$-2.9258700$

$-2.5156420$

$-0.9739750$

$-0.9022230$

$-1.4689290$

$-0.4325040$

0.1508560

$-0.0044120$

0.7226550

$-0.3171690$

1. 1148790

0.9543270

0.0822310

$-0.8739030$

0.7981540 


\begin{tabular}{|c|c|c|c|c|c|c|c|}
\hline $\mathrm{H}$ & -7.2850080 & 2.1361860 & 1.6659250 & C & 3.3299460 & 2.9688750 & 3.0239840 \\
\hline H & -6.7601980 & 6.0025480 & -0.1642070 & $\mathrm{H}$ & 3.1799760 & 4.0538680 & 3.1045710 \\
\hline $\mathrm{H}$ & -8.1768510 & 4.3950770 & 1.1057810 & $\mathrm{H}$ & 2.3443600 & 2.4994090 & 2.9602370 \\
\hline H & -1.9419600 & 1.4089980 & -0.2281370 & C & 4.1268330 & 2.4324760 & 4.2537560 \\
\hline $\mathrm{s}$ & -2.3083200 & 3.8233840 & -1.5582890 & $\mathrm{H}$ & 4.3482600 & 3.2441020 & 4.9598310 \\
\hline c & -0.1873790 & 3.7400560 & 1.0140520 & $\mathrm{H}$ & 3.5438730 & 1.6741570 & 4.7898440 \\
\hline c & 0.2431280 & 3.5975410 & -0.3813900 & $\mathrm{H}$ & 6.0301330 & 1.4005930 & 4.6177260 \\
\hline H & -0.9295320 & 3.0224810 & 1.3655630 & $\mathrm{H}$ & 3.5628830 & 3.0209810 & 0.8500440 \\
\hline$H$ & 0.1519160 & 2.6223720 & -0.8427700 & C & 6.2795240 & 2.9217090 & 3.0569020 \\
\hline 0 & 1.1717680 & 3.2113260 & 1.1501290 & $\mathrm{H}$ & 6.4552020 & 3.7428570 & 3.7647330 \\
\hline $\mathrm{H}$ & 1.0499800 & 2.1529350 & 1.2561660 & $\mathrm{H}$ & 7.2573090 & 2.5258650 & 2.7575530 \\
\hline c & 0.6777070 & 6.1228220 & 0.9957700 & C & 5.4831070 & 3.4257820 & 1.8127480 \\
\hline c & 0.6193370 & 6.1024120 & -0.5394290 & $\mathrm{H}$ & 5.2863270 & 4.5033690 & 1.8898010 \\
\hline C & 0.9391200 & 4.7058510 & -1.1015680 & $\mathrm{H}$ & 6.0547910 & 3.2568370 & 0.8922560 \\
\hline c & -0.3405070 & 5.1332400 & 1.5872440 & C & 4.4310440 & -1.0373090 & 0.7233830 \\
\hline $\mathrm{H}$ & 0.4644460 & 7.1315550 & 1.3690450 & c & 4.0848620 & -2.1523120 & -0.2370620 \\
\hline H & 1.3215920 & 6.8300220 & -0.9633470 & $\mathrm{C}$ & 5.5539360 & -2.9462430 & 1.7549530 \\
\hline $\mathrm{H}$ & 2.0170400 & 4.4921550 & -1.0407010 & $\mathrm{C}$ & 5.1964960 & -1.4817510 & 1.8188620 \\
\hline H & -1.3623670 & 5.4630290 & 1.3517550 & C & 5.4067170 & -2.7606600 & -0.7793950 \\
\hline $\mathrm{H}$ & -0.2543530 & 5.0745690 & 2.6795280 & $\mathrm{H}$ & 5.1644230 & -3.6184820 & -1.4213300 \\
\hline $\mathrm{H}$ & 1.6871890 & 5.8524470 & 1.3333570 & $\mathrm{H}$ & 5.9278500 & -2.0200660 & -1.3983370 \\
\hline$H$ & -0.3903850 & 6.3920550 & -0.8617970 & C & 6.3047270 & -3.2061590 & 0.4182080 \\
\hline $\mathrm{H}$ & 0.6777370 & 4.6316250 & -2.1632790 & $\mathrm{H}$ & 6.5440870 & -4.2754180 & 0.3455040 \\
\hline & & & & $\mathrm{H}$ & 7.2511470 & -2.6522910 & 0.4189820 \\
\hline & , S) $\mathrm{G}[\mathrm{B} 97-\mathrm{D}$ & ZVP] & 723 & $\mathrm{H}$ & 6.1776640 & -3.2380730 & 2.6086750 \\
\hline $\mathrm{P}$ & 0.1970130 & 0.4094340 & 1.0001840 & $\mathrm{H}$ & 3.4568190 & -1.7972960 & -1.0580960 \\
\hline 0 & -0.5386400 & 0.9438400 & 2.2007060 & C & 4.2395680 & -3.7723320 & 1.7250060 \\
\hline 0 & 0.6688040 & -1.0194510 & 1.0405590 & $\mathrm{H}$ & 4.4903090 & -4.8334600 & 1.5918100 \\
\hline 0 & 1.4034770 & 1.4597920 & 0.7324170 & $\mathrm{H}$ & 3.7196960 & -3.6711060 & 2.6850990 \\
\hline 0 & -0.7078210 & 0.5022830 & -0.3627580 & C & 3.3409430 & -3.2625740 & 0.5585300 \\
\hline c & 2.0465330 & 1.3771810 & -0.5084520 & $\mathrm{H}$ & 3.0922680 & -4.0820690 & -0.1283430 \\
\hline C & 1.3671280 & 1.8606550 & -1.6353990 & $\mathrm{H}$ & 2.4053150 & -2.8543280 & 0.9457840 \\
\hline c & 3.3194900 & 0.8028900 & -0.5781890 & C & 5.5535210 & -0.6012460 & 2.8397090 \\
\hline c & 3.8882930 & 0.6995520 & -1.8522660 & $\mathrm{H}$ & 6.1429050 & -0.9500670 & 3.6886830 \\
\hline H & 4.8825530 & 0.2644180 & -1.9376780 & C & -4.8613490 & -0.5669010 & -0.6387340 \\
\hline c & -0.9996350 & 1.7702730 & -0.8701750 & $\mathrm{C}$ & -5.1539420 & -1.7117620 & -1.5776800 \\
\hline C & -2.3124870 & 2.2492190 & -0.7918190 & C & -3.1847420 & -0.2103620 & -2.3616860 \\
\hline c & 0.0371740 & 2.5085330 & -1.4534780 & $\mathrm{C}$ & -3.7911990 & 0.2454960 & -1.0547880 \\
\hline c & -2.5331220 & 3.5567960 & -1.2304830 & c & -5.4906960 & -1.1062960 & -2.9707530 \\
\hline H & -3.5459280 & 3.9543090 & -1.1833520 & $\mathrm{H}$ & -5.6612150 & -1.9248190 & -3.6824480 \\
\hline c & 4.0464570 & 0.3124100 & 0.6209930 & H & -6.4174530 & -0.5244100 & -2.9024420 \\
\hline & -3.4246240 & 1.3862800 & -0.3152010 & C & -4.3052620 & -0.2052310 & -3.4399770 \\
\hline C & 4.4090960 & 1.1923840 & 1.6564950 & $\mathrm{H}$ & -3.8858510 & -0.5784160 & -4.3835900 \\
\hline c & 4.1243040 & 2.6749660 & 1.7215660 & $\mathrm{H}$ & -4.6438860 & 0.8235260 & -3.6105420 \\
\hline C & 5.4647530 & 1.8075210 & 3.7704940 & $\mathrm{H}$ & -2.3594150 & 0.4345520 & -2.6740800 \\
\hline C & 5.1509100 & 0.732107 & 2.7606120 & $\mathrm{H}$ & -5.9897780 & -2.3206250 & -1.2113990 \\
\hline
\end{tabular}




-2.6886910
-2.2659380
-1.8866710
-3.8747270
-4.0705430
-3.6324590
-5.2240460
-5.9003530
-3.9388470
-4.1544260
-4.8333840
-5.3088730
-4.4435050
-3.6777170
-3.6165610
-2.7132030
-3.1047070
-6.7219660
-5.2576650
-5.1267510
-5.4647250
-6.4309620
-6.8622590
-7.2314540
-5.5806110
-6.4147790
-0.1971390
0.9609370
0.6528210
-0.7012130
-1.8155840
-1.4990830
1.4574230
1.2230120
1.8494030
-0.6864210
-0.8954590
-2.7658070
-1.9816020
0.6183740
1.9283930
3.2143810
1.1001990
1.9435580
2.9391060
3.9069080

$-1.6693650$

$-2.0201840$

$-1.6817260$

$-2.5769650$

$-3.3560400$

$-3.0848920$

0.8762840

1.3537800

2. 8823490

1.6962480

1.4244100

1.7905840

0.4202590

2.3727610

3.2430660

1.8618790

3.5034400

0.6850130

3.7049600

4.5582690

4.1037190

2.7941680

3.1792560

2.7645790

$-0.2508250$

$-0.8770930$

3.8541820

4.7312590

6.2314070

6.5288980

5.8249170

4.3767850

6.8025480

4.5026760

4.4779640

6.1616690

7.6084630

5.8685050

6.3705090

6.5356880

1.6562810

1.0894530

1.8941890

2.0376590

0.8765730

0.9089620

-2.1897360
-3.1399140
-1.4503700
-1.7450880
-2.4934970
-0.8052670
1.2546490
2.5144580
1.7553380
0.8439540
3.6417610
4.5613700
3.8433010
3.1994570
3.8663260
3.2348690
1.4181530
2.7997170
1.7832730
2.4614460
0.7828180
2.2656810
3.1992170
1.5170730
0.5149090
0.8337920
-1.8161700
-2.2662410
-2.1668960
-2.8211500
-2.0368580
-1.7002190
-2.6473450
-3.3094640
-1.6741750
-3.8585910
-2.8574830
-2.5867620
-1.0941080
-1.1101890
-2.9147600
-3.0133820
-4.1675660
-5.4398270
-5.5453840
-4.3552460

\begin{abstract}
1.2839510
0.4352650

0.4348390

2. 3825660

3.5033520

4.5187220

4.6088770

2. 4969180

$-0.3282480$

0.3784150

$-0.2742970$

$-1.2598210$

0.7768310

$-1.1989530$

$-2.0762960$

0.8416760

1.5434890

$-0.1466430$

$-1.9745970$

1.6647310

$-0.0975810$

0.4379270

$-1.2107150$

$-1.3245010$

$-2.2610930$

$-0.2721300$

$-1.9636340$

$-1.9812170$

$-1.3796140$

$-3.1115190$

$-3.8090170$

$-3.6520280$

$-1.6070620$

$-3.2524760$

$-4.8763440$

$-4.3058470$

$-1.1306180$

$-1.1096340$

$-3.5579930$

$-3.3688050$
\end{abstract}

H $\quad-3.9550260$

2.0681120

1.0221000

2.7519690

$-0.0727720$

0.9258640

$-0.0026030$

1.7467360

2.9888020

$-3.7448740$

$-2.6605290$

$-4.6408670$

$-5.6187930$

$-4.5050190$

$-6.4368330$

$-5.7209410$

$-5.3350230$

$-3.7551720$

$-6.3009780$

$-7.1804670$

$-5.2277640$

$-6.9433820$

$-2.0077070$

$-4.1472500$

$-1.9640750$

$-1.9284550$

$-1.8536250$

$-1.3990560$

$-0.6586550$

0.0505000

$-3.1370970$

$-3.5391630$

$-2.4558300$

$-2.9205130$

$-3.9155000$

$-3.7243140$

$-1.5954540$

$-3.8747500$

$-2.5455780$

$-2.2122070$

$-4.4764240$

$-2.8150690$
$-4.2837810$

$-4.0462920$

$-5.5445940$

$-6.4857180$

$-4.3278870$

$-4.4976500$

$-5.4107510$

$-0.6531900$

$-0.8017480$

$-1.8410260$

$-2.0690120$

$-2.7712700$

$-3.1954980$

$-1.3605040$

$-3.8895290$

$-2.6041140$

$-4.1079720$

$-3.3636780$

$-4.5926000$

$-4.9845920$

0.0246300

0.7228940

3.2883860

2. 1613810

3.0310780

1.2645560

3.4662680

2.9525720

4.6638450

3. 3555540

2. 2751700

4.4274580

5.4231360

3.5255380

2.4763810

4.1614240

5.3306870

5.0531050

(2)

1.2860420

TS286(S,S) G[B97-D/def2-TZVP] $=-3864.563703$

$\begin{array}{lrrr}\mathrm{P} & 0.2069500 & -0.0670700 & 0.3751140 \\ \mathrm{O} & 0.7238310 & 0.7818670 & 1.5108640 \\ \mathrm{O} & -0.8090130 & 0.5228070 & -0.5666940\end{array}$




-0.2876060
1.4003080
-0.3869670
0.8033410
-1.6572540
-1.7176700
-2.6931910
2.4111780
3.6620820
2.1274050
4.6417000
5.6309800
-2.9075760
3.9323330
-3.3270470
-2.5998480
-4.8594910
-4.5584410
-2.3480300
-1.8788530
-1.6473050
-3.7021350
-3.8910370
-3.6849250
-5.8202940
-1.6542550
-4.8767450
-5.0261670
-5.7227650
-3.5281920
-3.0188530
-3.7015040
-3.7316360
-3.4348160
-5.7297020
-4.9661720
-4.5662790
-4.3917760
-4.5327610
-5.9489780
-6.4737070
-6.5877920
-6.6910520
-2.4613380
-4.8575870
-5.3774320

$-1.4423250$ $-0.5755050$ $-2.5902980$ $-3.2437470$ $-3.0233000$ $-4.1696210$ $-4.5242380$ $-1.3470850$ $-0.7545270$ $-2.6813770$ $-1.5583060$ $-1.1347870$ $-2.2933690$ 0.6588690 $-2.1285370$ $-2.5946500$ $-1.4251880$ $-1.5105420$ $-1.3628690$ $-1.7073310$ $-0.6843440$ $-0.6472150$ $-0.6052020$ 0.3829590 $-0.9266560$ $-3.0848980$ $-2.8674180$ $-2.8126720$ $-3.4208830$ $-3.5755680$ $-3.8949040$ $-4.4690360$ $-1.7940260$ $-1.8034820$ $-0.7284560$ $-1.1890180$ $-2.5874240$ $-2.5421090$ $-3.6426670$ $-1.9574720$ $-1.6301100$ $-2.6894420$ $-0.2820120$ $-2.2500420$ 0.2961350 0.5687710

1.0783470
-0.6031330
0.2852350
-0.0941760
-0.1097080
-0.9160170
-1.2429230
-0.0294900
0.1783630
0.3040080
0.7690230
0.9333090
0.2325740
-0.1957920
1.5638860
2.8027360
3.3302890
1.8552090
3.7157600
4.6469820
3.2212350
4.0157310
5.0968820
3.6399940
3.5090810
2.5594670
3.9094940
4.9961590
3.4848370
3.5721410
4.4909710
2.9603370
-0.7952160
-2.2779460
-1.7103860
-0.4941910
-2.9986470
-4.0818860
-2.7011920
-2.6376730
-3.5450960
-2.1289100
-1.4282570
-2.4938320
-2.4861240
-3.4143320

$\mathrm{H}$

\author{
$-4.7472540$ \\ $-3.4683700$ \\ $-3.2791250$ \\ $-2.6620400$ \\ $-5.3863390$ \\ $-6.3486210$ \\ 4.0534690 \\ 3. 8944130 \\ 3.4235840 \\ 3.7822710 \\ 4.8787750 \\ 4.7297820 \\ 5. 9109740 \\ 4. 6103820 \\ 4. 3571320 \\ 5. 5014020 \\ 3. 2322970 \\ 4.1017040 \\ 2. 1763990 \\ 1.9640850 \\ 1. 3102190 \\ 2. 4545370 \\ 2. 3579730 \\ 1.7334590 \\ 4.6518360 \\ 5. 1531770 \\ 4. 6046450 \\ 4. 3680650 \\ 4.0773440 \\ 4. 4628520 \\ 3. 1751380 \\ 3. 7517470 \\ 3. 9828920 \\ 2. 6910510 \\ 4. 3562710 \\ 5. 3765680 \\ 6.1001060 \\ 6.2818300 \\ 6.7347790 \\ 6.4303980 \\ 6.7764630 \\ 7.2267290 \\ 4.4887670 \\ 4. 7191120 \\ 4. 3867600 \\ 3. 1152480
}

1.2080660
-0.3376920
-0.3449010
0.2277260
-1.0578360
-0.5986490
2.4295340
2.7129860
0.2386520
1.0945350
1.7886650
1.9332390
2.0744630
0.3043250
-0.2953060
-0.1399790
-0.7988100
3.7667690
0.8419410
0.2545170
0.7559910
2.3313260
2.4917610
2.9899450
2.9197240
3.7711630
1.3312560
1.5896260
3.7880460
4.3620640
4.2881940
2.3230690
2.1637060
2.1007410
0.3047380
4.7905290
1.6304610
1.4852980
0.9202280
3.0979930
3.6796440
3.1153930
3.3430810
4.3718960
-2.8671430
-3.4366590

$-1.8925280$

$-2.7946580$

$-3.8763480$

-2. 3193020

0.8290690

1.0582080

$-1.8804590$

$-3.3539960$

$-2.7210630$

$-1.5273990$

$-4.1257220$

$-5.2040770$

$-3.8908730$

$-3.7251090$

$-4.6093590$

$-3.2657790$

$-2.4372230$

$-3.5780450$

$-3.4221280$

$-4.3252750$

$-2.7612570$

$-3.7907140$

$-4.8726120$

$-3.2958720$

0.3994680

1. 5391980

2. 2389670

0.7672490

2.6583930

3. 5117930

2. 2925580

3. 0773540

4.1389030

2. 9321190

2. 5174070

1. 2007420

2. 5386260

3. 6116940

1.9947600

2. 1179070

2. 9825390

1. 3643870

$-0.9189880$

$-1.1995870$

1.1904350

0.9781310 


$\begin{array}{rr}5.5155030 & -3.6488740 \\ 5.0309590 & -4.8406540 \\ 4.0101230 & -5.6541710 \\ 2.7776300 & -4.7967390 \\ 5.8868810 & -5.4615020 \\ 6.1822970 & -4.0248890 \\ 6.1202330 & -2.9663850 \\ 4.4721860 & -5.9890010 \\ 3.7027900 & -6.5517590 \\ 2.0903510 & -5.3441910 \\ 2.2261240 & -4.6184740 \\ 4.5539560 & -4.4772690 \\ 0.7015410 & -4.3570320 \\ 1.8803380 & -5.0537980 \\ 1.6869170 & -5.1230370 \\ 0.3029080 & -5.7121390 \\ -0.6010440 & -5.9902630 \\ -0.5669910 & -4.8244520 \\ 1.9548180 & -6.0808620 \\ 2.4993940 & -5.7187760 \\ 0.4331860 & -6.6436830 \\ -0.2262580 & -6.8916040 \\ 2.8208440 & -4.5506690 \\ 1.7856390 & -4.1052610 \\ -0.2168500 & -5.0057750 \\ -1.6289050 & -6.2011330 \\ -3.2098480 & 2.8973700 \\ -2.8212090 & 1.8120220 \\ -4.5736090 & 3.3374440 \\ -5.3948160 & 2.4660130 \\ -5.0753420 & 4.6038340 \\ -6.6826100 & 2.8511300 \\ -5.0257860 & 1.4816560 \\ -6.3616570 & 4.9862080 \\ -4.4464570 & 5.2811380 \\ -7.1710600 & 4.1114180 \\ -7.3074770 & 2.1636600 \\ -6.7347110 & 5.9687080 \\ -8.1764450 & 4.4110850 \\ -1.9450520 & 1.3479300 \\ -2.2827250 & 3.7204530 \\ -0.2147110 & 3.7087270 \\ 0.2490720 & 3.5393230 \\ -0.9577510 & 2.9919030 \\ 0.1764350 & 2.5530110 \\ 1.1460150 & 3.1975040\end{array}$

1.8454050
2.6805480
1.8776660
1.5672490
2.9755580
1.0524940
2.4574220
0.9363620
2.4296630
0.9199300
2.5049910
3.6030190
-0.9518110
-1.5889900
-3.1234200
-3.5294370
-2.3005900
-1.3459320
-1.1980900
-3.5577320
-4.0944200
-1.7908560
-1.3494820
-3.5207520
-4.1881910
-2.6187780
-0.4603800
0.1573390
-0.0608470
0.6848000
-0.4173560
1.0525010
0.9506300
-0.0425090
-0.9879410
0.6920890
1.6184920
-0.3225050
0.9803280
-0.1917950
-1.5979100
1.0080380
-0.3734850
1.3590670
1.18141450
-186360

$\begin{array}{lr}\mathrm{H} & 1.0357920 \\ \mathrm{C} & 0.6279360 \\ \mathrm{C} & 0.6045250 \\ \mathrm{C} & 0.9491620 \\ \mathrm{C} & -0.3938640 \\ \mathrm{H} & 0.3972410 \\ \mathrm{H} & 1.3097500 \\ \mathrm{H} & 2.0277570 \\ \mathrm{H} & -1.4135720 \\ \mathrm{H} & -0.3303640 \\ \mathrm{H} & 1.6319780 \\ \mathrm{H} & -0.4001600 \\ \mathrm{H} & 0.7107210 \\ \mathrm{H} & \end{array}$
2.1386830
6.0988650
6.0440070
4.6383510
5. 1127780
7.1135210
6.7685860
4.4364860
5.4280300
5.0790790
5.8459080
6.3169740
4.5378330

1. 3116550

0.9551510

$-0.5801090$

$-1.1040210$

1. 5462650

1. 3010300

$-1.0042820$

$-1.0180360$

1. 2827300

2. 6411650

1. 3214160

$-0.9313870$

15

TS287(S,S) G[B97-D/def2-TZVP $]=-3864.563396$

$\begin{array}{lrcr}\text { TS287(S, S) G }[\mathrm{B} 97-\mathrm{D} / \mathrm{def}-\mathrm{TZVP}]=-3864.563396 \\ \mathrm{P} & 0.3178190 & 0.6059820 & 1.1107960 \\ \mathrm{O} & -0.3409660 & 1.1925270 & 2.3301870 \\ \mathrm{O} & 0.8596650 & -0.7954380 & 1.2169540 \\ \mathrm{O} & 1.4428330 & 1.6708280 & 0.6349110 \\ \mathrm{O} & -0.7117350 & 0.5362700 & -0.1640050 \\ \mathrm{C} & 1.9842600 & 1.4256920 & -0.6376680 \\ \mathrm{C} & 1.2054950 & 1.7798480 & -1.7516640 \\ \mathrm{C} & 3.2317460 & 0.8007470 & -0.7399090 \\ \mathrm{C} & 3.6731200 & 0.4774030 & -2.0318050 \\ \mathrm{H} & 4.6325050 & -0.0238920 & -2.1431150 \\ \mathrm{C} & -1.0878280 & 1.7153760 & -0.8061080 \\ \mathrm{C} & -2.4244440 & 2.1221010 & -0.7356320 \\ \mathrm{C} & -0.1279770 & 2.4146110 & -1.5522890 \\ \mathrm{C} & -2.7484030 & 3.3389680 & -1.3380590 \\ \mathrm{H} & -3.7820870 & 3.6796020 & -1.2950270 \\ \mathrm{C} & 4.0643260 & 0.4664050 & 0.4445760 \\ \mathrm{C} & -3.4773420 & 1.2379990 & -0.1687150 \\ \mathrm{C} & 4.5263160 & 1.4772050 & 1.3078520 \\ \mathrm{C} & 4.2483790 & 2.9584490 & 1.1951880 \\ \mathrm{C} & 5.7951820 & 2.3671120 & 3.1961870 \\ \mathrm{C} & 5.3762330 & 1.1653900 & 2.3861490 \\ \mathrm{C} & 3.5914240 & 3.4379140 & 2.5186370 \\ \mathrm{H} & 3.4400560 & 4.5241990 & 2.4611810 \\ \mathrm{H} & 2.6089340 & 2.9693190 & 2.6247050 \\ \mathrm{C} & 4.5162140 & 3.0757320 & 3.7221610 \\ \mathrm{H} & 4.8110460 & 3.9785340 & 4.2737810 \\ \mathrm{H} & 3.9949760 & 2.4127400 & 4.4228450 \\ \mathrm{H} & 6.4458220 & 2.0737730 & 4.0290670 \\ \mathrm{H} & 3.6009090 & 3.1902860 & 0.3463670 \\ \mathrm{C} & 6.5322710 & 3.3560530 & 2.2498550 \\ \mathrm{H} & 6.7886390 & 4.2636130 & 2.8126390\end{array}$




$\begin{array}{lrrr}\text { H } & 7.4693640 & 2.9026520 & \\ \mathrm{C} & 5.6067660 & 3.6980710 & 1.9053120 \\ \mathrm{H} & 5.4152350 & 4.7783340 & 0.9413280 \\ \mathrm{H} & 6.0777010 & 3.4026750 & 0.0960340 \\ \mathrm{C} & 4.4605920 & -0.8612860 & 0.6948590 \\ \mathrm{C} & 4.0366140 & -2.0956760 & -0.0698560 \\ \mathrm{C} & 5.6890440 & -2.6195270 & 1.8663150 \\ \mathrm{C} & 5.3282420 & -1.1583550 & 1.7636370 \\ \mathrm{C} & 5.3095550 & -2.7749720 & -0.6456640 \\ \mathrm{H} & 5.0169950 & -3.7105670 & -1.1411200 \\ \mathrm{H} & 5.7653290 & -2.1263700 & -1.4039260 \\ \mathrm{C} & 6.3176900 & -3.0583160 & 0.5130200 \\ \mathrm{H} & 6.5605840 & -4.1281750 & 0.5620400 \\ \mathrm{H} & 7.2547060 & -2.5116110 & 0.3527420 \\ \mathrm{H} & 6.3908350 & -2.7956650 & 2.6906870 \\ \mathrm{H} & 3.3303270 & -1.8552880 & -0.8675420 \\ \mathrm{C} & 4.3862220 & -3.4380560 & 2.0682180 \\ \mathrm{H} & 4.6343280 & -4.5080940 & 2.0623700 \\ \mathrm{H} & 3.9524690 & -3.2027770 & 3.0475230 \\ \mathrm{C} & 3.3820800 & -3.0930000 & 0.9274640 \\ \mathrm{H} & 3.0903400 & -3.9974020 & 0.3779060 \\ \mathrm{H} & 2.4762750 & -2.6442150 & 1.3389350 \\ \mathrm{C} & 5.7843440 & -0.1491540 & 2.6112740 \\ \mathrm{H} & 6.4557890 & -0.3848830 & 3.4378820 \\ \mathrm{C} & -4.8629600 & -0.7650910 & -0.3973460 \\ \mathrm{C} & -5.1399170 & -1.9470430 & -1.2950120 \\ \mathrm{C} & -3.2271820 & -0.4194520 & -2.1633850 \\ \mathrm{C} & -3.8251770 & 0.0628950 & -0.8621030 \\ \mathrm{C} & -5.5163110 & -1.3959460 & -2.7006730 \\ \mathrm{H} & -5.6786470 & -2.2420020 & -3.3813620 \\ \mathrm{H} & -6.4561190 & -0.8353000 & -2.6336250 \\ \mathrm{C} & -4.3630140 & -0.4822360 & -3.2226750 \\ \mathrm{H} & -3.9483250 & -0.8788870 & -4.1589160 \\ \mathrm{H} & -4.7311300 & 0.5305120 & -3.4249790 \\ \mathrm{H} & -2.4249540 & 0.2364300 & -2.5105250 \\ \mathrm{H} & -5.9522070 & -2.5665490 & -0.8954780 \\ \mathrm{C} & -2.6873080 & -1.8563220 & -1.9482290 \\ \mathrm{H} & -2.2608360 & -2.2278910 & -2.8883790 \\ \mathrm{H} & -1.8808450 & -1.8195800 & -1.2142330 \\ \mathrm{C} & -3.8400570 & -2.7823970 & -1.4592150 \\ \mathrm{H} & -4.0267700 & -3.5864560 & -2.1829850 \\ \mathrm{H} & -3.5684920 & -3.2585430 & -0.5104540 \\ \mathrm{C} & -5.2212360 & 0.7269000 & 1.4591620 \\ \mathrm{C} & -5.8797570 & 1.2296010 & 2.7188750 \\ \mathrm{C} & -3.9827120 & 2.7871540 & 1.8576040 \\ \mathrm{C} & -4.1858520 & 1.5634550 & 0.9963920 \\ & & & \end{array}$

-4.7881260
-5.2522530
-4.3665080
-3.6698710
-3.6123750
-2.6922020
-3.1745540
-6.6743970
-5.3236150
-5.2005470
-5.5672470
-6.4574270
-6.8779120
-7.2741430
-5.5647200
-6.3734010
-1.7968110
-0.4744350
-2.2426080
-1.0802420
0.0197710
0.5911260
-1.4420270
-2.7823520
-2.9678700
-0.4043770
0.8291090
1.2834340
1.1943330
-0.6674280
1.6596270
0.8450370
0.7394490
2.1266960
3.2811030
2.8976100
1.3381380
0.2402010
2.3392970
3.4753080
-0.1532390
0.0944290
2.1092450
4.2032040
-0.0848310
0.5888310

3728480

1.7609500

0.3881590

2.3351160

3.2277260

1.8497860

3.4166760

0.5492030

3.5732490

4.4513980

3.9329900

2.6454890

3.0498780

2.5670270

$-0.4318690$

$-1.0701070$

4.1288060

3.6616650

5.4510390

6.4030140

5.6454620

4.5253070

7.2570440

5.2431450

5.9280050

5.2119820

. 3229770

3. 9085210

4.9764760

6.7993640

1. 3982650

1.5290010

0.1495020

$-0.5356980$

0.2835590

0.7413580

2.2357920

0.2769290

$-0.6851260$

1.1606030

1.9241660

$-0.4891750$

$-1.5277750$

$-0.3090160$

$-3.5280470$

$-2.4245430$
3.8153820

4.7317250

4.0483250

3. 3098080

3.9465490

3.3341760

1.4744190

3.0491620

1.8925580

2.5396890

0.8856990

2.4342700

3.3645680

1.7069950

0.7625720

1.1210180

$-1.9937590$

$-2.1203880$

$-2.5975250$

$-2.9038660$

$-3.6551210$

$-2.7760160$

$-3.4907640$

$-3.5356950$

$-1.9248670$

$-4.5738480$

$-3.9561580$

$-3.3541690$

$-1.9714280$

$-1.9641690$

$-3.0274840$

$-4.2910150$

$-4.9885410$

$-5.1766950$

$-4.5422190$

$-3.1584280$

$-4.9769340$

$-5.9571220$

$-6.2427470$

$-5.1791000$

$-4.0829520$

$-4.3728320$

$-4.7089870$

$-4.5140790$

$-0.4765930$

$-0.6274140$ 


\begin{tabular}{|c|c|c|c|c|c|c|c|}
\hline C & -0.0972940 & -4.3698920 & -1.7055840 & $c$ & 4.5730980 & -1.7728960 & 0.6736270 \\
\hline C & -1.0424170 & -5.3960030 & -1.8851490 & $\mathrm{H}$ & 5.5701630 & -1.3914910 & 0.8848750 \\
\hline C & 0.8356100 & -4.1180020 & -2.7327640 & $\mathrm{C}$ & -2.9907380 & -2.1632980 & 0.1913720 \\
\hline c & -1.0578860 & -6.1474010 & -3.0587660 & $\mathrm{C}$ & 3.9698950 & 0.4985830 & -0.2333000 \\
\hline $\mathrm{H}$ & -1.7697010 & -5.5857970 & -1.1015720 & $\mathrm{C}$ & -3.3602130 & -1.9870530 & 1.5356340 \\
\hline C & 0.8242800 & -4.8791630 & -3.9004440 & $\mathrm{C}$ & -2.6117730 & -2.4844290 & 2.7494410 \\
\hline $\mathrm{H}$ & 1.5678170 & -3.3271970 & -2.6059230 & $\mathrm{C}$ & -4.8088090 & -1.2339030 & 3.3517680 \\
\hline C & -0.1239580 & -5.8941360 & -4.0696520 & $\mathrm{C}$ & -4.5564310 & -1.3220940 & 1.8675600 \\
\hline $\mathrm{H}$ & -1.8020150 & -6.9301740 & -3.1872460 & $\mathrm{C}$ & -2.2854450 & -1.2661250 & 3.6554320 \\
\hline $\mathrm{H}$ & 1.5545180 & -4.6779830 & -4.6811700 & $\mathrm{H}$ & -1.7938880 & -1.6291980 & 4.5678540 \\
\hline $\mathrm{H}$ & -0.1360570 & -6.4831280 & -4.9842690 & $\mathrm{H}$ & -1.5807240 & -0.6107210 & 3.1359930 \\
\hline $\mathrm{H}$ & 0.6614580 & -1.7826470 & 0.2118280 & $\mathrm{C}$ & -3.6023240 & -0.5056310 & 4.0066490 \\
\hline $\mathrm{s}$ & -0.8674160 & -3.9964480 & 0.9403250 & $\mathrm{H}$ & -3.7549790 & -0.4700090 & 5.0937480 \\
\hline C & -1.0044450 & -1.7718030 & 3.5075660 & $\mathrm{H}$ & -3.5595530 & 0.5279370 & 3.6424150 \\
\hline C & -1.9618980 & -1.7793620 & 2.3979450 & $\mathrm{H}$ & -5.7442110 & -0.7006770 & 3.5624440 \\
\hline $\mathrm{H}$ & 0.0403070 & -1.6474200 & 3.2288200 & $\mathrm{H}$ & -1.6931450 & -3.0078840 & 2.4740550 \\
\hline $\mathrm{H}$ & -1.6897090 & -1.2704930 & 1.4821330 & C & -4.8627690 & -2.6780680 & 3.9241570 \\
\hline 0 & -1.6842170 & -0.4731530 & 3.6668010 & $\mathrm{H}$ & -4.9790130 & -2.6242380 & 5.0148800 \\
\hline $\mathrm{H}$ & -1.1191200 & 0.2455610 & 3.1350410 & $\mathrm{H}$ & -5.7401190 & -3.1976940 & 3.5208990 \\
\hline C & -2.7490600 & -2.9406820 & 4.9400850 & $\mathrm{C}$ & -3.5510570 & -3.4334850 & 3.5452260 \\
\hline $\mathrm{C}$ & -3.4596120 & -3.3899020 & 3.6542000 & $\mathrm{H}$ & -3.0258820 & -3.7734800 & 4.4476400 \\
\hline C & -3.3370740 & -2.3350710 & 2.5417130 & $\mathrm{H}$ & -3.7748600 & -4.3187780 & 2.9378980 \\
\hline C & -1.2523530 & -2.7036830 & 4.6744590 & C & -3.8257690 & -1.6271010 & -0.8081640 \\
\hline $\mathrm{H}$ & -2.8637150 & -3.7012020 & 5.7215850 & $\mathrm{C}$ & -3.5747140 & -1.6414980 & -2.2992540 \\
\hline $\mathrm{H}$ & -4.5208280 & -3.5882350 & 3.8459730 & $\mathrm{C}$ & -5.8047630 & -0.4747930 & -1.6573940 \\
\hline $\mathrm{H}$ & -4.0116240 & -1.4852830 & 2.7199350 & $\mathrm{C}$ & -5.0247530 & -0.9735530 & -0.4667270 \\
\hline $\mathrm{H}$ & -0.7634840 & -3.6558630 & 4.4236710 & $\mathrm{C}$ & -4.7594210 & -2.3737920 & -2.9877060 \\
\hline $\mathrm{H}$ & -0.7473450 & -2.2984090 & 5.5601750 & $\mathrm{H}$ & -4.6151660 & -2.3330290 & -4.0755440 \\
\hline $\mathrm{H}$ & -3.2058350 & -2.0141220 & 5.3128970 & $\mathrm{H}$ & -4.7626460 & -3.4302570 & -2.6929150 \\
\hline $\mathrm{H}$ & -3.0086830 & -4.3294680 & 3.3050330 & $\mathrm{C}$ & -6.1023250 & -1.6860560 & 5848730 \\
\hline $\mathrm{H}$ & -3.6381070 & -2.7300510 & 1.5656370 & $\mathrm{H}$ & -6.6369180 & -1.3293760 & 753180 \\
\hline 15 & & & & $\mathrm{H}$ & -6.7586080 & -2.3927890 & -2.0628670 \\
\hline TS & S) G[B97-I & [ZVP] & 183 & $\mathrm{H}$ & -6.7376540 & 0.0092290 & -1.3437010 \\
\hline $\mathrm{P}$ & 0.2348730 & -0.0673350 & 0.2550190 & $\mathrm{H}$ & -2.6275840 & -2.1268030 & -2.5466390 \\
\hline 0 & 0.8003380 & 0.7455900 & 1.3934630 & C & -4.9132260 & 0.5188150 & -2.4515850 \\
\hline 0 & -0.7694380 & 0.5738440 & -0.6657710 & $\mathrm{H}$ & -5.4488310 & 0.8227550 & -3.3608870 \\
\hline 0 & -0.3082510 & -1.4284550 & 0.9499040 & $\mathrm{H}$ & -4.7446030 & 1.4193170 & -1.8539760 \\
\hline 0 & 1.3965060 & -0.6158380 & -0.7400580 & $\mathrm{C}$ & -3.5633270 & -0.1725860 & -2.8087020 \\
\hline C & -0.4826470 & -2.5618500 & 0.1503260 & $\mathrm{H}$ & -3.4105360 & -0.1821090 & -3.8960890 \\
\hline C & 0.6557680 & -3.2698970 & -0.2751090 & $\mathrm{H}$ & -2.7180800 & 0.3553700 & -2.3584330 \\
\hline C & -1.7838100 & -2.9418960 & -0.1945460 & C & -5.3972180 & -0.8313560 & 0.8695950 \\
\hline C & -1.9137900 & -4.0827260 & -0.9923410 & $\mathrm{H}$ & -6.3318960 & -0.3340580 & 1.1307910 \\
\hline $\mathrm{H}$ & -2.9160710 & -4.3997300 & -1.2757800 & $\mathrm{C}$ & 4.2202460 & 2.3262840 & -1.8411920 \\
\hline C & 2.3655800 & -1.4481320 & -0.1760100 & $\mathrm{C}$ & 4.1315610 & 2.6710560 & -3.3071840 \\
\hline C & 3.6344080 & -0.9164910 & 0.0789650 & C & 3.5034270 & 0.2055350 & -2.7852540 \\
\hline & 2.0098720 & -2.7805270 & 0.1115490 & $\mathrm{C}$ & 3.8762860 & 0.9930910 & -1.5491070 \\
\hline
\end{tabular}




\begin{tabular}{|c|c|c|}
\hline $\begin{array}{l}5.0961220 \\
5.0150210 \\
6.1295090 \\
4.7158890 \\
4.4421850 \\
5.5630360 \\
3.2462390 \\
4.4030510 \\
2.3101500 \\
2.0694890 \\
1.4319310 \\
2.6919130 \\
2.6560810 \\
1.9889540 \\
4.7714970 \\
5.2873010 \\
4.6049690 \\
4.4195170 \\
4.1942960 \\
4.5989050 \\
3.3300420 \\
3.7762190 \\
3.9593220 \\
2.7114460 \\
4.3005760 \\
5.5668740 \\
6.1043050 \\
6.2467630 \\
6.7198880 \\
6.5187770 \\
6.8832440 \\
7.3250750 \\
4.6674580 \\
4.9547960 \\
2.9584350 \\
2.6609230 \\
3.0629130 \\
4.5400900 \\
5.1963640 \\
4.2441120 \\
3.2465110 \\
2.8974510 \\
5.1388590 \\
5.4624670 \\
1.6034470\end{array}$ & $\begin{array}{r}1.7220440 \\
1.9246690 \\
1.9300710 \\
0.2413220 \\
-0.2914490 \\
-0.2888620 \\
-0.8290920 \\
3.7194030 \\
0.9077160 \\
0.3577920 \\
0.8639970 \\
2.3830360 \\
2.5655290 \\
3.0762190 \\
2.6998850 \\
3.4809280 \\
1.0458230 \\
1.3707000 \\
3.5069140 \\
4.0139490 \\
4.0784650 \\
2.0451560 \\
1.8271330 \\
1.8869920 \\
0.0229040 \\
4.5005970 \\
1.2623040 \\
1.0768120 \\
0.5375990 \\
2.7226500 \\
3.2554230 \\
2.7222220 \\
3.1810680 \\
4.2072990 \\
-3.5865840 \\
-4.9533520 \\
-4.9906520 \\
-4.5535480 \\
-3.9859700 \\
-3.0772340 \\
-5.7153190 \\
-6.0018910 \\
-5.4012110 \\
-4.8317520 \\
-5.2074530 \\
-4.3181050\end{array}$ & $\begin{array}{r}-4.0738150 \\
-5.1500780 \\
-3.7720460 \\
-3.7594910 \\
-4.6797890 \\
-3.3083800 \\
-2.5476490 \\
-3.4830030 \\
-3.4866330 \\
-4.4060040 \\
-2.8371300 \\
-3.8143100 \\
-4.8965160 \\
-3.3416920 \\
0.4718470 \\
1.6552290 \\
2.2441040 \\
0.7780330 \\
2.7573520 \\
3.6436830 \\
2.4048950 \\
3.0978610 \\
4.1584510 \\
2.9064900 \\
2.4752550 \\
1.3625980 \\
2.5933070 \\
3.6661520 \\
2.0465900 \\
2.2275540 \\
3.1157980 \\
1.4844760 \\
-0.8326480 \\
-1.0663640 \\
0.7672070 \\
1.3319220 \\
2.8265440 \\
3.0564740 \\
1.7696990 \\
1.0356850 \\
0.7944610 \\
3.2184840 \\
3.4127100 \\
1.1165180 \\
1.2122830 \\
3.3715510\end{array}$ \\
\hline
\end{tabular}

\begin{abstract}
4.5761000
6.1273190

0.4880610

1.6923450

1. 3525740

0.1667100

$-1.0781570$

$-0.8142600$

2. 2331900

2. 1056700

2. 4839180

0.4153590

$-0.0368660$

$-1.8696670$

$-1.4840920$

1. 0901520

$-3.0733320$

$-2.7159040$

$-4.4058900$

$-5.2353790$

$-4.8699280$

$-6.4945670$

$-4.8961740$

$-6.1279060$

$-4.2340150$

$-6.9458160$

$-7.1266700$

$-6.4721510$

$-7.9290850$

$-1.8666460$

$-2.1382680$

$-0.0144760$

0.4059730

$-0.7711080$

0.2927740

1. 3322960

1.1831720

0.9038330

0.8423080

1. 1259720

$-0.1361030$

0.7141480

1.5618890

2. 1985300

-1.1502650
-0.0515360
\end{abstract}

3.8367770

2. 0123140

$-1.1402360$

$-1.7540050$

$-3.0423510$

$-2.7844780$

$-2.4448330$

$-1.4828370$

$-3.3779870$

$-1.0368960$

$-1.9327980$

$-1.9429300$

$-3.6552560$

$-2.0325470$

$-3.3763480$

$-3.8397300$

$-0.4614320$

0.1294780

$-0.0200950$

0.7553740

$-0.3654100$

1.1632380

1. 0134370

0.0486200

$-0.9586080$

0.8129750

1.7525860

$-0.2238990$

1.1322380

$-0.2481470$

$-1.6088590$

0.9546160

$-0.4422070$

1. 3125770

$-0.8979430$

1. 0901370

1. 2026770

0.9241790

$-0.6108090$

$-1.1685980$

1. 5206920

1. 2935490

$-1.0388420$

$-1.1104030$

1.2826010
2.6132760 


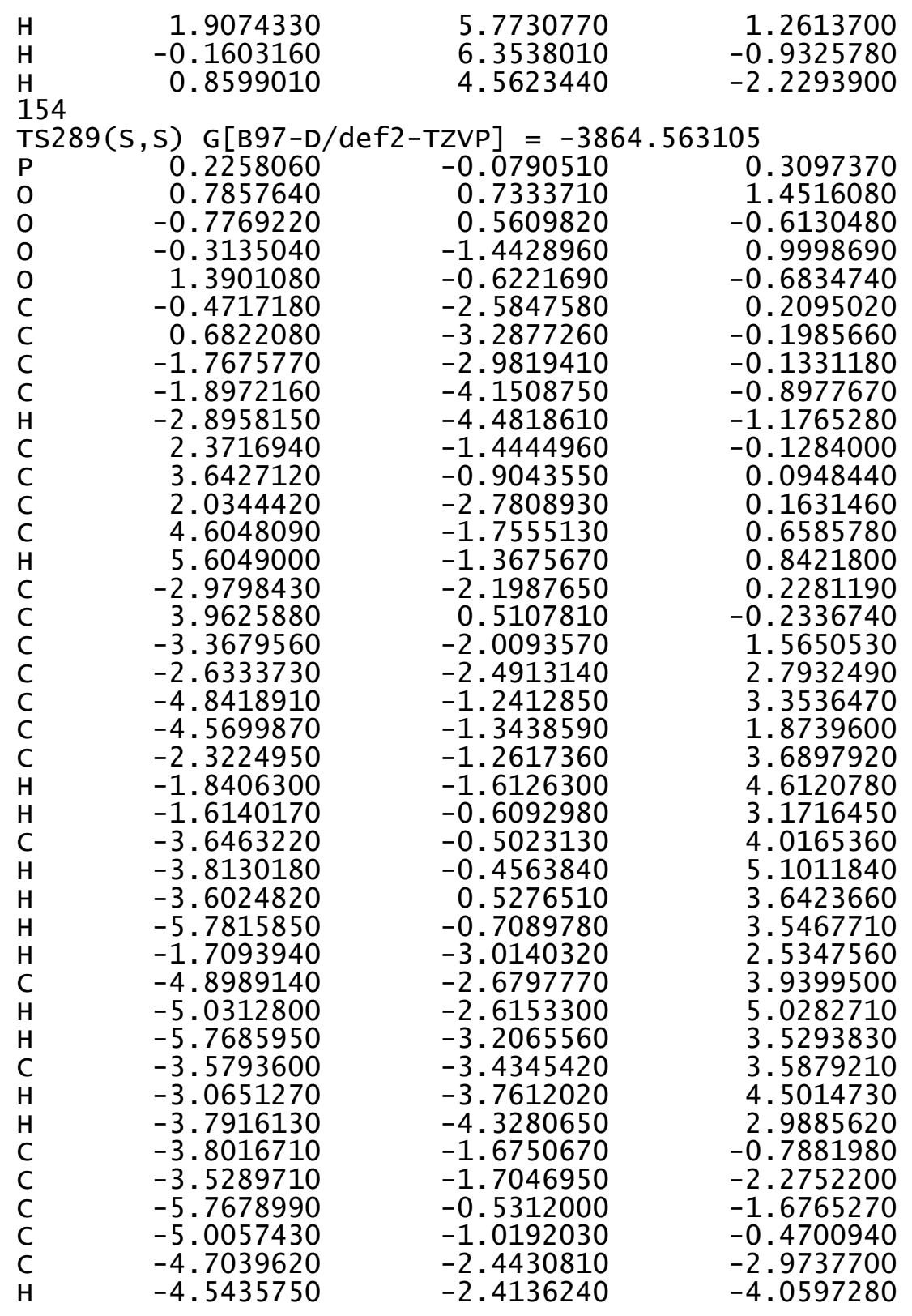

-4.7123330
-6.0522020
-6.5725380
-6.7172120
-6.7052940
-2.5785860
-4.8639300
-5.3860730
-4.7029840
-3.5095360
-3.3414410
-2.6706190
-5.3973940
-6.3364600
4.1753800
4.0573790
3.4537560
3.8441630
5.0128520
4.9097310
6.0506260
4.6478940
4.3604560
5.5066040
3.2070710
4.3197350
2.2437680
1.9904660
1.3776780
2.6103860
2.5532880
1.9117680
4.7621720
5.2874190
4.6353360
4.4237240
4.2070360
4.6144540
3.3310210
3.8117990
4.0173500
2.7452550
4.3435650
5.5539860
6.1380300
6.3000570
$-2.6683570$ $-2.5977880$ $-3.4995790$ $-2.0798150$ $-1.3815410$ $-2.5032740$ $-2.4662860$ $-3.3857730$ $-1.8737300$ $-2.7979490$ $-3.8829800$ $-2.3403370$ 0.8594160 1.1022370 $-1.8620950$ $-3.3292940$ $-2.7741790$ $-1.5522480$ $-4.1057930$ $-5.1820410$ $-3.8261600$ $-3.7710010$ $-4.6811780$ $-3.3308690$ $-2.5230890$ $-3.5193150$ $-3.4598990$ $-4.3702530$ $-2.7944260$ $-3.8068320$ $-4.8896400$ $-3.3274660$ 0.4384330 1. 6080950 2. 2269670 0.7618990 2. 7227040 3. 5980860 2. 3727530 3. 0862270 4. 1452860 2. 9169920 2. 4697490 1.3035700 2. 5505360 3. 6216020 


\begin{tabular}{|c|c|c|c|}
\hline $\mathrm{H}$ & 6.7508680 & 0.5916680 & 1.9966400 \\
\hline 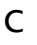 & 6.5326370 & 2.7756460 & 2.1715340 \\
\hline H & 6.9006190 & 3.3177510 & 3.0526570 \\
\hline $\mathrm{H}$ & 7.3313270 & 2.7794350 & 1.4202920 \\
\hline c & 4.6344380 & 3.1911830 & -0.8686430 \\
\hline $\mathrm{H}$ & 4.9114630 & 4.2170380 & -1.1160030 \\
\hline$C$ & 3.0115410 & -3.5867150 & 0.7781940 \\
\hline c & 2.7558890 & -4.9809230 & 1.2960750 \\
\hline C & 3.2295500 & -5.0927570 & 2.7662010 \\
\hline c & 4.6939190 & -4.6019020 & 2.9696490 \\
\hline C & 5.2931260 & -3.9840860 & 1.6807260 \\
\hline$C$ & 4.3004320 & -3.0683390 & 1.0115760 \\
\hline $\mathrm{H}$ & 3.3131730 & -5.7081060 & 0.6855740 \\
\hline $\mathrm{H}$ & 3.1256790 & -6.1341470 & 3.0954060 \\
\hline $\mathrm{H}$ & 5.3342090 & -5.4306510 & 3.2969920 \\
\hline $\mathrm{H}$ & 5.5475490 & -4.8038110 & 0.9903590 \\
\hline $\mathrm{H}$ & 1.6966850 & -5.2423960 & 1.2190450 \\
\hline $\mathrm{H}$ & 2.5514910 & -4.4907650 & 3.3833180 \\
\hline $\mathrm{H}$ & 4.7181720 & -3.8441740 & 3.7623350 \\
\hline $\mathrm{H}$ & 6.2241890 & -3.4501280 & 1.9055570 \\
\hline c & 0.5111400 & -4.4269580 & -1.0099510 \\
\hline C & 1.6419790 & -5.1828980 & -1.6657610 \\
\hline C & 1.3692170 & -5.3299820 & -3.1830130 \\
\hline c & -0.0340440 & -5.9299700 & -3.4912350 \\
\hline C & -0.8998850 & -6.0849860 & -2.2146990 \\
\hline $\mathrm{C}$ & -0.7870840 & -4.8660640 & -1.3362950 \\
\hline $\mathrm{H}$ & 1.7248160 & -6.1851910 & -1.2179870 \\
\hline $\mathrm{H}$ & 2.1571420 & -5.9499190 & -3.6286140 \\
\hline $\mathrm{H}$ & 0.0651130 & -6.9102740 & -3.9741030 \\
\hline $\mathrm{H}$ & -0.5392270 & -6.9638870 & -1.6577430 \\
\hline $\mathrm{H}$ & 2.6002050 & -4.6815650 & -1.5083630 \\
\hline $\mathrm{H}$ & 1.4501220 & -4.3332470 & -3.6343100 \\
\hline $\mathrm{H}$ & -0.5640570 & -5.2765700 & -4.1950270 \\
\hline $\mathrm{H}$ & -1.9463460 & -6.2765730 & -2.4800030 \\
\hline C & -3.0872520 & 3.0211570 & -0.4629520 \\
\hline 0 & -2.7364940 & 1.9161030 & 0.1429370 \\
\hline C & -4.4325540 & 3.5073950 & -0.0546970 \\
\hline C & -5.2768660 & 2.6655380 & 0.6991270 \\
\hline C & -4.8938310 & 4.7894460 & -0.4100140 \\
\hline $\mathrm{C}$ & -6.5477120 & 3.0947730 & 1.0765050 \\
\hline $\mathrm{H}$ & -4.9388950 & 1.6700830 & 0.9645850 \\
\hline C & -6.1635370 & 5.2156060 & -0.0262140 \\
\hline $\mathrm{H}$ & -4.2463890 & 5.4438020 & -0.9865730 \\
\hline C & -6.9960060 & 4.3701580 & 0.7169380 \\
\hline $\mathrm{H}$ & -7.1909830 & 2.4303410 & 1.6493460 \\
\hline $\mathrm{H}$ & -6.5056260 & 6.2094540 & -0.3056650 \\
\hline
\end{tabular}

$\begin{array}{lr}\mathrm{H} & -7.9882950 \\ \mathrm{H} & -1.8801160 \\ \mathrm{~S} & -2.1320490 \\ \mathrm{C} & -0.0490990 \\ \mathrm{C} & 0.3956140 \\ \mathrm{H} & -0.8109680 \\ \mathrm{H} & 0.2897010 \\ \mathrm{O} & 1.2959500 \\ \mathrm{H} & 1.1502250 \\ \mathrm{C} & 0.8685120 \\ \mathrm{C} & 0.8346240 \\ \mathrm{C} & 1.1266390 \\ \mathrm{C} & -0.1812920 \\ \mathrm{H} & 0.6719290 \\ \mathrm{H} & 1.5626050 \\ \mathrm{H} & 2.1982100 \\ \mathrm{H} & -1.1914490 \\ \mathrm{H} & -0.1151790 \\ \mathrm{H} & 1.8660610 \\ \mathrm{H} & -0.1616030 \\ \mathrm{H} & 0.8766290 \\ \mathrm{H} & \end{array}$

$\begin{array}{lr}4.7043910 & 1.0124770 \\ 1.4258740 & -0.2165720 \\ 3.8204010 & -1.5941790 \\ 3.6924430 & 1.0034770 \\ 3.5332420 & -0.3856130 \\ 2.9932370 & 1.3495640 \\ 2.5573240 & -0.8422950 \\ 3.1360490 & 1.1616570 \\ 2.0801920 & 1.2704780 \\ 6.0553850 & 0.9860530 \\ 6.0287490 & -0.5497860 \\ 4.6217560 & -1.1015810 \\ 5.0921990 & 1.5656770 \\ 7.0706380 & 1.3507460 \\ 6.7356600 & -0.9652590 \\ 4.3815620 & -1.0277440 \\ 5.4425610 & 1.3103350 \\ 5.0387580 & 2.6596110 \\ 5.7645030 & 1.3416740 \\ 6.3436960 & -0.8898880 \\ 4.5497290 & -2.1662010\end{array}$
TS290(S,S) G[B97-D/def2-TZVP] $=-3864.562939$

\begin{tabular}{|c|c|c|c|}
\hline \multicolumn{4}{|c|}{ s) $\mathrm{G}[\mathrm{B} 97-\mathrm{D} / \mathrm{c}$} \\
\hline \multicolumn{4}{|c|}{ D } \\
\hline 0 & -0.5187240 & 1.0835680 & 2.1766220 \\
\hline 0 & 0.6706830 & -0.9393580 & 1.1105140 \\
\hline 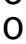 & 1.4055210 & 1.5137680 & 0.6460930 \\
\hline & -0.7168130 & 0.5052060 & -0.3579490 \\
\hline C & 2.0279490 & 1.3296770 & -0.5961250 \\
\hline & 1.3547550 & 1.7734280 & -1.7436970 \\
\hline & 3.2789080 & 0.7069100 & -0.6415630 \\
\hline & 3.8196670 & 0.4845180 & -1.9114960 \\
\hline $\mathrm{H}$ & 4.7899390 & -0.0049830 & -1.9785700 \\
\hline$c$ & -1.0087800 & 1.7391410 & -0.9417930 \\
\hline & -2.3232410 & 2.2134220 & -0.8858920 \\
\hline & 0.0278220 & 2.4333510 & -1.5927080 \\
\hline & -2.5693560 & 3.4890710 & -1.4114550 \\
\hline & -3.5822130 & 3.8862760 & -1.3706300 \\
\hline C & 4.0264730 & 0.3031260 & 0.5776810 \\
\hline C & -3.4291890 & 1.3632730 & -0.3708190 \\
\hline & 4.4550160 & 1.2688700 & 1.5062010 \\
\hline & 4.1981250 & 2.7567050 & 1.4437450 \\
\hline & 5.623 & 2.0574270 & 3.4993090 \\
\hline & 390 & 460 & 2.6137380 \\
\hline & 3.44 & $3.1 \xi$ & 2.7486700 \\
\hline & 3.3 & & 3405 \\
\hline
\end{tabular}




$\begin{array}{rrrr}\mathrm{H} & 2.4800580 & 2.7265490 & 2.7747800 \\ \mathrm{C} & 4.3217440 & 2.7523460 & 3.9849020 \\ \mathrm{H} & 4.5898000 & 3.6228180 & 4.5985120 \\ \mathrm{H} & 3.7545880 & 2.0599540 & 4.6182110 \\ \mathrm{H} & 6.2247070 & 1.7205790 & 4.3529240 \\ \mathrm{H} & 3.6001930 & 3.0317180 & 0.5715210 \\ \mathrm{C} & 6.4195090 & 3.0836770 & 2.6443420 \\ \mathrm{H} & 6.6408050 & 3.9643390 & 3.2620840 \\ \mathrm{H} & 7.3763630 & 2.6435070 & 2.3390860 \\ \mathrm{C} & 5.5716970 & 3.4838920 & 1.3971180 \\ \mathrm{H} & 5.3967270 & 4.5678910 & 1.3792880 \\ \mathrm{H} & 6.0943230 & 3.2165490 & 0.4707660 \\ \mathrm{C} & 4.3827240 & -1.0403670 & 0.7947210 \\ \mathrm{C} & 3.9631200 & -2.2334690 & -0.0337810 \\ \mathrm{C} & 5.5063400 & -2.8703340 & 1.9588720 \\ \mathrm{C} & 5.1891980 & -1.3970940 & 1.8928540 \\ \mathrm{C} & 5.2415690 & -2.9325140 & -0.5721750 \\ \mathrm{H} & 4.9460480 & -3.8416480 & -1.1131140 \\ \mathrm{H} & 5.7522510 & -2.2736650 & -1.2850130 \\ \mathrm{C} & 6.1834280 & -3.2855110 & 0.6224970 \\ \mathrm{H} & 6.3890550 & -4.3639070 & 0.6473360 \\ \mathrm{H} & 7.1441090 & -2.7659430 & 0.5243030 \\ \mathrm{H} & 6.1627850 & -3.0954880 & 2.8082580 \\ \mathrm{H} & 3.3052340 & -1.9409360 & -0.8561340 \\ \mathrm{C} & 4.1708080 & -3.6534580 & 2.0738090 \\ \mathrm{H} & 4.3859190 & -4.7302390 & 2.0442110 \\ \mathrm{H} & 3.6987980 & -3.4349120 & 3.0391920 \\ \mathrm{C} & 3.2321800 & -3.2411850 & 0.8998540 \\ \mathrm{H} & 2.9390610 & -4.1185990 & 0.3089670 \\ \mathrm{H} & 2.3218920 & -2.7757540 & 1.2824190 \\ \mathrm{C} & 5.6181570 & -0.4313870 & 2.8031860 \\ \mathrm{H} & 6.2409920 & -0.7128560 & 3.6532230 \\ \mathrm{C} & -4.8631850 & -0.6008660 & -0.6402580 \\ \mathrm{C} & -5.1460810 & -1.7783870 & -1.5413420 \\ \mathrm{C} & -3.1675220 & -0.3068870 & -2.3561010 \\ \mathrm{C} & -3.7884780 & 0.1965260 & -1.0735830 \\ \mathrm{C} & -5.4666460 & -1.2227120 & -2.9587940 \\ \mathrm{H} & -5.6303460 & -2.0663240 & -3.6422660 \\ \mathrm{H} & -6.3932540 & -0.6378020 & -2.9214330 \\ \mathrm{C} & -4.2747650 & -0.3406850 & -3.4472120 \\ \mathrm{H} & -3.8441580 & -0.7496920 & -4.3708050 \\ \mathrm{H} & -4.6096540 & 0.6813400 & -3.6604090 \\ \mathrm{H} & -2.3381360 & 0.3252550 & -2.6825440 \\ \mathrm{H} & -5.9866600 & -2.3732380 & -1.1632300 \\ \mathrm{C} & -2.6741100 & -1.7586590 & -2.1249620 \\ \mathrm{H} & -2.2389360 & -2.1435720 & -3.0561290\end{array}$

-1.8817450
-3.8660160
-4.0542720
-3.6360310
-5.2429360
-5.9311360
-3.9621870
-4.1691840
-4.8758980
-5.3611770
-4.4896070
-3.7144160
-3.6561180
-2.7518180
-3.1241990
-6.7554900
-5.2810280
-5.1564430
-5.4787390
-6.4590000
-6.8990770
-7.2525190
-5.5937080
-6.4314060
-0.2465630
0.8019950
0.7411620
-0.6939320
-1.7519590
-1.5476380
0.6085110
1.4448040
-0.7175690
-1.6434700
1.8019830
1.0877650
-0.9795750
-2.7651130
1.9099930
1.1488170
1.5950310
3.1197140
3.7787670
3.1532350
1.0995330
1.3022010
$-1.7444920$
$-2.6495930$
$-3.4532400$
$-3.1256940$
0.9072700
1. 4290700
2.9306310
1.7126950
1. 5419130
1.9441240
0.5462080
2.4719310
3. 3644520
1. 9601830
3.5384930
0.7707460
3.7536090
4.6303560
4.1167210
2.8599660
3.2777450
2. 8039970
$-0.2443890$
$-0.8587830$
3. 7220280
4.6529900
6. 0104200
6. 6172860
5.6579370
4.2514900
4.8311230
6.7079210
7.5647600
5.6701530
4. 2168480
5.8468250
6.8411660
6.0092960
1.4960760
1.8451920
1.0216540
1.0929300
0.4379650
0.8381620
1.4030680
2. 9079960

$-1.3750290$

$-1.6633250$

$-2.3872520$

$-0.7037310$

1.1981810

2.4338290

1.6394760

0.7695090

3.5687450

4.4679320

3.8152090

3. 1027840

3.7397380

3. 1634550

1. 2873430

2.7349930

1. 6260130

2. 2748070

0.6101980

2. 1291200

3. 0443540

1. 3745950

0.4942590

0.8267180

$-2.0820350$

$-2.6366400$

$-1.8935100$

$-1.8715150$

$-2.4764920$

$-1.9756380$

$-3.7059480$

$-2.3646410$

$-2.4243530$

$-3.5725880$

$-2.5531980$

$-0.8658080$

$-0.8365580$

$-2.2477540$

$-3.0153400$

$-4.2826000$

$-5.4978050$

$-5.6363140$

$-3.0883770$

-6.3998930
-4.5175720 


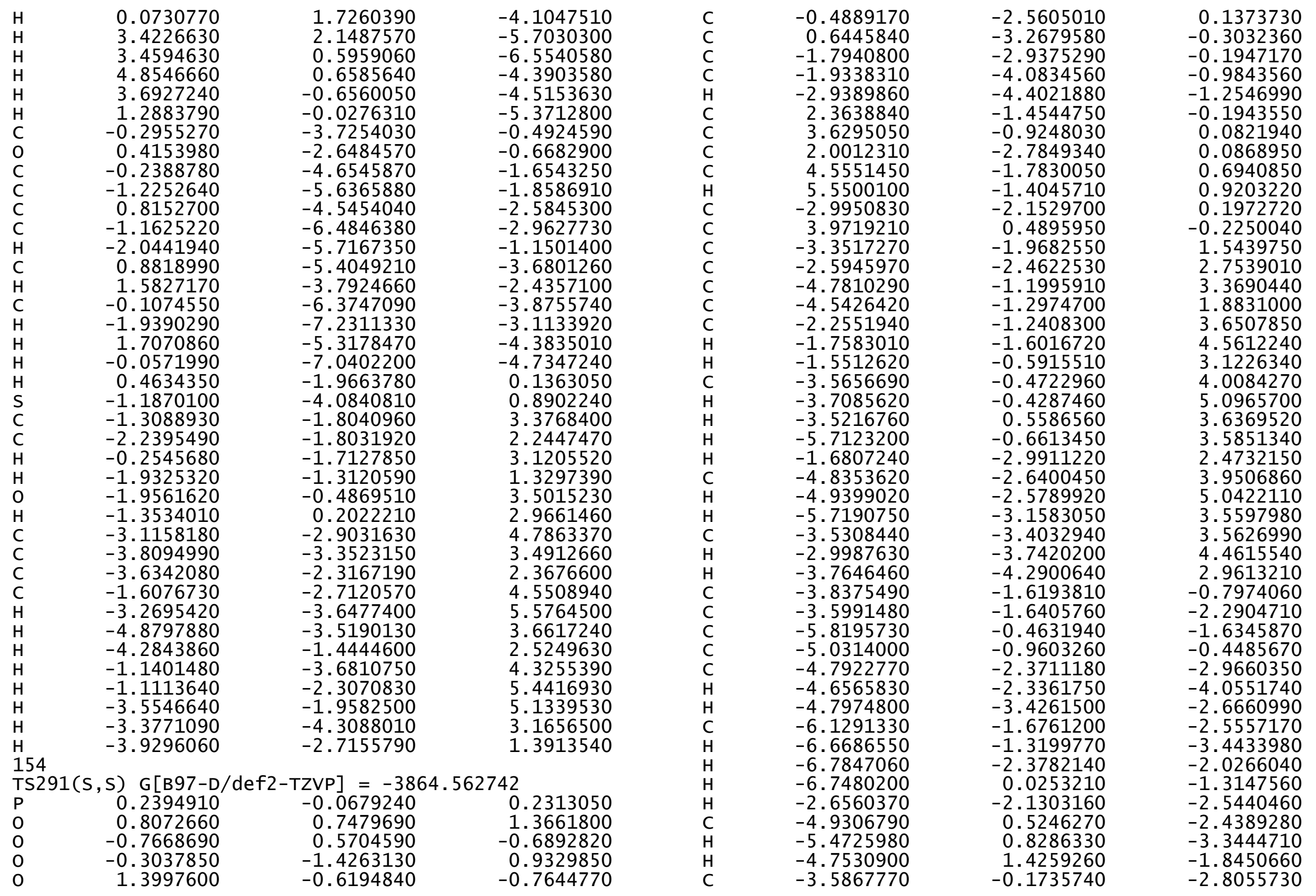




\begin{tabular}{|c|c|c|}
\hline $\begin{array}{l}-3.4438720 \\
-2.7354660 \\
-5.3911980 \\
-6.3215910 \\
4.2404730 \\
4.1682080 \\
3.5326030 \\
3.8917400 \\
5.1410460 \\
5.0720740 \\
6.1710300 \\
4.7567340 \\
4.4941620 \\
5.5981550 \\
3.2722710 \\
4.4421240 \\
2.3480770 \\
2.1178000 \\
1.4621920 \\
2.7344300 \\
2.7115890 \\
2.0262610 \\
4.7723910 \\
5.2826020 \\
4.5865860 \\
4.4143270 \\
4.1829130 \\
4.5848820 \\
3.3241200 \\
3.7550560 \\
3.9279380 \\
2.6913120 \\
4.2764310 \\
5.5680680 \\
6.0839980 \\
6.2170000 \\
6.7012020 \\
6.5073870 \\
6.8691680 \\
7.3178240 \\
4.6802240 \\
4.9717470 \\
2.9345250 \\
2.6270540 \\
3.0056230 \\
4.4748350\end{array}$ & $\begin{array}{r}-0.1879040 \\
0.3528030 \\
-0.8097360 \\
-0.3075790 \\
2.3186460 \\
2.6651060 \\
0.1995770 \\
0.9856660 \\
1.7163440 \\
1.9195000 \\
1.9238650 \\
0.2356680 \\
-0.2968610 \\
-0.2948190 \\
-0.8352560 \\
3.7135290 \\
0.9029670 \\
0.3539880 \\
0.8591730 \\
2.3783210 \\
2.5616400 \\
3.0714060 \\
2.6886160 \\
3.4668560 \\
1.0340480 \\
1.3601140 \\
3.4962900 \\
3.9990970 \\
4.0735940 \\
2.0360250 \\
1.8155230 \\
1.8830250 \\
0.0123560 \\
4.4856380 \\
1.2447430 \\
1.0594740 \\
0.5172630 \\
2.7032030 \\
3.2343290 \\
2.6989840 \\
3.1715050 \\
4.1973480 \\
-3.5918220 \\
-4.9661780 \\
-5.0245150 \\
-4.5778610\end{array}$ & $\begin{array}{r}-3.8942420 \\
-2.3649360 \\
0.8902710 \\
1.1573600 \\
-1.8287830 \\
-3.2952810 \\
-2.7827510 \\
-1.5413670 \\
-4.0516320 \\
-5.1286340 \\
-3.7381220 \\
-3.7422570 \\
-4.6659260 \\
-3.2809560 \\
-2.5496560 \\
-3.4668770 \\
-3.4976230 \\
-4.4202520 \\
-2.8585550 \\
-3.8195260 \\
-4.9019430 \\
-3.3546950 \\
0.4892170 \\
1.6768620 \\
2.2586090 \\
0.7911860 \\
2.7721090 \\
3.6620550 \\
2.4157420 \\
3.1065460 \\
4.1683430 \\
2.9055750 \\
2.4872030 \\
1.3868710 \\
2.6197450 \\
3.6938530 \\
2.0785510 \\
2.2564220 \\
3.1467440 \\
1.5178740 \\
-0.8154530 \\
-1.0456920 \\
0.7640180 \\
1.3080490 \\
2.8076110 \\
3.0702490\end{array}$ \\
\hline
\end{tabular}

5.1548470
4.2158920
3.2175400
2.8428830
5.0721250
5.4379680
1.5718160
2.3171230
4.4887180
6.0789110
0.4668120
-0.8404930
1.6602920
1.3670060
0.0688720
-1.1034530
2.2142180
1.9658680
2.5200380
0.1560060
-0.1132110
-2.0221120
-1.2990670
1.2660810
-3.0620700
-2.7048310
-4.3883160
-5.2132050
-4.8509140
-6.4665080
-4.8753970
-6.1030240
-4.2185160
-6.9164190
-7.0951600
-6.4462010
-7.8951370
-1.8596230
-2.1327100
0.0015800
0.4150450
-0.7540200
0.2994830
1.3483670
1.1977890
0.9219270

-3.9978810
-3.0858060
-5.7235330
-6.0434270
-5.4230190
-4.8373510
-5.2189230
-4.3670770
-3.8120350
-3.4708960
-4.3733380
-4.7937810
-5.0049150
-6.3785430
-6.3358180
-6.0012260
-6.6835630
-4.3134240
-5.0754340
-5.5641510
-7.2943110
-5.8313530
-6.8737730
-7.1271570
3.0415940
1.9298060
3.5411070
2.7109080
4.8251470
3.1534800
1.7138580
5.2644620
5.4707080
4.4304780
2.4980150
6.2595060
4.7747530
1.4367440
3.8364280
3.7031400
3.5419500
3.0057620
2.5653900
3.1435750
2.0909860
6.0649240
1.8018230

1. 0544400

0.7681900

3. 1802470

3.4347420

1. 1473440

1. 1678080

3. 3524470

3.8553040

2. 0679290

$-1.1686250$

$-1.4848570$

$-1.8709260$

$-2.4871470$

$-3.2995430$

$-2.3706220$

$-3.1149320$

$-2.6735120$

$-1.2036340$

$-4.0790560$

$-3.8026920$

$-2.9475170$

$-1.7260980$

$-1.6865430$

$-0.4723040$

0.1165700

$-0.0199790$

0.7676590

$-0.3665430$

1. 1861410

1. 0270410

0.0579820

$-0.9691330$

0.8344070

1.7849600

$-0.2158070$

1. 1619280

$-0.2664290$

$-1.6283600$

0.9254780

$-0.4730570$

1. 2885250

$-0.9260060$

1. 0564340

1.1712220

0.8856990 


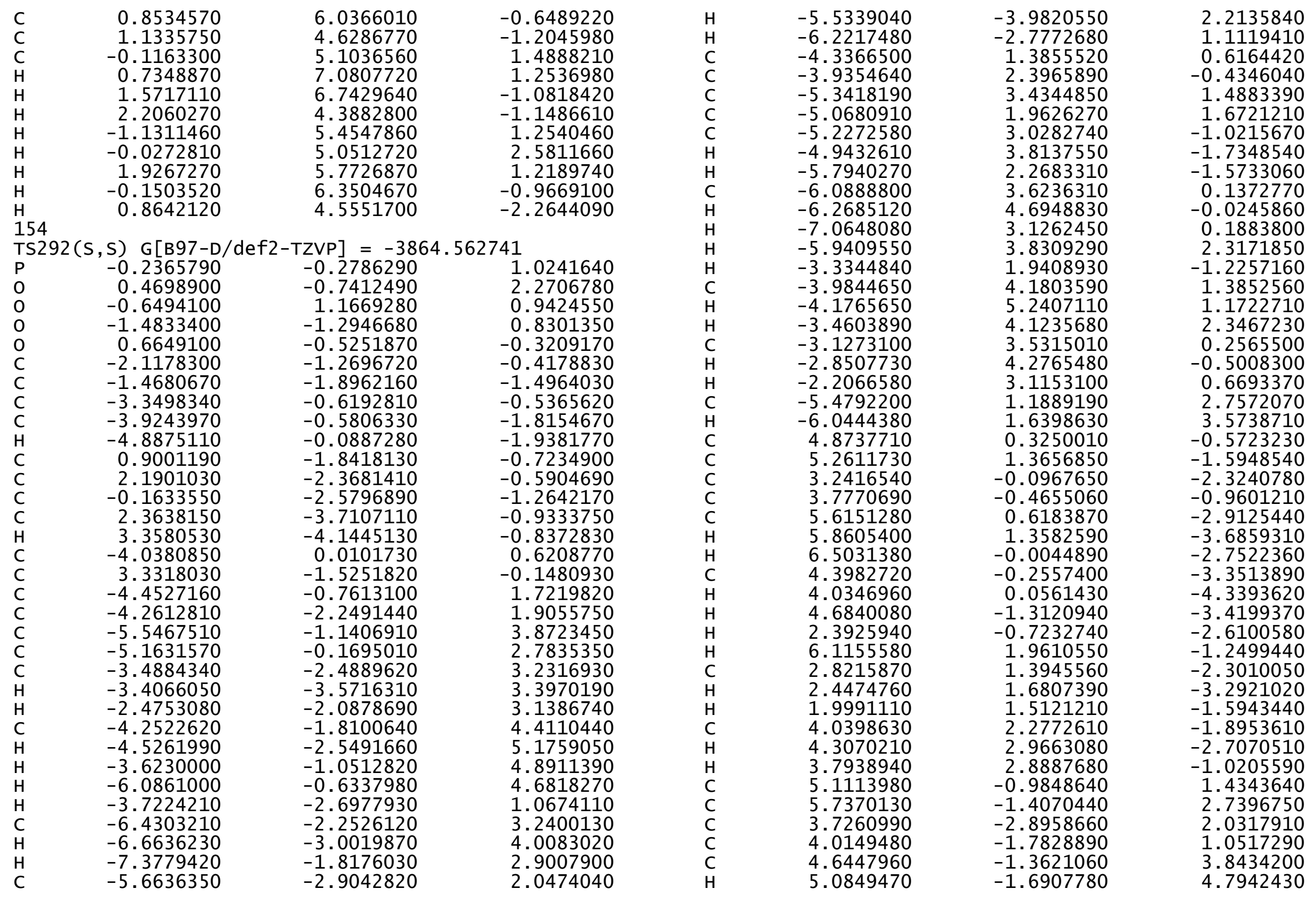




$\begin{array}{lrrr}\mathrm{H} & 4.2969510 & -0.3313660 & 3.9744600 \\ \mathrm{C} & 3.4568320 & -2.2848380 & 3.4343030 \\ \mathrm{H} & 3.3426740 & -3.1076070 & 4.1525120 \\ \mathrm{H} & 2.5158820 & -1.7306110 & 3.4136320 \\ \mathrm{H} & 2.8730520 & -3.5012980 & 1.7139010 \\ \mathrm{H} & 6.5813480 & -0.7574850 & 3.0021210 \\ \mathrm{C} & 5.0069710 & -3.7704860 & 2.1419490 \\ \mathrm{H} & 4.8261980 & -4.5733180 & 2.8686590 \\ \mathrm{H} & 5.2161770 & -4.2397410 & 1.1729220 \\ \mathrm{C} & 6.2097100 & -2.8814860 & 2.5925370 \\ \mathrm{H} & 6.6058640 & -3.2252140 & 3.5572570 \\ \mathrm{H} & 7.0247900 & -2.9328010 & 1.8609910 \\ \mathrm{C} & 5.5413200 & 0.0669650 & 0.6260210 \\ \mathrm{H} & 6.3977120 & 0.6742710 & 0.9218710 \\ \mathrm{C} & 0.0285040 & -3.9519250 & -1.5445200 \\ \mathrm{C} & -1.1450410 & -4.8108450 & -1.9846330 \\ \mathrm{C} & -0.9094770 & -6.3109630 & -1.7659020 \\ \mathrm{C} & 0.4460820 & -6.7126340 & -2.3577930 \\ \mathrm{C} & 1.5707860 & -5.9967410 & -1.5996890 \\ \mathrm{C} & 1.3061860 & -4.5174080 & -1.3693300 \\ \mathrm{H} & -1.7257820 & -6.8803150 & -2.2286670 \\ \mathrm{H} & -1.3344400 & -4.6497790 & -3.0538370 \\ \mathrm{H} & -2.0520420 & -4.4775100 & -1.4653950 \\ \mathrm{H} & 0.4715970 & -6.4257820 & -3.4201480 \\ \mathrm{H} & 0.5952940 & -7.7988110 & -2.3073740 \\ \mathrm{H} & 2.5293660 & -6.1150680 & -2.1232990 \\ \mathrm{H} & 1.6963820 & -6.4798180 & -0.6175200 \\ \mathrm{H} & -0.9180590 & -6.5380640 & -0.6894090 \\ \mathrm{C} & -3.2806760 & -1.1237490 & -2.9253520 \\ \mathrm{C} & -3.8650700 & -1.0518110 & -4.3147810 \\ \mathrm{C} & -3.7698130 & -2.4365060 & -4.9900140 \\ \mathrm{C} & -2.3057610 & -2.9482070 & -5.0455500 \\ \mathrm{C} & -1.3626870 & -2.2108040 & -4.0536280 \\ \mathrm{C} & -2.0445830 & -1.7826070 & -2.7750940 \\ \mathrm{H} & -3.3012960 & -0.3217910 & -4.9192640 \\ \mathrm{H} & -4.1975240 & -2.3968270 & -5.9996250 \\ \mathrm{H} & -1.8994280 & -2.8193900 & -6.0565970 \\ \mathrm{H} & -1.0186400 & -1.2882040 & -4.5472870 \\ \mathrm{H} & -4.9050120 & -0.7056730 & -4.2744310 \\ \mathrm{H} & -4.3817260 & -3.1356380 & -4.4053260 \\ \mathrm{H} & -2.2923510 & -4.0245950 & -4.8363550 \\ \mathrm{H} & -0.4679040 & -2.8060260 & -3.8557210 \\ \mathrm{C} & 0.5445650 & 3.6244980 & -1.0103100 \\ \mathrm{O} & -0.2358560 & 2.5828300 & -1.0545040 \\ \mathrm{C} & 0.6706600 & 4.3112330 & -2.3251820 \\ \mathrm{C} & 1.7324470 & 5.1944220 & -2.5932400\end{array}$

$\begin{array}{lr}\mathrm{C} & -0.2719350 \\ \mathrm{C} & 1.8522900 \\ \mathrm{H} & 2.4673170 \\ \mathrm{C} & -0.1559810 \\ \mathrm{H} & -1.0948740 \\ \mathrm{C} & 0.9077360 \\ \mathrm{H} & 2.6858180 \\ \mathrm{H} & -0.8958470 \\ \mathrm{H} & 1.0006980 \\ \mathrm{H} & -0.3663460 \\ \mathrm{~S} & 1.3388820 \\ \mathrm{C} & 1.3604970 \\ \mathrm{C} & 2.3040040 \\ \mathrm{H} & 0.3074790 \\ \mathrm{H} & 2.0021310 \\ \mathrm{O} & 1.9805870 \\ \mathrm{H} & 1.3555840 \\ \mathrm{C} & 3.1630400 \\ \mathrm{C} & 3.8769100 \\ \mathrm{C} & 3.7069070 \\ \mathrm{C} & 1.6560300 \\ \mathrm{H} & 3.3154090 \\ \mathrm{H} & 4.9463170 \\ \mathrm{H} & 4.3336620 \\ \mathrm{H} & 1.1995660 \\ \mathrm{H} & 1.1457310 \\ \mathrm{H} & 3.5892440 \\ \mathrm{H} & 3.4562520 \\ \mathrm{H} & 4.0346130 \\ \mathrm{H} & \\ \mathrm{H} & \\ \mathrm{H} & \end{array}$

$\begin{array}{lr}4.0540980 & -3.3420320 \\ 5.8002470 & -3.8424610 \\ 5.3859010 & -1.8172400 \\ 4.6724270 & -4.5859610 \\ 3.3737440 & -3.1476370 \\ 5.5443890 & -4.8426440 \\ 6.4711080 & -4.0377190 \\ 4.4720000 & -5.3577120 \\ 6.0204850 & -5.8164200 \\ 2.0390970 & -0.1588030 \\ 4.1791950 & 0.3682810 \\ 2.2075670 & 3.1182650 \\ 2.0664210 & 2.0045240 \\ 2.1036740 & 2.8612700 \\ 1.4738040 & 1.1496290 \\ 0.9032400 & 3.4017160 \\ 0.1762100 & 2.9464100 \\ 3.4467700 & 4.4146690 \\ 3.7312410 & 3.0841590 \\ 2.5655610 & 2.0954690 \\ 3.2415960 & 4.1840040 \\ 4.2780940 & 5.1132250 \\ 3.9103320 & 3.2474170 \\ 1.7084910 & 2.3803160 \\ 4.1815460 & 3.8426130 \\ 2.9454600 & 5.1090440 \\ 2.5456900 & 4.8756870 \\ 4.6427990 & 2.6368390 \\ 2.8326340 & 1.0855120\end{array}$
TS293(S,S) G[B97-D/def2-TZVP $]=-3864.562651$

$\begin{array}{lrrr}\text { TS293(S, S) G }[\mathrm{B} 97-\mathrm{D} / \mathrm{def}-\mathrm{TZVP}]=-3864.562651 \\ \mathrm{P} & 0.2380740 & -0.0414470 & 0.1830500 \\ \mathrm{O} & 0.7871430 & 0.7522780 & 1.3426150 \\ \mathrm{O} & -0.7700690 & 0.6093620 & -0.7274240 \\ \mathrm{O} & -0.2920730 & -1.4223580 & 0.8460590 \\ \mathrm{O} & 1.4087010 & -0.5568620 & -0.8182890 \\ \mathrm{C} & -0.4598540 & -2.5438040 & 0.0293820 \\ \mathrm{C} & 0.6889770 & -3.2345080 & -0.4117120 \\ \mathrm{C} & -1.7610170 & -2.9358030 & -0.3004520 \\ \mathrm{C} & -1.9000510 & -4.0828450 & -1.0956500 \\ \mathrm{H} & -2.9025070 & -4.4166350 & -1.3568300 \\ \mathrm{C} & 2.3832740 & -1.3964840 & -0.2742290 \\ \mathrm{C} & 3.6458670 & -0.8596420 & 0.0009890 \\ \mathrm{C} & 2.0428680 & -2.7415690 & -0.0309080 \\ \mathrm{C} & 4.5982540 & -1.7261710 & 0.5568550 \\ \mathrm{H} & 5.5911180 & -1.3397870 & 0.7789320\end{array}$




-2.9691670
3.9618760
-3.3238260
-2.5575910
-4.7553680
-4.5213240
-2.2284310
-1.7274780
-1.5308550
-3.5455330
-3.6855140
-3.5130020
-5.6917000
-1.6392040
-4.7916040
-4.8902830
-5.6721470
-3.4814780
-2.9425820
-3.7094310
-3.8212020
-3.5871740
-5.8169540
-5.0210950
-4.7753290
-4.6429880
-4.7693440
-6.1176990
-6.6635090
-6.7648090
-6.7488360
-2.6403480
-4.9389860
-5.4862440
-4.7679910
-3.5894130
-3.4499340
-2.7417520
-5.3786360
-6.3137120
4.1993110
4.1259590
3.5454620
3.8770370
5.1250020
5.0546170

$-2.1719240$

0.5717540

$-2.0187120$

$-2.5280640$

$-1.3012590$

$-1.3684440$

$-1.3215500$

$-1.6961170$

$-0.6554630$

$-0.5725640$

$-0.5488010$

0.4649110

$-0.7774990$

$-3.0416720$

$-2.7526560$

$-2.7128470$

$-3.2729690$

$-3.4950530$

$-3.8480690$

$-4.3708140$

$-1.6287510$

$-1.6194510$

$-0.4766030$

$-0.9898900$

$-2.3475090$

$-2.2889310$

$-3.4084050$

$-1.6739600$

$-1.3057120$

$-2.3927770$

$-0.0029080$

$-2.0957720$

0.5354640

0.8531500

1.4258660

$-0.1424890$

$-0.1334880$

0.3830560

$-0.8707320$

$-0.3846220$

2. 4675790

2.8689870

0.3708390

1. 1173630

1.9730130

2. 2137010

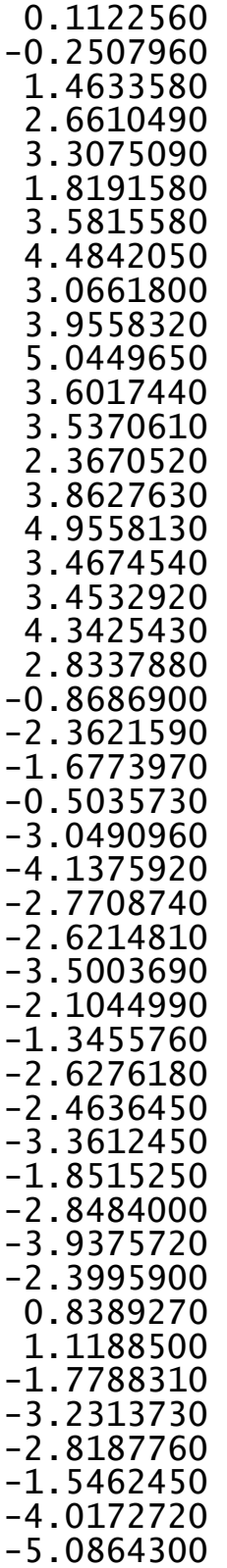

6.1483340

4.7770530

4.5375830

5.6277340

3. 3091690

4. 3758420

2. 3509300

2. 1449760

1.4597410

2.7022820

2.6803850

1.9743810

4.7193370

5.2150060

4.5608600

4. 3852980

4.1144020

4.5084810

3. 2474320

3.7089100

3.8796830

2.6491810

4.2691710

5.4832790

6.0535460

6.1869750

6.6848500

6.4524360

6.8057640

7.2624270

4.6199680

4.8925170

3.0077870

2.7435860

3.1772540

4.6350960

5.2664760

4.2898260

3.3213830

3.0671560

5.2680520

5.5388220

1.6890540

2. 4816680

4.6374280
6.1912380
2.1945570

0.4726630

$-0.0336750$

$-0.0515740$

$-0.6779220$

3.9293380

1.0718910

0.5552270

0.9793140

2.5686370

2.7959430

. 2237940

2.7541490

3.4912390

1.0248820

1.4084440

3.4569030

3.9269600

4.0360940

1.9767600

1.7151260

1.8145550

$-0.0113090$

4.5257960

1.2471110

1.0222840

0.5519420

. 7256560

3.2272200

2.7639200

. 2875930

4.3271650

$-3.5623690$

$-4.9703400$

$-5.1192100$

$-4.6314880$

$-3.9824540$

$-3.0501030$

$-5.6798250$

$-6.1688320$

$-5.4670550$

$-4.7849460$

$-4.5341170$

$-3.8925000$

$-3.4542990$
$-3.6918180$

$-3.7641020$

$-4.7085060$

$-3.3127470$

$-3.3613090$

$-3.5179040$

$-4.4645960$

$-2.8912970$

$-3.7757050$

$-4.8497350$

$-3.2873340$

0.5536970

1.7728770

2.2535330

0.8006550

2.8673090

3.7784190

2.5348220

3.1381360

4.1909130

2.9238330

2.4377260

1.5255440

2. 6276380

3.6941540

2.0609160

2.3224340

3.2330960

1.5843680

$-0.7307510$

$-0.9189470$

0.5833990

1.0575520

2.5361580

2.7894560

1. 5312480

0.8614080

0.4456620

2.8359660

3.1136520

0.8275450

0.9435860

3.1500170

3.5999880

1.7926560 


\begin{tabular}{|c|c|c|}
\hline $\begin{array}{l}-0.7950280 \\
-0.9255100 \\
0.0058100 \\
1.4776580 \\
1.6240980 \\
0.5070920 \\
-0.6392220 \\
-0.0509720 \\
2.1072080 \\
1.5689040 \\
-1.9674400 \\
-0.3672610 \\
1.8732090 \\
2.6090290 \\
-3.1055560 \\
-2.7248490 \\
-4.4388810 \\
-5.2336510 \\
-4.9379850 \\
-6.4921890 \\
-4.8684580 \\
-6.1955810 \\
-4.3300880 \\
-6.9782930 \\
-7.0965360 \\
-6.5669210 \\
-7.9610950 \\
-1.8722760 \\
-2.1946780 \\
-0.0564790 \\
0.3543370 \\
-0.8035340 \\
0.2485140 \\
1.2964520 \\
1.1585080 \\
0.8361390 \\
0.7601580 \\
1.0581930 \\
-0.1867130 \\
0.6386150 \\
1.4660080 \\
2.1337640 \\
-1.2071850 \\
-0.0894380 \\
1.8462910 \\
-0.2496160\end{array}$ & $\begin{array}{l}-4.7724720 \\
-5.9872220 \\
-7.1125200 \\
-6.6288130 \\
-5.0898770 \\
-4.3438670 \\
-5.7251360 \\
-7.9734400 \\
-7.0962320 \\
-4.8329690 \\
-6.3281340 \\
-7.4456450 \\
-6.9429890 \\
-4.7705090 \\
3.0345830 \\
1.9123590 \\
3.4947370 \\
2.6346580 \\
4.7708400 \\
3.0409580 \\
1.6431410 \\
5.1734130 \\
5.4396630 \\
4.3103360 \\
2.3627640 \\
6.1624890 \\
4.6259390 \\
1.4445450 \\
3.8829400 \\
3.7128480 \\
3.5944970 \\
2.9977260 \\
2.6294640 \\
3.1640750 \\
2.1070010 \\
6.0857850 \\
6.0974710 \\
4.7092120 \\
5.0956190 \\
7.0889080 \\
6.8255320 \\
4.4817910 \\
5.4412220 \\
5.0131850 \\
5.7970780 \\
6.4047200\end{array}$ & $\begin{array}{r}-1.5857810 \\
-2.4705670 \\
-1.9673950 \\
-1.8259690 \\
-1.9526230 \\
-1.2613970 \\
-3.5022870 \\
-2.6450820 \\
-2.5934840 \\
-3.0225290 \\
-2.5012990 \\
-0.9903760 \\
-0.8536820 \\
-1.6076490 \\
-0.4274300 \\
0.1252600 \\
0.0457340 \\
0.8323430 \\
-0.2793010 \\
1.2716340 \\
1.0751100 \\
0.1650440 \\
-0.8817540 \\
0.9412500 \\
1.8701620 \\
-0.0932040 \\
1.2850130 \\
-0.2753480 \\
-1.5602020 \\
0.9966170 \\
-0.4073810 \\
1.3423930 \\
-0.8868090 \\
1.1082190 \\
1.1906860 \\
1.0174960 \\
-0.5171100 \\
-1.1105070 \\
1.5998420 \\
1.4137850 \\
-0.9342190 \\
-1.0617250 \\
1.3822650 \\
2.6896450 \\
1.3372670 \\
-0.8223670\end{array}$ \\
\hline
\end{tabular}

154

TS294(S,S) G[B97-D/def2-TZVP $]=-3864.562587$

\begin{tabular}{|c|c|c|c|}
\hline & S) $\mathrm{G}[\mathrm{B} 97-\mathrm{D}$ & [VP] & \\
\hline $\mathrm{P}$ & 0.2126520 & 0.5057840 & 0.993830 \\
\hline 0 & -0.5002340 & 1.1290160 & 2.164364 \\
\hline 0 & 0.6858540 & -0.9160880 & 1.136770 \\
\hline 0 & 1.4112100 & 1.5271140 & 0.608629 \\
\hline 0 & -0.7191930 & 0.4875840 & 353453 \\
\hline$C$ & 2.0329820 & 1.3046360 & -0.62 \\
\hline C & 1.3505300 & 1.6973810 & -1.788 \\
\hline C & 3.2858020 & 0.6845260 & -0.654 \\
\hline C & 3.8273250 & 0.4285220 & -1.918 \\
\hline $\mathrm{H}$ & 4.8006410 & -0.0563920 & -1.9724690 \\
\hline C & -1.0079120 & 1.7075940 & -0.9682900 \\
\hline C & -2.3152240 & 2.2015400 & -0.904 \\
\hline C & 0.0275930 & 2.3706420 & -1.6494690 \\
\hline C & -2.5472920 & 3.4738780 & -1.4443100 \\
\hline $\mathrm{H}$ & -3.5528620 & 3.8883790 & -1.39 \\
\hline C & 4.0317680 & 0.3098420 & 0.57 \\
\hline C & -3.4272840 & 1.3690350 & -0.37 \\
\hline C & 4.4609210 & 1.2963440 & 1.48 \\
\hline C & 4.2053970 & 2.7823210 & 1.38 \\
\hline C & 5.6334530 & 2.1305130 & 3.45 \\
\hline C & 5.2468610 & 0.9507510 & 2.59 \\
\hline C & 3.4824520 & 3.2418590 & 2.67 \\
\hline y & 3.3483270 & 4.3311660 & 2.63 \\
\hline $\mathrm{H}$ & 2.4893060 & 2.7847180 & 2.71 \\
\hline C & 4.3332730 & 2.8392700 & 3.9 \\
\hline $\mathrm{H}$ & 4.6039540 & 3.7246030 & 4.51 \\
\hline H & 3.7666050 & 2.1638920 & 4.575269 \\
\hline H & 6.2351070 & 1.8135200 & 4.31 \\
\hline $\mathrm{H}$ & 3.6063800 & 3.0369900 & 0.505253 \\
\hline C & 6.4293010 & 3.1341560 & 2.571144 \\
\hline$\Pi$ & 6.6547420 & 4.0285330 & 3.167 \\
\hline 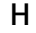 & 7.3839560 & 2.6838700 & 2.2 \\
\hline C & 5.5792090 & 3.5070610 & 1 \\
\hline $\mathrm{H}$ & 5.40482 & 05150 & 60 \\
\hline$\pi$ & & 40 & \\
\hline C & & 8900 & 0 \\
\hline C & 0 & 4500 & 9 \\
\hline C & 5. & 6590 & 5 \\
\hline C & J. & 500 & \\
\hline 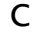 & 0 & -2 & \\
\hline n & & -3 & -1 \\
\hline $\mathrm{H}$ & 8 & -2 & -1 \\
\hline & & & \\
\hline
\end{tabular}




$\begin{array}{rrrr}\mathrm{H} & 6.3891310 & -4.3562960 & 0.7535910 \\ \mathrm{H} & 7.1460400 & -2.7623330 & 0.5956740 \\ \mathrm{H} & 6.1613870 & -3.0389010 & 2.8853690 \\ \mathrm{H} & 3.3086600 & -1.9667470 & -0.8074790 \\ \mathrm{C} & 4.1701220 & -3.6121530 & 2.1613670 \\ \mathrm{H} & 4.3848840 & -4.6893940 & 2.1572660 \\ \mathrm{H} & 3.6967590 & -3.3709600 & 3.1206830 \\ \mathrm{C} & 3.2334300 & -3.2269870 & 0.9766800 \\ \mathrm{H} & 2.9418640 & -4.1176440 & 0.4051090 \\ \mathrm{H} & 2.3223130 & -2.7532500 & 1.3468640 \\ \mathrm{C} & 5.6213750 & -0.3746850 & 2.8172000 \\ \mathrm{H} & 6.2440020 & -0.6369630 & 3.6734920 \\ \mathrm{C} & -4.8749380 & -0.5893600 & -0.6166680 \\ \mathrm{C} & -5.1675630 & -1.7762200 & -1.5024160 \\ \mathrm{C} & -3.1790520 & -0.3304490 & -2.3376760 \\ \mathrm{C} & -3.7945690 & 0.1943670 & -1.0608560 \\ \mathrm{C} & -5.4862770 & -1.2353630 & -2.9260310 \\ \mathrm{H} & -5.6581070 & -2.0859230 & -3.5988190 \\ \mathrm{H} & -6.4079870 & -0.6424480 & -2.8941980 \\ \mathrm{C} & -4.2880580 & -0.3692340 & -3.4269050 \\ \mathrm{H} & -3.8617630 & -0.7934270 & -4.3456820 \\ \mathrm{H} & -4.6147250 & 0.6527190 & -3.6527690 \\ \mathrm{H} & -2.3458430 & 0.2911400 & -2.6737300 \\ \mathrm{H} & -6.0121000 & -2.3597860 & -1.1157370 \\ \mathrm{C} & -2.6964270 & -1.7829340 & -2.0891670 \\ \mathrm{H} & -2.2643060 & -2.1824750 & -3.0156070 \\ \mathrm{H} & -1.9038790 & -1.7661180 & -1.3394030 \\ \mathrm{C} & -3.8947290 & -2.6590560 & -1.6163900 \\ \mathrm{H} & -4.0907860 & -3.4686550 & -2.3315410 \\ \mathrm{H} & -3.6671610 & -3.1271480 & -0.6522010 \\ \mathrm{C} & -5.2454680 & 0.9481050 & 1.1990600 \\ \mathrm{C} & -5.9310870 & 1.4922420 & 2.4264950 \\ \mathrm{C} & -3.9512000 & 2.9687250 & 1.6124970 \\ \mathrm{C} & -4.1658360 & 1.7400740 & 0.7597510 \\ \mathrm{C} & -4.8760980 & 1.6141050 & 3.5606750 \\ \mathrm{H} & -5.3594300 & 2.0329350 & 4.4533050 \\ \mathrm{H} & -4.4974050 & 0.6193570 & 3.8226430 \\ \mathrm{C} & -3.7076490 & 2.5290150 & 3.0823290 \\ \mathrm{H} & -3.6430320 & 3.4298510 & 3.7067810 \\ \mathrm{H} & -2.7489090 & 2.0109970 & 3.1506220 \\ \mathrm{H} & -3.1084230 & 3.5652190 & 1.2524500 \\ \mathrm{H} & -6.7602840 & 0.8440610 & 1.5361860 \\ \mathrm{C} & -5.2642350 & 3.8004350 & 2.2213660 \\ \mathrm{H} & -5.1342020 & 4.6859880 & 2.1011370 \\ \mathrm{H} & -5.4586560 & 4.1495190 & \\ \mathrm{C} & -6.4486680 & 2.9223950 & \\ & & & \end{array}$

-6.8860330
-7.2424240
-5.6035040
-6.4456160
-0.2324950
0.8163820
0.8168320
-0.6091930
-1.7053070
-1.5206520
0.5858210
1.4899320
-0.6470430
-1.6246700
1.8087990
1.2350430
-0.8443030
-2.7033350
1.8980910
3.1539030
1.0775090
1.8891740
2.8008450
3.8074330
1.2023710
0.2921060
0.5492070
2.1882830
3.3326930
4.3904190
4.5294980
2.5093200
-0.3088960
0.4130830
-0.2696470
-1.2604500
0.7713260
-1.2148340
-2.0694600
0.8205780
1.5421960
-0.1729930
-1.9945810
1.6354670
-0.1364120
0.4684470

3.3562790

2.8613260

$-0.2115110$

$-0.8151070$

3.6559730

4. 5472290

5. 9301710

6.5521270

5. 6165370

. 2113120

4.6964660

6.6026600

7.5117360

. 6175840

4.0898130

5.8018340

6.7555030

5. 9943470

1. 3701230

0.7338650

1.5672900

. 4541040

0.2228880

. 3701290

1.4090210

0.7938480

2. 5225100

$-0.6776840$

0.0978750

$-0.5516280$

1. 1587170

2. 3546730

$-3.7125870$

$-2.6442350$

$-4.6445880$

$-5.6250630$

$-4.5379150$

$-6.4739140$

$-5.7028820$

$-5.3976820$

$-3.7862180$

$-6.3659870$

$-7.2193060$

$-5.3119440$

$-7.0318540$

$-1.9562330$
3. 0101640

1. 3472560

0.5121280

0.8531210

$-2.1567980$

$-2.7747660$

$-2.0770100$

$-1.9749200$

$-2.5462630$

$-2.0338560$

$-3.8417410$

$-2.6231190$

$-2.5054370$

3. 6449850

$-2.7202050$

$-1.0712850$

$-0.9231250$

$-2.2942390$

$-3.0501820$

$-3.1045540$

$-4.3156720$

$-5.6129410$

$-5.5744510$

$-4.4273670$

$-6.4680330$

$-4.3171520$

$-4.2917740$

$-5.4165390$

$-6.5265520$

$-4.2996360$

$-4.6944860$

$-5.7392670$

$-0.4378810$

$-0.6204180$

$-1.5984340$

$-1.7881870$

$-2.5437450$

$-2.8925690$

$-1.0680620$

$-3.6399810$

$-2.4070660$

$-3.8206860$

$-4.3554170$

$-4.6802540$

0.1787520 


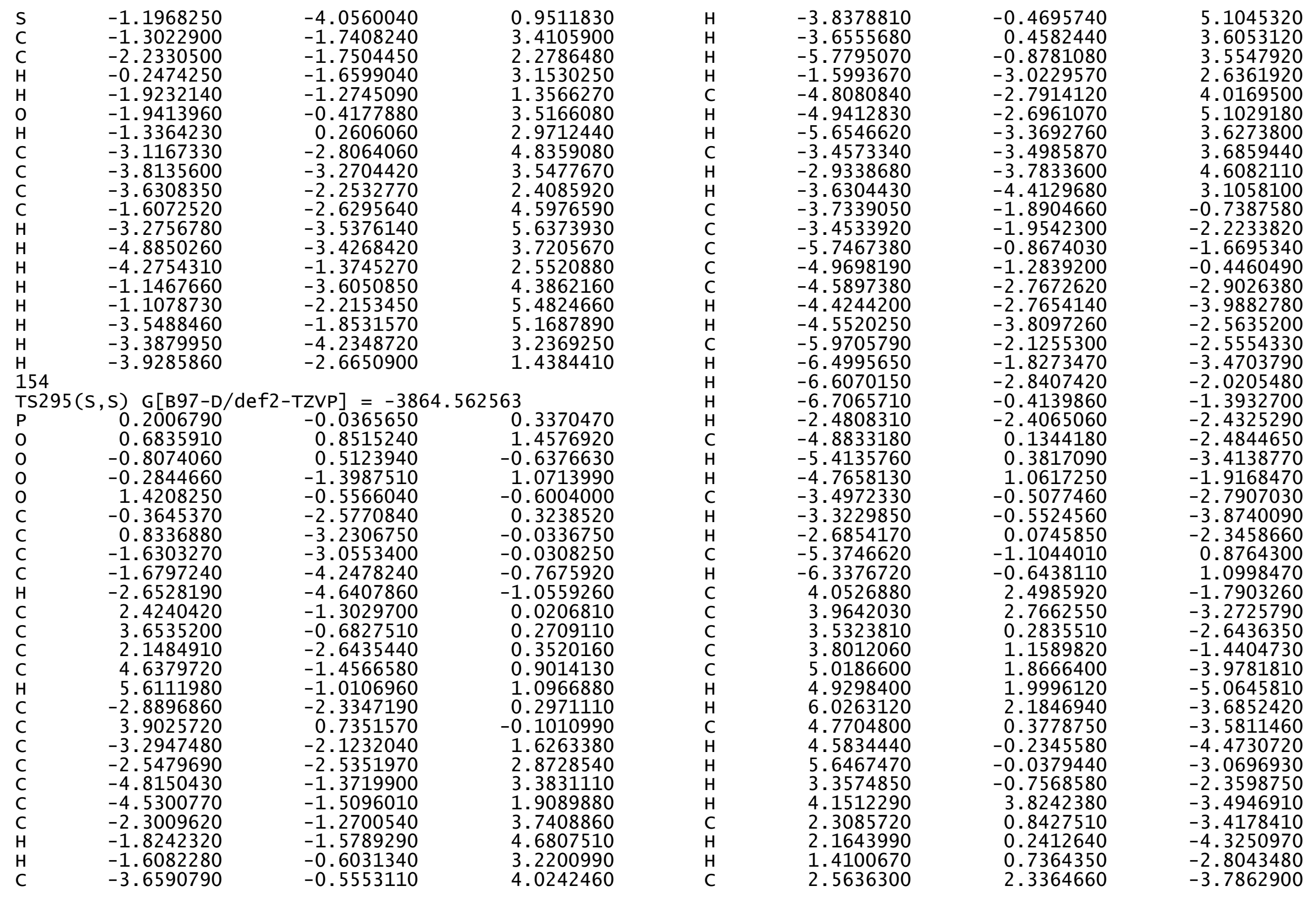




$\begin{array}{lrrr}\text { H } & 2.5249450 & 2.4831710 & -4.8737600 \\ \text { H } & 1.7962970 & 2.9778950 & -3.3413090 \\ \mathrm{C} & 4.5407670 & 3.0162190 & 0.5095380 \\ \mathrm{C} & 4.9897910 & 3.8819860 & 1.6599180 \\ \mathrm{C} & 4.4608270 & 1.4361940 & 2.3543740 \\ \mathrm{C} & 4.2724320 & 1.6822380 & 0.8737650 \\ \mathrm{C} & 3.8853970 & 3.8863160 & 2.7510230 \\ \mathrm{H} & 4.2462360 & 4.4601730 & 3.6152820 \\ \mathrm{H} & 2.9885750 & 4.3813920 & 2.3658320 \\ \mathrm{C} & 3.5627150 & 2.4171410 & 3.1576680 \\ \mathrm{H} & 3.7541020 & 2.2611550 & 4.2276830 \\ \mathrm{H} & 2.5115950 & 2.1804640 & 2.9711440 \\ \mathrm{H} & 4.2211330 & 0.4067750 & 2.6299400 \\ \mathrm{H} & 5.2062940 & 4.9033460 & 1.3229780 \\ \mathrm{C} & 5.9399070 & 1.7633380 & 2.7027220 \\ \mathrm{H} & 6.0859150 & 1.6322040 & 3.7830360 \\ \mathrm{H} & 6.6047440 & 1.0580200 & 2.1894270 \\ \mathrm{C} & 6.2612230 & 3.2318910 & 2.2776300 \\ \mathrm{H} & 6.5774750 & 3.8254790 & 3.1455850 \\ \mathrm{H} & 7.0758040 & 3.2534280 & 1.5439780 \\ \mathrm{C} & 4.4238000 & 3.4279760 & -0.8172740 \\ \mathrm{H} & 4.6453050 & 4.4593860 & -1.0955190 \\ \mathrm{C} & 4.3877670 & -2.7661880 & 1.3062200 \\ \mathrm{C} & 5.4205710 & -3.6040540 & 2.0183270 \\ \mathrm{C} & 5.5181320 & -4.9972220 & 1.3577660 \\ \mathrm{C} & 4.1347570 & -5.7039170 & 1.2826360 \\ \mathrm{C} & 2.9497890 & -4.7548490 & 1.6050510 \\ \mathrm{C} & 3.1415870 & -3.3675720 & 1.0405290 \\ \mathrm{H} & 5.1285270 & -3.7313420 & 3.0736370 \\ \mathrm{H} & 6.2365540 & -5.6199590 & 1.9052730 \\ \mathrm{H} & 4.0990310 & -6.5446510 & 1.9866770 \\ \mathrm{H} & 2.8908530 & -4.6507120 & 2.7000500 \\ \mathrm{H} & 6.3948680 & -3.1005240 & 2.0103250 \\ \mathrm{H} & 5.9162550 & -4.8600120 & 0.3441060 \\ \mathrm{H} & 3.9926790 & -6.1212810 & 0.2797540 \\ \mathrm{H} & 2.0056230 & -5.1985040 & 1.2821440 \\ \mathrm{C} & 0.7416740 & -4.3963540 & -0.8193940 \\ \mathrm{C} & 1.9271050 & -5.1102920 & -1.4222400 \\ \mathrm{C} & 1.7087570 & -5.2999890 & -2.9431070 \\ \mathrm{C} & 0.3468630 & -5.9769950 & -3.2771720 \\ \mathrm{C} & -0.5485290 & -6.1501100 & -2.0227160 \\ \mathrm{C} & -0.5226340 & -4.9099770 & -1.1675110 \\ \mathrm{H} & 2.0418230 & -6.0992220 & -0.9526300 \\ \mathrm{H} & 2.5396190 & -5.8874860 & -3.3533520 \\ \mathrm{H} & 0.5096930 & -6.9605990 & -3.7353070 \\ \mathrm{H} & -0.1620470 & -6.9992180 & -1.4375560 \\ & & & \\ & & & \end{array}$
2.8557610
1.7519710
$-0.1943890$
$-1.5758060$
$-3.2410020$
$-2.8397970$
$-4.6109540$
$-5.4273450$
$-5.1226970$
$-6.7202220$
$-5.0504420$
$-6.4139680$
$-4.4973620$
$-7.2185060$
$-7.3412570$
$-6.7945670$
$-8.2277400$
$-1.9566850$
$-2.3226630$
$-0.2671580$
0.1984550
$-1.0014710$
0.1400300
1.0996760
0.9976550
0.5454700
0.5216370
0.8862350
$-0.4630450$
0.3021940
1.2162950
1.9665710
$-1.4871230$
$-0.3971320$
1.5528760
$-0.4871180$
0.6534060

154

$\operatorname{TS} 296(\mathrm{~S}, \mathrm{~S}) \mathrm{G}[\mathrm{B} 97-\mathrm{D} / \mathrm{def} 2-\mathrm{TZVP}]=-3864.562392$

$\begin{array}{lrrr}\text { TS296(S, S } & \text { G }[\mathrm{B} 97-\mathrm{D} / \mathrm{def}-\mathrm{TZVP}]=-3864.562392 \\ \mathrm{P} & -0.2297130 & -0.2603560 & 1.0282180 \\ \mathrm{O} & 0.4846170 & -0.7020470 & 2.2778750 \\ \mathrm{O} & -0.6389240 & 1.1845100 & 0.9224950 \\ \mathrm{O} & -1.4814280 & -1.2754400 & 0.8637730 \\ \mathrm{O} & 0.6609480 & -0.5354590 & -0.3184950 \\ \mathrm{C} & -2.1197190 & -1.2803180 & -0.3825440 \\ \mathrm{C} & -1.4760360 & -1.9388360 & -1.4442580\end{array}$

$-1.2428910$

$-3.4121430$

$-4.0098800$

$-2.3144140$

0.0392380

$-0.2062950$

0.5393220

$-0.5705900$

0.8996310

0.8120330

$-0.2025980$

$-1.1409880$

0.5320410

1.4658070

0.8149230

$-0.2956690$

$-1.7447790$

0.8711240

$-0.5065790$

1.2377060

$-0.9251750$

1.0596940

1.2156980

0.7674890

$-0.7661770$

$-1.2589230$

1.3803080

1.0920540

$-1.2069220$

$-1.1629650$

2.4755730

1.1378030

$-1.1223760$

$-2.3222890$
$-0.5966760$

$-0.4878680$

1.1131450

-1.2803180
-1.9388360

$-1.4442580$

$-0.1620470$

$-6.9992180$

$-1.4375560$ 


-3.3507240
-3.9307860
-4.8951540
0.8982750
2.1934220
-0.1685520
2.3764530
3.3760440
-4.0367010
3.3329850
-4.4533910
-4.2624970
-5.5521310
-5.1664900
-3.4925970
-3.4115980
-2.4789900
-4.2583510
-4.5331500
-3.6300550
-6.0932840
-3.7217720
-6.4339270
-6.6677510
-7.3813850
-5.6652530
-5.5361440
-6.2213750
-4.3347990
-3.9278620
-5.3415090
-5.0692360
-5.2156230
-4.9267370
-5.7800380
-6.0829270
-6.2633110
-7.0584480
-5.9438010
-3.3242310
-3.9832480
-4.1737580
-3.4640250
-3.1207880
-2.8371370
-2.2041520

$-0.6296290$ $-0.6259790$ $-0.1388910$ $-1.8588520$ $-2.3735880$ $-2.6153980$ $-3.7166620$ $-4.1399460$ 0.0288970 $-1.5218470$ $-0.7159520$ $-2.1990450$ $-1.0437450$ $-0.0990330$ $-2.4069910$ $-3.4853830$ $-2.0089050$ $-1.6991800$ $-2.4191330$ $-0.9283220$ $-0.5175590$ $-2.6678050$ $-2.1710830$ $-2.9022840$ $-1.7450740$ $-2.8503910$ $-3.9241140$ $-2.7445710$ 1.4038070 2. 3899090 3.4730920

2. 0058200 3.0104020 3.7806070 2.2396760

3.6307150

4.6977560

3.1332890

3.8891730

1.9148480

4.2155500

5.2702990

4.1825680

3.5384230

4.2651410

3.1289380

-0.5158260
-1.7927940
-1.9245300
-0.6963770
-0.5694660
-1.2056050
-0.9060290
-0.8194140
0.6268570
-0.1382280
1.7454490
1.9649220
3.9019030
2.7909270
3.2981370
3.4899820
3.1971920
4.4592000
5.2417890
4.9209830
4.6977530
1.1391470
3.2947910
4.0802660
2.9438920
2.1191620
2.3103020
1.1798960
0.5892310
-0.4828350
1.4095240
1.6287980
-1.0901410
-1.8179580
-1.6290760
0.0512900
-0.1356450
0.1095370
2.2263710
-1.2605570
1.2947190
1.0543640
2.2599100
0.1869980
-0.5853600
0.6153350

\begin{abstract}
$-5.4831570$
$-6.0506450$

4.8745180

5.2611900

3.2383550

3.7766140

5.6114290

5.8569790

6.4985040

4.3923240

4.0270260

4.6764210

2. 3876510

6.1171270

2. 8201000

2.4433590

1.9998550

4.0404150

4.3066440

3.7969620

5.1154880

5.7436940

3.7305130

4.0180910

4.6539910

5.0961470

4. 3075960

3.4642270

3. 3498720

2. 5240060

2.8766680

6.5889460

5.0111990

4.8316630

5.2180430

6.2154340

6.6138920

7.0286510

5.5442000

6.4016490

1. 3220930

0.0327540

1.6153860

0.3669540

$-0.7495270$

$-1.1497670$
\end{abstract}

1.2582410

1.7285700

0.3228550

1.3508900

$-0.1179080$

$-0.4713360$

0.5872160

1. 3174590

$-0.0354020$

$-0.2897530$

0.0122560

$-1.3471790$

$-0.7467510$

1.9492140

1.3739970

1.6489400

1.5007240

2.2601570

2.9386350

2.8830730

$-0.9647770$

$-1.3737750$

$-2.8689470$

$-1.7665090$

$-1.3172870$

$-1.6373520$

$-0.2849640$

$-2.2427130$

$-3.0575320$

$-1.6874290$

$-3.4772720$

$-0.7220810$

$-3.7430650$

$-4.5379390$

$-4.2227770$

$-2.8498830$

$-3.1837150$

$-2.9091490$

0.0779080

0.6879500

$-4.5369850$

$-3.9897270$

$-5.9948810$

$-6.8854520$

$-6.2193940$

$-4.8875630$
2.7309560

3.5350090

$-0.5856140$

$-1.6212670$

$-2.3293200$

$-0.9625220$

$-2.9306040$

$-3.7130630$

$-2.7642650$

$-3.3574690$

$-4.3478880$

$-3.4156060$

$-2.6054460$

$-1.2854190$

$-2.3224770$

$-3.3157440$

$-1.6148380$

$-1.9305880$

$-2.7514770$

$-1.0632620$

1.4351780

2.7435190

2.0554290

1.0632070

3.8491780

4.8020040

3.9713160

3.4516780

4.1788650

3.4262470

1.7454780

2.9975480

2.1722590

2.9079850

1. 2078470

2.6104930

3. 5777380

1. 8774650

0.6143690

0.9013800

$-1.3225230$

$-1.4697290$

$-1.6408600$

$-1.6312780$

$-2.4428210$

$-1.7974930$ 


$\begin{array}{rrr}0.6122770 & -7.8756290 & -2.0365320 \\ 2.0749360 & -6.0464420 & -2.6413140 \\ 2.3678950 & -6.3742530 & -0.9367820 \\ -0.3939880 & -6.0399780 & -3.4690860 \\ -1.6290790 & -6.8725860 & -2.5105390 \\ -1.8733830 & -4.3653060 & -2.4256490 \\ -1.6755490 & -5.0958430 & -0.8512890 \\ 0.0217010 & -7.0289850 & -0.5965220 \\ -3.2936320 & -1.2048350 & -2.8887230 \\ -3.8880500 & -1.1946010 & -4.2755770 \\ -3.8278600 & -2.6159550 & -4.8788700 \\ -2.3810140 & -3.1835940 & -4.8827110 \\ -1.4068440 & -2.3698660 & -3.9877380 \\ -2.0580040 & -1.8595150 & -2.7235740 \\ -3.3142730 & -0.5099900 & -4.9217620 \\ -4.2381480 & -2.6091260 & -5.8963910 \\ -1.9789250 & -3.1906380 & -5.9035550 \\ -1.0810350 & -1.4876550 & -4.5609660 \\ -4.9205840 & -0.8260140 & -4.2474530 \\ -4.4753020 & -3.2630710 & -4.2732460 \\ -2.3973710 & -4.2261820 & -4.5435180 \\ -0.5080470 & -2.9534290 & -3.7739960 \\ 0.5456790 & 3.6218840 & -1.0600840 \\ -0.2303900 & 2.5765070 & -1.0926990 \\ 0.6639670 & 4.2979320 & -2.3811450 \\ 1.7228160 & 5.1807600 & -2.6618400 \\ -0.2838990 & 4.0313680 & -3.3906510 \\ 1.8344920 & 5.7773570 & -3.9162660 \\ 2.4616590 & 5.3794480 & -1.8914380 \\ -0.1761660 & 4.6406800 & -4.6397820 \\ -1.1043350 & 3.3508940 & -3.1863750 \\ 0.8845650 & 5.5125390 & -4.9089880 \\ 2.6657320 & 6.4481110 & -4.1213800 \\ -0.9200600 & 4.4332390 & -5.4057830 \\ 0.9710590 & 5.9815750 & -5.8867810 \\ -0.3585810 & 2.0430180 & -0.1909440 \\ 1.3417040 & 4.1925730 & 0.3110020 \\ 1.3739730 & 2.2567160 & 3.0827550 \\ 2.3160410 & 2.1032090 & 1.9695350 \\ 0.3208690 & 2.1471550 & 2.8284700 \\ 2.0139330 & 1.4985730 & 1.1231680 \\ 1.9977050 & 0.9576220 & 3.3820640 \\ 1.3724850 & 0.2239230 & 2.9378460 \\ 3.1750450 & 3.5175310 & 3.0249410 \\ 3.8861680 & 3.7861110 & 2.0523570 \\ 3.7180760 & 2.6066200 & \end{array}$

1.6682650
3.3260790
4.9553060
4.3467990
1.2081340
1.1606490
3.6046440
3.4623580
4.0448590

3.3048480

4. 3586630

3.9703410

1.7550240

4.2391420

3.0184460

2.6240590

4.6903980

2. 8606600

154

TS297(S, S) G[в97-D/def2-TZVP $]=-3864.562188$

$\begin{array}{lrrr}\text { P } & 0.2066680 & -0.0288940 & 0.2503060 \\ \mathrm{O} & 0.7273910 & 0.7860940 & 1.4087000 \\ \mathrm{O} & -0.8082820 & 0.5919180 & -0.6733770 \\ \mathrm{O} & -0.2978470 & -1.4180020 & 0.9186010 \\ \mathrm{O} & 1.3998730 & -0.5174820 & -0.7397420 \\ \mathrm{C} & -0.4268820 & -2.5437260 & 0.0996330 \\ \mathrm{C} & 0.7465380 & -3.1954710 & -0.3322970 \\ \mathrm{C} & -1.7113470 & -2.9654280 & -0.2585900 \\ \mathrm{C} & -1.8022020 & -4.0988480 & -1.0796830 \\ \mathrm{H} & -2.7897900 & -4.4567530 & -1.3645270 \\ \mathrm{C} & 2.3889450 & -1.3281340 & -0.1781460 \\ \mathrm{C} & 3.6394470 & -0.7634020 & 0.1007030 \\ \mathrm{C} & 2.0790810 & -2.6704550 & 0.0858470 \\ \mathrm{C} & 4.5832980 & -1.6036620 & 0.6983490 \\ \mathrm{H} & 5.5698710 & -1.2001040 & 0.9196780 \\ \mathrm{C} & -2.9454030 & -2.2384360 & 0.1428690 \\ \mathrm{C} & 3.9422970 & 0.6611980 & -0.1986690 \\ \mathrm{C} & -3.3193110 & -2.1010660 & 1.4907250 \\ \mathrm{C} & -2.5567530 & -2.6017720 & 2.6943550 \\ \mathrm{C} & -4.7863940 & -1.4240410 & 3.3221070 \\ \mathrm{C} & -4.5338940 & -1.4780460 & 1.8364250 \\ \mathrm{C} & -2.2631570 & -1.3944230 & 3.6263830 \\ \mathrm{H} & -1.7670040 & -1.7650940 & 4.5332760 \\ \mathrm{H} & -1.5722090 & -0.7113430 & 3.1246680 \\ \mathrm{C} & -3.5994240 & -0.6737850 & 3.9877580 \\ \mathrm{H} & -3.7526740 & -0.6569320 & 5.0752180 \\ \mathrm{H} & -3.5841030 & 0.3652510 & 3.6372120 \\ \mathrm{H} & -5.7357740 & -0.9207220 & 3.5434420 \\ \mathrm{H} & -1.6245320 & -3.0948100 & 2.4083880 \\ \mathrm{C} & -4.7992240 & -2.8788640 & 3.8691170 \\ \mathrm{H} & -4.9107910 & -2.8472770 & 4.9611910 \\ \mathrm{H} & -5.6645390 & -3.4148860 & 3.4613340 \\ \mathrm{C} & -3.4697710 & -3.5920690 & 3.4702510 \\ \mathrm{H} & -2.9339610 & -3.9392870 & 4.3635960 \\ \mathrm{H} & -3.6727540 & -4.4684920 & 2.8430430 \\ & & & \end{array}$

4.1349560

5.0473340

3.1841840

2.3489440

3.7831880

5.0645350

4.8328740

2.5659900

1.0388130
$-3.6727540$
$-0.0288940$

(1)

$-4.4684920$
2.8430430

$-4.8788700$

$-3.9877380$

$-2.7235740$

9217620

$-5.9035550$

(

474530

$-4.5435180$

(7739960

0600840

$-2.3811450$

.6618400

3906510

$-1.8914380$

6397820

$-4.1213800$

$-0.1909440$

1231680

9378460

0249410 


$\begin{array}{rrr}-3.7980020 & -1.7086870 & -0.8454150 \\ -3.5507810 & -1.6909400 & -2.3369000 \\ -5.8097530 & -0.5965570 & -1.6705470 \\ -5.0142540 & -1.0954500 & -0.4901540 \\ -4.7170760 & -2.4430240 & -3.0355630 \\ -4.5772190 & -2.3777840 & -4.1227490 \\ -4.6900340 & -3.5045160 & -2.7607300 \\ -6.0773960 & -1.8004030 & -2.6169630 \\ -6.6254590 & -1.4445030 & -3.4995170 \\ -6.7114280 & -2.5338000 & -2.1042830 \\ -6.7544530 & -0.1429130 & -1.3470000 \\ -2.5918640 & -2.1462370 & -2.5950940 \\ -4.9473670 & 0.4334960 & -2.4505580 \\ -5.4935670 & 0.7366940 & -3.3537750 \\ -4.8020620 & 1.3288770 & -1.8394500 \\ -3.5794790 & -0.2141870 & -2.8215790 \\ -3.4286840 & -0.2008090 & -3.9092160 \\ -2.7480450 & 0.3287920 & -2.3633840 \\ -5.3887820 & -0.9903210 & 0.8490670 \\ -6.3367750 & -0.5251070 & 1.1215500 \\ 4.1579040 & 2.5035330 & -1.7947840 \\ 4.0786020 & 2.8524590 & -3.2604100 \\ 3.5252030 & 0.3648150 & -2.7582970 \\ 3.8499810 & 1.1593750 & -1.5132300 \\ 5.0855650 & 1.9396730 & -4.0162390 \\ 5.0104730 & 2.1413070 & -5.0931170 \\ 6.1071740 & 2.1838530 & -3.7016920 \\ 4.7545360 & 0.4458410 & -3.7084870 \\ 4.5195660 & -0.0969870 & -4.6335130 \\ 5.6114520 & -0.0521270 & -3.2395710 \\ 3.2994960 & -0.6790640 & -2.5289910 \\ 4.3179110 & 3.9100110 & -3.4275890 \\ 2.3223860 & 1.0287510 & -3.4803520 \\ 2.1210490 & 0.4772690 & -4.4082550 \\ 1.4328740 & 0.9488710 & -2.8497920 \\ 2.6571720 & 2.5195040 & -3.7904380 \\ 2.6302290 & 2.7097190 & -4.8715400 \\ 1.9239620 & 3.1838050 & -3.3222300 \\ 4.6763390 & 2.8795480 & 0.5255260 \\ 5.1666230 & 3.6654370 & 1.7163940 \\ 4.5403740 & 1.2110150 & 2.2870130 \\ 4.3567120 & 1.5399200 & 0.8217560 \\ 4.0680230 & 3.6585110 & 2.8131300 \\ 4.4561520 & 4.1692390 & 2.7047020 \\ 3.1924060 & 4.2122690 & 3.1421130 \\ 3.6833630 & 2.1849310 & \end{array}$

2.6236730

4.2593270

5.4233610

6.0322660

6.1732910

6.6678900

6.4130770

6.7607710

7.2222440

4. 5708140

4.8329820

3.0280970

2. 6625490

3.5729410

5.0406960

5.3500580

4.2928810

3. 3493710

2.7326970

1. 6109500

5.2160490

5.7148460

6.3280490

5.4296440

3.3753950

$-0.6697140$

$-0.7576220$

0.2385950

1.6925880

1.7689800

0.6137970

$-0.5058940$

0.1758850

2.2436280

1.7198210

$-1.7824200$

$-0.0604250$

2.2087500

2.7336900

$-3.1735560$

$-2.7817170$

$-4.5265080$

$-5.3450270$

-5.0212120
-6.6234650
1.9646550

2.0028630

0.1794230

4.6932220

1.4610110

1.2730490

0.7542520

2.9330020

3.4694340

2. 9559600

3.3649910

4.3996810

$-3.4668830$

$-4.8693740$

$-5.3969400$

$-5.2055800$

$-3.7081610$

$-2.9231380$

$-6.4555550$

$-5.5620190$

$-4.8830220$

$-5.7001570$

$-5.6686160$

$-3.5501170$

$-3.2833080$

$-4.8504170$

$-4.7365720$

$-5.9192680$

$-7.0095110$

$-6.4707370$

$-4.9188100$

$-4.2784040$

$-5.6039900$

$-7.8782820$

$-6.7996390$

$-4.5444910$

$-6.3094600$

$-7.3465350$

$-6.9022700$

$-4.5941900$

2.9977700

1.8932290

3.4359920

2.5457820

. 7188050

2. 9295270
4.2023180

2. 9439820

2. 5095260

1.4313600

2.6458810

3.7184670

2.0986880

2. 2900910

3.1828330

1.5505220

$-0.7771990$

$-1.0030210$

0.7685210

1.2234040

2. 3400570

1. 9417290

1.8234650

1.0627110

2.5243550

0.3752350

1.5356930

0.9741730

2.6736300

1.3483850

2.8368690

3.2743550

$-1.5786080$

$-2.5119030$

$-2.0663200$

$-2.0209300$

$-1.9592590$

$-1.2230580$

$-3.5381790$

$-2.7335140$

$-2.9109450$

$-2.9940090$

$-2.5358890$

$-1.0651180$

$-1.1549980$

$-1.5625960$

$-0.4424820$

0.1378650

$-0.0060130$

0.7203220

$-0.3097730$

1. 1206800 


\begin{tabular}{|c|c|c|c|c|c|c|c|}
\hline & & & & & & & \\
\hline H & -4.9828120 & 1. 5485750 & 0.9449030 & C & -3.2976280 & -2.1293610 & 1.5829310 \\
\hline $\mathrm{H}$ & -4.3943880 & 5.4100830 & -0.8657610 & c & -4.8030800 & -1.4136310 & $\begin{array}{l}2.0143200 \\
3.3674610\end{array}$ \\
\hline C & -7.1048810 & 4.2064440 & 0.8130900 & $\mathrm{c}$ & -4.5288550 & -1.5185340 & 1.8884450 \\
\hline $\mathrm{H}$ & -7.2468140 & 2.2280160 & 1.6708950 & C & -2.2861170 & -1.3263910 & 3.7084250 \\
\hline $\mathrm{H}$ & -6.6656430 & 6.0947160 & -0.1414000 & $\mathrm{H}$ & -1.8018470 & -1.6570920 & 4.6369470 \\
\hline $\mathrm{H}$ & -8.1029140 & 4.5047290 & 1.1271590 & $\mathrm{H}$ & -1.5966460 & -0.6495480 & 3.1962860 \\
\hline $\mathrm{H}$ & -1.9204490 & 1.4274580 & -0.2451430 & c & -3.6401890 & -0.6156210 & 4.0195730 \\
\hline $\mathrm{S}$ & -2.2608410 & 3.8454350 & -1.5734660 & $\mathrm{H}$ & -3.8107130 & -0.5562280 & 5.1029640 \\
\hline C & -0.1323490 & 3.7378560 & 0.9896750 & $\mathrm{H}$ & -3.6373400 & 0.4080560 & 3.6259910 \\
\hline $\mathrm{C}$ & 0.2914520 & 3.5901150 & -0.4072300 & $\mathrm{H}$ & -5.7648020 & -0.9209220 & 3.5569980 \\
\hline $\mathrm{H}$ & -0.8806270 & 3.0283020 & 1.3444120 & $\mathrm{H}$ & -1.5978190 & -3.0563560 & 5605320 \\
\hline $\mathrm{H}$ & 0.1891630 & 2.6156150 & -0.8676940 & C & -4.7964650 & -2.8472430 & 3.9681030 \\
\hline 0 & 1.2214440 & 3.1948770 & 1.1219000 & $\mathrm{H}$ & -4.9227700 & -2.7769680 & 5.0567800 \\
\hline $\mathrm{H}$ & 1.0869380 & 2.1383630 & 1.2292610 & $\mathrm{H}$ & -5.6468990 & -3.4136460 & 3.5702170 \\
\hline C & 0.7576200 & 6.1114650 & 0.9671720 & C & -3.4496220 & -3.5499990 & 3.6123600 \\
\hline $\mathrm{C}$ & 0.6944260 & 6.0909620 & -0.5678490 & $\mathrm{H}$ & -2.9205780 & -3.8560990 & 4.5245490 \\
\hline $\mathrm{C}$ & 0.9972510 & 4.6906070 & -1.1301280 & $\mathrm{H}$ & -3.6286730 & -4.4510850 & 3.0135010 \\
\hline $\mathrm{C}$ & -0.2693860 & 5.1330300 & 1.5619900 & C & -3.7538090 & -1.8434060 & -0.7733890 \\
\hline $\mathrm{H}$ & 0.5561110 & 7.1225830 & 1.3405180 & $\mathrm{C}$ & -3.4869170 & -1.8784400 & -2.2614220 \\
\hline H & 1.4034350 & 6.8105130 & -0.9942590 & C & -5.7751640 & -0.8034870 & -1.6663650 \\
\hline H & 2.0729470 & 4.4656040 & -1.0718320 & C & -4.9864720 & -1.2413730 & -0.4578430 \\
\hline H & -1.2882690 & 5.4730060 & 1.3282730 & $\mathrm{C}$ & -4.6291030 & -2.6796990 & -2.9450700 \\
\hline $\mathrm{H}$ & -0.1813690 & 5.0744790 & 2.6541250 & $\mathrm{H}$ & -4.4751220 & -2.6551860 & -4.0320870 \\
\hline $\mathrm{H}$ & 1.7651880 & 5.8305610 & 1.3018530 & $\mathrm{H}$ & -4.5863700 & -3.7287660 & -2.6276680 \\
\hline $\mathrm{H}$ & -0.3129590 & 6.3916810 & -0.8873680 & C & -6.0070330 & -2.0470480 & 5706600 \\
\hline $\mathrm{H}$ & 0.7323610 & 4.6185430 & -2.1911510 & $\mathrm{H}$ & -6.5476620 & -1.7347800 & -3.4740240 \\
\hline 15 & & & & $\mathrm{H}$ & -6.6355110 & -2.7724410 & -2.0400890 \\
\hline & S) $\mathrm{G}[\mathrm{B} 97-\mathrm{D}$ & {$[\mathrm{VPP}]=-386$} & 070 & $\mathrm{H}$ & -6.7326530 & -0.3559820 & -1.3729830 \\
\hline $\mathrm{P}$ & 0.1978470 & -0.0240080 & 0.3037890 & $\mathrm{H}$ & -2.5159740 & -2.3251560 & -2.4885790 \\
\hline 0 & 0.6830150 & 0.8535070 & 1.4316850 & C & -4.9212300 & 0.2125530 & -2.4733950 \\
\hline 0 & -0.8142700 & 0.5330390 & -0.6625580 & $\mathrm{H}$ & -5.4606690 & 0.4729280 & -3.3938960 \\
\hline 0 & -0.2866430 & -1.3932170 & 1.0270900 & $\mathrm{H}$ & -4.7999880 & 1.1314490 & -1.8931280 \\
\hline 0 & 1.4171170 & -0.5312840 & -0.6436340 & C & -3.5370610 & -0.4222990 & -2.8027190 \\
\hline C & -0.3764350 & -2.5567640 & 0.2573030 & $\mathrm{H}$ & -3.3722860 & -0.4479260 & -3.8881040 \\
\hline C & 0.8109870 & -3.2035250 & -0.1257290 & H & -2.7220690 & 0.1536310 & 554190 \\
\hline C & -1.6449670 & -3.0229930 & -0.1065990 & C & -5.3801770 & -1.0888600 & 0.8713930 \\
\hline $\mathrm{C}$ & -1.6870230 & -4.1853890 & -0.8819970 & $\mathrm{H}$ & -6.3406230 & -0.6317660 & 1.1124410 \\
\hline $\mathrm{H}$ & -2.6615620 & -4.5733600 & -1.1738650 & C & 4.0866560 & 2.5172930 & -1.7814550 \\
\hline C & 2.4142900 & -1.2911170 & -0.0278080 & C & 4.0203190 & 2.7986120 & -3.2622310 \\
\hline $\mathrm{C}$ & 3.6431690 & -0.6799180 & 0.2464130 & $\mathrm{C}$ & 3.5600540 & 0.3144280 & -2.6604620 \\
\hline C & 2.1252870 & -2.6314930 & 0.2903240 & C & 3.8220390 & 1.1764160 & -1.4463070 \\
\hline C & 4.6088530 & -1.4646710 & 0.8920210 & C & 5.0776390 & 1.8967240 & -3.9604770 \\
\hline H & 5.5824670 & -1.0282500 & 1.1060100 & $\mathrm{H}$ & 5.0080170 & 2.0420500 & -5.0467120 \\
\hline C & -2.9028900 & -2.3125180 & 0.2463280 & $\mathrm{H}$ & 6.0831460 & 2.2022220 & 6475200 \\
\hline $\mathrm{C}$ & 3.9065880 & 0.7393760 & -0.1098650 & C & 4.8093210 & 0.4063110 & -3.5833290 \\
\hline
\end{tabular}




$\begin{array}{ll}\mathrm{H} & 4.6253440 \\ \mathrm{H} & 5.6754990 \\ \mathrm{H} & 3.3731840 \\ \mathrm{H} & 4.2191320 \\ \mathrm{C} & 2.3496960 \\ \mathrm{H} & 2.2066110 \\ \mathrm{H} & 1.4449100 \\ \mathrm{C} & 2.6239560 \\ \mathrm{H} & 2.6045250 \\ \mathrm{H} & 1.8555230 \\ \mathrm{C} & 4.5538040 \\ \mathrm{C} & 5.0002960 \\ \mathrm{C} & 4.4456100 \\ \mathrm{C} & 4.2726040 \\ \mathrm{C} & 3.8879770 \\ \mathrm{H} & 4.2476040 \\ \mathrm{H} & 2.9984530 \\ \mathrm{C} & 3.5490670 \\ \mathrm{H} & 3.7298690 \\ \mathrm{H} & 2.4975250 \\ \mathrm{H} & 4.1946170 \\ \mathrm{H} & 5.2279230 \\ \mathrm{C} & 5.9244240 \\ \mathrm{H} & 6.0598860 \\ \mathrm{H} & 6.5878610 \\ \mathrm{C} & 6.2615880 \\ \mathrm{H} & 6.5774510 \\ \mathrm{H} & 7.0810210 \\ \mathrm{C} & 4.4533150 \\ \mathrm{H} & 4.6850010 \\ \mathrm{C} & 4.3370160 \\ \mathrm{C} & 5.3488330 \\ \mathrm{C} & 5.4103290 \\ \mathrm{C} & 4.0263590 \\ \mathrm{C} & 2.8408940 \\ \mathrm{C} & 3.0918770 \\ \mathrm{H} & 5.0532550 \\ \mathrm{H} & 6.1576580 \\ \mathrm{H} & 3.9849930 \\ \mathrm{H} & 2.6936460 \\ \mathrm{H} & 6.3334520 \\ \mathrm{H} & 5.7430630 \\ \mathrm{H} & 3.8972280 \\ \mathrm{H} & 1.9116030 \\ \mathrm{C} & 0.7311940 \\ \mathrm{C} & 1.9945100\end{array}$

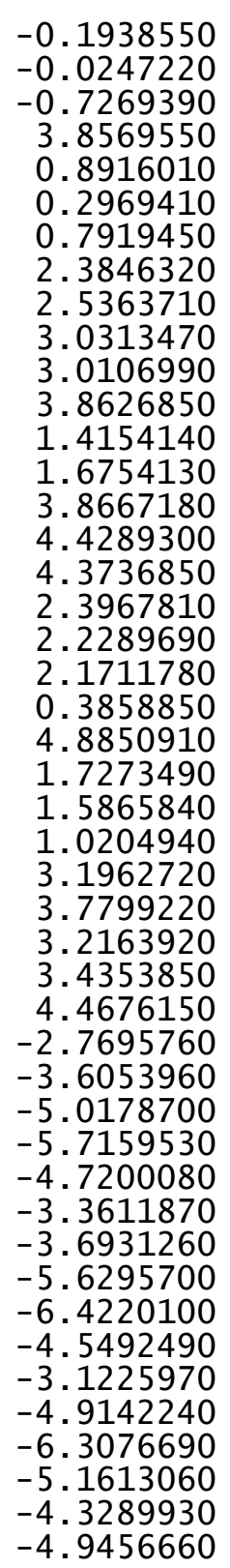

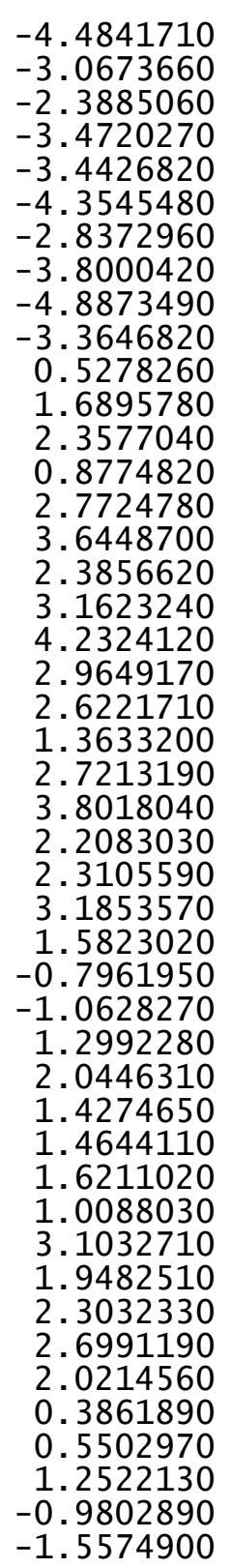

\begin{abstract}
1.7355760
0.6026690

$-0.6991220$

$-0.5344370$

2. 6582020

2. 4661000

2.7197980

0.8637970

0.4660360

$-1.4710660$

$-1.0877920$

1.4502030

$-3.2361410$

$-2.8302730$

$-4.6008580$

$-5.4107780$

$-5.1145170$

$-6.6993210$

$-5.0330420$

$-6.4013860$

$-4.4942550$

$-7.1995740$

$-7.3155900$

$-6.7834930$

$-8.2054590$

$-1.9534700$

$-2.3289710$

$-0.2389970$

0.2086160

$-0.9770540$

0.1342260

1. 1245410

1. 0124440

0.6008440

0.5596770

0.9004000

$-0.4134290$

0.3732820

1. 2590900

1.9795130

$-1.4362890$

$-0.3377520$

1. 6091120

$-0.4493880$

0.6531980
\end{abstract}

$\begin{array}{rr}-5.7928390 & -2.8098290 \\ -6.7870160 & -2.5327000 \\ -6.0193870 & -2.2736060 \\ -4.8302790 & -1.3392700 \\ -6.3143640 & -3.0952010 \\ -5.5901120 & -0.8054940 \\ -4.1500600 & -1.7683150 \\ -7.3893230 & -1.6493190 \\ -7.4797270 & -3.3730070 \\ -6.6894800 & -1.8706920 \\ -5.6486680 & -3.2353440 \\ -5.1422340 & -3.6499630 \\ 2.9021720 & -0.5663190 \\ 1.8254960 & 0.0552920 \\ 3.3296840 & -0.1568780 \\ 2.4487490 & 0.5900980 \\ 4.5934000 & -0.5055980 \\ 2.8225890 & 0.9666930 \\ 1.4660410 & 0.8503660 \\ 4.9645830 & -0.1213600 \\ 5.2777190 & -1.0773150 \\ 4.0805940 & 0.6144260 \\ 2.1280670 & 1.5334880 \\ 5.9453950 & -0.3949860 \\ 4.3712900 & 0.9099970 \\ 1.3647670 & -0.2959540 \\ 3.7278300 & -1.7174980 \\ 3.7636100 & 0.8695250 \\ 3.5685840 & -0.5137720 \\ 3.0525810 & 1.2424340 \\ 2.5737660 & -0.9344570 \\ 3.2570400 & 1.0413760 \\ 2.2029870 & 1.1940480 \\ 6.1530970 & 0.7630560 \\ 6.0695480 & -0.7704990 \\ 4.6545500 & -1.2714360 \\ 5.1773180 & 1.3835420 \\ 7.1738140 & 1.0928290 \\ 6.7866380 & -1.2164910 \\ 4.4553950 & -1.1886650 \\ 5.4871060 & 1.1257490 \\ 5.1639760 & 2.4781360 \\ 5.9072230 & 1.1224060 \\ 6.3349400 & -1.1150030 \\ 4.5338480 & -2.3322600 \\ -5 & \end{array}$




\begin{tabular}{|c|c|c|c|}
\hline \multicolumn{4}{|c|}{ TS299(S, S) G[B97-D/def2-TZVP $]=-3864.561791$} \\
\hline $\mathrm{P}$ & 0.2594520 & 0.3028840 & 1.0608460 \\
\hline 0 & -0.4534930 & 0.7250280 & 2.3181050 \\
\hline 0 & 0.7199280 & -1.1268180 & 0.9585650 \\
\hline 0 & 1.4692890 & 1.3643140 & 0.8736520 \\
\hline 0 & -0.6581850 & 0.5363870 & -0.2750950 \\
\hline C & 2.0928600 & 1.3918310 & -0.3791620 \\
\hline C & 1.4166590 & 2.0084150 & -1.4413440 \\
\hline $\mathrm{C}$ & 3.3524790 & 0.7999280 & -0.5216880 \\
\hline C & 3.9066300 & 0.8117190 & -1.8050680 \\
\hline $\mathrm{H}$ & 4.8877640 & 0.3611020 & -1.9461100 \\
\hline C & -0.9486770 & 1.8491670 & -0.6542940 \\
\hline C & -2.2576510 & 2.3178740 & -0.5035230 \\
\hline C & 0.0874970 & 2.6362220 & -1.1914000 \\
\hline $\mathrm{C}$ & -2.5009300 & 3.6576520 & -0.8322880 \\
\hline $\mathrm{H}$ & -3.5125440 & 4.0459980 & -0.7283250 \\
\hline C & 4.0816150 & 0.1750470 & 0.6124540 \\
\hline C & -3.3605490 & 1.4280160 & -0.0542410 \\
\hline C & 4.4750910 & 0.9355710 & 1.7285690 \\
\hline C & 4.2144940 & 2.4063510 & 1.9567690 \\
\hline C & 5.5742530 & 1.3061050 & 3.8775180 \\
\hline C & 5.2269770 & 0.3493000 & 2.7643280 \\
\hline C & 3.4460230 & 2.5702440 & 3.2971960 \\
\hline $\mathrm{H}$ & 3.3170940 & 3.6425350 & 3.4968560 \\
\hline $\mathrm{H}$ & 2.4509010 & 2.1268340 & 3.2012780 \\
\hline C & 4.2525350 & 1.8912010 & 4.4478240 \\
\hline $\mathrm{H}$ & 4.4947520 & 2.6165870 & 5.2361010 \\
\hline $\mathrm{H}$ & 3. 6661810 & 1.0856070 & 4.9056050 \\
\hline $\mathrm{H}$ & 6.1466110 & 0.8029540 & 4.6664490 \\
\hline $\mathrm{H}$ & 3.6452880 & 2.8522100 & 1.1374520 \\
\hline C & 6.3939630 & 2.4799030 & 3.2724400 \\
\hline $\mathrm{H}$ & 6.5938270 & 3.2183400 & 4.0604980 \\
\hline $\mathrm{H}$ & 7.3604010 & 2.1049800 & 2.9150220 \\
\hline C & 5.5852390 & 3.1244720 & 2.1037260 \\
\hline $\mathrm{H}$ & 5.405 & .1900950 & 2.2988000 \\
\hline $\mathrm{H}$ & 6.1392210 & 3.0474920 & 1.1604120 \\
\hline C & 4.4 & -1.1848350 & 5678380 \\
\hline C & 4.0 & -2.1882350 & 0.4974 \\
\hline C & 5. & -3.2087980 & 16890 \\
\hline C & 5. & -1.75 & 80 \\
\hline C & 5.3 & 56540 & -1 \\
\hline $\mathrm{H}$ & 5.0 & -3.5 & -1.8 \\
\hline $\mathrm{H}$ & 5. & -1.96 & -1.6 \\
\hline C & 6.2 & -3.3325630 & 19890 \\
\hline $\mathrm{H}$ & 6.503 & -4.3897830 & -0.1876390 \\
\hline $\mathrm{H}$ & 7.2 & -2.78 & 0.0450 \\
\hline
\end{tabular}

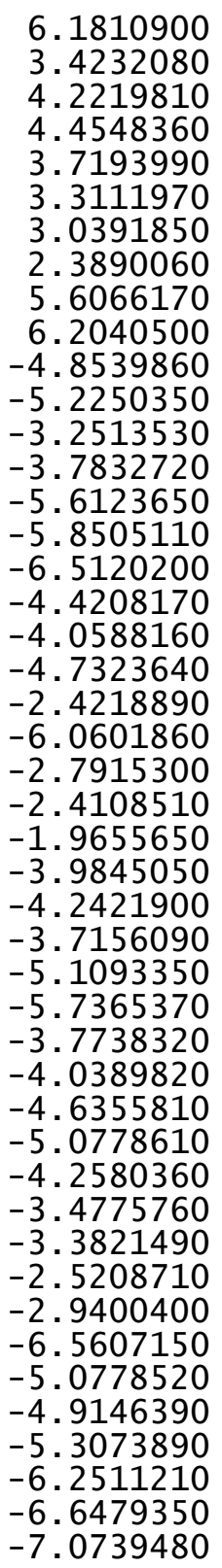
$-3.5982570$
$-1.7409570$
$-4.0098150$
$-5.0537820$
$-4.0049080$
$-3.3663690$
$-4.1039190$
$-2.9897410$
$-0.9913290$
$-1.4365800$
$-0.4614530$
$-1.5022040$
0.0185820
0.3619280
$-0.7506010$
$-1.4884800$
$-0.1494210$
0.1548450
$-0.1432630$
1. 2043620
0.6700570
$-2.1229560$
$-1.4608090$
$-1.7295200$
$-1.5615980$
$-2.3788510$
$-3.0604000$
$-2.9991550$
0.8276350
1. 2270480
2.7743360
1.6579140
1. 2078310
1. 5197200
0.1866860
2. 1659520
2. 9891580
1. 6398040
3. 4042800
0.5534880
3. 6122770
4. 4187000
4.0771430
2. 6884520
3. 0170920
2. 7196450

2.1785380 $-1.265$ 1. 2772750 1. 0275450

2. 2517780

0.1882250

$-0.5780130$

0.6350280

2. 6961980

3. 4928290

$-0.4767900$

$-1.5056180$

$-2.2408700$

$-0.8693460$

$-2.8118080$

$-3.5893280$

$-2.6345150$

$-3.2570780$

-4. 2498710

$-3.3175250$

$-2.5288140$

$-1.1582960$

$-2.2317140$

$-3.2251070$

$-1.5265120$

$-1.8304740$

$-2.6514620$

$-0.9686390$

1.5414030

2. 8531290

2.1368800

1.1540110

3. 9488960

4.9043830

4.0728480

3. 5350350

4. 2555140

3. 5056400

1.8158490

3. 1189850

2. 2584970

2. 9853730

1. 2919720

2.7159080

3.6855800

1.9919460 


$\begin{array}{rrr}-5.5173750 & -0.2302930 & 0.7294140 \\ -6.3544160 & -0.8628160 & 1.0278000 \\ -1.4780960 & 4.5007040 & -1.2641890 \\ -1.7117640 & 5.9571980 & -1.5797650 \\ -1.0631890 & 6.3027870 & -2.9370690 \\ 0.4567390 & 5.9928510 & -2.9411340 \\ 0.8857750 & 5.0023870 & -1.8210480 \\ -0.1781010 & 3.9934770 & -1.4539380 \\ -1.2582300 & 6.5868310 & -0.7964940 \\ -1.2360850 & 7.3576360 & -3.1844390 \\ 1.0291780 & 6.9194170 & -2.8083430 \\ 1.0788320 & 5.5937440 & -0.9121350 \\ -2.7861900 & 6.1773570 & -1.5915520 \\ -1.5629620 & 5.7016730 & -3.7081210 \\ 0.7383490 & 5.5885870 & -3.9207630 \\ 1.8305510 & 4.5151390 & -2.0753150 \\ 1.9744370 & 1.9421640 & -2.7391230 \\ 1.1936740 & 2.4602080 & -3.9350440 \\ 1.6887570 & 1.8937080 & -5.2719980 \\ 3.2102790 & 2.0494060 & -5.3685260 \\ 3.8822210 & 1.2021030 & -4.2813390 \\ 3.2369230 & 1.3432580 & -2.9117550 \\ 1.1855160 & 2.4129980 & -6.0976780 \\ 1.2737770 & 3.5539530 & -3.9766420 \\ 0.1280840 & 2.2422720 & -3.7918460 \\ 3.4713920 & 3.1094750 & -5.2292160 \\ 3.5790730 & 1.7481800 & -6.3574350 \\ 4.9505240 & 1.4461510 & -4.2030150 \\ 3.8311560 & 0.1423240 & -4.5786030 \\ 1.4276060 & 0.8271990 & -5.3449350 \\ -0.4168350 & -3.6000210 & -1.0106360 \\ 0.3380760 & -2.5393690 & -1.0480280 \\ -0.5419780 & -4.2698830 & -2.3343500 \\ -1.5853210 & -5.1746460 & -2.6029100 \\ 0.3803780 & -3.9732880 & -3.3590150 \\ -1.7068770 & -5.7633550 & -3.8601210 \\ -2.3050120 & -5.3962210 & -1.8207760 \\ 0.2631510 & -4.5745520 & -4.6111570 \\ 1.1881820 & -3.2751590 & -3.1643180 \\ -0.7823450 & -5.4683040 & -4.8681240 \\ -2.5262820 & -6.4513440 & -4.0555400 \\ 0.9874770 & -4.3436500 & -5.3891180 \\ -0.8766410 & -5.9309040 & -5.8482560 \\ 0.4591320 & -2.0010920 & -0.1483290 \\ -1.1820650 & -4.1944290 & 0.3680480 \\ -1.2318390 & -2.2612610 & 3.1428010\end{array}$
$-2.1934980$
$-0.1863110$
$-1.9232640$
$-1.8917330$
$-1.2985380$
$-2.9750780$
$-3.6959170$
$-3.5776790$
$-1.4790010$
$-3.0897180$
$-4.7566220$
$-4.2277620$
$-0.9960570$
$-0.9667460$
$-3.4253330$
$-3.2511980$
$-3.9112700$

154

TS300(S, S) G[B97-D/def2-TZVP $]=-3864.561644$

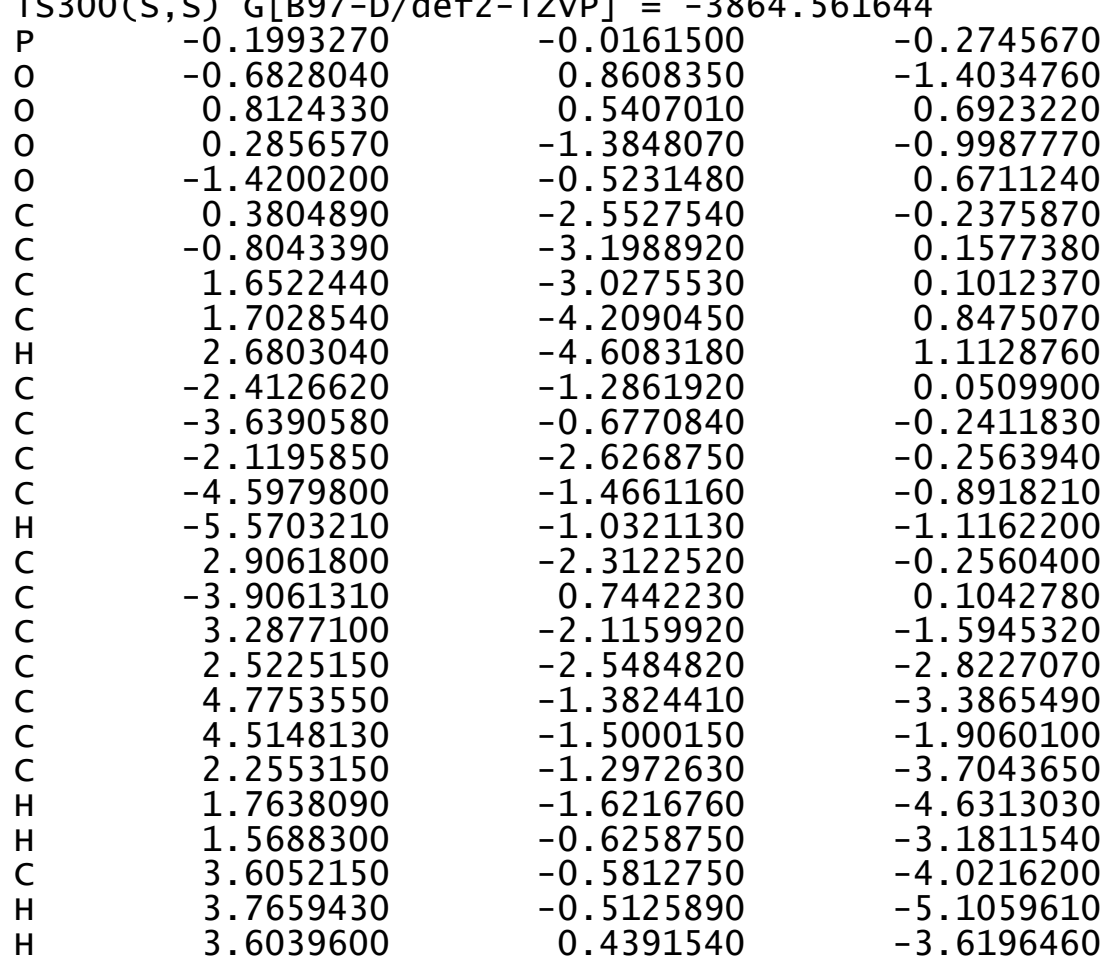

2.0426720

2.8742350

1.1920750

3.4514160

2.9960960

4.4448330

3.1195320

2. 1451600

4.1977820

5.1333250

3.2934640

2.4512230

3.8377040

5.1203200

4.9242750

2.6547890

1.1365200
$-3.0181740$

$-2.6986350$

$-4.7597300$

$-2.9486010$

$-0.2745670$

(

9987770

0.1577380

.1012370

1.1128760

(

0.8918210

(1)

0.1042780

(2)

4.6313030

$-3.6196460$ 


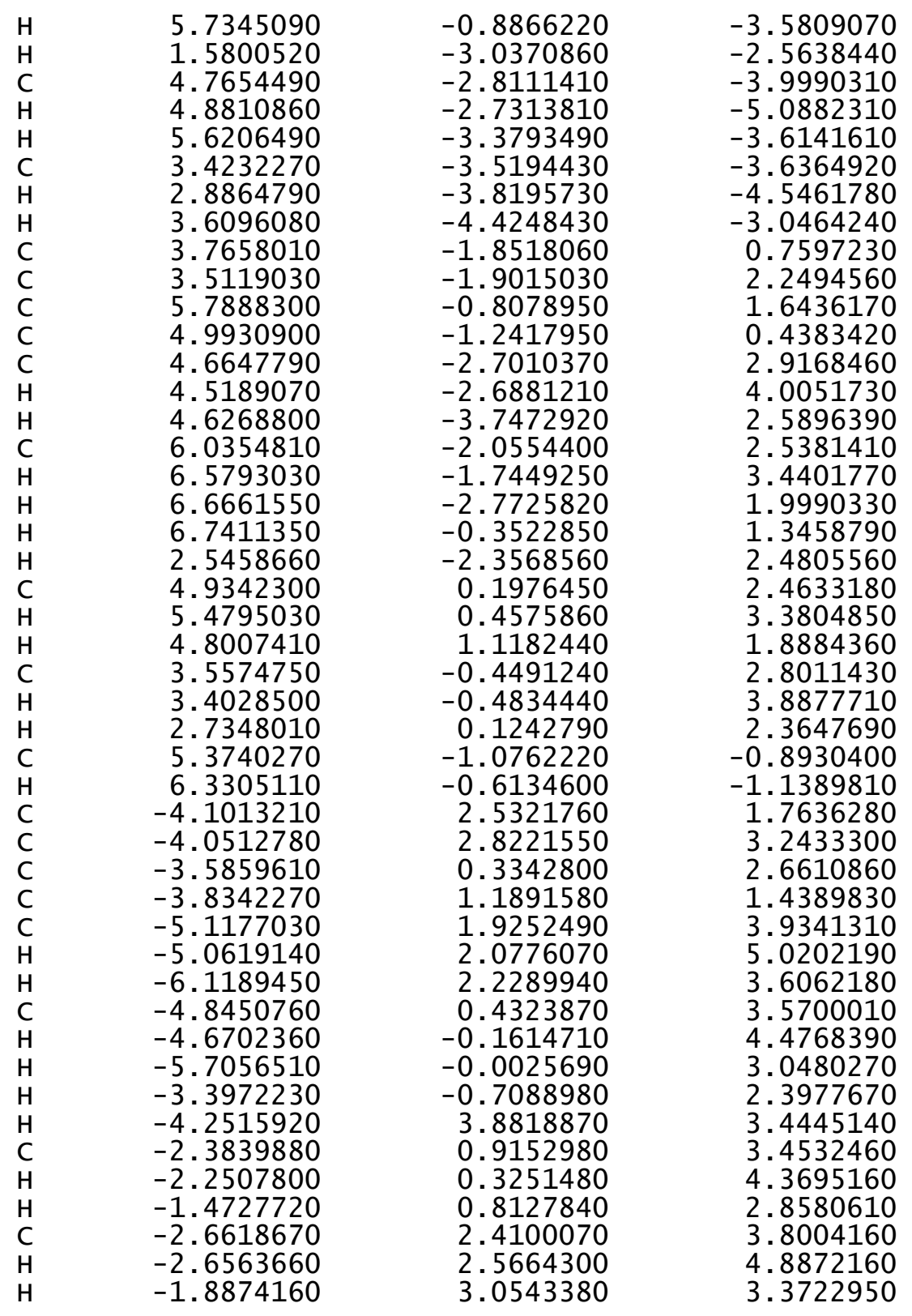

-4.5475770
-4.9855480
-4.4223730
-4.2629990
-3.8652830
-4.2202170
-2.9798870
-3.5195440
-3.6889580
-2.4696470
-4.1678490
-5.2170120
-5.8982470
-6.0235080
-6.5654810
-6.2412070
-6.5526060
-7.0653690
-4.4588040
-4.6929980
-4.3214940
-5.3259700
-5.4090140
-4.0222530
-2.8374880
-3.0782020
-5.0138990
-6.1310740
-3.9664460
-2.7126180
-6.3086120
-5.7921900
-3.8985590
-1.9060900
-0.7143790
0.5554970
-1.9561570
-1.7771480
-0.5055230
0.7206560
-2.6608570
-2.1890600
-2.8235780
-0.5767020
-0.3971690
1.6173400

0117850

3. 8564000

1.4059110

1.6745380

3.8555020

4.4101810

4. 3678120

2. 3836320

2. 2081500

1615510

0.3751150

4.8804230

1.7142720

1. 5686010

1. 0087290

3. 1844300

3. 7631620

3.2062350

3.4444170

. 4781590

$-2.7743200$

$-3.6261860$

$-5.0213220$

$-5.7190500$

$-4.7407090$

$-3.3605960$

$-3.7455900$

$-5.6489440$

$-6.5064640$

$-4.6200020$

$-3.1389280$

$-4.8873540$

$-6.2114180$

$-5.1727510$

$-4.8630060$

$-4.9228290$

$-6.3688170$

$-6.4897310$

$-6.1381080$

$-6.6785120$

$-4.2921310$

$-4.8450190$

$-5.7966610$

$-7.5027770$

$-6.0534010$
$-0.5525970$

$-1.7229850$

$-2.3719520$

$-0.8918180$

$-2.7977220$

$-3.6768490$

$-2.4083980$

$-3.1740600$

$-4.2447990$

$-2.9642780$

$-2.6278810$

$-1.4046010$

$-2.7505690$

3.8316420

$-2.2406760$

$-2.3493090$

$-3.2289620$

$-1.6264530$

0.7696480

1.0281220

$-1.2866470$

$-2.0231960$

$-1.3657370$

$-1.3134130$

$-1.5487110$

$-0.9821920$

$-3.0737560$

$-1.9028290$

$-2.0752470$

$-2.6361030$

$-2.0303380$

$-0.3456810$

$-0.3415040$

$-1.1738540$

0.9886960

1. 3034300

1. 6454680

2. 1208870

2. 9676690

2. 1163010

2.6937580

2. 5195830

0.9900320

3. 8191800

3. 3762750

2. 7445660 


\begin{tabular}{|c|c|c|c|c|c|c|c|}
\hline$H$ & & & & & & & \\
\hline${ }_{H}^{H}$ & $\begin{array}{r}0.9117610 \\
-1.6996230\end{array}$ & -6.9688400 & $\begin{array}{l}1.4176600 \\
1.493050\end{array}$ & $\mathrm{H}$ & -4.9290430 & -0.4386120 & -1.9033020 \\
\hline C & $-\frac{1}{3 .} 2295310$ & 2.9165670 & $\begin{array}{l}1.2493030 \\
0.5897140\end{array}$ & $\mathrm{C}$ & $\begin{array}{l}0.9491050 \\
2.2560760\end{array}$ & $\begin{array}{l}-1.8466830 \\
-2.3186080\end{array}$ & \\
\hline 0 & 2.8246780 & 1.8379540 & -0.0292160 & $\mathrm{c}$ & -0.0867300 & -2.6336370 & -1.1769200 \\
\hline C & 4.5932950 & 3.3450080 & 0.1781870 & $\mathrm{C}$ & 2.4976400 & -3.6612780 & -0.7978370 \\
\hline $\mathrm{C}$ & 5.4040220 & 2.4634550 & -0.5672630 & $\mathrm{H}$ & 3. 5079450 & -4.0512350 & -0.6875210 \\
\hline C & 5.1050490 & 4.6106120 & 0.5228540 & $\mathrm{C}$ & -4.0834580 & -0.1815810 & 0.6339220 \\
\hline C & 6.6913290 & 2.8387680 & -0.9466010 & $\mathrm{C}$ & 3.3595860 & -1.4271060 & -0.0356940 \\
\hline H & 5.0279180 & 1.4793590 & -0.8247380 & C & -4.4557620 & -0.9171970 & 1.7735160 \\
\hline $\mathrm{C}$ & 6.3907250 & 4.9831780 & 0.1359660 & $\mathrm{c}$ & -4.1764140 & -2.3787500 & 2.0362560 \\
\hline $\mathrm{H}$ & 4.4841380 & 5.2953810 & 1.0933280 & $\mathrm{C}$ & -5.5242820 & -1.2431900 & 3.9450000 \\
\hline C & 7.1896050 & 4.0987170 & -0.5984900 & C & -5.2010880 & -0.3120750 & 2.8033560 \\
\hline $\mathrm{H}$ & 7.3081750 & 2.1439650 & -1.5124250 & $\mathrm{C}$ & -3.3898500 & -2.4990500 & 3.3707490 \\
\hline $\mathrm{H}$ & 6.7713110 & 5.9654690 & 0.4063760 & $\mathrm{H}$ & -3.2470740 & -3.5641410 & 3.5975140 \\
\hline $\mathrm{H}$ & 8.1944910 & 4.3905640 & -0.8963130 & $\mathrm{H}$ & -2.4006290 & -2.0486280 & 3.2499260 \\
\hline $\mathrm{H}$ & 1.9491910 & 1.3762240 & 0.3238000 & C & -4.1883650 & -1.7972200 & 4.5133400 \\
\hline $\mathrm{S}$ & 2.3216350 & 3.7437560 & 1.7392500 & $\mathrm{H}$ & -4.4105870 & -2.5025530 & 5.3253410 \\
\hline C & 0.2338850 & 3.7724380 & -0.8501470 & $\mathrm{H}$ & -3.6050810 & -0.9723330 & 4.9397060 \\
\hline $\mathrm{C}$ & -0.2135380 & 3.5799930 & 0.5336860 & $\mathrm{H}$ & -6.0926620 & -0.7257480 & 4.7275320 \\
\hline $\mathrm{H}$ & 0.9727340 & 3.0613700 & -1.2214200 & $\mathrm{H}$ & -3.6121300 & -2.8395570 & 1.2218420 \\
\hline $\mathrm{H}$ & -0.1392080 & 2.5859650 & 0.9562990 & C & -6.3374160 & -2.4422910 & 3.3815260 \\
\hline 0 & -1.1290050 & 3.2645370 & -1.0218690 & $\mathrm{H}$ & -6.5171190 & -3.1624290 & 4.1910540 \\
\hline H & -1.0157360 & 2.2103070 & -1.1707110 & $\mathrm{H}$ & -7.3135020 & -2.0887940 & 3.0284090 \\
\hline C & -0.6080410 & 6.1614380 & -0.7489480 & C & -5.5372060 & -3.1071600 & 2.2185500 \\
\hline $\mathrm{C}$ & -0.5675450 & 6.0810620 & 0.7847980 & $\mathrm{H}$ & -5.3435860 & -4.1657210 & 2.4371640 \\
\hline $\mathrm{C}$ & -0.9075370 & 4.6667900 & 1.2882360 & $\mathrm{H}$ & -6.1041280 & -3.0590080 & 1.2810150 \\
\hline C & 0.4074130 & 5.1852900 & -1.3669060 & C & -4.4550990 & 1.1739950 & 0.5606550 \\
\hline $\mathrm{H}$ & -0.3812150 & 7.1816770 & -1.0807080 & $\mathrm{c}$ & -4.0888580 & 2.1557570 & -0.5300330 \\
\hline $\mathrm{H}$ & -1.2678080 & 6.7984520 & 1.2289670 & $\mathrm{C}$ & -5.5640090 & 3.2096950 & 1.3311000 \\
\hline $\mathrm{H}$ & -1.9863200 & 4.4665270 & 1.2034900 & $\mathrm{C}$ & -5.2206910 & 1.7629260 & 1.5850130 \\
\hline $\mathrm{H}$ & 1.4298510 & 5.4965090 & -1.1091770 & C & -5.3985050 & 2.7056860 & 579500 \\
\hline H & 0.3323630 & 5.1696490 & -2.4615150 & $\mathrm{H}$ & -5.1390170 & 3.4791530 & -1.8933270 \\
\hline H & -1.6159510 & 5.9139350 & -1.1081940 & $\mathrm{H}$ & -5.9205980 & 1.9023810 & -1.6918800 \\
\hline H & 0.4411040 & 6.3479510 & 1.1293630 & C & -6.3053570 & 3.2992220 & -0.0332690 \\
\hline H & -0.6621960 & 4.5485240 & 2.3497580 & $\mathrm{H}$ & -6.5444750 & 4.3503550 & -0.2427540 \\
\hline 15 & & & & $\mathrm{H}$ & -7.2517530 & 2.7490010 & 0.0311330 \\
\hline & , S) G[B97-D/ & [ZVP] & 325 & $\mathrm{H}$ & -6.1904530 & 3.6140040 & 2.1355820 \\
\hline$P$ & -0.2574830 & -0.2853860 & 1.0590530 & $\mathrm{H}$ & -3.4586900 & 1.6946830 & -1.2949350 \\
\hline 0 & 0.4576810 & -0.6916110 & 2.3202680 & C & -4.2426400 & 4.0149310 & 1.2055770 \\
\hline 0 & -0.7239190 & 1.1412380 & 0.9416750 & $\mathrm{H}$ & -4.4832030 & 5.0513160 & 0.9327170 \\
\hline 0 & -1.4625570 & -1.3546940 & 0.8853530 & $\mathrm{H}$ & -3.7314060 & 4.0363440 & 2.1753440 \\
\hline 0 & 0.6599100 & -0.5298520 & -0.2747890 & $\mathrm{C}$ & -3.3381230 & 3.3500150 & 0.1241570 \\
\hline C & -2.0959520 & -1.3998400 & -0.3615110 & $\mathrm{H}$ & -3.0726860 & 4.0716210 & -0.6594150 \\
\hline C & -1.4197560 & -2.0131130 & -1.4270310 & $\mathrm{H}$ & -2.4124020 & 2.9837550 & 0.5723920 \\
\hline C & -3.3644060 & -0.8257780 & -0.4958670 & $\mathrm{C}$ & -5.5931660 & 1.0232190 & 2.7071090 \\
\hline C & -3.9387220 & -0.8708400 & -1.7699660 & $\mathrm{H}$ & -6.1842730 & 1.4831900 & 3.5000790 \\
\hline
\end{tabular}




\begin{tabular}{|c|c|c|}
\hline $\begin{array}{l}.8584420 \\
.2348590 \\
.2612440 \\
.7873200 \\
.6257820 \\
.8677010 \\
.5239080 \\
.4344380 \\
.0766520 \\
.7443970 \\
.4319040 \\
.0697320 \\
.8040730 \\
.4284340 \\
.9750200 \\
.9969870 \\
.2589380 \\
.7255100 \\
.1054360 \\
.7289230 \\
.7637600 \\
.0340270 \\
.6259710 \\
.0658480 \\
.2510410 \\
.4661910 \\
.3670070 \\
.5109810 \\
.9290460 \\
.5542770 \\
.0655080 \\
.8990490 \\
.2957100 \\
.2402210 \\
.6344260 \\
.0643460 \\
.5177830 \\
.3554860 \\
.4747730 \\
.7031830 \\
.0949630 \\
.4117780 \\
.8733720 \\
.2376190 \\
.2360610\end{array}$ & $\begin{array}{r}0.4546600 \\
1.4840420 \\
-0.0412730 \\
-0.3705670 \\
0.7178840 \\
1.4470960 \\
0.1173500 \\
-0.1905160 \\
0.0973790 \\
-1.2411420 \\
-0.6944330 \\
2.1071560 \\
1.4388570 \\
1.6972830 \\
1.5490240 \\
2.3590190 \\
3.0316330 \\
2.9888350 \\
-0.8149030 \\
-1.2037540 \\
-2.7527740 \\
-1.6464050 \\
-1.1722170 \\
-1.4770410 \\
-0.1490100 \\
-2.1308570 \\
-2.9471730 \\
-1.6024210 \\
-3.3834900 \\
-0.5299040 \\
-3.5928230 \\
-4.3922270 \\
-4.0669930 \\
-2.6676420 \\
-2.9883910 \\
-2.7074780 \\
0.2341960 \\
0.8677780 \\
-4.5032150 \\
-5.9588610 \\
-6.2893270 \\
-5.9160680 \\
-4.9967800 \\
-3.9933820 \\
-6.5892430 \\
-7.3532380\end{array}$ & $\begin{array}{l}-0.4732120 \\
-1.5116800 \\
-2.2379810 \\
-0.8607000 \\
-2.8083490 \\
-3.5928770 \\
-2.6214200 \\
-3.2481150 \\
-4.2454750 \\
-3.2960400 \\
-2.5221430 \\
-1.1679550 \\
-2.2471180 \\
-3.2451770 \\
-1.5468430 \\
-1.8507370 \\
-2.6777510 \\
-0.9967370 \\
1.5585560 \\
2.8752720 \\
2.1693160 \\
1.1766480 \\
3.9687140 \\
4.9275830 \\
4.0833300 \\
3.5611780 \\
4.2889360 \\
3.5249500 \\
1.8522220 \\
3.1366890 \\
2.3011590 \\
3.0350250 \\
1.3393070 \\
2.7522770 \\
3.7256520 \\
2.0302060 \\
0.7372840 \\
1.0315430 \\
-1.2329030 \\
-1.5544090 \\
-2.9362700 \\
-3.0111770 \\
-1.8471290 \\
-1.4310260 \\
-0.7917750 \\
-3.1640280\end{array}$ \\
\hline
\end{tabular}

-1.0279270
-1.0728790
2.7745810
1.6531310
-0.6162350
-1.8182510
-1.9946900
-3.2768530
-1.1899970
-2.0488050
-3.0181260
-3.9785210
-1.3971160
-0.4870980
-0.5670080
-2.4415390
-3.5880630
-4.5782750
-4.6912250
-2.6236910
0.4226470
-0.3348160
0.5476160
1.5909100
-0.3748490
1.7123740
2.3103830
-0.2576040
-1.1825210
0.7879420
2.5316280
-0.9819560
0.8822650
-0.4575480
1.1910340
1.2351480
2.1994550
0.1903450
1.9322920
1.8955940
1.3033090
2.9734790
3.6970380
3.5828150
1.4783890
3.0851500
-6.8235410
-5.6350890
-6.1924090
-5.7200660
-5.4185360
-4.5088820
-1.9694610
-1.4084220
-2.3969240
-2.6710920
-1.5088610
-1.3838900
-2.8254660
-1.5790020
-3.2631780
-0.5780600
-1.6553760
-0.4674690
-2.2237230
-3.5982450
3.5900580
2.5307330
4.2421140
5.1432100
3.9315240
5.7146380
5.3759170
4.5154530
3.2360110
5.4055560
6.4000490
4.2738530
5.8545450
2.0044760
4.2005420
2.3023010
2.1671130
2.1561490
1.5426660
1.0277020
0.2696880
3.6365910
3.9123800
2.7170610
3.3717910
4.4906210
$-2.9907130$ $-0.9721590$ $-1.5345740$ $-3.6909320$ $-3.9666970$ $-2.1003750$ $-2.7178900$ $-2.8776080$ $-3.9353290$ $-5.1752520$ $-5.4155190$ $-4.2263410$ $-6.0450420$ $-4.1649560$ $-3.7096080$ $-5.5256220$ $-6.3421420$ $-4.3056110$ $-4.2635070$ $-5.0276310$ $-1.0576850$ $-1.0803440$ $-2.3903810$ $-2.6713480$ $-3.4108550$ $-3.9365380$ $-1.8922840$ $-4.6711730$ $-3.2065890$ $-4.9404150$ $-4.1413970$ $-5.4458600$ $-5.9268570$ $-0.1740750$ 0.3122060 3. 1101200 2. 0136800 2.8407550 1.1698410 3.4350700 2.9876000 4.4013040 3.0742900 2. 1137050 4.1538730 5.0798990 


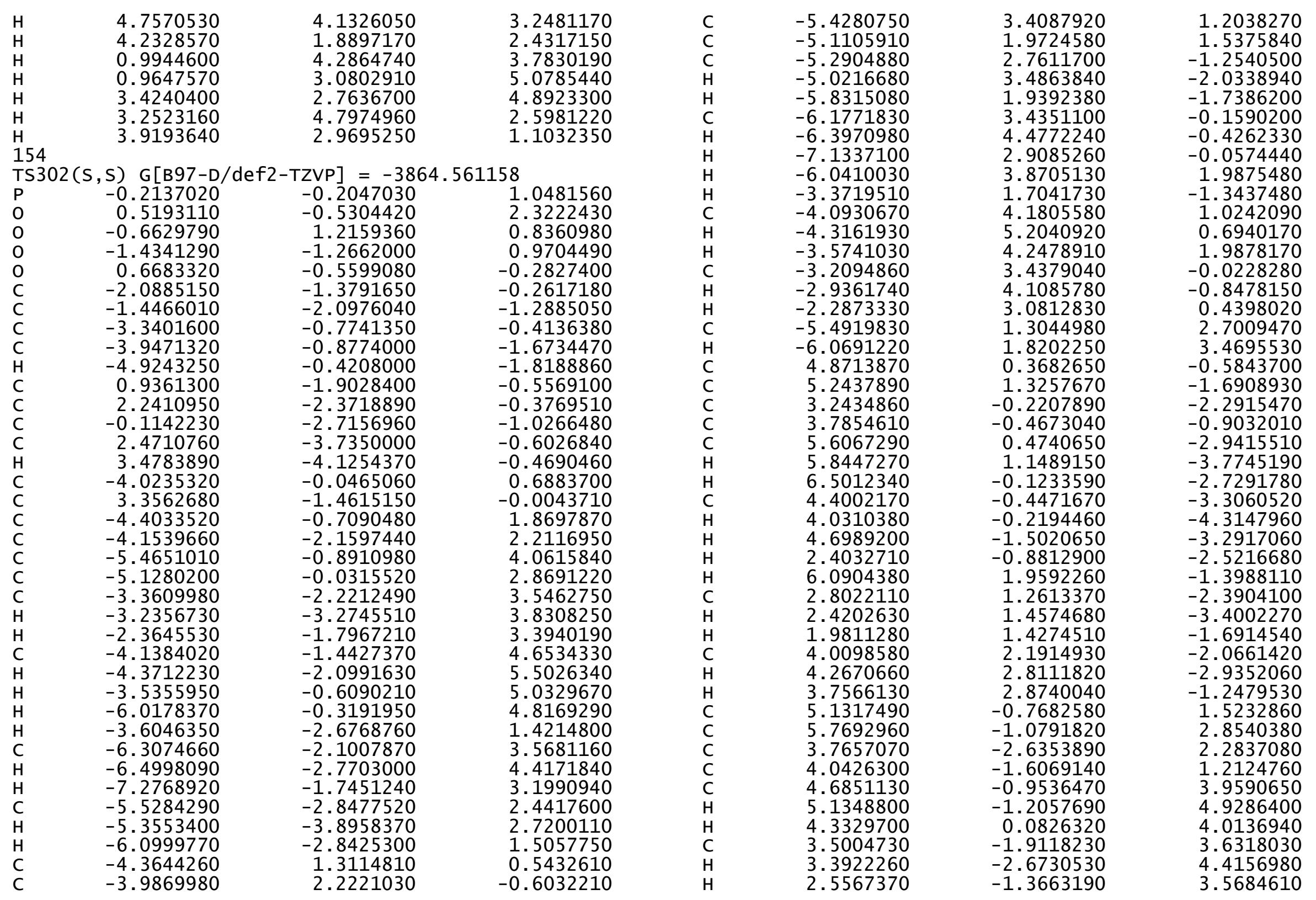




$\begin{array}{rrrr}\mathrm{H} & 2.9138220 & -3.2688710 & 2.0231680 \\ \mathrm{H} & 6.6108770 & -0.4053210 & 3.0568230 \\ \mathrm{C} & 5.0529120 & -3.4902750 & 2.4580680 \\ \mathrm{H} & 4.8821470 & -4.2298420 & 3.2513500 \\ \mathrm{H} & 5.2596230 & -4.0383940 & 1.5307790 \\ \mathrm{C} & 6.2519920 & -2.5578150 & 2.8238940 \\ \mathrm{H} & 6.6582470 & -2.8183220 & 3.8102470 \\ \mathrm{H} & 7.0620050 & -2.6632380 & 2.0924410 \\ \mathrm{C} & 5.5455850 & 0.2187530 & 0.6289100 \\ \mathrm{H} & 6.3961280 & 0.8583590 & 0.8682960 \\ \mathrm{C} & 1.4404380 & -4.5936840 & -0.9802730 \\ \mathrm{C} & 1.6525690 & -6.0713880 & -1.1918880 \\ \mathrm{C} & 1.0612840 & -6.5094530 & -2.5513200 \\ \mathrm{C} & -0.4132850 & -6.0441380 & -2.7285610 \\ \mathrm{C} & -0.8987070 & -5.1089400 & -1.5915730 \\ \mathrm{C} & 0.1455890 & -4.0879240 & -1.2091180 \\ \mathrm{H} & 1.1452310 & -6.6311400 & -0.3894240 \\ \mathrm{H} & 1.1336900 & -7.5996780 & -2.6499090 \\ \mathrm{H} & -1.0852640 & -6.9102950 & -2.7722440 \\ \mathrm{H} & -1.0997730 & -5.7280420 & -0.7029940 \\ \mathrm{H} & 2.7193070 & -6.3196590 & -1.1352800 \\ \mathrm{H} & 1.6808480 & -6.0723790 & -3.3446250 \\ \mathrm{H} & -0.5143080 & -5.5178180 & -3.6837080 \\ \mathrm{H} & -1.8451450 & -4.6365900 & -1.8653200 \\ \mathrm{C} & -3.3202300 & -1.5221220 & -2.7366060 \\ \mathrm{C} & -3.9344780 & -1.6063420 & -4.1113930 \\ \mathrm{C} & -3.8776990 & -3.0583010 & -4.6370340 \\ \mathrm{C} & -2.4434300 & -3.6564350 & -4.5510880 \\ \mathrm{C} & -1.4487290 & -2.7444600 & -3.7880610 \\ \mathrm{C} & -2.0665130 & -2.1379420 & -2.5521610 \\ \mathrm{H} & -3.3709840 & -0.9588850 & -4.8030640 \\ \mathrm{H} & -4.2455620 & -3.0911680 & -5.6700580 \\ \mathrm{H} & -2.0433120 & -3.8373650 & -5.5565260 \\ \mathrm{H} & -1.1646680 & -1.9157110 & -4.4561750 \\ \mathrm{H} & -4.9678090 & -1.2394060 & -4.0937830 \\ \mathrm{H} & -4.5637100 & -3.6634630 & -4.0310200 \\ \mathrm{H} & -2.4862400 & -4.6285730 & -4.0480450 \\ \mathrm{H} & -0.5304250 & -3.2889830 & -3.5584060 \\ \mathrm{C} & 0.4384900 & 3.5568520 & -1.3062810 \\ \mathrm{O} & -0.2975450 & 2.4831300 & -1.2678450 \\ \mathrm{C} & 0.4954970 & 4.1736490 & -2.6600620 \\ \mathrm{C} & 1.5269110 & 5.0603540 & -3.0198330 \\ \mathrm{C} & -0.4886040 & 3.8498060 & -3.6166090 \\ \mathrm{C} & 1.5754650 & 5.6072220 & -4.3007720 \\ \mathrm{H} & 2.2929930 & 5.3012170 & -2.2889870 \\ \mathrm{C} & -0.4444430 & 4.4104280 & -4.8922330\end{array}$

$\begin{array}{lrrr}\mathrm{H} & -1.2867950 & 3.1648430 & -3.3489860 \\ \mathrm{C} & 0.5886120 & 5.2877640 & -5.2401420 \\ \mathrm{H} & 2.3854430 & 6.2819390 & -4.5683380 \\ \mathrm{H} & -1.2160160 & 4.1612210 & -5.6173130 \\ \mathrm{H} & 0.6251980 & 5.7188460 & -6.2383610 \\ \mathrm{H} & -0.4021260 & 2.0039510 & -0.3332830 \\ \mathrm{~S} & 1.2452890 & 4.2272470 & 0.0121200 \\ \mathrm{C} & 1.3216780 & 2.5003670 & 2.9184960 \\ \mathrm{C} & 2.2724510 & 2.2980070 & 1.8208300 \\ \mathrm{H} & 0.2733020 & 2.3438460 & 2.6692490 \\ \mathrm{H} & 1.9906770 & 1.6267680 & 1.0188220 \\ \mathrm{O} & 1.9823490 & 1.2439310 & 3.3072890 \\ \mathrm{H} & 1.3800820 & 0.4638190 & 2.9141770 \\ \mathrm{C} & 3.0803910 & 3.8907180 & 4.1165980 \\ \mathrm{C} & 3.7918330 & 4.0896960 & 2.7691720 \\ \mathrm{C} & 3.6590880 & 2.8472600 & 1.8716900 \\ \mathrm{C} & 1.5811440 & 3.6235340 & 3.8998630 \\ \mathrm{H} & 3.2052820 & 4.7798050 & 4.7460560 \\ \mathrm{H} & 4.8551590 & 4.3107340 & 2.9201850 \\ \mathrm{H} & 4.3126220 & 2.0344820 & 2.2186710 \\ \mathrm{H} & 1.0978400 & 4.5201620 & 3.4867300 \\ \mathrm{H} & 1.0764480 & 3.3843860 & 4.8442770 \\ \mathrm{H} & 3.5302880 & 3.0429360 & 4.6504280 \\ \mathrm{H} & 3.3482650 & 4.9521990 & 2.2521840 \\ \mathrm{H} & 3.9788690 & 3.0505100 & 0.8446070 \\ \mathrm{H} & & & \end{array}$

TS303(S,S) G[B97-D/def2-TZVP $]=-3864.560128$

$\begin{array}{lrcr}\text { TS303(S, S) G[B97-D/def2-TZVP] }=-3864.560128 \\ \text { P } & 0.1643030 & 0.3375110 & 0.9392500 \\ \text { O } & -0.5803740 & 0.8601220 & 2.1394720 \\ \text { O } & 0.5790750 & -1.1093550 & 0.9396850 \\ \text { O } & 1.4121870 & 1.3490160 & 0.7382390 \\ \text { O } & -0.6894480 & 0.5210850 & -0.4438660 \\ \text { C } & 2.0876590 & 1.2911750 & -0.4858270 \\ \text { C } & 1.4755630 & 1.8836400 & -1.6062640 \\ \text { C } & 3.3357580 & 0.6630230 & -0.5339980 \\ \mathrm{C} & 3.9735020 & 0.6116280 & -1.7820350 \\ \text { H } & 4.9498770 & 0.1353650 & -1.8489060 \\ \mathrm{C} & -0.9267250 & 1.8181680 & -0.9041510 \\ \mathrm{C} & -2.2263680 & 2.3291000 & -0.8211230 \\ \mathrm{C} & 0.1485760 & 2.5433650 & -1.4527640 \\ \mathrm{C} & -2.4252000 & 3.6529190 & -1.2352570 \\ \mathrm{H} & -3.4262670 & 4.0760400 & -1.1726830 \\ \mathrm{C} & 3.9859010 & 0.0805310 & 0.6696650 \\ \mathrm{C} & -3.3624520 & 1.4879020 & -0.3589310 \\ \mathrm{C} & 4.3479280 & 0.8946070 & 1.7584520 \\ \mathrm{C} & 4.1306360 & 2.3848820 & 1.8826210\end{array}$




$\begin{array}{rrr}5.3595800 & 1.3644950 & 3.9309890 \\ 5.0256100 & 0.3500260 & 2.8655590 \\ 3.3074050 & 2.6587850 & 3.1712110 \\ 3.2012080 & 3.7450170 & 3.2938210 \\ 2.3054050 & 2.2358590 & 3.0570670 \\ 4.0402140 & 2.0373040 & 4.4008950 \\ 4.2806500 & 2.8098260 & 5.1437080 \\ 3.4047420 & 1.2903530 & 4.8913140 \\ 5.8763950 & 0.8939770 & 4.7763370 \\ 3.6159770 & 2.7960540 & 1.0108370 \\ 6.2522150 & 2.4634680 & 3.2886760 \\ 6.4537950 & 3.2413210 & 4.0373710 \\ 7.2143370 & 2.0268590 & 2.9953130 \\ 5.5178680 & 3.0650920 & 2.0502610 \\ 5.3674580 & 4.1454930 & 2.1770500 \\ 6.1093960 & 2.9167610 & 1.1387960 \\ 4.3042180 & -1.2889890 & 0.7229620 \\ 3.9573310 & -2.3402810 & -0.3073860 \\ 5.3027560 & -3.2921800 & 1.7021100 \\ 5.0019350 & -1.8192840 & 1.8251410 \\ 5.2784110 & -2.9737950 & -0.8232810 \\ 5.0315680 & -3.7884680 & -1.5175030 \\ 5.8552060 & -2.2259600 & -1.3811260 \\ 6.1037380 & -3.5148170 & 0.3874610 \\ 6.3056240 & -4.5878580 & 0.2696680 \\ 7.0695430 & -3.0003010 & 0.4581610 \\ 5.8753620 & -3.6515550 & 2.5658620 \\ 3.3810020 & -1.9193210 & -1.1354840 \\ 3.9613110 & -4.0617470 & 1.5726640 \\ 4.1767600 & -5.1248170 & 1.3996000 \\ 3.4006860 & -3.9839100 & 2.5117390 \\ 3.1381210 & -3.4612250 & 0.3932680 \\ 2.8960070 & -4.2351060 & -0.3466390 \\ 2.1996510 & -3.0411000 & -7585990 \\ 5.3602810 & -1.0037410 & 2.8981680 \\ 5.9002460 & -1.4183690 & -3.7504050 \\ -4.8681080 & -0.4050110 & -0.7244370 \\ -5.2124840 & -1.5071860 & -1.6959970 \\ -3.1875100 & -0.0629650 & -2.4462810 \\ -3.7695150 & 0.3775930 & -1.1230980 \\ -5.5340420 & -0.8411710 & -3.0647430 \\ -5.7491630 & -1.6262400 & -3.9616550 \\ -6.4324200 & -0.2202150 & \\ -4.3131320 & 0.0207680 & -3162700 \\ -3.9154870 & -0.3434030 & \\ -4.6080770 & 1.0675710 & \\ 5 & & \\ 5 & & -3556330\end{array}$

-2.3371620
-6.0705080
-2.7515130
-2.3431300
-1.9506440
-3.9727380
-4.2072610
-3.7475510
-5.1809120
-5.8485630
-3.8334080
-4.0826390
-4.7879870
-5.2581600
-4.4335010
-3.5981950
-3.5117660
-2.6519680
-2.9775310
-6.6932180
-5.1253060
-4.9721100
-5.3121480
-6.3314430
-6.7582930
-7.1258080
-5.5761050
-6.4346100
-0.0804400
1.0031820
0.9595000
-0.4653980
-1.5283070
-1.3712650
0.8447060
1.6747930
-0.4652330
-1.3876300
1.9902500
1.3031520
-0.7635920
-2.5389900
3.3812060
4.0394290
4.0048660
2.5655460

0.5564390 $-2.0923310$

$-1.5455840$

$-1.8821360$

$-1.6148780$

$-2.4192340$

$-3.1577460$

$-2.9750920$

1.0083750

1. 4886120

2.9629550

1.7986390

1. 5110190

1.8827550

0.4921790

2. 4252570

3. 2840510

1.8810450

3. 5615840

0.8428080

3. 8273850

4. 6661350

4. 2471930

2. 9483230

3. 3349000

2.9542590

$-0.0895140$

$-0.6902360$

3. 8804210

4.8249760

6. 1277230

6.7552260

5.8809760

4.4322120

5.0791440

6.8447090

7.7548950

5.9840440

4.3607210

5. 8885410

6.8757380

6.2379050

1.1375710

1.0773740

2. 4714630

3. 0624170
$-2.7428800$

$-1.3426540$

$-2.3229720$

$-3.2845070$

$-1.5858090$

$-1.9054830$

$-2.6833170$

$-0.9887170$

1. 1994090

2.4628010

1.7370810

0.8063170

3. 5978870

4. 5180730

3. 7901060

3. 1752770

3.8542300

3. 2084070

1.4138260

2.7334220

1.7687090

2.4603100

0.7728160

2. 2292370

3. 1642190

1.4735530

0.4360580

0.7390690

$-1.8256270$

$-2.2828230$

$-1.4469950$

$-1.3810120$

$-2.0939160$

$-1.7095950$

$-3.3421370$

$-1.8688050$

$-1.8331730$

$-3.1815560$

$-2.2087890$

$-0.4332530$

$-0.3324370$

$-1.8627340$

$-2.9278850$

$-4.2836170$

$-4.9514430$

$-4.9955830$ 


\begin{tabular}{|c|c|c|c|c|c|c|c|}
\hline C & 1.5484940 & 2.2362790 & -4.1658650 & 0 & -0.6682040 & 0.8528320 & -1.4188390 \\
\hline C & 2.1275220 & 1.7757710 & -2.8496300 & 0 & 0.8263870 & 0.5350910 & 0.6776850 \\
\hline $\mathrm{H}$ & 3.4957720 & 0.3651400 & -4.9257980 & 0 & 0.2453590 & -1.4090700 & -0.9735820 \\
\hline $\mathrm{H}$ & 4.4242350 & 2.4080600 & -5.9632720 & 0 & -1.4271230 & -0.4803910 & 0.6832560 \\
\hline $\mathrm{H}$ & 2.2051750 & 3.1117420 & -6.0307440 & C & 0.3192000 & -2.5560530 & -0.1775810 \\
\hline $\mathrm{H}$ & 1.2932600 & 1.3337850 & -4.7438350 & $\mathrm{C}$ & -0.8839670 & -3.1813120 & 0.2137150 \\
\hline $\mathrm{H}$ & 5.0703130 & 0.7137190 & -4.1954550 & $\mathrm{C}$ & 1.5837580 & -3.0268900 & 0.1914670 \\
\hline $\mathrm{H}$ & 4.6577180 & 3.1369450 & -4.3724630 & $\mathrm{C}$ & 1.6269720 & -4.1836510 & 0.9825170 \\
\hline $\mathrm{H}$ & 2.5802330 & 4.0889450 & -4.6125580 & $\mathrm{H}$ & 2.5979260 & -4.5766680 & 1.2778280 \\
\hline $\mathrm{H}$ & 0.6203160 & 2.7957900 & -4.0333150 & C & -2.4420480 & -1.2303020 & 0.0846270 \\
\hline C & -0.4789780 & -3.7660390 & -0.8199290 & $\mathrm{C}$ & -3.6538010 & -0.5912770 & -0.2033300 \\
\hline 0 & 0.2412060 & -2.6881250 & -0.9504160 & $\mathrm{C}$ & -2.1914930 & -2.5878350 & -0.1963430 \\
\hline C & -0.4345420 & -4.6445000 & -2.0209720 & $\mathrm{C}$ & -4.6440070 & -1.3612990 & -0.8285570 \\
\hline C & -1.4343830 & -5.6032610 & -2.2678220 & $\mathrm{H}$ & -5.6025090 & -0.8993460 & -1.0565100 \\
\hline C & 0.6235730 & -4.5125920 & -2.9437640 & C & 2.8475130 & -2.3340340 & -0.1775260 \\
\hline $\mathrm{C}$ & -1.3804100 & -6.4064260 & -3.4052720 & C & -3.8786700 & 0.8419840 & 0.1239710 \\
\hline $\mathrm{H}$ & -2.2560360 & -5.7028390 & -1.5649100 & $\mathrm{C}$ & 3.2426660 & -2.1819720 & -1.5178440 \\
\hline C & 0.6814530 & -5.3281710 & -4.0729670 & C & 2.4799830 & -2.6343010 & -2.7403700 \\
\hline $\mathrm{H}$ & 1.4009720 & -3.7773690 & -2.7621850 & $\mathrm{C}$ & 4.7557440 & -1.5209300 & -3.3171740 \\
\hline C & -0.3209290 & -6.2749760 & -4.3100990 & $\mathrm{C}$ & 4.4830990 & -1.5969030 & -1.8360930 \\
\hline $\mathrm{H}$ & -2.1669970 & -7.1349900 & -3.5879850 & $\mathrm{C}$ & 2.2396450 & -1.4015120 & -3.6553420 \\
\hline $\mathrm{H}$ & 1.5100540 & -5.2244780 & -4.7700170 & $\mathrm{H}$ & 1.7495820 & -1.7412750 & -4.5775410 \\
\hline $\mathrm{H}$ & -0.2772500 & -6.9059800 & -5.1952400 & $\mathrm{H}$ & 1.5606150 & -0.7057690 & -3.1546920 \\
\hline $\mathrm{H}$ & 0.3179800 & -2.0575980 & -0.1083120 & C & 3.6036330 & -0.7162550 & -3.9801720 \\
\hline $\mathrm{S}$ & -1.3677930 & -4.1786080 & 0.5487400 & $\mathrm{H}$ & 3.7728580 & -0.6768610 & -5.0646680 \\
\hline C & -1.4561940 & -2.0370080 & 3.1544290 & $\mathrm{H}$ & 3.6169890 & 0.3135400 & -3.6031350 \\
\hline $\mathrm{C}$ & -2.3789360 & -1.9654310 & 2.0174850 & $\mathrm{H}$ & 5.7243670 & -1.0459040 & -3.5166140 \\
\hline $\mathrm{H}$ & -0.3988630 & -1.9431440 & 2.9106480 & $\mathrm{H}$ & 1.5279600 & -3.1009290 & -2.4763100 \\
\hline $\mathrm{H}$ & -2.0595930 & -1.4281680 & 1.1330220 & C & 4.7266020 & -2.9636430 & -3.8940890 \\
\hline 0 & -2.0889860 & -0.7220460 & 3.3442390 & $\mathrm{H}$ & 4.8503370 & -2.9133980 & -4.9841800 \\
\hline $\mathrm{H}$ & -1.4610450 & -0.0179820 & 2.8526000 & $\mathrm{H}$ & 5.5698500 & -3.5360420 & -3.4895220 \\
\hline C & -3.2805620 & -3.1953240 & 4.4922940 & C & 3.3705560 & -3.6402020 & -3.5223200 \\
\hline C & -3.9720590 & -3.5620920 & 3.1703980 & $\mathrm{H}$ & 2.8349020 & -3.9554790 & -4.4274840 \\
\hline C & -3.7818010 & -2.4650890 & 2.1092050 & $\mathrm{H}$ & 3.5382660 & -4.5325200 & -2.9072390 \\
\hline C & -1.7696420 & -3.0050390 & 4.2755410 & C & 3.7077330 & -1.8593040 & 0.8318550 \\
\hline $\mathrm{H}$ & -3.4456650 & -3.9824800 & 5.2375780 & $\mathrm{C}$ & 3.4435050 & -1.8638500 & 2.3203890 \\
\hline $\mathrm{H}$ & -5.0447030 & -3.7278810 & 3.3264790 & $\mathrm{C}$ & 5.7534100 & -0.8493410 & 1.7035130 \\
\hline $\mathrm{H}$ & -4.4188510 & -1.5941350 & 2.3179540 & $\mathrm{C}$ & 4.9513160 & -1.2874390 & 0.5038320 \\
\hline $\mathrm{H}$ & -1.3084890 & -3.9641070 & 4.0000120 & C & 4.5694900 & -2.6782080 & 3.0156330 \\
\hline $\mathrm{H}$ & -1.2751480 & -2.6534320 & 5.1897110 & $\mathrm{H}$ & 4.4207470 & -2.6279690 & 4.1024830 \\
\hline $\mathrm{H}$ & -3.7128190 & -2.2677490 & 4.8910620 & $\mathrm{H}$ & 4.5005860 & -3.7321860 & 2.7199440 \\
\hline $\mathrm{H}$ & -3.5475290 & -4.5022400 & 2.7911530 & C & 5.9604470 & -2.0861320 & 2.6233560 \\
\hline $\mathrm{H}$ & -4.0823240 & -2.8032180 & 1.1118980 & $\mathrm{H}$ & 6.5154110 & -1.7757610 & 3.5186470 \\
\hline & & & & II & & -2.8343460 & 2.0981680 \\
\hline & -0.2013340 & -0.0151400 & -0.2760840 & $\begin{array}{l}\mathrm{H} \\
\mathrm{H}\end{array}$ & $\begin{array}{l}6.7197000 \\
2.4634370\end{array}$ & $\begin{array}{l}-0.4277690 \\
-2.2848550\end{array}$ & $\begin{array}{l}1.4008060 \\
2.5569690\end{array}$ \\
\hline
\end{tabular}




$\begin{array}{rrr}4.9264620 & 0.1959340 & 2.5009890 \\ 5.4734130 & 0.4506990 & 3.4186710 \\ 4.8286080 & 1.1129330 & 1.9132670 \\ 3.5269260 & -0.4004970 & 2.8378940 \\ 3.3599750 & -0.4041660 & 3.9232350 \\ 2.7265860 & 0.1874720 & 2.3798970 \\ 5.3453060 & -1.1669590 & -0.8284910 \\ 6.3142070 & -0.7338040 & -1.0795840 \\ -4.0236870 & 2.6535760 & 1.7626800 \\ -3.9582550 & 2.9595200 & 3.2386300 \\ -3.5508090 & 0.4556340 & 2.6831870 \\ -3.7881730 & 1.3011570 & 1.4526350 \\ -5.0403180 & 2.0945510 & 3.9456840 \\ -4.9759740 & 2.2590460 & 5.0295190 \\ -6.0363210 & 2.4158540 & 3.6185660 \\ -4.8015490 & 0.5918730 & 3.5989290 \\ -4.6329100 & 0.0060930 & 4.5121440 \\ -5.6745280 & 0.1683360 & 3.0882690 \\ -3.3870750 & -0.5944100 & 2.4311800 \\ -4.1339930 & 4.0256380 & 3.4286480 \\ -2.3310870 & 1.0192020 & 3.4605060 \\ -2.2046930 & 0.4370180 & 4.3827800 \\ -1.4262270 & 0.8898000 & 2.8607540 \\ -2.5748630 & 2.5234440 & 3.7921330 \\ -2.5590700 & 2.6919980 & 4.8770070 \\ -1.7895380 & 3.1461370 & 3.3518920 \\ -4.4746460 & 3.1150230 & -0.5562360 \\ -4.9024120 & 3.9544430 & -1.7340220 \\ -4.4001000 & 1.4838140 & -2.3559900 \\ -4.2216290 & 1.7677860 & -0.8809610 \\ -3.7896030 & 3.9145940 & -2.8157740 \\ -4.1356100 & 4.4695770 & -3.6982560 \\ -2.8891800 & 4.4077030 & -2.4363560 \\ -3.4839170 & 2.4305910 & -3.1794850 \\ -3.6709230 & 2.2474610 & -4.2459950 \\ -2.4371960 & 2.1853790 & -2.9802490 \\ -4.1708380 & 0.4442010 & -2.6002360 \\ -5.1082660 & 4.9873880 & -1.4270470 \\ -5.8719430 & 1.8200570 & -2.7258360 \\ -6.0106890 & 1.6615640 & -3.8034210 \\ -6.5503000 & 1.1376000 & -2.1994890 \\ -6.1775820 & 3.3035930 & -2.3431890 \\ -6.4807040 & 3.8772530 & -3.2290520 \\ -6.9965640 & 3.3550630 & -1.6159770 \\ -4.3680280 & 3.5614490 & 0.7601040 \\ -4.5790220 & 4.6028530 & 1.0076180\end{array}$

-4.4158480
-5.4493220
-5.6121560
-4.2499670
-3.0287350
-3.1910060
-5.1258780
-6.3067480
-4.2074210
-2.9399410
-6.4082590
-6.0705450
-4.1600470
-2.1073970
0.4664060
0.4960360
-0.4685740
-1.9003350
-1.9801360
-0.7965390
0.1798120
-0.5004440
-2.6088080
-1.9709900
1.5166640
-0.0607400
-2.2372820
-2.9285740
3.2982130
2.8702240
4.6639030
5.4459060
5.2060840
6.7351880
5.0458900
6.4936060
4.6075290
7.2641380
7.3297050
6.8977800
8.2706620
1.9847960
2.4160040
0.3271580
-0.1226530
1.0478600
$-1.1898230$

$-1.9118300$

$-1.2238950$

$-1.0432830$

$-1.3816380$

$-0.8776440$

$-2.9531500$

$-1.8009020$

$-1.6794350$

$-2.4783060$

$-1.9503420$

$-0.2411390$

$-0.0080580$

$-1.0148560$

1. 4291540

2. 3221750

1.7869820

1. 5486840

1.6871530

1.0572340

3. 3389850

2. 4841300

2.2581750

2.7609560

2.4001620

0.8403990

0.5454200

1. 2930200

0.5305380

$-0.0699730$

0.0994610

$-0.6595050$

0.4393070

$-1.0559370$

$-0.9132130$

0.0355320

1.0205790

$-0.7118530$

$-1.6319140$

0.3032150

$-1.0228090$

0.2922680

1. 6798210

$-0.9088420$

0.4781490 


$\begin{array}{lrrr}\text { H } & -0.0721670 & 2.5957550 & 0.9119860 \\ \mathrm{O} & -1.0479830 & 3.2747400 & -1.0727970 \\ \mathrm{H} & -0.9625030 & 2.2164300 & -1.2071290 \\ \mathrm{C} & -0.4506280 & 6.1628050 & -0.8325170 \\ \mathrm{C} & -0.4057920 & 6.0976060 & 0.7018780 \\ \mathrm{C} & -0.7863830 & 4.6997040 & 1.2210290 \\ \mathrm{C} & 0.5348620 & 5.1523940 & -1.4439090 \\ \mathrm{H} & -0.1971470 & 7.1727360 & -1.1763230 \\ \mathrm{H} & -1.0815710 & 6.8408970 & 1.1413560 \\ \mathrm{H} & -1.8704820 & 4.5291010 & 1.1388230 \\ \mathrm{H} & 1.5665830 & 5.4398970 & -1.1957670 \\ \mathrm{H} & 0.4532160 & 5.1251730 & -2.5378200 \\ \mathrm{H} & -1.4666360 & 5.9396630 & -1.1847690 \\ \mathrm{H} & 0.6122820 & 6.3356300 & 1.0395920 \\ \mathrm{H} & -0.5438510 & 4.5860390 & 2.2836510\end{array}$


M06-2X/6-31G(d) Optimized Cartesion Coordinates $(\AA)$ and absolute solution phase free energies $(G)$ in hartrees. Reported free energies were computed using the RRHO model at the M06-2X/6$31 \mathrm{G}(\mathrm{d})$ level of theory. Free energies computed with the QuasiRRHO model and/or at the M06-2X/6-311+G(d,p)//M06-2X/6$31 \mathrm{G}(\mathrm{d})$ level are given in the appropriate tables above.

\begin{tabular}{|c|c|c|c|}
\hline & $\mathrm{G}[\mathrm{M} 06-2 \mathrm{X} / 6$ & d) $]=-4124$. & \\
\hline 0 & -0.2116360 & -1.0773300 & -0.0034320 \\
\hline 0 & 1.7109760 & -0.2359410 & -1.3908550 \\
\hline C & -0.1208240 & -2.3204350 & -0.6049450 \\
\hline C & 1.1242830 & -2.8949730 & -0.7628080 \\
\hline C & -1.3364240 & -2.9522830 & -0.9826750 \\
\hline C & 1.2113170 & -4.1349700 & -1.4771300 \\
\hline C & -1.2439260 & -4.1833030 & -1.5840980 \\
\hline C & 0.0089820 & -4.7847730 & -1.8736190 \\
\hline $\mathrm{H}$ & -2.1512360 & -4.7164950 & -1.8561390 \\
\hline C & 2.5381490 & -0.8747090 & -0.4873170 \\
\hline C & 3.6347830 & -0.1319690 & 0.0303660 \\
\hline C & 2.3138540 & -2.2050040 & -0.1939780 \\
\hline C & 4.5246590 & -0.7919000 & 0.8406320 \\
\hline C & 3.2018140 & -2.8602620 & 0.7255570 \\
\hline $\mathrm{C}$ & 4.3281560 & -2.1452650 & 1.2222920 \\
\hline $\mathrm{H}$ & 5.3992800 & -0.2650410 & 1.2140220 \\
\hline $\mathrm{C}$ & 4.9864320 & -4.0621510 & 2.5586580 \\
\hline C & 3.8471840 & -4.7634550 & 2.0960450 \\
\hline C & 2.9788250 & -4.1806250 & 1.2068160 \\
\hline $\mathrm{C}$ & 5.2155540 & -2.7794400 & 2.1321720 \\
\hline $\mathrm{H}$ & 5.6658830 & -4.5369650 & 3.2590570 \\
\hline $\mathrm{H}$ & 3.6 & -5.7689870 & 2.4557960 \\
\hline $\mathrm{H}$ & 2.09 & -4.72 & 0.8794240 \\
\hline $\mathrm{H}$ & 6.0 & 3690 & 2.4941210 \\
\hline $\mathrm{C}$ & 70 & -5.9 & 1570 \\
\hline $\mathrm{C}$ & 1.2 & 1370 & 50410 \\
\hline C & 0.0812880 & -6.0217130 & 585160 \\
\hline C & 519770 & -4.7269030 & -1.8391840 \\
\hline $\mathrm{H}$ & 3.4468940 & -6.3472670 & -2.8002850 \\
\hline $\mathrm{H}$ & 1880 & -7.5212710 & -3.4216600 \\
\hline $\mathrm{H}$ & -0.8464600 & -6.5100200 & -2.8545410 \\
\hline $\mathrm{H}$ & 3.3754170 & -4.2206940 & -1.5777500 \\
\hline $\mathrm{C}$ & 340 & -2.2850860 & -0.6976700 \\
\hline C & & & \\
\hline
\end{tabular}

$$
\begin{array}{r}
-3.1104220 \\
-4.6171090 \\
-4.3263930 \\
-5.0940290 \\
-5.2088440 \\
-4.6974780 \\
3.8298850 \\
3.4405550 \\
4.4234600 \\
3.7049980 \\
4.6623030 \\
4.3189220 \\
3.4125390 \\
5.1297080 \\
-2.3430960 \\
-6.4161890 \\
-2.9717390 \\
2.7451520 \\
4.5800410 \\
4.8251850 \\
6.3499520 \\
6.7424070 \\
6.8303420 \\
6.6364270 \\
4.1451790 \\
3.0576230 \\
4.4710770 \\
4.3976950 \\
5.5208050 \\
5.7375880 \\
5.0648410 \\
6.4670310 \\
3.2689520 \\
2.5981020 \\
2.7474460 \\
3.4652820 \\
3.7503880 \\
4.4726670 \\
4.3061970 \\
3.2250530 \\
1.6899450 \\
2.1504990 \\
1.0094710 \\
1.1049570 \\
-2.5331060 \\
-3.5922680
\end{array}
$$

0.6340520

$-1.4446660$

0.8831560

$-0.1386630$

$-2.2540450$

1.9018060

$-0.3370200$

0.5581790

$-1.5673910$

0.2146660

$-1.8693450$

$-0.9915040$

0.9011950

$-2.8183380$

1.7771160

0.1709410

$-3.2047800$

1.8745690

$-1.3424760$

$-2.5763080$

$-2.7260640$

$-3.1068740$

$-1.7634030$

$-3.4280870$

$-3.9311930$

$-3.8183090$

$-4.3892780$

$-4.6209920$

$-0.3299560$

$-0.6246940$

0.6659800

$-0.2548500$

$-1.4586550$

$-2.1919660$

$-0.4954350$

$-1.7650090$

3. 0224270

2. 8241650

3. 1750910

3.9541360

2. 2604900

2. 5719180

1. 4280580

3.1102000

1.7470960

1.8870860 


$\begin{array}{rrr}-2.2047570 & -4.8244390 & 0.8008540 \\ -1.9658530 & -4.8578450 & 2.5575330 \\ -2.7288960 & -2.3442830 & 3.1643710 \\ -2.7134760 & -1.2513750 & 3.2164230 \\ -3.7286460 & -2.6884570 & 3.4524200 \\ -2.0219580 & -2.7285770 & 3.9067340 \\ -7.5871460 & -1.0714190 & -0.4946660 \\ -8.5382070 & -0.6003470 & -0.2259210 \\ -7.4932680 & -1.0405620 & -1.5858900 \\ -7.6222250 & -2.1210470 & -0.1881330 \\ -6.3969170 & 1.1362880 & -0.2393780 \\ -5.5590790 & 1.6656590 & 0.2284910 \\ -6.2956540 & 1.2309750 & -1.3266240 \\ -7.3275730 & 1.6328180 & 0.0543570 \\ -3.2067280 & -0.5589800 & -4.0094250 \\ -4.2740000 & -0.3443960 & -4.1332480 \\ -2.7278030 & 0.2999590 & -3.5362140 \\ -2.7817460 & -0.6721760 & -5.0120980 \\ -3.7056540 & -3.0059940 & -3.8844890 \\ -4.7861140 & -2.8202500 & -3.8873950 \\ -3.3766650 & -3.1133220 & -4.9234850 \\ -3.5334970 & -3.9556430 & -3.3709810 \\ 0.2112170 & 0.2049860 & -0.9283050 \\ -0.6100440 & 0.2620140 & -2.1867390 \\ 0.2380810 & 1.3828190 & 0.0003190 \\ -1.8967140 & -2.0418280 & -3.2234380 \\ -6.5601290 & -0.3870550 & 1.2576520 \\ -1.2771060 & -2.6452820 & 1.6334720 \\ 4.4925130 & -0.4095080 & -2.2028780 \\ 5.0721830 & 5.4635480 & -2.3231430 \\ 2.2228440 & 1.0527450 & 1.7376570 \\ -2.8351570 & 2.4455950 & -1.8560820 \\ -1.8847580 & 3.3890570 & -1.2602640 \\ -2.9492580 & 1.4863920 & -1.3415130 \\ -1.0394980 & 2.9725100 & -0.7205520 \\ -1.7664210 & 2.4131420 & -2.8289590 \\ -2.5423650 & 2.7240430 & 2.1273970 \\ -2.0225880 & 1.3461850 & 4.1599690 \\ -1.2318760 & 1.0563950 & 3.0503250 \\ -1.5610700 & 1.8379000 & 1.9522840 \\ -1.0013720 & 1.7385350 & 1.0795590 \\ -3.1738600 & 2.6067390 & 3.7570600 \\ -3.1246970 & 3.8394620 & -2.6195160 \\ -1.2059750 & 1.5747360 & 5.274650 \\ -0.8042850 & -0.2853470 & \\ -0.0139120 & -0.5716970 & \\ -1 & & \end{array}$

$-0.2193870$

$-1.8099650$

$-0.6060680$

0.3431570

$-2.4136700$

$-3.2240100$

$-1.8545010$

$-4.1136410$

$-4.8517220$

$-3.1026230$

$-1.1322580$

$-4.8273900$

$-4.5246610$

$-3.2616690$

$-3.8698240$

$-1.4427180$

0.9258720

1. 7013150

1. 0629500

2. 2762980

2. 3871340
0.0959800

0.6625360

$-0.8383330$

0.8697930

4.3562360

5. 3371020

4. 8254820

3.0011950

4.7544100

6. 3189310

4.8838700

3. 1083890

2.2637450

4. 2249620

5.4762300

5.4231330

$-1.5296870$

$-1.8712750$

$-2.2422580$

$-1.0085710$

$-2.6577310$
$-0.1093610$

3.0724970

5. 3603980

6. 3198260

2. 1696950

6.2369960

$-3.1128430$

$-2.1456900$

$-1.6922750$

$-2.4366510$

3.4518070

$-2.6115680$

$-2.5156410$

$-1.6115190$

$-3.1352280$

$-4.0005420$

$-1.2686820$

$-0.8754830$

4. 4438770

3. 3065980

2. 4943890

2. 9433240

3. 6226570

$\operatorname{TS} 2(R, R) \quad G[M 06-2 X / 6-31 G(d)]=-4124.881014$

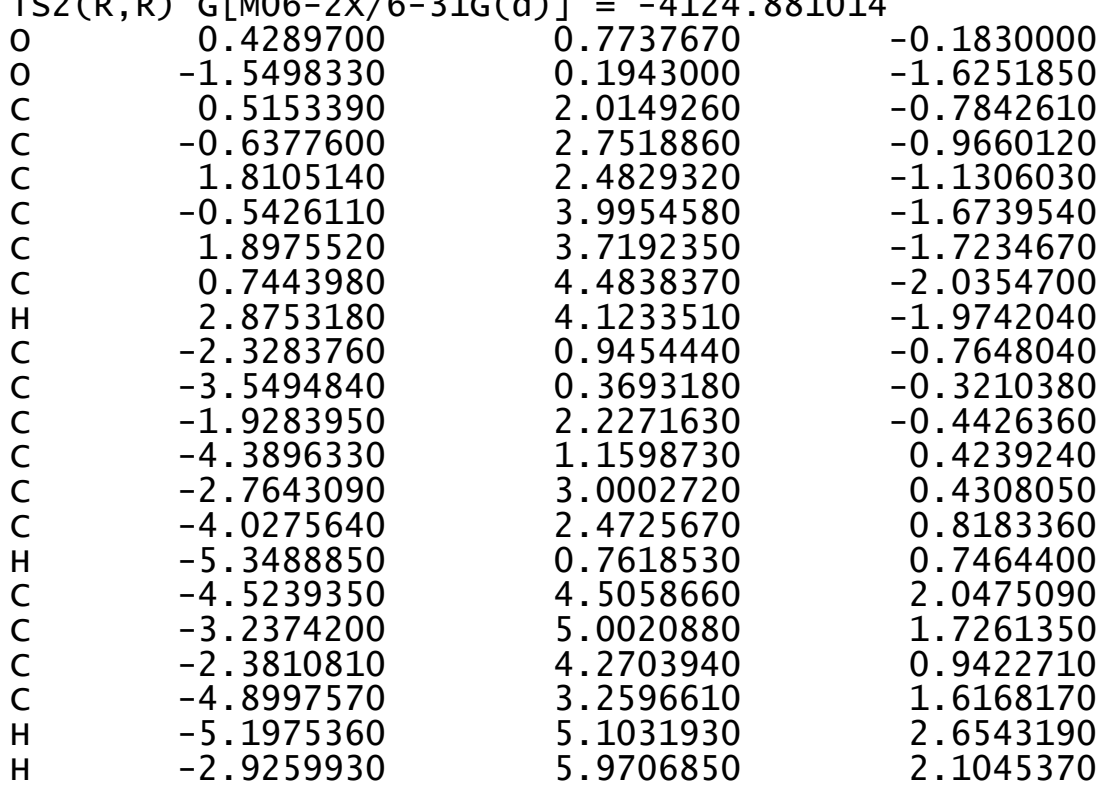




\begin{tabular}{lr}
$\mathrm{H}$ & -1.3965870 \\
$\mathrm{H}$ & -5.8698590 \\
$\mathrm{C}$ & -1.5469650 \\
$\mathrm{C}$ & -0.2636320 \\
$\mathrm{C}$ & 0.8546650 \\
$\mathrm{C}$ & -1.6841000 \\
$\mathrm{H}$ & -2.4311630 \\
$\mathrm{H}$ & -0.1712350 \\
$\mathrm{H}$ & 1.8462970 \\
$\mathrm{H}$ & -2.6732380 \\
$\mathrm{C}$ & 3.0282530 \\
$\mathrm{C}$ & 3.7926000 \\
$\mathrm{C}$ & 3.4330290 \\
$\mathrm{C}$ & 4.9736260 \\
$\mathrm{C}$ & 4.5992490 \\
$\mathrm{C}$ & 5.3930530 \\
$\mathrm{H}$ & 5.5871350 \\
$\mathrm{H}$ & 4.9081490 \\
$\mathrm{C}$ & -3.8917200 \\
$\mathrm{C}$ & -3.9323670 \\
$\mathrm{C}$ & -4.1967470 \\
$\mathrm{C}$ & -4.3166620 \\
$\mathrm{C}$ & -4.5662050 \\
$\mathrm{C}$ & -4.6401180 \\
$\mathrm{H}$ & -4.3612310 \\
$\mathrm{H}$ & -4.8116340 \\
$\mathrm{C}$ & 2.6701020 \\
$\mathrm{C}$ & 6.7112340 \\
$\mathrm{C}$ & 3.3953660 \\
$\mathrm{C}$ & -3.5692460 \\
$\mathrm{C}$ & -5.0562860 \\
$\mathrm{C}$ & -4.1750060 \\
$\mathrm{C}$ & -5.5862210 \\
$\mathrm{H}$ & -5.9846070 \\
$\mathrm{H}$ & -6.2738760 \\
$\mathrm{H}$ & -5.5718010 \\
$\mathrm{C}$ & -3.2022480 \\
$\mathrm{H}$ & -2.1949430 \\
$\mathrm{H}$ & -3.5220980 \\
$\mathrm{H}$ & -3.1600640 \\
$\mathrm{C}$ & -4.0705650 \\
$\mathrm{H}$ & -4.3560770 \\
$\mathrm{H}$ & -4.0604710 \\
$\mathrm{H}$ & -3.0532360 \\
$\mathrm{C}$ & -6.4824980 \\
$\mathrm{H}$ & -7.1957290 \\
& \\
\hline
\end{tabular}

$\begin{array}{rr}4.6610070 & 0.7087730 \\ 2.8482740 & 1.8833770 \\ 5.9323830 & -2.7442270 \\ 6.4364280 & -3.0660460 \\ 5.7228780 & -2.7221570 \\ 4.7472520 & -2.0663450 \\ 6.4866040 & -3.0429300 \\ 7.3787740 & -3.5963540 \\ 6.0850500 & -2.9799650 \\ 4.3660050 & -1.8365610 \\ 1.6653380 & -0.8405130 \\ 1.1412010 & -1.9012930 \\ 1.4563850 & 0.4976450 \\ 0.4558030 & -1.6008550 \\ 0.7298360 & 0.7431040 \\ 0.2330360 & -0.2923090 \\ 0.0742840 & -2.4160990 \\ 0.5616680 & 1.7692760 \\ -1.0493580 & -0.6390440 \\ -2.0017740 & 0.3983390 \\ -1.4294860 & -1.9618040 \\ -3.3104390 & 0.0916620 \\ -2.7492890 & -2.2165540 \\ -3.7052120 & -1.2027870 \\ -4.0498790 & 0.8890740 \\ -3.0380990 & -3.2372660 \\ 2.0826670 & 1.6601100 \\ -0.4748760 & -0.0326970 \\ 1.2994270 & -3.3625430 \\ -1.6713890 & 1.8401950 \\ -5.1323770 & -1.5045570 \\ -0.4436110 & -3.1203090 \\ -0.2383680 & -3.6856520 \\ -1.1730240 & -4.0956990 \\ 0.1095120 & -2.9082160 \\ 0.5035060 & -4.4908640 \\ -0.8883360 & -4.2181120 \\ -1.0210210 & -3.8134630 \\ -1.8335630 & -4.6705280 \\ -0.1366090 & -5.0132810 \\ -5.8102150 & -2.4630230 \\ -6.8533030 & -2.6340280 \\ -5.3013320 & -3.4332420 \\ -5.7907290 & -2.0613300 \\ -5.1916540 & -2.0635590 \\ -4.7271220 & -1.3761510\end{array}$
$-6.5458660$
$-6.7877940$
$-4.8096020$
$-5.5719160$
$-2.4965730$
$-2.8709590$
$-1.6173880$
-2. 1918980
2. 9921050
4. 0613450
2. 7404220
2. 4323880
2. 9476580
2.8879690
3. 9432840
2. 2130650
6.7562480
7.6593760
6.7850400
5.8843240
7.8685320
7.8829530
7.7615280
8.8305220
3. 4437620
4. 4694750
2. 8422690
3. 0493450
4. 2903630
5.3353440
3.9867140
4. 2453120
$-0.1663070$
0.6731150
$-0.3957570$
2. 3620900
6.8474300
1. 5977280
$-3.8302650$
$-5.0409380$
$-3.1527450$
3. 0035690
2. 0755680
2. 9059290

-4.6658800
-6.2298920
-1.6851810
-0.9838480
-2.6843790
-1.4088370
-2.6198620
-3.6464010
-2.6185000
-2.2989450
3.5818530
3.7209620
4.1165120
4.0350970
1.4144440
0.3243130
1.6714190
1.7509650
-1.2195150
-1.8345720
-0.5191550
-1.8692500
0.5304010
1.0313050
1.2973720
0.0279340
-0.0316940
-0.4052270
-0.7927470
0.1031940
2.3309040
1.9999890
2.4548750
3.3088360
-0.4606800
-0.7144030
-1.5600690
1.6561910
-1.2093970
1.9750180
0.5252810
-5.6847330
-0.6604940
-2.4677180
-3.4239000
-1.4246640

$-3.0227800$

$-2.2296160$

2.7419640

2. 3883550

2.7683090

3. 7666930

2. 3885280

2.4675320

1.7376320

3. 3914550

1. 7627070

1.9585130

0.8430050

2. 5877440

3.0084450

2.9365590

3. 3873390

3. 7476050

1. 3031330

1. 3594670

2. 1449940

1. 4385250

$-0.1216130$

$-1.0943130$

0.6536900

0.0238060

$-4.1209280$

$-4.2167190$

$-3.6184660$

$-5.1334340$

$-4.0628250$

$-4.0512440$

$-5.1075430$

$-3.5746910$

$-1.0780650$

$-2.2993350$

$-0.0817830$

$-3.3985800$

$-0.8381300$

1. 4649390

$-2.7480940$

$-0.5564580$

1. 8683700

$-1.1826070$

$-0.5692140$

$-0.8632190$ 


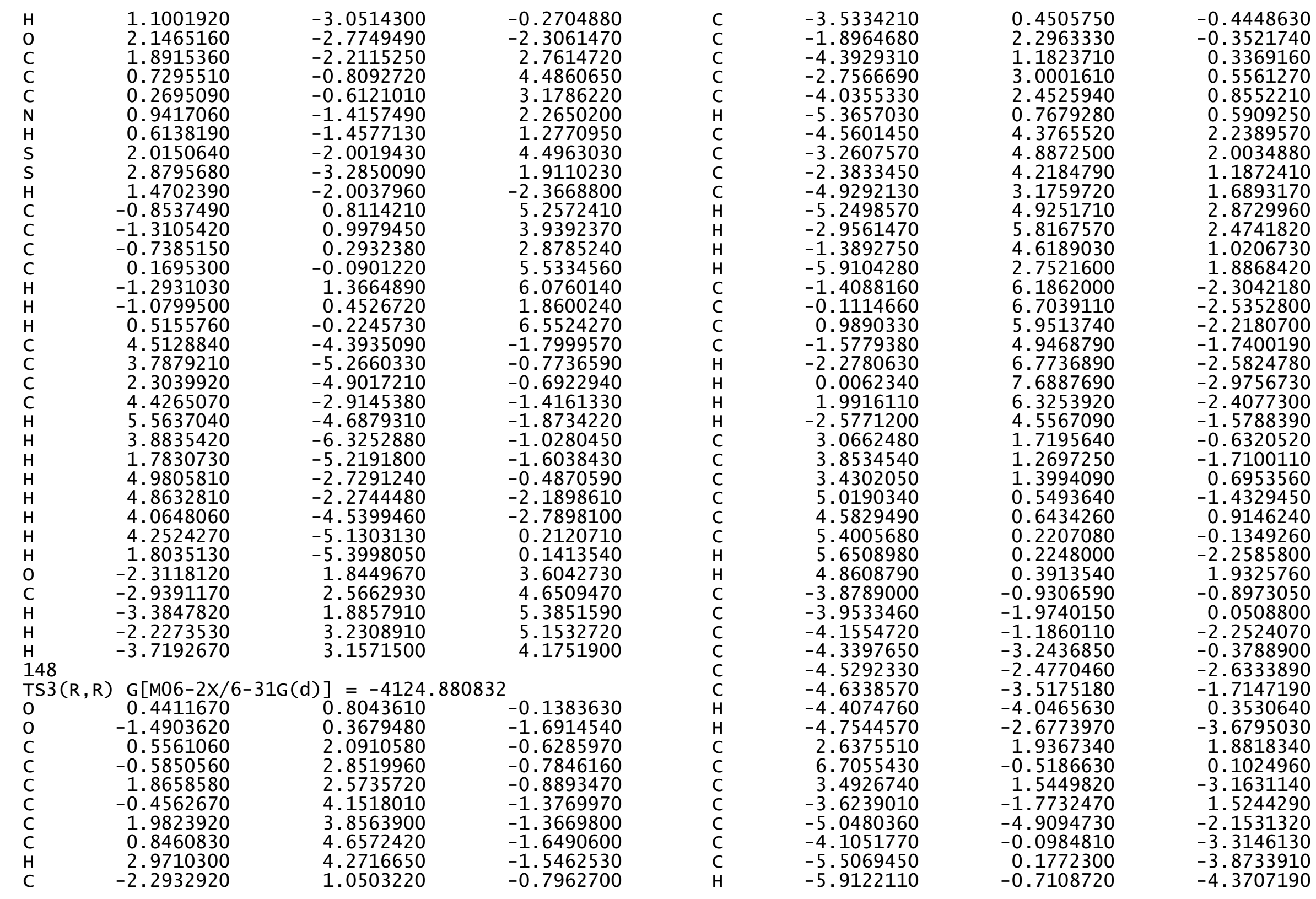




-6.1987460
-5.4738870
-3.1248890
-2.1240340
-3.4494610
-3.0645520
-6.3480970
-6.6603960
-6.2117080
-7.1558890
-3.9318230
-3.0054220
-3.7234720
-4.2234150
-4.8889720
-5.6300960
-5.3550980
-4.6416670
-2.5827280
-2.9734090
-1.6830600
-2.3063180
2.9797410
4.0437930
2.7681690
2.3994940
2.8560580
2.7867360
3.8395310
2.0979530
6.7058410
7.6049910
6.7101280
5.8286020
7.8712300
7.9186430
7.7452070
8.8251850
3.5805210
4.6153160
2.9894910
3.1971770
4.3900920
5.4394280
4.1154620
4.3149110

0.4623530

0.9891830

$-0.4509840$

$-0.6334450$

$-1.3452880$

0.3716980

$-5.3477570$

$-6.3324980$

$-5.4176280$

$-4.6357930$

$-5.9287930$

$-5.6329260$

$-6.0146330$

$-6.9186510$

$-1.8393400$

$-1.0974780$

$-2.8290410$

$-1.6540870$

$-2.7835570$

$-3.8068860$

$-2.7397760$

$-2.5561400$

3.4148930

3.5164520

4.0313310

3.8058950

1.1475810

0.0690920

1.3549480

1.4279050

$-1.3754020$

$-1.9980790$

$-0.7503650$

$-2.0297780$

0.4803760

1.0632670

1.1784330

$-0.0412140$

0.2844590

$-0.0583480$

$-0.5297570$

0.4973140

2.6418520

2. 3251400

2.8437530
3.5775550

-3.0743440
-4.6072860
-4.4385420
-4.0376250
-4.9817530
-5.1587270
-1.4693890
-1.8319700
-0.3844920
-1.6630760
-1.8975210
-2.3985160
-0.8252590
-2.2635060
2.3888400
2.0752030
2.3193170
3.4399670
2.0200990
1.9976640
1.3995270
3.0562400
2.1253710
2.3671050
1.2476460
2.9678960
3.1746900
3.0037670
3.6113670
3.9135450
1.3700780
1.4014280
2.2697060
1.4213230
0.1350570
-0.7897300
0.9702930
0.2653600
-4.0305990
-4.1426230
-3.6050950
-5.0338830
-3.7520360
-3.7342950
-4.7926430
-3.1908370

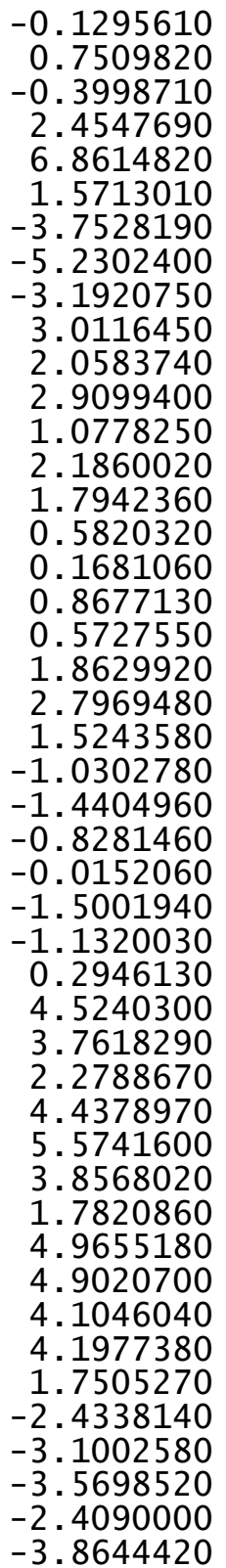

-0.3434720
-0.4994020
-1.5224900
1.8887700
-1.1827320
1.8673740
0.8267040
-4.8709950
-0.7761200
-2.3859140
-3.3833240
-1.3756960
-3.0284690
-2.5822630
-2.4597660
-1.1983980
-0.8878400
-1.6153180
-1.5680610
-2.3953460
-3.4726310
-1.7956820
0.3521650
0.6521030
0.0431200
-0.5706940
0.8341030
0.2910000
-0.7931180
-4.2628100
-5.2169730
-4.8463520
-2.8234470
-4.5606480
-6.2497260
-5.0759770
-2.7270120
-2.1212960
-4.3143830
-5.1761100
-5.4115540
1.5224100
2.1522990
1.4120460
2.7720970
2.7816970
$-1.1563660$

$-2.3652710$

$-0.2673070$

$-3.1942570$

$-0.7586040$

1.6411110

$-2.8497170$

$-3.2345520$

1.6481690

$-1.3282420$

$-0.8309040$

$-0.9186860$

$-0.5278320$

$-2.4991570$

2.5799410

4.3778420

3.0771580

2.1214130

1.1236950

4. 3294860

1. 6747310

$-2.5147980$

5.2297980

3.9175360

2. 8208800

5.4629170

6.0774610

1.8083750

6.4780470

$-2.0719190$

$-1.1505690$

$-1.0791070$

$-1.5590170$

$-2.1411900$

$-1.4973970$

$-2.0296620$

$-0.6014270$

$-2.2596110$

$-3.0836100$

$-0.1435640$

$-0.3077230$

3.6209630

4.7018590

5.3593310

5.2837510

4.2500310 
148

TS4 $(R, R) \quad G[M 06-2 X / 6-31 G(d)]=-4124.881554$

$\begin{array}{lrrr}\mathrm{O} & 0.2543670 & 1.0541660 & -0.0377690 \\ \mathrm{O} & -1.7338730 & 0.1890690 & -1.3133900 \\ \mathrm{C} & 0.1780520 & 2.2650460 & -0.7040850 \\ \mathrm{C} & -1.0560910 & 2.8634960 & -0.8577810 \\ \mathrm{C} & 1.3974390 & 2.8424430 & -1.1510680 \\ \mathrm{C} & -1.1335070 & 4.0641830 & -1.6371670 \\ \mathrm{C} & 1.3178160 & 4.0408470 & -1.8167210 \\ \mathrm{C} & 0.0722750 & 4.6589600 & -2.1032380 \\ \mathrm{H} & 2.2295230 & 4.5345370 & -2.1429510 \\ \mathrm{C} & -2.5118880 & 0.9016920 & -0.4212960 \\ \mathrm{C} & -3.6087730 & 0.2215080 & 0.1757690 \\ \mathrm{C} & -2.2436960 & 2.2399870 & -0.2135210 \\ \mathrm{C} & -4.4533040 & 0.9519060 & 0.9741400 \\ \mathrm{C} & -3.0815780 & 2.9715960 & 0.6954180 \\ \mathrm{C} & -4.2090170 & 2.3188960 & 1.2692830 \\ \mathrm{H} & -5.3274020 & 0.4715420 & 1.4068410 \\ \mathrm{C} & -4.7695310 & 4.3264870 & 2.5137160 \\ \mathrm{C} & -3.6280050 & 4.9670320 & 1.9747240 \\ \mathrm{C} & -2.8066490 & 4.3100130 & 1.0926780 \\ \mathrm{C} & -5.0475290 & 3.0288350 & 2.1695040 \\ \mathrm{H} & -5.4114890 & 4.8596080 & 3.2074760 \\ \mathrm{H} & -3.3946100 & 5.9852030 & 2.2696380 \\ \mathrm{H} & -1.9244240 & 4.8084290 & 0.7067440 \\ \mathrm{H} & -5.9065970 & 2.5137980 & 2.5910050 \\ \mathrm{C} & -2.3982540 & 5.8182220 & -2.7439350 \\ \mathrm{C} & -1.1960970 & 6.4299780 & -3.1736920 \\ \mathrm{C} & 0.0101500 & 5.8582340 & -2.8621480 \\ \mathrm{C} & -2.3692700 & 4.6684790 & -1.9952700 \\ \mathrm{H} & -3.3517250 & 6.2605210 & -3.0148290 \\ \mathrm{H} & -1.2348410 & 7.3427400 & -3.7594220 \\ \mathrm{H} & 0.9408260 & 6.3056740 & -3.2005850 \\ \mathrm{H} & -3.2967680 & 4.2017650 & -1.6801790 \\ \mathrm{C} & 2.6967360 & 2.1558630 & -0.8651250 \\ \mathrm{C} & 3.4052660 & 1.5159370 & -1.9020250 \\ \mathrm{C} & 3.1971230 & 2.1411950 & 0.4555410 \\ \mathrm{C} & 4.6069150 & 0.8697770 & -1.5944140 \\ \mathrm{C} & 4.3985000 & 1.4796130 & 0.7090780 \\ \mathrm{C} & 5.1174650 & 0.8379050 & -0.2982320 \\ \mathrm{H} & 5.1614040 & 0.3806780 & -2.3929210 \\ \mathrm{H} & 4.7960020 & 1.4582040 & 1.7197750 \\ \mathrm{C} & -3.8549120 & -1.2291940 & -0.0949720 \\ \mathrm{C} & -3.4574030 & -2.2012030 & 0.8437270 \\ \mathrm{C} & -4.5108800 & -1.6093480 & -1.2797290 \\ \mathrm{C} & -3.7781940 & -3.5376100 & \\ & & & \end{array}$

$-4.8039940$

$-4.4549900$

$-3.4843190$

$-5.3195910$

2.4849390

6.4268610

2. 9385240

$-2.6949470$

$-4.7818920$

$-4.9219340$

$-6.4491040$

$-6.8866600$

$-6.8816210$

$-6.7409880$

$-4.3077430$

$-3.2175380$

$-4.6784180$

$-4.5680900$

$-4.0491110$

$-4.2517710$

$-4.3806100$

$-2.9676830$

$-6.2927940$

$-6.8218580$

$-6.6928620$

$-6.5140040$

$-3.6413900$

$-4.3451500$

$-4.2201090$

$-3.0663150$

$-1.6575780$

$-2.1337180$

$-1.0180120$

$-1.0274580$

2. 7196710

3. 7896300

2. 3743910

2. 1930710

2.8976070

2. 8557210

3. 9140910

2. 2230410

7. 6016010

8.5459300

7.4821000

7.6706070
-2.9577130
-3.9362440
-4.2988200
-3.2504480
2.8878550
0.1394690
1.5640610
-1.8262140
-5.3974610
-0.5948490
-0.4922870
-1.4495650
-0.2161270
0.2627800
-0.9239790
-0.9817720
-1.8806490
-0.1494500
-5.9299980
-6.9970440
-5.4047860
-5.7917140
-5.6218210
-5.2613020
-5.0914030
-6.6866640
-1.5575170
-0.7494440
-2.4579160
-1.2785700
-2.8772710
-3.7858600
-3.1455600
-2.4784670
4.4014080
4.6191400
4.7910180
4.9367220
2.4421750
1.3552970
2.7752330
2.8894930
0.8096050
0.3253940
0.7333810
1.8703700

$-1.4863020$

$-0.5566210$

1. 3129810

$-2.3995470$

1.5777960

0.0151660

$-3.3506410$

2. 1069590

$-0.8011810$

$-2.3357070$

$-2.4267810$

$-2.7315810$

$-1.4597410$

$-3.1640280$

$-3.7007510$

$-3.6323440$

$-4.0844970$

$-4.4294960$

$-2.0381480$

$-2.1764400$

$-2.9408150$

$-1.9456420$

$-0.9283710$

$-0.0412140$

$-1.7995650$

$-1.0541530$

3. 2842810

3. 0669640

3. 5206940

4.1749780

2. 5127700

2.8973320

1.6676690

3. 3155170

1.4494830

1.5424400

0.4889640

2. 2467060

2. 9826070

3. 1011000

3. 2210390

3.7197770

$-0.7068750$

$-0.4376550$

$-1.7933980$

$-0.4481010$ 


\begin{tabular}{rrr}
6.3614150 & -1.3520830 & -0.3267560 \\
5.5197770 & -1.8380320 & 0.1800930 \\
6.2372510 & -1.4934310 & -1.4065470 \\
7.2848020 & -1.8589320 & -0.0277110 \\
3.1221540 & 0.2399630 & -4.0967060 \\
4.1802860 & -0.0084820 & -4.2349090 \\
2.6331100 & -0.5821700 & -3.5715370 \\
2.6757220 & 0.3158310 & -5.0935410 \\
3.6815720 & 2.6760710 & -4.1063410 \\
4.7564980 & 2.4617390 & -4.1290900 \\
3.3262530 & 2.7415110 & -5.1401330 \\
3.5482520 & 3.6537740 & -3.6358730 \\
-0.2274930 & -0.2623390 & -0.8819590 \\
0.5490150 & -0.3995120 & -2.1626090 \\
-0.2470690 & -1.3923580 & 0.1046010 \\
1.8681030 & 1.7894400 & -3.3522650 \\
6.5953040 & 0.2324030 & 1.0954180 \\
1.4088130 & 2.6963530 & 1.4780980 \\
-4.5437500 & 0.3877640 & -2.0371120 \\
-4.4280590 & -5.9619690 & 0.0707550 \\
-2.1482410 & -0.9025640 & 1.8871720 \\
2.7378400 & -2.6209670 & -1.7925500 \\
1.7860200 & -3.5031490 & -1.1115330 \\
2.8964520 & -1.6387190 & -1.3367230 \\
0.9718560 & -3.0347030 & -0.5666150 \\
1.6343650 & -2.6122270 & -2.7269370 \\
2.5780250 & -2.6671050 & 2.2106080 \\
2.1539460 & -1.1748090 & 4.1839950 \\
1.3338080 & -0.9246990 & 3.0860660 \\
1.6106860 & -1.7687190 & 2.0202550 \\
1.0234030 & -1.7043030 & 1.1624880 \\
3.2661510 & -2.4789590 & 3.8103970 \\
3.0974670 & -3.8551900 & 1.1258430 \\
1.1024470 & -1.7496770 & -2.5446000 \\
1.0081540 & 0.5431160 & 5.3846310 \\
0.1877210 & 0.7888270 & 4.2628740 \\
0.3415970 & 0.0568660 & 3.0907830 \\
1.9929580 & -0.4271740 & 5.3539720 \\
0.8501800 & 1.1452890 & 6.2725500 \\
-0.2454350 & 0.2282370 & 2.1966270 \\
2.6198950 & -0.6027900 & 6.2211750 \\
3.6972670 & -4.6274060 & -1.9760180 \\
3.0421900 & -5.5337520 & -2.35309950 \\
1.7040080 & -4.9602070 & \\
3.9790110 & -3.2434660 & -3257880 \\
4.6341410 & -5.0700680 & \\
\hline & &
\end{tabular}

\begin{tabular}{ll}
$\mathrm{H}$ & 2.8790090 \\
$\mathrm{H}$ & 0.9501290 \\
$\mathrm{H}$ & 4.7193310 \\
$\mathrm{H}$ & 4.3834910 \\
$\mathrm{H}$ & 3.0386520 \\
$\mathrm{H}$ & 3.7164780 \\
$\mathrm{H}$ & 1.3084320 \\
$\mathrm{O}$ & -0.7269060 \\
$\mathrm{C}$ & -1.5308690 \\
$\mathrm{H}$ & -0.9111130 \\
$\mathrm{H}$ & -2.1367800 \\
$\mathrm{H}$ & -2.1880760 \\
\hline
\end{tabular}
$-6.5367550$
54050
$-2.5577440$
$-4.5291100$
$-5.499$
1.7742220
2.0746380
2.3869680
1.2082770

$-2.2511350$

$-1.5817750$

$-3.1391700$

-1.0710300

$-0.5947900$

4.4113890

3.2822050

2.4318280

148

2.9835120

$\operatorname{TS} 5(R, R) \quad G[M 06-2 X / 6-31 G(d)]=-4124.884053$

$\begin{array}{lr}\text { O } & 0.1747090 \\ \mathrm{O} & -1.7717010 \\ \mathrm{C} & 0.0464980 \\ \mathrm{C} & -1.2133240 \\ \mathrm{C} & 1.2391080 \\ \mathrm{C} & -1.3437930 \\ \mathrm{C} & 1.1060650 \\ \mathrm{C} & -0.1651920 \\ \mathrm{H} & 1.9944600 \\ \mathrm{C} & -2.5842640 \\ \mathrm{C} & -3.6637140 \\ \mathrm{C} & -2.3750960 \\ \mathrm{C} & -4.5472240 \\ \mathrm{C} & -3.2548240 \\ \mathrm{C} & -4.3618620 \\ \mathrm{H} & -5.4074540 \\ \mathrm{C} & -5.0190240 \\ \mathrm{C} & -3.8978050 \\ \mathrm{C} & -3.0397670 \\ \mathrm{C} & -5.2397690 \\ \mathrm{H} & -5.6906510 \\ \mathrm{H} & -3.7091470 \\ \mathrm{H} & -2.1733510 \\ \mathrm{H} & -6.0825470 \\ \mathrm{C} & -2.6833320 \\ \mathrm{C} & -1.5090210 \\ \mathrm{C} & -0.2791860 \\ \mathrm{C} & -2.6044980 \\ \mathrm{H} & -3.6551530 \\ \mathrm{H} & -1.5872920 \\ \mathrm{H} & 0.6314600 \\ \mathrm{H} & -3.5110060 \\ & \\ & \end{array}$

$=-4124$

0.0071340

$0.2250290 \quad-1.3551370$

2. $3215210-0.5241680$

2. 8769170

$-0.6206950$

2.9933010

$-0.9088630$

.1498260

4.2537150

.8451660

$-1.2681400$

$-1.4372970$

$-1.6579270$

4. 8158960

$-1.7130310$

0.8152140

$-0.4070480$

0.0369070

0.0950120

.1355250

0.0590840

0.6460020

0.9505490

2.7347200

0.9063260

1.9826220

1. 3908550

1. 3142760

3.8257650

2.8275150

4.56299

2. 3769560

4.0341920

2. 5613420

1.4450940

2. 3457780

3. 5620960

. 2581030

2.7800840

4. 6041200

1. 9721140

5.9524140

6.6609380

6.1145800

4.7319370

6.3766270

7.6280130

6.6363820

1.1287080

2. 6970450

$-2.1948340$

$-2.5456470$

$-2.2854890$

$-1.5722760$

$-2.4266300$

$-3.0317980$

$-2.5672540$

4. 1927630

$-1.3179930$ 


$\begin{array}{rrr}2.5624160 & 2.3163070 & -0.7347230 \\ 3.2830600 & 1.8770380 & -1.8607740 \\ 3.0697750 & 2.0929340 & 0.5680440 \\ 4.4895840 & 1.1946960 & -1.6596510 \\ 4.2623280 & 1.3880600 & 0.7122460 \\ 4.9809300 & 0.9155390 & -0.3895640 \\ 5.0628580 & 0.8642060 & -2.5233680 \\ 4.6507340 & 1.2022090 & 1.7087850 \\ -3.8569170 & -1.3821860 & -0.3381290 \\ -3.4078500 & -2.4400260 & 0.4811660 \\ -4.5121020 & -1.6499540 & -1.5499920 \\ -3.6772280 & -3.7491260 & 0.0859220 \\ -4.7518250 & -2.9803130 & -1.9075070 \\ -4.3537510 & -4.0410550 & -1.1012120 \\ -3.3409790 & -4.5700910 & 0.7145120 \\ -5.2646030 & -3.1953500 & -2.8435410 \\ 2.3692150 & 2.6735810 & 1.7921130 \\ 6.2841140 & 0.1602480 & -0.2093930 \\ 2.8278390 & 2.1568310 & -3.2862190 \\ -2.6480270 & -2.1727520 & 1.7731390 \\ -4.6278130 & -5.4770290 & -1.5068660 \\ -4.9681750 & -0.5415230 & -2.4856890 \\ -6.4950440 & -0.5271530 & -2.6221520 \\ -6.8581740 & -1.4613080 & -3.0646240 \\ -6.9749740 & -0.4068080 & -1.6456340 \\ -6.8144830 & 0.2974530 & -3.2677050 \\ -4.2903630 & -0.6609440 & -3.8552750 \\ -3.2017150 & -0.6508770 & -3.7485840 \\ -4.5777410 & -1.5898470 & -4.3598670 \\ -4.5839820 & 0.1746110 & -4.4990660 \\ -5.5130200 & -6.1906300 & -0.4788290 \\ -5.7428780 & -7.2084820 & -0.8101970 \\ -5.0053930 & -6.2600340 & 0.4896310 \\ -6.4550570 & -5.6548280 & -0.3289460 \\ -3.3233440 & -6.2514320 & -1.7285340 \\ -2.6940290 & -5.7579160 & -2.4750240 \\ -2.7507730 & -6.3217280 & -0.7969990 \\ -3.5339440 & -7.2698400 & -2.0709500 \\ -3.5950560 & -2.0559650 & 2.9746370 \\ -4.3303530 & -1.2579770 & 2.8392800 \\ -4.1377320 & -2.9966460 & 3.1234320 \\ -3.0245100 & -1.8433400 & 3.8863500 \\ -1.5733250 & -3.2234360 & 2.0654920 \\ -2.0154720 & -4.1861500 & 2.3444380 \\ -0.9226990 & -3.3718870 & 1.1994110 \\ -0.9601580 & -2.8935050 & 2.9114320\end{array}$

3.6287560

2. 1828450

2.0438640

2.8360330

2.8106950

3.8570370

2.1837990

6.1756630

7.0895840

6.0444840

5. 3269730

7.4226280

7.5368330

7.2161900

8.3717320

3.0198320

4.0801560

2. 5565540

2. 5587350

3.5765460

4.6513250

3.2275680

3.4424040

$-0.2366670$

0.5230740

$-0.1625490$

1.7580720

6.5238070

1.2959020

$-4.6680030$

$-5.1708470$

$-2.1332670$

2.9511630

2.1219840

2.8685400

1.1892450

1.9649290

2.6393780

2. 1020550

1.2957240

1.6349880

1.0689310

3.2838460

3.2388820

1. 2874210
4.1979760

4.4325390

4.6926650

4.6169110

2.0778010

0.9842820

. 3970240

2. 4286750

$-0.9491000$

$-1.5516270$

$-0.5303440$

$-1.6135440$

1.1263430

1.8978020

1.6248410

0.5887520

0.9653380

0.7608320

0.0619140

1.1850330

3.3736260

3. 1636220

3.6026110

4.2648330

$-0.1829110$

$-0.1122170$

$-1.4250450$

2. 3832070

$-0.3074120$

2.4636770

0.4190290

$-5.4491460$

$-1.2135150$

$-2.0522280$

$-3.1988510$

$-1.1613330$

$-2.9872930$

$-2.0597810$

$-2.7938000$

$-1.5344650$

$-1.2077130$

$-1.9245600$

$-1.8007510$

$-2.7393450$

-3.8413320
-1.3116300
1.8436200

1.9383240

0.9468330

2.7123780

3.1217170

3.1184380

3. 3603220

3.9277000

0.8423520

0.8547320

1.8462230

0.6438300

0.1430150

$-0.6244240$

1.0969340

0.2387590

$-4.2288650$

$-4.4156630$

$-3.8280280$

$-5.1972900$

$-3.8507400$

$-3.9016520$

$-4.8631730$

$-3.2318010$

$-0.9879000$

$-2.2846170$

$-0.1505800$

$-3.2616860$

$-1.1737850$

1.6983970

$-2.0555000$

$-2.4600130$

1.6474220

$-1.8856720$

$-1.5005010$

$-1.2551270$

$-0.9863160$

$-2.9411440$

1.9345480

4.0348230

2. 9465190

1.8075170

0.9417120

3.5607190

0.7527530

$-2.7140020$ 


$\begin{array}{lrrr}\mathrm{C} & 0.8649240 & 0.0086260 & 5.3723290 \\ \mathrm{C} & 0.0573750 & 0.3326510 & 4.2612340 \\ \mathrm{C} & 0.2649450 & -0.2702810 & 3.0251780 \\ \mathrm{C} & 1.8888040 & -0.9145260 & 5.2688360 \\ \mathrm{H} & 0.6637140 & 0.5117040 & 6.3116440 \\ \mathrm{H} & -0.3090720 & -0.0294360 & 2.1385500 \\ \mathrm{H} & 2.5061740 & -1.1512830 & 6.1282720 \\ \mathrm{C} & 4.4031890 & -3.5951630 & -3.2484940 \\ \mathrm{C} & 3.8196390 & -4.7871550 & -2.4863250 \\ \mathrm{C} & 2.3526710 & -4.5490650 & -2.1172830 \\ \mathrm{C} & 4.3442460 & -2.3201220 & -2.4028960 \\ \mathrm{H} & 5.4409370 & -3.7975630 & -3.5281960 \\ \mathrm{H} & 3.8929620 & -5.6999620 & -3.0841410 \\ \mathrm{H} & 1.7233560 & -4.5907100 & -3.0140270 \\ \mathrm{H} & 5.0077540 & -2.4078770 & -1.5330540 \\ \mathrm{H} & 4.6691390 & -1.4469820 & -2.9785910 \\ \mathrm{H} & 3.8410530 & -3.4406160 & -4.1768690 \\ \mathrm{H} & 4.4049240 & -4.9547000 & -1.5731410 \\ \mathrm{H} & 1.9732060 & -5.3133780 & -1.4350270 \\ \mathrm{O} & -0.9025340 & 1.2574130 & 4.4882370 \\ \mathrm{C} & -1.6970640 & 1.6390000 & 3.3776610 \\ \mathrm{H} & -1.0759680 & 2.0696080 & 2.5816330 \\ \mathrm{H} & -2.2532630 & 0.7821660 & 2.9732310 \\ \mathrm{H} & -2.3995100 & 2.3892610 & 3.7415540 \\ \mathrm{I} 48 & & & \\ \mathrm{TS} 6(\mathrm{R}, \mathrm{R}) & \mathrm{G}[\mathrm{M} 06-2 \times / 6-31 \mathrm{G}(\mathrm{d})] & -1.410 & -4124.883243 \\ \mathrm{O} & 0.2085180 & 1.0456310 & -0.0168630 \\ \mathrm{O} & -1.7848440 & 0.1787350 & -1.2776600 \\ \mathrm{C} & 0.0802620 & 2.2849320 & -0.6210700 \\ \mathrm{C} & -1.1738300 & 2.8548080 & -0.7080830 \\ \mathrm{C} & 1.2683850 & 2.9115340 & -1.0873730 \\ \mathrm{C} & -1.3074080 & 4.0903320 & -1.4235510 \\ \mathrm{C} & 1.1363380 & 4.1415750 & -1.6832550 \\ \mathrm{C} & -0.1326590 & 4.7421090 & -1.8925680 \\ \mathrm{H} & 2.0229290 & 4.6711000 & -2.0221790 \\ \mathrm{C} & -2.5606990 & 0.8285980 & -0.3378390 \\ \mathrm{C} & -3.6289620 & 0.0902170 & 0.2415190 \\ \mathrm{C} & -2.3266950 & 2.1632090 & -0.0701060 \\ \mathrm{C} & -4.4792540 & 0.7556860 & 1.0887010 \\ \mathrm{C} & -3.1699270 & 2.8252070 & 0.8865570 \\ \mathrm{C} & -4.2680800 & 2.1131560 & 1.4463170 \\ \mathrm{H} & -5.3320140 & 0.2297090 & 1.5108770 \\ \mathrm{C} & -4.8644700 & 4.0413190 & 2.7952710 \\ \mathrm{C} & -3.7512890 & 4.7401850 & 2.2698160 \\ \mathrm{C} & -2.9268200 & 4.1505580 & 1.3441000 \\ \mathrm{C} & -5.1108950 & 2.7540480 & 2.3931110\end{array}$

-5.5094190
-3.5417620
-2.0655880
-5.9475390
-2.6509330
-1.4789600
-0.2492240
-2.5689810
-3.6237150
-1.5594850
0.6588150
-3.4742280
2.5852380
3.2514320
3.1384100
4.4517820
4.3225160
4.9878460
4.9838230
4.7453910
-3.8440130
-3.3948780
-4.5258680
-3.6931330
-4.7956470
-4.3977130
-3.3606680
-5.3325950
2.4967900
6.2819300
2.7450180
-2.6067890
-4.7078810
-4.9867900
-6.5175460
-6.9369240
-6.9371770
-6.8434000
-4.3921950
-3.2997750
-4.7470910
-4.6855220
-4.0283710
-4.2191270
-4.4136340
-2.9463850

4.5215290

5.7493400

4. 6925100

2.1943730

5.8599200

6.5277550

5.9759070

4.6753660

6.2866940

7.4673310

6.4655270

4. 1666740

2. 2201540

1.6933830

2. 0687550

0.9996830

1. 3497150

0.7924470

0.6017610

1. 2186230

$-1.3498890$

$-2.3600550$

$-1.6823170$

$-3.6889120$

$-3.0244690$

$-4.0419130$

$-4.4798420$

3.280

2. 7402550

0.0213690

1. 8911840

$-2.0310690$

$-5.4945950$

$-0.6239280$

$-0.5574320$

$-1.5080770$

$-0.3450280$

0.2280920

$-0.8639760$

$-0.8980510$

$-1.8082300$

$-0.0587410$

$-5.9455200$

$-7.0075710$

$-5.3803880$

$-5.7907240$
3. 5240150

2. 6101240

0.9696950

2. 8025780

$-2.4064380$

$-2.8359720$

$-2.5870900$

$-1.7183980$

$-2.6293530$

$-3.3730300$

$-2.9286360$

$-1.4038960$

$-0.9214030$

$-2.0435070$

0.3727970

$-1.8446500$

0.5169010

$-0.5787080$

$-2.7062640$

1. 5081210

$-0.1021090$

0.7701640

$-1.2865340$

0.4597190

$-1.5557470$

$-0.6897370$

1. 1274730

$-2.4676080$

1.5822640

$-0.3996290$

$-3.4655440$

2. 0303770

$-0.9986130$

$-2.2767470$

$-2.3328680$

$-2.6808730$

$-1.3442830$

$-3.0224910$

$-3.6688520$

$-3.6232450$

$-4.0958260$

$-4.3502420$

$-2.2967130$

$-2.4822270$

-3. 1526720

$-2.2498790$ 


\begin{tabular}{|c|c|c|}
\hline $\begin{array}{l}-6.2193060 \\
-6.7098380 \\
-6.6705970 \\
-6.4285030 \\
-3.5276690 \\
-4.2590620 \\
-4.0747550 \\
-2.9365440 \\
-1.5336790 \\
-1.9771190 \\
-0.9066270 \\
-0.8962850 \\
2.7274670 \\
3.8017070 \\
2.3262770 \\
2.2515380 \\
2.9976970 \\
2.9483110 \\
4.0333740 \\
2.3827070 \\
6.1908200 \\
7.0916800 \\
6.1065460 \\
5.3213900 \\
7.4521240 \\
7.5531510 \\
7.2920000 \\
8.3930500 \\
2.8833370 \\
3.9326660 \\
2.4227500 \\
2.3887110 \\
3.4888320 \\
4.5579600 \\
3.1055310 \\
3.3902660 \\
-0.2500960 \\
0.4808380 \\
-0.1824530 \\
1.6802360 \\
6.4758490 \\
1.4160800 \\
-4.6277170 \\
-4.3018490 \\
-2.0887970 \\
2.8683130\end{array}$ & $\begin{array}{r}-5.7416770 \\
-5.4415440 \\
-5.1710700 \\
-6.8019880 \\
-1.8462420 \\
-1.0492530 \\
-2.7733030 \\
-1.5940140 \\
-3.0743970 \\
-4.0155120 \\
-3.2780630 \\
-2.7039340 \\
4.2594990 \\
4.4718650 \\
4.7077880 \\
4.7441960 \\
2.2146810 \\
1.1238710 \\
2.5229040 \\
2.6290970 \\
-1.0187630 \\
-1.6405760 \\
-0.5366830 \\
-1.6739060 \\
0.9815060 \\
1.7011940 \\
1.5426780 \\
0.4295490 \\
0.6411830 \\
0.4081420 \\
-0.2286150 \\
0.8098460 \\
3.0582440 \\
2.8280840 \\
3.2294720 \\
3.9883020 \\
-0.2391770 \\
-0.2642230 \\
-1.4282340 \\
2.1363280 \\
-0.5098120 \\
2.5528270 \\
0.3510880 \\
-6.0977380 \\
-1.0833920 \\
-2.2344590\end{array}$ & $\begin{array}{r}-1.0647050 \\
-0.1339630 \\
-1.8839830 \\
-1.2397860 \\
3.2436220 \\
3.0838470 \\
3.4503550 \\
4.1317430 \\
2.3544880 \\
2.6975550 \\
1.4823600 \\
3.1650580 \\
1.5320060 \\
1.5736750 \\
0.6202090 \\
2.3909960 \\
2.9289730 \\
2.9931610 \\
3.1124350 \\
3.7340690 \\
0.7221330 \\
0.7381390 \\
1.7023510 \\
0.5959880 \\
-0.1497720 \\
-0.9676750 \\
0.7778620 \\
-0.0549790 \\
-4.3396560 \\
-4.5533580 \\
-3.8676270 \\
-5.3017570 \\
-4.1325160 \\
-4.2091700 \\
-5.1439660 \\
-3.5663260 \\
-0.9207890 \\
-2.2354000 \\
-0.0090970 \\
-3.4148760 \\
-1.3411520 \\
1.5393300 \\
-1.9329020 \\
-0.1767130 \\
1.8448060 \\
-1.8072280\end{array}$ \\
\hline
\end{tabular}

$$
\begin{array}{r}
2.0298110 \\
2.8240810 \\
1.1187000 \\
1.8473010 \\
2.6714260 \\
2.2369940 \\
1.3968270 \\
1.6798480 \\
1.0852580 \\
3.3761430 \\
3.2089650 \\
1.1946820 \\
1.0792890 \\
0.2363590 \\
0.3864680 \\
2.0814260 \\
0.9228390 \\
-0.2166870 \\
2.7259890 \\
4.2414550 \\
3.6604370 \\
2.2122660 \\
4.2375560 \\
5.2646730 \\
3.6943300 \\
1.5508340 \\
4.9281350 \\
4.5607910 \\
3.6511190 \\
4.2736970 \\
1.8426390 \\
-0.6981170 \\
-1.5279380 \\
-0.9305260 \\
-2.1135610 \\
-2.2032700
\end{array}
$$$$
148
$$$$
\text { TS7 }(R, R) \quad G[M 06-2 X / 6-31 G(d)]=-4124.881063
$$

$\begin{array}{rr}\mathrm{O} & 0.5211580 \\ \mathrm{O} & -1.5434040 \\ \mathrm{C} & 0.7506680 \\ \mathrm{C} & -0.3123720 \\ \mathrm{C} & 2.0918390 \\ \mathrm{C} & -0.0823220 \\ \mathrm{C} & 2.3124230 \\ \mathrm{C} & 1.2508090\end{array}$

0.2713940

1.8142200

2.6782180

. 1487560

3. 8961140

3. 3698590

4.2449080
$-1.3288270$ $-1.2276410$ $-0.7988430$ $-2.8278440$ 2.0522370

4.0845230

3.0071600

1.9053970

1.0541820

3.6476000

0.9194460

$-2.6222460$

5.3633980

4.2638230

3.0625810

5.2828840

6.2748230

2.1839250

6.1329340

$-3.1260120$

$-2.2775410$

$-1.8732220$

$-2.3530560$

$-3.4277770$

$-2.8240580$

$-2.7438710$

$-1.5017940$

$-2.9878310$

$-4.0426270$

$-1.3764730$

$-1.1347100$

4.4653570

3. 3624650

2. 5176570

3.0353530

3.7028940

$-2.2344590$

$-1.8072280$

$-0.2536790$

$-0.8421040$

$-1.0286710$

$-1.7473710$

$-1.7580830$

$-2.0988430$ 


$\begin{array}{lr}\mathrm{H} & 3.3318250 \\ \mathrm{C} & -2.1972570 \\ \mathrm{C} & -3.4412140 \\ \mathrm{C} & -1.6456160 \\ \mathrm{C} & -4.1469210 \\ \mathrm{C} & -2.3554490 \\ \mathrm{C} & -3.6339870 \\ \mathrm{H} & -5.1300150 \\ \mathrm{C} & -3.8804810 \\ \mathrm{C} & -2.5892760 \\ \mathrm{C} & -1.8406110 \\ \mathrm{C} & -4.3800300 \\ \mathrm{H} & -4.4609510 \\ \mathrm{H} & -2.1825560 \\ \mathrm{H} & -0.8444500 \\ \mathrm{H} & -5.3586230 \\ \mathrm{C} & -0.8722760 \\ \mathrm{C} & 0.4597210 \\ \mathrm{C} & 1.4956260 \\ \mathrm{C} & -1.1373300 \\ \mathrm{H} & -1.6912210 \\ \mathrm{H} & 0.6533280 \\ \mathrm{H} & 2.5217620 \\ \mathrm{H} & -2.1621270 \\ \mathrm{C} & 3.2547090 \\ \mathrm{C} & 4.0122920 \\ \mathrm{C} & 3.6750080 \\ \mathrm{C} & 5.2301310 \\ \mathrm{C} & 4.8892040 \\ \mathrm{C} & 5.7004760 \\ \mathrm{H} & 5.8335950 \\ \mathrm{H} & 5.2372660 \\ \mathrm{C} & -3.9801440 \\ \mathrm{C} & -3.9344660 \\ \mathrm{C} & -4.5642590 \\ \mathrm{C} & -4.5300670 \\ \mathrm{C} & -5.1376940 \\ \mathrm{C} & -5.1411830 \\ \mathrm{H} & -4.5139470 \\ \mathrm{H} & -5.5982310 \\ \mathrm{C} & 2.8832820 \\ \mathrm{C} & 7.0775320 \\ \mathrm{C} & 3.5570180 \\ \mathrm{C} & -3.2688840 \\ \mathrm{C} & -5.7815070 \\ \mathrm{C} & -4.6084090 \\ & \end{array}$

$\begin{array}{rr}3.6729390 & -1.9836010 \\ 1.0886440 & -0.7713500 \\ 0.6375910 & -0.2558100 \\ 2.3180400 & -0.4745860 \\ 1.5082600 & 0.5376270 \\ 3.1867460 & 0.4208810 \\ 2.7811710 & 0.8961500 \\ 1.2207470 & 0.9013070 \\ 4.8777030 & 2.0924350 \\ 5.2650120 & 1.6579340 \\ 4.4376890 & 0.8604040 \\ 3.6539550 & 1.7328920 \\ 5.5440930 & 2.7229970 \\ 6.2219360 & 1.9714260 \\ 4.7394370 & 0.5575160 \\ 3.3296820 & 2.0767680 \\ 5.9138600 & -2.8455030 \\ 6.2782140 & -3.1605180 \\ 5.4580910 & -2.7971850 \\ 4.7575850 & -2.1563390 \\ 6.5536780 & -3.1586990 \\ 7.1994490 & -3.7005070 \\ 5.7131700 & -3.0482770 \\ 4.4828360 & -1.9291940 \\ 1.2596340 & -0.8505950 \\ 0.7084200 & -1.9038740 \\ 1.0915690 & 0.4864350 \\ 0.0901050 & -1.6070870 \\ 0.4452070 & 0.7305690 \\ -0.0284030 & -0.3004780 \\ -0.2984510 & -2.4260590 \\ 0.3341100 & 1.7540760 \\ -0.7136720 & -0.6036000 \\ -1.7590580 & 0.3404940 \\ -0.9211220 & -1.8669700 \\ -2.9791210 & 0.0112640 \\ -2.1607590 & -2.1510830 \\ -3.1993520 & -1.2213150 \\ -3.7920280 & 0.7330660 \\ -2.3166930 & -3.1253430 \\ 1.6811690 & 1.6472770 \\ -0.5988530 & -0.0080950 \\ 0.7708720 & -3.3558240 \\ -1.5878600 & 1.7002110 \\ -4.5358200 & -1.5461630 \\ 0.1680220 & -2.9280290\end{array}$

-6.0532090
-6.6403800
-6.5420120
-6.0765170
-3.9041080
-2.8685560
-4.4138440
-3.9002660
-5.0833490
-5.5219850
-5.1919130
-4.0143600
-7.2826570
-7.7924090
-7.4564530
-7.7407170
-4.2878460
-4.8117760
-5.0368970
-3.7842200
-2.5082200
-3.1884560
-1.7910010
-1.9627580
3.2376600
4.2966220
3.0532110
2.6394900
3.0745660
2.9263490
4.0749140
2.3497090
8.1636290
9.1599220
8.0906460
8.0658970
7.2444210
6.5508950
7.0657630
8.2634980
3.5894620
4.6154010
3.0249530
3.1483430
4.4061150
5.4583240

0.5987080

$-0.2364120$

0.9495820

1. 4085540

$-0.2754820$

$-0.5616960$

$-1.1305000$

0.5404250

$-5.2161820$

$-6.2009980$

$-4.6174790$

$-5.3445760$

$-4.3845150$

$-3.9184780$

$-3.7600300$

$-5.3616390$

$-1.1927600$

$-0.2675280$

$-1.9838020$

$-1.0475900$

$-2.8380110$

$-3.6671570$

$-3.1645400$

$-2.6183030$

3. 1622800

3. 2648170

3.7435300

3. 5919160

0.9225410

$-0.1558310$

1. 0793410

1. 2810600

0.2990540

$-0.0667860$

0.3078960

1. 3299220

$-2.0421740$

$-2.7180880$

$-2.1182080$

$-2.3918730$

$-0.6132050$

$-0.9818970$

$-1.3422850$

$-0.5608610$

1.7553920

1. 4481800
$-3.2083790$

$-3.6063830$

$-2.2938050$

$-3.9448960$

$-4.2149170$

$-4.0101960$

$-4.6723940$

$-4.9451360$

$-2.7292060$

$-2.9204020$

$-3.6403730$

$-2.5354980$

$-1.8175050$

$-0.9689820$

$-2.7008910$

$-2.0023020$

2.7773930

2. 5249140

2.8996500

3.7396910

2. 1555390

2. 3768140

1. 3965960

3. 0811140

1.8437100

2. 1068770

0.9354790

2.6546910

2.9630980

2. 8301860

3. 3808730

3. 7020860

$-0.6151870$

$-0.3467550$

$-1.7084740$

$-0.2620540$

$-0.4953980$

0.0126140

$-1.5743450$

$-0.3008850$

$-4.0140070$

$-4.1246490$

$-3.4277820$

$-5.0147160$

-4.1699800
-4.1665530 


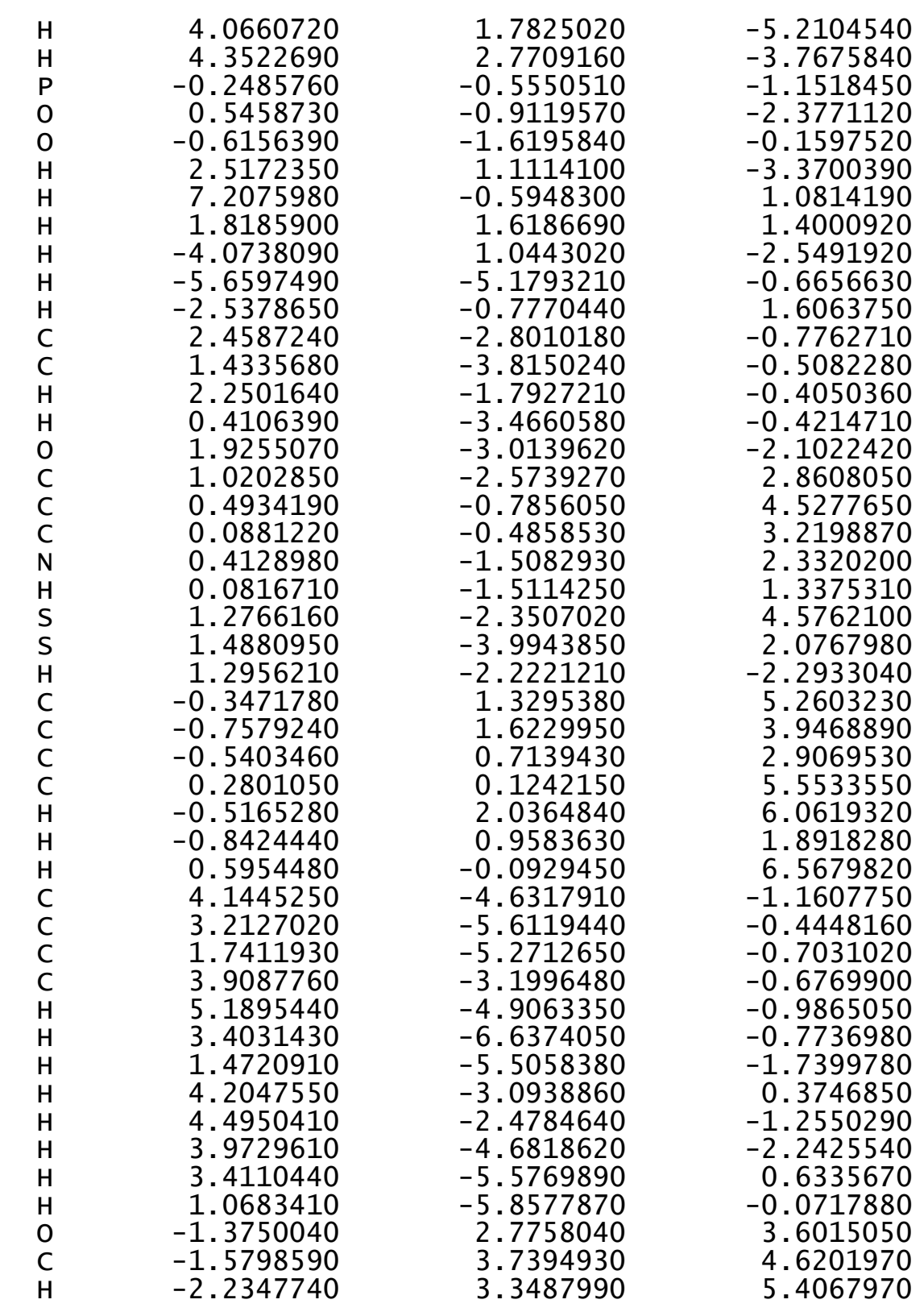

$\begin{array}{lrrr}\mathrm{H} & -0.6275010 & 4.0556430 & 5.0601680 \\ \mathrm{H} & -2.0589450 & 4.5869800 & 4.1345370 \\ \mathrm{I} 48 & & & \\ \mathrm{TS} 8(\mathrm{R}, \mathrm{R}) & \mathrm{G}[\mathrm{M} 06-2 \times / 6-31 \mathrm{G}(\mathrm{d})] & -4124.880371 & \\ \mathrm{O} & 0.2936190 & 0.8754320 & 0.0928200 \\ \mathrm{O} & -1.6857330 & 0.2659190 & -1.3359830 \\ \mathrm{C} & 0.3354210 & 2.1568710 & -0.4254330 \\ \mathrm{C} & -0.8487220 & 2.8561230 & -0.5642140 \\ \mathrm{C} & 1.6103400 & 2.6868760 & -0.7610960 \\ \mathrm{C} & -0.8035570 & 4.1457090 & -1.1920540 \\ \mathrm{C} & 1.6448180 & 3.9744650 & -1.2412250 \\ \mathrm{C} & 0.4639670 & 4.7166390 & -1.4943140 \\ \mathrm{H} & 2.6046630 & 4.4298930 & -1.4709540 \\ \mathrm{C} & -2.4721240 & 0.9682950 & -0.4463930 \\ \mathrm{C} & -3.6860010 & 0.3448100 & -0.0435060 \\ \mathrm{C} & -2.1216790 & 2.2542820 & -0.0794380 \\ \mathrm{C} & -4.5629610 & 1.0773510 & 0.7155840 \\ \mathrm{C} & -3.0012810 & 2.9710140 & 0.8053860 \\ \mathrm{C} & -4.2463440 & 2.3844390 & 1.1686020 \\ \mathrm{H} & -5.5189260 & 0.6416410 & 0.9965240 \\ \mathrm{C} & -4.7901620 & 4.3129740 & 2.5387760 \\ \mathrm{C} & -3.5329140 & 4.8792970 & 2.2193030 \\ \mathrm{C} & -2.6641060 & 4.2292460 & 1.3785630 \\ \mathrm{C} & -5.1325510 & 3.0880580 & 2.0270810 \\ \mathrm{H} & -5.4694100 & 4.8413620 & 3.1998720 \\ \mathrm{H} & -3.2486890 & 5.8335520 & 2.6513150 \\ \mathrm{H} & -1.6970730 & 4.6693610 & 1.1624870 \\ \mathrm{H} & -6.0814970 & 2.6242090 & 2.2823630 \\ \mathrm{C} & -1.8791270 & 6.1027070 & -2.1511310 \\ \mathrm{C} & -0.6159050 & 6.6901240 & -2.4042400 \\ \mathrm{C} & 0.5284880 & 6.0065550 & -2.0872840 \\ \mathrm{C} & -1.9720390 & 4.8674710 & -1.5609570 \\ \mathrm{H} & -2.7831790 & 6.6327980 & -2.4336840 \\ \mathrm{H} & -0.5593850 & 7.6714570 & -2.8640940 \\ \mathrm{H} & 1.5066060 & 6.4303880 & -2.2986830 \\ \mathrm{H} & -2.9463550 & 4.4248020 & -1.3845820 \\ \mathrm{C} & 2.8686610 & 1.8834070 & -0.6454590 \\ \mathrm{C} & 3.5905470 & 1.5690820 & -1.8126240 \\ \mathrm{C} & 3.3724810 & 1.4945530 & 0.6207770 \\ \mathrm{C} & 4.8329000 & 0.9281870 & -1.6938860 \\ \mathrm{C} & 4.5888450 & 0.8195020 & 0.6764960 \\ \mathrm{C} & 5.3550710 & 0.5503410 & -0.4637970 \\ \mathrm{H} & 5.3979710 & 0.7258580 & -2.5995680 \\ \mathrm{H} & 4.9810390 & 0.5116950 & 1.6416320 \\ \mathrm{C} & -4.0191700 & -1.0466960 & -0.4813840 \\ \mathrm{C} & -3.7252690 & -2.1391300 & \\ & & & \end{array}$




\begin{tabular}{|c|c|c|}
\hline $\begin{array}{l}-4.6582680 \\
-4.1309470 \\
-5.0423900 \\
-4.7971780 \\
-3.9184310 \\
-5.5470020 \\
2.6647770 \\
6.7244560 \\
3.0981170 \\
-2.9956100 \\
-5.2298230 \\
-4.9480520 \\
-6.4574050 \\
-6.9559950 \\
-6.9053890 \\
-6.6596030 \\
-4.3061540 \\
-3.2275430 \\
-4.7343050 \\
-4.4743490 \\
-4.5238620 \\
-4.8074200 \\
-4.8010350 \\
-3.4364840 \\
-6.7518640 \\
-7.2635860 \\
-7.0965970 \\
-7.0537410 \\
-3.9722220 \\
-4.6014360 \\
-4.6287480 \\
-3.4189690 \\
-2.0823020 \\
-2.6577590 \\
-1.3998760 \\
-1.4890520 \\
2.8810320 \\
3.9493800 \\
2.5143140 \\
2.3598530 \\
3.1095540 \\
3.1407070 \\
4.1070390 \\
2.4125350 \\
7.6435170 \\
8.6152630\end{array}$ & $\begin{array}{r}-1.2476130 \\
-3.4138670 \\
-2.5407520 \\
-3.6352690 \\
-4.2669000 \\
-2.6955790 \\
1.8865530 \\
-0.0935550 \\
1.9055370 \\
-1.9430000 \\
-5.0319300 \\
-0.0985310 \\
0.1377580 \\
-0.7446380 \\
0.3490600 \\
0.9850300 \\
-0.3332760 \\
-0.4878810 \\
-1.2112830 \\
0.5316540 \\
-5.4842080 \\
-6.5108210 \\
-4.8409900 \\
-5.4429040 \\
-5.1212060 \\
-4.8192550 \\
-4.4681490 \\
-6.1449920 \\
-1.6668320 \\
-0.7942720 \\
-2.5304610 \\
-1.4851340 \\
-3.1146920 \\
-4.0114120 \\
-3.3498000 \\
-2.8514100 \\
3.3792320 \\
3.5812040 \\
4.0140730 \\
3.6625590 \\
1.0657880 \\
-0.0051400 \\
1.3663870 \\
1.2202040 \\
0.8266690 \\
0.3498980\end{array}$ & $\begin{array}{l}-1.7171500 \\
-0.0427010 \\
-2.0748860 \\
-1.2471300 \\
0.5966950 \\
-3.0272780 \\
1.9135940 \\
-0.2961410 \\
-3.2153900 \\
1.6791220 \\
-1.6522960 \\
-2.6703280 \\
-2.7986140 \\
-3.2152110 \\
-1.8222610 \\
-3.4620030 \\
-4.0421700 \\
-3.9444830 \\
-4.5377480 \\
-4.6919970 \\
-2.9357060 \\
-3.1895530 \\
-3.7782620 \\
-2.8232360 \\
-1.8113450 \\
-0.8926660 \\
-2.6207510 \\
-2.0550980 \\
2.8298960 \\
2.6317000 \\
2.9869480 \\
3.7594390 \\
2.0460390 \\
2.3004810 \\
1.2255450 \\
2.9286520 \\
2.2097570 \\
2.3465440 \\
1.3989800 \\
3.1304960 \\
3.1275640 \\
2.9040770 \\
3.4677010 \\
3.9584120 \\
0.5226340 \\
0.6860850\end{array}$ \\
\hline
\end{tabular}

7.8091710

7.2141750

7.4144170

6.8110230

7.6299170

8.3670100

3. 1489520

4.1795000

2.6075630

2.6883360

3.9094310

4.9641120

3.5347680

3.8623790

$-0.2176500$

0.5778580

$-0.2851700$

2.0517920

6.5797000

1. 5909240

$-4.5057270$

$-4.9332440$

$-2.3521800$

2. 9551030

2. 1079780

2.8627670

1.1611100

2.0004660

2.3233830

1.4581660

0.8285030

1.3327720

0.8781460

2.7002640

3.1444870

1. 3204750

0.0867850

$-0.5353470$

$-0.1659050$

1.0859760

$-0.2362890$

$-0.5906310$

1.5677500

4.4422350

3.8279720
2.3499350
1.7659420

1.0689260

$-0.4536020$

$-1.1245170$

0.4471360

$-0.9505460$

0.6907650

0.3787820

$-0.1563850$

0.9426650

3.0572030

2.7740130

3.3001680

3.9626790

$-0.3028690$

$-0.3114660$

$-1.5359430$

2. 2151120

$-1.0186820$

1.7147690

0.8127240

$-5.7132770$

$-1.0644430$

$-2.1975490$

$-3.2971430$

$-3.0319730$

$-2.3002940$

$-2.7995690$

$-1.7338730$

$-1.3012670$

$-1.9257970$

$-1.7683710$

$-2.9047530$

$-3.7143000$

$-1.5414260$

$-0.2442070$

0.1955220

$-0.3232190$

$-1.1991400$

0.1963740

0.0106390

$-1.5201740$

$-3.8791750$

$-4.9815680$

$-4.7026580$
$-0.0167260$

1.4984740

$-1.6112410$

$-2.2291990$

$-1.4056310$

$-4.1491670$

$-4.3533260$

$-3.7226960$

$-5.1101400$

$-3.8249550$

$-3.9198300$

$-4.8247610$

$-3.2133490$

$-0.9178800$

$-2.1940080$

$-0.0662100$

$-3.1481210$

0.2804580

1.7814660

$-2.2556480$

$-0.8448740$

1.5573300

$-1.6699640$

$-1.1962510$

$-1.1329650$

$-0.7349540$

$-2.7498490$

2.2998850

4.3970370

3.2319580

2.0979440

1.1740960

4.0058050

1. 1428700

$-2.5886130$

5.6597490

4.4722570

3.2339930

5.6318930

6.5963390

2. 2941380

6.5486820

$-2.8193980$

$-1.9541680$

$-1.6681430$ 


$\begin{array}{lrrr}\mathrm{C} & 4.3579210 & -2.5194880 & -2.1215100 \\ \mathrm{H} & 5.4886330 & -4.1092940 & -3.0401960 \\ \mathrm{H} & 3.9190860 & -5.9540090 & -2.4461440 \\ \mathrm{H} & 1.7562400 & -4.8290490 & -2.5812740 \\ \mathrm{H} & 5.0026240 & -2.4994770 & -1.2329560 \\ \mathrm{H} & 4.6878380 & -1.7128860 & -2.7844740 \\ \mathrm{H} & 3.9125170 & -3.8271310 & -3.7778870 \\ \mathrm{H} & 4.3785170 & -5.0502770 & -1.0068350 \\ \mathrm{H} & 1.9383010 & -5.3944380 & -0.9297580 \\ \mathrm{O} & -1.4894500 & 1.1394660 & 4.6314760 \\ \mathrm{C} & -2.0451520 & 1.6824700 & 3.4462470 \\ \mathrm{H} & -1.2630450 & 2.1361250 & 2.8250080 \\ \mathrm{H} & -2.5661770 & 0.9114610 & 2.8632190 \\ \mathrm{H} & -2.7581360 & 2.4470880 & 3.7564230 \\ \mathrm{I} 48 & & & \\ \mathrm{TS} 9(\mathrm{R}, \mathrm{R}) & \mathrm{G}[\mathrm{M} 06-2 \mathrm{~N} / 6-31 \mathrm{G}(\mathrm{d})] & = & -4124.880564 \\ \mathrm{O} & 0.5294660 & 0.5985840 & -0.2492420 \\ \mathrm{O} & -1.4852530 & 0.4177350 & -1.7637080 \\ \mathrm{C} & 0.8039810 & 1.8579900 & -0.7518390 \\ \mathrm{C} & -0.2352000 & 2.7535130 & -0.9258320 \\ \mathrm{C} & 2.1629550 & 2.1847080 & -1.0009960 \\ \mathrm{C} & 0.0448270 & 4.0066270 & -1.5613210 \\ \mathrm{C} & 2.4281150 & 3.4334180 & -1.5130170 \\ \mathrm{C} & 1.3966080 & 4.3480080 & -1.8416310 \\ \mathrm{H} & 3.4608980 & 3.7286160 & -1.6810000 \\ \mathrm{C} & -2.1581420 & 1.1922370 & -0.8347220 \\ \mathrm{C} & -3.4277210 & 0.7355090 & -0.3919160 \\ \mathrm{C} & -1.5955840 & 2.3897680 & -0.4443020 \\ \mathrm{C} & -4.1469710 & 1.5710700 & 0.4266570 \\ \mathrm{C} & -2.3229090 & 3.2176400 & 0.4756230 \\ \mathrm{C} & -3.6251820 & 2.8098710 & 0.8795920 \\ \mathrm{H} & -5.1473700 & 1.2815230 & 0.7376080 \\ \mathrm{C} & -3.8769250 & 4.8344820 & 2.1931490 \\ \mathrm{C} & -2.5650130 & 5.2224150 & 1.8269730 \\ \mathrm{C} & -1.8026250 & 4.4297750 & 1.0074990 \\ \mathrm{C} & -4.3851400 & 3.6450870 & 1.7418630 \\ \mathrm{H} & -4.4676000 & 5.4726380 & 2.8430920 \\ \mathrm{H} & -2.1533530 & 6.1510160 & 2.2113370 \\ \mathrm{H} & -0.7909000 & 4.7293550 & 0.7589460 \\ \mathrm{H} & -5.3808970 & 3.3199920 & 2.0313690 \\ \mathrm{C} & -0.6652480 & 6.1015150 & -2.5655780 \\ \mathrm{C} & 0.6843050 & 6.4563150 & -2.8085070 \\ \mathrm{C} & 1.6902660 & 5.5950170 & -2.4565380 \\ \mathrm{C} & -0.9777200 & 4.9122730 & -1.9570970 \\ \mathrm{H} & -1.4596700 & 6.7755590 & -2.8699930 \\ \mathrm{H} & 0.9154000 & 7.4037660 & -3.2846190 \\ & & & \\ & & & \end{array}$

2.7298020
-2.0153070
3.2980380
4.0956120
3.6556480
5.2889850
4.8472420
5.6969840
5.9228050
5.1469310
-3.9749490
-3.9891980
-4.5066930
-4.5927390
-5.0928890
-5.1567910
-4.6187290
-5.5175260
2.8223220
7.0482840
3.7110100
-3.3767610
-5.8086190
-4.4891200
-5.9124280
-6.5157300
-6.4111230
-5.8926970
-3.7676510
-2.7503510
-4.2977050
-3.7111580
-7.0054370
-7.4956490
-6.6829410
-7.7426830
-4.7965130
-3.9493480
-4.4063130
-5.2684700
-4.4330900
-4.9320630
-5.1987050
-3.9676430
-2.6543800
-3.3563540

5.8433290

4.6457530

1.2586340

0.7612670

1.0018580

0.1034010

0.3193870

$-0.1051970$

$-0.2448870$

0.1388320

$-0.5837830$

$-1.6800100$

$-0.7127410$

$-2.8662900$

-1.9260560

$-3.0086880$

$-3.7118370$

$-2.0280870$

1.5340720

$-0.7220530$

0.9204830

$-1.5937410$

$-4.3124370$

0.4310250

0.9228870

0.1282490

1.2430390

1.7693020

0.0342380

$-0.3059680$

$-0.7704460$

0.8905610

$-4.6536210$

$-5.5694430$

$-4.8161310$

$-3.8452380$

$-5.4637660$

$-5.2368030$

$-5.6484750$

$-6.3869480$

$-1.2426200$

$-0.2952320$

$-2.0259200$

$-1.1624080$

$-2.8803560$

$-3.7072820$
$-2.6532800$

$-1.7849650$

$-0.6911240$

$-1.7416270$

0.6505340

$-1.4301660$

0.9071780

$-0.1142010$

$-2.2442760$

1.9359560

$-0.8356830$

0.0544990

$-2.1290590$

$-0.3606800$

$-2.5008350$

$-1.6303070$

0.3225330

$-3.4980660$

1.8102330

0.2028260

$-3.2064800$

1.4471640

$-2.0509250$

$-3.1312360$

$-3.4208580$

$-3.8737050$

$-2.5003360$

$-4.1152140$

$-4.4236090$

$-4.2100580$

$-4.9445630$

$-5.1035990$

$-1.1559270$

$-1.5018600$

$-0.1215180$

$-1.1568680$

$-2.0635010$

$-2.7173540$

$-1.0564900$

$-2.4153800$

2.5035020

2.2849770

2. 5489100

3.4922400

1.8617110

2. 0125230 


\begin{tabular}{|c|c|c|c|c|c|c|c|}
\hline H & -1.9150330 & -3.1795310 & 1.1128130 & $\mathrm{~s}$ & 1.2933830 & -4.1257090 & 1.8476560 \\
\hline $\mathrm{H}$ & -2.1406480 & -2.7211670 & 2.8173850 & $\mathrm{H}$ & 1.3318170 & -2.0976280 & -2.4119390 \\
\hline C & 3.1746610 & 3.0002610 & 2.1005000 & C & -0.5085330 & 1.0615010 & 5.2676380 \\
\hline $\mathrm{H}$ & 4.2227070 & 3.0818240 & 2.4105700 & $\mathrm{C}$ & -0.8542400 & 1.4444060 & 3.9584440 \\
\hline H & 3.0285550 & 3.6316340 & 1.2192160 & $\mathrm{C}$ & -0.6283800 & 0.5877240 & 2.8766560 \\
\hline H & 2.5463310 & 3.3884120 & 2.9097220 & C & 0.0650730 & -0.1805810 & 5.5136890 \\
\hline C & 2.9594060 & 0.7059530 & 3.0904040 & $\mathrm{H}$ & -0.6864900 & 1.7274660 & 6.1019080 \\
\hline H & 2.8076910 & -0.3626880 & 2.8965090 & $\mathrm{H}$ & -0.8818790 & 0.8997090 & 1.8667370 \\
\hline $\mathrm{H}$ & 3.9449630 & 0.8315280 & 3.5515700 & $\mathrm{H}$ & 0.3311950 & -0.4674600 & 6.5252100 \\
\hline $\mathrm{H}$ & 2.2108930 & 1.0327890 & 3.8204000 & C & 4.0929780 & -4.6150940 & -1.2971580 \\
\hline C & 8.1785440 & 0.1827250 & -0.3051140 & C & 3.1150470 & -5.6190610 & -0.6829470 \\
\hline $\mathrm{H}$ & 9.1540180 & -0.2206400 & -0.0148890 & C & 1.6620590 & -5.2388600 & -0.9867650 \\
\hline $\mathrm{H}$ & 8.1548960 & 0.2540760 & -1.3982670 & $\mathrm{C}$ & 3.8562930 & -3.2097420 & -0.7407710 \\
\hline $\mathrm{H}$ & 8.0867730 & 1.1943300 & 0.1012460 & $\mathrm{H}$ & 5.1247610 & -4.9174350 & -1.0925800 \\
\hline C & 7.2053440 & -2.1391700 & -0.3591780 & $\mathrm{H}$ & 3.3055390 & -6.6267060 & -1.0629650 \\
\hline $\mathrm{H}$ & 6.4766210 & -2.8260300 & 0.0802790 & $\mathrm{H}$ & 1.4376690 & -5.4078280 & -2.0467410 \\
\hline $\mathrm{H}$ & 7.0709040 & -2.1497550 & -1.4471530 & $\mathrm{H}$ & 4.1060400 & -3.1701840 & 0.3273320 \\
\hline H & 8.2075970 & -2.5232780 & -0.1432660 & $\mathrm{H}$ & 4.4782710 & -2.4662320 & -1.2491010 \\
\hline C & 3.7356880 & -0.4257600 & -3.9393140 & $\mathrm{H}$ & 3.9704980 & -4.5990840 & -2.3865960 \\
\hline $\mathrm{H}$ & 4.7544080 & -0.8215730 & -4.0183890 & $\mathrm{H}$ & 3.2640170 & -5.6505180 & 0.4034350 \\
\hline $\mathrm{H}$ & 3.1199500 & -1.1661770 & -3.4227650 & $\mathrm{H}$ & 0.9524220 & -5.8498830 & -0.4224690 \\
\hline $\mathrm{H}$ & 3.3484080 & -0.3049800 & -4.9562790 & 0 & -1.4125550 & 2.6386150 & 3.6565310 \\
\hline C & 4.6245220 & 1.9233270 & -3.9225350 & C & -1.6183400 & 3.5530890 & 4.7192840 \\
\hline $\mathrm{H}$ & 5.6663340 & 1.5840810 & -3.8913880 & $\mathrm{H}$ & -2.3199510 & 3.1489000 & 5.4572710 \\
\hline $\mathrm{H}$ & 4.3332040 & 2.0215100 & -4.9734590 & $\mathrm{H}$ & -0.6720930 & 3.8033140 & 5.2118110 \\
\hline $\mathrm{H}$ & 4.5812330 & 2.9149300 & -3.4629000 & $\mathrm{H}$ & -2.0425140 & 4.4457040 & 4.2641580 \\
\hline $\mathrm{P}$ & -0.2317040 & -0.4691160 & -1.2376250 & 14 & & & \\
\hline 0 & 0.5986200 & -0.7783040 & -2.4519940 & \multicolumn{4}{|c|}{$\begin{array}{l}140 \\
\text { TS10 ( R , R }\end{array}$} \\
\hline 0 & -0.6592840 & -1.5764260 & -0.3194850 & 0 & 0.2591370 & 0.8470600 & 0.1234260 \\
\hline $\mathrm{H}$ & 2.6835770 & 1.2940310 & -3.2491420 & 0 & -1.6665970 & 0.2516340 & -1.3919330 \\
\hline $\mathrm{H}$ & 7.1309170 & -0.7826690 & 1.2952800 & C & 0.2702550 & 2.1649260 & -0.2969360 \\
\hline $\mathrm{H}$ & 1.7676680 & 1.4914400 & 1.5194420 & $\mathrm{C}$ & -0.9353960 & 2.8240230 & -0.4329100 \\
\hline $\mathrm{H}$ & -3.9366050 & 1.2678130 & -2.6935370 & C & 1.5330570 & 2.7669880 & -0.5438570 \\
\hline $\mathrm{H}$ & -6.1793660 & -4.1767110 & -3.0747920 & C & -0.9232220 & 4.1533960 & -0.9736920 \\
\hline $\mathrm{H}$ & -2.6307650 & -0.7911210 & 1.4281080 & C & 1.5301040 & 4.0856550 & -0.9321990 \\
\hline C & 2.4182140 & -2.7812570 & -0.8812180 & C & 0.3288640 & 4.7946310 & -1.1849960 \\
\hline C & 1.3668130 & -3.7912650 & -0.7201260 & $\mathrm{H}$ & 2.4775370 & 4.5943290 & -1.0906050 \\
\hline $\mathrm{H}$ & 2.2082320 & -1.7927020 & -0.4608180 & C & -2.5077610 & 0.8848410 & -0.5011090 \\
\hline $\mathrm{H}$ & 0.3461110 & -3.4303640 & -0.6595540 & $\mathrm{C}$ & 57600 & 0.2354130 & -0.2239600 \\
\hline 0 & 1.9442240 & -2.9076190 & -2.2402530 & $\mathrm{C}$ & -2.2004960 & 2.1487330 & -0.0331750 \\
\hline C & 0.8343390 & -2.7401610 & 2.6966460 & C & -4.6530820 & 0.8916190 & 0.5678120 \\
\hline C & 0.2908680 & -1.0366050 & 4.4452440 & $\mathrm{C}$ & -3.1260020 & 2.7879220 & 0.8648640 \\
\hline $\mathrm{C}$ & -0.0515970 & -0.6487040 & 3.1426110 & C & -4.3720560 & 2.1596240 & 1.1392550 \\
\hline $\mathrm{N}$ & 0.2793370 & -1.6273890 & 2.2089370 & $\mathrm{H}$ & -5.6101950 & 0.4219990 & 0.7822780 \\
\hline $\mathrm{H}$ & -0.0138250 & -1.5643540 & 1.2045360 & C & -4.9918560 & 3.9675570 & 2.6349890 \\
\hline $\mathrm{S}$ & 1.0256180 & -2.6255780 & 4.4312110 & C & -3.7359160 & 4.5758520 & 2.3993000 \\
\hline
\end{tabular}




$\begin{array}{rrr}-2.8299030 & 4.0051060 & 1.5403600 \\ -5.2969160 & 2.7805790 & 2.0202080 \\ -5.7003470 & 4.4334410 & 3.3121860 \\ -3.4821900 & 5.4988760 & 2.9108610 \\ -1.8646010 & 4.4753520 & 1.3904670 \\ -6.2455710 & 2.2846950 & 2.2073550 \\ -2.0454400 & 6.1233290 & -1.8484200 \\ -0.8003010 & 6.7777040 & -2.0100280 \\ 0.3598560 & 6.1225870 & -1.6900950 \\ -2.1072430 & 4.8491570 & -1.3428960 \\ -2.9600450 & 6.6332240 & -2.1341720 \\ -0.7689250 & 7.7886980 & -2.4032280 \\ 1.3257830 & 6.5995530 & -1.8330950 \\ -3.0674620 & 4.3560180 & -1.2354440 \\ 2.8188710 & 2.0080030 & -0.4360460 \\ 3.5985660 & 1.8161890 & -1.5925180 \\ 3.2886630 & 1.5403120 & 0.8166030 \\ 4.8611260 & 1.2169130 & -1.4716190 \\ 4.5286000 & 0.9096050 & 0.8706420 \\ 5.3491470 & 0.7610510 & -0.2539680 \\ 5.4690270 & 1.1096040 & -2.3657900 \\ 4.8951110 & 0.5406730 & 1.8243320 \\ -4.0627090 & -1.1074360 & -0.8039150 \\ -3.6692600 & -2.2688760 & -0.1002270 \\ -4.7739940 & -1.2155490 & -2.0122730 \\ -4.0105480 & -3.5177330 & -0.6150270 \\ -5.0851470 & -2.4964340 & -2.4856430 \\ -4.7204900 & -3.6542190 & -1.8091760 \\ -3.7108140 & -4.4120470 & -0.0750430 \\ -5.6350530 & -2.5852730 & -3.4213900 \\ 2.5145360 & 1.8008080 & 2.1046910 \\ 6.7341340 & 0.1530550 & -0.0805940 \\ 3.1465180 & 2.2424870 & -2.9842590 \\ -2.9269890 & -2.1709560 & 1.2262260 \\ -5.0745030 & -5.0243870 & -2.3553220 \\ -5.3027110 & -0.0479010 & -2.8448020 \\ -4.3645080 & 1.1482060 & -3.0544980 \\ -4.3687610 & 1.8279030 & -2.1974470 \\ -3.3337580 & 0.8310930 & -3.2372770 \\ -4.7067510 & 1.7190690 & -3.9238840 \\ -6.6656880 & 0.4275920 & -2.3214230 \\ -7.3677670 & -0.4067760 & -2.2293240 \\ -6.5604670 & 0.8989120 & -1.3391260 \\ -7.0961430 & 1.1675690 & -3.0047670 \\ -5.9823870 & -5.7937750 & -1.3889990 \\ -6.2700020 & -6.7590300 & -1.8181790\end{array}$
$-5.4661480$
$-6.8927060$
$-3.8159290$
$-3.1717520$
$-3.2350560$
$-4.0848750$
$-3.9046440$
$-4.5516330$
$-4.5435630$
$-3.3512800$
$-2.0010050$
$-2.5696620$
$-1.3343940$
$-1.3899540$
2. 6468720
3.6978660
2. 2827480
2. 0761290
2.9508920
3.0397430
3. 9198570
2. 2163900
7.5824260
8.5634100
7.7358530
7.1033890
7.4915790
6.9407890
7.6959060
8.4533550
3.2757840
4.3238860
2.7476020
2.8445980
3.9365250
5.0035180
3.5892210
3.8349500
$-0.1701720$
0.6464020
$-0.1763520$
2.0877800
6.6002600
1.4561400
$-5.4809670$
$-5.6281890$

$-0.4423820$

$-1.1675980$

$-2.6792310$

$-3.3839500$

$-1.7707820$

$-3.1187290$

2. 3985530

2.2745760

2.4851130

3.3388560

1.4993000

1.7137130

0.6537860

2.3832790

2. 5199440

2.7178890

1.7433850

3.4355740

3.2718170

2.9712370

3.6739190

4.0822830

0.8559380

1.0217350

0.4082030

1.8285470

$-1.3897580$

$-2.0901230$

$-1.8850760$

$-1.1856940$

$-4.0043530$

$-4.1884890$

$-3.6667510$

$-4.9618170$

$-3.4726950$

$-3.5484210$

$-4.4646800$

$-2.7960680$

$-0.9982020$

$-2.2530330$

$-0.2642150$

$-2.9343440$

0.4061510

1.9155030

$-3.8398150$

$-0.4739910$

$-4.8705140$

$-3.2901440$ 


\begin{tabular}{|c|c|c|c|}
\hline & & & \\
\hline $\mathrm{H}$ & -2.2949930 & -1.2775570 & 1.1773640 \\
\hline C & 3.1014560 & -1.9486960 & -1.7736320 \\
\hline C & 2.3007850 & -3.1240380 & -1.4158620 \\
\hline $\mathrm{H}$ & 2.9486700 & -1.0536490 & -1.1615320 \\
\hline $\mathrm{H}$ & 1.3309710 & -2.9449830 & -0.9618470 \\
\hline 0 & 2.1790950 & -2.0072910 & -2.8847510 \\
\hline C & 2.3882260 & -2.8945490 & 2.1091640 \\
\hline C & 1.3795060 & -2.0507250 & 4.2436310 \\
\hline C & 0.7805090 & -1.5515020 & 3.0890930 \\
\hline $\mathrm{N}$ & 1.3644660 & -2.0540120 & 1.9330620 \\
\hline $\mathrm{H}$ & 0.9453670 & -1.8378480 & 1.0043200 \\
\hline $\mathrm{S}$ & 2.6939870 & -3.1268440 & 3.8168130 \\
\hline $\mathrm{S}$ & 3.3055930 & -3.6639860 & 0.9193270 \\
\hline $\mathrm{H}$ & 1.4629610 & -1.2903360 & -2.6926850 \\
\hline C & -0.1150190 & -0.7318910 & 5.5564350 \\
\hline C & -0.7047210 & -0.2225620 & 4.3803260 \\
\hline C & -0.2593980 & -0.6227700 & 3.1233630 \\
\hline C & 0.9284320 & -1.6372060 & 5.4986620 \\
\hline $\mathrm{H}$ & -0.4980440 & -0.3841670 & 6.5093790 \\
\hline $\mathrm{H}$ & -0.6592840 & -0.2314170 & 2.1950450 \\
\hline $\mathrm{H}$ & 1.3845370 & -2.0115500 & 6.4083630 \\
\hline C & 4.7003700 & -3.4566780 & -3.0117270 \\
\hline C & 4.1235940 & -4.6549050 & -2.2552240 \\
\hline C & 2.6269360 & -4.4729370 & -1.9893780 \\
\hline C & 4.5297450 & -2.1649330 & -2.2087360 \\
\hline $\mathrm{H}$ & 5.7622570 & -3.6157630 & -3.2209430 \\
\hline $\mathrm{H}$ & 4.2763250 & -5.5786300 & -2.8203430 \\
\hline $\mathrm{H}$ & 2.0621960 & -4.5554200 & -2.9 \\
\hline $\mathrm{H}$ & 5.1485950 & -2.1887830 & -1.3021350 \\
\hline $\mathrm{H}$ & 4.8377680 & -1.2917620 & -2.7929270 \\
\hline $\mathrm{H}$ & 4.1917370 & -3.3525860 & -3.9774070 \\
\hline $\mathrm{H}$ & 4.6539900 & -4.7727660 & -1.3012330 \\
\hline $\mathrm{H}$ & 6830 & -5.2405920 & -1.3 \\
\hline 0 & -1.7 & 0.6651200 & 4.5 \\
\hline C & -2.2 & 1.2991860 & 3.4 \\
\hline $\mathrm{H}$ & -1.4 & 4650 & 2.8 \\
\hline $\mathrm{H}$ & -2.6 & 850 & 80 \\
\hline $\mathrm{H}$ & -2.97 & 2.0075360 & \\
\hline & 106 & d) $]=-4124$ & \\
\hline 0 & 0.4843730 & 0.6968070 & -0.1628440 \\
\hline 0 & -1.4232020 & 0.2805700 & -1.7536230 \\
\hline C & 0.6525890 & 1.9578460 & -0.7071080 \\
\hline C & -0.4591100 & 2.7519630 & -0.9112330 \\
\hline C & 1.9786340 & 2.3809620 & -0.9859930 \\
\hline$c$ & -0 . & 4.020243 & \\
\hline
\end{tabular}

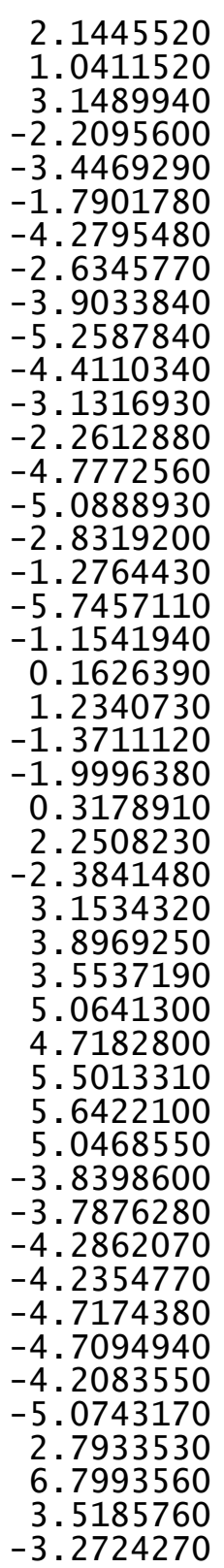

3.6466670

4.4767690

4.0142570

0.9988170

0.4231010

2. 2534880

1.1871340

3. 0094990

2. 4743600

0.8015530

4.4729630

4.9895140

4.2723790

3. 2342050

5.0528490

5. 9581730

4.6717270

2. 8116970

6.0396030

6.5119870

5. 7431040

.8296450

6.6387580

7.4746070

6.0806540

4.4733070

1.4852770

1. 0178880

1.1630890

0.2794090

0.4224260

$-0.0124120$

$-0.0664360$

0.1779320

$-0.9446610$

$-2.0358620$

$-1.1287780$

$-3.2803540$

$-2.3979720$

$-3.4823500$

$-4.1239390$

$-2.5445320$

1. 6933740

$-0.7510700$

1. 3175320
$-1.4953860$

$-1.8176560$

$-1.6906530$

$-0.8687970$

$-0.4749760$

$-0.4719640$

0.3052500

0.4087510

0.7672440

0.5765710

2. 0460770

1.7266860

0.9465560

1. 5903230

2. 6649370

2. 1156910

0.7328570

1.8446580

$-2.5854660$

$-2.8053080$

$-2.4329870$

$-1.9761300$

$-2.9085510$

$-3.2817720$

$-2.6154590$

$-1.8219230$

$-0.7566060$

$-1.8553880$

0.5622090

$-1.6171110$

0.7434830

$-0.3315230$

$-2.4694810$

1.7514730

$-0.9347040$

$-0.0401470$

$-2.2537350$

$-0.4813350$

$-2.6500650$

$-1.7792710$

0.2041570

$-3.6681040$

1.7712350

$-0.0486530$

$-3.3010290$

1. 3857320 


$\begin{array}{rrr}-5.1901620 & -4.8495220 & -2.2281520 \\ -4.3384970 & 0.0101980 & -3.2604380 \\ -5.7884730 & 0.3364640 & -3.6385340 \\ -6.2681630 & -0.5205670 & -4.1242160 \\ -6.3761050 & 0.5950100 & -2.7517630 \\ -5.8230330 & 1.1813720 & -4.3341420 \\ -3.4999570 & -0.3010870 & -4.5048080 \\ -2.4638970 & -0.5171050 & -4.2289040 \\ -3.8995970 & -1.1643730 & -5.0478670 \\ -3.5053690 & 0.5542860 & -5.1884070 \\ -6.3945230 & -5.3170220 & -1.4030240 \\ -6.7610800 & -6.2814480 & -1.7694940 \\ -6.1188630 & -5.4412100 & -0.3499190 \\ -7.2135800 & -4.5934950 & -1.4547090 \\ -4.0607730 & -5.8846590 & -2.1719070 \\ -3.2044590 & -5.5667240 & -2.7738400 \\ -3.7151710 & -6.0268270 & -1.1419990 \\ -4.4078750 & -6.8532070 & -2.5464750 \\ -4.4234050 & -1.6461800 & 2.3725570 \\ -5.0079230 & -0.7593760 & 2.1153640 \\ -5.1008970 & -2.5080210 & 2.3777280 \\ -4.0318270 & -1.5138510 & 3.3872830 \\ -2.4438680 & -3.0853530 & 1.8522140 \\ -3.0617360 & -3.9824460 & 1.9653560 \\ -1.6324100 & -3.3030630 & 1.1519800 \\ -2.0098370 & -2.8723240 & 2.8364960 \\ 3.2449180 & 3.1268580 & 2.0868100 \\ 4.3094750 & 3.1379390 & 2.3468800 \\ 3.0958150 & 3.7895100 & 1.2289340 \\ 2.6801080 & 3.5287240 & 2.9346990 \\ 2.9331240 & 0.8146260 & 3.0170780 \\ 2.7296800 & -0.2393480 & 2.7901530 \\ 3.9371570 & 0.8790590 & 3.4501910 \\ 2.2223390 & 1.1442760 & 3.7825240 \\ 7.8870080 & 0.2498210 & 0.3678400 \\ 8.8154580 & -0.2711770 & 0.6232690 \\ 8.0964580 & 0.9410370 & -0.4560410 \\ 7.5724570 & 0.8401570 & 1.2335880 \\ 7.2936860 & -1.6114210 & -1.2121730 \\ 6.5211530 & -2.2990080 & -1.5747500 \\ 7.6122160 & -0.9896530 & -2.0558300 \\ 8.1573570 & -2.2042580 & -0.8969890 \\ 3.4730450 & 0.0591090 & -4.1755540 \\ 4.4487190 & -0.4351370 & -4.2297840 \\ 2.7394970 & -0.6555930 & -3.7991500 \\ 3.1865190 & 0.3333620 & -1963360\end{array}$

\begin{abstract}
4. 4926750
5.5073870

4. 1947640

4. 5304330

$-0.1097350$

0.8089100

$-0.4634550$

2. 5159420

6. 6124080

1. 7279480

$-3.9128030$

$-5.5116010$

$-2.6163640$

1.9618660

2. 6273730

0.8816170

2. 1692680

2. 6725320

0.9369010

0.2073590

$-0.1569990$

0.2767290

$-0.0025710$

1.0986680

1.5456590

1.9983780

$-0.8353440$

$-1.2051220$

$-0.8607240$

$-0.1279130$

$-1.1000170$

$-1.1312130$

0.1526770

3.9738460

4. 4120210

4.0452920

2. 4570560

4.2724440

5. 4929680

4.6626570

1.9447610

2. 1436600

4.4737130

3. 9290110

4.2167240

$-1.9013580$
\end{abstract}

2. 3371680

1.9241440

2. 5883740

3. 2621940

$-0.4681990$

$-0.7070850$

$-1.5962870$

1.7535480

$-1.4138570$

1.7224330

0.9046170

$-4.7558960$

$-1.0056150$

$-3.4171410$

$-2.6758440$

$-3.2844000$

$-1.7462440$

$-2.3998940$

$-2.5074840$

$-0.8311430$

$-0.5062420$

$-1.4676970$

$-1.4644930$

$-2.3373580$

$-3.8391040$

$-1.6565520$

1.1781470

1. 4954640

0.6550650

0.0149210

1.8341850

0.9175050

$-0.2213030$

$-4.9331980$

$-4.4502580$

$-2.9804270$

$-4.8094130$

$-5.9744940$

$-4.5665130$

$-2.3411110$

$-5.4957270$

$-5.0702340$

$-4.3375750$

$-5.0623450$

$-2.6477590$

2. 6069680
$-3.9085240$

-3.9437670

$-4.9317430$

$-3.3255020$

$-2.3194740$

$-0.2316720$

$-3.3109220$

0.8083940

1. 5227900

$-2.7957450$

$-3.2732280$

1. 4054430

$-1.1169920$

$-0.0437950$

$-1.1667010$

0.2871220

$-1.8677440$

2.8554480

4.5595690

3. 2450560

2. 3381720

1. 3232300

4. 5862900

2. 0121520

$-2.0670650$

5. 3293260

4.0088360

2. 9462840

5. 6075310

6. 1481950

1. 9267320

6.6281330

$-1.2618930$

0.1224670

0.3323610

$-1.4266370$

$-1.4124480$

0.2482150

$-0.3081230$

$-0.7406760$

$-2.4422050$

$-2.0354330$

0.8947800

1. 3600750

3. 6791250 


\begin{tabular}{|c|c|c|c|c|c|c|c|}
\hline & & & & & & & \\
\hline C & -2.2437100 & 3.4996850 & 4.7251160 & C & -3.9084710 & -1.2261450 & 0.0216170 \\
\hline H & -1.3478000 & $\begin{array}{l}3.0163910 \\
3.8772720\end{array}$ & $\begin{array}{l}5.456 / 090 \\
5.2301460\end{array}$ & $\begin{array}{l}\mathrm{C} \\
\mathrm{C}\end{array}$ & $\begin{array}{l}-3.4698200 \\
-4.6250540\end{array}$ & $\begin{array}{l}-2.1916600 \\
-1.6127480\end{array}$ & $\begin{array}{r}0.9484520 \\
-1.1249840\end{array}$ \\
\hline $\mathrm{H}$ & -2.7714670 & 4.3243740 & 4.2506860 & c & -3.8094230 & -3.5283620 & 0.7284270 \\
\hline 14 & & & & C & -4.9374510 & -2.9609750 & -1.3026760 \\
\hline TS & 5) $G[M 06-2 X]$ & d) $]=-412$ & & c & -4.5471340 & -3.9329760 & -0.3825880 \\
\hline 0 & 0.2363660 & 0.9984480 & -0.0683710 & $\mathrm{H}$ & -3.4838410 & -4.2846340 & 1.4383240 \\
\hline 0 & -1.8022140 & 0.1381970 & -1.2634720 & $\mathrm{H}$ & -5.5012080 & -3.2590230 & -2.1852330 \\
\hline C & & 2.2039540 & -0.7433950 & C & 2.5973930 & 2.7766220 & 1.4291240 \\
\hline C & -1.0726870 & 2.8182730 & -0.8635630 & C & 6.3473300 & -0.1420540 & -0.3263670 \\
\hline $\mathrm{C}$ & 1.3704220 & 2.7538250 & -1.2407010 & C & 2.7800410 & 1.4478300 & -3.5128270 \\
\hline C & -1.1562290 & 4.0145740 & -1.6497390 & C & -2.6494360 & -1.8048630 & 2.1710020 \\
\hline $\mathrm{C}$ & 1.2882840 & 3.9520340 & -1.9059650 & c & -4.8987380 & -5.3935800 & -0.5942620 \\
\hline $\mathrm{C}$ & 0.0439320 & 4.5894240 & -2.1536050 & C & -5.0789010 & -0.6047620 & -2.1695020 \\
\hline $\mathrm{H}$ & 2.1953820 & 4.4300170 & -2.2657780 & C & -6.6072840 & -0.4834860 & -2.1816820 \\
\hline C & -2.5428150 & 0.8788900 & -0.3633370 & $\mathrm{H}$ & -7.0717230 & -1.4389850 & -2.4501810 \\
\hline $\mathrm{C}$ & -3.6378850 & 0.2249700 & 0.2657450 & $\mathrm{H}$ & -6.9849340 & -0.1895160 & -1.1970580 \\
\hline C & -2.2502240 & 2.2163040 & -0.1807340 & $\mathrm{H}$ & -6.9283350 & 0.2658230 & -2.9126560 \\
\hline $\mathrm{C}$ & -4.4557760 & 0.9785860 & 1.0697910 & C & -4.5411380 & -0.9565030 & -3.5609220 \\
\hline $\mathrm{C}$ & -3.0577140 & 2.9715690 & 0.7370980 & $\mathrm{H}$ & -3.4498390 & -1.0309450 & -3.5479290 \\
\hline C & -4.1843470 & 2.3453070 & 1.3411710 & $\mathrm{H}$ & -4.9453110 & -1.9109280 & -3.9152880 \\
\hline $\mathrm{H}$ & -5.3269270 & 0.5166530 & 1.5279470 & $\mathrm{H}$ & -4.8268410 & -0.1850250 & -4.2833500 \\
\hline C & -4.6843810 & 4.3754400 & 2.5747560 & C & -4.2463420 & -5.9433770 & -1.8680450 \\
\hline C & -3.5422260 & 4.9892610 & 2.0069060 & $\mathrm{H}$ & -4.4660060 & -7.0097360 & -1.9837440 \\
\hline $\mathrm{C}$ & -2.7511610 & 4.3087460 & 1.1151140 & $\mathrm{H}$ & -4.6272940 & -5.4234550 & -2.7540960 \\
\hline C & -4.9923690 & 3.0796110 & 2.2497120 & $\mathrm{H}$ & -3.1602270 & -5.8140610 & -1.8421470 \\
\hline $\mathrm{H}$ & -5.3025940 & 4.9271800 & 3.2754770 & C & -6.4165010 & -5.6052960 & -0.6284010 \\
\hline H & -3.2841110 & 6.0056450 & 2.2870100 & $\mathrm{H}$ & -6.8880560 & -5.2326540 & 0.2857840 \\
\hline $\mathrm{H}$ & -1.8681160 & 4.7872850 & 0.7065810 & $\mathrm{H}$ & -6.8633730 & -5.0780320 & -1.4785470 \\
\hline $\mathrm{H}$ & -5.8517730 & 2.5843820 & 2.6937000 & $\mathrm{H}$ & -6.6542220 & -6.6690240 & -0.7314780 \\
\hline C & -2.4268660 & 5.7799040 & -2.7318100 & C & -3.5429950 & -1.4952500 & 3.3793440 \\
\hline C & -1.2289060 & 6.3730160 & -3.1976230 & $\mathrm{H}$ & -4.2475090 & -0.6853560 & 3.1705750 \\
\hline $\mathrm{C}$ & -0.0225740 & 5.7857710 & -2.9166970 & $\mathrm{H}$ & -4.1200860 & -2.3830220 & 3.6627930 \\
\hline $\mathrm{C}$ & -2.3929250 & 4.6337850 & -1.9777240 & $\mathrm{H}$ & -2.9283070 & -1.2000840 & 4.2380010 \\
\hline $\mathrm{H}$ & -3.3814190 & 6.2335410 & -2.9791260 & C & -1.6109290 & -2.8615440 & 2.5573620 \\
\hline $\mathrm{H}$ & -1.2712580 & 7.2832010 & -3.7870920 & $\mathrm{H}$ & -2.0837840 & -3.7564180 & 2.9767310 \\
\hline H & 0.9044210 & 6.2176440 & -3.2842620 & $\mathrm{H}$ & -1.0060640 & -3.1520840 & 1.6947320 \\
\hline H & -3.3177670 & 4.1817960 & -1.6348050 & $\mathrm{H}$ & -0.9471560 & -2.4557910 & 3.3288700 \\
\hline C & 2.6598260 & 2.0262320 & -1.0147760 & C & 2.8948360 & 4.2753030 & 1.2583950 \\
\hline C & 3.2950830 & 1.3651550 & -2.0825840 & $\mathrm{H}$ & 3.9772780 & 4.4429660 & 1.2913090 \\
\hline C & 3.2166630 & 1.9807480 & 0.2851170 & $\mathrm{H}$ & 2.5178510 & 4.6654790 & 0.3103900 \\
\hline C & 4.4737520 & 0.6543350 & -1.8238510 & $\mathrm{H}$ & 2.4371250 & 4.8482330 & 2.0717700 \\
\hline C & 4.3881190 & 1.2543530 & 0.4899650 & C & 3.0645880 & 2.3413160 & 2.8194150 \\
\hline $\mathrm{C}$ & 5.0315610 & 0.5799890 & -0.5515030 & $\mathrm{H}$ & 2.9816650 & 1.2608240 & 2.9647020 \\
\hline $\mathrm{H}$ & 4.9806850 & 0.1493300 & -2.6437700 & $\mathrm{H}$ & 4.1067570 & 2.6315960 & 2.9951130 \\
\hline H & 40 & 1.2200180 & 1.4845360 & $\mathrm{H}$ & 2.4526250 & 2.8336190 & 3.5818830 \\
\hline
\end{tabular}




$\begin{array}{rrr}6.3313280 & -1.0430540 & 0.9117170 \\ 7.2654450 & -1.6099260 & 0.9792180 \\ 6.2389080 & -0.4522030 & 1.8296410 \\ 5.4983890 & -1.7540630 & 0.8829750 \\ 7.4961280 & 0.8718310 & -0.2372890 \\ 7.5432420 & 1.4944510 & -1.1357630 \\ 7.3528470 & 1.5319790 & 0.6257010 \\ 8.4565890 & 0.3596310 & -0.1185560 \\ 2.9332870 & 0.1369070 & -4.2886240 \\ 3.9825810 & -0.0742130 & -4.5247790 \\ 2.5277310 & -0.7053610 & -3.7268480 \\ 2.3963990 & 0.2062990 & -5.2400480 \\ 3.5060850 & 2.5666440 & -4.2752540 \\ 4.5788740 & 2.3477000 & -4.3299660 \\ 3.1228310 & 2.6430120 & -5.2983490 \\ 3.3901160 & 3.5403730 & -3.7931980 \\ -0.2799580 & -0.3155080 & -0.8960210 \\ 0.4411500 & -0.4412110 & -2.2101390 \\ -0.2415310 & -1.4504650 & 0.0829400 \\ 1.7117860 & 1.6803000 & -3.4725730 \\ 6.5269200 & -0.7741180 & -1.2065730 \\ 1.5096020 & 2.6333890 & 1.3889680 \\ -4.6728610 & 0.3758420 & -1.9021960 \\ -4.4978380 & -5.9535640 & 0.2600390 \\ -2.0990480 & -0.8946410 & 1.9085640 \\ 1.5478340 & -3.4857670 & -1.6801430 \\ 2.7222560 & -2.6597940 & -1.3895200 \\ 0.6822950 & -3.3141700 & -1.0393130 \\ 2.5521800 & -1.6727910 & -0.9610810 \\ 1.5612520 & -2.6105250 & -2.8307180 \\ 2.7483440 & -2.5150960 & 2.0731910 \\ 2.2887720 & -1.0900030 & 4.0814940 \\ 1.4230410 & -0.8713230 & 3.0112480 \\ 1.7132230 & -1.6867150 & 1.9262440 \\ 1.0807320 & -1.6828740 & 1.0955850 \\ 3.4749300 & -2.3054440 & 3.6495270 \\ 3.3146900 & -3.6383800 & 0.9449350 \\ 1.0148720 & -1.7617050 & -2.6216220 \\ 1.1107030 & 0.5685110 & 5.3315990 \\ 0.2492540 & 0.7902730 & 4.2356290 \\ 0.3938040 & 0.0700710 & 3.0546930 \\ 2.1326070 & -0.3599130 & 5.2629260 \\ 0.9559640 & 1.1586850 & 6.2280730 \\ -0.2252880 & 0.2255980 & 6.1797630 \\ 2.7956690 & -0.5132890 & -2.9341160 \\ 3.0425740 & -5.0820110 & \\ & & \end{array}$

$\begin{array}{ll}\mathrm{C} & 4.2604070 \\ \mathrm{C} & 4.0634280 \\ \mathrm{C} & 1.7836230 \\ \mathrm{H} & 3.2185490 \\ \mathrm{H} & 5.1615550 \\ \mathrm{H} & 4.1241700 \\ \mathrm{H} & 1.8771000 \\ \mathrm{H} & 0.8959610 \\ \mathrm{H} & 2.8855270 \\ \mathrm{H} & 4.4305110 \\ \mathrm{H} & 4.8355550 \\ \mathrm{O} & -0.6957320 \\ \mathrm{C} & -1.5110850 \\ \mathrm{H} & -0.8990940 \\ \mathrm{H} & -2.1104340 \\ \mathrm{H} & -2.1746300 \\ \mathrm{H} & \end{array}$
$-4.4652640$
$-2.9682940$
$-4.9238960$
$-6.1440600$
$-4.6100760$
$-2.4051600$
$-5.5064360$
$-5.2860780$
$-4.5980490$
$-4.9759930$
$-2.5574240$
1. 7400650
2. 0517640
2. 3852170
1.1850440
1.1850440
2.8582140

148

-2.2413260
-1.9878130
-2.0766610
-3.1270120
-2.8436370
-2.9280320
-1.1522530
-2.6045040
-3.9049680
-1.2845560
-1.3306630
4.4185640
3.3008420
2.4530260
2.9908020
3.6146230

$\operatorname{TS} 13(\mathrm{~S}, \mathrm{~S}) \mathrm{G}[\mathrm{M} 06-2 \mathrm{X} / 6-31 \mathrm{G}(\mathrm{d})]=-4124.881003$

$\begin{array}{lrrr}\text { TS } 13(\mathrm{~S}, \mathrm{~S}) \mathrm{G}[\mathrm{M} 06-2 \mathrm{X} / 6-31 \mathrm{G}(\mathrm{d})]=-4124.881003 \\ \mathrm{O} & -0.2346920 & -1.0596910 & -0.0502090 \\ \mathrm{O} & 1.7463980 & -0.2210830 & -1.3572970 \\ \mathrm{C} & -0.1734160 & -2.2734730 & -0.7108920 \\ \mathrm{C} & 1.0544340 & -2.8822730 & -0.8728310 \\ \mathrm{C} & -1.4023260 & -2.8453050 & -1.1382960 \\ \mathrm{C} & 1.1142010 & -4.0882210 & -1.6455480 \\ \mathrm{C} & -1.3390710 & -4.0436880 & -1.8054690 \\ \mathrm{C} & -0.1006790 & -4.6721260 & -2.1024260 \\ \mathrm{H} & -2.2582240 & -4.5327890 & -2.1181220 \\ \mathrm{C} & 2.5300310 & -0.9295250 & -0.4667180 \\ \mathrm{C} & 3.6355670 & -0.2495550 & 0.1148010 \\ \mathrm{C} & 2.2527270 & -2.2630560 & -0.2433290 \\ \mathrm{C} & 4.4812220 & -0.9777160 & 0.9138800 \\ \mathrm{C} & 3.0901640 & -2.9898010 & 0.6694500 \\ \mathrm{C} & 4.2271350 & -2.3392180 & 1.2271790 \\ \mathrm{H} & 5.3627190 & -0.5002780 & 1.3345000 \\ \mathrm{C} & 4.7754790 & -4.3345580 & 2.4966640 \\ \mathrm{C} & 3.6242550 & -4.9715260 & 1.9743070 \\ \mathrm{C} & 2.8044750 & -4.3195330 & 1.0870440 \\ \mathrm{C} & 5.0645540 & -3.0449400 & 2.1317030 \\ \mathrm{H} & 5.4164030 & -4.8639010 & 3.1942720 \\ \mathrm{H} & 3.3827110 & -5.9828550 & 2.2857380 \\ \mathrm{H} & 1.9153730 & -4.8144740 & 0.7121910 \\ \mathrm{H} & 5.9313960 & -2.5327350 & 2.5405640 \\ \mathrm{C} & 2.3541640 & -5.8616220 & -2.7494540 \\ \mathrm{C} & 1.1433690 & -6.4610510 & -3.1719570 \\ \mathrm{C} & -0.0550860 & -5.8746550 & -2.8571200 \\ \mathrm{C} & 2.3413970 & -4.7082690 & -2.0057800\end{array}$




$\begin{array}{rrrr}\mathrm{H} & 3.3013580 & -6.3163390 & -3.0218310 \\ \mathrm{H} & 1.1691530 & -7.3765680 & -3.7540920 \\ \mathrm{H} & -0.9921940 & -6.3136500 & -3.1888260 \\ \mathrm{H} & 3.2753970 & -4.2509060 & -1.6961190 \\ \mathrm{C} & -2.6954780 & -2.1632700 & -0.8141480 \\ \mathrm{C} & -3.4214490 & -1.4941990 & -1.8190200 \\ \mathrm{C} & -3.1742000 & -2.1884730 & 0.5142270 \\ \mathrm{C} & -4.6248600 & -0.8696170 & -1.4754990 \\ \mathrm{C} & -4.3790550 & -1.5483010 & 0.8044750 \\ \mathrm{C} & -5.1205030 & -0.8866220 & -0.1734400 \\ \mathrm{H} & -5.1934060 & -0.3607620 & -2.2512200 \\ \mathrm{H} & -4.7619730 & -1.5619370 & 1.8210650 \\ \mathrm{C} & 3.8796090 & 1.1980100 & -0.1749240 \\ \mathrm{C} & 3.4894670 & 2.1831930 & 0.7580050 \\ \mathrm{C} & 4.5093720 & 1.5650380 & -1.3746020 \\ \mathrm{C} & 3.7857320 & 3.5168010 & 0.4811160 \\ \mathrm{C} & 4.7816320 & 2.9163250 & -1.6083960 \\ \mathrm{C} & 4.4345400 & 3.9046550 & -0.6936930 \\ \mathrm{H} & 3.4905560 & 4.2812250 & 1.1958410 \\ \mathrm{H} & 5.2773790 & 3.2070180 & -2.5330370 \\ \mathrm{C} & -2.4320580 & -2.9489840 & 1.6069780 \\ \mathrm{C} & -6.4427060 & -0.2299750 & 0.1758350 \\ \mathrm{C} & -2.9629330 & -1.4749640 & -3.2704090 \\ \mathrm{C} & 2.7601480 & 1.8187250 & 2.0437030 \\ \mathrm{C} & 4.7248210 & 5.3668300 & -0.9751680 \\ \mathrm{C} & 4.9164510 & 0.5395430 & -2.4211660 \\ \mathrm{C} & 6.4434290 & 0.4489480 & -2.5280410 \\ \mathrm{H} & 6.8687950 & 1.4055570 & -2.8514670 \\ \mathrm{H} & 6.8891580 & 0.1903060 & -1.5621650 \\ \mathrm{H} & 6.7336170 & -0.3140050 & -3.2578230 \\ \mathrm{C} & 4.2848220 & 0.8433680 & -3.7840840 \\ \mathrm{H} & 3.1951500 & 0.8958190 & -3.7042340 \\ \mathrm{H} & 4.6457170 & 1.7955540 & -4.1874910 \\ \mathrm{H} & 4.5415540 & 0.0586100 & -4.5031010 \\ \mathrm{C} & 5.6284500 & 5.9804550 & 0.0999440 \\ \mathrm{H} & 5.8680780 & 7.0197390 & -0.1472280 \\ \mathrm{H} & 5.1314580 & 5.9748050 & 1.0762530 \\ \mathrm{H} & 6.5650290 & 5.4228900 & 0.1943310 \\ \mathrm{C} & 3.4259850 & 6.1701190 & -1.1145420 \\ \mathrm{H} & 2.7861010 & 5.7515680 & -1.8973530 \\ \mathrm{H} & 2.8616140 & 6.1595300 & -0.1754030 \\ \mathrm{H} & 3.6430270 & 7.2136170 & -1.3651680 \\ \mathrm{C} & 3.7384130 & 1.5721290 & 3.1997910 \\ \mathrm{H} & 4.4440520 & 0.7679160 & 2.9747120 \\ \mathrm{H} & 4.3146940 & 2.4804870 & 4.1083520 \\ \mathrm{H} & 3.1889590 & 1.2992410 & \\ & & & \end{array}$

\begin{abstract}
1.7235560 2.2005380

1.0629600

1. 1145300

$-2.6706620$

$-3.7380100$

$-2.3501330$

$-2.1241090$

$-2.8040560$

$-2.7724520$

$-3.8080900$

$-2.1002560$

$-7.6103620$

$-8.5648440$

$-7.5183830$

$-7.6349130$

$-6.4443980$

$-5.6094680$

$-6.3587490$

$-7.3783950$

$-3.1771850$

$-4.2400670$

$-2.7474260$

$-2.6913520$

$-3.6847570$

$-4.7663570$

$-3.3466070$

$-3.5085940$

0.2552480

$-0.5437120$

0.3053060

$-1.8882290$

$-6.5798850$

$-1.3590950$

4. 5503640

5. 2572640

2. 2162430

$-1.3072710$

$-2.5994940$

$-0.5018520$

$-2.5874340$

$-1.3928880$

$-2.6728250$

$-2.2014400$

$-1.3487980$

$-1.6458660$
\end{abstract}

2.8646790

3.7800960

3.1199580

2.4663960

$-4.4609230$

$-4.6793910$

$-4.8410730$

$-5.0041100$

$-2.5137790$

$-1.4267380$

$-2.8596650$

$-2.9534860$

$-0.9419600$

$-0.4918290$

$-0.8620700$

$-2.0044310$

1. 2641310

1.7808690

1.4154930

1.7322890

$-0.1191620$

0.0915680

0.6888640

$-0.1162570$

$-2.5672440$

$-2.3885990$

$-2.5646640$

$-3.5637280$

0.2518670

0.4085920

1.3758440

$-1.6785680$

$-0.3311320$

$-2.7567230$

$-0.4418650$

5.4158880

0.8884590

3. 5207280

2.8578530

3. 1996990

1.8369480

2.7031290

2.5768660

1. 1556280

0.9267700

1.7402350
2.4641540

2.8315030

1.6316860

3. 2832780

1. 4702350

1. 5883320

0.4973640

2. 2485270

3. 0262230

3. 1443900

3. 2970760

3. 7404480

$-0.5177440$

$-0.2260200$

$-1.6067310$

$-0.2579080$

$-0.1612550$

0.3255390

$-1.2436850$

0.1661450

$-3.9494360$

$-4.1126210$

$-3.3547660$

$-4.9304630$

$-4.0727830$

$-4.0657680$

$-5.1144450$

$-3.6589220$

$-0.8978220$

$-2.1621140$

0.0929480

$-3.2858950$

1. 2598200

1. 4787890

$-2.1040600$

$-1.9334370$

1.8479310

$-1.6374120$

$-1.4374630$

$-0.9765130$

$-1.0598610$

$-2.8304680$

2. 0562300

4.0677070

2. 9894310

1.9040660 


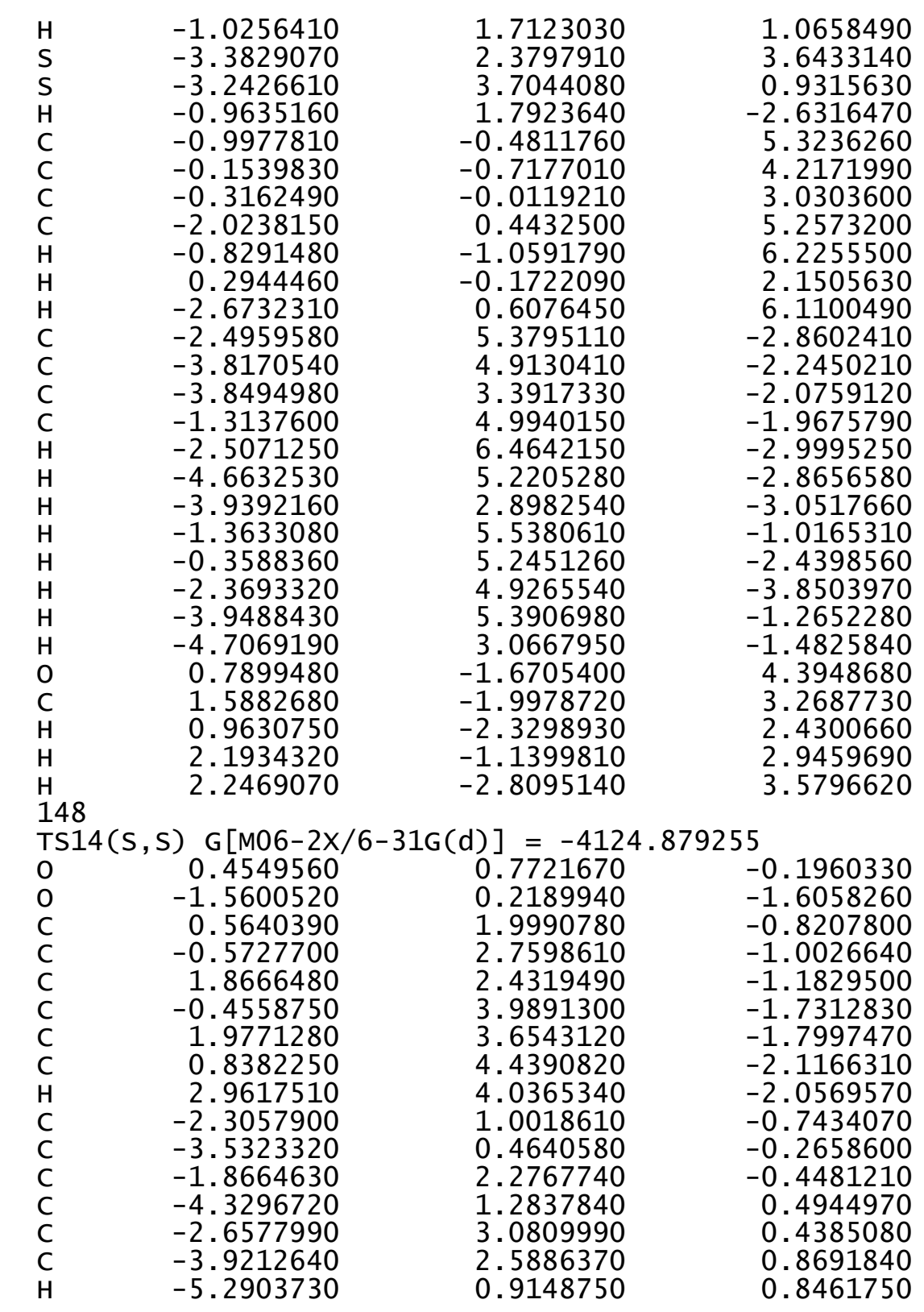

-4.3148850
-3.0304050
-2.2252220
-4.7431260
-4.9478430
-2.6794190
-1.2412640
-5.7151270
-1.4261430
-0.1352190
0.9697130
-1.5835430
-2.2999670
-0.0265920
1.9663010
-2.5782860
3.0670660
3.7955460
3.4868570
4.9646390
4.6502630
5.4160340
5.5496790
4.9771420
-3.9292860
-3.9882140
-4.2725740
-4.4240510
-4.6932600
-4.7825690
-4.4823100
-4.9681860
2.7377360
6.7436530
3.3696690
-3.5924010
-5.2511380
-4.2457100
-5.6656980
-6.1167700
-6.3106000
-5.6481530
-3.3301770
-2.3161420
-3.7038490
-3.2833030

4.6325570

5. 0960510

4. 3440360

3.4005000

5. 2453760

6.0558970

4.7098020

3. 0166460

5.9350020

6. 3996650

5.6635010

4.7644200

6.5080820

7.3306200

5.9965860

4.4127350

1.5989160

.9823400

1. 4658190

0.2886700

0.7443030

0.1662080

$-0.1704920$

0.6434180

$-0.9437170$

$-1.8910720$

$-1.3163370$

$-3.1859740$

$-2.6226780$

$-3.5730670$

$-3.9209320$

$-2.9045570$

2. 1607740

$-0.5182680$

1.0634520

$-1.5707190$

$-4.9863400$

$-0.3328590$

$-0.0692000$

$-0.9875390$

0.3091030

0.6695920

$-0.8166030$

$-0.9921120$

$-1.7467790$

$-0.0659700$
2.1186490

1.7463390

0.9278250

1.6953250

2.7529050

2. 1120510

0.6552560

1.9950970

$-2.8158020$

$-3.1651450$

$-2.8251490$

$-2.1172740$

$-3.1093960$

$-3.7122290$

$-3.1024670$

$-1.8649910$

$-0.8630120$

$-1.8984330$

0.4795200

$-1.5722850$

0.7536240

$-0.2603700$

$-2.3679010$

1.7834500

$-0.5698150$

0.4715510

$-1.8857030$

0.1751450

$-2.1299510$

$-1.1122200$

0.9758060

$-3.1450540$

1.6113170

0.0168360

$-3.3574460$

1. 9069170

$-1.4022750$

$-3.0463390$

$-3.5637450$

$-3.9557660$

$-2.7641250$

$-4.3717410$

$-4.1761270$

$-3.8061950$

$-4.6182120$

$-4.9720130$ 


$\begin{array}{rrr}-4.3004740 & -5.7008120 & -2.3696480 \\ -4.6224160 & -6.7346260 & -2.5320990 \\ -4.2857940 & -5.1966230 & -3.3422820 \\ -3.2780470 & -5.7131730 & -1.9806890 \\ -6.6853380 & -5.0002530 & -1.9434060 \\ -7.3741480 & -4.5091470 & -1.2495870 \\ -6.7431980 & -4.4771120 & -2.9043890 \\ -7.0271610 & -6.0284310 & -2.1006260 \\ -4.8166350 & -1.5455930 & 2.8301110 \\ -5.5652470 & -0.8247710 & 2.4862070 \\ -5.2943680 & -2.5312170 & 2.8724550 \\ -4.5176250 & -1.2701590 & 3.8477310 \\ -2.5400500 & -2.5513730 & 2.4383040 \\ -2.9394990 & -3.5692110 & 2.5073080 \\ -1.6637750 & -2.5625970 & 1.7832260 \\ -2.2221830 & -2.2495050 & 3.4430410 \\ 3.0899920 & 3.6559070 & 1.6501930 \\ 4.1620990 & 3.7808650 & 1.8397280 \\ 2.8484530 & 4.1563770 & 0.7089290 \\ 2.5408050 & 4.1550860 & 2.4555560 \\ 2.9987060 & 1.5449730 & 2.9876220 \\ 2.9253410 & 0.4543020 & 2.9593500 \\ 3.9955390 & 1.8036470 & 3.3620680 \\ 2.2650440 & 1.9201740 & 3.7085820 \\ 6.8200000 & -1.1897600 & 1.3895140 \\ 7.7392220 & -1.7781280 & 1.4673670 \\ 6.8400730 & -0.4450800 & 2.1925940 \\ 5.9671520 & -1.8537130 & 1.5702530 \\ 7.8886990 & 0.4920230 & -0.1458300 \\ 7.8812280 & 0.9400900 & -1.1440090 \\ 7.7861680 & 1.2982080 & 0.5892020 \\ 8.8580820 & 0.0072660 & 0.0099430 \\ 3.4562630 & -0.2926680 & -4.0651490 \\ 4.4956090 & -0.6104850 & -4.2047040 \\ 2.9312750 & -1.0649270 & -3.4995810 \\ 3.0007200 & -0.2231980 & -5.0581070 \\ 4.2139650 & 2.0956460 & -4.1173340 \\ 5.2718220 & 1.8087370 & -4.0973830 \\ 3.8979680 & 2.1520560 & -5.1642980 \\ 4.1304900 & 3.0947980 & -3.6812530 \\ -0.1857220 & -0.4609690 & -1.0637350 \\ 0.6399080 & -0.7539370 & -2.2859620 \\ -0.4254670 & -1.5345090 & -0.0440070 \\ 2.3223000 & 1.3780260 & -3.3845150 \\ 6.8758670 & -1.2929350 & -0.7513150 \\ 1.6637280 & 2.0656560 & 1.4169150\end{array}$

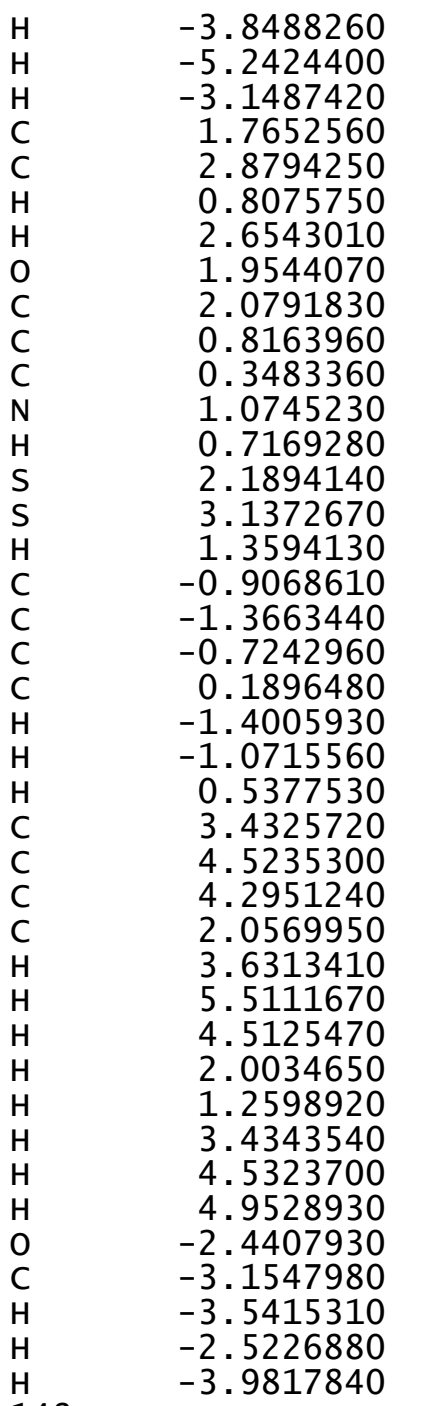
0.6208970 $-3.5651270$ $-2.6610030$ $-3.3050730$ $-1.6107320$ $-2.9272110$ $-2.0963630$ $-0.8259030$ $-0.6269250$ $-1.3614020$ $-1.4368960$ $-1.9179980$ $-3.0780440$ $-2.0921200$ 0.6267990
0.8185080
0.2062860 $-0.1911930$ 1. 1122030

$\begin{array}{lrcr}\text { TS15(S, S }) \text { G }[M 06-2 X / 6-31 G(d)]=-4124.875450 \\ \text { O } & 0.4919140 & 0.6803780 & -0.2149570 \\ \text { O } & -1.5060400 & 0.3429170 & -1.7279640 \\ \text { C } & 0.7074830 & 1.9369330 & -0.7452940 \\ \text { C } & -0.3722850 & 2.7727200 & -0.9492320\end{array}$




$\begin{array}{lrrr}\text { C } & 2.0519140 & 2.3234170 & -0.9829250 \\ \mathrm{C} & -0.1461990 & 4.0240160 & -1.6105980 \\ \mathrm{C} & 2.2631580 & 3.5561070 & -1.5528260 \\ \mathrm{C} & 1.1899040 & 4.4107270 & -1.9119810 \\ \mathrm{H} & 3.2816410 & 3.8961750 & -1.7246930 \\ \mathrm{C} & -2.2329810 & 1.1299460 & -0.8539310 \\ \mathrm{C} & -3.5134710 & 0.6578530 & -0.4544560 \\ \mathrm{C} & -1.7192620 & 2.3530390 & -0.4754420 \\ \mathrm{C} & -4.2830970 & 1.4929360 & 0.3173680 \\ \mathrm{C} & -2.4887180 & 3.1659400 & 0.4226570 \\ \mathrm{C} & -3.8006670 & 2.7443860 & 0.7768200 \\ \mathrm{H} & -5.2807480 & 1.1760980 & 0.6124820 \\ \mathrm{C} & -4.1014430 & 4.7423680 & 2.1221210 \\ \mathrm{C} & -2.7734540 & 5.1310820 & 1.8244950 \\ \mathrm{C} & -1.9893190 & 4.3671310 & 0.9964770 \\ \mathrm{C} & -4.5972520 & 3.5689700 & 1.6146020 \\ \mathrm{H} & -4.7156820 & 5.3649350 & 2.7652300 \\ \mathrm{H} & -2.3716250 & 6.0424150 & 2.2560190 \\ \mathrm{H} & -0.9713010 & 4.6744830 & 0.7822850 \\ \mathrm{H} & -5.6055350 & 3.2411490 & 1.8552660 \\ \mathrm{C} & -0.9450670 & 6.0679930 & -2.6529970 \\ \mathrm{C} & 0.3869450 & 6.4662340 & -2.9219300 \\ \mathrm{C} & 1.4284740 & 5.6517490 & -2.5614780 \\ \mathrm{C} & -1.2058590 & 4.8822080 & -2.0137030 \\ \mathrm{H} & -1.7677280 & 6.7052690 & -2.9616580 \\ \mathrm{H} & 0.5770710 & 7.4101110 & -3.4226210 \\ \mathrm{H} & 2.4556510 & 5.9355120 & -2.7743870 \\ \mathrm{H} & -2.2308860 & 4.5818940 & -1.8238240 \\ \mathrm{C} & 3.2039050 & 1.4722890 & -0.5492240 \\ \mathrm{C} & 4.0766310 & 0.9159540 & -1.5046410 \\ \mathrm{C} & 3.4736190 & 1.3248720 & 0.8312770 \\ \mathrm{C} & 5.2437450 & 0.2791740 & -1.0659950 \\ \mathrm{C} & 4.6511770 & 0.6807790 & 1.2141250 \\ \mathrm{C} & 5.5670080 & 0.1817300 & 0.2862380 \\ \mathrm{H} & 5.9340260 & -0.1198840 & -1.8082900 \\ \mathrm{H} & 4.8903640 & 0.5903190 & 2.2699470 \\ \mathrm{C} & -3.9931440 & -0.7008200 & -0.8485050 \\ \mathrm{C} & -4.1544740 & -1.6962830 & 0.1394770 \\ \mathrm{C} & -4.3066740 & -0.9807470 & -2.1912560 \\ \mathrm{C} & -4.6603260 & -2.9403020 & -0.2392030 \\ \mathrm{C} & -4.8016420 & -2.2449710 & -2.5195840 \\ \mathrm{C} & -4.9918590 & -3.2364130 & -1.5611340 \\ \mathrm{H} & -4.7937190 & -3.7052470 & 0.5237350 \\ \mathrm{H} & -5.0552870 & -2.4628080 & -3.5556280 \\ \mathrm{C} & 2.5603420 & 1.9313570 & 1.8936500 \\ \mathrm{C} & 6.9075010 & -0.3655150 & 0.7501670 \\ & & & \end{array}$

3.8197950
-3.7935630
-5.5364430
-4.1699020
-5.5479030
-6.0405170
-6.1990180
-5.4499440
-3.2436200
-2.2580280
-3.6584870
-3.1177510
-6.8678800
-7.2726210
-6.7326060
-7.6067810
-4.5188400
-3.5709080
-4.3148040
-4.9028010
-5.0471620
-5.7386940
-5.5836580
-4.7700850
-2.8153930
-3.2692460
-1.9146200
-2.5239570
2.7654690
3.8003300
2.5580870
2.1043480
2.7411020
2.7675700
3.6701920
1.9104230
8.0560840
9.0164600
8.0970610
7.9316790
7.1101270
6.3665990
7.0387140
8.1024910
3.8551120
4.8480670
-5

1.0296580

$-1.4760530$

$-4.5996420$

0.0560680

0.4404300

$-0.4239110$

0.8180610

1.2180320

$-0.4295160$

$-0.6894520$

$-1.3093730$

0.3557930

$-4.8873080$

$-5.8520600$

$-4.9268460$

$-4.1111460$

$-5.7082800$

$-5.5186640$

$-5.7738560$

$-6.6794650$

$-1.4300080$

$-0.6460010$

$-2.3853770$

$-1.2330150$

$-2.5413990$

$-3.5386850$

$-2.5612140$

$-2.3161720$

3. 4508190

3.6678680

3.9629790

3.8662950

1.3075970

0.2151640

1.6435080

1.6026410

0.4728340

0.1349980

0.3788860

1.5321280

$-1.8470230$

$-2.4739450$

$-2.0223530$

$-2.1753320$

$-0.3337830$

$-0.7937470$
$-3.0014450$

1.6028970

$-1.9435100$

$-3.2960830$

$-3.8501030$

$-4.3090160$

$-3.0552240$

$-4.6147940$

$-4.4162510$

$-4.0202100$

$-4.9200430$

$-5.1689700$

$-1.2403750$

$-1.5631170$

$-0.1537970$

$-1.4607990$

$-1.6508670$

$-2.1629770$

$-0.5763470$

$-1.9797540$

2. 4848460

2.1598920

2.4521930

3. 5265960

2. 1129060

2.1048890

1.4920520

3.1455040

2.0004660

2. 2885840

1.0577410

2.7684940

3.2795960

3.2307730

3.7536940

3.9292990

0.1742040

0.5766190

$-0.9166480$

0.4175010

0.4171000

0.9203030

$-0.6631610$

0.7428060

$-3.6977250$

$-3.6336750$ 


$\begin{array}{rrr}3.1232920 & -1.0143600 & -3.2561250 \\ 3.6141640 & -0.2180360 & -4.7595070 \\ 4.8251900 & 1.9847080 & -3.6581120 \\ 5.8463390 & 1.6003860 & -3.5536510 \\ 4.6114370 & 2.0891340 & -4.7268080 \\ 4.7958970 & 2.9797070 & -3.2040260 \\ -0.1999950 & -0.4536030 & -1.1796000 \\ 0.6467870 & -0.7318930 & -2.3908810 \\ -0.5334550 & -1.5634320 & -0.2282120 \\ 2.8145590 & 1.4363540 & -3.1439790 \\ 6.9341560 & -0.2649080 & 1.8423120 \\ 1.5226710 & 1.7443440 & 1.5955070 \\ -3.7260690 & 0.9613880 & -2.8723540 \\ -5.7206570 & -4.5862610 & -3.0252080 \\ -3.2968320 & -0.5067100 & 1.6947300 \\ 1.6206580 & -3.6390500 & -1.2294900 \\ 2.7044260 & -2.7188360 & -0.8710230 \\ 0.6437660 & -3.4075680 & -0.8056160 \\ 2.4497590 & -1.6789280 & -0.6707310 \\ 1.8376180 & -2.9750350 & -2.4980190 \\ 1.8256020 & -2.2715840 & 2.5714830 \\ 0.4869860 & -1.1098350 & 4.3454950 \\ 0.1117850 & -0.7863560 & 3.0360860 \\ 0.8729500 & -1.4686670 & 2.0937890 \\ 0.5668560 & -1.4773060 & 1.1004800 \\ 1.8231530 & -2.2469780 & 4.3234120 \\ 2.9179750 & -3.1980650 & 1.6787040 \\ 1.2672670 & -2.1250580 & -2.5332910 \\ -1.2220140 & 0.3533260 & 5.1638110 \\ -1.5861530 & 0.6730770 & 3.8428080 \\ -0.9042010 & 0.1164390 & 2.7595570 \\ -0.1801210 & -0.5339060 & 5.4184380 \\ -1.7467330 & 0.7939850 & 6.0018010 \\ -1.1762730 & 0.3732420 & 1.7403120 \\ 0.0962170 & -0.7684510 & 6.4405180 \\ 3.3623720 & -5.3579780 & -1.8596870 \\ 4.4064210 & -4.5692400 & -1.0653770 \\ 4.1352410 & -3.0656780 & -1.1409370 \\ 1.9544060 & -5.1104210 & -1.3103260 \\ 3.5842180 & -6.4281010 & -1.8176490 \\ 5.4136880 & -4.7690650 & -1.4425420 \\ 4.3665270 & -2.6822470 & -2.1425910 \\ 1.8668340 & -5.5179910 & -0.2960120 \\ 1.1958900 & -5.6013150 & -1.9278560 \\ 3.4003640 & -5.0601510 & -0.0168180 \\ 4.3878520 & -4.8941990 & \end{array}$

$\begin{array}{lr}\mathrm{H} & 4.7527660 \\ \mathrm{O} & -2.6000800 \\ \mathrm{C} & -3.3522540 \\ \mathrm{H} & -3.8172000 \\ \mathrm{H} & -2.7244790 \\ \mathrm{H} & -4.1223660\end{array}$
$-2.4916080$
1. 5120190
2. 0700600
1.2854290
2.7030040
2. 6790210
$-0.4498150$
3.5239270
4.5877720
5.1949710
5.2247950
4.1176250

148

$\operatorname{TS} 16(\mathrm{~S}, \mathrm{~S}) \mathrm{G}[\mathrm{M} 06-2 \mathrm{X} / 6-31 \mathrm{G}(\mathrm{d})]=-4124.880380$

\begin{tabular}{|c|c|c|c|}
\hline & & & \\
\hline 0 & 0.2851020 & 1.0222930 & -0.0869820 \\
\hline 0 & -1.7692330 & 0.1936740 & -1.2851260 \\
\hline C & 0.2490060 & 2.2030560 & -0.8068150 \\
\hline C & -0.9602760 & 2.8481990 & -0.9691460 \\
\hline C & 1.4855220 & 2.7076580 & -1.2930390 \\
\hline $\mathrm{C}$ & -0.9984750 & 4.0121560 & -1.8049990 \\
\hline C & 1.4462350 & 3.8692330 & -2.0240270 \\
\hline $\mathrm{C}$ & 0.2230450 & 4.5247420 & -2.3251820 \\
\hline $\mathrm{H}$ & 2.3732930 & 4.3065630 & -2.3861540 \\
\hline C & -2.4957290 & 0.9801440 & -0.4120380 \\
\hline C & -3.6058620 & 0.3768800 & 0.2406930 \\
\hline $\mathrm{C}$ & -2.1614110 & 2.3119840 & -0.2725090 \\
\hline C & -4.3987480 & 1. 1812440 & 1.0205920 \\
\hline C & -2.9419900 & 3.1206950 & 0.6216980 \\
\hline C & -4.0851020 & 2.5474020 & 1.2474580 \\
\hline $\mathrm{H}$ & -5.2830960 & 0.7621890 & 1.4941230 \\
\hline C & -4.5187100 & 4.6301620 & 2.4168950 \\
\hline C & -3.3603940 & 5.1910320 & 1.8274050 \\
\hline C & -2.5933630 & 4.4591380 & 0.9554040 \\
\hline C & -4.8675130 & 3.3347880 & 2.1336920 \\
\hline $\mathrm{H}$ & -5.1176990 & 5.2224560 & 3.1010160 \\
\hline $\mathrm{H}$ & -3.0712080 & 6.2076250 & 2.0744240 \\
\hline $\mathrm{H}$ & -1.6978770 & 4.8970730 & 0.5283060 \\
\hline $\mathrm{H}$ & -5.7405290 & 2.8809000 & 2.5950220 \\
\hline C & -2.2052780 & 5.7667120 & -2.9738020 \\
\hline C & -0.9863240 & 6.2962390 & -3.4612920 \\
\hline C & 0.1989660 & 5.6843520 & -3.1451430 \\
\hline C & -2.2125210 & 4.6567110 & -2.1667580 \\
\hline $\mathrm{H}$ & -3.1429960 & 5660 & -3.2463620 \\
\hline $\mathrm{H}$ & -0.9959040 & 7.1 & 34010 \\
\hline $\mathrm{H}$ & 1.1413670 & 4580 & -3.5253060 \\
\hline $\mathrm{H}$ & -3.1530020 & 22730 & -1.8070780 \\
\hline C & 2.7626180 & 1.9998290 & -0.9602540 \\
\hline C & 5960 & 0400 & -1.9427600 \\
\hline C & 3.27 & 5120 & 0.3 \\
\hline C & 4.6312840 & 0.6054910 & $-1 \cdot 5$ \\
\hline C & 4.4614700 & 1.4134440 & 0.6528150 \\
\hline C & 5.1565420 & 0.6734560 & -0.3031200 \\
\hline
\end{tabular}




\begin{tabular}{|c|c|c|c|}
\hline $\begin{array}{l}\mathrm{H} \\
\mathrm{H} \\
\mathrm{C} \\
\mathrm{C} \\
\mathrm{C} \\
\mathrm{C} \\
\mathrm{C} \\
\mathrm{C} \\
\mathrm{H} \\
\mathrm{H} \\
\mathrm{C} \\
\mathrm{C} \\
\mathrm{C} \\
\mathrm{C} \\
\mathrm{C} \\
\mathrm{C} \\
\mathrm{C} \\
\mathrm{H} \\
\mathrm{H} \\
\mathrm{H} \\
\mathrm{C} \\
\mathrm{H} \\
\mathrm{H} \\
\mathrm{H} \\
\mathrm{C} \\
\mathrm{H} \\
\mathrm{H} \\
\mathrm{H} \\
\mathrm{C} \\
\mathrm{H} \\
\mathrm{H} \\
\mathrm{H} \\
\mathrm{C} \\
\mathrm{H} \\
\mathrm{H} \\
\mathrm{H} \\
\mathrm{C} \\
\mathrm{H} \\
\mathrm{H} \\
\mathrm{H} \\
\mathrm{C} \\
\mathrm{H} \\
\mathrm{H} \\
\mathrm{H} \\
\mathrm{C} \\
\mathrm{H}\end{array}$ & $\begin{array}{r}5.1653190 \\
4.8688890 \\
-3.9114560 \\
-3.5320600 \\
-4.5950360 \\
-3.8971120 \\
-4.9325140 \\
-4.6009600 \\
-3.6169780 \\
-5.4688630 \\
2.5870870 \\
6.4661560 \\
2.9536380 \\
-2.7440090 \\
-4.9725380 \\
-4.9866970 \\
-6.5109740 \\
-6.9789390 \\
-6.9272070 \\
-6.7868530 \\
-4.3936760 \\
-3.3053120 \\
-4.7967850 \\
-4.6356080 \\
-4.2545740 \\
-4.4900970 \\
-4.5678960 \\
-3.1694080 \\
-6.4892240 \\
-7.0083780 \\
-6.8726210 \\
-6.7417140 \\
-3.6694330 \\
-4.3529320 \\
-4.2703540 \\
-3.0772310 \\
-1.7307330 \\
-2.2259620 \\
-1.1024140 \\
-1.0862140 \\
2.8742040 \\
3.9513130 \\
2.5409740 \\
2.3680720 \\
2.9827800 \\
2.9194390\end{array}$ & $\begin{array}{r}0.0386070 \\
1.4688050 \\
-1.0753670 \\
-2.0126990 \\
-1.4924030 \\
-3.3488000 \\
-2.8394310 \\
-3.7818740 \\
-4.0827070 \\
-3.1599530 \\
2.9301250 \\
-0.0049270 \\
1.1811740 \\
-1.6007210 \\
-5.2429890 \\
-0.5188570 \\
-0.3760500 \\
-1.3332760 \\
-0.0414690 \\
0.3513880 \\
-0.9318580 \\
-1.0203470 \\
-1.8938570 \\
-0.1854240 \\
-5.8592840 \\
-6.9250970 \\
-5.3691080 \\
-5.7502100 \\
-5.4288760 \\
-5.0055380 \\
-4.9350030 \\
-6.4919540 \\
-1.2471100 \\
-0.4311590 \\
-2.1180000 \\
-0.9412280 \\
-2.6581230 \\
-3.5368790 \\
-2.9766270 \\
-2.2383750 \\
4.4218630 \\
4.6078490 \\
4.7557430 \\
5.0291870 \\
2.5677940 \\
1.4916570\end{array}$ & $\begin{array}{r}-2.3517210 \\
1.6583990 \\
0.0492030 \\
1.0303640 \\
-1.1072240 \\
0.8509630 \\
-1.2434150 \\
-0.2710820 \\
1.6026160 \\
-2.1351150 \\
1.4173220 \\
0.0524050 \\
-3.3822230 \\
2.2661380 \\
-0.4407370 \\
-2.2080970 \\
-2.2932130 \\
-2.5490030 \\
-1.3375380 \\
-3.0636480 \\
-3.5596680 \\
-3.4947470 \\
-3.8943670 \\
-4.3232530 \\
-1.6472380 \\
-1.7331110 \\
-2.5757520 \\
-1.5579490 \\
-0.5599940 \\
0.3049850 \\
-1.4596590 \\
-0.6287880 \\
3.4377740 \\
3.1876600 \\
3.7240990 \\
4.3082170 \\
2.7135350 \\
3.1410120 \\
1.8780520 \\
3.4938550 \\
1.1841740 \\
1.2625820 \\
0.1987320 \\
1.9421120 \\
2.8504520 \\
3.0351450\end{array}$ \\
\hline
\end{tabular}

\begin{abstract}
4.0045240
2. 3132350

7.6393160

8.5872480

7. 5261090

7.6946180

6.4260800

5.5873290

6. 3199650

7. 3539270

3. 0947750

4. 1442610

2. 6349260

2. 5963240

3.7052230

4.7773960

3. 3424480

3. 5850110

$-0.2811020$

0.4668040

$-0.3360980$

1. 8889330

6.6240100

1. 5049420

$-4.5779720$

$-4.6364030$

$-2.1734950$

1. 1700740

2. 4821140

0.3940290

2. 5045960

1. 2352490

2. 6736790

2. 3063800

1. 4290680

1. 6682350

1.0211880

3. 4382200

3. 1742060

0.8344070

1. 1872040

0.3194200

0.4260950

2. 1837740

1.0613620
-0.2053100
\end{abstract}

3.0743640

3. 5543070

$-0.6882710$

$-0.3940710$

$-1.7714570$

$-0.4724140$

$-0.2228590$

0.2975590

$-1.2966310$

0.1094820

$-3.9949820$

$-4.1586860$

$-3.3558210$

$-4.9692540$

$-4.2516350$

$-4.2595950$

$-5.2843600$

$-3.8817450$

$-0.8508060$

$-2.1315620$

0.1975780

$-3.3874680$

1. 1290370

1.3272290

$-1.9605930$

0.4597550

1.9951140

$-1.4675760$

$-1.3431810$

$-0.7999610$

$-1.0200660$

$-2.7046630$

2. 1286790

4.0775510

3. 0137110

1. 9633910

1.1453470

3. 6817460

1.0488370

$-2.5409840$

5. 2857320

4.1925310

3.0374840

5. 2358840

6.1629910

2. 1684690 


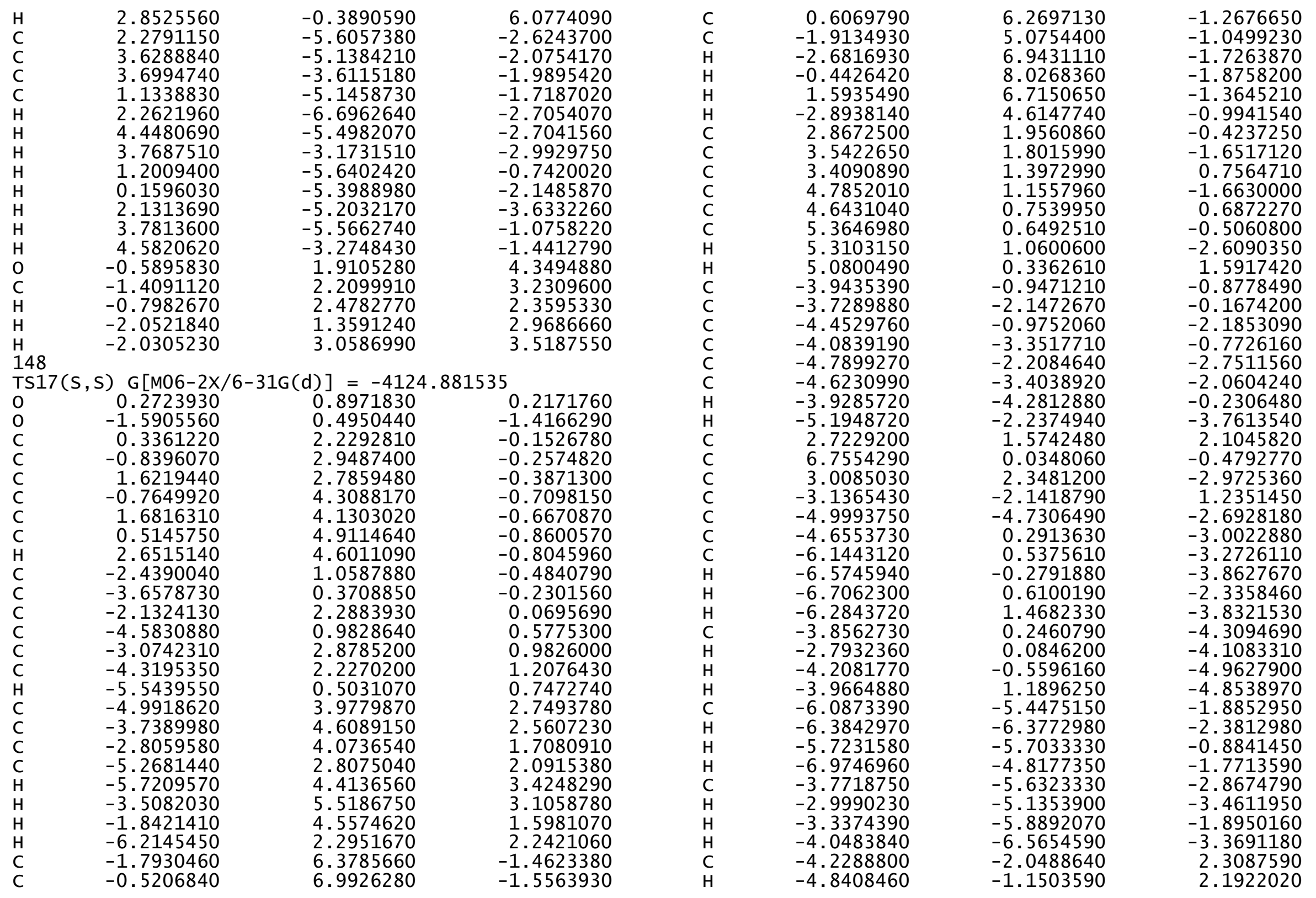




$\begin{array}{rrr}-4.8909040 & -2.9207210 & 2.2528030 \\ -3.7768470 & -2.0270910 & 3.3071830 \\ -2.2471510 & -3.3568670 & 1.5127890 \\ -2.8365580 & -4.2766120 & 1.5940000 \\ -1.4998590 & -3.4881380 & 0.7250540 \\ -1.7312010 & -3.2228070 & 2.4702410 \\ 3.0893750 & 2.9388280 & 2.7057310 \\ 4.1695980 & 2.9952130 & 2.8815750 \\ 2.8094340 & 3.7569800 & 2.0350530 \\ 2.5779100 & 3.0870480 & 3.6627700 \\ 3.0470570 & 0.4554350 & 3.0996690 \\ 2.9414720 & -0.5333000 & 2.6369950 \\ 4.0691070 & 0.5405760 & 3.4845920 \\ 2.3660060 & 0.5062780 & 3.9562780 \\ 7.7504630 & 1.0334310 & 0.1305800 \\ 8.7492800 & 0.5904780 & 0.2001570 \\ 7.8150640 & 1.9295830 & -0.4964250 \\ 7.4392600 & 1.3429860 & 1.1326350 \\ 7.2582070 & -0.4348840 & -1.8449300 \\ 6.5477930 & -1.1057680 & -2.3412840 \\ 7.4373040 & 0.4155360 & -2.5118360 \\ 8.2065400 & -0.9679290 & -1.7299040 \\ 3.0249770 & 1.3102000 & -4.1015290 \\ 4.0406350 & 0.9697740 & -4.3292120 \\ 2.4146920 & 0.4412970 & -3.8525900 \\ 2.6187020 & 1.7606940 & -5.0133470 \\ 3.8105030 & 3.5835170 & -3.4080150 \\ 4.8563330 & 3.3129730 & -3.5941840 \\ 3.3996680 & 3.9983080 & -4.3343420 \\ 3.8012600 & 4.3698070 & -2.6481090 \\ -0.1664050 & -0.1519950 & -0.9598040 \\ 0.7342650 & -0.0223000 & -2.1563030 \\ -0.3241070 & -1.4764790 & -0.2766180 \\ 1.9661350 & 2.6437020 & -2.8258840 \\ 6.7124940 & -0.8380910 & 0.1874590 \\ 1.6407620 & 1.5551770 & 1.9417890 \\ -4.2818970 & 1.1403440 & -2.4212760 \\ -5.4047470 & -4.5141180 & -3.6893180 \\ -2.5028490 & -1.2505330 & 1.3133770 \\ 2.2245910 & -2.8110850 & -1.9378100 \\ 2.9545290 & -2.4207770 & -0.7298960 \\ 1.1375870 & -2.7926660 & -1.8481370 \\ 2.4669950 & -1.7223800 & -0.0529010 \\ 2.7598050 & -1.5260820 & -2.3314020 \\ 1.6919110 & -3.3852670 & 2.1089320 \\ 1.0587380 & -2.3002820 & 4.2739230\end{array}$

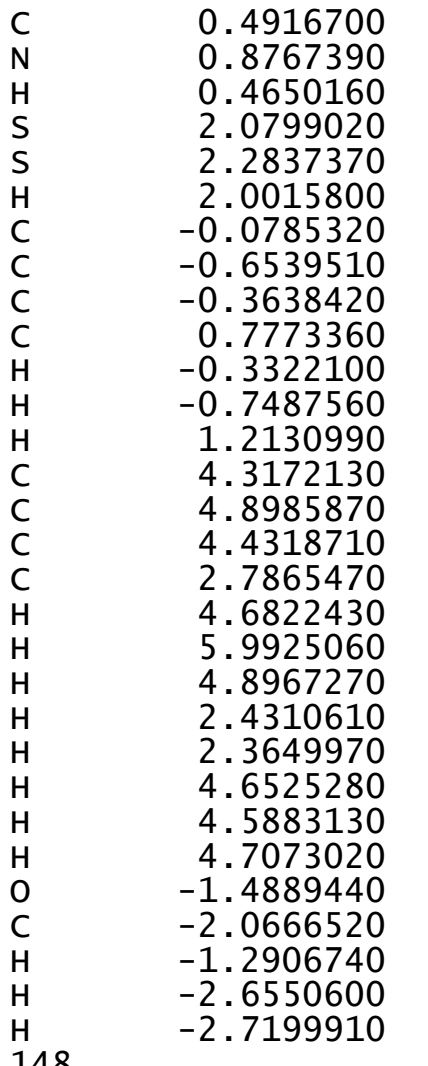
$-1.7073210$
$-2.2336430$
$-3.9205290$
$-3.8885620$
$-2.6474930$
$-3.9444510$
$-4.7998060$
$-3.8933470$
$-1.7480490$
$-4.8813210$
$-3.8829230$
$-3.0371460$
$-4.7898140$
$-2.6803000$
0.9101140
1. 5210010
1. 9511350
0.7972390
2. 3123010

1.9732180

$-2.0528300 \quad 1.0518750$

$\begin{array}{ll}-3.6396420 & 3.7932360\end{array}$

$-4.3492080 \quad 0.8540160$

$-0.8394050 \quad-2.2253220$

$-0.7107540 \quad 5.6429490$

$-0.1255310 \quad 4.4943540$

$-0.6083940 \quad 3.2214100$

$-1.7916590 \quad 5.5440500$

$-0.2886900 \quad 6.6089980$

$-0.1655870 \quad 2.3094470$

6.4331820

$-2.7978940$

$-1.3821530$

$-0.6193230$

$-2.7624870$

$-3.3361720$

$-1.4147140$

$-1.0366310$

$-2.3155940$

$-3.7704400$

$-3.3547480$

$-0.8382790$

0.4384280

4.7257320

3. 5850680

2.9395870

3. 0051850

148

3. 9516440

TS18(S , S ) G[M06-2X/6-31G(d) $]=-4124.882187$

$\begin{array}{lrcr}\text { TS18(S, S }) \text { G }[\mathrm{M} 06-2 \times / 6-31 G(\mathrm{~d})]=-4124.882187 \\ \text { O } & 0.1922990 & 1.0162010 & -0.0422650 \\ \text { O } & -1.7852270 & 0.1873130 & -1.3556880 \\ \text { C } & 0.1039620 & 2.2583540 & -0.6453980 \\ \text { C } & -1.1364300 & 2.8512500 & -0.7682810 \\ \text { C } & 1.3184970 & 2.8640260 & -1.0687080 \\ \text { C } & -1.2239940 & 4.0903710 & -1.4847540 \\ \text { C } & 1.2282830 & 4.0974690 & -1.6649920 \\ \text { C } & -0.0227950 & 4.7205310 & -1.9149950 \\ \text { H } & 2.1344140 & 4.6159630 & -1.9665550 \\ \text { C } & -2.5723990 & 0.8517270 & -0.4360460 \\ \text { C } & -3.6680490 & 0.1324720 & 0.1160540 \\ \text { C } & -2.3204560 & 2.1820250 & -0.1630700 \\ \text { C } & -4.5320480 & 0.8153450 & 0.9348070 \\ \text { C } & -3.1784960 & 2.8601330 & 0.7690840\end{array}$




-4.3075250
-5.4049290
-4.9064470
-3.7624830
-2.9222240
-5.1666760
-5.5640240
-3.5424360
-2.0390210
-6.0268970
-2.5021680
-1.3048750
-0.0941940
-2.4642990
-3.4591730
-1.3506730
0.8331740
-3.3883580
2.6176770
3.3056470
3.1320210
4.4937570
4.3151920
5.0106790
5.0410650
4.7168760
-3.8871160
-3.4614680
-4.5366570
-3.7481890
-4.7968420
-4.4200010
-3.4288690
-5.3081880
2.4531660
6.3369020
2.8363790
-2.7061770
-4.7088060
-4.9735950
-6.5027130
-6.9159830
-6.9428400
-6.8124570
-4.3503070
-3.2592080

2.1702420

0.3052420

4.1103330

4.7856840

4.1796790

2.8285000

4.6036230

5.7898640

4.7039550

2.2859330

5.8880290

6.5340940

5.9580740

4.7004550

6.3341660

7.4764170

6.4313160

4.2086120

2. 1537400

1.5765350

2.0402140

0.8802290

1.3316620

0.7398340

0.4407970

1.2453160

$-1.3082750$

$-2.3165210$

$-1.6454670$

$-3.6446200$

$-2.9923830$

$-4.0037360$

$-4.4271730$

$-3.2608510$

2.7462590

0.0372980

1.7358270

$-1.9780700$

$-5.4592860$

$-0.5928180$

$-0.5241310$

$-1.4761770$

$-0.3051590$

0.2578050

$-0.8416650$

$-0.8786810$

1.2945970
1.3348300
2.6253910
2.1365680
1.2359660
2.2144410
3.3338440
2.4848440
0.8883520
2.5972120
-2.5031010
-2.8941680
-2.6088760
-1.8163800
-2.7545560
-3.4304820
-2.9203530
-1.5310840
-0.8429280
-1.9263720
0.4702620
-1.6715140
0.6704360
-0.3876230
-2.5032260
1.6754480
-0.2229010
0.6684260
-1.4204120
0.3575490
-1.6906640
-0.8136760
1.0415510
-2.6135310
1.6389640
-0.1618990
-3.3654800
1.9461750
-1.1290680
-2.4269880
-2.5161340
-2.8674550
-1.5379550
-3.2171910
-3.8048950
-3.7360200

$-4.6992630$

$-4.6265290$

$-5.6107840$

$-5.8491670$

$-5.1123870$

$-6.5480500$

$-3.4117070$

$-2.7692520$

$-2.8486350$

$-3.6314110$

$-3.6587630$

$-4.3849040$

$-4.2123720$

$-3.0912110$

$-1.6438840$

$-2.0974960$

$-0.9901810$

$-1.0320190$

2.7077400

3.7828500

2. 3469930

2. 2082240

2.8923600

2.8428310

3. 9182190

2. 2409420

6.3053880

7.2505210

6.1712110

5.4910820

7.4561870

7.5156090

7.2693610

8.4251530

3.0379170

4.0982550

2.6261620

2. 5343430

3.5668360

4.6443580

3.2143960

3.4197630

$-0.2668350$

0.4977310

$-0.2348720$

1.7637190
-1.7865740
-0.0386780
-6.0955300
-7.1302260
-6.1074160
-5.5412270
-6.2610310
-5.8225900
-6.2792950
-7.2964320
-1.7764130
-0.9809240
-2.7002400
-1.5133450
-3.0190290
-3.9579360
-3.2259040
-2.6423910
4.2608370
4.4582300
4.6989050
4.7674540
2.2385080
1.1487240
2.5502940
2.6593140
-0.9352850
-1.4840230
-0.4017740
-1.6618180
1.0709820
1.7460250
1.6760440
0.5754320
0.4756730
0.2934920
-0.4040130
0.5939320
2.9096520
2.7105300
3.0426790
3.8503760
-0.2568720
-0.2914710
-1.4461020
1.9495090

$-4.2350590$

$-4.4960150$

$-0.0655640$

$-0.3325110$

0.9100430

0.0406220

$-1.2891260$

$-2.0586320$

$-0.3494250$

$-1.5692150$

3.1319140

2. 9426690

3. 3358180

4.0323520

2. 3100500

2.6460990

1. 4588560

3.1372500

1.5704450

1.6480510

0.6372020

2.4030170

3.0133070

3.0835640

3.2407140

3.7857410

1.0205430

1.0842830

1.9679520

0.9244760

0.0233380

$-0.8356970$

0.9177770

0.1436510

$-4.2111500$

$-4.4205800$

$-3.7150940$

$-5.1758830$

$-4.0353620$

$-4.0687920$

$-5.0637130$

$-3.4990710$

$-0.9630320$

$-2.2578880$

$-0.0505470$

$-3.3465340$ 


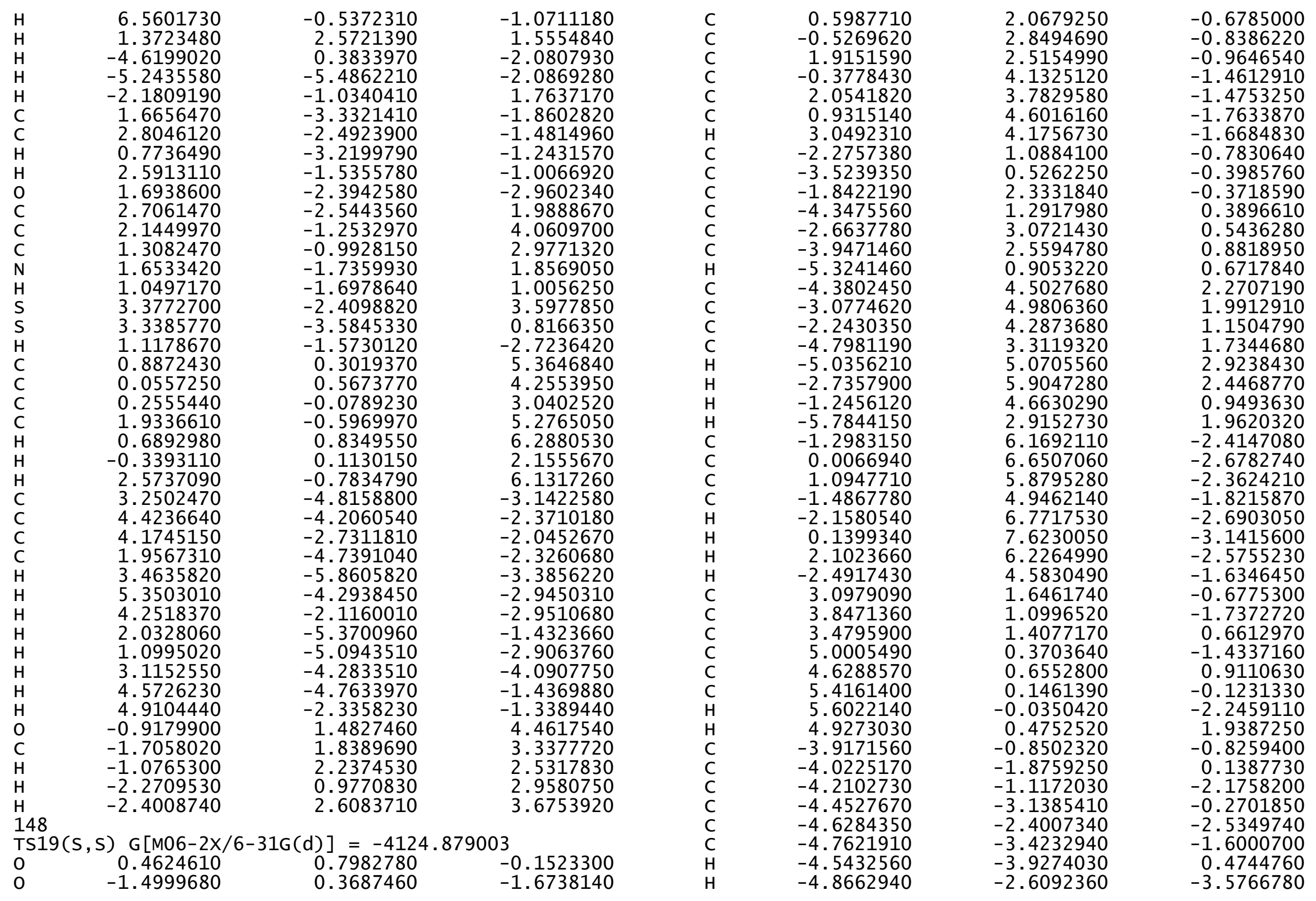




\begin{tabular}{|c|c|c|}
\hline $\begin{array}{l}2.7063520 \\
6.7299650 \\
3.4594780 \\
-3.6787690 \\
-5.2235220 \\
-4.1339290 \\
-5.5313290 \\
-5.9702170 \\
-6.2056570 \\
-5.4787660 \\
-3.1772320 \\
-2.1789610 \\
-3.5367600 \\
-3.0967100 \\
-6.5329490 \\
-6.8789330 \\
-6.3925700 \\
-7.3185170 \\
-4.1389490 \\
-3.2061450 \\
-3.9278110 \\
-4.4636490 \\
-4.9367860 \\
-5.6677250 \\
-5.4216090 \\
-4.6751950 \\
-2.6513520 \\
-3.0562240 \\
-1.7503900 \\
-2.3718850 \\
3.0826870 \\
4.1500710 \\
2.8800480 \\
2.5162130 \\
2.9119760 \\
2.8259610 \\
3.8987460 \\
2.1591040 \\
6.7659980 \\
7.6757670 \\
6.7765310 \\
5.9009070 \\
7.8884670 \\
7.9097400 \\
7.7759740 \\
8.8489680\end{array}$ & $\begin{array}{r}2.0232560 \\
-0.5708770 \\
1.2950860 \\
-1.6674170 \\
-4.8074730 \\
-0.0484730 \\
0.2648540 \\
-0.6167600 \\
0.5844050 \\
1.0642740 \\
-0.4507900 \\
-0.6603200 \\
-1.3418210 \\
0.3583230 \\
-5.1935350 \\
-6.1728780 \\
-5.2519270 \\
-4.4587430 \\
-5.8581720 \\
-5.5988380 \\
-5.9354260 \\
-6.8433210 \\
-1.7023340 \\
-0.9508880 \\
-2.6841280 \\
-1.5084910 \\
-2.6932050 \\
-3.7109880 \\
-2.6584190 \\
-2.4725550 \\
3.5030160 \\
3.5916100 \\
4.0819190 \\
3.9463520 \\
1.2908890 \\
0.2077760 \\
1.5010530 \\
1.6153220 \\
-1.3358630 \\
-1.9409310 \\
-0.6478150 \\
-1.9985280 \\
0.4350980 \\
0.9511980 \\
1.1895750 \\
-0.0703880\end{array}$ & $\begin{array}{r}1.8222080 \\
0.1371650 \\
-3.1963320 \\
1.6080880 \\
-2.0153580 \\
-3.2557250 \\
-3.8056990 \\
-4.2857610 \\
-3.0047370 \\
-4.5521340 \\
-4.3832180 \\
-3.9890890 \\
-4.9094630 \\
-5.1167030 \\
-1.3182310 \\
-1.6645190 \\
-0.2331690 \\
-1.5180920 \\
-1.7505720 \\
-2.2599390 \\
-0.6781990 \\
-2.1010860 \\
2.4843800 \\
2.1691370 \\
2.4324630 \\
3.5306390 \\
2.1015600 \\
2.0751950 \\
1.4818180 \\
3.1382880 \\
1.9935020 \\
2.2254140 \\
1.0886370 \\
2.8193110 \\
3.1499900 \\
3.0263360 \\
3.5776970 \\
3.8757740 \\
1.4618290 \\
1.5198820 \\
2.3141250 \\
1.5780150 \\
0.0724340 \\
-0.8920900 \\
0.8590870 \\
0.2169760\end{array}$ \\
\hline
\end{tabular}

3.5742490

4.6196280

3.0494600

3.1356530

4. 3177340

5.3763830

4.0309600

4.2154790

$-0.1509300$

0.7193420

$-0.4370350$

2.4108530

6.8738780

1.6377600

$-3.7465320$

$-5.4106930$

$-3.2303450$

1.7752430

2.8900630

0.8048390

2.6746000

2.0211590

1. 9674780

0.6618350

0.2341340

0.9826600

0.6576060

2.0260590

3.0469950

1.4362360

$-1.0693490$

$-1.4886330$

$-0.8223980$

0.0105360

$-1.5819140$

$-1.1375400$

0.3272590

3.4399050

4.5134470

4. 3100170

2. 0470540

3.6209770

5.5121330

4.5698730

1.9487400

1. 2650450
0.0005840

$-0.2942470$

$-0.8202140$

0.1444850

2. 3873250

2. 1040520

2. 5229840

3. 3494530

$-0.3617830$

$-0.5705090$

$-1.5066090$

1.6063720

$-1.2918900$

1.9662680

0.8718180

$-4.7790740$

$-0.6767680$

$-3.4741940$

$-2.6158820$

$-3.2330390$

$-1.5786820$

$-2.7432560$

$-2.3075080$

$-1.1491940$

$-0.8570530$

$-1.5324720$

$-1.5316490$

$-2.2511900$

$-3.2330890$

$-1.9000930$

0.2665790

0.5518210

0.0050090

$-0.5803320$

0.7009170

0.2360490

$-0.7913260$

$-5.2157610$

$-4.5251560$

$-3.0084900$

$-4.9500350$

$-6.2938560$

$-4.7403190$

$-2.5553810$

$-5.4168250$

$-5.3690280$
$-4.0078660$

$-4.1529560$

$-3.5154080$

$-5.0004360$

$-3.8494130$

$-3.8213540$

$-4.8975490$

$-3.3404060$

$-1.1329720$

$-2.3414330$

$-0.2070240$

$-3.2268700$

$-0.6795220$

1.5874030

$-2.8095730$

$-3.0962570$

1.7218210

$-1.2977250$

$-0.8820520$

$-0.8643300$

$-0.6242120$

$-2.5226300$

2.5472030

4.3481820

3.0476150

2. 0896190

1.1001130

4.2971030

1.6362970

$-2.5201000$

5.2028870

3.8903740

2.7930510

5.4348780

6.0516800

1.7804890

6.4503200

$-2.0445340$

$-1.2015830$

$-1.1662480$

$-1.4692020$

$-2.0769010$

1.5919620

$-2.1320230$

$-0.4817460$

$-2.1099990$ 


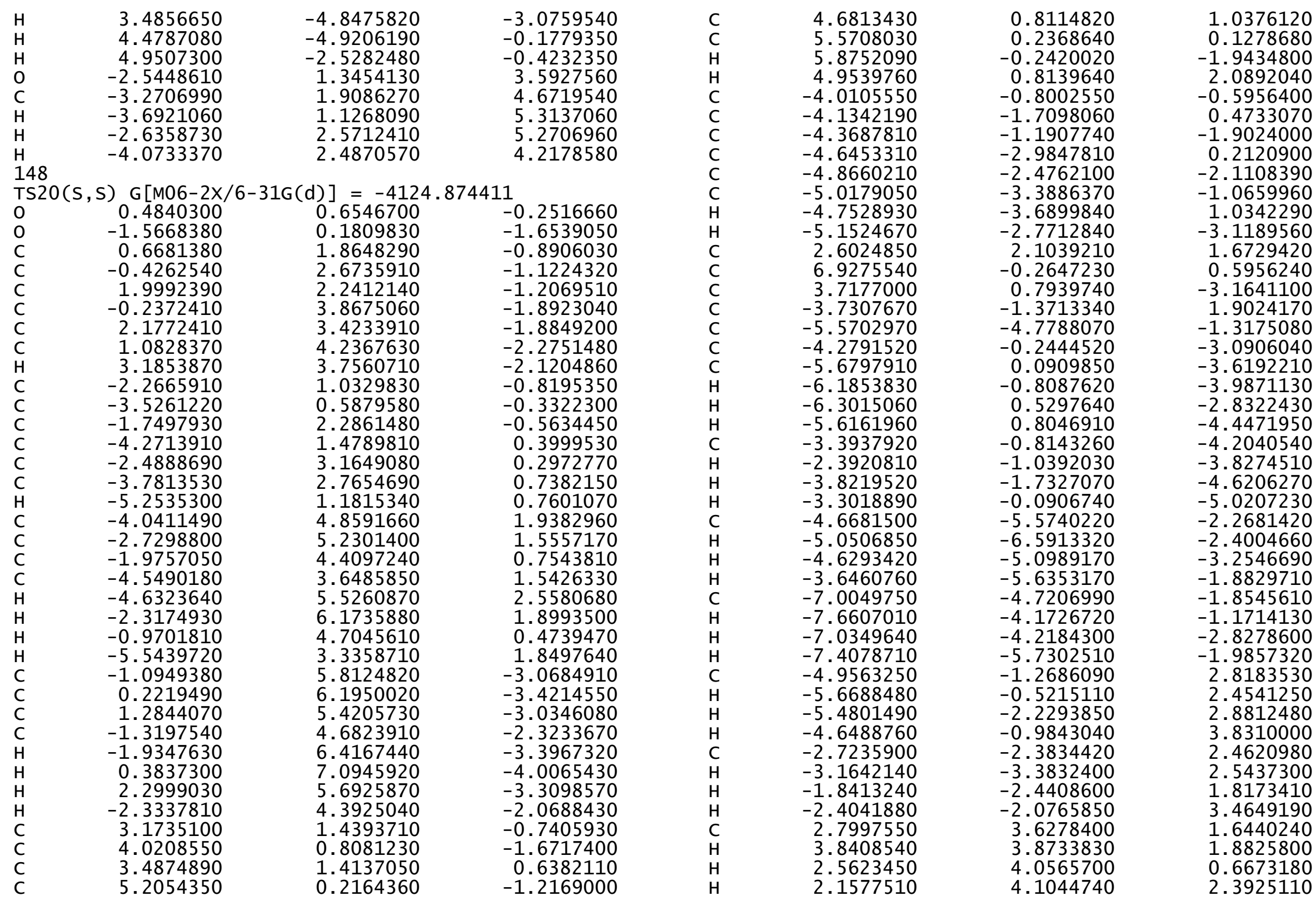




$\begin{array}{rr}\mathrm{C} & 2.8293640 \\ \mathrm{H} & 2.8639950 \\ \mathrm{H} & 3.7689890 \\ \mathrm{H} & 2.0153700 \\ \mathrm{C} & 8.0537810 \\ \mathrm{H} & 9.0277780 \\ \mathrm{H} & 8.0619950 \\ \mathrm{H} & 7.9310260 \\ \mathrm{C} & 7.1288090 \\ \mathrm{H} & 6.3978350 \\ \mathrm{H} & 7.0358000 \\ \mathrm{H} & 8.1289330 \\ \mathrm{C} & 3.7477150 \\ \mathrm{H} & 4.7467370 \\ \mathrm{H} & 3.0349530 \\ \mathrm{H} & 3.4763870 \\ \mathrm{C} & 4.6927460 \\ \mathrm{H} & 5.7204310 \\ \mathrm{H} & 4.4456250 \\ \mathrm{H} & 4.6671940 \\ \mathrm{P} & -0.2317230 \\ \mathrm{O} & 0.5714850 \\ \mathrm{O} & -0.5168660 \\ \mathrm{H} & 2.7040560 \\ \mathrm{H} & 6.9853180 \\ \mathrm{H} & 1.5577290 \\ \mathrm{H} & -3.8247780 \\ \mathrm{H} & -5.5906620 \\ \mathrm{H} & -3.2442370 \\ \mathrm{C} & 1.6135730 \\ \mathrm{C} & 2.7065950 \\ \mathrm{H} & 0.6539510 \\ \mathrm{H} & 2.4552510 \\ \mathrm{O} & 1.7750350 \\ \mathrm{C} & 1.9550630 \\ \mathrm{C} & 0.6676500 \\ \mathrm{C} & 0.2360090 \\ \mathrm{~N} & 0.9713700 \\ \mathrm{H} & 0.6296660 \\ \mathrm{~S} & 2.0207880 \\ \mathrm{~S} & 3.0256670 \\ \mathrm{H} & 1.1984910 \\ \mathrm{C} & -1.0320340 \\ \mathrm{C} & -1.4538240 \\ \mathrm{C} & -0.8054490 \\ \mathrm{C} & 0.0339990\end{array}$

$\begin{array}{rr}1.6003020 & 3.1002750 \\ 0.5078720 & 3.1418870 \\ 1.9805730 & 3.5165650 \\ 1.9433170 & 3.7475330 \\ 0.5270560 & -0.0814550 \\ 0.2260090 & 0.3174300 \\ 0.3447080 & -1.1618010 \\ 1.6023180 & 0.0784060 \\ -1.7680550 & 0.3807680 \\ -2.3528980 & 0.9489900 \\ -2.0314560 & -0.6798030 \\ -2.0667360 & 0.7112260 \\ -0.6218940 & -3.7463940 \\ -1.0671270 & -3.6729640 \\ -1.2693940 & -3.2301840 \\ -0.5958850 & -4.8068430 \\ 1.6995490 & -3.9280250 \\ 1.3328490 & -3.8239470 \\ 1.7140000 & -4.9945360 \\ 2.7282280 & -3.5563460 \\ -0.5582160 & -1.0952350 \\ -0.9256600 & -2.3123440 \\ -1.5904340 & -0.0457650 \\ 1.1785610 & -3.3083090 \\ -0.0738860 & 1.6744460 \\ 1.8872580 & 1.4238050 \\ 0.6929680 & -2.7579020 \\ -5.3018080 & -0.3530130 \\ -0.3926530 & 1.9019740 \\ -3.7223780 & -0.9540380 \\ -2.7737970 & -0.7182990 \\ -3.4575390 & -0.5107940 \\ -1.7209610 & -0.5966040 \\ -3.1662100 & -2.2814720 \\ -2.0486360 & 2.7090970 \\ -0.7640110 & 4.4357030 \\ -0.5535790 & 3.1205810 \\ -1.3001330 & 2.2067770 \\ -1.3905080 & 1.2291620 \\ -1.8809140 & 4.4518200 \\ -3.0330010 & 1.8523140 \\ -2.3237800 & -2.3643420 \\ 0.7376910 & 5.2017680 \\ 0.9427570 & 3.8751840 \\ 0.3095470 & 2.8134900 \\ -0.1114800 & 5.4848510\end{array}$

$\begin{array}{lr}\mathrm{H} & -1.5306610 \\ \mathrm{H} & -1.1234070 \\ \mathrm{H} & 0.3547340 \\ \mathrm{C} & 3.3371340 \\ \mathrm{C} & 4.4076570 \\ \mathrm{C} & 4.1270770 \\ \mathrm{C} & 1.9505390 \\ \mathrm{H} & 3.5650030 \\ \mathrm{H} & 5.4004420 \\ \mathrm{H} & 4.3160740 \\ \mathrm{H} & 1.9039300 \\ \mathrm{H} & 1.1707550 \\ \mathrm{H} & 3.3329530 \\ \mathrm{H} & 4.4307930 \\ \mathrm{H} & 4.7697360 \\ \mathrm{O} & -2.4950910 \\ \mathrm{C} & -3.2116790 \\ \mathrm{H} & -3.6393580 \\ \mathrm{H} & -2.5682100 \\ \mathrm{H} & -4.0096890 \\ \mathrm{I} & \end{array}$
1. 2384580
0.4781440
$-0.2578190$
$-5.4862250$
$-4.6324010$
$-3.1410690$
$-5.1949580$
$-6.5488640$
$-4.8624620$
$-2.8458310$
$-5.5168340$
$-5.7362420$
$-5.2774170$
$-4.8674150$
$-2.5092360$
1.7371200
2. 3732550
1.6367600
3. 0647760
2. 9316360

148

6.0217860

1.7891050

6.5104410

$-1.5065630$

$-0.8225300$

$-1.0153240$

$-0.9253910$

$-1.3840330$

$-1.2202210$

$-2.0548980$

0.1218850

$-1.4700680$

$-2.5827380$

0.2496480

$-0.4017420$

3. 5310940

4.5753720

5. 2647120

5.1305110

TS21(S, S) G $[M 06-2 X / 6-31 G(d)]=-4124.880282$

$\begin{array}{lrrr}\mathrm{O} & -0.1387640 & -1.0847320 & -0.0567350 \\ \mathrm{O} & 1.7679600 & -0.0834330 & -1.3581580 \\ \mathrm{C} & 0.0430790 & -2.3020610 & -0.6884450 \\ \mathrm{C} & 1.3244710 & -2.7945360 & -0.8308050 \\ \mathrm{C} & -1.1235280 & -2.9959720 & -1.1103680 \\ \mathrm{C} & 1.5028980 & -4.0055610 & -1.5775050 \\ \mathrm{C} & -0.9427020 & -4.2003150 & -1.7440090 \\ \mathrm{C} & 0.3516300 & -4.7143570 & -2.0219160 \\ \mathrm{H} & -1.8087640 & -4.7822830 & -2.0482330 \\ \mathrm{C} & 2.6060670 & -0.7026090 & -0.4511790 \\ \mathrm{C} & 3.6407680 & 0.0868020 & 0.1227530 \\ \mathrm{C} & 2.4552010 & -2.0533040 & -0.2082020 \\ \mathrm{C} & 4.5486850 & -0.5460890 & 0.9342440 \\ \mathrm{C} & 3.3560180 & -2.6843530 & 0.7160510 \\ \mathrm{C} & 4.4245130 & -1.9210310 & 1.2659890 \\ \mathrm{H} & 5.3788750 & 0.0193990 & 1.3502060 \\ \mathrm{C} & 5.1590610 & -3.8374600 & 2.5626310 \\ \mathrm{C} & 4.0753060 & -4.5885350 & 2.0479240 \\ \mathrm{C} & 3.1983930 & -4.0297370 & 1.1516760 \\ \mathrm{C} & 5.3236870 & -2.5310210 & 2.1807710 \\ \mathrm{H} & 5.8464970 & -4.2937960 & 3.2675070 \\ \mathrm{H} & 3.9309550 & -5.6141110 & 2.3726410 \\ \mathrm{H} & 2.3614160 & -4.6127740 & 0.7836840 \\ \mathrm{H} & 6.1366740 & -1.9327450 & 2.5833150\end{array}$




\begin{tabular}{rrr}
2.9113900 & -5.6735050 & -2.6432840 \\
1.7654530 & -6.3969320 & -3.0522400 \\
0.5151770 & -5.9230680 & -2.7498800 \\
2.7851850 & -4.5111300 & -1.9246320 \\
3.8988310 & -6.0393040 & -2.9064530 \\
1.8810650 & -7.3178630 & -3.6146030 \\
-0.3743360 & -6.4574150 & -3.0726600 \\
3.6698620 & -3.9586530 & -1.6254650 \\
-2.4746020 & -2.4174660 & -0.8227560 \\
-3.2334680 & -1.8340900 & -1.8508270 \\
-2.9734590 & -2.4468240 & 0.5016880 \\
-4.4837180 & -1.2787680 & -1.5391360 \\
-4.2242040 & -1.8944880 & 0.7555330 \\
-4.9968270 & -1.2989920 & -0.2472480 \\
-5.0674280 & -0.8341030 & -2.3394770 \\
-4.6257150 & -1.9252780 & 1.7650950 \\
3.7501900 & 1.5462700 & -0.1888040 \\
3.2436890 & 2.5037690 & 0.7165900 \\
4.3694930 & 1.9525510 & -1.3810460 \\
3.4130380 & 3.8550080 & 0.4200790 \\
4.5127310 & 3.3200150 & -1.6352170 \\
4.0477230 & 4.2846120 & -0.7479480 \\
3.0272150 & 4.5989670 & 1.1126780 \\
4.9991710 & 3.6423830 & -2.5542450 \\
-2.1934930 & -3.1234960 & 1.6228170 \\
-6.3453030 & -0.7035220 & 0.1269380 \\
-2.7686040 & -1.8390210 & -3.3001540 \\
2.5277310 & 2.0882840 & 1.9943810 \\
4.2006160 & 5.7634000 & -1.0501110 \\
4.9001910 & 0.9532550 & -2.3970540 \\
6.4314330 & 1.0033920 & -2.4596210 \\
6.7755660 & 1.9909190 & -2.7867930 \\
6.8705090 & 0.8008000 & -1.4774240 \\
6.8122800 & 0.2605890 & -3.1681900 \\
4.2832890 & 1.1752170 & -3.7821900 \\
3.1914270 & 1.1315560 & -3.7319670 \\
4.5691050 & 2.1485610 & -4.1951070 \\
4.6288530 & 0.4039340 & -4.4781590 \\
5.0352860 & 6.4735940 & 0.0214770 \\
5.1767310 & 7.5278690 & -0.2376440 \\
4.5348130 & 6.4321720 & 0.9952000 \\
6.0199110 & 6.0085920 & -1.1276290 \\
2.8335680 & 6.4396810 & -1.9839920 \\
2.2381500 & 5.9477650 & -1.4802310 \\
2.2686160 & 6.3962790 & \\
2.9541930 & 7.4935030 & \\
-2 & & \\
\hline & &
\end{tabular}

3. 5049190

4. 2852250

3. 9915020

2. 9671970

1. 3962690

1.7844680

0.7243560

0.8171330

$-2.3087970$

$-3.3573010$

$-1.9432790$

$-1.7337550$

$-2.6235980$

$-2.6795940$

$-3.6020880$

$-1.9005240$

$-7.2034030$

$-8.1837160$

$-6.7409430$

$-7.3557810$

$-6.1560950$

$-5.6036840$

$-5.5899700$

$-7.1244960$

$-3.1061470$

$-4.1806270$

$-2.7850310$

$-2.5964710$

$-3.3809120$

$-4.4742690$

$-3.0372410$

$-3.1185890$

0.2281440

$-0.5545210$

0.1365250

$-1.6795420$

$-6.8893470$

$-1.1369730$

4. 6169250

4. 7332200

2.0713190

$-1.7467190$

$-2.9088040$

$-0.8729080$

-2.7217530
-1.7614120
1.9394770

1. 2021730

2.8983830

1. 6228310

3. 0420260

4. 0018870

3.2250370

2. 6023030

$-4.6520900$

$-4.9523090$

$-5.0251150$

$-5.1342690$

$-2.6902750$

$-1.6017920$

$-3.1088190$

$-3.0589810$

$-0.3082980$

0.0386080

0.5093860

$-1.1486480$

0.4963770

.2173850

1. 2911150

0.9062860

$-0.5462830$

$-0.4602830$

0.3304550

$-0.5354230$

$-3.0305080$

$-2.9503500$

- 3.0451370

$-3.9866150$

0.2459470

0.2805160

1.3989410

$-1.9412390$

$-1.4767930$

$-2.8487910$

$-0.0526900$

5.8484470

1.1114480

3. 2976040

2.4628880

3. 1484520

1.4869130

2. 3970780
3. 1681080

2.9607720

3. 3808520

4.0695260

2. 3884200

2.7467510

1. 5458840

3. 2080210

1. 5154760

1.6224030

0.5558100

2. 3131150

3. 0263620

3. 1222820

3.2880500

3. 7611400

$-1.0732270$

$-0.7339020$

$-1.6383780$

$-1.7575170$

1.0657290

1.9683400

0.5657780

1. 3709240

$-4.0477950$

$-4.2460000$

$-3.4834960$

$-5.0165030$

$-4.0514430$

$-4.0559300$

$-5.0912510$

$-3.5913380$

$-0.9368450$

$-2.2206680$

0.0168640

$-3.3014280$

0.6870770

1. 5063930

$-2.0720460$

$-2.0057700$

1.8000300

$-1.7567650$

$-1.4398190$

$-1.1218650$

$-0.9945880$

$-2.8893300$ 


$\begin{array}{lrrr}\mathrm{C} & -2.8703530 & 2.3715840 & 2.0321450 \\ \mathrm{C} & -2.2767760 & 1.0242040 & 4.0607910 \\ \mathrm{C} & -1.4273640 & 0.8301960 & 2.9730370 \\ \mathrm{~N} & -1.7954670 & 1.5977670 & 1.8769110 \\ \mathrm{H} & -1.1848980 & 1.6050850 & 1.0304710 \\ \mathrm{~S} & -3.5389890 & 2.1644010 & 3.6350690 \\ \mathrm{~S} & -3.5235820 & 3.4352330 & 0.8915790 \\ \mathrm{H} & -1.1893700 & 1.5693860 & -2.6712240 \\ \mathrm{C} & -0.9462380 & -0.5013510 & 5.3283960 \\ \mathrm{C} & -0.0999280 & -0.6965110 & 4.2157900 \\ \mathrm{C} & -0.3296060 & -0.0309590 & 3.0169490 \\ \mathrm{C} & -2.0350920 & 0.3478000 & 5.2600300 \\ \mathrm{H} & -0.7245270 & -1.0474010 & 6.2386910 \\ \mathrm{H} & 0.2784660 & -0.1673680 & 2.1312610 \\ \mathrm{H} & -2.6823560 & 0.4838450 & 6.1192840 \\ \mathrm{C} & -3.2683790 & 4.8547560 & -3.0279830 \\ \mathrm{C} & -4.4724820 & 4.2360890 & -2.3136510 \\ \mathrm{C} & -4.2565530 & 2.7456040 & -2.0382360 \\ \mathrm{C} & -2.0012640 & 4.7250930 & -2.1782690 \\ \mathrm{H} & -3.4581980 & 5.9112910 & -3.2372630 \\ \mathrm{H} & -5.3812730 & 4.3610840 & -2.9088160 \\ \mathrm{H} & -4.3155820 & 2.1675590 & -2.9692790 \\ \mathrm{H} & -2.0942370 & 5.3225740 & -1.2633150 \\ \mathrm{H} & -1.1209720 & 5.0873320 & -2.7185050 \\ \mathrm{H} & -3.1143010 & 4.3559650 & -3.9917840 \\ \mathrm{H} & -4.6383330 & 4.7599080 & -1.3631510 \\ \mathrm{H} & -5.0229310 & 2.3400280 & -1.3725220 \\ \mathrm{O} & 0.9171210 & -1.5688070 & 4.4011430 \\ \mathrm{C} & 1.7409700 & -1.8372750 & 3.2782590 \\ \mathrm{H} & 1.1494270 & -2.2428190 & 2.4473990 \\ \mathrm{H} & 2.2593540 & -0.9301930 & 2.9387690 \\ \mathrm{H} & 2.4755610 & -2.5758470 & 3.6006580 \\ \mathrm{H} & & & \\ \mathrm{TS} & & & \end{array}$

148

TS22(S, S) G[M06-2X/6-31G(d) $]=-4124.876034$

$\begin{array}{lrll}\text { O } & 0.4501210 & 0.7817470 & -0.1982150 \\ \mathrm{O} & -1.5170650 & 0.3093450 & -1.7095950 \\ \mathrm{C} & 0.5742500 & 2.0491040 & -0.7322010 \\ \mathrm{C} & -0.5610880 & 2.8103480 & -0.9204760 \\ \mathrm{C} & 1.8872460 & 2.5207870 & -0.9913470 \\ \mathrm{C} & -0.4256060 & 4.0796820 & -1.5722380 \\ \mathrm{C} & 2.0112520 & 3.7710610 & -1.5472170 \\ \mathrm{C} & 0.8788970 & 4.5579790 & -1.8813250 \\ \mathrm{H} & 3.0025760 & 4.1779420 & -1.7314140 \\ \mathrm{C} & -2.2955220 & 1.0359420 & -0.8273610 \\ \mathrm{C} & -3.5348010 & 0.4678920 & -0.4235490 \\ \mathrm{C} & -1.8710130 & 2.2912050 & -0.4420260\end{array}$

-4.3574980
-2.6903310
-3.9639520
-5.3267710
-4.3962590
-3.1034690
-2.2744550
-4.8102070
-5.0474380
-2.7652080
-1.2842470
-5.7892550
-1.3699010
-0.0703410
1.0270080
-1.5442390
-2.2364270
0.0514130
2.0305590
-2.5447630
3.0855510
3.9330800
3.4013990
5.1132640
4.5843850
5.4706450
5.7733710
4.8509340
-3.9168100
-4.0061570
-4.2135950
-4.4231310
-4.6183650
-4.7352100
-4.5010150
-4.8590090
2.5167300
6.8001170
3.6436280
-3.6596310
-5.1821330
-4.1557680
-5.5595180
-5.9890750
-6.2344770
-5.5204010

.2375630

3.0372480

2. 5161630

0.8456960

4.4711200

4.9598140

4. 2656590

3.2704920

5.0393960

5.8931610

4.6495050

. 8669460

6.0759320

6.5654030

5.8189740

4.8686380

6.6597100

7.5249430

6.1736150

4.4979570

1.7148990

1.1754040

1. 5620710

0.5307700

0.9148360

0.4056180

0.1409990

0.8288090

$-0.9188890$

$-1.9308130$

$-1.2099900$

$-3.2045730$

$-2.5036190$

$-3.5134200$

$-3.9828330$

$-2.7306760$

2. 1600290

$-0.1869090$

1.3087640

$-1.6959690$

$-4.9090470$

$-0.1574300$

0.1293490

$-0.7650750$

0.4525750

0.9177610
0.3617220

0.4694520

0.8310630

0.6612080

2.2035530

1. 8979320

1.0525370

1.6864510

2. 8604130

2. 3368940

0.8317730

1.9327010

$-2.5850080$

$-2.8611670$

$-2.5189620$

$-1.9565510$

$-2.8791180$

$-3.3533350$

$-2.7383270$

$-1.7600830$

$-0.6016890$

$-1.5822740$

0.7709090

$-1.1811980$

1.1146140

0.1573140

$-1.9509170$

2.1640390

$-0.8269400$

0.1538200

$-2.1711900$

$-0.2338440$

$-2.5086050$

$-1.5576710$

0.5233230

$-3.5457820$

1.8606010

0.6148340

$-3.0710070$

1. 6184770

$-1.9497660$

$-3.2680470$

$-3.8164350$

$-4.2809530$

$-3.0174990$

$-4.5753010$ 


$\begin{array}{rrr}-3.1992160 & -0.5652160 & -4.3937350 \\ -2.1967330 & -0.7569100 & -4.0012620 \\ -3.5504760 & -1.4683560 & -4.9047240 \\ -3.1318090 & 0.2335700 & -5.1397540 \\ -6.4837930 & -5.2996820 & -1.2407580 \\ -6.8198080 & -6.2881260 & -1.5705880 \\ -6.3379040 & -5.3399390 & -0.1556010 \\ -7.2787980 & -4.5772170 & -1.4480800 \\ -4.0841800 & -5.9429860 & -1.6744330 \\ -3.1570690 & -5.6808800 & -2.1926480 \\ -3.8667200 & -6.0012550 & -0.6021200 \\ -4.3992600 & -6.9370730 & -2.0080620 \\ -4.9126820 & -1.7412410 & 2.5012690 \\ -5.6581320 & -1.0069010 & 2.1796210 \\ -5.3806410 & -2.7319020 & 2.4653770 \\ -4.6494290 & -1.5287850 & 3.5435610 \\ -2.6108950 & -2.6955810 & 2.1207480 \\ -2.9952610 & -3.7215450 & 2.1052150 \\ -1.7115570 & -2.6486840 & 1.4993890 \\ -2.3345900 & -2.4587490 & 3.1548180 \\ 2.7337390 & 3.6769830 & 1.9745780 \\ 3.7751830 & 3.8847360 & 2.2452420 \\ 2.5137810 & 4.1950720 & 1.0378180 \\ 2.0893760 & 4.0933490 & 2.7561990 \\ 2.7264270 & 1.5191810 & 3.2345860 \\ 2.7574170 & 0.4279590 & 3.1684240 \\ 3.6631340 & 1.8528210 & 3.6952830 \\ 1.9073100 & 1.7998510 & 3.9048670 \\ 7.6948240 & -0.6524770 & -0.5334950 \\ 8.6436540 & -1.0260400 & -0.1376220 \\ 7.2253070 & -1.4690800 & -1.0957530 \\ 7.9163580 & 0.1582830 & -1.2337800 \\ 6.6232940 & -1.3223970 & 1.6332970 \\ 5.9766800 & -1.0341620 & 2.4665310 \\ 6.1816550 & -2.2096850 & 1.1666900 \\ 7.5967570 & -1.6108330 & 2.0426430 \\ 3.7706840 & -0.0248600 & -3.8129700 \\ 4.8046070 & -0.3886460 & -3.8195840 \\ 3.1351550 & -0.7865550 & -3.3567950 \\ 3.4613000 & 0.0981630 & -4.8559540 \\ 4.5681290 & 2.3527870 & -3.7118650 \\ 5.6154770 & 2.0419220 & -3.6217120 \\ 4.3389480 & 2.4650380 & -4.7766820 \\ 4.4690100 & 3.3322150 & -3.2350450 \\ -0.1559460 & -0.3950700 & -1.1670050 \\ 0.7088700 & -0.6049360 & -2.3793210\end{array}$
$-0.4140040$
2. 6088250
7. 3285840
1.4716870
$-3.7787220$
$-5.3745890$
$-3.2294320$
1.8534600
2. 9198560
0.8783520
2. 6443850
2. 0308910
2. 0277500
0.6630900
0.2381410
1. 0194890
0.6950120
2. 0685420
3. 1434070
1.4201450
$-1.1214970$
$-1.5367160$
$-0.8428520$
$-0.0164000$
$-1.6560330$
$-1.1553330$
0.2981960
3. 6206150
4.6589890
4. 3554410
2. 2180170
3. 8680600
5.6643360
4.5624610
2. 1574470
1. 4592850
3.6337410
4.6632400
4. 9750560
$-2.6138380$
$-3.3770930$
$-3.7761380$
$-2.7783830$
$-4.1956300$
$-1.5324000$
1.6443450
0.6257440
1. 9818890
0.7748220
$-4.8991750$
$-0.6958130$
$-3.4607830$
$-2.5191200$
$-3.2608440$
$-1.4882490$
$-2.7705700$
$-2.1577550$
$-1.0646840$
$-0.7749590$
$-1.4139230$
$-1.4367750$
$-2.1144770$
$-3.0394240$
$-1.9474380$
0.2861000
0.5708410
0.0552340
$-0.5272840$
0.6956030
0.2855390
$-0.7362010$
$-5.1286590$
$-4.3303310$
$-2.8314990$
$-4.9226700$
$-6.1937810$
$-4.5016340$
$-2.4224340$
$-5.3493790$
$-5.4192630$
$-4.8114780$
$-4.6730230$
$-2.2584790$
1. 3349260
1. 8506860
1.0420450
2. 5282770
2. 4043460

148

$\operatorname{TS} 23(\mathrm{~S}, \mathrm{~S}) \mathrm{G}[\mathrm{M} 06-2 \mathrm{X} / 6-31 \mathrm{G}(\mathrm{d})]=-4124.875146$
$-0.2243840$

$-3.1852320$

1.1337600

1.5831270

$-2.8382490$

$-3.0300760$

1.7162770

$-1.2767620$

$-0.9209380$

$-0.8330050$

$-0.6999800$

$-2.5371160$

2.5309780

4.3302440

3.0281640

2. 0717280

1.0837530

4.2827430

1.6224450

$-2.5501240$

5.1804080

3.8665990

2.7708520

5.4153010

6.0279870

1.7570830

6.4318730

$-1.9661110$

$-1.1746510$

$-1.2180770$

$-1.3881060$

$-1.9469350$

$-1.5701550$

$-2.2156460$

$-0.3797420$

$-2.0007900$

$-3.0153590$

$-0.1315120$

$-0.5252260$

3.5661410

4.6431150

5.2656370

5.2619600

4.1864010

0.7088700

$-2.3793210$ 


$\begin{array}{lrrr} & & & \\ \mathrm{O} & 0.2470420 & 0.8682090 & \\ \mathrm{O} & -1.6776520 & 0.1703900 & -1.3609290 \\ \mathrm{C} & 0.2380270 & 2.1564870 & -0.3882870 \\ \mathrm{C} & -0.9756160 & 2.7952390 & -0.5407850 \\ \mathrm{C} & 1.4914880 & 2.7483050 & -0.6967520 \\ \mathrm{C} & -0.9839000 & 4.0927880 & -1.1539890 \\ \mathrm{C} & 1.4723650 & 4.0389720 & -1.1679590 \\ \mathrm{C} & 0.2599150 & 4.7252880 & -1.4333110 \\ \mathrm{H} & 2.4120030 & 4.5447080 & -1.3738110 \\ \mathrm{C} & -2.5233690 & 0.8367720 & -0.4987080 \\ \mathrm{C} & -3.7541300 & 0.1890250 & -0.1869900 \\ \mathrm{C} & -2.2268440 & 2.1242950 & -0.0920230 \\ \mathrm{C} & -4.6651580 & 0.8731380 & 0.5765000 \\ \mathrm{C} & -3.1508890 & 2.7914180 & 0.7861500 \\ \mathrm{C} & -4.3910020 & 2.1661560 & 1.0928660 \\ \mathrm{H} & -5.6178440 & 0.4060430 & 0.8148190 \\ \mathrm{C} & -5.0114440 & 4.0274630 & 2.5211950 \\ \mathrm{C} & -3.7605880 & 4.6334380 & 2.2541590 \\ \mathrm{C} & -2.8575710 & 4.0359860 & 1.4103240 \\ \mathrm{C} & -5.3143850 & 2.8164110 & 1.9539700 \\ \mathrm{H} & -5.7181090 & 4.5150830 & 3.1848640 \\ \mathrm{H} & -3.5093840 & 5.5769780 & 2.7281220 \\ \mathrm{H} & -1.8968420 & 4.5061340 & 1.2316600 \\ \mathrm{H} & -6.2592290 & 2.3233950 & 2.1663050 \\ \mathrm{C} & -2.1372630 & 6.0072390 & -2.1082050 \\ \mathrm{C} & -0.8998240 & 6.6548750 & -2.3396340 \\ \mathrm{C} & 0.2712120 & 6.0222700 & -2.0137180 \\ \mathrm{C} & -2.1800560 & 4.7631360 & -1.5307340 \\ \mathrm{H} & -3.0613520 & 6.4977280 & -2.3973810 \\ \mathrm{H} & -0.8831060 & 7.6421460 & -2.7898650 \\ \mathrm{H} & 1.2308740 & 6.4934490 & -2.2086160 \\ \mathrm{H} & -3.1352000 & 4.2742790 & -1.3715980 \\ \mathrm{C} & 2.7769950 & 1.9948990 & -0.5504010 \\ \mathrm{C} & 3.5255270 & 1.6823580 & -1.7000960 \\ \mathrm{C} & 3.2669990 & 1.6369410 & 0.7302130 \\ \mathrm{C} & 4.7802600 & 1.0734310 & -1.5506180 \\ \mathrm{C} & 4.4998580 & 0.9954670 & 0.8180860 \\ \mathrm{C} & 5.2927530 & 0.7313650 & -0.3056630 \\ \mathrm{H} & 5.3643880 & 0.8704600 & -2.4435530 \\ \mathrm{H} & 4.8811880 & 0.7103200 & 1.7943600 \\ \mathrm{C} & -4.0566010 & -1.1839900 & -0.7005960 \\ \mathrm{C} & -3.6503690 & -2.3050030 & 0.0595560 \\ \mathrm{C} & -4.7630830 & -1.3596380 & -1.9037620 \\ \mathrm{C} & -3.9726410 & -3.5816230 & -0.3957620 \\ \mathrm{C} & -5.0556230 & -2.6659070 & -2.3156790 \\ \mathrm{C} & -4.6763800 & -3.7848670 & -1.5840710 \\ & & & \end{array}$

-3.6618460
-5.6017380
2.5179000
6.6756400
3.0474670
-2.9158660
-5.0080830
-5.3061900
-4.3818770
-4.3905530
-3.3484780
-4.7332500
-6.6735270
-7.3662120
-6.5721360
-7.1131400
-5.9099090
-6.1805610
-5.3963680
-6.8298690
-3.7360390
-3.0957180
-3.1575710
-3.9883590
-3.9017760
-4.5625090
-4.5265610
-3.3551790
-1.9732930
-2.5284550
-1.2997780
-1.3681860
2.6569480
3.7124120
2.2710080
2.1109690
2.9792290
3.0733680
3.9520310
2.2574190
7.5437600
8.5241320
7.6963140
7.0825270
7.4171200
6.8570090
-
0.1873070 $-3.2472030$ 2.0017250 $-0.1120200$ $-2.0657850$ $-2.7913120$ $-3.0608920$ $-2.2368550$ $-3.2325400$ $-3.9549450$ $-2.2886510$ $-2.1550290$ $-1.3304030$ $-3.0059760$ $-1.0680440$ $-1.4514880$ $-0.1104600$ $-0.8789290$ $-2.3446380$ $-3.0704990$ $-1.4250860$ $-2.7394050$ 2.5413530 2. 3708570 2.6702140 3.4769560 1.7196210 1.9789040

0.8876040

2.5922330 2. 2730620 2.4201600 1.4481500 3.1825740 3.2417790 3.0436710 3.5948190 4.0544960 0.7641770 0.9378790 0.2627830 1.7358510 $-1.4165600$ $-2.0815400$ 


\begin{tabular}{|c|c|c|}
\hline $\begin{array}{l}7.6209220 \\
8.3775920 \\
3.2092420 \\
4.2638500 \\
2.7537660 \\
2.7234950 \\
.7925930 \\
4.8669020 \\
3.4332390 \\
.6635250 \\
0.1779100 \\
.6416520 \\
0.1686500 \\
1.9797340 \\
6.5417970 \\
1.4560550 \\
5.4812730 \\
5.5589450 \\
2.2955910 \\
2.0233650 \\
.0852080 \\
1.1010520 \\
2.7933990 \\
2.0251020 \\
2.6578750 \\
1.5428760 \\
0.9097710 \\
1.5495940 \\
1.0902040 \\
2.9674720 \\
.6700050 \\
1.3838390 \\
0.0746960 \\
0.6908600 \\
0.2052010 \\
1.0432670 \\
0.4931950 \\
0.6301460 \\
1.5229500 \\
3.7491170 \\
4.8484360 \\
4.4876300 \\
2.4280510 \\
5.0403020 \\
4.5645290\end{array}$ & $\begin{array}{r}0.7568600 \\
-0.6466650 \\
0.7950760 \\
0.5829130 \\
-0.1003120 \\
1.0073970 \\
3.2029580 \\
2.9936260 \\
3.4210930 \\
4.1016810 \\
-0.3086370 \\
-0.2351480 \\
-1.5639630 \\
2.2253610 \\
-0.8254520 \\
1.7946970 \\
-0.7160680 \\
-5.0833800 \\
-1.2346690 \\
-3.1593690 \\
-2.2322620 \\
-3.1018170 \\
-1.2768360 \\
-2.2426050 \\
-2.5039300 \\
-1.7521000 \\
-1.3251220 \\
-1.7844750 \\
-1.6659160 \\
-2.6889920 \\
-3.1699250 \\
-1.4664510 \\
-0.5554660 \\
-0.1068030 \\
-0.4871330 \\
-1.3683300 \\
-0.2290180 \\
-0.1455970 \\
-1.6895580 \\
-4.5532040 \\
-3.8538030 \\
-2.3957680 \\
-4.5506320 \\
-5.5844680 \\
-3.8826970 \\
-1.7814120\end{array}$ & $\begin{array}{l}-1.9624500 \\
-1.1977690 \\
-4.0542760 \\
-4.2639840 \\
-3.6285440 \\
-5.0121810 \\
-3.6953290 \\
-3.7568010 \\
-4.7064900 \\
-3.0862610 \\
-0.9384850 \\
-2.1976200 \\
-0.1197050 \\
-3.0670010 \\
0.4311410 \\
1.8533920 \\
-3.7640460 \\
-3.0094570 \\
1.2919870 \\
-1.7496800 \\
-1.3466720 \\
-1.1717940 \\
-0.9111300 \\
-2.8693690 \\
2.1719440 \\
4.2874150 \\
3.1218790 \\
1.9767510 \\
1.0494620 \\
3.8846770 \\
0.9959360 \\
-2.6626280 \\
5.5715140 \\
4.3843320 \\
3.1365860 \\
5.5335770 \\
6.5170330 \\
2.2001460 \\
6.4514430 \\
-2.9497480 \\
-2.1478230 \\
-1.8531480 \\
-2.1764220 \\
-3.1684230 \\
-2.6856530 \\
-2.7600480\end{array}$ \\
\hline
\end{tabular}

$\begin{array}{lrrr}\mathrm{H} & 2.5186880 & -5.1575050 & -1.2675480 \\ \mathrm{H} & 1.6164080 & -4.9748730 & -2.7755730 \\ \mathrm{H} & 3.6108890 & -4.0441070 & -3.9105290 \\ \mathrm{H} & 5.0001580 & -4.3889150 & -1.2013050 \\ \mathrm{H} & 5.1641510 & -1.9483090 & -1.1211170 \\ \mathrm{O} & -1.7566490 & 0.7087870 & 4.5519300 \\ \mathrm{C} & -2.2648190 & 1.3356250 & 3.3856800 \\ \mathrm{H} & -1.4763050 & 1.9053460 & 2.8785880 \\ \mathrm{H} & -2.6826030 & 0.5996520 & 2.6866810 \\ \mathrm{H} & -3.0549780 & 2.0119200 & 3.7142630 \\ \mathrm{1} & & & \end{array}$

148

$\begin{array}{lrcr}\text { TS24 }(\mathrm{S}, \mathrm{S}) \mathrm{G}[\mathrm{M} 06-2 \times / 6-31 \mathrm{G}(\mathrm{d})]=-4124.874582 \\ \mathrm{O} & 0.2411810 & 0.8613510 & 0.2216870 \\ \mathrm{O} & -1.6111270 & 0.3516620 & -1.4016730 \\ \mathrm{C} & 0.2485680 & 2.1965640 & -0.1438010 \\ \mathrm{C} & -0.9585270 & 2.8575890 & -0.2603480 \\ \mathrm{C} & 1.5084380 & 2.8133770 & -0.3672980 \\ \mathrm{C} & -0.9462540 & 4.2183910 & -0.7176990 \\ \mathrm{C} & 1.5050820 & 4.1594870 & -0.6452940 \\ \mathrm{C} & 0.3038010 & 4.8826790 & -0.8532030 \\ \mathrm{H} & 2.4521960 & 4.6771420 & -0.7720150 \\ \mathrm{C} & -2.4902630 & 0.9057840 & -0.4929090 \\ \mathrm{C} & -3.7173620 & 0.2123100 & -0.2858180 \\ \mathrm{C} & -2.2241950 & 2.1448640 & 0.0607450 \\ \mathrm{C} & -4.6586310 & 0.7989980 & 0.5209550 \\ \mathrm{C} & -3.1909100 & 2.7146640 & 0.9618450 \\ \mathrm{C} & -4.4253100 & 2.0393330 & 1.1692430 \\ \mathrm{H} & -5.6100420 & 0.2973120 & 0.6811130 \\ \mathrm{C} & -5.1440190 & 3.7593040 & 2.7235980 \\ \mathrm{C} & -3.9025090 & 4.4165650 & 2.5502640 \\ \mathrm{C} & -2.9530100 & 3.9092180 & 1.6986680 \\ \mathrm{C} & -5.3926770 & 2.5910800 & 2.0509170 \\ \mathrm{H} & -5.8862820 & 4.1736460 & 3.3980950 \\ \mathrm{H} & -3.6936840 & 5.3250230 & 3.1063060 \\ \mathrm{H} & -1.9983480 & 4.4133900 & 1.6009310 \\ \mathrm{H} & -6.3303280 & 2.0590740 & 2.1874250 \\ \mathrm{C} & -2.0646990 & 6.2326950 & -1.4908240 \\ \mathrm{C} & -0.8230500 & 6.9085630 & -1.5694840 \\ \mathrm{C} & 0.3348870 & 6.2429200 & -1.2633740 \\ \mathrm{C} & -2.1265940 & 4.9263430 & -1.0753890 \\ \mathrm{H} & -2.9763080 & 6.7519170 & -1.7692580 \\ \mathrm{H} & -0.7917190 & 7.9444950 & -1.8912650 \\ \mathrm{H} & 1.2995990 & 6.7359690 & -1.3487790 \\ \mathrm{H} & -3.0839220 & 4.4182040 & -1.0310200 \\ \mathrm{C} & 2.7933110 & 2.0461430 & -0.3984850 \\ \mathrm{C} & 3.4864050 & 1.9395980 & -1.6214390\end{array}$




$\begin{array}{rrr}3.3528130 & 1.5035550 & 0.7815920 \\ 4.7620910 & 1.3602130 & -1.6270550 \\ 4.6182460 & 0.9238520 & 0.7169720 \\ 5.3564880 & 0.8716430 & -0.4700460 \\ 5.3007860 & 1.3043320 & -2.5686050 \\ 5.0675990 & 0.5192250 & 1.6212590 \\ -3.9823680 & -1.0999480 & -0.9544460 \\ -3.5988840 & -2.2934330 & -0.3003950 \\ -4.6286250 & -1.1490610 & -2.2026850 \\ -3.8757920 & -3.5150000 & -0.9111780 \\ -4.8775580 & -2.4044490 & -2.7712450 \\ -4.5141420 & -3.5930170 & -2.1499450 \\ -3.5828790 & -4.4349080 & -0.4124480 \\ -5.3768800 & -2.4475620 & -3.7380250 \\ 2.6476920 & 1.6368210 & 2.1252820 \\ 6.7780830 & 0.3321710 & -0.4336570 \\ 2.9389430 & 2.4742690 & -2.9417520 \\ -2.9473030 & -2.2642770 & 1.0767900 \\ -4.7965760 & -4.9342970 & -2.7996750 \\ -5.1598490 & 0.0544740 & -2.9809650 \\ -4.2510600 & 1.2865240 & -3.0839630 \\ -4.3007610 & 1.9087680 & -2.1857200 \\ -3.2068340 & 1.0098350 & -3.2542390 \\ -4.5844180 & 1.9029260 & -3.9253020 \\ -6.5521860 & 0.4595450 & -2.4757610 \\ -7.2333150 & -0.3968740 & -2.4622450 \\ -6.4922070 & 0.8675490 & -1.4617490 \\ -6.9803840 & 1.2317130 & -3.1240300 \\ -5.7308690 & -5.7885400 & -1.9352350 \\ -5.9659270 & -6.7314570 & -2.4395240 \\ -5.2600620 & -6.0298680 & -0.9758250 \\ -6.6683810 & -5.2636070 & -1.7290280 \\ -3.4981090 & -5.6903640 & -3.1038420 \\ -2.8346870 & -5.0953980 & -3.7383200 \\ -2.9599510 & -5.9251160 & -2.1788230 \\ -3.7136220 & -6.6338420 & -3.6158710 \\ -4.0036280 & -2.1832650 & 2.1882900 \\ -4.6456830 & -1.3048690 & 2.0832290 \\ -4.6411220 & -3.0747280 & 2.1692570 \\ -3.5149760 & -2.1340260 & 3.1687650 \\ -2.0291430 & -3.4589200 & 1.3445090 \\ -2.6006790 & -4.3870150 & 1.4546550 \\ -1.2968280 & -3.5875020 & 0.5432150 \\ -1.4944600 & -3.3019120 & 2.2877440 \\ 2.9148800 & 3.0255970 & 2.7239450 \\ 3.9875740 & 3.1565610 & 2.9060400\end{array}$

2. 5831100

2. 3889200

3.0425920

3.0129170

4.0523740

2. 3515800

7.6981430

8.7171710

7.7321310

7.3418250

7.3479940

6.6999030

7.4874830

8.3264370

3. 0178780

4.0510240

2. 4462930

2.6022250

3.6833880

4.7420660

3.2588840

3.6324460

$-0.1355510$

0.7418670

$-0.1847510$

1. 8816350

6.7652120

1.5704880

$-5.2929810$

$-5.3039430$

$-2.3278570$

2.3568980

3.1210820

1. 2761980

2. 6302070

2. 8026660

1. 9570330

1. 1961670

0.6152470

1.0705440

0.6538860

2. 3232580

2.6478090

2.0199500

$-0.0851330$

$-0.6724870$
3.8213200

3.1399370

0.5495110

$-0.4473120$

0.7107320

0.5567920

1.3462890

0.9536390

2.2786530

1. 5820070

$-0.0278230$

$-0.7160450$

0.8684570

$-0.5028830$

1.4528350

1.1629290

0.5532080

1.8956820

3.7522080

3. 5341990

4.1579290

4.5282510

$-0.1911380$

0.0258210

$-1.5389700$

2.7150050

$-0.5790700$

1.5412130

$-0.3129780$

$-4.7355200$

$-1.3615280$

$-2.7329790$

$-2.2863220$

$-2.7716990$

$-1.6048250$

$-1.4272330$

$-3.3196620$

$-2.3104090$

$-1.7451080$

$-2.3315200$

$-2.0705320$

$-3.5661550$

$-4.2256760$

$-0.7729610$

$-0.8239890$

$-0.2658450$
2.0502030

3.6777800

3.1294800

2. 6737040

3.5225500

3.9796000

0.2627970

0.3403110

$-0.3115830$

1. 2698190

$-1.8059940$

$-2.3600620$

$-2.4203540$

$-1.6895070$

$-4.0827900$

$-4.3016310$

$-3.8530210$

$-4.9941120$

$-3.3565210$

$-3.5390870$

$-4.2806900$

$-2.5879290$

$-0.9720560$

$-2.1734050$

$-0.3189280$

$-2.8026740$

0.1808660

1.9558480

$-4.0059050$

$-3.7521670$

1.1259980

$-1.9805070$

$-0.8134760$

$-1.8390740$

$-0.1216380$

$-2.4149330$

2.0728200

4.2340550

3.1002640

1.9287260

1.0035460

3.7634820

0.8255710

$-2.2828540$

5.5923450

4.4364750 


\begin{tabular}{|c|c|c|c|c|c|c|c|}
\hline C & -0.3173620 & -0.7102970 & 3.1661110 & H & -2.3495070 & 3.8176190 & 1.8662180 \\
\hline C & 0.8481950 & -1.8398410 & 5.5023550 & $\mathrm{H}$ & -5.8361380 & 0.4426250 & 2.9314970 \\
\hline $\mathrm{H}$ & -0.3915880 & -0.4341750 & 6.5566460 & C & -2.9703150 & 5.8894360 & -1.0552830 \\
\hline $\mathrm{H}$ & -0.7102130 & -0.2831600 & 2.2503880 & C & -1.8474850 & 6.7222980 & -1.2780510 \\
\hline $\mathrm{H}$ & 1.2930120 & -2.2608470 & 6.3971610 & $\mathrm{c}$ & -0.5827040 & 6.2043080 & -1.1794310 \\
\hline C & 4.4639000 & -3.7444180 & -2.9252200 & C & -2.8062870 & 4.5688740 & -0.7217590 \\
\hline C & 5.1061900 & -3.6616770 & -1.5382410 & $\mathrm{H}$ & -3.9704170 & 6.2992440 & -1.1556270 \\
\hline C & 4.6125050 & -2.4327950 & -0.7727530 & $\mathrm{H}$ & -1.9931300 & 7.7661370 & -1.5368490 \\
\hline C & 2.9398430 & -3.8461200 & -2.8183090 & $\mathrm{H}$ & 0.2883460 & 6.8277140 & -1.3626000 \\
\hline $\mathrm{H}$ & 4.8488930 & -4.6118010 & -3.4688840 & $\mathrm{H}$ & -3.6729090 & 3.9353080 & -0.5623790 \\
\hline $\mathrm{H}$ & 6.1963640 & -3.6135350 & -1.6203270 & C & 2.5047050 & 2.3720950 & -0.4607170 \\
\hline $\mathrm{H}$ & 5.0093570 & -1.5180540 & -1.2260810 & $\mathrm{C}$ & 3.2992130 & 2.3518120 & -1.6257460 \\
\hline $\mathrm{H}$ & 2.6545150 & -4.7941820 & -2.3454960 & $\mathrm{C}$ & 3.0091520 & 1.8676170 & 0.7570660 \\
\hline $\mathrm{H}$ & 2.4697030 & -3.8194050 & -3.8061230 & C & 4.6556580 & 1.8843320 & -1.5472480 \\
\hline $\mathrm{H}$ & 4.7282790 & -2.8531790 & -3.5070680 & $\mathrm{C}$ & 4.3525830 & 1.3610130 & 0.8098670 \\
\hline $\mathrm{H}$ & 4.8659910 & -4.5684050 & -0.9683480 & $\mathrm{C}$ & 5.1500660 & 1.4071000 & -0.3338110 \\
\hline $\mathrm{H}$ & 4.9392080 & -2.4330740 & 0.2706510 & $\mathrm{C}$ & -3.5222130 & -1.7818290 & -0.9595950 \\
\hline 0 & -1.5848080 & 0.7050950 & 4.6575370 & $\mathrm{C}$ & -3.1291270 & -2.9769420 & -0.3260330 \\
\hline C & -2.1624770 & 1.3023290 & 3.5094660 & C & -3.9900460 & -1.7960140 & -2.2881380 \\
\hline $\mathrm{H}$ & -1.3934850 & 1.7902790 & 2.8976030 & $\mathrm{C}$ & -3.2237780 & -4.2184800 & -1.0425230 \\
\hline $\mathrm{H}$ & -2.6883940 & 0.5569110 & 2.8971160 & $\mathrm{C}$ & -4.0767900 & -3.0427610 & -2.9940940 \\
\hline $\mathrm{H}$ & -2.8734560 & 2.0461460 & 3.8688980 & C & -3.6967270 & -4.2225540 & -2.3539080 \\
\hline & & & & $\mathrm{C}$ & 3.3857480 & -1.5245390 & -1.3643810 \\
\hline & 2) $G[M 06-2 x$ & (d) $]=-3917$ & & $\mathrm{C}$ & 2.6038160 & -2.7276770 & -1.0574010 \\
\hline 0 & 0.2167820 & 0.8173310 & 0.0149090 & $\mathrm{H}$ & 3.0659400 & -0.6072190 & -0.8591300 \\
\hline 0 & -1.6318810 & 0.2303610 & -1.5854230 & $\mathrm{H}$ & 1.5542480 & -2.5957860 & -0.8103690 \\
\hline C & -0.0013730 & 2.1470290 & -0.2639810 & 0 & 2.7056390 & -1.7186750 & -2.6215540 \\
\hline C & -1.2915500 & 2.6355280 & -0.2452280 & C & 2.0466250 & -2.1760610 & 2.4373960 \\
\hline C & 1.1365000 & 2.9586370 & -0.5108440 & C & 0.7388040 & -1.1368410 & 4.3130420 \\
\hline C & -1.5070670 & 4.0073670 & -0.5920430 & C & 0.1885710 & -0.9397920 & 3.0425310 \\
\hline C & 0.9273120 & 4.2894180 & -0.7804260 & $\mathrm{~N}$ & 0.9403650 & -1.5454920 & 2.0444170 \\
\hline C & -0.3792520 & 4.8383010 & -0.8468920 & $\mathrm{H}$ & 0.6026350 & -1.5505650 & 1.0531970 \\
\hline $\mathrm{H}$ & 1.7805710 & 4.9378370 & -0.9654640 & $\mathrm{~s}$ & 2.2131940 & -2.0740670 & 4.1829990 \\
\hline C & -2.5014270 & 0.4995150 & -0.5472940 & $\mathrm{~S}$ & 3.1701330 & -2.9901400 & 1.4781460 \\
\hline C & -3.4634710 & -0.4931150 & -0.2120650 & $\mathrm{P}$ & -0.1434350 & -0.2831740 & -1.1529310 \\
\hline C & -2.3899920 & 1.7019910 & 0.1230830 & 0 & 0.7422100 & -0.1119610 & -2.3488130 \\
\hline C & -4.3190970 & -0.2300550 & 0.8296290 & 0 & -0.1955420 & -1.5834060 & -0.4055670 \\
\hline C & -3.2507020 & 1.9440280 & 1.2446240 & $\mathrm{H}$ & 1.8902100 & -1.0995380 & -2.6147200 \\
\hline C & -4.2328470 & 0.9693310 & 1.5823530 & C & -1.0340480 & 0.1455960 & 5.2665230 \\
\hline $\mathrm{H}$ & -5.0652140 & -0.9714250 & 1.1055820 & C & -1.5829520 & 0.3408070 & 3.9912550 \\
\hline C & -4.9583400 & 2.3215140 & 3.4641650 & $\mathrm{C}$ & -0.9786360 & -0.1975650 & 2.8629370 \\
\hline C & -3.9559420 & 3.2722940 & 3.1552540 & C & 0.1309700 & -0.5928570 & 5.4425470 \\
\hline C & -3.1233090 & 3.0887620 & 2.0796100 & $\mathrm{H}$ & -1.5240840 & 0.5783880 & 6.1322760 \\
\hline C & -5.0863100 & 1.1930600 & 2.6961610 & $\mathrm{H}$ & -1.3773890 & -0.0455610 & 1.8648520 \\
\hline $\mathrm{H}$ & -5.6128610 & 2.4828990 & 4.3147320 & $\mathrm{H}$ & 0.5560750 & -0.7408680 & 6.4295680 \\
\hline $\mathrm{H}$ & -3.8393260 & 4.1517140 & 3.7807390 & C & 5.2734100 & -3.0077510 & -2.1441920 \\
\hline
\end{tabular}




$\begin{array}{rrr}4.6369220 & -4.1833590 & -1.4012810 \\ 3.1102600 & -4.0886040 & -1.4347620 \\ 4.8832950 & -1.6738930 & -1.5020710 \\ 6.3631930 & -3.1047910 & -2.1452950 \\ 4.9483010 & -5.1350720 & -1.8416760 \\ 2.7380950 & -4.2843330 & -2.4478410 \\ 5.3117570 & -1.5912970 & -0.4947960 \\ 5.2562000 & -0.8310940 & -2.0920400 \\ 4.9463920 & -3.0139260 & -3.1906760 \\ 4.9806560 & -4.1855990 & -0.3586410 \\ 2.6378320 & -4.8249710 & -0.7802480 \\ -2.4923320 & 0.9240860 & 3.8794700 \\ -3.7663100 & -5.1658340 & -2.8918510 \\ 6.1758710 & 1.0467190 & -0.2817290 \\ -2.2223190 & -4.1866940 & 1.5855820 \\ -2.6047540 & -3.0128630 & 1.0083040 \\ -2.8204780 & -5.4313300 & -0.3962660 \\ -2.3363890 & -5.4202150 & 0.8765350 \\ -1.8182000 & -4.1852990 & 2.5936500 \\ -2.5017850 & -2.0828140 & 1.5558130 \\ -2.9063520 & -6.3611180 & -0.9526760 \\ -2.0292320 & -6.3445370 & 1.3558510 \\ -4.8557500 & -0.6538060 & -4.2570890 \\ -4.4031330 & -0.6063430 & -2.9714600 \\ -4.5581820 & -3.0497010 & -4.3418040 \\ -4.9346200 & -1.8940830 & -4.9573440 \\ -5.1598370 & 0.2603500 & -4.7572760 \\ -4.3525540 & 0.3441540 & -2.4500020 \\ -4.6149330 & -4.0025970 & -4.8614660 \\ -5.2968170 & -1.9092750 & -5.9804770 \\ 3.6052190 & 2.7350780 & -4.0124140 \\ 2.8035440 & 2.7570090 & -2.9095830 \\ 5.4691060 & 1.8949070 & -2.7259130 \\ 4.9641780 & 2.3101940 & -3.9208590 \\ 3.2045530 & 3.0323440 & -4.9762710 \\ 1.7659090 & 3.0582560 & -2.9980360 \\ 6.4984210 & 1.5545750 & -2.6416310 \\ 5.5863410 & 2.3086740 & -4.8102970 \\ 4.0833010 & 0.8135150 & 3.1624520 \\ 4.8544880 & 0.8273400 & 2.0398430 \\ 2.2403770 & 1.8485740 & 1.9676290 \\ 2.7576520 & 1.3415100 & 3.1236030 \\ 4.4702850 & 0.4050950 & 4.0907090 \\ 5.8679480 & 0.4349090 & 2.0536640 \\ 1.2299020 & 2.2444080 & 1.9615930 \\ 2.1533140 & 1.3378160 & 4.0266110\end{array}$

$\begin{array}{lrrr}\text { C14 } & & & \\ \text { TS26(S }, \mathrm{S}) \mathrm{G}[\mathrm{M} 06-2 \times / 6-31 \mathrm{G}(\mathrm{d})] & =-3917.748198 \\ \mathrm{O} & -1.6728520 & 0.0261340 & -1.5508040 \\ \mathrm{O} & 0.1791770 & 0.8045410 & -0.0349100 \\ \mathrm{C} & -2.5306090 & 0.4009990 & -0.5367470 \\ \mathrm{C} & -2.4274690 & 1.6731560 & -0.0073690 \\ \mathrm{C} & -3.4765080 & -0.5573670 & -0.0792630 \\ \mathrm{C} & -3.2746980 & 2.0291990 & 1.0930740 \\ \mathrm{C} & -4.3198730 & -0.1896630 & 0.9405220 \\ \mathrm{C} & -4.2374300 & 1.0863200 & 1.5543690 \\ \mathrm{H} & -5.0525510 & -0.9040410 & 1.3082870 \\ \mathrm{C} & -0.0515580 & 2.0894880 & -0.4696320 \\ \mathrm{C} & 1.0769380 & 2.8605720 & -0.8460620 \\ \mathrm{C} & -1.3439100 & 2.5686010 & -0.4952200 \\ \mathrm{C} & 0.8591380 & 4.1401460 & -1.2938330 \\ \mathrm{C} & -1.5715810 & 3.8838540 & -1.0114940 \\ \mathrm{C} & -0.4521620 & 4.6731020 & -1.4030910 \\ \mathrm{H} & 1.7052450 & 4.7579530 & -1.5853270 \\ \mathrm{C} & -1.9375000 & 6.4814260 & -2.0477500 \\ \mathrm{C} & -3.0517530 & 5.6868360 & -1.6862130 \\ \mathrm{C} & -2.8753040 & 4.4229760 & -1.1824630 \\ \mathrm{C} & -0.6688040 & 5.9813600 & -1.9118000 \\ \mathrm{H} & -2.0928970 & 7.4807630 & -2.4414310 \\ \mathrm{H} & -4.0552630 & 6.0800200 & -1.8151390 \\ \mathrm{H} & -3.7353880 & 3.8163680 & -0.9173590 \\ \mathrm{H} & 0.1953140 & 6.5744130 & -2.1990470 \\ \mathrm{C} & -3.9658370 & 3.5530690 & 2.8568610 \\ \mathrm{C} & -4.9480050 & 2.6293930 & 3.2889780 \\ \mathrm{C} & -5.0742490 & 1.4216310 & 2.6527060 \\ \mathrm{C} & -3.1503810 & 3.2620720 & 1.7918390 \\ \mathrm{H} & -3.8518810 & 4.4982720 & 3.3782460 \\ \mathrm{H} & -5.5891380 & 2.8757430 & 4.1293050 \\ \mathrm{H} & -5.8095250 & 0.6931620 & 2.9836960 \\ \mathrm{H} & -2.3920030 & 3.9726350 & 1.4820360 \\ \mathrm{C} & -3.5334500 & -1.9212570 & -0.6782710 \\ \mathrm{C} & -3.1236210 & -3.0358210 & 0.0791710 \\ \mathrm{C} & -4.0206330 & -2.0862360 & -1.9896040 \\ \mathrm{C} & -3.2242350 & -4.3506340 & -0.4913140 \\ \mathrm{C} & -4.1118000 & -3.4048210 & -2.5495100 \\ \mathrm{C} & -3.7172630 & -4.5037950 & -1.7862250 \\ \mathrm{C} & 2.4439360 & 2.2815330 & -0.7256370 \\ \mathrm{C} & 3.2010900 & 2.0187890 & -1.8840750 \\ \mathrm{C} & 2.9766390 & 2.0131490 & 0.5530340 \\ \mathrm{C} & 4.5615840 & 1.5765990 & -1.7527710 \\ \mathrm{C} & 4.3285390 & 1.5416670 & -0.4820680 \\ \mathrm{C} & 5.0984720 & 1.3740620 & \end{array}$




$\begin{array}{rr}-0.1623990 & -0.4047490 \\ -0.1565410 & -1.6338340 \\ 0.6980000 & -0.2934150 \\ 6.1324060 & 1.0469970 \\ -3.7908560 & -5.5019710 \\ 3.4166260 & 1.8688600 \\ 2.6574050 & 2.1426810 \\ 5.3315260 & 1.3240560 \\ 4.7783720 & 1.4633390 \\ 2.9805130 & 1.9482760 \\ 1.6162100 & 2.4238190 \\ 6.3648840 & 1.0067350 \\ 5.3654310 & 1.2579610 \\ 2.7651880 & 1.9038770 \\ 2.2236930 & 2.1936990 \\ 4.8605420 & 1.2506820 \\ 4.1041800 & 1.4213580 \\ 2.1700940 & 2.0385590 \\ 1.2038310 & 2.5598030 \\ 5.8832030 & 0.8881000 \\ 4.5129350 & 1.1920020 \\ -2.1807060 & -4.0200350 \\ -2.5771470 & -2.9195950 \\ -2.8066950 & -5.4818470 \\ -2.3026000 & -5.3260400 \\ -1.7590300 & -3.9038200 \\ -2.4681380 & -1.9336210 \\ -2.8975820 & -6.4686570 \\ -1.9837600 & -6.1895820 \\ -5.0036270 & -2.4871760 \\ -4.6128130 & -3.5649780 \\ -4.4495380 & -0.9823820 \\ -4.9205440 & -1.1757370 \\ -5.3804070 & -2.6185950 \\ -4.6722070 & -4.5705550 \\ -4.3962590 & 0.0210800 \\ -5.2364820 & -0.3246610 \\ 2.5648940 & -2.6686740 \\ 3.3942500 & -1.7243220 \\ 1.5108160 & -2.6923950 \\ 2.9209440 & -0.8155080 \\ 2.8457640 & -1.6745960 \\ 2.1935890 & -1.7865900 \\ 0.8983510 & -0.5841080 \\ 0.3017170 & -0.5635960 \\ 1.0503150 & -1.2553830\end{array}$

-1.0989480
-0.2411340
-2.3198550
-0.3872810
-2.2130650
-4.3044930
-3.2054620
-2.9335060
-4.1705300
-5.2950780
-3.3162990
-2.8153640
-5.0601330
2.9782830
1.7601000
1.9667580
3.0870300
3.8771980
1.6999170
2.0334790
4.0660690
2.0993790
1.3999680
0.2813660
1.5367460
3.0933380
1.8372720
-0.1650570
2.1120670
-4.6164610
-3.8804610
-2.7961440
-4.0613390
-5.6259230
-4.2886570
-2.3862060
-4.6564630
-1.4772590
-0.7194080
-1.2017730
-0.3485000
-2.4927890
2.5694480
4.3483440
3.0832160
2.1389200

$\begin{array}{lr}\mathrm{H} & 0.6784970 \\ \mathrm{~S} & 2.4099730 \\ \mathrm{~S} & 3.3147790 \\ \mathrm{H} & 2.0343320 \\ \mathrm{C} & -0.9030390 \\ \mathrm{C} & -1.5002440 \\ \mathrm{C} & -0.9069320 \\ \mathrm{C} & 0.2999040 \\ \mathrm{H} & -1.3861710 \\ \mathrm{H} & -1.3464050 \\ \mathrm{H} & 0.7616640 \\ \mathrm{C} & 4.6477810 \\ \mathrm{C} & 5.3916130 \\ \mathrm{C} & 4.8936760 \\ \mathrm{C} & 3.1510170 \\ \mathrm{H} & 5.0421110 \\ \mathrm{H} & 6.4682480 \\ \mathrm{H} & 5.1929330 \\ \mathrm{H} & 2.9722280 \\ \mathrm{H} & 2.5918000 \\ \mathrm{H} & 4.8027460 \\ \mathrm{H} & 5.2450340 \\ \mathrm{H} & 5.3153730 \\ \mathrm{H} & -2.4403500 \\ \mathrm{H} & \end{array}$
$-1.4217940$
$-1.4641340$
$-2.6773820$
$-1.0559930$
0.7227870
0.7398510
0.0981160
0.0615200
1. 2336300
0.1115810
0.0484120
$-3.9439210$
$-3.2460340$
$-1.8106270$
$-4.0185820$
$-4.9532060$
$-3.2258570$
$-1.1953780$
$-4.6725810$
$-4.4310380$
$-3.3908410$
$-3.8089440$
$-1.3407850$
1. 2641330

1.1706480

4.2795000

1. 6731000

$-2.5159880$

5. 2102440

3. 9415030

2.8628920

5.4286230

6.0364390

1. 8709540

6.4102020

$-2.1206180$

$-0.9809740$

$-0.7974560$

$-1.8160290$

$-2.2711360$

$-1.1745320$

$-1.6549580$

$-0.9536190$

$-2.6616090$

$-3.0546250$

$-0.0497320$

0.0941590

3. 7956740

$\operatorname{TS} 27(\mathrm{~S}, \mathrm{~S}) \mathrm{G}[\mathrm{M} 06-2 \mathrm{X} / 6-31 \mathrm{G}(\mathrm{d})]=-3917.744935$

$\begin{array}{lrrr}\mathrm{O} & 0.3020880 & 1.3923640 & 0.5723090 \\ \mathrm{O} & -1.6708970 & 0.4643540 & -0.7135190 \\ \mathrm{C} & 0.2606940 & 2.4793850 & -0.2815090 \\ \mathrm{C} & -0.9495470 & 3.1103080 & -0.5018850 \\ \mathrm{C} & 1.4822680 & 2.9170820 & -0.8639940 \\ \mathrm{C} & -0.9955380 & 4.1939200 & -1.4383320 \\ \mathrm{C} & 1.4360510 & 3.9947840 & -1.7137100 \\ \mathrm{C} & 0.2158520 & 4.6406790 & -2.0372900 \\ \mathrm{H} & 2.3584900 & 4.3584530 & -2.1600030 \\ \mathrm{C} & -2.4927700 & 1.2669210 & 0.0481030 \\ \mathrm{C} & -3.6361830 & 0.6637640 & 0.6355970 \\ \mathrm{C} & -2.1717120 & 2.6001670 & 0.1826760 \\ \mathrm{C} & -4.4599510 & 1.4644970 & 1.3873270 \\ \mathrm{C} & -3.0036790 & 3.4149360 & 1.0173690 \\ \mathrm{C} & -4.1652510 & 2.8361450 & 1.6054720 \\ \mathrm{H} & -5.3485550 & 1.0376050 & 1.8463940 \\ \mathrm{C} & -4.6948910 & 4.9496730 & 2.6757860 \\ \mathrm{C} & -3.5229990 & 5.5162280 & 2.1202890 \\ \mathrm{C} & -2.6993630 & 4.7711160 & 1.3147880 \\ \mathrm{C} & -5.0027490 & 3.6379150 & 2.4259830\end{array}$




$$
\begin{array}{r}
-5.3393610 \\
-3.2712300 \\
-1.7984180 \\
-5.8899190 \\
-2.2125220 \\
-1.0019570 \\
0.1832210 \\
-2.2115790 \\
-3.1520610 \\
-1.0188120 \\
1.1196270 \\
-3.1475690 \\
2.7986490 \\
3.3640820 \\
3.5017440 \\
4.6645080 \\
4.8178660 \\
5.3680260 \\
-3.9080270 \\
-3.7907390 \\
-4.2861540 \\
-4.1065020 \\
-4.5727270 \\
-4.4905210 \\
-0.2898180 \\
0.5594880 \\
-0.5453650 \\
-4.7195160 \\
6.3520140 \\
-3.2031910 \\
-3.3201320 \\
-3.9825180 \\
-3.5486240 \\
-2.8321580 \\
-3.0283320 \\
-4.2353350 \\
-3.4519020 \\
-4.7606030 \\
-4.4061540 \\
-4.9423890 \\
-5.0298830 \\
-4.8394020 \\
-4.2080120 \\
-5.1538250 \\
-5.3093070 \\
3.2337270
\end{array}
$$

3. 3069390

2. 3386550

0.9018760

2. 8591840

$-2.7340420$

$-3.3009530$

$-2.9616260$

$-1.8296550$

$-3.0236230$

$-4.0114940$

$-3.4009930$

$-1.4126040$

$-0.5187170$

$-1.3330490$

0.6037960

$-1.0105120$

0.8871290

0.0738160

0.4281760

1.5065230

$-0.8480150$

1. 3080390

$-1.0392300$

0.0449890

$-0.0014620$

$-1.1188440$

1. 2045160

$-0.1021170$

0.3120570

3.8172420

2.8002550

2.4061970

3. 6240350

4.7844520

2. 9510670

2. 2382060

4.4497150

$-3.2057470$

$-1.9797820$

$-2.3420310$

$-3.3947630$

$-4.0500920$

$-1.8515420$

$-2.4676230$

$-4.3790650$

$-3.2031790$
2.6881540

5.2011770

4.5052230

2.6911220

1.7094040

6.1737330

4.9101130

4.9592430

5.5229060

2. 9485120

3.6486710

5. 5003580

6.5170100

1. 9530270

3.2076660

0.0815720

$-0.1642580$

0.8341310

$-0.0159780$

$-1.2281430$

3.0385480

4.5017560

3. 3833770

2.6399920

1.8326570

4.5184650

2. 2103950

$-1.0923000$

5.0967640

3.9544030

3.0826510

5.3823750

5.7659780

2. 2078400

6.2557360

$-0.9888810$

$-0.8013540$

$-0.9522870$

0.0717000

$-0.9252590$

$-1.5272370$

$-1.9997830$

1.0705670

$-0.0993360$

$-1.9850580$

0.1981970
0.7562270

$-0.3021150$

$-0.8093650$

$-0.6747530$

1.1453350

$-0.7033060$

$-1.6326370$

3.7072550

2.7774060

. .7573350

4.2007680

4.0760240

2. 3849430

4.1416560

4.9375540

$-3.8781300$

$-3.5521010$

$-2.5242640$

$-3.2856350$

$-3.1008850$

$-2.3524310$

$-1.5416620$

$-1.9704190$

$-1.4126370$

$-3.6747060$

$-3.9271710$

$-2.3141700$

$-0.9885080$

$-0.2038690$

$-0.4659800$

$-2.0731760$

$-0.7538610$

0.1526000

$-2.6929170$

$-6.1247360$

$-5.9516610$

$-4.4844270$

$-5.3332900$

$-7.1822150$

$-6.5551600$

$-4.1640620$

$-5.7404640$

$-5.3864510$

$-5.7689040$

$-6.3038360$
$-2.4780130$

$-1.7976190$

$-2.8538320$

$-4.0471230$

$-2.7339480$

$-1.5227960$

$-3.4348820$

2.8495090

2.0273890

1.4918990

2.5759520

3.7153340

2.2268430

1.2935380

3.2400560

0.4829150

$-0.9298610$

0.9904040

$-1.2456630$

0.5874270

$-0.7946230$

1.1029100

0.8792250

$-0.2128500$

$-0.5989400$

$-0.0500870$

$-2.0128610$

0.8711670

2.9676520

2.7598500

1.7132490

2.1453500

3.7889700

1.5355330

2. 3182850

0.1191130

$-1.3897140$

$-1.7971280$

0.8882050

0.3919960

$-1.9426360$

$-1.7072680$

0.6865770

1. 9681060

0.4120340

$-1.6737360$

.




\begin{tabular}{|c|c|c|c|}
\hline $\begin{array}{l}\mathrm{H} \\
\mathrm{H} \\
14\end{array}$ & $\begin{array}{r}-0.6655800 \\
3.7562670\end{array}$ & $\begin{array}{r}-4.3121330 \\
0.6405890\end{array}$ & $\begin{array}{r}-2.8377260 \\
3.4124510\end{array}$ \\
\hline \multicolumn{4}{|c|}{$\begin{array}{l}144 \\
\text { TS92 }(R, R) G[M 06-2 X / 6-31 G(d)]=-3310.354565\end{array}$} \\
\hline 0 & 0.4063580 & 0.9334260 & 0.3813130 \\
\hline 0 & -1.7080870 & 0.9253960 & -0.9799850 \\
\hline C & 0.3656680 & 2. 3123150 & 0.2981310 \\
\hline C & -0.7952660 & 2.9780250 & 0.6376460 \\
\hline $\mathrm{C}$ & 1.5602080 & 2.9736320 & -0.0918850 \\
\hline $\mathrm{C}$ & -0.8077240 & 4.4100800 & 0.54 \\
\hline C & 1.5709050 & 4.3451510 & -0.05 \\
\hline $\mathrm{C}$ & 0.4030800 & 5.0923080 & 0.2 \\
\hline $\mathrm{H}$ & 2.4863120 & 4.8785950 & -0.31 \\
\hline C & -2.4204430 & 1.1748110 & 0.1834550 \\
\hline C & -3.5688830 & 0.3752290 & 0.4304340 \\
\hline C & -1.9995100 & 2.1908100 & 1.0161110 \\
\hline C & -4.3128250 & 0.6707210 & 1.5452750 \\
\hline $\mathrm{C}$ & -2.7232710 & 2.4132090 & 2.2346040 \\
\hline $\mathrm{C}$ & -3.9087340 & 1.6637960 & 2.4748510 \\
\hline $\mathrm{H}$ & -5.2254700 & 0.1138350 & 1.7400070 \\
\hline $\mathrm{C}$ & -4.2232770 & 2.8042230 & 4.5941300 \\
\hline $\mathrm{C}$ & -3.0204980 & 3.5178790 & 4.3785210 \\
\hline $\mathrm{C}$ & -2.2902400 & 3.3285690 & 3.2322690 \\
\hline $\mathrm{C}$ & -4.6513410 & 1.8941980 & 3.6632760 \\
\hline $\mathrm{H}$ & -4.7940700 & 2.9693440 & 5.5024010 \\
\hline $\mathrm{H}$ & -2.6694250 & 4.2176500 & 5.1304690 \\
\hline $\mathrm{H}$ & -1.3645530 & 3.8738980 & 3.0831940 \\
\hline $\mathrm{H}$ & -5.5602940 & 1.3204160 & 3.8240590 \\
\hline $\mathrm{C}$ & -1.9511380 & 6.5524780 & 0.6553050 \\
\hline $\mathrm{C}$ & -0.7355090 & 7.2298310 & 0.3981900 \\
\hline $\mathrm{C}$ & 0.4127190 & 6.5114600 & 0.1906780 \\
\hline $\mathrm{C}$ & -1.98 & 5.1828790 & 0.7286560 \\
\hline $\mathrm{H}$ & -2.8652630 & 7.1224640 & 7887540 \\
\hline $\mathrm{H}$ & -0.7 & 8.3139780 & 3510 \\
\hline $\mathrm{H}$ & 20 & 7.013 & 46570 \\
\hline $\mathrm{H}$ & -2 & 6766620 & 47440 \\
\hline C & 2.7 & 2.1897020 & 29500 \\
\hline $\mathrm{C}$ & 2. & 2.0695030 & $\lcm{2450}$ \\
\hline $\mathrm{C}$ & 3.60 & 1.590 & 09470 \\
\hline C & 4. & 1.390 & 8030 \\
\hline C & 4.74 & 0.924 & 2320 \\
\hline C & 5.0 & 20 & 740 \\
\hline $\mathrm{H}$ & 4. & 30 & -3 \\
\hline $\mathrm{H}$ & 0 & 0.467 & 80 \\
\hline C & -3 & -0.740 & 20 \\
\hline C & -3.8210 & -2 & \\
\hline
\end{tabular}

-4.4391600
-4.2499390
-4.8459750
-4.7698630
-4.1668950
-5.2386560
3.3525040
6.3021740
2.0708900
-3.2420550
-5.2215250
-4.5717700
-6.0445580
-6.4968950
-6.6220020
-6.1352630
-3.7516300
-2.7010080
-4.1306830
-3.8111140
-6.3617660
-6.7105070
-6.0270030
-7.2087460
-4.0502570
-3.2415920
-3.6440380
-4.3762880
-4.3576070
-5.0496630
-4.9382400
-3.9338590
-2.2542500
-2.7644560
-1.4956020
-1.7511080
4.4663620
5.4308000
4.5695060
4.2485830
3.1836750
2.3185850
4.0748250
3.0228270
7.4257880
8.3531920
$-1.7748530$

$-0.9113990$

$-2.5937630$

$-2.1795030$

$-0.5786300$

$-3.5854750$

1.8784390

$-1.9067400$

$-2.9795660$

1. 2990840

$-3.0849400$

$-2.3017280$

$-2.5266220$

$-3.2879530$

$-1.6035030$

$-2.8671540$

$-3.5811960$

$-3.4111050$

$-4.3956830$

$-3.9091890$

$-2.4518600$

$-3.1371890$

$-1.5304840$

$-2.2013440$

$-3.4546710$

$-3.9263170$

$-2.5614980$

$-4.1467410$

2. 2952690

2. 4409540

1. 9322060

3. 2696240

1. 2161050

0.9926100

0.4492840

2. 1813560

2.5895220

2. 4846550

2. 1718160

3. 6586700

2.4802740

2. 0435620

2. 3151760

3. 5620610

$-2.1096390$ 


$\begin{array}{rrr}7.1450560 & 1.8693720 & -2.8894430 \\ 7.6192880 & 1.7129350 & -1.1900840 \\ 6.0935320 & -0.7029460 & -3.1765950 \\ 5.1989550 & -1.3294960 & -3.1002250 \\ 5.9730150 & -0.0582500 & -4.0537770 \\ 6.9608270 & -1.3449120 & -3.3597460 \\ 1.9221830 & 1.8141260 & -4.2338850 \\ 2.8358310 & 1.8172940 & -4.8386980 \\ 1.6708160 & 0.7843680 & -3.9743820 \\ 1.1182700 & 2.2142030 & -4.8597910 \\ 2.5601680 & 4.0796200 & -3.3847550 \\ 3.5680910 & 4.0169100 & -3.8114220 \\ 1.8967760 & 4.5118080 & -4.1414050 \\ 2.5963290 & 4.7641030 & -2.5335480 \\ -0.3520930 & 0.0568310 & -0.7753310 \\ 0.4073780 & 0.0641930 & -2.0642250 \\ -0.6664960 & -1.2617250 & -0.1107170 \\ 1.0768890 & 2.7755280 & -2.5294570 \\ 6.6146410 & -0.5522080 & -1.1017120 \\ 2.4154900 & 2.1988170 & 2.0430850 \\ -4.1782910 & 1.6568130 & -1.5494670 \\ -5.6004940 & -3.5117790 & -4.0097950 \\ -2.6883010 & -1.5896550 & 1.6793430 \\ 2.5755050 & -1.9666970 & -0.8771230 \\ 1.5311450 & -2.9663860 & -1.1305040 \\ 2.2898920 & -1.1250710 & -0.2360670 \\ 0.5060340 & -2.6120900 & -1.1457980 \\ 2.3591810 & -1.6725740 & -2.2714650 \\ 1.6645550 & -0.9394140 & -2.3045380 \\ 4.3029540 & -3.7103370 & -1.4649100 \\ 3.2494090 & -4.8062100 & -1.3021170 \\ 1.8637800 & -4.3010950 & -1.7086520 \\ 3.9629170 & -2.4964520 & -0.5969270 \\ 5.2919820 & -4.0883330 & -1.1888340 \\ 3.5041630 & -5.6834900 & -1.9035930 \\ 1.7917430 & -4.1877190 & -2.7972420 \\ 3.9922480 & -2.7736150 & 0.4658460 \\ 4.6786350 & -1.6844460 & -0.7434800 \\ 4.3568970 & -3.4053540 & -2.5168940 \\ 3.2133500 & -5.1243760 & -0.2545330 \\ 1.0709450 & -4.9930270 & -1.4126400 \\ 1.2059480 & -3.0494660 & 1.8721200 \\ 0.4991130 & -1.9642780 & 1.9868210 \\ 1.9254570 & -3.4507010 & 3.1124320 \\ 2.7097510 & -4.6060970 & 4.2700240 \\ 1.8375010 & -2.6726700 & \end{array}$

3.4066930
2.7575920
2.5392750
1.2240810
3.3221820
4.0150610
2.4751640
3.8679410
0.0094430
1.3115300

$$
\begin{aligned}
& -4.9837720 \\
& -5.1985060 \\
& -3.0526500 \\
& -1.7783060 \\
& -4.2060680 \\
& -5.8823400 \\
& -2.4501900 \\
& -4.5006350 \\
& -1.6933110 \\
& -3.7178990
\end{aligned}
$$
4.2394430
2. 1898920
5.4089450
4. 2650820
5. 3937890
4. 2314950
6. 3091910
6.2849190
1.1112110
0.8339710 144

TS93 $(R, R) \quad G[M 06-2 X / 6-31 G(d)]=-3310.354504$

$\begin{array}{lrcr}\text { TS93 }(\mathrm{R}, \mathrm{R}) \mathrm{G}[\mathrm{M} 06-2 \mathrm{X} / 6-31 \mathrm{G}(\mathrm{d})]=-3310.354504 \\ \mathrm{O} & 0.4062880 & 0.9332620 & 0.3812290 \\ \mathrm{O} & -1.7082270 & 0.9254150 & -0.9799910 \\ \mathrm{C} & 0.3657630 & 2.3121540 & 0.2979870 \\ \mathrm{C} & -0.7950930 & 2.9780100 & 0.6374820 \\ \mathrm{C} & 1.5603760 & 2.9733260 & -0.0920520 \\ \mathrm{C} & -0.8074170 & 4.4100580 & 0.5497070 \\ \mathrm{C} & 1.5711950 & 4.3448500 & -0.0586090 \\ \mathrm{C} & 0.4034430 & 5.0921460 & 0.2428940 \\ \mathrm{H} & 2.4866570 & 4.8781840 & -0.3019570 \\ \mathrm{C} & -2.4204960 & 1.1749560 & 0.1834730 \\ \mathrm{C} & -3.5690100 & 0.3755150 & 0.4305540 \\ \mathrm{C} & -1.9994060 & 2.1909520 & 1.0160490 \\ \mathrm{C} & -4.3128590 & 0.6711510 & 1.5454200 \\ \mathrm{C} & -2.7230770 & 2.4135060 & 2.2345660 \\ \mathrm{C} & -3.9086110 & 1.6642360 & 2.4749170 \\ \mathrm{H} & -5.2255610 & 0.1143850 & 1.7402240 \\ \mathrm{C} & -4.2229230 & 2.8048260 & 4.5941430 \\ \mathrm{C} & -3.0200760 & 3.5183360 & 4.3784310 \\ \mathrm{C} & -2.2898970 & 3.3288800 & 3.2321530 \\ \mathrm{C} & -4.6511340 & 1.8947920 & 3.6633650 \\ \mathrm{H} & -4.7936530 & 2.9700650 & 5.5024320 \\ \mathrm{H} & -2.6688890 & 4.2181140 & 5.1303200 \\ \mathrm{H} & -1.3641580 & 3.8740990 & 3.0829980 \\ \mathrm{H} & -5.5601430 & 1.3211220 & 3.8242270 \\ \mathrm{C} & -1.9506320 & 6.5525680 & 0.6548720 \\ \mathrm{C} & -0.7349480 & 7.2297870 & 0.3976590 \\ \mathrm{C} & 0.4132100 & 6.5112930 & 0.1901870 \\ \mathrm{C} & -1.9875640 & 5.1829800 & 0.7283510 \\ \mathrm{H} & -2.8647000 & 7.1226510 & 0.7882950 \\ \mathrm{H} & -0.7201350 & 8.3139280 & 0.3487180 \\ \mathrm{H} & 1.3503050 & 7.0136870 & -0.0352130 \\ \mathrm{H} & -2.9284300 & 4.6768660 & 0.9145070 \\ \mathrm{C} & 2.7465190 & 2.1893050 & -0.5529430 \\ \mathrm{C} & 2.9970930 & 2.0691580 & -1.9361890 \\ \mathrm{C} & & & \end{array}$




$\begin{array}{rrr}3.6079880 & 1.5903540 & 0.3811060 \\ 4.1428510 & 1.3898710 & -2.3505440 \\ 4.7442840 & 0.9238960 & -0.0838690 \\ 5.0345890 & 0.8205750 & -1.4424550 \\ 4.3410610 & 1.2978850 & -3.4144850 \\ 5.4253460 & 0.4669230 & 0.6338910 \\ -3.9447780 & -0.7398590 & -0.4907300 \\ -3.8214120 & -2.0792610 & -0.0595710 \\ -4.4395360 & -0.4532610 & -1.7746360 \\ -4.2505360 & -3.0962020 & -0.9110860 \\ -4.8465430 & -1.5096590 & -2.5934640 \\ -4.7705570 & -2.8347620 & -2.1791500 \\ -4.1675630 & -4.1287710 & -0.5782890 \\ -5.2392810 & -1.2916410 & -3.5851530 \\ 3.3522560 & 1.6569220 & 1.8785570 \\ 6.3028140 & 0.1276230 & -1.9060460 \\ 2.0715340 & 2.6812960 & -2.9796780 \\ -3.2423280 & -2.4534810 & 1.2992590 \\ -5.2223820 & -3.9648690 & -3.0845230 \\ -4.5720150 & 0.9674100 & -2.3015640 \\ -6.0447690 & 1.3316870 & -2.5264500 \\ -6.4971830 & 0.6866620 & -3.2877570 \\ -6.6222130 & 1.2196820 & -1.6033200 \\ -6.1353720 & 2.3684930 & -2.8670110 \\ -3.7518760 & 1.1672810 & -3.5810540 \\ -2.7012620 & 0.9166090 & -3.4109510 \\ -4.1309620 & 0.5399750 & -4.3954900 \\ -3.8113200 & 2.2104350 & -3.9091250 \\ -6.3623860 & -4.7702250 & -2.4512010 \\ -6.7112700 & -5.5497380 & -3.1365190 \\ -6.0273320 & -5.2595960 & -1.5299790 \\ -7.2093380 & -4.1249220 & -2.2003700 \\ -4.0511260 & -4.8821400 & -3.4546120 \\ -3.2426650 & -4.3162380 & -3.9264990 \\ -3.6445890 & -5.3697250 & -2.5615560 \\ -4.3772770 & -5.6657120 & -4.1465850 \\ -4.3577850 & -2.7987930 & 2.2956270 \\ -5.0496730 & -1.9648260 & 2.4413920 \\ -4.9386200 & -3.6547980 & 1.9326790 \\ -3.9339320 & -3.0648600 & 3.2699240 \\ -2.2547050 & -3.6245100 & 1.2162140 \\ -2.7650820 & -4.5679160 & 0.9928820 \\ -1.4961550 & -3.4493340 & 0.4492560 \\ -1.7514220 & -3.7497310 & 2.1813920 \\ 4.4659130 & 2.4349220 & 2.4850650 \\ 5.4304300 & 1.9257980 & \end{array}$

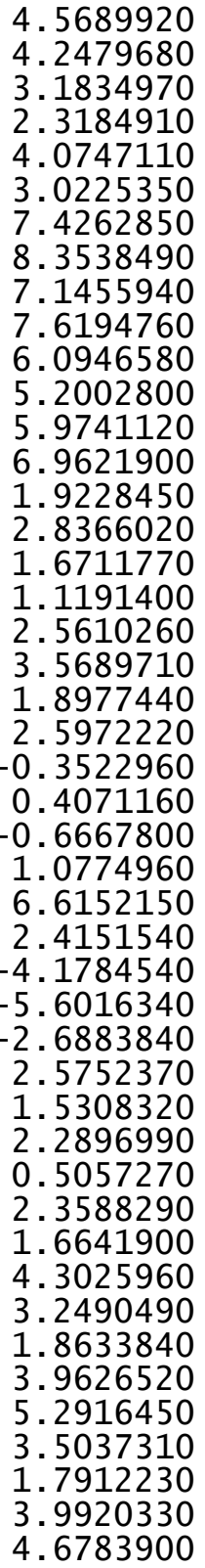

3.4412990

2. 5253180

0.2567740

$-0.2520340$

$-0.3605650$

0.3227460

1. 1528190

0.6584450

1. 8694760

1. 7131180

$-0.7030300$

$-1.3298850$

$-0.0583470$

$-1.3447200$

1.8136300

1.8164780

0.7839720

2.2138080

4.0791570

4. 0162700

4.5113530

4.7637260

0.0567410

0.0640700

$-1.2618000$

2. 7753490

$-0.5522100$

2. 1982900

1.6570890

$-3.5114310$

$-1.5894230$

$-1.9668760$

$-2.9665220$

$-1.1252770$

$-2.6122000$

$-1.6726840$

$-0.9395230$

$-3.7105050$

$-4.8063780$

$-4.3012090$

$-2.4966920$

$-4.0885410$

$-5.6835990$

$-4.1877820$

$-2.7739470$

$-1.6846980$
2. 1720580

3. 6588940

2.4804100

2. 0436310

2. 3154200

3. 5621800

$-2.1086880$

$-2.4163640$

$-2.8885500$

$-1.1890870$

$-3.1759620$

$-3.0997980$

$-4.0531500$

$-3.3589590$

$-4.2338990$

$-4.8385480$

$-3.9742920$

$-4.8600070$

$-3.3849860$

$-3.8115770$

$-4.1417280$

$-2.5338500$

$-0.7753870$

$-2.0643180$

$-0.1107870$

$-2.5296890$

$-1.1009460$

2. 0430480

$-1.5493380$

$-4.0092520$

1.6793780

$-0.8773090$

$-1.1306630$

$-0.2361840$

$-1.1458630$

$-2.2716190$

$-2.3046220$

$-1.4653450$

$-1.3025610$

$-1.7089090$

$-0.5972280$

$-1.1893930$

$-2.7974850$

0.4655200

$-0.7437480$ 


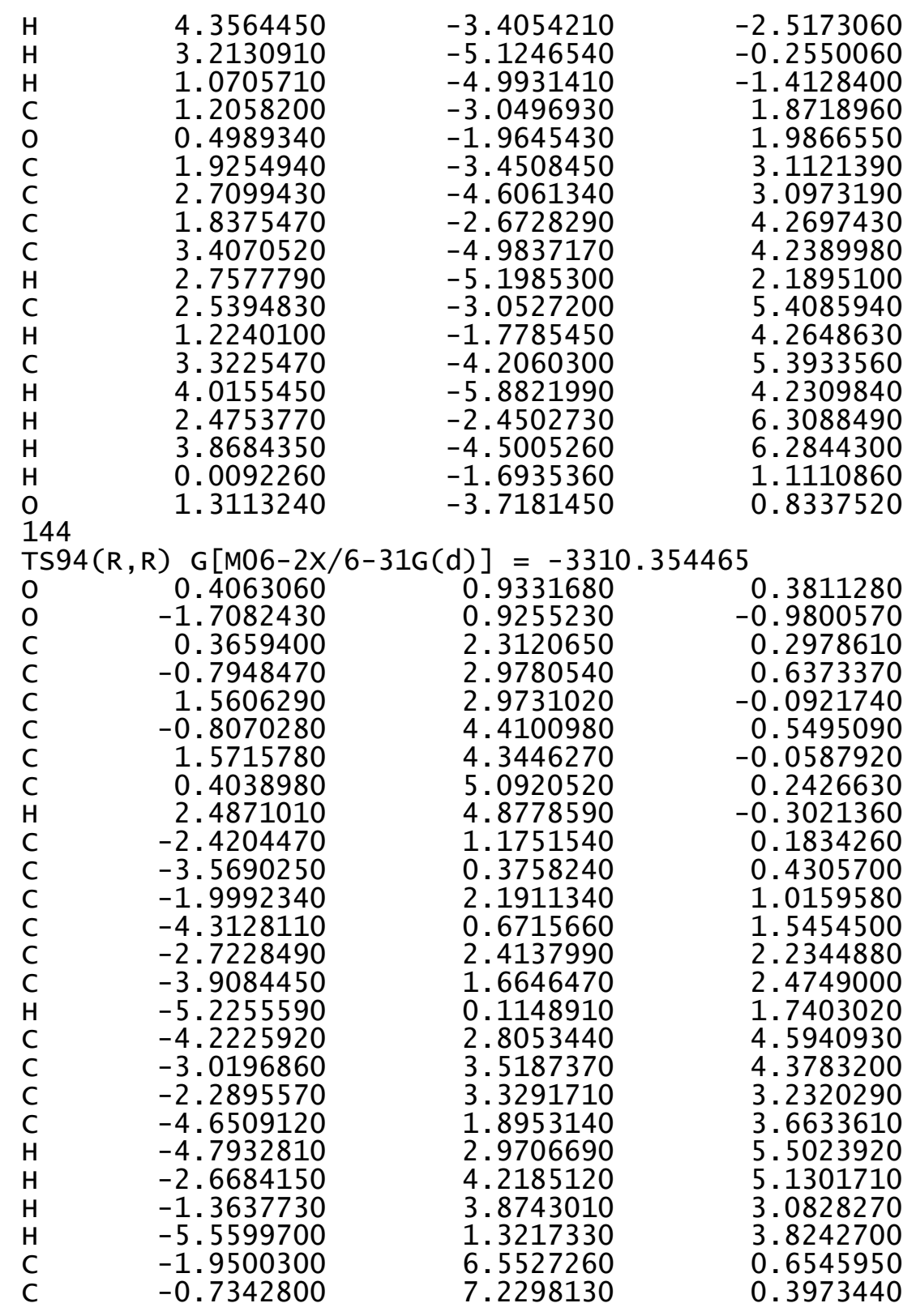

0.4138060
-1.9870980
-2.8640400
-0.7193600
1.3509490
-2.9280110
2.7467490
2.9974620
3.6081070
4.1432540
4.7444330
5.0348940
4.3415710
5.4254090
-3.9449230
-3.8216270
-4.4397220
-4.2508510
-4.8468340
-4.7709090
-4.1679290
-5.2396040
3.3522270
6.3032060
2.0720230
-3.2425130
-5.2228440
-4.5721350
-6.0448790
-6.4973910
-6.6222650
-6.1354330
-3.7520690
-2.7014640
-4.1312610
-3.8114490
-6.3628990
-6.7118570
-6.0278670
-7.2097970
-4.0516700
-3.2431700
-3.6451580
-4.3778980
-4.3579580
-5.0497550

6. 5111970

5. 1831450

7. 1229050

8. 3139500

7.0134890

4. 6771310

2. 1889710

2. 0687700

1. 5900180

1. 3894510

0.9235240

0.8201770

1.2974420

0.4665510

$-0.7395460$

$-2.0789470$

$-0.4529430$

$-3.0958770$

$-1.5093300$

$-2.8344310$

$-4.1284450$

$-1.2913080$

1.6566340

0.1272490

2. 6808910

$-2.4531720$

$-3.9645280$

0.9677280

1. 3321040

0.6870900

1.2201610

2. 3689080

1.1675160

0.9167590

0.5402310

2. 2106700

$-4.7697710$

$-5.5492830$

$-5.2591330$

$-4.1243920$

$-4.8819060$

$-4.3160830$

$-5.3695040$

$-5.6654680$

$-2.7983380$

-1. 9642970
0.1898960

0.7281320

0.7880010

0.3483570

$-0.0355290$

0.9143170

$-0.5529400$

$-1.9361530$

0.3812160

$-2.3503730$

$-0.0836240$

$-1.4421770$

$-3.4142930$

0.6342180

$-0.4906660$

$-0.0594830$

$-1.7745530$

$-0.9109580$

$-2.5933420$

$-2.1790060$

$-0.5781460$

$-3.5850180$

1.8786380

$-1.9055750$

$-2.9797580$

1. 2993340

$-3.0843360$

$-2.3015010$

$-2.5262920$

$-3.2875500$

$-1.6031180$

$-2.8668710$

$-3.5810500$

$-3.4110150$

$-4.3954520$

$-3.9091290$

$-2.4509640$

$-3.1362460$

$-1.5297290$

$-2.2001420$

$-3.4544220$

$-3.9263360$

$-2.5613610$

4.1463700

2. 2957670

2.4415380 


$\begin{array}{rrr}-4.9388960 & -3.6542970 & 1.9328750 \\ -3.9340840 & -3.0644150 & 3.2700530 \\ -2.2550090 & -3.6243010 & 1.2162900 \\ -2.7654900 & -4.5676680 & 0.9930300 \\ -1.4964830 & -3.4492360 & 0.4492810 \\ -1.7516860 & -3.7495250 & 2.1814470 \\ 4.4658410 & 2.4346070 & 2.5899600 \\ 5.4303520 & 1.9254530 & 2.4853420 \\ 4.5689890 & 3.4409770 & 2.1722380 \\ 4.2477950 & 2.5250200 & 3.6590520 \\ 3.1833460 & 0.2565050 & 2.4804960 \\ 2.3183830 & -0.2522810 & 2.0436050 \\ 4.0745630 & -0.3608650 & 2.3156390 \\ 3.0222370 & 0.3225030 & 3.5622430 \\ 7.4267330 & 1.1524640 & -2.1078280 \\ 8.3543700 & 0.6581220 & -2.4153340 \\ 7.1462310 & 1.8692090 & -2.8876780 \\ 7.6197010 & 1.7126590 & -1.1881170 \\ 6.0953710 & -0.7032490 & -3.1756420 \\ 5.2010710 & -1.3302450 & -3.0997320 \\ 5.9749050 & -0.0584640 & -4.0527650 \\ 6.9630170 & -1.3448030 & -3.3585660 \\ 1.9233970 & 1.8131460 & -4.2339310 \\ 2.8371970 & 1.8159240 & -4.8385140 \\ 1.6716850 & 0.7835100 & -3.9742780 \\ 1.1197470 & 2.2133010 & -4.8601240 \\ 2.5616210 & 4.0786990 & -3.3851200 \\ 3.5696050 & 4.0157350 & -3.8116080 \\ 1.8984330 & 4.5108720 & -4.1419570 \\ 2.5977660 & 4.7633270 & -2.5340290 \\ -0.3523790 & 0.0567390 & -0.7754930 \\ 0.4070130 & 0.0640440 & -2.0644370 \\ -0.6669620 & -1.2617940 & -0.1109230 \\ 1.0779520 & 2.7750250 & -2.5298590 \\ 6.6154120 & -0.5526860 & -1.1004850 \\ 2.4151300 & 2.1980430 & 2.0430240 \\ -4.1784720 & 1.6573920 & -1.5493140 \\ -5.6020780 & -3.5110880 & -4.0090710 \\ -2.6884700 & -1.5891510 & 1.6793940 \\ 2.5749450 & -1.9670950 & -0.8775070 \\ 1.5305010 & -2.9667180 & -1.1307500 \\ 2.2894800 & -1.1254530 & -0.2364050 \\ 0.5054080 & -2.6123630 & -1.1459130 \\ 2.3584300 & -1.6729850 & -2.2718190 \\ 1.6638340 & -0.9397800 & -2.3048100 \\ 4.3022260 & -3.7107650 & -1.4656170\end{array}$

$\begin{array}{ll}\mathrm{C} & 3.2486710 \\ \mathrm{C} & 1.8629830 \\ \mathrm{C} & 3.9623720 \\ \mathrm{H} & 5.2912900 \\ \mathrm{H} & 3.5032930 \\ \mathrm{H} & 1.7907330 \\ \mathrm{H} & 3.9918310 \\ \mathrm{H} & 4.6781090 \\ \mathrm{H} & 4.3559940 \\ \mathrm{H} & 3.2127900 \\ \mathrm{H} & 1.0701840 \\ \mathrm{C} & 1.2055690 \\ \mathrm{O} & 0.4987400 \\ \mathrm{C} & 1.9251670 \\ \mathrm{C} & 2.7095820 \\ \mathrm{C} & 1.8371780 \\ \mathrm{C} & 3.4066180 \\ \mathrm{H} & 2.7574510 \\ \mathrm{C} & 2.5390400 \\ \mathrm{H} & 1.2236650 \\ \mathrm{C} & 3.3220730 \\ \mathrm{H} & 4.0150860 \\ \mathrm{H} & 2.4749010 \\ \mathrm{H} & 3.8679040 \\ \mathrm{H} & 0.0090500 \\ \mathrm{O} & 1.3110820 \\ \mathrm{H} & \end{array}$
$-4.8066140$
$-4.3014370$
$-2.4969210$
$-4.0888120$
$-5.6838620$
$-4.1880640$
$-2.7741360$
$-1.6849420$
$-3.4057070$
$-5.1248510$
$-4.9933400$
$-3.0497580$
$-1.9645650$
$-3.4509190$
$-4.6062320$
$-2.6728940$
$-4.9838260$
$-5.1986370$
$-3.0527970$
$-1.7785940$
$-4.2061280$
$-5.8823260$
$-2.4503410$
$-4.5006330$
$-1.6935550$
$-3.7182460$

$-1.3027140$

$-1.7089740$

$-0.5975100$

$-1.1897350$

$-1.9042910$

$-2.7975480$

0.4652470

$-0.7441140$

$-2.5175890$

$-0.2551450$

$-1.4128020$

1.8718140

1.9865180

3. 1120990

3.0973320

4.2696930

4.2390510

2.1895300

5.4085860

4.2647750

5. 3933990

4. 2310770

6.3088330

6. 2845050

1. 1109410

144

0.8336940

$\operatorname{TS} 95(R, R) \quad G[M 06-2 X / 6-31 G(d)]=-3310.352734$

$\begin{array}{lrrr}\text { TS95 }(\mathrm{R}, \mathrm{R}) \mathrm{G}[\mathrm{M} 06-2 X / 6-31 \mathrm{G}(\mathrm{d})]=-3310.352734 \\ \mathrm{O} & -0.0304590 & 1.1236220 & 0.2613430 \\ \mathrm{O} & -2.0780920 & 0.2150190 & -0.8767460 \\ \mathrm{C} & -0.3256630 & 2.3876350 & -0.2096950 \\ \mathrm{C} & -1.6148080 & 2.8597680 & -0.0920750 \\ \mathrm{C} & 0.7596260 & 3.1524110 & -0.7204740 \\ \mathrm{C} & -1.9060760 & 4.1552700 & -0.6351030 \\ \mathrm{C} & 0.4884120 & 4.4295060 & -1.1394420 \\ \mathrm{C} & -0.8360530 & 4.9460420 & -1.1413500 \\ \mathrm{H} & 1.2965950 & 5.0671360 & -1.4873140 \\ \mathrm{C} & -2.7947060 & 0.6967330 & 0.2020960 \\ \mathrm{C} & -3.7178760 & -0.1956320 & 0.8107780 \\ \mathrm{C} & -2.6299650 & 2.0106410 & 0.5909110 \\ \mathrm{C} & -4.5139810 & 0.2993870 & 1.8121200 \\ \mathrm{C} & -3.3912060 & 2.4841000 & 1.7126270 \\ \mathrm{C} & -4.3653370 & 1.6253880 & 2.2961680 \\ \mathrm{H} & -5.2657800 & -0.3427240 & 2.2642640 \\ \mathrm{C} & -4.9540230 & 3.3468390 & 3.9031120 \\ \mathrm{C} & -3.9526580 & 4.1842510 & 3.3577000\end{array}$




$\begin{array}{rrr}-3.1923800 & 3.7662810 & 2.2945060 \\ -5.1476730 & 2.0930740 & 3.3850030 \\ -5.5527440 & 3.6936200 & 4.7393500 \\ -3.7794560 & 5.1652180 & 3.7891090 \\ -2.4201020 & 4.4149650 & 1.8964530 \\ -5.8945770 & 1.4264190 & 3.8080560 \\ -3.4635010 & 5.9214500 & -1.2317020 \\ -2.3947740 & 6.7216500 & -1.6994660 \\ -1.1120650 & 6.2396770 & -1.6569450 \\ -3.2265940 & 4.6732480 & -0.7127520 \\ -4.4805150 & 6.2964610 & -1.2893900 \\ -2.5955760 & 7.7094080 & -2.1020040 \\ -0.2831650 & 6.8353810 & -2.0302740 \\ -4.0530470 & 4.0625830 & -0.3643710 \\ 2.1264970 & 2.5445010 & -0.7079300 \\ 2.7419170 & 2.1213800 & -1.9023370 \\ 2.7691220 & 2.3486380 & 0.5299760 \\ 4.0051770 & 1.5301450 & -1.8250730 \\ 4.0191730 & 1.7269090 & 0.5578910 \\ 4.6587580 & 1.3159980 & -0.6109150 \\ 4.5041490 & 1.2232170 & -2.7428800 \\ 4.5096420 & 1.5748450 & 1.5162170 \\ -3.8143100 & -1.6153470 & 0.3499380 \\ -3.2006760 & -2.6398930 & 1.0961520 \\ -4.5216010 & -1.9186820 & -0.8273920 \\ -3.3595620 & -3.9604570 & 0.6701960 \\ -4.6478220 & -3.2525760 & -1.2146010 \\ -4.0822680 & -4.2892980 & -0.4740640 \\ -2.8976690 & -4.7621760 & 1.2420210 \\ -5.2021770 & -3.4867620 & -2.1221280 \\ 2.1756510 & 2.8899060 & 1.8228530 \\ 6.0618750 & 0.7340480 & -0.6061880 \\ 2.0899530 & 2.3303450 & -3.2609460 \\ -2.3871650 & -2.3461100 & 2.3492440 \\ -4.2298380 & -5.7334700 & -0.9146540 \\ -5.1552390 & -0.8379040 & -1.6894490 \\ -6.6824880 & -0.9722250 & -1.7037950 \\ -6.9880560 & -1.9277690 & -2.1445500 \\ -7.0898710 & -0.9240960 & -0.6889190 \\ -7.1335380 & -0.1689430 & -2.2956860 \\ -4.5837830 & -0.8542070 & -3.1115460 \\ -3.4967210 & -0.7369700 & -3.0924890 \\ -4.8208920 & -1.7938170 & -3.6228760 \\ -5.0104310 & -0.0362710 & -3.7015370 \\ -3.5462530 & -5.9729380 & -2.2658510 \\ -3.6153020 & -7.0273440 & -2.5534280\end{array}$

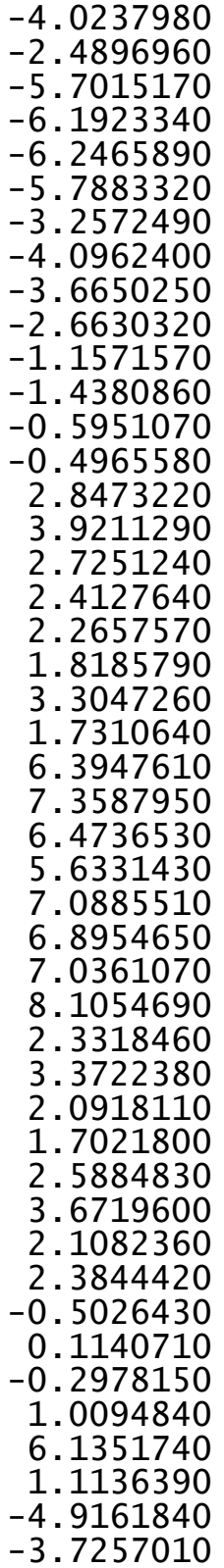

-5.3776590
-5.6915510
-6.1579920
-6.0049930
-5.5755630
-7.2157550
-2.4688360
-1.7681710
-3.4832180
-2.2688100
-3.2515440
-4.2684500
-3.3037910
-2.8570380
4.2251470
4.0820970
4.9481370
4.6515400
1.9080120
0.9472710
1.7395920
2.3096050
-0.0679650
-0.5731160
0.5822890
-0.8245170
1.8576200
2.4071480
2.5692070
1.4530280
1.1704420
1.1401440
0.2089770
1.2942430
3.6349260
3.5852880
3.7941740
4.5052830
-0.1256020
-0.0718920
-1.3447830
2.4043570
0.0570450
3.0925110
0.1367040
-6.3571410

$-3.0519550$ $-2.2275110$ $-0.9692550$ $-0.0036940$ $-1.7202160$ $-1.2387590$

3.6079650

3.5919860

3.6882490

4.5061760

2.4886240

2.7852750

1.5530560

3.2680500

2.1713080

2. 3380000

1. 3583010

3.0816520

2.9911560

2.7194710

3.2982440

3.8579070

0.6528610

0.5350650

1.5311730

0.8682360

$-0.8098160$

$-1.7359690$

0.0218450

$-0.8513840$

$-4.2304510$

$-4.5739550$

$-3.7724850$

$-5.1171310$

$-3.8996510$

$-4.0594870$

$-4.8709420$

$-3.2710310$

$-0.6746140$

$-2.0414390$

0.1895670

$-3.1028920$

$-1.4684610$

1.6614460

$-1.2529050$

$-0.1652440$ 


\begin{tabular}{|c|c|c|c|c|c|c|}
\hline & & & & & & \\
\hline-2.0263890 & -1.3148270 & 2.2749770 & C & -3.5389500 & 0.2009900 & 0.5744360 \\
\hline 2.5461660 & -1.8476270 & $\begin{array}{l}-1.6138560 \\
-1\end{array}$ & C & $\begin{array}{l}-2.0298840 \\
-4\end{array}$ & $\begin{array}{l}2.0815390 \\
0.4674170\end{array}$ & $\begin{array}{l}1.1108470 \\
1.7110960\end{array}$ \\
\hline 2.3763210 & -0.9296310 & -1.0414400 & c & -2.7253600 & 2.2762510 & 2.3503270 \\
\hline 0.7358300 & -2.7998110 & -0.8548650 & $\mathrm{C}$ & -3.8701750 & 1.4780670 & 2.6274420 \\
\hline 1.7017530 & -1.9135620 & -2.7781030 & $\mathrm{H}$ & -5.1426930 & -0.1269410 & 1.9338240 \\
\hline 0.9689920 & -1.2223300 & -2.6053620 & C & -4.1669890 & 2.6082570 & 4.7547180 \\
\hline 4.2066510 & -3.4118900 & -2.6836050 & c & -3.0030120 & 3.3720990 & 4.5013350 \\
\hline 3.5895770 & -4.5733470 & -1.9020740 & C & -2.3011610 & 3.2115180 & 3.3331800 \\
\hline 2.0838640 & -4.3773700 & -1.7117190 & $\mathrm{C}$ & -4.5847540 & 1.6791230 & 3.8381640 \\
\hline 4.0003530 & -2.0894400 & -1.9421060 & $\mathrm{H}$ & -4.7157970 & 2.7506850 & 5.6802270 \\
\hline 5.2762710 & -3.5833930 & -2.8363040 & $\mathrm{H}$ & -2.6590590 & 4.0878770 & 5.2414200 \\
\hline 3.7671220 & -5.5231150 & -2.4147170 & $\mathrm{H}$ & -1.4047510 & 3.7955840 & 3.1546500 \\
\hline 1.5503150 & -4.5149290 & -2.6596040 & $\mathrm{H}$ & -5.4631750 & 1.0677150 & 4.0274970 \\
\hline 4.5578640 & -2.0902150 & -0.9944640 & C & -2.1808610 & 6.4408310 & 0.7441090 \\
\hline 4.3584410 & -1.2388880 & -2.5317280 & C & -1.0051450 & 7.1688440 & 0.4436190 \\
\hline 3.7439320 & -3.3448020 & -3.6752740 & c & 0.1648540 & 6.4995970 & 0.1971990 \\
\hline 4.0633210 & -4.6411710 & -0.9160340 & C & -2.1562350 & 5.0711490 & 0.8209470 \\
\hline 1.6634730 & -5.0969370 & -1.0047010 & $\mathrm{H}$ & -3.1134590 & 6.9714840 & 0.9083450 \\
\hline 2.5982080 & -2.3993260 & 1.5021850 & $\mathrm{H}$ & -1.0385870 & 8.2524650 & 0.3924730 \\
\hline 1.7727740 & -1.4074860 & 1.6788840 & $\mathrm{H}$ & 1.0709610 & 7.0409960 & -0.0620340 \\
\hline 3.8359040 & -2.3311130 & 2.3234140 & $\mathrm{H}$ & -3.0673590 & 4.5256780 & 1.0407180 \\
\hline 4.8445650 & -3.2716550 & 2.1003550 & C & 2.6469220 & 2.2763780 & -0.6323480 \\
\hline 4.0213060 & -1.3058240 & 3.2535160 & C & 2.8417340 & 2.1684730 & -2.0258420 \\
\hline 6.0444480 & -3.1759860 & 2.7956590 & $\mathrm{C}$ & 3.5665580 & 1.7038650 & 0.2615890 \\
\hline 4.6768020 & -4.0636110 & 1.3778660 & $\mathrm{C}$ & 3.9884040 & 1.5253430 & -2.4911640 \\
\hline 5.2224310 & -1.2143050 & 3.9476090 & C & 4.7017600 & 1.0734400 & -0.2542240 \\
\hline 3.2277980 & -0.5867720 & 3.4171460 & $\mathrm{C}$ & 4.9344430 & 0.9796600 & -1.6243070 \\
\hline 6.2350370 & -2.1442300 & 3.7144960 & $\mathrm{H}$ & 4.1445500 & 1.4428170 & -3.5629720 \\
\hline 6.8322180 & -3.9013260 & 2.6202690 & $\mathrm{H}$ & 5.4277350 & 0.6377970 & 0.4320920 \\
\hline 5.3705750 & -0.4158130 & 4.6678180 & C & -3.8966050 & -0.9311250 & -0.3334360 \\
\hline 7.1747630 & -2.0668670 & 4.2526750 & $\mathrm{C}$ & -3.7135510 & -2.2607440 & 0.0970390 \\
\hline 0.9311290 & -1.4389900 & 1.0863180 & $\mathrm{C}$ & -4.4387360 & -0.6670430 & -1.6062840 \\
\hline 2.4237050 & -3.3288000 & 0.7018090 & C & -4.1328950 & -3.2968610 & -0.7409920 \\
\hline & & & C & & & -2.4087790 \\
\hline G $\mathrm{G}[\mathrm{M} 06-2 \mathrm{X}$ & $(d)]=-3310$ & & C & -4.6982840 & -3.0602360 & -1.9906020 \\
\hline 0.4080520 & 0.9278490 & 0.4052170 & $\mathrm{H}$ & -4.0088620 & -4.3268060 & -0.4129960 \\
\hline-1.7436110 & 0.8216210 & -0.8902730 & $\mathrm{H}$ & -5.2603280 & -1.5297090 & -3.3879700 \\
\hline 0.3032680 & 2.3030800 & 0.3171540 & C & 3.3760470 & 1.7633850 & 1.7691050 \\
\hline-0.8733380 & 2.9190640 & 0.6939340 & $\mathrm{C}$ & 6.1975550 & 0.3220110 & -2.1485090 \\
\hline 1.4523210 & 3.0136520 & -0.1200740 & $\mathrm{C}$ & 1.8561200 & 2.7570000 & -3.0273150 \\
\hline 07520 & 4.3489550 & 0.6031980 & $\mathrm{C}$ & -3.0815160 & -2.6113960 & 1.4382440 \\
\hline 2710 & 4.3844000 & -0.0903600 & $\mathrm{C}$ & -5.1468070 & -4.2076680 & -2.8755840 \\
\hline 1120 & 5.0815230 & 0.2527470 & C & -4.6368750 & 0.7466430 & -2.1320130 \\
\hline 6040 & 4.9559070 & -0.3702030 & C & -6.1279500 & 1.0584550 & -2.3106950 \\
\hline-2 . & 1.0462290 & 0.2926880 & $\mathrm{H}$ & -6 & 0.3940070 & -3.0543400 \\
\hline
\end{tabular}




-6.6712090
-6.2658310
-3.8651070
-2.8017190
-4.2485310
-3.9698530
-4.3579100
-4.6553100
-4.5443640
-3.2821330
-6.6536370
-7.2246120
-6.9089380
-6.9737040
-4.1525880
-4.8750190
-4.7062990
-3.6902710
-2.0471270
-2.5223950
-1.3178910
-1.5127880
4.4938920
5.4693080
4.5436700
4.3219560
3.2826570
2.4157980
4.1855820
3.1701940
7.2701390
8.1939610
6.9180140
7.4992050
5.9405030
5.0677950
5.7531750
6.8128190
1.6714760
2.5609060
1.4437550
0.8390200
2.2953380
3.2825760
1.5871900
2.3586830

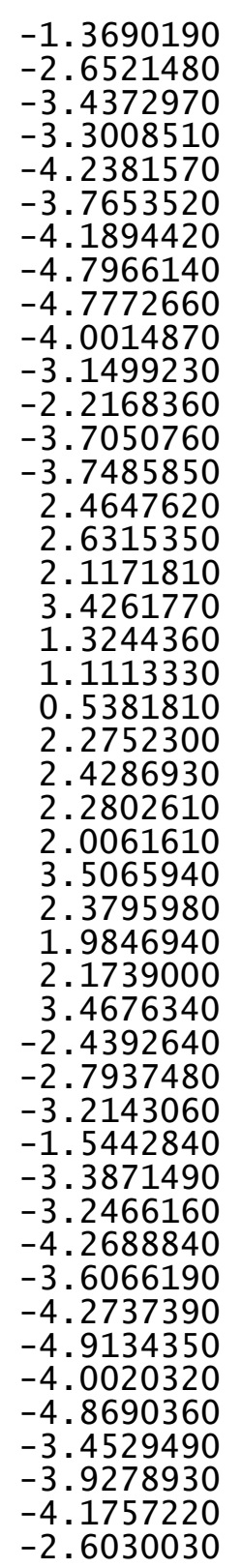

$-0.3476360$

0.3725130

$-0.5888350$

0.8806450

6.5812950

2.4296500

$-4.2441860$

$-4.9403180$

$-2.5549610$

2.6499330

1.6363660

2. 3520350

0.5989810

2. 3805510

1.6617330

4.4229890

3.4153970

2. 0011820

5.4325170

3.6847060

1.8942050

4.1361350

4.7441530

4.4363490

3.4212180

1.2430860

1.4000220

0.6529410

2. 1690090

2.9874930

2.0920810

3.7290520

3.0260970

2. 8389880

1.4520040

3.6555170

4.3635850

2.7837810

4.2361650

0.1311490

1. 5039230
4.0640850
0.0100940
0.0348420
$-1.3138890$
2. 8300340
$-0.3292230$
2. 2732680
1.4523890
$-5.1392190$
$-1.7246750$
$-1.9039890$
$-2.9442280$
$-1.0664010$
$-2.6278940$
$-1.6336660$
$-0.9242500$
$-3.5908000$
$-4.7218290$
$-4.2725960$
$-2.3783470$
$-3.9291100$
$-5.5969560$
$-2.6399760$
$-1.5424560$
$-3.2985820$
$-3.0128620$
$-1.9568840$
$-3.3793860$
$-4.5105900$
$-2.5926840$
$-4.8557550$
$-5.1104330$
$-2.9396390$
$-1.7171660$
$-4.0692960$
$-5.7359090$
$-2.3301640$
$-4.3384040$
$-1.7092110$
$-3.6814690$

144

$\operatorname{TS} 97(R, R) \quad G[M 06-2 X / 6-31 G(d)]=-3310.353566$
O
0.4080690
0.9277730
0.8216190
0.4051760
-0.8903030
$-1.7435980$

$-0.7207080$

$-2.0316240$

$-0.0365920$

$-1.5346420$

1.9755980

$-1.3945350$

$-2.3330250$

$-0.9014770$

$-1.1126420$

$-0.2608850$

$-1.0996980$

$-2.2914930$

$-1.5211180$

$-1.3142100$

$-1.6860570$

$-0.6586120$

$-1.2688120$

$-1.9124800$

0.4060040

$-0.8379200$

$-2.5779760$

$-0.2623440$

$-1.3600410$

1.9038730
2.0345650

3.1251020

3.0972010

4.2775720

4.2209580

2.1938430

5.3981420

5.3701140

4.2030880

6.2942460

6.2471130

1.1705330

0.8656840

4.5844580 


\begin{tabular}{|c|c|c|}
\hline $\begin{array}{l}0.3033510 \\
-0.8732210 \\
1.4524310 \\
-0.9505610 \\
1.4054560 \\
0.2183390 \\
2.2868160 \\
-2.4319740 \\
-3.5389520 \\
-2.0298030 \\
-4.2605550 \\
-2.7252620 \\
-3.8701120 \\
-5.1427000 \\
-4.1668600 \\
-3.0028490 \\
-2.3010120 \\
-4.5846750 \\
-4.7156560 \\
-2.6588560 \\
-1.4045750 \\
-5.4631230 \\
-2.1805560 \\
-1.0048020 \\
0.1651600 \\
-2.1560040 \\
-3.1131240 \\
-1.0381860 \\
1.0712950 \\
-3.0671570 \\
2.6469820 \\
2.8417210 \\
3.5666420 \\
3.9883560 \\
4.7018090 \\
4.9344300 \\
4.1444510 \\
5.4278080 \\
-3.8966530 \\
-3.7136520 \\
-4.4387680 \\
-4.1330360 \\
-4.8318500 \\
-4.6984100 \\
-4.0090450 \\
-2603860\end{array}$ & $\begin{array}{r}2.3030080 \\
2.9190590 \\
3.0135140 \\
4.3489540 \\
4.3842640 \\
5.0814550 \\
4.9557200 \\
1.0462870 \\
0.2011020 \\
2.0815980 \\
0.4675880 \\
2.2763680 \\
1.4782420 \\
-0.1267290 \\
2.6084880 \\
3.3722690 \\
3.2116340 \\
1.6793570 \\
2.7509600 \\
4.0880440 \\
3.7956530 \\
1.0679930 \\
6.4408980 \\
7.1688450 \\
6.4995310 \\
5.0712150 \\
6.9716040 \\
8.2524680 \\
7.0408780 \\
4.5257990 \\
2.2761790 \\
2.1682150 \\
1.7036750 \\
1.5250440 \\
1.0732100 \\
0.9793810 \\
1.4424740 \\
0.6375790 \\
-0.9310250 \\
-2.2606370 \\
-0.6669600 \\
-3.2967640 \\
-1.7371780 \\
-3.0601540 \\
-4.3267040 \\
-1.5296510\end{array}$ & $\begin{array}{r}0.3170770 \\
0.6938560 \\
-0.1201830 \\
0.6031000 \\
-0.0904800 \\
0.2526350 \\
-0.3703440 \\
0.2926570 \\
0.5744330 \\
1.1107920 \\
1.7110900 \\
2.3502720 \\
2.6274110 \\
1.9338380 \\
4.7546670 \\
4.5012620 \\
3.3331050 \\
3.8381320 \\
5.6801760 \\
5.2413310 \\
3.1545590 \\
4.0274830 \\
0.7439940 \\
0.4434900 \\
0.1970720 \\
0.8208480 \\
0.9082280 \\
0.3923320 \\
-0.0621730 \\
1.0406310 \\
-0.6324830 \\
-2.0259830 \\
0.2614330 \\
-2.4913340 \\
-0.2544090 \\
-1.6244990 \\
-3.5631460 \\
0.4318900 \\
-0.3334050 \\
0.0971130 \\
-1.6062630 \\
-0.7408870 \\
-2.4087260 \\
-1.9905070 \\
-0.4128580 \\
-3.3879260\end{array}$ \\
\hline
\end{tabular}

\begin{abstract}
3. 3761930
6. 1975260

1.8560690

$-3.0816470$

$-5.1469660$

$-4.6368480$

$-6.1279060$

$-6.5817860$

$-6.6712090$

$-6.2657400$

$-3.8650230$

$-2.8016450$

$-4.2484290$

$-3.9697380$

$-4.3579870$

$-4.6554180$

$-4.5443270$

$-3.2822270$

$-6.6537740$

$-7.2248070$

$-6.9089790$

$-6.9738630$

$-4.1527480$

$-4.8751450$

$-4.7064970$

$-3.6904510$

$-2.0473080$

$-2.5226190$

$-1.3180510$

$-1.5129860$

4.4940660

5.4694790

4.5438140

4. 3221880

3. 2828250

2. 4159520

4. 1857440

3. 1703990

7. 2701100

8. 1939240

6.9179750

7.4991940

5.9404600

5.0677620

5.7531180

6.8127780
\end{abstract}

1.7632550

0.3217210

2. 7567300

$-2.6112730$

$-4.2075940$

0.7467180

1. 0585720

0.3941310

0.9309920

2.0898480

0.9703900

0.7553260

0.3282750

2. 0099540

$-4.2412810$

$-5.1027030$

$-3.3357710$

$-4.3032660$

$-4.1446640$

$-4.1401040$

$-3.2352630$

$-5.0038660$

$-3.0050900$

$-2.2014690$

$-3.8850470$

$-3.2533360$

$-3.7385820$

$-4.7023670$

$-3.5285490$

$-3.8440040$

2. 5799860

1035620

3. 5883940

2. 6651720

0.3597590

$-0.1791260$

$-0.2275660$

0.4230800

1.3795350

0.9101460

2.0693970

1. 9656080

$-0.5424270$

$-1.1883590$

0.0798110

$-1.1660250$
1.7689540

$-2.1487290$

$-3.0274260$

1.4383370

$-2.8754620$

$-2.1320370$

$-2.3107850$

$-3.0544440$

$-1.3691320$

$-2.6522560$

$-3.4372980$

$-3.3008010$

$-4.2381510$

$-3.7653940$

$-4.1892680$

$-4.7964310$

$-4.7771350$

$-4.0012390$

$-3.1498990$

$-2.2168470$

$-3.7051110$

$-3.7485400$

2. 4648570

2.6315970

2. 1172990

3. 4262840

1. 3245800

1. 1115010

0.5383280

2. 2753840

2. 4284590

2. 2799890

2. 0058950

3. 5063670

2. 3795120

1.9846650

2. 1738140

3.4675480

$-2.4394930$

$-2.7939970$

$-3.2145210$

$-1.5445100$

$-3.3873740$

$-3.2468410$

-4.2691010
-3.6068600 


$\begin{array}{rrr}1.6713890 & 1.8848390 & -4.2738420 \\ 2.5607920 & 1.8991800 & -4.9135760 \\ 1.4437000 & 0.8527200 & -4.0021280 \\ 0.8389000 & 2.2733400 & -4.8691030 \\ 2.2952610 & 4.1652690 & -3.4530790 \\ 3.2824880 & 4.1244350 & -3.9280460 \\ 1.5870920 & 4.5841770 & -4.1758380 \\ 2.3586180 & 4.8494260 & -2.6031400 \\ -0.3476620 & 0.0100270 & -0.7207240 \\ 0.3724960 & 0.0347140 & -2.0316380 \\ -0.5889250 & -1.3139380 & -0.0365960 \\ 0.8806090 & 2.8297540 & -2.5347190 \\ 6.5812770 & -0.3295190 & -1.3509400 \\ 2.4298050 & 2.2731460 & 1.9754640 \\ -4.2441670 & 1.4524720 & -1.3945620 \\ -4.9405780 & -5.1391360 & -2.3328500 \\ -2.5550560 & -1.7245640 & 1.8047990 \\ 2.6498470 & -1.9041370 & -0.9014870 \\ 1.6362620 & -2.9443490 & -1.1126030 \\ 2.3519840 & -1.0664830 & -0.2609690 \\ 0.5988790 & -2.6280000 & -1.0996470 \\ 2.3803710 & -1.6339670 & -2.2915260 \\ 1.6615860 & -0.9245100 & -2.3103450 \\ 4.4228630 & -3.5910150 & -1.5210520 \\ 3.4152530 & -4.7220070 & -1.3140260 \\ 2.0010380 & -4.2727860 & -1.6858920 \\ 4.0640010 & -2.3784890 & -0.6586310 \\ 5.4323910 & -3.9293230 & -1.2687430 \\ 3.6845350 & -5.5971980 & -1.9122170 \\ 1.8940220 & -4.1747910 & -2.7731210 \\ 4.1360650 & -2.6400370 & 0.4060040 \\ 4.7440810 & -1.5426210 & -0.8380110 \\ 4.4362020 & -3.2988800 & -2.5779340 \\ 3.4210870 & -5.0276140 & -0.2621300 \\ 1.2429370 & -4.9896700 & -1.3597550 \\ 1.3999610 & -3.0127350 & 1.9041020 \\ 0.6528340 & -1.9567670 & 2.0346600 \\ 2.1689390 & -3.3790800 & 3.1253950 \\ 2.9876230 & -4.5101410 & 3.0975880 \\ 2.0918030 & -2.5923510 & 4.2778340 \\ 3.7291760 & -4.8551340 & 4.2214030 \\ 3.0263930 & -5.1100080 & 2.1942530 \\ 2.8386970 & -2.9391380 & 5.3984650 \\ 1.4515720 & -1.7169460 & 5.3705280 \\ 3.6554290 & -4.0686500 & 4.2036020 \\ 4.3638700 & -5.7351740 & \end{array}$

$\mathrm{H}$

144

2.7833220

4. 2360700

0.1310290

1. 5039160

$-2.3296430$

$-4.3376240$

$-1.7092020$

$-3.6814510$

6.2945450

6.2475730

1.1705930

$\operatorname{TS} 98(R, R) \quad G[M 06-2 X / 6-31 G(d)]=-3310.351343$

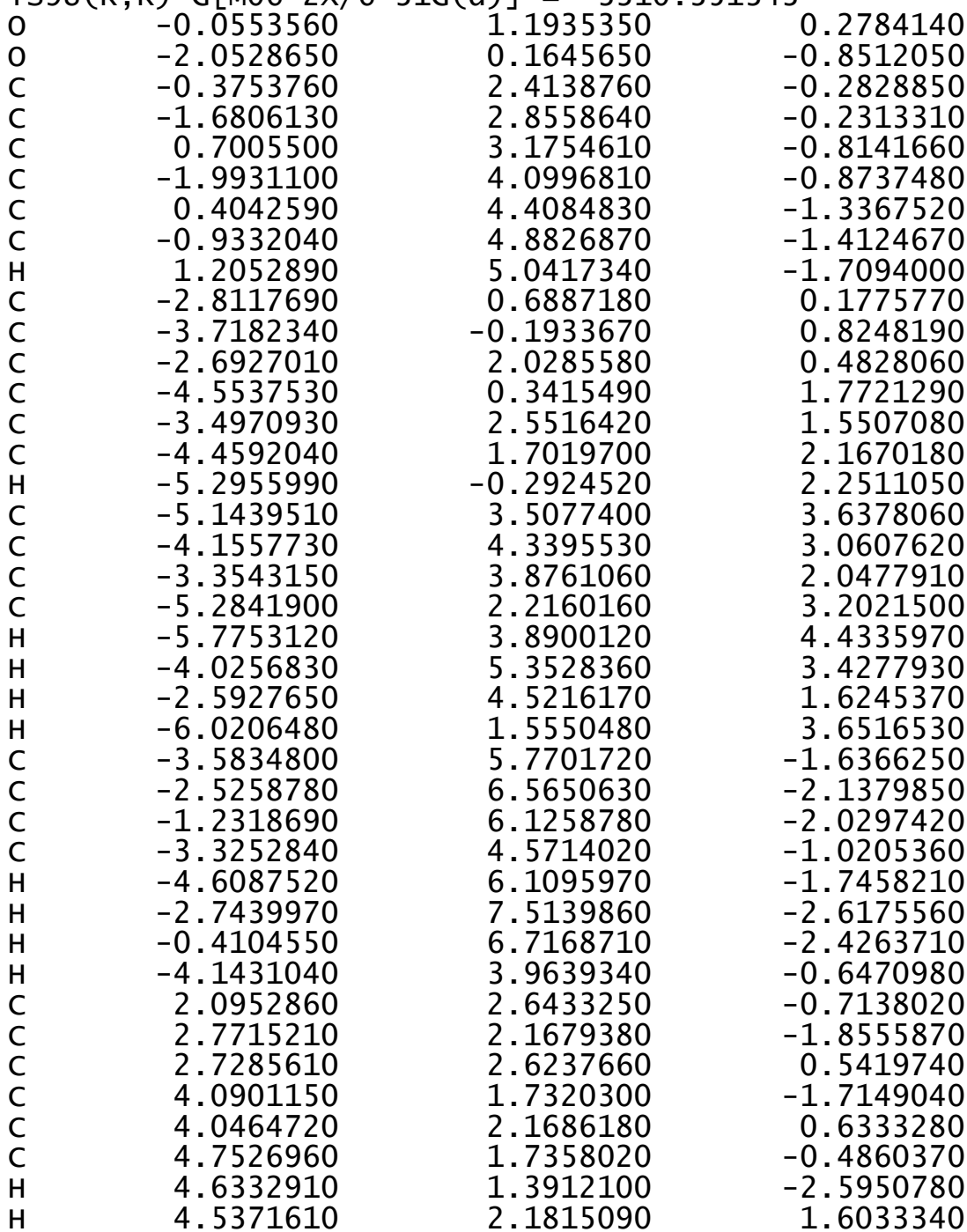




\begin{tabular}{|c|c|c|}
\hline $\begin{array}{l}-3.7469300 \\
-3.1346080 \\
-4.3806550 \\
-3.2215820 \\
-4.4337370 \\
-3.8686430 \\
-2.7604610 \\
-4.9286700 \\
2.0497280 \\
6.1948030 \\
2.1212260 \\
-2.3941710 \\
-3.9291620 \\
-5.0106910 \\
-6.5262160 \\
-6.7595340 \\
-6.9977310 \\
-6.9763610 \\
-4.3522070 \\
-3.2754020 \\
-4.5125910 \\
-4.7798210 \\
-3.1281610 \\
-3.1361420 \\
-3.5590230 \\
-2.0885800 \\
-5.3740850 \\
-5.9489950 \\
-5.8767660 \\
-5.3981020 \\
-3.3168440 \\
-4.1809510 \\
-3.6881970 \\
-2.7733790 \\
-1.1378670 \\
-1.3923910 \\
-0.5308190 \\
-0.5300790 \\
2.6285280 \\
3.6993000 \\
2.5050590 \\
2.1274050 \\
2.1444100 \\
1.7658350 \\
3.1783530 \\
1.5526810\end{array}$ & $\begin{array}{l}-1.6442850 \\
-2.5921480 \\
-2.0539920 \\
-3.9431890 \\
-3.4144170 \\
-4.3761800 \\
-4.6856890 \\
-3.7295630 \\
3.1612640 \\
1.2603540 \\
2.1645700 \\
-2.1869820 \\
-5.8505820 \\
-1.0608200 \\
-1.2725310 \\
-2.2660110 \\
-1.1857020 \\
-0.5287710 \\
-1.1351840 \\
-0.9608570 \\
-2.1157670 \\
-0.3783320 \\
-6.1456570 \\
-7.2175420 \\
-5.6181450 \\
-5.8204700 \\
-6.3407930 \\
-6.1435530 \\
-5.8361830 \\
-7.4172880 \\
-2.2686740 \\
-1.6061650 \\
-3.2923400 \\
-1.9895210 \\
-3.0300840 \\
-4.0369260 \\
-3.1177920 \\
-2.5597440 \\
4.5350260 \\
4.4570950 \\
5.2412430 \\
4.9457230 \\
2.1935300 \\
1.2051900 \\
2.0887480 \\
2.5682700\end{array}$ & $\begin{array}{r}0.4617560 \\
1.3048020 \\
-0.7254270 \\
0.9618180 \\
-1.0287110 \\
-0.1929230 \\
1.6092740 \\
-1.9460290 \\
1.7934100 \\
-0.4138130 \\
-3.2312220 \\
2.5720080 \\
-0.5450040 \\
-1.6895040 \\
-1.7854030 \\
-2.1843540 \\
-0.8014050 \\
-2.4511310 \\
-3.0716570 \\
-2.9952260 \\
-3.5336530 \\
-3.7375810 \\
-1.8186940 \\
-2.0428670 \\
-2.6768850 \\
-1.7129430 \\
-0.6875990 \\
0.2218600 \\
-1.5199340 \\
-0.8864320 \\
3.7961350 \\
3.6994820 \\
3.9223090 \\
4.7053080 \\
2.8242010 \\
3.1742780 \\
1.9200360 \\
3.6044040 \\
2.1555950 \\
2.3762060 \\
1.3282570 \\
3.0385060 \\
2.9756070 \\
2.6970820 \\
3.3259270 \\
3.8170090\end{array}$ \\
\hline
\end{tabular}

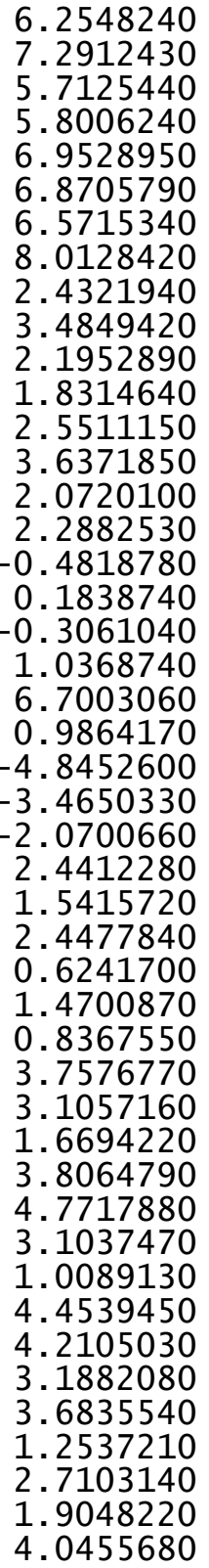

-0.2718640
-0.6244790
-0.6978540
-0.6515800
1.7772470
2.8641330
1.3179040
1.5169240
0.8985090
0.8558520
-0.0008100
0.8846250
3.4004500
3.3961200
3.4050170
4.3301840
-0.1334490
-0.1884580
-1.2756690
2.1984210
1.6435620
3.3013890
-0.0507000
-6.4033790
-1.1485880
-2.1680070
-3.1524340
-1.1769070
-2.7749940
-2.2456550
-1.4706630
-4.0619400
-5.0266550
-4.6058250
-2.6479390
-4.3937390
-6.0470060
-4.7916500
-2.6270060
-1.9287280
-4.0500470
-5.0368700
-5.1628770
-2.2494840
-1.2400350
-2.0770800

$-0.4632040$

$-0.4200820$

0.3890540

$-1.3832850$

0.8085100

0.9063940

1.7270930

0.7291670

$-4.0344560$

$-4.3358410$

$-3.4622620$

$-4.9494950$

$-4.0339320$

$-4.1833680$

$-5.0187320$

$-3.5221960$

$-0.5672700$

$-1.9100350$

0.4032990

$-3.0873860$

$-1.3106650$

1. 5815830

$-1.3024770$

0.2818910

2. 4478580

$-1.6512950$

$-1.0401670$

$-1.1861140$

$-0.6006740$

$-2.7131340$

$-2.5345330$

$-2.6652300$

$-1.6725780$

$-1.3547670$

$-2.0829900$

$-2.9062180$

$-2.0663770$

$-2.2106700$

$-1.1961610$

$-2.8041150$

$-3.6019850$

$-0.7411080$

$-0.5108470$

1.4917430

1.6563740

2. 1233110 


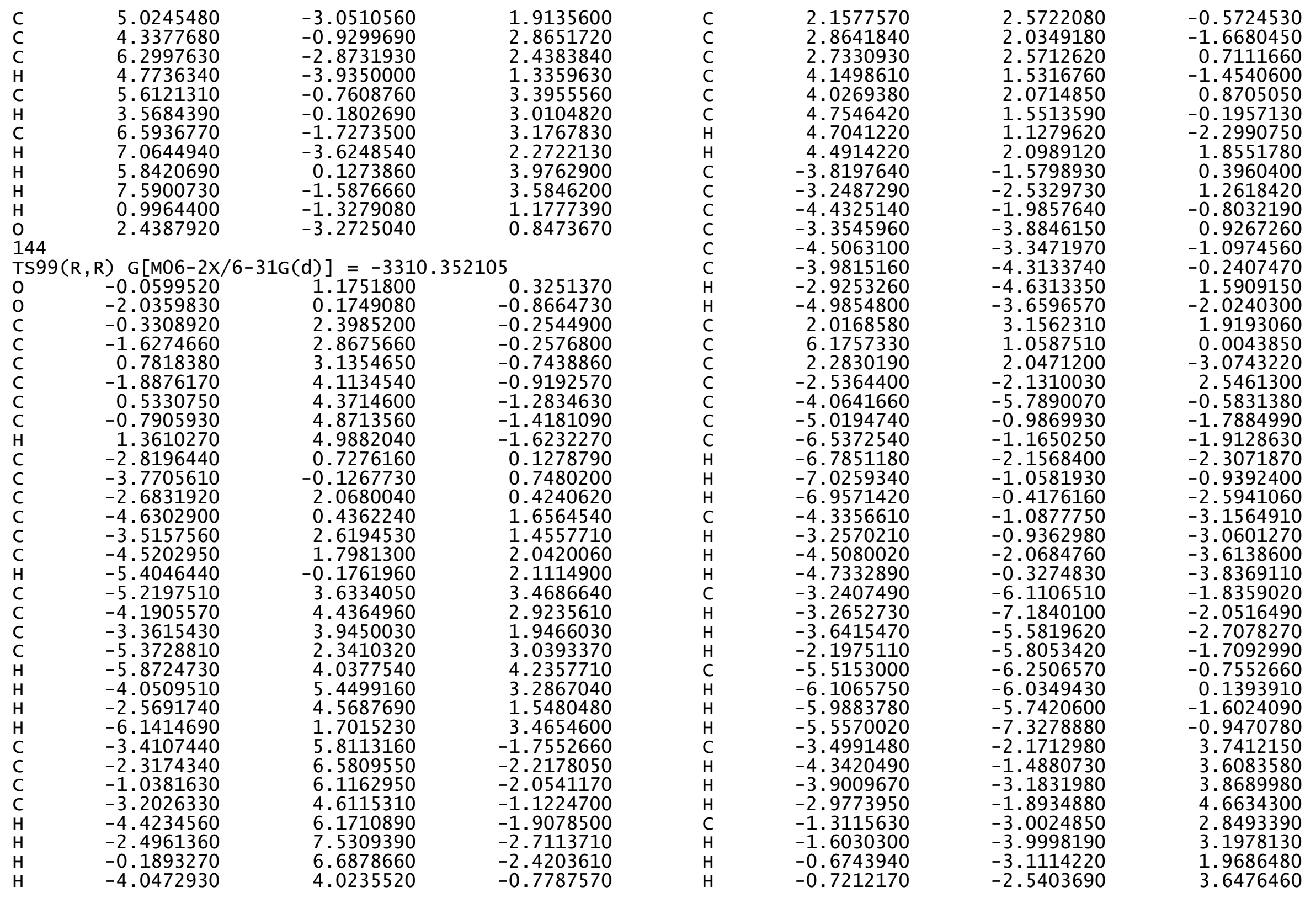




$\begin{array}{rrr}2.6201890 & 4.5188230 & 2.2826720 \\ 3.6773530 & 4.4141520 & 2.5522450 \\ 2.5542140 & 5.2129310 & 1.4387000 \\ 2.0934300 & 4.9600720 & 3.1353460 \\ 2.0315200 & 2.2072040 & 3.1208810 \\ 1.6417270 & 1.2230860 & 2.8426470 \\ 3.0446440 & 2.0823640 & 3.5217260 \\ 1.4112400 & 2.6105960 & 3.9277240 \\ 7.1723880 & 1.9198810 & -0.7805720 \\ 8.1999200 & 1.5931160 & -0.5893730 \\ 6.9888220 & 1.8391610 & -1.8577500 \\ 7.0864500 & 2.9746340 & -0.5033260 \\ 6.3276010 & -0.4195800 & -0.3631500 \\ 5.6314380 & -1.0370080 & 0.2132100 \\ 6.1288020 & -0.5792820 & -1.4294940 \\ 7.3455340 & -0.7667310 & -0.1552400 \\ 2.6043980 & 0.7820670 & -3.8735460 \\ 3.6686120 & 0.7198500 & -4.1280770 \\ 2.3227540 & -0.1175520 & -3.3231610 \\ 2.0462720 & 0.7893610 & -4.8151690 \\ 2.7826000 & 3.2778850 & -3.8447600 \\ 3.8743450 & 3.2466180 & -3.9399500 \\ 2.3530120 & 3.3012260 & -4.8519080 \\ 2.5183560 & 4.2103740 & -3.3391520 \\ -0.4792980 & -0.1468400 & -0.5324130 \\ 0.2275180 & -0.2135160 & -1.8535660 \\ -0.3473510 & -1.2890350 & 0.4451760 \\ 1.1939570 & 2.1063360 & -2.9849310 \\ 6.4065670 & 1.1596980 & 1.0729750 \\ 0.9686610 & 3.3230380 & 1.6567480 \\ -4.8384750 & 0.0223840 & -1.4064820 \\ -3.6305870 & -6.3446220 & 0.2583160 \\ -2.1803520 & -1.1034840 & 2.4187590 \\ 2.4421290 & -2.2194810 & -1.5248600 \\ 1.5227730 & -3.1990750 & -0.9332090 \\ 2.4398130 & -1.2265140 & -1.0631110 \\ 0.5936340 & -2.8163320 & -0.5237870 \\ 1.5014680 & -2.2956710 & -2.6135040 \\ 0.8717560 & -1.5136740 & -2.4566730 \\ 3.7789490 & -4.1179910 & -2.4996690 \\ 3.0981610 & -5.0782290 & -1.5218480 \\ 1.6550900 & -4.6532270 & -1.2418460 \\ 3.8177410 & -2.7036910 & -1.9176700 \\ 4.7975910 & -4.4543690 & -2.7136640 \\ 3.1034660 & -6.0997560 & -1.9125800 \\ 1.0167700 & -4.8382290 & -2.1146400\end{array}$
4. 4416820
4. 2448730
3. 2337540
3. 6520590
1. 2158480
2. 6518030
1.8631030
4.0055070
4. 9415820
4. 3613690
6. 2368760
4.6409840
5. 6586260
3. 6241490
6. 5958230
6.9677960
5. 9403970
7.6092060
0.9498970
2. 3507650

144

$$
\begin{array}{r}
-2.6859590 \\
-1.9847950 \\
-4.1056510 \\
-5.0871730 \\
-5.2082880 \\
-2.2682240 \\
-1.2408920 \\
-2.0846270 \\
-3.1146860 \\
-0.8733880 \\
-2.9298260 \\
-4.0468780 \\
-0.6934270 \\
-0.0823030 \\
-1.7179110 \\
-3.7260220 \\
0.2476890 \\
-1.5725500 \\
-1.3376400 \\
-3.3150890
\end{array}
$$

$-1.0143130$

$-2.6256340$

$-3.4507520$

$-0.5758790$

$-0.4086610$

1. 5731400

1. 7058130

2. 1591640

2.0360490

2. 7572900

2. 5052790

1. 5685210

3. 2252550

2. 8351810

3.0955140

2. 4090080

3. 6882010

3. 4570230

1. 2388480

0.9812650

$\operatorname{TS} 100(R, R) \quad G[M 06-2 X / 6-31 G(d)]=-3310.354240$

$\begin{array}{lrlr}\mathrm{O} & 0.3929780 & 0.8633010 & 0.2026130 \\ \mathrm{O} & -1.8550320 & 0.8501880 & -0.9415100 \\ \mathrm{C} & 0.3937620 & 2.2388610 & 0.0730620 \\ \mathrm{C} & -0.7236000 & 2.9562240 & 0.4529370 \\ \mathrm{C} & 1.5936500 & 2.8477750 & -0.3820260 \\ \mathrm{C} & -0.7085850 & 4.3801200 & 0.2821040 \\ \mathrm{C} & 1.6330340 & 4.2185910 & -0.4366630 \\ \mathrm{C} & 0.4949040 & 5.0115210 & -0.1386760 \\ \mathrm{H} & 2.5545550 & 4.7139820 & -0.7323240 \\ \mathrm{C} & -2.4307700 & 1.1778190 & 0.2754700 \\ \mathrm{C} & -3.5593260 & 0.4214410 & 0.6914460 \\ \mathrm{C} & -1.9064820 & 2.2321550 & 0.9915190 \\ \mathrm{C} & -4.1770690 & 0.8034480 & 1.8559530 \\ \mathrm{C} & -2.4962740 & 2.5508090 & 2.2600840 \\ \mathrm{C} & -3.6630280 & 1.8465010 & 2.6697980 \\ \mathrm{H} & -5.0740200 & 0.2821200 & 2.1796450 \\ \mathrm{C} & -3.7407440 & 3.1330790 & 4.7265540 \\ \mathrm{C} & -2.5547940 & 3.8024170 & 4.3422590 \\ \mathrm{C} & -1.9485570 & 3.5200620 & 3.1440010 \\ \mathrm{C} & -4.2761770 & 2.1727740 & 3.9084610 \\ \mathrm{H} & -4.2132380 & 3.3719370 & 5.6740400 \\ \mathrm{H} & -2.1172820 & 4.5425550 & 5.0049220 \\ \mathrm{H} & -1.0341290 & 4.0327800 & 2.8660970 \\ \mathrm{H} & -5.1733120 & 1.6324700 & 4.1992050\end{array}$




-1.7978060
-0.5877970
0.5303650
-1.8579710
-2.6887430
-0.5540420
1.4618190
-2.7938910
2.7893470
3.0936890
3.6360630
4.2820920
4.8180630
5.1709620
4.5331400
5.4754580
-4.0490280
-3.8805320
-4.6946940
-4.4166340
-5.2029050
-5.0842700
-4.3043710
-5.7094830
3.3264940
6.4830000
2.1787400
-3.1436900
-5.6551310
-4.8780330
-6.3639070
-6.9117340
-6.8248460
-6.4868840
-4.2182380
-3.1569380
-4.6979290
-4.3084510
-4.9864500
-5.3712300
-5.1862860
-3.9017860
-7.1764720
-7.6629870
-7.4429350
-7.5819430

6.5518560

7.1813520

6.4239640

5.1908250

7.1518950

8.2596900

6.8891370

4.7189890

2.0282760

1.8001470

1.5376530

1.1423690

0.8824920

0.6970820

0.9668540

0.5200480

$-0.7361870$

$-2.0506060$

$-0.5075540$

$-3.1049230$

$-1.5950980$

$-2.9016870$

$-4.1231350$

$-1.4143050$

1. 7158160

0.0582570

2.2790960

$-2.3623990$

$-4.0683000$

0.8879900

1.2627620

0.5873170

1.2048530

2. 2826230

1.0129760

0.7560480

0.3510650

2.0391980

$-4.1986360$

$-5.0729060$

$-3.3138480$

$-4.3005420$

$-3.9516140$

$-3.8768810$

$-3.0597540$

$-4.8236240$
0.3278500

$-0.0496840$

$-0.2817780$

0.4900850

0.4842700

$-0.1681560$

$-0.5937460$

0.7692820

$-0.7509960$

$-2.1101220$

0.2558380

$-2.4226710$

$-0.1080490$

$-1.4408670$

$-3.4672040$

0.6781500

$-0.1174970$

0.3615850

$-1.3478210$

$-0.3818940$

$-2.0568520$

$-1.5862870$

$-0.0157690$

$-3.0034720$

1.7340700

$-1.8674890$

$-3.2282360$

1.6579940

$-2.3700080$

$-1.9246960$

$-1.9863900$

$-2.6528750$

$-0.9952490$

$-2.3656550$

$-3.3024660$

$-3.2479530$

$-4.0321240$

$-3.6738830$

$-3.7432610$

$-4.2787530$

$-4.3576270$

$-3.6453250$

$-2.5172830$

$-1.5403390$

$-3.0952170$

$-3.0409660$
$-4.1320320$

$-4.7777830$

$-4.7754300$

$-3.5946760$

$-2.2003600$

$-2.7555990$

$-1.5452380$

$-1.5791460$

4. 3729480

5.3668440

4.4329130

4.1173920

3.2083280

2. 4116840

4.1454290

2. 9695780

7. 1424310

8.0155830

7.4898290

6.4557190

7.4561810

7.0219950

7.6940410

8.3902190

2. 0992010

3. 0155650

1.9329670

1.2710560

2. 6291110

3.6545330

1.9786820

2. 6034660

$-0.4901920$

0.1244990

$-0.7300710$

1.1702810

6.2615400

2. 3580670

$-4.3889200$

$-5.4395260$

$-2.5284750$

2. 3416820

1. 3240890

2. 1449480

0.2976360
1.8307600
-2.6160780
-1.7528400
-3.4715950
-2.8415820
-3.5650720
-4.5046010
-3.4597390
-3.6447860
2.6095630
2.1501000
3.5847450
2.7704340
0.3623520
-0.2394910
-0.2039000
0.5068060
-0.7952770
-1.3114770
-0.1751040
-1.5480110
1.1436380
1.7335450
1.8265610
0.6974100
1.2862870
1.2975480
0.2676860
1.5592870
3.6489970
3.5882450
3.9748500
4.4150900
-0.0208360
-0.0295010
-1.3286250
2.3773630
-0.5938940
2.2169940
1.6071760
-4.9815920
-1.4933090
-2.0707440
-3.1281660
-1.1862940
-2.8111840
-1.9206490

2.8047830

2. 9865940

2.5684010

3.7322130

1.5262000

1.4313160

0.6575300

2.4249030

2.4100790

2. 3714600

1. 9165280

3.4624880

2.4435030

1.9960220

2. 3894040

3.5030050

$-0.7843730$

$-1.1943780$

0.0492360

$-0.3831680$

$-2.3502250$

$-3.1626860$

$-1.5272050$

$-2.7072860$

$-4.3925470$

$-4.9936200$

$-4.0368930$

$-5.0534270$

$-3.7548540$

$-4.1378540$

$-4.5735260$

$-2.9756160$

$-0.8564540$

$-2.2222520$

$-0.1423150$

$-2.8138850$

$-2.7246770$

1.8263150

$-1.2613310$

$-1.8006130$

1. 9124300

$-1.3066700$

$-1.2657110$

$-0.6901930$

$-1.1221620$

$-2.6455820$ 


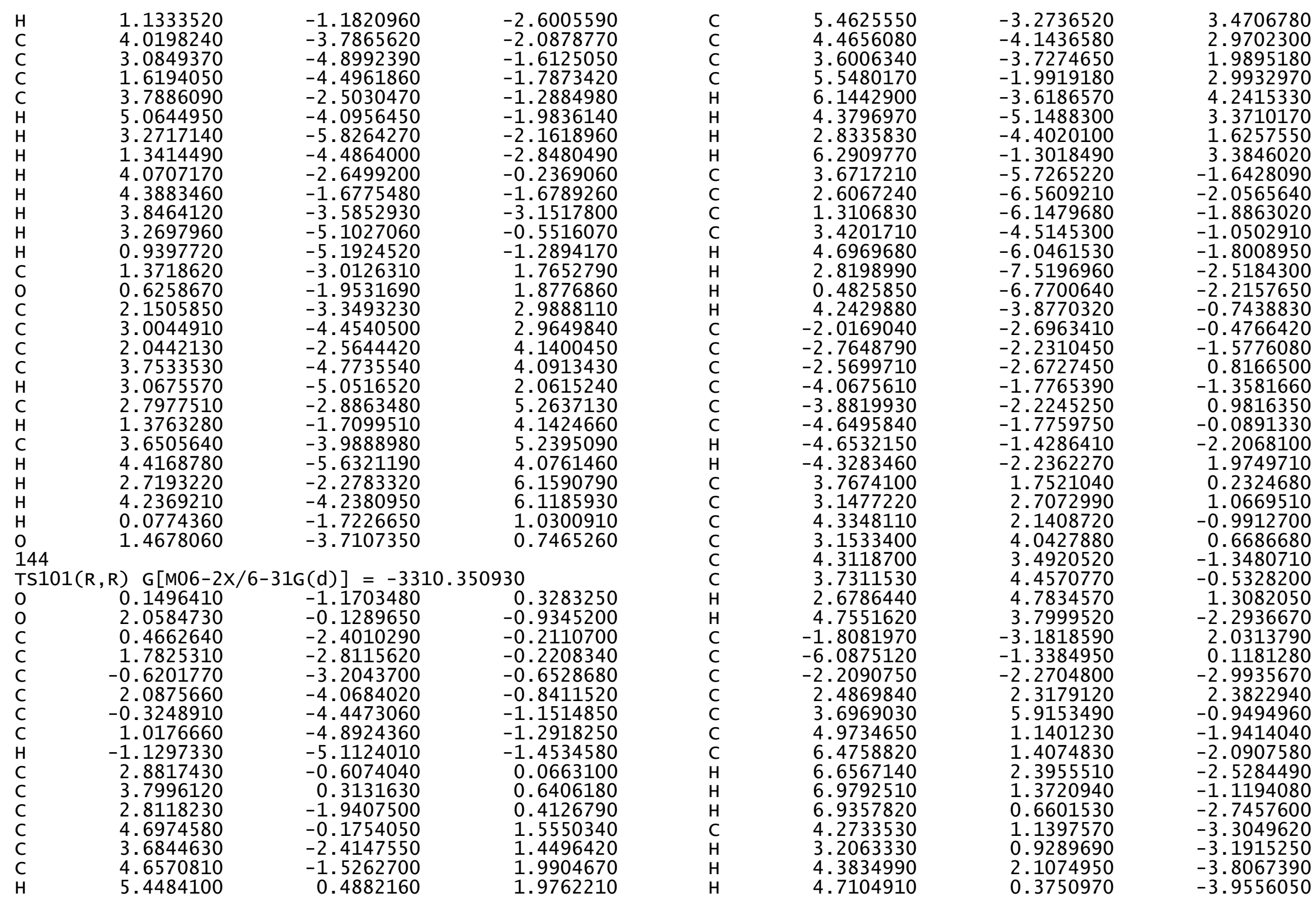




$\begin{array}{rrr}4.4113350 & 6.8119640 & 0.0674710 \\ 4.4214930 & 7.8519050 & -0.2751550 \\ 3.9020320 & 6.7839950 & 1.0370030 \\ 5.4447560 & 6.4878670 & 0.2208790 \\ 2.2559330 & 6.3901690 & -1.1731500 \\ 1.7495270 & 5.7680550 & -1.9175200 \\ 1.6825630 & 6.3358360 & -0.2406730 \\ 2.2390370 & 7.4290540 & -1.5190110 \\ 3.4738510 & 2.4542960 & 3.5504230 \\ 4.3491470 & 1.8124210 & 3.4216410 \\ 3.8231960 & 3.4903060 & 3.6302600 \\ 2.9894970 & 2.1844820 & 4.4951240 \\ 1.2252710 & 3.1352410 & 2.6857140 \\ 1.4726840 & 4.1556700 & 3.0000940 \\ 0.5663080 & 3.1874540 & 1.8158250 \\ 0.6745740 & 2.6681170 & 3.5089780 \\ -2.3391950 & -4.5593810 & 2.4476150 \\ -3.3963040 & -4.4967210 & 2.7301480 \\ -2.2515010 & -5.2766820 & 1.6252730 \\ -1.7795350 & -4.9467610 & 3.3054890 \\ -1.8509950 & -2.1961940 & 3.2024480 \\ -1.5117970 & -1.2042770 & 2.8877770 \\ -2.8630690 & -2.1060270 & 3.6150770 \\ -1.2010000 & -2.5441350 & 4.0117210 \\ -7.0580350 & -2.2729910 & -0.6138240 \\ -8.0957180 & -1.9838060 & -0.4169450 \\ -6.8959470 & -2.2277710 & -1.6965230 \\ -6.9210830 & -3.3109930 & -0.2967700 \\ -6.3103350 & 0.1157940 & -0.3046380 \\ -5.6297840 & 0.7846310 & 0.2314030 \\ -6.1393530 & 0.2402230 & -1.3804530 \\ -7.3378950 & 0.4280430 & -0.0890080 \\ -2.5990120 & -1.0503150 & -3.8312530 \\ -3.6693320 & -1.0426260 & -4.0669100 \\ -2.3463900 & -0.1199340 & -3.3192150 \\ -2.0583260 & -1.0686350 & -4.7828540 \\ -2.6694430 & -3.5486510 & -3.7093860 \\ -3.7630230 & -3.5662440 & -3.7851850 \\ -2.2566760 & -3.5912570 & -4.7229150 \\ -2.3576180 & -4.4502030 & -3.1755130 \\ 0.4947580 & 0.1355790 & -0.5848320 \\ -0.2359170 & 0.1213710 & -1.8943020 \\ 0.3272700 & 1.3061440 & 0.3529280 \\ -1.1170820 & -2.2806140 & -2.9235640 \\ -6.2955860 & -1.4065820 & 1.7570450 \\ -0.7570930 & -3.3061950 & \end{array}$

\begin{abstract}
4.8597090
4. 2306300

2. 1841760

$-2.5264420$

$-1.6404110$

$-2.4737410$

$-0.6888460$

$-1.6084020$

$-0.9441850$

$-3.9606350$

$-3.3059890$

$-1.8412110$

$-3.9280730$

$-4.9964260$

$-3.3624190$

$-1.2264160$

$-4.5356210$

$-4.3350740$

$-3.4314170$

$-3.8435410$

$-1.4111210$

$-2.6900340$

$-1.8581780$

$-4.0249110$

$-5.0059960$

$-4.3188100$

$-6.2842700$

$-4.7532390$

$-5.5990880$

$-3.5475400$

$-6.5813080$

$-7.0499850$

$-5.8325310$

$-7.5813630$

$-0.9569770$

$-2.4416490$
\end{abstract}

144

$\operatorname{TS} 102(R, R) G[M 06-2 X / 6-31 G(d)]=-3310.350929$

$\begin{array}{lrrr}\mathrm{O} & 0.1496300 & -1.1703480 & 0.3283230 \\ \mathrm{O} & 2.0584750 & -0.1289630 & -0.9345020 \\ \mathrm{C} & 0.4662650 & -2.4010230 & -0.2110810 \\ \mathrm{C} & 1.7825330 & -2.8115550 & -0.2208310 \\ \mathrm{C} & -0.6201700 & -3.2043640 & -0.6528960 \\ \mathrm{C} & 2.0875760 & -4.0683900 & -0.8411550 \\ \mathrm{C} & -0.3248760 & -4.4472950 & -1.1515190 \\ \mathrm{C} & 1.0176820 & -4.8924220 & -1.2918480\end{array}$

\begin{tabular}{|c|c|}
\hline $\begin{array}{l}0.1386150 \\
5.9956570 \\
1.2688440 \\
2.0470630 \\
3.0852550 \\
1.0724340 \\
2.7572400 \\
2.1234610 \\
1.3741970 \\
3.8522060 \\
4.8736730 \\
4.5207870 \\
2.4580830 \\
4.1368360 \\
5.8801280 \\
4.7032540 \\
2.4441990 \\
1.6977540 \\
3.8309940 \\
4.8911750 \\
5.1223170 \\
2.1903230 \\
1.1998750 \\
1.9713470 \\
2.9561620 \\
0.7684500 \\
2.7338340 \\
3.8829620 \\
0.5511570 \\
0.0120220 \\
1.5298830 \\
3.4946340 \\
0.3836830 \\
1.3550580 \\
1.3209320 \\
3.2289460\end{array}$ & $\begin{array}{r}-1.5152090 \\
-1.9051560 \\
2.3017100 \\
-1.5954680 \\
-1.0548820 \\
-1.0990890 \\
-0.6494940 \\
-2.7032560 \\
-2.5314900 \\
-2.6086820 \\
-1.6760560 \\
-1.4090170 \\
-1.9794350 \\
-2.8153070 \\
-2.1003810 \\
-2.2990940 \\
-1.0648950 \\
-2.6552490 \\
-3.5685820 \\
-0.7208020 \\
-0.6036330 \\
1.5015200 \\
1.6514830 \\
2.1179060 \\
1.9759880 \\
2.7645150 \\
2.4747450 \\
1.4709220 \\
3.2624010 \\
2.8558210 \\
3.1136660 \\
2.3638860 \\
3.7633640 \\
3.4984980 \\
1.1669990 \\
0.8720050\end{array}$ \\
\hline $\begin{array}{l}=-331 \\
03480\end{array}$ & $\begin{array}{r}929 \\
0.3283230 \\
-0.9345020 \\
-0.2110810 \\
-0.2208310 \\
-0.6528960 \\
-0.8411550 \\
-1.1515190 \\
-1.2918480\end{array}$ \\
\hline
\end{tabular}




$\begin{array}{lrrr}\mathrm{H} & -1.1297150 & -5.1123890 & -1.4535050 \\ \mathrm{C} & 2.8817470 & -0.6074020 & 0.0663260 \\ \mathrm{C} & 3.7996200 & 0.3131620 & 0.6406320 \\ \mathrm{C} & 2.8118210 & -1.9407470 & 0.4126940 \\ \mathrm{C} & 4.6974600 & -0.1754090 & 1.5550530 \\ \mathrm{C} & 3.6844540 & -2.4147550 & 1.4496610 \\ \mathrm{C} & 4.6570730 & -1.5262740 & 1.9904880 \\ \mathrm{H} & 5.4484160 & 0.4882070 & 1.9762390 \\ \mathrm{C} & 5.4625340 & -3.2736590 & 3.4707030 \\ \mathrm{C} & 4.4655870 & -4.1436620 & 2.9702500 \\ \mathrm{C} & 3.6006180 & -3.7274660 & 1.9895350 \\ \mathrm{C} & 5.5480030 & -1.9919250 & 2.9933220 \\ \mathrm{H} & 6.1442650 & -3.6186660 & 4.2415610 \\ \mathrm{H} & 4.3796710 & -5.1488340 & 3.3710370 \\ \mathrm{H} & 2.8335670 & -4.4020080 & 1.6257670 \\ \mathrm{H} & 6.2909650 & -1.3018590 & 3.3846300 \\ \mathrm{C} & 3.6717420 & -5.7265020 & -1.6428050 \\ \mathrm{C} & 2.6067520 & -6.5608990 & -2.0565810 \\ \mathrm{C} & 1.3107090 & -6.1479490 & -1.8863310 \\ \mathrm{C} & 3.4201840 & -4.5145150 & -1.0502810 \\ \mathrm{H} & 4.6969920 & -6.0461310 & -1.8008810 \\ \mathrm{H} & 2.8199340 & -7.5196710 & -2.5184510 \\ \mathrm{H} & 0.4826150 & -6.7700440 & -2.2158090 \\ \mathrm{H} & 4.2429970 & -3.8770190 & -0.7438580 \\ \mathrm{C} & -2.0169010 & -2.6963420 & -0.4766750 \\ \mathrm{C} & -2.7648670 & -2.2310280 & -1.5776390 \\ \mathrm{C} & -2.5699790 & -2.6727740 & 0.8166120 \\ \mathrm{C} & -4.0675540 & -1.7765330 & -1.3582010 \\ \mathrm{C} & -3.8820050 & -2.2245630 & 0.9815940 \\ \mathrm{C} & -4.6495880 & -1.7759960 & -0.0891730 \\ \mathrm{H} & -4.6532010 & -1.4286200 & -2.2068430 \\ \mathrm{H} & -4.3283640 & -2.2362800 & 1.9749270 \\ \mathrm{C} & 3.7674280 & 1.7521000 & 0.2324720 \\ \mathrm{C} & 3.1477650 & 2.7073120 & 1.0669540 \\ \mathrm{C} & 4.3348080 & 2.1408480 & -0.9912840 \\ \mathrm{C} & 3.1533830 & 4.0427950 & 0.6686510 \\ \mathrm{C} & 4.3118680 & 3.4920220 & -1.3481030 \\ \mathrm{C} & 3.7311710 & 4.4570630 & -0.5328560 \\ \mathrm{H} & 2.6787030 & 4.7834760 & 1.3081860 \\ \mathrm{H} & 4.7551420 & 3.7999060 & -2.2937130 \\ \mathrm{C} & -1.8082110 & -3.1818910 & 2.0313430 \\ \mathrm{C} & -6.0875190 & -1.3385230 & 0.1180860 \\ \mathrm{C} & -2.2090460 & -2.2704290 & -2.9935920 \\ \mathrm{C} & 2.4870470 & 2.3179510 & 2.3823150 \\ \mathrm{C} & 3.6969090 & 5.9153260 & -1.9414150 \\ \mathrm{C} & 4.9734410 & 1.1400830 & \end{array}$

\begin{abstract}
6.4758600
6.6566970

6.9792380

6.9357460

4. 2733170

3. 2062950

4. 3834740

4. 7104360

4.4113440

4.4214920

3. 9020480

5. 4447680

2. 2559330

1. 7495250

1. 6825730

2. 2390250

3.4739340

4. 3492280

3. 8232790

2.9895960

1. 2253400

1. 4727580

0.5663650

0.6746540

$-2.3392370$

$-3.3963420$

$-2.2515660$

$-1.7795790$

$-1.8509820$

$-1.5117730$

$-2.8630510$

$-1.2009840$

$-7.0580350$

$-8.0957200$

$-6.8959390$

$-6.9210820$

$-6.3103420$

$-5.6297960$

$-6.1393550$

$-7.3379040$

$-2.5989860$

$-3.6693030$

$-2.3463790$

$-2.0582910$

$-2.6693880$

$-3.7629670$
\end{abstract}

$\begin{array}{rr}1.4074280 & -2.0907870 \\ 2.3954920 & -2.5284850 \\ 1.3720390 & -1.1194410 \\ 0.6600890 & -2.7457880 \\ 1.1397130 & -3.3049670 \\ 0.9289440 & -3.1915170 \\ 2.1074420 & -3.8067570 \\ 0.3750370 & -3.9556050 \\ 6.8119690 & 0.0673820 \\ 7.8519010 & -0.2752670 \\ 6.7840190 & 1.0369180 \\ 6.4878810 & 0.2207900 \\ 6.3901300 & -1.1732110 \\ 5.7679940 & -1.9175610 \\ 6.3358120 & -0.2407270 \\ 7.4290070 & -1.5190950 \\ 2.4543570 & 3.5504250 \\ 1.8124810 & 3.4216410 \\ 3.4903690 & 3.6302370 \\ 2.1845610 & 4.4951390 \\ 3.1352870 & 2.6857380 \\ 4.1557210 & 3.0000970 \\ 3.1874850 & 1.8158570 \\ 2.6681770 & 3.5090190 \\ -4.5593930 & 2.4476080 \\ -4.4967060 & 2.7301490 \\ -5.2767120 & 1.6252780 \\ -4.9467690 & 3.3054840 \\ -2.1962020 & 3.2023930 \\ -1.2042960 & 2.8876950 \\ -2.1060120 & 3.6150280 \\ -2.5441340 & 4.0116670 \\ -2.2730080 & -0.6138900 \\ -1.9838290 & -0.4170120 \\ -2.2277670 & -1.6965870 \\ -3.3110160 & -0.2968540 \\ 0.1157730 & -0.3046540 \\ 0.7846020 & 0.2314030 \\ 0.2402230 & -1.3804660 \\ 0.4280160 & -0.0890240 \\ -1.0502530 & -3.8312590 \\ -1.0425700 & -4.0669270 \\ -0.1198780 & -3.3192030 \\ -1.0685500 & -4.7828550 \\ -3.5485920 & -3.7094420 \\ -3.5661950 & -3.7852580\end{array}$




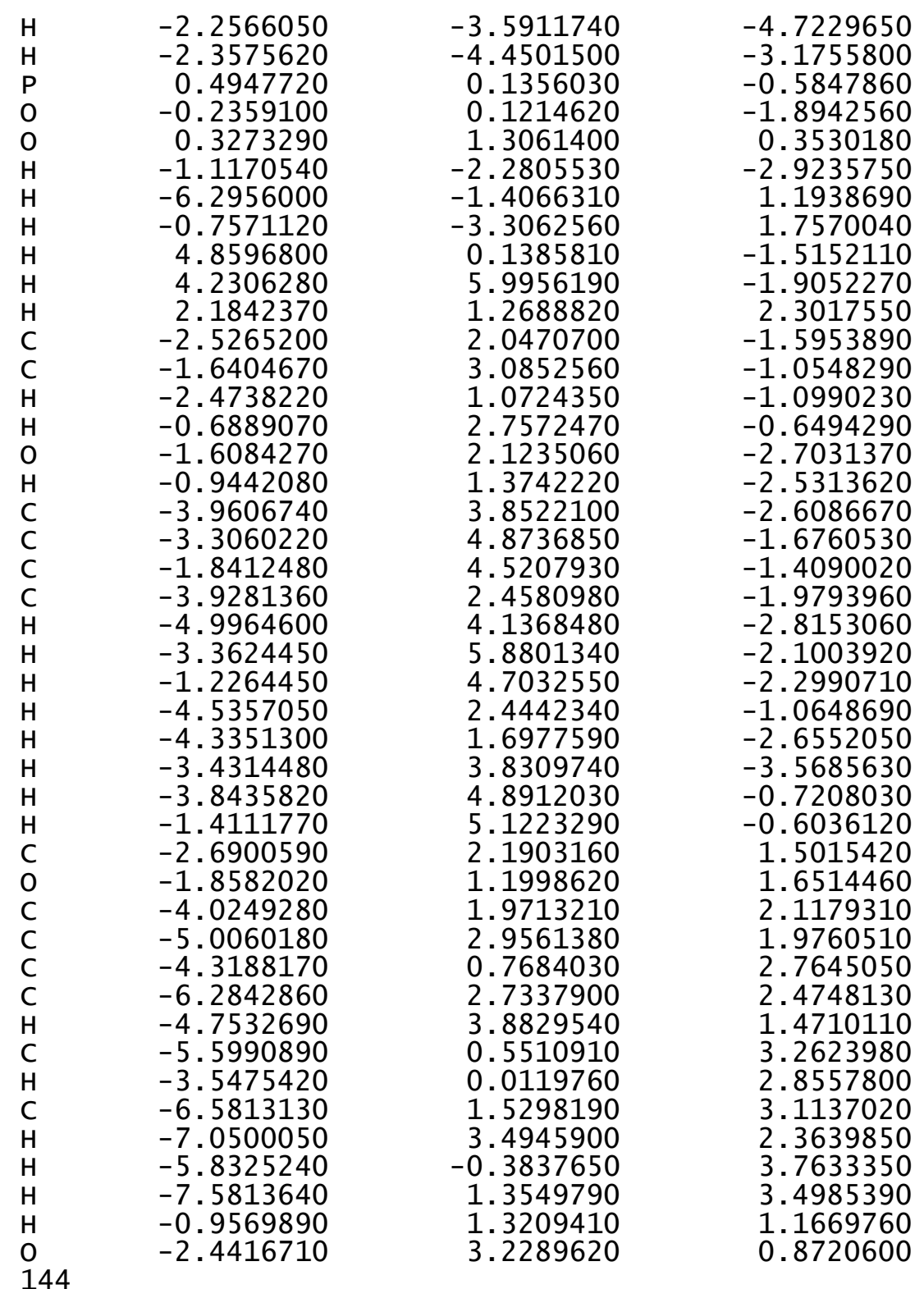

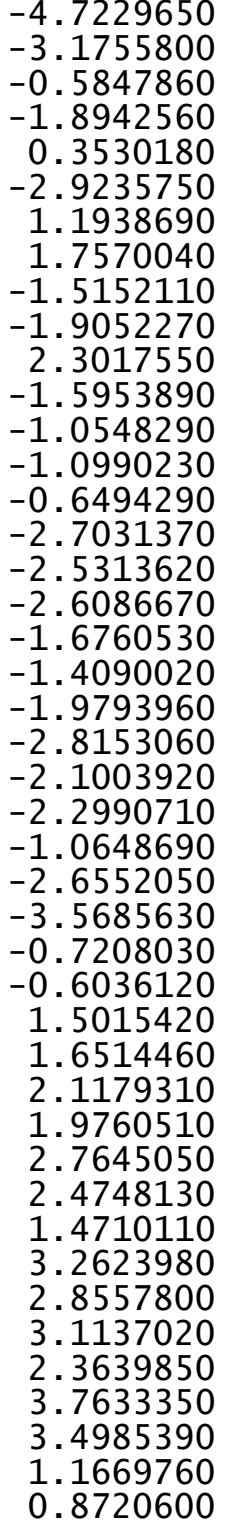

0.2479720 $-0.8849060$ $-0.2464810$ $-0.1572500$ $-0.7514210$ $-0.7362270$ $-1.2103820$ $-1.2499360$ $-1.5584850$

0.1753770

0.7929300

0.5355500

1.7747070

1.6377170

2.2306220

2.2326310

3.7901380

3.2355250

2.1907700

3.2999020

4.6114930

3.6451080

1.7855010

3.7305890

$-1.4000160$

$-1.8766010$

$-1.8045650$

$-0.8437960$

$-1.4806000$

$-2.3088220$

$-2.1830230$

$-0.4890940$

$-0.6759960$

$-1.8285810$

0.5858930

$-1.6978410$

0.6637370

$-0.4642880$

$-2.5946230$

1.6338510

0.3622400

1.1348290

$-0.8113560$

0.7390470

$-1.1684780$

$-0.4010870$ 


\begin{tabular}{|c|c|c|}
\hline $\begin{array}{r}-2.8603680 \\
-5.1548480 \\
2.0947070 \\
6.1660490 \\
2.1873260 \\
-2.3715420 \\
-4.1744230 \\
-5.1288790 \\
-6.6542880 \\
-6.9446290 \\
-7.0748570 \\
-7.1061480 \\
-4.5393700 \\
-3.4542590 \\
-4.7576120 \\
-4.9690330 \\
-3.4864640 \\
-3.5468750 \\
-3.9671600 \\
-2.4321850 \\
-5.6428270 \\
-6.1362780 \\
-6.1909100 \\
-5.7214600 \\
-3.2455050 \\
-4.0897450 \\
-3.6458060 \\
-2.6564970 \\
-1.1351710 \\
-1.4091260 \\
-0.5702670 \\
-0.4803380 \\
2.7498910 \\
3.8097110 \\
2.6843410 \\
2.2587820 \\
2.1199060 \\
1.6669790 \\
3.1446840 \\
1.5624580 \\
6.8754720 \\
7.9087040 \\
6.3810590 \\
6.8946420 \\
6.1965630 \\
5.7617850\end{array}$ & $\begin{array}{r}-4.7668450 \\
-3.5860750 \\
3.0267300 \\
1.0354980 \\
2.2882710 \\
-2.3207650 \\
-5.7972170 \\
-0.9272630 \\
-1.0782920 \\
-2.0448920 \\
-1.0156420 \\
-0.2909580 \\
-0.9660110 \\
-0.8339350 \\
-1.9196040 \\
-0.1665900 \\
-6.0627330 \\
-7.1240660 \\
-5.4897900 \\
-5.7723210 \\
-6.2338520 \\
-6.0630840 \\
-5.6721130 \\
-7.2979350 \\
-2.4194790 \\
-1.7258780 \\
-3.4347230 \\
-2.1927060 \\
-3.2128430 \\
-4.2259170 \\
-3.2775060 \\
-2.7966860 \\
4.3445380 \\
4.1915320 \\
5.0941110 \\
4.7445450 \\
1.9981530 \\
1.0569590 \\
1.7938540 \\
2.3761870 \\
0.7350280 \\
0.4306420 \\
-0.0855730 \\
1.6071910 \\
-0.2010280 \\
-0.0049320\end{array}$ & $\begin{array}{r}1.3316620 \\
-2.0736040 \\
1.8542500 \\
-0.2947040 \\
-3.2135870 \\
2.3837490 \\
-0.8086770 \\
-1.7020120 \\
-1.7325660 \\
-2.1592170 \\
-0.7238980 \\
-2.3448930 \\
-3.1163380 \\
-3.0861810 \\
-3.6099030 \\
-3.7290800 \\
-2.1527650 \\
-2.4157130 \\
-2.9534070 \\
-2.1193060 \\
-0.8559820 \\
0.1052530 \\
-1.6204100 \\
-1.1020710 \\
3.6417530 \\
3.6050840 \\
3.7453960 \\
4.5370150 \\
2.5477690 \\
2.8633920 \\
1.6147310 \\
3.3204570 \\
2.2873940 \\
2.5207320 \\
1.4921840 \\
3.1807200 \\
2.9873800 \\
2.6620310 \\
3.3190010 \\
3.8507310 \\
-1.6137470 \\
-1.4224780 \\
-2.1472310 \\
-2.2742040 \\
0.6133720 \\
1.5985610\end{array}$ \\
\hline
\end{tabular}

5.6238940
7.2247270
2.4693330
3.5289620
2.1712400
1.9022420
2.6850960
3.7721630
2.2227190
2.4584680
-0.4763370
0.1438180
-0.2573810
1.1017960
6.7311080
1.0444320
-4.9055870
-3.6670490
-2.0177260
2.5831090
1.8267280
2.4710800
0.8729130
1.6047470
0.8957180
4.1099120
3.6130350
2.1383400
3.9856820
5.1530440
3.7431790
1.4965490
4.6554950
4.2624240
3.5220910
4.2039130
1.8163930
2.7229160
1.7744800
3.8661860
4.9849870
3.8455980
6.0898180
4.9790890
4.9529850
2.9670850
$-1.0184020$
$-0.5490720$
1.0908510
1.0256490
0.1526820
1. 1944280
3. 5720480
3. 5299610
3. 6888260
4.4627150
$-0.1391660$
$-0.0931330$
$-1.3463620$
2. 3528930
1.8298190
3.2412570
0.0583810
$-6.3995560$
$-1.2889410$
$-1.8445770$
$-2.9653950$
$-0.8827810$
$-2.7295560$
$-2.0088100$
$-1.3043280$
$-3.4948690$
$-4.5796060$
$-4.3728610$
$-2.1140330$
$-3.6785720$
$-5.5736540$
$-4.6007910$
$-2.0454500$
$-1.3137130$
$-3.5173560$
$-4.5484400$
$-5.0242990$
$-2.2458470$
$-1.3781990$
$-2.1414110$
$-2.9518530$
$-1.2155050$
$-2.8259880$
$-3.6635480$
$-1.0930300$
$-0.5979890$

0.1619760

0.7598470

$-4.1236900$

$-4.3968220$

$-3.6523030$

$-5.0543280$

$-3.8938110$

$-4.0300020$

$-4.8796720$

$-3.3019600$

$-0.6724380$

$-2.0378090$

0.2051350

$-3.0903600$

0.2134590

1.6391020

$-1.2821060$

$-0.0441390$

2. 2861440

$-1.8049490$

$-1.2372220$

$-1.2940890$

$-0.7791610$

$-2.8526420$

$-2.6490110$

$-2.9421620$

$-1.9839160$

$-1.6317240$

$-2.2949360$

$-3.2157720$

$-2.4212440$

$-2.4910400$

$-1.4290140$

$-2.9905680$

$-3.8672650$

$-1.0608330$

$-0.8153330$

1.5358190

1.7429220

2. 4829200

2. 2805710

3. 5282580

3. 1143170

1. 4611920

4. 3612500

3. 6757890 


\begin{tabular}{|c|c|c|c|c|c|c|c|}
\hline & & & & & & & \\
\hline C & 6.0751260 & -1.8937170 & 4.1515390 & $\mathrm{H}$ & -4.5697470 & -1.6753640 & -2.5143690 \\
\hline 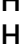 & $\begin{array}{l}0.9633160 \\
4.9409730\end{array}$ & $\begin{array}{l}-3.4500350 \\
-0.3729920\end{array}$ & $\begin{array}{l}2.9554500 \\
5.1733100\end{array}$ & ${ }^{H}$ & $\begin{array}{r}-4.3988050 \\
3.6983810\end{array}$ & -2.3426910 & $\begin{array}{l}1.1030280 \\
0.2944450\end{array}$ \\
\hline $\mathrm{H}$ & 6.9400400 & -1.7930520 & 4.7999980 & $\mathrm{C}$ & 3.0478350 & 2.7647760 & 1.1030600 \\
\hline $\mathrm{H}$ & 0.9765920 & -1.4218930 & 1.0971820 & $\mathrm{c}$ & 4.2924350 & 2.1911810 & -0.9185650 \\
\hline 0 & 2.7301010 & -3.0977780 & 0.6374320 & $\mathrm{C}$ & 3.0516200 & 4.0967760 & 0.6929520 \\
\hline & & & & C & 4.2655590 & 3.5387150 & -1.2884870 \\
\hline & R) $\mathrm{G}[\mathrm{M} 06-2$ & $G(d)]=-33$ & 384 & $\mathrm{C}$ & 3.6565340 & 4.5059460 & -0.4968340 \\
\hline 0 & 0.1335500 & -1.1925360 & 0.2859430 & $\mathrm{H}$ & 2.5538140 & 4.8384270 & 1.3136410 \\
\hline 0 & 2.0673690 & -0.1216540 & -0.9137280 & $\mathrm{H}$ & 4.7293580 & 3.8420020 & -2.2257010 \\
\hline $\mathrm{C}$ & 0.4999680 & -2.4166120 & -0.2374930 & C & -1.8705070 & -3.2111520 & 1.8926350 \\
\hline C & 1.8239940 & -2.7991820 & -0.1881560 & $\mathrm{C}$ & -6.1119740 & -1.5436120 & -0.3191200 \\
\hline C & -0.5476230 & -3.2425680 & $-0 . \overline{7277820}$ & $\mathrm{C}$ & -2.0340120 & -2.3647530 & -3.1570330 \\
\hline $\mathrm{C}$ & 2.1831700 & -4.0510280 & -0.7890880 & c & 2.3524760 & 2.3823790 & 2.4024010 \\
\hline $\mathrm{C}$ & -0.2036390 & -4.4799540 & -1.2093140 & c & 3.6214080 & 5.9602210 & -0.9271680 \\
\hline C & 1.1523320 & -4.8984290 & -1.2850090 & $\mathrm{C}$ & 4.9631550 & 1.1888430 & -1.8446710 \\
\hline $\mathrm{H}$ & -0.9803530 & -5.1608160 & -1.5478780 & $\mathrm{C}$ & 6.4640460 & 1.4758510 & -1.9709320 \\
\hline C & 2.8640380 & -0.5712140 & 0.1217490 & H & 6.6392160 & 2.4601870 & -2.4191290 \\
\hline C & 3.7402060 & 0.3742390 & 0.7200420 & $\mathrm{H}$ & 6.9504170 & 1.4607260 & -0.9904560 \\
\hline $\mathrm{C}$ & 2.8078680 & -1.9019810 & 0.4797760 & $\mathrm{H}$ & 6.9459050 & 0.7262690 & -2.6072900 \\
\hline C & 4.6109080 & -0.0865700 & 1.6744570 & C & 4.2870030 & 1.1631580 & -3.2201510 \\
\hline C & 3.6490180 & -2.3476280 & 1.5542890 & $\mathrm{H}$ & 3.2214160 & 0.9377770 & -3.1224950 \\
\hline C & 4.5808510 & -1.4333690 & 2.1231110 & H & 4.3919370 & 2.1271310 & -3.7303000 \\
\hline $\mathrm{H}$ & 5.3317050 & 0.5964210 & 2.1168240 & $\mathrm{H}$ & 4.7465860 & 0.3980750 & -3.8546580 \\
\hline C & 5.3644700 & -3.1494060 & 3.6509600 & C & 4.3004740 & 6.8716500 & 0.1007740 \\
\hline C & 4.4066810 & -4.0454610 & 3.1207360 & $\mathrm{H}$ & 4.3111050 & 7.9086460 & -0.2506460 \\
\hline C & 3.5719700 & -3.6569700 & 2.1031640 & H & 3.7653870 & 6.8476040 & 1.0564270 \\
\hline C & 5.4414980 & -1.8705570 & 3.1645130 & $\mathrm{H}$ & 5.3320570 & 6.5578460 & 0.2848240 \\
\hline $\mathrm{H}$ & 6.0228480 & -3.4726730 & 4.4509890 & C & 2.1832730 & 6.4208930 & -1.1942300 \\
\hline $\mathrm{H}$ & 4.3269070 & -5.0486570 & 3.5276860 & $\mathrm{H}$ & 1.7028190 & 5.7882870 & -1.9468850 \\
\hline $\mathrm{H}$ & 2.8343910 & -4.3510850 & 1.7158680 & $\mathrm{H}$ & 1.5849820 & 6.3695210 & -0.2773810 \\
\hline $\mathrm{H}$ & 6.1540630 & -1.1612830 & 3.5774270 & $\mathrm{H}$ & 2.1672320 & 7.4566460 & -1.5493860 \\
\hline C & 3.8360790 & -5.6784000 & -1.5112470 & C & 3.2960180 & 2.5583880 & 3.6005330 \\
\hline $\mathrm{C}$ & 2.8084840 & -6.5361770 & -1.9694970 & $\mathrm{H}$ & 4.1877830 & 1.9324730 & 3.5123350 \\
\hline C & 1.4978930 & -6.1495250 & -1.8604500 & $\mathrm{H}$ & 3.6220110 & 3.6024430 & 3.6744320 \\
\hline C & 3.5329270 & -4.4699100 & -0.9360000 & H & 2.7853170 & 2.2943980 & 4.5329330 \\
\hline $\mathrm{H}$ & 4.8738760 & -5.9770610 & -1.6212700 & C & 1.0648630 & 3.1787220 & 2.6490100 \\
\hline $\mathrm{H}$ & 3.0622600 & -7.4917750 & -2.4172090 & $\mathrm{H}$ & 1.2813790 & 4.2074090 & 2.9592000 \\
\hline $\mathrm{H}$ & 0.6985070 & -6.7894330 & -2.2247990 & $\mathrm{H}$ & 0.4378690 & 3.2092010 & 1.7546450 \\
\hline $\mathrm{H}$ & 4.3277340 & -3.8144080 & -0.5956210 & $\mathrm{H}$ & 0.4921830 & 2.7109610 & 3.4567880 \\
\hline C & -1.9633030 & -2.7682480 & -0.6275810 & C & -2.3904590 & -4.5941890 & 2.3051040 \\
\hline C & -2.6702580 & -2.3545290 & -1.7748640 & $\mathrm{H}$ & -3.4607950 & -4.5510460 & 2.5370860 \\
\hline C & -2.5843380 & -2.7400910 & 0.6339470 & $\mathrm{H}$ & -2.2491200 & -5.3210200 & 1.4987450 \\
\hline c & -4.0042800 & -1.9695540 & -1.6314490 & $\mathrm{H}$ & -1.8632150 & -4.9567420 & 3.1938590 \\
\hline c & -3.9192210 & -2.3376760 & 0.7274290 & C & -1.9898110 & -2.2105390 & 3.0449170 \\
\hline C & 5260 & 531 & 953900 & $\mathrm{H}$ & -1.6519850 & -1.2180700 & 2.7308850 \\
\hline
\end{tabular}




$\begin{array}{rrr}-3.0234230 & -2.1334540 & 3.4033050 \\ -1.3756050 & -2.5355410 & 3.8908930 \\ -6.2301820 & -0.0165770 & -0.4087110 \\ -7.2784450 & 0.2984210 & -0.3602100 \\ -5.6928650 & 0.4523640 & 0.4239490 \\ -5.8027290 & 0.3544560 & -1.3449720 \\ -6.8364280 & -2.0550690 & 0.9257130 \\ -6.7112140 & -3.1348920 & 1.0522070 \\ -6.4632140 & -1.5563810 & 1.8269810 \\ -7.9064010 & -1.8377590 & 0.8518060 \\ -2.4170930 & -1.1450380 & -3.9998920 \\ -3.4718610 & -1.1702630 & -4.2961610 \\ -2.2277050 & -0.2150670 & -3.4597380 \\ -1.8219810 & -1.1294220 & -4.9185300 \\ -2.4070200 & -3.6477870 & -3.9129070 \\ -3.4933090 & -3.7047840 & -4.0493020 \\ -1.9384780 & -3.6606790 & -4.9027240 \\ -2.0905280 & -4.5448430 & -3.3740120 \\ 0.4906440 & 0.1218160 & -0.6099250 \\ -0.2012230 & 0.1052280 & -1.9400960 \\ 0.2876970 & 1.2880470 & 0.3261110 \\ -0.9480860 & -2.3381730 & -3.0239460 \\ -6.6122930 & -1.9699420 & -1.1992580 \\ -0.8050300 & -3.3160940 & 1.6713460 \\ 4.8563970 & 0.1907210 & -1.4089370 \\ 4.1802440 & 6.0367720 & -1.8686770 \\ 2.0741690 & 1.3262160 & 2.3293030 \\ -2.5271050 & 2.0062570 & -1.7043940 \\ -1.6549270 & 3.0427590 & -1.1410400 \\ -2.4873320 & 1.0311020 & -1.2081910 \\ -0.7156430 & 2.7142430 & -0.7084940 \\ -1.5798490 & 2.0852910 & -2.7874200 \\ -0.9142410 & 1.3414080 & -2.5965440 \\ -3.9335520 & 3.8171370 & -2.7489230 \\ -3.3016370 & 4.8358070 & -1.7981180 \\ -1.8446360 & 4.4797020 & -1.4960770 \\ -3.9173040 & 2.4205830 & -2.1242450 \\ -4.9639140 & 4.1024080 & -2.9803940 \\ -3.3461270 & 5.8430440 & -2.2220000 \\ -1.2077040 & 4.6640990 & -2.3699600 \\ -4.5479970 & 2.4015260 & -1.2253650 \\ -4.3054890 & 1.6651390 & -2.8164440 \\ -3.3809690 & 3.7984070 & -3.6956100 \\ -3.8624890 & 4.8524840 & -0.8564270 \\ -1.4338310 & 5.0778350 & -0.6781640 \\ -2.7387020 & 2.1742350 & 1.4456190\end{array}$

$\begin{array}{ll}\mathrm{O} & -1.8832030 \\ \mathrm{C} & -4.0599910 \\ \mathrm{C} & -5.0839340 \\ \mathrm{C} & -4.2947880 \\ \mathrm{C} & -6.3461580 \\ \mathrm{H} & -4.8775890 \\ \mathrm{C} & -5.5566660 \\ \mathrm{H} & -3.4913270 \\ \mathrm{C} & -6.5826500 \\ \mathrm{H} & -7.1454570 \\ \mathrm{H} & -5.7420460 \\ \mathrm{H} & -7.5688960 \\ \mathrm{H} & -0.9904650 \\ \mathrm{O} & -2.5222150 \\ \mathrm{H} & \end{array}$
1. 2130540
0.6164680
0.1171050
1. 5302410

$\begin{array}{ll}0.8264310 & 2.8740540 \\ 2.6643900 & 2.4030010\end{array}$

$3.7586420 \quad 1.2551430$

3. 4196160

3.1790990

2.2195760

4. 0291970

1.3584120 3.5991900

$\begin{array}{ll}1.3190890 & 1.1388040\end{array}$

$\operatorname{TS} 105(R, R) \quad G[M 06-2 X / 6-31 G(d)]=-3310.354233$

$\begin{array}{lrrr}\text { O } & 0.3930790 & 0.8633290 & 0.2028440 \\ \mathrm{O} & -1.8548600 & 0.8503870 & -0.9414070 \\ \mathrm{C} & 0.3938540 & 2.2389070 & 0.0735110 \\ \mathrm{C} & -0.7235240 & 2.9561970 & 0.4534770 \\ \mathrm{C} & 1.5937430 & 2.8479050 & -0.3814600 \\ \mathrm{C} & -0.7085020 & 4.3801270 & 0.2829210 \\ \mathrm{C} & 1.6331360 & 4.2187310 & -0.4358180 \\ \mathrm{C} & 0.4950050 & 5.0116080 & -0.1376910 \\ \mathrm{H} & 2.5546580 & 4.7141780 & -0.7313840 \\ \mathrm{C} & -2.4306840 & 1.1778220 & 0.2755830 \\ \mathrm{C} & -3.5592940 & 0.4214000 & 0.6913290 \\ \mathrm{C} & -1.9064330 & 2.2320230 & 0.9918590 \\ \mathrm{C} & -4.1771130 & 0.8032010 & 1.8558630 \\ \mathrm{C} & -2.4963060 & 2.5504470 & 2.2604430 \\ \mathrm{C} & -3.6631020 & 1.8460860 & 2.6699450 \\ \mathrm{H} & -5.0740990 & 0.2818320 & 2.1793940 \\ \mathrm{C} & -3.7409180 & 3.1322690 & 4.7269460 \\ \mathrm{C} & -2.5549280 & 3.8016540 & 4.3428560 \\ \mathrm{C} & -1.9486220 & 3.5195170 & 3.1445820 \\ \mathrm{C} & -4.2763210 & 2.1721340 & 3.9086320 \\ \mathrm{H} & -4.2134660 & 3.3709530 & 5.6744480 \\ \mathrm{H} & -2.1174390 & 4.5416510 & 5.0056910 \\ \mathrm{H} & -1.0341660 & 4.0322660 & 2.8668330 \\ \mathrm{H} & -5.1734870 & 1.6317940 & 4.1992160 \\ \mathrm{C} & -1.7977160 & 6.5518600 & 0.3290620 \\ \mathrm{C} & -0.5876900 & 7.1814260 & -0.0482990 \\ \mathrm{C} & 0.5304770 & 6.4240800 & -0.2805050 \\ \mathrm{C} & -1.8578920 & 5.1907960 & 0.4910180 \\ \mathrm{H} & -2.6886560 & 7.1518690 & 0.4855710 \\ \mathrm{H} & -0.5539260 & 8.2597880 & -0.1665510 \\ & & & \\ & & & \end{array}$




$\begin{array}{lr}\mathrm{H} & 1.4619440 \\ \mathrm{H} & -2.7938260 \\ \mathrm{C} & 2.7894170 \\ \mathrm{C} & 3.0936440 \\ \mathrm{C} & 3.6362200 \\ \mathrm{C} & 4.2820170 \\ \mathrm{C} & 4.8182120 \\ \mathrm{C} & 5.1709920 \\ \mathrm{H} & 4.5329660 \\ \mathrm{H} & 5.4756860 \\ \mathrm{C} & -4.0489440 \\ \mathrm{C} & -3.8805200 \\ \mathrm{C} & -4.6944720 \\ \mathrm{C} & -4.4165520 \\ \mathrm{C} & -5.2026210 \\ \mathrm{C} & -5.0840490 \\ \mathrm{H} & -4.3043400 \\ \mathrm{H} & -5.7090980 \\ \mathrm{C} & 3.3267450 \\ \mathrm{C} & 6.4829930 \\ \mathrm{C} & 2.1786270 \\ \mathrm{C} & -3.1438420 \\ \mathrm{C} & -5.6548460 \\ \mathrm{C} & -4.8777340 \\ \mathrm{C} & -6.3635930 \\ \mathrm{H} & -6.9114070 \\ \mathrm{H} & -6.8245810 \\ \mathrm{H} & -6.4865220 \\ \mathrm{C} & -4.2178640 \\ \mathrm{H} & -3.1565820 \\ \mathrm{H} & -4.6975580 \\ \mathrm{H} & -4.3079950 \\ \mathrm{C} & -4.9861040 \\ \mathrm{H} & -5.3708300 \\ \mathrm{H} & -5.1859480 \\ \mathrm{H} & -3.9014390 \\ \mathrm{C} & -7.1761830 \\ \mathrm{H} & -7.6627410 \\ \mathrm{H} & -7.4426350 \\ \mathrm{H} & -7.5816150 \\ \mathrm{C} & -4.1323420 \\ \mathrm{H} & -4.7781540 \\ \mathrm{H} & -4.7756730 \\ \mathrm{H} & -3.5951150 \\ \mathrm{C} & -2.2004730 \\ \mathrm{H} & -2.7556820\end{array}$

$\begin{array}{rr}6.8893110 & -0.5923470 \\ 4.7189090 & 0.7700860 \\ 2.0284700 & -0.7506460 \\ 1.8005510 & -2.1098360 \\ 1.5377000 & 0.2560410 \\ 1.1428160 & -2.4225820 \\ 0.8826350 & -0.1080450 \\ 0.6974200 & -1.4409210 \\ 0.9674390 & -3.4671610 \\ 0.5200970 & 0.6780440 \\ -0.7360590 & -0.1178870 \\ -2.0505740 & 0.3609490 \\ -0.5071750 & -1.3482380 \\ -3.1047390 & -0.3827970 \\ -1.5945710 & -2.0575390 \\ -2.9012570 & -1.5872250 \\ -4.1230240 & -0.0168580 \\ -1.4135810 & -3.0041750 \\ 1.7155860 & 1.7343260 \\ 0.0586580 & -1.8677610 \\ 2.2797180 & -3.2278030 \\ -2.3626340 & 1.6573870 \\ -4.0677050 & -2.3712370 \\ 0.8884850 & -1.9248580 \\ 1.2633130 & -1.9865560 \\ 0.5879980 & -2.6531850 \\ 1.2052430 & -0.9954480 \\ 2.2832440 & -2.3656480 \\ 1.0137080 & -3.3025690 \\ 0.7567040 & -3.2480540 \\ 0.3519740 & -4.0323850 \\ 2.0400100 & -3.6737850 \\ -4.1977240 & -3.7444890 \\ -5.0718950 & -4.2801810 \\ -3.3128160 & -4.3586800 \\ -4.2996110 & -3.6465310 \\ -3.9510160 & -2.5185520 \\ -3.8765140 & -1.5416120 \\ -3.0590280 & -3.0962950 \\ -4.8229130 & -3.0424520 \\ -2.6165690 & 2.8039840 \\ -1.7533900 & 2.9858620 \\ -3.4720650 & 2.5673440 \\ -2.8422290 & 3.7314500 \\ -3.5652620 & 1.5254700 \\ -4.5047780 & 1.4302740\end{array}$
$-1.5452020$
$-1.5794170$
4. 3731890
5. 3671110
4.4330500
4. 1176990
3. 2087260
2. 4121140
4.1458760
2. 9700140
7. 1426920
8.0158170
7. 4901810
6.4561240
7.4560150
7.0216410
7. 6939900
8. 3900110
2. 0990650
3. 0153960
1. 9328880
1.2708750
2. 6289500
3. 6543580
1. 9784840
2. 6033190
$-0.4900290$
0.1247400
$-0.7299250$
1.1701790
6. 2614100
2. 3582910
$-4.3886310$
$-5.4392480$
$-2.5286760$
2. 3419470
1. 3243250
2. 1451030
0.2978470
1. 8313810
1.1339370
4.0202230
3. 0852050
1. 6197210
3. 7888590
5.0648640

-3.4597170
-3.6451800
2.6092850
2.1498930
3.5845540
2.7699590
0.3619880
-0.2398520
-0.2041690
0.5062390
-0.7947720
-1.3109120
-0.1745300
-1.5475540
1.1440750
1.7339160
1.8270530
0.6978850
1.2871860
1.2986320
0.2684920
1.5603180
3.6497490
3.5891000
3.9757700
4.4156700
-0.0206570
-0.0291280
-1.3285480
2.3778690
-0.5935550
2.2166820
1.6075300
-4.9811210
-1.4935900
-2.0704230
-3.1278390
-1.1861110
-2.8108580
-1.9199680
-1.1814710
-3.7860930
-4.8988660
-4.4957310
-2.5027700
-4.0952260

0.6569390

2. 4242670

2.4104160

2. 3716440

1. 9170250

3. 4628720

2.4435320

1. 9959870

2. 3892850

3. 5030690

$-0.7847270$

$-1.1948670$

0.0487930

$-0.3833650$

$-2.3507390$

$-3.1631480$

$-1.5277990$

$-2.7079560$

$-4.3923490$

$-4.9934690$

$-4.0369400$

$-5.0531160$

$-3.7541310$

$-4.1371840$

$-4.5727070$

$-2.9747240$

$-0.8564000$

$-2.2221600$

$-0.1424500$

$-2.8133960$

$-2.7248710$

1.8267300

$-1.2613310$

$-1.8020360$

1. 9120930

$-1.3065230$

$-1.2660140$

$-0.6898800$

$-1.1226350$

$-2.6455220$

$-2.6004930$

$-2.0877540$

$-1.6128650$

$-1.7879210$

$-1.2881100$

$-1.9833370$ 


\begin{tabular}{|c|c|c|c|c|c|c|c|}
\hline $\mathrm{H}$ & 3.2720770 & -5.8259210 & -2.1624480 & $\mathrm{H}$ & -2.2670170 & 4.2979810 & 2.1085570 \\
\hline $\mathrm{H}$ & 1.3419990 & -4.4856580 & -2.8486880 & $\mathrm{H}$ & -5.8554410 & 1. 3120520 & 3.8023220 \\
\hline $\mathrm{H}$ & 4.0707310 & -2.6499060 & -0.2364920 & C & -3.3184670 & 6.0272110 & -0.9308060 \\
\hline $\mathrm{H}$ & 4.3887040 & -1.6771930 & -1.6782030 & C & -2.2372800 & 6.8327220 & -1.3593190 \\
\hline $\mathrm{H}$ & 3.8470520 & -3.5845560 & -3.1516470 & $\mathrm{c}$ & -0.9638730 & 6.3251640 & -1.3525590 \\
\hline $\mathrm{H}$ & 3.2698260 & -5.1026000 & -0.5519780 & C & -3.1029410 & 4.7468070 & -0.4863540 \\
\hline $\mathrm{H}$ & 0.9399560 & -5.1921080 & -1.2903320 & $\mathrm{H}$ & -4.3283770 & 6.4241200 & -0.9598630 \\
\hline C & 1.3715710 & -3.0131180 & 1.7649750 & $\mathrm{H}$ & -2.4214330 & 7.8454490 & -1.7035300 \\
\hline 0 & 0.6259100 & -1.9534320 & 1.8774770 & $\mathrm{H}$ & -0.1255400 & 6.9255960 & -1.6959510 \\
\hline C & 2.1498690 & -3.3503990 & 2.9886180 & $\mathrm{H}$ & -3.9394660 & 4.1331820 & -0.1688100 \\
\hline C & 3.0032240 & -4.4555520 & 2.9648010 & C & 2.2131650 & 2.5362420 & -0.6132830 \\
\hline C & 2.0435920 & -2.5656860 & 4.1399760 & C & 2.8540110 & 2.2284070 & -1.8281190 \\
\hline C & 3.7516220 & -4.7756570 & 4.0912980 & C & 2.8305610 & 2.2208680 & 0.6150860 \\
\hline $\mathrm{H}$ & 3.0662160 & -5.0530220 & 2.0612480 & C & 4.1073020 & 1.6071690 & -1.7840670 \\
\hline C & 2.7966700 & -2.8881930 & 5.2637810 & $\mathrm{c}$ & 4.0679080 & 1.5805390 & 0.6055840 \\
\hline $\mathrm{H}$ & 1.3761300 & -1.7108650 & 4.1423960 & C & 4.7206580 & 1.2560040 & -0.5836950 \\
\hline C & 3.6489250 & -3.9911730 & 5.2395900 & $\mathrm{H}$ & 4.6192080 & 1.3765320 & -2.7164660 \\
\hline $\mathrm{H}$ & 4.4147100 & -5.6345600 & 4.0761120 & $\mathrm{H}$ & 4.5410480 & 1.3379490 & 1.5546790 \\
\hline $\mathrm{H}$ & 2.7183100 & -2.2803130 & 6.1592460 & C & -3.9005020 & -1.5407030 & 0.1202340 \\
\hline $\mathrm{H}$ & 4.2349120 & -4.2408470 & 6.1187860 & $\mathrm{C}$ & -3.3643790 & -2.6563700 & 0.7964660 \\
\hline $\mathrm{H}$ & 0.0775320 & -1.7227200 & 1.0299080 & C & -4.6041890 & -1.7102700 & -1.0827010 \\
\hline 0 & 1.46 & -3.7109960 & 0.7460690 & C & -3.5994050 & -3.9278870 & 0.2755920 \\
\hline & & & & C & -4.8071140 & -3.0045830 & -1.5686500 \\
\hline & R) $\mathrm{G}[\mathrm{M} 06$ & j(d)] & 369 & C & -4.3235890 & -4.1248310 & -0.9014150 \\
\hline 0 & 0.0219130 & 1.0766600 & 0.2411390 & $\mathrm{H}$ & -3.1965950 & -4.7928410 & 0.7979630 \\
\hline 0 & -2.0549480 & 0.2909850 & -0.9437710 & $\mathrm{H}$ & -5.3592670 & -3.1432640 & -2.4967600 \\
\hline C & -0.2451160 & 2.3779820 & -0.1378350 & C & 2.2268030 & 2.6268820 & 1.9519860 \\
\hline C & -1.5239080 & 2.8679270 & 0.0125280 & $\mathrm{C}$ & 6.0769980 & 0.5762950 & -0.5673230 \\
\hline C & 0.8537360 & 3.1571930 & -0.5951000 & C & 2.2413400 & 2.5806590 & -3.1755900 \\
\hline C & -1.79 & 4.1999900 & -0.4477530 & C & -2.5502800 & -2.5045770 & 2.0740980 \\
\hline C & 0.60 & 4.4599030 & -0.9428600 & C & -4.5605390 & -5.5210450 & -1.4454080 \\
\hline C & -0.7100770 & 4.9992630 & -0.9129800 & C & -5.1557950 & -0.5331740 & -1.8713850 \\
\hline $\mathrm{H}$ & 1.42 & 5.0997750 & -1.2593480 & $\mathrm{C}$ & -6.6875570 & -0.5749610 & -1.9228260 \\
\hline C & -2.76 & 0.7227270 & 0.1600170 & $\mathrm{H}$ & -7.0385320 & -1.4746210 & -2.4404080 \\
\hline C & -3.73 & -0.1697760 & 0.6934700 & $\mathrm{H}$ & -7.1137080 & -0.5783700 & -0.9145270 \\
\hline C & -2.5 & 2.0002590 & 0.6395490 & $\mathrm{H}$ & -7.0777490 & 0.2958640 & -2.4598960 \\
\hline C & -4.51 & 0.2819800 & 1.7264620 & C & -4.5548150 & -0.4787170 & -3.2803580 \\
\hline C & -3.30 & 2.4208620 & 1.7904100 & $\mathrm{H}$ & -3.4634160 & -0.4298870 & -3.2330530 \\
\hline C & -4.31 & 1.5603220 & 2.3097960 & $\mathrm{H}$ & -4.8362440 & -1.3617530 & -3.8647500 \\
\hline $\mathrm{H}$ & -5.25 & -0.3591460 & 2.1234300 & $\mathrm{H}$ & -4.9187890 & 0.4052460 & -3.8144480 \\
\hline C & -4.84 & 3.1842900 & 4.0343240 & C & -5.3644920 & -6.3770310 & -0.4603300 \\
\hline C & -3.81 & 4.0211270 & 3.5496470 & $\mathrm{H}$ & -5.5712380 & -7.3645250 & -0.8859970 \\
\hline C & -3.0 & 3.6514490 & 2.4601940 & $\mathrm{H}$ & -4.8068110 & -6.5235590 & 0.4713020 \\
\hline C & -5.0 & & 3.4285640 & $\mathrm{H}$ & -6.3171910 & -5.9009870 & -0.2105670 \\
\hline $\mathrm{H}$ & -5.2 & 3.4930020 & 4.8926500 & C & -3.2397710 & -6.2093050 & -1.8086580 \\
\hline $\mathrm{H}$ & -3. & 4. & 4.0489130 & $\mathrm{H}$ & -2.6696650 & -5.6124100 & -2.5265550 \\
\hline
\end{tabular}




-2.6180470
-3.4256880
-3.4342600
-4.2435380
-3.8848430
-2.8395950
-1.3605100
-1.6859340
-0.7884340
-0.6963790
3.0004990
4.0452210
2.9925610
2.5563230
2.1781390
1.6338370
3.1834960
1.6737830
6.1076920
7.0445160
6.0373750
5.2721930
7.1802810
7.1937180
7.0133130
8.1639190
2.3936410
3.4400830
1.9995420
1.8384430
2.8578890
3.9329650
2.3961750
2.7328430
-0.4847790
0.1234140
-0.3032160
1.1690150
6.2720790
1.1970270
-4.8705330
-5.1514910
-2.1440070
2.6422110
1.9648200
2.5114770

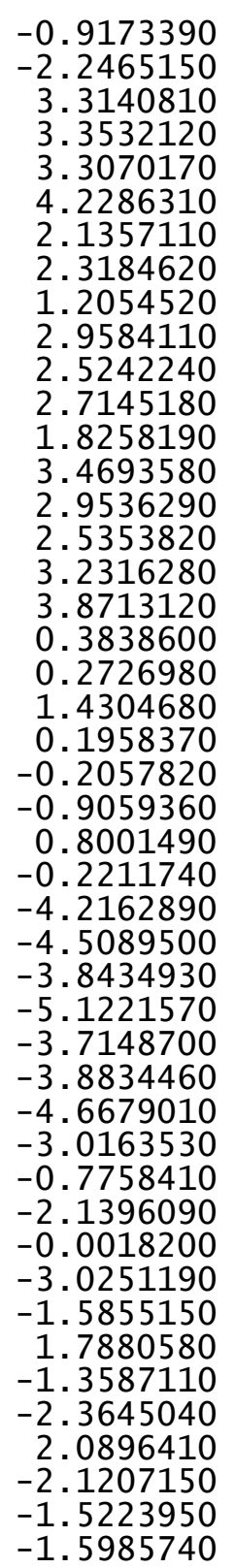

1.0174330
1.6207990
0.8895320
4.1942530
3.7977240
2.3276350
4.0273810
5.2320910
3.9665020
1.6697640
4.7420770
4.2160070
3.5673050
4.4189790
2.0631810
2.6610940
1.5485230
3.6245750
4.8264190
3.3401250
5.7500540
5.0244060
4.2686400
2.3985520
5.4724120
6.6852110
4.0522860
6.1953360
0.8624580
2.9498280
$-2.5438840$
$-1.7830310$
$-1.1261830$
$-3.1668010$
$-4.2604970$
$-4.1263170$
$-1.7834580$
$-3.3004040$
$-5.2537630$
$-4.3862510$
$-1.6566680$
$-0.9845280$
$-3.2357910$
$-4.1787630$
$-4.7913620$
$-2.3142840$
$-1.7043860$
$-2.3963250$
$-3.0844800$
$-1.7898800$
$-3.1520910$
$-3.5544410$
$-1.8577030$
$-1.2684170$
$-2.5350840$
$-3.6842320$
$-1.3830910$
$-2.5855360$
$-1.5924290$
$-2.8067310$

$-1.0302400$

$-3.1235910$

$-2.8666980$

$-3.3192630$

$-2.3254910$

$-1.9239650$

$-2.6864160$

$-3.6383360$

$-2.7512810$

$-2.7620450$

$-1.8647010$

$-3.4118890$

$-4.2161760$

$-1.4254630$

$-1.0979220$

1.4210670

1.7115400

2.5546750

2. 3722130

3.7805150

3.4082530

1.4142730

4.8143010

3.9125770

4.6278230

3.2671770

5.7658800

5.4362410

0.9552650

0.3227260

TS107 $(R, R) \quad G[M 06-2 X / 6-31 G(d)]=-3310.351911$

3.8792780

3.7460390

4.1580760

4.7114120

$-0.0956740$

0.0388450

$-1.3780890$

2.7378830

0.2128130

2.9553540

0.3905670

$-5.4179010$

$-1.4877040$

$-1.5800020$

$-0.6281860$

$-1.1301260$

$-0.1552150$

$-2.3936010$

$-2.7874150$

$-3.2365910$

$-4.0838480$

$-4.5102900$

$-4.9512670$

$-5.2033400$

$-0.5499020$

0.4226690

48820

0.0149800
-2.2396370
0.2725200

$-0.9516460$

$-0.1564690$

$-0.0557010$

$-0.6107780$

$-0.5597920$

$-0.9932890$

$-1.0107840$

$-1.3012000$

0.1287610

0.6859310

0.5683940

1.6823230
1.6857550 


$\begin{array}{lr}\mathrm{C} & 4.5474470 \\ \mathrm{H} & 5.3160290 \\ \mathrm{C} & 5.2848250 \\ \mathrm{C} & 4.3253390 \\ \mathrm{C} & 3.5132650 \\ \mathrm{C} & 5.3850100 \\ \mathrm{H} & 5.9253950 \\ \mathrm{H} & 4.2258720 \\ \mathrm{H} & 2.7741180 \\ \mathrm{H} & 6.0983960 \\ \mathrm{C} & 3.8108500 \\ \mathrm{C} & 2.7787210 \\ \mathrm{C} & 1.4720090 \\ \mathrm{C} & 3.5154510 \\ \mathrm{H} & 4.8459820 \\ \mathrm{H} & 3.0259260 \\ \mathrm{H} & 0.6699260 \\ \mathrm{H} & 4.3137120 \\ \mathrm{C} & -1.9486880 \\ \mathrm{C} & -2.6145560 \\ \mathrm{C} & -2.5726370 \\ \mathrm{C} & -3.9055880 \\ \mathrm{C} & -3.8538480 \\ \mathrm{C} & -4.5415980 \\ \mathrm{H} & -4.4418350 \\ \mathrm{H} & -4.3295370 \\ \mathrm{C} & 3.7268370 \\ \mathrm{C} & 3.0422340 \\ \mathrm{C} & 4.3759170 \\ \mathrm{C} & 3.0689290 \\ \mathrm{C} & 4.3697710 \\ \mathrm{C} & 3.7286810 \\ \mathrm{H} & 2.5454580 \\ \mathrm{H} & 4.8757510 \\ \mathrm{C} & -1.9213320 \\ \mathrm{C} & -5.9745950 \\ \mathrm{C} & -1.9879230 \\ \mathrm{C} & 2.2884860 \\ \mathrm{C} & 3.7188570 \\ \mathrm{C} & 5.0839780 \\ \mathrm{C} & 6.5911390 \\ \mathrm{H} & 6.7943140 \\ \mathrm{H} & 7.0286260 \\ \mathrm{H} & 7.0988580 \\ \mathrm{C} & 4.4765830 \\ \mathrm{H} & 3.4063180 \\ & \end{array}$

$\begin{array}{rr}-1.3007950 & 2.2128330 \\ 0.7192290 & 2.0952630 \\ -2.9230920 & 3.8612700 \\ -3.8406690 & 3.3726450 \\ -3.5096950 & 2.3171200 \\ -1.6791260 & 3.2953220 \\ -3.2012150 & 4.6921230 \\ -4.8143900 & 3.8420690 \\ -4.2195840 & 1.9630060 \\ -0.9521280 & 3.6745660 \\ -5.7800660 & -1.1350530 \\ -6.6555620 & -1.5467100 \\ -6.2450340 & -1.4883100 \\ -4.5291790 & -0.6539150 \\ -6.0990350 & -1.2061800 \\ -7.6443780 & -1.9197330 \\ -6.8993750 & -1.8196300 \\ -3.8606120 & -0.3488110 \\ -2.7051200 & -0.5886320 \\ -2.3711700 & -1.7841850 \\ -2.4880400 & 0.6555930 \\ -1.8441820 & -1.7006300 \\ -1.9337460 & 0.6882220 \\ -1.6101790 & -0.4810020 \\ -1.6069050 & -2.6181590 \\ -1.7647660 & 1.6511470 \\ 1.8294270 & 0.1772300 \\ 2.8287420 & 0.9019540 \\ 2.1448860 & -1.0266160 \\ 4.1363330 & 0.4209080 \\ 3.4702280 & -1.4704190 \\ 4.4794230 & -0.7607580 \\ 4.9113120 & 0.9762200 \\ 3.7216720 & -2.4011280 \\ -2.9403410 & 1.9548810 \\ -1.1062260 & -0.4681540 \\ -2.6104970 & -3.1495090 \\ 2.5153320 & 2.1874200 \\ 5.9085170 & -1.2695230 \\ 1.0914430 & -1.8638560 \\ 1.3650050 & -1.9305760 \\ 2.3243570 & -2.4193000 \\ 1.3984790 & -0.9277450 \\ 0.5816660 & -2.5029770 \\ 0.9940010 & -3.2678870 \\ 0.7766080 & -3.2124150\end{array}$

\begin{abstract}
4. 6109620
4. 9635420

4. 3669510

4. 3965970

3. 7978000

5. 3893370

2. 2949440

1.8343180

1. 6645020

2. 3009350

3. 1857180

4.0680920

3. 5277880

2. 6325090

1.0063740

1.2288500

0.4174200

0.3933520

$-2.5054880$

$-3.5821890$

$-2.3575800$

$-2.0276640$

$-2.0449460$

$-1.6576670$

$-3.0857390$

$-1.4722800$

$-6.3266720$

$-7.3227560$

$-6.3445130$

$-5.6089710$

$-6.9402890$

$-6.7342560$

$-6.8318330$

$-7.9789740$

$-2.2906030$

$-3.3411960$

$-2.0630770$

$-1.6843010$

$-2.4581010$

$-3.5456410$

$-1.9928000$

$-2.2144710$

0.5220840

$-0.1194980$

0.2642150

$-0.9022980$
\end{abstract}

1.9289780
0.1951250
6.8694560
7.8866900
6.8974950
6.5611860
6.3625340
5.6914420
6.3684140
7.3763790
2.7279390
2.0833560
3.7685880
2.5142310
3.3417190
4.3791840
3.3443670
2.9185840
-4.2976660
-4.2108230
-5.0440620
-4.6614070
-1.9203950
-0.9471900
-1.7987020
-2.2582900
-0.2686530
0.1706370
-0.8811450
0.5423180
-2.2930120
-2.8729430
-2.9617160
-1.9477110
-1.4961880
-1.5065450
-0.5124730
-1.6372860
-3.9549390
-3.9456250
-4.1402110
-4.7914310
0.1026210
-0.0446550
1.3432840
-2.6452230

$-3.8230400$

$-3.8369690$

$-0.2668860$

$-0.6711100$

0.6688170

$-0.0296240$

$-1.6129580$

$-2.3441830$

$-0.7166420$

$-2.0268530$

3. 4148470

3. 3910920

3.4565000

4. 3358230

2. 3467290

2.6213150

1.4262320

3. 1495630

2. 3698440

2. 5554990

1. 5826610

3. 2856700

3. 0870450

2.7711760

3. 4096960

3.9566990

0.7619880

0.6467930

1.6702850

0.9230690

$-0.5991480$

$-1.5037220$

0.2621490

$-0.6367210$

$-4.1548090$

$-4.4669150$

$-3.7400630$

$-5.0551320$

$-3.7237990$

$-3.8622170$

$-4.6977430$

$-3.0636010$

$-0.7263260$

$-2.0744380$

0.0917970
-3.0164250 


\begin{tabular}{|c|c|c|c|c|c|c|}
\hline-6.1036450 & -0.4734030 & -1.3570390 & C & -0.2166280 & -4.5103090 & -0.9932670 \\
\hline-0.8527930 & -3.0871120 & 1.7771250 & $\mathrm{C}$ & 1.1348340 & -4.9512780 & -1.0107330 \\
\hline 4.9484150 & 0.1187850 & -1.3809400 & $\mathrm{H}$ & -0.9949960 & -5.2033690 & -1.3011800 \\
\hline 4.3123880 & 5.9315430 & -2.1924330 & C & 2.8748180 & -0.5498830 & 0.1287800 \\
\hline 1.9947530 & 1.4611980 & 2.1487020 & C & 3.7483830 & 0.4227030 & 0.6859510 \\
\hline-2.6235990 & 1.6266370 & -1.6708820 & C & 2.7994160 & -1.8558600 & 0.5684240 \\
\hline-1.8675870 & 2.8437330 & -1.3486420 & $\mathrm{c}$ & 4.5980960 & 0.0150300 & 1.6823510 \\
\hline-2.3931770 & 0.7436420 & -1.0659800 & $\mathrm{C}$ & 3.6156200 & -2.2395970 & 1.6857950 \\
\hline-0.8546570 & 2.7062590 & -0.9875700 & C & 4.5474380 & -1.3007420 & 2.2128710 \\
\hline-1.8052620 & 1.6898220 & -2.8538170 & $\mathrm{H}$ & 5.3160030 & 0.7192880 & 2.0952910 \\
\hline-1.0353510 & 1.0440460 & -2.6686240 & C & 5.2848160 & -2.9230170 & 3.8613300 \\
\hline-4.3870230 & 3.0525060 & -2.7683360 & C & 4.3253400 & -3.8406060 & 3.3727090 \\
\hline-3.8206290 & 4.2769920 & -2.0465640 & C & 3.5132720 & -3.5096490 & 2.3171730 \\
\hline-2.3031640 & 4.1712970 & -1.8787910 & C & 5.3849950 & -1.6790550 & 3.2953700 \\
\hline-4.0946790 & 1.7749430 & -1.9785090 & $\mathrm{H}$ & 5.9253820 & -3.2011260 & 4.6921920 \\
\hline-5.4670280 & 3.1594850 & -2.9059240 & $\mathrm{H}$ & 4.2258770 & -4.8143220 & 3.8421430 \\
\hline-4.0596770 & 5.1938360 & -2.5930380 & $\mathrm{H}$ & 2.7741330 & -4.2195470 & 1.9630630 \\
\hline-1.7947790 & 4.2989380 & -2.8418390 & $\mathrm{H}$ & 6.0983730 & -0.9520480 & 3.6746120 \\
\hline-4.6337990 & 1.7851260 & -1.0203520 & C & 3.8109070 & -5.7800600 & -1.1349560 \\
\hline-4.4159950 & 0.8820030 & -2.5252570 & C & 2.7787890 & -6.6555710 & -1.5466090 \\
\hline-3.9392940 & 2.9697070 & -3.7657200 & C & 1.4720730 & -6.2450510 & -1.4882320 \\
\hline-4.2797270 & 4.3592060 & -1.0546760 & $\mathrm{C}$ & 3.5154930 & -4.5291660 & -0.6538430 \\
\hline-1.9099070 & 4.9410680 & -1.2098590 & $\mathrm{H}$ & 4.8460430 & -6.0990220 & -1.2060660 \\
\hline-2.6564080 & 2.2947310 & 1.4181560 & $\mathrm{H}$ & 3.0260060 & -7.6443920 & -1.9196110 \\
\hline-1.7843410 & 1.3465300 & 1.6091300 & $\mathrm{H}$ & 0.6700000 & -6.8994040 & -1.8195510 \\
\hline-3.8720480 & 2.2006380 & 2.2691620 & $\mathrm{H}$ & 4.3137460 & -3.8605880 & -0.3487420 \\
\hline-4.9280010 & 3.0846170 & 2.0339550 & C & -1.9486530 & -2.7051260 & -0.5886940 \\
\hline-3.9893270 & 1.2049120 & 3.2416090 & $\mathrm{C}$ & -2.6144670 & -2.3711650 & -1.7842730 \\
\hline-6.1068700 & 2.9615560 & 2.7601630 & $\mathrm{C}$ & -2.5726450 & -2.4880290 & 0.6555080 \\
\hline-4.8127150 & 3.8545830 & 1.2781770 & $\mathrm{C}$ & -3.9054890 & -1.8441430 & -1.7007690 \\
\hline-5.1693020 & 1.0865450 & 3.9672220 & $\mathrm{C}$ & -3.8538430 & -1.9337030 & 0.6880880 \\
\hline-3.1600580 & 0.5293290 & 3.4134360 & C & -4.5415410 & -1.6101270 & -0.4811660 \\
\hline-6.2289550 & 1.9594710 & 3.7224730 & $\mathrm{H}$ & -4.4416950 & -1.6068540 & -2.6183190 \\
\hline-6.9313070 & 3.6424900 & 2.5754640 & $\mathrm{H}$ & -4.3295620 & -1.7647000 & 1.6509920 \\
\hline-5.2645140 & 0.3112220 & 4.7209410 & C & 3.7268000 & 1.8294570 & 0.1772390 \\
\hline-7.1520120 & 1.8607270 & 4.2854340 & $\mathrm{C}$ & 3.0421930 & 2.8287700 & 0.9019620 \\
\hline-0.9536790 & 1.4000240 & 1.0020090 & C & 4.3758530 & 2.1449120 & -1.0266230 \\
\hline-2.5399340 & 3.2029780 & 0.5834970 & C & 3.0688540 & 4.1363540 & 0.4208960 \\
\hline R) $\mathrm{G}[\mathrm{M} 06$ & (d) $]=-3$ & 9 & c & $\begin{array}{l}4.3696 / 100 \\
3.7285780\end{array}$ & $\begin{array}{l}3.4702480 \\
4.4794400\end{array}$ & $\begin{array}{l}-1.4704440 \\
-0.7607860\end{array}$ \\
\hline 0.1459460 & -1.1301300 & 0.2725070 & $\mathrm{H}$ & 2.5453780 & 4.9113310 & 0.9762070 \\
\hline 2.1093910 & -0.1552140 & -0.9516350 & $\mathrm{H}$ & 4.8756360 & 3.7216880 & -2.4011640 \\
\hline 0.5016790 & -2.3936000 & -0.1564720 & $\mathrm{C}$ & -1.9213910 & -2.9403790 & 1.9548050 \\
\hline 1.8185500 & -2.7874050 & -0.0556760 & $\mathrm{C}$ & -5.9745360 & -1.1061610 & -0.4683840 \\
\hline-0.5503720 & -3.2366040 & -0.6107870 & $\mathrm{C}$ & -1.9877910 & -2.6105270 & -3.1495720 \\
\hline 2.169789 & -4.0838430 & -0.5597430 & $\mathrm{C}$ & 2.2884780 & 2.5153670 & 2.1874500 \\
\hline
\end{tabular}




\begin{tabular}{|c|c|c|}
\hline $\begin{array}{l}3.7187140 \\
5.0839200 \\
6.5910750 \\
6.7942290 \\
7.0285760 \\
7.0988000 \\
4.4765090 \\
3.4062480 \\
4.6108680 \\
4.9634740 \\
4.3667840 \\
4.3964030 \\
3.7976340 \\
5.3891780 \\
2.2947880 \\
1.8341830 \\
1.6643450 \\
2.3007470 \\
3.1857340 \\
4.0681220 \\
3.5277810 \\
2.6325500 \\
1.0063540 \\
1.2288160 \\
0.4173620 \\
0.3933700 \\
2.5054030 \\
3.5821330 \\
2.3573180 \\
2.0276190 \\
2.0452340 \\
1.6580540 \\
3.0860670 \\
1.4725920 \\
6.3266970 \\
7.3227700 \\
6.3446080 \\
5.6090050 \\
6.9402270 \\
6.7341360 \\
6.8318350 \\
7.9789080 \\
2.2904490 \\
3.3410250 \\
2.0629770\end{array}$ & $\begin{array}{r}5.9085260 \\
1.0914700 \\
1.3650570 \\
2.3244080 \\
1.3985490 \\
0.5817200 \\
0.9940030 \\
0.7765940 \\
1.9289760 \\
0.1951280 \\
6.8695000 \\
7.8867280 \\
6.8975410 \\
6.5612620 \\
6.3624960 \\
5.6913840 \\
6.3683650 \\
7.3763380 \\
2.7280140 \\
2.0834510 \\
3.7686710 \\
2.5143090 \\
3.3417320 \\
4.3792100 \\
3.3443390 \\
2.9186080 \\
-4.2978310 \\
-4.2111490 \\
-5.0441180 \\
-4.6616110 \\
-1.9205890 \\
-0.9472940 \\
-1.7990700 \\
-2.2585190 \\
-0.2686610 \\
0.1706390 \\
-0.8812110 \\
0.5422970 \\
-2.2929340 \\
-2.8728120 \\
-2.9616900 \\
-1.9476240 \\
-1.4962520 \\
-1.5066470 \\
-0.5125210 \\
-1.6373550\end{array}$ & $\begin{array}{r}-1.2695740 \\
-1.8638610 \\
-1.9306040 \\
-2.4193400 \\
-0.9277790 \\
-2.5030040 \\
-3.2678830 \\
-3.2123960 \\
-3.8230490 \\
-3.8369610 \\
-0.2669550 \\
-0.6711970 \\
0.6687490 \\
-0.0296890 \\
-1.6130120 \\
-2.3442330 \\
-0.7166970 \\
-2.0269170 \\
3.4148520 \\
3.3910880 \\
3.4564810 \\
4.3358450 \\
2.3467760 \\
2.6213250 \\
1.4263030 \\
3.1496460 \\
2.3695590 \\
2.5551180 \\
1.5823070 \\
3.2853900 \\
3.0870830 \\
2.7713690 \\
3.4096730 \\
3.9567400 \\
0.7617830 \\
0.6465420 \\
1.6700400 \\
0.9229710 \\
-0.5995160 \\
-1.5041110 \\
0.2617490 \\
-0.6371400 \\
-4.1549150 \\
-4.4670760 \\
-3.7401790 \\
-5.0552030\end{array}$ \\
\hline
\end{tabular}

-2.4579480
-3.5454850
-1.9926220
-2.2143310
0.5220910
-0.1194700
0.2642050
-0.9021710
-6.1035220
-0.8528160
4.9483800
4.3122410
1.9947650
-2.6234850
-1.8673470
-2.3930780
-0.8544600
-1.8052330
-1.0352930
-4.3868980
-3.8204200
-2.3029470
-4.0945730
-5.4669050
-4.0594780
-1.7945960
-4.6336330
-4.4159610
-3.9392240
-4.2794360
-1.9096160
-2.6563710
-1.7843350
-3.8721410
-4.9280360
-3.9895910
-6.1070160
-4.8126130
-5.1696740
-3.1603690
-6.2292690
-6.9314070
-5.2650140
-7.1524090
-0.9537380
-2.5397120
$-3.7238380$ $-3.8622830$ $-4.6977660$ $-3.0636120$ $-0.7262960$ $-2.0744310$ 0.0918280 $-3.0164550$ $-1.3572400$ 1. 7771250 $-1.3809330$ $-2.1924870$ 2. 1487570 $-1.6708220$ $-1.3487640$ $-1.0658420$ $-0.9875770$ $-2.8538680$ $-2.6685780$ $-2.7681730$ $-2.0464110$ $-1.8787410$ $-1.9783610$ $-2.9057060$ $-2.5928390$ $-2.8418010$ $-1.0201720$ $-2.5250840$ $-3.7655810$ $-1.0544840$ $-1.2097580$

1. 4184750

1.6094960 2. 2692970 2.0340450 3.2416110 2. 7600660 1. 2783800 3.9670430 3. 4134840 3. 7222440 2. 5753270 4.7206600 4. 2850610 1.0024010 0.5839450 
144

$\operatorname{TS} 109(R, R) \quad G[M 06-2 X / 6-31 G(d)]=-3310.350254$

$\begin{array}{lrrr}\mathrm{O} & -0.0921150 & 1.0969210 & 0.2962140 \\ \mathrm{O} & -2.0926640 & 0.1325510 & -0.8775270 \\ \mathrm{C} & -0.3196840 & 2.3378290 & -0.2661450 \\ \mathrm{C} & -1.6034770 & 2.8366190 & -0.2789730 \\ \mathrm{C} & 0.8193380 & 3.0499650 & -0.7354800 \\ \mathrm{C} & -1.8268730 & 4.0909730 & -0.9384210 \\ \mathrm{C} & 0.6045450 & 4.2910860 & -1.2775200 \\ \mathrm{C} & -0.7063190 & 4.8215770 & -1.4259410 \\ \mathrm{H} & 1.4487450 & 4.8857410 & -1.6171400 \\ \mathrm{C} & -2.8479330 & 0.7213400 & 0.1170600 \\ \mathrm{C} & -3.8213390 & -0.0988670 & 0.7487250 \\ \mathrm{C} & -2.6783550 & 2.0617330 & 0.4015560 \\ \mathrm{C} & -4.6635580 & 0.4927160 & 1.6549320 \\ \mathrm{C} & -3.4928830 & 2.6422050 & 1.4327700 \\ \mathrm{C} & -4.5177520 & 1.8539470 & 2.0286280 \\ \mathrm{H} & -5.4497360 & -0.0979880 & 2.1189250 \\ \mathrm{C} & -5.1639600 & 3.7170780 & 3.4438930 \\ \mathrm{C} & -4.1139720 & 4.4870610 & 2.8909150 \\ \mathrm{C} & -3.3011720 & 3.9662620 & 1.9154570 \\ \mathrm{C} & -5.3529250 & 2.4264430 & 3.0241130 \\ \mathrm{H} & -5.8035760 & 4.1447060 & 4.2094070 \\ \mathrm{H} & -3.9449130 & 5.4985540 & 3.2468820 \\ \mathrm{H} & -2.4927340 & 4.5659390 & 1.5126530 \\ \mathrm{H} & -6.1373940 & 1.8107000 & 3.4562860 \\ \mathrm{C} & -3.2998020 & 5.8256720 & -1.7876900 \\ \mathrm{C} & -2.1837010 & 6.5674240 & -2.2406650 \\ \mathrm{C} & -0.9176320 & 6.0715540 & -2.0648760 \\ \mathrm{C} & -3.1269820 & 4.6212120 & -1.1527790 \\ \mathrm{H} & -4.3018840 & 6.2103620 & -1.9494140 \\ \mathrm{H} & -2.3342690 & 7.5211590 & -2.7363810 \\ \mathrm{H} & -0.0517850 & 6.6222020 & -2.4231710 \\ \mathrm{H} & -3.9886690 & 4.0539990 & -0.8163040 \\ \mathrm{C} & 2.1685640 & 2.4305930 & -0.5554830 \\ \mathrm{C} & 2.9262810 & 1.9908580 & -1.6537180 \\ \mathrm{C} & 2.6584780 & 2.2474490 & 0.7568070 \\ \mathrm{C} & 4.1655790 & 1.3759420 & -1.4199360 \\ \mathrm{C} & 3.8828170 & 1.6141000 & 0.9369250 \\ \mathrm{C} & 4.6607440 & 1.1644820 & -0.1354430 \\ \mathrm{H} & 4.7529130 & 1.0605130 & -2.2772540 \\ \mathrm{H} & 4.2544130 & 1.4717870 & 1.9478470 \\ \mathrm{C} & -3.9157820 & -1.5536680 & 0.4147920 \\ \mathrm{C} & -3.3086350 & -2.5057210 & 1.2560830 \\ \mathrm{C} & -4.6133550 & -1.9617580 & -0.7355390 \\ \mathrm{C} & -3.4641180 & -3.8593190 & 0.9503180\end{array}$

-4.7353930
-4.1765210
-3.0076520
-5.2800880
1.9337500
5.9945800
2.4691120
-2.5112040
-4.3192240
-5.2329900
-6.7578490
-7.0439920
-7.1959000
-7.1966660
-4.6182950
-3.5339240
-4.8265710
-5.0366680
-3.6213540
-3.6877550
-4.0903140
-2.5651210
-5.7900800
-6.2909320
-6.3276540
-5.8736740
-3.4082940
-4.2297240
-3.8410310
-2.8271770
-1.3036480
-1.6109380
-0.7233040
-0.6524450
2.5309370
3.5901940
2.4573710
2.0061220
1.9493470
1.5836610
2.9559490
1.3057470
6.8857570
7.8163570
7.1397880
6.3996950

$-1.0026720$

$-0.1665070$

1. 5961120

$-1.8913400$

1.9650300

0.1451780

$-3.0883090$

2.4863190

$-0.4762650$

$-1.6989380$

$-1.7470630$

$-2.1191340$

$-0.7517120$

$-2.4141120$

$-3.0973770$

$-3.0551220$

$-3.5332610$

$-3.7673380$

$-1.7929330$

$-1.9844340$

$-2.6348560$

$-1.7689560$

$-0.5074310$

0.4351980

$-1.3135480$

$-0.6808270$

3.7315380

3. 6203300

3. 9125120

4.6154170

2. 7489070

3. 1438630

1.8369040

3.4977330

2. 3030780

2. 5670090

1. 4480200

3.1509970

3. 1872570

2. 9262850

3. 6128030

3. 9706200

1.0476680

1. 2775310

0.5438800

1.9958070 


$\begin{array}{rrr}6.7748490 & 0.1019780 & -1.1130730 \\ 6.2106290 & -0.5691140 & -1.7665400 \\ 7.0412360 & 0.9942000 & -1.6915410 \\ 7.7033980 & -0.4055130 & -0.8343610 \\ 2.6861540 & 0.9897400 & -3.9861660 \\ 3.7510350 & 0.7811750 & -4.1417980 \\ 2.2134140 & 0.1001900 & -3.5649810 \\ 2.2455970 & 1.1726110 & -4.9716360 \\ 3.1853440 & 3.4277440 & -3.6903070 \\ 4.2656500 & 3.2467210 & -3.7370740 \\ 2.8288680 & 3.6208390 & -4.7076310 \\ 3.0277140 & 4.3305410 & -3.0932600 \\ -0.5181630 & -0.1805830 & -0.6229110 \\ 0.1315100 & -0.1425870 & -1.9753190 \\ -0.3130230 & -1.3793710 & 0.2693840 \\ 1.3937720 & 2.4102890 & -3.0707070 \\ 5.7726690 & -0.4436790 & 0.6977240 \\ 0.8845820 & 2.9830730 & 1.7048150 \\ -5.0139360 & 0.0472510 & -1.3358690 \\ -3.8226670 & -6.3214340 & 0.3315940 \\ -2.1260660 & -1.0845660 & 2.3083040 \\ 2.4970250 & -1.9556570 & -1.6761950 \\ 1.6946820 & -3.0807830 & -1.1839820 \\ 2.3726570 & -1.0087020 & -1.1403820 \\ 0.7263010 & -2.8406290 & -0.7584370 \\ 1.5811350 & -2.0616200 & -2.7826240 \\ 0.8783990 & -1.3462400 & -2.5911730 \\ 4.0704960 & -3.5952000 & -2.7633130 \\ 3.4858010 & -4.7090260 & -1.8922870 \\ 1.9981910 & -4.4784140 & -1.6160130 \\ 3.9251440 & -2.2362310 & -2.0756020 \\ 5.1268250 & -3.7908300 & -2.9702810 \\ 3.6156950 & -5.6834300 & -2.3718970 \\ 1.4015850 & -4.6563160 & -2.5186230 \\ 4.5336900 & -2.2004970 & -1.1604410 \\ 4.2605540 & -1.4204130 & -2.7252470 \\ 3.5505490 & -3.5673380 & -3.7281350 \\ 4.0198400 & -4.7435480 & -0.9358660 \\ 1.6154500 & -5.1539860 & -0.8468160 \\ 2.7204400 & -2.3049290 & 1.4373290 \\ 1.8525430 & -1.3558280 & 1.6450970 \\ 4.0225530 & -2.1150760 & 2.1304750 \\ 5.0873050 & -2.9599960 & 1.8072960 \\ 4.2031370 & -1.0721410 & 3.0412750 \\ 6.3376800 & -2.7468520 & 2.3777160 \\ 4.9230890 & -3.7702960 & 1.1043660\end{array}$
5.4538360
3. 3651130
6.5218650
7.1683190
7.4994610
0.9773510
2. 5331250

144

TS110 $(R, R) \quad G[M 06-2 x / 6-31 G(d)]=-3310.355067$

$\begin{array}{lll}\mathrm{O} & 0.3949640 & 0 \\ \mathrm{O} & -1.8137570 & 0 \\ \mathrm{C} & 0.4291680 & 2 . \\ \mathrm{C} & -0.6836750 & \end{array}$

0.8820550

0.9364260

2. 2590060

2.9948450

2. 8477990

4.4205570

4.2180520

5.0313860

4.6974580

1. 2589990

0.5251540

2. 2887180

0.9025460

2. 6002540

1. 9179750

0.3993170

3. 1709870

3.8174370

3. 5407100

2. 2382800

3. 4051790

4. 5351340

4.0353180

1.7154790

6.6151940

7.2232800

6.4447680

5.2534900

7.2324580

8.3023020

6.8937430

4.7986350

2.0065430

1.7948230

1. 4770330

1. 1124230
3. 6125900

3. 2834600

3. 2752440

2. 1218700

4. 3176490

3. 7158780

1.1152450

0.7042470

0.1018020

0.4585640

$-0.3094810$

0.3113000

$-0.3407850$

$-0.0630260$

$-0.6021510$

0.1951830

0.5583530

0.9463100

1.7079680

2. 1996320

2. 5576090

1. 9914390

4.6332880

4. 3005000

3. 1181430

3.7809000

5.5687690

4.9904330

2.8797020

4.0321550

0.3595900

0.0288840

$-0.1819630$

0.4976540

0.4989560

$-0.0709220$

$-0.4583870$

0.7405390

$-0.6604190$

$-2.0148990$

0.3597790
-2.3070070 


$\begin{array}{rrr}4.8219280 & 0.7991080 & 0.0161110 \\ 5.2068430 & 0.6286310 & -1.3097920 \\ 4.6311640 & 0.9490580 & -3.3473920 \\ 5.4482390 & 0.4066080 & 0.8131780 \\ -4.0747430 & -0.6023930 & -0.2920040 \\ -3.9502310 & -1.9336040 & 0.1618440 \\ -4.6729010 & -0.3316240 & -1.5344700 \\ -4.4820650 & -2.9575240 & -0.6206880 \\ -5.1816290 & -1.3943100 & -2.2853820 \\ -5.1064860 & -2.7107000 & -1.8438800 \\ -4.3990660 & -3.9838200 & -0.2694090 \\ -5.6549770 & -1.1882470 & -3.2438440 \\ 3.3002620 & 1.6352290 & 1.8321550 \\ 6.5149370 & -0.0334120 & -1.7129200 \\ 2.3070020 & 2.3196270 & -3.1487730 \\ -3.2657660 & -2.2878630 & 1.4761040 \\ -5.6724040 & -3.8474010 & -2.6737870 \\ -4.8094020 & 1.0800300 & -2.0839620 \\ -6.2844890 & 1.4903610 & -2.1726160 \\ -6.8304860 & 0.8428400 & -2.8676150 \\ -6.7707290 & 1.4206980 & -1.1943950 \\ -6.3751050 & 2.5211290 & -2.5309800 \\ -4.1135920 & 1.2217300 & -3.4422270 \\ -3.0603030 & 0.9376650 & -3.3692450 \\ -4.5913140 & 0.5901950 & -4.1994200 \\ -4.1701750 & 2.2586440 & -3.7897990 \\ -6.7769690 & -4.5961550 & -1.9197390 \\ -7.2110360 & -5.3800150 & -2.5491740 \\ -6.3770640 & -5.0734990 & -1.0182920 \\ -7.5765500 & -3.9154040 & -1.6133150 \\ -4.5692210 & -4.8139260 & -3.1198290 \\ -3.7875950 & -4.2886280 & -3.6763520 \\ -4.1014470 & -5.2939030 & -2.2529940 \\ -4.9811620 & -5.6021850 & -3.7586770 \\ -4.2997490 & -2.5376090 & 2.5828460 \\ -4.9307480 & -1.6621580 & 2.7580330 \\ -4.9547640 & -3.3725040 & 2.3076400 \\ -3.8012630 & -2.7928400 & 3.5241730 \\ -2.3479700 & -3.5111060 & 1.3541930 \\ -2.9222050 & -4.4348750 & 1.2232640 \\ -1.6605530 & -3.4069860 & 0.5106850 \\ -1.7604840 & -3.6221820 & 2.2720400 \\ 4.3537780 & 2.4810780 & 2.5570430 \\ 5.3334050 & 1.9907920 & 2.5388400 \\ 4.4594490 & 3.4632960 & 2.0856350 \\ 4.0715020 & 2.6293830 & \\ -4 & & \\ -4 & -6044570\end{array}$

3.1188790

139970

4. 0377360

2. 8580930

7.1345220

8.0070860

7.4742730

6.4257780

7. 5193700

7.1144280

7.7532330

8.4517110

2. 2334740

3. 1679180

2. 0250410

1.4350730

2. 8103100

3. 8439450

2. 1925520

2.7845650

$-0.4725400$

0.1860390

$-0.7661810$

1. 2902200

6. 2992920

2. 3457080

$-4.3203830$

$-6.1177440$

$-2.6388070$

2. 3329340

1. 2855550

2. 1407390

0.2639210

1.8718160

1.1897260

3. 9869570

3. 0064580

1. 5589690

3. 7657170

5. 0186040

3. 1848760

1. 3145430

4.0083990

4.4013500

3. 8540550

3. 1513490
0.2715730
-0.2923530
-0.3246950
0.4009010
-0.9065270
-1.4356370
-0.2984430
-1.6495780
1.0356340
1.6395740
1.7081670
0.5732010
1.3523610
1.3469420
0.3327230
1.6640570
3.6859190
3.6030120
4.0459150
4.4374950
0.0330360
0.0296000
-1.2784010
2.4390910
-0.6754220
2.1650430
1.7723420
-3.4046250
-1.4393630
-2.0732390
-3.1019640
-1.1962930
-2.7591210
-1.8808700
-1.1278770
-3.8190210
-4.9149610
-4.4664060
-2.5475300
-4.1602060
-5.8343200
-4.4262800
-2.7261260
-1.7314870
-3.5890160
-5.1478940

2. 5080910

2. 0272620

2. 4640610

3. 5643600

$-0.6220190$

$-1.0164840$

0.2235730

$-0.2415920$

$-2.1673620$

$-2.9845980$

$-1.3346980$

$-2.5078350$

$-4.3347790$

$-4.9073850$

$-4.0053980$

$-5.0148750$

$-3.6355090$

3. 9913140

$-4.4651250$

$-2.8423140$

$-0.8886060$

$-2.2336450$

$-0.2017040$

$-2.7612330$

$-2.5792590$

1. 9076430

$-1.3924510$

$-3.5738950$

1.7683810

$-1.2892590$

$-1.3039420$

$-0.6605000$

$-1.1867870$

$-2.6406080$

$-2.6015990$

$-2.0545530$

$-1.6354590$

$-1.8464890$

$-1.2333450$

$-1.9241870$

$-2.2005670$

$-2.9146900$

0.1768520

$-1.5847430$

$-3.1184510$

$-0.5744180$ 


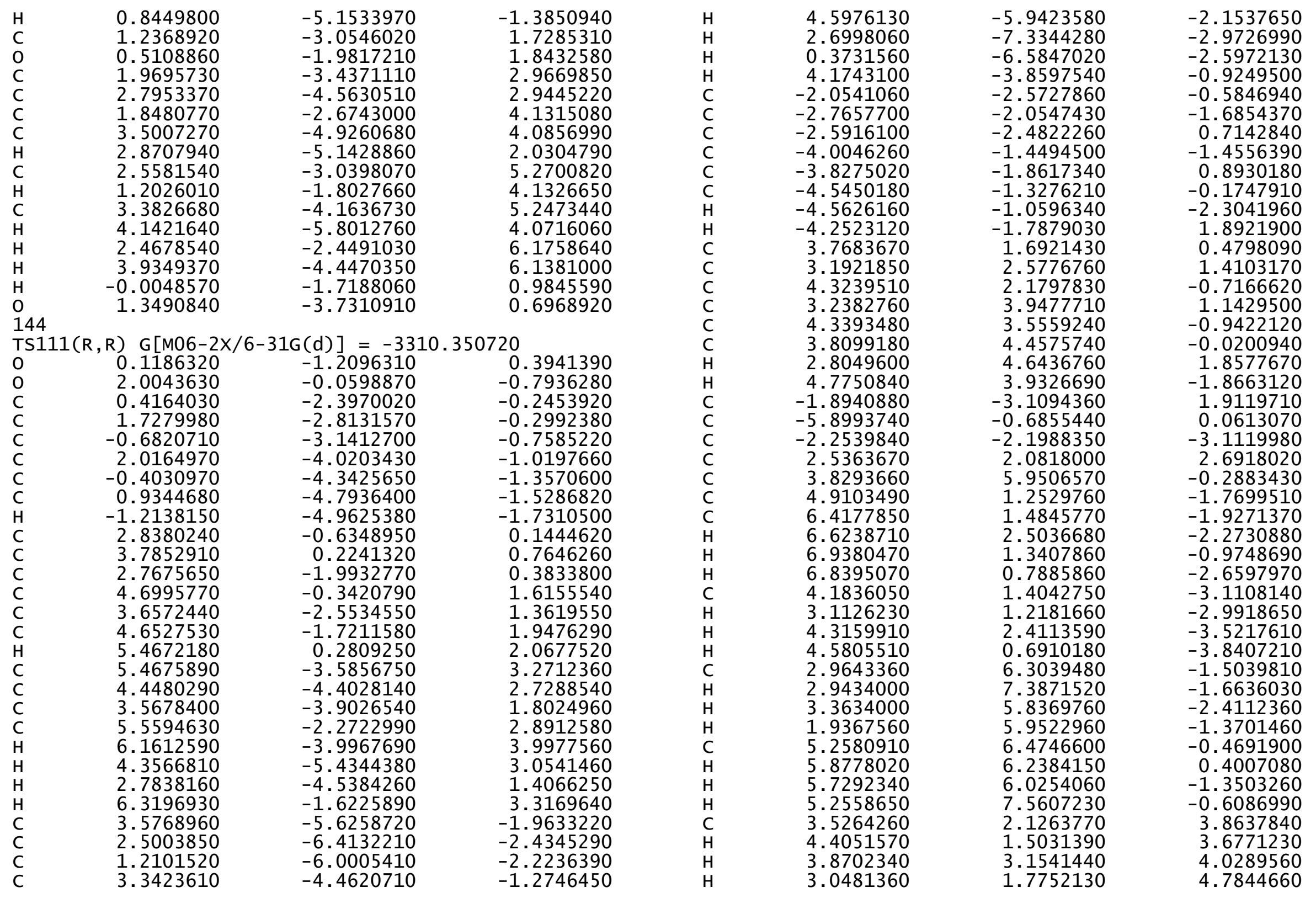




$\begin{array}{rrr}1.2703980 & 2.8656370 & 3.0549500 \\ 1.5078290 & 3.8752550 & 3.4085920 \\ 0.5993980 & 2.9381580 & 2.1965870 \\ 0.7410930 & 2.3535990 & 3.8652630 \\ -2.5152910 & -4.4816100 & 2.2059830 \\ -3.5768470 & -4.3758440 & 2.4573790 \\ -2.4380750 & -5.1402560 & 1.3348650 \\ -2.0097410 & -4.9632660 & 3.0497230 \\ -1.9153840 & -2.2189250 & 3.1558720 \\ -1.5407610 & -1.2175460 & 2.9216410 \\ -2.9271310 & -2.1286140 & 3.5687030 \\ -1.2842160 & -2.6537050 & 3.9373890 \\ -7.0272980 & -1.6589070 & -0.3035820 \\ -8.0069720 & -1.2127390 & -0.1017420 \\ -6.9809780 & -1.9132910 & -1.3686630 \\ -6.9450450 & -2.5881890 & 0.2679390 \\ -6.0562360 & 0.6330200 & -0.6978340 \\ -5.2205480 & 1.3049570 & -0.4776200 \\ -6.0899820 & 0.4695330 & -1.7813770 \\ -6.9848210 & 1.1359390 & -0.4068860 \\ -2.4731360 & -0.9585010 & -3.9819070 \\ -3.5369300 & -0.7352280 & -4.1217750 \\ -1.9788570 & -0.0856370 & -3.5520950 \\ -2.0467560 & -1.1292980 & -4.9758440 \\ -2.9172670 & -3.4144640 & -3.7768940 \\ -3.9984970 & -3.2541290 & -3.8628090 \\ -2.5153210 & -3.5726860 & -4.7832130 \\ -2.7653340 & -4.3294380 & -3.1973760 \\ 0.4254610 & 0.1441530 & -0.4570460 \\ -0.2839350 & 0.1508750 & -1.7791090 \\ 0.1942750 & 1.2762480 & 0.5133680 \\ -1.1742330 & -2.3689200 & -3.0652810 \\ -5.9727910 & -0.4630040 & 1.1350540 \\ -0.8441580 & -3.2759750 & 1.6577810 \\ 4.7701570 & 0.2199060 & -1.4371900 \\ 3.3963190 & 6.4462140 & 0.5900950 \\ 2.2369030 & 1.0406910 & 2.5300440 \\ -2.5562880 & 2.0744630 & -2.0455630 \\ -1.8164800 & 2.9379450 & -1.1215850 \\ -2.6933490 & 1.0413330 & -1.7130060 \\ -1.0092240 & 2.4857980 & -0.5567760 \\ -1.3486940 & 2.2342790 & -2.8240020 \\ -0.7648910 & 1.4540610 & -2.5463050 \\ -3.5366920 & 4.1404820 & -3.1097510 \\ -3.1779430 & 4.9293530 & -1.8487020 \\ -1.8663080 & 4.4242830 & -1.2454160\end{array}$

-3.7469660
-4.4468110
-3.0879270
-1.0120310
-4.6202250
-3.9305500
-2.7336850
-3.9772140
-1.6757170
-3.0561830
-2.0328090
-4.2703160
-5.4301790
-4.2619520
-6.5876380
-5.4137530
-5.4213760
-3.3505750
-6.5847120
-7.4916080
-5.4181790
-7.4900330
-1.1789730
-3.0789250

$$
\begin{array}{r}
2.6641530 \\
4.5427280 \\
5.9971560 \\
4.7062110 \\
2.5594470 \\
2.0624630 \\
4.2360960 \\
4.8134880 \\
4.8436180 \\
1.9270620 \\
1.1910130 \\
1.6770080 \\
2.4111420 \\
0.7005770 \\
2.1516530 \\
3.1700600 \\
0.4433380 \\
0.1461670 \\
1.1639110 \\
2.7172390 \\
-0.3194450 \\
0.9590560 \\
1.3001080 \\
2.7710630
\end{array}
$$

$-2.7657800$

$-3.5641540$ $-2.0673780$ $-1.8736530$ $-2.1133040$ $-3.6626730$ $-3.8499940$ $-1.1065640$ $-0.2538730$

1.4573600

1.7867610

2. 2840990

2.0285830

3. 2827450

2.7535720

1. 2529380

4.0067480

3.4745340

3.7383690

2.5518080

4.7790250

4. 3015240

1.2331280

0.5519390

$\operatorname{TS} 112(\mathrm{R}, \mathrm{R}) \mathrm{G}[\mathrm{M} 06-2 \mathrm{X} / 6-31 \mathrm{G}(\mathrm{d})]=-3310.352437$

$\begin{array}{lrcr}\text { TS112 }(\mathrm{R}, \mathrm{R}) \mathrm{G}[\mathrm{M} 06-2 \mathrm{X} / 6-31 \mathrm{G}(\mathrm{d})]=-3310.352437 \\ \mathrm{O} & 0.0912170 & 1.0589300 & 0.2199390 \\ \mathrm{O} & -2.0422110 & 0.2894910 & -0.8717010 \\ \mathrm{C} & -0.1531040 & 2.3511010 & -0.2026580 \\ \mathrm{C} & -1.4125460 & 2.8831660 & -0.0321350 \\ \mathrm{C} & 0.9510590 & 3.0807440 & -0.7242900 \\ \mathrm{C} & -1.6589700 & 4.2035910 & -0.5361620 \\ \mathrm{C} & 0.7267830 & 4.3760450 & -1.1142850 \\ \mathrm{C} & -0.5707080 & 4.9535760 & -1.0649710 \\ \mathrm{H} & 1.5534820 & 4.9799550 & -1.4794540 \\ \mathrm{C} & -2.7058640 & 0.7825480 & 0.2345550 \\ \mathrm{C} & -3.6824230 & -0.0615410 & 0.8284170 \\ \mathrm{C} & -2.4507180 & 2.0704640 & 0.6590400 \\ \mathrm{C} & -4.4195450 & 0.4499520 & 1.8660610 \\ \mathrm{C} & -3.1527020 & 2.5550330 & 1.8140290 \\ \mathrm{C} & -4.1674350 & 1.7428920 & 2.3942680 \\ \mathrm{H} & -5.2079630 & -0.1532410 & 2.3093410 \\ \mathrm{C} & -4.6033910 & 3.4443450 & 4.0694820 \\ \mathrm{C} & -3.5632020 & 4.2333890 & 3.5244690 \\ \mathrm{C} & -2.8575480 & 3.8026500 & 2.4291070 \\ \mathrm{C} & -4.8913940 & 2.2236910 & 3.5171890\end{array}$




$\begin{array}{rrr}-5.1581520 & 3.8013710 & 4.9313330 \\ -3.3167340 & 5.1867410 & 3.9813700 \\ -2.0551060 & 4.4132760 & 2.0301080 \\ -5.6711210 & 1.5938360 & 3.9374420 \\ -3.1496010 & 6.0532520 & -1.0444710 \\ -2.0608390 & 6.8112270 & -1.5358350 \\ -0.8020800 & 6.2685260 & -1.5477130 \\ -2.9550550 & 4.7852310 & -0.5569860 \\ -4.1489220 & 6.4768730 & -1.0590550 \\ -2.2281490 & 7.8150110 & -1.9132490 \\ 0.0410690 & 6.8316950 & -1.9390650 \\ -3.7977380 & 4.2078060 & -0.1914850 \\ 2.2928000 & 2.4230970 & -0.7577180 \\ 2.8830430 & 2.0401020 & -1.9768770 \\ 2.9441800 & 2.1512280 & 0.4631670 \\ 4.1232870 & 1.3927210 & -1.9446440 \\ 4.1656340 & 1.4811890 & 0.4434980 \\ 4.7698740 & 1.0852650 & -0.7495630 \\ 4.5972570 & 1.1048590 & -2.8812170 \\ 4.6641680 & 1.2708670 & 1.3872500 \\ -3.9094950 & -1.4456710 & 0.3104440 \\ -3.3973430 & -2.5526140 & 1.0126110 \\ -4.6545170 & -1.6332680 & -0.8681380 \\ -3.6965810 & -3.8347380 & 0.5456310 \\ -4.9210070 & -2.9326880 & -1.2979620 \\ -4.4606930 & -4.0475730 & -0.5988530 \\ -3.3161180 & -4.6990560 & 1.0853850 \\ -5.5055380 & -3.0768430 & -2.2052900 \\ 2.3943900 & 2.6300920 & 1.7991640 \\ 6.1098370 & 0.3736610 & -0.7451350 \\ 2.2275420 & 2.3367650 & -3.3176540 \\ -2.5415310 & -2.3876870 & 2.2608780 \\ -4.7714340 & -5.4524870 & -1.0797220 \\ -5.1834830 & -0.4647150 & -1.6851320 \\ -6.7165690 & -0.4471180 & -1.6901440 \\ -7.1180210 & -1.3508750 & -2.1620060 \\ -7.1113010 & -0.3961380 & -0.6704020 \\ -7.0892050 & 0.4179030 & -2.2486360 \\ -4.6237070 & -0.4866100 & -3.1117870 \\ -3.5303070 & -0.4768210 & -3.0974940 \\ -4.9540800 & -1.3801410 & -3.6531350 \\ -4.9711010 & 0.3893660 & -3.6695440 \\ -4.1692270 & -5.7147380 & -2.4647540 \\ -4.3571170 & -6.7468770 & -2.7786580 \\ -4.6142710 & -5.0499640 & -3.2132590 \\ -3.0889120 & -5.5428480 & -2.4636860\end{array}$

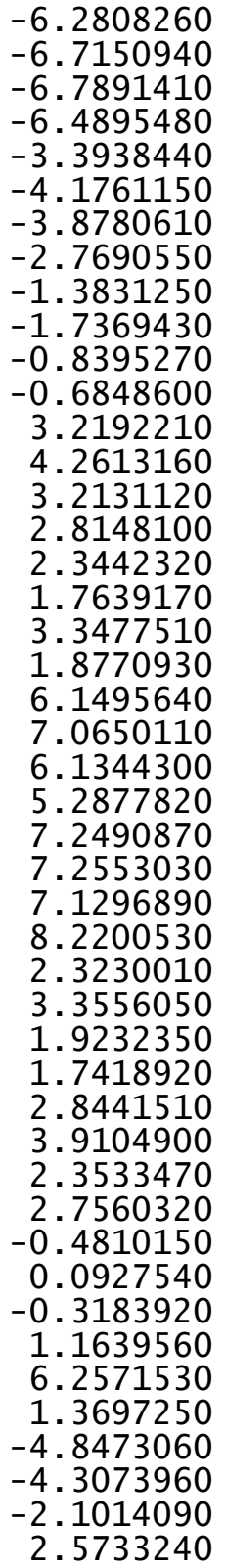

-5.7195570
-5.5523740
-5.0548600
-6.7515710
-2.5092670
-1.7464200
-3.4920270
-2.4027660
-3.3899150
-4.4022570
-3.4100340
-3.1028270
3.8240100
3.5299120
4.6384390
4.2039820
1.5168630
0.6661830
1.1621050
1.8881400
-0.7730100
-1.3625110
-0.3949240
-1.4398200
1.3668380
2.1654280
1.8287590
0.8609000
1.1721130
0.9919340
0.2519630
1.4035400
3.5961160
3.4365720
3.8347600
4.4639600
-0.1406560
-0.0879190
-1.3918370
2.5193100
-0.0507980
2.9819570
0.4656090
-6.1507590
-1.3852790
-1.7657490

$-1.0860780$ $-0.0960410$ $-1.7933400$ $-1.3868160$

3. 5312830

3.5699320

3.5695260

4.4246530

2.3188100

2. 5441850

1. 3713880

3.1116450

2. 2984090

2.4677140

1. 5668720

3.2424700

2.8478520

2.4802130

3.1094980

3.7663860

0.2713420

0.1526150

1.2997220

0.1587270

$-0.4828430$

$-1.2304810$

0.5036150

$-0.5065480$

$-4.3068150$

$-4.6272960$

$-3.8755590$

$-5.2052600$

$-3.9428660$

$-4.1414900$

$-4.8922440$

$-3.2834070$

$-0.7284820$

$-2.1126910$

0.0991400

$-3.1362030$

$-1.7476120$

1. 6524570

$-1.2172690$

$-0.3712910$

2.2315370
-2.0710880 


$\begin{array}{lll}1.8867880 & -2.8687420 & -1.3936480 \\ 2.4775080 & -0.7842850 & -1.5986620 \\ 0.9566000 & -2.6306270 & -0.8900380 \\ 1.5214180 & -1.9992570 & -3.0353880 \\ 0.8121330 & -1.3126690 & -2.7968610 \\ 4.0578110 & -3.4513050 & -3.2191450 \\ 3.6651250 & -4.4792780 & -2.1561880 \\ 2.2096270 & -4.2895610 & -1.7249080 \\ 3.9382420 & -2.0321510 & -2.6592960 \\ 5.0833850 & -3.6261730 & -3.5572940 \\ 3.8010220 & -5.4981590 & -2.5299880 \\ 1.5239380 & -4.5811700 & -2.5295970 \\ 4.6764960 & -1.8781360 & -1.8635250 \\ 4.1257010 & -1.2782480 & -3.4317550 \\ 3.4052200 & -3.5547510 & -4.0941810 \\ 4.3123130 & -4.3620400 & -1.2786340 \\ 1.9536310 & -4.9012360 & -0.8560200 \\ 2.6522750 & -2.3213870 & 1.5134600 \\ 1.5494400 & -1.6922120 & 1.8010670 \\ 3.6354910 & -2.3692780 & 2.6320370 \\ 4.8284340 & -3.0739420 & 2.4538810 \\ 3.3769320 & -1.7179110 & 3.8405230 \\ 5.7690080 & -3.1133260 & 3.4761320 \\ 5.0057730 & -3.5799600 & 1.5104030 \\ 4.3220030 & -1.7581930 & 4.8605770 \\ 2.4422390 & -1.1834880 & 3.9698730 \\ 5.5169170 & -2.4521590 & 4.6779070 \\ 6.6972090 & -3.6583280 & 3.3382450 \\ 4.1256210 & -1.2490150 & 5.7985010 \\ 6.2527900 & -2.4810790 & 5.4756260 \\ 0.8548330 & -1.5951900 & 1.0507870 \\ 2.9170890 & -2.8569950 & 0.4294990\end{array}$

144

2. 9170890

$-2.8569950$

4. 7252710

3. 6714730

4.6579490

5.4832860

5.4195070

4.4080620

3. 5579130

5.5332180

6.0893480

4. 2987780

2. 7796260

6.2870740

3. 5958810

2. 5126120

1. 2257130

3. 3710930

4.6137870

2. 7043350

0.3840970

4. 2075580

$-2.0176060$

$-2.7932080$

$-2.5104590$

$-4.0556430$

$-3.7594050$

$-4.5569660$

$-4.6559470$

$-4.1334810$

3. 8432970

3. 1792840

4. 4888320

3. 2198570

4.4975340

3.8749710

2. 7124430

4.9993200

$-1.7537040$

$-5.9162360$

$-2.3257790$

2. 4403850

3. 8819270

5. 1698060

6.6770780

6. 8776180

7. 1391670

0.6537500

0.4000110

7. 1635160

$-0.5988830$

$-1.9346520$
$-0.2142020$
$-2.4317580$
$-1.5671540$
0.4341660
$-4.1857990$
$-3.7458870$
$-2.0568950$
$-3.7035560$
$-5.1914990$
$-4.4028440$
$-1.3840890$
$-5.7415370$
$-6.5528930$
$-6.1136490$
$-4.5251120$
$-6.0822320$
$-7.5152700$
$-6.7185170$
$-3.9047310$
$-2.5714720$
$-2.3828850$
$-1.6114150$
$-1.7967720$
$-1.3987330$
$-1.3328550$
$-1.6492000$
1.7405570
2.6883150
2.1407650
4. 0291160
3. 4971020
4.4552050
4.7649690
3.8143720
$-2.8972750$
$-0.7641630$
$-2.4035720$
2. 2798710
5.9202570
1.1453360
1.4120530
2.4005670
1.3742230
0.6656360

1. 5698370

1.4399940

1.9939440

2. 0025120

3.4672540

2. 9549660

1.9717060

2. 9990880

4. 2398950

3. 3486980

1. 6001090

3. 3995040

$-1.6625080$

$-2.0739530$

$-1.8987230$

$-1.0679750$

$-1.8240060$

$-2.5377350$

$-2.2260370$

$-0.7631710$

$-0.4817340$

$-1.5857550$

0.8282420

$-1.3596370$

1.0005900

$-0.0775010$

$-2.2206140$

2. 0099210

0.2605650

1.0686250

$-0.9191950$

0.6918920

$-1.2574170$

$-0.4654070$

1. 3113990

$-2.1701700$

2. 0449950

0.1940330

$-3.0164150$

2. 3359290

$-0.8590010$

$-1.8449610$

$-1.9302760$

$-2.3581790$

$-0.9387100$

$-2.5668560$ 


\begin{tabular}{|c|c|c|}
\hline $\begin{array}{l}4.5265190 \\
.4565750 \\
4.6523340 \\
4.9931480 \\
4.5603630 \\
4.6019120 \\
4.0048580 \\
5.5804630 \\
2.4615690 \\
1.9794900 \\
1.8434180 \\
2.4797180 \\
3.3706000 \\
4.2306510 \\
3.7468160 \\
2.8310340 \\
1.1889270 \\
1.4492110 \\
0.5803840 \\
0.5816330 \\
2.2888550 \\
3.3496420 \\
2.1916400 \\
1.7395950 \\
1.7998000 \\
1.4786470 \\
2.8065640 \\
1.1338580 \\
6.7693770 \\
7.7198820 \\
6.9868380 \\
6.2714600 \\
6.7147620 \\
6.1798130 \\
6.9459370 \\
7.6624190 \\
2.6175290 \\
3.6930220 \\
2.2037570 \\
2.1628520 \\
2.9682930 \\
4.0578260 \\
2.6051510 \\
2.7528940 \\
0.5290160 \\
0.1469410\end{array}$ & $\begin{array}{r}1.1532270 \\
0.9376290 \\
2.1263200 \\
0.3961830 \\
6.7871090 \\
7.8330410 \\
6.7483900 \\
6.4442120 \\
6.4233140 \\
5.8205770 \\
6.3678660 \\
7.4667930 \\
2.3482920 \\
1.6821450 \\
3.3696700 \\
2.0653710 \\
3.1248190 \\
4.1264100 \\
3.2244540 \\
2.6478620 \\
-4.2848370 \\
-4.2245200 \\
-4.9841390 \\
-4.6914350 \\
-1.9461150 \\
-0.9411960 \\
-1.8850590 \\
-2.3089850 \\
-1.6439940 \\
-1.1469540 \\
-2.6038020 \\
-1.8473680 \\
-0.4431240 \\
0.2364270 \\
-1.3573660 \\
0.0320830 \\
-1.2202420 \\
-1.0840880 \\
-0.2928080 \\
-1.3975690 \\
-3.6752520 \\
-3.5611370 \\
-3.8673720 \\
-4.5541200 \\
0.1590310 \\
0.0533080\end{array}$ & $\begin{array}{r}-3.2363600 \\
-3.1684740 \\
-3.7239410 \\
-3.8753590 \\
0.2074240 \\
-0.1140160 \\
1.1509170 \\
0.4034130 \\
-1.1436150 \\
-1.9192100 \\
-0.2405040 \\
-1.4752820 \\
3.5553380 \\
3.4487180 \\
3.6853450 \\
4.4656770 \\
2.6014590 \\
2.9625750 \\
1.6996350 \\
3.3779350 \\
2.4247680 \\
2.6939320 \\
1.5879400 \\
3.2806070 \\
3.2419470 \\
2.9507710 \\
3.6726210 \\
4.0311800 \\
1.1185270 \\
1.3400290 \\
0.6365760 \\
2.0700990 \\
-1.0690350 \\
-1.7381100 \\
-1.6279340 \\
-0.7978610 \\
-3.9423960 \\
-4.1051890 \\
-3.5417110 \\
-4.9224260 \\
-3.5890680 \\
-3.6332250 \\
-4.6042360 \\
-2.9748090 \\
-0.6608040 \\
-1.9964940\end{array}$ \\
\hline
\end{tabular}

0.2903510

$-1.2400230$

$-5.7297750$

$-0.7005640$

5.0370200

4.4630030

2. 1116950

$-2.5543200$

$-1.7854610$

$-2.3950870$

$-0.8065310$

$-1.6501100$

$-0.9262610$

$-4.1888660$

$-3.6355040$

$-2.1387790$

$-3.9934860$

$-5.2525040$

$-3.8023030$

$-1.5554250$

$-4.5949840$

$-4.3044250$

$-3.6751300$

$-4.1624980$

$-1.7714390$

$-2.7592830$

$-1.8508000$

$-4.0470710$

$-5.1507100$

$-4.1747850$

$-5.0277760$

$-5.4109150$

$-3.3074750$

$-6.5173270$

$-7.2471850$

$-5.5130560$

$-7.4833090$

$-0.9859230$

$-2.6184520$

\begin{tabular}{rr}
1.3721580 & 0.2033060 \\
-2.5371540 & -2.9960930 \\
0.1811570 & 0.7253230 \\
-3.0172890 & 1.7794520 \\
0.1419150 & -1.4286050 \\
6.0074890 & -1.7860200 \\
1.2428040 & 2.2070020 \\
1.8082380 & -1.7215120 \\
2.9677850 & -1.2567680 \\
0.8766580 & -1.1686220 \\
2.7675990 & -0.8346770 \\
1.9213740 & -2.8371870 \\
1.2306510 & -2.6385340 \\
3.3754760 & -2.8246970 \\
4.5225370 & -1.9763610 \\
4.3466780 & -1.7091000 \\
2.0336380 & -2.1159210 \\
3.5325710 & -3.0268550 \\
5.4841290 & -2.4701630 \\
4.5287060 & -2.6196860 \\
1.9925940 & -1.1964450 \\
1.1960920 & -2.7501950 \\
3.3488080 & -3.7928840 \\
4.5542850 & -1.0159040 \\
5.0476190 & -0.9550100 \\
2.1982630 & 1.3913400 \\
1.2905950 & 1.6109250 \\
1.9667230 & 2.0984210 \\
2.7554750 & 1.7640700 \\
0.9379070 & 3.0339680 \\
2.5000280 & 2.3487540 \\
3.5555770 & 1.0412310 \\
0.6880280 & 3.6200220 \\
0.3379250 & 3.2838970 \\
1.4628420 & 3.2720000 \\
3.1056080 & 2.0842760 \\
-0.1139650 & 4.3448760 \\
1.2598770 & 3.7241990 \\
1.4039130 & 1.0687120 \\
3.1712430 & 0.6381630 \\
& \\
\hline & -10
\end{tabular}

$\begin{array}{lllr}\mathrm{O} & -0.0921210 & 1.0969250 & 0.2962010 \\ \mathrm{O} & -2.0926690 & 0.1325530 & -0.8775390 \\ \mathrm{C} & -0.3196950 & 2.3378330 & -0.2661530 \\ \mathrm{C} & -1.6034900 & 2.8366190 & -0.2789780\end{array}$




0.8193240
-1.8268910
0.6045260
-0.7063400
1.4487240
-2.8479350
-3.8213290
-2.6783630
-4.6635460
-3.4928880
-4.5177480
-5.4497160
-5.1639600
-4.1139810
-3.3011830
-5.3529180
-5.8035740
-3.9449260
-2.4927520
-6.1373800
-3.2998270
-2.1837290
-0.9176580
-3.1270020
-4.3019100
-2.3343010
-0.0518140
-3.9886870
2.1685520
2.9262700
2.6584640
4.1655680
3.8828030
4.6607320
4.7529030
4.2543970
-3.9157620
-3.3085960
-4.6133440
-3.4640680
-4.7353690
-4.1764780
-3.0075860
-5.2800700
1.9337340
5.9945670

3.0499750

4.0909750

4.2910960

4.8215830

4.8857560

0.7213340

$-0.0988810$

2.0617270

0.4926940

2.6421920

1.8539260

$-0.0980160$

3.7170510

4.4870420

3.9662500

2.4264150

4.1446740

5.4985350

4.5659330

1.8106660

5.8256710

6.5674270

6.0715610

4.6212100

6.2103580

7.5211630

6.6222130

4.0539930

2.4306070

1.9908700

2. 2474650

1. 3759550

1.6141180

1.1644980

1.0605240

1.4718060

$-1.5536830$

$-2.5057300$

$-1.9617800$

$-3.8593300$

$-3.3253880$

$-4.2904220$

$-4.6059050$

$-3.6406860$

2.8246180

0.4817040

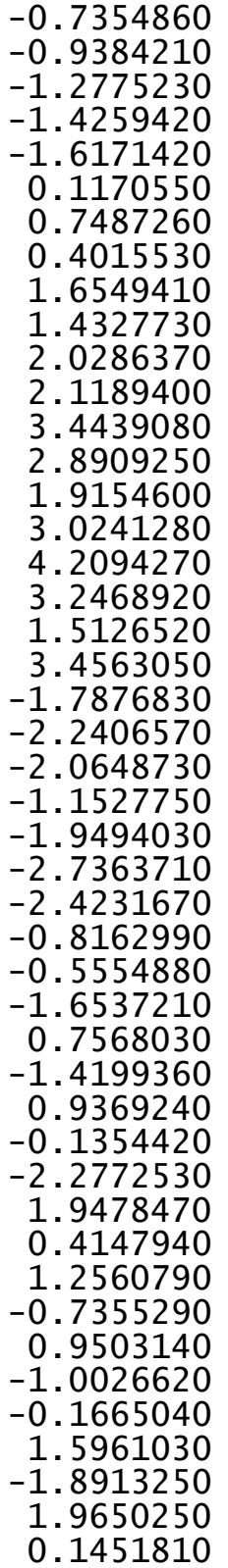

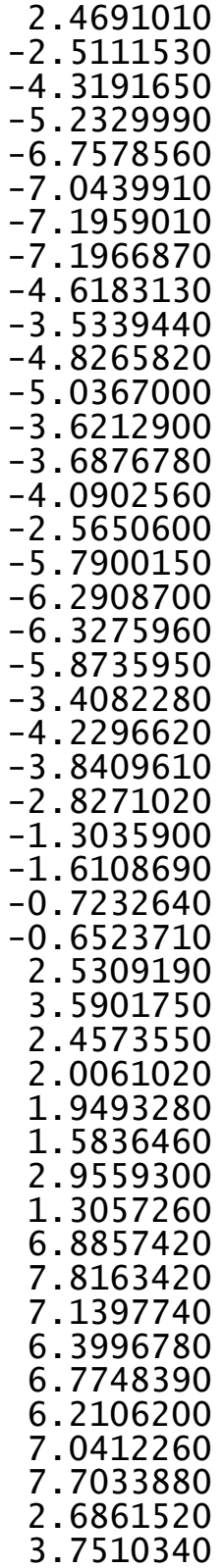

\author{
2. 2102100 \\ $-2.0945280$ \\ $-5.7687240$ \\ $-0.9617580$ \\ $-1.1119370$ \\ $-2.1020120$ \\ $-0.9870380$ \\ $-0.3626310$ \\ $-1.0883310$ \\ $-0.9531670$ \\ $-2.0718590$ \\ $-0.3298900$ \\ $-6.1290470$ \\ $-7.2052200$ \\ $-5.6078770$ \\ $-5.8447490$ \\ $-6.1984370$ \\ $-5.9592360$ \\ $-5.6871110$ \\ $-7.2763790$ \\ $-2.0602980$ \\ $-1.3475640$ \\ $-3.0511050$ \\ $-1.7758270$ \\ $-3.0016030$ \\ $-3.9766000$ \\ $-3.1586590$ \\ $-2.5384150$ \\ 4.1977420 \\ 4.0992670 \\ 4.8776320 \\ 4.6506120 \\ 1.9053510 \\ 0.9074700 \\ 1.8135130 \\ 2. 3178290 \\ 1. 3466480 \\ 0.8168150 \\ 2.2860450 \\ 1.5907290 \\ 0.1019930 \\ $-0.5690990$ \\ 0.9942160 \\ $-0.4054970$ \\ 0.9897510 \\ 0.7811920
}

$-3.0883130$ 2. 4863040 $-0.4762630$ $-1.6989210$ $-1.7470340$ $-2.1191050$ $-0.7516800$ $-2.4140780$ $-3.0973650$ $-3.0551180$ $-3.5332490$ $-3.7673210$ $-1.7929330$ $-1.9844370$ $-2.6348540$ $-1.7689540$ $-0.5074310$

0.4351980

$-1.3135480$

$-0.6808270$

3.7315340

3.6203350

3.9125140

4.6154060

2.7488810

3.1438600

1.8368690

3.4976860

2.3030750

2.5670080

1.4480160

3.1509930

3.1872510

2.9262770

3.6128000

3.9706130

1.0476740

1.2775380

0.5438870

1. 9958130

$-1.1130680$

$-1.7665360$

$-1.6915350$

$-0.8343540$

$-3.9861700$

$-4.1418020$ 


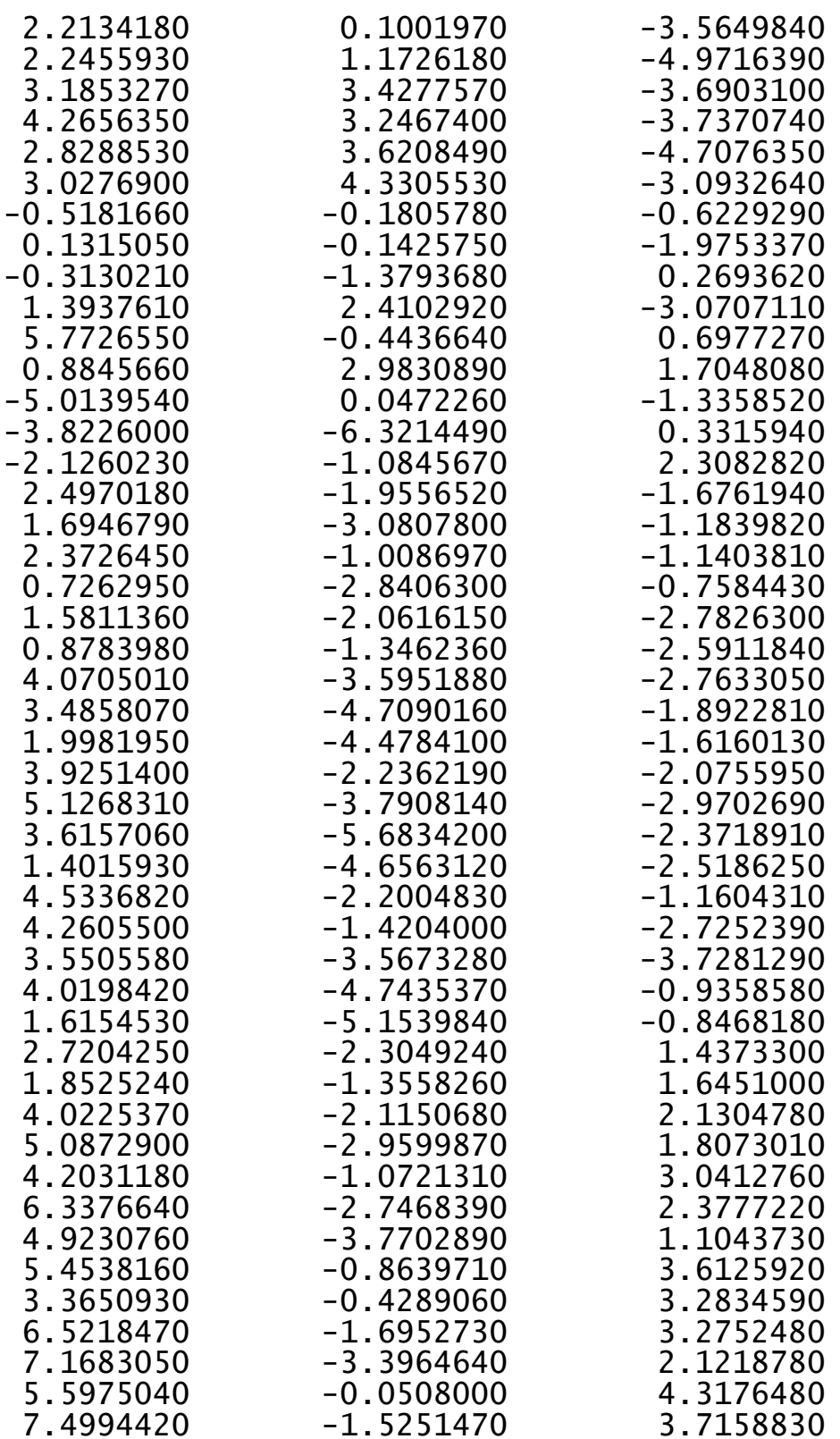

$\begin{array}{llll}\mathrm{H} & 0.9773380 & -1.4447580 & 1.1152400 \\ \mathrm{O} & 2.5331130 & -3.2854210 & 0.7042450\end{array}$

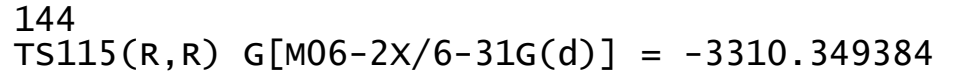

$\begin{array}{lrrr}\mathrm{TS} 115(\mathrm{R}, \mathrm{R}) \mathrm{G}[\mathrm{M} 06-2 \mathrm{~V} / 6-31 \mathrm{G}(\mathrm{d})]=-3310.349384 \\ \mathrm{O} & 0.1807030 & -1.1080580 & 0.3034850 \\ \mathrm{O} & 2.1102780 & -0.0907330 & -0.9402130 \\ \mathrm{C} & 0.4647300 & -2.3531820 & -0.2224160 \\ \mathrm{C} & 1.7718220 & -2.7875550 & -0.2361120 \\ \mathrm{C} & -0.6427410 & -3.1347120 & -0.6551580 \\ \mathrm{C} & 2.0490710 & -4.0518950 & -0.8547410 \\ \mathrm{C} & -0.3735100 & -4.3822750 & -1.1562830 \\ \mathrm{C} & 0.9603230 & -4.8533740 & -1.3016070 \\ \mathrm{H} & -1.1910340 & -5.0289510 & -1.4643530 \\ \mathrm{C} & 2.9093990 & -0.5988210 & 0.0645920 \\ \mathrm{C} & 3.8421720 & 0.2972400 & 0.6537620 \\ \mathrm{C} & 2.8147150 & -1.9345940 & 0.4000130 \\ \mathrm{C} & 4.7252780 & -0.2140880 & 1.5698470 \\ \mathrm{C} & 3.6715490 & -2.4316780 & 1.4399940 \\ \mathrm{C} & 4.6579970 & -1.5670450 & 1.9939480 \\ \mathrm{H} & 5.4832720 & 0.4343020 & 2.0025270 \\ \mathrm{C} & 5.4196100 & -3.3396350 & 3.4672510 \\ \mathrm{C} & 4.4081920 & -4.1857010 & 2.9549600 \\ \mathrm{C} & 3.5580300 & -3.7458120 & 1.9717010 \\ \mathrm{C} & 5.5332810 & -2.0567620 & 2.9990900 \\ \mathrm{H} & 6.0894630 & -3.7034110 & 4.2398900 \\ \mathrm{H} & 4.2989400 & -5.1914060 & 3.3486880 \\ \mathrm{H} & 2.7797630 & -4.4027920 & 1.6001010 \\ \mathrm{H} & 6.2871160 & -1.3839340 & 3.3995090 \\ \mathrm{C} & 3.5960410 & -5.7414540 & -1.6625180 \\ \mathrm{C} & 2.5127930 & -6.5528340 & -2.0739710 \\ \mathrm{C} & 1.2258830 & -6.1136210 & -1.8987440 \\ \mathrm{C} & 3.3712210 & -4.5250370 & -1.0679810 \\ \mathrm{H} & 4.6139550 & -6.0821230 & -1.8240140 \\ \mathrm{H} & 2.7045410 & -7.5152050 & -2.5377560 \\ \mathrm{H} & 0.3842820 & -6.7185080 & -2.2260640 \\ \mathrm{H} & 4.2076700 & -3.9046370 & -0.7631710 \\ \mathrm{C} & -2.0175270 & -2.5715260 & -0.4817580 \\ \mathrm{C} & -2.7931230 & -2.1800180 & -1.5857810 \\ \mathrm{C} & -2.5103980 & -2.3829700 & 0.8282160 \\ \mathrm{C} & -4.0555710 & -1.6114910 & -1.3596670 \\ \mathrm{C} & -3.7593580 & -1.7968840 & 1.0005600 \\ \mathrm{C} & -4.5569130 & -1.3988420 & -0.0775340 \\ \mathrm{H} & -4.6558700 & -1.3329250 & -2.2206460 \\ \mathrm{H} & -4.1334470 & -1.6493340 & 2.0098900 \\ \mathrm{C} & 3.8432300 & 1.7406480 & 0.2605800 \\ \mathrm{C} & 3.1791870 & 2.6883800 & 1.0686470\end{array}$




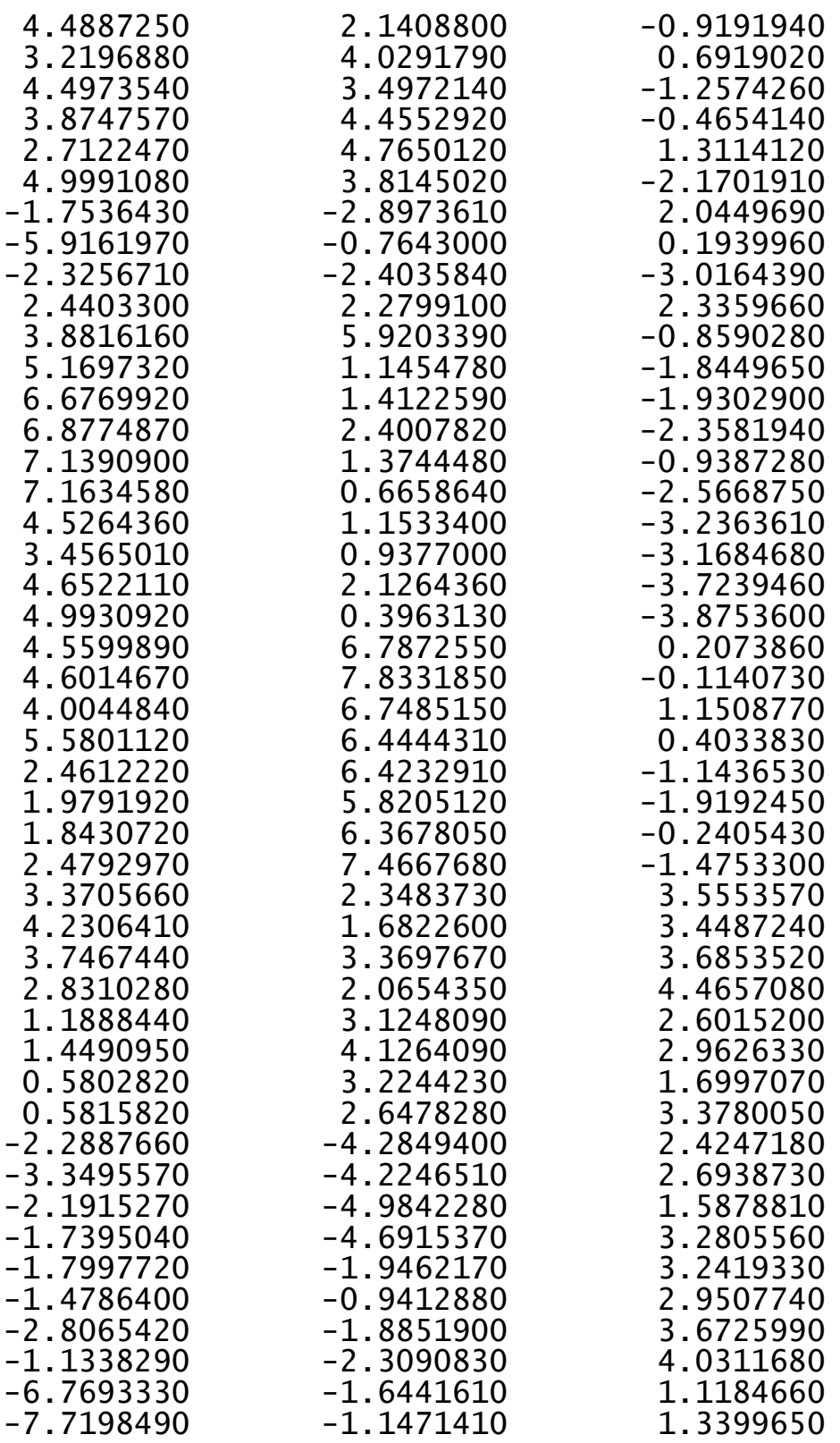

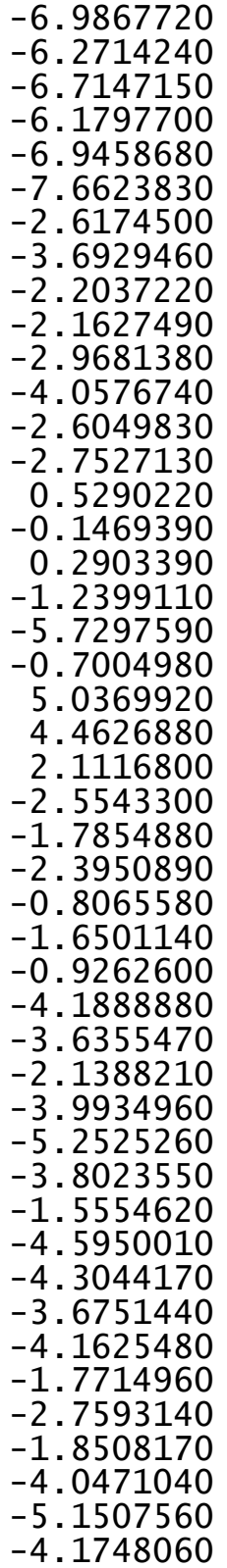

-2.6039650
-1.8475400
-0.4432560
0.2363150
-1.3574930
0.0319300
-1.2202500
-1.0841350
-0.2928070
-1.3975440
-3.6752760
-3.5611970
-3.8673670
-4.5541480
0.1590380
0.0533190
1.3721480
-2.5371310
0.1810150
-3.0173460
0.1420510
6.0075980
1.2428290
1.8082220
2.9677700
0.8766340
2.7675890
1.9213880
1.2306690
3.3754580
4.5225110
4.3466670
2.0336100
3.5325440
5.4841100
4.5287210
1.9925410
1.1960720
3.3488160
4.5542320
5.0475980
2.1981790
1.2905240
1.9666010
2.7553350
0.9377680
0.6364980 2. 0700410 $-1.0690760$ $-1.7381340$ $-1.6279930$ $-0.7979050$ $-3.9424050$ $-4.1052160$ $-3.5416980$ $-4.9224310$ $-3.5891180$ $-3.6332810$ $-4.6042860$ $-2.9748720$ $-0.6607850$ $-1.9964720$ 0.2033430 $-2.9961070$

0.7253020

1.7794330 $-1.4286070$ $-1.7860470$ 2. 2070490 $-1.7214840$ $-1.2567140$ $-1.1686090$ $-0.8346210$ $-2.8371510$ $-2.6385060$ $-2.8246530$ $-1.9762900$ $-1.7090220$ $-2.1159000$ $-3.0268150$ $-2.4700750$ $-2.6196000$ $-1.1964300$ $-2.7501930$ $-3.7928370$ $-1.0158360$ $-0.9549160$ 1. 3913740 1. 6109500 2. 0984380 1. 7640880 3.0339670 


\begin{tabular}{|c|c|c|c|c|c|c|c|}
\hline$C$ & 63865610 & 2100051 & 23187560 & & 36166070 & 1977070 & \\
\hline $\mathrm{H}$ & $\begin{array}{l}-0.5 \\
-5.0278310\end{array}$ & $\begin{array}{l}2.4998540 \\
3.5554510\end{array}$ & $\begin{array}{l}2.3487560 \\
1.0412630\end{array}$ & c & $\begin{array}{l}3.6466070 \\
4.3559390\end{array}$ & $\begin{array}{l}1.4170 / 20 \\
1.1124950\end{array}$ & $\begin{array}{r}0.3597700 \\
-2.3070090\end{array}$ \\
\hline C & -5.4109370 & 0.6878550 & 3.6200050 & c & 4.8218890 & 0.7991470 & 0.0161120 \\
\hline $\mathrm{H}$ & -3.3074870 & 0.3377990 & 3.2838960 & $\mathrm{C}$ & 5.2068260 & 0.6286900 & -1.3097870 \\
\hline C & -6.5173620 & 1.4626510 & 3.2719850 & $\mathrm{H}$ & 4.6311810 & 0.9491490 & -3.3473920 \\
\hline $\mathrm{H}$ & -7.2472420 & 3.1054200 & 2.0842780 & $\mathrm{H}$ & 5.4481870 & 0.4066380 & 0.8131850 \\
\hline $\mathrm{H}$ & -5.5130680 & -0.1141510 & 4.3448470 & C & -4.0747360 & -0.6024510 & -0.2920010 \\
\hline H & -7.4833440 & 1.2596600 & 3.7241710 & $\mathrm{C}$ & -3.9502130 & -1.9336670 & 0.1618280 \\
\hline $\mathrm{H}$ & -0.9859370 & 1.4038670 & 1.0687450 & $\mathrm{C}$ & -4.6728930 & -0.3316680 & -1.5344650 \\
\hline 0 & -2.6184930 & 3.1711770 & 0.6382180 & $\mathrm{c}$ & -4.4820330 & -2.9575810 & -0.6207220 \\
\hline & & & & $\mathrm{C}$ & -5.1816070 & -1.3943480 & -2.2853940 \\
\hline & R) $\mathrm{G}[\mathrm{M06}-2$ & (d) $]=-33$ & 58 & $\mathrm{C}$ & -5.1064520 & -2.7107440 & -1.8439130 \\
\hline 0 & 0.3949640 & 0.8820530 & 0.2110090 & $\mathrm{H}$ & -4.3990270 & -3.9838810 & -0.2694580 \\
\hline 0 & -1.8137530 & 0.9363770 & -1.0063400 & $\mathrm{H}$ & -5.6549550 & -1.1882750 & -3.2438550 \\
\hline C & 0.4291400 & 2.2590050 & 0.1018140 & C & 3.3001960 & 1.6352470 & 1.8321430 \\
\hline $\mathrm{C}$ & -0.6837160 & 2.9948250 & 0.4585740 & $\mathrm{C}$ & 6.5149360 & -0.0333290 & -1.7129000 \\
\hline C & 1.6545210 & 2.8478210 & -0.3094990 & $\mathrm{C}$ & 2.3070040 & 2.3196890 & -3.1487940 \\
\hline $\mathrm{C}$ & -0.6326140 & 4.4205360 & 0.3112880 & $\mathrm{C}$ & -3.2657550 & -2.2879390 & 1.4760880 \\
\hline C & 1.7254090 & 4.2180750 & -0.3408290 & C & -5.6723580 & -3.8474370 & -2.6738390 \\
\hline $\mathrm{C}$ & 0.5965840 & 5.0313880 & -0.0630660 & $\mathrm{C}$ & -4.8094090 & 1.0799930 & -2.0839340 \\
\hline $\mathrm{H}$ & 2.6655210 & 4.6974980 & -0.6022160 & $\mathrm{C}$ & -6.2845010 & 1.4903070 & -2.1725850 \\
\hline C & -2.4233130 & 1.2589470 & 0.1952020 & $\mathrm{H}$ & -6.8304870 & 0.8427920 & -2.8675980 \\
\hline $\mathrm{C}$ & -3.5844140 & 0.5250910 & 0.5583700 & $\mathrm{H}$ & -6.7707430 & 1.4206200 & -1.1943670 \\
\hline C & -1.8990540 & 2.2886760 & 0.9463270 & $\mathrm{H}$ & -6.3751290 & 2.5210800 & -2.5309310 \\
\hline $\mathrm{C}$ & -4.2322310 & 0.9024750 & 1.7079850 & C & -4.1135980 & 1.2217260 & -3.4421950 \\
\hline $\mathrm{C}$ & -2.5238370 & 2.6002070 & 2.1996460 & $\mathrm{H}$ & -3.0603060 & 0.9376720 & -3.3692180 \\
\hline $\mathrm{C}$ & -3.7203820 & 1.9179100 & 2.5576250 & H & -4.5913110 & 0.5901990 & -4.1994010 \\
\hline $\mathrm{H}$ & -5.1528450 & 0.3992370 & 1.9914540 & $\mathrm{H}$ & -4.1701930 & 2.2586450 & -3.7897490 \\
\hline C & -3.8359210 & 3.1709290 & 4.6333000 & C & -6.7769250 & -4.5962050 & -1.9198090 \\
\hline C & -2.6220140 & 3.8173960 & 4.3005100 & $\mathrm{H}$ & -7.2109820 & -5.3800610 & -2.5492560 \\
\hline $\mathrm{C}$ & -1.9830110 & 3.5406750 & 3.1181540 & $\mathrm{H}$ & -6.3770250 & -5.0735570 & -1.0183640 \\
\hline C & -4.3666710 & 32110 & 3.7809150 & $\mathrm{H}$ & -7.5765110 & -3.9154620 & -1.6133840 \\
\hline $\mathrm{H}$ & -4.3339870 & 3.4051170 & 5.5687800 & C & -4.5691660 & -4.8139520 & -3.1198850 \\
\hline $\mathrm{H}$ & -2.1892770 & 4.5351030 & 4.9904400 & $\mathrm{H}$ & -3.7875380 & -4.2886440 & -3.6763940 \\
\hline $\mathrm{H}$ & -1.0477360 & 4.0352960 & 2.8797090 & $\mathrm{H}$ & -4.1013980 & -5.2939390 & -2.2530530 \\
\hline $\mathrm{H}$ & -5.2858250 & 1.7153980 & 4.0321730 & H & -4.9810990 & -5.6022040 & -3.7587470 \\
\hline C & -1.6749740 & 6.6151500 & 0.3595610 & C & -4.2997480 & -2.5377120 & 2.5828140 \\
\hline $\mathrm{C}$ & -0.4405880 & 7.2232590 & 0.0288280 & $\mathrm{H}$ & -4.9307580 & -1.6622680 & 2.7580060 \\
\hline $\mathrm{C}$ & 0.6672190 & 47690 & -0.1820240 & $\mathrm{H}$ & -4.9547520 & -3.3726080 & 2.3075890 \\
\hline C & -1.7698090 & 5.25344 & 0.4976460 & $\mathrm{H}$ & -3.8012720 & -2.7929510 & 3.5241440 \\
\hline $\mathrm{H}$ & -2.5569410 & $7.2 .25-9$ & 0.4989310 & C & -2.3479450 & -3.5111700 & 1.3541680 \\
\hline $\mathrm{H}$ & -0.3796480 & 8.30228 & -0.0709950 & $\mathrm{H}$ & -2.9221690 & -4.4349420 & 1.2232140 \\
\hline $\mathrm{H}$ & 1.6176360 & 6.8937620 & -0.4584690 & $\mathrm{H}$ & -1.6605170 & -3.4070260 & 0.5106720 \\
\hline $\mathrm{H}$ & -2.7240340 & 85730 & 0.7405510 & $\mathrm{H}$ & -1.7604700 & -3.6222550 & 2.2720210 \\
\hline C & 2.8404160 & 65 & -0.6604330 & C & 4.3537400 & 2.4810130 & 2.5570870 \\
\hline C & 3.1763440 & 1. & -2.0149100 & $\mathrm{H}$ & 5.3333450 & 1.9906830 & 2.5388840 \\
\hline
\end{tabular}




$\begin{array}{rr}\mathrm{H} & 4.4594660 \\ \mathrm{H} & 4.0714500 \\ \mathrm{C} & 3.1187120 \\ \mathrm{H} & 2.3138160 \\ \mathrm{H} & 4.0375380 \\ \mathrm{H} & 2.8578840 \\ \mathrm{C} & 7.1344940 \\ \mathrm{H} & 8.0070680 \\ \mathrm{H} & 7.4742220 \\ \mathrm{H} & 6.4257390 \\ \mathrm{C} & 7.5193740 \\ \mathrm{H} & 7.1144530 \\ \mathrm{H} & 7.7532030 \\ \mathrm{H} & 8.4517310 \\ \mathrm{C} & 2.2334620 \\ \mathrm{H} & 3.1679160 \\ \mathrm{H} & 2.0249880 \\ \mathrm{H} & 1.4350840 \\ \mathrm{C} & 2.8103380 \\ \mathrm{H} & 3.8439710 \\ \mathrm{H} & 2.1925850 \\ \mathrm{H} & 2.7846130 \\ \mathrm{P} & -0.4725190 \\ \mathrm{O} & 0.1860680 \\ \mathrm{O} & -0.7661500 \\ \mathrm{H} & 1.2902230 \\ \mathrm{H} & 6.2993240 \\ \mathrm{H} & 2.3456710 \\ \mathrm{H} & -4.3204010 \\ \mathrm{H} & -6.1176920 \\ \mathrm{H} & -2.6388100 \\ \mathrm{C} & 2.3330140 \\ \mathrm{C} & 1.2856300 \\ \mathrm{H} & 2.1408610 \\ \mathrm{H} & 0.2640050 \\ \mathrm{O} & 1.8718070 \\ \mathrm{H} & 1.1897230 \\ \mathrm{C} & 3.9869790 \\ \mathrm{C} & 3.0065030 \\ \mathrm{C} & 1.5590030 \\ \mathrm{C} & 3.7658010 \\ \mathrm{H} & 5.0186330 \\ \mathrm{H} & 3.1848810 \\ \mathrm{H} & 1.3145120 \\ \mathrm{H} & 4.0085600 \\ \mathrm{H} & \\ & \end{array}$

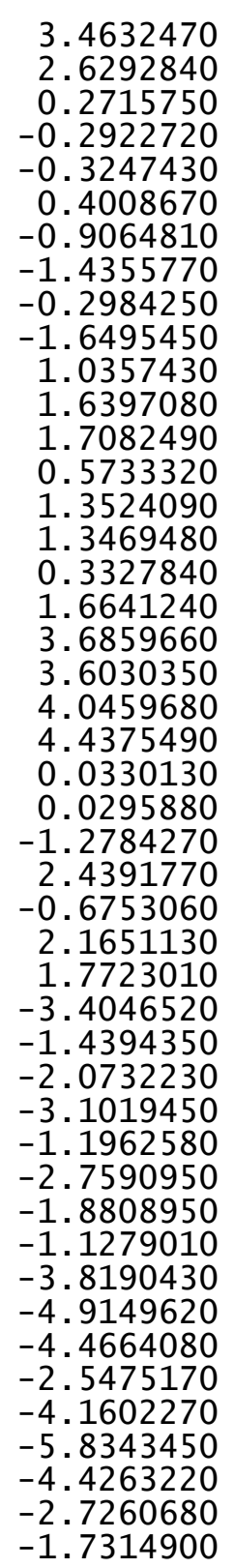
2. 0857250
3.6045030
2. 5080170
2. 0271200
2. 4639970
3.5642790
$-0.6220140$
$-1.0164740$
0.2236080
$-0.2416320$
$-2.1672710$
$-2.9844980$
$-1.3345750$
$-2.5077290$
$-4.3347870$
$-4.9073770$
$-4.0053940$
$-5.0149020$
$-3.6355440$
$-3.9913500$
$-4.4651610$
$-2.8423550$
$-0.8885710$
$-2.2336050$
$-0.2016700$
$-2.7612580$
$-2.5792710$
1.9076260
$-1.3924110$
$-3.5739450$
1.7683850
$-1.2893540$
$-1.3039360$
$-0.6606100$
$-1.1867300$
$-2.6406790$
$-2.6016390$
$-2.0546940$
$-1.6354910$
$-1.8464490$
$-1.2335240$
$-1.9243820$
$-2.2005710$
$-2.9146370$
$-0.1770420$
$-1.5850030$

$\begin{array}{lr}\mathrm{H} & 3.8540070 \\ \mathrm{H} & 3.1514590 \\ \mathrm{H} & 0.8450380 \\ \mathrm{C} & 1.2369730 \\ \mathrm{O} & 0.5108330 \\ \mathrm{C} & 1.9696530 \\ \mathrm{C} & 2.7956110 \\ \mathrm{C} & 1.8479700 \\ \mathrm{C} & 3.5010100 \\ \mathrm{H} & 2.8712160 \\ \mathrm{C} & 2.5580490 \\ \mathrm{H} & 1.2023470 \\ \mathrm{C} & 3.3827600 \\ \mathrm{H} & 4.1426020 \\ \mathrm{H} & 2.4676020 \\ \mathrm{H} & 3.9350340 \\ \mathrm{H} & -0.0048750 \\ \mathrm{O} & 1.3492900 \\ \mathrm{H} & \end{array}$
$-3.5890820$
$-5.1478520$
$-5.1533780$
$-3.0545510$
$-1.9817630$
$-3.4370250$
$-4.5628210$
$-2.6743150$
$-4.9257950$
$-5.1425770$
$-3.0397810$
$-1.8028890$
$-4.1635010$
$-5.8008880$
$-2.4491560$
$-4.4468290$
$-1.7188500$
$-3.7309800$

144

TS117 (S, S ) G[M06-2X/6-31G(d)] = -3310.352436

$\begin{array}{lrcr}\text { TS117(S , S }) \text { G }[\mathrm{M} 06-2 \mathrm{X} / 6-31 \mathrm{G}(\mathrm{d})]=-3310.352436 \\ \mathrm{O} & 0.4985480 & 0.8565230 & 0.3710110 \\ \mathrm{O} & -1.6338650 & 0.9823010 & -0.9607460 \\ \mathrm{C} & 0.5125200 & 2.2381080 & 0.3567330 \\ \mathrm{C} & -0.6229460 & 2.9255830 & 0.7337850 \\ \mathrm{C} & 1.7303620 & 2.8721490 & -0.0059810 \\ \mathrm{C} & -0.5889850 & 4.3590290 & 0.7030720 \\ \mathrm{C} & 1.7840370 & 4.2407300 & 0.0738150 \\ \mathrm{C} & 0.6402720 & 5.0127030 & 0.4063560 \\ \mathrm{H} & 2.7164180 & 4.7543480 & -0.1456240 \\ \mathrm{C} & -2.3078130 & 1.1895410 & 0.2324560 \\ \mathrm{C} & -3.4699690 & 0.4072700 & 0.4701140 \\ \mathrm{C} & -1.8436900 & 2.1571280 & 1.0993370 \\ \mathrm{C} & -4.1779970 & 0.6649630 & 1.6173230 \\ \mathrm{C} & -2.5343640 & 2.3418970 & 2.3427830 \\ \mathrm{C} & -3.7292760 & 1.6062540 & 2.5798290 \\ \mathrm{H} & -5.0995430 & 0.1215760 & 1.8083380 \\ \mathrm{C} & -3.9701700 & 2.6609460 & 4.7526150 \\ \mathrm{C} & -2.7586650 & 3.3599560 & 4.5379080 \\ \mathrm{C} & -2.0594900 & 3.2054440 & 3.3673280 \\ \mathrm{C} & -4.4384770 & 1.7998530 & 3.7949220 \\ \mathrm{H} & -4.5160190 & 2.7982990 & 5.6806450 \\ \mathrm{H} & -2.3759440 & 4.0203530 & 5.3097690 \\ \mathrm{H} & -1.1265380 & 3.7385250 & 3.2192110 \\ \mathrm{H} & -5.3553400 & 1.2380730 & 3.9532730 \\ \mathrm{C} & -1.6618770 & 6.5301830 & 0.9043330 \\ \mathrm{C} & -0.4287020 & 7.1775570 & 0.6537740\end{array}$

$-3.1185930$ $-0.5744490$

$-1.3849840$

1.7285940

2.9670590

2. 9445590

4.0857460

2.0304790

4.0716220

6.1760330

6.1382010

0.9846220

0.6969290

0.7337850

0.7030720

0.0738150

0.1456240

.2324560

(

1.6173230

.3427830

(2)

4.7526150

(1)

5.6806450

. 2192110

0.9043330

7.1775570

0.6537740 


$\begin{array}{rrr}0.6937500 & 6.4318380 & 0.4050370 \\ -1.7413510 & 5.1606630 & 0.9282380 \\ -2.5550750 & 7.1236530 & 1.0724940 \\ -0.3800470 & 8.2617670 & 0.6437950 \\ 1.6433390 & 6.9132380 & 0.1863060 \\ -2.6946670 & 4.6763880 & 1.1100570 \\ 2.8950890 & 2.0510110 & -0.4571000 \\ 3.2071240 & 1.9735350 & -1.8306640 \\ 3.6710960 & 1.3580730 & 0.4888240 \\ 4.3233770 & 1.2324980 & -2.2213910 \\ 4.7771130 & 0.6289710 & 0.0468570 \\ 5.1237840 & 0.5583120 & -1.3007500 \\ 4.5675020 & 1.1721540 & -3.2780010 \\ 5.3910600 & 0.0976990 & 0.7742470 \\ -3.9039100 & -0.6409340 & -0.5032690 \\ -3.8053550 & -2.0050140 & -0.1631620 \\ -4.4353610 & -0.2581840 & -1.7503760 \\ -4.2965440 & -2.9534940 & -1.0638240 \\ -4.9016100 & -1.2447060 & -2.6179840 \\ -4.8516400 & -2.5983900 & -2.2896090 \\ -4.2388990 & -4.0088640 & -0.8067640 \\ -5.3207860 & -0.9449190 & -3.5770570 \\ -0.2820620 & 0.0895920 & -0.8512280 \\ 0.4620740 & 0.2131530 & -2.1426170 \\ -0.5768270 & -1.2795320 & -0.2908440 \\ 3.3634050 & 1.3997340 & 1.9781560 \\ 2.4524380 & 1.9876040 & 2.1262300 \\ 6.3582730 & -0.2121170 & -1.7330020 \\ 6.5589240 & -0.9649220 & -0.9572470 \\ 2.3839710 & 2.6976340 & -2.8878870 \\ 1.3818140 & 2.8587390 & -2.4778620 \\ -4.5466330 & 1.1975070 & -2.1784840 \\ -4.0993040 & 1.8260790 & -1.4032290 \\ -5.3749550 & -3.6535340 & -3.2455440 \\ -5.2325210 & -4.6298860 & -2.7647370 \\ -3.1938210 & -2.4831880 & 1.1480420 \\ -2.5967030 & -1.6635220 & 1.5611880 \\ -2.2536520 & -3.6804170 & 0.9579720 \\ -1.7464710 & -3.9010660 & 1.9038570 \\ -2.8031230 & -4.5822470 & 0.6672670 \\ -1.4981020 & -3.4707260 & 0.1960580 \\ -4.2868950 & -2.8505010 & 2.1612720 \\ -4.9084000 & -3.6646330 & 1.7704980 \\ -3.8399330 & -3.1870760 & 3.1029960 \\ -4.9442540 & -2.0044010 & 2.5491190 \\ 3.0971130 & -0.0010690 & \\ -4 & & \\ -4 & & \\ -4 & -300230\end{array}$

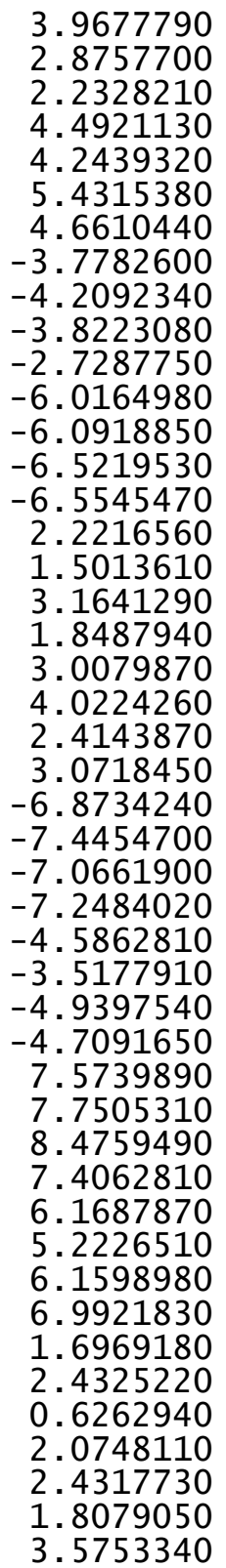

-0.6553080
0.0497230
-0.4546080
2.0978570
2.1708870
1.5411300
3.1080760
1.4586620
0.8948740
2.5219220
1.1708480
1.6152100
2.6737290
1.0320950
1.4596010
1.8948300
2.3996130
1.8232360
0.8883270
4.0625490
3.9291640
4.5831450
4.7049280
-3.4726050
-3.4895910
-2.5148340
-4.2687070
-3.6546800
-3.8016750
-4.4523130
-2.7018670
0.7219660
1.2052010
0.1688300
1.5071830
-0.9412020
-1.4925550
-0.2348850
-1.6411550
-2.6187370
-2.1750950
-2.4265940
-1.2832290
-1.5171170
-0.7276250
-4.2344080
2.4122170
3.6113700
2.0453060
2.7465130
3.8103140
2.6567580
2.3608510
-3.4786290
-4.3134600
-3.7370570
-3.3724250
-2.3105100
-2.5807230
-3.0883500
-1.3700170
-4.1830730
-4.8345930
-4.7370280
-3.9847800
-3.2131540
-3.6068600
-3.9720330
-2.3312540
-3.5128170
-2.5805060
-4.0087180
-4.1644930
-4.5600360
-4.3779830
-5.2218690
-5.0866970
-1.7961830
-0.8306430
-2.0787470
-2.5416720
-3.0666070
-3.0909470
-3.9036300
-3.2390100
-1.8297150
-0.6427510
-1.8025090
-0.1338420
-2.4209470
-2.3864450
-2.3360020
-2 


\begin{tabular}{|c|c|c|c|c|c|c|c|}
\hline & & & & & & & \\
\hline C & 4.0672190 & -4.0719930 & -0.8964040 & $\mathrm{C}$ & 3.2656460 & -4.0091330 & 1.9208280 \\
\hline C & 3.8274070 & -2.6477890 & -0.3917850 & $\mathrm{C}$ & 5.3089790 & -2.4449620 & 3.0117900 \\
\hline C & 2.0744760 & -3.9534110 & -2.4263260 & $\mathrm{H}$ & 5.7761230 & -4.1510870 & 4.2079760 \\
\hline $\mathrm{H}$ & 3.7794680 & -5.2478770 & -2.6938630 & $\mathrm{H}$ & 3.9266960 & -5.5273770 & 3.2602360 \\
\hline $\mathrm{H}$ & 5.1346460 & -4.3011460 & -0.8236460 & $\mathrm{H}$ & 2.4608510 & -4.6172650 & 1.5229150 \\
\hline $\mathrm{H}$ & 4.4807960 & -1.9372690 & -0.9096780 & $\mathrm{H}$ & 6.0905880 & -1.8208220 & 3.4370140 \\
\hline $\mathrm{H}$ & 1.5164270 & -4.7173250 & -1.8712330 & C & 3.2800050 & -5.8696980 & -1.7900810 \\
\hline $\mathrm{H}$ & 1.7247040 & -3.9779570 & -3.4630250 & C & 2.1726820 & -6.6194870 & -2.2518060 \\
\hline $\mathrm{H}$ & 4.1180780 & -3.5456040 & -2.9942600 & $\mathrm{C}$ & 0.9017390 & -6.1343140 & -2.0825360 \\
\hline $\mathrm{H}$ & 3.5322780 & -4.7742490 & -0.2467860 & C & 3.0937760 & -4.6685000 & -1.1531360 \\
\hline $\mathrm{H}$ & 4.0358980 & -2.5433890 & 0.6771050 & $\mathrm{H}$ & 4.2861210 & -6.2459180 & -1.9466190 \\
\hline C & 1.0868670 & -2.9894830 & 1.8924140 & $\mathrm{H}$ & 2.3340830 & -7.5707120 & -2.7489340 \\
\hline 0 & 0.2361390 & -2.0093730 & 1.9465790 & $\mathrm{H}$ & 0.0424150 & -6.6908520 & -2.4474380 \\
\hline C & 1.4036820 & -3.6135520 & 3.2053790 & $\mathrm{H}$ & 3.9490700 & -4.0955910 & -0.8102070 \\
\hline C & 2.3962250 & -4.5940750 & 3.2667910 & C & -2.2326700 & -2.5550890 & -0.5570750 \\
\hline C & 0.7260500 & -3.2208980 & 4.3626020 & C & -2.9356120 & -1.9743390 & -1.6319720 \\
\hline C & 2.7122900 & -5.1819260 & 4.4857770 & $\mathrm{C}$ & -2.8028960 & -2.5864940 & 0.7282920 \\
\hline $\mathrm{H}$ & 2.9071470 & -4.8838200 & 2.3541180 & $\mathrm{C}$ & -4.2173480 & -1.4708770 & -1.3996370 \\
\hline c & 1.0447210 & -3.8129660 & 5.5798490 & C & -4.0912580 & -2.0779560 & 0.9079470 \\
\hline $\mathrm{H}$ & -0.0407930 & -2.4567730 & 4.2964960 & $\mathrm{C}$ & -4.8183890 & -1.5225110 & -0.1406790 \\
\hline C & 2.0360880 & -4.7913190 & 5.6412250 & $\mathrm{H}$ & -4.7692050 & -1.0359560 & -2.2303650 \\
\hline H & 3.4834330 & -5.9436970 & 4.5375550 & $\mathrm{H}$ & -4.5499030 & -2.1244080 & 1.8947690 \\
\hline $\mathrm{H}$ & 0.5206310 & -3.5120500 & 6.4811250 & C & 3.8463270 & 1.4988210 & 0.3463840 \\
\hline $\mathrm{H}$ & 2.2828450 & -5.2515240 & 6.5931060 & C & 3.2593010 & 2.4789500 & 1.1751380 \\
\hline $\mathrm{H}$ & -0.0706430 & -1.6842610 & 1.0027050 & C & 4.5048330 & 1.8706190 & -0.8362210 \\
\hline 0 & 1.6255670 & -3.3993890 & 0.8529080 & C & 3.3860150 & 3.8190170 & 0.8135870 \\
\hline & & & & $\mathrm{C}$ & 4.6051120 & 3.2275980 & -1.1557430 \\
\hline & S) $\mathrm{G}[\mathrm{M06}-2 \mathrm{x}$ & $G(d)]=-33$ & 398 & $\mathrm{C}$ & 4.0587780 & 4.2154300 & -0.3439600 \\
\hline 0 & 0.0150340 & -1.1809550 & 0.3051530 & $\mathrm{H}$ & 2.9361140 & 4.5785900 & 1.4486420 \\
\hline 0 & 2.0091200 & -0.2129160 & -0.8889600 & $\mathrm{H}$ & 5.1207790 & 3.5224810 & -2.0682940 \\
\hline C & 0.2623670 & -2.4100280 & -0.2715490 & C & -2.0825540 & -3.2004820 & 1.9192320 \\
\hline C & 1.5510770 & -2.8998460 & -0.2807330 & C & -6.2348840 & -1.0232200 & 0.0751490 \\
\hline C & -0.8647080 & -3.1305280 & -0.7520310 & c & -2.3469060 & -1.9241960 & -3.0339740 \\
\hline C & 1.7878860 & -4.1490150 & -0.9449230 & $\mathrm{C}$ & 2.5160240 & 2.1096190 & 2.4514780 \\
\hline c & -0.6386830 & -4.3681670 & -1.2976680 & c & 4.1599770 & 5.6810420 & -0.7222990 \\
\hline $\mathrm{c}$ & 0.6766690 & -4.8875760 & -1.4418270 & $\mathrm{C}$ & 5.1110950 & 0.8453770 & -1.7815510 \\
\hline $\mathrm{H}$ & -1.4787110 & -4.9717400 & -1.6319940 & $\mathrm{C}$ & 6.6338820 & 1.0037820 & -1.8626710 \\
\hline C & 2.7845370 & -0.7816230 & 0.1027560 & $\mathrm{H}$ & 6.9057450 & 1.9838490 & -2.2701040 \\
\hline C & 3.7573880 & 0.0521330 & 0.7175230 & $\mathrm{H}$ & 7.0910410 & 0.9121660 & -0.8723280 \\
\hline C & 2.6221230 & -2.1191530 & 0.3993980 & $\mathrm{H}$ & 7.0654220 & 0.2371520 & -2.5146500 \\
\hline C & 4.6054970 & -0.5269940 & 1.6265480 & C & 4.4711160 & 0.9250320 & -3.1720630 \\
\hline C & 3.4452240 & -2.6867350 & 1.4300120 & $\mathrm{H}$ & 3.3880830 & 0.7884580 & -3.1066570 \\
\hline C & 4.4665290 & -1.8854780 & 2.0150360 & $\mathrm{H}$ & 4.6692430 & $\begin{array}{l}1.8938470 \\
\text {. }\end{array}$ & -3.6438510 \\
\hline $\mathrm{H}$ & 5.3948020 & 0.0690690 & 2.0778750 & $\mathrm{H}$ & 4.8800690 & 0.1459970 & -3.8239850 \\
\hline C & 5.1308250 & -3.7340400 & 3.4413890 & c & 4.8370820 & 6.5086200 & 0.3753350 \\
\hline C & 4.0857220 & -4.5168210 & 2.8970770 & $\mathrm{H}$ & 4.9531480 & 7.5495110 & 0.0559870 \\
\hline
\end{tabular}




$\begin{array}{lrrr}\mathrm{H} & 4.2383470 & 6.5059710 & 1.2926400 \\ \mathrm{H} & 5.8259170 & 6.1084640 & 0.6175580 \\ \mathrm{C} & 2.7786340 & 6.2543920 & -1.0611690 \\ \mathrm{H} & 2.3095650 & 5.6879450 & -1.8713620 \\ \mathrm{H} & 2.1165710 & 6.2070880 & -0.1890510 \\ \mathrm{H} & 2.8573640 & 7.3021350 & -1.3699780 \\ \mathrm{C} & 3.4662600 & 2.1253740 & 3.6571920 \\ \mathrm{H} & 4.2879290 & 1.4144050 & 3.5370670 \\ \mathrm{H} & 3.8990300 & 3.1245260 & 3.7842480 \\ \mathrm{H} & 2.9254420 & 1.8692420 & 4.5747020 \\ \mathrm{C} & 1.3166960 & 3.0222520 & 2.7341440 \\ \mathrm{H} & 1.6370950 & 4.0072100 & 3.0929800 \\ \mathrm{H} & 0.7005340 & 3.1562230 & 1.8418790 \\ \mathrm{H} & 0.6956020 & 2.5784670 & 3.5194730 \\ \mathrm{C} & -2.7121490 & -4.5504700 & 2.2842650 \\ \mathrm{H} & -3.7617320 & -4.4229880 & 2.5728860 \\ \mathrm{H} & -2.6772010 & -5.2403990 & 1.4350520 \\ \mathrm{H} & -2.1816390 & -5.0097060 & 3.1249960 \\ \mathrm{C} & -2.0560630 & -2.2578830 & 3.1260960 \\ \mathrm{H} & -1.6484990 & -1.2810780 & 2.8469170 \\ \mathrm{H} & -3.0604800 & -2.1122920 & 3.5417640 \\ \mathrm{H} & -1.4334400 & -2.6796610 & 3.9216630 \\ \mathrm{C} & -7.2408940 & -1.8778150 & -0.7051780 \\ \mathrm{H} & -8.2658940 & -1.5481890 & -0.5054890 \\ \mathrm{H} & -7.0639290 & -1.7939200 & -1.7832570 \\ \mathrm{H} & -7.1564900 & -2.9338010 & -0.4322640 \\ \mathrm{C} & -6.3843630 & 0.4571180 & -0.2862010 \\ \mathrm{H} & -5.6911100 & 1.0702580 & 0.2976620 \\ \mathrm{H} & -6.1822500 & 0.6230790 & -1.3511010 \\ \mathrm{H} & -7.4025190 & 0.8042770 & -0.0798970 \\ \mathrm{C} & -2.6788520 & -0.6265480 & -3.7760390 \\ \mathrm{H} & -3.7340380 & -0.5837150 & -4.0694210 \\ \mathrm{H} & -2.4507700 & 0.2473020 & -3.1617480 \\ \mathrm{H} & -2.0834070 & -0.5613590 & -4.6922010 \\ \mathrm{C} & -2.8239230 & -3.1264370 & -3.8605430 \\ \mathrm{H} & -3.9163570 & -3.1144680 & -3.9519820 \\ \mathrm{H} & -2.3968000 & -3.0934820 & -4.8685190 \\ \mathrm{H} & -2.5363950 & -4.0750760 & -3.3994470 \\ \mathrm{P} & 0.4557480 & 0.1318460 & -0.5579630 \\ \mathrm{O} & -0.2512260 & 0.2044820 & -1.8785570 \\ \mathrm{O} & 0.3299370 & 1.2799850 & 0.4106640 \\ \mathrm{H} & -1.2580880 & -1.9717490 & -2.9381420 \\ \mathrm{H} & -6.4570170 & -1.1266050 & 1.1454530 \\ \mathrm{H} & -1.0430380 & -3.3903000 & -1.6378300 \\ \mathrm{H} & 4.9063930 & -0.1536160 & -1.6246320 \\ \mathrm{H} & 4.7816870 & 5.7437940 & \\ & & & \end{array}$

\begin{tabular}{|c|c|}
\hline & \\
\hline $\mathrm{H}$ & 2.1290490 \\
\hline $\mathrm{C}$ & -2.6068260 \\
\hline 0 & -1.7751120 \\
\hline C & -3.9471630 \\
\hline C & -4.8650850 \\
\hline C & -4.3150690 \\
\hline C & -6.1526570 \\
\hline $\mathrm{H}$ & -4.5555610 \\
\hline C & -5.6076400 \\
\hline $\mathrm{H}$ & -3.5916180 \\
\hline C & -6.5247070 \\
\hline $\mathrm{H}$ & -6.8680850 \\
\hline $\mathrm{H}$ & -5.9016570 \\
\hline $\mathrm{H}$ & -7.5333940 \\
\hline $\mathrm{H}$ & -0.8866430 \\
\hline 0 & -2.3447750 \\
\hline C & -0.9881360 \\
\hline C & -2.1978560 \\
\hline $\mathrm{H}$ & -0.0926880 \\
\hline $\mathrm{H}$ & -2.0606860 \\
\hline 0 & -1.1496760 \\
\hline $\mathrm{H}$ & -0.6906600 \\
\hline C & -2.4664490 \\
\hline C & -3.6362890 \\
\hline C & -3.5565150 \\
\hline C & -1.1331610 \\
\hline $\mathrm{H}$ & -2.5568590 \\
\hline $\mathrm{H}$ & -4.5931360 \\
\hline $\mathrm{H}$ & -3.7787770 \\
\hline $\mathrm{H}$ & -1.0548890 \\
\hline $\mathrm{H}$ & 20 \\
\hline $\mathrm{H}$ & 2100 \\
\hline $\mathrm{H}$ & -3.60 \\
\hline $\mathrm{H}$ & -4.2800840 \\
\hline
\end{tabular}

$\begin{array}{rr}1.0932890 & 2.3255730 \\ 2.1796340 & 1.5247190 \\ 1.2223880 & 1.8043870 \\ 2.0569560 & 2.1511370 \\ 3.0987660 & 1.9909980 \\ 0.8851280 & 2.8172820 \\ 2.9679060 & 2.4981880 \\ 3.9975370 & 1.4666840 \\ 0.7550090 & 3.3134460 \\ 0.0839730 & 2.9209900 \\ 1.7938170 & 3.1531360 \\ 3.7749620 & 2.3788600 \\ -0.1572460 & 3.8238050 \\ 1.6883790 & 3.5406830 \\ 1.3010230 & 1.2709920 \\ 3.1197050 & 0.7542670 \\ 3.2375080 & -1.6329730 \\ 2.5150090 & -1.2307420 \\ 2.9726600 & -1.0713650 \\ 1.4794010 & -0.9311330 \\ 2.4410450 & -2.8306460 \\ 1.5622750 & -2.6452310 \\ 5.0437330 & -2.5975860 \\ 4.5024860 & -1.7727790 \\ 2.9809300 & -1.6385150 \\ 4.7128780 & -1.9222290 \\ 6.1274610 & -2.7156140 \\ 4.7752100 & -2.2269100 \\ 2.4889890 & -2.5944550 \\ 5.2378050 & -0.9624370 \\ 5.0264290 & -2.5400870 \\ 4.6053750 & -3.6023940 \\ 4.9451860 & -0.7699370 \\ 2.5912240 & -0.9154100\end{array}$

$\mathrm{TS} 119(\mathrm{~S}, \mathrm{~S}) \mathrm{G}[\mathrm{M} 06-2 \mathrm{X} / 6-31 \mathrm{G}(\mathrm{d})]=-3310.352923$

$\begin{array}{lrrr}\text { TS119(S, S) } & \text { G }[\mathrm{M} 06-2 \mathrm{X} / 6-31 \mathrm{G}(\mathrm{d})]=-3310.352923 \\ \mathrm{O} & 0.5280210 & 0.8619060 & 0.3505850 \\ \mathrm{O} & -1.5461050 & 1.1035290 & -1.0548030 \\ \mathrm{C} & 0.6177310 & 2.2404730 & 0.3397400 \\ \mathrm{C} & -0.4907330 & 2.9902270 & 0.6764510 \\ \mathrm{C} & 1.8822420 & 2.8057030 & 0.0258040 \\ \mathrm{C} & -0.3747430 & 4.4196700 & 0.6517020 \\ \mathrm{C} & 2.0104800 & 4.1686880 & 0.1142920 \\ \mathrm{C} & 0.9000910 & 5.0032970 & 0.4060400 \\ \text { H } & 2.9781930 & 4.6291430 & -0.0671680 \\ \mathrm{C} & -2.2507090 & 1.3498440 & 0.1131270\end{array}$




-3.4622510
-1.7652150
-4.1968840
-2.4896150
-3.7320710
-5.1541030
-3.9938890
-2.7374910
-2.0050280
-4.4741100
-4.5654780
-2.3469320
-1.0386030
-5.4265710
-1.3299600
-0.0532010
1.0339760
-1.4877700
-2.1940460
0.0573770
2.0172120
-2.4733210
3.0135700
3.3639170
3.7176590
4.4471830
4.7950480
5.1787000
4.7200970
5.3547050
-3.9180150
-3.9017870
-4.3858860
-4.4113260
-4.8748590
-4.9069040
-4.4138470
-5.2465530
3.3623920
6.3787910
2.6175410
-3.3579650
-5.4514660
-4.4025820
-5.8409500
-6.3472970

0.6333580

2.2918170

0.9334410

2. 5172370

1.8506590

0.4433280

2.9221210

3.5507390

3.3544090

2.0868140

3.0923200

4.1900030

3.8336310

1.5781100

6.6479210

7.2248070

6.4170410

5.2850080

7.2906560

8.3045120

6.8441770

4.8551830

1.9207080

1.8243950

1.1849390

1.0195920

0.3951090

0.3024710

0.9431520

$-0.1691260$

$-0.3919080$

$-1.7617300$

0.0152280

$-2.6837360$

$-0.9499800$

$-2.3006030$

$-3.7387420$

$-0.6376590$

1.2435790

$-0.5392490$

2. 5944620

$-2.2672660$

$-3.3295200$

1.4725780

1.9695890

1.4118690

0.3083490
0.9963730
1.4282140
2.2137940
2.4062670
1.5851140
4.5684140
4.3991730
3.2555240
3.5939250
5.4752520
5.1847880
3.1423560
3.7179050
0.8209170
0.6218500
0.4135570
0.8352410
0.9570550
0.6191120
0.2344080
0.9779360
-0.3876250
-1.7507310
0.5817960
-2.1065470
0.1749580
-1.1612940
-3.1550680
0.9211100
-0.6797050
-0.3362610
-1.9411660
-1.2496920
-2.8247310
-2.4962750
-0.9846280
-3.7991450
2.0598740
-1.5558750
-2.8322860
0.9946840
-3.4688190
-2.3764260
-2.5664970
-3.3620480

$-6.4226870$

$-5.8471280$

$-3.5719560$

$-2.5464480$

$-4.0052300$

$-6.6667300$

$-7.0796560$

$-6.3861960$

$-7.4529380$

$-4.3674900$

$-3.5047170$

$-4.0171850$

$-4.7590070$

$-4.4998780$

$-5.1149440$

$-5.1540010$

$-4.1009630$

-2. 4818100

$-3.0718730$

$-1.6916530$

$-2.0185190$

4.4919930

5.4122900

4.7096520

4.2148610

3. 0229090

2. 1641260

3.8734450

2.7591490

7.6464680

8.5226900

7.5422220

7.8254180

6.1823210

5.2065860

6.2364050

6.9678620

2.4574600

3.4128070

2.0293820

1.7846220

3. 3246970

4.3435190

2.7864300
3.3922430
1.8485240

3.0293780

1.6848870

1. 3343480

1.1464840

2.7484010

$-4.0605600$

$-4.7646690$

$-4.6297000$

$-3.3548970$

$-4.3287690$

$-3.8143740$

$-4.9060770$

$-5.0347150$

$-2.5676480$

$-1.6851150$

$-3.3475430$

$-2.9241410$

$-3.5166800$

$-4.3873940$

$-3.3531680$

$-3.7620730$

1.3085400

2. 9029260

1.9829820

$-0.1433340$

$-0.5689770$

$-0.8295250$

$-0.0777200$

0.3243790

$-0.2806350$

1.1122480

0.8041060

$-1.2671120$

$-1.7627410$

$-0.5684460$

$-2.0150530$

1.8055070

1.6890600

0.8179450

2.3477450

3.9248660

3.7381200

4.4786010

4.5604900
$-1.6471880$

$-2.8421240$

$-3.6470160$

$-3.5022340$

$-4.4972230$

$-3.9064680$

$-2.8872640$

$-3.6171920$

$-1.9940590$

$-2.6033250$

$-3.8888100$

$-4.3217770$

$-3.0260020$

$-4.6287640$

1.9754540

2. 1707150

1. 5683680

2.9313380

0.8372690

0.5313780

0.0994890

1.7994310

2.8628730

2.8005500

2.4827260

3.9187830

2. 6167620

2.0886310

2.5284900

3.6775890

$-1.5932990$

$-1.8492170$

$-2.3475270$

$-0.6264500$

$-2.8892900$

$-2.9350430$

$-3.7309930$

$-3.0349520$

$-4.1361560$

$-4.6596400$

$-3.9547620$

$-4.8079340$

$-3.4886740$

$-3.9059960$

$-2.2430290$ 


$\begin{array}{rrr}-0.2536410 & 0.1341250 & -0.8946200 \\ 0.5401350 & 0.2044660 & -2.1600380 \\ -0.6461170 & -1.2113450 & -0.3371870 \\ 1.6117540 & 2.8083110 & -2.4563460 \\ 6.5163350 & -1.2961600 & -0.7704290 \\ 2.4705340 & 1.8668510 & 2.1759400 \\ -3.9516130 & 2.0789470 & -1.5857720 \\ -5.7791350 & -2.7894750 & -4.3663670 \\ -2.7273480 & -1.4811490 & 1.4229420 \\ 1.5994640 & -2.6935030 & -1.7841880 \\ 2.3211540 & -2.2707070 & -0.5810690 \\ 0.5399640 & -2.4455910 & -1.7929900 \\ 1.9963200 & -1.3529510 & -0.0967220 \\ 2.4085920 & -1.6418720 & -2.3689300 \\ 1.8277870 & -0.8193360 & -2.3616360 \\ 3.4051310 & -4.4114600 & -2.2105460 \\ 3.8624620 & -4.2532520 & -0.7590430 \\ 3.6804260 & -2.8119810 & -0.2789910 \\ 1.9241020 & -4.0553710 & -2.3495900 \\ 3.5670630 & -5.4393170 & -2.5484020 \\ 4.9143650 & -4.5346900 & -0.6515400 \\ 4.3876110 & -2.1422140 & -0.7805550 \\ 1.3112130 & -4.7805810 & -1.8004720 \\ 1.6039260 & -4.0785520 & -3.3958360 \\ 4.0017570 & -3.7613590 & -2.8614520 \\ 3.2739630 & -4.9187870 & -0.1170940 \\ 3.8578800 & -2.7050710 & 0.7952800 \\ 0.8389920 & -2.9904030 & 1.9178590 \\ 0.0381980 & -1.9678020 & 1.9345190 \\ 1.0613490 & -3.6297090 & 3.2429830 \\ 2.0043670 & -4.6541420 & 3.3502830 \\ 0.3443950 & -3.2091210 & 4.3661240 \\ 2.2316340 & -5.2580460 & 4.5811370 \\ 2.5475070 & -4.9645780 & 2.4634100 \\ 0.5739470 & -3.8175570 & 5.5953450 \\ -0.3830240 & -2.4110400 & 4.2646520 \\ 1.5159410 & -4.8397580 & 5.7025610 \\ 2.9643070 & -6.0537880 & 4.6685220 \\ 0.0188390 & -3.4952220 & 6.4702500 \\ 1.6931330 & -5.3124690 & 6.6637550 \\ -0.2112060 & -1.6310080 & 0.9781930 \\ 1.4037830 & -3.4289510 & 0.9043410\end{array}$

TS120(S, S) G[M06-2X/6-31G(d)] = -3310.352437

$\begin{array}{rrrrrr}\text { TS120(S, S }) & \text { G }[M 06-2 X / 6-31 G(d)]= & -3310.352437 & \mathrm{C} & -4.8515010 \\ \mathrm{O} & 0.4984540 & 0.8565760 & 0.3709870 & \mathrm{H} & -4.2386420 \\ \mathrm{O} & -1.6339890 & 0.9821260 & -0.9607460 & \mathrm{H} & -5.3207730\end{array}$

0.5123370
-0.6231670
1.7301320
-0.5892960
1.7837220
0.6399150
2.7160640
-2.3079330
-3.4700350
-1.8438570
-4.1780640
-2.5345240
-3.7293870
-5.0995720
-3.9703130
-2.7588540
-2.0596880
-4.4385820
-4.5161570
-2.3761620
-1.1267710
-5.3554070
-1.6623210
-0.4291910
0.6933040
-1.7417090
-2.5555540
-0.3806040
1.6428580
-2.6949920
2.8948920
3.2069040
3.6709460
4.3231770
4.7769760
5.1236250
4.5672870
5.3909600
-3.9039100
-3.8052460
-4.4353980
-4.2963680
-4.9015740
-4.8515010
-4.2386420
-5.3207730

2. 2381600

2.9255890

2. 8722530

4.3590350

4.2408440

5.0127660

4.7545060

1. 1894060

0.4070760

2.1570810

0.6648040

2. 3418920

1.6061900

0.1213710

2. 6610110

3. 3600810

3. 2055350

1.7998270

2.7983910

. 0205520

3.7386630

1.2380000

6. 5301360

7.1775700

6.4319050

5. 1606130

.

8.2617820

6.9133500

4.6762900

2.0511560

1.9736320

1. 3582890

1.2326210

0.6292020

0.5585000

1. 1722470

0.0979880

$-0.6412310$

$-2.0052800$

$-0.2586120$

$-2.9538620$

$-1.2452330$

$-2.5988900$

$-4.0092080$

$-0.9455480$
0.3566110

0.7336340

$-0.0061730$

0.7028230

0.0735280

0.4060410

$-0.1459690$

0.2324510

0.4701800

1.0992570

1. 6173810

2. 3427020

2. 5798150

1.8084490

4.7525350

4.5377630

3. 3671830

3.7949050

5.6805640

5.3095750

3. 2190170

3. 9533070

0.9039570

0.6533350

0.4046280

0.9279550

1.0720920

0.6432830

0.1858490

1. 1098200

$-0.4572770$

$-1.8308430$

0.4886620

$-2.2215600$

0.0467040

$-1.3009070$

$-3.2781730$

0.7741040

$-0.5031210$

$-0.1629180$

$-1.7502540$

$-1.0635080$

$-2.6177890$

$-2.2893150$

$-0.8063730$

$-3.5768850$ 


$\begin{array}{rrr}-0.2821270 & 0.0895110 & -0.8511870 \\ 0.4619760 & 0.2130410 & -2.1425970 \\ -0.5767980 & -1.2795940 & -0.2907080 \\ 3.3632980 & 1.4000270 & 1.9780000 \\ 2.4522940 & 1.9878400 & 2.1260650 \\ 6.3581390 & -0.2118900 & -1.7331550 \\ 6.5588600 & -0.9646350 & -0.9573600 \\ 2.3837080 & 2.6976560 & -2.8880830 \\ 1.3815630 & 2.8587780 & -2.4780350 \\ -4.5467900 & 1.1970400 & -2.1784620 \\ -4.0995090 & 1.8257020 & -1.4032530 \\ -5.3747010 & -3.6541480 & -3.2451860 \\ -5.2323790 & -4.6304380 & -2.7642190 \\ -3.1936760 & -2.4833160 & 1.1483190 \\ -2.5966130 & -1.6635800 & 1.5614070 \\ -2.2534270 & -3.6804960 & 0.9583340 \\ -1.7462220 & -3.9010350 & 1.9042320 \\ -2.8028410 & -4.5823860 & 0.6677050 \\ -1.4978980 & -3.4708150 & 0.1963960 \\ -4.2867260 & -2.8506300 & 2.1615750 \\ -4.9081730 & -3.6648340 & 1.7708590 \\ -3.8397420 & -3.1871040 & 3.1033250 \\ -4.9441450 & -2.0045610 & 2.3793570 \\ 3.0971260 & -0.0007540 & 2.5399740 \\ 3.9678580 & -0.6549230 & 2.4122600 \\ 2.8757320 & 0.0501060 & 3.6113070 \\ 2.2328970 & -0.4544160 & 2.0452600 \\ 4.4919810 & 2.0982890 & 2.7462680 \\ 4.2438650 & 2.1713100 & 3.8100850 \\ 5.4314620 & 1.5416670 & 2.6564560 \\ 4.6607710 & 3.1085200 & 2.3605780 \\ -3.7784470 & 1.4581700 & -3.4786290 \\ -4.2093800 & 0.8942900 & -4.3134190 \\ -3.8225860 & 2.5214090 & -3.7371290 \\ -2.7289370 & 1.1704520 & -3.3724120 \\ -6.0166910 & 1.6146120 & -2.3105080 \\ -6.0921660 & 2.6731070 & -2.5807940 \\ -6.5221010 & 1.0314020 & -3.0883050 \\ -6.5547210 & 1.4590250 & -1.3700010 \\ 2.2213610 & 1.8947690 & -4.1832130 \\ 1.5010350 & 2.3994990 & -4.8347410 \\ 3.1638160 & 1.8231550 & -4.7371970 \\ 1.8485190 & 0.8882740 & -3.9848410 \\ 3.0077000 & 4.0625580 & -3.2134530 \\ 4.0221370 & 3.9291610 & -3.6071580 \\ 2.4140850 & 4.5830910 & -3.9723630\end{array}$

3. 0715560 $-6.8731180$ $-7.4453250$ $-7.0657750$ $-7.2480060$ $-4.5857930$ $-3.5173460$ $-4.9392000$ $-4.7085170$ 7.5738030 7.7503570 8.4757810 7.4060220 6.1686460 5. 2225310 6.1597080 6.9920640 1. 6969450 2. 4325740 0.6263150 2. 0748490 2. 4317320 1. 8078260 3. 5754030 4.0673310 3. 8274850 2. 0745310 3. 7795660 5.1347680 4.4808270 1. 5165320 1. 7247230 4.1180970 3. 5324350 4.0360170 1. 0871350 0.2363110 1. 4042370 2. 3968260 0.7268490 2.7131760 2. 9075550 1. 0458080 $-0.0400340$ 2. 0372170
3.4843510
4. 7050000 $-3.4732210$ $-3.4900110$ $-2.5155430$ $-4.2694370$ $-3.6554940$ $-3.8025410$ $-4.4531740$ $-2.7027250$

0.7222550

1. 2055540

0.1691520

1. 5074210

$-0.9410650$

$-1.4924560$

$-0.2347990$

$-1.6409970$

$-2.6187610$

$-2.1750410$

$-2.4266590$

$-1.2831620$

$-1.5171440$

$-0.7276790$

$-4.2343820$

$-4.0718890$

$-2.6476700$

$-3.9534490$

$-5.2478570$

$-4.3010020$

$-1.9371520$

$-4.7173610$

$-3.9780540$

$-3.5455820$

$-4.7741360$

$-2.9892880$

$-2.0092620$

$-3.6131310$

-4.5936120

$-3.2202940$

$-5.1812400$

$-4.8835000$

$-3.8121350$

$-2.4562070$

$-4.7904480$

$-5.9429800$
$-2.3315990$

$-3.5127490$

$-2.5805340$

$-4.0088720$

$-4.1643370$

$-4.5595370$

$-4.3772760$

$-5.2213490$

$-5.0863170$

$-1.7964370$

$-0.8309310$

$-2.0790050$

$-2.5419630$

$-3.0667100$

$-3.0909930$

- 3.9037750

$-3.2390930$

$-1.8296310$

$-0.6427110$

$-1.8023890$

$-0.1338350$

$-2.4209460$

$-2.3864720$

$-2.3359130$

$-0.8963390$

$-0.3917740$

$-2.4261940$

$-2.6937390$

$-0.8236050$

$-0.9097280$

$-1.8710480$

$-3.4628800$

$-2.9942180$

$-0.2466730$

0.6771020

1.8925660

1. 9467290

3.2055680

3. 2669360

4. 3628710

4.4859550

2. 3542010

5.5801520

4. 2968000

5.6414830

4.5376970 


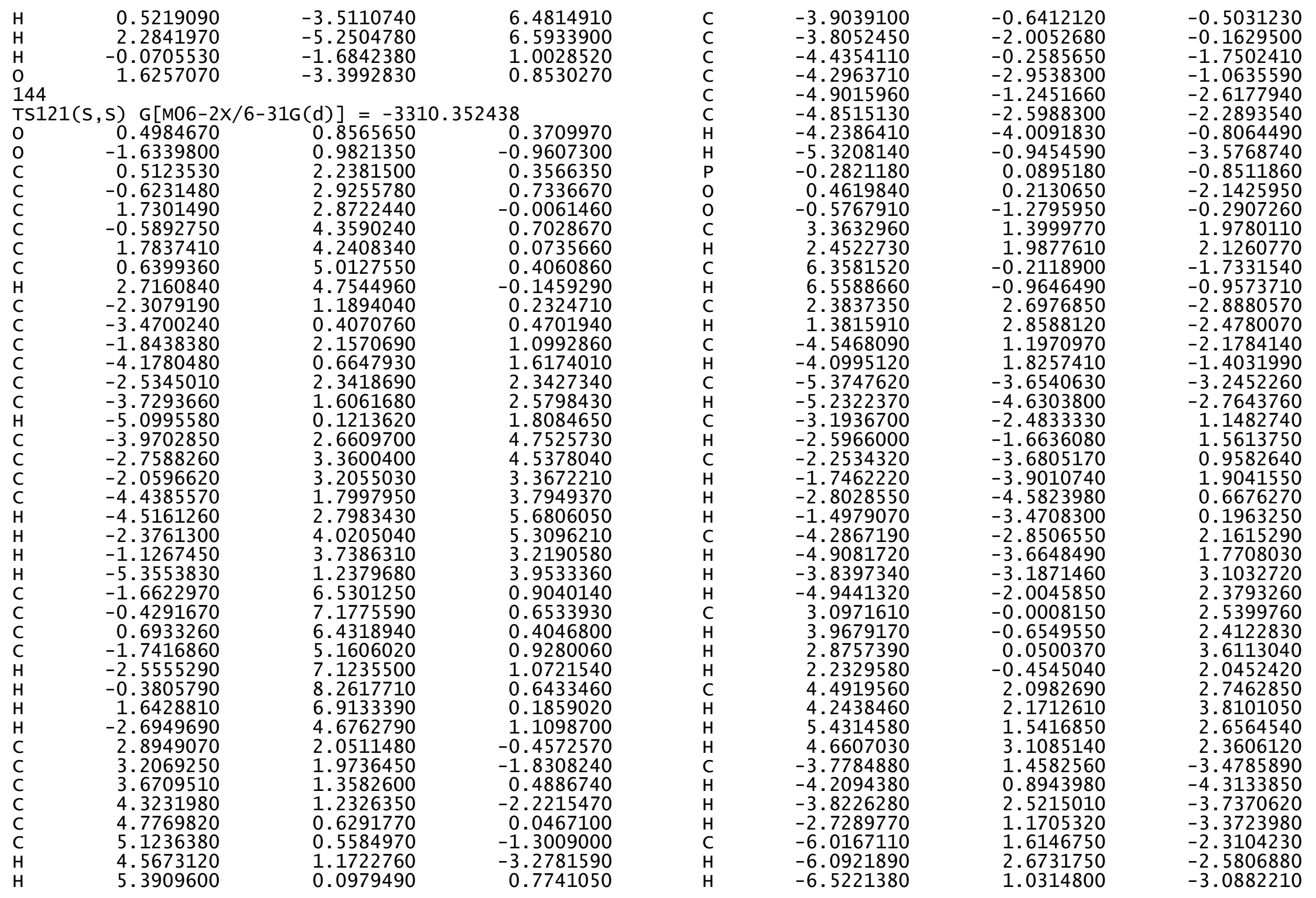




$\begin{array}{rr}-6.5547230 & 1.4590700 \\ 2.2213820 & 1.8948110 \\ 1.5010650 & 2.3995570 \\ 3.1638370 & 1.8231880 \\ 1.8485240 & 0.8883200 \\ 3.0077390 & 4.0625850 \\ 4.0221700 & 3.9291820 \\ 2.4141210 & 4.5831360 \\ 3.0716140 & 4.7050140 \\ -6.8732540 & -3.4732700 \\ -7.4452740 & -3.4902550 \\ -7.0661100 & -2.5155390 \\ -7.2481830 & -4.2694350 \\ -4.5861210 & -3.6552090 \\ -3.5176150 & -3.8021090 \\ -4.9395450 & -4.4529020 \\ -4.7090930 & -2.7024310 \\ 7.5738190 & 0.7222520 \\ 7.7503710 & 1.2055330 \\ 8.4757970 & 0.1691510 \\ 7.4060440 & 1.5074320 \\ 6.1686630 & -0.9410420 \\ 5.2225440 & -1.4924250 \\ 6.1597390 & -0.2347620 \\ 6.9920770 & -1.6409780 \\ 1.6969520 & -2.6187380 \\ 2.4325860 & -2.1750380 \\ 0.6263230 & -2.4266320 \\ 2.0748650 & -1.2831660 \\ 2.4317430 & -1.5171130 \\ 1.8078410 & -0.7276470 \\ 3.5754030 & -4.2343600 \\ 4.0673340 & -4.0718890 \\ 3.8274970 & -2.6476750 \\ 2.0745330 & -3.9534170 \\ 3.7795600 & -5.2478320 \\ 5.1347690 & -4.3010090 \\ 4.4808380 & -1.9371550 \\ 1.5165310 & -4.7173360 \\ 1.7247240 & -3.9780030 \\ 4.1181020 & -3.5455550 \\ 3.5324340 & -4.7741410 \\ 4.0360350 & -2.5432260 \\ 1.0871430 & -2.9893290 \\ 0.2363030 & -2.0093170 \\ 1.4042430 & -3.6131920\end{array}$

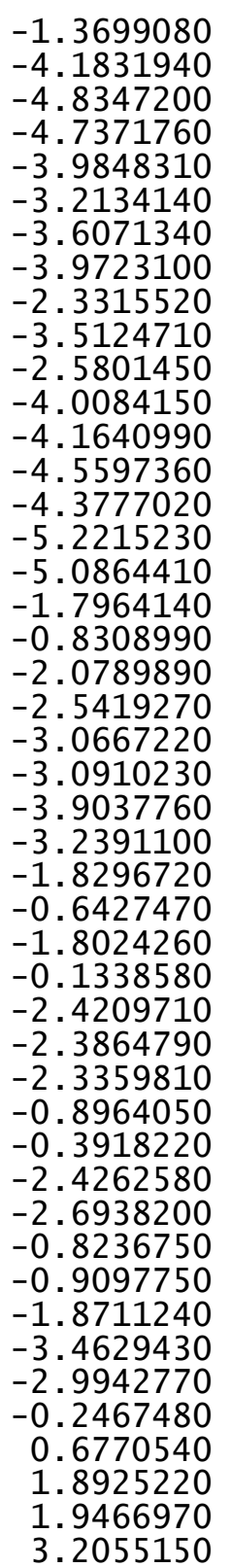

2.3968730
0.7268120
2.7132230
2.9076350
1.0457700
-0.0401020
2.0372210
3.4844330
0.5218370
2.2842010
-0.0705510
1.6257290

$$
\begin{aligned}
& -4.5936310 \\
& -3.2204150 \\
& -5.1812760 \\
& -4.8834740 \\
& -3.8122750 \\
& -2.4563590 \\
& -4.7905460 \\
& -5.9429820 \\
& -3.5112630 \\
& -5.2505900 \\
& -1.6842700 \\
& -3.3992960
\end{aligned}
$$
3.2668780
4. 3628130
4.4858900
2. 3541470
5.5800860
4. 2967450
5.6414130
4.5376290
6.4814200
6.5933130
1. 0028250
1.0028250
0.8529800

TS122(S, S) G[M06-2X/6-31G(d) $]=-3310.353364$

$\begin{array}{lrcr}\text { TS } 122(\mathrm{~S}, \mathrm{~S}) \mathrm{G}[\mathrm{M} 06-2 \mathrm{X} / 6-31 \mathrm{G}(\mathrm{d})]=-3310.353364 \\ \mathrm{O} & 0.4726920 & 0.7813040 & 0.1980620 \\ \mathrm{O} & -1.7621440 & 0.9425300 & -0.9642470 \\ \mathrm{C} & 0.5277660 & 2.1614110 & 0.1705780 \\ \mathrm{C} & -0.5644780 & 2.8885350 & 0.5997690 \\ \mathrm{C} & 1.7501040 & 2.7563810 & -0.2408220 \\ \mathrm{C} & -0.4949470 & 4.3195380 & 0.5387500 \\ \mathrm{C} & 1.8421130 & 4.1247110 & -0.1902400 \\ \mathrm{C} & 0.7335830 & 4.9346620 & 0.1685930 \\ \mathrm{H} & 2.7821700 & 4.6070590 & -0.4458630 \\ \mathrm{C} & -2.3360920 & 1.1959800 & 0.2712820 \\ \mathrm{C} & -3.4997430 & 0.4576240 & 0.6167280 \\ \mathrm{C} & -1.7772850 & 2.1681620 & 1.0729780 \\ \mathrm{C} & -4.1100540 & 0.7667130 & 1.8066310 \\ \mathrm{C} & -2.3639500 & 2.4058020 & 2.3602310 \\ \mathrm{C} & -3.5600800 & 1.7167340 & 2.7064910 \\ \mathrm{H} & -5.0305740 & 0.2586250 & 2.0816620 \\ \mathrm{C} & -3.6015250 & 2.8334550 & 4.8612710 \\ \mathrm{C} & -2.3880660 & 3.4852010 & 4.5371440 \\ \mathrm{C} & -1.7852200 & 3.2779120 & 3.3220570 \\ \mathrm{C} & -4.1683310 & 1.9647710 & 3.9655650 \\ \mathrm{H} & -4.0709010 & 3.0127100 & 5.8233630 \\ \mathrm{H} & -1.9268890 & 4.1513870 & 5.2595630 \\ \mathrm{H} & -0.8496260 & 3.7750620 & 3.0901780 \\ \mathrm{H} & -5.0878890 & 1.4385090 & 4.2079580 \\ \mathrm{C} & -1.5006560 & 6.5212430 & 0.7515140 \\ \mathrm{C} & -0.2660910 & 7.1310150 & 0.4245010 \\ \mathrm{C} & 0.8231650 & 6.3515620 & 0.1350950 \\ \mathrm{C} & -1.6133560 & 5.1550900 & 0.8079010 \\ \mathrm{H} & -2.3685280 & 7.1412370 & 0.9532820 \\ \mathrm{H} & -0.1906990 & 8.2131680 & 0.3896550 \\ \mathrm{H} & 1.7725880 & 6.8035550 & -0.1397600 \\ \mathrm{H} & -2.5675590 & 4.6991600 & 1.0490510\end{array}$




$\begin{array}{rrr}2.9061910 & 1.9101210 & -0.6709240 \\ 3.1923410 & 1.7477240 & -2.0441910 \\ 3.7248940 & 1.3090730 & 0.2988840 \\ 4.3267260 & 1.0225890 & -2.4036890 \\ 4.8619470 & 0.6046070 & -0.1124850 \\ 5.1899220 & 0.4640080 & -1.4573430 \\ 4.5555570 & 0.8856440 & -3.4585440 \\ 5.4976110 & 0.1550930 & 0.6463440 \\ -4.0337350 & -0.6003560 & -0.2942140 \\ -3.9330400 & -1.9587810 & 0.0662320 \\ -4.6552020 & -0.2317930 & -1.5032240 \\ -4.5090510 & -2.9155110 & -0.7733720 \\ -5.2055350 & -1.2260150 & -2.3107380 \\ -5.1521750 & -2.5738460 & -1.9594300 \\ -4.4492370 & -3.9665330 & -0.4996260 \\ -5.6939250 & -0.9373600 & -3.2400770 \\ 3.4239700 & 1.4021290 & 1.7874460 \\ 6.4413070 & -0.2572500 & -1.9365610 \\ 2.3282330 & 2.3862190 & -3.1233870 \\ -3.2326400 & -2.4204420 & 1.3380750 \\ -5.7676890 & -3.6372280 & -2.8490040 \\ -4.7713470 & 1.2171630 & -1.9520600 \\ -6.2387830 & 1.6619030 & -1.9840570 \\ -6.8107860 & 1.0749400 & -2.7112500 \\ -6.7090730 & 1.5343470 & -1.0038750 \\ -6.3140610 & 2.7165520 & -2.2690160 \\ -4.0970470 & 1.4373140 & -3.3107030 \\ -3.0488190 & 1.1284270 & -3.2769420 \\ -4.6008630 & 0.8673990 & -4.0993140 \\ -4.1386580 & 2.4962380 & -3.5867480 \\ -5.0946350 & -3.6711850 & -4.2258050 \\ -5.5124450 & -4.4756680 & -4.8401510 \\ -5.2515540 & -2.7263810 & -4.7577770 \\ -4.0162010 & -3.8286200 & -4.1332260 \\ -7.2811960 & -3.4394340 & -2.9904210 \\ -7.7716190 & -3.4342230 & -2.0125530 \\ -7.5032140 & -2.4865550 & -3.4835200 \\ -7.7205880 & -4.2399600 & -3.5947320 \\ -4.2530820 & -2.7343950 & 2.4410190 \\ -4.8706660 & -1.8660110 & 2.6859390 \\ -4.9225180 & -3.5408480 & 2.1197250 \\ -3.7434520 & -3.0599050 & 3.3543850 \\ -2.3378250 & -3.6448170 & 1.1080830 \\ -2.9288550 & -4.5408450 & 0.8898460 \\ -1.6439880 & -3.4731570 & 0.2810330 \\ -1.7585830 & -3.8504850 & 2.0149010\end{array}$

4. 5619470

5. 4895640

4.7591080

4.3029630

3. 1262860

2. 2567320

3. 9843390

2. 8993920

7. 1440070

7.9809940

7. 5500640

6.4717020

7.4266740

6.9659940

7. 7560200

8. 3096640

2. 1907860

3. 1267510

1.8894630

1. 4287220

2. 8904100

3. 9092710

2. 2723440

2. 9247400

$-0.4312410$

0.2064720

$-0.7090850$

1. 3242160

6.1308550

2. 5246780

$-4.2558510$

$-5.5970680$

$-2.5864820$

1. 4802820

2. 2975030

0.4069650

1. 9496440

2. 0960310

1. 4454360

3. 3952940

4. 0060010

3. 7322890

1.8774730

3. 6287110

5. 0873260

4.2913420
2.0926940

1. 5130710

3. 0882040

2. 1999490

0.0161410

$-0.4259160$

$-0.6573440$

0.0857720

$-1.0782580$

$-1.6312650$

$-0.4286470$

$-1.8002590$

0.7512010

1. 3211200

1. 4616350

0.2393860

1.5237840

1.4879220

0.5018180

1. 9562020

3. 7606540

3.6538360

4.2166960

4. 4465520

0.0162220

0.0981900

$-1.3332250$

2. 5232880

$-0.9445650$

2. 0111800

1.8512170

$-4.6074710$

$-1.6062610$

$-2.7513400$

$-2.1961330$

$-2.6113740$

$-1.2861350$

$-1.6624980$

$-0.8913880$

$-4.2908230$

$-4.0213980$

$-2.5830720$

$-4.1023130$

$-5.3079790$

$-4.1905090$

$-1.8739640$
2.5477480

2.4875910

2. 1380620

3.6059880

2. 3722690

1.8761310

2. 2566060

3. 4415080

$-0.8555980$

$-1.2918450$

$-0.0722150$

$-0.3798970$

$-2.5454420$

$-3.3571410$

$-1.7793090$

$-2.9417750$

$-4.3819130$

$-4.9504380$

$-4.1453200$

$-5.0373700$

$-3.5155820$

$-3.9058910$

$-4.2961770$

$-2.6655940$

$-0.9401050$

$-2.2914910$

$-0.3269820$

$-2.7093840$

$-2.7375060$

1.9205170

$-1.2249250$

$-2.3650240$

1.6827310

$-1.9294280$

$-0.8467390$

$-1.8121860$

$-0.3633350$

$-2.6584010$

$-2.6013490$

$-2.5197870$

$-1.1434000$

$-0.7030690$

$-2.4763660$

$-2.8478130$

$-1.1576530$

$-1.3242930$ 


\begin{tabular}{|c|c|c|c|c|c|c|c|}
\hline $\mathrm{H}$ & 1.4238540 & -4.8580500 & -1.8235970 & C & -1.5006650 & 6.5212410 & 0.7515530 \\
\hline $\mathrm{H}$ & 1.4324330 & -4.2140270 & -3.4699610 & C & -0.2661000 & 7.1310160 & 0.4245450 \\
\hline $\mathrm{H}$ & 3.8282740 & -3.6040050 & -3.2571770 & C & 0.8231570 & 6.3515660 & 0.1351370 \\
\hline H & 3.5725210 & -4.7098190 & -0.4086670 & C & -1.6133630 & 5.1550870 & 0.8079330 \\
\hline $\mathrm{H}$ & 4.0338920 & -2.3943710 & 0.3319020 & $\mathrm{H}$ & -2.3685380 & 7.1412330 & 0.9533240 \\
\hline C & 1.1835090 & -2.8998930 & 1.8316770 & $\mathrm{H}$ & -0.1907100 & 8.2131690 & 0.3897050 \\
\hline 0 & 0.2634020 & -1.9842710 & 1.8804250 & $\mathrm{H}$ & 1.7725800 & 6.8035620 & -0.1397150 \\
\hline C & 1.6307270 & -3.4029010 & 3.1587030 & $\mathrm{H}$ & -2.5675660 & 4.6991550 & 1.0490790 \\
\hline C & 2.6996190 & -4.2998500 & 3.2155230 & C & 2.9061900 & 1.9101320 & -0.6709010 \\
\hline C & 1.0032260 & -2.9763400 & 4.3320040 & $\mathrm{C}$ & 3.1923510 & 1.7477500 & -2.0441670 \\
\hline C & 3.1410410 & -4.7717020 & 4.4459790 & C & 3.7248860 & 1.3090720 & 0.2989070 \\
\hline H & 3.1707970 & -4.6162220 & 2.2904350 & $\mathrm{C}$ & 4.3267390 & 1.0226170 & -2.4036640 \\
\hline C & 1.4475460 & -3.4523190 & 5.5607900 & C & 4.8619410 & 0.6046100 & -0.1124620 \\
\hline $\mathrm{H}$ & 0.1768810 & -2.2767120 & 4.2695680 & C & 5.1899270 & 0.4640250 & -1.4573180 \\
\hline C & 2.5142610 & -4.3482750 & 5.6175000 & $\mathrm{H}$ & 4.5555780 & 0.8856850 & -3.4585190 \\
\hline $\mathrm{H}$ & 3.9716590 & -5.4683990 & 4.4940620 & $\mathrm{H}$ & 5.4976000 & 0.1550880 & 0.6463680 \\
\hline $\mathrm{H}$ & 0.9626970 & -3.1249720 & 6.4747160 & C & -4.0337310 & -0.6003550 & -0.2942260 \\
\hline H & 2.8590740 & -4.7176910 & 6.5784550 & C & -3.9330340 & -1.9587830 & 0.0662090 \\
\hline $\mathrm{H}$ & -0.1059020 & -1.7123230 & 0.9452870 & C & -4.6551980 & -0.2317830 & -1.5032330 \\
\hline 0 & 1.6875030 & -3.3424040 & 0.7883660 & $\mathrm{C}$ & -4.5090420 & -2.9155060 & -0.7734040 \\
\hline & & & & C & -5.2055290 & -1.2259990 & -2.3107560 \\
\hline & S) G[M06-2 & $G(d)]=-33$ & 365 & C & -5.1521660 & -2.5738330 & -1.9594600 \\
\hline 0 & 0.472 & 0.7813070 & 0.1980740 & $\mathrm{H}$ & -4.4492260 & -3.9665310 & -0.4996670 \\
\hline 0 & -1.7621410 & 0.9425390 & -0.9642420 & $\mathrm{H}$ & -5.6939180 & -0.9373370 & -3.2400930 \\
\hline C & 0.5277640 & 2.1614150 & 0.1705980 & $\mathrm{P}$ & -0.4312350 & 0.0162340 & -0.9401050 \\
\hline C & -0.5644820 & 2.8885340 & 0.5997900 & 0 & 0.2064820 & 0.0982180 & -2.2914880 \\
\hline C & 1.7501020 & 2.7563880 & -0.2407980 & 0 & -0.7090780 & -1.3332200 & -0.3269970 \\
\hline C & -0.4949520 & 4.3195380 & 0.5387780 & C & 3.4239530 & 1.4021160 & 1.7874670 \\
\hline C & 1.8421090 & 4.1247180 & -0.1902090 & $\mathrm{H}$ & 2.5246530 & 2.0111550 & 1.9205380 \\
\hline C & 0.73 & 4.9346650 & 0.1686270 & C & 6.4413130 & -0.2572310 & -1.9365350 \\
\hline $\mathrm{H}$ & $2.7 \varepsilon$ & 4.6070690 & -0.4458270 & $\mathrm{H}$ & 6.1308630 & -0.9445430 & -2.7374830 \\
\hline C & -2.3360920 & 1.1959790 & 0.2712880 & C & 2.3282520 & 2.3862570 & -3.1233640 \\
\hline C & -3.4997430 & 0.4576190 & 0.6167260 & $\mathrm{H}$ & 1.3242350 & 2.5233310 & -2.7093640 \\
\hline C & -1.7 & 2.1681570 & 1.0729910 & C & -4.7713460 & 1.2171760 & -1.9520570 \\
\hline C & -4.1 & 0.7666990 & 1.8066290 & $\mathrm{H}$ & -4.2558520 & 1.8512260 & -1.2249160 \\
\hline C & -2.36 & 2.4057880 & 2.3602440 & C & -5.7676780 & -3.6372090 & -2.8490430 \\
\hline C & -3.56 & 1.7167150 & 2.7064960 & $\mathrm{H}$ & -5.5970540 & -4.6074550 & -2.3650720 \\
\hline $\mathrm{H}$ & -5.0 & 0.2586070 & 2.0816540 & C & -3.2326340 & -2.4204530 & 1.3380480 \\
\hline C & -3.6 & 34210 & 4.8612840 & $\mathrm{H}$ & -2.5864760 & -1.6062740 & 1.6827100 \\
\hline C & -2.3 & 1710 & 4.5371650 & C & -2.3378200 & -3.6448260 & 1.1080470 \\
\hline C & -1.78 & 78920 & 3.3220780 & $\mathrm{H}$ & -1.7585770 & -3.8505000 & 2.0148640 \\
\hline C & -4.1683410 & 1.9647420 & 3.9655710 & $\mathrm{H}$ & -2.9288500 & -4.5408530 & 0.8898060 \\
\hline $\mathrm{H}$ & -4.07 & 26680 & 5.8233760 & $\mathrm{H}$ & -1.6439830 & -3.4731610 & 0.2809980 \\
\hline $\mathrm{H}$ & -1.9269060 & 13540 & 5.2595890 & C & -4.2530760 & -2.7344130 & 2.4409900 \\
\hline $\mathrm{H}$ & -0.8 & 50450 & 3.0902030 & $\mathrm{H}$ & -4.9225120 & -3.5408630 & 2.1196900 \\
\hline $\mathrm{H}$ & -5.0 & 84770 & 4.2079580 & $\mathrm{H}$ & -3.7434460 & -3.0599300 & 3.3543540 \\
\hline
\end{tabular}




-4.8706590
3.1262840
3.9843460
2.8993870
2.2567370
4.5619190
4.3029310
5.4895440
4.7590660
-4.0970420
-4.6008540
-4.1386550
-3.0488130
-6.2387820
-6.3140610
-6.8107820
-6.7090740
2.1908050
1.4287520
3.1267730
1.8894660
2.8904410
3.9093030
2.2723810
2.9247740
-7.2811850
-7.7716080
-7.5032060
-7.7205750
-5.0946240
-4.0161890
-5.5124320
-5.2515440
7.1440100
6.4717040
7.9810000
7.5500620
7.4266830
6.9660060
7.7560280
8.3096730
1.4803060
2.2975080
0.4069870
1.9496390
2.0960700

$-1.8660310$

0.0161210

$-0.6573520$

0.0857470

$-0.4259450$

2.0926930

2. 1999370

1.5130830

3.0882080

1.4373400

0.8674290

2.4962660

1.1284560

1.6619150

2.7165650

1.0749560

1.5343490

1. 5238310

1.9562620

1.4879600

0.5018680

3.7606910

3.6538680

4.2167430

4.4465820

$-3.4394170$

$-3.4342170$

$-2.4865340$

$-4.2399390$

$-3.6711520$

$-3.8285850$

$-4.4756310$

$-2.7263440$

$-1.0782430$

$-1.8002480$

$-1.6312470$

$-0.4286340$

0.7512210

1. 3211440

1.4616520

0.2394060

$-2.7513040$

$-2.1961260$

$-2.6113400$

$-1.2861400$

$-1.6624440$

2.6859170
2.3722850
2.2566220
3.4415230
1.8761430
2.5477760
3.6060170
2.4876170
2.1380970
-3.3106970
-4.0993140
-3.5867340
-3.2769360
-1.9840560
-2.2690060
-2.7112550
-1.0038760
-4.3818960
-5.0373560
-4.9504130
-4.1453100
-3.5155470
-3.9058540
-4.2961410
-2.6655540
-2.9904580
-2.0125900
-3.4835480
-3.5947760
-4.2258440
-4.1332670
-4.8401980
-4.7578080
-0.8555730
-0.3798780
-1.2918180
-0.0721850
-2.5454100
-3.3571080
-1.7792730
-2.9417430
-1.9294530
-0.8467350
-1.8122280
-0.3633160
-2.6583870

$-2.6583870$

\begin{abstract}
1. 4454730
3.3953270

4.0060100

3.7322910
1.8775050

1. 8775050

3.6287500

5.0873340

4.2913520

1.4238750

1.4324830

3.8283220

3. 5725160

4.0338790

1. 1834920

0.2634030

1.6307010

2. 6995850

1.0032010

3.1409990

3.1707630

1. 4475130

0.1768620

2. 5142190

3.9716100

0.9626630

2.8590250

-0.1059000
1.687480
\end{abstract}

144

$\operatorname{TS} 124(\mathrm{~S}, \mathrm{~S})$ G[M06-2X/6-31G(d) $]=-3310.352435$

$\begin{array}{lrrr}\text { O } & 0.4984930 & 0.8565880 & 0.3710100 \\ \mathrm{O} & -1.6339320 & 0.9821950 & -0.9607470 \\ \mathrm{C} & 0.5123910 & 2.2381730 & 0.3566710 \\ \mathrm{C} & -0.6231100 & 2.9256060 & 0.7336950 \\ \mathrm{C} & 1.7302020 & 2.8722610 & -0.0060690 \\ \mathrm{C} & -0.5892190 & 4.3590530 & 0.7029250 \\ \mathrm{C} & 1.7838110 & 4.2408480 & 0.0736790 \\ \mathrm{C} & 0.6400070 & 5.0127760 & 0.4061900 \\ \mathrm{H} & 2.7161650 & 4.7545040 & -0.1457800 \\ \mathrm{C} & -2.3078900 & 1.1894550 & 0.2324450 \\ \mathrm{C} & -3.4700060 & 0.4071340 & 0.4701370 \\ \mathrm{C} & -1.8438150 & 2.1571050 & 1.0992810 \\ \mathrm{C} & -4.1780480 & 0.6648430 & 1.6173340 \\ \mathrm{C} & -2.5344990 & 2.3418940 & 2.3427190 \\ \mathrm{C} & -3.7293750 & 1.6062020 & 2.5797970 \\ \mathrm{H} & -5.0995660 & 0.1214180 & 1.8083760\end{array}$

$-2.6013320$

$-2.5198180$

$-2.4764190$

$-2.8478650$

$-1.1576510$

$-1.3242510$

$-1.8236790$

$-3.4700260$

$-3.2571830$

$-0.4087040$

0.3319290

1.8316450

1.8804040

3.1586660

3.2154760

4.3319710

4.4459270

2.2903850

5.5607510

4.2695410

5.6174520

4.4940030

6.4746810

6.5784020

0.9452680

0.7883290
$-1.1434120$

$-0.7030510$

$-5.0995660$

0.1214180

1.8083760 


-3.9703230
-2.7588530
-2.0596690
-4.4385870
-4.5161800
-2.3761650
-1.1267430
-5.3554210
-1.6622210
-0.4290770
0.6934140
-1.7416270
-2.5554510
-0.3804760
1.6429800
-2.6949200
2.8949560
3.2069770
3.6709810
4.3232360
4.7770020
5.1236620
4.5673510
5.3909670
-3.9038810
-3.8052430
-4.4353420
-4.2963610
-4.9015140
-4.8514640
-4.2386540
-5.3206930
3.3633100
6.3581640
2.3837990
-3.1937050
-5.3746660
-4.5467090
-6.0166030
-6.5220230
-6.5546350
-6.0920620
-3.7783570
-2.7288570
-4.2093090
-3.8224600

2.6609750

3.3600360

3.2055080

1.7998180

2.7983410

4.0204840

3.7386290

1.2379980

6.5301620

7.1775870

6.4319140

5.1606390

7.1235930

8.2617990

6.9133530

4.6763230

2.0511570

1. 9736470

1. 3582510

1. 2326140

0.6291510

0.5584650

1.1722460

0.0979110

$-0.6411410$

$-2.0052010$

$-0.2584790$

$-2.9537500$

$-1.2450690$

$-2.5987350$

$-4.0091050$

$-0.9453510$

1. 3999650

$-0.2119460$

2.6977010

-2. 4832820

$-3.6539590$

1. 1971870

1. 6147850

1.0315940

1.4591900

2.6732850

1.4583430

1.1705870

0.8945130

2.5215950

4.7525370
4.5378000
3.3672280
3.7948800
5.6805610
5.3096330
3.2190900
3.9532540
0.9040950
0.6535190
0.4048170
0.9280540
1.0722260
0.6434970
0.1860730
1.1098830
-0.4571740
-1.8307390
0.4887600
-2.2214580
0.0468010
-1.3008070
-3.2780690
0.7741990
-0.5031980
-0.1630310
-1.7503290
-1.0636580
-2.6179020
-2.2894660
-0.8065510
-3.5769960
1.9780940
-1.7330550
-2.8879710
1.1482040
-3.2453740
-2.1784970
-2.3105340
-3.0883400
-1.3700290
-2.5808040
-3.4786530
-3.3724480
-4.3134670
-3.7371090

-4.5857770
-4.9391770
-4.7085290
-3.5173240
-6.8730880
-7.4452820
-7.0657580
-7.2479790
-4.2867820
-4.9442110
-4.9082140
-3.8398220
-2.2534720
-2.8028930
-1.4979220
-1.7462940
4.4919550
5.4314690
4.6606800
4.2438440
3.0971860
2.2330100
3.9679610
2.8757260
7.5738570
8.4758240
7.4061150
7.7504040
6.1686780
5.2225450
6.1597850
6.9920770
2.2214870
3.1639520
1.8486660
1.5011590
3.0077850
4.0222200
2.4141630
3.0716450
-0.2820830
0.4620370
-0.5767780
1.3816440
6.5588450
2.4522780

-3.6552460
-4.4529120
-2.7024640
-3.8022750
-3.4730300
-3.4898700
-2.5153290
-4.2692170
-2.8506060
-2.0045410
-3.6648110
-3.1870830
-3.6804720
-4.5823440
-3.4707790
-3.9010490
2.0982730
1.5417100
3.1085260
2.1712480
-0.0008280
-0.4545410
-0.6549510
0.0500290
0.7221650
0.1690460
1.5073650
1.2054220
-0.9410680
-1.4924250
-0.2347720
-1.6410220
1.8948560
1.8232820
0.8883460
2.3995930
4.0626200
3.9292440
4.5831860
4.7050230
0.0895590
0.2131010
-1.2795540
2.8588000
-0.9647260
1.9877380

$-4.5597370$

$-5.2215690$

$-5.0864860$

$-4.3774960$

$-3.5129090$

$-2.5806870$

$-4.0089810$

$-4.1645310$

2. 1614290

2.3791970

1.7706910

3.1031890

0.9582050

0.6675360

0.1962920

1.9041080

2.7463760

2.6565370

2. 3607130

3.8101980

2.5400590

2. 0452980

2. 4124010

3.6113790

$-1.7962750$

$-2.0788460$

$-2.5417750$

$-0.8307470$

$-3.0666400$

$-3.0909640$

$-3.9036810$

$-3.2390280$

$-4.1831320$

$-4.7371050$

$-3.9848000$

$-4.8346520$

$-3.2132830$

$-3.6070020$

$-3.9721660$

$-2.3314010$

$-0.8511890$

$-2.1425890$

$-0.2907410$

$-2.4779390$

-0.9572830
2.1261510 


\begin{tabular}{|c|c|c|c|c|c|c|c|}
\hline & & & & & & & \\
\hline $\mathrm{H}$ & -4.0994200 & 1.8258200 & -1.4032680 & H & 3.3667040 & 4.2982560 & -0.0220690 \\
\hline $\mathrm{H}$ & -5.2323300 & -4.6302670 & -2.7644500 & C & -2.1415660 & 1.5043910 & 0.2027300 \\
\hline $\mathrm{H}$ & -2.5966370 & -1.6635680 & 1.5613270 & C & -3.4061440 & 0.8945540 & 0.4206430 \\
\hline C & 1.6969580 & -2.6187220 & -1.8296630 & C & -1.5226950 & 2.3361360 & 1.1113670 \\
\hline c & 2.4325660 & -2.1750560 & -0.6427100 & $\mathrm{C}$ & -4.0484140 & 1.1842950 & 1.5986310 \\
\hline $\mathrm{H}$ & 0.6263310 & -2.4266000 & -1.8024420 & C & -2.1580790 & 2.5482730 & 2.3792640 \\
\hline $\mathrm{H}$ & 2.0748440 & -1.2831880 & -0.1338130 & $\mathrm{c}$ & -3.4466870 & 1.9868640 & 2.6029920 \\
\hline 0 & 2.4317820 & -1.5170990 & -2.4209220 & $\mathrm{H}$ & -5.0398490 & 0.7775270 & 1.7796600 \\
\hline H & 1.8078950 & -0.7276210 & -2.3864290 & C & -3.4920120 & 2.9368510 & 4.8362150 \\
\hline c & 3.5753980 & -4.2343610 & -2.3359540 & $\mathrm{C}$ & -2.1928490 & 3.4591780 & 4.6314780 \\
\hline C & 4.0673010 & -4.0719220 & -0.8963650 & C & -1.5430710 & 3.2706620 & 3.4377610 \\
\hline C & 3.8274620 & -2.6477190 & -0.3917510 & C & -4.0994340 & 2.2126780 & 3.8438410 \\
\hline C & 2.0745340 & -3.9533970 & -2.4262610 & $\mathrm{H}$ & -3.9966280 & 3.0997350 & 5.7832520 \\
\hline H & 3.7795500 & -5.2478280 & -2.6938070 & $\mathrm{H}$ & -1.7037170 & 4.0095400 & 5.4291000 \\
\hline $\mathrm{H}$ & 5.1347340 & -4.3010470 & -0.8236210 & $\mathrm{H}$ & -0.5433740 & 3.6670820 & 3.2963720 \\
\hline $\mathrm{H}$ & 4.4808290 & -1.9371900 & -0.9096580 & $\mathrm{H}$ & -5.0878990 & 1.7860660 & 3.9930020 \\
\hline H & 1.5165090 & -4.7173170 & -1.8711520 & C & -0.7126670 & 6.6352870 & 1.1325700 \\
\hline $\mathrm{H}$ & 1.7247490 & -3.9779620 & -3.4629550 & C & 0.6000340 & 7.1107880 & 0.9004160 \\
\hline $\mathrm{H}$ & 4.1181200 & -3.5455520 & -2.9942270 & $\mathrm{C}$ & 1.6024740 & 6.2254810 & 0.6020980 \\
\hline H & 3.5323870 & -4.7741860 & -0.2467330 & C & -0.9890410 & 5.2922480 & 1.0876090 \\
\hline $\mathrm{H}$ & 4.0359620 & -2.5433060 & 0.6771350 & $\mathrm{H}$ & -1.5099890 & 7.3415520 & 1.3417140 \\
\hline C & 1.0870280 & -2.9893950 & 1.8925010 & $\mathrm{H}$ & 0.8043920 & 8.1757750 & 0.9450650 \\
\hline 0 & 0.2362730 & -2.0093100 & 1.9466880 & $\mathrm{H}$ & 2.6111150 & 6.5758540 & 0.3991980 \\
\hline C & 1.4039930 & -3.6133730 & 3.2054730 & $\mathrm{H}$ & -2.0013580 & 4.9409900 & 1.2559260 \\
\hline C & 2.3964870 & -4.5939510 & 3.2668240 & C & 3.2024950 & 1.6418010 & -0.4232810 \\
\hline C & 0.7265620 & -3.2205720 & 4.3627620 & c & 3.5904810 & 1.6009190 & -1.7766740 \\
\hline C & 2.712 & -5.1817120 & 4.4858150 & C & 3.8991650 & 0.8843520 & 0.5384710 \\
\hline H & 2.90 & 38060 & 2.3541000 & $\mathrm{c}$ & 4.7252780 & 0.8617630 & -2.1230100 \\
\hline C & 1.0453840 & -3.8125460 & 5.5800150 & $\mathrm{C}$ & 5.0275670 & 0.1639070 & 0.1425130 \\
\hline $\mathrm{H}$ & -0.0402470 & -2.4564090 & 4.2967030 & $\mathrm{C}$ & 5.4755900 & 0.1637640 & -1.1799950 \\
\hline C & 2.0366970 & -4.7909570 & 5.6413300 & $\mathrm{H}$ & 5.0512140 & 0.8475870 & -3.1605190 \\
\hline $\mathrm{H}$ & 3.4838000 & -5.9435290 & 4.5375440 & $\mathrm{H}$ & 5.5868500 & -0.3921510 & 0.8946680 \\
\hline $\mathrm{H}$ & 0.5214510 & -3.5115140 & 6.4813440 & C & -4.0105570 & -0.0040230 & -0.6098700 \\
\hline H & 2.28 & 10910 & 6.5932140 & C & -4.1094570 & -1.3921270 & -0.3706120 \\
\hline $\mathrm{H}$ & -0.0705550 & -1.6842320 & 1.0028200 & $\mathrm{C}$ & -4.4990280 & 0.5437330 & -1.8089420 \\
\hline 0 & 1.625 & -3.3993430 & 0.8529660 & $\mathrm{c}$ & -4.7499560 & -2.1876690 & -1.3198800 \\
\hline & & & & $\mathrm{C}$ & -5.1210280 & -0.2991470 & -2.7330470 \\
\hline & S) $\mathrm{G}[\mathrm{M06}-2$ & $G(d)]=-3$ & & C & -5.2666470 & -1.6628740 & -2.5046260 \\
\hline 0 & 0.5915950 & 0.7468190 & 0.2638970 & $\mathrm{H}$ & -4.8414650 & -3.2555570 & -1.1346550 \\
\hline 0 & -1.5221870 & 1.2745130 & -1.0154580 & $\mathrm{H}$ & -5.5085480 & 0.1223730 & -3.6589480 \\
\hline C & 0.8157310 & 2.1082080 & 0.3359870 & C & 3.4998990 & 0.8862450 & 2.0072740 \\
\hline C & -0.2054180 & 2.9339360 & 0.7620280 & C & 6.7666250 & -0.5326370 & -1.5754120 \\
\hline C & 2.1179200 & 2.5748170 & 0.0168700 & $\mathrm{C}$ & 2.8491310 & 2.3829910 & -2.8528360 \\
\hline C & 0.0349150 & 4.3 & 0.8071170 & C & -3.5535060 & -2.0489130 & 0.8873400 \\
\hline C & 2.3 & 70 & 0.1639320 & C & -5.9523080 & -2.5574240 & -3.5198400 \\
\hline C & & & 0.5326050 & $\mathrm{C}$ & -4.3991910 & 2.0269940 & -2.1315980 \\
\hline
\end{tabular}




$\begin{array}{rrr}-5.7922420 & 2.6647690 & -2.2027250 \\ -6.3862540 & 2.2216880 & -3.0096720 \\ -6.3379670 & 2.5215330 & -1.2647130 \\ -5.7122330 & 3.7397990 & -2.3951660 \\ -3.6150990 & 2.2665590 & -3.4266540 \\ -2.6183250 & 1.8216600 & -3.3638850 \\ -4.1340510 & 1.8340970 & -4.2892110 \\ -3.5049690 & 3.3408340 & -3.6085630 \\ -7.1932930 & -3.2322330 & -2.9249280 \\ -7.7058110 & -3.8342290 & -3.6825310 \\ -6.9156700 & -3.8976210 & -2.0999870 \\ -7.8978680 & -2.4909070 & -2.5366890 \\ -4.9838960 & -3.6036570 & -4.0835160 \\ -4.1050950 & -3.1273960 & -4.5276930 \\ -4.6375110 & -4.2771180 & -3.2917400 \\ -5.4755340 & -4.2112320 & -4.8505590 \\ -4.6693270 & -2.3114700 & 1.9081740 \\ -5.1817580 & -1.3908680 & 2.2003000 \\ -5.4181200 & -2.9913560 & 1.4852610 \\ -4.2605040 & -2.7764950 & 2.8120020 \\ -2.8166940 & -3.3608820 & 0.5903330 \\ -3.5031430 & -4.1400470 & 0.2420850 \\ -2.0419110 & -3.2137560 & -0.1670030 \\ -2.3474620 & -3.7333220 & 1.5080340 \\ 4.5841820 & 1.5520310 & 2.8633680 \\ 5.5240540 & 0.9909590 & 2.8142100 \\ 4.7818890 & 2.5712320 & 2.5168920 \\ 4.2723640 & 1.5977980 & 3.9118120 \\ 3.1860450 & -0.5249530 & 2.5149750 \\ 2.3461550 & -0.9590750 & 1.9617300 \\ 4.0538450 & -1.1874670 & 2.4172060 \\ 2.9049500 & -0.4968770 & 3.5727330 \\ 6.7025650 & -2.0516280 & -1.3822250 \\ 7.6551920 & -2.5137770 & -1.6609160 \\ 6.5014780 & -2.3067520 & -0.3342670 \\ 5.9160870 & -2.4968790 & -2.0006710 \\ 7.9587700 & 0.0556120 & -0.8107520 \\ 8.0210910 & 1.1383670 & -0.9523200 \\ 7.8668520 & -0.1387940 & 0.2635680 \\ 8.8961910 & -0.3940050 & -1.1537730 \\ 2.6910970 & 1.5975180 & -4.1594600 \\ 3.6495410 & 1.4766030 & -4.6762000 \\ 2.2610920 & 0.6087360 & -3.9889680 \\ 2.0234150 & 2.1429420 & -4.8335400 \\ 3.5580650 & 3.7136030 & -3.1429000 \\ 4.5808710 & 3.5305750 & -3924580\end{array}$
3.0258030
3.6131590
$-0.3149140$
0.4316550
$-0.7905460$
1. 8433250
6.9238930
2. 5867490
$-3.8554570$
$-6.2800690$
$-2.8263920$
0.4712010
$-0.1664370$
0.5016890
1.2950160
$-0.2537160$
1. 3371550
1.8688930
$-0.2106130$
$-0.8658930$
0.5841930
1.9541210
$-0.7965890$
0.6162750
$-0.3746130$
1. 0321700
1.4459810
2.0686240
0.3847280
1.6857420
2.2790430
1. 6844860
3. 3366460
3.6932410
3.4101900
1.8507420
3.5723060
4.7491010
4.1270780
1.2383660
1.5953180
3.9344560
3. 0997930
3.4893990

144

TS126(S,S) G[M06-2X/6-31G(d)] = -3310.353784
-3.9239730
-2.2548280
-0.9897790
-2.2824880
-0.5134470
-2.4775380
-2.6439650
2.1167320
-1.3238070
-4.3498210
1.3346850
1.6013130
1.7108680
2.8415880
2.8721620
3.9583790
4.0214650
1.9905940
5.1052690
3.9163200
5.1369420
4.0497370
5.9746160
6.0340310
0.7885810
0.5688070
-2.0122400
-0.7250530
-2.0771410
-0.1792160
-2.4272630
-2.4106290
-2.4834600
-0.9954390
-0.3669400
-2.6812390
-2.9238420
-0.8472670
-0.7128150
-2.2418090
-3.7432980
-3.0150990
-0.4751480
0.7237800

0.3027260

$-1.1567020$

2. 5957280

$-0.3396750$

1.4784980

$-1.9184160$

$-1.3629490$

$-3.2475050$

4.0684040

$-5.2172700$

$-3.7012910$

4840090

$-3.94258$

$-5.2232650$

$-4.4897730$

.1226970

$-4.7393270$

$-3.8757230$

0021690 


\begin{tabular}{|c|c|c|c|}
\hline & & & \\
\hline 0 & 0.4723920 & 0.8060050 & 0.1971950 \\
\hline 0 & -1.7060930 & 1.0437100 & -1.0546520 \\
\hline$C$ & 0.5784880 & 2.1830950 & 0.1671430 \\
\hline C & -0.5026210 & 2.9506050 & 0.5517060 \\
\hline C & 1.8356820 & 2.7311580 & -0.2021500 \\
\hline C & -0.3783340 & 4.3778410 & 0.4905400 \\
\hline$C$ & 1.9761370 & 4.0952870 & -0.1498380 \\
\hline C & 0.8854350 & 4.9461600 & 0.1667070 \\
\hline $\mathrm{H}$ & 2.9417390 & 4.5424110 & -0.3720780 \\
\hline C & -2.3171170 & 1.3179800 & 0.1584080 \\
\hline C & -3.5145180 & 0.6160110 & 0.4629400 \\
\hline C & -1.7576780 & 2.2745270 & 0.9786270 \\
\hline C & -4.1594750 & 0.9498260 & 1.6276860 \\
\hline C & -2.3849320 & 2.5360390 & 2.2418050 \\
\hline C & -3.6149240 & 1.8869690 & 2.5441730 \\
\hline H & -5.1048850 & 0.4715770 & 1.8697610 \\
\hline C & -3.7030890 & 3.0143150 & 4.6920220 \\
\hline C & -2.4574400 & 3.6249160 & 4.4129520 \\
\hline C & -1.8154210 & 3.3929040 & 3.2226930 \\
\hline C & -4.2628400 & 2.1602650 & 3.7779970 \\
\hline $\mathrm{H}$ & -4.2030700 & 3.2129990 & 5.6346720 \\
\hline $\mathrm{H}$ & -2.0029460 & 4.2789410 & 5.1505530 \\
\hline H & -0.8558830 & 3.8583930 & 3.0255710 \\
\hline $\mathrm{H}$ & -5.2075980 & 1.6650220 & 3.9864190 \\
\hline C & -1.3093830 & 6.6157110 & 0.6617410 \\
\hline C & -0.0414610 & 7.1785950 & 0.3816070 \\
\hline C & 1.0284600 & 6.3586810 & 0.1350240 \\
\hline C & -1.4745990 & 5.2547580 & 0.7153790 \\
\hline $\mathrm{H}$ & -2.1607930 & 7.2678170 & 0.8295650 \\
\hline H & 0.0752630 & 8.2571370 & 0.3484480 \\
\hline $\mathrm{H}$ & 2.0037210 & 6.7746340 & -0.1039230 \\
\hline H & -2.4534530 & 4.8348410 & 0.9206300 \\
\hline C & 2.9718010 & 1.8404020 & -0.5940420 \\
\hline C & 3.2998720 & 1.6698560 & -1.9572250 \\
\hline$C$ & 3.7300770 & 1.2036460 & 0.4014680 \\
\hline 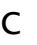 & 4.4154500 & 0.8995600 & -2.2790630 \\
\hline$C$ & 4.8518780 & 0.4549060 & 0.0278050 \\
\hline C & 5.2210100 & 0.3033850 & -1.3051420 \\
\hline H & 4.6748010 & 0.7552520 & -3.3257880 \\
\hline $\mathrm{H}$ & 5.4423080 & -0.0203560 & 0.8072460 \\
\hline C & -4.0454170 & -0.4313760 & -0.4624800 \\
\hline$\sigma$ & -3.9945540 & -1.7932070 & -0.0921920 \\
\hline C & -4.6105500 & -0.0534920 & -1.6925840 \\
\hline$C$ & -4.5638330 & -2.7374870 & -0.9454310 \\
\hline & -5.1586660 & -1.039 & -2.5161400 \\
\hline & -5.15 & & \\
\hline
\end{tabular}

-4.5378780
-5.6062650
-0.4079760
0.2881710
-0.7558900
3.3850520
2.4893560
6.4565160
6.1406990
2.5011670
1.4897070
-4.6715580
-4.1649960
-5.7600690
-6.1717760
-3.3530460
-2.6941670
-2.4897790
-1.9506310
-3.1008020
-1.7618290
-4.4204260
-5.1032210
-3.9534480
-5.0187500
3.0545310
3.9072150
2.7961520
2.1950130
4.5097210
4.2264050
5.4331140
4.7255180
-3.9442590
-4.4354210
-3.9475520
-2.9064140
-6.1243690
-6.1607630
-6.6857830
-6.6323880
2.3724460
1.6578090
3.3270230
2.0135120
3.1328100
-3.7867260
-0.7504730
0.0743620
0.1393670
-1.2664080
1.3115960
1.9319050
-0.4700900
-1.1558840
2.3454850
2.5222640
1.3949500
2.0188550
-3.4366990
-2.9165430
-2.2647550
-1.4677570
-3.5186540
-3.7342910
-4.3979460
-3.3778270
-2.5371750
-3.3250250
-2.8706370
-1.6481530
-0.0641100
-0.7490530
0.0213090
-0.5030480
1.9971510
2.1136540
1.4086620
2.9881790
1.5893160
1.0291220
2.6475180
1.2529220
1.8787700
2.9335630
1.3037730
1.7692410
1.4930310
1.9629520
1.4145150
0.4860190
3.6964040
$-0.6595790$

$-3.4654050$

$-0.9800740$

$-2.3031730$

$-0.3840230$

1.8794430

1.9808570

$-1.7430090$

$-2.5433120$

$-3.0640830$

$-2.6847560$

$-2.1529350$

$-1.4108810$

$-3.0673140$

$-3.9417000$

1.2070730

1.5675630

1. 0190630

1.9480970

0.7878760

0.2157590

2.2761170

1.9371600

3.2091740

2.4929990

2.4702100

2. 3893860

3.5311160

1.9541560

2.6639510

3.7149920

2.6285920

2.2526660

$-3.4879940$

$-4.2912940$

$-3.7698800$

$-3.4161760$

$-2.2391130$

$-2.5310570$

$-2.9838100$

$-1.2757270$

$-4.3304590$

$-5.0130570$

$-4.8625640$

-4.1107570
-3.4317310 


$\begin{array}{rrr}4.1585770 & 3.5482280 & -3.7890820 \\ 2.5602950 & 4.1802160 & -4.2301410 \\ 3.1680680 & 4.3779820 & -2.5783650 \\ -4.6949590 & -4.4219840 & -3.5622590 \\ -3.8820950 & -3.8984350 & -4.0737140 \\ -4.2619830 & -4.9775870 & -2.7230360 \\ -5.1325180 & -5.1475870 & -4.2560010 \\ -6.9082550 & -4.1811960 & -2.3768390 \\ -7.6807990 & -3.4858470 & -2.0357150 \\ -7.3679720 & -4.9016690 & -3.0614210 \\ -6.5436210 & -4.7342380 & -1.5041710 \\ 7.1005640 & -1.3022220 & -0.6343260 \\ 6.3916890 & -1.9959030 & -0.1697280 \\ 7.9298340 & -1.8881330 & -1.0413910 \\ 7.5066660 & -0.6575910 & 0.1531770 \\ 7.4952010 & 0.4921480 & -2.3380100 \\ 7.0784840 & 1.0692250 & -3.1681390 \\ 7.8321920 & 1.1989540 & -1.5718470 \\ 8.3675140 & -0.0583010 & -2.7050930 \\ 1.4496450 & -2.7418280 & -1.9191690 \\ 2.2242580 & -2.2509450 & -0.7759220 \\ 0.3786130 & -2.5554890 & -1.8610560 \\ 1.8869080 & -1.3383830 & -0.2902600 \\ 2.1561470 & -1.6637110 & -2.5794790 \\ 1.5337190 & -0.8688890 & -2.5462460 \\ 3.3258870 & -4.3539820 & -2.4357930 \\ 3.8623220 & -4.1462530 & -1.0183110 \\ 3.6293140 & -2.7067050 & -0.5590500 \\ 1.8183910 & -4.0952730 & -2.4795140 \\ 3.5326090 & -5.3728330 & -2.7761670 \\ 4.9333920 & -4.3667330 & -0.9714580 \\ 4.2533850 & -2.0120150 & -1.1323850 \\ 1.2911740 & -4.8455170 & -1.8777530 \\ 1.4313180 & -4.1600540 & -3.5011430 \\ 3.8344770 & -3.6708250 & -3.1269480 \\ 3.3526170 & -4.8296510 & -0.3292310 \\ 3.8799560 & -2.5540170 & 0.4953980 \\ 0.9828270 & -2.9169250 & 1.8266700 \\ 0.1278410 & -1.9390750 & 1.8539720 \\ 1.3824690 & -3.4321460 & 3.1636610 \\ 2.3827520 & -4.4036680 & 3.2415150 \\ 0.7809960 & -2.9424460 & 4.3259970 \\ 2.7813850 & -4.8869650 & 4.4820820 \\ 2.8345310 & -4.7683320 & 2.3245510 \\ 1.1825380 & -3.4298040 & 5.5648910 \\ 0.0083740 & -2.1854580 & 4.2470350\end{array}$

2.1806720
3.5584860
0.7180220
2.4922360
-0.2113420
1.4655890

$-4.4002200$

$-5.6416590$

$-3.0532640$

$-4.7783780$

$-1.6596800$

4.5466530

6.4703750

6.6114540

0.9088130

0.7922370

$\operatorname{TS} 127(\mathrm{~S}, \mathrm{~S}) \mathrm{G}[\mathrm{M06}-2 \mathrm{X} / 6-31 \mathrm{G}(\mathrm{d})]=-3310.350628$

$\begin{array}{lrrr}\text { TS127(S, S }) \text { G }[\mathrm{M} 06-2 \times / 6-31 G(\mathrm{~d})]=-3310.350628 \\ \mathrm{O} & 1.5597180 & -0.2210540 & -0.7614820 \\ \mathrm{O} & 0.3616100 & -1.6291200 & 0.9198420 \\ \mathrm{C} & 2.7471050 & -0.7415720 & -0.2896260 \\ \mathrm{C} & 2.8781540 & -2.1099060 & -0.1658730 \\ \mathrm{C} & 3.7851050 & 0.1828050 & 0.0049530 \\ \mathrm{C} & 4.1198740 & -2.6262400 & 0.3334510 \\ \mathrm{C} & 5.0027320 & -0.3316940 & 0.3743160 \\ \mathrm{C} & 5.1986610 & -1.7248990 & 0.5599450 \\ \mathrm{H} & 5.8304330 & 0.3439240 & 0.5753410 \\ \mathrm{C} & 0.4803930 & -2.6900020 & 0.0402800 \\ \mathrm{C} & -0.6936150 & -3.4482620 & -0.2190340 \\ \mathrm{C} & 1.7166100 & -2.9786460 & -0.5025970 \\ \mathrm{C} & -0.5647740 & -4.5573830 & -1.0174150 \\ \mathrm{C} & 1.8105740 & -4.0748860 & -1.4237090 \\ \mathrm{C} & 0.6637560 & -4.8889980 & -1.6444300 \\ \mathrm{H} & -1.4368890 & -5.1763080 & -1.2156100 \\ \mathrm{C} & 1.9151250 & -6.2668350 & -3.2027510 \\ \mathrm{C} & 3.0421460 & -5.4303450 & -3.0224120 \\ \mathrm{C} & 2.9923390 & -4.3649300 & -2.1592400 \\ \mathrm{C} & 0.7517830 & -5.9945330 & -2.5319910 \\ \mathrm{H} & 1.9711880 & -7.1100980 & -3.8837770 \\ \mathrm{H} & 3.9530490 & -5.6296370 & -3.5781880 \\ \mathrm{H} & 3.8596810 & -3.7249600 & -2.0401330 \\ \mathrm{H} & -0.1313300 & -6.6109680 & -2.6783410 \\ \mathrm{C} & 5.5237200 & -4.4539350 & 1.1059850 \\ \mathrm{C} & 6.6088370 & -3.5632670 & 1.2844570 \\ \mathrm{C} & 6.4436070 & -2.2284220 & 1.0226960 \\ \mathrm{C} & 4.3139080 & -3.9997690 & 0.6448900 \\ \mathrm{H} & 5.6480830 & -5.5059300 & 1.3429930 \\ \mathrm{H} & 7.5620530 & -3.9378460 & 1.6437720 \\ \mathrm{H} & 7.2585610 & -1.5266100 & 1.1796500 \\ \mathrm{H} & 3.4869980 & -4.6908920 & 0.5230870 \\ \mathrm{C} & 3.5166430 & 1.6527970 & -0.0187370 \\ \mathrm{C} & 3.5291560 & 2.3746240 & 1.1926180 \\ \mathrm{C} & 3.2389100 & 2.3184850 & -1.2297070 \\ \mathrm{C} & 3.3167090 & 3.7555030 & 1.1586870 \\ \mathrm{C} & 3.0143300 & 3.6944780 & -1.2057180 \\ \mathrm{C} & 3.0487810 & 4.4352670 & -0.0243630 \\ & & & \end{array}$




\begin{tabular}{|c|c|c|c|}
\hline $\begin{array}{l}\mathrm{H} \\
\mathrm{H} \\
\mathrm{C} \\
\mathrm{C} \\
\mathrm{C} \\
\mathrm{C} \\
\mathrm{C} \\
\mathrm{C} \\
\mathrm{H} \\
\mathrm{H} \\
\mathrm{P} \\
\mathrm{O} \\
\mathrm{O} \\
\mathrm{C} \\
\mathrm{H} \\
\mathrm{C} \\
\mathrm{H} \\
\mathrm{C} \\
\mathrm{H} \\
\mathrm{C} \\
\mathrm{H} \\
\mathrm{C} \\
\mathrm{H} \\
\mathrm{C} \\
\mathrm{H} \\
\mathrm{C} \\
\mathrm{C} \\
\mathrm{H} \\
\mathrm{H} \\
\mathrm{O} \\
\mathrm{H} \\
\mathrm{C} \\
\mathrm{C} \\
\mathrm{C} \\
\mathrm{C} \\
\mathrm{H} \\
\mathrm{H} \\
\mathrm{H} \\
\mathrm{H} \\
\mathrm{H} \\
\mathrm{H} \\
\mathrm{H} \\
\mathrm{H} \\
\mathrm{C} \\
\mathrm{H} \\
\mathrm{H}\end{array}$ & $\begin{array}{r}3.3297530 \\
2.8072950 \\
-2.0159770 \\
-3.0296240 \\
-2.2539150 \\
-4.2726710 \\
-3.5037040 \\
-4.5308890 \\
-5.0660700 \\
-3.6841970 \\
0.3069400 \\
0.5619730 \\
-0.8913110 \\
3.2090150 \\
3.3629190 \\
2.7927760 \\
1.9231890 \\
3.7452790 \\
3.6640270 \\
-1.2149800 \\
-0.2847980 \\
-5.8834440 \\
-6.5129740 \\
-2.8533820 \\
-1.8038490 \\
-1.1689930 \\
-0.8567250 \\
-1.5269500 \\
-0.5275210 \\
0.2685250 \\
0.6061340 \\
-1.3600540 \\
-1.4789830 \\
-0.6520620 \\
-1.8611280 \\
-1.9345500 \\
-1.1508380 \\
0.4241700 \\
-2.9370490 \\
-1.7110240 \\
-0.3124750 \\
-2.5288060 \\
-0.8759790 \\
-3.1898290 \\
-3.1084800 \\
-4.2114150\end{array}$ & $\begin{array}{r}4.3024970 \\
4.2124610 \\
-3.0100850 \\
-2.5964020 \\
-3.0120340 \\
-2.2327710 \\
-2.6179970 \\
-2.2283220 \\
-1.9348600 \\
-2.6270770 \\
-0.1372330 \\
0.8288320 \\
0.0557180 \\
1.6014590 \\
0.5324910 \\
5.9318940 \\
6.0897010 \\
1.7162040 \\
0.6333400 \\
-3.4794450 \\
-3.6994530 \\
-1.8098340 \\
-1.5364790 \\
-2.5468750 \\
-2.7426340 \\
3.2560820 \\
3.0998770 \\
2.3557610 \\
2.1221090 \\
3.3670950 \\
2.4411810 \\
5.7420910 \\
5.4963010 \\
4.2832720 \\
4.5310900 \\
6.6279040 \\
6.3756060 \\
4.4983960 \\
4.3915920 \\
4.6680140 \\
5.9379420 \\
5.3153340 \\
3.9739230 \\
-1.1704560 \\
-1.2069000 \\
-0.8577310\end{array}$ & $\begin{array}{r}2.0962690 \\
-2.1426380 \\
0.3209760 \\
-0.5668400 \\
1.7117740 \\
-0.0405490 \\
2.1848150 \\
1.3256440 \\
-0.7244380 \\
3.2584010 \\
0.2795070 \\
1.3971320 \\
-0.6146880 \\
-2.5709500 \\
-2.3984640 \\
-0.0697690 \\
-0.7265400 \\
2.5495970 \\
2.4216520 \\
2.7206780 \\
2.1901890 \\
1.8703150 \\
1.0116050 \\
-2.0800480 \\
-2.3140680 \\
0.7900450 \\
-0.6325240 \\
1.2920420 \\
-0.9622120 \\
0.8626080 \\
1.0803630 \\
0.4202450 \\
-1.0849460 \\
-1.5154690 \\
1.2122340 \\
0.7066240 \\
-1.6471900 \\
-1.4775650 \\
1.0445280 \\
2.2876360 \\
0.6786450 \\
-1.3436330 \\
-2.5399510 \\
-2.6686030 \\
-3.7603980 \\
-2.4221610\end{array}$ \\
\hline
\end{tabular}

-2.4935340
-3.7005830
-4.7683600
-3.5296280
-3.4641110
1.8541560
1.6431470
1.8504010
1.0473220
4.3519680
4.3582080
4.2419590
5.3216150
-0.9064780
-1.7868150
-0.1117610
-0.5777660
-1.6708100
-0.9050340
-2.5952530
-1.8587850
2.6767810
2.8115520
2.7506280
1.6751910
5.1437550
5.2594280
5.3015300
5.9319150
-6.5754790
-6.6929340
-5.9884280
-7.5657780
-5.7594710
-5.2359300
-6.7487960
-5.1950730
3.9878470
4.2082090
3.7915630
4.8806490
2.4603440
1.6668490
3.3399610
2.1341730
-3.5790420
$-0.4156340$
$-3.6366620$
$-3.4581200$
$-3.6450520$
$-4.6303040$
1.7648200
2.8189530
1. 2253160
1.3615190
2. 0900860
1. 5383400
3.1553370
1.9503430
$-2.3989330$
$-2.1782040$
$-2.7402160$
$-1.4740810$
$-4.7796890$
$-5.1392210$
$-4.6238670$
$-5.5625510$
2.1284120
1. 5565730
3. 1901850
1.9263240
2.0347740
3. 1146880
1.5415760
1.7080080
$-2.9645860$
$-3.8331680$
$-3.2758370$
$-2.6597990$
$-0.5844110$
0.2354850
$-0.2303910$
$-0.8357530$
6.6607030
6.2767840
7.7351880
6.5190310
6.5362410
.9742770
7.5743550
1.9019940

$-2.2977350$

$-2.7513450$

$-2.5802000$

$-3.8328750$

$-3.2692430$

$-3.4848700$

$-4.2220750$

$-2.6498870$

$-3.4701790$

$-4.4158920$

$-3.7020860$

$-2.9824370$

3. 7630200

4.3770900

4.4346880

3. 2815640

3. 3958550

4.0912090

3. 9631160

2. 6543050

3. 5702520

4.4943800

3.8294240

3. 1841150

3. 0945750

3. 2435510

4.0594510

2. 4096600

2.6035560

1. 9491250

3.4745740

2.9578620

2. 7841930

2. 2828010

3.0932400

3. 6889790

$-0.6998110$

$-1.7002970$

$-0.7777850$

$-0.0809510$

1. 2940360

1.9475290

1.1779710 


$\begin{array}{llll}\mathrm{O} & -3.2329730 & 0.8659390 & -0.1087240 \\ \mathrm{C} & -5.0475900 & 2.1271820 & -0.8962990 \\ \mathrm{C} & -5.5318400 & 3.3185170 & -1.4393610 \\ \mathrm{C} & -5.9320330 & 1.1519890 & -0.4306490 \\ \mathrm{C} & -6.9029470 & 3.5385410 & -1.5041210 \\ \mathrm{H} & -4.8264020 & 4.0571270 & -1.8060750 \\ \mathrm{C} & -7.3027130 & 1.3744650 & -0.4989470 \\ \mathrm{H} & -5.5289300 & 0.2300460 & -0.0249500 \\ \mathrm{C} & -7.7870530 & 2.5680030 & -1.0327740 \\ \mathrm{H} & -7.2845910 & 4.4645060 & -1.9218630 \\ \mathrm{H} & -7.9927990 & 0.6181380 & -0.1375740 \\ \mathrm{H} & -8.8574080 & 2.7421150 & -1.0847150 \\ \mathrm{H} & -2.2543300 & 0.5746580 & -0.2497370 \\ \mathrm{O} & -2.7898960 & 2.6676030 & -1.3885230\end{array}$

144

2. 6676030

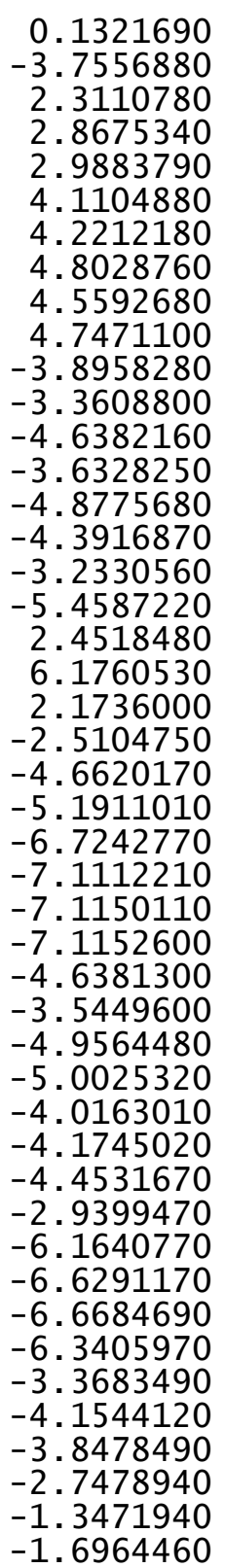

6.7939930

4. 2554500

2. 3718150

1. 9139720

2. 1608910

1. 2739110

1. 5090260

1.0608460

0.9300120

1. 3612010

$-1.4074900$

$-2.4838550$

$-1.6425100$

$-3.7839150$

$-2.9584290$

$-4.0436810$

$-4.6251620$

$-3.1395890$

2. 6728150

0.4141160

2. 1297640

$-2.2661750$

$-5.4674540$

$-0.5070650$

$-0.5168130$

$-1.4414450$

$-0.4427970$

0.3243740

$-0.5588890$

$-0.5311150$

$-1.4719780$

0.2957450

$-5.7514210$

$-6.7945260$

$-5.1134980$

$-5.5572100$

$-5.7696270$

$-5.5844780$

$-6.8145630$

$-2.3418150$

$-1.5819970$

$-2.1977460$

$-3.2586300$

$-4.2624090$
$-2.0539160$

$-0.2886080$

$-0.7935450$

$-2.0036590$

0.4240300

$-1.9665820$

0.4095320

$-0.7752690$

$-2.8967580$

1. 3504510

0.3736540

1.1063360

$-0.7977470$

0.6739890

$-1.1921630$

$-0.4644480$

1. 2355930

$-2.0950460$

1.7529800

$-0.7793420$

$-3.3405030$

2. 3504670

$-0.9131130$

$-1.6449310$

$-1.6424420$

$-2.0851040$

$-0.6225840$

$-2.2243390$

$-3.0734370$

$-3.0653190$

$-3.5886990$

$-3.6530340$

$-2.2743170$

$-2.5677640$

$-3.0505720$

$-2.2474250$

$-0.9521130$

0.0205810

$-1.6921580$

$-1.2277170$

3. 6208780

3. 6268790

3. 6951450

4. 5120110

2.4547810

2.7220770 


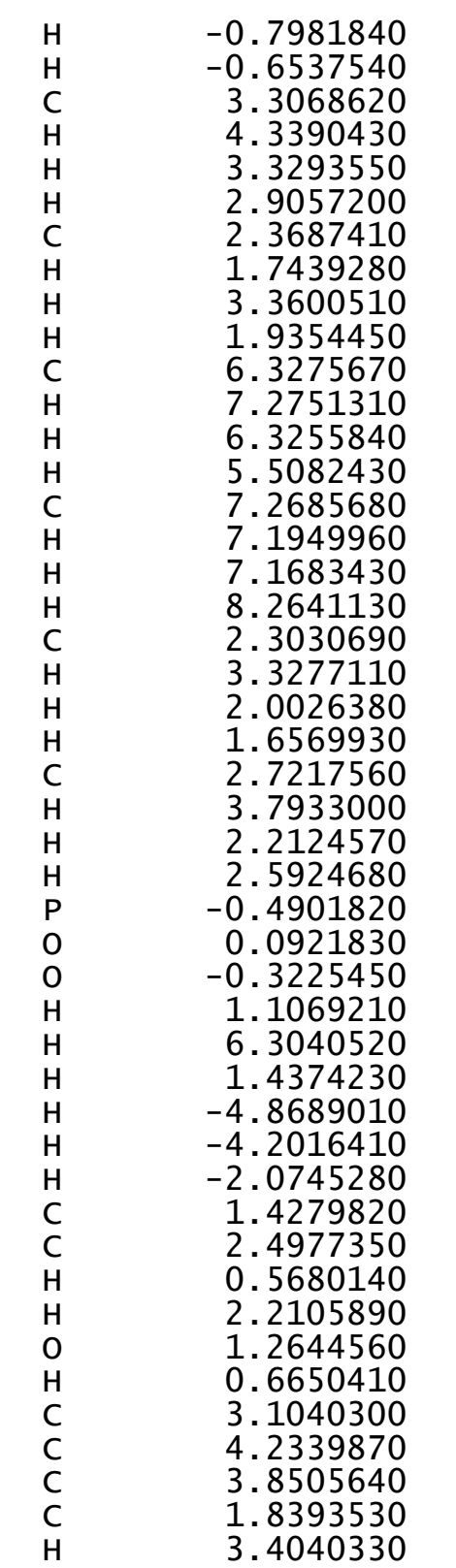

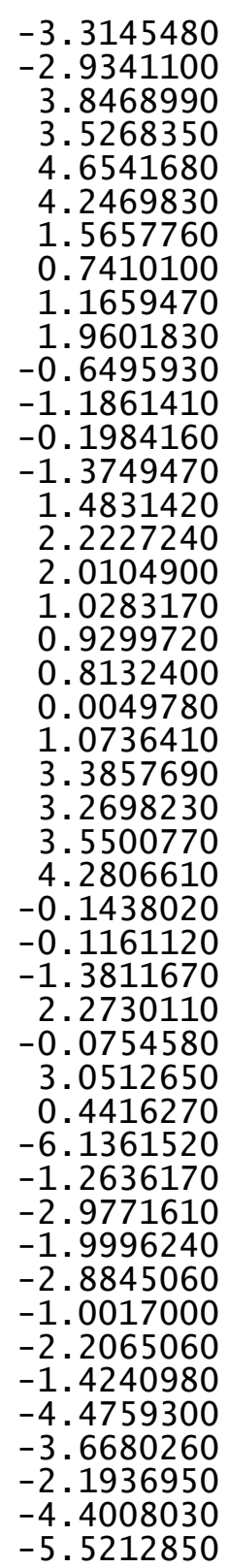

1.5123660
3.2373710
2.2474110
2.4292720
1.5083780
3.1844240
2.8076990
2.4540100
3.0510230
3.7331390
0.3127200
0.1970870
1.3110940
0.2818160
-0.6474490
-1.4501690
0.3078060
-0.6830470
-4.2829150
-4.6546150
-3.7871710
-5.1546150
-4.0327260
-4.2332710
-4.9881840
-3.4184210
-0.7183730
-2.1005590
0.1257460
-3.1423780
-1.7548700
1.5992360
-1.2047480
-0.1744620
2.2832410
-1.8366580
-1.6155630
-1.1748830
-1.2919540
-3.0503130
-2.8199890
-2.9887180
-2.3477050
-2.2167080
-2.1307110
-3.1068170

5.1532600
3.8233510
2.0106310
0.9977430
2.8897440
4.4460460
4.5642110
2.7613610
1.5996900
3.6684510
4.8754590
3.3202680
5.7398910
5.1213890
4.1913600
2.3752990
5.3995790
6.6770970
3.9268940
6.0766150
0.9070600
3.1382990
$-3.7503810$
$-1.7024240$
$-4.8921490$
$-4.9073790$
$-4.0843990$
$-4.0650210$
$-1.6315660$
$-2.1355120$
$-1.6314600$
$-2.2826170$
$-2.9682200$
$-1.7434720$
$-3.1022460$
$-3.3918860$
$-1.8739330$
$-1.2223210$
$-2.5509540$
$-3.6362100$
$-1.4490510$
$-2.6525440$
$-1.5405530$
$-2.4871570$

144

3.1979730

$-1.1650830$

$-2.6135120$

9900970

$-1.3476050$

$-1.6106390$

1.4499780

1.7412640

2.6238790

2.4689660

3. 8651010

3.5490260

1. 5006430

4.9415990

3. 9741640

4.7837970

3.4301900

5.9044280

5.6264050

0.9826490

0.3238640

$\operatorname{TS} 129(\mathrm{~S}, \mathrm{~S}) \mathrm{G}[\mathrm{M} 06-2 \mathrm{X} / 6-31 \mathrm{G}(\mathrm{d})]=-3310.349216$

\begin{tabular}{lrrr}
\multicolumn{2}{c}{ TS129(S, S $)$ G $[\mathrm{M} 06-2 \times / 6-31 G(\mathrm{~d})]=-3310.349216$} \\
$\mathrm{O}$ & -0.0002330 & 1.0686900 & 0.2385610 \\
$\mathrm{O}$ & -2.0593490 & 0.2465300 & -0.9578840 \\
$\mathrm{C}$ & -0.2595300 & 2.3619190 & -0.1692520 \\
$\mathrm{C}$ & -1.5428090 & 2.8526850 & -0.0643520 \\
$\mathrm{C}$ & 0.8511480 & 3.1328250 & -0.6075860 \\
$\mathrm{C}$ & -1.7997940 & 4.1718100 & -0.5659240 \\
$\mathrm{C}$ & 0.6102480 & 4.4261670 & -0.9948110 \\
$\mathrm{C}$ & -0.7060190 & 4.9613900 & -1.0207060 \\
$\mathrm{H}$ & 1.4386660 & 5.0621870 & -1.2959050 \\
$\mathrm{C}$ & -2.7831740 & 0.7094290 & 0.1234400 \\
$\mathrm{C}$ & -3.7539810 & -0.1722710 & 0.6707390 \\
$\mathrm{C}$ & -2.5899960 & 2.0020640 & 0.5655020 \\
$\mathrm{C}$ & -4.5570870 & 0.3081990 & 1.6734250 \\
$\mathrm{C}$ & -3.3614500 & 2.4554940 & 1.6885550 \\
$\mathrm{C}$ & -4.3755190 & 1.6071470 & 2.2161870 \\
$\mathrm{H}$ & -5.3421020 & -0.3250690 & 2.0792980 \\
$\mathrm{C}$ & -4.9458160 & 3.2832730 & 3.8767840 \\
$\mathrm{C}$ & -3.9072330 & 4.1088390 & 3.3856260 \\
$\mathrm{C}$ & -3.1366650 & 3.7079150 & 2.3233150 \\
$\mathrm{C}$ & -5.1670810 & 2.0573290 & 3.3057020 \\
$\mathrm{H}$ & -5.5520480 & 3.6166180 & 4.7130730 \\
$\mathrm{H}$ & -3.7137160 & 5.0667200 & 3.8582950
\end{tabular}




$\begin{array}{rrr}-2.3363680 & 4.3468410 & 1.9668780 \\ -5.9442750 & 1.3995010 & 3.6858500 \\ -3.3148930 & 5.9817480 & -1.1398250 \\ -2.2233240 & 6.7783650 & -1.5587240 \\ -0.9496450 & 6.2746950 & -1.5019210 \\ -3.1098490 & 4.7142480 & -0.6554410 \\ -4.3242380 & 6.3752440 & -1.2080180 \\ -2.3994820 & 7.7811790 & -1.9346860 \\ -0.1029510 & 6.8683180 & -1.8365140 \\ -3.9537160 & 4.1068810 & -0.3452320 \\ 2.2199330 & 2.5330820 & -0.5484430 \\ 2.9104510 & 2.1776480 & -1.7204870 \\ 2.8169360 & 2.3264440 & 0.7132090 \\ 4.2012240 & 1.6415190 & -1.6089950 \\ 4.1019810 & 1.7950010 & 0.7708490 \\ 4.8208440 & 1.4489320 & -0.3755110 \\ 4.7366360 & 1.3894370 & -2.5200530 \\ 4.5745710 & 1.6713550 & 1.7436060 \\ -3.8914800 & -1.5646240 & 0.1429390 \\ -3.3263310 & -2.6438830 & 0.8540710 \\ -4.5835360 & -1.7887470 & -1.0574460 \\ -3.5181500 & -3.9356670 & 0.3670180 \\ -4.7440560 & -3.1016790 & -1.5081900 \\ -4.2278830 & -4.1870120 & -0.8086330 \\ -3.0911490 & -4.7731000 & 0.9142430 \\ -5.2861400 & -3.2831980 & -2.4348230 \\ 2.1386360 & 2.7292870 & 2.0149250 \\ 6.2442710 & 0.9265950 & -0.2202990 \\ 2.3222360 & 2.4213770 & -3.1029510 \\ -2.5299360 & -2.4291310 & 2.1339770 \\ -4.4129750 & -5.6040910 & -1.3175280 \\ -5.1643880 & -0.6503380 & -1.8811370 \\ -6.6941440 & -0.7365990 & -1.9368120 \\ -7.0172980 & -1.6600700 & -2.4300760 \\ -7.1245310 & -0.7238180 & -0.9303930 \\ -7.1063570 & 0.1073960 & -2.4996690 \\ -4.5597270 & -0.6180310 & -3.2892240 \\ -3.4703030 & -0.5368120 & -3.2402680 \\ -4.8136750 & -1.5245450 & -3.8497340 \\ -4.9466330 & 0.2399560 & -3.8489580 \\ -5.1951960 & -6.4615170 & -0.3163760 \\ -5.3640480 & -7.4664170 & -0.7173060 \\ -4.6407950 & -6.5640380 & 0.6230390 \\ -6.1659220 & -6.0125410 & -0.0866370 \\ -3.0661600 & -6.2552870 & -1.6527620 \\ -2.5103560 & -5.6570110 & -2.3808140\end{array}$

-2.4478040
-3.2142230
-3.4293170
-4.2502360
-3.8642090
-2.8497590
-1.3274500
-1.6404760
-0.7375850
-0.6831830
2.8442880
3.8833580
2.8522910
2.3362780
2.0753160
1.5541730
3.0776060
1.5412840
6.9712500
8.0029950
6.4938040
6.9973800
6.2802010
5.8535310
5.7035080
7.3108200
2.5476250
3.6074840
2.1747930
2.0115170
2.9006940
3.9852550
2.4574150
2.7173860
-0.4872320
0.1367790
-0.2784940
1.2406720
6.8006010
1.1088340
-4.9069290
-4.9994730
-2.1376900
2.7951610
1.6037920
3.6720510
-6.3511720
-7.2580270
-2.5807150
-1.8586390
-3.5866330
-2.4338750
-3.3717360
-4.3992510
-3.3679190
-3.0455410
3.9473910
3.7094730
4.7876130
4.2668450
1.5682370
0.7121740
1.2421700
1.8776610
0.6940580
0.3848760
-0.0979890
1.6004950
-0.3407060
-0.1674130
-1.1509760
-0.6869780
1.2555170
1.1326710
0.3204030
1.4415360
3.7097940
3.6145150
3.9085500
4.5767270
-0.1175040
0.0142690
-1.3877370
2.5419230
1.7034650
3.0259690
0.2948610
-5.5451690
-1.4066540
-2.0483210
-1.6110510
-2.2283870
$-0.7533430$

$-2.0672150$

3. 3684510

3. 3647170

3. 3972170

4. 2862490

2. 2528420

2. 4704040

1. 3337430

3. 0757540

2.6246810

2.8786480

1. 9229810

3. 5405730

3. 0116200

2. 5740920

3. 3132830

3. 9164500

$-1.5443480$

$-1.3530650$

$-2.1334750$

$-2.1565210$

0.6461510

1. 6382250

0.1861480

0.7788670

$-4.0685490$

$-4.3207800$

$-3.6469690$

$-5.0046050$

$-3.7051120$

$-3.8342320$

$-4.6865370$

$-3.0642930$

$-0.7734600$

$-2.1313950$

0.0098900

$-2.9902280$

0.3233410

1. 7979600

$-1.3932850$

$-2.2433680$

2. 1127970

1. 4005760

1. 6830240

2. 5923770 


\begin{tabular}{|c|c|c|c|c|c|c|c|}
\hline$c$ & 10082300 & 2 ᄀ & & & 1 & & \\
\hline$c$ & $\begin{array}{l}4.9083200 \\
3.2697220\end{array}$ & $\begin{array}{l}-2.8590560 \\
-1.7709940\end{array}$ & $\begin{array}{l}2.4357350 \\
3.8497220\end{array}$ & $\mathrm{C}$ & $\begin{array}{l}-4.2721580 \\
-5.2908150\end{array}$ & $\begin{array}{r}1.6893100 \\
-0.2154560\end{array}$ & $\begin{array}{l}2.3034070 \\
2.1624680\end{array}$ \\
\hline C & 5.7467420 & -3.0218850 & 3.5320830 & C & -4.7646470 & 3.3569690 & 3.9971070 \\
\hline H & 5.1982790 & -3.2142030 & 1.4521530 & $\mathrm{c}$ & -3.7153310 & 4.1623160 & 3.4952260 \\
\hline C & 4.1155280 & -1.9286280 & 4.9428870 & $\mathrm{C}$ & -2.9776860 & 3.7563250 & 2.4116200 \\
\hline $\mathrm{H}$ & 2.3028170 & -1.2918610 & 3.9591160 & $\mathrm{C}$ & -5.0293400 & 2.1449150 & 3.4148940 \\
\hline C & 5.3519440 & -2.5523130 & 4.7844790 & $\mathrm{H}$ & -5.3447230 & 3.6943120 & 4.8501720 \\
\hline $\mathrm{H}$ & 6.7067180 & -3.5135290 & 3.4121550 & $\mathrm{H}$ & -3.4872810 & 5.1083070 & 3.9763420 \\
\hline $\mathrm{H}$ & 3.8088680 & -1.5672820 & 5.9190720 & $\mathrm{H}$ & -2.1685910 & 4.3790630 & 2.0463390 \\
\hline $\mathrm{H}$ & 6.0088080 & -2.6759820 & 5.6400200 & $\mathrm{H}$ & -5.8153590 & 1.5022050 & 3.8027320 \\
\hline $\mathrm{H}$ & 0.9314150 & -1.5174370 & 0.9082710 & C & -3.1804590 & 6.0821400 & -1.0109490 \\
\hline 0 & 3.2196120 & -2.3172320 & 0.2695050 & $\mathrm{C}$ & -2.0813430 & 6.8541840 & -1.4551460 \\
\hline $\mathrm{C}$ & 1.6183900 & -2.7932280 & -1.9709050 & $\mathrm{C}$ & -0.8211610 & 6.3147500 & -1.4469840 \\
\hline C & 2.6027000 & -1.7546180 & -1.6571240 & C & -2.9951070 & 4.8031790 & -0.5492310 \\
\hline $\mathrm{H}$ & 0.7321750 & -2.8029690 & -1.3377530 & $\mathrm{H}$ & -4.1802510 & 6.5037850 & -1.0414290 \\
\hline $\mathrm{H}$ & 2.2315780 & -0.8029860 & -1.2851360 & $\mathrm{H}$ & -2.2415750 & 7.8663770 & -1.8126240 \\
\hline 0 & 1.4400900 & -1.9639330 & -3.1437400 & $\mathrm{H}$ & 0.0302430 & 6.8889570 & -1.8028260 \\
\hline $\mathrm{H}$ & 0.7884750 & -1.2309140 & -2.8916490 & $\mathrm{H}$ & -3.8452090 & 4.2153110 & -0.2192660 \\
\hline C & 3.4460310 & -4.0905850 & -3.1396810 & C & 2.2620560 & 2.4550870 & -0.6827630 \\
\hline $\mathrm{C}$ & 4.4886580 & -3.2383390 & -2.4126830 & $\mathrm{C}$ & 2.8512710 & 2.0558200 & -1.8981860 \\
\hline $\mathrm{C}$ & 3.9847640 & -1.8086860 & -2.2164680 & $\mathrm{C}$ & 2.9183170 & 2.2073380 & 0.5394220 \\
\hline $\mathrm{C}$ & 2.1493630 & -4.1615390 & -2.3294090 & $\mathrm{C}$ & 4.1057180 & 1.4386190 & -1.8604360 \\
\hline $\mathrm{H}$ & 3.8292960 & -5.1019960 & -3.3035880 & $\mathrm{C}$ & 4.1636710 & 1.5796470 & 0.5249460 \\
\hline $\mathrm{H}$ & 5.4302850 & -3.2160150 & -2.9692210 & $\mathrm{C}$ & $4 . \overline{7781720}$ & 1.1912850 & -0.6643390 \\
\hline $\mathrm{H}$ & 3.9546100 & -1.2645560 & -3.1692600 & $\mathrm{H}$ & 4.5795920 & 1.1398690 & -2.7937520 \\
\hline $\mathrm{H}$ & 2.3244090 & -4.6944490 & -1.3868200 & $\mathrm{H}$ & 4.6726260 & 1.4030620 & 1.4701410 \\
\hline $\mathrm{H}$ & 1.3678610 & -4.7017540 & -2.8729670 & C & -3.9091670 & -1.4673580 & 0.1820800 \\
\hline $\mathrm{H}$ & 3.2370310 & -3.6590600 & -4.1256350 & $\mathrm{C}$ & -3.3782680 & -2.5713360 & 0.8817320 \\
\hline $\mathrm{H}$ & 4.6981750 & -3.6755090 & -1.4289530 & $\mathrm{C}$ & -4.6064890 & -1.6576750 & -1.0214190 \\
\hline $\mathrm{H}$ & 4.6245150 & -1.2247980 & -1.5518260 & C & -3.6084070 & -3.8513080 & 0.3799160 \\
\hline & & & & $\mathrm{C}$ & & -2.9600150 & -1.4871470 \\
\hline & S) $\mathrm{G}[\mathrm{M06}-$ & $G(d)]=-331$ & 395 & C & -4.3225590 & -4.0682450 & -0.7996860 \\
\hline 0 & 0.0461540 & 1.0717090 & 0.2231880 & $\mathrm{H}$ & -3.2072060 & -4.7070580 & 0.9182620 \\
\hline 0 & -2.0552640 & 0.3117230 & -0.9333970 & $\mathrm{H}$ & -5.3513080 & -3.1146580 & -2.4163700 \\
\hline C & -0.1954260 & 2.3699370 & -0.1787380 & C & 2.3446280 & 2.6539930 & 1.8764230 \\
\hline $\mathrm{C}$ & -1.4610620 & 2.8933630 & -0.0274370 & $\mathrm{C}$ & 6.1637910 & 0.5717100 & -0.6659290 \\
\hline C & 0.9179550 & 3.1104760 & -0.6612600 & C & 2.1787840 & 2.3089070 & -3.2395050 \\
\hline $\mathrm{C}$ & -1.6983420 & 4.2240840 & -0.5082540 & $\mathrm{C}$ & -2.5738760 & -2.3979350 & 2.1629160 \\
\hline $\mathrm{C}$ & 0.7013720 & 4.4152900 & -1.0228060 & $\mathrm{C}$ & -4.5452030 & -5.4730610 & -1.3271650 \\
\hline C & -0.5986680 & 4.9888920 & -0.9905690 & $\mathrm{C}$ & -5.1554230 & -0.4943250 & -1.8320930 \\
\hline $\mathrm{H}$ & 1.5356010 & 5.0308660 & -1.3487000 & C & -6.6874910 & -0.5332030 & -1.8789030 \\
\hline C & -2.7471710 & 0.7794540 & 0.1670450 & $\mathrm{H}$ & -7.0414710 & -1.4424760 & -2.3773380 \\
\hline $\mathrm{C}$ & -3.7279190 & -0.0853360 & 0.7235970 & $\mathrm{H}$ & -7.1111280 & -0.5155560 & -0.8696980 \\
\hline $\mathrm{C}$ & -2.5123100 & 2.0614270 & 0.6204000 & $\mathrm{H}$ & -7.0771430 & 0.3275130 & -2.4324390 \\
\hline C & -4.4980600 & 0.4025340 & 1.7483680 & C & -4.5585770 & -0.4679620 & -3.2435850 \\
\hline 6 & 480700 & 2.51890 & 1.7654950 & $\mathrm{H}$ & -3.4668030 & -0.4233050 & -3.2008580 \\
\hline
\end{tabular}




-4.8460180
-4.9202780
-5.3470540
-5.5419970
-4.7937980
-6.3056810
-3.2155760
-2.6458600
-2.5987250
-3.3892380
-3.4718220
-4.2761620
-3.9295970
-2.8855670
-1.3932430
-1.7292880
-0.8092770
-0.7364150
3.1624500
4.1982150
3.1800430
2.7343250
2.2665720
1.6688580
3.2617650
1.8049290
6.3181730
7.2763280
6.2947270
5.5117600
7.2322560
7.1572830
7.1051230
8.2372340
2.3599090
3.3933930
2.0805230
1.7235530
2.7060640
3.7845600
2.2133180
2.5379640
-0.4953650
0.1155200
-0.3266400
1.1047530

1. 1047530

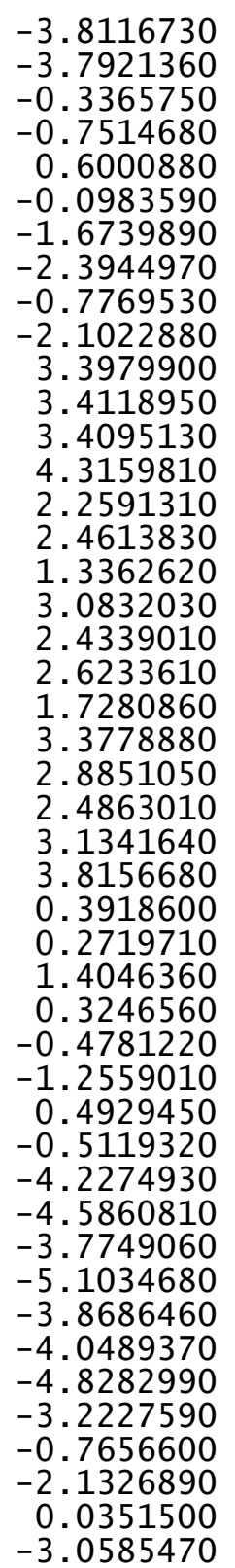

$\begin{array}{rr}-1.3596290 & -3.8116730 \\ 0.4080470 & -3.7921360 \\ -6.3245170 & -0.3365750 \\ -7.3189720 & -0.7514680 \\ -6.4546720 & 0.6000880 \\ -5.8544880 & -0.0983590 \\ -6.1531530 & -1.6739890 \\ -5.5587420 & -2.3944970 \\ -6.2770700 & -0.7769530 \\ -7.1459290 & -2.1022880 \\ -2.5527590 & 3.3979900 \\ -1.8124280 & 3.4118950 \\ -3.5488200 & 3.4095130 \\ -2.4369470 & 4.3159810 \\ -3.3705590 & 2.2591310 \\ -4.3939160 & 2.4613830 \\ -3.3657900 & 1.3362620 \\ -3.0736520 & 3.0832030 \\ 3.8263460 & 2.4339010 \\ 3.5226570 & 2.6233610 \\ 4.6629580 & 1.7280860 \\ 4.1793180 & 3.3778880 \\ 1.5044430 & 2.8851050 \\ 0.6802050 & 2.4863010 \\ 1.1180510 & 3.1341640 \\ 1.8518120 & 3.8156680 \\ -0.5257160 & 0.3918600 \\ -1.0420760 & 0.2719710 \\ -0.1085870 & 1.4046360 \\ -1.2629680 & 0.3246560 \\ 1.6565830 & -0.4781220 \\ 2.4222280 & -1.2559010 \\ 2.1479980 & 0.4929450 \\ 1.2228420 & -0.5119320 \\ 1.1533240 & -4.2274930 \\ 1.0826810 & -4.5860810 \\ 0.1999510 & -3.7749060 \\ 1.3132150 & -5.1034680 \\ 3.6063160 & -3.8686460 \\ 3.5276930 & -4.0489370 \\ 3.7948840 & -4.8282990 \\ 4.4719470 & -3.2227590 \\ -0.1094740 & -0.7656600 \\ -0.0206640 & -2.1326890 \\ -1.3750750 & 0.0351500 \\ 2.4147430 & -3.0585470\end{array}$

2. 4147430

\begin{abstract}
6. 3182560
1. 3248370

$-4.8663940$

$-5.1314290$

$-2.1582240$

2. 7392560

1. 5584800

3.6201690

4.8477950

3.2269050

5.6874530

5.1290770

4.0730810

2. 2665910

5. 3016360

6.6405950

3.7731660

5.9590120

0.8832080

3.1531440

1. 5011070

2. 5465900

0.6236970

2. 2327280

1. 3521120

0.7326590

3.2317530

4.3308650

3.9166160

1. 9460990

3.5544060

5.2647760

3.9037230

2. 1039060

1. 1256610

3.0334720

4.5276690

4.6046890
\end{abstract}

144

$\operatorname{TS} 131(\mathrm{~S}, \mathrm{~S}) \mathrm{G}[\mathrm{M} 06-2 \mathrm{X} / 6-31 \mathrm{G}(\mathrm{d})]=-3310.350342$

$\begin{array}{lrrr}\mathrm{O} & 1.3562170 & -0.5625410 & -0.7249310 \\ \mathrm{O} & -0.2489270 & -1.6574600 & 0.8707170 \\ \mathrm{C} & 2.3284610 & -1.4343000 & -0.2757040 \\ \mathrm{C} & 2.0449670 & -2.7841760 & -0.2327350 \\ \mathrm{C} & 3.5889330 & -0.8824080 & 0.0769940 \\ \mathrm{C} & 3.0593970 & -3.6763080 & 0.2498230\end{array}$

$-1.6551460$

0.1185040

3.0154100

0.4382250

$-1.3847020$

1259340

$-2.3038750$

$-2.9520660$

$-1.8308620$

$-3.1148090$

$-3.3247020$

$-1.9899760$

$-1.3382240$

$-2.6297860$

$-3.6199840$

$-1.6167790$

$-2.7542140$

$-1.5511350$

$-1.8735700$

$-2.8091430$

$-0.8937240$

$-2.0494190$

$-1.2872370$

$-4.2865760$

$-3.4821060$

$-2.0207630$

$-5.3212670$

$-3.5259590$

$-1.4936320$

$-4.7696310$

$-3.8628910$

$-3.9117290$

$-1.4689630$

$-1.9445660$

$-4.1007920$
$-5.3865390$

$-2.8614570$
1.7173120

$-1.3377170$

$-2.2510050$

2.1592630

1.4029890

1.6863060

2. 5925730

2.4389470

3.8472710

3.5345580

1.4593320

4.9396490

3.9547490

4.7836570

3.4166240

5.9134520

5.6386860

0.9147870

0.2727590

$-1.6618520$

$-1.3015590$

$-1.3093850$

$-3.1329370$

$-2.8882880$

$-3.1092450$

$-2.4126170$

$-2.2368430$

$-2.2798660$

$-3.2576140$

$-2.9803910$

$-3.1995730$

$-1.3287440$

$-2.8005140$

$-1.4229400$

$-1.5929110$
-0.8824080
-3.6763080

0.2498230 


$\begin{array}{lrrr}\mathrm{C} & 4.5842290 & -1.7588640 & \\ \mathrm{C} & 4.3498630 & -3.1531540 & 0.4327620 \\ \mathrm{H} & 5.5728520 & -1.3740510 & 0.5449130 \\ \mathrm{C} & -0.4229040 & -2.6693300 & -0.6700040 \\ \mathrm{C} & -1.7558260 & -3.0900940 & -0.3137300 \\ \mathrm{C} & 0.6889090 & -3.2557470 & -0.6264430 \\ \mathrm{C} & -1.9291720 & -4.1429060 & -1.1779140 \\ \mathrm{C} & 0.4851810 & -4.2814700 & -1.6079830 \\ \mathrm{C} & -0.8378780 & -4.7429010 & -1.8568720 \\ \mathrm{H} & -2.9340370 & -4.5086880 & -1.3765290 \\ \mathrm{C} & -0.0007340 & -6.3131110 & -3.5077410 \\ \mathrm{C} & 1.3104710 & -5.8255540 & -3.2934860 \\ \mathrm{C} & 1.5479460 & -4.8384950 & -2.3706030 \\ \mathrm{C} & -1.0493440 & -5.7771170 & -2.8072890 \\ \mathrm{H} & -0.1725310 & -7.0991900 & -4.2361620 \\ \mathrm{H} & 2.1353430 & -6.2324460 & -3.8700090 \\ \mathrm{H} & 2.5558050 & -4.4656520 & -2.2234100 \\ \mathrm{H} & -2.0652910 & -6.1245240 & -2.9756050 \\ \mathrm{C} & 3.8307500 & -5.8788100 & 0.9290290 \\ \mathrm{C} & 5.1256440 & -5.3642490 & 1.1770030 \\ \mathrm{C} & 5.3737650 & -4.0293120 & 0.9938090 \\ \mathrm{C} & 2.8259290 & -5.0600800 & 0.4790470 \\ \mathrm{H} & 3.6289890 & -6.9310310 & 1.1035560 \\ \mathrm{H} & 5.9120810 & -6.0254740 & 1.5267120 \\ \mathrm{H} & 6.3553600 & -3.6124680 & 1.2037430 \\ \mathrm{H} & 1.8353650 & -5.4655220 & 0.3043870 \\ \mathrm{C} & 3.8231320 & 0.5946800 & 0.0981040 \\ \mathrm{C} & 4.0515710 & 1.2404360 & 1.3351710 \\ \mathrm{C} & 3.8797720 & 1.3308090 & -1.0978520 \\ \mathrm{C} & 4.4072610 & 2.5874910 & 1.3280320 \\ \mathrm{C} & 4.2277030 & 2.6858020 & -1.0487480 \\ \mathrm{C} & 4.5138400 & 3.3290430 & 0.1517370 \\ \mathrm{H} & 4.5974860 & 3.0893970 & 2.2754850 \\ \mathrm{H} & 4.2905610 & 3.2393920 & -1.9832460 \\ \mathrm{C} & -2.9183260 & -2.4064420 & 0.3308140 \\ \mathrm{C} & -3.8481600 & -1.6982810 & -0.4607550 \\ \mathrm{C} & -3.1078420 & -2.5018910 & 1.7231650 \\ \mathrm{C} & -4.9679010 & -1.1361900 & 0.1571580 \\ \mathrm{C} & -4.2418590 & -1.9195690 & 2.2909720 \\ \mathrm{C} & -5.1912350 & -1.2445890 & 1.5289270 \\ \mathrm{H} & -5.6964080 & -0.6133150 & -0.4608480 \\ \mathrm{H} & -4.4021800 & -2.0107180 & 3.3639580 \\ \mathrm{P} & 0.1588340 & -0.1859990 & 0.3180840 \\ \mathrm{O} & 0.6799830 & 0.5879940 & 1.4925470 \\ \mathrm{O} & -0.9124210 & 0.4117730 & -0.5580100 \\ \mathrm{C} & 3.6214350 & 0.6979740 & -2.4568190 \\ & & & \end{array}$

\begin{abstract}
3. 3628270
4.9808060

4.5065400

3.9225620

3.4370970

$-2.1574420$

$-1.3161830$

$-6.4603060$

$-6.3206510$

$-3.7089780$

$-2.7380510$

0.0411290

0.2128130

$-0.6357210$

0.1063250

1. 3984720

1. 3348980

0.9011000

0.6251580

0.8579460

$-0.0401700$

0.7746980

1.2603880

1.9276990

$-1.0790170$

0.1898150

1.9402240

$-0.4160530$

0.4906640

$-3.7366270$

$-3.6614720$

$-4.6657110$

$-2.8952190$

$-4.7991970$

$-5.7937360$

$-4.6646910$

$-4.7829470$

2.4369210

2.6354780

2.2482240

1.5280460

4.8808790

4.7004480

5.1780180

5.7201180

$-1.5859040$
\end{abstract}

-2.3075700
0.2319040
1.1190440
2.6746980
2.5008700
2.6343940
2.0393390
2.1739420
3.2608760
-1.9713390
-2.2685730
0.9433200
-0.4930220
1.4509260
-0.8503840
0.9448980
1.1558210
0.6244780
-0.8758410
-1.3666700
1.4179990
0.9545400
-1.4438400
-1.3723650
1.2948870
2.4876830
0.8333610
-1.0786810
-2.3849420
-2.4250170
-3.5165700
-2.1315770
-1.9943930
-2.6909100
-2.4536030
-3.7761930
-2.3957770
-3.1659310
-3.3596750
-4.1295940
-2.5618300
-3.3313050
-4.2851130
-3.5497500
-2.8318100
3.7354380

\begin{tabular}{rr}
-0.3539510 & -2.3075700 \\
4.7724570 & 0.2319040 \\
5.2146060 & 1.1190440 \\
0.5253250 & 2.6746980 \\
-0.4393110 & 2.5008700 \\
-3.2650780 & 2.6343940 \\
-3.6298750 & 2.0393390 \\
-0.7182270 & 2.1739420 \\
-0.7767800 & 3.2608760 \\
-1.5531290 & -1.9713390 \\
-1.9577390 & -2.2685730 \\
3.5029060 & 0.9433200 \\
3.2746760 & -0.4930220 \\
2.8133540 & 1.4509260 \\
2.2576710 & -0.8503840 \\
3.0130130 & 0.9448980 \\
2.0258310 & 1.1558210 \\
5.8452440 & 0.6244780 \\
5.7265520 & -0.8758410 \\
4.2963080 & -1.3666700 \\
4.9349120 & 1.4179990 \\
6.8804470 & 0.9545400 \\
6.4133250 & -1.4438400 \\
4.0457480 & -1.3723650 \\
5.2666930 & 1.2948870 \\
4.9583480 & 2.4876830 \\
5.5596390 & 0.8333610 \\
6.0042450 & -1.0786810 \\
4.1430270 & -2.3849420 \\
-0.0893850 & -2.4250170 \\
-0.0379290 & -3.5165700 \\
0.4126610 & -2.1315770 \\
0.4557100 & -1.9943930 \\
-2.3587080 & -2.6909100 \\
-1.9638490 & -2.4536030 \\
-2.3030140 & -3.7761930 \\
-3.4127160 & -2.3957770 \\
1.3636310 & -3.1659310 \\
2.4244690 & -3.3596750 \\
0.8792680 & -4.1295940 \\
1.2788480 & -2.5618300 \\
0.7387760 & -3.3313050 \\
0.2323860 & -4.2851130 \\
1.7704110 & -3.5497500 \\
0.2449280 & -2.8318100 \\
-2.3650940 & 3.7354380 \\
& \\
\hline
\end{tabular}




$\begin{array}{rrr}-2.3774510 & -2.0044520 & 4.4017650 \\ -0.8680780 & -2.9246170 & 4.3444250 \\ -1.0730680 & -1.5007960 & 3.3051610 \\ -2.8537310 & -4.4939870 & 3.2336020 \\ -2.1536980 & -5.0665140 & 3.8510490 \\ -3.6974800 & -4.1989180 & 3.8670690 \\ -3.2367790 & -5.1510290 & 2.4463640 \\ 3.0415630 & 1.3007970 & 3.6620660 \\ 2.9128210 & 0.7146880 & 4.5779640 \\ 3.4953300 & 2.2568240 & 3.9445660 \\ 2.0533100 & 1.4899530 & 3.2381490 \\ 5.3022630 & 0.2702640 & 3.2958630 \\ 5.8172840 & 1.2190760 & 3.4864330 \\ 5.2015810 & -0.2575240 & 4.2499940 \\ 5.9402040 & -0.3290310 & 2.6397740 \\ -6.7515220 & 0.7415630 & 1.8172480 \\ -5.9063120 & 1.3892300 & 2.0689470 \\ -6.9585980 & 0.8469980 & 0.7454250 \\ -7.6339630 & 1.0987820 & 2.3583120 \\ -7.6526210 & -1.6093100 & 1.8019220 \\ -7.4701830 & -2.6494690 & 2.0873200 \\ -8.5656050 & -1.2669020 & 2.3005360 \\ -7.8250670 & -1.5826130 & 0.7199200 \\ 4.6007380 & 5.6239790 & -0.9786570 \\ 3.5249650 & 5.5879580 & -1.1807600 \\ 4.8775150 & 6.6682270 & -0.8046960 \\ 5.1271110 & 5.2912480 & -1.8799510 \\ 6.5009910 & 4.8133860 & 0.4471900 \\ 6.7898980 & 4.2513900 & 1.3401700 \\ 7.0133950 & 4.3669730 & -0.4122820 \\ 6.8513630 & 5.8449190 & 0.5586180 \\ -2.7504970 & 3.1623990 & -0.6661230 \\ -2.7690590 & 2.0307950 & -0.0176950 \\ -4.0719120 & 3.8406220 & -0.7398430 \\ -4.1430240 & 5.1566920 & -1.1995500 \\ -5.2301080 & 3.1598730 & -0.3587440 \\ -5.3753280 & 5.7966000 & -1.2657030 \\ -3.2305540 & 5.6619370 & -1.4994750 \\ -6.4607930 & 3.8030360 & -0.4277090 \\ -5.1495230 & 2.1335680 & -0.0169830 \\ -6.5324260 & 5.1205870 & -0.8785250 \\ -5.4364560 & 6.8210420 & -1.6183770 \\ -7.3634130 & 3.2779230 & -0.1300970 \\ -7.4936410 & 5.6226180 & -0.9310280 \\ -1.9500410 & 1.4255500 & -0.1749090 \\ -1.7463160 & 3.6726070 & -1.1774050\end{array}$

\begin{tabular}{|c|c|c|c|}
\hline \multicolumn{4}{|c|}{$\operatorname{TS} 132(\mathrm{~S}, \mathrm{~S}) \mathrm{G}[\mathrm{M} 06-2 \mathrm{X} / 6-31 \mathrm{G}(\mathrm{d})]=-3310.348483$} \\
\hline 0 & 0.0585250 & 1.1745260 & 0.3030930 \\
\hline 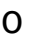 & -1.9899020 & 0.2548740 & -0.8 \\
\hline C & -0.1437360 & 2.3938200 & -0.3116740 \\
\hline C & -1.4105960 & 2.9377870 & -0.3220550 \\
\hline C & 1.0054870 & 3.0503390 & -0.8302930 \\
\hline C & -1.6042250 & 4.1735050 & -1.0240350 \\
\hline C & 0.8239000 & 4.2785220 & -1.4128230 \\
\hline C & -0.4700740 & 4.8485620 & -1.5576860 \\
\hline $\mathrm{H}$ & 1.6840470 & 4.8347770 & -1.7767300 \\
\hline C & -2.7264730 & 0.8880730 & 0.1466580 \\
\hline C & -3.7232220 & 0.1166820 & 0.8025200 \\
\hline C & -2.5041920 & 2.2264370 & 0.3964700 \\
\hline C & -4.5329200 & 0.7606460 & 1.7029940 \\
\hline C & -3.2876530 & 2.8621590 & 1.4180300 \\
\hline C & -4.3324840 & 2.1242140 & 2.0434690 \\
\hline $\mathrm{H}$ & -5.3381720 & 0.2128230 & 2.1859980 \\
\hline$c$ & -4.8983570 & 4.0447200 & 3.4160290 \\
\hline C & -3.8300860 & 4.7646920 & 2.8314450 \\
\hline C & -3.0462980 & 4.1913690 & 1.8617740 \\
\hline C & -5.1360490 & 2.7507970 & 3.0323590 \\
\hline $\mathrm{H}$ & -5.5143030 & 4.5135060 & 4.1768490 \\
\hline $\mathrm{H}$ & -3.6240620 & 5.7791570 & 3.1582800 \\
\hline $\mathrm{H}$ & -2.2232460 & 4.7521530 & 1.4329 \\
\hline $\mathrm{H}$ & -5.9362040 & 2.1740270 & 38600 \\
\hline C & 346090 & 5.9274270 & -1.9066100 \\
\hline C & 031760 & 6.6154390 & -2.4048000 \\
\hline C & -0.6514240 & 6.0828590 & -2.2 \\
\hline C & 901320 & 4.7402090 & -1.2 \\
\hline $\mathrm{H}$ & 262180 & 6.34 & -2 \\
\hline $\mathrm{H}$ & -2.031 & 7.55 & -2 \\
\hline $\mathrm{H}$ & 0 & 6.5 & -2 \\
\hline $\mathrm{H}$ & -3 & 4 & -0 \\
\hline C & 2.3 & 2 & -0 \\
\hline C & 3 & 1.7 & -1 \\
\hline C & 2.9430660 & 2.5 & 0 \\
\hline C & 4.2795870 & 1.2406 & -1 \\
\hline C & 4.2126110 & 1.91849 & 0.8 \\
\hline C & 4.9013790 & 1.3048200 & -0. \\
\hline $\mathrm{H}$ & 4.8015950 & 0.76101 & -2.3 \\
\hline $\mathrm{H}$ & 4.6882340 & 1.9760950 & 1.7 \\
\hline C & -3.874 & 1.3387480 & 0.4 \\
\hline C & -3.3548000 & -2.3040350 & 1. \\
\hline C & 440 & -1.7347440 & -0 \\
\hline & 00410 & -3.0541800 & \\
\hline
\end{tabular}




-4.7086280
-4.2378170
-3.1710120
-5.2258460
2.2681960
6.3005260
2.4042450
-2.5923710
-4.4351210
-5.0648020
-6.5937620
-6.9225570
-7.0531490
-6.9706070
-4.4176760
-3.3287080
-4.6747900
-4.7673500
-3.6726270
-3.7830700
-4.0562240
-2.6065400
-5.9202530
-6.4688500
-6.3787570
-6.0452880
-3.5310780
-4.3324840
-3.9925670
-2.9743740
-1.4180040
-1.7644180
-0.8110680
-0.7811060
2.9604160
4.0080850
2.9413980
2.4634080
2.2204430
1.7656440
3.2241470
1.6298010
7.3268850
8.3409230
7.1287600
7.2898330

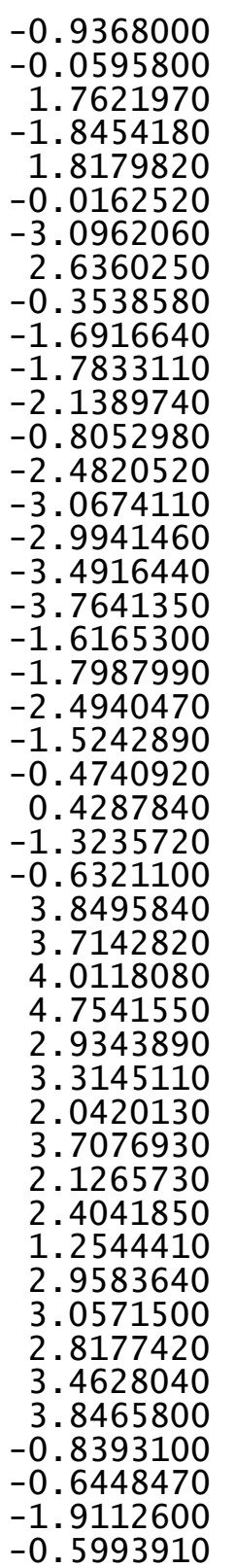

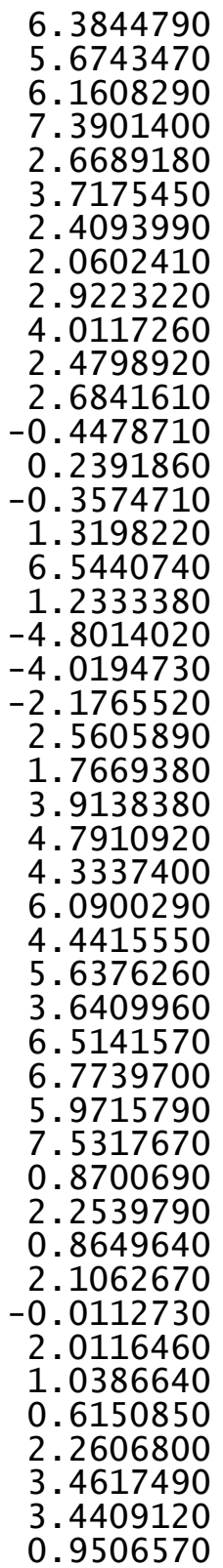

-0.7391570
-1.3029350
-0.9313670
-1.1216210
0.3876860
0.2886480
-0.4558760
0.3215830
2.8747670
2.8109780
2.8301660
3.8492250
-0.1449290
-0.2835470
-1.2690290
1.8196480
0.8859930
3.3812120
0.2785610
-6.1071490
-0.9166500
-2.2474890
-1.2547710
-2.1567360
-3.2353970
-0.9813800
-3.1375790
-4.1365060
-0.8845410
-0.1522690
-1.9600760
-3.9732100
0.0302370
-1.8807980
-1.3138630
-3.1966910
-3.3293370
-2.6421800
-3.0142500
-1.5942190
-2.5744270
-1.6741840
-5.2147010
-4.6961940
-3.1697640
-4.8165260
$-0.3302550$

0.2823010

$-1.3864320$

$-0.1250960$

$-3.8015570$

$-4.1046970$

$-3.1581870$

$-4.7089360$

$-3.9658020$

$-4.0708530$

$-4.9666920$

$-3.5311200$

$-0.5122600$

$-1.8382310$

0.4882080

$-2.9884170$

1.0466040

1. 5451910

$-1.3463790$

0.4930430

2.4761460

1.5902370

1.8578520

2.1938060

2.0502270

2.8216670

2.5354050

1.5559760

3.2952730

2.9141060

3.1513380

2.4288790

3.7756550

3.5213940

1. 3374000

0.8480930

$-1.5178440$

$-1.1516460$

$-0.9518880$

$-0.8798420$

$-2.7400910$

$-2.5735510$

$-2.4518430$

$-1.6577140$

$-1.5628560$

$-1.7671440$ 


\begin{tabular}{|c|c|c|c|c|c|c|c|}
\hline & & & & & & & \\
\hline $\mathrm{H}$ & 2.3092420 & -6.3037690 & -2.5420270 & C & 3.5211020 & 3.4114810 & -1.1081370 \\
\hline $\mathrm{H}$ & 4.4010750 & -5.0166380 & -2.1172150 & $\mathrm{C}$ & 3.6575750 & 4.1339210 & 0.0733440 \\
\hline $\mathrm{H}$ & 3.6693340 & -2.7111650 & -2.5338530 & $\mathrm{H}$ & 3.8009310 & 3.9742200 & 2.2011000 \\
\hline $\mathrm{H}$ & 0.8686680 & -5.3117880 & -0.7921570 & $\mathrm{H}$ & 3.4500050 & 3.9404260 & -2.0562130 \\
\hline $\mathrm{H}$ & 0.0832950 & -5.1145020 & -2.3644670 & C & -2.3573540 & -2.8429690 & 0.2258380 \\
\hline $\mathrm{H}$ & 2.2817570 & -4.8039100 & -3.4681580 & $\mathrm{C}$ & -3.3005170 & -2.2770930 & -0.6555870 \\
\hline $\mathrm{H}$ & 3.4317410 & -5.1111460 & -0.6431140 & $\mathrm{C}$ & -2.6219250 & -2.8846620 & 1.6108560 \\
\hline $\mathrm{H}$ & 4.1876370 & -2.7889000 & -0.8588380 & $\mathrm{C}$ & -4.5033980 & -1.7973200 & -0.1295480 \\
\hline & & & & C & -3.8279430 & -2.3730850 & 2.0852480 \\
\hline & S) $\mathrm{G}[\mathrm{M06}-2$ & $G(d)]=-33$ & 730 & $\mathrm{C}$ & -4.7851370 & -1.8253900 & 1.2317360 \\
\hline 0 & 1.4643360 & -0.3582410 & -0.6943670 & $\mathrm{H}$ & -5.2445920 & -1.3785000 & -0.8083610 \\
\hline 0 & 0.1227610 & -1.7225250 & 0.9236000 & $\mathrm{H}$ & -4.0289570 & -2.4103800 & 3.1545070 \\
\hline C & 2.5946860 & -0.9968650 & -0.2248490 & $\mathrm{P}$ & 0.2174090 & -0.2080090 & 0.3481300 \\
\hline C & 2.6007320 & -2.3753740 & -0.1437650 & 0 & 0.5696800 & 0.6791370 & 1.5049470 \\
\hline C & 3.7073060 & -0.1805170 & 0.1117130 & 0 & -0.9537770 & 0.1381820 & -0.5345850 \\
\hline C & 3.7778810 & -3.0176980 & 0.3642080 & $\mathrm{C}$ & 3.3766220 & 1.3039930 & -2.4625030 \\
\hline $\mathrm{C}$ & 4.8633550 & -0.8163310 & 0.4920030 & $\mathrm{H}$ & 3.3653530 & 0.2246770 & -2.2874880 \\
\hline C & 4.9270650 & -2.2253860 & 0.6438710 & C & 3.7818300 & 5.6474190 & 0.1161480 \\
\hline $\mathrm{H}$ & 5.7473700 & -0.2257460 & 0.7185220 & $\mathrm{H}$ & 3.2231860 & 5.9901470 & 0.9980810 \\
\hline $\mathrm{C}$ & 0.1597660 & -2.7514760 & -0.0000880 & C & 3.7272710 & 1.3366590 & 2.6700820 \\
\hline $\mathrm{C}$ & -1.0734070 & -3.3856600 & -0.3120780 & $\mathrm{H}$ & 3.4612550 & 0.2854150 & 2.5262160 \\
\hline C & 1.3754130 & -3.1268550 & -0.5342430 & C & -1.6607490 & -3.5136500 & 2.6088260 \\
\hline C & -1.0293240 & -4.4676090 & -1.1554300 & $\mathrm{H}$ & -0.7460670 & -3.7990520 & 2.0822250 \\
\hline C & 1.3900870 & -4.1911220 & -1.4963800 & C & -6.0839650 & -1.2631130 & 1.7767490 \\
\hline C & 0.1784060 & -4.8865850 & -1.7713890 & $\mathrm{H}$ & -6.6686650 & -0.8995610 & 0.9198440 \\
\hline $\mathrm{H}$ & -1.9496210 & -4.9946350 & -1.3965350 & C & -3.0896640 & -2.1820780 & -2.1618170 \\
\hline C & 1.3352930 & -6.3165320 & -3.3557890 & $\mathrm{H}$ & -2.0572360 & -2.4634610 & -2.3853730 \\
\hline C & 2.5299900 & -5.5958040 & -3.1200900 & C & -0.7594400 & 3.3500390 & 0.8867040 \\
\hline C & 2.5575150 & -4.5623040 & -2.2178990 & $\mathrm{C}$ & -0.5360090 & 3.1390330 & -0.5453760 \\
\hline C & 0.1859690 & -5.9618360 & -2.6993980 & $\mathrm{H}$ & -1.2574890 & 2.5297690 & 1.4072140 \\
\hline $\mathrm{H}$ & 1.3300100 & -7.1362960 & -4.0671260 & $\mathrm{H}$ & -0.3972790 & 2.1182140 & -0.8787410 \\
\hline $\mathrm{H}$ & 3.4320180 & -5.8591750 & -3.6635590 & 0 & 0.6750760 & 3.1997370 & 0.9057560 \\
\hline $\mathrm{H}$ & 3.4769560 & -4.0105190 & -2.0557260 & $\mathrm{H}$ & 0.8506370 & 2.2314720 & 1.1373820 \\
\hline $\mathrm{H}$ & -0.7461050 & -6.4882250 & -2.8874770 & C & -0.4785600 & 5.8217260 & 0.5160970 \\
\hline $\mathrm{C}$ & 4.9912230 & -4.9880640 & 1.1071600 & $\mathrm{C}$ & -0.7080550 & 5.6109550 & -0.9821090 \\
\hline C & 6.1470220 & -4.2050900 & 1.3398480 & C & -0.1491210 & 4.2634450 & -1.4424770 \\
\hline C & 6.1096280 & -2.8535000 & 1.1179340 & $\mathrm{C}$ & -1.1838840 & 4.7315430 & 1.3279150 \\
\hline C & 3.8393700 & -4.4123410 & 0.6337690 & $\mathrm{H}$ & -0.8485390 & 6.8043850 & 0.8227700 \\
\hline $\mathrm{H}$ & 5.0147520 & -6.0537850 & 1.3121140 & $\mathrm{H}$ & -0.2448620 & 6.4143660 & -1.5633280 \\
\hline $\mathrm{H}$ & 7.0530100 & -4.6754890 & 1.7084900 & $\mathrm{H}$ & 0.9498410 & 4.2657170 & -1.4481800 \\
\hline $\mathrm{H}$ & 6.9802750 & -2.2335940 & 1.3156110 & $\mathrm{H}$ & -2.2706770 & 4.8041380 & 1.1929670 \\
\hline $\mathrm{H}$ & 2.9567710 & -5.0212630 & 0.4705930 & $\mathrm{H}$ & -0.9757370 & 4.8330560 & 2.3974460 \\
\hline C & 3.6124170 & 1.3124770 & 0.0927860 & $\mathrm{H}$ & 0.5969970 & 5.7948790 & 0.7335870 \\
\hline C & 3.6902350 & 2.0257840 & 1.3114500 & $\mathrm{H}$ & -1.7838330 & 5.6362270 & -1.1930880 \\
\hline C & 3.4931520 & 2.011788 & -1.1208980 & $\mathrm{H}$ & -0.4695520 & 4.0057960 & -2.4556210 \\
\hline C & 3.72 & 3.41772 & 1.2680150 & $\mathrm{C}$ & 380830 & -0.7589540 & 994 \\
\hline
\end{tabular}




$\begin{array}{rrr}-3.1748020 & -0.7582370 & -3.7890340 \\ -4.2864030 & -0.3685270 & -2.4700800 \\ -2.5425230 & -0.0833110 & -2.2759460 \\ -4.0189100 & -3.1620460 & -2.8914560 \\ -5.0689260 & -2.8939230 & -2.7271920 \\ -3.8301970 & -3.1412070 & -3.9698950 \\ -3.8803970 & -4.1884880 & -2.5372010 \\ 2.0667450 & 1.6663240 & -3.1713620 \\ 2.0185500 & 2.7394770 & -3.3912810 \\ 1.9859780 & 1.1284590 & -4.1216980 \\ 1.2054130 & 1.3924380 & -2.5539390 \\ 4.5875590 & 1.6093870 & -3.3530070 \\ 4.5198520 & 1.0534410 & -4.2938300 \\ 4.6411380 & 2.6761810 & -3.5965640 \\ 5.5206690 & 1.3304500 & -2.8538010 \\ -1.2665910 & -2.5326010 & 3.7182480 \\ -2.1324620 & -2.2587040 & 4.3313300 \\ -0.5247650 & -2.9914460 & 4.3802930 \\ -0.8364070 & -1.6198070 & 3.2978090 \\ -2.2649250 & -4.7951730 & 3.1978510 \\ -1.5587070 & -5.2710910 & 3.8863290 \\ -3.1828300 & -4.5750280 & 3.7543500 \\ -2.5134720 & -5.5113460 & 2.4082660 \\ 2.7043240 & 1.9294750 & 3.6471370 \\ 2.7023690 & 1.3471140 & 4.5744170 \\ 2.9477900 & 2.9644740 & 3.9101050 \\ 1.6981920 & 1.8991240 & 3.2242370 \\ 5.1323940 & 1.4049510 & 3.2829340 \\ 5.4288860 & 2.4482000 & 3.4429110 \\ 5.1535870 & 0.8949350 & 4.2516980 \\ 5.8828920 & 0.9411940 & 2.6363330 \\ -6.9159200 & -2.3388480 & 2.4836120 \\ -7.1312770 & -3.1755970 & 1.8127460 \\ -6.3795090 & -2.7335120 & 3.3534180 \\ -7.8655670 & -1.9230910 & 2.8363680 \\ -5.8185040 & -0.0779700 & 2.7135400 \\ -5.1902720 & 0.6765070 & 2.2299790 \\ -6.7588130 & 0.3926630 & 3.0201890 \\ -5.2992650 & -0.4137220 & 3.6180580 \\ 3.2116620 & 6.3614170 & -1.1085820 \\ 2.1682590 & 6.0846960 & -1.2930730 \\ 3.2527990 & 7.4452560 & -0.9636360 \\ 3.7897210 & 6.1291840 & -2.0096800 \\ 5.2534280 & 6.0396970 & 1.2163390 \\ 5.6680150 & 5.5786160 & \\ 5.8500440 & 5.7033240 & \\ -4 & & \\ -2.5401590\end{array}$

$\begin{array}{lr}\mathrm{H} & 5.3590860 \\ \mathrm{C} & -3.4115260 \\ \mathrm{O} & -3.1925920 \\ \mathrm{C} & -4.8468570 \\ \mathrm{C} & -5.1925720 \\ \mathrm{C} & -5.8378140 \\ \mathrm{C} & -6.5316860 \\ \mathrm{H} & -4.4062830 \\ \mathrm{C} & -7.1762340 \\ \mathrm{H} & -5.5422880 \\ \mathrm{C} & -7.5220570 \\ \mathrm{H} & -6.8057460 \\ \mathrm{H} & -7.9490240 \\ \mathrm{H} & -8.5671720 \\ \mathrm{H} & -2.2528330 \\ \mathrm{O} & -2.5385000 \\ \mathrm{H} & \end{array}$

7.1262170

2. 3300630

1.2236000

2. 7144070

3.9886740

1. 8036820

4.3559620

4.6763050

2.1734710

0.8151880

3.4495500

5. 3470530

1.4676560

3.7386710

0.8279020

3.0376540
0.4018900 $-0.7069730$ $-0.0519140$ $-0.7826320$ $-1.2356330$ $-0.4099420$ $-1.3019190$ $-1.5299420$ $-0.4806310$ $-0.0737450$ $-0.9233030$ $-1.6486310$ $-0.1918720$ $-0.9764090$ $-0.1835890$ $-1.2241060$

$\operatorname{TS} 134(\mathrm{~S}, \mathrm{~S}) \mathrm{G}[\mathrm{M} 06-2 \mathrm{X} / 6-31 \mathrm{G}(\mathrm{d})]=-3310.347479$

$\begin{array}{lrlr}\text { TS } & 0.0624150 & 1.1974590 & 0.2607340 \\ \text { O } & -2.0031690 & 0.2461140 & -0.8195040 \\ \text { C } & -0.1923040 & 2.4188380 & -0.3308430 \\ \text { C } & -1.4712000 & 2.9323990 & -0.2844990 \\ \text { C } & 0.9186980 & 3.1072830 & -0.8893550 \\ \text { C } & -1.7219920 & 4.1716540 & -0.9617190 \\ \text { C } & 0.6844670 & 4.3377100 & -1.4483980 \\ \text { C } & -0.6264110 & 4.8801590 & -1.5312320 \\ \text { H } & 1.5158700 & 4.9159940 & -1.8435250 \\ \text { C } & -2.7197000 & 0.8450890 & 0.1986750 \\ \text { C } & -3.6722000 & 0.0388560 & 0.8778430 \\ \text { C } & -2.5202440 & 2.1842760 & 0.4626580 \\ \text { C } & -4.4635790 & 0.6487180 & 1.8175980 \\ \text { C } & -3.2812030 & 2.7847560 & 1.5217550 \\ \text { C } & -4.2841320 & 2.0115130 & 2.1725530 \\ \text { H } & -5.2368520 & 0.0735750 & 2.3207070 \\ \text { C } & -4.8461430 & 3.8962960 & 3.5953080 \\ \text { C } & -3.8182420 & 4.6517410 & 2.9836990 \\ \text { C } & -3.0565630 & 4.1128700 & 1.9773500 \\ \text { C } & -5.0659890 & 2.6027420 & 3.1999150 \\ \text { H } & -5.4450470 & 4.3378970 & 4.3854440 \\ \text { H } & -3.6256160 & 5.6661500 & 3.3187640 \\ \text { H } & -2.2639920 & 4.7005090 & 1.5275360 \\ \text { H } & -5.8345270 & 1.9993560 & 3.6759130 \\ \text { C } & -3.2272880 & 5.9015490 & -1.7636130 \\ \text { C } & -2.1330560 & 6.6230010 & -2.2964880 \\ \text { C } & -0.8634450 & 6.1184210 & -2.1841290 \\ \text { C } & -3.0282800 & 4.7097460 & -1.1131090\end{array}$




$\begin{array}{rr}-4.2338250 & 6.2921750 \\ -2.3042440 & 7.5674410 \\ -0.0150680 & 6.6522470 \\ -3.8741190 & 4.1585550 \\ 2.2871550 & 2.5125810 \\ 2.9196500 & 1.9402510 \\ 2.9393790 & 2.5316810 \\ 4.2172820 & 1.4493610 \\ 4.2350020 & 2.0163160 \\ 4.8994010 & 1.4862570 \\ 4.7261840 & 1.0300750 \\ 4.7396910 & 2.0547800 \\ -3.7996100 & -1.4153640 \\ -3.2301950 & -2.3803230 \\ -4.4898010 & -1.8110230 \\ -3.4175720 & -3.7307970 \\ -4.6443590 & -3.1718650 \\ -4.1250210 & -4.1481390 \\ -2.9904090 & -4.4860480 \\ -5.1853440 & -3.4761060 \\ 2.3034370 & 3.1577890 \\ 6.3126600 & 0.9313020 \\ 2.2398020 & 1.8769520 \\ -2.4316570 & -1.9935530 \\ -4.3025860 & -5.6235320 \\ -5.0767070 & -0.8015990 \\ -6.6063930 & -0.8990300 \\ -6.9283360 & -1.8844020 \\ -7.0329870 & -0.7442880 \\ -7.0235510 & -0.1449240 \\ -4.4768350 & -0.9668770 \\ -3.3875310 & -0.8763520 \\ -4.7293020 & -1.9440520 \\ -4.8677040 & -0.1974190 \\ -3.5711680 & -6.0124730 \\ -3.6667990 & -7.0863970 \\ -3.9912140 & -5.4777500 \\ -2.5072530 & -5.7644800 \\ -5.7833820 & -6.0106040 \\ -6.3094230 & -5.7475390 \\ -6.2780330 & -5.4929940 \\ -5.8919640 & -7.0874380 \\ -3.3262890 & -1.9794960 \\ -4.1459750 & -1.2624050 \\ -3.7624600 & -2.9717460 \\ -2.7423350 & -1.7135090\end{array}$

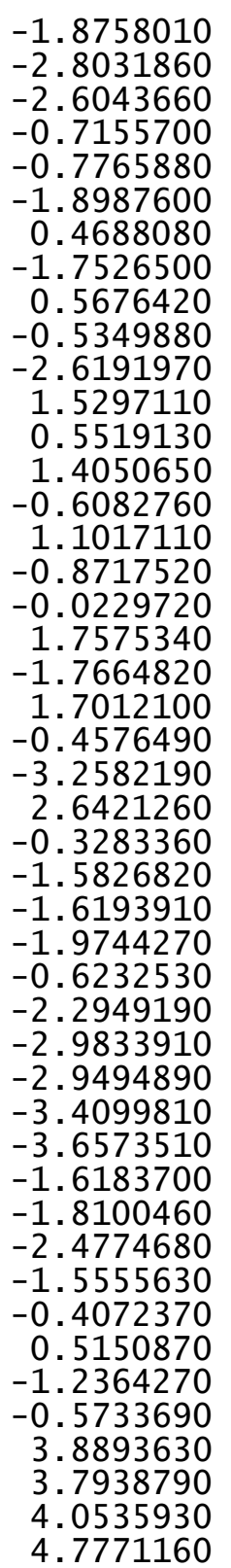
$-1.2281370$
$-1.5402720$
$-0.6540260$
$-0.5693620$
2. 9694660
4.0343170
2. 8864840
2. 4997340
2. 3443870
1. 8999220
3. 3727910
1.7861600
6.2825310
7. 2969720
5.7330820
5.7901540
7. 1061480
7.0943310
6. 6980170
8. 1469860
2. 4966340
3.5306390
2. 2894120
1.8444090
2.6858940
3.7694190
2. 1930730
2. 4488060
$-0.4438770$
0.2038770
$-0.3039880$
1. 1607120
6.8346760
1. 2498830
$-4.8208990$
$-3.8502170$
$-2.0421240$
2. 6232790
1. 8183090
3.9606510
4.8788260
4. 3242100
6.1632920
4.5725010
5. 6106020
3. 6013770
$-2.9126930$
$-3.8906670$
$-3.0628020$
$-2.4646200$
4. 5037850
4. 3666310
5. 1874070
4.9754100
2. 2264100
1.2558060
2. 0658770
2. 6671910
$-0.6028840$
$-1.0150060$
$-0.9729410$
$-0.9790920$
1. 4299090
2. 5220320
1.0204470
1. 1001990
0.5528320
0.4787540
$-0.2987140$
0.4775300
3. 0479010
3.0099670
2.9999270
4. 0137840
$-0.1244990$
$-0.2385450$
$-1.2565290$
1.9512780
1. 2625840
3. 3571690
0.2039340
$-6.1853410$
$-0.9831580$
$-2.2428480$
$-1.2650960$
$-2.1679130$
$-3.1951550$
$-1.0538540$
$-3.1060890$
$-4.0506200$
$-0.9685670$
$-0.2622310$

2.8848330

3.2686750

1.9675240

3. 6362620

2. 0135310

2. 2338000

1.1625530

2.8831300

2.9157370

2.6742420

3. 2611940

3. 7479820

$-0.4694910$

$-0.4341860$

0.4040010

$-1.3721130$

0.7493840

0.8186520

1.6802540

0.6756850

$-3.9845920$

$-4.3402430$

$-3.3324960$

$-4.8604830$

$-4.1443310$

$-4.3067670$

$-5.1212170$

$-3.6900490$

$-0.5499190$

$-1.8975060$

0.4360200

$-3.0934330$

$-1.3656710$

1.4869070

$-1.2349370$

0.4989320

2. 4812260

1. 5111130

1.7973400

2. 1528030

1. 9196550

2. 9137350

2. 4443530

1. 3256940

3. 4347670

3.0760050 


\begin{tabular}{|c|c|c|c|c|c|c|c|}
\hline C & 6.5297830 & -1.9909160 & 3.1969370 & $\mathrm{H}$ & 6.0907840 & -4.0496740 & 3.9852210 \\
\hline $\mathrm{H}$ & 6.8793760 & -3.9013120 & 2.2648300 & $\mathrm{H}$ & 4.2380590 & -5.4400270 & 3.0636860 \\
\hline $\mathrm{H}$ & 5.8990440 & -0.1030850 & 4.0235630 & $\mathrm{H}$ & 2.6978910 & -4.5196600 & 1.4017610 \\
\hline $\mathrm{H}$ & 7.5348510 & -1.9181670 & 3.6009000 & H & 6.3273700 & -1.6925810 & 3.2674630 \\
\hline $\mathrm{H}$ & 0.9249070 & -1.3120140 & 1.2671000 & C & 3.4167010 & -5.7130250 & -1.9343660 \\
\hline 0 & 2.3409140 & -3.1669950 & 0.7291700 & $\mathrm{C}$ & 2.3055670 & -6.4811390 & -2.3541280 \\
\hline C & 0.8894600 & -3.2799150 & -1.6110230 & C & 1.0335990 & -6.0241180 & -2.1237230 \\
\hline C & 2.1291210 & -2.5856400 & -1.2534270 & c & 3.2341580 & -4.5218340 & -1.2774780 \\
\hline $\mathrm{H}$ & 0.0179070 & -2.9823590 & -1.0287440 & H & 4.4223530 & -6.0666580 & -2.1390480 \\
\hline $\mathrm{H}$ & 2.0292360 & -1.5435970 & -0.9626400 & $\mathrm{H}$ & 2.4638380 & -7.4241630 & -2.8676130 \\
\hline 0 & 1.0411550 & -2.5027780 & -2.8225350 & $\mathrm{H}$ & 0.1707040 & -6.5952620 & -2.4561530 \\
\hline $\mathrm{H}$ & 0.6047880 & -1.6110710 & -2.6385360 & $\mathrm{H}$ & 4.0915740 & -3.9340020 & -0.9664140 \\
\hline c & 2.2861860 & -5.1358440 & -2.5999730 & C & -2.0892230 & -2.4795750 & -0.4538810 \\
\hline C & 3.4948030 & -4.6194720 & -1.8161970 & $\mathrm{C}$ & -2.8816210 & -2.0134870 & -1.5213740 \\
\hline C & 3.4622630 & -3.0950880 & -1.6938170 & c & -2.5415450 & -2.3442920 & 0.8749830 \\
\hline $\mathrm{C}$ & 0.9842520 & -4.7617570 & -1.8871540 & $\mathrm{C}$ & -4.1184950 & -1.4220210 & -1.2343250 \\
\hline $\mathrm{H}$ & 2.3434350 & -6.2226970 & -2.7096110 & $\mathrm{C}$ & -3.7818770 & -1.7543660 & 1.1086860 \\
\hline $\mathrm{H}$ & 4.4296660 & -4.9231400 & -2.2958350 & $\mathrm{C}$ & -4.5887200 & -1.2855160 & 0.0725400 \\
\hline $\mathrm{H}$ & 3.6748930 & -2.6170340 & -2.6588920 & $\mathrm{H}$ & -4.7417270 & -1.0866000 & -2.0607370 \\
\hline $\mathrm{H}$ & 0.9224220 & -5.2746320 & -0.9197460 & $\mathrm{H}$ & -4.1478050 & -1.6643910 & 2.1302840 \\
\hline $\mathrm{H}$ & 0.1098240 & -5.0570950 & -2.4754260 & C & 3.8706320 & 1.6614810 & 0.3888400 \\
\hline $\mathrm{H}$ & 2.2867380 & -4.7075180 & -3.6092270 & $\mathrm{C}$ & 3.1870400 & 2.5555520 & 1.2404380 \\
\hline H & 3.4843940 & -5.0525320 & -0.8087570 & $\mathrm{C}$ & 4.5461990 & 2.1342310 & -0.7455230 \\
\hline$H$ & 4.2135680 & -2.7227630 & -0.9903060 & $\mathrm{C}$ & 3.2339420 & 3.9173770 & 0.9500340 \\
\hline & S) $\mathrm{G}[\mathrm{M06}-2 \mathrm{X}$ & $G(d)]=-33$ & 796 & $\mathrm{c}$ & 3.9174980 & 4.4150780 & -0.1618300 \\
\hline 0 & 0.1540140 & -1.1019510 & 0.3157230 & $\mathrm{H}$ & 2.7106560 & 4.6131910 & 1.6015080 \\
\hline 0 & 2.0874570 & -0.0482970 & -0.8862640 & $\mathrm{H}$ & 5.0858370 & 3.8839800 & -1.8747050 \\
\hline c & 0.3996620 & -2.3329800 & -0.2582080 & C & -1.7579410 & -2.8986410 & 2.0558340 \\
\hline C & 1.6960490 & -2.7915440 & -0.3235620 & C & -5.9767600 & -0.7589220 & 0.3926660 \\
\hline $\mathrm{c}$ & -0.7356540 & -3.0701040 & -0.6933890 & C & -2.4692040 & -2.2199460 & -2.9722980 \\
\hline C & 1.9289100 & -4.0318460 & -1.0066770 & C & 2.4316000 & 2.0618850 & 2.4672880 \\
\hline $\mathrm{c}$ & -0.5086550 & -4.2983870 & -1.2580730 & C & 3.9298280 & 5.9016300 & -0.4642610 \\
\hline $\mathrm{c}$ & 0.8111690 & -4.7885160 & -1.4610650 & C & 5.2442240 & 1.1968600 & -1.7177210 \\
\hline H & -1.3475430 & -4.9122810 & -1.5763680 & $\mathrm{C}$ & 6.7501520 & 1.4768560 & -1.7763340 \\
\hline C & 2.8854060 & -0.6403210 & 0.0712820 & $\mathrm{H}$ & 6.9485870 & 2.4878280 & -2.1490800 \\
\hline C & 3.8533390 & 0.1967520 & 0.6899310 & $\mathrm{H}$ & 7.2049000 & 1.3883440 & -0.7846230 \\
\hline $\mathrm{c}$ & 2.7692060 & -1.9919860 & 0.3310700 & $\mathrm{H}$ & 7.2460390 & 0.7685310 & -2.4481860 \\
\hline $\mathrm{C}$ & 4.7478130 & -0.3850490 & 1.5504940 & C & 4.6109070 & 1.2789110 & -3.1114130 \\
\hline C & 3.6376790 & -2.5655390 & 1.3221930 & $\mathrm{H}$ & 3.5418330 & 1.0534320 & -3.0629740 \\
\hline C & 4.6594000 & -1.7581970 & 1.8977120 & $\mathrm{H}$ & 4.7328130 & 2.2794020 & -3.5412180 \\
\hline $\mathrm{H}$ & 5.5287780 & 0.2221850 & 2.0020040 & $\mathrm{H}$ & 5.0866700 & 0.5626060 & -3.7894850 \\
\hline C & 5.4116060 & -3.6272800 & 3.2515430 & C & 4.5611770 & 6.7060270 & 0.6772770 \\
\hline C & 4.3650920 & -4.4176870 & 2.7214910 & $\mathrm{H}$ & 4.6090470 & 7.7692020 & 0.4196000 \\
\hline C & 3.5027730 & -3.9033900 & 1.7859710 & $\mathrm{H}$ & 3.9697730 & 6.6103370 & 1.5944660 \\
\hline C & 68070 & 8750 & 2.8509390 & $\mathrm{H}$ & 5.5749140 & 6.3558450 & 0.8925760 \\
\hline
\end{tabular}




\begin{tabular}{|c|c|c|}
\hline $\begin{array}{l}2.5173580 \\
2.0707660 \\
1.8655190 \\
2.5392190 \\
3.3581140 \\
4.2108020 \\
3.7454360 \\
2.8115260 \\
1.1914030 \\
1.4636680 \\
0.5776740 \\
0.5860850 \\
2.3776200 \\
3.4109760 \\
2.3887600 \\
1.8091640 \\
1.6619610 \\
1.2878260 \\
2.6366510 \\
0.9799320 \\
6.9990030 \\
8.0000630 \\
7.0388010 \\
6.7284820 \\
6.4014490 \\
5.6617260 \\
6.5239340 \\
7.3614680 \\
2.6952260 \\
3.7548150 \\
2.1245010 \\
2.3566180 \\
3.2181660 \\
4.2961680 \\
2.8842010 \\
3.0638050 \\
0.4966860 \\
0.1559360 \\
0.2056790 \\
1.3965690 \\
5.9594870 \\
0.7361440 \\
5.1141540 \\
4.5448760 \\
2.0871820 \\
1.3736440\end{array}$ & $\begin{array}{r}6.4132100 \\
5.8567700 \\
6.2969900 \\
7.4754550 \\
2.0185930 \\
1.3531300 \\
3.0216440 \\
1.6690790 \\
2.8998970 \\
3.8760470 \\
3.0513280 \\
2.3821920 \\
-4.2278810 \\
-4.0776460 \\
-4.9531110 \\
-4.6558680 \\
-1.9124540 \\
-0.9419640 \\
-1.7645540 \\
-2.3011820 \\
-1.8995410 \\
-1.5506650 \\
-2.2808490 \\
-2.7311740 \\
0.4216770 \\
1.2257690 \\
0.1242660 \\
0.8209600 \\
-0.9944060 \\
-0.7205330 \\
-0.1396220 \\
-1.2097830 \\
-3.4280710 \\
-3.2299980 \\
-3.6337290 \\
-4.3290380 \\
0.1773520 \\
0.0723580 \\
1.3828030 \\
-2.4325690 \\
-0.4132200 \\
-3.1115330 \\
0.1712290 \\
6.0479680 \\
1.0427520 \\
2.9716500\end{array}$ & $\begin{array}{r}-0.7719500 \\
-1.6016000 \\
0.1010900 \\
-1.0372750 \\
3.6909050 \\
3.5327700 \\
3.9044640 \\
4.5734420 \\
2.7928550 \\
3.2105160 \\
1.9028750 \\
3.5441420 \\
2.5077250 \\
2.8406700 \\
1.6876930 \\
3.3401890 \\
3.2224230 \\
2.8813690 \\
3.7021330 \\
3.9853300 \\
0.2888910 \\
0.5638670 \\
-0.7378460 \\
0.9463180 \\
-0.4794560 \\
-0.4282810 \\
-1.5276500 \\
-0.1355920 \\
-3.8596990 \\
-3.9209470 \\
-3.4919650 \\
-4.8784360 \\
-3.5541000 \\
-3.5810270 \\
-4.5764950 \\
-2.9530650 \\
-0.6347080 \\
-1.9832570 \\
0.2209740 \\
-2.9902970 \\
1.4358700 \\
1.7325250 \\
-1.3583090 \\
-1.3614340 \\
2.2568070 \\
-1.9006470\end{array}$ \\
\hline
\end{tabular}
$-2.4755710$
$-0.5886270$
$-2.2310110$
$-1.1006680$
$-0.5602020$
$-2.9119270$
$-4.1068340$
$-3.7507090$
$-1.7271490$
$-3.1831790$
$-4.9691570$
$-3.6048860$
$-1.9702130$
$-0.8359610$
$-2.6184250$
$-4.3998710$
$-4.5336240$
$-3.1028520$
$-1.9882810$
$-4.1954460$
$-5.3890510$
$-4.0442820$
$-6.4409820$
$-5.4815400$
$-5.1011880$
$-3.1053180$
$-6.3002430$
$-7.3701160$
$-4.9897660$
$-7.1246840$
$-1.1808780$
$-3.3009270$

144

$\operatorname{TS} 136(\mathrm{~S}, \mathrm{~S}) \mathrm{G}[\mathrm{M} 06-2 \mathrm{X} / 6-31 \mathrm{G}(\mathrm{d})]=-3310.351521$

$\begin{array}{lrcr}\text { O O } & 0.5908940 & 0.7313440 & 0.2998570 \\ \mathrm{O} & -1.5759440 & 1.1899790 & -0.9158210 \\ \mathrm{C} & 0.7506150 & 2.0997420 & 0.4031980 \\ \mathrm{C} & -0.2977190 & 2.8624840 & 0.8778200 \\ \mathrm{C} & 2.0192110 & 2.6382170 & 0.0630400 \\ \mathrm{C} & -0.1243500 & 4.2832780 & 0.9582410 \\ \mathrm{C} & 2.2062810 & 3.9863990 & 0.2434950 \\ \mathrm{C} & 1.1530500 & 4.8379820 & 0.6658630 \\ \mathrm{H} & 3.1823750 & 4.4208140 & 0.0422840 \\ \mathrm{C} & -2.1772320 & 1.3580390 & 0.3216810 \\ \mathrm{C} & -3.4077720 & 0.6853800 & 0.5495470 \\ \mathrm{C} & -1.5758820 & 2.1918730 & 1.2402630\end{array}$

$2.0104560 \quad-1.8064100$

$2.8569990 \quad-1.1546600$

$1.0073410-1.4653700$

$2.2050440-3.1001730$

$1.4019670-2.8117950$

$4.5248180 \quad-3.1762830$

$3.7202040 \quad-2.6597720$

$2.2374080 \quad-2.5506250$

$4.4063030-2.2147800$

$5.5782850 \quad-3.2920530$

$3.8365700 \quad-3.3225380$

$1.8028160 \quad-3.5468080$

$4.8893300-1.2604150$

$4.9003490 \quad-2.6146700$

$4.1549410 \quad-4.1659420$

$4.0887370 \quad-1.6694100$

$1.6494960 \quad-2.0675940$

$1.8708420 \quad 1.1521880$

$1.3146150 \quad 1.5267100$

$1.7806180 \quad 2.1609110$

$2.4691750 \quad 1.9362690$

$0.9884520 \quad 3.3012720$

$\begin{array}{ll}2.3433980 & 2.8366800\end{array}$

$3.0915030 \quad 1.0520640$

$0.8577980 \quad 4.1957770$

$0.4728020 \quad 3.4670450$

$1.5302520 \quad 3.9608850$

$\begin{array}{ll}2.8764060 & 2.6624730\end{array}$

$0.2332540 \quad 5.0764780$

$1.4260980 \quad 4.6595650$

1.3918570

0.0693660 


-4.0330240
-2.1893230
-3.4440710
-5.0000050
-3.4783170
-2.2110130
-1.5831440
-4.0755460
-3.9662130
-1.7287560
-0.6072860
-5.0387940
-0.9716140
0.3106160
1.3460590
-1.1847010
-1.7960770
0.4650200
2.3313120
-2.1744780
3.1341440
3.4761280
3.8997570
4.6326490
5.0475690
5.4482600
4.9219540
5.6591850
-3.9997200
-4.0390950
-4.5408150
-4.6731780
-5.1520740
-5.2389930
-4.7236690
-5.5775800
-0.3199580
0.3892190
-0.7222400
3.5503420
2.6160280
6.7563040
6.8652270
2.6584170
1.6527600
-4.5070300

0.9096370

2.3375260

1.7090140

0.4518690

2.5897850

3.1799230

3.0579520

1.8667850

2.7004500

3.7297920

3.5061570

1.3891730

6.5231530

7.0679920

6.2410710

5.1701020

7.1838740

8.1399300

6.6454350

4.7657670

1.7716270

1.7774880

1.0306730

1.1021630

0.3749410

0.4231180

1.1239560

$-0.1677590$

$-0.2060780$

$-1.6006020$

0.3595060

$-2.3919780$

$-0.4756660$

$-1.8537960$

$-3.4683050$

$-0.0344450$

0.1645850

0.3391910

$-1.2152150$

0.9815170

1. 5294510

$-0.2019830$

0.0169770

2. 5420430

2. 6825940

1.8552920

1.7507590
2.5282430
2.7651070
1.9420750
5.0266660
4.8071430
3.5931610
4.0272840
5.9898700
5.6092750
3.4402970
4.1865180
1.3689810
1.1194320
0.7707060
1.2911920
1.6179710
1.1906450
0.5537250
1.4739280
-0.4336660
-1.8000600
0.4872170
-2.2005550
0.0379110
-1.2987580
-3.2487160
0.7583910
-0.4942480
-0.2969000
-1.6657040
-1.2581880
-2.5994760
-2.4100180
-1.1101840
-3.4992970
-0.9450000
-2.2515360
-0.4908740
1.9678070
2.1208450
-1.7528470
-2.8223980
-2.8327160
-2.4242530
-1.9422500

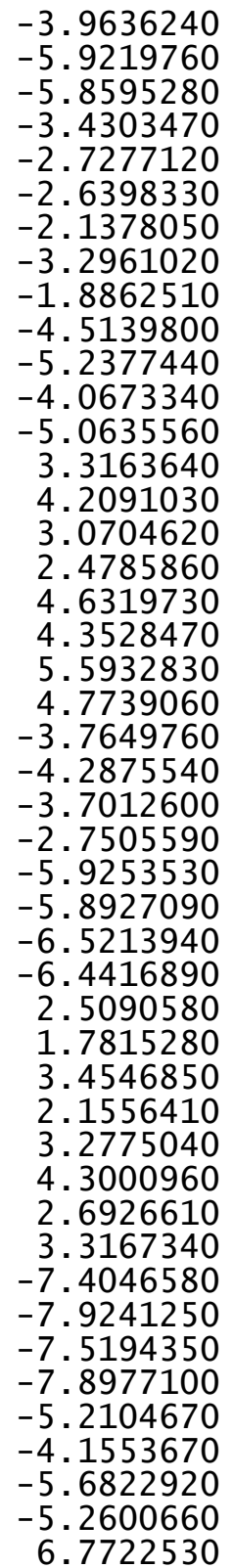

2.3496700

$-2.7410630$

$-3.7730830$

$-2.2751750$

$-1.5725220$

$-3.5394620$

$-3.9246370$

$-4.3353380$

$-3.3269640$

$-2.6252680$

$-3.3253770$

$-3.1007010$

$-1.7402210$

$-0.4534350$

$-1.0735000$

$-0.4612810$

$-0.9133170$

1.6754500

1.6839040

1.1577580

2.7098900

2.1657060

1.7392610

3.2485280

1.7587240

2.4386700

3.5216210

1.9977670

2.2436830

1.7836480

2.2990110

1.7451440

0.7624340

3.9203670

3.8088160

4.4586850

4.5355490

$-2.3814190$

$-2.4420940$

$-1.3605460$

$-3.0594720$

$-2.6797470$

$-3.3624620$

$-1.6686830$

$-1.7252990$
$-1.1319500$

$-3.4333350$

$-3.0649040$

0.9263200

1. 3866430

0.5674090

1.4622040

0.1995550

$-0.1957540$

1.9555200

1. 5222250

2.8357930

2.2876410

2.4514500

2. 3097260

3. 5182660

1. 9158520

2.8046460

3.8632140

2.7126700

2.4765070

$-3.2469860$

$-4.1104610$

$-3.3976440$

$-3.2215710$

$-1.9599330$

$-2.1189110$

$-2.7666740$

$-1.0146320$

$-4.1563470$

$-4.7909170$

$-4.7083450$

$-4.0016100$

$-3.1048830$

$-3.4839030$

$-3.8581790$

$-2.2022320$

$-3.5824550$

$-2.6215910$

$-3.9633770$

$-4.2869050$

$-4.7896360$

$-5.5041040$

$-5.2086440$

$-1.5863460$ 


\begin{tabular}{|c|c|c|c|c|c|c|c|}
\hline $\mathrm{H}$ & 5.9842950 & -2.1970320 & -2.1827920 & C & -0.7413360 & -3.1953190 & -0.8274690 \\
\hline $\mathrm{H}$ & 7.7342430 & -2.1361430 & -1.9097060 & $\mathrm{C}$ & 1.9502730 & -4.1253420 & -0.8660510 \\
\hline $\mathrm{H}$ & 6.6244360 & -2.0077410 & -0.5365210 & C & -0.4443560 & -4.4284770 & -1.3489760 \\
\hline C & 7.9454750 & 0.4303830 & -1.0194670 & $\mathrm{C}$ & 0.8927210 & -4.9060270 & -1.4131700 \\
\hline $\mathrm{H}$ & 7.9506310 & 1.5171960 & -1.1425480 & $\mathrm{H}$ & -1.2443910 & -5.0595580 & -1.7278510 \\
\hline $\mathrm{H}$ & 7.9013620 & 0.2131220 & 0.0535050 & C & 2.7749570 & -0.7142190 & 0.1722720 \\
\hline $\mathrm{H}$ & 8.8905550 & 0.0329420 & -1.4034770 & $\mathrm{C}$ & 3.6943500 & 0.1644500 & 0.8065460 \\
\hline C & 1.5450750 & -2.5732780 & -2.0656590 & C & 2.6470430 & -2.0510990 & 0.4871430 \\
\hline $\mathrm{C}$ & 2.1821870 & -2.2562080 & -0.7860510 & $\mathrm{C}$ & 4.5233890 & -0.3680000 & 1.7607270 \\
\hline $\mathrm{H}$ & 0.4726410 & -2.3875890 & -2.1013720 & $\mathrm{c}$ & 3.4466380 & -2.5707610 & 1.5601910 \\
\hline $\mathrm{H}$ & 1.7713320 & -1.4320930 & -0.2118320 & $\mathrm{C}$ & 4.4137700 & -1.7229050 & 2.1711570 \\
\hline 0 & 2.3145630 & -1.4147580 & -2.4733250 & $\mathrm{H}$ & 5.2743550 & 0.2623560 & 2.2303690 \\
\hline $\mathrm{H}$ & 1.6826310 & -0.6274150 & -2.4276600 & C & 5.0814300 & -3.5212740 & 3.6588840 \\
\hline C & 3.4993310 & -4.0652820 & -2.6183730 & $\mathrm{C}$ & 4.0892250 & -4.3510880 & 3.0859030 \\
\hline $\mathrm{C}$ & 3.8923970 & -4.0715550 & -1.1395480 & $\mathrm{C}$ & 3.2937520 & -3.8903310 & 2.0670150 \\
\hline C & 3.5562230 & -2.7383070 & -0.4702440 & C & 5.2322300 & -2.2342790 & 3.2127260 \\
\hline $\mathrm{C}$ & 1.9955610 & -3.8288210 & -2.7731730 & $\mathrm{H}$ & 5.7079100 & -3.9012860 & 4.4596050 \\
\hline $\mathrm{H}$ & 3.7722160 & -5.0154000 & -3.0868310 & $\mathrm{H}$ & 3.9513800 & -5.3605010 & 3.4606920 \\
\hline $\mathrm{H}$ & 4.9629750 & -4.2688900 & -1.0229900 & $\mathrm{H}$ & 2.5288570 & -4.5337380 & 1.6464070 \\
\hline $\mathrm{H}$ & 4.2279610 & -1.9414390 & -0.8097320 & $\mathrm{H}$ & 5.9726030 & -1.5751370 & 3.6584410 \\
\hline $\mathrm{H}$ & 1.4345140 & -4.6642660 & -2.3371240 & C & 3.5422300 & -5.8014780 & -1.6135580 \\
\hline $\mathrm{H}$ & 1.7105280 & -3.7520790 & -3.8270140 & $\mathrm{C}$ & 2.4867430 & -6.5938470 & -2.1231480 \\
\hline $\mathrm{H}$ & 4.0450440 & -3.2739780 & -3.1468850 & C & 1.1931650 & -6.1506110 & -2.0266620 \\
\hline $\mathrm{H}$ & 3.3519280 & -4.8742530 & -0.6240290 & $\mathrm{C}$ & 3.2822550 & -4.6012130 & -1.0011060 \\
\hline $\mathrm{H}$ & 3.6590700 & -2.7845470 & 0.6176330 & $\mathrm{H}$ & 4.5674250 & -6.1440080 & -1.7134160 \\
\hline C & 0.6571500 & -3.3119990 & 1.5423450 & $\mathrm{H}$ & 2.7061730 & -7.5439870 & -2.5997010 \\
\hline 0 & -0.0255690 & -2.2134900 & 1.6935400 & $\mathrm{H}$ & 0.3734500 & -6.7396870 & -2.4296100 \\
\hline C & 0.7091860 & -4.1829180 & 2.7476360 & $\mathrm{H}$ & 4.0987770 & -3.9957640 & -0.6216820 \\
\hline C & 1.5528920 & -5.2957410 & 2.7432880 & C & -2.1363660 & -2.6625310 & -0.7312020 \\
\hline $\mathrm{C}$ & -0.0806020 & -3.8994770 & 3.8650290 & $\mathrm{c}$ & -2.7883250 & -2.1365320 & -1.8647050 \\
\hline $\mathrm{C}$ & 1.6110330 & -6.1228160 & 3.8585430 & $\mathrm{C}$ & -2.7905690 & -2.6863410 & 0.5131020 \\
\hline $\mathrm{H}$ & 2.1530390 & -5.4978420 & 1.8620410 & $\mathrm{C}$ & -4.1055800 & -1.6971950 & -1.7300210 \\
\hline C & -0.0218050 & -4.7321540 & 4.9773690 & $\mathrm{C}$ & -4.1066880 & -2.2234700 & 0.6001050 \\
\hline $\mathrm{H}$ & -0.7319880 & -3.0320250 & 3.8499290 & $\mathrm{C}$ & -4.7887500 & -1.7402290 & -0.5132010 \\
\hline C & 0.8233470 & -5.8408960 & 4.9743680 & $\mathrm{H}$ & -4.6285540 & -1.3134700 & -2.6045480 \\
\hline $\mathrm{H}$ & 2.2672190 & -6.9871370 & 3.8597770 & $\mathrm{H}$ & -4.6121260 & -2.2651210 & 1.5616060 \\
\hline $\mathrm{H}$ & -0.6349020 & -4.5174890 & 5.8465700 & $\mathrm{C}$ & 3.7506060 & 1.6046330 & 0.4046360 \\
\hline $\mathrm{H}$ & 0.8676790 & -6.4887630 & 5.8444450 & $\mathrm{C}$ & 3.1182800 & 2.5866320 & 1.1970500 \\
\hline $\mathrm{H}$ & -0.2595910 & -1.7592840 & 0.7885180 & $\mathrm{C}$ & 4.4215180 & 1.9679330 & -0.7740270 \\
\hline 0 & 1.2400790 & -3.6398040 & 0.4997420 & $\mathrm{C}$ & 3.2116580 & 3.9208790 & 0.8044530 \\
\hline \multicolumn{4}{|c|}{$-2 \times / 6-31 G(d) 1=-3310348253$} & C & 4.4884290 & 3.3192080 & -1.1244300 \\
\hline \multicolumn{4}{|c|}{$\operatorname{TS} 137(\mathrm{~S}, \mathrm{~S}) \quad \mathrm{G}[\mathrm{M} 06-2 \mathrm{X} / 6-31 \mathrm{G}(\mathrm{d})]=-3310.348253$} & $\mathrm{C}$ & 3.8961920 & 4.3095150 & -0.3486720 \\
\hline 0 & 0.0108840 & -1.2150980 & 0.2702230 & $\mathrm{H}$ & 2.7255760 & 4.6813820 & 1.4111520 \\
\hline 0 & 2.0155600 & -0.1913390 & -0.8566180 & $\mathrm{H}$ & 5.0139790 & 3.6075180 & -2.0334170 \\
\hline C & 0.3320450 & -2.4356180 & -0.2883960 & C & -2.1313450 & -3.2633890 & 1.7571960 \\
\hline C & 1.6360880 & -2.8796650 & -0.2277360 & C & -6.2224070 & -1.2388570 & -0.4473870 \\
\hline
\end{tabular}




\begin{tabular}{|c|c|c|}
\hline $\begin{array}{l}-2.1068210 \\
2.3600020 \\
3.9583110 \\
5.0775630 \\
6.5967640 \\
6.8497490 \\
7.0332980 \\
7.0649660 \\
4.4681460 \\
3.3878680 \\
4.6519200 \\
4.9129760 \\
4.5593580 \\
4.6509320 \\
3.9241420 \\
5.5511850 \\
2.5702520 \\
2.1593250 \\
1.8750840 \\
2.6187960 \\
3.2802220 \\
4.1318910 \\
3.6705100 \\
2.7285130 \\
1.1211810 \\
1.3965490 \\
0.5208260 \\
0.4989440 \\
-2.7275640 \\
-3.7998640 \\
-2.6020970 \\
-2.2403900 \\
-2.2297250 \\
-1.8314120 \\
-3.2675450 \\
-1.6572780 \\
-6.2497970 \\
-7.2789580 \\
-5.7174310 \\
-5.7683880 \\
-7.0004570 \\
-6.9459680 \\
-6.6123910 \\
-8.0531700 \\
-2.4338530 \\
-3.4651300\end{array}$ & $\begin{array}{r}-2.0689180 \\
2.2276930 \\
5.7671880 \\
0.9401840 \\
1.1408370 \\
2.1192310 \\
1.0843760 \\
0.3729410 \\
0.9716750 \\
0.8073630 \\
1.9346970 \\
0.1897270 \\
6.6515670 \\
7.6859060 \\
6.6549210 \\
6.2960750 \\
6.2752160 \\
5.6705600 \\
6.2202210 \\
7.3177310 \\
2.3175050 \\
1.6368070 \\
3.3364380 \\
2.0676510 \\
3.1026970 \\
4.1095290 \\
3.1873770 \\
2.6603390 \\
-4.6393910 \\
-4.5560420 \\
-5.3265960 \\
-5.0764770 \\
-2.3231410 \\
-1.3342060 \\
-2.2099550 \\
-2.7272810 \\
0.2951460 \\
0.6696570 \\
0.6959540 \\
0.6782450 \\
-1.7511360 \\
-2.8408250 \\
-1.3125070 \\
-1.4637380 \\
-0.7802520 \\
-0.7791970\end{array}$ & $\begin{array}{r}-3.2230560 \\
2.4673790 \\
-0.7641760 \\
-1.6828130 \\
-1.7332040 \\
-2.1565400 \\
-0.7310020 \\
-2.3578200 \\
-3.0889620 \\
-3.0455450 \\
-3.5780830 \\
-3.7133200 \\
0.3332150 \\
-0.0140610 \\
1.2255930 \\
0.6272370 \\
-1.1729140 \\
-1.9871490 \\
-0.3272550 \\
-1.5048070 \\
3.6929750 \\
3.6132650 \\
3.7987470 \\
4.6057090 \\
2.6946720 \\
3.0293350 \\
1.7855240 \\
3.4799310 \\
2.0797410 \\
2.2907010 \\
1.2368700 \\
2.9577580 \\
2.9614990 \\
2.7137080 \\
3.2973900 \\
3.8026300 \\
-0.4786870 \\
-0.4515250 \\
0.3919330 \\
-1.3843340 \\
0.7639900 \\
0.8490790 \\
1.6901630 \\
0.6821190 \\
-3.9844450 \\
-4.3554670\end{array}$ \\
\hline
\end{tabular}

-2.2895530
-1.7749520
-2.4819630
-3.5654930
-1.9904980
-2.1895120
0.4458150
-0.2244420
0.2717430
-1.0260900
-6.7284830
-1.0670630
4.8924870
4.6121070
2.0152290
-2.6760740
-1.8274660
-4.0097430
-4.9746810
-4.3227200
-6.2551430
-4.7073500
-5.6050440
-3.5642640
-6.5708160
-7.0074080
-5.8539650
-7.5725300
-0.9413440
-2.4350260
-1.0194080
-2.2269210
-0.1282510
-2.0837430
-1.1572380
-0.6855340
-2.5002720
-3.6767590
-3.5839770
-1.1748800
-2.5999620
-4.6294700
-3.7897600
-1.1165910
-0.3212770
-2.4995990
$-3.3508500$

$-4.8534030$

$-4.0806690$

$-4.2448690$

$-5.0580080$

$-3.6027010$

$-0.5684280$

$-1.9077690$

0.4051260

$-3.0525350$

$-1.3525330$

1.5521880

$-1.2685800$

$-1.6436040$

2. 3644390

1. 5001700

1.7965980

2. 1409500

1.8951630

2. 9128570

2. 4175170

1.2932860

3.4318850

3.0853990

3. 1809150

2. 2278650

4. 0292670

3. 5831010

1. 2569760

0.7086930

$-1.6529790$

$-1.2709970$

$-1.0805430$

$-0.9721320$

$-2.8543310$

$-2.6662480$

$-2.6381620$

$-1.8322930$

$-1.6982370$

$-1.9420840$

$-2.7559920$

$-2.3003840$

$-2.6569310$

$-0.9805200$

$-2.5459950$

$-3.6437200$ 


\begin{tabular}{|c|c|c|c|}
\hline $\begin{array}{l}\mathrm{H} \\
\mathrm{H} \\
14\end{array}$ & $\begin{array}{l}-3.6680690 \\
-4.3119340\end{array}$ & $\begin{array}{l}4.8811330 \\
2.5232600\end{array}$ & $\begin{array}{l}-0.8286980 \\
-0.9827010\end{array}$ \\
\hline \multicolumn{4}{|c|}{$\operatorname{TS} 138(\mathrm{~S}, \mathrm{~S}) \quad \mathrm{G}[\mathrm{M} 06-2 \mathrm{X} / 6-31 \mathrm{G}(\mathrm{d})]=-3310.352430$} \\
\hline 0 & 0.4984800 & 0.8565860 & 0.3710240 \\
\hline 0 & -1.6339290 & 0.9822030 & -0.9607570 \\
\hline C & 0.5123960 & 2.2381690 & 0.3566780 \\
\hline C & -0.6231010 & 2.9256190 & 0.7336800 \\
\hline $\mathrm{C}$ & 1.7302230 & 2.8722360 & -0.0060440 \\
\hline C & -0.5891900 & 4.3590650 & 0.7028970 \\
\hline C & 1.7838510 & 4 . 2408230 & 0.0737010 \\
\hline $\mathrm{C}$ & 0.6400510 & 5.0127690 & 0.4061820 \\
\hline $\mathrm{H}$ & 2.7162220 & 4.7544610 & -0.1457280 \\
\hline C & -2.3078940 & 1.1894810 & 0.2324270 \\
\hline C & -3.4700250 & 0.4071800 & 0.4701130 \\
\hline C & -1.8438220 & 2.1571370 & 1.0992570 \\
\hline $\mathrm{C}$ & -4.1780840 & 0.6649170 & 1.6172930 \\
\hline $\mathrm{C}$ & -2.5345260 & 2.3419560 & 2.3426810 \\
\hline $\mathrm{C}$ & -3.7294170 & 1.6062860 & 2.5797480 \\
\hline $\mathrm{H}$ & -5.0996150 & 0.1215100 & 1.8083230 \\
\hline $\mathrm{C}$ & -3.9703960 & 2.6611070 & 4.7524620 \\
\hline $\mathrm{C}$ & -2.7589100 & 3.3601450 & 4.5377380 \\
\hline $\mathrm{C}$ & -2.0597040 & 3.2055830 & 3.3671830 \\
\hline $\mathrm{C}$ & -4.4386510 & 1.7999360 & 3.7948130 \\
\hline $\mathrm{H}$ & -4.5162710 & 2.7985000 & 5.6804710 \\
\hline $\mathrm{H}$ & -2.3762290 & 4.0206040 & 5.3095650 \\
\hline $\mathrm{H}$ & -1.1267680 & 3.7386880 & 3.2190530 \\
\hline $\mathrm{H}$ & -5.3554970 & 1.2381330 & 3.9531790 \\
\hline $\mathrm{C}$ & -1.6621670 & 6.5301900 & 0.9040290 \\
\hline $\mathrm{C}$ & -0.4290080 & 7.1775960 & 0.6534750 \\
\hline $\mathrm{C}$ & 0.6934780 & 6.4319060 & 0.4048020 \\
\hline $\mathrm{C}$ & 15920 & 5.1606680 & 9279950 \\
\hline $\mathrm{H}$ & $\$ 3920$ & 7.1236350 & 0721360 \\
\hline $\mathrm{H}$ & 3920 & 8.2618070 & 6434490 \\
\hline $\mathrm{H}$ & 50 & 33300 & 1860760 \\
\hline $\mathrm{H}$ & -2 & 763650 & 1098080 \\
\hline $\mathrm{C}$ & 2. & 511170 & 4570950 \\
\hline $\mathrm{C}$ & 0 & 35430 & 3306510 \\
\hline $\mathrm{C}$ & 3. & 2800 & 4888860 \\
\hline C & 0 & 450 & 3130 \\
\hline C & 4.7 & 2040 & 0469830 \\
\hline $\mathrm{C}$ & 5.1 & 34770 & 006140 \\
\hline $\mathrm{H}$ & 4. & & 779170 \\
\hline $\mathrm{H}$ & 5.3 & 980130 & 4170 \\
\hline C & -3 & 411020 & 032120 \\
\hline C & -3.8 & 2 & \\
\hline
\end{tabular}

-4.4353740
-4.2963960
-4.9015520
-4.8514980
-4.2386860
-5.3207360
3.3633000
6.3583190
2.3838060
-3.1937280
-5.3747380
-4.5467280
-6.0166170
-6.5220300
-6.5546630
-6.0920640
-3.7783560
-2.7288550
-4.2092850
-3.8224660
-4.5861160
-4.9395300
-4.7091220
-3.5176030
-6.8732360
-7.4452450
-7.0661100
-7.2481620
-4.2867950
-4.9441850
-4.9082680
-3.8398250
-2.2535230
-2.8029770
-1.4980180
-1.7462890
4.4919030
5.4314280
4.6606290
4.2437460
3.0971970
2.2330390
3.9679900
2.8757230
7.5740000
8.4759990
$-1.7503430$

$-1.0636520$

$-2.6179060$

$-2.2894630$

$-0.8065380$

$-3.5769990$

1. 9782100

$-1.7327930$

$-2.8879390$

1.1482030

$-3.2453490$

$-2.1785240$

$-2.3105850$

$-3.0883970$

$-1.3700880$

$-2.5808580$

$-3.4786730$

$-3.3724390$

$-4.3134800$

$-3.7371560$

$-4.5598710$

$-5.2216600$

$-5.0865650$

$-4.3778560$

$-3.5125710$

$-2.5802390$

$-4.0084900$

$-4.1642130$

2. 1614540

2.3792380

1. 7707330

3. 1032040

0.9582160

0.6676380

0.1962420

1. 9041010

2.7465120

2. 6567330

2. 3608170

3. 8103200

2. 5401960

2. 0454330

2. 4125580

3. 6115120

-1.7958960
-2.0784110 


\begin{tabular}{rrr}
7.4063010 & 1.5074500 & -2.5413880 \\
7.7504660 & 1.2054690 & -0.8303420 \\
6.1689480 & -0.9409910 & -3.0664100 \\
5.2228220 & -1.4923570 & -3.0908240 \\
6.1601170 & -0.2346760 & -3.9034350 \\
6.9923670 & -1.6409340 & -3.2387470 \\
2.2216900 & 1.8945750 & -4.1830980 \\
3.1641730 & 1.8232430 & -4.7370730 \\
1.8491290 & 0.8879640 & -3.9847840 \\
1.5012300 & 2.3991260 & -4.8346140 \\
3.0075960 & 4.0624720 & -3.2132520 \\
4.0221050 & 3.9292500 & -3.6068320 \\
2.4139810 & 4.5828850 & -3.9722440 \\
3.0712220 & 4.7049430 & -2.3314030 \\
-0.2820760 & 0.0895720 & -0.8511980 \\
0.4620520 & 0.2131330 & -2.1425890 \\
-0.5767640 & -1.2795490 & -0.2907650 \\
1.3816030 & 2.8584080 & -2.4779620 \\
6.5589570 & -0.9646970 & -0.9570250 \\
2.4522480 & 1.9877780 & 2.1262230 \\
-4.0994440 & 1.8258520 & -1.4032950 \\
-5.2321900 & -4.6302550 & -2.7645180 \\
-2.5966370 & -1.6635110 & 1.5612950 \\
1.0869610 & -2.9894900 & 1.8923960 \\
0.2362980 & -2.0093280 & 1.9466400 \\
1.4038570 & -3.6135900 & 3.2053270 \\
2.3960840 & -4.5944450 & 3.2665780 \\
0.7266180 & -3.2206340 & 4.3626770 \\
2.7122190 & -5.1823300 & 4.4855290 \\
2.9066980 & -4.8844230 & 2.3538080 \\
1.0453710 & -3.8127240 & 5.5798930 \\
-0.0399870 & -2.4562610 & 4.2966940 \\
2.0364160 & -4.7914150 & 5.6411060 \\
3.4831080 & -5.9443670 & 4.5371800 \\
0.5215920 & -3.5115680 & 6.4812690 \\
2.2832330 & -5.2516420 & 6.5929600 \\
-0.0705340 & -1.6842130 & 1.0027790 \\
1.6255380 & -3.3994140 & 0.8528330 \\
1.6971360 & -2.6185670 & -1.8297590 \\
2.4326830 & -2.1750000 & -0.6427320 \\
0.6265090 & -2.4264430 & -1.8025840 \\
2.0749210 & -1.2831850 & -0.1337690 \\
2.4320020 & -1.5168930 & -2.4208760 \\
1.8081440 & -0.7274020 & -3.3363470 \\
3.5756100 & -4.2341550 & \\
4.0674460 & -4.0718270 & \\
\hline & &
\end{tabular}

\begin{tabular}{ll}
$\mathrm{C}$ & 3.8275710 \\
$\mathrm{C}$ & 2.0747490 \\
$\mathrm{H}$ & 3.7797820 \\
$\mathrm{H}$ & 5.1348760 \\
$\mathrm{H}$ & 4.4809540 \\
$\mathrm{H}$ & 1.5166990 \\
$\mathrm{H}$ & 1.7250150 \\
$\mathrm{H}$ & 4.1183610 \\
$\mathrm{H}$ & 3.5325040 \\
$\mathrm{H}$ & 4.0360200 \\
\hline
\end{tabular}
$-2.6476680$
$-3.9531890$
$-5.2475940$
$-4.3009540$
$-1.9370860$
$-4.7171540$
$-3.9776750$
$-3.5452950$
$-4.7741450$
$-2.5433420$
$-0.3917430$
$-2.4264490$
$-2.6940110$
$-0.8236880$
$-0.9095580$
$-1.8714270$
$-3.4631620$
$-2.9942840$
$-0.2469150$
0.6771630

$\operatorname{TS} 139(\mathrm{~S}, \mathrm{~S}) \mathrm{G}[\mathrm{M} 06-2 \mathrm{X} / 6-31 \mathrm{G}(\mathrm{d})]=-3310.349082$

$\begin{array}{lrrr}\text { TS139(S, S) } \mathrm{G}[\mathrm{M} 06-2 \mathrm{X} / 6-31 \mathrm{G}(\mathrm{d})]=-3310.349082 \\ \mathrm{O} & 1.4413130 & -0.4904820 & -0.7731010 \\ \mathrm{O} & -0.0545970 & -1.6378470 & 0.8814310 \\ \mathrm{C} & 2.4809470 & -1.2802450 & -0.3261600 \\ \mathrm{C} & 2.2929780 & -2.6441570 & -0.2415230 \\ \mathrm{C} & 3.7061160 & -0.6305690 & -0.0179070 \\ \mathrm{C} & 3.3805850 & -3.4490870 & 0.2350900 \\ \mathrm{C} & 4.7707360 & -1.4241930 & 0.3301810 \\ \mathrm{C} & 4.6385260 & -2.8288750 & 0.4795590 \\ \mathrm{H} & 5.7334020 & -0.9644660 & 0.5398230 \\ \mathrm{C} & -0.1747570 & -2.6843040 & -0.0149580 \\ \mathrm{C} & -1.4816950 & -3.1960280 & -0.2394900 \\ \mathrm{C} & 0.9631630 & -3.2152890 & -0.5899070 \\ \mathrm{C} & -1.6012260 & -4.2824990 & -1.0704300 \\ \mathrm{C} & 0.8091560 & -4.2792340 & -1.5398170 \\ \mathrm{C} & -0.4852290 & -4.8313120 & -1.7518940 \\ \mathrm{H} & -2.5840000 & -4.7159380 & -1.2416580 \\ \mathrm{C} & 0.4221920 & -6.3893320 & -3.3768420 \\ \mathrm{C} & 1.7024870 & -5.8124750 & -3.2008730 \\ \mathrm{C} & 1.8917570 & -4.7872690 & -2.3089340 \\ \mathrm{C} & -0.6463070 & -5.9026140 & -2.6706000 \\ \mathrm{H} & 0.2887810 & -7.2041820 & -4.0812930 \\ \mathrm{H} & 2.5416600 & -6.1806250 & -3.7827850 \\ \mathrm{H} & 2.8757040 & -4.3462370 & -2.1924150 \\ \mathrm{H} & -1.6407440 & -6.3182360 & -2.8104990 \\ \mathrm{C} & 4.3228160 & -5.5727850 & 0.9489770 \\ \mathrm{C} & 5.5844680 & -4.9628050 & 1.1456670 \\ \mathrm{C} & 5.7330000 & -3.6192790 & 0.9211190 \\ \mathrm{C} & 3.2512240 & -4.8385720 & 0.5071650 \\ \mathrm{H} & 4.2001940 & -6.6311780 & 1.1565920 \\ \mathrm{H} & 6.4246990 & -5.5576940 & 1.4893650 \\ \mathrm{H} & 6.6882000 & -3.1297240 & 1.0921340 \\ \mathrm{H} & 2.2870870 & -5.3163900 & 0.3722280 \\ \mathrm{C} & 3.8038220 & 0.8606320 & -0.0155080 \\ \mathrm{C} & 4.0128120 & 1.5368100 & 1.2040380 \\ & & & \end{array}$




\begin{tabular}{|c|c|c|}
\hline $\begin{array}{l}3.7028460 \\
4.1764780 \\
3.8524420 \\
4.0952830 \\
4.3427930 \\
3.7844380 \\
2.6777270 \\
3.6683500 \\
2.8410250 \\
4.8193320 \\
4.0086370 \\
5.0173880 \\
5.5938010 \\
4.1488120 \\
0.2464080 \\
0.7321430 \\
0.8746820 \\
3.4782380 \\
3.3608670 \\
4.2590140 \\
3.4354310 \\
4.0545330 \\
3.7092360 \\
1.8251350 \\
0.9682380 \\
6.3196490 \\
6.1532430 \\
3.5647230 \\
2.5778730 \\
0.2908350 \\
0.0366490 \\
0.8761340 \\
0.0143770 \\
1.1235690 \\
1.1974340 \\
0.2031650 \\
0.0136190 \\
0.4790050 \\
0.6070560 \\
0.1041430 \\
0.5654060 \\
1.5732330 \\
1.6806540 \\
0.4214650 \\
1.2654540 \\
1.0473470\end{array}$ & $\begin{array}{r}1.5929150 \\
2.9251090 \\
2.9783110 \\
3.6674460 \\
3.4351290 \\
3.5449470 \\
-2.5718020 \\
-1.9580100 \\
-2.6400930 \\
-1.4635020 \\
-2.1297440 \\
-1.5519300 \\
-1.0167100 \\
-2.2039420 \\
-0.1562090 \\
0.6815230 \\
0.3487070 \\
0.9282160 \\
-0.1477340 \\
5.1778890 \\
5.5780270 \\
0.8258190 \\
-0.2016400 \\
-3.3041460 \\
-3.6261310 \\
-1.1235000 \\
-1.1190300 \\
-1.8527810 \\
-2.2106120 \\
3.4951960 \\
3.3044700 \\
2.7107580 \\
2.2849770 \\
3.2092390 \\
2.2196180 \\
5.9484540 \\
5.7929740 \\
4.4159430 \\
4.8961210 \\
6.9481520 \\
6.5678900 \\
4.3309560 \\
5.0606630 \\
4.9522920 \\
5.8396860 \\
5.9120550\end{array}$ & $\begin{array}{r}-1.2158790 \\
1.1882090 \\
-1.1732900 \\
0.0154650 \\
2.1320490 \\
-2.1024780 \\
0.4038740 \\
-0.3928080 \\
1.8014070 \\
0.2264270 \\
2.3694550 \\
1.6035520 \\
-0.3955470 \\
3.4465900 \\
0.2848350 \\
1.4295690 \\
-0.5877450 \\
-2.5656860 \\
-2.4097820 \\
-0.0166980 \\
-0.6286220 \\
2.5512330 \\
2.4075620 \\
2.7194490 \\
2.1223150 \\
2.2554060 \\
3.3401750 \\
-1.9099910 \\
-2.2144870 \\
0.9023710 \\
-0.5272770 \\
1.3847220 \\
-0.8899700 \\
0.9532420 \\
1.1431470 \\
0.6095130 \\
-0.9004100 \\
-1.3775800 \\
1.3712560 \\
0.9302560 \\
-1.4409420 \\
-1.3464970 \\
1.2120530 \\
2.4483060 \\
0.8593660 \\
-1.1496240\end{array}$ \\
\hline
\end{tabular}

0.1747950

$-3.6841890$

$-3.6635250$

$-4.6204450$

$-2.8493720$

$-4.6190260$

$-5.6307700$

$-4.5023510$

$-4.5405580$

2. 1991210

2.2546830

2.0510500

1. 3240200

4.6958110

4.5518670

4.8533760

5.6055550

$-1.3050300$

$-2.1109730$

$-0.5383620$

$-0.8644520$

$-2.4210740$

$-1.6718460$

$-3.2744070$

$-2.7667180$

3.1153080

3.1139910

3.4365970

2. 0929570

5.4855820

5.8628400

5.5124990

6.1725290

$-6.7680890$

$-5.9925370$

$-7.0035530$

$-7.6742870$

$-7.4209520$

$-7.1257310$

$-8.3542930$

$-7.6162820$

5.5767000

5.6359170

5.6790050

6.4249020

4.1770020
4. 2125180

$-0.4086550$

$-0.3926790$

0.0610320

0.1892870

$-2.7430920$

$-2.3949120$

$-2.7209900$

$-3.7828020$

. 4379680

2. 5161360

0.9376360

1.2335560

1.1282730

0.6056380

2.1901140

0.7435150

$-2.3300670$

$-2.0077120$

$-2.8153750$

$-1.4445910$

$-4.5604860$

$-5.0609060$

$-4.3077330$

$-5.2683200$

1.4748190

0.8847370

. 4892190

1.5141090

0.7819530

1.7973040

0.2486120

0.2830600

0.2807710

1.0239400

0.3186340

0.5660130

$-2.1479080$

3.1498740

$-1.8788660$

$-2.1875300$

5.5581950

5.1410670

6.6461570

5.1700500

5.8341700
$-2.4077760$

$-2.4099510$

$-3.5050280$

$-2.0869540$

$-2.0407660$

$-2.5820160$

$-2.3445720$

$-3.6705270$

$-2.2483720$

$-3.2392520$

$-3.4309990$

$-4.2018980$

$-2.6149540$

$-3.4773190$

$-4.4285890$

$-3.6968770$

$-3.0061830$

3.7822990

4.4510600

4.3953870

3. 3165120

3. 3678560

3.9902030

4.0066710

2.6078170

3.5760220

4.4985290

3.8361170

3.1938070

3.1028010

3.2714070

4.0587660

2. 4126100

1.8454050

2.0533900

0.7749770

2. 3899180

1.9512720

2.2761860

2.4571800

0.8735680

$-0.7067130$

$-1.7162480$

$-0.7766870$

$-0.1322230$

1. 3608810 


\begin{tabular}{|c|c|c|c|c|c|c|c|}
\hline $\mathrm{H}$ & 3.2771840 & 5.5251580 & 1.9024630 & $c$ & 0.2638960 & 6.2066980 & -1.5419760 \\
\hline $\mathrm{H}$ & 5.0465890 & 5.5694230 & 1.9728910 & $\mathrm{C}$ & -2.1344650 & 5.0864010 & -0.6405540 \\
\hline $\mathrm{H}$ & 4.1644150 & 6.9235670 & 1.2593540 & $\mathrm{H}$ & -3.0137580 & 6.9632080 & -1.1331540 \\
\hline C & -2.9692660 & 2.8640800 & -0.7504180 & $\mathrm{H}$ & -0.8729970 & 7.9767600 & -1.9067110 \\
\hline 0 & -2.8895580 & 1.7612560 & -0.0590580 & $\mathrm{H}$ & 1.2004210 & 6.6275110 & -1.8985380 \\
\hline C & -4.3434850 & 3.4236480 & -0.8479170 & $\mathrm{H}$ & -3.0719930 & 4.6518290 & -0.3100510 \\
\hline C & -4.5262930 & 4.7102030 & -1.3576930 & C & 2.6692200 & 1.9141010 & -0.7561620 \\
\hline $\mathrm{C}$ & -5.4393160 & 2.6629430 & -0.4353760 & c & 3.2097920 & 1.4393830 & -1.9638410 \\
\hline C & -5.8083330 & 5.2408180 & -1.4427380 & $\mathrm{C}$ & 3.3027480 & 1.6205320 & 0.4695760 \\
\hline $\mathrm{H}$ & -3.6602220 & 5.2782250 & -1.6817860 & $\mathrm{c}$ & 4.4149670 & 0.7259930 & -1.9292860 \\
\hline C & -6.7200220 & 3.1968850 & -0.5228970 & $\mathrm{C}$ & 4.4767070 & 0.8705290 & 0.4551910 \\
\hline $\mathrm{H}$ & -5.2707720 & 1.6615180 & -0.0536900 & $\mathrm{C}$ & 5.0694960 & 0.4385980 & -0.7340070 \\
\hline C & -6.9034300 & 4.4852280 & -1.0238260 & $\mathrm{H}$ & 4.8451840 & 0.3893850 & -2.8681820 \\
\hline $\mathrm{H}$ & -5.9564560 & 6.2419390 & -1.8344270 & $\mathrm{H}$ & 4.9689720 & 0.6402310 & 1.3991990 \\
\hline $\mathrm{H}$ & -7.5747630 & 2.6100950 & -0.2002780 & $\mathrm{C}$ & -4.1617960 & -0.8856060 & 0.0953030 \\
\hline $\mathrm{H}$ & -7.9037470 & 4.9019340 & -1.0904650 & $\mathrm{C}$ & -3.8752740 & -2.0724650 & 0.8022840 \\
\hline $\mathrm{H}$ & -2.0152410 & 1.2328020 & -0.2065710 & C & -4.8703600 & -0.9345300 & -1.1160590 \\
\hline 0 & -2.0108650 & 3.4383570 & -1.2815600 & $\mathrm{C}$ & -4.3581580 & -3.2800720 & 0.3003820 \\
\hline & & & & C & -5.3269170 & -2.1704350 & -1.5813890 \\
\hline & , S) $\mathrm{G}[\mathrm{M} 06-2 \mathrm{x}$ & (d) $]=-33$ & 223 & $\mathrm{C}$ & -5.0904120 & -3.3510900 & -0.8858100 \\
\hline 0 & 0.2220270 & 0.8838380 & 0.1253130 & $\mathrm{H}$ & -4.1474100 & -4.1977060 & 0.8450910 \\
\hline 0 & -1.9987100 & 0.5264140 & -1.0222820 & $\mathrm{H}$ & -5.8838430 & -2.2138120 & -2.5159010 \\
\hline C & 0.2077630 & 2.2051250 & -0.2749040 & C & 2.8027580 & 2.1837440 & 1.7926390 \\
\hline C & -0.9465690 & 2.9439080 & -0.1199380 & $\mathrm{C}$ & 6.4108160 & -0.2793720 & -0.6686240 \\
\hline $\mathrm{C}$ & 1.4312780 & 2.7524830 & -0.7489220 & $\mathrm{C}$ & 2.5419320 & 1.7109170 & -3.3040970 \\
\hline $\mathrm{C}$ & -0.9546930 & 4.2951010 & -0.6014080 & c & -3.0664720 & -2.0661820 & 2.0925770 \\
\hline C & 1.43 & 4.0754240 & -1.1115520 & $\mathrm{C}$ & -5.5949430 & -4.6819120 & -1.4109270 \\
\hline $\mathrm{C}$ & 4230 & 4.8624600 & -1.0849150 & $\mathrm{C}$ & -5.1655520 & 0.3127810 & -1.9342540 \\
\hline $\mathrm{H}$ & 2.3695960 & 4.5386100 & -1.4300120 & $\mathrm{C}$ & -6.6734920 & 0.5834870 & -1.9964210 \\
\hline C & -2.59 & 1.1049930 & 0.0814620 & $\mathrm{H}$ & -7.1989600 & -0.2368240 & -2.4978480 \\
\hline $\mathrm{C}$ & -3.7 & 0.4336130 & 0.6409080 & $\mathrm{H}$ & -7.0945040 & 0.6891490 & -0.9915370 \\
\hline C & -2.12 & 2. 3179910 & 0.5371540 & $\mathrm{H}$ & -6.8758720 & 1.5036650 & -2.5544490 \\
\hline C & -4.3747770 & 1.0516110 & 1.6744440 & C & -4.5620600 & 0.2129870 & -3.3396410 \\
\hline $\mathrm{C}$ & -2.75 & 2.9006920 & 1.6880190 & $\mathrm{H}$ & -3.4841610 & 0.0373960 & -3.2855120 \\
\hline $\mathrm{C}$ & -3.91 & 12620 & 2.2327170 & $\mathrm{H}$ & -5.0175010 & -0.6048090 & -3.9089920 \\
\hline $\mathrm{H}$ & -5.2663550 & 0.5906980 & 2.0919150 & $\mathrm{H}$ & -4.7348780 & 1.1417860 & -3.8933880 \\
\hline C & -4.07 & 3.9955730 & 3.9333080 & C & -6.5618540 & -5.3451530 & -0.4238840 \\
\hline C & $-2.8 \mathrm{~s}$ & 4.5973290 & 3.4227870 & $\mathrm{H}$ & -6.9558680 & -6.2791420 & -0.8379640 \\
\hline C & -2.25 & 4.0663670 & 2.3324110 & $\mathrm{H}$ & -6.0535290 & -5.5839500 & 0.5168300 \\
\hline C & -4.56 & 41970 & 3.3519840 & $\mathrm{H}$ & -7.4039320 & -4.6860420 & -0.1932970 \\
\hline $\mathrm{H}$ & -4.57 & 07110 & 4.7920970 & C & -4.4326030 & -5.6235060 & -1.7466770 \\
\hline H & -2.49 & 5.4846700 & 3.9028190 & $\mathrm{H}$ & -3.7484910 & -5.1629880 & -2.4653930 \\
\hline $\mathrm{H}$ & -1.35 & 4.5318150 & 1.9603940 & $\mathrm{H}$ & -3.8596610 & -5.8678190 & -0.8452480 \\
\hline $\mathrm{H}$ & -5.4 & 51730 & 3.7458430 & $\mathrm{H}$ & -4.8052170 & -6.5610510 & -2.1725430 \\
\hline C & -2.10 & 78150 & -1.1031700 & C & -3.9895650 & -2.0568120 & 3.3187230 \\
\hline C & $-0.8 \varepsilon$ & 215 & -1.5489660 & $\mathrm{H}$ & -4.6376710 & -1.1765530 & 3.3345660 \\
\hline
\end{tabular}




$\begin{array}{rrr}-4.6298310 & -2.9467050 & 3.3165310 \\ -3.4009520 & -2.0634710 & 4.2424980 \\ -2.0972900 & -3.2499560 & 2.1888710 \\ -2.6272590 & -4.1916490 & 2.3710980 \\ -1.5102830 & -3.3475250 & 1.2726940 \\ -1.4086570 & -3.0953370 & 3.0258530 \\ 3.7467800 & 3.2971690 & 2.2663390 \\ 4.7495760 & 2.8987980 & 2.4587220 \\ 3.8359340 & 4.0840400 & 1.5103650 \\ 3.3758960 & 3.7477580 & 3.1928070 \\ 2.6309150 & 1.1093180 & 2.8677380 \\ 1.9065650 & 0.3559580 & 2.5473920 \\ 3.5760920 & 0.6050770 & 3.1000160 \\ 2.2641410 & 1.5614890 & 3.7956020 \\ 7.4903180 & 0.6765660 & -0.1377600 \\ 8.4499140 & 0.1587480 & -0.0387370 \\ 7.6219310 & 1.5135220 & -0.8321500 \\ 7.2204140 & 1.0891660 & 0.8382330 \\ 6.8711890 & -0.8783070 & -1.9972010 \\ 6.1368670 & -1.5704190 & -2.4207260 \\ 7.0578870 & -0.0906030 & -2.7355450 \\ 7.8077160 & -1.4254150 & -1.8545490 \\ 2.5736800 & 0.4947610 & -4.2343910 \\ 3.5837910 & 0.2984750 & -4.6122060 \\ 2.2146270 & -0.4017090 & -3.7247890 \\ 1.9310650 & 0.6743760 & -5.1018510 \\ 3.1916560 & 2.9123580 & -4.0039390 \\ 4.2541620 & 2.7149270 & -4.1884650 \\ 2.7083900 & 3.1008200 & -4.9683940 \\ 3.1189250 & 3.8225060 & -3.4027690 \\ -0.5608780 & -0.1994400 & -0.8196400 \\ 0.0635960 & -0.2961450 & -2.1791160 \\ -0.6667060 & -1.4331750 & 0.0402100 \\ 1.4902640 & 1.9433500 & -3.1099810 \\ 6.3069860 & -1.0985520 & 0.0589460 \\ 1.8205100 & 2.6366020 & 1.6302200 \\ -4.6998870 & 1.1704360 & -1.4392390 \\ -6.1448400 & -4.4798600 & -2.3390510 \\ -2.4625740 & -1.1526400 & 2.1024500 \\ 2.1204120 & -2.3374850 & 1.7645830 \\ 0.9448470 & -1.8132610 & 1.9478100 \\ 2.9696920 & -2.3815370 & 2.9876020 \\ 4.3165100 & -2.7243690 & 2.8555010 \\ 2.4457690 & -2.0471560 & 3.2385250 \\ 5.1425280 & -2.7260890 & 1.8728440 \\ 4.7005640 & -2.9797790 & \end{array}$

3.2736130
1.3996710
4.6204950
6.1914820
2.8704670
5.2656910
0.3520920
2.5571480
1.1549730
2.2566150
0.2153020
2.0131540
1.2460190
0.6742830
2.8542560
3.9373970
3.6664260
1.4857260
3.0785990
4.9262280
3.8466120
1.4687470
0.6871140
2.8278440
3.9588410
4.3123110
$-2.0574530$
$-1.7728490$
$-2.3930810$
$-2.9856600$
$-1.7991050$
$-2.3941960$
$-1.7076650$
$-2.7757550$
$-3.2079380$
$-2.3135330$
$-3.0255110$
$-1.2845260$
$-2.4623980$
$-1.6366040$
$-4.8674510$
$-4.1414560$
$-2.6382830$
$-4.6678620$
$-5.9363920$
$-4.3113400$
$-2.1610220$
$-5.1432970$
$-5.1192150$
$-4.4822910$
$-4.5332990$
$-2.1278190$

144

TS141(S, S) G[M06-2X/6-31G(d) $]=-3310.348230$

$\begin{array}{lrrr}\text { TS141(S, S) G[M06-2X/6-31G(d) }]=-3310.348230 \\ \text { O } & 0.0351730 & 1.0771160 & 0.2310110 \\ \text { O } & -2.0515290 & 0.2135710 & -0.8823210 \\ \text { C } & -0.2217300 & 2.3497370 & -0.2385650 \\ \text { C } & -1.4982230 & 2.8578510 & -0.1329310 \\ \text { C } & 0.8864160 & 3.0845570 & -0.7406490 \\ \text { C } & -1.7529650 & 4.1528750 & -0.6951170 \\ \text { C } & 0.6502800 & 4.3602510 & -1.1848670 \\ \text { C } & -0.6610190 & 4.9078080 & -1.2093380 \\ \text { H } & 1.4781220 & 4.9721170 & -1.5335790 \\ \text { C } & -2.7497050 & 0.7373610 & 0.1881200 \\ \text { C } & -3.7126530 & -0.1092090 & 0.8003350 \\ \text { C } & -2.5398950 & 2.0497480 & 0.5592040 \\ \text { C } & -4.4944620 & 0.4277890 & 1.7909300 \\ \text { C } & -3.2878170 & 2.5658140 & 1.6714460 \\ \text { C } & -4.2966840 & 1.7524790 & 2.2605580 \\ \text { H } & -5.2737910 & -0.1791520 & 2.2451000 \\ \text { C } & -4.8285500 & 3.5156930 & 3.8416510 \\ \text { C } & -3.7941210 & 4.3078620 & 3.2905340\end{array}$

5.3557030

4.3227760

5.2231340

3.8736810

6.3294620

6.0963160

1.1092250

$-1.6862100$

$-1.3200740$

$-1.1670900$

$-1.0671480$

$-2.9233260$

$-2.7983920$

$-2.5445980$

$-1.7449210$

$-1.6865720$

$-1.8898700$

$-2.6049360$

$-2.1819270$

$-2.6588230$

$-0.9015400$

$-2.4868280$

$-3.5707260$

$-0.7209850$

$-0.9672920$
0.6933870 


$\begin{array}{rrr}-3.0446860 & 3.8476940 & 2.2370020 \\ -5.0663410 & 2.2632450 & 3.3389630 \\ -5.4180850 & 3.8957550 & 4.6699240 \\ -3.5867850 & 5.2875500 & 3.7096180 \\ -2.2471460 & 4.4621500 & 1.8343500 \\ -5.8400160 & 1.6307090 & 3.7662950 \\ -3.2621960 & 5.9475540 & -1.3294060 \\ -2.1715600 & 6.7118300 & -1.8068070 \\ -0.9017290 & 6.1986100 & -1.7493140 \\ -3.0594880 & 4.7033700 & -0.7870670 \\ -4.2690660 & 6.3472050 & -1.3983460 \\ -2.3456700 & 7.6968880 & -2.2279450 \\ -0.0562170 & 6.7666350 & -2.1284120 \\ -3.9027830 & 4.1202620 & -0.4320070 \\ 2.2487680 & 2.4699070 & -0.6830300 \\ 2.9026710 & 2.0369800 & -1.8501130 \\ 2.8746100 & 2.3245050 & 0.5728790 \\ 4.1873820 & 1.4860630 & -1.7405290 \\ 4.1522910 & 1.7753060 & 0.6288100 \\ 4.8357070 & 1.3533220 & -0.5136890 \\ 4.6955370 & 1.1741640 & -2.6486300 \\ 4.6478100 & 1.6979460 & 1.5949710 \\ -3.8639870 & -1.5279840 & 0.3530870 \\ -3.2851990 & -2.5658630 & 1.1077510 \\ -4.5871420 & -1.8163210 & -0.8181300 \\ -3.4939580 & -3.8840720 & 0.6962420 \\ -4.7633060 & -3.1484200 & -1.1912720 \\ -4.2325250 & -4.1974940 & -0.4422130 \\ -3.0594300 & -4.6965540 & 1.2740810 \\ -5.3290000 & -3.3715760 & -2.0945880 \\ 2.2343890 & 2.8092690 & 1.8658860 \\ 6.2543180 & 0.8167020 & -0.3642330 \\ 2.2804650 & 2.2128120 & -3.2282110 \\ -2.4563960 & -2.2853990 & 2.3538890 \\ -4.4324000 & -5.6395180 & -0.8689610 \\ -5.1827840 & -0.7209230 & -1.6886890 \\ -6.7138390 & -0.8003140 & -1.7013680 \\ -7.0539330 & -1.7481090 & -2.1334640 \\ -7.1185150 & -0.7285860 & -0.6868040 \\ -7.1361120 & 0.0133770 & -2.3002490 \\ -4.6129930 & -0.7698800 & -3.1106980 \\ -3.5224130 & -0.6912040 & -3.0924380 \\ -4.8831510 & -1.7050960 & -3.6136300 \\ -5.0104290 & 0.0574150 & -3.7080060 \\ -3.7607740 & -5.9151580 & -2.2193180 \\ -3.8672180 & -6.9691570 & -2.4967990\end{array}$

-4.2192870
-2.6949840
-5.9182600
-6.4010440
-6.4443770
-6.0429250
-3.3258080
-4.1398440
-3.7686660
-2.7218070
-1.2589510
-1.5745710
-0.6956490
-0.5885740
2.9710160
4.0127670
2.9729620
2.4900180
2.1799060
1.6376990
3.1850710
1.6716860
6.9464190
7.9779350
6.4440170
6.9706670
6.2909810
5.8905510
5.6911540
7.3187560
2.4836810
3.5365070
2.1261640
1.9210920
2.8423920
3.9238280
2.3761280
2.6724710
-0.4786150
0.1189770
-0.2621980
1.2015180
6.8348820
1.2036260
-4.9083290
-3.9486790
-5.3109480
-5.6703480
-6.0122940
-5.8334580
-5.4178330
-7.0687130
-2.3576060
-1.6282090
-3.3557150
-2.1649440
-3.2309440
-4.2397110
-3.2902930
-2.8607720
4.0487930
3.8100390
4.8486620
4.4259510
1.7065360
0.8348650
1.3839500
2.0733950
0.5052500
0.1904600
-0.3083900
1.3786710
-0.4049480
-0.1742150
-1.2280910
-0.7617310
1.0026570
0.8704900
0.0876120
1.1427650
3.4701930
3.3688540
3.6192760
4.3680360
-0.1538120
-0.0933230
-1.3862280
2.3369550
1.6111240
3.1085580
0.2480790
-6.2737490
$-3.0099510$

$-2.1858010$

$-0.9165420$

0.0486540

$-1.6715920$

$-1.1762680$

3. 6166690

3. 5921290

3.7136570

4. 5099160

2. 4978600

2.7873260

1.5641810

3. 2802940

2. 3897810

2. 6317600

1. 6423750

3. 2982710

2.9271820

2. 5501160

3. 2229820

3.8253770

$-1.6907070$

$-1.5074730$

$-2.2272230$

$-2.3492420$

0.5652860

1. 5566950

0.1607650

0.6922730

$-4.1427820$

$-4.4186660$

$-3.6674390$

$-5.0712550$

$-3.9069170$

$-4.0561430$

$-4.8864990$

$-3.3065630$

$-0.7115910$

$-2.0865230$

0.1282390

$-3.0945390$

0.1260340

1.6571950

$-1.2603970$

$-0.1150030$ 


\begin{tabular}{|c|c|c|c|c|c|c|c|}
\hline & & & & & & & \\
\hline $\mathrm{H}$ & -2.0564220 & -1.2695490 & 2.2639760 & C & -3.8192770 & -0.0518040 & 0.7950270 \\
\hline C & 2.8332620 & -2.0313400 & $\begin{array}{l}1.4632860 \\
1.7554770\end{array}$ & C & -2.6814380 & $\begin{array}{l}2.0879160 \\
0.5979260\end{array}$ & 0.3223170 \\
\hline $\mathrm{C}$ & 3.7504050 & -2.1570350 & 2.6312170 & $\mathrm{c}$ & -3.5209680 & 2.7299520 & 1.2961130 \\
\hline c & 4.9592600 & -2.8386660 & 2.4736140 & $\mathrm{C}$ & -4.5556080 & $\begin{array}{l}1.9777060 \\
\text {. }\end{array}$ & 1.9212430 \\
\hline $\mathrm{C}$ & 3.4146080 & -1.5958570 & 3.8656750 & $\mathrm{H}$ & -5.4766700 & 0.0291440 & 2.1280340 \\
\hline $\mathrm{C}$ & 5.8371230 & -2.9488710 & 3.5452400 & C & -5.2399990 & 3.9266970 & 3.1960590 \\
\hline $\mathrm{H}$ & 5.1974320 & -3.2731860 & 1.5081460 & $\mathrm{C}$ & -4.1806710 & 4.6635070 & 2.6166570 \\
\hline C & 4.3002720 & -1.7006610 & 4.9334190 & C & -3.3449140 & 4.0837230 & 1.6953290 \\
\hline $\mathrm{H}$ & 2.4679540 & -1.0782450 & 3.9767130 & $\mathrm{C}$ & -5.4146690 & 2.6102600 & 2.8582040 \\
\hline C & 5.5095190 & -2.3751520 & 4.7734760 & $\mathrm{H}$ & -5.8978280 & 4.4005140 & 3.9177030 \\
\hline $\mathrm{H}$ & 6.7759660 & -3.4796540 & 3.4248040 & $\mathrm{H}$ & -4.0224630 & 5.6968800 & 2.9091090 \\
\hline $\mathrm{H}$ & 4.0459120 & -1.2582700 & 5.8910950 & $\mathrm{H}$ & -2.5298280 & 4.6599880 & 1.2724280 \\
\hline $\mathrm{H}$ & 6.1977420 & -2.4568980 & 5.6091600 & $\mathrm{H}$ & -6.2056060 & 2.0198580 & 3.3133630 \\
\hline $\mathrm{H}$ & 0.9713940 & -1.4781000 & 0.9998960 & $\mathrm{C}$ & -3.2561940 & 5.7158540 & -2.1078260 \\
\hline 0 & 3.2027350 & -2.3965620 & 0.3397930 & $\mathrm{C}$ & -2.1301070 & 6.4287830 & -2.5815860 \\
\hline C & 1.5404710 & -2.9251530 & -1.8397110 & $\mathrm{C}$ & -0.8685640 & 5.9444110 & -2.3497920 \\
\hline $\mathrm{C}$ & 2.5544680 & -1.8972560 & -1.5935180 & $\mathrm{c}$ & -3.0978840 & 4.5525690 & -1.3968440 \\
\hline $\mathrm{H}$ & 0.6702120 & -2.8873070 & -1.1858550 & $\mathrm{H}$ & -4.2542910 & 6.0897760 & -2.3134680 \\
\hline H & 2.2124640 & -0.9230790 & -1.2532520 & $\mathrm{H}$ & -2.2690570 & 7.3506180 & -3.1374020 \\
\hline 0 & 1.3524320 & -2.1422920 & -3.0425440 & $\mathrm{H}$ & 0.0050660 & 6.4722750 & -2.7231440 \\
\hline $\mathrm{H}$ & 0.7234920 & -1.3842000 & -2.8074280 & $\mathrm{H}$ & -3.9669220 & 4.0068170 & -1.0441740 \\
\hline C & 3.3097060 & -4.3115150 & -2.9955470 & C & 2.1761910 & 2.3893180 & -0.5637340 \\
\hline C & 4.3884330 & -3.4536520 & -2.3304090 & C & 2.9331990 & 1.8489800 & -1.6219690 \\
\hline C & 3.9211110 & -2.0057560 & -2.1823000 & C & 2.6504360 & 2.3026670 & 0.7613660 \\
\hline $\mathrm{C}$ & 2.0323970 & -4.3191410 & -2.1521000 & $\mathrm{C}$ & 4.1568250 & 1.2333840 & -1.3293640 \\
\hline $\mathrm{H}$ & 3.6668750 & -5.3372790 & -3.1256780 & $\mathrm{C}$ & 3.8765130 & 1.6855530 & 1.0006520 \\
\hline $\mathrm{H}$ & 5.3160710 & -3.4760890 & -2.9098950 & $\mathrm{C}$ & 4.6482890 & 1.1433740 & -0.0262780 \\
\hline H & 3.8795580 & -1.5009820 & -3.1560870 & $\mathrm{H}$ & 4.7532170 & 0.8405460 & -2.1502080 \\
\hline $\mathrm{H}$ & 2.2192300 & -4.8158270 & -1.1921640 & $\mathrm{H}$ & 4.2588970 & 1.6326150 & 2.0189430 \\
\hline $\mathrm{H}$ & 1.2262270 & -4.8636760 & -2.6537030 & C & -3.8775300 & -1.5279980 & 0.5637000 \\
\hline $\mathrm{H}$ & 3.0854670 & -3.9168470 & -3.9935100 & $\mathrm{C}$ & -3.2341490 & -2.3989450 & 1.4638350 \\
\hline H & 4.6127670 & -3.8542640 & -1.3345260 & $\mathrm{C}$ & -4.5557520 & -2.0350090 & -0.5579140 \\
\hline $\mathrm{H}$ & 4.5900240 & -1.4099220 & -1.5579120 & C & -3.3300300 & -3.7740300 & 1.2418550 \\
\hline & & & & $\mathrm{C}$ & -4.6192100 & -3.4165710 & -0.7403330 \\
\hline & S) $\mathrm{G}[\mathrm{M06}-2$ & $G(d)]=-33$ & 77 & $\mathrm{C}$ & -4.0187990 & -4.3028500 & 0.1522760 \\
\hline 0 & -0.0929600 & 1.1252150 & 0.3208740 & $\mathrm{H}$ & -2.8432650 & -4.4596670 & 1.9313610 \\
\hline 0 & -2.0682840 & 0.0722110 & -0.8109160 & $\mathrm{H}$ & -5.1465170 & -3.8092520 & -1.6084210 \\
\hline C & -0.3120290 & 2.3315560 & -0.3137260 & C & 1.9063940 & 2.9317670 & 1.9301560 \\
\hline C & -1.5954930 & 2.8247390 & -0.3831160 & $\mathrm{C}$ & 6.0255250 & 0.5884510 & 0.2927490 \\
\hline C & 0.8366300 & 3.0096100 & -0.8064130 & $\mathrm{C}$ & 2.5001700 & 1.9977980 & -3.0739780 \\
\hline C & -1.8033090 & 4.0366200 & -1.1229150 & $\mathrm{C}$ & -2.4679250 & -1.8743500 & 2.6707560 \\
\hline C & 0.6362440 & 4.2148240 & -1.4280000 & $\mathrm{C}$ & -4.0853690 & -5.8018350 & -0.0722870 \\
\hline C & -0.6715940 & 4.7356970 & -1.6317820 & C & -5.2091520 & -1.1224170 & -1.5836000 \\
\hline $\mathrm{H}$ & 1.4872260 & 4.7854420 & -1.7915640 & $\mathrm{C}$ & -6.7257190 & -1.3400570 & -1.6328770 \\
\hline C & $-\overline{2}$ & 95580 & 0.1268820 & $\mathrm{H}$ & -6.9663050 & -2.3618850 & -1.94679 \\
\hline
\end{tabular}




\begin{tabular}{|c|c|c|}
\hline $\begin{array}{l}-7.1779920 \\
-7.1896640 \\
-4.5769610 \\
-3.4992210 \\
-4.7412930 \\
-5.0190990 \\
-3.3383910 \\
-3.3472750 \\
-3.8111870 \\
-2.2980930 \\
-5.5320080 \\
-6.0676330 \\
-6.0739300 \\
-5.5578710 \\
-3.3901090 \\
-4.2317560 \\
-3.7942290 \\
-2.8355600 \\
-1.2408940 \\
-1.5276610 \\
-0.6294920 \\
-0.6282780 \\
2.5929500 \\
3.6207640 \\
2.6316990 \\
2.0527850 \\
1.7754690 \\
1.3562710 \\
2.7472100 \\
1.1181110 \\
7.0792570 \\
8.0741130 \\
7.1126860 \\
6.8450510 \\
6.3994560 \\
5.6380260 \\
6.5112660 \\
7.3536190 \\
2.6685280 \\
3.7170500 \\
2.0758590 \\
2.3193380 \\
3.2778790 \\
4.3480680 \\
2.9320640 \\
3.1644720\end{array}$ & $\begin{array}{r}-1.1748940 \\
-0.6513920 \\
-1.3072670 \\
-1.1261900 \\
-2.3229650 \\
-0.6081520 \\
-6.2006080 \\
-7.2877710 \\
-5.7505250 \\
-5.8625970 \\
-6.3060610 \\
-6.0370710 \\
-5.8721280 \\
-7.3952540 \\
-1.7704780 \\
-1.0968390 \\
-2.7579630 \\
-1.3978830 \\
-2.7215050 \\
-3.6807800 \\
-2.9072930 \\
-2.1908690 \\
4.2426640 \\
4.0537690 \\
4.9412720 \\
4.7225680 \\
1.9861890 \\
1.0235200 \\
1.8102920 \\
2.4276310 \\
1.6919870 \\
1.3249070 \\
2.0274810 \\
2.5589310 \\
-0.6399480 \\
-1.4191420 \\
-0.3907620 \\
-1.0528100 \\
0.7222810 \\
0.4092540 \\
-0.0926780 \\
0.8981780 \\
3.1492780 \\
2.9136890 \\
3.3150230 \\
4.0838380\end{array}$ & $\begin{array}{l}-0.6498490 \\
-2.3466860 \\
-2.9677890 \\
-2.9259590 \\
-3.3446040 \\
-3.6853800 \\
-1.3505860 \\
-1.4820360 \\
-2.2305810 \\
-1.3190260 \\
-0.1102410 \\
0.8048410 \\
-0.9577100 \\
-0.2197590 \\
3.8937820 \\
3.7121900 \\
4.1454420 \\
4.7617850 \\
3.0220570 \\
3.4677870 \\
2.1369890 \\
3.7583660 \\
2.3363080 \\
2.6666720 \\
1.4943270 \\
3.1592450 \\
3.1267230 \\
2.8177100 \\
3.6029920 \\
3.8826270 \\
0.1233080 \\
0.3971440 \\
-0.9195270 \\
0.7482640 \\
-0.5355790 \\
-0.4376660 \\
-1.5974090 \\
-0.1914700 \\
-3.9020740 \\
-3.9664560 \\
-3.4822100 \\
-4.9248380 \\
-3.7284780 \\
-3.7635170 \\
-4.7541860 \\
-3.1711260\end{array}$ \\
\hline
\end{tabular}

$$
\begin{array}{r}
-0.4833950 \\
0.1599570 \\
-0.2234860 \\
1.4351790 \\
6.0170600 \\
0.8930830 \\
-5.0354770 \\
-3.5822920 \\
-2.1068330 \\
3.0991060 \\
2.0126920 \\
4.2171330 \\
5.3842910 \\
4.1158630 \\
6.4600830 \\
5.4377960 \\
5.1965730 \\
3.1964810 \\
6.3694580 \\
7.3688970 \\
5.1244430 \\
7.2127550 \\
1.1862820 \\
3.2525800 \\
1.2639100 \\
2.4045050 \\
0.5015490 \\
2.2068880 \\
0.9937620 \\
0.4902650 \\
2.7073240 \\
3.9455350 \\
3.6522260 \\
1.5526600 \\
2.9337100 \\
4.7862470 \\
3.5013810 \\
1.8007920 \\
0.6329940 \\
2.4039920 \\
4.2475350 \\
4.4694490
\end{array}
$$

144

$\operatorname{TS} 143(\mathrm{~S}, \mathrm{~S}) \mathrm{G}[\mathrm{M06}-2 \mathrm{X} / 6-31 \mathrm{G}(\mathrm{d})]=-3310.347937$
0
0.5356740
$-1.6659890$
0.7467440
0.8530590
0.1209550
$-1.0992670$

$-0.5580290$

$-1.9151180$

0.3563890

$-3.0844500$

1. 3498350

1.6086090

$-1.2805170$

0.7767620

2. 4231700

1.2217640

2. 2002990

1. 9848110

3. 3016410

2. 8545260

1.1312580

4.1652580

3.4600710

3.9387850

2. 6874800

5. 0150650

4. 6130190

0.9883080

0.1668460

$-1.7343420$

$-1.7101950$

$-0.9780580$

$-1.4117210$

$-2.9632350$

2.9691760

$-2.5178870$

$-1.9870490$

3. 0420910

- 3.4812020

$-1.0167520$

$-2.3425910$

$-3.9678520$

$-1.5182620$
$-2.4694980$

3.3150230
4.0838380 


$\begin{array}{rrr}0.6154960 & 2.1197160 & 0.0111770 \\ -0.4771380 & 2.8905170 & 0.3601530 \\ 1.8593800 & 2.6699830 & -0.3936350 \\ -0.3833090 & 4.3125860 & 0.2053430 \\ 1.9647050 & 4.0368680 & -0.4526040 \\ 0.8624170 & 4.8841170 & -0.1779270 \\ 2.9178460 & 4.4874710 & -0.7179470 \\ -2.2706540 & 1.2067180 & 0.0978540 \\ -3.4528630 & 0.5096160 & 0.4665460 \\ -1.7152700 & 2.2233090 & 0.8451270 \\ -4.0899470 & 0.9149620 & 1.6126430 \\ -2.3301000 & 2.5601260 & 2.0971200 \\ -3.5480840 & 1.9186370 & 2.4571070 \\ -5.0238080 & 0.4390600 & 1.8998410 \\ -3.6235290 & 3.1819500 & 4.5282480 \\ -2.3882700 & 3.7860550 & 4.1944530 \\ -1.7582100 & 3.4845090 & 3.0132710 \\ -4.1843760 & 2.2643770 & 3.6787160 \\ -4.1142460 & 3.4360180 & 5.4624400 \\ -1.9318740 & 4.4907010 & 4.8826070 \\ -0.8063780 & 3.9467160 & 2.7743690 \\ -5.1204570 & 1.7730820 & 3.9310420 \\ -1.3594920 & 6.5378490 & 0.2398670 \\ -0.1083130 & 7.1068240 & -0.0994890 \\ 0.9746280 & 6.2943780 & -0.3083320 \\ -1.4947730 & 5.1810330 & 0.3885320 \\ -2.2221570 & 7.1822480 & 0.3771390 \\ -0.0158010 & 8.1828110 & -0.2073610 \\ 1.9371270 & 6.7124270 & -0.5913840 \\ -2.4616590 & 4.7570170 & 0.6368250 \\ 3.0063030 & 1.7788890 & -0.7499250 \\ 3.2222670 & 1.4763050 & -2.1128590 \\ 3.8694620 & 1.2629470 & 0.2390630 \\ 4.3058040 & 0.6694620 & -2.4647910 \\ 4.9423890 & 0.4677600 & -0.1747460 \\ 5.1812080 & 0.1576630 & -1.5113970 \\ 4.4639450 & 0.4336360 & -3.5125830 \\ 5.6158430 & 0.0669620 & 0.5835930 \\ -3.9722080 & -0.6184890 & -0.3649570 \\ -3.9080730 & -1.9391200 & 0.1235510 \\ -4.5417790 & -0.3557680 & -1.6259170 \\ -4.4682050 & -2.9618100 & -0.6459010 \\ -5.0792470 & -1.4132860 & -2.3582020 \\ -5.0615920 & -2.7231980 & -1.8821040 \\ -4.4360020 & -3.9835140 & -0.2737630 \\ -5.5284380 & -1.2056820 & -3.3280210\end{array}$

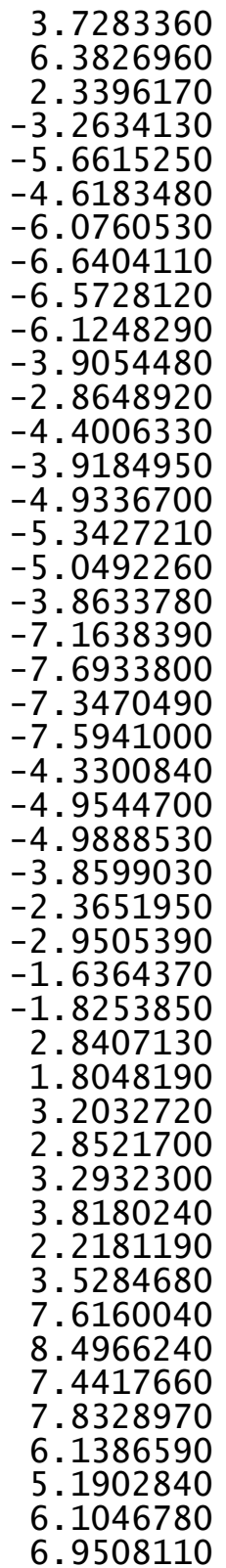

3.7283360

2. 3396170

$-5.2634130$

$-4.6183480$

$-6.0760530$

-3.9054480

$-2.8648920$

$-4.4006330$

.

$-5.3427210$

5.0492260

.

$-7.6933800$

$-3470490$

$-4.9544700$

$-4.9888530$

$-3.8599030$

$-2.3651950$

$-1.6364370$

$-1.8253850$

2. 8407130

1.8048190

2.8521700

3. 2932300

3.8180240

5284680

6160040

8.4966240

7.4417660

6.1386590

5. 1902840

6.9508110
1. 4441060

$-0.6900040$

2. 0513500

$-2.2924930$

$-3.8562100$

1.0468880

1. 5101090

0.8655330

1. 4839530

2.5339580

1.1298510

0.8063260

0.4999410

2. 1598010

$-4.0308980$

$-4.8823790$

$-3.1376480$

$-4.1969110$

- 3.6490380

$-3.5423750$

$-2.7446090$

$-4.4968030$

$-2.5108560$

$-1.6249360$

$-3.3416970$

$-2.7572670$

$-3.5331120$

$-4.4445250$

$-3.4348350$

$-3.6590010$

0.3464570

0.4416690

$-0.6503930$

0.4042240

2.8215100

3. 6322160

2.9863970

2.8860760

0.1996610

$-0.4049930$

0.8967410

0.7872870

$-1.5624910$

$-2.1069170$

$-0.9558770$

$-2.2859310$
1.7523020

$-1.8906580$

$-3.2150100$

1.4582930

$-2.6928630$

$-2.2099370$

$-2.3229990$

$-3.0060740$

$-1.3479440$

$-2.7084060$

$-3.5641320$

$-3.4747720$

$-4.3114840$

$-3.9359980$

$-4.0306070$

$-4.5845430$

$-4.6543080$

$-3.8779910$

$-2.9163430$

$-1.9650370$

$-3.5069830$

$-3.4597140$

2. 5403480

2.6847110

2. 2619330

3. 4984910

1. 3720690

1. 2086880

0.5636210

2. 3171430

2. 3585900

2. 0218310

2.0754920

3. 4528290

2.2677360

1.7534940

2. 1572540

3. 3353080

$-2.0964950$

$-2.3375550$

$-2.9235650$

$-1.1995280$

$-3.1249040$

$-3.0550650$

-4.0361320
-3.2444890 


$\begin{array}{rrr}2.1703380 & 1.1144210 & -4.4159690 \\ 3.0858220 & 1.0577120 & -5.0155370 \\ 1.8881480 & 0.1055120 & -4.1105160 \\ 1.3799570 & 1.5006090 & -5.0666930 \\ 2.9061520 & 3.3913630 & -3.7088930 \\ 3.9179990 & 3.2481630 & -4.1056250 \\ 2.2800850 & 3.7958550 & -4.5112920 \\ 2.9581110 & 4.1357220 & -2.9110610 \\ -0.3694280 & -0.1104250 & -0.9469920 \\ 0.3050420 & -0.2116210 & -2.2772930 \\ -0.7082990 & -1.3678800 & -0.1862320 \\ 1.3438720 & 2.2253990 & -2.7951050 \\ 6.5906470 & -1.3543540 & -1.0394590 \\ 4.7402870 & 1.2821070 & 2.1448760 \\ -4.1102110 & 1.7370320 & -1.5303070 \\ -5.5299720 & -4.7787800 & -2.1129590 \\ -2.6273200 & -1.4542150 & 1.7601480 \\ 1.5429910 & -2.9682450 & -1.4742240 \\ 2.3550990 & -2.2957590 & -0.4545670 \\ 0.4753570 & -2.7667090 & -1.4172490 \\ 2.0362020 & -1.3114920 & -0.1162640 \\ 2.2294760 & -2.0200800 & -2.3302270 \\ 1.6061100 & -1.2341180 & -2.4137180 \\ 3.3876560 & -4.6748820 & -1.7605240 \\ 3.9684160 & -4.2298920 & -0.4174170 \\ 3.7630560 & -2.7296400 & -0.2053920 \\ 1.8840170 & -4.4013170 & -1.8073460 \\ 3.5711190 & -5.7414900 & -1.9200560 \\ 5.0381790 & -4.4537180 & -0.3634600 \\ 4.3798460 & -2.1516030 & -0.9019590 \\ 1.3661180 & -5.0292190 & -1.0719530 \\ 1.4649820 & -4.6328140 & -2.7915100 \\ 3.8839470 & -4.1363160 & -2.5768770 \\ 3.4737960 & -4.7761000 & 0.3942200 \\ 4.0507050 & -2.4094750 & 0.8002180 \\ 1.1077350 & -2.5497140 & 2.2637390 \\ 0.1598280 & -1.6723900 & 2.1368740 \\ 1.5340940 & -2.8065650 & 3.6668300 \\ 2.6056230 & -3.6713660 & 3.8991660 \\ 0.8882130 & -2.1795540 & 4.7353160 \\ 3.0309450 & -3.9111400 & 5.2004370 \\ 3.0914520 & -4.1463280 & 3.0526990 \\ 1.3173180 & -2.4223750 & 6.0356390 \\ 0.0604910 & -1.5075930 & 6.5365060 \\ 2.3863900 & -3.2867320 & 5.3846670 \\ 3.8631140 & -4.5828930 & \end{array}$

$\begin{array}{rrrr}\mathrm{H} & 0.8188460 & -1.9375520 & 6.8687120 \\ \mathrm{H} & 2.7190570 & -3.4740400 & 7.2841320 \\ \mathrm{H} & -0.1696940 & -1.5552020 & 1.1555980 \\ \mathrm{O} & 1.6512560 & -3.1487320 & 1.3228270\end{array}$

144

0.169694

$-1.5552020$

1. 3228270

$\operatorname{TS} 144(\mathrm{~S}, \mathrm{~S}) \mathrm{G}[\mathrm{M} 06-2 \mathrm{X} / 6-31 \mathrm{G}(\mathrm{d})]=-3310.348610$

\begin{tabular}{lrrr}
$\mathrm{TS} 144(\mathrm{~S}, \mathrm{~S})$ & \multicolumn{2}{c}{$\mathrm{G}[\mathrm{M} 06-2 \mathrm{X} / 6-31 \mathrm{G}(\mathrm{d})]=-3310.348610$} \\
$\mathrm{O}$ & 1.8002590 & -0.4217070 & -0.7616070 \\
$\mathrm{O}$ & 0.0916240 & -1.3658790 & 0.8156380 \\
$\mathrm{C}$ & 2.7748880 & -1.0260440 & 0.0109500 \\
$\mathrm{C}$ & 2.6364100 & -2.3615370 & 0.3256710 \\
$\mathrm{C}$ & 3.8803810 & -0.2277540 & 0.4098800 \\
$\mathrm{C}$ & 3.6463310 & -2.9730180 & 1.1388780 \\
$\mathrm{C}$ & 4.8895600 & -0.8513690 & 1.1003560 \\
$\mathrm{C}$ & 4.7975300 & -2.2128750 & 1.4911460 \\
$\mathrm{H}$ & 5.7698960 & -0.2835130 & 1.3902170 \\
$\mathrm{C}$ & 0.1799620 & -2.5367010 & 0.0837570 \\
$\mathrm{C}$ & -1.0335470 & -3.1292040 & -0.3566050 \\
$\mathrm{C}$ & 1.4265660 & -3.0843520 & -0.1537730 \\
$\mathrm{C}$ & -0.9456560 & -4.3401640 & -1.0000780 \\
$\mathrm{C}$ & 1.5069180 & -4.2916180 & -0.9224950 \\
$\mathrm{C}$ & 0.3008860 & -4.9390880 & -1.3108900 \\
$\mathrm{H}$ & -1.8585830 & -4.8433980 & -1.3081110 \\
$\mathrm{C}$ & 1.5692400 & -6.6888880 & -2.4149660 \\
$\mathrm{C}$ & 2.7694910 & -6.0218430 & -2.0712910 \\
$\mathrm{C}$ & 2.7409440 & -4.8567800 & -1.3474710 \\
$\mathrm{C}$ & 0.3635460 & -6.1526440 & -2.0466490 \\
$\mathrm{H}$ & 1.6083780 & -7.6131280 & -2.9826690 \\
$\mathrm{H}$ & 3.7221020 & -6.4331400 & -2.3902890 \\
$\mathrm{H}$ & 3.6677100 & -4.3499490 & -1.1018050 \\
$\mathrm{H}$ & -0.5688290 & -6.6376190 & -2.3239850 \\
$\mathrm{C}$ & 4.5319500 & -4.8506260 & 2.4016360 \\
$\mathrm{C}$ & 5.6956550 & -4.1081060 & 2.7136700 \\
$\mathrm{C}$ & 5.8190470 & -2.8168720 & 2.2715660 \\
$\mathrm{C}$ & 3.5347740 & -4.2995960 & 1.6370660 \\
$\mathrm{H}$ & 4.4249340 & -5.8637070 & 2.7765180 \\
$\mathrm{H}$ & 6.4791070 & -4.5589030 & 3.3145230 \\
$\mathrm{H}$ & 6.6956300 & -2.2253650 & 2.5226500 \\
$\mathrm{H}$ & 2.6425760 & -4.8742210 & 1.4124290 \\
$\mathrm{C}$ & 3.8956320 & 1.2381820 & 0.1187150 \\
$\mathrm{C}$ & 3.7811000 & 2.1607560 & 1.1803850 \\
$\mathrm{C}$ & 4.0070040 & 1.6993740 & -1.2076420 \\
$\mathrm{C}$ & 3.8365930 & 3.5255500 & 0.8885270 \\
$\mathrm{C}$ & 4.0438280 & 3.0735010 & -1.4463770 \\
$\mathrm{C}$ & 3.9739870 & 4.0039160 & -0.4114360 \\
$\mathrm{H}$ & 3.7619050 & 4.2450220 & 1.7011790 \\
$\mathrm{H}$ & 4.1380020 & 3.4241990 & -2.4725860
\end{tabular}




$$
\begin{array}{r}
-2.3719710 \\
-3.0895460 \\
-2.9642730 \\
-4.4217560 \\
-4.2919490 \\
-5.0516170 \\
-4.9787820 \\
-4.7633660 \\
0.4436830 \\
0.7127650 \\
-0.5872110 \\
4.1246710 \\
4.0279620 \\
3.9982460 \\
4.0809700 \\
3.6115190 \\
3.3291270 \\
-2.2131770 \\
-1.2064280 \\
-6.5305920 \\
-6.6623070 \\
-2.4652080 \\
-1.3827350 \\
-0.8777090 \\
-0.8708550 \\
-1.1056650 \\
-0.5713500 \\
0.5470970 \\
0.9102050 \\
-1.2334060 \\
-1.6742700 \\
-0.9153160 \\
-1.4987630 \\
-1.7691300 \\
-1.5186660 \\
0.1290050 \\
-2.5795470 \\
-1.1198350 \\
-0.1647570 \\
-2.7466150 \\
-1.3492410 \\
-2.7078450 \\
-2.1522970 \\
-3.7669910 \\
-2.3691560 \\
-2.9883060
\end{array}
$$

$\begin{array}{rr}-2.4867850 & -0.1634010 \\ -2.0329110 & -1.2894770 \\ -2.4334980 & 1.1124450 \\ -1.6404690 & -1.1299110 \\ -2.0203120 & 1.2208710 \\ -1.6507650 & 0.1114750 \\ -1.3200920 & -2.0067670 \\ -2.0035390 & 2.2039050 \\ 0.0155440 & 0.0241100 \\ 1.0648350 & 1.0590510 \\ 0.2792470 & -1.0477420 \\ 0.7545350 & -2.3942780 \\ -0.2732590 & -2.0336880 \\ 5.4948070 & -0.6878610 \\ 6.0038170 & 0.2809050 \\ 1.7338220 & 2.6329290 \\ 0.6772810 & 2.6465130 \\ -2.8185880 & 2.3767630 \\ -3.1427060 & 2.0989910 \\ -1.3513490 & 0.2808900 \\ -0.9339020 & 1.2880570 \\ -1.9428870 & -2.6765660 \\ -2.0601460 & -2.5681520 \\ 3.4985830 & 0.6709050 \\ 3.2944390 & -0.7824080 \\ 2.6100170 & 1.2608570 \\ 2.3166430 & -1.1454270 \\ 3.6208250 & 0.4558670 \\ 2.7026950 & 0.6363760 \\ 5.9661990 & 0.2771700 \\ 5.6529000 & -1.1540620 \\ 4.4506300 & -1.7203930 \\ 4.7716370 & 1.1953050 \\ 6.8421130 & 0.6545480 \\ 6.5170740 & -1.8061490 \\ 4.7080820 & -1.9380320 \\ 4.5942680 & 1.2745450 \\ 4.9496320 & 2.2064820 \\ 6.2112630 & 0.2945210 \\ 5.4246830 & -1.1613280 \\ 4.0938020 & -2.6581470 \\ -0.5724590 & -3.3226350 \\ -0.5060250 & -4.2640850 \\ -0.4174010 & -3.5557990 \\ 0.2367610 & -2.6718080 \\ -3.0504640 & -3.6003880\end{array}$

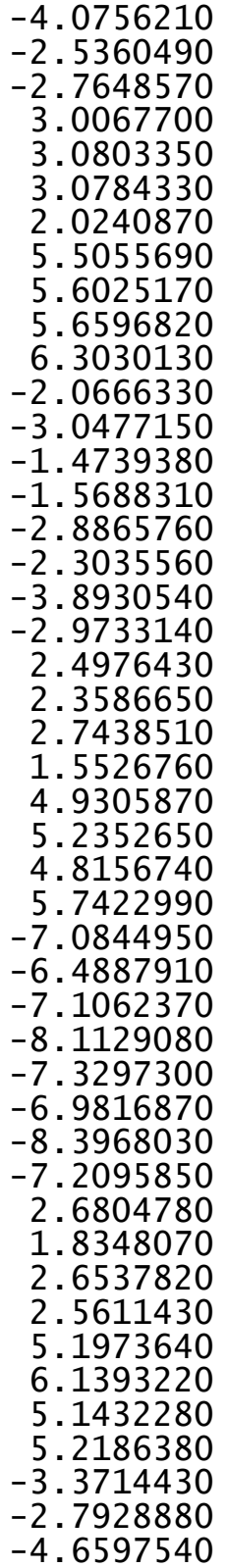
$-2.9712500$
$-2.9662470$
$-4.0468100$
0.9896840
1. 9885010
0.2591860
0.8874390
0.8798240
0.1694030
1.8878600
0.6793150
$-1.6047650$
$-1.2569390$
$-1.8654400$
$-0.7808550$
$-3.9901960$
$-4.2803380$
$-3.7220800$
$-4.8605030$
2. 5062700
2. 0978340
3. 5678400
2.4147500
1.8961100
2. 9490700
1. 5625410
1. 3223030
$-0.33$
0.5800470
$-0.7543490$
$-0.0729390$
$-2.6610940$
$-3.3768580$
$-2.4760340$
$-3.1227440$
5.9348850
5.6366660
7.0204950
5.4596130
5.9124590
5.5980860
5.4667820
7.0001070
1. 9499120
0.8455090
2. 2355500

-3.7156530
-4.5942540
-3.2086410
-3.4163260
-3.8611980
-4.2288610
-2.9478470
-3.0504920
-3.8781010
-3.4514170
-2.3281430
3.3022000
3.6472630
4.1853790
2.7824980
3.1007080
3.9809530
3.4401190
2.4427710
3.3501320
4.3566100
3.4597560
2.8109090
3.4010270
3.4152090
4.4377750
2.9446780
-0.7213540
-0.7401770
-1.7345610
-0.4553340
0.2104480
0.9613130
0.3744900
-0.7760660
-1.3362560
-0.7088240
-1.4791790
-2.3177550
-1.5440410
-1.0853490
-2.5431110
-1.6676480
-0.2210520
0.1562830
0.4615980
-3 


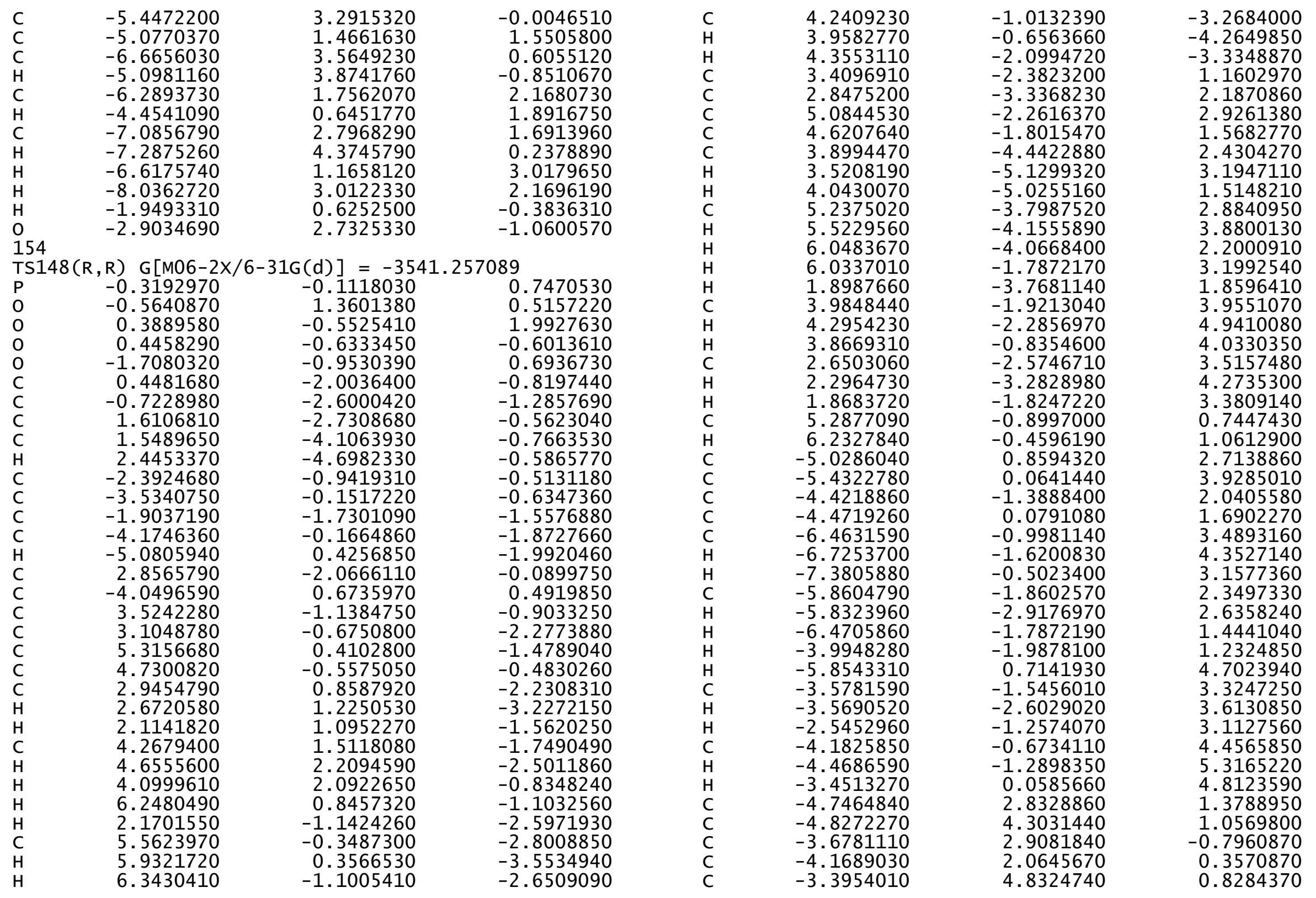




$\begin{array}{rrr}-3.4525950 & 5.8738610 & 0.4904150 \\ -2.8457970 & 4.8294420 & 1.7746130 \\ -2.6833450 & 3.9437550 & -0.2230590 \\ -2.2844480 & 4.5499510 & -1.0444280 \\ -1.8456250 & 3.4061550 & 0.2261030 \\ -3.1831370 & 2.3016280 & -1.5585420 \\ -5.3229060 & 4.8561880 & 1.8619240 \\ -4.8766430 & 3.6772750 & -1.3929800 \\ -4.5075800 & 4.3618880 & -2.1652470 \\ -5.5621490 & 2.9790670 & -1.8845840 \\ -5.6056770 & 4.4593780 & -0.2678470 \\ -5.6805120 & 5.5232660 & -0.5194030 \\ -6.6253820 & 4.0854930 & -0.1342790 \\ -5.1797790 & 2.2335230 & 2.5563370 \\ -5.6260830 & 2.8329120 & 3.3485090 \\ -3.6791700 & -0.8777100 & -2.9667070 \\ -2.5141320 & -1.6472400 & -2.8228180 \\ -4.4403670 & -0.8193970 & -4.2784730 \\ -3.5949830 & -1.2104770 & -5.4889780 \\ -2.8225750 & -2.4944000 & -5.1946670 \\ -1.8489480 & -2.2588140 & -4.0426980 \\ -4.2371680 & -1.3293280 & -6.3675780 \\ -5.2975800 & -1.5039150 & -4.2099100 \\ -4.8612550 & 0.1835210 & -4.4097660 \\ -3.5283340 & -3.2929590 & -4.9269490 \\ -2.2737870 & -2.8312790 & -6.0800130 \\ -1.3155710 & -3.1755070 & -3.7853080 \\ -1.0731040 & -1.5537100 & -4.3762480 \\ -2.8805070 & -0.4088460 & -5.7168670 \\ -0.7850230 & -4.0046100 & -1.3963920 \\ -2.1095360 & -4.6859890 & -1.6966710 \\ -2.1001680 & -6.1724170 & -1.3426280 \\ -0.8582970 & -6.8354590 & -1.9318360 \\ 0.3861070 & -6.2698100 & -1.2527020 \\ 0.3734360 & -4.7559560 & -1.1525630 \\ -3.0153790 & -6.6432070 & -1.7150390 \\ -2.3592960 & -4.5857200 & -2.7616350 \\ -2.9057580 & -4.1645440 & -1.1539500 \\ -0.8197610 & -6.6344090 & -3.0107870 \\ -0.8903800 & -7.9223040 & -1.8065630 \\ 1.2942410 & -6.5872750 & -1.7779290 \\ 0.4583340 & -6.6857630 & -0.2383330 \\ -2.0928230 & -6.2960120 & -0.2518670 \\ 1.6558580 & 3.5229480 & -0.7417420 \\ 0.5353140 & 2.9601160 & -1.0853080 \\ 2.3259090 & 4.2776620 & -1.8373910\end{array}$

3.4803100
1.8358930
4.1439780
3.8475470
2.5067620
0.9419140
3.6582750
5.0404400
2.1325450
4.1799040
0.1330600
2.1807900
2.7996110
1.7741530
2.7154110
0.7785980
2.0980590
1.3872940
4.2916590
3.4193420
1.9549530
4.2214170
5.3315110
3.5020410
1.5275870
4.6726680
4.7733910
3.9542130
3.7586300
1.3362260

5.0083530

4. 2199960

5.6834380

5.0311510

4.8921720

3. 6424010

5. 6227780

6.2534130

4.8453460

6.1462040

2. 3075830

3. 4644670

1. 3874780

2.4358010

0.6952050

2. 1797710

0.9059630

0.2756260

2.8267890

4.0379980

3.6252380

1. 7849840

3.1344400

4.8030430

3. 3499490

2. 1804670

0.8791090

2.3733980

4.4870630

4.4336460
$-1.5487680$

$-3.1439440$

$-2.5660040$

$-0.5278160$

$-4.1604640$

$-3.3519030$

$-3.8720740$

$-2.3443680$

$-5.1779750$

$-4.6674950$

$-0.3929460$

0.3783840

2.0666540

2. 1456690

1. 2207810

1.8001160

3.2325670

2.8744120

3. 5130490

3. 1819760

3.0324230

2. 3946440

3. 6583710

3.9594010

4.0041290

1.4751040

2. 6607590

4.4531030

2. 2412490

2.6347380

$\begin{array}{lrcr}\text { TS149(R, R) } \text { G }[\mathrm{M} 06-2 \times / 6-31 G(\mathrm{~d})]=-3541.257143 \\ \text { P } & -0.3307210 & -0.1365740 & 0.7862870 \\ \mathrm{O} & -0.5956850 & 1.3299460 & 0.5438900 \\ \mathrm{O} & 0.3825640 & -0.5579500 & 2.0358300 \\ \mathrm{O} & 0.4447800 & -0.6559280 & -0.5572040 \\ \mathrm{O} & -1.7090160 & -0.9955700 & 0.7358780 \\ \mathrm{C} & 0.4803170 & -2.0262240 & -0.7692040 \\ \mathrm{C} & -0.6839120 & -2.6596920 & -1.2106400 \\ \mathrm{C} & 1.6691680 & -2.7167800 & -0.5393250 \\ \mathrm{C} & 1.6507110 & -4.0927490 & -0.7581020 \\ \mathrm{H} & 2.5713830 & -4.6537840 & -0.6052280 \\ \mathrm{C} & -2.3784540 & -1.0047320 & -0.4801080 \\ \mathrm{C} & -3.5132390 & -0.2085420 & -0.6371200 \\ \mathrm{C} & -1.8782400 & -1.8140200 & -1.4990640 \\ \mathrm{C} & -4.1180720 & -0.2272910 & -1.8915270\end{array}$




\begin{tabular}{|c|c|c|c|}
\hline $\begin{array}{l}\mathrm{H} \\
\mathrm{C} \\
\mathrm{C} \\
\mathrm{C} \\
\mathrm{C} \\
\mathrm{C} \\
\mathrm{C} \\
\mathrm{C} \\
\mathrm{H} \\
\mathrm{H} \\
\mathrm{C} \\
\mathrm{H} \\
\mathrm{H} \\
\mathrm{H} \\
\mathrm{H} \\
\mathrm{C} \\
\mathrm{H} \\
\mathrm{H} \\
\mathrm{C} \\
\mathrm{H} \\
\mathrm{H} \\
\mathrm{C} \\
\mathrm{C} \\
\mathrm{C} \\
\mathrm{C} \\
\mathrm{C} \\
\mathrm{H} \\
\mathrm{H} \\
\mathrm{C} \\
\mathrm{H} \\
\mathrm{H} \\
\mathrm{H} \\
\mathrm{H} \\
\mathrm{C} \\
\mathrm{H} \\
\mathrm{H} \\
\mathrm{C} \\
\mathrm{H} \\
\mathrm{H} \\
\mathrm{C} \\
\mathrm{H} \\
\mathrm{C} \\
\mathrm{C} \\
\mathrm{C} \\
\mathrm{C} \\
\mathrm{C}\end{array}$ & $\begin{array}{r}-5.0141920 \\
2.9017330 \\
-4.0607590 \\
3.5349090 \\
3.0851370 \\
5.2764170 \\
4.7296700 \\
2.8846240 \\
2.5856390 \\
2.0576580 \\
4.1962260 \\
4.5546880 \\
4.0262490 \\
6.2017180 \\
2.1588470 \\
5.5259040 \\
5.8682330 \\
6.3272720 \\
4.2161640 \\
3.9110620 \\
4.3579940 \\
3.4776420 \\
2.9520120 \\
5.1670860 \\
4.6770420 \\
4.0353580 \\
3.6831940 \\
4.1839640 \\
5.3608930 \\
5.6674270 \\
6.1706280 \\
6.1060500 \\
2.0123010 \\
4.0695260 \\
4.3997050 \\
3.9244120 \\
2.7475150 \\
2.4172010 \\
1.9467130 \\
5.3103040 \\
6.2465640 \\
-5.1009840 \\
-5.5296800 \\
-4.4559780 \\
-4.5087300 \\
-6.5389400\end{array}$ & $\begin{array}{r}0.3729530 \\
-2.0178320 \\
0.6198190 \\
-1.0740140 \\
-0.6227030 \\
0.5213800 \\
-0.4604060 \\
0.9062250 \\
1.2659580 \\
1.1187940 \\
1.5944830 \\
2.3010900 \\
2.1714280 \\
0.9818590 \\
-1.1151980 \\
-0.2316720 \\
0.4826140 \\
-0.9632350 \\
-0.9298730 \\
-0.5792520 \\
-2.0126750 \\
-2.3168730 \\
-3.2837120 \\
-2.1475560 \\
-1.7029440 \\
-4.3601020 \\
-5.0547810 \\
-4.9425200 \\
-3.6801830 \\
-4.0270840 \\
-3.9278720 \\
-1.6473930 \\
-3.7417710 \\
-1.8354050 \\
-2.1919900 \\
-0.7530400 \\
-2.5225840 \\
-3.2363450 \\
-1.7918520 \\
-0.7852670 \\
-0.3190980 \\
0.8058300 \\
0.0128430 \\
-1.4395570 \\
0.0264980 \\
-1.0608530\end{array}$ & $\begin{array}{r}-2.0419080 \\
-0.0822750 \\
0.4721390 \\
-0.9046050 \\
-2.2729630 \\
-1.5035010 \\
-0.4994940 \\
-2.2224450 \\
-3.2138540 \\
-1.5404290 \\
-1.7600570 \\
-2.5182780 \\
-0.8439910 \\
-1.1403970 \\
-2.5797230 \\
-2.8283230 \\
-3.5854920 \\
-2.6878790 \\
-3.2794360 \\
-4.2716100 \\
-3.3491810 \\
1.1615640 \\
2.1957210 \\
2.9089870 \\
1.5550640 \\
2.4312340 \\
3.2017790 \\
1.5159250 \\
2.8680520 \\
3.8612040 \\
2.1750820 \\
3.1715150 \\
1.8785750 \\
3.9492780 \\
4.9316580 \\
4.0294000 \\
3.5238070 \\
4.2869500 \\
3.3934100 \\
0.7223630 \\
1.0275580 \\
2.6662220 \\
3.8737040 \\
2.0206950 \\
1.6619610 \\
3.4126850\end{array}$ \\
\hline
\end{tabular}

-6.8175490
-7.4520720
-5.8985420
-5.8694480
-6.4844380
-4.0028710
-5.9780550
-3.6464660
-3.6373350
-2.6103700
-4.2879720
-4.5910600
-3.5728600
-4.8055030
-4.8963440
-3.6766710
-4.1908890
-3.4659660
-3.5276640
-2.9440660
-2.7117070
-2.2915650
-1.8841420
-3.1519380
-5.4222560
-4.8654610
-4.4806600
-5.5271060
-5.6384000
-5.7218620
-6.6558220
-5.2637420
-5.7389320
-2.4577240
-1.8015010
-2.2772270
-3.8019910
-4.2814940
-3.6007260
-1.8941680
-2.0097260
-0.7138730
-4.2062330
-4.1746670
-5.3669000
-4.0829350
4. 2717110

3. 0552620

2. 2928820

2. 5833630

1. 3708060

1. 2279040

4. 6325960

3. 3281740

3. 6219660

3. 1430130

4.4387330

5. 2944320

4.8093250

1. 3287420

0.9970060

$-0.8149250$

0.3272990

0.8060920

0.4573610

1.7677390

$-0.2163970$

$-1.0279900$

0.2635870

$-1.5599110$

1. 7845820

$-1.4503780$

$-2.2122440$

$-1.9615960$

$-0.3502840$

$-0.6086480$

$-0.2445650$

2. 4971020

3. 2733290

$-2.7825540$

$-3.9534060$

$-5.3053910$

$-5.3284810$

$-4.3168740$

$-2.9668730$

$-6.1057020$

$-3.9116260$

$-3.8616270$

$-5.0710000$

$-6.3264550$

$-4.1790500$

$-4.7155480$ 


\begin{tabular}{|c|c|c|}
\hline $\begin{array}{l}-1.8728160 \\
-0.7002770 \\
0.4912710 \\
-2.0097310 \\
-1.8178900 \\
-0.7943950 \\
0.5651870 \\
-2.7776350 \\
-2.4844650 \\
-2.7069760 \\
-1.1367640 \\
-0.7028220 \\
1.2602130 \\
1.0022850 \\
-1.4647660 \\
1.5579330 \\
0.4534660 \\
2.2018900 \\
3.3386400 \\
1.7059650 \\
3.9787660 \\
3.7110080 \\
2.3532520 \\
0.8260140 \\
3.4871880 \\
4.8615570 \\
1.9744500 \\
3.9905340 \\
0.0716110 \\
2.0877020 \\
2.7632540 \\
1.7171720 \\
2.6851210 \\
0.7241610 \\
2.0848300 \\
1.3826130 \\
4.2388670 \\
3.3378810 \\
1.8812550 \\
4.1795730 \\
5.2733700 \\
3.4116000 \\
1.4682120 \\
4.6111600 \\
4.7550920 \\
3.9208610\end{array}$ & $\begin{array}{r}-0.9205040 \\
-4.0621780 \\
-4.7768520 \\
-4.8050760 \\
-6.2086660 \\
-6.9705500 \\
-6.2790160 \\
-6.7349310 \\
-4.8875520 \\
-4.2264960 \\
-6.9975530 \\
-8.0083020 \\
-6.7104350 \\
-6.4708250 \\
-6.1434390 \\
3.5458630 \\
2.9504220 \\
4.3205600 \\
5.0801210 \\
4.2538430 \\
5.7748790 \\
5.1095840 \\
4.9460780 \\
3.6539520 \\
5.7053610 \\
6.3671280 \\
4.8924980 \\
6.2442400 \\
2.2891620 \\
3.5020330 \\
1.4359280 \\
2.4627440 \\
0.7429610 \\
2.1862690 \\
0.9386010 \\
0.2933600 \\
2.9051920 \\
4.0982380 \\
3.6549050 \\
1.8643260 \\
3.2346610 \\
4.8631240 \\
3.3698660 \\
2.2720390 \\
0.9708400 \\
2.4428630\end{array}$ & $\begin{array}{l}-5.4747200 \\
-1.3265420 \\
-1.1268770 \\
-1.5214130 \\
-2.0901930 \\
-1.2514500 \\
-1.3303920 \\
-2.1074510 \\
-0.5324760 \\
-2.1295600 \\
-0.2089430 \\
-1.5877900 \\
-0.6017510 \\
-2.3205460 \\
-3.1287770 \\
-0.7290740 \\
-1.0695230 \\
-1.8264200 \\
-1.5422730 \\
-3.1303160 \\
-2.5613150 \\
-0.5233470 \\
-4.1486450 \\
-3.3349480 \\
-3.8647460 \\
-2.3432200 \\
-5.1641220 \\
-4.6616210 \\
-0.3740090 \\
0.3894310 \\
2.0698780 \\
2.1599190 \\
1.2238170 \\
1.8228760 \\
3.2425980 \\
2.8938930 \\
3.5028450 \\
3.1830330 \\
3.0460020 \\
2.3829410 \\
3.6382080 \\
3.9615120 \\
4.0211100 \\
1.4593100 \\
2.6404450 \\
4.4453400\end{array}$ \\
\hline
\end{tabular}

$\begin{array}{llll}\mathrm{H} & 3.6587300 & 4.5565010 & 2.2402880 \\ \mathrm{H} & 1.2421280 & 4.4504250 & 2.6545850\end{array}$

154

$\begin{array}{llll}1 & \text { TS150 (R , R ) } & G[M 06-2 X / 6-31 G(d)]= & -3541.256893 \\ P & -0.3230130 & -0.1268540 & 0.7637960\end{array}$

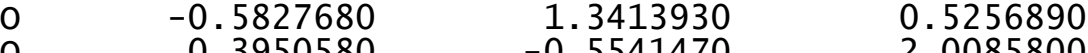

$\begin{array}{rrrr}0 & 0.3950580 & -0.5541470 & 2.0085800 \\ 0 & 0.4401930 & -0.6481260 & -0.5858110\end{array}$

$0-1.7039240 \quad-0.9816290 \quad 0.7192220$

C $\quad 0.4614520 \quad-2.0196850 \quad-0.7937910$

C $\quad-0.7054810 \quad-2.6394460 \quad-1.2440040$

C $\quad 1.6382990 \quad-2.7242250 \quad-0.5415440$

$\begin{array}{llll}1.6011770 & -4.1018420 & -0.7403470\end{array}$

$2.5096320-4.6756600 \quad-0.5638130$

C $\quad-2.3854000 \quad-0.9814900 \quad-0.4897390$

$\begin{array}{llll}\mathrm{C} & -3.5199590 & -0.1820940 & -0.6255560\end{array}$

$\mathrm{C}-1.8965110 \quad-1.7831440 \quad-1.5224070$

$-4.1461670 \quad-0.1953080 \quad-1.8699080$

$\begin{array}{lll}-5.0427470 & 0.4080180 & -2.0030650\end{array}$

$2.8758760-2.0371070 \quad-0.0800500$

$-4.0438400$

3.5250540

3.0899590

5.2898650

4.7255380

2.9085160

2.6249370

2. 0770660

4. 2238680

4.5960040

4.0530630

6.2183090

2. 1600430

5.5384480

5.8911040

6.3321710

4. 2242390

3.9294870

4. 3547810

3. 4420650

2.9005480

5. 1287270

4.6482220

3. 9689500

3.6055780

-2.0371070
0.6494180

0.4927770

$-1.1055870$

$-0.9042660$

$-0.6580250$

$-2.2787270$

0.4647610

$-1.5024090$

$-0.5050100$

0.8736490

$-0.4963720$

$-2.2416860$

1. 2290850

1.1026000

1.5489800

2. 2491850

2. 1307310

0.9162850

$-1.1411340$

$-0.2983860$

0.4082290

$-1.0366870$

$-0.9867200$

$-0.6422820$

$-2.0715980$

$-2.3367710$

$-3.2925460$

$-2.1836850$

$-1.7373120$

$-4.3826670$

$-5.0708720$

3.2391680

$-1.5705340$

$-1.7710800$

$-2.5286450$

$-0.8581630$

$-1.1360890$

$-2.5889400$

$-2.8216300$

$-3.5812730$

$-2.6732550$

$-3.2748240$

$-4.2722790$

$-3.3333720$

1. 1682930

2. 2047340

2. 9201700

1.5634660

2. 4457920

3. 2169430

$\mathrm{H} \quad 4.1128930$

$-4.9691910$

1. 5323270 


5.3019670
5.6003540
6.1107050
6.0737200
1.9552080
4.0329480
4.3563010
3.9010950
2.7037890
2.3651870
1.9110930
5.2967780
6.2380720
-5.0444610
-5.4561800
-4.4233950
-4.4746670
-6.4792900
-6.7465910
-7.3956750
-5.8633480
-5.8345630
-6.4651980
-3.9875000
-5.8875230
-3.5911720
-3.5814270
-2.5572570
-4.2086990
-4.5001970
-3.4832850
-4.7608860
-4.8489650
-3.6692510
-4.1685430
-3.4190850
-3.4810420
-2.8803760
-2.6878440
-2.2787140
-1.8550140
-3.1611440
-5.3577950
-4.8645460
-4.4890940
-5.5389710

2.8849740
3.8803620
2.1957060
3.1832300
1.8869800
3.9562980
4.9413740
4.0300580
3.5309140
4.2950890
3.3986170
0.7293560
1.0360970
2.7045180
3.9202370
2.0502240
1.6907270
3.4782280
4.3428100
3.1359840
2.3487760
2.6405360
1.4371380
1.2501400
4.6862440
3.3430980
3.6378560
3.1389540
4.4640840
5.3252980
4.8217620
1.3599410
1.0280490
-0.8032390
0.3487240
0.8113420
0.4623960
1.7637060
-0.2230430
-1.0425910
0.2429970
-1.5565460
1.8240240
-1.4187780
-2.1878120
-1.9179240

-5.6139440
-5.6964220
-6.6313330
-5.2020170
-5.6597390
-3.6488850
-2.4998500
-4.3889600
-3.5297650
-2.8111670
-1.8499960
-4.1542470
-5.2626830
-4.7838680
-3.5526080
-2.2589290
-1.3916330
-1.0176010
-2.7835610
-0.7388470
0.4366710
-2.0529560
-1.8663540
-0.8845530
0.4894290
-2.8354020
-2.5971160
-2.6909380
-1.2528720
-0.8074190
1.1632040
0.9401250
-1.4782790
1.5969030
0.4863360
2.2515330
3.3948290
1.7589500
4.0448230
3.7644060
2.4161670
0.8738200
3.5565950
4.9326630
2.0399840
4.0676140
4.4198600

5. 4816100

4.0365210

2. 2158840

2.8175260

$-0.9204900$

$-1.7090080$

$-0.8463280$

$-1.2559770$

$-2.5671580$

$-2.3759710$

$-1.3484470$

$-1.5111240$

0.1663530

$-3.3379840$

$-2.9257890$

$-3.3279550$

$-1.7331620$

$-0.4772770$

$-4.0439870$

$-4.7733200$

$-4.7645340$

$-6.1928380$

$-6.9424220$

$-6.2807880$

$-6.7010170$

$-4.7919340$

$-4.1974050$

$-6.9195080$

$-7.9944730$

$-6.7036670$

$-6.5080460$

$-6.1731690$

3.5307720

. 9453570

4.2902920

5.0409920

4.2175740

5.7209980

5.0752390

4.8948580

3.6245770

5.6453870

6. 3064370

4.8364940

6.1726840
$-0.3061470$

$-0.5642390$

$-0.1820160$

2. 5368940

3.3207940

$-2.9547480$

$-2.7933530$

$-4.2774600$

$-5.4712150$

$-5.1644700$

$-3.9935810$

$-6.3655710$

$-4.2252730$

$-4.4144540$

$-4.9121300$

$-6.0390130$

$-3.7154950$

$-4.3160510$

$-5.6760230$

$-1.3527360$

$-1.1190700$

$-1.6012360$

$-2.1076190$

$-1.2111310$

$-1.2844820$

$-2.1391700$

$-0.6457190$

$-2.2829570$

$-0.1773500$

$-1.5037590$

$-0.5313010$

$-2.2609250$

$-3.1355790$

$-0.7537710$

$-1.0920200$

$-1.8553950$

$-1.5739950$

$-3.1602270$

$-2.5967450$

$-0.5542060$

$-4.1822520$

$-3.3626140$

$-2.3807770$

$-5.1984390$

$-4.7008310$ 


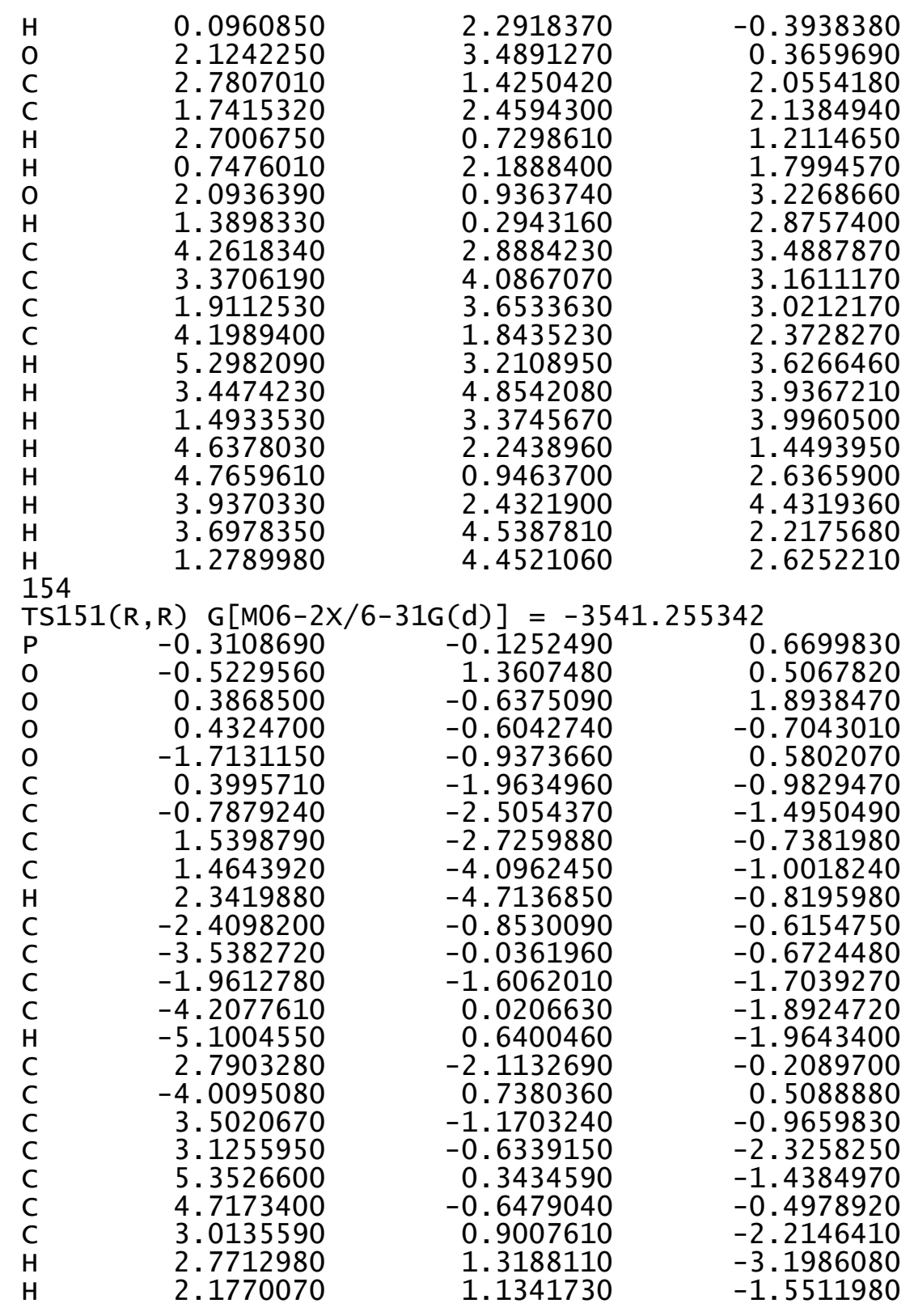

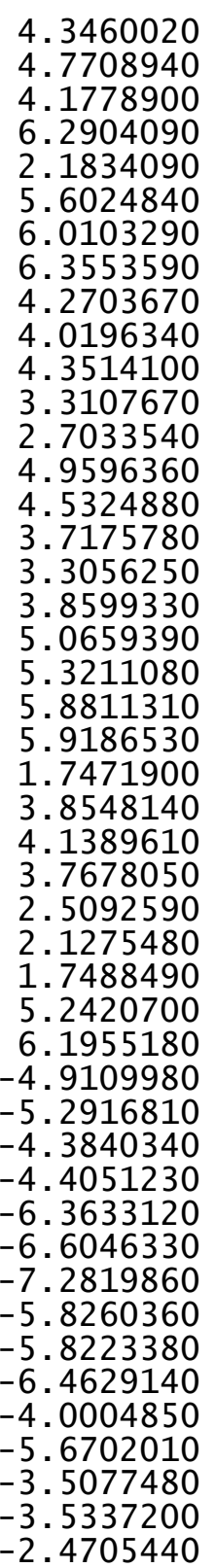

4. 3460020

. 7708940

6. 2904090

2.1834090

. 6024840

6.3553590

4.2703670

4. 0196340

3. 3107670

.7033540

3. 7175780

(1)

.8599330

5. 3211080

(11310

3. 8548140

2. 5092590

2. 1275480

1.4889160

2. 2049310

2. 0347350

0.7319210

$-1.0565330$

$-0.3652390$

0.3594290

$-1.1481220$

$-0.9650440$

$-0.5556140$

$-2.0504650$

$-2.5042900$

$-3.4910730$

$-2.5251530$

$-1.9835280$

$-4.6398190$

$-5.3555340$

$-5.1774240$

$-4.0624060$

$-4.4768570$

$-4.3219970$

$-2.0950860$

$-3.8758760$

$-2.2007310$

$-2.6190850$

$-1.1164690$

$-2.7935900$

$-3.5310860$

$-2.0171990$

$-1.0654040$

$-0.6727490$

0.8215930

$-0.0301580$

$-1.3992670$

0.0873560

$-1.0429040$

$-1.7100610$

$-0.5100930$

$-1.8472190$

$-2.9212600$

$-1.6976600$

$-1.9625870$

0.5858110

$-1.6556390$

$-2.7265770$

$-1.3983700$
$-1.6800060$

$-2.3937060$

$-0.7447910$

$-1.0264170$

$-2.6832400$

$-2.7876040$

$-3.5012360$

$-2.6555920$

$-3.3092360$

$-4.2941050$

$-3.4220420$

1.0342230

2. 0035020

2. 8285490

1. 4890140

2. 2035300

2. 9235470

1. 2601680

2. 7121770

3.6939370

2. 0300380

3. 1385470

1. 6415890

3. 8566810

4.8290400

3. 9825910

3. 3678520

4.0830560

3. 2638080

0.7209540

1.0728740

2. 7696970

3.9531100

1.9536360

1.6874220

3.4944870

4. 3298390

3. 2307630

2.2821210

2.4995110

1.4047450

1.0995360

4.7759010

3.1992950

3. 4312800

2.9696990 


$\begin{array}{lrr}-4.0426680 & -0.8236580 & 4.3944330 \\ -4.3102850 & -1.4729190 & 5.2359300 \\ -3.2783860 & -0.1277060 & 4.7531490 \\ -4.6351160 & 2.8583320 & 1.5321380 \\ -4.6994490 & 4.3448440 & 1.2916920 \\ -3.6491890 & 3.0314690 & -0.6757950 \\ -4.1089700 & 2.1357770 & 0.4506820 \\ -3.2674300 & 4.8606560 & 1.0357730 \\ -3.3165450 & 5.9204280 & 0.7592040 \\ -2.6817420 & 4.7941390 & 1.9576730 \\ -2.6146540 & 4.0204250 & -0.0914400 \\ -2.2415210 & 4.6650010 & -0.8955250 \\ -1.7674290 & 3.4479160 & 0.2918740 \\ -3.1961660 & 2.4583840 & -1.4883840 \\ -5.1529900 & 4.8624140 & 2.1437110 \\ -4.8572300 & 3.8485650 & -1.1830310 \\ -4.5080530 & 4.5649340 & -1.9354700 \\ -5.5748990 & 3.1867350 & -1.6790280 \\ -5.5251620 & 4.5850360 & 0.0087840 \\ -5.5869040 & 5.6618560 & -0.1845520 \\ -6.5466470 & 4.2244680 & 0.1634990 \\ -5.0382170 & 2.2048710 & 2.6912570 \\ -5.4441920 & 2.7691500 & 3.5295830 \\ -3.7625840 & -0.6630520 & -3.0250050 \\ -2.6188020 & -1.4726260 & -2.9432160 \\ -4.5570570 & -0.5262370 & -4.3109010 \\ -3.7593200 & -0.9052380 & -5.5560100 \\ -3.0518740 & -2.2379540 & -5.3273400 \\ -2.0366640 & -2.1041130 & -4.1949080 \\ -4.4245800 & -0.9548590 & -6.4239900 \\ -5.4401310 & -1.1773230 & -4.2448590 \\ -4.9384570 & 0.4975650 & -4.3922390 \\ -3.7945350 & -3.0049370 & -5.0676350 \\ -2.5452280 & -2.5746210 & -6.2375500 \\ -1.5872910 & -3.0724580 & -3.9713780 \\ -1.2075250 & -1.4647830 & -4.5321450 \\ -3.0090200 & -0.1319810 & -5.7663580 \\ -0.8445710 & -3.8917970 & -1.7034760 \\ -2.0956450 & -4.6464440 & -2.0832950 \\ -2.3226090 & -5.7915580 & -1.0793280 \\ -1.0865740 & -6.7140990 & -0.9413910 \\ 0.1455720 & -6.1631260 & -1.6875370 \\ 0.2904620 & -4.6814120 & -1.4612380 \\ -1.9870940 & -5.0721660 & -3.0915130 \\ -3.2010060 & -6.3687510 & -1.3833280 \\ -1.3100250 & -7.7158270 & -1.3211420\end{array}$

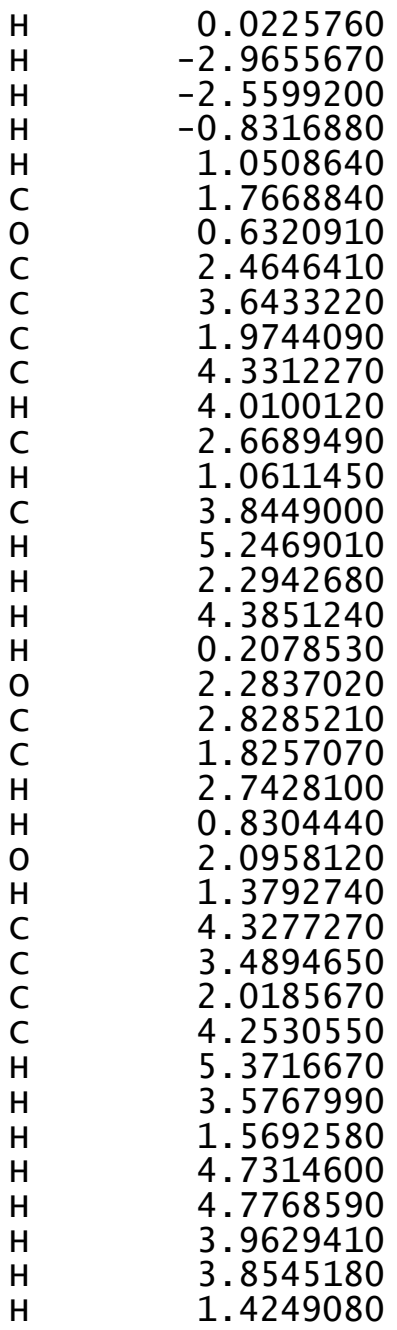

$\begin{array}{rr}-6.3527090 & -2.7625760 \\ -3.9846510 & -2.1085610 \\ -5.3420380 & -0.1100240 \\ -6.8281850 & 0.1164300 \\ -6.6928220 & -1.3760000 \\ 3.5234620 & -0.6255620 \\ 3.0116140 & -1.0008640 \\ 4.3109640 & -1.6801220 \\ 4.9848170 & -1.3535380 \\ 4.3405450 & -2.9875910 \\ 5.6900040 & -2.3336210 \\ 4.9400740 & -0.3331310 \\ 5.0432750 & -3.9668890 \\ 3.8063990 & -3.2256110 \\ 5.7168150 & -3.6405710 \\ 6.2154470 & -2.0824140 \\ 5.0643430 & -4.9850970 \\ 6.2638640 & -4.4071850 \\ 2.3353840 & -0.3452860 \\ 3.3945690 & 0.4924490 \\ 1.2370640 & 2.1032510 \\ 2.3046040 & 2.2092100 \\ 0.5824500 & 1.2282550 \\ 2.0857960 & 1.8381260 \\ 0.7243220 & 3.2363580 \\ 0.1259620 & 2.8378830 \\ 2.5801120 & 3.6322000 \\ 3.8240510 & 3.3362820 \\ 3.4522330 & 3.1472530 \\ 1.5856590 & 2.4717190 \\ 2.8566720 & 3.8075540 \\ 4.5552670 & 4.1451080 \\ 3.1474240 & 4.1000630 \\ 2.0052520 & 1.5769370 \\ 0.6558940 & 2.7118360 \\ 2.0975850 & 4.5471720 \\ 4.3022670 & 2.4198720 \\ 4.2904870 & 2.7738930\end{array}$

TS152(R,R) G[M06-2X/6-31G(d) $]=-3541.257723$

$\begin{array}{lrrr}\text { TS152(R, R) } & \text { G }[\mathrm{M} 06-2 X / 6-31 G(\mathrm{~d})]=-3541.257723 \\ \mathrm{P} & -0.3339190 & -0.1625250 & 0.7497100 \\ \mathrm{O} & -0.6011260 & 1.3116080 & 0.5607630 \\ \mathrm{O} & 0.3722840 & -0.6281840 & 1.9873150 \\ \mathrm{O} & 0.4489300 & -0.6294980 & -0.6089400 \\ \mathrm{O} & -1.7084170 & -1.0237030 & 0.6553020 \\ \mathrm{C} & 0.4746690 & -1.9929910 & -0.8651470\end{array}$




$\begin{array}{rrr}-0.6845700 & -2.5977020 & -1.3556750 \\ 1.6474510 & -2.7048400 & -0.6149250 \\ 1.6088680 & -4.0778610 & -0.8410240 \\ 2.5117090 & -4.6591990 & -0.6589200 \\ -2.3715270 & -0.9702360 & -0.5631280 \\ -3.4996890 & -0.1592080 & -0.6812000 \\ -1.8679710 & -1.7274600 & -1.6225010 \\ -4.0933600 & -0.0983790 & -1.9395820 \\ -4.9800870 & 0.5216910 & -2.0632930 \\ 2.8791780 & -2.0303680 & -0.1206520 \\ -4.0460690 & 0.6097170 & 0.4704550 \\ 3.5349530 & -1.0728850 & -0.9092700 \\ 3.1144190 & -0.5854560 & -2.2746300 \\ 5.3014650 & 0.5198900 & -1.4396350 \\ 4.7290380 & -0.4817210 & -0.4699570 \\ 2.9273830 & 0.9438050 & -2.1941540 \\ 2.6541630 & 1.3277610 & -3.1838970 \\ 2.0875350 & 1.1501750 & -1.5260640 \\ 4.2349800 & 1.6085130 & -1.6883760 \\ 4.6136220 & 2.3319400 & -2.4204260 \\ 4.0511830 & 2.1623730 & -0.7607940 \\ 6.2240580 & 0.9630360 & -1.0491270 \\ 2.1897180 & -1.0619110 & -2.6100480 \\ 5.5678200 & -0.2025690 & -2.7781570 \\ 5.9267510 & 0.5275650 & -3.5121910 \\ 6.3622450 & -0.9426160 & -2.6428090 \\ 4.2611560 & -0.8808260 & -3.2669520 \\ 3.9767310 & -0.5073460 & -4.2569180 \\ 4.3959010 & -1.9630640 & -3.3566100 \\ 3.4325070 & -2.3662720 & 1.1243730 \\ 2.8826710 & -3.3539260 & 2.1262900 \\ 5.1010470 & -2.2632930 & 2.8974030 \\ 4.6331430 & -1.7766720 & 1.5502460 \\ 3.9509300 & -4.4490350 & 2.3444460 \\ 3.5815000 & -5.1613070 & 3.0904670 \\ 4.1054620 & -5.0069980 & 1.4149510 \\ 5.2779680 & -3.7963880 & 2.8171910 \\ 5.5670060 & -4.1736470 & 3.8045170 \\ 6.0942340 & -4.0346000 & 2.1285770 \\ 6.0423960 & -1.7813520 & 3.1843490 \\ 1.9409410 & -3.7911110 & 1.7866060 \\ 3.9945400 & -1.9659710 & 3.9321710 \\ 4.3089680 & -2.3501410 & 4.9093050 \\ 3.8595100 & -0.8844170 & 4.0371230 \\ 2.6714250 & -2.6288870 & 4.2120970 \\ 2.3276870 & -3.3618640 & \end{array}$

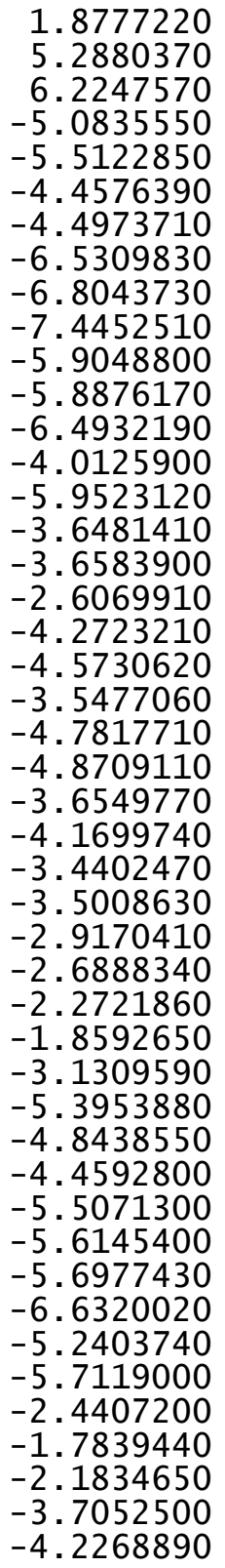

-1.8884880
-0.8444490
-0.3969100
0.6796980
-0.1759340
-1.5306940
-0.0461360
-1.2161910
-1.8853620
-0.7069010
-2.0123780
-3.0850160
-1.8825610
-2.0891000
0.4343870
-1.7591790
-2.8294480
-1.4743280
-0.9368010
-1.5899220
-0.2211320
2.7181090
4.2015430
2.9103350
2.0054700
4.7571200
5.8125230
4.7159410
3.9263170
4.5745460
3.3754680
2.3479260
4.7089050
3.6977430
4.4249850
3.0201520
4.4136320
5.4875940
4.0202410
2.0588540
2.6156660
-1.5819520
-2.2159670
-1.5273190
-1.4448410
-0.5261440
3. 3563510

0.7512420

1.0818730

2. 6728140

3. 8367770

1. 9011090

1.6258730

3. 3230350

4.1467870

3. 0034780

2. 1485160

2. 3720340

1. 2348740

1.0740440

4. 6327140

3. 1964180

3. 4323620

3. 0249810

4. 3543900

5. 1815770

4.7541990

1.4443890

1.1924390

$-0.6930570$

0.4020810

1. 0298540

0.7391350

1.9900230

$-0.0409900$

$-0.8203220$

0.4070830

$-1.4699840$

2. 0091190

$-1.2857780$

$-2.0100480$

$-1.8334010$

$-0.1444840$

$-0.3453850$

$-0.0583220$

2. 5793000

3. 3877560

$-2.9021330$

$-4.1146800$

$-5.4186770$

$-5.5010250$

$-4.3986760$ 


$\begin{array}{rrr}-3.5711590 & -0.7641070 & -3.0503820 \\ -1.7714390 & -2.0816290 & -6.2678800 \\ -2.0682560 & -3.2719560 & -4.1941250 \\ -0.6966770 & -2.2021130 & -3.9816260 \\ -4.1245680 & -2.4522070 & -5.3791850 \\ -4.0326020 & -1.0760370 & -6.4782140 \\ -5.3134000 & -0.6256610 & -4.2934800 \\ -4.0437680 & 0.5175700 & -4.6888210 \\ -1.7598460 & -0.5150910 & -5.4534290 \\ -0.7199360 & -4.0001550 & -1.4951820 \\ -2.0257830 & -4.7077550 & -1.8082600 \\ -2.0087560 & -6.1749630 & -1.3827340 \\ -0.7728000 & -6.8555690 & -1.9644830 \\ 0.4829620 & -6.2530820 & -1.3389500 \\ 0.4467710 & -4.7384240 & -1.2447990 \\ -2.9263870 & -6.6659510 & -1.7217760 \\ -2.2287770 & -4.6746900 & -2.8852320 \\ -2.8488590 & -4.1695510 & -1.3258750 \\ -0.7599670 & -6.7041880 & -3.0518920 \\ -0.7929280 & -7.9359490 & -1.7902330 \\ 1.3773280 & -6.5583160 & -1.8944110 \\ 0.6030770 & -6.6557220 & -0.3239090 \\ -1.9855960 & -6.2477210 & -0.2876550 \\ 1.5744370 & 3.5425980 & -0.6434960 \\ 0.4673480 & 2.9650280 & -1.0059300 \\ 2.2340500 & 4.3360920 & -1.7179600 \\ 3.3746940 & 5.0794160 & -1.4076910 \\ 1.7485780 & 4.3026170 & -3.0270600 \\ 4.0291670 & 5.7910930 & -2.4057720 \\ 3.7388640 & 5.0830030 & -0.3853970 \\ 2.4101280 & 5.0116990 & -4.0244290 \\ 0.8654920 & 3.7147860 & -3.2521060 \\ 3.5480050 & 5.7547250 & -3.7144200 \\ 4.9150800 & 6.3706960 & -2.1673020 \\ 2.0394040 & 4.9837790 & -5.0439140 \\ 4.0625880 & 6.3067490 & -4.4949680 \\ 0.0750590 & 2.2900510 & -0.3292000 \\ 2.0949600 & 3.4689440 & 0.4777900 \\ 2.7485790 & 1.3619100 & 2.1158700 \\ 1.7037490 & 2.3887400 & 2.2195670 \\ 2.6795210 & 0.6913890 & 1.2512410 \\ 0.7141420 & 2.1233530 & 1.8644140 \\ 2.0532780 & 0.8357160 & 3.2661710 \\ 1.3562700 & 0.2009390 & 2.8899280 \\ 4.2094590 & 2.7907820 & 3.6041230 \\ 3.3160240 & 3.9940130 & 3.3016900\end{array}$

\begin{abstract}
1.8600890
4.1618070

5.2430450

3.3823420

1.4349230

4.6080140

4.7301890

3.8776350

3.6495440

1. 2272540
\end{abstract}

154

TS153 (R, R) G[M06-2X/6-31G(d) $]=-3541.255974$

$\begin{array}{lrrr}\text { TS153(R, R) G[M06-2X/6-31G(d) }]=-3541.255974 \\ \text { P } & -0.3206390 & -0.1512720 & 0.6895980 \\ \text { O } & -0.5495450 & 1.3327420 & 0.5316480 \\ \text { O } & 0.3782450 & -0.6602290 & 1.9142310 \\ \text { O } & 0.4346070 & -0.6150610 & -0.6834230 \\ \text { O } & -1.7133280 & -0.9783860 & 0.5882310 \\ \text { C } & 0.4240490 & -1.9723050 & -0.9716430 \\ \text { C } & -0.7560640 & -2.5339680 & -1.4830090 \\ \text { C } & 1.5811120 & -2.7132700 & -0.7435130 \\ \text { C } & 1.5337460 & -4.0815210 & -1.0254470 \\ \text { H } & 2.4255240 & -4.6820770 & -0.8561500 \\ \text { C } & -2.3971270 & -0.8931100 & -0.6153990 \\ \text { C } & -3.5209790 & -0.0709840 & -0.6884820 \\ \text { C } & -1.9379090 & -1.6488800 & -1.6966420 \\ \text { C } & -4.1623610 & 0.0010740 & -1.9224780 \\ \text { H } & -5.0471220 & 0.6300240 & -2.0097610 \\ \text { C } & 2.8214580 & -2.0813680 & -0.2131720 \\ \text { C } & -4.0173640 & 0.6912730 & 0.4902700 \\ \text { C } & 3.5141550 & -1.1206420 & -0.9651010 \\ \text { C } & 3.1222900 & -0.5792310 & -2.3183780 \\ \text { C } & 5.3341330 & 0.4316950 & -1.4303230 \\ \text { C } & 4.7203360 & -0.5788240 & -0.4957150 \\ \text { C } & 2.9806070 & 0.9515400 & -2.1896860 \\ \text { H } & 2.7208530 & 1.3758870 & -3.1664550 \\ \text { H } & 2.1456790 & 1.1601340 & -1.5159320 \\ \text { C } & 4.3057120 & 1.5603530 & -1.6597740 \\ \text { H } & 4.7137440 & 2.2872690 & -2.3723180 \\ \text { H } & 4.1333780 & 2.0985960 & -0.7209710 \\ \text { H } & 6.2659600 & 0.8341340 & -1.0182560 \\ \text { H } & 2.1866620 & -1.0164780 & -2.6757280 \\ \text { C } & 5.5922920 & -0.2615020 & -2.7858520 \\ \text { H } & 5.9870500 & 0.4754270 & -3.4942400 \\ \text { H } & 6.3577630 & -1.0334040 & -2.6617980 \\ \text { C } & 4.2687880 & -0.8780600 & -3.3099080 \\ \text { H } & 4.0069960 & -0.4609280 & -4.2886460 \\ & & & \end{array}$

3.1366560

2.4588230

3.7609670

4.0989650

4.0995260

1.5514210

2.7029860

4.5309550

2.3740630

2.7575210

$\begin{array}{ll}2.3069760 & 4.5309550 \\ 4.4736460 & 2.3740630\end{array}$

4.3646370

0.6895980

16480

$-0.6834230$

-

0.7435130

(1)

(

97610

0.4902700

(1)

(

59320

$-2.3723180$

$-4.2886460$ 


\begin{tabular}{|c|c|c|c|}
\hline $\begin{array}{l}\mathrm{H} \\
\mathrm{C} \\
\mathrm{C} \\
\mathrm{C} \\
\mathrm{C} \\
\mathrm{C} \\
\mathrm{H} \\
\mathrm{H} \\
\mathrm{C} \\
\mathrm{H} \\
\mathrm{H} \\
\mathrm{H} \\
\mathrm{H} \\
\mathrm{C} \\
\mathrm{H} \\
\mathrm{H} \\
\mathrm{C} \\
\mathrm{H} \\
\mathrm{H} \\
\mathrm{C} \\
\mathrm{H} \\
\mathrm{C} \\
\mathrm{C} \\
\mathrm{C} \\
\mathrm{C} \\
\mathrm{C} \\
\mathrm{H} \\
\mathrm{H} \\
\mathrm{C} \\
\mathrm{H} \\
\mathrm{H} \\
\mathrm{H} \\
\mathrm{H} \\
\mathrm{C} \\
\mathrm{H} \\
\mathrm{H} \\
\mathrm{C} \\
\mathrm{H} \\
\mathrm{H} \\
\mathrm{C} \\
\mathrm{C} \\
\mathrm{C} \\
\mathrm{C} \\
\mathrm{C} \\
\mathrm{H} \\
\mathrm{H}\end{array}$ & $\begin{array}{r}4.3684250 \\
3.3509490 \\
2.7618530 \\
5.0016860 \\
4.5635610 \\
3.7960810 \\
3.3977010 \\
3.9461130 \\
5.1351070 \\
5.4000180 \\
5.9531750 \\
5.9532230 \\
1.8122350 \\
3.8922740 \\
4.1843840 \\
3.7869930 \\
2.5563470 \\
2.1860920 \\
1.7838870 \\
5.2549320 \\
6.2013400 \\
-4.9646710 \\
-5.3604310 \\
-4.4073360 \\
-4.4324680 \\
-6.4162120 \\
-6.6657330 \\
-7.3347700 \\
-5.8523770 \\
-5.8463940 \\
-6.4740990 \\
-4.0052090 \\
-5.7578460 \\
-3.5525490 \\
-3.5772240 \\
-2.5126860 \\
-4.1134930 \\
-4.3905780 \\
-3.3603880 \\
-4.6809150 \\
-4.7554720 \\
-3.6445380 \\
-4.1248140 \\
-3.3237870 \\
-3.3785300 \\
-2.7601050\end{array}$ & $\begin{array}{r}-1.9604100 \\
-2.4707110 \\
-3.4731860 \\
-2.4718410 \\
-1.9304480 \\
-4.6049110 \\
-5.3303340 \\
-5.1364180 \\
-4.0062490 \\
-4.4216020 \\
-4.2473510 \\
-2.0265440 \\
-3.8727040 \\
-2.1730740 \\
-2.5933380 \\
-1.0913610 \\
-2.7850880 \\
-3.5315040 \\
-2.0202050 \\
-0.9946860 \\
-0.5864420 \\
0.7499250 \\
-0.1133780 \\
-1.4605920 \\
0.0280310 \\
-1.1304840 \\
-1.8067420 \\
-0.6028400 \\
-1.9197940 \\
-2.9958770 \\
-1.7652020 \\
-2.0154760 \\
0.4938520 \\
-1.7215190 \\
-2.7942340 \\
-1.4573600 \\
-0.9008720 \\
-1.5572820 \\
-0.2017630 \\
2.7984050 \\
4.2859960 \\
2.9982770 \\
2.0889470 \\
4.8194490 \\
5.8795790 \\
4.7564580\end{array}$ & $\begin{array}{r}-3.4365630 \\
1.0265410 \\
1.9908950 \\
2.8189680 \\
1.4828760 \\
2.1849610 \\
2.9028920 \\
1.2393570 \\
2.6937640 \\
3.6725520 \\
2.0082860 \\
3.1305060 \\
1.6274670 \\
3.8500770 \\
4.8192490 \\
3.9837950 \\
3.3583420 \\
4.0702850 \\
3.2572210 \\
0.7194420 \\
1.0726100 \\
2.7332530 \\
3.9032800 \\
1.9102730 \\
1.6552360 \\
3.4185250 \\
4.2440880 \\
3.1442270 \\
2.2084080 \\
2.4153550 \\
1.3210980 \\
1.0592970 \\
4.7236520 \\
3.1698900 \\
3.3936710 \\
2.9611610 \\
4.3609250 \\
5.1937540 \\
4.7367280 \\
1.5172680 \\
1.2863330 \\
-0.6648380 \\
0.4421020 \\
1.0681020 \\
0.7939210 \\
2.0038780\end{array}$ \\
\hline
\end{tabular}

-2.6340200
-2.2425830
-1.7940920
-3.1649890
-5.2350760
-4.8459370
-4.4829040
-5.5438790
-5.5528280
-5.6248820
-6.5726100
-5.1023460
-5.5305590
-2.5733430
-2.0103690
-2.4824790
-4.0029200
-4.4173810
-3.6992590
-2.1476940
-2.3204060
-0.9165000
-4.4452920
-4.3841230
-5.4996480
-4.2001690
-2.0324170
-0.7841460
-2.0260840
-2.2017230
-0.9516790
0.2561000
0.3703730
-1.9312220
-3.0852300
-1.1700720
0.1204990
-2.9105760
-2.4045990
-0.6732450
1.1783040
1.6953170
0.5709120
2.3780400
3.5441800
1.8867930

.9909090

4.6429000

3.4217820

2.4363310

4.7920950

3. 8101020

4. 5383530

3. 1481220

4.5266130

5. 6041550

4. 1505790

2.1325750

2.6867780

$-1.5098220$

$-2.2034490$

$-1.5641120$

$-1.4333410$

$-0.4521680$

$-0.6787470$

$-2.1704710$

$-3.2560800$

$-2.2088290$

$-2.4193170$

$-1.0942250$

$-0.4930790$

0.5701840

$-0.5692500$

$-3.9174260$

$-4.6976790$

$-5.8487910$

$-6.7575960$

$-6.1671410$

$-4.6855250$

$-5.1200810$

$-6.4347960$

$-7.7502660$

$-6.3449400$

$-4.0551590$

$-5.4043300$

$-6.9004170$

$-6.6795890$

3. 5416210

3. 0104080

4. 3500160

5.0429840

4.3808570
$-0.0454160$

$-0.8346480$

0.3582700

$-1.4701360$

2.1309210

$-1.1958180$

$-1.9301060$

$-1.7189060$

$-0.0143440$

$-0.1998550$

0.1122460

2. 6626580

3.4966230

$-2.9476490$

$-4.1741450$

$-5.4788910$

$-5.4621270$

$-4.3685680$

$-3.0505900$

$-6.3265280$

$-4.1792010$

$-4.1154720$

$-5.2677600$

$-6.4305520$

$-4.2006060$

$-4.7076360$

$-5.5929220$

$-1.7093550$

$-2.0632910$

$-1.0554940$

$-0.9714140$

$-1.7281990$

$-1.4854140$

$-3.0736430$

$-1.3260870$

$-1.3771440$

$-2.8036310$

$-2.0617290$

$-0.0760070$

0.0769070

$-1.4382800$

$-0.5930100$

$-0.9726620$

$-1.6415600$

$-1.3100050$

$-2.9486280$ 


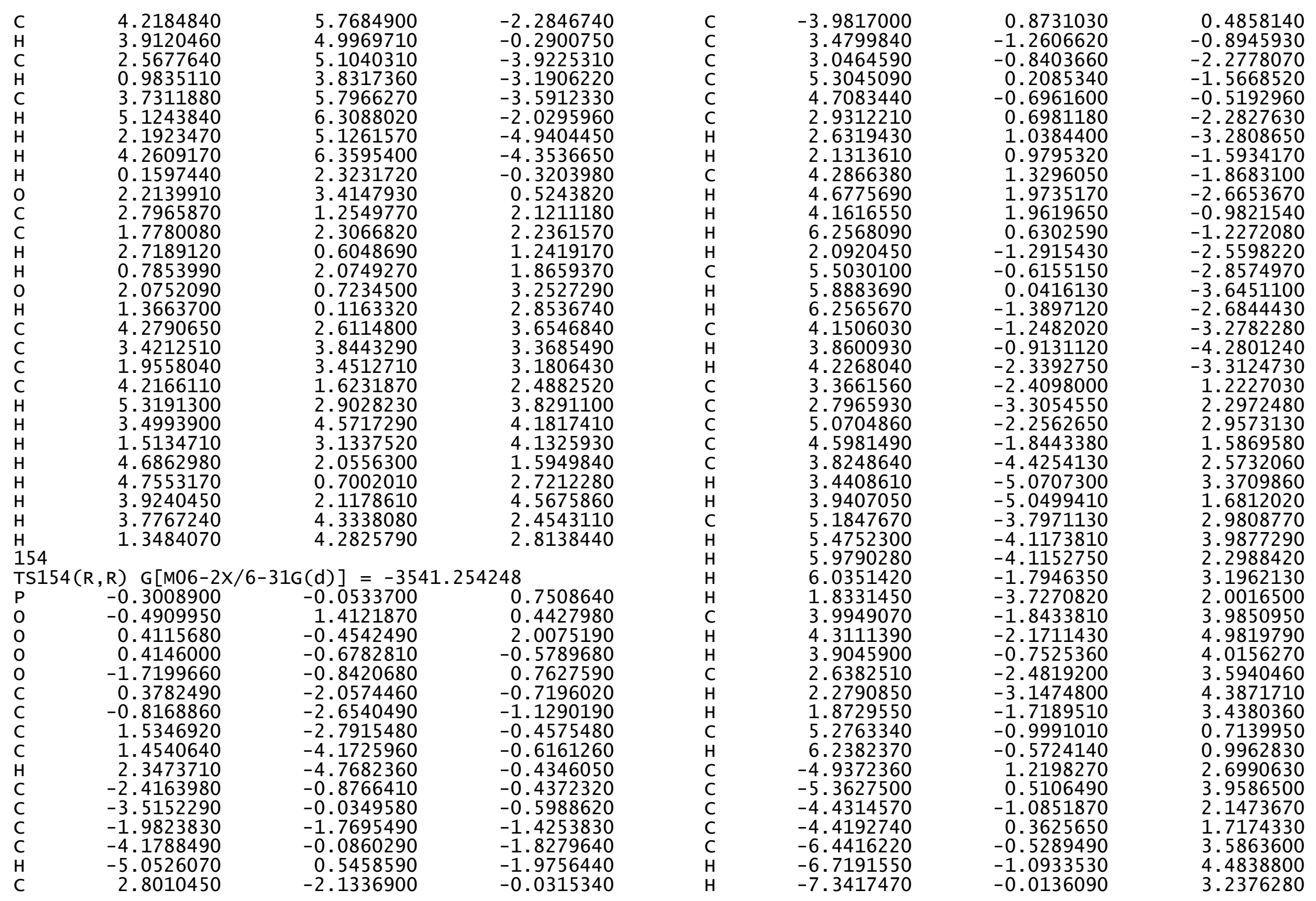




-5.8876840
-5.9057390
-6.5007610
-4.0384950
-5.7497800
-3.5869840
-3.6274860
-2.5427230
-4.1405280
-4.4432240
-3.3745730
-4.5932810
-4.6286250
-3.5333760
-4.0497300
-3.1828400
-3.2110650
-2.6295550
-2.5015370
-2.0732050
-1.6892120
-3.0615780
-5.1026910
-4.7043780
-4.3104460
-5.4120610
-5.4089630
-5.4588910
-6.4368700
-5.0392490
-5.4585400
-3.7366120
-4.4291880
-4.6427800
-3.3050230
-2.1600920
-2.6302980
-3.8164460
-5.1833600
-2.9951650
-1.7021940
-5.3826400
-5.2809000
-3.4408980
-1.3700250
-0.8892960

2.4882700
2.8272250
1.5834040
1.3712590
4.6980300
3.4343830
3.7813380
3.2023790
4.5184290
5.4154070
4.8245250
1.2537370
0.8480920
-0.9244020
0.2745970
0.5827700
0.1755910
1.5257400
-0.4078290
-1.2604940
0.0805060
-1.6522680
1.6213650
-1.5629600
-2.3665580
-2.0225730
-0.4808560
-0.7906900
-0.3234840
2.4653240
3.2244480
-2.8610090
-4.1981530
-4.5719360
-4.6503570
-3.8854770
-2.6696660
-4.9706370
-5.5199820
-5.6943360
-4.5604190
-4.1622880
-3.8022330
-4.2615290
-3.6340440
-1.2055850

0.2675750 $-2.2210520$ $-2.0705860$ $-1.1101810$ 0.2801850 $-3.0518730$ $-2.7511920$ $-2.8562850$ $-1.4840970$ $-1.0562390$ 0.9340930 0.7372610 $-1.6795040$ 1.7644290 0.6223020 2. 4371670 3.6227390 1. 9167930 4.2873210 4.0129670 2. 5879740 0.9986120 3.7707200 5.2083230 2. 1898240 4.2925870 0.2154480 2. 3063480 2.8843110 1.8901130 2.7724310 0.8844690 2.1813430 1. 4484000 4.4310450 3.5899080 2. 1130640 4. 3199800 5.4806240 3.7014600 1.6881150 4.7730110 4.8486900 4.0906800 3.9327030 1. 5130820

\begin{tabular}{ll}
-4.8175520 & -0.9696220 \\
-4.7507230 & -1.4342800 \\
-6.1792460 & -1.9502890 \\
-6.9563950 & -1.0537000 \\
-6.3282370 & -1.1163270 \\
-6.6634330 & -1.9865850 \\
-4.7731420 & -0.4707100 \\
-4.1665540 & -2.1017410 \\
-6.9294320 & -0.0220710 \\
-8.0087580 & -1.3502660 \\
-6.7605110 & -0.3509960 \\
-6.5766280 & -2.0845750 \\
-6.1621120 & -2.9774660 \\
3.4630540 & -0.9290780 \\
2.9219880 & -1.2345060 \\
4.1585420 & -2.0615870 \\
4.8556290 & -1.8200390 \\
4.0789860 & -3.3552360 \\
5.4752370 & -2.8713740 \\
4.8957830 & -0.8081930 \\
4.6962700 & -4.4057240 \\
3.5279390 & -3.5264450 \\
5.3932240 & -4.1643390 \\
6.0185560 & -2.6864890 \\
4.6326730 & -5.4131860 \\
5.8735320 & -4.9863450 \\
2.3050870 & -0.5133890 \\
3.4280430 & 0.1839450 \\
1.4127770 & 1.9627490 \\
2.4930780 & 2.0007980 \\
0.6844540 & 1.1511170 \\
2.2497850 & 1.6759660 \\
1.0061490 & 3.1558000 \\
0.3813130 & 2.8342460 \\
2.8741220 & 3.3275150 \\
4.0934160 & 2.9486470 \\
3.7160740 & 2.8304450 \\
1.7841020 & 2.2594790 \\
3.1582050 & 3.4490430 \\
4.8901590 & 3.6897790 \\
3.4971470 & 3.8174520 \\
2.1230440 & 1.3184780 \\
0.8760460 & 2.5629440 \\
2.4744500 & 4.2906290 \\
4.4898170 & 1.9857490 \\
4.5229440 & 2.4020990 \\
& \\
\hline
\end{tabular}


154

$\operatorname{TS} 155(R, R) \quad G[M 06-2 X / 6-31 G(d)]=-3541.254563$

$\begin{array}{lrrr}\mathrm{P} & -0.3020180 & -0.0585350 & 0.7346960 \\ \mathrm{O} & -0.4941410 & 1.4114460 & 0.4490580 \\ \mathrm{O} & 0.4050770 & -0.4772760 & 1.9885020 \\ \mathrm{O} & 0.4214150 & -0.6610100 & -0.6015520 \\ \mathrm{O} & -1.7190080 & -0.8511450 & 0.7267480 \\ \mathrm{C} & 0.3790830 & -2.0383960 & -0.7607490 \\ \mathrm{C} & -0.8127410 & -2.6206190 & -1.1963130 \\ \mathrm{C} & 1.5244790 & -2.7851310 & -0.4841220 \\ \mathrm{C} & 1.4282040 & -4.1652170 & -0.6381650 \\ \mathrm{H} & 2.3101720 & -4.7722710 & -0.4389360 \\ \mathrm{C} & -2.4139020 & -0.8615860 & -0.4744860 \\ \mathrm{C} & -3.5161260 & -0.0218550 & -0.6164880 \\ \mathrm{C} & -1.9736990 & -1.7283020 & -1.4848090 \\ \mathrm{C} & -4.1780870 & -0.0465470 & -1.8475450 \\ \mathrm{H} & -5.0530600 & 0.5866660 & -1.9819060 \\ \mathrm{C} & 2.7925380 & -2.1395620 & -0.0449770 \\ \mathrm{C} & -3.9839550 & 0.8629880 & 0.4865900 \\ \mathrm{C} & 3.4816130 & -1.2627780 & -0.8965290 \\ \mathrm{C} & 3.0625270 & -0.8324040 & -2.2812350 \\ \mathrm{C} & 5.3181600 & 0.2030420 & -1.5439610 \\ \mathrm{C} & 4.7102120 & -0.7075270 & -0.5083240 \\ \mathrm{C} & 2.9547970 & 0.7066390 & -2.2820400 \\ \mathrm{H} & 2.6739200 & 1.0510080 & -3.2840990 \\ \mathrm{H} & 2.1450100 & 0.9906010 & -1.6054780 \\ \mathrm{C} & 4.3066000 & 1.3300790 & -1.8443650 \\ \mathrm{H} & 4.7080440 & 1.9826240 & -2.6290560 \\ \mathrm{H} & 4.1726870 & 1.9521160 & -0.9521580 \\ \mathrm{H} & 6.2691020 & 0.6186700 & -1.1931710 \\ \mathrm{H} & 2.1090120 & -1.2779060 & -2.5750390 \\ \mathrm{C} & 5.5243320 & -0.6114220 & -2.8395300 \\ \mathrm{H} & 5.9140620 & 0.0517870 & -3.6198820 \\ \mathrm{H} & 6.2770670 & -1.3867770 & -2.6681570 \\ \mathrm{C} & 4.1744830 & -1.2410730 & -3.2727980 \\ \mathrm{H} & 3.8933560 & -0.9042250 & -4.2767650 \\ \mathrm{H} & 4.2488610 & -2.3322540 & -3.3075770 \\ \mathrm{C} & 3.3484870 & -2.4293360 & 1.2104880 \\ \mathrm{C} & 2.7688160 & -3.3319010 & 2.2740300 \\ \mathrm{C} & 5.0443710 & -2.3007530 & 2.9557390 \\ \mathrm{C} & 4.5812250 & -1.8735350 & 1.5869920 \\ \mathrm{C} & 3.7899880 & -4.4597860 & 2.5444300 \\ \mathrm{H} & 3.3986960 & -5.1105670 & 3.3341880 \\ \mathrm{H} & 3.9073030 & -5.0765700 & 1.6472480 \\ \mathrm{C} & 5.1509830 & -3.8422750 & 2.9648450 \\ \mathrm{H} & 5.4349270 & -4.1738440 & 3.9698950\end{array}$

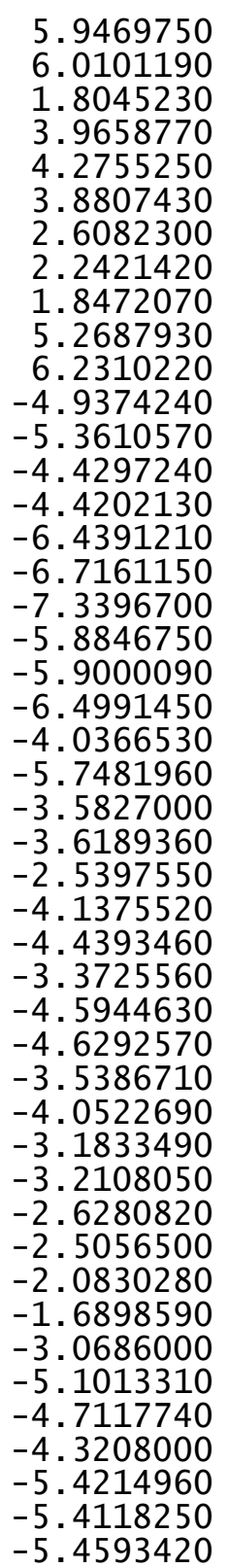

5.9469750
$-4.1576750$
$-1.8462600$
$-3.7455880$
$-1.8924010$
$-2.2312900$
$-0.8014760$
$-2.5205050$
$-3.1923360$
$-1.7525700$
$-1.0238500$
$-0.6044680$
1.1646390
0.4297800
$-1.1283620$
0.3276180
$-0.6032810$
$-1.1851290$
$-0.0819540$
$-1.5263820$
$-2.5744790$
$-1.4554200$
$-1.7727330$
1.1236050
$-1.2778270$
$-2.3236290$
$-1.0443750$
$-0.3398090$
$-0.9115140$
0.3734540
3. 0771130
4. 5364750
3. 0363570
2. 2520100
5.0109570
6.0378500
5. 0324960
4. 0557610
4.6137970
3. 5044610
2. 3864800
5.1346450
3.8264330
4.4736540
3.1373370
4.6677220
5.7236980

2. 2835490

3.2037550

1.9701550

3. 9821390

4. 9773700

4. 0227260

3. 5781520

4.3627830

3. 4261950

0.7258450

1. 0178150

2. 7072440

3.9526310

2. 1088000

1.7078880

3. 5604000

4.4468660

3. 2218010

2. 4442440

2.7637070

1. 5413980

1. 3189280

4.7065400

3. 3915920

3. 7173550

3. 1623000

4.4958870

5.3809750

4.8170200

1. 3002180

0.9245790

$-0.8812610$

0.3033860

0.6669120

0.2837630

1. 6095320

$-0.3477760$

$-1.1910950$

0.1243020

$-1.6236050$

1. 7112350

$-1.5010250$

$-2.2945000$

$-1.9704440$

$-0.4005700$

$-0.6893460$ 


$\begin{array}{rrr}-6.4404030 & 4.3268220 & -0.2480000 \\ -5.0393460 & 2.5367920 & 2.5015080 \\ -5.4574400 & 3.1800220 & 3.2744580 \\ -3.7291260 & -0.8354390 & -2.9003350 \\ -4.4110550 & -0.8584970 & -4.2447850 \\ -4.5924850 & -2.3162050 & -4.6891820 \\ -3.2377570 & -3.0420380 & -4.8084210 \\ -2.1190670 & -2.3760840 & -3.9722220 \\ -2.6192960 & -1.6784160 & -2.7287360 \\ -3.8022020 & -0.3240670 & -4.9886160 \\ -5.1340780 & -2.3641020 & -5.6388220 \\ -2.9121570 & -3.0594350 & -5.8534200 \\ -1.6537770 & -1.5976760 & -4.5929690 \\ -5.3744390 & -0.3420160 & -4.1908360 \\ -5.2155040 & -2.8169170 & -3.9400140 \\ -3.3611490 & -4.0872860 & -4.5081690 \\ -1.3236590 & -3.0886890 & -3.7427480 \\ -0.9017590 & -4.0255140 & -1.2741330 \\ -2.2309830 & -4.6881810 & -1.5861030 \\ -2.2685270 & -6.1587600 & -1.1741990 \\ -1.0375140 & -6.8765130 & -1.7203990 \\ 0.2147020 & -6.3138310 & -1.0531380 \\ 0.2380360 & -4.7972370 & -1.0046130 \\ -3.1908220 & -6.6188780 & -1.5427000 \\ -2.4314090 & -4.6280870 & -2.6631590 \\ -3.0356390 & -4.1267330 & -1.0987940 \\ -0.9839750 & -6.7230680 & -2.8064440 \\ -1.0978310 & -7.9559560 & -1.5496180 \\ 1.1186360 & -6.6711830 & -1.5595580 \\ 0.2706570 & -6.6943600 & -0.0239360 \\ -2.2802320 & -6.2399670 & -0.0794930 \\ 1.7790150 & 3.4625250 & -0.8932590 \\ 0.6355780 & 2.9298770 & -1.2084270 \\ 2.4621150 & 4.1614910 & -2.0174310 \\ 3.6480350 & 4.8536940 & -1.7638360 \\ 1.9513450 & 4.0892570 & -3.3152990 \\ 4.3225470 & 5.4757660 & -2.8073560 \\ 4.0307440 & 4.8881650 & -0.7489130 \\ 2.6325670 & 4.7087700 & -4.3580000 \\ 1.0328040 & 3.5418070 & -3.4958640 \\ 3.8156450 & 5.4008760 & -4.1045960 \\ 5.2437880 & 6.0153900 & -2.6130570 \\ 2.2419540 & 4.6507070 & -5.3687450 \\ 4.3453420 & 5.8829940 & -4.9205110 \\ 0.2211880 & 2.3095530 & -0.4941220 \\ 2.3146090 & 3.4175210 & 0.2224670\end{array}$

2.8789440
1.8869350
2.7696940
0.8823650
2.1681190
1.4358730
4.4217370
3.5854690
2.1083850
4.3138020
5.4713160
3.6951170
1.6779470
4.7727110
4.8386590
4.0752550
3.9340580
1.5124160

1. 3837420

2. 4657540

0.6640890

2. 2283620

0.9665930

0.3470420

2. 8274340

4.0524730

3.6797750

1. 7485140

3. 1077150

4.8415490

3. 4518990

2. 0957570

0.8361600

2.4188620

4.4575680

4.4922900
1. 9831450

2. 0275090

1.1634810

1.6951760

3. 1680520

2.8351660

3. 3710730

3. 0001180

2. 8707030

2. 2915230

3. 5010900

3.7496900

3.8532830

1. 3564080

2. 5887050

4. 3282520

2. 0429410

2. 4474520

154

$\operatorname{TS} 156(R, R) \quad G[M 06-2 X / 6-31 G(d)]=-3541.254491$

$\begin{array}{lrrr}\text { TS156(R, R) }(\mathrm{G}[\mathrm{M} 06-2 X / 6-31 \mathrm{G}(\mathrm{d})]=-3541.254491 \\ \mathrm{P} & -0.3166890 & -0.0595090 & 0.7816990 \\ \mathrm{O} & -0.5847260 & 1.3827960 & 0.4249080 \\ \mathrm{O} & 0.4087830 & -0.3803990 & 2.0536330 \\ \mathrm{O} & 0.4369370 & -0.6891320 & -0.5242900 \\ \mathrm{O} & -1.6914600 & -0.9225440 & 0.8156090 \\ \mathrm{C} & 0.4596960 & -2.0732780 & -0.6193940 \\ \mathrm{C} & -0.7089340 & -2.7266530 & -1.0318650 \\ \mathrm{C} & 1.6384620 & -2.7486530 & -0.3086450 \\ \mathrm{C} & 1.6221220 & -4.1422360 & -0.4047250 \\ \mathrm{H} & 2.5351650 & -4.6928340 & -0.1859970 \\ \mathrm{C} & -2.3899890 & -1.0261620 & -0.3782290 \\ \mathrm{C} & -3.5425000 & -0.2598690 & -0.5486730 \\ \mathrm{C} & -1.9146410 & -1.9069630 & -1.3527830 \\ \mathrm{C} & -4.2100170 & -0.3962550 & -1.7638010 \\ \mathrm{H} & -5.1229200 & 0.1766070 & -1.9190370 \\ \mathrm{C} & 2.8715160 & -2.0186160 & 0.0980300 \\ \mathrm{C} & -4.0482200 & 0.6582570 & 0.5091550 \\ \mathrm{C} & 3.5188940 & -1.1525140 & -0.7963370 \\ \mathrm{C} & 3.0812720 & -0.8112460 & -2.2002850 \\ \mathrm{C} & 5.2836530 & 0.3658290 & -1.5172670 \\ \mathrm{C} & 4.7197750 & -0.5216420 & -0.4377250 \\ \mathrm{C} & 2.9006990 & 0.7190470 & -2.2788730 \\ \mathrm{H} & 2.6106860 & 0.9981880 & -3.2985230 \\ \mathrm{H} & 2.0736050 & 0.9982870 & -1.6214510 \\ \mathrm{C} & 4.2187470 & 1.4271730 & -1.8682330 \\ \mathrm{H} & 4.5896450 & 2.0637330 & -2.6805880\end{array}$




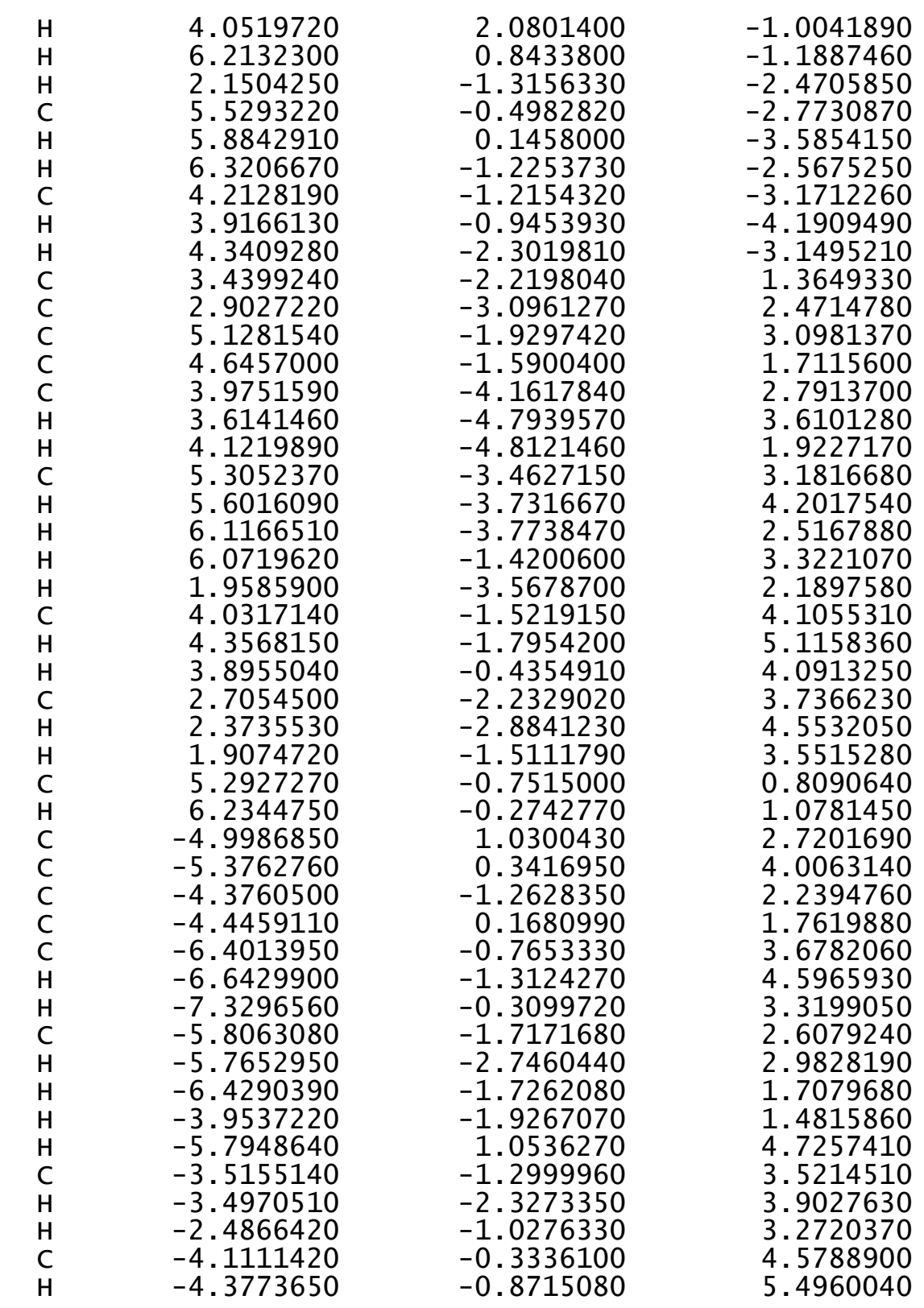

-3.3821580
-4.7633640
-4.8722320
-3.7269470
-4.1893540
-3.4530630
-3.5332120
-2.8906280
-2.7397040
-2.3536660
-1.8929720
-3.2334690
-5.3653010
-4.9434250
-4.5926120
-5.6233250
-5.6724410
-5.7728070
-6.6822380
-5.1712920
-5.6155260
-3.7394740
-2.5684680
-4.5397490
-3.7410330
-2.9802980
-1.9615040
-4.4133110
-5.4076430
-4.9450820
-3.6891310
-2.4698090
-1.4516910
-1.1748210
-3.0214040
0.4648230
0.4135000
-0.2633900
-1.6946070
-1.9403700
-0.7109420
-0.1600310
-0.2777570
-2.4270230
-2.2302920
1.4219600

0.4343450

2.8792600

4. 3132710

2.7732210

2.0298060

4.8416140

5. 8448110

4. 9382870

3.8681150

4.4004270

3.3830230

2. 1075810

4.9293310

3. 4710910

4. 0923220

2. 7238560

4.3363050

5. 3718410

3. 9561750

2. 3824470

3. 0445400

$-1.2160300$

$-1.9648510$

$-1.2953930$

$-1.8446520$

$-3.0976980$

$-2.7425450$

$-2.0559670$

$-1.9469840$

$-0.3051740$

$-3.8412670$

$-3.5558000$

$-3.6386290$

$-2.1155460$

$-1.0907770$

$-4.8322570$

$-6.3351890$

$-6.7208420$

$-6.1508590$

$-4.9744150$

$-4.1299370$

$-6.7554430$

$-7.8084720$

$-6.9326520$

$-5.3923270$

$-6.7559750$
4.8543910

1. 2099550

0.7582730

$-0.9789120$

0.2521680

0.4591960

0.0241890

1. 3928500

$-0.5130250$

$-1.3898200$

$-0.0230600$

$-1.6914850$

1. 5177640

$-1.6249590$

$-2.4570530$

$-2.0473360$

$-0.5625750$

$-0.9065420$

$-0.3799920$

2. 4428030

3. 1846160

$-2.7911080$

$-2.5993940$

$-4.0785440$

$-5.2585110$

$-4.8322210$

$-3.7527690$

$-6.0962210$

$-3.9047700$

$-4.3142100$

$-4.4412190$

$-5.6856540$

$-3.3954300$

$-4.1978400$

$-5.6032720$

$-0.7487270$

$-0.8543690$

$-2.1780630$

$-2.2705750$

$-1.2972300$

$-1.0544580$

$-0.0157180$

$-2.2970890$

$-2.0461050$

$-0.3233380$

$-0.7940890$ 


\begin{tabular}{|c|c|c|c|c|c|c|c|}
\hline & & & & & & & \\
\hline $\mathrm{H}$ & 0.3486920 & -6.3190460 & -2.9929890 & $\mathrm{C}$ & 1.6989290 & -4.0505490 & -0.8867320 \\
\hline $\mathrm{H}$ & -1.8945760 & -5.8292730 & -3.2983300 & $\mathrm{H}$ & 2.6170250 & -4.6073350 & -0.7045770 \\
\hline $\mathrm{H}$ & -2.7869230 & -4.3695300 & -1.6304880 & $\mathrm{C}$ & -2.3706420 & -1.0421190 & -0.5829080 \\
\hline C & 1.5847680 & 3.4782170 & -1.0211640 & $\mathrm{C}$ & -3.5213550 & -0.2649600 & -0.6909400 \\
\hline 0 & 0.4704540 & 2.8723830 & -1.3074240 & $\mathrm{C}$ & -1.8512520 & -1.8007090 & -1.6423660 \\
\hline C & 2.2331060 & 4.1522390 & -2.1805910 & $\mathrm{C}$ & -4.1518070 & -0.2316820 & -1.9386410 \\
\hline C & 3.3813210 & 4.9167750 & -1.9640050 & $\mathrm{H}$ & -5.0541680 & 0.3661480 & -2.0518250 \\
\hline C & 1.7294790 & 3.9862780 & -3.4726780 & $\mathrm{C}$ & 2.8972730 & -1.9847380 & -0.1045270 \\
\hline C & 4.0251050 & 5.5177640 & -3.0388550 & $\mathrm{C}$ & -4.0643770 & 0.5015880 & 0.4648110 \\
\hline $\mathrm{H}$ & 3.7594950 & 5.0236360 & -0.9524450 & $\mathrm{C}$ & 3.5460150 & -1.0099960 & -0.8773040 \\
\hline C & 2.3801830 & 4.5850050 & -4.5465570 & $\mathrm{C}$ & 3.1334290 & -0.5197630 & -2.2439230 \\
\hline $\mathrm{H}$ & 0.8408960 & 3.3833090 & -3.6240420 & $\mathrm{C}$ & 5.2908490 & 0.6168060 & -1.3739840 \\
\hline C & 3.5254310 & 5.3495520 & -4.3301500 & $\mathrm{C}$ & 4.7228100 & -0.3999430 & -0.4176010 \\
\hline $\mathrm{H}$ & 4.9168440 & 6.1136130 & -2.8733760 & $\mathrm{C}$ & 2.9178430 & 1.0049840 & -2.1511140 \\
\hline $\mathrm{H}$ & 1.9953680 & 4.4541470 & -5.5527200 & $\mathrm{H}$ & 2.6419050 & 1.3936250 & -3.1382850 \\
\hline $\mathrm{H}$ & 4.0312550 & 5.8152390 & -5.1704080 & $\mathrm{H}$ & 2.0712320 & 1.1884610 & -1.4848430 \\
\hline $\mathrm{H}$ & 0.0869890 & 2.2687960 & -0.5623080 & $\mathrm{C}$ & 4.2099500 & 1.6891120 & -1.6314780 \\
\hline 0 & 2.1200480 & 3.5181270 & 0.0948980 & $\mathrm{H}$ & 4.5848450 & 2.4190170 & -2.3590000 \\
\hline C & 2.7858900 & 1.6017130 & 1.9479510 & $\mathrm{H}$ & 4.0082440 & 2.2388980 & -0.7052520 \\
\hline C & 1.7407560 & 2.6334270 & 1.9442230 & $\mathrm{H}$ & 6.2008050 & 1.0729930 & -0.9690840 \\
\hline $\mathrm{H}$ & 2.7121940 & 0.8397310 & 1.1632170 & $\mathrm{H}$ & 2.2211810 & -1.0094410 & -2.5937530 \\
\hline $\mathrm{H}$ & 0.7490670 & 2.3303510 & 1.6266630 & $\mathrm{C}$ & 5.5866970 & -0.0938110 & -2.7126090 \\
\hline 0 & 2.0984070 & 1.2059040 & 3.1536700 & $\mathrm{H}$ & 5.9472390 & 0.6452370 & -3.4368500 \\
\hline $\mathrm{H}$ & 1.3985120 & 0.5344150 & 2.8533920 & $\mathrm{H}$ & 6.3885110 & -0.8243470 & -2.5693310 \\
\hline C & 4.2548900 & 3.1847750 & 3.2615990 & $\mathrm{C}$ & 4.2965380 & -0.7858840 & -3.2252310 \\
\hline C & 3.3578680 & 4.3474540 & 2.8359260 & $\mathrm{H}$ & 4.0183820 & -0.4050110 & -4.2141790 \\
\hline C & 1.9012440 & 3.8956850 & 2.7282390 & $\mathrm{H}$ & 4.4487910 & -1.8648260 & -3.3260360 \\
\hline $\mathrm{c}$ & 4.2009750 & 20910 & 2.2342220 & C & 3.4390440 & -2.3185720 & 1.1458240 \\
\hline H & 5.2890690 & 3.5234280 & 3.3750950 & $\mathrm{C}$ & 2.8945140 & -3.3249960 & 2.1315010 \\
\hline $\mathrm{H}$ & 3.4281930 & 5.1753630 & 3.5473450 & $\mathrm{C}$ & 5.0798520 & -2.1973320 & 2.9430360 \\
\hline $\mathrm{H}$ & 1.4827400 & 3.6936520 & 3.7215760 & $\mathrm{C}$ & 4.6222470 & -1.7096040 & 1.5927810 \\
\hline $\mathrm{H}$ & 4.6412270 & 2.3781960 & 1.2827010 & $\mathrm{C}$ & 3.9810840 & -4.4006870 & 2.3559740 \\
\hline $\mathrm{H}$ & 4.7712900 & 1.1821420 & 2.5722170 & $\mathrm{H}$ & 3.6149670 & -5.1271760 & 3.0898070 \\
\hline $\mathrm{H}$ & 3.9302310 & 2.8049410 & 4.2380520 & $\mathrm{H}$ & 4.1606250 & -4.9466230 & 1.4238790 \\
\hline $\mathrm{H}$ & 3.6851970 & 4.7241200 & 1.8598090 & $\mathrm{C}$ & 5.2875370 & -3.7261590 & 2.8546830 \\
\hline $\mathrm{H}$ & 1.2653490 & 4.6562530 & 2.2681870 & $\mathrm{H}$ & 5.5679670 & -4.1050270 & 3.8438830 \\
\hline & & & & $\mathrm{H}$ & 6.1192080 & -3.9436260 & 2.1777150 \\
\hline & , R) $G[M 06-2 x$ & $G(d)]=-35$ & +98 & $\mathrm{H}$ & 6.0074250 & -1.6995470 & 3.2473280 \\
\hline $\mathrm{P}$ & -0.3527750 & -0.1852800 & 0.7392700 & $\mathrm{H}$ & 1.9667220 & -3.7777480 & 1.7739680 \\
\hline 0 & -0.6464170 & 1.2833710 & 0.5477030 & C & 3.9526290 & -1.9287300 & 3.9631340 \\
\hline 0 & 0.3517680 & -0.6368120 & 1.9831670 & $\mathrm{H}$ & 4.2604840 & -2.3119730 & 4.9427150 \\
\hline 0 & 0.4431380 & -0.6398200 & -0.6140690 & $\mathrm{H}$ & 3.7944890 & -0.8506540 & 4.0716720 \\
\hline 0 & -1.7048930 & -1.0764250 & 0.6340030 & $\mathrm{C}$ & 2.6499740 & -2.6157450 & 3.4815170 \\
\hline C & 0.4999480 & -2.0007350 & -0.8766230 & $\mathrm{H}$ & 2.3126960 & -3.3622000 & 4.2095960 \\
\hline C & -0.6355600 & -2.6293000 & -1.3946950 & $\mathrm{H}$ & 1.8420130 & -1.8917090 & 3.3598450 \\
\hline C & 1.6894390 & 834270 & -0.6248090 & $\mathrm{C}$ & 5.2711940 & -0.7603380 & 0.8091240 \\
\hline
\end{tabular}




$\begin{array}{ll}\mathrm{H} & 6.1951740 \\ \mathrm{C} & -5.0568250 \\ \mathrm{C} & -5.4392450 \\ \mathrm{C} & -4.3972980 \\ \mathrm{C} & -4.4762910 \\ \mathrm{C} & -6.4448520 \\ \mathrm{H} & -6.6825120 \\ \mathrm{H} & -7.3783680 \\ \mathrm{C} & -5.8279230 \\ \mathrm{H} & -5.7825320 \\ \mathrm{H} & -6.4390870 \\ \mathrm{H} & -3.9586130 \\ \mathrm{H} & -5.8751440 \\ \mathrm{C} & -3.5551530 \\ \mathrm{H} & -3.5399620 \\ \mathrm{H} & -2.5233600 \\ \mathrm{C} & -4.1703650 \\ \mathrm{H} & -4.4362430 \\ \mathrm{H} & -3.4530010 \\ \mathrm{C} & -4.8309580 \\ \mathrm{C} & -4.9604870 \\ \mathrm{C} & -3.7562560 \\ \mathrm{C} & -4.2249090 \\ \mathrm{C} & -3.5472430 \\ \mathrm{H} & -3.6389970 \\ \mathrm{H} & -3.0021910 \\ \mathrm{C} & -2.8001230 \\ \mathrm{H} & -2.4153710 \\ \mathrm{H} & -1.9486750 \\ \mathrm{H} & -3.2365710 \\ \mathrm{H} & -5.4785660 \\ \mathrm{C} & -4.9763670 \\ \mathrm{H} & -4.6255200 \\ \mathrm{H} & -5.6355830 \\ \mathrm{C} & -5.7382260 \\ \mathrm{H} & -5.8507700 \\ \mathrm{H} & -6.7441240 \\ \mathrm{C} & -5.2483720 \\ \mathrm{H} & -5.7149380 \\ \mathrm{C} & -2.4822590 \\ \mathrm{C} & -1.9388670 \\ \mathrm{C} & -1.8405680 \\ \mathrm{C} & -3.1888900 \\ \mathrm{C} & -4.2477050 \\ \mathrm{C} & -3.6381750 \\ \mathrm{H} & -2.6110040 \\ & \end{array}$

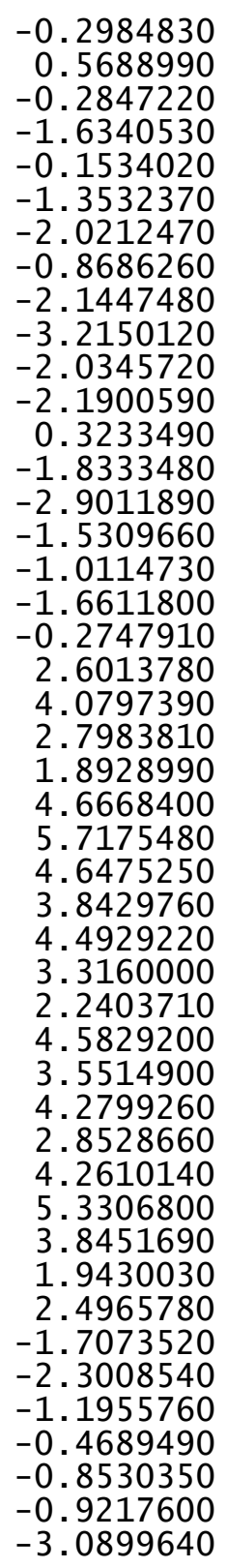

1.1555270
2.6881940
3.8696580
1.9235110
1.6356010
3.3893330
4.2250570
3.0875210
2.2069190
2.4376990
1.3057610
1.0917290
4.6696070
3.2028450
3.4494540
3.0061060
4.3659880
5.2076940
4.7399670
1.4342010
1.1701840
-0.7294210
0.3857590
0.9704180
0.6709620
1.9190690
-0.1087470
-0.9029420
0.3265200
-1.5124020
1.9935130
-1.3029340
-2.0429630
-1.8286130
-0.1517730
-0.3611810
-0.0391870
2.5853870
3.3989330
-2.8905210
-4.1664100
-5.2341290
-5.4591220
-4.4050070
-3.0297780
-4.5320860

\author{
$-1.4765770$ \\ $-3.5850580$ \\ $-4.6621810$ \\ $-0.9595070$ \\ $-1.0833000$ \\ $-3.0319780$ \\ $-5.0820890$ \\ $-0.6144430$ \\ $-1.8743920$ \\ $-1.8219050$ \\ $-0.5274620$ \\ 0.6625620 \\ 0.5707890 \\ $-2.6973550$ \\ $-2.0215640$ \\ $-2.7419960$ \\ $-0.4553770$ \\ $-0.5144400$ \\ 1. 6022410 \\ 0.7246320 \\ $-1.8618460$ \\ 1. 5009370 \\ 0.4084540 \\ 2. 1522160 \\ 3. 2794940 \\ 1. 6719390 \\ 3.9258700 \\ 3. 6398950 \\ 2. 3251120 \\ 0.7991660 \\ 3. 4497550 \\ 4.8015680 \\ 1.9582510 \\ 3.9579440 \\ 0.0238720 \\ 2. 0143070 \\ 2. 6847420 \\ 1. 6192510 \\ 2.6405530 \\ 0.6400310 \\ 1.9831890 \\ 1. 3041930 \\ 4.0966000 \\ 3. 1846800 \\ 1. 7398030 \\ 4.0848220
}

$\begin{array}{rr}-1.6282220 & -6.1709680 \\ -0.6939970 & -6.4541850 \\ -1.8369660 & -4.6631780 \\ -2.7588790 & -4.0029830 \\ -0.4787070 & -4.9011970 \\ 0.6131590 & -5.4206220 \\ -0.1455440 & -4.4242340 \\ -4.0252240 & -1.5956570 \\ -4.7578300 & -2.0179890 \\ -6.2507860 & -1.6984080 \\ -6.8448410 & -2.2463550 \\ -6.2397820 & -1.5062020 \\ -4.7333240 & -1.3445740 \\ -6.7481890 & -2.1277540 \\ -4.6462070 & -3.0996820 \\ -4.2878160 & -1.5424860 \\ -6.6199800 & -3.3186860 \\ -7.9345080 & -2.1437860 \\ -6.4910440 & -2.0113450 \\ -6.6895780 & -0.5057300 \\ -6.4028950 & -0.6119590 \\ 3.5664000 & -0.6182330 \\ 2.9699830 & -0.9942870 \\ 4.3824780 & -1.6807340 \\ 5.1404070 & -1.3571820 \\ 4.3572460 & -2.9919740 \\ 5.8746730 & -2.3441110 \\ 5.1375130 & -0.3335540 \\ 5.0894320 & -3.9780350 \\ 3.7582110 & -3.2273990 \\ 5.8468330 & -3.6547920 \\ 6.4653740 & -2.0953820 \\ 5.0682990 & -4.9990790 \\ 6.4167350 & -4.4266230 \\ 2.2817420 & -0.3269850 \\ 3.4930540 & 0.5063330 \\ 1.3950680 & 2.1501030 \\ 2.4017860 & 2.2394970 \\ 0.7241710 & 1.2841580 \\ 2.1178720 & 1.8699920 \\ 0.8546270 & 3.2898430 \\ 0.2070850 & 2.9023500 \\ 2.8492020 & 3.6608450 \\ 4.0355780 & 3.3472500 \\ 3.5723470 & 3.1606600 \\ 1.8363290 & 2.5139190\end{array}$




\begin{tabular}{|c|c|c|c|c|c|c|}
\hline 5.1214520 & 3.1915160 & 3.8327740 & C & 2.9733470 & -3.2738130 & 2.1535820 \\
\hline 3.2252820 & 4.7811570 & 4.1466040 & C & 5.1402870 & -2.0967400 & 2.9443040 \\
\hline 1.3069880 & 3.2543720 & 4.1168290 & C & 4.6637610 & -1.6259130 & 1.5945020 \\
\hline 4.5362150 & 2.2735480 & 1.6135570 & C & 4.0833850 & -4.3255060 & 2.3765950 \\
\hline 4.6662320 & 0.9448430 & 2.7656740 & $\mathrm{H}$ & 3.7373440 & -5.0552130 & 3.1169500 \\
\hline 3.7610590 & 2.3578450 & 4.5823460 & $\mathrm{H}$ & 4.2676280 & -4.8729010 & 1.4462660 \\
\hline 3.5222910 & 4.5233200 & 2.4253500 & C & 5.3790700 & -3.6214590 & 2.8623900 \\
\hline 1.0971600 & 4.3673580 & 2.7739570 & $\mathrm{H}$ & 5.6744160 & -3.9891720 & 3.8514540 \\
\hline R) $\mathrm{G}[\mathrm{M06}-2$ & $\xi(d)]=-35$ & 385 & $\begin{array}{l}\mathrm{H} \\
\mathrm{H}\end{array}$ & $\begin{array}{l}0.210<070 \\
6.0593410\end{array}$ & $\begin{array}{l}-3.8251 / 10 \\
-1.5783490\end{array}$ & $\begin{array}{l}2.1805050 \\
3.2399180\end{array}$ \\
\hline-0.3566260 & -0.2022840 & 0.7674270 & $\mathrm{H}$ & 2.0530760 & -3.7478610 & 1.8043200 \\
\hline-0.6708180 & 1.2606920 & 0.5654170 & C & 4.0144840 & -1.8464530 & 3.9707100 \\
\hline 0.3559000 & -0.6348800 & 2.0133720 & $\mathrm{H}$ & 4.3366340 & -2.2190700 & 4.9497930 \\
\hline 0.4467940 & -0.6541370 & -0.5828010 & $\mathrm{H}$ & 3.8350820 & -0.7713900 & 4.0759390 \\
\hline-1.6986780 & -1.1101760 & 0.6682140 & C & 2.7227610 & -2.5618140 & 3.5009890 \\
\hline 0.5291960 & -2.0149890 & -0.8373810 & $\mathrm{H}$ & 2.4042620 & -3.3102540 & 4.2354380 \\
\hline-0.5994260 & -2.6708070 & -1.3375870 & $\mathrm{H}$ & 1.9001390 & -1.8542560 & 3.3799960 \\
\hline 1.7359240 & -2.6707110 & -0.5973250 & C & 5.2878660 & -0.6682610 & 0.8008990 \\
\hline 1.7749150 & -4.0370240 & -0.8626780 & $\mathrm{H}$ & 6.2046260 & -0.1857400 & 1.1381900 \\
\hline 2.7085270 & -4.5714520 & -0.6940320 & C & -5.1131510 & 0.5346360 & 2.6537090 \\
\hline-2.3553240 & -1.0894420 & -0.5547600 & C & -5.5181320 & -0.3152920 & 3.8302790 \\
\hline-3.5013280 & -0.3080920 & -0.6866470 & $\mathrm{C}$ & -4.4171880 & -1.6668880 & 1.9185830 \\
\hline-1.8245050 & -1.8588720 & -1.5988260 & $\mathrm{C}$ & -4.5013290 & -0.1886370 & 1.6196010 \\
\hline-4.1092040 & -0.2788510 & -1.9454170 & C & -6.5031010 & -1.3942980 & 3.3312970 \\
\hline-5.0068630 & 0.3221000 & -2.0776320 & $\mathrm{H}$ & -6.7579040 & -2.0584420 & 4.1650380 \\
\hline 2.9328360 & -1.9453330 & -0.0886580 & $\mathrm{H}$ & -7.4317370 & -0.9185010 & 3.0017180 \\
\hline-4.0667830 & 0.4632140 & 0.4551250 & C & -5.8494500 & -2.1890710 & 2.1710250 \\
\hline 3.5563660 & -0.9624790 & -0.8716470 & $\mathrm{H}$ & -5.7993710 & -3.2569690 & 2.4116900 \\
\hline 3.1241520 & -0.4890250 & -2.2381530 & $\mathrm{H}$ & -6.4391220 & -2.0923570 & 1.2541430 \\
\hline 5.2649120 & 0.6952060 & -1.3908200 & $\mathrm{H}$ & -3.9537140 & -2.2246470 & 1.1017130 \\
\hline 4.7239440 & -0.3263720 & -0.4241140 & $\mathrm{H}$ & -5.9787180 & 0.2938290 & 4.6154420 \\
\hline 2.8793040 & 1.0317640 & -2.1529560 & C & -3.6039160 & -1.8494770 & 3.2189000 \\
\hline 2.5886460 & 1.4089400 & -3.1403650 & $\mathrm{H}$ & -3.5810110 & -2.9157130 & 3.4716980 \\
\hline 2.0340500 & 1.2024530 & -1.4817220 & $\mathrm{H}$ & -2.5 & -1.5346780 & 3.0452180 \\
\hline 4.1612350 & 1.7444100 & -1.6465450 & C & -4.2566140 & -1.0291660 & 4.3625340 \\
\hline 4.5163370 & 2.4775240 & -2.3807570 & $\mathrm{H}$ & -4.5391860 & -1.6784130 & 5.1991440 \\
\hline 3.9553880 & 2.2952110 & -0.7218070 & $\mathrm{H}$ & -3.5557760 & -0.2851350 & 4.7528950 \\
\hline 6.1686640 & 1.1716610 & -0.9954630 & C & -4.8733160 & 2.5619140 & 1.3942130 \\
\hline 2.2190320 & -0.9986280 & -2.5780590 & C & -5.0105650 & 4.0374340 & 1.1183780 \\
\hline 5.5649620 & -0.0177040 & -2.7272980 & C & -3.7429740 & 2.7573490 & -0.7408050 \\
\hline 5.9054820 & 0.7239040 & -3.4585830 & C & -4.2346430 & 1.8531090 & 0.3654710 \\
\hline 6.3821650 & -0.7312820 & -2.5855460 & $\mathrm{C}$ & -3.5987310 & 4.6382050 & 0.9527600 \\
\hline 4.2851580 & -0.7384000 & -3.2262760 & $\mathrm{H}$ & -3.6934230 & 5.6851760 & 0.6413070 \\
\hline 3.9924390 & -0.3694120 & -4.2155270 & $\mathrm{H}$ & -3.0798290 & 4.6333120 & 1.9161350 \\
\hline 4.4580150 & -1.8147490 & -3.3214450 & C & -2.8136670 & 3.8129340 & -0.0977230 \\
\hline 3.4902820 & -2.2615340 & 1.1592510 & $\mathrm{H}$ & -2.4085250 & 4.4601800 & -0.8838980 \\
\hline
\end{tabular}




$\begin{array}{rrr}-1.9726670 & 3.2945660 & 0.3671550 \\ -3.1966310 & 2.2003060 & -1.5062570 \\ -5.5558220 & 4.5397410 & 1.9245160 \\ -4.9522430 & 3.4986080 & -1.3516850 \\ -4.5857530 & 4.2290380 & -2.0821170 \\ -5.5895070 & 2.7937620 & -1.8957650 \\ -5.7541600 & 4.2031800 & -0.2250350 \\ -5.8745960 & 5.2703860 & -0.4424480 \\ -6.7573540 & 3.7748550 & -0.1379340 \\ -5.3143370 & 1.9062630 & 2.5380660 \\ -5.8067400 & 2.4600740 & 3.3360780 \\ -2.4336620 & -1.7706530 & -2.8589120 \\ -1.8853140 & -2.4000130 & -4.1162410 \\ -1.7559850 & -1.3228710 & -5.2088400 \\ -3.0813860 & -0.5578490 & -5.4477030 \\ -4.1649210 & -0.9162380 & -4.4104530 \\ -3.5803880 & -0.9784320 & -3.0239480 \\ -2.5659770 & -3.1873440 & -4.4715580 \\ -1.4089210 & -1.7888780 & -6.1360150 \\ -3.4696250 & -0.7654860 & -6.4496110 \\ -4.5895260 & -1.8965250 & -4.6664520 \\ -0.9175690 & -2.8745470 & -3.9316360 \\ -0.9751680 & -0.6227840 & -4.8952740 \\ -2.8955070 & 0.5193060 & -5.3996590 \\ -4.9878550 & -0.1963620 & -4.4521270 \\ -0.5497500 & -4.0656090 & -1.5332550 \\ 0.6577500 & -4.7451310 & -1.3078120 \\ -1.8124860 & -4.8389710 & -1.8686500 \\ -1.5356340 & -6.2288150 & -2.4370140 \\ -0.5415640 & -6.9716020 & -1.5491310 \\ 0.7960220 & -6.2363870 & -1.5523590 \\ -2.4755890 & -6.7832240 & -2.5237220 \\ -2.3922600 & -4.9405520 & -0.9394640 \\ -2.4484110 & -4.2723460 & -2.5497440 \\ -0.9373190 & -7.0185380 & -0.5263370 \\ -0.4014280 & -8.0024660 & -1.8897890 \\ 1.4745300 & -6.6627320 & -0.8054100 \\ 1.2801600 & -6.3868640 & -2.5276380 \\ -1.1200370 & -6.1366980 & -3.4499150 \\ 1.4239200 & 3.5738550 & -0.6275590 \\ 0.3441790 & 2.9510530 & -0.9973890 \\ 2.0535030 & 4.3988460 & -1.6962630 \\ 3.1662310 & 5.1813420 & -1.3809490 \\ 1.5675570 & 4.3587240 & -3.0050380 \\ 3.7922970 & 5.9253540 & -2.3736330 \\ 3.5314670 & 5.1897400 & -0.3590570\end{array}$

2.2003900
0.7063340
3.3104530
4.6566750
1.8290630
3.8027520
-0.0247270
1.9419730
2.6594540
1.5770300
2.6219240
0.6008210
1.9752320
1.3046960
4.0544450
3.1199140
1.6824030
4.0536450
5.0741340
3.1519830
1.2606130
4.4905980
4.6532430
3.7333360
3.4433790
1.0235270

5.1008950

3.7407600

5.8828710

6.5351090

5.0684950

6.4605780

2. 2608750

3. 5179600

1.4405800

2.4289820

0.7642780

2.1258940

0.8939080

0.2323300

2. 9278640

4.0963600

3. 6066820

1.9092130

3. 2890310

4.8465110

3. 2865250

2. 3502540

1.0299560

2.4352300

4.5853640

4. 3879450
$-3.9968460$

$-3.2339770$

$-3.6818290$

$-2.1313620$

$-5.0159790$

$-4.4581320$

$-0.3229220$

0.4958990

2.1478660

2. 2384360

1. 2857750

1.8763210

3. 2951140

2. 9169810

3. 6418820

3. 3280020

3. 1522120

2. 5000250

3. 8057660

4. 1234510

4.1125940

1.5944220

2.7519270

4.5678510

2.4017050

2.7647820

154

$\operatorname{TS} 159(R, R) \quad G[M 06-2 X / 6-31 G(d)]=-3541.254586$

$\begin{array}{lrrr}\text { TS159(R, R) } \text { G }[\mathrm{M} 06-2 \mathrm{~V} / 6-31 \mathrm{G}(\mathrm{d})]=-3541.254586 \\ \mathrm{P} & -0.3266970 & -0.0918800 & 0.7937820 \\ \mathrm{O} & -0.6110400 & 1.3526540 & 0.4589810 \\ \mathrm{O} & 0.3992460 & -0.4237450 & 2.0626280 \\ \mathrm{O} & 0.4389900 & -0.6911930 & -0.5199070 \\ \mathrm{O} & -1.6919290 & -0.9711330 & 0.8073920 \\ \mathrm{C} & 0.4819640 & -2.0730240 & -0.6375850 \\ \mathrm{C} & -0.6786310 & -2.7390340 & -1.0572390 \\ \mathrm{C} & 1.6716430 & -2.7342110 & -0.3411070 \\ \mathrm{C} & 1.6756040 & -4.1266840 & -0.4561320 \\ \mathrm{H} & 2.5968150 & -4.6665740 & -0.2449870 \\ \mathrm{C} & -2.3764740 & -1.0578380 & -0.3965170 \\ \mathrm{C} & -3.5224290 & -0.2830090 & -0.5754830 \\ \mathrm{C} & -1.8885910 & -1.9253070 & -1.3754190 \\ \mathrm{C} & -4.1561000 & -0.3827140 & -1.8115970 \\ \mathrm{H} & -5.0591000 & 0.2026880 & -1.9783690 \\ \mathrm{C} & 2.8944580 & -1.9926280 & 0.0749360 \\ \mathrm{C} & -4.0531270 & 0.6072530 & 0.4937360 \\ \mathrm{C} & 3.5278690 & -1.1033010 & -0.8063770\end{array}$




\begin{tabular}{|c|c|c|}
\hline $\begin{array}{l}3.0837590 \\
5.2674620 \\
4.7189030 \\
2.8782810 \\
2.5826520 \\
2.0474180 \\
4.1853170 \\
4.5453640 \\
4.0091350 \\
6.1895120 \\
2.1607370 \\
5.5258910 \\
5.8696840 \\
6.3290190 \\
4.2207250 \\
3.9193460 \\
4.3663330 \\
3.4669270 \\
2.9436840 \\
5.1512150 \\
4.6629820 \\
4.0322790 \\
3.6812260 \\
4.1881900 \\
5.3520060 \\
5.6538820 \\
6.1671850 \\
6.0871740 \\
2.0069140 \\
4.0491380 \\
4.3786680 \\
3.8967560 \\
2.7333520 \\
2.4101770 \\
1.9254700 \\
5.2961690 \\
6.2303010 \\
5.0447330 \\
5.4392750 \\
4.3944410 \\
4.4681930 \\
6.4493550 \\
6.7042230 \\
7.3740280 \\
5.8265620 \\
5\end{array}$ & $\begin{array}{r}-0.7472220 \\
0.4540410 \\
-0.4592840 \\
0.7809540 \\
1.0715820 \\
1.0360680 \\
1.5035640 \\
2.1582690 \\
2.1405480 \\
0.9413000 \\
-1.2623730 \\
-0.3860560 \\
0.2764210 \\
-1.1034610 \\
-1.1179750 \\
-0.8368360 \\
-2.2025220 \\
-2.2049410 \\
-3.1058800 \\
-1.9150700 \\
-1.5619310 \\
-4.1596060 \\
-4.8082310 \\
-4.7957730 \\
-3.4461770 \\
-3.7258810 \\
-3.7346910 \\
-1.3943700 \\
-3.5881520 \\
-1.5395740 \\
-1.8243530 \\
-0.4552150 \\
-2.2640490 \\
-2.9314970 \\
-1.5510510 \\
-0.6993920 \\
-0.2113690 \\
0.9201010 \\
0.1995420 \\
-1.3558490 \\
0.0850370 \\
-0.9074110 \\
-1.4764730 \\
-0.4508640 \\
-1.8307170 \\
-2.8668660\end{array}$ & $\begin{array}{l}-2.2045060 \\
-1.5043070 \\
-0.4385370 \\
-2.2586810 \\
-3.2734810 \\
-1.5962460 \\
-1.8379940 \\
-2.6407450 \\
-0.9639200 \\
-1.1689020 \\
-2.4818140 \\
-2.7738160 \\
-3.5760850 \\
-2.5803050 \\
-3.1824000 \\
-4.1975850 \\
-3.1776810 \\
1.3382100 \\
2.4316170 \\
3.0751860 \\
1.6940110 \\
2.7361690 \\
3.5463380 \\
1.8586320 \\
3.1354410 \\
4.1510010 \\
2.4649930 \\
3.3066730 \\
2.1431900 \\
4.0890460 \\
5.0947900 \\
4.0923490 \\
3.7090270 \\
4.5159290 \\
3.5334330 \\
0.8043160 \\
1.0805110 \\
2.6961440 \\
3.9593530 \\
2.1746840 \\
1.7281070 \\
3.5874560 \\
4.4887370 \\
3.2214610 \\
2.5082640 \\
2.8621450\end{array}$ \\
\hline
\end{tabular}

-6.4333240
-3.9535850
-5.8764490
-3.5552670
-3.5315690
-2.5255820
-4.1792370
-4.4579970
-3.4623300
-4.7998830
-4.9148530
-3.7243780
-4.2016460
-3.4961760
-3.5787200
-2.9511370
-2.7564660
-2.3603480
-1.9137670
-3.2113270
-5.4274030
-4.9358600
-4.5762060
-5.6011880
-5.6918610
-5.7960600
-6.7010120
-5.2246280
-5.6875940
-2.5108160
-1.9045570
-2.3979500
-3.9222100
-4.3749590
-3.6616790
-2.0373990
-2.1564280
-0.8121820
-4.3295420
-4.3119650
-5.4568330
-4.1851160
-1.9856900
0.5276250
0.4975870
-0.2027740
1. 5974510

1. 4100470

4.6870370

3. 4700190

3. 8276130

3. 2441540

4. 5392420

5.4376740

4.8473960

1. 2330370

0.8129130

$-0.9385350$

0.2662090

0.5514600

0.1382620

1.4968960

$-0.4300640$

$-1.2875680$

0.0637140

$-1.6572750$

1. 5776870

$-1.5900520$

$-2.4009600$

$-2.0419530$

$-0.5210580$

$-0.8426650$

$-0.3653930$

2. 4468100

3. 1956630

$-2.6399050$

$-3.7713620$

$-5.1461440$

$-5.1443380$

$-4.1870320$

$-2.8481220$

$-5.9098990$

$-3.6454710$

$-3.7113390$

$-4.8222720$

$-6.1482260$

$-4.0198310$

$-4.6523120$

$-5.3873420$

$-0.8028360$

$-0.9056990$

$-2.2114630$ 


\begin{tabular}{|c|c|c|c|}
\hline 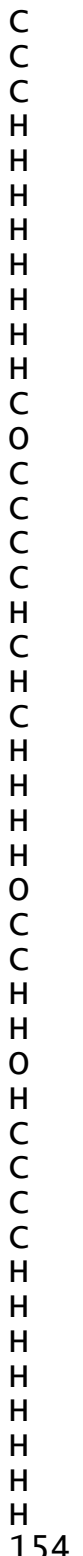 & $\begin{array}{r}-1.6474730 \\
-1.8840460 \\
-0.6600430 \\
-0.0501180 \\
-0.1962370 \\
-2.3529940 \\
-2.1482180 \\
1.5139390 \\
0.3777250 \\
-1.8879630 \\
-2.7444240 \\
1.5237680 \\
0.4215870 \\
2.1625660 \\
3.2974810 \\
1.6639110 \\
3.9329170 \\
3.6721340 \\
2.3063220 \\
0.7857830 \\
3.4382950 \\
4.8143280 \\
1.9254080 \\
3.9376970 \\
0.0462880 \\
2.0552710 \\
2.7478970 \\
1.6890660 \\
2.6827630 \\
0.7013140 \\
2.0681740 \\
1.3775450 \\
4.1973880 \\
3.2841480 \\
1.8336290 \\
4.1572020 \\
5.2270850 \\
3.3443040 \\
1.4187570 \\
4.5907610 \\
4.7404240 \\
3.8794770 \\
3.6049410 \\
1.1869970\end{array}$ & $\begin{array}{l}-6.1959770 \\
-5.0011070 \\
-4.1409710 \\
-6.7599070 \\
-7.8202760 \\
-6.9876730 \\
-5.4055640 \\
-6.7370490 \\
-6.3151030 \\
-5.9096420 \\
-4.4088370 \\
3.5004510 \\
2.8785390 \\
4.2036040 \\
4.9836160 \\
4.0502510 \\
5.6125830 \\
5.0803310 \\
4.6770300 \\
3.4350980 \\
5.4569560 \\
6.2204290 \\
4.5560740 \\
5.9445100 \\
2.2595140 \\
3.5334440 \\
1.5970910 \\
2.6145490 \\
0.8473350 \\
2.3031560 \\
1.1722030 \\
0.4953030 \\
3.1786350 \\
4.3359010 \\
3.8662800 \\
2.0623160 \\
3.5293190 \\
5.1529660 \\
3.6431050 \\
2.4102820 \\
1.1951080 \\
2.7784900 \\
4.7327780 \\
4.6252620\end{array}$ & $\begin{array}{l}-2.2646750 \\
-1.3111380 \\
-1.0992560 \\
-0.0529950 \\
-2.3328400 \\
-1.9933280 \\
-0.3240870 \\
-0.8663260 \\
-3.0413110 \\
-3.2932970 \\
-1.6301470 \\
-0.9569390 \\
-1.2553320 \\
-2.1043290 \\
-1.8732520 \\
-3.3998930 \\
-2.9369910 \\
-0.8593640 \\
-4.4627060 \\
-3.5628120 \\
-4.2317940 \\
-2.7601650 \\
-5.4715880 \\
-5.0634430 \\
-0.5187180 \\
0.1611430 \\
1.9828460 \\
1.9972990 \\
1.1856260 \\
1.6756100 \\
3.1830370 \\
2.8737680 \\
3.3197480 \\
2.9143450 \\
2.8008630 \\
2.2739650 \\
3.4373600 \\
3.6391000 \\
3.7912140 \\
1.3271260 \\
2.5964710 \\
4.2902970 \\
1.9441010 \\
2.3532710\end{array}$ \\
\hline
\end{tabular}

TS160 (R, R) G[M06-2X/6-31G(d)] = -3541.253451

$P \quad-0.2930490$

$0 \quad-0.4515630$

0.4039760

0.4083420

$-1.7247670$

0.3309640

$-0.8782670$

1.4576750

1.3519850

2.2200620

$-2.4314030$

$-3.5198490$

$-2.0310760$

$-4.2162790$

$-5.0807380$

2.7297400

$-3.9413620$

3.4609080

3.0839880

5.3516270

4.6966030

3.0202640

2.7720870

2.2035620

4.3791650

4.8162340

4.2434330

6.3062550

2. 1244050

5.5624720

5.9873050

6.2892940

4.2049530

3.9554060

4.2489980

3.2515420

2.6266460

4.9181710

4.4928850

3.6104150

3.1860480

3.7282560

4.9794070

5.2316870

5.7804920

5.8921980

\begin{tabular}{rr}
-0.0678270 & 0.6715260 \\
1.4143550 & 0.4321270 \\
-0.5415190 & 1.9111550 \\
-0.6478590 & -0.6843940 \\
-0.8298010 & 0.6406840 \\
-2.0175790 & -0.8907370 \\
-2.5527600 & -1.3603490 \\
-2.7957000 & -0.6339820 \\
-4.1720110 & -0.8520110 \\
-4.8004840 & -0.6621930 \\
-0.7851350 & -0.5520950 \\
0.0793350 & -0.6460060 \\
-1.6350690 & -1.5940030 \\
0.0964630 & -1.8577930 \\
0.7506700 & -1.9541240 \\
-2.1934810 & -0.1448030 \\
0.9434930 & 0.4920280 \\
-1.3085140 & -0.9511920 \\
-0.8219750 & -2.3292230 \\
0.1231820 & -1.5120830 \\
-0.8026340 & -0.5196570 \\
0.7187530 & -2.2830880 \\
1.1024100 & -3.2794700 \\
1.0048030 & -1.6156160 \\
1.2881120 & -1.7965820 \\
1.9532590 & -2.5510270 \\
1.8850410 & -0.8877080 \\
0.4997840 & -1.1286770 \\
-1.2300420 & -2.6558660 \\
-0.6550370 & -2.8291710 \\
0.0214160 & -3.5792690 \\
-1.4568200 & -2.6678310 \\
-1.2313210 & -3.3103950 \\
-0.8549230 & -4.3086480 \\
-2.3225790 & -3.3786590 \\
-2.5422450 & 1.1101860 \\
-3.4675820 & 2.1276240 \\
-2.5312660 & 2.8877520 \\
-2.0381770 & 1.5291010 \\
-4.6350250 & 2.3681120 \\
-5.3074910 & 3.1218040 \\
-5.2161690 & 1.4474710 \\
-4.0746860 & 2.8396030 \\
-4.4521320 & 3.8369150 \\
-4.3879710 & 2.1633190 \\
-2.1157110 & 3.1694800 \\
& \\
\hline
\end{tabular}




\begin{tabular}{|c|c|c|c|}
\hline $\begin{array}{l}\mathrm{H} \\
\mathrm{C} \\
\mathrm{H} \\
\mathrm{H} \\
\mathrm{C} \\
\mathrm{H} \\
\mathrm{H} \\
\mathrm{C} \\
\mathrm{H} \\
\mathrm{C} \\
\mathrm{C} \\
\mathrm{C} \\
\mathrm{C} \\
\mathrm{C} \\
\mathrm{H} \\
\mathrm{H} \\
\mathrm{C} \\
\mathrm{H} \\
\mathrm{H} \\
\mathrm{H} \\
\mathrm{H} \\
\mathrm{C} \\
\mathrm{H} \\
\mathrm{H} \\
\mathrm{C} \\
\mathrm{H} \\
\mathrm{H} \\
\mathrm{C} \\
\mathrm{C} \\
\mathrm{C} \\
\mathrm{C} \\
\mathrm{C} \\
\mathrm{H} \\
\mathrm{H} \\
\mathrm{C} \\
\mathrm{H} \\
\mathrm{H} \\
\mathrm{H} \\
\mathrm{H} \\
\mathrm{C} \\
\mathrm{H} \\
\mathrm{H} \\
\mathrm{C} \\
\mathrm{H} \\
\mathrm{H} \\
\mathrm{C}\end{array}$ & $\begin{array}{l}1.6562700 \\
3.8337180 \\
4.1166270 \\
3.7778590 \\
2.4672470 \\
2.0750430 \\
1.7263630 \\
5.2216200 \\
6.1904480 \\
-4.8247280 \\
-5.2311410 \\
-4.3904050 \\
-4.3561360 \\
-6.3450480 \\
-6.6111990 \\
-7.2416730 \\
-5.8449570 \\
-5.8774600 \\
-6.4824870 \\
-4.0356260 \\
-5.5797370 \\
-3.5121800 \\
-3.5661950 \\
-2.4702550 \\
-4.0123230 \\
-4.3030710 \\
-3.2211310 \\
-4.4755550 \\
-4.4832130 \\
-3.4909910 \\
-3.9839830 \\
-3.0335790 \\
-3.0441030 \\
-2.4480830 \\
-2.4157390 \\
-2.0111780 \\
-1.5963420 \\
-3.0627390 \\
-4.9136840 \\
-4.6647550 \\
-4.2859820 \\
-5.4080850 \\
-5.3034670 \\
-5.3282800 \\
-6.3364670 \\
-4.8979280\end{array}$ & $\begin{array}{l}-3.8403910 \\
-2.1291890 \\
-2.5096300 \\
-1.0382080 \\
-2.7064900 \\
-3.4018560 \\
-1.9153350 \\
-1.1788970 \\
-0.8001800 \\
1.2046760 \\
0.4454020 \\
-1.0818160 \\
0.3843230 \\
-0.5500550 \\
-1.1500950 \\
0.0019460 \\
-1.4539330 \\
-2.5100840 \\
-1.3424300 \\
-1.7129330 \\
1.1268670 \\
-1.2880090 \\
-2.3414100 \\
-1.0742050 \\
-0.3682870 \\
-0.9568700 \\
0.3172540 \\
3.1473430 \\
4.6173860 \\
3.1430830 \\
2.3380640 \\
5.0609810 \\
6.1005840 \\
5.0354960 \\
4.1222350 \\
4.6962620 \\
3.5388710 \\
2.5034470 \\
5.2043880 \\
3.9772130 \\
4.6340250 \\
3.3175130 \\
4.8080160 \\
5.8725120 \\
4.4933740 \\
2.5841970\end{array}$ & $\begin{array}{r}1.7915960 \\
3.9099270 \\
4.8980850 \\
3.9857730 \\
3.4620630 \\
4.2129270 \\
3.3327040 \\
0.7123780 \\
1.0364060 \\
2.7467310 \\
3.9832140 \\
2.0711660 \\
1.7102840 \\
3.5938170 \\
4.4714480 \\
3.2958510 \\
2.4369230 \\
2.7273390 \\
1.5543840 \\
1.2531370 \\
4.7666320 \\
3.3248070 \\
3.6226110 \\
3.0726460 \\
4.4696910 \\
5.3473900 \\
4.7877450 \\
1.3834920 \\
1.0505920 \\
-0.8313800 \\
0.3480760 \\
0.7605720 \\
0.4125220 \\
1.6846500 \\
-0.3064380 \\
-1.1479310 \\
0.1184070 \\
-1.6069920 \\
1.8689080 \\
-1.3889780 \\
-2.1804560 \\
-1.8485120 \\
-0.2440930 \\
-0.5029120 \\
-0.0670620 \\
2.5823280\end{array}$ \\
\hline
\end{tabular}

-5.2767540
-3.8206840
-4.5497660
-4.8297060
-3.5310090
-2.3308270
-2.7244400
-3.9378150
-5.3619890
-3.2621980
-1.9135920
-5.4814040
-5.5009120
-3.6996910
-1.5323810
-0.9608140
-2.2247030
-2.4675080
-1.2416470
-0.0058880
0.1632880
-2.1270460
-3.3487640
-1.4766200
-0.1373940
-3.0856010
-2.7092180
-0.9854530
0.8931770
1.8913170
0.7370270
2.6014700
3.8035060
2.0980880
4.5015740
4.1798050
2.8026230
1.1665330
4.0018470
5.4354670
2.4175240
4.5497760
0.3003160
2.4161780
2.9052980
1.9387120

3. 2152140

$-0.6886760$

$-0.6892310$

$-2.1390720$

$-2.9724740$

$-2.2933920$

$-1.5576820$

$-0.1968610$

$-2.1572060$

$-3.1469680$

$-1.5465790$

$-0.1204180$

$-2.5787330$

$-3.9576860$

$-3.0148860$

$-3.9423550$

$-4.6821590$

$-5.8161620$

$-6.7486320$

$-6.2295840$

$-4.7465300$

$-5.1133140$

$-6.3881210$

$-7.7573240$

$-6.4357040$

$-4.0087120$

$-5.3551910$

$-6.8382500$

$-6.7672690$

3.4525010

2. 9615390

4.1725700

4.8230140

4.1619470

5.4648420

4.8102170

4.8013200

3.6464500

5.4516840

5.9719760

4.7910890

5.9494280

2. 3266210

3. 3540450

1. 2582110

2. 3621080
3. 3850490 $-2.9340150$ $-4.2530790$ $-4.6768180$ $-4.7652880$ $-4.0692790$

$-2.8112030$ $-5.0223630$ $-5.6326600$ $-5.8118640$ $-4.7592490$ $-4.1747080$ $-3.9314210$ $-4.3199790$ $-3.8854690$ $-1.5325880$ $-1.8947880$ $-0.8816870$ $-0.7223690$ $-1.4836550$ $-1.2847910$ $-2.9020570$ $-1.1873590$ $-1.0757030$ $-2.5547390$ $-1.9193340$ 0.0810300 0.3375060 $-1.1677350$ $-0.8148090$ $-1.1570700$ $-1.9084140$ $-1.6221400$ $-3.2111770$ $-2.6378650$ $-0.6043220$ $-4.2260310$ $-3.4173320$ $-3.9399940$ $-2.4181180$ $-5.2405110$ $-4.7342120$ $-0.4694640$

0.3025500 2. 0118570 2. 0720460 


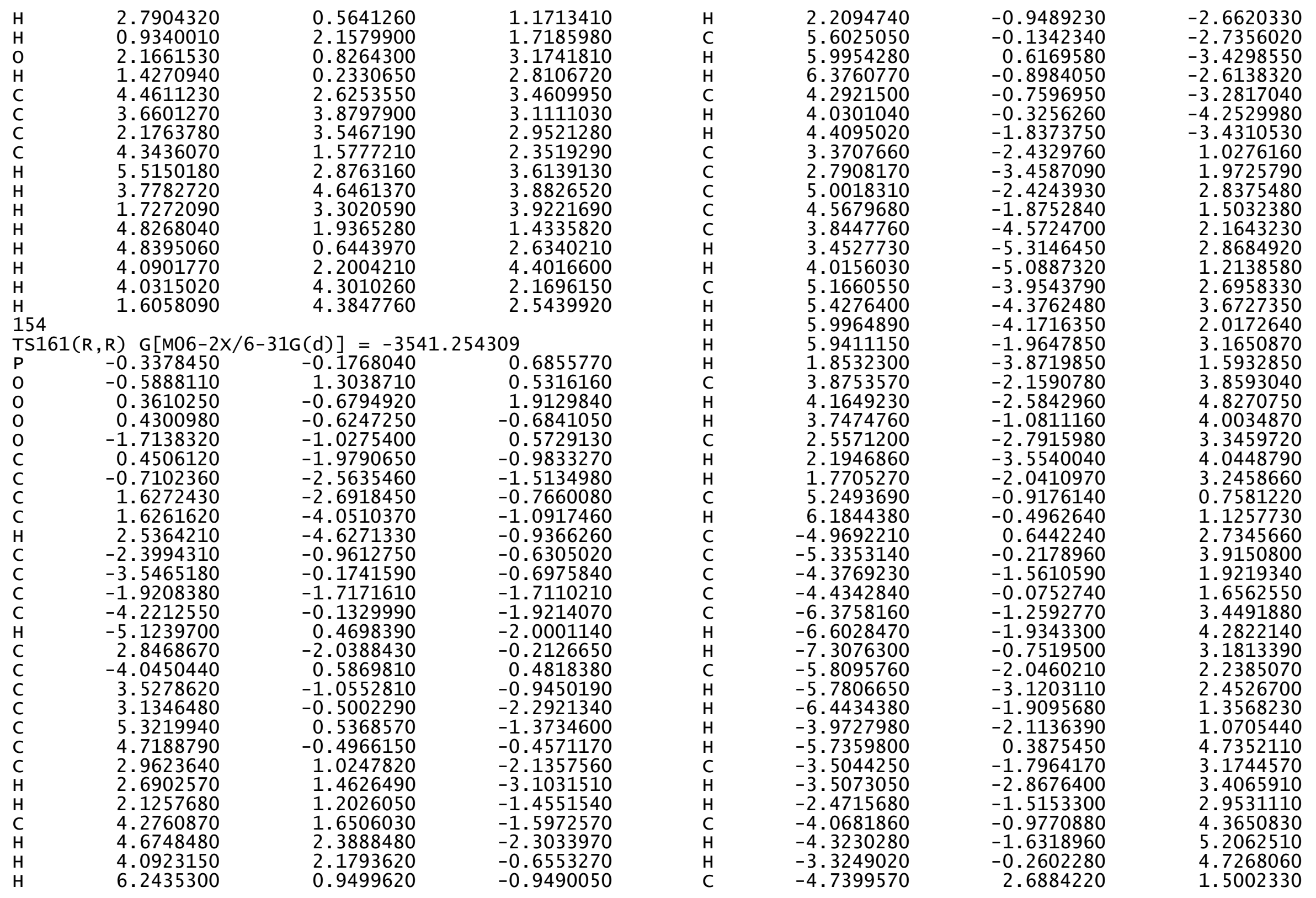




$\begin{array}{lr}-4.8457070 & 4.1724340 \\ -3.7340020 & 2.8905990 \\ -4.1825720 & 1.9814560 \\ -3.4272350 & 4.7312750 \\ -3.5057400 & 5.7877820 \\ -2.8511390 & 4.6870930 \\ -2.7348560 & 3.9066750 \\ -2.3641160 & 4.5593400 \\ -1.8801760 & 3.3564340 \\ -3.2546190 & 2.3311200 \\ -5.3244020 & 4.6761850 \\ -4.9572850 & 3.6749820 \\ -4.6174680 & 4.4040580 \\ -5.6485900 & 2.9953850 \\ -5.6634440 & 4.3873690 \\ -5.7583830 & 5.4618180 \\ -6.6742350 & 3.9929750 \\ -5.1349490 & 2.0233460 \\ -5.5645210 & 2.5753510 \\ -2.6022710 & -1.6244200 \\ -2.1373280 & -2.2653520 \\ -2.0973010 & -1.2091660 \\ -3.4332170 & -0.4410660 \\ -4.4386490 & -0.7854060 \\ -3.7587710 & -0.8335470 \\ -2.8287200 & -3.0712910 \\ -1.8253670 & -1.6951030 \\ -3.8937290 & -0.6556130 \\ -4.8801630 & -1.7686960 \\ -1.1509330 & -2.7204560 \\ -1.2920890 & -0.5062720 \\ -3.2405750 & 0.6357250 \\ -5.2630430 & -0.0659950 \\ -0.6889180 & -3.9380330 \\ -1.8934290 & -4.7369660 \\ -2.0505930 & -5.9676020 \\ -0.7628550 & -6.8223200 \\ 0.4380310 & -6.1435240 \\ 0.4896150 & -4.6746680 \\ -1.7663920 & -5.0682730 \\ -2.8908490 & -6.5724910 \\ -0.9219550 & -7.8041660 \\ 0.3363820 & -6.2631370 \\ -2.8021910 & -4.1312000 \\ -2.3202210 & -5.6073800 \\ -0.5101600 & -7.0008270\end{array}$

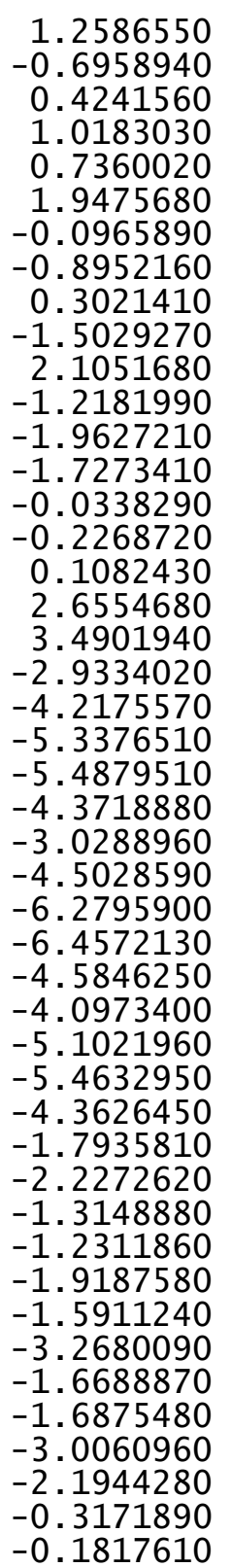

1.3725230
1.6256780
0.5142140
2.2985650
3.4517900
1.8108780
4.1167520
3.8171060
2.4823040
0.9176180
3.6328760
5.0126840
2.1095530
4.1552250
0.1125860
2.1398390
2.7436820
1.7054930
2.6866400
0.7211800
2.0202120
1.3259480
4.1855420
3.3087650
1.8526210
4.1530850
5.2183080
3.3651960
1.4061220
4.6249050
4.7051660
3.8299460
3.6652120
1.2345110
$-6.6364230$
3. 5740610
3. 0272270
4. 4122650
5.1184810
4.4592600
5.8732410
5.0597670
5. 2121320
3.8998340
5.9178500
6.4237620
5.2472390
6.5038800
2. 3216600
3.4393180
1. 2731380
2. 3057440
0.6298080
2.0597740
0.7187700
0.1031350
2.6405230
3.8606410
3.4436390
1. 6624940
2. 9487550
4. 5809970
3. 1085430
2. 1114160
0.7467440
2.1318440
4. 3657320
4.2678850

$-1.6341090$

$-0.5400480$

$-0.9353140$

$-1.5713000$

$-2.8792600$

$-2.1816280$

$-0.2027170$

$-3.1344830$

$-3.4889820$

$-1.9135200$

$-4.2389610$

$-0.2975330$

0.5784700

2. 1593380

2. 2728110

1. 2735610

1.8896780

3. 2783610

2. 8662200

3.7215060

3.4381940

3.2306530

2. 5452010

3. 9097410

4. 2594240

4.1745710

1.6612500

2.7756400

4.6258700

2. 5328710

2.8658710

$\operatorname{TS} 162(R, R) \quad G[M 06-2 X / 6-31 G(d)]=-3541.253567$

$\begin{array}{lrrr}\text { TS162 (R, R) } & \text { G }[\mathrm{M} 06-2 \mathrm{X} / 6-31 \mathrm{G}(\mathrm{d})]=-3541.253567 \\ \mathrm{P} & -0.3513540 & -0.1394730 & 0.7951380 \\ \mathrm{O} & -0.6859650 & 1.2961740 & 0.4688420 \\ \mathrm{O} & 0.3718620 & -0.4540390 & 2.0697880 \\ \mathrm{O} & 0.4456440 & -0.6998870 & -0.5160460 \\ \mathrm{O} & -1.6790250 & -1.0712180 & 0.7847190 \\ \mathrm{C} & 0.5399880 & -2.0768630 & -0.6557050 \\ \mathrm{C} & -0.5865830 & -2.7806080 & -1.1086330 \\ \mathrm{C} & 1.7547520 & -2.6941670 & -0.3656760 \\ \mathrm{C} & 1.8261150 & -4.0802220 & -0.5263270 \\ \mathrm{H} & 2.7688100 & -4.5834280 & -0.3197310\end{array}$




$\begin{array}{lr}\mathrm{C} & -2.3561030 \\ \mathrm{C} & -3.5271040 \\ \mathrm{C} & -1.8341550 \\ \mathrm{C} & -4.1803950 \\ \mathrm{H} & -5.0999920 \\ \mathrm{C} & 2.9417890 \\ \mathrm{C} & -4.0750690 \\ \mathrm{C} & 3.5538060 \\ \mathrm{C} & 3.1132590 \\ \mathrm{C} & 5.2450610 \\ \mathrm{C} & 4.7155190 \\ \mathrm{C} & 2.8532700 \\ \mathrm{H} & 2.5494220 \\ \mathrm{H} & 2.0122670 \\ \mathrm{C} & 4.1319470 \\ \mathrm{H} & 4.4783720 \\ \mathrm{H} & 3.9266140 \\ \mathrm{H} & 6.1453140 \\ \mathrm{H} & 2.2120840 \\ \mathrm{C} & 5.5488950 \\ \mathrm{H} & 5.8849690 \\ \mathrm{H} & 6.3702770 \\ \mathrm{C} & 4.2731020 \\ \mathrm{H} & 3.9742910 \\ \mathrm{H} & 4.4540400 \\ \mathrm{C} & 3.5056390 \\ \mathrm{C} & 3.0024340 \\ \mathrm{C} & 5.1538530 \\ \mathrm{C} & 4.6720820 \\ \mathrm{C} & 4.1262210 \\ \mathrm{H} & 3.7888260 \\ \mathrm{H} & 4.3186130 \\ \mathrm{C} & 5.4117840 \\ \mathrm{H} & 5.7089760 \\ \mathrm{H} & 6.2472690 \\ \mathrm{H} & 6.0660700 \\ \mathrm{H} & 2.0891840 \\ \mathrm{C} & 4.0239810 \\ \mathrm{H} & 4.3499830 \\ \mathrm{H} & 3.8300960 \\ \mathrm{C} & 2.7422440 \\ \mathrm{H} & 2.4347340 \\ \mathrm{H} & 1.9097440 \\ \mathrm{C} & 5.2848230 \\ \mathrm{H} & 6.1967870 \\ \mathrm{C} & -5.0562550 \\ & \end{array}$

$\begin{array}{rr}-1.1684340 & -0.4223540 \\ -0.4324000 & -0.5897750 \\ -2.0193520 & -1.4075470 \\ -0.5462270 & -1.8206480 \\ 0.0142610 & -1.9774880 \\ -1.9126560 & 0.0810770 \\ 0.4371870 & 0.4888370 \\ -0.9874480 & -0.7778300 \\ -0.6245380 & -2.1751360 \\ 0.6411290 & -1.4297540 \\ -0.3062930 & -0.3842750 \\ 0.8958810 & -2.2037950 \\ 1.1946310 & -3.2137980 \\ 1.1078510 & -1.5388440 \\ 1.6573560 & -1.7649190 \\ 2.3293930 & -2.5592670 \\ 2.2816470 & -0.8881180 \\ 1.1551470 & -1.0755860 \\ -1.1671150 & -2.4711580 \\ -0.1721530 & -2.7066890 \\ 0.5117630 & -3.4940870 \\ -0.8677240 & -2.5100180 \\ -0.9372330 & -3.1463970 \\ -0.6443540 & -4.1590380 \\ -2.0163230 & -3.1628160 \\ -2.1246580 & 1.3479850 \\ -3.0632040 & 2.4187870 \\ -1.8001640 & 3.1126800 \\ -1.4435780 & 1.7302540 \\ -4.0796760 & 2.7221410 \\ -4.7548400 & 3.5162570 \\ -4.6944800 & 1.8366570 \\ -3.3233110 & 3.1525420 \\ -3.6074110 & 4.1682680 \\ -3.5701180 & 2.4904780 \\ -1.2483250 & 3.3658860 \\ -3.5761960 & 2.1086150 \\ -1.4821410 & 4.1156040 \\ -1.7687570 & 5.1219700 \\ -0.4045080 & 4.1317400 \\ -2.2508350 & 3.7062480 \\ -2.9431820 & 4.4981520 \\ -1.5666650 & 3.5304120 \\ -0.5445060 & 0.8626640 \\ -0.0272050 & 1.1589480 \\ 0.7099750 & 2.7017040\end{array}$

-5.4138790
-4.3413740
-4.4628020
-6.3931500
-6.6176640
-7.3367480
-5.7552780
-5.6766350
-6.3717430
-3.8903020
-5.8638910
-3.4874430
-3.4312980
-2.4683950
-4.1266090
-4.3767370
-3.4298650
-4.8883470
-5.0575900
-3.8311400
-4.2712050
-3.6617550
-3.7836350
-3.1118070
-2.8975440
-2.5261770
-2.0355760
-3.3018570
-5.5841480
-5.0714480
-4.7408860
-5.7142500
-5.8474910
-5.9919970
-6.8403760
-5.2833370
-5.7601420
-2.4960800
-1.9891720
-1.9497130
-3.2992830
-4.3227330
-3.6741670
-2.6583540
-1.6523250
-3.7312960
3.9666140

2.1645490

1.7247090

3. 6024160

4. 5040920

3. 2509060

2. 5105460

2.8569110

1. 6062640

1. 3924310

4.7016230

3.4509520

3.8046910

3. 2160870

4. 5296090

5.4304220

4.8301270

1. 2431310

0.8286780

$-0.9375890$

0.2682990

0.5565790

0.1462140

1.4975390

$-0.4328870$

$-1.2913000$

0.0540270

$-1.6627630$

1.5997210

$-1.5767410$

$-2.3876270$

$-2.0267060$

$-0.4983040$

$-0.8154320$

$-0.3352230$

2. 4591600

3. 2146270

$-2.6422670$

$-3.8432720$

$-5.0672040$

$-5.3161760$

$-4.1948220$

$-2.8400950$

$-4.0578340$

$-5.9486470$

$-6.2714770$ 


\begin{tabular}{|c|c|c|}
\hline $\begin{array}{l}-4.7287900 \\
-0.9967630 \\
-1.1630050 \\
-3.1322370 \\
-5.1680780 \\
0.7203330 \\
0.7616910 \\
0.1527970 \\
-1.2872850 \\
-1.6494670 \\
-0.4938150 \\
0.1874370 \\
0.1705330 \\
-2.0109500 \\
-1.9141490 \\
1.7902550 \\
0.7931360 \\
-1.4091440 \\
-2.5397370 \\
1.3845870 \\
0.3074630 \\
2.0004180 \\
3.1100580 \\
1.5045380 \\
3.7230020 \\
3.4830650 \\
2.1241200 \\
0.6459180 \\
3.2309710 \\
4.5848850 \\
1.7450660 \\
3.7127430 \\
-0.0502420 \\
1.9104840 \\
2.6511950 \\
1.5558870 \\
2.6242830 \\
0.5842250 \\
1.9719610 \\
1.3090010 \\
4.0246480 \\
3.0752920 \\
1.6444200 \\
4.0386360 \\
5.0393360 \\
3.0965080\end{array}$ & $\begin{array}{l}-2.4666500 \\
-3.2593930 \\
-1.1647020 \\
-0.1087490 \\
-0.7633960 \\
-4.8202160 \\
-6.3161350 \\
-6.6846770 \\
-6.1427790 \\
-5.0897040 \\
-4.1765960 \\
-6.8052470 \\
-7.7698580 \\
-6.9608750 \\
-5.6194210 \\
-6.6817710 \\
-6.2550290 \\
-5.7048000 \\
-4.5308470 \\
3.5366370 \\
2.8802410 \\
4.2858830 \\
5.0960920 \\
4.1494760 \\
5.7717600 \\
5.1795230 \\
4.8236780 \\
3.5113110 \\
5.6334410 \\
6.4028230 \\
4.7165410 \\
6.1579010 \\
2.2368530 \\
3.5671610 \\
1.6426820 \\
2.6209030 \\
0.8965020 \\
2.2761190 \\
1.1850990 \\
0.4876190 \\
3.2670450 \\
4.3928290 \\
3.8714610 \\
2.1562850 \\
3.6539920 \\
5.2074210\end{array}$ & $\begin{array}{l}-4.3184500 \\
-3.6569310 \\
-4.8949030 \\
-5.3856730 \\
-4.2761690 \\
-0.9265490 \\
-1.1037680 \\
-2.4653270 \\
-2.6167930 \\
-1.5469200 \\
-1.2160000 \\
-0.3040960 \\
-2.6041760 \\
-2.5467180 \\
-0.6211120 \\
-1.0263260 \\
-3.2429810 \\
-3.6122280 \\
-1.8408210 \\
-0.9001790 \\
-1.2153990 \\
-2.0308240 \\
-1.7812840 \\
-3.3293920 \\
-2.8294740 \\
-0.7656030 \\
-4.3765660 \\
-3.5070250 \\
-4.1271810 \\
-2.6382770 \\
-5.3877030 \\
-4.9466780 \\
-0.4908820 \\
0.2206270 \\
2.0382670 \\
2.0449530 \\
1.2354860 \\
1.7085750 \\
3.2266120 \\
2.9034310 \\
3.4043380 \\
2.9933380 \\
2.8586300 \\
2.3519440 \\
3.5375740 \\
3.7230420\end{array}$ \\
\hline
\end{tabular}

$\begin{array}{cc}\mathrm{H} & 1.2257510 \\ \mathrm{H} & 4.4731450 \\ \mathrm{H} & 4.6473690 \\ \mathrm{H} & 3.7088020 \\ \mathrm{H} & 3.3935990 \\ \mathrm{H} & 0.9759820\end{array}$

154

$\operatorname{TS} 163(\mathrm{R}, \mathrm{R}) \mathrm{G}[\mathrm{M06}-2 \mathrm{X} / 6-31 \mathrm{G}(\mathrm{d})]=-3541.252296$

\begin{tabular}{|c|c|c|c|}
\hline & , R) $\mathrm{G}[\mathrm{MO}$ & (d) & \\
\hline $\mathrm{P}$ & -0.3079120 & -0.0179540 & 0.7688220 \\
\hline 0 & -0.5360080 & 1.4199180 & 0.3684570 \\
\hline 0 & 0.4117440 & -0.3190970 & 2.0487350 \\
\hline 0 & 0.4188000 & -0.7088720 & -0.5207820 \\
\hline 0 & -1.7037360 & -0.8432100 & 0.8278100 \\
\hline $\mathrm{C}$ & 0.4023870 & -2.0953440 & -0.5740870 \\
\hline C & -0.7818100 & -2.7324770 & -0.9751300 \\
\hline C & 1.5645980 & -2.7879300 & 2406370 \\
\hline $\mathrm{C}$ & 1.5189940 & -4.1829500 & -0.2 \\
\hline $\mathrm{H}$ & 2.4165940 & -4.7468930 & 320 \\
\hline $\mathrm{C}$ & -2.4044030 & -0.9481350 & -( \\
\hline C & -3.5132680 & -0.1268470 & -( \\
\hline C & -1.9677900 & -1.8885020 & -1.3 \\
\hline $\mathrm{C}$ & -4.1892360 & -0.2458010 & -1.7 \\
\hline $\mathrm{H}$ & -5.0657750 & 0.3754380 & -1.9 \\
\hline C & 2.8140190 & -2.0762700 & 0.1 \\
\hline C & -3.9791170 & 0.8314390 & C \\
\hline C & 3.4910680 & -1.2622910 & - \\
\hline $\mathrm{C}$ & 3.0724990 & -0.9628260 & 98860 \\
\hline $\mathrm{C}$ & 5.3024070 & 0.1779340 & -1.5347490 \\
\hline C & 4.7081570 & -0.6541880 & -0.4278530 \\
\hline C & 2.9362530 & 0.5675060 & -2.3 \\
\hline $\mathrm{H}$ & 2.6677310 & 0.8138270 & -3.36 \\
\hline $\mathrm{H}$ & 2.1090150 & 0.8957940 & -1.6961520 \\
\hline C & 4.2685550 & 1.2545640 & -1.9293940 \\
\hline $\mathrm{H}$ & 4.6623600 & 1.8559820 & -2.7575740 \\
\hline $\mathrm{H}$ & 4.1112790 & 1.9381590 & $-1.0 \varepsilon$ \\
\hline $\mathrm{H}$ & 6.2429030 & 0.6414830 & -1.2174280 \\
\hline $\mathrm{H}$ & 2.1297170 & -1.4511850 & -2.447580 \\
\hline $\mathrm{C}$ & 5.5310890 & -0.7368040 & -2 \\
\hline $\mathrm{H}$ & 5.9039690 & -0.1309600 & -3.5910090 \\
\hline $\mathrm{H}$ & 6.3041300 & -1.4747160 & -2.5229010 \\
\hline $\mathrm{C}$ & 4.1990330 & -1.4361730 & -3.1350930 \\
\hline $\mathrm{H}$ & 3.9163840 & -1.2015900 & -4.1673410 \\
\hline $\mathrm{H}$ & 4.2988400 & -2.5237890 & -3.0669620 \\
\hline $\mathrm{C}$ & 3.3706630 & -2.2497760 & 1.4260340 \\
\hline $\mathrm{C}$ & 2.8053300 & -3.0743570 & 2.558057 \\
\hline & 5.0629400 & -1.9548200 & \\
\hline
\end{tabular}

$\begin{array}{lll}1.2257510 & 3.6262990 & 3.8421610 \\ 4.4731450 & 2.5245490 & 1.4132820 \\ 4.6473690 & 1.3081910 & 2.6783350 \\ 3.7088020 & 2.8497650 & 4.3683110 \\ 3.3935990 & 4.8064780 & 2.0293140 \\ 0.9759820 & 4.6094410 & 2.4079510\end{array}$

2.6262990

1. 3081910

2.8497650

4. 3683110

2.4079510

$-2.7879300$

0.3754380

.076270

0.9628260

0.1779340

0382670

. 2354860

1.7085750

266120

.4043380

(1)

3. 7230420

3. 1544880 


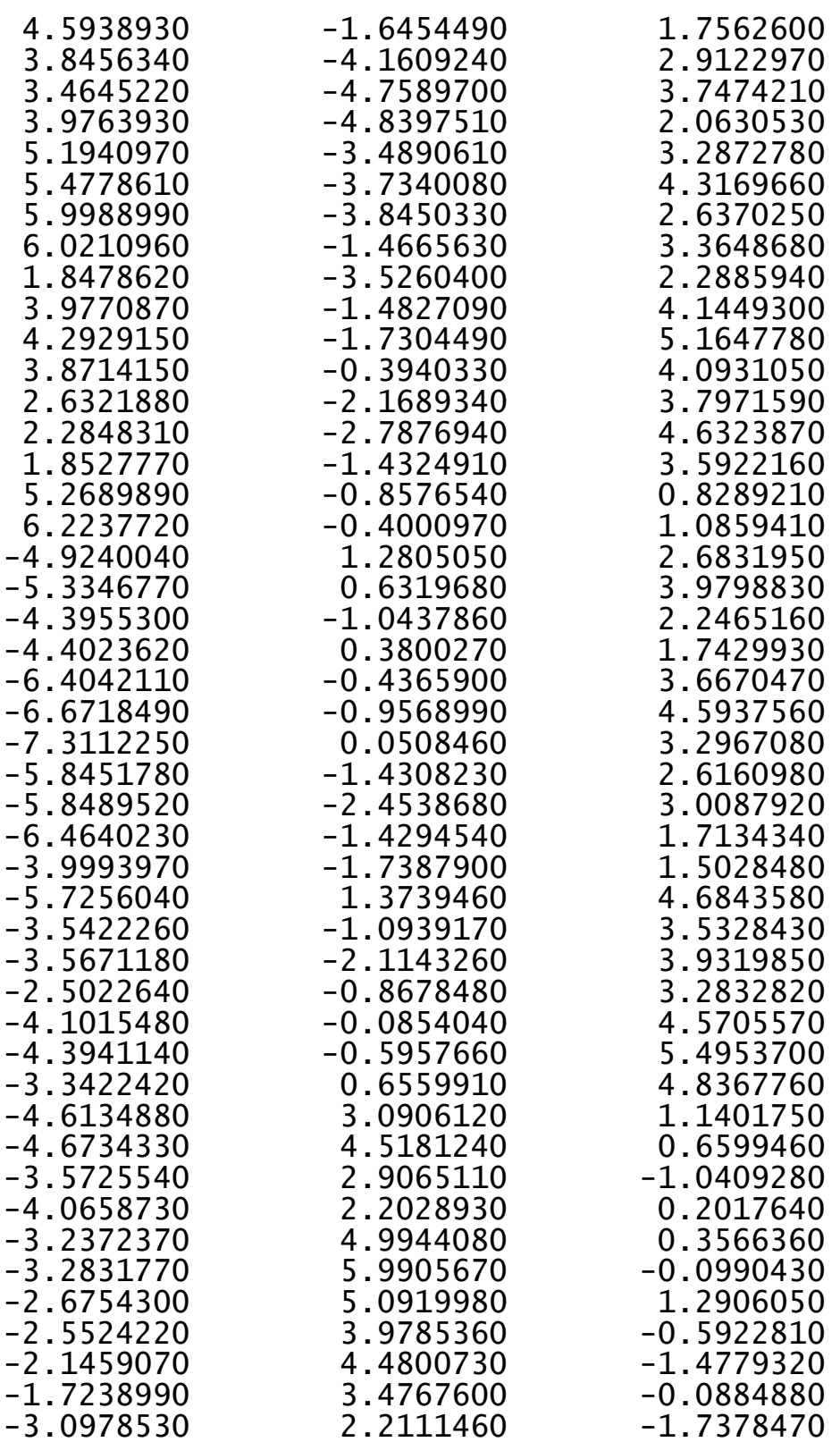

-5.1493760
-4.7622440
-4.3867980
-5.4647190
-5.4675060
-5.5340200
-6.4893740
-5.0444330
-5.4675730
-3.7561550
-4.4501920
-4.7059420
-3.4067140
-2.2232350
-2.6449040
-3.8214310
-5.1806370
-3.1108780
-1.8108830
-5.3891120
-5.4219010
-3.5930560
-1.4145230
0.3517950
0.2604980
-0.3771490
-1.7418960
-2.0292910
-0.8047630
-0.3554540
-0.4872330
-2.5561620
-2.3463510
1.2517950
0.3161070
-1.7688020
-2.8678120
1.6850230
0.5581880
2.3521070
3.5172010
1.8484310
4.1779360
3.8949090
2.5160400
0.9464110

5.1646940

3.6319590

4. 2256880

2. 9003540

4.5404230

5. 5716170

4. 1957150

2. 6328900

3. 3247480

$-1.1219740$

$-1.2560340$

$-2.7405630$

$-3.5778400$

$-2.8068860$

-1.9502200

$-0.8274230$

$-2.8467860$

$-3.9060470$

$-2.1347160$

$-0.6938460$

$-3.1122260$

$-4.4876780$

$-3.4900170$

$-4.8550790$

$-6.3584400$

$-6.8033690$

$-6.1165750$

-4. 9719040

$-4.1356580$

$-6.7164430$

$-7.8918350$

$-6.8446210$

$-5.4164630$

$-6.8058840$

$-6.5457350$

$-5.7234750$

$-4.3617520$

3.4005660

2.8091950

4.0132910

4. 7591020

3.8073530

5.3011780

4. 8979120

4.3470080

3. 2196010
1.4048650 $-1.7067610$ $-2.5481220$ $-2.1192820$ $-0.6646430$ $-1.0292510$ $-0.4790140$

2. 3802110

3. 1070430

$-2.7631920$

$-4.0939140$

$-4.3956580$

$-4.3290010$

$-3.7058390$

$-2.5375500$

$-4.8873050$

$-5.3757080$

$-5.3305410$

$-4.4713470$

$-4.0941640$

$-3.6553460$

$-3.7491560$

$-3.4357510$

$-0.6376870$

$-0.6800630$

$-2.0052920$

$-2.2521970$

$-1.2577760$

$-0.9790980$

0.1570140

$-2.0185900$

$-2.1805240$

$-0.3040750$

$-0.5586390$

$-2.8125610$

$-3.2737980$

$-1.6010290$

$-1.1532470$

-1. 4199020

$-2.3358790$

$-2.1457070$

$-3.6221760$

$-3.2413800$

$-1.1378510$

$-4.7167770$

$-3.7523720$ 


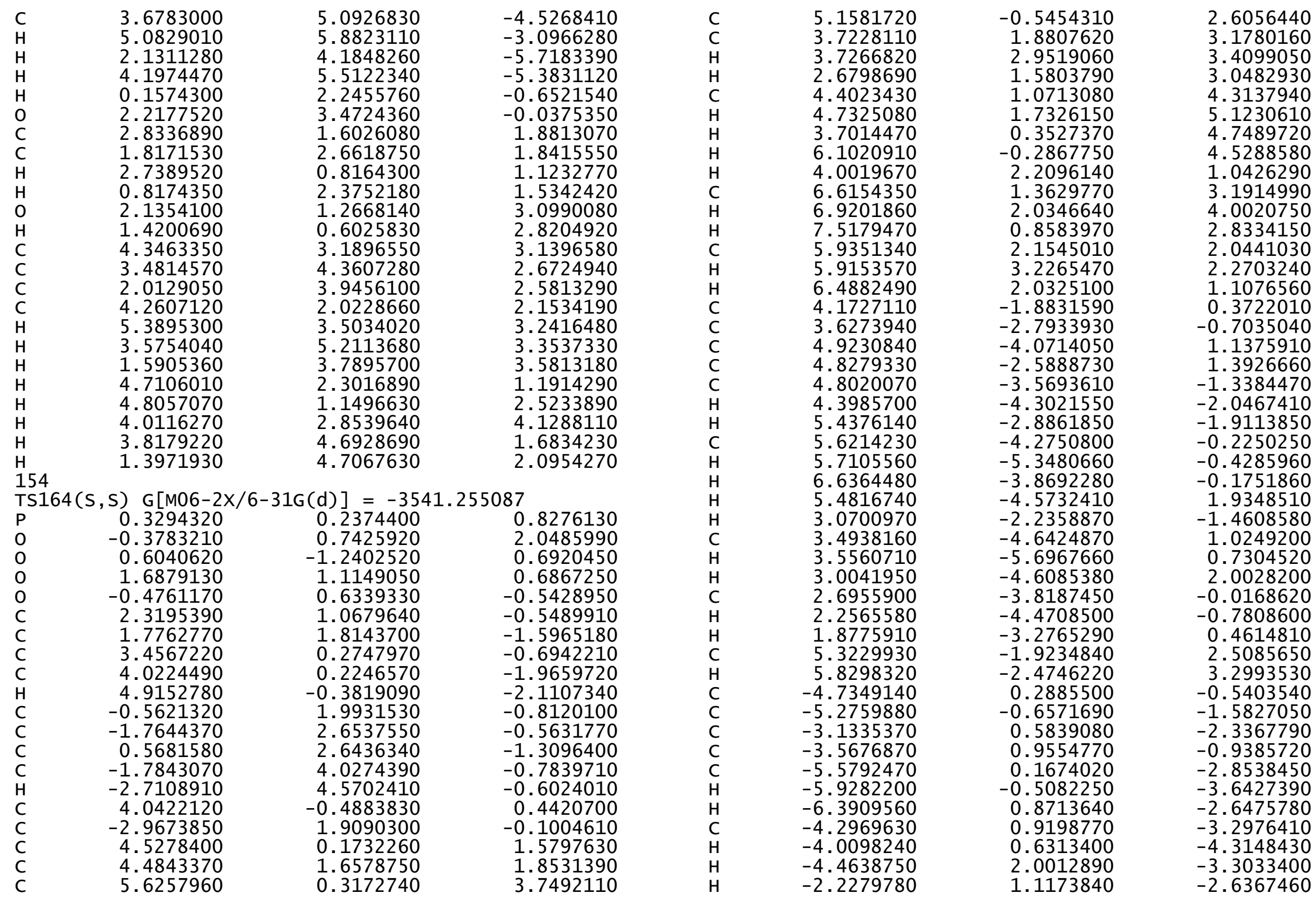




$\begin{array}{rrr}-6.1805020 & -1.1619940 & -1.2257100 \\ -2.8958640 & -0.9392930 & -2.3863980 \\ -2.6166000 & -1.2268380 & -3.4063790 \\ -2.0442560 & -1.1789890 & -1.7445380 \\ -4.1801310 & -1.6849950 & -1.9383350 \\ -4.5473870 & -2.3440100 & -2.7336150 \\ -3.9732340 & -2.3230230 & -1.0734170 \\ -4.7268520 & 1.5045430 & 1.5322530 \\ -5.2480330 & 1.9329400 & 2.8795010 \\ -3.0904680 & 3.1791030 & 2.1722820 \\ -3.5522810 & 2.1709690 & 1.1464530 \\ -4.1428180 & 1.6816220 & 3.9268140 \\ -4.5051850 & 1.9941100 & 4.9127160 \\ -3.9224260 & 0.6108110 & 3.9854090 \\ -2.8714310 & 2.4720940 & 3.5277620 \\ -2.6405580 & 3.2364400 & 4.2785980 \\ -1.9989150 & 1.8205520 & 3.4566460 \\ -2.1713360 & 3.6801790 & 1.8593580 \\ -6.1621160 & 1.3877140 & 3.1393040 \\ -4.2380750 & 4.1973240 & 2.3620280 \\ -3.9331580 & 4.9449110 & 3.1025250 \\ -4.4202750 & 4.7288220 & 1.4221220 \\ -5.5197250 & 3.4539740 & 2.8265390 \\ -5.8270470 & 3.7936220 & 3.8220430 \\ -6.3525240 & 3.6543990 & 2.1458140 \\ -5.3208610 & 0.5688690 & 0.6916030 \\ -6.2390020 & 0.0643470 & 0.9915850 \\ 0.5438530 & 4.0462930 & -1.4491860 \\ 1.8157920 & 4.8059650 & -1.7782370 \\ 1.7460340 & 6.2701850 & -1.3479230 \\ 0.4768640 & 6.9027000 & -1.9122670 \\ -0.7466590 & 6.2483290 & -1.2749400 \\ -0.6499370 & 4.7358620 & -1.1877690 \\ 2.6390080 & 6.7982320 & -1.6969390 \\ 2.0031010 & 4.7838310 & -2.8584200 \\ 2.6667690 & 4.2997210 & -1.3099040 \\ 0.4572780 & 6.7551490 & -3.0000980 \\ 0.4557920 & 7.9823430 & -1.7336270 \\ -1.6588900 & 6.5206510 & -1.8183400 \\ -0.8703280 & 6.6415380 & -0.2566480 \\ 1.7338540 & 6.3389460 & -0.2524380 \\ 2.3247790 & 1.6826950 & -2.8882220 \\ 1.6332950 & 2.3091500 & -4.0851830 \\ 2.0154770 & 1.6298480 & -5.3991750 \\ 3.5362110 & 1.5730070 & -5.5147800 \\ 4.0973180 & 0.6620420 & -4.4254910\end{array}$

\begin{abstract}
3.4662570
1. 5760190

1.9014560

0.5492680

3.9410460

3.8483280

5.1838330

3.9269320

1.6077630

$-1.5107290$

$-0.4183660$

$-1.9691660$

$-3.1354590$

$-1.2590060$

$-3.5911880$

$-3.6733880$

$-1.7197320$

$-0.3571800$

$-2.8832250$

$-4.4970030$

$-1.1721070$

$-3.2405250$

$-0.0760780$

$-2.1730250$

$-1.4793190$

$-2.4799140$

$-0.4442330$

$-2.2338480$

$-1.9563410$

$-1.3513870$

$-3.2373700$

$-4.0909200$

$-3.9157530$

$-1.7562860$

$-3.3995440$

$-5.1495500$

$-4.3657630$

$-1.4260520$

$-1.1329950$

$-3.5312230$

$-3.7930170$

$-4.4053140$
\end{abstract}

154

$\operatorname{TS} 165(\mathrm{~S}, \mathrm{~S}) \mathrm{G}[\mathrm{M} 06-2 \mathrm{X} / 6-31 \mathrm{G}(\mathrm{d})]=-3541.254707$
$\mathrm{P}$
$\mathrm{O}$
0.3273450
-0.3688590
0.2260930
0.7734800
0.7782300
1.9877120

$-3.0629170$

$-6.2378540$

$-4.1671820$

$-3.9298750$

$-6.4994000$

$-4.3431020$

38150

(1) - n

$-2.4726190$

$-0.5468080$

.0298870

$-3.6681550$

4.4422410

$-0.1964940$

0.4173870

2.8003450

2.4670280

1.0070980

2. 9227610

4.1074650

2.8407460

1.9899810

3.7420660

4.7280700

2. 4789120

3.2396340

4.6320190

4.7033150

2. 2483580

1.0164770 


\begin{tabular}{|c|c|c|}
\hline $\begin{array}{l}0.5641590 \\
1.7044740 \\
-0.4588230 \\
2.3499010 \\
1.8442790 \\
3.4817270 \\
4.0871690 \\
4.9772690 \\
-0.5110010 \\
-1.6984680 \\
0.6386350 \\
-1.7114810 \\
-2.6277110 \\
4.0239870 \\
-2.9112460 \\
4.4815550 \\
4.4578440 \\
5.4999240 \\
5.0614170 \\
3.6541720 \\
3.6812130 \\
2.6081590 \\
4.2684430 \\
4.5759910 \\
3.5356230 \\
5.9342830 \\
4.0175430 \\
6.5289530 \\
6.8087060 \\
7.4383140 \\
5.9111390 \\
5.9074490 \\
6.4966250 \\
4.1358440 \\
3.6158090 \\
4.8169940 \\
4.7407210 \\
4.7998430 \\
4.4114590 \\
5.4700100 \\
5.5614600 \\
5.6351170 \\
6.5823620 \\
5.3354290 \\
3.0987730 \\
3.3817290\end{array}$ & $\begin{array}{r}-1.2605670 \\
1.0685370 \\
0.6201020 \\
0.9916710 \\
1.7401830 \\
0.1854870 \\
0.1273450 \\
-0.4892720 \\
1.9749030 \\
2.6639760 \\
2.5845610 \\
4.0339220 \\
4.5952790 \\
-0.5761140 \\
1.9560350 \\
0.0880800 \\
1.5762400 \\
0.2332050 \\
-0.6317660 \\
1.8312450 \\
2.9039340 \\
1.5607880 \\
1.0131590 \\
1.6708690 \\
0.3095790 \\
-0.3720090 \\
2.1287640 \\
1.2567370 \\
1.9337740 \\
0.7345040 \\
2.0439800 \\
3.1198150 \\
1.8938900 \\
-1.9729770 \\
-2.8831590 \\
-4.1668530 \\
-2.6804150 \\
-3.6849250 \\
-4.4150590 \\
-3.0170970 \\
-4.3976070 \\
-5.4740620 \\
-4.0128760 \\
-4.6713590 \\
-2.3224500 \\
-4.7112090\end{array}$ & $\begin{array}{r}0.6730300 \\
0.6242670 \\
-0.6020470 \\
-0.6016050 \\
-1.6670860 \\
-0.7119460 \\
-1.9648540 \\
-2.0808250 \\
-0.9008290 \\
-0.6681420 \\
-1.4240260 \\
-0.9423390 \\
-0.7693240 \\
0.4468340 \\
-0.1718900 \\
1.5947990 \\
1.8517480 \\
3.8026250 \\
2.6491980 \\
3.1457300 \\
3.3692410 \\
2.9802660 \\
4.3120050 \\
5.1330140 \\
4.7182050 \\
4.6055430 \\
1.0182470 \\
3.2756680 \\
4.0908250 \\
2.9630740 \\
2.0906520 \\
2.2989400 \\
1.1782610 \\
0.3925860 \\
-0.6954840 \\
1.2044910 \\
1.4424620 \\
-1.2783800 \\
-1.9977240 \\
-1.8296150 \\
-0.1289700 \\
-0.3200250 \\
-0.0442230 \\
2.0267820 \\
-1.4784320 \\
1.0439940\end{array}$ \\
\hline
\end{tabular}

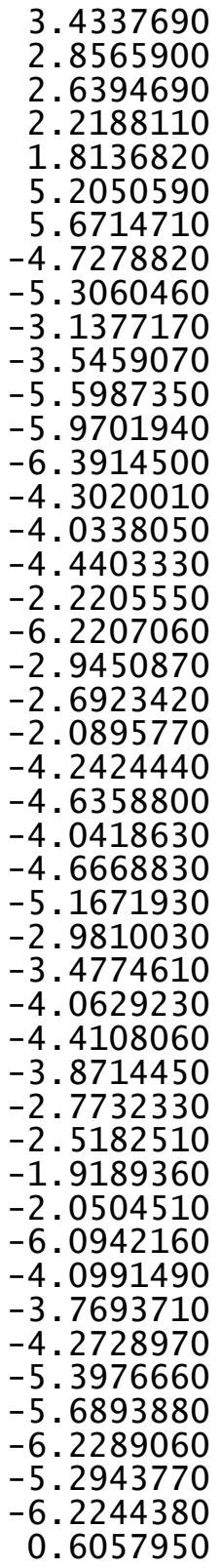

-5.7698980
-4.6559890
-3.8844490
-4.5370620
-3.3226930
-2.0134200
-2.5659420
0.3767230
-0.5754780
0.5885780
1.0008550
0.2286140
-0.4554370
0.9566330
0.9393830
0.6267140
2.0246560
1.0895350
-1.0465740
-0.9415320
-1.2566250
-1.1942700
-1.6411010
-2.3125390
-2.2598250
1.6399310
2.1192990
3.2857140
2.2632760
1.8676220
2.2172250
0.7932720
2.6120490
3.3914310
1.9355180
3.7518630
1.6069060
4.3401060
5.0991680
4.8503720
3.6452410
4.0209170
3.8492990
0.7017130
0.2317010
3.9681800
0.7637670 2. 0023080 $-0.0363940$ $-0.8102130$ 0.4044550

2. 5704800

3. 3848220 $-0.5585440$ $-1.5747270$ $-2.3767160$ $-0.9821890$ $-2.8613840$ $-3.6325830$ $-2.6650060$ $-3.3311990$ $-4.3464290$ $-3.3530050$ $-2.6964910$ $-1.1979970$ $-2.3995250$ $-3.4183260$ $-1.7680580$ $-1.9157270$ $-2.6878210$ $-1.0354090$

1.4844980

2. 8224860

2.0681510

1.0732960

3.8704040

4.8490610

3.9576770

3. 4424280

4. 1696660

3. 3867130

1. 7354520

3. 1025670

2. 2359580

2.9537700

1.2827730

2. 7281460

3.7153820

2. 0465880

0.6714400

0.9901510 


$\begin{array}{rrr}1.8087220 & 4.7970260 & -2.0241650 \\ 1.9571450 & 5.9554130 & -1.0203190 \\ 0.6708010 & 6.8091430 & -0.9070370 \\ -0.5247100 & 6.1733460 & -1.6462100 \\ -0.5761320 & 4.6875690 & -1.4068930 \\ 1.6784200 & 5.2140380 & -3.0328170 \\ 2.8077700 & 6.5790500 & -1.3113320 \\ 0.8392090 & 7.8131400 & -1.3086510 \\ -0.4141090 & 6.3596680 & -2.7231010 \\ 2.7177050 & 4.1896790 & -2.0389140 \\ 2.2017040 & 5.5212940 & -0.0458370 \\ 0.4055350 & 6.9323900 & 0.1471380 \\ -1.4615730 & 6.6479820 & -1.3394430 \\ 2.4461730 & 1.6178830 & -2.9361800 \\ 1.8365250 & 2.3064980 & -4.1430250 \\ 2.2831410 & 1.6823020 & -5.4639580 \\ 3.8054440 & 1.5780830 & -5.4902430 \\ 4.2671920 & 0.5988180 & -4.4140790 \\ 3.5827400 & 0.8065330 & -3.0752740 \\ 1.9142600 & 2.2870550 & -6.2984960 \\ 2.1272100 & 3.3646950 & -4.1506970 \\ 0.7447170 & 2.2917780 & -4.0541490 \\ 4.2359440 & 2.5706130 & -5.3028020 \\ 4.1652840 & 1.2505390 & -6.4707350 \\ 5.3528680 & 0.6576030 & -4.2762670 \\ 4.0580650 & -0.4254600 & -4.7523810 \\ 1.8474140 & 0.6803010 & -5.5709720 \\ -1.6242890 & -3.4713980 & -0.5752340 \\ -0.5172570 & -2.8709830 & -0.8812500 \\ -2.1287890 & -4.4035190 & -1.6230200 \\ -3.3008040 & -5.1239380 & -1.3828440 \\ -1.4591480 & -4.5326520 & -2.8420630 \\ -3.8021600 & -5.9751080 & -2.3601950 \\ -3.8075860 & -5.0019730 & -0.4307780 \\ -1.9663890 & -5.3831070 & -3.8188700 \\ -0.5529640 & -3.9625540 & -3.0143260 \\ -3.1351120 & -6.1034730 & -3.5783500 \\ -4.7121530 & -6.5368340 & -2.1758600 \\ -1.4509540 & -5.4834460 & -4.7685250 \\ -3.5287100 & -6.7663020 & -4.3428680 \\ -0.1484790 & -2.1852720 & -0.1862470 \\ -2.2662940 & -3.3186730 & 0.4756850 \\ -1.5263670 & -2.0113180 & 2.8198990 \\ -2.5280260 & -1.7319320 & 1.7845630 \\ -0.4928150 & -1.9820400 & 2.4773900 \\ -2.2703920 & -1.0123450 & 1.0081140\end{array}$
O $\quad-1.9692230$
$-1.3522340$
$-3.3032810$
$-4.1681540$
$-3.9700260$
$-1.8228590$
$-3.4813950$
$-5.2272710$
$-4.3956160$
$-1.5179100$
$-1.1900230$
$-3.5721470$
$-3.8954310$
$-4.4686470$
$-0.7292930$
$-0.0473800$
$-3.1938720$
$-3.2766390$
$-2.0440080$
$-3.1303050$
$-4.0608650$
$-3.3632150$
$-1.1490620$
$-4.0670420$
$-2.9918900$
$-2.3005780$
$-4.1707780$
$-2.1404800$
3.3154730
2.8850230
4.1693680
2. 9109560
2. 0283760
3.7910740
4.8123790
3. 1709180
2. 4990120
3. 3087980
4.6732310
4.7460390
2.3379410
1.0605090

TS166 (S, S ) G[M06-2X/6-31G(d)] = -3541.256050

\begin{tabular}{|c|c|c|c|}
\hline \multicolumn{4}{|c|}{$\operatorname{TS} 166(\mathrm{~S}, \mathrm{~S}) \quad \mathrm{G}[\mathrm{M06}-2 \mathrm{X} / 6-31 \mathrm{G}(\mathrm{d})]=-3541.256050$} \\
\hline $\mathrm{P}$ & -0.3195310 & -0.1712810 & 0.8174930 \\
\hline 0 & 0.3829710 & -0.6597330 & 2.0486180 \\
\hline 0 & -0.5585380 & 1.3081610 & 0.6412920 \\
\hline 0 & -1.6989300 & -1.0208210 & 0.7167060 \\
\hline 0 & 0.4678010 & -0.6279560 & -0.5444440 \\
\hline C & -2.3544720 & -1.0200610 & -0.5059600 \\
\hline C & -1.8273630 & -1.7977220 & -1.5 \\
\hline C & -3.5095380 & -0.2541500 & -0.6517060 \\
\hline C & -4.1255710 & -0.2846940 & -1.9018920 \\
\hline $\mathrm{H}$ & -5.0408420 & 0.2887150 & -2.0401200 \\
\hline $\mathrm{C}$ & 0.5219190 & -1.9956610 & -0.7763850 \\
\hline $\mathrm{C}$ & 1.7097290 & -2.6803070 & -0.5195030 \\
\hline $\mathrm{C}$ & -0.6246240 & -2.6310880 & -1.2495710 \\
\hline $\mathrm{C}$ & 1.6978320 & -4.0570850 & -0.7202480 \\
\hline $\mathrm{H}$ & 2.6148090 & -4.6166510 & -0.5412180 \\
\hline C & -4.0607990 & 0.5652040 & 0.4623820 \\
\hline C & 2.9321810 & -1.9566870 & -0.0745920 \\
\hline C & -4.4991640 & -0.0331440 & 1.6527780 \\
\hline C & 4408710 & -1.5004500 & 2.00 \\
\hline C & -5.5062170 & -0.0579240 & 80 \\
\hline C & 0859410 & 0.7414000 & 20 \\
\hline C & 8000 & -1.6505250 & 00 \\
\hline 11 & 60100 & -2.7075730 & 920 \\
\hline $\mathrm{H}$ & 889330 & -1.3568690 & 100 \\
\hline C & -4.2597460 & -0.7813870 & $\$ 210$ \\
\hline n & -4.55 & -1.3983360 & 5120 \\
\hline $\mathrm{H}$ & -3.54 & -0.0408920 & \\
\hline ח & -5.9 & 0.5876450 & \\
\hline & -3 & -2.0945610 & 1 \\
\hline & & & \\
\hline
\end{tabular}




$\begin{array}{lrrr}\mathrm{H} & -6.7876140 & -1.7574570 & 4.2678060 \\ \mathrm{H} & -7.4325390 & -0.6465790 & 3.0608960 \\ \mathrm{C} & -5.8812380 & -1.9860680 & 2.2818350 \\ \mathrm{H} & -5.8495430 & -3.0437980 & 2.5664650 \\ \mathrm{H} & -6.4725610 & -1.9166000 & 1.3635450 \\ \mathrm{C} & -4.1942640 & 1.9541720 & 0.3227830 \\ \mathrm{C} & -3.6945870 & 2.8012600 & -0.8239980 \\ \mathrm{C} & -4.8883820 & 4.1862230 & 1.0082100 \\ \mathrm{C} & -4.7993650 & 2.7170450 & 1.3326930 \\ \mathrm{C} & -4.8919600 & 3.5560100 & -1.4410250 \\ \mathrm{H} & -4.5186230 & 4.2415830 & -2.2104090 \\ \mathrm{H} & -5.5629860 & 2.8489360 & -1.9399320 \\ \mathrm{C} & -5.6453970 & 4.3347380 & -0.3297560 \\ \mathrm{H} & -5.7231540 & 5.3977140 & -0.5842750 \\ \mathrm{H} & -6.6647200 & 3.9541850 & -0.2130760 \\ \mathrm{H} & -5.4028040 & 4.7351770 & 1.8041520 \\ \mathrm{H} & -3.1817230 & 2.1987670 & -1.5778400 \\ \mathrm{C} & -3.4574910 & 4.7282450 & 0.8040550 \\ \mathrm{H} & -3.5182240 & 5.7695620 & 0.4664440 \\ \mathrm{H} & -2.9236180 & 4.7286180 & 1.7590910 \\ \mathrm{C} & -2.7197460 & 3.8472970 & -0.2363730 \\ \mathrm{H} & -2.3150720 & 4.4582160 & -1.0516820 \\ \mathrm{H} & -1.8829520 & 3.3192590 & 0.2253020 \\ \mathrm{C} & -5.2488610 & 2.1137270 & 2.5019780 \\ \mathrm{H} & -5.7171920 & 2.7086980 & 3.2846910 \\ \mathrm{C} & 4.7431890 & -0.3960890 & -0.5513580 \\ \mathrm{C} & 5.3097350 & 0.5093320 & -1.6157200 \\ \mathrm{C} & 3.1278530 & -0.6821950 & -2.3372740 \\ \mathrm{C} & 3.5564880 & -1.0378260 & -0.9333220 \\ \mathrm{C} & 5.5836170 & -0.3519680 & -2.8690380 \\ \mathrm{H} & 5.9531160 & 0.2946540 & -3.6727410 \\ \mathrm{H} & 6.3722470 & -1.0774610 & -2.6483000 \\ \mathrm{C} & 4.2767770 & -1.0716600 & -3.2952990 \\ \mathrm{H} & 3.9952000 & -0.7926060 & -4.3167110 \\ \mathrm{H} & 4.4097380 & -2.1577130 & -3.2820930 \\ \mathrm{H} & 2.2053890 & -1.1939410 & -2.6229850 \\ \mathrm{H} & 6.2304000 & 0.9939170 & -1.2722460 \\ \mathrm{C} & 2.9357720 & 0.8463120 & -2.4159620 \\ \mathrm{H} & 2.6577140 & 1.1221620 & -3.4395030 \\ \mathrm{H} & 2.0962660 & 1.1238700 & -1.7732570 \\ \mathrm{C} & 4.2447010 & 1.5620490 & -1.9910610 \\ \mathrm{H} & 4.6281290 & 2.1905110 & -2.8033070 \\ \mathrm{H} & 4.0619480 & 2.2262970 & -1.1406650 \\ \mathrm{C} & 4.7029510 & -1.5635040 & 1.5487010 \\ \mathrm{C} & 5.2112400 & -1.9740610 & 2.9064050 \\ \mathrm{C} & 3.0219930 & -3.1786210 & 2.2250910\end{array}$

\begin{abstract}
3. 5110320
4.1117540

4.4648110
3.9197470

2. 8201830

2. 5689850

1. 9651840

2. 0906780

6.1390920

4.1420110

3. 8165810

4. 3108710

5. 4423990

5.7390070

6. 2705880

5. 3222920

6. 2544170

$-0.6367830$

$-1.9349530$

$-1.8782800$

$-0.6092910$

0.6093260

0.5452490

$-2.7734830$

$-2.1788180$

$-2.7527640$

$-0.5680290$

$-0.6055540$

1. 5323020

0.6859360

$-1.8766540$

$-2.4152530$

$-3.5945080$

$-1.7124680$

$-2.6567190$

$-3.4525350$

$-4.3311420$

$-2.0822170$

$-0.9451030$

$-1.1651240$

$-2.7524590$

$-4.0738010$

$-4.7740400$

$-5.1723080$

-3.3490700
1.6306410
\end{abstract}

-2.2067280
-1.6691350
-1.9676680
-0.5917710
-2.4349980
-3.1754700
-1.7624840
-3.6622310
-1.4472420
-4.2222020
-4.9443410
-4.7796760
-3.5026350
-3.8268090
-3.7412000
-0.6644610
-0.1790390
-4.0371000
-4.7627970
-6.2477810
-6.8688320
-6.2603970
-4.7476100
-6.7497710
-4.6711090
-4.2682010
-6.6681470
-7.9559610
-6.5488640
-6.6711550
-6.3701150
-1.7307800
-0.9879470
-2.3281080
-2.5850340
-1.3192630
-0.9488870
-2.9085880
-1.6065540
-3.2333340
-0.5010300
-1.4525220
0.0432680
-1.6544200
-3.3999250
3.4616090

3.9456560

4.9392320

3.9787110

3. 5635170

4. 3315540

3. 4761600

1. 9212110

3. 1551470

2. 4400170

3.1968490

1. 5127650

2.8896300

3.8935090

2. 2156130

0.6865150

0.9746410

$-1.3596050$

$-1.6702420$

$-1.3147380$

$-1.8918970$

$-1.2029390$

$-1.1070130$

$-1.6949300$

$-2.7373870$

$-1.1345630$

$-2.9708240$

$-1.7650570$

$-1.7189590$

$-0.1867940$

$-0.2238350$

$-2.8163180$

$-2.9840840$

$-4.0221750$

$-5.1938200$

$-5.5037220$

$-4.3104890$

$-6.0677430$

$-4.3400380$

$-3.7538530$

$-5.7168670$

$-6.3951930$

$-4.2583170$

$-4.9408070$

$-0.7170970$ 


\begin{tabular}{|c|c|c|c|}
\hline & & & \\
\hline 0 & 0.5128950 & 2.8606800 & -0.9806110 \\
\hline $\begin{array}{l}\mathrm{C} \\
\mathrm{C}\end{array}$ & $\begin{array}{l}2.1180990 \\
3.3081250\end{array}$ & $\begin{array}{l}4.3582790 \\
5.0649070\end{array}$ & $\begin{array}{l}-1.8032340 \\
-1.6163520\end{array}$ \\
\hline 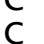 & 1.4118860 & 4.4717450 & -3.0031020 \\
\hline $\mathrm{C}$ & 3.7914300 & 5.8860220 & -2.6279740 \\
\hline H & 3.8425420 & 4.9565010 & -0.6778640 \\
\hline C & 1.9003660 & 5.2930750 & -4.0137900 \\
\hline $\mathrm{H}$ & 0.4914270 & 3.9135410 & -3.1336460 \\
\hline C & 3.0874730 & 5.9991820 & -3.8266510 \\
\hline $\mathrm{H}$ & 4.7156760 & 6.4364710 & -2.4851970 \\
\hline $\mathrm{H}$ & 1.3559560 & 5.3819300 & -4.9483010 \\
\hline $\mathrm{H}$ & 3.4663830 & 6.6391060 & -4.6176570 \\
\hline H & 0.1534070 & 2.2009750 & -0.2576720 \\
\hline 0 & 2.2944530 & 3.3367650 & 0.3237240 \\
\hline C & 1.5575160 & 2.1542570 & 2.7344600 \\
\hline C & 2.5516650 & 1.8167500 & 1.7092650 \\
\hline H & 0.5217290 & 2.1128590 & 2.3996220 \\
\hline H & 2.2853610 & 1.0605770 & 0.971552 \\
\hline 0 & 1.9969400 & 0.8970580 & 3.292648 \\
\hline $\mathrm{H}$ & 1.3742890 & 0.1973940 & 2.900315 \\
\hline C & 3.3483870 & 3.3938690 & 4.012190 \\
\hline C & 4.2074800 & 3.4062950 & 2.7470330 \\
\hline C & 3.9968410 & 2.1319230 & 1.9292910 \\
\hline C & 1.8656170 & 3.3202590 & 3.6448810 \\
\hline $\mathrm{H}$ & 3.5350290 & $4.2 \mathrm{~s}$ & 4.6089320 \\
\hline $\mathrm{H}$ & 5.2684160 & 3.4986680 & 2.997392 \\
\hline H & 4.4202970 & 1.2592800 & 2.4418260 \\
\hline H & 1.5639730 & 4.2326170 & 3.116025 \\
\hline H & 1.2365700 & 3.2320210 & 4.536134 \\
\hline $\mathrm{H}$ & 3.61458 & 2.5296270 & 4.632722 \\
\hline H & 3.9379600 & 4.2720240 & 2.130502 \\
\hline $\mathrm{H}$ & 4.4902090 & 2.1766470 & 0.9550670 \\
\hline $\begin{array}{l}15 \\
\text { TS }\end{array}$ & & 84 & \\
\hline $\mathrm{P}$ & -0.3317190 & -0.1972600 & 0.8558750 \\
\hline 0 & 0.3759140 & -0.6593270 & 2.0940030 \\
\hline 0 & -0.5986390 & 1.2754400 & 0.6635470 \\
\hline 0 & -1.6956410 & -1.0716500 & 0.7550640 \\
\hline 0 & 0.4706280 & -0.6492990 & -0.4989870 \\
\hline C & -2.3319130 & -1.0836900 & -0.4787260 \\
\hline C & -1.7912830 & -1.8782190 & -1.4886130 \\
\hline C & -3.4751310 & -0.3054820 & -0.6598390 \\
\hline $\mathrm{C}$ & -4.0506180 & -0.3305490 & -1.927837 \\
\hline H & -4.9524590 & 0.2557560 & -2.0976790 \\
\hline C & 0.5596530 & -2.0155480 & -0.7257260 \\
\hline$C$ & 1.7714730 & -2.6622080 & -0.49026 \\
\hline
\end{tabular}

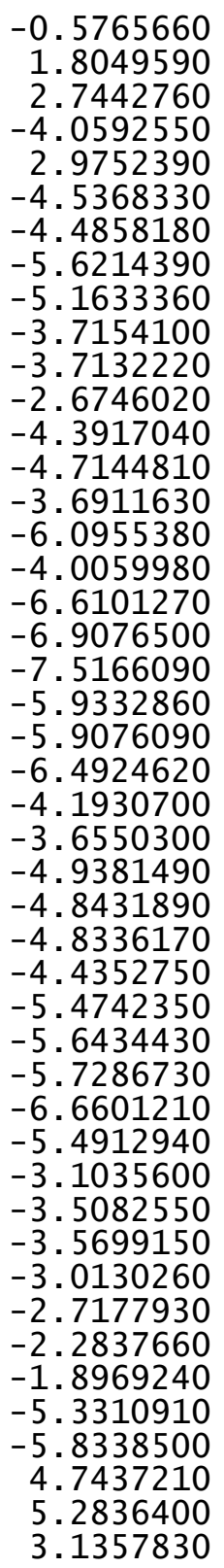
$-2.6876720$
$-4.0375340$
$-4.5653220$
0.5114140
$-1.9039090$
$-0.0943120$
$-1.5631810$
$-0.1331470$
0.6727940
$-1.7161520$
$-2.7743360$
$-1.4184260$
$-0.8535480$
$-1.4747870$
$-0.1114700$
0.5075500
$-2.1529660$
$-1.2092450$
$-1.8402680$
$-0.7266520$
$-2.0551190$
$-3.1142730$
$-1.9827960$
1.9005990
2.7568610
4.1235350
2.6548790
3. 5014580
4. 1966360
2.7905070
4.2640350
5. 3263900
3.8662590
4.6639240
2. 1624210
4. 6853640
5.7251450
4.6951460
3.8127000
4.4278500
3. 2926970
2. 0442480
2.6327700
$-0.2989250$
0.6154050
$-0.6416140$

$-1.1808080$

0.5498220

0.4393400

$-0.0516720$

1. 6110610

1. 9580750

3.7915730

3. 2878600

3. 5731430

3. 1367450

4.3858240

5. 2291170

4.7802910

4. 5427750

1. 1733140

3. 2929200

4.1381170

2. 9151050

2. 1829120

2. 4628500

1. 2449330

0.3018140

$-0.8205280$

0.9648370

1. 2902010

$-1.4846070$

$-2.2325620$

$-2.0167030$

$-0.4021010$

$-0.6567930$

$-0.3271790$

1.7404190

$-1.5535150$

0.8176050

0.4753780

1.7932110

$-0.1900470$

$-0.9868730$

0.3075780

2. 4400280

3.2060920

$-0.5399990$

-1.6106490
-2.3228380 


\begin{tabular}{|c|c|c|}
\hline $\begin{array}{l}3.5747680 \\
5.5809610 \\
5.9317290 \\
6.3897610 \\
4.2945870 \\
4.0048580 \\
4.4580170 \\
2.2277920 \\
6.1904490 \\
2.9003270 \\
2.6134930 \\
2.0542380 \\
4.1890150 \\
4.5545020 \\
3.9881090 \\
4.7371430 \\
5.2577000 \\
3.1004900 \\
3.5619470 \\
4.1515860 \\
4.5136670 \\
3.9305960 \\
2.8805770 \\
2.6504440 \\
2.0076550 \\
2.1822380 \\
6.1714390 \\
4.2485700 \\
3.9436790 \\
4.4310980 \\
5.5295570 \\
5.8354480 \\
6.3633740 \\
5.3311350 \\
6.2500990 \\
0.6723480 \\
0.5410410 \\
0.8002430 \\
0.5361860 \\
1.5722370 \\
1.8210700 \\
0.4043580 \\
1.2591080 \\
1.4979100 \\
1.2053450 \\
5.5177620\end{array}$ & $\begin{array}{r}-0.9751780 \\
-0.2462930 \\
0.4050370 \\
-0.9478730 \\
-1.0053070 \\
-0.7411630 \\
-2.0870900 \\
-1.1817460 \\
1.1282070 \\
0.8800650 \\
1.1414020 \\
1.1377910 \\
1.6350650 \\
2.2679600 \\
2.3002770 \\
-1.4550960 \\
-1.8451210 \\
-3.1113330 \\
-2.1314180 \\
-1.5642930 \\
-1.8477680 \\
-0.4923140 \\
-2.3667330 \\
-3.1096360 \\
-1.7178830 \\
-3.6216780 \\
-1.2924900 \\
-4.1233450 \\
-4.8508810 \\
-4.6797730 \\
-3.3670230 \\
-3.6781090 \\
-3.5867130 \\
-0.5444940 \\
-0.0324510 \\
-4.7620000 \\
-4.0896380 \\
-6.2610800 \\
-6.9981610 \\
-6.2721360 \\
-4.8762660 \\
-8.0324190 \\
-6.4391040 \\
-6.6678720 \\
-6.1944390 \\
-6.8313480\end{array}$ & $\begin{array}{l}-0.9169870 \\
-2.8584650 \\
-3.6667460 \\
-2.6334830 \\
-3.2790600 \\
-4.3021500 \\
-3.2585010 \\
-2.6034150 \\
-1.2713260 \\
-2.4116270 \\
-3.4365260 \\
-1.7694560 \\
-1.9927020 \\
-2.8097410 \\
-1.1471200 \\
1.5666670 \\
2.9257030 \\
2.2534770 \\
1.2007980 \\
3.9647480 \\
4.9594760 \\
3.9922780 \\
3.5885340 \\
4.3608000 \\
3.4989140 \\
1.9530530 \\
3.1706200 \\
2.4713710 \\
3.2315770 \\
1.5460900 \\
2.9162570 \\
3.9214950 \\
2.2427570 \\
0.6987320 \\
0.9830560 \\
-1.0848660 \\
-1.2992490 \\
-1.2850050 \\
-1.2258340 \\
-2.0808470 \\
-1.5153010 \\
-1.5595080 \\
-2.2678440 \\
-0.5448260 \\
-3.1137660 \\
-2.1129080\end{array}$ \\
\hline
\end{tabular}

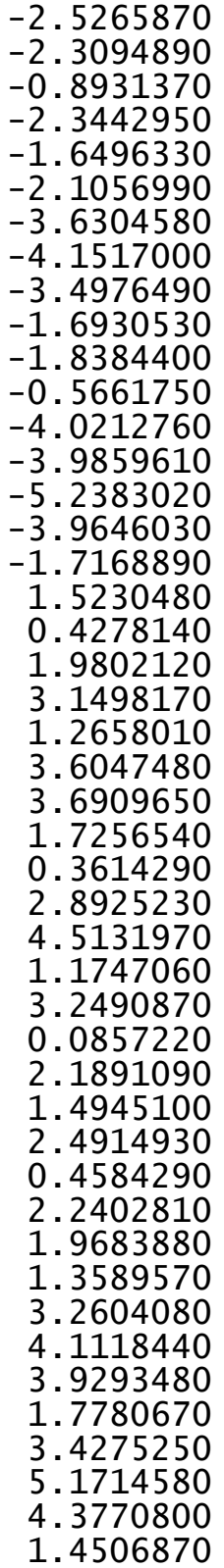

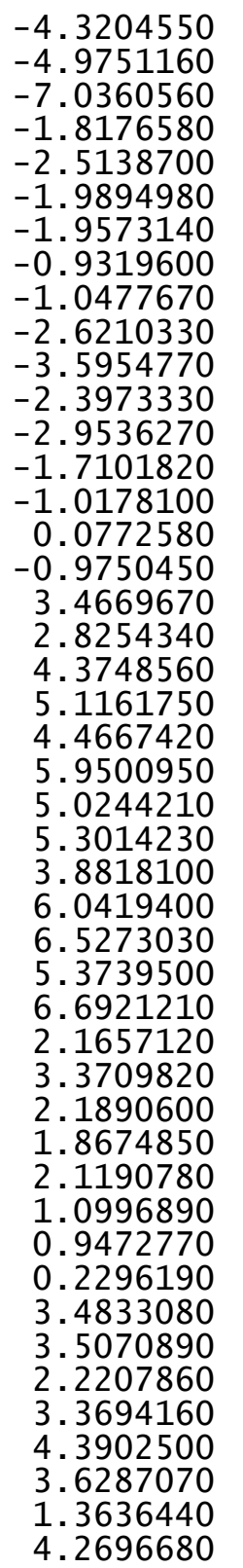

$-2.1352720$ $-0.5346360$ $-0.1885800$ $-2.7841640$ $-3.9422000$ $-5.3030890$ $-5.3595010$ $-4.3561100$ $-2.9919270$ $-6.0961600$ $-3.9072050$ $-3.8268390$ $-5.1134820$ $-6.3647620$ $-4.2416030$ $-4.7483330$ $-5.4612650$ $-0.7245860$ $-0.9863330$ $-1.8146380$ $-1.6333820$ $-3.0114860$ $-2.6476520$ $-0.6969810$ $-4.0246900$ $-3.1376910$ $-3.8432070$ $-2.5092360$ $-4.9567830$ $-4.6362250$ $-0.2539360$ 0.3179050 2.7421160 1.7145790

2.4132280

0.9836710

3. 3073110

2. 9275890

4.0003030

2.7301650

1.9244160

3.6418500

4.5888140

2.9735010

2. 4423900

3.1075810 


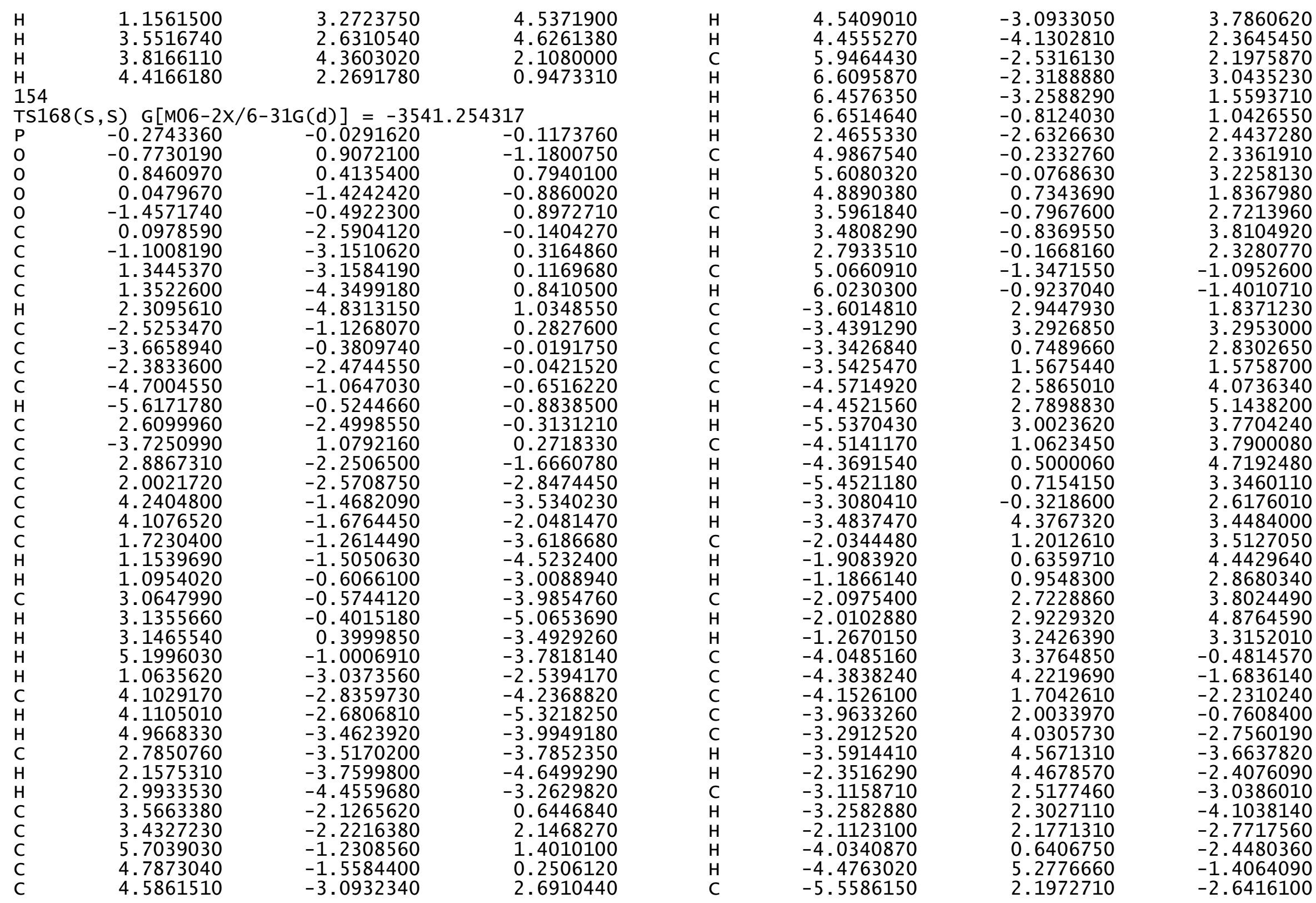




-5.6822660
-6.3263670
-5.7146330
-5.9821070
-6.5125810
-3.8605570
-3.9301140
-3.4065570
-3.1908100
-4.1711160
-5.5941190
-5.7343140
-4.5848390
-4.0702220
-3.2980590
-2.1563330
-5.8003950
-6.3302920
-6.6776500
-5.7825670
-3.9307430
-1.0478540
0.1896400
-2.2764420
-2.1976420
-0.8886860
0.2989010
-3.0582030
-2.3457280
-3.1913300
-0.8365860
-0.8446560
1.2370690
0.3708100
-2.2444120
3.2917650
3.0171260
4.6892520
5.5955850
5.0821810
6.8966330
5.2676950
6.3838370
4.3625040
7.2900990
7.6043230

2. 0519480

3.6958150

4.2910080

3.8298850

3.8501490

4.9167100

$-3.1113120$

$-4.5089150$

$-4.8532320$

$-4.5278430$

$-3.0188710$

$-2.4007690$

$-5.9108470$

$-5.2599140$

$-4.5923100$

$-5.0404740$

$-4.8854280$

$-2.7745110$

$-2.5432020$

$-4.2707180$

$-4.2902790$

$-4.9161730$

$-4.7523370$

$-6.2169670$

$-6.4680750$

$-6.2125030$

$-6.4675310$

$-4.1298340$

$-4.5519040$

$-5.7942650$

$-7.4916680$

$-6.2176990$

$-7.0412070$

$-6.8632680$

2. 6678700

1.4641850

3.1030780

2. 2274010

4.3961130

2.6493920

1.2261690

4.8136540

5.0566530

3.9405170

1.9716590

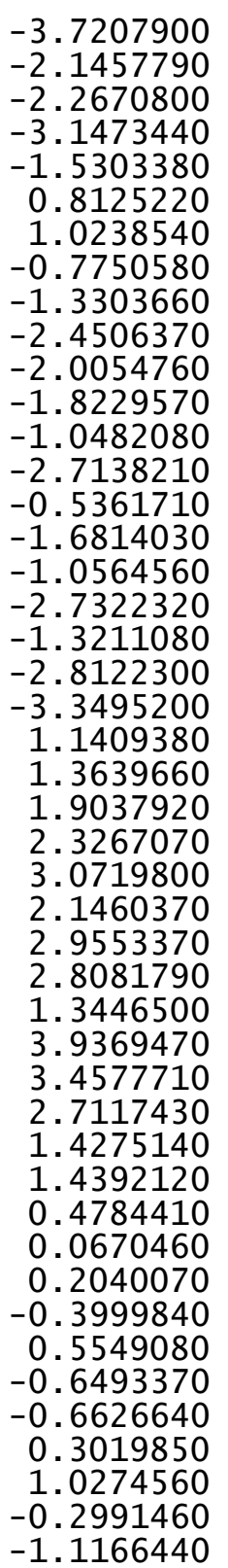
6.6940730
8.3067290
2.0957140
2.4952640
0.5112390
0.4541040
0.9395810
0.3364150
$-0.9148600$
$-1.0965350$
0.3005460
0.6976550
0.1707140
0.8903090
0.6505230
0.3159510
$-0.9207200$
1.9862960
0.5565830
$-0.7927860$
1.7906340
0.5927690

154

TS169(S, S) G[M06-2X/6-31G(d) $]=-3541.254601$

$\begin{array}{lrrr}\text { TS169(S, S) G[M06-2X/6-31G(d) }]=-3541.254601 \\ \text { P } & 0.3486340 & 0.2530350 & 0.8090280 \\ \text { O } & -0.3584640 & 0.7433150 & 2.0366040 \\ \text { O } & 0.6445520 & -1.2199340 & 0.6677280 \\ \text { O } & 1.6876700 & 1.1557630 & 0.6632920 \\ \text { O } & -0.4650430 & 0.6450440 & -0.5558020 \\ \text { C } & 2.3245580 & 1.1353310 & -0.5695070 \\ \text { C } & 1.7673700 & 1.8878980 & -1.6139600 \\ \text { C } & 3.4861950 & 0.3798790 & -0.7068600 \\ \text { C } & 4.0916590 & 0.3668520 & -1.9674040 \\ \text { H } & 5.0018210 & -0.2141870 & -2.1031810 \\ \text { C } & -0.5785030 & 2.0020650 & -0.8242160 \\ \text { C } & -1.7945680 & 2.6351840 & -0.5713930 \\ \text { C } & 0.5292500 & 2.6750760 & -1.3442990 \\ \text { C } & -1.8587730 & 4.0020650 & -0.8236500 \\ \text { H } & -2.7985410 & 4.5211830 & -0.6410340 \\ \mathrm{C} & 4.0646630 & -0.3885640 & 0.4302900 \\ \mathrm{C} & -2.9738780 & 1.8687720 & -0.0828110 \\ \mathrm{C} & 4.5005190 & 0.2622450 & 1.5944250 \\ \mathrm{C} & 4.4135350 & 1.7398420 & 1.8946930 \\ \mathrm{C} & 5.5240680 & 0.3885260 & 3.8013020 \\ \mathrm{C} & 5.1165840 & -0.4613620 & 2.6255730 \\ \mathrm{C} & 3.6055190 & 1.9209590 & 3.1985400\end{array}$

$\begin{array}{rr}5.8173060 & 0.5740930 \\ 4.2678990 & -0.4942180 \\ 1.0880160 & 0.3530580 \\ 3.4305420 & 1.0440910 \\ 3.5453280 & -0.8555120 \\ 3.4755590 & 0.6069710 \\ 2.6699810 & -1.3468400 \\ 2.4927290 & 1.0508440 \\ 3.4085820 & -0.6885610 \\ 2.4187620 & -0.8256540 \\ 6.0500080 & -0.7155280 \\ 5.9800790 & 0.7600600 \\ 4.7012330 & 1.4146460 \\ 4.8656940 & -1.4843330 \\ 6.9864410 & -1.1596890 \\ 6.8471530 & 1.3067210 \\ 4.7410880 & 1.5292060 \\ 4.9276530 & -1.4882740 \\ 4.8590300 & -2.5270500 \\ 6.0348790 & -0.8059750 \\ 5.9949100 & 0.8442880 \\ 4.5498590 & 2.4117070\end{array}$

5740930

$\begin{array}{ll}1.0880160 & 0.3530580\end{array}$

$-1.0440910$

$3.4755590 \quad 0.6069710$

$2.6699810 \quad-1.3468400$

$3.4085820-0.6885610$

$2.4187620 \quad-0.8256540$

6.0500080

9864410

4.7410880

. 9276530

.8590300

. 0348790

5.9949100

2.4117070

0.7600600

1.4843330

1.3067210

1.5292060

.8059750

0.3584640

0.6445520

0.4650430

4.0020650

1.9209590

3.1985400 


$\begin{array}{rrrr}\mathrm{H} & 3.5842010 & 2.9869420 & 3.4525010 \\ \mathrm{H} & 2.5722340 & 1.6071180 & 3.0295700 \\ \mathrm{C} & 4.2629100 & 1.0993240 & 4.3385710 \\ \mathrm{H} & 4.5467250 & 1.7473370 & 5.1757080 \\ \mathrm{H} & 3.5644710 & 0.3532600 & 4.7293400 \\ \mathrm{H} & 5.9888090 & -0.2200440 & 4.5844310 \\ \mathrm{H} & 3.9451960 & 2.2960980 & 1.0793250 \\ \mathrm{C} & 6.5048690 & 1.4701770 & 3.2995410 \\ \mathrm{H} & 6.7610230 & 2.1342830 & 4.1328930 \\ \mathrm{H} & 7.4334580 & 0.9967290 & 2.9664750 \\ \mathrm{C} & 5.8457670 & 2.2646500 & 2.1419590 \\ \mathrm{H} & 5.7949040 & 3.3323320 & 2.3833610 \\ \mathrm{H} & 6.4323550 & 2.1692070 & 1.2229550 \\ \mathrm{C} & 4.2338420 & -1.7778100 & 0.3378340 \\ \mathrm{C} & 3.7453080 & -2.6793290 & -0.7719190 \\ \mathrm{C} & 5.0093910 & -3.9630710 & 1.0868100 \\ \mathrm{C} & 4.8741180 & -2.4877910 & 1.3647410 \\ \mathrm{C} & 4.9572950 & -3.4166290 & -1.3819560 \\ \mathrm{H} & 4.5943680 & -4.1425340 & -2.1186770 \\ \mathrm{H} & 5.5967610 & -2.7079600 & -1.9185040 \\ \mathrm{C} & 5.7541610 & -4.1282510 & -0.2560720 \\ \mathrm{H} & 5.8695730 & -5.1954530 & -0.4762470 \\ \mathrm{H} & 6.7594090 & -3.7052600 & -0.1665260 \\ \mathrm{H} & 5.5530000 & -4.4675260 & 1.8927300 \\ \mathrm{H} & 3.2007460 & -2.1200010 & -1.5369490 \\ \mathrm{C} & 3.5964440 & -4.5610490 & 0.9193220 \\ \mathrm{H} & 3.6895740 & -5.6090020 & 0.6107540 \\ \mathrm{H} & 3.0754660 & -4.5524670 & 1.8814460 \\ \mathrm{C} & 2.8147490 & -3.7368030 & -0.1346650 \\ \mathrm{H} & 2.4156050 & -4.3849940 & -0.9235070 \\ \mathrm{H} & 1.9698730 & -3.2209420 & 0.3253440 \\ \mathrm{C} & 5.3175520 & -1.8330030 & 2.5081960 \\ \mathrm{H} & 5.8115820 & -2.3875150 & 3.3047100 \\ \mathrm{C} & -4.7215310 & 0.2181110 & -0.4872650 \\ \mathrm{C} & -5.2648420 & -0.7388200 & -1.5180900 \\ \mathrm{C} & -3.1536490 & 0.5322870 & -2.3103660 \\ \mathrm{C} & -3.5716820 & 0.9019130 & -0.9068630 \\ \mathrm{C} & -5.6008040 & 0.0771810 & -2.7865650 \\ \mathrm{H} & -5.9526840 & -0.6060190 & -3.5676190 \\ \mathrm{H} & -6.4193000 & 0.7698510 & -2.5691600 \\ \mathrm{C} & -4.3371510 & 0.8469280 & -3.2537640 \\ \mathrm{H} & -4.0620660 & 0.5586730 & -4.2743850 \\ \mathrm{H} & -4.5202660 & 1.9257310 & -3.2608460 \\ \mathrm{H} & -2.2610070 & 1.0782440 & -2.6259610 \\ \mathrm{H} & -6.1557700 & -1.2561700 & -1.1452150 \\ \mathrm{C} & -2.8936090 & -0.9872640 & -2.3582730\end{array}$

-2.6255990
-2.0287240
-4.1592270
-4.5284880
-3.9298770
-4.6997850
-5.2075960
-3.0831340
-3.5430270
-4.0824280
-4.4352950
-3.8428020
-2.8310240
-2.6019510
-1.9487110
-2.1781460
-6.1079500
-4.2455120
-3.9426060
-4.4510860
-5.5067950
-5.8051750
-6.3530510
-5.2918190
-6.1972330
0.4530490
1.6818090
1.5749940
0.2538990
-0.9055550
-0.7570190
2.4269280
1.8264870
2.5691790
0.1805650
0.2004330
-1.8590720
-0.9731970
1.6187230
2.3749730
1.7927170
1.6965630
3.0550550
4.1267220
3.5439640
2.4395130
$-3.3814250$

$-1.7287260$

$-1.8875790$

$-2.6745880$

$-1.0231780$

1. 5799030

2.9322540

2.1835380

1.1719170

3. 9643100

4.9532520

4.0274390

3.5402360

4. 2822410

3.4597420

1.8524010

3.2097510

2. 3839960

3. 1148160

1.4434680

2.8728640

3.8705130

2. 2033530

0.7530250

1.0694710

$-1.5383530$

$-1.9666190$

$-1.6431980$

$-2.1779510$

$-1.4289770$

$-1.2772230$

$-2.0788810$

$-3.0494540$

$-1.4972380$

$-3.2502460$

$-2.0719860$

$-1.9228140$

$-0.4253350$

$-0.5567820$

$-2.8749530$

$-4.1359510$

$-5.2083920$

$-4.4302620$

$-3.0426240$

$-4.5099640$ 


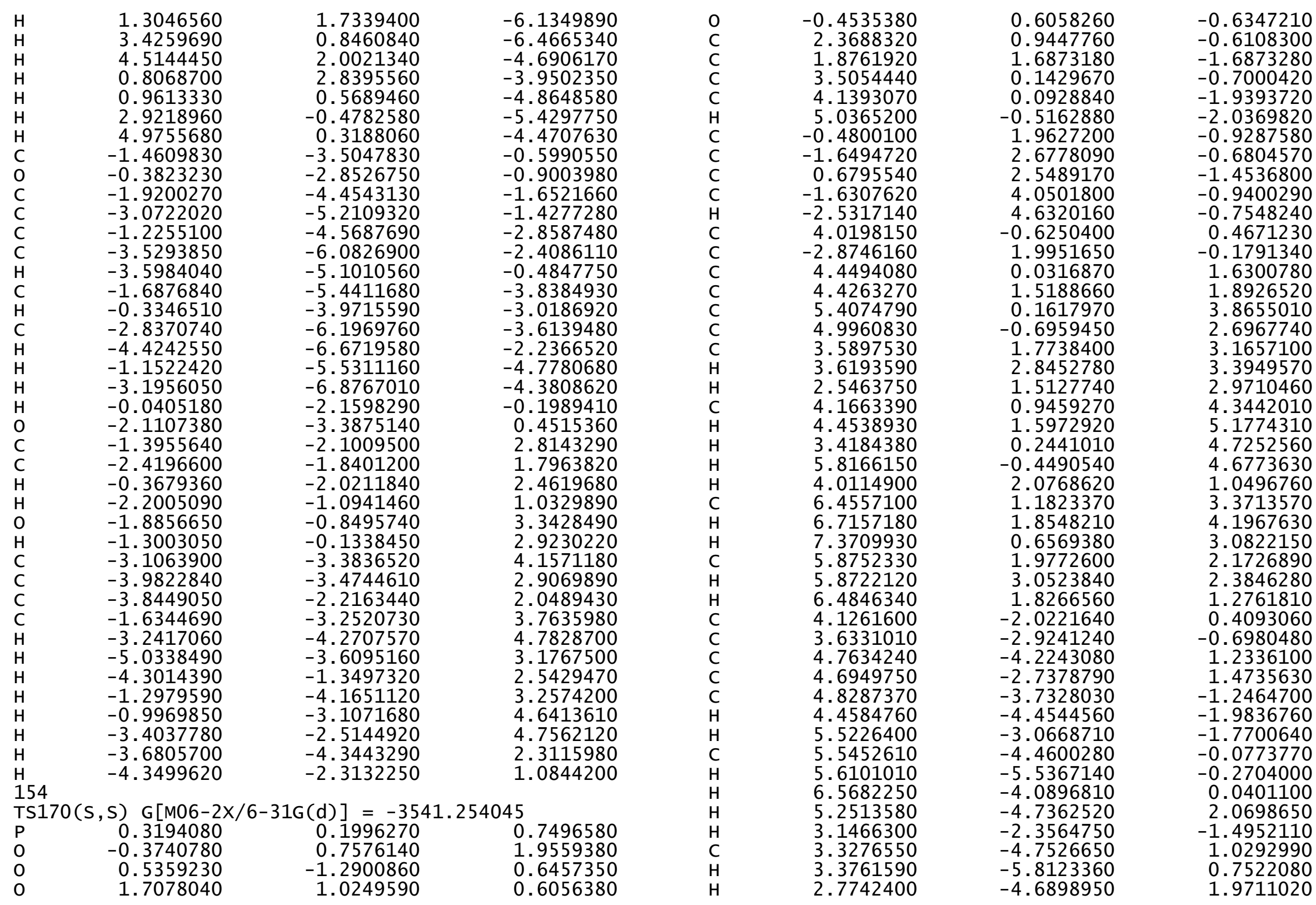




$\begin{array}{rrr}2.6279290 & -3.9194620 & -0.0746260 \\ 2.2305480 & -4.5685930 & -0.8635380 \\ 1.7907700 & -3.3530070 & 0.3381180 \\ 5.1318260 & -2.0783660 & 2.6168970 \\ 5.5702330 & -2.6373590 & 3.4423150 \\ -4.7274340 & 0.4563200 & -0.5584090 \\ -5.3323990 & -0.4809510 & -1.5730240 \\ -3.1386080 & 0.6299160 & -2.3814890 \\ -3.5330180 & 1.0534050 & -0.9863970 \\ -5.6098720 & 0.3318250 & -2.8575880 \\ -6.0045540 & -0.3408680 & -3.6272200 \\ -6.3807890 & 1.0815580 & -2.6561080 \\ -4.2964220 & 1.0065390 & -3.3340410 \\ -4.0392670 & 0.6824400 & -4.3485600 \\ -4.4066420 & 2.0949060 & -3.3605580 \\ -2.2106300 & 1.1080320 & -2.7045330 \\ -6.2571240 & -0.9294200 & -1.1932820 \\ -2.9823900 & -0.9043410 & -2.4011060 \\ -2.7333670 & -1.2278540 & -3.4181680 \\ -2.1352320 & -1.1752240 & -1.7658190 \\ -4.2971930 & -1.5723140 & -1.9199640 \\ -4.7087270 & -2.2288450 & -2.6954430 \\ -4.1119150 & -2.2010950 & -1.0434780 \\ -4.6309320 & 1.7182110 & 1.4839480 \\ -5.1162220 & 2.2086790 & 2.8233900 \\ -2.9074140 & 3.3262310 & 2.0615850 \\ -3.4296000 & 2.3150730 & 1.0681820 \\ -4.0145070 & 1.9326180 & 3.8677510 \\ -4.3514160 & 2.2895630 & 4.8476000 \\ -3.8466200 & 0.8542760 & 3.9540670 \\ -2.7102630 & 2.6486110 & 3.4355350 \\ -2.4364190 & 3.4227150 & 4.1615690 \\ -1.8710420 & 1.9536620 & 3.3778490 \\ -1.9680100 & 3.7718830 & 1.7259960 \\ -6.0533800 & 1.7167480 & 3.1063860 \\ -4.0014260 & 4.4051850 & 2.2329240 \\ -3.6525430 & 5.1567820 & 2.9495640 \\ -4.1670840 & 4.9191930 & 1.2802830 \\ -5.3133420 & 3.7392500 & 2.7295230 \\ -5.5933740 & 4.1214240 & 3.7176520 \\ -6.1421520 & 3.9615180 & 2.0507070 \\ -5.2822200 & 0.7942380 & 0.6733530 \\ -6.2217330 & 0.3456780 & -1.6619630 \\ 0.6793740 & 3.9363930 & -0585020 \\ 1.8957120 & 4.7371410 & -85110\end{array}$

0.8253040 $-0.3949670$ $-0.4821680$ 1.7565540 2. 9436370 1.0041180 $-0.2927280$ 2. 7886470 2. 3673430 0.5807820 $-1.3150170$ 2. 5006730 3.6531030 1.8716800 2. 8450520 3. 5628540 4. 4089610 2. 3021260 1.0344660 1. 4253430 2. 8172210 4.1963690 4.8076290 5.2805080 3.5859200 $-1.7064230$ $-0.5816710$ $-2.2327110$ $-3.4234200$ $-1.5645480$ $-3.9450420$ $-3.9285130$ $-2.0920090$ $-0.6435040$ $-3.2794840$ $-4.8696730$ $-1.5777410$ $-3.6888350$ $-0.1994390$ $-2.3483080$ $-1.5716790$ $-2.5734180$ $-0.5395280$ $-2.3067980$ $-1.9873290$ $-1.3625420$
6.7614720

6.1681050

4. 6813300

5. 1596700

6.5014070

.7.7738520

6.3656950

4. 1073060

5.4487380

6.8579450

6632060

1. 5638490

0.7703390

2.1844940

2. 3189080

0.9939950

0.6401110

2.6396370

1.5387740

3. 1519570

0.2076940

1. 0444190

$-0.3766290$

1.3090160

3. 0982350

$-3.4579180$

$-2.8894060$

$-4.3775930$

$-5.0672790$

$-4.5247240$

$-5.9056990$

-4 9317520

$-5.3623140$

$-3.9785330$

$-6.0519270$

$-6.4434370$

$-5.4764920$

$-6.7047200$

$-2.2082950$

$-3.2855460$

$-2.0014960$

$-1.6998100$

$-0.9812410$

$-0.7136180$

$-0.0421530$
$-0.8949310$

$-1.6279100$

$-1.4048620$

$-3.0642670$

$-1.2700060$

$-2.7037020$

$-2.0976180$

$-0.0924220$

0.1670460

$-1.3030320$

$-2.9443850$

$-3.0585720$

$-4.1788810$

$-5.3475930$

$-5.5882520$

$-4.3680340$

$-6.2425030$

$-4.4826050$

$-3.9454630$

$-5.7641110$

$-6.4796030$

$-4.4546280$

$-4.3357330$

$-5.1221280$

$-0.5948490$

$-0.8975140$

$-1.6428970$

$-1.4043490$

$-2.8607050$

$-2.3821230$

$-0.4532080$

$-3.8379280$

$-3.0315930$

-3. 5990620

$-2.1991030$

$-4.7866510$

$-4.3639470$

$-0.2062780$

0.4529900

2.7974560

1.7684310

2. 4494900

0.9940190

3. 3009080

2.8654990 


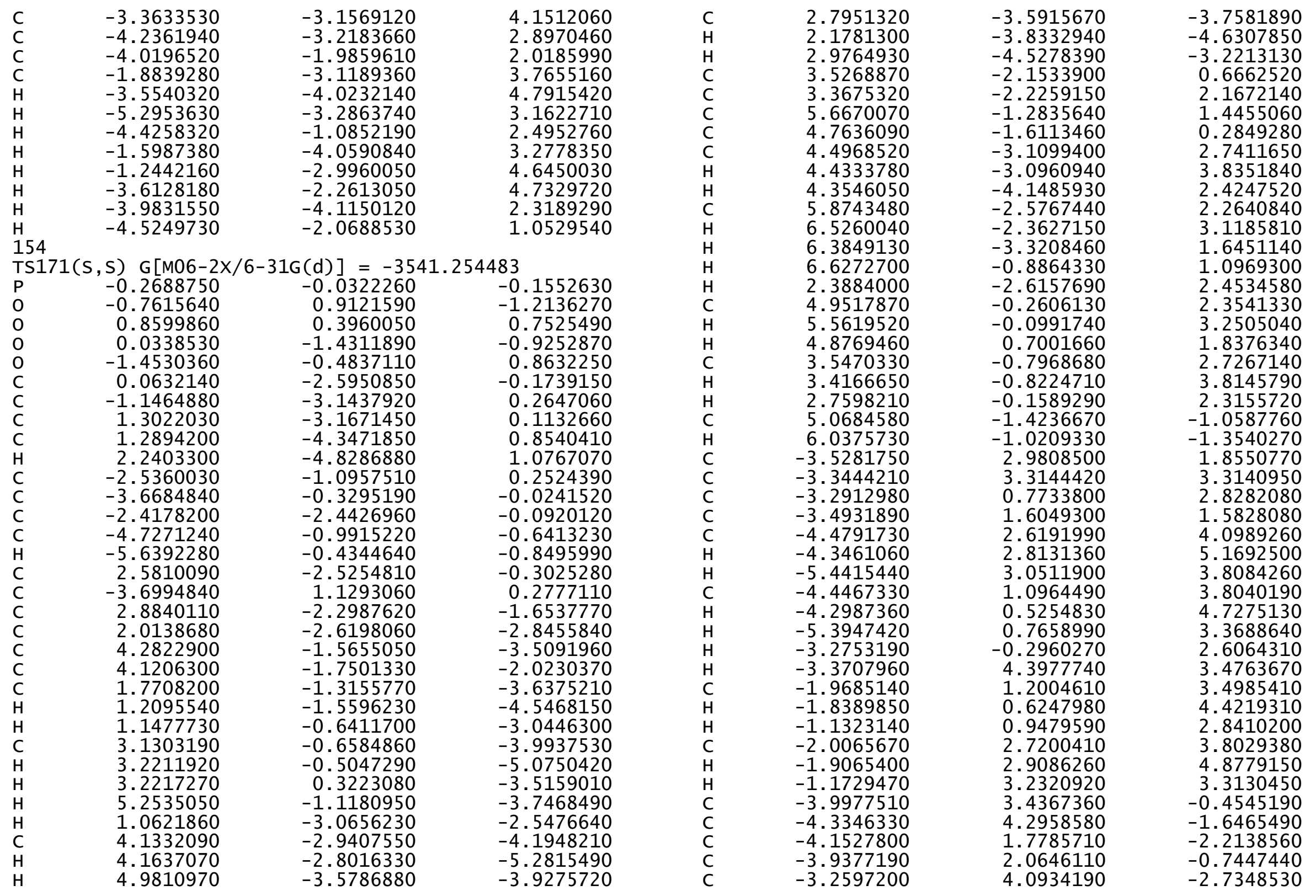




\begin{tabular}{|c|c|c|}
\hline $\begin{array}{l}-3.5626560 \\
-2.3081440 \\
-3.1141360 \\
-3.2757790 \\
-2.1125650 \\
-4.0561910 \\
-4.4055980 \\
-5.5560980 \\
-5.6976920 \\
-6.3265050 \\
-5.6817600 \\
-5.9505620 \\
-6.4676010 \\
-3.7863720 \\
-3.8372590 \\
-4.6413720 \\
-3.4648710 \\
-5.8422570 \\
-5.4890170 \\
-4.5994260 \\
-3.2785420 \\
-6.4045800 \\
-6.5674970 \\
-6.3420640 \\
-5.1150240 \\
-4.4033300 \\
-2.6606020 \\
-2.7031940 \\
-4.9521050 \\
-1.1174200 \\
0.1132050 \\
-2.3765180 \\
-2.2882840 \\
-1.0020790 \\
0.2067260 \\
-3.1672000 \\
-2.5246880 \\
-3.2609230 \\
-0.9784620 \\
-0.9623900 \\
1.1317900 \\
0.2953670 \\
-2.2975010 \\
3.3288670 \\
3.0390820 \\
4.7321640\end{array}$ & $\begin{array}{r}4.6417330 \\
4.5119090 \\
2.5799220 \\
2.3752950 \\
2.2203310 \\
0.7146350 \\
5.3510970 \\
2.2975400 \\
2.1608720 \\
1.7040340 \\
3.7961090 \\
4.4012590 \\
3.9390720 \\
3.8977830 \\
4.9636600 \\
-2.3258300 \\
-3.0540640 \\
-2.9526210 \\
-4.1820980 \\
-5.1207810 \\
-4.4307530 \\
-4.6879070 \\
-3.2456300 \\
-2.1979840 \\
-5.4000170 \\
-6.0467160 \\
-5.0674870 \\
-4.2986090 \\
-3.8737740 \\
-4.2807950 \\
-4.9036870 \\
-4.7527390 \\
-6.2002350 \\
-6.4113570 \\
-6.1813160 \\
-6.4414850 \\
-4.0988300 \\
-4.6091080 \\
-5.7039410 \\
-7.4191410 \\
-6.1695140 \\
-7.0287970 \\
-6.8767650 \\
2.6272430 \\
1.4308690 \\
3.0465550\end{array}$ & $\begin{array}{l}-3.6346320 \\
-2.3960260 \\
-3.0308430 \\
-4.0953560 \\
-2.7815520 \\
-2.4392960 \\
-1.3613340 \\
-2.6011630 \\
-3.6792610 \\
-2.0977270 \\
-2.2155300 \\
-3.0885700 \\
-1.4675240 \\
0.8404360 \\
1.0605340 \\
-1.0472550 \\
-0.8095360 \\
-1.7318700 \\
-2.5657290 \\
-1.7548050 \\
-1.4233640 \\
-2.8888160 \\
-0.9595840 \\
-2.3490460 \\
-0.8253050 \\
-2.3048790 \\
-0.7861580 \\
-2.3513580 \\
-3.4721380 \\
1.0965200 \\
1.3583870 \\
1.8043260 \\
2.2818110 \\
3.0758570 \\
2.1721480 \\
2.8882330 \\
2.6763290 \\
1.1801030 \\
3.9149130 \\
3.5016170 \\
2.7589330 \\
1.4778020 \\
1.4158230 \\
0.4470330 \\
0.0247270 \\
0.1776840\end{array}$ \\
\hline
\end{tabular}

5.1382240

6.9380260

5.2932710

6.4455120

4.4240680

7.3444320

7.6402370

6.7658730

8.3655270

2. 1150290

2. 5418770

0.5519620

0.4993510

0.9708510

0.3741570

$-0.8745360$

$-1.0660220$

0.3624130

0.7653950

0.2307170

0.9395410

0.7177000

0.3933720

$-0.8597480$

2.0359580

0.6015680

$-0.7314390$

1.8588440

0.6569610
2.1603620

4.3350020

2. 5675600

1.1626370

4.7378630

5.0036440

3. 8543490

1.8816950

5.7380490

4.1701520

1.0618950

3.3950890

3.5367140

3.4614650

2.6597750

2.4778720

3.4116910

. 4245290

6.0422950

.9633060

4.6863650

4.8563770

6.9777180

6.8313540

4.7349260

4.9094570

4.8565590

6.0359780

5.9688040

4.5274250
$-0.4219690$

0.5303570

$-0.6655180$

$-0.6854020$

0.2827920

0.9998730

$-0.3141690$

$-1.1291780$

0.5559190

$-0.5049560$

0.3105050

1.0188090

$-0.8737010$

0.5886770

$-1.3702130$

1.0288160

$-0.7017040$

$-0.8448730$

$-0.7229260$

0.7505670

1.4025100

$-1.4988100$

$-1.1649900$

1. 3023360

1.5232800

$-1.5069670$

$-2.5401800$

$-0.8084440$

0.8300140

2. 3966030

$\operatorname{TS} 172(\mathrm{~S}, \mathrm{~S}) \mathrm{G}[\mathrm{M} 06-2 \mathrm{X} / 6-31 \mathrm{G}(\mathrm{d})]=-3541.255402$

$\begin{array}{lrrr}\text { TS172(S, S) G[M06-2X/6-31G(d) }]=-3541.255402 \\ \text { P } & -0.3219730 & -0.1886300 & 0.8336650 \\ \text { O } & 0.3908540 & -0.6596460 & 2.0655160 \\ O & -0.5810650 & 1.2863590 & 0.6484070 \\ \text { O } & -1.6906080 & -1.0563080 & 0.7396880 \\ \text { O } & 0.4662160 & -0.6425490 & -0.5287140 \\ \text { C } & -2.3409120 & -1.0607870 & -0.4864010 \\ \text { C } & -1.8122890 & -1.8482160 & -1.5102990 \\ \text { C } & -3.4861680 & -0.2817580 & -0.6456160 \\ \text { C } & -4.0846070 & -0.3032160 & -1.9034980 \\ \text { H } & -4.9884080 & 0.2846990 & -2.0553250 \\ \text { C } & 0.5403350 & -2.0103690 & -0.7531480 \\ \text { C } & 1.7413850 & -2.6718320 & -0.5001260 \\ \text { C } & -0.6001670 & -2.6684140 & -1.2143800 \\ \text { C } & 1.7552220 & -4.0493340 & -0.6986040\end{array}$




$\begin{array}{lr}\mathrm{H} & 2.6832730 \\ \mathrm{C} & -4.0474030 \\ \mathrm{C} & 2.9521290 \\ \mathrm{C} & -4.5046020 \\ \mathrm{C} & -4.4517800 \\ \mathrm{C} & -5.5466290 \\ \mathrm{C} & -5.1087570 \\ \mathrm{C} & -3.6558760 \\ \mathrm{H} & -3.6511260 \\ \mathrm{H} & -2.6173770 \\ \mathrm{C} & -4.3080480 \\ \mathrm{H} & -4.6165260 \\ \mathrm{H} & -3.5975400 \\ \mathrm{H} & -6.0038970 \\ \mathrm{H} & -3.9891980 \\ \mathrm{C} & -6.5484360 \\ \mathrm{H} & -6.8317830 \\ \mathrm{H} & -7.4603680 \\ \mathrm{C} & -5.8961260 \\ \mathrm{H} & -5.8684660 \\ \mathrm{H} & -6.4732410 \\ \mathrm{C} & -4.1791960 \\ \mathrm{C} & -3.6594870 \\ \mathrm{C} & -4.8991350 \\ \mathrm{C} & -4.8059980 \\ \mathrm{C} & -4.8473010 \\ \mathrm{H} & -4.4606740 \\ \mathrm{H} & -5.5026250 \\ \mathrm{C} & -5.6305940 \\ \mathrm{H} & -5.7140510 \\ \mathrm{H} & -6.6481080 \\ \mathrm{H} & -5.4336260 \\ \mathrm{H} & -3.1264500 \\ \mathrm{C} & -3.4690940 \\ \mathrm{H} & -3.5314590 \\ \mathrm{H} & -2.9545280 \\ \mathrm{C} & -2.7038590 \\ \mathrm{H} & -2.2829820 \\ \mathrm{H} & -1.8757590 \\ \mathrm{C} & -5.2737840 \\ \mathrm{H} & -5.7584630 \\ \mathrm{C} & 4.7381920 \\ \mathrm{C} & 5.2890980 \\ \mathrm{C} & 3.1266940 \\ \mathrm{C} & 3.5614120 \\ \mathrm{C} & 5.5765880 \\ & \end{array}$

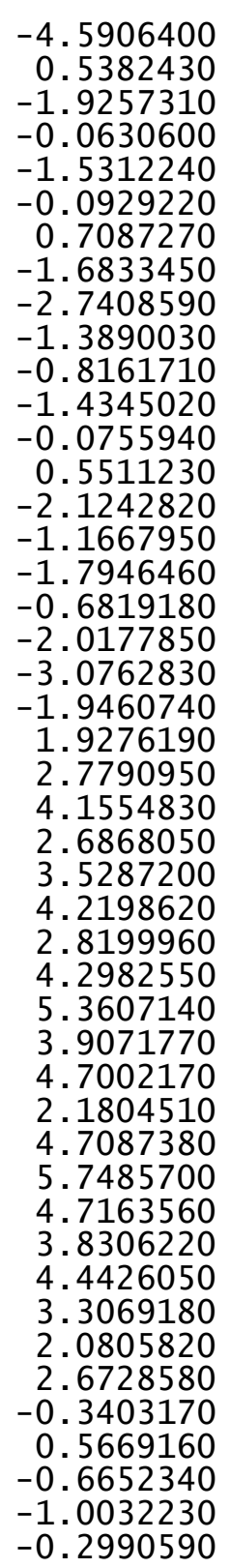

-0.5213150
0.4631150
-0.0602110
1.6451240
1.9946290
3.8464040
2.6479530
3.3095020
3.5972480
3.1373800
4.4184300
5.2691810
4.7976290
4.6051420
1.2020150
3.3695450
4.2219490
3.0081130
2.2489270
2.5311540
1.3218220
0.3248760
-0.8097390
0.9985580
1.3243800
-1.4512750
-2.2090430
-1.9683460
-0.3542710
-0.6091210
-0.2584260
1.7841160
-1.5527280
0.8222400
0.4803140
1.7878040
-0.2002210
-1.0065000
0.2812990
2.4848990
3.2596660
-0.5492280
-1.6203050
-2.3320810
-0.9257170
-2.8674410

\begin{abstract}
5. 9348920
6. 3772460

4. 2815770

3. 9952450

4.4323080

2. 2125630

6.2018420

2. 9096800

2. 6270260

2. 0659710

4. 2068500

4. 5800890

4. 0133480

4.7198640

5. 2379000

3. 0650100

3. 5367840

4. 1369720

4. 4973030

3. 9288710

2. 8561010

2. 6187660

1. 9905020

2. 1402050

6.1584900

4. 2012340

3. 8888850

4. 3758610

5. 4919000

5.7961150

6. 3217310

5. 3235940

6.2488300

0.6156270

$-0.5825440$

0.7212080

$-0.6304220$

$-1.6268170$

$-1.8683420$

$-0.5142390$

1. 1915270

1. 3993620

$-1.2273320$

$-2.5772090$

$-2.5165840$

-2. 4226630
\end{abstract}

0.3476020
-1.0097280
-1.0434680
-0.7771670
-2.1270610
-1.1942660
1.0690880
0.8591500
1.1227140
1.1277980
1.5992200
2.2274970
2.2670600
-1.4966770
-1.8934550
-3.1337480
-2.1595410
-1.6003000
-1.8887900
-0.5258150
-2.3874740
-3.1284270
-1.7286610
-3.6327350
-1.3517490
-4.1594900
-4.8832340
-4.7182720
-3.4184620
-3.7337040
-3.6475250
-0.5927880
-0.0917160
-4.7609660
-4.0730520
-6.2659200
-6.9735900
-6.2574320
-4.8372330
-8.0222620
-6.4782090
-6.6655140
-6.2229840
-6.7988350
-4.2900950
-4.8848150

$-3.6761740$

$-2.6418560$

$-3.2874730$

$-4.3109700$

$-3.2656620$

$-2.6132360$

$-1.2809590$

$-2.4224860$

$-3.4480000$

$-1.7816270$

$-2.0032990$

$-2.8204000$

$-1.1580820$

1. 5572690

2. 9153460

2. 2456780

1.1922330

3.9563870

4.9503170

3. 9848510

3. 5815710

4. 3535450

3.4941640

1.9466740

3. 1590350

2. 4618040

3. 2226070

1.5363840

2. 9045610

3.9090110

2. 2292660

0.6890490

0.9725820

$-1.0822460$

$-1.3259710$

$-1.2459110$

$-1.1887510$

$-2.0964420$

$-1.5906030$

$-1.4805660$

$-2.2164420$

$-0.4840060$

$-3.1196190$

$-2.1400350$

$-2.2792400$

$-0.6418090$ 


$\begin{array}{rrr}-1.0112450 & -6.9637410 & -0.1593150 \\ -2.3903240 & -1.7840620 & -2.7934230 \\ -3.5512530 & -1.0180290 & -2.9779410 \\ -1.7009540 & -2.4341660 & -3.9808150 \\ -2.6311400 & -2.6394340 & -5.1741090 \\ -3.3697010 & -1.3421240 & -5.4919420 \\ -4.2638870 & -0.9558640 & -4.3162240 \\ -2.0517730 & -2.9826790 & -6.0371970 \\ -0.8735190 & -1.7746010 & -4.2817610 \\ -1.2312720 & -3.3785500 & -3.6958240 \\ -2.6355540 & -0.5471200 & -5.6766950 \\ -3.9718320 & -1.4437070 & -6.4005230 \\ -4.6782220 & 0.0482330 & -4.4587960 \\ -5.1234520 & -1.6401540 & -4.2852250 \\ -3.3619410 & -3.4265210 & -4.9419290 \\ 1.5629520 & 3.4638570 & -0.7359100 \\ 0.4592550 & 2.8357670 & -0.9950320 \\ 2.0284050 & 4.3660390 & -1.8271290 \\ 3.2073990 & 5.0931040 & -1.6488880 \\ 1.3122790 & 4.4662660 & -3.0222950 \\ 3.6699830 & 5.9211430 & -2.6645290 \\ 3.7496470 & 4.9950490 & -0.7137620 \\ 1.7797900 & 5.2950130 & -4.0368490 \\ 0.4005150 & 3.8924230 & -3.1461200 \\ 2.9560340 & 6.0213100 & -3.8583970 \\ 4.5857360 & 6.4872740 & -2.5285100 \\ 1.2275010 & 5.3739770 & -4.9676210 \\ 3.3185870 & 6.6668770 & -4.6524720 \\ 0.1122410 & 2.1773300 & -0.2643380 \\ 2.2303430 & 3.3598220 & 0.3049350 \\ 1.5252790 & 2.1783560 & 2.7260910 \\ 2.5204870 & 1.8520210 & 1.6983140 \\ 0.4888480 & 2.1170060 & 2.3965060 \\ 2.2640290 & 1.0874970 & 0.9657240 \\ 1.9894500 & 0.9318190 & 3.2886070 \\ 1.3764980 & 0.2194030 & 2.9045140 \\ 3.3000260 & 3.4565380 & 3.9883960 \\ 4.1524980 & 3.4768730 & 2.7188800 \\ 3.9608850 & 2.1937810 & 1.9101470 \\ 1.8170910 & 3.3544460 & 3.6288040 \\ 3.4734960 & 4.3608620 & 4.5790940 \\ 5.2128200 & 3.5899330 & 2.9632640 \\ 4.4013950 & 1.3321170 & 2.4267860 \\ 1.4966990 & 4.2583750 & 4.5965070 \\ 1.1939570 & 3.2599100 & 45630 \\ 3.5845860 & 2.6007290 & \\ -1.6124700\end{array}$

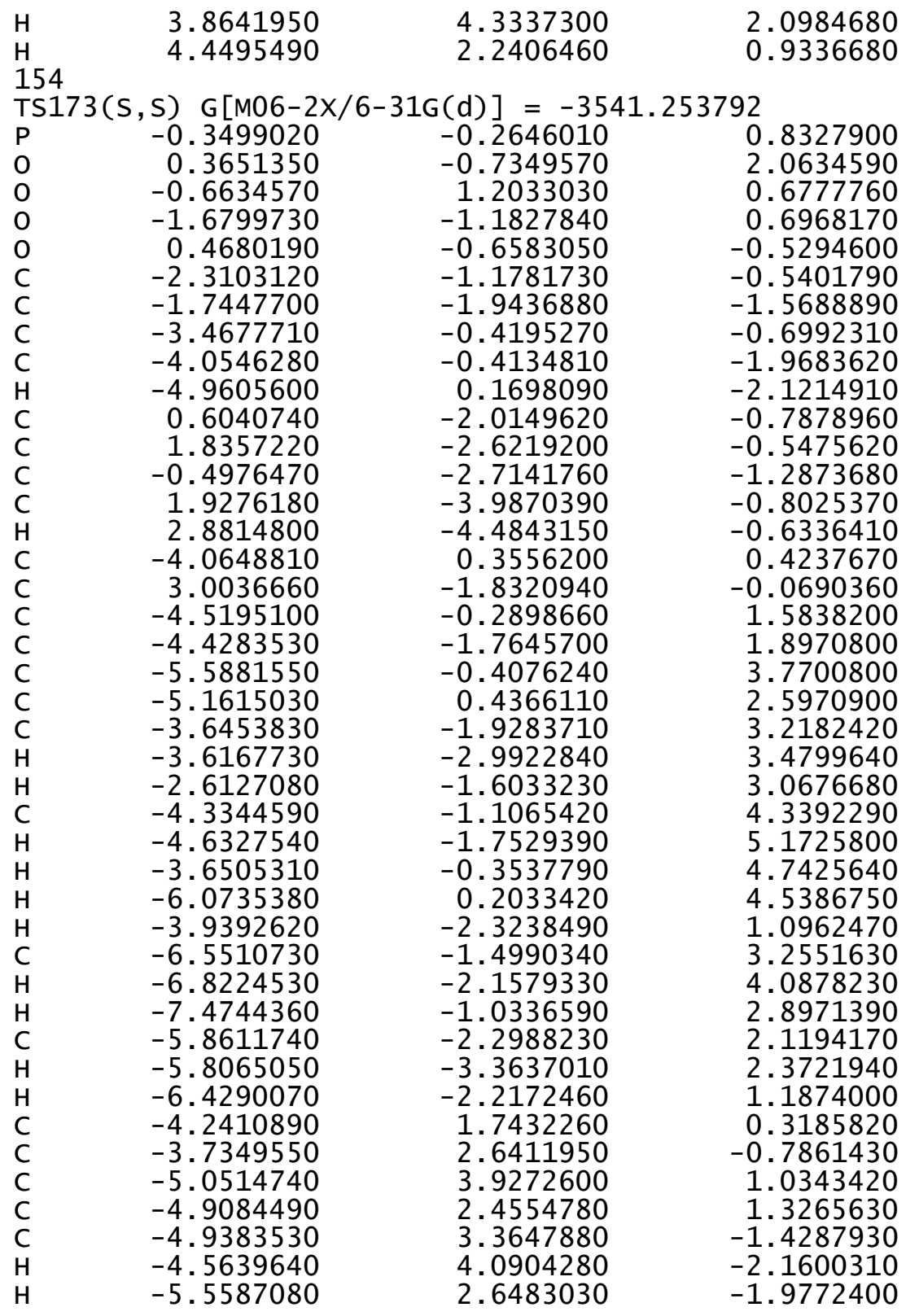




$\begin{array}{rrr}-5.7680790 & 4.0747710 & -0.3258740 \\ -5.8907700 & 5.1390440 & -0.5560760 \\ -6.7701660 & 3.6407650 & -0.2555600 \\ -5.6175750 & 4.4324500 & 1.8241590 \\ -3.1680060 & 2.0813880 & -1.5344350 \\ -3.6411890 & 4.5377940 & 0.8929150 \\ -3.7376410 & 5.5817270 & 0.5719490 \\ -3.1418350 & 4.5438860 & 1.8664590 \\ -2.8282600 & 3.7111070 & -0.1350040 \\ -2.4146830 & 4.3558150 & -0.9192470 \\ -1.9910560 & 3.2053560 & 0.3495630 \\ -5.3707990 & 1.8056070 & 2.4652690 \\ -5.8862620 & 2.3618810 & 3.2468450 \\ 4.7217360 & -0.1553220 & -0.4915980 \\ 5.2444120 & 0.8036580 & -1.5312540 \\ 3.1500870 & -0.5065350 & -2.3048650 \\ 3.5817150 & -0.8609050 & -0.9017500 \\ 5.5874570 & -0.0156460 & -2.7957500 \\ 5.9270000 & 0.6673060 & -3.5824500 \\ 6.4163390 & -0.6953330 & -2.5766930 \\ 4.3331690 & -0.8061860 & -3.2535660 \\ 4.0481550 & -0.5254590 & -4.2735700 \\ 4.5328270 & -1.8820660 & -3.2582930 \\ 2.2647270 & -1.0690930 & -2.6119360 \\ 6.1286400 & 1.3378590 & -1.1663250 \\ 2.8641200 & 1.0080180 & -2.3580500 \\ 2.5810310 & 1.2861010 & -3.3796510 \\ 2.0019500 & 1.2200780 & -1.7206530 \\ 4.1209200 & 1.7952670 & -1.9028860 \\ 4.4751540 & 2.4607640 & -2.6986710 \\ 3.8872790 & 2.4311990 & -1.0433100 \\ 4.7295550 & -1.3676930 & 1.5831560 \\ 5.2495720 & -1.7788350 & 2.9361790 \\ 3.1442010 & -3.0981530 & 2.2039330 \\ 3.5826720 & -2.0734780 & 1.1844360 \\ 4.1244710 & -1.5669000 & 3.9709550 \\ 4.4861650 & -1.8681100 & 4.9606080 \\ 3.8670540 & -0.5042600 & 4.0280570 \\ 2.8853240 & -2.4000320 & 3.5569780 \\ 2.6718880 & -3.1721300 & 4.3050410 \\ 1.9922150 & -1.7782790 & 3.4754270 \\ 2.2471500 & -3.6310280 & 1.8793860 \\ 6.1413210 & -1.2027030 & 3.2066790 \\ 4.3242310 & -4.0762410 & 2.4060950 \\ 4.0369910 & -4.8345570 & 3.1427310 \\ 4.5349570 & -4.6002350 & 1.4679440\end{array}$

5.5741820
5.8820190
6.4209000
5.3020590
6.1999740
0.8365910
-0.3945240
1.0306270
-0.2792700
-1.2944680
-1.6260480
-0.0989810
1.5282590
1.7178500
-0.8761490
-2.2125200
-2.2780320
-2.2071180
-0.6798920
-2.3349100
-1.7522020
-1.6281570
-2.9658280
-4.0581710
-3.4952460
-2.4095020
-1.2548690
-3.3328340
-4.4573380
-0.7773500
-0.8685130
-2.8045030
-4.8956070
1.3958370
0.3292860
1.8323600
2.9744440
1.1265070
3.4103560
3.5096180
1.5670960
0.2436830
2.7065310
4.2974750
1.0226020
3.0481590

-3.2895360
-3.6193700
-3.4603970
-0.4139850
0.1204430
-4.7397190
-4.1073520
-6.2264780
-7.0101900
-6.3082020
-4.9276820
-8.0365120
-6.3648150
-6.6235430
-6.2043690
-6.8975870
-4.3864030
-5.0473050
-7.0676750
-1.8800730
-2.5078950
-1.4375280
-0.7044680
-1.0766780
-1.1137690
-3.3132380
-1.9035220
-0.9306140
-2.0682250
-2.9592690
-0.7175040
0.3769660
-0.3751990
3.5101320
2.8358560
4.4655030
5.2398410
4.5695310
6.1187240
5.1381060
5.4495610
3.9587110
6.2228940
6.7215510
5.5318180
6.9084950
2.8853000

3.8839430

2. 2137220

0.7477840

1. 0570530

$-1.2414370$

$-1.4749880$

$-1.4755210$

$-1.4783950$

$-2.3756850$

$-1.8141020$

$-1.8137910$

$-2.4457860$

$-0.7204380$

$-3.3863410$

$-2.4660210$

$-2.5009360$

$-0.8879770$

$-0.4580570$

$-2.8394510$

$-4.0820520$

$-5.1818780$

$-5.4501200$

$-4.4270090$

$-3.0306970$

$-4.4410400$

$-6.0988090$

$-6.4560030$

$-4.6804770$

$-3.8779320$

$-4.8612750$

$-5.4101710$

$-4.4886070$

$-0.6160920$

$-0.9115800$

$-1.6735130$

$-1.4580410$

$-2.8744490$

$-2.4422430$

$-0.5192350$

$-3.8573470$

$-3.0275630$

$-3.6417770$

$-2.2772520$

$-4.7924170$ 


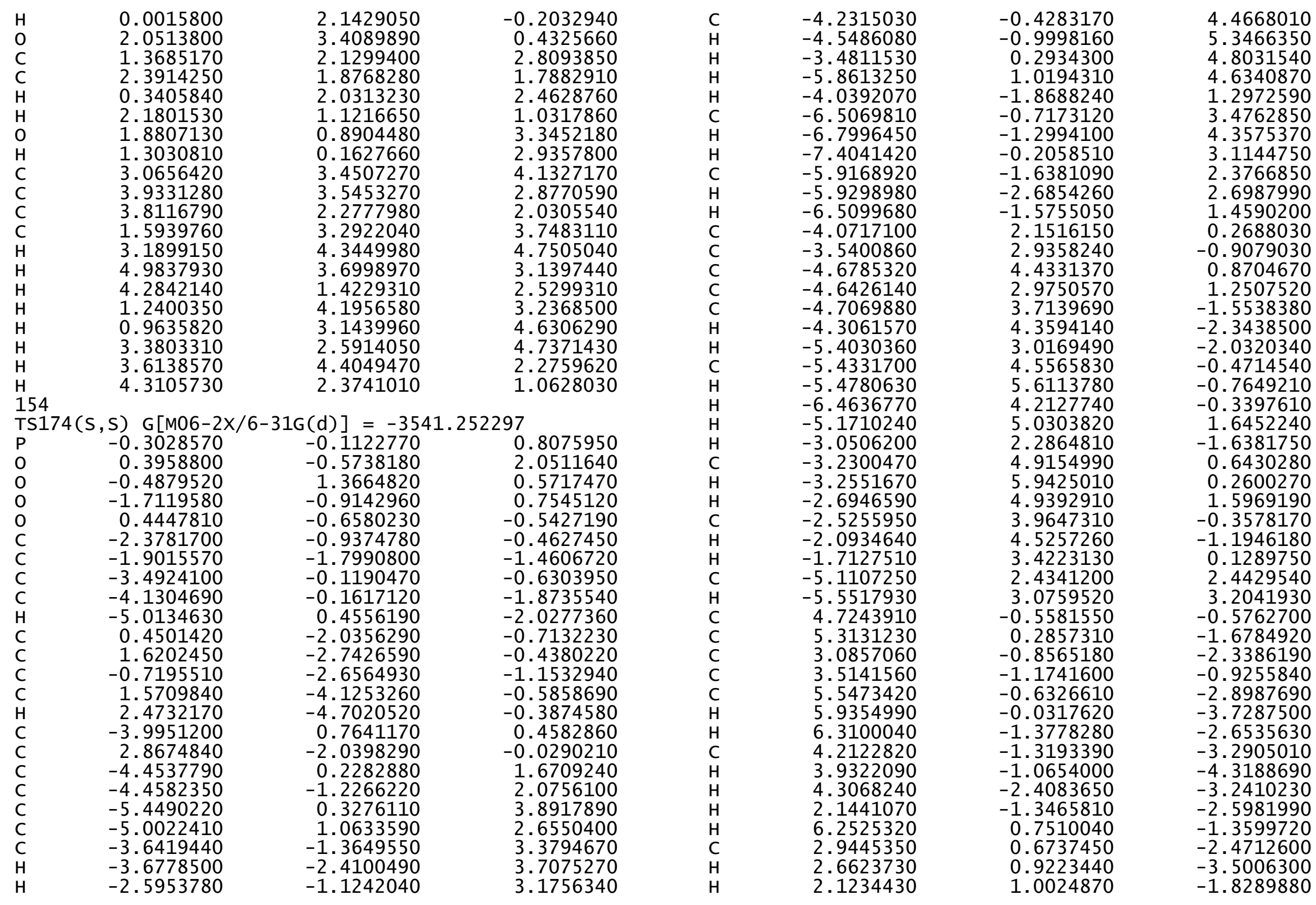




$\begin{array}{rrr}4.2821460 & 1.3593990 & -2.0879040 \\ 4.6791270 & 1.9397280 & -2.9289690 \\ 4.1319440 & 2.0643200 & -1.2643340 \\ 4.6641610 & -1.6455180 & 1.5656900 \\ 5.1709190 & -2.0224850 & 2.9336880 \\ 2.9393420 & -3.1811200 & 2.3124310 \\ 3.4494980 & -2.2635510 & 1.2266240 \\ 4.0902380 & -1.6459650 & 3.9691580 \\ 4.4415770 & -1.9210910 & 4.9700860 \\ 3.9338550 & -0.5623340 & 3.9657050 \\ 2.7713830 & -2.3821870 & 3.6232820 \\ 2.5010050 & -3.0845220 & 4.4200740 \\ 1.9389320 & -1.6849310 & 3.5151330 \\ 1.9915280 & -3.6468710 & 2.0328320 \\ 6.1167550 & -1.5163670 & 3.1562220 \\ 4.0279140 & -4.2508330 & 2.5582550 \\ 3.6861500 & -4.9331610 & 3.3442470 \\ 4.1709960 & -4.8479750 & 1.6515850 \\ 5.3542640 & -3.5570760 & 2.9706350 \\ 5.6501260 & -3.8540860 & 3.9831080 \\ 6.1681440 & -3.8457250 & 2.2987620 \\ 5.3047190 & -0.7990950 & 0.6666420 \\ 6.2545570 & -0.3342230 & 0.9296790 \\ -0.7616930 & -4.0639060 & -1.2272580 \\ -2.0670820 & -4.7694180 & -1.5454670 \\ -2.0610540 & -6.2382520 & -1.1258570 \\ -0.8043120 & -6.9196550 & -1.6600990 \\ 0.4256030 & -6.3138820 & -0.9889150 \\ 0.4010840 & -4.7971570 & -0.9491400 \\ -2.9657660 & -6.7293710 & -1.4978860 \\ -2.2610670 & -4.7210060 & -2.6243980 \\ -2.8924790 & -4.2309730 & -1.0669790 \\ -0.7488950 & -6.7711120 & -2.7467300 \\ -0.8315100 & -7.9994310 & -1.4830560 \\ 1.3433530 & -6.6457650 & -1.4877850 \\ 0.4872100 & -6.6862650 & 0.0429270 \\ -2.0775270 & -6.3138460 & -0.0308310 \\ -3.6482260 & -0.9485960 & -2.9128900 \\ -4.3036220 & -0.9913540 & -4.2699520 \\ -4.4511470 & -2.4544130 & -4.7098190 \\ -3.0828230 & -3.1603110 & -4.7925330 \\ -1.9908420 & -2.4636370 & -3.9467260 \\ -2.5254860 & -1.7688490 & -2.7161590 \\ -3.6897690 & -0.4502970 & -5.0048020 \\ -4.9693370 & -2.5156470 & -5.6716470 \\ -2.7371800 & -3.1896070 & -5.8307890\end{array}$
$-1.5341170$
$-5.2767730$
$-5.0842180$
$-3.1951930$
$-1.1835210$
1.7562090
0.6177790
2. 2659710
3. 4804520
1. 5559530
3.9842100
4. 0174070
2. 0647660
0.6167130
3. 2762000
4.9274760
1. 5173090
3. 6708980
0.2432670
2.4222760
1.6646850
2. 6419990
0.6263820
2. 3479840
2. 0681700
1. 4211900
3. 4994720
4. 3522000
4.0975380
2. 0133390
3.7175140
5.4167600
4.4960980
1.7378690
1. 3863130
3. 7410790
4.1067790
4. 5868110

154

$\operatorname{TS} 175(\mathrm{~S}, \mathrm{~S}) \mathrm{G}[\mathrm{M} 06-2 \mathrm{X} / 6-31 \mathrm{G}(\mathrm{d})]=-3541.251869$

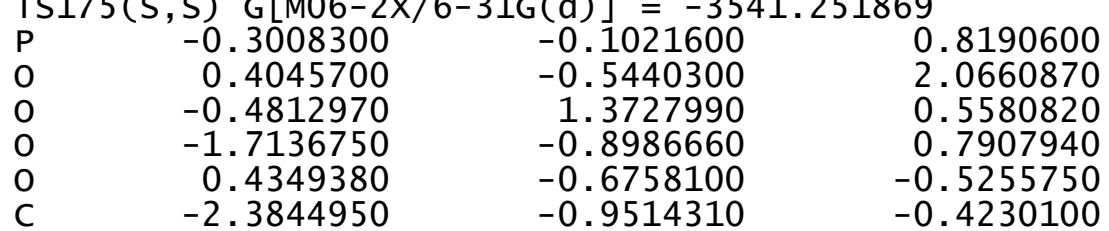

$-4.5670240$

$-1.6797690$

$-3.9729200$

$-4.4769560$

$-3.7007410$

$-0.8746290$

$-1.1100810$

$-1.9956840$

$-3.1950460$

$-2.8857410$

$-0.9029290$

$-4.2380820$

$-3.2998890$

$-4.0837540$

$-2.7686190$

$-5.1721960$

$-4.8998990$

$-0.3625080$

0.1666750

2.6214160

1. 6049050

2. 2926680

0.8970820

3. 2245610

2. 8620120

3.8452170

2. 5771590

1.8069950

3.4865200

4.4077230

2. 8197900

2. 3491300

2. 9253110

4.3828370

4.4961740

1.9302730

0.8296190
$-2.3844950$

$-0.6758100$

$-0.4230100$

H $\quad-2.7371800$

$-3.1896070$

$-5.8307890$ 


\begin{tabular}{|c|c|c|}
\hline $\begin{array}{l}-1.9165570 \\
-3.4968970 \\
-4.1416230 \\
-5.0248910 \\
0.4444890 \\
1.6248150 \\
-0.7294990 \\
1.5900920 \\
2.5029360 \\
-3.9931480 \\
2.8699670 \\
-4.4457320 \\
-4.4530500 \\
-5.4282130 \\
-4.9867340 \\
-3.6309730 \\
-3.6716140 \\
-2.5838990 \\
-4.2096600 \\
-4.5228200 \\
-3.4544570 \\
-5.8338630 \\
-4.0409360 \\
-6.4919610 \\
-6.7801800 \\
-7.3901830 \\
-5.9118550 \\
-5.9276910 \\
-6.5096210 \\
-4.0696840 \\
-3.5446130 \\
-4.6694620 \\
-4.6330280 \\
-4.7139700 \\
-4.3163970 \\
-5.4136260 \\
-5.4329950 \\
-5.4798070 \\
-6.4626080 \\
-5.1559360 \\
-3.0612770 \\
-3.2219170 \\
-3.2484270 \\
-2.6804450 \\
-2.5247040 \\
-2.0945180\end{array}$ & $\begin{array}{r}-1.8427740 \\
-0.1333020 \\
-0.2095520 \\
0.4041310 \\
-2.0557680 \\
-2.7500660 \\
-2.6913320 \\
-4.1334810 \\
-4.6987540 \\
0.7792310 \\
-2.0355860 \\
0.2760580 \\
-1.1680540 \\
0.4354830 \\
1.1376250 \\
-1.2771650 \\
-2.3131760 \\
-1.0485020 \\
-0.3093270 \\
-0.8556650 \\
0.4183720 \\
1.1476150 \\
-1.8315760 \\
-0.6162580 \\
-1.1755060 \\
-0.1113580 \\
-1.5657060 \\
-2.6049340 \\
-1.5223430 \\
2.1612860 \\
2.9136970 \\
4.4588860 \\
3.0111840 \\
3.6774290 \\
4.3023430 \\
2.9698850 \\
4.5479440 \\
5.5947840 \\
4.2078160 \\
5.0769730 \\
2.2447870 \\
4.9329900 \\
5.9489970 \\
4.9827140 \\
3.9540430 \\
4.4913140\end{array}$ & $\begin{array}{r}-1.3969230 \\
-0.6094030 \\
-1.8472030 \\
-2.0137740 \\
-0.6723700 \\
-0.4104920 \\
-1.0821570 \\
-0.5594400 \\
-0.3780220 \\
0.4579060 \\
-0.0149520 \\
1.6869370 \\
2.1287960 \\
3.9101660 \\
2.6521100 \\
3.4317740 \\
3.7870390 \\
3.2168970 \\
4.4973030 \\
5.3943760 \\
4.8090860 \\
4.6367610 \\
1.3649650 \\
3.5280320 \\
4.4254140 \\
3.1596990 \\
2.4475110 \\
2.7947330 \\
1.5317950 \\
0.2323150 \\
-0.9678510 \\
0.7779760 \\
1.1960200 \\
-1.6264210 \\
-2.4344010 \\
-2.0834170 \\
-0.5615950 \\
-0.8819980 \\
-0.4142510 \\
1.5400750 \\
-1.6843900 \\
0.5281980 \\
0.1169690 \\
1.4776320 \\
-0.4502000 \\
-1.3034000\end{array}$ \\
\hline
\end{tabular}

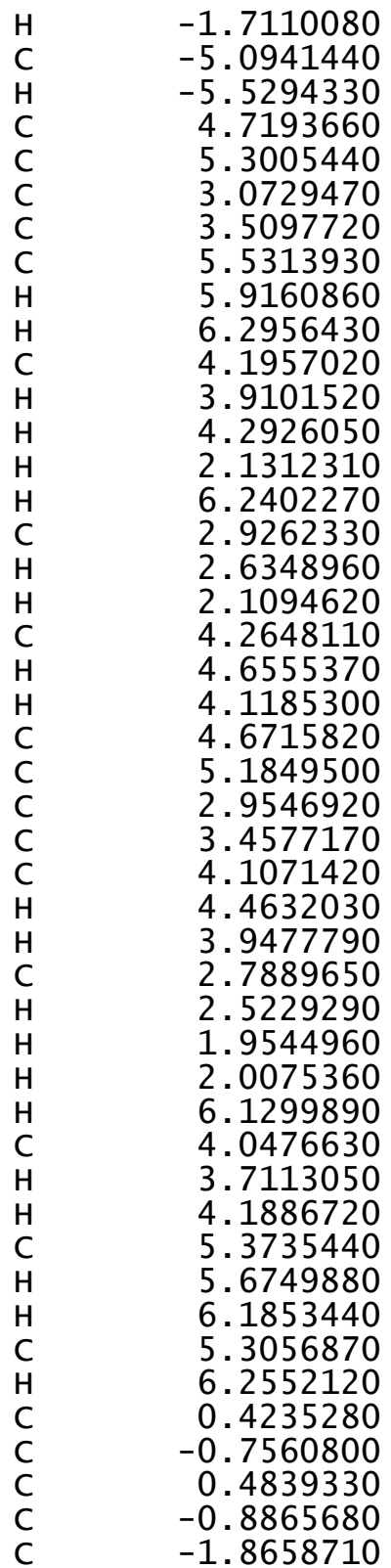

3.4225450
2.5025790
3.1649240
-0.5539660
0.2786050
-0.8770780
-1.1780260
-0.6536110
-0.0620510
-1.3951320
-1.3461780
-1.1017090
-2.4345590
-1.3726940
0.7500780
0.6513030
0.8892700
0.9837490
1.3446270
1.9142390
2.0603550
-1.6171170
-1.9774960
-3.1502030
-2.2429920
-1.5933790
-1.8572880
-0.5102530
-2.3368050
-3.0305240
-1.6428070
-3.6223330
-1.4659220
-4.2135850
-4.8878040
-4.8206930
-3.5110120
-3.7959900
-3.8042490
-0.7788830
-0.3081860
-4.8182150
-4.0988230
-6.3288310
-6.9996680
-6.2585300

0.0471590

2. 4050000

3. 1518580

$-0.5864510$

$-1.7012230$

$-2.3375880$

$-0.9236910$

$-2.9116520$

$-3.7499030$

$-2.6603870$

$-3.2908670$

$-4.3200250$

$-3.2324040$

$-2.5862010$

$-1.3927850$

$-2.4845480$

$-3.5138570$

$-1.8387010$

$-2.1185420$

$-2.9698030$

$-1.3036350$

1.5679380

2.9379260

2. 3383530

1. 2406810

3.9736390

4. 9759220

3.9597980

3. 6405810

4.4463270

3. 5268980

2. 0672720

3. 1511930

2. 5919820

3. 3872480

1. 6916190

2. 9907140

4.0049980

2. 3183020

0.6566520

0.9103010

$-0.9067740$

$-1.1508190$

$-1.0418070$

$-0.9830610$ 


$\begin{array}{rrr}-2.0642530 & -4.8327280 & -1.3824620 \\ -0.7980230 & -8.0516110 & -1.2727050 \\ 0.9551420 & -6.5700790 & -2.0050640 \\ 1.1457430 & -6.7347380 & -0.2688760 \\ -1.4695240 & -6.2347020 & -2.9147300 \\ -2.8310520 & -6.7738280 & -1.9287350 \\ -2.7132680 & -4.2716640 & -2.0566800 \\ -2.5988620 & -4.8666850 & -0.4217710 \\ -1.2669880 & -6.9781450 & 0.0463010 \\ -3.6689760 & -1.0307330 & -2.8650310 \\ -4.3411160 & -1.1299750 & -4.2108310 \\ -4.5176180 & -2.6121380 & -4.5735950 \\ -3.1632100 & -3.3498770 & -4.6229850 \\ -2.0442160 & -2.6109080 & -3.8524190 \\ -2.5473920 & -1.8461930 & -2.6501690 \\ -3.7282460 & -0.6371820 & -4.9793680 \\ -5.0409600 & -2.7119960 & -5.5293680 \\ -2.8363450 & -3.4688340 & -5.6608510 \\ -1.5957650 & -1.8706940 & -4.5294380 \\ -5.3063580 & -0.6145680 & -4.1933970 \\ -5.1588370 & -3.0659160 & -3.8100750 \\ -3.2832870 & -4.3618380 & -4.2215100 \\ -1.2408130 & -3.3013940 & -3.5848460 \\ 1.7504400 & 3.3998460 & -0.9170830 \\ 0.6139950 & 2.8191470 & -1.1431950 \\ 2.2527160 & 4.2305330 & -2.0479130 \\ 3.4663620 & 4.9068000 & -1.9054980 \\ 1.5367720 & 4.3114710 & -3.2446060 \\ 3.9633750 & 5.6653830 & -2.9584330 \\ 4.0079870 & 4.8240820 & -0.9685410 \\ 2.0387650 & 5.0706100 & -4.2965060 \\ 0.5983670 & 3.7765390 & -3.3405050 \\ 3.2493790 & 5.7466110 & -4.1537180 \\ 4.9060110 & 6.1920520 & -2.8503300 \\ 1.4866550 & 5.1342880 & -5.2285460 \\ 3.6387510 & 6.3379670 & -4.9768000 \\ 0.2446920 & 2.2023430 & -0.3880700 \\ 2.4202650 & 3.3048060 & 0.1230880 \\ 1.6738330 & 2.2602550 & 2.5913380 \\ 2.6479560 & 1.8452250 & 1.5757240 \\ 0.6343220 & 2.2322880 & 2.2668700 \\ 2.3524710 & 1.0626760 & 0.8776820 \\ 2.0827190 & 1.0199770 & 3.2072210 \\ 1.4345310 & 0.3209390 & 2.8569250 \\ 3.5105150 & 3.5065640 & 3.7940570 \\ 4.3588230 & 3.4326740 & \\ -2.5237320\end{array}$

$\begin{array}{ll}\mathrm{C} & 4.1038010 \\ \mathrm{C} & 2.0231870 \\ \mathrm{H} & 3.7290100 \\ \mathrm{H} & 5.4241160 \\ \mathrm{H} & 4.5059510 \\ \mathrm{H} & 1.7440990 \\ \mathrm{H} & 1.3994470 \\ \mathrm{H} & 3.7558680 \\ \mathrm{H} & 4.1095120 \\ \mathrm{H} & 4.5894010 \\ \mathrm{H} & \end{array}$

2.1275190
3.4598660
4.4258870
3.5052940
1.2680120
4.3540420
3.4336980
2.6654110
4.2754310
2.1111850

1.7691440 3. 4411910 4.3453620 2. 7617520 2. 3196650 2.8705500 4. 3401190 4.3401190
4.4537070 4. 4537070 0.7900170 15 $\operatorname{TS} 176(\mathrm{~S}, \mathrm{~S}) \mathrm{G}[\mathrm{M06}-2 \mathrm{X} / 6-31 \mathrm{G}(\mathrm{d})]=-3541.254577$

$\begin{array}{lrrr}\text { TS176(S , S }) ~ G[M 06-2 X / 6-31 G(d)]=-3541.254577 \\ \text { P } & -0.2693000 & -0.0638400 & -0.1646430 \\ \text { O } & -0.7765110 & 0.8802560 & -1.2164710 \\ \text { O } & 0.8561820 & 0.3730270 & 0.7433910 \\ \text { O } & 0.0495340 & -1.4572590 & -0.9381200 \\ \text { O } & -1.4468880 & -0.5394660 & 0.8515970 \\ \mathrm{C} & 0.1097920 & -2.6096160 & -0.1696010 \\ \mathrm{C} & -1.0864340 & -3.1910700 & 0.2677380 \\ \mathrm{C} & 1.3655510 & -3.1289390 & 0.1428750 \\ \mathrm{C} & 1.3878860 & -4.2808020 & 0.9253300 \\ \mathrm{H} & 2.3535720 & -4.7149440 & 1.1806340 \\ \mathrm{C} & -2.5129560 & -1.1731310 & 0.2320940 \\ \mathrm{C} & -3.6518760 & -0.4226920 & -0.0610740 \\ \mathrm{C} & -2.3710900 & -2.5211140 & -0.1055620 \\ \mathrm{C} & -4.6823260 & -1.0924660 & -0.7147820 \\ \mathrm{H} & -5.5930370 & -0.5439870 & -0.9514210 \\ \mathrm{C} & 2.6271290 & -2.4589680 & -0.2808370 \\ \mathrm{C} & -3.7180300 & 1.0315750 & 0.2567060 \\ \mathrm{C} & 2.9319000 & -2.2546150 & -1.6351480 \\ \mathrm{C} & 2.0757440 & -2.6206060 & -2.8242470 \\ \mathrm{C} & 4.3228600 & -1.5291330 & -3.4994930 \\ \mathrm{C} & 4.1579470 & -1.6864540 & -2.0105730 \\ \mathrm{C} & 1.8070350 & -1.3383700 & -3.6430770 \\ \mathrm{H} & 1.2538730 & -1.6123930 & -4.5487670 \\ \mathrm{H} & 1.1681310 & -0.6659160 & -3.0650110 \\ \mathrm{C} & 3.1531280 & -0.6590580 & -4.0084840 \\ \mathrm{H} & 3.2453590 & -0.5273100 & -5.0925630 \\ \mathrm{H} & 3.2203350 & 0.3339050 & -3.5522930 \\ \mathrm{H} & 5.2849030 & -1.0647870 & -3.7419700 \\ \mathrm{H} & 1.1328710 & -3.0818820 & -2.5220850 \\ \mathrm{C} & 4.2087440 & -2.9214700 & -4.1567220 \\ \mathrm{H} & 4.2422280 & -2.8045580 & -5.2459780 \\ \mathrm{H} & 5.0692710 & -3.5342140 & -3.8715470 \\ \mathrm{C} & 2.8832560 & -3.5928400 & -3.7131850 \\ \mathrm{H} & 2.2761670 & -3.8658040 & -4.5835220 \\ & & & \end{array}$




\begin{tabular}{|c|c|c|c|}
\hline & & & \\
\hline $\mathrm{H}$ & 3.0825290 & -4.5138470 & -3.1566070 \\
\hline $\begin{array}{l}\mathrm{C} \\
\mathrm{C}\end{array}$ & $\begin{array}{l}3.5595630 \\
3.3968860\end{array}$ & $\begin{array}{l}-2.0441670 \\
-2.0892350\end{array}$ & $\begin{array}{l}0.6843430 \\
2.1864130\end{array}$ \\
\hline c & 5.6839910 & -1.1267230 & $\begin{array}{l}.4529630 \\
1.456\end{array}$ \\
\hline $\mathrm{C}$ & 4.7878340 & -1.4878750 & 0.2966100 \\
\hline C & 4.5374350 & -2.9467890 & 2.7788230 \\
\hline $\mathrm{H}$ & 4.4719170 & -2.9130490 & 3.8723080 \\
\hline $\mathrm{H}$ & 4.4112040 & -3.9933250 & 2.4822580 \\
\hline C & 5.9079830 & -2.4029240 & 2.2936900 \\
\hline $\mathrm{H}$ & 6.5558480 & -2.1656150 & 3.1449180 \\
\hline $\mathrm{H}$ & 6.4292190 & -3.1503860 & 1.6877900 \\
\hline $\mathrm{H}$ & 6.6391850 & -0.7223390 & 1.0990680 \\
\hline H & 2.4222730 & -2.4865180 & 2.4779560 \\
\hline C & 4.9533290 & -0.0989380 & 2.343072 \\
\hline H & 5.5599900 & 0.0871010 & 3.2370430 \\
\hline $\mathrm{H}$ & 4.8661420 & 0.8513940 & 1.809653 \\
\hline C & 3.5563840 & -0.6493570 & 2.7234630 \\
\hline $\mathrm{H}$ & 3.4270770 & -0.6615830 & 3.8117120 \\
\hline $\mathrm{H}$ & 2.7590660 & -0.0293230 & 2.3051460 \\
\hline C & 5.0949460 & -1.3218960 & -1.0494970 \\
\hline $\mathrm{H}$ & 6.0577730 & -0.9073280 & -1.3488960 \\
\hline C & -3.6165750 & 2.8658430 & 1.8603210 \\
\hline $\mathrm{C}$ & -3.4616620 & 3.1848500 & 3.3259790 \\
\hline C & -3.3398580 & 0.6525280 & 2.8086760 \\
\hline C & -3.5433450 & 1.4949650 & 1.570862 \\
\hline C & -4.5904320 & 2.4525180 & 4.085126 \\
\hline $\mathrm{H}$ & -4.4776490 & 2.6355810 & 5.1596830 \\
\hline $\mathrm{H}$ & -5.5586850 & 2.8649970 & 3.7859220 \\
\hline C & -4.5168000 & 0.9350280 & 3.771316 \\
\hline $\mathrm{H}$ & -4.3683350 & 0.3556820 & 4.6894850 \\
\hline $\mathrm{H}$ & -5.4501150 & 0.5875350 & 3.3179810 \\
\hline $\mathrm{H}$ & -3.2950400 & -0.4132890 & 2.5743130 \\
\hline $\mathrm{H}$ & -3.5166850 & 4.2650200 & 3.5014250 \\
\hline C & -2.0376110 & 1.1024580 & 3.503537 \\
\hline $\mathrm{H}$ & -1.9067870 & 0.5177000 & 4.4209830 \\
\hline $\mathrm{H}$ & -1.1866200 & 0.8790100 & 2.8545940 \\
\hline C & -2.1170370 & 2.6165320 & 3.827082 \\
\hline $\mathrm{H}$ & -2.0378100 & 2.7928420 & 4.905870 \\
\hline $\mathrm{H}$ & -1.2888060 & 3.1550260 & 3.3568560 \\
\hline C & -4.0669490 & 3.3400730 & -0.4494420 \\
\hline C & -4.4136310 & 4.2059610 & -1.6337520 \\
\hline C & -4.1529240 & 1.7030840 & -2.2332770 \\
\hline C & -3.9651860 & 1.9741660 & -0.7572370 \\
\hline C & -3.3206580 & 4.0500340 & -2.7114220 \\
\hline $\mathrm{H}$ & -3.6279060 & 4.6032670 & 606699 \\
\hline $\mathrm{H}$ & -2 & 4.4896850 & 354180 \\
\hline
\end{tabular}

-3.1295470
-3.2758990
-2.1204610
-4.0211470
-4.5184430
-5.5660120
-5.6909120
-6.3253360
-5.7389760
-6.0144700
-6.5374790
-3.8853100
-3.9676810
-3.3984560
-3.2086440
-4.0965140
-5.5459380
-5.6996610
-4.5665240
-3.9829990
-3.4633800
-2.1511510
-5.8181000
-6.2319300
-6.6528450
-5.7404880
-3.7856590
-1.0239830
-2.2773430
-1.9702240
-0.9350330
0.3865430
0.2281290
-2.8923490
-2.7901880
-2.9750000
-1.3061710
-0.7789160
1.0575130
0.8974630
-1.5786370
3.2591390
2.9925240
4.6430600
5.5529850
5.0203730

2.5459020

2.3524590

2.2107760

0.6459150

5.2545780

2.1869620

2. 0613840

1.5651030

3.6758790

4.2852830

3.7859730

3.7890920

4.8501860

$-3.1452110$

$-4.5399940$

$-4.7965460$

$-4.4925510$

$-3.0024010$

$-2.4208550$

$-5.8354050$

$-5.2919870$

$-4.6907610$

$-5.0823310$

$-4.7825170$

$-2.8059940$

$-2.4523390$

$-4.1558680$

$-4.3073240$

$-4.8455780$

$-5.5991410$

$-6.6829450$

$-6.0348750$

$-4.8611290$

$-6.0320160$

$-5.5435210$

$-4.0207080$

$-7.3217280$

$-7.3282960$

$-6.7755160$

$-5.6719820$

$-4.9063690$

2.6781790

1.4819430

3.1416040

2.2883010

4.4367920
$-3.0277110$

$-4.0965280$

$-2.7750610$

$-2.4727460$

$-1.3348520$

$-2.6306900$

$-3.7121980$

$-2.1449730$

$-2.2262420$

$-3.0942210$

$-1.4861530$

0.8541900

1.0874480

$-0.8432500$

$-1.4082850$

$-2.6245810$

$-2.2556770$

$-1.9578640$

$-1.1302770$

$-2.9502550$

$-0.6522960$

$-1.6499700$

$-1.3705960$

$-3.0577840$

$-1.4533230$

$-2.9079240$

$-3.4599450$

1.1277310

1.7919310

3.0847960

2.7967980

2.3897780

1.4389140

3.4854080

1.1199680

1.9714120

1.9847130

3.6669760

1.9393490

3.2920790

3.8409540

0.4266150

$-0.0111300$

0.1311260

$-0.4989010$

0.4910980 


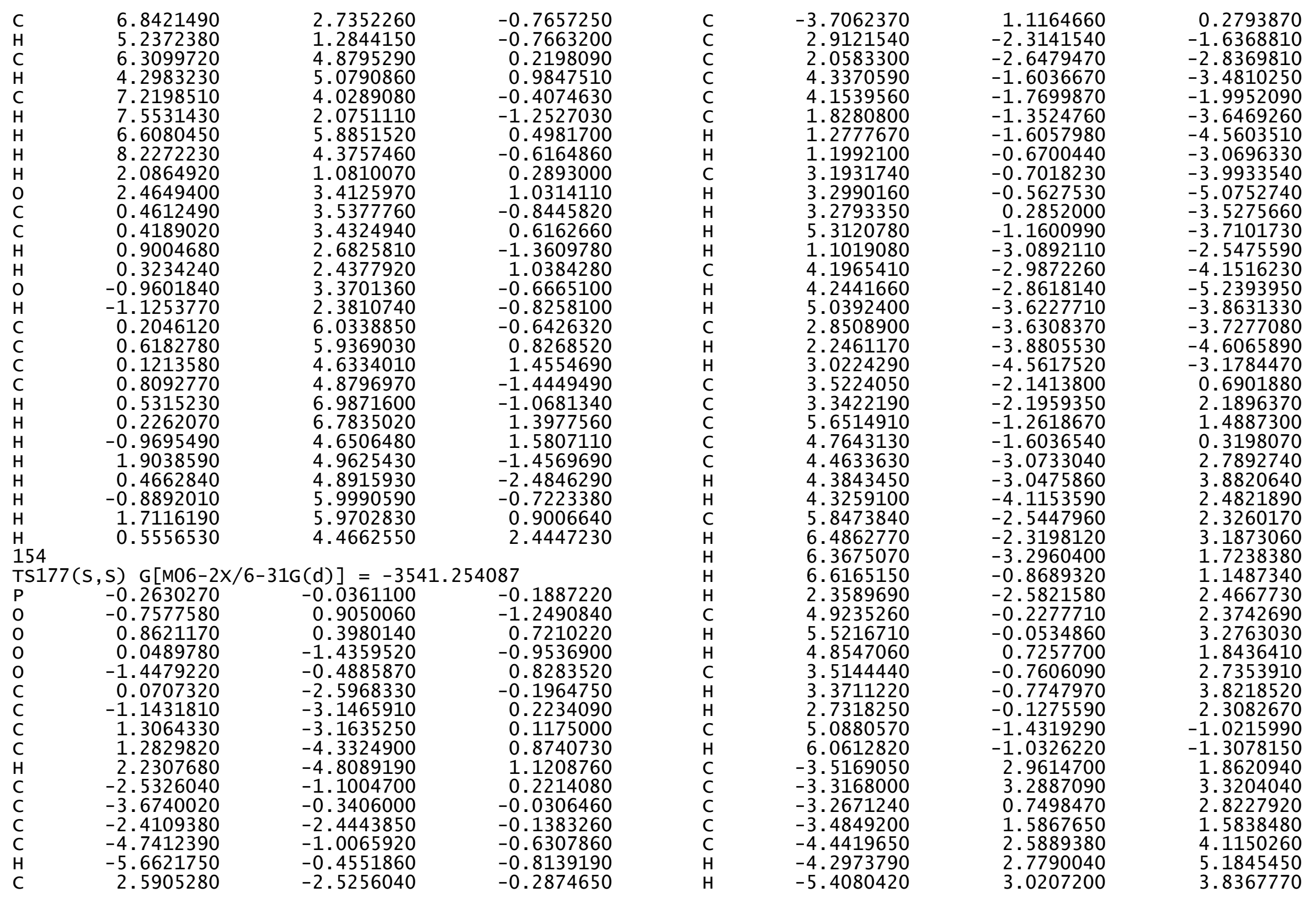




$\begin{array}{lrr}-4.4108790 & 1.0672840 & 3.8140930 \\ -4.2504420 & 0.4931250 & 4.7335120 \\ -5.3637790 & 0.7368970 & 3.3896160 \\ -3.2527480 & -0.3185140 & 2.5954230 \\ -3.3421520 & 4.3712780 & 3.4878930 \\ -1.9366630 & 1.1752100 & 3.4788900 \\ -1.7949550 & 0.5949920 & 4.3976090 \\ -1.1083740 & 0.9273360 & 2.8097110 \\ -1.9730470 & 2.6931620 & 3.7914980 \\ -1.8615170 & 2.8764020 & 4.8662740 \\ -1.1453580 & 3.2085850 & 3.2951850 \\ -4.0111610 & 3.4267770 & -0.4406120 \\ -4.3592260 & 4.2903900 & -1.6261510 \\ -4.1904360 & 1.7744040 & -2.2034050 \\ -3.9567060 & 2.0555800 & -0.7363370 \\ -3.2972000 & 4.0881610 & -2.7272450 \\ -3.6090080 & 4.6398460 & -3.6219110 \\ -2.3407530 & 4.5031970 & -2.3981380 \\ -3.1587050 & 2.5751420 & -3.0295630 \\ -3.3323350 & 2.3742750 & -4.0928970 \\ -2.1552780 & 2.2122270 & -2.7922340 \\ -4.1003050 & 0.7107630 & -2.4329200 \\ -4.4239580 & 5.3449990 & -1.3371480 \\ -5.5967800 & 2.2982950 & -2.5725290 \\ -5.7515000 & 2.1650390 & -3.6492430 \\ -6.3624890 & 1.7051090 & -2.0616320 \\ -5.7140960 & 3.7960250 & -2.1811870 \\ -5.9911880 & 4.4044380 & -3.0493490 \\ -6.4909940 & 3.9388780 & -1.4238640 \\ -3.7851640 & 3.8826040 & 0.8537330 \\ -3.8324760 & 4.9476990 & 1.0784560 \\ -4.6525170 & -2.3356860 & -1.0502340 \\ -3.4621940 & -3.0536050 & -0.8477560 \\ -5.8653180 & -2.9749400 & -1.7020600 \\ -5.5272280 & -4.1980440 & -2.5526700 \\ -4.5831560 & -5.1246280 & -1.7894150 \\ -3.2666430 & -4.4028980 & -1.5139300 \\ -6.4478410 & -4.7189180 & -2.8350020 \\ -6.5611450 & -3.2790780 & -0.9073490 \\ -6.3948540 & -2.2247890 & -2.2996130 \\ -5.0494890 & -5.4231640 & -0.8402210 \\ -4.3920690 & -6.0402120 & -2.3583110 \\ -2.5817640 & -5.0314840 & -0.9426660 \\ -2.7608940 & -4.2175800 & -2.4730150 \\ -5.0383250 & -3.8793170 & -3.4822890 \\ -1.1283700 & -4.2736010 & 1.0718730\end{array}$
$-2.4139880$
$-2.1588310$
$-1.1560450$
0.1975320
0.0991980
$-3.1048740$
$-3.0222100$
$-3.0163710$
$-1.5186010$
$-1.0529720$
0.8793820
0.6637770
$-1.7561450$
3. 3271280
3. 0417450
4.7314410
5. 6364190
5.1325940
6. 9442980
5. 3023760
6.4408760
4. 4138130
7. 3457330
7.6511880
6.7573780
8. 3675970
2. 1174170
2. 5359890
0.5426860
0.4913470
0.9659190
0.3713140
$-0.8831510$
$-1.0698470$
0.3401820
0.7456080
0.2185320
0.9226710
0.6898450
0. 3699610
$-0.8719070$
2. 0187610
0.5834610
$-0.7537380$
1.8391410
0.6479640

$\begin{array}{rr}-4.7582010 & 1.7215370 \\ -5.6543080 & 2.9324340 \\ -6.7437140 & 2.5630550 \\ -6.1037940 & 2.2667450 \\ -4.8841110 & 1.3688310 \\ -6.0857020 & 3.2743920 \\ -5.3208290 & 1.0007210 \\ -3.8881870 & 2.0050350 \\ -7.2807090 & 1.6764400 \\ -7.4797060 & 3.3665910 \\ -6.8306550 & 1.8104770 \\ -5.8010410 & 3.2144740 \\ -5.0571640 & 3.7610030 \\ 2.6332110 & 0.4224200 \\ 1.4360240 & -0.0007010 \\ 3.0541830 & 0.1608240 \\ 2.1667980 & -0.4279580 \\ 4.3449740 & 0.5104880 \\ 2.5752700 & -0.6638420 \\ 1.1671140 & -0.6888550 \\ 4.7491480 & 0.2704370 \\ 5.0144430 & 0.9717050 \\ 3.8645120 & -0.3157790 \\ 1.8885330 & -1.1189930 \\ 5.7512350 & 0.5410920 \\ 4.1813300 & -0.5006730 \\ 1.0652090 & 0.2813680 \\ 3.4002080 & 0.9894370 \\ 3.5359150 & -0.9007040 \\ 3.4589220 & 0.5616110 \\ 2.6616570 & -1.3982150 \\ 2.4742810 & 1.0008330 \\ 3.4032600 & -0.7281360 \\ 2.4157200 & -0.8741010 \\ 6.0402810 & -0.7471160 \\ 5.9619360 & 0.7257060 \\ 4.6816120 & 1.3771630 \\ 4.8582220 & -1.5248800 \\ 6.9780290 & -1.1887330 \\ 6.8275030 & 1.2789140 \\ 4.7243790 & 1.5007780 \\ 4.9171240 & -1.5341940 \\ 4.8576590 & -2.5658350 \\ 6.0281880 & -0.8311170 \\ 5.9729650 & 0.8035990 \\ 4.5236420 & 2.3700430 \\ -6 & \end{array}$


154

TS178(S, S) G[M06-2X/6-31G(d)] = -3541.254139

$\begin{array}{lrrr}\mathrm{P} & -0.2922130 & -0.0625790 & -0.1123570 \\ \mathrm{O} & -0.8292380 & 0.8560690 & -1.1718120 \\ \mathrm{O} & 0.8282410 & 0.4142160 & 0.7814250 \\ \mathrm{O} & 0.0486520 & -1.4546350 & -0.8769840 \\ \mathrm{O} & -1.4461310 & -0.5591860 & 0.9176860 \\ \mathrm{C} & 0.1391260 & -2.6120540 & -0.1209610 \\ \mathrm{C} & -1.0377030 & -3.2231650 & 0.3285350 \\ \mathrm{C} & 1.4084450 & -3.1255980 & 0.1438690 \\ \mathrm{C} & 1.4656220 & -4.3029330 & 0.8855410 \\ \mathrm{H} & 2.4428820 & -4.7335740 & 1.0993670 \\ \mathrm{C} & -2.5102890 & -1.2204960 & 0.3256120 \\ \mathrm{C} & -3.6706130 & -0.4974780 & 0.0561330 \\ \mathrm{C} & -2.3445770 & -2.5758710 & 0.0055790 \\ \mathrm{C} & -4.7267400 & -1.1954070 & -0.5365690 \\ \mathrm{H} & -5.6536270 & -0.6660070 & -0.7490110 \\ \mathrm{C} & 2.6489290 & -2.4281220 & -0.2981070 \\ \mathrm{C} & -3.7429360 & 0.9653900 & 0.3331900 \\ \mathrm{C} & 2.9139210 & -2.1882510 & -1.6551420 \\ \mathrm{C} & 2.0356420 & -2.5489980 & -2.8297270 \\ \mathrm{C} & 4.2398770 & -1.3927850 & -3.5377280 \\ \mathrm{C} & 4.1175630 & -1.5860330 & -2.0489520 \\ \mathrm{C} & 1.7174420 & -1.2579480 & -3.6162630 \\ \mathrm{H} & 1.1522950 & -1.5285450 & -4.5156150 \\ \mathrm{H} & 1.0738670 & -0.6131590 & -3.0123070 \\ \mathrm{C} & 3.0381510 & -0.5383140 & -3.9964660 \\ \mathrm{H} & 3.1006830 & -0.3774030 & -5.0787150 \\ \mathrm{H} & 3.0939810 & 0.4442710 & -3.5167300 \\ \mathrm{H} & 5.1846730 & -0.9015550 & -3.7945810 \\ \mathrm{H} & 1.1115270 & -3.0378690 & -2.5133960 \\ \mathrm{C} & 4.1390740 & -2.7727140 & -4.2227890 \\ \mathrm{H} & 4.1389860 & -2.6311650 & -5.3096420 \\ \mathrm{H} & 5.0211160 & -3.3712440 & -3.9757080 \\ \mathrm{C} & 2.8424300 & -3.4846630 & -3.7578230 \\ \mathrm{H} & 2.2192800 & -3.7565070 & -4.6170880 \\ \mathrm{H} & 3.0786690 & -4.4104930 & -3.2241560 \\ \mathrm{C} & 3.5986730 & -2.0175400 & 0.6512340 \\ \mathrm{C} & 3.4763270 & -2.1018270 & 2.1550510 \\ \mathrm{C} & 5.7188990 & -1.0646450 & 1.3872090 \\ \mathrm{C} & 4.8031740 & -1.4233080 & 0.2452580 \\ \mathrm{C} & 4.6531710 & -2.9414220 & 2.6998520 \\ \mathrm{H} & 4.6151760 & -2.9313850 & 3.7951160 \\ \mathrm{H} & 4.5448290 & -3.9845630 & 2.3848900 \\ \mathrm{C} & 5.9967800 & -2.3535310 & 2.1915380 \\ \mathrm{H} & 6.6615290 & -2.1197250 & 3.0306180\end{array}$

6.5190470
6.6541030
2.5206830
4.9853580
5.6083470
4.8634650
3.6101830
3.5018270
2.7906900
5.0709930
6.0160790
-3.6122090
-3.4175100
-3.2906230
-3.5362570
-4.5173790
-4.3738000
-5.4973390
-4.4415760
-4.2628150
-5.3849420
-3.2438490
-3.4759100
-1.9724900
-1.8113910
-1.1381470
-2.0541800
-1.9431120
-1.2451060
-4.1294000
-4.5123070
-4.2466130
-4.0259600
-3.4452450
-3.7815890
-2.5062820
-3.2447900
-3.4055610
-2.2288620
-4.1136130
-4.6178260
-5.6710300
-5.8165020
-6.4156510
-5.8476360
-6.1517800

-3.0739970
-0.6270670
-2.5323640
-0.0773400
0.0983490
0.8847610
-0.6691350
-0.7036570
-0.0609940
-1.2209800
-0.7781220
2.8523300
3.2207370
0.6734500
1.4730140
2.5059340
2.7246520
2.9019440
0.9794890
0.4313080
0.6115590
-0.3989430
4.3057790
1.1552490
0.6029020
0.9161350
2.6788290
2.8928930
3.2064900
3.2468340
4.0706850
1.5510200
1.8722650
3.8865620
4.4031490
4.3488640
2.3737870
2.1409830
2.0578040
0.4871980
5.1278430
2.0131000
1.8550330
1.4001900
3.5118790
4.0923330

1. 0193750

2.4616480

2.3203490

3. 2051710

1. 8158220

2. 7175230

3.8075330

2. 3244360

$-1.1041970$

$-1.4192800$

1.8720140

3. 3212650

2.8841990

1. 6259580

4.1373830

5. 2015580

3.8544830

3. 8707270

4.8025310

3.4559340

2.6826450

3. 4626440

3. 5259910

4.4585800

2.8610470

3.8003170

4.8694220

3. 2864120

$-0.4385850$

$-1.6414780$

$-2.1646390$

$-0.7034230$

$-2.7405630$

$-3.6472500$

$-2.4243840$

$-3.0063830$

$-4.0652300$

$-2.7563490$

$-2.3719920$

$-1.3746780$

$-2.5468670$

$-3.6215480$

$-2.0281370$

$-2.1827090$

$-3.0609020$ 


-6.6274070
-3.9154890
-3.9989170
-3.4022820
-3.3162110
-3.7430640
-5.1545630
-5.6869360
-4.5992290
-3.9870080
-3.6987990
-5.8637410
-6.0597210
-2.3032370
-3.0055730
-5.1272380
-6.5374720
-0.9403390
-2.1801890
-1.8545760
-0.7890300
0.5148030
0.3256160
-2.7663510
-2.7087710
-2.8721780
-1.1344680
-0.6213400
1.2247560
0.9896400
-1.4830730
3.2083610
2.9648120
4.5912620
5.5181190
4.9499690
6.8054690
5.2164380
6.2380250
4.2150630
7.1648050
7.5291510
6.5216370
8.1708280
2.0560580
2.3941800

3.6370810

4.8078110

$-3.2260040$

$-4.6101950$

$-4.5494940$

$-3.9421350$

$-3.2945570$

$-2.5325690$

$-5.2958580$

$-5.5537420$

$-4.7092880$

$-4.0871090$

$-5.0126600$

-3. 9419410

$-3.1835190$

$-2.6420470$

$-4.3755380$

$-4.9755900$

$-5.8453310$

$-6.8689160$

$-6.1468070$

$-4.9238160$

$-6.3374060$

$-5.5998310$

$-4.1710630$

$-7.4367370$

$-7.5895850$

$-6.8293300$

$-5.8248120$

$-5.2195270$

2.7388070

1.5270180

3.2127120

2. 3615050

4.5169340

2. 8189960

1. 3510580

4.9700700

5.1579470

4.1213200

2.1602600

5.9825420

4.4764660

1.1256570

3.4803170

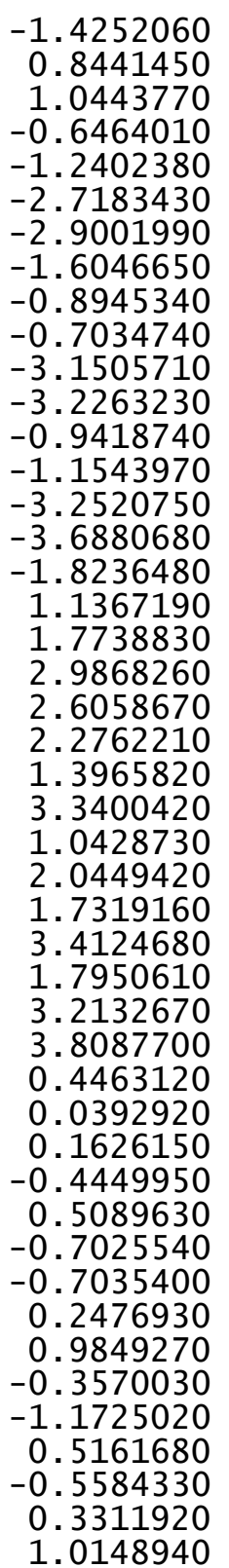

$$
\begin{array}{r}
0.4108130 \\
0.3560060 \\
0.8542720 \\
0.2565680 \\
-1.0119850 \\
-1.1758500 \\
0.1510930 \\
0.5489820 \\
0.0494420 \\
0.7649720 \\
0.4826100 \\
0.1494790 \\
-1.0421060 \\
1.8595690 \\
0.4324180 \\
-0.9416070 \\
1.6414420 \\
0.4785900
\end{array}
$$

154

TS179(S, S) G[M06-2X/6-31G(d) $]=-3541.253660$

$\begin{array}{lrrr}\text { TS } & 0.3412180 & 0.2469480 & 0.7635380 \\ \mathrm{P} & -0.3599840 & 0.7905490 & 1.9721970 \\ \mathrm{O} & 0.5957410 & -1.2371010 & 0.6657440 \\ \mathrm{O} & 1.7040280 & 1.1091830 & 0.6066410 \\ \mathrm{O} & -0.4471660 & 0.6282840 & -0.6171040 \\ \mathrm{C} & 2.3595530 & 1.0616130 & -0.6145890 \\ \mathrm{C} & 1.8385780 & 1.8128560 & -1.6787380 \\ \mathrm{C} & 3.5217260 & 0.3008490 & -0.7144630 \\ \mathrm{C} & 4.1722300 & 0.2886750 & -1.9520920 \\ \mathrm{H} & 5.0862990 & -0.2929190 & -2.0555950 \\ \mathrm{C} & -0.5293550 & 1.9801220 & -0.9212430 \\ \mathrm{C} & -1.7356640 & 2.6395640 & -0.6997820 \\ \mathrm{C} & 0.6021300 & 2.6141320 & -1.4556860 \\ \mathrm{C} & -1.7936980 & 3.9998570 & -1.0129280 \\ \mathrm{H} & -2.7278160 & 4.5354200 & -0.8545920 \\ \mathrm{C} & 4.0563770 & -0.4691210 & 0.4430390 \\ \mathrm{C} & -2.9277230 & 1.9111720 & -0.1824210 \\ \mathrm{C} & 4.4600300 & 0.1805820 & 1.6192950 \\ \mathrm{C} & 4.3918530 & 1.6617940 & 1.9064120 \\ \mathrm{C} & 5.4032460 & 0.3017400 & 3.8616350 \\ \mathrm{C} & 5.0239580 & -0.5480110 & 2.6763540 \\ \mathrm{C} & 3.5430250 & 1.8722260 & 3.1795460 \\ \mathrm{H} & 3.5434630 & 2.9399590 & 3.4272760 \\ \mathrm{H} & 2.5075780 & 1.5876190 & 2.9756390 \\ \mathrm{C} & 4.1364290 & 1.0397250 & 4.3463980 \\ \mathrm{H} & 4.3990110 & 1.6835070 & 5.1936560\end{array}$

$\begin{array}{rr}3.5181020 & -0.8886130 \\ 3.4742590 & 0.5751520 \\ 2.6419560 & -1.3648220 \\ 2.4973780 & 1.0367550 \\ 3.3588300 & -0.7170040 \\ 2.3632200 & -0.8358860 \\ 6.0200620 & -0.7967100 \\ 5.9861640 & 0.6798370 \\ 4.7095220 & 1.3599210 \\ 4.8333180 & -1.5423800 \\ 6.9546810 & -1.2585200 \\ 6.8554260 & 1.2100660 \\ 4.7288680 & 1.4785380 \\ 4.9169950 & -1.5469770 \\ 4.8000470 & -2.5849430 \\ 5.9813090 & -0.8872290 \\ 6.0253390 & 0.7631500 \\ 4.5866770 & 2.3578250\end{array}$

886130

$2.6419560 \quad-1.3648220$

$-1.0367550$

$2.3632200-0.8358860$

$6.0200620-0.7967100$

$4.7095220 \quad 1.3599210$

$-1.5423800$

6.9546810

0.7631500

2.3578250

0.6282840

0.2886750

. 9998570

1.6835070

5.1936560 


\begin{tabular}{|c|c|c|c|c|c|c|c|}
\hline & & & & & & & \\
\hline $\mathrm{H}$ & 3.4074190 & 0.3088240 & 4.7090650 & $\mathrm{H}$ & -3.9996790 & -2.3370680 & -0.9828360 \\
\hline $\mathrm{H}$ & 5.8263260 & -0.3103120 & 4.6654160 & C & -4.6631690 & 1.5853370 & 1.4931880 \\
\hline $\mathrm{H}$ & 3.9650440 & 2.2208620 & 1.0704010 & C & -5.1606140 & 2.0736300 & 2.8290330 \\
\hline C & 6.4228060 & 1.3615370 & 3.3908060 & C & -3.0020740 & 3.2686590 & 2.0401720 \\
\hline $\mathrm{H}$ & 6.6568220 & 2.0284250 & 4.2284660 & C & -3.4885740 & 2.2241700 & 1.0636480 \\
\hline $\mathrm{H}$ & 7.3555920 & 0.8690330 & 3.0997120 & C & -4.0431860 & 1.8561440 & 3.8706280 \\
\hline C & 5.8256850 & 2.1572100 & 2.2008890 & $\mathrm{H}$ & -4.3889420 & 2.2134200 & 4.8472730 \\
\hline $\mathrm{H}$ & 5.7908930 & 3.2285360 & 2.4285560 & $\mathrm{H}$ & -3.8320740 & 0.7866440 & 3.9713520 \\
\hline $\mathrm{H}$ & 6.4434310 & 2.0373190 & 1.3054630 & C & -2.7703410 & 2.6168210 & 3.4211360 \\
\hline C & 4.2089980 & -1.8606470 & 0.3614760 & $\mathrm{H}$ & -2.5221560 & 3.4098110 & 4.1359050 \\
\hline C & 3.7532100 & -2.7577600 & -0.7655400 & $\mathrm{H}$ & -1.9053000 & 1.9540730 & 3.3658340 \\
\hline $\mathrm{C}$ & 4.9102720 & -4.0559850 & 1.1516560 & $\mathrm{H}$ & -2.0829540 & 3.7467700 & 1.6926960 \\
\hline c & 4.7936920 & -2.5768310 & 1.4167580 & $\mathrm{H}$ & -6.0759610 & 1.5487020 & 3.1239650 \\
\hline $\mathrm{C}$ & 4.9770260 & -3.5197280 & -1.3187190 & C & -4.1374330 & 4.3050940 & 2.2039690 \\
\hline $\mathrm{H}$ & 4.6340640 & -4.2400280 & -2.0703410 & $\mathrm{H}$ & -3.8152570 & 5.0791170 & 2.9091360 \\
\hline $\mathrm{H}$ & 5.6532650 & -2.8240950 & -1.8265040 & $\mathrm{H}$ & -4.3280330 & 4.7997750 & 1.2458330 \\
\hline C & 5.7084760 & -4.2439640 & -0.1571080 & $\mathrm{C}$ & -5.4192440 & 3.5938240 & 2.7161120 \\
\hline $\mathrm{H}$ & 5.8090270 & -5.3145090 & -0.3681690 & $\mathrm{H}$ & -5.7098500 & 3.9775960 & 3.7005650 \\
\hline $\mathrm{H}$ & 6.7182490 & -3.8432810 & -0.0257080 & $\mathrm{H}$ & -6.2594180 & 3.7736640 & 2.0386830 \\
\hline $\mathrm{H}$ & 5.4079800 & -4.5671240 & 1.9826210 & C & -5.2815390 & 0.6267600 & 0.6970050 \\
\hline $\mathrm{H}$ & 3.2553050 & -2.1916440 & -1.5567150 & $\mathrm{H}$ & -6.2011540 & 0.1455590 & 1.0290540 \\
\hline C & 3.4933900 & -4.6253790 & 0.9267260 & C & 0.5211160 & 3.9881480 & -1.7275380 \\
\hline $\mathrm{H}$ & 3.5774680 & -5.6772720 & 0.6292290 & $\mathrm{C}$ & 1.6866200 & 4.8363700 & -2.1730460 \\
\hline $\mathrm{H}$ & 2.9318460 & -4.5987700 & 1.8654010 & $\mathrm{C}$ & 1.8075540 & 6.0696650 & -1.2585890 \\
\hline C & 2.7747090 & -3.7931090 & -0.1656150 & $\mathrm{C}$ & 0.4853760 & 6.8669550 & -1.1460840 \\
\hline $\mathrm{H}$ & 2.3986360 & -4.4390850 & -0.9674810 & C & -0.6951570 & 6.1465510 & -1.8263780 \\
\hline $\mathrm{H}$ & 1.9202100 & -3.2586160 & 0.2537880 & $\mathrm{C}$ & -0.6846580 & 4.6743190 & -1.5099380 \\
\hline C & 5.2028410 & -1.9240160 & 2.5740780 & $\mathrm{H}$ & 1.5318030 & 5.1659830 & -3.2105520 \\
\hline $\mathrm{H}$ & 5.6537840 & -2.4832150 & 3.3925720 & $\mathrm{H}$ & 2.6133650 & 6.7116850 & -1.6269340 \\
\hline C & -4.7197120 & 0.2960990 & -0.5335550 & $\mathrm{H}$ & 0.5958280 & 7.8607570 & -1.5906990 \\
\hline C & -5.2901780 & -0.6773120 & -1.5337700 & $\mathrm{H}$ & -0.6106480 & 6.2787400 & -2.9137730 \\
\hline C & -3.1453900 & 0.5096550 & -2.3656340 & $\mathrm{H}$ & 2.6190150 & 4.2667700 & -2.1557920 \\
\hline C & -3.5517750 & 0.9345580 & -0.9746250 & $\mathrm{H}$ & 2.1124110 & 5.7189100 & -0.2677800 \\
\hline C & -5.6039220 & 0.1062740 & -2.8280670 & $\mathrm{H}$ & 0.2396390 & 7.0202140 & -0.0910530 \\
\hline $\mathrm{H}$ & -5.9731370 & -0.5922650 & -3.5872670 & $\mathrm{H}$ & -1.6451470 & 6.5997160 & -1.5273780 \\
\hline $\mathrm{H}$ & -6.4042420 & 0.8265710 & -2.6340290 & C & 2.4963640 & 1.7486380 & -2.9156360 \\
\hline C & -4.3205130 & 0.8274560 & -3.3182500 & $\mathrm{C}$ & 1.9862620 & 2.3849010 & -4.1850210 \\
\hline $\mathrm{H}$ & -4.0541850 & 0.5014200 & -4.3297720 & C & 1.9527910 & 1.3350340 & -5.3112570 \\
\hline $\mathrm{H}$ & -4.4745650 & 1.9101230 & -3.3575970 & C & 3.3082880 & 0.6108180 & -5.4984810 \\
\hline $\mathrm{H}$ & -2.2380290 & 1.0204900 & -2.6973530 & C & 4.3253290 & 0.9729060 & -4.3986070 \\
\hline $\mathrm{H}$ & -6.1949150 & -1.1575760 & -1.1448500 & $\mathrm{C}$ & 3.6713990 & 0.9898120 & -3.0421260 \\
\hline C & -2.9279840 & -1.0173280 & -2.3661470 & $\mathrm{H}$ & 2.6477420 & 3.2126260 & -4.4787930 \\
\hline $\mathrm{H}$ & -2.6716380 & -1.3439960 & -3.3803950 & $\mathrm{H}$ & 1.6429870 & 1.8175240 & -6.2432230 \\
\hline $\mathrm{H}$ & -2.0674270 & -1.2461220 & -1.7323560 & $\mathrm{H}$ & 3.7419860 & 0.8517720 & -6.4738920 \\
\hline C & -4.2127600 & -1.7307530 & -1.8688030 & $\mathrm{H}$ & 4.7346540 & 1.9702440 & -4.6101900 \\
\hline $\mathrm{H}$ & -4.6000440 & -2.4157060 & -2.6320810 & $\mathrm{H}$ & 0.9897270 & 2.8099950 & -4.0411690 \\
\hline
\end{tabular}




\begin{tabular}{|c|c|c|c|c|c|c|c|}
\hline & & & & & & & \\
\hline $\mathrm{H}$ & 1.1761110 & 0.6055220 & -5.0610600 & C & -1.3030050 & -4.3645120 & -0.8024080 \\
\hline $\mathrm{H}$ & 3.1494620 & -0.4716680 & -5.4842560 & $\mathrm{H}$ & -2.2574550 & -4.8241010 & -1.0520400 \\
\hline $\mathrm{H}$ & 5.1697300 & 0.2771700 & -4.4125180 & C & 2.5372330 & -1.1358580 & -0.1895870 \\
\hline C & -1.5737520 & -3.5107040 & -0.5188220 & C & 3.6740750 & -0.3634380 & 0.0466660 \\
\hline 0 & -0.4781740 & -2.8978140 & -0.8407260 & $\mathrm{C}$ & 2.4320090 & -2.4805790 & 0.1747480 \\
\hline C & -2.0665650 & -4.4699980 & -1.5473780 & C & 4.7541780 & -1.0141620 & 0.6380010 \\
\hline $\mathrm{C}$ & -3.2292830 & -5.2003440 & -1.2923610 & $\mathrm{H}$ & 5.66888870 & -0.4504060 & 0.8144410 \\
\hline C & -1.3951410 & -4.6152570 & -2.7636380 & C & -2.5800340 & -2.5215400 & 0.3335260 \\
\hline C & -3.7197100 & -6.0772860 & -2.2522960 & $\mathrm{C}$ & 3.6929630 & 1.0917230 & -0.2738850 \\
\hline $\mathrm{H}$ & -3.7375750 & -5.0657680 & -0.3428000 & $\mathrm{C}$ & -2.9063660 & -2.2964930 & 1.6795910 \\
\hline C & -1.8913030 & -5.4918050 & -3.7228590 & C & -2.0596300 & -2.6242570 & 2.8863860 \\
\hline $\mathrm{H}$ & -0.4960860 & -4.0374570 & -2.9473160 & $\mathrm{C}$ & -4.3351930 & -1.5618340 & 3.5113120 \\
\hline C & -3.0508970 & -6.2218300 & -3.4676650 & C & -4.1472630 & -1.7434650 & 2.0278850 \\
\hline $\mathrm{H}$ & -4.6225460 & -6.6466450 & -2.0565490 & C & -1.8255260 & -1.3225490 & 3.6850110 \\
\hline $\mathrm{H}$ & -1.3744280 & -5.6049440 & -4.6702890 & $\mathrm{H}$ & -1.2805920 & -1.5700920 & 4.6032400 \\
\hline $\mathrm{H}$ & -3.4359660 & -6.9049450 & -4.2185400 & $\mathrm{H}$ & -1.1901680 & -0.6492550 & 3.1039970 \\
\hline $\mathrm{H}$ & -0.1167050 & -2.1917300 & -0.1629730 & C & -3.1884740 & -0.6609740 & 4.0191280 \\
\hline 0 & -2.2140530 & -3.3491550 & 0.5317010 & $\mathrm{H}$ & -3.2978930 & -0.5102210 & 5.0991310 \\
\hline C & -1.4613040 & -2.0013020 & 2.8466970 & $\mathrm{H}$ & -3.2674600 & 0.3216560 & 3.5428520 \\
\hline $\mathrm{c}$ & -2.4802300 & -1.7492310 & 1.8214900 & $\mathrm{H}$ & -5.3086990 & -1.1108600 & 3.7323570 \\
\hline $\mathrm{H}$ & -0.4326720 & -1.9600120 & 2.4904920 & $\mathrm{H}$ & -1.1046260 & -3.0733740 & 2.6050810 \\
\hline $\mathrm{H}$ & -2.2428570 & -1.0351840 & 1.0334260 & C & -4.2046250 & -2.9394520 & 4.1959290 \\
\hline 0 & -1.9179700 & -0.7208840 & 3.3333340 & $\mathrm{H}$ & -4.2551430 & -2.8031000 & 5.2822670 \\
\hline $\mathrm{H}$ & -1.3164050 & -0.0344280 & 2.8873710 & $\mathrm{H}$ & -5.0499350 & -3.5731150 & 3.9109220 \\
\hline C & -3.2022560 & -3.1929340 & 4.2336790 & C & -2.8612590 & -3.5944780 & 3.7827990 \\
\hline $\mathrm{C}$ & -4.0829490 & -3.3031460 & 2.9883950 & $\mathrm{H}$ & -2.2612250 & -3.8399810 & 4.6661140 \\
\hline $\mathrm{C}$ & -3.9144490 & -2.0790710 & 2.0876980 & $\mathrm{H}$ & -3.0361150 & -4.5291950 & 3.2410720 \\
\hline $\mathrm{C}$ & -1.7281070 & -3.1132960 & 3.8344470 & C & -3.5067420 & -2.1425500 & -0.6510630 \\
\hline $\mathrm{H}$ & -3.3595270 & -4.0544870 & 4.8893140 & $\mathrm{C}$ & -3.3215280 & -2.2138290 & -2.1492610 \\
\hline $\mathrm{H}$ & -5.1370920 & -3.4013210 & 3.2640100 & $\mathrm{C}$ & -5.6311070 & -1.2671660 & -1.4663390 \\
\hline $\mathrm{H}$ & -4.3476340 & -1.1844720 & 2.5519570 & $\mathrm{C}$ & -4.7482830 & -1.5974670 & -0.2907930 \\
\hline $\mathrm{H}$ & -1.4164630 & -4.0513130 & 3.3589950 & C & -4.4427560 & -3.0950300 & -2.7434000 \\
\hline $\mathrm{H}$ & -1.0855060 & -2.9555950 & 4.7062560 & $\mathrm{H}$ & -4.3608970 & -3.0798160 & -3.8361920 \\
\hline $\mathrm{H}$ & -3.4756170 & -2.2965110 & 4.8033140 & $\mathrm{H}$ & -4.3083450 & -4.1344720 & -2.4262930 \\
\hline $\mathrm{H}$ & -3.8053870 & -4.2003960 & 2.4226400 & C & -5.8270370 & -2.5597120 & -2.2887750 \\
\hline $\mathrm{H}$ & -4.4231520 & -2.1957200 & 1.1272200 & $\mathrm{H}$ & -6.4642680 & -2.3443910 & -3.1537650 \\
\hline & & & & $\mathrm{H}$ & -6.3488480 & -3.3032190 & -1.6785110 \\
\hline & , S) $\mathrm{G}[\mathrm{M} 06-2\rangle$ & $G(d)]=-3$ & 578 & $\mathrm{H}$ & -6.5963770 & -0.8683880 & -1.1338930 \\
\hline $\mathrm{P}$ & 0.2704600 & -0.0625570 & 0.2172990 & $\mathrm{H}$ & -2.3380740 & -2.6050220 & -2.4187570 \\
\hline 0 & 0.7727810 & 0.8876180 & 1.2660100 & C & -4.8989410 & -0.2455050 & -2.3626960 \\
\hline 0 & -0.8563780 & 0.3671620 & -0.6921400 & $\mathrm{H}$ & -5.4936800 & -0.0828860 & -3.2691650 \\
\hline 0 & -0.0368410 & -1.4551620 & 0.9952810 & $\mathrm{H}$ & -4.8316250 & 0.7150810 & -1.8447990 \\
\hline 0 & 1.4478190 & -0.5347610 & -0.7983130 & C & -3.4891080 & -0.7843240 & -2.7116190 \\
\hline C & -0.0622830 & -2.6234380 & 0.2490800 & $\mathrm{H}$ & -3.3411530 & -0.8107080 & -3.7972390 \\
\hline C & 1.1582960 & -3.1891910 & -0.1554900 & $\mathrm{H}$ & -2.7073000 & -0.1479800 & -2.2880950 \\
\hline C & -1.3 & -3.1795570 & -0.0602220 & C & -5.0766660 & -1.4119590 & 1.0476920 \\
\hline
\end{tabular}




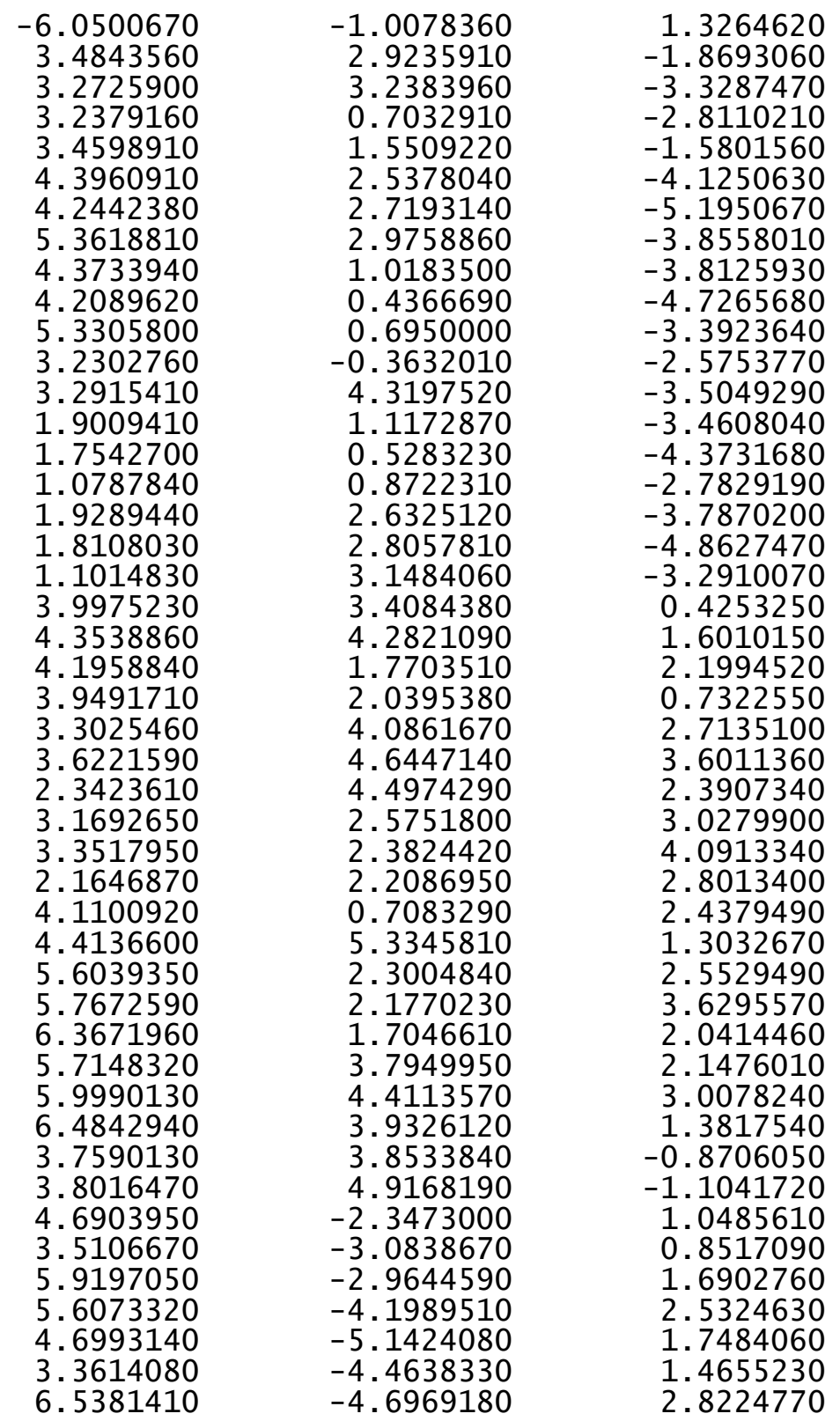

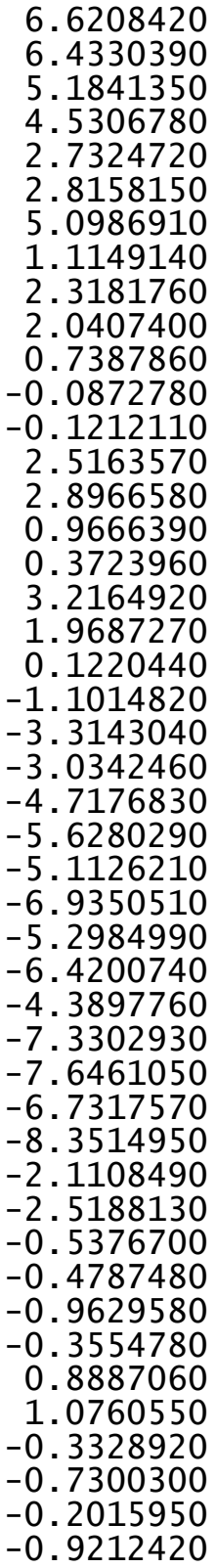
$-3.2488520$
$-2.2059960$
$-5.4122230$
$-6.0725330$
$-5.1029850$
$-4.3432000$
$-3.8983580$
$-4.3471550$
$-4.9749400$
$-5.1408000$
$-5.9306380$
$-6.1743050$
$-4.9397170$
$-5.6314220$
$-6.8963650$
$-6.9954130$
$-4.1399640$
$-5.3779430$
$-6.4966910$
2. 6172700
1. 4251010
3. 0470160
2. 1702850
4.3350660
2. 5865670
1.1726340
4.7471570
4.9961380
3.8731130
1. 9080400
5.7471570
4. 1961060
1.0467580
3. 3735220
3. 5134240
3.4320870
2. 6406180
2. 4460020
3. 3813420
2. 3942270
. 0169360
5.9352650
4.6526090
4.8374760

0.8929320

2. 2914700

0.7999740

2. 3005850

0.8451360

2. 4128740

3. 4575960

$-0.9467470$

$-1.6085730$

$-3.1137620$

$-3.3930700$

$-2.1134480$

$-1.2518720$

$-1.1718170$

$-3.5869830$

$-3.8547170$

$-1.5468300$

$-1.4519490$

$-3.5509330$

$-4.1080870$

$-2.3677180$

$-0.4313170$

0.0092040

$-0.1795630$

0.4167260

$-0.5460820$

0.6433950

0.6905900

$-0.3151330$

$-1.0130040$

0.2786410

1. 1043350

$-0.5987850$

0.4563840

$-0.2646760$

$-1.0065970$

0.8959940

$-0.5658990$

1. 3943610

$-1.0012040$

0.7307420

0.8819150

0.7370850

$-0.7377510$

$-1.3835380$

1. 5143170 


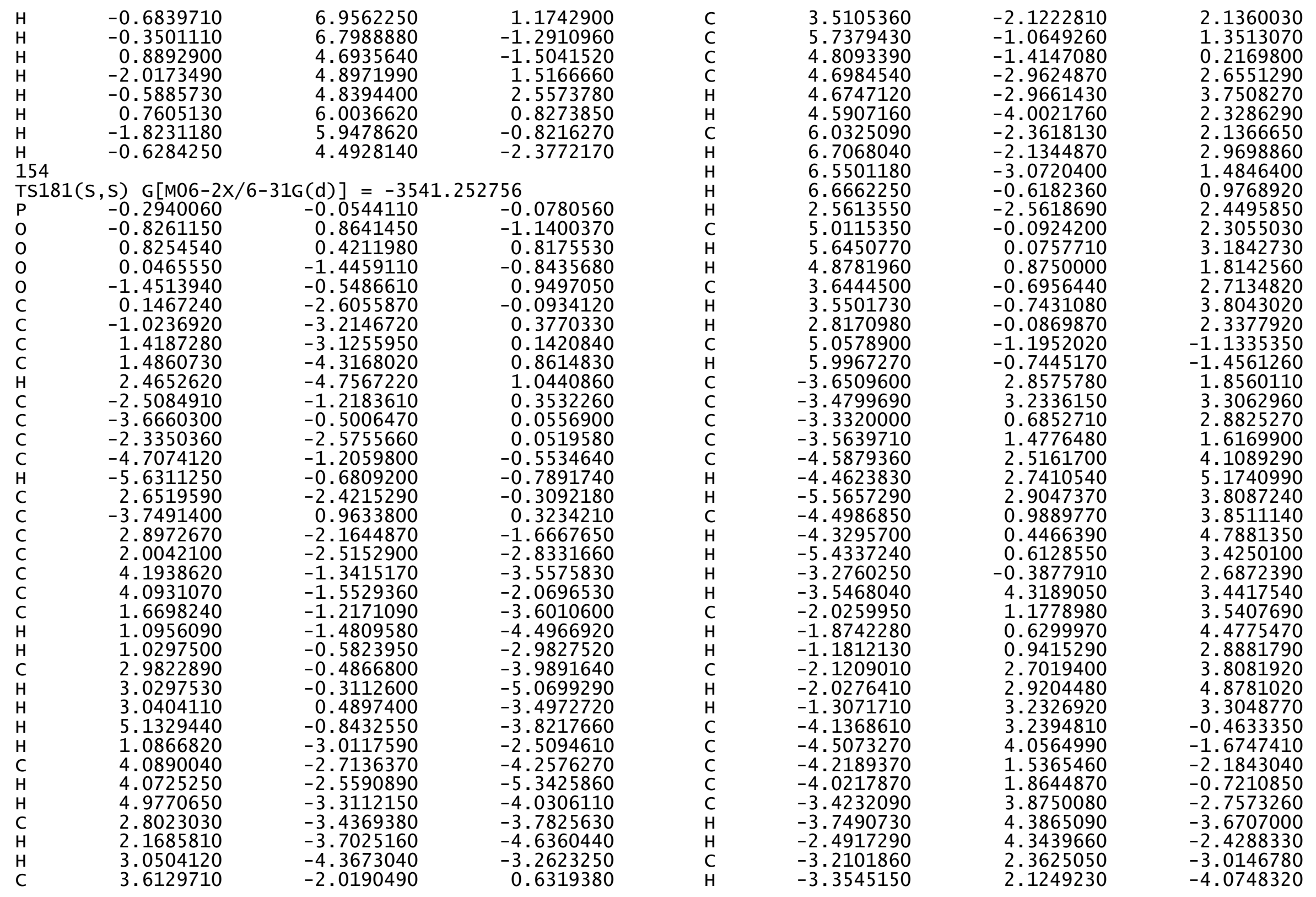




\begin{tabular}{|c|c|c|}
\hline $\begin{array}{l}-2.1961810 \\
-4.0759780 \\
-4.6232510 \\
-5.6404000 \\
-5.7693940 \\
-6.3888520 \\
-5.8311910 \\
-6.1258990 \\
-6.6227020 \\
-3.9441180 \\
-4.0362360 \\
-3.3785030 \\
-3.2868180 \\
-3.6805880 \\
-5.0724610 \\
-5.6397010 \\
-4.5695350 \\
-3.9725980 \\
-3.6527460 \\
-5.7846930 \\
-6.0331930 \\
-2.2799360 \\
-2.9167840 \\
-5.0001170 \\
-6.4819940 \\
-0.9131030 \\
0.3538730 \\
-2.1258070 \\
-1.9508180 \\
-0.6365220 \\
0.5342830 \\
-2.8010990 \\
-2.2893430 \\
-3.0280320 \\
-0.6279910 \\
-0.5336400 \\
1.4727660 \\
0.6486350 \\
-1.9464660 \\
3.2018220 \\
2.9598780 \\
4.5827410 \\
5.5077110 \\
4.9412180 \\
6.7929500 \\
5.2062580\end{array}$ & $\begin{array}{r}2.0534390 \\
0.4728740 \\
5.1139320 \\
1.9883930 \\
1.8252850 \\
1.3731060 \\
3.4874840 \\
4.0627210 \\
3.6108970 \\
3.7392840 \\
4.8073610 \\
-3.2349510 \\
-4.6369530 \\
-4.6220690 \\
-3.9867250 \\
-3.3185120 \\
-2.5465240 \\
-5.3036240 \\
-5.6432830 \\
-4.7408550 \\
-4.0993970 \\
-5.0449400 \\
-4.0547350 \\
-3.2330340 \\
-2.6678220 \\
-4.3720300 \\
-4.9400670 \\
-4.9302540 \\
-6.3831500 \\
-6.5477620 \\
-6.2313380 \\
-6.6859550 \\
-4.3084620 \\
-4.8205120 \\
-5.8619850 \\
-7.5628010 \\
-6.1854560 \\
-7.0551670 \\
-7.0348550 \\
2.7494160 \\
1.5356360 \\
3.2255640 \\
2.3768630 \\
4.5298530 \\
2.8368000 \\
1.3664910\end{array}$ & $\begin{array}{l}-2.7490070 \\
-2.3856250 \\
-1.4134380 \\
-2.5889080 \\
-3.6649350 \\
-2.0785550 \\
-2.2332720 \\
-3.1180970 \\
-1.4877590 \\
0.8201760 \\
1.0146060 \\
-0.6154540 \\
-1.1673390 \\
-2.6555270 \\
-2.8929640 \\
-1.6235440 \\
-0.8974720 \\
-0.6243630 \\
-3.0474060 \\
-3.2418550 \\
-0.9587010 \\
-1.0426500 \\
-3.1963610 \\
-3.6827620 \\
-1.8772190 \\
1.1737790 \\
1.3857230 \\
1.8970270 \\
2.3320140 \\
3.0896340 \\
2.1626950 \\
2.9515710 \\
2.7895230 \\
1.2953870 \\
3.9467580 \\
3.4865820 \\
2.7260710 \\
1.4440740 \\
1.4473770 \\
0.4734590 \\
0.0713930 \\
0.1839820 \\
-0.4299950 \\
0.5303820 \\
-0.6936590 \\
-0.6891260\end{array}$ \\
\hline
\end{tabular}
6.2272380
4.2077690
7. 1521510
7. 5150410
6.5107090
8.1565570
2. 0519960
2.3873560
0.4071280
0.3499190
0.8518600
0.2507720
$-1.0159810$
$-1.1784430$
0.1468530
0.5417230
0.0404980
0.7621030
0.4793710
0.1410410
$-1.0514600$
1.8567120
0.4314650
$-0.9456550$
1.6339830
0.4659730

154

TS182(S, S) G[M06-2X/6-31G(d) $]=-3541.251516$

$\begin{array}{lrrr}\text { TS182(S, S) G[M06-2X/6-31G(d) }]=-3541.251516 \\ \text { P } & -0.3613090 & -0.1836540 & 0.8755330 \\ \text { O } & 0.3489610 & -0.5273730 & 2.1500510 \\ \text { O } & -0.6952370 & 1.2585410 & 0.5824120 \\ \text { O } & -1.6736140 & -1.1324860 & 0.8157010 \\ \text { O } & 0.4690520 & -0.6911740 & -0.4396670 \\ \text { C } & -2.3092640 & -1.2458070 & -0.4125350 \\ \text { C } & -1.7403830 & -2.0908130 & -1.3764460 \\ \text { C } & -3.4870320 & -0.5320740 & -0.6204780 \\ \text { C } & -4.1019560 & -0.6675140 & -1.8688190 \\ \text { H } & -5.0260140 & -0.1244150 & -2.0572910 \\ \text { C } & 0.6174040 & -2.0640140 & -0.5805460 \\ \text { C } & 1.8518450 & -2.6333110 & -0.2778150 \\ \text { C } & -0.4765250 & -2.8099850 & -1.0437490 \\ \text { C } & 1.9768200 & -4.0164840 & -0.4257380 \\ \text { H } & 2.9360720 & -4.4829070 & -0.2094060 \\ \text { C } & -4.0785360 & 0.3392130 & 0.4332820 \\ \text { C } & 3.0051120 & -1.7901180 & 0.1442610 \\ \text { C } & -4.5044470 & -0.1960960 & 1.6582190\end{array}$

$\begin{array}{lr}4.9853520 & 0.2632540 \\ 5.1690240 & 1.0110510 \\ 4.1390430 & -0.3476980 \\ 2.1799820 & -1.1687320 \\ 5.9978010 & 0.5319550 \\ 4.4960780 & -0.5538070 \\ 1.1340870 & 0.3656470 \\ 3.4909670 & 1.0416170 \\ 3.5281010 & -0.8647460 \\ 3.4830400 & 0.5988840 \\ 2.6524940 & -1.3407570 \\ 2.5057820 & 1.0596960 \\ 3.3678720 & -0.6959580 \\ 2.3718500 & -0.8126680 \\ 6.0300350 & -0.7708440 \\ 5.9946890 & 0.7065250 \\ 4.7173370 & 1.3840440 \\ 4.8440300 & -1.5165900 \\ 6.9650900 & -1.2310430 \\ 6.8634120 & 1.2367430 \\ 4.7366260 & 1.4985140 \\ 4.9277320 & -1.5191140 \\ 4.8118010 & -2.5598010 \\ 5.9914430 & -0.8636760 \\ 6.0337020 & 0.7922570 \\ 4.5935160 & 2.3834060\end{array}$

0.2632540

.5319550

0.3656470

1.8647460

0.5988840

1.3407570

$-0.6959580$

- n - n

0.7065250

.3840440

1.2367430

4985140

8010

3834060
$-0.3476980$ 


$\begin{array}{rrr}-4.3885380 & -1.6326560 & 2.1095030 \\ -5.5328270 & -0.1146840 & 3.8650790 \\ -5.1369710 & 0.6178280 & 2.6091230 \\ -3.5799700 & -1.6593260 & 3.4251410 \\ -3.5302540 & -2.6928380 & 3.7864860 \\ -2.5552490 & -1.3359070 & 3.2244030 \\ -4.2615270 & -0.7434050 & 4.4756220 \\ -4.5385020 & -1.3117540 & 5.3709120 \\ -3.5805030 & 0.0512010 & 4.7948170 \\ -6.0125070 & 0.5618730 & 4.5805110 \\ -3.9064600 & -2.2600860 & 1.3564480 \\ -6.4911690 & -1.2601970 & 3.4736000 \\ -6.7423910 & -1.8383550 & 4.3701240 \\ -7.4251130 & -0.8403460 & 3.0877440 \\ -5.8101930 & -2.1590250 & 2.4088730 \\ -5.7371770 & -3.1935000 & 2.7629370 \\ -6.3948820 & -2.1751840 & 1.4838420 \\ -4.2761940 & 1.7077060 & 0.1973910 \\ -3.8021900 & 2.4993780 & -0.9988520 \\ -5.1025530 & 3.9412470 & 0.7129530 \\ -4.9337100 & 2.5069140 & 1.1446060 \\ -5.0267230 & 3.1427470 & -1.6853680 \\ -4.6754930 & 3.7992330 & -2.4898100 \\ -5.6460280 & 2.3688430 & -2.1506760 \\ -5.8471450 & 3.9451540 & -0.6403150 \\ -5.9915070 & 4.9799300 & -0.9706420 \\ -6.8404950 & 3.5056540 & -0.5075130 \\ -5.6608250 & 4.5143710 & 1.4608810 \\ -3.2423330 & 1.8753720 & -1.7001620 \\ -3.7044200 & 4.5527570 & 0.4833140 \\ -3.8226310 & 5.5575720 & 0.0608460 \\ -3.1874150 & 4.6637860 & 1.4412360 \\ -2.8976380 & 3.6373120 & -0.4718470 \\ -2.5034760 & 4.2058130 & -1.3221910 \\ -2.0478560 & 3.1903230 & 0.0474780 \\ -5.3661230 & 1.9653310 & 2.3498930 \\ -5.8740320 & 2.5889450 & 3.0841510 \\ 4.7198940 & -0.1469160 & -0.4054250 \\ 5.2535380 & 0.7156020 & -1.5214620 \\ 3.1784350 & -0.6747760 & -2.2013820 \\ 3.5897400 & -0.8946380 & -0.7652860 \\ 5.6202370 & -0.2145810 & -2.6997330 \\ 5.9668380 & 0.3953380 & -3.5414520 \\ 6.4501190 & -0.8647200 & -2.4071240 \\ 4.3778340 & -1.0537470 & -3.0998550 \\ 4.1072910 & -0.8722350 & -4.1459390\end{array}$

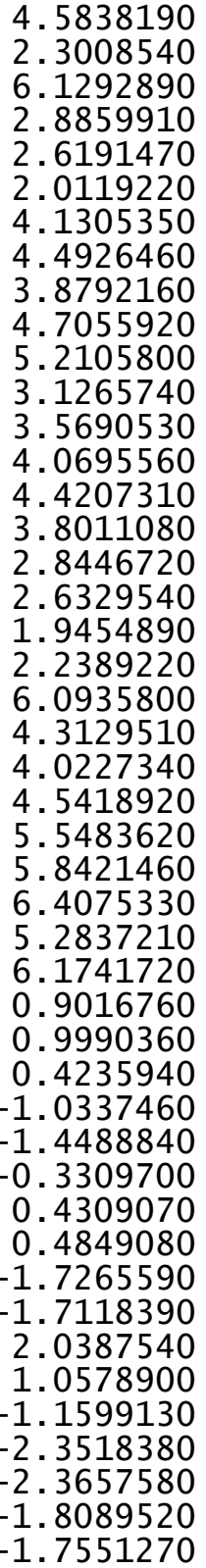

-2.1238260
-1.2692680
1.2869430
0.8262320
1.0065260
1.0903160
1.6611470
2.2545880
2.3711660
-1.1661140
-1.4517860
-2.8504650
-1.9136690
-1.1603030
-1.3662230
-0.0998150
-2.0417520
-2.7493730
-1.4404160
-3.4183510
-0.8454290
-3.7954870
-4.4923640
-4.3941140
-2.9579010
-3.1959120
-3.1789780
-0.2872450
0.2792510
-4.7993900
-6.2937330
-6.6899040
-6.2043700
-5.1566180
-4.2022800
-6.7991100
-7.7740040
-7.0488300
-5.6881930
-6.6220260
-6.2382720
-5.7803540
-4.6292210
-2.1762050
-2.9326570
-2.0071830

$-3.0001630$ $-2.4677400$ $-1.1943290$ $-2.4000080$ $-3.4475710$ $-1.7994800$ $-1.9992090$ $-2.8468640$ $-1.2051140$

1. 7704740

3.1608340

2. 5203360

1.4213880

4. 1579630

5.1754310

4.1144790

3. 8056050

4.6153910

3. 6625580

2. 2311770

3. 3909710

2. 8198220

3. 6137070

1. 9319650

3. 2475960

4. 2761670

2. 6071960

0.8602600

1.1317640

$-0.8301490$

$-0.9979130$

$-2.3664130$

$-2.5398740$

$-1.4840920$

$-1.1404430$

$-0.2040410$

$-2.5011260$

$-2.4709160$

$-0.5589040$

$-0.9038460$

$-3.1365780$

$-3.5407890$

$-1.7973420$

$-2.6285690$

-3.8087280
-5.0383470 


$\begin{array}{rrr}-3.1131560 & -1.3235420 & -5.3329730 \\ -4.1610710 & -1.5992400 & -4.2367480 \\ -3.5525900 & -1.4645960 & -2.8657420 \\ -2.4531930 & -3.7952010 & -4.0346240 \\ -1.4177810 & -2.5821510 & -5.9060370 \\ -3.5115680 & -1.6584390 & -6.2956000 \\ -4.5434500 & -2.6215900 & -4.3614310 \\ -0.8131070 & -3.3288100 & -3.5917700 \\ -0.9916970 & -1.2462260 & -4.8482030 \\ -2.9686040 & -0.2418510 & -5.4117450 \\ -5.0175070 & -0.9273370 & -4.3475300 \\ 1.3617420 & 3.4395460 & -0.9138720 \\ 0.2995680 & 2.7362880 & -1.1523260 \\ 1.8205770 & 4.2706470 & -2.0628130 \\ 2.9382270 & 5.0923090 & -1.9014700 \\ 1.1651430 & 4.2056820 & -3.2945470 \\ 3.3996290 & 5.8506080 & -2.9706750 \\ 3.4352240 & 5.1210140 & -0.9369710 \\ 1.6324440 & 4.9637900 & -4.3631460 \\ 0.3016830 & 3.5588150 & -3.4041900 \\ 2.7470510 & 5.7849980 & -4.2015100 \\ 4.2674400 & 6.4904550 & -2.8477180 \\ 1.1282460 & 4.9133930 & -5.3227480 \\ 3.1095330 & 6.3752380 & -5.0375780 \\ -0.0331000 & 2.1161530 & -0.3816080 \\ 1.9977200 & 3.4553470 & 0.1514910 \\ 1.3071950 & 2.4072350 & 2.6358230 \\ 2.3421270 & 2.0664260 & 1.6530040 \\ 0.2837200 & 2.2648850 & 2.2911670 \\ 2.1452630 & 1.2386590 & 0.9725420 \\ 1.8246570 & 1.2322090 & 3.2968810 \\ 1.2626320 & 0.4610010 & 2.9514940 \\ 2.9798990 & 3.8698450 & 3.8356570 \\ 3.8585180 & 3.8487700 & 2.5841240 \\ 3.7561970 & 2.5029270 & 1.8663220 \\ 1.5133420 & 3.6591320 & 3.4565610 \\ 3.0900790 & 4.8218540 & 4.3632710 \\ 4.9051660 & 4.0389890 & 2.8393100 \\ 4.2312820 & 1.7061280 & 2.4520310 \\ 1.1559430 & 4.5034090 & 2.8544730 \\ 0.8761600 & 3.5933000 & 4.3439670 \\ 3.2967490 & 3.0773750 & 4.5243960 \\ 3.5371810 & 4.6417630 & 0.8982040 \\ 4.2635060 & 2.5071620 & \end{array}$

154

$\operatorname{TS} 183(\mathrm{~S}, \mathrm{~S}) \mathrm{G}[\mathrm{M} 06-2 \mathrm{X} / 6-31 \mathrm{G}(\mathrm{d})]=-3541.252259$

-0.2958810
-0.8362760
0.8248920
0.0421670
-1.4452920
0.1238960
-1.0641780
1.3888130
1.4529000
2.4288950
-2.5193780
-3.6809290
-2.3710630
-4.7592150
-5.6882280
2.6308170
-3.7355100
2.8997470
2.0219100
4.2322990
4.1059580
1.7103660
1.1427820
1.0708710
3.0348940
3.0993980
3.0950150
5.1795380
1.0953780
4.1277620
4.1331610
5.0059040
2.8257500
2.2032970
3.0551890
3.5788670
3.4497800
5.6958610
4.7851310
4.6244960
4.5821160
4.5171640
5.9702300
6.6324210
6.4939380
6.6327590
$-0.1442160$

$-1.2006590$

0.7488040

$-0.9127010$

0.8866070

0.2651290

0.0660340

0.7636400

0.9550800

0.3018320

0.0589490

$-0.0204850$

$-0.4984110$

$-0.6855940$

$-0.3592470$

0.3367530

$-1.7099140$

$-2.8934260$

$-3.5715490$

$-2.0875330$

$-3.6497690$

$-4.5545280$

$-3.0304310$

$-4.0145490$

$-5.0934010$

$-3.5157980$

$-3.8158260$

$-2.5888780$

$-4.2862500$

$-5.3698540$

$-4.0481440$

$-3.8421240$

$-4.7093370$

$-3.3307480$

0.6008100

2. 1017830

1. 3645270

0.2115510

2.6356760

3. 7307820

2. 3011330

2.1436900

2. 9894720

1.4940740

1.0100300 


$\begin{array}{rrrr}\mathrm{H} & 2.4930300 & -2.6191270 & 2.3949060 \\ \mathrm{C} & 4.9581640 & -0.1612490 & 2.3148940 \\ \mathrm{H} & 5.5765140 & -0.0062020 & 3.2068150 \\ \mathrm{H} & 4.8401010 & 0.8120120 & 1.8312360 \\ \mathrm{C} & 3.5798270 & -0.7593410 & 2.6914210 \\ \mathrm{H} & 3.4630570 & -0.8126960 & 3.7797950 \\ \mathrm{H} & 2.7640730 & -0.1437610 & 2.3018910 \\ \mathrm{C} & 5.0574590 & -1.2342890 & -1.1325800 \\ \mathrm{H} & 6.0042030 & -0.7866000 & -1.4355910 \\ \mathrm{C} & -3.5636770 & 2.8576680 & 1.8726200 \\ \mathrm{C} & -3.3456030 & 3.2239460 & 3.3190700 \\ \mathrm{C} & -3.2497570 & 0.6754110 & 2.8806470 \\ \mathrm{C} & -3.5047820 & 1.4776160 & 1.6260510 \\ \mathrm{C} & -4.4412620 & 2.5198220 & 4.1501720 \\ \mathrm{H} & -4.2807000 & 2.7367920 & 5.2122790 \\ \mathrm{H} & -5.4210440 & 2.9257050 & 3.8810000 \\ \mathrm{C} & -4.3844570 & 0.9927000 & 3.8823510 \\ \mathrm{H} & -4.1990720 & 0.4424970 & 4.8116700 \\ \mathrm{H} & -5.3368710 & 0.6344580 & 3.4799110 \\ \mathrm{H} & -3.2161720 & -0.3972610 & 2.6779160 \\ \mathrm{H} & -3.3912830 & 4.3095090 & 3.4613420 \\ \mathrm{C} & -1.9186160 & 1.1440040 & 3.5050490 \\ \mathrm{H} & -1.7512070 & 0.5904780 & 4.4358440 \\ \mathrm{H} & -1.0951050 & 0.8962900 & 2.8297390 \\ \mathrm{C} & -1.9813650 & 2.6684010 & 3.7796580 \\ \mathrm{H} & -1.8532940 & 2.8818730 & 4.8469760 \\ \mathrm{H} & -1.1742480 & 3.1876920 & 3.2543500 \\ \mathrm{C} & -4.1096400 & 3.2566710 & -0.4307040 \\ \mathrm{C} & -4.5001490 & 4.0833120 & -1.6291850 \\ \mathrm{C} & -4.2719600 & 1.5598940 & -2.1523520 \\ \mathrm{C} & -4.0252940 & 1.8808620 & -0.6956250 \\ \mathrm{C} & -3.4512970 & 3.8845450 & -2.7433470 \\ \mathrm{H} & -3.7947150 & 4.4035810 & -3.6459760 \\ \mathrm{H} & -2.5025060 & 4.3362490 & -2.4417320 \\ \mathrm{C} & -3.2722200 & 2.3690140 & -3.0095480 \\ \mathrm{H} & -3.4501620 & 2.1366420 & -4.0657320 \\ \mathrm{H} & -2.2567110 & 2.0416260 & -2.7725430 \\ \mathrm{H} & -4.1560050 & 0.4939040 & -2.3590980 \\ \mathrm{H} & -4.5889860 & 5.1420970 & -1.3627460 \\ \mathrm{C} & -5.6962370 & 2.0385320 & -2.5143170 \\ \mathrm{H} & -5.8593750 & 1.8805790 & -3.5864660 \\ \mathrm{H} & -6.4401520 & 1.4350870 & -1.9836460 \\ \mathrm{C} & -5.8497480 & 3.5398170 & -2.1500860 \\ \mathrm{H} & -6.1596060 & 4.1225920 & -3.0247320 \\ \mathrm{H} & -6.6169700 & 3.6754330 & -1.3816220 \\ \mathrm{C} & -3.8722960 & 3.7481590 & 0.8486050 \\ & & & \end{array}$

-3.9419030
-3.4570170
-3.4137500
-3.9208090
-5.3172530
-5.7837430
-4.6566660
-4.0499980
-3.9337070
-6.0620180
-6.1391810
-2.4005240
-3.1919600
-5.2910980
-6.6333300
-0.9566510
-2.1169100
-1.7741830
-0.4425990
0.3402200
0.3063670
-2.3226330
-2.5985690
-0.6264540
-0.1176300
-3.0284470
-1.7121260
0.1850260
1.3722820
3.2217270
2.9795640
4.6131760
5.5465430
4.9731870
6.8416080
5.2435790
6.2690550
4.2329550
7.2022800
7.5702170
6.5536960
8.2143280
2.0627790
2.4004210
0.4367430
0.3674950
4.8168160
-3.2282030
-4.6362420
-4.6443070
-3.9944970
-3.2962640
-2.5284590
-5.2921380
-5.6734100
-4.7444760
-4.0601110
-5.0434220
-4.0991390
-3.2564230
-2.6377990
-4.4265740
-5.1181120
-5.3747540
-6.1416560
-6.2812240
-4.9987110
-6.0764490
-5.9182080
-7.1402510
-7.0801850
-4.5199610
-4.4023720
-5.6160250
-6.5873470
2.7173730
1.5084790
3.1839200
2.3311400
4.4832900
2.7821900
1.3246690
4.9299040
5.1256540
4.0795740
2.1222800
5.9385250
4.4296290
1.1118250
3.4619970
3.4999070
3.4669010
1.0494700

$-0.6266540$

$-1.1670120$

$-2.6208800$

$-2.7723160$

$-1.4794840$

$-0.8402090$

$-0.5554560$

$-2.9924360$

$-3.0561560$

$-0.7744750$

$-1.1221860$

$-3.2285810$

$-3.5795580$

$-1.6841890$

1.0048780

1.6783920

3.1575630

3. 3410200

2. 0206230

1. 2318930

1.1802020

3.6290930

3. 7493060

1.4216150

1.6065260

3.6561440

4.0669180

2. 2165270

0.4650100

0.0487300

0.2124980

$-0.3827640$

0.5752850

$-0.6115410$

$-0.6549440$

0.3431380

1. 0410340

$-0.2493170$

$-1.0721220$

0.6246970

$-0.4282030$

0.3205420

1.0189600

$-0.9077460$

0.5557590 


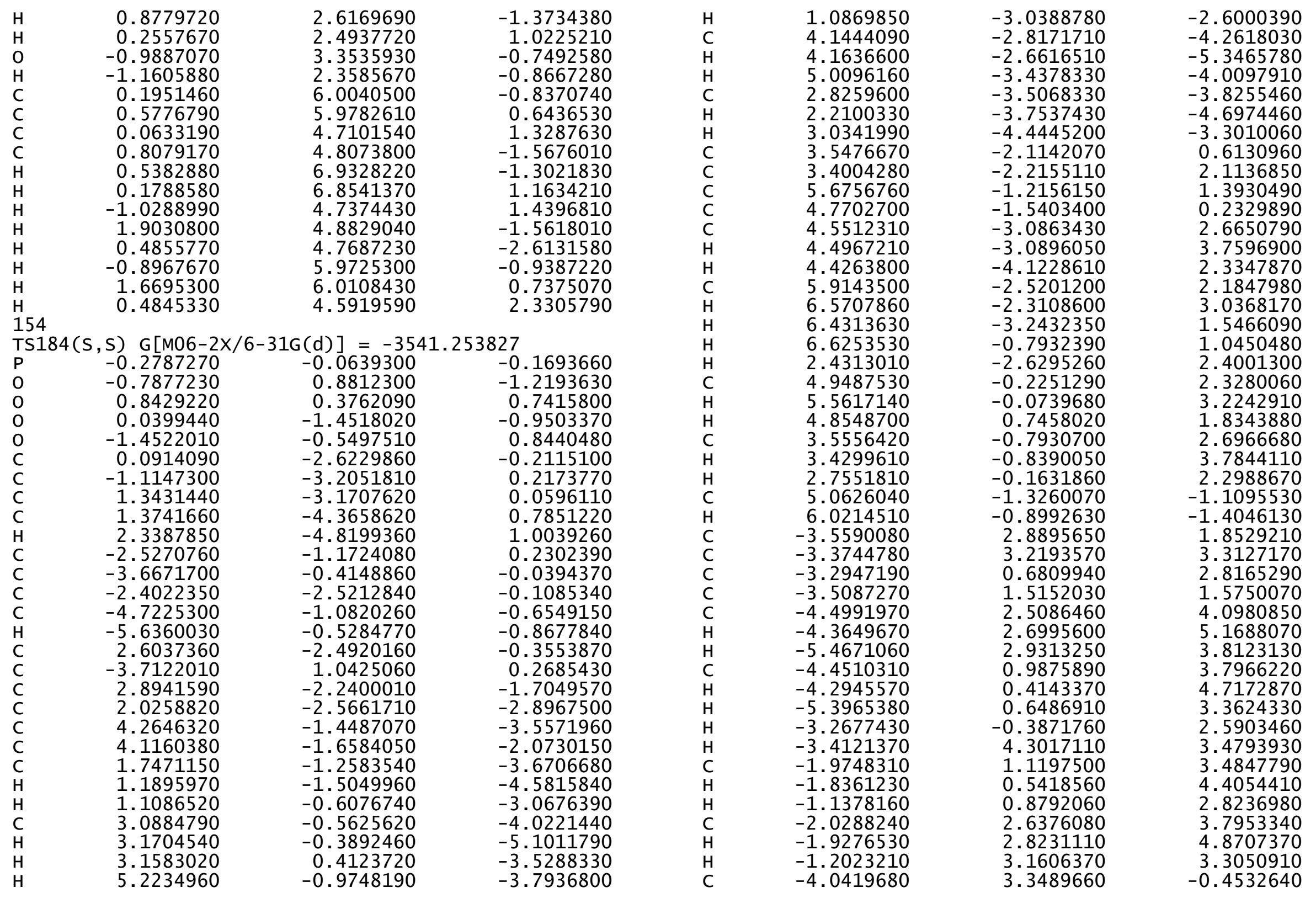




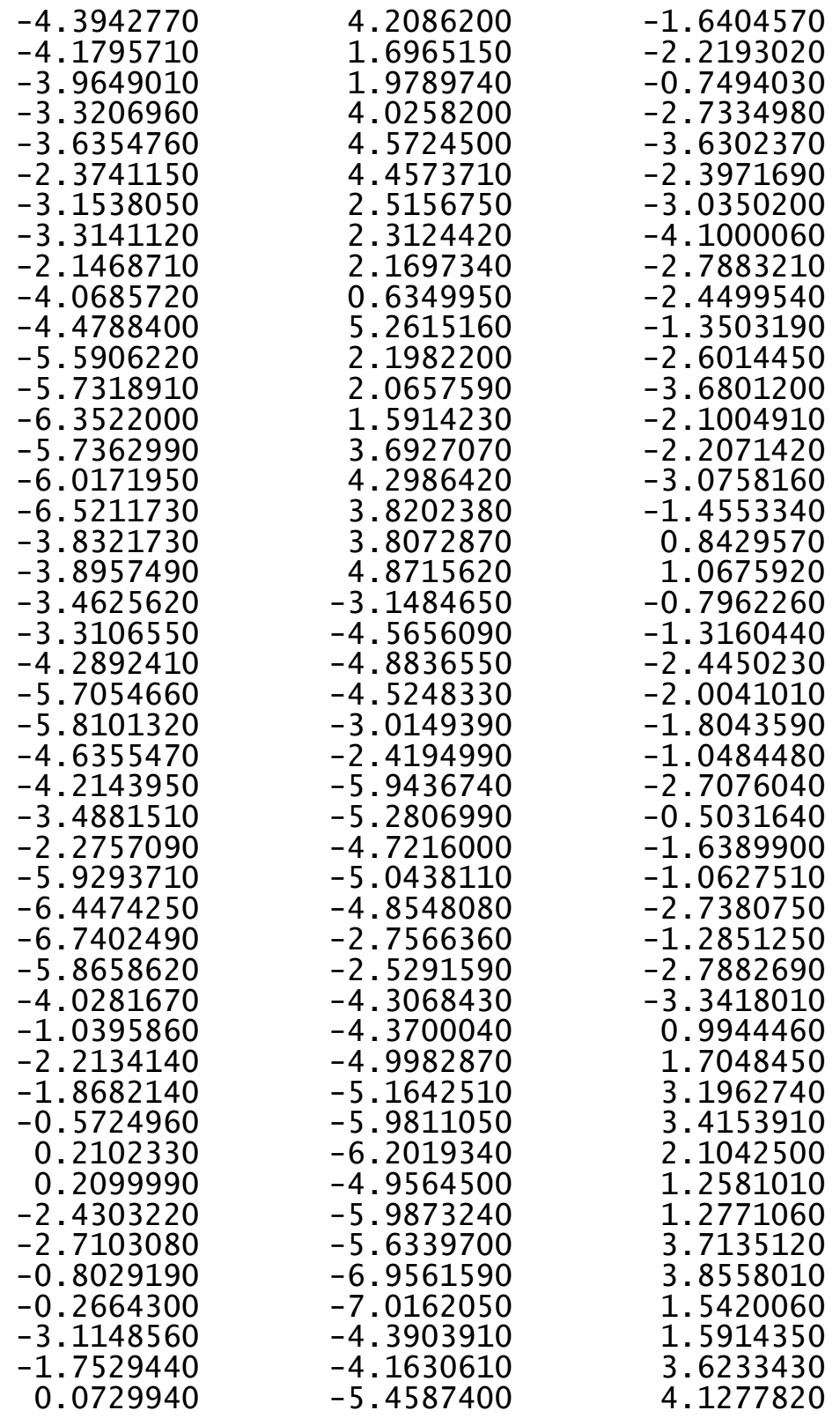

\begin{abstract}
1.2336460
3. 2739480

3. 0083080

4.6696840

5. 5858050

5. 0513220

6.8853220

5. 2663660

6. 3514390

4. 3242110

7.2674670

7.6006920

6.6528590

8. 2829070

2. 0890010

2.4705740

0.4970780

0.4336020

0.9306060

0.3168660

$-0.9288140$

$-1.1074680$

0.2734990

0.6642190

0.1421170

0.8735130

0.6202620

0.2752360

$-0.9496980$

1. 9691940

0.5453790

$-0.8193080$

1.7567440

0.5640520
\end{abstract}

154

$\operatorname{TS} 185(\mathrm{~S}, \mathrm{~S}) \mathrm{G}[\mathrm{M06}-2 \mathrm{X} / 6-31 \mathrm{G}(\mathrm{d})]=-3541.252660$

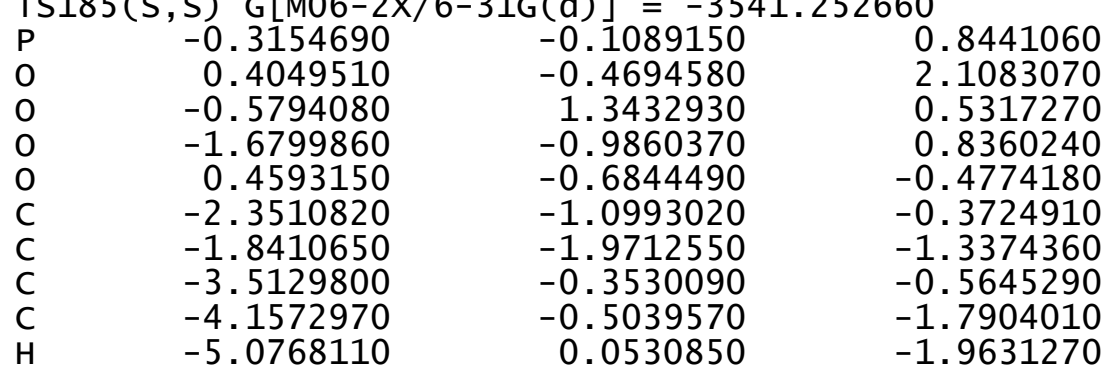

2.3179410

$-6.5245200$

2. 6555080

1.4571580

3.1039640

2. 2415720

4. 3956170

2.6754290

1.2411340

4.8251900

. 0455820

3. 9652930

2. 0079800

5.8278820

4. 3019940

1. 0694300

3.4038280

3. 5185350

. 4416830

2.6472250

.4561510

3. 3762230

. 3865360

6.0211570

5.9471950

4.6627760

4.8432780

6. 9613790

6.8098120

4. 6961110

4.9108550

4.8393670

6. 0001640

5. 9680960

4. 5098100

0.0388160

0.2097500

$-0.3984310$

0.5778340

$-0.6349660$

$-0.6740320$

0.3374310

1. 0536320

$-0.2679420$

$-1.1053370$

0.6226740

$-0.4531160$

0.3149750

1.0460940

$-0.8645670$

0.5973890

$-1.3585390$

1.0357260

$-0.7037750$

$-0.8490130$

$-0.7150930$

0.7619110

1. 4094550

$-1.4857700$

$-1.1537430$

1.5230410

$-1.4836520$

$-2.5302700$

$-0.8106260$

0.8509230

2.4062910
1. 3105060

0.0530850

$-1.9631270$

$-5.4587400$

4.1277820 


$\begin{array}{rr}0.5276850 & -2.0673070 \\ 1.7258150 & -2.7037120 \\ -0.6169180 & -2.7565660 \\ 1.7543200 & -4.0969930 \\ 2.6830340 & -4.6182500 \\ -4.0478840 & 0.5609230 \\ 2.9328820 & -1.9200290 \\ -4.4612260 & 0.0680150 \\ -4.3886490 & -1.3624990 \\ -5.4305450 & 0.2342750 \\ -5.0357570 & 0.9258220 \\ -3.5507840 & -1.3946340 \\ -3.5329820 & -2.4219990 \\ -2.5192050 & -1.1162460 \\ -4.1708740 & -0.4322950 \\ -4.4489070 & -0.9719530 \\ -3.4519390 & 0.3406470 \\ -5.8666860 & 0.9428460 \\ -3.9481300 & -2.0227030 \\ -6.4419020 & -0.8799690 \\ -6.6954900 & -1.4291580 \\ -7.3670190 & -0.4310390 \\ -5.8217930 & -1.8272450 \\ -5.7800000 & -2.8559410 \\ -6.4288310 & -1.8403310 \\ -4.1953370 & 1.9311140 \\ -3.7240310 & 2.6768760 \\ -4.8987940 & 4.2103460 \\ -4.7885750 & 2.7768410 \\ -4.9386430 & 3.3650430 \\ -4.5839940 & 3.9851680 \\ -5.6100740 & 2.6119180 \\ -5.6826570 & 4.2300200 \\ -5.7807290 & 5.2649940 \\ -6.6939530 & 3.8481530 \\ -5.4043140 & 4.8237010 \\ -3.2189940 & 2.0138870 \\ -3.4783700 & 4.7454700 \\ -3.5575750 & 5.7499650 \\ -2.9274000 & 4.8408380 \\ -2.7489790 & 3.7785060 \\ -2.3602420 & 4.3150670 \\ -1.9020290 & 3.3002620 \\ -5.2121980 & 2.2772350 \\ -5.6717720 & 2.9365200 \\ 4.7230610 & -0.3802610\end{array}$

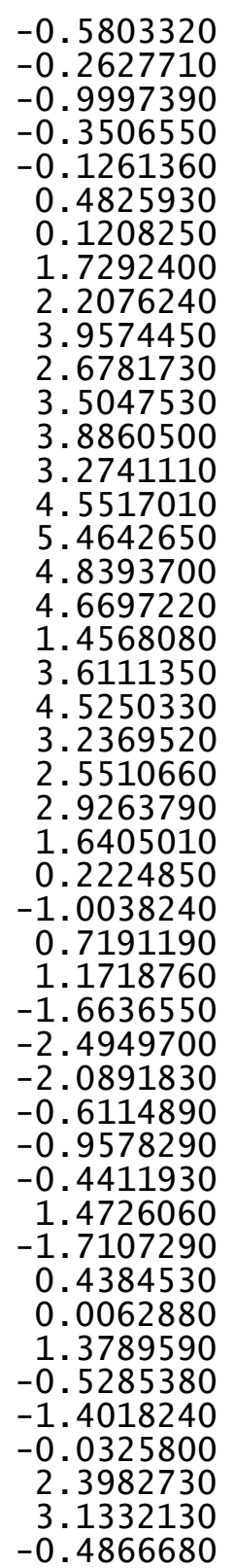

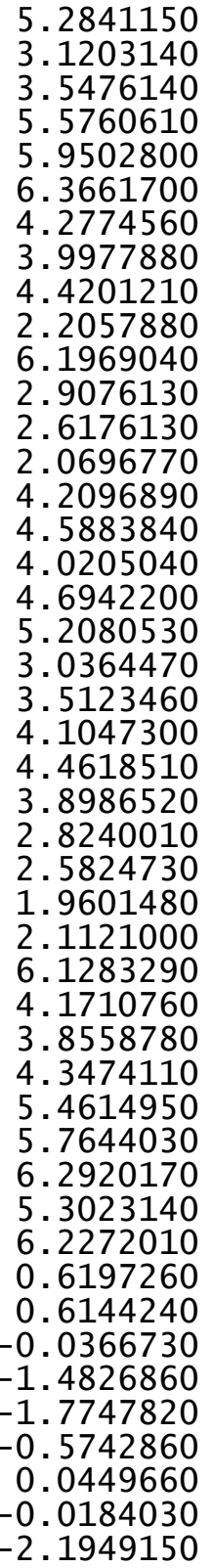

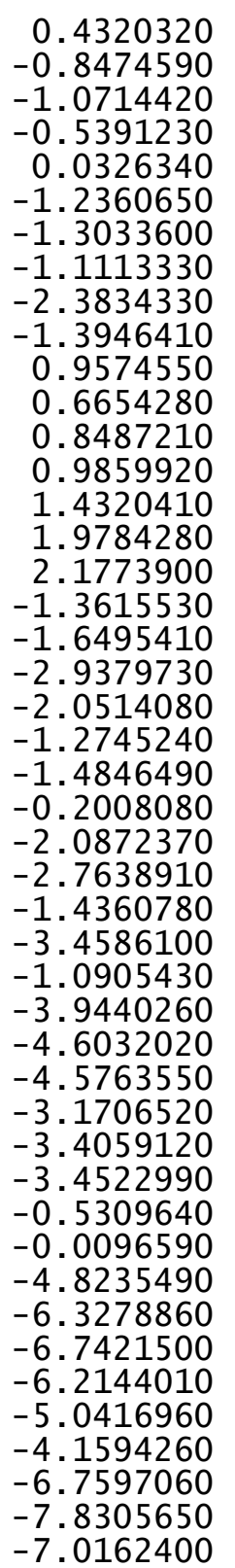

$-1.6266690$ $-2.2448900$ $-0.8139190$ $-2.7926790$ $-3.6492050$ $-2.4976470$ $-3.1630020$ $-4.2048330$ $-3.0607110$ $-2.4866520$ $-1.3246630$ $-2.4556420$ $-3.4964240$ $-1.8311280$ $-2.1050910$ $-2.9767380$ $-1.3263720$

1. 7063410

3.0929800

2. 5171210

1. 3906090

4.1045100

5.1190570

4.0477190

3. 7884970

4. 6162110

3. 6450670

2. 2561570

3. 2953450

2. 8184100

3.6346670

1. 9419470

3. 2029590

4.2294950

2. 5486900

0.7707840

1.0164770

$-0.6975090$

$-0.7942050$

$-2.1222300$

$-2.2347250$

$-1.2696130$

$-1.0175390$

0.0413020

-2.2331510
-2.0162200 


$\begin{array}{rrr}-2.0632940 & -5.4636990 & -0.2970930 \\ 1.6342260 & -6.7181170 & -0.7208140 \\ 0.5725630 & -6.3288250 & -2.9334920 \\ -1.6790010 & -5.9025550 & -3.2661850 \\ -2.6349960 & -4.4634930 & -1.6152890 \\ -2.4735040 & -2.0447860 & -2.5940970 \\ -3.6555490 & -1.3189730 & -2.8066460 \\ -1.8321960 & -2.8122980 & -3.7356500 \\ -2.8250870 & -3.1881770 & -4.8321220 \\ -3.6037890 & -1.9511310 & -5.2724720 \\ -4.4331090 & -1.4174130 & -4.1065840 \\ -2.2909200 & -3.6362930 & -5.6763000 \\ -1.0494980 & -2.1712900 & -4.1677400 \\ -1.3122580 & -3.6983680 & -3.3680100 \\ -2.8940000 & -1.1830320 & -5.6060420 \\ -4.2575440 & -2.1768120 & -6.1210240 \\ -4.8558050 & -0.4365200 & -4.3505020 \\ -5.2894770 & -2.0873190 & -3.9457010 \\ -3.5252440 & -3.9457710 & -4.4526870 \\ 1.5697610 & 3.4058520 & -1.0163550 \\ 0.4587670 & 2.7710550 & -1.2229250 \\ 2.0425290 & 4.2124380 & -2.1769290 \\ 3.2240380 & 4.9471500 & -2.0552000 \\ 1.3318420 & 4.2143460 & -3.3793920 \\ 3.6944090 & 5.6848500 & -3.1348440 \\ 3.7621000 & 4.9257300 & -1.1127960 \\ 1.8072720 & 4.9526820 & -4.4581010 \\ 0.4184240 & 3.6352070 & -3.4584480 \\ 2.9859410 & 5.6868010 & -4.3360590 \\ 4.6120080 & 6.2568370 & -3.0427800 \\ 1.2594690 & 4.9548250 & -5.3948310 \\ 3.3545650 & 6.2617420 & -5.1800550 \\ 0.1134810 & 2.1684070 & -0.4456940 \\ 2.2384750 & 3.3786780 & 0.0283910 \\ 1.5227190 & 2.4076050 & 2.5365910 \\ 2.5269970 & 1.9993900 & 1.5476080 \\ 0.4890590 & 2.3115440 & 2.2064050 \\ 2.2807330 & 1.1708750 & 0.8840170 \\ 1.9883680 & 1.2189330 & 3.2112390 \\ 1.3829470 & 0.4730730 & 2.8835780 \\ 3.2806200 & 3.8032260 & 3.6935070 \\ 4.1429220 & 3.7168690 & 2.4334460 \\ 3.9638960 & 2.3666050 & 1.7390480 \\ 1.8010700 & 3.6607540 & 4.3337480 \\ 3.4447150 & 4.7571960 & 2.6746950 \\ 5.2007240 & 3.8569530 & \end{array}$

$\begin{array}{ll}\mathrm{H} & 4.4034720 \\ \mathrm{H} & 1.4796680 \\ \mathrm{H} & 1.1717590 \\ \mathrm{H} & 3.5648110 \\ \mathrm{H} & 3.8551500 \\ \mathrm{H} & 4.4601960\end{array}$

154

TS186(S, S) G[M06-2X/6-31G(d)] $=-3541.252839$

$\begin{array}{lrrr}\text { TS186(S }, \mathrm{S}) & \mathrm{G}[\mathrm{M} 06-2 \mathrm{X} / 6-31 \mathrm{G}(\mathrm{d})]=-3541.252839 \\ \mathrm{P} & -0.3378880 & -0.1375600 & 0.8703250 \\ \mathrm{O} & 0.3725800 & -0.4970400 & 2.1403070 \\ \mathrm{O} & -0.6262150 & 1.3128990 & 0.5697930 \\ \mathrm{O} & -1.6845970 & -1.0409450 & 0.8313310 \\ \mathrm{O} & 0.4662990 & -0.6791810 & -0.4488650 \\ \mathrm{C} & -2.3298540 & -1.1373570 & -0.3933790 \\ \mathrm{C} & -1.7948500 & -1.9927880 & -1.3580960 \\ \mathrm{C} & -3.4837310 & -0.3833590 & -0.6047220 \\ \mathrm{C} & -4.0808580 & -0.4957500 & -1.8577510 \\ \mathrm{H} & -4.9888450 & 0.0735680 & -2.0510050 \\ \mathrm{C} & 0.5633670 & -2.0582390 & -0.5756860 \\ \mathrm{C} & 1.7740440 & -2.6735560 & -0.2668960 \\ \mathrm{C} & -0.5655350 & -2.7658130 & -1.0118910 \\ \mathrm{C} & 1.8318320 & -4.0647540 & -0.3744290 \\ \mathrm{H} & 2.7701180 & -4.5696890 & -0.1523950 \\ \mathrm{C} & -4.0559680 & 0.4998140 & 0.4490720 \\ \mathrm{C} & 2.9642830 & -1.8711090 & 0.1300600 \\ \mathrm{C} & -4.5026480 & -0.0291220 & 1.6693640 \\ \mathrm{C} & -4.4305990 & -1.4708050 & 2.1135330 \\ \mathrm{C} & -5.5419420 & 0.0704590 & 3.8701980 \\ \mathrm{C} & -5.1149810 & 0.7982580 & 2.6217460 \\ \mathrm{C} & -3.6316580 & -1.5273330 & 3.4341640 \\ \mathrm{H} & -3.6120150 & -2.5640400 & 3.7891230 \\ \mathrm{H} & -2.5974010 & -1.2302880 & 3.2419560 \\ \mathrm{C} & -4.2950010 & -0.6000920 & 4.4863100 \\ \mathrm{H} & -4.5964320 & -1.1672280 & 5.3744440 \\ \mathrm{H} & -3.5936650 & 0.1713780 & 4.8179870 \\ \mathrm{H} & -6.0066950 & 0.7566280 & 4.5863040 \\ \mathrm{H} & -3.9612650 & -2.1081150 & 1.3604730 \\ \mathrm{C} & -6.5312620 & -1.0432990 & 3.4641010 \\ \mathrm{H} & -6.8088260 & -1.6174740 & 4.3553800 \\ \mathrm{H} & -7.4479990 & -0.5929290 & 3.0714790 \\ \mathrm{C} & -5.8684590 & -1.9580600 & 2.4014640 \\ \mathrm{H} & -5.8267160 & -2.9950640 & 2.7531750 \\ \mathrm{H} & -6.4470300 & -1.9555060 & 1.4724900 \\ \mathrm{C} & -4.2086850 & 1.8752430 & 0.2204590 \\ \mathrm{C} & -3.7067790 & 2.6583900 & -0.9699690 \\ \mathrm{C} & -4.9605280 & 4.1319320 & \\ & & & \end{array}$

2.3335240

2.7215800

4.2289700

4.3926930

1.7380420

0.7659460
4.5122310

.0075300

4.5142470
.5863040

0.7494940

$-3.1881770 \quad-4.8321220$

2724720

$-4.1065840$

$-5.6763000$

1400

$-5.6060420$

$-6.1210240$

3505020

010

$-1.0163550$

$-1.2229250$

52000

.1800550

.5365910

2. 2064050

0170

.6935070

2.6746950 


$\begin{array}{rrr}-4.8431900 & 2.6894050 & 1.1707160 \\ -4.9088000 & 3.3457160 & -1.6536000 \\ -4.5357880 & 3.9918320 & -2.4565990 \\ -5.5546350 & 2.5946700 & -2.1203120 \\ -5.7003160 & 4.1730950 & -0.6057110 \\ -5.8029000 & 5.2148620 & -0.9296720 \\ -6.7106230 & 3.7723030 & -0.4785960 \\ -5.5003300 & 4.7186260 & 1.5004510 \\ -3.1674160 & 2.0205500 & -1.6747140 \\ -3.5406240 & 4.6950630 & 0.5291360 \\ -3.6213680 & 5.7086050 & 0.1189380 \\ -3.0220930 & 4.7754730 & 1.4892850 \\ -2.7654920 & 3.7627780 & -0.4361610 \\ -2.3570950 & 4.3257080 & -1.2834800 \\ -1.9280200 & 3.2853220 & 0.0760550 \\ -5.2991420 & 2.1541260 & 2.3701310 \\ -5.7901510 & 2.7891740 & 3.1060780 \\ 4.7290360 & -0.2944970 & -0.4560360 \\ 5.2798590 & 0.5377150 & -1.5866440 \\ 3.1501100 & -0.7858850 & -2.2293490 \\ 3.5695190 & -1.0060830 & -0.7955610 \\ 5.6012450 & -0.4173850 & -2.7582260 \\ 5.9613630 & 0.1706080 & -3.6098060 \\ 6.4098320 & -1.0941070 & -2.4662710 \\ 4.3253470 & -1.2149560 & -3.1372640 \\ 4.0496420 & -1.0326550 & -4.1818630 \\ 4.4941860 & -2.2908380 & -3.0305500 \\ 2.2496560 & -1.3521650 & -2.4805980 \\ 6.1784790 & 1.0814400 & -1.2747970 \\ 2.9074550 & 0.7224040 & -2.4396370 \\ 2.6332800 & 0.9017470 & -3.4854220 \\ 2.0509740 & 1.0226620 & -1.8306360 \\ 4.1856180 & 1.5168410 & -2.0634990 \\ 4.5589530 & 2.0862220 & -2.9226870 \\ 3.9689410 & 2.2453940 & -1.2759980 \\ 4.7047030 & -1.2906690 & 1.7304050 \\ 5.2153760 & -1.5792290 & 3.1180860 \\ 3.0742740 & -2.9072450 & 2.5179010 \\ 3.5381730 & -2.0006670 & 1.4024220 \\ 4.0979240 & -1.2349500 & 4.1250530 \\ 4.4533580 & -1.4431160 & 5.1405810 \\ 3.8682260 & -0.1657970 & 4.0731360 \\ 2.8373940 & -2.0739100 & 3.7964080 \\ 2.6086600 & -2.7639630 & 4.6166600 \\ 1.9592550 & -1.4415440 & 3.6561710 \\ 2.1626830 & -3.4448240 & 2.2462770\end{array}$

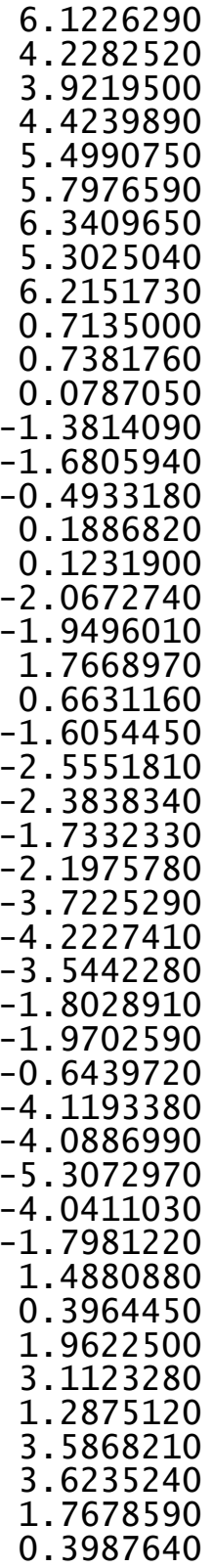

-1.0031590
-3.8921660
-4.5673480
-4.5103370
-3.0957180
-3.3330620
-3.3550290
-0.4419280
0.0959340
-4.8096670
-6.3138540
-6.7446720
-6.2553780
-5.0703810
-4.1662060
-6.7553390
-7.8321280
-7.0701060
-5.4841630
-6.6821590
-6.3142250
-5.9747650
-4.5092850
-2.0346550
-2.8343830
-2.3974080
-2.3516900
-1.2613160
-1.2839840
-3.0871880
-3.8989260
-2.7564780
-3.3270630
-2.1634380
-1.3359220
-0.2807160
-1.4012230
3.4060560
2.7425210
4.2108090
4.9906000
4.1625120
5.7238520
5.0069530
4.8954180
3.5481170

3.3310360
2.8157360
3.6222300
1.9333160
3.2179170
4.2452490
2.5689790
0.8044870
1.0599880
-0.7320130
-0.8276620
-2.1449350
-2.2332680
-1.2851610
-1.0509030
0.0163630
-2.2578300
-1.9804870
-0.3033120
-0.7658700
-2.9654420
-3.2671450
-1.6217060
-2.6380340
-3.7515150
-5.1397310
-5.1794110
-4.2354190
-2.8780080
-5.8924980
-3.6303460
-3.6625960
-4.8680240
-6.1935900
-4.0965420
-4.6966930
-5.3707660
-0.9712360
-1.1897370
-2.1326160
-1.9926810
-3.3546570
-3.0735480
-1.0353380
-4.4349510
-3.4476790




\begin{tabular}{|c|c|c|c|c|c|c|c|}
\hline C & 2.9148210 & 5.6749670 & -4.2946470 & C & -4.9427660 & 1.0649540 & 2.6740500 \\
\hline $\mathrm{H}$ & 4.4798140 & 6.3312200 & -2.9672540 & C & -3.6164510 & -1.4066820 & 3.3129590 \\
\hline $\mathrm{H}$ & 1.2486580 & 4.8577600 & -5.3871040 & $\mathrm{H}$ & -3.6681580 & -2.4597640 & 3.6122420 \\
\hline $\mathrm{H}$ & 3.2873260 & 6.2457100 & -5.1397900 & $\mathrm{H}$ & -2.5681110 & -1.1796350 & 3.1028020 \\
\hline $\mathrm{H}$ & 0.0524400 & 2.1401880 & -0.4103390 & C & -4.1751170 & -0.4900940 & 4.4332020 \\
\hline 0 & 2.1390340 & 3.4053830 & 0.0851980 & $\mathrm{H}$ & -4.4907300 & -1.0797870 & 5.3014900 \\
\hline C & 1.4419380 & 2.4020200 & 2.5871760 & $\mathrm{H}$ & -3.4077460 & 0.2091100 & 4.7786510 \\
\hline $\mathrm{C}$ & 2.4540940 & 2.0199900 & 1.5957040 & $\mathrm{H}$ & -5.7772890 & 0.9798390 & 4.6622640 \\
\hline $\mathrm{H}$ & 0.4105120 & 2.2902640 & 2.2553230 & $\mathrm{H}$ & -4.0474200 & -1.8434410 & 1.2224470 \\
\hline $\mathrm{H}$ & 2.2234830 & 1.1931180 & 0.9246180 & C & -6.4675010 & -0.7123780 & 3.4649050 \\
\hline 0 & 1.9283950 & 1.2159340 & 3.2520640 & $\mathrm{H}$ & -6.7580500 & -1.3149050 & 4.3330410 \\
\hline $\mathrm{H}$ & 1.3361090 & 0.4618230 & 2.9199890 & $\mathrm{H}$ & -7.3607310 & -0.1759490 & 3.1305560 \\
\hline $\mathrm{c}$ & 3.1740370 & 3.8200180 & 3.7562200 & C & -5.9077930 & -1.6104570 & 2.3311300 \\
\hline C & 4.0376390 & 3.7599020 & 2.4954990 & $\mathrm{H}$ & -5.9363140 & -2.6665400 & 2.6221850 \\
\hline C & 3.8837950 & 2.4120620 & 1.7907610 & $\mathrm{H}$ & -6.5106400 & -1.5098140 & 1.4232480 \\
\hline C & 1.6973870 & 3.6531660 & 3.3950170 & C & -4.0245400 & 2.2037780 & 0.3068530 \\
\hline $\mathrm{H}$ & 3.3204360 & 4.7725790 & 4.2739250 & $\mathrm{C}$ & -3.4960720 & 3.0116100 & -0.8551060 \\
\hline $\mathrm{H}$ & 5.0926700 & 3.9179380 & 2.7377860 & $\mathrm{C}$ & -4.5913840 & 4.4765850 & 0.9764110 \\
\hline $\mathrm{H}$ & 4.3380830 & 1.6053180 & 2.3792850 & $\mathrm{C}$ & -4.5711520 & 3.0085680 & 1.3176840 \\
\hline $\mathrm{H}$ & 1.3603390 & 4.5035260 & 2.7897490 & C & -4.6594800 & 3.8228650 & -1.4656350 \\
\hline $\mathrm{H}$ & 1.0684200 & 3.6150070 & 4.2898730 & $\mathrm{H}$ & -4.2589120 & 4.4841380 & -2.2425820 \\
\hline $\mathrm{H}$ & 3.4729890 & 3.0238980 & 4.4487590 & $\mathrm{H}$ & -5.3706440 & 3.1486780 & -1.9541790 \\
\hline $\mathrm{H}$ & 3.7348040 & 4.5571850 & 1.8064210 & C & -5.3610950 & 4.6454310 & -0.3520270 \\
\hline $\mathrm{H}$ & 4.3812470 & 2.3911610 & 0.8178100 & $\mathrm{H}$ & -5.3960440 & 5.7081550 & -0.6168520 \\
\hline 15 & & & & $\mathrm{H}$ & -6.3941920 & 4.3111340 & -0.2162170 \\
\hline TS & S) $\mathrm{G}[\mathrm{M} 06-2$ & $(d)]=-35$ & 38 & $\mathrm{H}$ & -5.0658560 & 5.0597920 & 1.7728060 \\
\hline $\mathrm{P}$ & -0.3008170 & -0.1344670 & 0.7720850 & H & -3.0249270 & 2.3753430 & -1.6085670 \\
\hline 0 & 0.3855930 & -0.6452030 & 2.0031320 & C & -3.1394430 & 4.9453340 & 0.7429600 \\
\hline 0 & -0.4658890 & 1.3535470 & 0.5844740 & $\mathrm{H}$ & -3.1552770 & 5.9820210 & 0.3864820 \\
\hline 0 & -1.7171730 & -0.9176010 & 0.6847330 & $\mathrm{H}$ & -2.5918780 & 4.9376250 & 1.6901920 \\
\hline 0 & 0.4407760 & -0.6465680 & -0.5930810 & C & -2.4604490 & 4.0110750 & -0.2903480 \\
\hline C & -2.3857670 & -0.8878760 & -0.5306960 & $\mathrm{H}$ & -2.0292060 & 4.5873870 & -1.1171310 \\
\hline C & -1.9330970 & -1.7292330 & -1.5578150 & $\mathrm{H}$ & -1.6505940 & 3.4442720 & 0.1729910 \\
\hline $\mathrm{C}$ & -3.4907920 & -0.0501170 & -0.6611330 & C & -5.0324630 & 2.4425100 & 2.5008130 \\
\hline $\mathrm{C}$ & -4.1521420 & -0.0549300 & -1.8927580 & $\mathrm{H}$ & -5.4542380 & 3.0698490 & 3.2847460 \\
\hline $\mathrm{H}$ & -5.0283000 & 0.5786340 & -2.0169430 & C & 4.7217690 & -0.5956140 & -0.6004790 \\
\hline C & 0.4277140 & -2.0165690 & -0.8164820 & C & 5.3331500 & 0.2731950 & -1.6705640 \\
\hline C & 1.5893540 & -2.7428310 & -0.5651850 & C & 3.0968770 & -0.8167050 & -2.3865780 \\
\hline $\mathrm{C}$ & -0.7522970 & -2.6024420 & -1.2970980 & $\mathrm{C}$ & 3.5058190 & -1.1819990 & -0.9794440 \\
\hline C & 1.5462820 & -4.1222690 & -0.7809120 & $\mathrm{C}$ & 5.5663830 & -0.6099720 & -2.9168550 \\
\hline $\mathrm{H}$ & 2.4425930 & -4.7109590 & -0.5949680 & H & 5.9673640 & 0.0119030 & -3.7250170 \\
\hline C & -3.9663840 & 0.8107280 & 0.4577510 & $\mathrm{H}$ & 6.3189620 & -1.3708610 & -2.6894490 \\
\hline C & 2.8382110 & -2.0659020 & -0.1166540 & $\mathrm{C}$ & 4.2268250 & -1.2691460 & -3.3393560 \\
\hline $\mathrm{C}$ & -4.4198060 & 0.2489240 & 1.6605070 & $\mathrm{H}$ & 3.9604230 & -0.9853480 & -4.3635310 \\
\hline C & -4.4454780 & -1.2166350 & 2.0238540 & $\mathrm{H}$ & 4.3070540 & -2.3602540 & -3.3169100 \\
\hline C & -5.3864550 & 0.3023350 & 3.8955390 & $\mathrm{H}$ & 2.1510360 & -1.2850900 & -2.6693220 \\
\hline
\end{tabular}




\begin{tabular}{lrrr} 
H & 6.2758500 & 0.7148670 & -1.3289590 \\
$\mathrm{C}$ & 2.9796010 & 0.7184720 & -2.4772840 \\
$\mathrm{H}$ & 2.7169550 & 0.9997440 & -3.5034220 \\
$\mathrm{H}$ & 2.1535540 & 1.0414340 & -1.8385760 \\
$\mathrm{C}$ & 4.3209820 & 1.3735630 & -2.0550900 \\
$\mathrm{H}$ & 4.7349590 & 1.9776800 & -2.8708270 \\
$\mathrm{H}$ & 4.1698220 & 2.0512050 & -1.2090740 \\
$\mathrm{C}$ & 4.6273070 & -1.7512250 & 1.5039800 \\
$\mathrm{C}$ & 5.1197040 & -2.1845680 & 2.8604450 \\
$\mathrm{C}$ & 2.8763860 & -3.2885550 & 2.1836610 \\
$\mathrm{C}$ & 3.4064270 & -2.3392160 & 1.1353790 \\
$\mathrm{C}$ & 4.0377940 & -1.8314000 & 3.9026000 \\
$\mathrm{H}$ & 4.3794290 & -2.1465080 & 4.8950660 \\
$\mathrm{H}$ & 3.8954780 & -0.7464420 & 3.9365640 \\
$\mathrm{C}$ & 2.7116740 & -2.5375510 & 3.5231250 \\
$\mathrm{H}$ & 2.4289980 & -3.2663890 & 4.2913770 \\
$\mathrm{H}$ & 1.8885460 & -1.8261910 & 3.4384450 \\
$\mathrm{H}$ & 1.9240300 & -3.7302990 & 1.8809180 \\
$\mathrm{H}$ & 6.0709880 & -1.7002650 & 3.1070540 \\
$\mathrm{C}$ & 3.9488830 & -4.3816390 & 2.3957190 \\
$\mathrm{H}$ & 3.5929870 & -5.0883940 & 3.1533600 \\
$\mathrm{H}$ & 4.0900700 & -4.9462090 & 1.4681060 \\
$\mathrm{C}$ & 5.2817050 & -3.7219190 & 2.8419800 \\
$\mathrm{H}$ & 5.5663020 & -4.0600760 & 3.8447300 \\
$\mathrm{H}$ & 6.0962420 & -3.9970830 & 2.1652660 \\
$\mathrm{C}$ & 5.2877630 & -0.8852760 & 0.6385690 \\
$\mathrm{H}$ & 6.2425510 & -0.4443180 & 0.9239200 \\
$\mathrm{C}$ & -0.7723970 & -3.9942570 & -1.4706070 \\
$\mathrm{C}$ & -1.9994370 & -4.7860140 & -1.8485380 \\
$\mathrm{C}$ & -2.2064420 & -5.9314740 & -0.8402980 \\
$\mathrm{C}$ & -0.9418000 & -6.8071660 & -0.6616640 \\
$\mathrm{C}$ & 0.2785000 & -6.2379800 & -1.4117670 \\
$\mathrm{C}$ & 0.3832570 & -4.7488430 & -1.2135180 \\
$\mathrm{H}$ & -1.8714610 & -5.2108440 & -2.8551050 \\
$\mathrm{H}$ & -3.0553280 & -6.5426260 & -1.1613150 \\
$\mathrm{H}$ & -1.1274460 & -7.8273550 & -1.0112700 \\
$\mathrm{H}$ & 0.1665210 & -6.4509160 & -2.4837780 \\
$\mathrm{H}$ & -2.8872250 & -4.1487020 & -1.8829860 \\
$\mathrm{H}$ & -2.4848970 & -5.4839200 & 0.1187460 \\
$\mathrm{H}$ & -0.6938950 & -6.8791270 & 0.4014880 \\
$\mathrm{H}$ & 1.1960300 & -6.7371980 & -1.0859970 \\
$\mathrm{C}$ & -3.7079640 & -0.8359360 & -2.9527730 \\
$\mathrm{C}$ & -4.3975220 & -0.8606960 & -4.2925710 \\
$\mathrm{C}$ & -4.6280700 & -2.3185980 & -4.7189660 \\
$\mathrm{C}$ & -3.3081460 & -3.1226820 & -4.7570330 \\
$\mathrm{C}$ & -2.1453670 & -2.4047280 & -4.0377390 \\
& & & \\
\hline
\end{tabular}
$-2.5930870$
$-3.7757040$
$-5.1251870$
$-3.0050330$
$-1.7338750$
$-5.3452660$
$-5.3155690$
$-3.4662700$
$-1.3309380$
1.8119200
0.6693630
2. 3423050
3.5580430
1.6506040
4.0811270
4.0808280
2.1790850
0.7102130
3. 3915590
5.0253000
1. 6461040
3.8015660
0.2807300
2.4663980
1.6831040
2.6612310
0.6466020
2. 3621440
2.0670840
1.4146670
3.5264320
4.3845460
4.1187150
2.0416370
3.7518890
5.4486300
4.5043440
1.7791380
1.4099440
3.7556620
4.1519660
4.6128160

154

TS188(S, S) G[M06-2X/6-31G(d) $]=-3541.251603$

$\begin{array}{llcr}\mathrm{T} \\ \mathrm{P} & -0.2583050 & -0.0072800 & -0.1773040 \\ \mathrm{O} & -0.6754670 & 0.9807900 & -1.2282580\end{array}$
$-2.7943900$

$-1.6751170$

$-5.0453450$

$-5.6931390$

$-5.7923740$

$-4.7221870$

$-4.2444950$

$-3.9964420$

$-4.2998220$

$-3.8304990$

$-0.7883520$

$-1.0495350$

$-1.8824630$

$-1.6962520$

$-3.0898260$

$-2.7158230$

$-0.7520930$

$-4.1086130$

$-3.2199480$

-3.9219660

$-2.5735450$

$-5.0490220$

$-4.7191620$

$-0.3221140$

0.2560700

2. 6726860

1.6491200

2. 3377630

0.9162020

3.2384850

2. 8497410

3.9428270

2.6773290

1.8666470

3.5764590

4.5340940

2.9267080

3.0425690

4.4684290

4.5685330

2.0558870

0.8918330
2. 3838240

$-2.1226820$

$-4.0377390$ 


$\begin{array}{rrr}0.8780600 & 0.3392750 & 0.7553160 \\ -0.0290860 & -1.4076460 & -0.9670120 \\ -1.4828670 & -0.4089480 & 0.8124460 \\ -0.0650040 & -2.5869350 & -0.2415550 \\ -1.3033190 & -3.0870550 & 0.1781420 \\ 1.1423030 & -3.2314970 & 0.0255300 \\ 1.0687510 & -4.4400690 & 0.7149270 \\ 1.9935300 & -4.9781980 & 0.9170910 \\ -2.5866950 & -0.9578410 & 0.1784900 \\ -3.6698180 & -0.1275680 & -0.1070600 \\ -2.5363980 & -2.3146600 & -0.1632940 \\ -4.7678760 & -0.7185610 & -0.7372790 \\ -5.6442790 & -0.1086200 & -0.9484770 \\ 2.4527100 & -2.6362860 & -0.3607210 \\ -3.6205880 & 1.3281180 & 0.2097740 \\ 2.7776070 & -2.3780130 & -1.7011520 \\ 1.9053100 & -2.6207840 & -2.9099540 \\ 4.2240730 & -1.6463550 & -3.5196970 \\ 4.0408000 & -1.8734460 & -2.0419820 \\ 1.7273390 & -1.2827620 & -3.6617460 \\ 1.1680050 & -1.4742360 & -4.5847310 \\ 1.1258440 & -0.6004670 & -3.0556780 \\ 3.1176110 & -0.6734380 & -3.9816460 \\ 3.2265270 & -0.4849590 & -5.0557460 \\ 3.2462210 & 0.2846050 & -3.4675170 \\ 5.2161710 & -1.2346190 & -3.7345670 \\ 0.9324600 & -3.0336570 & -2.6340400 \\ 4.0209520 & -2.9898500 & -4.2528260 \\ 4.0658960 & -2.8154990 & -5.3339670 \\ 4.8382770 & -3.6728590 & -4.0023070 \\ 2.6525220 & -3.5962040 & -3.8469940 \\ 2.0335640 & -3.7843370 & -4.7313260 \\ 2.7893470 & -4.5554620 & -3.3382340 \\ 3.4052660 & -2.3400450 & 0.6272660 \\ 3.2289470 & -2.4561600 & 2.1235640 \\ 5.5760940 & -1.5982010 & 1.4524570 \\ 4.6686400 & -1.8420140 & 0.2742420 \\ 4.3109830 & -3.4106990 & 2.6755560 \\ 4.2400380 & -3.4283570 & 3.7690750 \\ 4.1220110 & -4.4308940 & 2.3254120 \\ 5.7156670 & -2.9288290 & 2.2239250 \\ 6.3720430 & -2.7782970 & 3.0882940 \\ 6.1927220 & -3.6739390 & 1.5799190 \\ 6.5568110 & -1.2341710 & 2.3852100 \\ 2.2305370 & -2.8104720 & 2.3943340 \\ 4.9022990 & -0.5770940 & \end{array}$

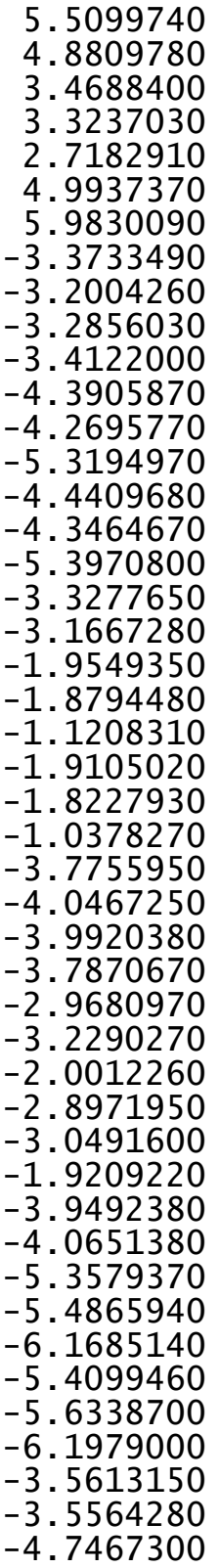

-0.4826370
0.4061310
-1.0568060
-1.1119700
-0.3683340
-1.6234310
-1.2565420
3.1527300
3.4617970
0.9265640
1.7795540
2.8275310
3.0037720
3.3179240
1.3081950
0.7213860
1.0377730
-0.1397730
4.5433280
1.2689740
0.6784390
0.9736480
2.7854640
2.9584740
3.2517310
3.6554300
4.5418900
2.0233580
2.2847830
4.2910810
4.8608410
4.6572530
2.7741160
2.5862450
2.3603840
0.9574470
5.5969410
2.6215910
2.5046860
2.0651150
4.1203580
4.7483740
4.2963980
4.0921560
5.1571430
-2.0532310

3. 3019110

1. 9167080

2.7330480

3. 8179280

2. 3348960

$-1.0600020$

$-1.3342790$

1.8083760

3.2741130

2. 7635960

1. 5230110

4. 0279690

5.1027930

3. 7216380

3.7182400

4.6388230

3.2599100

2. 5310000

3. 4468750

3. 4662250

4. 3862160

2. 8239980

3.7849680

4.8636340

3. 3179280

$-0.5042390$

$-1.6930460$

$-2.2818760$

$-0.8068400$

$-2.7675010$

$-3.6671230$

$-2.4120280$

$-3.0733040$

$-4.1423090$

$-2.8082090$

$-2.5147460$

$-1.3991210$

$-2.6880620$

$-3.7700870$

$-2.2059790$

$-2.2865920$

$-3.1561440$

$-1.5479130$

0.7987040

1. 0288050

$-1.1304260$ 


\begin{tabular}{|c|c|c|}
\hline $\begin{array}{l}-5.9122170 \\
-6.1883180 \\
-4.9621870 \\
-3.6394860 \\
-3.6231000 \\
-5.6828620 \\
-7.0622230 \\
-5.0904590 \\
-3.5178680 \\
-6.7955310 \\
-6.4367190 \\
-4.8880080 \\
-2.7836010 \\
-1.3327500 \\
-0.1366610 \\
-2.6164780 \\
-2.6100290 \\
-1.3504130 \\
-0.1129270 \\
-3.5108440 \\
-2.7296220 \\
-3.4875990 \\
-1.3134090 \\
-1.3643980 \\
0.7975370 \\
-0.0386850 \\
-2.6349810 \\
3.4633050 \\
3.1260610 \\
4.8914510 \\
5.7596400 \\
5.3514660 \\
7.0898840 \\
5.3797560 \\
6.6820170 \\
4.6599810 \\
7.5502460 \\
7.7683280 \\
7.0442060 \\
8.5895250 \\
2.1770710 \\
2.7007930 \\
0.7781860 \\
0.6814320 \\
1.1590820 \\
0.4882640\end{array}$ & $\begin{array}{l}-2.7146400 \\
-4.0690130 \\
-5.0012690 \\
-4.2365910 \\
-2.8534830 \\
-2.8740870 \\
-4.5484320 \\
-5.7203680 \\
-4.1012000 \\
-2.0698930 \\
-3.8758520 \\
-5.5906330 \\
-4.8314990 \\
-4.2637390 \\
-4.9611990 \\
-4.7150380 \\
-6.1974820 \\
-6.5295850 \\
-6.2881090 \\
-6.4412480 \\
-4.1247820 \\
-4.4716510 \\
-5.8902320 \\
-7.5675310 \\
-6.3495650 \\
-7.0930050 \\
-6.8031970 \\
2.4493660 \\
1.2759040 \\
2.8043050 \\
1.8903930 \\
4.0621310 \\
2.2391050 \\
0.9176470 \\
4.4064990 \\
4.7528840 \\
3.4951530 \\
1.5316450 \\
5.3826390 \\
3.7650070 \\
0.9492690 \\
3.2455880 \\
3.5190920 \\
3.4301770 \\
2.6251490 \\
2.4501980\end{array}$ & $\begin{array}{r}-1.8222750 \\
-1.1527810 \\
-1.2354870 \\
-1.4775150 \\
-0.8693230 \\
-2.8858500 \\
-1.6042490 \\
-2.0506940 \\
-2.5609850 \\
-1.7797550 \\
-0.1033430 \\
-0.3152140 \\
-1.1498650 \\
0.9527560 \\
1.1859790 \\
1.6257080 \\
1.9885510 \\
2.7844720 \\
1.9229800 \\
2.5612430 \\
2.5470330 \\
1.0164130 \\
3.6759600 \\
3.1321040 \\
2.5292460 \\
1.1781590 \\
1.0716140 \\
0.5559790 \\
0.1059960 \\
0.3278360 \\
-0.2755780 \\
0.7222520 \\
-0.4806190 \\
-0.5730540 \\
0.5133530 \\
1.1936420 \\
-0.0871070 \\
-0.9472410 \\
0.8192350 \\
-0.2478740 \\
0.3592980 \\
1.1221990 \\
-0.8259060 \\
0.6336300 \\
-1.3227860 \\
1.0571110 \\
-1\end{array}$ \\
\hline
\end{tabular}

$\begin{array}{lr}\mathrm{O} & -0.6571020 \\ \mathrm{H} & -0.9006700 \\ \mathrm{C} & 0.7263740 \\ \mathrm{C} & 1.0819000 \\ \mathrm{C} & 0.4589670 \\ \mathrm{C} & 1.2578930 \\ \mathrm{H} & 1.1463840 \\ \mathrm{H} & 0.7433340 \\ \mathrm{H} & -0.6299590 \\ \mathrm{H} & 2.3553480 \\ \mathrm{H} & 0.9505980 \\ \mathrm{H} & -0.3631380 \\ \mathrm{H} & 2.1712670 \\ \mathrm{H} & 0.8492540\end{array}$
3.4750320
5.9112900
4.6591930
4.8213030
6.9479230
6.7926920
4.7672880
4.8120100
4.8523380
6.0857900
5.8546820
4.4659300

$-0.6935540$

$-0.8518410$

$-0.6487060$

0.8343610

1.4554930

$-1.4230850$

$-1.0686860$

1.3865270

1. 5485090

$-1.3999530$

$-2.4734430$

$-0.7652500$

0.9441050

154

2. 4581860

TS189(S, S) G[M06-2X/6-31G(d) $]=-3541.251298$

$\begin{array}{lrrr}\text { TS189(S, S) } \mathrm{G}[\mathrm{M} 06-2 \mathrm{~V} / 6-31 \mathrm{G}(\mathrm{d})]=-3541.251298 \\ \mathrm{P} & -0.2663450 & -0.0112450 & -0.1396550 \\ \mathrm{O} & -0.7253230 & 0.9259570 & -1.2193120 \\ \mathrm{O} & 0.8855830 & 0.3917280 & 0.7502010 \\ \mathrm{O} & -0.0328840 & -1.4407460 & -0.8742140 \\ \mathrm{O} & -1.4605790 & -0.3886730 & 0.8957960 \\ \mathrm{C} & -0.0553020 & -2.5875060 & -0.0961530 \\ \mathrm{C} & -1.2954420 & -3.0772360 & 0.3429940 \\ \mathrm{C} & 1.1586430 & -3.2040480 & 0.2013030 \\ \mathrm{C} & 1.1080240 & -4.3769370 & 0.9600950 \\ \mathrm{H} & 2.0387100 & -4.8936440 & 1.1871100 \\ \mathrm{C} & -2.5795860 & -0.9604450 & 0.3125440 \\ \mathrm{C} & -3.6803340 & -0.1457000 & 0.0468750 \\ \mathrm{C} & -2.5368630 & -2.3202060 & -0.0026080 \\ \mathrm{C} & -4.7920940 & -0.7686460 & -0.5151060 \\ \mathrm{H} & -5.6814430 & -0.1711390 & -0.7093680 \\ \mathrm{C} & 2.4610460 & -2.6227630 & -0.2318100 \\ \mathrm{C} & -3.6285280 & 1.3209440 & 0.3062030 \\ \mathrm{C} & 2.7700610 & -2.4383020 & -1.5883260 \\ \mathrm{C} & 1.8842280 & -2.7463990 & -2.7720810 \\ \mathrm{C} & 4.1941030 & -1.8048650 & -3.4612060 \\ \mathrm{C} & 4.0282700 & -1.9518100 & -1.9713270 \\ \mathrm{C} & 1.6955300 & -1.4495920 & -3.5906550 \\ \mathrm{H} & 1.1217240 & -1.6879420 & -4.4935980 \\ \mathrm{H} & 1.1042500 & -0.7357950 & -3.0113790 \\ \mathrm{C} & 3.0811070 & -0.8594910 & -3.9629250 \\ \mathrm{H} & 3.1759530 & -0.7322900 & -5.0473250 \\ \mathrm{H} & 3.2159460 & 0.1261830 & -3.5058250 \\ \mathrm{H} & 5.1830870 & -1.4045100 & -3.7093360 \\ \mathrm{H} & 0.9152180 & -3.1447670 & -2.4635470 \\ \mathrm{C} & 3.9849900 & -3.1861950 & -4.1178210 \\ & & & \end{array}$




\begin{tabular}{|c|c|c|c|}
\hline $\mathrm{H}$ & 4.0190960 & -3.0713330 & -5.2072820 \\
\hline $\mathrm{H}$ & 4.8052230 & -3.8542500 & -3.8381710 \\
\hline C & 2.6209500 & -3.7694580 & -3.6656920 \\
\hline $\mathrm{H}$ & 1.9918980 & -4.0021750 & -4.5321360 \\
\hline $\mathrm{H}$ & 2.7629950 & -4.7015030 & -3.1099300 \\
\hline C & 3.4254110 & -2.2720690 & 0.7266800 \\
\hline C & 3.2682710 & -2.3075000 & 2.2291220 \\
\hline C & 5.6053530 & -1.4844170 & 1.4831410 \\
\hline C & 4.6838670 & -1.7934360 & 0.3316300 \\
\hline C & 4.3586990 & $-\overline{3} .2297910$ & 2.8180750 \\
\hline $\mathrm{H}$ & 4.3003300 & -3.1901110 & 3.9117420 \\
\hline $\mathrm{H}$ & 4. 1681730 & -4.2673070 & 2.5244330 \\
\hline C & 5.7570270 & -2.7700320 & 2.3254800 \\
\hline $\mathrm{H}$ & 6.4220310 & -2.5702790 & 3.1731270 \\
\hline $\mathrm{H}$ & 6.2295870 & -3.5485100 & 1.7187050 \\
\hline $\mathrm{H}$ & 6.5812770 & -1.1377820 & 1.1242640 \\
\hline $\mathrm{H}$ & 2.2736130 & -2.6474330 & 2.5255760 \\
\hline C & 4.9408200 & -0.4134340 & 2.3745870 \\
\hline $\mathrm{H}$ & 5.5603220 & -0.2646210 & 3.2667330 \\
\hline $\mathrm{H}$ & 4.9091530 & 0.5403950 & 1.8412210 \\
\hline C & 3.5139050 & -0.8771650 & 2.7594440 \\
\hline $\mathrm{H}$ & 3.3846210 & -0.8747410 & 3.8477320 \\
\hline $\mathrm{H}$ & 2.7559160 & -0.2124190 & 2.336 \\
\hline C & 4.9924920 & -1.6473200 & -1.0162680 \\
\hline $\mathrm{H}$ & 5.9776480 & -1.2946100 & -1.3222000 \\
\hline C & -3.3285830 & 3.2069350 & 1.82 \\
\hline C & -3.0993330 & 3.5732490 & 3.2665340 \\
\hline C & -3.1937580 & 1.0196040 & 2.8586050 \\
\hline C & -3.3720620 & 1.8235640 & 1.5918900 \\
\hline C & -4.2553740 & 2.9651090 & 4.0917250 \\
\hline $\mathrm{H}$ & -4.0908240 & 3.1817950 & 5.1532840 \\
\hline $\mathrm{H}$ & -5.1978230 & 3.4418670 & 3.8059530 \\
\hline C & -4.3132080 & 1.4350590 & 3.8414560 \\
\hline $\mathrm{H}$ & -4.1823300 & 0.8834940 & 4.7791990 \\
\hline $\mathrm{H}$ & -5.2852430 & 1.1462890 & 3.4302160 \\
\hline $\mathrm{H}$ & -3.2403420 & -0.0550280 & 2.6689050 \\
\hline $\mathrm{H}$ & -3.0635570 & 4.6607600 & 3.3959860 \\
\hline C & -1.8387390 & 1.3926000 & 3.4958350 \\
\hline $\mathrm{H}$ & -1.7273750 & 0.8405430 & 4.4358720 \\
\hline $\mathrm{H}$ & -1.0281480 & 1.0722020 & 2.8357760 \\
\hline C & -1.7868830 & 2.9209280 & 3.7504470 \\
\hline $\mathrm{H}$ & -1.6548470 & 3.1380850 & 4.8165220 \\
\hline $\mathrm{H}$ & -0.9363180 & 3.3695820 & 3.2286060 \\
\hline C & -3.8194780 & 3.6178720 & -0.4918190 \\
\hline C & -4.1358470 & 4.4568940 & -1.7035580 \\
\hline C & -4.0965760 & 1.9173450 & -2.1951080 \\
\hline
\end{tabular}

-3.8377670
-3.0959420
-3.3905750
-2.1175060
-3.0327070
-3.2224490
-2.0464930
-4.0607170
-4.1460010
-5.4778690
-5.6462050
-6.2685920
-5.5186390
-5.7748490
-6.2798740
-3.5581960
-3.5482250
-4.7903070
-3.6424870
-6.0532110
-5.8146720
-4.9291220
-3.5589500
-6.7733940
-6.7613450
-6.5321160
-5.4006970
-4.8137940
-2.9271240
-3.0444100
-5.3155440
-0.0935010
-0.1767220
-1.3092580
-2.6679290
-2.5230450
-1.3000580
-0.3819560
-1.3619900
-3.2464330
-2.4079600
0.7790670
-1.0624890
-3.2570290
-3.4365390
3.4528620

4.1652800

4.6990600

4.5455680

2. 6375310

2.4077680

2.2355160

0.8432420

5. 5225810

4979810

2. 3380380

1.9601740

4. 0114100

4. 6043560

4. 2161710

4.1056140

5.1788430

$-2.1181780$

$-2.8971530$

$-2.7070820$

$-4.0026540$

$-4.9411140$

$-4.3030460$

$-4.4732190$

$-2.9078440$

$-1.9587260$

$-5.1370690$

$-5.9066230$

$-4.9387920$

$-4.2376020$

$-3.7805970$

$-4.8715920$

$-6.1284040$

$-7.0119820$

$-6.2824600$

$-4.7453660$

$-4.2132470$

$-5.8783500$

7.9523590

$-6.6189150$

$-4.4775930$

$-6.6610880$

$-7.2682310$

$-6.5480650$

$-4.2509230$

2. 5010270
$-0.7400190$

$-2.8056660$

$-3.7168810$

$-2.5001250$

$-4.1090320$

$-2.8084640$

$-2.3880750$

$-1.4503550$

$-2.5738090$

$-3.6448450$

$-2.0402030$

$-2.2304850$

$-3.1155910$

$-1.4714750$

0.7841890

0.9719880

$-0.8752170$

$-0.6581290$

$-1.4777710$

$-2.2504610$

$-1.4342700$

$-1.2229360$

$-2.4908680$

$-0.6614250$

$-2.1188050$

$-0.4608350$

$-1.9375740$

$-0.6010610$

$-2.1928550$

$-3.2024820$

1.4534670

2. 2823860

1.7392690

1.7867240

1.8785380

1. 1671580

3. 3333500

2. 2962820

2. 6529210

2.9379410

2. 2620370

0.7031250

0.9023360

1.5404570

0.4053870 


\begin{tabular}{|c|c|c|c|c|c|c|c|}
\hline 0 & 3.1119870 & 1.3098440 & 0.0074480 & C & -2.5036500 & -2.3563010 & -0.2122800 \\
\hline $\mathrm{C}$ & 4.8751720 & 2.8518430 & 0.1376520 & $\mathrm{C}$ & -4.7564750 & -0.7817670 & -0.7648520 \\
\hline C & 5.7388770 & 1.9157100 & -0.4375400 & $\mathrm{H}$ & -5.6402350 & -0.1804910 & -0.9701890 \\
\hline C & 5.3347160 & 4.1279450 & 0.4687030 & $\mathrm{C}$ & 2.4940700 & -2.6017690 & -0.3378860 \\
\hline C & 7.0640910 & 2.2609730 & -0.6781500 & $\mathrm{C}$ & -3.6371270 & 1.2684360 & 0.2060650 \\
\hline $\mathrm{H}$ & 5.3602980 & 0.9283620 & -0.6843230 & $\mathrm{C}$ & 2.8285450 & -2.3656250 & -1.6802720 \\
\hline C & 6.6601480 & 4.4686990 & 0.2242720 & $\mathrm{C}$ & 1.9700790 & -2.6401380 & -2.8922610 \\
\hline $\mathrm{H}$ & 4.6469830 & 4.8356720 & 0.9199400 & C & 4.2850090 & -1.6557630 & -3.5000200 \\
\hline C & 7.5238450 & 3.5353910 & -0.3482330 & $\mathrm{C}$ & 4.0901050 & -1.8559770 & -2.0199160 \\
\hline $\mathrm{H}$ & 7.7390990 & 1.5362790 & -1.1227170 & $\mathrm{C}$ & 1.7865670 & -1.3184050 & -3.6709540 \\
\hline $\mathrm{H}$ & 7.0219420 & 5.4590770 & 0.4808590 & $\mathrm{H}$ & 1.2363270 & -1.5321730 & -4.5945010 \\
\hline $\mathrm{H}$ & 8.5592270 & 3.8023040 & -0.5367050 & $\mathrm{H}$ & 1.1743640 & -0.6304670 & -3.0823400 \\
\hline $\mathrm{H}$ & 2.1713030 & 0.9894960 & 0.2969530 & $\mathrm{C}$ & 3.1739410 & -0.7026470 & -3.9911330 \\
\hline 0 & 2.6974700 & 3.3157530 & 0.9544420 & $\mathrm{H}$ & 3.2906090 & -0.5349230 & -5.0678630 \\
\hline C & 0.7257830 & 3.4852410 & -0.9569370 & $\mathrm{H}$ & 3.2892110 & 0.2666890 & -3.4954150 \\
\hline C & 0.6645090 & 3.4674590 & 0.5070790 & $\mathrm{H}$ & 5.2752120 & -1.2388330 & -3.7136340 \\
\hline $\mathrm{H}$ & 1.0999490 & 2.5699040 & -1.4186460 & $\mathrm{H}$ & 0.9985810 & -3.0571900 & -2.6181840 \\
\hline $\mathrm{H}$ & 0.4871490 & 2.5082910 & 0.9821030 & $\mathrm{C}$ & 4.1014880 & -3.0151630 & -4.2084860 \\
\hline 0 & -0.7055760 & 3.4408910 & -0.7883750 & $\mathrm{H}$ & 4.1552600 & -2.8616090 & -5.2923700 \\
\hline $\mathrm{H}$ & -0.9474270 & 2.4601820 & -0.8977060 & $\mathrm{H}$ & 4.9226330 & -3.6853780 & -3.9367840 \\
\hline C & 0.6630790 & 6.0008230 & -0.9022310 & C & 2.7349000 & -3.6261450 & -3.8036970 \\
\hline C & 1.0543790 & 5.9578600 & 0.5758890 & $\mathrm{H}$ & 2.1258020 & -3.8364350 & -4.6898810 \\
\hline C & 0.4556280 & 4.7342430 & 1.2731270 & $\mathrm{H}$ & 2.8754270 & -4.5744170 & -3.2757080 \\
\hline C & 1.1834420 & 4.7586890 & -1.6286950 & $\mathrm{C}$ & 3.4359520 & -2.2787970 & 0.6524420 \\
\hline $\mathrm{H}$ & 1.0676570 & 6.8996510 & -1.3766860 & $\mathrm{C}$ & 3.2506050 & -2.3709490 & 2.1495170 \\
\hline $\mathrm{H}$ & 0.7230090 & 6.8634180 & 1.0921980 & C & 5.5953270 & -1.5054830 & 1.4801770 \\
\hline $\mathrm{H}$ & -0.6312680 & 4.8406980 & 1.3900230 & $\mathrm{C}$ & 4.6979850 & -1.7766800 & 0.3003060 \\
\hline $\mathrm{H}$ & 2.2811770 & 4.7559210 & -1.6313400 & $\mathrm{C}$ & 4.3361230 & -3.3078710 & 2.7244240 \\
\hline $\mathrm{H}$ & 0.8510770 & 4.7362420 & -2.6715980 & $\mathrm{H}$ & 4.2571590 & -3.3088820 & 3.8175380 \\
\hline $\mathrm{H}$ & -0.4291740 & 6.0463740 & -0.9953070 & $\mathrm{H}$ & 4.1584890 & -4.3350530 & 2.3891660 \\
\hline $\mathrm{H}$ & 2.1464580 & 5.9132570 & 0.6617780 & C & 5.7401270 & -2.8214110 & 2.2755500 \\
\hline $\mathrm{H}$ & 0.8732730 & 4.5921660 & 2.2733790 & $\mathrm{H}$ & 6.3882670 & -2.6503170 & 3.1423050 \\
\hline & & & & $\mathrm{H}$ & 6.2287640 & -3.5732420 & 1.6482800 \\
\hline & , S) G[M06- & id) $]=-354$ & 382 & $\mathrm{H}$ & 6.5753460 & -1.1395060 & 1.1531780 \\
\hline $\mathrm{P}$ & -0.2554980 & -0.0206640 & -0.2042710 & $\mathrm{H}$ & 2.2528760 & $-\overline{2} .7274520$ & 2.4144360 \\
\hline 0 & -0.6870940 & 0.9635920 & -1.2530610 & $\mathrm{C}$ & 4.9071020 & -0.4735920 & 2.3992140 \\
\hline 0 & 0.8731840 & 0.3429770 & 0.7314280 & $\mathrm{H}$ & 5.5082650 & -0.3566010 & 3.3084840 \\
\hline 0 & -0.0019760 & -1.4176260 & -0.9936640 & $\mathrm{H}$ & 4.8802120 & 0.5003640 & 1.9031520 \\
\hline 0 & -1.4750530 & -0.4441570 & 0.7828730 & $\mathrm{C}$ & 3.4761010 & -0.9602400 & 2.7378830 \\
\hline C & -0.0277250 & -2.5921750 & -0.2585480 & $\mathrm{H}$ & 3.3255210 & -0.9996030 & 3.8227070 \\
\hline C & -1.2636760 & -3.1124310 & 0.1404500 & $\mathrm{H}$ & 2.7218410 & -0.2849700 & 2.3250390 \\
\hline C & 1.1872800 & -3.2061620 & 0.0462530 & $\mathrm{C}$ & 5.0322640 & -1.5791570 & -1.0349170 \\
\hline C & 1.1237690 & -4.3918480 & 0.7738720 & $\mathrm{H}$ & 6.0205930 & -1.2084580 & -1.3074830 \\
\hline $\mathrm{H}$ & 2.0551430 & -4.9014120 & 1.0168700 & $\mathrm{C}$ & -3.4148860 & 3.0779350 & 1.8253630 \\
\hline C & -2.5717310 & -1.0026500 & 0.1453300 & $\mathrm{C}$ & -3.2438920 & 3.3722120 & 3.2944230 \\
\hline C & -3.6660700 & -0.1839960 & -0.1274250 & $\mathrm{C}$ & -3.2875020 & 0.8422300 & 2.7536810 \\
\hline
\end{tabular}




$\begin{array}{lrr}-3.4324490 & 1.7077750 & 1.5239290 \\ -4.4218980 & 2.7090970 & 4.0425670 \\ -4.3030950 & 2.8762770 & 5.1190750 \\ -5.3596150 & 3.1860780 & 3.7419270 \\ -4.4454830 & 1.1924380 & 3.7170590 \\ -4.3360240 & 0.5978100 & 4.6309470 \\ -5.3985020 & 0.9091340 & 3.2600520 \\ -3.3121710 & -0.2217960 & 2.5082320 \\ -3.2279110 & 4.4519790 & 3.4802460 \\ -1.9599360 & 1.1988930 & 3.4549570 \\ -1.8686320 & 0.5966490 & 4.3658550 \\ -1.1240610 & 0.9283530 & 2.8041180 \\ -1.9420260 & 2.7112210 & 3.7953100 \\ -1.8574910 & 2.8702200 & 4.8763950 \\ -1.0775550 & 3.1993010 & 3.3354580 \\ -3.8304250 & 3.6011030 & -0.4804680 \\ -4.1176570 & 4.4969770 & -1.6584250 \\ -4.0279880 & 1.9864730 & -2.2763170 \\ -3.8215340 & 2.2340570 & -0.7989920 \\ -3.0385940 & 4.2741580 & -2.7386310 \\ -3.3097550 & 4.8513230 & -3.6304630 \\ -2.0758440 & 4.6490620 & -2.3811360 \\ -2.9477540 & 2.7622500 & -3.0635060 \\ -3.1026280 & 2.5854510 & -4.1339900 \\ -1.9642930 & 2.3588940 & -2.8088640 \\ -3.9701720 & 0.9241080 & -2.5217640 \\ -4.1505590 & 5.5482630 & -1.3524720 \\ -5.4042590 & 2.5688330 & -2.6703990 \\ -5.5361460 & 2.4611780 & -3.7529860 \\ -6.2044510 & 1.9956880 & -2.1904900 \\ -5.4762880 & 4.0625200 & -2.2531920 \\ -5.7110570 & 4.6963170 & -3.1156640 \\ -6.2648710 & 4.2198030 & -1.5109610 \\ -3.6200840 & 4.0259410 & 0.8270510 \\ -3.6316040 & 5.0881360 & 1.0695500 \\ -4.7165350 & -2.1093740 & -1.1768840 \\ -5.8672580 & -2.7667920 & -1.8964080 \\ -6.1260380 & -4.1460090 & -1.2753130 \\ -4.8861860 & -5.0559040 & -1.3860770 \\ -3.5721200 & -4.2623110 & -1.5770860 \\ -3.5835380 & -2.9007630 & -0.9228910 \\ -5.6281830 & -2.8870080 & -2.9631620 \\ -6.9910890 & -4.6230880 & -1.7459080 \\ -4.9948920 & -5.7373190 & -2.2358550 \\ -3.4423830 & -4.0821800 & -2.6533190 \\ -6.7604700 & -2.1369530 & -1.8391830\end{array}$

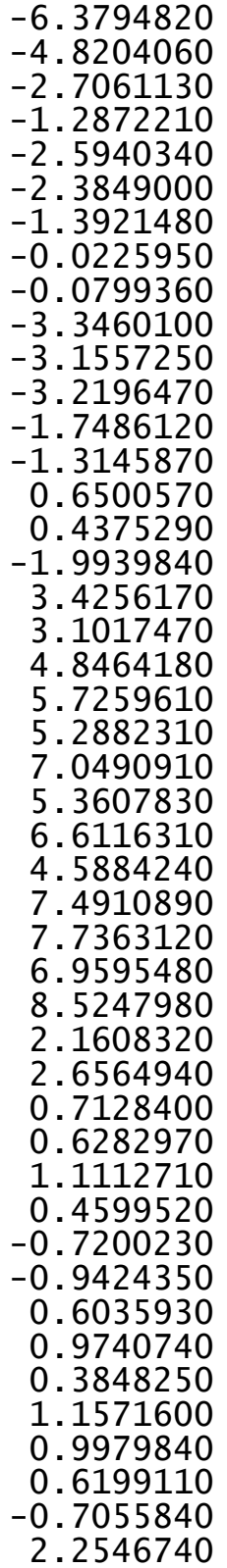

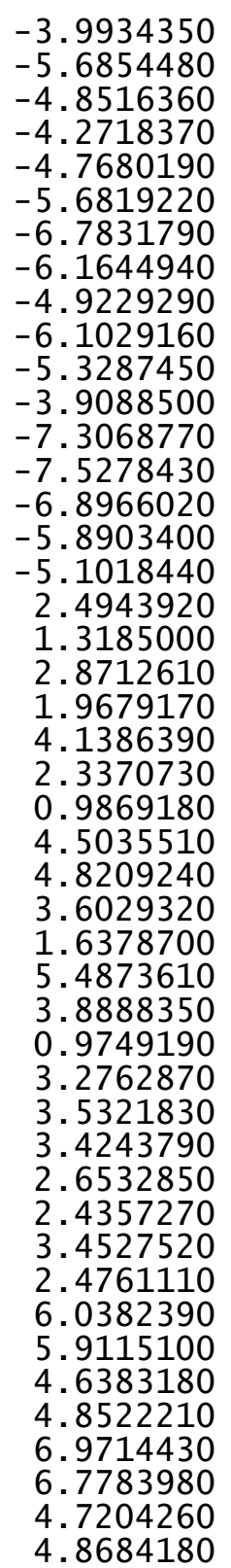

$-0.2204840$

$-0.4932600$

$-1.2679280$

0.9432210

1.5343710

2. 7402190

2. 3788720

2. 1111060

1. 2394310

3.0520890

0.7771560

1.7996410

1. 4819800

3.1773190

1. 6497000

3.0704570

3. 5863450

0.5225920

0.0688950

0.2837120

$-0.3193020$

0.6682040

$-0.5342470$

$-0.6079950$

0.4490150

1.1396490

$-0.1512770$

$-1.0004420$

0.7467760

$-0.3200120$

0.3294580

1.0996430

$-0.8287490$

0.6302450

$-1.3385440$

1.0439560

$-0.6870050$

$-0.8565970$

$-0.6213350$

0.8573160

1.4681400

$-1.4140580$

$-1.0339490$

1.4225110

1.5704250 


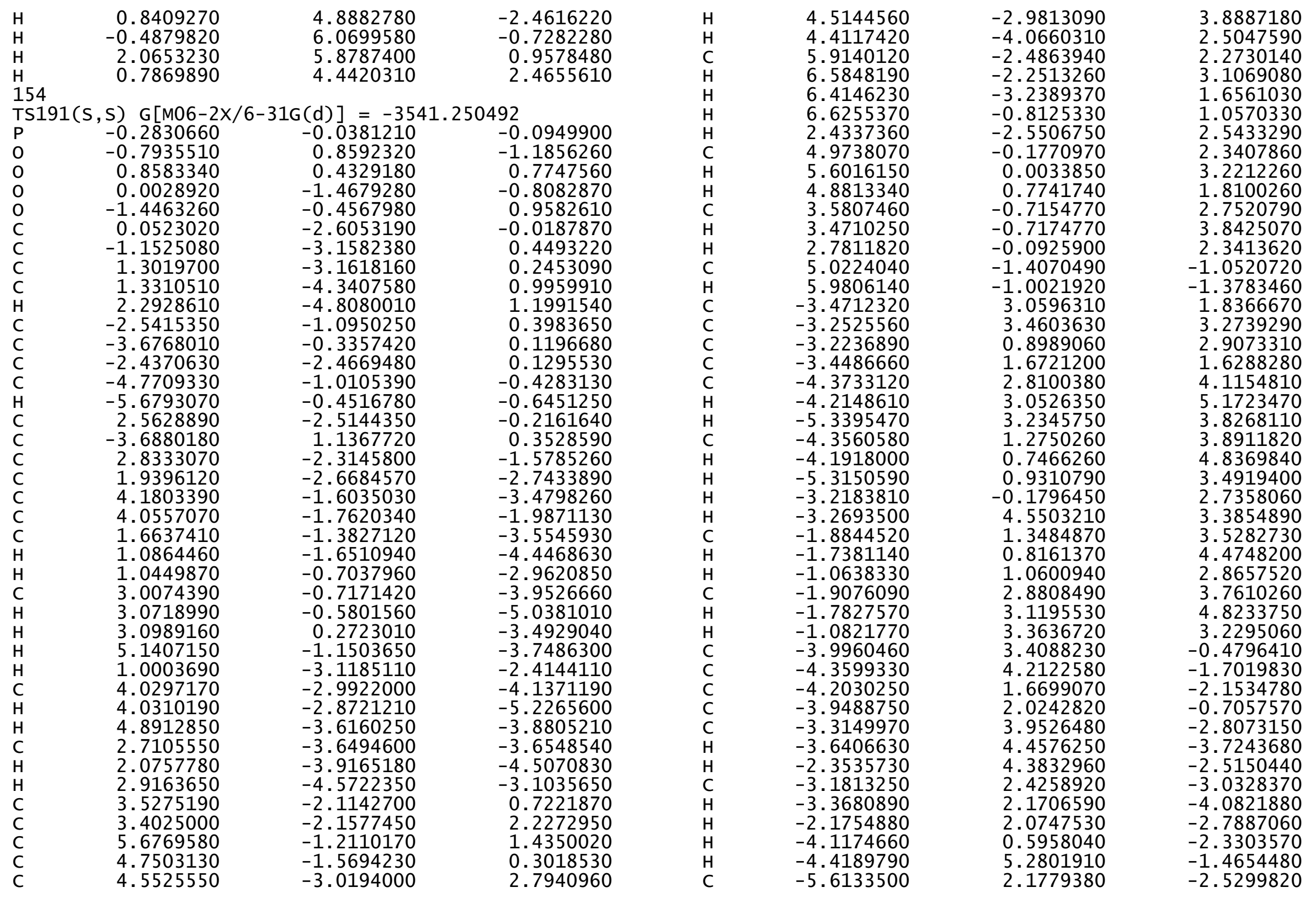




-5.7821130
-6.3737460
-5.7234720
-6.0131090
-6.4887600
-3.7509220
-3.7925760
-3.5369890
-3.5381580
-4.0108930
-5.3705140
-5.8458810
-4.7078030
-4.2188250
-4.0667710
-6.1394620
-6.2442930
-2.5447930
-3.2427380
-5.2836780
-6.6673210
0.1681610
0.1679610
-0.9088980
-2.3085430
-2.2519280
-1.0783070
-0.0462750
-0.9178650
-2.9174520
-2.1247560
1.1549680
-0.6309020
-2.8282470
-3.1990620
3.3137580
3.0298080
4.7145700
5.6164010
5.1152430
6.9206590
5.2824390
6.4200250
4.3991190
7.3216880
7.6249870

1.9920410

1.6125940

3.6934240

4.2584040

3.8748220

3.9292310

5.0045510

$-3.0991480$

$-4.5272450$

$-4.5738350$

$-3.8638570$

$-3.1158780$

$-2.3670880$

$-5.1316180$

$-5.6165590$

$-4.5848750$

$-3.8472950$

$-4.9753760$

$-4.0919120$

$-3.1446460$

$-2.4357080$

$-4.9050990$

$-6.1708160$

$-7.1240460$

$-6.4687090$

$-4.9353550$

$-4.3087550$

$-5.9389930$

$-8.0517300$

$-6.8804260$

$-4.7189020$

$-6.6427450$

$-7.3929060$

$-6.7104550$

$-4.4774040$

2.6651650

1.4539790

3.0831790

2.1856170

4.3825670

2. 5923730

1.1797110

4.7847950

5.0599860

3.8899940

1.8977970

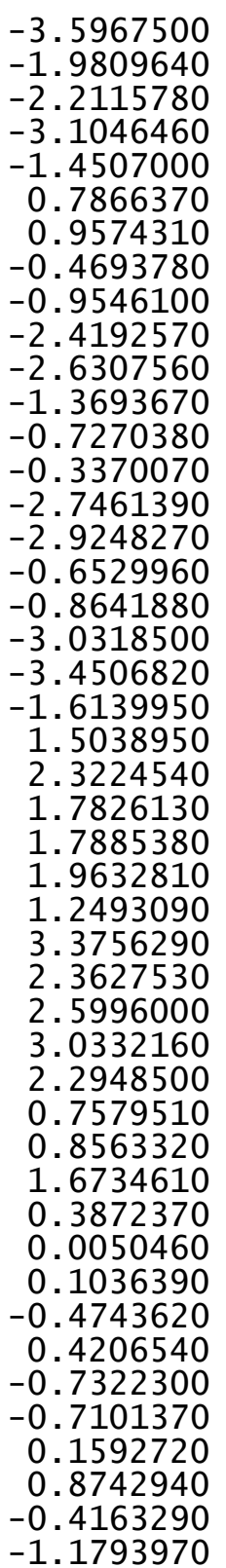
6.7362570
8.3407760
2. 1081800
2. 5226870
0.5393640
0.4841020
0.9559800
0.3550650
$-0.8872860$
$-1.0821940$
0.3538020
0.7517110
0.9322940
0.7118910
0.3781570
$-0.8741410$
2. 0288540
0.5982630
$-0.7396840$
1.8449770
0.6443080

154

TS192(S, S) G[M06-2X/6-31G(d) $]=-3541.250672$

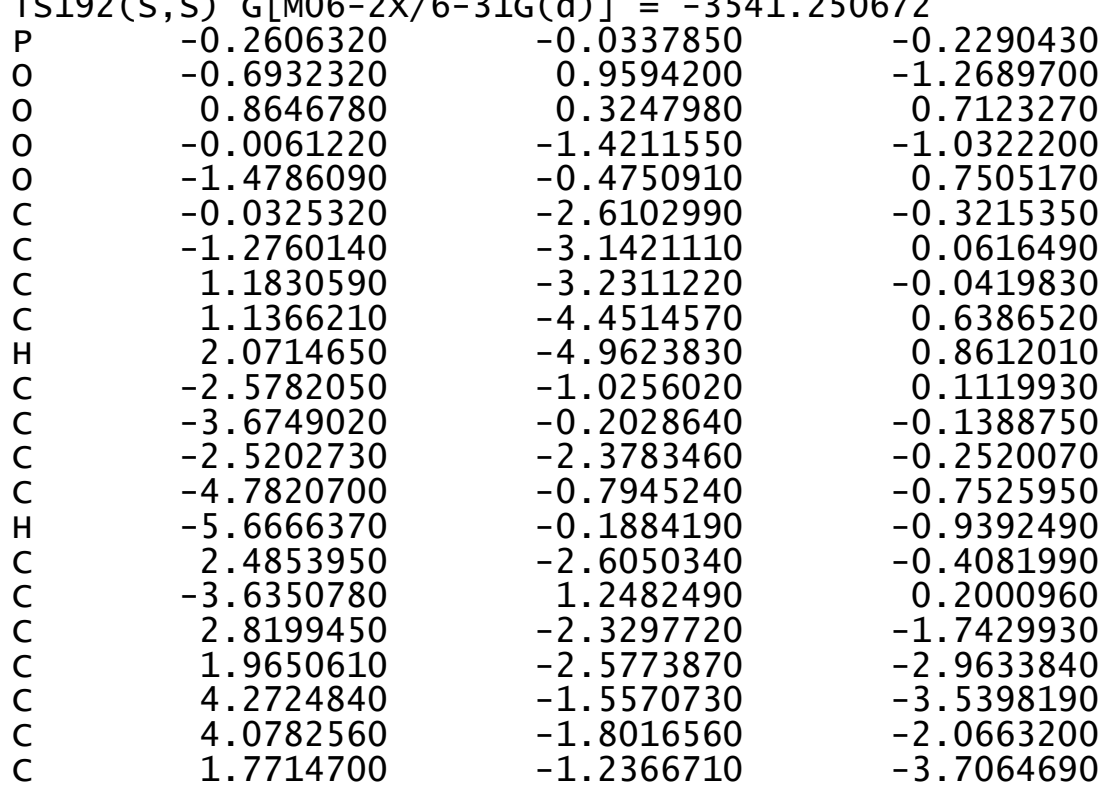

$\begin{array}{rr}5.7933870 & 0.4049020 \\ 4.2054380 & -0.6181170 \\ 1.0902800 & 0.3046090 \\ 3.4474720 & 0.9331000 \\ 3.4889190 & -0.9729510 \\ 3.4917700 & 0.4915530 \\ 2.5856320 & -1.4219980 \\ 2.5330110 & 0.9827850 \\ 3.3780570 & -0.7972750 \\ 2.3848260 & -0.8893580 \\ 5.9988790 & -0.9580720 \\ 5.9990370 & 0.5188710 \\ 4.7590640 & 1.2381380 \\ 4.7722500 & -1.6666990 \\ 6.9087730 & -1.4485290 \\ 6.8955200 & 1.0220200 \\ 4.8137760 & 1.3584620 \\ 4.8232110 & -1.6735340 \\ 4.7168050 & -2.7078490 \\ 5.9889210 & -1.0474520 \\ 6.0091330 & 0.6008190 \\ 4.6540530 & 2.2384980\end{array}$

4049020

0.9331000

$-1.4219980$

$-0.7972750$

8893580

0.5188710

2381380

1.0220200

(

(

0.6008190

.2384980
$-0.3215350$

0.6386520

2010

$-3.7064690$ 


\begin{tabular}{|c|c|c|c|}
\hline $\mathrm{H}$ & 1.2284500 & -1 4312710 & -4.6385190 \\
\hline$n$ & $\begin{array}{l}1.1484740 \\
1.04\end{array}$ & -0.5712340 & -3.1033490 \\
\hline C & 3.1539610 & -0.5983680 & -4.0027140 \\
\hline $\mathrm{H}$ & 3.2714520 & -0.3943320 & -5.0730580 \\
\hline $\mathrm{H}$ & 3.2595270 & 0.3551770 & -3.4750900 \\
\hline $\mathrm{H}$ & 5.2594080 & -1.1262040 & -3.7405400 \\
\hline $\mathrm{H}$ & 0.9968200 & -3.0097320 & -2.7019500 \\
\hline C & 4.0997130 & -2.8962760 & -4.2884190 \\
\hline $\mathrm{H}$ & 4.1500520 & -2.7095720 & -5.3672730 \\
\hline $\mathrm{H}$ & 4.9274200 & -3.5669380 & -4.0385820 \\
\hline C & 2.7395500 & -3.5315160 & -3.9001430 \\
\hline $\mathrm{H}$ & 2.1327310 & -3.7250460 & -4.7916820 \\
\hline $\mathrm{H}$ & 2.8898350 & -4.4916260 & -3.3968100 \\
\hline C & 3.4237510 & -2.3035600 & 0.5917330 \\
\hline C & 3.2357070 & -2.4393240 & 2.0849760 \\
\hline C & 5.5754860 & -1.5383330 & 1.4454210 \\
\hline C & 4.6824770 & -1.7823620 & 0.2562010 \\
\hline C & 4.3272430 & -3.3832690 & 2.6366430 \\
\hline $\mathrm{H}$ & 4.2471670 & -3.4131180 & 3.7292780 \\
\hline $\mathrm{H}$ & 4.1569980 & -4.4026440 & 2.2747890 \\
\hline C & 5.7283410 & -2.8758920 & 2.2022430 \\
\hline $\mathrm{H}$ & 6.3755480 & -2.7263680 & 3.0736670 \\
\hline $\mathrm{H}$ & 6.2212110 & -3.6062690 & 1.5532860 \\
\hline $\mathrm{H}$ & 6.5534700 & -1.1557880 & 1.1313610 \\
\hline $\mathrm{H}$ & 2.2402700 & -2.8113290 & 2.3371390 \\
\hline C & 4.8782490 & -0.5391430 & 2.3937000 \\
\hline $\mathrm{H}$ & 5.4758010 & -0.4481410 & 3.3083330 \\
\hline $\mathrm{H}$ & 4.8477610 & 0.4499280 & 1.928858 \\
\hline C & 3.4486370 & -1.0434110 & 2.7120740 \\
\hline $\mathrm{H}$ & 3.2928400 & -1.1121150 & 3.7946970 \\
\hline $\mathrm{H}$ & 2.6924250 & -0.3620540 & 2.3127080 \\
\hline C & 5.0172640 & -1.5458490 & -1.0725860 \\
\hline $\mathrm{H}$ & 6.0034750 & -1.1614410 & -1.3335300 \\
\hline c & -3.3891700 & 3.0499830 & 1.8243500 \\
\hline C & -3.2047640 & 3.3370080 & 3.2932350 \\
\hline $\mathrm{C}$ & -3.2672730 & 0.8094710 & 2.7424920 \\
\hline C & -3.4167920 & 1.6811480 & 1.5177180 \\
\hline $\mathrm{C}$ & -4.3807930 & 2.6775540 & 4.0478010 \\
\hline $\mathrm{H}$ & -4.2525670 & 2.8394130 & 5.1240460 \\
\hline $\mathrm{H}$ & -5.3180040 & 3.1613270 & 3.756575 \\
\hline C & -4.4159260 & 1.1624050 & 3.716033 \\
\hline $\mathrm{H}$ & -4.3032830 & 0.5632550 & 4.626571 \\
\hline $\mathrm{H}$ & -5.3740180 & 0.8868260 & 3.264944 \\
\hline $\mathrm{H}$ & -3.2998080 & -0.2532030 & 2.492256 \\
\hline $\mathrm{H}$ & -3.1808880 & 4.4158790 & 3.483549 \\
\hline C & -1.9325340 & 1.1553590 & 3.4353480 \\
\hline
\end{tabular}

-1.8382630
-1.1028950
-1.9030460
-1.8088180
-1.0393860
-3.8219810
-4.1152120
-4.0455950
-3.8238310
-3.0482140
-3.3262100
-2.0801080
-2.9673820
-3.1308110
-1.9841360
-3.9967540
-4.1391380
-5.4218110
-5.5633690
-6.2210830
-5.4820330
-5.7222590
-6.2622500
-3.5977500
-3.6013470
-4.7619590
-5.9379090
-6.2094490
-4.9722280
-3.6690020
-3.6263940
-5.7224600
-7.0715660
-5.1116200
-3.6175890
-6.8201640
-6.4794700
-4.8615770
-2.7938910
-1.2788870
-2.5090050
-2.2515210
-0.9761810
-0.1537200
-0.0681690
-2.7466680

.5491430

0.8823330

2.6662120

2. 8207000

3.1507730

3.5843500

4.4857960

1.9768300

2. 2184910

4.2604010

4.8406510

4.6302770

2.7487730

2.5753540

2. 3401030

0.9147680

5.5362490

2. 5686800

2.4664390

1.9978060

4.0608830

4.6995370

4.2189450

4.0029820

5.0643220

$-2.1262530$

$-2.7913090$

$-4.1416100$

$-5.0654410$

$-4.3059530$

$-2.9213990$

$-2.9571480$

$-4.6321760$

$-5.8470030$

$-4.1801920$

$-2.1456680$

$-3.9395260$

$-5.5768710$

$-4.8963490$

$-4.3435040$

$-4.9661530$

$-5.2547510$

$-6.1008790$

$-6.2897100$

$-5.0032560$

$-5.9091720$
4.3433230

2.7775170

3.7812660

4.8622020

3.3163470

$-0.4758400$

$-1.6480670$

$-2.2752560$

$-0.7993350$

$-2.7397690$

$-3.6274920$

$-2.3917340$

$-3.0689630$

$-4.1387210$

$-2.8220480$

$-2.5242570$

$-1.3384580$

$-2.6551030$

$-3.7369940$

$-2.1709910$

$-2.2309030$

$-3.0882730$

$-1.4800290$

0.8314430

1.0780490

$-1.1521340$

$-1.8213300$

$-1.1410910$

$-1.1895640$

$-1.5231650$

$-0.9234890$

$-2.8866780$

$-1.6030200$

$-1.9430870$

$-2.6136390$

$-1.7716360$

$-0.0989840$

$-0.2284600$

$-1.2456460$

0.7860520

1. 3993220

2.8897360

3.1226150

1.8324740

1.0544040

0.8853070 


\begin{tabular}{rrr}
-3.1270160 & -5.7534880 & 3.3164130 \\
-1.2353980 & -7.0855600 & 3.5236290 \\
-0.6415400 & -7.0527450 & 1.2107020 \\
-3.3804280 & -4.3154310 & 1.2876660 \\
-2.1558420 & -4.2924650 & 3.4022930 \\
-0.3450640 & -5.6126680 & 3.8712460 \\
0.8461900 & -6.6671940 & 2.0667890 \\
3.4180610 & 2.4792790 & 0.5668390 \\
3.1044080 & 1.3061820 & 0.0990730 \\
4.8429200 & 2.8592430 & 0.3591500 \\
5.7325920 & 1.9648130 & -0.2420620 \\
5.2782880 & 4.1204750 & 0.7704120 \\
7.0594280 & 2.3362280 & -0.4282550 \\
5.3715460 & 0.9890650 & -0.5526340 \\
6.6055240 & 4.4877070 & 0.5801990 \\
4.5705810 & 4.7960000 & 1.2397790 \\
7.4951200 & 3.5957730 & -0.0181880 \\
7.7544330 & 1.6438220 & -0.8930860 \\
6.9485370 & 5.4666330 & 0.8990200 \\
8.5317450 & 3.8835800 & -0.1643000 \\
2.1583900 & 0.9607510 & 0.3372170 \\
2.6370600 & 3.2566650 & 1.1338950 \\
0.7238690 & 3.5164300 & -0.8259830 \\
0.6189700 & 3.4113310 & 0.6320030 \\
1.1240320 & 2.6345000 & -1.3292790 \\
0.4393070 & 2.4239560 & 1.0442270 \\
-0.7109810 & 3.4459970 & -0.7035590 \\
-0.9372170 & 2.4699970 & -0.8743960 \\
0.6239380 & 6.0230810 & -0.6250680 \\
0.9724390 & 5.8977040 & 0.8589800 \\
0.3702310 & 4.6280280 & 1.4646510 \\
1.1837400 & 4.8329810 & -1.4071620 \\
1.0285940 & 6.9536670 & -1.0336440 \\
0.6136870 & 6.7671160 & 1.4173780 \\
-0.7209310 & 4.7142310 & 1.5550560 \\
2.2809720 & 4.8440100 & -1.3761520 \\
0.8833030 & 4.8684810 & -2.4593560 \\
-0.4657410 & 6.0594460 & -0.7480440 \\
2.0620570 & 5.8610730 & 0.9749180 \\
0.7609750 & 4.4323250 & 2.4666680 \\
\hline & &
\end{tabular}

154

TS193 (S , S) G[M06-2X/6-31G(d) $]=-3541.251305$

\begin{tabular}{|c|c|c|c|}
\hline & -0.2707160 & -0.0227530 & -0.1151160 \\
\hline 0 & 7455390 & 0.9071900 & -1.1942990 \\
\hline 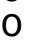 & 0.8760000 & 3987990 & 0.7 \\
\hline 0 & -0.0163090 & -1.4482080 & - \\
\hline
\end{tabular}

-1.4579430
-0.0082620
-1.2344060
1.2210290
1.2034060
2.1473380
-2.5611590
-3.6694620
-2.4916220
-4.7502640
-5.6413600
2.5064390
-3.6543490
2.7967600
1.9051690
4.1861270
4.0397970
1.6789420
1.1009820
1.0780700
3.0471150
3.1273240
3.1650680
5.1632710
0.9486120
4.0001660
4.0194530
4.8380570
2.6545370
2.0213750
2.8234540
3.4735660
3.3343070
5.6444230
4.7169030
4.4510820
4.4042760
4.2798700
5.8334630
6.5033890
6.3160390
6.6084780
2.3506350
4.9671080
5.5933640
4.9087420

$-2.5942040$

$-3.1080080$

$-3.1853400$

$-4.3565330$

$-4.8521510$

$-1.0179040$

$-0.2226880$

$-2.3795370$

$-0.8646190$

$-0.2819380$

$-2.5773670$

1. 2444660

$-2.3834270$

$-2.7083200$

$-1.7144050$

$-1.8685520$

$-1.4141080$

$-1.6630860$

$-0.7152180$

$-0.7925950$

$-0.6606850$

0.1947280

$-1.2917220$

$-3.1288610$

$-3.0983730$

$-2.9799320$

$-3.7488610$

$-3.7127890$

$-3.9576240$

$-4.6426250$

$-2.2085790$

$-2.2522450$

$-1.3761490$

$-1.7015900$

$-3.1527400$

$-3.1182390$

$-4.1931040$

$-2.6609910$

$-2.4496600$

$-3.4269390$

$-1.0072310$

$-2.6141790$

$-0.3229500$

$-0.1640510$

0.6319550
0.9211570 0.0717760

0.3838730

0.2112470

0.9744150

1. 1936790

0.3317120

0.0364600

0.0334220

$-0.5611710$

$-0.7903990$

$-0.2354320$

0.2978030

$-1.5947020$

$-2.7695700$

$-3.4811840$

$-1.9899730$

3.5827450

$-4.4801530$

$-2.9952820$

$-3.9682830$

5.0532980

$-3.5100680$

$-3.7389740$

$-2.4515280$

$-4.1392630$

$-5.2287050$

$-3.8707460$

$-3.6735980$

$-4.5336720$

$-3.1216590$

0.7136060

2. 2176910

1. 4483990

0.3061960

2. 7908080

3.8852070

2.4953340

2. 2843370

3. 1252670

1. 6695640

1.0797470

2. 5244370

2. 3513750

3. 2370490

1.8222840 


$\begin{array}{rrr}3.5550140 & -0.8188370 & 2.7504790 \\ 3.4379630 & -0.8229670 & 3.8401460 \\ 2.7781000 & -0.1691680 & 2.3382890 \\ 5.0074120 & -1.5451380 & -1.0445810 \\ 5.9811090 & -1.1699000 & -1.3602590 \\ -3.4224130 & 3.1342350 & 1.8212670 \\ -3.2222120 & 3.5036690 & 3.2694610 \\ -3.2453710 & 0.9493880 & 2.8560430 \\ -3.4276280 & 1.7506430 & 1.5879510 \\ -4.3734050 & 2.8645890 & 4.0778200 \\ -4.2295440 & 3.0841650 & 5.1417820 \\ -5.3238710 & 3.3168870 & 3.7791490 \\ -4.3874610 & 1.3339900 & 3.8252970 \\ -4.2535100 & 0.7847550 & 4.7639860 \\ -5.3463160 & 1.0199410 & 3.4018490 \\ -3.2618320 & -0.1258330 & 2.6650980 \\ -3.2162250 & 4.5915020 & 3.4008400 \\ -1.9083560 & 1.3562490 & 3.5104430 \\ -1.7931230 & 0.8041250 & 4.4499600 \\ -1.0820490 & 1.0597450 & 2.8587660 \\ -1.9001390 & 2.8844740 & 3.7702510 \\ -1.7894080 & 3.1013720 & 4.8388120 \\ -1.0541120 & 3.3571120 & 3.2623230 \\ -3.8927720 & 3.5380500 & -0.4977900 \\ -4.2138350 & 4.3727450 & -1.7112780 \\ -4.1025960 & 1.8369050 & -2.2093300 \\ -3.8720040 & 2.1572200 & -0.7495550 \\ -3.1515780 & 4.1117880 & -2.7995440 \\ -3.4461750 & 4.6420920 & -3.7127930 \\ -2.1873120 & 4.5146910 & -2.4783150 \\ -3.0471930 & 2.5872280 & -3.0529890 \\ -3.2190830 & 2.3568980 & -4.1106040 \\ -2.0542580 & 2.2087740 & -2.7973230 \\ -4.0355180 & 0.7649720 & -2.4058340 \\ -4.2549030 & 5.4369110 & -1.4548300 \\ -5.4934720 & 2.3826980 & -2.6046030 \\ -5.6438260 & 2.2211080 & -3.6780600 \\ -6.2771660 & 1.8238580 & -2.0823810 \\ -5.5772780 & 3.8938530 & -2.2588050 \\ -5.8358260 & 4.4822010 & -3.1462940 \\ -6.3541900 & 4.0775080 & -1.5103610 \\ -3.6613750 & 4.0291860 & 0.7826260 \\ -3.6818540 & 5.1018100 & -0.9728770 \\ -3.5704620 & -2.9802570 & -1.1377990 \\ -3.4647000 & -4.4123300 & -2517730 \\ -4.4612890 & -4.7270720 & \end{array}$

-5.8612640
-5.9088760
-4.7140400
-4.4264190
-3.6531280
-2.4373190
-6.0995610
-6.6182270
-6.8294000
-5.9436880
-4.1840510
0.0193940
-0.0207330
-1.1547370
-2.5218190
-2.3992290
-1.2055060
-0.1978410
-1.1747760
-3.0586350
-2.2536550
0.9425250
-0.9385940
-3.1375500
-3.3283620
3.3956520
3.0762130
4.8071640
5.6840680
5.2431400
6.9987930
5.3236050
6.5581660
4.5457730
7.4349870
7.6840000
6.9016980
8.4622190
2.1453690
2.6284720
0.6486450
0.5918780
1.0411510
0.4365730
-0.7808650
-1.0016700
$-1.8165870$

$-1.6639610$

$-0.9213780$

$-2.4838730$

$-0.3081040$

$-1.4651530$

$-0.8583980$

$-2.5371680$

$-1.1557680$

$-2.6625560$

$-3.1661460$

1.4868460

2. 3401320

1.8499060

1.9300950

1. 9690520

1. 2113970

3. 3919700

2. 4239650

2.8295800

3. 0167460

2. 3013570

0.8104160

1.0792300

1. 6509190

0.4153920

0.0152170

0.1344510

$-0.4552240$

0.4670170

$-0.7089250$

$-0.7029160$

0.2095010

0.9295690

$-0.3775700$

$-1.1648300$

0.4672020

$-0.5762400$

0.3121340

0.9757970

$-0.9281370$

0.5359140

$-1.3956210$

1.0068310

$-0.7555880$

$-0.8683750$ 


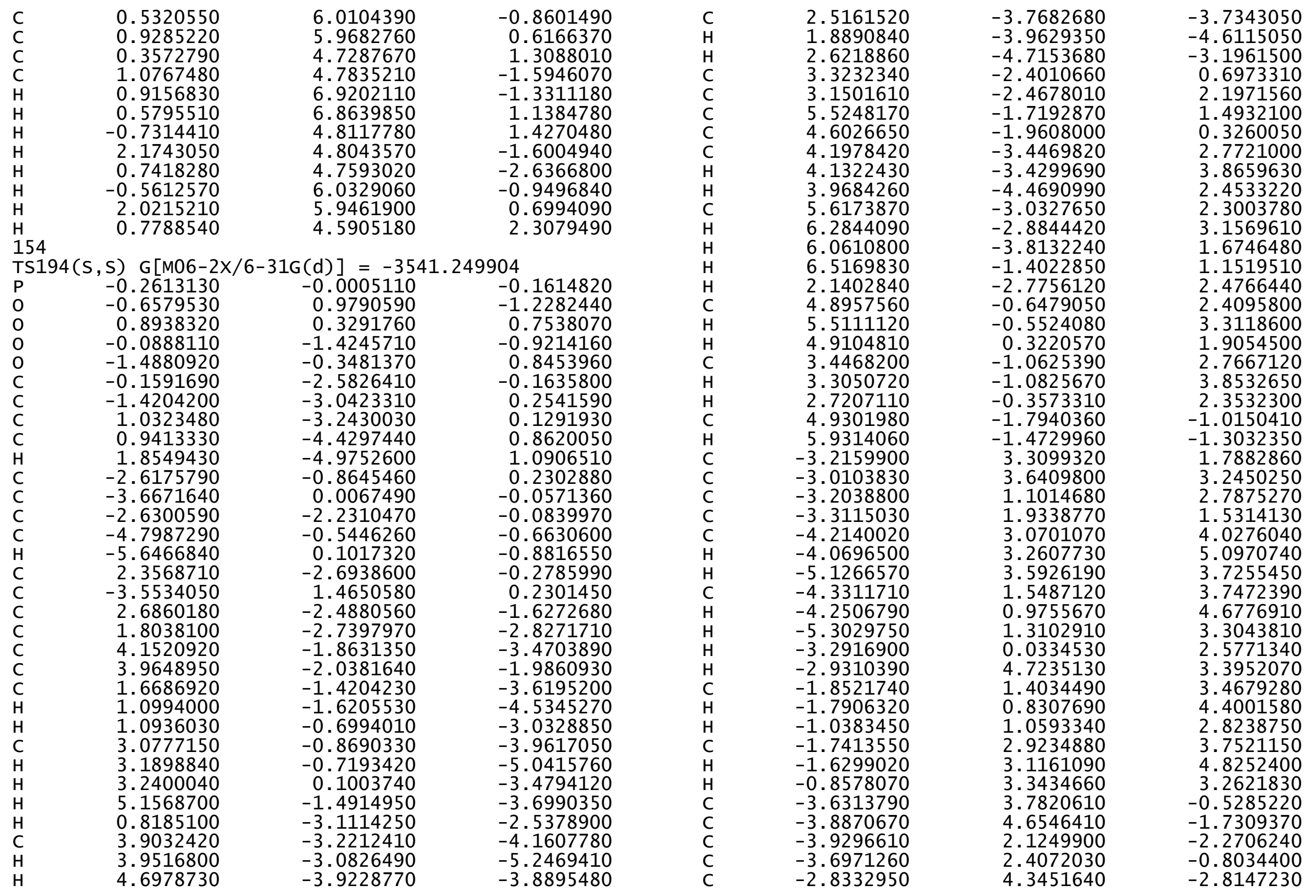




$\begin{array}{rrr}-3.0879390 & 4.9050880 & -3.7222990 \\ -1.8493320 & 4.6848680 & -2.4806790 \\ -2.8188250 & 2.8208270 & -3.0895620 \\ -2.9898350 & 2.6163430 & -4.1526190 \\ -1.8545200 & 2.3787820 & -2.8265770 \\ -3.9276670 & 1.0540670 & -2.4834360 \\ -3.8640790 & 5.7151130 & -1.4575190 \\ -5.2782190 & 2.7640900 & -2.6728020 \\ -5.4231650 & 2.6326340 & -3.7511120 \\ -6.1027280 & 2.2456650 & -2.1722650 \\ -5.2725010 & 4.2705650 & -2.2980500 \\ -5.4869050 & 4.8905930 & -3.1756920 \\ -6.0431360 & 4.4871210 & -1.5519070 \\ -3.3829250 & 4.2357930 & 0.7625260 \\ -3.3352090 & 5.3041770 & 0.9710840 \\ -4.8457540 & -1.8878080 & -1.0191250 \\ -6.0416220 & -2.5053200 & -1.6977140 \\ -6.4195150 & -3.8114200 & -0.9834850 \\ -5.2314030 & -4.7993550 & -0.9216650 \\ -3.8820680 & -4.1411740 & -1.2828540 \\ -3.7597890 & -2.7362270 & -0.7461770 \\ -5.8048480 & -2.7214720 & -2.7493020 \\ -7.2782770 & -4.2755930 & -1.4776570 \\ -5.3953480 & -5.6376460 & -1.6063110 \\ -3.8160650 & -4.0758110 & -2.3778130 \\ -6.8835940 & -1.8062020 & -1.6988150 \\ -6.7387490 & -3.5548470 & 0.0320670 \\ -5.1713010 & -5.2295850 & 0.0834070 \\ -3.0457570 & -4.7690480 & -0.9671540 \\ -0.2795470 & -4.9027280 & 1.3252300 \\ -0.4059090 & -6.1638030 & 2.1407430 \\ -1.5280400 & -7.0403330 & 1.5646030 \\ -2.8694780 & -6.2754870 & 1.4747280 \\ -2.7103630 & -4.7550830 & 1.6894060 \\ -1.4651680 & -4.2067900 & 1.0362080 \\ -0.6415840 & -5.9115470 & 3.1845240 \\ -1.6430030 & -7.9464370 & 2.1669790 \\ -3.5761880 & -6.6530130 & 2.2206830 \\ -2.6176540 & -4.5705080 & 2.7687950 \\ 0.5436220 & -6.7077800 & 2.1522600 \\ -1.2193930 & -7.3619180 & 0.5645090 \\ -3.3256960 & -6.4598010 & 0.4965770 \\ -3.6073960 & -4.2213590 & 1.3681050 \\ 3.5453540 & 2.3468500 & 0.5034760 \\ 3.1690490 & 1.1782160 & \\ 4.9808790 & 2.6573410 & \\ -6 & & \\ -6 & & \\ -6 & -2580770\end{array}$

5.8176160
5.4796990
7.1554100
5.4077700
6.8176580
4.8120230
7.6545100
7.8095870
7.2099590
8.6996390
2.2124200
2.8116950
0.8992950
0.8016600
1.2430970
0.5700890
-0.5360450
-0.8190960
0.9468920
1.2990240
0.6296120
1.4296450
1.4025190
0.9954960
-0.4540900
2.5259280
1.1228330
-0.1395870
2.3855980
1.0159760

1.7103930

3.9071630

2.0181100

0.7444960

4.2104980

4.6237850

3.2661430

1.2848840

5.1802690

3.5038230

0.8867420

3.1721590

3.4653220

3.4218890

2.5433060

2.4626360

3.4827080

2. 5158570

5.9796240

5.8905630

4.6817990

4.7297320

6.8684440

6.7999560

4.8341240

4.6781280

4.7423640

6.0758320

5.7952690

4.5023990
$-0.3385730$

0.6302240

$-0.5594200$

$-0.6177600$

0.4055710

1.0972690

$-0.1882900$

$-1.0207680$

0.6940700

$-0.3614840$

0.3381010

1.0661560

$-0.8884040$

0.5732270

$-1.3603300$

1.0241440

$-0.7543740$

$-0.8859940$

$-0.7839090$

0.7019840

1.3597060

$-1.5228290$

$-1.2301390$

1.2285770

1.4528300

$-1.4992320$

$-2.5737000$

$-0.9021790$

0.8129950

2. 3664800

154

$\begin{array}{lrrr}\text { TS195(S, S) } G[M 06-2 X / 6-31 G(d)]=-3541.250316 \\ \text { P } & -0.2982430 & -0.0635130 & 0.8223930 \\ O & 0.4193100 & -0.4159740 & 2.0905980 \\ O & -0.5044900 & 1.3892370 & 0.4712150 \\ O & -1.6950080 & -0.8866460 & 0.8491400 \\ O & 0.4334640 & -0.7100880 & -0.4895370 \\ \text { C } & -2.3776360 & -1.0060040 & -0.3528990 \\ \text { C } & -1.9189820 & -1.9471590 & -1.2868270 \\ \text { C } & -3.4970620 & -0.2033770 & -0.5611170 \\ \text { C } & -4.1625650 & -0.3432680 & -1.7821100 \\ \text { H } & -5.0464440 & 0.2639380 & -1.9678210 \\ \text { C } & 0.4523350 & -2.0969990 & -0.5461700 \\ \text { C } & 1.6323600 & -2.7581730 & -0.2128890 \\ \text { C } & -0.7166570 & -2.7635670 & -0.9414470 \\ \text { C } & 1.6217180 & -4.1537220 & -0.2580010\end{array}$




$\begin{array}{lrrr}\mathrm{H} & 2.5337660 & -4.6944060 & -0.0122810 \\ \mathrm{C} & -3.9810230 & 0.7595810 & 0.4674620 \\ \mathrm{C} & 2.8634000 & -2.0013930 & 0.1492200 \\ \mathrm{C} & -4.4122680 & 0.3162290 & 1.7268780 \\ \mathrm{C} & -4.4049140 & -1.1039670 & 2.2403320 \\ \mathrm{C} & -5.3626170 & 0.5814900 & 3.9542270 \\ \mathrm{C} & -4.9438620 & 1.2220430 & 2.6561420 \\ \mathrm{C} & -3.5631840 & -1.1439550 & 3.5346830 \\ \mathrm{H} & -3.5924280 & -2.1612440 & 3.9414590 \\ \mathrm{H} & -2.5206670 & -0.9208230 & 3.2933870 \\ \mathrm{C} & -4.1315780 & -0.1273260 & 4.5594180 \\ \mathrm{H} & -4.4285920 & -0.6298840 & 5.4870680 \\ \mathrm{H} & -3.3758980 & 0.6185490 & 4.8234400 \\ \mathrm{H} & -5.7614110 & 1.3271460 & 4.6503440 \\ \mathrm{H} & -4.0003140 & -1.8025230 & 1.5044210 \\ \mathrm{C} & -6.4261290 & -0.4930650 & 3.6410020 \\ \mathrm{H} & -6.6980190 & -1.0083260 & 4.5692970 \\ \mathrm{H} & -7.3324580 & -0.0114500 & 3.2615080 \\ \mathrm{C} & -5.8566900 & -1.4922280 & 2.6002530 \\ \mathrm{H} & -5.8616710 & -2.5130260 & 2.9987280 \\ \mathrm{H} & -6.4682310 & -1.4970990 & 1.6926330 \\ \mathrm{C} & -4.0700990 & 2.1282520 & 0.1741140 \\ \mathrm{C} & -3.5716570 & 2.8228230 & -1.0716140 \\ \mathrm{C} & -4.6796610 & 4.4463400 & 0.6128990 \\ \mathrm{C} & -4.6247820 & 3.0219370 & 1.1026680 \\ \mathrm{C} & -4.7591100 & 3.5424120 & -1.7474740 \\ \mathrm{H} & -4.3813840 & 4.1272790 & -2.5940170 \\ \mathrm{H} & -5.4611680 & 2.8069800 & -2.1539660 \\ \mathrm{C} & -5.4660550 & 4.4620420 & -0.7163060 \\ \mathrm{H} & -5.5254650 & 5.4907780 & -1.0890090 \\ \mathrm{H} & -6.4906790 & 4.1236670 & -0.5342170 \\ \mathrm{H} & -5.1585490 & 5.0985660 & 1.3509800 \\ \mathrm{H} & -3.0943940 & 2.1221500 & -1.7613870 \\ \mathrm{C} & -3.2403720 & 4.9176060 & 0.3155900 \\ \mathrm{H} & -3.2811420 & 5.9126900 & -0.1429070 \\ \mathrm{H} & -2.6832760 & 5.0159760 & 1.2521820 \\ \mathrm{C} & -2.5527550 & 3.8973930 & -0.6268180 \\ \mathrm{H} & -2.1455070 & 4.3951020 & -1.5145930 \\ \mathrm{H} & -1.7237900 & 3.3993920 & -0.1203000 \\ \mathrm{C} & -5.0638310 & 2.5721960 & 2.3427920 \\ \mathrm{H} & -5.4924990 & 3.2686380 & 3.0619660 \\ \mathrm{C} & 4.7060790 & -0.5433470 & -0.5013450 \\ \mathrm{C} & 5.2946340 & 0.2150660 & -1.6641680 \\ \mathrm{C} & 3.0890220 & -1.0082960 & -2.2471940 \\ \mathrm{C} & 3.5072510 & -1.2026830 & -0.8093000 \\ \mathrm{C} & 5.5538680 & -0.7992330 & -2.8007890 \\ & & & \end{array}$

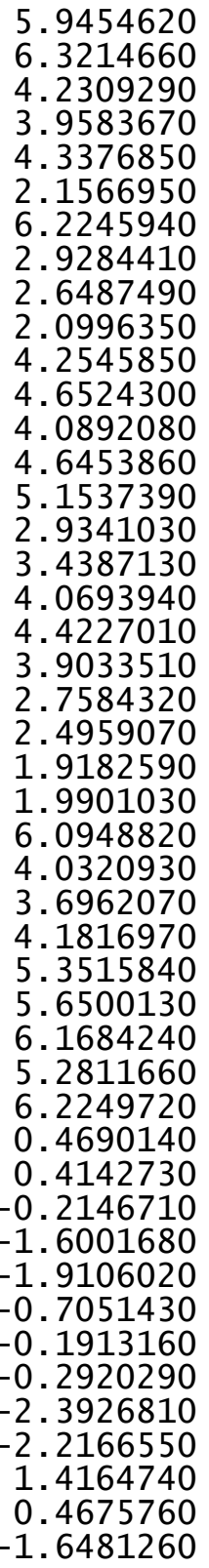

. 9454620

3.9583670

4.3376850

. 1566950

2. 9284410

2.6487490

2. 0996350

4.2545850

4.0892080

4.6453860

5.1537390

3. 9033510

2.7584320

1. 9901030

6. 0948820

3.6962070

4.1816970

5.3515840

6.

5. 2811660

6.2249720

0.4690140

$-0.2146710$

$-1.6001680$

$-1.9106020$

-0. 1951430

$-0.2920290$

$-2.3926810$

1. 4164740

$-1.6481260$
$-3.6739180$

$-2.4853630$

$-3.1482170$

$-4.1962190$

$-3.0103850$

$-2.4734270$

$-1.3767360$

$-2.5053590$

$-3.5527170$

$-1.8951730$

$-2.1726140$

$-3.0576540$

$-1.4143170$

1. 7180940

3. 1101220

2. 5734150

1. 4226350

4.1155640

5. 1338430

4.0326840

3. 8237570

4.6687460

3. 6674220

2. 3286280

3. 2952480

2. 8968200

3.7305720

2.0362430

3. 2574810

4.2885990

2. 6077780

0.7596500

0.9898990

$-0.5911420$

$-0.6253220$

$-1.9476340$

$-2.1895160$

$-1.2059380$

$-0.9359420$

0.2144960

$-1.9601510$

$-2.1004180$

$-0.2474260$

$-0.5038740$

$\begin{array}{ll}-6.5484660 & -2.7575500 \\ -5.8028000 & -3.2157340\end{array}$ 


\begin{tabular}{|c|c|c|}
\hline $\begin{array}{l}-2.7626120 \\
-3.7102660 \\
-4.3936390 \\
-4.6250330 \\
-3.3148670 \\
-2.1463100 \\
-2.5873370 \\
-3.7662560 \\
-5.0880900 \\
-3.0083190 \\
-1.7428530 \\
-5.3409830 \\
-5.3432990 \\
-3.4921580 \\
-1.3273210 \\
1.7015810 \\
0.5733740 \\
2.1991290 \\
3.4042910 \\
1.4879410 \\
3.8976160 \\
3.9422990 \\
1.9862490 \\
0.5561670 \\
3.1884400 \\
4.8336750 \\
1.4379900 \\
3.5748780 \\
0.2097370 \\
2.3674180 \\
1.6275810 \\
2.6159250 \\
0.5906930 \\
2.3411580 \\
2.0575510 \\
1.4284680 \\
3.4314530 \\
4.2876220 \\
4.0644040 \\
1.9473600 \\
3.6271050 \\
5.3498960 \\
4.4790660 \\
1.6519130 \\
1.3197490 \\
3.6916740\end{array}$ & $\begin{array}{l}-4.4445740 \\
-1.2229150 \\
-1.3810980 \\
-2.8723540 \\
-3.6906080 \\
-2.8930010 \\
-2.0311400 \\
-0.9508870 \\
-2.9950500 \\
-4.0318450 \\
-2.2227640 \\
-0.8333860 \\
-3.2466760 \\
-4.5927980 \\
-3.5595620 \\
3.3404870 \\
2.7313830 \\
4.0971010 \\
4.7957920 \\
4.0871800 \\
5.4859840 \\
4.7840010 \\
4.7780940 \\
3.5359460 \\
5.4764250 \\
6.0297980 \\
4.7709350 \\
6.0143070 \\
2.1624240 \\
3.3264720 \\
2.4384030 \\
1.9760530 \\
2.3675130 \\
1.1409370 \\
1.2510730 \\
0.5166110 \\
3.8027960 \\
3.6607330 \\
2.3013420 \\
3.6996340 \\
4.7622830 \\
3.7726590 \\
1.4908070 \\
4.5470900 \\
3.7205580 \\
3.0144220\end{array}$ & $\begin{array}{l}-1.5535420 \\
-2.7580620 \\
-4.0917310 \\
-4.3804770 \\
-4.2919720 \\
-3.6746750 \\
-2.5174950 \\
-4.8853230 \\
-5.3641500 \\
-5.2859000 \\
-4.4465840 \\
-4.1037320 \\
-3.6437970 \\
-3.6974690 \\
-3.3943620 \\
-1.1351730 \\
-1.3248800 \\
-2.3187960 \\
-2.2184600 \\
-3.5209360 \\
-3.3190170 \\
-1.2758580 \\
-4.6205040 \\
-3.5835720 \\
-4.5197850 \\
-3.2435990 \\
-5.5569430 \\
-5.3800730 \\
-0.5303330 \\
-0.0882330 \\
2.4438470 \\
1.4627880 \\
2.1177720 \\
0.8190920 \\
3.1440420 \\
2.8350300 \\
3.5663840 \\
2.3073070 \\
1.6436470 \\
3.2121790 \\
4.0541860 \\
2.5435570 \\
2.2557240 \\
2.5815860 \\
4.1085540\end{array}$ \\
\hline
\end{tabular}

$\begin{array}{llll}\mathrm{H} & 4.0235380 & 4.4508640 & 1.5944240 \\ \mathrm{H} & 4.5572380 & 2.2267810 & 0.6708380 \\ 144 & & \end{array}$

144

$\operatorname{TS} 196(R, R) \quad G[M 06-2 X / 6-31 G(d)]=-3633.306812$

$\begin{array}{lrrr}\text { TS196(R, R }) \text { G }[M 06-2 X / 6-31 G(\mathrm{~d})]=-3633.306812 \\ \mathrm{O} & 0.6251710 & 0.7243460 & 0.2554950 \\ \mathrm{O} & -1.4583210 & 1.1872110 & -1.0900160 \\ \mathrm{C} & 0.8391610 & 2.0907000 & 0.2970310 \\ \mathrm{C} & -0.1992820 & 2.9247790 & 0.6588120 \\ \mathrm{C} & 2.1545670 & 2.5503680 & 0.0267650 \\ \mathrm{C} & 0.0340580 & 4.3397530 & 0.6633390 \\ \mathrm{C} & 2.3908350 & 3.8989230 & 0.1330980 \\ \mathrm{C} & 1.3516930 & 4.8202650 & 0.4231300 \\ \mathrm{H} & 3.3975060 & 4.2778680 & -0.0235130 \\ \mathrm{C} & -2.1170150 & 1.4691720 & 0.0984680 \\ \mathrm{C} & -3.3904970 & 0.8729080 & 0.3015390 \\ \mathrm{C} & -1.5247810 & 2.3368740 & 0.9904950 \\ \mathrm{C} & -4.0687170 & 1.2142040 & 1.4454310 \\ \mathrm{C} & -2.1964460 & 2.5999100 & 2.2303370 \\ \mathrm{C} & -3.4943940 & 2.0533870 & 2.4357590 \\ \mathrm{H} & -5.0667720 & 0.8181620 & 1.6127220 \\ \mathrm{C} & -3.6018850 & 3.0863900 & 4.6295270 \\ \mathrm{C} & -2.2932750 & 3.5936300 & 4.4454750 \\ \mathrm{C} & -1.6087940 & 3.3582190 & 3.2794560 \\ \mathrm{C} & -4.1841480 & 2.3288020 & 3.6467000 \\ \mathrm{H} & -4.1334650 & 3.2872090 & 5.5541310 \\ \mathrm{H} & -1.8249620 & 4.1695560 & 5.2373930 \\ \mathrm{H} & -0.6024050 & 3.7432460 & 3.1552730 \\ \mathrm{H} & -5.1792320 & 1.9132910 & 3.7814930 \\ \mathrm{C} & -0.7344580 & 6.6354060 & 0.8735530 \\ \mathrm{C} & 0.5848390 & 7.1088900 & 0.6749920 \\ \mathrm{C} & 1.6020800 & 6.2183590 & 0.4499240 \\ \mathrm{C} & -1.0038510 & 5.2898620 & 0.8686890 \\ \mathrm{H} & -1.5417400 & 7.3450300 & 1.0248050 \\ \mathrm{H} & 0.7829550 & 8.1758080 & 0.6879060 \\ \mathrm{H} & 2.6167130 & 6.5659530 & 0.2746170 \\ \mathrm{H} & -2.0210120 & 4.9407800 & 1.0105960 \\ \mathrm{C} & 3.2590770 & 1.6096020 & -0.3346900 \\ \mathrm{C} & 3.7484420 & 1.5801020 & -1.6536950 \\ \mathrm{C} & 3.8585240 & 0.8104790 & 0.6595970 \\ \mathrm{C} & 4.8929020 & 0.8249370 & -1.9334220 \\ \mathrm{C} & 4.9888030 & 0.0645380 & 0.3294190 \\ \mathrm{C} & 5.5427070 & 0.0810220 & -0.9520460 \\ \mathrm{H} & 5.2770700 & 0.8197670 & -2.9493090 \\ \mathrm{H} & 5.4631890 & -0.5494300 & 1.0945360 \\ \mathrm{C} & -3.9654620 & -0.0742980 & -0.3884940 \\ \mathrm{C} & -4.0918720 & -1.4429880 & \end{array}$




$\begin{array}{lr}-4.4048270 & 0.4109090 \\ -4.7048670 & -2.2863550 \\ -4.9996410 & -0.4743670 \\ -5.1687940 & -1.8257480 \\ -4.8218520 & -3.3417360 \\ -5.3470990 & -0.0947320 \\ 3.3121590 & 0.7239060 \\ 6.8193830 & -0.7007290 \\ 3.0719980 & 2.3432610 \\ -3.5969970 & -2.0331470 \\ -5.8310280 & -2.7670980 \\ -4.2813510 & 1.8747550 \\ -5.6661720 & 2.5162930 \\ -6.2355570 & 2.0338940 \\ -6.2441880 & 2.4269980 \\ -5.5710230 & 3.5789020 \\ -3.4524340 & 2.0413640 \\ -2.4620780 & 1.5929420 \\ -3.9458380 & 1.5686400 \\ -3.3265590 & 3.1038300 \\ -5.0297600 & -2.8614780 \\ -5.4917130 & -3.5805530 \\ -4.9961770 & -1.8902820 \\ -4.0001450 & -3.1775430 \\ -7.2786650 & -2.3501290 \\ -7.8634940 & -2.2993570 \\ -7.3121020 & -1.3638840 \\ -7.7595570 & -3.0649740 \\ -4.7599000 & -2.2468340 \\ -5.2915940 & -1.3159150 \\ -5.4825860 & -2.9566750 \\ -4.3921200 & -2.6568740 \\ -2.8462060 & -3.3564170 \\ -3.5139180 & -4.1537950 \\ -2.0325130 & -3.2497720 \\ -2.4212150 & -3.6808000 \\ 4.3360470 & 1.2135560 \\ 5.2335810 & 0.5855940 \\ 4.6419980 & 2.2432090 \\ 3.9092690 & 1.1795070 \\ 2.8522160 & -0.7063860 \\ 2.1158320 & -1.0336530 \\ 3.6952880 & -1.4068790 \\ 2.3868630 & -0.7615520 \\ 8.0215380 & 0.0542260 \\ 8.9435070 & -0.5202370\end{array}$

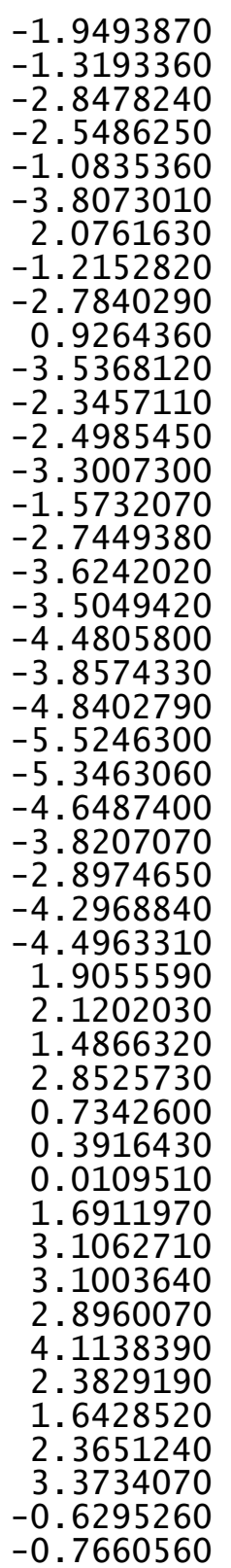

\begin{abstract}
8.1407630
7.8883230

7.0640700

6.2059880

7. 2693210

7.9353600

2. 9322880

3.9001570

2. 4945890

2. 2835920

3.8250050

4.8539770

3. 3357450

3. 8669730

$-0.2492720$

0.5011480

$-0.7521510$

2. 0607220

6. 7284680

2.4348560

$-3.7616900$

$-5.8493780$

$-2.8931010$

2.4160070

1.2663530

2. 2937160

0.2807430

1. 9949720

1. 3873240

3. 9045780

2. 8080160

1. 4215770

3.7948100

4.8925440

2. 9085960

1. 2313360

4. 0152500

4. 5069530

3.8226890

2. 9052070

0.6207270

0.3089320

$-0.3188920$

0.2870920

1.1843820
-0.6611100
\end{abstract}

1.0196430

0.2448130

$-1.0177710$

$-1.5189870$

$-0.1057560$

$-1.6714910$

1. 4906220

1. 3310650

0.5163520

2. 0003570

3.6395540

3. 4149520

4. 1686740

4. 3133850

0.1116670

0.1499370

$-1.2145550$

2. 6014500

$-1.6534890$

1.3733290

2. 4114510

$-3.7635510$

$-1.3221670$

$-2.2125720$

$-3.1186790$

-2. 6862860

$-1.9148890$

$-1.1044760$

$-4.0811480$

$-5.0870530$

$-4.4836350$

$-2.8262650$

$-4.5299180$

$-5.9978860$

$-4.3704880$

- 3.0617820

$-2.0621300$

$-3.7998130$

$-5.3797750$

$-5.1203900$

$-3.0387620$

$-1.9358160$

$-3.4328890$

-4.3755110
-2.8415510
$-1.1337310$

0.4395580

$-2.6906530$

$-3.1517650$

$-3.2617170$

$-2.7916580$

$-4.0507010$

$-4.5382570$

$-3.8220590$

$-4.7700470$

$-3.1104970$

$-3.4145110$

$-3.9350770$

$-2.2502750$

$-0.9818800$

$-2.2775790$

$-0.4607700$

$-2.4536020$

$-0.6742760$

2. 1512240

$-1.5468150$

$-3.0774870$

1. 3721140

$-1.4067000$

$-1.5229680$

$-0.7429180$

$-1.3967800$

$-2.7589000$

$-2.6932160$

$-2.2329840$

1.8825120

$-2.1186210$

$-1.3644600$

$-2.0945220$

$-2.4790080$

$-3.1934860$

$-0.3147530$

$-1.6825500$

$-3.2899680$

$-0.8291870$

$-1.7329430$

2. 1030500

1.8720500

3.5338430

4.0493440

4.3807750 


$\begin{array}{lrrr}\mathrm{C} & 1.1329020 & -4.7217390 & 5.3937680 \\ \mathrm{H} & 1.9232360 & -4.8239440 & 3.3940430 \\ \mathrm{C} & -0.7156580 & -3.2007340 & 5.7224120 \\ \mathrm{H} & -1.3542370 & -2.1115340 & 3.9771100 \\ \mathrm{C} & 0.1816110 & -4.1385750 & 6.2306680 \\ \mathrm{H} & 1.8361690 & -5.4464600 & 5.7904340 \\ \mathrm{H} & -1.4578270 & -2.7482810 & 6.3718160 \\ \mathrm{H} & 0.1409720 & -4.4147790 & 7.2796580 \\ \mathrm{H} & -0.4279280 & -1.6665690 & 0.8674350 \\ \mathrm{~S} & 1.0689040 & -3.9877380 & 0.9400180 \\ \mathrm{I} 44 & & & \\ \mathrm{TS} 197(\mathrm{R}) \mathrm{R}) & \mathrm{G}[\mathrm{M} 06-2 \mathrm{~N} / 6-31 \mathrm{G}(\mathrm{d})] & -35633.304023 \\ \mathrm{O} & 0.6752430 & 0.1476660 & -0.4459510 \\ \mathrm{O} & -1.5818410 & -0.1001370 & -1.5737650 \\ \mathrm{C} & 0.9541940 & 1.1626430 & -1.3408730 \\ \mathrm{C} & 0.0169640 & 2.1498350 & -1.5671030 \\ \mathrm{C} & 2.2531510 & 1.1889160 & -1.9159170 \\ \mathrm{C} & 0.3074940 & 3.1701500 & -2.5293730 \\ \mathrm{C} & 2.5471390 & 2.2085890 & -2.7883140 \\ \mathrm{C} & 1.5926080 & 3.1971380 & -3.1380360 \\ \mathrm{H} & 3.5431020 & 2.2641100 & -3.2220720 \\ \mathrm{C} & -2.0542100 & 1.0015370 & -0.8619490 \\ \mathrm{C} & -3.3045630 & 0.8843990 & -0.1942130 \\ \mathrm{C} & -1.2643730 & 2.1267190 & -0.8159270 \\ \mathrm{C} & -3.7252160 & 1.9703160 & 0.5338130 \\ \mathrm{C} & -1.6653870 & 3.2112060 & 0.0313180 \\ \mathrm{C} & -2.9172600 & 3.1253160 & 0.7014190 \\ \mathrm{H} & -4.6852710 & 1.9301220 & 1.0416230 \\ \mathrm{C} & -2.4997110 & 5.2573020 & 1.7847920 \\ \mathrm{C} & -1.2430170 & 5.3321920 & 1.1370000 \\ \mathrm{C} & -0.8360430 & 4.3380460 & 0.2815160 \\ \mathrm{C} & -3.3168080 & 4.1768720 & 1.5688640 \\ \mathrm{H} & -2.8084220 & 6.0515720 & 2.4570720 \\ \mathrm{H} & -0.5918060 & 6.1802210 & 1.3254390 \\ \mathrm{H} & 0.1364000 & 4.3977150 & -0.1955650 \\ \mathrm{H} & -4.2772200 & 4.0980520 & 2.0711750 \\ \mathrm{C} & -0.3280010 & 5.1107590 & -3.8451140 \\ \mathrm{C} & 0.9609140 & 5.1509730 & -4.4302090 \\ \mathrm{C} & 1.8971290 & 4.2115070 & -4.0846750 \\ \mathrm{C} & -0.6473390 & 4.1491360 & -2.9198400 \\ \mathrm{H} & -1.0720110 & 5.8453780 & -4.1366140 \\ \mathrm{H} & 1.1991690 & 5.9206030 & -5.1572880 \\ \mathrm{H} & 2.8865780 & 4.2208980 & -4.5338830 \\ \mathrm{H} & -1.6416460 & 4.1220250 & -2.4863950 \\ \mathrm{C} & 3.3028990 & 0.1941270 & -1.5333590 \\ \mathrm{C} & 3.4430770 & -1.0019760 & -2.2563550 \\ & & & \end{array}$

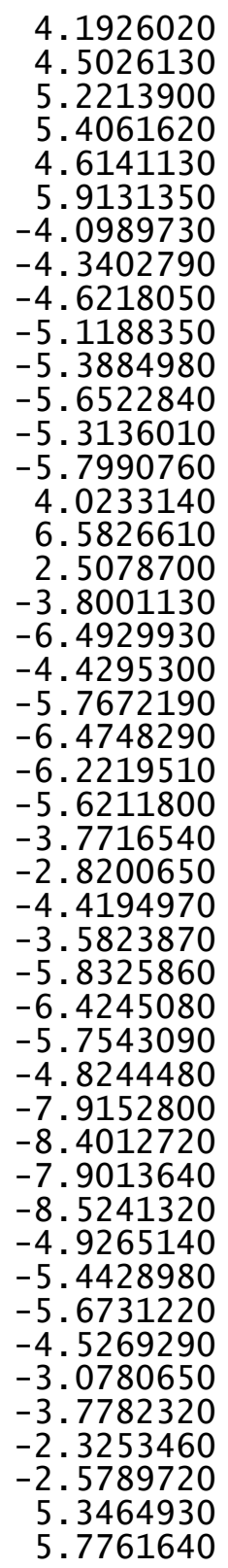

4. 1926020

5.2213900

5.4061620

4.6141130

5.9131350

-4.0989730
-4.3402790

$-4.6218050$

$-5.1188350$

$-5.6522840$

$-5.3136010$

$-5.7990760$

4.0233140
6.5826610

2. 5078700

$-3.8001130$

$-6.4929930$

$-4.4295300$

$-6.2219510$

$-5.6211800$

$-3.7716540$

-4.4194970
-3.5823870

$-5.8325860$

$-6.4245080$

$-5.7543090$

$-7.9152800$

$-8.4012720$

(

$-4.9265140$

4428980

$-3.0780650$

.7782320

$-2.5789720$

5. 7761640
0.5043150
-1.8612520
-0.3913880
-1.5691960
-2.7806490
-0.1718000
-0.3793360
-1.1078060
-0.8372270
-2.2660680
-2.0018090
-2.7313070
-2.8292820
-2.3451810
1.7678350
-2.4685400
-1.3424460
-0.6905620
-3.9929670
-0.0836140
0.4786590
-0.3291470
1.1337090
1.0554110
-0.9623100
-1.3644500
-1.8012080
-0.3765230
-5.0712100
-5.9921770
-4.7380240
-5.3014350
-3.6993980
-2.9447340
-3.3267360
-4.6091230
-0.1478690
0.6991280
-0.9275580
0.1792520
-1.8346740
-2.6123730
-2.2917650
-1.4476060
2.3326320
1.6967880

$-0.4814030$

$-1.9427700$

$-0.1967450$

$-0.9250980$

$-2.5089680$

0.6137910

$-0.2474660$

0.9372240

$-1.4750600$

0.8646050

$-1.4923560$

$-0.3335230$

1.7753140

$-2.4403740$

0.3522770

$-0.5804310$

$-3.4064060$

2. 2990140

$-0.3788390$

$-2.7833650$

$-3.2820640$

$-3.4991730$

$-2.5322000$

$-4.2013120$

$-3.8529590$

$-3.4947680$

$-4.1298550$

$-4.7585390$

$-1.2456030$

$-1.2312580$

$-2.2864180$

$-0.8887390$

$-0.8698160$

$-0.2443000$

$-1.9000630$

$-0.8507310$

3. 1898380

2. 7281280

3. 3796950

4.1556190

3. 0229370

3. 3472990

2. 3766020

3. 9186440

0.8764640

1.6577680 


\begin{tabular}{|c|c|c|c|}
\hline & & & \\
\hline $\mathrm{H}_{\mathrm{H}}^{\mathrm{H}}$ & $\begin{array}{l}6.0857750 \\
5.1758370\end{array}$ & $\begin{array}{l}2.4371480 \\
3.3186540\end{array}$ & $\begin{array}{l}0.0762690 \\
1.3190770\end{array}$ \\
\hline$\vec{c}$ & 3.0580390 & $\begin{array}{l}1.5182030 \\
1.5182030\end{array}$ & 1.5206620 \\
\hline H & 2.0710240 & 1. 1980850 & 1.1707010 \\
\hline $\mathrm{H}$ & 3.4511450 & 0.7322910 & 2.1785350 \\
\hline H & 2.9317290 & 2.4286870 & 2.1177140 \\
\hline C & 7.8895500 & -1.8353560 & -1.0797080 \\
\hline H & 8.7506970 & -2.4468150 & -0.7913630 \\
\hline H & 7.8750190 & -1.7567130 & -2.1724590 \\
\hline $\mathrm{H}$ & 8.0274140 & -0.8303400 & -0.6700450 \\
\hline C & 6.4541950 & -3.8960610 & -1.1117170 \\
\hline H & 5.5141700 & -4.3673190 & -0.8045500 \\
\hline H & 6.5002000 & -3.9153360 & -2.2060770 \\
\hline H & 7.2798570 & -4.5081940 & -0.7371480 \\
\hline C & 2.2580280 & -2.8463090 & -3.5496810 \\
\hline H & 3.1271650 & -3.3588880 & -3.9770410 \\
\hline $\mathrm{H}$ & 2.0254770 & -3.3039660 & -2.5849570 \\
\hline H & 1.4114180 & -3.0176970 & -4.2209980 \\
\hline C & 3.0520680 & -0.7738520 & -4.7245230 \\
\hline $\mathrm{H}$ & 4.0358110 & -1.2047290 & -4.9437960 \\
\hline $\mathrm{H}$ & 2.3796450 & -1.0188440 & -5.5535330 \\
\hline $\mathrm{H}$ & 3.1592200 & 0.3137970 & -4.6805050 \\
\hline $\mathrm{P}$ & -0.5078670 & -0.9586500 & -0.710109 \\
\hline 0 & -0.0029040 & -2.1142800 & -1.511543 \\
\hline 0 & -1.0748010 & -1.2337830 & 0.6663520 \\
\hline H & 1.5410060 & -0.8711250 & -3.2006710 \\
\hline H & 6.6341380 & -2.5191930 & 0.5167570 \\
\hline H & 3.5738930 & 2.5325400 & -0.2905180 \\
\hline H & -3.7683750 & 0.7689250 & -2.6058190 \\
\hline $\mathrm{H}$ & -6.5613510 & -4.3773310 & 0.6467420 \\
\hline $\mathrm{H}$ & -3.0688270 & 0.1070970 & 2.1487040 \\
\hline C & 2.0882170 & -2.6730380 & 0.9338840 \\
\hline C & 0.9345960 & -3.0677570 & 1.7548430 \\
\hline H & 2.0541630 & -1.6730500 & 0.4918780 \\
\hline H & -0.0308950 & -2.6601650 & 1.4827200 \\
\hline 0 & 1.5026590 & -3.6771260 & 0.0645290 \\
\hline H & 0.9086460 & -3.1955700 & -0.5900780 \\
\hline C & 3.4579880 & -4.4226780 & 2.1498570 \\
\hline C & 2.4231740 & -4.4245170 & 3.2748020 \\
\hline C & 1.0178190 & -4.2126980 & 2.7094570 \\
\hline C & 3.4607040 & -3.0800900 & 1.4166170 \\
\hline H & 4.4578970 & -4.6165940 & 2.548753 \\
\hline H & 2.4494730 & -5.3652640 & 3.8311730 \\
\hline H & 0.6915260 & -5.0986460 & 2.1483310 \\
\hline H & 3.8200710 & -2.2819970 & 2.079542 \\
\hline H & 1234480 & -3.1018450 & 0.5492 \\
\hline
\end{tabular}

-5.2276290
-3.6214590
-4.0568550
0.3621850
0.5408490
1.6092210
1.6290680
2.8003930
2.8213440
0.7089220
3.9899970
2.7909070
4.0033100
2.8301090
4.9070490
4.9340300
-0.2774920
-1.1147090

1.4397070

3.9863490

3.4914150

3.2136600

2. 3081780

3.9493850

4.9584620

3.5876730

5. 5971600

5.2315410

4.2291600

2.8008880

5.2332640

6.3775260

3.9401850

5.7317330

1.6798400

3.5567930

TS198 $(R, R) \quad G[M 06-2 X / 6-31 G(d)]=-3633.302754$

$\begin{array}{lrrr}\text { TS198(R, R) } \mathrm{G}[\mathrm{M} 06-2 \mathrm{X} / 6-31 \mathrm{G}(\mathrm{d})]=-3633.302754 \\ \mathrm{O} & 0.5687320 & 0.3147370 & -0.2317510 \\ \mathrm{O} & -1.6010910 & 0.3160760 & -1.5367590 \\ \mathrm{C} & 0.9025030 & 1.4719270 & -0.9126760 \\ \mathrm{C} & -0.0147670 & 2.5005900 & -0.9937580 \\ \mathrm{C} & 2.2183190 & 1.5670030 & -1.4367450 \\ \mathrm{C} & 0.3284310 & 3.6725520 & -1.7437480 \\ \mathrm{C} & 2.5604240 & 2.7220200 & -2.0972960 \\ \mathrm{C} & 1.6377060 & 3.7815680 & -2.2883920 \\ \mathrm{H} & 3.5700280 & 2.8307840 & -2.4869680 \\ \mathrm{C} & -2.1134700 & 1.2502870 & -0.6425380 \\ \mathrm{C} & -3.4020290 & 1.0151010 & -0.0914360 \\ \mathrm{C} & -1.3405980 & 2.3490720 & -0.3404010 \\ \mathrm{C} & -3.8978470 & 1.9686040 & 0.7646200 \\ \mathrm{C} & -1.8226620 & 3.2766310 & 0.6398900 \\ \mathrm{C} & -3.1289140 & 3.0897090 & 1.1713640 \\ \mathrm{H} & -4.8938150 & 1.8428410 & 1.1810170 \\ \mathrm{C} & -2.8478790 & 5.0408950 & 2.5875280 \\ \mathrm{C} & -1.5322100 & 5.2037840 & 2.0904170 \\ \mathrm{C} & -1.0328660 & 4.3491910 & 1.1388630 \\ \mathrm{C} & -3.6251770 & 4.0052420 & 2.1377200 \\ \mathrm{H} & -3.2312970 & 5.7295730 & 3.3334030 \\ \text { H } & -0.9126990 & 6.0120460 & 2.4666290 \\ \text { H } & -0.0206660 & 4.4790380 & 0.7690500 \\ \text { H } & -4.6290860 & 3.8551230 & 2.5257100 \\ \mathrm{C} & -0.2257500 & 5.8274630 & -2.7197780 \\ \mathrm{C} & 1.0887270 & 5.9481580 & -3.2319080\end{array}$




$\begin{array}{rrr}1.9966950 & 4.9430570 & -3.0232000 \\ -0.5973170 & 4.7224300 & -1.9960260 \\ -0.9484370 & 6.6152320 & -2.9076240 \\ 1.3683040 & 6.8308840 & -3.7978990 \\ 3.0045600 & 5.0117380 & -3.4237090 \\ -1.6114040 & 4.6372870 & -1.6204650 \\ 3.2272640 & 0.4782130 & -1.2539140 \\ 3.4942740 & -0.4132180 & -2.3046810 \\ 3.9584630 & 0.4024580 & -0.0472740 \\ 4.5559930 & -1.3174220 & -2.1706450 \\ 4.9942200 & -0.5238330 & 0.0428500 \\ 5.3291950 & -1.3736490 & -1.0163190 \\ 4.7691030 & -1.9951840 & -2.9916960 \\ 5.5680480 & -0.5974390 & 0.9638430 \\ -4.1650280 & -0.2325790 & -0.3976350 \\ -4.4218210 & -1.1643430 & 0.6344250 \\ -4.6431190 & -0.4728150 & -1.6984610 \\ -5.1850510 & -2.2939780 & 0.3419300 \\ -5.3953080 & -1.6255480 & -1.9407110 \\ -5.6838180 & -2.5444070 & -0.9369820 \\ -5.3916090 & -3.0063470 & 1.1384960 \\ -5.7758170 & -1.8091480 & -2.9439740 \\ 3.6569520 & 1.3390170 & 1.1157030 \\ 6.5100080 & -2.3174800 & -0.8527000 \\ 2.6733100 & -0.4101540 & -3.5857320 \\ -3.9026690 & -0.9900050 & 2.0561050 \\ -6.5111640 & -3.7830190 & -1.2236220 \\ -4.4096020 & 0.4934450 & -2.8501010 \\ -5.7326040 & 1.1191320 & -3.3101230 \\ -6.4160600 & 0.3533670 & -3.6931380 \\ -6.2315860 & 1.6347520 & -2.4834770 \\ -5.5548120 & 1.8435630 & -4.1117220 \\ -3.6886280 & -0.1872590 & -4.0189560 \\ -2.7465560 & -0.6330280 & -3.6878820 \\ -4.3081770 & -0.9753060 & -4.4608380 \\ -3.4698630 & 0.5431650 & -4.8048920 \\ -7.8116660 & -3.7847110 & -0.4118570 \\ -8.4214870 & -4.6583730 & -0.6639660 \\ -7.5984510 & -3.8228290 & 0.6622330 \\ -8.4006220 & -2.8837060 & -0.6075120 \\ -5.7091620 & -5.0632660 & -0.9635860 \\ -4.7876820 & -5.0778850 & -1.5529840 \\ -5.4350060 & -5.1414710 & 0.0943650 \\ -6.3009590 & -5.9470900 & -1.2231090 \\ -5.0373840 & -0.5877310 & 3.0085300 \\ -5.5271160 & 0.3386600 & 2.6944160\end{array}$
$-5.8016980$
$-4.6527950$
$-3.2098570$
$-3.9290460$
$-2.4591550$
$-2.7146100$
4. 3786520
5.4635080
4.1043210
4.1303850
4.0162410
3. 5665220
5.1011560
3.6504890
7.8231410
8.6779980
7.9569710
7.8279810
6.5665970
5.6380290
6.7510550
7.3857980
2. 3829920
3.2761150
2. 0164450
1.6196810
3. 3681400
4.3552880
2.7776260
3.5049870
$-0.5452620$
0.0403910
$-1.1775740$
1. 7090400
6.4117370
2.5757050
$-3.7707610$
$-6.7763690$
$-3.1590300$
2.0154180
0.8345940
2.0668230
$-0.0979590$
1. 3427840
0.7926100
3.2298940

-1.3727540
-0.4445110
-2.2520760
-3.0571470
-2.6160210
-2.0273830
2.6829550
2.5267520
3.1744910
3.3565440
0.7503760
-0.2379330
0.6608350
1.4133970
-1.5211290
-2.1829910
-1.0340530
-0.7447750
-3.4347350
-4.0144400
-3.0301930
-4.1206920
-1.8267780
-2.2901530
-2.4668950
-1.7900050
0.4063920
-0.0166500
0.3880660
1.4499830
-0.7135140
-1.5861390
-1.3658280
0.0582130
-2.7827550
1.5309840
1.3088820
-3.7599170
-0.1884350
-2.9843460
-3.4720150
-1.9053380
-2.9406410
-3.6786520
-2.9840470
-5.0921010

3.0435700

4.0238620

2.5884580

2.7750870

1.8825960

3.5397150

0.9378010

0.9376540

0.0008630

1.7654050

2.4812120

2.6280220

2.6051820

3.2718660

$-0.8660800$

$-0.6937160$

$-1.8383240$

$-0.0954770$

$-1.8938290$

$-1.9295240$

$-2.8951910$

$-1.6593540$

$-4.0934770$

$-4.5270800$

$-3.2874110$

$-4.8767800$

$-4.6834160$

$-4.9030380$

$-5.6055360$

$-4.3863350$

$-0.8599170$

$-1.9232710$

0.3514030

$-3.3616440$

0.1389500

1.1192120

$-2.4994390$

$-2.2881770$

2.0531140

0.1392820

0.8700010

$-0.0361890$

0.7248780

$-0.9401520$

$-1.4190200$

0.8493090 


\begin{tabular}{|c|c|c|c|c|c|c|c|}
\hline & & & & & & & \\
\hline C & 2.2051310 & -5.2827410 & 1. 9671550 & $\mathrm{C}$ & -1.6088930 & $\begin{array}{l}3.3583220 \\
3\end{array}$ & $\begin{array}{l}3.2794920 \\
3\end{array}$ \\
\hline c & $\begin{array}{l}0.821 / 820 \\
3.3490030\end{array}$ & $\begin{array}{l}-4.8231210 \\
-3.6159330\end{array}$ & $\begin{array}{l}1.5054430 \\
0.4658010\end{array}$ & $\begin{array}{l}\mathrm{C} \\
\mathrm{H}\end{array}$ & $\begin{array}{l}-4.1842560 \\
-4.1336310\end{array}$ & $\begin{array}{l}2.3289040 \\
3.2873330\end{array}$ & $\begin{array}{l}3.046067<0 \\
5.5540920\end{array}$ \\
\hline $\mathrm{H}$ & 4.2106470 & -5.4627130 & 1.1610180 & $\mathrm{H}$ & -1.8251220 & 4.1696850 & 5.2374100 \\
\hline $\mathrm{H}$ & 2.1522170 & -6.3299540 & 2.2767010 & $\mathrm{H}$ & -0.6025030 & 3.7433520 & 3.1553310 \\
\hline $\mathrm{H}$ & 0.4230280 & -5.5093780 & 0.7461460 & $\mathrm{H}$ & -5.1793440 & 1.9133950 & 3.7814400 \\
\hline H & 3.7885750 & -3.0384570 & 1.2899260 & $\mathrm{C}$ & -0.7344350 & 6.6354620 & 0.8735410 \\
\hline $\mathrm{H}$ & 3.9981800 & -3.4827010 & -0.4030330 & $\mathrm{c}$ & 0.5848740 & 7.1089280 & 0.6750150 \\
\hline $\mathrm{H}$ & 2.9280050 & -5.6756900 & -0.0289870 & C & 1.6021130 & 6.2183820 & 0.4500050 \\
\hline $\mathrm{H}$ & 2.5089480 & -4.7008360 & 2.8470870 & $\mathrm{c}$ & -1.0038430 & 5.2899210 & 0.8686990 \\
\hline $\mathrm{H}$ & 0.0893020 & -4.8095350 & 2.3170830 & $\mathrm{H}$ & -1.5417160 & 7.3450980 & 1.0247440 \\
\hline C & 0.4098880 & -0.4883920 & 3.0854580 & $\mathrm{H}$ & 0.7829990 & 8.1758450 & 0.6879060 \\
\hline 0 & -0.6383640 & -0.1417300 & 2.4208820 & $\mathrm{H}$ & 2.6167550 & 6.5659610 & 0.2747210 \\
\hline C & 0.9519460 & 0.6111170 & 3.9191710 & $\mathrm{H}$ & -2.0210140 & 4.9408560 & 1.0105740 \\
\hline $\mathrm{C}$ & 1.6991810 & 0.3710240 & 5.0762310 & $\mathrm{C}$ & 3.2590690 & 1.6096070 & -0.3345680 \\
\hline $\mathrm{C}$ & 0.7377150 & 1.9258680 & 3.4832960 & $\mathrm{C}$ & 3.7484570 & 1.5801960 & -1.6535670 \\
\hline $\mathrm{C}$ & 2.2234920 & 1.4391380 & 5.7942450 & $\mathrm{C}$ & 3.8584820 & 0.8103880 & 0.6596660 \\
\hline H & 1.8600370 & -0.6498950 & 5.4071240 & C & 4.8928990 & 0.8250180 & -1.9333370 \\
\hline C & 1.2890050 & 2.9890280 & 4.1896280 & C & 4.9887360 & 0.0644310 & 0.3294390 \\
\hline $\mathrm{H}$ & 0.1592660 & 2.1010860 & 2.5807980 & $\mathrm{C}$ & 5.5426660 & 0.0810030 & -0.9520140 \\
\hline C & 2.0278860 & 2.7469990 & 5.3474380 & $\mathrm{H}$ & 5.2770850 & 0.8199250 & -2.9492180 \\
\hline $\mathrm{H}$ & 2.7915970 & 1.2532060 & 6.6997780 & $\mathrm{H}$ & 5.4630910 & -0.5496140 & 1.0945140 \\
\hline $\mathrm{H}$ & 1.1343180 & 4.0046180 & 3.8382900 & C & -3.9654700 & -0.0742450 & -0.7015320 \\
\hline H & 2.4508670 & 3.5774000 & 5.9041990 & C & -4.0920800 & -1.4428920 & -0.3883750 \\
\hline $\mathrm{H}$ & -0.8899900 & -0.7619840 & 1.6138420 & C & -4.4046540 & 0.4108880 & -1.9494750 \\
\hline $\mathrm{s}$ & 1.1487720 & -2.0025510 & 3.0374810 & $\mathrm{C}$ & -4.7050630 & -2.2862820 & -1.3192060 \\
\hline & & & & $\mathrm{C}$ & -4.9994730 & -0.4744080 & -2.8478890 \\
\hline & R) $\mathrm{G}[\mathrm{M} 06-2$ & $G(d)]$ & 323 & C & -5.1688050 & -1.8257430 & -2.5485880 \\
\hline 0 & 0.6251480 & 0.7243760 & 0.2556290 & $\mathrm{H}$ & -4.8221930 & -3.3416260 & -1.0833090 \\
\hline 0 & -1.4582830 & 1.1872910 & -1.0899520 & $\mathrm{H}$ & -5.3467940 & -0.0948240 & -3.8074350 \\
\hline C & 0.8391550 & 2.0907300 & 0.2971630 & C & 3.3120950 & 0.7237000 & 2.0762170 \\
\hline C & -0.1992910 & 2.9248250 & 0.6589030 & C & 6.8193380 & -0.7007420 & -1.2152890 \\
\hline C & 2.1545720 & 2.5503770 & 0.0269160 & $\mathrm{C}$ & 3.0720750 & 2.3434640 & -2.7838650 \\
\hline $\mathrm{C}$ & 0.0340630 & 4.3397960 & 0.6634100 & C & -3.5974520 & -2.0330240 & 0.9266580 \\
\hline C & 2.3908500 & 3.8989330 & 0.1332400 & C & -5.8310440 & -2.7671130 & -3.5367510 \\
\hline $\mathrm{C}$ & 1.3517120 & 4.8202900 & 0.4232330 & $\mathrm{C}$ & -4.2809900 & 1.8746770 & -2.3459460 \\
\hline H & 3.3975260 & 4.2778660 & -0.0233650 & $\mathrm{C}$ & -5.6657280 & 2.5163360 & -2.4990180 \\
\hline C & -2.1170290 & 1.4692500 & 0.0985050 & $\mathrm{H}$ & -6.2350630 & 2.0339110 & -3.3012220 \\
\hline C & -3.3905190 & 0.8729880 & 0.3015340 & $\mathrm{H}$ & -6.2438670 & 2.4271950 & -1.5737410 \\
\hline C & -1.5248120 & 2.3369450 & 0.9905490 & $\mathrm{H}$ & -5.5704430 & 3.5789090 & -2.7455100 \\
\hline C & -4.0687610 & 1.2142850 & 1.4454160 & $\mathrm{C}$ & -3.4519110 & 2.0410480 & -3.6243640 \\
\hline C & -2.1965130 & 2.5999970 & 2.2303650 & $\mathrm{H}$ & -2.4616240 & 1.5925140 & -3.5049500 \\
\hline C & -3.4944670 & 2.0534750 & 2.4357530 & $\mathrm{H}$ & -3.9452830 & 1.5682950 & -4.4807440 \\
\hline $\mathrm{H}$ & 8110 & 0.8182250 & 1.6126930 & $\mathrm{H}$ & -3.3258780 & 3.1034730 & -3.8576950 \\
\hline C & -3.60 & 3.08650 & 4.6295070 & C & -5.0296920 & -2.8616620 & -4.8401550 \\
\hline C & 34090 & 374 & 4.4454880 & $\mathrm{H}$ & -5.4916650 & -3.5807460 & -5.5244830 \\
\hline
\end{tabular}




$\begin{array}{rr}-4.9959820 & -1.8905090 \\ -4.0001210 & -3.1778110 \\ -7.2786260 & -2.3500420 \\ -7.8635140 & -2.2991600 \\ -7.3119480 & -1.3638250 \\ -7.7595330 & -3.0648910 \\ -4.7605560 & -2.2467700 \\ -5.2923700 & -1.3158830 \\ -5.4830980 & -2.9566780 \\ -4.3929530 & -2.6567490 \\ -2.8465560 & -3.3562580 \\ -3.5141760 & -4.1536670 \\ -2.0327550 & -3.2495770 \\ -2.4216990 & -3.6806110 \\ 4.3360670 & 1.2129560 \\ 5.2334360 & 0.5847590 \\ 4.6423000 & 2.2425540 \\ 3.9092230 & 1.1788890 \\ 2.8518600 & -0.7065540 \\ 2.1153360 & -1.0335030 \\ 3.6947840 & -1.4072210 \\ 2.3865870 & -0.7618260 \\ 8.0214830 & 0.0541380 \\ 8.9434490 & -0.5203240 \\ 8.1407360 & 1.0196040 \\ 7.8882390 & 0.2446170 \\ 7.0640720 & -1.0176330 \\ 6.2060050 & -1.5187950 \\ 7.2693510 & -0.1055610 \\ 7.9353610 & -1.6713490 \\ 2.9322440 & 1.4908660 \\ 3.9000900 & 1.3311760 \\ 2.4943990 & 0.5166590 \\ 2.2836250 & 2.0007090 \\ 3.8252270 & 3.6396770 \\ 4.8541660 & 3.4149650 \\ 3.3360120 & 4.1688760 \\ 3.8672920 & 4.3134800 \\ -0.2492830 & 0.1117050 \\ 0.5011840 & 0.1499870 \\ -0.7522320 & -1.2144990 \\ 2.0608360 & 2.6017740 \\ 6.7283930 & -1.6535560 \\ 2.4349290 & 1.3732990 \\ -3.7613710 & 2.4114160 \\ -5.8495110 & -3.7635290\end{array}$

-5.3462520
-4.6485180
-3.8207760
-2.8975770
-4.2970190
-4.4963840
1.9055300
2.1200230
1.4864720
2.8526390
0.7346210
0.3918950
0.0114390
1.6916260
3.1064320
3.1005070
2.8963190
4.1139720
2.3827220
1.6426540
2.3647040
3.3732420
-0.6294190
-0.7659820
-1.1335220
0.4396800
-2.6906850
-3.1518830
-3.2616470
-2.7917260
-4.0505530
-4.5381080
-3.8219260
-4.7698920
-3.1103150
-3.4143560
-3.9348700
-2.2500750
-0.9817560
-2.2774430
-0.4606920
-2.4534160
-0.6743840
2.1513800
-1.5470520
-3.0773520

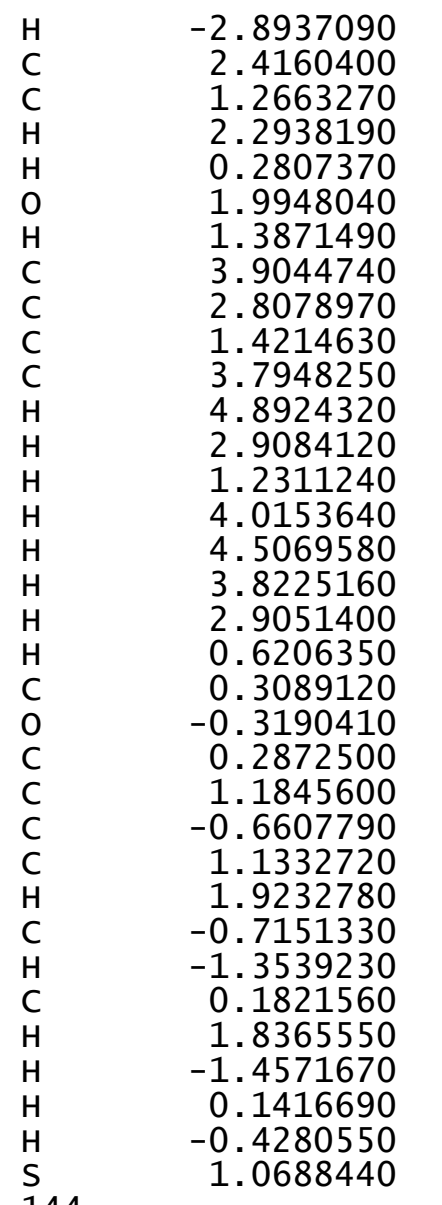
$-1.3220070$ $-2.2125190$ $-3.1185340$ $-1.3505230$ $-2.6861580$ $-1.9147330$ $-1.1042680$ $-4.0810570$ $-5.0869490$ $-4.4834690$ $-2.8262560$ $-4.5298780$ $-5.9977420$ $-4.3703000$ $-3.0618860$ $-2.0621090$ $-3.7996180$ $-5.3797510$ $-5.1202080$ $-3.0389330$ $-1.9360310$ $-3.4330930$ $-4.3757820$ $-2.8417110$ $-4.7220330$ $-4.8242510$ $-3.2009130$ $-2.1116410$ $-4.1388210$ $-5.4468100$ $-2.7484200$ $-4.4150400$ $-1.6667140$ $-3.9877650$

1.3725090 $-1.4068160$ $-1.5232230$ $-0.7429590$ $-1.3968280$ $-2.7589610$ $-2.6931070$ $-2.2334470$ $-1.8829860$ $-2.1189680$ $-1.3647920$ $-2.0951020$ $-2.4795530$ $-3.1938100$ $-0.3151290$ $-1.6828720$ $-3.2903980$ $-0.8296870$ $-1.7332170$ 2. 1030460 1.8721370 3. 5338350 4. 0491770 4. 3809310 5. 3936020 3. 3937480 5.7225710 3.9773940 6.2306670 5.7901410 6.3721030 7.2796590 0.8675590 0.9398850

TS200(R,R) G[M06-2X/6-31G(d)] = -3633.303633

$\begin{array}{lrcr}\text { TS200(R, R) } \text { G }[\mathrm{M} 06-2 \mathrm{X} / 6-31 \mathrm{G}(\mathrm{d})]=-3633.303633 \\ \mathrm{O} & 0.5459570 & 0.2436350 & -0.2762610 \\ \mathrm{O} & -1.6294120 & 0.3302780 & -1.5789520 \\ \mathrm{C} & 0.9124710 & 1.4170390 & -0.9090260 \\ \mathrm{C} & 0.0082260 & 2.4592520 & -0.9753960 \\ \mathrm{C} & 2.2434160 & 1.5210930 & -1.3916820 \\ \mathrm{C} & 0.3687130 & 3.6439490 & -1.6947920 \\ \mathrm{C} & 2.5975540 & 2.6856070 & -2.0318190 \\ \mathrm{C} & 1.6832020 & 3.7510290 & -2.2258860 \\ \mathrm{H} & 3.6174400 & 2.7998410 & -2.3923450 \\ \mathrm{C} & -2.1171780 & 1.2446860 & -0.6517570\end{array}$




$$
\begin{array}{r}
-3.4063930 \\
-1.3192550 \\
-3.8736190 \\
-1.7737070 \\
-3.0776290 \\
-4.8676650 \\
-2.7433890 \\
-1.4313170 \\
-0.9585710 \\
-3.5450050 \\
-3.1050540 \\
-0.7931250 \\
0.0521320 \\
-4.5467370 \\
-0.1570580 \\
1.1618070 \\
2.0588520 \\
-0.5446770 \\
-0.8699510 \\
1.4536190 \\
3.0705190 \\
-1.5617280 \\
3.2964900 \\
3.6971700 \\
4.0055340 \\
4.8784360 \\
5.1699380 \\
5.6484690 \\
5.2057660 \\
5.7467370 \\
-4.1976080 \\
-4.4781520 \\
-4.6787490 \\
-5.2641820 \\
-5.4548780 \\
-5.7646430 \\
-5.4884390 \\
-5.8377770 \\
3.5584400 \\
6.9502350 \\
2.8917980 \\
-3.9642130 \\
-6.6187750 \\
-4.4264200 \\
-5.7370340 \\
-6.4365690
\end{array}
$$

$\begin{array}{rr}1.0185770 & -0.0991930 \\ 2.3165400 & -0.3231900 \\ 1.9526680 & 0.7936270 \\ 3.2237130 & 0.6886440 \\ 3.0439580 & 1.2288510 \\ 1.8332900 & 1.2166140 \\ 4.9422910 & 2.7043250 \\ 5.0984130 & 2.1954790 \\ 4.2657620 & 1.2112970 \\ 3.9361650 & 2.2308680 \\ 5.6129640 & 3.4769840 \\ 5.8838100 & 2.5887240 \\ 4.3882350 & 0.8348330 \\ 3.7917290 & 2.6265190 \\ 5.8232580 & -2.6299500 \\ 5.9397190 & -3.1323820 \\ 4.9223540 & -2.9365240 \\ 4.7085270 & -1.9300470 \\ 6.6225220 & -2.8063200 \\ 6.8301770 & -3.6797880 \\ 4.9894140 & -3.3275800 \\ 4.6262860 & -1.5615800 \\ 0.4756910 & -1.1805950 \\ -0.3399420 & -2.2516730 \\ 0.4462580 & 0.0403920 \\ -1.0824920 & -2.1331880 \\ -0.3179090 & 0.1133000 \\ -1.0547870 & -0.9735170 \\ -1.6800670 & -2.9803670 \\ -0.3275830 & 1.0354670 \\ -0.2008370 & -0.4444130 \\ -1.1581260 & 0.5574920 \\ -0.3893030 & -1.7529760 \\ -2.2618000 & 0.2282610 \\ -1.5174320 & -2.0322920 \\ -2.4615270 & -1.0586750 \\ -2.9937580 & 1.0020410 \\ -1.6607440 & -3.0411600 \\ 1.2861830 & 1.2307860 \\ -1.8273670 & -0.8368460 \\ -0.4146870 & -3.5396410 \\ -1.0378370 & 1.9864560 \\ -3.6717250 & -1.3852060 \\ 0.6088670 & -2.8731830 \\ 1.2766500 & -3.3091690 \\ 0.5384390 & -3.7164800\end{array}$

-6.2241940
-5.5453820
-3.7222700
-2.7900910
-4.3603110
-3.4881110
-7.9239310
-8.5528230
-7.7181760
-8.4900690
-5.8490280
-4.9247380
-5.5830240
-6.4602850
-5.1008000
-5.5833820
-5.8703570
-4.7200410
-3.2838580
-4.0089060
-2.5277120
-2.7974860
4.1569240
5.2500840
3.8902690
3.7967090
3.8967790
3.4987320
4.9783170
3.4602790
7.7170710
8.7044670
7.1947370
7.8518970
6.7079260
6.1928750
6.0912930
7.6576670
2.6608160
3.5882780
2.2619920
1.9431820
3.5632870
4.5665200
2.9787070
3.6604430

1.7742850

2. 0238960

$-0.0475260$

$-0.5259380$

$-0.8053740$

0.7044980

$-3.6651200$

$-4.5165150$

$-3.7383070$

$-2.7451050$

$-4.9775750$

$-4.9978670$

$-5.0919590$

$-0.6590110$

0.2798640

$-1.4395950$

$-0.5501130$

$-2.3232470$

$-3.1313820$

$-2.6631340$

$-2.1393420$

2. 6986610

2. 6458670

3. 2118750

3. 2996340

0.6506950

$-0.3674030$

0.6160350

1. 2509020

$-1.9620830$

-2. 3961020

$-2.6245810$

$-0.9915780$

$-3.2115680$

$-3.1330100$

$-3.8255540$

$-3.7329490$

$-2.3330490$

$-2.4641810$

$-1.8975760$

0.3818400

$-0.0113600$

0.3089120
1.4401170
$-2.4646350$

$-4.0862600$

$-4.0659970$

$-3.7528890$

$-4.5336230$

$-4.8267170$

$-0.5808640$

$-0.8607700$

0.4928600

$-0.7541990$

$-1.1572570$

$-1.7420310$

$-0.1005490$

$-1.4445500$

2. 9462390

2. 6588190

2.9534040

3.9672440

2. 4764760

2.6228480

1.7644040

3.4410120

1.1653960

1. 2254970

0.2374440

2. 0077950

2. 5809230

2.6601380

2.7503740

3. 3856720

$-2.1537070$

$-1.9694600$

$-2.8521920$

$-2.6407230$

$-0.2191820$

0.7441450

$-0.8866900$

$-0.0606670$

$-3.9760730$

$-4.3247110$

$-3.1524410$

$-4.8018000$

$-4.6648250$

$-4.8661410$

$-5.5878800$

$-4.4043370$ 


\begin{tabular}{rrr}
-0.5866790 & -0.7401390 & -0.9511380 \\
-0.0127430 & -1.5663510 & -2.0578760 \\
-1.2119200 & -1.4432540 & 0.2337400 \\
1.9076060 & 0.0239740 & -3.3438570 \\
7.5796540 & -1.2612430 & -0.1378030 \\
2.4653450 & 1.3780650 & 1.1835710 \\
-3.7705700 & 1.3994540 & -2.4980960 \\
-6.8769320 & -3.6121020 & -2.4500690 \\
-3.2145070 & -0.2428330 & 2.0164050 \\
1.8904340 & -2.8735370 & 0.0748750 \\
0.7893900 & -3.5356380 & 0.7872720 \\
1.8015400 & -1.7936620 & -0.0594050 \\
-0.2006490 & -3.1119620 & 0.6723400 \\
1.3067010 & -3.6114580 & -1.0279420 \\
0.7226510 & -2.9538830 & -1.5213360 \\
3.3868490 & -4.8171100 & 0.6728470 \\
2.4068570 & -5.1975360 & 1.7840820 \\
0.9648780 & -4.9015740 & 1.3633130 \\
3.2994740 & -3.3212260 & 0.3675350 \\
4.4109520 & -5.0691650 & 0.9647310 \\
2.4948030 & -6.2571820 & 2.0385400 \\
0.6407390 & -5.6019870 & 0.5818270 \\
3.6578870 & -2.7327830 & 1.2225410 \\
3.9147740 & -3.0434560 & -0.4926560 \\
3.1583460 & -5.3912970 & -0.2333580 \\
2.6454360 & -4.6270940 & 2.6910120 \\
0.2554640 & -5.0188010 & 2.1872050 \\
0.2773890 & -0.6189730 & 3.0602730 \\
-0.7211410 & -0.2422530 & 2.3370660 \\
0.7843950 & 0.4565530 & 3.9456940 \\
1.4986350 & 0.1822410 & 5.1162240 \\
0.5750890 & 1.7844540 & 3.5484030 \\
1.9976680 & 1.2282010 & 5.8831560 \\
1.6559700 & -0.8481020 & 5.4180510 \\
1.0998340 & 2.8260630 & 4.3051930 \\
0.0204920 & 1.9885930 & 2.6372860 \\
1.8082910 & 2.5492660 & 5.4741870 \\
2.5414540 & 1.0146460 & 6.7974780 \\
0.9486300 & 3.8517990 & 3.9828340 \\
2.2118730 & 3.3626080 & 6.0692530 \\
-0.9469590 & -0.8532110 & 1.5182930 \\
0.9871080 & -2.1469250 & \\
-0 & & \\
\hline & -0290280
\end{tabular}

$\operatorname{TS} 201(R, R) \quad G[M 06-2 X / 6-31 G(d)]=-3633.306259$

$\begin{array}{lrrrrr}T S 201(\mathrm{R}, \mathrm{R}) & \mathrm{G}[\mathrm{M} 06-2 \mathrm{~N} / 6-31 \mathrm{G}(\mathrm{d})]=-3633.306259 & \mathrm{C} & -5.2476690 \\ \mathrm{O} & 0.6014550 & 0.7322550 & 0.1892430 & \mathrm{H} & -4.8134650 \\ \mathrm{O} & -1.5395940 & 1.1612350 & -1.0771090 & \mathrm{H} & -5.5064790\end{array}$

0.7996310
-0.2361710
2.1007230
-0.0202250
2.3237130
1.2829280
3.3208070
-2.1551460
-3.4088420
-1.5411030
-4.0471520
-2.1678630
-3.4484690
-5.0308090
-3.4869470
-2.1942710
-1.5517490
-4.0950220
-3.9854380
-1.7045270
-0.5569710
-5.0776110
-0.8104020
0.4959470
1.5166110
-1.0629030
-1.6213570
0.6809180
2.5207580
-2.0706730
3.2085610
3.6488430
3.8625190
4.7946770
5.0043620
5.5060810
5.1539100
5.5106970
-4.0045820
-4.0921630
-4.5011710
-4.7261890
-5.1148820
-5.2476690
-4.8134650
-5.5064790

2.1013250

2.9256570

2. 5752320

4. 3433200

3.9272030

4.8382430

4. 3168590

1. 4415410

0.8255070

2. 3251040

1. 1676520

2. 5899960

2.0262480

0.7566220

3. 0787130

3. 6035210

3. 3669280

2. 3030070

3. 2805200

4.1940280

3. 7658770

1.8738080

6.6307750

1190120

6.2394570

5. 2820330

7. 3314670

8.1883640

6.5982270

4. 9215630

1.6474230

1. 6034580

0.8838590

0.8688440

0.1554500

0.1616170

0.8420860

$-0.4226320$

$-0.1462760$

$-1.5123440$

0.3116080

$-2.3805860$

$-0.5977130$

$-1.9470470$

$-3.4342840$

$-0.2390990$
0.2175360

0.6091770

$-0.0947640$

0.5961990

$-0.0041740$

0.3110480

$-0.1928170$

0.1345710

0.3936360

0.9954410

1. 5600660

2. 2583130

2. 5188550

1.7708040

4.7056830

4.4664750

3.2770600

3.7530830

5.6483260

5. 2343240

3. 1107210

3. 9300840

0.8151800

0.5715830

0.3203630

0.8281670

0.9870360

0.5707510

0.1105550

1.0044850

$-0.4803030$

$-1.8202630$

0.5015260

$-2.1214300$

0.1491120

$-1.1491490$

$-3.1489300$

0.9178150

$-0.5732860$

$-0.2362890$

$-1.8098370$

$-1.1293600$

$-2.6708530$

$-2.3453290$

$-0.8738830$

$-3.6213850$ 


\begin{tabular}{|c|c|c|}
\hline $\begin{array}{l}3.3676750 \\
6.7906700 \\
2.9159730 \\
-3.5320770 \\
-5.9308550 \\
-4.4179830 \\
-5.8180660 \\
-6.4097370 \\
-6.3586880 \\
-5.7499810 \\
-3.6415570 \\
-2.6408580 \\
-4.1605310 \\
-3.5406840 \\
-5.1760210 \\
-5.6520420 \\
-5.1745870 \\
-4.1357550 \\
-7.3943180 \\
-7.9456090 \\
-7.4611180 \\
-7.8882420 \\
-4.6483480 \\
-5.1875580 \\
-5.3749280 \\
-4.2332830 \\
-2.7652420 \\
-3.4321540 \\
-1.9869530 \\
-2.2912380 \\
4.4148450 \\
5.3226430 \\
4.6960410 \\
4.0200410 \\
2.9431380 \\
2.1941390 \\
3.7980850 \\
2.5055800 \\
7.2285220 \\
8.0944520 \\
7.5246850 \\
6.4315970 \\
7.9156850 \\
7.6426800 \\
8.1193980 \\
8.8370930\end{array}$ & $\begin{array}{r}0.8095250 \\
-0.5483960 \\
2.3360790 \\
-2.0748560 \\
-2.9152650 \\
1.7706750 \\
2.3874850 \\
1.8829400 \\
2.3043100 \\
3.4472740 \\
1.9294360 \\
1.4969320 \\
1.4362430 \\
2.9898030 \\
-3.0202390 \\
-3.7568070 \\
-2.0570770 \\
-3.3190060 \\
-2.5255560 \\
-2.4669440 \\
-1.5488160 \\
-3.2598800 \\
-2.2959080 \\
-1.3719020 \\
-3.0242680 \\
-2.6863600 \\
-3.3870400 \\
-4.2022330 \\
-3.2770430 \\
-3.6872370 \\
1.3310020 \\
0.7185490 \\
2.3626650 \\
1.3041420 \\
-0.6245930 \\
-0.9737510 \\
-1.3101650 \\
-0.6753070 \\
-1.6384150 \\
-2.1730740 \\
-1.2088440 \\
-2.3664600 \\
0.4812170 \\
1.2427980 \\
0.9864300 \\
-0.0072990\end{array}$ & $\begin{array}{r}1.9374270 \\
-1.5446420 \\
-2.9349940 \\
1.0646280 \\
-3.2924600 \\
-2.2329360 \\
-2.3423080 \\
-3.1141800 \\
-1.3940910 \\
-2.6090150 \\
-3.5449100 \\
-3.4574880 \\
-4.3741790 \\
-3.7989540 \\
-4.6226080 \\
-5.2780960 \\
-5.1448570 \\
-4.4635840 \\
-3.5299930 \\
-2.5867850 \\
-4.0219440 \\
-4.1745890 \\
2.0949760 \\
2.3202480 \\
1.7165400 \\
3.0306280 \\
0.8591200 \\
0.5595230 \\
0.0983370 \\
1.8007760 \\
2.9275470 \\
2.9031710 \\
2.6937940 \\
3.9482820 \\
2.2760490 \\
1.5591950 \\
2.2438180 \\
3.2794230 \\
-0.5670930 \\
-0.9683410 \\
0.3962300 \\
-0.3839210 \\
-1.7267930 \\
-2.4632400 \\
-0.7761020 \\
-2.0600540\end{array}$ \\
\hline
\end{tabular}

3.7148890

2. 3568160

2.0733620

3.6245740

4.6481810

3.0947650

3.6781290

$-0.3139180$

0.3840910

$-0.7802210$

1. 9100230

6.6117720

2.4815200

$-3.8770330$

$-5.9167330$

$-2.8233690$

2. 3730850

1.2341780

2.2732890

0.2494200

1.8724840

1.2558840

3.8468920

2.7863330

1. 3803470

3.7619860

4.8479930

2.8705390

1.1348960

4.0371670

4. 4469620

3.7066100

2.9424120

0.6100580

0.4597700

$-0.2182150$

0.5416510

1.4983470

$-0.3640740$

1. 5471370

2. 2044020

$-0.3179310$

$-1.1031500$

0.6379700

2. 2960990
1.4686080

1. 3316390

0.4843600

1.9534150

3.6481040

3.4461150

4.1575190

4.3304310

0.1016190

0.1413010

$-1.2269580$

2. 5717340

$-1.0202880$

1.4442570

2. 3282030

$-3.9037440$

$-1.3452790$

$-2.1737700$

$-3.1006680$

$-1.3026790$

$-2.6822500$

$-1.9065510$

$-1.1048110$

$-4.0365670$

$-5.0514280$

$-2.7650720$

$-4.4668990$

$-5.9721350$

$-4.3813290$

$-2.9774230$

$-1.9975490$

$-3.7797880$

$-5.3221570$

$-5.1150720$

$-2.9802880$

$-1.9047480$

$-3.3363310$

$-4.2390600$

$-2.7462030$

$-4.5474910$

$-4.6860070$

$-3.0674140$

$-2.0467720$

-3.9657540
-5.2411420
$-4.1890400$

4.7057630

$-3.9387670$

$-4.8913390$

$-3.2966970$

$-3.6330530$

$-4.1085340$

$-2.4438790$

$-1.0093790$

$-2.3341420$

$-0.4607070$

$-2.5731720$

$-2.5212540$

2.0328480

$-1.4629110$

$-2.8160760$

1.4706470

$-1.5528890$

$-1.5908410$

$-0.8969760$

$-1.4191470$

$-2.8838970$

$-2.7950070$

$-2.4223150$

$-1.9957750$

$-2.1714260$

$-1.5754040$

$-2.3250650$

$-2.5795250$

$-3.2370810$

$-0.5336440$

$-1.9443680$

$-3.4791980$

$-0.9436940$

$-1.7354400$

2.0768380

1.8590450

3.5153950

3.9942870

4.4084190

5.3479900

3.3027720

5.7599280

4.0332970

6.2314180

5.7154750 


\begin{tabular}{|c|c|c|c|c|c|c|c|}
\hline $\mathrm{H}$ & -1.0271880 & -2.6158700 & 6.4457440 & C & 3.5987900 & 1.8793360 & 0.1851790 \\
\hline $\mathrm{H}$ & 0.6760280 & -4.2120460 & 7.2879310 & C & 2.8434690 & 2.8522480 & 0.8756620 \\
\hline $\mathrm{H}$ & -0.3857120 & -1.6578620 & 0.8569770 & C & 4.2989510 & 2.2207270 & -0.9825990 \\
\hline $\mathrm{S}$ & 1.1788860 & -3.9331580 & 0.8910870 & C & 2.8488520 & 4.1615580 & 0.3973470 \\
\hline & & & & C & 4.2701210 & 3.5467090 & -1.4248290 \\
\hline & , R) G[M06-2> & $G(d)]=-36$ & 142 & C & 3.5570620 & 4.5308990 & -0.7485710 \\
\hline 0 & 0.1195410 & -1.1906140 & 0.1472690 & $\mathrm{H}$ & 2.2718190 & 4.9172540 & 0.9251200 \\
\hline 0 & 2.0972770 & -0.1636280 & -1.0098330 & $\mathrm{H}$ & 4.8169680 & 3.8193030 & -2.3259190 \\
\hline C & 0.5257970 & -2.4546170 & -0.2448650 & C & -1.9127030 & -3.1112550 & 1.8434370 \\
\hline C & 1.8513850 & -2.8012590 & -0.1011540 & $\mathrm{c}$ & -5.8178950 & -1.0124210 & -0.5573790 \\
\hline C & -0.4850190 & -3.3400020 & -0.7135210 & C & -1.9240540 & -2.7641560 & -3.2654020 \\
\hline C & 2.2616630 & -4.0892750 & -0.5820490 & $\mathrm{C}$ & 2.0396010 & 2.5084560 & 2.1233470 \\
\hline C & -0.0964690 & -4.6033960 & -1.0777650 & C & 3.5253320 & 5.9608510 & -1.2543080 \\
\hline C & 1.2706260 & -4.9950640 & -1.0564890 & C & 5.0929240 & 1.1955240 & -1.7766950 \\
\hline H & -0.8403210 & -5.3283730 & -1.3969590 & $\mathrm{C}$ & 6.5936410 & 1.5053240 & -1.7220050 \\
\hline C & 2.8292760 & -0.5286260 & 0.1067200 & $\mathrm{H}$ & 6.8084620 & 2.4782000 & -2.1780100 \\
\hline C & 3.6464570 & 0.4739230 & 0.6949440 & $\mathrm{H}$ & 6.9511520 & 1.5305870 & -0.6876180 \\
\hline C & 2.7784360 & -1.8343390 & 0.5502310 & $\mathrm{H}$ & 7.1632010 & 0.7445300 & -2.2657890 \\
\hline C & 4.4695100 & 0.0974190 & 1.7257500 & C & 4.6003150 & 1.1034740 & -3.2250380 \\
\hline C & 3.5606000 & -2.1874800 & 1.7019450 & $\mathrm{H}$ & 3.5325330 & 0.8682090 & -3.2563300 \\
\hline C & 4.4407150 & -1.2173870 & 2.2602920 & $\mathrm{H}$ & 4.7613240 & 2.0454580 & -3.7604510 \\
\hline $\mathrm{H}$ & 5.1470260 & 0.8256940 & 2.1643590 & $\mathrm{H}$ & 5.1438910 & 0.3184780 & -3.7609530 \\
\hline C & 5.1609750 & -2.8068590 & 3.9477080 & C & 4.0948820 & 6.9372480 & -0.2191820 \\
\hline C & 4.2504230 & -3.7566390 & 3.4268660 & $\mathrm{H}$ & 4.1100330 & 7.9562150 & -0.6194140 \\
\hline C & 3.4718390 & -3.4570350 & 2.3369010 & $\mathrm{H}$ & 3.4816770 & 6.9429740 & 0.6887120 \\
\hline C & 5.2458030 & -1.5630310 & 3.3782600 & $\mathrm{H}$ & 5.1151490 & 6.6619100 & 0.0640260 \\
\hline $\mathrm{H}$ & 5.7752910 & -3.0607900 & 4.8055530 & C & 2.1053600 & 6.3718040 & -1.6624530 \\
\hline $\mathrm{H}$ & 4.1620030 & -4.7300670 & 3.8989150 & $\mathrm{H}$ & 1.6991600 & 5.6905960 & -2.4163650 \\
\hline $\mathrm{H}$ & 2.7696210 & -4.1918590 & 1.9593280 & $\mathrm{H}$ & 1.4338510 & 6.3567670 & -0.7966130 \\
\hline $\mathrm{H}$ & 5.9200390 & -0.8120280 & 3.7812740 & $\mathrm{H}$ & 2.1010630 & 7. 3865280 & -2.0737570 \\
\hline C & 3.9773850 & -5.7303010 & -1.0965920 & C & 2.8732970 & 2.7341720 & 3.3927100 \\
\hline C & 2.9884960 & -6.6437970 & -1.5323330 & $\mathrm{H}$ & 3.7747680 & 2.1163000 & 3.4049210 \\
\hline C & 1.6665790 & -6.2794830 & -1.5149090 & $\mathrm{H}$ & 3.1809420 & 3.7842070 & 3.4587280 \\
\hline C & 3.6244670 & -4.4875810 & -0.6324530 & $\mathrm{H}$ & 2.2841480 & 2.4959630 & 4.2848590 \\
\hline $\mathrm{H}$ & 5.0244260 & -6.0137660 & -1.1350920 & C & 0.7310190 & 3.3005920 & 2.2287450 \\
\hline $\mathrm{H}$ & 3.2811770 & -7.6254950 & -1.8906480 & $\mathrm{H}$ & 0.9148720 & 4.3453670 & 2.5028170 \\
\hline $\mathrm{H}$ & 0.8982360 & -6.9641730 & -1.8638260 & $\mathrm{H}$ & 0.1782480 & 3.2826680 & 1.2855560 \\
\hline $\mathrm{H}$ & 4.3899120 & -3.7898850 & -0.3086200 & $\mathrm{H}$ & 0.0980420 & 2.8671980 & 3.0110260 \\
\hline C & -1.8967320 & -2.8461560 & -0.7082640 & C & -2.6664790 & -4.3418090 & 2.3658350 \\
\hline C & -2.5478190 & -2.4917370 & -1.9052650 & $\mathrm{H}$ & -3.7114420 & -4.0922420 & 2.5821590 \\
\hline C & -2.5332730 & -2.6462490 & 0.5334730 & $\mathrm{H}$ & -2.6577700 & -5.1502950 & 1.6277020 \\
\hline C & -3.8199260 & -1.9152320 & -1.8271150 & $\mathrm{H}$ & -2.2069930 & -4.7109670 & 3.2886700 \\
\hline C & -3.7944680 & -2.0533030 & 0.5601040 & C & -1.8682850 & -2.0047910 & 2.8995980 \\
\hline C & -4.4475680 & -1.6615420 & -0.6084650 & $\mathrm{H}$ & -1.3338990 & -1.1299410 & 2.5198990 \\
\hline $\mathrm{H}$ & -4 & -1.6446060 & -2.7444880 & $\mathrm{H}$ & -2.8755530 & -1.6871000 & 3.1919710 \\
\hline $\mathrm{H}$ & -4.2 & -1.8930 & 1.5208600 & $\mathrm{H}$ & -1.3610040 & -2.3653360 & 3.8009820 \\
\hline
\end{tabular}




$\begin{array}{rrr}-5.8722010 & 0.1375090 & 0.4541610 \\ -6.8294730 & 0.6651350 & 0.3861560 \\ -5.7696980 & -0.2296570 & 1.4814290 \\ -5.0668570 & 0.8608510 & 0.2821300 \\ -6.9002390 & -2.0541400 & -0.2466970 \\ -6.8967520 & -2.8589350 & -0.9878520 \\ -6.7264590 & -2.5004040 & 0.7390510 \\ -7.8930500 & -1.5923530 & -0.2406380 \\ -2.2257980 & -1.6825080 & -4.3052790 \\ -3.2797370 & -1.6907780 & -4.6053000 \\ -1.9803380 & -0.6871780 & -3.9291460 \\ -1.6323320 & -1.8635130 & -5.2071830 \\ -2.3961240 & -4.1259120 & -3.7967220 \\ -3.4827640 & -4.1161080 & -3.9413760 \\ -1.9263790 & -4.3464230 & -4.7611170 \\ -2.1599510 & -4.9396220 & -3.1059210 \\ 0.4907590 & 0.0261790 & -0.8687940 \\ -0.0739580 & -0.1840380 & -2.2444790 \\ 0.1363970 & 1.2845610 & -0.1149130 \\ -0.8382000 & -2.7978830 & -3.1315550 \\ -6.0213330 & -0.5996830 & -1.5545910 \\ -0.8810240 & -3.4201650 & 1.6519550 \\ 4.9434120 & 0.2132650 & -1.3177930 \\ 4.1605200 & 6.0058390 & -2.1480150 \\ 1.7750340 & 1.4466850 & 2.0641540 \\ -2.6464930 & 1.5102420 & -2.1172580 \\ -1.9095180 & 2.7322670 & -1.7768120 \\ -2.4934270 & 0.6535140 & -1.4536810 \\ -0.9599560 & 2.6094450 & -1.2690830 \\ -1.6680310 & 1.5485730 & -3.1812090 \\ -0.9265560 & 0.9023120 & -2.8891900 \\ -4.2250890 & 2.8895520 & -3.5166020 \\ -3.7557970 & 4.1414210 & -2.7723750 \\ -2.2721000 & 4.0439180 & -2.4066760 \\ -4.0556030 & 1.6439150 & -2.6429730 \\ -5.2753700 & 2.9912710 & -3.8038660 \\ -3.9160630 & 5.0361530 & -3.3801900 \\ -1.6491320 & 4.1349680 & -3.3049060 \\ -4.7293520 & 1.6945020 & -1.7783120 \\ -4.3001170 & 0.7310040 & -3.1969120 \\ -3.6461260 & 2.7690180 & -4.4397110 \\ -4.3494760 & 4.2626560 & -1.8574570 \\ -1.9591780 & 4.8489220 & -1.7360260 \\ -2.6745050 & 2.0311070 & 1.6476200 \\ -1.7136030 & 1.1683190 & 1.5864200 \\ -3.5407080 & 1.8559610 & 2.8374720\end{array}$

$\begin{array}{ll}\mathrm{C} & -4.8321910 \\ \mathrm{C} & -3.0544270 \\ \mathrm{C} & -5.6287460 \\ \mathrm{H} & -5.2097740 \\ \mathrm{C} & -3.8516060 \\ \mathrm{H} & -2.0507390 \\ \mathrm{C} & -5.1399110 \\ \mathrm{H} & -6.6329830 \\ \mathrm{H} & -3.4671820 \\ \mathrm{H} & -5.7637950 \\ \mathrm{H} & -1.0108000 \\ \mathrm{~S} & -2.9434290\end{array}$
2. 3957280
1. 1249840
2. 1967510
2. 9527850
0.9382260
0.7165940
1.4693820
2. 6065160
0.3782130
1. 3174540
1.2888110
3. 2619510

2.8846880

3.9299680

4.0052360

2. 0336210

5.0533890

3.8936930

5.0905670

4.0315720

5.8995770

5. 9657710

0.8352250

144

0.5345250

$\operatorname{TS} 203(\mathrm{R}, \mathrm{R}) \mathrm{G}[\mathrm{M} 06-2 \mathrm{X} / 6-31 \mathrm{G}(\mathrm{d})]=-3633.306804$

$\begin{array}{lrcr}\text { TS203(R, R }) \text { G }[M 06-2 X / 6-31 G(\mathrm{~d})]=-3633.306804 \\ \mathrm{O} & 0.6449830 & 0.7349840 & 0.2343960 \\ \mathrm{O} & -1.3672020 & 1.2621800 & -1.1927840 \\ \mathrm{C} & 0.9250720 & 2.0898720 & 0.2483980 \\ \mathrm{C} & -0.0818380 & 2.9832580 & 0.5540760 \\ \mathrm{C} & 2.2696950 & 2.4771040 & 0.0100180 \\ \mathrm{C} & 0.2224760 & 4.3846920 & 0.5334640 \\ \mathrm{C} & 2.5703820 & 3.8142190 & 0.0949990 \\ \mathrm{C} & 1.5703430 & 4.7927200 & 0.3295890 \\ \mathrm{H} & 3.5993250 & 4.1389380 & -0.0369030 \\ \mathrm{C} & -2.0500730 & 1.6077230 & -0.0352780 \\ \mathrm{C} & -3.3569000 & 1.0781910 & 0.1395200 \\ \mathrm{C} & -1.4456170 & 2.4700970 & 0.8540200 \\ \mathrm{C} & -4.0554540 & 1.4839570 & 1.2496620 \\ \mathrm{C} & -2.1447430 & 2.7993080 & 2.0625930 \\ \mathrm{C} & -3.4742970 & 2.3222880 & 2.2366220 \\ \mathrm{H} & -5.0764970 & 1.1405790 & 1.3928130 \\ \mathrm{C} & -3.6049740 & 3.4197970 & 4.3975680 \\ \mathrm{C} & -2.2671700 & 3.8569960 & 4.2467750 \\ \mathrm{C} & -1.5560340 & 3.5563590 & 3.1120850 \\ \mathrm{C} & -4.1903250 & 2.6645280 & 3.4148230 \\ \mathrm{H} & -4.1571000 & 3.6720180 & 5.2972150 \\ \mathrm{H} & -1.7978020 & 4.4308090 & 5.0396100 \\ \mathrm{H} & -0.5279840 & 3.8879350 & 3.0140450 \\ \mathrm{H} & -5.2088490 & 2.3019200 & 3.5250240 \\ \mathrm{C} & -0.4345150 & 6.7206640 & 0.6638820 \\ \mathrm{C} & 0.9134850 & 7.1223660 & 0.5035130 \\ \mathrm{C} & 1.8909680 & 6.1765890 & 0.3349100 \\ \mathrm{C} & -0.7720320 & 5.3905630 & 0.6792040 \\ \mathrm{H} & -1.2093830 & 7.4734030 & 0.7694710 \\ \mathrm{H} & 1.1653900 & 8.1779560 & 0.5002550 \\ \mathrm{H} & 2.9276280 & 6.4681120 & 0.1888060 \\ \mathrm{H} & -1.8101350 & 5.0968220 & 0.7916840\end{array}$




$\begin{array}{rrr}3.3355390 & 1.4751690 & -0.2996460 \\ 3.8562380 & 1.3909640 & -1.6043050 \\ 3.8686850 & 0.6708020 & 0.7274280 \\ 4.9675650 & 0.5730500 & -1.8370990 \\ 4.9669980 & -0.1393330 & 0.4433010 \\ 5.5534980 & -0.1800820 & -0.8230030 \\ 5.3767990 & 0.5256150 & -2.8420270 \\ 5.3895160 & -0.7588800 & 1.2339560 \\ -3.9453160 & 0.1306620 & -0.8559320 \\ -4.1498660 & -1.2231170 & -0.5075210 \\ -4.3219790 & 0.5981510 & -2.1274800 \\ -4.7772030 & -2.0616760 & -1.4286880 \\ -4.9357360 & -0.2861170 & -3.0186980 \\ -5.1823450 & -1.6143050 & -2.6870580 \\ -4.9514540 & -3.1011840 & -1.1595900 \\ -5.2384380 & 0.0742220 & -4.0003450 \\ 3.2831890 & 0.6454920 & 2.1305800 \\ 6.7962130 & -1.0293860 & -1.0349600 \\ 3.2486490 & 2.1615710 & -2.7682480 \\ -3.7213070 & -1.7966370 & 0.8379690 \\ -5.8640740 & -2.5514800 & -3.6659460 \\ -4.1157360 & 2.0411120 & -2.5627700 \\ -5.4626930 & 2.7466190 & -2.7642850 \\ -6.0385960 & 2.2703780 & -3.5654160 \\ -6.0636450 & 2.7130470 & -1.8499150 \\ -5.3084980 & 3.7955830 & -3.0377940 \\ -3.2535360 & 2.1283280 & -3.8270420 \\ -2.2918390 & 1.6298300 & -3.6760700 \\ -3.7549530 & 1.6612170 & -4.6817150 \\ -3.0646320 & 3.1758400 & -4.0840790 \\ -7.1926620 & -3.0726870 & -3.1062720 \\ -7.6973450 & -3.7075340 & -3.8417820 \\ -7.0256110 & -3.6719120 & -2.2043110 \\ -7.8618120 & -2.2476460 & -2.8445220 \\ -4.9470290 & -3.7162310 & -4.0561680 \\ -4.0042860 & -3.3526160 & -4.4753900 \\ -4.7124880 & -4.3349690 & -3.1828840 \\ -5.4333380 & -4.3559060 & -4.7998570 \\ -4.9199210 & -1.9238770 & 1.7886190 \\ -5.4099760 & -0.9619580 & 1.9616010 \\ -5.6655810 & -2.6090260 & 1.3687530 \\ -4.5996860 & -2.3234810 & 2.7571110 \\ -3.0318090 & -3.1600310 & 0.7049880 \\ -3.7280210 & -3.9337460 & 0.3646990 \\ -2.1938410 & -3.1138850 & 0.0032200 \\ -2.6506620 & -3.4769170 & 1.6826250\end{array}$

4.3077010
5.1652280
4.6812620
3.8530750
2.7307030
1.9924910
3.5300440
2.2408530
8.0174850
8.9129260
8.1996260
7.8634330
7.0676150
6.1996570
7.3346610
7.9078300
3.1045280
4.0767340
2.6205940
2.4964660
4.0705640
5.0962630
3.6305300
4.1189640
-0.2175840
0.5756640
-0.8026430
2.2419200
6.6422130
2.4447830
-3.5860370
-6.0822260
-2.9955180
2.3457440
1.1533160
2.2533640
0.1886930
1.9723980
1.4054060
3.7539260
2.5983600
1.2512990
3.6894530
4.7136580
2.6653690
1.0932290

1.0938770

0.4130150

2. 0986020

1. 1065280

$-0.7485740$

$-1.0432490$

$-1.4988060$

$-0.7580830$

$-0.3244670$

$-0.9465330$

0.6243220

$-0.1076540$

$-1.3851820$

$-1.8518990$

$-0.4949270$

$-2.0828230$

1. 2911680

1.0815490

0.3406800

1.8136040

3.4145700

3. 1362090

3. 9476720

. 1042460

0.1319930

0.0995880

$-1.1534870$

2.4732990

$-1.9663630$

1.3473330

2. 5740730

$-1.9739240$

$-1.1080060$

$-2.3294110$

$-3.1780350$

$-1.4448950$

$-2.6924030$

$-2.0461320$

$-1.2045810$

$-4.2937040$

$-5.2320300$

$-4.5643570$

$-3.0116330$

$-4.7888360$

$-6.1624440$

$-4.4698460$
3.1786390

3. 2100920

2.9573420

4.1744480

2. 4506740

1. 6988920

2.4637430

3. 4308160

$-0.4267440$

$-0.5261380$

$-0.9435600$

0.6345050

$-2.4967560$

$-2.9752510$

$-3.0765390$

$-2.5603660$

$-4.0223360$

4.4815230

$-3.7869360$

$-4.7675640$

$-3.0978080$

$-3.3664110$

$-3.9472060$

$-2.2505920$

$-1.0157800$

$-2.2858360$

$-0.4790500$

$-2.4718950$

$-0.4807770$

2.1702070

$-1.7680380$

$-4.5731440$

1. 2835170

$-1.3173110$

$-1.4389350$

$-0.6787200$

$-1.3489330$

$-2.6864340$

$-2.6543870$

$-2.0584900$

$-1.7099040$

$-1.9961250$

$-1.2259190$

$-1.8841970$

$-2.2803360$

$-3.0779680$ 


\begin{tabular}{|c|c|c|c|c|c|c|c|}
\hline $\mathrm{H}$ & 3.8740200 & -3.2290450 & -0.1654350 & C & -0.1466520 & 5.8777380 & -2.4980390 \\
\hline $\mathrm{H}$ & 4.4469660 & -2.2937410 & -1.5469850 & $\mathrm{c}$ & 1.1545590 & 5.9840150 & -3.0463720 \\
\hline $\mathrm{H}$ & 3.7115130 & -4.0373600 & -3.1240980 & $\mathrm{C}$ & 2.0393430 & 4.9464300 & -2.9092640 \\
\hline $\mathrm{H}$ & 2.6551160 & -5.5013120 & -0.6473990 & $\mathrm{C}$ & -0.5282570 & 4.7526470 & -1.8114540 \\
\hline $\mathrm{H}$ & 0.4093260 & -5.1487320 & -1.6151400 & $\mathrm{H}$ & -0.8511700 & 6.6931140 & -2.6282940 \\
\hline C & 0.1049360 & -2.9588240 & 2.1572940 & $\mathrm{H}$ & 1.4422370 & 6.8827200 & -3.5823710 \\
\hline 0 & -0.4753280 & -1.8401600 & 1.8821070 & $\mathrm{H}$ & 3.0368740 & 5.0054690 & -3.3362940 \\
\hline C & 0.0391610 & -3.3107530 & 3.5976850 & $\mathrm{H}$ & -1.5324050 & 4.6789900 & -1.4075930 \\
\hline C & 0.8954710 & -4.2654140 & 4.1587770 & C & 3.2402810 & 0.4212670 & -1.3176670 \\
\hline C & -0.9065910 & -2.6654630 & 4.4069800 & C & 3.5374720 & -0.4115250 & -2.4087850 \\
\hline C & 0.8059690 & -4.5703930 & 5.5111500 & $\mathrm{C}$ & 4.0027070 & 0.3438200 & -0.1313220 \\
\hline $\mathrm{H}$ & 1.6329740 & -4.7552610 & 3.5322550 & c & 4.6660920 & -1.2361180 & -2.3369890 \\
\hline C & -0.9999520 & -2.9838500 & 5.7568000 & $\mathrm{C}$ & 5.1114170 & -0.5020170 & -0.1044510 \\
\hline $\mathrm{H}$ & -1.5673460 & -1.9257690 & 3.9680610 & $\mathrm{C}$ & 5.4802200 & -1.2747070 & -1.2088700 \\
\hline c & -0.1432890 & -3.9339400 & 6.3105610 & $\mathrm{H}$ & 4.9232930 & -1.8606200 & -3.1903300 \\
\hline $\mathrm{H}$ & 1.4779700 & -5.3044560 & 5.9432500 & $\mathrm{H}$ & 5.7195200 & -0.5465860 & 0.7951720 \\
\hline H & -1.7404650 & -2.4897800 & 6.3771210 & C & -4.2224960 & -0.1155320 & -0.3170790 \\
\hline $\mathrm{H}$ & -0.2139110 & -4.1779330 & 7.3659280 & C & -4.4803950 & -1.1005410 & 0.6637180 \\
\hline $\mathrm{H}$ & -0.5442370 & -1.5914620 & 0.8687920 & $\mathrm{C}$ & -4.7635350 & -0.2486830 & -1.6086810 \\
\hline S & 0.8522430 & -3.9688920 & 1.0381960 & $\mathrm{C}$ & -5.3042900 & -2.1756370 & 0.3326750 \\
\hline \multirow{2}{*}{\multicolumn{4}{|c|}{$\begin{array}{l}144 \\
\text { TS204 }(R, R) G[M 06-2 X / 6-31 G(d)]=-3633.305020\end{array}$}} & C & -5.5762240 & -1.3500030 & -1.8912460 \\
\hline & & & & $\mathrm{C}$ & -5.8646210 & -2.3202660 & -0.9369890 \\
\hline 0 & 0.5430760 & 0.2257940 & -0.3130730 & $\mathrm{H}$ & -5.5104630 & -2.9293280 & 1.0904330 \\
\hline 0 & -1.6810340 & 0.3890530 & -1.5260680 & $\mathrm{H}$ & -6.0048260 & -1.4508180 & -2.8867820 \\
\hline c & 0.9028190 & 1.4108770 & -0.9285440 & C & 3.6691160 & 1.2100720 & 1.0771420 \\
\hline C & 0.0171830 & 2.4708350 & -0.9327560 & C & 6.7508080 & -2.1058110 & -1.1925780 \\
\hline C & 2.2155270 & 1.5037960 & -1.4611310 & $\mathrm{C}$ & 2.6802050 & -0.4176790 & -3.6648750 \\
\hline C & 0.3734830 & 3.6667450 & -1.6363560 & C & -3.9023430 & -1.0373130 & 2.0716720 \\
\hline C & 2.5692750 & 2.6772860 & -2.0835110 & C & -6.7570220 & -3.5016510 & -1.2669430 \\
\hline $\mathrm{C}$ & 1.6688270 & 3.7642680 & -2.2142360 & $\mathrm{C}$ & -4.5336250 & 0.7804890 & -2.7054070 \\
\hline $\mathrm{H}$ & 3.5760530 & 2.7812480 & -2.4819680 & $\mathrm{C}$ & -5.8459280 & 1.4851440 & -3.0726170 \\
\hline C & -2.1181870 & 1.2897470 & -0.5605400 & $\mathrm{H}$ & -6.5731090 & 0.7731830 & -3.4782460 \\
\hline C & -3.3915260 & 1.0758620 & 0.0324380 & $\mathrm{H}$ & -6.2939060 & 1.9634470 & -2.1958540 \\
\hline C & -1.2876540 & 2.3372930 & -0.2348650 & $\mathrm{H}$ & -5.6677040 & 2.2538170 & -3.8317830 \\
\hline C & -3.8077140 & 1.9963140 & 0.9636730 & C & -3.8849110 & 0.1499290 & -3.9426520 \\
\hline C & -1.6880190 & 3.2289980 & 0.8131540 & $\mathrm{H}$ & -2.9489090 & -0.3495760 & -3.6781530 \\
\hline $\mathrm{C}$ & -2.9752700 & 3.0614650 & 1.3955240 & $\mathrm{H}$ & -4.5510670 & -0.5854190 & -4.4070350 \\
\hline $\mathrm{H}$ & -4.7891330 & 8190 & 1.4175370 & $\mathrm{H}$ & -3.6682530 & 0.9205650 & -4.6898200 \\
\hline C & -2.5535450 & 4.9204420 & 2.8983260 & C & -8.0185280 & -3.5082140 & -0.3959050 \\
\hline C & -1.2575870 & 5.0637950 & 2.3464100 & $\mathrm{H}$ & -8.6761960 & -4.3369920 & -0.6775400 \\
\hline C & -0.8355170 & 43490 & 1.3286920 & $\mathrm{H}$ & -7.7592730 & -3.6299560 & 0.6616980 \\
\hline $\mathrm{C}$ & -3.3900960 & 3.9395390 & 2.4325210 & $\mathrm{H}$ & -8.5762130 & -2.5728600 & -0.5012930 \\
\hline $\mathrm{H}$ & -2.8752110 & 07270 & 3.6971740 & $\mathrm{C}$ & -6.0000670 & -4.8279560 & -1.1333830 \\
\hline $\mathrm{H}$ & -0.5913330 & 89040 & 2.7329780 & $\mathrm{H}$ & -5.1083860 & -4.8387510 & -1.7670110 \\
\hline $\mathrm{H}$ & 0.1632490 & 4.3573110 & 0.9189970 & $\mathrm{H}$ & -5.6810150 & -4.9899650 & -0.0978210 \\
\hline $\mathrm{H}$ & -4.3798940 & 046630 & 2.8602770 & $\mathrm{H}$ & -6.6410540 & -5.6672260 & -1.4223770 \\
\hline
\end{tabular}




$\begin{array}{rr}-4.9842040 & -0.6511550 \\ -5.4467960 & 0.3110580 \\ -5.7782780 & -1.4066780 \\ -4.5573030 & -0.5858370 \\ -3.2432510 & -2.3558690 \\ -3.9871330 & -3.1440100 \\ -2.5305410 & -2.7010350 \\ -2.7092490 & -2.2116200 \\ 4.3411670 & 2.5868270 \\ 5.4311570 & 2.4713760 \\ 4.0479920 & 3.1160390 \\ 4.0687830 & 3.2064980 \\ 4.0486150 & 0.5624360 \\ 3.6245810 & -0.4432400 \\ 5.1355440 & 0.4949250 \\ 3.6682250 & 1.1748580 \\ 6.8129050 & -3.0798330 \\ 7.7450690 & -3.6529820 \\ 6.7842710 & -2.5454340 \\ 5.9790160 & -3.7875590 \\ 7.9819360 & -1.1898370 \\ 7.9622440 & -0.5046470 \\ 8.0142340 & -0.5892990 \\ 8.9025640 & -1.7802260 \\ 2.3863770 & -1.8418400 \\ 3.2800270 & -2.3167120 \\ 2.0154410 & -2.4663600 \\ 1.6249200 & -1.8186860 \\ 3.3349210 & 0.3996980 \\ 4.3168160 & -0.0171480 \\ 2.7146540 & 0.3787190 \\ 3.4754410 & 1.4440680 \\ -0.6407270 & -0.7164140 \\ -0.1350430 & -1.5428530 \\ -1.2427210 & -1.4179960 \\ 1.7190780 & 0.0442900 \\ 6.7674120 & -2.6953730 \\ 2.5817010 & 1.3624630 \\ -3.8494420 & 1.5466000 \\ -7.0686660 & -3.3937280 \\ -3.1268370 & -0.2669510 \\ 1.8477070 & -3.0150930 \\ 0.7351260 & -3.5754250 \\ 1.8485730 & -1.9303400 \\ -0.2193410 & -3.0675860 \\ 1.1290290 & -3.6934660\end{array}$

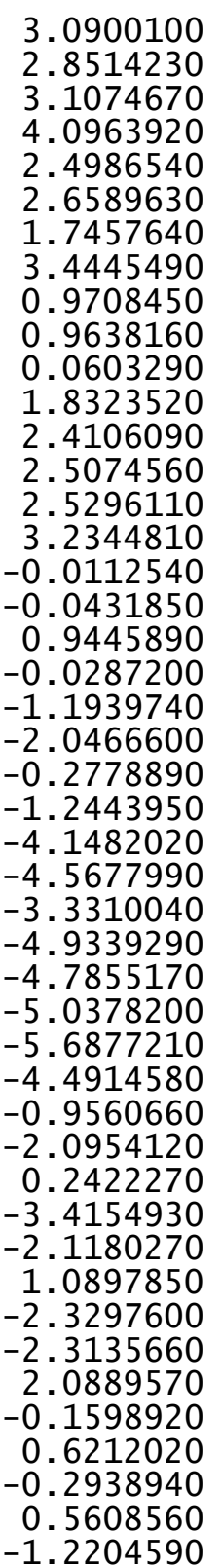

$\begin{array}{lr}\mathrm{H} & 0.5586240 \\ \mathrm{C} & 3.1883640 \\ \mathrm{C} & 2.2464940 \\ \mathrm{C} & 0.8169210 \\ \mathrm{C} & 3.2240920 \\ \mathrm{H} & 4.1998270 \\ \mathrm{H} & 2.2553060 \\ \mathrm{H} & 0.3943050 \\ \mathrm{H} & 3.6954290 \\ \mathrm{H} & 3.8067120 \\ \mathrm{H} & 2.8529600 \\ \mathrm{H} & 2.5857060 \\ \mathrm{H} & 0.1425050 \\ \mathrm{C} & 0.4329300 \\ \mathrm{O} & -0.6154940 \\ \mathrm{C} & 0.9925240 \\ \mathrm{C} & 1.7566740 \\ \mathrm{C} & 0.7758610 \\ \mathrm{C} & 2.2969630 \\ \mathrm{H} & 1.9187680 \\ \mathrm{C} & 1.3424760 \\ \mathrm{H} & 0.1824500 \\ \mathrm{C} & 2.0994980 \\ \mathrm{H} & 2.8786870 \\ \mathrm{H} & 1.1853590 \\ \mathrm{H} & 2.5347730 \\ \mathrm{H} & -0.8950420 \\ \mathrm{~S} & 1.1524780 \\ \mathrm{H} & \end{array}$
$-2.9871720$
$-5.0924910$
$-5.3693760$
$-4.9503490$
$-3.5979030$
$-5.4377900$
$-6.4293560$
$-5.6219450$
$-3.0365740$
$-3.3945760$
$-5.6525800$
$-4.8108300$
$-5.0008280$
$-0.6823040$
$-0.2840770$
0.3583740
0.0382080
1.7004680
1.0532830
$-1.0032300$
2. 7113800
1. 9397180
2. 3890580
0.8042320
3. 7488560
3. 1784640
$-0.8655650$
$-2.1988240$

144

$-1.6590220$

0.3733770

1. 5456060

1. 1970000

0.0477060

0.6083320

1.8131650

0.4376020

0.8655270

$-0.8558220$

$-0.5079120$

2. 4276580

2. 0561270

2. 9817450

2. 3459070

3.8771060

5.0034470

3.5362070

5.7838520

5. 2616020

4.3043570

2. 6586410

5.4304700

6.6652580

4. 0253890

6.0353250

1.5217110

2. 8374070

$\operatorname{TS} 205(R, R) G[M 06-2 X / 6-31 G(d)]=-3633.303644$

$\begin{array}{lrcr}\text { O } & 0.2633790 & 0.9844530 & -0.0278530 \\ \text { O } & -1.9995210 & 0.4945090 & -1.0236870 \\ \text { C } & 0.1771490 & 2.2900820 & -0.4778170 \\ \text { C } & -0.9959450 & 2.9887420 & -0.2870050 \\ \text { C } & 1.3542000 & 2.8605410 & -1.0374060 \\ \text { C } & -1.0824890 & 4.3173000 & -0.8212620 \\ \text { C } & 1.2904880 & 4.1656890 & -1.4538080 \\ \text { C } & 0.0791950 & 4.9068440 & -1.3947200 \\ \text { H } & 2.1818320 & 4.6536390 & -1.8390370 \\ \text { C } & -2.5466730 & 1.0924600 & 0.0991030 \\ \text { C } & -3.5830090 & 0.3898390 & 0.7707630 \\ \text { C } & -2.1055650 & 2.3455080 & 0.4685960 \\ \text { C } & -4.2005040 & 1.0245660 & 1.8191110 \\ \text { C } & -2.6836130 & 2.9507390 & 1.6351550 \\ \text { C } & -3.7652090 & 2.2926420 & 2.2859730 \\ \text { H } & -5.0343870 & 0.5410240 & 2.3213420\end{array}$




-3.9012440
-2.7944040
-2.2015680
-4.3691170
-4.3645270
-2.4064990
-1.3460970
-5.2018740
-2.3348280
-1.1701040
0.0078110
-2.2928860
-3.2706210
-1.2164700
0.9061590
-3.1932040
2.6057400
3.0697890
3.2872370
4.2118250
4.4083530
4.8805770
4.5927540
4.9296600
-3.9917950
-3.5794330
-4.8020660
-4.0359400
-5.2274820
-4.8627200
-3.7341840
-5.8630620
2.8806650
6.1177930
2.3868150
-2.6754230
-5.3430270
-5.2436840
-6.7611510
-7.2955970
-7.0743560
-7.0690380
-4.7935330
-3.7085220
-5.2666710
-5.0711810

4.0837920

4.7155860

4.1662680

2.8950280

4.5341850

5.6409470

4.6561100

2. 3833460

6.3325560

6.9309490

6.2295840

5.0617690

6.8824720

7.9388870

6.6693870

4.6079420

2.0420920

1.4466360

1.8330760

0.6420670

1.0031460

0.3879850

0.1888480

0.8397560

$-0.9777700$

$-2.1119520$

$-1.1211630$

$-3.3673930$

$-2.3965970$

$-3.5323420$

$-4.2509160$

$-2.5047000$

2.5353840

$-0.4901220$

1.6988700

$-2.0059190$

$-4.9097510$

0.0725630

0.2721890

$-0.5948990$

0.4074860

1.1543350

$-0.0642370$

$-0.1893340$

$-0.9262790$

0.8298980

3.9186080
3.3033770
2.1939810
3.4216870
4.7905720
3.7174300
1.7421200
3.8968060
-1.3429430
-1.8798570
-1.9064770
-0.8258410
-1.3454860
-2.2794700
-2.3313560
-0.4246920
-1.0523650
-2.2408430
0.1627940
-2.1809900
0.1748850
-0.9836900
-3.0937930
1.1156330
0.3238900
1.0510500
-0.8177900
0.6414570
-1.1893870
-0.4675600
1.1990470
-2.0668270
1.4508240
-0.9594510
-3.5768350
2.2728030
-0.8842010
-1.6506050
-1.5560320
-1.9599200
-0.5158090
-2.1269020
-3.1091050
-3.1687590
-3.5919610
-3.6768550

-4.8292490
-5.1408780
-5.2298510
-3.7372870
-6.8715990
-7.2451840
-7.3336020
-7.2012190
-3.5043280
-4.1867130
-4.1027770
-2.8478690
-1.6570450
-2.1390080
-1.1229600
-0.9250330
3.9036190
4.8920140
3.9968890
3.6025380
2.7013780
1.9532840
3.6370920
2.3707360
6.1273180
6.9537200
6.2614820
5.1887790
7.3865490
7.4124720
7.4202330
8.2835130
2.3883900
3.3951560
1.9900440
1.7643830
3.0480230
4.0998560
2.5464200
3.0157370
-0.5194300
0.0106630
-0.5329450
1.3416560
6.1152000
1.9178970

-5.2813480
-6.2963620
-4.5969620
-5.2310500
-5.0096710
-4.7657850
-4.3169090
-6.0220790
-1.9621460
-1.1084060
-2.8755810
-1.8933800
-3.1490670
-4.0969500
-3.2817900
-2.9288610
3.6275430
3.1894670
4.3476720
4.1684470
1.5622960
0.8025720
1.0502530
2.1015440
-1.4606580
-2.1719070
-0.9274960
-2.0230660
0.3727150
1.0366680
0.9939250
-0.2551650
0.4798250
0.2492030
-0.4046230
0.6824500
2.8841220
2.6572380
3.0838290
3.7961570
-0.1584580
-0.2894780
-1.3699650
1.9518970
-1.0818410
3.0296360

$-2.2799050$

$-2.5471790$

$-3.0359260$

$-2.3244470$

$-0.8255960$

0.1733620

$-1.5377660$

$-1.0807750$

3.5643610

3.5827190

3.6597990

4.4385260

2. 3635270

2.6263310

1.4184740

3.1479680

1. 7908270

1.9703110

0.9713690

2. 6940290

2. 6181290

2.3762400

2.8716630

3.5127000

0.2264670

0.1294390

1.1743560

0.2932230

$-0.9572600$

$-1.8265250$

$-0.0550480$

$-0.9731180$

$-4.5020170$

$-4.8682940$

$-3.9999550$

$-5.3782940$

$-4.2953320$

$-4.5048700$

$-5.2481150$

$-3.6937910$

$-0.8875690$

$-2.2845580$

0.0146980

$-3.3710220$

$-1.8846270$

1.2901870 


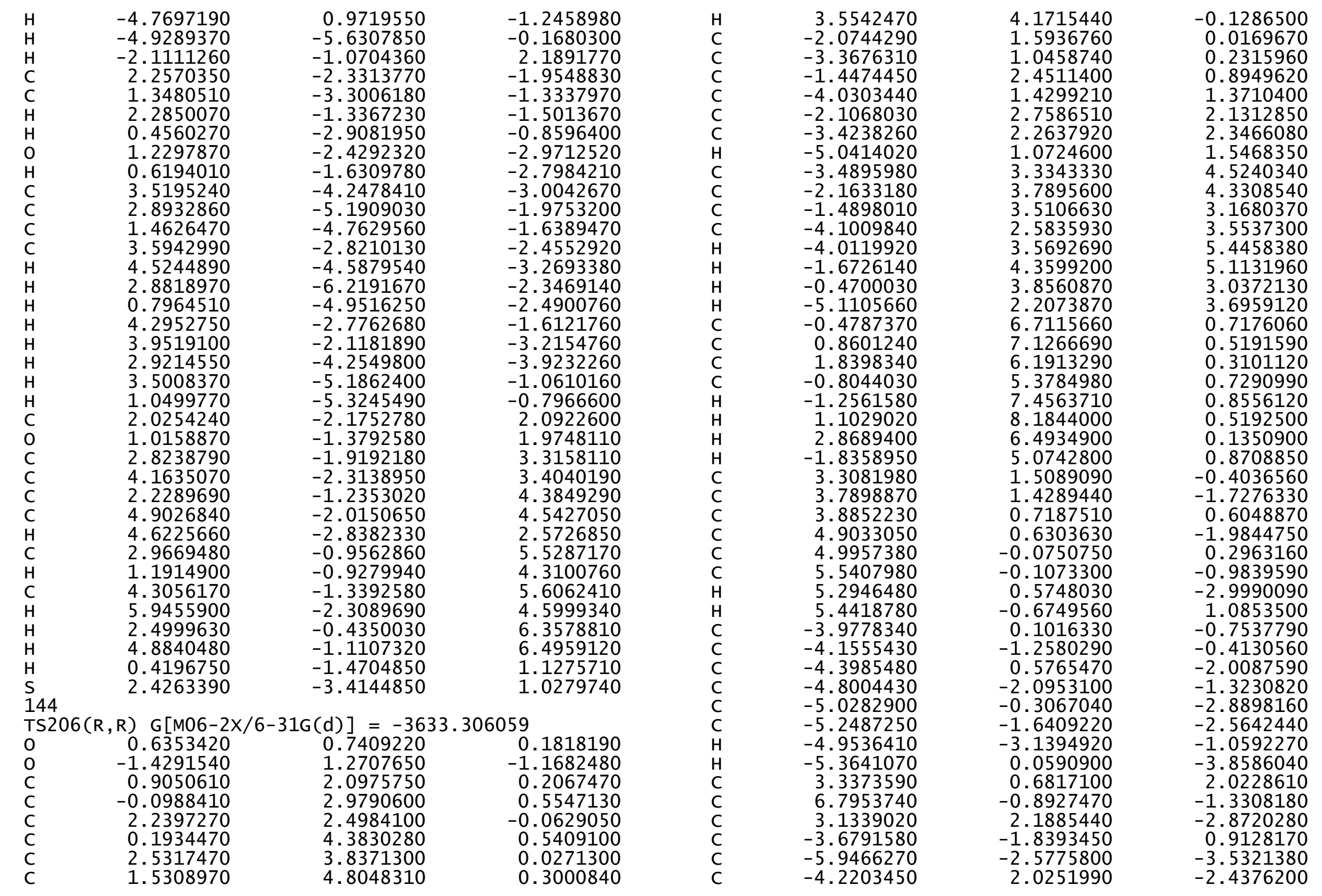




$\begin{array}{rrr}-5.5795870 & 2.7155560 & -2.6063160 \\ -6.1660340 & 2.2390080 & -3.3995380 \\ -6.1615020 & 2.6677120 & -1.6803610 \\ -5.4434630 & 3.7684040 & -2.8744450 \\ -3.3858520 & 2.1311140 & -3.7190630 \\ -2.4152920 & 1.6434150 & -3.5912450 \\ -3.8998100 & 1.6630810 & -4.5657950 \\ -3.2152010 & 3.1824620 & -3.9732740 \\ -7.2482980 & -3.1269380 & -2.9373590 \\ -7.7660110 & -3.7608580 & -3.6645590 \\ -7.0430920 & -3.7344420 & -2.0489240 \\ -7.9217820 & -2.3161770 & -2.6440260 \\ -5.0237190 & -3.7227170 & -3.9644950 \\ -4.1006300 & -3.3389620 & -4.4087570 \\ -4.7518750 & -4.3469360 & -3.1060270 \\ -5.5228190 & -4.3621200 & -4.6998950 \\ -4.8466990 & -1.9910830 & 1.8979640 \\ -5.3442070 & -1.0375780 & 2.0945910 \\ -5.5954840 & -2.6825340 & 1.4943170 \\ -4.4920530 & -2.3949620 & 2.8525920 \\ -2.9776990 & -3.1929070 & 0.7455650 \\ -3.6754260 & -3.9722590 & 0.4215510 \\ -2.1637730 & -3.1309890 & 0.0172350 \\ -2.5609370 & -3.5130040 & 1.7075120 \\ 4.3812610 & 1.1345510 & 3.0494760 \\ 5.2453330 & 0.4614730 & 3.0600240 \\ 4.7407980 & 2.1435770 & 2.8245610 \\ 3.9484830 & 1.1389490 & 4.0550420 \\ 2.8070660 & -0.7204470 & 2.3462250 \\ 2.0567310 & -1.0184380 & 1.6078410 \\ 3.6148200 & -1.4618000 & 2.3370280 \\ 2.3385010 & -0.7416630 & 3.3365840 \\ 7.1399060 & -1.9936010 & -0.3284180 \\ 7.9869660 & -2.5804370 & -0.6956130 \\ 7.4285710 & -1.5695480 & 0.6396240 \\ 6.2981170 & -2.6740360 & -0.1637910 \\ 7.9820260 & 0.0696300 & -1.4874290 \\ 7.7757650 & 0.8365280 & -2.2398720 \\ 8.1843000 & 0.5738710 & -0.5359260 \\ 8.8841800 & -0.4739840 & -1.7862580 \\ 2.9782470 & 1.3205320 & -4.1259300 \\ 3.9434530 & 1.1360870 & -4.6100140 \\ 2.5234680 & 0.3573940 & -3.8836170 \\ 2.3394050 & 1.8313840 & -4.8531650 \\ 3.9181580 & 3.4616940 & -3.2161860 \\ 4.9427640 & 3.2076030 & -3.5116970\end{array}$
3.4446030
3.9722870
$-0.2664780$
0.4812640
$-0.8238780$
2.1282490
6.6209780
2. 4927190
$-3.6809190$
$-6.2023900$
$-2.9488130$
2. 3042210
1. 1189060
2. 2272820
0.1527240
1.8688210
1.2957950
3.7062070
2. 5767000
1.2117590
3.6588500
4.6780350
2.6325960
1.0119870
3.8836630
4.3973660
3. 6197850
2. 6779770
0.3918320
0.2067580
$-0.4010850$
0.1979540
1.0850000
$-0.7253210$
1.0476170
1.8051530
$-0.7661850$
$-1.4100340$
0.1205550
1.7430160
$-1.4893310$
0.0906720
$-0.5096120$

144

$\operatorname{TS} 207(R, R) G[M 06-2 X / 6-31 G(d)]=-3633.303497$
-4.0515540
-2.3671870
-1.0451520
-2.3430010
-0.5028870
-2.5479640
-2.3077280
2.0871980
-1.6503300
-4.4251650
1.3430430
-1.4730520
-1.5457560
-0.8291980
-1.4140900
-2.8242210
-2.7644890
-2.2693770
-1.8747710
-2.1087730
-1.4377540
-2.1302980
-2.4468720
-3.1837460
-0.3845240
-1.7877750
-3.3330510
-0.8151600
-1.6971350
2.0902670
1.8415940
3.5302840
4.0553540
4.3765210
5.4084810
3.4002860
5.7271860
3.9655590
6.2448460
5.8123330
6.3763700
7.3007710
0.8330110
0.9397920

$0.1472140-1.0451520$

$0.1357940 \quad-2.3430010$

4753650

$-1.3657500$

1.3742630

$-1.9934150$

$-1.1458310$

$-2.2778960$

1.3956330

$-2.6695660$

$-5.1780830$

$-4.5266250$

$-2.9431130$

$-4.7093620$

$-6.1082040$

$-4.4326830$

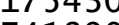

3.9741890

$-4.7383520$

$-3.0082030$

$-1.9487300$

.9509580

$-1.5911890$

3.4616940
3.2076030

$-3.5116970$ 


$\begin{array}{lr}\text { O } & 0.0358590 \\ \mathrm{O} & -2.0695060 \\ \mathrm{C} & -0.2610560 \\ \mathrm{C} & -1.5418850 \\ \mathrm{C} & 0.8145810 \\ \mathrm{C} & -1.8415450 \\ \mathrm{C} & 0.5380350 \\ \mathrm{C} & -0.7836440 \\ \mathrm{H} & 1.3381930 \\ \mathrm{C} & -2.7282870 \\ \mathrm{C} & -3.6215730 \\ \mathrm{C} & -2.5362630 \\ \mathrm{C} & -4.3695700 \\ \mathrm{C} & -3.2419710 \\ \mathrm{C} & -4.1943090 \\ \mathrm{H} & -5.1010580 \\ \mathrm{C} & -4.7000510 \\ \mathrm{C} & -3.7176910 \\ \mathrm{C} & -3.0080930 \\ \mathrm{C} & -4.9250830 \\ \mathrm{H} & -5.2586550 \\ \mathrm{H} & -3.5187190 \\ \mathrm{H} & -2.2494340 \\ \mathrm{H} & -5.6566280 \\ \mathrm{C} & -3.4064400 \\ \mathrm{C} & -2.3485010 \\ \mathrm{C} & -1.0680630 \\ \mathrm{C} & -3.1604220 \\ \mathrm{H} & -4.4218450 \\ \mathrm{H} & -2.5562730 \\ \mathrm{H} & -0.2484810 \\ \mathrm{H} & -3.9789170 \\ \mathrm{C} & 2.1741050 \\ \mathrm{C} & 2.7574010 \\ \mathrm{C} & 2.8245290 \\ \mathrm{C} & 3.9777580 \\ \mathrm{C} & 4.0291850 \\ \mathrm{C} & 4.6146080 \\ \mathrm{H} & 4.4464230 \\ \mathrm{H} & 4.5253850 \\ \mathrm{C} & -3.7338920 \\ \mathrm{C} & -3.0819310 \\ \mathrm{C} & -4.4921750 \\ \mathrm{C} & -3.2509510 \\ \mathrm{C} & -4.6259630 \\ \mathrm{C} & -4.0199580\end{array}$

$\begin{array}{rr}1.1627820 & 0.1015000 \\ 0.2901750 & -0.9557300 \\ 2.4431410 & -0.3328960 \\ 2.9228910 & -0.1666470 \\ 3.2086330 & -0.8638260 \\ 4.2256190 & -0.6878680 \\ 4.4892690 & -1.2678260 \\ 5.0123120 & -1.2256220 \\ 5.1269930 & -1.6333120 \\ 0.7679760 & 0.1640240 \\ -0.1245220 & 0.8150120 \\ 2.0782570 & 0.5513930 \\ 0.3710450 & 1.8527720 \\ 2.5513520 & 1.7094740 \\ 1.6955670 & 2.3322850 \\ -0.2692240 & 2.3392560 \\ 3.4123060 & 3.9721860 \\ 4.2460990 & 3.3871570 \\ 3.8290610 & 2.2886770 \\ 2.1621420 & 3.4573300 \\ 3.7588620 & 4.8356740 \\ 5.2231810 & 3.8160410 \\ 4.4751520 & 1.8612910 \\ 1.4981470 & 3.9100770 \\ 6.0065990 & -1.2177460 \\ 6.8024080 & -1.7175830 \\ 6.3116200 & -1.7231530 \\ 4.7531680 & -0.7150500 \\ 6.3896190 & -1.2372490 \\ 7.7942730 & -2.1059600 \\ 6.9047610 & -2.1199580 \\ 4.1463890 & -0.3416040 \\ 2.5855620 & -0.8688290 \\ 2.1190020 & -2.0622540 \\ 2.3875360 & 0.3659150 \\ 1.4395480 & -1.9864490 \\ 1.6866140 & 0.3915380 \\ 1.1880600 & -0.7719530 \\ 1.0841220 & -2.9015630 \\ 1.5291760 & 1.3469540 \\ -1.5461910 & 0.3646080 \\ -2.5649000 & 1.0872740 \\ -1.8575430 & -0.7785310 \\ -3.8877730 & 0.6711970 \\ -3.1934600 & -1.1576790 \\ -4.2244610 & -0.4408740\end{array}$

-2.7596180
-5.2187040
2.2863150
5.9282250
2.1189190
-2.2156720
-4.1689880
-5.1775350
-6.7025770
-7.0313140
-7.0463400
-7.1910220
-4.6969550
-3.6105760
-4.9652660
-5.1598830
-3.5149930
-3.5842960
-4.0151770
-2.4590490
-5.6380610
-6.1087480
-6.2050800
-5.7202920
-3.0321840
-3.8710250
-3.4356000
-2.3995520
-0.9852860
-1.2607470
-0.4537710
-0.2978340
3.1637330
4.1869370
3.2088510
2.7656280
2.1719490
1.5442440
3.1530430
1.7320370
5.9282340
6.8326130
5.9057150
5.0561510
7.1015690
7.1356690

$-4.6857490$

$-3.4336960$

2.9697930

0.4306050

2. 3784290

$-2.2636900$

$-5.6703720$

$-0.7832620$

$-0.9200030$

$-1.8804820$

$-0.8626890$

$-0.1230180$

$-0.8082220$

$-0.6911780$

$-1.7500200$

0.0067580

$-5.9045780$

$-6.9593580$

$-5.3120620$

$-5.6178910$

$-6.1066550$

$-5.9561390$

$-5.5325370$

$-7.1659840$

$-2.3730600$

$-1.6723610$

$-3.3867160$

$-2.1662870$

$-3.1739940$

$-4.1910890$

$-3.2258550$

$-2.7871170$

4.1494000

3.8146800

4.9178600

4.6046570

1. 9241930

1. 0882580

1. 5238720

2. 3745400

$-0.6579910$

$-1.2710720$

- . 2226780

$-1.3155250$

1. 3962730

2. 1525390
1. 2229540

$-2.0389060$

1.6654940

$-0.7232000$

$-3.4184060$

2. 3035730

$-0.8754830$

$-1.6087270$

$-1.5274190$

$-1.9398050$

$-0.4896500$

$-2.0975830$

$-3.0638930$

$-3.1143590$

$-3.5550070$

$-3.6299540$

$-2.2424230$

$-2.5279870$

$-3.0166150$

$-2.2281240$

$-0.8960370$

0.0800410

$-1.6371020$

$-1.1600700$

3. 5990780

3. 6133990

3. 7054860

4.4690240

2.4000570

2. 6997690

1. 4459470

3. 1604590

2. 1060490

2. 3110430

1. 3275550

3. 0189020

2.7767430

2.4574020

3. 0561190

3. 6732390

0.3556670

0.2821080

1.3608960

0.2625860

-0.5128730
-1.3027720 


$\begin{array}{lrrr}\text { H } & 6.9997550 & 1.9138540 & 0.4477440 \\ \text { H } & 8.0537410 & 0.8555860 & -0.5089050 \\ \mathrm{C} & 2.3093530 & 1.2312770 & -4.4133880 \\ \mathrm{H} & 3.3522790 & 1.1447000 & -4.7384920 \\ \mathrm{H} & 1.9991870 & 0.2754380 & -3.9858270 \\ \mathrm{H} & 1.7068540 & 1.4168000 & -5.3083260 \\ \mathrm{C} & 2.6780370 & 3.6740640 & -4.0251860 \\ \mathrm{H} & 3.7566960 & 3.5745110 & -4.1934970 \\ \mathrm{H} & 2.2012940 & 3.8844860 & -4.9883950 \\ \mathrm{H} & 2.5214650 & 4.5348180 & -3.3698820 \\ \mathrm{P} & -0.4872260 & -0.0568560 & -0.8426770 \\ \mathrm{O} & 0.0569960 & 0.0254220 & -2.2396340 \\ \mathrm{O} & -0.2435260 & -1.3066760 & -0.0320600 \\ \mathrm{H} & 1.0423890 & 2.5005810 & -3.2610330 \\ \mathrm{H} & 6.0612860 & -0.0556580 & -1.6988490 \\ \mathrm{H} & 1.2818080 & 3.3628170 & 1.4836780 \\ \mathrm{H} & -4.9131740 & 0.1945910 & -1.1944520 \\ \mathrm{H} & -3.6424270 & -6.2892580 & -0.1379780 \\ \mathrm{H} & -1.8540060 & -1.2338490 & 2.2059180 \\ \mathrm{C} & 2.4963600 & -1.8484170 & -2.0716280 \\ \mathrm{C} & 1.6749870 & -2.9824840 & -1.6341990 \\ \mathrm{H} & 2.4276810 & -0.9401030 & -1.4653640 \\ \mathrm{H} & 0.7499830 & -2.7511660 & -1.1189550 \\ \mathrm{O} & 1.4917010 & -1.8785540 & -3.1118170 \\ \mathrm{H} & 0.8091940 & -1.1617270 & -2.8461050 \\ \mathrm{C} & 3.9301180 & -3.4412590 & -3.3997630 \\ \mathrm{C} & 3.3830320 & -4.5975230 & -2.5603460 \\ \mathrm{C} & 1.9200500 & -4.3583790 & -2.1772750 \\ \mathrm{C} & 3.8772860 & -2.1284560 & -2.6141700 \\ \mathrm{H} & 4.9625360 & -3.6447180 & -3.6975150 \\ \mathrm{H} & 3.4601720 & -5.5423640 & -3.1053360 \\ \mathrm{H} & 1.2718510 & -4.4611530 & -3.0562280 \\ \mathrm{H} & 4.5661170 & -2.1704460 & -1.7609840 \\ \mathrm{H} & 4.1772910 & -1.2785030 & -3.2366640 \\ \mathrm{H} & 3.3410790 & -3.3402660 & -4.3188910 \\ \mathrm{H} & 3.9861550 & -4.7009020 & -1.6493470 \\ \mathrm{H} & 1.5610810 & -5.0882980 & -1.4465890 \\ \mathrm{C} & 2.5152340 & -2.1548240 & 1.7638000 \\ \mathrm{O} & 1.5973470 & -1.2492880 & 1.6782100 \\ \mathrm{C} & 3.3901490 & -1.9879130 & 2.9487900 \\ \mathrm{C} & 4.6568950 & -2.5819990 & 3.0095890 \\ \mathrm{C} & 2.9344080 & -1.2148150 & 4.0256370 \\ \mathrm{C} & 5.4599440 & -2.3951430 & 4.1277430 \\ \mathrm{H} & 5.0104920 & -3.1735460 & 2.1715770 \\ \mathrm{C} & 3.7367820 & -1.0417830 & 5.1474650 \\ \mathrm{H} & 1.9499830 & -0.7626650 & 3.9786070 \\ & & & \\ & & & \end{array}$

$\begin{array}{llll}\mathrm{C} & 5.0008240 & -1.6273730 & 5.1980320 \\ \mathrm{H} & 6.4455080 & -2.8472480 & 4.1643390 \\ \mathrm{H} & 3.3754760 & -0.4498260 & 5.9819920 \\ \mathrm{H} & 5.6289810 & -1.4862520 & 6.0719880 \\ \mathrm{H} & 0.8924830 & -1.3519690 & 0.9254630 \\ \mathrm{~S} & 2.7200630 & -3.4333820 & 0.6911220\end{array}$
144 TS208(R,R) G[M06-2X/6-31G(d)] = -3633.303497

$\begin{array}{lrrr}\text { TS208(R, R) G[M06-2X/6-31G(d)] }=-3633.303497 \\ \text { O } & 0.0358870 & 1.1627680 & 0.1014860 \\ \text { O } & -2.0694990 & 0.2902040 & -0.9557380 \\ \text { C } & -0.2610050 & 2.4431310 & -0.3329140 \\ \text { C } & -1.5418230 & 2.9229070 & -0.1666600 \\ \text { C } & 0.8146450 & 3.2085990 & -0.8638540 \\ \text { C } & -1.8414590 & 4.2256400 & -0.6878850 \\ \text { C } & 0.5381230 & 4.4892390 & -1.2678570 \\ \text { C } & -0.7835450 & 5.0123100 & -1.2256470 \\ \text { H } & 1.3382920 & 5.1269460 & -1.6333490 \\ \text { C } & -2.7282680 & 0.7680190 & 0.1640190 \\ \text { C } & -3.6215680 & -0.1244620 & 0.8150110 \\ \text { C } & -2.5362150 & 2.0782960 & 0.5513860 \\ \text { C } & -4.3695510 & 0.3711210 & 1.8527740 \\ \text { C } & -3.2419100 & 2.5514060 & 1.7094680 \\ \text { C } & -4.1942630 & 1.6956410 & 2.3322850 \\ \text { H } & -5.1010500 & -0.2691320 & 2.3392610 \\ \text { C } & -4.6999650 & 3.4123920 & 3.9721850 \\ \text { C } & -3.7175920 & 4.2461650 & 3.3871500 \\ \text { C } & -3.0080060 & 3.8291120 & 2.2886690 \\ \text { C } & -4.9250240 & 2.1622310 & 3.4573310 \\ \text { H } & -5.2585600 & 3.7589600 & 4.8356740 \\ \text { H } & -3.5186000 & 5.2232440 & 3.8160320 \\ \text { H } & -2.2493360 & 4.4751870 & 1.8612780 \\ \text { H } & -5.6565790 & 1.4982520 & 3.9100820 \\ \text { C } & -3.4063220 & 6.0066500 & -1.2177600 \\ \text { C } & -2.3483690 & 6.8024360 & -1.7176060 \\ \text { C } & -1.0679410 & 6.3116210 & -1.7231810 \\ \text { C } & -3.1603260 & 4.7532160 & -0.7150610 \\ \text { H } & -4.4217180 & 6.3896900 & -1.2372590 \\ \text { H } & -2.5561240 & 7.7943030 & -2.1059860 \\ \text { H } & -0.2483490 & 6.9047450 & -2.1199930 \\ \text { H } & -3.9788310 & 4.1464540 & -0.3416080 \\ \text { C } & 2.1741570 & 2.5855000 & -0.8688620 \\ \text { C } & 2.7574330 & 2.1189190 & -2.0622890 \\ \text { C } & 2.8245870 & 2.3874740 & 0.3658770 \\ \text { C } & 3.9777790 & 1.4394460 & -1.9864890 \\ \text { C } & 4.0292310 & 1.6865280 & 0.3914960 \\ \text { C } & 4.6146360 & 1.1879580 & -0.7719960\end{array}$




$\begin{array}{lr}\mathrm{H} & 4.4464300 \\ \mathrm{H} & 4.5254360 \\ \mathrm{C} & -3.7339160 \\ \mathrm{C} & -3.0819770 \\ \mathrm{C} & -4.4922060 \\ \mathrm{C} & -3.2510240 \\ \mathrm{C} & -4.6260220 \\ \mathrm{C} & -4.0200370 \\ \mathrm{H} & -2.7597080 \\ \mathrm{H} & -5.2187680 \\ \mathrm{C} & 2.2863940 \\ \mathrm{C} & 5.9282430 \\ \mathrm{C} & 2.1189410 \\ \mathrm{C} & -2.2157150 \\ \mathrm{C} & -4.1690930 \\ \mathrm{C} & -5.1775470 \\ \mathrm{C} & -6.7025910 \\ \mathrm{H} & -7.0313490 \\ \mathrm{H} & -7.0463520 \\ \mathrm{H} & -7.1910220 \\ \mathrm{C} & -4.6969710 \\ \mathrm{H} & -3.6105900 \\ \mathrm{H} & -4.9653010 \\ \mathrm{H} & -5.1598840 \\ \mathrm{C} & -3.5150960 \\ \mathrm{H} & -3.5844170 \\ \mathrm{H} & -4.0152640 \\ \mathrm{H} & -2.4591460 \\ \mathrm{C} & -5.6381750 \\ \mathrm{H} & -6.1088650 \\ \mathrm{H} & -6.2051790 \\ \mathrm{H} & -5.7204250 \\ \mathrm{C} & -3.0322340 \\ \mathrm{H} & -3.8710610 \\ \mathrm{H} & -3.4356700 \\ \mathrm{H} & -2.3996010 \\ \mathrm{C} & -0.9853490 \\ \mathrm{H} & -1.2608320 \\ \mathrm{H} & -0.4538300 \\ \mathrm{H} & -0.2978930 \\ \mathrm{C} & 3.1638310 \\ \mathrm{H} & 4.1870350 \\ \mathrm{H} & 3.2089490 \\ \mathrm{H} & 2.7657430 \\ \mathrm{C} & 2.1720300 \\ \mathrm{H} & 1.5443090 \\ & \end{array}$

$\begin{array}{rr}1.0840040 & -2.9016040 \\ 1.5290900 & 1.3469090 \\ -1.5461290 & 0.3646080 \\ -2.5648520 & 1.0872740 \\ -1.8574650 & -0.7785300 \\ -3.8877210 & 0.6711970 \\ -3.1933800 & -1.1576800 \\ -4.2243930 & -0.4408750 \\ -4.6857070 & 1.2229530 \\ -3.4336030 & -2.0389060 \\ 2.9697540 & 1.6654550 \\ 0.4304860 & -0.7232510 \\ 2.3783400 & -3.4184370 \\ -2.2636610 & 2.3035760 \\ -5.6703000 & -0.8754870 \\ -0.7831710 & -1.6087260 \\ -0.9198810 & -1.5274150 \\ -1.8803540 & -1.9397990 \\ -0.8625600 & -0.4896440 \\ -0.1228870 & -2.0975770 \\ -0.8081400 & -3.0638920 \\ -0.6911170 & -3.1143610 \\ -1.7499320 & -3.5550060 \\ 0.0068500 & -3.6299520 \\ -5.9045180 & -2.2424230 \\ -6.9592960 & -2.5279900 \\ -5.3119900 & -3.0166170 \\ -5.6178510 & -2.2281180 \\ -6.1065560 & -0.8960490 \\ -5.9560310 & 0.0800260 \\ -5.5324260 & -1.6371170 \\ -7.1658830 & -1.1600840 \\ -2.3730180 & 3.5990780 \\ -1.6723010 & 3.6133990 \\ -3.3866660 & 3.7054830 \\ -2.1662590 & 4.4690270 \\ -3.1739900 & 2.4000630 \\ -4.1910820 & 2.6997710 \\ -3.2258600 & 1.4459560 \\ -2.7871310 & 3.1604690 \\ 4.1493570 & 2.1059810 \\ 3.8146290 & 2.3109670 \\ 4.9178050 & 1.3274760 \\ 4.6046320 & 3.0188330 \\ 1.9241720 & 2.7767200 \\ 1.0882410 & 2.4574000 \\ -5 & \end{array}$

\begin{abstract}
3. 1531220
1.7321350

5.9282560

6.8326240

5.9057620

5. 0561610

7. 1016020

7.1357020

6.9998090

8.0537680

2. 3093490

3. 3522710

1.9991730

1.7068450

2. 6780680

3.7567240

2. 2013180

2. 5215150

$-0.4872290$

0.0569990

$-0.2435630$

1. 0424130

6. 0612840

1. 2818900

$-4.9131670$

$-3.6425490$

$-1.8540280$

2. 4963240

1.6749440

2. 4276580

0.7499460

1. 4916520

0.8091580

3. 9300540

3. 3829650

1. 9199880

3. 8772410

4. 9624670

3. 4600910

1. 2717800

4. 5660800

4. 1772480

3. 3410070

3.9860950

1.5610200
2.5151760
\end{abstract}

3.0560920

3. 6732160

0.3556330

0.2820670

1. 3608560

0.2625780

$-0.5129550$

$-1.3028670$

0.4476550

$-0.5089920$

$-4.4134090$

$-4.7385210$

$-3.9858350$

$-5.3083430$

$-4.0252370$

$-4.1935590$

$-4.9884440$

$-3.3699420$

$-0.8426800$

$-2.2396370$

$-0.0320480$

$-3.2610540$

$-1.6988940$

1.4836450

$-1.1944510$

$-0.1379790$

2. 2059250

$-2.0716300$

$-1.6341570$

$-1.4653960$

$-1.1189110$

$-3.1118080$

$-2.8461080$

$-3.3997270$

$-2.5602650$

$-2.1771880$

$-2.6141770$

$-3.6974810$

$-3.1052240$

$-3.0561320$

$-1.7609960$

$-3.2367020$

$-4.3188520$

$-1.6492680$

$-1.4464740$

1.7638540 


$\begin{array}{llll}\mathrm{O} & 1.5972700 & -1.2492900 & 1.6782620 \\ \mathrm{C} & 3.3900760 & -1.9878850 & 2.9488540 \\ \mathrm{C} & 4.6568330 & -2.5819460 & 3.0096620 \\ \mathrm{C} & 2.9343080 & -1.2148050 & 4.0257030 \\ \mathrm{C} & 5.4598660 & -2.3950840 & 4.1278260 \\ \mathrm{H} & 5.0104500 & -3.1734810 & 2.1716500 \\ \mathrm{C} & 3.7366660 & -1.0417670 & 5.1475400 \\ \mathrm{H} & 1.9498750 & -0.7626740 & 3.9786660 \\ \mathrm{C} & 5.0007200 & -1.6273320 & 5.1981160 \\ \mathrm{H} & 6.4454390 & -2.8471700 & 4.1644280 \\ \mathrm{H} & 3.3753400 & -0.4498240 & 5.9820680 \\ \mathrm{H} & 5.6288650 & -1.4862060 & 6.0720800 \\ \mathrm{H} & 0.8924240 & -1.3519730 & 0.9254970 \\ \mathrm{~S} & 2.7200430 & -3.4333530 & 0.6911700\end{array}$

TS209 $(R, R) \quad G[M 06-2 X / 6-31 G(d)]=-3633.306810$

$\begin{array}{lrlr}\mathrm{O} & 0.6450150 & 0.7349480 & 0.2342920 \\ \mathrm{O} & -1.3671560 & 1.2621840 & -1.1928950 \\ \mathrm{C} & 0.9251600 & 2.0898260 & 0.2482450 \\ \mathrm{C} & -0.0817120 & 2.9832650 & 0.5538930 \\ \mathrm{C} & 2.2698000 & 2.4769940 & 0.0098610 \\ \mathrm{C} & 0.2226600 & 4.3846850 & 0.5332330 \\ \mathrm{C} & 2.5705430 & 3.8140980 & 0.0948010 \\ \mathrm{C} & 1.5705450 & 4.7926510 & 0.3293500 \\ \mathrm{H} & 3.5995010 & 4.1387690 & -0.0370970 \\ \mathrm{C} & -2.0500090 & 1.6077910 & -0.0353970 \\ \mathrm{C} & -3.3568410 & 1.0782900 & 0.1394440 \\ \mathrm{C} & -1.4455110 & 2.4701740 & 0.8538660 \\ \mathrm{C} & -4.0553570 & 1.4840960 & 1.2495950 \\ \mathrm{C} & -2.1446070 & 2.7994450 & 2.0624380 \\ \mathrm{C} & -3.4741650 & 2.3224510 & 2.2365130 \\ \mathrm{H} & -5.0763980 & 1.1407310 & 1.3927870 \\ \mathrm{C} & -3.6047800 & 3.4200390 & 4.3974230 \\ \mathrm{C} & -2.2669720 & 3.8572120 & 4.2465860 \\ \mathrm{C} & -1.5558640 & 3.5565230 & 3.1118930 \\ \mathrm{C} & -4.1901630 & 2.6647430 & 3.4147180 \\ \mathrm{H} & -4.1568830 & 3.6723010 & 5.2970720 \\ \mathrm{H} & -1.7975790 & 4.4310450 & 5.0393910 \\ \mathrm{H} & -0.5278100 & 3.8880770 & 3.0138220 \\ \mathrm{H} & -5.2086900 & 2.3021550 & 3.5249530 \\ \mathrm{C} & -0.4342370 & 6.7206880 & 0.6635710 \\ \mathrm{C} & 0.9137810 & 7.1223290 & 0.5031940 \\ \mathrm{C} & 1.8912260 & 6.1765070 & 0.3346260 \\ \mathrm{C} & -0.7718080 & 5.3906010 & 0.6789360 \\ \mathrm{H} & -1.2090740 & 7.4734620 & 0.7691310 \\ \mathrm{H} & 1.1657280 & 8.1779090 & 0.4999030\end{array}$

2.9278980
-1.8099230
3.3356110
3.8563000
3.8687200
4.9675860
4.9669940
5.5534860
5.3768170
5.3894840
-3.9453030
-4.1499440
-4.3219250
-4.7773320
-4.9357210
-5.1824260
-4.9516510
-5.2383770
3.2832400
6.7961570
3.2487440
-3.7214190
-5.8641640
-4.1155870
-5.4624900
-6.0381920
-6.0636810
-5.3082110
-3.2531340
-2.2914060
-3.7543230
-3.0643130
-7.1926640
-7.6973730
-7.0254950
-7.8618450
-4.9470370
-4.0043780
-4.7123370
-5.4333560
-4.9200530
-5.4101500
-5.6656770
-4.5998270
-3.0319220
-3.7281280

5.0969050

1. 4750000

1. 3906660

0.6706910

0.5726770

$-0.1395200$

$-0.1804000$

0.5251390

$-0.7590240$

0.1307370

$-1.2230060$

0.5981630

$-2.0615860$

$-0.2861300$

$-1.6142770$

$-3.1010640$

0.0741590

0.6455410

$-1.0297840$

2. 1612260

$-1.7964780$

$-2.5514870$

2. 0410800

2.7465920

2.2703600

2.7130330

3.7955520

2. 1281430

1.6298000

1. 6607830

3.1756250

$-3.0728290$

$-3.7076410$

$-3.6721300$

$-2.2478570$

$-3.7161460$

$-3.3524360$

$-4.3348390$

$-4.3558860$

$-1.9236810$

$-0.9617660$

$-2.3231890$

$-3.1598800$

$-3.9336000$
0.1885180

0.7914230

$-0.2997310$

$-1.6043860$

0.7274070

$-1.8371160$

0.4433430

$-0.8229600$

$-2.8420400$

1. 2340460

$-0.8559590$

$-0.5074600$

$-2.1275430$

$-1.4285720$

$-3.0187100$

$-2.6869790$

$-1.1594050$

$-4.0003900$

2. 1305680

$-1.0348540$

$-2.7683770$

0.8380620

$-3.6658280$

$-2.5629390$

$-2.7648060$

$-3.5660860$

$-1.8505930$

$-3.0382800$

$-3.8270500$

$-3.6757700$

$-4.6817230$

$-4.0842720$

$-3.1060740$

$-3.8415960$

$-2.2041860$

$-2.8441870$

$-4.0561360$

$-4.4754630$

$-3.1828620$

$-4.7997620$

1.7886920

1. 9615760

1. 3688680

2. 7572270

0.7051450

0.3648540 


$\begin{array}{rrr}-2.1939330 & -3.1137610 & 0.0034010 \\ -2.6508080 & -3.4767390 & 1.6828030 \\ 4.3077820 & 1.0940120 & 3.1785620 \\ 5.1652600 & 0.4130930 & 3.2101180 \\ 4.6814150 & 2.0986770 & 2.9571160 \\ 3.8531550 & 1.1068430 & 4.1743690 \\ 2.7307200 & -0.7484760 & 2.4508200 \\ 1.9924580 & -1.0431920 & 1.6991030 \\ 3.5300320 & -1.4987370 & 2.4639160 \\ 2.2409240 & -0.7578740 & 3.4309890 \\ 8.0174630 & -0.3248930 & -0.4266730 \\ 8.9128680 & -0.9470200 & -0.5260090 \\ 8.1996680 & 0.6238470 & -0.9435580 \\ 7.8634100 & -0.1079910 & 0.6345570 \\ 7.0675520 & -1.3856890 & -2.4966250 \\ 6.1995670 & -1.8523730 & -2.9751030 \\ 7.3346690 & -0.4954880 & -3.0764590 \\ 7.9077170 & -2.0833930 & -2.5601810 \\ 3.1046210 & 1.2907790 & -4.0224320 \\ 4.0768410 & 1.0809750 & -4.4815050 \\ 2.6205020 & 0.3403860 & -3.7870300 \\ 2.4967270 & 1.8132760 & -4.7677540 \\ 4.0706830 & 3.4141990 & -3.0979760 \\ 5.0963350 & 3.1357960 & -3.3667170 \\ 3.6305740 & 3.9473660 & -3.9472930 \\ 4.1192260 & 4.1038300 & -2.2507320 \\ -0.2175950 & 0.1319490 & -1.0158490 \\ 0.5756360 & 0.0994570 & -2.2859210 \\ -0.8027190 & -1.1534830 & -0.4790700 \\ 2.2420120 & 2.4729770 & -2.4720560 \\ 6.6421020 & -1.9667180 & -0.4806120 \\ 2.4448520 & 1.3474070 & 2.1701330 \\ -3.5860460 & 2.5741180 & -1.7681530 \\ -6.0824330 & -1.9739330 & -4.5729990 \\ -2.9956400 & -1.1078350 & 1.2836050 \\ 2.3456070 & -2.3295670 & -1.3172020 \\ 1.1531590 & -3.1781730 & -1.4387560 \\ 2.2532620 & -1.4450080 & -0.6786640 \\ 0.1885430 & -2.6925290 & -1.3487500 \\ 1.9721630 & -2.0463930 & -2.6863240 \\ 1.4052090 & -1.2047890 & -2.6542940 \\ 3.7537200 & -4.2938840 & -2.0584020 \\ 2.5981760 & -5.2322050 & -1.7097250 \\ 1.2510900 & -4.5645400 & -1.9958550 \\ 3.6893000 & -3.0118200 & -1.2258200 \\ 4.7134670 & -4.7890150 & -1.8841910\end{array}$

2.6651450
1.0928760
3.8738540
4.4468380
3.7112170
2.6550070
0.4091670
0.1047560
-0.4754490
0.0389390
0.8951810
-0.9067880
0.8056440
1.6326620
-1.0001840
-1.5674950
-0.1435850
1.4775960
-1.7406770
-0.2142340
-0.5443740
0.8520440

$$
\begin{aligned}
& -6.1626310 \\
& -4.4701340 \\
& -3.2292530 \\
& -2.2939500 \\
& -4.0375310 \\
& -5.5014670 \\
& -5.1488810 \\
& -2.9585910 \\
& -1.8399270 \\
& -3.3103420 \\
& -4.2649880 \\
& -2.6649000 \\
& -4.5697970 \\
& -4.7549560 \\
& -2.9831160 \\
& -1.9252220 \\
& -3.9331890 \\
& -5.3038480 \\
& -2.4889270 \\
& -4.1770470 \\
& -1.5913220 \\
& -3.9688280
\end{aligned}
$$

$\begin{array}{lrrr}\text { TS210(R, R) } & \text { G }[\mathrm{M} 06-2 \mathrm{X} / 6-31 \mathrm{G}(\mathrm{d})]=-3633.303404 \\ \mathrm{O} & -0.0748400 & 1.2605010 & 0.3370810 \\ \mathrm{O} & -2.0316250 & 0.1994160 & -0.8350470 \\ \mathrm{C} & -0.3815830 & 2.4748770 & -0.2509550 \\ \mathrm{C} & -1.6897650 & 2.9095510 & -0.2461560 \\ \mathrm{C} & 0.7069810 & 3.2349230 & -0.7594060 \\ \mathrm{C} & -1.9884090 & 4.1444850 & -0.9136480 \\ \mathrm{C} & 0.4216820 & 4.4617380 & -1.3021090 \\ \mathrm{C} & -0.9153960 & 4.9278900 & -1.4252010 \\ \mathrm{H} & 1.2298100 & 5.0973080 & -1.6543270 \\ \mathrm{C} & -2.8372640 & 0.7452110 & 0.1469450 \\ \mathrm{C} & -3.7908820 & -0.1199660 & 0.7471100 \\ \mathrm{C} & -2.7248250 & 2.0880110 & 0.4408200 \\ \mathrm{C} & -4.6663850 & 0.4304340 & 1.6485790 \\ \mathrm{C} & -3.5736900 & 2.6268340 & 1.4661910 \\ \mathrm{C} & -4.5732680 & 1.7916550 & 2.0415610 \\ \mathrm{H} & -5.4382180 & -0.1912070 & 2.0950170 \\ \mathrm{C} & -5.3045680 & 3.6140290 & 3.4690810 \\ \mathrm{C} & -4.2810590 & 4.4315880 & 2.9337190 \\ \mathrm{C} & -3.4392930 & 3.9531990 & 1.9607900 \\ \mathrm{C} & -5.4399360 & 2.3210430 & 3.0346220 \\ \mathrm{H} & -5.9672710 & 4.0083190 & 4.2327070 \\ \mathrm{H} & -4.1564680 & 5.4452340 & 3.3014140\end{array}$




$\begin{array}{rrr}-2.6514740 & 4.5877140 & 1.5699350 \\ -6.2042400 & 1.6715200 & 3.4528980 \\ -3.5613080 & 5.7968980 & -1.7498920 \\ -2.4914760 & 6.5932760 & -2.2229390 \\ -1.1990840 & 6.1632140 & -2.0656950 \\ -3.3176870 & 4.6063400 & -1.1115790 \\ -4.5840090 & 6.1288550 & -1.8983750 \\ -2.6986530 & 7.5357390 & -2.7195480 \\ -0.3679110 & 6.7547320 & -2.4403050 \\ -4.1453960 & 3.9989590 & -0.7606130 \\ 2.0969290 & 2.7025880 & -0.6075170 \\ 2.7879000 & 2.1568380 & -1.7092600 \\ 2.6970730 & 2.7288290 & 0.6642600 \\ 4.0777430 & 1.6601030 & -1.5105570 \\ 3.9956690 & 2.2345200 & 0.8082720 \\ 4.7023650 & 1.6937080 & -0.2621240 \\ 4.6169800 & 1.2414700 & -2.3576120 \\ 4.4774310 & 2.2774870 & 1.7839950 \\ -3.8129590 & -1.5733400 & 0.3971600 \\ -3.3233280 & -2.5209030 & 1.3168310 \\ -4.3152200 & -1.9887040 & -0.8509910 \\ -3.4017700 & -3.8762490 & 0.9850770 \\ -4.3650610 & -3.3522740 & -1.1389000 \\ -3.9219990 & -4.3135670 & -0.2303250 \\ -3.0374400 & -4.6180830 & 1.6923570 \\ -4.7619350 & -3.6726180 & -2.1009070 \\ 1.9990140 & 3.3300840 & 1.8752090 \\ 6.1160600 & 1.1753280 & -0.0780920 \\ 2.1824900 & 2.1449590 & -3.1051450 \\ -2.7188480 & -2.1159490 & 2.6543920 \\ -3.9849010 & -5.7916920 & -0.5659630 \\ -4.8172900 & -1.0002420 & -1.8922610 \\ -6.3238600 & -1.1679900 & -2.1242800 \\ -6.5503840 & -2.1646770 & -2.5190880 \\ -6.8812070 & -1.0396490 & -1.1909230 \\ -6.6854230 & -0.4289960 & -2.8468150 \\ -4.0391350 & -1.1296580 & -3.2065600 \\ -2.9666580 & -0.9983130 & -3.0361930 \\ -4.1988500 & -2.1112600 & -3.6662550 \\ -4.3725190 & -0.3694080 & -3.9206350 \\ -3.0887500 & -6.1261380 & -1.7641920 \\ -3.1023410 & -7.2018640 & -1.9677780 \\ -3.4372480 & -5.6086550 & -2.6649430 \\ -2.0540990 & -5.8214630 & -1.5792300 \\ -5.4249800 & -6.2495500 & -0.8228160 \\ -6.0678290 & -6.0309410 & 0.0350180\end{array}$

-5.8446660
-5.4575110
-3.7416070
-4.6370730
-4.0533940
-3.3039300
-1.4436980
-1.6610010
-0.7341870
-0.9662300
2.6190810
3.6769700
2.5531620
2.1034200
2.0179430
1.6099030
3.0351430
1.4154980
7.1193730
8.1405580
6.9121130
7.0704080
6.2212740
5.5019070
6.0229340
7.2266980
2.5289670
3.5841420
2.3141120
1.9353630
2.6312350
3.7218500
2.1870460
2.3446200
-0.4654820
0.2250070
-0.2869000
1.0938860
6.3693700
0.9498580
-4.6555740
-3.6049410
-2.4417380
2.3804500
1.3793170
2.4140960
$-1.6980700$

$-1.0138010$

3. 7869570

3. 6174040

3.8665920

4.7457960

2. 9877530

3. 2182040

2. 1577730

3.8729040

2. 2234420

2. 4860390

1.3748780

3. 0769120

3. 0847880

2. 8223730

3.4705320

3.8972810

$-0.8984900$

$-0.7216370$

$-1.9703140$

$-0.6371460$

$-0.4212710$

0.1591970

$-1.4861920$

$-0.2028320$

$-3.9036820$

$-4.1984430$

$-3.3319900$

$-4.8230490$

$-3.8918680$

$-4.0030430$

$-4.8929860$

$-3.3903380$

$-0.5078850$

$-1.8405570$

0.4625310

$-2.9959160$

0.9834570

1.6213260

$-1.5145080$

0.3045140

2. 5891350

$-1.5480520$

$-1.1085770$

$-0.9873200$ 


\begin{tabular}{|c|c|c|c|c|c|c|c|}
\hline & & & & & & & \\
\hline $\mathrm{H}$ & 0.4712910 & -2.8680620 & -0.6572860 & C & -3.0699900 & 3.0623760 & 1.3308960 \\
\hline $\mathrm{H}$ & $\begin{array}{l}1.4462640 \\
0.8498730\end{array}$ & -2.1946050 & -2.6455760 & $\mathrm{H}$ & -4.8534730 & 1.8420970 & 1.3263700 \\
\hline$c$ & $\begin{array}{l}0.8498730 \\
3.6313720\end{array}$ & $\begin{array}{l}-1.3912960 \\
-4.1082640\end{array}$ & -2.4303200 & C & -2.7309570 & 4.9504890 & 2.8181240 \\
\hline c & 2.8653780 & -5.1414990 & -1.9035770 & C & $\begin{array}{l}-1.4232390 \\
-0.9560200\end{array}$ & $\begin{array}{l}3.1144590 \\
4.2916980\end{array}$ & $\begin{array}{l}2.3008150 \\
1.3058560\end{array}$ \\
\hline C & 1.4401410 & -4.6692690 & -1.6011340 & $\mathrm{C}$ & -3.5327910 & 3.9451700 & 2.3432370 \\
\hline $\mathrm{C}$ & 3.7348840 & -2.7788310 & -1.9817550 & $\mathrm{H}$ & -3.0888510 & 5.6139300 & 3.5987580 \\
\hline $\mathrm{H}$ & 4.6355890 & -4.4766370 & -2.9603440 & $\mathrm{H}$ & -0.7840650 & 5.8979160 & 2.6961340 \\
\hline $\mathrm{H}$ & 2.8186480 & -6.0996860 & -2.4284030 & $\mathrm{H}$ & 0.0506660 & 4.4216620 & 0.9212950 \\
\hline $\mathrm{H}$ & 0.8245900 & -4.7007590 & -2.5088820 & $\mathrm{H}$ & -4.5307920 & 3.7938730 & 2.7457340 \\
\hline $\mathrm{H}$ & 4.3466070 & -2.8980410 & -1.0774140 & C & -0.2086760 & 5.9135480 & -2.5031460 \\
\hline $\mathrm{H}$ & 4.2083310 & -2.0055280 & -2.5964710 & C & 1.0986880 & 6.0408380 & -3.0316160 \\
\hline $\mathrm{H}$ & 3.1204250 & -3.9467570 & -3.6891070 & C & 1.9980020 & 5.0179090 & -2.8795750 \\
\hline $\mathrm{H}$ & 3.3969470 & -5.3138650 & -0.9598970 & $\mathrm{C}$ & -0.5814200 & 4.7837660 & -1.8192790 \\
\hline $\mathrm{H}$ & 0.9398330 & -5.3129280 & -0.8725480 & $\mathrm{H}$ & -0.9251030 & 6.7162610 & -2.6464660 \\
\hline C & 2.7123000 & -2.1910700 & 1.7032220 & $\mathrm{H}$ & 1.3794720 & 6.9430340 & 5654060 \\
\hline 0 & 1.9808610 & -1.1314610 & 1.5635690 & $\mathrm{H}$ & 3.0001250 & 5.0918530 & -3.2933470 \\
\hline C & 4.0575290 & -1.9098170 & 2.2486640 & $\mathrm{H}$ & -1.5903220 & 4.6943910 & -1.4309640 \\
\hline C & 5.0731390 & -2.8745320 & 2.2102980 & C & 3.2081140 & 0.4737510 & -1.3166380 \\
\hline C & 4.3332470 & -0.6306980 & 2.7526940 & C & 3.4341180 & -0.3909500 & -2.4044010 \\
\hline $\mathrm{C}$ & 6.3479100 & -2.5590960 & 2.6634120 & $\mathrm{C}$ & 3.9793960 & 0.3627750 & -0.1434750 \\
\hline $\mathrm{H}$ & 4.8590710 & -3.8594140 & 1.8090200 & $\mathrm{C}$ & 4.4929570 & -1.2965680 & -2.3256660 \\
\hline C & 5.6071910 & -0.3243590 & 3.2138750 & C & 5.0255300 & -0.5641070 & -0.1113030 \\
\hline $\mathrm{H}$ & 3.5455280 & 0.1127280 & 2.7733810 & $\mathrm{C}$ & 5.3148050 & -1.3828210 & -1.2017580 \\
\hline C & 6.6172670 & -1.2849970 & 3.1636130 & $\mathrm{H}$ & 4.6886800 & -1.9623260 & -3.1645120 \\
\hline $\mathrm{H}$ & 7.1343170 & -3.3052720 & 2.6220850 & $\mathrm{H}$ & 5.6265610 & -0.6464710 & 0.7884660 \\
\hline $\mathrm{H}$ & 5.8141850 & 0.6654900 & 3.6100040 & C & -4.1875490 & -0.1794600 & -0.3462850 \\
\hline $\mathrm{H}$ & 7.6149260 & -1.0414160 & 3.5154200 & $\mathrm{C}$ & -4.4372220 & -1.1471360 & 0.6540640 \\
\hline $\mathrm{H}$ & 1.0328930 & -1.2775570 & 1.1665340 & C & -4.6970780 & -0.3609200 & -1.6445920 \\
\hline$c$ & 2.2041170 & -3.7379160 & 1.2750160 & $\mathrm{C}$ & -5.2253470 & -2.2520930 & 0.3347880 \\
\hline & & & & C & -5.4 & -1.4906460 & 145570 \\
\hline & R) G[M06- & $G(d)]=-3$ & 168 & $\mathrm{C}$ & -5.7567510 & -2.4434130 & -0.9412270 \\
\hline 0 & 0.5606660 & 0.2928760 & -0.2591640 & $\mathrm{H}$ & -5.4261540 & -2.9921420 & 1.1072430 \\
\hline 0 & -1.6379610 & 0.3771540 & -1.5150180 & $\mathrm{H}$ & -5.8794820 & -1.6285280 & -2.9155240 \\
\hline C & 0.8960600 & 1.4736480 & -0.8971160 & C & 3.7198080 & 1.2647420 & 1.0565100 \\
\hline C & -0.0089490 & 2.5161940 & -0.9188440 & $\mathrm{C}$ & 6.4909200 & -2.3452310 & -1.2188730 \\
\hline C & 2.2048130 & 1.5766420 & -1.4376410 & $\mathrm{C}$ & 2.5724690 & -0.3426350 & -3.6572210 \\
\hline c & 0.3358560 & 3.7141190 & -1.6259500 & $\mathrm{C}$ & -3.8860960 & -1.0356940 & 2.0701550 \\
\hline C & 2.5502110 & 2.7545610 & -2.0545710 & C & -6.6118080 & -3.6557570 & -1.2572970 \\
\hline C & 1.6371530 & 3.8312010 & -2.1873200 & C & -4.4698710 & 0.6445260 & -2.7634690 \\
\hline $\mathrm{H}$ & 3.5545780 & 2.8677920 & -2.4563900 & $\mathrm{C}$ & -5.7904680 & 1.3090580 & -3.1731530 \\
\hline C & -2.1191750 & 1.2828470 & -0.5751250 & $\mathrm{H}$ & -6.4941970 & 0.5699890 & -3.5716090 \\
\hline C & -3.3998050 & 1.0442250 & -0.0075790 & $\mathrm{H}$ & -6.2646330 & 1.8007570 & -2.3178160 \\
\hline C & -1.3237200 & 2.3570740 & -0.2454730 & $\mathrm{H}$ & -5.6155820 & 2.0607230 & -3.9499400 \\
\hline$C$ & $-3.864 C$ & 1.9699620 & 0.8952570 & C & -3.7837670 & -0.0032770 & -3.9714370 \\
\hline$c$ & -1.7717 & 20 25117 & 0.7807860 & $\mathrm{H}$ & -2.8446440 & -0.4797310 & -3.67647 \\
\hline
\end{tabular}




$\begin{array}{rrr}-4.4268710 & -0.7614530 & -4.4315840 \\ -3.5654580 & 0.7532180 & -4.7324470 \\ -7.8925600 & -3.6694190 & -0.4148210 \\ -8.5233830 & -4.5217710 & -0.6873760 \\ -7.6547180 & -3.7552020 & 0.6513270 \\ -8.4701280 & -2.7514900 & -0.5590800 \\ -5.8260470 & -4.9583740 & -1.0687090 \\ -4.9191670 & -4.9640940 & -1.6804680 \\ -5.5283710 & -5.0837250 & -0.0217440 \\ -6.4390220 & -5.8211980 & -1.3489450 \\ -4.9945110 & -0.6517990 & 3.0604780 \\ -5.4759750 & 0.2928550 & 2.7912750 \\ -5.7702910 & -1.4259760 & 3.0826600 \\ -4.5869750 & -0.5523260 & 4.0720770 \\ -3.2040520 & -2.3276420 & 2.5408650 \\ -3.9322370 & -3.1286890 & 2.7089230 \\ -2.4706760 & -2.6746920 & 1.8089190 \\ -2.6896960 & -2.1473430 & 3.4914290 \\ 4.4726110 & 2.5955300 & 0.9146270 \\ 5.5530520 & 2.4131740 & 0.8877400 \\ 4.1932330 & 3.1268440 & 0.0009380 \\ 4.2568340 & 3.2448070 & 1.7702990 \\ 4.0813200 & 0.6164160 & 2.3946010 \\ 3.6113790 & -0.3668650 & 2.5082860 \\ 5.1650850 & 0.4983650 & 2.5038290 \\ 3.7380540 & 1.2558620 & 3.2143420 \\ 6.9726890 & -2.7691690 & 0.1684510 \\ 7.7407850 & -3.5427110 & 0.0757540 \\ 7.4194000 & -1.9267630 & 0.7076590 \\ 6.1563550 & -3.1691000 & 0.7787430 \\ 7.6498830 & -1.7312510 & -2.0178720 \\ 7.3374260 & -1.4711290 & -3.0336140 \\ 8.0017040 & -0.8171370 & -1.5266490 \\ 8.4895450 & -2.4309680 & -2.0824430 \\ 2.2689080 & -1.7392360 & -4.2095100 \\ 3.1495310 & -2.1829210 & -4.6868990 \\ 1.9284140 & -2.4110390 & -3.4177770 \\ 1.4818390 & -1.6750120 & -4.9670870 \\ 3.2326300 & 0.5173160 & -4.7433160 \\ 4.2123190 & 0.1040280 & -5.0098830 \\ 2.6132260 & 0.5347000 & -5.6462960 \\ 3.3787300 & 1.5485410 & -4.4097380 \\ -0.5865200 & -0.6926600 & -0.8971170 \\ -0.0404860 & -1.5387130 & -2.0021740 \\ -1.2037110 & -1.3748650 & 0.3055030 \\ 1.6149650 & 0.1150580 & -3.3865470\end{array}$
6.1612060
2. 6442000
$-3.8103940$
$-6.9017180$
$-3.1290540$
1.9619350
0.7966140
2. 0149780
$-0.1355160$
1.2591350
0.7023230
3. 1848470
2.1825840
0.7915840
3.2993470
4.1712370
2. 1319370
0.3742130
3.7495210
3.9374290
2. 8647240
2. 5068810
0.0766490
0.4607830
$-0.5985850$
1. 0355850
1. 8062050
0.8265880
2. 3589470
1.9626400
1.4059910
0.2293790
2.1681480
2.9452500
1. 2549260
2.6130670
$-0.8778130$
1.1800070

144

$\operatorname{TS} 212(R, R) \quad G[M 06-2 X / 6-31 G(d)]=-3633.302923$

$\begin{array}{lrrr}\mathrm{O} & 0.5295550 & 0.1918960 & -0.3093550 \\ \mathrm{O} & -1.7420410 & 0.2317270 & -1.4415900 \\ \mathrm{C} & 0.8501620 & 1.3247790 & -1.0353150 \\ \mathrm{C} & -0.0492240 & 2.3715180 & -1.0919750 \\ \mathrm{C} & 2.1391890 & 1.3860450 & -1.6268530 \\ \mathrm{C} & 0.2614620 & 3.5072460 & -1.9077220\end{array}$

$-1.7525980$

$-3.2475990$

1.4839150

1.4355230

$-3.5849970$

$-0.2468180$

$-3.0190540$

$-1.9359040$

$-2.9803790$

$-3.6784970$

$-2.9654110$

$-5.1492140$

$-5.3693260$

$-4.8915390$

$-3.6632630$

$-5.5293040$

$-6.4251160$

$-3.1094930$

$-3.5057480$

$-5.7081720$

$-4.8149850$

$-4.8997890$

$-0.6077420$

$-0.2243120$

0.4524090

0.1588420

1.7855170

1.1920410

$-0.8760490$

2.8139890

2.0025100

2. 5185640

0.9643030

3.8442840

3. 3217950

$-0.8139990$

$-2.1270550$
1.0834630

$-2.3955260$

$-2.3132910$

$-0.0492480$

0.6956210

$-0.1953160$

0.5906810

$-1.1311440$

$-1.5739990$

0.5764860

1.7091640

1.2906730

0.2315590

0.8583480

1.9884920

0.5199330

1.0661570

$-0.6422850$

$-0.3112840$

2. 5994890

2.1178530

3.0248250

2. 3991570

3.8870470

5.0161240

3.5084560

5.7633160

5.3027540

4.2430840

2.6275800

5.3730770

6.6474570

3.9357380

5.9521530

1.5780600

2. 9017120
0.2614620

3. 5072460

$-1.9077220$

1.6149650

1.3748650
0.1150580

$-3.3865470$ 


$\begin{array}{lrrr}\text { C } & 2.4500300 & 2.5053840 & -2.3620050 \\ \mathrm{C} & 1.5299070 & 3.5676590 & -2.5471080 \\ \mathrm{H} & 3.4384040 & 2.5856530 & -2.8090180 \\ \mathrm{C} & -2.1513360 & 1.2037800 & -0.5349790 \\ \mathrm{C} & -3.3958670 & 1.0262940 & 0.1274110 \\ \mathrm{C} & -1.3226950 & 2.2832630 & -0.3318060 \\ \mathrm{C} & -3.7848810 & 2.0156540 & 0.9981660 \\ \mathrm{C} & -1.6911700 & 3.2537280 & 0.6556850 \\ \mathrm{C} & -2.9495600 & 3.1209870 & 1.3054750 \\ \mathrm{H} & -4.7440960 & 1.9330330 & 1.5025590 \\ \mathrm{C} & -2.4891650 & 5.1012700 & 2.6319470 \\ \mathrm{C} & -1.2203260 & 5.2125140 & 2.0134650 \\ \mathrm{C} & -0.8313220 & 4.3167150 & 1.0482780 \\ \mathrm{C} & -3.3313040 & 4.0768890 & 2.2848260 \\ \mathrm{H} & -2.7852600 & 5.8213070 & 3.3879230 \\ \mathrm{H} & -0.5487730 & 6.0137430 & 2.3064710 \\ \mathrm{H} & 0.1470390 & 4.4058310 & 0.5866240 \\ \mathrm{H} & -4.2997920 & 3.9674450 & 2.7653090 \\ \mathrm{C} & -0.3235100 & 5.6324350 & -2.9285840 \\ \mathrm{C} & 0.9512260 & 5.7033820 & -3.5411130 \\ \mathrm{C} & 1.8546100 & 4.6896270 & -3.3557320 \\ \mathrm{C} & -0.6608730 & 4.5660980 & -2.1337320 \\ \mathrm{H} & -1.0430830 & 6.4277180 & -3.0955940 \\ \mathrm{H} & 1.2036710 & 6.5558180 & -4.1634670 \\ \mathrm{H} & 2.8319520 & 4.7215550 & -3.8297990 \\ \mathrm{H} & -1.6454140 & 4.5178850 & -1.6806790 \\ \mathrm{C} & 3.1878660 & 0.3346950 & -1.4318640 \\ \mathrm{C} & 3.4659420 & -0.5773960 & -2.4627860 \\ \mathrm{C} & 3.9921300 & 0.3673050 & -0.2709510 \\ \mathrm{C} & 4.6164320 & -1.3690930 & -2.3677930 \\ \mathrm{C} & 5.1200220 & -0.4511300 & -0.2174140 \\ \mathrm{C} & 5.4698140 & -1.3002770 & -1.2709010 \\ \mathrm{H} & 4.8586000 & -2.0540930 & -3.1780620 \\ \mathrm{H} & 5.7587510 & -0.4129340 & 0.6610910 \\ \mathrm{C} & -4.2219290 & -0.1998880 & -0.0870590 \\ \mathrm{C} & -4.4296290 & -1.0984270 & 0.9807680 \\ \mathrm{C} & -4.8061470 & -0.4514100 & -1.3452450 \\ \mathrm{C} & -5.2485180 & -2.2108770 & 0.7700770 \\ \mathrm{C} & -5.6094730 & -1.5800870 & -1.5044450 \\ \mathrm{C} & -5.8493470 & -2.4705370 & -0.4582780 \\ \mathrm{H} & -5.4210940 & -2.9033610 & 1.5917630 \\ \mathrm{H} & -6.0685080 & -1.7648760 & -2.4742240 \\ \mathrm{C} & 3.6784290 & 1.3188510 & 0.8773950 \\ \mathrm{C} & 6.7582170 & -2.1026370 & -1.2320920 \\ \mathrm{C} & 2.5627090 & -0.7066820 & -3.6794570 \\ \mathrm{C} & -3.8070190 & -0.9085430 & 2.3579290 \\ & & & \end{array}$

-6.7343780
-4.6328900
-5.9669720
-6.7057860
-6.3779280
-5.8314500
-4.0339800
-3.0841060
-4.7143630
-3.8550760
-6.1478410
-6.7704530
-6.1006980
-5.1346030
-8.1642840
-8.5976200
-8.1791230
-8.8034250
-4.8635600
-5.3590600
-5.6365440
-4.4045900
-3.1031470
-3.8211950
-2.3981220
-2.5516960
4.3071530
5.3989740
3.9668130
4.0484210
4.1239260
3.7293820
5.2159250
3.7595150
6.8567710
7.8034350
6.8208970
6.0416280
7.9709170
7.9231080
8.0085280
8.9016190
2.2822720
3.1699570
1.9557520
1.4919950
-3.6865830
0.4850940
1.1496970
0.4017630
1.6983410
1.8520140
-0.2412250
-0.7154460
-1.0137950
0.4673470
-4.6385740
-5.5338760
-4.1532060
-4.9490300
-3.2817610
-2.6164190
-2.7586230
-4.1661790
-0.4615670
0.4654590
-1.2308100
-0.2996550
-2.1733350
-2.9656670
-2.5565490
-1.9427480
2.6993000
2.6109720
3.1472000
3.3780790
0.7894590
-0.2142200
0.7561380
1.4580010
-3.0083790
-3.5581430
-2.4226010
-3.7372720
-1.1654450
-0.5274620
-0.5161660
-1.7408570
-2.1727460
-2.6657260
-2.7310890
-2.2324560

$-0.6552740$

$-2.5321840$

$-2.8957760$

$-3.2043090$

$-2.0423670$

$-3.7248460$

$-3.7417700$

$-3.4798810$

$-4.1164610$

$-4.5572260$

$-1.7039920$

$-1.8018090$

$-2.6851430$

$-1.4324710$

$-1.0314300$

$-0.2787750$

$-1.9940150$

$-1.1208250$

3.3779790

3.0745920

3.4888010

4. 3590090

2. 8677700

3.1057930

2.1260120

3.7861230

0.6382510

0.5991760

$-0.2990220$

1.4584370

2.2422290

2.4359860

2.3231960

3.0287610

0.0001900

$-0.0090170$

0.9252930

0.0289350

$-1.3048710$

$-2.1923530$

$-0.4229370$

$-1.3409980$

$-4.0261400$

$-4.4373720$

$-3.1445730$

$-4.7809020$ 


\begin{tabular}{|c|c|c|}
\hline $\begin{array}{l}3.1564080 \\
4.1371930 \\
2.5033010 \\
3.2844440 \\
0.6629370 \\
-0.1874230 \\
-1.2071120 \\
1.6015080 \\
6.7717150 \\
2.5881680 \\
-3.9399000 \\
-6.7743770 \\
-3.0491050 \\
1.8906270 \\
0.8196300 \\
1.8655520 \\
0.1460090 \\
1.1450960 \\
0.5466200 \\
3.2900950 \\
2.3995760 \\
0.9499090 \\
3.2849690 \\
4.3161630 \\
2.4390290 \\
0.5112850 \\
3.7769460 \\
3.8280100 \\
2.9313010 \\
2.7617390 \\
0.3101350 \\
0.5548430 \\
-0.5234020 \\
1.1288100 \\
1.9365950 \\
0.8794650 \\
2.4878580 \\
2.1235550 \\
1.4568920 \\
0.2524560 \\
2.2575800 \\
3.1037370 \\
1.2739360 \\
2.7015240 \\
-0.8230090 \\
1.2953540\end{array}$ & $\begin{array}{r}0.0218780 \\
-0.3966050 \\
-0.0896530 \\
1.0904060 \\
-0.8130080 \\
-1.7173440 \\
-1.4279450 \\
-0.2434000 \\
-2.7423480 \\
1.4438590 \\
1.2832620 \\
-4.2205240 \\
-0.1240530 \\
-3.0026540 \\
-3.5175820 \\
-1.9321780 \\
-3.0316730 \\
-3.7746780 \\
-3.1148050 \\
-5.0096110 \\
-5.2083520 \\
-4.8411670 \\
-3.5448700 \\
-5.3193250 \\
-6.2436850 \\
-5.5772090 \\
-2.9138730 \\
-3.4037360 \\
-5.6423070 \\
-4.5767330 \\
-4.8345590 \\
-0.4497360 \\
-0.1203550 \\
0.6696730 \\
0.4550670 \\
1.9754310 \\
1.5389600 \\
-0.5589130 \\
3.0543530 \\
2.1329570 \\
2.8375820 \\
1.3724080 \\
4.0625500 \\
3.6805450 \\
-0.7701120 \\
-1.9622410\end{array}$ & $\begin{array}{r}-4.8914650 \\
-5.1453650 \\
-5.7633350 \\
-4.6947430 \\
-0.8305650 \\
-1.9226420 \\
0.4407020 \\
-3.4327730 \\
-2.1236150 \\
0.9194250 \\
-2.2521360 \\
0.3026030 \\
2.2859010 \\
0.0501170 \\
0.9159900 \\
-0.1689740 \\
0.8536980 \\
-0.9247120 \\
-1.3965080 \\
0.6898390 \\
1.9168330 \\
1.5939380 \\
0.2475660 \\
0.9105120 \\
2.2657770 \\
0.9067510 \\
0.9997780 \\
-0.6918040 \\
-0.1310370 \\
2.7384250 \\
2.4805590 \\
3.0479150 \\
2.4228470 \\
3.8322140 \\
4.9531150 \\
3.3877310 \\
5.6258510 \\
5.2914020 \\
4.0477080 \\
2.5147810 \\
5.1689440 \\
6.5034980 \\
3.6885590 \\
5.6893340 \\
1.6595010 \\
2.9997250\end{array}$ \\
\hline
\end{tabular}

$\begin{array}{lrrr}\text { TS213(R, R }) \text { G }[\mathrm{M} 06-2 \times / 6-31 G(\mathrm{~d})]=-3633.303954 \\ \mathrm{O} & 0.5479200 & 0.2234060 & -0.2855360 \\ \mathrm{O} & -1.6295820 & 0.3052220 & -1.5891470 \\ \mathrm{C} & 0.9235920 & 1.3835270 & -0.9376100 \\ \mathrm{C} & 0.0266400 & 2.4300300 & -1.0297540 \\ \mathrm{C} & 2.2593940 & 1.4731820 & -1.4101980 \\ \mathrm{C} & 0.3977120 & 3.5969020 & -1.7727520 \\ \mathrm{C} & 2.6232860 & 2.6204030 & -2.0750640 \\ \mathrm{C} & 1.7149270 & 3.6849720 & -2.3003160 \\ \mathrm{H} & 3.6467310 & 2.7226490 & -2.4291160 \\ \mathrm{C} & -2.1098180 & 1.2425010 & -0.6811170 \\ \mathrm{C} & -3.4002250 & 1.0389880 & -0.1225400 \\ \mathrm{C} & -1.3021360 & 2.3135000 & -0.3754290 \\ \mathrm{C} & -3.8576190 & 1.9951900 & 0.7517810 \\ \mathrm{C} & -1.7467710 & 3.2454410 & 0.6180690 \\ \mathrm{C} & -3.0512680 & 3.0879270 & 1.1638510 \\ \mathrm{H} & -4.8519660 & 1.8933630 & 1.1786020 \\ \mathrm{C} & -2.6974240 & 5.0112610 & 2.6018230 \\ \mathrm{C} & -1.3850330 & 5.1457870 & 2.0876920 \\ \mathrm{C} & -0.9214220 & 4.2901420 & 1.1189740 \\ \mathrm{C} & -3.5088820 & 4.0035040 & 2.1491710 \\ \mathrm{H} & -3.0515240 & 5.6998630 & 3.3621120 \\ \mathrm{H} & -0.7392000 & 5.9329290 & 2.4646390 \\ \mathrm{H} & 0.0897870 & 4.3960550 & 0.7389640 \\ \mathrm{H} & -4.5111340 & 3.8756140 & 2.5491680 \\ \mathrm{C} & -0.1114350 & 5.7587710 & -2.7562340 \\ \mathrm{C} & 1.2098050 & 5.8562960 & -3.2563100 \\ \mathrm{C} & 2.1001590 & 4.8382060 & -3.0348460 \\ \mathrm{C} & -0.5082840 & 4.6616630 & -2.0339420 \\ \mathrm{H} & -0.8187600 & 6.5584140 & -2.9522120 \\ \mathrm{H} & 1.5089590 & 6.7329690 & -3.8217030 \\ \mathrm{H} & 3.1137150 & 4.8910880 & -3.4231750 \\ \mathrm{H} & -1.5270040 & 4.5935230 & -1.6671320 \\ \mathrm{C} & 3.3063280 & 0.4321350 & -1.1539690 \\ \mathrm{C} & 3.6994220 & -0.4391560 & -2.1860620 \\ \mathrm{C} & 4.0097440 & 0.4517460 & 0.0677900 \\ \mathrm{C} & 4.8655620 & -1.1928150 & -2.0250060 \\ \mathrm{C} & 5.1651610 & -0.3250110 & 0.1830740 \\ \mathrm{C} & 5.6336100 & -1.1196520 & -0.8632650 \\ \mathrm{H} & 5.1868080 & -1.8427560 & -2.8366860 \\ \mathrm{H} & 5.7383500 & -0.2958290 & 1.1063470 \\ \mathrm{C} & -4.2029450 & -0.1800190 & -0.4414130 \\ \mathrm{C} & -4.4907420 & -1.1137310 & 0.5804910 \\ \mathrm{C} & -4.6881380 & -0.3907550 & -1.7450460 \\ \mathrm{C} & -5.2882550 & -2.2161110 & 0.2753140\end{array}$




$\begin{array}{rrr}-5.4752660 & -1.5170670 & -1.9999830 \\ -5.7925780 & -2.4377280 & -1.0065490 \\ -5.5181490 & -2.9298030 & 1.0643120 \\ -5.8610450 & -1.6775620 & -3.0051670 \\ 3.5737580 & 1.3518850 & 1.2173860 \\ 6.9334270 & -1.8925470 & -0.7222540 \\ 2.9002430 & -0.5527970 & -3.4749230 \\ -3.9719430 & -0.9698160 & 2.0055080 \\ -6.6588790 & -3.6461120 & -1.3067130 \\ -4.4283220 & 0.5818050 & -2.8858340 \\ -5.7332250 & 1.2533680 & -3.3329500 \\ -6.4409280 & 0.5138280 & -3.7233710 \\ -6.2135530 & 1.7731510 & -2.4978980 \\ -5.5359640 & 1.9824240 & -4.1257520 \\ -3.7328070 & -0.1057560 & -4.0660500 \\ -2.8045640 & -0.5862540 & -3.7445800 \\ -4.3787400 & -0.8671650 & -4.5167690 \\ -3.4930030 & 0.6280600 & -4.8426260 \\ -7.9643700 & -3.6079970 & -0.5038440 \\ -8.6016960 & -4.4591800 & -0.7646800 \\ -7.7598560 & -3.6586660 & 0.5714100 \\ -8.5210460 & -2.6864980 & -0.6986240 \\ -5.9029730 & -4.9545370 & -1.0489470 \\ -4.9780120 & -4.9971650 & -1.6314640 \\ -5.6399310 & -5.0487650 & 0.0105190 \\ -6.5226790 & -5.8159220 & -1.3185580 \\ -5.1016140 & -0.5590970 & 2.9602730 \\ -5.5745920 & 0.3790930 & 2.6552750 \\ -5.8797320 & -1.3308090 & 2.9852040 \\ -4.7168230 & -0.4338710 & 3.9778910 \\ -3.3046120 & -2.2525080 & 2.5200450 \\ -4.0382190 & -3.0493210 & 2.6846770 \\ -2.5541930 & -2.6150650 & 1.8131970 \\ -2.8139580 & -2.0541400 & 3.4794670 \\ 4.1974680 & 2.7494190 & 1.0916760 \\ 5.2896510 & 2.6789790 & 1.1514700 \\ 3.9375070 & 3.2288640 & 0.1441210 \\ 3.8512510 & 3.3914200 & 1.9092410 \\ 3.8999060 & 0.7709870 & 2.5950800 \\ 3.5031810 & -0.2439900 & 2.7097420 \\ 4.9800790 & 0.7448420 & 2.7749630 \\ 3.4555300 & 1.4017960 & -1.9110030 \\ 7.8705490 & -1.6539570 & -2.8345500 \\ 8.8341800 & -2.1438140 & \\ 7.4498340 & -2.0658680 & -0669920 \\ 8.0485260 & -0.5858740 & \end{array}$

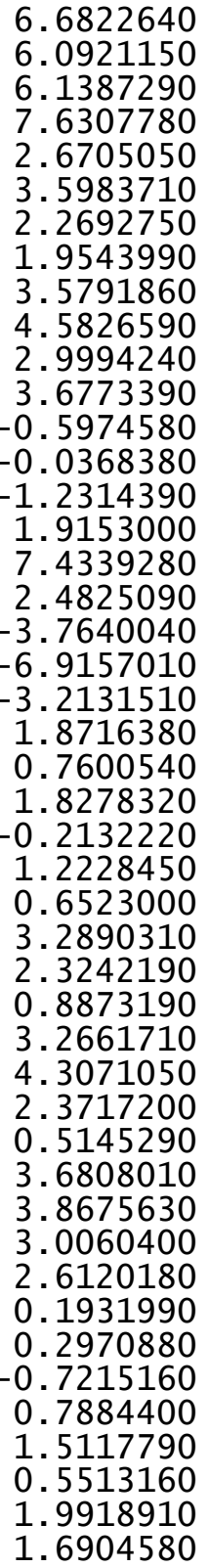

-3.3926710
-3.5801780
-3.8096660
-3.9317310
-2.0139920
-2.4902030
-2.5934680
-2.0661400
0.2126580
-0.1880640
0.1166070
1.2771650
-0.7609950
-1.6201110
-1.4278690
-0.1080530
-1.5187680
1.4606290
1.3733890
-3.6076230
-0.1829900
-2.9453060
-3.5285080
-1.8680840
-3.0668780
-3.6960960
-3.0291870
-4.9355920
-5.2211300
-4.8776910
-3.4508850
-5.2279880
-6.2715220
-5.5928140
-2.8445260
-3.2435130
-5.5342700
-4.6228890
-4.9296680
-0.5507430
-0.1990910
0.5366640
0.2787220
1.8592850
1.3358630
-0.7477420
$-0.5275180$

0.3749680

$-1.3839260$

$-0.4343390$

$-3.8747970$

$-4.2102260$

$-3.0385760$

$-4.7008460$

$-4.6173240$

$-4.8019480$

$-5.5413110$

$-4.3842570$

$-0.9370670$

$-2.0254950$

0.2642190

$-3.2971410$

0.1799450

1.1630570

$-2.5281470$

$-2.3728750$

2. 0170510

0.0691190

0.8335410

$-0.1062230$

0.7236690

$-0.9879780$

$-1.4846470$

0.7075060

1.8594430

1.4597290

0.3401010

0.9817740

2. 1589740

0.7140960

1.1564850

$-0.5496900$

$-0.1667900$

2. 7337010

2. 3029930

3. 0554190

2. 3475860

3. 9353020

5.1038890

3. 5363110

5.8675880

5.4070950 


\begin{tabular}{|c|c|c|c|c|c|c|c|}
\hline & & & & & & $0-1511400$ & 02171100 \\
\hline H & -0.0106610 & $\begin{array}{l}2.91<3560 \\
2.0509360\end{array}$ & $\begin{array}{l}4.2893320 \\
2.6269420\end{array}$ & C & $\begin{array}{l}5.1200320 \\
5.4698270\end{array}$ & $\begin{array}{l}-0.4511480 \\
-1.3002040\end{array}$ & $\begin{array}{l}-0.21 / 4400 \\
-1.2709970\end{array}$ \\
\hline C & 1.7749120 & 2.6520930 & 5.4568860 & $\mathrm{H}$ & 4.8586120 & -2.0538630 & -3.1782180 \\
\hline $\mathrm{H}$ & 2.5423770 & 1.1348780 & 6.7807530 & $\mathrm{H}$ & 5.7587540 & -0.4130240 & 0.6610720 \\
\hline $\mathrm{H}$ & 0.8857440 & 3.9344240 & 3.9651120 & $\mathrm{C}$ & -4.2219370 & -0.1998680 & -0.0871230 \\
\hline $\mathrm{H}$ & 2.1638620 & 3.4743840 & 6.0494070 & $\mathrm{C}$ & -4.4296400 & -1.0984330 & 0.9806830 \\
\hline $\mathrm{H}$ & -0.9487530 & -0.8187390 & 1.5360530 & C & -4.8061320 & -0.4513710 & -1.3453220 \\
\hline $\mathrm{s}$ & 1.0483350 & -2.0580760 & 3.0123380 & $\mathrm{C}$ & -5.2485160 & -2.2108850 & 0.7699590 \\
\hline & & & & $\mathrm{C}$ & -5.6094440 & -1.5800540 & -1.5045570 \\
\hline & R) $\mathrm{G}[\mathrm{M06}-2$ & $G(d)]=-36$ & 924 & $\mathrm{C}$ & -5.8493250 & -2.4705270 & -0.4584110 \\
\hline 0 & 0.5295410 & 0.1919180 & -0.3093560 & $\mathrm{H}$ & -5.4210980 & -2.9033870 & 1.5916280 \\
\hline 0 & -1.7420350 & 0.2318550 & -1.4416260 & $\mathrm{H}$ & -6.0684650 & -1.7648270 & -2.4743460 \\
\hline $\mathrm{C}$ & 0.8501630 & 1.3248550 & -1.0352250 & $\mathrm{C}$ & 3.6784360 & 1.3187380 & 0.8775170 \\
\hline C & -0.0492150 & 2.3716070 & -1.0918280 & $\mathrm{C}$ & 6.7582290 & -2.1025700 & -1.2322790 \\
\hline $\mathrm{C}$ & 2.1391990 & 1. 3861490 & -1.6267430 & $\mathrm{C}$ & 2.5627220 & -0.7064350 & -3.6795020 \\
\hline C & 0.2614920 & 3.5073850 & -1.9074980 & $\mathrm{C}$ & -3.8070490 & -0.9085720 & 2.3578580 \\
\hline C & 2.4500580 & 2.5055350 & -2.3618170 & $\mathrm{C}$ & -6.7343500 & -3.6865710 & -0.6554390 \\
\hline C & 1.5299470 & 3.5678300 & -2.5468610 & $\mathrm{C}$ & -4.6328680 & 0.4851600 & -2.5322380 \\
\hline $\mathrm{H}$ & 3.4384380 & 2.5858240 & -2.8088120 & $\mathrm{C}$ & -5.9669490 & 1.1497660 & -2.8958270 \\
\hline $\mathrm{C}$ & -2.1513510 & 1.2038460 & -0.5349610 & $\mathrm{H}$ & -6.7057550 & 0.4018360 & -3.2043880 \\
\hline C & -3.3958960 & 1.0263200 & 0.1273920 & $\mathrm{H}$ & -6.3779180 & 1.6983830 & -2.0424070 \\
\hline C & -1.3227100 & 2.2833130 & -0.3316990 & $\mathrm{H}$ & -5.8314220 & 1.8521060 & -3.7248750 \\
\hline $\mathrm{C}$ & -3.7849400 & 2.0156410 & 0.9981790 & $\mathrm{C}$ & -4.0339430 & -0.2411280 & -3.7418340 \\
\hline C & -1.6912160 & 3.2537290 & 0.6558290 & $\mathrm{H}$ & -3.0840710 & -0.7153530 & -3.4799460 \\
\hline $\mathrm{C}$ & -2.9496330 & 3.1209640 & 1.3055620 & $\mathrm{H}$ & -4.7143190 & -1.0136910 & -4.1165520 \\
\hline $\mathrm{H}$ & -4.7441680 & 1.9329920 & 1.5025410 & $\mathrm{H}$ & -3.8550310 & 0.4674640 & -4.5572710 \\
\hline C & -2.4892930 & 5.1011930 & 2.6321320 & $\mathrm{C}$ & -6.1478420 & -4.6385090 & -1.7042220 \\
\hline C & -1.2204270 & 5.2124590 & 2.0137090 & $\mathrm{H}$ & -6.7704420 & -5.5338180 & -1.8020470 \\
\hline C & -0.8313840 & 4.3166990 & 1.0485010 & $\mathrm{H}$ & -6.1007490 & -4.1531030 & -2.6853560 \\
\hline C & -3.3314180 & 4.0768280 & 2.2849350 & $\mathrm{H}$ & -5.1345870 & -4.9489580 & -1.4327570 \\
\hline $\mathrm{H}$ & -2.7854190 & 5.8212020 & 3.3881230 & $\mathrm{C}$ & -8.1642690 & -3.2817380 & -1.0315310 \\
\hline $\mathrm{H}$ & -0.5488850 & 6.0136740 & 2.3067760 & $\mathrm{H}$ & -8.5975930 & -2.6164530 & -0.2788170 \\
\hline $\mathrm{H}$ & 0.1469940 & 4.4058350 & 0.5868890 & $\mathrm{H}$ & -8.1791370 & -2.7585320 & -1.9940780 \\
\hline $\mathrm{H}$ & -4.2999270 & 3.9673660 & 2.7653720 & $\mathrm{H}$ & -8.8034050 & -4.1661560 & -1.1209710 \\
\hline C & -0.3234460 & 5.6326450 & -2.9282330 & C & -4.8636120 & -0.4616420 & 3.3779050 \\
\hline C & 0.9513010 & 5.7036240 & -3.5407350 & $\mathrm{H}$ & -5.3591690 & 0.4653490 & 3.0745060 \\
\hline C & 1.8546730 & 4.6898480 & -3.3554060 & $\mathrm{H}$ & -5.6365500 & -1.2309290 & 3.4887420 \\
\hline C & -0.6608300 & 4.5662590 & -2.1334570 & $\mathrm{H}$ & -4.4046480 & -0.2996890 & 4.3589310 \\
\hline $\mathrm{H}$ & -1.0430100 & 6.4279440 & -3.0952040 & C & -3.1031540 & -2.1733590 & 2.8676800 \\
\hline $\mathrm{H}$ & 1.2037640 & 6.5560990 & -4.1630290 & $\mathrm{H}$ & -3.8211800 & -2.9657410 & 3.1055990 \\
\hline $\mathrm{H}$ & 2.8320230 & 4.7217990 & -3.8294550 & $\mathrm{H}$ & -2.3980490 & -2.5564940 & 2.1259570 \\
\hline $\mathrm{H}$ & -1.6453810 & 4.5180250 & -1.6804270 & $\mathrm{H}$ & -2.5517890 & -1.9427960 & 3.7860900 \\
\hline C & 3.1878740 & 0.3347810 & -1.4318280 & $\mathrm{C}$ & 4.3071720 & 2.6992030 & 0.6385070 \\
\hline c & 3.4659540 & -0.5772280 & -2.4628230 & $\mathrm{H}$ & 5.3989940 & 2.6108760 & 0.5994730 \\
\hline C & 3.9921390 & 0.3672930 & -0.2709120 & $\mathrm{H}$ & 3.9668740 & 3.1471770 & -0.2987460 \\
\hline C & 4.6164440 & -1.36892 & -2.3678940 & $\mathrm{H}$ & 4.0484070 & 3.3779190 & 1.4587350 \\
\hline
\end{tabular}




$\begin{array}{rrr}4.1239150 & 0.7892120 & 2.2423080 \\ 3.7293220 & -0.2144640 & 2.4359830 \\ 5.2159120 & 0.7558320 & 2.3232700 \\ 3.7595380 & 1.4577050 & 3.0288970 \\ 6.8569530 & -3.0082080 & 0.0000630 \\ 7.8035640 & -3.5580630 & -0.0093000 \\ 6.8213390 & -2.4223490 & 0.9251240 \\ 6.0417460 & -3.7370210 & 0.0290450 \\ 7.9709180 & -1.1653810 & -1.3053030 \\ 7.9230100 & -0.5275060 & -2.1928580 \\ 8.0086180 & -0.5159950 & -0.4234520 \\ 8.9016190 & -1.7407920 & -1.3414610 \\ 2.2822990 & -2.1724810 & -4.0262680 \\ 3.1700020 & -2.6654440 & -4.4374780 \\ 1.9557380 & -2.7308650 & -3.1447420 \\ 1.4920620 & -2.2321590 & -4.7810730 \\ 3.1564190 & 0.0222000 & -4.8914660 \\ 4.1371930 & -0.3962840 & -5.1454080 \\ 2.5032990 & -0.0892550 & -5.7633350 \\ 3.2844790 & 1.0907110 & -4.6946690 \\ -0.6629650 & -0.8129370 & -0.8306420 \\ -0.1874680 & -1.7172290 & -1.9227620 \\ -1.2071740 & -1.4279210 & 0.4405910 \\ 1.6015170 & -0.2431740 & -3.4327930 \\ 6.7716110 & -2.7423570 & -2.1237480 \\ 2.5881760 & 1.4437480 & 0.9195510 \\ -3.9398830 & 1.2833240 & -2.2521650 \\ -6.7743160 & -4.2205600 & 0.3024130 \\ -3.0491490 & -0.1240660 & 2.2858570 \\ 1.8906370 & -3.0025630 & 0.0499910 \\ 0.8195780 & -3.5175650 & 0.9157410 \\ 1.8655380 & -1.9320800 & -0.1690590 \\ -0.1460690 & -3.0316820 & 0.8533890 \\ 1.1452150 & -3.7745760 & -0.9249340 \\ 0.5467880 & -3.1147050 & -1.3967770 \\ 3.2901310 & -5.0094760 & 0.6898000 \\ 2.3994990 & -5.2082680 & 1.9167050 \\ 0.9498460 & -4.8411730 & 1.5936450 \\ 3.2849830 & -3.5447290 & 0.2475500 \\ 4.3161910 & -5.3191480 & 0.9105660 \\ 2.4389800 & -6.2435950 & 2.2656610 \\ 0.5113680 & -5.5772100 & 0.9063580 \\ 3.7768630 & -2.9137270 & -0.6917670 \\ 3.8281020 & -3.4035510 & 2.7383300 \\ 2.9314390 & -5.6421770 & \end{array}$

$\begin{array}{lr}\mathrm{H} & 0.3099570 \\ \mathrm{C} & 0.5547780 \\ \mathrm{O} & -0.5234400 \\ \mathrm{C} & 1.1288090 \\ \mathrm{C} & 1.9365060 \\ \mathrm{C} & 0.8796210 \\ \mathrm{C} & 2.4878320 \\ \mathrm{H} & 2.1233450 \\ \mathrm{C} & 1.4571170 \\ \mathrm{H} & 0.2526830 \\ \mathrm{C} & 2.2577120 \\ \mathrm{H} & 3.1036390 \\ \mathrm{H} & 1.2742930 \\ \mathrm{H} & 2.7017070 \\ \mathrm{H} & -0.8230690 \\ \mathrm{~S} & 1.2952150 \\ \mathrm{I} & \end{array}$
$-4.8346550$
$-0.4500040$
$-0.1205030$
0.6692860
0.4545070
1.9751040
1.5382900
$-0.5595190$
3.0539150
2.1327590
2.8369720
1. 3716060
4.0621600
3.6798490
$-0.7701770$
$-1.9625440$

144

2.4801810
3.0478750
2.4228270
3.8322960
4.9532270
3.3879040
5.6260880
5.2914410
4.0480030
2.5149270
5.1692730
6.5037610
3.6889240
5.6897600
1.6594100
2.9995440

$\operatorname{TS} 215(\mathrm{R}, \mathrm{R}) \mathrm{G}[\mathrm{M} 06-2 \mathrm{X} / 6-31 \mathrm{G}(\mathrm{d})]=-3633.304233$

$\begin{array}{lrrr}\mathrm{TS} 215(\mathrm{R}, \mathrm{R}) \mathrm{G}[\mathrm{M} 06-2 \mathrm{X} / 6-31 \mathrm{G}(\mathrm{d})]=-3633.304233 \\ \mathrm{O} & 0.2277430 & -1.2236530 & 0.3203200 \\ \mathrm{O} & 2.0867070 & -0.0842300 & -0.9322060 \\ \mathrm{C} & 0.5975700 & -2.4434590 & -0.2196630 \\ \mathrm{C} & 1.9319170 & -2.7897710 & -0.2373610 \\ \mathrm{C} & -0.4512190 & -3.2974610 & -0.6583820 \\ \mathrm{C} & 2.2952230 & -4.0276640 & -0.8655050 \\ \mathrm{C} & -0.0976790 & -4.5234970 & -1.1615900 \\ \mathrm{C} & 1.2642350 & -4.9017680 & -1.3121000 \\ \mathrm{H} & -0.8700050 & -5.2273500 & -1.4603260 \\ \mathrm{C} & 2.9348420 & -0.5395670 & 0.0608880 \\ \mathrm{C} & 3.8220780 & 0.4117920 & 0.6325010 \\ \mathrm{C} & 2.9237390 & -1.8772850 & 0.3963140 \\ \mathrm{C} & 4.7526530 & -0.0469780 & 1.5293620 \\ \mathrm{C} & 3.8258070 & -2.3230670 & 1.4214790 \\ \mathrm{C} & 4.7713830 & -1.4015270 & 1.9548280 \\ \mathrm{H} & 5.4803310 & 0.6431200 & 1.9486150 \\ \mathrm{C} & 5.6608610 & -3.1239590 & 3.4160500 \\ \mathrm{C} & 4.6898560 & -4.0282480 & 2.9244590 \\ \mathrm{C} & 3.7974940 & -3.6404710 & 1.9561830 \\ \mathrm{C} & 5.6927040 & -1.8375150 & 2.9439750 \\ \mathrm{H} & 6.3646370 & -3.4470380 & 4.1763810 \\ \mathrm{H} & 4.6460910 & -5.0371750 & 3.3224290 \\ \mathrm{H} & 3.0512050 & -4.3419870 & 1.6002250 \\ \mathrm{H} & 6.4141470 & -1.1218940 & 3.3292890 \\ \mathrm{C} & 3.9536550 & -5.6009550 & -1.6882580 \\ \mathrm{C} & 2.9279600 & -6.4863030 & -2.0965620 \\ \mathrm{C} & 1.6139860 & -6.1393830 & -1.9143170 \\ \mathrm{C} & 3.6469770 & -4.4053970 & -1.0875830\end{array}$




$\begin{array}{rrrr}\mathrm{H} & 4.9920530 & -5.8682300 & -1.8568700 \\ \mathrm{H} & 3.1852130 & -7.4313250 & -2.5640450 \\ \mathrm{H} & 0.8153050 & -6.8005500 & -2.2396400 \\ \mathrm{H} & 4.4401220 & -3.7291980 & -0.7859110 \\ \mathrm{C} & -1.8704530 & -2.8593540 & -0.4767580 \\ \mathrm{C} & -2.6443480 & -2.4316410 & -1.5754780 \\ \mathrm{C} & -2.4186130 & -2.8607640 & 0.8188850 \\ \mathrm{C} & -3.9604240 & -2.0230480 & -1.3484710 \\ \mathrm{C} & -3.7452850 & -2.4595900 & 0.9916860 \\ \mathrm{C} & -4.5321810 & -2.0336930 & -0.0746890 \\ \mathrm{H} & -4.5637570 & -1.6972820 & -2.1932880 \\ \mathrm{H} & -4.1847630 & -2.4851510 & 1.9877330 \\ \mathrm{C} & 3.7077950 & 1.8539980 & 0.2554140 \\ \mathrm{C} & 3.0527980 & 2.7523070 & 1.1252520 \\ \mathrm{C} & 4.2240270 & 2.3011600 & -0.9710900 \\ \mathrm{C} & 2.9662450 & 4.0938890 & 0.7557660 \\ \mathrm{C} & 4.1131710 & 3.6559960 & -1.2967110 \\ \mathrm{C} & 3.4913110 & 4.5669420 & -0.4488230 \\ \mathrm{H} & 2.4603120 & 4.7905700 & 1.4200910 \\ \mathrm{H} & 4.5176500 & 4.0108210 & -2.2432430 \\ \mathrm{C} & -1.6333690 & -3.3456460 & 2.0289240 \\ \mathrm{C} & -5.9750430 & -1.6183050 & 0.1414290 \\ \mathrm{C} & -2.1007540 & -2.4614700 & -2.9963400 \\ \mathrm{C} & 2.4543370 & 2.2949410 & 2.4488470 \\ \mathrm{C} & 3.3491430 & 6.0257090 & -0.8403770 \\ \mathrm{C} & 4.9035340 & 1.3597100 & -1.9527570 \\ \mathrm{C} & 6.3925030 & 1.6988090 & -2.0922200 \\ \mathrm{H} & 6.5266290 & 2.7092100 & -2.4943130 \\ \mathrm{H} & 6.8980760 & 1.6512190 & -1.1224370 \\ \mathrm{H} & 6.8846000 & 0.9965750 & -2.7731330 \\ \mathrm{C} & 4.2054060 & 1.3707470 & -3.3172090 \\ \mathrm{H} & 3.1462000 & 1.1181610 & -3.2128770 \\ \mathrm{H} & 4.2800940 & 2.3544320 & -3.7934500 \\ \mathrm{H} & 4.6714510 & 0.6405420 & -3.9869470 \\ \mathrm{C} & 3.9678240 & 6.9619770 & 0.2029990 \\ \mathrm{H} & 3.9031290 & 8.0028400 & -0.1301710 \\ \mathrm{H} & 3.4404300 & 6.8847790 & 1.1600220 \\ \mathrm{H} & 5.0204460 & 6.7196530 & 0.3766380 \\ \mathrm{C} & 1.8772560 & 6.3819060 & -1.0846380 \\ \mathrm{H} & 1.4413420 & 5.7382030 & -1.8550760 \\ \mathrm{H} & 1.2921530 & 6.2545570 & -0.1664660 \\ \mathrm{H} & 1.7789780 & 7.4238230 & -1.4067860 \\ \mathrm{C} & 3.4574370 & 2.4887990 & 3.5948740 \\ \mathrm{H} & 4.3800820 & 1.9259490 & 3.4304650 \\ \mathrm{H} & 3.7205020 & 3.5488520 & 3.6885640 \\ \mathrm{H} & 3.0218890 & 2.1613600 & 4.5450020 \\ & & & \end{array}$

\begin{abstract}
1. 1415470
1. 3122560

0.4525870

0.6607430

$-2.1336940$

$-3.1873370$

$-2.0444650$

$-1.5547810$

$-1.6824810$

$-1.3610240$

$-2.6927530$

$-1.0206480$

$-6.9391650$

$-7.9781980$

$-6.7820870$

$-6.7933750$

$-6.2136100$

$-5.5224750$

$-6.0693900$

$-7.2371190$

$-2.5495520$

$-3.6198910$

$-2.3403370$

$-2.0129860$

$-2.5151450$

$-3.6076980$

$-2.1096940$

$-2.1634660$

0.5042410

$-0.1960600$

0.2384600

$-1.0085700$

$-6.1768120$

$-0.5832660$

4.8341650

3. 8912590

2. 2319380

$-2.5137740$

$-1.6058740$

$-2.4398680$

$-0.6534210$

$-1.6023440$

$-0.9343570$

$-3.9723990$

$-3.2951000$

$-1.8226830$
\end{abstract}

3.0071030

4.0481480

2. 9944760

2. 5051150

$-4.7289830$

$-4.6825820$

$-5.4516860$

$-5.0984910$

$-2.3521570$

$-1.3570450$

$-2.2741770$

$-2.6831630$

$-2.5605610$

$-2.2800670$

$-2.5121470$

$-3.5978260$

$-0.1647640$

0.5088360

$-0.0423750$

0.1427470

$-1.2661960$

$-1.3093340$

$-0.3218000$

$-1.2662100$

$-3.7639050$

$-3.8248340$

$-3.7988780$

$-4.6477430$

0.0829300

$-0.0124100$

1.2706370

$-2.4271990$

$-1.6913280$

$-3.4537660$

0.3413030

6.1653460

1. 2259400

1. 9714200

3. 0765030

1. 0758820

2. 8146080

1.9118670

1.1740930

3.5852590

4.7456860

4.4404610
2. 7919110

3.0883950

1.9442980

3.6383860

2.4638470

2.7616250

1.6463470

3. 3167070

3. 1931950

2.8689000

3. 6120450

3. 9999260

$-0.5887870$

$-0.3871770$

$-1.6721290$

$-0.2726880$

$-0.2782150$

0.2385360

$-1.3581240$

$-0.0384380$

$-3.8405590$

$-4.0707710$

$-3.3342180$

$-4.7948530$

$-3.6966400$

$-3.7632160$

$-4.7134290$

$-3.1578670$

$-0.6114480$

$-1.9367100$

0.2819070

$-2.9367770$

1. 2177250

1. 7433520

$-1.5577960$

$-1.7842400$

2. 3601480

$-1.6864400$

$-1.3464130$

$-1.0634510$

$-0.8975650$

$-2.8041690$

$-2.5627250$

$-2.9547240$

$-2.2221080$

$-1.9309910$ 


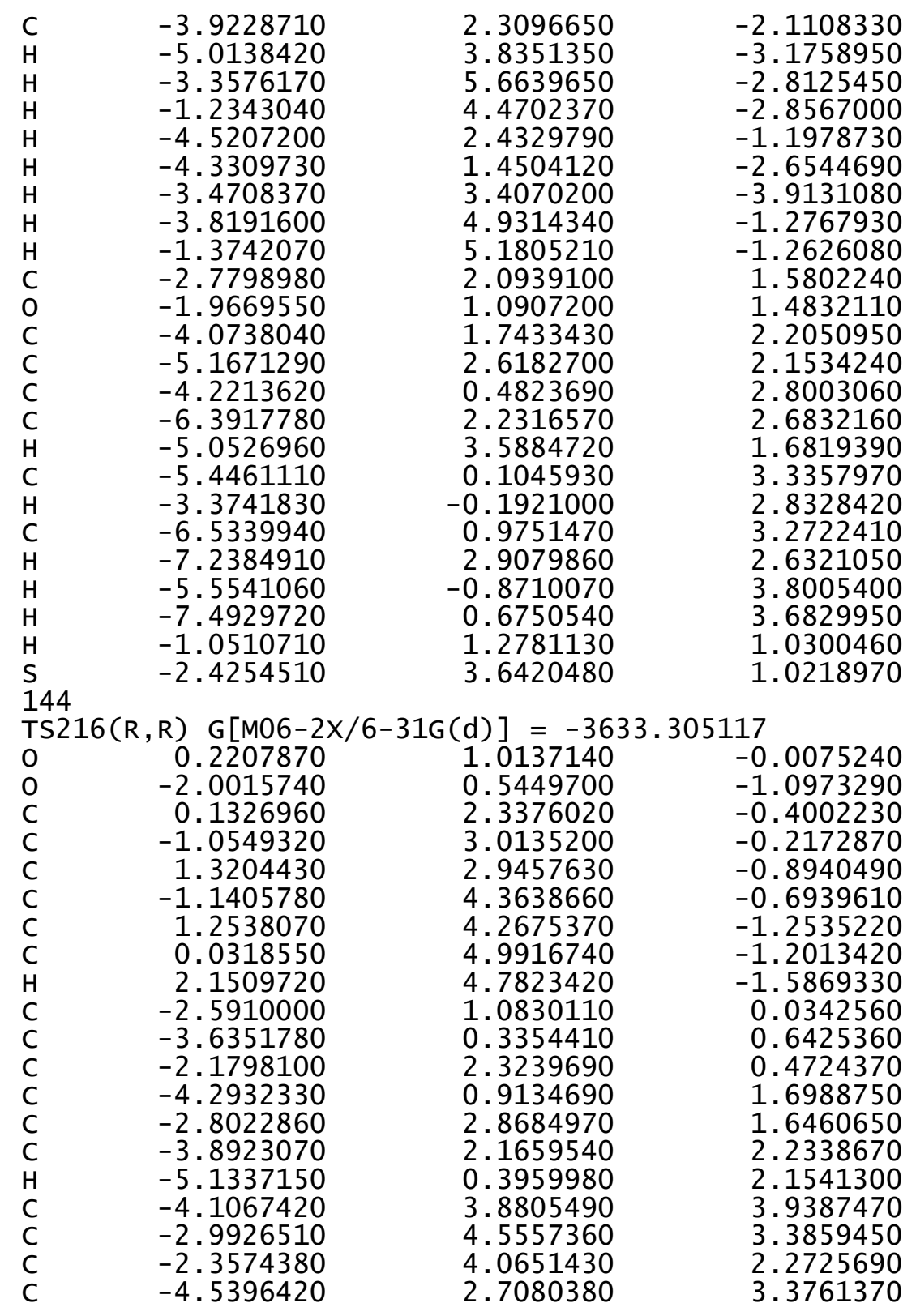

-4.6032970
-2.6329130
-1.4969510
-5.3776220
-2.4005720
-1.2264300
-0.0394640
-2.3595130
-3.3426960
-1.2724060
0.8667470
-3.2666500
2.5816380
3.0957990
3.2212230
4.2441580
4.3502260
4.8714410
4.6636060
4.8389090
-4.0041410
-3.5956140
-4.7640170
-4.0058970
-5.1445260
-4.7836280
-3.7016130
-5.7407760
2.7624620
6.1174490
2.4618940
-2.7371870
-5.2067900
-5.1989260
-6.7235290
-7.2187150
-7.0847920
-7.0275810
-4.6842120
-3.5950790
-5.1175080
-4.9577980
-6.0804670
-6.4190370
-5.5177760
-6.9605480

.2848160

5.4677920

4. 5874620

2. 1630360

6. 3865410

7. 0216930

6.3354530

5.0941370

6.9254640

8.0462230

6.8042600

4.6123390

2. 1421540

1.6119640

1. 8791080

0.8163530

1. 0601680

0.5084730

0.4142480

0.8546540

$-1.0176120$

$-2.1776900$

$-1.1251050$

$-3.4203510$

$-2.3941180$

$-3.5513940$

$-4.3180240$

$-2.4829060$

2. 5158580

$-0.3570280$

1. 9255850

$-2.1076890$

$-4.9189260$

0.0941800

0.2551520

$-0.6041640$

0.3360590

1.1553170

0.0329360

$-0.0668310$

$-0.8155800$

0.9461200

$-5.6480180$

$-6.6104690$

$-5.8440880$

$-5.0535780$
4.8149840

3. 8517370

1.8693570

3.8025180

$-1.1661220$

$-1.6360690$

$-1.6548960$

$-0.7055530$

$-1.1752760$

$-1.9910150$

$-2.0289620$

$-0.3556660$

$-0.9042900$

$-2.1029490$

0.3233400

$-2.0409940$

0.3355880

$-0.8340120$

$-2.9608530$

1. 2854770

0.1226180

0.8163910

$-1.0537430$

0.3348640

$-1.4994360$

$-0.8175420$

0.8680760

$-2.4059970$

1.6278200

$-0.8073490$

$-3.4501140$

2. 0732470

$-1.3199640$

$-1.8519580$

$-1.8156920$

$-2.2813710$

$-0.7854650$

$-2.3598920$

$-3.2942770$

$-3.3126610$

$-3.8348650$

$-3.8329880$

$-0.2929540$

$-0.6902000$

0.6264740

$-0.0302110$ 


$\begin{array}{rrr}-3.9914960 & -5.7728290 & -1.7002550 \\ -3.3721000 & -5.2666930 & -2.4466890 \\ -3.3673250 & -5.9720380 & -0.8220240 \\ -4.3112590 & -6.7362810 & -2.1104930 \\ -3.6107020 & -2.1310230 & 3.3355740 \\ -4.3085100 & -1.2902390 & 3.3649320 \\ -4.1957040 & -3.0573470 & 3.3721420 \\ -2.9862040 & -2.0878090 & 4.2344160 \\ -1.7027170 & -3.2372600 & 2.1529110 \\ -2.1764910 & -4.2021530 & 2.3637520 \\ -1.1371490 & -3.3255720 & 1.2208910 \\ -0.9998000 & -3.0366610 & 2.9685480 \\ 3.7528900 & 3.6112810 & 2.0450650 \\ 4.7443930 & 3.1842120 & 2.2338600 \\ 3.8548460 & 4.3686870 & 1.2609730 \\ 3.4154260 & 4.1057860 & 2.9619040 \\ 2.5685540 & 1.4900720 & 2.7463880 \\ 1.8454360 & 0.7265640 & 2.4480930 \\ 3.5066130 & 0.9868690 & 3.0085060 \\ 2.1979990 & 1.9834560 & 3.6516980 \\ 6.0955880 & -1.3855670 & 0.3286240 \\ 6.9333390 & -2.0827190 & 0.2267240 \\ 6.1892390 & -0.8991650 & 1.3060580 \\ 5.1619950 & -1.9600770 & 0.3333220 \\ 7.3746040 & 0.5179510 & -0.7151960 \\ 7.4239320 & 1.2248380 & -1.5488580 \\ 7.3677220 & 1.0934810 & 0.2173890 \\ 8.2792370 & -0.0988710 & -0.7290450 \\ 2.5015190 & 0.7510030 & -4.4305810 \\ 3.5220560 & 0.5367700 & -4.7673740 \\ 2.0849300 & -0.1555670 & -3.9860450 \\ 1.9121690 & 0.9946000 & -5.3203200 \\ 3.1461050 & 3.1444710 & -4.0860730 \\ 4.2054660 & 2.9288420 & -4.2677680 \\ 2.6790840 & 3.3900120 & -5.0456910 \\ 3.0898440 & 4.0261600 & -3.4423620 \\ -0.5182710 & -0.0965070 & -0.9451020 \\ 0.0570020 & -0.1539280 & -2.3290530 \\ -0.5431910 & -1.3498440 & -0.1022050 \\ 1.4090940 & 2.1681390 & -3.2726390 \\ 6.1562280 & -0.9016420 & -1.7602300 \\ 1.7950170 & 2.9984150 & 1.4598310 \\ -4.7647400 & 0.9849410 & -1.3878280 \\ -5.8070720 & -4.7630400 & -2.2252460 \\ -2.1868500 & -1.1605150 & -2.0476810 \\ 2.3175810 & -2.1841650 & \\ -30270240\end{array}$

\begin{abstract}
1. 3996680
2. 3195430

0.4880480

1. 3254860

0.6998630

3. 6342280

2. 9851230

1.5397680

3. 6756920

4.6509230

2. 9969490

0.9035180

4. 3484310

4.0501770

3.0664660

3. 5625750

1. 1058460

1.9586110

0.9524590

2.7144010

4. 0501820

2. 0821280

4.7486230

4.5383540

2. 7794050

1.0477320

4.1144160

5.7887690

2. 2834850

4.6610270

0.3815540

2. 3967570
\end{abstract}

$-1.4843740$

$-1.5252190$

$-1.0218350$

$-3.0806150$

$-2.8886290$

$-3.1270390$

$-2.1674030$

$-1.8576680$

$-2.5062700$

$-3.3753430$

$-2.5897450$

$-2.7385240$

$-1.6393610$

$-3.2189090$

$-4.0644430$

$-1.2344460$

$-1.0583670$

2. 0122260

1.8982790

3. 2731680

3. 3891480

4. 3528340

4. 5664080

2. 5499390

5. 5340640

4.2568210

5.6399580

4.6460490

6.3707220

6.5596400

1.0311560

0.9041970 144

$\operatorname{TS} 217(R, R) G[M 06-2 X / 6-31 G(d)]=-3633.303403$

$\begin{array}{llcr}\text { TS } 217(\mathrm{R}, \mathrm{R}) & \mathrm{G}[\mathrm{M} 06-2 \mathrm{X} / 6-31 \mathrm{G}(\mathrm{d})]=-3633.303403 \\ \mathrm{O} & -0.0747760 & 1.2606020 & 0.3370620 \\ \mathrm{O} & -2.0315670 & 0.1996330 & -0.8351810 \\ \mathrm{C} & -0.3814950 & 2.4749810 & -0.2509780 \\ \mathrm{C} & -1.6896740 & 2.9096790 & -0.2462150 \\ \mathrm{C} & 0.7070870 & 3.2350350 & -0.7593770 \\ \mathrm{C} & -1.9882870 & 4.1446140 & -0.9137110 \\ \mathrm{C} & 0.4218140 & 4.4618470 & -1.3021010 \\ \mathrm{C} & -0.9152550 & 4.9280080 & -1.4252410 \\ \mathrm{H} & 1.2299610 & 5.0974030 & -1.6543020 \\ \mathrm{C} & -2.8372160 & 0.7453680 & 0.1468460 \\ \mathrm{C} & -3.7907680 & -0.1198480 & 0.7470570 \\ \mathrm{C} & -2.7247630 & 2.0881590 & 0.4407440\end{array}$




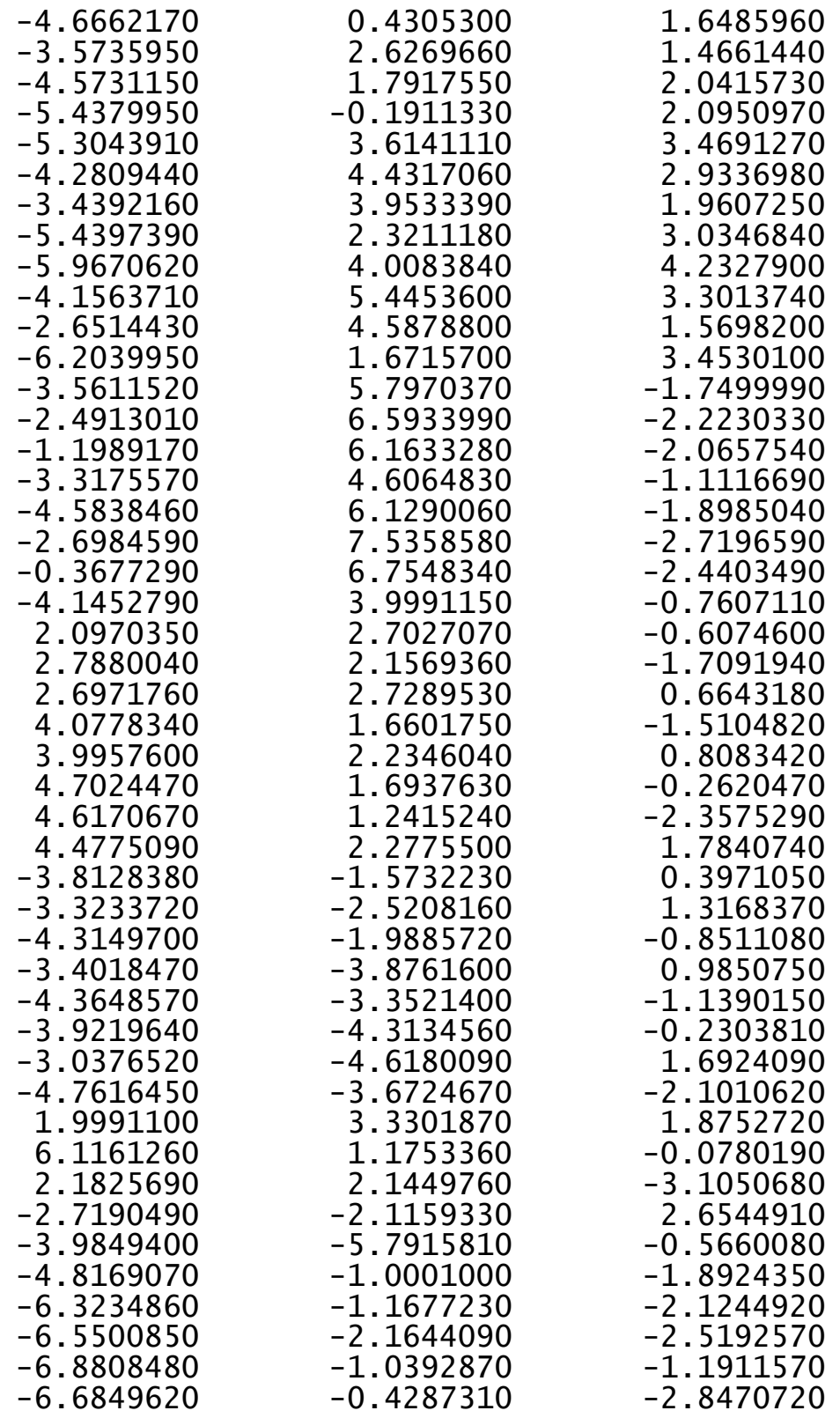

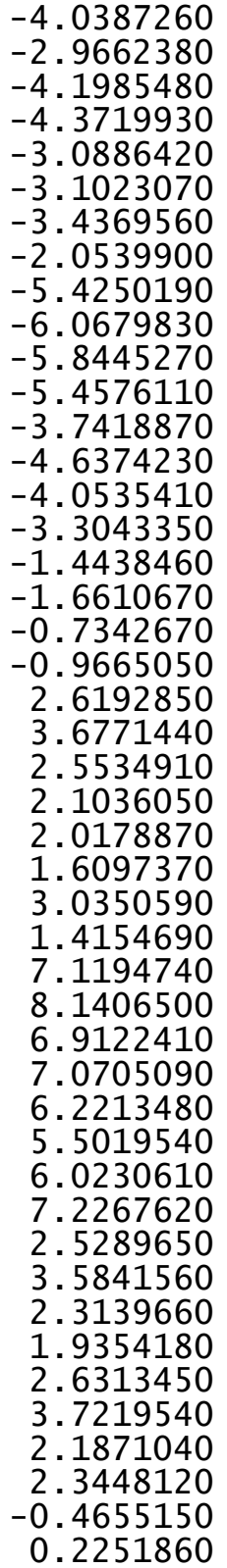

-1.1296320
-0.9984180
-2.1112190
-0.3693500
-6.1261200
-7.2018460
-5.6086060
-5.8215440
-6.2493220
-6.0306300
-5.7411820
-7.3268940
-2.2796160
-1.6750970
-3.3276150
-1.9820550
-2.8988240
-3.9476320
-2.8617660
-2.4650040
4.6892150
4.5754010
5.3778710
5.1416590
2.3910830
1.4095450
2.2545500
2.8097890
1.9945560
1.6412380
1.9007590
3.0558530
-0.3139260
-0.9009380
-0.4829930
-0.6895710
0.8855160
0.8690130
-0.0196650
0.8568340
3.3850720
3.3862400
3.3859410
4.3131340
-0.0753560
-0.1240560

$-3.2067070$

$-3.0363090$

$-3.6663970$

$-3.9208010$

$-1.7641000$

$-1.9676820$

$-2.6649040$

$-1.5789840$

$-0.8230700$

0.0346580

$-1.6984060$

$-1.0140230$

3.7869510

3.6173720

3.8664750

4.7458540

2.9878740

3. 2181770

2. 1579610

3.8731230

2.2236560

2.4863220

1. 3751430

3.0771240

3.0847670

2. 8222450

3.4705230

3.8972830

$-0.8982790$

$-0.7214420$

$-1.9701200$

$-0.6367950$

$-0.4213820$

0.1589760

$-1.4863340$

$-0.2029430$

$-3.9035130$

$-4.1982030$

$-3.3317820$

$-4.8229170$

$-3.8918950$

$-4.0031280$

$-4.8929900$

$-3.3904050$

-0.5077710
-1.8403180 


\begin{tabular}{|c|c|c|c|c|c|c|c|}
\hline & & & & & & & \\
\hline $\begin{array}{l}\mathrm{O} \\
\mathrm{H}\end{array}$ & $\begin{array}{r}-0.2872930 \\
1.0939670\end{array}$ & $\begin{array}{r}-1.2183060 \\
2.1735060\end{array}$ & $\begin{array}{r}0.4628700 \\
-2.9958230\end{array}$ & C & 2.3380020 & $\begin{array}{l}1.4546320 \\
3.5708640\end{array}$ & $\begin{array}{l}-1.6761370 \\
-1.9537640\end{array}$ \\
\hline $\mathrm{H}$ & 6.3693980 & 1.2908940 & 0.9835520 & c & $\begin{array}{l}0.46047220 \\
2.684720\end{array}$ & 2.6134310 & $\begin{array}{l}-1.9273120 \\
-2.32730\end{array}$ \\
\hline $\mathrm{H}$ & 0.9499850 & 3.5060980 & 1.6213390 & C & 1.7647360 & $\begin{array}{l}2.0134510 \\
3.6767950\end{array}$ & -2.5103300 \\
\hline $\mathrm{H}$ & -4.6551140 & 0.0139990 & -1.5147210 & $\mathrm{H}$ & 3.6973240 & 2.7234700 & -2.7092480 \\
\hline $\mathrm{H}$ & -3.6051570 & -6.3411960 & 0.3045330 & C & -2.0110640 & 1.2080880 & -0.7814380 \\
\hline $\mathrm{H}$ & -2.4420520 & -1.0586460 & 2.5893800 & $\mathrm{C}$ & -3.2774230 & 1.0073940 & -0.1659190 \\
\hline C & 2.3804560 & -2.2717120 & -1.5478660 & $\mathrm{C}$ & -1.1862040 & 2.2734350 & -0.5064490 \\
\hline C & 1.3791210 & -3.2544270 & -1.1082930 & C & -3.6779120 & 1.9475830 & 0.7517650 \\
\hline $\mathrm{H}$ & 2.4142470 & -1.3334580 & -0.9873220 & $\mathrm{C}$ & -1.5699510 & 3.1889080 & 0.5277390 \\
\hline $\mathrm{H}$ & 0.4711560 & -2.8678820 & -0.6570770 & C & -2.8381430 & 3.0205420 & 1.1496970 \\
\hline 0 & 1.4461360 & -2.1950420 & -2.6453020 & $\mathrm{H}$ & -4.6486730 & 1.8439040 & 1.2294430 \\
\hline $\mathrm{H}$ & 0.8498270 & -1.3916590 & -2.4300980 & C & -2.3783230 & 4.8920610 & 2.6261210 \\
\hline C & 3.6309360 & -4.1089140 & -2.7325140 & C & -1.1056790 & 5.0443060 & 2.0249110 \\
\hline C & 2.8647490 & -5.1419300 & -1.9032470 & C & -0.7111310 & 4.2172450 & 1.0022450 \\
\hline C & 1.4396220 & -4.6693940 & -1.6007650 & C & -3.2236550 & 3.9011740 & 2.1956750 \\
\hline C & 3.7347570 & -2.7794520 & -1.9816140 & $\mathrm{H}$ & -2.6772370 & 5.5548860 & 3.4320610 \\
\hline $\mathrm{H}$ & 4.6350720 & -4.4775160 & -2.9601010 & $\mathrm{H}$ & -0.4321760 & 5.8170570 & 2.3825920 \\
\hline $\mathrm{H}$ & 2.8178000 & -6.1001390 & -2.4280160 & $\mathrm{H}$ & 0.2735410 & 4.3320400 & 0.5618170 \\
\hline H & 0.8240140 & -4.7008550 & -2.5084650 & $\mathrm{H}$ & -4.1969680 & 3.7619470 & 2.6585230 \\
\hline $\mathrm{H}$ & 4.3464940 & -2.8987320 & -1.0772910 & C & -0.0861520 & 5.7463560 & -2.8862700 \\
\hline $\mathrm{H}$ & 4.2083390 & -2.0062840 & -2.5963930 & C & 1.2213680 & 5.8602770 & -3.4177490 \\
\hline $\mathrm{H}$ & 3.1200060 & -3.9473660 & -3.6888720 & $\mathrm{C}$ & 2.1233750 & 4.8446020 & -3.2351810 \\
\hline H & 3.3963360 & -5.3143550 & -0.9595880 & $\mathrm{C}$ & -0.4577620 & 4.6345610 & -2.1730300 \\
\hline $\mathrm{H}$ & 0.9392800 & -5.3129020 & -0.8720730 & $\mathrm{H}$ & -0.8027320 & 6.5449660 & -3.0502720 \\
\hline C & 2.7120870 & -2.1911240 & 1.7029760 & $\mathrm{H}$ & 1.5010700 & 6.7478750 & -3.9760370 \\
\hline 0 & 1.9807420 & -1.1314720 & 1.5632340 & $\mathrm{H}$ & 3.1266410 & 4.9110350 & -3.6473630 \\
\hline C & 4.0573330 & -1.9099640 & 2.2484060 & $\mathrm{H}$ & -1.4659310 & 4.5536120 & 02560 \\
\hline C & 5.072 & -2.8747790 & 2.2101250 & C & 3.3530730 & 0.3794590 & -1.4497480 \\
\hline C & 4.3331530 & -0.6308410 & 2.7523760 & $\mathrm{C}$ & 3.4754050 & -0.6829330 & -2.3658100 \\
\hline c & 6.347 & -2.5594380 & 2.6632650 & C & 4.2224110 & 0.4758610 & -0.3452710 \\
\hline $\mathrm{H}$ & 4.85 & -3.8596640 & 1.8088950 & C & 4.4958060 & -1.6144080 & -2.1740560 \\
\hline C & 5.607 & -0.3246000 & 3.2135860 & $\mathrm{C}$ & 5.2205460 & -0.4911960 & -0.1889120 \\
\hline $\mathrm{H}$ & 3.54 & 0.1126580 & 2.7729930 & C & 5.3826010 & -1.5329520 & -1.0995180 \\
\hline C & 6.61 & -1.2853390 & 3.1634100 & $\mathrm{H}$ & 4.6100280 & -2.4348740 & -2.8797020 \\
\hline $\mathrm{H}$ & 7.1339740 & -3.3056910 & 2.6220090 & $\mathrm{H}$ & 5.8912150 & -0.4205260 & 0.6616250 \\
\hline $\mathrm{H}$ & 5.81 & 0.6652510 & 3.6096720 & C & -4.1105500 & -0.1954680 & -0.4668060 \\
\hline $\mathrm{H}$ & 7.61 & -1.0418350 & 3.5152410 & C & -4.4039700 & -1.1209620 & 0.5622470 \\
\hline $\mathrm{H}$ & 1.0326590 & -1.2775110 & 1.1664060 & $\mathrm{C}$ & -4.6214150 & -0.4003050 & -1.7624880 \\
\hline 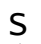 & 2.2037300 & -3.7379570 & 1.2748650 & $\mathrm{C}$ & -5.2232640 & -2.2109020 & 0.2696990 \\
\hline & & & & $\mathrm{C}$ & -5. & -1.5133520 & -2.0032080 \\
\hline & R) $\mathrm{G}[\mathrm{M06}-2$ & (d) $]=-3$ & 38 & C & -5.7485810 & -2.4278570 & -1.0042570 \\
\hline 0 & 0.6860800 & 0.1881890 & -0.4947500 & $\mathrm{H}$ & -5.4545590 & -2.9184440 & 1.0638870 \\
\hline 0 & -1.5551880 & 0.2480210 & -1.6840290 & $\mathrm{H}$ & -5.8359920 & -1.6679920 & -3.0018820 \\
\hline C & 1.017 & 1.3520410 & -1.1613590 & C & 4.0690910 & 1.5885010 & 0.6845000 \\
\hline C & 0.113 & 2.393780 & -1.2148170 & $\mathrm{C}$ & 6.5027090 & -2.5529490 & -0.9828760 \\
\hline
\end{tabular}




2.5639170
-3.8718440
-6.6378610
-4.3714710
-5.6757770
-6.4042660
-6.1278790
-5.4854890
-3.7162410
-2.7904040
-4.3866050
-3.4803470
-7.9310830
-8.5851060
-7.7121180
-8.4761080
-5.8988150
-4.9827490
-5.6227570
-6.5355190
-4.9930790
-5.4755790
-5.7670860
-4.5974970
-3.1986330
-3.9285400
-2.4506680
-2.7022440
5.3876110
5.7520180
6.1688580
5.2363260
3.0402780
2.0579350
3.3693250
2.9226420
6.9802670
7.6913920
7.4940840
6.1486440
7.6808620
7.3658120
8.0907820
8.4794800
2.2910520
3.1655360

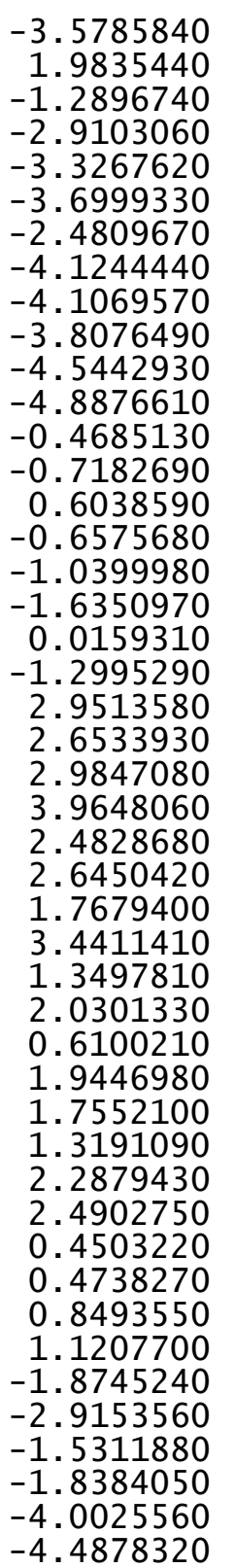

2.0148400
1.4663380
3.1534930
4.1342640
2.4985710
3.2798370
-0.5357010
-0.0611220
-1.1466980
1.6005070
6.1130640
3.6829440
-3.6866630
-6.9090400
-3.1123330
1.9355150
0.7581060
1.9592870
-0.1815060
1.2964260
0.7401300
3.2039750
2.1638310
0.7752820
3.2818940
4.1904150
2.1384840
0.4127430
3.6867320
3.9424890
2.9405700
2.4312440
0.0276010
0.2420280
-0.6391040
0.5895710
1.5595010
-0.0464430
1.8887630
2.0561190
0.2876440
-0.7945600
1.2547030
2.6430370
-0.2082560
1.5157030
$-2.8508700$
$-2.2554640$
0.0035920
$-0.4027830$
$-0.0681400$
1.0615650
$-0.7977090$
$-1.8118010$
$-1.2912800$
$-0.3381800$
$-3.5043250$
2. 4716640
1. 3486580
$-3.5823970$
$-0.2009170$
$-2.9223870$
$-3.3788700$
$-1.8638110$
$-2.8799070$
$-3.7364220$
$-3.1155230$
$-4.9132550$
$-5.0311300$
$-4.6641740$
$-3.4780340$
$-5.2167400$
$-6.0453760$
$-5.4324230$
$-2.8094510$
$-3.4078690$
$-5.5900630$
$-4.3580300$
$-4.5983080$
$-0.2229130$
0.1481300
0.8497040
0.6585840
2. 0952290
1.6961740
$-0.3021460$
3. 1293080
2.2505050
2.9328480
1.5420110
4.0900880
3.7427610

$-3.1456520$ $-4.7208570$ $-4.7560840$ $-5.0287780$ $-5.6308810$ $-4.5089270$ $-0.9751540$ $-1.9644790$ 0.3191530 $-3.3119720$ $-1.3710150$ 0.1642630 $-2.5703210$ $-2.3521960$

1.9935710

0.3275380

1.0806090

0.0535720

0.8806240 $-0.6896980$ $-1.2537870$

1.2522590

2. 3657570

1.8397090

0.7279960

1.6154410

2.7730690

1.1434790

1.4993230

$-0.1398330$

0.4303770

3.1906270

2. 6351480

3.1203760

2.2557420

4.0885360

5.0796700

3.9729020

5.9428650

5.1620850

4.8390340

3.2019850

5.8238260

6.7074580

4.7405710

6.4980060 


\begin{tabular}{|c|c|c|c|}
\hline $\begin{array}{l}\mathrm{H} \\
\mathrm{S} \\
144\end{array}$ & $\begin{array}{r}-0.8438690 \\
0.9473870\end{array}$ & $\begin{array}{l}-0.5364380 \\
-1.7548630\end{array}$ & $\begin{array}{l}1.4862900 \\
3.1810990\end{array}$ \\
\hline \multicolumn{4}{|c|}{$\operatorname{TS} 219(R, R) \quad G[M 06-2 X / 6-31 G(d)]=-3633.303440$} \\
\hline 0 & 0.0324100 & 1.1221330 & 0.1120150 \\
\hline 0 & -2.0620380 & 0.3591410 & -1.0484270 \\
\hline C & -0.2218600 & 2.4307890 & -0.2579050 \\
\hline C & -1.4961440 & 2.9311180 & -0.1023760 \\
\hline C & 0.8881970 & 3.1993200 & -0.7069080 \\
\hline $\mathrm{C}$ & -1.7510000 & 4.2667520 & -0.5596480 \\
\hline C & 0.6507760 & 4.5055180 & -1.0516130 \\
\hline C & -0.6602670 & 5.0557020 & -1.0234760 \\
\hline $\mathrm{H}$ & 1.4753380 & 5.1411670 & -1.3636990 \\
\hline C & -2.7417480 & 0.7908640 & 0.077 \\
\hline C & -3.6743770 & -0.1130550 & 80 \\
\hline C & -2.5301020 & 1540 & 460 \\
\hline C & -4.4396400 & 4640 & 1.6960900 \\
\hline C & -3.2568780 & 2.5003600 & 1.7031050 \\
\hline C & -4.2464520 & 1.6362640 & 700 \\
\hline $\mathrm{H}$ & -5.2009950 & -0.3016750 & 2.12 \\
\hline C & -4.7580260 & 3.2748780 & 3.9686710 \\
\hline C & -3.7401190 & 4.1140870 & 3.4566660 \\
\hline C & -3.0096940 & 3.7390030 & 2.3567730 \\
\hline C & -4.9977830 & 2.0601460 & 3.3806880 \\
\hline $\mathrm{H}$ & -5.3326940 & 3.5884760 & 4.8342160 \\
\hline $\mathrm{H}$ & -3.5306110 & 5.0615480 & 3.9431110 \\
\hline $\mathrm{H}$ & -2.2245170 & 4.3881530 & 1.9856090 \\
\hline $\mathrm{H}$ & -5.7576300 & 1.3915570 & 3.7764810 \\
\hline C & -3.2601290 & 6.1083810 & -1.0411720 \\
\hline C & -2.1708090 & 6.9034350 & -1.4697750 \\
\hline C & -0.9015680 & 6.38 & -1.4627250 \\
\hline C & -3.0566040 & 4.8256610 & -0.5967460 \\
\hline $\mathrm{H}$ & -4.2659180 & 6.5154520 & -1.0698370 \\
\hline $\mathrm{H}$ & -2.3454740 & 7.9178760 & -1.8135190 \\
\hline $\mathrm{H}$ & -0.0578950 & 776920 & -1.8049480 \\
\hline $\mathrm{H}$ & 987370 & 98400 & -0.2783700 \\
\hline C & 7890 & 2.5500380 & -0.7191280 \\
\hline C & 2.85 & 2.1857290 & -1.9275250 \\
\hline C & 9880 & 2.2375840 & 0.5167960 \\
\hline C & 4.07 & 1.5044370 & -1.8726920 \\
\hline C & 4.0460970 & 1.5346020 & 0.5190290 \\
\hline C & 50820 & -480360 & 6664640 \\
\hline $\mathrm{H}$ & 3680 & 8530 & -2.8005300 \\
\hline $\mathrm{H}$ & 4.5078250 & 08030 & 1.4690320 \\
\hline C & -3.8 & 26500 & 0.1169470 \\
\hline C & -3.2030980 & $-2.50<5+50$ & 0.7940660 \\
\hline
\end{tabular}

-4.5436810
-3.3903740
-4.6986100
-4.1371140
-2.9299460
-5.2726070
2.2514840
5.9956680
2.2575090
-2.3658740
-4.3102510
-5.1792760
-6.7088110
-7.0460540
-7.0840140
-7.1606840
-4.6539880
-3.5638280
-4.9318010
-5.0775430
-5.0320200
-5.1926210
-4.4385620
-6.0039090
-2.9623890
-2.4495800
-2.3071370
-3.1043270
-3.2256150
-4.0430710
-3.6624890
-2.6147960
-1.1674340
-1.4832800
-0.6024880
-0.4977300
2.7659030
3.8561110
2.5127610
2.3291390
2.5240300
2.2316100
3.5807720
1.9493320
6.0130650
6.9119900
$-1.0599570$

0.2950180

$-1.5232540$

$-0.8586760$

0.8135850

$-2.4309020$

1.8299570

$-0.6435230$

$-3.2794330$

2.0503390

$-1.3822690$

$-1.8434740$

$-1.8167140$

$-2.2969600$

$-0.7883080$

$-2.3517130$

$-3.2825170$

$-3.2936950$

$-3.8379100$

$-3.8109290$

$-0.3651350$

$-0.7768490$

0.5498510

$-0.0909680$

$-1.7796000$

$-2.5201570$

$-0.9065140$

$-2.2036560$

3. 3129300

3. 3420180

3. 3497290

4. 2115910

2. 1329990

2. 3603760

1.1966400

2.9394410

2. 1189750

2. 2306240

1. 3043730

3. 0453870

3. 0121800

2. 7716260

3. 3014950

3. 8839010

0.3942200

0.2758810 


$\begin{array}{lrrr}\text { H } & 6.0188410 & -0.3284880 & \\ \text { H } & 5.1342880 & -1.3747050 & 0.2982110 \\ \mathrm{C} & 7.1575140 & 1.3733840 & -0.3996590 \\ \mathrm{H} & 7.1831700 & 2.1559260 & -1.1638980 \\ \mathrm{H} & 7.0476920 & 1.8569840 & -.5775910 \\ \mathrm{H} & 8.1161320 & 0.8442820 & -0.4126020 \\ \mathrm{C} & 2.4205540 & 1.4387590 & -4.3260970 \\ \mathrm{H} & 3.4665680 & 1.3172150 & -4.6291700 \\ \mathrm{H} & 2.0542510 & 0.4801360 & -3.9523230 \\ \mathrm{H} & 1.8513180 & 1.6953800 & -5.2252820 \\ \mathrm{C} & 2.8816350 & 3.8445150 & -3.8063270 \\ \mathrm{H} & 3.9585440 & 3.7094560 & -3.9607620 \\ \mathrm{H} & 2.4331880 & 4.1255150 & -4.7650620 \\ \mathrm{H} & 2.7473220 & 4.6750230 & -3.1079090 \\ \mathrm{P} & -0.4931550 & -0.0343640 & -0.9067960 \\ \mathrm{O} & 0.0925770 & 0.1005500 & -2.2819400 \\ \mathrm{O} & -0.3030560 & -1.3241880 & -0.1452930 \\ \mathrm{H} & 1.1841730 & 2.7042800 & -3.1370970 \\ \mathrm{H} & 6.1341800 & -0.0461950 & -1.6367920 \\ \mathrm{H} & 1.1647970 & 2.7913600 & 1.7192930 \\ \mathrm{H} & -4.9064670 & 0.3577350 & -1.3629980 \\ \mathrm{H} & -4.9337070 & -5.4737290 & -2.2833920 \\ \mathrm{H} & -1.9695290 & -1.3550600 & 2.0216070 \\ \mathrm{C} & 2.5423650 & -1.7336230 & -2.1401270 \\ \mathrm{C} & 1.7189480 & -2.8799570 & -1.7390430 \\ \mathrm{H} & 2.4508950 & -0.8339390 & -1.5246760 \\ \mathrm{H} & 0.7771140 & -2.6632880 & -1.2484040 \\ \mathrm{O} & 1.5674460 & -1.7536440 & -3.2092430 \\ \mathrm{H} & 0.8690670 & -1.0576240 & -2.9399080 \\ \mathrm{C} & 4.0266790 & -3.3005590 & -3.4445240 \\ \mathrm{C} & 3.4665290 & -4.4698920 & -2.6322110 \\ \mathrm{C} & 1.9907790 & -4.2468370 & -2.2910990 \\ \mathrm{C} & 3.9395130 & -1.9965600 & -2.6475960 \\ \mathrm{H} & 5.0688990 & -3.4918380 & -3.7149730 \\ \mathrm{H} & 3.5686910 & -5.4090250 & -3.1828930 \\ \mathrm{H} & 1.3708670 & -4.3424510 & -3.1911270 \\ \mathrm{H} & 4.6037930 & -2.0416820 & -1.7753380 \\ \mathrm{H} & 4.2495130 & -1.1367410 & -3.2514520 \\ \mathrm{H} & 3.4628210 & -3.1958850 & -4.3789640 \\ \mathrm{H} & 4.0436120 & -4.5763960 & -1.7046470 \\ \mathrm{H} & 1.6140720 & -4.9884690 & -1.5814160 \\ \mathrm{C} & 2.3473440 & -2.1930680 & 1.7789200 \\ \mathrm{O} & 1.3823180 & -1.3354170 & 1.7197920 \\ \mathrm{C} & 3.1371650 & -2.0960700 & 3.0300560 \\ \mathrm{C} & 4.4519400 & -2.5720040 & 3.1002890 \\ \mathrm{C} & 2.5491590 & -1.5116050 & 4.1606350 \\ & & & \\ & & & \end{array}$

$\begin{array}{ll}\mathrm{C} & 5.1733510 \\ \mathrm{H} & 4.9054570 \\ \mathrm{C} & 3.2700030 \\ \mathrm{H} & 1.5271330 \\ \mathrm{C} & 4.5835860 \\ \mathrm{H} & 6.1967530 \\ \mathrm{H} & 2.8071380 \\ \mathrm{H} & 5.1481050 \\ \mathrm{H} & 0.7428190 \\ \mathrm{~S} & 2.6977780 \\ \mathrm{1} & \end{array}$

144

TS220(R, R) G[M06-2X/6-31G(d)] $=-3633.303397$

$\begin{array}{lrrr}\text { TS220(R, R) G[M06-2X/6-31G(d) }]=-3633.303397 \\ \text { O } & -0.0747370 & 1.2604420 & 0.3368840 \\ \text { O } & -2.0316790 & 0.1995460 & -0.8351490 \\ \text { C } & -0.3814410 & 2.4748510 & -0.2510970 \\ \text { C } & -1.6895930 & 2.9096180 & -0.2462090 \\ \text { C } & 0.7071510 & 3.2348520 & -0.7595530 \\ \text { C } & -1.9881840 & 4.1445940 & -0.9136440 \\ \text { C } & 0.4219120 & 4.4617050 & -1.3022000 \\ \text { C } & -0.9151430 & 4.9279440 & -1.4252250 \\ \text { H } & 1.2300670 & 5.0972470 & -1.6544070 \\ \text { C } & -2.8372160 & 0.7453540 & 0.1469190 \\ \text { C } & -3.7908290 & -0.1197900 & 0.7471410 \\ \text { C } & -2.7246700 & 2.0881380 & 0.4408180 \\ \text { C } & -4.6662170 & 0.4306360 & 1.6487060 \\ \text { C } & -3.5734210 & 2.6269860 & 1.4662690 \\ \text { C } & -4.5729920 & 1.7918450 & 2.0417080 \\ \text { H } & -5.4380390 & -0.1909740 & 2.0952060 \\ \text { C } & -5.3040770 & 3.6142270 & 3.4693270 \\ \text { C } & -4.2805720 & 4.4317460 & 2.9338950 \\ \text { C } & -3.4389130 & 3.9533330 & 1.9608840 \\ \text { C } & -5.4395470 & 2.3212580 & 3.0348540 \\ \text { H } & -5.9666950 & 4.0085360 & 4.2330180 \\ \text { H } & -4.1558970 & 5.4453770 & 3.3015990 \\ \text { H } & -2.6510940 & 4.5878160 & 1.5699760 \\ \text { H } & -6.2038480 & 1.6717650 & 3.4531810 \\ \text { C } & -3.5610140 & 5.7971430 & -1.7497500 \\ \text { C } & -2.4911520 & 6.5934630 & -2.2228260 \\ \text { C } & -1.1987810 & 6.1633090 & -2.0656630 \\ \text { C } & -3.3174410 & 4.6065480 & -1.1114870 \\ \text { H } & -4.5837000 & 6.1291760 & -1.8981670 \\ \text { H } & -2.6982900 & 7.5359570 & -2.7193940 \\ \text { H } & -0.3675870 & 6.7547840 & -2.4402940 \\ \text { H } & -4.1451720 & 3.9992120 & -0.7604960 \\ \text { C } & 2.0970730 & 2.7024510 & -0.6076400 \\ \text { C } & 2.7879960 & 2.1565310 & -1.7093300 \\ & & & \end{array}$

4.2817110

2.2235400

5.3442260

4.1045820

5.4043700

4. 3268950

6.2193290

6.3271490

0.9043950

0.6119660

\section{$-1.4059140$ \\ $-1.8692040$ \\ 0.9618 \\ $-1.7792440$ \\ $-1.3960710$}

$-3.3514560$

-

(1)

0.9151430

2.7246700

$-5.3040770$

2.7879960
$-1.7093300$

2.8816350

0.0925770

. 3030560

1.1647970

. 9695290

. 7189480

.8690670

. 9395130

.

1. 3708670

2. 5491590

$-1.5116050$ 


$\begin{array}{lrrr}\mathrm{C} & 2.6972290 & 2.7287960 & \\ \mathrm{C} & 4.0778110 & 1.6597380 & -1.5641260 \\ \mathrm{C} & 3.9958070 & 2.2344490 & 0.8081730 \\ \mathrm{C} & 4.7024600 & 1.6934750 & -0.2621690 \\ \mathrm{H} & 4.6170080 & 1.2409640 & -2.3575970 \\ \mathrm{H} & 4.4775840 & 2.2775040 & 1.7838860 \\ \mathrm{C} & -3.8130130 & -1.5731520 & 0.3971510 \\ \mathrm{C} & -3.3235220 & -2.5207890 & 1.3168200 \\ \mathrm{C} & -4.3152480 & -1.9884370 & -0.8510400 \\ \mathrm{C} & -3.4020840 & -3.8761200 & 0.9850270 \\ \mathrm{C} & -4.3652090 & -3.3519940 & -1.1389860 \\ \mathrm{C} & -3.9222940 & -4.3133560 & -0.2304110 \\ \mathrm{H} & -3.0378700 & -4.6180080 & -1.6923110 \\ \mathrm{H} & -4.7620680 & -3.6722740 & -2.1010200 \\ \mathrm{C} & 1.9992000 & 3.3301570 & 1.8750390 \\ \mathrm{C} & 6.1161470 & 1.1750830 & -0.0780990 \\ \mathrm{C} & 2.1825490 & 2.1445600 & -3.1051970 \\ \mathrm{C} & -2.7190560 & -2.1159520 & 2.6544230 \\ \mathrm{C} & -3.9853350 & -5.7914660 & -0.5660890 \\ \mathrm{C} & -4.8171920 & -0.9999060 & -1.8923070 \\ \mathrm{C} & -6.3237860 & -1.1674640 & -2.1243140 \\ \mathrm{H} & -6.5504380 & -2.1641270 & -2.5191080 \\ \mathrm{H} & -6.8811090 & -1.0390420 & -1.1909530 \\ \mathrm{H} & -6.6852600 & -0.4284310 & -2.8468530 \\ \mathrm{C} & -4.0390660 & -1.1294150 & -3.2066140 \\ \mathrm{H} & -2.9665680 & -0.9982320 & -3.0362530 \\ \mathrm{H} & -4.1989310 & -2.1109830 & -3.6663290 \\ \mathrm{H} & -4.3723430 & -0.3691010 & -3.9206710 \\ \mathrm{C} & -3.0891480 & -6.1259770 & -1.7642730 \\ \mathrm{H} & -3.1028470 & -7.2016960 & -1.9678910 \\ \mathrm{H} & -3.4375330 & -5.6084280 & -2.6650290 \\ \mathrm{H} & -2.0544740 & -5.8214250 & -1.5792390 \\ \mathrm{C} & -5.4254480 & -6.2491630 & -0.8230410 \\ \mathrm{H} & -6.0683270 & -6.0304990 & 0.0347570 \\ \mathrm{H} & -5.8450230 & -5.7409660 & -1.6983120 \\ \mathrm{H} & -5.4580830 & -7.3267230 & -1.0140470 \\ \mathrm{C} & -3.7418070 & -2.2795640 & 3.7869700 \\ \mathrm{H} & -4.6373070 & -1.6749710 & 3.6174670 \\ \mathrm{H} & -4.0535380 & -3.3275390 & 3.8665100 \\ \mathrm{H} & -3.3041510 & -1.9820450 & 4.7458380 \\ \mathrm{C} & -1.4438680 & -2.8989170 & 2.9876840 \\ \mathrm{H} & -1.6611190 & -3.9477240 & 3.2179650 \\ \mathrm{H} & -0.7343480 & -2.8618600 & 2.1577200 \\ \mathrm{H} & -0.9664390 & -2.4651510 & 3.8729120 \\ \mathrm{C} & 2.6194050 & 4.6892070 & 2.2232850 \\ \mathrm{H} & 3.6772730 & 4.5754010 & 2.4859160 \\ & & & \end{array}$

\begin{abstract}
2. 5535850
2.1037660

2.0179920

1. 6098790

3.0351650

1.4155510

7.1194630

8.1406460

6.9121910

7.0705100

6.2213500

5.5019890

6.0229970

7.2267760

2. 5290740

3. 5842410

2. 3142860

1. 9354450

2.6311950

3.7218070

2. 1869780

2.3445350

$-0.4655590$

0.2248540

$-0.2870780$

1.0939460

6.3694620

0.9500720

$-4.6553460$

$-3.6054860$

$-2.4419970$

2. 3803360

1. 3791950

2.4138990

0.4711110

1.4462340

0.8498270

3.6313850

2.8653480

1.4400820

3.7348150

4.6356270

2.8186690

0.8245980

4.3464620

4.2083090
\end{abstract}

1. 3747170

3.0767370

3.0846230

2.8221930

3.4704060

3.8970920

$-0.8985690$

$-0.7216960$

$-1.9703830$

$-0.6373050$

$-0.4211540$

0.1593740

$-1.4860580$

$-0.2026960$

$-3.9037040$

$-4.1984940$

$-3.3319840$

$-4.8230530$

$-3.8919790$

$-4.0031850$

$-4.8930850$

$-3.3904770$

$-0.5080260$

$-1.8407310$

0.4624480

$-2.9959320$

0.9834390

1.6211190

$-1.5145570$

0.3043970

2. 5892860

$-1.5479200$

$-1.1084610$

$-0.9872510$

$-0.6572960$

$-2.6455210$

$-2.4303710$

$-2.7324540$

$-1.9033080$

$-1.6009730$

$-1.9814890$

$-2.9599580$

$-2.4281140$

$-2.5087640$

$\begin{array}{ll}-2.8980970 & -1.0770930 \\ -2.0056190 & -2.5961800\end{array}$ 


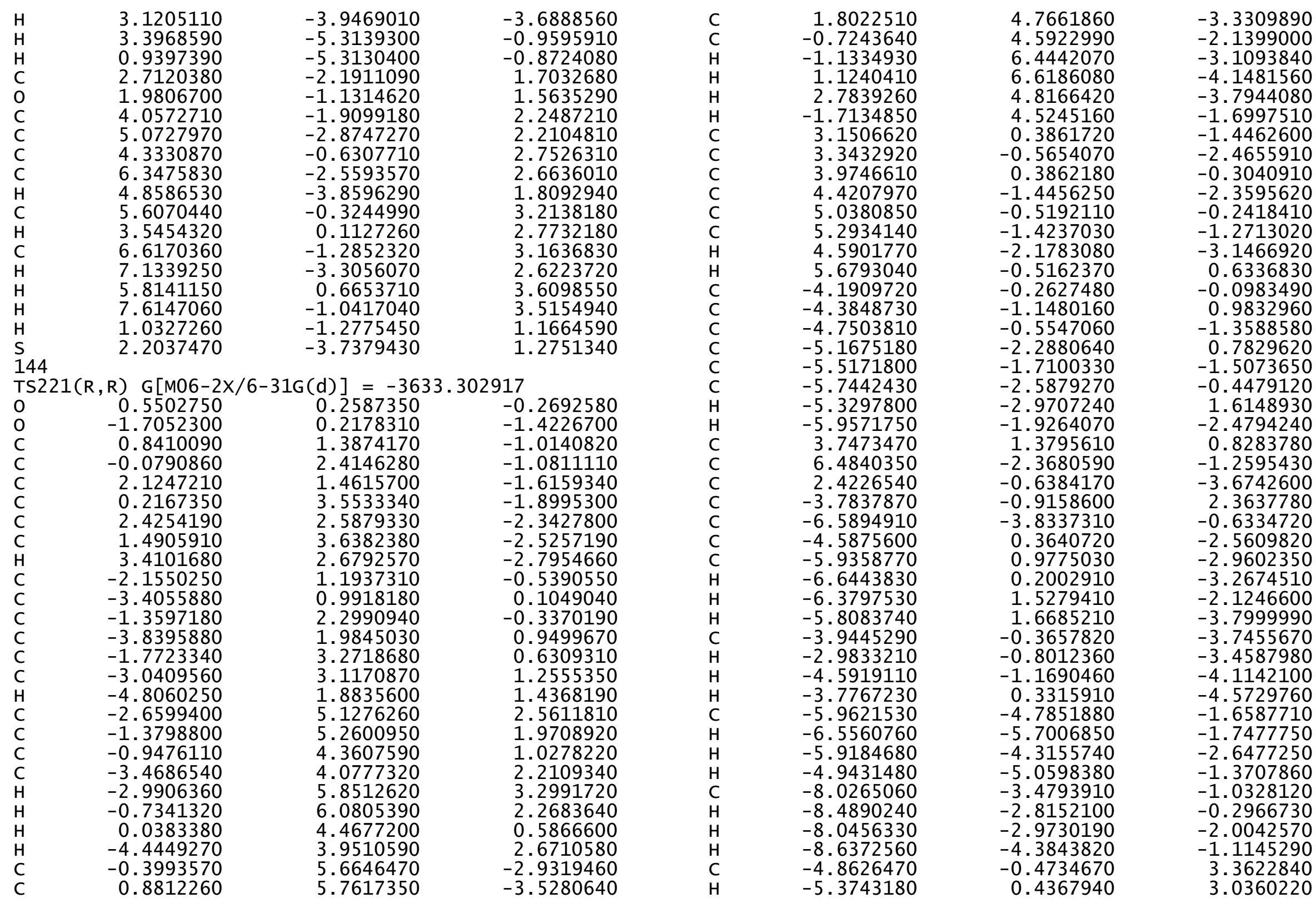




\begin{tabular}{|c|c|c|}
\hline $\begin{array}{l}-5.6199180 \\
-4.4197800 \\
-3.0576690 \\
-3.7609640 \\
-2.3383650 \\
-2.5189650 \\
4.4713320 \\
5.5530010 \\
4.1488210 \\
4.2764410 \\
4.1710630 \\
3.7212980 \\
5.2599770 \\
3.8504630 \\
7.0286910 \\
7.8039940 \\
7.4843490 \\
6.2440980 \\
7.5989110 \\
7.2408730 \\
7.9547780 \\
8.4471300 \\
2.1251760 \\
2.9908210 \\
1.8423750 \\
1.2987950 \\
3.0096130 \\
3.9849400 \\
2.3476900 \\
3.1470630 \\
-0.6113170 \\
-0.1042180 \\
-1.1629710 \\
1.4690360 \\
6.1480650 \\
2.6699000 \\
-3.9257250 \\
-6.6244770 \\
-3.0430850 \\
2.0091420 \\
0.8846590 \\
2.0356100 \\
-0.0610690 \\
1.2758530 \\
0.6884540 \\
3.2977220\end{array}$ & $\begin{array}{r}-1.2575360 \\
-0.2838120 \\
-2.1536210 \\
-2.9551470 \\
-2.5386100 \\
-1.8903130 \\
2.7046770 \\
2.5361060 \\
3.1553990 \\
3.4185200 \\
0.8468130 \\
-0.1296100 \\
0.7513030 \\
1.5468880 \\
-2.6700340 \\
-3.4395240 \\
-1.7807280 \\
-3.0274580 \\
-1.8061070 \\
-1.6348230 \\
-0.8498820 \\
-2.4971520 \\
-2.0817260 \\
-2.5457240 \\
-2.6930450 \\
-2.0961680 \\
0.1403570 \\
-0.2747020 \\
0.0698750 \\
1.1985650 \\
-0.7872300 \\
-1.7152540 \\
-1.3752690 \\
-0.1787560 \\
-3.3155450 \\
1.5850370 \\
1.1897620 \\
-4.3520990 \\
-0.1154850 \\
-2.9954020 \\
-3.4549250 \\
-1.9269580 \\
-2.9383800 \\
-3.7519210 \\
-3.0872720 \\
-5.0469440\end{array}$ & $\begin{array}{r}3.4777010 \\
4.3457560 \\
2.9080940 \\
3.1590450 \\
2.1811370 \\
3.8252290 \\
0.5501010 \\
0.4963750 \\
-0.3922140 \\
1.3580230 \\
2.1988390 \\
2.4107790 \\
2.2735380 \\
2.9771640 \\
0.1364380 \\
0.0754660 \\
0.5852790 \\
0.8113210 \\
-2.1537690 \\
-3.1733170 \\
-1.7541110 \\
-2.1961770 \\
-4.0939160 \\
-4.5794320 \\
-3.2333220 \\
-4.8111300 \\
-4.8590710 \\
-5.1385410 \\
-5.7286900 \\
-4.6195840 \\
-0.7702970 \\
-1.8270770 \\
0.5111660 \\
-3.3936390 \\
-1.7036680 \\
0.8802920 \\
-2.2851000 \\
0.3331090 \\
2.2846910 \\
0.1641490 \\
0.9946800 \\
-0.0700440 \\
0.8855750 \\
-0.8307760 \\
-1.3081970 \\
0.9099230\end{array}$ \\
\hline
\end{tabular}

2.3467310
0.9307510
3.3691590
4.3019250
2.3286740
0.4968150
3.8428620
3.9673980
2.9526420
2.6955510
0.2493190
0.5982150
-0.4889690
1.1921050
2.0157640
0.9454420
2.5841640
2.2010600
1.5401800
0.3062490
2.3556720
3.2119080
1.3596070
2.8128880
-0.7931660
1.3306970
$-5.1883520$
$-4.7704890$
$-3.5930700$
$-5.3865680$
$-6.2176970$
$-5.5009310$
$-2.9650100$
$-3.4984940$
$-5.6826910$
$-4.5559490$
$-4.7224370$
$-0.3596880$
$-0.0407670$
0.7749160
0.5837750
2. 0702870
1.6809110
$-0.4223810$
3.1620430
2.2082030
2. 9688600
1. 5329530
4.1620670
3.8219660
$-0.7019690$
$-1.8775400$

2.0979990

1.6994760

0.4395230

1.1803310

2.4659210

1.0033020

1.2057070

$-0.4708000$

0.0854320

2. 9248210

2. 5531630

3.0857630

2.4715670

3.8325730

4.9461640

3.3575340

5.5821360

5.3079680

3.9801660

2. 4902320

5.0949340

6.4546940

3.5978240

5.5865350

1.7171490

3.0632200

TS222(R,R) G[M06-2X/6-31G(d)] = -3633.302522

$\begin{array}{lrcr}\text { TS222(R, R) } G[M 06-2 X / 6-31 G(d)]=-3633.302522 \\ \text { O } & 0.5575510 & 0.2847370 & -0.2500240 \\ \text { O } & -1.6751770 & 0.1565800 & -1.4400740 \\ \text { C } & 0.8429320 & 1.3847650 & -1.0388780 \\ \text { C } & -0.0926060 & 2.3922710 & -1.1621560 \\ \text { C } & 2.1320950 & 1.4519230 & -1.6285900 \\ \text { C } & 0.1954060 & 3.4989520 & -2.0253110 \\ \text { C } & 2.4228770 & 2.5480510 & -2.4043000 \\ \text { C } & 1.4742680 & 3.5745470 & -2.6430110 \\ \text { H } & 3.4117910 & 2.6357810 & -2.8483900 \\ \text { C } & -2.1532710 & 1.1564650 & -0.5991880 \\ \text { C } & -3.4080360 & 0.9566860 & 0.0373700 \\ \text { C } & -1.3807490 & 2.2834140 & -0.4302740 \\ \text { C } & -3.8681420 & 1.9710030 & 0.8420120 \\ \text { C } & -1.8211710 & 3.2836540 & 0.4963350 \\ \text { C } & -3.0930000 & 3.1281700 & 1.1142860 \\ \text { H } & -4.8383780 & 1.8708330 & 1.3214650 \\ \text { C } & -2.7609130 & 5.1912490 & 2.3493980 \\ \text { C } & -1.4778130 & 5.3260230 & 1.7661910\end{array}$




-1.0200340
-3.5471980
-3.1117770
-0.8501150
-0.0319590
-4.5254040
-0.4402890
0.8443630
1.7775030
-0.7577610
-1.1833150
1.0809650
2.7627490
-1.7497490
3.1701300
3.4214990
3.9437510
4.5099610
5.0039520
5.3237060
4.7102140
5.6103480
-4.1728780
-4.3660350
-4.7141410
-5.1278670
-5.4604910
-5.6847560
-5.2886990
-5.8860290
3.6566870
6.5312310
2.5513590
-3.7851010
-6.5053420
-4.5528120
-5.9069550
-6.5963280
-6.3726320
-5.7810160
-3.8805500
-2.9135260
-4.5060110
-3.7181180
-5.8493500
-6.4248660

4.4011310

4.1146820

5.9348970

6.1686750

4.5099120

3.9868660

5.5542990

5.6433210

4.6709070

4.5130070

6.3150020

6.4757250

4.7156020

4.4501410

0.4019000

$-0.5692750$

0.4428650

$-1.4352090$

$-0.4504720$

$-1.3777790$

$-2.1748910$

$-0.4355000$

$-0.3151200$

$-1.1707240$

$-0.6523430$

$-2.3289240$

$-1.8239930$

$-2.6746460$

$-2.9894430$

$-2.0755590$

1.4641500

$-2.2799760$

$-0.6967280$

$-0.8881570$

$-3.9404650$

0.2339530

0.8097780

0.0108500

1. 3745380

1.4794970

$-0.5176230$

$-0.9222290$

$-1.3459420$

0.1574960

$-4.9091200$

$-5.8380730$

0.8606110
2.0302530
3.0575770
2.0392080
0.4247730
2.4859280
-3.1538750
-3.7426050
-3.4939610
-2.3186000
-3.3715710
-4.3974700
-3.9503410
-1.8841060
-1.3914190
-2.3728470
-0.2093280
-2.2041850
-0.0810230
-1.0783150
-2.9734670
0.8216440
-0.1351060
0.9700050
-1.3922730
0.7947970
-1.5151750
-0.4331080
1.6446920
-2.4851780
0.8844940
-0.8786940
-3.6144080
2.3494820
-0.5915970
-2.6187340
-3.0527340
-3.3474800
-2.2388650
-3.9098020
-3.7730490
-3.4618470
-4.1236400
-4.6197790
-1.5822940
-1.6503550

\begin{abstract}
$-5.8041310$
$-4.8284240$

$-7.9441170$

$-8.4268060$

$-7.9618530$

$-8.5372410$

$-4.8818890$

$-5.4003140$

$-5.6308600$

$-4.4538480$

$-3.0486820$

$-3.7448270$

$-2.3200050$

$-2.5197890$

4. 3201630

5.4092190

3.9908370

4.0798700

4.0934930

3.6844120

5.1851350

3.7392310

7.8241270

8.6990740

7.9181830

7.8330560

6.5839650

5.6694770

6.7273050

7.4268580

2.2680650

3.1514310

1.9482840

1.4718910

3.1857750

4.1720720

2. 5601780

3.3127280

$-0.5693730$

$-0.0214640$

$-1.1305530$

1. 5878950

6.4732100

2. 5698990

$-3.9102440$

$-6.5415060$
\end{abstract}

\begin{tabular}{rr}
-4.4678150 & -2.5841360 \\
-5.1555660 & -1.2762060 \\
-3.6244120 & -1.0159950 \\
-2.9484800 & -0.3038790 \\
-3.1460840 & -2.0015860 \\
-4.5425750 & -1.0783530 \\
-0.4312330 & 3.3215040 \\
0.4628570 & 2.9628040 \\
-1.2212010 & 3.4500000 \\
-0.2079540 & 4.3044870 \\
-2.0988670 & 2.9391460 \\
-2.8997700 & 3.2112020 \\
-2.4994300 & 2.2300750 \\
-1.7998330 & 3.8510380 \\
2.8121360 & 0.5655980 \\
2.6931410 & 0.5323720 \\
3.2141760 & -0.3958620 \\
3.5435870 & 1.3450500 \\
1.0057940 & 2.2770900 \\
0.0207130 & 2.5274770 \\
0.9606680 & 2.3590060 \\
1.7231700 & 3.0243290 \\
-1.4592160 & -0.9948830 \\
-2.0869360 & -0.7974320 \\
-1.0480670 & -2.0060900 \\
-0.6242350 & -0.2882610 \\
-3.4741370 & -1.8306430 \\
-4.0754470 & -1.7904450 \\
-3.1455300 & -2.8659500 \\
-4.1208490 & -1.5700870 \\
-2.1589720 & -3.9762510 \\
-2.6468490 & -4.4029120 \\
-2.7270980 & -3.0992930 \\
-2.2075940 & -4.7254720 \\
0.0253400 & -4.8102480 \\
-0.3995290 & -5.0301370 \\
-0.0884320 & -5.7018920 \\
1.0941700 & -4.6163410 \\
-0.8029690 & -0.7398270 \\
-1.7487850 & -1.7599590 \\
-1.3655190 & 0.5489130 \\
-0.2252110 & -3.3941800 \\
-2.6678480 & 0.1488130 \\
1.6187200 & 0.9199670 \\
1.0799120 & -2.3589310 \\
-4.4315350 & 0.3890880 \\
& \\
\hline
\end{tabular}




\begin{tabular}{|c|c|c|c|c|c|c|c|}
\hline & & & & & & & \\
\hline $\begin{array}{ll}\mathrm{H} \\
\mathrm{C}\end{array}$ & -3.0552670 & $\begin{array}{l}-0.0796170 \\
-2.9479670\end{array}$ & 2.2543910 & $\mathrm{C}$ & -3.4196870 & 0.9515360 & $\begin{array}{r}0.0114310 \\
-0.4297610\end{array}$ \\
\hline c & 0.9373190 & -3.3931390 & 1.1423710 & $\mathrm{c}$ & -3.8615700 & 1.9613880 & 0.8318870 \\
\hline $\mathrm{H}$ & 2.0990140 & -1.8854780 & 0.0602710 & $\mathrm{C}$ & -1.7891450 & 3.2404450 & 0.5096860 \\
\hline $\mathrm{H}$ & -0.0096700 & -2.8871110 & 1.0008620 & $\mathrm{C}$ & -3.0644180 & 3.0987120 & 1.1240450 \\
\hline 0 & 1.3635950 & -3.7346590 & -0.6670870 & $\mathrm{H}$ & -4.8345860 & 1.8725920 & 1.3079890 \\
\hline H & 0.7800840 & -3.0886040 & -1.1740290 & $\mathrm{C}$ & -2.6956890 & 5.1358910 & 2.3914840 \\
\hline C & 3.3571610 & -4.9760980 & 1.1357320 & $\mathrm{C}$ & -1.4104140 & 5.2572190 & 1.8099330 \\
\hline C & 2.3892940 & -5.0905850 & 2.3131050 & $\mathrm{C}$ & -0.9684930 & 4.3381640 & 0.8905690 \\
\hline C & 0.9780060 & -4.6892150 & 1.8825970 & $\mathrm{C}$ & -3.5010160 & 4.0787870 & 2.0554210 \\
\hline c & 3.4324990 & -3.5337380 & 0.6314780 & $\mathrm{H}$ & -3.0333680 & 5.8745110 & 3.1112270 \\
\hline H & 4.3577630 & -5.3071610 & 1.4282860 & $\mathrm{H}$ & -0.7683140 & 6.0848970 & 2.0952780 \\
\hline $\mathrm{H}$ & 2.3697020 & -6.1100350 & 2.7075830 & $\mathrm{H}$ & 0.0222020 & 4.4353410 & 0.4577090 \\
\hline H & 0.5571550 & -5.4397190 & 1.1998760 & $\mathrm{H}$ & -4.4816250 & 3.9616280 & 2.5087520 \\
\hline $\mathrm{H}$ & 3.9000700 & -2.8866950 & 1.3854680 & $\mathrm{C}$ & -0.3718690 & 5.5217520 & -3.1216550 \\
\hline $\mathrm{H}$ & 4.0381140 & -3.4613240 & -0.2752700 & $\mathrm{C}$ & 0.9195860 & 5.6035670 & -3.6969590 \\
\hline H & 3.0247150 & -5.6321790 & 0.3219960 & $\mathrm{C}$ & 1.8424170 & 4.6223860 & -3.4447300 \\
\hline $\mathrm{H}$ & 2.7233700 & -4.4352500 & 3.1281100 & $\mathrm{C}$ & -0.7069530 & 4.4777960 & -2.2967830 \\
\hline H & 0.2835650 & -4.6210970 & 2.7243750 & $\mathrm{H}$ & -1.1058240 & 6.2905660 & -3.3415880 \\
\hline C & 0.5580730 & -0.2491900 & 3.1376010 & $\mathrm{H}$ & 1.1696350 & 6.4383250 & -4.3437710 \\
\hline 0 & -0.5191320 & 0.0306530 & 2.4877150 & $\mathrm{H}$ & 2.8332580 & 4.6625950 & -3.8892190 \\
\hline C & 1.1074890 & 0.9164740 & 3.8706600 & $\mathrm{H}$ & -1.7038280 & 4.4202180 & -1.8727170 \\
\hline C & 1.9160800 & 0.7701790 & 5.0020220 & $\mathrm{C}$ & 3.2382200 & 0.3897400 & -1.3183270 \\
\hline C & 0.8364130 & 2.1951350 & 3.3641760 & $\mathrm{C}$ & 3.6092270 & -0.5179540 & -2.3242750 \\
\hline C & 2.4462870 & 1.8944400 & 5.6232970 & $\mathrm{C}$ & 3.9990150 & 0.4886630 & -0.1328270 \\
\hline H & 2.1205090 & -0.2229670 & 5.3886110 & $\mathrm{C}$ & 4.8092130 & -1.2266170 & -2.1883540 \\
\hline C & 1.3927210 & 3.3144870 & 3.9732130 & $\mathrm{C}$ & 5.1811200 & -0.2458120 & -0.0398220 \\
\hline $\mathrm{H}$ & 0.2094400 & 2.2995080 & 2.4834130 & C & 5.6276930 & -1.0750830 & -1.0720900 \\
\hline C & 2.1941420 & 3.1655990 & 5.1047900 & $\mathrm{H}$ & 5.1118400 & -1.8978920 & -2.9883110 \\
\hline $\mathrm{H}$ & 3.0627010 & 1.7808670 & 6.5089810 & $\mathrm{H}$ & 5.7968210 & -0.1579410 & 0.8524980 \\
\hline $\mathrm{H}$ & 1.1937270 & 4.3012840 & 3.5665240 & $\mathrm{C}$ & -4.2081610 & -0.3018300 & -0.1872720 \\
\hline H & 2.6218 & 4.0401100 & 5.5851810 & C & -4.4273770 & -1.1710100 & 0.9020900 \\
\hline $\mathrm{H}$ & -0.7928600 & -0.6551410 & 1.7442390 & $\mathrm{C}$ & -4.7462850 & -0.6076710 & -1.4540250 \\
\hline & 1.3223950 & -1.7510570 & 3.1722130 & $\mathrm{C}$ & -5.2106370 & -2.3108930 & 0.7022980 \\
\hline & & & & $\mathrm{C}$ & -5.5148430 & -1.7617670 & -1.6014790 \\
\hline & , R) $G[M 06-2\rangle$ & $g(d)]=-363$ & 574 & $\mathrm{C}$ & -5.7647050 & -2.6252500 & -0.5351560 \\
\hline 0 & 0.5332710 & 0.2124450 & -0.2667690 & $\mathrm{H}$ & -5.3916910 & -2.9812860 & 1.5403260 \\
\hline 0 & -1.6977720 & 0.1434860 & -1.4737390 & $\mathrm{H}$ & -5.9383370 & -1.9887040 & -2.5784110 \\
\hline C & 0.8526690 & 1.3222430 & -1.0276330 & $\mathrm{C}$ & 3.5859510 & 1.4303440 & 0.9919860 \\
\hline C & -0.0713210 & 2.3412170 & -1.1534350 & C & 6.9493820 & -1.8102910 & -0.9221880 \\
\hline C & 2.1576730 & 1.3943450 & -1.5814190 & $\mathrm{c}$ & 2.7514370 & -0.7280290 & -3.5626150 \\
\hline C & 0.2346680 & 3.4533250 & -2.0020730 & C & -3.8539810 & -0.9209800 & 2.2906380 \\
\hline C & 2.4613190 & 2.4940150 & -2.3495990 & C & -6.6116040 & -3.8699390 & -0.7204200 \\
\hline C & 1.5208380 & 3.5232870 & -2.6039490 & C & -4.5604440 & 0.2959450 & -2.6643250 \\
\hline $\mathrm{H}$ & 3.46128 & 2.5848510 & -2.7678480 & C & -5.9000770 & 0.9094090 & -3.0919090 \\
\hline C & 590880 & 1.1371060 & -0.6170430 & $\mathrm{H}$ & -6.6065720 & 0.1311010 & -3.4009700 \\
\hline
\end{tabular}




-6.3548690
-5.7568000
-3.9015090
-2.9462440
-4.5455340
-3.7186160
-5.9725270
-6.5684610
-5.9125380
-4.9589520
-8.0408920
-8.5119640
-8.0429310
-8.6540080
-4.9520220
-5.4559960
-5.7122680
-4.5284620
-3.1382150
-3.8465500
-2.4103810
-2.6117600
4.1446820
5.2404470
3.8215840
3.8094140
4.0012470
3.6353880
5.0898120
3.5847070
7.6745880
8.6760210
7.1425040
7.7737380
6.7569250
6.2766640
6.1276080
7.7217160
2.5292950
3.4499620
2.1763370
1.7786760
3.3598950
4.3607130
2.7380200
3.4485260

1.4712860

1.5899620

$-0.4500660$

$-0.8846830$

$-1.2558280$

0.2367540

$-4.8347860$

$-5.7490940$

$-4.3764880$

$-5.1098700$

$-3.5142010$

$-2.8405580$

$-3.0178800$

$-4.4175870$

$-0.4664800$

0.4403360

$-1.2484730$

$-0.2654740$

$-2.1520630$

$-2.9492180$

$-2.5481550$

$-1.8783130$

2.8422600

2.8181700

3.2592740

3.5128060

0.9348120

$-0.0800220$

0.9415070

1.5990270

$-2.0291030$

$-2.4311310$

$-2.7502410$

$-1.0949880$

$-3.1478780$

$-3.0049230$

$-3.8203850$

$-3.6402140$

$-2.2173680$

$-2.7028200$

$-2.7396520$

$-2.3386580$

$-0.0344540$

$-0.4310090$

-0.2044330
1.0452580

-2.2699150
-3.9376530
-3.8298640
-3.5228100
-4.1988990
-4.6628710
-1.7257400
-1.8134140
-2.7191290
-1.4196420
-1.1455590
-0.4235630
-2.1223830
-1.2272960
3.2622500
2.9149210
3.3719390
4.2519570
2.8623490
3.1131520
2.1500300
3.7835730
0.7645890
0.7755500
-0.1931120
1.5636390
2.3788600
2.5723510
2.4999270
3.1428480
-2.2514750
-2.0702900
-2.8810510
-2.8125710
-0.1943730
0.7794060
-0.7898050
-0.0338970
-3.8480120
-4.1900070
-2.9545870
-4.6352350
-4.7873860
-4.9941390
-5.6725210
-4.6340630

$-0.6069660$

$-0.0636990$

$-1.1668940$

1.7682500

7.5893890

2. 4897480

$-3.9001410$

$-6.6634910$

$-3.1124980$

1.9536430

0.8979540

1.8413590

$-0.1031150$

1. 3307010

0.7144960

3. 5083630

2. 5874820

1. 1229000

3.3820180

4. 5489860

2.7049090

0.7755040

3.7704370

3.9516040

3. 2474360

2.8579910

0.4540540

0.4310640

$-0.6081750$

0.9544180

1.7214640

0.7036300

2. 2316030

1.9103850

1.2390070

0.1088070

2. 0001450

2.8163920

1.0548160

2. 4121930

$-0.8578070$

1.1715140
$-0.8475670$
$-1.7623520$
$-1.4492200$
$-0.2870670$
$-1.1810840$
1.4940430
1.1226920
$-4.3777450$
$-0.1211430$
$-2.8427070$
$-3.4479560$
$-1.7814150$
$-3.0489810$
$-3.6876620$
$-3.0859480$
$-4.7029370$
$-4.9878890$
$-4.7512460$
$-3.2440490$
$-4.9138180$
$-6.0173550$
$-5.5262080$
$-2.5726140$
$-3.0421100$
$-5.3634150$
$-4.3309370$
$-4.7993530$
$-0.3395820$
$-0.0511100$
0.8242480
0.6744500
2.1046460
1.7964750
$-0.3196760$
3.2217810
2. 2126980
3.0691790
1.6799680
4.2095980
3.9418820
$-0.7398160$
$-1.8510130$

144

TS224(S, S) G[M06-2X/6-31G(d)] = -3633.305920
0
0.6548870
0.7065170
1.2287160
0.2306280
$-1.0761350$

1.8490910

0.4723690

- .3669720

0.9883870

$-2.3879940$

0.2509880

2. 2162050

0.3055010

1.1287460

0.0750700

1.0221050

0.6941920

1.2190120
1.0113290

2. 1989780

1.8209710

0.5706100

1. 2761960

2. 5478830

1. 3478850

$-0.3407210$

0.1754090

3.0357030

2.6847470

2. 4330590

3.8938680

5.0535000

3.3809190

5.6952650
5.4453370

4.0122400

2. 4785340

5. 1708790

6.6017690

3.6008120

(

1.6867230
3.2191630 


$\begin{array}{lrrr}\text { C } & 0.8763530 & 2.0694100 & \\ \text { C } & -0.1569310 & 2.8879630 & 0.3288480 \\ \text { C } & 2.1903800 & 2.5364570 & 0.7357920 \\ \text { C } & 0.0810250 & 4.3005820 & 0.8642460 \\ \text { C } & 2.4330050 & 3.8773100 & 0.2312970 \\ \text { C } & 1.3984110 & 4.7872970 & 0.5717450 \\ \text { H } & 3.4400530 & 4.2605030 & 0.0886570 \\ \text { C } & -2.0831530 & 1.4575890 & 0.1340200 \\ \text { C } & -3.3551800 & 0.8550750 & 0.3258590 \\ \text { C } & -1.4790410 & 2.2855000 & 1.0558720 \\ \text { C } & -4.0168670 & 1.1412410 & 1.4942580 \\ \text { C } & -2.1341760 & 2.4914500 & 2.3152670 \\ \text { C } & -3.4284540 & 1.9337200 & 2.5141890 \\ \text { H } & -5.0125930 & 0.7371640 & 1.6567290 \\ \mathrm{C} & -3.5056900 & 2.8649020 & 4.7542990 \\ \mathrm{C} & -2.2007920 & 3.3831580 & 4.5745910 \\ \mathrm{C} & -1.5327550 & 3.2025620 & 3.3894300 \\ \mathrm{C} & -4.1008710 & 2.1516530 & 3.7464190 \\ \mathrm{H} & -4.0239740 & 3.0218880 & 5.6947890 \\ \mathrm{H} & -1.7219160 & 3.9234280 & 5.3850900 \\ \mathrm{H} & -0.5287270 & 3.5948400 & 3.2690310 \\ \mathrm{H} & -5.0931620 & 1.7280060 & 3.8763240 \\ \mathrm{C} & -0.6777380 & 6.5860010 & 1.1253690 \\ \mathrm{C} & 0.6413670 & 7.0639510 & 0.9369390 \\ \mathrm{C} & 1.6536070 & 6.1817780 & 0.6615200 \\ \mathrm{C} & -0.9517360 & 5.2428870 & 1.0606650 \\ \mathrm{H} & -1.4810630 & 7.2902850 & 1.3171420 \\ \mathrm{H} & 0.8434190 & 8.1284740 & 0.9976680 \\ \mathrm{H} & 2.6677760 & 6.5343180 & 0.4937430 \\ \mathrm{H} & -1.9685010 & 4.8900500 & 1.1964190 \\ \mathrm{C} & 3.2802260 & 1.5931600 & -0.3349530 \\ \mathrm{C} & 3.7201760 & 1.5449900 & -1.6753760 \\ \mathrm{C} & 3.9020170 & 0.7982060 & 0.6428370 \\ \mathrm{C} & 4.8170570 & 0.7425420 & -1.9858940 \\ \mathrm{C} & 5.0083860 & 0.0207200 & 0.2842280 \\ \mathrm{C} & 5.4931650 & -0.0077370 & -1.0204240 \\ \mathrm{H} & 5.1675390 & 0.6985440 & -3.0152970 \\ \mathrm{H} & 5.4932550 & -0.5785830 & 1.0507720 \\ \mathrm{C} & -3.9470500 & -0.0394280 & -0.7147830 \\ \mathrm{C} & -4.0714500 & -1.4216690 & -0.4695770 \\ \mathrm{C} & -4.4052470 & 0.5093790 & -1.9291930 \\ \mathrm{C} & -4.7026610 & -2.2156130 & -1.4313720 \\ \mathrm{C} & -5.0172000 & -0.3279610 & -2.8611190 \\ \mathrm{C} & -5.1858400 & -1.6924390 & -2.6275020 \\ \mathrm{H} & -4.8195220 & -3.2811630 & -1.2468330 \\ \mathrm{H} & -5.3798800 & 0.1005710 & -3.7939610 \\ & & & \end{array}$

\begin{abstract}
3.4236540
6.7178770

3.0599750

$-3.5579620$

$-5.8680630$

$-4.2870690$

$-5.6738050$

$-6.2636400$

$-6.2291360$

$-5.5823180$

$-3.4893210$

$-2.4970910$

$-4.0038240$

$-3.3672740$

$-5.0862260$

$-5.5621090$

$-5.0544950$

$-4.0558340$

$-7.3173140$

$-7.8881670$

$-7.3524160$

$-7.8120890$

$-4.7086960$

$-5.2442090$

$-5.4311820$

$-4.3279730$

$-2.7970440$

$-3.4583630$

$-1.9826640$

$-2.3734600$

4. 5051730

5.3920430

4.8151840

4.1288180

2.9597130

2.1522240

3.7826490

2. 5792940

7.1774770

8.0017810

7. 5408390

6.3739930

7.8741730

7.5886080

8.1683260

8.7452070
\end{abstract}

0.7614690

$-0.8105430$

2. 3741410

$-2.0791590$

$-2.5811320$

1.9918040

. 6419120

2.2028540

2.5044740

.7159590

2.2253030

1.7708250

1.7987840

3.2985540

$-2.6160640$

$-3.2988270$

$-1.6215790$

$-2.9462770$

$-2.1438350$

$-2.1341910$

$-1.1359560$

$-2.8234800$

$-2.3565350$

$-1.4427680$

$-3.0472020$

$-2.8168680$

$-3.3815510$

$-4.1600640$

$-3.2214840$

$-3.7649590$

1.2714720

0.6290500

2.2872790

1.2821910

$-0.6477940$

$-0.9810720$

$-1.3695070$

$-0.6602460$

$-1.8308400$

$-2.4270000$

$-1.3320940$

$-2.5157310$

0.1391840

0.8503120

0.7103220

$-0.4243310$
2.0868090

$-1.4338470$

$-2.7688680$

0.8055600

$-3.6502570$

$-2.2511200$

$-2.3358060$

$-3.1480690$

$-1.4025000$

$-2.5280310$

$-3.5388210$

$-3.4679740$

$-4.4068910$

$-3.7185070$

$-4.9684030$

$-5.6798660$

$-5.4271400$

$-4.8074250$

$-3.8921590$

$-2.9588740$

$-4.3205230$

$-4.5935830$

1.7829190

2.0550360

1. 3327740

2.7015320

0.5302530

0.1357890

$-0.1820180$

1.4660750

3.0462590

3.0198790

2.7816480

4.0741870

2. 4743810

1. 8144040

2.4128870

3.5014610

$-0.3930070$

$-0.7950100$

0.5123950

$-0.1020570$

$-1.7799990$

$-2.5604500$

$-0.8924640$

$-2.1295440$ 


\begin{tabular}{rrr}
2.9215210 & 1.6202000 & -4.0956250 \\
3.8946080 & 1.4572080 & -4.5711510 \\
2.4344760 & 0.6522550 & -3.9618290 \\
2.3157280 & 2.2113740 & -4.7895880 \\
3.8404550 & 3.6753770 & -3.0042660 \\
4.8644260 & 3.4485610 & -3.3233010 \\
3.3632830 & 4.2705740 & -3.7899570 \\
3.8970470 & 4.2871120 & -2.1001640 \\
-0.2203530 & 0.1646330 & -1.0418460 \\
0.5284990 & 0.2973850 & -2.3325040 \\
-0.6813750 & -1.2037340 & -0.5988350 \\
2.0502030 & 2.6286490 & -2.4310870 \\
6.4502760 & -1.3569700 & -2.3499930 \\
2.5603890 & 1.4265870 & 2.1840720 \\
-3.7469440 & 2.4850580 & -1.4379530 \\
-5.8852610 & -3.5986080 & -3.2395760 \\
-2.8557710 & -1.3878810 & 1.2840320 \\
1.4580350 & -2.7524370 & -2.1646020 \\
2.2329340 & -2.3749710 & -0.9781450 \\
0.3946510 & -2.5250160 & -2.1165000 \\
1.8978300 & -1.5189920 & -0.3993920 \\
2.2324870 & -1.6383310 & -2.6825050 \\
1.6478120 & -0.8140150 & -2.5967660 \\
3.2998850 & -4.3647240 & -2.7963140 \\
3.8131220 & -4.3041160 & -1.3564700 \\
3.6168280 & -2.9055010 & -0.7695780 \\
1.8022710 & -4.0532970 & -2.8496530 \\
3.4802400 & -5.3551190 & -3.2234480 \\
4.8756850 & -4.5613970 & -1.3121860 \\
4.2845460 & -2.1889980 & -1.2629610 \\
1.2347940 & -4.8435960 & -2.3425510 \\
1.4406430 & -4.0041130 & -3.8813250 \\
3.8470240 & -3.6408960 & -3.4122780 \\
3.2781310 & -5.0416680 & -0.7443110 \\
3.8531940 & -2.8581950 & 0.2977070 \\
0.2527170 & -3.0415740 & 1.9707540 \\
-0.3295130 & -1.9142320 & 1.7386170 \\
0.0387410 & -3.5419740 & 3.3502290 \\
0.8540500 & -4.5351960 & 3.9052650 \\
-1.0166000 & -3.0078460 & 4.1038150 \\
0.6163240 & -4.9879510 & 5.1969910 \\
1.6746170 & -4.9391980 & 3.3222320 \\
-1.2567190 & -3.4727020 & 5.3914150 \\
-1.6450710 & -2.2379530 & 5.93946350 \\
-0.4400900 & -4.4608480 & \\
1.2558420 & -5.7520550 & \\
\hline & &
\end{tabular}

$\begin{array}{llll}\mathrm{H} & -2.0803810 & -3.0641280 & 5.9675610 \\ \mathrm{H} & -0.6259870 & -4.8202190 & 6.9469210 \\ \mathrm{H} & -0.3957390 & -1.6320000 & 0.7265500 \\ \mathrm{~S} & 1.1519240 & -3.9069490 & 0.8412500\end{array}$

TS225(S, S ) G[M06-2X/6-31G(d)] $=-3633.308242$

$\begin{array}{lrrr}\mathrm{O} & 0.7121210 & 0.7309150 & 0.3061720 \\ \mathrm{O} & -1.2260260 & 1.2930250 & -1.2075680 \\ \mathrm{C} & 1.0008190 & 2.0840420 & 0.3506740 \\ \mathrm{C} & -0.0123000 & 2.9755140 & 0.6353900 \\ \mathrm{C} & 2.3533550 & 2.4669430 & 0.1542110 \\ \mathrm{C} & 0.3018250 & 4.3749660 & 0.6580900 \\ \mathrm{C} & 2.6614070 & 3.7981870 & 0.2829120 \\ \mathrm{C} & 1.6593930 & 4.7769780 & 0.5120920 \\ \mathrm{H} & 3.6952830 & 4.1203890 & 0.1907440 \\ \mathrm{C} & -1.9576410 & 1.6225260 & -0.0745270 \\ \mathrm{C} & -3.2784520 & 1.1083390 & 0.0244580 \\ \mathrm{C} & -1.3895410 & 2.4605290 & 0.8620610 \\ \mathrm{C} & -4.0230200 & 1.4899380 & 1.1130630 \\ \mathrm{C} & -2.1408660 & 2.7652120 & 2.0454560 \\ \mathrm{C} & -3.4797290 & 2.2940850 & 2.1484930 \\ \mathrm{H} & -5.0531230 & 1.1542680 & 1.1992730 \\ \mathrm{C} & -3.6982610 & 3.3358200 & 4.3297610 \\ \mathrm{C} & -2.3527110 & 3.7678860 & 4.2487000 \\ \mathrm{C} & -1.5944110 & 3.4916320 & 3.1386630 \\ \mathrm{C} & -4.2446530 & 2.6106360 & 3.3029850 \\ \mathrm{H} & -4.2876180 & 3.5683780 & 5.2108800 \\ \mathrm{H} & -1.9153200 & 4.3180460 & 5.0758240 \\ \mathrm{H} & -0.5611160 & 3.8184630 & 3.0939270 \\ \mathrm{H} & -5.2693170 & 2.2529370 & 3.3589600 \\ \mathrm{C} & -0.3431960 & 6.7120580 & 0.8228810 \\ \mathrm{C} & 1.0124250 & 7.1067440 & 0.7215500 \\ \mathrm{C} & 1.9887740 & 6.1577410 & 0.5641100 \\ \mathrm{C} & -0.6902310 & 5.3845860 & 0.7926090 \\ \mathrm{H} & -1.1160930 & 7.4679980 & 0.9195770 \\ \mathrm{H} & 1.2717890 & 8.1600310 & 0.7540500 \\ \mathrm{H} & 3.0318660 & 6.4450550 & 0.4621850 \\ \mathrm{H} & -1.7336860 & 5.0962080 & 0.8608300 \\ \mathrm{C} & 3.3963480 & 1.4433120 & -0.1592890 \\ \mathrm{C} & 3.9019690 & 1.3322880 & -1.4701260 \\ \mathrm{C} & 3.8894970 & 0.6141550 & 0.8661120 \\ \mathrm{C} & 4.9358700 & 0.4249210 & -1.7155730 \\ \mathrm{C} & 4.9238790 & -0.2742280 & 0.5706770 \\ \mathrm{C} & 5.4687750 & -0.3777340 & -0.7089910 \\ \mathrm{H} & 5.3310810 & 0.3435570 & -2.7240420 \\ \mathrm{H} & 5.3210610 & -0.9119910 & 1.3602150 \\ & & & \end{array}$




-3.8354250
-4.0734750
-4.1528040
-4.6703930
-4.7386190
-5.0146840
-4.8701390
-4.9955810
3.3486800
6.6264310
3.3808300
-3.7164740
-5.6641030
-3.9147890
-5.2462780
-5.7972780
-5.8804200
-5.0715620
-3.0055110
-2.0532960
-3.4767810
-2.8006760
-7.0203880
-7.4998200
-6.8978720
-7.6916810
-4.7429900
-3.7795660
-4.5520710
-5.2035350
-4.9639440
-5.4621570
-5.6865590
-4.6958850
-3.0197530
-3.6893870
-2.1328100
-2.7122890
4.4372050
5.2568380
4.8557830
4.0245310
2.7365560
1.9093210
3.4830890
2.3395780

0.2057220

0.7264530

$-1.9509960$

$-0.1150410$

$-1.4515860$

$-2.9968790$

0.2861520

0.6664230

$-1.3277790$

2. 1937120

$-1.7832450$

$-2.3422710$

2. 1831870

2. 9118920

2.4747750

2. 8484560

3. 9691600

2. 3121740

1.8012810

1.8815720

3. 3672700

$-2.8691560$

$-3.4687530$

$-3.5051610$

$-2.0471710$

$-3.5017380$

$-3.1332820$

$-4.1564100$

$-4.1063580$

$-1.9430940$

$-0.9866970$

$-2.6109950$

$-2.3788980$

$-3.1403560$

$-3.8936350$

$-3.0597120$

$-3.5083100$

1. 1033950

0.3772320

2. 0741680

1.1869370

$-0.6773780$

$-0.9452300$

$-1.4798060$

$-0.6221460$

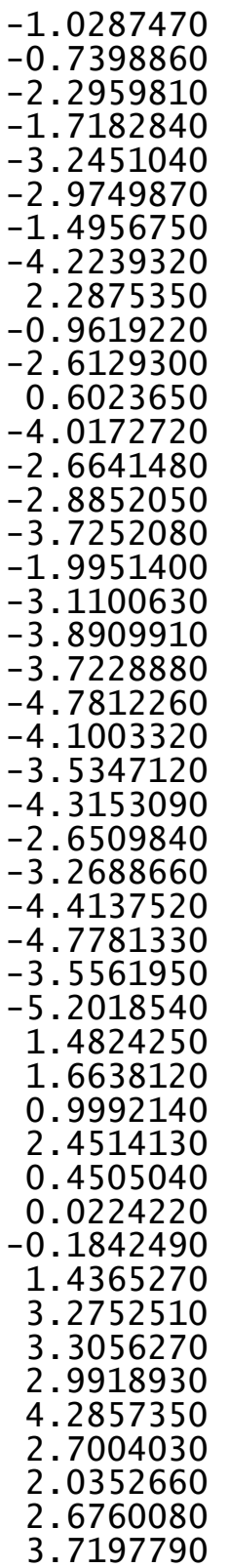

\begin{abstract}
7.9602420
8.8022200

8.0753220

8.0073990

6.6073550

5. 6269390

6.8447980

7.3570360

3. 2380460

4. 2132510

2. 6512830

2. 7280630

4. 2922690

5. 3044490

3. 9136020

4. 3630810

$-0.0784970$

0.7860820

$-0.6636280$

2. 3840640

6.5429920

2. 5510390

$-3.4094040$

$-5.8378190$

$-3.0147940$

1. 4838210

2. 1901820

0.4508210

1.8981930

2.4136360

1. 9057280

3. 1849070

3. 5854800

3. 4871740

1.7408540

3. 2890180

4.6096390

4. 2670540

1.0500920

1. 4726030

3.8554270

2. 9317990

3.6354550

$-0.1094820$

$-0.5782370$

$-0.4922690$
\end{abstract}

-0.6107090
-1.2960570
0.2240550
-0.2094490
-1.9499960
-2.3760630
-1.2064250
-2.7437790
1.4226750
1.1554160
0.5113440
2.0513030
3.4097650
3.0803090
4.0291730
4.0340240
0.1635730
0.1743420
-1.1472880
2.5504760
-2.1409980
1.4143280
2.6769440
-1.7277060
-1.1152250
-2.8855860
-2.5493190
-2.5476570
-1.6431380
-1.8738700
-0.9993180
-4.7068560
-4.6444760
-3.2146750
-4.2387350
-5.7275260
-5.0033330
-2.5879110
-4.9442110
-4.1895470
-4.0732500
-5.3045020
-3.1617430
-2.9426370
-1.7827120
-3.3851960

$-0.7118910$

$-0.8542370$

$-1.4123210$

0.3049070

$-2.3602760$

$-2.6008190$

$-3.1284490$

$-2.4300430$

$-3.9298880$

$-4.3507440$

$-3.7981930$

$-4.6668030$

$-2.8318210$

$-3.0938850$

$-3.6517280$

$-1.9373010$

$-1.0086610$

$-2.2308040$

$-0.5378580$

$-2.3344730$

$-0.2271030$

2. 3282940

$-1.8292390$

$-4.9096260$

1.1132720

$-1.8721440$

$-0.6316500$

$-1.9250130$

$-0.1079200$

$-2.3465430$

$-2.3405050$

$-2.2999380$

$-0.8247560$

$-0.2893480$

$-2.4899080$

$-2.6784710$

$-0.6863450$

$-0.7358090$

$-2.0113170$

$-3.5496750$

$-2.8927630$

$-0.2400090$

0.7929600

2.1525350

1.8397400

3. 5155100 


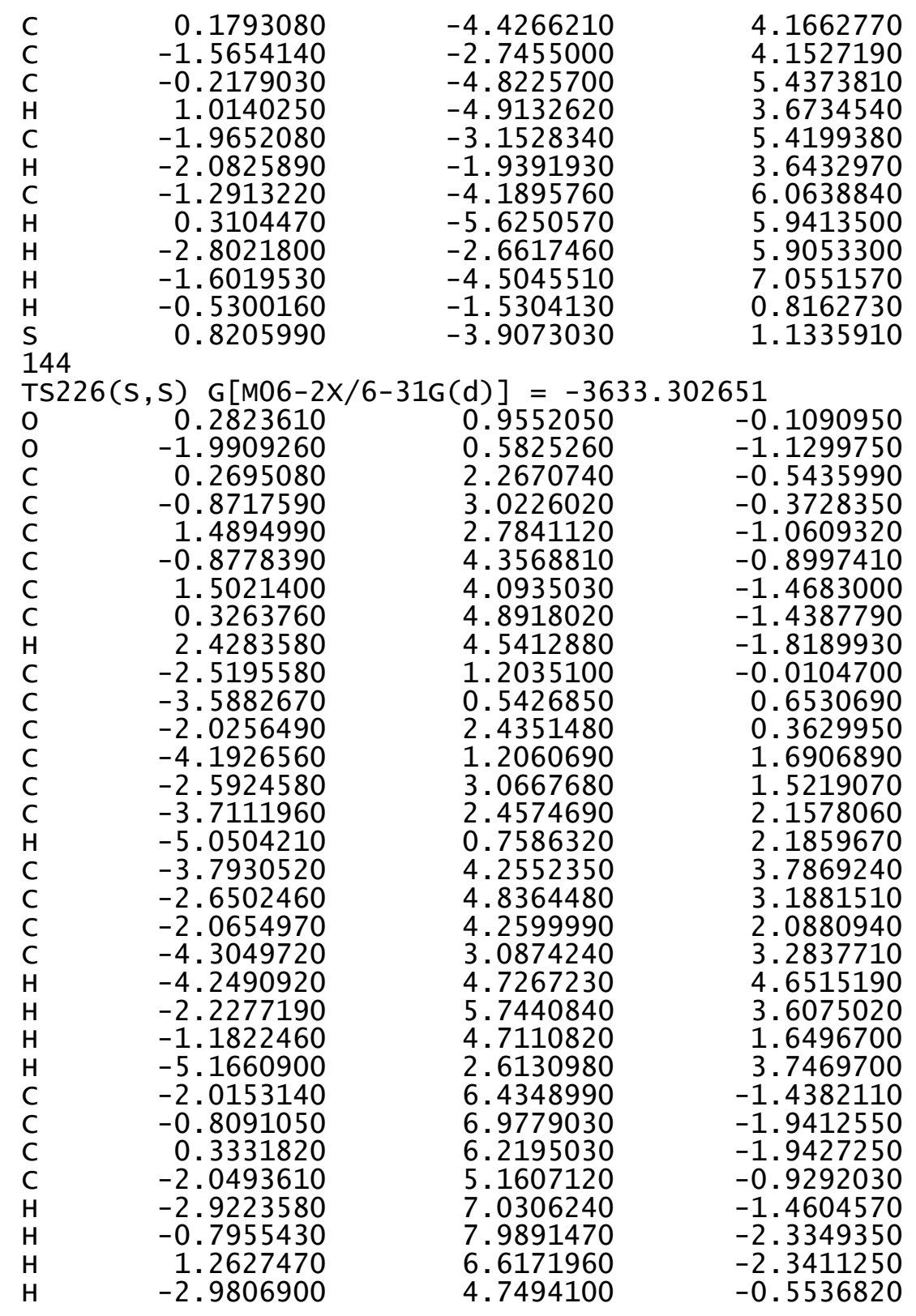

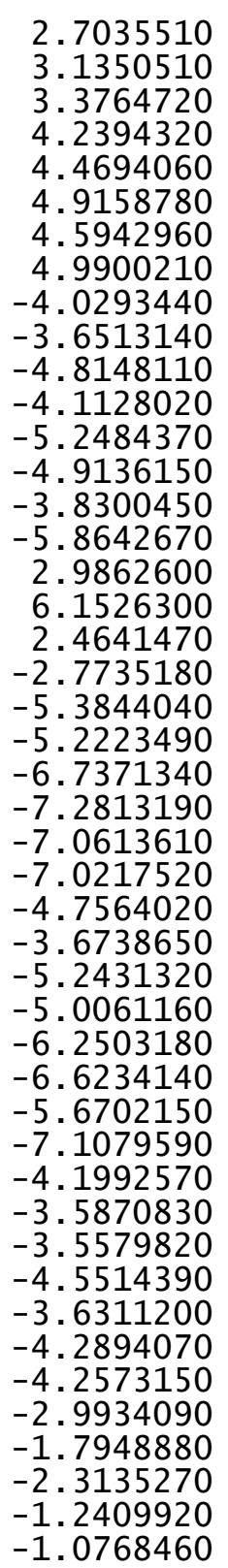

1. 9095510

1. 2238620

1.7385460

0.3738130

0.8713390

0.1774760

$-0.1517510$

0.7466890

$-0.8157530$

$-1.9584950$

$-0.9466870$

$-3.2050920$

$-2.2191880$

$-3.3581380$

-4. 0894470

$-2.3255530$

2. 5064630

$-0.7017780$

1. 4420820

$-1.8649860$

$-4.7316380$

0.2524870

0.4796670

$-0.3792030$

0.6238950

1. 3653270

0.1049790

$-0.0449280$

$-0.7455360$

1. 0053170

$-5.4003930$

$-6.3668710$

$-5.5792120$

$-4.7738680$

$-5.6290800$

$-5.1671630$

$-5.8089960$

$-6.5996370$

$-1.7787650$

$-0.9063640$

$-2.6737170$

$-1.7169280$

3. 0384450

$-3.9665260$

-3.2009160
-2.8300430
$-1.0267680$

$-2.1788380$

0.1980180

$-2.0735260$

0.2548720

$-0.8681520$

$-2.9579080$

1. 2021830

0.2088160

0.9478750

$-0.9479290$

0.5271840

$-1.3310310$

$-0.6065620$

1. 0931210

$-2.2224650$

1.4533630

$-0.8162380$

$-3.5266700$

2. 1901670

$-1.0462110$

$-1.7896670$

$-1.7154000$

$-2.1237210$

$-0.6797750$

$-2.2929010$

$-3.2421060$

$-3.2876690$

$-3.7315950$

$-3.8130090$

0.0272730

$-0.3269090$

0.9393370

0.2897230

$-1.4223790$

$-2.2028560$

$-0.5522870$

$-1.7868660$

3.4608520

3.4529890

3. 5535850

4. 3493400

2.3220890

2. 5860920

1. 3933420

3. 1223750 


$\begin{array}{rrr}4.0831700 & 3.5145810 & 1.8194770 \\ 5.0218890 & 3.0009760 & 2.0556470 \\ 4.2747100 & 4.2049260 & 0.9916200 \\ 3.7881120 & 4.0999060 & 2.6965060 \\ 2.6780880 & 1.5732870 & 2.6273370 \\ 1.8697050 & 0.8827720 & 2.3711990 \\ 3.5540730 & 0.9793550 & 2.9140450 \\ 2.3719660 & 2.1541990 & 3.5042940 \\ 6.2030810 & -1.5994940 & 0.4238200 \\ 7.0566230 & -2.2818620 & 0.3616640 \\ 6.3213820 & -1.0069660 & 1.3377700 \\ 5.2895220 & -2.1954990 & 0.5294010 \\ 7.4162480 & 0.1653850 & -0.9002440 \\ 7.4125170 & 0.7795940 & -1.8056540 \\ 7.4718470 & 0.8362510 & -0.0354780 \\ 8.3158060 & -0.4588410 & -0.9071600 \\ 2.4770670 & 0.1992230 & -4.4188920 \\ 3.4815300 & -0.0123260 & -4.8029330 \\ 2.1207530 & -0.6808460 & -3.8797150 \\ 1.8253530 & 0.3577770 & -5.2837170 \\ 3.1307740 & 2.6093760 & -4.2692470 \\ 4.1882000 & 2.3850940 & -4.4512190 \\ 2.6456720 & 2.7737380 & -5.2372950 \\ 3.0771830 & 3.5397540 & -3.6983100 \\ -0.5514100 & -0.1480250 & -0.9744260 \\ -0.0120930 & -0.3220170 & -2.3625270 \\ -0.6189440 & -1.3489440 & -0.0615290 \\ 1.4171220 & 1.6997900 & -3.3369430 \\ 6.1317630 & -1.3475930 & -1.7047650 \\ 2.0765500 & 3.0769500 & 1.2432710 \\ -4.7372360 & 1.1437060 & -1.3799760 \\ -6.0023460 & -4.5941090 & -1.9425510 \\ -2.1770170 & -0.9492580 & 2.1084820 \\ 0.8461520 & -3.3647710 & -1.8247860 \\ 2.0446740 & -2.5675160 & -1.5460470 \\ -0.0388530 & -3.0947010 & -1.2521340 \\ 1.9110440 & -1.5295390 & -1.2536530 \\ 0.9544830 & -2.6065430 & -3.0577500 \\ 0.4667630 & -1.7281900 & -2.9115480 \\ 2.3312530 & -5.1639760 & -2.7952110 \\ 3.5237690 & -4.5443840 & -2.0639790 \\ 3.3992290 & -3.0209730 & -1.9961170 \\ 1.0252130 & -4.8448010 & -2.0640530 \\ 2.4526150 & -6.2484650 & -2.8659460 \\ 4.4628740 & -4.8044560 & -2.5600230 \\ 3.5636190 & -2.5726820 & -2.9852380\end{array}$
1. 0133490
0.1556870
2. 2832440
3. 5761870
4. 1453850
1.8909070
0.8457290
2. 5776770
3. 9108540
1.8838440
4.5444570
4.4475210
2. 5175910
0.8525140
3. 8493840
5.5821200
1. 9747740
4. 3460050
0.2920260
2. 4494980

$-1.0816360$

$-2.6196590$

$-3.8193110$

$-1.0449320$

$-1.3345390$

2. 0809130

1. 9403440

3. 3802760

3. 5655500

4.4250860

4.7772830

2.7530200

5.6390120

4.2752000

5.8148520

4. 9114090

6.4472270

6.7607820

1. 0566310

0.9519820

$\operatorname{TS} 227(\mathrm{~S}, \mathrm{~S}) \mathrm{G}[\mathrm{M} 06-2 \mathrm{X} / 6-31 \mathrm{G}(\mathrm{d})]=-3633.304299$

$\begin{array}{lrcr}\text { TS227(S , S }) \text { G }[\mathrm{M} 06-2 X / 6-31 \mathrm{G}(\mathrm{d})]=-3633.304299 \\ \mathrm{O} & 0.6514250 & 0.6553670 & 0.2010230 \\ \mathrm{O} & -1.4563340 & 1.2555840 & -1.0625350 \\ \mathrm{C} & 0.9070610 & 2.0103890 & 0.3240110 \\ \mathrm{C} & -0.1023590 & 2.8440410 & 0.7609900 \\ \mathrm{C} & 2.2266010 & 2.4545020 & 0.0501280 \\ \mathrm{C} & 0.1646310 & 4.2500820 & 0.8476330 \\ \mathrm{C} & 2.4972720 & 3.7882890 & 0.2360220 \\ \mathrm{C} & 1.4877170 & 4.7138840 & 0.6056600 \\ \mathrm{H} & 3.5100050 & 4.1526620 & 0.0840050 \\ \mathrm{C} & -2.0723470 & 1.4732500 & 0.1622990 \\ \mathrm{C} & -3.3563850 & 0.8996860 & 0.3620010 \\ \mathrm{C} & -1.4330530 & 2.2662860 & 1.0907540 \\ \mathrm{C} & -3.9924820 & 1.1798250 & 1.5461230 \\ \mathrm{C} & -2.0631380 & 2.4640840 & 2.3639670 \\ \mathrm{C} & -3.3685040 & 1.9363770 & 2.5719970 \\ \mathrm{H} & -4.9954550 & 0.7976490 & 1.7165420 \\ \mathrm{C} & -3.3882610 & 2.8260920 & 4.8300700 \\ \mathrm{C} & -2.0732780 & 3.3144580 & 4.6399970 \\ \mathrm{C} & -1.4277120 & 3.1393660 & 3.4416570 \\ \mathrm{C} & -4.0166480 & 2.1476710 & 3.8183140 \\ \mathrm{H} & -3.8883400 & 2.9784270 & 5.7811210 \\ \mathrm{H} & -1.5688980 & 3.8272680 & 5.4528600 \\ \mathrm{H} & -0.4159950 & 3.5086410 & 3.3130120 \\ \mathrm{H} & -5.0176370 & 1.7473350 & 3.9553010 \\ & & & \end{array}$




$\begin{array}{rrr}-0.5422290 & 6.5446830 & 1.2195130 \\ 0.7834520 & 6.9990240 & 1.0183310 \\ 1.7731950 & 6.1012810 & 0.7135400 \\ -0.8447820 & 5.2086810 & 1.1377410 \\ -1.3279610 & 7.2619120 & 1.4347160 \\ 1.0079380 & 8.0581720 & 1.0926830 \\ 2.7917620 & 6.4357390 & 0.5357430 \\ -1.8664870 & 4.8745300 & 1.2831030 \\ 3.3129190 & 1.5180670 & -0.3780690 \\ 3.7835940 & 1.5492260 & -1.7046040 \\ 3.9479500 & 0.7036410 & 0.5815520 \\ 4.9446870 & 0.8356590 & -2.0187300 \\ 5.1029330 & 0.0089600 & 0.2185600 \\ 5.6399850 & 0.0912520 & -1.0686980 \\ 5.3387980 & 0.8846270 & -3.0316140 \\ 5.6115930 & -0.5938120 & 0.9701220 \\ -3.9854140 & 0.0359120 & -0.6826200 \\ -4.1477520 & -1.3445790 & -0.4498530 \\ -4.4410640 & 0.6103440 & -1.8861080 \\ -4.8115810 & -2.1101570 & -1.4126510 \\ -5.0862010 & -0.1995650 & -2.8198630 \\ -5.2912340 & -1.5611480 & -2.5985040 \\ -4.9563960 & -3.1739100 & -1.2377270 \\ -5.4457620 & 0.2486940 & -3.7445980 \\ -0.2551120 & 0.1683390 & -1.0737100 \\ 0.4848370 & 0.3269970 & -2.3674010 \\ -0.7343210 & -1.2038530 & -0.6647830 \\ 3.4516620 & 0.6146160 & 2.0180200 \\ 2.5317350 & 1.2001720 & 2.1037580 \\ 6.9561470 & -0.5814800 & -1.4203960 \\ 7.2107000 & -0.2770970 & -2.4432190 \\ 3.1018330 & 2.3775890 & -2.7855820 \\ 2.0920720 & 2.6164470 & -2.4368950 \\ -4.2860340 & 2.0923810 & -2.1937410 \\ -3.7241430 & 2.5620900 & -1.3813980 \\ -6.0057400 & -2.4201230 & -3.6245910 \\ -6.0505260 & -3.4402410 & -3.2226610 \\ -3.6346440 & -2.0313710 & 0.8097250 \\ -2.9107350 & -1.3633800 & 1.2889850 \\ -2.9064290 & -3.3454240 & 0.5043070 \\ -2.4767020 & -3.7515960 & 1.4276490 \\ -3.5902860 & -4.1037670 & 0.1089470 \\ -2.1005100 & -3.1920740 & -0.2189180 \\ -4.7783030 & -2.2977920 & 1.7981700 \\ -5.5208250 & -2.9669820 & 1.3482200 \\ -4.3954070 & -2.7785580 & \\ -4 & & \\ -4 & & \\ -4 & -7053190\end{array}$

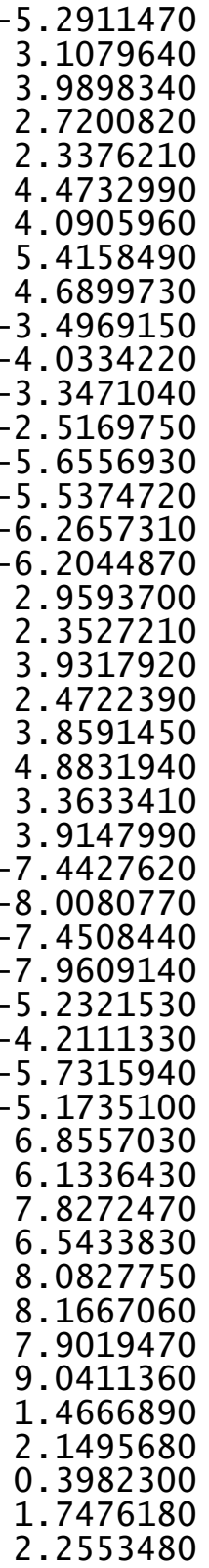

-1.3769870
-0.8261590
-1.4752800
-0.8611890
-1.2376750
1.2212680
1.1964270
0.6632230
2.2615590
2.3183740
1.9164570
3.3898220
1.8357370
2.7802140
3.8534960
2.3660120
2.6473460
1.6176120
2.2051890
1.4492480
0.6504710
3.6903840
3.4823980
4.2778480
4.3025860
-1.9385680
-1.9198180
-0.9267510
-2.5978560
-2.4663300
-2.8281690
-3.1284340
-1.4690610
-2.1110330
-2.4702030
-2.5604620
-2.4716840
-0.1067190
0.9839440
-0.4245630
-0.5303190
-2.7087190
-2.3807500
-2.4996460
-1.5693700
-1.5560120

2. 4113670

2. 3652900

3.4348610

1.7499900

2. 9874810

4.0128440

2. 9645250

2.7247370

$-3.4881190$

$-4.3546100$

$-3.6572490$

$-3.4346090$

$-2.2560420$

$-2.4377230$

$-3.0664320$

$-1.3182300$

$-4.1091720$

$-4.8055200$

$-4.5841490$

$-3.9688090$

$-3.0292710$

$-3.3603550$

$-3.8091570$

$-2.1251740$

$-3.8546860$

$-2.9181790$

$-4.2750250$

$-4.5586400$

$-4.9473300$

$-4.7948030$

$-5.6621560$

$-5.3973110$

$-1.4018860$

$-2.1430380$

$-1.6313150$

$-0.4139130$

$-0.4949030$

$-0.5030330$

0.5377360

$-0.8115700$

$-2.2209760$

$-0.9661800$

$-2.2366060$

-0.3681710
-2.6205360 


\begin{tabular}{|c|c|c|c|c|c|c|c|}
\hline 4 & & 0 745528 & & & 27780000 & & \\
\hline c & $\begin{array}{l}1.6467390 \\
3.3883990\end{array}$ & $\begin{array}{l}-0.7455280 \\
-4.2342800\end{array}$ & $\begin{array}{l}-2.5565510 \\
-2.8146920\end{array}$ & C & $\begin{array}{l}-3.7289000 \\
-2.4102510\end{array}$ & $\begin{array}{l}2.9818050 \\
3.4850700\end{array}$ & $\begin{array}{l}4.5552080 \\
4.4473950\end{array}$ \\
\hline C & 3.8073470 & -4.2411450 & -1.3432890 & $\mathrm{C}$ & -1.6731280 & 3.2788070 & 3.3082550 \\
\hline C & 3.5265130 & -2.8892090 & -0.6863290 & $\mathrm{C}$ & -4.2687680 & 2.2574410 & 3.5244250 \\
\hline$c$ & 1.8878270 & -3.9627450 & -2.9482310 & $\mathrm{H}$ & -4.3021190 & 3.1594630 & 5.4594400 \\
\hline $\mathrm{H}$ & 3.6276200 & -5.1931420 & -3.2828040 & $\mathrm{H}$ & -1.9763770 & 4.0344880 & 5.2768620 \\
\hline $\mathrm{H}$ & 4.8728130 & -4.4704080 & -1.2433430 & $\mathrm{H}$ & -0.6597210 & 3.6599530 & 3.2427290 \\
\hline $\mathrm{H}$ & 4.2040910 & -2.1203380 & -1.0750430 & $\mathrm{H}$ & -5.2716160 & 1.8456620 & 3.6000640 \\
\hline $\mathrm{H}$ & 1.3141080 & -4.7935800 & -2.5188710 & C & -0.6636070 & 6.6216630 & 1.0450180 \\
\hline $\mathrm{H}$ & 1.5904140 & -3.8691420 & -3.9971600 & $\mathrm{C}$ & 0.6655690 & 7.0900820 & 0.9124170 \\
\hline $\mathrm{H}$ & 3.9483010 & -3.4606930 & -3.3547040 & $\mathrm{C}$ & 1.6846290 & 6.1984780 & 0.6996780 \\
\hline $\mathrm{H}$ & 3.2589400 & -5.0288210 & -0.8102640 & $\mathrm{C}$ & -0.9417070 & 5.2789240 & 0.9896190 \\
\hline $\mathrm{H}$ & 3.6822850 & -2.9109940 & 0.3958370 & $\mathrm{H}$ & -1.4713280 & 7.3329160 & 1.1858620 \\
\hline C & 0.1590210 & -3.1099060 & 1.8760030 & $\mathrm{H}$ & 0.8705010 & 8.1544850 & 0.9651330 \\
\hline 0 & -0.3618700 & -1.9463370 & 1.6694810 & $\mathrm{H}$ & 2.7074410 & 6.5432730 & 0.5732900 \\
\hline C & -0.0457120 & -3.6095570 & 3.2564750 & $\mathrm{H}$ & -1.9659460 & 4.9339520 & 1.0816850 \\
\hline C & 0.7273870 & -4.6522730 & 3.7803870 & C & 3.3121430 & 1.5847570 & -0.1921770 \\
\hline C & -1.0487070 & -3.0246520 & 4.0431180 & $\mathrm{C}$ & 3.7836010 & 1.5340400 & -1.5193830 \\
\hline C & 0.5000050 & -5.1034640 & 5.0745040 & $\mathrm{C}$ & 3.8795950 & 0.7587530 & 0.7964480 \\
\hline $\mathrm{H}$ & 1.5076090 & -5.0954940 & 3.1710490 & $\mathrm{C}$ & 4.8586730 & 0.6927630 & -1.8171650 \\
\hline C & -1.2792880 & -3.4878330 & 5.3330400 & $\mathrm{C}$ & 4.9529790 & -0.0622310 & 0.4493360 \\
\hline $\mathrm{H}$ & -1.6449290 & -2.2170230 & 3.6313640 & $\mathrm{C}$ & 5.4650400 & -0.1030070 & -0.8472940 \\
\hline C & -0.5044400 & -4.5251730 & 5.8503830 & $\mathrm{H}$ & 5.2279320 & 0.6581050 & -2.8381350 \\
\hline $\mathrm{H}$ & 1.1070060 & -5.9062700 & 5.4796920 & $\mathrm{H}$ & 5.4073170 & -0.6963560 & 1.2104360 \\
\hline $\mathrm{H}$ & -2.0628950 & -3.0397530 & 5.9350780 & $\mathrm{C}$ & -3.8681610 & -0.0113510 & -0.8794080 \\
\hline $\mathrm{H}$ & -0.6826550 & -4.8832380 & 6.8595200 & C & -4.0358770 & -1.3846750 & -0.6109950 \\
\hline $\mathrm{H}$ & -0.4458860 & -1.6589800 & 0.6635020 & $\mathrm{C}$ & -4.2417750 & 0.5173960 & -2.1311990 \\
\hline$\because$ & 0.9706190 & -4.0110410 & 0.7103210 & $\mathrm{C}$ & -4.6245680 & -2.1889990 & -1.5909700 \\
\hline & & & & C & -4.8131730 & -0.3301920 & -3.0794790 \\
\hline & S) $\mathrm{G}[\mathrm{M0}$ & $G(d)]$ & L26 & C & -5.0235780 & -1.6853350 & -2.8258490 \\
\hline 0 & 0.6827340 & 0.7204370 & 0.3287920 & $\mathrm{H}$ & -4.7741560 & -3.2474770 & -1.3898110 \\
\hline 0 & -1.3276480 & 1.2227360 & -1.1093110 & $\mathrm{H}$ & -5.1101020 & 0.0829490 & -4.0420510 \\
\hline C & 0.9030880 & 2.0850540 & 0.4044480 & C & 3.3787480 & 0.7461970 & 2.2332170 \\
\hline C & -0.1449710 & 2.9142030 & 0.7449650 & $\mathrm{C}$ & 6.6646990 & -0.9806390 & -1.1588420 \\
\hline $\mathrm{C}$ & 2.2272000 & 2.5435320 & 0.1787160 & $\mathrm{C}$ & 5330 & 2.3896510 & -2.6246360 \\
\hline C & 0.0968160 & 4.3270490 & 0.7971980 & $\mathrm{C}$ & -3.6078370 & -2.0227350 & 0.7048250 \\
\hline C & 2.4699980 & 3.8853260 & 0.3350410 & C & -5.6613430 & -2.5848050 & -3.8676600 \\
\hline C & 1.4262690 & 4.8039270 & 0.6207250 & C & -4.0761510 & 1.9896140 & -2.4783610 \\
\hline $\mathrm{H}$ & 3.4825740 & 4.2633910 & 0.2213030 & $\mathrm{C}$ & -5.4426080 & 2.6607270 & -2.6660120 \\
\hline C & -2.0389610 & 1.4815550 & 0.0545340 & $\mathrm{H}$ & -5.9875320 & 2.2129520 & -3.5044290 \\
\hline C & -3.3273380 & 0.8958950 & 0.1778140 & $\mathrm{H}$ & -6.0585180 & 2.5541670 & -1.7673230 \\
\hline C & -1.48 & 2.3220490 & 0.9978480 & $\mathrm{H}$ & -5.3195850 & 3.7283540 & -2.8755980 \\
\hline C & -4.05 & 1.2090980 & 1.2980150 & C & -3.1943650 & 2.1788670 & -3.7177160 \\
\hline C & -2.2 & 2.5550020 & 2.2110620 & $\mathrm{H}$ & -2.2160820 & 1.7117530 & -3.5730090 \\
\hline C & -3.5 & 2.0124740 & 2.3394850 & $\mathrm{H}$ & -3.6600740 & 1.7385260 & -4.6062520 \\
\hline $\mathrm{H}$ & & 171630 & 1.4047350 & $\mathrm{H}$ & -3.0435480 & 3.2453690 & -3.9149260 \\
\hline
\end{tabular}




$\begin{array}{rrr}-4.8094400 & -2.6568970 & -5.1400260 \\ -5.2555300 & -3.3480340 & -5.8626040 \\ -4.7389020 & -1.6727370 & -5.6163060 \\ -3.7940770 & -2.9970070 & -4.9163650 \\ -7.0885520 & -2.1321420 & -4.1967980 \\ -7.7093010 & -2.0969540 & -3.2965710 \\ -7.0849120 & -1.1320310 & -4.6443980 \\ -7.5544870 & -2.8178730 & -4.9119320 \\ -4.8173400 & -2.2719330 & 1.6164100 \\ -5.3598760 & -1.3488700 & 1.8389250 \\ -5.5181110 & -2.9653090 & 1.1370130 \\ -4.4962630 & -2.7176170 & 2.5645650 \\ -2.8442020 & -3.3358340 & 0.4970310 \\ -3.4867130 & -4.1142480 & 0.0725410 \\ -1.9854120 & -3.1921860 & -0.1647830 \\ -2.4835170 & -3.7089360 & 1.4628760 \\ 4.4617630 & 1.2474820 & 3.1961010 \\ 5.3353730 & 0.5865610 & 3.1838880 \\ 4.7946160 & 2.2531540 & 2.9207440 \\ 4.0776010 & 1.2792500 & 4.2207080 \\ 2.8814290 & -0.6459780 & 2.6394180 \\ 2.0641130 & -0.9698930 & 1.9870440 \\ 3.6866320 & -1.3880850 & 2.5894650 \\ 2.5022990 & -0.6338300 & 3.6669020 \\ 7.9638720 & -0.1903050 & -0.9512490 \\ 8.8375790 & -0.8240060 & -1.1355350 \\ 8.0031250 & 0.6577090 & -1.6441110 \\ 8.0281090 & 0.2013770 & 0.0683420 \\ 6.6256230 & -1.5862280 & -2.5642270 \\ 5.6640870 & -2.0693190 & -2.7708550 \\ 6.7851460 & -0.8206050 & -3.3308250 \\ 7.4187950 & -2.3315650 & -2.6756450 \\ 3.0440490 & 1.6423200 & -3.9560700 \\ 4.0208230 & 1.4477460 & -4.4117350 \\ 2.5204200 & 0.6922770 & -3.8316400 \\ 2.4732870 & 2.2544530 & -4.6616290 \\ 4.0078600 & 3.6639050 & -2.8386860 \\ 5.0306770 & 3.4032340 & -3.1346880 \\ 3.5698410 & 4.2764290 & -3.6338210 \\ 4.0646920 & 4.2718190 & -1.9320060 \\ -0.1168590 & 0.1509820 & -0.9799980 \\ 0.7083100 & 0.2433870 & -2.2258380 \\ -0.6173360 & -1.2029750 & -0.5338860 \\ 2.1704450 & 2.6777910 & -2.3094570 \\ 6.6576430 & -1.8061140 & -0.4331840 \\ 2.5289520 & 1.4309700 & 2.3107880\end{array}$
$-3.5798450$
$-5.7161980$
$-2.9279470$
1. 5820770
2.2986780
0.5316090
1.9691600
2. 4449370
1.8900500
3.3676560
3.7981460
3.6368420
1. 8968560
3.5173880
4.8427290
4.3710930
1.2554180
1. 6028050
3.9897220
3.1937910
3.8074490
0.1090610
$-0.4218000$
$-0.2026950$
0.5403180
$-1.2802530$
0.2095130
1.3779950
$-1.6134220$
$-1.8526290$
$-0.8682410$
0.7930980
$-2.4538190$
$-1.1266840$
$-0.4224260$
1.0430020

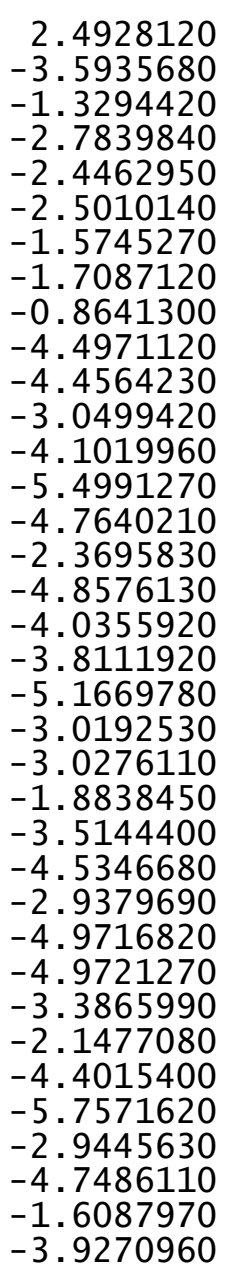

$-1.6436900$

$-3.4391820$

1. 2117370

$-1.9691420$

$-0.7351790$

$-1.9882590$

$-0.1767500$

$-2.4315070$

$-2.3896650$

$-2.4887460$

$-1.0212180$

$-0.4411320$

$-2.6341040$

$-2.9003990$

$-0.9158930$

$-0.8863620$

$-2.1635410$

$-3.6860610$

$-3.0761300$

$-0.4426070$

0.6387910

2.0931630

1.8234760

3.4591410

4.0641560

4.1472830

5.3408660

3.5317940

5.4197350

3.6728810

6.0182600

5.8094790

5.9447500

7.0138050

0.8044200

1.0193820

TS229(S, S) G[M06-2X/6-31G(d) $]=-3633.303686$

$\begin{array}{lrcr}\text { TS229(S, S) G[M06-2X/6-31G(d)] }=-3633.303686 \\ \text { O } & 0.5728400 & 0.1887340 & -0.3669380 \\ \text { O } & -1.6817400 & 0.0631610 & -1.5223650 \\ \text { C } & 0.8824680 & 1.2627070 & -1.1783690 \\ \text { C } & -0.0400070 & 2.2757430 & -1.3419730 \\ \text { C } & 2.1929170 & 1.3139150 & -1.7229490 \\ \text { C } & 0.2776430 & 3.3579620 & -2.2254620 \\ \text { C } & 2.5153790 & 2.3945210 & -2.5072870 \\ \text { C } & 1.5744250 & 3.4135750 & -2.8066520\end{array}$




$\begin{array}{lr}\mathrm{H} & 3.5213260 \\ \mathrm{C} & -2.1241970 \\ \mathrm{C} & -3.3699270 \\ \mathrm{C} & -1.3249470 \\ \mathrm{C} & -3.7860470 \\ \mathrm{C} & -1.7228010 \\ \mathrm{C} & -2.9774570 \\ \mathrm{H} & -4.7448310 \\ \mathrm{C} & -2.5662700 \\ \mathrm{C} & -1.3030700 \\ \mathrm{C} & -0.8913340 \\ \mathrm{C} & -3.3820920 \\ \mathrm{H} & -2.8791450 \\ \mathrm{H} & -0.6523570 \\ \mathrm{H} & 0.0845410 \\ \mathrm{H} & -4.3463250 \\ \mathrm{C} & -0.3157990 \\ \mathrm{C} & 0.9845930 \\ \mathrm{C} & 1.9062310 \\ \mathrm{C} & -0.6611780 \\ \mathrm{H} & -1.0479530 \\ \mathrm{H} & 1.2434070 \\ \mathrm{H} & 2.9042030 \\ \mathrm{H} & -1.6640200 \\ \mathrm{C} & 3.2381100 \\ \mathrm{C} & 3.5078090 \\ \mathrm{C} & 4.0275020 \\ \mathrm{C} & 4.6181750 \\ \mathrm{C} & 5.1383240 \\ \mathrm{C} & 5.4626390 \\ \mathrm{H} & 4.8433400 \\ \mathrm{H} & 5.7773380 \\ \mathrm{C} & -4.1676100 \\ \mathrm{C} & -4.3783420 \\ \mathrm{C} & -4.7232930 \\ \mathrm{C} & -5.1649800 \\ \mathrm{C} & -5.4955360 \\ \mathrm{C} & -5.7328680 \\ \mathrm{H} & -5.3378960 \\ \mathrm{H} & -5.9324440 \\ \mathrm{C} & 3.7393560 \\ \mathrm{C} & 6.7116960 \\ \mathrm{C} & 2.6558280 \\ \mathrm{C} & -3.7962540 \\ \mathrm{C} & -6.5833950 \\ \mathrm{C} & -4.5564400 \\ & \end{array}$

$\begin{array}{rr}2.4754140 & -2.9127810 \\ 1.1037660 & -0.7117310 \\ 0.9493210 & -0.0452430 \\ 2.2181830 & -0.5973190 \\ 1.9919440 & 0.7464550 \\ 3.2536310 & 0.3107490 \\ 3.1362580 & 0.9715930 \\ 1.9250860 & 1.2540140 \\ 5.2144870 & 2.1569550 \\ 5.3158860 & 1.5255630 \\ 4.3640250 & 0.6253050 \\ 4.1471680 & 1.8841180 \\ 5.9769760 & 2.8628900 \\ 6.1533570 & 1.7582010 \\ 4.4462450 & 0.1584670 \\ 4.0456650 & 2.3747800 \\ 5.3903970 & -3.4152440 \\ 5.4585650 & -3.9709740 \\ 4.4883870 & -3.6736440 \\ 4.3702780 & -2.5647690 \\ 6.1501280 & -3.6699550 \\ 6.2741060 & -4.6385160 \\ 4.5192170 & -4.1026130 \\ 4.3218440 & -2.1532220 \\ 0.2964210 & -1.3837360 \\ -0.7733070 & -2.2587330 \\ 0.4963340 & -0.2353280 \\ -1.5812940 & -2.0056600 \\ -0.3280850 & -0.0337750 \\ -1.3571850 & -0.9159460 \\ -2.3951110 & -2.6914490 \\ -0.1607760 & 0.8311210 \\ -0.3071750 & -0.1751590 \\ -1.1242810 & 0.9554800 \\ -0.6681490 & -1.4201660 \\ -2.2709380 & 0.8160530 \\ -1.8258940 & -1.5060770 \\ -2.6407190 & -0.3993300 \\ -2.9017300 & 1.6859230 \\ -2.0946900 & -2.4663120 \\ 1.6301070 & 0.7399110 \\ -2.1962560 & -0.7116010 \\ -1.0116390 & -3.4962920 \\ -0.8115450 & 2.3274520 \\ -3.8910040 & -0.5179300 \\ 0.1818520 & -2.6715170\end{array}$

-5.9024980
-6.6152340
-6.3422430
-5.7728710
-3.9201580
-2.9609810
-4.5728480
-3.7499490
-5.9602600
-6.5587220
-5.9141220
-4.9427690
-8.0184820
-8.4782950
-8.0344530
-8.6335740
-4.8923320
-5.4119980
-5.6410400
-4.4633350
-3.0629910
-3.7598160
-2.3243820
-2.5445020
4.6547650
5.7049400
4.5270710
4.4347690
3.8610860
3.2324190
4.8939970
3.5343910
7.7042810
8.6308770
7.2813900
7.9505540
6.3955850
5.7595300
5.8802990
7.3190240
2.4583380
3.3753750
2.1531630
1.6830550
3.2641750
4.2717920

0.7798830

$-0.0092060$

1. 3765510

1.4241790

$-0.6148360$

$-1.0395210$

$-1.4329830$

0.0356840

$-4.8999610$

$-5.8159840$

$-4.4858020$

$-5.1634600$

$-3.5516200$

$-2.8470020$

$-3.0975060$

$-4.4567170$

$-0.3272050$

0.5557070

$-1.1130720$

$-0.0753310$

$-2.0100530$

$-2.8086370$

$-2.4184070$

$-1.6952610$

2.8267790

2. 5429960

3.1833770

3.6540420

1. 2020890

0.3305310

0.9547050

2. 0166690

$-1.9741760$

$-2.5288430$

$-2.3218600$

$-0.9137640$

$-3.6885840$

$-3.8777920$

$-4.0746530$

$-4.2625850$

$-2.4976510$

$-2.9505370$

$-3.0610930$

$-2.6130960$

$-0.3028390$

$-0.6872170$
$-3.1011420$

3. 3652640

$-2.2956540$

$-3.9769560$

$-3.8158250$

$-3.5072010$

$-4.1394300$

$-4.6800730$

$-1.4893240$

$-1.5281720$

$-2.5026340$

$-1.1861390$

$-0.9370090$

$-0.2376600$

$-1.9340430$

$-0.9709550$

3. 2868860

2. 9026440

3.4395140

4.2625010

2. 9438150

3. 2213010

2. 2498850

3. 8566240

0.4507130

0.5839810

$-0.5761540$

1.1335740

2. 2053150

2. 4206340

2.4732720

2.8617360

$-1.8599650$

$-1.6806290$

$-2.8090450$

$-1.9680360$

$-0.5594000$

0.3112730

$-1.4456790$

$-0.4321950$

$-3.8099110$

$-4.2027970$

$-2.9249850$

$-4.5732300$

$-4.7145500$

$-4.9108170$ 


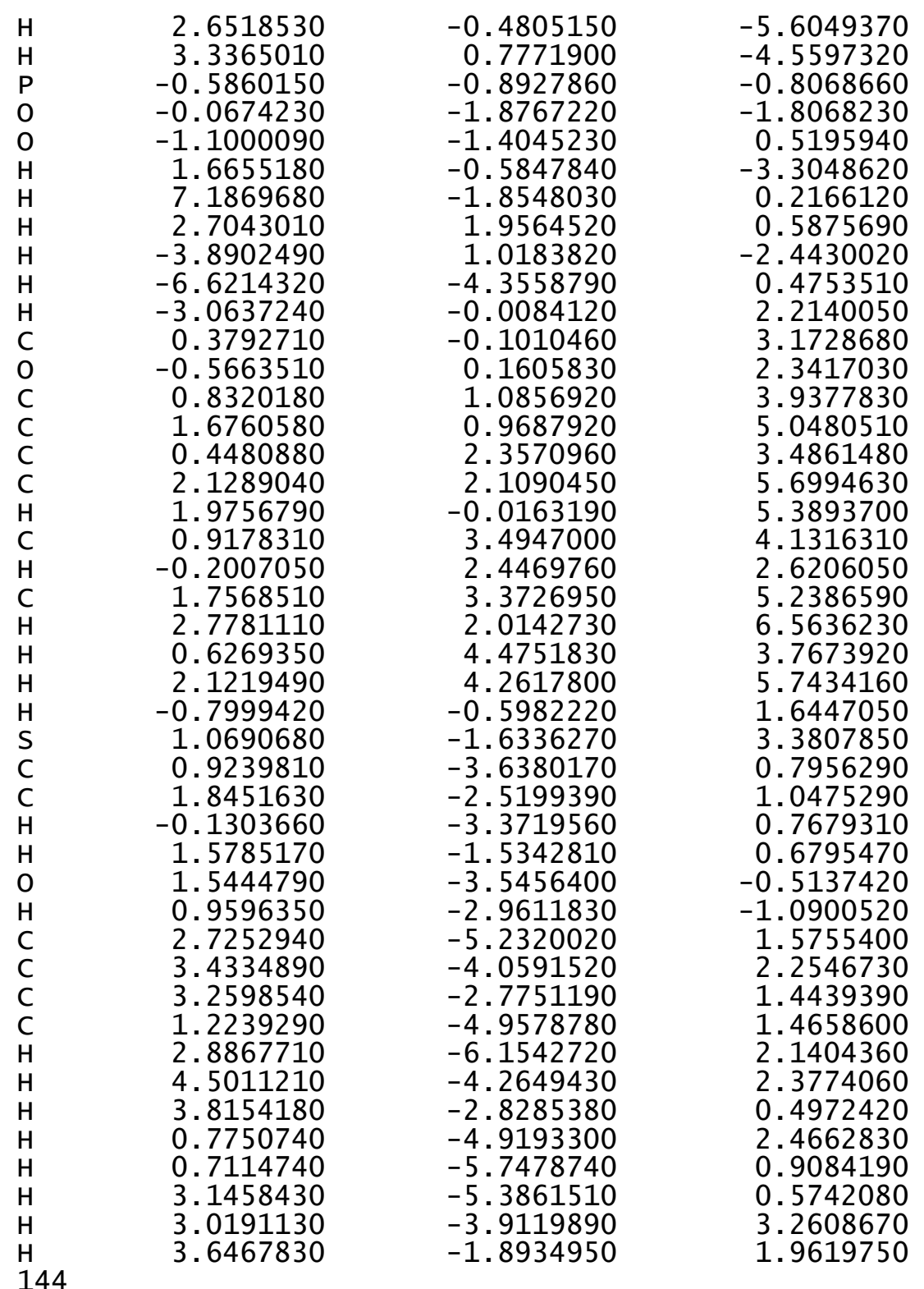

$\operatorname{TS} 230(\mathrm{~S}, \mathrm{~S}) \mathrm{G}[\mathrm{M} 06-2 \mathrm{X} / 6-31 \mathrm{G}(\mathrm{d})]=-3633.303128$

0.6888880
-1.5131950
1.0308140
0.1043100
2.3771820
0.4600510
2.7288230
1.7902980
3.7594330
-2.0084050
-3.2872240
-1.2184410
-3.7445540
-1.6589530
-2.9463810
-4.7277240
-2.5840120
-1.2890240
-0.8384210
-3.3916570
-2.9284120
-0.6434740
0.1620730
-4.3802160
-0.0899310
1.2408700
2.1575600
-0.4711630
-0.8162190
1.5275300
3.1796630
-1.4959980
3.4126150
3.6476910
4.2098250
4.7047790
5.2615780
5.5338900
4.9007710
5.8986780
-4.0734920
-4.3592050
-4.5453480
-5.1303300
-5.3088690
-5.6159790

0.1586950

0.0572510

1.2734840

2. 2801500

1. 3742270

3. 4029800

2. 4883630

3. 5014130

2.6042640

1.0758980

0.8960710

2.1844410

1.9062840

3. 1768950

3.0350860

1. 8193440

5.0437940

5.1663580

4.2608340

3. 9987660

5.7710810

5.9800590

4. 3564860

3. 8793760

5.4780440

5.5858700

4. 6162740

4. 4182000

6.2394160

6.4324420

4.6791060

4. 3398410

0.3634310

$-0.7624320$

0.6051970

$-1.6154080$

$-0.2728860$

$-1.3782680$

$-2.4755780$

$-0.0914400$

$-0.3563070$

$-1.1970460$

$-0.6922440$

$-2.3395870$

-1.8515530
-2.6869830
$-0.5012890$

$-1.7572810$

$-1.2379150$

$-1.4175400$

$-1.6794300$

$-2.2320360$

$-2.4025770$

$-2.7261530$

7307890

$-0.9459000$

$-0.3513390$

0.746

0.4588770

0.1897470

0.7786960

0.9140960

2.0927940

1. 5337190

0.6053080

1.7230820

2.8214150

1.8494500

0.1973340

2.1582330

$-3.3678210$

$-3.5261990$

$-2.5841250$

$-3.6342070$

$-3.8898290$

$-2.2361480$

$-1.2918080$

$-0.1530350$

$-1.7799720$

0.1226700

$-0.6830160$

$-2.4156410$

0.9845460

$-0.5611310$

0.5397730

0.3274820

$-2.0023730$

$-0.9331370$ 


\begin{tabular}{|c|c|c|c|}
\hline $\begin{array}{l}\mathrm{H} \\
\mathrm{H} \\
\mathrm{C} \\
\mathrm{C} \\
\mathrm{C} \\
\mathrm{C} \\
\mathrm{C} \\
\mathrm{C} \\
\mathrm{C} \\
\mathrm{H} \\
\mathrm{H} \\
\mathrm{H} \\
\mathrm{C} \\
\mathrm{H} \\
\mathrm{H} \\
\mathrm{H} \\
\mathrm{C} \\
\mathrm{H} \\
\mathrm{H} \\
\mathrm{H} \\
\mathrm{C} \\
\mathrm{H} \\
\mathrm{H} \\
\mathrm{H} \\
\mathrm{C} \\
\mathrm{H} \\
\mathrm{H} \\
\mathrm{H} \\
\mathrm{C} \\
\mathrm{H} \\
\mathrm{H} \\
\mathrm{H} \\
\mathrm{C} \\
\mathrm{H} \\
\mathrm{H} \\
\mathrm{H} \\
\mathrm{C} \\
\mathrm{H} \\
\mathrm{H} \\
\mathrm{H} \\
\mathrm{C} \\
\mathrm{H} \\
\mathrm{H} \\
\mathrm{H} \\
\mathrm{C} \\
\mathrm{H}\end{array}$ & $\begin{array}{r}-5.3559710 \\
-5.6846030 \\
3.9318380 \\
6.7235980 \\
2.8345150 \\
-3.8753750 \\
-6.4520360 \\
-4.3047400 \\
-5.6232050 \\
-6.3193940 \\
-6.1090210 \\
-5.4421830 \\
-3.6033270 \\
-2.6675760 \\
-4.2391390 \\
-3.3765560 \\
-7.7549260 \\
-8.3699470 \\
-7.5456470 \\
-8.3369880 \\
-5.6593000 \\
-4.7362250 \\
-5.3886860 \\
-6.2562450 \\
-5.0427670 \\
-5.5563320 \\
-5.7826010 \\
-4.6845930 \\
-3.1622530 \\
-3.8549680 \\
-2.3539730 \\
-2.7334290 \\
5.1739530 \\
5.5104430 \\
6.0049870 \\
4.9390440 \\
2.8286780 \\
1.8992260 \\
3.1539700 \\
2.6053300 \\
7.7742000 \\
8.6595190 \\
7.3705090 \\
8.0847530 \\
6.3201000 \\
5.6345200\end{array}$ & $\begin{array}{r}-2.9823670 \\
-2.1076320 \\
1.7901250 \\
-2.2770990 \\
-0.9993030 \\
-0.9096360 \\
-3.9369780 \\
0.1826620 \\
0.7913780 \\
0.0087900 \\
1.3726220 \\
1.4528250 \\
-0.5911090 \\
-1.0298250 \\
-1.3967020 \\
0.0785720 \\
-3.8706760 \\
-4.7562980 \\
-3.8319330 \\
-2.9824230 \\
-5.1998980 \\
-5.2634960 \\
-5.2019990 \\
-6.0965280 \\
-0.4681390 \\
0.4110350 \\
-1.2721810 \\
-0.2270800 \\
-2.1102210 \\
-2.9381390 \\
-2.4685320 \\
-1.8159860 \\
2.3007180 \\
1.5920130 \\
2.4810900 \\
3.2399700 \\
1.4535400 \\
1.1415060 \\
0.6422590 \\
2.3249060 \\
-2.1502660 \\
-2.7492760 \\
-2.5058710 \\
-1.1094760 \\
-3.7439230 \\
-3.8677280\end{array}$ & $\begin{array}{r}1.1764120 \\
-2.9913670 \\
0.7656700 \\
-0.3945900 \\
-3.3675180 \\
1.9555330 \\
-1.1311220 \\
-3.0655470 \\
-3.5608590 \\
-3.8819340 \\
-2.7707340 \\
-4.4145310 \\
-4.1871790 \\
-3.8303990 \\
-4.5702810 \\
-5.0233320 \\
-0.3261670 \\
-0.5162350 \\
0.7486300 \\
-0.5892050 \\
-0.7753540 \\
-1.3589350 \\
0.2863570 \\
-0.9715060 \\
2.8493800 \\
2.4483470 \\
2.9376480 \\
3.8558480 \\
2.5903870 \\
2.7781940 \\
1.9486820 \\
3.5551870 \\
1.5000520 \\
2.2643140 \\
0.8112950 \\
2.0096020 \\
1.7818350 \\
1.2937000 \\
2.4461630 \\
2.4080300 \\
-1.5053890 \\
-1.2690750 \\
-2.4599320 \\
-1.6363830 \\
-0.2063570 \\
0.6382220\end{array}$ \\
\hline
\end{tabular}

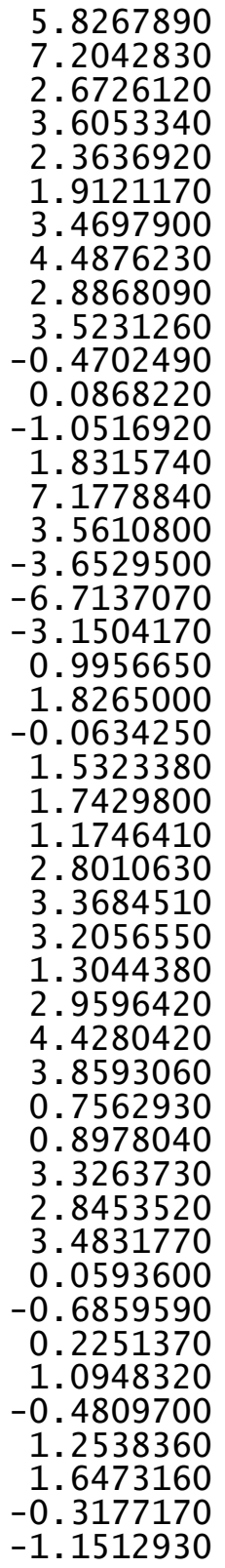

5. 8267890

2. 6726120

2. 6053340

3.4697900

4.4876230

2.8868090

3.5231260

0.0868220

$-1.0516920$

1.8315740

3. 5610800

$-3.6529500$

.7137070

$-3.1504170$

0.9956650

1.8265000

1.7429800

1.1746410

.8010630

3684510

1. 3044380

.9596420

.4280420

0.7562930

8040

3. 3263730

2. 8453520

0.0593600

$-0.6859590$

0.4809700

1.2538360

-0.3177170
-1.1512930
$-1.1022780$ $-0.0143170$ $-3.7208220$ $-4.1104410$ $-2.8579150$ $-4.4993850$ $-4.5521060$ $-4.7248010$ $-5.4640370$ $-4.3727200$ $-0.9765950$ $-1.9199370$ 0.3360190

$-3.2010310$

0.5414750

0.1423220 $-2.7811040$ $-2.1954760$

1. 9129070

0.8397430

1. 1701920

0.7043040

0.7893490 $-0.4003470$ $-1.0484700$

1.7901860

2. 5366780

1.7091600

1. 5314900

2. 3676670

2. 7681430

0.8266380

2. 4819550

0.9236570

0.8348630

3.4948500

2. 2644480

3. 1572320

2. 1402650

4.0737510

5. 1695680

3.8087650

5. 9914220

5.3643470

4.6351060

2. 9561620 


\begin{tabular}{|c|c|c|c|c|c|c|c|}
\hline & & & & & & & \\
\hline C & 0.5484440 & 3.3832160 & 5.7259620 & $\mathrm{H}$ & 4.5615650 & -0.3365430 & -3.0424920 \\
\hline H & 1.9313140 & 2.1606770 & 6.8375170 & $\mathrm{H}$ & 5.0497840 & 0.6988450 & 1.0759220 \\
\hline $\mathrm{H}$ & -0.8678580 & 4.3577450 & 4.4229490 & C & -4.0130010 & -0.7701200 & 0.3890500 \\
\hline $\mathrm{H}$ & 0.6759790 & 4.2489780 & 6.3684510 & C & -3.6499540 & -1.8848430 & 1.1712070 \\
\hline $\mathrm{H}$ & -0.8230490 & -0.5100500 & 1.4435720 & $\mathrm{c}$ & -4.8354530 & -0.9320920 & -0.7414510 \\
\hline $\mathrm{S}$ & 0.7826110 & -1.4994480 & 3.4375050 & C & -4.1696630 & -3.1354630 & 0.8274650 \\
\hline & S) $G[\mathrm{MO} 6-2$ & $-(d) 1--36$ & & C & -5.3227130 & -2.2028380 & \\
\hline 0 & S) G[MO6-2 & $\begin{array}{r}\text { (d) }\rfloor=-36 \\
0.9166390\end{array}$ & $\begin{array}{l}36 \\
-0.1332990\end{array}$ & $\begin{array}{l}\mathrm{C} \\
\mathrm{H}\end{array}$ & $\begin{array}{l}-5.0095430 \\
-3.9069550\end{array}$ & $\begin{array}{l}-3.3164970 \\
-4.0035630\end{array}$ & $\begin{array}{r}-0.2690880 \\
1.4272770\end{array}$ \\
\hline 0 & -1.9926280 & 0.5406620 & -1.0698630 & $\mathrm{H}$ & -5.9659220 & -2.3245550 & -1.9172560 \\
\hline $\mathrm{C}$ & 0.3097710 & 2.2112640 & -0.6172690 & C & 3.0699800 & 2.4890130 & 1.3051450 \\
\hline $\mathrm{C}$ & -0.8152210 & 2.9904830 & -0.4449030 & C & 6.1619870 & -0.8236000 & -0.9174250 \\
\hline $\mathrm{C}$ & 1.5226210 & 2.6912430 & -1.1844670 & $\mathrm{c}$ & 2.4286780 & 1.2511950 & -3.6199150 \\
\hline $\mathrm{C}$ & -0.8173370 & 4.3033550 & -1.0230760 & C & -2.7270480 & -1.7650490 & 2.3777690 \\
\hline $\mathrm{C}$ & 1.5425030 & 3.9835720 & -1.6427130 & C & -5.5483600 & -4.6907990 & -0.6192130 \\
\hline $\mathrm{C}$ & 0.3789020 & 4.7993600 & -1.6144560 & $\mathrm{C}$ & -5.2257020 & 0.2378450 & -1.6317330 \\
\hline $\mathrm{H}$ & 2.4655670 & 4.4040000 & -2.0332900 & C & -6.7301490 & 0.5182450 & -1.5333690 \\
\hline C & -2.4766870 & 1.2131460 & 0.0400570 & $\mathrm{H}$ & -7.3115470 & -0.3412060 & -1.8854740 \\
\hline C & -3.5322830 & 0.5956340 & 0.7628890 & $\mathrm{H}$ & -7.0234070 & 0.7234830 & -0.4988000 \\
\hline $\mathrm{C}$ & -1.9543170 & 2.4510430 & 0.3482100 & $\mathrm{H}$ & -7.0009090 & 1.3835320 & -2.1472600 \\
\hline $\mathrm{C}$ & -4.0917430 & 1.3072960 & 1.7936880 & C & -4.8009850 & 0.0039780 & -3.0855810 \\
\hline $\mathrm{C}$ & -2.4742320 & 3.1369670 & 1. 4983640 & $\mathrm{H}$ & -3.7248360 & -0.1809760 & -3.1497390 \\
\hline $\mathrm{C}$ & -3.5785730 & 2.5687240 & 2.1948800 & $\mathrm{H}$ & -5.3247010 & -0.8546830 & -3.5197400 \\
\hline $\mathrm{H}$ & -4.9382210 & 0.8916700 & 2.3340590 & $\mathrm{H}$ & -5.0385950 & 0.8819180 & -3.6952380 \\
\hline C & -3.5853800 & 4.4310580 & 3.7516630 & C & -5.0108960 & -5.1668730 & -1.9739010 \\
\hline C & -2.4564780 & 4.9727930 & 3.0925660 & $\mathrm{H}$ & -5.3664230 & -6.1783740 & -2.1959920 \\
\hline C & -1.9149880 & 4.3450930 & 1.9984470 & $\mathrm{H}$ & -5.3508180 & -4.5059960 & -2.7790280 \\
\hline $\mathrm{C}$ & -4.1270740 & 07490 & 3.3135190 & $\mathrm{H}$ & -3.9167700 & -5.1740840 & -1.9831300 \\
\hline H & -4.0069310 & 4.9429700 & 4.6107440 & C & -7.0808180 & -4.7157320 & -0.6051560 \\
\hline H & -2.0099420 & 5.8911930 & 3.4604850 & $\mathrm{H}$ & -7.4722790 & -4.3916100 & 0.3636990 \\
\hline $\mathrm{H}$ & -1.0417240 & 4.7669780 & 1.5135500 & $\mathrm{H}$ & -7.4883780 & -4.0503790 & -1.3742780 \\
\hline $\mathrm{H}$ & -4.9778480 & 2.8066110 & 3.8234070 & $\mathrm{H}$ & -7.4505500 & -5.7262570 & -0.8071780 \\
\hline C & -1.9403810 & 6.3757570 & -1.6111190 & C & -3.5351270 & -1.6187660 & 3.6749100 \\
\hline C & -0.7410330 & 6.8810820 & -2.1670990 & $\mathrm{H}$ & -4.1758210 & -0.7333330 & 3.6604340 \\
\hline $\mathrm{C}$ & 0.3902850 & 6.1064230 & -2.1696170 & $\mathrm{H}$ & -4.1750710 & -2.4965520 & 3.8217230 \\
\hline $\mathrm{C}$ & -1.9780340 & 5.1227700 & -1.0521980 & $\mathrm{H}$ & -2.8638380 & -1.5402130 & 4.5369640 \\
\hline $\mathrm{H}$ & -2.8393920 & 6.9835800 & -1.6321260 & C & -1.7651570 & -2.9521390 & 2.5120400 \\
\hline $\mathrm{H}$ & -0.7243850 & 61520 & -2.5999220 & $\mathrm{H}$ & -2.2907640 & -3.8627470 & 2.8192190 \\
\hline $\mathrm{H}$ & 1.3140880 & 6.4747320 & -2.6076760 & $\mathrm{H}$ & -1.2459060 & -3.1499810 & 1.5702960 \\
\hline $\mathrm{H}$ & -2.9043260 & 4.7400270 & -0.6361740 & $\mathrm{H}$ & -1.0168570 & -2.7340980 & 3.2814390 \\
\hline C & 2.7268500 & 1.8041280 & -1.1443810 & C & 4.1842780 & 3.4998400 & 1.6058570 \\
\hline C & 3.1283770 & 1.0755740 & -2.2805800 & $\mathrm{H}$ & 5.1236190 & 2.9864910 & 1.8401400 \\
\hline C & 3.4240290 & 00690 & 0.0714330 & $\mathrm{H}$ & 4.3625070 & 4.1554820 & 0.7473810 \\
\hline C & 4.2290940 & 0.2216530 & -2.1694410 & $\mathrm{H}$ & 3.9151640 & 4.1217570 & 2.4658800 \\
\hline $\mathrm{C}$ & 4.5104130 & 53150 & 0.1358510 & C & 2.7812190 & 1.6058980 & 2.5217640 \\
\hline C & 4.9293220 & 513880 & -0.9722990 & $\mathrm{H}$ & 1.9595250 & 0.9155960 & 2.3120870 \\
\hline
\end{tabular}




$\begin{array}{rrrr}\mathrm{H} & 3.6578690 & 1.0141620 & 2.8108820 \\ \mathrm{H} & 2.5021080 & 2.2241260 & 3.3820610 \\ \mathrm{C} & 6.2390730 & -1.6748560 & 0.3535770 \\ \mathrm{H} & 7.0882350 & -2.3630310 & 0.2960020 \\ \mathrm{H} & 6.3821990 & -1.0495120 & 1.2417190 \\ \mathrm{H} & 5.3262820 & -2.2624600 & 0.5032360 \\ \mathrm{C} & 7.4278490 & 0.0317460 & -1.0654650 \\ \mathrm{H} & 7.4045370 & 0.6118300 & -1.9928300 \\ \mathrm{H} & 7.5090960 & 0.7338970 & -0.2280170 \\ \mathrm{H} & 8.3234460 & -0.5981600 & -1.0717040 \\ \mathrm{C} & 2.4012200 & -0.0263870 & -4.4612560 \\ \mathrm{H} & 3.3935330 & -0.2717500 & -4.8565920 \\ \mathrm{H} & 2.0406540 & -0.8772250 & -3.8797080 \\ \mathrm{H} & 1.7345250 & 0.1091680 & -5.3185420 \\ \mathrm{C} & 3.0943760 & 2.3774290 & -4.4240200 \\ \mathrm{H} & 4.1435680 & 2.1292860 & -4.6220250 \\ \mathrm{H} & 2.5883140 & 2.5112720 & -5.3860060 \\ \mathrm{H} & 3.0688450 & 3.3303140 & -3.8894000 \\ \mathrm{P} & -0.5598570 & -0.2063600 & -0.9292150 \\ \mathrm{O} & -0.0660190 & -0.4426060 & -2.3251590 \\ \mathrm{O} & -0.6179180 & -1.3699070 & 0.0313880 \\ \mathrm{H} & 1.3905060 & 1.5329800 & -3.4158390 \\ \mathrm{H} & 6.1155060 & -1.5020830 & -1.7802940 \\ \mathrm{H} & 2.1607690 & 3.0600020 & 1.0944140 \\ \mathrm{H} & -4.7027440 & 1.1318740 & -1.2789680 \\ \mathrm{H} & -5.1915790 & -5.3873660 & 0.1500260 \\ \mathrm{H} & -2.1172080 & -0.8645560 & 2.2430470 \\ \mathrm{C} & 0.8025040 & -3.4621920 & -1.6874720 \\ \mathrm{C} & 2.0106220 & -2.6596050 & -1.4723510 \\ \mathrm{H} & -0.0665980 & -3.1648880 & -1.1040760 \\ \mathrm{H} & 1.8884660 & -1.6098740 & -1.2196140 \\ \mathrm{O} & 0.8816930 & -2.7547420 & -2.9523500 \\ \mathrm{H} & 0.3994620 & -1.8697610 & -2.8291330 \\ \mathrm{C} & 2.2556570 & -5.3060030 & -2.6221190 \\ \mathrm{C} & 3.4686340 & -4.6631960 & -1.9470470 \\ \mathrm{C} & 3.3514950 & -3.1378210 & -1.9377150 \\ \mathrm{C} & 0.9699910 & -4.9514700 & -1.8714990 \\ \mathrm{H} & 2.3709900 & -6.3930470 & -2.6520650 \\ \mathrm{H} & 4.3938610 & -4.9473370 & -2.4558470 \\ \mathrm{H} & 3.4930560 & -2.7302060 & -2.9477670 \\ \mathrm{H} & 0.9812370 & -5.4000250 & -0.8704100 \\ \mathrm{H} & 0.0854550 & -5.3340390 & -2.3901890 \\ \mathrm{H} & 2.1828250 & -4.9588830 & -3.6595040 \\ \mathrm{H} & 3.5456410 & -5.0276870 & -0.9141520 \\ \mathrm{H} & 4.1158040 & -2.6667940 & -1.3136220 \\ \mathrm{C} & 1.9416720 & -1.9899970 & 2.1408660\end{array}$

$\begin{array}{ll}\mathrm{O} & 0.8912530 \\ \mathrm{C} & 2.6532770 \\ \mathrm{C} & 3.9903590 \\ \mathrm{C} & 1.9791780 \\ \mathrm{C} & 4.6468450 \\ \mathrm{H} & 4.5122160 \\ \mathrm{C} & 2.6359890 \\ \mathrm{H} & 0.9449460 \\ \mathrm{C} & 3.9711860 \\ \mathrm{H} & 5.6872530 \\ \mathrm{H} & 2.1083800 \\ \mathrm{H} & 4.4856580 \\ \mathrm{H} & 0.3204090 \\ \mathrm{~S} & 2.4830180 \\ \mathrm{H} & \end{array}$
$-1.2571180$
$-1.7492580$
$-2.1257470$
$-1.0915800$
$-1.8399040$
$-2.6264350$
$-0.8206380$
$-0.7960090$
$-1.1894830$
$-2.1224440$
$-0.3176810$
$-0.9689610$
$-1.3869410$
$-3.1392960$

$\begin{array}{lrcr}\text { TS232(S, S }) \text { G }[\mathrm{M} 06-2 \times / 6-31 G(\mathrm{~d})]=-3633.305815 \\ \mathrm{O} & 0.6935820 & 0.6608830 & 0.1977590 \\ \mathrm{O} & -1.3323100 & 1.3353550 & -1.1587240 \\ \mathrm{C} & 1.0146800 & 2.0040630 & 0.2936650 \\ \mathrm{C} & 0.0362060 & 2.8986010 & 0.6767830 \\ \mathrm{C} & 2.3625630 & 2.3735260 & 0.0489670 \\ \mathrm{C} & 0.3733620 & 4.2907570 & 0.7411850 \\ \mathrm{C} & 2.6968810 & 3.6954110 & 0.2147500 \\ \mathrm{C} & 1.7262510 & 4.6797230 & 0.5336870 \\ \mathrm{H} & 3.7311070 & 4.0038740 & 0.0852200 \\ \mathrm{C} & -1.9807140 & 1.6179500 & 0.0360730 \\ \mathrm{C} & -3.3018870 & 1.1221670 & 0.1985690 \\ \mathrm{C} & -1.3335850 & 2.3977960 & 0.9705100 \\ \mathrm{C} & -3.9634440 & 1.4615660 & 1.3528290 \\ \mathrm{C} & -1.9973910 & 2.6578700 & 2.2151160 \\ \mathrm{C} & -3.3354900 & 2.2043140 & 2.3863410 \\ \mathrm{H} & -4.9918530 & 1.1392860 & 1.4930090 \\ \mathrm{C} & -3.3868190 & 3.1430000 & 4.6239860 \\ \mathrm{C} & -2.0421020 & 3.5581030 & 4.4714860 \\ \mathrm{C} & -1.3652970 & 3.3232640 & 3.3009220 \\ \mathrm{C} & -4.0146470 & 2.4764600 & 3.6040260 \\ \mathrm{H} & -3.9109020 & 3.3419910 & 5.5532430 \\ \mathrm{H} & -1.5400790 & 4.0618760 & 5.2914310 \\ \mathrm{H} & -0.3316080 & 3.6364550 & 3.2012940 \\ \mathrm{H} & -5.0396530 & 2.1321850 & 3.7128440 \\ \mathrm{C} & -0.2235470 & 6.6269770 & 1.0394920 \\ \mathrm{C} & 1.1301640 & 7.0071620 & 0.8742090 \\ \mathrm{C} & 2.0806390 & 6.0525490 & 0.6219170 \\ \mathrm{C} & -0.5931620 & 5.3069600 & 0.9758940 \\ \mathrm{H} & -0.9771040 & 7.3888390 & 1.2122130 \\ \mathrm{H} & 1.4074210 & 8.0546950 & 0.9337110\end{array}$

(2)

6797230

6179500

1221670

21850

8.0546950
0.9337110
1.9995390

3. 4199940

3.5907600

4.7821170

2.7824030

5.6525130

4.3196920

5.8139740

4.9046860

6.4562270

6.7440440

1.1311090

1.0348650

2. 3774290

2. 5112720

3303140

0.2063600

3. 0600020

5.3873

$-0.8645560$

$-3.4621920$

$-3.1648880$

. 7547420

$-5.4000250$

$-1.9899970$

-1.3136220
2.1408660 


$\begin{array}{lr}\mathrm{H} & 3.1205840 \\ \mathrm{H} & -1.6353650 \\ \mathrm{C} & 3.4080990 \\ \mathrm{C} & 3.9089200 \\ \mathrm{C} & 3.9749320 \\ \mathrm{C} & 5.0340870 \\ \mathrm{C} & 5.0965480 \\ \mathrm{C} & 5.6648560 \\ \mathrm{H} & 5.4526100 \\ \mathrm{H} & 5.5543440 \\ \mathrm{C} & -3.9439990 \\ \mathrm{C} & -4.1886860 \\ \mathrm{C} & -4.3311340 \\ \mathrm{C} & -4.8631270 \\ \mathrm{C} & -4.9940180 \\ \mathrm{C} & -5.2781970 \\ \mathrm{H} & -5.0673430 \\ \mathrm{H} & -5.3058850 \\ \mathrm{C} & 3.4431790 \\ \mathrm{C} & 6.9500240 \\ \mathrm{C} & 3.3006100 \\ \mathrm{C} & -3.7539980 \\ \mathrm{C} & -6.0083880 \\ \mathrm{C} & -4.0884520 \\ \mathrm{C} & -5.4165300 \\ \mathrm{H} & -6.0405000 \\ \mathrm{H} & -5.9807800 \\ \mathrm{H} & -5.2368670 \\ \mathrm{C} & -3.2735970 \\ \mathrm{H} & -2.3259680 \\ \mathrm{H} & -3.8242640 \\ \mathrm{H} & -3.0559460 \\ \mathrm{C} & -7.3383610 \\ \mathrm{H} & -7.8770130 \\ \mathrm{H} & -7.1682500 \\ \mathrm{H} & -7.9779530 \\ \mathrm{C} & -5.1326760 \\ \mathrm{H} & -4.1892240 \\ \mathrm{H} & -4.8969270 \\ \mathrm{H} & -5.6523960 \\ \mathrm{C} & -4.9420510 \\ \mathrm{H} & -5.4123010 \\ \mathrm{H} & -5.7055880 \\ \mathrm{H} & -4.6162300 \\ \mathrm{C} & -3.0908170 \\ \mathrm{H} & -3.8001700 \\ & \end{array}$

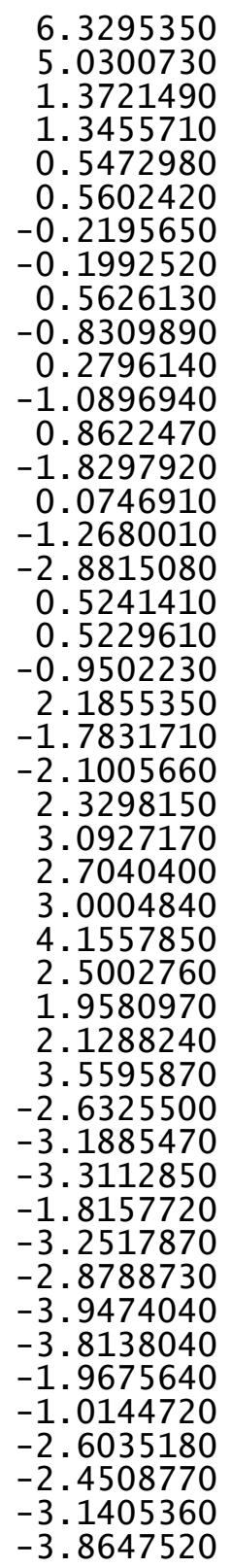
0.4710070
1.0932400
$-0.3307550$
$-1.6463750$
0.6619170
$-1.9167890$
0.3420670
$-0.9342900$
$-2.9210050$
1. 1189810
$-0.8556090$
$-0.6129760$
$-2.0755140$
$-1.5840400$
$-3.0202190$
$-2.7920290$
$-1.3954480$
$-3.9613760$
2. 0882070
$-1.2395920$
$-2.7617920$
0.6725100
$-3.8288600$
$-2.3939290$
$-2.4771250$
$-3.2895250$
$-1.5435570$
$-2.6683460$
$-3.6806270$
$-3.6150360$
$-4.5516560$
$-3.8521390$
$-3.2829250$
$-4.0571640$
$-2.4397830$
$-2.9353630$
$-4.3367090$
$-4.7459690$
$-3.5237030$
$-5.1195390$
1.6267890
1.8843820
1.1638500
2. 5546980
0.4122780
$-0.0019260$

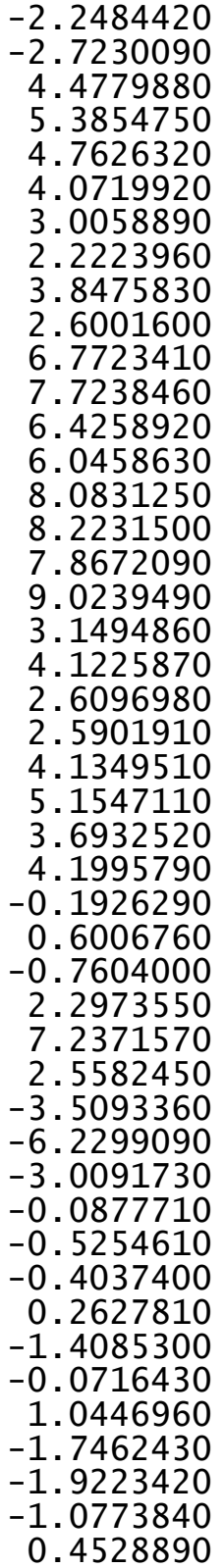
$-3.0428770$
$-3.5574170$
1.0926270
0.4789170
2. 1108970
1. 1174540
$-0.8843580$
$-1.2653920$
$-1.5861430$
$-0.8704360$
$-2.4713390$
$-2.9757210$
$-2.7834080$
$-2.8187750$
$-0.5013670$
0.5826700
$-0.7729410$
$-0.9839960$
1.4051120
1.1770560
0.4677340
2. 0075950
3. 4493820
3. 1787060
4.0443300
4.0788140
0.1851940
0.2645990
$-1.1464080$
2. 4875970
$-0.6934080$
1. 1638370
2.7737190
$-1.4430710$
$-1.1485680$
$-3.0491670$
$-1.8598430$
$-3.5176890$
$-4.6047260$
$-2.8560260$
$-5.0237180$
$-5.1084240$
$-2.0145610$
$-4.3682270$
$-5.8617530$

-0.2783870
1.3573590
3.0661550
3.0793830
2.7828120
4.0825170
2.5086550
1.8442440
2.4941060
3.5256870
-1.1732390
-1.3701260
-0.1801180
-1.9156880
-0.3089710
-0.3527930
0.7301420
-0.5919980
-4.0725420
-4.5204270
-3.9235070
-4.7953070
-3.0129810
-3.3103300
-3.8192990
-2.1214010
-1.0947360
-2.3635670
-0.6651770
-2.4447970
-2.2668320
2.1388370
-1.5791310
-4.6790460
1.1648500
1.9393430
1.6916190
3.3100340
3.8872860
4.0310480
5.1690330
3.3291930
5.3086120
3.5782240
5.8792980
5.6158820
-1 


\begin{tabular}{|c|c|c|c|c|c|c|c|}
\hline & & & & & & & \\
\hline H & -2.5307970 & -2.7778150 & 5.8592850 & $\mathrm{H}$ & 2.6428720 & -4.6165290 & 1.4585320 \\
\hline H & -1.3392510 & -4.7012150 & 6.8787440 & $\mathrm{H}$ & 6.2042830 & -1.7426170 & 3.3886880 \\
\hline $\mathrm{H}$ & -0.5462280 & -1.5845460 & 0.6781560 & C & 3.5078790 & -5.7817850 & -1.8809900 \\
\hline S & 0.7353430 & -4.0116950 & 0.8321300 & C & 2.4266160 & -6.5689470 & -2.3434250 \\
\hline C & 1.4020420 & -2.8118810 & -2.1119380 & $\mathrm{c}$ & 1.1385820 & -6.1376900 & -2.1558410 \\
\hline C & 2.0597570 & -2.4921490 & -0.8416150 & $\mathrm{C}$ & 3.2800950 & -4.5980000 & -1.2245120 \\
\hline $\mathrm{H}$ & 0.3486560 & -2.5424030 & -2.1702280 & $\mathrm{H}$ & 4.5264510 & -6.1154860 & -2.0521680 \\
\hline $\mathrm{H}$ & 1.6853170 & -1.6438980 & -0.2781420 & $\mathrm{H}$ & 2.6213830 & -7.5059240 & -2.8552050 \\
\hline 0 & 2.2697280 & -1.7175570 & -2.5109950 & $\mathrm{H}$ & 0.2987150 & -6.7230320 & -2.5206590 \\
\hline $\mathrm{H}$ & 1.7086800 & -0.8713170 & -2.4857980 & $\mathrm{H}$ & 4.1156970 & -3.9968680 & -0.8812320 \\
\hline C & 3.2520600 & -4.4567870 & -2.6038330 & C & -2.1216650 & -2.7061070 & -0.5589510 \\
\hline C & 3.6226620 & -4.4500030 & -1.1193850 & $\mathrm{C}$ & -2.8624800 & -2.1635310 & -1.6284250 \\
\hline C & 3.3950990 & -3.0697150 & -0.5016550 & $\mathrm{C}$ & -2.6740440 & -2.7528590 & 0.7342310 \\
\hline C & 1.7741520 & -4.1061020 & -2.7940200 & C & -4.1566710 & -1.7017580 & -1.3806060 \\
\hline H & 3.4521910 & -5.4389030 & -3.0411000 & $\mathrm{C}$ & -3.9757970 & -2.2851320 & 0.9295520 \\
\hline $\mathrm{H}$ & 4.6703160 & -4.7337800 & -0.9788480 & $\mathrm{C}$ & -4.7344040 & -1.7559850 & -0.1111760 \\
\hline $\mathrm{H}$ & 4.1278800 & -2.3481610 & -0.8807750 & $\mathrm{H}$ & -4.7345180 & -1.2902160 & -2.2053800 \\
\hline $\mathrm{H}$ & 1.1409520 & -4.8922930 & -2.3641530 & $\mathrm{H}$ & -4.4187210 & -2.3407600 & 1.9229270 \\
\hline $\mathrm{H}$ & 1.5163830 & -4.0234940 & -3.8542950 & C & 3.7995980 & 1.5661710 & 0.4102630 \\
\hline $\mathrm{H}$ & 3.8713440 & -3.7290870 & -3.1427210 & C & 3.1583800 & 2.4785590 & 1.2756850 \\
\hline $\mathrm{H}$ & 3.0153180 & -5.1940530 & -0.5875630 & $\mathrm{C}$ & 4.4335630 & 2.0182270 & -0.7572490 \\
\hline $\mathrm{H}$ & 3.5109890 & -3.0764240 & 0.5856090 & $\mathrm{C}$ & 3.2036530 & 3.8362540 & 0.9633760 \\
\hline & & & & $\mathrm{C}$ & 4.4519250 & 3.3898370 & -1.0276650 \\
\hline & S) $\mathrm{G}[\mathrm{M} 06-2$ & $G(d)]=-363$ & 322 & C & 3.8476950 & 4.3132340 & -0.1808710 \\
\hline 0 & 0.0818090 & -1.2449100 & 0.2955040 & $\mathrm{H}$ & 2.7095370 & 4.5445940 & 1.6240800 \\
\hline 0 & 2.0397310 & -0.1843250 & -0.8793710 & $\mathrm{H}$ & 4.9466790 & 3.7481780 & -1.9289640 \\
\hline C & 0.3687320 & -2.4657910 & -0.2897500 & C & -1.9216720 & -3.3459610 & 1.9165370 \\
\hline C & 1.6752380 & -2.9040980 & -0.3144100 & $\mathrm{C}$ & -6.1531520 & -1.2732510 & 0.1256010 \\
\hline $\mathrm{C}$ & -0.7337680 & -3.2242350 & -0.7686760 & $\mathrm{C}$ & -2.3005030 & -2.1016210 & -3.0408040 \\
\hline C & 1.9562760 & -4.1344030 & -0.9974010 & $\mathrm{C}$ & 2.4402170 & 2.0150650 & 2.5360060 \\
\hline C & -0.4629070 & -4.4449640 & -1.3323110 & $\mathrm{C}$ & 3.8512780 & 5.7939560 & -0.5109080 \\
\hline C & 0.8707450 & -4.9095010 & -1.4951700 & C & 5.0970250 & 1.0634690 & -1.7369810 \\
\hline $\mathrm{H}$ & -1.2806340 & -5.0773290 & -1.6682740 & $\mathrm{C}$ & 6.6096560 & 1.3049240 & -1.8022960 \\
\hline C & 2.8348600 & -0.7478490 & 0.1022990 & $\mathrm{H}$ & 6.8298910 & 2.3135630 & -2.1691900 \\
\hline C & 3.7822370 & 0.1053080 & 0.7297780 & $\mathrm{H}$ & 7.0668840 & 1.1976650 & -0.8134850 \\
\hline C & 2.7202700 & -2.0957070 & 0.3730280 & $\mathrm{H}$ & 7.0836600 & 0.5887180 & -2.4814530 \\
\hline C & 4.6595620 & -0.4632380 & 1.6175930 & C & 4.4610520 & 1.1627960 & -3.1280950 \\
\hline C & 3.5685040 & -2.6551510 & 1.3883850 & $\mathrm{H}$ & 3.3849130 & 0.9736840 & -3.0749470 \\
\hline C & 4.5699380 & -1.8332670 & 1.9791320 & $\mathrm{H}$ & 4.6153650 & 2.1559530 & -3.5639930 \\
\hline $\mathrm{H}$ & 5.4298780 & 0.1504320 & 2.0778870 & $\mathrm{H}$ & 4.9095640 & 0.4277770 & -3.8045080 \\
\hline C & 5.3027840 & -3.6842500 & 3.3682200 & C & 4.4595690 & 6.6313430 & 0.6190160 \\
\hline C & 4.2762590 & -4.4891210 & 2.8197510 & $\mathrm{H}$ & 4.5049030 & 7.6867840 & 0.3317260 \\
\hline C & 3.4328180 & -3.9905360 & 1.8584440 & $\mathrm{H}$ & 3.8544940 & 6.5594350 & 1.5292780 \\
\hline C & 5.4385420 & -2.3830840 & 2.9592230 & $\mathrm{H}$ & 5.4725590 & 6.2948760 & 0.8589440 \\
\hline $\mathrm{H}$ & 5.9668170 & -4.0945560 & 4.1221820 & C & 2.4350560 & 6.2789840 & -0.8448960 \\
\hline $\mathrm{H}$ & 4.1504320 & 9414 & 3.1681490 & $\mathrm{H}$ & 2.0135960 & 5.7090550 & -1.6786860 \\
\hline
\end{tabular}




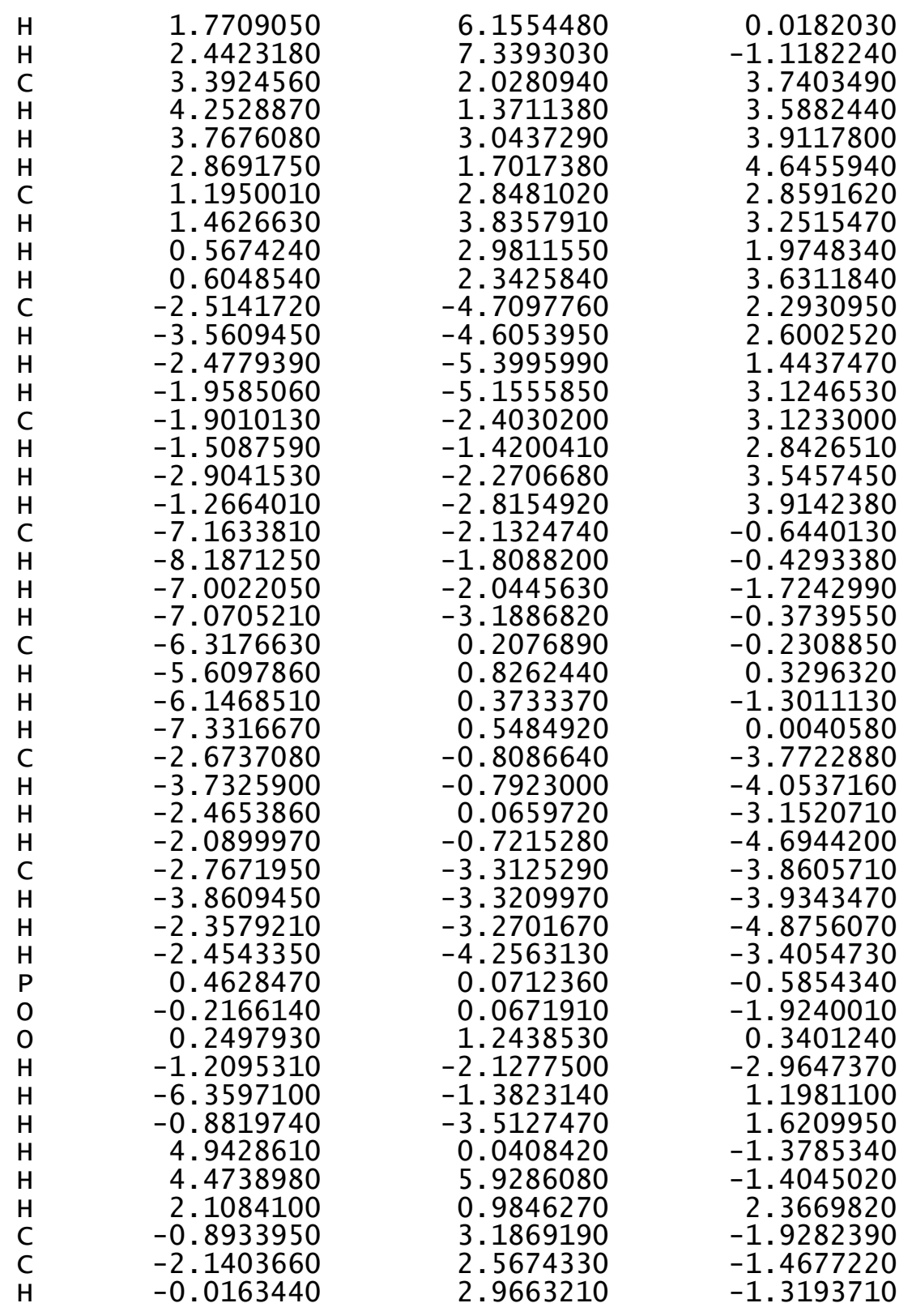

$\begin{array}{lrrr}\mathrm{H} & -2.0509730 & 1.5997210 & -0.9820880 \\ \mathrm{O} & -1.0978850 & 2.2189600 & -2.9849520 \\ \mathrm{H} & -0.6618030 & 1.3492080 & -2.6844780 \\ \mathrm{C} & -2.2710230 & 4.8673450 & -3.2119490 \\ \mathrm{C} & -3.4880670 & 4.4976870 & -2.3609620 \\ \mathrm{C} & -3.4736520 & 3.0146740 & -1.9846590 \\ \mathrm{C} & -0.9700820 & 4.6023140 & -2.4491390 \\ \mathrm{H} & -2.3159870 & 5.9226370 & -3.4952560 \\ \mathrm{H} & -4.4159160 & 4.7230780 & -2.8937480 \\ \mathrm{H} & -3.6928970 & 2.3897650 & -2.8603040 \\ \mathrm{H} & -0.8932680 & 5.2727630 & -1.5838080 \\ \mathrm{H} & -0.0951510 & 4.7842870 & -3.0810630 \\ \mathrm{H} & -2.2760910 & 4.2825040 & -4.1391210 \\ \mathrm{H} & -3.4872600 & 5.0986750 & -1.4427060 \\ \mathrm{H} & -4.2340450 & 2.7733640 & -1.2363810 \\ \mathrm{C} & -2.7041130 & 2.1425200 & 1.5973670 \\ \mathrm{O} & -1.9079820 & 1.1259170 & 1.5887340 \\ \mathrm{C} & -4.0021780 & 1.8808810 & 2.2558690 \\ \mathrm{C} & -5.0234900 & 2.8402800 & 2.2602990 \\ \mathrm{C} & -4.2328900 & 0.6197770 & 2.8247820 \\ \mathrm{C} & -6.2586460 & 2.5380340 & 2.8196240 \\ \mathrm{H} & -4.8442690 & 3.8117810 & 1.8128110 \\ \mathrm{C} & -5.4696860 & 0.3231970 & 3.3830810 \\ \mathrm{H} & -3.4425890 & -0.1210120 & 2.8120130 \\ \mathrm{C} & -6.4852500 & 1.2793540 & 3.3768440 \\ \mathrm{H} & -7.0482870 & 3.2819400 & 2.8160830 \\ \mathrm{H} & -5.6435100 & -0.6553830 & 3.8208130 \\ \mathrm{H} & -7.4535660 & 1.0445340 & 3.8077780 \\ \mathrm{H} & -0.9914560 & 1.2691860 & 1.0964030 \\ \mathrm{~S} & -2.3190450 & 3.6194830 & 0.8719330 \\ \mathrm{H} & - & & \end{array}$

TS234(S, S) G[M06-2X/6-31G(d)] = -3633.303247

\begin{tabular}{lrcr} 
TS234(S, S $)$ & \multicolumn{2}{c}{ G $[\mathrm{M} 06-2 \mathrm{X} / 6-31 \mathrm{G}(\mathrm{d})]=-3633.303247$} \\
$\mathrm{O}$ & 0.6611790 & 0.1512330 & -0.5473690 \\
O & -1.5858110 & 0.1869890 & -1.7227250 \\
$\mathrm{C}$ & 1.0043280 & 1.3035300 & -1.2226750 \\
$\mathrm{C}$ & 0.0999120 & 2.3430110 & -1.2977240 \\
$\mathrm{C}$ & 2.3342350 & 1.3982270 & -1.7121800 \\
$\mathrm{C}$ & 0.4562080 & 3.5096050 & -2.0479600 \\
$\mathrm{C}$ & 2.6893570 & 2.5498020 & -2.3723950 \\
$\mathrm{C}$ & 1.7691210 & 3.6076090 & -2.5866990 \\
H & 3.7088910 & 2.6600240 & -2.7354070 \\
$\mathrm{C}$ & -2.0262070 & 1.1613900 & -0.8294210 \\
$\mathrm{C}$ & -3.2871970 & 0.9717500 & -0.2009510 \\
$\mathrm{C}$ & -1.2002360 & 2.2342540 & -0.5855280 \\
$\mathrm{C}$ & -3.6883910 & 1.9353010 & 0.6918190 \\
$\mathrm{C}$ & -1.5810100 & 3.1711450 & 0.4308300
\end{tabular}




$\begin{array}{rrr}-2.8498900 & 3.0203470 & 1.0567130 \\ -4.6567550 & 1.8411830 & 1.1763810 \\ -2.3884080 & 4.9270950 & 2.4869130 \\ -1.1118950 & 5.0571270 & 1.8884450 \\ -0.7185190 & 4.2054610 & 0.8859980 \\ -3.2355690 & 3.9284150 & 2.0792740 \\ -2.6877420 & 5.6116890 & 3.2744360 \\ -0.4347510 & 5.8328780 & 2.2323010 \\ 0.2684590 & 4.3054060 & 0.4472300 \\ -4.2104670 & 3.8034970 & 2.5427900 \\ -0.0764550 & 5.6722850 & -3.0165280 \\ 1.2383130 & 5.7783930 & -3.5315980 \\ 2.1377560 & 4.7655810 & -3.3223580 \\ -0.4582530 & 4.5704910 & -2.2932960 \\ -0.7901500 & 6.4689600 & -3.2011220 \\ 1.5259300 & 6.6581850 & -4.0981320 \\ 3.1467020 & 4.8268860 & -3.7212330 \\ -1.4713890 & 4.4946900 & -1.9122370 \\ 3.3547260 & 0.3343350 & -1.4450620 \\ 3.5260950 & -0.7271300 & -2.3496800 \\ 4.2110510 & 0.4711900 & -0.3281680 \\ 4.5886400 & -1.6150710 & -2.1493870 \\ 5.2553120 & -0.4408670 & -0.1729390 \\ 5.4759660 & -1.4763880 & -1.0859240 \\ 4.7464900 & -2.4240250 & -2.8586440 \\ 5.9295460 & -0.3338330 & 0.6728240 \\ -4.1127440 & -0.2453040 & -0.4626270 \\ -4.3841660 & -1.1467370 & 0.5929860 \\ -4.6370400 & -0.4871700 & -1.7465020 \\ -5.1958090 & -2.2518530 & 0.3379100 \\ -5.4391730 & -1.6131490 & -1.9491290 \\ -5.7347450 & -2.5053070 & -0.9234640 \\ -5.4112310 & -2.9408200 & 1.1525640 \\ -5.8552750 & -1.7962660 & -2.9381930 \\ 4.0064850 & 1.5943970 & 0.6835720 \\ 6.6559340 & -2.4205010 & -0.9312400 \\ 2.6468330 & -0.8575590 & -3.5842920 \\ -3.8411680 & -0.9655190 & 2.0048970 \\ -6.6165280 & -3.7150960 & -1.1675470 \\ -4.4111280 & 0.4547630 & -2.9200040 \\ -5.7262670 & 1.1295610 & -3.3313370 \\ -6.4563720 & 0.3882440 & -3.6742320 \\ -6.1674090 & 1.6750610 & -2.4910960 \\ -5.5537180 & 1.8365840 & -4.1494840 \\ -3.7703490 & -0.2674960 & -4.1103740 \\ -2.8384050 & -0.7566520 & -3.8137360\end{array}$

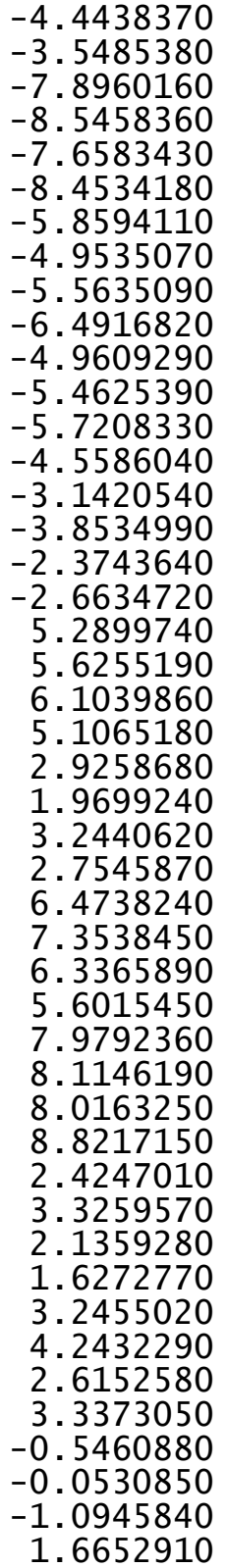

-1.0273450
0.4466400
-3.6502370
-4.5032540
-3.6773230
-2.7300310
-5.0213760
-5.0830940
-5.0919520
-5.8852900
-0.5523590
0.3654150
-1.3397910
-0.3888890
-2.2252810
-3.0419340
-2.5657480
-2.0068030
2.0081850
1.2351460
2.2091200
2.9155870
1.2281490
0.9800510
0.3653530
2.0634010
-3.3674180
-4.0073140
-2.8003140
-4.0153900
-1.6558360
-0.9690260
-1.0705470
-2.3544060
-2.3076730
-2.7303200
-2.9476680
-2.3465980
-0.0544550
-0.4354860
-0.1461170
1.0069590
-0.8518790
-1.8251770
-1.3854180
-0.4365330
$-4.5215380$ $-4.9102720$ $-0.3255430$ $-0.5464990$ 0.7437280 $-0.5243710$ $-0.9024040$ $-1.5124780$ 0.1501960 $-1.1318180$

2.9703390

2.6484860 3. 0346240

3.9757400

2. 5315480

2. 6972860

1.8327940

3.4930190

1. 4075050

2.1072670

0.7042370

1.9905420

1. 7133240

1. 2402840

2. 3128990

2. 4019970

0.2612270

0.3827600

1. 1903480

0.1231010

$-0.8135200$

$-1.6541350$

0.1116140

$-0.7982850$

$-4.0225380$

$-4.4804390$

$-3.1852110$

$-4.7702970$

$-4.7484310$

$-4.9949140$

$-5.6393450$

$-4.5021340$

$-1.0395180$

$-2.0617770$

0.2639590
-3.3422210 


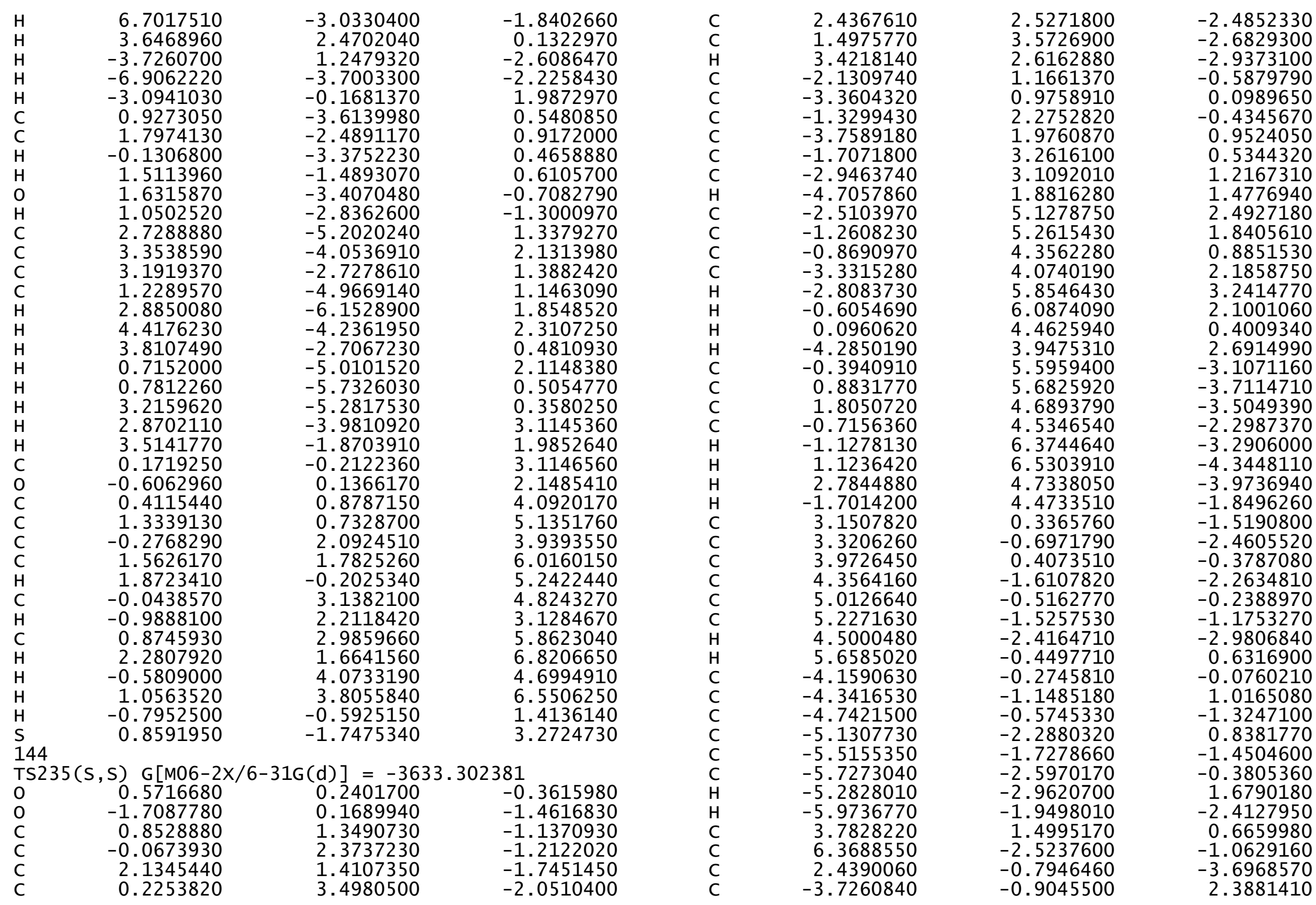




$\begin{array}{lr}-6.5800500 & -3.8410110 \\ -4.6028160 & 0.3354670 \\ -5.9578550 & 0.9524060 \\ -6.6766160 & 0.1764190 \\ -6.3794840 & 1.5095510 \\ -5.8474880 & 1.6380050 \\ -3.9920480 & -0.4039870 \\ -3.0264340 & -0.8430340 \\ -4.6521890 & -1.2055510 \\ -3.8407580 & 0.2883940 \\ -5.9777900 & -4.8001390 \\ -6.5764110 & -5.7141450 \\ -5.9539650 & -4.3364770 \\ -4.9537270 & -5.0764890 \\ -8.0239100 & -3.4833980 \\ -8.4686140 & -2.8143300 \\ -8.0615000 & -2.9811970 \\ -8.6393660 & -4.3865640 \\ -4.7989650 & -0.4702490 \\ -5.3289280 & 0.4292370 \\ -5.5429200 & -1.2643640 \\ -4.3466810 & -0.2655100 \\ -2.9779080 & -2.1311080 \\ -3.6666760 & -2.9466520 \\ -2.2490480 & -2.4983170 \\ -2.4454340 & -1.8623370 \\ 4.6922560 & 2.6965880 \\ 5.7440090 & 2.3903160 \\ 4.4906620 & 3.1050320 \\ 4.5427260 & 3.4928500 \\ 4.0161900 & 1.0116000 \\ 3.4228350 & 0.1168440 \\ 5.0707550 & 0.7795480 \\ 3.7217820 & 1.7916860 \\ 6.9509400 & -2.6489920 \\ 7.6823570 & -3.4618370 \\ 7.4681730 & -1.7302450 \\ 6.1774900 & -2.8588190 \\ 7.4776350 & -2.1633070 \\ 7.0925300 & -2.1213840 \\ 7.8985720 & -1.1816450 \\ 8.2854800 & -2.9014290 \\ 2.1609130 & -2.2374360 \\ 3.0491560 & -2.7029780 \\ 1.8307710 & -2.8548300 \\ 1.3742710 & -2.2480660\end{array}$

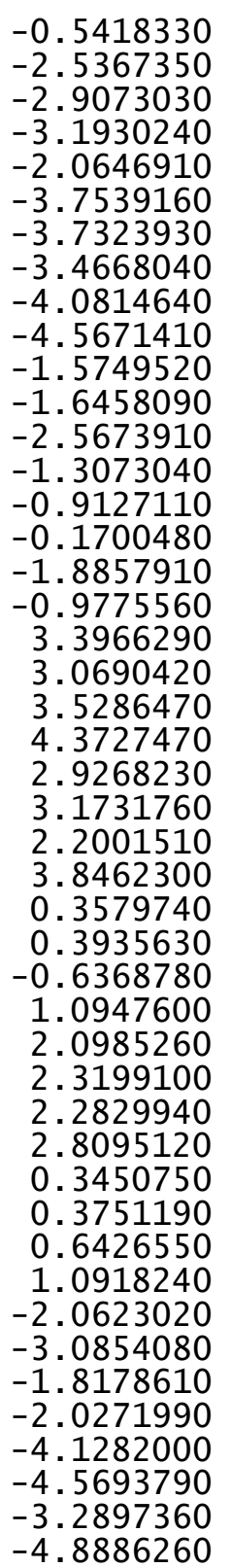
3. 0690590
4. 0519340
2. 4370930
3. 2018710
$-0.5948770$
$-0.1022730$
$-1.0853040$
1. 4751720
5.9666740
2. 7433530
$-3.9315740$
$-6.5964870$
$-2.9962640$
0.5287770
$-0.4734240$
1. 0507840
1. 9251260
0.6967800
2. 4366680
2.2002770
1. 2271520
0.0236620
2. 0952270
3. 1080380
0.9596810
2. 5068950
$-0.7470100$
1. 2185180
0.8811550
1. 8848690
$-0.1507390$
1. 6963350
1. 4941050
0.9422460
2. 5523430
3. 3439680
3. 2765740
1.0785240
2. 6369100
4. 3921240
3. 8230740
0.6246840
0.5114970
2. 9676480
2.9354860
3. 7390100
$-0.0237520$
$-0.4442570$
$-0.0981750$
1.0352530
$-0.8211540$
$-1.7554970$
$-1.3954220$
$-0.3339480$
$-3.5049830$
1.8441070
1.1604270
$-4.3540870$
$-0.0958720$
$-0.2571900$
0.0504430
0.8871310
0.7085290
2. 1830400
1. 8135100
$-0.2959030$
3. 2850290
2. 3184150
3. 1020990
1. 6717910
4. 2849680
3.9630810
$-0.6651180$
$-1.8031940$
$-3.6846780$
$-2.6488900$
$-3.3420130$
$-1.6325010$
$-3.5843080$
$-2.9292530$
$-5.4426440$
$-4.3507750$
$-3.0352670$
$-5.0486920$
$-4.6415140$
$-3.1196550$
$-5.0144450$
$-5.7747250$
$-5.5969510$
$-4.2033880$
$-2.2013630$

$-4.8659080$

$-5.1081080$

$-5.7572090$

$-4.6292750$

$-0.8223850$

$-1.8800450$

0.4882470

$-3.4552540$

$-1.3515460$

0.6110090

$-2.2826730$

0.4280340

2. 2977040

3. 1096260

2. 3678730

3. 8945680

4.9723390

3. 4915240

5.6410940

5. 2761180

4.1517310

2.6506850

5. 2276120

6.4815860

3.8242460

5.7450860

1. 6338210

3.1969520

0.5335590

0.8248590

0.5279600

0.4883520

$-0.7790150$

$-1.3060080$

1. 2607140

1. 9812260

1. 2058290

1.1515900

1.7958990

2. 0985850

0.2564830

2. 1499140

0.5609290

0.2575080

2.9897130

1. 7417920 
144

$\operatorname{TS} 236(\mathrm{~S}, \mathrm{~S}) \mathrm{G}[\mathrm{M} 06-2 \mathrm{X} / 6-31 \mathrm{G}(\mathrm{d})]=-3633.300184$

$\begin{array}{lrrr}\mathrm{O} & 0.1086920 & -1.2071920 & 0.1671870 \\ \mathrm{O} & 2.0784710 & -0.0830640 & -0.9121210 \\ \mathrm{C} & 0.4180990 & -2.4196710 & -0.4236870 \\ \mathrm{C} & 1.7300480 & -2.8379340 & -0.4360730 \\ \mathrm{C} & -0.6725750 & -3.1862090 & -0.9212880 \\ \mathrm{C} & 2.0338290 & -4.0531190 & -1.1364950 \\ \mathrm{C} & -0.3807700 & -4.3928280 & -1.5021300 \\ \mathrm{C} & 0.9632750 & -4.8328480 & -1.6601590 \\ \mathrm{H} & -1.1834010 & -5.0352210 & -1.8544180 \\ \mathrm{C} & 2.8384830 & -0.6713480 & 0.0823270 \\ \mathrm{C} & 3.7354800 & 0.1779590 & 0.7845990 \\ \mathrm{C} & 2.7443360 & -2.0315440 & 0.2995140 \\ \mathrm{C} & 4.5906160 & -0.4031660 & 1.6855350 \\ \mathrm{C} & 3.5666340 & -2.6094790 & 1.3267900 \\ \mathrm{C} & 4.5241980 & -1.7884760 & 1.9875520 \\ \mathrm{H} & 5.3215080 & 0.2122080 & 2.2046190 \\ \mathrm{C} & 5.2492550 & -3.6727240 & 3.3353350 \\ \mathrm{C} & 4.2632020 & -4.4787540 & 2.7188520 \\ \mathrm{C} & 3.4446870 & -3.9637800 & 1.7446430 \\ \mathrm{C} & 5.3680960 & -2.3543790 & 2.9800000 \\ \mathrm{H} & 5.8941430 & -4.0959420 & 4.0987140 \\ \mathrm{H} & 4.1479100 & -5.5137560 & 3.0250070 \\ \mathrm{H} & 2.6851230 & -4.5927880 & 1.2943680 \\ \mathrm{H} & 6.1004840 & -1.7125100 & 3.4623250 \\ \mathrm{C} & 3.6172880 & -5.6645710 & -2.0290640 \\ \mathrm{C} & 2.5518710 & -6.4562240 & -2.5190720 \\ \mathrm{C} & 1.2559500 & -6.0450000 & -2.3392530 \\ \mathrm{C} & 3.3658630 & -4.4957990 & -1.3542610 \\ \mathrm{H} & 4.6420420 & -5.9825500 & -2.1930590 \\ \mathrm{H} & 2.7646440 & -7.3811470 & -3.0454110 \\ \mathrm{H} & 0.4285820 & -6.6349690 & -2.7245040 \\ \mathrm{H} & 4.1885150 & -3.8900020 & -0.9880320 \\ \mathrm{C} & -2.0486290 & -2.6494490 & -0.6795380 \\ \mathrm{C} & -2.7693460 & -2.0015130 & -1.7022160 \\ \mathrm{C} & -2.5624520 & -2.6995850 & 0.6303440 \\ \mathrm{C} & -3.9803150 & -1.3810900 & -1.3791820 \\ \mathrm{C} & -3.7831060 & -2.0806080 & 0.8999180 \\ \mathrm{C} & -4.4978350 & -1.3979010 & -0.0822460 \\ \mathrm{H} & -4.5357140 & -0.8742190 & -2.1642380 \\ \mathrm{H} & -4.1910930 & -2.1161210 & 1.9091350 \\ \mathrm{C} & 3.7300900 & 1.6517730 & 0.5284740 \\ \mathrm{C} & 2.9759170 & 2.5045360 & 1.3638070 \\ \mathrm{C} & 4.4665910 & 2.1758740 & -0.5445210 \\ \mathrm{C} & 3.0118820 & 3.8762970 & 1.1198400\end{array}$

4.4741470
3.7584620
2.4331010
5.0508520
-1.8470970
-5.8352950
-2.2902700
2.1566810
3.7632780
5.2556920
6.7613580
7.0075510
7.0976260
7.3250790
4.7924590
3.7200670
4.9866720
5.3281540
4.2785890
4.3265830
3.6128540
5.2777800
2.3697630
2.0114220
1.6478660
2.3881190
3.0054990
3.8798910
3.3575150
2.4110340
0.8827320
1.1110120
0.3320980
0.2311330
-2.5845000
-3.6032430
-2.6524620
-2.0628010
-1.6813190
-1.1952780
-2.6494510
-1.0680730
-6.9737060
-7.9407950
-7.0064330
-6.8329710

3.5596300

4.4248750

4.5397550

3.9740540

$-3.4415980$

$-0.7690300$

$-2.0396990$

1.9615190

5. 9212960

1.2858470

1. 5420000

2.5739840

1.3760660

0.8741810

1.4625000

1.2663580

2.4792330

0.7692040

6.6979570

7.7691630

6.5635180

6.3599080

6.4145550

. 8859540

6.2464570

7.4872830

1.9012620

1. 2564230

2. 9049830

1. 5174690

2.7696530

3.7263440

.9663840

2. 2101610

$-4.7493200$

$-4.5433330$

$-5.3853370$

$-5.3064330$

$-2.5888660$

$-1.6365880$

$-2.3799230$

$-3.1192630$

$-1.7555380$

$-1.3293100$

$-1.9883440$

$-2.6934320$
$-0.7465870$

0.0738370

1.7579020

$-1.5718910$

1.7501430

0.2641970

$-3.1456840$

2. 5272140

$-0.1759250$

$-1.4916070$

$-1.3580460$

$-1.6319270$

$-0.3295630$

$-2.0176710$

$-2.9420120$

$-3.0330630$

$-3.3003080$

$-3.5986460$

1. 0406960

0.8197090

1. 9002740

1. 3310080

$-0.5838510$

$-1.4724100$

0.2233040

$-0.8025610$

3.8055380

3.6844490

4.0704570

4.6416750

2. 7995840

3.2829110

1.8765430

3.4791350

2. 0665000

2. 4141160

1.1780720

2. 8517330

3. 0109510

2. 7752690

3.4806100

3. 7465290

$-0.0304230$

0.2566390

$-1.1009670$

0.5154020 


$\begin{array}{rrr}-6.0827860 & 0.5607390 & -0.4495530 \\ -5.2505120 & 1.2543400 & -0.2877770 \\ -6.2109390 & 0.4199760 & -1.5290120 \\ -6.9941820 & 1.0316150 & -0.0664500 \\ -2.6331550 & -0.7832170 & -3.9470100 \\ -3.7109190 & -0.6915980 & -4.1229060 \\ -2.2825850 & 0.1147670 & -3.4353110 \\ -2.1474010 & -0.8267930 & -4.9270670 \\ -2.8761580 & -3.2715110 & -3.8523310 \\ -3.9700170 & -3.2045720 & -3.8802270 \\ -2.5116380 & -3.3338730 & -4.8832170 \\ -2.6121410 & -4.1997330 & -3.3384330 \\ 0.4772230 & 0.1118750 & -0.7118490 \\ -0.1233120 & 0.0679040 & -2.0879210 \\ 0.1541160 & 1.2851530 & 0.1797010 \\ -1.1998110 & -2.1292350 & -3.1311200 \\ -5.8287620 & -0.5722750 & 1.3454000 \\ -0.8434670 & -3.7085470 & 1.4069350 \\ 5.0727190 & 0.2428510 & -1.2146100 \\ 4.4472340 & 6.1109330 & -1.0128900 \\ 1.8489140 & 0.9420970 & 2.2680420 \\ -1.0232640 & 3.1399720 & -2.0205190 \\ -2.2560430 & 2.3728880 & -1.8160320 \\ -0.2317530 & 2.9499670 & -1.2971360 \\ -2.1560180 & 1.3821130 & -1.3787700 \\ -0.9699940 & 2.2364240 & -3.1540810 \\ -0.5147100 & 1.3809810 & -2.8414510 \\ -2.3419030 & 4.7955990 & -3.4003070 \\ -3.6304580 & 4.2514000 & -2.7806890 \\ -3.5263070 & 2.7472540 & -2.5200260 \\ -1.1533400 & 4.5783540 & -2.4606570 \\ -2.4457330 & 5.8633110 & -3.6128310 \\ -4.4861850 & 4.4426740 & -3.4339690 \\ -3.5335800 & 2.1917980 & -3.4666620 \\ -1.2713020 & 5.1841920 & -1.5534170 \\ -0.2128670 & 4.8792270 & -2.9325910 \\ -2.1505060 & 4.2922960 & -4.3552280 \\ -3.8269350 & 4.7694280 & -1.8328650 \\ -4.3718530 & 2.3725030 & -1.9383380 \\ -2.9302750 & 1.8331130 & 1.4922090 \\ -1.9461960 & 0.9992740 & 1.5175350 \\ -4.0323940 & 1.4742040 & 2.4151380 \\ -5.2635030 & 2.1412850 & 2.3815810 \\ -3.8438200 & 0.4170760 & 3.3164130 \\ -6.2943550 & 1.7444590 & 3.2244400 \\ -5.4103950 & 2.9562440 & 1.6809230\end{array}$

$\begin{array}{ll}\mathrm{C} & -4.8757150 \\ \mathrm{H} & -2.8875400 \\ \mathrm{C} & -6.1039970 \\ \mathrm{H} & -7.2487780 \\ \mathrm{H} & -4.7224910 \\ \mathrm{H} & -6.9121220 \\ \mathrm{H} & -1.1162580 \\ \mathrm{~S} & -2.9803540\end{array}$
0.0257170 $-0.0911120$
0.6849140
2. 2590680
$-0.7934240$
0.3754130
1. 2221680
3. 2083000

4. 1606580

3. 3432790

4.1117860

3. 1866950

4.8563040

4.7671720

144

0.9282510

$\operatorname{TS} 237(\mathrm{~S}, \mathrm{~S}) \mathrm{G}[\mathrm{M} 06-2 \mathrm{X} / 6-31 \mathrm{G}(\mathrm{d})]=-3633.303608$

$\begin{array}{lrcr}\text { TS237(S }(\mathrm{S}) \mathrm{G}[\mathrm{M} 06-2 \mathrm{X} / 6-31 \mathrm{G}(\mathrm{d})]=-3633.303608 \\ \mathrm{O} & -1.4649260 & 0.5768920 & -0.6943950 \\ \mathrm{O} & 0.2350090 & 1.6333680 & 0.8301020 \\ \mathrm{C} & -2.4045480 & 1.4379040 & -0.1551680 \\ \mathrm{C} & -2.1151900 & 2.7860730 & -0.0966080 \\ \mathrm{C} & -3.6387140 & 0.8768330 & 0.2678790 \\ \mathrm{C} & -3.0923170 & 3.6661700 & 0.4761460 \\ \mathrm{C} & -4.6056600 & 1.7446950 & 0.7128010 \\ \mathrm{C} & -4.3606360 & 3.1357700 & 0.8452740 \\ \mathrm{H} & -5.5790070 & 1.3565440 & 1.0012880 \\ \mathrm{C} & 0.3563710 & 2.6639930 & -0.0886110 \\ \mathrm{C} & 1.6717760 & 3.0705400 & -0.4392350 \\ \mathrm{C} & -0.7878100 & 3.2659030 & -0.5705220 \\ \mathrm{C} & 1.7941900 & 4.1341740 & -1.2990930 \\ \mathrm{C} & -0.6450920 & 4.3081430 & -1.5455710 \\ \mathrm{C} & 0.6622020 & 4.7590080 & -1.8834540 \\ \mathrm{H} & 2.7853800 & 4.4903710 & -1.5698820 \\ \mathrm{C} & -0.2744700 & 6.3661650 & -3.4429030 \\ \mathrm{C} & -1.5727510 & 5.8896420 & -3.1407350 \\ \mathrm{C} & -1.7543540 & 4.8895550 & -2.2186720 \\ \mathrm{C} & 0.8165780 & 5.8070990 & -2.8301150 \\ \mathrm{H} & -0.1472060 & 7.1623800 & -4.1692830 \\ \mathrm{H} & -2.4322250 & 6.3158670 & -3.6484760 \\ \mathrm{H} & -2.7534850 & 4.5257700 & -2.0042890 \\ \mathrm{H} & 1.8214640 & 6.1461330 & -3.0671490 \\ \mathrm{C} & -3.8109190 & 5.8514850 & 1.2603380 \\ \mathrm{C} & -5.0870480 & 5.3307900 & 1.5835890 \\ \mathrm{C} & -5.3503230 & 4.0005930 & 1.3847700 \\ \mathrm{C} & -2.8405290 & 5.0438930 & 0.7223330 \\ \mathrm{H} & -3.5963940 & 6.8991330 & 1.4463520 \\ \mathrm{H} & -5.8462840 & 5.9835810 & 2.0021230 \\ \mathrm{H} & -6.3162220 & 3.5791970 & 1.6503210 \\ \mathrm{H} & -1.8636600 & 5.4537950 & 0.4899400 \\ \mathrm{C} & -3.8777090 & -0.5999970 & 0.2567720 \\ \mathrm{C} & -3.9868580 & -1.2938450 & 1.4838100 \\ \mathrm{C} & -4.0583860 & -1.2858390 & -0.9561610 \\ \mathrm{C} & -4.3509910 & -2.6387160 & 1.4584610\end{array}$




$\begin{array}{rrr}-4.4118210 & -2.6401790 & -0.9276890 \\ -4.5808670 & -3.3309440 & 0.2689670 \\ -4.4510940 & -3.1786320 & 2.3986930 \\ -4.5717410 & -3.1552570 & -1.8724780 \\ 2.8670710 & 2.3564310 & 0.1040750 \\ 3.7128630 & 1.6359060 & -0.7632950 \\ 3.1679180 & 2.4329370 & 1.4805290 \\ 4.8610880 & 1.0380790 & -0.2346090 \\ 4.3227020 & 1.8144640 & 1.9577870 \\ 5.1895380 & 1.1178020 & 1.1159880 \\ 5.5300100 & 0.4965760 & -0.9021800 \\ 4.5616240 & 1.8976790 & 3.0162870 \\ -0.1993140 & 0.1800370 & 0.2524520 \\ -0.6278640 & -0.6474000 & 1.4282550 \\ 0.8203440 & -0.3712580 & -0.7150720 \\ -3.9273730 & -0.5972380 & -2.3059530 \\ -3.6256120 & 0.4406240 & -2.1388510 \\ -5.0479460 & -4.7750570 & 0.3352610 \\ -4.4935380 & -5.2549100 & 1.1533990 \\ -3.7217590 & -0.6277640 & 2.8284770 \\ -3.2046610 & 0.3184660 & 2.6407770 \\ 2.3116000 & 3.2165210 & 2.4649710 \\ 1.4315440 & 3.5975650 & 1.9401880 \\ 6.4835860 & 0.5197610 & 1.6371500 \\ 6.8959080 & -0.1092450 & 0.8359190 \\ 3.4649720 & 1.5124780 & -2.2616720 \\ 2.4824520 & 1.9364720 & -2.4864130 \\ 3.4416670 & 0.0549530 & -2.7376750 \\ 3.3350690 & 0.0223800 & -3.8272880 \\ 4.3647380 & -0.4771390 & -2.4786410 \\ 2.5972370 & -0.4781730 & -2.2974770 \\ 4.5170310 & 2.3130990 & -3.0419880 \\ 5.5176610 & 1.8955440 & -2.8821700 \\ 4.3052670 & 2.2797470 & -4.1156530 \\ 4.5404020 & 3.3615510 & -2.7280960 \\ -2.8432980 & -1.2562700 & -3.1655380 \\ -3.0951530 & -2.2982660 & -3.3943760 \\ -2.7341460 & -0.7237580 & -4.1159340 \\ -1.8767490 & -1.2359120 & -2.6516030 \\ -5.2728730 & -0.5693910 & -3.0415990 \\ -5.1789200 & -0.0315600 & -3.9905940 \\ -5.6222560 & -1.5839310 & -3.2625200 \\ -6.0384750 & -0.0722090 & -2.4376480 \\ 1.8151020 & 2.3307250 & 3.6128760 \\ 2.6501390 & 1.9605760 & 4.2178640 \\ 1.1551470 & 2.9033940 & 4.2728260\end{array}$

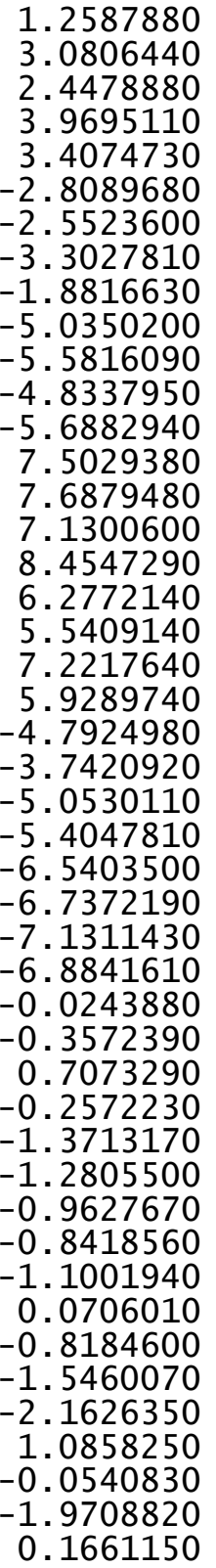

1.4714920

4. 4312690

5.0176210

4.1179520

5.0807870

$-1.4725000$

$-0.9050630$

$-2.3946530$

$-1.7334350$

$-0.3292890$

$-1.2596520$

0.1517120

0.3284560

1.6289460

2. 2374060

2. 2896120

1.2028240

$-0.3549030$

$-1.1452960$

$-0.8240940$

0.2441960

$-5.5754970$

$-5.5295250$

$-6.6262070$

$-5.2066820$

$-4.8201670$

$-4.2966670$

$-4.3373640$

$-5.8547610$

$-3.5522990$

$-3.2236870$

$-2.9008930$

$-2.1895530$

$-3.0451210$

$-2.0723360$

$-5.8585170$

$-5.6508630$

$-4.1903410$

$-5.0104750$

$-6.9130010$

$-6.2923020$

$-3.9344170$

$-5.3484070$

$-5.0928160$

$-5.5787940$

$-5.9361370$
3.2282440

3.0006540

3.6749730

3.5593610

2.1823590

3.7260690

4.6266290

4.0502900

3.2113170

3.5643010

3.7577170

4.5272730

2.9842480

1.9290640

1.0389260

2.7199030

2.2628320

2.8782940

2.7018030

3.1733500

3.7263550

$-0.9410630$

$-1.2475040$

$-0.7824660$

$-1.7712010$

0.6957050

1.6359960

$-0.0908450$

0.7971900

0.6297300

$-0.7599990$

1.1099980

$-1.0664930$

0.7820030

1.0480980

0.2635690

$-1.2476670$

$-1.6255680$

1. 0106340

0.5146020

$-1.7861190$

$-1.5023540$

0.7679940

2.0946380

0.5962990
-1.5734580 


$\begin{array}{lrrr}\mathrm{H} & -0.8553830 & -3.9816150 & -2.6709970 \\ \mathrm{C} & 3.0442830 & -2.8429190 & -0.7612190 \\ \mathrm{O} & 2.7744510 & -1.7904760 & -0.0571600 \\ \mathrm{C} & 4.3984010 & -3.3862720 & -0.5063430 \\ \mathrm{C} & 4.7631010 & -4.6726030 & -0.9210370 \\ \mathrm{C} & 5.3273080 & -2.5857750 & 0.1731920 \\ \mathrm{C} & 6.0407730 & -5.1508580 & -0.6577060 \\ \mathrm{H} & 4.0385240 & -5.2921130 & -1.4385070 \\ \mathrm{C} & 6.6043610 & -3.0684560 & 0.4322100 \\ \mathrm{H} & 5.0346740 & -1.5874780 & 0.4818080 \\ \mathrm{C} & 6.9623230 & -4.3508900 & 0.0178050 \\ \mathrm{H} & 6.3176860 & -6.1506680 & -0.9751640 \\ \mathrm{H} & 7.3215400 & -2.4463570 & 0.9597880 \\ \mathrm{H} & 7.9592450 & -4.7283650 & 0.2226640 \\ \mathrm{H} & 1.9206110 & -1.2613260 & -0.3340490 \\ \mathrm{~S} & 1.9867110 & -3.5385330 & -1.8680750 \\ \mathrm{H} 44 & & & \\ \mathrm{TS} 238(\mathrm{~S}) \mathrm{S}) & \mathrm{G}[\mathrm{M} 06-2 \mathrm{~N} & & \\ \mathrm{O} & 0.5739100 & 0.2896190 & -0.3291900 \\ \mathrm{O} & -1.6435670 & 0.3541300 & -1.5505000 \\ \mathrm{C} & 0.9062970 & 1.4627630 & -0.9805380 \\ \mathrm{C} & 0.0006960 & 2.5032610 & -1.0044570 \\ \mathrm{C} & 2.2166910 & 1.5612380 & -1.5182310 \\ \mathrm{C} & 0.3471430 & 3.6975860 & -1.7167680 \\ \mathrm{C} & 2.5671090 & 2.7386350 & -2.1320930 \\ \mathrm{C} & 1.6510470 & 3.8130840 & -2.2732890 \\ \mathrm{H} & 3.5740470 & 2.8547510 & -2.5262340 \\ \mathrm{C} & -2.1033540 & 1.2686870 & -0.6083340 \\ \mathrm{C} & -3.3699770 & 1.0316560 & -0.0095550 \\ \mathrm{C} & -1.3035200 & 2.3490500 & -0.3089120 \\ \mathrm{C} & -3.8126610 & 1.9599780 & 0.9013150 \\ \mathrm{C} & -1.7288500 & 3.2490310 & 0.7225240 \\ \mathrm{C} & -3.0099490 & 3.0560480 & 1.3107660 \\ \mathrm{H} & -4.7895600 & 1.8312090 & 1.3599090 \\ \mathrm{C} & -2.6360010 & 4.9478350 & 2.7850770 \\ \mathrm{C} & -1.3450760 & 5.1169810 & 2.2286370 \\ \mathrm{C} & -0.9030260 & 4.2941660 & 1.2221210 \\ \mathrm{C} & -3.4468050 & 3.9385150 & 2.3349890 \\ \mathrm{H} & -2.9736860 & 5.6108250 & 3.5750160 \\ \mathrm{H} & -0.6982770 & 5.9045470 & 2.6030470 \\ \mathrm{H} & 0.0922320 & 4.4271130 & 0.8105550 \\ \mathrm{H} & -4.4318590 & 3.7831940 & 2.7667300 \\ \mathrm{C} & -0.1954330 & 5.8948310 & -2.5993310 \\ \mathrm{C} & 1.1132370 & 6.0205380 & -3.1245200 \\ \mathrm{C} & 2.0128820 & 4.9984340 & -2.9669990 \\ \mathrm{C} & -0.5696090 & 4.7664330 & -1.9137790 \\ & & & \end{array}$

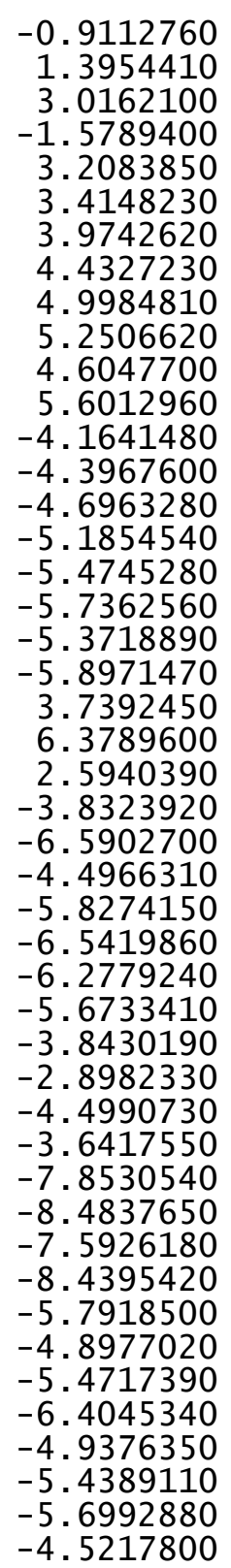

6.6974240

6.9213620

5.0725360

4.6771100

0.4524840

$-0.4743720$

0.3865220

$-1.4179560$

$-0.5607860$

$-1.4638240$

$-2.1419110$

$-0.6004000$

$-0.1934120$

$-1.1572920$

$-0.3797590$

$-2.2665240$

$-1.5124460$

$-2.4641420$

$-3.0041570$

$-1.6537490$

1. 3585900

$-2.4816990$

$-0.4260040$

$-1.0361340$

$-3.6815410$

0.6256750

1. 2910220

0.5518540

1.7833840

2. 0421620

$-0.0212390$

$-0.5008020$

$-0.7768130$

0.7363630

$-3.7043320$

$-4.5605690$

$-3.7897150$

$-2.7901020$

$-4.9788680$

$-4.9780480$

$-5.1024380$

$-5.8457800$

$-0.6671990$

0.2671640

$-1.4548210$

$-0.5548750$
$-2.7460780$

$-3.6598610$

$-3.3777680$

$-1.5260770$

$-1.3591770$

$-2.3993650$

$-0.1795990$

$-2.2552350$

$-0.0937130$

$-1.1249550$

$-3.0493160$

0.8084970

$-0.3279850$

0.6798530

$-1.6169360$

0.3763730

$-1.8704330$

$-0.8901100$

1. 1546930

$-2.8635930$

0.9697670

$-1.0704130$

$-3.6799290$

2. 0895300

$-1.1890020$

$-2.7411160$

$-3.1152060$

$-3.4937090$

$-2.2475430$

$-3.8969050$

$-3.9672160$

$-3.6967180$

$-4.4130480$

$-4.7317760$

$-0.3201070$

$-0.5804450$

0.7407680

$-0.4513020$

$-1.0174680$

$-1.6477140$

0.0230750

$-1.2854910$

3.0891300

2. 8194660

3. 1235400

4.0959800 


$\begin{array}{rrr}-3.1251130 & -2.3162720 & 2.5538850 \\ -3.8375460 & -3.1322960 & 2.7177740 \\ -2.3833460 & -2.6452130 & 1.8219570 \\ -2.6150650 & -2.1305300 & 3.5058870 \\ 4.6238690 & 2.6028590 & 0.8117320 \\ 5.6818940 & 2.3184140 & 0.8418840 \\ 4.4350360 & 3.1116230 & -0.1379940 \\ 4.4367790 & 3.3112990 & 1.6257390 \\ 3.9610370 & 0.7314100 & 2.3486910 \\ 3.3892490 & -0.1963600 & 2.4669850 \\ 5.0186940 & 0.5102920 & 2.5290830 \\ 3.6334650 & 1.4277020 & 3.1285610 \\ 6.8926090 & -2.7684060 & 0.3405040 \\ 7.6168250 & -3.5878140 & 0.3142500 \\ 7.4006150 & -1.8940900 & 0.7619260 \\ 6.0833830 & -3.0507610 & 1.0226070 \\ 7.5364070 & -2.0260670 & -1.9709770 \\ 7.2008300 & -1.8676340 & -3.0000940 \\ 7.9511170 & -1.0820010 & -1.6003480 \\ 8.3372260 & -2.7725660 & -1.9798000 \\ 2.3173270 & -1.8109780 & -4.2717360 \\ 3.2201940 & -2.2469620 & -4.7132710 \\ 1.9338350 & -2.5039800 & -3.5196680 \\ 1.5719940 & -1.7272350 & -5.0683850 \\ 3.2920120 & 0.4486180 & -4.7314870 \\ 4.2795150 & 0.0364550 & -4.9691850 \\ 2.7036420 & 0.4783310 & -5.6546800 \\ 3.4280940 & 1.4750160 & -4.3807030 \\ -0.5705370 & -0.7075450 & -0.9585240 \\ -0.0266000 & -1.5315900 & -2.0808060 \\ -1.1335800 & -1.4089860 & 0.2578230 \\ 1.6257940 & 0.0254780 & -3.4388110 \\ 5.9846830 & -3.4202660 & -1.4844330 \\ 2.6916910 & 1.6818380 & 0.9298940 \\ -3.8273330 & 1.4161580 & -2.3903960 \\ -6.9024370 & -3.6121670 & -2.2387290 \\ -3.0883090 & -0.2353110 & 2.0940460 \\ 0.3998550 & -0.5654380 & 3.0319810 \\ -0.5842160 & -0.1827780 & 2.3018500 \\ 0.9184220 & 0.5009800 & 3.9210860 \\ 1.7170220 & 0.2169390 & 5.0340250 \\ 0.6410170 & 1.8331920 & 3.5822100 \\ 2.2284150 & 1.2551790 & 5.8026920 \\ 1.9326760 & -0.8157760 & 5.2872260 \\ 1.1744180 & 2.8682720 & 2.7132980 \\ 0.0264140 & 2.0478270 & \end{array}$

$$
\begin{array}{r}
1.9654000 \\
2.8392320 \\
0.9680040 \\
2.3779990 \\
-0.8379340 \\
1.0747470 \\
0.8046640 \\
1.8148110 \\
-0.2194530 \\
1.6612740 \\
1.4732150 \\
0.9593940 \\
2.4094160 \\
3.1928710 \\
3.1822600 \\
0.9494000 \\
2.4535400 \\
4.2296770 \\
3.7642700 \\
0.4563890 \\
0.3921570 \\
2.8617530 \\
2.7478070 \\
3.6391170
\end{array}
$$$$
144
$$

2. 5807500

1.0327680

3.8974580

3. 3890560

$-0.8163270$

$-2.1201460$

$-3.7234570$

$-2.7446770$

$-3.3587010$

$-1.6961730$

$-3.5102790$

$-2.7899510$

$-5.5834530$

$-4.5868560$

$-3.1997410$

$-5.1457320$

$-6.5831080$

$-3.2023080$

$-5.1983750$

$-5.7965960$

$-5.6480460$

$-4.5308220$

$-2.4342260$
5.4531990
6.6714320
4.0637950
6.0489850
1.4876730
2.9984030
0.1522530
0.5855430
0.1395730
0.3415350
-1.1185360
-1.5961610
0.7654490
1.6204710
0.9785800
0.6399070
1.2065270
1.7474410
0.0466920
1.6186370
-0.0408050
-0.2315060
2.6226760
1.6121790

$\operatorname{TS} 239(\mathrm{~S}, \mathrm{~S}) \mathrm{G}[\mathrm{M} 06-2 \mathrm{X} / 6-31 \mathrm{G}(\mathrm{d})]=-3633.302986$

$\begin{array}{lrlr}\mathrm{O} & 1.5631230 & -0.3909560 & -0.7390890 \\ \mathrm{O} & 0.1739500 & -1.6597390 & 0.9117060 \\ \mathrm{C} & 2.6894100 & -1.0211390 & -0.2469000 \\ \mathrm{C} & 2.6744550 & -2.3925460 & -0.1013030 \\ \mathrm{C} & 3.8126520 & -0.2023530 & 0.0430480 \\ \mathrm{C} & 3.8446380 & -3.0259200 & 0.4348550 \\ \mathrm{C} & 4.9616490 & -0.8341410 & 0.4504180 \\ \mathrm{C} & 5.0051480 & -2.2355090 & 0.6720590 \\ \mathrm{H} & 5.8544750 & -0.2468980 & 0.6495310 \\ \mathrm{C} & 0.2218830 & -2.7332540 & 0.0392350 \\ \mathrm{C} & -1.0066720 & -3.3914320 & -0.2391270 \\ \mathrm{C} & 1.4408430 & -3.1431240 & -0.4644060 \\ \mathrm{C} & -0.9596770 & -4.5148480 & -1.0270030 \\ \mathrm{C} & 1.4580820 & -4.2517560 & -1.3759170 \\ \mathrm{C} & 0.2481930 & -4.9604200 & -1.6222350 \\ \mathrm{H} & -1.8783920 & -5.0573050 & -1.2384500 \\ \mathrm{C} & 1.4088250 & -6.4616250 & -3.1360560 \\ \mathrm{C} & 2.6026280 & -5.7291570 & -2.9311750 \\ \mathrm{C} & 2.6278400 & -4.6554480 & -2.0768090 \\ \mathrm{C} & 0.2575020 & -6.0781480 & -2.4993260\end{array}$




\begin{tabular}{|c|c|c|c|}
\hline $\begin{array}{l}\mathrm{H} \\
\mathrm{H} \\
\mathrm{H} \\
\mathrm{H} \\
\mathrm{C} \\
\mathrm{C} \\
\mathrm{C} \\
\mathrm{C} \\
\mathrm{H} \\
\mathrm{H} \\
\mathrm{H} \\
\mathrm{H} \\
\mathrm{C} \\
\mathrm{C} \\
\mathrm{C} \\
\mathrm{C} \\
\mathrm{C} \\
\mathrm{C} \\
\mathrm{H} \\
\mathrm{H} \\
\mathrm{C} \\
\mathrm{C} \\
\mathrm{C} \\
\mathrm{C} \\
\mathrm{C} \\
\mathrm{C} \\
\mathrm{H} \\
\mathrm{H} \\
\mathrm{P} \\
\mathrm{O} \\
\mathrm{O} \\
\mathrm{C} \\
\mathrm{H} \\
\mathrm{C} \\
\mathrm{H} \\
\mathrm{C} \\
\mathrm{H} \\
\mathrm{C} \\
\mathrm{H} \\
\mathrm{C} \\
\mathrm{H} \\
\mathrm{C} \\
\mathrm{H} \\
\mathrm{C} \\
\mathrm{H} \\
\mathrm{H}\end{array}$ & $\begin{array}{r}1.4061820 \\
3.5056330 \\
3.5468550 \\
-0.6737800 \\
5.0330780 \\
6.1997490 \\
6.1805360 \\
3.8878900 \\
5.0431860 \\
7.0999920 \\
7.0601670 \\
2.9973080 \\
3.7141180 \\
3.8376610 \\
3.5113720 \\
3.8282580 \\
3.4883240 \\
3.6638860 \\
3.9438210 \\
3.3456180 \\
-2.2991350 \\
-3.2633480 \\
-2.5660090 \\
-4.4891680 \\
-3.7970740 \\
-4.7778330 \\
-5.2474220 \\
-4.0033030 \\
0.3410510 \\
0.7401020 \\
-0.8095830 \\
3.3659590 \\
3.3882510 \\
3.7167690 \\
3.1318000 \\
3.9880430 \\
3.7828450 \\
-1.5821800 \\
-0.6594950 \\
-6.1226280 \\
-6.6925530 \\
-3.0521140 \\
-2.0240290 \\
-3.2284540 \\
-3.1227410 \\
-4.2182010\end{array}$ & $\begin{array}{r}-7.3130600 \\
-6.0159180 \\
-4.0961390 \\
-6.6132240 \\
-4.9729480 \\
-4.1945670 \\
-2.8550500 \\
-4.4061340 \\
-6.0272240 \\
-4.6581540 \\
-2.2384170 \\
-5.0110550 \\
1.2862970 \\
2.0621290 \\
1.9197240 \\
3.4548750 \\
3.3144230 \\
4.1021840 \\
4.0480570 \\
3.8095760 \\
-2.8517060 \\
-2.3603340 \\
-2.8540420 \\
-1.9158770 \\
-2.3836130 \\
-1.9148460 \\
-1.5600380 \\
-2.3994010 \\
-0.1589630 \\
0.7119690 \\
0.2456990 \\
1.1526150 \\
0.0814190 \\
5.6139950 \\
5.8862840 \\
1.4515990 \\
0.3799920 \\
-3.4041870 \\
-3.6829600 \\
-1.4400190 \\
-1.0689880 \\
-2.3057280 \\
-2.6066790 \\
-0.8879670 \\
-0.9022000 \\
-0.4768650\end{array}$ & $\begin{array}{r}-3.8089990 \\
-3.4608740 \\
-1.9404950 \\
-2.6646860 \\
1.2736400 \\
1.4652240 \\
1.1754700 \\
0.7729190 \\
1.5316870 \\
1.8556310 \\
1.3400070 \\
0.6406020 \\
-0.0471160 \\
1.1265250 \\
-1.2902290 \\
1.0183480 \\
-1.3397210 \\
-0.2035050 \\
1.9217050 \\
-2.2998880 \\
0.2806010 \\
-0.6218460 \\
1.6666170 \\
-0.1161230 \\
2.1197940 \\
1.2447560 \\
-0.8122660 \\
3.1885970 \\
0.3092880 \\
1.4638560 \\
-0.5751270 \\
-2.5965490 \\
-2.3790690 \\
-0.3290910 \\
-1.2187460 \\
2.5151350 \\
2.4430650 \\
2.6890820 \\
2.1731830 \\
1.7623470 \\
0.8991220 \\
-2.1298560 \\
-2.3475040 \\
-2.6885210 \\
-3.7785800 \\
-2.4552010\end{array}$ \\
\hline
\end{tabular}

-2.4687360
-3.9911370
-5.0389990
-3.7969030
-3.8630590
2.0298630
1.9493580
1.9439140
1.1868420
4.5439660
4.4627490
4.5627510
5.4979780
-1.2204380
-2.0959230
-0.4663870
-0.8173890
-2.1397470
-1.4130360
-3.0605960
-2.3680180
2.9859170
3.0985360
3.1525780
1.9589060
5.4185830
5.6521200
5.5307350
6.1602850
-6.9164520
-7.0576810
-6.3878790
-7.9010650
-5.9722510
-5.3819910
-6.9547460
-5.4707650
5.1616810
5.5827390
5.2076510
5.7890790
3.1147590
2.1361410
3.7619310
2.9982380
-0.8411290
$-0.2177860$
$-3.2881110$
$-3.0060320$
$-3.2895040$
$-4.3089980$
1.4589140
2.5222470
0.8891700
1.1864830
1.4504950
0.8553700
2. 5076790
1.2140030
$-2.3579410$
$-2.0799560$
$-2.7601300$
$-1.4545050$
$-4.6759700$
$-5.0947660$
$-4.4612120$
$-5.4365280$
2.0343610
1.5298320
3.1037060
1.8890580
1.6360390
2. 7015710
1.1648600
1.2002370
$-2.5980930$
$-3.4075950$
$-3.0071110$
$-2.2558660$
$-0.2931330$
0.5303590
0.0957590
$-0.6400780$
6.0732990
5.6023020
7.1600770
5.8004360
6.3386820
.9242310
6.2498130
7.4050450
3.2358550

-2.2797000
-2.8424980
-2.6898000
-3.9199620
-2.4684400
-3.2840740
-3.5374380
-4.2153250
-2.6415430
-3.5337410
-4.4491130
-3.8202230
-3.0521870
3.7489310
4.3461350
4.4335420
3.2831050
3.3417810
4.0456630
3.8952900
2.5883280
3.5197860
4.4852130
3.6868790
3.1778620
3.0396940
3.1474340
4.0216960
2.3637320
2.3791230
1.6569850
3.2473610
2.7139990
2.7688440
2.3547090
3.0562640
3.6789160
-0.5677490
-1.4610610
-0.6936170
0.2881960
0.8766220
1.1403930
1.7557670
0.6611110
0.9070860 


$\begin{array}{lrrr}\mathrm{C} & -0.2551810 & 3.3252610 & -0.4344410 \\ \mathrm{H} & -1.2710340 & 2.2731320 & 1.1788950 \\ \mathrm{H} & 0.1297280 & 2.4123100 & -0.8746500 \\ \mathrm{O} & 0.5728340 & 3.2832050 & 1.2226900 \\ \mathrm{H} & 0.8513040 & 2.3149230 & 1.3271580 \\ \mathrm{C} & -0.9666530 & 5.7526640 & 0.9975650 \\ \mathrm{C} & -0.7731200 & 5.8012890 & -0.5199700 \\ \mathrm{C} & 0.1109750 & 4.6542650 & -1.0194940 \\ \mathrm{C} & -1.5999030 & 4.4298880 & 1.4328510 \\ \mathrm{H} & -1.6023650 & 6.5821540 & 1.3199570 \\ \mathrm{H} & -0.3202460 & 6.7517070 & -0.8176990 \\ \mathrm{H} & 1.1585570 & 4.8181140 & -0.7381740 \\ \mathrm{H} & -2.6259200 & 4.3554170 & 1.0491660 \\ \mathrm{H} & -1.6538670 & 4.3538280 & 2.5229900 \\ \mathrm{H} & -0.0024410 & 5.8737780 & 1.5036600 \\ \mathrm{H} & -1.7522700 & 5.7402320 & -1.0100380 \\ \mathrm{H} & 0.1005600 & 4.5722390 & -2.1104010 \\ \mathrm{C} & -3.4256170 & 2.1558530 & -0.9334860 \\ \mathrm{O} & -3.0835290 & 1.1816080 & -0.1522670 \\ \mathrm{C} & -4.8679660 & 2.4824760 & -0.8719680 \\ \mathrm{C} & -5.3619640 & 3.7091970 & -1.3312600 \\ \mathrm{C} & -5.7488800 & 1.5382720 & -0.3274480 \\ \mathrm{C} & -6.7209350 & 3.9856010 & -1.2437760 \\ \mathrm{H} & -4.6745030 & 4.4406320 & -1.7428760 \\ \mathrm{C} & -7.1075250 & 1.8171210 & -0.2469720 \\ \mathrm{H} & -5.3520980 & 0.5896720 & 0.0177720 \\ \mathrm{C} & -7.5944500 & 3.0415810 & -0.7035520 \\ \mathrm{H} & -7.1000920 & 4.9401740 & -1.5932360 \\ \mathrm{H} & -7.7876200 & 1.0814570 & 0.1720070 \\ \mathrm{H} & -8.6553300 & 3.2616240 & -0.6375600 \\ \mathrm{H} & -2.1162670 & 0.8226860 & -0.3061840 \\ \mathrm{~S} & -2.3560990 & 2.9847440 & -1.9317190 \\ 144 & & & \end{array}$

TS240(S, S) G[M06-2X/6-31G(d) $]=-3633.302642$

$\begin{array}{lrcr}\text { TS240(S, S }) & \text { G }[\mathrm{M} 06-2 \mathrm{X} / 6-31 \mathrm{G}(\mathrm{d})]=-3630.302642 \\ \mathrm{O} & 0.5636150 & 0.2508450 & -0.3570750 \\ \mathrm{O} & -1.6420380 & 0.2740820 & -1.6072210 \\ \mathrm{C} & 0.9085480 & 1.4088690 & -1.0260170 \\ \mathrm{C} & -0.0049270 & 2.4404790 & -1.1066610 \\ \mathrm{C} & 2.2375740 & 1.5107970 & -1.5147750 \\ \mathrm{C} & 0.3479930 & 3.6141080 & -1.8487260 \\ \mathrm{C} & 2.5900470 & 2.6709540 & -2.1611080 \\ \mathrm{C} & 1.6649150 & 3.7252810 & -2.3738960 \\ \mathrm{H} & 3.6095800 & 2.7891240 & -2.5211170 \\ \mathrm{C} & -2.1136120 & 1.2149170 & -0.6976500 \\ \mathrm{C} & -3.3844470 & 0.9910990 & -0.1029290 \\ \mathrm{C} & -1.3190030 & 2.3054650 & -0.4251790\end{array}$

-3.8367970
-1.7562420
-3.0407640
-4.8160920
-2.6858860
-1.3926460
-0.9391890
-3.4884750
-3.0319770
-0.7525560
0.0585150
-4.4755900
-0.1938720
1.1263110
2.0324020
-0.5740740
-0.9134160
1.4126910
3.0453400
-1.5916690
3.2721670
3.5876060
4.0244440
4.7135390
5.1457140
5.5263060
4.9729670
5.7609230
-4.1703430
-4.4150000
-4.6820240
-5.1935260
-5.4499400
-5.7222730
-5.3896480
-5.8566510
3.6815940
6.7845630
2.7758740
-3.8744210
-6.5673480
-4.4737260
-5.8038880
-6.5094420
-6.2677020
-5.6447560

3.2361000

3.0551590

1.8254660

4.9923300

5.1517320

4. 3002980

3.9655490

5.6770800

5.9542900

4.4256400

3.8181180

5.7736460

. 8948890

4.8894300

4.6658250

6.5632250

6.7795090

4.9608760

4.5789300

0.4559050

$-0.4837740$

0.5118940

$-1.2981350$

$-0.3082180$

$-2.0011200$

$-0.2444950$

$-0.2466570$

$-1.1762260$

$-0.4796200$

$-2.2994350$

$-1.6248140$

$-2.5439660$

$-3.0102800$

$-1.8021510$

1.5017090

$-2.0340610$

$-0.5766200$

$-1.0046990$

$-3.7743600$

0.4895130

1.1337630

0.3780770

1.6514350

1.8598300
0.7768150

0.5737780

1.1588240

1.2330750

2. 5780260

2.0241810

1.0468480

2.1535140

3.3454400

2. 3778410

0.6392010

2.5832860

$-2.8193980$

$-3.3163770$

$-3.0998620$

$-2.1043220$

$-3.0115590$

$-3.8759610$

$-3.4868100$

$-1.7379970$

$-1.2665940$

$-2.2619850$

$-0.0736880$

$-2.0884560$

0.0459580

$-0.9609800$

$-2.8746910$

0.9421720

$-0.3908440$

0.6459820

$-1.6807830$

0.3684850

$-1.9073090$

$-0.8991140$

1.1690460

$-2.9013430$

1.0325510

$-0.7772450$

$-3.5454990$

2.0596530

$-1.1687240$

$-2.8350000$

$-3.2465920$

$-3.6090030$

$-2.4009520$

$-4.0506400$ 


-3.8013100
-2.8561670
-4.4466840
-3.5966680
-7.8512320
-8.4750200
-7.6166630
-8.4346860
-5.7734340
-4.8635890
-5.4798570
-6.3790990
-4.9995610
-5.5091420
-5.7511940
-4.6005770
-3.1568060
-3.8595010
-2.3981130
-2.6657110
4.5266530
5.5910250
4.3784710
4.2609780
3.8399820
3.2628990
4.8877270
3.4750720
7.3875210
8.3565030
6.7443390
7.5351420
6.5290350
6.1328230
5.8056500
7.4562390
2.5946210
3.5251370
2.2654390
1.8433630
3.4181210
4.4341580
2.8360940
3.4779050
-0.5720430
-0.0108090

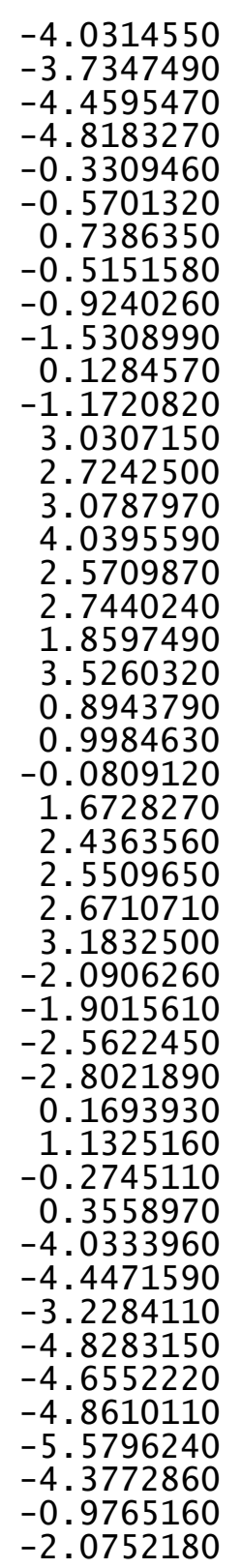

$\begin{array}{rr}-0.1926980 & -4.0314550 \\ -0.6558120 & -3.7347490 \\ -0.9674680 & -4.4595470 \\ 0.5407690 & -4.8183270 \\ -3.7623250 & -0.3309460 \\ -4.6297400 & -0.5701320 \\ -3.8022000 & 0.7386350 \\ -2.8553320 & -0.5151580 \\ -5.0625920 & -0.9240260 \\ -5.0864690 & -1.5308990 \\ -5.1437450 & 0.1284570 \\ -5.9402890 & -1.1720820 \\ -0.6204760 & 3.0307150 \\ 0.2980300 & 2.7242500 \\ -1.4169350 & 3.0787970 \\ -0.4709840 & 4.0395590 \\ -2.2607080 & 2.5709870 \\ -3.0832900 & 2.7440240 \\ -2.5962220 & 1.8597490 \\ -2.0421640 & 3.5260320 \\ 2.7750460 & 0.8943790 \\ 2.5353640 & 0.9984630 \\ 3.2483800 & -0.0809120 \\ 3.4980800 & 1.6728270 \\ 0.9111980 & 2.4363560 \\ -0.0134490 & 2.5509650 \\ 0.6947880 & 2.6710710 \\ 1.6251720 & 3.1832500 \\ -2.5327280 & -2.0906260 \\ -3.0040730 & -1.9015610 \\ -3.2836330 & -2.5622450 \\ -1.7146610 & -2.8021890 \\ -3.2149740 & 0.1693930 \\ -2.8754770 & 1.1325160 \\ -3.9104940 & -0.2745110 \\ -3.7664620 & 0.3558970 \\ -2.0174100 & -4.0333960 \\ -2.4216330 & -4.4471590 \\ -2.6786940 & -3.2284110 \\ -2.0453140 & -4.8283150 \\ 0.2673350 & -4.6552220 \\ -0.0889850 & -4.8610110 \\ 0.1894850 & -5.5796240 \\ 1.3232220 & -4.3772860 \\ -0.7659790 & -0.9765160 \\ -1.6116450 & -2.0752180\end{array}$

1.1336210
1.7780180
7.5239520
2.6276380
-3.8138550
-6.8535630
-3.1406520
0.2976090
-0.6468760
0.7511870
1.5312000
0.4338810
1.9855600
1.7786400
0.9088610
-0.1659400
1.6823490
2.5831630
0.6717140
2.0501560
-0.8658410
0.9868580
0.8779430
1.8015620
-0.1736540
1.5511380
1.5262410
0.9643700
2.6502620
3.3586270
3.2067950
1.1535520
2.7941320
4.4229510
3.7806410
0.6874770
0.6439260
3.0856050
2.9318730
3.5892180

$-1.1336210$

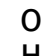
TS241(S, S ) G[M06-2X/6-31G(d)] = -3633.306056

$\begin{array}{lrcr}\text { TS241(S, S) } & \text { G }[\mathrm{M} 06-2 \mathrm{X} / 6-31 \mathrm{G}(\mathrm{d})]=-3633.306056 \\ \mathrm{O} & 0.6878800 & 0.7130670 & 0.2089400 \\ \mathrm{O} & -1.3265290 & 1.3085240 & -1.1919190 \\ \mathrm{C} & 0.9817000 & 2.0639210 & 0.2741170 \\ \mathrm{C} & -0.0171720 & 2.9507290 & 0.6195910\end{array}$

0.2528320

$-1.4431900$

$-0.1765350$

$-1.3816590$

1. 7837150

1.2955120

$-3.7487710$

$-0.1946530$

$-0.4699450$

$-0.1189370$

0.6274560

0.3850850

1.9454160

1.4489640

$-0.6361650$

3. 0067560

2. 1304220

2. 7602160

1.2578040

4. 0246850

3.5892380

$-0.7878360$

$-2.0160010$

$-3.7011670$

$-2.6288390$

$-3.4232980$

$-1.6045940$

$-3.4677180$

$-2.8070290$

$-5.3925370$

4.3133910

5.0916570

$-6.3750070$

$-4.5423750$

$-2.8847660$

$-5.1617970$

$-5.8077590$

$-5.4361820$

$-4.2812590$
$-3.3365490$

$-0.2938880$

0.9215280

$-2.5023040$

$-2.2277610$

2. 0517150

3. 0672520

2. 2687530

3.9542090

5.0903630

3.5942500

5.8595420

5.3602590

4. 3557300

2. 7085100

5.4895620

6.7447420

4.0620090

6.0862390

1. 4818660

3. 1120940

0.3106060

0.7102270

0.2920060

0.4524240

$-0.9670920$

$-1.4802090$

0.9336200

1.7534940

1.0979740

0.8318300

1. 3917420

1. 8620540

0.1622680

1.8226130

0.1799660

$-0.0721630$

2.7648250

1.7238260
$-0.0171720$

0.6195910 


\begin{tabular}{|c|c|c|}
\hline $\begin{array}{l}2.3282650 \\
0.2995730 \\
2.6422460 \\
1.6506920 \\
3.6739170 \\
1.9979070 \\
3.3079910 \\
1.3823940 \\
3.9955590 \\
2.0713660 \\
3.4015990 \\
5.0176110 \\
3.5091860 \\
2.1710870 \\
1.4708570 \\
4.1060110 \\
4.0524170 \\
1.6926440 \\
0.4422250 \\
5.1249540 \\
0.3358650 \\
1.0151150 \\
1.9835010 \\
0.6852910 \\
1.1033570 \\
1.2766210 \\
3.0220790 \\
1.7254000 \\
3.3727680 \\
3.8401960 \\
3.9231170 \\
4.8943210 \\
4.9908500 \\
5.5029760 \\
5.2638940 \\
5.4218460 \\
3.9099060 \\
4.1231060 \\
4.2890620 \\
4.7589320 \\
4.9119530 \\
5.1652710 \\
4.9396650 \\
5.2168160 \\
3.4083040 \\
6.6868580\end{array}$ & $\begin{array}{r}2.4483630 \\
4.3490330 \\
3.7770870 \\
4.7530810 \\
4.0986270 \\
1.6109780 \\
1.0852550 \\
2.4325800 \\
1.4443220 \\
2.7130360 \\
2.2342570 \\
1.1006280 \\
3.2361030 \\
3.6749140 \\
3.4216400 \\
2.5268970 \\
3.4504030 \\
4.2117190 \\
3.7531120 \\
2.1634270 \\
6.6824720 \\
7.0785440 \\
6.1324690 \\
5.3559850 \\
7.4367450 \\
8.1309620 \\
6.4213460 \\
5.0662390 \\
1.4340460 \\
1.3193740 \\
0.6343420 \\
0.4448630 \\
-0.2159340 \\
-0.3129990 \\
0.3474370 \\
-0.8184690 \\
0.1912590 \\
-1.1758700 \\
0.7227350 \\
-1.9650850 \\
-0.1132150 \\
-1.4547080 \\
-3.0150390 \\
0.2964400 \\
0.6688700 \\
-1.1987290\end{array}$ & $\begin{array}{r}0.0426490 \\
0.6568990 \\
0.1851150 \\
0.4655260 \\
0.0682380 \\
-0.0150110 \\
0.1448000 \\
0.9052410 \\
1.2776090 \\
2.1318840 \\
2.2962560 \\
1.4132890 \\
4.5044400 \\
4.3611090 \\
3.2082620 \\
3.4947060 \\
5.4191880 \\
5.1741670 \\
3.1162570 \\
3.5978770 \\
0.8926230 \\
0.7444440 \\
0.5305040 \\
0.8510610 \\
1.0346370 \\
0.7864890 \\
0.3931220 \\
0.9556710 \\
-0.2997180 \\
-1.6269090 \\
0.7159550 \\
-1.8861110 \\
0.4084250 \\
-0.8823200 \\
-2.9050260 \\
1.2041390 \\
-0.8909320 \\
-0.6074220 \\
-2.1365220 \\
-1.5656900 \\
-3.0668110 \\
-2.7992560 \\
-1.3459410 \\
-4.0282300 \\
2.1473780 \\
-1.2422260\end{array}$ \\
\hline
\end{tabular}

3.2559890
-3.6974450
-5.8553490
-4.0786240
-5.4240040
-6.0103590
-6.0157570
-5.2673430
-3.2287020
-2.2669590
-3.7396960
-3.0393060
-7.1825790
-7.6934500
-7.0126240
-7.8475310
-4.9436080
-4.0017420
-4.7068030
-5.4354120
-4.8997480
-5.3960730
-5.6396660
-4.5817690
-2.9996790
-3.6859520
-2.1484170
-2.6376380
4.4923880
5.3441530
4.8622440
4.0931440
2.8600760
2.0495020
3.6436990
2.4580980
7.0732210
7.8722820
7.4458960
6.2304000
7.9002380
7.6678090
8.2129990
8.7429810
3.1192280
4.0965410

2.1517430

$-1.8150820$

$-2.3392760$

2. 1848340

2.9057900

. 4732110

2.8286120

3.9665890

2. 3329380

1.8252200

1.9104990

3. 3915590

$-2.8845490$

$-3.4787580$

$-3.5304650$

$-2.0721780$

$-3.4853770$

$-3.1037060$

$-4.1478360$

$-4.0853370$

$-1.9941920$

$-1.0439960$

$-2.6627260$

$-2.4382060$

$-3.1656180$

$-3.9206420$

$-3.0745550$

$-3.5384850$

1.1517120

0.4630340

2.1407810

1.2151910

$-0.7004560$

$-1.0116180$

$-1.4668430$

$-0.6596450$

$-2.1999280$

$-2.8530280$

$-1.6851910$

$-2.8311140$

$-0.3299890$

0.3640690

0.2589300

$-0.9551790$

1.3712530
1.1570190
$-2.7605670$

0.7089180

$-3.8203300$

$-2.5003290$

$-3.4699630$

$-1.7340810$

$-2.8733710$

$-3.7671170$

$-3.6510690$

$-4.6391400$

$-3.9732140$

$-3.2807910$

$-4.0454010$

$-2.4122030$

$-2.9727700$

$-4.2732930$

$-4.6781380$

$-3.4333330$

$-5.0459150$

1.6464010

1.8622450

1.1910590

2.5963850

0.5094110

0.1117280

$-0.1711860$

1.4749010

3.1179810

3.1336110

2.8300010

4.1353070

2. 5669600

1.8998030

2.5467090

3.5850200

$-0.1540420$

$-0.5165890$

0.7385410

0.1471080

$-1.6054580$

$-2.4181880$

$-0.7361060$

$-1.9174490$

$-4.0722410$

$-4.5180020$ 


$\begin{array}{rr}\mathrm{H} & 2.5895860 \\ \mathrm{H} & 2.5562550 \\ \mathrm{C} & 4.1082930 \\ \mathrm{H} & 5.1294130 \\ \mathrm{H} & 3.6876600 \\ \mathrm{H} & 4.1658610 \\ \mathrm{P} & -0.1714680 \\ \mathrm{O} & 0.6292920 \\ \mathrm{O} & -0.7271060 \\ \mathrm{H} & 2.2510190 \\ \mathrm{H} & 6.4027530 \\ \mathrm{H} & 2.5800050 \\ \mathrm{H} & -3.5385370 \\ \mathrm{H} & -6.0763210 \\ \mathrm{H} & -2.9771000 \\ \mathrm{C} & 1.3805690 \\ \mathrm{C} & 2.1263630 \\ \mathrm{H} & 0.3323950 \\ \mathrm{H} & 1.8202910 \\ \mathrm{O} & 2.2447210 \\ \mathrm{H} & 1.7078830 \\ \mathrm{C} & 3.1414270 \\ \mathrm{C} & 3.5957590 \\ \mathrm{C} & 3.4648350 \\ \mathrm{C} & 1.6707230 \\ \mathrm{H} & 3.2758000 \\ \mathrm{H} & 4.6369160 \\ \mathrm{H} & 4.1934920 \\ \mathrm{H} & 1.0321400 \\ \mathrm{H} & 1.3581460 \\ \mathrm{H} & 3.7600000 \\ \mathrm{H} & 2.9891790 \\ \mathrm{H} & 3.6636580 \\ \mathrm{C} & -0.0017160 \\ \mathrm{O} & -0.4987910 \\ \mathrm{C} & -0.3038880 \\ \mathrm{C} & 0.4130120 \\ \mathrm{C} & -1.3457550 \\ \mathrm{C} & 0.0912620 \\ \mathrm{H} & 1.2235920 \\ \mathrm{C} & -1.6700710 \\ \mathrm{H} & -1.8981210 \\ \mathrm{C} & -0.9513040 \\ \mathrm{H} & 0.6545890 \\ \mathrm{H} & -1.4830420 \\ \mathrm{H} & \\ & \end{array}$

$\begin{array}{rr}0.4273410 & -3.9281720 \\ 1.9692160 & -4.7957540 \\ 3.4056050 & -3.0041660 \\ 3.1193010 & -3.2817930 \\ 4.0007910 & -3.8215810 \\ 4.0397730 & -2.1157640 \\ 0.1773140 & -1.0763550 \\ 0.2196240 & -2.3414630 \\ -1.1454220 & -0.6042000 \\ 2.4658260 & -2.4606560 \\ -1.7662210 & -2.1404960 \\ 1.3816180 & 2.2015570 \\ 2.6742550 & -1.6848330 \\ -1.7161280 & -4.6961360 \\ -1.1467530 & 1.1927390 \\ -2.8599450 & -2.0382040 \\ -2.4957810 & -0.8290790 \\ -2.5663950 & -2.0439540 \\ -1.6046910 & -0.2887780 \\ -1.8108110 & -2.5505520 \\ -0.9512010 & -2.5154300 \\ -4.6031850 & -2.5422760 \\ -4.5296800 & -1.0832840 \\ -3.1032260 & -0.5474190 \\ -4.1995210 & -2.6716390 \\ -5.6157850 & -2.9326510 \\ -4.8513240 & -0.9846540 \\ -2.4466070 & -1.0373030 \\ -4.9369110 & -2.1694300 \\ -4.1586820 & -3.7195780 \\ -3.9348120 & -3.1533400 \\ -5.2122060 & -0.4740740 \\ -3.0345130 & 0.5262720 \\ -2.9429730 & 2.0559970 \\ -1.7885730 & 1.7662350 \\ -3.3829750 & 3.4396670 \\ -4.4145890 & 4.0568650 \\ -2.7495910 & 4.1328240 \\ -4.8070400 & 5.3502400 \\ -4.8961290 & 3.5206090 \\ -3.1534730 & 5.4224190 \\ -1.9507380 & 3.6492670 \\ -4.1803270 & 6.0327390 \\ -5.6018000 & 5.8278830 \\ -2.6673090 & 5.9515580 \\ -4.4925690 & 7.0415040\end{array}$

$\begin{array}{lrrr}\mathrm{H} & -0.5120460 & -1.5385470 & 0.7430230 \\ \mathrm{~S} & 0.8819890 & -3.9003050 & 0.9900380 \\ 144 & & \end{array}$

144

$\operatorname{TS} 242(\mathrm{~S}, \mathrm{~S}) \mathrm{G}[\mathrm{M06}-2 \mathrm{X} / 6-31 \mathrm{G}(\mathrm{d})]=-3633.303137$

$\begin{array}{lrcr}\mathrm{TS} 242(\mathrm{~S}, \mathrm{~S}) \mathrm{G}[\mathrm{M} 06-2 \mathrm{X} / 6-31 \mathrm{G}(\mathrm{d})]=-3633.303137 \\ \mathrm{O} & 0.5466070 & 0.2390740 & -0.3543010 \\ \mathrm{O} & -1.6880970 & 0.3547940 & -1.5464840 \\ \mathrm{C} & 0.8959280 & 1.4171940 & -0.9858610 \\ \mathrm{C} & 0.0017130 & 2.4685520 & -0.9956250 \\ \mathrm{C} & 2.2091210 & 1.5117420 & -1.5165870 \\ \mathrm{C} & 0.3556130 & 3.6651790 & -1.6995300 \\ \mathrm{C} & 2.5656160 & 2.6913100 & -2.1245360 \\ \mathrm{C} & 1.6578270 & 3.7728130 & -2.2607520 \\ \mathrm{H} & 3.5745030 & 2.8031240 & -2.5151600 \\ \mathrm{C} & -2.1189000 & 1.2644630 & -0.5863870 \\ \mathrm{C} & -3.3820040 & 1.0432990 & 0.0253790 \\ \mathrm{C} & -1.2971000 & 2.3277040 & -0.2869710 \\ \mathrm{C} & -3.7963120 & 1.9681620 & 0.9529200 \\ \mathrm{C} & -1.6945590 & 3.2254650 & 0.7576250 \\ \mathrm{C} & -2.9703040 & 3.0469050 & 1.3620110 \\ \mathrm{H} & -4.7693930 & 1.8510490 & 1.4226770 \\ \mathrm{C} & -2.5483910 & 4.9214280 & 2.8454730 \\ \mathrm{C} & -1.2633320 & 5.0766350 & 2.2717390 \\ \mathrm{C} & -0.8469560 & 4.2549610 & 1.2534380 \\ \mathrm{C} & -3.3797330 & 3.9272190 & 2.3993840 \\ \mathrm{H} & -2.8654450 & 5.5831730 & 3.6449360 \\ \mathrm{H} & -0.6003360 & 5.8524660 & 2.6423610 \\ \mathrm{H} & 0.1443430 & 4.3770900 & 0.8291740 \\ \mathrm{H} & -4.3608380 & 3.7827820 & 2.8436800 \\ \mathrm{C} & -0.1713010 & 5.8727220 & -2.5649750 \\ \mathrm{C} & 1.1358380 & 5.9897090 & -3.0963620 \\ \mathrm{C} & 2.0272560 & 4.9589690 & -2.9492150 \\ \mathrm{C} & -0.5523600 & 4.7437480 & -1.8843290 \\ \mathrm{H} & -0.8804500 & 6.6827310 & -2.7032060 \\ \mathrm{H} & 1.4232550 & 6.8911830 & -3.6278140 \\ \mathrm{H} & 3.0293970 & 5.0267480 & -3.3639530 \\ \mathrm{H} & -1.5604470 & 4.6610940 & -1.4918710 \\ \mathrm{C} & 3.2219930 & 0.4185020 & -1.3655460 \\ \mathrm{C} & 3.4812270 & -0.4591220 & -2.4318680 \\ \mathrm{C} & 4.0045660 & 0.3696350 & -0.1915460 \\ \mathrm{C} & 4.5865960 & -1.3125870 & -2.3423270 \\ \mathrm{C} & 5.1005470 & -0.4927960 & -0.1533110 \\ \mathrm{C} & 5.4269810 & -1.3200910 & -1.2317000 \\ \mathrm{H} & 4.8153370 & -1.9750060 & -3.1743290 \\ \mathrm{H} & 5.7253920 & -0.5137830 & 0.7369160 \\ \mathrm{C} & -4.2034810 & -0.1611730 & -0.3013820 \\ \mathrm{C} & -4.4394080 & -1.1378130 & 0.6928940\end{array}$




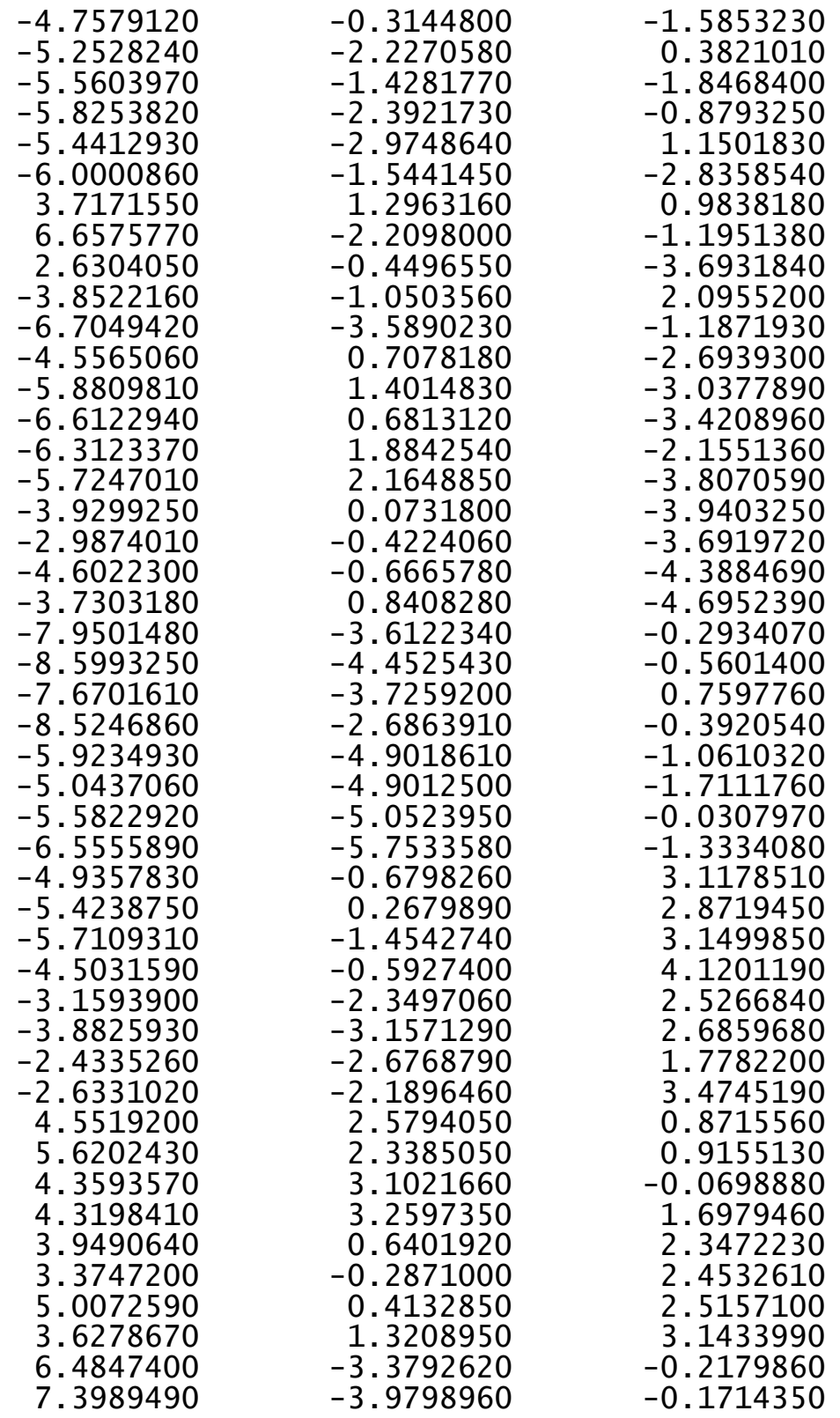

6.2691150

5.6645280

7.9220310

8.0486910

7.8810070

8.8066910

2. 3873240

3.2940180

2. 0496910

1.6197720

3.2680910

4.2646630

2.6563010

3.3723160

$-0.6192520$

$-0.0968360$

$-1.1638520$

1.6528570

6.7832080

2.6578390

$-3.8695980$

$-7.0371260$

$-3.0948090$

0.3771850

$-0.5962590$

0.9044340

1.6897350

0.6521930

2.2129410

1.8858160

1.1979680

0.0479960

1.9754760

2. 8132390

1.0114040

2. 3975250

$-0.8560630$

1.0284800

0.8162800

1.7740830

$-0.2284120$

1. 5459390

1.4420020

0.8830620

2.5568730

3.3063140
-3.0122410
-4.0364180
-1.4111840
-0.5660200
-1.0184010
-2.0513880
-1.8541660
-2.2676410
-2.5459420
-1.8119900
0.4345790
0.0578270
0.4282490
1.4705570
-0.7303160
-1.5290730
-1.4582880
-0.0310080
-2.6301210
1.5783730
1.4808290
-3.4914400
-0.2621470
-0.6393520
-0.2422730
0.4211750
0.1262790
1.7570870
1.1574950
-0.9093770
2.7847650
1.9803610
2.4864980
0.9268470
3.8165810
3.2891270
-0.8720210
-2.2024090
-3.7460780
-2.7175350
-3.4436430
-1.6757190
-3.4634290
-2.7655280
-5.5104980
-4.4904080

0.7932060

$-0.5262080$

$-0.8591130$

$-1.5419850$

0.1624970

$-0.9329900$

$-4.2542990$

$-4.7094870$

$-3.4790910$

$-5.0329390$

$-4.7736650$

$-5.0314230$

$-5.6819380$

$-4.4398030$

$-0.9934830$

$-2.1450130$

0.2145170

$-3.4315430$

$-2.2007390$

0.9415830

$-2.3381110$

$-2.2285030$

2.1018250

3.0126230

2. 2732460

3.9029120

5.0225410

3.5588960

5.7926560

5.2796190

4.3192400

2.6848930

5.4378650

6.6665490

4.0376060

6. 0346910

1.4649020

2.9805580

0.1017380

0.5358670

0.1211330

0.3316070

$-1.1771750$

$-1.6412460$

0.6072960

1.4651270 


\begin{tabular}{|c|c|c|c|c|c|c|c|}
\hline C & 3.1777970 & -3.0856820 & 0.8777910 & $c$ & 3.9880490 & 0.3758930 & -0.3687450 \\
\hline $\mathrm{C}$ & 1.0669720 & -5.1673140 & 0.5476990 & $\mathrm{C}$ & 4.4941700 & -1.4870790 & -2.3857630 \\
\hline $\mathrm{H}$ & 2.6827250 & -6.5175650 & 1.0144570 & $\mathrm{C}$ & 5.0920930 & -0.4740280 & -0.3015470 \\
\hline $\mathrm{H}$ & 4.3666540 & -4.7488050 & 1.5435880 & $\mathrm{C}$ & 5.3799590 & -1.3924530 & -1.3155080 \\
\hline $\mathrm{H}$ & 3.7310990 & -3.0016360 & -0.0677420 & $\mathrm{H}$ & 4.6937670 & -2.2186480 & -3.1657550 \\
\hline $\mathrm{H}$ & 0.6166440 & -5.2753540 & 1.5423290 & $\mathrm{H}$ & 5.7545710 & -0.4125420 & 0.5590790 \\
\hline $\mathrm{H}$ & 0.5279110 & -5.8372830 & -0.1290420 & $\mathrm{C}$ & -4.1971830 & -0.2446700 & -0.0521450 \\
\hline $\mathrm{H}$ & 2.9738410 & -5.5160520 & -0.4072310 & $\mathrm{C}$ & -4.3828780 & -1.1350620 & 1.0260580 \\
\hline H & 2.8979620 & -4.4963000 & 2.4845260 & $\mathrm{C}$ & -4.7936400 & -0.5140840 & -1.3013520 \\
\hline $\mathrm{H}$ & 3.5980740 & -2.3159460 & 1.5305300 & $\mathrm{C}$ & -5.1897770 & -2.2598930 & 0.8344650 \\
\hline & & & & $\mathrm{C}$ & -5.5844090 & -1.6538470 & -1.4411100 \\
\hline & S) $G[M 06-2 x$ & $G(d)]=-36$ & 568 & $\mathrm{C}$ & -5.8004870 & -2.5384030 & -0.3845950 \\
\hline 0 & 0.5394880 & 0.2035600 & -0.3498400 & $\mathrm{H}$ & -5.3446420 & -2.9465940 & 1.6644460 \\
\hline 0 & -1.7483700 & 0.1839670 & -1.4408930 & $\mathrm{H}$ & -6.0530130 & -1.8525240 & -2.4035170 \\
\hline C & 0.8390940 & 1.3223920 & -1.1032750 & $\mathrm{C}$ & 3.7425840 & 1.4037550 & 0.7295550 \\
\hline C & -0.0704620 & 2.3585990 & -1.1634690 & $\mathrm{c}$ & 6.6205440 & -2.2668090 & -1.2531420 \\
\hline $\mathrm{C}$ & 2.1221820 & 1.3812610 & -1.7075870 & $\mathrm{C}$ & 2.4750040 & -0.7588330 & -3.7224430 \\
\hline $\mathrm{C}$ & 0.2294100 & 3.4891390 & -1.9907980 & $\mathrm{C}$ & -3.7514450 & -0.9227130 & 2.3956300 \\
\hline $\mathrm{C}$ & 2.4289840 & 2.5023540 & -2.4403800 & $\mathrm{C}$ & -6.6723280 & -3.7670740 & -0.5608110 \\
\hline $\mathrm{C}$ & 1.4991600 & 3.5572650 & -2.6276580 & $\mathrm{C}$ & -4.6499000 & 0.4159340 & -2.4975230 \\
\hline $\mathrm{H}$ & 3.4146070 & 2.5877310 & -2.8921670 & $\mathrm{C}$ & -5.9978040 & 1.0621490 & -2.8432500 \\
\hline C & -2.1497510 & 1.1721250 & -0.5481140 & $\mathrm{H}$ & -6.7322150 & 0.3033520 & -3.1353230 \\
\hline $\mathrm{C}$ & -3.3804300 & 0.9915840 & 0.1385690 & $\mathrm{H}$ & -6.4016160 & 1.6108510 & -1.9864780 \\
\hline $\mathrm{C}$ & -1.3318520 & 2.2668820 & -0.3828120 & $\mathrm{H}$ & -5.8843490 & 1.7610320 & -3.6785140 \\
\hline C & -3.7637640 & 1.9875500 & 1.0038500 & $\mathrm{C}$ & -4.0624290 & -0.3104400 & -3.7126490 \\
\hline $\mathrm{C}$ & -1.6943410 & 3.2481120 & 0.5969010 & $\mathrm{H}$ & -3.1014270 & -0.7695650 & -3.4649470 \\
\hline C & -2.9354900 & 3.1067700 & 1.2779720 & $\mathrm{H}$ & -4.7383930 & -1.0948270 & -4.0703060 \\
\hline $\mathrm{H}$ & -4.7116150 & 1.9009930 & 1.5287480 & $\mathrm{H}$ & -3.9080520 & 0.3951380 & -4.5356830 \\
\hline C & -2.4734180 & 5.1103190 & 2.5685880 & C & -6.0889360 & -4.7185610 & -1.6116940 \\
\hline C & -1.2216430 & 5.2318740 & 1.9182720 & $\mathrm{H}$ & -6.7013860 & -5.6223630 & -1.6942780 \\
\hline C & -0.84 & 4.3287040 & 0.9562250 & $\mathrm{H}$ & -6.0624320 & -4.2394960 & -2.5967150 \\
\hline C & -3.3081030 & 4.0695270 & 2.2540930 & $\mathrm{H}$ & -5.0679570 & -5.0142180 & -1.3530150 \\
\hline $\mathrm{H}$ & -2.7622040 & 5.8356710 & 3.3222870 & C & -8.1125810 & -3.3828100 & -0.9187330 \\
\hline $\mathrm{H}$ & -0.5553880 & 6.0468020 & 2.1844860 & $\mathrm{H}$ & -8.5436290 & -2.7185920 & -0.1637710 \\
\hline $\mathrm{H}$ & 0.12 & 4.4257820 & 0.4728180 & $\mathrm{H}$ & -8.1478170 & -2.8655830 & -1.8839870 \\
\hline $\mathrm{H}$ & -4.2634260 & 3.9521250 & 2.7584270 & $\mathrm{H}$ & -8.7415200 & -4.2758320 & -0.9938310 \\
\hline C & -0.3740450 & 5.6022840 & -3.0251820 & C & -4.8079570 & -0.4866340 & 3.4203790 \\
\hline $\mathrm{C}$ & 0.9013310 & 5.6818340 & -3.6348770 & $\mathrm{H}$ & -5.3249990 & 0.4269230 & 3.1116840 \\
\hline $\mathrm{C}$ & 1.8143290 & 4.6779820 & -3.4414600 & $\mathrm{H}$ & -5.5642180 & -1.2700830 & 3.5460680 \\
\hline C & -0.7025500 & 4.5372700 & -2.2246970 & $\mathrm{H}$ & -4.3439520 & -0.3053270 & 4.3956180 \\
\hline $\mathrm{H}$ & -1.1008640 & 6.3895640 & -3.1984960 & C & -3.0185500 & -2.1694810 & 2.9078000 \\
\hline H & 1.1470640 & 6.5328730 & -4.2618000 & $\mathrm{H}$ & -3.7182350 & -2.9775270 & 3.1479840 \\
\hline H & 2.7921190 & 4.7168730 & -3.9140780 & $\mathrm{H}$ & -2.3030330 & -2.5372620 & 2.1682090 \\
\hline $\mathrm{H}$ & -1.6870220 & 4.4817050 & -1.7720490 & $\mathrm{H}$ & -2.4730120 & -1.9242150 & 3.8260920 \\
\hline C & 3.1556130 & 0.3157790 & -1.5076430 & C & 4.5514460 & 2.6799160 & 0.4588860 \\
\hline $\mathrm{C}$ & 3.3782190 & -0.6506360 & -2.5028770 & $\mathrm{H}$ & 5.6242620 & 2.4559720 & 0.4741740 \\
\hline
\end{tabular}




$\begin{array}{rrrr}\mathrm{H} & 4.3073560 & 3.1102110 & -0.5167880 \\ \mathrm{H} & 4.3473210 & 3.4318820 & 1.2286480 \\ \mathrm{C} & 4.0486370 & 0.8801600 & 2.1348120 \\ \mathrm{H} & 3.4929930 & -0.0385170 & 2.3544990 \\ \mathrm{H} & 5.1167860 & 0.6803460 & 2.2717760 \\ \mathrm{H} & 3.7561800 & 1.6292290 & 2.8789910 \\ \mathrm{C} & 6.5104960 & -3.3356640 & -0.1587670 \\ \mathrm{H} & 7.4338190 & -3.9209770 & -0.0989780 \\ \mathrm{H} & 6.3392890 & -2.8732180 & 0.8213120 \\ \mathrm{H} & 5.6851920 & -4.0269500 & -0.3600820 \\ \mathrm{C} & 7.8900410 & -1.4294860 & -1.0602530 \\ \mathrm{H} & 7.9736490 & -0.6556710 & -1.8289560 \\ \mathrm{H} & 7.8926190 & -0.9370110 & -0.0818680 \\ \mathrm{H} & 8.7779050 & -2.0673550 & -1.1131900 \\ \mathrm{C} & 2.2096880 & -2.2100930 & -4.1355660 \\ \mathrm{H} & 3.0965860 & -2.6680500 & -4.5871460 \\ \mathrm{H} & 1.9058310 & -2.8222350 & -3.2833470 \\ \mathrm{H} & 1.4088680 & -2.2407480 & -4.8805970 \\ \mathrm{C} & 3.0662210 & 0.0168400 & -4.9076340 \\ \mathrm{H} & 4.0516200 & -0.3854060 & -5.1697410 \\ \mathrm{H} & 2.4172130 & -0.0734410 & -5.7850820 \\ \mathrm{H} & 3.1834300 & 1.0794330 & -4.6776320 \\ \mathrm{P} & -0.6402660 & -0.8336590 & -0.8389080 \\ \mathrm{O} & -0.1599050 & -1.7331020 & -1.9327730 \\ \mathrm{O} & -1.1191490 & -1.4495410 & 0.4562000 \\ \mathrm{H} & 1.5094200 & -0.3127390 & -3.4618390 \\ \mathrm{H} & 6.7018400 & -2.7830800 & -2.2177760 \\ \mathrm{H} & 2.6783640 & 1.6695920 & 0.7129740 \\ \mathrm{H} & -3.9623730 & 1.2244620 & -2.2342550 \\ \mathrm{H} & -6.6915640 & -4.2949590 & 0.4010250 \\ \mathrm{H} & -3.0092470 & -0.1246190 & 2.3108480 \\ \mathrm{C} & 0.5287550 & -0.3568140 & 3.0914710 \\ \mathrm{O} & -0.4806190 & -0.0368800 & 2.3627900 \\ \mathrm{C} & 1.0833550 & 0.7857060 & 3.8546510 \\ \mathrm{C} & 1.9252390 & 0.6014910 & 4.9567810 \\ \mathrm{C} & 0.7958920 & 2.0823580 & 3.4042250 \\ \mathrm{C} & 2.4698280 & 1.7033800 & 5.6043230 \\ \mathrm{H} & 2.1476890 & -0.4045280 & 5.2967110 \\ \mathrm{C} & 1.3622750 & 3.1800960 & 4.0416910 \\ \mathrm{H} & 0.1472000 & 2.2197910 & 2.5443750 \\ \mathrm{C} & 2.1966120 & 2.9922800 & 5.1432450 \\ \mathrm{H} & 3.1143020 & 1.5588580 & 6.4651410 \\ \mathrm{H} & 1.1474880 & 4.1802520 & 3.6779180 \\ \mathrm{H} & 2.6351700 & 3.8499140 & 5.6439250 \\ \mathrm{H} & -0.7678540 & -0.7422270 & 1.6305380 \\ \mathrm{~S} & 1.1937960 & -1.9122220 & 3.1773840\end{array}$

$\begin{array}{lr}\mathrm{C} & 0.8759600 \\ \mathrm{C} & 1.8388350 \\ \mathrm{H} & -0.1705830 \\ \mathrm{H} & 1.5882820 \\ \mathrm{O} & 1.4414560 \\ \mathrm{H} & 0.8539990 \\ \mathrm{C} & 2.6592350 \\ \mathrm{C} & 3.4339350 \\ \mathrm{C} & 3.2608600 \\ \mathrm{C} & 1.1638630 \\ \mathrm{H} & 2.8166790 \\ \mathrm{H} & 4.4999740 \\ \mathrm{H} & 3.7712340 \\ \mathrm{H} & 0.7602970 \\ \mathrm{H} & 0.6032880 \\ \mathrm{H} & 3.0292150 \\ \mathrm{H} & 3.0728310 \\ \mathrm{H} & 3.6984230 \\ \mathrm{H} & \end{array}$

$$
\begin{aligned}
& -3.7229930 \\
& -2.6501560 \\
& -3.4285260 \\
& -1.6342040 \\
& -3.5568450 \\
& -2.9103920 \\
& -5.4177230 \\
& -4.3162560 \\
& -2.9722590 \\
& -5.0944370 \\
& -6.3816120 \\
& -4.5572390 \\
& -2.9680290 \\
& -5.1137530 \\
& -5.8287140 \\
& -5.5134340 \\
& -4.2317610 \\
& -2.1426410
\end{aligned}
$$

$$
\begin{array}{r}
0.4673790 \\
0.7606390 \\
0.5050950 \\
0.4705490 \\
-0.8589250 \\
-1.3607390 \\
1.0525550 \\
1.7770600 \\
1.0710560 \\
1.0312180 \\
1.5444130 \\
1.8292880 \\
0.0980410 \\
2.0512960 \\
0.4446680 \\
0.0243890 \\
2.8106900 \\
1.6327410
\end{array}
$$

$\begin{array}{lrcr}\text { TS244(S, S }) \text { G }[\mathrm{M} 06-2 \mathrm{~N} / 6-31 \mathrm{G}(\mathrm{d})]=-3633.302634 \\ \mathrm{O} & 0.5841810 & 0.2520720 & -0.3421680 \\ \mathrm{O} & -1.6011720 & 0.2591540 & -1.6251950 \\ \mathrm{C} & 0.9394430 & 1.3963690 & -1.0296870 \\ \mathrm{C} & 0.0320780 & 2.4306110 & -1.1342070 \\ \mathrm{C} & 2.2727070 & 1.4831650 & -1.5100540 \\ \mathrm{C} & 0.4003710 & 3.5937710 & -1.8854100 \\ \mathrm{C} & 2.6410470 & 2.6345060 & -2.1625380 \\ \mathrm{C} & 1.7244760 & 3.6924850 & -2.3952290 \\ \mathrm{H} & 3.6649690 & 2.7423730 & -2.5131230 \\ \mathrm{C} & -2.0844280 & 1.2154330 & -0.7379100 \\ \mathrm{C} & -3.3645430 & 1.0027810 & -0.1588540 \\ \mathrm{C} & -1.2909510 & 2.3076350 & -0.4682430 \\ \mathrm{C} & -3.8246400 & 1.9661960 & 0.7056940 \\ \mathrm{C} & -1.7375770 & 3.2495310 & 0.5157560 \\ \mathrm{C} & -3.0292310 & 3.0775420 & 1.0876890 \\ \mathrm{H} & -4.8102220 & 1.8553710 & 1.1503310 \\ \mathrm{C} & -2.6834650 & 5.0246640 & 2.4954850 \\ \mathrm{C} & -1.3839540 & 5.1759790 & 1.9541200 \\ \mathrm{C} & -0.9225110 & 4.3150850 & 0.9888400 \\ \mathrm{C} & -3.4848130 & 3.9971180 & 2.0702540 \\ \mathrm{H} & -3.0356180 & 5.7164340 & 3.2538050 \\ \mathrm{H} & -0.7453380 & 5.9796180 & 2.3080170 \\ \mathrm{H} & 0.0799360 & 4.4338610 & 0.5912100 \\ \mathrm{H} & -4.4770180 & 3.8562160 & 2.4903790 \\ \mathrm{C} & -0.1178910 & 5.7462560 & -2.8842050 \\ \mathrm{C} & 1.2089680 & 5.8554700 & -3.3657750\end{array}$




2.1069520
-0.5128110
-0.8307050
1.5070680
3.1248370
-1.5351760
3.2875100
3.5843210
4.0216740
4.6679740
5.1082200
5.4598550
4.9143040
5.7054980
-4.1516050
-4.4219880
-4.6387540
-5.1998820
-5.4065670
-5.7032850
-5.4157150
-5.7940930
3.7021090
6.6854520
2.7931680
-3.9107300
-6.5478610
-4.4059500
-5.7264530
-6.4274810
-6.2042110
-5.5502990
-3.7139870
-2.7754120
-4.3535590
-3.4935560
-7.8488820
-8.4710430
-7.6369110
-8.4246470
-5.7647370
-4.8426930
-5.4932770
-6.3689530
-5.0552290
-5.5567280

4.8474520

4.6475360

6.5376900

6.7329060

4.9098190

4.5696360

0.4161050

$-0.5559530$

0.4721520

$-1.4100560$

$-0.3919340$

$-1.3250590$

$-2.1478970$

$-0.3349310$

$-0.2338930$

$-1.1512380$

$-0.4781820$

$-2.2739680$

$-1.6226950$

$-2.5301130$

$-2.9751670$

$-1.8086640$

1. 4989340

$-2.2055590$

$-0.6387680$

$-0.9672230$

$-3.7598690$

0.4788170

1.1228640

0.3658280

1.6498030

1.8408640

$-0.2168120$

$-0.6790160$

$-0.9942450$

0.5083770

$-3.7335940$

$-4.6012190$

$-3.7624580$

$-2.8264820$

$-5.0486340$

$-5.0828490$

$-5.1197140$

$-5.9264510$

$-0.5713030$

0.3447970

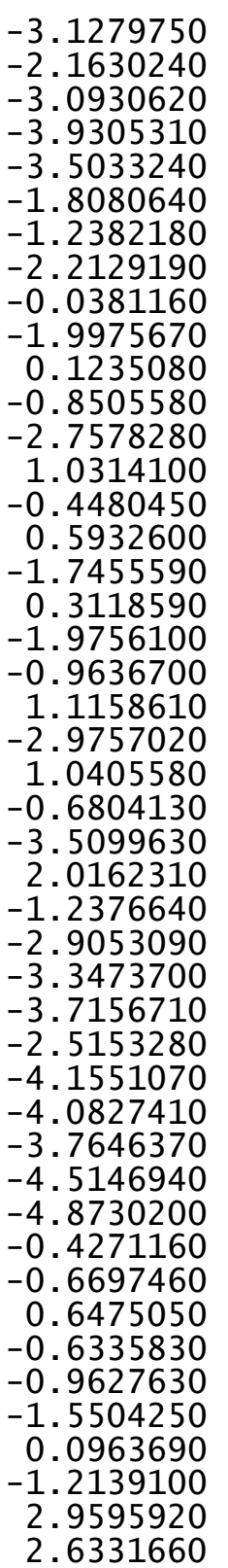

-5.8091660
-4.6771180
-3.2068760
-3.9147450
-2.4328570
-2.7365560
4.6379680
5.6793320
4.5563760
4.3958890
3.7599490
3.1153710
4.7787600
3.4136400
7.7509030
8.6594080
7.3844340
8.0128790
6.3510410
5.6527650
5.8998730
7.2602550
2.5924370
3.5235790
2.2277470
1.8607350
3.4726120
4.4837660
2.9045510
3.5513490
-0.5456710
0.0297660
-1.1315720
1.7999990
7.1026320
2.6765860
-3.7500940
-6.8118850
-3.1750530
0.2181880
-0.6896360
0.6508390
1.4350120
0.3117580
1.8725460
1.7003370
-1.3659870
-0.4121770
-2.2197260
-3.0395870
-2.5628350
-1.9934070
2.7084880
2.3991840
3.1736040
3.4612340
0.9180100
0.0370460
0.6309480
1.6638360
-1.8487430
-2.4385070
-2.0583620
-0.7878200
-3.7000180
-3.9935660
-3.9598270
-4.2985820
-2.0760780
-2.5012910
-2.7286690
-2.0884690
0.1895570
-0.1896620
0.1233740
1.2441980
-0.7729910
-1.6446620
-1.4222240
-0.2178550
-1.9971770
1.8516180
1.2864120
-3.7445610
-0.1589250
-0.4252060
-0.0708820
0.6750860
0.4383660
1.9905350
1.5040530
-0.5802290
3.0001100

3.9749800

2.5545850

2.7193300

1.8637800

3.5182670

0.9176570

1.0628430

$-0.0696770$

1.6751940

2.4562430

2.5540240

2.7381630

3.1807520

$-1.7250130$

$-1.5661540$

$-2.7361270$

$-1.6736620$

$-0.7486410$

0.0418580

$-1.7123330$

$-0.6329090$

$-3.9995840$

$-4.3901720$

$-3.2028840$

$-4.8129400$

$-4.6095800$

$-4.7976130$

$-5.5435210$

$-4.3319680$

$-0.9565020$

$-2.0269460$

0.2768040

$-3.3203250$

0.3129090

0.8821320

$-2.5686830$

$-2.3026550$

2. 0161190

3.1203960

2. 2820360

4.0149130

5.1496670

3.6647720

5.9259780

5.4121570 


\begin{tabular}{|c|c|c|c|c|c|c|c|}
\hline C & 0.7672960 & 3.0537770 & 4.4351400 & $\mathrm{C}$ & 1.8224630 & -4.8435500 & -2.2791270 \\
\hline $\mathrm{H}$ & -0.2908740 & 2.1732830 & 2.7806230 & $\mathrm{C}$ & -0.7329830 & -5.9134250 & -2.6644270 \\
\hline C & 1.5460480 & 2.8124730 & 5.5664430 & $\mathrm{H}$ & 0.1910090 & -7.2270330 & -4.0715650 \\
\hline $\mathrm{H}$ & 2.4748450 & 1.3164670 & 6.8087540 & $\mathrm{H}$ & 2.4586290 & -6.2437620 & -3.7528600 \\
\hline $\mathrm{H}$ & 0.5122130 & 4.0695800 & 4.1492930 & $\mathrm{H}$ & 2.8133890 & -4.4206410 & -2.1549610 \\
\hline $\mathrm{H}$ & 1.8999130 & 3.6433170 & 6.1689870 & $\mathrm{H}$ & -1.7333060 & -6.3111270 & -2.8131030 \\
\hline $\mathrm{H}$ & -0.8923060 & -0.7492040 & 1.4973770 & C & 4.2013210 & -5.6910450 & 1.0063020 \\
\hline s & 0.8834340 & -1.9806190 & 3.2045020 & C & 5.4777550 & -5.1110220 & 1.2013030 \\
\hline C & 0.8712470 & -3.6870420 & 0.4050710 & C & 5.6611080 & -3.7731700 & 0.9668540 \\
\hline C & 1.7824810 & -2.6160800 & 0.8362530 & $\mathrm{c}$ & 3.1490410 & -4.9333730 & 0.5567900 \\
\hline $\mathrm{H}$ & -0.1780000 & -3.4061440 & 0.3446930 & $\mathrm{H}$ & 4.0524050 & -6.7445650 & 1.2210270 \\
\hline $\mathrm{H}$ & 1.5451200 & -1.5924350 & 0.5630430 & $\mathrm{H}$ & 6.3019700 & -5.7244750 & 1.5507730 \\
\hline 0 & 1.5700440 & -3.4582720 & -0.8467430 & $\mathrm{H}$ & 6.6283830 & -3.3072060 & 1.1350160 \\
\hline $\mathrm{H}$ & 1.0234160 & -2.8054790 & -1.3853170 & $\mathrm{H}$ & 2.1734910 & -5.3880860 & 0.4230910 \\
\hline C & 2.6110830 & -5.3828870 & 1.1053590 & C & 3.8608920 & 0.7450330 & -0.0516310 \\
\hline $\mathrm{C}$ & 3.2869210 & -4.3036340 & 1.9521100 & $\mathrm{C}$ & 4.1493130 & 1.4535910 & 1.1351590 \\
\hline C & 3.1692030 & -2.9342340 & 1.2834950 & $\mathrm{C}$ & 3.7486800 & 1.4378030 & -1.2750010 \\
\hline $\mathrm{C}$ & 1.1210780 & -5.0770470 & 0.9405480 & $\mathrm{C}$ & 4.3879890 & 2.8285330 & 1.0594450 \\
\hline $\mathrm{H}$ & 2.7324090 & -6.3649080 & 1.5708300 & C & 3.9816100 & 2.8134010 & -1.2923400 \\
\hline H & 4.3447210 & -4.5353980 & 2.1093470 & c & 4.3227070 & 3.5261260 & -0.1435680 \\
\hline $\mathrm{H}$ & 3.7785840 & -2.8897970 & 0.3704820 & $\mathrm{H}$ & 4.6298330 & 3.3658300 & 1.9729290 \\
\hline H & 0.6139180 & -5.1434040 & 1.9112150 & $\mathrm{H}$ & 3.9130010 & 3.3515070 & -2.2374260 \\
\hline $\mathrm{H}$ & 0.6365820 & -5.7930140 & 0.2696490 & C & -2.7297190 & -2.5617330 & 0.4069690 \\
\hline $\mathrm{H}$ & 3.0882400 & -5.4294380 & 0.1187000 & $\mathrm{C}$ & -3.7074570 & -1.9309230 & -0.3921950 \\
\hline $\mathrm{H}$ & 2.8170490 & -4.2661070 & 2.9438800 & C & -2.9044190 & -2.6400990 & 1.8032050 \\
\hline $\mathrm{H}$ & 3.5326380 & -2.1220260 & 1.9186720 & $\mathrm{C}$ & -4.8558330 & -1.4235450 & 0.2233240 \\
\hline \multirow{2}{*}{\multicolumn{4}{|c|}{$\begin{array}{l}144 \\
\text { TS245(S,S) G[M06-2x/6-31G(d) }]=-3633.300229\end{array}$}} & $\mathrm{C}$ & -4.0692500 & -2.1189520 & 2.3672180 \\
\hline & & & & $\mathrm{C}$ & -5.0653580 & -1.5210630 & 1.5986540 \\
\hline 0 & 1.4437020 & -0.5464920 & -0.7308690 & $\mathrm{H}$ & -5.6207300 & -0.9641750 & -0.4011860 \\
\hline 0 & -0.0944040 & -1.6670180 & 0.9064150 & $\mathrm{H}$ & -4.2204370 & -2.2030290 & 3.4421470 \\
\hline c & 2.4700740 & -1.3617250 & -0.2941340 & $\mathrm{P}$ & 0.2821790 & -0.1859640 & 0.3507280 \\
\hline C & 2.2472430 & -2.7193790 & -0.2044240 & 0 & 0.8238700 & 0.5741310 & 1.5251400 \\
\hline C & 3.7137630 & -0.7411670 & -0.0048550 & 0 & -0.8129120 & 0.4102730 & -0.4943300 \\
\hline C & 3.3140730 & -3.5492580 & 0.2764830 & $\mathrm{C}$ & 3.4387540 & 0.7454380 & -2.5947630 \\
\hline C & 4.7574440 & -1.5599240 & 0.3502320 & $\mathrm{H}$ & 3.2775060 & -0.3190270 & -2.4049820 \\
\hline C & 4.5870540 & -2.9587850 & 0.5174450 & C & 4.6575630 & 5.0037640 & -0.2420400 \\
\hline $\mathrm{H}$ & 5.7345490 & -1.1261700 & 0.5479850 & $\mathrm{H}$ & 4.1241920 & 5.3995870 & -1.1176640 \\
\hline c & -0.2252490 & -2.7071370 & 0.0023730 & C & 4.2285250 & 0.7874990 & 2.5040420 \\
\hline $\mathrm{C}$ & -1.5383340 & -3.1985100 & -0.2320460 & $\mathrm{H}$ & 3.8696720 & -0.2407290 & 2.4086780 \\
\hline C & 0.9074990 & -3.2597700 & -0.5626370 & C & -1.9072290 & -3.3321320 & 2.7213890 \\
\hline C & -1.6707450 & -4.2810520 & -1.0666300 & $\mathrm{H}$ & -1.0486760 & -3.6569510 & 2.1281040 \\
\hline C & 0.7425600 & -4.3184960 & -1.5171410 & C & -6.3703780 & -1.0932220 & 2.2457460 \\
\hline C & -0.5594930 & -4.8473640 & -1.7413270 & $\mathrm{H}$ & -6.2032560 & -1.0699980 & 3.3299580 \\
\hline $\mathrm{H}$ & -2.6594940 & -4.6974690 & -1.2460890 & C & -3.5944880 & -1.8178120 & -1.9083470 \\
\hline C & 0.3327300 & -6.4167080 & -3.3637030 & $\mathrm{H}$ & -2.6267460 & -2.2231330 & -2.2142670 \\
\hline C & 1.6218560 & 62695 & -3.1759680 & C & -0.2611850 & 3.3414940 & 1.0169560 \\
\hline
\end{tabular}




$\begin{array}{lrrr}\text { C } & 0.3549830 & 3.3588600 & -0.3142100 \\ \mathrm{H} & -0.8539140 & 2.4624700 & 1.2622060 \\ \mathrm{H} & 0.5913180 & 2.4045880 & -0.7723320 \\ \mathrm{O} & 1.1351190 & 3.1350120 & 1.3474570 \\ \mathrm{H} & 1.2362850 & 2.1307490 & 1.4298970 \\ \mathrm{C} & 0.0491650 & 5.8392940 & 1.1702090 \\ \mathrm{C} & 0.2644310 & 5.8887480 & -0.3441300 \\ \mathrm{C} & 0.9511200 & 4.6205040 & -0.8596360 \\ \mathrm{C} & -0.8088440 & 4.6363050 & 1.5657050 \\ \mathrm{H} & -0.4356240 & 6.7586170 & 1.5105590 \\ \mathrm{H} & 0.8743290 & 6.7553750 & -0.6163900 \\ \mathrm{H} & 2.0043760 & 4.6004230 & -0.5556660 \\ \mathrm{H} & -1.8272870 & 4.7498500 & 1.1715760 \\ \mathrm{H} & -0.8896450 & 4.5442650 & 2.6529530 \\ \mathrm{H} & 1.0147110 & 5.7766020 & 1.6849330 \\ \mathrm{H} & -0.7053210 & 6.0036930 & -0.8432370 \\ \mathrm{H} & 0.9508930 & 4.5661490 & -1.9523660 \\ \mathrm{C} & -3.6378400 & -0.3635540 & -2.3917880 \\ \mathrm{H} & -3.6112900 & -0.3340740 & -3.4862620 \\ \mathrm{H} & -4.5512190 & 0.1480530 & -2.0658900 \\ \mathrm{H} & -2.7749040 & 0.1885790 & -2.0133580 \\ \mathrm{C} & -4.6870600 & -2.6486810 & -2.5947190 \\ \mathrm{H} & -5.6827200 & -2.2545880 & -2.3620220 \\ \mathrm{H} & -4.5607420 & -2.6215450 & -3.6818830 \\ \mathrm{H} & -4.6590390 & -3.6941650 & -2.2710070 \\ \mathrm{C} & 2.1603820 & 1.2996370 & -3.2355450 \\ \mathrm{H} & 2.2561780 & 2.3704740 & -3.4492920 \\ \mathrm{H} & 1.9603070 & 0.7893250 & -4.1835470 \\ \mathrm{H} & 1.2951350 & 1.1477180 & -2.5824860 \\ \mathrm{C} & 4.6271380 & 0.8593490 & -3.5589900 \\ \mathrm{H} & 4.4210640 & 0.3105320 & -4.4836100 \\ \mathrm{H} & 4.8223390 & 1.9040830 & -3.8243730 \\ \mathrm{H} & 5.5370510 & 0.4490320 & -3.1099360 \\ \mathrm{C} & -1.3848530 & -2.3839450 & 3.8062510 \\ \mathrm{H} & -2.1935690 & -2.0566250 & 4.4689260 \\ \mathrm{H} & -0.6357740 & -2.8925080 & 4.4221500 \\ \mathrm{H} & -0.9218190 & -1.4994700 & 3.3600950 \\ \mathrm{C} & -2.5282380 & -4.5898240 & 3.3434040 \\ \mathrm{H} & -1.7926320 & -5.1110280 & 3.9647800 \\ \mathrm{H} & -3.3841590 & -4.3341690 & 3.9774970 \\ \mathrm{H} & -2.8758380 & -5.2802170 & 2.5682690 \\ \mathrm{C} & 3.3358570 & 1.4812450 & 3.5408130 \\ \mathrm{H} & 3.4092070 & 0.9567660 & 4.4993990 \\ \mathrm{H} & 3.6402910 & 2.5197170 & 3.7087130 \\ \mathrm{H} & 2.2897430 & 1.4696350 & 3.2270400 \\ \mathrm{C} & 5.6786280 & 0.7453060 & 3.0051210 \\ & & & \end{array}$

$\begin{array}{rrr}6.0665120 & 1.7607420 & 3.1457000 \\ 5.7365600 & 0.2260840 & 3.9673300 \\ 6.3382650 & 0.2327650 & 2.2983640 \\ -6.8370420 & 0.2971380 & 1.8117760 \\ -6.0794070 & 1.0582460 & 2.0220180 \\ -7.0584710 & 0.3169420 & 0.7380640 \\ -7.7555160 & 0.5722220 & 2.3403590 \\ -7.4594330 & -2.1360170 & 1.9584450 \\ -7.1520210 & -3.1294850 & 2.2983060 \\ -8.3942460 & -1.8700770 & 2.4628200 \\ -7.6564360 & -2.1926230 & 0.8817680 \\ 6.1608580 & 5.1839910 & -0.4945300 \\ 6.4742030 & 4.6567140 & -1.4005750 \\ 6.4116730 & 6.2440890 & -0.6050900 \\ 6.7344990 & 4.7819490 & 0.3481290 \\ 4.2203230 & 5.8088230 & 0.9833420 \\ 3.1738660 & 5.6121540 & 1.2390930 \\ 4.8301840 & 5.5622540 & 1.8589050 \\ 4.3365310 & 6.8801380 & 0.7937270 \\ -2.9603790 & 2.8201410 & -0.8949260 \\ -2.8203520 & 1.8210500 & -0.0824230 \\ -4.3215470 & 3.3992570 & -0.8902250 \\ -4.5650980 & 4.6959700 & -1.3580900 \\ -5.3806080 & 2.6266880 & -0.3942720 \\ -5.8543770 & 5.2130650 & -1.3280870 \\ -3.7386810 & 5.2926080 & -1.7298130 \\ -6.6690480 & 3.1463250 & -0.3731930 \\ -5.1791690 & 1.6210170 & -0.0397480 \\ -6.9065730 & 4.4395670 & -0.8381900 \\ -6.0390990 & 6.2214840 & -1.6830710 \\ -7.4895540 & 2.5455220 & 0.0066590 \\ -7.9129080 & 4.8459690 & -0.8176170 \\ -1.9425850 & 1.2726150 & -0.2217390 \\ -1.7307020 & 3.4176130 & -1.8715820\end{array}$

S246(S,S) G[M06-2X/6-31G(d)] = -3633.300617

$\begin{array}{lrcr}\text { TS246(S, S) } \text { G[M06-2X/6-31G(d) }]=-3633.300617 \\ \text { O } & -1.3599850 & 0.6285040 & -0.6722240 \\ \text { O } & 0.3999110 & 1.6115920 & 0.8467380 \\ \text { C } & -2.2333200 & 1.5919140 & -0.1958840 \\ \text { C } & -1.8271610 & 2.9103570 & -0.1914580 \\ \text { C } & -3.5239730 & 1.1673840 & 0.2180320 \\ \text { C } & -2.7301840 & 3.9017870 & 0.3180100 \\ \text { C } & -4.4155170 & 2.1387710 & 0.6027100 \\ \text { C } & -4.0479140 & 3.5063290 & 0.6815990 \\ \text { H } & -5.4267590 & 1.8530750 & 0.8806820 \\ \text { C } & 0.6260310 & 2.5944910 & -0.1042190\end{array}$




$\begin{array}{rrr}1.9770170 & 2.9145640 & -0.4059430 \\ -0.4550390 & 3.2532280 & -0.6530570 \\ 2.1996340 & 3.9239500 & -1.3107230 \\ -0.2094410 & 4.2341220 & -1.6697800 \\ 1.1353420 & 4.5815820 & -1.9788660 \\ 3.2217210 & 4.2127920 & -1.5450380 \\ 0.3626420 & 6.1637230 & -3.6493280 \\ -0.9745770 & 5.7911480 & -3.3711100 \\ -1.2542850 & 4.8540140 & -2.4084750 \\ 1.3923520 & 5.5668330 & -2.9697830 \\ 0.5676980 & 6.9110380 & -4.4090920 \\ -1.7856970 & 6.2474260 & -3.9297040 \\ -2.2823570 & 4.5691130 & -2.2119300 \\ 2.4251840 & 5.8269820 & -3.1853260 \\ -3.2619250 & 6.1775150 & 0.9853030 \\ -4.5860810 & 5.7898530 & 1.3022310 \\ -4.9653100 & 4.4808460 & 1.1580890 \\ -2.3588690 & 5.2615510 & 0.5069460 \\ -2.9564540 & 7.2092030 & 1.1284550 \\ -5.2906040 & 6.5274820 & 1.6727580 \\ -5.9704170 & 4.1610120 & 1.4198230 \\ -1.3445890 & 5.5710510 & 0.2799160 \\ -3.9169440 & -0.2754630 & 0.2458270 \\ -4.1139140 & -0.9193960 & 1.4882520 \\ -4.1765810 & -0.9623050 & -0.9521770 \\ -4.6328530 & -2.2129840 & 1.4928760 \\ -4.6907490 & -2.2626210 & -0.8935950 \\ -4.9408560 & -2.9004100 & 0.3186990 \\ -4.8007560 & -2.7138150 & 2.4449100 \\ -4.9126490 & -2.7762240 & -1.8267110 \\ 3.1129290 & 2.2013530 & 0.2528370 \\ 4.0063690 & 1.4247490 & -0.5162770 \\ 3.3320780 & 2.3644340 & 1.6354490 \\ 5.1206880 & 0.8611010 & 0.1117030 \\ 4.4614600 & 1.7821910 & 2.2128730 \\ 5.3785310 & 1.0431150 & 1.4704590 \\ 5.8236790 & 0.2875120 & -0.4906900 \\ 4.6494930 & 1.9314030 & 3.2746830 \\ -0.1475280 & 0.1751980 & 0.3217720 \\ -0.6787230 & -0.5591960 & 1.5177050 \\ 0.8435240 & -0.5037440 & -0.5918180 \\ -3.9675840 & -0.3207600 & -2.3154460 \\ -3.5442140 & 0.6770030 & -2.1688230 \\ -5.5771040 & -4.2765890 & 1.2626160 \\ -5.1042180 & -4.7905950 & \\ -3.7887610 & -0.2495520 & \\ -3.8175680\end{array}$

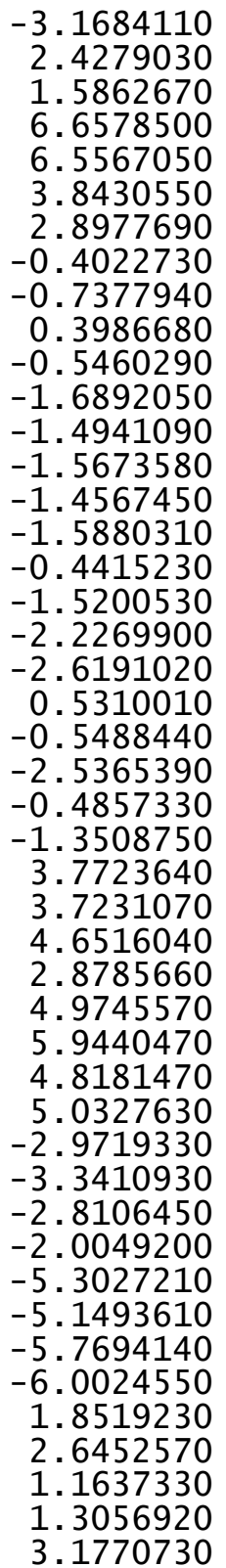

0.6281130

3.2082340

3.5710550

0.5399190

0.6837900

1.2127810

1.6644750

$-3.5118040$

$-3.1733900$

$-2.9284540$

$-2.1593730$

$-2.8693440$

$-1.9097350$

$-5.7224640$

$-5.5532740$

$-4.0814750$

$-4.9651360$

$-6.7812400$

$-6.1355640$

$-3.7279900$

$-5.4041610$

$-5.0153410$

$-5.3400970$

$-5.9358450$

$-3.9138200$

$-0.2715670$

$-0.3780130$

$-0.8251060$

$-0.7293970$

1.9096810

1.4587630

1.8156160

2.9743530

$-1.1184370$

$-2.1306920$

$-0.6243250$

$-1.1950000$

$-0.1470030$

0.3606130

$-1.1170240$

0.4463930

2.3872090

2.0371820

3.0001360

1.5169700

4. 4424150
2.6105140

2. 5227730

1.9274480

2.1137620

3.1967570

$-2.0169310$

$-2.3290590$

0.7144770

$-0.6724540$

1.1698610

$-1.0013950$

0.8814150

1.1384730

0.4125610

$-1.1039760$

$-1.5031320$

1. 1218050

0.6818430

$-1.6185680$

$-1.3602440$

0.8653990

2.2096040

0.7588480

$-1.4420080$

$-2.5576440$

$-2.3940450$

$-3.4829120$

$-2.0439630$

$-1.9662980$

$-2.7849290$

$-2.5446500$

$-3.8644190$

$-2.5369720$

$-3.1638730$

$-3.3654280$

$-4.1274220$

$-2.6560330$

$-3.0499110$

$-4.0077200$

$-3.2534900$

$-2.4529460$

3.6816610

4.3512370

4.2730270

3.3076670

3.0421690 


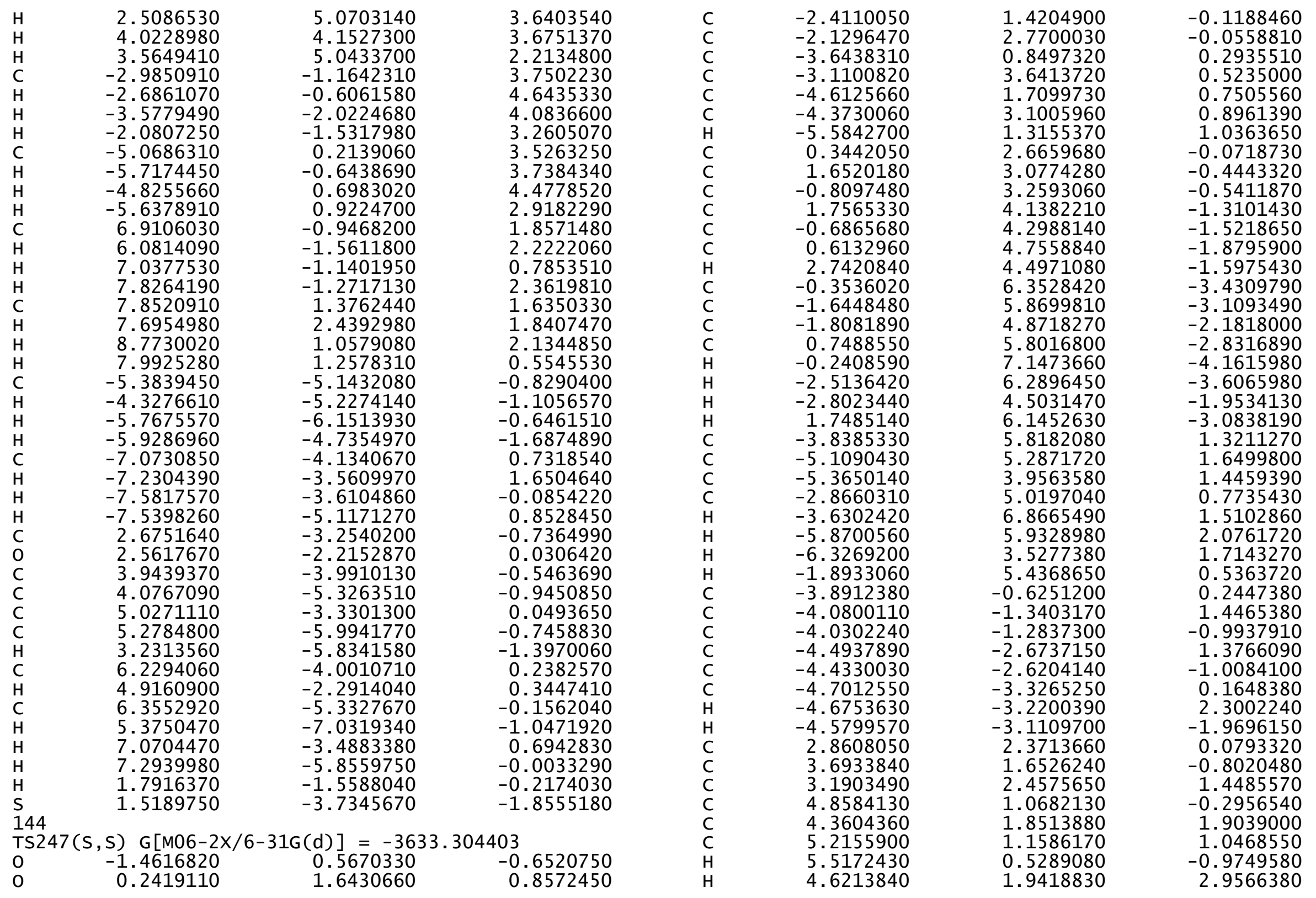




$\begin{array}{rrr}-0.2075820 & 0.1813030 & 0.3133520 \\ -0.6535240 & -0.6065670 & 1.5098820 \\ 0.8064620 & -0.4084350 & -0.6359340 \\ -3.8204260 & -0.5783740 & -2.3255950 \\ -3.5192750 & 0.4546650 & -2.1322100 \\ -5.2894400 & -4.7256310 & 0.1267190 \\ -5.2858240 & -5.1100390 & 1.1545040 \\ -3.8723180 & -0.7149650 & 2.8206380 \\ -3.3836460 & 0.2543100 & 2.6841690 \\ 2.3477150 & 3.2385330 & 2.4466160 \\ 1.4553620 & 3.6118040 & 1.9372180 \\ 6.5254550 & 0.5743370 & 1.5435440 \\ 6.9305430 & -0.0485250 & 0.7338330 \\ 3.4119730 & 1.5138400 & -2.2930870 \\ 2.4248490 & 1.9361030 & -2.5001640 \\ 0.0549540 & -3.4840350 & 0.7917210 \\ -0.3514930 & -3.2571190 & -0.5982210 \\ 0.7607280 & -2.7620150 & 1.2041370 \\ -0.3212010 & -2.2387940 & -0.9662190 \\ -1.3147630 & -3.0479180 & 0.9723790 \\ -1.2626280 & -2.0594250 & 1.1883660 \\ -0.7483290 & -5.8590270 & 0.6052880 \\ -0.7259610 & -5.7328160 & -0.9204310 \\ -1.0745530 & -4.3118930 & -1.3728090 \\ 0.2642100 & -4.9077770 & 1.2469680 \\ -0.5221310 & -6.8871040 & 0.9015560 \\ -1.4279210 & -6.4361840 & -1.3787060 \\ -2.1440970 & -4.1015890 & -1.2279680 \\ 1.2862990 & -5.1936900 & 0.9685980 \\ 0.2003380 & -4.9355880 & 2.3389620 \\ -1.7491520 & -5.6237130 & 0.9878980 \\ 0.2745020 & -5.9923410 & -1.2877070 \\ -0.8765460 & -4.1581120 & -2.4376340 \\ 3.3770670 & 0.0503560 & -2.7501530 \\ 3.2487280 & 0.0036530 & -3.8368940 \\ 4.3043700 & -0.4796970 & -2.5019650 \\ 2.5411130 & -0.4760440 & -2.2860380 \\ 4.4454920 & 2.3051020 & -3.1067850 \\ 5.4499000 & 1.8900500 & -2.9655000 \\ 4.2088820 & 2.2588150 & -4.1747530 \\ 4.4750900 & 3.3572830 & -2.8060770 \\ -2.6978350 & -1.2405440 & -3.1321970 \\ -2.9360010 & -2.2863200 & -3.3586110 \\ -2.5516980 & -0.7176400 & -4.0830280 \\ -1.7535690 & -1.2084480 & -2.5794180 \\ -5.1235960 & -0.5275740 & -3.1333960\end{array}$

-4.9737360
-5.4665930
-5.9188150
1.8790520
2.7279980
1.2273400
1.3219580
3.1180280
2.4937430
4.0192910
3.4249940
-2.9545930
-2.7838750
-3.4015210
-1.9858630
-5.2133690
-5.7279990
-5.0537270
-5.8791470
7.5376510
7.7004440
7.1717240
8.4996610
6.3502510
5.6189740
7.3045830
6.0109100
-4.4854920
-3.4646180
-4.9573240
-4.4313750
-6.7472160
-7.3391570
-6.7971920
-7.2050060
3.0582960
2.8117250
4.4258500
4.7818300
5.3766420
6.0727600
4.0406520
6.6666380
5.0896300
7.0159870
6.3432970
0.0300110
-1.5340660
-0.0381790
2.3523590
1.9897930
2.9218780
1.4880910
4.4601430
5.0440510
4.1548300
5.1095030
-1.5650240
-1.0511950
-2.5387420
-1.7280850
-0.4855910
-1.4392610
-0.0156520
0.1566670
1.6939390
2.3060350
2.3490760
1.2774830
-0.3051610
-1.1025660
-0.7654560
0.2887110
-5.6974030
-5.8115250
-6.6852090
-5.3550310
-4.6649030
-3.9932150
-4.2951260
-5.6591160
-2.8243620
-1.7670680
-3.3567470
-4.6439710
-2.5446830
-5.1114210
-5.2725590
-3.0165650
-1.5461730
-4.2999300
-6.1119050
$-4.0635540$

$-3.3966410$

$-2.5623130$

3. 6058290

4. 1958820

4.2765830

3.2337690

2.9644820

3. 6487350

3. 5075470

2. 1384110

3.7083240

4.6601150

3. 9349340

3. 2314070

3.5307570

3. 6951050

4.5067820

2. 9471110

1.8198900

0.9279100

2. 6185230

2. 1360660

2. 7861580

2. 6208820

3. 0631760

3. 6415070

$-0.7409640$

$-0.3657580$

$-0.7421970$

$-1.7807690$

$-0.3500580$

0.2787130

$-1.3805600$

$-0.3233030$

$-0.7355880$

$-0.0309590$

$-0.5351000$

$-0.9544150$

0.0987410

$-0.7407560$

$-1.4362200$

0.3085530

0.4115340

$-0.1103380$

$-1.0615760$ 


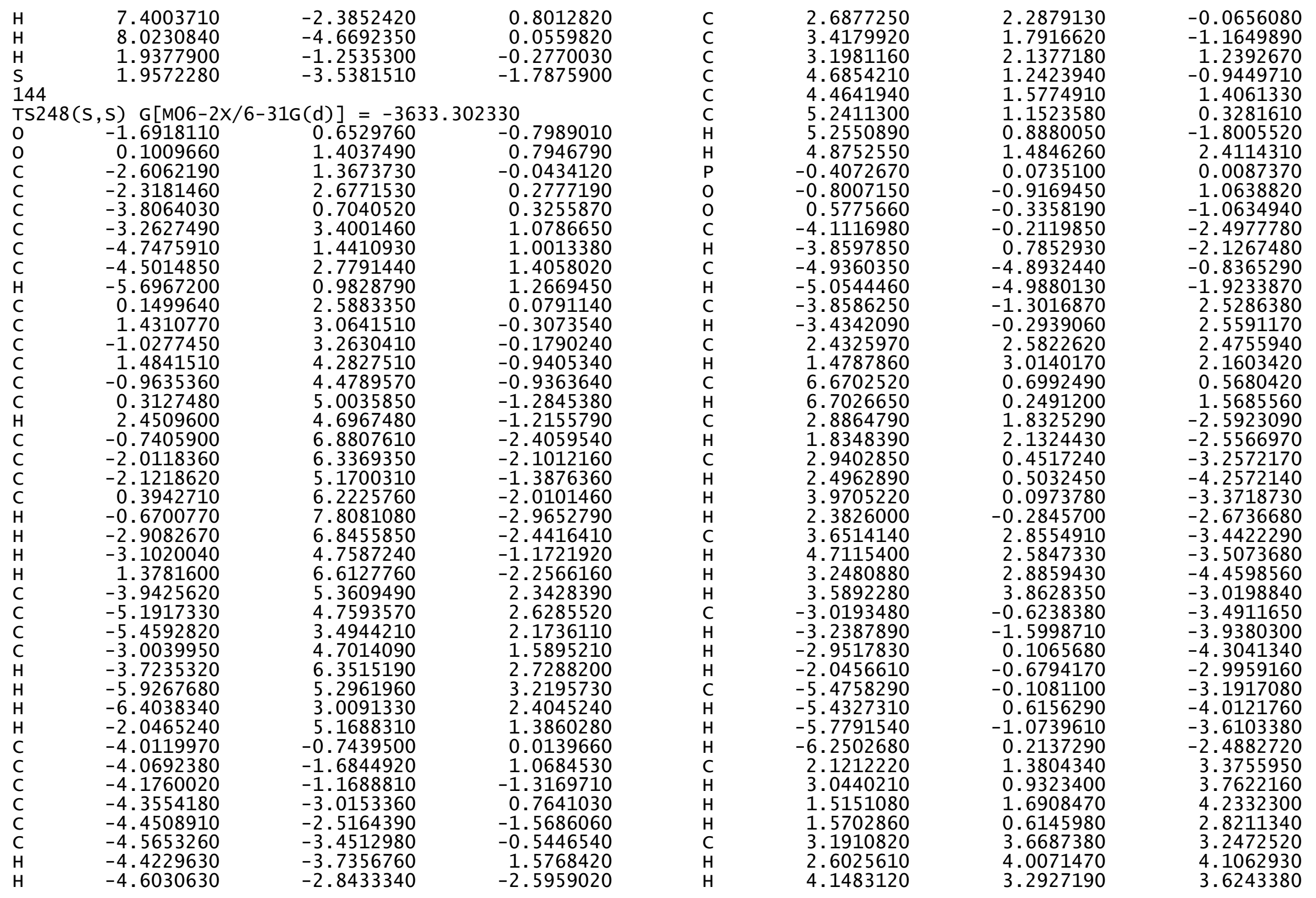




$\begin{array}{rr}\mathrm{H} & 3.3958410 \\ \mathrm{C} & -2.8712450 \\ \mathrm{H} & -2.7093080 \\ \mathrm{H} & -3.2527530 \\ \mathrm{H} & -1.9069770 \\ \mathrm{C} & -5.1954000 \\ \mathrm{H} & -5.6399480 \\ \mathrm{H} & -5.0462030 \\ \mathrm{H} & -5.9170680 \\ \mathrm{C} & 7.1744770 \\ \mathrm{H} & 6.4925650 \\ \mathrm{H} & 7.2916930 \\ \mathrm{H} & 8.1558200 \\ \mathrm{C} & 7.5985250 \\ \mathrm{H} & 7.2829580 \\ \mathrm{H} & 8.6300980 \\ \mathrm{H} & 7.5827820 \\ \mathrm{C} & -6.2740470 \\ \mathrm{H} & -7.0684500 \\ \mathrm{H} & -6.5649890 \\ \mathrm{H} & -6.2004700 \\ \mathrm{C} & -3.8373760 \\ \mathrm{H} & -2.8965720 \\ \mathrm{H} & -3.6524670 \\ \mathrm{H} & -4.1248340 \\ \mathrm{C} & 0.3566130 \\ \mathrm{C} & 0.1760140 \\ \mathrm{H} & 0.8476260 \\ \mathrm{H} & 0.0788450 \\ \mathrm{O} & -1.0774260 \\ \mathrm{H} & -1.2132450 \\ \mathrm{C} & 0.0264020 \\ \mathrm{C} & 0.2798070 \\ \mathrm{C} & -0.2116560 \\ \mathrm{C} & 0.7272490 \\ \mathrm{H} & 0.3857260 \\ \mathrm{H} & -0.2148010 \\ \mathrm{H} & -1.3075610 \\ \mathrm{H} & 1.8166340 \\ \mathrm{H} & 0.4807730 \\ \mathrm{H} & -1.0492840 \\ \mathrm{H} & 1.3557650 \\ \mathrm{H} & 0.1462110 \\ \mathrm{C} & 3.2308900 \\ \mathrm{C} & 2.6538610 \\ & 4.4259790 \\ & \end{array}$

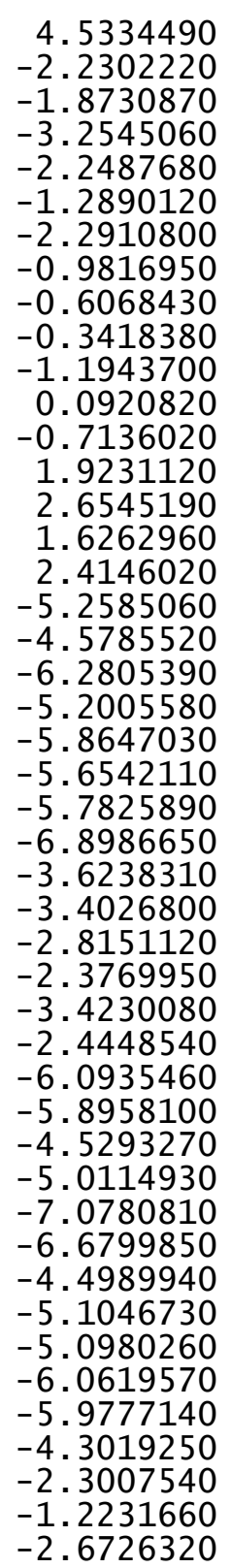
2. 6081730
3.2461030
4.2685800
3. 3137720
2. 7345480
3.2833330
3.2865270
4.3237180
2.8248980
$-0.4314560$
$-0.5047390$
$-1.4308060$
$-0.1196590$
0.5758370
1.3262100
0.7931640
$-0.4036090$
$-0.1822610$
$-0.5037440$
$-0.4456970$
0.9093570
$-0.3944420$
$-0.9115530$
0.6826890
$-0.6114690$
0.5051190
$-0.9348890$
1.0476350
$-1.2752410$
0.4558100
0.6655330
0.1451990
$-1.3515950$
$-1.8394670$
0.9689610
0.4577070
$-1.9321370$
$-1.8967730$
0.8743980
2.0316490
0.3510820
$-1.5477610$
$-2.8472680$
$-0.3753440$
0.0420750
0.4137440

$\begin{array}{ll}\mathrm{C} & 5.2757590 \\ \mathrm{C} & 4.7124520 \\ \mathrm{C} & 6.4090790 \\ \mathrm{H} & 5.0471930 \\ \mathrm{C} & 5.8370570 \\ \mathrm{H} & 4.0553770 \\ \mathrm{C} & 6.6910950 \\ \mathrm{H} & 7.0721760 \\ \mathrm{H} & 6.0519850 \\ \mathrm{H} & 7.5749050 \\ \mathrm{H} & 1.8290150 \\ \mathrm{~S} & 2.6601740 \\ \mathrm{H} & \end{array}$
$-3.7078570$
$-1.9626010$
$-4.0132460$
$-4.2576840$
$-2.2875580$
$-1.1573630$
$-3.3042630$
$-4.8060430$
$-1.7409730$
$-3.5481400$
$-0.9022280$
$-3.2231240$

0.0025490

1. 5888910

0.7449530

$-0.9040070$

2. 3382140

1.8989430

1.9130210

0.4151640

3.2511920

2.4942710

$-0.5057100$

$\operatorname{TS} 249(\mathrm{~S}, \mathrm{~S}) \mathrm{G}[\mathrm{M} 06-2 \mathrm{X} / 6-31 \mathrm{G}(\mathrm{d})]=-3633.302470$

$\begin{array}{lrlr}\mathrm{O} & -1.6394810 & 0.6074170 & -0.7015270 \\ \mathrm{O} & 0.1145430 & 1.5023260 & 0.8578370 \\ \mathrm{C} & -2.5792410 & 1.3465230 & -0.0049630 \\ \mathrm{C} & -2.3252510 & 2.6807680 & 0.2382600 \\ \mathrm{C} & -3.7687740 & 0.6776730 & 0.3870220 \\ \mathrm{C} & -3.2974690 & 3.4276010 & 0.9817310 \\ \mathrm{C} & -4.7357490 & 1.4324970 & 1.0050480 \\ \mathrm{C} & -4.5259790 & 2.7978600 & 1.3303900 \\ \mathrm{H} & -5.6772680 & 0.9684770 & 1.2873710 \\ \mathrm{C} & 0.1460990 & 2.6373800 & 0.0649480 \\ \mathrm{C} & 1.4207800 & 3.1016410 & -0.3550730 \\ \mathrm{C} & -1.0433020 & 3.2693910 & -0.2409760 \\ \mathrm{C} & 1.4564680 & 4.2807960 & -1.0596220 \\ \mathrm{C} & -0.9957490 & 4.4378960 & -1.0711150 \\ \mathrm{C} & 0.2738900 & 4.9624530 & -1.4445100 \\ \mathrm{H} & 2.4163830 & 4.6896060 & -1.3647470 \\ \mathrm{C} & -0.8049760 & 6.7508450 & -2.6812540 \\ \mathrm{C} & -2.0684070 & 6.2034950 & -2.3518690 \\ \mathrm{C} & -2.1627190 & 5.0796000 & -1.5701520 \\ \mathrm{C} & 0.3385640 & 6.1373450 & -2.2410530 \\ \mathrm{H} & -0.7469530 & 7.6443750 & -3.2944530 \\ \mathrm{H} & -2.9713070 & 6.6745210 & -2.7274840 \\ \mathrm{H} & -3.1369660 & 4.6644640 & -1.3363540 \\ \mathrm{H} & 1.3170550 & 6.5291700 & -2.5057890 \\ \mathrm{C} & -4.0397250 & 5.4439920 & 2.1167380 \\ \mathrm{C} & -5.2784810 & 4.8308610 & 2.4229420 \\ \mathrm{C} & -5.5105630 & 3.5349820 & 2.0417440 \\ \mathrm{C} & -3.0759490 & 4.7631050 & 1.4162150 \\ \mathrm{H} & -3.8489520 & 6.4608410 & 2.4450320 \\ \mathrm{H} & -6.0337010 & 5.3844650 & 2.9715480 \\ \mathrm{H} & -6.4468060 & 3.0420220 & 2.2896610 \\ \mathrm{H} & -2.1268260 & 5.2401140 & 1.1963620\end{array}$




-3.9443780
-4.0295130
-4.0732040
-4.3254130
-4.3509720
-4.5094540
-4.4225920
-4.4797890
2.6793700
3.4001020
3.1914910
4.6607750
4.4469150
5.2160350
5.2233120
4.8586250
-0.3439770
-0.7108790
0.6496130
-3.9684260
-3.7400640
-4.9277530
-4.9361780
-3.8305000
-3.4237540
2.4454260
1.4914430
6.6374390
6.6395760
2.8698750
1.8198150
2.9170880
2.4738360
3.9454250
2.3554070
3.6425190
4.7003130
3.2389950
3.5895400
-2.8309050
-3.0190910
-2.7397220
-1.8770120
-5.3016910
-5.2288110
-5.5785570

0.1676630
1.2806970
-1.1363440
1.0630570
-1.2994990
-0.2123160
1.9191510
-2.3080070
-0.0835300
-1.1624460
1.2256680
-0.9214730
1.4145920
0.3536530
-1.7623360
2.4222390
0.1463640
1.2534470
-0.9065860
-2.3816550
-2.0772810
-0.4081930
0.5810030
2.7152170
2.6857040
2.4413570
2.1113290
0.6149960
1.5927690
-2.5910260
-2.5695800
-3.2044630
-4.2060100
-3.3062840
-2.5944380
-3.4779550
-3.5333720
-4.4959780
-3.0925780
-3.2930940
-3.6626920
-4.1622430
-2.7573490
-3.1394970
-4.0062820
-3.5023120

-6.1091580
2.1358640
3.0590360
1.5482890
1.5661900
3.2261050
2.6559060
4.1869160
3.4267140
-2.8239120
-2.6685870
-3.1845730
-1.8596230
-5.1655920
-5.5949440
-5.0186760
-5.8984850
7.1757230
6.4959540
7.3309330
8.1435270
7.5647270
7.2278800
8.5895590
7.5770320
-3.9530760
-2.9503440
-4.2878090
-3.8834060
-6.3495670
-7.0584790
-6.3867780
-6.6781610
0.3216500
0.0937340
0.8834730
0.0447750
-1.0986840
-1.1775620
-0.1927890
0.0036850
-0.4026910
0.6250620
0.1045070
-0.5755340
-1.4952980
$-0.0463150$
1.6317350
1.1996960
2.0100180
0.8357250
3.8930710
4.2933360
3.5331680
4.7113570
$-2.0505780$
$-1.6373270$
$-3.0775470$
$-2.0757820$
$-1.1474800$
$-2.1540040$
$-0.7781370$
$-0.5036260$
$-1.1197770$
0.2142640
$-0.6673170$
1.9341600
2.6260940
1.6249570
2.4759140
$-5.7563970$
$-5.7721840$
$-6.7918320$
$-5.3099220$
$-5.0499940$
$-4.5136510$
$-4.6010100$
$-6.0908980$
$-3.5932360$
$-3.3453290$
$-2.8213340$
$-2.3127580$
$-3.3133850$
$-2.3304160$
$-6.0266670$
$-5.8345260$
$-4.4304230$
$-5.0073400$
$-7.0386160$
$-6.5740150$
$-4.3186410$

-2.4948870
3.4271420
3.8300740
4.2703020
2.9382170
3.1261690
3.9709020
3.5100900
2.4273780
3.4800650
4.4822500
3.6001560
2.9683240
3.4714230
3.5348000
4.4917240
2.9765690
-0.4198510
-0.5665410
-1.3882870
-0.0920050
0.7138790
1.4918700
0.9452540
-0.2386540
-1.2955370
-0.8578990
-1.4157000
-2.2941880
-0.9799890
-0.3423390
-1.9789400
-1.0649540
0.6512590
-0.7769330
1.1792250
-1.1041080
0.6658920
0.8864560
0.2875220
-1.2188890
-1.6767830
1.0833810
0.5764850
-1.7803060
-1.6946210 


\begin{tabular}{|c|c|c|c|c|c|c|c|}
\hline $\mathrm{H}$ & 16987430 & 51730530 & 0 & & -41894550 & 8162020 & 2015010100 \\
\hline $\mathrm{H}$ & $\begin{array}{l}1.0901430 \\
0.4284630\end{array}$ & $\begin{array}{l}-5.1730530 \\
-5.0914490\end{array}$ & $\begin{array}{l}0.9296600 \\
2.1565440\end{array}$ & C & $\begin{array}{l}-4.1894550 \\
-5.3615550\end{array}$ & $\begin{array}{l}4.8162930 \\
4.0917020\end{array}$ & $\begin{array}{l}2.8420450 \\
3\end{array}$ \\
\hline $\mathrm{H}$ & -1.2517260 & -59148840 & 0.5487480 & c & -5.5622930 & 2.8452920 & 2.10001030 \\
\hline $\mathrm{H}$ & 1.0600100 & -5.9979880 & -1.4650620 & $\mathrm{c}$ & -3.2621070 & 4.2951780 & $\begin{array}{l}1.9749210 \\
1.9\end{array}$ \\
\hline $\mathrm{H}$ & -0.0632420 & -4.2173330 & -2.6942300 & $\mathrm{H}$ & -4.0204730 & 5.7908850 & 3.2890570 \\
\hline C & 3.2479610 & -2.3666430 & -0.3581430 & $\mathrm{H}$ & -6.0893560 & 4.5195710 & 3.8480050 \\
\hline 0 & 2.7294950 & -1.2766760 & 0.0982390 & $\mathrm{H}$ & -6.4451190 & 2.2662390 & 2.8897610 \\
\hline C & 4.4965270 & -2.7581500 & 0.3354790 & $\mathrm{H}$ & -2.3628640 & 4.8553890 & 1.7419830 \\
\hline C & 5.3148000 & -3.7804710 & -0.1617290 & C & -3.9323300 & -1.0897200 & 0.0546280 \\
\hline $\mathrm{C}$ & 4.8633970 & -2.0815630 & 1.5078460 & $\mathrm{c}$ & -3.7861890 & -2.1036290 & 1.0297050 \\
\hline $\mathrm{C}$ & 6.4929830 & -4.1102470 & 0.4957360 & $\mathrm{c}$ & -4.1522320 & -1.4336270 & -1.2909480 \\
\hline $\mathrm{H}$ & 5.0257290 & -4.3024690 & -1.0675610 & $\mathrm{C}$ & -3.9109620 & -3.4350000 & 0.6341630 \\
\hline C & 6.0306630 & -2.4347950 & 2.1751870 & $\mathrm{C}$ & -4.2643620 & -2.7838120 & -1.6358400 \\
\hline H & 4.2340940 & -1.2805480 & 1.8813490 & $\mathrm{C}$ & -4.1561530 & -3.7983060 & -0.6907810 \\
\hline $\mathrm{C}$ & 6.8505200 & -3.4409470 & 1.6664860 & $\mathrm{H}$ & -3.8094000 & -4.2167630 & 1.3838000 \\
\hline H & 7.1311450 & -4.8926460 & 0.0987080 & $\mathrm{H}$ & -4.4497050 & -3.0523400 & -2.6744710 \\
\hline $\mathrm{H}$ & 6.3060960 & -1.9178100 & 3.0890750 & $\mathrm{C}$ & 2.4261380 & 2.4241040 & -0.3921100 \\
\hline $\mathrm{H}$ & 7.7675310 & -3.7061330 & 2.1834370 & $\mathrm{C}$ & 2.9313220 & 1.8549530 & -1.5837320 \\
\hline $\mathrm{H}$ & 1.8763930 & -0.9264790 & -0.3922940 & C & 3.1697010 & 2.3482900 & 0.7947320 \\
\hline $\mathrm{s}$ & 2.5610330 & -3.2879220 & -1.5941850 & $\mathrm{C}$ & 4.1897880 & 1.2595120 & -1.5519880 \\
\hline & & & & $\mathrm{C}$ & 4.4338990 & 1.7455260 & 0.7740380 \\
\hline & , S) G[M06-2 & $=-36$ & 571 & $\mathrm{C}$ & 4.9694160 & 1.2069050 & -0.3919460 \\
\hline 0 & -1.8176930 & 0.5594890 & -0.8125370 & $\mathrm{H}$ & 4.5855840 & 0.8154830 & -2.4631510 \\
\hline 0 & 0.0219050 & 1.3411190 & 0.7086020 & $\mathrm{H}$ & 5.0049940 & 1.7015120 & 1.6982820 \\
\hline C & -2.7280180 & 1.1365210 & 0.0574720 & $\mathrm{P}$ & -0.4483580 & 0.0170110 & -0.1228510 \\
\hline C & -2.5208910 & 2.4389660 & 0.4613140 & 0 & -0.7165200 & -1.0623610 & 0.8830650 \\
\hline $\mathrm{C}$ & -3.8383380 & 0.3464570 & 0.4603500 & 0 & 0.5167960 & -0.2545200 & 2522850 \\
\hline C & -3.4558160 & 3.0178740 & 1.3816730 & $\mathrm{C}$ & -4.3062690 & -0.3910940 & -2.3878620 \\
\hline $\mathrm{C}$ & -4.7814530 & 0.9496180 & 1.2550620 & $\mathrm{H}$ & -4.1389500 & 0.5988560 & -1.9541850 \\
\hline $\mathrm{C}$ & -4.6127230 & 2.2716080 & 1.7446220 & C & -4.2983090 & -5.2559220 & -1.0850490 \\
\hline $\mathrm{H}$ & -5.6679120 & 0.3945480 & 1.5501640 & $\mathrm{H}$ & -4.4853360 & -5.2860400 & -2.1659950 \\
\hline C & -0.0799380 & 2.5624730 & 0.0627110 & C & -3.5167690 & -1.8012970 & 2.4986580 \\
\hline $\mathrm{C}$ & 1.1185800 & 3.1535630 & -0.4234820 & $\mathrm{H}$ & -3.1706010 & -0.7662130 & 2.5736410 \\
\hline C & -1.3209350 & 3.1611090 & -0.0439160 & C & 2.6535560 & 2.9351560 & 2.0992600 \\
\hline C & 1.0309710 & 4.4181550 & -0.9513880 & $\mathrm{H}$ & 1.6538280 & 3.3429950 & 1.9196690 \\
\hline $\mathrm{C}$ & -1.4124130 & 4.4294300 & -0.7078330 & C & 6.3573170 & 0.5860820 & -0.4703570 \\
\hline C & -0.21 & 5.0767990 & -1.1199610 & $\mathrm{H}$ & 6.2334750 & -0.4248880 & -0.8877950 \\
\hline $\mathrm{H}$ & 1.9360730 & 4.9211310 & -1.2819540 & C & 2.1637070 & 1.9098080 & -2.8986390 \\
\hline C & $-1.4 \mathrm{C}$ & 6.9465000 & -1.9880320 & $\mathrm{H}$ & 1.1049630 & 2.0569490 & -2.6615820 \\
\hline C & 4150 & 6.2822680 & -1.6250970 & C & 2.2736070 & 0.6148850 & -3.7108020 \\
\hline C & 9250 & 5.0592940 & -1.0037180 & $\mathrm{H}$ & 1.5834730 & 0.6571250 & -4.5601380 \\
\hline C & 1140 & 6.3504600 & -1.7453230 & $\mathrm{H}$ & 3.2810840 & 0.4778030 & -4.1190160 \\
\hline $\mathrm{H}$ & -1.54 & 7.9166750 & -2.4721870 & $\mathrm{H}$ & 2.0196510 & -0.2549780 & -3.1011250 \\
\hline $\mathrm{H}$ & -3.6 & 6.74275 & -1.8467420 & C & 2.6425270 & 3.0902550 & -3.7561560 \\
\hline H & -3.5 & 73480 & -0.7421470 & $\mathrm{H}$ & 3.7079850 & 2.9787170 & -3.9891110 \\
\hline $\mathrm{H}$ & 0.6 & 6.8 & -2.0415160 & $\mathrm{H}$ & 2.0901970 & 3.1251660 & -4.7013890 \\
\hline
\end{tabular}




$\begin{array}{lr}\mathrm{H} & 2.5077960 \\ \mathrm{C} & -3.2707340 \\ \mathrm{H} & -3.4264430 \\ \mathrm{H} & -3.3549530 \\ \mathrm{H} & -2.2556860 \\ \mathrm{C} & -5.7314250 \\ \mathrm{H} & -5.8486380 \\ \mathrm{H} & -5.9572270 \\ \mathrm{H} & -6.4698760 \\ \mathrm{C} & 2.5191880 \\ \mathrm{H} & 3.4939330 \\ \mathrm{H} & 2.1081490 \\ \mathrm{H} & 1.8494480 \\ \mathrm{C} & 3.5429560 \\ \mathrm{H} & 3.1386570 \\ \mathrm{H} & 4.5587140 \\ \mathrm{H} & 3.6096280 \\ \mathrm{C} & -2.4207810 \\ \mathrm{H} & -2.1984580 \\ \mathrm{H} & -2.7344750 \\ \mathrm{H} & -1.5024610 \\ \mathrm{C} & -4.8026170 \\ \mathrm{H} & -5.1673720 \\ \mathrm{H} & -4.6140890 \\ \mathrm{H} & -5.6014570 \\ \mathrm{C} & 7.2449500 \\ \mathrm{H} & 6.8031130 \\ \mathrm{H} & 7.3879480 \\ \mathrm{H} & 8.2290020 \\ \mathrm{C} & 7.0578040 \\ \mathrm{H} & 6.4556850 \\ \mathrm{H} & 8.0114460 \\ \mathrm{H} & 7.2715800 \\ \mathrm{C} & -5.4918650 \\ \mathrm{H} & -6.4194300 \\ \mathrm{H} & -5.6130140 \\ \mathrm{H} & -5.3440500 \\ \mathrm{C} & -3.0098300 \\ \mathrm{H} & -2.1590200 \\ \mathrm{H} & -2.7814300 \\ \mathrm{H} & -3.1079960 \\ \mathrm{C} & 0.8458010 \\ \mathrm{C} & 0.8502740 \\ \mathrm{H} & 1.1427990 \\ \mathrm{H} & 0.6243490 \\ \mathrm{O} & -0.5823220 \\ & \end{array}$

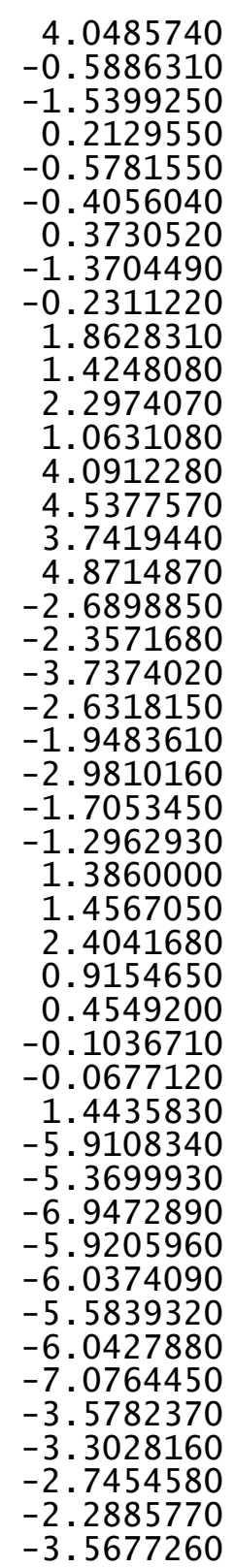
$-3.2477450$
$-3.5008060$
$-4.0214880$
$-4.2420440$
$-3.0936180$
$-2.9551320$
$-3.7160080$
$-3.4225020$
$-2.1662050$
3. 1848210
3.4307170
4.1020670
2.8526390
2. 5719780
3.4863340
2.7879800
1.8070850
3.0994830
4.1188000
3. 1606320
2. 5118070
3. 3248420
3. 2756340
4. 3759110
2. 9608270
$-1.4358630$
$-2.4335770$
$-1.0568410$
$-1.5308400$
0.8796950
1. 6012060
0.7561770
1. 3031280
$-0.3802780$
$-0.5901240$
$-0.7116530$
0.7053060
$-0.8062820$
$-1.3232760$
0.2656510
$-1.1376400$
0.3512870
$-1.0952450$
0.9896680
-1.4122870
0.1261500

\begin{tabular}{lr}
$\mathrm{H}$ & -0.8780080 \\
$\mathrm{C}$ & 0.9563910 \\
$\mathrm{C}$ & 1.4118320 \\
$\mathrm{C}$ & 0.7713240 \\
$\mathrm{C}$ & 1.3456250 \\
$\mathrm{H}$ & 1.4060340 \\
$\mathrm{H}$ & 1.1576890 \\
$\mathrm{H}$ & -0.2987340 \\
$\mathrm{H}$ & 2.4382140 \\
$\mathrm{H}$ & 0.9539360 \\
$\mathrm{H}$ & -0.1315200 \\
$\mathrm{H}$ & 2.5031720 \\
$\mathrm{H}$ & 1.2056600 \\
$\mathrm{C}$ & 3.4092700 \\
$\mathrm{O}$ & 2.5487350 \\
$\mathrm{C}$ & 4.3810190 \\
$\mathrm{C}$ & 5.4979500 \\
$\mathrm{C}$ & 4.1512750 \\
$\mathrm{C}$ & 6.3758180 \\
$\mathrm{H}$ & 5.6707680 \\
$\mathrm{C}$ & 5.0193890 \\
$\mathrm{H}$ & 3.2893930 \\
$\mathrm{C}$ & 6.1332160 \\
$\mathrm{H}$ & 7.2458090 \\
$\mathrm{H}$ & 4.8294460 \\
$\mathrm{H}$ & 6.8142240 \\
$\mathrm{H}$ & 1.8673580 \\
$\mathrm{~S}$ & 3.3536110 \\
$\mathrm{H}$ & \\
\hline
\end{tabular}
$-2.6338870$
$-6.0471480$
$-5.7193790$
$-4.4237630$
$-4.9263420$
$-6.9868100$
$-6.5330580$
$-4.5705880$
$-4.8566550$
$-5.1107360$
$-6.1833560$
$-5.6101870$
$-4.0851790$
$-1.7407480$
$-0.8136430$
$-1.9732110$
$-2.7980630$
$-1.3805980$
-3. 0222140
$-3.2551700$
$-1.6280830$
$-0.7370300$
$-2.4460260$
$-3.6530510$
$-1.1798880$
$-2.6339550$
$-0.6111560$
$-2.6601560$

0.3618290

$-0.1528800$

$-1.5764340$

$-2.0804490$

0.8134040

0.1799950

$-2.2613330$

$-2.2787110$

0.8933760

1.8186060

$-0.1326440$

$-1.5924400$

$-3.0248660$

$-0.0599620$

0.2066280

1. 0292200

0.8457890

2.2796310

1.8992870

$-0.1230200$

3. 3364920

2. 4117570

3. 1466610

1.7506310

4. 3065630

3. 9708280

$-0.5332400$

$-1.4703750$

TS251(S, S) G[M06-2X/6-31G(d)] $=-3633.299620$

\begin{tabular}{lrcr}
\multicolumn{2}{c}{ TS251(S, S $)$ G $[\mathrm{M} 06-2 X / 6-31 \mathrm{G}(\mathrm{d})]=-3633.299620$} \\
$\mathrm{O}$ & -1.8476700 & 0.4772150 & -0.7444900 \\
$\mathrm{O}$ & -0.0081400 & 1.3526820 & 0.7220750 \\
$\mathrm{C}$ & -2.7610180 & 1.0654220 & 0.1148540 \\
$\mathrm{C}$ & -2.5779660 & 2.3852400 & 0.4709910 \\
$\mathrm{C}$ & -3.8499330 & 0.2669390 & 0.5574390 \\
$\mathrm{C}$ & -3.5146880 & 2.9757200 & 1.3820860 \\
$\mathrm{C}$ & -4.7969870 & 0.8770540 & 1.3421170 \\
$\mathrm{C}$ & -4.6512410 & 2.2183520 & 1.7841590 \\
H & -5.6671940 & 0.3132970 & 1.6676260 \\
$\mathrm{C}$ & -0.1461270 & 2.5466990 & 0.0325210 \\
$\mathrm{C}$ & 1.0305950 & 3.1443710 & -0.4970300 \\
$\mathrm{C}$ & -1.4012680 & 3.1150400 & -0.0755350 \\
$\mathrm{C}$ & 0.9071940 & 4.3880030 & -1.0661730 \\
$\mathrm{C}$ & -1.5311150 & 4.3564720 & -0.7824620 \\
$\mathrm{C}$ & -0.3538400 & 5.0145930 & -1.2360730 \\
$\mathrm{H}$ & 1.7957760 & 4.8967910 & -1.4309730
\end{tabular}




-1.6885250
-2.8642460
-2.7895590
-0.4622020
-1.7637400
-3.8350480
-3.6994280
0.4497600
-4.2698150
-5.4224020
-5.6025960
-3.3415180
-4.1166370
-6.1513320
-6.4699130
-2.4572150
-3.9136100
-3.7469670
-4.1215470
-3.8430810
-4.1967740
-4.0722360
-3.7278480
-4.3638670
2.3544100
2.8412420
3.1324310
4.1161520
4.4122430
4.9295000
4.4986230
5.0105950
-0.4592910
-0.6932850
0.4988840
-4.3041870
-4.1561270
-4.1289280
-4.1875680
-3.4790150
-3.1635770
2.6352660
1.6150330
6.3311430
6.2232120
2.0358400

6.8244040

4.9483860

6.2634920

7.7756210

6.5790040

4.4360120

6.7556820

4.8076490

4.0702320

2.8022060

4.2764780

5.8004500

4.5060040

2.2141170

4.8470080

$-1.1846810$

$-2.1555320$

$-1.5849400$

$-3.5050450$

$-2.9465810$

$-3.9234730$

$-4.2612710$

$-3.2498500$

2. 4445150

1.8451210

2.4320030

1.2852300

1.8637940

1.2972080

0.8186270

1.8701200

$-0.0119400$

$-1.0600250$

$-0.3049170$

$-0.5870190$

0.4219680

$-5.4008090$

$-5.9494830$

$-1.7961130$

$-0.7492820$

3.0508990

3.4126240

0.7107480

$-0.3175580$

1.8316160

-2.1488470
-1.7443110
-1.0812190
-1.9045150
-2.6659070
-1.9671360
-0.7885700
-2.2320180
2.7888130
3.1515820
2.6630670
1.9288400
3.2000010
3.8272930
2.9510940
1.6656240
0.2062940
1.2173220
-1.1283470
0.8701240
-1.4244000
-0.4381280
1.6437650
-2.4562510
-0.4731890
-1.6578080
0.6935100
-1.6412390
0.6575710
-0.5033280
-2.5468030
1.5653990
-0.0525660
0.9943040
-1.1823050
-2.2623170
-1.8674550
-0.7766500
0.1719190
2.6733630
2.7114930
1.9904520
1.8280420
-0.5992740
-0.9768680
-2.9511640

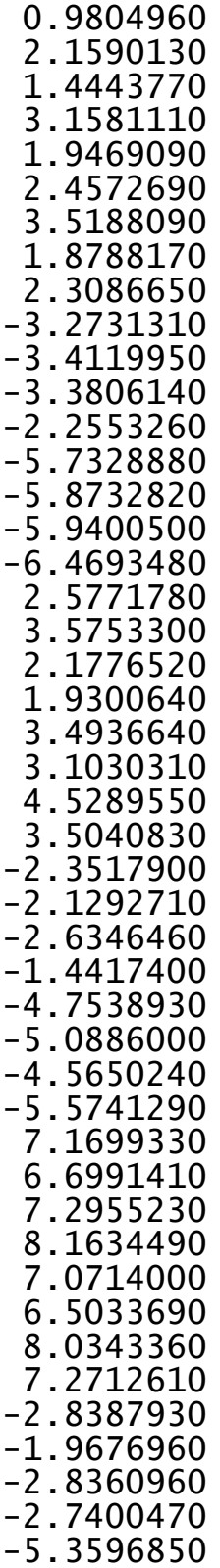

1. 9584210

0.5117370

0.5048130

0.3873770

$-0.3420440$

2.9926060

. 9019690

2.9771540

3.9649030

$-0.8019380$

$-1.7737540$

$-0.0289580$

$-0.7553480$

$-0.6546300$

0.0914200

$-1.6418530$

$-0.4666030$

2. 0191900

1.6287190

2.4746570

1.1810930

4.2577420

4.7245340

3.9556360

5.0090430

$-2.6315210$

$-2.2577630$

$-3.6847960$

$-2.5666710$

$-1.9533030$

$-2.9971260$

$-1.6697340$

$-1.3372490$

1.4981840

1.5213480

2.5327540

1.0504950

0.6465640

0.1002030

0.1432440

1.6550120

$-5.8290340$

$-5.5789700$

$-6.9064820$

$-5.3072620$

$-5.7679280$
$-2.6886480$

$-3.7200360$

$-4.5499220$

$-4.1523060$

$-3.8636600$

$-4.1221700$

$-4.7936680$

$-3.3866770$

$-3.3761200$

$-3.8625530$

$-4.1442470$

$-2.9786480$

$-2.8170760$

$-3.6061780$

$-3.2449250$

$-2.0293780$

3.1212160

3.3527330

4.0333690

2.8431240

2. 3875090

3.2976810

2. 5808920

1.5914170

3.2928740

4.2978400

3. 3925010

2. 6929440

3.5142720

3.5029130

4.5550730

3.1351680

$-1.6171950$

$-2.6038460$

$-1.2785300$

$-1.7234040$

0.7343060

1.4919670

0.6029290

1.1157950

$-1.4865190$

$-0.8725730$

$-1.6824110$

$-2.4462170$

$-1.6106580$ 


\begin{tabular}{|c|c|c|c|c|c|c|c|}
\hline & & & & & & & \\
\hline $\mathrm{H}$ & -6.2829230 & -5.4573160 & -1.1125770 & C & -2.4964270 & 3.5906510 & -1.3857800 \\
\hline $\mathrm{H}$ & -5.3279080 & -5.2889010 & -2.5950910 & $\mathrm{H}$ & -3.5094430 & 3.9794910 & -1.3018000 \\
\hline $\mathrm{H}$ & -5.4011820 & -6.8499890 & -1.7710890 & C & 2.0611950 & 1.4267440 & -0.6200970 \\
\hline C & 0.9282250 & -3.5459370 & 0.5113190 & $\mathrm{C}$ & 3.3018770 & 0.7955100 & -0.6322350 \\
\hline C & 0.8974680 & -3.3330260 & -0.9450970 & C & 1.4526680 & 1.9558210 & -1.7673640 \\
\hline $\mathrm{H}$ & 1.2096840 & -2.6777270 & 1.1078310 & C & 3.9542960 & 0.7023610 & -1.8659800 \\
\hline $\mathrm{H}$ & 0.6398850 & -2.3389750 & -1.2997560 & $\mathrm{H}$ & 4.9304910 & 0.2231020 & -1.9044770 \\
\hline 0 & -0.5040620 & -3.5868350 & 0.3129470 & C & -3.2992920 & 1.4526260 & -0.3207490 \\
\hline $\mathrm{H}$ & -0.8244740 & -2.6543260 & 0.5205410 & C & 3.9308800 & 0.2499360 & 0.6038660 \\
\hline c & 1.1131040 & -6.0305180 & 0.1100440 & C & -3.7070670 & 0.2794370 & -0.9745600 \\
\hline C & 1.5294250 & -5.7488450 & -1.3352250 & C & -3.1303560 & -0.2926480 & -2.2467230 \\
\hline c & 0.8392360 & -4.4959040 & -1.8807150 & C & -5.0823070 & -1.7071250 & -1.3017730 \\
\hline C & 1.4789140 & -4.8566040 & 1.0207140 & $\mathrm{C}$ & -4.7579660 & -0.4967040 & -0.4634160 \\
\hline $\mathrm{H}$ & 1.6033400 & -6.9381780 & 0.4730540 & $\mathrm{C}$ & -2.6323850 & -1.7145120 & -1.9157590 \\
\hline $\mathrm{H}$ & 1. 2875080 & -6.5984390 & -1.9797990 & $\mathrm{H}$ & -2.1586670 & -2.1588400 & -2.7991330 \\
\hline $\mathrm{H}$ & -0.2286630 & -4.6851200 & -2.0537900 & $\mathrm{H}$ & -1.8622470 & -1.6274190 & -1.1458720 \\
\hline H & 2.5697360 & -4.7478140 & 1.0776160 & C & -3.8155040 & -2.5836050 & -1.4199210 \\
\hline $\mathrm{H}$ & 1.1109120 & -5.0107150 & 2.0397890 & $\mathrm{H}$ & -4.0150040 & -3.4066660 & -2.1165500 \\
\hline $\mathrm{H}$ & 0.0321180 & -6.2078240 & 0.1588980 & $\mathrm{H}$ & -3.5770310 & -3.0373680 & -0.4513560 \\
\hline $\mathrm{H}$ & 2.6162950 & -5.6075530 & -1.3780620 & $\mathrm{H}$ & -5.9112050 & -2.2763710 & -0.8673670 \\
\hline $\mathrm{H}$ & 1.2473800 & -4.1833660 & -2.8456290 & $\mathrm{H}$ & -2.3080960 & 0.3150670 & -2.6318400 \\
\hline c & 3.4549190 & -1.6721840 & -0.0089870 & C & -5.4446580 & -1.2128490 & -2.7192720 \\
\hline 0 & 2.5749790 & -0.7619110 & 0.2548600 & $\mathrm{H}$ & -5.6723590 & -2.0762630 & -3.3541890 \\
\hline C & 4.4658500 & -1.8409580 & 1.0563320 & $\mathrm{H}$ & -6.3489530 & -0.5987430 & -2.6698910 \\
\hline $\mathrm{c}$ & 5.5992480 & -2.6411910 & 0.8649570 & C & -4.2576180 & -0.3991240 & -3.2977650 \\
\hline $\mathrm{C}$ & 4.2590760 & -1.2121520 & 2.2929280 & $\mathrm{H}$ & -3.8551420 & -0.8838700 & -4.1942770 \\
\hline $\mathrm{c}$ & 6.5157020 & -2.8051030 & 1.8964780 & $\mathrm{H}$ & -4.5813810 & 0.6038660 & -3.5918160 \\
\hline $\mathrm{H}$ & 5.7543770 & -3.1268020 & -0.0928900 & C & -3.9531980 & 1.8209580 & 0.8635150 \\
\hline C & 5.1668990 & -1.3988080 & 3.3286960 & $\mathrm{C}$ & -3.6655720 & 3.0315630 & 1.7191180 \\
\hline $\mathrm{H}$ & 3.3840710 & -0.5881500 & 2.4314820 & C & -5.6114140 & 1.5870070 & 2.6327110 \\
\hline c & 6.2967490 & -2.1924350 & 3.1307080 & $\mathrm{C}$ & -5.0101130 & 1.0432130 & 1.3628140 \\
\hline $\mathrm{H}$ & 7.3977730 & -3.4172920 & 1.7411370 & $\mathrm{C}$ & -4.9557370 & 3.8795800 & 1.7830870 \\
\hline $\mathrm{H}$ & 4.9952990 & -0.9221330 & 4.2885630 & $\mathrm{H}$ & -4.7746900 & 4.7551590 & 2.4162320 \\
\hline H & 7.0085810 & -2.3328990 & 3.9380920 & $\mathrm{H}$ & -5.2043600 & 4.2503300 & 0.7832020 \\
\hline $\mathrm{H}$ & 1.8701500 & -0.6021590 & -0.4731920 & C & -6.1167100 & 3.0200290 & 2.3517090 \\
\hline $\mathrm{s}$ & 3.3 & -2.6371550 & -1.3873860 & $\mathrm{H}$ & -6.4995920 & 3.4509500 & 3.2836120 \\
\hline & & & & $\mathrm{H}$ & -6.9523750 & 2.9817780 & 1.6464570 \\
\hline & , R) G[M06- & $G(d)]=-3 \xi$ & & $\mathrm{H}$ & -6.4317570 & 0.9510310 & 2.9835230 \\
\hline$P$ & 0.2051850 & 0.4596150 & 0.8289360 & $\mathrm{H}$ & -2.8343270 & 3.6182660 & 1.3203480 \\
\hline 0 & 0.7550690 & -0.9500590 & 0.8436850 & C & -4.4933650 & 1.6745570 & 3.6936090 \\
\hline 0 & -0.5886820 & 0.9359670 & 2.0073870 & $\mathrm{H}$ & -4.9093510 & 2.0912960 & 4.6179180 \\
\hline 0 & -0.6284470 & 0.5625460 & -0.5678500 & $\mathrm{H}$ & -4.1233210 & 0.6705950 & 3.9272710 \\
\hline 0 & 1.3839120 & 1.5422460 & 0.5883900 & C & -3.3413250 & 2.5597730 & 3.1538980 \\
\hline C & -0.9219880 & 1.8367750 & -1.0349510 & $\mathrm{H}$ & -3.2021380 & 3.4437620 & 3.7865650 \\
\hline C & 0.1039 & 2.5772140 & -1.6409980 & $\mathrm{H}$ & -2.3948870 & 2.0154140 & 3.1442590 \\
\hline C & & 2. & -0.908778 & C & -5.4212840 & -0.1070210 & 0.6963270 \\
\hline
\end{tabular}




$\begin{array}{rrrr}\mathrm{H} & -6.2506110 & -0.6996680 & 1.0812640 \\ \mathrm{C} & 4.9704370 & 0.5942420 & 2.7792970 \\ \mathrm{C} & 5.2648550 & 1.6352920 & 3.8281790 \\ \mathrm{C} & 4.0149000 & 2.5795270 & 1.7686200 \\ \mathrm{C} & 4.2802710 & 1.0963910 & 1.6662970 \\ \mathrm{C} & 6.1136240 & 2.7492030 & 3.1782860 \\ \mathrm{H} & 6.2874710 & 3.5419420 & 3.9147590 \\ \mathrm{H} & 7.0923210 & 2.3476290 & 2.8986050 \\ \mathrm{C} & 5.3700020 & 3.3029450 & 1.9350430 \\ \mathrm{H} & 5.1865740 & 4.3782690 & 2.0390740 \\ \mathrm{H} & 5.9703010 & 3.1643430 & 1.0305030 \\ \mathrm{H} & 3.4894890 & 2.9625780 & 0.8908780 \\ \mathrm{H} & 5.7931060 & 1.1961870 & 4.6812310 \\ \mathrm{C} & 3.1778060 & 2.8275600 & 3.0425620 \\ \mathrm{H} & 3.0145370 & 3.9056860 & 3.1525570 \\ \mathrm{H} & 2.1963180 & 2.3606460 & 2.9239350 \\ \mathrm{C} & 3.9243990 & 2.2567980 & 4.2771220 \\ \mathrm{H} & 4.1243680 & 3.0441800 & 5.0128160 \\ \mathrm{H} & 3.3190200 & 1.4942400 & 4.7761570 \\ \mathrm{C} & 4.9835660 & -1.5945790 & 1.7973230 \\ \mathrm{C} & 5.3200570 & -3.0612140 & 1.7030710 \\ \mathrm{C} & 3.8723810 & -2.2083740 & -0.2675340 \\ \mathrm{C} & 4.2564940 & -1.1116090 & 0.6987900 \\ \mathrm{C} & 4.0070920 & -3.8688790 & 1.6456550 \\ \mathrm{H} & 4.2461190 & -4.9208800 & 1.4508340 \\ \mathrm{H} & 3.5009240 & -3.8269690 & 2.6147760 \\ \mathrm{C} & 3.1022270 & -3.2873090 & 0.5314490 \\ \mathrm{H} & 2.7702580 & -4.0740190 & -0.1570530 \\ \mathrm{H} & 2.2094800 & -2.8324970 & 0.9660530 \\ \mathrm{H} & 3.2460540 & -1.8306810 & -1.0809050 \\ \mathrm{H} & 5.9373090 & -3.3786510 & 2.5502050 \\ \mathrm{C} & 5.1613150 & -2.8614290 & -0.8138520 \\ \mathrm{H} & 4.8853020 & -3.7298580 & -1.4231980 \\ \mathrm{H} & 5.6881070 & -2.1627000 & -1.4719050 \\ \mathrm{C} & 6.0689930 & -3.2881900 & 0.3708580 \\ \mathrm{H} & 6.3462730 & -4.3448850 & 0.2868150 \\ \mathrm{H} & 6.9970110 & -2.7083030 & 0.3753870 \\ \mathrm{C} & 5.3415310 & -0.7451060 & 2.8383960 \\ \mathrm{H} & 5.9023930 & -1.1243000 & 3.6913870 \\ \mathrm{C} & 2.1216710 & 1.8185790 & -2.9918280 \\ \mathrm{C} & 1.5430890 & 2.2159570 & -4.3272030 \\ \mathrm{C} & 1.6471050 & 1.0330240 & -5.3079280 \\ \mathrm{C} & 3.0827440 & 0.4633210 & -5.4136290 \\ \mathrm{C} & 4.0407260 & 1.0870410 & -4.3798630 \\ \mathrm{C} & 3.3812770 & 1.1983320 & -3.0306380 \\ \mathrm{H} & 2.0969400 & 3.0754820 & -4.7315680\end{array}$

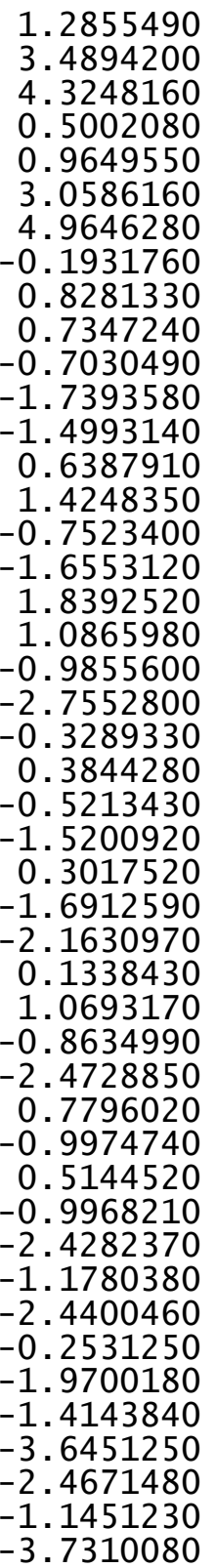

1. 2855490

4. 3248160

. 502080

3. 0586160

4. 9646280

$-0.1931760$

0.8281330

$-0.7030490$

$-1.7393580$

$-1.4993140$

0.7523400

$-1.6553120$

1.8392520

1.0865980

$-0.9855600$

2.7552800

$-0.5213430$

$-1.5200920$

. 3017520

$-1.6912590$

0.1338430

1.0693170

0.7796020

$-0.9974740$

$-2.4282370$

1780380

$-1.9700180$

1.4143840

$-3.7310080$
1. 3486350

0.6277400

2. 0914690

2. 5273270

0.2519890

$-0.6195680$

0.5047620

3. 8737530

4.8354350

6.1787920

6.7530430

5.7757510

4.3739740

.0009970

6.8928160

7.6956120

5.7876010

4.4291740

6.0147900

6.9799240

6.1026870

$-3.6652670$

$-2.6284580$

$-4.5582670$

$-5.5382830$

$-4.3867110$

$-6.3363750$

$-5.6594840$

$-5.1956010$

$-3.6210850$

$-6.1692900$

$-7.0889740$

$-5.0648620$

$-6.7964330$

$-1.9295350$

$-4.0463100$

$-1.5792240$

$-2.2598120$

$-1.0768890$

$-1.8412730$

$-0.7076670$

0.0170010

$-3.0146820$

-3. 9894270

$-3.2407390$

$-2.2554500$
$-6.2912380$

$-6.4161950$

$-4.7222410$

$-4.2290620$

$-5.2581790$

$-4.3122140$

$-2.0837060$

$-2.6379960$

$-1.8904570$

$-1.8620710$

$-2.4502700$

$-1.9551890$

$-3.7083980$

$-2.3498710$

$-2.4157470$

$-3.5456680$

$-2.5531850$

$-0.8672980$

$-0.8297370$

$-2.2089890$

$-0.6510600$

$-0.9160400$

$-1.8239330$

$-1.8425490$

$-2.9452970$

$-2.9672150$

$-0.9774880$

$-4.0625990$

$-2.9273220$

$-4.0762620$

$-2.9792360$

$-4.9248980$

$-4.9522650$

$-0.1325600$

0.8493060

2.3370030

2. 7074180

1. 3625790

2. 3276710

3. 3931280

2. 9359890

4.0250340

4. 0213720

3. 8412180

2. 6989260 


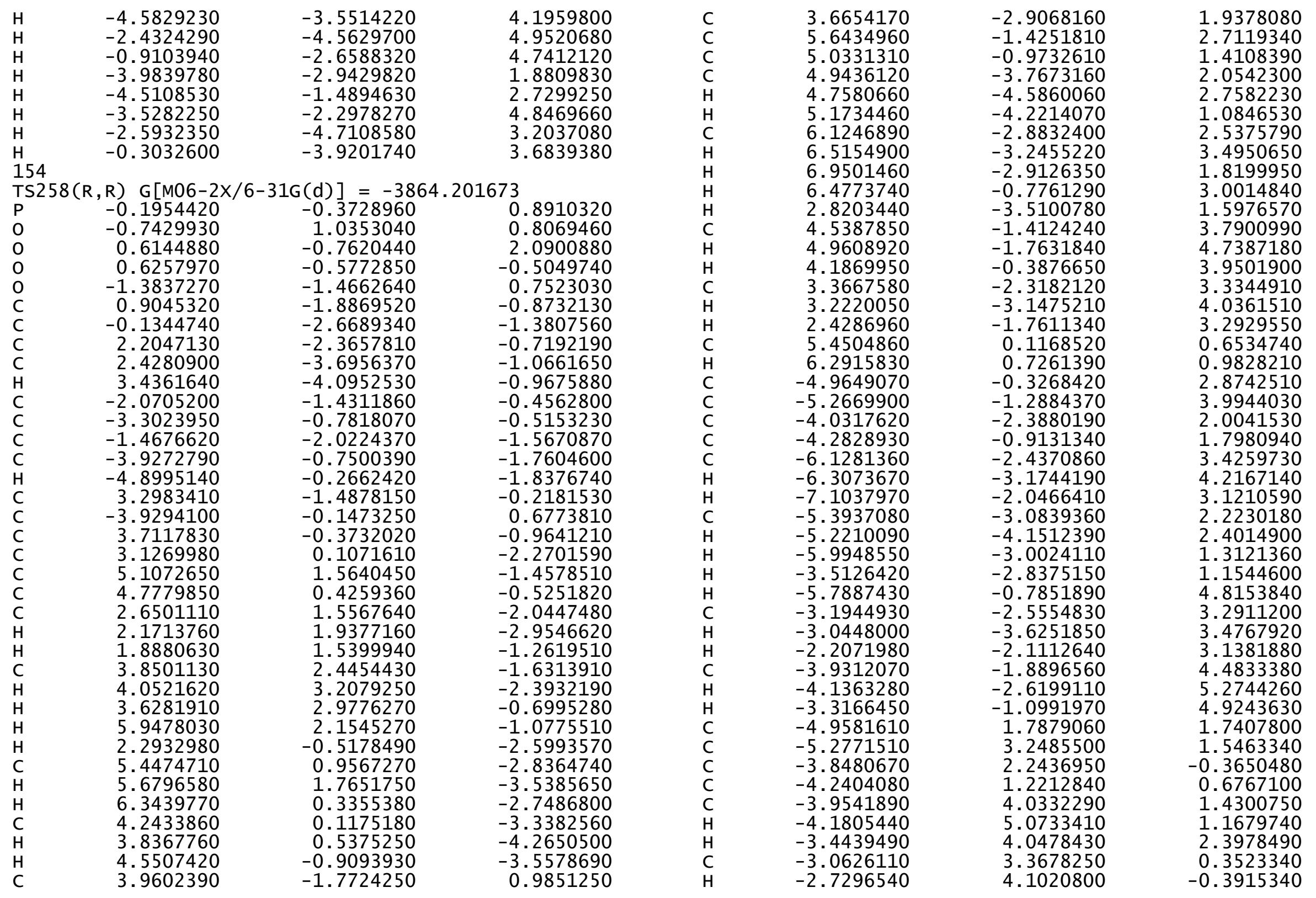




$\begin{array}{rrr}-2.1700700 & 2.9364730 & 0.8106610 \\ -3.2296630 & 1.8032750 & -1.1522910 \\ -5.8871380 & 3.6313120 & 2.3714690 \\ -5.1324890 & 2.8706820 & -0.9510880 \\ -4.8501350 & 3.6892190 & -1.6233350 \\ -5.6710680 & 2.1320430 & -1.5538310 \\ -6.0284220 & 3.3929370 & 0.2039770 \\ -6.2900920 & 4.4453090 & 0.0472200 \\ -6.9651900 & 2.8293300 & 0.2521790 \\ -5.3215610 & 1.0173460 & 2.8398720 \\ -5.8750770 & 1.4615700 & 3.6658410 \\ -3.3402900 & -1.2806040 & -2.9109800 \\ -2.0850800 & -1.9028060 & -2.8272610 \\ -4.0932180 & -1.1752510 & -4.2241030 \\ -3.1885680 & -1.3061560 & -5.4469570 \\ -2.2759840 & -2.5188290 & -5.2854950 \\ -1.3490840 & -2.3229430 & -4.0879510 \\ -3.7967140 & -1.3887340 & -6.3534340 \\ -4.8499000 & -1.9717430 & -4.2565540 \\ -4.6447780 & -0.2290240 & -4.2494810 \\ -2.8895200 & -3.4178020 & -5.1347510 \\ -1.6795630 & -2.6839540 & -6.1884540 \\ -0.7529060 & -3.2221520 & -3.9159460 \\ -0.6229050 & -1.5307240 & -4.3229670 \\ -2.5720410 & -0.4037880 & -5.5508860 \\ 0.1012510 & -4.0322640 & -1.6433450 \\ 1.4022370 & -4.5385520 & -1.5006090 \\ -1.0565900 & -4.9619970 & -1.9619960 \\ -0.6126020 & -6.2631750 & -2.6261480 \\ 0.5188030 & -6.8998120 & -1.8232770 \\ 1.7383900 & -5.9812720 & -1.8296920 \\ -1.4658750 & -6.9438460 & -2.7087360 \\ -1.5579680 & -5.1986300 & -1.0121430 \\ -1.8102330 & -4.4567750 & -2.5710550 \\ 0.1804540 & -7.0602210 & -0.7914600 \\ 0.7885840 & -7.8792790 & -2.2308990 \\ 2.4991860 & -6.3437320 & -1.1297950 \\ 2.1999740 & -6.0117630 & -2.8267520 \\ -0.2631700 & -6.0572880 & -3.6474610 \\ 0.3554970 & 3.6046880 & -0.9229900 \\ -0.3741920 & 2.5588110 & -1.0899850 \\ 0.5286420 & 4.4012910 & -2.1658900 \\ 1.5552900 & 5.3433740 & -2.2945710 \\ -0.3441770 & 4.1760790 & -3.2389370 \\ 1.7046620 & 6.0517220 & -3.4808650 \\ 2.2362150 & 5.5052010 & -1.4658190\end{array}$

-0.1982690
-1.1336870
0.8269060
2.5078330
-0.8830360
0.9433090
-0.5003660
1.0613300
2.4847010
1.2456190
2.4810720
0.3118540
2.0270240
1.4582690
3.7342620
2.5666100
1.2352110
3.7982920
4.6794160
2.5480930
1.0051870
4.0505990
4.5699540
3.6183810
2.6911180
0.3984830

$\begin{array}{lr}4.8968830 & -4.4179900 \\ 3.4401700 & -3.1347080 \\ 5.8332030 & -4.5415620 \\ 6.7748450 & -3.5784990 \\ 4.7265660 & -5.2424600 \\ 6.3913120 & -5.4654850 \\ 1.9294600 & -0.2495650 \\ 4.0961090 & 0.5267250 \\ 1.7438490 & 2.2004690 \\ 2.4667300 & 2.5253150 \\ 1.1609890 & 1.2718530 \\ 2.0290650 & 2.1913600 \\ 0.9694040 & 3.3303620 \\ 0.2169560 & 2.9395610 \\ 3.3024210 & 3.7502990 \\ 4.2866100 & 3.6751520 \\ 3.5400260 & 3.5728690 \\ 2.4327510 & 2.4925430 \\ 3.8409880 & 3.8661560 \\ 4.9378100 & 4.5537620 \\ 3.0388680 & 4.5213410 \\ 3.0455840 & 1.6170840 \\ 1.6629620 & 2.5807510 \\ 2.6591390 & 4.6311360 \\ 4.9342460 & 2.7976560 \\ 4.2129150 & 3.3675950\end{array}$

154

4. 2129150

$\begin{array}{lrrr}\text { TS259(R, R) } \mathrm{G}[\mathrm{M} 06-2 \mathrm{X} / 6-31 \mathrm{G}(\mathrm{d})]=-3864.200922 \\ \mathrm{P} & 0.2146040 & 0.4630700 & 0.8782210 \\ \mathrm{O} & 0.8075950 & -0.9290900 & 0.8522930 \\ \mathrm{O} & -0.5798990 & 0.8851720 & 2.0764860 \\ \mathrm{O} & -0.6420990 & 0.5687290 & -0.5061800 \\ \mathrm{O} & 1.3595630 & 1.5853200 & 0.6488240 \\ \mathrm{C} & -0.9731140 & 1.8451830 & -0.9405340 \\ \mathrm{C} & 0.0209870 & 2.6275420 & -1.5325220 \\ \mathrm{C} & -2.2876240 & 2.2827350 & -0.7867890 \\ \mathrm{C} & -2.5716830 & 3.5782550 & -1.2097200 \\ \mathrm{H} & -3.5912510 & 3.9465950 & -1.1061220 \\ \mathrm{C} & 2.0209690 & 1.5024560 & -0.5720070 \\ \mathrm{C} & 3.2684680 & 0.8859990 & -0.6178940 \\ \mathrm{C} & 1.3751630 & 2.0249860 & -1.7015360 \\ \mathrm{C} & 3.8833600 & 0.7939200 & -1.8709920 \\ \text { H } & 4.8615130 & 0.3218530 & -1.9380510 \\ \mathrm{C} & -3.3379280 & 1.3904490 & -0.2217150 \\ \mathrm{C} & 3.9333920 & 0.3390870 & 0.5982390 \\ \mathrm{C} & -3.7215900 & 0.2280810 & -0.9085290\end{array}$




-3.1434710
-5.0487910
-4.7478970
-2.6060760
-2.1339330
-1.8275930
-3.7608160
-3.9466230
-3.4994570
-5.8582120
-2.3411500
-5.4379020
-5.6478070
-6.3582480
-4.2792070
-3.8741540
-4.6325540
-3.9903160
-3.7264750
-5.6256530
-5.0226280
-5.0378230
-4.8738090
-5.3057530
-6.1702770
-6.5536550
-7.0124480
-6.4258360
-2.9144290
-4.5000090
-4.9178620
-4.1007850
-3.3776350
-3.2585190
-2.4162970
-5.4101980
-6.2202290
5.0029910
5.2962420
3.9949870
4.2848630
6.1140890
6.2863680
7.0948360
5.3397560
5.1388040

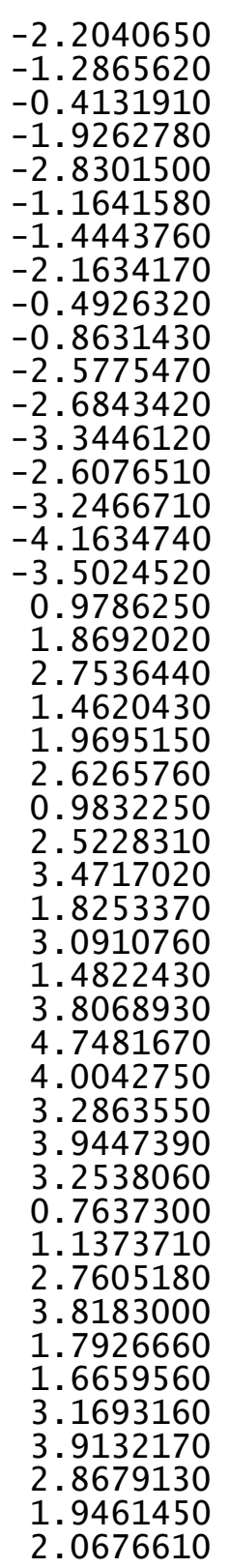

$\begin{array}{rr}-0.2875850 & -2.2040650 \\ -1.7816560 & -1.2865620 \\ -0.5899260 & -0.4131910 \\ -1.7063600 & -1.9262780 \\ -2.1094290 & -2.8301500 \\ -1.6252250 & -1.1641580 \\ -2.6203190 & -1.4443760 \\ -3.4271310 & -2.1634170 \\ -3.0963970 & -0.4926320 \\ -2.3861140 & -0.8631430 \\ 0.3534780 & -2.5775470 \\ -1.2525880 & -2.6843420 \\ -2.1013810 & -3.3446120 \\ -0.6657360 & -2.6076510 \\ -0.3881630 & -3.2466710 \\ -0.8309530 & -4.1634740 \\ 0.6153280 & -3.5024520 \\ 1.7059850 & 0.9786250 \\ 2.8967550 & 1.8692020 \\ 1.3740780 & 2.7536440 \\ 0.8863290 & 1.4620430 \\ 3.7079650 & 1.9695150 \\ 4.5691550 & 2.6265760 \\ 4.1009800 & 0.9832250 \\ 2.8015990 & 2.5228310 \\ 3.1932910 & 3.4717020 \\ 2.7638260 & 1.8253370 \\ 0.7060160 & 3.0910760 \\ 3.5170780 & 1.4822430 \\ 1.4571400 & 3.8068930 \\ 1.8319160 & 4.7481670 \\ 0.4565220 & 4.0042750 \\ 2.3905430 & 3.2863550 \\ 3.2585360 & 3.9447390 \\ 1.8739830 & 3.2538060 \\ -0.2531000 & 0.7637300 \\ -0.8787350 & 1.1373710 \\ 0.6743980 & 2.7605180 \\ 1.7067030 & 3.8183000 \\ 2.6548390 & 1.7926660 \\ 1.1780950 & 1.6659560 \\ 2.8439290 & 3.1693160 \\ 3.6300520 & 3.9132170 \\ 2.4636250 & 2.8679130 \\ 3.4003030 & 1.9461450 \\ 4.4706500 & 2.0676610\end{array}$

5.9270660

3.4484690

5.8465370

3.1754420

2.9953030

2. 2005270

3.9527840

4.1511480

3. 3695290

5.0412280

5.4041730

3.9095030

4.2868560

4.1061730

4. 3621720

3. 6136870

3.1742540

2.8524390

2.2763750

3.2631980

6.0406780

5.2028420

4.9348060

5.7064710

6.1362190

6.4307260

7.0538600

5.4000300

5.9818270

2.0004930

1. 3541720

1.4201410

2.8634780

3.8606220

3.2631420

1.8862320

0.9803920

3.2073040

4.1096830

0.3157290

0.7901730

2.8802480

4.7966510

$-0.2825140$

0.8105580
0.4456100
3.2845340

3. 0402880

1.2660350

2.8715290

3.9450780

2. 3888030

2980940

3.0792000

1.5183120

$-1.5005940$

$-2.9591400$

$-2.1092050$

$-1.0174750$

$-3.7911960$

$-4.8364440$

$-3.7686080$

$-3.2157260$

$-4.0005880$

$-2.7882060$

$-1.7339000$

$-3.2752660$

$-2.7282400$

$-3.5927140$

$-2.0096830$

$-3.1547210$

$-2.5585450$

$-0.6582350$

$-1.0379560$

1.8760230

2.2051500

0.9670750

0.4355500

1.1145480

1.2649190

3.0363820

1.2101450

0.5864840

2.1090940

2. 5193980

0.1915360

$-0.6433820$

0.5497680

3.9529210

4.8686100

6.3472140
1.0299560

0.9288850

4.6564740

3.0835900

3.2105370

2.9752910

4.2977270

5.0405110

4.7965030

1.7487970

1.6301280

$-0.3060470$

0.6689260

1. 5830080

1.3739730

2.5598130

0.4879870

$-0.2075320$

0.9393780

$-1.1045680$

2.4633980

$-0.8809940$

$-1.4994660$

$-1.5358620$

0.2836840

0.1809320

0.2819730

2.7954010

3.6340290

$-2.9466030$

$-4.2692460$

$-5.1828230$

$-5.3570610$

$-4.3953760$

$-3.0214940$

$-4.7532690$

$-6.1548090$

$-6.3849940$

$-4.7892750$

$-4.1343240$

$-4.7352150$

$-5.1749780$

$-1.9033160$

$-2.4216950$

$-2.3010040$ 


$\begin{array}{lrrr}\mathrm{C} & -0.9344500 & 6.5849520 & -2.9077950 \\ \mathrm{C} & -1.9870690 & 5.8489550 & -2.0827550 \\ \mathrm{C} & -1.5943150 & 4.4239170 & -1.7390060 \\ \mathrm{H} & 1.2066440 & 6.9546260 & -2.8009280 \\ \mathrm{H} & 1.0044090 & 4.6488670 & -3.4794420 \\ \mathrm{H} & 1.7436830 & 4.6524130 & -1.8903400 \\ \mathrm{H} & -0.9415750 & 6.2130010 & -3.9408880 \\ \mathrm{H} & -1.1728130 & 7.6524680 & -2.9476880 \\ \mathrm{H} & -2.9520550 & 5.8412360 & -2.6022450 \\ \mathrm{H} & -2.1499910 & 6.3970510 & -1.1445450 \\ \mathrm{H} & 0.4349750 & 6.6447210 & -1.2443890 \\ \mathrm{C} & -0.2167850 & -3.6097200 & -0.7446780 \\ \mathrm{O} & 0.4668220 & -2.5440430 & -0.9699710 \\ \mathrm{C} & -0.3831980 & -4.4630280 & -1.9507000 \\ \mathrm{C} & -1.3516900 & -5.4714700 & -2.0073860 \\ \mathrm{C} & 0.4347300 & -4.2247650 & -3.0637100 \\ \mathrm{C} & -1.4977420 & -6.2320740 & -3.1612650 \\ \mathrm{H} & -1.9907580 & -5.6447670 & -1.1482330 \\ \mathrm{C} & 0.2920950 & -4.9960930 & -4.2106500 \\ \mathrm{H} & 1.1787800 & -3.4374200 & -3.0160880 \\ \mathrm{C} & -0.6748610 & -5.9987460 & -4.2620420 \\ \mathrm{H} & -2.2557560 & -7.0074550 & -3.2024470 \\ \mathrm{H} & 0.9340470 & -4.8137780 & -5.0664430 \\ \mathrm{H} & -0.7889630 & -6.5968880 & -5.1608520 \\ \mathrm{H} & 0.5834270 & -1.8737440 & -0.1589690 \\ \mathrm{~S} & -0.8737830 & -4.0651770 & 0.7397340 \\ \mathrm{C} & -2.3540170 & -1.6899490 & 2.3310660 \\ \mathrm{C} & -1.0852340 & -2.3532130 & 2.6679620 \\ \mathrm{H} & -2.3856910 & -1.1538460 & 1.3752440 \\ \mathrm{H} & -0.1734150 & -1.8992580 & 2.2974660 \\ \mathrm{O} & -1.9073660 & -0.8459290 & 3.4145350 \\ \mathrm{H} & -1.3742970 & -0.0913700 & 2.9804970 \\ \mathrm{C} & -3.5226240 & -3.2155170 & 3.9741800 \\ \mathrm{C} & -2.3222940 & -4.1612830 & 3.9243930 \\ \mathrm{C} & -1.0200060 & -3.3746940 & 3.7637470 \\ \mathrm{C} & -3.6376170 & -2.4092060 & 2.6783950 \\ \mathrm{H} & -4.4461760 & -3.7802630 & 4.1318960 \\ \mathrm{H} & -2.2665810 & -4.7691860 & 4.8319580 \\ \mathrm{H} & -0.7922710 & -2.8214590 & 4.6834530 \\ \mathrm{H} & -3.8821590 & -3.0713540 & 1.8372840 \\ \mathrm{H} & -4.4345270 & -1.6632310 & 2.7440930 \\ \mathrm{H} & -3.4151810 & -2.5276930 & 4.8218230 \\ \mathrm{H} & -2.4378190 & -4.8535130 & 3.0803230 \\ \mathrm{H} & -0.1632450 & -4.0270280 & 3.5750920 \\ \mathrm{H} & & & \end{array}$

$\operatorname{TS} 260(R, R) G[M 06-2 X / 6-31 G(d)]=-3864.201752$
0.9123390

0.8166260

2.1193390

$-0.4760790$

0.7719630

$-0.8424380$

$-1.3407420$

$-0.7066370$

$-1.0685250$

$-0.9895160$

$-0.4442400$

$-0.5251350$

$-1.5404220$

$-1.7793210$

$-1.8744540$

$-0.2114510$

0.6559420

$-0.9596050$

$-2.2633790$

$-1.4596720$

$-0.5241690$

$-2.0356960$

$-2.9423130$

$-1.2475660$

$-1.6306120$

$-2.3960590$

$-0.6997190$

$-1.0832410$

$-2.5879240$

$-2.8384990$

$-3.5422070$

$-2.7522540$

$-3.3362870$

$-4.2608000$

$-3.5584790$

0.9902860

1.9438850

2. 7170160

1. 4142050

2. 0628750

2. 7675250

1.0939810

2. 5465220

3. 5054710

1.8305280

3.0065310 


\begin{tabular}{|c|c|c|c|}
\hline $\begin{array}{l}\mathrm{H} \\
\mathrm{C} \\
\mathrm{H} \\
\mathrm{H} \\
\mathrm{C} \\
\mathrm{H} \\
\mathrm{H} \\
\mathrm{C} \\
\mathrm{H} \\
\mathrm{C} \\
\mathrm{C} \\
\mathrm{C} \\
\mathrm{C} \\
\mathrm{C} \\
\mathrm{H} \\
\mathrm{H} \\
\mathrm{C} \\
\mathrm{H} \\
\mathrm{H} \\
\mathrm{H} \\
\mathrm{H} \\
\mathrm{C} \\
\mathrm{H} \\
\mathrm{H} \\
\mathrm{C} \\
\mathrm{H} \\
\mathrm{H} \\
\mathrm{C} \\
\mathrm{C} \\
\mathrm{C} \\
\mathrm{C} \\
\mathrm{C} \\
\mathrm{H} \\
\mathrm{H} \\
\mathrm{C} \\
\mathrm{H} \\
\mathrm{H} \\
\mathrm{H} \\
\mathrm{H} \\
\mathrm{C} \\
\mathrm{H} \\
\mathrm{H} \\
\mathrm{C} \\
\mathrm{H} \\
\mathrm{H} \\
\mathrm{C}\end{array}$ & $\begin{array}{l}2.8721460 \\
4.5570030 \\
4.9829920 \\
4.1904470 \\
3.3981290 \\
3.2636550 \\
2.4525510 \\
5.4505940 \\
6.2835450 \\
-5.0042880 \\
-5.3170420 \\
-4.0491340 \\
-4.3043560 \\
-6.1646510 \\
-6.3506740 \\
-7.1381260 \\
-5.4108440 \\
-5.2357990 \\
-5.9997890 \\
-3.5162240 \\
-5.8525210 \\
-3.2292470 \\
-3.0777080 \\
-2.2417870 \\
-3.9852380 \\
-4.1976210 \\
-3.3804730 \\
-4.9929670 \\
-5.3160860 \\
-3.8555630 \\
-4.2576670 \\
-3.9951630 \\
-4.2223960 \\
-3.4987910 \\
-3.0853760 \\
-2.7451470 \\
-2.1973660 \\
-3.2241630 \\
-5.9393510 \\
-5.1343440 \\
-4.8459880 \\
-5.6609960 \\
-6.0489330 \\
-6.3132500 \\
-6.9836430 \\
-5.3673990\end{array}$ & $\begin{array}{l}-3.4932120 \\
-1.3666810 \\
-1.7098680 \\
-0.3468290 \\
-2.2897410 \\
-3.1191720 \\
-1.7454100 \\
0.1690130 \\
0.7902440 \\
-0.3491560 \\
-1.3081620 \\
-2.4086290 \\
-0.9356470 \\
-2.4630750 \\
-3.1987050 \\
-2.0787030 \\
-3.1100810 \\
-4.1760580 \\
-3.0339220 \\
-2.8588370 \\
-0.8044650 \\
-2.5679220 \\
-3.6363220 \\
-2.1200930 \\
-1.9010130 \\
-2.6291310 \\
-1.1058950 \\
1.7615820 \\
3.2200900 \\
2.2163280 \\
1.1950520 \\
4.0104470 \\
5.0487440 \\
4.0302530 \\
3.3459420 \\
4.0796780 \\
2.9199550 \\
1.7764920 \\
3.6023030 \\
2.8359310 \\
3.6539720 \\
2.0933690 \\
3.3572430 \\
4.4079240 \\
2.7893580 \\
0.9930030\end{array}$ & $\begin{array}{r}1.6029590 \\
3.7938240 \\
4.7434700 \\
3.9520860 \\
3.3388030 \\
4.0423440 \\
3.2945880 \\
0.6544180 \\
0.9821520 \\
2.8389180 \\
3.9583450 \\
1.9892260 \\
1.7744500 \\
3.3823420 \\
4.1730960 \\
3.0630570 \\
2.1914850 \\
2.3754590 \\
1.2722050 \\
1.1485140 \\
4.7701630 \\
3.2883620 \\
3.4799080 \\
3.1476090 \\
4.4678050 \\
5.2589400 \\
4.9139660 \\
1.6980680 \\
1.4948190 \\
-0.3932240 \\
0.6459210 \\
1.3950580 \\
1.1266000 \\
2.3699140 \\
0.3321720 \\
-0.4089620 \\
0.8042980 \\
-1.1704450 \\
2.3102190 \\
-0.9991320 \\
-1.6694700 \\
-1.6074570 \\
0.1416320 \\
-0.0218370 \\
0.1781490 \\
2.7947760\end{array}$ \\
\hline
\end{tabular}

-5.9344640
-2.0418500
-1.2977720
-1.8618260
-3.3780640
-4.0006820
-3.2924070
-1.3931120
-1.3410390
-0.2362620
-3.6237930
-3.7950220
-5.0609820
-3.9663290
-1.6171130
0.1435280
1.4561220
-1.0030310
-0.5577980
0.6125190
1.8121240
-1.3981130
-1.4323730
-1.8102090
0.3062390
0.8936390
2.5933640
2.2554250
-0.2479200
0.3219130
-0.3952770
0.4848420
1.5031650
-0.3893190
1.6431190
2.1851310
-0.2530750
-1.1723170
0.7639560
2.4400220
-0.9389140
0.8729500
-0.5161260
1.0226510
2.4620650
1.2191260
1.4372360
-1.9455240
-2.4198120
-1.8257630
-1.9966410
-1.1599860
-1.3196360
-2.3105360
-3.5147980
-2.1705870
-3.0568110
-1.6992270
-1.4073630
-0.1001640
-0.7570020
-4.0509440
-4.5356510
-5.0074130
-6.2799060
-6.9116050
-5.9662060
-6.9778550
-5.2832410
-4.5116200
-7.1119590
-7.8716270
-6.3277140
-5.9668940
-6.0393790
3.6000140
2.5457310
4.4000000
5.3509040
4.1694920
6.0626250
5.5168100
4.8938350
3.4267330
5.8388410
6.7925040
4.7194870
6.3996030
1.9161860
4.0985090
1.7585990
2.4752160
3. 6114990 $-2.8128280$ $-4.0494500$ $-5.3393710$ $-5.3659840$ $-4.2515160$ $-2.9191090$ $-6.2015070$ $-4.1255640$ $-3.9413040$ $-5.2195150$ $-6.3332720$ $-4.1260330$ $-4.5395230$ $-5.3957070$ $-1.6075590$ $-1.4934290$ $-1.8792200$ $-2.5954480$ $-1.8450130$ $-1.8543570$ $-2.6662750$ $-0.9047860$ $-2.4214810$ $-0.8102100$ $-2.2896130$ $-1.1768460$ $-2.8601500$ $-3.6219060$ $-0.9163890$ $-1.0840430$ $-2.1584600$ $-2.2883520$ $-3.2292700$ $-3.4737770$ $-1.4612500$ $-4.4073250$ $-3.1239910$ $-4.5322020$ $-3.5725110$ $-5.2300610$ $-5.4554210$ $-0.2427390$ 0.5333000 2.2123830
2.5361380 


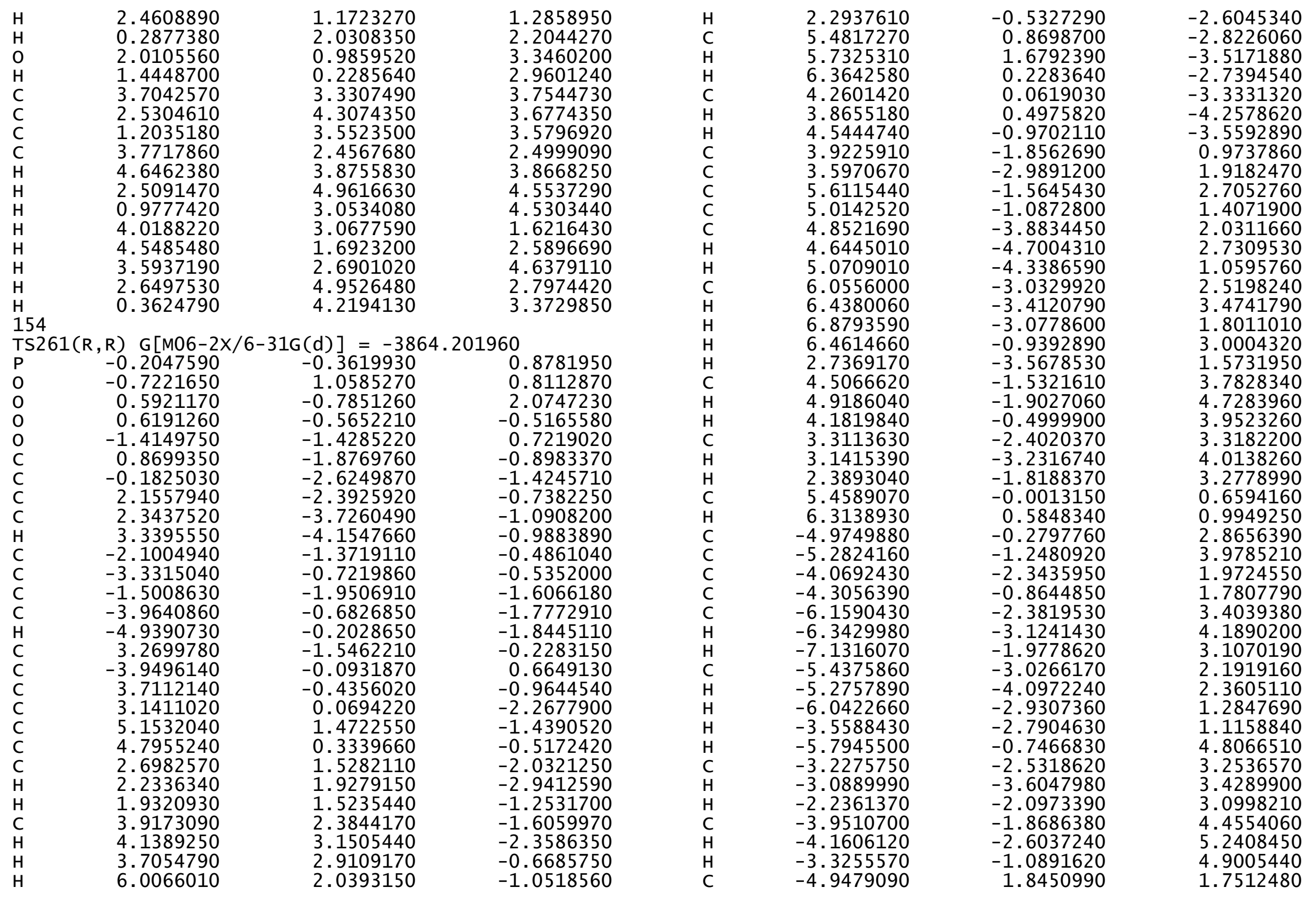




$\begin{array}{rrr}-5.2458650 & 3.3121850 & 1.5728590 \\ -3.8482520 & 2.3039000 & -0.3597790 \\ -4.2453550 & 1.2785330 & 0.6771170 \\ -3.9112990 & 4.0768390 & 1.4531620 \\ -4.1232770 & 5.1234480 & 1.2053230 \\ -3.3922820 & 4.0717670 & 2.4163460 \\ -3.0399170 & 3.4102560 & 0.3594290 \\ -2.7050840 & 4.1470840 & -0.3808870 \\ -2.1486210 & 2.9620750 & 0.8036830 \\ -3.2440190 & 1.8612810 & -1.1566530 \\ -5.8427560 & 3.6968450 & 2.4066450 \\ -5.1292800 & 2.9529310 & -0.9287620 \\ -4.8422130 & 3.7710830 & -1.5994110 \\ -5.6842350 & 2.2251660 & -1.5298800 \\ -6.0057740 & 3.4813360 & 0.2383380 \\ -6.2495700 & 4.5396050 & 0.0928150 \\ -6.9522440 & 2.9344950 & 0.2899160 \\ -5.3142890 & 1.0692720 & 2.8456860 \\ -5.8563240 & 1.5131860 & 3.6793970 \\ -3.3801920 & -1.1990530 & -2.9348690 \\ -2.1185800 & -1.8114540 & -2.8626910 \\ -4.1441310 & -1.0997100 & -4.2424030 \\ -3.2517770 & -1.2173550 & -5.4763340 \\ -2.3033900 & -2.4042080 & -5.3236270 \\ -1.3737550 & -2.1745170 & -4.1346560 \\ -3.8703570 & -1.3201450 & -6.3736620 \\ -4.8902090 & -1.9064110 & -4.2675230 \\ -4.7092410 & -0.1613890 & -4.2621670 \\ -2.8874330 & -3.3214910 & -5.1662930 \\ -1.7112600 & -2.5500070 & -6.2327610 \\ -0.7178420 & -3.0326030 & -3.9789280 \\ -0.7035980 & -1.3337150 & -4.3680550 \\ -2.6598110 & -0.3004820 & -5.5947090 \\ 0.0136760 & -3.9950010 & -1.6910160 \\ -1.1695950 & -4.8720040 & -2.0634650 \\ -0.8793690 & -6.3606950 & -1.8777220 \\ 0.4508780 & -6.7135080 & -2.5374730 \\ 1.5843870 & -6.0049410 & -1.8005640 \\ 1.2969770 & -4.5390250 & -1.5354320 \\ -1.6997020 & -6.9493380 & -2.3001390 \\ -1.4566630 & -4.7030590 & -3.1102040 \\ -2.0356890 & -4.5692060 & -1.4646050 \\ 0.4290700 & -6.3925160 & -3.5874680 \\ 0.6218420 & -7.7945500 & -2.5335720 \\ 2.5244960 & -6.0917130 & -2.3572850 \\ 1.7516920 & -6.5070010 & -0.8377120\end{array}$

$$
\begin{array}{r}
-0.8266780 \\
0.4512660 \\
-0.3069830 \\
0.6581690 \\
1.7033390 \\
-0.2010320 \\
1.8842060 \\
2.3737820 \\
-0.0230420 \\
-1.0048820 \\
1.0202040 \\
2.7012990 \\
-0.6970360 \\
1.1613700 \\
-0.4530960 \\
1.1590680 \\
2.5185130 \\
1.2969970 \\
2.5020950 \\
0.3533310 \\
2.0401030 \\
1.4540870 \\
3.8033080 \\
2.6603310 \\
1.3109760 \\
3.8478270 \\
4.7612010 \\
2.6560410 \\
1.0659600 \\
4.1149600 \\
4.6011760 \\
3.6704110 \\
2.8027270 \\
0.4919030
\end{array}
$$

6.5995850
3.6199500
2.5965490
4.4183370
5.3423870
4.2124120
6.0521580
5.4892990
4.9339810
3.4902660
5.8525790
6.7614990
4.7781870
6.4116040
1.9620690
4.0810360
1.6751280
2.4240260
1.1040530
2.0139060
0.8982150
0.1643840
3.1814590
4.1951070
3.4833690
2.3285360
3.6949590
4.8335970
2.9754610
2.9475270
1.5397900
2.5286250
4.8523060
4.1796770

$-0.8075970$ $-0.8860620$ $-1.0654290$ -2. 1227450 $-2.2317080$ $-3.2106250$ $-3.4127390$ $-1.3917580$ $-4.3847310$ $-3.1218950$ $-4.4883500$ $-3.4946130$ $-5.2208670$ $-5.4082540$ $-0.2326520$ 0.5727890 2. 2186560 2. 5508870 1. 2828660 2. 2098300 3. 3381660 2. 9369210 3.7906580 3. 7281400 3. 6126210 2. 5207520 3. 9153310 4.6162090 4.5537100 1. 6540590 2. 5983800 4.6620270 2.8605350 154

3. 4145260

$\operatorname{TS} 262(R, R) G[M 06-2 X / 6-31 G(d)]=-3864.200253$

$\begin{array}{lrcr}\text { TS262 (R, R) } \quad \text { G }[\mathrm{M} 06-2 X / 6-31 G(\mathrm{~d})]=-3864.200253 \\ \mathrm{P} & 0.1977020 & 0.4197680 & 0.8302670 \\ \mathrm{O} & 0.7173130 & -1.0014380 & 0.8277800 \\ \mathrm{O} & -0.6023990 & 0.8946250 & 2.0051510 \\ \mathrm{O} & -0.6151350 & 0.5636870 & -0.5763120 \\ \mathrm{O} & 1.4042080 & 1.4805340 & 0.6245450 \\ \mathrm{C} & -0.8684880 & 1.8556040 & -1.0184440 \\ \mathrm{C} & 0.1879250 & 2.5833220 & -1.5870440 \\ \mathrm{C} & -2.1590560 & 2.3607930 & -0.8861660 \\ \mathrm{C} & -2.3789230 & 3.6744020 & -1.3103560 \\ \mathrm{H} & -3.3784600 & 4.0948730 & -1.2176050\end{array}$




\begin{tabular}{|c|c|c|}
\hline $\begin{array}{l}2.0900920 \\
3.3057830 \\
1.5142580 \\
3.9397870 \\
4.8967680 \\
-3.2625960 \\
3.9138590 \\
-3.6957090 \\
-3.1188150 \\
-5.1261920 \\
-4.7765230 \\
-2.6691390 \\
-2.1955510 \\
-1.9088110 \\
-3.8855860 \\
-4.1003480 \\
-3.6751890 \\
-5.9781420 \\
-2.2731600 \\
-5.4536080 \\
-5.6998180 \\
-6.3386510 \\
-4.2337250 \\
-3.8336460 \\
-4.5218960 \\
-3.9204470 \\
-3.6068110 \\
-5.6114520 \\
-5.0077740 \\
-4.8697710 \\
-4.6707110 \\
-5.0906010 \\
-6.0667770 \\
-6.4521600 \\
-6.8907980 \\
-6.4569980 \\
-2.7506920 \\
-4.5085470 \\
-4.9257950 \\
-4.1746740 \\
-3.3202970 \\
-3.1618450 \\
-2.3920390 \\
-5.4443690 \\
-6.2967720 \\
4.9575840\end{array}$ & $\begin{array}{r}1.3588100 \\
0.6776980 \\
1.9132420 \\
0.5607600 \\
0.0430010 \\
1.5264710 \\
0.1064300 \\
0.3801180 \\
-0.1828240 \\
-1.5559880 \\
-0.3745570 \\
-1.6261050 \\
-2.0658560 \\
-1.5791450 \\
-2.4687500 \\
-3.2690680 \\
-2.9515150 \\
-2.1083330 \\
0.4071150 \\
-1.0201090 \\
-1.8619430 \\
-0.3787020 \\
-0.2323120 \\
-0.7108530 \\
0.7861840 \\
1.8875910 \\
3.0679780 \\
1.6667940 \\
1.1323380 \\
3.9559810 \\
4.8094740 \\
4.3591570 \\
3.1210680 \\
3.5430480 \\
3.1248530 \\
1.0497870 \\
3.6362560 \\
1.6953640 \\
2.1059250 \\
0.6753330 \\
2.5536300 \\
3.4184020 \\
1.9789390 \\
0.0102400 \\
-0.5648550 \\
0.4085100\end{array}$ & $\begin{array}{l}-0.5786410 \\
-0.5907940 \\
-1.7237190 \\
-1.8255760 \\
-1.8680660 \\
-0.3326600 \\
0.6433640 \\
-1.0171260 \\
-2.2933830 \\
-1.4059260 \\
-0.5369810 \\
-1.9859280 \\
-2.8716130 \\
-1.2028070 \\
-1.5267740 \\
-2.2450490 \\
-0.5657230 \\
-0.9949670 \\
-2.6547840 \\
-2.8167910 \\
-3.4734060 \\
-2.7655520 \\
-3.3617690 \\
-4.2626680 \\
-3.6390930 \\
0.8521190 \\
1.7405860 \\
2.5919680 \\
1.3192520 \\
1.8060150 \\
2.4634840 \\
0.8121350 \\
2.3354510 \\
3.2704060 \\
1.6156320 \\
2.9157220 \\
1.3689720 \\
3.6716590 \\
4.5981660 \\
3.8896180 \\
3.1687870 \\
3.8231190 \\
3.1606100 \\
0.6217100 \\
0.9820310 \\
2.8229380\end{array}$ \\
\hline
\end{tabular}

4.07171

4. 2877620

6.1699480

6. 3662010

7.1363430

5.4498280

5. 3025640

6.0481420

3. 5620270

5. 7937120

3. 2398120

3. 1142740

2. 2427960

3.9620370

4. 1848980

3. 3296990

4.8992950

5. 1867710

3. 7748350

4.1915670

3. 8475090

4. 0514600

3. 3389100

2. 9680380

2. 6195040

2. 0848400

3. 1634170

5.7905350

5.0443980

4.7432950

5. 5970430

5.9316920

6.1707480

6.8802160

5. 2832560

5.8288990

2. 1569070

1.4975900

2. 0476720

3. 5715000

4.1233650

3. 3859530

1. 6278290

1.6593480

0.4137080
1.4355490

2.4280350

0.9371610

2. 5235690

3. 3079770

0914990

3. 1049100

4.1856030

2.9479400

8319110

0.9762840

2.7002560

3.7830760

2. 2688530

2. 0989140

2. 8755870

1.3543030

$-1.7765190$

$-3.2533140$

$-2.3458810$

$-1.2659480$

$-4.0160000$

$-5.0756330$

$-3.9562200$

$-3.4044030$

$-4.1788550$

$-2.9263680$

$-1.9445790$

$-3.5940510$

$-3.0369720$

$-3.8916330$

$-2.3510870$

$-3.5013400$

$-4.5670360$

$-2.9557430$

$-0.9428240$

$-1.3434610$

1.7368250

2.2143220

1.5146980

1.5968000

0.8010890

1.0602290

1.9784470

3. 2935360

2. 0756240
3.8764660

1.8165070

1.7098820

3.2333620

3. 9731090

2.9564000

1.9889340

2. 0948270

1.0860940

0.9386990

4.7295830

3.0888930

3. 2026000

2. 9651110

4.3234480

5.0639220

4.8160730

1.8337040

1. 7353230

$-0.2366770$

0.7349870

1.6718340

1. 4785880

2. 6387610

0.5532690

$-0.1410320$

0.9826010

$-1.0500380$

2. 5831330

$-0.7816980$

$-1.3985900$

$-1.4320410$

0.4044750

0.3168990

0.4147180

2.8783150

3.7313660

$-2.9662650$

4.2465010

$-5.4881640$

$-5.4871020$

$-4.3070310$

$-3.0056580$

$-6.3863930$

$-4.3636380$

$-4.1688050$ 


$\begin{array}{rrr}3.8737260 & 2.6490120 & -5.4032090 \\ 3.9916660 & 1.2145470 & -6.4227350 \\ 5.1897770 & 1.0092890 & -4.1638120 \\ 4.0504780 & -0.2711160 & -4.5359060 \\ 1.7391770 & 0.4610810 & -5.4917230 \\ -0.0569400 & 3.9104170 & -1.9642530 \\ 1.0110730 & 4.8761640 & -2.4127300 \\ 0.9387360 & 6.1471460 & -1.5461520 \\ -0.4704420 & 6.7879550 & -1.5506090 \\ -1.5244120 & 5.8892780 & -2.2297830 \\ -1.3465340 & 4.4500560 & -1.8244000 \\ 0.8517990 & 5.1517660 & -3.4648120 \\ 1.6880620 & 6.8646790 & -1.8936900 \\ -0.4510230 & 7.7552250 & -2.0620330 \\ -1.4095670 & 5.9713430 & -3.3191400 \\ 2.0046860 & 4.4253030 & -2.3419380 \\ 1.2167940 & 5.8691590 & -0.5247530 \\ -0.7866220 & 6.9826060 & -0.5215400 \\ -2.5344930 & 6.2366490 & -1.9929220 \\ -0.4281540 & -3.6579270 & -0.7328390 \\ 0.3211660 & -2.6390500 & -0.9671890 \\ -0.6251040 & -4.5253350 & -1.9237980 \\ -1.6647790 & -5.4600060 & -1.9849500 \\ 0.2370120 & -4.3750770 & -3.0183990 \\ -1.8378190 & -6.2345840 & -3.1256710 \\ -2.3375120 & -5.5639330 & -1.1404450 \\ 0.0671260 & -5.1614040 & -4.1514220 \\ 1.0364790 & -3.6444200 & -2.9670480 \\ -0.9711030 & -6.0897710 & -4.2077860 \\ -2.6510810 & -6.9515470 & -3.1712170 \\ 0.7433040 & -5.0483810 & -4.9926390 \\ -1.1064470 & -6.6989680 & -5.0961600 \\ 0.4596310 & -1.9587390 & -0.1697880 \\ -1.1351670 & -4.0444820 & 0.7477200 \\ -2.5119320 & -1.5692190 & 2.2702910 \\ -1.2851400 & -2.2917370 & 2.6393900 \\ -2.4993460 & -1.0455650 & 1.3071480 \\ -0.3447010 & -1.8919300 & 2.2778270 \\ -2.0396540 & -0.7340040 & 3.3496200 \\ -1.4587590 & -0.0169320 & 2.9123010 \\ -3.7864770 & -3.0052180 & 3.9148330 \\ -2.6358860 & -4.0121200 & 3.9027440 \\ -1.2917720 & -3.2968320 & 2.7526490 \\ -3.8366190 & -2.2163980 & 4.0644330 \\ -4.7405740 & -3.5191260 & \\ -2.6274550 & -4.6061010 & \\ -1.6211330\end{array}$

$\begin{array}{ll}\mathrm{H} & -1.0512630 \\ \mathrm{H} & -4.0989370 \\ \mathrm{H} & -4.5957080 \\ \mathrm{H} & -3.6590830 \\ \mathrm{H} & -2.7728750 \\ \mathrm{H} & -0.4673280\end{array}$

154

$\operatorname{TS} 263(\mathrm{R}, \mathrm{R}) \mathrm{G}[\mathrm{M06}-2 \mathrm{X} / 6-31 \mathrm{G}(\mathrm{d})]=-3864.201643$

$\begin{array}{lrcr}\text { TS263(R, R }) & \text { G }[M 06-2 X / 6-31 G(d)] & -3864.201643 \\ \mathrm{P} & 0.2010780 & 0.4181170 & 0.8651700 \\ \mathrm{O} & 0.7559670 & -0.9893880 & 0.8303650 \\ \mathrm{O} & -0.6050010 & 0.8468560 & 2.0533990 \\ \mathrm{O} & -0.6260660 & 0.5676580 & -0.5341990 \\ \mathrm{O} & 1.3824780 & 1.5118530 & 0.6774480 \\ \mathrm{C} & -0.9110510 & 1.8638970 & -0.9433150 \\ \mathrm{C} & 0.1208480 & 2.6314730 & -1.4886590 \\ \mathrm{C} & -2.2107810 & 2.3432290 & -0.7880610 \\ \mathrm{C} & -2.4377630 & 3.6668360 & -1.1546540 \\ \mathrm{H} & -3.4433780 & 4.0706620 & -1.0461860 \\ \mathrm{C} & 2.0591030 & 1.4248550 & -0.5347130 \\ \mathrm{C} & 3.2843840 & 0.7616660 & -0.5743360 \\ \mathrm{C} & 1.4512050 & 1.9747440 & -1.6650680 \\ \mathrm{C} & 3.8888830 & 0.6515720 & -1.8243590 \\ \mathrm{H} & 4.8508580 & 0.1455460 & -1.8902360 \\ \mathrm{C} & -3.2985720 & 1.4745640 & -0.2587260 \\ \mathrm{C} & 3.9231320 & 0.1878730 & 0.6426660 \\ \mathrm{C} & -3.7066190 & 0.3345300 & -0.9687740 \\ \mathrm{C} & -3.1223050 & -0.1832590 & -2.2607720 \\ \mathrm{C} & -5.0910600 & -1.6262600 & -1.3979480 \\ \mathrm{C} & -4.7672510 & -0.4568500 & -0.5029510 \\ \mathrm{C} & -2.6359030 & -1.6223660 & -1.9922370 \\ \mathrm{H} & -2.1600610 & -2.0288800 & -2.8925220 \\ \mathrm{H} & -1.8696390 & -1.5767280 & -1.2148170 \\ \mathrm{C} & -3.8284110 & -2.5045310 & -1.5444350 \\ \mathrm{H} & -4.0269630 & -3.2949870 & -2.2781290 \\ \mathrm{H} & -3.6004770 & -3.0017160 & -0.5948380 \\ \mathrm{H} & -5.9270700 & -2.2094140 & -0.9969130 \\ \mathrm{H} & -2.2932910 & 0.4360370 & -2.6118640 \\ \mathrm{C} & -5.4377730 & -1.0663990 & -2.7948230 \\ \mathrm{H} & -5.6637080 & -1.8990210 & -3.4702100 \\ \mathrm{H} & -6.3395020 & -0.4504020 & -2.7260020 \\ \mathrm{C} & -4.2414110 & -0.2335710 & -3.3248360 \\ \mathrm{H} & -3.8341150 & -0.6779630 & -4.2398620 \\ \mathrm{H} & -4.5570800 & 0.7840540 & -3.5739180 \\ \mathrm{C} & -3.9605880 & 1.7924910 & 0.9364480 \\ \mathrm{C} & -3.6720020 & 2.9578730 & 1.8532330 \\ \mathrm{C} & -5.6373150 & 1.4870520 & 2.6778640 \\ & & & \end{array}$

4.6673480

1.7692110

2.6430740

3. 0683810

3. 5896910
4.7530260
$-2.8793620$

$-1.4302490$

$-2.3092630$

$-4.7121230$

$-3.9959920$
86590

1.1546540

0.5743360

1.8243590

8902360

.2587260

0.9687740

2607720

1.5444350 20 80

2. 9578730

2.6778640

$-3.5191260$

4.8211330 


$\begin{array}{lr}-5.0272220 & 0.9999300 \\ -4.9561040 & 3.8124220 \\ -4.7745420 & 4.6543840 \\ -5.1920830 & 4.2339100 \\ -6.1293170 & 2.9356920 \\ -6.5190870 & 3.3243980 \\ -6.9575900 & 2.9375530 \\ -6.4657700 & 0.8412440 \\ -2.8321540 & 3.5565930 \\ -4.5296590 & 1.5147940 \\ -4.9517820 & 1.8906620 \\ -4.1695900 & 0.4980050 \\ -3.3657520 & 2.4150680 \\ -3.2259490 & 3.2668330 \\ -2.4233500 & 1.8643560 \\ -5.4391770 & -0.1157320 \\ -6.2754200 & -0.7201020 \\ 4.9855020 & 0.4792960 \\ 5.3017990 & 1.4967450 \\ 4.0454180 & 2.4943970 \\ 4.2916120 & 1.0102530 \\ 6.1581630 & 2.6126720 \\ 6.3474510 & 3.3894790 \\ 7.1296840 & 2.2050860 \\ 5.4107530 & 3.1980620 \\ 5.2414430 & 4.2734450 \\ 6.0014540 & 3.0687620 \\ 3.5172220 & 2.9009720 \\ 5.8321020 & 1.0353370 \\ 3.2229170 & 2.7291740 \\ 3.0751530 & 3.8071110 \\ 2.2342800 & 2.2776750 \\ 3.9727910 & 2.1253810 \\ 4.1884030 & 2.8957310 \\ 3.3623750 & 1.3604620 \\ 4.9624440 & -1.6913390 \\ 5.2795820 & -3.1601850 \\ 3.8272530 & -2.2545680 \\ 4.2317110 & -1.1797840 \\ 3.9558550 & -3.9498730 \\ 4.1796720 & -5.0022590 \\ 3.4567750 & -3.9139600 \\ 3.0518400 & -3.3415340 \\ 2.7131190 & -4.1132410 \\ 2.1628820 & -2.8890090 \\ 3.1984580 & -1.8553020\end{array}$

1.3893750
1.9461790
2.6232700
0.9634390
2.4608450
3.4083310
1.7459140
2.9894240
1.4929170
3.7527570
4.6916940
3.9424350
3.2666970
3.9419860
3.2403150
0.6671040
1.0172440
2.8147910
3.8803050
1.8501720
1.7176310
3.2438460
3.9933620
2.9483300
2.0175260
2.1427690
1.1053480
0.9845450
4.7201850
3.1360830
3.2677350
3.0173810
4.3528130
5.1019900
4.8419770
1.7929140
1.6700450
-0.2730190
0.7098440
1.6101470
1.4000530
2.5831960
0.5095270
-0.1926730
0.9542960
-1.0738850

5. 8996310

5. 1048760

4.8146360

5. 6362280

6.0147880

6. 2751400

6.9517020

5. 3402000

5.9036130

2. 0543450

1. 3339150

1.8272110

3. 3460990

3. 9866200

3. 2909210

1.3468770

1. 4921320

0.2551600

3. 6209730

3. 7258490

5.0454390

3. 9631090

1. 5451450

$-0.1208050$

1. 0224160

0.7135490

$-0.6223630$

$-1.7469370$

$-1.4166420$

1. 5223870

1. 2297820

1.9334150

$-0.5863240$

$-0.8178830$

$-2.6729130$

$-1.9634380$

0.6603860

$-0.3338880$

0.3922220

$-0.5105770$

$-1.5262970$

0.3471420

$-1.6798120$

$-2.1957650$

0.1969640

1. 1280620

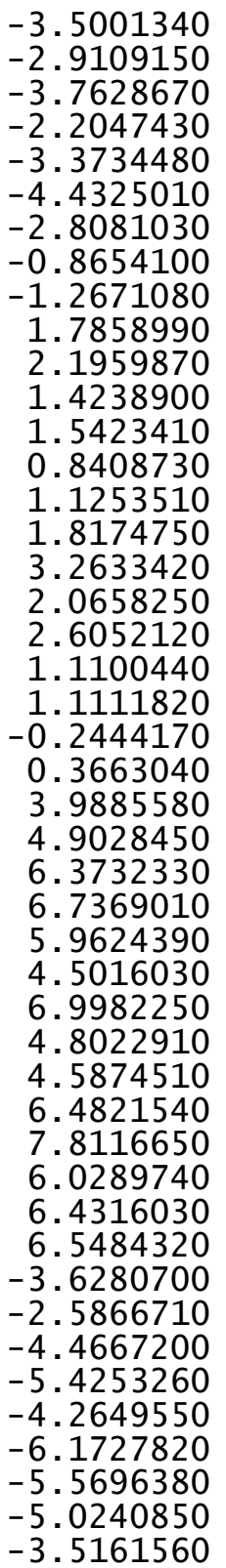
$-0.8416410$

$-1.4674320$

$-1.4880570$

0.3279210

0.2222880

0.3359040

2.8459230

3. 6868450

$-2.9251580$

$-4.1958020$

$-5.4182180$

$-5.5083040$

$-4.3127280$

$-2.9933780$

$-6.3195870$

$-4.3916180$

$-4.0574130$

$-5.5110520$

$-6.4393980$

$-4.2274520$

$-4.4820370$

$-5.3331030$

$-1.7811330$

$-2.1799390$

$-1.9032780$

$-2.5455950$

$-1.8620380$

$-1.6138870$

$-2.2947060$

$-3.2519510$

$-1.6600270$

$-3.6129400$

$-2.4778900$

$-2.4448050$

$-0.8925820$

$-0.8209640$

$-0.7995190$

$-1.0052990$

$-2.0141150$

-2. 1012150

$-3.1039620$

$-3.2627030$

$-1.2600200$

$-4.2580900$

$-3.0324670$ 


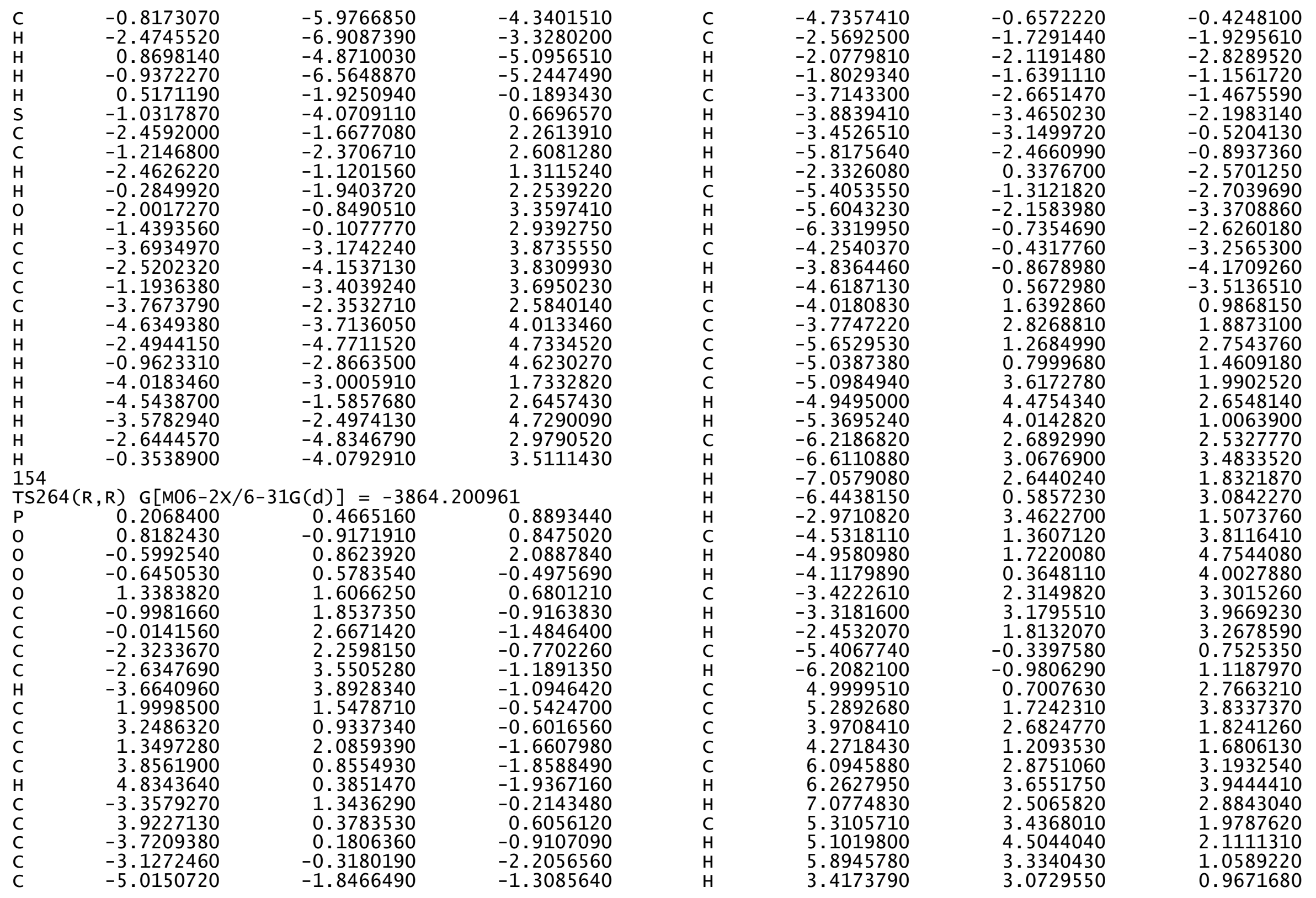




$\begin{array}{lrrr}\text { H } & 5.8470760 & 1.2793080 & 4.6646650 \\ \mathrm{C} & 3.1560280 & 2.8797040 & 3.1211260 \\ \mathrm{H} & 2.9691140 & 3.9505960 & 3.2602610 \\ \mathrm{H} & 2.1839450 & 2.3914620 & 3.0122620 \\ \mathrm{C} & 3.9431130 & 2.2990270 & 4.3254550 \\ \mathrm{H} & 4.1381190 & 3.0732690 & 5.0762670 \\ \mathrm{H} & 3.3684290 & 1.5088160 & 4.8177270 \\ \mathrm{C} & 5.0545700 & -1.4625350 & 1.7308540 \\ \mathrm{C} & 5.4323790 & -2.9157590 & 1.5944740 \\ \mathrm{C} & 3.9169880 & -2.0611020 & -0.3234240 \\ \mathrm{C} & 4.2894670 & -0.9755060 & 0.6602510 \\ \mathrm{C} & 4.1432460 & -3.7612370 & 1.5461710 \\ \mathrm{H} & 4.4090460 & -4.8011420 & 1.3231640 \\ \mathrm{H} & 3.6569680 & -3.7554520 & 2.5263260 \\ \mathrm{C} & 3.1981050 & -3.1830240 & 0.4641140 \\ \mathrm{H} & 2.8788160 & -3.9636930 & -0.2373720 \\ \mathrm{H} & 2.2994290 & -2.7691080 & 0.9266050 \\ \mathrm{H} & 3.2618000 & -1.6842480 & -1.1139190 \\ \mathrm{H} & 6.0773830 & -3.2337300 & 2.4204700 \\ \mathrm{C} & 5.2125770 & -2.6616450 & -0.9127950 \\ \mathrm{H} & 4.9489100 & -3.5233000 & -1.5371150 \\ \mathrm{H} & 5.7045200 & -1.9323730 & -1.5646930 \\ \mathrm{C} & 6.1582280 & -3.0889860 & 0.2416180 \\ \mathrm{H} & 6.4644670 & -4.1346680 & 0.1262040 \\ \mathrm{H} & 7.0687200 & -2.4819420 & 0.2404090 \\ \mathrm{C} & 5.4102760 & -0.6281220 & 2.7847840 \\ \mathrm{H} & 6.0002110 & -1.0110580 & 3.6162400 \\ \mathrm{C} & 1.9693170 & 1.9522980 & -2.9114210 \\ \mathrm{C} & 1.3271670 & 2.3255030 & -4.2250050 \\ \mathrm{C} & 1.3768390 & 1.1153300 & -5.1759010 \\ \mathrm{C} & 2.8090900 & 0.5507270 & -5.3445600 \\ \mathrm{C} & 3.8186110 & 1.2061040 & -4.3802080 \\ \mathrm{C} & 3.2294120 & 1.3399440 & -3.0008820 \\ \mathrm{H} & 1.8701470 & 3.1642460 & -4.6843880 \\ \mathrm{H} & 0.9597280 & 1.4012580 & -6.1461690 \\ \mathrm{H} & 3.1594890 & 0.6912890 & -6.3717020 \\ \mathrm{H} & 4.0756670 & 2.2038380 & -4.7609710 \\ \mathrm{H} & 0.2941870 & 2.6542230 & -4.0818780 \\ \mathrm{H} & 0.7197610 & 0.3424200 & -4.7644560 \\ \mathrm{H} & 2.8008400 & -0.5278940 & -5.1598140 \\ \mathrm{H} & 4.7489250 & 0.6309930 & -4.3501520 \\ \mathrm{C} & -0.3453210 & 3.9884920 & -1.8437210 \\ \mathrm{C} & -1.6727630 & 4.4227390 & -1.7020700 \\ \mathrm{C} & 0.7397570 & 4.9585470 & -2.2744700 \\ \mathrm{C} & 0.1957350 & 6.1859190 & -3.0019810 \\ \mathrm{C} & -0.9478340 & 6.8054660 & -2.2037770 \\ & & & \\ & & & \end{array}$

5.8163260

6.9084650

5. 2844680

4.4557610

7.0500670

7.7394140

6.1768890

5. 7521710

5.8931950

$-3.6017950$

$-2.5192300$

$-4.4553910$

$-5.4771000$

$-4.2052890$

$-6.2393430$

$-5.6591980$

$-4.9787760$

$-3.4076930$

$-5.9947150$

$-7.0249700$

$-4.7878030$

$-6.5944280$

$-4.0786040$

$-1.7425860$

$-2.3886430$

$-1.1971050$

$-1.9160920$

$-0.9027300$

$-0.1354750$

$-3.3036290$

$-4.2294550$

$-3.4203470$

$-2.4857590$

$-3.8847060$

$-4.8459780$

$-2.8731610$

$-3.1428870$

$-1.7533090$

$-2.6230250$

$-4.9144630$

$-4.0567630$
$-2.1166220$

$-3.1567640$

$-1.3678390$

$-2.8878270$

$-1.1947420$

$-2.6620770$

$-1.4208590$

$-3.1004310$

$-3.9953720$

$-0.7758290$

$-0.9926190$

$-1.9853670$

$-2.0580490$

$-3.0845080$

$-3.2142000$

$-1.2094990$

$-4.2334090$

$-3.0243910$

$-4.3008680$

$-3.2681590$

$-5.0782140$

$-5.2013080$

$-0.1759530$

0.7014200

2. 3139390

2. 6461280

1. 3637750

2. 2818180

3. 4068350

2.9815420

3.9391680

3.8813830

3. 7312980

2.6517040

4.0895960

4.7825580

4. 6571380

1.8033980

2.7238210

4.7940570

3. 0298140

3. 5374170

154 -2.3671040
-0.1069080

$\operatorname{TS} 265(R, R) \quad G[M 06-2 X / 6-31 G(d)]=-3864.197897$
$\mathrm{P}$
0.2157870
0.3923600
-0.9799610
0.9574000
0.7874190 


$\begin{array}{rrr}-0.5795780 & 0.6775110 & 2.1949540 \\ -0.6462580 & 0.6278530 & -0.4065850 \\ 1.3396980 & 1.5514510 & 0.8389890 \\ -1.0022890 & 1.9355460 & -0.7086210 \\ -0.0201040 & 2.7917430 & -1.2281780 \\ -2.3256840 & 2.3222870 & -0.5135950 \\ -2.6592240 & 3.6434490 & -0.8225310 \\ -3.6893810 & 3.9687800 & -0.6907700 \\ 1.9990440 & 1.6062740 & -0.3841630 \\ 3.2559940 & 1.0168400 & -0.4929650 \\ 1.3493090 & 2.2433510 & -1.4506360 \\ 3.8798160 & 1.0803660 & -1.7430320 \\ 4.8663180 & 0.6353900 & -1.8567940 \\ -3.3509880 & 1.3507160 & -0.0386390 \\ 3.9275280 & 0.3500200 & 0.6583500 \\ -3.7135420 & 0.2586580 & -0.8423280 \\ -3.1246890 & -0.1119540 & -2.1818080 \\ -5.0039910 & -1.7254410 & -1.4281280 \\ -4.7236060 & -0.6257930 & -0.4354230 \\ -2.5616670 & -1.5415070 & -2.0443230 \\ -2.0728000 & -1.8416190 & -2.9787260 \\ -1.7927980 & -1.5234670 & -1.2685180 \\ -3.7021530 & -2.5212790 & -1.6704490 \\ -3.8728250 & -3.2474480 & -2.4742800 \\ -3.4355290 & -3.0942930 & -0.7751820 \\ -5.8029940 & -2.3840300 & -1.0708700 \\ -2.3336330 & 0.5778200 & -2.4855570 \\ -5.4024000 & -1.0606070 & -2.7638890 \\ -5.6020560 & -1.8393200 & -3.5083210 \\ -6.3302470 & -0.4969930 & -2.6267770 \\ -4.2559850 & -0.1275390 & -3.2339100 \\ -3.8415100 & -0.4722820 & -4.1879030 \\ -4.6243360 & 0.8906080 & -3.3920180 \\ -4.0072290 & 1.5265220 & 1.1877240 \\ -3.7652500 & 2.6241460 & 2.1958260 \\ -5.6375190 & 0.9853840 & 2.9149160 \\ -5.0241400 & 0.6424350 & 1.5821590 \\ -5.0909600 & 3.3976230 & 2.3750540 \\ -4.9435560 & 4.1886660 & 3.1185290 \\ -5.3641790 & 3.8862800 & 1.4340050 \\ -6.2081010 & 2.4189090 & 2.8273770 \\ -6.6020810 & 2.7052470 & 3.8089660 \\ -7.0469300 & 2.4366120 & 2.1250600 \\ -6.4258630 & 0.2723620 & 3.1805570 \\ -2.9635250 & 3.2944150 & 3.9754550 \\ -4.5157550 & 0.9826200 & \end{array}$
$-4.9424420$
$-4.0991260$
$-3.4088380$
$-3.3039580$
$-2.4395020$
$-5.3914970$
$-6.1899600$
4. 9946590
5. 2720150
3. 9540320
4. 2670370
6.0700210
6. 2274200
7.0578000
5. 2872490
5.0703960
5.8759590
3. 4006020
5.8302400
3. 1327760
2. 9384040
2. 1638490
3. 9189550
4. 1041310
3. 3483460
5.0659340
5. 4527530
3.9395210
4. 3019580
4. 1690360
4.4420760
3. 6793280
3. 2238950
2. 9108230
2. 3214550
3. 2857930
6.0964680
5. 2412110
4.9855990
5.7307710
6.1849030
6.4975940
7. 0918950
5. 4134440
6.0028130
1. 9911540

1. 2546190

$-0.0254460$

1.9824740

2. 7807910

1.4872030

$-0.4250690$

$-1.1005790$

0.4663440

1.3821930

2. 5235280

1.0740070

2. 5972000

3.3012440

2. 2690300

3. 2685890

4. 3169250

3.2590370

2.9913870

0.8619440

2. 5866790

3. 6375090

2. 1061920

1.8949830

2. 5920400

1.0552470

$-1.5854170$

$-3.0162800$

$-1.9869610$

$-1.0005430$

$-3.8602060$

$-4.8716050$

$-3.9534870$

$-3.1840450$

$-3.8943730$

$-2.8223850$

$-1.5380170$

$-3.4095910$

$-2.5195620$

$-1.7264820$

$-3.0525190$

$-4.0802270$

$-2.4434310$

$-0.8555300$

$-1.3142970$

2. 2611880
4.9475800

4.0740600

3. 5548940

4.2983380

3.4719450

0.7688500

1.0743250

2.8447610

4. 0087910

2. 0967300

1.8109690

3. 4884640

4. 3134680

3. 1508860

2. 3302640

2. 5642070

1.4077110

1. 2795930

4.7944830

3. 4032250

3. 6462840

3.2425320

4.5481500

5. 3733860

4.9559340

1.6035600

1. 3278090

$-0.5040170$

0.5823390

1. 1914580

0.8685270

2. 1654520

0.1677120

$-0.6074730$

0.6656340

$-1.2572400$

2. 1217570

$-1.1434800$

$-1.8507790$

$-1.7180660$

$-0.0322230$

$-0.2480510$

0.0298690

2.7350180

3.5275690
-2.6968850 


$\begin{array}{rrr}1.3713430 & 2.7891300 & -3.9663260 \\ 1.4666140 & 1.7216520 & -5.0723590 \\ 2.9061370 & 1.1841160 & -5.2649620 \\ 3.8840160 & 1.7270370 & -4.2044440 \\ 3.2636670 & 1.6833490 & -2.8329210 \\ 1.9040070 & 3.6952780 & -4.2907710 \\ 1.0837490 & 2.1396330 & -6.0082500 \\ 3.2834080 & 1.4412590 & -6.2594490 \\ 4.1352790 & 2.7671910 & -4.4530090 \\ 0.3274870 & 3.0738480 & -3.8089730 \\ 0.7985900 & 0.8991490 & -4.7978270 \\ 2.8996310 & 0.0915300 & -5.2039210 \\ 4.8216420 & 1.1633870 & -4.2232480 \\ -1.7034340 & 4.5411850 & -1.2843760 \\ -2.0271510 & 5.9720780 & -1.6283770 \\ -1.4664420 & 6.2992060 & -3.0212590 \\ 0.0544360 & 6.0345020 & -3.1073280 \\ 0.5879760 & 5.1954140 & -1.9257880 \\ -0.3794160 & 4.1213000 & -1.4923830 \\ -1.5772910 & 6.6477230 & -0.8871200 \\ -1.6897550 & 7.3381160 & -3.2819180 \\ 0.6044720 & 6.9804400 & -3.1279970 \\ 0.7316440 & 5.8648940 & -1.0662810 \\ -3.1083210 & 6.1381080 & -1.5962710 \\ -1.9926380 & 5.6701220 & -3.7470370 \\ 0.2827360 & 5.5223880 & -4.0471310 \\ 1.5724320 & 4.7839440 & -2.1577020 \\ -0.1431470 & -3.5098440 & -1.0624510 \\ 0.5160150 & -2.4126980 & -1.1892410 \\ -0.2887670 & -4.2553610 & -2.3400200 \\ -1.2617510 & -5.2481360 & -2.5004800 \\ 0.5534860 & -3.9299310 & -3.4117230 \\ -1.3884730 & -5.9077350 & -3.7170560 \\ -1.9196610 & -5.4870210 & -1.6717890 \\ 0.4314250 & -4.6026060 & -4.6216060 \\ 1.3007370 & -3.1546810 & -3.2822280 \\ -0.5403400 & -5.5899280 & -4.7766890 \\ -2.1505720 & -6.6705270 & -3.8390700 \\ 1.0930960 & -4.3552740 & -5.4454420 \\ -0.6383990 & -6.1102540 & -5.7244820 \\ 0.6217090 & -1.8221630 & -0.3179260 \\ -0.7904840 & -4.1146370 & 0.3716480 \\ -2.3080410 & -1.9387060 & 2.1935800 \\ -1.0267660 & -2.6092460 & 2.4612840 \\ -2.3518960 & -1.3097090 & 1.2967590 \\ -0.1241050 & -2.1042350 & 2.1372920\end{array}$

$\begin{array}{ll}\mathrm{O} & -1.8733860 \\ \mathrm{H} & -1.3540890 \\ \mathrm{C} & -3.4450020 \\ \mathrm{C} & -2.2280960 \\ \mathrm{C} & -0.9406540 \\ \mathrm{C} & -3.5775360 \\ \mathrm{H} & -4.3579870 \\ \mathrm{H} & -2.1594800 \\ \mathrm{H} & -0.7211490 \\ \mathrm{H} & -3.8125320 \\ \mathrm{H} & -4.3876440 \\ \mathrm{H} & -3.3478750 \\ \mathrm{H} & -2.3329690 \\ \mathrm{H} & -0.0725530 \\ \mathrm{H} & \end{array}$
$-1.2000660$
$-4.5574680$
$-3.7349940$
$-2.7125520$
$-4.2375740$
$-5.2529560$
$-3.2735350$
$-3.2902390$
$-1.9919150$
$-3.0425490$
$-5.1626200$
$-4.3491100$

3.6747780

3. 5290760

3.4482550

2. 4673350

3.7753760

4.3704480

4.4191410

1.5635800

2. 6088880

4. 5873260

2. 6192610

3.1943470

TS266 $(R, R) \quad G[M 06-2 X / 6-31 G(d)]=-3864.199180$

$\begin{array}{lrrr}\text { TS266(R, R }(\mathrm{G}[\mathrm{M} 06-2 \mathrm{C} / \mathrm{r}-31 \mathrm{G}(\mathrm{d})]=-3864.199180 \\ \mathrm{P} & -0.1935610 & -0.2896640 & 0.8837370 \\ \mathrm{O} & -0.6651440 & 1.1403560 & 0.7333030 \\ \mathrm{O} & 0.6022490 & -0.6641250 & 2.0968810 \\ \mathrm{O} & 0.6025220 & -0.6078330 & -0.5045770 \\ \mathrm{O} & -1.4377920 & -1.3232120 & 0.7997770 \\ \mathrm{C} & 0.8079390 & -1.9477960 & -0.8060670 \\ \mathrm{C} & -0.2690050 & -2.6954080 & -1.2865100 \\ \mathrm{C} & 2.0807720 & -2.4866890 & -0.6228620 \\ \mathrm{C} & 2.2342060 & -3.8419280 & -0.9014050 \\ \mathrm{H} & 3.2184410 & -4.2902740 & -0.7742440 \\ \mathrm{C} & -2.1305300 & -1.3061190 & -0.4059950 \\ \mathrm{C} & -3.3244260 & -0.5939680 & -0.4815040 \\ \mathrm{C} & -1.5710400 & -1.9943660 & -1.4910710 \\ \mathrm{C} & -3.9766080 & -0.5834690 & -1.7182800 \\ \mathrm{H} & -4.9222340 & -0.0518390 & -1.8047980 \\ \mathrm{C} & 3.2208400 & -1.6434270 & -0.1668360 \\ \mathrm{C} & -3.8994250 & 0.1205800 & 0.6927560 \\ \mathrm{C} & 3.6902300 & -0.5910220 & -0.9684610 \\ \mathrm{C} & 3.1242290 & -0.1431180 & -2.2941730 \\ \mathrm{C} & 5.1868550 & 1.2401720 & -1.5619200 \\ \mathrm{C} & 4.8015060 & 0.1686370 & -0.5735100 \\ \mathrm{C} & 2.7292180 & 1.3398390 & -2.1376010 \\ \mathrm{H} & 2.2658470 & 1.7021600 & -3.0629480 \\ \mathrm{H} & 1.9727550 & 1.4032970 & -1.3518590 \\ \mathrm{C} & 3.9789320 & 2.1801580 & -1.7729760 \\ \mathrm{H} & 4.2177560 & 2.8923280 & -2.5718940 \\ \mathrm{H} & 3.7931770 & 2.7683840 & -0.8673140 \\ \mathrm{H} & 6.0611100 & 1.8009420 & -1.2141120 \\ \mathrm{H} & 2.2553550 & -0.7363170 & -2.5889270 \\ \mathrm{C} & 5.4839540 & 0.5489640 & -2.9105490\end{array}$




$\begin{array}{lrrr}\text { H } & 5.7557940 & 1.3083260 & -3.6521240 \\ \mathrm{H} & 6.3454450 & -0.1159310 & -2.7961130 \\ \mathrm{C} & 4.2321400 & -0.2454500 & -3.3661120 \\ \mathrm{H} & 3.8427850 & 0.1516480 & -4.3102520 \\ \mathrm{H} & 4.4811090 & -1.2970480 & -3.5375370 \\ \mathrm{C} & 3.8735950 & -1.9037410 & 1.0468690 \\ \mathrm{C} & 3.5231740 & -2.9713360 & 2.0561620 \\ \mathrm{C} & 5.5862720 & -1.5666080 & 2.7468740 \\ \mathrm{C} & 4.9920190 & -1.1455110 & 1.4281490 \\ \mathrm{C} & 4.7521480 & -3.8955680 & 2.2092190 \\ \mathrm{H} & 4.5264390 & -4.6663260 & 2.9543750 \\ \mathrm{H} & 4.9490830 & -4.4096390 & 1.2626460 \\ \mathrm{C} & 5.9843430 & -3.0560190 & 2.6419700 \\ \mathrm{H} & 6.3624930 & -3.3924480 & 3.6138540 \\ \mathrm{H} & 6.8008140 & -3.1663290 & 1.9220030 \\ \mathrm{H} & 6.4572700 & -0.9518820 & 2.9996460 \\ \mathrm{H} & 2.6428760 & -3.5418520 & 1.7505210 \\ \mathrm{C} & 4.4925470 & -1.4391820 & 3.8284800 \\ \mathrm{H} & 4.9015670 & -1.7662350 & 4.7911980 \\ \mathrm{H} & 4.1994840 & -0.3896940 & 3.9392620 \\ \mathrm{C} & 3.2687270 & -2.2991500 & 3.4234920 \\ \mathrm{H} & 3.0829900 & -3.0836430 & 4.1658620 \\ \mathrm{H} & 2.3629930 & -1.6932570 & 3.3586170 \\ \mathrm{C} & 5.4639060 & -0.1188330 & 0.6161680 \\ \mathrm{H} & 6.3398340 & 0.4580210 & 0.9113960 \\ \mathrm{C} & -4.9269440 & 0.0803050 & 2.9005240 \\ \mathrm{C} & -5.2782820 & -0.8219190 & 4.0550950 \\ \mathrm{C} & -4.1290400 & -2.0628840 & 2.0969850 \\ \mathrm{C} & -4.2907480 & -0.5832490 & 1.8412930 \\ \mathrm{C} & -6.2131620 & -1.9336370 & 3.5321260 \\ \mathrm{H} & -6.4312490 & -2.6309600 & 4.3489730 \\ \mathrm{H} & -7.1652740 & -1.4941850 & 3.2195250 \\ \mathrm{C} & -5.5294550 & -2.6656430 & 2.3480760 \\ \mathrm{H} & -5.4212470 & -3.7347690 & 2.5626320 \\ \mathrm{H} & -6.1317310 & -2.5786140 & 1.4384300 \\ \mathrm{H} & -3.6454750 & -2.5721830 & 1.2601020 \\ \mathrm{H} & -5.7614910 & -0.2596180 & 4.8612460 \\ \mathrm{C} & -3.2930330 & -2.2372010 & 3.3838230 \\ \mathrm{H} & -3.2064970 & -3.3070270 & 3.6053580 \\ \mathrm{H} & -2.2823530 & -1.8584660 & 3.2095450 \\ \mathrm{C} & -3.9783440 & -1.4876620 & 4.5568490 \\ \mathrm{H} & -4.2221100 & -2.1770400 & 5.3732620 \\ \mathrm{H} & -3.3130110 & -0.7224200 & 4.9676420 \\ \mathrm{C} & -4.8009380 & 2.1502510 & 1.6940150 \\ \mathrm{C} & -5.0360550 & 3.6191360 & 1.4486400 \\ \mathrm{C} & -3.6878330 & 2.4635600 & -0.4362030 \\ & & & \end{array}$

-4.1269050
-3.6712300
-3.8394380
-3.1494970
-2.8328520
-2.4742240
-1.9565880
-3.1038970
-5.6140860
-4.9418680
-4.6221260
-5.5291800
-5.7918300
-5.9913010
-6.7603000
-5.2015470
-5.7209470
-3.4334680
-4.1044480
-4.1081270
-2.6773280
-1.6463510
-2.2216200
-3.5623130
-4.6188220
-2.3465860
-1.3125730
-5.1235630
-4.6902500
-2.6813360
-0.7521870
-0.1001340
-1.2918210
-1.0548600
0.2748120
1.4187700
1.1682000
-1.8853770
-1.5144120
-2.1777480
0.2767350
0.4149900
2.3609980
1.5657810
-1.0301930
0.5580370

.5045980

4. 3205200

5. 3631470

4. 3362440

3.5693710

4.2570820

3. 1094760

1.9591110

4.0665300

. 1394040

3.9126520

2.4092570

3.7582030

4.8178950

3.2546920

1.4417350

1.9470190

$-1.2118090$

$-1.1940200$

$-2.6149620$

$-3.1773570$

$-2.4243970$

$-1.9149060$

$-0.5231030$

$-2.6298020$

$-3.1171200$

$-1.5397050$

$-0.8046860$

$-3.2489280$

$-4.2402380$

$-3.0303300$

$-4.0783070$

$-4.9415300$

$-6.4241940$

$-6.8556660$

$-6.1329870$

$-4.6476610$

$-7.0114510$

$-4.8263510$

$-4.5798400$

$-6.6037090$

$-7.9384660$

$-6.2744710$

$-6.5839220$

$-6.5995630$

3.5705210
0.6464880

1.2926920

0.9988080

2. 2543440

0.2286710

$-0.5470880$

0.6905840

$-1.2113600$

2. 2642200

$-1.0339290$

$-1.7420620$

$-1.6002260$

0.1082110

$-0.0863240$

0.1860250

2.8215200

3.6344530

$-2.8327940$

$-4.1826550$

$-4.7636600$

$-4.9022310$

$-4.0303000$

$-2.7302950$

$-4.8648080$

$-5.7312270$

$-5.9439060$

$-4.5904130$

$-4.0961350$

$-4.0863560$

$-4.6412010$

$-3.8683890$

$-1.4979240$

$-1.8681610$

$-1.5870120$

$-2.1995020$

$-1.4927280$

$-1.3086390$

$-1.9912980$

$-2.9365820$

$-1.3348340$

$-3.2682980$

$-2.1230720$

$-2.0343680$

$-0.5016730$

$-0.5036470$

$-1.1123060$ 


\begin{tabular}{|c|c|c|c|c|c|c|c|}
\hline 0 & & 25561530 & 12305760 & & 15316090 & 18506100 & \\
\hline c & $\begin{array}{r}-0.2242410 \\
0.7661690\end{array}$ & $\begin{array}{l}2.5564530 \\
4.3004240\end{array}$ & $\begin{array}{l}-1.2305760 \\
-2.3903310\end{array}$ & $\mathrm{C}$ & $\begin{array}{l}1.5346090 \\
3.9646650\end{array}$ & $\begin{array}{l}1.8596100 \\
0.5122400\end{array}$ & $\begin{array}{l}-1.7512950 \\
-1.8199580\end{array}$ \\
\hline C & 1.8364850 & 5.1851950 & -2.5626270 & $\mathrm{H}$ & 4.9222950 & -0.0047910 & -1.8494190 \\
\hline C & -0.1199030 & 4.0693480 & -3.4511830 & C & -3.2421120 & 1.5615300 & -0.3367000 \\
\hline $\mathrm{C}$ & 2.0159350 & 5.8311800 & -3.7798810 & $\mathrm{C}$ & 3.9142690 & 0.0739070 & 0.6518360 \\
\hline $\mathrm{H}$ & 2.5270780 & 5.3513610 & -1.7428050 & C & -3.6939730 & 0.4201790 & -1.0179680 \\
\hline C & 0.0568350 & 4.7281160 & -4.6618600 & C & -3.1311690 & -0.1527700 & -2.2963350 \\
\hline $\mathrm{H}$ & -0.9437110 & 3.3781090 & -3.3127000 & $\mathrm{C}$ & -5.1543580 & -1.4954690 & -1.3986320 \\
\hline C & 1.1253740 & 5.6074380 & -4.8287530 & C & -4.7846150 & -0.3174120 & -0.5335580 \\
\hline $\mathrm{H}$ & 2.8526040 & 6.5096190 & -3.9110530 & $\mathrm{C}$ & -2.7017620 & -1.6027650 & -1.9912240 \\
\hline $\mathrm{H}$ & -0.6379650 & 4.5539180 & -5.4770640 & $\mathrm{H}$ & -2.2429600 & -2.0500870 & -2.8808960 \\
\hline $\mathrm{H}$ & 1.2655280 & 6.1169760 & -5.7771050 & $\mathrm{H}$ & -1.9340000 & -1.5676150 & -1.2147490 \\
\hline $\mathrm{H}$ & -0.3791730 & 1.9725850 & -0.3626700 & C & -3.9274490 & -2.4261170 & -1.5217530 \\
\hline $\mathrm{S}$ & 1.2932810 & 4.0908310 & 0.3124530 & $\mathrm{H}$ & -4.1571870 & -3.2267610 & -2.2349700 \\
\hline C & 2.5998180 & 1.7457410 & 2.0849400 & $\mathrm{H}$ & -3.7188500 & -2.9070670 & -0.5593400 \\
\hline $\mathrm{C}$ & 1.4013870 & 2.5447990 & 2.3815790 & $\mathrm{H}$ & -6.0125330 & -2.0346090 & -0.9831120 \\
\hline $\mathrm{H}$ & 2.5605440 & 1.1237710 & 1.1830180 & $\mathrm{H}$ & -2.2788000 & 0.4240270 & -2.6628660 \\
\hline $\mathrm{H}$ & 0.4447990 & 2.1412080 & 2.0699750 & C & -5.4795790 & -0.9580910 & -2.8094150 \\
\hline 0 & 2.1103770 & 1.0459800 & 3.2495110 & $\mathrm{H}$ & -5.7377260 & -1.7980750 & -3.4638230 \\
\hline $\mathrm{H}$ & 1.5015040 & 0.3070360 & 2.8945670 & $\mathrm{H}$ & -6.3567950 & -0.3061360 & -2.7567600 \\
\hline C & 3.9359950 & 3.3027020 & 3.5612910 & C & -4.2519980 & -0.1858240 & -3.3592320 \\
\hline C & 2.8198830 & 4.3417450 & 3.4489050 & $\mathrm{H}$ & -3.8629020 & -0.6682540 & -4.2628630 \\
\hline $\mathrm{C}$ & 1.4511230 & 3.6614490 & 3.3814120 & $\mathrm{H}$ & -4.5277670 & 0.8369220 & -3.6335120 \\
\hline C & 3.9484250 & 2.3788630 & 2.3411420 & C & -3.8917930 & 1.9346720 & 0.8489600 \\
\hline $\mathrm{H}$ & 4.9080290 & 3.7964810 & 3.6512960 & C & -3.5588360 & 3.1117690 & 1.7347670 \\
\hline $\mathrm{H}$ & 2.8391970 & 5.0284520 & 4.3000460 & C & -5.5843980 & 1.7441360 & 2.5910760 \\
\hline H & 1.1994320 & 3.2141010 & 4.3510530 & C & -4.9898950 & 1.1971540 & 1.3193460 \\
\hline $\mathrm{H}$ & 4.2253110 & 2.9408090 & 1.4393840 & $\mathrm{C}$ & -4.8076860 & 4.0196560 & 1.7987860 \\
\hline $\mathrm{H}$ & 4.6810380 & 1.5755500 & 2.4584260 & $\mathrm{H}$ & -4.5949570 & 4.8714720 & 2.4541460 \\
\hline $\mathrm{H}$ & 3.7922220 & 2.7034260 & 4.4686680 & $\mathrm{H}$ & -5.0229990 & 4.4239150 & 0.8041160 \\
\hline $\mathrm{H}$ & 2.9731160 & 4.9458390 & 2.5452690 & C & -6.0172370 & 3.2046670 & 2.3309640 \\
\hline $\mathrm{H}$ & 0.6494570 & 4.3674270 & 3.1490040 & $\mathrm{H}$ & -6.3955670 & 3.6350370 & 3.2649760 \\
\hline & & & & $\mathrm{H}$ & -6.8414340 & 3.2193450 & 1.6114900 \\
\hline & R) $\mathrm{G}[\mathrm{M06}-2$ & (d) $]=-386$ & 455 & $\mathrm{H}$ & -6.4392740 & 1.1412080 & 2.9168090 \\
\hline $\mathrm{P}$ & 0.2008620 & 0.4136310 & 0.8167180 & $\mathrm{H}$ & -2.6938100 & 3.6654090 & 1.3617440 \\
\hline 0 & 0.7099460 & -1.0113760 & 0.8252960 & C & -4.4806750 & 1.7583520 & 3.6700980 \\
\hline 0 & -0.5949860 & 0.9047440 & 1.9878710 & $\mathrm{H}$ & -4.8909950 & 2.1775020 & 4.5958560 \\
\hline 0 & -0.6117920 & 0.5520420 & -0.5910320 & $\mathrm{H}$ & -4.1623270 & 0.7338490 & 3.8902460 \\
\hline 0 & 1.4154750 & 1.4639510 & 0.6034440 & C & -3.2798750 & 2.5970170 & 3.1643120 \\
\hline C & -0.8482030 & 1.8447340 & -1.0413510 & $\mathrm{H}$ & -3.1081400 & 3.4612600 & 3.8159910 \\
\hline C & 0.2160260 & 2.5485870 & -1.6220690 & $\mathrm{H}$ & -2.3604070 & 2.0085090 & 3.1575270 \\
\hline C & -2.1271580 & 2.3764720 & -0.8959890 & C & -5.4443980 & 0.0800040 & 0.6254100 \\
\hline C & -2.3222040 & 3.6959540 & -1.3130630 & $\mathrm{H}$ & -6.3047440 & -0.4813250 & 0.9885430 \\
\hline $\mathrm{H}$ & -3.3111660 & 4.1381980 & -1.2088600 & C & 4.9419910 & 0.3822500 & 2.8379910 \\
\hline C & 2.1064660 & 242030 & -0.5945950 & $\mathrm{C}$ & 5.2668570 & 1.4141060 & 3.8870250 \\
\hline C & 3.32041 & 07140 & -0.5911650 & C & 4.0804400 & 2.4018040 & 1.8102650 \\
\hline
\end{tabular}




\begin{tabular}{|c|c|c|}
\hline $\begin{array}{l}4.2859760 \\
6.1674880 \\
6.3639690 \\
7.1326510 \\
5.4621450 \\
5.3223220 \\
6.0667030 \\
3.5809590 \\
5.7669230 \\
3.2400060 \\
3.1214530 \\
2.2409240 \\
3.9474630 \\
4.1703550 \\
3.3053150 \\
4.8728500 \\
5.1475860 \\
3.7617170 \\
4.1790450 \\
3.8018400 \\
3.9975980 \\
3.2847450 \\
2.9387440 \\
2.5922970 \\
2.0543580 \\
3.1614690 \\
5.7405720 \\
5.0306790 \\
4.7286930 \\
5.5955310 \\
5.9020410 \\
6.1305710 \\
6.8561930 \\
5.2554740 \\
5.7903680 \\
3.4177340 \\
2.1817840 \\
4.1919570 \\
3.3134020 \\
2.4483840 \\
1.4942370 \\
3.9384530 \\
4.9734270 \\
4.7139780 \\
3.0959460\end{array}$ & $\begin{array}{r}0.9087130 \\
2.4907750 \\
3.2786100 \\
2.0492650 \\
3.0693870 \\
4.1518500 \\
2.9018490 \\
2.8031040 \\
0.9569230 \\
2.6889150 \\
3.7733790 \\
2.2639270 \\
2.0908590 \\
2.8710820 \\
1.3547390 \\
-1.8091480 \\
-3.2891690 \\
-2.3827930 \\
-1.3001360 \\
-4.0398410 \\
-5.1029940 \\
-3.9668310 \\
-3.4297770 \\
-4.2065100 \\
-2.9426470 \\
-1.9816020 \\
-3.6295670 \\
-3.0874300 \\
-3.9424800 \\
-2.4091720 \\
-3.5534320 \\
-4.6221380 \\
-3.0178800 \\
-0.9714330 \\
-1.3708180 \\
0.9963880 \\
1.6624130 \\
0.7916430 \\
0.8761780 \\
2.1311670 \\
2.0499860 \\
0.8775040 \\
1.5617480 \\
-0.1705760 \\
3.0121040 \\
2.2641570\end{array}$ & $\begin{array}{r}1.7157150 \\
3.2437590 \\
3.9797960 \\
2.9775910 \\
1.9896080 \\
2.0872030 \\
1.0928420 \\
0.9253810 \\
4.7475330 \\
3.0737350 \\
3.1789930 \\
2.9445580 \\
4.3184060 \\
5.0551310 \\
4.8111020 \\
1.8635060 \\
1.7777570 \\
-0.2130720 \\
0.7552970 \\
1.7077220 \\
1.5256470 \\
2.6692370 \\
0.5755200 \\
-0.1172190 \\
0.9919190 \\
-1.0346780 \\
2.6332780 \\
-0.7419480 \\
-1.3578230 \\
-1.3899040 \\
0.4553950 \\
0.3768070 \\
0.4710060 \\
2.9054770 \\
3.7658910 \\
-3.0091510 \\
-2.9869450 \\
-4.2979280 \\
-5.5432520 \\
-5.4733380 \\
-4.2837510 \\
-6.4419540 \\
-4.3633580 \\
-4.2554240 \\
-5.3666880 \\
-6.3953540\end{array}$ \\
\hline
\end{tabular}

0.9525490
0.7265930
2.6638110
-0.0032130
1.0783520
1.0624650
-0.3358470
-1.4246100
-1.2773880
0.8998910
1.8081890
-0.3136860
-1.3265200
2.0612970
1.3786090
-0.6206210
-2.4206760
-0.4794690
0.2879700
-0.6987730
-1.7563410
0.1594200
-1.9509180
-2.4258270
-0.0321530
0.9726750
-1.0880790
-2.7778360
0.6409950
-1.2401310
0.4386660
-1.1868880
-2.5299260
-1.3110770
-2.5124000
-0.3664090
-2.0460870
-1.4577200
-3.8188770
-2.6800250
-1.3279030
-3.8615870
-4.7786680
-2.6773340
-1.0799140
-4.1326840
2. 9901440

1. 2895270

$-0.0070070$

3. 8828960

4.8198750

6. 0965290

6.7608350

5.8971550

4.4511300

5.0965730

6.7990600

7.7401850

5.9851550

4. 3424760

5.8211570

6.9346930

6.2694770

$-3.6566200$

$-2.6520320$

$-4.5203640$

$-5.4351630$

$-4.3862560$

$-6.2062730$

$-5.5262880$

$-5.1692190$

$-3.6706530$

-6.0778400

$-6.9077510$

$-5.0690040$

$-6.6843850$

$-1.9717960$

$-4.0296330$

$-1.5335860$

$-2.2682990$

$-1.0147980$

-1.8813190

$-0.6987310$

0.0089890

$-2.9464840$

$-3.9667140$

$-3.2677480$

$-2.1636090$

-3.4485390

$-4.5561510$

$-2.7100600$

$-2.8275710$
$-4.1721740$ $-4.4899350$ $-5.6003640$ $-1.9921480$ $-2.4702910$

$-1.6094210$

$-1.5593200$

$-2.2281850$

$-1.8342430$

$-3.5195000$

$-1.9933540$

$-2.0470210$

$-3.3188010$

$-2.4280760$

$-0.5985270$

$-0.5174070$

$-1.9706640$

$-0.7243390$

$-0.9624120$

$-1.9140270$

$-1.9689280$

$-3.0137340$

$-3.1085100$

$-1.1203950$

$-4.1456670$

$-2.9672220$

$-4.1957440$

$-3.1490890$

$-4.9909290$

$-5.0832330$

$-0.1674970$

0.7594880

2. 2719070

2. 6431360

1.3062170

2. 2786060

3. 3464160

2. 9037570

3. 9251650

3. 9166990

3. 7614490

2. 6108570

4.0785350

4.8380460

4.6730850

1.7793490 


$\begin{array}{lrrr}\mathrm{H} & -4.6114480 & -1.3685240 & 2.6466580 \\ \mathrm{H} & -3.6823300 & -2.2479310 & 4.7597560 \\ \mathrm{H} & -2.8261500 & -4.6692300 & 3.0860050 \\ \mathrm{H} & -0.5118120 & -3.9771920 & 3.6009600 \\ \mathrm{I} 54 & & & \\ \mathrm{TS} 268(\mathrm{R}, \mathrm{R}) & \mathrm{G}[\mathrm{M} 06-2 \mathrm{X} / 6-31 \mathrm{G}(\mathrm{d})]=-3864.197819 \\ \mathrm{P} & -0.1869090 & -0.3091360 & 0.8341990 \\ \mathrm{O} & -0.6191650 & 1.1377900 & 0.7378860 \\ \mathrm{O} & 0.5999870 & -0.7503670 & 2.0307000 \\ \mathrm{O} & 0.5934270 & -0.6007560 & -0.5669840 \\ \mathrm{O} & -1.4571430 & -1.3054350 & 0.7151750 \\ \mathrm{C} & 0.7634400 & -1.9329240 & -0.9207450 \\ \mathrm{C} & -0.3378570 & -2.6299330 & -1.4392310 \\ \mathrm{C} & 2.0229590 & -2.5054410 & -0.7619770 \\ \mathrm{C} & 2.1639090 & -3.8512850 & -1.1112900 \\ \mathrm{H} & 3.1380400 & -4.3231520 & -0.9980700 \\ \mathrm{C} & -2.1576500 & -1.2305870 & -0.4832500 \\ \mathrm{C} & -3.3396740 & -0.4958660 & -0.5189550 \\ \mathrm{C} & -1.6308250 & -1.9024760 & -1.5953970 \\ \mathrm{C} & -4.0198440 & -0.4481440 & -1.7397800 \\ \mathrm{H} & -4.9576510 & 0.1012890 & -1.7934520 \\ \mathrm{C} & 3.1782500 & -1.7064900 & -0.2642510 \\ \mathrm{C} & -3.8828590 & 0.1962740 & 0.6841420 \\ \mathrm{C} & 3.6771310 & -0.6352980 & -1.0218140 \\ \mathrm{C} & 3.1273820 & -0.1204770 & -2.3299820 \\ \mathrm{C} & 5.2213280 & 1.1800320 & -1.5372610 \\ \mathrm{C} & 4.8056770 & 0.0797680 & -0.5939160 \\ \mathrm{C} & 2.7691420 & 1.3643990 & -2.1141800 \\ \mathrm{H} & 2.3179770 & 1.7754610 & -3.0249910 \\ \mathrm{H} & 2.0121080 & 1.4147610 & -1.3280450 \\ \mathrm{C} & 4.0380350 & 2.1576750 & -1.7125510 \\ \mathrm{H} & 4.2969610 & 2.8962910 & -2.4806440 \\ \mathrm{H} & 3.8640660 & 2.7121020 & -0.7835520 \\ \mathrm{H} & 6.1081030 & 1.7040070 & -1.1645910 \\ \mathrm{H} & 2.2449860 & -0.6790730 & -2.6505360 \\ \mathrm{C} & 5.5058600 & 0.5367000 & -2.9119750 \\ \mathrm{H} & 5.7989710 & 1.3183490 & -3.6216010 \\ \mathrm{H} & 6.3501700 & -0.1535790 & -2.8224540 \\ \mathrm{C} & 4.2362450 & -0.2071450 & -3.4023030 \\ \mathrm{H} & 3.8602960 & 0.2373290 & -4.3306220 \\ \mathrm{H} & 4.4595440 & -1.2569090 & -3.6153990 \\ \mathrm{C} & 3.8202330 & -2.0317120 & 0.9393580 \\ \mathrm{C} & 3.4391640 & -3.1303980 & 1.9030020 \\ \mathrm{C} & 5.5351250 & -1.8077300 & 2.6554110 \\ \mathrm{C} & 4.9562640 & -1.3183120 & 1.3534700 \\ \mathrm{C} & 4.6438530 & -4.0909750 & 2.0213070 \\ & & & \end{array}$

4.3961260
4.8312440
5.8953960
6.2610800
6.7115800
6.4206130
2.5456420
4.4410280
4.8383120
4.1739730
3.1971910
2.9893150
2.3074370
5.4567400
6.3461660
-4.8914400
-5.2536260
-4.1530040
-4.2807310
-6.2198400
-6.4464070
-7.1642560
-5.5655440
-5.4820320
-6.1738380
-3.6893480
-5.7156170
-3.3101890
-3.2486240
-2.2919600
-3.9655230
-4.2181460
-3.2776820
-4.7284810
-4.9325990
-3.6247690
-4.0782030
-3.5537600
-3.7010500
-3.0248890
-2.7396490
-2.3683010
-1.8725310
-3.0582750
-5.4944360
-4.8669040
$-4.8856520$

$-4.5707430$

$-3.3014520$

$-3.6864090$

$-3.4035830$

$-1.2263820$

$-3.6652970$

$-1.6966360$

$-2.0723000$

$-0.6454120$

$-2.5082090$

$-3.3173240$

$-1.8772220$

$-0.2719930$

0.2699130

0.1113300

$-0.8184490$

$-2.0239490$

$-0.5334950$

$-1.8905810$

$-2.6083800$

$-1.4190630$

$-2.6001550$

$-3.6780620$

$-2.4687000$

$-2.5172500$

-0.2707620

$-2.2596670$

$-3.3378040$

$-1.9009550$

$-1.5306200$

$-2.2389930$

$-0.7948240$

2.2139870

3.6944440

2. 5668270

1.5856780

4.3700060

5.4239840

4. 3470560

3. 6314010

4. 3334780

3. 1366900

2. 0726660

4. 1298030

3. 2893430
2.7336250

1.0549750

2.4911810

3.4497510

1. 7696650

2.9353830

1.5719530

3.7384920

4.6883190

3. 8905920

3. 2961010

4.0054710

3. 2536060

0.5846960

0.9047450

2. 8993200

4.0283630

2. 0206870

1.8138590

3.4800950

4.2766440

3. 1918170

2. 2661240

2. 4447960

1. 3658670

1.1632940

4.8567100

3. 2930790

3.4800910

3. 1206840

4.4957680

5. 2930070

4.9229580

1.7555670

1. 5562690

$-0.3735490$

0.6828010

1.4100460

1.1470370

2. 3676420

0.3188140

$-0.4378080$

0.7615120

$-1.1681260$

2. 3894860

$-0.9406700$ 


$\begin{array}{rrr}-4.5343390 & 4.0772600 & -1.6263600 \\ -5.4741600 & 2.5908160 & -1.5254530 \\ -5.6949610 & 3.8906280 & 0.2266880 \\ -5.8729640 & 4.9598190 & 0.0662600 \\ -6.6734370 & 3.4056740 & 0.2967530 \\ -5.1354350 & 1.4804710 & 2.8646380 \\ -5.6362240 & 1.9720400 & 3.6974180 \\ -3.5199530 & -1.0751270 & -2.8749040 \\ -4.2303970 & -1.0454200 & -4.2038330 \\ -4.3114350 & -2.4715540 & -4.7709480 \\ -2.9150790 & -3.1253000 & -4.8918340 \\ -1.8211640 & -2.3487250 & -4.1280450 \\ -2.3180790 & -1.7995160 & -2.8133560 \\ -3.6819330 & -0.4045260 & -4.9088490 \\ -4.8150100 & -2.4608810 & -5.7422610 \\ -2.6152960 & -3.1924760 & -5.9423180 \\ -1.5169800 & -1.4899930 & -4.7427510 \\ -5.2302170 & -0.6144670 & -4.0934570 \\ -4.9387010 & -3.0624680 & -4.0953770 \\ -2.9587590 & -4.1517990 & -4.5147600 \\ -0.9277540 & -2.9630330 & -4.0018710 \\ -0.1693220 & -3.9862220 & -1.7511550 \\ -1.2849140 & -4.8996450 & -2.1931580 \\ -1.3056160 & -6.1594240 & -1.3075200 \\ 0.0741340 & -6.8585210 & -1.2275430 \\ 1.1843440 & -6.0538210 & -1.9314670 \\ 1.0871830 & -4.5910800 & -1.5865550 \\ -1.1278920 & -5.1978020 & -3.2403090 \\ -2.0655430 & -6.8501280 & -1.6850610 \\ 0.0257830 & -7.8575320 & -1.6714190 \\ 1.0754980 & -6.1748150 & -3.0180280 \\ -2.2522670 & -4.3915980 & -2.1492750 \\ -1.6248690 & -5.8566730 & -0.3055580 \\ 0.3551410 & -6.9936440 & -0.1788470 \\ 2.1700580 & -6.4489130 & -1.6679570 \\ 0.6645450 & 3.6139680 & -1.0003720 \\ -0.1441620 & 2.6270780 & -1.1632510 \\ 0.8972460 & 4.3909440 & -2.2459130 \\ 1.9950300 & 5.2482800 & -2.3796650 \\ 0.0063840 & 4.2329850 & -3.3160600 \\ 2.1965020 & 5.9400680 & -3.5679490 \\ 2.6893000 & 5.3568170 & -1.5533460 \\ 0.2053420 & 4.9375870 & -4.4971160 \\ -0.8383630 & 3.5619320 & -3.2077670 \\ 1.3008730 & 5.7897130 & -4.6255700 \\ 3.0542770 & 6.5968570 & -3.6696750\end{array}$

$\begin{array}{lr}\mathrm{H} & -0.4932480 \\ \mathrm{H} & 1.4582270 \\ \mathrm{H} & -0.3146420 \\ \mathrm{~S} & 1.4075390 \\ \mathrm{C} & 2.6506030 \\ \mathrm{C} & 1.4718730 \\ \mathrm{H} & 2.5983730 \\ \mathrm{H} & 0.5066010 \\ \mathrm{O} & 2.1393670 \\ \mathrm{H} & 1.5144120 \\ \mathrm{C} & 4.0213580 \\ \mathrm{C} & 2.9326610 \\ \mathrm{C} & 1.5470210 \\ \mathrm{C} & 4.0142770 \\ \mathrm{H} & 5.0054220 \\ \mathrm{H} & 2.9667410 \\ \mathrm{H} & 1.2805010 \\ \mathrm{H} & 4.3094490 \\ \mathrm{H} & 4.7251070 \\ \mathrm{H} & 3.8589300 \\ \mathrm{H} & 3.1045740 \\ \mathrm{H} & 0.7646620 \\ \mathrm{H} & \end{array}$

$\begin{array}{lr}4.8203300 & -5.3192180 \\ 6.3351600 & -5.5509740 \\ 2.0097170 & -0.3221270 \\ 4.0551830 & 0.4470050 \\ 1.6082740 & 2.1248420 \\ 2.4251620 & 2.4507050 \\ 1.0247670 & 1.1982660 \\ 2.0590870 & 2.1201190 \\ 0.8745710 & 3.2586540 \\ 0.1655730 & 2.8713400 \\ 3.0680830 & 3.6668400 \\ 4.1394370 & 3.5945590 \\ 3.4981470 & 3.4958540 \\ 2.1949200 & 2.4100140 \\ 3.5324770 & 3.7795840 \\ 4.7899380 & 4.4732260 \\ 3.0179600 & 4.4456070 \\ 2.7855190 & 1.5326880 \\ 1.3684190 & 2.4969240 \\ 2.4361450 & 4.5485350 \\ 4.7758430 & 2.7168320 \\ 4.2336550 & 3.2908630\end{array}$

154

$\begin{array}{lrrr}\text { TS269(R, R) } G[\mathrm{M} 06-2 \mathrm{X} / 6-31 \mathrm{G}(\mathrm{d})]=-3864.199119 \\ \mathrm{P} & -0.1884540 & -0.2763350 & 0.9082930 \\ \mathrm{O} & -0.6661400 & 1.1480680 & 0.7274770 \\ \mathrm{O} & 0.6121180 & -0.6201040 & 2.1274170 \\ \mathrm{O} & 0.6067680 & -0.6217510 & -0.4737100 \\ \mathrm{O} & -1.4289700 & -1.3160940 & 0.8505750 \\ \mathrm{C} & 0.8254020 & -1.9644880 & -0.7508130 \\ \mathrm{C} & -0.2477250 & -2.7364760 & -1.2022930 \\ \mathrm{C} & 2.1092320 & -2.4806040 & -0.5814720 \\ \mathrm{C} & 2.2848810 & -3.8333440 & -0.8611500 \\ \mathrm{H} & 3.2803150 & -4.2609520 & -0.7521210 \\ \mathrm{C} & -2.1204630 & -1.3358520 & -0.3559830 \\ \mathrm{C} & -3.3189570 & -0.6329830 & -0.4546420 \\ \mathrm{C} & -1.5574250 & -2.0532270 & -1.4182840 \\ \mathrm{C} & -3.9701360 & -0.6645280 & -1.6913100 \\ \mathrm{H} & -4.9206420 & -0.1445400 & -1.7935510 \\ \mathrm{C} & 3.2407440 & -1.6158850 & -0.1444250 \\ \mathrm{C} & -3.9028750 & 0.1090050 & 0.6979120 \\ \mathrm{C} & 3.6938450 & -0.5720880 & -0.9659510 \\ \mathrm{C} & 3.1140160 & -0.1522940 & -2.2946840 \\ \mathrm{C} & 5.1663170 & 1.2650540 & -1.5989880 \\ \mathrm{C} & 4.7983500 & 0.2068080 & -0.5898680 \\ \mathrm{C} & 2.7036420 & 1.3284180 & -2.1579010\end{array}$




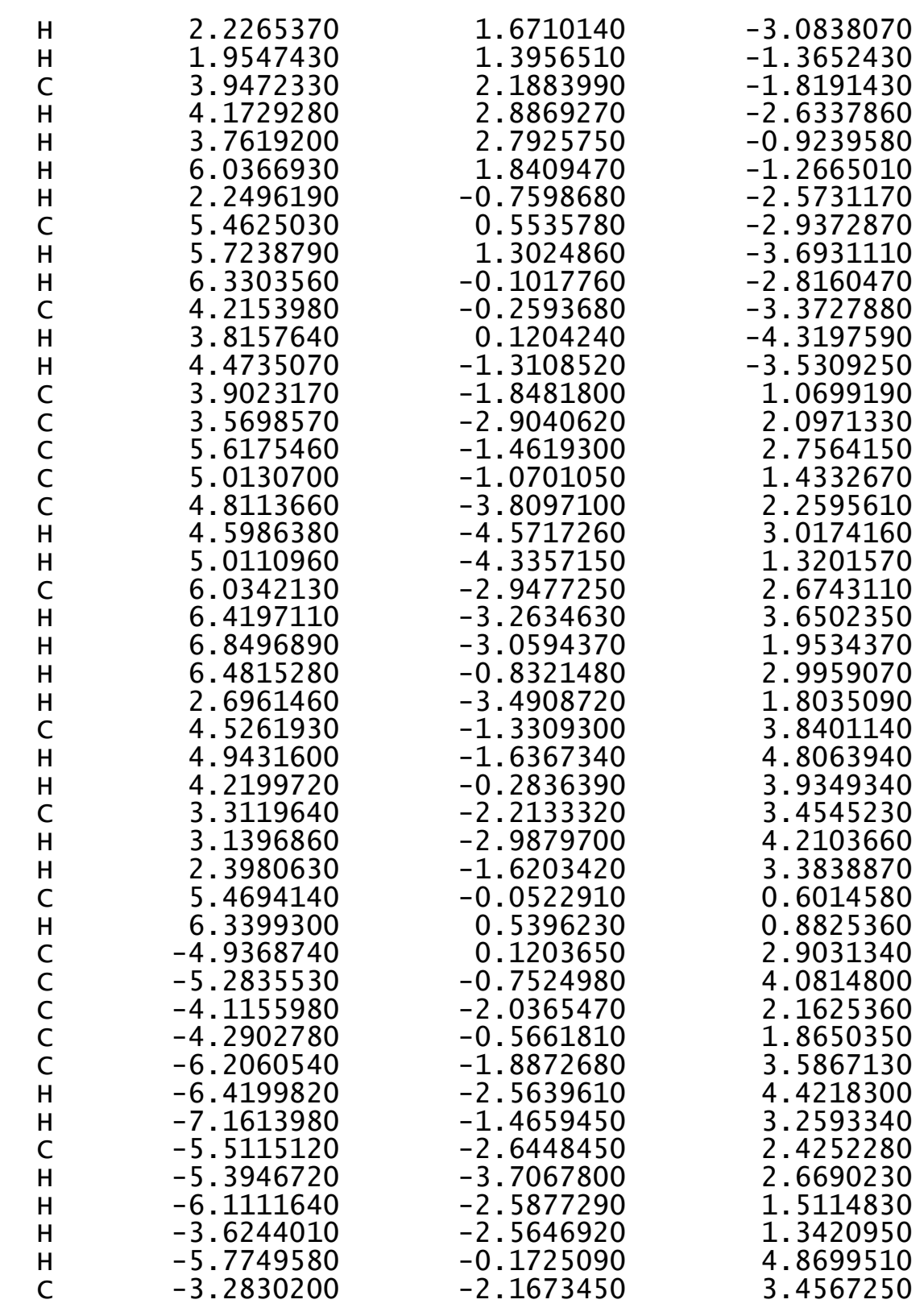

-3.1879810
-2.2749870
-3.9792470
-4.2192910
-3.3225130
-4.8294530
-5.0801020
-3.7133260
-4.1457870
-3.7229120
-3.9022880
-3.2050940
-2.8715420
-2.5122960
-1.9953800
-3.1220060
-5.6654300
-4.9716700
-4.6566150
-5.5484370
-5.8336120
-6.0468920
-6.7953610
-5.2256870
-5.7530630
-3.4233010
-4.1014470
-4.1080710
-2.6775920
-1.6399440
-2.2070520
-3.5634910
-4.6248300
-2.3555940
-1.3074220
-5.1203760
-4.6863810
-2.6726780
-0.7486800
-0.0560920
1.2288920
-1.2406740
-0.8378060
0.2710100
1.5188840
-1.7115010
3.7084130

3. 2756740

4. 6059210

5.4407800

4.9969740

1. 6386390

1. 3506510

$-0.4965640$

0.6114970

1.1774990

0.8494440

2. 1399040

0.1412770

$-0.6512440$

0.6223110

$-1.2556450$

2. 1514850

$-1.1170190$

$-1.8433380$

$-1.6671390$

0.0048350

$-0.2198750$

0.0940710

2.7847890

3. 5815210

$-2.7858000$

$-4.1312580$

$-4.6381700$

$-4.7560660$

$-3.9371400$

$-2.6608600$

$-4.8501040$

$-5.6004530$

$-5.8021630$

$-4.5471070$

$-4.0590840$

$-3.9252610$

$-4.4338190$

$-3.7485060$

$-1.4073220$

$-1.2522960$

$-1.6915980$

$-2.3387090$

$-1.5221100$

$-1.5338870$

$-2.4139290$ 


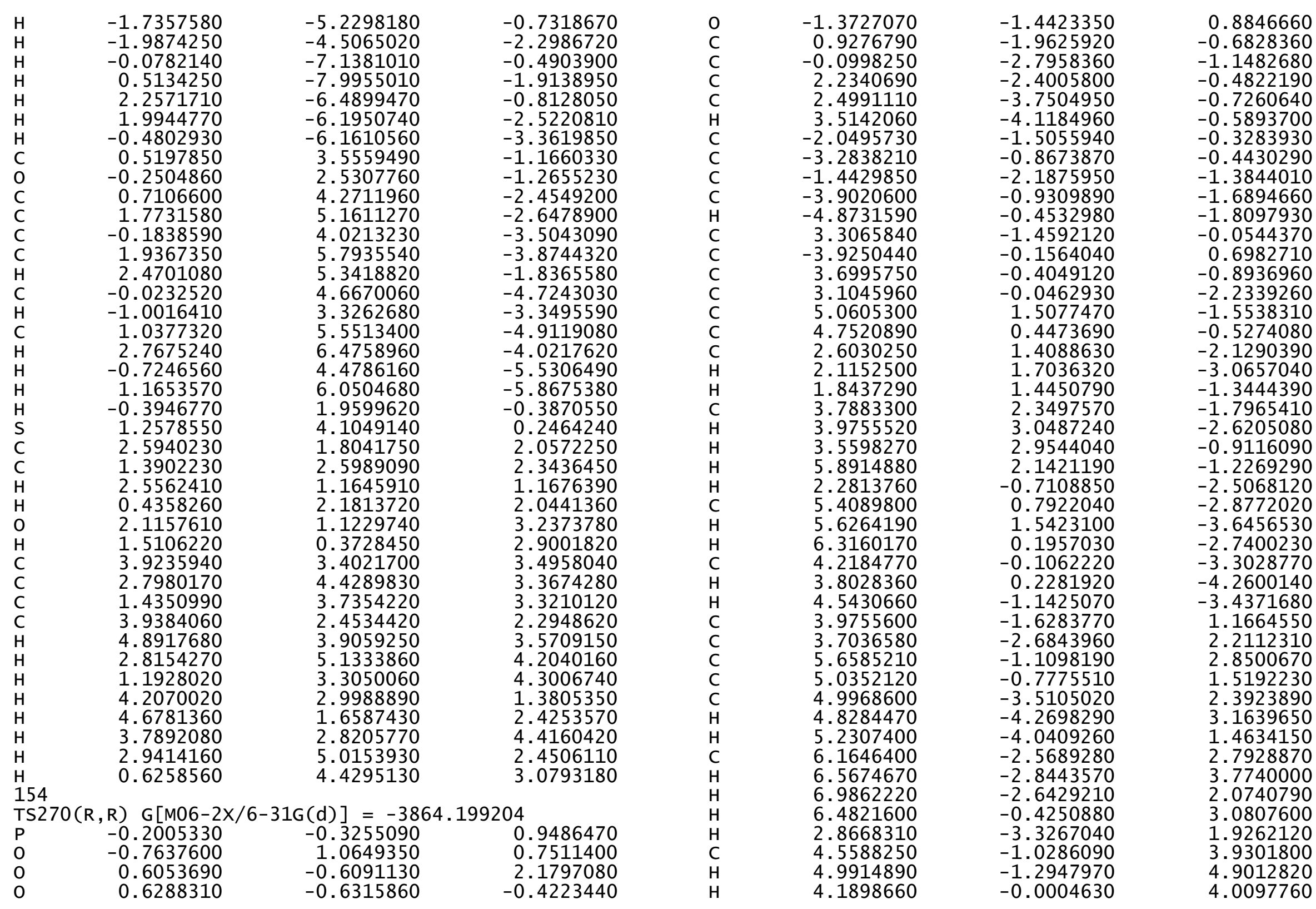




$\begin{array}{rr}3.4004060 & -1.9874980 \\ 3.2708260 & -2.7581840 \\ 2.4538780 & -1.4501120 \\ 5.4319850 & 0.2514830 \\ 6.2630790 & 0.9008540 \\ -4.9820050 & -0.1978590 \\ -5.2873690 & -1.0847390 \\ -4.0261670 & -2.3051400 \\ -4.2843150 & -0.8488030 \\ -6.1347380 & -2.2757880 \\ -6.3128580 & -2.9613470 \\ -7.1117790 & -1.9151710 \\ -5.3866660 & -2.9933650 \\ -5.2105740 & -4.0463800 \\ -5.9800370 & -2.9721950 \\ -3.4959380 & -2.8063980 \\ -5.8203600 & -0.5318230 \\ -3.2012370 & -2.3826440 \\ -3.0505260 & -3.4369950 \\ -2.2135930 & -1.9465310 \\ -3.9516230 & -1.6410880 \\ -4.1580680 & -2.3162310 \\ -3.3458600 & -0.8174790 \\ -4.9814700 & 1.8360340 \\ -5.3149320 & 3.2761160 \\ -3.8577250 & 2.1595790 \\ -4.2476750 & 1.2061570 \\ -4.0006020 & 4.0671120 \\ -4.2368860 & 5.0839690 \\ -3.4987230 & 4.1542070 \\ -3.0921220 & 3.3407330 \\ -2.7631400 & 4.0264710 \\ -2.1973460 & 2.9545920 \\ -3.2275920 & 1.6737240 \\ -5.9365080 & 3.7068980 \\ -5.1445980 & 2.7286150 \\ -4.8661550 & 3.5018100 \\ -5.6694720 & 1.9434240 \\ -6.0562560 & 3.3188890 \\ -6.3281970 & 4.3548260 \\ -6.9870880 & 2.7490470 \\ -5.3495830 & 1.1376570 \\ -5.9156890 & 1.6312770 \\ -2.0661760 & -2.1839950 \\ -1.3659250 & -2.7931810 \\ -1.9190850 & -2.2797680\end{array}$

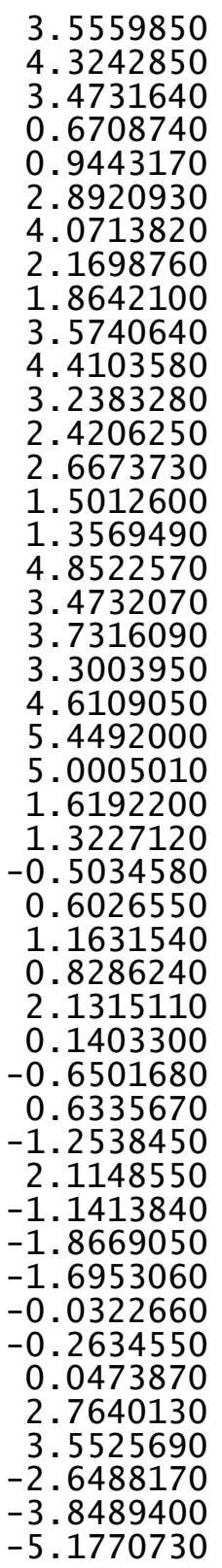
$-3.4394240$
$-4.0348770$
$-3.3134430$
$-1.4702310$
$-1.4823650$
$-0.2894690$
$-3.7099010$
$-3.8560970$
$-5.0972220$
$-3.9885740$
$-1.6444970$
1. 4924570
1.7435890
1.1008000
$-0.4190390$
$-0.8556140$
0.1862420
1. 3062290
1. 3034350
$-0.9649350$
$-1.0116710$
2. 8183140
1.5797160
$-0.7219130$
$-1.8211430$
0.2916360
$-0.4222490$
0.4526860
1. 4713060
$-0.4232150$
1.6101100
2. 1543660
$-0.2884580$
$-1.2064570$
0.7290400
2. 4073810
$-0.9758680$
0.8368190
$-0.5373730$
0.9905560
2. 4370910
1.1860130
2.4446030
0.2606100
1. 9886350
1. 4320410

-2.4119970
-1.4885080
-1.5572740
-2.8409950
-3.8842200
-2.6032340
-3.4538480
-2.1688400
-1.7093080
-0.4528170
-1.2255820
-4.6270180
-6.0926770
-6.4952870
-6.2293690
-5.2099270
-4.1564010
-6.6927590
-7.5478790
-7.1614120
-5.7582600
-6.2990820
-5.9066200
-5.8788840
-4.7635660
3.5221180
2.4563270
4.2350870
5.1744340
3.9328820
5.8038620
5.3957380
4.5759760
3.1994860
5.5095550
6.5246860
4.3471200
6.0065060
1.8843960
4.1225740
1.9277790
2.6525470
1.2774940
2.1763640
1.2325470
0.4444650

$-5.1832730$

$-4.1236120$

$-2.7899630$

$-6.0027320$

$-3.8294260$

$-3.7749570$

$-4.9664010$

$-6.1657700$

$-3.9694820$

$-4.4876740$

$-5.3134280$

$-1.1152510$

$-1.3620050$

$-2.6966810$

$-2.6983270$

$-1.6204960$

$-1.3285980$

$-0.5509930$

$-2.9162490$

$-2.5213960$

$-0.6810430$

$-1.3675030$

$-3.4866480$

$-3.6898510$

$-1.8691980$

$-1.1562300$

$-1.2507760$

$-2.4503770$

$-2.6458770$

$-3.5016160$

$-3.8769820$

$-1.8327810$

$-4.7261500$

$-3.3447190$

$-4.9163400$

$-4.0262380$

$-5.5340850$

$-5.8755460$

$-0.3688740$

0.2550670

2. 1026250

2. 3706030

1.2201350

2. 0675580

3. 2865570

2.9519110 


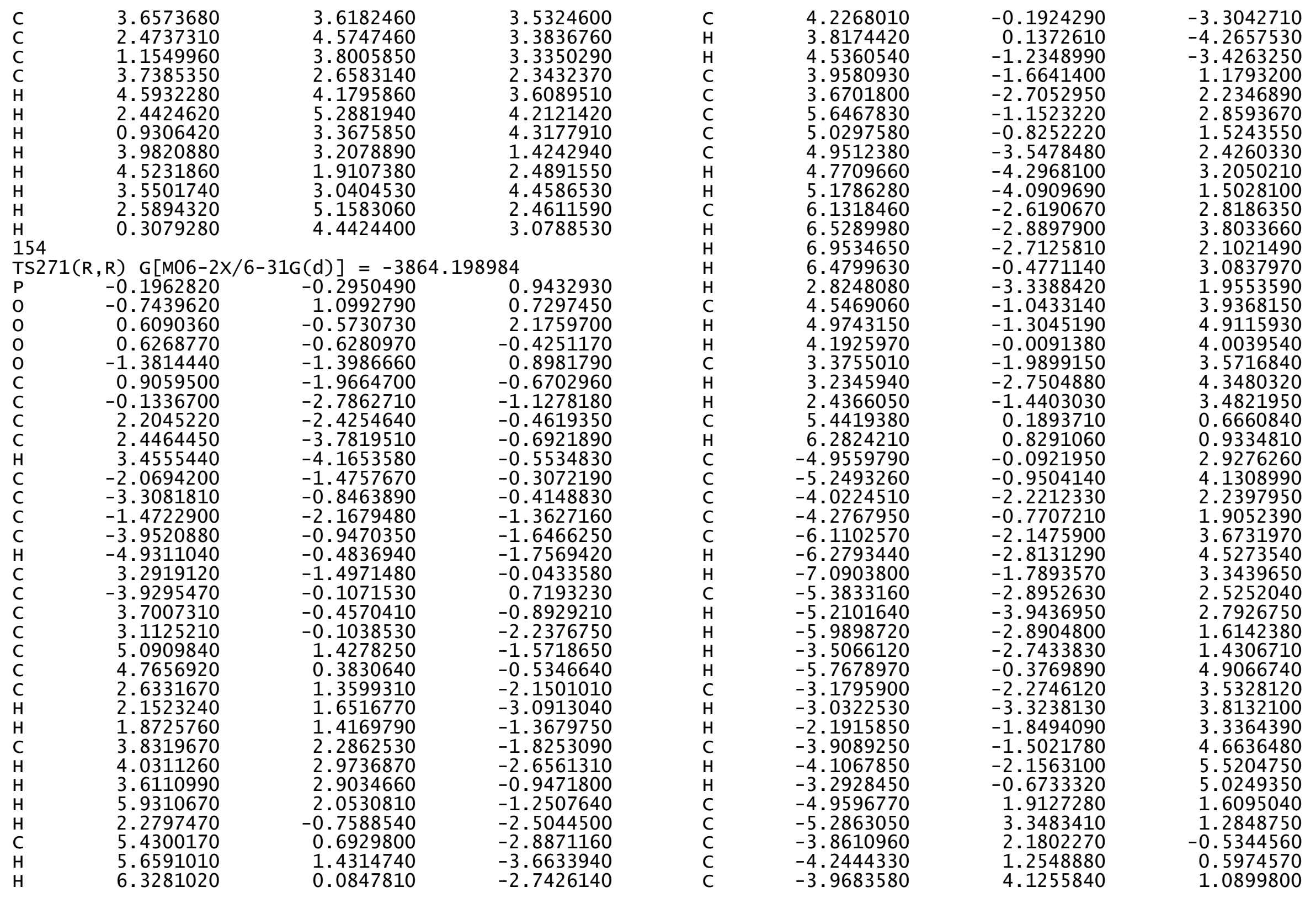




$\begin{array}{rrr}-4.2018210 & 5.1354980 & 0.7331250 \\ -3.4540410 & 4.2331690 & 2.0497060 \\ -3.0772760 & 3.3677830 & 0.0746980 \\ -2.7492170 & 4.0324340 & -0.7339080 \\ -2.1818260 & 2.9831680 & 0.5678750 \\ -3.2449060 & 1.6725080 & -1.2819640 \\ -5.8941940 & 3.8018550 & 2.0749410 \\ -5.1511130 & 2.7461840 & -1.1685650 \\ -4.8755930 & 3.5023380 & -1.9129030 \\ -5.6881640 & 1.9538210 & -1.7002210 \\ -6.0450020 & 3.3659210 & -0.0610890 \\ -6.3141300 & 4.3981380 & -0.3114380 \\ -6.9778760 & 2.8034090 & 0.0430700 \\ -5.3167760 & 1.2426170 & 2.7745290 \\ -5.8685250 & 1.7576860 & 3.5595430 \\ -3.3802150 & -1.5954520 & -2.7425920 \\ -2.1163540 & -2.1924120 & -2.6149820 \\ -4.1643880 & -1.6535830 & -4.0407680 \\ -3.2955890 & -1.9478920 & -5.2615610 \\ -2.3648620 & -3.1208860 & -4.9644520 \\ -1.4072820 & -2.7517300 & -3.8346790 \\ -3.9307360 & -2.1572490 & -6.1282140 \\ -4.9249000 & -2.4418010 & -3.9495350 \\ -4.7133750 & -0.7148410 & -4.1738910 \\ -2.9616250 & -3.9960360 & -4.6714000 \\ -1.7934670 & -3.4033330 & -5.8546120 \\ -0.7837600 & -3.6061960 & -3.5671630 \\ -0.7095750 & -1.9809940 & -4.1944920 \\ -2.6907850 & -1.0657280 & -5.5087080 \\ 1.4250520 & -4.6454190 & -1.0750020 \\ 1.6515700 & -6.1143140 & -1.3268720 \\ 1.0128560 & -6.4954320 & -2.6708710 \\ -0.5023580 & -6.2032900 & -2.6832190 \\ -0.9311170 & -5.1870980 & -1.5988780 \\ 0.1278240 & -4.1545770 & -1.2906980 \\ 1.1960690 & -6.7117320 & -0.5241110 \\ 1.1993800 & -7.5496370 & -2.8969070 \\ -1.0656270 & -7.1275490 & -2.5207300 \\ -1.1114960 & -5.7387620 & -0.6660420 \\ 2.7222440 & -6.3407050 & -1.3251730 \\ 1.5093710 & -5.9092190 & -3.4518940 \\ -0.7955460 & -5.8357600 & -3.6724520 \\ -1.8832090 & -4.7198770 & -1.8624370 \\ 0.3458100 & 3.5171750 & -1.2081720 \\ -0.3834450 & 2.4607100 & -1.2890640 \\ 0.5183160 & 4.2103130 & -2.5115940\end{array}$

1.5487910
-0.3590260
1.6978160
2.2328220
-0.2138120
-1.1516040
0.8154120
2.5041270
-0.9023110
0.9312740
-0.5065620
1.0525640
2.4734490
1.2320660
2.4714960
0.3002890
2.0175230
1.4501700
3.7168930
2.5457810
1.2169110
3.7846430
4.6600730
2.5244430
0.9878010
4.0350550
4.5592390
3.6027010
2.6684380
0.3781220
1.95

$\begin{array}{lr}5.1343290 & -2.7177850 \\ 3.9043800 & -3.5605700 \\ 5.7449950 & -3.9570990 \\ 5.3585590 & -1.9063150 \\ 4.5287410 & -4.7935540 \\ 3.1829980 & -3.3953360 \\ 5.4471220 & -4.9943100 \\ 6.4539360 & -4.1144890 \\ 4.2971260 & -5.5997730 \\ 5.9294520 & -5.9600090 \\ 1.9019640 & -0.3998810 \\ 4.1259070 & 0.1958030 \\ 1.9355360 & 2.0693100 \\ 2.6797630 & 2.3290790 \\ 1.2746100 & 1.1947820 \\ 2.2117840 & 2.0328920 \\ 1.2605150 & 3.2619260 \\ 0.4760920 & 2.9369810 \\ 3.6268720 & 3.4778010 \\ 4.5969160 & 3.3179750 \\ 3.8396380 & 3.2795730 \\ 2.6516060 & 2.3002260 \\ 4.1767830 & 3.5470280 \\ 5.3207590 & 4.1376920 \\ 3.4214170 & 4.2676320 \\ 3.1868460 & 1.3747350 \\ 1.8952960 & 2.4548560 \\ 3.0618020 & 4.4109730 \\ 5.1676360 & 2.3883380 \\ 4.4894500 & 3.0164180\end{array}$

54

$\begin{array}{lrrr}\text { TS272 (R, R) } & \text { G }[\mathrm{M} 06-2 \mathrm{X} / 6-31 \mathrm{G}(\mathrm{d})]=-3864.196734 \\ \mathrm{P} & -0.1851650 & -0.2302930 & 0.9341640 \\ \mathrm{O} & -0.6898790 & 1.1697220 & 0.6601100 \\ \mathrm{O} & 0.6255290 & -0.4773470 & 2.1699680 \\ \mathrm{O} & 0.6058550 & -0.6584420 & -0.4259130 \\ \mathrm{O} & -1.4038770 & -1.2961640 & 0.9504840 \\ \mathrm{C} & 0.8433750 & -2.0153340 & -0.6034700 \\ \mathrm{C} & -0.2206300 & -2.8294620 & -1.0195920 \\ \mathrm{C} & 2.1308340 & -2.4960230 & -0.3773870 \\ \mathrm{C} & 2.3409000 & -3.8668450 & -0.5467940 \\ \mathrm{H} & 3.3397480 & -4.2681960 & -0.3864980 \\ \mathrm{C} & -2.1040990 & -1.4028310 & -0.2459690 \\ \mathrm{C} & -3.3111690 & -0.7202340 & -0.3735470 \\ \mathrm{C} & -1.5461530 & -2.1869090 & -1.2655410 \\ \mathrm{C} & -3.9815910 & -0.8419020 & -1.5943240\end{array}$




\begin{tabular}{|c|c|c|c|}
\hline $\begin{array}{l}\mathrm{H} \\
\mathrm{C} \\
\mathrm{C} \\
\mathrm{C} \\
\mathrm{C} \\
\mathrm{C} \\
\mathrm{C} \\
\mathrm{C} \\
\mathrm{H} \\
\mathrm{H} \\
\mathrm{C} \\
\mathrm{H} \\
\mathrm{H} \\
\mathrm{H} \\
\mathrm{H} \\
\mathrm{C} \\
\mathrm{H} \\
\mathrm{H} \\
\mathrm{C} \\
\mathrm{H} \\
\mathrm{H} \\
\mathrm{C} \\
\mathrm{C} \\
\mathrm{C} \\
\mathrm{C} \\
\mathrm{C} \\
\mathrm{H} \\
\mathrm{H} \\
\mathrm{C} \\
\mathrm{H} \\
\mathrm{H} \\
\mathrm{H} \\
\mathrm{H} \\
\mathrm{C} \\
\mathrm{H} \\
\mathrm{H} \\
\mathrm{C} \\
\mathrm{H} \\
\mathrm{H} \\
\mathrm{C} \\
\mathrm{H} \\
\mathrm{C} \\
\mathrm{C} \\
\mathrm{C} \\
\mathrm{C} \\
\mathrm{C}\end{array}$ & $\begin{array}{r}-4.9355520 \\
3.2440400 \\
-3.8867790 \\
3.6846440 \\
3.1044540 \\
5.1324530 \\
4.7769670 \\
2.6719000 \\
2.1956820 \\
1.9173730 \\
3.9005270 \\
4.1186630 \\
3.7022380 \\
5.9930570 \\
2.2503160 \\
5.4439910 \\
5.6941150 \\
6.3227230 \\
4.2119470 \\
3.8102980 \\
4.4875670 \\
3.9067200 \\
3.5885810 \\
5.6157990 \\
5.0061510 \\
4.8426910 \\
4.6407730 \\
5.0505000 \\
6.0530310 \\
6.4416260 \\
6.8708950 \\
6.4710580 \\
2.7223560 \\
4.5228550 \\
4.9445510 \\
4.2008040 \\
3.3220330 \\
3.1630460 \\
2.3991060 \\
5.4495190 \\
6.3114110 \\
-4.8922260 \\
-5.2099960 \\
-4.0470570 \\
-4.2482980 \\
-6.1210110\end{array}$ & $\begin{array}{r}-0.3333600 \\
-1.5798140 \\
0.0950830 \\
-0.5936630 \\
-0.2832140 \\
1.2130980 \\
0.2271800 \\
1.1974030 \\
1.4626120 \\
1.3123480 \\
2.0989070 \\
2.7413970 \\
2.7619160 \\
1.8249380 \\
-0.9224740 \\
0.4096030 \\
1.1048290 \\
-0.2198210 \\
-0.4548020 \\
-0.1555640 \\
-1.5110120 \\
-1.7114010 \\
-2.6940080 \\
-1.1792940 \\
-0.8933170 \\
-3.5681430 \\
-4.2771050 \\
-4.1571720 \\
-2.6613470 \\
-2.8998580 \\
-2.8138530 \\
-0.5220430 \\
-3.3118890 \\
-0.9854760 \\
-1.2132040 \\
0.0611540 \\
-1.9117790 \\
-2.6332670 \\
-1.3392290 \\
0.0666360 \\
0.6897410 \\
0.2516940 \\
-0.5378750 \\
-1.9409260 \\
-0.4988850 \\
-1.7190940\end{array}$ & $\begin{array}{r}-1.7188550 \\
-0.0004880 \\
0.7330860 \\
-0.8968720 \\
-2.2553720 \\
-1.6621100 \\
-0.5782870 \\
-2.2316490 \\
-3.1829570 \\
-1.4498890 \\
-1.9516610 \\
-2.8131390 \\
-1.1019940 \\
-1.3708650 \\
-2.4903440 \\
-2.9435940 \\
-3.7526360 \\
-2.7732690 \\
-3.3187520 \\
-4.2933420 \\
-3.3946270 \\
1.2285950 \\
2.3303680 \\
2.8815820 \\
1.5337730 \\
2.5562210 \\
3.3666910 \\
1.6568310 \\
2.9074720 \\
3.9038940 \\
2.1968400 \\
3.0740730 \\
2.0813240 \\
3.9541200 \\
4.9397390 \\
3.9722110 \\
3.6355420 \\
4.4450180 \\
3.5260180 \\
0.6293600 \\
0.8661260 \\
2.9461890 \\
4.1896980 \\
2.3527010 \\
1.9515540 \\
3.7917560\end{array}$ \\
\hline
\end{tabular}

-6.3133370
-7.0870780
-5.4298050
-5.2932480
-6.0422020
-3.5584500
-5.7001260
-3.1956410
-3.0797520
-2.1963270
-3.8892930
-4.1080400
-3.2400310
-4.8352840
-5.1167910
-3.7467800
-4.1527610
-3.7760140
-3.9793260
-3.2507700
-2.9182960
-2.5711290
-2.0345540
-3.1540420
-5.7025520
-5.0207930
-4.7238640
-5.5882160
-5.8860960
-6.1261010
-6.8345090
-5.2057820
-5.7325620
-3.4501760
-4.1401770
-4.1886570
-2.7856400
-1.7115440
-2.2281160
-3.5907380
-4.6472440
-2.4627370
-1.4251650
-5.1490770
-4.8445290
-2.8354550
4.6767360

3. 4482640

2. 6785590

2.9969800

1.7716660

1. 5662720

4.9405530

3. 6409510

3. 9676320

3.4188860

4.7407580

5.6250150

5.0649180

1. 5353620

1.1447380

$-0.6260670$

0.5504730

0.9028790

0.4967950

1.8526250

$-0.0777890$

$-0.9186830$

0.4305260

$-1.3524930$

1.9163760

$-1.2784040$

$-2.0562280$

$-1.7727640$

$-0.1951170$

$-0.4917910$

$-0.0580680$

2.7332760

3.4967260

$-2.6439200$

$-3.9764730$

$-4.3864160$

$-4.3721020$

$-3.7213120$

$-2.4891250$

$-4.7395300$

$-5.3749560$

$-5.3905530$

$-3.9309560$

$-3.6810190$

$-3.8334000$ 


$\begin{array}{rrr}-0.8037220 & -3.5271630 & -3.5135460 \\ 1.3009020 & -4.7189800 & -0.8999580 \\ 1.4876860 & -6.2035950 & -1.0774240 \\ 0.8758460 & -6.6412690 & -2.4172630 \\ -0.6113260 & -6.2325970 & -2.5358290 \\ -1.0496630 & -5.2300820 & -1.4466640 \\ 0.0155580 & -4.2064730 & -1.1405740 \\ 0.9921130 & -6.7430250 & -0.2578560 \\ 0.9873560 & -7.7221780 & -2.5446450 \\ -1.2565800 & -7.1140790 & -2.4671510 \\ -1.2380590 & -5.7902120 & -0.5202350 \\ 2.5496730 & -6.4639080 & -1.0332700 \\ 1.4534090 & -6.1667490 & -3.2174200 \\ -0.7891990 & -5.7969670 & -3.5246340 \\ -1.9982730 & -4.7576700 & -1.7126360 \\ 0.4554300 & 3.4652600 & -1.3915320 \\ -0.3008930 & 2.4253210 & -1.4215400 \\ 0.6366390 & 4.0952360 & -2.7254760 \\ 1.6903270 & 4.9804150 & -2.9792060 \\ -0.2569280 & 3.7677290 & -3.7540370 \\ 1.8463380 & 5.5314180 & -4.2453090 \\ 2.3865910 & 5.2209400 & -2.1829820 \\ -0.1042870 & 4.3327410 & -5.0144270 \\ -1.0676510 & 3.0765630 & -3.5517070 \\ 0.9481070 & 5.2125190 & -5.2624920 \\ 2.6705470 & 6.2100670 & -4.4393420 \\ -0.8051110 & 4.0848360 & -5.8050300 \\ 1.0697860 & 5.6481480 & -6.2494360 \\ -0.4353640 & 1.9110140 & -0.5074790 \\ 1.1867030 & 4.1174800 & -0.0200720 \\ 2.5559210 & 1.9748160 & 1.9466530 \\ 1.3371390 & 2.7651160 & 2.1759800 \\ 2.5324720 & 1.2765600 & 1.1019350 \\ 0.3915070 & 2.3108320 & 1.9036660 \\ 2.0849790 & 1.3664150 & 3.1688020 \\ 1.4960430 & 0.5835930 & 2.8803790 \\ 3.8521860 & 3.6909150 & 3.2756600 \\ 2.7090740 & 4.6860900 & 3.0737430 \\ 1.3588830 & 3.9665780 & 3.0732540 \\ 3.8880780 & 2.6618010 & 2.1433810 \\ 4.8110890 & 4.2157450 & 3.3182210 \\ 2.7114500 & 5.4473980 & 3.8590780 \\ 1.1215950 & 3.6002420 & 4.0798260 \\ 4.1520050 & 3.1471340 & 2.3313910 \\ 4.6402190 & 1.8905420 & \\ 3.7244600 & 3.1723260 & \\ -1.2337240\end{array}$

$\begin{array}{llll}\mathrm{H} & 2.8449280 & 5.2093290 & 2.1183570 \\ \mathrm{H} & 0.5380230 & 4.6277070 & 2.7830770\end{array}$

154

$\operatorname{TS} 273(\mathrm{~S}, \mathrm{~S}) \mathrm{G}[\mathrm{M} 06-2 \mathrm{X} / 6-31 \mathrm{G}(\mathrm{d})]=-3864.195548$

\begin{tabular}{|c|c|c|c|}
\hline & -0.1984690 & -0.4454840 & 1.0538 \\
\hline & 0.5953480 & -0.7767600 & 2.28121 \\
\hline & -0.8020400 & 0.9365830 & 0.93431 \\
\hline 0 & -1.3114260 & -1.6065800 & 0.880826 \\
\hline 0 & 0.6740260 & -0.6090290 & -0.3172 \\
\hline C & -1.9356380 & -1.6554410 & -0.3612 \\
\hline & -1.2469560 & -2.2692870 & -1.41682 \\
\hline c & -3.1991210 & -1.0868570 & -0.49760 \\
\hline & -3.7884450 & -1.1472480 & -1.76463 \\
\hline $\mathrm{H}$ & -4.7787760 & -0.7170510 & -1.8998 \\
\hline C & 1.0806740 & -1.8970970 & -0.6404 \\
\hline & 2.4187280 & -2.2337 & -0.45441 \\
\hline C & 0.1325210 & -2.7817200 & -1.17290 \\
\hline $\mathrm{C}$ & 2.8012780 & -3.5394180 & -0.76916 \\
\hline $\mathrm{H}$ & 3.8436820 & -3.8262370 & -0.6445 \\
\hline C & -3.9130170 & -0.4419560 & 0.6405 \\
\hline c & 3.4035350 & -1.2069690 & -0.01146 \\
\hline C & -4.2833810 & -1.1839820 & 1.7715 \\
\hline C & -3.9654190 & -2.6340470 & \\
\hline c & -5.3609930 & -1.5317170 & 3.92796 \\
\hline C & -5.0520810 & -0.5968900 & 872 \\
\hline C & -3.1898910 & -2.7073850 & 3.3816 \\
\hline H & -2.9925250 & -3.7594200 & 3.6 \\
\hline $\mathrm{H}$ & -2.2212140 & -2.2145780 & 2618 \\
\hline C & -4.0230120 & -2.0400580 & 4.5076 \\
\hline $\mathrm{H}$ & -4.2306 & -2.7511 & \\
\hline $\mathrm{H}$ & -3.4754910 & -1.2014240 & 4.94800 \\
\hline $\mathrm{H}$ & -5.9515150 & -1.0281210 & 4.7007 \\
\hline $\mathrm{H}$ & -3.3785410 & -3.0844450 & 2444 \\
\hline C & -6.1285870 & -2.7462150 & 3.362 \\
\hline $\mathrm{H}$ & -6.3095310 & -3.46 & 1717 \\
\hline H & -7.1063140 & -2.4217090 & 2.99365 \\
\hline C & -5.2984050 & -3.3951550 & 2.224 \\
\hline $\mathrm{H}$ & -5 & -4.4442740 & 20 \\
\hline H & -5.8540120 & -3.3793590 & 1.2817 \\
\hline C & 2979690 & 0.9056580 & \\
\hline$c$ & -3.9070980 & 1.9085560 & -0.4923 \\
\hline C & -5.4944720 & 2.9001220 & 17 \\
\hline C & 00 & 9840 & 171 \\
\hline c & -5.1898870 & 2.4374620 & -1.1715 \\
\hline & -4.91618 & 3.2478010 & -1 \\
\hline & -5.650 & & \\
\hline
\end{tabular}




-6.1784730
-6.4957230
-7.0796250
-6.1698840
-3.2220640
-4.2153420
-4.4873900
-3.7611630
-3.2274580
-2.8957740
-2.3391550
-5.4809120
-6.1022830
4.6827620
4.9545860
3.1644390
3.7203930
5.4336130
5.6400560
6.3721330
4.3391640
3.9552350
4.7467190
2.4108810
5.7146100
2.5493560
2.0969790
1.7426840
3.6409570
3.8315790
3.3109790
5.0435000
5.6979980
3.9129150
4.0685770
4.5977090
5.0578700
4.1108360
3.5627040
3.5651430
2.5485300
3.1452830
6.4483290
5.2898790
5.2116480
5.5726980

2.9452100

3.9718770

2. 3247510

3.2777110

1.4743350

3.7582080

4.7704880

3.8448290

3.1036450

3.8269560

2.7455920

0.7220690

1.1644110

0.8113740

1.8417170

0.1006250

$-0.1392820$

1.0773290

1.7947920

0.5617750

0.0658130

0.3112840

$-0.9483260$

$-0.6428860$

2.5593120

1.5138600

1.7084050

1. 5401570

2. 5634050

3.2090700

3.2188220

$-0.3551820$

$-0.6353550$

$-2.3960990$

$-1.3026980$

$-0.6771000$

$-0.8823850$

0.3010190

$-1.7714560$

$-2.5700670$

$-1.3693210$

$-3.1167730$

0.1265900

$-3.0838590$

-3.8859100
-3.5487790

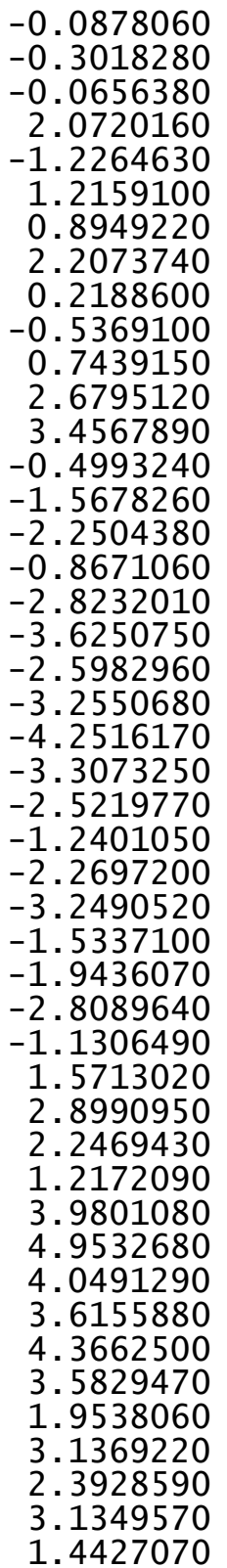

6.3501700
6.7645340
7.1855480
5.3528090
6.1192450
1.8772240
2.2521040
1.7128540
0.1842590
-0.3873520
0.5403830
1.8200480
1.9747800
-0.3315390
-0.5202410
3.3380290
2.2212840
-0.0558210
-1.3812690
-1.8551450
-1.1923570
-1.2742730
-2.7149510
-3.7137700
-3.1336360
-1.7015450
-0.8648750
-3.0614940
-3.9378210
-0.1485910
-0.6221650
-2.7243060
-4.6607930
0.0489820
-0.5508240
-0.0022640
0.8893170
-0.9583060
0.8233860
1.6337970
-1.0279460
-1.6427100
-0.1365280
1.5221860
-1.7766420
-0.1887050

-2.0352280
-2.2844570
-2.0143300
0.6984290
1.4226060
-4.4697060
-5.8861600
-6.2218500
-6.0108070
-5.1965050
-4.0954060
-6.5818640
-7.2499840
-6.9756120
-5.8757890
-6.0157020
-5.5683650
-5.5031210
-4.8131400
-2.2851090
-2.7868690
-1.7073600
-1.1833780
-1.7621300
-1.7278710
-3.6972740
-2.1105150
-1.4220430
-2.8024830
-3.0569280
-0.8806200
-0.0924300
-1.2153870
3.4129770
2.2788310
4.1689940
5.2128490
3.8064340
5.8863910
5.4813470
4.4919020
2.9927740
5.5304520
6.6898620
4.2155590
6.0617320
2.8255870

3.8089760

2.1193270

0.7171370

0.9921900

$-1.2334240$

$-1.5846260$

$-2.9839880$

$-3.0789630$

$-1.8976140$

$-1.4459690$

$-0.8514070$

$-3.2513570$

$-3.1074490$

$-1.0441340$

$-1.5457030$

$-3.7008760$

$-4.0182140$

$-2.1381550$

$-2.6796940$

$-3.9380160$

$-5.0337230$

$-5.2540970$

$-4.2329380$

$-2.8437620$

$-4.2871710$

$-5.9648690$

$-6.2642370$

$-4.5054230$

$-3.7563710$

$-4.7343780$

$-5.1676580$

$-4.2705300$

$-1.0370490$

$-1.0941350$

$-2.3146940$

$-2.5850400$

$-3.2735590$

$-3.7988700$

$-1.8431340$

$-4.4801160$

$-3.0582070$

$-4.7450760$

$-4.0076810$

$-5.2155340$

$-5.6904320$ 


\begin{tabular}{|c|c|c|c|c|c|c|c|}
\hline & & & & & & & \\
\hline H & -0.6099740 & 1.7178030 & -0.1868060 & C & -3.3777800 & -0.5033760 & 3.8714470 \\
\hline s & 0.8212020 & 4.0454800 & 0.3242750 & $\mathrm{H}$ & -3.5305560 & -0.3724730 & 4.9487780 \\
\hline c & 0.9855800 & 2.2752970 & 2.9910830 & $\mathrm{H}$ & -3.3819990 & 0.4952840 & 3.4218650 \\
\hline C & 1.9296110 & 2.2883610 & 1.8682750 & $\mathrm{H}$ & -5.5026560 & -0.8387800 & 3.4727310 \\
\hline $\mathrm{H}$ & -0.0211990 & 1.9439520 & 2.7437760 & $\mathrm{H}$ & -1.3504820 & -2.9962340 & 2.5188000 \\
\hline $\mathrm{H}$ & 1.7975620 & 1.5539110 & 1.0770080 & C & -4.5144840 & -2.7264340 & 3.9635840 \\
\hline 0 & 1.8497390 & 1.1744270 & 3.3639050 & $\mathrm{H}$ & -4.6137680 & -2.6016500 & 5.0479320 \\
\hline H & 1.4093280 & 0.3313940 & 2.9915080 & $\mathrm{H}$ & -5.3733290 & -3.3143010 & 3.6260900 \\
\hline $\mathrm{C}$ & 2.4826530 & 3.8946860 & 4.2232670 & C & -3.1841830 & -3.4405500 & 3.6083610 \\
\hline c & 3.1592390 & 4.2388210 & 2.8954610 & $\mathrm{H}$ & -2.6398710 & -3.7209160 & 4.5170310 \\
\hline C & 3.2380970 & 3.0113300 & 1.9859870 & $\mathrm{H}$ & -3.3763530 & -4.3617740 & 3.0497550 \\
\hline c & 1.0511620 & 3.4111670 & 3.9842700 & C & -3.5439780 & -1.9053400 & -0.8331890 \\
\hline $\mathrm{H}$ & 2.4689120 & 4.7682690 & 4.8814850 & $\mathrm{C}$ & -3.2807030 & -1.9405550 & -2.3208250 \\
\hline $\mathrm{H}$ & 4.1692680 & 4.6245500 & 3.0617810 & C & -5.6060130 & -0.9663740 & -1.7338370 \\
\hline$H$ & 3.9512910 & 2.2779180 & 2.3838160 & $\mathrm{C}$ & -4.7860910 & -1.3310900 & -0.5237870 \\
\hline H & 0.4388490 & 4.2278480 & 3.5818100 & $\mathrm{C}$ & -4.3848240 & -2.7824010 & -2.9987160 \\
\hline$H$ & 0.5816550 & 3.0786240 & 4.9153190 & $\mathrm{H}$ & -4.2510260 & -2.7282010 & -4.0851400 \\
\hline$H$ & 3.0526920 & 3.1103170 & 4.7358490 & $\mathrm{H}$ & -4.2812420 & -3.8350770 & -2.7155620 \\
\hline $\mathrm{H}$ & 2.5919850 & 5.0323020 & 2.3913760 & C & -5.7803950 & -2.2405940 & -2.5892600 \\
\hline$H$ & 3.5957790 & 3.2594880 & 0.9831610 & $\mathrm{H}$ & -6.3803550 & -2.0024190 & -3.4746360 \\
\hline & & & & $\mathrm{H}$ & -6.3335180 & -2.9899580 & -2.0146620 \\
\hline & S) $\mathrm{G}[\mathrm{M06}-2\rangle$ & $g(d)]=-386$ & 245 & $\mathrm{H}$ & -6.5799690 & -0.5601080 & -1.4405340 \\
\hline $\mathrm{P}$ & 0.2985530 & -0.0791400 & 0.1921960 & $\mathrm{H}$ & -2.2903780 & -2.3423630 & -2.5479040 \\
\hline 0 & 0.7772370 & 0.8793130 & 1.2447840 & C & -4.8156680 & 0.0590440 & -2.5731630 \\
\hline 0 & -0.7702080 & 0.3741530 & -0.7793910 & $\mathrm{H}$ & -5.3567790 & 0.2421700 & -3.5088620 \\
\hline 0 & -0.1069370 & -1.4407640 & 0.9734530 & $\mathrm{H}$ & -4.7707100 & 1.0152430 & -2.0428010 \\
\hline 0 & 1.5054760 & -0.6088350 & -0.7550310 & C & -3.3957530 & -0.4927470 & -2.8531590 \\
\hline c & -0.1757840 & -2.6036750 & 0.2178570 & $\mathrm{H}$ & -3.1861330 & -0.4933860 & -3.9287020 \\
\hline C & 1.0245830 & -3.2404810 & -0.1404750 & $\mathrm{H}$ & -2.6290470 & 0.1236160 & -2.3752250 \\
\hline c & -1.4322600 & -3.0739590 & -0.1530340 & C & -5.1708040 & -1.1498260 & 0.7991420 \\
\hline c & -1.4743540 & -4.2541490 & -0.9028800 & $\mathrm{H}$ & -6.1381960 & -0.7073970 & 1.0347330 \\
\hline $\mathrm{H}$ & -2.4436880 & -4.6497940 & -1.1996500 & C & 3.8585700 & 2.6589740 & -1.7715510 \\
\hline c & 2.5195450 & -1.2730710 & -0.0792360 & C & 3.8054900 & 2.9311450 & -3.2539990 \\
\hline C & 3.6769240 & -0.5647550 & 0.2427420 & C & 3.5597460 & 0.4241520 & -2.6623610 \\
\hline c & 2.3175190 & -2.6114310 & 0.2625320 & $\mathrm{c}$ & 3.7216710 & 1.3020610 & -1.4435650 \\
\hline c & 4.6633040 & -1.2678470 & 0.9289560 & $\mathrm{C}$ & 4.9545840 & 2.1348550 & -3.9122940 \\
\hline $\mathrm{H}$ & 5.5876600 & -0.7525430 & 1.1861150 & $\mathrm{H}$ & 4.9221800 & 2.2881240 & -4.9968370 \\
\hline c & -2.6853500 & -2.3427100 & 0.1882680 & $\mathrm{H}$ & 5.9152430 & 2.5211650 & -3.5586680 \\
\hline C & 3.8111730 & 0.8770300 & -0.1081530 & C & 4.8016180 & 0.6314010 & -3.5609190 \\
\hline c & -3.0654010 & -2.1200560 & 1.5202830 & $\mathrm{H}$ & 4.6831640 & 0.0307320 & -4.4696830 \\
\hline c & -2.2943800 & -2.5029670 & 2.7610450 & $\mathrm{H}$ & 5.6921900 & 0.2618600 & -3.0435870 \\
\hline C & -4.5425470 & -1.3364080 & 3.2922640 & $\mathrm{H}$ & 3.4618230 & -0.6306110 & -2.3957690 \\
\hline c & -4.2959060 & -1.5159670 & 1.8167130 & $\mathrm{H}$ & 3.9098650 & 4.0019270 & -3.4616840 \\
\hline $\mathrm{C}$ & -2.0334 & -1.2219960 & 3.5833930 & C & 2.3188090 & 0.8941230 & -3.4494910 \\
\hline $\mathrm{H}$ & -1.5361640 & 14410 & 4.5191970 & $\mathrm{H}$ & 2.2169480 & 0.2779000 & -4.3497840 \\
\hline $\mathrm{H}$ & -1.3477 & 1234 & 3.0345110 & $\mathrm{H}$ & 1.4232720 & 0.7299190 & -2.8439610 \\
\hline
\end{tabular}




\begin{tabular}{|c|c|c|}
\hline $\begin{array}{l}4220 \\
4920 \\
6020 \\
4430 \\
3380 \\
6090 \\
7790 \\
8120 \\
2260 \\
9160 \\
2040 \\
5450 \\
8560 \\
8900 \\
7870 \\
8480 \\
8600 \\
2450 \\
2420 \\
5750 \\
6090 \\
0330 \\
4860 \\
3250 \\
6850 \\
2870 \\
6300 \\
8060 \\
7040 \\
4170 \\
4070 \\
1390 \\
1470 \\
8460 \\
5360 \\
6400 \\
3280 \\
4080\end{array}$ & $\begin{array}{r}2.3894100 \\
2.5234880 \\
2.9744180 \\
3.1935090 \\
4.0833440 \\
1.6070860 \\
1.8416580 \\
3.9900790 \\
4.5635170 \\
4.4428110 \\
2.5023020 \\
2.3388750 \\
2.1886650 \\
0.5606900 \\
5.1194560 \\
2.0604850 \\
1.9596600 \\
1.4041550 \\
3.5326740 \\
4.1574510 \\
3.6007260 \\
3.6054410 \\
4.6549890 \\
-3.2764200 \\
-4.6836960 \\
-5.0413800 \\
-4.7384180 \\
-3.2337750 \\
-2.5949160 \\
-6.0977490 \\
-5.4076950 \\
-4.7927740 \\
-5.2653020 \\
-5.0976120 \\
-3.0117440 \\
-2.7501680 \\
-4.4554510 \\
-4.3909070 \\
-5.0726540 \\
-5.2048250 \\
-5.9494060 \\
-6.1378510 \\
-4.9037420 \\
-6.0754580 \\
-5.7127170 \\
-6.9323520\end{array}$ & $\begin{array}{r}-3.8251130 \\
-4.9128050 \\
-3.4369870 \\
0.5447270 \\
1.7197650 \\
2.3770450 \\
0.8877300 \\
2.7475030 \\
3.6391650 \\
2.3327890 \\
3.1007260 \\
4.1802830 \\
2.8146840 \\
2.6370700 \\
1.3976490 \\
2.8235700 \\
3.9121990 \\
2.3867870 \\
2.3898740 \\
3.2544880 \\
1.6875700 \\
-0.7801720 \\
-1.0412340 \\
1.0142290 \\
1.5263900 \\
2.7079200 \\
2.3502280 \\
2.1608270 \\
1.3316390 \\
2.9626360 \\
0.7238630 \\
1.7920500 \\
1.4220070 \\
3.1251560 \\
1.7031770 \\
3.1473210 \\
3.5901540 \\
-0.9370820 \\
-1.5705500 \\
-3.0848970 \\
-3.4082540 \\
-2.1616770 \\
-1.2990460 \\
-1.1409210 \\
-3.5474830 \\
-3.8407960\end{array}$ \\
\hline
\end{tabular}
0.0671940
3. 0530960
1.8555460
0.0087760
$-1.3405310$
$-3.3254040$
$-2.8542480$
$-4.6341140$
$-5.1832210$
$-5.3258810$
$-6.4066030$
$-4.6461630$
$-6.5514020$
$-4.8973940$
$-7.0932970$
$-6.8240490$
$-7.0853970$
$-8.0501880$
$-1.9631250$
$-2.5505880$
$-0.3469320$
$-0.1219950$
$-0.8829170$
$-0.0437810$
1.0757110
1.1742400
0.0979690
$-0.1441240$
0.3350650
$-0.6726010$
$-0.2135260$
0.3713620
1.4330880
$-1.7534120$
$-0.4480870$
1.1699630
$-1.2163280$
0.0132910

154

$\operatorname{TS} 275(\mathrm{~S}, \mathrm{~S}) \mathrm{G}[\mathrm{M} 06-2 \mathrm{X} / 6-31 \mathrm{G}(\mathrm{d})]=-3864.199417$

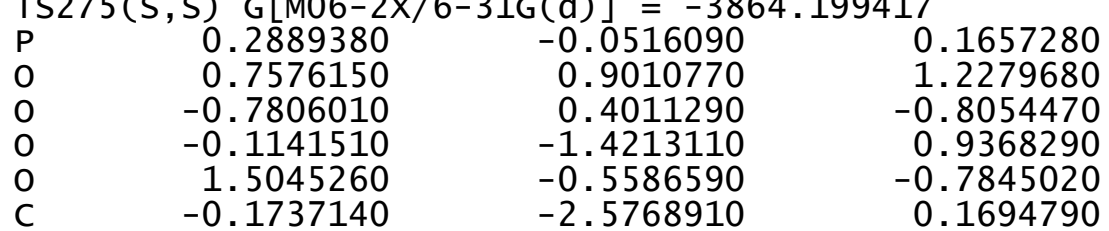

$-1.5769910$

$-4.5133310$

$-4.1942460$

$-5.3883700$

$-6.3993440$

2.4669820

.8851630

2.1414730

3.9977620

2. 5065960

1. 2771380

4. 3547020

4. 5729480

3. 6114970

1. 9266520

5.2149130

3.8953610

1.0016290

3.3380390

3. 5684580

.4055480

2.7541640

3928150

3. 3223340

. 3282780

6.0344220

5.9194190

4. 5685020

4.9468610

7. 0190690

6.7203270

4. 5184190

5.0986790

4.9673240

5.9335190

6.0284570

4.4074480
$-1.3851700$

$-3.5044070$

$-4.1611130$

$-0.4369030$

0.0988020

0.1271050

1. 1805940

$-0.3671390$

1. 7291340

1. 5527910

0.1808690

$-1.1807060$

1. 2294100

2. 5466980

$-0.2093730$

1. 6566750

$-0.3162270$

$-1.6569960$

0.6343650

$-0.8066740$

1. 1238710

$-1.1876360$

0.6083180

0.8196440

0.4070680

$-1.0988680$

$-1.6368630$

1.1588260

0.7675510

$-1.6367520$

$-1.6392820$

1.0440690

2. 2302810

0.6182890

$-1.3027140$
$-0.1737140$

0.1694790

$-6.9323520$

$-3.8407960$ 


1.0250640
-1.4242900
-1.4361330
-2.3986080
2.5190490
3.6755160
2.3085770
4.6564170
5.5843020
-2.6885260
3.8113900
-3.0702600
-2.2916400
-4.5558100
-4.3081660
-2.0481390
-1.5439580
-1.3739000
-3.4024980
-3.5549540
-3.4230970
-5.5227250
-1.3409890
-4.5066760
-4.6077830
-5.3566220
-3.1659840
-2.6165090
-3.3444810
-3.5523590
-3.2887760
-5.6239220
-4.8008740
-4.3839480
-4.2493110
-4.2704970
-5.7850490
-6.3840580
-6.3342090
-6.6021240
-2.2943670
-4.8428970
-5.3867920
-4.8042870
-3.4191620
-3.2136470

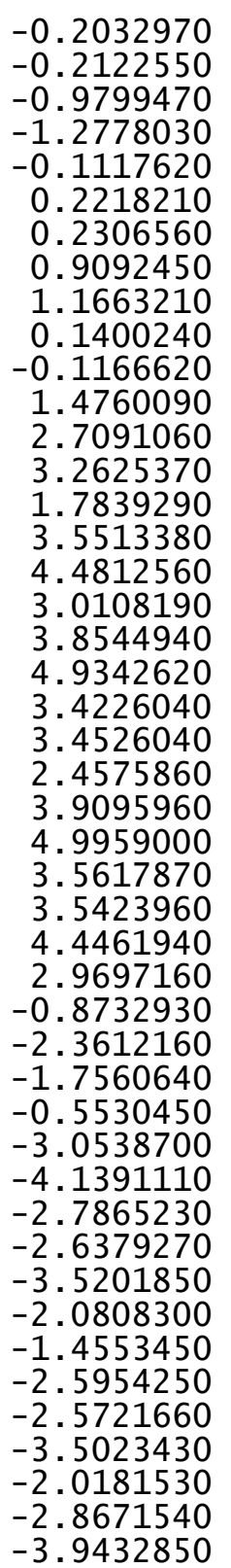

\author{
$-2.6562250$ \\ $-5.1873660$ \\ $-6.1597150$ \\ 3.8632320 \\ 3.8177640 \\ 3.5736350 \\ 3.7276890 \\ 4.9719400 \\ 4.9452490 \\ 5.9299100 \\ 4.8198890 \\ 4.7073500 \\ 5.7083710 \\ 3.4763000 \\ 3.9213260 \\ 2. 3360780 \\ 2. 2402650 \\ 1.4377510 \\ 2. 4942360 \\ 2. 5027500 \\ 1. 6526590 \\ 4.1908970 \\ 4.4976280 \\ 4.1371710 \\ 4.0317790 \\ 3. 3447960 \\ 3.6166620 \\ 2. 4410700 \\ 3.0990590 \\ 3.1879780 \\ 2. 0946430 \\ 3.9651900 \\ 4.6466410 \\ 5.5416780 \\ 5.6108800 \\ 6. 3059120 \\ 5.7745900 \\ 6.0178130 \\ 6.6149330 \\ 4.1000400 \\ 4.2314000 \\ 4.4728370 \\ 3.2766600 \\ 5.5927430 \\ 5.1231170 \\ 4.2411300
}

0.1581000

$-1.2018470$

$-0.7747880$

2.7184380

3.0023370

0.4902480

1.3586800

2. 2134420

2.3754000

2.5986200

0.7068950

0.1134720

0.3347410

$-0.5668500$

4.0749170

0.9642840

0.3551290

0.7935140

2.4628190

. 6054260

3.0432540

3.2352200

4.1164480

1.6338730

1.8801920

4.0114420

4.5787450

4.4645660

2. 5201630

2. 3484560

2.2057590

0.5849030

5.1555820

2.0877750

1.9781080

1.4372590

3.5642010

4.1826450

3.6406530

3.6575830

4.7095640

$-2.5556530$

3. 2229060

$-3.2399480$

$-4.4458690$

$-5.3416420$
-2.3814420
0.7731690
1.0169050
-1.7654550
-3.2459360
-2.6756740
-1.4490630
-3.9042250
-4.9876610
-3.5421840
-3.5659120
-4.4801810
-3.0468740
-2.4180190
-3.4444690
-3.4657360
-4.3714620
-2.8662890
-3.8286230
-4.9152020
-3.4405190
0.5568610
1.7408570
2.3761200
0.8881770
2.7608950
3.6587220
2.3442250
3.1009270
4.1796860
2.8065910
2.6265890
1.4280260
2.8342480
3.9224030
2.3961660
2.4140810
3.2852690
1.7173900
-0.7651450
-1.0170100
1.3188950
1.0106340
2.0811010
2.8915710
2.0256340 


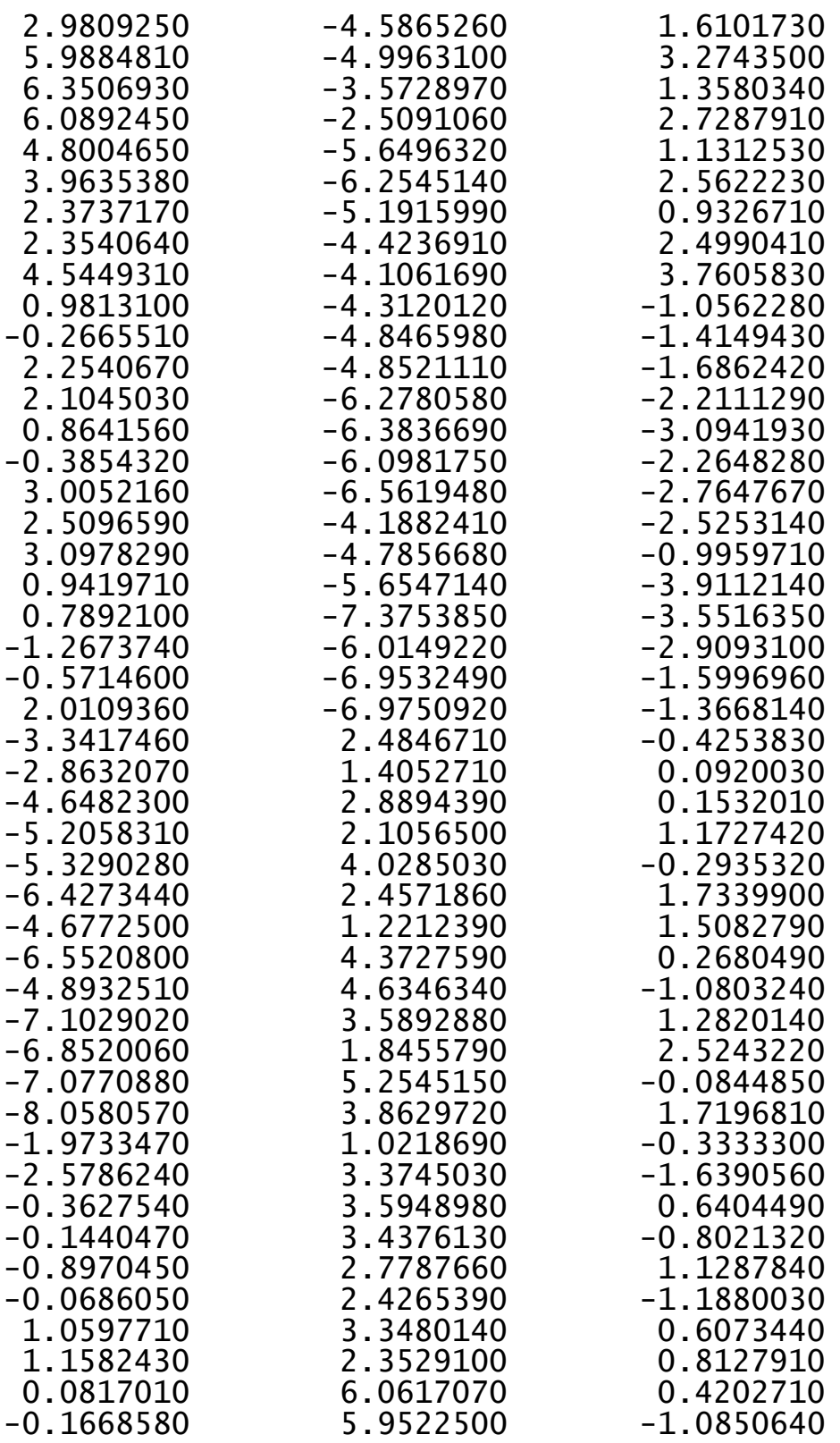

$\begin{array}{lr}\mathrm{C} & 0.3106350 \\ \mathrm{C} & -0.6855250 \\ \mathrm{H} & -0.2281260 \\ \mathrm{H} & 0.3461680 \\ \mathrm{H} & 1.4086890 \\ \mathrm{H} & -1.7668610 \\ \mathrm{H} & -0.4560980 \\ \mathrm{H} & 1.1546370 \\ \mathrm{H} & -1.2399430 \\ \mathrm{H} & -0.0136680 \\ \mathrm{H} & \end{array}$
4. 6033640
4.9714130
7.0450290
6.7552550
4.5535940
5. 1236860
4.9879020
5.9599760
6.0617640
4.4457020
$-1.6295940$
1.1715530
0.7857710
$-1.6221590$
$-1.6347660$
1. 0623630
2. 2420380
0.6263240
$-1.2840340$
$-2.6620290$

15

$\begin{array}{lccc}\text { TS276(S , S ) } & \text { G }[M 06-2 X / 6-31 G(d)]= & -3864.199196 \\ P & 0.2914180 & -0.0771890 & 0.1625060\end{array}$

$\begin{array}{llll}0 & 0.7756860 & 0.8724310 & 1.2206630\end{array}$

$\begin{array}{llll}0 & -0.7741540 & 0.3883080 & -0.8070330\end{array}$

$0-0.1244930 \quad-1.4405830 \quad 0.9374380$

$0-1.4970370 \quad-0.6081650 \quad-0.7877830$

C $\quad-0.2083830 \quad-2.5903000 \quad 0.1629300$

C $\quad 0.9770940 \quad-3.2335700 \quad-0.2111360$

C $\quad-1.4709880 \quad-3.0342940 \quad-0.2256080$

C $\quad-1.5114810 \quad-4.1760240 \quad-1.0224840$

$\mathrm{H}-2.4834330 \quad-4.5513600 \quad-1.3393000$

C $\quad 2.4975770 \quad-1.2898650 \quad-0.1080140$

$\begin{array}{llrr}\mathrm{C} & 2.4975770 & -1.2898650 & -0.1080140 \\ \mathrm{C} & 3.6561560 & -0.5946270 & 0.2379590\end{array}$

C $\quad 2.2711990 \quad-2.6260410 \quad 0.2298380$

$\mathrm{C} \quad 4.6136030 \quad-1.3058060 \quad 0.9558380$

$-1.3058060$

$-2.3080950$

$\begin{array}{rrr}-2.7196570 & -2.3080950 & 0.1389690 \\ 3.8183150 & 0.8448320 & -0.1101660\end{array}$

$-3.0911040 \quad-2.1141590 \quad 1.4779980$

$\begin{array}{lll}-2.3143030 & -2.5258450 & 2.7059830\end{array}$

$-4.5549540 \quad-1.3631010 \quad 3.2754510$

$-4.3175910 \quad-1.5120890 \quad 1.7950960$

$-2.0439120 \quad-1.2627750 \quad 3.5526740$

$-1.5418670 \quad-1.5630540 \quad 4.4794680$

$\begin{array}{lll}-1.3592810 & -0.6035010 & 3.0126390\end{array}$

$-3.3838880 \quad-0.5455760 \quad 3.8637440$

$-3.5292240 \quad-0.4356310 \quad 4.9444280$

$-3.3883710 \quad 0.4616260 \quad 3.4336410$

$\begin{array}{lll}-5.5121410 & -0.8661230 & 3.4720110\end{array}$

$-1.3736560 \quad-3.0176760 \quad 2.4485250$

$\begin{array}{lll}-4.5274820 & -2.7667530 & 3.9177080\end{array}$

$-4.6190680-2.6638610 \quad 5.0050140$

$\begin{array}{lll}-5.3906680 & -3.3446360 & 3.5741050\end{array}$

$\begin{array}{lll}-3.2022910 & -3.4778890 & 3.5388980\end{array}$

$\begin{array}{ll}-3.4778890 & 3.5388980 \\ -3.7794650 & 4.4378290\end{array}$ 


$\begin{array}{rrr}-3.4014440 & -4.3863060 & 2.9620670 \\ -3.5823130 & -1.8440030 & -0.8677540 \\ -3.3286500 & -1.8454180 & -2.3577780 \\ -5.6460520 & -0.8758790 & -1.7339350 \\ -4.8203270 & -1.2722660 & -0.5379090 \\ -4.4400300 & -2.6676140 & -3.0476500 \\ -4.3117290 & -2.5905140 & -4.1333360 \\ -4.3402030 & -3.7267150 & -2.7878980 \\ -5.8307680 & -2.1281610 & -2.6190290 \\ -6.4331330 & -1.8653230 & -3.4957430 \\ -6.3856880 & -2.8881580 & -2.0603630 \\ -6.6166400 & -0.4731950 & -1.4249470 \\ -2.3409490 & -2.2439460 & -2.6009020 \\ -4.8561340 & 0.1670140 & -2.5515660 \\ -5.4028060 & 0.3790570 & -3.4778360 \\ -4.8014550 & 1.1077680 & -1.9950490 \\ -3.4419160 & -0.3858080 & -2.8566110 \\ -3.2417130 & -0.3636490 & -3.9336970 \\ -2.6675910 & 0.2154860 & -2.3723160 \\ -5.1965280 & -1.1192820 & 0.7911350 \\ -6.1606000 & -0.6779950 & 1.0423380 \\ 3.9215670 & 2.6263120 & -1.7718920 \\ 3.8967510 & 2.8997300 & -3.2548740 \\ 3.5938630 & 0.3980270 & -2.6681100 \\ 3.7549710 & 1.2720970 & -1.4464500 \\ 5.0411830 & 2.0819270 & -3.8946790 \\ 5.0310780 & 2.2377090 & -4.9792980 \\ 6.0028890 & 2.4483690 & -3.5231990 \\ 4.8519420 & 0.5813220 & -3.5491760 \\ 4.7330940 & -0.0147420 & -4.4609270 \\ 5.7274570 & 0.1918410 & -3.0209080 \\ 3.4719900 & -0.6547960 & -2.4039020 \\ 4.0248430 & 3.9683390 & -3.4603330 \\ 2.3731860 & 0.8924730 & -3.4716350 \\ 2.2688880 & 0.2766090 & -4.3718270 \\ 1.4673610 & 0.7486710 & -2.8763710 \\ 2.5686550 & 2.3832430 & -3.8480830 \\ 2.5940140 & 2.5138250 & -4.9359190 \\ 1.7352550 & 2.9862480 & -3.4771100 \\ 4.2415690 & 3.1538050 & 0.5489650 \\ 4.5585660 & 4.0381410 & 1.7279300 \\ 4.1402080 & 1.5696060 & 2.3805010 \\ 4.0508110 & 1.8051620 & 0.8894690 \\ 3.3966840 & 3.9666710 & 2.7404750 \\ 3.6739210 & 4.5363950 & 3.6351100 \\ 2.5056260 & 4.4347080 & 2.3131360\end{array}$

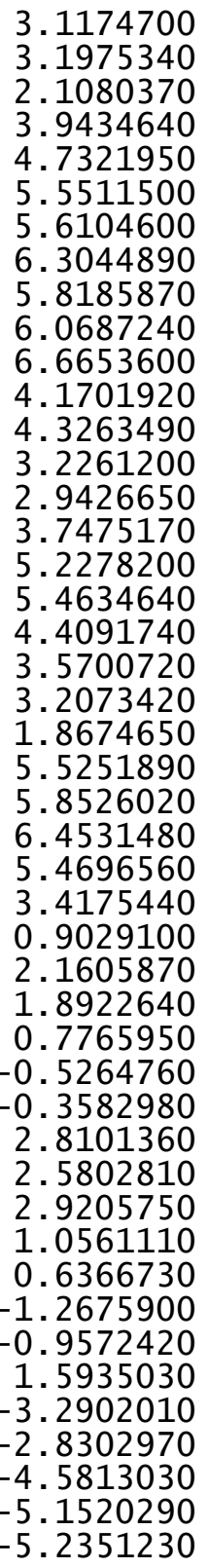

2. 4840890

2. 3198040

2. 1884700

0.5271170

5.0711150

1.9965900

1.8940720

1.3266630

3.4643640

4.0843560

3. 5172760

3. 5678640

4.6147700

$-3.2930450$

$-4.6770890$

$-4.9726940$

$-4.7325060$

$-3.2510560$

$-2.6225680$

$-6.0056040$

$-5.4385920$

$-4.7814850$

$-5.3350630$

$-5.0506750$

$-3.0966110$

$-2.7013720$

$-4.3192540$

$-4.3425110$

$-4.9551590$

$-5.6788980$

$-6.6989630$

$-5.9748410$

$-4.8195360$

$-6.1650040$

$-5.6896860$

$-4.1763480$

$-7.3666650$

$-7.3257140$

$-6.6768300$

$-5.5744500$

$-4.9575490$

2. 5310240

1.4435170

2. 9669490

2. 1926780

4.1258450
3. 0918540

4. 1724620

2. 7954580

2.6393270

1.4075000

2.8448730

3.9343060

2. 4172970

2. 4149270

3. 2830440

1.7238620

$-0.7768140$

$-1.0355140$

1.0244430

1. 5756350

2. 8400220

2. 5559120

2. 2689050

1.3742980

3. 1557430

0.8325540

1.7560210

1. 6877270

3. 3963930

1.8236050

3. 2200290

3. 6579800

$-1.0791550$

$-1.6654400$

$-2.9837460$

$-2.7745090$

$-2.4426570$

$-1.4711000$

$-3.3292630$

$-0.9680180$

$-1.7890770$

$-1.9491180$

$-3.6608430$

$-2.0432830$

$-3.3704980$

$-3.7553730$

$-0.4204420$

0.0970830

0.1698460

1.1894270

$-0.2662000$ 


\begin{tabular}{|c|c|c|c|c|c|c|c|}
\hline C & -6.3603730 & 2.5726440 & 1.7605290 & C & -3.4539960 & 1.1681750 & -0.2993470 \\
\hline $\mathrm{H}$ & -4.6441930 & 1.2931910 & 1.5169370 & $\mathrm{C}$ & 4.1979680 & 1.3653200 & 1.7343640 \\
\hline c & -6.4448310 & 4.4989520 & 0.3057730 & $\mathrm{C}$ & 3.8643490 & 2.8335820 & 1.8498520 \\
\hline H & -4.7889230 & 4.7247190 & -1.0527100 & C & 5.2348740 & 1.9499800 & 3.8590850 \\
\hline c & -7.0092010 & 3.7244310 & 1.3191290 & $\mathrm{c}$ & 4.9526180 & 0.8963520 & 2.8194790 \\
\hline $\mathrm{H}$ & -6.7957410 & 1.9678670 & 2.5502870 & C & 3.0648300 & 3.0403930 & 3.1546140 \\
\hline $\mathrm{H}$ & -6.9487990 & 5.3962360 & -0.0382060 & $\mathrm{H}$ & 2.8541380 & 4.1092930 & 3.2731740 \\
\hline $\mathrm{H}$ & -7.9540080 & 4.0204470 & 1.7646670 & $\mathrm{H}$ & 2.1032890 & 2.5268850 & 3.0712700 \\
\hline H & -1.9526640 & 1.0385020 & -0.3327100 & C & 3.8834520 & 2.5051950 & 4.3590410 \\
\hline $\mathrm{S}$ & -2.5210960 & 3.3980370 & -1.6467080 & $\mathrm{H}$ & 4.0721440 & 3.3008180 & 5.0887790 \\
\hline c & -0.2893950 & 3.5862400 & 0.6165160 & $\mathrm{H}$ & 3.3347060 & 1.7137620 & 4.8781620 \\
\hline $\mathrm{C}$ & -0.0819070 & 3.4125920 & -0.8257980 & H & 5.8155010 & 1.5370600 & 4.6908630 \\
\hline $\mathrm{H}$ & -0.8391520 & 2.7862560 & 1.1143190 & $\mathrm{H}$ & 3.2883330 & 3.1913950 & 0.9930330 \\
\hline $\mathrm{H}$ & -0.0313030 & 2.3971300 & -1.2040260 & C & 6.0035660 & 3.1041730 & 3.1801970 \\
\hline 0 & 1.1268650 & 3.3070410 & 0.5774800 & $\mathrm{H}$ & 6.1661910 & 3.9041110 & 3.9114130 \\
\hline $\mathrm{H}$ & 1.2044180 & 2.3117310 & 0.7915890 & $\mathrm{H}$ & 6.9894600 & 2.7507770 & 2.8632400 \\
\hline C & 0.2088570 & 6.0406630 & 0.3737980 & C & 5.1881460 & 3.6220050 & 1.9667880 \\
\hline c & -0.0503660 & 5.9245050 & -1.1292770 & $\mathrm{H}$ & 4.9568330 & 4.6871020 & 2.0800480 \\
\hline C & 0.3950230 & 4.5611210 & -1.6647470 & $\mathrm{H}$ & 5.7600270 & 3.5129450 & 1.0400700 \\
\hline $\mathrm{C}$ & -0.5783040 & 4.9738790 & 1.1381660 & C & 4.2474870 & -0.8401290 & 0.7605850 \\
\hline $\mathrm{H}$ & -0.0767630 & 7.0336140 & 0.7329050 & $\mathrm{C}$ & 3.8818750 & -1.9534560 & -0.1943310 \\
\hline H & 0.4770910 & 6.7117720 & -1.6756280 & C & 5.4370690 & -2.7376070 & 1.7218480 \\
\hline $\mathrm{H}$ & 1.4916820 & 4.4874630 & -1.6729910 & $\mathrm{C}$ & 5.0368160 & -1.2881870 & 1.8305220 \\
\hline H & -1.6565730 & 5.1494710 & 1.0335630 & $\mathrm{C}$ & 5.1795500 & -2.5440980 & -0.7881350 \\
\hline $\mathrm{H}$ & -0.3426940 & 4.9938560 & 2.2072730 & $\mathrm{H}$ & 4.9218600 & -3.4227010 & -1.3917850 \\
\hline H & 1.2804760 & 5.9165220 & 0.5746680 & $\mathrm{H}$ & 5.6510310 & -1.8199700 & -1.4606290 \\
\hline$H$ & -1.1218870 & 6.0556690 & -1.3235570 & C & 6.1460990 & -2.9327270 & 0.3628860 \\
\hline H & 0.0635050 & 4.4020150 & -2.6946670 & $\mathrm{H}$ & 6.4638530 & -3.9769690 & 0.2675750 \\
\hline & & & & $\mathrm{H}$ & 7.0488080 & -2.3148070 & 0.3346550 \\
\hline & , S) G[M06-2> & $(d)]=-38$ & 790 & $\mathrm{H}$ & 6.0988250 & -3.0258420 & 2.5454930 \\
\hline $\mathrm{P}$ & 0.0931490 & 0.4923060 & 0.9459570 & $\mathrm{H}$ & 3.2095130 & -1.6039120 & -0.9834740 \\
\hline 0 & -0.7640270 & 0.9175780 & 2.0996990 & C & 4.1600170 & -3.6024480 & 1.7138110 \\
\hline 0 & 0.6997690 & -0.8929990 & 0.9674590 & $\mathrm{H}$ & 4.4380440 & -4.6446700 & 1.5184940 \\
\hline 0 & 1.2180900 & 1.6406250 & 0.7425230 & $\mathrm{H}$ & 3.6854720 & -3.5739270 & 2.6991240 \\
\hline 0 & -0.7119980 & 0.5424090 & -0.4759910 & C & 3.1933390 & -3.0706410 & 0.6265040 \\
\hline c & 1.8881020 & 1.5990190 & -0.4763180 & $\mathrm{H}$ & 2.8893530 & -3.8765000 & -0.0538020 \\
\hline & 1.2377140 & 2.1121410 & -1.6008010 & $\mathrm{H}$ & 2.2876240 & -2.6666830 & 1.0843470 \\
\hline c & 3.1561100 & 1.0229040 & -0.5274630 & C & 5.3907640 & -0.4233140 & 2.8603810 \\
\hline c & 3.7562960 & 0.9542910 & -1.7826720 & $\mathrm{H}$ & 6.0000440 & -0.7759380 & 3.6912660 \\
\hline H & 4.7498540 & 0.5147060 & -1.8561780 & C & -4.6956270 & -0.8938230 & -0.6955810 \\
\hline c & -1.1063460 & 1.8073240 & -0.8924370 & $\mathrm{C}$ & -4.9059800 & -2.0069270 & -1.6923710 \\
\hline & -2.4470120 & 2.1636570 & -0.7601860 & C & -3.0696610 & -0.3255470 & -2.3998730 \\
\hline c & -0.1393210 & 2.6636850 & -1.4204510 & $\mathrm{C}$ & -3.7144550 & 0.0302740 & -1.0813190 \\
\hline c & -2.7859590 & 56560 & -1.1155060 & C & -5.3049840 & -1.3448020 & -3.0308340 \\
\hline $\mathrm{H}$ & -3.8258580 & 66160 & -1.0262450 & $\mathrm{H}$ & -5.4764470 & -2.1235110 & -3.7821880 \\
\hline & 3.85262 & 4261 & 0.6826550 & $\mathrm{H}$ & -6.2472020 & -0.8037840 & -2.9015200 \\
\hline
\end{tabular}




$\begin{array}{rrr}-4.1742620 & -0.3824210 & -3.4805160 \\ -3.7260560 & -0.7230020 & -4.4206990 \\ -4.5676150 & 0.6232180 & -3.6567940 \\ -2.2981890 & 0.3936620 & -2.6853640 \\ -5.6876200 & -2.6960670 & -1.3546360 \\ -2.4582370 & -1.7338640 & -2.2549460 \\ -1.9316290 & -2.0061010 & -3.1767520 \\ -1.7105590 & -1.7007790 & -1.4583780 \\ -3.5759660 & -2.7558960 & -1.9315150 \\ -3.7161060 & -3.4566330 & -2.7629480 \\ -3.3034910 & -3.3574970 & -1.0584010 \\ -5.1753250 & 0.4281090 & 1.2536440 \\ -5.8965600 & 0.7979480 & 2.5237070 \\ -4.0866190 & 2.5186370 & 1.8367570 \\ -4.1873740 & 1.3543370 & 0.8798740 \\ -4.8509210 & 0.9222850 & 3.6518620 \\ -5.3588020 & 1.1964970 & 4.5834380 \\ -4.3676440 & -0.0462580 & 3.8159810 \\ -3.7993180 & 1.9907380 & 3.2595690 \\ -3.8335390 & 2.8391110 & 3.9526380 \\ -2.7851690 & 1.5886660 & 3.2991450 \\ -3.3077970 & 3.2226720 & 1.5337420 \\ -6.6554540 & 0.0497870 & 2.7775330 \\ -5.4702730 & 3.2071390 & 1.8692980 \\ -5.4296220 & 4.0573710 & 2.5589460 \\ -5.7076990 & 3.6062620 & 0.8776200 \\ -6.5484360 & 2.1845660 & 2.3194170 \\ -7.0139220 & 2.4992490 & 3.2602200 \\ -7.3460040 & 2.1087270 & 1.5742830 \\ -5.4342940 & -0.6902260 & 0.4678820 \\ -6.2125430 & -1.3964900 & 0.7559980 \\ -0.4965020 & 3.9963300 & -1.7073870 \\ 0.5662150 & 5.0063090 & -2.0969000 \\ 0.1299310 & 6.4444820 & -1.8228400 \\ -1.2307910 & 6.6908240 & -2.4690040 \\ -2.2860030 & 5.8214280 & -1.7889230 \\ -1.8337950 & 4.3923300 & -1.5489270 \\ 0.8825410 & 7.1371640 & -2.2124130 \\ 0.7894110 & 4.9230660 & -3.1673110 \\ 1.4970130 & 4.7700100 & -1.5700340 \\ -1.1701240 & 6.4406310 & -3.5363400 \\ -1.5188590 & 7.7445870 & -2.4015000 \\ -3.2147060 & 5.8130580 & -2.3710380 \\ -2.5400580 & 6.2660360 & -0.8170100 \\ 0.0585060 & 6.6150720 & -0.7408820 \\ 1.8438130 & 1.9693380 & -2.8655820\end{array}$

1.0918000

1.6217390

3.1297520

3.8213970

3.1198580

1.1113330

1.1865030

0.0233200

3.3391500

3. 5279140

4.8623460

3.8627050

1. 4052890

0.0365770

0.5985040

0.2857220

$-0.5359770$

1. 3720650

$-0.2722830$

$-1.3811330$

1. 6394220

2. 0018750

0.8169080

$-0.9172880$

2.4888390

1.0236620

0.5710680

$-0.8816020$

$-1.1809770$

$-2.0704140$

$-0.1642110$

$-1.9025670$

$-2.0642670$

$-1.6076090$

$-2.7330560$

$-3.3451100$

$-3.3815940$

$-1.2921140$

$-2.7503300$

$-4.3616860$

$-4.1147100$

$-0.6607800$

$-0.8679030$

$-3.3274470$

-2.7539680
-3.6901380
2. 3407680

1.6042400

1. 8157240

1.1525800

1.3900310

1.9708610

3. 4165110

2. 1472590

2.8934040

4090300

1.4868720

0.0682490

0.5312200

$-3.5875530$

$-2.4428890$

$-4.5218550$

$-5.6303100$

$-4.2731410$

$-6.4802850$

$-5.8111930$

$-5.1345230$

$-3.4089980$

$-6.2369320$

$-7.3339560$

$-4.9458420$

$-6.9064210$

$-1.7782490$

$-4.0593660$

$-2.0604230$

$-2.1764100$

$-1.7453920$

$-1.5119060$

$-0.9368740$

$-0.1272980$

$-3.5725960$

$-4.0365080$

$-2.8947110$

$-3.1052170$

$-4.3841710$

$-4.4109020$

$-2.1335160$

$-3.9511800$

$-2.6893720$

$-2.7485150$

$-4.8689830$

$-3.2330340$
$-4.1298030$

$-5.3588210$

$-5.4601800$

$-4.2712770$

$-6.2550190$

$-4.3214070$

$-3.9856920$

$-5.4627400$

$-6.3951050$

$-4.1932840$

$-4.4432620$

$-5.2755620$

$-0.7661240$

$-0.9214760$

$-1.8938730$

$-2.1246750$

$-2.7438880$

$-3.1919380$

$-1.4691210$

$-3.8010410$

$-2.5593910$

$-4.0275510$

$-3.3728060$

$-4.4495850$

$-4.8568330$

$-0.0872380$

0.5684260

3.0530400

1.8922660

2.8256900

1.0479360

3.2865710

2. 8618240

4.3507590

3.0281580

2. 0105700

4.1380360

5.0839690

3.1798540

2. 3067960

3.8387290

5.0572710

4.7637190

2.6245550

1.0178910 
154

TS278(S, S ) G[M06-2X/6-31G(d)] = -3864.198592

$\begin{array}{lrrr}\mathrm{P} & 0.2782800 & -0.0548750 & 0.1469090 \\ \mathrm{O} & 0.7624250 & 0.8750120 & 1.2225650 \\ \mathrm{O} & -0.7755430 & 0.4331770 & -0.8244450 \\ \mathrm{O} & -0.1602630 & -1.4228760 & 0.9015770 \\ \mathrm{O} & 1.4885990 & -0.5756770 & -0.8031190 \\ \mathrm{C} & -0.2467680 & -2.5725620 & 0.1285100 \\ \mathrm{C} & 0.9380610 & -3.2052040 & -0.2635810 \\ \mathrm{C} & -1.5085030 & -3.0387140 & -0.2328560 \\ \mathrm{C} & -1.5485350 & -4.2050910 & -0.9964050 \\ \mathrm{H} & -2.5214980 & -4.6078730 & -1.2730660 \\ \mathrm{C} & 2.4806870 & -1.2785830 & -0.1326230 \\ \mathrm{C} & 3.6514450 & -0.6101010 & 0.2272490 \\ \mathrm{C} & 2.2348130 & -2.6135210 & 0.1812330 \\ \mathrm{C} & 4.5994560 & -1.3552100 & 0.9229120 \\ \mathrm{H} & 5.5362610 & -0.8756260 & 1.2030380 \\ \mathrm{C} & -2.7582680 & -2.3181660 & 0.1390030 \\ \mathrm{C} & 3.8358660 & 0.8320670 & -0.0968810 \\ \mathrm{C} & -3.1250190 & -2.1329130 & 1.4800910 \\ \mathrm{C} & -2.3373420 & -2.5424100 & 2.7014160 \\ \mathrm{C} & -4.5812450 & -1.3935520 & 3.2875140 \\ \mathrm{C} & -4.3511000 & -1.5343860 & 1.8053900 \\ \mathrm{C} & -2.0689850 & -1.2782160 & 3.5474960 \\ \mathrm{H} & -1.5580490 & -1.5761890 & 4.4701660 \\ \mathrm{H} & -1.3925830 & -0.6142790 & 3.0027230 \\ \mathrm{C} & -3.4112940 & -0.5698460 & 3.8694160 \\ \mathrm{H} & -3.5494360 & -0.4628580 & 4.9513350 \\ \mathrm{H} & -3.4266180 & 0.4375470 & 3.4401280 \\ \mathrm{H} & -5.5398940 & -0.9031130 & 3.4920480 \\ \mathrm{H} & -1.3950840 & -3.0270430 & 2.4352580 \\ \mathrm{C} & -4.5411870 & -2.7989950 & 3.9252200 \\ \mathrm{H} & -4.6288340 & -2.6997020 & 5.0131610 \\ \mathrm{H} & -5.4020980 & -3.3816010 & 3.5838440 \\ \mathrm{C} & -3.2131800 & -3.5010030 & 3.5391680 \\ \mathrm{H} & -2.6569480 & -3.7986500 & 4.4350140 \\ \mathrm{H} & -3.4091890 & -4.4108400 & 2.9634710 \\ \mathrm{C} & -3.6220020 & -1.8458350 & -0.8619970 \\ \mathrm{C} & -3.3701410 & -1.8405810 & -2.3518160 \\ \mathrm{C} & -5.6779120 & -0.8538210 & -1.7171520 \\ \mathrm{C} & -4.8568320 & -1.2714610 & -0.5252050 \\ \mathrm{C} & -4.4902170 & -2.6494760 & -3.0430570 \\ \mathrm{H} & -4.3592410 & -2.5759150 & -4.1286110 \\ \mathrm{H} & -4.4023100 & -3.7089880 & -2.7806310 \\ \mathrm{C} & -5.8753350 & -2.0931380 & -2.6175630 \\ \mathrm{H} & -6.4673360 & -1.8104410 & -3.4951670\end{array}$

$-6.4462450$

$-6.6442760$

$-2.3872930$

$-4.8751310$

$-5.4226550$

$-4.8031530$

$-3.4704990$

$-3.2815920$

$-2.6840030$

$-5.2308090$

$-6.1938530$

3.9691930

3.9588700

3. 6201120

3. 7848820

5. 0981170

5.0998600

6.0611610

4.8860010

4.7644330

5.7527970

3.4828750

4. 1018230

2. 4110200

2. 3028130

1. 5001700

2. 6294520

2. 6667710

1.8005260

4. 2786920

4. 5948970

4.1516000

4.0730170

3. 4253110

3. 7009990

2. 5410190

3. 1312870

3. 2018440

2. 1216490

3. 9460520

4.7791250

5. 5630740

5. 6149550

6.3130440

5.8453180
6.0939250
-2.8507840
-0.4437520
-2.2490720
0.1910740
0.4291580
1.1194870
-0.3772520
-0.3529990
0.2127270
-1.1305250
-0.6898950
2.6366090
2.9316760
0.4256590
1.2798540
2.1087900
2.2816970
2.4564680
0.6055360
0.0260300
0.1953140
-0.6292660
4.0014170
0.9476000
0.3451170
0.8087120
2.4401580
2.5837780
3.0493440
3.1259580
3.9888670
1.5137920
1.7741610
3.9107310
4.4638550
4.3928350
2.4250940
2.2432700
2.1428430
0.4684620
5.0253820
1.9215250
1.8004420
1.2522060
3.3938790
3.9977020

$-2.0719940$

$-1.4044470$

-2.5982170

$-2.5202100$

$-3.4395260$

$-1.9451290$

$-2.8425390$

$-3.9216250$

$-2.3638860$

0.8062390

1.0631000

$-1.7310560$

$-3.2100820$

$-2.6621740$

$-1.4269530$

$-3.8527180$

$-4.9347730$

$-3.4669630$

$-3.5327650$

$-4.4547930$

$-3.0058430$

$-2.4142030$

$-3.3992210$

$-3.4656320$

$-4.3743770$

$-2.8768720$

$-3.8214730$

$-4.9073180$

$-3.4508830$

0.5997140

1.7946200

2. 4053390

0.9182870

2. 7979350

3. 7034040

2. 3721200

3. 1240770

4.2025180

2.8153960

2.6453740

1. 4920400

2. 8851020

3.9730000

2. 4507800

2. 4811710

3. 3610400 


$\begin{array}{lr}\mathrm{H} & 6.6975630 \\ \mathrm{C} & 4.2206760 \\ \mathrm{H} & 4.3894060 \\ \mathrm{C} & 3.1702600 \\ \mathrm{C} & 2.8342770 \\ \mathrm{C} & 3.7235980 \\ \mathrm{C} & 5.1880600 \\ \mathrm{C} & 5.4309060 \\ \mathrm{C} & 4.3727270 \\ \mathrm{H} & 3.5426470 \\ \mathrm{H} & 2.9400720 \\ \mathrm{H} & 1.7777990 \\ \mathrm{H} & 5.4197610 \\ \mathrm{H} & 5.8556690 \\ \mathrm{H} & 6.4173780 \\ \mathrm{H} & 5.4443640 \\ \mathrm{H} & 3.4660430 \\ \mathrm{C} & 0.8651060 \\ \mathrm{C} & -0.3950420 \\ \mathrm{C} & 2.1110030 \\ \mathrm{C} & 1.9554580 \\ \mathrm{C} & 0.6903530 \\ \mathrm{C} & -0.5401190 \\ \mathrm{H} & 2.8395240 \\ \mathrm{H} & 2.2989430 \\ \mathrm{H} & 2.9914630 \\ \mathrm{H} & 0.7508080 \\ \mathrm{H} & 0.6005210 \\ \mathrm{H} & -1.4328120 \\ \mathrm{H} & -0.7229760 \\ \mathrm{H} & 1.8890520 \\ \mathrm{C} & -3.2582130 \\ \mathrm{O} & -2.8151090 \\ \mathrm{C} & -4.5549660 \\ \mathrm{C} & -5.1818010 \\ \mathrm{C} & -5.1592060 \\ \mathrm{C} & -6.3982250 \\ \mathrm{H} & -4.7101820 \\ \mathrm{C} & -6.3757600 \\ \mathrm{H} & -4.6689020 \\ \mathrm{C} & -6.9971480 \\ \mathrm{H} & -6.8791200 \\ \mathrm{H} & -6.8406490 \\ \mathrm{H} & -7.9477060 \\ \mathrm{H} & -1.9398170 \\ \mathrm{~S} & -2.4638970 \\ & \end{array}$

\begin{abstract}
3.4512450
3. 5605400

4. 6095270

$-3.3131070$

$-4.6954200$

$-5.1047280$

$-4.8653210$

$-3.3671090$

$-2.6781300$

$-6.1553120$

$-5.4487040$

$-4.7142320$

$-5.3858100$

$-5.2702810$

$-3.1788900$

$-2.8986180$

$-4.5128570$

$-4.3181500$

$-4.8432790$

$-4.8513560$

$-6.2919910$

$-6.4205460$

$-6.1052830$

$-6.5877120$

$-4.2084880$

$-4.7414420$

$-5.7181840$

$-7.4255060$

$-6.0246370$

$-6.9465870$

$-6.9666610$

2. 6141680

1.5201670

3.0565430

2. 2429730

4.2579370

2. 6262010

1. 3115690

4.6357340

4.8863720

3. 8216890

1. 9906100

5. 5672800

4.1207740

1. 1040600

3. 4790900
\end{abstract}

1.7972100

$-0.7200980$

$-0.9619230$

0.9707770

1.5034870

2. 6766850

2. 3197960

2. 1575380

1.3157280

2. 9245440

0.7111830

1.7930820

1. 3809780

3. 0867380

1.7184070

3. 1512810

3. 5646040

$-1.1208360$

$-1.4518690$

$-1.8050200$

$-2.2853790$

$-3.1306830$

$-2.2824640$

$-2.8593250$

$-2.6778160$

$-1.1704080$

$-3.9720410$

$-3.5554350$

$-2.9123080$

$-1.5991540$

$-1.4206150$

$-0.4475000$

0.0714040

0.1245260

1.0785610

$-0.2649700$

1. 6297410

1. 3699410

0.2892620

$-1.0005270$

1. 2359650

2. 3671280

$-0.0165900$

1. 6669860

$-0.3542430$

$-1.6582790$

$\begin{array}{lr}\mathrm{C} & -0.2572460 \\ \mathrm{C} & -0.0342520 \\ \mathrm{H} & -0.8235550 \\ \mathrm{H} & 0.0069100 \\ \mathrm{O} & 1.1555550 \\ \mathrm{H} & 1.2166070 \\ \mathrm{C} & 0.2767400 \\ \mathrm{C} & 0.0336450 \\ \mathrm{C} & 0.4677810 \\ \mathrm{C} & -0.5336180 \\ \mathrm{H} & 0.0003510 \\ \mathrm{H} & 0.5775520 \\ \mathrm{H} & 1.5634310 \\ \mathrm{H} & -1.6081960 \\ \mathrm{H} & -0.3109260 \\ \mathrm{H} & 1.3441870 \\ \mathrm{H} & -1.0337590 \\ \mathrm{H} & 0.1463330 \\ \mathrm{H} & \end{array}$
3. 6099040
3. 4512670
2. 8115700
2. 4401390
3. 3114640
2. 3126670
6.0602640
5.9661410
4.6037960
4. 9947930
7.0524410
6.7530930
4.5161350
5. 1860030
4.9983780
5.9189890
6.1136300
4.4620920

154

TS279(S, S) G[M06-2X/6-31G(d) $]=-3864.200090$

$\begin{array}{lrcr}\text { TS279(S, S) G }[\mathrm{M} 06-2 X / 6-31 \mathrm{G}(\mathrm{d})]=-3864.200090 \\ \mathrm{P} & 0.2837350 & -0.0510900 & 0.1900080 \\ \mathrm{O} & 0.7525620 & 0.8977990 & 1.2556750 \\ \mathrm{O} & -0.7811990 & 0.4077370 & -0.7833980 \\ \mathrm{O} & -0.1267900 & -1.4217710 & 0.9554730 \\ \mathrm{O} & 1.5004580 & -0.5579370 & -0.7585000 \\ \mathrm{C} & -0.1785720 & -2.5759140 & 0.1847180 \\ \mathrm{C} & 1.0230660 & -3.1911800 & -0.1724730 \\ \mathrm{C} & -1.4247240 & -3.0565760 & -0.2154200 \\ \mathrm{C} & -1.4252050 & -4.2115990 & -0.9940960 \\ \mathrm{H} & -2.3831880 & -4.6229000 & -1.3087300 \\ \mathrm{C} & 2.5169110 & -1.2253890 & -0.0900910 \\ \mathrm{C} & 3.6825210 & -0.5276190 & 0.2213350 \\ \mathrm{C} & 2.3032900 & -2.5587220 & 0.2646210 \\ \mathrm{C} & 4.6737220 & -1.2434960 & 0.8903930 \\ \mathrm{H} & 5.6108810 & -0.7408190 & 1.1243030 \\ \mathrm{C} & -2.6935810 & -2.3573480 & 0.1315860 \\ \mathrm{C} & 3.8181570 & 0.9159110 & -0.1208740 \\ \mathrm{C} & -3.0853790 & -2.1724240 & 1.4660560 \\ \mathrm{C} & -2.3157330 & -2.5719090 & 2.7024670 \\ \mathrm{C} & -4.5855370 & -1.4538210 & 3.2462320 \\ \mathrm{C} & -4.3261660 & -1.5930090 & 1.7685980 \\ \mathrm{C} & -2.0805030 & -1.3062030 & 3.5557640 \\ \mathrm{H} & -1.5833930 & -1.5992140 & 4.4875320 \\ \mathrm{H} & -1.4027510 & -0.6319070 & 3.0255940 \\ \mathrm{C} & -3.4380150 & -0.6167450 & 3.8532130 \\ \mathrm{H} & -3.5990380 & -0.5152770 & 4.9324930\end{array}$

0.6378560

$-0.8039080$

1.1192800

$-1.1945580$

0.6113410

0.8146270

0.4317490

$-1.0754830$

$-1.6226280$

1.1732810

0.8000870

$-1.6059170$

$-1.6189240$

1.0579610

2. 2453170

0.6432440

$-1.2805940$

$-2.6582320$ 


$\begin{array}{lrr}-3.4565350 & 0.3924690 & 3.4282730 \\ -5.5545120 & -0.9759380 & 3.4321070 \\ -1.3625050 & -3.0445950 & 2.4552650 \\ -4.5397530 & -2.8594130 & 3.8835450 \\ -4.6496090 & -2.7629120 & 4.9697350 \\ -5.3861430 & -3.4526850 & 3.5246910 \\ -3.1953270 & -3.5431050 & 3.5220320 \\ -2.6525730 & -3.8348110 & 4.4280570 \\ -3.3681400 & -4.4545400 & 2.9415010 \\ -3.5505090 & -1.9062050 & -0.8852100 \\ -3.2768100 & -1.9016880 & -2.3713250 \\ -5.6166490 & -0.9677330 & -1.7768390 \\ -4.8017860 & -1.3550160 & -0.5704610 \\ -4.3666110 & -2.7397430 & -3.0763190 \\ -4.2243170 & -2.6615110 & -4.1601680 \\ -4.2545840 & -3.7969330 & -2.8139450 \\ -5.7709050 & -2.2202560 & -2.6675040 \\ -6.3634940 & -1.9632690 & -3.5525780 \\ -6.3238880 & -2.9891370 & -2.1191660 \\ -6.5971280 & -0.5797720 & -1.4804950 \\ -2.2804680 & -2.2866050 & -2.6011000 \\ -4.8306350 & 0.0886300 & -2.5809900 \\ -5.3682990 & 0.2961180 & -3.5135310 \\ -4.7961750 & 1.0280940 & -2.0207120 \\ -3.4047220 & -0.4430620 & -2.8696620 \\ -3.1920500 & -0.4165840 & -3.9442600 \\ -2.6453930 & 0.1692440 & -2.3752740 \\ -5.1980340 & -1.2118200 & 0.7538360 \\ -6.1724970 & -0.7870890 & 0.9931700 \\ 3.8513070 & 2.7108130 & -1.7703740 \\ 3.7890130 & 2.9940680 & -3.2503490 \\ 3.5510470 & 0.4821710 & -2.6763880 \\ 3.7197710 & 1.3511350 & -1.4522490 \\ 4.9353700 & 2.2046160 & -3.9215480 \\ 4.8965270 & 2.3664890 & -5.0046190 \\ 5.8975240 & 2.5894440 & -3.5703710 \\ 4.7866750 & 0.6980810 & -3.5814590 \\ 4.6629060 & 0.1047200 & -4.4943030 \\ 5.6811930 & 0.3255980 & -3.0731170 \\ 3.4564430 & -0.5747310 & -2.4164370 \\ 3.8904030 & 4.0665390 & -3.4506150 \\ 2.3043080 & 0.9561140 & -3.4519140 \\ 2.1975670 & 0.3465410 & -4.3561230 \\ 1.4130220 & 0.7860510 & -2.8418330 \\ 2.4587540 & 2.4544010 & -3.8174220 \\ 2.4547250 & 2.5964560 & -4.9041000\end{array}$

1. 6218340 4. 2024340 4.5188890 4.1712660 4. 0490890 3. 3764940 3. 6558840 2. 4673960 3. 1379550 3. 2376970 2. 1314670 4. 0051890 4. 6618880 5. 5793060 5. 6604570 6.3402450 5.8039890 6.0540320 6.6372130 4. 0974510 4. 2248960 4.4885530 3. 2776760 5. 6235370 5.1717560 4.2337630 2. 9740900 6.0456800 6.3539520 6. 1488610 4.7436160 3. 9627130 2. 2976190 2.4174220 4. 6409310 0.9932130 2. 2852970 2. 0539240 0.9615100 $-0.3651780$ $-0.2490380$ 2. 9910200 2.8088070 2. 9580140 1. 2298400 0.8649330

3.0351970
3.2284150
4.1099780
1.6262350
1.8730830
4.0013950
4.5690200
4.4521780
2.5093270
2.3376200
2.1923410
0.5765710
5.1496730
2.0836250
1.9732750
1.4352020
3.5609180
4.1795110
3.6401860
3.6504270
4.7025500
-2.5629580
-3.2194780
-3.2610440
-4.4664010
-5.3446930
-4.5586470
-5.0322310
-3.5981950
-2.5378000
-5.6639810
-6.2507440
-5.1509590
-4.3518870
-4.1244340
-4.3044070
-4.8611290
-5.7246530
-6.7512440
-6.0288050
-4.8306550
-6.2122210
-5.4694790
-4.0276310
-7.3214910
-7.4689460

$-3.4199310$

0.5484500

1.7296030

2. 3677820

0.8812910

2. 7611020

3. 6564070

2.3537310

3. 1031810

4. 1809970

2. 8184290

2.6195530

1.4158230

2.8111970

3.8984550

2. 3643890

2. 3897540

3. 2589040

1.6848780

$-0.7726520$

$-1.0260170$

1. 3094840

1. 0342470

2.0366210

2.8592800

2.0340040

1. 6798140

3. 1976590

1. 2876180

2. 6699550

1.1144480

2. 5853540

1.0614060

2. 6057010

3.7570890

$-1.0377140$

$-1.6117640$

$-2.8508270$

$-2.5651840$

$-2.3471180$

$-1.4227550$

$-3.1374060$

$-0.8620400$

$-1.8420760$

-1.6659270
-3.3858450 


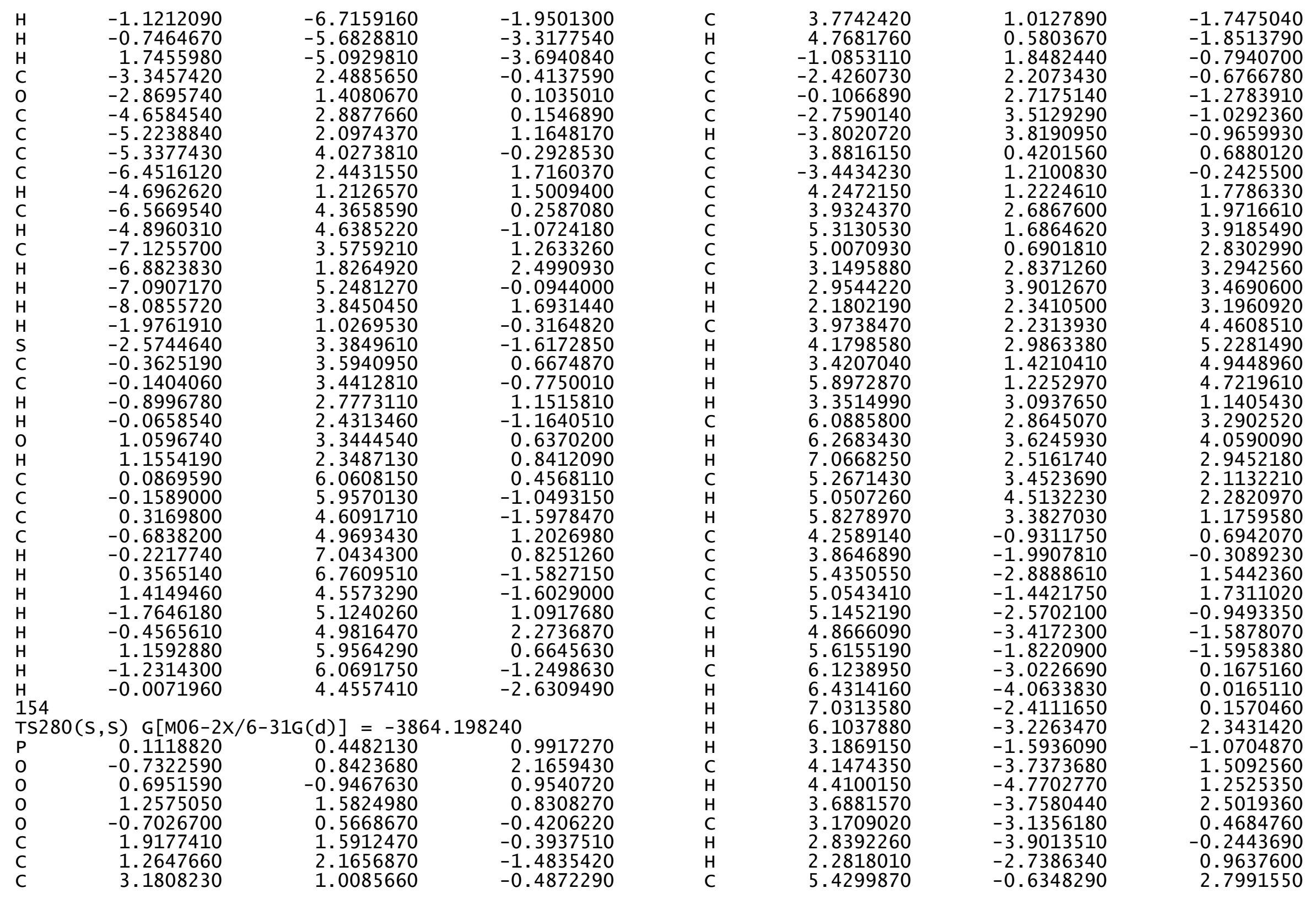




$\begin{array}{lrrr}\mathrm{H} & 6.0440150 & -1.0360560 & 3.6040950 \\ \mathrm{C} & -4.7129310 & -0.8224690 & -0.6960150 \\ \mathrm{C} & -4.9423840 & -1.8996960 & -1.7274420 \\ \mathrm{C} & -3.0858440 & -0.2212130 & -2.3887510 \\ \mathrm{C} & -3.7219890 & 0.1008870 & -1.0578220 \\ \mathrm{C} & -5.3371490 & -1.1888080 & -3.0421000 \\ \mathrm{H} & -5.5244250 & -1.9402390 & -3.8170730 \\ \mathrm{H} & -6.2701280 & -0.6376550 & -2.8905020 \\ \mathrm{C} & -4.1938310 & -0.2299740 & -3.4671890 \\ \mathrm{H} & -3.7532980 & -0.5493330 & -4.4183830 \\ \mathrm{H} & -4.5730820 & 0.7859730 & -3.6126140 \\ \mathrm{H} & -2.3053860 & 0.4967980 & -2.6525680 \\ \mathrm{H} & -5.7321660 & -2.5888330 & -1.4092710 \\ \mathrm{C} & -2.4928580 & -1.6411800 & -2.2886700 \\ \mathrm{H} & -1.9688910 & -1.8917770 & -3.2180970 \\ \mathrm{H} & -1.7459430 & -1.6417630 & -1.4909420 \\ \mathrm{C} & -3.6235050 & -2.6583390 & -1.9966500 \\ \mathrm{H} & -3.7757930 & -3.3279470 & -2.8513100 \\ \mathrm{H} & -3.3566120 & -3.2932970 & -1.1455730 \\ \mathrm{C} & -5.1650810 & 0.4433070 & 1.2967300 \\ \mathrm{C} & -5.8738950 & 0.7831650 & 2.5820240 \\ \mathrm{C} & -4.0473820 & 2.5024620 & 1.9358350 \\ \mathrm{C} & -4.1680410 & 1.3685430 & 0.9457610 \\ \mathrm{C} & -4.8197020 & 0.8608950 & 3.7064350 \\ \mathrm{H} & -5.3183910 & 1.1120700 & 4.6493860 \\ \mathrm{H} & -4.3469530 & -0.1177680 & 3.8374850 \\ \mathrm{C} & -3.7578660 & 1.9288620 & 3.3404090 \\ \mathrm{H} & -3.7786890 & 2.7561750 & 4.0589860 \\ \mathrm{H} & -2.7480970 & 1.5143690 & 3.3619660 \\ \mathrm{H} & -3.2624940 & 3.2060960 & 1.6475600 \\ \mathrm{H} & -6.6401120 & 0.0367510 & 2.8183930 \\ \mathrm{C} & -5.4225820 & 3.2058250 & 1.9975440 \\ \mathrm{H} & -5.3676910 & 4.0346920 & 2.7117570 \\ \mathrm{H} & -5.6606880 & 3.6368480 & 1.0195130 \\ \mathrm{C} & -6.5101180 & 2.1830730 & 2.4240590 \\ \mathrm{H} & -6.9654980 & 2.4746400 & 3.3771690 \\ \mathrm{H} & -7.3134820 & 2.1393870 & 1.6825880 \\ \mathrm{C} & -5.4424360 & -0.6466370 & 0.4778180 \\ \mathrm{H} & -6.2279620 & -1.3518600 & 0.7481220 \\ \mathrm{C} & -1.7990930 & 4.4441740 & -1.4337270 \\ \mathrm{C} & -0.4545300 & 4.0550480 & -1.5414140 \\ \mathrm{C} & -2.2526710 & 5.8482900 & -1.7896930 \\ \mathrm{C} & -1.1211730 & 6.8745790 & -1.7820700 \\ \mathrm{C} & 0.0912160 & 6.3249000 & -2.5304140 \\ \mathrm{C} & 0.6229700 & 5.0891080 & -1.8093480 \\ \mathrm{H} & -1.4684290 & 7.8117790 & -2.2285730 \\ & & & \end{array}$

-2.6987840
-3.0543710
-0.1999720
0.8803410
1.4647320
1.0279640
-0.8301100
1.8562350
1.0820820
1.6586950
3.1653980
3.8360380
3.1336960
1.1600970
1.0802950
0.0325320
3.3649170
3.5854380
4.8849770
3.8477180
1.4611350
-0.0299190
0.5511060
0.1763140
-0.6706240
1.2451630
-0.4493430
-1.5020560
1.4699700
1.8947660
0.6223470
-1.1138420
2.3057520
0.7959640
0.5456370
-0.9298930
-1.1945380
-2.0897530
-0.1746660
-1.9159180
-2.0622430
-1.5947820
-2.7607880
-3.3844270
-3.4098050
-1.3147220
5.8206670

6.1541620

6.0598910

7.0800960

4. 6486160

5. 3968810

7.0979520

2. 0865410

2. 5308170

1.9625250

2. 2009660

1. 3941110

1. 5200200

2. 4262160

3. 6268450

2. 2383790

3. 2712150

1. 9213410

1. 6899530

0.3338260

0.8839800

$-3.5573250$

$-2.4174930$

$-4.4424920$

$-5.5250060$

$-4.1719640$

$-6.3276530$

$-5.7226240$

$-4.9864180$

$-3.3280690$

$-6.0629430$

$-7.1609670$

$-4.7812640$

$-6.6954360$

$-1.7883110$

$-4.0719150$

$-2.1592520$

$-2.2222340$

$-1.8494200$

$-1.5307240$

$-1.0335460$

$-0.2152900$

$-3.6966310$

$-4.1046880$

$-2.9271660$

$-3.2409540$
$-2.7938480$

$-1.1086900$

$-3.5563090$

$-2.6026670$

$-2.3471460$

$-0.8341410$

$-0.7476150$

$-2.7611330$

$-3.9903670$

$-5.2862780$

$-5.3283450$

$-4.2197620$

$-2.8805410$

$-6.1432310$

$-4.0648000$

$-3.8739600$

$-5.1852060$

$-6.2995900$

$-4.1040170$

$-4.5078010$

$-5.3406290$

$-0.8795950$

$-0.9940900$

$-2.0546020$

$-2.3157420$

$-2.9200140$

$-3.4283140$

$-1.6476090$

$-4.0232740$

$-2.7119860$

$-4.2798690$

$-3.6321050$

$-4.6844000$

$-5.1450160$

$-0.1321540$

0.4518110

3. 0065950

1. 8461410

2.7862530

1.0250590

3. 2840160

2. 8889520

4.2564460

2. 9208490

1.9444020

4.0536900 


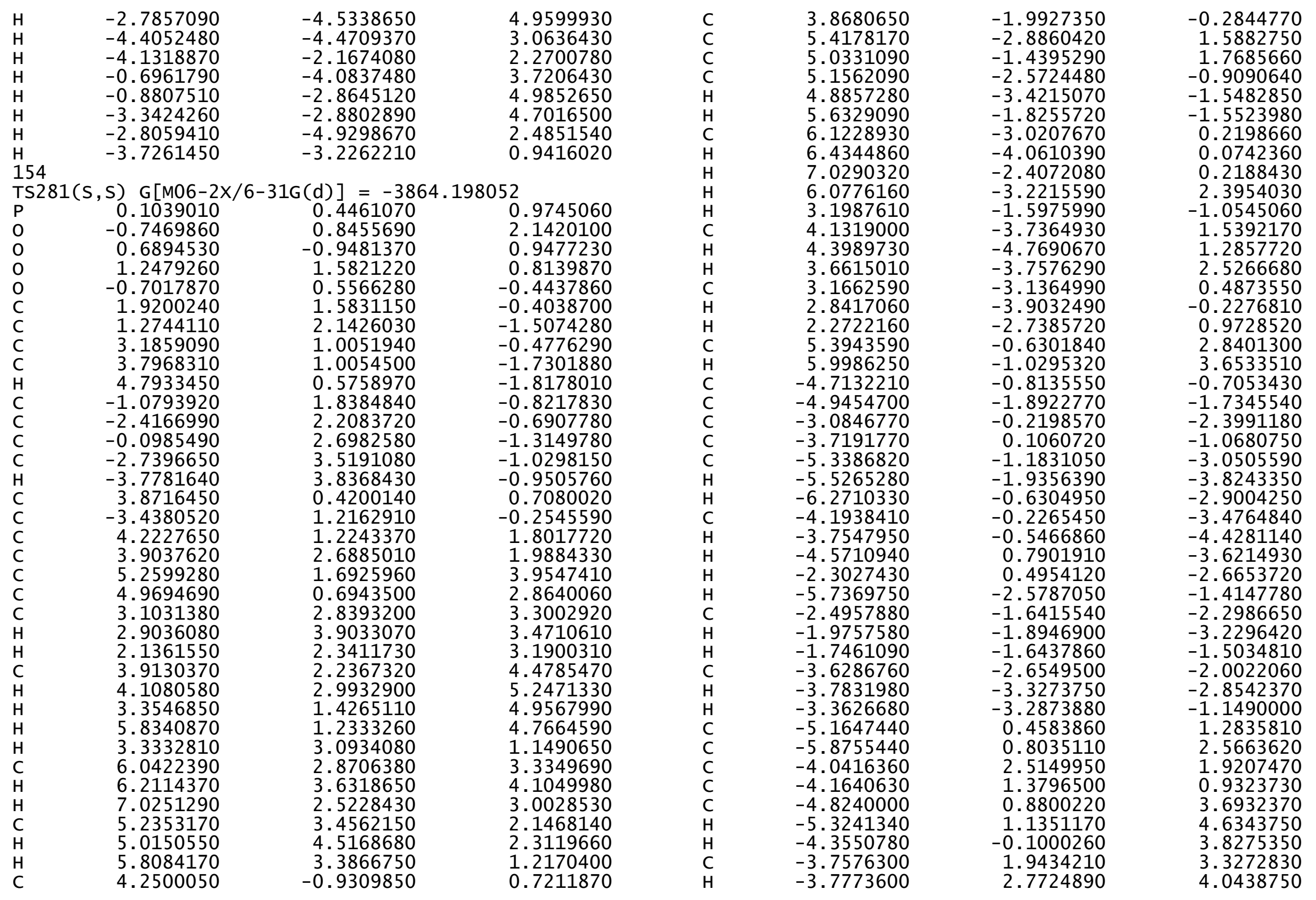




$\begin{array}{rrr}-2.7493490 & 1.5255890 & 3.3522940 \\ -3.2534330 & 3.2151890 & 1.6333360 \\ -6.6449340 & 0.0602500 & 2.8023500 \\ -5.4144770 & 3.2233740 & 1.9778410 \\ -5.3583470 & 4.0533070 & 2.6907220 \\ -5.6489580 & 3.6535090 & 0.9985180 \\ -6.5066100 & 2.2051980 & 2.4036110 \\ -6.9636560 & 2.5004240 & 3.3547970 \\ -7.3080470 & 2.1625530 & 1.6599970 \\ -5.4439240 & -0.6327540 & 0.4669790 \\ -6.2321580 & -1.3348240 & 0.7376120 \\ -1.7746460 & 4.4433810 & -1.4408990 \\ -0.4371920 & 4.0404930 & -1.5741950 \\ -2.2174920 & 5.8581190 & -1.7659500 \\ -1.0707730 & 6.8665520 & -1.7576930 \\ 0.1089160 & 6.3171820 & -2.5557460 \\ 0.6480120 & 5.0555990 & -1.8860660 \\ -1.4133500 & 7.8220320 & -2.1675230 \\ -2.6808140 & 5.8561750 & -2.7626700 \\ -3.0029150 & 6.1609940 & -1.0649440 \\ -0.2200030 & 6.0825050 & -3.5776760 \\ 0.9075950 & 7.0612610 & -2.6371920 \\ 1.4415250 & 4.6070430 & -2.4892260 \\ 1.1238260 & 5.3317570 & -0.9338270 \\ -0.7487650 & 7.0537640 & -0.7251860 \\ 1.8843960 & 2.0592800 & -2.7742980 \\ 3.1695330 & 1.5038850 & -2.8733790 \\ 1.1129010 & 2.4410810 & -4.0260460 \\ 2.0127660 & 2.6795290 & -5.2364270 \\ 2.9846700 & 1.5152300 & -5.4067090 \\ 3.9108180 & 1.4370080 & -4.1955800 \\ 1.3965300 & 2.8103920 & -6.1316550 \\ 0.4225630 & 1.6142780 & -4.2501430 \\ 0.4766650 & 3.3111560 & -3.8480690 \\ 2.4147560 & 0.5815420 & -5.4992790 \\ 3.5755980 & 1.6259260 & -6.3215340 \\ 4.5129110 & 0.5222850 & -4.2261320 \\ 4.6222490 & 2.2736610 & -4.2392400 \\ 2.5810060 & 3.6094760 & -5.0967780 \\ -0.0350480 & -3.5652290 & -0.8783040 \\ 0.5503450 & -2.4277550 & -0.9943440 \\ 0.1728740 & -4.4550890 & -2.0494670 \\ -0.6759100 & -5.5363900 & -2.3096970 \\ 1.2452160 & -4.1901990 & -2.9122840 \\ -0.4529570 & -6.3433980 & -3.4187730 \\ -1.5100530 & -5.7296590 & -1.6436790\end{array}$

1.4715570
1.8962200
0.6221350
-1.1188440
2.3099340
0.7969730
0.5427000
-0.9421480
-1.2083950
-2.1023290
-0.1881540
-1.9278510
-2.0759700
-1.6088320
-2.7764840
-3.3988460
-3.4227670
-1.3300750
-2.8023890
-4.4199610
-4.1446550
-0.7114200
-0.8969920
-3.3583640
-2.8202270
-3.7384450
$-5.0089060$
$-1.7952000$
$-4.0723280$
$-2.1507270$
$-2.2176850$
$-4.9228240$
$-3.2241810$

$-4.0120770$

$-3.3472290 \quad-2.7051090$

$-6.0842170 \quad-4.2677550$

$-7.1757720 \quad-3.6218730$

$-4.8079510-4.6711970$

$-6.7200710 \quad-5.1301920$

$-0.1350800$

0.4511860

2.9990100

1.8377570

2.7787610

1.0146590

3.2716660

2.8721610

4. 2526010

2. 9178100

1.9372230

4.0497580

4.9590850

3. 0608160

2.2597340

3.7203380

4.9804620

4.6942970

2.4856780

0.9352220 154

$\operatorname{TS} 282(\mathrm{~S}, \mathrm{~S}) \mathrm{G}[\mathrm{M} 06-2 \mathrm{X} / 6-31 \mathrm{G}(\mathrm{d})]=-3864.197856$

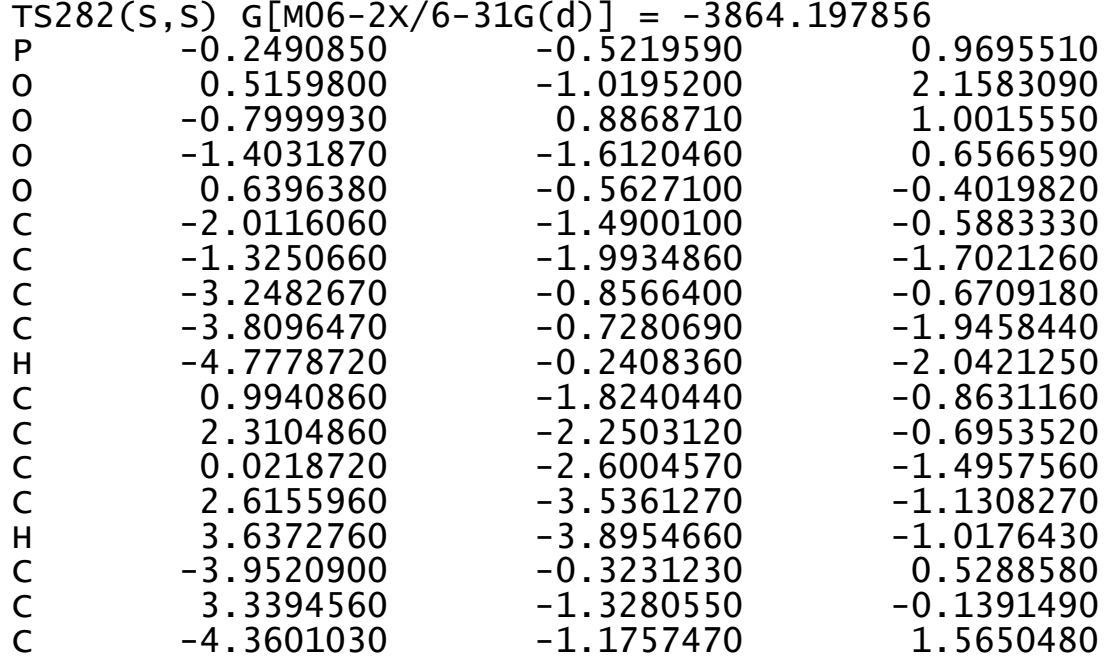




$\begin{array}{rrr}-4.1055370 & -2.6605660 & 1.6713410 \\ -5.4662880 & -1.7262970 & 3.6641770 \\ -5.1107100 & -0.6790320 & 2.6408250 \\ -3.3441630 & -2.9239790 & 2.9889170 \\ -3.1967890 & -4.0042800 & 3.1000440 \\ -2.3532940 & -2.4656550 & 2.9303100 \\ -4.1549230 & -2.3551800 & 4.1831830 \\ -4.3977440 & -3.1447690 & 4.9034260 \\ -3.5749140 & -1.5970130 & 4.7177630 \\ -6.0403610 & -1.2915080 & 4.4893630 \\ -3.5328160 & -3.0392830 & 0.8215430 \\ -6.2804950 & -2.8328440 & 2.9597440 \\ -6.4960760 & -3.6309550 & 3.6791910 \\ -7.2415900 & -2.4266700 & 2.6301760 \\ -5.4712270 & -3.3789140 & 1.7545720 \\ -5.2987250 & -4.4560450 & 1.8585600 \\ -6.0193450 & -3.2288540 & 0.8192760 \\ -4.2817850 & 1.0384570 & 0.6131580 \\ -3.8496950 & 2.1395220 & -0.3278480 \\ -5.3952980 & 2.9840100 & 1.5700340 \\ -5.0660290 & 1.5163840 & 1.6738010 \\ -5.1105610 & 2.7925030 & -0.9364160 \\ -4.8049800 & 3.6612390 & -1.5311350 \\ -5.6047400 & 2.0940450 & -1.6197180 \\ -6.0754800 & 3.2184330 & 0.2025890 \\ -6.3441040 & 4.2765410 & 0.1087790 \\ -7.0048130 & 2.6423630 & 0.1578780 \\ -6.0545410 & 3.2980240 & 2.3862380 \\ -3.1829180 & 1.7656070 & -1.1100230 \\ -4.0804610 & 3.7905410 & 1.5837150 \\ -4.3090810 & 4.8445300 & 1.3872570 \\ -3.6222590 & 3.7389540 & 2.5758080 \\ -3.1220820 & 3.2181590 & 0.5099300 \\ -2.7644970 & 4.0093660 & -0.1608520 \\ -2.2468960 & 2.7688100 & 0.9838120 \\ -5.4814740 & 0.6608690 & 2.6883240 \\ -6.0871210 & 1.0358260 & 3.5120760 \\ 4.7093140 & 0.6728530 & -0.3977140 \\ 5.0380420 & 1.7981340 & -1.3475030 \\ 3.2120350 & 0.1976890 & -2.2446730 \\ 3.7198220 & -0.1957300 & -0.8778690 \\ 5.5216760 & 1.1547590 & -2.6672990 \\ 5.7549700 & 1.9459420 & -3.3884370 \\ 6.4463270 & 0.5993950 & -1936670 \\ 4.4147980 & 0.2157130 & \\ 4.0678400 & 0.5568420 & \\ -4 & & -1975670\end{array}$

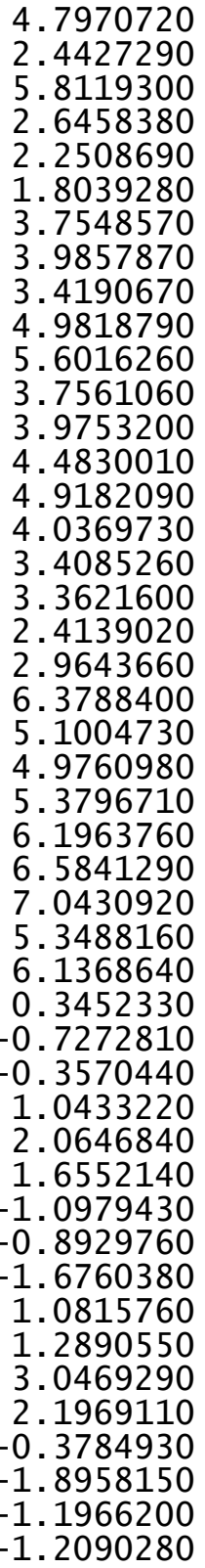
$-0.8015030$
$-0.4893380$
2.4525910
1.6276820
1.9360980
1.6100690
2. 5977940
3. 3387240
3. 1583220
$-0.7139720$
$-1.1567680$
$-2.7623900$
$-1.5776190$
$-1.2626970$
$-1.5887900$
$-0.2768070$
$-2.2661710$
$-1.8169960$
$-3.4159270$
$-0.4560990$
$-3.5203810$
$-4.3896470$
$-3.8978480$
$-2.5683230$
$-2.9349130$
-2. 5108960
0.4086120
1.0688890
$-3.9159780$
$-4.8246950$
$-6.3038620$
$-6.5195150$
$-5.7979810$
$-4.3814600$
$-6.9026690$
$-4.5790530$
$-4.6264860$
$-6.1230690$
$-7.5845620$
$-5.7772110$
$-6.3647630$
$-6.6276600$
$-1.8077200$
$-2.1091710$
$-0.8456670$

$-3.3440520$

$-2.6065320$

$-0.9317920$

$-2.1474730$

$-3.1225130$

$-1.4518950$

$-1.6675000$

$-2.4418430$

$-0.7882790$

1. 5468500

2.8468450

1. 9936440

1.0834050

3.9042900

4.8557090

4.0702350

3.4144310

4.0786720

3. 4089560

1. 6186290

3. 1710430

2. 0854670

2. 7404820

1.0962760

2. 6367660

3. 5939060

1.9462060

0.8117340

1.1730000

$-1.8842250$

$-2.4548590$

$-2.3597820$

$-2.9272280$

$-2.0515440$

$-1.6930990$

$-2.8987900$

$-3.5118740$

$-1.9445940$

$-3.9504970$

$-2.9845170$

$-2.5372250$

$-1.1196250$

$-1.3111200$

$-2.9680650$

$-4.2702300$

$-5.1509640$ 


$\begin{array}{lrrr}\mathrm{C} & -2.6370610 & -0.2896040 & -5.3700200 \\ \mathrm{C} & -3.6829880 & -0.9850670 & -4.4741500 \\ \mathrm{C} & -3.1476750 & -1.1799850 & -3.0804440 \\ \mathrm{H} & -1.7161880 & -2.9207010 & -4.7992080 \\ \mathrm{H} & -0.7320910 & -1.0681130 & -6.1102630 \\ \mathrm{H} & -2.9377310 & -0.4029530 & -6.4161590 \\ \mathrm{H} & -3.9239000 & -1.9650540 & -4.9074930 \\ \mathrm{H} & -0.1684100 & -2.4394800 & -4.0998460 \\ \mathrm{H} & -0.5886770 & -0.0911640 & -4.6560530 \\ \mathrm{H} & -2.6487730 & 0.7832780 & -5.1538400 \\ \mathrm{H} & -4.6143260 & -0.4112450 & -4.4552120 \\ \mathrm{C} & 0.2452810 & 3.4869320 & -0.7099790 \\ \mathrm{O} & -0.4319850 & 2.4087240 & -0.8758120 \\ \mathrm{C} & 0.3512540 & 4.3094510 & -1.9426340 \\ \mathrm{C} & 1.2983920 & 5.3326460 & -2.0605210 \\ \mathrm{C} & -0.4994860 & 4.0244160 & -3.0199030 \\ \mathrm{C} & 1.3921290 & 6.0616850 & -3.2400670 \\ \mathrm{H} & 1.9613180 & 5.5411030 & -1.2276640 \\ \mathrm{C} & -0.4081040 & 4.7630240 & -4.1930490 \\ \mathrm{H} & -1.2269950 & 3.2257730 & -2.9236260 \\ \mathrm{C} & 0.5384340 & 5.7806040 & -4.3054660 \\ \mathrm{H} & 2.1332910 & 6.8492660 & -3.3290150 \\ \mathrm{H} & -1.0740080 & 4.5443900 & -5.0216680 \\ \mathrm{H} & 0.6121530 & 6.3533890 & -5.2248120 \\ \mathrm{H} & -0.5562430 & 1.7761600 & -0.0228790 \\ \mathrm{~S} & 0.9599790 & 3.9755430 & 0.7405740 \\ \mathrm{C} & 1.0189670 & 1.9272850 & 3.2015140 \\ \mathrm{C} & 1.9750230 & 2.0201920 & 2.0923940 \\ \mathrm{H} & 0.0009290 & 1.6720410 & 2.9130210 \\ \mathrm{H} & 1.8198350 & 1.3820970 & 1.2251380 \\ \mathrm{O} & 1.8271630 & 0.7538510 & 3.4591230 \\ \mathrm{H} & 1.3558540 & -0.0236610 & 2.9937860 \\ \mathrm{C} & 2.5745640 & 3.3314810 & 4.6119010 \\ \mathrm{C} & 3.2810570 & 3.7870810 & 3.3342670 \\ \mathrm{C} & 3.3135510 & 2.6643700 & 2.2959410 \\ \mathrm{C} & 1.1250570 & 2.9446260 & 4.3128720 \\ \mathrm{H} & 2.5937960 & 4.1271810 & 5.3622740 \\ \mathrm{H} & 4.3057880 & 4.1045130 & 3.5481970 \\ \mathrm{H} & 3.9884470 & 1.8593750 & 2.6144040 \\ \mathrm{H} & 0.5554460 & 3.8280270 & 3.9982510 \\ \mathrm{H} & 0.6304760 & 2.5350940 & 5.1991780 \\ \mathrm{H} & 3.1024240 & 2.4697370 & 5.0381510 \\ \mathrm{H} & 2.7566420 & 4.6565520 & 2.9165300 \\ \mathrm{H} & 3.6925240 & 3.0042650 & 1.3283910 \\ \mathrm{I} & & & \\ \mathrm{H} 4 & & & \\ & & & \end{array}$

154 TS283(S, S ) G[M06-2X/6-31G(d)] = -3864.196761

\begin{abstract}
0.1779060
$-0.6288700$

0.7214600

1.3434210

$-0.6516700$

2. 0020860

1. 3647570

3.2572090

3.8954990

4.8823110

$-0.9969550$

$-2.3203220$

$-0.0021020$

$-2.6418360$

$-3.6700040$

3.9143380

$-3.3515060$

4.2681560

3.9825090

5.2769310

4.9826200

3.1616300

2.9865090

2.1842970

3.9338330

4.1322790

3. 3469580

5.8243340

3.4382610

6.0988920

6.2706160

7.0797690

5. 3294080

5.1321050

5.9183680

4.2598770

3.8718910

5. 3653260

5.0113750

5.1581710

4.8811720

5.6635730

6.0933250

6.3844180

7.0126570
6.0016750
\end{abstract}

0.8872010

2.0347370

0.9718560

0.5990370

$-0.5185730$

$-0.6189220$

$-1.7640950$

$-0.6408870$

$-1.8821730$

$-1.9280690$

$-1.0122230$

$-0.8931900$

$-1.6313890$

$-1.3842370$

$-1.3070300$

0.5923070

$-0.3166840$

1.6374070

1.7239720

3.7765670

2.7457990

3. 0072840

3.1061490

2. 9098090

4.2374640

4.9613020

4.7535590

4.6272280

0.8491200

3.0999290

3.8233790

2.8116560

1.8601750

1.9520830

0.9488610

0.7002370

$-0.2434560$

1.7123760

1.7928510

$-0.8039240$

$-1.3938140$

$-1.4831980$

0.3700060

0.2960250

0.3490340

2.5524590 


$\begin{array}{rrr}3.2267050 & -1.7448750 & -1.0505450 \\ 4.0621590 & -3.7358830 & 1.6893620 \\ 4.3114980 & -4.7869970 & 1.5028230 \\ 3.5731770 & -3.6885100 & 2.6668230 \\ 3.1288860 & -3.1799630 & 0.5854020 \\ 2.7930640 & -3.9806880 & -0.0854900 \\ 2.2395880 & -2.7296320 & 1.0314530 \\ 5.3738840 & -0.5789780 & 2.8164980 \\ 5.9531030 & -0.9387920 & 3.6656000 \\ -4.7142450 & -0.7125600 & -0.5113680 \\ -5.0271520 & -1.8773790 & -1.4181340 \\ -3.1621950 & -0.3331310 & -2.3347560 \\ -3.7107140 & 0.1315800 & -1.0068970 \\ -5.4694600 & -1.2880490 & -2.7771410 \\ -5.7028640 & -2.1072970 & -3.4661770 \\ -6.3864780 & -0.7071830 & -2.6398930 \\ -4.3323990 & -0.3967270 & -3.3432250 \\ -3.9573930 & -0.8009060 & -4.2901850 \\ -4.6968190 & 0.6148640 & -3.5461140 \\ -2.3766550 & 0.3299380 & -2.7056600 \\ -5.8182650 & -2.5058840 & -0.9948450 \\ -2.6089860 & -1.7589180 & -2.1423380 \\ -2.1677080 & -2.1145710 & -3.0804880 \\ -1.8024360 & -1.7118280 & -1.4071830 \\ -3.7447210 & -2.7012440 & -1.6710720 \\ -3.9611930 & -3.4611120 & -2.4311760 \\ -3.4441160 & -3.2401830 & -0.7663080 \\ -5.0335810 & 0.7645020 & 1.3579340 \\ -5.6851680 & 1.2682570 & 2.6195490 \\ -3.8195480 & 2.8333400 & 1.7356830 \\ -4.0171290 & 1.6066450 & 0.8773310 \\ -4.5932620 & 1.4263200 & 3.6980770 \\ -5.0517850 & 1.7991520 & 4.6209740 \\ -4.1531910 & 0.4496350 & 3.9233570 \\ -3.5057530 & 2.4045610 & 3.1860920 \\ -3.4732600 & 3.3055620 & 3.8093390 \\ -2.5120920 & 1.9548370 & 3.2247150 \\ -3.0190070 & 3.4692200 & 1.3492270 \\ -6.4700640 & 0.5828510 & 2.9576090 \\ -5.1653880 & 3.5939910 & 1.7605170 \\ -5.0565430 & 4.4918070 & 2.3787710 \\ -5.4203490 & 3.9267970 & 0.7489660 \\ -6.2750320 & 2.6670310 & 2.3268740 \\ -6.6889130 & 3.0780070 & 1.6171650 \\ -7.1026490 & 2.5749150 & \\ -5.3839310 & -0.3912600 & \\ -5 & & \\ -5 & -6674720\end{array}$

-6.1829890
-0.3514440
0.6297110
0.4905940
-0.9686010
-1.9700160
-1.6753920
0.4276440
1.1477120
-1.0586090
-1.8906780
1.6557490
0.8581450
-1.2526900
-2.9964860
2.0208290
1.4135030
1.5360230
2.9861430
3.9400880
3.2955460
1.9380090
1.1478480
3.3731080
4.1925660
0.3639150
0.8842840
2.9963300
4.8802200
-0.2250360
0.4312200
-0.2127540
-1.1596760
0.7613400
-1.1303450
-1.9179010
0.7940610
1.4886410
-0.1521170
-1.8718560
1.5568120
-0.1284650
0.5076420
-1.0276230
-1.1440590
-2.0811530
1.0384120 $-2.0899220$ $-2.6636770$ $-1.9341930$ $-1.8996210$ $-2.4726860$ $-1.9624910$ $-3.7349350$ $-2.4106570$ $-2.4597390$ $-3.5685240$ $-2.5801670$ $-0.9123540$ $-0.8669650$ $-2.2305800$ $-2.9956340$ $-4.3280010$ $-5.2926250$ $-5.4152870$ $-4.4007830$ $-3.0442830$ $-4.7521230$ $-6.2740430$ $-6.4249490$ $-4.7579330$ $-4.2196860$ $-4.9175090$ $-5.2514430$ $-4.3386640$ $-0.6016470$ $-0.8278930$ $-1.7459790$ $-1.8574470$ $-2.7415890$ $-2.9500150$ $-1.0893190$ $-3.8250140$ $-2.6502970$ $-3.9316270$ $-3.0365910$ $-4.5880550$ $-4.7815100$ $-0.0199030$

0.8351170 3. 1954690 2. 0762130 


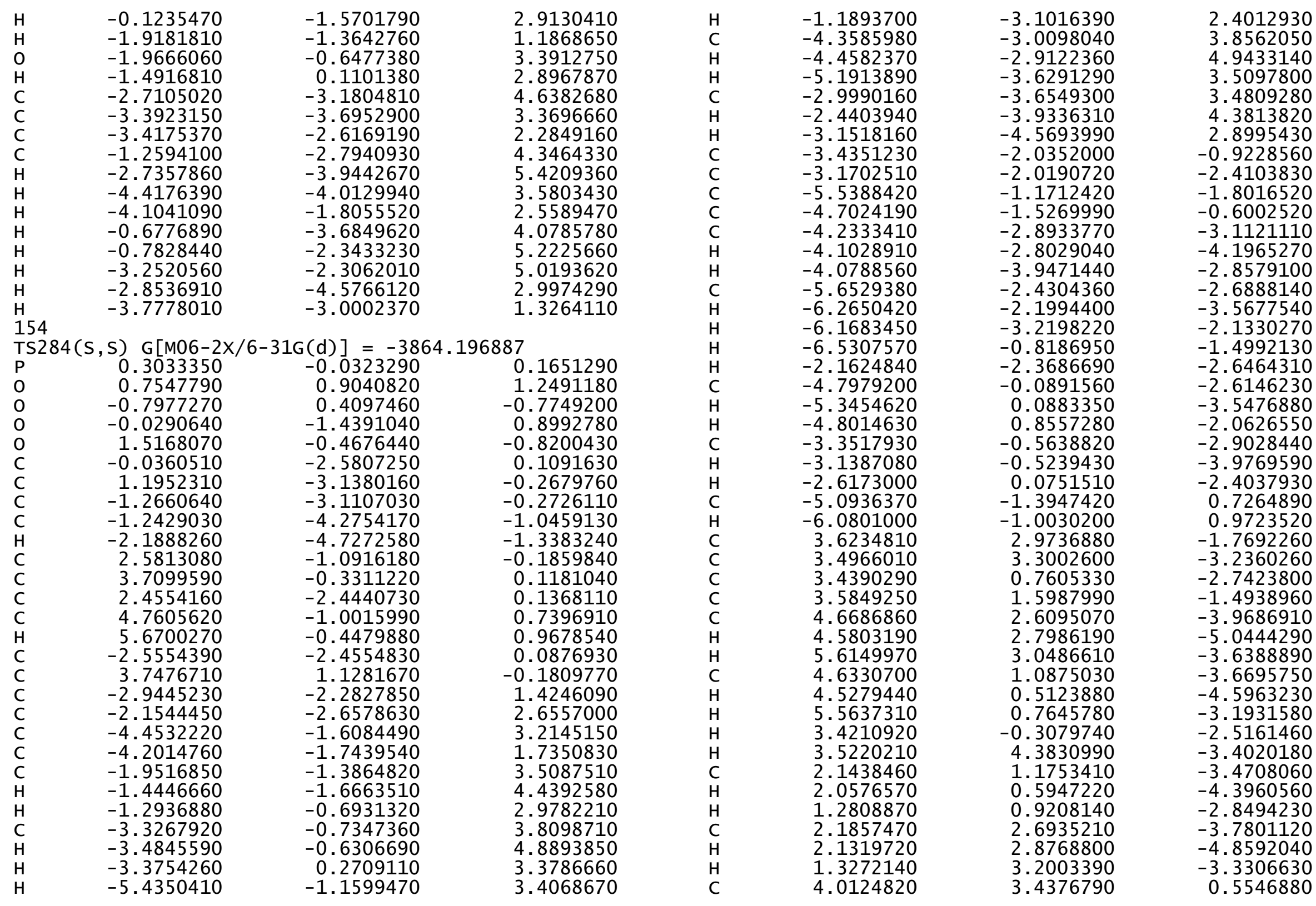




\begin{tabular}{|c|c|c|}
\hline $\begin{array}{l}4.3115250 \\
4.1259980 \\
3.9486640 \\
3.2083890 \\
3.4827970 \\
2.2634910 \\
3.0640760 \\
3.2016730 \\
2.0702950 \\
4.0287300 \\
4.3852580 \\
5.5171110 \\
5.6337240 \\
6.3018200 \\
5.6448650 \\
5.8849530 \\
6.4509650 \\
3.8433630 \\
3.9009340 \\
4.6728820 \\
3.4996710 \\
5.8740370 \\
5.5344220 \\
4.6458260 \\
3.3201180 \\
6.4560860 \\
6.6113050 \\
6.3561720 \\
5.1543720 \\
4.4595700 \\
2.6904120 \\
2.7611940 \\
5.0003080 \\
0.0470680 \\
0.0081100 \\
1.0469710 \\
2.4487050 \\
2.4062930 \\
1.1793000 \\
0.2929610 \\
1.0783680 \\
3.0683210 \\
2.3695020 \\
0.9785710 \\
0.7167940\end{array}$ & $\begin{array}{r}4.2993260 \\
1.7811040 \\
2.0658220 \\
4.0921870 \\
4.6448330 \\
4.5036950 \\
2.5785310 \\
2.3776200 \\
2.2134600 \\
0.7162460 \\
5.3547980 \\
2.3063700 \\
2.1687190 \\
1.7171470 \\
3.8061570 \\
4.4118420 \\
3.9546550 \\
3.8943430 \\
4.9597380 \\
-2.3452250 \\
-3.0691770 \\
-2.9905810 \\
-4.2682230 \\
-5.1683820 \\
-4.4664250 \\
-4.7829430 \\
-3.2304450 \\
-2.2622480 \\
-5.3929060 \\
-6.1239990 \\
-5.0731080 \\
-4.3725600 \\
-4.0160450 \\
-4.8428320 \\
-6.0931120 \\
-7.0541270 \\
-6.4104510 \\
-4.8676570 \\
-4.2650560 \\
-5.8437810 \\
-7.9858350 \\
-6.7804330 \\
-4.5906870 \\
-6.5641750 \\
-7.3130210 \\
-6.7131780\end{array}$ & $\begin{array}{l}1.7549670 \\
2.3201480 \\
0.8461690 \\
2.8137450 \\
3.7199190 \\
2.4486130 \\
3.1102980 \\
4.1788550 \\
2.8390370 \\
2.5421370 \\
1.4713520 \\
2.7417990 \\
3.8227140 \\
2.2558210 \\
2.3613150 \\
3.2422830 \\
1.6363500 \\
-0.7480930 \\
-0.9697320 \\
1.1122870 \\
0.8456260 \\
1.7796530 \\
2.5446380 \\
1.6895050 \\
1.4086870 \\
2.8343910 \\
1.0005510 \\
2.4409740 \\
0.7412350 \\
2.1903030 \\
0.7560210 \\
2.3512950 \\
3.4698110 \\
-1.4695470 \\
-2.3105080 \\
-1.7136390 \\
-1.6561980 \\
-1.7477040 \\
-1.1033760 \\
-3.3428670 \\
-2.2864920 \\
-2.4791840 \\
-2.8103630 \\
-2.3569960 \\
-0.7017830 \\
-0.7321450\end{array}$ \\
\hline
\end{tabular}

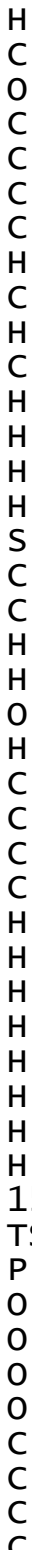

C
O
C
C
C
C
H
C
H
C
H
H
H
H
S
C
C
H
H
O
H
C
C
C
C
H
H
H
H
H
H
H
H
I

3.3277390

$-3.4686900$

$-2.9434150$

$-4.8012460$

$-5.3028160$

$-5.5604700$

$-6.5453690$

$-4.7131160$

$-6.8052600$

$-5.1683180$

$-7.2991280$

$-6.9254150$

$-7.3916400$

$-8.2709850$

$-2.0270600$

$-2.7333560$

$-0.5596420$

$-0.3193930$

$-1.0364850$

$-0.1644610$

0.8770490

1.0460190

$-0.2959190$

$-0.5226980$

0.0517920

$-0.9874440$

$-0.6808550$

$-0.0632310$

1. 1506680

$-2.0761330$

$-0.7687960$

0.7795250

$-1.5990790$

$-0.2606150$

154

$\operatorname{TS} 285(\mathrm{~S}, \mathrm{~S}) \mathrm{G}[\mathrm{M} 06-2 \mathrm{X} / 6-31 \mathrm{G}(\mathrm{d})]=-3864.198881$

$\begin{array}{lrrr}\mathrm{P} & -0.1848340 & -0.4272400 & 0.9694910 \\ \mathrm{O} & 0.6132700 & -0.8939080 & 2.1495420 \\ \mathrm{O} & -0.6938160 & 0.9969950 & 0.9538020 \\ \mathrm{O} & -1.3841520 & -1.4964750 & 0.7651070 \\ \mathrm{O} & 0.6499040 & -0.5599980 & -0.4308090 \\ \mathrm{C} & -2.0356990 & -1.4428800 & -0.4618110 \\ \mathrm{C} & -1.3965590 & -2.0073500 & -1.5676910 \\ \mathrm{C} & -3.2752260 & -0.8131900 & -0.5420390 \\ \mathrm{C} & -3.8747990 & -0.7789080 & -1.8006410 \\ \mathrm{H} & -4.8549850 & -0.3140030 & -1.8921630\end{array}$

$-4.4324920$

$-0.3808730$

$1.2608570 \quad 0.1265410$

$2.6541770 \quad 0.1849680$

$\begin{array}{ll}2.8735480 & 1.2354530\end{array}$

. $-0.3242160 \quad-1.787770$

$\begin{array}{ll}1.1616940 & 1.7870690\end{array}$

$1.0425600 \quad 1.6042550$

$4.0030230 \quad 0.2456650$

4.3278310

$3.2243040-1.2921390$

$1.5549260 \quad 2.6033870$

$4.8299890-0.1412670$

$3.4474230 \quad-1.7216450$

$0.9361200 \quad-0.2935690$

$3.2712910 \quad-1.5675530$

$3.5281900 \quad 0.7519310$

$.4664900-0.6944560$

$2.6503570-1.1899000$

$2.4858920-1.1319050$

$3.3875610 \quad 0.7227930$

0.8847210

$6.0294030 \quad 0.6733300$

$5.9860980 \quad-0.8386480$

$4.7065360-1.4528830$

$4.8458990 \quad 1.3535120$

$\begin{array}{ll}6.9656460 & 1.0877830\end{array}$

$6.8517580-1.3244940$

$4.7341290-1.4579190$

$4.9249400 \quad 1.2395460$

$\begin{array}{ll}4.8191320 & 2.4259980\end{array}$

0.8879530

$\begin{array}{lr}5.9956180 & 0.8879530 \\ 6.0312850 & -1.0444960 \\ 4.5849750 & -2.4938630\end{array}$

4.5849750 


\begin{tabular}{|c|c|c|}
\hline $\begin{array}{l}0.9576110 \\
2.2659270 \\
0.0617340 \\
2.5091880 \\
3.5230860 \\
-3.9392890 \\
3.3409130 \\
-4.3323180 \\
4.0903060 \\
5.3919470 \\
5.0495240 \\
-3.2988720 \\
-3.1578970 \\
-2.3059040 \\
-4.0749780 \\
4.3088230 \\
-3.4743660 \\
5.9410440 \\
-3.5418520 \\
6.2347250 \\
6.4424290 \\
7.1985990 \\
5.4602850 \\
5.2962100 \\
6.0294260 \\
-4.2446740 \\
-3.8104890 \\
5.3013950 \\
-4.9967650 \\
-5.0696270 \\
-4.7586450 \\
-5.5896370 \\
6.0037960 \\
6.2551710 \\
6.9440620 \\
5.9381450 \\
-3.1675550 \\
-3.9708480 \\
-4.1825920 \\
-3.4950580 \\
-3.0444470 \\
-2.6819580 \\
-5.1707760 \\
5.9811300 \\
4.7475660\end{array}$ & $\begin{array}{l}-1.8554140 \\
-2.3142330 \\
-2.6415640 \\
-3.6384430 \\
-4.0243660 \\
-0.2017370 \\
-1.3957360 \\
-0.9890950 \\
-2.4682220 \\
-1.4072890 \\
-0.4228980 \\
-2.6652120 \\
-3.7389660 \\
-2.2206760 \\
-2.0222680 \\
-2.7684040 \\
-1.2429820 \\
-0.9205900 \\
-2.8995720 \\
-2.5417340 \\
-3.2951560 \\
-2.1427480 \\
-3.1653070 \\
-4.2366350 \\
-3.0627810 \\
1.1674780 \\
2.2083200 \\
3.1813280 \\
1.7152340 \\
2.8553060 \\
3.6867680 \\
2.1330520 \\
3.3575650 \\
4.4143160 \\
2.7977250 \\
3.5510680 \\
1.7791040 \\
3.9603050 \\
5.0067560 \\
3.9524550 \\
3.3118890 \\
4.0600460 \\
2.8663850 \\
0.9232080 \\
1.3523010 \\
0.5569860\end{array}$ & $\begin{array}{r}-0.8278070 \\
-0.6766820 \\
-1.3610090 \\
-1.0276660 \\
-0.9331420 \\
0.6425140 \\
-0.2088200 \\
1.7340580 \\
1.9168840 \\
3.8860830 \\
2.7978460 \\
3.2282650 \\
3.3964020 \\
3.1192170 \\
4.4080010 \\
5.1759600 \\
4.8864760 \\
4.6991510 \\
1.0758560 \\
3.2638110 \\
4.0320720 \\
2.9335390 \\
2.0733460 \\
2.2350300 \\
1.1441890 \\
0.6595340 \\
-0.3465450 \\
1.5340460 \\
1.7096900 \\
-0.9644220 \\
-1.6078910 \\
-1.6023090 \\
0.1690950 \\
0.0256150 \\
0.1717880 \\
2.3447510 \\
-1.1204710 \\
1.4806140 \\
1.2318720 \\
2.4656790 \\
0.4220410 \\
-0.2941310 \\
0.9026700 \\
2.7789940 \\
3.5944910 \\
-0.6060350\end{array}$ \\
\hline
\end{tabular}

5.0647820

3.1418130

3.7128550

5.4584550

5.6941470

6.3643210

4.2863070

3.8956520

4.6212460

2. 3326530

5.8806

2.6260550

2. 1671860

1.8394390

3.7954430

4.0084040

3.5325960

5.0700890

5.7289500

3.8124030

4.0247790

4.6492000

5.1120010

4.2346520

3. 5315780

3.4879520

2. 5490910

2. 9906800

6.5353270

5.1395040

5.0185990

5. 3705900

6.2794690

6.6942720

7.1002820

5.4337390

6.2534520

0.1907850

$-0.9551780$

$-0.6091720$

0.7398640

1.8385680

1.4939910

$-1.4021030$

$-1.2410640$

$-1.8363190$

\begin{tabular}{rr}
1.6342460 & -1.6136460 \\
0.0676190 & -2.3544760 \\
-0.3003180 & -1.0056220 \\
0.9246280 & -2.9291050 \\
1.6783370 & -3.6885490 \\
0.3331210 & -2.7656930 \\
0.0201970 & -3.3929680 \\
0.3568780 & -4.3598060 \\
-1.0133200 & -3.5226970 \\
-0.6038000 & -2.6522730 \\
2.2751840 & -1.2621660 \\
1.5179050 & -2.2653160 \\
1.8072360 & -3.2177140 \\
1.5499680 & -1.5079950 \\
2.4667230 & -1.9019370 \\
3.1565370 & -2.7270870 \\
3.0847020 & -1.0367150 \\
-0.7577460 & 1.3799940 \\
-1.1594420 & 2.6741760 \\
-2.7508590 & 1.9648040 \\
-1.6134370 & 0.9943820 \\
-1.1874760 & 3.7763350 \\
-1.4822050 & 4.7249510 \\
-0.1835850 & 3.9132770 \\
-2.1823390 & 3.3730150 \\
-3.0205490 & 4.0778180 \\
-1.7068130 & 3.3835760 \\
-3.3989460 & 1.6503830 \\
-0.4661290 & 2.9371480 \\
-3.5399970 & 2.0408080 \\
-4.3755700 & 2.7389080 \\
-3.9685780 & 1.0600470 \\
-2.5943590 & 2.5068330 \\
-2.9282880 & 3.4645770 \\
-2.5905920 & 1.7834230 \\
0.3232940 & 0.5836440 \\
0.9757900 & 0.8832870 \\
-4.0020880 & -1.6286210 \\
-4.9222850 & -2.0126030 \\
-6.3995190 & -1.8306910 \\
-6.6979010 & -2.4791840 \\
-5.9483700 & -1.7300200 \\
-4.4944630 & -1.4676330 \\
-7.0175400 & -2.2633280 \\
-4.7592350 & -3.0606930 \\
-4.6553130 & -1.4186760 \\
& \\
\hline
\end{tabular}




$\begin{array}{rrr}0.7153040 & -6.3745930 & -3.5284240 \\ 0.9526530 & -7.7714990 & -2.4767830 \\ 2.7870770 & -6.0000340 & -2.2767330 \\ 2.0139930 & -6.4445740 & -0.7655770 \\ -0.5572620 & -6.6410590 & -0.7611510 \\ -1.9837070 & -1.8754470 & -2.8392130 \\ -3.2528600 & -1.2832970 & -2.9433390 \\ -1.1994530 & -2.2213220 & -4.0922560 \\ -2.0924150 & -2.4593520 & -5.3073900 \\ -3.0559680 & -1.2876720 & -5.4803200 \\ -3.9832150 & -1.1920510 & -4.2704780 \\ -1.4731440 & -2.5904910 & -6.2005280 \\ -0.5356190 & -1.3695830 & -4.3034350 \\ -0.5356630 & -3.0707130 & -3.9232090 \\ -2.4766000 & -0.3603690 & -5.5779530 \\ -3.6481840 & -1.3960890 & -6.3946140 \\ -4.5648710 & -0.2642100 & -4.3014700 \\ -4.7132780 & -2.0122150 & -4.3190740 \\ -2.6651360 & -3.3870150 & -5.1708260 \\ 0.3267640 & 3.5428800 & -0.8476380 \\ -0.3766610 & 2.4754220 & -0.9726970 \\ 0.3397110 & 4.3859810 & -2.0702940 \\ 1.3320140 & 5.3495270 & -2.2823820 \\ -0.6552890 & 4.1892820 & -3.0375670 \\ 1.3277200 & 6.1074020 & -3.4470910 \\ 2.1055650 & 5.4889260 & -1.5348860 \\ -0.6627600 & 4.9599390 & -4.1937770 \\ -1.4179420 & 3.4367770 & -2.8678170 \\ 0.3292690 & 5.9173030 & -4.4010560 \\ 2.1044490 & 6.8471140 & -3.6113930 \\ -1.4414960 & 4.8131150 & -4.9351900 \\ 0.3251550 & 6.5145040 & -5.3077080 \\ -0.4647740 & 1.8536860 & -0.1104830 \\ 1.1634290 & 4.0093650 & 0.5424180 \\ 1.2359120 & 2.0514450 & 3.0672360 \\ 2.1621940 & 2.0727350 & 1.9300560 \\ 0.2037200 & 1.8132470 & 2.8166800 \\ 1.9650970 & 1.4049710 & 1.0942320 \\ 2.0202160 & 0.8674760 & 3.3488210 \\ 1.5123880 & 0.0886080 & 2.9242320 \\ 2.8676440 & 3.4642740 & 4.3784450 \\ 3.5504100 & 3.8497600 & 3.0652440 \\ 3.5226620 & 2.6879200 & 2.0703520 \\ 1.4001910 & 3.1083250 & 4.1335680 \\ 2.9299940 & 4.2875560 & 3.2388290 \\ 4.5892460 & 4.1452390 & \end{array}$

$\begin{array}{ll}\mathrm{H} & 4.1859600 \\ \mathrm{H} & 0.8470770 \\ \mathrm{H} & 0.9189370 \\ \mathrm{H} & 3.3828570 \\ \mathrm{H} & 3.0390820 \\ \mathrm{H} & 3.8812560\end{array}$

154

TS2 $86(\mathrm{~S}, \mathrm{~S}) \mathrm{G}[\mathrm{M06}-2 \mathrm{X} / 6-31 \mathrm{G}(\mathrm{d})]=-3864.197729$

$\begin{array}{lrrr}\text { TS286(S }, \mathrm{S}) & \mathrm{G}[\mathrm{M} 06-2 \mathrm{X} / 6-31 \mathrm{G}(\mathrm{d})]=-3864.197729 \\ \mathrm{P} & 0.2974850 & -0.0743570 & 0.2222930 \\ \mathrm{O} & 0.7707580 & 0.8885880 & 1.2732570 \\ \mathrm{O} & -0.7769950 & 0.3695990 & -0.7471670 \\ \mathrm{O} & -0.0943370 & -1.4399550 & 1.0042300 \\ \mathrm{O} & 1.5059760 & -0.5923600 & -0.7291380 \\ \mathrm{C} & -0.1404900 & -2.6045720 & 0.2487610 \\ \mathrm{C} & 1.0715560 & -3.2254960 & -0.0927800 \\ \mathrm{C} & -1.3861380 & -3.0886340 & -0.1426210 \\ \mathrm{C} & -1.4024710 & -4.2642850 & -0.8999490 \\ \mathrm{H} & -2.3622890 & -4.6701000 & -1.2134080 \\ \mathrm{C} & 2.5366130 & -1.2357920 & -0.0593750 \\ \mathrm{C} & 3.6912200 & -0.5108240 & 0.2318330 \\ \mathrm{C} & 2.3544320 & -2.5715200 & 0.3062210 \\ \mathrm{C} & 4.7053000 & -1.1982560 & 0.8941180 \\ \mathrm{H} & 5.6313710 & -0.6714180 & 1.1187120 \\ \mathrm{C} & -2.6529160 & -2.3754400 & 0.1861000 \\ \mathrm{C} & 3.7953780 & 0.9319560 & -0.1251940 \\ \mathrm{C} & -3.0499910 & -2.1614260 & 1.5147080 \\ \mathrm{C} & -2.2875190 & -2.5385780 & 2.7626440 \\ \mathrm{C} & -4.5561500 & -1.4028530 & 3.2731650 \\ \mathrm{C} & -4.2915250 & -1.5747740 & 1.7997950 \\ \mathrm{C} & -2.0520960 & -1.2575490 & 3.5924510 \\ \mathrm{H} & -1.5619190 & -1.5342130 & 4.5328490 \\ \mathrm{H} & -1.3682910 & -0.5961810 & 3.0538060 \\ \mathrm{C} & -3.4084800 & -0.5570090 & 3.8677870 \\ \mathrm{H} & -3.5747740 & -0.4315250 & 4.9437490 \\ \mathrm{H} & -3.4201050 & 0.4430320 & 3.4214300 \\ \mathrm{H} & -5.5245090 & -0.9182210 & 3.4448310 \\ \mathrm{H} & -1.3348880 & -3.0191210 & 2.5293650 \\ \mathrm{C} & -4.5174670 & -2.7946750 & 3.9403470 \\ \mathrm{H} & -4.6297800 & -2.6748250 & 5.0239880 \\ \mathrm{H} & -5.3651050 & -3.3922870 & 3.5918070 \\ \mathrm{C} & -3.1744890 & -3.4905290 & 3.5968630 \\ \mathrm{H} & -2.6365190 & -3.7675320 & 4.5103350 \\ \mathrm{H} & -3.3488980 & -4.4119280 & 3.0327610 \\ \mathrm{C} & -3.5073360 & -1.9479430 & -0.8430870 \\ \mathrm{C} & -3.2288160 & -1.9759830 & -2.3280890 \\ \mathrm{C} & -5.5740630 & -1.0378180 & -1.7626640\end{array}$

2. 3999550

3.7987300

5.0483480

4.8241640

2. 6289780

1. 0797590
3.9947150

2.6047270

4.7178990

2.9805190
2. 7485770
27800

(2)

(626440

.8677870

.

.5103350

$-1.7626640$ 


$\begin{array}{rrr}-4.7606260 & -1.3921420 & -0.5451250 \\ -4.3133770 & -2.8327110 & -3.0188170 \\ -4.1699980 & -2.7733940 & -4.1037570 \\ -4.1966130 & -3.8845150 & -2.7375600 \\ -5.7208950 & -2.3133110 & -2.6213770 \\ -6.3164120 & -2.0831910 & -3.5118690 \\ -6.2674900 & -3.0720210 & -2.0528240 \\ -6.5568250 & -0.6467080 & -1.4782970 \\ -2.2303570 & -2.3623980 & -2.5459740 \\ -4.7912290 & 0.0004370 & -2.5931020 \\ -5.3253640 & 0.1755710 & -3.5343350 \\ -4.7668370 & 0.9569760 & -2.0620250 \\ -3.3600100 & -0.5289010 & -2.8585570 \\ -3.1386380 & -0.5238310 & -3.9317390 \\ -2.6081040 & 0.0981010 & -2.3709500 \\ -5.1610600 & -1.2185840 & 0.7741190 \\ -6.1368940 & -0.7902570 & 1.0006830 \\ 3.7819750 & 2.7111760 & -1.7918320 \\ 3.6958920 & 2.9793680 & -3.2734340 \\ 3.5088280 & 0.4691120 & -2.6734280 \\ 3.6754290 & 1.3526560 & -1.4594520 \\ 4.8466520 & 2.2034330 & -3.9527390 \\ 4.7887340 & 2.3521920 & -5.0368440 \\ 5.8066820 & 2.6097390 & -3.6204670 \\ 4.7307770 & 0.6984190 & -3.5936320 \\ 4.6097610 & 0.0919570 & -4.4981900 \\ 5.6373900 & 0.3495380 & -3.0899550 \\ 3.4359560 & -0.5866720 & -2.4026690 \\ 3.7759170 & 4.0515220 & -3.4849040 \\ 2.2457520 & 0.9132260 & -3.4400430 \\ 2.1424490 & 0.2952450 & -4.3389780 \\ 1.3633880 & 0.7304810 & -2.8205990 \\ 2.3677050 & 2.4115670 & -3.8175080 \\ 2.3448390 & 2.5463910 & -4.9048550 \\ 1.5262660 & 2.9795820 & -3.4112010 \\ 4.1540890 & 3.2561880 & 0.5174770 \\ 4.4733780 & 4.1533430 & 1.6860590 \\ 4.1658790 & 1.6717770 & 2.3523240 \\ 4.0231040 & 1.9022710 & 0.8650420 \\ 3.3453450 & 4.0404010 & 2.7327300 \\ 3.6284690 & 4.6204270 & 3.6188650 \\ 2.4254710 & 4.4753390 & 2.3324690 \\ 3.1306310 & 2.5488490 & 3.0922030 \\ 3.2466790 & 2.3890390 & 4.1702340 \\ 2.1248720 & 2.2158640 & 2.8238440 \\ 4.0158670 & 0.6226530 & 2.6162180\end{array}$

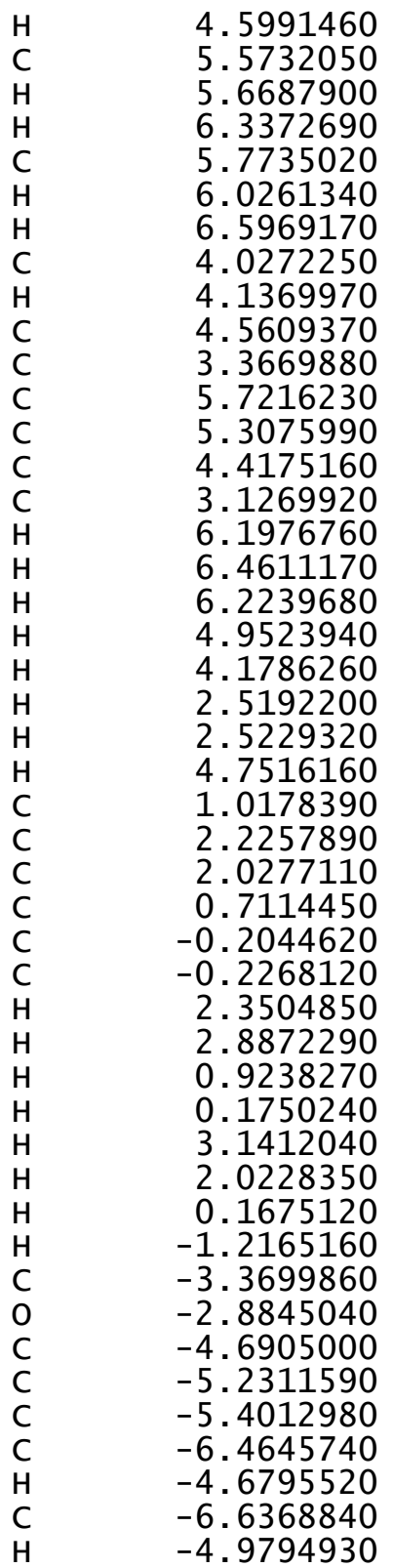

5.1916300
2.1515010
2.0531050
1.5086470
3.6272950
4.2576760
3.7103040
3.6637780
4.7150670
-2.5234950
-3.2141280
-3.1840700
-4.4008920
-5.3134280
-4.5869480
-4.9322440
-3.4994320
-2.4426200
-5.6052320
-6.2341830
-5.2030500
-4.4467710
-4.0757390
-4.3737770
-5.0544880
-5.1934200
-5.9232580
-6.1274730
-4.8980240
-6.0559580
-5.7155030
-6.8993930
-6.9742500
-4.4964730
-4.1857730
-5.3445670
-6.3944110
2.4150550
1.3499260
2.8103940
2.0635580
3.9053920
2.4086310
1.2125900
4.2419630
4.4829940

1.3604250

2.7742000

3. 8614310

2. 3246740

2. 3355410

3. 1954330

1.6196550

$-0.8063000$

$-1.0713180$

1. 3107700

1.0454260

2. 0319910

2.8559180

2. 0168250

1. 6462280

3. 2077610

1. 2824100

2. 6629040

1. 1024740

2. 5586090

0.9824630

2. 5545340

3. 7447670

$-0.8964210$

$-1.4938360$

$-3.0142040$

$-3.3758130$

$-2.1513290$

$-1.2820470$

$-1.0567540$

$-3.4452830$

$-3.8226970$

$-1.5635180$

$-1.2791570$

$-3.4417380$

$-4.1283170$

$-2.4700900$

$-0.4219290$

0.1178150

0.1311000

1. 1867150

$-0.3753090$

1.7257680

1. 5682980

0.1628770

$-1.1906550$ 


$\begin{array}{lrrr}\mathrm{C} & -7.1700170 & 3.4960310 & 1.2139780 \\ \mathrm{H} & -6.8751650 & 1.8267300 & 2.5453900 \\ \mathrm{H} & -7.1855440 & 5.0884250 & -0.2369760 \\ \mathrm{H} & -8.1347380 & 3.7640860 & 1.6337750 \\ \mathrm{H} & -1.9833210 & 0.9743270 & -0.2902260 \\ \mathrm{~S} & -2.6006750 & 3.3000420 & -1.6354740 \\ \mathrm{C} & -0.4058210 & 3.5576220 & 0.6625790 \\ \mathrm{C} & -0.1748500 & 3.4067430 & -0.7788010 \\ \mathrm{H} & -0.9282960 & 2.7312520 & 1.1464220 \\ \mathrm{H} & -0.0770560 & 2.3975710 & -1.1647270 \\ \mathrm{O} & 1.0210020 & 3.3372810 & 0.6385510 \\ \mathrm{H} & 1.1372010 & 2.3443720 & 0.8473860 \\ \mathrm{C} & -0.0042190 & 6.0323320 & 0.4504640 \\ \mathrm{C} & -0.2409800 & 5.9219460 & -1.0565910 \\ \mathrm{C} & 0.2615150 & 4.5824940 & -1.6020480 \\ \mathrm{C} & -0.7572580 & 4.9270060 & 1.1940130 \\ \mathrm{H} & -0.3339220 & 7.0092050 & 0.8159020 \\ \mathrm{H} & 0.2620380 & 6.7345520 & -1.5887060 \\ \mathrm{H} & 1.3602420 & 4.5507980 & -1.6062670 \\ \mathrm{H} & -1.8402830 & 5.0604060 & 1.0774200 \\ \mathrm{H} & -0.5356540 & 4.9452620 & 2.2660930 \\ \mathrm{H} & 1.0688730 & 5.9492660 & 0.6636870 \\ \mathrm{H} & -1.3144370 & 6.0142970 & -1.2620750 \\ \mathrm{H} & -0.0585930 & 4.4222910 & -2.6353800 \\ \mathrm{H} & & & \end{array}$

TS287(S,S) G[M06-2X/6-31G(d) ] = -3864.197633

$\begin{array}{lrrr}\text { TS287(S, S }) & \text { G }[\mathrm{M} 06-2 X / 6-31 G(\mathrm{~d})]=-3864.197633 \\ \mathrm{P} & -0.2469380 & -0.5232040 & 0.9897090 \\ \mathrm{O} & 0.5242330 & -0.9955400 & 2.1848950 \\ \mathrm{O} & -0.8088990 & 0.8815490 & 1.0008050 \\ \mathrm{O} & -1.3936390 & -1.6266690 & 0.6949060 \\ \mathrm{O} & 0.6407280 & -0.5774050 & -0.3821330 \\ \mathrm{C} & -1.9969700 & -1.5273130 & -0.5551120 \\ \mathrm{C} & -1.3049190 & -2.0516030 & -1.6544320 \\ \mathrm{C} & -3.2306600 & -0.8895420 & -0.6573480 \\ \mathrm{C} & -3.7778620 & -0.7720890 & -1.9391180 \\ \mathrm{H} & -4.7423920 & -0.2808770 & -2.0509310 \\ \mathrm{C} & 1.0118660 & -1.8406280 & -0.8238190 \\ \mathrm{C} & 2.3369270 & -2.2406690 & -0.6605020 \\ \mathrm{C} & 0.0473380 & -2.6446180 & -1.4344830 \\ \mathrm{C} & 2.6638720 & -3.5242740 & -1.0865140 \\ \mathrm{H} & 3.6933780 & -3.8620870 & -0.9788910 \\ \mathrm{C} & -3.9471920 & -0.3437090 & 0.5293150 \\ \mathrm{C} & 3.3530800 & -1.2951550 & -0.1201780 \\ \mathrm{C} & -4.3633930 & -1.1868390 & 1.5702800 \\ \mathrm{C} & -4.1058080 & -2.6698260 & 1.6951460 \\ \mathrm{C} & -5.4924560 & -1.7192350 & 3.6621540\end{array}$

-5.1284630
-3.3595390
-3.2116580
-2.3687620
-4.1853040
-4.4339240
-3.6136290
-6.0774360
-3.5222530
-6.2950280
-6.5158100
-7.2538240
-5.4705450
-5.2964970
-6.0081290
-4.2840950
-3.8463930
-5.4182010
-5.0827220
-5.1030020
-4.7942800
-5.5858330
-6.0834850
-6.3566090
-7.0091390
-6.0885910
-3.1684240
-4.1077570
-4.3392420
-3.6609870
-3.1341210
-2.7728410
-2.2620660
-5.5058350
-6.1228420
4.6951490
5.0051950
3.1966490
3.7155300
5.4915510
5.7115440
6.4241060
4.3946890
4.0390120
4.7896900
2.4347260

-0.6817190
-2.9178620
-3.9967010
-2.4585060
-2.3382940
-3.1205710
-1.5724380
-1.2775770
-3.0561090
-2.8357600
-3.6273100
-2.4363790
-3.3910170
-4.4666600
-3.2512830
1.0172310
2.1102330
2.9676700
1.5030060
2.7506940
3.6147500
2.0430450
3.1836260
4.2391870
2.6020390
3.2870840
1.7311730
3.7810700
4.8316600
3.7427550
3.2017090
3.9874210
2.7623850
0.6568240
1.0378800
0.7195990
1.8347070
0.1973860
-0.1691130
1.1783580
1.9618770
0.6377460
0.2172110
0.5397720
-0.7966710
-0.5049200
2. 6318990

3. 0242960

3. 1483890

2.9725270

4. 2029700

4. 9291860

4.7355760

4.4759530

0.8563140

2.9600620

3.6851660

2.6158060

1.7695520

1.8857410

0.8265790

0.5950240

$-0.3527530$

1. 5175400

1. 6412910

$-0.9831470$

$-1.5831060$

$-1.6651080$

0.1397830

0.0313920

0.0904720

2. 3224880

$-1.1226950$

1.5377370

1.3269640

2. 5356320

0.4816260

$-0.1937350$

0.9705980

2. 6604010

3.4728460

$-0.4136590$

$-1.3814870$

$-2.2470630$

$-0.8769870$

$-2.6938590$

$-3.4274380$

$-2.5062080$

$-3.2238900$

$-4.2088220$

$-3.3394480$

$-2.5951560$ 


\begin{tabular}{lrrr} 
H & 5.7721910 & 2.5052930 & -0.9789180 \\
C & 2.6118880 & 1.6209780 & -2.1673700 \\
H & 2.2077010 & 1.9100820 & -3.1445390 \\
H & 1.7740200 & 1.6019200 & -1.4670600 \\
C & 3.7101560 & 2.6126590 & -1.7071670 \\
H & 3.9276640 & 3.3449900 & -2.4934870 \\
H & 3.3713540 & 3.1816080 & -0.8345130 \\
$\mathrm{C}$ & 4.9922750 & -0.6337480 & 1.5508100 \\
$\mathrm{C}$ & 5.6216210 & -1.0490490 & 2.8553180 \\
$\mathrm{C}$ & 3.7962560 & -2.6924980 & 2.0310060 \\
$\mathrm{C}$ & 3.9961980 & -1.5178320 & 1.1035980 \\
$\mathrm{C}$ & 4.5075410 & -1.1556830 & 3.9176040 \\
$\mathrm{H}$ & 4.9501830 & -1.4622380 & 4.8720830 \\
$\mathrm{H}$ & 4.0481960 & -0.1739340 & 4.0712300 \\
$\mathrm{C}$ & 3.4456660 & -2.1808870 & 3.4455030 \\
$\mathrm{H}$ & 3.4130880 & -3.0423390 & 4.1223500 \\
$\mathrm{H}$ & 2.4447860 & -1.7457350 & 3.4363460 \\
$\mathrm{H}$ & 3.0128140 & -3.3622300 & 1.6672720 \\
$\mathrm{H}$ & 6.3898430 & -0.3331030 & 3.1673640 \\
$\mathrm{C}$ & 5.1514170 & -3.4301860 & 2.1295060 \\
$\mathrm{H}$ & 5.0412150 & -4.2915890 & 2.7973220 \\
$\mathrm{H}$ & 5.4328900 & -3.8179100 & 1.1449220 \\
$\mathrm{C}$ & 6.2355010 & -2.4550750 & 2.6636180 \\
$\mathrm{H}$ & 6.6311190 & -2.8024710 & 3.6246850 \\
$\mathrm{H}$ & 7.0792880 & -2.3957590 & 1.9696470 \\
$\mathrm{C}$ & 5.3420430 & 0.4822440 & 0.7974010 \\
$\mathrm{H}$ & 6.1223780 & 1.1583240 & -1.1459530 \\
$\mathrm{C}$ & 1.7157050 & -4.3932270 & -1.6325340 \\
$\mathrm{C}$ & 0.3929290 & -3.9583130 & -1.8083830 \\
$\mathrm{C}$ & 2.1634510 & -5.7808480 & -2.0529840 \\
$\mathrm{C}$ & 1.0067530 & -6.7659920 & -2.2004640 \\
$\mathrm{C}$ & -0.1057860 & -6.1306620 & -3.0296690 \\
$\mathrm{C}$ & -0.6762540 & -4.9166370 & -2.3000470 \\
$\mathrm{H}$ & 1.3629740 & -7.6924750 & -2.6619140 \\
$\mathrm{H}$ & 2.6872010 & -5.7017950 & -3.0160600 \\
$\mathrm{H}$ & 2.9008110 & -6.1544510 & -1.3341130 \\
$\mathrm{H}$ & 0.2986150 & -5.8195980 & -4.0027180 \\
$\mathrm{H}$ & -0.9071100 & -6.8493440 & -3.2285830 \\
$\mathrm{H}$ & -1.3985480 & -4.3982650 & -2.9334240 \\
$\mathrm{H}$ & -1.2440110 & -5.2586200 & -1.4225650 \\
$\mathrm{H}$ & 0.6129340 & -7.0273640 & -1.2097710 \\
$\mathrm{C}$ & -1.8629350 & -1.8785600 & -2.9287280 \\
$\mathrm{C}$ & -1.1641630 & -2.2277210 & -4.2196770 \\
$\mathrm{C}$ & -1.1453030 & -0.9898670 & -5.1357540 \\
$\mathrm{C}$ & -2.5570380 & -0.3917390 & -5.3552960 \\
$\mathrm{C}$ & -3.6247240 & -1.0585000 & -4.4640380 \\
& & & \\
\hline
\end{tabular}

-3.1069990
-1.6989590
-0.6875010
-2.8584280
-3.8774140
-0.1452610
-0.4942800
-2.5385020
-4.5461700
0.1989220
-0.4614730
0.2857100
1.2204470
-0.5710470
1.2961860
1.8880420
-0.4979920
-1.2888370
0.4364290
2.0279840
-1.1687180
0.4959730
-0.5760360
0.9119770
0.9990670
1.9512390
-0.0167200
1.8002090
1.8213610
1.3566450
2.5424680
3.2401050
3.2828610
1.0966860
2.5546000
4.2616450
3.9680910
0.5159780
0.6093850
3.0814270
2.7044850
3.6551710

$-1.2415610$

$-3.0426190$

$-1.2562360$

$-0.4910450$

$-2.0399190$

$-2.5798830$

$-0.2424450$

0.6799450

$-0.4685480$

3.4742180

2. 3834870

4.2855800

5.3179320

3. 9810410

6.0366310

5.5415370

4.7096490

3.1753770

5.7362660

6.8312470

4.4761280

6.3011040

1.7597110

3.9899190

1.9732550

2.0626190

1.7029210

1.4129120

0.8121620

0.0230570

3.4126130

3.8600950

2.7246540

3.0053460

. 2178700

4.1921080

1.9318730

3.8781100

2.6012330

2. 5624610

4.7180620

3.0565120
$-3.0619470$ $-4.7287800$ $-6.0931440$ $-6.4026190$ $-4.8875560$ $-4.0367710$ $-4.6705160$ $-5.1334690$ $-4.4632250$ $-0.7448480$ $-0.8965760$ $-1.9863230$ $-2.1219020$ $-3.0533710$ $-3.3090340$ $-1.2967470$ $-4.2340660$ $-2.9430900$ $-4.3642410$ $-3.4118690$ $-5.0546970$ $-5.2895080$ $-0.0357950$

0.6967710 3.1833330 2.0706960 2.9007550

1. 2114560 3.4525010 2.9998850 4.5715980 3.2862630 2. 2622510 4.2817640

5.3118540

3.4932090

2.5890100 3.9578840 5.1745670 5.0071130 2.8590780 1.2893150 TS288(S, S) G[M06-2X/6-31G(d)] = -3864.199778
$\mathrm{P}$
0.3119590
$-0.083770$
0.1559980 


$\begin{array}{rrr}-0.7570340 & 0.4116020 & -0.7943370 \\ -0.1149830 & -1.4501990 & 0.9170570 \\ 1.4960870 & -0.6234980 & -0.8138210 \\ -0.2220190 & -2.5961070 & 0.1406970 \\ 0.9489450 & -3.2620770 & -0.2381850 \\ -1.4946900 & -3.0270070 & -0.2290700 \\ -1.5617130 & -4.1879910 & -0.9960940 \\ -2.5428020 & -4.5559510 & -1.2925980 \\ 2.5006910 & -1.3259250 & -0.1624230 \\ 3.6778080 & -0.6522510 & 0.1556450 \\ 2.2597960 & -2.6688150 & 0.1615590 \\ 4.6659880 & -1.3841930 & 0.8204030 \\ 5.6030730 & -0.8926670 & 1.0748670 \\ -2.7276110 & -2.2719630 & 0.1311020 \\ 3.8396250 & 0.7926500 & -0.1721200 \\ -3.0916580 & -2.0550990 & 1.4684360 \\ -2.3217710 & -2.4726210 & 2.6988200 \\ -4.5351190 & -1.2550360 & 3.2606600 \\ -4.3043380 & -1.4238000 & 1.7812190 \\ -2.0218140 & -1.2090510 & 3.5344300 \\ -1.5255920 & -1.5126750 & 4.4632800 \\ -1.3230400 & -0.5695640 & 2.9887080 \\ -3.3448610 & -0.4593000 & 3.8406960 \\ -3.4865970 & -0.3370880 & 4.9205480 \\ -3.3259430 & 0.5443650 & 3.4028410 \\ -5.4806460 & -0.7349510 & 3.4543960 \\ -1.3928200 & -2.9874100 & 2.4438630 \\ -4.5379270 & -2.6529740 & 3.9159170 \\ -4.6250220 & -2.5381160 & 5.0024030 \\ -5.4145310 & -3.2144150 & 3.5791350 \\ -3.2296290 & -3.3971730 & 3.5413670 \\ -2.6860900 & -3.7033340 & 4.4421390 \\ -3.4502300 & -4.3057820 & 2.9727020 \\ -3.5825540 & -1.8014620 & -0.8786940 \\ -3.3298790 & -1.8222230 & -2.3684680 \\ -5.6270000 & -0.8004440 & -1.7523800 \\ -4.8078270 & -1.2005370 & -0.5530090 \\ -4.4582650 & -2.6266290 & -3.0515240 \\ -4.3306510 & -2.5578320 & -4.1378690 \\ -4.3777710 & -3.6859600 & -2.7862160 \\ -5.8375600 & -2.0585040 & -2.6232970 \\ -6.4389070 & -1.7946790 & -3.5004190 \\ -6.4032090 & -2.8026530 & -2.0541330 \\ -6.5889270 & -0.3750140 & -1.4468540 \\ -2.3510930 & -2.2443680 & -2.6079770 \\ -4.8176430 & 0.2170570 & -2.5832430\end{array}$

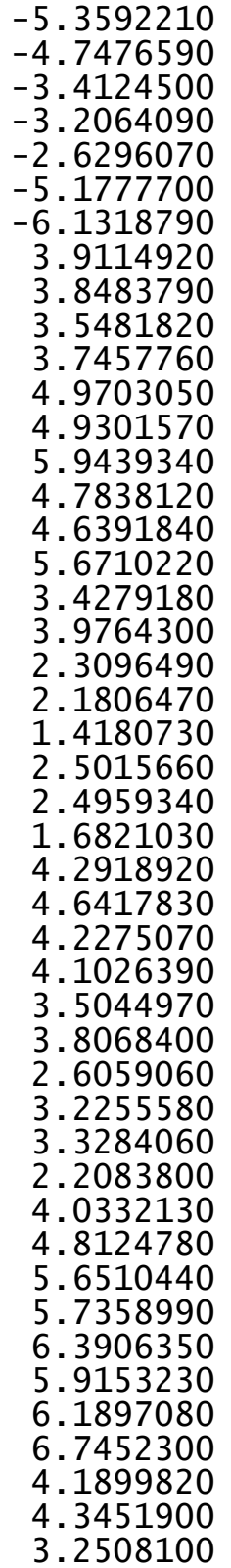

0.4241350

1.1654760

$-0.3639780$

$-0.3520490$

0.2255820

$-1.0248590$

$-0.5610870$

2. 6028700

2. 9032420

0.3927610

1. 2438400

2. 0916830

2. 2661680

2.4475900

0.5861770

0.0059990

0.1846850

$-0.6638910$

3. 9748480

0.9071420

0.3095110

0.7547370

2.4042550

2. 5567800

3. 0029970

3. 0874180

3.9488340

1.4704740

1.7338080

3.8639240

4.4148640

4. 3445840

2. 3761880

2.1915160

2. 0911470

0.4239030

4.9867200

1.8833210

1.7611800

1. 2176760

3. 3572560

3. 9605070

3.4186050

3. 5254200

4.5760230

$-3.3548410$
$-3.5136850$

$-2.0415730$

$-2.8777250$

$-3.9538740$

$-2.3922520$

0.7748050

1.0230160

$-1.8041360$

$-3.2806850$

$-2.7312760$

$-1.4986800$

$-3.9665140$

$-5.0475890$

$-3.6164340$

$-3.6413840$

$-4.5596280$

$-3.1424190$

$-2.4824310$

$-3.4701620$

$-3.4938640$

$-4.4031210$

$-2.8794300$

$-3.8459220$

$-4.9311810$

$-3.4387520$

0.5168240

1. 7033390

2. 3213130

0.8377090

2. 7425160

3. 6407910

2. 3461370

3. 0725320

4.1479160

2. 7925310

2. 5656120

1. 3975560

2.7592340

3.8449580

2. 3019980

2. 3490030

3. 2215810

1.6384470

$-0.7992710$

$-1.0432290$

0.8770420 


\begin{tabular}{|c|c|c|}
\hline $\begin{array}{l}3.0738760 \\
3.4157940 \\
4.8327840 \\
5.4694240 \\
4.4589190 \\
3.7459630 \\
3.3143970 \\
5.4931970 \\
5.8497910 \\
2.0519100 \\
2.6679020 \\
4.7806360 \\
6.3305590 \\
0.8485280 \\
2.0967080 \\
1.8162030 \\
0.6648640 \\
0.6155030 \\
0.4239210 \\
2.7220240 \\
2.5293380 \\
2.8535450 \\
0.9063120 \\
0.5182360 \\
1.4012400 \\
0.9939260 \\
1.5486310 \\
3.2641810 \\
2.8152650 \\
4.5596250 \\
5.1146120 \\
5.2333480 \\
6.3258510 \\
4.5912720 \\
6.4471290 \\
4.8003240 \\
6.9948280 \\
6.7478160 \\
6.9673600 \\
7.9423810 \\
1.9355250 \\
2.4761490 \\
0.2533800 \\
0.0410590 \\
0.8024060 \\
0.0143130\end{array}$ & $\begin{array}{l}-4.7317070 \\
-4.6850000 \\
-4.1224510 \\
-3.5075760 \\
-2.7085520 \\
-5.4452230 \\
-5.6876240 \\
-4.9085690 \\
-4.3197070 \\
-5.0926330 \\
-4.0549460 \\
-3.3538850 \\
-2.8878300 \\
-4.4002360 \\
-5.0669510 \\
-5.9076030 \\
-6.8678220 \\
-6.0710050 \\
-4.8703870 \\
-6.4513010 \\
-5.7259300 \\
-4.3029400 \\
-7.4623780 \\
-7.5705940 \\
-6.7135190 \\
-5.7112300 \\
-5.2549550 \\
2.5494380 \\
1.4578040 \\
2.9906060 \\
2.2530600 \\
4.1190110 \\
2.6397280 \\
1.3766980 \\
4.4972910 \\
4.6895890 \\
3.7601010 \\
2.0645080 \\
5.3695180 \\
4.0607290 \\
1.0533920 \\
3.4194160 \\
3.5644860 \\
3.4241770 \\
2.7518840 \\
2.4174070\end{array}$ & $\begin{array}{r}1.4665420 \\
2.9671610 \\
3.2334550 \\
1.9695930 \\
1.1895990 \\
0.9691410 \\
3.3933560 \\
3.6123510 \\
1.3353360 \\
1.3217730 \\
3.4585880 \\
4.0103320 \\
2.2368750 \\
-1.0649810 \\
-1.6127740 \\
-2.8570730 \\
-2.5727300 \\
-2.3365080 \\
-1.4271060 \\
-3.1435610 \\
-0.8495580 \\
-1.8206470 \\
-1.6818120 \\
-3.3989450 \\
-1.9229350 \\
-3.3032210 \\
-3.6982810 \\
-0.4029980 \\
0.1154940 \\
0.1739830 \\
1.2285950 \\
-0.3093250 \\
1.7892470 \\
1.5919900 \\
0.2503240 \\
-1.1237240 \\
1.3000850 \\
2.6078130 \\
-0.1317490 \\
1.7366010 \\
-0.3106020 \\
-1.6153960 \\
0.6644540 \\
-0.7808510 \\
1.1422290 \\
-1.1810590\end{array}$ \\
\hline
\end{tabular}

1.1638790
1.2439270
0.2372940
-0.0164350
0.4332260
-0.5491210
-0.0529020
0.5110200
1.5300650
-1.6275680
-0.3174360
1.3084730
-1.0875960
0.1018880
1.1638790

$\mathrm{H} \quad 1.2439270$

$\begin{array}{lr}\text { C } & 0.2372940 \\ \text { C } & -0.0164350\end{array}$

C $\quad 0.4332260$

C $\quad-0.5491210$

$\mathrm{H} \quad-0.0529020$

$\mathrm{H}$

$\mathrm{H} \quad-1.6275680$

$\mathrm{H} \quad-0.3174360$

$\mathrm{H} \quad 1.3084730$

H $\quad 0.1018880$

154

TS289(S, S) G[M06-2X/6-31G(d) $]=-3864.197612$

$\begin{array}{lrrr}\text { TS289(S, S } \text { G G M06-2X/6-31G(d)] }=-3864.197612 \\ \text { P } & 0.3174240 & -0.0886860 & 0.1832590 \\ \mathrm{O} & 0.8233590 & 0.8481340 & 1.2426160 \\ \mathrm{O} & -0.7557080 & 0.3939090 & -0.7688590 \\ \mathrm{O} & -0.0967660 & -1.4536550 & 0.9517870 \\ \mathrm{O} & 1.5021670 & -0.6270010 & -0.7845710 \\ \mathrm{C} & -0.1918340 & -2.6123790 & 0.1933830 \\ \mathrm{C} & 0.9926730 & -3.2720120 & -0.1763020 \\ \mathrm{C} & -1.4605400 & -3.0679230 & -0.1533590 \\ \mathrm{C} & -1.5333520 & -4.2629260 & -0.8768500 \\ \mathrm{H} & -2.5135090 & -4.6486530 & -1.1501930 \\ \mathrm{C} & 2.5200930 & -1.3137800 & -0.1382600 \\ \mathrm{C} & 3.6956300 & -0.6270380 & 0.1563380 \\ \mathrm{C} & 2.3030690 & -2.6598770 & 0.1874080 \\ \mathrm{C} & 4.7102650 & -1.3501560 & 0.7898650 \\ \mathrm{H} & 5.6474810 & -0.8481400 & 1.0224660 \\ \mathrm{C} & -2.6966800 & -2.3094010 & 0.1909060 \\ \mathrm{C} & 3.8310320 & 0.8203720 & -0.1726730 \\ \mathrm{C} & -3.0636170 & -2.0672570 & 1.5229650 \\ \mathrm{C} & -2.2923030 & -2.4550330 & 2.7619250 \\ \mathrm{C} & -4.5132340 & -1.2388160 & 3.2968770 \\ \mathrm{C} & -4.2799590 & -1.4360970 & 1.8213370 \\ \mathrm{C} & -2.0003770 & -1.1728070 & 3.5716800 \\ \mathrm{H} & -1.5029190 & -1.4542530 & 4.5068210 \\ \mathrm{H} & -1.3049770 & -0.5403920 & 3.0134250 \\ \mathrm{C} & -3.3280780 & -0.4247950 & 3.8617900 \\ \mathrm{H} & -3.4714760 & -0.2817450 & 4.9388700 \\ \mathrm{H} & -3.3144530 & 0.5700030 & 3.4039350 \\ \mathrm{H} & -5.4619620 & -0.7203570 & 3.4792280 \\ \mathrm{H} & -1.3601410 & -2.9690690 & 2.5174680 \\ \mathrm{C} & -4.5087270 & -2.6232260 & 3.9802740\end{array}$

$\begin{array}{lr}3.2909450 & 0.6248800 \\ 2.2912500 & 0.8171810 \\ 6.0253860 & 0.4794540 \\ 5.9429640 & -1.0266640 \\ 4.5932380 & -1.5924520 \\ 4.9389220 & 1.2162630 \\ 7.0089310 & 0.8601330 \\ 6.7436340 & -1.5531720 \\ 4.5219210 & -1.6030660 \\ 5.1132400 & 1.1115520 \\ 4.9353500 & 2.2863710 \\ 5.9001350 & 0.6813830 \\ 6.0761390 & -1.2214530 \\ 4.4575800 & -2.6257770\end{array}$

$-1.4536550$

$-3.2720120$

0.8203720

0.4247950

$-2.6232260$

3. 9802740 


$\begin{array}{lrrr}\mathrm{H} & -4.5982900 & -2.4870690 & 5.0641010 \\ \mathrm{H} & -5.3814560 & -3.1965220 & 3.6535270 \\ \mathrm{C} & -3.1953660 & -3.3671790 & 3.6228550 \\ \mathrm{H} & -2.6510720 & -3.6509130 & 4.5304970 \\ \mathrm{H} & -3.4097840 & -4.2889670 & 3.0733520 \\ \mathrm{C} & -3.5526150 & -1.8640830 & -0.8290040 \\ \mathrm{C} & -3.2971440 & -1.9155230 & -2.3172910 \\ \mathrm{C} & -5.6001780 & -0.8917060 & -1.7263780 \\ \mathrm{C} & -4.7812280 & -1.2626200 & -0.5175240 \\ \mathrm{C} & -4.4209710 & -2.7391730 & -2.9849570 \\ \mathrm{H} & -4.2924220 & -2.6922250 & -4.0723670 \\ \mathrm{H} & -4.3353190 & -3.7923300 & -2.6977160 \\ \mathrm{C} & -5.8036680 & -2.1693870 & -2.5700650 \\ \mathrm{H} & -6.4057670 & -1.9278590 & -3.4530820 \\ \mathrm{H} & -6.3657050 & -2.9040620 & -1.9852260 \\ \mathrm{H} & -6.5644310 & -0.4638890 & -1.4316470 \\ \mathrm{H} & -2.3163930 & -2.3394670 & -2.5458110 \\ \mathrm{C} & -4.7943650 & 0.1108040 & -2.5788760 \\ \mathrm{H} & -5.3352860 & 0.2929620 & -3.5149120 \\ \mathrm{H} & -4.7308480 & 1.0720080 & -2.0595820 \\ \mathrm{C} & -3.3851250 & -0.4685620 & -2.8573540 \\ \mathrm{H} & -3.1764090 & -0.4781710 & -3.9330230 \\ \mathrm{H} & -2.6068560 & 0.1356680 & -2.3822740 \\ \mathrm{C} & -5.1541240 & -1.0621910 & 0.8059560 \\ \mathrm{H} & -6.1112020 & -0.5990190 & 1.0434650 \\ \mathrm{C} & 3.8489070 & 2.6322650 & -1.8039430 \\ \mathrm{C} & 3.7583360 & 2.9321880 & -3.2791550 \\ \mathrm{C} & 3.5124480 & 0.4160870 & -2.7275810 \\ \mathrm{C} & 3.7113150 & 1.2703750 & -1.4974320 \\ \mathrm{C} & 4.8841970 & 2.1415630 & -3.9827890 \\ \mathrm{H} & 4.8228060 & 2.3142570 & -5.0631550 \\ \mathrm{H} & 5.8565190 & 2.5163500 & -3.6491740 \\ \mathrm{C} & 4.7321210 & 0.6329520 & -3.6537790 \\ \mathrm{H} & 4.5875310 & 0.0488790 & -4.5695460 \\ \mathrm{H} & 5.6335330 & 0.2500910 & -3.1657290 \\ \mathrm{H} & 3.4151160 & -0.6426020 & -2.4772870 \\ \mathrm{H} & 3.8637570 & 4.0060830 & -3.4697630 \\ \mathrm{C} & 2.2548140 & 0.9073250 & -3.4739650 \\ \mathrm{H} & 2.1276310 & 0.3097030 & -4.3835250 \\ \mathrm{H} & 1.3733020 & 0.7353430 & -2.8501930 \\ \mathrm{C} & 2.4123300 & 2.4089230 & -3.8238930 \\ \mathrm{H} & 2.3851460 & 2.5650870 & -4.9082860 \\ \mathrm{H} & 1.5884800 & 2.9901290 & -3.4005960 \\ \mathrm{C} & 4.2531900 & 3.1218630 & 0.5121110 \\ \mathrm{C} & 4.6037380 & 3.9878080 & 1.6951170 \\ \mathrm{C} & 4.2408430 & 1.5015590 & 2.3151780 \\ & & & \end{array}$

\begin{abstract}
4.0918280
3. 4819190

3. 7872120

2. 5702170

3. 2319830

3. 3494690

2. 2168860

4.0684110

4.7521490

5. 6623030

5. 7629170

6.4074020

5. 8957850

6.1715330

6.7148580

4.1254430

4. 2588780

3. 3267550

3. 1999210

3. 6140640

5. 0159770

5. 5903690

4.5351480

3.8502530

3.5752460

5. 7175280

5.9647890

2. 1778110

2.8640390

4.9569680

6. 4470130

0.8817440

2. 0527350

1.8084580

0.4577300

$-0.4299820$

$-0.3889550$

2. 1726270

2. 6375120

0.6217610

$-0.0620840$

2. 9851210

1.8317020

$-0.0853360$

$-1.4587850$

$-3.2901720$
\end{abstract}

1.7648890

3. 8815420

4.4353680

4. 3481010

2. 3885990

2.2039340

2. 0867980

0.4511260

5.0289420

1.9391880

1. 8180410

1. 2865670

3.4176470

4.0249840

3. 4936870

3. 5588310

4. 6121830

$-3.3422810$

$-4.7460730$

$-4.7696390$

$-4.1586990$

$-3.4855610$

$-2.6837790$

$-5.4246470$

$-5.7991560$

$-4.9269800$

$-4.2657250$

$-5.1174930$

$-4.2051560$

$-3.4126150$

$-2.8553920$

$-4.4449590$

$-5.1876830$

$-5.4125670$

$-6.1092750$

$-6.2181590$

$-4.9471450$

$-6.1595600$

$-5.9922190$

$-7.1132270$

$-7.0457340$

$-4.6371430$

$-4.4332990$

$-5.5476530$

$-6.4667020$

2. 4988510
0.8340310

2.7491250

3.6446260

2. 3661290

3. 0794060

4.1532890

2. 8094240

2.5598360

1. 3887380

2.7354310

3. 8199240

2. 2685140

2. 3229620

3. 1922730

1.6013330

$-0.8021220$

$-1.0472610$

0.8604200

1. 3974790

2. 8803440

3. 1211620

1.8586940

1.1432090

0.8268820

3. 2487990

3. 4604690

1. 1818870

1. 2876320

3. 4429460

3. 9192370

2. 1161450

$-0.9368830$

$-1.5323120$

$-3.0358920$

$-3.3273660$

$-2.0717780$

$-1.2650960$

$-1.0321830$

3.4529450

$-3.7308450$

$-1.4500410$

$-1.3859050$

$-3.5243220$

$-4.0933470$

$-2.3492610$

$-0.4274430$ 


$\begin{array}{lrrr}\mathrm{O} & -2.8340690 & 1.4206310 & 0.1119030 \\ \mathrm{C} & -4.6000800 & 2.9297550 & 0.1240350 \\ \mathrm{C} & -5.1468870 & 2.2174670 & 1.1999510 \\ \mathrm{C} & -5.2955350 & 4.0239580 & -0.4049090 \\ \mathrm{C} & -6.3713210 & 2.5958820 & 1.7372620 \\ \mathrm{H} & -4.6062530 & 1.3672770 & 1.5986580 \\ \mathrm{C} & -6.5229030 & 4.3929870 & 0.1306640 \\ \mathrm{H} & -4.8689570 & 4.5747760 & -1.2361240 \\ \mathrm{C} & -7.0621200 & 3.6815610 & 1.2023550 \\ \mathrm{H} & -6.7865580 & 2.0411480 & 2.5732050 \\ \mathrm{H} & -7.0604060 & 5.2378580 & -0.2872910 \\ \mathrm{H} & -8.0201830 & 3.9751640 & 1.6203080 \\ \mathrm{H} & -1.9433410 & 1.0226350 & -0.2991150 \\ \mathrm{~S} & -2.4949220 & 3.3649180 & -1.6381000 \\ \mathrm{C} & -0.3080040 & 3.5450570 & 0.6782640 \\ \mathrm{C} & -0.0741160 & 3.4177400 & -0.7649960 \\ \mathrm{H} & -0.8449890 & 2.7181240 & 1.1448210 \\ \mathrm{H} & 0.0096910 & 2.4144770 & -1.1692710 \\ \mathrm{O} & 1.1152530 & 3.3030300 & 0.6557570 \\ \mathrm{H} & 1.2143640 & 2.3041950 & 0.8445940 \\ \mathrm{C} & 0.1320430 & 6.0169490 & 0.5149790 \\ \mathrm{C} & -0.0993380 & 5.9387730 & -0.9948650 \\ \mathrm{C} & 0.3839950 & 4.6020410 & -1.5639820 \\ \mathrm{C} & -0.6411030 & 4.9093400 & 1.2339790 \\ \mathrm{H} & -0.1845730 & 6.9916400 & 0.8973960 \\ \mathrm{H} & 0.4190440 & 6.7530840 & -1.5093290 \\ \mathrm{H} & 1.4820870 & 4.5527400 & -1.5655270 \\ \mathrm{H} & -1.7214950 & 5.0612930 & 1.1153450 \\ \mathrm{H} & -0.4241890 & 4.9038740 & 2.3071350 \\ \mathrm{H} & 1.2026270 & 5.9134480 & 0.7314620 \\ \mathrm{H} & -1.1702010 & 6.0520300 & -1.2032780 \\ \mathrm{H} & 0.0649140 & 4.4673090 & -2.6012460 \\ \mathrm{H} & & & \end{array}$

154 TS290(S, S) G[M06-2X/6-31G(d)] = -3864.198073

$\begin{array}{lrrr}\text { TS290(S, S) } \mathrm{G}[\mathrm{M} 06-2 \mathrm{X} / 6-31 \mathrm{G}(\mathrm{d})]=-3864.198073 \\ \text { P } & 0.1581440 & 0.4789610 & 0.8894620 \\ \mathrm{O} & -0.6584710 & 0.9969750 & 2.0348400 \\ \mathrm{O} & 0.6753570 & -0.9407800 & 0.9517720 \\ \mathrm{O} & 1.3485160 & 1.5482320 & 0.6468340 \\ \mathrm{O} & -0.6480940 & 0.5432790 & -0.5297490 \\ \mathrm{C} & 2.0197610 & 1.4373080 & -0.5651950 \\ \mathrm{C} & 1.4133820 & 1.9679990 & -1.7059670 \\ \mathrm{C} & 3.2583520 & 0.7995030 & -0.5879440 \\ \mathrm{C} & 3.8838120 & 0.7005250 & -1.8287200 \\ \mathrm{H} & 4.8578120 & 0.2162980 & -1.8788720 \\ \mathrm{C} & -0.9611980 & 1.8151460 & -0.9925350 \\ \mathrm{C} & -2.2758270 & 2.2576970 & -0.8731800\end{array}$

0.0622040

$-2.5559330$

$-3.5752000$

3.8991860

$-3.3362950$

4.2630160

4.0056040

5.2701280

4.9618480

3. 1824720

3.0293680

2.1958390

3.9359080

4.1448210

3.3310430

5.8041790

3.4736560

6.1172120

6.2988460

7.0926360

5. 3660070

5.1894330

5.9576770

4.2171240

3. 8115940

5.2770890

4.9530620

5.0872800

4.7952300

5.6109750

6.0072170

6.2761540

6.9391540

5.9024860

3.1782030

3.9567870

4.1847830

3.4634930

3.0420030

2.6952230

2.1584780

5.3266380

5.8936970

$-4.7335450$

$-5.0494170$

$-3.1295610$
2. 5855100

3. 5568830

3.9295290

0.2538480

1. 3549530

1.1008080

2.5854090

1.6387910

0.5973050

2. 8414380

3.9210510

2. 3830380

2. 2658710

3.0515360

1.5053100

1.1990130

2.9692230

2.7487060

3.5425340

2. 3437750

3. 3021520

4. 3788360

3. 1568620

$-1.1093470$

$-2.2063830$

$-3.0634740$

$-1.5940190$

$-2.8729960$

$-3.7425190$

$-2.1823930$

$-3.3010590$

$-4.3599550$

$-2.7275290$

$-3.3831080$

$-1.8255150$

$-3.8604040$

$-4.9148830$

$-3.8112770$

$-3.2749310$

$-4.0622960$

$-2.8112950$

$-0.7440090$

$-1.1245180$

$-0.6259530$

$-1.7634430$

$-0.2413080$
$-1.5633220$

$-1.3040680$

$-1.2222570$

0.6411710

$-0.3435010$

1.6982090

1.7997010

3.8494210

2.8049760

3.0811360

3.1913240

2. 9723880

4.3093020

5.0443180

4.8119690

4.6984750

0.9259960

3.1904580

3.9238680

2.9043330

1.9515490

2.0532520

1.0423990

0.7357360

$-0.2215300$

1.7339000

1.8276210

$-0.7818290$

$-1.3825080$

$-1.4509150$

0.3930030

0.3099330

0.3829020

2. 5741950

$-1.0280840$

1.6960820

1. 5014550

2. 6712880

0.5919700

$-0.0891390$

1.0360690

2.8627750

3.7110570

$-0.6146890$

$-1.5546000$

$-2.3907210$ 


$\begin{array}{rrr}-3.7023680 & 0.2088080 & -1.0680970 \\ -5.4459750 & -1.1341250 & -2.9097000 \\ -5.6857360 & -1.9318010 & -3.6214560 \\ -6.3499640 & -0.5316270 & -2.7793980 \\ -4.2732620 & -0.2621290 & -3.4310460 \\ -3.8818670 & -0.6613990 & -4.3734610 \\ -4.6081180 & 0.7606220 & -3.6286240 \\ -2.3214160 & 0.4119710 & -2.7287570 \\ -5.8638980 & -2.3833790 & -1.1642660 \\ -2.6104400 & -1.6815590 & -2.2094230 \\ -2.1432780 & -2.0275040 & -3.1385400 \\ -1.8295030 & -1.6649630 & -1.4456350 \\ -3.7796150 & -2.6098400 & -1.7966570 \\ -3.9924400 & -3.3420690 & -2.5844610 \\ -3.5160810 & -3.1817540 & -0.9006940 \\ -5.0635430 & 0.8117360 & 1.2829300 \\ -5.7277700 & 1.2937230 & 2.5464840 \\ -3.8158700 & 2.8437680 & 1.7395850 \\ -4.0215740 & 1.6452280 & 0.8438070 \\ -4.6517780 & 1.3979770 & 3.6474080 \\ -5.1184870 & 1.7524700 & 4.5734170 \\ -4.2346320 & 0.4064640 & 3.8506600 \\ -3.5367970 & 2.3687400 & 3.1826200 \\ -3.4985710 & 3.2512920 & 3.8314330 \\ -2.5526910 & 1.8988590 & 3.2262180 \\ -2.9959210 & 3.4737750 & 1.3860610 \\ -6.5320930 & 0.6153180 & 2.8513640 \\ -5.1463530 & 3.6309130 & 1.7621410 \\ -5.0304150 & 4.5094540 & 2.4062500 \\ -5.3769720 & 3.9958630 & 0.7558210 \\ -6.2839470 & 2.7121040 & 2.2843110 \\ -6.7043470 & 3.1055100 & 3.2166850 \\ -7.1016450 & 2.6573400 & 1.5594210 \\ -5.4221640 & -0.3193980 & 0.5569800 \\ -6.2406570 & -0.9539070 & 0.8960310 \\ -0.2433000 & 3.8980250 & -1.9483510 \\ 0.7797860 & 4.9087140 & -2.4020290 \\ 0.6499290 & 6.1795440 & -1.5417890 \\ -0.7876180 & 6.7541730 & -1.5474290 \\ -1.7985020 & 5.8064940 & -2.2254170 \\ -1.5565810 & 4.3783060 & -1.8140040 \\ 0.6074920 & 5.1716370 & -3.4552860 \\ 1.3646990 & 6.9291240 & -1.8943800 \\ -0.8132430 & 7.7205770 & -2.0601870 \\ -1.6850970 & 5.8897620 & -3.3148530 \\ 1.7928470 & 4.5033680 & -2.3300450\end{array}$

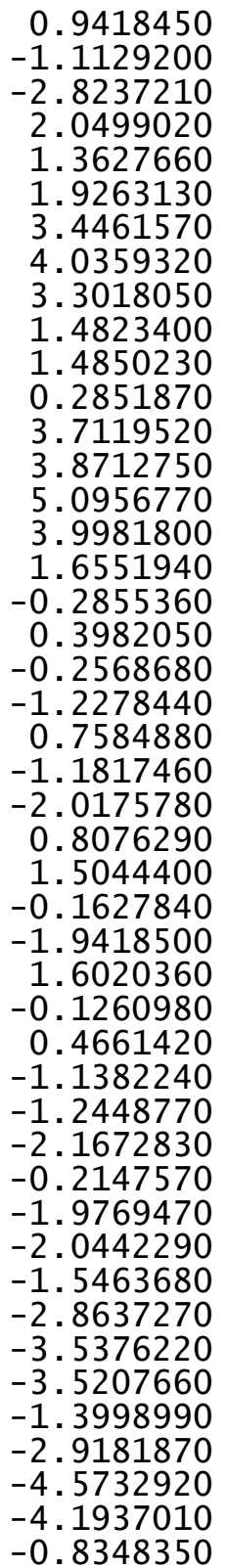

5.9202600
6.9351390
6.1087860
1.8104910
2.2617960
1.5805090
1.7164500
0.9421670
1.1775580
2.0281340
3.3459040
2.0847170
2.7787220
1.3479860
1.1875500
-0.1322510
0.5166720
-3.6108050
-2.5408920
-4.5460210
-5.5411750
-4.4087620
-6.3897290
-5.6344170
-5.2692160
-3.6314620
-6.2584430
-7.1543760
-5.1677160
-6.9262080
-1.8606930
-3.9891910
-1.8912000
-1.9879400
-1.6546950
-1.3647170
-0.7032260
0.0553480
-3.2488850
-3.7156450
-2.6105410
-2.8888340
-4.0314770
-4.0138360
-1.7908540
-3.7857380
$-0.5193920$

$-0.5187240$

$-1.9918060$

$-2.9542430$

$-4.2293970$

$-5.4751840$

$-5.4878110$

$-4.3119240$

$-3.0042340$

$-6.3699900$

$-4.3490930$

$-4.1422950$

$-5.4076190$

$-6.4267260$

$-4.1785120$

$-4.5389140$

$-5.4750520$

$-0.6868290$

$-0.8811560$

$-1.8403360$

$-1.9978080$

$-2.7966450$

$-3.0972400$

$-1.2600990$

$-3.8866840$

$-2.6694510$

$-4.0394230$

$-3.2199260$

$-4.6190110$

$-4.8946690$

$-0.0626210$

0.7200080

3. 1261270

1.9897410

2.8653270

1. 1187120

3. 3394190

2. 8688320

4. 5101230

3. 2189250

2. 1612110

4.2495330

5. 2724650

3. 4062080

2. 4441840

3. 9665740 


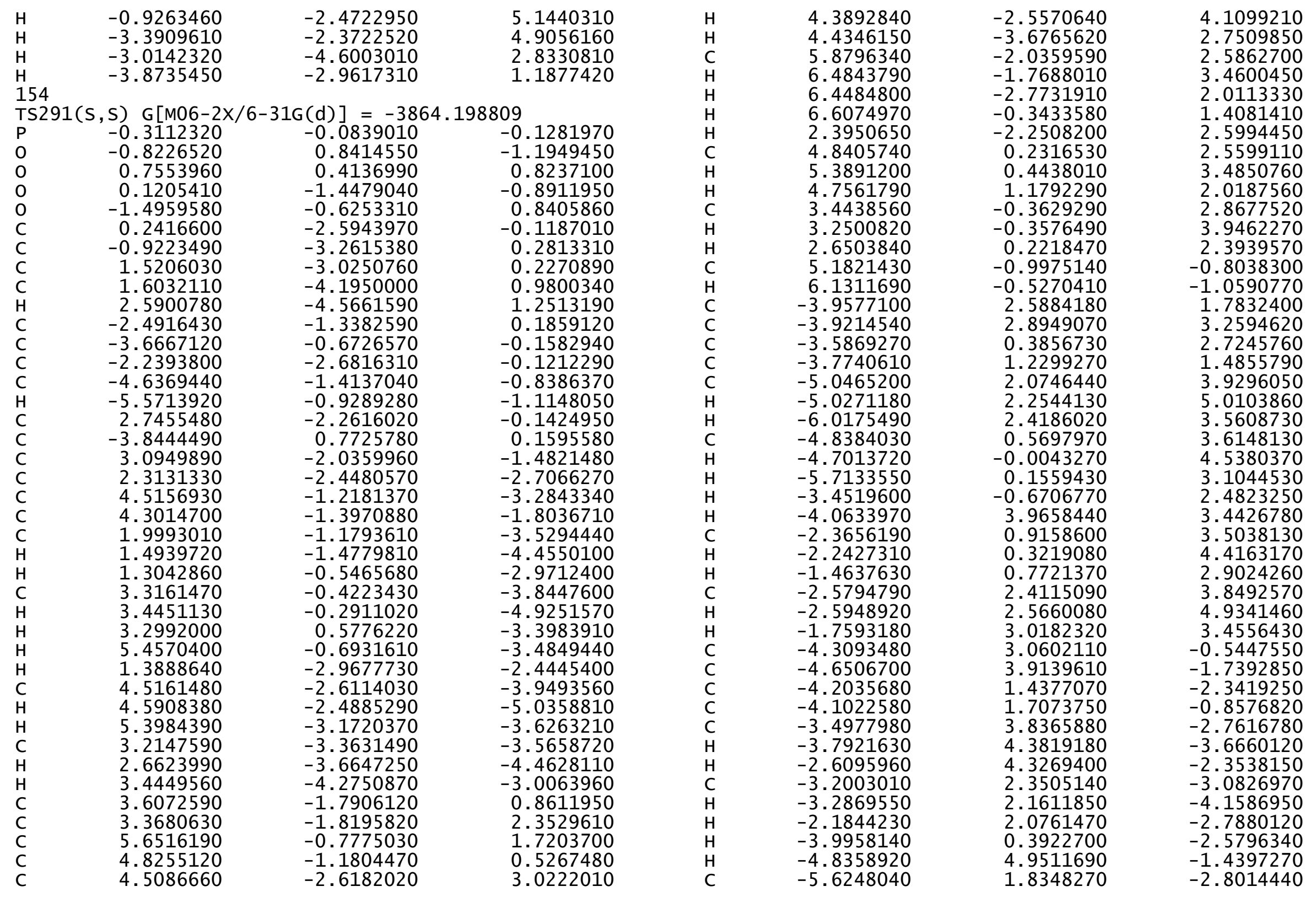




\begin{tabular}{|c|c|c|}
\hline $\begin{array}{l}-5.6931650 \\
-6.3643530 \\
-5.9090160 \\
-6.1763930 \\
-6.7497230 \\
-4.2305550 \\
-4.3996560 \\
-3.2123160 \\
-3.0251280 \\
-3.3298200 \\
-4.7279850 \\
-5.4027030 \\
-4.4151960 \\
-3.7120910 \\
-3.2402960 \\
-5.3852780 \\
-5.7995770 \\
-2.0101520 \\
-2.5569250 \\
-4.6351000 \\
-6.2568810 \\
-0.8053640 \\
0.4741420 \\
-2.0365090 \\
-1.8045130 \\
-0.5432430 \\
0.6711720 \\
-2.6793080 \\
-2.3233350 \\
-2.8854650 \\
-0.6381720 \\
-0.4102090 \\
1.5643390 \\
0.8790150 \\
-1.6933860 \\
3.2355950 \\
2.7977200 \\
4.5212890 \\
5.0896420 \\
5.1725970 \\
6.2928600 \\
4.5828880 \\
6.3780060 \\
4.7286180 \\
6.9397230 \\
6.7257720\end{array}$ & $\begin{array}{l}1.7075430 \\
1.1639160 \\
3.3077430 \\
3.9049930 \\
3.3638910 \\
3.5043340 \\
4.5542480 \\
-3.3778730 \\
-4.7676670 \\
-4.7548190 \\
-4.1695600 \\
-3.5478790 \\
-2.7394080 \\
-5.4692030 \\
-5.7711910 \\
-4.9438500 \\
-4.3559360 \\
-5.1317880 \\
-4.1554200 \\
-3.3983280 \\
-2.9321640 \\
-4.3986320 \\
-4.8805750 \\
-5.0176490 \\
-6.4389840 \\
-6.4926460 \\
-6.1385760 \\
-6.7724770 \\
-4.3776010 \\
-4.9955500 \\
-5.7759810 \\
-7.4839550 \\
-6.0240560 \\
-6.9745630 \\
-7.1216630 \\
2.5830210 \\
1.4867370 \\
3.0422040 \\
2.3013290 \\
4.1905100 \\
2.7037250 \\
1.4102780 \\
4.5851830 \\
4.7638050 \\
3.8440480 \\
2.1253350\end{array}$ & $\begin{array}{l}-3.8877380 \\
-2.3518820 \\
-2.4010060 \\
-3.2798680 \\
-1.7028150 \\
0.7708550 \\
1.0083510 \\
-0.8531140 \\
-1.4101730 \\
-2.9193620 \\
-3.2362310 \\
-1.9962160 \\
-1.1964960 \\
-0.9148550 \\
-3.3144260 \\
-3.6436960 \\
-1.3667690 \\
-1.2277300 \\
-3.4102790 \\
-4.0066850 \\
-2.2935430 \\
1.1047210 \\
1.4272160 \\
1.7421700 \\
2.2496700 \\
3.1067350 \\
2.2525370 \\
2.8170800 \\
2.5895580 \\
1.0585160 \\
3.9327950 \\
3.5516960 \\
2.8764400 \\
1.5698480 \\
1.3960710 \\
0.4296460 \\
-0.0883040 \\
-0.1546810 \\
-1.1998820 \\
0.3121170 \\
-1.7665760 \\
-1.5510380 \\
-0.2543400 \\
1.1186730 \\
-1.2938360 \\
-2.5771710\end{array}$ \\
\hline
\end{tabular}

$\begin{array}{lr}\mathrm{H} & 6.8807530 \\ \mathrm{H} & 7.8808870 \\ \mathrm{H} & 1.9242670 \\ \mathrm{~S} & 2.4434250 \\ \mathrm{C} & 0.2285110 \\ \mathrm{C} & 0.0128910 \\ \mathrm{H} & 0.7850340 \\ \mathrm{H} & -0.0362550 \\ \mathrm{O} & -1.1866950 \\ \mathrm{H} & -1.2584000 \\ \mathrm{C} & -0.2810020 \\ \mathrm{C} & -0.0321190 \\ \mathrm{C} & -0.4742850 \\ \mathrm{C} & 0.5160540 \\ \mathrm{H} & 0.0031790 \\ \mathrm{H} & -0.5673920 \\ \mathrm{H} & -1.5706080 \\ \mathrm{H} & 1.5928590 \\ \mathrm{H} & 0.2884150 \\ \mathrm{H} & -1.3505010 \\ \mathrm{H} & 1.0373040 \\ \mathrm{H} & -0.1475080 \\ \mathrm{H} & \end{array}$
5.4732590
6.0274760
5.9444800
4.5905870
4.9480420
7.0137640
6.7403990
4.5113900
5.1300650
4.9445280
5.8944730
6.0850530
4.4557320

0.1144090

$3.4389960 \quad 1.6493250$

$3.5705410 \quad-0.6397450$

$3.4264780 \quad 0.8047940$

$2.7628690 \quad-1.1171350$

$2.4188070 \quad 1.2035060$

$3.2863400 \quad-0.6050660$

$2.2859240 \quad-0.7967930$

$-0.4525770$

1.0543420

1. 6160240

$-1.1882650$

$-0.8306850$

1. 5801660

1.6203030

$-1.0793330$

$-2.2592510$

$-0.6584970$

1. 2533970

154

$\begin{array}{lrrr}\text { TS292(S , S }) & \text { G }[\mathrm{M} 06-2 \times / 6-31 G(\mathrm{~d})] & = & -3864.197441 \\ \mathrm{P} & -0.1788840 & -0.3317420 & 0.9719230 \\ \mathrm{O} & 0.6164760 & -0.7545830 & 2.1700710 \\ \mathrm{O} & -0.6274640 & 1.1093130 & 0.8712040 \\ \mathrm{O} & -1.4225040 & -1.3604900 & 0.8481530 \\ \mathrm{O} & 0.6235530 & -0.5951150 & -0.4273490 \\ \mathrm{C} & -2.0839040 & -1.3593770 & -0.3751420 \\ \mathrm{C} & -1.4882320 & -2.0462660 & -1.4419540 \\ \mathrm{C} & -3.2892840 & -0.6716720 & -0.4834350 \\ \mathrm{C} & -3.9131480 & -0.6821200 & -1.7349090 \\ \mathrm{H} & -4.8660360 & -0.1684510 & -1.8464720 \\ \mathrm{C} & 0.8779830 & -1.9265510 & -0.7318690 \\ \mathrm{C} & 2.1701720 & -2.4182420 & -0.5515870 \\ \mathrm{C} & -0.1717320 & -2.7111030 & -1.2107640 \\ \mathrm{C} & 2.3698620 & -3.7695930 & -0.8159970 \\ \mathrm{H} & 3.3699000 & -4.1823860 & -0.6920620 \\ \mathrm{C} & -3.9087740 & 0.0390980 & 0.6701770 \\ \mathrm{C} & 3.2783870 & -1.5099910 & -0.1447210 \\ \mathrm{C} & -4.3266190 & -0.6656420 & 1.8084440 \\ \mathrm{C} & -4.1512080 & -2.1416090 & 2.0752750 \\ \mathrm{C} & -5.3841960 & -0.9105280 & 3.9885440 \\ \mathrm{C} & -5.0082590 & -0.0074190 & 2.8423870 \\ \mathrm{C} & -3.3573950 & -2.2994990 & 3.3904950\end{array}$




$\begin{array}{rrr}-3.2647120 & -3.3671570 & 3.6197890 \\ -2.3461370 & -1.9087840 & 3.2490280 \\ -4.0915480 & -1.5539280 & 4.5361790 \\ -4.3513960 & -2.2425330 & 5.3482480 \\ -3.4514500 & -0.7764680 & 4.9637490 \\ -5.9028330 & -0.3528570 & 4.7756490 \\ -3.6316050 & -2.6461950 & 1.2572620 \\ -6.2836020 & -2.0390080 & 3.4393950 \\ -6.5168940 & -2.7369910 & 4.2514540 \\ -7.2320200 & -1.6161020 & 3.0944210 \\ -5.5503700 & -2.7641010 & 2.2810380 \\ -5.4342360 & -3.8310420 & 2.5021920 \\ -6.1230170 & -2.6878210 & 1.3514870 \\ -4.1563440 & 1.4187900 & 0.6088220 \\ -3.6919880 & 2.3793640 & -0.4616670 \\ -5.1203640 & 3.5235500 & 1.3702780 \\ -4.8738950 & 2.0590690 & 1.6304100 \\ -4.9310660 & 3.0394460 & -1.1058380 \\ -4.5941460 & 3.8170860 & -1.8013970 \\ -5.4876610 & 2.3025330 & -1.6940130 \\ -5.8306370 & 3.6471570 & 0.0037010 \\ -6.0400180 & 4.7030030 & -0.2009130 \\ -6.7930280 & 3.1282040 & 0.0487330 \\ -5.7323690 & 3.9663920 & 2.1632160 \\ -3.0753490 & 1.8790720 & -1.2140600 \\ -3.7602660 & 4.2425380 & 1.2584410 \\ -3.9320350 & 5.2802790 & 0.9496530 \\ -3.2737560 & 4.2732530 & 2.2378030 \\ -2.8733450 & 3.4948460 & 0.2318770 \\ -2.4895570 & 4.1834490 & -0.5313020 \\ -2.0131370 & 3.0436090 & 0.7307490 \\ -5.3009970 & 1.3493350 & 2.7473860 \\ -5.8546050 & 1.8502490 & 3.5401910 \\ 4.7573350 & 0.3566720 & -0.6717100 \\ 5.1093760 & 1.3545410 & -1.7471520 \\ 3.1267820 & -0.1842490 & -2.3816080 \\ 3.6878650 & -0.4835010 & -1.0117200 \\ 5.4698380 & 0.5492160 & -3.0162090 \\ 5.7234480 & 1.2447410 & -3.8238920 \\ 6.3577720 & -0.0593810 & -2.8203950 \\ 4.2659150 & -0.3448280 & -3.4144090 \\ 3.8847290 & -0.0645310 & -4.4027400 \\ 4.5646260 & -1.3959630 & -3.4702580 \\ 2.2916990 & -0.8425180 & -2.6333320 \\ 5.9501120 & 1.9858080 & -1.4393890 \\ 2.6668090 & 1.2872100 & -2.3911190\end{array}$

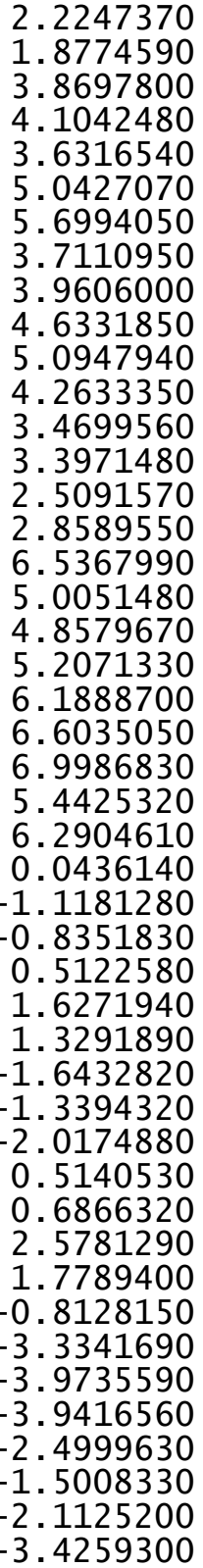

1.5299600

1. 3989870

2.2134560

2.8486320

2.8871820

$-0.8415820$

$-1.1898840$

$-2.7409480$

$-1.6767420$

$-1.1062380$

$-1.3642230$

$-0.0790440$

$-2.0742640$

$-2.8646670$

$-1.5567460$

$-3.3716730$

$-0.5161090$

$-3.5789330$

$-4.3620040$

$-4.0791800$

$-2.6540220$

$-2.9455080$

$-2.7283310$

0.1717630

0.8079100

$-4.0893330$

$-4.9922120$

$-6.4649280$

$-6.8566030$

$-6.0932010$

$-4.6149060$

$-7.0809820$

$-4.8895790$

$-4.6558540$

$-6.6110990$

$-7.9338390$

$-6.2103580$

$-6.5308700$

$-6.6335510$

$-1.3092530$

$-1.3123590$

$-2.7372200$

$-3.2816490$

$-2.4997720$

$-1.9894370$

$-0.6378210$
$-3.3642080$

$-1.6444510$

$-2.0822470$

$-2.9446180$

$-1.2521800$

1.3921660

2.7027880

2.1098990

1.0677490

3.8148990

4.7747520

3.8959260

3.4820080

4.2378130

3.4701730

1.8448700

2.9147820

2.2248230

2.9767090

1.2719510

2. 6182270

3.5897920

1.8862740

0.5270500

0.7797350

$-1.4103280$

$-1.7809040$

$-1.4899990$

$-2.0903420$

$-1.3799820$

$-1.2114840$

$-1.8968480$

$-2.8509760$

$-1.2534710$

-3.1606620

$-2.0061230$

$-1.9121320$

$-0.3837090$

$-0.4055360$

$-2.8315140$

$-4.1965910$

$-4.7673020$

$-3.9786960$

$-2.6958100$

$-4.8707200$ 


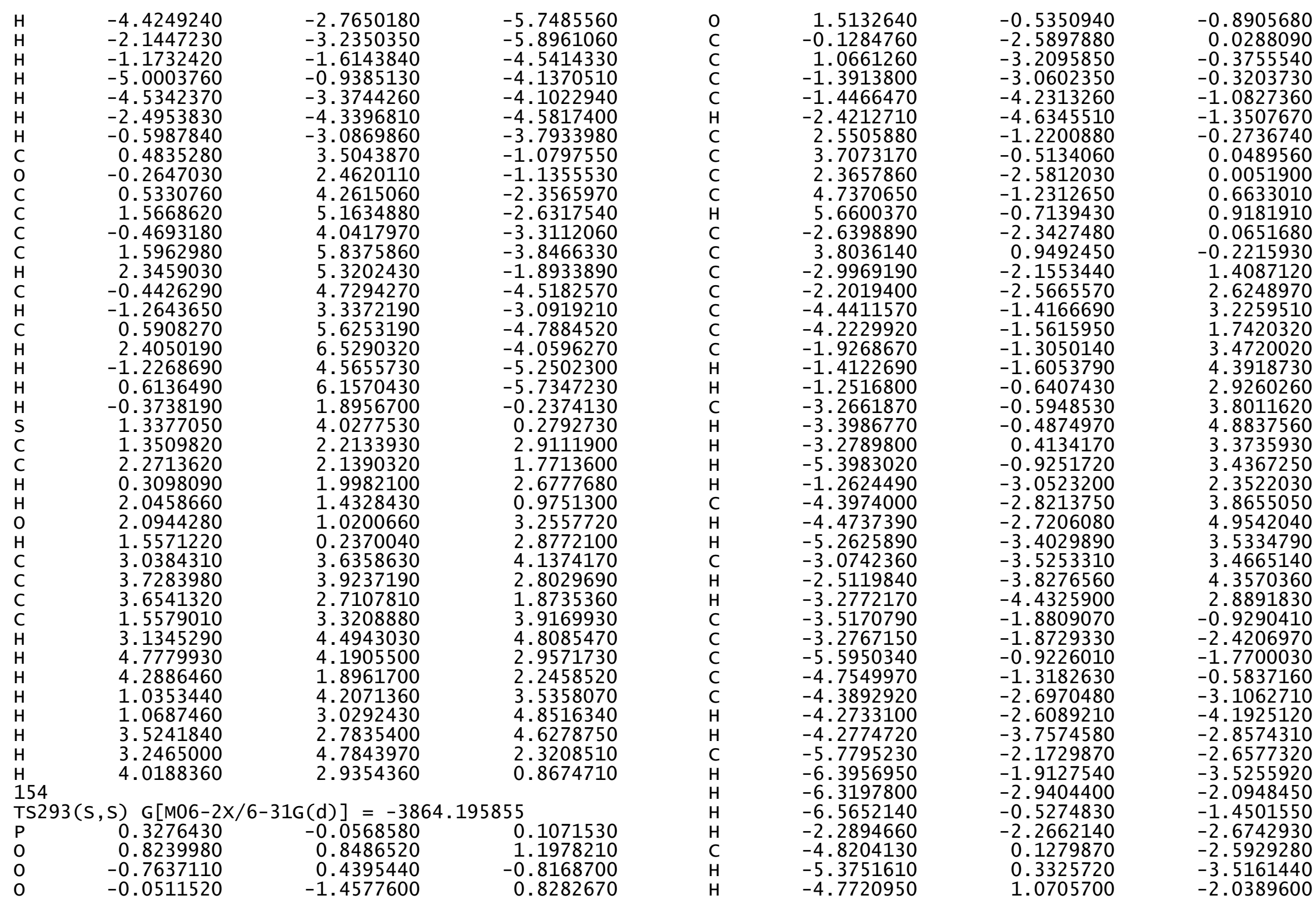




$\begin{array}{ll}\mathrm{C} & -3.4019370 \\ \mathrm{H} & -3.2047190 \\ \mathrm{H} & -2.6319950 \\ \mathrm{C} & -5.1169880 \\ \mathrm{H} & -6.0808850 \\ \mathrm{C} & 3.7564780 \\ \mathrm{C} & 3.6412520 \\ \mathrm{C} & 3.4767490 \\ \mathrm{C} & 3.6602960 \\ \mathrm{C} & 4.7820520 \\ \mathrm{H} & 4.7012330 \\ \mathrm{H} & 5.7464920 \\ \mathrm{C} & 4.6815100 \\ \mathrm{H} & 4.5492640 \\ \mathrm{H} & 5.5985790 \\ \mathrm{H} & 3.4139280 \\ \mathrm{H} & 3.7123620 \\ \mathrm{C} & 2.1988340 \\ \mathrm{H} & 2.0851940 \\ \mathrm{H} & 1.3273100 \\ \mathrm{C} & 2.3052220 \\ \mathrm{H} & 2.2582480 \\ \mathrm{H} & 1.4694960 \\ \mathrm{C} & 4.1693200 \\ \mathrm{C} & 4.5064450 \\ \mathrm{C} & 4.2111870 \\ \mathrm{C} & 4.0467870 \\ \mathrm{C} & 3.3960040 \\ \mathrm{H} & 3.6958420 \\ \mathrm{H} & 2.4703790 \\ \mathrm{C} & 3.1839500 \\ \mathrm{H} & 3.3095940 \\ \mathrm{H} & 2.1758770 \\ \mathrm{H} & 4.0665680 \\ \mathrm{H} & 4.6262480 \\ \mathrm{C} & 5.6232130 \\ \mathrm{H} & 5.7317760 \\ \mathrm{H} & 6.3826580 \\ \mathrm{C} & 5.8172220 \\ \mathrm{H} & 6.0854050 \\ \mathrm{H} & 6.6280810 \\ \mathrm{C} & 4.0172030 \\ \mathrm{H} & 4.1191430 \\ \mathrm{C} & 3.4025250 \\ \mathrm{C} & 3.7092180 \\ \mathrm{C} & \\ & \end{array}$

$\begin{array}{rr}-0.4097160 & -2.9066020 \\ -0.3755010 & -3.9839180 \\ 0.1942190 & -2.4175620 \\ -1.1724460 & 0.7498670 \\ -0.7385810 & 1.0137060 \\ 2.8294370 & -1.7732580 \\ 3.1909360 & -3.2328640 \\ 0.6466370 & -2.7903230 \\ 1.4521040 & -1.5250440 \\ 2.4664390 & -3.9819090 \\ 2.6816890 & -5.0533270 \\ 2.8577480 & -3.6444040 \\ 0.9414860 & -3.7144110 \\ 0.3910360 & -4.6525940 \\ 0.5687560 & -3.2481520 \\ -0.4244900 & -2.5863400 \\ 4.2749180 & -3.3767250 \\ 1.1309710 & -3.5061790 \\ 0.5726790 & -4.4421480 \\ 0.9018080 & -2.8868980 \\ 2.6516460 & -3.7862390 \\ 2.8580810 & -4.8614850 \\ 3.1857680 & -3.3255560 \\ 3.2304530 & 0.5579840 \\ 4.0555510 & 1.7735820 \\ 1.5374460 & 2.2916180 \\ 1.8572930 & 0.8230210 \\ 3.8773040 & 2.8298300 \\ 4.3986770 & 3.7464420 \\ 4.3383610 & 2.4744780 \\ 2.3660040 & 3.0960000 \\ 2.1380330 & 4.1605980 \\ 2.0511220 & 2.8151370 \\ 0.4740330 & 2.4942350 \\ 5.1118480 & 1.5094560 \\ 1.9940980 & 2.7233800 \\ 1.8331310 & 3.8019220 \\ 1.3797430 & 2.2284610 \\ 3.4931090 & 2.3687720 \\ 4.0720060 & 3.2595950 \\ 3.6179710 & 1.6446500 \\ 3.7195320 & -0.7350630 \\ 4.7857340 & -0.9357000 \\ -3.2577590 & 0.6639650 \\ -4.6798300 & 1.1573820 \\ -4.7367550 & 2.6446700 \\ & \end{array}$

5.0820070
5.6576800
4.5920520
3.9900880
3.6941710
5.8003870
6.0587920
2.3008250
2.9283300
4.9861370
6.4960110
-0.2920320
-0.3186380
0.6598930
2.0944760
2.1450890
0.9712960
-0.0236870
0.6520230
2.7364780
2.0964730
-1.3327750
0.2963690
2.5269260
3.0993140
-3.3426210
-2.8575880
-4.6503170
-5.1685030
-5.3704070
-6.3885700
-4.6099520
-6.5932850
-4.9656230
-7.1036350
-6.7814290
-7.1496780
-8.0581260
-1.9630950
-2.5820220
-0.3900620
-0.1598200
-0.8964690
-0.0431390
1.0402410
1.1723020
2.9222280

1. 6831640

0.9755560

0.5790470

2. 9798610

3. 2605980

0.9935370

1. 0179110

3. 2142570

3.7320140

1.9670450

$-1.5177810$

$-2.3407940$

$-1.7385650$

$-1.6416300$

$-1.8258810$

$-1.1840990$

$-3.3768890$

$-2.3269810$

$-2.4021260$

$-2.9021990$

$-2.3785760$

$-0.7384130$

$-0.6713350$

$-1.4797320$

$-0.3645370$

0.1262960

0.2238720

1.2791240

$-0.2487960$

1.8517330

1.6347460

0.3218530

$-1.0640030$

1.3730640

2. 6715260

$-0.0525770$

1. 8188100

$-0.3114470$

$-1.5537100$

0.7591620

$-0.6894980$

1. 1862000

$-1.1430980$

0.7206310

0.8620510 


$\begin{array}{lrrr}\mathrm{C} & -0.0353530 & 6.0181060 & 0.7184610 \\ \mathrm{C} & -0.2691840 & 6.0071350 & -0.7930380 \\ \mathrm{C} & 0.2547300 & 4.7169450 & -1.4298990 \\ \mathrm{C} & -0.7671400 & 4.8500780 & 1.3830290 \\ \mathrm{H} & -0.3842230 & 6.9611370 & 1.1491330 \\ \mathrm{H} & 0.2205270 & 6.8626010 & -1.2672530 \\ \mathrm{H} & 1.3538420 & 4.7037130 & -1.4389140 \\ \mathrm{H} & -1.8526800 & 4.9704810 & 1.2749160 \\ \mathrm{H} & -0.5457100 & 4.7985530 & 2.4540280 \\ \mathrm{H} & 1.0388210 & 5.9414990 & 0.9281170 \\ \mathrm{H} & -1.3438440 & 6.0956450 & -0.9935360 \\ \mathrm{H} & -0.0655270 & 4.6238410 & -2.4714240 \\ \mathrm{I} 54 & & & \\ \mathrm{TS} 294(\mathrm{~S} & \mathrm{S}) & \mathrm{G}[\mathrm{M} 06-2 \mathrm{X} & \\ \mathrm{P} & 0.1546330 & 0.4760230 & 0.8722500 \\ \mathrm{O} & -0.6631880 & 1.0080060 & 2.0103000 \\ \mathrm{O} & 0.6676100 & -0.9445240 & 0.9494770 \\ \mathrm{O} & 1.3489590 & 1.5400720 & 0.6245670 \\ \mathrm{O} & -0.6478510 & 0.5288070 & -0.5500520 \\ \mathrm{C} & 2.0301030 & 1.4148780 & -0.5802320 \\ \mathrm{C} & 1.4285100 & 1.9227650 & -1.7344670 \\ \mathrm{C} & 3.2711950 & 0.7823550 & -0.5839160 \\ \mathrm{C} & 3.9112330 & 0.6757290 & -1.8170710 \\ \mathrm{H} & 4.8889140 & 0.1980450 & -1.8513640 \\ \mathrm{C} & -0.9497180 & 1.7999010 & -1.0234650 \\ \mathrm{C} & -2.2570150 & 2.2627670 & -0.8955550 \\ \mathrm{C} & 0.0803380 & 2.5518380 & -1.6036440 \\ \mathrm{C} & -2.5185370 & 3.5668810 & -1.3222810 \\ \mathrm{H} & -3.5308750 & 3.9563110 & -1.2329750 \\ \mathrm{C} & 3.8979660 & 0.2435170 & 0.6552420 \\ \mathrm{C} & -3.3266760 & 1.3752260 & -0.3590230 \\ \mathrm{C} & 4.2502570 & 1.0948430 & 1.7124480 \\ \mathrm{C} & 3.9919760 & 2.5798440 & 1.8042770 \\ \mathrm{C} & 5.2321300 & 1.6418070 & 3.8728550 \\ \mathrm{C} & 4.9356020 & 0.5957080 & 2.8295830 \\ \mathrm{C} & 3.1536620 & 2.8411700 & 3.0747730 \\ \mathrm{H} & 2.9987210 & 3.9211730 & 3.1784030 \\ \mathrm{H} & 2.1685920 & 2.3817630 & 2.9564300 \\ \mathrm{C} & 3.8928530 & 2.2714240 & 4.3142910 \\ \mathrm{H} & 4.0935780 & 3.0606360 & 5.0477900 \\ \mathrm{H} & 3.2820160 & 1.5135120 & 4.8137270 \\ \mathrm{H} & 5.7560670 & 1.2057070 & 4.7300740 \\ \mathrm{H} & 3.4702410 & 2.9595680 & 0.9226100 \\ \mathrm{C} & 6.0871920 & 2.7485710 & 3.2188720 \\ \mathrm{H} & 6.2608590 & 2.3453790 & 2.9456070 \\ \mathrm{H} & 7.0656110 & & \end{array}$

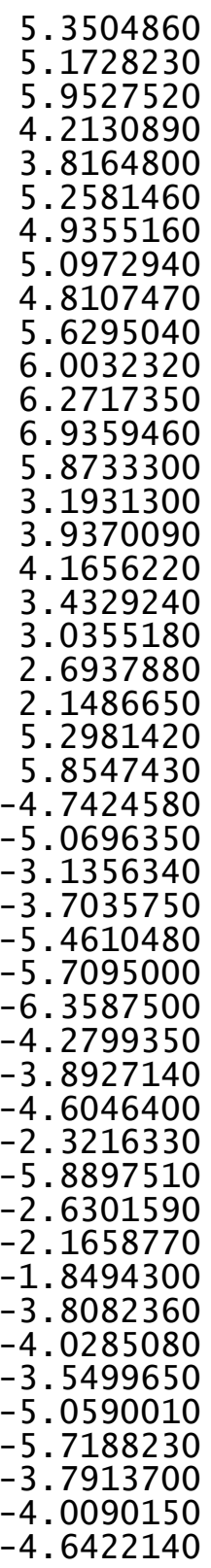

3.2971910

4. 3742700

3. 1483130

$-1.1195950$

$-2.2204110$

$-3.0705860$

$-1.6001650$

$-2.8917590$

$-3.7637500$

$-2.2051330$

$-3.3153990$

$-4.3750040$

$-2.7430510$

$-1.8422830$

$-3.8656820$

$-4.9212690$

$-3.8119140$

$-3.2833120$

$-4.0728520$

$-2.8150760$

0.7457530

$-1.1229970$

$-0.5946830$

$-1.7361550$

$-0.2388330$

0.2269440

$-1.1129730$

$-1.9133810$

$-0.5003690$

$-0.2568610$

$-0.6676170$

0.7675020

0.4038030

$-2.3453430$

$-1.6825700$

$-2.0399240$

$-1.6676670$

$-2.5965540$

$-3.3317390$

$-3.1652490$

0.8604500

1. 3579320

2. 8830330

1. 6806090

1. 4589300
1.9691590

2. 0645860

1.0675790

0.7597220

$-0.1969990$

1.7789680

1.8622920

$-0.7396170$

$-1.3393170$

$-1.4061710$

0.4476170

0.3725610

0.4454970

2. 6278170

$-1.0125310$

1.7295800

1. 5417530

2. 6990090

0.6129410

$-0.0681450$

1. 0453410

2. 8978000

3.7544220

$-0.6152190$

$-1.5464620$

$-2.3945480$

$-1.0750580$

$-2.9058400$

$-3.6115240$

$-2.7792350$

$-3.4343320$

$-4.3735450$

$-3.6402300$

$-2.7385460$

$-1.1510330$

$-2.2022500$

$-3.1285270$

$-1.4381250$

$-1.7832590$

$-2.5662550$

$-0.8837650$

1. 2714220

2. 5312370

1. 7141740

0.8263200

3.6318080 


$\begin{array}{rrr}-5.1055450 & 1.8242960 & 4.5552840 \\ -4.2351020 & 0.4646670 & 3.8417360 \\ -3.5176540 & 2.4154090 & 3.1606790 \\ -3.4713420 & 3.3022030 & 3.8031600 \\ -2.5380400 & 1.9366210 & 3.2082720 \\ -2.9649140 & 3.5022350 & 1.3569390 \\ -6.5299960 & 0.6897250 & 2.8404620 \\ -5.1138640 & 3.6837170 & 1.7309060 \\ -4.9892340 & 4.5654140 & 2.3690550 \\ -5.3407200 & 4.0441140 & 0.7220790 \\ -6.2607190 & 2.7799350 & 2.2589700 \\ -6.6775310 & 3.1839930 & 3.1883930 \\ -7.0786670 & 2.7282720 & 1.5341350 \\ -5.4282240 & -0.2727250 & 0.5540210 \\ -6.2527520 & -0.8968500 & 0.8976920 \\ -0.2056700 & 3.8710520 & -1.9831040 \\ 0.8291660 & 4.8595990 & -2.4607440 \\ 0.7427010 & 6.1375960 & -1.6060170 \\ -0.6880180 & 6.7296970 & -1.5682860 \\ -1.7268380 & 5.8080120 & -2.2392340 \\ -1.5084570 & 4.3734420 & -1.8365650 \\ 0.6423190 & 5.1221210 & -3.5121240 \\ 1.4540790 & 6.8756270 & -1.9884890 \\ -0.7128830 & 7.7062810 & -2.0613550 \\ -1.6261550 & 5.8954020 & -3.3296690 \\ 1.8349170 & 4.4329330 & -2.4113280 \\ 1.0656260 & 5.8831620 & -0.5918110 \\ -0.9881350 & 6.8941880 & -0.5291800 \\ -2.7422830 & 6.1302500 & -1.9900140 \\ 2.0746680 & 1.7481810 & -2.9741010 \\ 3.3369520 & 1.1335370 & -3.0034630 \\ 1.3633420 & 2.1031820 & -4.2674930 \\ 2.3052510 & 2.2193690 & -5.4639890 \\ 3.2208840 & 1.0010520 & -5.5364080 \\ 4.1103530 & 0.9570230 & -4.2968620 \\ 1.7188090 & 2.3255910 & -6.3822470 \\ 0.6257760 & 1.3114700 & -4.4659600 \\ 0.7849430 & 3.0210550 & -4.1553820 \\ 2.6081490 & 0.0915580 & -5.5868210 \\ 3.8393770 & 1.0257780 & -6.4392900 \\ 4.6722460 & 0.0175020 & -4.2548720 \\ 4.8587170 & 1.7586960 & -4.3695690 \\ 2.9165780 & 3.1266340 & -5.3646230 \\ -0.3159740 & -3.6191200 & -0.6710640 \\ -0.3799580 & -2.5580120 & -0.8701480 \\ -2951330 & -4.5611370 & -1.8192410\end{array}$
$-1.2748550$
0.7219120
$-1.2356530$
$-2.0657810$
0.7639420
1.4747390
$-0.2151320$
$-2.0024470$
1. 5595650
$-0.1839810$
0.4521480
$-1.1758270$
$-1.2656910$
$-2.1898050$
$-0.2343760$
$-1.9970720$
$-2.0572670$
$-1.5559670$
$-2.8914350$
$-3.5699160$
$-3.5470970$
$-1.4256680$
$-2.9499680$
$-4.6072970$
$-4.2141870$
$-0.8666650$
$-3.4125610$
$-3.0528050$
$-3.9034050$

154

TS295(S, S) G[M06-2X/6-31G(d) $]=-3864.195694$

$\begin{array}{lrrr}\mathrm{P} & 0.2954100 & -0.0445230 & 0.2472420 \\ \mathrm{O} & 0.7123300 & 0.9588750 & 1.2840880 \\ \mathrm{O} & -0.7820980 & 0.3342780 & -0.7464220 \\ \mathrm{O} & -0.0471500 & -1.4080570 & 1.0535900 \\ \mathrm{O} & 1.5358620 & -0.5347450 & -0.6749760 \\ \mathrm{C} & -0.0386450 & -2.5946770 & 0.3329740 \\ \mathrm{C} & 1.1991160 & -3.1808440 & 0.0190740 \\ \mathrm{C} & -1.2625930 & -3.1453210 & -0.0375280 \\ \mathrm{C} & -1.2317830 & -4.3585270 & -0.7325130 \\ \mathrm{H} & -2.1741830 & -4.8172140 & -1.0252760 \\ \mathrm{C} & 2.5832810 & -1.1207150 & 0.0214300 \\ \mathrm{C} & 3.6981070 & -0.3383010 & 0.3146280 \\ \mathrm{C} & 2.4538200 & -2.4650480 & 0.3967280 \\ \mathrm{C} & 4.7470720 & -0.9622490 & 0.9949100\end{array}$

$-1.9720070$

$-5.5484290$

$-4.4380490$

$-5.6307550$

$-3.6668540$

$-6.2859360$

$-7.1617470$

$-5.2139110$

$-6.9585270$

$-1.8724740$

$-1.8641410$

$-1.9629210$

$-1.6357820$

$-0.6698280$

0.0815840

$-3.2023950$

$-3.6733940$

$-2.5755850$

$-2.8534290$

$-3.9795610$

$-3.9634950$

$-1.7495180$

$-3.7557300$

$-2.4340670$

$-2.3198060$

$-2.3198060$

$-4.5640520$

$-2.7756830$

$-3.0668970$

$-1.2342740$

$-3.8611840$

$-2.6523000$

$-4.0092410$

$-3.1859200$

$-4.5936080$

$-4.8609190$

$-0.0565740$

0.7361810

3.1277290

1.9928600

2.8644270

1.1177900

3. 3342420

2.8561280

4.5224930

3.2352410

2.1700650

4.2578040

5.2900700

3.4257390

2.4483080

3.9800200

5.1489950

4.9127900

2.8547780

1.1994460
$-6.4032250$

$-3.9797700$

$-1.3459650$

0.0445230

0.6749760

(1)

4.7470720

$-0.9622490$

0.9949100

2. 2193690

1. 3114700

3.0210550

0.0175020

1.7586960

$-2.5580120$

$-1.8192410$

6. 4392900

$-4.2548720$

$-0.2951330$ 


$\begin{array}{lrrr}\mathrm{H} & 5.6446590 & -0.3886280 & 1.2183140 \\ \mathrm{C} & -2.5572590 & -2.4658140 & 0.2534280 \\ \mathrm{C} & 3.7367070 & 1.1022560 & -0.0662650 \\ \mathrm{C} & -2.9763240 & -2.2163230 & 1.5689000 \\ \mathrm{C} & -2.2137840 & -2.5170900 & 2.8374110 \\ \mathrm{C} & -4.5261030 & -1.4433120 & 3.2823070 \\ \mathrm{C} & -4.2407300 & -1.6634680 & 1.8191990 \\ \mathrm{C} & -2.0321000 & -1.1975320 & 3.6187630 \\ \mathrm{H} & -1.5449460 & -1.4207550 & 4.5747950 \\ \mathrm{H} & -1.3640640 & -0.5343130 & 3.0628840 \\ \mathrm{C} & -3.4146090 & -0.5336430 & 3.8510420 \\ \mathrm{H} & -3.5966880 & -0.3678490 & 4.9189630 \\ \mathrm{H} & -3.4555830 & 0.4452040 & 3.3615660 \\ \mathrm{H} & -5.5125040 & -0.9868360 & 3.4266130 \\ \mathrm{H} & -1.2426320 & -2.9721990 & 2.6311700 \\ \mathrm{C} & -4.4444770 & -2.8048200 & 4.0057800 \\ \mathrm{H} & -4.5696080 & -2.6451000 & 5.0828610 \\ \mathrm{H} & -5.2681130 & -3.4453190 & 3.6763700 \\ \mathrm{C} & -3.0754740 & -3.4667080 & 3.7000670 \\ \mathrm{H} & -2.5371660 & -3.6905460 & 4.6277780 \\ \mathrm{H} & -3.2132130 & -4.4141170 & 3.1699190 \\ \mathrm{C} & -3.4147120 & -2.1078950 & -0.7992390 \\ \mathrm{C} & -3.1176140 & -2.1816270 & -2.2787540 \\ \mathrm{C} & -5.5003510 & -1.3054740 & -1.7744000 \\ \mathrm{C} & -4.6896300 & -1.5849590 & -0.5354390 \\ \mathrm{C} & -4.1638460 & -3.1000300 & -2.9489120 \\ \mathrm{H} & -4.0107840 & -3.0741400 & -4.0338610 \\ \mathrm{H} & -4.0127820 & -4.1362640 & -2.6289730 \\ \mathrm{C} & -5.5929860 & -2.6174540 & -2.5840070 \\ \mathrm{H} & -6.1874950 & -2.4436600 & -3.4878390 \\ \mathrm{H} & -6.1175860 & -3.3729260 & -1.9909840 \\ \mathrm{H} & -6.4991480 & -0.9378810 & -1.5157170 \\ \mathrm{H} & -2.1043980 & -2.5424260 & -2.4709370 \\ \mathrm{C} & -4.7440240 & -0.2736180 & -2.6371770 \\ \mathrm{H} & -5.2711730 & -0.1574900 & -3.5914120 \\ \mathrm{H} & -4.7622480 & 0.7043070 & -2.1464680 \\ \mathrm{C} & -3.2907040 & -0.7597930 & -2.8632910 \\ \mathrm{H} & -3.0535510 & -0.7849430 & -3.9328070 \\ \mathrm{H} & -2.5689910 & -0.0885280 & -2.3882640 \\ \mathrm{C} & -5.1107020 & -1.3765720 & 0.7722560 \\ \mathrm{H} & -6.1032180 & -0.9744320 & 0.9728590 \\ \mathrm{C} & 3.6613610 & 2.8536650 & -1.7605780 \\ \mathrm{C} & 3.5797950 & 3.0952750 & -3.2470380 \\ \mathrm{C} & 3.5090470 & 0.5879410 & -2.6106450 \\ \mathrm{C} & 3.6156850 & 1.4966080 & -1.4084810 \\ \mathrm{C} & 4.7747340 & 2.3669430 & -3.9021570 \\ & & & \end{array}$

4.7203790
5.7099200
4.7303770
4.6533980
5.6463200
3.4851440
3.6092930
2.2359780
2.1770740
1.3551140
2.2872400
2.2685400
1.4144700
3.9769010
4.2383380
4.0364780
3.9043620
3.1027880
3.3474390
2.1695250
2.9515230
3.0594320
1.9661290
3.9324080
4.3206100
5.4141220
5.4986510
6.2133870
5.5523410
5.7640520
6.3807590
3.8484160
3.9119040
4.6533210
5.7655100
6.0245410
4.7529170
3.4621770
3.5022660
5.4855070
6.8392500
4.8223180
3.3561370
6.6723220
6.3624330
4.6753160

2. 4946980

2.8262200

0.8641920

0.2373510

0.5705190

$-0.4658850$

4.1668260

0.9570150

0.3230210

0.7377250

2.4545630

2.5742200

2. 9849160

.4507690

4. 3791970

1.8963410

2.0976700

. 2297680

4.8348530

4.6164670

2.7352370

.5966590

2.3523220

0.8451720

5.4172230

2.4470640

2. 3697780

1.8335380

3.9237090

4.5781160

4.0337690

3. 8318530

4.8830170

$-2.2863770$

$-2.9876020$

$-4.3529040$

$-5.2304300$

$-4.4230680$

$-3.0417590$

$-3.1358740$

$-4.8710180$

$-6.0149100$

$-4.2905290$

$-2.3750210$

$-4.1699930$

$-5.7397280$
$-4.9891370$

$-3.5680780$

$-3.5185870$

$-4.4140310$

$-2.3242720$

$-3.4742350$

$-3.4000880$

$-4.2918370$

$-2.7903080$

$-3.7963390$

$-4.8855250$

$-3.4055370$

0.5441880

1. 7026290

2.4032280

0.9109470

2.7364720

3. 6172980

2. 3182350

3. 1155900

4.1973940

2. 8384420

2.6808050

1. 3625520

2. 8365060

3.9263450

2. 4077380

2. 3771070

3. 2300920

1.6706730

$-0.7873720$

$-1.0676220$

1.4097670

2.1472030

1. 4921940

1.4738140

1.7353440

1.1287130

3. 1999820

2. 0069640

2. 2338970

2. 8210360

2. 1420030

0.4665190

0.5083070 


\begin{tabular}{|c|c|c|c|}
\hline $\begin{array}{l}\mathrm{H} \\
\mathrm{C} \\
\mathrm{C} \\
\mathrm{C} \\
\mathrm{C} \\
\mathrm{C} \\
\mathrm{C} \\
\mathrm{H} \\
\mathrm{H} \\
\mathrm{H} \\
\mathrm{H} \\
\mathrm{H} \\
\mathrm{H} \\
\mathrm{H} \\
\mathrm{H} \\
\mathrm{C} \\
\mathrm{O} \\
\mathrm{C} \\
\mathrm{C} \\
\mathrm{C} \\
\mathrm{C} \\
\mathrm{H} \\
\mathrm{C} \\
\mathrm{H} \\
\mathrm{C} \\
\mathrm{H} \\
\mathrm{H} \\
\mathrm{H} \\
\mathrm{H} \\
\mathrm{S} \\
\mathrm{C} \\
\mathrm{C} \\
\mathrm{H} \\
\mathrm{H} \\
\mathrm{O} \\
\mathrm{H} \\
\mathrm{C} \\
\mathrm{C} \\
\mathrm{C} \\
\mathrm{C} \\
\mathrm{H} \\
\mathrm{H} \\
\mathrm{H} \\
\mathrm{H} \\
\mathrm{H} \\
\mathrm{H}\end{array}$ & $\begin{array}{r}2.5822920 \\
1.1909000 \\
2.4268380 \\
2.2553320 \\
0.9582150 \\
0.0403400 \\
-0.0314320 \\
2.5831280 \\
3.1316280 \\
1.1946330 \\
0.4436230 \\
3.3196320 \\
2.2417390 \\
0.4053350 \\
-0.9594350 \\
-3.4640170 \\
-2.9472800 \\
-4.8092700 \\
-5.3311950 \\
-5.5615160 \\
-6.5873200 \\
-4.7466930 \\
-6.8197100 \\
-5.1539260 \\
-7.3341510 \\
-6.9834140 \\
-7.4006130 \\
-8.3167110 \\
-2.0217650 \\
-2.7052150 \\
-0.5921580 \\
-0.3152030 \\
-1.0800850 \\
-0.1496560 \\
0.8446740 \\
1.0106120 \\
-0.3252400 \\
-0.5128440 \\
0.0762980 \\
-1.0353820 \\
-0.7200860 \\
-0.0403230 \\
1.1749090 \\
-2.1205520 \\
-0.8461720 \\
0.7442170\end{array}$ & $\begin{array}{l}-4.9824600 \\
-4.3757720 \\
-5.0546560 \\
-5.3207720 \\
-6.1002580 \\
-6.2533770 \\
-4.9697750 \\
-6.0111010 \\
-5.8597620 \\
-7.0950720 \\
-7.0449830 \\
-4.4482890 \\
-4.3514680 \\
-5.5776080 \\
-6.5759930 \\
2.2641130 \\
1.2371580 \\
2.6243150 \\
1.9063760 \\
3.6586220 \\
2.2200740 \\
1.1023170 \\
3.9631380 \\
4.2141130 \\
3.2462570 \\
1.6612650 \\
4.7618560 \\
3.4892090 \\
0.8895470 \\
3.1406670 \\
3.5476760 \\
3.3929740 \\
2.6992700 \\
2.3863110 \\
3.4077980 \\
2.4258790 \\
6.0387320 \\
5.8979070 \\
4.5819520 \\
4.9009220 \\
6.9993660 \\
6.7308140 \\
4.6101630 \\
4.9708980 \\
4.9441390 \\
6.0196200\end{array}$ & $\begin{array}{r}1.4116190 \\
-0.7149300 \\
-1.2518440 \\
-2.7586480 \\
-3.0843310 \\
-1.8550600 \\
-1.0709120 \\
-0.7313550 \\
-3.1312140 \\
-3.4742020 \\
-1.2089100 \\
-1.0772900 \\
-3.2667910 \\
-3.8705890 \\
-2.1608550 \\
-0.5290270 \\
0.0534700 \\
-0.0132300 \\
1.0713940 \\
-0.5833110 \\
1.5761980 \\
1.5026680 \\
-0.0795220 \\
-1.4209500 \\
1.0006910 \\
2.4186750 \\
-0.5289580 \\
1.3935370 \\
-0.3260050 \\
-1.7554770 \\
0.5985540 \\
-0.8344290 \\
1.0800220 \\
-1.2036420 \\
0.6146460 \\
0.8409360 \\
0.3652820 \\
-1.1461270 \\
-1.6613990 \\
1.1019220 \\
0.7085180 \\
-1.6747930 \\
-1.6396690 \\
0.9533180 \\
2.1794470\end{array}$ \\
\hline
\end{tabular}

$\begin{array}{llll}\mathrm{H} & -1.5835470 & 5.9287290 & -1.3821110 \\ \mathrm{H} & -0.2091220 & 4.3932000 & -2.7000510 \\ 154 & & \end{array}$

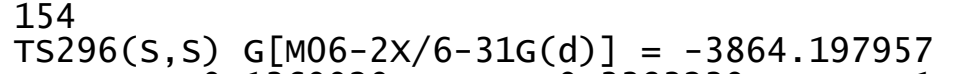

$\begin{array}{lrrr}\text { TS296(S }, \mathrm{S}) \mathrm{G}[\mathrm{M} 06-2 \mathrm{X} / 6-31 \mathrm{G}(\mathrm{d})]=-3864.197957 \\ \mathrm{P} & -0.1360020 & -0.3383230 & 1.0004170 \\ \mathrm{O} & 0.6860820 & -0.6997670 & 2.2004780 \\ \mathrm{O} & -0.6414020 & 1.0813080 & 0.8694480 \\ \mathrm{O} & -1.3395740 & -1.4169610 & 0.9109540 \\ \mathrm{O} & 0.6634230 & -0.5992110 & -0.4007810 \\ \mathrm{C} & -2.0092300 & -1.4635520 & -0.3073600 \\ \mathrm{C} & -1.3993690 & -2.1554100 & -1.3610170 \\ \mathrm{C} & -3.2359330 & -0.8147520 & -0.4239580 \\ \mathrm{C} & -3.8681060 & -0.8765190 & -1.6694810 \\ \mathrm{H} & -4.8390240 & -0.3989120 & -1.7853870 \\ \mathrm{C} & 0.9697180 & -1.9220920 & -0.6915640 \\ \mathrm{C} & 2.2885740 & -2.3479850 & -0.5430170 \\ \mathrm{C} & -0.0534350 & -2.7615520 & -1.1358810 \\ \mathrm{C} & 2.5520310 & -3.6861780 & -0.8199540 \\ \mathrm{H} & 3.5758000 & -4.0452970 & -0.7273810 \\ \mathrm{C} & -3.8736680 & -0.1010460 & 0.7181270 \\ \mathrm{C} & 3.3600830 & -1.3862850 & -0.1605690 \\ \mathrm{C} & -4.2653350 & -0.7980030 & 1.8705360 \\ \mathrm{C} & -4.0377890 & -2.2615080 & 2.1653850 \\ \mathrm{C} & -5.3112480 & -1.0379220 & 4.0569130 \\ \mathrm{C} & -4.9692410 & -0.1446090 & 2.8926000 \\ \mathrm{C} & -3.2373470 & -2.3656260 & 3.4819140 \\ \mathrm{H} & -3.1070810 & -3.4246910 & 3.7319220 \\ \mathrm{H} & -2.2404480 & -1.9428810 & 3.3305100 \\ \mathrm{C} & -3.9956080 & -1.6238280 & 4.6142490 \\ \mathrm{H} & -4.2293360 & -2.3047930 & 5.4405640 \\ \mathrm{H} & -3.3828740 & -0.8156380 & 5.0246260 \\ \mathrm{H} & -5.8483790 & -0.4837730 & 4.8340480 \\ \mathrm{H} & -3.5014750 & -2.7632990 & 1.3566020 \\ \mathrm{C} & -6.1705390 & -2.2083230 & 3.5316700 \\ \mathrm{H} & -6.3771460 & -2.8985580 & 4.3574870 \\ \mathrm{H} & -7.1341940 & -1.8263510 & 3.1811630 \\ \mathrm{C} & -5.4139780 & -2.9285940 & 2.3854030 \\ \mathrm{H} & -5.2604090 & -3.9865980 & 2.6258040 \\ \mathrm{H} & -5.9901110 & -2.8892530 & 1.4557060 \\ \mathrm{C} & -4.1698720 & 1.2676670 & 0.6308130 \\ \mathrm{C} & -3.7384860 & 2.2243740 & -0.4568020 \\ \mathrm{C} & -5.2129290 & 3.3494370 & 1.3505040 \\ \mathrm{C} & -4.9114290 & 1.9008370 & 1.6396460 \\ \mathrm{C} & -4.9990590 & 2.8263140 & -1.1159800 \\ \mathrm{H} & -4.6887650 & 3.6037330 & -1.8243420 \\ \mathrm{H} & -5.5259700 & 2.0590140 & -1.6925100\end{array}$




-5.9241240
-6.1738340
-6.8657520
-5.8433340
-3.1013480
-3.8810100
-4.0909480
-3.3986400
-2.9639090
-2.6044580
-2.0885520
-5.3120130
-5.8839230
4.7535610
5.0527680
3.1084530
3.7131660
5.4165220
5.6520290
6.3156440
4.2240000
3.8100860
4.5460080
2.2905510
5.8781960
2.5967790
2.0934230
1.8466320
3.7812170
3.9772580
3.5448660
5.1150420
5.7973180
3.8694670
4.0649390
4.7374050
5.2169090
4.3267660
3.6120920
3.5783230
2.6304780
3.0421710
6.6080590
5.1977990
5.0893630
5.4118850

3.4195490

4.4624030

2.8644980

3.7839070

1.7329780

4.1170490

5.1409540

4.1862260

3. 3825800

4.0711910

2.9733320

1.1981800

1.6939550

0.5327740

1.5270050

$-0.0828440$

$-0.3530000$

0.7124620

1.4011980

0.1207500

$-0.2058830$

0.0757080

$-1.2488270$

$-0.7709370$

2.1892620

1. 3716580

1. 5931520

1.4620130

2. 3401420

2. 9446640

3.0419040

$-0.6268640$

$-0.9284230$

$-2.5689400$

$-1.5101850$

$-0.8707330$

$-1.0938940$

0.1418740

$-1.8910320$

$-2.6713750$

$-1.4137440$

$-3.2382300$

$-0.2177910$

$-3.3510610$

$-4.1280240$

$-3.8570360$

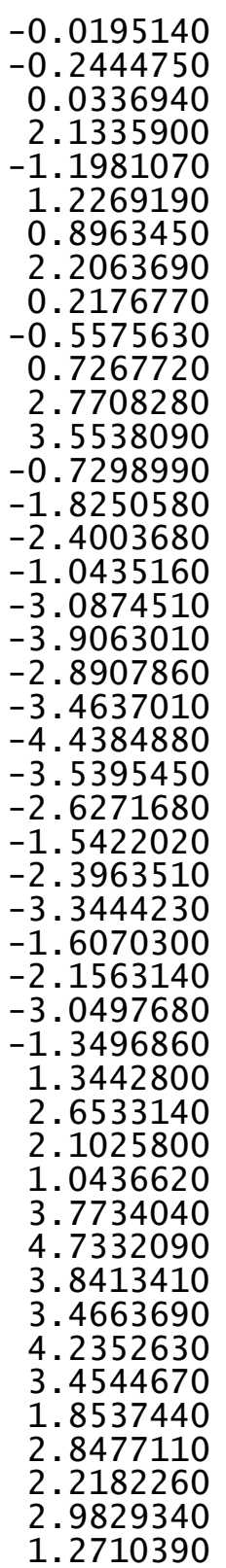

\begin{abstract}
6.3455740
6.7799070

7.1517580

5.4626140

6.2874730

1. 5472130

0.2283850

1.9306360

0.7423050

$-0.4085380$

$-0.8956550$

1.0480020

2. 4098020

2.6912200

$-0.0652040$

$-1.2374990$

$-1.6742720$

$-1.3753970$

0.4043180

$-3.2789180$

$-3.9363580$

$-3.8732380$

$-2.4184280$

$-1.4276310$

$-2.0346420$

$-3.4169690$

$-4.3694420$

$-2.0811740$

$-1.1314260$

$-4.9723400$

$-4.4394750$

$-2.3742900$

$-0.5101320$

0.2795210

$-0.3892990$

0.1829780

1.1252210

$-0.8754550$

1.0080760

1.9477850

$-0.9954390$

$-1.5993270$

$-0.0533050$

1.7459520

$-1.8231480$

$-0.1451390$
\end{abstract}

$\begin{array}{rr}-2.3724290 & 2.5868350 \\ -2.6312400 & 3.5590520 \\ -2.4250920 & 1.8490080 \\ 0.3901120 & 0.4608970 \\ 1.0630310 & 0.6938410 \\ -4.5821790 & -1.1945290 \\ -4.1261530 & -1.3420400 \\ -6.0240070 & -1.4742660 \\ -6.9831220 & -1.4607320 \\ -6.3973120 & -2.2751650 \\ -5.1049850 & -1.6254440 \\ -7.9582130 & -1.8531060 \\ -6.0671940 & -2.4624730 \\ -6.3422990 & -0.7529050 \\ -6.1904290 & -3.2986430 \\ -7.1084460 & -2.3500630 \\ -4.6374100 & -2.2311120 \\ -5.3465070 & -0.6657370 \\ -7.1412230 & -0.4284920 \\ -1.5163180 & -2.7542340 \\ -1.5974180 & -4.1083720 \\ -3.0475320 & -4.6118910 \\ -3.5576230 & -4.7024640 \\ -2.7026510 & -3.8796800 \\ -2.1501020 & -2.6116060 \\ -0.9411830 & -4.8215190 \\ -3.1345930 & -5.5831710 \\ -3.5575170 & -5.7437190 \\ -1.8390430 & -4.4911200 \\ -1.2495650 & -4.0507730 \\ -3.6674410 & -3.9085700 \\ -4.5988600 & -4.3654400 \\ -3.2614760 & -3.6807500 \\ 3.4961270 & -1.1429170 \\ 2.4000960 & -1.1752850 \\ 4.2803020 & -2.4007420 \\ 5.2615410 & -2.7277070 \\ 4.0130460 & -3.2797780 \\ 5.9676100 & -3.9189670 \\ 5.4558780 & -2.0476630 \\ 4.7320330 & -4.4627720 \\ 3.2477520 & -3.0203350 \\ 5.7076890 & -4.7847310 \\ 6.7215660 & -4.1730940 \\ 4.5308060 & -5.1351560 \\ 6.2650340 & -5.7118910 \\ & \end{array}$




\begin{tabular}{|c|c|c|c|c|c|c|c|}
\hline $\mathrm{H}$ & -0.4378930 & 1.8395820 & -0.2683300 & C & -3.3161210 & -0.6815200 & 3.8231010 \\
\hline$S$ & 1.1786340 & 4.0600900 & 0.1694230 & $\mathrm{H}$ & -3.4587840 & -0.5877830 & 4.9056680 \\
\hline c & 1.3067460 & 2.3177900 & 2.8521850 & $\mathrm{H}$ & -3.3530070 & 0.3299310 & 3.4046000 \\
\hline C & 2.2148830 & 2.2495300 & 1.7021230 & $\mathrm{H}$ & -5.4359880 & -1.0629390 & 3.4386230 \\
\hline H & 0.2726310 & 2.0530280 & 2.6396280 & $\mathrm{H}$ & -1.2391110 & -3.0763800 & 2.3720080 \\
\hline H & 2.0093140 & 1.5128390 & 0.9288200 & C & -4.3907850 & -2.9367860 & 3.8607910 \\
\hline 0 & 2.1043070 & 1.1661500 & 3.2185580 & $\mathrm{H}$ & -4.4800930 & -2.8472440 & 4.9494690 \\
\hline H & 1.5952520 & 0.3514470 & 2.8705860 & $\mathrm{H}$ & -5.2373520 & -3.5378290 & 3.5155480 \\
\hline C & 2.9482870 & 3.8443100 & 4.0144390 & C & -3.0461250 & -3.6028670 & 3.4688590 \\
\hline c & 3.6070500 & 4.1233930 & 2.6625440 & $\mathrm{H}$ & -2.4832520 & -3.8952670 & 4.3622980 \\
\hline C & 3.5728970 & 2.8822170 & 1.7687050 & $\mathrm{H}$ & -3.2200960 & -4.5118660 & 2.8848420 \\
\hline c & 1.4798960 & 3.4602490 & 3.8246510 & C & -3.5005390 & -1.9430540 & -0.9196000 \\
\hline $\mathrm{H}$ & 3.0162090 & 4.7247780 & 4.6599820 & C & -3.2537200 & -1.9250990 & -2.4102690 \\
\hline H & 4.6460370 & 4.4395110 & 2.7936600 & C & -5.5966910 & -1.0316300 & -1.7682120 \\
\hline $\mathrm{H}$ & 4.2467170 & 2.1062790 & 2.1543160 & $\mathrm{C}$ & -4.7535590 & -1.4116570 & -0.5790380 \\
\hline H & 0.9144170 & 4.3124450 & 3.4274170 & C & -4.3431550 & -2.7747940 & -3.1019460 \\
\hline$H$ & 1.0165360 & 3.1738640 & 4.7739450 & $\mathrm{H}$ & -4.2233020 & -2.6840290 & -4.1875400 \\
\hline $\mathrm{H}$ & 3.4770190 & 3.0278890 & 4.5211680 & $\mathrm{H}$ & -4.2084560 & -3.8323740 & -2.8523380 \\
\hline H & 3.0816800 & 4.9483410 & 2.1637000 & C & -5.7476190 & -2.2829710 & -2.6610460 \\
\hline$H$ & 3.9145290 & 3.0929350 & 0.7517420 & $\mathrm{H}$ & -6.3636040 & -2.0334160 & -3.5321640 \\
\hline & & & & $\mathrm{H}$ & -6.2745880 & -3.0639970 & -2.1042960 \\
\hline & S) G[M06- & (d) & 573 & $\mathrm{H}$ & -6.5774440 & -0.6605700 & -1.4518120 \\
\hline $\mathrm{P}$ & 0.3056000 & -0.0423890 & 0.1240120 & $\mathrm{H}$ & -2.2561080 & -2.2934710 & -2.6604850 \\
\hline 0 & 0.7674280 & 0.8916480 & 1.2056420 & C & -4.8432290 & 0.0397760 & -2.5836230 \\
\hline 0 & -0.7824030 & 0.4148840 & -0.8241970 & $\mathrm{H}$ & -5.3996480 & 0.2372240 & -3.5073480 \\
\hline 0 & -0.0579560 & -1.4387920 & 0.8638760 & $\mathrm{H}$ & -4.8174950 & 0.9799260 & -2.0241880 \\
\hline 0 & 1.5174500 & -0.5077170 & -0.8502710 & C & -3.4124610 & -0.4650700 & -2.8957830 \\
\hline C & -0.1021680 & -2.5783920 & 0.0719470 & $\mathrm{H}$ & -3.2153160 & -0.4267600 & -3.9729810 \\
\hline $\mathrm{C}$ & 1.1113930 & -3.1688710 & -0.3179050 & $\mathrm{H}$ & -2.6567660 & 0.1567940 & -2.4072090 \\
\hline c & -1.3492810 & -3.0721 & -0.3006960 & C & -5.1274140 & -1.2816340 & 0.7530100 \\
\hline C & -1.3650780 & -4.2300940 & -1.0846080 & $\mathrm{H}$ & -6.1030250 & -0.8719880 & 1.0127970 \\
\hline $\mathrm{H}$ & -2.3257190 & -4.6513600 & -1.3746110 & C & 3.7535060 & 2.8685240 & -1.7510900 \\
\hline c & 2.5540190 & -1.1641090 & -0.2013710 & $\mathrm{C}$ & 3.6718970 & 3.1956640 & -3.2209870 \\
\hline C & 3.6906980 & -0.4325280 & 0.1436650 & C & 3.5246780 & 0.6602450 & -2.7249210 \\
\hline c & 2.3887460 & -2.5161420 & 0.1015230 & C & 3.6650960 & 1.4962030 & -1.4740300 \\
\hline C & 4.6958910 & -1.1277860 & 0.8098680 & C & 4.8392420 & 2.4678470 & -3.9244780 \\
\hline H & 5.6059940 & -0.5953570 & 1.0824680 & $\mathrm{H}$ & 4.7816670 & 2.6562010 & -5.0024460 \\
\hline c & -2.6184820 & -2.3885590 & 0.0781090 & $\mathrm{H}$ & 5.7904560 & 2.8793850 & -3.5738280 \\
\hline C & 3.7798270 & 1.0243260 & -0.1562390 & C & 4.7508370 & 0.9486170 & -3.6225470 \\
\hline c & -2.9890630 & 77590 & 1.4204780 & $\mathrm{H}$ & 4.6518690 & 0.3739030 & -4.5502150 \\
\hline C & -2.1927870 & 63380 & 2.6400570 & $\mathrm{H}$ & 5.6591810 & 0.5996960 & -3.1220210 \\
\hline c & -4.4650470 & 83790 & 3.2321710 & $\mathrm{H}$ & 3.4688450 & -0.4068750 & -2.4986720 \\
\hline $\mathrm{c}$ & -4.2315590 & 63610 & 1.7490810 & $\mathrm{H}$ & 3.7347000 & 4.2768470 & -3.3876240 \\
\hline $\mathrm{C}$ & -1.9 & -1 & 3.4972500 & C & 2.2613200 & 1.1127630 & -3.4862540 \\
\hline$H$ & -1.4 & 66950 & 4.4181870 & $\mathrm{H}$ & 2.1816350 & 0.5337530 & -4.4131070 \\
\hline $\mathrm{H}$ & -1.2 & -0 & 2.9595620 & $\mathrm{H}$ & 1.3757010 & 0.8846230 & -2.8870170 \\
\hline
\end{tabular}




\begin{tabular}{|c|c|c|}
\hline $\begin{array}{l}0 \\
0 \\
0 \\
0 \\
0 \\
0 \\
0 \\
0 \\
0 \\
0 \\
0 \\
0 \\
0 \\
0 \\
0 \\
0 \\
0 \\
0 \\
0 \\
0 \\
0 \\
0 \\
0 \\
0 \\
0 \\
0 \\
0 \\
0 \\
0 \\
0 \\
0\end{array}$ & $\begin{array}{r}2.6287050 \\
2.8122790 \\
3.1619430 \\
3.3264320 \\
4.1817900 \\
1.6737520 \\
1.9584060 \\
4.0179430 \\
4.5634450 \\
4.4618330 \\
2.5114620 \\
2.3092410 \\
2.1808760 \\
0.6140940 \\
5.2328390 \\
2.1520240 \\
2.0163160 \\
1.5334160 \\
3.6443740 \\
4.2456270 \\
3.7602300 \\
3.7845560 \\
4.8469320 \\
-3.1717060 \\
-4.5938280 \\
-4.9580870 \\
-4.6129730 \\
-3.1004440 \\
-2.4687970 \\
-6.0228010 \\
-5.2978620 \\
-4.7300040 \\
-5.1109590 \\
-4.9750490 \\
-2.8447720 \\
-2.6404860 \\
-4.3994910 \\
-4.8245340 \\
-6.0536250 \\
-7.0574180 \\
-6.4498610 \\
-4.9049830 \\
-4.2879470 \\
-5.7823850 \\
-7.9698370 \\
-6.8137370\end{array}$ & $\begin{array}{r}-3.7951640 \\
-4.8753220 \\
-3.3657630 \\
0.5803600 \\
1.7848680 \\
2.3530490 \\
0.8737460 \\
2.8175890 \\
3.7286660 \\
2.4289900 \\
3.1140210 \\
4.1861520 \\
2.8188650 \\
2.5760350 \\
1.4996790 \\
2.8084230 \\
3.8926400 \\
2.3460440 \\
2.4245560 \\
3.3082610 \\
1.7178910 \\
-0.7270100 \\
-0.9493070 \\
0.8434170 \\
1.3268720 \\
2.4987090 \\
2.1472520 \\
1.9943760 \\
1.1790010 \\
2.7290840 \\
0.5096650 \\
1.5913060 \\
1.2062070 \\
2.9126430 \\
1.5429900 \\
2.9921840 \\
3.3944060 \\
-1.5257200 \\
-2.3986740 \\
-1.8493050 \\
-1.7904230 \\
-1.8421220 \\
-1.1608380 \\
-3.4288990 \\
-2.4531920 \\
-2.6285580\end{array}$ \\
\hline
\end{tabular}

2.1993400 $-1.1803140$ 0.5205180 2. 7606870 3. 1939710 -3. 3917840 85630 $-4.7019010$ $-5.2184870$ $-5.4254620$ $-6.4410880$ $-4.6568650$ $-6.6501460$ $-7.1594010$ $-6.8332220$ $-7.2088190$ $-8.1155820$ $-1.9899020$ $-2.6517340$ $-0.4559870$ $-0.2272820$ $-0.9592910$ $-0.1095000$ 0.9748750 1.1107740 $-0.1083010$ $-0.3448840$ 0.1841770 $-0.8350820$ $-0.4590520$ 0.1405720 1.2833650 $-1.9211900$ $-0.6109510$ 0.9666800 $-1.4202330$ $-0.1331410$

154 TS298(S, S ) G $[M 06-2 X / 6-31 G(d)]=-3864.196670$

$\begin{array}{lrrr}\mathrm{P} & 0.2881120 & -0.0350130 & 0.2166470 \\ \mathrm{O} & 0.7019530 & 0.9586580 & 1.2641080 \\ \mathrm{O} & -0.7914180 & 0.3502390 & -0.7725240 \\ \mathrm{O} & -0.0534880 & -1.4080270 & 1.0093090 \\ \mathrm{O} & 1.5317410 & -0.5082900 & -0.7122020 \\ \mathrm{C} & -0.0491700 & -2.5798100 & 0.2645870\end{array}$

$-2.8976720$ $-2.4404930$ $-0.8418730$ $-0.8790920$ $-1.4643990$ $-0.3905270$

0.1042700 0.2029130 1.2426600 $-0.2496300$

1.8190920

1. 5827870

0.3258400

$-1.0525120$

1.3608840

2. 6261170 $-0.0321810$

1.8100950 $-0.3318140$ $-1.5908010$ 0.7085590 $-0.7381870$

1. 1622390

$-1.1628420$

0.6729320

0.8405100

0.5900920

$-0.9199670$

$-1.5163680$

1. 2922780

0.9924410

$-1.4210980$

$-1.5217420$

1. 1832880

2.3638560

0.7992900

$-1.1212920$

$\begin{array}{lr}5.9797390 & 0.7992900 \\ 6.0663210 & -1.1212920 \\ 4.5539450 & -2.5551810\end{array}$




1.1798760
-1.2740710
-1.2286860
-2.1691120
2.5736770
3.6868510
2.4308610
4.7157170
5.6122530
-2.5708680
3.7406270
-2.9807280
-2.2086090
-4.5196890
-4.2445500
-2.0239350
-1.5312800
-1.3596560
-3.4059130
-3.5809540
-3.4518990
-5.5059950
-1.2380970
-4.4297980
-4.5461490
-5.2551220
-3.0624450
-2.5167410
-3.2030190
-3.4370770
-3.1522070
-5.5323410
-4.7110690
-4.2018190
-4.0569300
-4.0467290
-5.6289980
-6.2297420
-6.1489000
-6.5297720
-2.1397580
-4.7851740
-5.3212090
-4.7997570
-3.3337660
-3.1078650

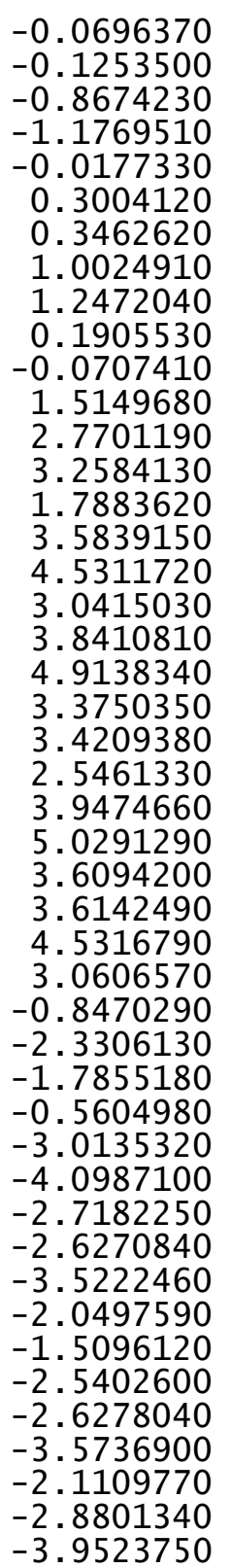

$-2.6075510$

$-5.1225720$

$-6.1142600$

3.6980190

3. 6362240

3.5424820

3.6391680

4.8344320

4.7932210

5.7683420

4.7772480

4.7089980

5.6845880

3.5087100

3.6752430

2. 2821140

2. 2321440

1. 3922680

2. 3463060

2. 3408590

1.4723230

3.9888640

4.2430590

4.0163020

3.9027380

3.0946270

3. 3323580

2. 1686200

2.9301520

3.0272390

1.9448700

3.9013300

4. 3363460

5.3930490

5.4655630

6.1926730

5.5460880

5.7519920

6.3834220

3.8789540

3.9522620

4.5985080

5.6805830

5.9240270

4. 6448420
3.3544820
$-0.0166550$
$-1.3865770$
$-0.9912010$
2. 8718500
3.1229600
0.6124390
1.5130930
2. 3911660
2.5247710
.8433440
0.8867600
0.2648700
0.5856950
$-0.4430940$
4.1956490
0.9939120
0.3664490
0.7747650
2.4939620
. 6212280
3.0268890
3.4534060
4.3736020
1. 8884020
2.0987210
4.2264170
4.8261550
4.6204050
2.7311980
2.5866940
2.3560130
0.8366510
5.4128800
2.4268610
2.3421810
1. 8106590
3.9053900
4.5531420
. 0144320
3.8431210
4.8954640
$-2.2858710$
$-2.9832560$
$-4.3733080$
$-5.2350460$
$-4.3930400$

-2.3979970
0.7550910
0.9727270
-1.7555550
-3.2413880
-2.6211470
-1.4120690
-3.8865170
-4.9733760
-3.5394750
-3.5113240
-4.4109110
-2.9788900
-2.3418460
-3.4615310
-3.4249990
-4.3218850
-2.8285800
-3.8099630
-4.8984390
-3.4252790
0.5563500
1.7229460
2.4072100
0.9144870
2.7427360
3.6290910
2.3151920
3.1131430
4.1952000
2.8254160
2.6780160
1.3895750
2.8584710
3.9486270
2.4342090
2.4099230
3.2693880
1.7139340
-0.7743230
-1.0475580
1.4239460
2.2089700
1.6053390
1.6329770
1.7661190 


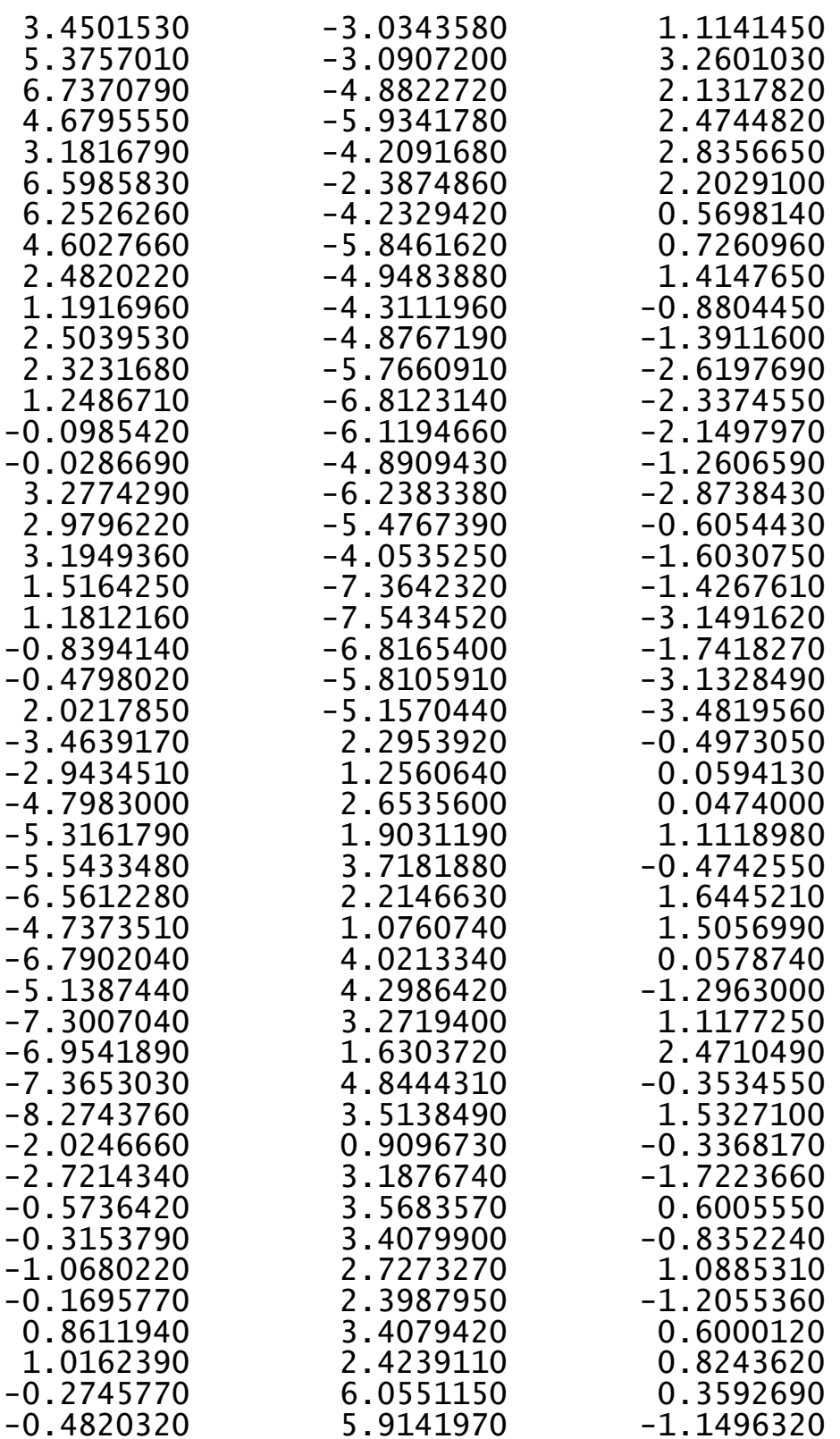

$\begin{array}{lr}\mathrm{C} & 0.0847520 \\ \mathrm{C} & -0.9913110 \\ \mathrm{H} & -0.6518940 \\ \mathrm{H} & -0.0050270 \\ \mathrm{H} & 1.1837890 \\ \mathrm{H} & -2.0771330 \\ \mathrm{H} & -0.7880250 \\ \mathrm{H} & 0.7974980 \\ \mathrm{H} & -1.5549450 \\ \mathrm{H} & -0.2111180 \\ \mathrm{H} & \end{array}$
4. 5897910
4.9285970
7.0217310
6.7400200
4.6041580
5. 0135170
4.9710870
6.0216520
5. 9583520
4.4022470
$-1.6681580$
1.1069570
0.7054380
$-1.6853320$
$-1.6545410$
0.9717620
2. 1819780
0.5906520
$-1.3731870$
$-2.7041360$

154

$\begin{array}{lrcr}\text { TS299(S , S }) \text { G }[\mathrm{M} 06-2 \times / 6-31 G(\mathrm{~d})]=-3864.197309 \\ \mathrm{P} & -0.1442030 & -0.3806430 & 1.0305560 \\ \mathrm{O} & 0.6842710 & -0.6876940 & 2.2413010 \\ \mathrm{O} & -0.7277210 & 1.0072680 & 0.8851830 \\ \mathrm{O} & -1.2835580 & -1.5269390 & 0.9367840 \\ \mathrm{O} & 0.6800910 & -0.6052410 & -0.3623310 \\ \mathrm{C} & -1.9426420 & -1.6109350 & -0.2848630 \\ \mathrm{C} & -1.2987070 & -2.2671750 & -1.3352480 \\ \mathrm{C} & -3.2003320 & -1.0234930 & -0.4113630 \\ \mathrm{C} & -3.8049170 & -1.1137460 & -1.6629560 \\ \mathrm{H} & -4.7935700 & -0.6760400 & -1.7918520 \\ \mathrm{C} & 1.0559170 & -1.9131350 & -0.6414700 \\ \mathrm{C} & 2.3901340 & -2.2699020 & -0.4662860 \\ \mathrm{C} & 0.0741860 & -2.8040890 & -1.0963590 \\ \mathrm{C} & 2.7326880 & -3.6011400 & -0.7129320 \\ \mathrm{H} & 3.7705930 & -3.9071680 & -0.5963060 \\ \mathrm{C} & -3.8831090 & -0.3379990 & 0.7214930 \\ \mathrm{C} & 3.4046150 & -1.2496500 & -0.0794690 \\ \mathrm{C} & -4.2422500 & -1.0463100 & 1.8775430 \\ \mathrm{C} & -3.9408670 & -2.4940560 & 2.1835440 \\ \mathrm{C} & -5.2802390 & -1.3238000 & 4.0632740 \\ \mathrm{C} & -4.9825070 & -0.4229760 & 2.8926270 \\ \mathrm{C} & -3.1401970 & -2.5487730 & 3.5030370 \\ \mathrm{H} & -2.9601900 & -3.5984160 & 3.7617840 \\ \mathrm{H} & -2.1640190 & -2.0803050 & 3.3513570 \\ \mathrm{C} & -3.9367350 & -1.8353680 & 4.6273290 \\ \mathrm{H} & -4.1356570 & -2.5187740 & 5.4607090 \\ \mathrm{H} & -3.3668360 & -0.9923230 & 5.0292040 \\ \mathrm{H} & -5.8470200 & -0.7921840 & 4.8350790 \\ \mathrm{H} & -3.3777700 & -2.9736360 & 1.3795760 \\ \mathrm{C} & -6.0759630 & -2.5418970 & 3.5463590 \\ \mathrm{H} & -6.2454660 & -3.2363130 & 4.3771040 \\ \mathrm{H} & -7.0585790 & -2.2143760 & 3.1934850 \\ \mathrm{C} & -5.2819120 & -3.2288010 & 2.4049400 \\ \mathrm{H} & -5.0753630 & -4.2764140 & 2.6510330 \\ & & & \end{array}$




\begin{tabular}{|c|c|c|}
\hline $\begin{array}{l}-5.8577230 \\
-4.2485270 \\
-3.8607570 \\
-5.4004080 \\
-5.0262190 \\
-5.1469830 \\
-4.8730970 \\
-5.6307370 \\
-6.1073620 \\
-6.4101620 \\
-7.0188250 \\
-6.0562900 \\
-3.1953370 \\
-4.1091270 \\
-4.3698830 \\
-3.6361090 \\
-3.1501600 \\
-2.8230320 \\
-2.2569590 \\
-5.3943020 \\
-5.9938740 \\
4.7094070 \\
4.9759300 \\
3.1189260 \\
3.7171050 \\
5.3955540 \\
5.6087520 \\
6.3193570 \\
4.2539400 \\
3.8408730 \\
4.6266950 \\
2.3375720 \\
5.7648680 \\
2.5389200 \\
2.0410600 \\
1.7727710 \\
3.6719710 \\
3.8515270 \\
3.3906720 \\
5.1011120 \\
5.7822230 \\
3.9451990 \\
4.0996130 \\
4.7078290 \\
5.1864320 \\
4.2445750\end{array}$ & $\begin{array}{r}-3.2233470 \\
1.0133470 \\
1.9848760 \\
3.0432270 \\
1.6138310 \\
2.5178350 \\
3.3065930 \\
1.7215790 \\
3.0682670 \\
4.0952670 \\
2.4648950 \\
3.4493100 \\
1.5225860 \\
3.8776770 \\
4.8873500 \\
3.9776520 \\
3.1853610 \\
3.8869860 \\
2.8254380 \\
0.8991660 \\
1.3701520 \\
0.7319050 \\
1.7309540 \\
0.0255060 \\
-0.2054480 \\
0.9249050 \\
1.6176280 \\
0.3788580 \\
-0.0530090 \\
0.1998280 \\
-1.0798640 \\
-0.7024750 \\
2.4340240 \\
1.4540640 \\
1.6430450 \\
1.5159530 \\
2.4798800 \\
3.0871360 \\
3.1746830 \\
-0.3946790 \\
-0.6547460 \\
-2.3924220 \\
-1.3303650 \\
-0.6435100 \\
-0.8339190 \\
0.3469380\end{array}$ & $\begin{array}{r}1.4742490 \\
0.6233740 \\
-0.4676920 \\
1.3253810 \\
1.6249630 \\
-1.1367780 \\
-1.8476250 \\
-1.7119270 \\
-0.0483770 \\
-0.2809230 \\
0.0036240 \\
2.1027480 \\
-1.2028000 \\
1.2034520 \\
0.8653790 \\
2.1848070 \\
0.2035310 \\
-0.5744090 \\
0.7191390 \\
2.7596910 \\
3.5372560 \\
-0.6450520 \\
-1.7443220 \\
-2.3321180 \\
-0.9651670 \\
-2.9948380 \\
-3.8164330 \\
-2.7811810 \\
-3.3789660 \\
-4.3619680 \\
-3.4405450 \\
-2.5638400 \\
-1.4559170 \\
-2.3487560 \\
-3.3066760 \\
-1.5722680 \\
-2.0994950 \\
-2.9945430 \\
-1.3013280 \\
1.4417140 \\
2.7600770 \\
2.1969810 \\
1.1343620 \\
3.8674090 \\
4.8346830 \\
3.9215810\end{array}$ \\
\hline
\end{tabular}

3.6409740
3.6432340
2.6352440
3.1546170
6.5542170
5.3099180
5.2321030
5.5604500
6.4024990
6.8359930
7.2202130
5.4094560
6.1978500
1.7725770
2.1061010
1.4695470
-0.0634060
-0.5453680
0.4383320
1.7159660
1.7330510
-0.5472320
-0.6632400
3.1909400
1.8992180
-0.4032510
-1.5360190
-1.9124330
-1.1796310
-1.7475010
-3.2609570
-3.8986810
-3.1836560
-1.2714840
-1.2473380
-0.1130120
-3.4896140
-3.6823180
-4.9526150
-3.8899520
-1.5162710
0.0861940
-0.5228320
-0.0393370
0.8575230
-1.0769600
-1.7234310
-2.5016950
-1.2998030
-3.1028270
0.0965710
-3.1064810
-3.8857690
-3.6037250
-2.0699610
-2.3020590
-2.0859430
0.6322930
1.3459740
-4.5376050
-5.9865780
-6.4297120
-6.2581900
-5.2570880
-4.1456920
-6.6091110
-7.4681430
-7.2205520
-5.8070590
-6.1294040
-5.8135060
-5.9372990
-4.8653310
-2.2937310
-2.8786570
-2.3996490
-2.5947660
-1.6877080
-1.7198700
-2.9484710
-3.9736970
-2.6404430
-3.6449320
-2.3770900
-1.9484520
-0.6535750
-1.3361540
3.4506510
2.3203190
4.2213100
5.2444770
3.8987570

3. 5556830

4. 3274450

3. 5347840

1.9427140

2.9592520

2. 3302170

3. 0962590

1. 3873530

2.7086000

3. 6879260

1. 9818390

0.5553810

0.7931410

$-1.0824020$

$-1.3298940$

$-2.6546740$

$-2.6333900$

$-1.5573590$

$-1.2795600$

$-0.5117270$

$-2.8770710$

$-2.4390770$

$-0.6133500$

$-1.3499690$

$-3.4520000$

$-3.6229500$

$-1.7996920$

$-2.6041000$

$-3.7969160$

$-5.1316680$

$-5.1440120$

$-4.0949390$

$-2.7564470$

$-5.9504170$

$-3.7696770$

$-3.7192560$

$-4.9193050$

$-6.1304720$

$-3.9456500$

$-4.4662110$

$-5.2756850$

$-1.1383250$

$-1.1694640$

$-2.4019260$

$-2.7278480$

- 3.2871240 


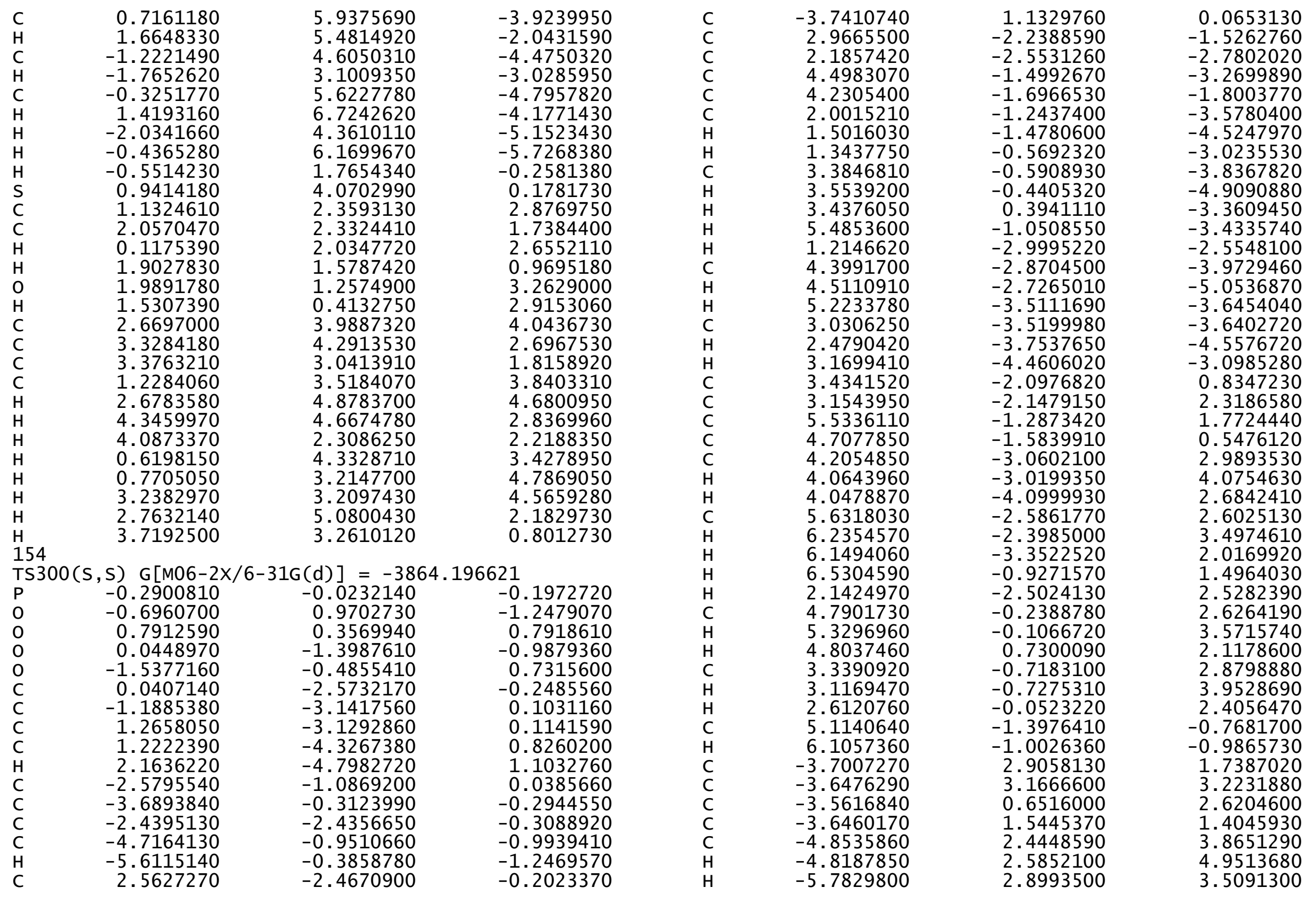




$\begin{array}{lrr}-4.8013860 & 0.9378380 & 3.4999360 \\ -4.7427830 & 0.3213970 & 4.4039490 \\ -5.7063550 & 0.6378980 & 2.9628690 \\ -3.5309980 & -0.4059830 & 2.3487430 \\ -3.6831020 & 4.2409880 & 3.4357980 \\ -2.3053020 & 1.0325460 & 3.4308100 \\ -2.2652350 & 0.4113180 & 4.3325390 \\ -1.4122310 & 0.8044910 & 2.8426560 \\ -2.3645180 & 2.5356010 & 3.8045920 \\ -2.3653360 & 2.6706510 & 4.8921430 \\ -1.4855350 & 3.0615050 & 3.4216250 \\ -3.9747610 & 3.4732000 & -0.5787200 \\ -4.2176300 & 4.3866720 & -1.7529610 \\ -3.9984530 & 1.8957460 & -2.4191150 \\ -3.8928830 & 2.1157380 & -0.9273170 \\ -3.0636400 & 4.2271850 & -2.7645970 \\ -3.2930780 & 4.8219270 & -3.6564900 \\ -2.1384890 & 4.6198850 & -2.3339650 \\ -2.9038180 & 2.7286540 & -3.1237460 \\ -2.9943420 & 2.5772250 & -4.2054220 \\ -1.9223070 & 2.3510010 & -2.8266400 \\ -3.8871140 & 0.8415210 & -2.6818560 \\ -4.3080700 & 5.4285950 & -1.4271710 \\ -5.3698440 & 2.4376370 & -2.8823710 \\ -5.4361470 & 2.3459190 & -3.9723570 \\ -6.1748710 & 1.8280080 & -2.4588330 \\ -5.5186100 & 3.9198720 & -2.4447370 \\ -5.7162480 & 4.5627560 & -3.3097810 \\ -6.3596560 & 4.0375460 & -1.7546490 \\ -3.8708200 & 3.8713580 & 0.7499230 \\ -3.9405710 & 4.9258430 & 1.0156550 \\ -4.6018100 & -2.2779640 & -1.3979520 \\ -5.6889860 & -2.9912580 & -2.1615670 \\ -5.9387480 & -4.3615340 & -1.5143080 \\ -4.6648920 & -5.2318870 & -1.5196760 \\ -3.3692940 & -4.4033400 & -1.6853950 \\ -3.4562130 & -3.0234530 & -1.0763720 \\ -5.3872480 & -3.1325090 & -3.2094550 \\ -6.7568020 & -4.8810720 & -2.0223650 \\ -4.7080590 & -5.9577560 & -2.3377630 \\ -3.1915190 & -4.2598050 & -2.7600100 \\ -6.6039430 & -2.3909260 & -2.1712690 \\ -6.2636140 & -4.1869820 & -0.4827160 \\ -4.6168020 & -5.8154870 & -0.5940480 \\ -2.5055280 & -4.9559170 & -1.3075600 \\ -1.1966790 & -4.3066250 & 0.8947370\end{array}$

0.0234450 $-2.4927110$ $-2.4151600$ $-1.1926640$ 0.0813920 $-3.3347060$ $-2.7114530$ $-3.3312830$ $-1.2548140$ $-1.1640310$ 0.9523050 0.2537370 $-2.3407800$ 3. 4716520 2. 9481320 4. 8082820 5. 3233690 5. 5582890 6. 5707650 4.7405060 6.8074390 5. 1557630 7. 3152770 6.9616880 7. 3864550 8. 2908140 2. 0271860 2. 7297470 0.5954460 0.3299880 1. 0848750 0.1742200 $-0.8406970$ $-1.0026460$ 0.3172920

0.5161630 $-0.0643870$ 1. 0274290 0.7050360 0.0436690 $-1.1632050$ 2. 1133460 0.8296730 $-0.7538500$ 1.5883010
0.2244870

$\begin{array}{rr}-4.9192940 & 1.2233830 \\ -4.8282200 & 1.4883490 \\ -6.2981090 & 1.8921180 \\ -6.5290460 & 2.7766880 \\ -6.2275870 & 1.9907140 \\ -6.5866590 & 2.4117140 \\ -4.2248460 & 2.3817980 \\ -4.6596610 & 0.8116510 \\ -5.8682430 & 3.6509660 \\ -7.5574480 & 3.1507140 \\ -6.2190290 & 2.6553030 \\ -7.0406640 & 1.2715060 \\ -6.9253090 & 0.9925610 \\ 2.2853840 & 0.5183900 \\ 1.2492040 & -0.0413440 \\ 2.6399660 & -0.0229830 \\ 1.8930910 & -1.0913010 \\ 3.6976160 & 0.5057330 \\ 2.2010260 & -1.6205420 \\ 1.0716180 & -1.4907800 \\ 3.9971320 & -0.0230530 \\ 4.2753740 & 1.3306930 \\ 3.2511530 & -1.0865910 \\ 1.6194180 & -2.4499270 \\ 4.8146740 & 0.3938190 \\ 3.4901600 & -1.4988710 \\ 0.9070210 & 0.3542740 \\ 3.1779180 & 1.7436910 \\ 3.5721540 & -0.5912750 \\ 3.4174510 & 0.8438510 \\ 2.7258510 & -1.0750880 \\ 2.4104650 & 1.2160410 \\ 3.4238430 & -0.5971460 \\ 2.4402230 & -0.8179520 \\ 6.0621310 & -0.3570490 \\ 5.9228720 & 1.1531800 \\ 4.6045320 & 1.6719810 \\ 4.9275050 & -1.0987480 \\ 7.0245070 & -0.7034970 \\ 6.7539830 & 1.6846760 \\ 4.6281240 & 1.6526280 \\ 5.0029970 & -0.9586070 \\ 4.9693560 & -2.1748130 \\ 6.0378550 & -0.5937070 \\ 5.9582700 & 1.3819140 \\ 4.4169720 & 2.7099430\end{array}$


154

TS301 (S , S ) G[M06-2X/6-31G(d) $]=-3864.196984$

$\begin{array}{lrrr}\mathrm{P} & -0.1494740 & -0.3495160 & 1.0238320 \\ \mathrm{O} & 0.6723730 & -0.6527230 & 2.2399670 \\ \mathrm{O} & -0.7141690 & 1.0440800 & 0.8601080 \\ \mathrm{O} & -1.3036180 & -1.4817310 & 0.9452890 \\ \mathrm{O} & 0.6740340 & -0.6037650 & -0.3644130 \\ \mathrm{C} & -1.9717620 & -1.5762590 & -0.2699630 \\ \mathrm{C} & -1.3368570 & -2.2426240 & -1.3201800 \\ \mathrm{C} & -3.2314970 & -0.9932130 & -0.3902810 \\ \mathrm{C} & -3.8579780 & -1.1155710 & -1.6290990 \\ \mathrm{H} & -4.8520070 & -0.6881150 & -1.7492920 \\ \mathrm{C} & 1.0260310 & -1.9216490 & -0.6283120 \\ \mathrm{C} & 2.3515900 & -2.3056210 & -0.4403580 \\ \mathrm{C} & 0.0296640 & -2.7951330 & -1.0804180 \\ \mathrm{C} & 2.6672100 & -3.6457280 & -0.6731980 \\ \mathrm{H} & 3.6981890 & -3.9720390 & -0.5504460 \\ \mathrm{C} & -3.8938300 & -0.2786410 & 0.7366470 \\ \mathrm{C} & 3.3841060 & -1.3009390 & -0.0604710 \\ \mathrm{C} & -4.2412080 & -0.9577790 & 1.9133970 \\ \mathrm{C} & -3.9450130 & -2.4002510 & 2.2475970 \\ \mathrm{C} & -5.2455890 & -1.1770300 & 4.1209310 \\ \mathrm{C} & -4.9607230 & -0.3055150 & 2.9251830 \\ \mathrm{C} & -3.1234920 & -2.4307300 & 3.5550480 \\ \mathrm{H} & -2.9482360 & -3.4755650 & 3.8356420 \\ \mathrm{H} & -2.1458180 & -1.9746390 & 3.3769440 \\ \mathrm{C} & -3.8961370 & -1.6844640 & 4.6747210 \\ \mathrm{H} & -4.0855510 & -2.3459980 & 5.5277480 \\ \mathrm{H} & -3.3139630 & -0.8359190 & 5.0462970 \\ \mathrm{H} & -5.7961220 & -0.6238820 & 4.8893640 \\ \mathrm{H} & -3.3980690 & -2.9017170 & 1.4457580 \\ \mathrm{C} & -6.0574290 & -2.4015640 & 3.6458840 \\ \mathrm{H} & -6.2166450 & -3.0754980 & 4.4953330 \\ \mathrm{H} & -7.0441140 & -2.0761510 & 3.3025350 \\ \mathrm{C} & -5.2874580 & -3.1197930 & 2.5072460 \\ \mathrm{H} & -5.0845990 & -4.1632540 & 2.7732370 \\ \mathrm{H} & -5.8785890 & -3.1305010 & 1.5862460 \\ \mathrm{C} & -4.2484680 & 1.0733870 & 0.6140140 \\ \mathrm{C} & -3.8657230 & 2.0169570 & -0.5029650 \\ \mathrm{C} & -5.3688860 & 3.1297950 & 1.2880710 \\ \mathrm{C} & -5.0052930 & 1.7032850 & 1.6134290 \\ \mathrm{C} & -5.1553880 & 2.5495460 & -1.1654950 \\ \mathrm{H} & -4.8833700 & 3.3202220 & -1.8965140 \\ \mathrm{H} & -5.6549680 & 1.7463590 & -1.7170820 \\ \mathrm{C} & -6.0949220 & 3.1326930 & -0.0759400 \\ \mathrm{H} & -6.3907900 & 4.1575530 & -0.3262080\end{array}$

$-7.0115060$

$-3.2161810$

$-4.0708810$

$-4.3261150$

$-3.5836480$

$-3.1323100$

$-2.8052490$

$-2.2380230$

$-5.3629720$

$-5.9460010$

4. 7224860

5.0091970

3. 1302780

3. 7177110

5.4232450

5.6499970

6. 3382690

4. 2696900

3. 8655310

4. 6278880

2. 3398230

5.8068450

2. 5714870

2. 0822340

1.8016420

3. 7180820

3. 9109270

3. 4430090

5.0871360

5.7586930

3.8960330

4.0724730

4. 6804660

5. 1522230

4.2346990

3. 5959270

3. 5786930

2. 5984770

3.0953170

6.5419600

5. 2481870

5.1542200

5. 4942560

6.3563050
6.7837490
2. 5399210

3.5596510

1.5316830

3.9477060

4.9518920

4.0655710

3. 2226380

3.9032900

2.8620260

1.0170960

1. 5108890

0.6535620

1.6365610

$-0.0478040$

$-0.2721850$

0.8111200

1.4918190

0.2545290

$-0.1547010$

0.0925210

$-1.1874450$

$-0.7668550$

2. 3311650

1. 3888780

1.5747520

1.4700760

2. 4006170

2. 9962800

3. 1073670

$-0.4559210$

$-0.7121090$

$-2.4261130$

$-1.3793090$

$-0.6714860$

$-0.8612270$

0.3275020

$-1.7354300$

$-2.5040690$

$-1.2935210$

$-3.1269270$

0.0288280

$-3.1607030$

$-3.9283500$

$-3.6745410$

-2.1375490
-2.3653190
0.0017050

2. 0654620

$-1.2373720$

1.1297230

0.7716450

2. 1020910

0.1334840

$-0.6627460$

0.6466050

2. 7689360

3. 5449360

$-0.6419680$

$-1.7506080$

$-2.3295050$

$-0.9566690$

$-2.9902120$

$-3.8182160$

$-2.7662570$

$-3.3690760$

$-4.3572090$

$-3.4167680$

$-2.5578560$

$-1.4656420$

$-2.3652800$

$-3.3282820$

$-1.5941870$

$-2.1203390$

$-3.0204120$

$-1.3305510$

1.4588610

2.7828630

2.2314500

1.1572000

3.8857840

4.8565620

3.9290590

3. 5801090

4. 3612590

3. 5468260

1. 9821920

2. 9768250

2. 3799760

3. 1559110

1.4448660

2.7493270

3. 7323220 


\begin{tabular}{|c|c|c|c|}
\hline & & 21792 & \\
\hline $\mathrm{H}$ & $\begin{array}{l}7.1753320 \\
5.4149650\end{array}$ & -2.1749240 & $\begin{array}{l}2.0248240 \\
0.5631110\end{array}$ \\
\hline $\mathrm{H}$ & 6.2129640 & 1.2609730 & 0.7969630 \\
\hline$C$ & 1.6893460 & -4.5653220 & -1.0416360 \\
\hline$C$ & 1.9933690 & -6.0202190 & -1.2930950 \\
\hline C & 1.3643640 & -6.4391010 & -2.6304630 \\
\hline$C$ & -0.1646930 & -6.2311950 & -2.6297140 \\
\hline$C$ & -0.6394020 & -5.2340850 & -1.5465780 \\
\hline$C$ & 0.3655550 & -4.1470120 & -1.2466020 \\
\hline $\mathrm{H}$ & 1.5784230 & -6.6395350 & -0.4849560 \\
\hline $\mathrm{H}$ & 1.6065730 & -7.4819770 & -2.8562480 \\
\hline $\mathrm{H}$ & -0.6743070 & -7.1843230 & -2.4573310 \\
\hline $\mathrm{H}$ & -0.7870150 & -5.7905660 & -0.6108820 \\
\hline $\mathrm{H}$ & 3.0747940 & -6.1878050 & -1.3007560 \\
\hline $\mathrm{H}$ & 1.8206690 & -5.8283130 & -3.4171910 \\
\hline $\mathrm{H}$ & -0.4867670 & -5.8860320 & -3.6180270 \\
\hline $\mathrm{H}$ & -1.6152980 & -4.8165390 & -1.8069220 \\
\hline C & -1.9674480 & -2.2914810 & -2.5786110 \\
\hline C & -3.2517400 & -1.7425370 & -2.7187880 \\
\hline C & -1.2255550 & -2.8221140 & -3.7917270 \\
\hline c & -2.1562580 & -3.2219180 & -4.9336140 \\
\hline C & -3.1273570 & -2.0839420 & -5.2371370 \\
\hline C & -4.0199270 & -1.8286960 & -4.0248720 \\
\hline $\mathrm{H}$ & -1.5646300 & -3.4792990 & -5.8181120 \\
\hline $\mathrm{H}$ & -0.5509830 & -2.0264000 & -4.1413530 \\
\hline $\mathrm{H}$ & -0.5758980 & -3.6553510 & -3.5196280 \\
\hline $\mathrm{H}$ & -2.5540760 & -1.1782600 & -5.4740790 \\
\hline $\mathrm{H}$ & -3.7443860 & -2.3139690 & -6.1115870 \\
\hline $\mathrm{H}$ & -4.6051400 & -0.9125650 & -4.1610090 \\
\hline $\mathrm{H}$ & -4.7486520 & -2.6474270 & -3.9435710 \\
\hline $\mathrm{H}$ & -2.7229360 & -4.1199640 & -4.650411 \\
\hline C & 0.1579890 & 3.4462590 & -1.1916530 \\
\hline 0 & -0.4783870 & 2.3308220 & -1.2078670 \\
\hline C & 0.0670510 & 4.1936490 & -2.4720980 \\
\hline C & 0.9879390 & 5.1934960 & -2.8036030 \\
\hline $\mathrm{C}$ & -0.9609360 & 3.8711080 & -3.3684950 \\
\hline C & 0.8797830 & 5.8633890 & -4.0163540 \\
\hline $\mathrm{H}$ & 1.7874760 & 5.4305440 & -2.1098480 \\
\hline C & -1.0725030 & 4.5540610 & -4.5734760 \\
\hline $\mathrm{H}$ & -1.6682880 & 3.0918170 & -3.1053730 \\
\hline C & & 5.5485510 & -4.8996920 \\
\hline $\mathrm{H}$ & 1.601340 & 6.6319770 & -4.2734610 \\
\hline $\mathrm{H}$ & -1.8769320 & 4.3100210 & -5.2597610 \\
\hline $\mathrm{H}$ & -0.2369310 & 6.0775880 & -5.84390 \\
\hline $\mathrm{H}$ & -0.5221850 & 1.7890620 & -0.289510 \\
\hline 3 & 1.0130050 & 4.0706680 & 0.1230590 \\
\hline
\end{tabular}

1.1643540
2.0936350
0.1456580
1.9321640
2.0020980
1.5326040
2.7205080
3.3906740
3.4234190
1.2731030
2.7399550
4.4132940
4.1209020
0.6791940
0.8059290
3.2742650
2.8404910
3.7746020

$\begin{array}{ll}\text { C } & 1.1643540 \\ \text { C } & 2.0936350\end{array}$

$\mathrm{H} \quad 0.1456580$
2. 3906830
2. 3363240
2. 0789320
1.5748820
1. 2811740
0.4402390
4.0103770
4.2865130
3.0255820
3. 5600920
4.9073890
4.6483960
2. 2866200
4.3786950
3. 2750180
3. 2289970
5.0776730
3. 2279180

2.8434280

1.7096130

2. 6204740

0.9497800

3.2478660

2.9073690

3.9979740

2. 6510120

1.7851180

3.7928960

4.6237160

2. 7920610

2.2002460

3.3674930

4.7407120

4.5324410

2. 1248720

0.7697090

TS302 (, S ) G $[M 06-2 X / 6-31 G(d)]=-3864.195526$

$\begin{array}{lrrr}\text { TS302(S, S }(\mathrm{G}[\mathrm{M} 06-2 \mathrm{X} / 6-31 \mathrm{G}(\mathrm{d})]=-3864.195526 \\ \mathrm{P} & -0.1500420 & -0.2724450 & 1.0288680 \\ \mathrm{O} & 0.6666490 & -0.5408830 & 2.2566140 \\ \mathrm{O} & -0.6662160 & 1.1299110 & 0.7949170 \\ \mathrm{O} & -1.3403670 & -1.3677670 & 1.0089640 \\ \mathrm{O} & 0.6536870 & -0.6294260 & -0.3474670 \\ \mathrm{C} & -2.0133340 & -1.4987390 & -0.2006800 \\ \mathrm{C} & -1.4087850 & -2.2621200 & -1.2095170 \\ \mathrm{C} & -3.2446210 & -0.8658000 & -0.3483370 \\ \mathrm{C} & -3.8917920 & -1.0176520 & -1.5783680 \\ \mathrm{H} & -4.8634600 & -0.5481330 & -1.7181200 \\ \mathrm{C} & 0.9651250 & -1.9693210 & -0.5412080 \\ \mathrm{C} & 2.2812620 & -2.3758270 & -0.3365660 \\ \mathrm{C} & -0.0551320 & -2.8388540 & -0.9523500 \\ \mathrm{C} & 2.5664750 & -3.7320690 & -0.5069700 \\ \mathrm{H} & 3.5888500 & -4.0766200 & -0.3640590 \\ \mathrm{C} & -3.8720190 & -0.0723330 & 0.7461480 \\ \mathrm{C} & 3.3423960 & -1.3824840 & -0.0077850 \\ \mathrm{C} & -4.2428460 & -0.6808080 & 1.9543210 \\ \mathrm{C} & -3.9981090 & -2.1147280 & 2.3598590 \\ \mathrm{C} & -5.2555540 & -0.7567860 & 4.1683310 \\ \mathrm{C} & -4.9379730 & 0.0450430 & 2.9326550 \\ \mathrm{C} & -3.1775780 & -2.1080030 & 3.6683100 \\ \mathrm{H} & -3.0333060 & -3.1429710 & 3.9986300 \\ \mathrm{H} & -2.1870340 & -1.6896020 & 3.4700140 \\ \mathrm{C} & -3.9267690 & -1.2862460 & 4.7503420 \\ \mathrm{H} & -4.1423510 & -1.9022480 & 5.6308200\end{array}$




$\begin{array}{lrr}-3.3160680 & -0.4432140 & 5.0870520 \\ -5.7865080 & -0.1473730 & 4.9074070 \\ -3.4691880 & -2.6745930 & 1.5852450 \\ -6.1113670 & -1.9718870 & 3.7502280 \\ -6.2989920 & -2.5966290 & 4.6309000 \\ -7.0837970 & -1.6269690 & 3.3858040 \\ -5.3647130 & -2.7736350 & 2.6525420 \\ -5.1976940 & -3.8081640 & 2.9728820 \\ -5.9547220 & -2.8123220 & 1.7315640 \\ -4.1791650 & 1.2839550 & 0.5583070 \\ -3.7654330 & 2.1582130 & -0.6031730 \\ -5.2277950 & 3.4084710 & 1.1301500 \\ -4.9122810 & 1.9881220 & 1.5254820 \\ -5.0359470 & 2.7033930 & -1.2919800 \\ -4.7370780 & 3.4299490 & -2.0569350 \\ -5.5627980 & 1.8938540 & -1.8075300 \\ -5.9553800 & 3.3679960 & -0.2324490 \\ -6.2195350 & 4.3881800 & -0.5323760 \\ -6.8898670 & 2.8085360 & -0.1260880 \\ -5.8523190 & 3.8976640 & 1.8851560 \\ -3.1334130 & 1.6157070 & -1.3124830 \\ -3.9035020 & 4.1736510 & 0.9310760 \\ -4.1256310 & 5.1658200 & 0.5211240 \\ -3.4117920 & 4.3256860 & 1.8964110 \\ -2.9903560 & 3.3671900 & -0.0251270 \\ -2.6381850 & 3.9954730 & -0.8530850 \\ -2.1103390 & 3.0009800 & 0.5082450 \\ -5.2931540 & 1.3719220 & 2.7124620 \\ -5.8583740 & 1.9226840 & 3.4628960 \\ 4.7434990 & 0.4927520 & -0.6939110 \\ 5.0590990 & 1.4055850 & -1.8534510 \\ 3.1244540 & -0.2444350 & -2.3399220 \\ 3.7069900 & -0.4137760 & -0.9573210 \\ 5.4424520 & 0.5024490 & -3.0479320 \\ 5.6875220 & 1.1305820 & -3.9115150 \\ 6.3403790 & -0.0699440 & -2.7963200 \\ 4.2577340 & -0.4453890 & -3.3723070 \\ 3.8597700 & -0.2408730 & -4.3726310 \\ 4.5825150 & -1.4901800 & -3.3622450 \\ 2.3102710 & -0.9481150 & -2.5285000 \\ 5.8799190 & 2.0877130 & -1.6066520 \\ 2.6133070 & 1.2056000 & -2.4531820 \\ 2.1290190 & 1.3557160 & -3.4248470 \\ 1.8477490 & 1.3530160 & -1.6877630 \\ 3.7926610 & 2.1909840 & -2.2619930 \\ 4.0026760 & 2.7324060 & -3.1919870\end{array}$

3.5421500

5.0799080

5.7502880

3.8295230

4.0333860

4.6809190

5.1524920

4.2692780

3.5591030

3. 5186180

2. 5776620

3.0059440

6.5582520

5.1577590

5.0428780

5. 3812610

6.3012990

6.7283760

7.1132320

5.4378170

6.2603590

1. 5696130

1.8370020

1.2391490

$-0.2707070$

$-0.7547600$

0.2564430

1. 3791560

1. 4138600

$-0.8612910$

$-0.9112090$

2.9121990

1.7790930

$-0.4863620$

$-1.7271450$

- 3.3168940

$-3.9824380$

$-3.9658640$

$-2.5392290$

$-1.5105840$

$-2.0704690$

$-3.4458290$

$-4.4015990$

$-2.1921720$

-1.2503600
-5.0079550
2.9463900

$-0.5176700$

$-0.7284930$

$-2.4055310$

$-1.4207570$

$-0.5979320$

$-0.7566650$

0.4165200

$-1.6378470$

$-2.3647770$

$-1.1634780$

$-3.0922500$

$-0.0046500$

$-3.8984020$

$-3.7455320$

$-2.1724720$

$-2.3644740$

$-2.2729180$

0.4352590

1.1230550

$-4.6415000$

$-6.1136720$

$-6.5843240$

$-6.2655340$

$-5.2800410$

$-4.2010630$

$-6.6794870$

$-7.6562390$

$-7.1830880$

$-5.8439600$

$-6.3142840$

$-6.0735480$

$-5.8518120$

$-4.8575090$

$-1.7401770$

$-1.9108170$

$-3.3937540$

$-3.9932190$

$-3.0475000$

$-2.3634310$

$-1.3228640$

$-3.5120180$

$-4.2329100$

$-2.2650570$

$-1.5300950$
$-1.5099940$

1.4607710

2.7934620

2. 3370870

1.2103810

3.8981120

4.8745390

3.8955840

3.6498110

4.4690690

3.5965470

2.1266160

2.9462670

2. 5179500

3.3332200

1. 6100250

2. 8288120

3.8195710

2. 1023260

0.5125060

0.7070730

$-0.8443090$

$-1.0227890$

$-2.3576290$

$-2.4599990$

$-1.3740860$

$-1.0754260$

$-0.1990940$

$-2.4899010$

$-2.3743630$

$-0.4440460$

$-0.9877120$

$-3.1618200$

$-3.4506580$

$-1.6387360$

$-2.6166820$

$-3.9578710$

$-4.3617280$

$-4.3181720$

$-3.6642420$

$-2.4420250$

$-4.7159920$

$-5.3582830$

$-5.3282770$

-4.3907800
-3.9281220 


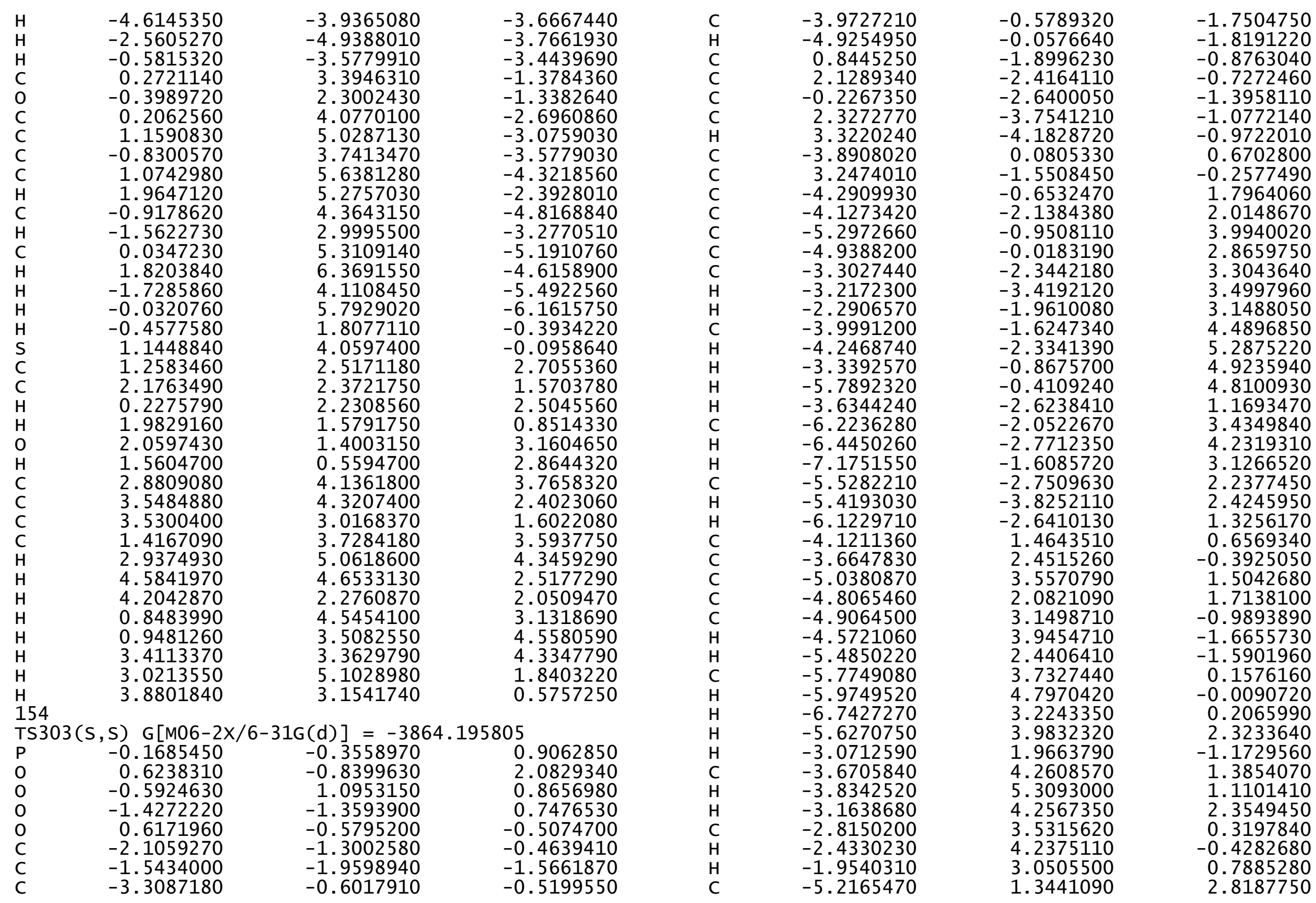




\begin{tabular}{|c|c|c|c|}
\hline & & & \\
\hline $\mathrm{H}$ & -5.7444890 & 1.8273910 & 3.6395300 \\
\hline $\begin{array}{l}\mathrm{C} \\
\mathrm{C}\end{array}$ & $\begin{array}{l}4.7750690 \\
5.1660000\end{array}$ & $\begin{array}{l}0.3064110 \\
1.3490550\end{array}$ & $\begin{array}{l}-0.6657710 \\
-1.6842080\end{array}$ \\
\hline c & 3.1495710 & -0.0998300 & -2.4176140 \\
\hline $\mathrm{C}$ & 3.6902580 & -0.4882830 & -1.0623500 \\
\hline C & 5.5199700 & 0.5982880 & -2.9882310 \\
\hline $\mathrm{H}$ & 5.8109230 & 1.3249060 & -3.7549190 \\
\hline $\mathrm{H}$ & 6.3829310 & -0.0505800 & -2.8107320 \\
\hline C & 4.2927830 & -0.2311850 & -3.4501740 \\
\hline $\mathrm{H}$ & 3.9291180 & 0.1220710 & -4.4216360 \\
\hline $\mathrm{H}$ & 4.5598050 & -1.2855160 & -3.5698550 \\
\hline $\mathrm{H}$ & 2.2998030 & -0.7210850 & -2.7105980 \\
\hline H & 6.0197980 & 1.9404360 & -1.3358550 \\
\hline C & 2.7274870 & 1.3809480 & -2.3450610 \\
\hline $\mathrm{H}$ & 2.2863300 & 1.6892560 & -3.2998460 \\
\hline $\mathrm{H}$ & 1.9455480 & 1.4710460 & -1.5875880 \\
\hline C & 3.9548950 & 2.2575850 & -1.9921730 \\
\hline $\mathrm{H}$ & 4.2154500 & 2.9173330 & -2.8282520 \\
\hline $\mathrm{H}$ & 3.7293810 & 2.9069280 & -1.1395360 \\
\hline C & 5.0095240 & -1.0104770 & 1.3311690 \\
\hline C & 5.6454740 & -1.4480940 & 2.6249030 \\
\hline C & 3.6284540 & -2.9165280 & 1.9276290 \\
\hline C & 3.9120510 & -1.8003330 & 0.950250 \\
\hline C & 4.5707990 & -1.4027690 & 3.7308010 \\
\hline $\mathrm{H}$ & 5.0169840 & -1.7239930 & 1670700 \\
\hline $\mathrm{H}$ & 4.2234380 & -0.3733780 & 3.865157 \\
\hline C & 3.3899500 & -2.3246620 & 3.3343430 \\
\hline $\mathrm{H}$ & 3.2949690 & -3.1556450 & 4.0427020 \\
\hline $\mathrm{H}$ & 2.4401870 & -1.7872200 & 3.345515 \\
\hline $\mathrm{H}$ & 2.7652480 & -3.5117830 & 1 \\
\hline $\mathrm{H}$ & 6.4958360 & -0.8069360 & 2.881747 \\
\hline C & 4.9023820 & -3.7892290 & 2.004772 \\
\hline $\mathrm{H}$ & 4.7304210 & -4.6106360 & 2.708993 \\
\hline $\mathrm{H}$ & 5.1023660 & -4.2385430 & 1.026412 \\
\hline C & 6.1026490 & -2.9158540 & 2.4606810 \\
\hline $\mathrm{H}$ & 6.5010160 & -3.2717820 & 3.4175000 \\
\hline $\mathrm{H}$ & 6.9177990 & -2.9668790 & 1.7326510 \\
\hline C & 5.4422850 & 0.0394880 & 0.5276600 \\
\hline $\mathrm{H}$ & 6.3028350 & 0.6392960 & 0.8229360 \\
\hline C & -0.0015110 & -3.9882100 & -1.7077940 \\
\hline $\mathrm{C}$ & -1.0787760 & -4.9470060 & -2.1487510 \\
\hline C & -1.0475050 & -6.2054830 & -1.2615160 \\
\hline $\mathrm{C}$ & 0.3607270 & -6.8451150 & -1.178829 \\
\hline C & 1.4355310 & -5.9979920 & -1.8877960 \\
\hline C & 1.2794660 & -4.5398930 & -1.5451660 \\
\hline $\mathrm{H}$ & -0.9094980 & -5.2401370 & -3.19538 \\
\hline
\end{tabular}
$-1.7766980$
0.3544200
1. 3287250
$-2.0659360$
$-1.3809240$
0.6479590
2. 4371080
$-3.4392470$
$-4.1316430$
$-4.1635600$
$-2.7478250$
$-1.6871950$
$-2.2165710$
$-3.5923100$
$-4.6495250$
$-2.4310510$
$-1.4049400$
$-5.1450160$
$-4.7868620$
$-2.7682180$
$-0.7758850$
0.5606960
$-0.2040050$
0.6170750
1.6631490
$-0.3928890$
1.6967310
2.4477400
$-0.3616970$
$-1.1974160$
0.6834670
2. 5146760
$-1.1517150$
0.7093860
$-0.3222230$
1.4293580
1. 3978310
2. 3269460
0.3557710
2. 1002540
2.1209920
1. 5766210
3.0933410
3.7980120
3.7160500
1.6108960

\begin{tabular}{ll}
-6.9282390 & -1.6396550 \\
-7.8477420 & -1.6171060 \\
-6.1257690 & -2.9737950 \\
-4.4783990 & -2.1057550 \\
-5.9157820 & -0.2603560 \\
-6.9622850 & -0.1296670 \\
-6.3521960 & -1.6260260 \\
-1.1959440 & -2.8753470 \\
-1.1930460 & -4.2139320 \\
-2.6231010 & -4.7759930 \\
-3.2392530 & -4.8687420 \\
-2.4229600 & -4.0996390 \\
-1.8828970 & -2.7938340 \\
-0.5392530 & -4.9140950 \\
-2.6295030 & -5.7562580 \\
-3.3117130 & -5.9138560 \\
-1.5585340 & -4.7168230 \\
-0.7909620 & -4.1193650 \\
-3.2283560 & -4.1095740 \\
-4.2614930 & -4.4783280 \\
-3.0073680 & -3.9608710 \\
3.5568190 & -0.9675020 \\
2.5309870 & -1.0784550 \\
4.3852330 & -2.1989000 \\
5.2864890 & -2.4258560 \\
4.2375280 & -3.1593780 \\
6.0317650 & -3.5982270 \\
5.3876460 & -1.6837030 \\
4.9958270 & -4.3232870 \\
3.5328650 & -2.9784290 \\
5.8913600 & -4.5451930 \\
6.7228000 & -3.7740800 \\
4.8873760 & -5.0593570 \\
6.4788230 & -5.4578200 \\
1.9229630 & -0.2095280 \\
3.9887840 & 0.4144580 \\
2.0674310 & 2.9658430 \\
2.0252380 & 1.8314590 \\
1.8770000 & 2.7155250 \\
1.3526250 & 1.0068430 \\
0.8506980 & 3.2696370 \\
0.0940950 & 2.8508660 \\
3.4190690 & 4.2603410 \\
3.7491910 & 2.9435560 \\
2.5739550 & 1.9674310 \\
3.1317860 & 4.0160400 \\
& \\
\hline
\end{tabular}




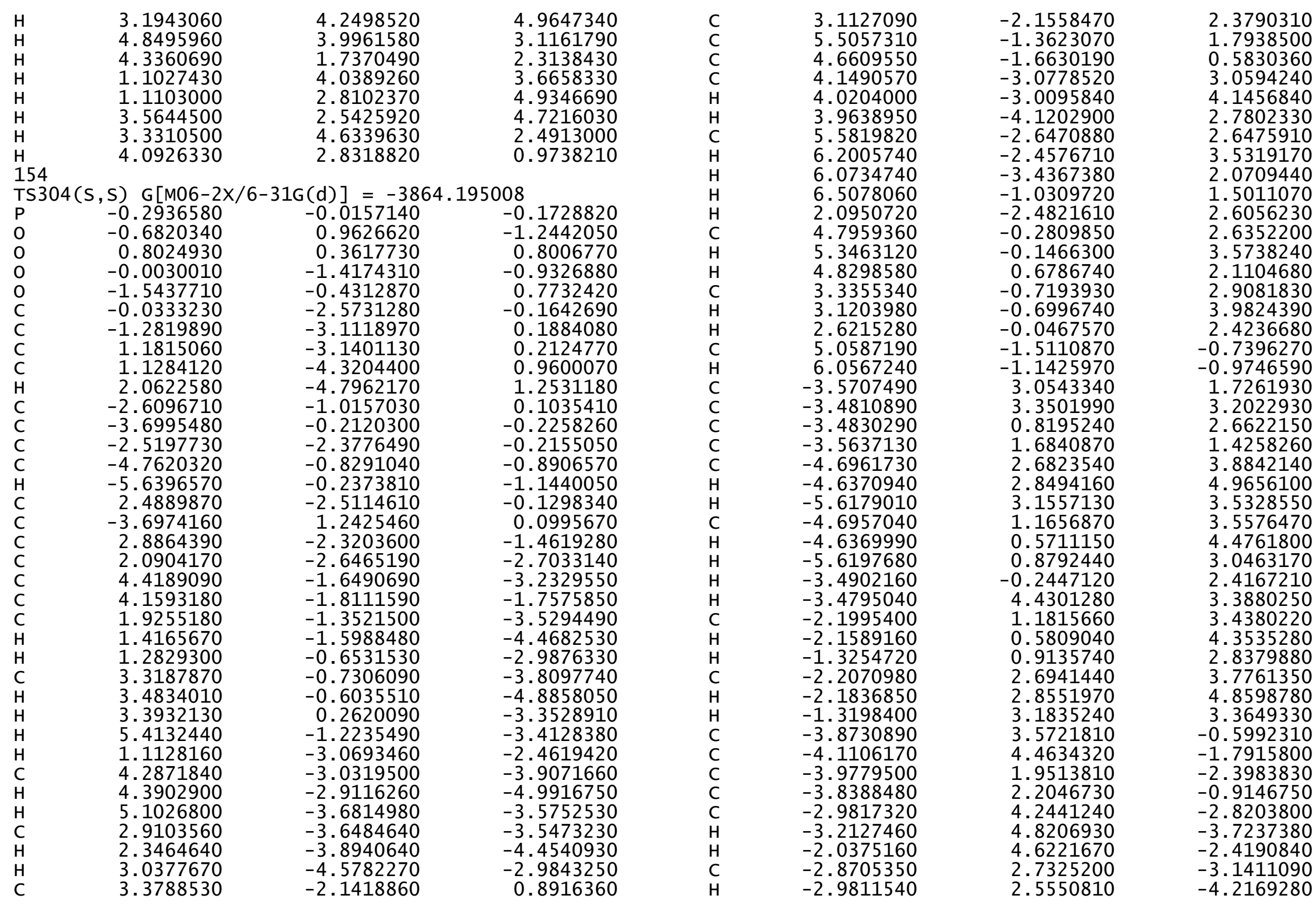




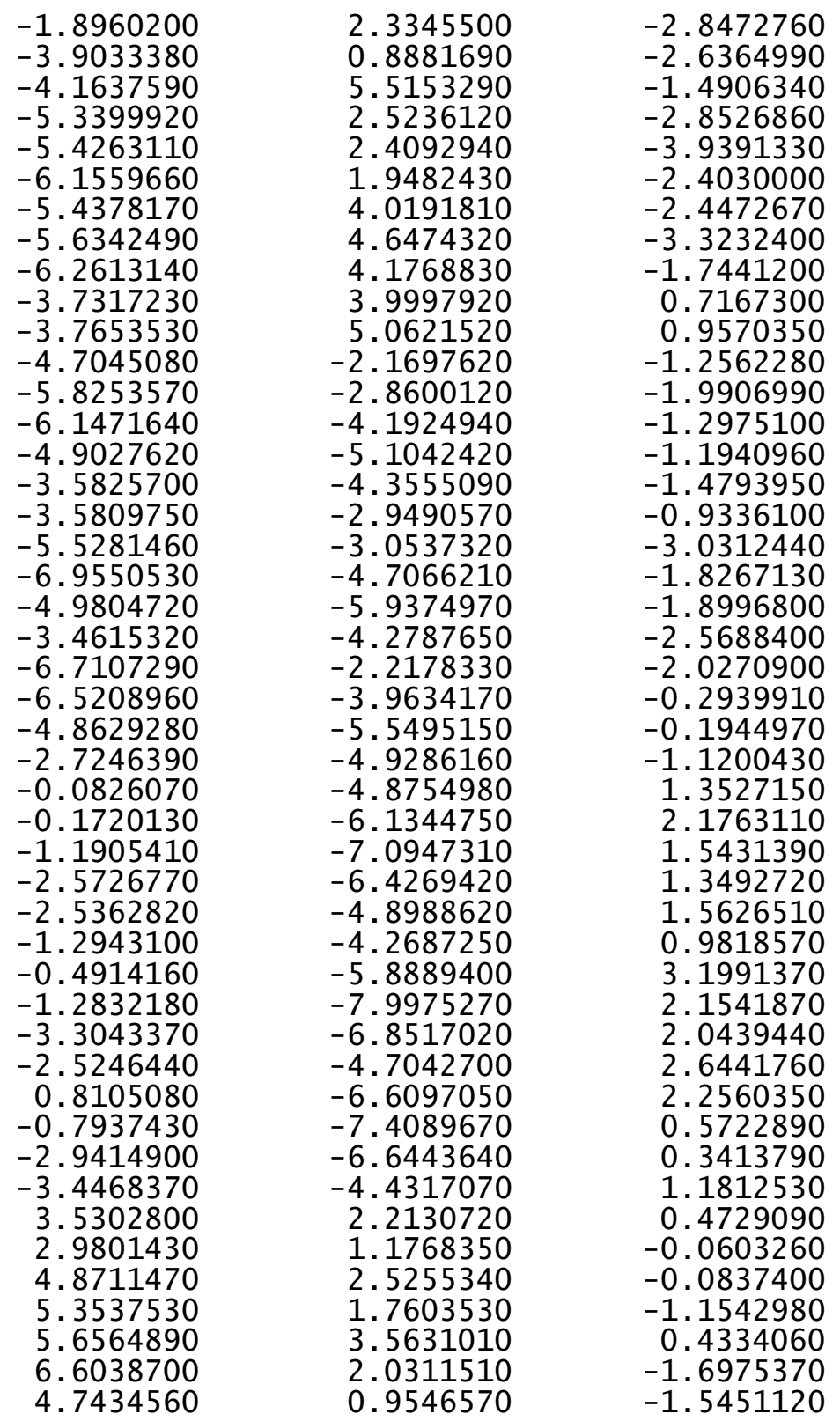

\begin{abstract}
6.9085170
5.2787540

7. 3837330

6.9694900

7.5151560

8.3614590

2.0523900

2.8149210

0.6951440

0.4264180

1. 1549980

0.2354370

$-0.7450590$

$-0.9388460$

0.5008940

0.6971910

0.0748540

1.1717180

0.9201510

0.2529560

$-1.0226650$

2.2597850

0.9739960

$-0.5707770$
\end{abstract}

$\begin{array}{ll}\mathrm{H} & 1.7702420 \\ \mathrm{H} & 0.3622490\end{array}$
3.8245760
4.1550590
3.0610290
1.4363930
4.6261740
3.2705130
0.8669580
3.1505440
3.5378150
3.4402370
2.6599390
2.4521440
3.4377060
2.4529670
6.0423260
5.9476550
4.6674590
4.8600570
6.9788990
6.8113160
4.7266520
4.9037930
4.8718930
6.0460380
5.9556780
4.5060570

$-0.1084920$

1.2600500

$-1.1746010$

$-2.5291570$

0.2999860

$-1.5976090$

0.3460220

1.6805570

$-0.6685980$

0.7709410

$-1.1243430$

1.1763250

$-0.6693290$

$-0.8578800$

$-0.5188040$

0.9951170

1.5592340

$-1.2215560$

$-0.8976920$

1.4983560

1.5433310

$-1.0844630$

$-2.2983760$

$-0.7541850$

1.2221200

2.6019640 
wB97XD/6-31G(d) Optimized Cartesion Coordinates $(\AA)$ and absolute solution phase free energies $(G)$ in hartrees. Reported free energies were computed using the RRHO model at the

wB97XD/6-31G(d) level of theory. Free energies computed with the Quasi-RRHO model and/or at the wB97XD/6-

$311+\mathrm{G}(\mathrm{d}, \mathrm{p}) / / \mathrm{wB} 97 \mathrm{XD} / 6-31 \mathrm{G}(\mathrm{d})$ level are given in the appropriate tables above.

\begin{tabular}{lrrr} 
C48 & \multicolumn{4}{c}{} \\
$\mathrm{TS} 1(\mathrm{R}, \mathrm{R})$ & $\mathrm{G}[\mathrm{WB} 97 \mathrm{XD} / 6-31 \mathrm{G}(\mathrm{d})]=-4125.319776$ & \\
$\mathrm{O}$ & -0.1667490 & -1.0749870 & -0.0449580 \\
$\mathrm{O}$ & 1.7354170 & -0.1143820 & -1.3868250 \\
$\mathrm{C}$ & -0.0129700 & -2.3085080 & -0.6459370 \\
$\mathrm{C}$ & 1.2582660 & -2.8157670 & -0.8156500 \\
$\mathrm{C}$ & -1.1948310 & -3.0059480 & -1.0106690 \\
$\mathrm{C}$ & 1.4037470 & -4.0416740 & -1.5440420 \\
$\mathrm{C}$ & -1.0429560 & -4.2304670 & -1.6087640 \\
$\mathrm{C}$ & 0.2353450 & -4.7584670 & -1.9218700 \\
$\mathrm{H}$ & -1.9232640 & -4.8107300 & -1.8698240 \\
$\mathrm{C}$ & 2.5908820 & -0.7415030 & -0.5084780 \\
$\mathrm{C}$ & 3.6677990 & 0.0290040 & 0.0068360 \\
$\mathrm{C}$ & 2.4186490 & -2.0833020 & -0.2391720 \\
$\mathrm{C}$ & 4.5995740 & -0.6154840 & 0.7777020 \\
$\mathrm{C}$ & 3.3406140 & -2.7201080 & 0.6591170 \\
$\mathrm{C}$ & 4.4567340 & -1.9794580 & 1.1377770 \\
$\mathrm{H}$ & 5.4634280 & -0.0664560 & 1.1420430 \\
$\mathrm{C}$ & 5.2064020 & -3.8925640 & 2.4287140 \\
$\mathrm{C}$ & 4.0753250 & -4.6185490 & 1.9903490 \\
$\mathrm{C}$ & 3.1685800 & -4.0503030 & 1.1317930 \\
$\mathrm{C}$ & 5.3863830 & -2.5991230 & 2.0135000 \\
$\mathrm{H}$ & 5.9183710 & -4.3557270 & 3.1046430 \\
$\mathrm{H}$ & 3.9174070 & -5.6326750 & 2.3442940 \\
$\mathrm{H}$ & 2.2964340 & -4.6159830 & 0.8256600 \\
$\mathrm{H}$ & 6.2373000 & -2.0197420 & 2.3616570 \\
$\mathrm{C}$ & 2.7625160 & -5.7384280 & -2.6286520 \\
$\mathrm{C}$ & 1.6008430 & -6.4708980 & -2.9661640 \\
$\mathrm{C}$ & 0.3658290 & -5.9862100 & -2.6230310 \\
$\mathrm{C}$ & 2.6679990 & -4.5578850 & -1.9367310 \\
$\mathrm{H}$ & 3.7377230 & -6.1124760 & -2.9251860 \\
$\mathrm{H}$ & 1.6914470 & -7.4082830 & -3.5060820 \\
$\mathrm{H}$ & -0.5365470 & -6.5280190 & -2.8937880 \\
$\mathrm{H}$ & 3.5668230 & -4.0023030 & -1.6921260 \\
$\mathrm{C}$ & -2.5343410 & -2.4059290 & -0.7184420 \\
$\mathrm{C}$ & -3.3078910 & -1.8599840 & -1.7616980
\end{tabular}

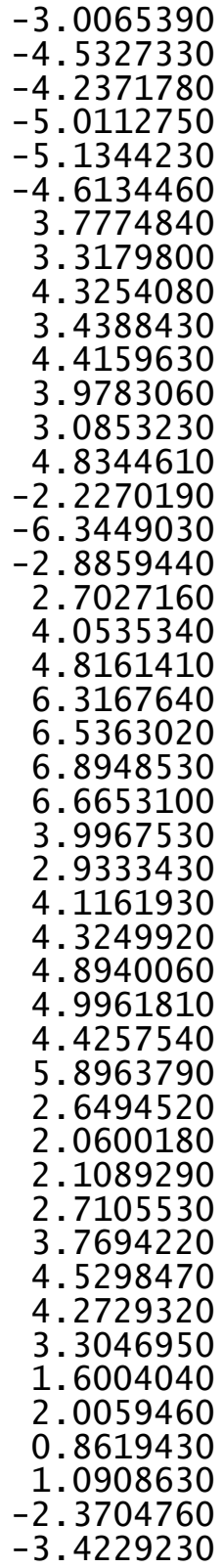

0.6109070

$-1.4482280$

0.8677800

$-0.1426020$

$-2.2500830$

1.8857240

$-0.3138660$

0.6104240

$-1.5416650$

0.2818540

$-1.8281810$

$-0.9332110$

0.9863730

$-2.7817270$

1. 7444510

0.1776500

$-3.2221160$

1.9446960

$-1.2804430$

$-2.5708390$

$-2.8438150$

$-3.2865330$

$-1.9178330$

$-3.5425350$

$-3.8653430$

$-3.6584030$

$-4.3531100$

$-4.5725960$

$-0.2655670$

$-0.5777660$

0.7254260

$-0.1677100$

$-1.4186410$

$-2.1666500$

$-0.4659660$

$-1.7202410$

3. 0404030

2. 7778860

3.2092830

3.9833660

2.4208460

2.7405850

1.6339170

3. 2862400

1.6814250

1. 7982720 


$\begin{array}{rrr}-2.0102850 & -4.9705670 & 0.7333360 \\ -1.8004770 & -5.0319480 & 2.4916320 \\ -2.6259760 & -2.5519480 & 3.1425920 \\ -2.6400030 & -1.4610560 & 3.2153950 \\ -3.6148350 & -2.9286200 & 3.4291700 \\ -1.9068090 & -2.9280600 & 3.8772960 \\ -7.5074470 & -1.2883980 & -0.5052970 \\ -8.4656410 & -0.8417900 & -0.2176480 \\ -7.4230340 & -1.2253310 & -1.5965010 \\ -7.5240720 & -2.3477710 & -0.2288990 \\ -6.3457470 & 0.9330460 & -0.1900850 \\ -5.5154800 & 1.4609350 & 0.2927390 \\ -6.2472110 & 1.0636190 & -1.2740070 \\ -7.2829290 & 1.4086540 & 0.1195200 \\ -3.1789630 & -0.7030760 & -4.0440010 \\ -4.2541520 & -0.5322940 & -4.1707160 \\ -2.7357540 & 0.1816890 & -3.5860990 \\ -2.7495050 & -0.8121090 & -5.0458820 \\ -3.5693750 & -3.1708950 & -3.8827950 \\ -4.6575370 & -3.0341770 & -3.8927090 \\ -3.2319310 & -3.2843460 & -4.9192180 \\ -3.3573550 & -4.1037340 & -3.3526630 \\ 0.2042350 & 0.2359940 & -0.9518890 \\ -0.5778650 & 0.2689770 & -2.2342970 \\ 0.1336460 & 1.4020800 & -0.0097530 \\ -1.8042360 & -2.1183210 & -3.2504920 \\ -6.4930850 & -0.6324080 & 1.2622700 \\ -1.1670200 & -2.7870900 & 1.6097260 \\ 4.6744070 & -0.1257260 & -2.1590260 \\ 4.5487760 & 5.7761860 & -2.2564570 \\ 2.2362990 & 1.0631520 & 1.7988660 \\ -2.9226850 & 2.3871830 & -1.9041170 \\ -1.9949300 & 3.3298980 & -1.2745220 \\ -3.0305000 & 1.4186670 & -1.4118200 \\ -1.1549840 & 2.9103600 & -0.7286670 \\ -1.8459420 & 2.3866280 & -2.8711220 \\ -2.6486970 & 2.5185460 & 2.2052400 \\ -2.0382560 & 1.1719080 & 4.2337320 \\ -1.2592870 & 0.9058740 & 3.1116420 \\ -1.6398400 & 1.6648350 & 2.0139780 \\ -1.0919400 & 1.5996580 & 1.1340200 \\ -3.2458480 & 2.3849880 & 3.8461890 \\ -3.2904300 & 3.6115120 & -2.0890880 \\ -1.2619040 & 1.5764180 & 5.4725050 \\ -0.7306860 & -0.3938110 & \\ 0.0486150 & -0.6585700 & \\ -1.3277320\end{array}$

$\begin{array}{lr}\mathrm{C} & -0.2102740 \\ \mathrm{C} & -1.7753160 \\ \mathrm{H} & -0.4944900 \\ \mathrm{H} & 0.3407890 \\ \mathrm{H} & -2.3678130 \\ \mathrm{C} & -4.0104840 \\ \mathrm{C} & -3.3670600 \\ \mathrm{C} & -1.9985980 \\ \mathrm{C} & -4.2037850 \\ \mathrm{H} & -4.9802350 \\ \mathrm{H} & -3.2488680 \\ \mathrm{H} & -1.2474820 \\ \mathrm{H} & -4.9331360 \\ \mathrm{H} & -4.5855900 \\ \mathrm{H} & -3.3817390 \\ \mathrm{H} & -4.0283630 \\ \mathrm{H} & -1.6250810 \\ \mathrm{O} & 1.0301290 \\ \mathrm{C} & 1.8098720 \\ \mathrm{H} & 1.1856790 \\ \mathrm{H} & 2.3494010 \\ \mathrm{H} & 2.5285230 \\ \mathrm{H} & \end{array}$
$-0.0117190$
0.5104890
$-0.9253440$
$-0.2035950$
0.7013590
4. 3196350
5.2830810
4.7794410
2.9339130
4.7095570
6.2763890
4.8998620
2. 9881900
2. 2147100
4.2378460
5. 3965690
5. 3455820
$-1.5715660$
$-1.9006900$
$-2.3167780$
$-1.0254880$

$\operatorname{TS} 2(R, R) \quad G[W B 97 X D / 6-31 G(d)]=-4125.319953$

\begin{tabular}{lrcr} 
TS2 $(\mathrm{R}, \mathrm{R})$ & \multicolumn{4}{c}{$\mathrm{G}[\mathrm{WB} 97 \times \mathrm{D} / 6-31 \mathrm{G}(\mathrm{d})]=-4125.319953$} \\
$\mathrm{O}$ & 0.4394240 & 0.7609910 & -0.2673810 \\
$\mathrm{O}$ & -1.6254910 & 0.0956340 & -1.5593400 \\
$\mathrm{C}$ & 0.4703500 & 1.9903120 & -0.8903520 \\
$\mathrm{C}$ & -0.7068010 & 2.6902580 & -1.0491200 \\
$\mathrm{C}$ & 1.7404650 & 2.5002460 & -1.2662130 \\
$\mathrm{C}$ & -0.6713080 & 3.9114520 & -1.7986010 \\
$\mathrm{C}$ & 1.7733230 & 3.7283840 & -1.8765640 \\
$\mathrm{C}$ & 0.5883420 & 4.4386560 & -2.1940900 \\
$\mathrm{H}$ & 2.7308250 & 4.1672370 & -2.1420810 \\
$\mathrm{C}$ & -2.3641280 & 0.8736460 & -0.6926340 \\
$\mathrm{C}$ & -3.5620820 & 0.3147310 & -0.1736560 \\
$\mathrm{C}$ & -1.9604870 & 2.1680450 & -0.4414970 \\
$\mathrm{C}$ & -4.3673720 & 1.1266770 & 0.5826860 \\
$\mathrm{C}$ & -2.7544110 & 2.9687160 & 0.4461570 \\
$\mathrm{C}$ & -3.9909540 & 2.4507780 & 0.9183520 \\
$\mathrm{H}$ & -5.3117730 & 0.7399490 & 0.9557480 \\
$\mathrm{C}$ & -4.4343060 & 4.5231870 & 2.1002720 \\
$\mathrm{C}$ & -3.1746700 & 5.0159500 & 1.6871390 \\
$\mathrm{C}$ & -2.3561570 & 4.2590940 & 0.8887040 \\
$\mathrm{C}$ & -4.8223110 & 3.2600520 & 1.7365510 \\
$\mathrm{H}$ & -5.0780670 & 5.1376390 & 2.7224130 \\
$\mathrm{H}$ & -2.8502660 & 6.0004260 & 2.0105800
\end{tabular}




$\begin{array}{lr}\mathrm{H} & -1.3896940 \\ \mathrm{H} & -5.7720980 \\ \mathrm{C} & -1.7690550 \\ \mathrm{C} & -0.5131230 \\ \mathrm{C} & 0.6380690 \\ \mathrm{C} & -1.8469530 \\ \mathrm{H} & -2.6797660 \\ \mathrm{H} & -0.4658310 \\ \mathrm{H} & 1.6089450 \\ \mathrm{H} & -2.8162080 \\ \mathrm{C} & 2.9875410 \\ \mathrm{C} & 3.7193820 \\ \mathrm{C} & 3.4341530 \\ \mathrm{C} & 4.8932320 \\ \mathrm{C} & 4.6012280 \\ \mathrm{C} & 5.3449820 \\ \mathrm{H} & 5.4738060 \\ \mathrm{H} & 4.9441690 \\ \mathrm{C} & -3.9289210 \\ \mathrm{C} & -3.7971360 \\ \mathrm{C} & -4.4174370 \\ \mathrm{C} & -4.1835930 \\ \mathrm{C} & -4.7802810 \\ \mathrm{C} & -4.6727060 \\ \mathrm{H} & -4.0932010 \\ \mathrm{H} & -5.1587250 \\ \mathrm{C} & 2.6958080 \\ \mathrm{C} & 6.6391240 \\ \mathrm{C} & 3.2938580 \\ \mathrm{C} & -3.2600700 \\ \mathrm{C} & -5.0493960 \\ \mathrm{C} & -4.5816170 \\ \mathrm{C} & -6.0636350 \\ \mathrm{H} & -6.5160120 \\ \mathrm{H} & -6.6285590 \\ \mathrm{H} & -6.1759840 \\ \mathrm{C} & -3.7678780 \\ \mathrm{H} & -2.7126800 \\ \mathrm{H} & -4.1351910 \\ \mathrm{H} & -3.8414900 \\ \mathrm{C} & -4.1213180 \\ \mathrm{H} & -4.3525390 \\ \mathrm{H} & -4.2371160 \\ \mathrm{H} & -3.0720860 \\ \mathrm{C} & -6.5204270 \\ \mathrm{H} & -7.1862950 \\ & \end{array}$

$\begin{array}{rr}4.6496710 & 0.5916600 \\ 2.8536510 & 2.0741780 \\ 5.7751770 & -2.9044830 \\ 6.3203050 & -3.2588750 \\ 5.6617080 & -2.9138730 \\ 4.6053970 & -2.1927390 \\ 6.2851130 & -3.2034350 \\ 7.2520430 & -3.8140120 \\ 6.0593590 & -3.1974250 \\ 4.1921840 & -1.9362860 \\ 1.7307530 & -0.9687210 \\ 1.1403280 & -2.0152160 \\ 1.6124340 & 0.3656120 \\ 0.4492350 & -1.7041730 \\ 0.8960770 & 0.6217060 \\ 0.3027770 & -0.3974310 \\ 0.0022190 & -2.5082410 \\ 0.7971040 & 1.6456040 \\ -1.1056140 & -0.4567460 \\ -2.0762430 & 0.5533480 \\ -1.4641220 & -1.7265140 \\ -3.3881680 & 0.2728380 \\ -2.7887240 & -1.9599860 \\ -3.7671500 & -0.9734160 \\ -4.1453730 & 1.0482020 \\ -3.0643440 & -2.9421350 \\ 2.2980130 & 1.5114420 \\ -0.4408170 & -0.1163410 \\ 1.2528280 & -3.4734860 \\ -1.7364420 & 1.9375830 \\ -5.2106640 & -1.2551050 \\ -0.4448440 & -2.8448000 \\ -0.2381200 & -3.1856790 \\ -1.1609230 & -3.5672090 \\ 0.0737890 & -2.3005080 \\ 0.5343820 & -3.9547770 \\ -0.8287380 & -4.0868040 \\ -0.9630650 & -3.8313720 \\ -1.7592340 & -4.5349870 \\ -0.0425780 & -4.8465950 \\ -5.8262160 & -2.3113280 \\ -6.8870800 & -2.4590420 \\ -5.3196010 & -3.2766180 \\ -5.7401570 & -2.0107510 \\ -5.3505760 & -1.6670930 \\ -4.9324050 & -0.9051180\end{array}$
$-6.7179010$
$-6.7792390$
$-4.3991850$
$-5.1316040$
$-4.9261780$
$-4.0006650$
$-2.2141590$
$-2.6557260$
$-1.4184480$
$-1.7699700$
3. 0097920
4.0815440
2. 7337580
2. 4621240
2. 9887520
2.9294730
3. 9860200
2. 2581120
6.6184020
7.4985120
6.6423380
5. 7217840
7.8281410
7.8883170
7.7239600
8. 7714440
3.3766770
4. 4122440
2. 8259760
2. 9403680
4. 1282040
5. 1888980
3.7986750
4.0446630
$-0.1896340$
0.5617200
$-0.3211030$
2. 2468100
6.7801630
1. 6210950
$-4.1985730$
$-4.9103980$
$-2.7636690$
2. 9718490
2. 0413240
2. 8980990

-4.8299460
-6.4057540
-1.6029860
-0.8507390
-2.5572680
-1.3095130
-2.7413410
-3.7227660
-2.8631130
-2.3791230
3.8025650
3.9625690
4.2920200
4.2913020
1.6890620
0.5976080
1.9636660
2.0517390
-1.2284140
-1.8769140
-0.5601770
-1.8526010
0.5312520
1.0501510
1.2878940
-0.0021070
-0.0786210
-0.4117310
-0.8644700
0.0362840
2.3195800
2.0407810
2.4233910
3.2983010
-0.5085780
-0.7840050
-1.5875560
1.5673290
-1.1610040
2.1807690
0.5173210
-5.7710370
-0.7649270
-2.5674100
-3.5211440
-1.5346070

$-2.6113090$

$-1.8088830$

2.9578340

2. 6489880

3. 0774630

3. 9358880

2.4344820

2.6413520

1. 6955030

3. 3687220

1. 5377310

1.7045590

0.5996320

2. 3515620

2.8855910

2.8606530

3. 2489190

3. 6161800

1. 1984500

1. 2619940

2. 0668610

1. 2843970

$-0.1469960$

$-1.1093330$

0.6397490

0.0158320

$-4.2287320$

$-4.3631590$

$-3.7081680$

$-5.2269890$

$-4.1988540$

$-4.2055690$

$-5.2387440$

$-3.7169320$

$-1.0904300$

$-2.3623000$

$-0.0541890$

$-3.4955990$

$-0.9332290$

1. 3395190

$-2.4932040$

$-0.3217090$

1.8731070

$-1.2904470$

$-0.6790290$

$-0.9452900$ 


\begin{tabular}{|c|c|c|c|c|c|c|c|}
\hline $\mathrm{H}$ & 1.0847440 & -3.1318710 & -0.3433580 & C & -3.5642790 & 0.4465930 & -0.3492940 \\
\hline 0 & 2.0913120 & -2.8320190 & -2.4079380 & $\mathrm{C}$ & -1.8956800 & 2.2579860 & -0.3475160 \\
\hline C & 1.9870790 & -2.2162810 & 2.7466060 & C & -4.3870490 & 1.2112670 & 0.4377510 \\
\hline C & 0.9905590 & -0.7082640 & 4.4866980 & $\mathrm{C}$ & -2.7104060 & 2.9939640 & 0.5758980 \\
\hline C & 0.4647880 & -0.5326990 & 3.2031710 & $\mathrm{C}$ & -3.9888240 & 2.4804270 & 0.9246570 \\
\hline $\mathrm{N}$ & 1.0463570 & -1.3918380 & 2.2779280 & $\mathrm{H}$ & -5.3612710 & 0.8224210 & 0.7215760 \\
\hline $\mathrm{H}$ & 0.6683190 & -1.4524710 & 1.3111410 & C & -4.4296190 & 4.4411190 & 2.2845210 \\
\hline $\mathrm{S}$ & 2.2261340 & -1.9540750 & 4.4617120 & $\mathrm{C}$ & -3.1295280 & 4.9190600 & 1.9985660 \\
\hline $\mathrm{s}$ & 2.8630650 & -3.3738740 & 1.8862980 & $\mathrm{C}$ & -2.2924950 & 4.2140490 & 1.1725650 \\
\hline $\mathrm{H}$ & 1.4145190 & -2.0697060 & -2.4355170 & $\mathrm{C}$ & -4.8396940 & 3.2379770 & 1.7722030 \\
\hline C & -0.4527470 & 1.0221530 & 5.2894790 & $\mathrm{H}$ & -5.0879150 & 5.0173650 & 2.9275980 \\
\hline $\mathrm{C}$ & -0.9778430 & 1.1878350 & 3.9954410 & $\mathrm{H}$ & -2.7894440 & 5.8499810 & 2.4418710 \\
\hline C & -0.5082040 & 0.4159960 & 2.9319170 & $\mathrm{H}$ & -1.2957290 & 4.5906000 & 0.9734870 \\
\hline $\mathrm{C}$ & 0.5359280 & 0.0761100 & 5.5369300 & $\mathrm{H}$ & -5.8225870 & 2.8398810 & 2.0101720 \\
\hline $\mathrm{H}$ & -0.8098770 & 1.6286100 & 6.1123330 & C & -1.5050550 & 6.0803380 & -2.4301350 \\
\hline $\mathrm{H}$ & -0.8923220 & 0.5681150 & 1.9299550 & $\mathrm{C}$ & -0.2217100 & 6.6228550 & -2.6731390 \\
\hline $\mathrm{H}$ & 0.9388180 & -0.0390240 & 6.5372480 & $\mathrm{C}$ & 0.8965150 & 5.9025480 & -2.3436480 \\
\hline C & 4.4564160 & -4.5007830 & -1.9553500 & C & -1.6437200 & 4.8500710 & -1.8401130 \\
\hline $\mathrm{C}$ & 3.7324350 & -5.3797380 & -0.9324720 & $\mathrm{H}$ & -2.3888890 & 6.6412270 & -2.7185440 \\
\hline $\mathrm{C}$ & 2.2549820 & -4.9949120 & -0.8072880 & $\mathrm{H}$ & -0.1267670 & 7.6013550 & -3.1335390 \\
\hline $\mathrm{C}$ & 4.3847070 & -3.0237030 & -1.5586240 & $\mathrm{H}$ & 1.8886030 & 6.2992140 & -2.5423550 \\
\hline $\mathrm{H}$ & 5.5051370 & -4.8024140 & -2.0356980 & $\mathrm{H}$ & -2.6335070 & 4.4412210 & -1.6698980 \\
\hline $\mathrm{H}$ & 3.8048080 & -6.4352530 & -1.2105780 & C & 3.0646490 & 1.7462150 & -0.6889730 \\
\hline $\mathrm{H}$ & 1.6975330 & -5.3108570 & -1.6978370 & $\mathrm{C}$ & 3.8570980 & 1.2832780 & -1.7555780 \\
\hline $\mathrm{H}$ & 4.9573840 & -2.8505680 & -0.6401400 & $\mathrm{C}$ & 3.4328380 & 1.4628290 & 0.6452950 \\
\hline $\mathrm{H}$ & 4.8118840 & -2.3805230 & -2.3338830 & $\mathrm{C}$ & 5.0113970 & 0.5522770 & -1.4635370 \\
\hline $\mathrm{H}$ & 4.0054260 & -4.6371770 & -2.9454570 & $\mathrm{C}$ & 4.5815970 & 0.7095130 & 0.8779930 \\
\hline $\mathrm{H}$ & 4.2190610 & -5.2741940 & 0.0455900 & C & 5.3856280 & 0.2432440 & -0.1610930 \\
\hline $\mathrm{H}$ & 1.7730120 & -5.4871580 & 0.0413270 & $\mathrm{H}$ & 5.6423420 & 0.2078080 & -2.2804220 \\
\hline 0 & -1.9461190 & 2.0749740 & 3.6824240 & $\mathrm{H}$ & 4.8652990 & 0.4849230 & 1.8998190 \\
\hline C & -2.4772470 & 2.8848790 & 4.7152600 & C & -3.9569610 & -0.9294620 & -0.7765470 \\
\hline $\mathrm{H}$ & -2.9263130 & 2.2746900 & 5.5077300 & $\mathrm{C}$ & -3.9527550 & -1.9779900 & 0.1655430 \\
\hline $\mathrm{H}$ & -1.7081670 & 3.5374460 & 5.1450280 & C & -4.3499570 & -1.1710130 & -2.1033020 \\
\hline $\mathrm{H}$ & -3.2471080 & 3.4921160 & 4.2422390 & C & -4.3743240 & -3.2432380 & -0.2398520 \\
\hline 148 & & & & C & -4.7529380 & -2.4581360 & \\
\hline $\operatorname{TS} 3(R, R)$ & $\mathrm{G}[\mathrm{WB} 97 \mathrm{XD} /$ & d) $]=-4125$ & & C & -4.7777780 & -3.5060140 & -1.5477640 \\
\hline 0 & 0.4584340 & 0.7727090 & -0.2065710 & $\mathrm{H}$ & -4.3858750 & -4.0503570 & 0.4888120 \\
\hline 0 & -1.5610740 & 0.2916000 & -1.6452730 & $\mathrm{H}$ & -5.0647070 & -2.6501260 & -3.4867870 \\
\hline C & 0.5494660 & 2.0576520 & -0.6989390 & C & 2.6362340 & 2.0162960 & 1.8240310 \\
\hline C & -0.6023930 & 2.8014010 & -0.8420740 & $\mathrm{C}$ & 6.6689060 & -0.5267750 & 0.0981710 \\
\hline C & 1.8466890 & 2.5674100 & -0.9646430 & C & 3. 5258610 & 1.5738020 & -3.2139860 \\
\hline C & -0.5040950 & 4.0887310 & -1.4643160 & $\mathrm{C}$ & -3.5150890 & -1.7727520 & 1.6102360 \\
\hline C & 1.9359790 & 3.8467920 & -1.4525430 & $\mathrm{C}$ & -5.2353180 & -4.8919150 & -1.9657550 \\
\hline C & 0.7844140 & 4.6170920 & -1.7504680 & C & -4.3827960 & -0.0687410 & -3.1522990 \\
\hline $\mathrm{H}$ & 2.9154800 & 4.2796980 & -1.6355920 & $\mathrm{C}$ & -5.8250670 & 0.2348980 & -3.5808040 \\
\hline C & -2.3218630 & 1.0084110 & -0.7457600 & $\mathrm{H}$ & -6.2908000 & -0.6378510 & -4.0530230 \\
\hline
\end{tabular}




-6.4377310
-5.8448680
-3.4978390
-2.4673810
-3.8621980
-3.4940500
-6.5074350
-6.8678160
-6.3167970
-7.3054820
-4.1244500
-3.2181380
-3.8631230
-4.4504850
-4.7238850
-5.4448750
-5.2435890
-4.4015120
-2.4835510
-2.9169830
-1.6384350
-2.1092520
2.9231920
3.9855790
2.6707170
2.3386030
2.8820990
2.8327040
3.8621940
2.1207670
6.6032780
7.4858280
6.5870430
5.7100170
7.8544130
7.9462590
7.7182650
8.7937210
3.5719910
4.5862120
2.9185300
3.2350010
4.4670250
5.5074290
4.2114190
4.4117710

0.5191810

$-0.4054450$

$-0.5914600$

$-1.2930630$

0.4277970

$-5.3095700$

$-6.2794770$

$-5.4016870$

$-4.5727910$

$-5.9351140$

$-5.6426660$

$-6.0548690$

$-6.9111910$

$-1.7611310$

$-0.9863490$

$-2.7265520$

$-1.5711290$

$-2.8135980$

$-3.8185680$

$-2.8375160$

$-2.5576760$

3.5135530

3.6697430

4.0981040

3.9029910

1.2732010

0.1892010

1.5147190

1. 5586660

$-1.4336070$

$-2.0807950$

$-0.8503380$

$-2.0687000$

0.4451620

1.0507060

1.1261160

$-0.0996770$

0.3192720

$-0.0881270$

$-0.4609400$

0.5640860

2.6497130

2. 3039320

2. 8778800

3.5788440

-2.7183250
-4.3031290
-4.3588550
-4.0419810
-4.8885030
-5.0704900
-1.2159630
-1.5769030
-0.1403650
-1.3548870
-1.7863600
-2.3265060
-0.7285620
-2.1628200
2.5558680
2.2756760
2.5349410
3.5861000
2.0621280
2.1210030
1.3693170
3.0599180
2.0230440
2.2444840
1.1341530
2.8641400
3.1402540
3.0053730
3.5677580
3.8736600
1.3312530
1.3688570
2.2590260
1.3216970
0.2013330
-0.7063450
1.0498180
0.3482580
-4.0941200
-4.1775580
-3.6999350
-5.1074630
-3.7759420
-3.7516220
-4.8167560
-3.1999100

\author{
$-0.1567650$ \\ 0.6424290 \\ $-0.3473570$ \\ 2. 5039110 \\ 6.8428590 \\ 1. 5715640 \\ $-3.9835960$ \\ $-5.4782360$ \\ $-3.0347610$ \\ 3.0051680 \\ 2.0459470 \\ 2. 9174570 \\ 1.0736390 \\ 2. 1801560 \\ 1.8289030 \\ 0.7470850 \\ 0.2983420 \\ 0.9222080 \\ 0.5975950 \\ 1.9688990 \\ 2.7479190 \\ 1. 5048560 \\ $-0.7165290$ \\ $-1.1603120$ \\ $-0.6437160$ \\ 0.2416580 \\ $-1.1137250$ \\ $-0.9667430$ \\ 0.5816520 \\ 4.5168660 \\ 3.7431420 \\ 2. 2626210 \\ 4.4284510 \\ 5.5676860 \\ 3.8242310 \\ 1.7465340 \\ 4.9589590 \\ 4.8909780 \\ 4.1130480 \\ 4.1854830 \\ 1.7403850 \\ $-2.0900480$ \\ $-2.6648570$ \\ $-3.1825440$ \\ -1.9072000
-3.3839410
}

-0.3885790
-0.5449000
-1.5694730
1.9593790
-1.1660830
1.9040250
0.8457530
-4.8455640
-0.7935440
-2.4457990
-3.4550020
-1.4559910
-3.0991060
-2.5805140
-2.5429420
-1.2103920
-0.8937140
-1.6618780
-1.6132070
-2.4674990
-3.6074740
-1.8174530
0.4571800
0.7676490
0.0966840
-0.5299110
0.9812710
0.3556020
-0.7550360
-4.3034410
-5.2868580
-4.9067290
-2.8792380
-4.6016410
-6.3033760
-5.1154970
-2.8099430
-2.1575730
-4.3251300
-5.2998430
-5.4883120
1.7074310
2.4211150
1.7461580
2.9955540
3.1031670

$-1.1847130$

$-2.4476960$

$-0.2760460$

$-3.2617960$

$-0.7775460$

1.5967610

$-2.7048100$

$-3.0350800$

1.6838970

$-1.4445150$

$-0.9837760$

$-0.9925360$

$-0.6555730$

$-2.6253740$

2. 5461080

4.3766500

3.0911050

2. 1153970

1.1292750

4.2902540

1. 6134920

$-2.6037610$

5.2708110

3.9728880

2. 8642800

5.4750690

6.1309390

1.8627680

6.4799790

$-2.2495870$

$-1.3678590$

$-1.2665770$

$-1.6950350$

$-2.3137580$

$-1.7639740$

$-2.2119410$

$-0.7383980$

$-2.3751050$

$-3.2686920$

$-0.3636560$

$-0.5026180$

3.6980480

4.7773830

5.4691090

5.3232610

4.3269490 
148

TS4 $(R, R) \quad G[W B 97 X D / 6-31 G(d)]=-4125.319003$

$\begin{array}{lrrr}\mathrm{O} & -0.2418390 & -1.0212610 & -0.0738950 \\ \mathrm{O} & 1.7634880 & -0.1180670 & -1.3058690 \\ \mathrm{C} & -0.1533970 & -2.2326960 & -0.7309500 \\ \mathrm{C} & 1.0870810 & -2.8106960 & -0.9007240 \\ \mathrm{C} & -1.3671950 & -2.8387160 & -1.1490070 \\ \mathrm{C} & 1.1740780 & -4.0033320 & -1.6900790 \\ \mathrm{C} & -1.2767860 & -4.0405460 & -1.8035870 \\ \mathrm{C} & -0.0268710 & -4.6282340 & -2.1237000 \\ \mathrm{H} & -2.1848790 & -4.5543340 & -2.1049450 \\ \mathrm{C} & 2.5438350 & -0.8506000 & -0.4397610 \\ \mathrm{C} & 3.6518840 & -0.1877030 & 0.1533670 \\ \mathrm{C} & 2.2741520 & -2.1898490 & -0.2531680 \\ \mathrm{C} & 4.5050460 & -0.9346260 & 0.9235830 \\ \mathrm{C} & 3.1164660 & -2.9377410 & 0.6371400 \\ \mathrm{C} & 4.2577080 & -2.3031040 & 1.2010220 \\ \mathrm{H} & 5.3880180 & -0.4661340 & 1.3491930 \\ \mathrm{C} & 4.8254130 & -4.3351580 & 2.4009260 \\ \mathrm{C} & 3.6694180 & -4.9565360 & 1.8752790 \\ \mathrm{C} & 2.8383520 & -4.2784870 & 1.0203790 \\ \mathrm{C} & 5.1065820 & -3.0344690 & 2.0730100 \\ \mathrm{H} & 5.4772510 & -4.8855280 & 3.0720430 \\ \mathrm{H} & 3.4334110 & -5.9782440 & 2.1564860 \\ \mathrm{H} & 1.9465280 & -4.7657990 & 0.6444540 \\ \mathrm{H} & 5.9783150 & -2.5356580 & 2.4879660 \\ \mathrm{C} & 2.4519670 & -5.7202140 & -2.8382350 \\ \mathrm{C} & 1.2558420 & -6.3635550 & -3.2326610 \\ \mathrm{C} & 0.0447590 & -5.8243900 & -2.8854680 \\ \mathrm{C} & 2.4135740 & -4.5734290 & -2.0867850 \\ \mathrm{H} & 3.4089310 & -6.1362830 & -3.1380140 \\ \mathrm{H} & 1.3014390 & -7.2755190 & -3.8198410 \\ \mathrm{H} & -0.8819390 & -6.2974980 & -3.1991680 \\ \mathrm{H} & 3.3379430 & -4.0843210 & -1.7992050 \\ \mathrm{C} & -2.6766300 & -2.1785980 & -0.8508410 \\ \mathrm{C} & -3.4054120 & -1.5540710 & -1.8823690 \\ \mathrm{C} & -3.1708370 & -2.1789250 & 0.4710010 \\ \mathrm{C} & -4.6082370 & -0.9194940 & -1.5640420 \\ \mathrm{C} & -4.3784930 & -1.5340390 & 0.7325520 \\ \mathrm{C} & -5.1089740 & -0.8941350 & -0.2654780 \\ \mathrm{H} & -5.1760850 & -0.4397180 & -2.3572580 \\ \mathrm{H} & -4.7735560 & -1.5247500 & 1.7439430 \\ \mathrm{C} & 3.8808280 & 1.2690220 & -0.0924090 \\ \mathrm{C} & 3.4776710 & 2.2189050 & 0.8651260 \\ \mathrm{C} & 4.4943380 & 1.6806290 & -1.2880220 \\ \mathrm{C} & 3.7305260 & 3.5674680 & \\ & & & \end{array}$

4.7119950

4.3414670

3. 4293530

5.1809200

$-2.4426490$

$-6.4262100$

$-2.9637120$

2.7929130

4.5650530

4. 9231940

6.4396480

6.7561840

6.9819940

6.7396020

4. 1507450

3. 0731720

4. 3597400

4. 4379140

3. 6872220

3.8155350

3.9550420

2. 6283220

6.0431400

6.6686710

6.4051570

6.1852120

3. 8141540

4.5551800

4. 3475870

3. 3068420

1. 7177030

2. 1514960

1.0038010

1. 1727190

$-2.6543550$

- 3.7201040

$-2.2990460$

$-2.1177530$

$-2.8430270$

$-2.8059500$

$-3.8537790$

$-2.1554720$

$-7.6002390$

$-8.5499010$

-7.5020780
-7.6466450
3.0407460

4.0004140

4. 3117460

3. 3591970

$-2.9205780$

$-0.2143740$

$-1.6188060$

1.8050000

5. 4796110

0.6861780

0.7320370

1.7062270

0.5502150

$-0.0318060$

0.9041180

0.8253930

1.8915790

0.1506550

5. 9724610

7. 0501170

5.4668510

5.7751410

5.8017490

5.4654790

5. 3129920

6.8810910

1.5806150

0.8228820

2. 5120920

1.2506160

2.7921750

3.7392250

2. 9982570

2. 3636040

$-4.4386630$

$-4.6787910$

$-4.8240960$

$-4.9644630$

$-2.4776150$

$-1.3911360$

$-2.8167370$

$-2.9168250$

$-0.8996870$

$-0.4361750$

-0.8115790
-1.9648230
$-1.5011960$

$-0.5624610$

1.3437390

$-2.4298520$

1. 5886990

0.0613240

$-3.3395100$

2. 1614920

$-0.8218080$

$-2.3571340$

$-2.5863390$

$-2.9765390$

$-1.6522960$

$-3.3122590$

$-3.6646880$

$-3.4926780$

$-4.0922700$

$-4.4066770$

$-1.9807330$

$-2.1317990$

$-2.9158060$

$-1.7833920$

$-1.0753200$

$-0.2419140$

$-1.9871000$

$-1.1983880$

3. 2860640

3.0174070

3. 5099060

4. 2003220

2. 6265740

2. 9669120

1.8255390

3.4745370

1. 4616010

1.5536260

0.5025470

2. 2596160

2. 9990010

3. 1156220

3. 2542000

3.7289230

$-0.6509980$

$-0.3616690$

$-1.7393290$

$-0.4016130$ 


$\begin{array}{rrr}-6.3879640 & 1.2833870 & -0.2630270 \\ -5.5487090 & 1.7760800 & 0.2411240 \\ -6.2791160 & 1.4442420 & -1.3418080 \\ -7.3156700 & 1.7717990 & 0.0549770 \\ -3.1593520 & -0.3088790 & -4.1089760 \\ -4.2185820 & -0.0589700 & -4.2387270 \\ -2.6616710 & 0.5231850 & -3.6103860 \\ -2.7261150 & -0.4052280 & -5.1105440 \\ -3.7073160 & -2.7521760 & -4.0651030 \\ -4.7845510 & -2.5477650 & -4.0900540 \\ -3.3549230 & -2.8424490 & -5.0988240 \\ -3.5654280 & -3.7183540 & -3.5727690 \\ 0.2385890 & 0.3021000 & -0.9100120 \\ -0.4909270 & 0.4309150 & -2.2176070 \\ 0.1984100 & 1.4317870 & 0.0771180 \\ -1.8934710 & -1.8407870 & -3.3573440 \\ -6.5859070 & -0.3177440 & 1.1420800 \\ -1.3704620 & -2.7189920 & 1.4807620 \\ 4.6836980 & -0.3211140 & -2.0036270 \\ 4.2572710 & 6.0202730 & 0.0823840 \\ 2.2880860 & 0.8528220 & 1.9694530 \\ -2.7766090 & 2.5976940 & -1.8275450 \\ -1.8404510 & 3.4984200 & -1.1513930 \\ -2.9127210 & 1.6151880 & -1.3709840 \\ -1.0195360 & 3.0428250 & -0.6054290 \\ -1.6838160 & 2.6089710 & -2.7762500 \\ -2.5900240 & 2.5769560 & 2.2780650 \\ -2.0820900 & 1.1316560 & 4.2657560 \\ -1.2914290 & 0.8719790 & 3.1502050 \\ -1.6133300 & 1.6887680 & 2.0748760 \\ -1.0505950 & 1.6327640 & 1.2037090 \\ -3.2256880 & 2.4113200 & 3.9009040 \\ -3.1693460 & 3.7328250 & 1.1919000 \\ -1.1245270 & 1.7750690 & -2.5912220 \\ -0.8824510 & -0.5441670 & 5.4685270 \\ -0.0928900 & -0.8046450 & 4.3298680 \\ -0.2897190 & -0.0973020 & 3.1490550 \\ -1.8789610 & 0.4130740 & 5.4450980 \\ -0.6933260 & -1.1217560 & 6.3665900 \\ 0.2728770 & -0.2808640 & 2.2432010 \\ -2.4814290 & 0.5996840 & 6.3271710 \\ -3.8050050 & 4.5895520 & -2.9850130 \\ -3.1575180 & 5.5066540 & -1.9433110 \\ -1.8082770 & 4.9589790 & -1.4664190 \\ -4.0368690 & 3.1901530 & -3.3108550 \\ -4.7610340 & 5.0102670 & \end{array}$

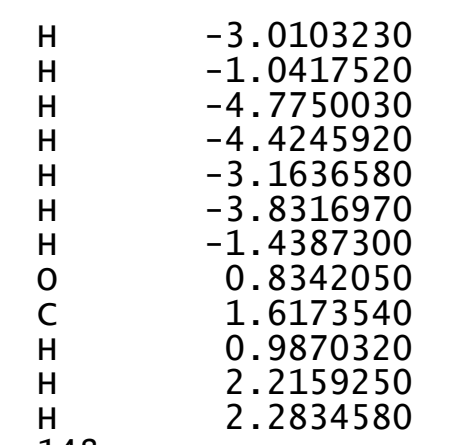
6.5103330
5.0886110
3. 2356650
2. 5018000
4. 5221940
5.6080510
5.4900650
$-1.7757230$
$-2.1039800$
$-2.4477380$
$-1.2472420$
$-2.9097530$

$\operatorname{TS} 5(R, R) \quad G[W B 97 X D / 6-31 G(d)]=-4125.322072$

\begin{tabular}{lrcr} 
TS5 $(\mathrm{R}, \mathrm{R})$ & \multicolumn{3}{c}{$\mathrm{G}[\mathrm{WB} 97 \times \mathrm{X} / 6-31 \mathrm{G}(\mathrm{d})]=-4125.322072$} \\
$\mathrm{O}$ & 0.1818510 & 1.0440530 & -0.0355330 \\
$\mathrm{O}$ & -1.7761370 & 0.1891570 & -1.3678600 \\
$\mathrm{C}$ & 0.0400780 & 2.3141270 & -0.5596090 \\
$\mathrm{C}$ & -1.2245630 & 2.8540790 & -0.6640570 \\
$\mathrm{C}$ & 1.2252300 & 3.0078950 & -0.9239910 \\
$\mathrm{C}$ & -1.3666820 & 4.1223100 & -1.3169790 \\
$\mathrm{C}$ & 1.0800220 & 4.2702100 & -1.4396710 \\
$\mathrm{C}$ & -0.1953130 & 4.8400970 & -1.6823930 \\
$\mathrm{H}$ & 1.9633610 & 4.8474950 & -1.6959490 \\
$\mathrm{C}$ & -2.5926190 & 0.7903830 & -0.4361100 \\
$\mathrm{C}$ & -3.6787700 & 0.0208710 & 0.0599470 \\
$\mathrm{C}$ & -2.3841960 & 2.1122560 & -0.0989830 \\
$\mathrm{C}$ & -4.5758800 & 0.6424660 & 0.8885620 \\
$\mathrm{C}$ & -3.2711930 & 2.7204330 & 0.8534710 \\
$\mathrm{C}$ & -4.3928590 & 1.9803790 & 1.3190390 \\
$\mathrm{H}$ & -5.4433590 & 0.0925690 & 1.2431440 \\
$\mathrm{C}$ & -5.0707850 & 3.8370960 & 2.7262460 \\
$\mathrm{C}$ & -3.9336990 & 4.5609540 & 2.2998100 \\
$\mathrm{C}$ & -3.0591490 & 4.0198250 & 1.3917650 \\
$\mathrm{C}$ & -5.2886510 & 2.5717710 & 2.2478450 \\
$\mathrm{H}$ & -5.7573650 & 4.2787930 & 3.4416590 \\
$\mathrm{H}$ & -3.7455240 & 5.5512600 & 2.7029000 \\
$\mathrm{H}$ & -2.1822300 & 4.5831000 & 1.0950510 \\
$\mathrm{H}$ & -6.1445510 & 1.9928020 & 2.5842520 \\
$\mathrm{C}$ & -2.7208080 & 5.9009140 & -2.2681880 \\
$\mathrm{C}$ & -1.5546710 & 6.6323200 & -2.5922680 \\
$\mathrm{C}$ & -0.3206650 & 6.1081670 & -2.3087660 \\
$\mathrm{C}$ & -2.6305900 & 4.6810830 & -1.6474690 \\
$\mathrm{H}$ & -3.6960550 & 6.3075100 & -2.5177430 \\
$\mathrm{H}$ & -1.6415680 & 7.5995590 & -3.0772820 \\
$\mathrm{H}$ & 0.5845170 & 6.6489220 & -2.5721490 \\
$\mathrm{H}$ & -3.5332430 & 4.1270230 & -1.4142870
\end{tabular}

$-2.3527180$

$-2.2407440$

$-1.6005970$

$-3.1672150$

0

4.4771430

3. 3425430

2.5140570

3.0095120

3. 6502680

2. 2880860

$-1.0195360$

1.044053

0.1891570

3. 0078950

223100

8400970

.8474950

1122560

6424660

.7204330

0925690

8370960

. 5717710

. 9928020

4. 6810830

4.1270230 


$\begin{array}{lr}\mathrm{C} & 2.5564980 \\ \mathrm{C} & 3.2780610 \\ \mathrm{C} & 3.0660190 \\ \mathrm{C} & 4.4726480 \\ \mathrm{C} & 4.2546780 \\ \mathrm{C} & 4.9621310 \\ \mathrm{H} & 5.0386640 \\ \mathrm{H} & 4.6471160 \\ \mathrm{C} & -3.8521940 \\ \mathrm{C} & -3.4080230 \\ \mathrm{C} & -4.4721760 \\ \mathrm{C} & -3.6304040 \\ \mathrm{C} & -4.6607590 \\ \mathrm{C} & -4.2541810 \\ \mathrm{H} & -3.2959350 \\ \mathrm{H} & -5.1379530 \\ \mathrm{C} & 2.3677470 \\ \mathrm{C} & 6.2523830 \\ \mathrm{C} & 2.8374960 \\ \mathrm{C} & -2.7071640 \\ \mathrm{C} & -4.4607160 \\ \mathrm{C} & -4.9360820 \\ \mathrm{C} & -6.4492690 \\ \mathrm{H} & -6.7329470 \\ \mathrm{H} & -6.9999290 \\ \mathrm{H} & -6.7716400 \\ \mathrm{C} & -4.1510850 \\ \mathrm{H} & -3.0777820 \\ \mathrm{H} & -4.3210320 \\ \mathrm{H} & -4.4633660 \\ \mathrm{C} & -5.3453720 \\ \mathrm{H} & -5.5305760 \\ \mathrm{H} & -4.8642970 \\ \mathrm{H} & -6.3110830 \\ \mathrm{C} & -3.1185130 \\ \mathrm{H} & -2.4935600 \\ \mathrm{H} & -2.5623740 \\ \mathrm{H} & -3.2776070 \\ \mathrm{C} & -3.7076980 \\ \mathrm{H} & -4.4590990 \\ \mathrm{H} & -4.2302110 \\ \mathrm{H} & -3.1838280 \\ \mathrm{C} & -1.6140460 \\ \mathrm{H} & -2.0338110 \\ \mathrm{H} & -0.9168320 \\ \mathrm{H} & -1.0533330 \\ & \end{array}$

$\begin{array}{rr}2.3505850 & -0.7373850 \\ 1.8906200 & -1.8544470 \\ 2.1574360 & 0.5673700 \\ 1.1961450 & -1.6390470 \\ 1.4512260 & 0.7242340 \\ 0.9402000 & -0.3646120 \\ 0.8375650 & -2.4952380 \\ 1.2890120 & 1.7224780 \\ -1.4099700 & -0.3374170 \\ -2.4374800 & 0.5205790 \\ -1.7209050 & -1.5565740 \\ -3.7598410 & 0.1450950 \\ -3.0633520 & -1.8936580 \\ -4.0956020 & -1.0570010 \\ -4.5569310 & 0.8030960 \\ -3.3109280 & -2.8398680 \\ 2.7627810 & 1.7824040 \\ 0.1649900 & -0.1690270 \\ 2.1769660 & -3.2845180 \\ -2.1291840 & 1.8381290 \\ -5.5470420 & -1.4508830 \\ -0.6422620 & -2.5245740 \\ -0.7162600 & -2.7665870 \\ -1.6586450 & -3.2488200 \\ -0.6406020 & -1.8228200 \\ 0.1026980 & -3.4191630 \\ -0.7053090 & -3.8413000 \\ -0.6036260 & -3.6549080 \\ -1.6556820 & -4.3606430 \\ 0.1034710 & -4.5115730 \\ -6.2908290 & -0.4420050 \\ -7.3173810 & -0.7772100 \\ -6.3430950 & 0.5415230 \\ -5.7896900 & -0.3189700 \\ -6.2670220 & -1.6417830 \\ -5.7449540 & -2.3736450 \\ -6.3172550 & -0.6983680 \\ -7.2928160 & -1.9926140 \\ -2.0174460 & 2.9974700 \\ -1.2440760 & 2.8162210 \\ -2.9701600 & 3.1445680 \\ -1.7698360 & 3.9283820 \\ -3.1415050 & 2.1955230 \\ -4.1169630 & 2.4658730 \\ -3.2772700 & 1.3650970 \\ -2.7809920 & 3.0645950\end{array}$

2. 5681700

3.6351680

2. 1835760

2.0533700

2. 8184520

2.7788250

3.8406980

2. 1623020

6.1103390

7.0228360

5.9472830

5.2689930

7.4059660

7.5353920

7. 2100230

8. 3474150

3.0576510

4.1219940

2. 6095030

2. 5962550

3. 5664000

4. 6458050

3. 2129610

3. 4142650

$-0.2318260$

0.4983270

$-0.1299920$

1.7641990

6.4967690

1. 2936800

$-4.7326180$

$-4.9810160$

$-2.2152520$

2. 9942870

2. 1203240

2. 9410920

1.1945490

2. 0269340

2. 6009540

2. 0756380

1. 2830810

1. 6186570

1. 0614880

3. 2366960

3.1819040
1.3459560
4.2877100

4. 5241440

4.7726890

4.7199560

2.1761670

1.0837780

2. 4848070

2. 5401870

$-0.9516300$

$-1.5568370$

$-0.5427460$

$-1.6140570$

1.1100390

1.8851200

1. 6070830

0.5570670

1.0071160

0.8224600

0.0876480

1. 2366120

3. 4199670

3.2340580

3.6653050

4. 2956960

$-0.1993110$

$-0.1379080$

$-1.4327760$

2. 3848820

$-0.3051160$

2. 5631370

0.3333130

$-5.5522600$

$-1.1580310$

$-2.0863320$

-3. 2020670

$-1.1982770$

$-2.9549980$

$-2.0452560$

$-2.7592260$

$-1.4871180$

$-1.1462760$

$-1.8646260$

$-1.7416750$

$-2.7159710$

$-3.8282930$

$-1.3191600$
1.8151810

1. 9020470

0.9147770

2.6805960

3. 1228710

3. 1296410

3. 3715150

3. 9197450

0.8728340

0.9084670

1.8765290

0.6413690

0.1955960

$-0.5670550$

1.1530630

0.2881550

$-4.2493080$

$-4.4359890$

$-3.8710070$

$-5.2158760$

$-3.8212850$

$-3.8766910$

$-4.8291370$

$-3.1842590$

$-1.0173030$

$-2.3294180$

$-0.1684210$

$-3.2715300$

$-1.1306150$

1.6869900

$-2.0731150$

2. 4171970

1.7210210

$-1.9297620$

$-1.5567110$

$-1.2974810$

$-1.0458570$

$-3.0039130$

1.9918010

4.0879270

2. 9955970

1.8561450

0.9872240

3. 6213020

0.8218540

$-2.7667650$ 


\begin{tabular}{|c|c|c|c|c|c|c|c|}
\hline C & 0.8480080 & 0.0605460 & 5.4261890 & $\mathrm{H}$ & -5.3958110 & 4.6975780 & 3.4173120 \\
\hline C & 0.0512920 & 0.3984210 & 4.3130820 & $\mathrm{H}$ & -3.3667230 & 5.8270930 & 2.5128280 \\
\hline $\mathrm{C}$ & 0.2639390 & -0.1990660 & 3.0755230 & $\mathrm{H}$ & -1.9213360 & 4.6884200 & 0.9070290 \\
\hline C & 1.8617530 & -0.8729580 & 5.3229740 & H & -5.9206310 & 2.3820480 & 2.7267550 \\
\hline $\mathrm{H}$ & 0.6491900 & 0.5574300 & 6.3692630 & C & -2.5500090 & 5.8280070 & -2.4933240 \\
\hline $\mathrm{H}$ & -0.2999940 & 0.0535170 & 2.1872860 & $\mathrm{C}$ & -1.3684630 & 6.4989300 & -2.8857020 \\
\hline $\mathrm{H}$ & 2.4687650 & -1.1222970 & 6.1862920 & C & -0.1457850 & 5.9467230 & -2.6066210 \\
\hline C & 4.4242850 & -3.6867420 & -3.2587460 & c & -2.4852110 & 4.6403780 & -1.8103670 \\
\hline C & 3.7790480 & -4.8552800 & -2.5082660 & H & -3.5170020 & 6.2562740 & -2.7386770 \\
\hline C & 2.3189290 & -4.5629990 & -2.1455370 & $\mathrm{H}$ & -1.4351650 & 7.4427460 & -3.4178400 \\
\hline C & 4.3842830 & -2.4022010 & -2.4253010 & $\mathrm{H}$ & 0.7696150 & 6.4408210 & -2.9212070 \\
\hline $\mathrm{H}$ & 5.4639610 & -3.9266360 & -3.5012930 & $\mathrm{H}$ & -3.3989630 & 4.1318710 & -1.5224810 \\
\hline $\mathrm{H}$ & 3.8195200 & -5.7673480 & -3.1111320 & C & 2.6431450 & 2.1853180 & -0.8974000 \\
\hline $\mathrm{H}$ & 1.6835150 & -4.6076440 & -3.0383520 & C & 3.3308630 & 1.6560930 & -2.0047920 \\
\hline H & 5.0294400 & -2.5009580 & -1.5441660 & $\mathrm{C}$ & 3.1757350 & 2.0443560 & 0.4049610 \\
\hline $\mathrm{H}$ & 4.7469730 & -1.5445380 & -3.0008130 & $\mathrm{C}$ & 4.5179820 & 0.9513090 & -1.7804240 \\
\hline $\mathrm{H}$ & 3.9019230 & -3.5222410 & -4.2084080 & $\mathrm{C}$ & 4.3542870 & 1.3239880 & 0.5716140 \\
\hline $\mathrm{H}$ & 4.3475970 & -5.0549130 & -1.5913520 & $\mathrm{C}$ & 5.0308940 & 0.7497880 & -0.5055430 \\
\hline $\mathrm{H}$ & 1.9151600 & -5.3032800 & -1.4500250 & $\mathrm{H}$ & 5.0587640 & 0.5407390 & -2.6294800 \\
\hline 0 & -0.9011010 & 1.3277480 & 4.5424710 & $\mathrm{H}$ & 4.7647650 & 1.2021950 & 1.5684520 \\
\hline C & -1.7040220 & 1.7188390 & 3.4424240 & C & -3.9191490 & -1.2884350 & -0.0941080 \\
\hline $\mathrm{H}$ & -1.0932110 & 2.1586580 & 2.6453660 & $\mathrm{C}$ & -3.4934240 & -2.2944290 & 0.7930890 \\
\hline $\mathrm{H}$ & -2.2675990 & 0.8692200 & 3.0373420 & $\mathrm{C}$ & -4.6045010 & -1.6233060 & -1.2742520 \\
\hline $\begin{array}{l}\mathrm{H} \\
148\end{array}$ & -2.4011360 & 2.4665430 & 3.8196850 & C & $\begin{array}{l}-3.8025450 \\
-4.8782930\end{array}$ & $\begin{array}{l}-3.6213270 \\
-2.9645090\end{array}$ & 0.4914990 \\
\hline TS6 $(R, R$ & $\mathrm{G}[\mathrm{WB} 97 \mathrm{XD} / 6$ & I) $]=-4125$ & & $\mathrm{c}$ & -4.4923110 & -3.9784950 & -0.6642950 \\
\hline 0 & 0.2388850 & 0.9953940 & -0.0743320 & $\mathrm{H}$ & -3.4861040 & -4.4083820 & 1.1708470 \\
\hline 0 & -1.8043990 & 0.1402740 & -1.2806720 & $\mathrm{H}$ & -5.4047630 & -3.2233860 & -2.4536770 \\
\hline C & 0.1298310 & 2.2402680 & -0.6632330 & C & 2.5155070 & 2.7219960 & 1.6030730 \\
\hline $\mathrm{C}$ & -1.1164750 & 2.8213220 & -0.7639180 & $\mathrm{C}$ & 6.3129720 & -0.03 & -0.2964840 \\
\hline C & 1.3281210 & 2.8716110 & -1.0909740 & $\mathrm{C}$ & 2.8624790 & 1.8778720 & -3.4376460 \\
\hline C & -1.2324390 & 4.0551860 & -1.4834650 & C & -2.7177850 & -1.9619670 & 2.0615150 \\
\hline $\mathrm{C}$ & 1.2130970 & 4.1090530 & -1.6713720 & $\mathrm{C}$ & -4.7819900 & -5.4358440 & -0.9764230 \\
\hline C & -0.0473710 & 4.7096410 & -1.9164240 & C & -5.0514860 & -0.5662360 & -2.2737940 \\
\hline $\mathrm{H}$ & 2.1092930 & 4.6406030 & -1.9770980 & $\mathrm{C}$ & -6.5797170 & -0.5310440 & -2.4041390 \\
\hline C & -2.5586980 & 0.8342610 & -0.3610490 & $\mathrm{H}$ & -6.9685510 & -1.4752450 & -2.8028800 \\
\hline C & -3.6584480 & 0.1491400 & 0.2219840 & $\mathrm{H}$ & -7.0520690 & -0.3561250 & -1.4315110 \\
\hline C & -2.2823270 & 2.1632780 & -0.1153960 & $\mathrm{H}$ & -6.8871420 & 0.2707520 & -3.0847060 \\
\hline C & -4.4901810 & 0.8586290 & 1.0489740 & C & -4.3754720 & -0.7656760 & -3.6364680 \\
\hline C & -3.1007810 & 2.8677430 & 0.8314550 & $\mathrm{H}$ & -3.2864560 & -0.7546960 & -3.5308430 \\
\hline C & -4.2310440 & 2.2099630 & 1.3900060 & $\mathrm{H}$ & -4.6663550 & -1.7194240 & -4.0916220 \\
\hline $\mathrm{H}$ & -5.3649770 & 0.3706380 & 1.4699170 & $\mathrm{H}$ & -4.6637700 & 0.0354740 & -4.3261990 \\
\hline C & -4.7621620 & 4.1800060 & 2.7039480 & C & -3.9982310 & -5.8997370 & -2.2121860 \\
\hline C & -3.6152980 & 4.8227410 & 2.1838640 & $\mathrm{H}$ & -4.1655550 & -6.9666370 & -2.3977000 \\
\hline C & -2.8075370 & 4.1862640 & 1.2764630 & $\mathrm{H}$ & -4.3143570 & -5.3487210 & -3.1056880 \\
\hline $\mathrm{C}$ & -5.0565490 & 2.8985440 & 2.3175280 & $\mathrm{H}$ & -2.9238030 & -5.7368040 & -2.0787530 \\
\hline
\end{tabular}




-6.2834940
-6.8418230
-6.6864260
-6.4688250
-3.6555190
-4.3846560
-4.2066920
-3.0783250
-1.6505060
-2.0957780
-1.0080700
-1.0257510
2.7510020
3.8244890
2.3592980
2.2630000
2.9792320
2.9160340
4.0125070
2.3467190
6.1608190
7.0671720
6.0030950
5.3119820
7.4791350
7.6128400
7.2948050
8.4147940
3.0070060
4.0576290
2.5166960
2.5434570
3.6229570
4.6924890
3.2487360
3.5231550
-0.2599580
0.4173450
-0.1631180
1.7990870
6.5481390
1.4356570
-4.7392580
-4.4344640
-2.1933560
2.8833240

-1.1454490
-0.2583800
-2.0093880
-1.3058630
3.2594060
3.0736500
3.4781370
4.1515940
2.4082320
2.7465440
1.5478930
3.2269030
1.5614880
1.6173140
0.6474400
2.4159170
2.9607110
3.0188960
3.1765040
3.7522080
0.7736130
0.8240430
1.7665410
0.5612580
0.0419850
-0.7363530
0.9906930
0.1403550
-4.3361970
-4.5247440
-3.9008170
-5.3087300
-4.0658000
-4.1403990
-5.0757880
-3.4767450
-0.9694640
-2.3109940
-0.0593040
-3.4097910
-1.2472190
1.5378090
-1.8997340
-0.1204630
1.8789420
-1.9086880

$-1.9086880$

$\begin{array}{r}2.0208160 \\ 2.8590270 \\ 1.1187150 \\ 1.8681100 \\ 2.6169260 \\ 2.1594930 \\ 1.3431220 \\ 1.6384020 \\ 1.0602910 \\ 3.2931180 \\ 3.1656940 \\ 1.2075290 \\ 0.9966180 \\ 0.1781290 \\ 0.3447440 \\ 1.9895490 \\ 0.8312220 \\ -0.2376770 \\ 2.6152210 \\ 4.2567200 \\ 3.6383990 \\ 2.1929050 \\ 4.2498580 \\ 5.2863590 \\ 3.6551310 \\ 1.5263540 \\ 4.9339740 \\ 4.5849270 \\ 3.6999630 \\ 4.2404650 \\ 1.8124610 \\ -0.7471450 \\ -1.5632520 \\ -0.9580210 \\ -2.1614150 \\ -2.2289480 \\ \hline\end{array}$

2.0208160

C

$-5.383$

$-5.1590830$

$-6.7677680$

$-1.7554150$

$-0.9616940$

$-2.6777720$

$-1.4844720$

$-3.0049240$

$-3.2078540$

$-2.6316390$

4.2417140

4.4572020

4.6946010

2.1869340

1.0971730

2. 4830880

(2)

-1.7371430

$-0.6870910$

$-1.7829000$

0.9039930

1.6625520

1. 4218410

0.6451730

0.3950040

$-0.2262600$

0.8431000

3.0572140

2.8263850

3.2588360

$-0.2823830$

$-0.3175870$

$-1.4715750$

2.1300510

$-0.5316810$

2.5439560

0.4132010

$-6.0284660$

-1.0185630
-2.2728020

$\begin{array}{lrrr}\mathrm{O} & 0.5183590 & 0.5813490 & -0.2419430 \\ \mathrm{O} & -1.5750230 & 0.2561110 & -1.6290490 \\ \mathrm{C} & 0.7268040 & 1.8295560 & -0.7910060 \\ \mathrm{C} & -0.3497500 & 2.6781160 & -0.9600770 \\ \mathrm{C} & 2.0633800 & 2.2026690 & -1.0839090 \\ \mathrm{C} & -0.1375950 & 3.9174290 & -1.6470600 \\ \mathrm{C} & 2.2658590 & 3.4523350 & -1.6166690 \\ \mathrm{C} & 1.1924810 & 4.3115860 & -1.9536530\end{array}$

$-1.4284800$ $-1.3367900$ $-0.8960040$ $-2.9391400$ 2.0839820

4.1386720

1.0789790

3.6943290

0.9431630

$-2.7202270$

5.4388450

4.3323380

3.1177530

6.3643780

2.2337740

$-3.1835070$

$-2.3295640$

$-1.9350980$

$-2.4416230$

$-3.4470630$

2.8681520

(

$-3.0926370$

$-4.1226720$

$-1.1796890$

4.5442920

3.4456760

2.6166920

3.0926280

3.8036370
3.0536340

1.9376580

$-1.4227220$

1. 1924810

4. 3115860

$-1.9536530$ 


$\begin{array}{lr}\mathrm{H} & 3.2815510 \\ \mathrm{C} & -2.2351160 \\ \mathrm{C} & -3.5019790 \\ \mathrm{C} & -1.6820490 \\ \mathrm{C} & -4.2287940 \\ \mathrm{C} & -2.4056780 \\ \mathrm{C} & -3.7109480 \\ \mathrm{H} & -5.2286160 \\ \mathrm{C} & -3.9625150 \\ \mathrm{C} & -2.6428730 \\ \mathrm{C} & -1.8810070 \\ \mathrm{C} & -4.4743620 \\ \mathrm{H} & -4.5557390 \\ \mathrm{H} & -2.2230320 \\ \mathrm{H} & -0.8633190 \\ \mathrm{H} & -5.4755930 \\ \mathrm{C} & -0.9526040 \\ \mathrm{C} & 0.3752280 \\ \mathrm{C} & 1.4227350 \\ \mathrm{C} & -1.2034420 \\ \mathrm{H} & -1.7809220 \\ \mathrm{H} & 0.5577960 \\ \mathrm{H} & 2.4475090 \\ \mathrm{H} & -2.2271000 \\ \mathrm{C} & 3.2457920 \\ \mathrm{C} & 4.0022980 \\ \mathrm{C} & 3.6853270 \\ \mathrm{C} & 5.2310850 \\ \mathrm{C} & 4.9114580 \\ \mathrm{C} & 5.7176260 \\ \mathrm{H} & 5.8342440 \\ \mathrm{H} & 5.2734930 \\ \mathrm{C} & -4.0453670 \\ \mathrm{C} & -3.9888720 \\ \mathrm{C} & -4.6479410 \\ \mathrm{C} & -4.5830980 \\ \mathrm{C} & -5.2139860 \\ \mathrm{C} & -5.2023510 \\ \mathrm{H} & -4.5610760 \\ \mathrm{H} & -5.6837640 \\ \mathrm{C} & 2.8934370 \\ \mathrm{C} & 7.1134190 \\ \mathrm{C} & 3.5360600 \\ \mathrm{C} & -3.3193940 \\ \mathrm{C} & -5.8431470 \\ \mathrm{C} & -4.7078040 \\ & \end{array}$

$\begin{array}{rr}3.7876220 & -1.8053300 \\ 1.0753250 & -0.7364220 \\ 0.6425040 & -0.2663600 \\ 2.2973590 & -0.4179560 \\ 1.5210660 & 0.4949050 \\ 3.1617210 & 0.4710430 \\ 2.7803400 & 0.8851830 \\ 1.2438310 & 0.8175530 \\ 4.8632680 & 2.1023930 \\ 5.2207440 & 1.7395080 \\ 4.3885570 & 0.9606220 \\ 3.6588030 & 1.6984450 \\ 5.5332540 & 2.7172080 \\ 6.1572020 & 2.0947860 \\ 4.6685280 & 0.7160880 \\ 3.3555120 & 1.9928980 \\ 5.9430270 & -2.7112750 \\ 6.3539990 & -2.9749560 \\ 5.5515990 & -2.6066610 \\ 4.7605150 & -2.0637920 \\ 6.5683660 & -3.0301810 \\ 7.2971880 & -3.4804120 \\ 5.8427190 & -2.8217720 \\ 4.4549890 & -1.8773230 \\ 1.3290530 & -0.7986950 \\ 0.8189860 & -1.8724370 \\ 1.1388890 & 0.5278790 \\ 0.2136680 & -1.6038450 \\ 0.5068310 & 0.7419320 \\ 0.0701760 & -0.3073190 \\ -0.1384230 & -2.4376600 \\ 0.3778870 & 1.7580400 \\ -0.7054790 & -0.6153720 \\ -1.7501050 & 0.3271320 \\ -0.9091910 & -1.8687810 \\ -2.9703000 & 0.0020540 \\ -2.1515610 & -2.1507650 \\ -3.1917520 & -1.2250080 \\ -3.7813820 & 0.7251190 \\ -2.3082760 & -3.1195200 \\ 1.6818320 & 1.7130980 \\ -0.4707910 & -0.0413010 \\ 0.9274760 & -3.3196960 \\ -1.5716370 & 1.6847170 \\ -4.5315100 & -1.5404050 \\ 0.1870290 & -2.9228350\end{array}$

-6.1575440
-6.6997660
-6.6958840
-6.1818220
-3.9287360
-2.8880260
-4.3711510
-3.9379260
-5.1501990
-5.5895340
-5.2609160
-4.0798240
-7.3498500
-7.8477780
-7.5436670
-7.8087820
-4.3291060
-4.8338070
-5.0949920
-3.8202760
-2.5586830
-3.2364270
-1.8524410
-1.9996240
3.1965060
4.2561720
2.9685520
2.5988370
3.1199470
3.0004050
4.1182590
2.3913380
8.1665640
9.1771780
8.0983600
8.0249490
7.3519600
6.6776680
7.2028960
8.3806510
3.6245960
4.6640090
3.1133170
3.1552970
4.3246640
5.3927870

0.5652210

$-0.2791120$

0.8789720

1. 3906190

$-0.2094710$

$-0.4398480$

$-1.0893120$

0.6098020

$-5.2248410$

$-6.2131220$

$-4.6404400$

$-5.3526610$

$-4.3878320$

$-3.9123630$

$-3.7763270$

$-5.3694230$

$-1.1488820$

$-0.2146400$

$-1.9223040$

$-1.0018200$

$-2.8185760$

$-3.6445840$

$-3.1565090$

$-2.5867020$

3. 1711700

3. 3129860

3.7672670

3.5582630

0.9015350

$-0.1765340$

1. 0768190

1.2227360

0.5215110

0.1748520

0.6293850

1.5114670

$-1.8669230$

$-2.6059280$

$-1.8777180$

$-2.1882180$

$-0.4143960$

$-0.7241920$

$-1.2072660$

$-0.3265070$

1. 9984240

1. 7510820
$-3.2540580$

$-3.6949750$

$-2.3532110$

$-3.9742230$

$-4.1834210$

$-3.9372240$

$-4.6649120$

$-4.9111050$

$-2.7208260$

$-2.8969280$

$-3.6416260$

$-2.5296690$

$-1.7933170$

$-0.9418460$

$-2.6822390$

$-1.9559700$

2. 7609660

2. 5007950

2.8957480

3. 7207560

2. 1499170

2. 3929660

1. 3870830

3. 0638390

1.9387490

2. 1820140

1.0501410

2. 7718670

3. 0116090

2. 8582140

3. 4282520

3. 7631120

$-0.5576430$

$-0.3147650$

$-1.6464870$

$-0.1117420$

$-0.6284090$

$-0.1864480$

$-1.7143820$

$-0.4318990$

$-4.0575770$

$-4.2160070$

$-3.5070540$

$-5.0435070$

$-4.0871840$

$-4.1101380$ 


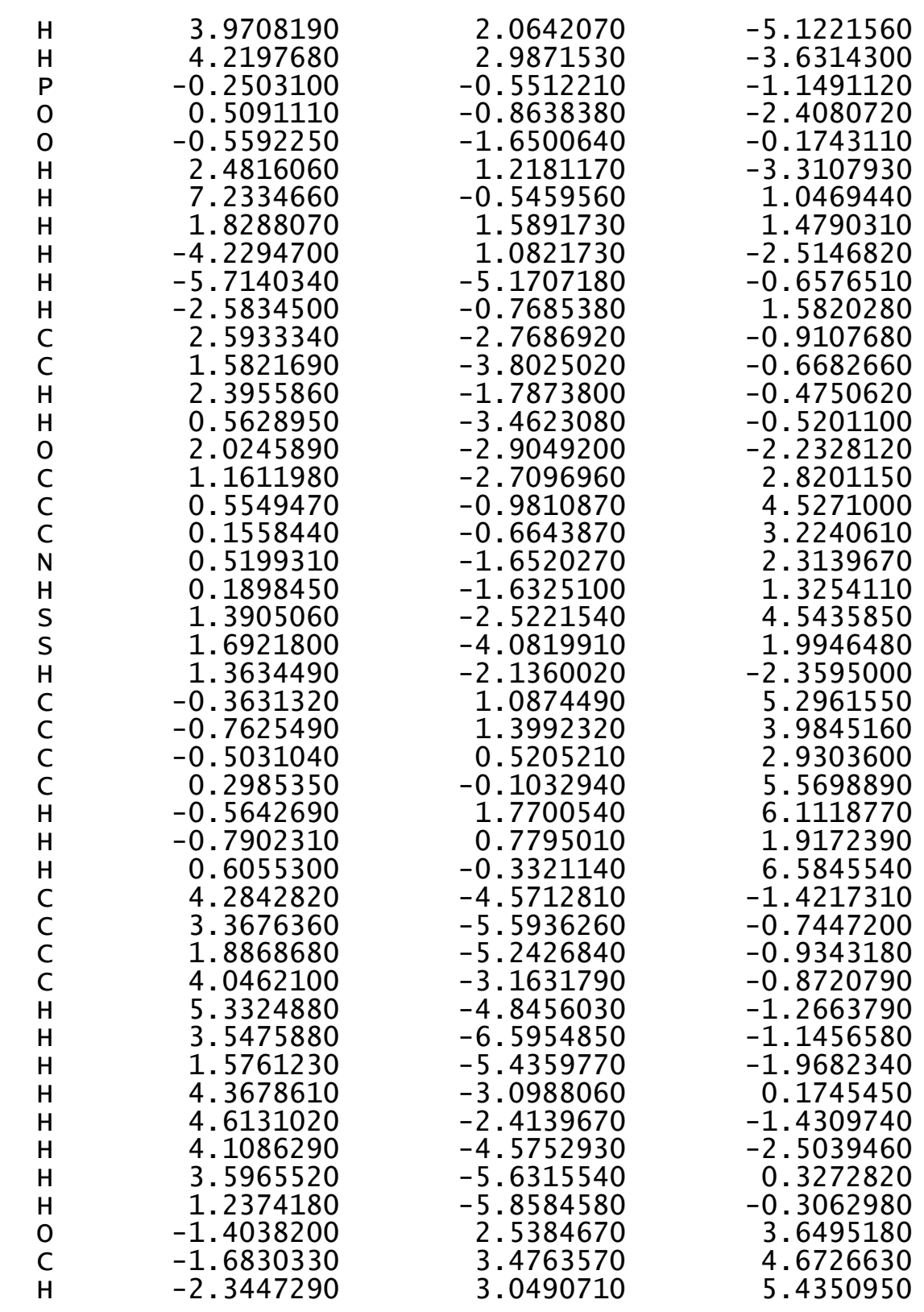

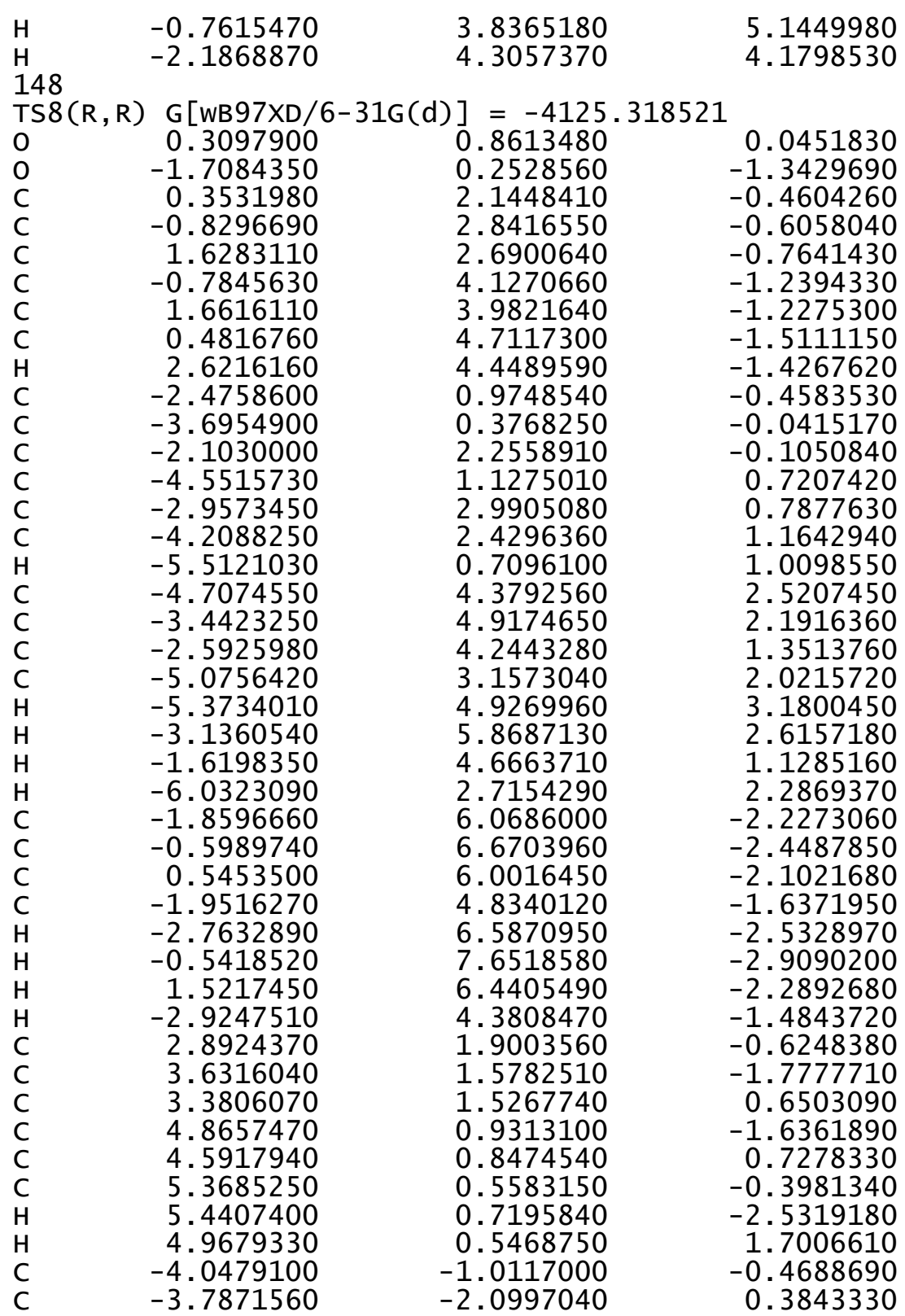




$\begin{array}{rrr}-4.6544570 & -1.2153890 & -1.7197610 \\ -4.1810270 & -3.3741820 & -0.0248430 \\ -5.0247560 & -2.5086300 & -2.0857440 \\ -4.8012250 & -3.6007320 & -1.2509300 \\ -3.9928260 & -4.2247550 & 0.6247200 \\ -5.4976440 & -2.6666710 & -3.0526550 \\ 2.6495130 & 1.9234290 & 1.9302910 \\ 6.7251130 & -0.1097930 & -0.2100600 \\ 3.1685090 & 1.9283560 & -3.1881230 \\ -3.1100320 & -1.9031610 & 1.7346840 \\ -5.2023450 & -5.0039780 & -1.6693670 \\ -4.9137320 & -0.0674090 & -2.6842950 \\ -6.4175240 & 0.1516550 & -2.8946680 \\ -6.8866790 & -0.7265850 & -3.3532540 \\ -6.9213660 & 0.3434410 & -1.9411520 \\ -6.5922540 & 1.0088550 & -3.5544480 \\ -4.1838300 & -0.2787280 & -4.0169970 \\ -3.1105610 & -0.4149630 & -3.8519190 \\ -4.5632960 & -1.1599910 & -4.5466900 \\ -4.3251930 & 0.5897100 & -4.6699510 \\ -4.4416350 & -5.4515420 & -2.9249950 \\ -4.6819900 & -6.4920660 & -3.1703730 \\ -4.7091000 & -4.8323550 & -3.7892490 \\ -3.3597290 & -5.3733220 & -2.7768490 \\ -6.7185390 & -5.1238910 & -1.8714620 \\ -7.2606030 & -4.8316790 & -0.9660470 \\ -7.0590080 & -4.4806120 & -2.6911730 \\ -6.9925690 & -6.1555800 & -2.1186640 \\ -4.1403470 & -1.6670950 & 2.8481120 \\ -4.7754440 & -0.8021060 & 2.6353940 \\ -4.7890850 & -2.5435080 & 2.9630150 \\ -3.6335660 & -1.4902410 & 3.8043520 \\ -2.1727560 & -3.0513850 & 2.1196940 \\ -2.7205290 & -3.9784990 & 2.3223960 \\ -1.4413080 & -3.2373240 & 1.3292840 \\ -1.6328630 & -2.7902460 & 3.0362750 \\ 2.8396330 & 3.4209520 & 2.2235330 \\ 3.9018820 & 3.6428680 & 2.3797470 \\ 2.4765850 & 4.0483470 & 1.4051080 \\ 2.2964940 & 3.7003020 & 3.1335890 \\ 3.0709940 & 1.1120680 & 3.1593390 \\ 3.0899940 & 0.0387360 & 2.9504830 \\ 4.0658910 & 1.4041360 & 3.9155320 \\ 2.3628680 & 1.2830690 & 0.6449900 \\ 7.6453790 & 0.7778580 & \\ 8.6075110 & 0.2819920 & \\ -4 & & \\ -4 & -136480\end{array}$

7.8359350

7.2051560

7.4370200

6.8332060

7.6868110

8.3740200

3.2294620

4.2598940

2.6700330

2.7915310

3.9854640

5.0417340

3.6139550

3.9350200

$-0.2303560$

0.5250830

$-0.2758310$

2. 1217480

6.5501560

1. 5813520

$-4.5139300$

$-4.9211360$

$-2.4904960$

2.9570090

2. 0691300

2. 8768010

1.1218840

2.0371000

2.2324290

1.4254130

0.7955360

1.2677710

0.8200630

2.6244910

3.0148280

1. 3503910

0.1191000

$-0.5085570$

$-0.1713670$

1.0878930

$-0.1741090$

$-0.6057090$

1. 5716870

4.4546990

3.7768040

2.2984180
1.7292480

1.0017110

$-0.4750210$

$-1.1308110$

0.4224830

$-0.9955490$

0.7291050

0.4055310

$-0.1191370$

1.0016830

3.0947620

2.8171910

3.3657470

3.9842610

$-0.3140300$

$-0.3271130$

$-1.5494400$

2. 2375980

$-1.0405890$

1.7388430

0.8495850

$-5.6810580$

$-1.0041040$

$-2.2418570$

$-3.3349400$

$-1.3141440$

$-3.0597570$

$-2.2729240$

$-2.8747360$

$-1.7832250$

$-1.3317430$

$-1.9682310$

$-1.7938340$

$-2.9944710$

$-3.8140950$

$-1.5359160$

$-0.2560570$

0.2008650

$-0.3289920$

$-1.2400380$

0.1909110

0.0144130

$-1.5766780$

$-3.9244120$

$-5.0392650$

$-4.7303010$
0.1346920

1.6211000

$-1.5139570$

$-2.1479380$

$-2.0922900$

$-1.2916060$

$-4.1422040$

$-4.3302580$

$-3.7446510$

$-5.1087760$

$-3.7640560$

$-3.8632260$

$-4.7586210$

$-3.1286600$

$-0.9564480$

$-2.2558340$

$-0.1048500$

$-3.1361290$

0.3483380

1.7797360

$-2.2417560$

$-0.8526700$

1.6541690

$-1.7109890$

$-1.3042400$

$-1.1408690$

$-0.8503430$

$-2.8266050$

2.3076820

4.4144310

3.2582480

2.1166630

1.1949040

4.0081890

1. 1455720

$-2.6511450$

5.6980730

4.5214190

3.2789540

5.6539220

6.6414600

2. 3487160

6.5640470

$-2.8566020$

-2.0559110
-1.7946630 


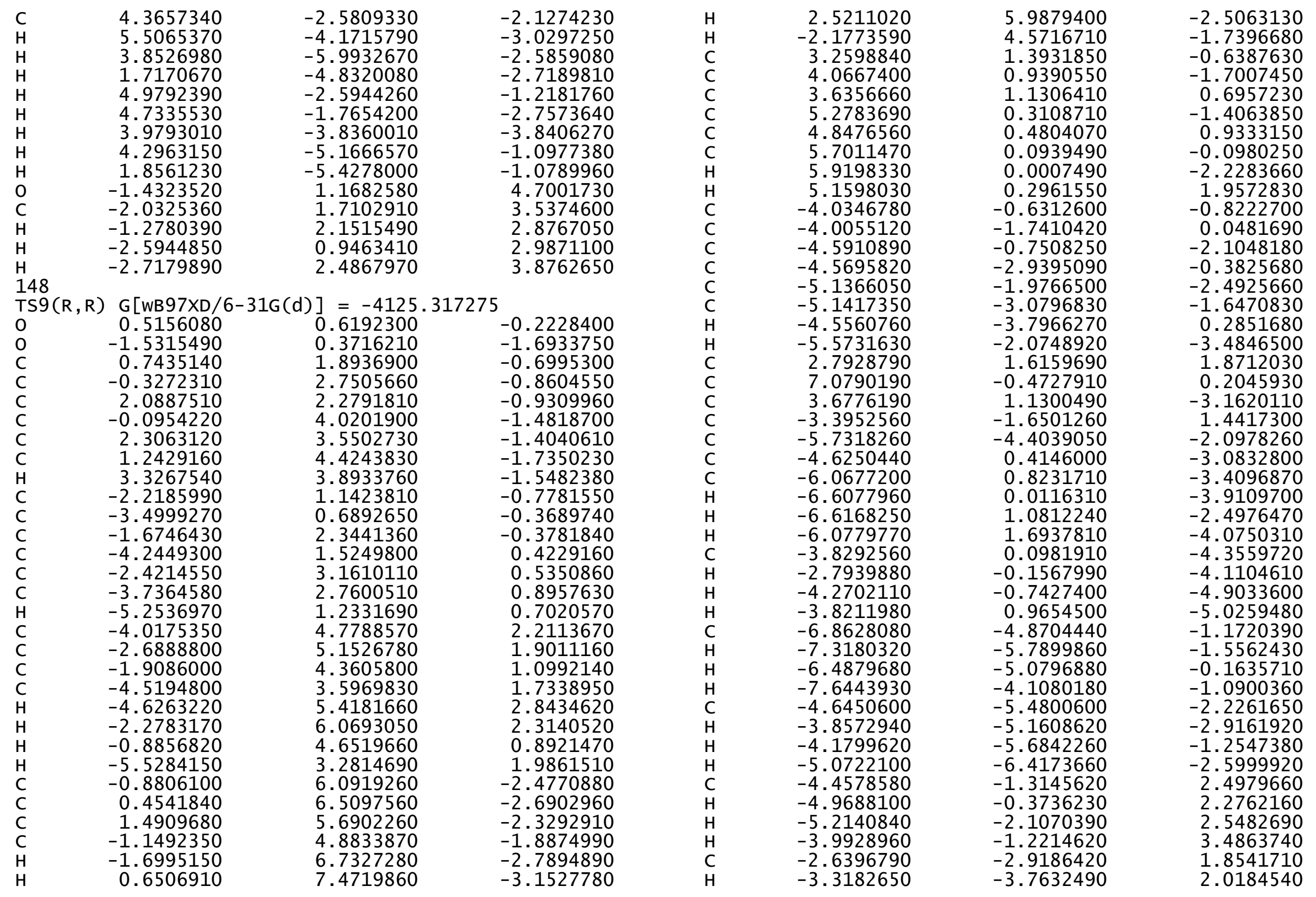




\begin{tabular}{|c|c|c|c|c|c|c|c|}
\hline $\mathrm{H}$ & -1.9034470 & -3.2026210 & 1.0978830 & $\mathrm{~s}$ & 1.6396340 & -4.1688220 & 1.7746380 \\
\hline $\mathrm{H}$ & -2.1170260 & -2.7420740 & 2.8011990 & $\mathrm{H}$ & 1.4158630 & -1.9887360 & -2.4739960 \\
\hline C & 3.0774490 & 3.0953270 & 2.1742190 & C & -0.5854610 & 0.7757070 & 5.3025230 \\
\hline $\mathrm{H}$ & 4.1253970 & 3.2310130 & 2.4666180 & $\mathrm{C}$ & -0.9442780 & 1.1605080 & 3.9985410 \\
\hline $\mathrm{H}$ & 2.8814970 & 3.7292500 & 1.3043620 & C & -0.6322910 & 0.3518630 & 2.9032580 \\
\hline $\mathrm{H}$ & 2.4438780 & 3.4425700 & 2.9983920 & $\mathrm{C}$ & 0.0845850 & -0.4206320 & 5.5277700 \\
\hline C & 2.9701840 & 0.7799090 & 3.1423290 & $\mathrm{H}$ & -0.8266380 & 1.4035870 & 6.1507460 \\
\hline $\mathrm{H}$ & 2.8678900 & -0.2911890 & 2.9368390 & $\mathrm{H}$ & -0.8879290 & 0.6680050 & 1.8979660 \\
\hline $\mathrm{H}$ & 3.9475970 & 0.9442160 & 3.6100600 & $\mathrm{H}$ & 0.3598520 & -0.7062600 & 6.5370970 \\
\hline $\mathrm{H}$ & 2.2057940 & 1.0606250 & 3.8743850 & C & 4.2964050 & -4.4932470 & -1.6021430 \\
\hline C & 8.1652360 & 0.5257940 & -0.2229260 & $\mathrm{C}$ & 3.3580350 & -5.5450490 & -1.0045100 \\
\hline $\mathrm{H}$ & 9.1587860 & 0.1589090 & 0.0576470 & $\mathrm{C}$ & 1.8838580 & -5.1741820 & -1.2067980 \\
\hline $\mathrm{H}$ & 8.1538440 & 0.6722490 & -1.3093420 & C & 4.0531250 & -3.1164180 & -0.9802320 \\
\hline $\mathrm{H}$ & 8.0111770 & 1.5011310 & 0.2501450 & $\mathrm{H}$ & 5.3390110 & -4.7832890 & -1.4381420 \\
\hline C & 7.3273750 & -1.8459150 & -0.4308380 & $\mathrm{H}$ & 3.5411610 & -6.5242420 & -1.4568120 \\
\hline $\mathrm{H}$ & 6.6275610 & -2.5943660 & -0.0487190 & $\mathrm{H}$ & 1.5941460 & -5.3110130 & -2.2557020 \\
\hline $\mathrm{H}$ & 7.2220570 & -1.8077910 & -1.5213490 & $\mathrm{H}$ & 4.3450850 & -3.1151060 & 0.0769540 \\
\hline $\mathrm{H}$ & 8.3437590 & -2.1881060 & -0.2073020 & $\mathrm{H}$ & 4.6403380 & -2.3407870 & -1.4792740 \\
\hline C & 3.7697060 & -0.1786130 & -3.9570710 & $\mathrm{H}$ & 4.1460040 & -4.4348050 & -2.6865560 \\
\hline $\mathrm{H}$ & 4.8076330 & -0.5105440 & -4.0762910 & $\mathrm{H}$ & 3.5625730 & -5.6454050 & 0.0683920 \\
\hline $\mathrm{H}$ & 3.2106590 & -0.9804040 & -3.4697860 & $\mathrm{H}$ & 1.2177170 & -5.8171790 & -0.6253890 \\
\hline $\mathrm{H}$ & 3.3538110 & -0.0342630 & -4.9602170 & 0 & -1.5948420 & 2.3070040 & 3.7095430 \\
\hline C & 4.5322230 & 2.2135860 & -3.8350820 & $\mathrm{C}$ & -1.9295010 & 3.1753870 & 4.7767030 \\
\hline $\mathrm{H}$ & 5.5942960 & 1.9416860 & -3.8097250 & $\mathrm{H}$ & -2.6068850 & 2.6890880 & 5.4883230 \\
\hline $\mathrm{H}$ & 4.2400500 & 2.3340720 & -4.8842170 & $\mathrm{H}$ & -1.0325340 & 3.5241340 & 5.3019230 \\
\hline $\mathrm{H}$ & 4.4239530 & 3.1836960 & -3.3412540 & $\mathrm{H}$ & -2.4343110 & 4.0226940 & 4.3167800 \\
\hline $\mathrm{P}$ & -0.2200220 & -0.4603090 & -1.2171620 & \multirow{2}{*}{\multicolumn{4}{|c|}{$\begin{array}{l}148 \\
\text { TS10 }\end{array}$}} \\
\hline 0 & 0.5814440 & -0.7014050 & -2.4657130 & & & & \\
\hline 0 & -0.5586330 & -1.6129420 & -0.3172510 & 0 & 0.2535470 & 0.8686110 & 0.0850700 \\
\hline $\mathrm{H}$ & 2.6322890 & 1.4501950 & -3.1942260 & 0 & -1.6795650 & 0.2153580 & -1.4016180 \\
\hline $\mathrm{H}$ & 7.1507180 & -0.5948180 & 1.2928570 & $\mathrm{C}$ & 0.2343700 & 2.1784310 & -0.3505690 \\
\hline $\mathrm{H}$ & 1.7392190 & 1.5291350 & 1.5909840 & $\mathrm{C}$ & -0.9835330 & 2.8103230 & -0.4946720 \\
\hline $\mathrm{H}$ & -4.1476170 & 1.2770550 & -2.6091590 & $\mathrm{C}$ & 1.4829810 & 2.8085010 & -0.5934550 \\
\hline $\mathrm{H}$ & -6.1635540 & -4.2463620 & -3.0946110 & $\mathrm{C}$ & -0.9990500 & 4.1270510 & -1.0628610 \\
\hline $\mathrm{H}$ & -2.6671780 & -0.8339560 & 1.4293750 & $\mathrm{C}$ & 1.4516330 & 4.1214200 & -0.9936830 \\
\hline C & 2.6040100 & -2.7101710 & -1.0341720 & $\mathrm{C}$ & 0.2376220 & 4.7948210 & -1.2740200 \\
\hline $\mathrm{C}$ & 1.5814370 & -3.7481850 & -0.8712340 & $\mathrm{H}$ & 2.3874660 & 4.6507590 & -1.1460960 \\
\hline $\mathrm{H}$ & 2.4005140 & -1.7523320 & -0.5515540 & $\mathrm{C}$ & -2.5275380 & 0.8505410 & -0.5257100 \\
\hline $\mathrm{H}$ & 0.5608980 & -3.4090050 & -0.7288230 & $\mathrm{C}$ & -3.7529700 & 0.1871750 & -0.2384960 \\
\hline 0 & 2.0670650 & -2.7697800 & -2.3752810 & $\mathrm{C}$ & -2.2361740 & 2.1230110 & -0.0770870 \\
\hline C & 1.0712200 & -2.8501040 & 2.6603770 & $\mathrm{C}$ & -4.6691030 & 0.8415800 & 0.5402890 \\
\hline C & 0.3902440 & -1.2304470 & 4.4441490 & $\mathrm{C}$ & -3.1673940 & 2.7596590 & 0.8151460 \\
\hline $\mathrm{c}$ & 0.0327780 & -0.8404320 & 3.1490020 & $\mathrm{C}$ & -4.4046640 & 2.1184430 & 1.0953080 \\
\hline $\mathrm{N}$ & 0.4391090 & -1.7686270 & 2.1954260 & $\mathrm{H}$ & -5.6216230 & 0.3652080 & 0.7555970 \\
\hline $\mathrm{H}$ & 0.1442300 & -1.6907660 & 1.1984630 & $\mathrm{C}$ & -5.0558390 & 3.9416110 & 2.5574190 \\
\hline $\mathrm{S}$ & 1.2405190 & -2.7627740 & 4.3989330 & C & -3.8092310 & 4.5632640 & 2.3165860 \\
\hline
\end{tabular}




$$
\begin{aligned}
& -2.8925080 \\
& -5.3406610 \\
& -5.7741400 \\
& -3.5713060 \\
& -1.9349610 \\
& -6.2842400 \\
& -2.1608330 \\
& -0.9320680 \\
& 0.2402740 \\
& -2.1951880 \\
& -3.0853150 \\
& -0.9209650 \\
& 1.1943450 \\
& -3.1448390 \\
& 2.7851660 \\
& 3.5687620 \\
& 3.2599300 \\
& 4.8307710 \\
& 4.5035140 \\
& 5.3217010 \\
& 5.4375360 \\
& 4.8711030 \\
& -4.0282460 \\
& -3.6375090 \\
& -4.6715790 \\
& -3.8912710 \\
& -4.8930570 \\
& -4.5101860 \\
& -3.5878620 \\
& -5.3832360 \\
& 2.4753050 \\
& 6.7051760 \\
& 3.1228750 \\
& -2.9872790 \\
& -4.7399470 \\
& -5.2171800 \\
& -4.2677740 \\
& -4.2032670 \\
& -3.2576710 \\
& -4.6478470 \\
& -6.5617990 \\
& -7.2692000 \\
& -6.4310590 \\
& -7.0051890 \\
& -5.6493530 \\
& -5.8414530
\end{aligned}
$$

\begin{tabular}{rr}
3.9911090 & 1.4713210 \\
2.7407340 & 1.9618830 \\
4.4081860 & 3.2241840 \\
5.4994460 & 2.8121240 \\
4.4744560 & 1.3168730 \\
2.2371960 & 2.1540820 \\
6.0480900 & -1.9914100 \\
6.7290640 & -2.1544090 \\
6.1114390 & -1.8067090 \\
4.7846510 & -1.4589730 \\
6.5282260 & -2.2972150 \\
7.7311080 & -2.5718250 \\
6.6112630 & -1.9512470 \\
4.2729240 & -1.3496300 \\
2.0820540 & -0.4579380 \\
1.8539030 & -1.6041270 \\
1.6719250 & 0.8108660 \\
1.2638490 & -1.4591660 \\
1.0539970 & 0.8904310 \\
0.8587170 & -0.2263520 \\
1.1228020 & -2.3479210 \\
0.7271100 & 1.8581690 \\
-1.1757630 & -0.7890950 \\
-2.3059400 & -0.0368890 \\
-1.3361870 & -2.0297070 \\
-3.5758570 & -0.5482250 \\
-2.6363360 & -2.4964290 \\
-3.7648000 & -1.7823100 \\
-4.4465110 & 0.0253690 \\
-2.7676210 & -3.4595500 \\
1.9665220 & 2.0864890 \\
0.2500490 & -0.0331940 \\
2.2486120 & -3.0079730 \\
-2.1573260 & 1.3347190 \\
-5.1576200 & -2.3404330 \\
-0.2069960 & -2.9064350 \\
0.9598120 & -3.2139510 \\
1.6697440 & -2.3849230 \\
0.6105480 & -3.4446010 \\
1.5090890 & -4.0824880 \\
0.3152290 & -2.3767230 \\
-0.5045480 & -2.2130210 \\
0.8495020 & -1.4306970 \\
1.0127140 & -3.0967370 \\
-5.9946490 & -1.4314510 \\
-6.9755690 & -1.8800840 \\
& \\
\hline
\end{tabular}

3.9911090

4.4081860

2.0820540

$-1.1757630$

$-4.4465110$

$-2.7676210$

2. 2486120

0.9598120

1.6697440

0.3152290

$-0.504$

$-6.9755690$
-6.1598790
-5.4966940
-5.8769860
-5.2870330
-6.0443420
-6.8530100
-2.0183480
-1.1742930
-2.9281830
-1.8674690
-3.2928420
-4.2393040
-3.4349810
-3.0478010
3.4549950
3.7204550
4.1032620
3.6626160
1.1140230
0.0559460
1.4396930
1.2021210
1.1494150
0.6900490
2.1234380
1.3235980
-0.0340770
-0.6913020
0.8947070
-0.5207380
1.1124290
0.8790090
0.2033130
1.4066080
3.4949020
3.2877700
3.7998870
4.3413660
-0.2713110
-0.1541310
-1.5599220
2.4845030
-0.7071550
1.7399310
-0.6817200
-5.0425400
$-0.4527800$ $-1.2683540$ $-2.5977480$ $-3.2580960$ $-1.6596710$ $-3.0648690$ 2.4391860 2.2604330 2. 5004970 3. 4113280

1.6900470

1.8580730

0.9054490

2.6217720

2.4577790

2.6524850

1.6604760

3. 3654930

3.2860790

3.0228750

3.6919790

4.0857540

0.8701970

1.0460450

0.3931190

1.8420220

$-1.3337560$

$-2.0039960$

$-1.8731700$

$-1.1105170$

$-4.0240980$

$-4.2057030$

$-3.6880370$

$-4.9842130$

$-3.4949700$

$-3.5795560$

$-4.4828460$

$-2.8126780$

$-1.0056680$

$-2.2786290$

$-0.2366800$

$-2.9722230$

0.4889150

1.8950740

$-3.8722130$

$-3.3062200$ 


\begin{tabular}{|c|c|c|c|}
\hline & & & \\
\hline $\mathrm{H}$ & -2.3962020 & -1.2361220 & 1. 3153210 \\
\hline C & 3.1106450 & -2.0017360 & -1.7735760 \\
\hline C & 2.2712900 & -3.1578330 & -1.4461530 \\
\hline $\mathrm{H}$ & 2.9729480 & -1.1133270 & -1.1541520 \\
\hline $\mathrm{H}$ & 1.3028030 & -2.9545150 & -0.9995230 \\
\hline 0 & 2.2157070 & -2.0131110 & -2.9095030 \\
\hline C & 2.3319990 & -2.8843550 & 2.18 \\
\hline C & 1.3937060 & -1.9584180 & 4.3176250 \\
\hline C & 0.7763870 & -1.4813520 & 3.1650450 \\
\hline $\mathrm{N}$ & 1.3233930 & -2.0243290 & 2.0090100 \\
\hline $\mathrm{H}$ & 0.8956980 & -1.8245790 & 1.0827420 \\
\hline $\mathrm{S}$ & 2.6771950 & -3.0729140 & 3.8901360 \\
\hline $\mathrm{S}$ & 3.1984080 & -3.7154090 & 1.0001040 \\
\hline $\mathrm{H}$ & 1.4959820 & -1.3142010 & -2.7133000 \\
\hline C & -0.0523680 & -0.5903200 & 5.6313650 \\
\hline C & -0.6670680 & -0.1073250 & 4.4582410 \\
\hline C & -0.2525210 & -0.5430460 & 3.2029550 \\
\hline C & 0.9791450 & -1.5077440 & 5.5709510 \\
\hline $\mathrm{H}$ & -0.4072260 & -0.2170630 & 6.5856550 \\
\hline $\mathrm{H}$ & -0.6733110 & -0.1736520 & 2.2765820 \\
\hline $\mathrm{H}$ & 1.4511620 & -1.8648150 & 6.4794880 \\
\hline C & 4.7115510 & -3.5438700 & -2.9752840 \\
\hline C & 4.0762890 & -4.7323810 & -2.2490790 \\
\hline C & 2.5797030 & -4.5113990 & -2.0039460 \\
\hline C & 4.5422500 & -2.2483510 & -2.1765050 \\
\hline $\mathrm{H}$ & 5.7771730 & -3.7299930 & -3.1400390 \\
\hline $\mathrm{H}$ & 4.2104430 & -5.6520510 & -2.8260830 \\
\hline $\mathrm{H}$ & 2.0208200 & -4.5932080 & -2.9440810 \\
\hline $\mathrm{H}$ & 5.1370370 & -2.2831090 & -1.2554970 \\
\hline $\mathrm{H}$ & 4.8812310 & -1.3814150 & -2.7517780 \\
\hline $\mathrm{H}$ & 5380 & -3.4253920 & -3.962 \\
\hline $\mathrm{H}$ & 4.58 & -4.8845040 & -1.28 \\
\hline $\mathrm{H}$ & 2.15 & -5.2659710 & -1.3 \\
\hline 0 & -1.65 & 3720 & 4.6 \\
\hline C & -2.2 & 1.3646880 & 3.4 \\
\hline $\mathrm{H}$ & $-1.4 \varepsilon$ & 1.8861840 & 2 . \\
\hline $\mathrm{H}$ & -2.7 & 0.6057090 & \\
\hline $\mathrm{H}$ & -2.9 & 2.0807920 & \\
\hline & $\mathrm{G}[\mathrm{wB} 9$ & d) $]=-412$ & \\
\hline 0 & 0.5017140 & 0.6884700 & -0.1495860 \\
\hline 0 & -1.4771340 & 0.3291530 & -1.6812430 \\
\hline C & 0.6576510 & 1.9777650 & -0.6153060 \\
\hline C & -0.4605020 & 2.7709640 & -0.7689170 \\
\hline C & 1.9773970 & 2.4376660 & -0.8560790 \\
\hline$c$ & -0 . & 4.069464 & \\
\hline
\end{tabular}

\begin{abstract}
2. 1271130
1. 0157450

3. 1267840

$-2.2381700$

$-3.5012680$

$-1.7857630$

$-4.3165240$

$-2.6047060$

$-3.8990510$

$-5.3127670$

$-4.3381140$

$-3.0303190$

$-2.1831650$

$-4.7524980$

$-4.9997410$

$-2.6892250$

$-1.1775600$

$-5.7438940$

$-1.1971660$

0.1116420

1. 1924220

$-1.3979140$

$-2.0503750$

0.2551820

2. 2038350

$-2.4061260$

3. 1713950

3. 9215130

3. 5884030

5. 1068220

4.7700880

5.5581130

5. 6894140

5. 1100080

$-3.9536480$

$-3.8832290$

$-4.4833920$

$-4.3815230$

$-4.9658670$

$-4.9313180$

$-4.3374240$

$-5.3864790$

2. 8157810

6.8790810

3.5291920
-3.3025980
\end{abstract}

3.7352430

4. 5623220

4.1297390

1.0360170

0.4973500

2. 2617800

1.2632850

3.0051100

2. 5122220

0.9022700

4.4713510

4.9382520

4. 2192700

3. 2739380

5.0535570

5. 8682110

4.5825110

2.8868050

6.1194700

6.6266280

5.8606770

4.8780620

6.7169730

7.6137490

6.2281930

4.4973900

1. 5553980

1.1795860

1.1616700

0.4589630

0.4391720

0.0941790

0.1874370

0.1397130

$-0.8330750$

$-1.9702770$

$-0.9328190$

$-3.1782330$

$-2.1678280$

$-3.2990810$

$-4.0576890$

$-2.2500590$

1. 5843540

$-0.6234530$

1.5648100
-1.8980540
$-1.2796700$

$-1.5739990$

$-1.4384710$

$-0.7711380$

$-0.4115580$

$-0.3274760$

0.3822910

0.5863790

0.9036640

0.6227390

2. 2651720

1.9957090

1.1933470

1.7452560

2.8993670

2.4407680

1.0181070

1. 9652920

$-2.2963510$

$-2.4725840$

$-2.1224120$

$-1.7496770$

$-2.6028430$

$-2.9008970$

$-2.2746120$

$-1.6293890$

$-0.6815300$

$-1.8095730$

0.6112500

$-1.6240050$

0.7378650

$-0.3636140$

$-2.4989620$

1.7258960

$-0.9212490$

$-0.0898700$

$-2.2167510$

$-0.5722620$

$-2.6554100$

$-1.8487470$

0.0642770

$-3.6557900$

1.8564770

$-0.1310300$

$-3.2323590$

1. 3176210 


-5.4658150
-4.5629120
-6.0218480
-6.5412140
-6.5671790
-6.0689300
-3.7711260
-2.7263650
-4.1917770
-3.7937030
-6.6013280
-7.0215730
-6.2409100
-7.4064170
-4.3447030
-3.5533890
-3.8923020
-4.7365270
-4.3968240
-4.9388130
-5.1228920
-3.9537360
-2.5016200
-3.1452300
-1.7360420
-2.0088130
3.1793690
4.2453380
2.9699390
2.6030600
3.0117600
2.8620940
4.0127020
2.2883580
7.9306620
8.8738600
8.1278620
7.5893840
7.4112170
6.6689890
7.7088080
8.2970200
3.4813780
4.4573070
2.7546830
3.1800880

$-4.6292780$

0.2658670

0.6386820

$-0.1726030$

0.8458980

1.5320090

0.0268190

$-0.2081080$

$-0.8014890$

0.9227570

$-5.1534020$

$-6.0756150$

$-5.3768890$

$-4.4160690$

$-5.6688050$

$-5.3098650$

$-5.8839760$

$-6.6085500$

$-1.6413860$

$-0.7125910$

$-2.4630830$

$-1.5670170$

$-3.1465540$

$-4.0255930$

$-3.3693690$

$-2.9826600$

3.0243750

3.0956020

3.7275140

3.3363680

0.6477920

$-0.4007860$

0.7418410

0.8974500

0.3728590

$-0.1385110$

1.1413160

0.8749470

$-1.3766350$

$-2.0688980$

$-0.6863500$

$-1.9571890$

0.3636350

$-0.1248370$

$-0.3757000$

0.6988380

-2.3487950
-3.1512590
-3.4471520
-3.9703930
-2.5199740
-4.0801000
-4.4429560
-4.2177160
-5.0244760
-5.0737050
-1.4597650
-1.8762490
-0.4490670
-1.3752050
-2.4810140
-3.1470870
-1.5060360
-2.8861790
2.3637820
2.1660790
2.3705840
3.3637280
1.7035440
1.8207560
0.9558650
2.6686180
2.2506010
2.4972680
1.4387430
3.1290590
3.0527330
2.7720440
3.4891980
3.8359690
0.3828400
0.6048040
-0.3737430
1.2937850
-1.3526340
-1.7661200
-2.1500770
-1.0752180
-4.1846630
-4.2826600
-3.8448470
-5.1835450

\author{
4.4813500 \\ 5.5013750 \\ 4.1588360 \\ 4.5184970 \\ $-0.1281790$ \\ 0.7320410 \\ $-0.4208630$ \\ 2. 5221340 \\ 6.7081540 \\ 1.7488340 \\ $-4.1083580$ \\ $-5.8812880$ \\ $-2.6091830$ \\ 2.0244620 \\ 2.7265070 \\ 0.9391400 \\ 2. 2536310 \\ 2. 6344930 \\ 1.0835920 \\ 0.3173290 \\ $-0.0377190$ \\ 0.4124430 \\ 0.1290760 \\ 1.2265450 \\ 1.7232080 \\ 1.9295080 \\ $-0.7526060$ \\ $-1.1081550$ \\ $-0.7461560$ \\ $-0.0378760$ \\ $-1.0312900$ \\ $-1.0027820$ \\ 0.2322240 \\ 4.0827100 \\ 4.5882490 \\ 4.1635370 \\ 2. 5542170 \\ 4.4052250 \\ 5.6798270 \\ 4.7233320 \\ 2. 1051570 \\ 2. 1952070 \\ 4.5196560 \\ 4.1942370 \\ 4.3654290 \\ $-1.8019720$
}

2.6378550

2. 2450230

2. 9612170

3.5189100

$-0.4277500$

$-0.6010030$

$-1.6093620$

1.9883040

$-1.3612430$

1.5620250

1.1251940

$-4.4585030$

$-1.0519610$

$-3.4125170$

$-2.7190490$

$-3.3301210$

$-1.8420900$

$-2.8152380$

$-1.2513800$

$-0.8542470$

$-1.7535060$

$-1.6784700$

$-2.7487950$

$-4.0776550$

$-1.5947350$

0.6984300

1.0906790

0.3149630

$-0.4727460$

1. 3021500

0.6347360

$-0.7641390$

$-4.8332140$

$-4.4204370$

$-2.9929290$

$-4.7559460$

$-5.8533700$

$-4.4857580$

$-2.2664990$

$-5.5115100$

$-4.9531710$

$-5.1121500$

$-2.7412030$

2. 2139370
$-3.7824920$

$-3.8697040$

$-4.7784970$

$-3.1339510$

$-1.1751040$

$-2.3951290$

$-0.2985650$

$-3.2083660$

0.6655370

1.6186530

$-2.6502460$

$-3.3502880$

1. 3424840

$-1.3776380$

$-0.2963570$

$-1.3497480$

0.1317610

$-2.1067360$

2. 7143740

4.5113470

3.2172750

2. 2568640

1.2526140

4.4541970

1.7939000

$-2.2225280$

5.3859890

4.0829620

2. 9792280

5.6031620

6.2402470

1.9749700

6.6122120

$-1.7430150$

$-0.3580780$

$-0.0085700$

$-1.8175180$

$-1.9717510$

$-0.3123110$

$-0.6070380$

$-1.1612460$

$-2.8323950$

$-2.5060300$

0.3969840

1.0357590

3.8045200 


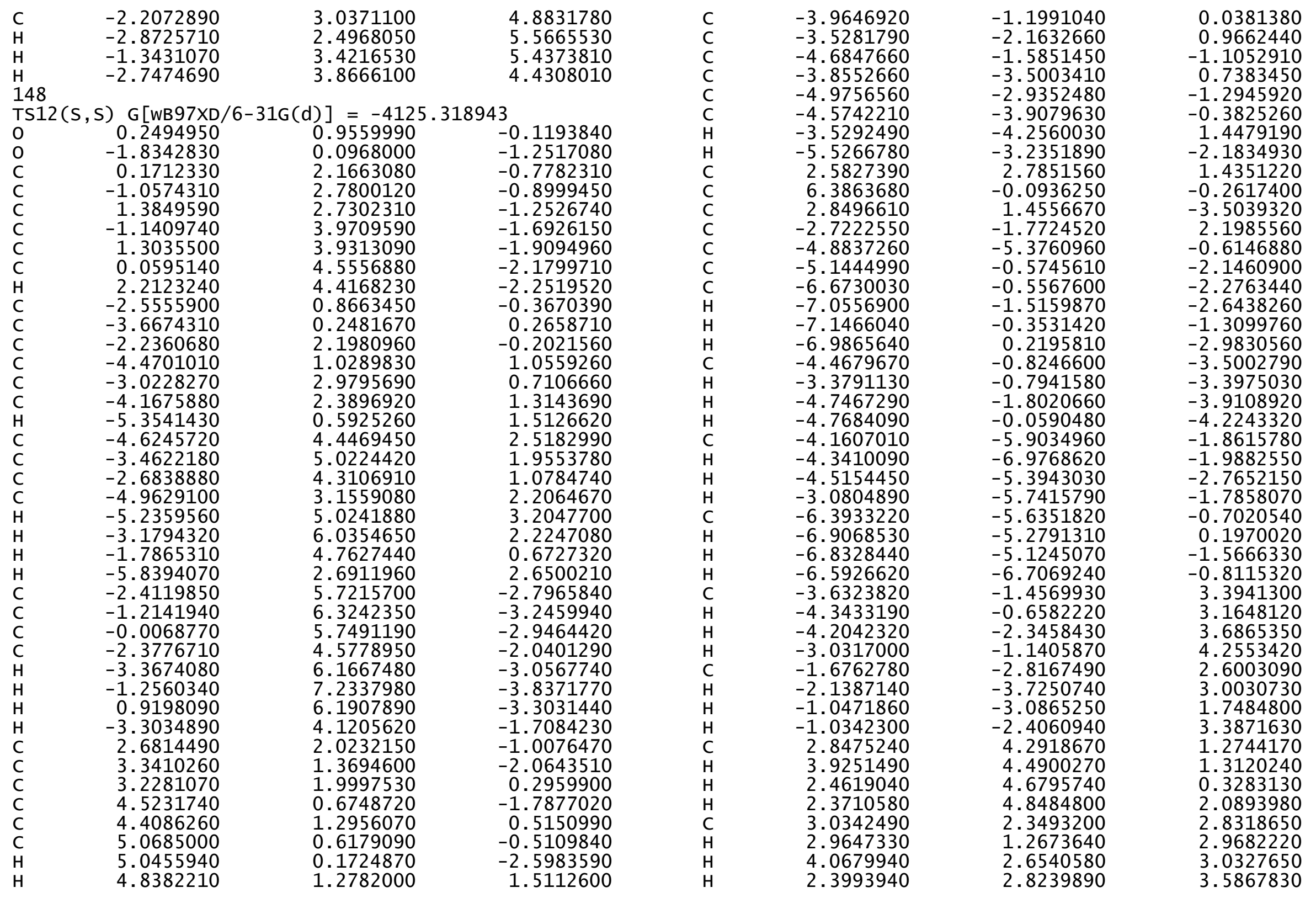




$\begin{array}{rrr}6.3309980 & -1.0517870 & 0.9335020 \\ 7.2782570 & -1.5937420 & 1.0290830 \\ 6.1661140 & -0.5110360 & 1.8724580 \\ 5.5255750 & -1.7862780 & 0.8256250 \\ 7.5183800 & 0.9297220 & -0.0863110 \\ 7.5850030 & 1.5959700 & -0.9526660 \\ 7.3437160 & 1.5474420 & 0.8024870 \\ 8.4828250 & 0.4244740 & 0.0367130 \\ 3.0059540 & 0.1505280 & -4.2901470 \\ 4.0582080 & -0.0833590 & -4.4906860 \\ 2.5588610 & -0.6899130 & -3.7595950 \\ 2.5068310 & 0.2437020 & -5.2606310 \\ 3.5821300 & 2.5841500 & -4.2483270 \\ 4.6548580 & 2.3652860 & -4.3124350 \\ 3.1946410 & 2.6833610 & -5.2686200 \\ 3.4710780 & 3.5501040 & -3.7486880 \\ -0.2992440 & -0.3541310 & -0.9358580 \\ 0.3596610 & -0.4858710 & -2.2806380 \\ -0.2157710 & -1.4884490 & 0.0410290 \\ 1.7818320 & 1.6895760 & -3.4789280 \\ 6.6116020 & -0.6870670 & -1.1580780 \\ 1.4996030 & 2.6234290 & 1.3821080 \\ -4.8405450 & 0.4222340 & -1.8130480 \\ -4.5007970 & -5.9310570 & 0.2512520 \\ -2.1745010 & -0.8597670 & 1.9434680 \\ 1.5876540 & -3.5094150 & -1.7442310 \\ 2.7531460 & -2.6685520 & -1.4679230 \\ 0.7295260 & -3.3474570 & -1.0930690 \\ 2.5812640 & -1.6898520 & -1.0302310 \\ 1.5672130 & -2.6376230 & -2.8996470 \\ 2.7141500 & -2.5197790 & 2.1091280 \\ 2.2426390 & -1.1196220 & 4.1321180 \\ 1.3940810 & -0.8750230 & 3.0555600 \\ 1.6913590 & -1.6737780 & 1.9591580 \\ 1.0743320 & -1.6570690 & 1.1209140 \\ 3.4186420 & -2.3448420 & 3.6987070 \\ 3.2890070 & -3.6370210 & 0.9811190 \\ 1.0019440 & -1.8136180 & -2.6868950 \\ 1.0630780 & 0.5200790 & 5.4014760 \\ 0.2171180 & 0.7699800 & 4.3013680 \\ 0.3735720 & 0.0729660 & 3.1080270 \\ 2.0782290 & -0.4139070 & 5.3250350 \\ 0.9032180 & 1.0886270 & 6.3108860 \\ -0.2324540 & 0.2523950 & 2.2299330 \\ 2.7288410 & -0.5895080 & -2.9795280 \\ 3.1039270 & -5.1097730 & \end{array}$

$\begin{array}{ll}\mathrm{C} & 4.3147480 \\ \mathrm{C} & 4.0982240 \\ \mathrm{C} & 1.8362670 \\ \mathrm{H} & 3.2910340 \\ \mathrm{H} & 5.2143250 \\ \mathrm{H} & 4.1750070 \\ \mathrm{H} & 1.9228820 \\ \mathrm{H} & 0.9547340 \\ \mathrm{H} & 2.9491780 \\ \mathrm{H} & 4.5014800 \\ \mathrm{H} & 4.8562740 \\ \mathrm{O} & -0.7223360 \\ \mathrm{C} & -1.5432080 \\ \mathrm{H} & -0.9402260 \\ \mathrm{H} & -2.1467800 \\ \mathrm{H} & -2.2040290 \\ \mathrm{H} & \end{array}$
$-4.4680500$
(10

$-2.1336930$

-3. 1472790

$-2.9040380$

$-2.9769430$

$-1.2040830$

$-2.6651070$

$-3.9634490$

$-1.3392650$

$-1.3738420$

4. 4971950

3. 3922070

2. 5495150

3.0678970

148

3. 7293550

$\operatorname{TS} 13(\mathrm{~S}, \mathrm{~S}) \mathrm{G}[\mathrm{WB} 97 \mathrm{XD} / 6-31 \mathrm{G}(\mathrm{d})]=-4125.317091$

$\begin{array}{lrrr}\text { TS13(S, S } r \text { G }[\text { WB } 97 X D / 6-31 G(\mathrm{~d})] & =-4125.317091 \\ \mathrm{O} & -0.1942660 & -1.0289640 & -0.1022160 \\ \mathrm{O} & 1.7975750 & -0.1093500 & -1.3463840 \\ \mathrm{C} & -0.0911940 & -2.2406760 & -0.7539120 \\ \mathrm{C} & 1.1560260 & -2.8030120 & -0.9270840 \\ \mathrm{C} & -1.2995620 & -2.8655320 & -1.1591740 \\ \mathrm{C} & 1.2541180 & -3.9962510 & -1.7141490 \\ \mathrm{C} & -1.1975570 & -4.0614240 & -1.8223250 \\ \mathrm{C} & 0.0589810 & -4.6321660 & -2.1491530 \\ \mathrm{H} & -2.1009050 & -4.5876030 & -2.1173130 \\ \mathrm{C} & 2.5899540 & -0.8242490 & -0.4765110 \\ \mathrm{C} & 3.6859270 & -0.1402680 & 0.1160470 \\ \mathrm{C} & 2.3365140 & -2.1652110 & -0.2824780 \\ \mathrm{C} & 4.5470660 & -0.8703550 & 0.8932440 \\ \mathrm{C} & 3.1851350 & -2.8946880 & 0.6166200 \\ \mathrm{C} & 4.3159200 & -2.2402990 & 1.1796350 \\ \mathrm{H} & 5.4220750 & -0.3878820 & 1.3196820 \\ \mathrm{C} & 4.9016110 & -4.2538650 & 2.4020070 \\ \mathrm{C} & 3.7556560 & -4.8942180 & 1.8771600 \\ \mathrm{C} & 2.9211800 & -4.2349000 & 1.0109990 \\ \mathrm{C} & 5.1693950 & -2.9532980 & 2.0622600 \\ \mathrm{H} & 5.5565090 & -4.7895770 & 3.0819630 \\ \mathrm{H} & 3.5303670 & -5.9157280 & 2.1677050 \\ \mathrm{H} & 2.0373000 & -4.7361080 & 0.6341870 \\ \mathrm{H} & 6.0332400 & -2.4403680 & 2.4764930 \\ \mathrm{C} & 2.5487590 & -5.7029900 & -2.8587010 \\ \mathrm{C} & 1.3590130 & -6.3558840 & -3.2563210 \\ \mathrm{C} & 0.1426520 & -5.8269860 & -2.9115380 \\ \mathrm{C} & 2.4991240 & -4.5562680 & -2.1076950\end{array}$




\begin{tabular}{|c|c|c|c|}
\hline $\begin{array}{l}\mathrm{H} \\
\mathrm{H} \\
\mathrm{H} \\
\mathrm{H} \\
\mathrm{C} \\
\mathrm{C} \\
\mathrm{C} \\
\mathrm{C} \\
\mathrm{C} \\
\mathrm{C} \\
\mathrm{H} \\
\mathrm{H} \\
\mathrm{C} \\
\mathrm{C} \\
\mathrm{C} \\
\mathrm{C} \\
\mathrm{C} \\
\mathrm{C} \\
\mathrm{H} \\
\mathrm{H} \\
\mathrm{C} \\
\mathrm{C} \\
\mathrm{C} \\
\mathrm{C} \\
\mathrm{C} \\
\mathrm{C} \\
\mathrm{C} \\
\mathrm{H} \\
\mathrm{H} \\
\mathrm{H} \\
\mathrm{C} \\
\mathrm{H} \\
\mathrm{H} \\
\mathrm{H} \\
\mathrm{C} \\
\mathrm{H} \\
\mathrm{H} \\
\mathrm{H} \\
\mathrm{C} \\
\mathrm{H} \\
\mathrm{H} \\
\mathrm{H} \\
\mathrm{C} \\
\mathrm{H} \\
\mathrm{H} \\
\mathrm{H}\end{array}$ & $\begin{array}{r}3.5098640 \\
1.4135230 \\
-0.7793070 \\
3.4188120 \\
-2.6165960 \\
-3.3712440 \\
-3.0933370 \\
-4.5883410 \\
-4.3159200 \\
-5.0775890 \\
-5.1768400 \\
-4.6980600 \\
3.8779660 \\
3.4233180 \\
4.4784950 \\
3.5953790 \\
4.6194660 \\
4.1808630 \\
3.2428790 \\
5.0755340 \\
-2.3268890 \\
-6.4172920 \\
-2.9344830 \\
2.7651140 \\
4.2999810 \\
4.9688590 \\
6.4793800 \\
6.7339010 \\
7.0326410 \\
6.8254060 \\
4.1843110 \\
3.1130370 \\
4.3371400 \\
4.5133710 \\
5.1163950 \\
5.2451610 \\
4.6148660 \\
6.1083000 \\
2.9129770 \\
2.3444300 \\
2.3346960 \\
3.0042390 \\
3.8064700 \\
4.5609460 \\
4.3212600 \\
3.3190300\end{array}$ & $\begin{array}{l}-6.1116210 \\
-7.2675070 \\
-6.3084810 \\
-4.0594460 \\
-2.2403730 \\
-1.5881020 \\
-2.3067020 \\
-1.0034400 \\
-1.7084630 \\
-1.0511280 \\
-0.5062030 \\
-1.7541430 \\
1.3205670 \\
2.2683120 \\
1.7400350 \\
3.6205600 \\
3.1087260 \\
4.0632840 \\
4.3569030 \\
3.4403950 \\
-3.0630420 \\
-0.4368730 \\
-1.5619440 \\
1.8426150 \\
5.5458270 \\
0.7497050 \\
0.8848020 \\
1.8678260 \\
0.7576050 \\
0.1252560 \\
0.8873470 \\
0.7530060 \\
1.8733480 \\
0.1317690 \\
6.2879430 \\
7.3400230 \\
6.2596740 \\
5.8396490 \\
6.1819610 \\
5.6737480 \\
6.1178460 \\
7.2404880 \\
1.6470970 \\
0.9049240 \\
2.5918950 \\
1.3108770\end{array}$ & $\begin{array}{r}-3.1555010 \\
-3.8432550 \\
-3.2264380 \\
-1.8182060 \\
-0.8194960 \\
-1.8132840 \\
0.5071450 \\
-1.4567620 \\
0.8082820 \\
-0.1546660 \\
-2.2235570 \\
1.8238000 \\
-0.1398450 \\
0.8012170 \\
-1.3365080 \\
0.5185410 \\
-1.5775650 \\
-0.6680280 \\
1.2348390 \\
-2.5084170 \\
1.5883690 \\
0.2094870 \\
-3.2724520 \\
2.1076350 \\
-0.9722970 \\
-2.3825980 \\
-2.6156450 \\
-3.0281400 \\
-1.6790370 \\
-3.3254760 \\
-3.6938990 \\
-3.5176680 \\
-4.1481560 \\
-4.4161950 \\
0.0934510 \\
-0.1837180 \\
1.0676050 \\
0.2123470 \\
-1.1462280 \\
-1.9323220 \\
-0.2170580 \\
-1.4143580 \\
3.2192140 \\
2.9452680 \\
3.4303860 \\
4.1419890\end{array}$ \\
\hline
\end{tabular}

\begin{abstract}
1. 6723340
2. 0885680

0.9493320

1.1419520

$-2.5108970$

$-3.5695110$

$-2.1688000$

$-1.9470400$

$-2.7048800$

$-2.7087780$

$-3.6942090$

$-1.9813510$

$-7.5705040$

$-8.5360190$

$-7.4967020$

$-7.5585450$

$-6.4623810$

$-5.6385300$

$-6.3899040$

$-7.4059830$

$-3.1889440$

$-4.2581510$

$-2.7561300$

$-2.7250960$

$-3.6332910$

$-4.7190430$

$-3.2893890$

$-3.4394640$

0.2741030

$-0.4681390$

0.2296620

$-1.8558090$

$-6.5498060$

$-1.2614100$

4. 7924520

4.8319150

2. 2809300

$-1.4251150$

$-2.6813990$

$-0.6157890$

$-2.6210710$

$-1.4396170$

$-2.7230140$

$-2.1797150$

-1.3472780
-1.6862320
\end{abstract}

2.8031440

3.7565280

2. 9988320

2. 3565430

$-4.5814460$

$-4.8451760$

$-4.9362260$

$-5.1156020$

$-2.6626580$

$-1.5780290$

$-3.0462940$

$-3.0873610$

$-1.1697740$

$-0.7579540$

$-1.0622040$

$-2.2391940$

1.0654200

1.5936620

1. 2473760

1.5008380

$-0.2180130$

$-0.0226650$

0.6041400

$-0.2195570$

$-2.6852310$

$-2.5302890$

$-2.7003820$

3.6691770

0.3005400

0.4369250

1.4213180

$-1.7372260$

$-0.5623440$

$-2.8371700$

$-0.2614720$

5.6427270

0.8784020

3.5229420

2. 7894620

3.2364390

1.7711240

2. 7215570

2.4335150

1.0457470

0.8205830

1.6097500
2. 5867090

2.9318900

1.7915530

3.4346900

1. 4281680

1. 5365710

0.4526720

2. 2014280

3. 0175460

3. 1534000

3.2930980

3. 7209290

$-0.4900400$

$-0.1759020$

$-1.5786130$

$-0.2552790$

$-0.0930080$

0.3999550

$-1.1720200$

0.2537180

$-3.9629280$

$-4.1053560$

$-3.3916350$

$-4.9550950$

$-4.0548720$

$-4.0578600$

$-5.0953480$

$-3.6183510$

$-0.9361360$

$-2.2356500$

0.0589090

$-3.3039380$

1. 2915910

1. 4621720

$-2.0038090$

$-1.9273600$

1. 9239120

$-1.6595610$

$-1.4847680$

$-0.9894870$

$-1.1132400$

$-2.8684890$

2. 1168720

4.1332220

3.0404390

1. 9490720 


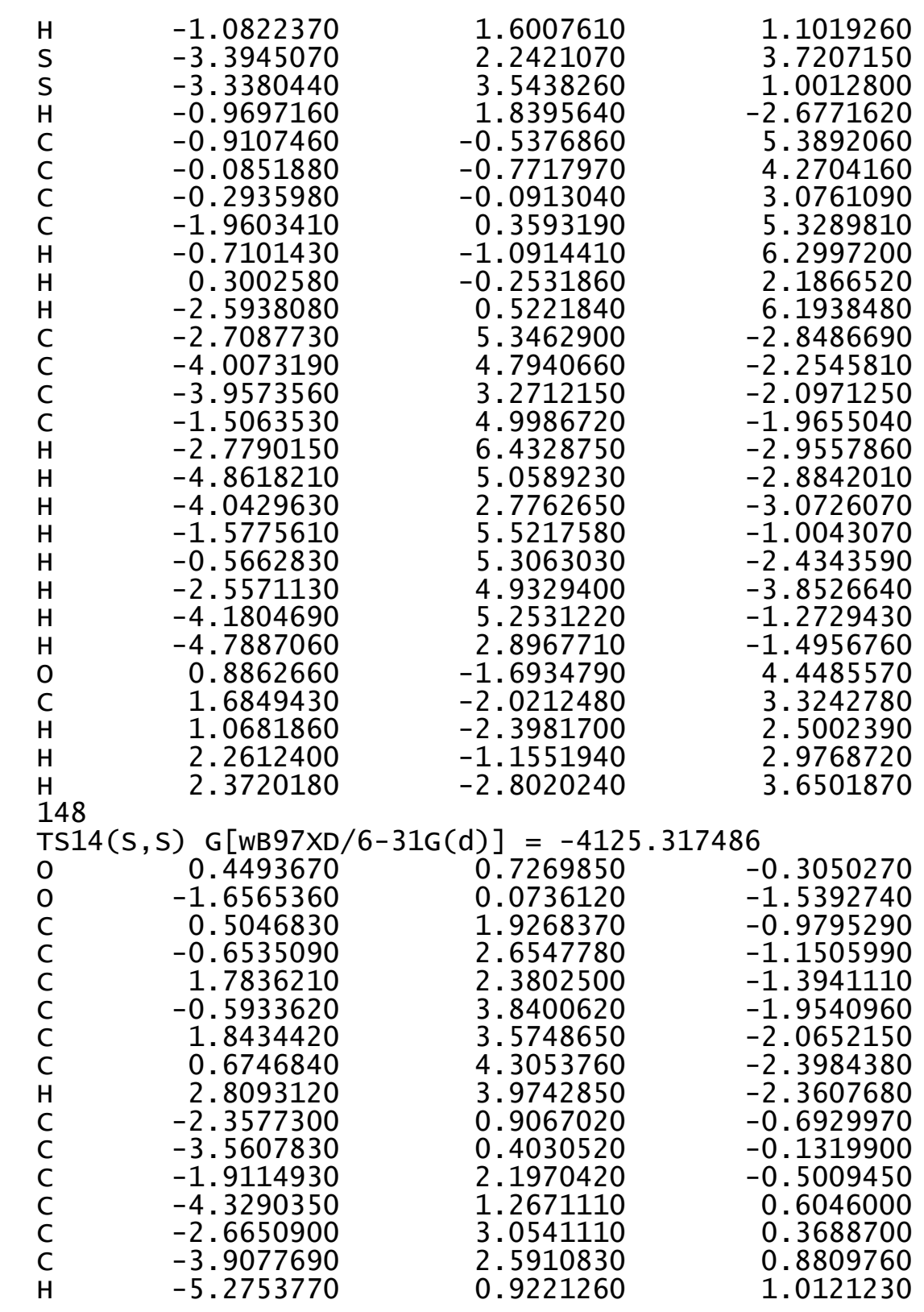

-4.2644990
-2.9985380
-2.2198570
-4.6990290
-4.8770430
-2.6380810
-1.2493830
-5.6543670
-1.6519510
-0.3867730
0.7490750
-1.7532990
-2.5515650
-0.3207620
1.7263340
-2.7302810
3.0126070
3.7068490
3.4808000
4.8728870
4.6477670
5.3630600
5.4257840
5.0154560
-3.9721830
-3.8609360
-4.4892780
-4.2981060
-4.9053710
-4.8215740
-4.2261250
-5.3117130
2.7610640
6.6769550
3.2504320
-3.3013380
-5.2871230
-4.6318970
-6.1090130
-6.5872330
-6.6627160
-6.2029160
-3.8323880
-2.7796200
-4.2227810
-3.8905350

. 7144490

5.1534740

4. 3464210

3.4534400

5. 3683090

6. 1378040

4.6966320

3. 0886140

5.6866830

6.1704550

5.4902500

4.5551470

6.2128280

7.0719520

5.8403080

4.1887300

1. 6000450

0.8890620

1.5991170

0. 2061700

0.8997130

0.2008800

$-0.3351970$

0.9040850

$-1.0170530$

$-1.9403350$

$-1.4209740$

$-3.2496620$

$-2.7404570$

$-3.6713650$

$-3.9698210$

$-3.0492160$

2. 3838200

$-0.4940280$

0.8739400

$-1.5521420$

$-5.1033140$

$-0.4535080$

$-0.2232880$

$-1.1489660$

0.1416670

0.5183570

$-0.9165650$

$-1.0623070$

$-1.8588370$

$-0.1658050$
1.9996260

1. 5472800

0.7577400

1.6841560

2. 6130040

1. 8304420

0.4246590

2. 0522490

$-3.1250080$

$-3.5314070$

$-3.1772600$

$-2.3565430$

$-3.4294660$

$-4.1326570$

$-3.4994250$

$-2.0611030$

$-1.0484060$

$-2.0438050$

0.2836820

$-1.6880780$

0.5849740

$-0.3863730$

$-2.4525500$

1. 6052960

$-0.3439350$

0.7119830

$-1.5886200$

0.5021170

$-1.7500850$

$-0.7160920$

1. 3140600

$-2.7109880$

1.3768380

$-0.0710190$

$-3.4969820$

2. 0743920

$-0.9094660$

$-2.7547040$

$-3.1017630$

$-3.4424350$

-2. 2299740

$-3.9029820$

$-3.7197130$

$-4.3809340$

$-4.7748540$ 


$\begin{array}{rrr}-4.4805720 & -5.8152910 & -2.0034950 \\ -4.7840600 & -6.8649370 & -2.0854650 \\ -4.6396560 & -5.3436740 & -2.9801890 \\ -3.4080930 & -5.7824260 & -1.7856610 \\ -6.7917730 & -5.1699660 & -1.2036530 \\ -7.3695120 & -4.6832500 & -0.4110050 \\ -7.0297580 & -4.6707860 & -2.1501450 \\ -7.1235600 & -6.2113160 & -1.2821500 \\ -4.4253530 & -1.3750590 & 3.1046410 \\ -5.1590000 & -0.6318370 & 2.7765250 \\ -4.9547650 & -2.3213220 & 3.2686900 \\ -4.0120500 & -1.0450580 & 4.0647960 \\ -2.2524960 & -2.5447410 & 2.5892820 \\ -2.6946040 & -3.5187360 & 2.8279900 \\ -1.4661070 & -2.6880050 & 1.8442600 \\ -1.7961960 & -2.1560570 & 3.5069140 \\ 3.0904830 & 3.8826280 & 1.2875590 \\ 4.1654740 & 4.0428500 & 1.4314790 \\ 2.8089310 & 4.3058120 & 0.3196960 \\ 2.5565660 & 4.4351490 & 2.0690850 \\ 3.0590060 & 1.8804630 & 2.7920810 \\ 3.0033930 & 0.7906470 & 2.8515140 \\ 4.0566990 & 2.1841350 & 3.1304860 \\ 2.3297030 & 2.2945240 & 3.4963350 \\ 6.7004910 & -1.1777500 & 1.3004550 \\ 7.6141340 & -1.7718220 & 1.4075080 \\ 6.6925810 & -0.4430160 & 2.1133610 \\ 5.8393010 & -1.8400780 & 1.4429490 \\ 7.8393900 & 0.5030440 & -0.1958160 \\ 7.8608370 & 0.9623680 & -1.1893980 \\ 7.7353660 & 1.3039180 & 0.5457850 \\ 8.7998420 & 0.0036730 & -0.0256810 \\ 3.3705600 & -0.5069220 & -4.1510250 \\ 4.4161250 & -0.7919690 & -4.3161460 \\ 2.8948260 & -1.2772770 & -3.5421610 \\ 2.8798390 & -0.4958740 & -5.1300240 \\ 4.0345170 & 1.9057310 & -4.3222680 \\ 5.1042000 & 1.6636270 & -4.3217930 \\ 3.6874130 & 1.9082780 & -5.3617040 \\ 3.9218100 & 2.9180800 & -3.9245390 \\ -0.2341060 & -0.5561750 & -1.0636540 \\ 0.4911230 & -0.9082280 & -2.3320220 \\ -0.3760190 & -1.5826020 & 0.0213980 \\ 2.1916630 & 1.1471250 & -3.5177730 \\ 6.8247010 & -1.2690590 & -2211850 \\ 1.6836570 & 2.2653390 & \end{array}$

\begin{tabular}{lr}
$\mathrm{H}$ & -4.2223680 \\
$\mathrm{H}$ & -5.1091770 \\
$\mathrm{H}$ & -2.8022230 \\
$\mathrm{C}$ & 1.7021880 \\
$\mathrm{C}$ & 2.8274250 \\
$\mathrm{H}$ & 0.7655260 \\
$\mathrm{H}$ & 2.6128700 \\
$\mathrm{O}$ & 1.8371750 \\
$\mathrm{C}$ & 2.1489020 \\
$\mathrm{C}$ & 1.1427500 \\
$\mathrm{C}$ & 0.5720060 \\
$\mathrm{~N}$ & 1.1541670 \\
$\mathrm{H}$ & 0.7269980 \\
$\mathrm{~S}$ & 2.4314730 \\
$\mathrm{~S}$ & 3.0486630 \\
$\mathrm{H}$ & 1.2400950 \\
$\mathrm{C}$ & -0.3364790 \\
$\mathrm{C}$ & -0.9026600 \\
$\mathrm{C}$ & -0.4390160 \\
$\mathrm{C}$ & 0.6911250 \\
$\mathrm{H}$ & -0.6901890 \\
$\mathrm{H}$ & -0.8561740 \\
$\mathrm{H}$ & 1.1269680 \\
$\mathrm{C}$ & 3.3535770 \\
$\mathrm{C}$ & 4.4673450 \\
$\mathrm{C}$ & 4.2315810 \\
$\mathrm{C}$ & 1.9882180 \\
$\mathrm{H}$ & 3.5500680 \\
$\mathrm{H}$ & 5.4390520 \\
$\mathrm{H}$ & 4.4368160 \\
$\mathrm{H}$ & 1.9465570 \\
$\mathrm{H}$ & 1.1801960 \\
$\mathrm{H}$ & 3.3358510 \\
$\mathrm{H}$ & 4.5211190 \\
$\mathrm{H}$ & 4.8979900 \\
$\mathrm{O}$ & -1.9075150 \\
$\mathrm{C}$ & -2.4203050 \\
$\mathrm{H}$ & -2.8354470 \\
$\mathrm{H}$ & -1.6491800 \\
$\mathrm{H}$ & -3.2134180 \\
$\mathrm{H}$ & \\
\hline
\end{tabular}
0.5140600
$-5.6339530$
$-0.5859110$
$-3.7071710$
$-2.8053900$
$-3.4373550$
$-1.7587840$
$-3.0862610$
$-2.0590430$
$-0.5429350$
$-0.4275390$
$-1.2966670$
$-1.4227170$
$-1.7341610$
$-3.1992520$
$-2.2611460$
1.1625340
1. 2706100
0.4798710
0.2584970
1.7831950
0.5859540
0.1894970
$-5.4969120$
$-4.7120950$
$-3.2010360$
$-5.1887860$
$-6.5706800$
$-4.9425970$
$-2.8224260$
$-5.5655000$
$-5.6740960$
$-5.2467000$
$-5.0150870$
$-2.6462000$
2. 1161580
2. 9612180
2. 3793840
3. 6429050
3.5365310

$-2.4515450$

1.9690060

$-1.0677970$

$-0.8083450$

$-0.5823920$

$-0.6124070$

$-2.3693740$

2. 7012150

4.4278480

3. 1568280

2. 2406870

1. 3004800

4. 3983920

1.8411210

$-2.4011800$

5.2200730

3.9373080

2.8857740

5.4668290

6.0339240

1.8916700

6.4574650

$-1.7462330$

$-1.0484120$

$-1.1270590$

$-1.1240700$

$-1.6713960$

$-1.4947920$

$-2.1362490$

$-0.0950190$

$-1.6805530$

$-2.8134240$

0.0049740

$-0.4633960$

3.6233060

4.6369340

5.4682450

5.0146200

148

4.1620670

$\operatorname{TS} 15(\mathrm{~S}, \mathrm{~S}) \mathrm{G}[\mathrm{WB} 97 \mathrm{XD} / 6-31 \mathrm{G}(\mathrm{d})]=-4125.315458$

$\begin{array}{lrrr}\mathrm{O} & -0.4339430 & -0.8294300 & -0.3536170 \\ \mathrm{O} & 1.6777170 & -0.1922290 & -1.5869610 \\ \mathrm{C} & -0.4763860 & -2.0337660 & -1.0197380 \\ \mathrm{C} & 0.6856430 & -2.7552120 & -1.1906000\end{array}$




$\begin{array}{rr}\mathrm{C} & -1.7548870 \\ \mathrm{C} & 0.6325230 \\ \mathrm{C} & -1.8066150 \\ \mathrm{C} & -0.6302850 \\ \mathrm{H} & -2.7701860 \\ \mathrm{C} & 2.3739250 \\ \mathrm{C} & 3.5478360 \\ \mathrm{C} & 1.9337210 \\ \mathrm{C} & 4.2959080 \\ \mathrm{C} & 2.6651590 \\ \mathrm{C} & 3.8773230 \\ \mathrm{H} & 5.2227060 \\ \mathrm{C} & 4.2090520 \\ \mathrm{C} & 2.9775820 \\ \mathrm{C} & 2.2241400 \\ \mathrm{C} & 4.6391370 \\ \mathrm{H} & 4.8005090 \\ \mathrm{H} & 2.6218540 \\ \mathrm{H} & 1.2777220 \\ \mathrm{H} & 5.5693370 \\ \mathrm{C} & 1.7073290 \\ \mathrm{C} & 0.4482340 \\ \mathrm{C} & -0.6928600 \\ \mathrm{C} & 1.7976870 \\ \mathrm{H} & 2.6107930 \\ \mathrm{H} & 0.3905090 \\ \mathrm{H} & -1.6653620 \\ \mathrm{H} & 2.7698550 \\ \mathrm{C} & -2.9807880 \\ \mathrm{C} & -3.6815420 \\ \mathrm{C} & -3.4187620 \\ \mathrm{C} & -4.8237690 \\ \mathrm{C} & -4.5651830 \\ \mathrm{C} & -5.2842880 \\ \mathrm{H} & -5.3753170 \\ \mathrm{H} & -4.9177170 \\ \mathrm{C} & 3.9251230 \\ \mathrm{C} & 3.7398400 \\ \mathrm{C} & 4.4408770 \\ \mathrm{C} & 4.0845480 \\ \mathrm{C} & 4.7660500 \\ \mathrm{C} & 4.5900280 \\ \mathrm{H} & 3.9397180 \\ \mathrm{H} & 5.1648990 \\ \mathrm{C} & -2.6963180 \\ \mathrm{C} & -6.5479110 \\ & \end{array}$

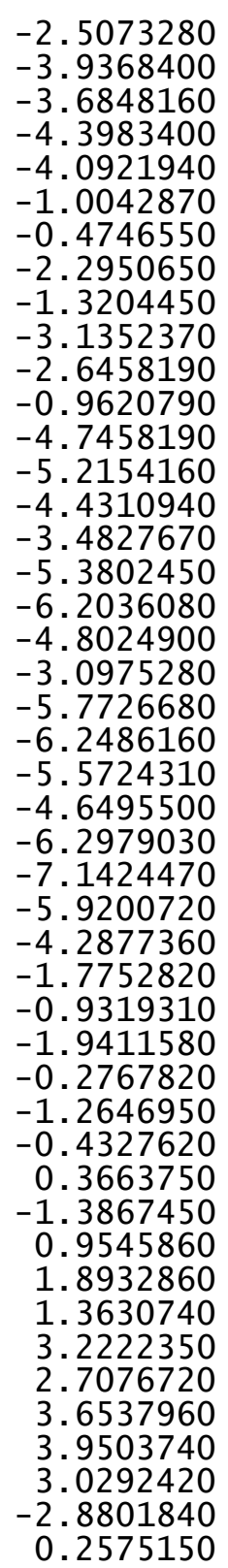

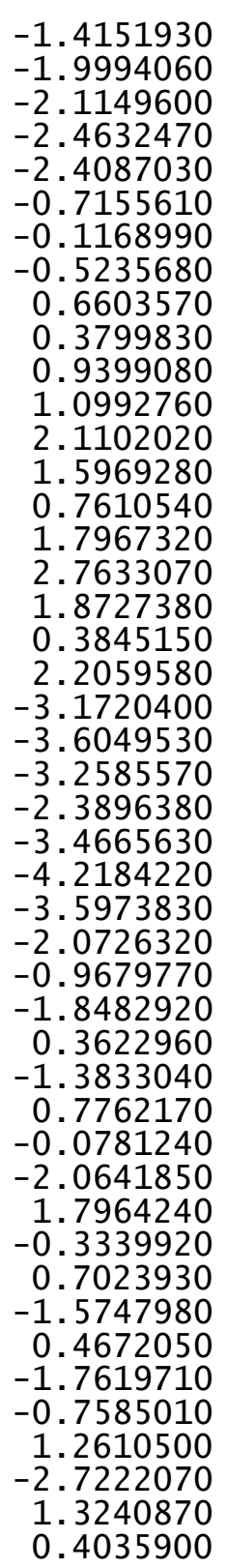

\begin{abstract}
$-3.2546030$
3. 1894810

4. 9015060

4.6722710

6.1701750

6.6023170

6.7185250

6.3318330

3. 8855250

2.8202630

4. 2419620

4.0022150

5. 9266400

6.1736660

5. 5338660

6.8513120

3. 6157560

2. 8980620

3. 1342650

3.8349000

4. 3248280

5. 0547640

4.8567260

3. 9221860

2. 1536960

2. 6115830

1. 3802910

1.6779430

$-3.1282840$

$-4.2012360$

$-2.9337790$

$-2.5858240$

$-2.8880430$

$-2.6872600$

$-3.9047460$

-2.1996180

$-7.7834290$

$-8.6938930$

$-7.7152930$

$-7.8848490$

$-6.4442510$

$-5.5665600$

$-6.3646740$

$-7.3354800$

$-3.4126420$

$-4.4655030$
\end{abstract}
$-0.7683380$
1.4974410
5. 1190730
0.3824630
0.2339830
1.1807500
$-0.0818560$
$-0.5144740$
0.7734840
0.8749320
1.7224150
0.0061800
5.6674470
6.7085050
5.6433780
5. 0813170
5. 9587320
5.5896870
5.9173980
7.0086450
1. 3157170
0.5716930
2. 2622350
0.9893510
2. 4892660
3.4420480
2. 6845160
2. 0713170
$-4.3364620$
$-4.4501760$
$-4.6565880$
$-5.0088180$
$-2.5235670$
$-1.4660980$
$-2.7467410$
$-3.1132710$
$-0.2574010$
0.2126060
$-0.0278130$
$-1.3423860$
1.7837420
2. 1543580
2. 1022260
2. 2591680
0.6629250
0.9497260

$-3.3005940$

2. 0669740

$-1.0035530$

$-2.7153930$

$-3.3593780$

$-2.1208750$

$-3.7989930$

$-3.9725710$

$-3.7458340$

$-4.3899430$

$-4.7462770$

$-0.0028120$

$-0.2387370$

1. 0201840

$-0.0232850$

$-0.9897340$

$-1.7301130$

$-0.0057070$

$-1.2143280$

3. 0851140

2. 7522410

3.2397520

4.0509520

2. 6094930

2. 8990070

1.8636730

3. 5039190

1.0874310

1. 2824820

0.0603520

1. 7617610

2. 8006130

2. 9909670

3.1445830

3. 4144160

$-0.3473210$

0.0408390

$-1.4171070$

$-0.2411540$

0.3035770

0.8450290

$-0.7427890$

0.7276640

$-3.8238900$

$-3.9285750$ 


$\begin{array}{rrr}-2.9193880 & 1.3787590 & -3.1639190 \\ -2.9522800 & 0.7479730 & -4.8139000 \\ -4.0338470 & -1.7414680 & -4.1985200 \\ -5.1102420 & -1.5405870 & -4.1372250 \\ -3.7258990 & -1.6299650 & -5.2443260 \\ -3.8699280 & -2.7827510 & -3.9058880 \\ 0.2695370 & 0.4542530 & -1.0922420 \\ -0.4585300 & 0.8594140 & -2.3423560 \\ 0.4571550 & 1.4569200 & 0.0082570 \\ -2.1907960 & -1.0153570 & -3.3658590 \\ -6.6734350 & 0.0044580 & 1.4639730 \\ -1.6219360 & -2.8119020 & 1.1200170 \\ 4.3113620 & -0.6012540 & -2.4018360 \\ 5.3409450 & 5.1982820 & -2.0062000 \\ 2.6841850 & 0.5341990 & 1.9576050 \\ -1.1294520 & 3.8668670 & -1.1445060 \\ -2.4311440 & 3.2178600 & -0.9606780 \\ -0.2965040 & 3.4054350 & -0.6171280 \\ -2.4404460 & 2.1485150 & -0.7746430 \\ -1.3202980 & 3.2898950 & -2.4621830 \\ -2.1331710 & 2.2588350 & 2.5394160 \\ -1.4410600 & 0.5638540 & 4.2563660 \\ -0.7721430 & 0.4543680 & 3.0334930 \\ -1.1888890 & 1.4157340 & 2.1210110 \\ -0.6936220 & 1.5045590 & 1.2086500 \\ -2.6024340 & 1.8792940 & 4.1835250 \\ -2.8128400 & 3.5424460 & 1.6746420 \\ -0.9108770 & 2.3606850 & -2.4718930 \\ -0.1846430 & -1.2992840 & 5.0807240 \\ 0.4782070 & -1.4018230 & 3.8445840 \\ 0.1854730 & -0.5210110 & 2.8045170 \\ -1.1484770 & -0.3183170 & 5.2881050 \\ 0.0421170 & -1.9854580 & 5.8872880 \\ 0.6810590 & -0.6169600 & 1.8466380 \\ -1.6572560 & -0.2517280 & 6.2433970 \\ -2.3292020 & 5.9727360 & -1.8699520 \\ -3.6166870 & 5.4280020 & -1.2466410 \\ -3.6998740 & 3.9022140 & -1.3558490 \\ -1.0933720 & 5.3748980 & -1.1911480 \\ -2.3007390 & 7.0626530 & -1.7774710 \\ -4.4946580 & 5.8669870 & -1.7293510 \\ -3.9089990 & 3.5977770 & -2.3891160 \\ -1.0241540 & 5.7248490 & -0.1540690 \\ -0.1730800 & 5.6813800 & -1.6985430 \\ -2.3085430 & 5.7398470 & -2.9411110 \\ -3.6578780 & 5.7159460 & -0.1884410\end{array}$

$\begin{array}{lr}\mathrm{H} & -4.5130080 \\ \mathrm{O} & 1.4222710 \\ \mathrm{C} & 1.7573880 \\ \mathrm{H} & 2.1627360 \\ \mathrm{H} & 0.8891010 \\ \mathrm{H} & 2.5212970\end{array}$
3.4945910
$-2.3264130$
$-3.2754860$
$-2.7891060$
3.8858080

4.5590140

5.4540520

4.8350370

4.1113830

TS16(S , S ) G[WB97XD/6-31G(d)] = -4125.317721

\begin{tabular}{|c|c|c|c|}
\hline & & & \\
\hline 0 & -0.2499560 & -1.0190410 & -0.1250720 \\
\hline 0 & 1.8065470 & -0.1081950 & -1.2607850 \\
\hline C & -0.1654160 & -2.1879280 & -0.8536970 \\
\hline C & 1.0652250 & -2.7895230 & -1.0166010 \\
\hline C & -1.3795410 & -2.7347370 & -1.3474180 \\
\hline C & 1.1481380 & -3.9342810 & -1.8749410 \\
\hline C & -1.2977490 & -3.8902640 & -2.0808000 \\
\hline C & -0.0520820 & -4.4911680 & -2.3954050 \\
\hline H & -2.2076080 & -4.3580120 & -2.4458920 \\
\hline C & 2.5508530 & -0.8961350 & -0.4122560 \\
\hline C & 3.6499490 & -0.2819260 & 0.2451400 \\
\hline C & 2.2476650 & -2.2359510 & -0.3017200 \\
\hline C & 4.4665160 & -1.0828020 & 0.9998380 \\
\hline C & 3.0491180 & -3.0433360 & 0.5738220 \\
\hline C & 4. 1847860 & -2.4585050 & 1.2005520 \\
\hline$H$ & 5.3437930 & -0.6540440 & 1.4757410 \\
\hline C & 4.6780120 & -4.5573090 & 2.3156390 \\
\hline C & 3.5269000 & -5.1299420 & 1.7272690 \\
\hline C & 2.7349130 & -4.39 & 0.8819610 \\
\hline C & 4.9934850 & -3.24 & 2.05 \\
\hline$H$ & 5.299 & -5.1520770 & 2.977 \\
\hline$H$ & 3.264 & -6.1592980 & 2620 \\
\hline$H$ & 1.8464860 & -4.84 & 0.4 \\
\hline$H$ & 5.8623250 & -2.7881980 & 2.5 \\
\hline C & 2.4211100 & -5.6136580 & -3.0 \\
\hline C & 1.2240520 & -6.1889190 & -3.5687360 \\
\hline C & 0.0160470 & -5.6351050 & -3.23 \\
\hline C & 2.3853830 & -4.5179940 & -2.2587270 \\
\hline H & 3.3770360 & - 6.0407140 & -3.3706820 \\
\hline $\mathrm{H}$ & 1.2666120 & -7.0593070 & -4.2161910 \\
\hline$H$ & -0.9100150 & -6.0562590 & -3.6157460 \\
\hline$H$ & 3.3109800 & -4.0804000 & -1.9007860 \\
\hline C & -2.6830220 & -2.0756520 & -1.0192200 \\
\hline C & -3.3602900 & -1.3119540 & -1.9894570 \\
\hline C & -3.2211610 & -2.2191120 & 0.2766730 \\
\hline C & -4.5625890 & -0.6950120 & -1.6378020 \\
\hline C & -4.4236710 & -1.5805760 & 0.5759610 \\
\hline $\mathrm{C}$ & -5.10 & -0.8128290 & -0.3623990 \\
\hline
\end{tabular}




$\begin{array}{rrrr}\mathrm{H} & -5.0942640 & -0.1128100 & -2.3858350 \\ \mathrm{H} & -4.8509320 & -1.6818440 & 1.5693180 \\ \mathrm{C} & 3.8898230 & 1.1850820 & 0.0885630 \\ \mathrm{C} & 3.4985480 & 2.0751860 & 1.1070340 \\ \mathrm{C} & 4.4834190 & 1.6706770 & -1.0897110 \\ \mathrm{C} & 3.7424010 & 3.4376540 & 0.9299770 \\ \mathrm{C} & 4.6918110 & 3.0421200 & -1.2248710 \\ \mathrm{C} & 4.3308230 & 3.9429410 & -0.2260360 \\ \mathrm{H} & 3.4490380 & 4.1364440 & 1.7090570 \\ \mathrm{H} & 5.1432760 & 3.4174790 & -2.1405760 \\ \mathrm{C} & -2.5434900 & -3.1010750 & 1.3207250 \\ \mathrm{C} & -6.4309590 & -0.1591380 & -0.0044370 \\ \mathrm{C} & -2.8486590 & -1.1933610 & -3.4191780 \\ \mathrm{C} & 2.8324970 & 1.5882490 & 2.3876070 \\ \mathrm{C} & 4.5274040 & 5.4376170 & -0.4063870 \\ \mathrm{C} & 4.9043460 & 0.7465090 & -2.2232710 \\ \mathrm{C} & 6.4234330 & 0.7885900 & -2.4371950 \\ \mathrm{H} & 6.7544930 & 1.7849430 & -2.7523530 \\ \mathrm{H} & 6.9558660 & 0.5328260 & -1.5147810 \\ \mathrm{H} & 6.7205010 & 0.0752100 & -3.2139400 \\ \mathrm{C} & 4.1471540 & 1.0642150 & -3.5193150 \\ \mathrm{H} & 3.0667400 & 1.0025710 & -3.3596740 \\ \mathrm{H} & 4.3846440 & 2.0697130 & -3.8855780 \\ \mathrm{H} & 4.4216800 & 0.3499100 & -4.3036190 \\ \mathrm{C} & 3.5618840 & 5.9868330 & -1.4665240 \\ \mathrm{H} & 3.6746120 & 7.0718950 & -1.5696110 \\ \mathrm{H} & 3.7586900 & 5.5317140 & -2.4443330 \\ \mathrm{H} & 2.5217460 & 5.7714910 & -1.1995270 \\ \mathrm{C} & 5.9794530 & 5.7968630 & -0.7448640 \\ \mathrm{H} & 6.6684690 & 5.4152310 & 0.0157430 \\ \mathrm{H} & 6.2783970 & 5.3766240 & -1.7118450 \\ \mathrm{H} & 6.1013580 & 6.8839990 & -0.8045040 \\ \mathrm{C} & 3.8670830 & 1.3437870 & 3.4955950 \\ \mathrm{H} & 4.6242110 & 0.6153190 & 3.1924370 \\ \mathrm{H} & 4.3806480 & 2.2780650 & 3.7518890 \\ \mathrm{H} & 3.3757040 & 0.9665340 & 4.4000860 \\ \mathrm{C} & 1.7350180 & 2.5284290 & 2.8974270 \\ \mathrm{H} & 2.1484280 & 3.4639430 & 3.2912120 \\ \mathrm{H} & 1.0246820 & 2.7627550 & 2.1014060 \\ \mathrm{H} & 1.1909190 & 2.0448810 & 3.7162840 \\ \mathrm{C} & -2.8324510 & -4.5860130 & 1.0451440 \\ \mathrm{H} & -3.9093280 & -4.7790920 & 1.1160140 \\ \mathrm{H} & -2.4959180 & -4.8903350 & 0.0505930 \\ \mathrm{H} & -2.3244660 & -5.2162640 & 1.7839280 \\ \mathrm{C} & -2.9329590 & -2.7703590 & 2.7642390 \\ \mathrm{H} & -2.8587820 & -1.6999030 & \\ & & & \\ \mathrm{H} & -2.9714710\end{array}$

-3.9561660
-2.2649010
-7.5911650
-8.5481140
-7.4747100
-7.6342670
-6.4088410
-5.5791560
-6.2996480
-7.3438730
-3.0441340
-4.1007850
-2.6385640
-2.5218740
-3.5214930
-4.6033020
-3.1228970
-3.3635840
0.2985730
-0.4088440
0.2921420
-1.7728090
-6.5984320
-1.4612480
4.6499760
4.2808500
2.3504440
-1.2315890
-2.5293160
-0.4649540
-2.5354010
-1.2396010
-2.7226510
-2.3371410
-1.4419570
-1.6948920
-1.0528280
-3.4961210
-3.2442940
-0.8206850
-1.1905270
-0.2998010
-0.4122220
-2.2115270
-1.0646980
0.2398780
2.9891900

3.4544950

$-0.7679960$

$-0.4702330$

$-1.8486480$

$-0.5737320$

$-0.2333240$

0.3073440

$-1.2992440$

0.1110050

$-4.0267440$

$-4.2177290$

$-3.3737000$

$-4.9874340$

$-4.3180540$

$-4.3655910$

$-5.3372830$

$-3.9490160$

$-0.8450390$

$-2.1435830$

0.2310500

$-3.4040820$

1. 0666760

1. 2371020

$-1.9442760$

0.5491740

2. 1580830

$-1.4028700$

$-1.3251930$

$-0.7249660$

$-1.0260980$

$-2.6633990$

2. 2323240

4.1436580

3.0878180

2.0542350

1.2368530

3.7693290

1.1864880

$-2.5147560$

5. 3223310

4. 2403160

3. 1050320

5. 2817880

6. 1854530

2. 2466930 


$\begin{array}{lrrr}\mathrm{H} & -2.8974980 & 0.1206650 & 6.1157430 \\ \mathrm{C} & -2.3703180 & 5.6525530 & -2.5136180 \\ \mathrm{C} & -3.7181820 & 5.1348170 & -2.0044900 \\ \mathrm{C} & -3.7540370 & 3.6043950 & -1.9472190 \\ \mathrm{C} & -1.2243120 & 5.1845230 & -1.6110520 \\ \mathrm{H} & -2.3791140 & 6.7460450 & -2.5506010 \\ \mathrm{H} & -4.5320900 & 5.4853540 & -2.6458190 \\ \mathrm{H} & -3.8290590 & 3.1780520 & -2.9554410 \\ \mathrm{H} & -1.3075800 & 5.6488160 & -0.6211250 \\ \mathrm{H} & -0.2518080 & 5.4696920 & -2.0247810 \\ \mathrm{H} & -2.2000190 & 5.2970910 & -3.5365020 \\ \mathrm{H} & -3.9057460 & 5.5391260 & -1.0016560 \\ \mathrm{H} & -4.6264130 & 3.2379350 & -1.4015510 \\ \mathrm{O} & 0.6340800 & -2.0280000 & 4.3910780 \\ \mathrm{C} & 1.4789780 & -2.2941720 & 3.2845600 \\ \mathrm{H} & 0.8929680 & -2.5636900 & 2.3981800 \\ \mathrm{H} & 2.1139340 & -1.4317640 & 3.0475060 \\ \mathrm{H} & 2.1096630 & -3.1350840 & 3.5728110 \\ \mathrm{I} 48 & & & \\ \mathrm{TS} 17(\mathrm{~S}, \mathrm{~S}) & \mathrm{G}[\mathrm{WB} 97 \mathrm{XD} / 6-31 \mathrm{G}(\mathrm{d})] & -1.4125 .317160 \\ \mathrm{O} & 0.3498110 & 0.8627660 & 0.1413770 \\ \mathrm{O} & -1.5956350 & 0.5619220 & -1.4290490 \\ \mathrm{C} & 0.4400910 & 2.2013340 & -0.1821460 \\ \mathrm{C} & -0.7167870 & 2.9540170 & -0.2418190 \\ \mathrm{C} & 1.7346190 & 2.7429980 & -0.3965990 \\ \mathrm{C} & -0.6169240 & 4.3210020 & -0.6636260 \\ \mathrm{C} & 1.8212710 & 4.0927420 & -0.6343430 \\ \mathrm{C} & 0.6718750 & 4.9013890 & -0.8073530 \\ \mathrm{H} & 2.8001620 & 4.5462950 & -0.7608800 \\ \mathrm{C} & -2.3985480 & 1.1371260 & -0.4684180 \\ \mathrm{C} & -3.6427330 & 0.5040730 & -0.2064940 \\ \mathrm{C} & -2.0220770 & 2.3329700 & 0.1101830 \\ \mathrm{C} & -4.5164570 & 1.1356060 & 0.6392770 \\ \mathrm{C} & -2.9067010 & 2.9343830 & 1.0705220 \\ \mathrm{C} & -4.1760420 & 2.3381300 & 1.3060590 \\ \mathrm{H} & -5.4968660 & 0.7001360 & 0.8114350 \\ \mathrm{C} & -4.7274340 & 4.0685160 & 2.9153240 \\ \mathrm{C} & -3.4532360 & 4.6458100 & 2.7123840 \\ \mathrm{C} & -2.5675110 & 4.0938610 & 1.8216360 \\ \mathrm{C} & -5.0742680 & 2.9342770 & 2.2297200 \\ \mathrm{H} & -5.4189220 & 4.5158450 & 3.6224350 \\ \mathrm{H} & -3.1670660 & 5.5291290 & 3.2751830 \\ \mathrm{H} & -1.5877670 & 4.5401180 & 1.7004380 \\ \mathrm{H} & -6.0410740 & 2.4644940 & 2.3892370 \\ \mathrm{C} & -1.6096900 & 6.4265880 & -1.3598380 \\ \mathrm{C} & -0.3274350 & 7.0171160 & -1.4475370\end{array}$

0.7876590
-1.7517190
-2.4896180
-0.2301720
1.7804450
-2.7402060
2.9687970
3.6468250
3.4951280
4.8652460
4.7111660
5.4242570
5.3889180
5.1366380
-4.0205080
-3.8867590
-4.5508290
-4.3303250
-4.9730790
-4.8801710
-4.2394150
-5.3906790
2.8015610
6.7881050
3.1393750
-3.2833670
-5.3585370
-4.6801380
-6.1484730
-6.5999230
-6.7364150
-6.2293040
-3.8332370
-2.7851080
-4.1882380
-3.8858430
-6.5110480
-6.8859570
-6.1818550
-7.3412300
-4.2094020
-3.3965260
-3.7958500
-4.5624840
-4.3565600
-4.8944120

6.2665600

5.1172730

7. 0138340

8.0575350

6. 6987170

4. 6751270

1.8975270

1.7568960

1. 3064030

1.0712670

0.6339260

0.5198550

0.9785510

0.1869220

$-0.7788690$

$-2.0008770$

$-0.7492410$

$-3.1658770$

$-1.9463560$

$-3.1611110$

$-4.1122080$

$-1.9303160$

1.4571990

$-0.1533660$

2. 3580110

$-2.0642600$

$-4.4472280$

0.5452660

0.8679960

0.1035350

0.9236910

1.8307900

0.5077510

0.2999000

$-0.2637890$

1. 4718870

$-5.0811260$

$-5.9735970$

5.3835950

$-4.3767230$

$-5.4460340$

$-5.0033040$

$-5.7621320$

$-6.3420440$

$-1.9181250$

$-0.9701550$
$-1.1823240$ $-0.9779190$

$-1.6043470$

$-1.7417190$

$-1.2755890$

$-0.9237430$

$-0.4424550$

$-1.6695630$

0.7277520

$-1.6938180$

0.6455070

$-0.5495860$

$-2.6403830$

1. 5398710

$-0.8736970$

$-0.1829180$

$-2.1722080$

$-0.8038990$

$-2.7547640$

$-2.0864210$

$-0.2786350$

$-3.7596730$

2. 0773680

$-0.5419420$

$-2.9775920$

1. 2161840

$-2.7356760$

$-2.9612900$

$-3.2676020$

$-3.9103990$

$-2.3450010$

$-3.7844390$

$-4.2398730$

$-4.0037280$

$-4.9328300$

$-4.7579460$

$-1.9449370$

$-2.4580550$

$-0.9439810$

$-1.8280180$

$-2.9272840$

$-3.5119420$

$-1.9626040$

$-3.4498580$

2. 3047950

2. 2223330 


$\begin{array}{lr}\mathrm{H} & -5.0888800 \\ \mathrm{H} & -3.8950060 \\ \mathrm{C} & -2.4706950 \\ \mathrm{H} & -3.1111210 \\ \mathrm{H} & -1.7321150 \\ \mathrm{H} & -1.9452780 \\ \mathrm{C} & 3.1290300 \\ \mathrm{H} & 4.2056830 \\ \mathrm{H} & 2.8315410 \\ \mathrm{H} & 2.6044980 \\ \mathrm{C} & 3.1274350 \\ \mathrm{H} & 2.9996480 \\ \mathrm{H} & 4.1541770 \\ \mathrm{H} & 2.4576860 \\ \mathrm{C} & 7.8645000 \\ \mathrm{H} & 8.8443610 \\ \mathrm{H} & 7.9380290 \\ \mathrm{H} & 7.6266190 \\ \mathrm{C} & 7.1740670 \\ \mathrm{H} & 6.3977360 \\ \mathrm{H} & 7.3507400 \\ \mathrm{H} & 8.1007040 \\ \mathrm{C} & 3.0679720 \\ \mathrm{H} & 4.0496000 \\ \mathrm{H} & 2.3889150 \\ \mathrm{H} & 2.6908640 \\ \mathrm{C} & 4.0078220 \\ \mathrm{H} & 5.0356880 \\ \mathrm{H} & 3.6065150 \\ \mathrm{H} & 4.0510250 \\ \mathrm{P} & -0.1933580 \\ \mathrm{O} & 0.6601610 \\ \mathrm{O} & -0.3928340 \\ \mathrm{H} & 2.1215040 \\ \mathrm{H} & 6.7520110 \\ \mathrm{H} & 1.7220880 \\ \mathrm{H} & -4.2955780 \\ \mathrm{H} & -5.7424760 \\ \mathrm{H} & -2.5893890 \\ \mathrm{C} & 2.2042560 \\ \mathrm{C} & 2.7698260 \\ \mathrm{H} & 1.1158210 \\ \mathrm{H} & 2.1784170 \\ \mathrm{O} & 1.3845950 \\ \mathrm{C} & 1.0069340 \\ \mathrm{C} & \\ & \end{array}$

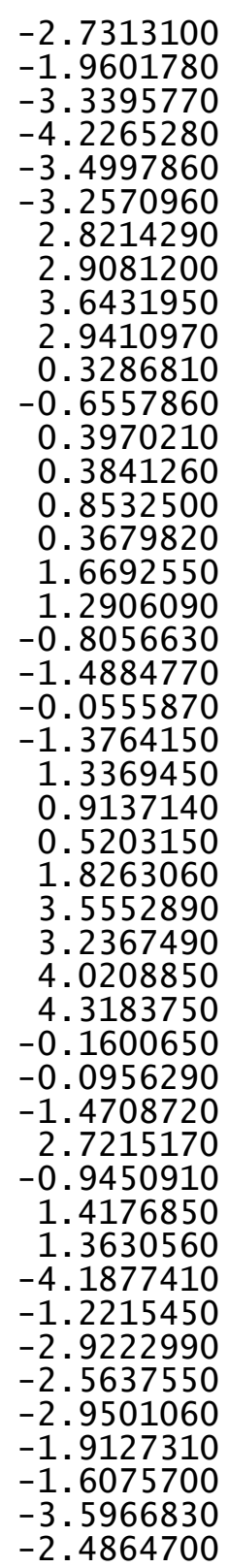

2.2345510
3.2983000
1.4647040
1.5282120
0.6743080
2.4225380
2.7031810
2.8915440
2.0443050
3.6580290
3.0617920
2.5975400
3.4389500
3.9261870
-0.1065340
-0.0361620
-0.8350340
0.8684610
-1.8725100
-2.2347200
-2.6514990
-1.7535100
-4.1201810
-4.3595600
-3.8712290
-5.0252980
-3.3938840
-3.6044610
-4.3008610
-2.6106080
-1.0201860
-2.2552310
-0.3231030
-2.8181470
0.2191240
1.9063000
-2.3452400
-3.7306150
1.3083600
-2.0702650
-0.7692470
-2.1096310
-0.1367630
-2.3660560
2.0067970
4.2189390

0.4374610
0.6794770
0.2695430
1.8371470
1.8055270
1.9567620
0.1669260
-0.4115720
-0.2799210
0.8749680
0.0400710
-0.6790390
1.3157920
4.4231740
4.8236070
4.2107340
2.9004920
4.8734550
5.9125760
4.7063370
2.5131040
2.6138350
4.8080130
4.4900490
4.3259310
-1.0769520
-1.6560370
-0.8915940
-2.4039410
-2.1400850
-1
$-1.8169900$
$-2.4634110$
$-2.1160610$
$-3.9262050$
$-4.5828480$
$-0.9462280$
$-0.7942100$
$-0.1270900$
$-0.6303890$
$-1.9685790$
$-0.3582170$
$-0.1348040$
$-2.4696230$
$-3.9502810$
$-3.9580130$
$-2.7790270$
$-4.0041740$
$-4.8053190$
$-3.9171660$
$-1.8400490$
$-4.9674410$
$-3.9049700$
$-3.0454340$
$-4.8948440$
$-2.8785090$
1.0054440
1. 7291900
2. 0641980
1.1273390
2. 5961780

3.1385710

1. 9362130

1.0405800

3. 6606560

0.7014820

$-2.2937200$

5.6733920

4.5731560

3. 2820850

5.5077920

6.6580720

2. 4049570

6.3623470

$-2.6960460$

$-1.2178360$

$-0.4592170$

$-2.8610110$

$-3.2090300$

$-1.1150420$

$-0.7271630$

$-2.5069370$

$-3.1815740$

$-0.7549620$

0.6236550

4.8762000

3.8055380

3.0938700

3. 2755330

4.2515330

TS18 (S, S) G[WB97XD/6-31G(d)] = -4125.318531

\begin{tabular}{|c|c|c|c|}
\hline \multicolumn{4}{|c|}{ S) $\mathrm{G}$} \\
\hline 0 & 0.2275880 & 0.9769610 & -0.087335 \\
\hline 0 & -1.8046900 & 0.1738740 & -1.349713 \\
\hline C & 0.1575590 & 2.2283160 & -0.665224 \\
\hline C & -1.0739870 & 2.8349080 & -0.792072 \\
\hline C & 1. 3799450 & 2.8393850 & -1.051168 \\
\hline C & -1.1448070 & 4.0774290 & -1.502288 \\
\hline C & 1. 3062980 & 4.0846240 & -1.621154 \\
\hline C & 0.0646860 & 4.7118570 & -1.896091 \\
\hline $\mathrm{H}$ & 2.2208010 & 4.6047280 & -1.889971 \\
\hline C & -2.5665480 & 0.8748460 & -0.442119 \\
\hline C & -3.6918930 & 0.2057000 & 00 \\
\hline C & -2.26 & 2.1957930 & -0.1 \\
\hline C & -4.52 & 0.9244 & 22288 \\
\hline C & -3 & & \\
\hline
\end{tabular}




$\begin{array}{rrr}-4.2519240 & 2.2675540 & 1.2811980 \\ -5.4225330 & 0.4498070 & 1.3198100 \\ -4.7716300 & 4.2338320 & 2.6054770 \\ -3.5988110 & 4.8570180 & 2.1207300 \\ -2.7842310 & 4.2139680 & 1.2241350 \\ -5.0845740 & 2.9637760 & 2.1965880 \\ -5.4106510 & 4.7569210 & 3.3099600 \\ -3.3358830 & 5.8512790 & 2.4686670 \\ -1.8793320 & 4.7010930 & 0.8806520 \\ -5.9688700 & 2.4614510 & 2.5794710 \\ -2.3991150 & 5.8853900 & -2.5301500 \\ -1.1941750 & 6.5360790 & -2.8833390 \\ 0.0093690 & 5.9571340 & -2.5761540 \\ -2.3762850 & 4.6905840 & -1.8569930 \\ -3.3504170 & 6.3346160 & -2.7983220 \\ -1.2273330 & 7.4856950 & -3.4082740 \\ 0.9424980 & 6.4365690 & -2.8598870 \\ -3.3070690 & 4.1965010 & -1.6003020 \\ 2.6764910 & 2.1300880 & -0.8132370 \\ 3.3731130 & 1.5541540 & -1.8912990 \\ 3.1842020 & 2.0225170 & 0.5015480 \\ 4.5530300 & 0.8515510 & -1.6264970 \\ 4.3640250 & 1.3133550 & 0.7087030 \\ 5.0599610 & 0.7110370 & -0.3403140 \\ 5.1028830 & 0.4074760 & -2.4528140 \\ 4.7628300 & 1.2301190 & 1.7143750 \\ -3.9657140 & -1.2262590 & -0.2211800 \\ -3.5648300 & -2.2442380 & 0.6683310 \\ -4.6309800 & -1.5458890 & -1.4137880 \\ -3.8774740 & -3.5639800 & 0.3533400 \\ -4.9102990 & -2.8861750 & -1.6911870 \\ -4.5479160 & -3.9076590 & -0.8213570 \\ -3.5785720 & -4.3530680 & 1.0377030 \\ -5.4238990 & -3.1412670 & -2.6161250 \\ 2.4987870 & 2.7272500 & 1.6694240 \\ 6.3743620 & -0.0104540 & -0.1009140 \\ 2.9220130 & 1.7311130 & -3.3356830 \\ -2.8122030 & -1.9243780 & 1.9536100 \\ -4.8612980 & -5.3574780 & -1.1450930 \\ -5.0509640 & -0.4782540 & -2.4134880 \\ -6.5763890 & -0.4279060 & -2.5692830 \\ -6.9671040 & -1.3655780 & -2.9812440 \\ -7.0630030 & -0.2553590 & -1.6032840 \\ -6.8650350 & 0.3815670 & -3.2489920 \\ -4.3538650 & -0.6735480 & -3.7660860 \\ -3.2666220 & -0.6728010 & -3.6424310\end{array}$

-4.6453420
-4.6237280
-5.8344340
-6.0878050
-5.3895150
-6.7617210
-3.5833100
-2.8930250
-3.0600710
-3.8232820
-3.7733700
-4.4940140
-4.3342280
-3.2134570
-1.7607660
-2.2196340
-1.0962920
-1.1565070
2.7687460
3.8443910
2.4161960
2.2647330
2.9023540
2.8252030
3.9297150
2.2441750
6.2913910
7.2369840
6.1033070
5.4901700
7.4976020
7.5812910
7.3003320
8.4611730
3.1205970
4.1811960
2.6767650
2.6416040
3.6576070
4.7359170
3.3042690
3.5098170
-0.2778240
0.4327350
-0.2211890
1.8505640

$-4.2335040$ $-4.4542340$ $-0.1234880$ $-0.3942440$ 0.8782340 $-0.0714630$ $-1.2512410$ $-1.9895460$ $-0.2892120$ $-1.5498640$

3.1315430

2. 9299010 3. 3392970 4. 0354660

2. 3197060

2. 6381700

1. 4750440

3.1579780

1.6226840

1.7110210

0.6929490

2. 4572150

3. 0468140

3. 1053970

3. 3024170

3. 8115390

1.0298680

1. 1142710

1.9965250

0.8555330

0.1661970

$-0.6529490$

1.0893490

0.2784040

$-4.2018430$

$-4.3918580$

$-3.7371620$

$-5.1756860$

$-3.9822620$

$-4.0210570$

$-5.0079410$

$-3.4288220$

$-1.0035540$

$-2.3276670$

-0.0971290
-3.3280400 


\begin{tabular}{|c|c|c|c|c|c|c|}
\hline & & & & & & \\
\hline 6.6241480 & -0.5466850 & -1.0263550 & $\mathrm{C}$ & 0.5710040 & 2.0078010 & -0.7958890 \\
\hline 1.4181950 & 2.5717130 & 1.5675930 & $\mathrm{C}$ & -0.5708500 & 2.7638850 & -0.9502190 \\
\hline-4.7362050 & 0.4954290 & -2.0267070 & $\mathrm{C}$ & 1.8715250 & 2.4847650 & -1.1019000 \\
\hline-5.3541040 & -5.3749220 & -2.1255510 & $\mathrm{C}$ & -0.4626640 & 4.0220480 & -1.6283040 \\
\hline-2.2754340 & -0.9858780 & 1.7834330 & $\mathrm{C}$ & 1.9738950 & 3.7409680 & -1.6430080 \\
\hline 1.6819460 & -3.3615940 & -1.9489600 & C & 0.8292140 & 4.5161940 & -1.9573100 \\
\hline 2.8242080 & -2.5244000 & -1.5803830 & $\mathrm{H}$ & 2.9567040 & 4.1568630 & -1.8452270 \\
\hline 0.8005910 & -3.2547140 & -1.3176650 & $\mathrm{C}$ & -2.3206450 & 1.0103790 & -0.7470480 \\
\hline 2.6230600 & -1.5786150 & -1.0864000 & C & -3.5695100 & 0.4922930 & -0.3117010 \\
\hline 1.6844580 & -2.4169880 & -3.0458100 & $\mathrm{C}$ & -1.8632290 & 2.2658940 & -0.4079580 \\
\hline 2.6588980 & -2.6203470 & 1.9941400 & C & -4.3628880 & 1.3047550 & 0.4571660 \\
\hline 2.1012270 & -1.3579920 & 4.0854370 & C & -2.6446060 & 3.0526240 & 0.5024460 \\
\hline 1.2848590 & -1.0560950 & 2.9986080 & $\mathrm{C}$ & -3.9271420 & 2.5805880 & 0.8924750 \\
\hline 1.6282910 & -1.7804510 & 1.8646860 & $\mathrm{H}$ & -5.3398410 & 0.9481870 & 0.7726520 \\
\hline 1.0384820 & -1.7163420 & 1.0094750 & $\mathrm{C}$ & -4.2899790 & 4.5879440 & 2.2068640 \\
\hline $\begin{array}{l}3.3089400 \\
2.2853020\end{array}$ & -2.5377060 & 3.6146090 & $\mathrm{C}$ & -2.9862480 & 5.0262510 & 1.8788320 \\
\hline 3.2853920 & -3.6557370 & 0.8169840 & C & -2.1862320 & 4.2809040 & 1.0508380 \\
\hline 1.1010120 & -1.6155120 & -2.7983500 & $\mathrm{C}$ & -4.7421570 & 3.3837850 & 1.7327760 \\
\hline 0.8586360 & 0.1851660 & 5.4144700 & $\mathrm{H}$ & -4.9194960 & 5.1960700 & 2.8493550 \\
\hline 0.0461980 & 0.4943630 & 4.3041780 & $\mathrm{H}$ & -2.6137510 & 5.9598790 & 2.2892700 \\
\hline 0.2509860 & -0.1245190 & 3.0756510 & $\mathrm{H}$ & -1.1858880 & 4.6283380 & 0.8188090 \\
\hline 1.8881620 & -0.7307570 & 5.3140410 & $\mathrm{H}$ & -5.7296090 & 3.0177630 & 2.0018640 \\
\hline 0.6624760 & 0.6946790 & 6.3513460 & $\mathrm{C}$ & -1.4479020 & 5.9904040 & -2.6558370 \\
\hline-0.3288560 & 0.1011420 & 2.1905860 & $\mathrm{C}$ & -0.1603690 & 6.5008310 & -2.9417820 \\
\hline 2.5122910 & -0.9523930 & 6.1726440 & $\mathrm{C}$ & 0.9516310 & 5.7749660 & -2.6027890 \\
\hline 3.2616310 & -4.8547600 & -3.2376180 & $\mathrm{C}$ & -1.5960900 & 4.7868060 & -2.0149900 \\
\hline 4.4415630 & -4.2397110 & -2.4793090 & $\mathrm{H}$ & -2.3273530 & 6.5544530 & -2.9513670 \\
\hline 4.1911330 & -2.7695990 & -2.1304700 & $\mathrm{H}$ & -0.0573890 & 7.4593610 & -3.4408240 \\
\hline 1.9668200 & -4.7667100 & -2.4223200 & $\mathrm{H}$ & 1.9464930 & 6.1463680 & -2.8341410 \\
\hline 3.4721710 & -5.9039530 & -3.4657900 & $\mathrm{H}$ & -2.5893720 & 4.4016640 & -1.8124950 \\
\hline 5.3589030 & -4.3142000 & -3.0706070 & $\mathrm{C}$ & 3.0796350 & 1.6629570 & -0.7829990 \\
\hline 4.2869240 & -2.1341880 & -3.0196860 & $\mathrm{C}$ & 3.8191980 & 1.0594070 & -1.8161660 \\
\hline 2.0351690 & -5.3999580 & -1.5297930 & $\mathrm{C}$ & 3.4874600 & 1.5214550 & 0.5606650 \\
\hline 1.1083700 & -5.1163830 & -3.0043420 & $\mathrm{C}$ & 4.9740720 & 0.3474570 & -1.4829810 \\
\hline 3.1268960 & -4.3385570 & -4.1952840 & $\mathrm{C}$ & 4.6429480 & 0.7933010 & 0.8391350 \\
\hline 4.6138460 & -4.8045530 & -1.5545280 & $\mathrm{C}$ & 5.4064720 & 0.2051940 & -0.1681920 \\
\hline 4.9211910 & -2.3885250 & -1.4108940 & $\mathrm{H}$ & 5.5633100 & -0.1094930 & -2.2750920 \\
\hline-0.9113500 & 1.4201030 & 4.5270540 & $\mathrm{H}$ & 4.9616030 & 0.6869660 & 1.8702340 \\
\hline-1.6927840 & 1.8240200 & 3.4158830 & C & -3.9961390 & -0.8908690 & -0.6792460 \\
\hline-1.0602170 & 2.2372180 & 2.6220020 & $\mathrm{C}$ & -4.0192580 & -1.8979170 & 0.3069200 \\
\hline-2.2779550 & 0.9881100 & 3.0139090 & C & -4.3909850 & -1.1806490 & -1.9963360 \\
\hline-2.3713620 & 2.5959050 & 3.7786560 & C & -4.4620900 & -3.1712550 & -0.0472280 \\
\hline & & & $C$ & -4.81699 & $2.41>0$ & -2.3021 \\
\hline G [WB97XD, & d) $]=-4125$ & & C & -4.8639150 & -3.4819540 & -1.3449160 \\
\hline 0.471886 & 0.7503140 & -0.2420210 & $\mathrm{H}$ & -4.4916670 & -3.9466580 & 0.7146660 \\
\hline-1 & 0.2407 & -1.6235000 & $\mathrm{H}$ & -5.1301980 & -2.7023080 & -3.3190 \\
\hline
\end{tabular}




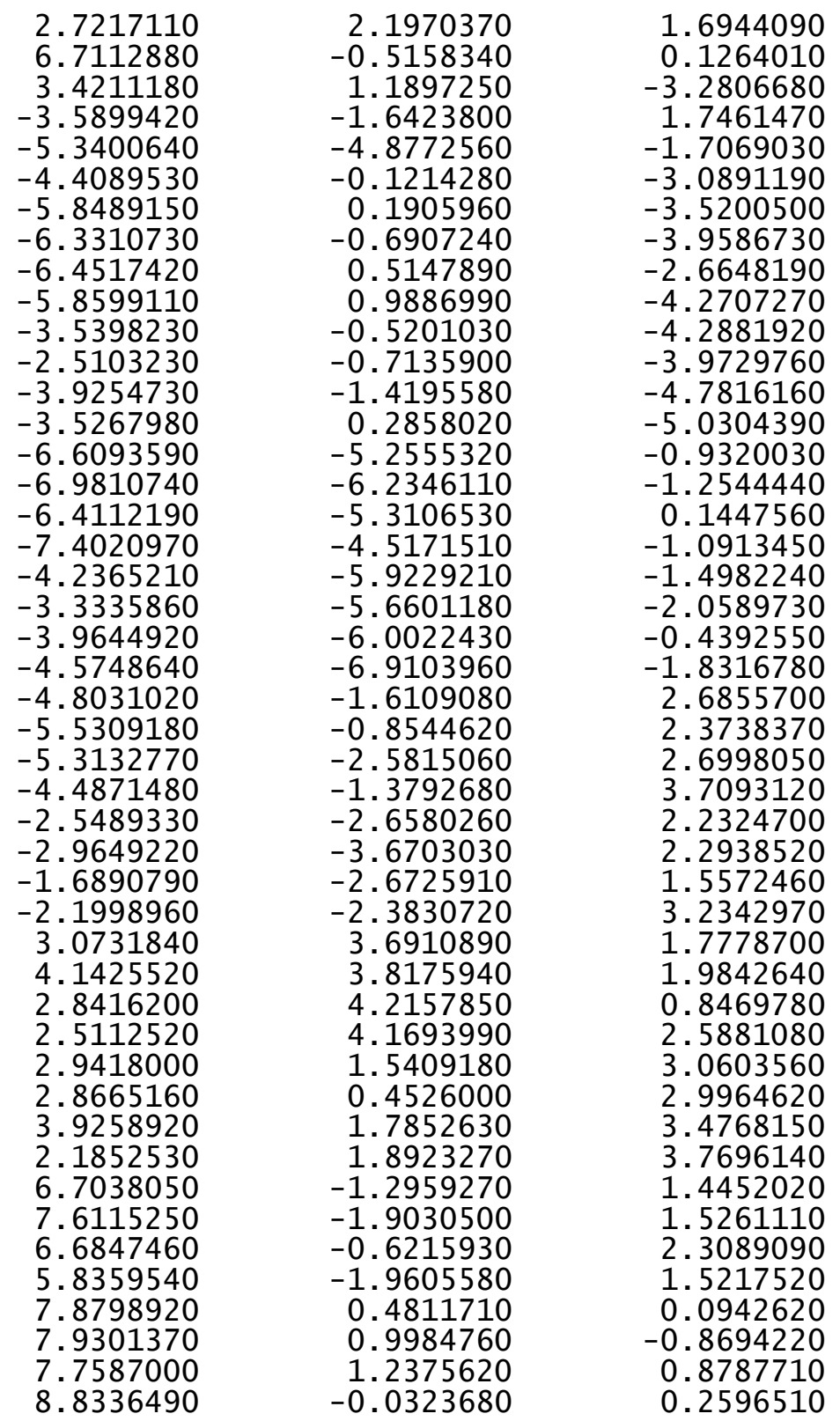

3.5540210

4.6026410

3.0449020

3.1047060

4.2438670

5.3119490

3.9432890

4.1162100

$-0.1893650$

0.5884760

$-0.3846230$

2. 3663440

6.8738280

1.6522130

$-3.9911110$

$-5.5917710$

$-3.1208020$

1.7718180

2. 8825230

0.8176030

2.6549820

1. 9444810

2.0509700

0.8920290

0.3925910

1.0594290

0.6888990

2.2215130

3.0373730

1.3444960

$-0.7126920$

$-1.2081080$

$-0.6415750$

0.3420490

$-1.1453100$

$-1.0090880$

0.7205850

3.4533920

4.5405240

4.2988020

2. 0683050

3.6547530

5.5267280

4.5381370

1.9971170

1.2808760
-0.1246160
-0.4076100
-0.9428230
-0.0133410
2.2866930
2.0388150
2.3866650
3.2596930
-0.4463570
-0.6875040
-1.5688730
1.4772570
-1.2363040
2.1137710
0.8030190
-4.8685630
-0.6567830
-3.5840200
-2.7032990
-3.3620570
-1.6774120
-2.8527830
-2.2928640
-0.9930170
-0.7646690
-1.5147540
-1.5501040
-2.1376290
-3.3091550
-2.0291850
0.5547310
0.7738350
0.1247690
-0.3259450
1.0658950
0.3102470
-0.4819900
-5.2990420
-4.5769640
-3.0658990
-5.0538630
-6.3743220
-4.7631420
-2.5950430
-5.5218430
-5.4891780
-

$-4.0582060$

$-4.2066840$

$-3.5460610$

$-5.0508410$

$-3.9734370$

$-3.9488180$

$-5.0224530$

$-3.4906910$

$-1.1468600$

$-2.4100890$

$-0.1701100$

$-3.3159730$

$-0.6869690$

1.4749450

$-2.6809470$

$-2.7752250$

1.7924160

$-1.3394630$

$-0.9681350$

$-0.8640910$

$-0.6926540$

$-2.5778990$

2. 5620380

4.3687360

3.0833040

2. 1212910

1.1506990

4.2980940

1. 6442410

$-2.5598420$

5.2376800

3.9398830

2.8434020

5.4542910

6.0885670

1. 8416550

6.4584820

$-2.1235720$

$-1.3236740$

$-1.2722420$

$-1.5176130$

$-2.1437760$

$-1.7589280$

$-2.2339340$

$-0.5286260$

$-2.1408990$ 


\begin{tabular}{|c|c|c|c|c|c|c|}
\hline & & & & & & \\
\hline 3.4653950 & -4.9498400 & -3.1626590 & C & 4.6589170 & 1.0130970 & 0.9022020 \\
\hline 4.5635740 & -4.9751360 & -0.3012550 & $\mathrm{c}$ & 5.4886790 & 0.3174880 & 0.0248660 \\
\hline 4.9377600 & -2.5734140 & -0.5362920 & $\mathrm{H}$ & 5.7179510 & -0.3365550 & -2.0038110 \\
\hline-2.2400890 & 1.5966800 & 3.6527210 & $\mathrm{H}$ & 4.9643300 & 1.0961640 & 1.9409330 \\
\hline-2.8784870 & 2.2758260 & 4.7185130 & C & -4.0413380 & -0.8326660 & -0.3833970 \\
\hline-3.3005590 & 1.5696420 & 5.4432960 & C & -4.0353710 & -1.7355900 & 0.6960580 \\
\hline-2.1863220 & 2.9558230 & 5.2287810 & C & -4.5407590 & -1.2313490 & -1.6377130 \\
\hline-3.6795030 & 2.8524250 & 4.2584550 & $\mathrm{c}$ & -4.5563800 & -3.0162750 & 0.4987250 \\
\hline & & & C & -5.0419940 & -2.5224740 & -1.7851070 \\
\hline 5) $\mathrm{G}[\mathrm{wB} 97 \mathrm{XD}$ & (d) $]=-412$ & & C & -5.0631970 & -3.4310720 & -0.7282390 \\
\hline 0.4793890 & 0.6867410 & -0.3083940 & $\mathrm{H}$ & -4.5654450 & -3.7182120 & 1.3299340 \\
\hline-1.6432310 & 0.1129790 & -1.5684920 & $\mathrm{H}$ & -5.4356930 & -2.8257030 & -2.7529650 \\
\hline 0.6051480 & 1.8767210 & -0.9902680 & $\mathrm{C}$ & 2.6378940 & 2.4123990 & 1.4892220 \\
\hline-0.5174880 & 2.6450950 & -1.2099030 & C & 6.8207740 & -0.2306300 & 0.5087670 \\
\hline 1.9131840 & 2.2843310 & -1.3549240 & $\mathrm{C}$ & 3.5756530 & 0.7109430 & -3.2571010 \\
\hline-0.3919440 & 3.8058300 & -2.0394190 & C & -3.4947600 & -1.3647240 & 2.0709710 \\
\hline 2.0343850 & 3.4448480 & -2.0769640 & $\mathrm{C}$ & -5.6259720 & -4.8295770 & -0.9074350 \\
\hline 0.9028820 & 4.2008990 & -2.4751410 & $\mathrm{C}$ & -4.5829840 & -0.2859660 & -2.8298590 \\
\hline 3.02 & 3.8034200 & -2.3482460 & C & -6.0316370 & 0.0371800 & -3.2212500 \\
\hline-2.3131840 & 0.9928780 & -0.7434920 & $\mathrm{H}$ & -6.5647650 & -0.8620120 & -3.5511960 \\
\hline-3.5459260 & 0.5621800 & -0.1856920 & $\mathrm{H}$ & -6.5792560 & 0.4625250 & -2.3733550 \\
\hline-1.8024240 & 2.2605460 & -0.5666590 & $\mathrm{H}$ & -6.0538570 & 0.7623260 & -4.0424460 \\
\hline-4.2660560 & 1.4746630 & 0.5418180 & C & -3.7881800 & -0.8345210 & -4.0210560 \\
\hline-2.5068670 & 3.1658140 & 0.2947570 & $\mathrm{H}$ & -2.7531290 & -1.0397900 & -3.7326800 \\
\hline-3.7711810 & 2.7737300 & 0.8129930 & $\mathrm{H}$ & -4.2295970 & -1.7603240 & -4.4080360 \\
\hline-5.2293890 & 1.1850510 & 0.9526580 & $\mathrm{H}$ & -3.7792810 & -0.1039280 & -4.8376620 \\
\hline-3.996 & 4.9108620 & 1.9393650 & C & -4.8345710 & -5.6260590 & -1.9533200 \\
\hline-2.7 & 92290 & 1.4769840 & $\mathrm{H}$ & -5.2187800 & -6.6494450 & -2.0290040 \\
\hline-1.9878 & 4.4326220 & 0.6768180 & $\mathrm{H}$ & -4.9170460 & -5.1641170 & -2.9439230 \\
\hline-4.5075330 & 3.6795460 & 1.6205150 & $\mathrm{H}$ & -3.7724880 & -5.6744670 & -1.6919140 \\
\hline-4.5659 & 5.5959920 & 2.5601940 & C & -7.1190170 & -4.7918560 & -1.2601340 \\
\hline-2.2942 & 6.2406340 & 1.7595050 & $\mathrm{H}$ & -7.6891090 & -4.2449680 & -0.5019230 \\
\hline-1.0018010 & 4.7277500 & 0.3357110 & $\mathrm{H}$ & -7.2819040 & -4.2983800 & -2.2254000 \\
\hline-5.4808720 & 3.3708150 & 1.9929830 & $\mathrm{H}$ & -7.5253700 & -5.8069900 & -1.3309260 \\
\hline-1.3497880 & 5.6616480 & -3.2789030 & C & -4.6326750 & -1.1745400 & 3.0830500 \\
\hline-0.0585670 & 6.0716780 & -3.6849920 & $\mathrm{H}$ & -5.3510260 & -0.4211240 & 2.7434170 \\
\hline 1.040 & 5.3539090 & -3.2921470 & $\mathrm{H}$ & -5.1781960 & -2.1128740 & 3.2393670 \\
\hline-1.51 & 4.5614500 & -2.4762490 & $\mathrm{H}$ & -4.2300330 & -0.8506730 & 4.0497540 \\
\hline-2.219 & 6.2200400 & -3.6106670 & C & -2.4729600 & -2.3837880 & 2.5897520 \\
\hline 0.0560 & 6.9482160 & -4.3151160 & $\mathrm{H}$ & -2.9318760 & -3.3617210 & 2.7751830 \\
\hline 2.037 & 500410 & -3.6091310 & $\mathrm{H}$ & -1.6614100 & -2.5072680 & 1.8679400 \\
\hline-2.50 & 509280 & -2.1801790 & $\mathrm{H}$ & -2.0460520 & -2.0328470 & 3.5361080 \\
\hline 3.11 & 316640 & -0.8751950 & C & 2.8751780 & 3.9244470 & 1.3441560 \\
\hline 3.91 & 30380 & -1.7742990 & $\mathrm{H}$ & 3.9266590 & 4.1623830 & 1.5440730 \\
\hline 3.4 & 02650 & 0.4874280 & $\mathrm{H}$ & 2.6292970 & 4.2836840 & 0.3415480 \\
\hline 5 . & 4313 & -1.3028470 & $\mathrm{H}$ & 2.258 & 4.4750260 & 2.0635580 \\
\hline
\end{tabular}




\begin{tabular}{|c|c|c|c|}
\hline & & & \\
\hline C & 2.8740030 & 2.0011240 & 2.9457820 \\
\hline $\mathrm{H}$ & $\begin{array}{l}2.8894690 \\
3.82 ? 7340\end{array}$ & 0.9146670 & $\begin{array}{l}3.0616780 \\
3.3304590\end{array}$ \\
\hline H & 2.0713680 & $\begin{array}{l}2.3936 / 30 \\
2.3950350\end{array}$ & $\begin{array}{l}3.3304590 \\
3.5781430\end{array}$ \\
\hline C & 7.9884730 & 0.4770500 & -0.1933320 \\
\hline H & 8.9465490 & 0.1293070 & 0.2087460 \\
\hline $\mathrm{H}$ & 7.9808410 & 0.2724680 & -1.2703010 \\
\hline H & 7.9298480 & 1.5616700 & -0.0558190 \\
\hline C & 6.9318490 & -1.7508930 & 0.3550690 \\
\hline H & 6.1242800 & -2.2582530 & 0.8942040 \\
\hline $\mathrm{H}$ & 6.8866900 & -2.0463380 & -0.7001320 \\
\hline H & 7.8857450 & -2.1089090 & 0.7578640 \\
\hline C & 3.6293950 & -0.7217930 & -3.7962040 \\
\hline $\mathrm{H}$ & 4.6368150 & -1.1496210 & -3.7320220 \\
\hline H & 2.9396530 & -1.3669050 & -3.2494810 \\
\hline $\mathrm{H}$ & 3.3351840 & -0.7346590 & -4.8513360 \\
\hline C & 4.5014170 & 1.6227500 & -4.0760380 \\
\hline H & 5.5453860 & 1. 3012150 & -3.9796520 \\
\hline $\mathrm{H}$ & 4.2331140 & 1.5897000 & -5.1378820 \\
\hline $\mathrm{H}$ & 4.4416760 & 2.6631730 & -3.7415490 \\
\hline $\mathrm{P}$ & -0.2516020 & -0.5687030 & -1.0714870 \\
\hline 0 & 0.4782680 & -0.9623630 & -2.3253780 \\
\hline 0 & -0.4493250 & -1.5810440 & 0.0183040 \\
\hline H & 2.5479580 & 1.0598780 & -3.3923120 \\
\hline $\mathrm{H}$ & 6.8928890 & -0.0044630 & 1.5800780 \\
\hline H & 1.5823610 & 2.2144800 & 1.2765650 \\
\hline H & -4.1142960 & 0.6581860 & -2.5394790 \\
\hline H & -5.5234330 & -5.3482220 & 0.0543470 \\
\hline H & -2.9774040 & -0.4065070 & 1.9848200 \\
\hline C & 1.4823340 & -3.8244460 & -1.0209740 \\
\hline $\mathrm{C}$ & 2.6797690 & -2.9902580 & -0.8884230 \\
\hline H & 0.5965430 & -3.4611510 & -0.5025380 \\
\hline H & 2.5436890 & -1.9223530 & -0.7478480 \\
\hline 0 & 1.5771540 & -3.2904990 & -2.3659600 \\
\hline C & 2.1718590 & -2.0870360 & 2.6386910 \\
\hline C & 1.1038600 & -0.6418870 & 4.3926840 \\
\hline C & 0.5461190 & -0.5103840 & 3.1180250 \\
\hline $\mathrm{N}$ & 1.1606180 & -1.3412770 & 2.1883360 \\
\hline H & 0.7353740 & -1.4559760 & 1.2481230 \\
\hline $\mathrm{s}$ & 2.4289880 & -1.7937460 & 4.3473030 \\
\hline $\mathrm{S}$ & 3.1094730 & -3.1811660 & 1.7598150 \\
\hline H & 1.0573040 & -2.4186150 & -2.4070290 \\
\hline C & -0.4426380 & 0.9930900 & 5.2074170 \\
\hline C & -0.9915170 & 1.1193760 & 3.9189710 \\
\hline C & -0.4855180 & 0.3744520 & 2.8544570 \\
\hline C & 0.6116150 & 0.1167540 & 5.44541 \\
\hline
\end{tabular}

$\begin{array}{lrr}\mathrm{H} & -0.8302180 & 1.5765710 \\ \mathrm{H} & -0.8940990 & 0.4879730 \\ \mathrm{H} & 1.0345550 & 0.0344580 \\ \mathrm{C} & 2.9702980 & -5.7678550 \\ \mathrm{C} & 4.1712560 & -5.0165570 \\ \mathrm{C} & 4.0292570 & -3.5028340 \\ \mathrm{C} & 1.6653250 & -5.3227520 \\ \mathrm{H} & 3.0992720 & -6.8451750 \\ \mathrm{H} & 5.0988060 & -5.3464040 \\ \mathrm{H} & 4.1848420 & -3.2241770 \\ \mathrm{H} & 1.6566860 & -5.6278390 \\ \mathrm{H} & 0.7963920 & -5.7842210 \\ \mathrm{H} & 2.9089020 & -5.5891490 \\ \mathrm{H} & 4.2634560 & -5.2465060 \\ \mathrm{H} & 4.7748990 & -2.9446130 \\ \mathrm{O} & -2.0212830 & 1.9370210 \\ \mathrm{C} & -2.5958160 & 2.7220660 \\ \mathrm{H} & -3.0053920 & 2.0925710 \\ \mathrm{H} & -1.8649170 & 3.4223510 \\ \mathrm{H} & -3.4004420 & 3.2813290 \\ \mathrm{I} & & \end{array}$

6.0333370
1.8580920
6.4406240
-1.6647310
-1.0841120
-1.2667490
-0.9968830
-1.5240350
-1.5610780
-2.3159750
0.0564710
-1.4763890
-2.7446520
-0.0152040
-0.6986220
3.6093480
4.6379050
5.4365500
5.0591140
4.1622070

TS21(S, S) G[WB97XD/6-31G(d)] = -4125.318843

$\begin{array}{lrrr}\text { TS21(S, S) G }[\mathrm{WB} 97 \times \mathrm{D} / 6-31 \mathrm{G}(\mathrm{d})]=-4125.318843 \\ \mathrm{O} & -0.1361120 & -1.0423580 & -0.0981520 \\ \mathrm{O} & 1.7962750 & -0.0235250 & -1.3572410 \\ \mathrm{C} & 0.0475560 & -2.2630990 & -0.7152680 \\ \mathrm{C} & 1.3292980 & -2.7480080 & -0.8698640 \\ \mathrm{C} & -1.1163770 & -2.9756660 & -1.1072050 \\ \mathrm{C} & 1.5075370 & -3.9570880 & -1.6185120 \\ \mathrm{C} & -0.9343960 & -4.1861020 & -1.7250010 \\ \mathrm{C} & 0.3576580 & -4.6857730 & -2.0291510 \\ \mathrm{H} & -1.8000130 & -4.7796280 & -2.0046140 \\ \mathrm{C} & 2.6308590 & -0.6675500 & -0.4718150 \\ \mathrm{C} & 3.6841450 & 0.0982920 & 0.0962290 \\ \mathrm{C} & 2.4652570 & -2.0176590 & -0.2444290 \\ \mathrm{C} & 4.5965110 & -0.5562040 & 0.8817940 \\ \mathrm{C} & 3.3651490 & -2.6690620 & 0.6657950 \\ \mathrm{C} & 4.4563950 & -1.9305620 & 1.2016190 \\ \mathrm{H} & 5.4414940 & -0.0079710 & 1.2891390 \\ \mathrm{C} & 5.1804470 & -3.8692350 & 2.4689900 \\ \mathrm{C} & 4.0726830 & -4.5941560 & 1.9731040 \\ \mathrm{C} & 3.1905310 & -4.0125520 & 1.0983120 \\ \mathrm{C} & 5.3616400 & -2.5639310 & 2.0930700 \\ \mathrm{H} & 5.8736350 & -4.3432060 & 3.1568330 \\ \mathrm{H} & 3.9135340 & -5.6190280 & 2.2939510 \\ \mathrm{H} & 2.3368890 & -4.5787020 & 0.7442610 \\ \mathrm{H} & 6.1941460 & -1.9858540 & 2.4850410\end{array}$




\begin{tabular}{rrr}
2.9128530 & -5.6133710 & -2.7057940 \\
1.7694710 & -6.3587790 & -3.0756860 \\
0.5206640 & -5.8994310 & -2.7477170 \\
2.7868620 & -4.4459170 & -1.9968360 \\
3.8992290 & -5.9665190 & -2.9908570 \\
1.8848560 & -7.2860470 & -3.6282080 \\
-0.3676610 & -6.4513310 & -3.0434070 \\
3.6720430 & -3.8789260 & -1.7297420 \\
-2.4707740 & -2.4138380 & -0.8057820 \\
-3.2430160 & -1.8342030 & -1.8257650 \\
-2.9637040 & -2.4610320 & 0.5185620 \\
-4.4877100 & -1.2802850 & -1.5006210 \\
-4.2140560 & -1.9146780 & 0.7830750 \\
-4.9914080 & -1.3072580 & -0.2062940 \\
-5.0787620 & -0.8345980 & -2.2936270 \\
-4.6108760 & -1.9607790 & 1.7931300 \\
3.7925870 & 1.5615980 & -0.1913970 \\
3.2881120 & 2.5018590 & 0.7313050 \\
4.3854280 & 1.9887660 & -1.3891200 \\
3.4148640 & 3.8564390 & 0.4354620 \\
4.4789010 & 3.3589210 & -1.6445290 \\
4.0017080 & 4.3070330 & -0.7475910 \\
3.0298220 & 4.5875070 & 1.1404610 \\
4.9316470 & 3.6967830 & -2.5747570 \\
-2.1782930 & -3.1487930 & 1.6312870 \\
-6.3425430 & -0.7215640 & 0.1806030 \\
-2.8028080 & -1.8619850 & -3.2838520 \\
2.6238480 & 2.0658070 & 2.0317100 \\
4.0911320 & 5.7906380 & -1.0573510 \\
4.9146030 & 1.0035250 & -2.4210000 \\
6.4179540 & 1.1930240 & -2.6608780 \\
6.6339050 & 2.1787240 & -3.0885830 \\
6.9783180 & 1.1005380 & -1.7243990 \\
6.7905420 & 0.4368750 & -3.3608690 \\
4.1207230 & 1.0915710 & -3.7310020 \\
3.0554210 & 0.9228640 & -3.5477470 \\
4.2375510 & 2.0749670 & -4.2014540 \\
4.4730800 & 0.3363120 & -4.4426190 \\
4.9082470 & 6.5448360 & -0.0002420 \\
5.0181430 & 7.5990290 & -0.2776020 \\
4.4174460 & 6.5071560 & 0.9791090 \\
5.9079410 & 6.1112490 & 0.1075150 \\
2.6946130 & 6.4081990 & -1.2174310 \\
2.1219240 & 5.8895910 & -1.9935120 \\
2.1286920 & 6.3431060 & -1.4920500 \\
2.7701170 & 7.4662920 & \\
\hline & &
\end{tabular}

3.6488500 4.4324140

4.1283180

3. 1544540

1.4825200

1. 8517810

0.7651270

0.9571800

$-2.2931290$

$-3.3406990$

-1.9211930

$-1.7182250$

$-2.5912970$

$-2.6447740$

$-3.5662000$

$-1.8594110$

$-7.2124120$

$-8.1938310$

$-6.7644870$

$-7.3658890$

$-6.1660210$

$-5.6005020$

$-5.6227460$

$-7.1398530$

$-3.1425800$

$-4.2216240$

$-2.7741460$

$-2.6722190$

$-3.4266650$

$-4.5196220$

$-3.0756390$

$-3.1781600$

0.2432010

$-0.4915330$

0.1038200

$-1.7148810$

$-6.8812800$

$-1.1224450$

4.7780360

4. 6123690

2. 1819710

$-1.8313850$

$-2.9568580$

$-0.9566090$

-2.7359800
-1.7939730
1.9264920

2044930

2. 8913760

1.5907970

2. 9868270

.

3. 1389330

2. 5328290

$-4.6779190$

$-4.9848050$

$-5.0467200$

$-5.1630850$

$-2.7209310$

$-1.6337270$

$-3.1394500$

$-3.0920940$

$-0.3116740$

0.0208360

0.5209760

$-1.1411100$

0.4600690

0.1720750

1. 2810590

0.8423520

$-0.5851860$

$-0.4739490$

0.3001490

$-0.6182000$

$-3.0714880$

$-2.9813080$

$-3.1276050$

$-4.0146960$

0.2896090

0.3230150

1.4389420

$-1.9688460$

$-1.5097210$

$-2.8793290$

$-0.0079250$

5.8937880

1.0803390

3. 3063920

2. 4236520

3. 1929990

1.4615330

2. 4127130
3.1664940

2.9212480

3. 3698310

4.0857730

2. 4745300

2.8097680

1. 6646280

3. 3217480

1. 5163180

1.6183970

0.5570470

2. 3133210

3. 0428760

3. 1450880

3. 3190270

3. 7674770

$-1.0086910$

$-0.6555160$

$-1.5643980$

$-1.7069180$

1.1465020

2.0385900

0.6644950

1. 4721920

$-4.0582460$

$-4.2174960$

$-3.5399480$

$-5.0467940$

$-3.9990520$

$-4.0148660$

$-5.0357430$

$-3.5048400$

$-0.9732170$

$-2.2839730$

$-0.0206070$

$-3.3032200$

0.7253220

1. 5086540

$-2.0268320$

$-2.0176430$

1.8529460

$-1.8028070$

$-1.4912490$

$-1.1638780$

$-1.0395170$

$-2.9420550$ 


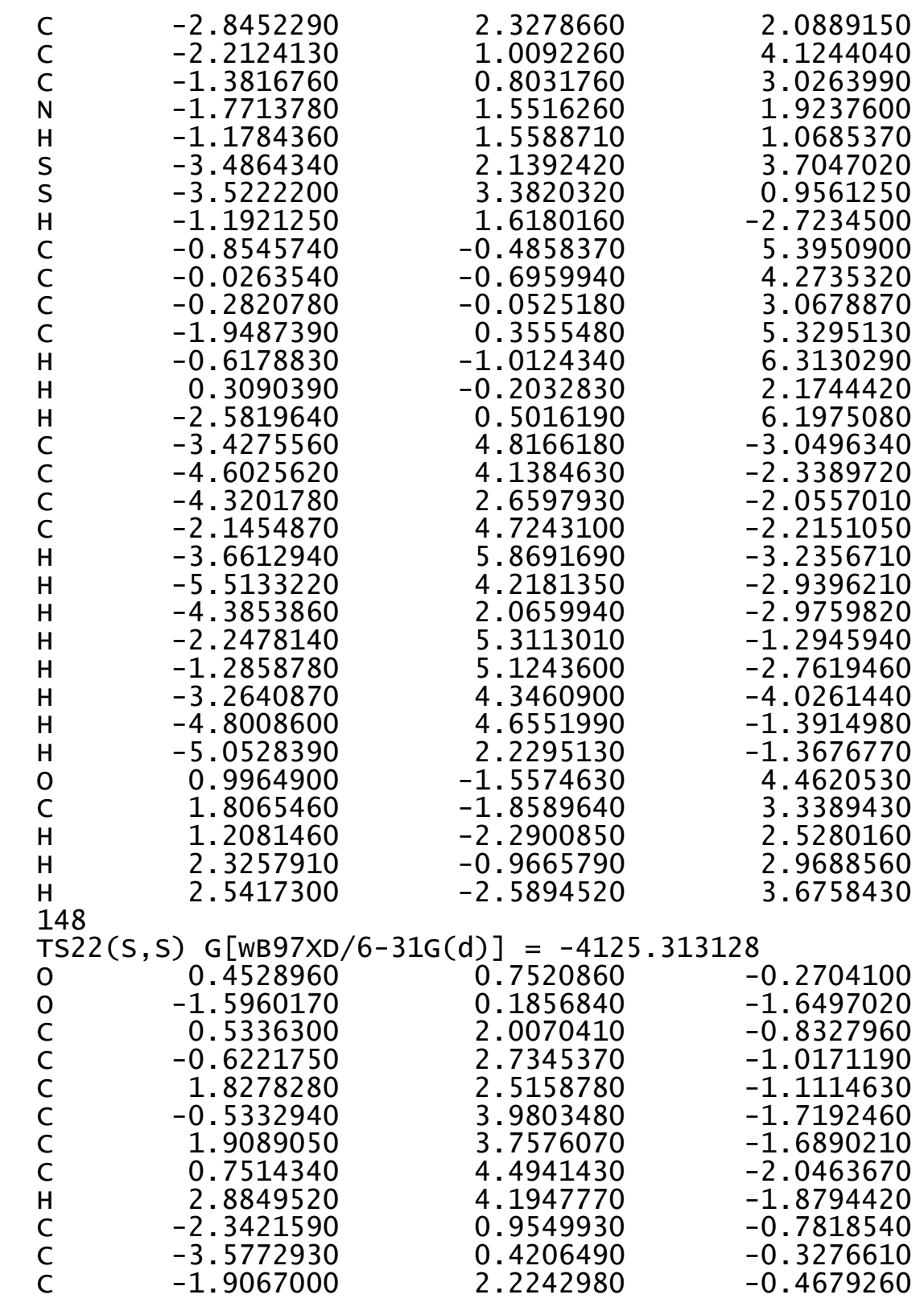

148

-4.3776610
-2.6948200
-3.9624590
-5.3438150
-4.3477690
-3.0592910
-2.2554940
-4.7819160
-4.9802660
-2.7016070
-1.2675420
-5.7574510
-1.5495640
-0.2701030
0.8535970
-1.6789370
-2.4375260
-0.1824260
1.8425690
-2.6657190
3.0521890
3.8814590
3.4013400
5.0636100
4.5905470
5.4462720
5.7073670
4.8809760
-3.9841230
-3.9814890
-4.3883630
-4.4091940
-4.7981170
-4.8198910
-4.4190450
-5.1183130
2.5353340
6.7663900
3.5692770
-3.5393450
-5.2791800
-4.4324820
-5.8805180
-6.3540540
-6.4801980
-5.9095840

3.0119180

2. 5200660

0.8587550

4. 5385320

5.0001820

4. 2590680

3. 3187660

5. 1425990

5.9483730

4.6252440

2. 9363960

5.9043390

6.4335800

5.7392360

4.7129260

6.4441250

7. 3825130

6. 1265990

4. 3119540

1.7594930

1.1638290

1.7034890

0.5369680

1.0797760

0.4937830

0.0964310

1. 0638400

$-0.9740730$

$-1.9695450$

$-1.2857280$

$-3.2533510$

$-2.5886920$

$-3.5857780$

$-4.0199850$

$-2.8336840$

2. 3618940

$-0.1034330$

1. 2369450

$-1.6915030$

$-4.9923210$

$-0.2405710$

0.0458660

$-0.8470000$

0.3711510

0.8352960
0.4378970

0.4356420

0.8490730

0.7698370

2.1399200

1.7846550

0.9564830

1.6893170

2.7833070

2. 1744170

0.7004860

1.9784510

$-2.7982530$

$-3.0864290$

$-2.7211360$

$-2.1310940$

$-3.1129040$

$-3.6063210$

$-2.9513850$

$-1.9270030$

$-0.7040980$

$-1.6664430$

0.6654470

$-1.2492680$

1. 0234400

0.0860980

$-2.0038030$

2.0694090

$-0.6732970$

0.3247090

$-1.9825930$

$-0.0096760$

$-2.2687370$

$-1.2991930$

0.7615480

$-3.2796040$

1.7363700

0.5637850

$-3.1561670$

1.7559290

$-1.6387860$

$-3.0880660$

$-3.5093650$

$-3.9337940$

$-2.6523370$

$-4.2686720$ 


$\begin{array}{rrr}-3.5683460 & -0.6419660 & -4.2897870 \\ -2.5345370 & -0.8212220 & -3.9802620 \\ -3.9477900 & -1.5505810 & -4.7711540 \\ -3.5695730 & 0.1564100 & -5.0402000 \\ -6.5375760 & -5.3767170 & -0.8491690 \\ -6.8970940 & -6.3661680 & -1.1531960 \\ -6.3313800 & -5.4115140 & 0.2269640 \\ -7.3419240 & -4.6524190 & -1.0147300 \\ -4.1601350 & -6.0201330 & -1.4243490 \\ -3.2658600 & -5.7540440 & -1.9973160 \\ -3.8773740 & -6.0796900 & -0.3668820 \\ -4.4881460 & -7.0170460 & -1.7394640 \\ -4.7414600 & -1.6638200 & 2.7094890 \\ -5.4822500 & -0.9201760 & 2.3975630 \\ -5.2393680 & -2.6403200 & 2.7423260 \\ -4.4157070 & -1.4154270 & 3.7262430 \\ -2.4796670 & -2.6881850 & 2.2412880 \\ -2.8800280 & -3.7060790 & 2.3125950 \\ -1.6245050 & -2.6945540 & 1.5598600 \\ -2.1274280 & -2.4014710 & 3.2386650 \\ 2.7594420 & 3.8821260 & 1.7772230 \\ 3.8039990 & 4.1028240 & 2.0270310 \\ 2.5308570 & 4.3555080 & 0.8187340 \\ 2.1218610 & 4.3382570 & 2.5429920 \\ 2.7474070 & 1.7786430 & 3.1369200 \\ 2.7800030 & 0.6865240 & 3.1165030 \\ 3.6819610 & 2.1335690 & 3.5870660 \\ 1.9263090 & 2.0811920 & 3.7952860 \\ 7.6675880 & -0.6081420 & -0.5646960 \\ 8.6153750 & -0.9673770 & -0.1511230 \\ 7.2053610 & -1.4449970 & -1.1027170 \\ 7.8944320 & 0.1790970 & -1.2908970 \\ 6.5581470 & -1.2106100 & 1.6091850 \\ 5.9330600 & -0.8777520 & 2.4430640 \\ 6.0725980 & -2.0879530 & 1.1685360 \\ 7.5230150 & -1.5309490 & 2.0171790 \\ 3.7423460 & -0.1034550 & -3.8772280 \\ 4.7895950 & -0.4275730 & -3.9008980 \\ 3.1468690 & -0.8846610 & -3.4024820 \\ 3.4086790 & -0.0102640 & -4.9163720 \\ 4.4319070 & 2.3126580 & -3.8333590 \\ 5.4962610 & 2.0619970 & -3.7497540 \\ 4.1844880 & 2.3908100 & -4.8979190 \\ 4.2855510 & 3.2963800 & -3.3774800 \\ -0.1826340 & -0.4629500 & -1.1713950 \\ 0.5998370 & -0.6899870 & -2.4347850\end{array}$

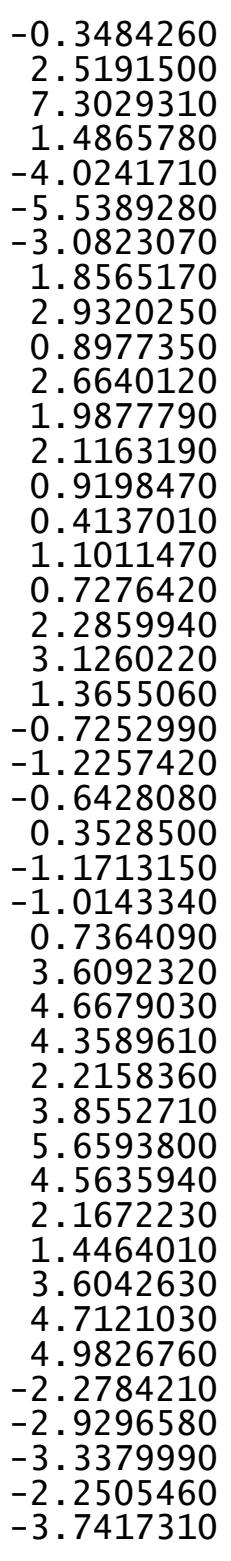
$-1.5853300$
1.5215930
0.7133420
2. 1825300
0.6949360
$-5.0018340$
$-0.6996700$
$-3.5532490$
$-2.6199870$
$-3.3623850$
$-1.6007430$
$-2.8380490$
$-2.1979160$
$-0.9311910$
$-0.7186450$
$-1.4504320$
$-1.5065730$
$-2.0322580$
$-3.1904950$
$-2.0317170$
0.5745690
0.7795350
0.1436300
$-0.2773840$
1.0750800
0.3201530
$-0.4213780$
$-5.2131280$
$-4.4254020$
$-2.9263260$
$-5.0122000$
$-6.2792660$
$-4.5807610$
$-2.4788740$
$-5.4615630$
$-5.4945480$
$-4.8913760$
$-4.7948790$
$-2.3754290$
1.5751360
2. 2459570
1. 5346480
2. 9410970
2. 8052010

$-0.1893200$

$-3.2660870$

1.0677550

1. 4781120

$-2.6957650$

$-2.7051880$

1.7857830

$-1.3462540$

$-0.8665810$

$-0.7394230$

$-2.5994770$

2. 5392480

4.3457290

3.0602150

2. 0988770

1. 1308010

4.2751370

1. 6227130

$-2.5870250$

5. 2131880

3.9151630

2. 8194940

5.4303970

6.0635400

1.8175990

6.4344710

$-2.1012330$

$-1.3245910$

$-1.3151020$

$-1.4968600$

$-2.0939460$

$-1.7601210$

$-2.2956250$

$-0.4978960$

$-2.1078500$

$-3.1492710$

$-0.2922430$

$-0.6081980$

3. 6262580

4.6894870

5.4171170

5.1968600

4.2272370

148

$\operatorname{TS} 23(\mathrm{~S}, \mathrm{~S}) \mathrm{G}[\mathrm{WB} 97 \mathrm{XD} / 6-31 \mathrm{G}(\mathrm{d})]=-4125.309014$ 


$\begin{array}{lrrr} & & & \\ \mathrm{O} & 0.2445110 & 0.8898330 & \\ \mathrm{O} & -1.6967740 & 0.1195380 & -1.35132830 \\ \mathrm{C} & 0.1981630 & 2.1643210 & -0.4634110 \\ \mathrm{C} & -1.0305100 & 2.7698960 & -0.6195650 \\ \mathrm{C} & 1.4332410 & 2.7874000 & -0.7805380 \\ \mathrm{C} & -1.0756930 & 4.0472510 & -1.2698300 \\ \mathrm{C} & 1.3779170 & 4.0664130 & -1.2748470 \\ \mathrm{C} & 0.1480960 & 4.7082550 & -1.5638490 \\ \mathrm{H} & 2.3029000 & 4.5940420 & -1.4874070 \\ \mathrm{C} & -2.5451270 & 0.7913160 & -0.5110610 \\ \mathrm{C} & -3.7593490 & 0.1304830 & -0.1746740 \\ \mathrm{C} & -2.2634450 & 2.0896190 & -0.1361100 \\ \mathrm{C} & -4.6722710 & 0.8149730 & 0.5819620 \\ \mathrm{C} & -3.1857350 & 2.7588680 & 0.7403110 \\ \mathrm{C} & -4.4123840 & 2.1201790 & 1.0695540 \\ \mathrm{H} & -5.6166500 & 0.3409400 & 0.8348980 \\ \mathrm{C} & -5.0556420 & 4.0031730 & 2.4571800 \\ \mathrm{C} & -3.8175370 & 4.6222930 & 2.1701560 \\ \mathrm{C} & -2.9102290 & 4.0206090 & 1.3352610 \\ \mathrm{C} & -5.3405960 & 2.7749500 & 1.9203530 \\ \mathrm{H} & -5.7673040 & 4.4945690 & 3.1131450 \\ \mathrm{H} & -3.5794710 & 5.5806050 & 2.6212500 \\ \mathrm{H} & -1.9592460 & 4.5034770 & 1.1426050 \\ \mathrm{H} & -6.2771390 & 2.2738710 & 2.1498490 \\ \mathrm{C} & -2.2845540 & 5.8959550 & -2.2815320 \\ \mathrm{C} & -1.0678140 & 6.5728240 & -2.5290090 \\ \mathrm{C} & 0.1208190 & 5.9868560 & -2.1812670 \\ \mathrm{C} & -2.2898420 & 4.6695520 & -1.6674380 \\ \mathrm{H} & -3.2227460 & 6.3495550 & -2.5860610 \\ \mathrm{H} & -1.0793290 & 7.5464360 & -3.0088930 \\ \mathrm{H} & 1.0649260 & 6.4838930 & -2.3878610 \\ \mathrm{H} & -3.2303380 & 4.1585800 & -1.4934220 \\ \mathrm{C} & 2.7388810 & 2.0756960 & -0.6053310 \\ \mathrm{C} & 3.4864140 & 1.7104970 & -1.7387080 \\ \mathrm{C} & 3.2392080 & 1.7944850 & 0.6881140 \\ \mathrm{C} & 4.7403370 & 1.1126100 & -1.5611270 \\ \mathrm{C} & 4.4733480 & 1.1633750 & 0.8054210 \\ \mathrm{C} & 5.2584350 & 0.8332270 & -0.3040970 \\ \mathrm{H} & 5.3202730 & 0.8651380 & -2.4438580 \\ \mathrm{H} & 4.8560610 & 0.9337560 & 1.7946670 \\ \mathrm{C} & -4.0266350 & -1.2603010 & -0.6557620 \\ \mathrm{C} & -3.6177790 & -2.3503140 & 0.1444730 \\ \mathrm{C} & -4.6783330 & -1.4851870 & -1.8820520 \\ \mathrm{C} & -3.8614090 & -3.6456100 & -0.3049030 \\ \mathrm{C} & -4.8880710 & -2.8079610 & -2.2862070 \\ \mathrm{C} & -4.4867280 & -3.8981000 & -1.5242090 \\ & & & \end{array}$

-3.5448730
-5.3841850
2.4856450
6.6338230
3.0053490
-2.9622880
-4.7055710
-5.2485030
-4.3207080
-4.2583520
-3.3077850
-4.7187450
-6.5943810
-7.2874740
-6.4599450
-7.0569560
-5.6033760
-5.7909710
-5.1309710
-6.5674040
-3.3687010
-2.7300490
-2.8244910
-3.5340470
-4.0195490
-4.7132020
-4.6049120
-3.5355190
-1.9670650
-2.4682720
-1.2254110
-1.4407370
2.6267470
3.6808130
2.2483230
2.0706770
2.9194270
2.9961940
3.8914260
2.1866820
7.5140900
8.4882030
7.6823790
7.0575750
7.3832160
6.8207310
-
0.3071420 $-3.2382580$ 1.9403420 $-0.0907020$ 1. 5037190 $-2.0164110$ $-2.8030950$ $-3.1743360$ $-2.3792770$ $-3.4002530$ $-4.0626560$ $-2.2806280$ $-2.0710010$ $-1.3609890$ $-3.0250660$ $-1.0662920$

$-1.4710110$ $-0.0843600$ $-0.9187770$ $-2.2473390$ $-2.9306500$ $-1.3042830$ $-2.6766560$ 2.6068360

2. 3954120

2.7091710

3.5696690

1.8999760

2.1186540

1.1141020

2. 8114450

2.1530450

2. 3002850

1.3000780

3.0446560

3.2146180

3.0573020

3.5749430

4.0100090

0.7852150

0.9609130

0.2865350

1.7585940

-1.3842940
-2.0392520 


\begin{tabular}{|c|c|c|}
\hline $\begin{array}{l}7.6077770 \\
8.3344370 \\
3.1972220 \\
4.2560790 \\
2.7613440 \\
.7056650 \\
3.7095840 \\
4.7900980 \\
3.3325020 \\
3.5563480 \\
.1879200 \\
0.5976850 \\
.1511100 \\
1.9318790 \\
6.4798810 \\
1.4245970 \\
5.4648430 \\
5.2192560 \\
2.3928400 \\
2.0117760 \\
3.0843830 \\
1.1073950 \\
2.8075490 \\
1.9747740 \\
2.6600600 \\
1.5993380 \\
0.9307420 \\
1.5399940 \\
1.0680430 \\
3.0176670 \\
3.6504540 \\
1.3418540 \\
0.0135540 \\
0.6433930 \\
.1897140 \\
1.1359250 \\
0.3767760 \\
.6473840 \\
1.6444740 \\
3.7127430 \\
4.8316430 \\
4.4764650 \\
2.4028320 \\
3.9971230 \\
5.7661790 \\
4.5569380\end{array}$ & $\begin{array}{r}0.7909370 \\
-0.6075470 \\
0.7465060 \\
0.5519010 \\
-0.1495150 \\
0.9245050 \\
3.1826770 \\
3.0085350 \\
3.3703230 \\
4.0895440 \\
-0.3291570 \\
-0.3032520 \\
-1.5522380 \\
2.1654190 \\
-0.7302520 \\
2.0104240 \\
-0.9249390 \\
-5.2509220 \\
-1.1997330 \\
-3.2154190 \\
-2.2793830 \\
-3.1353330 \\
-1.3163160 \\
-2.3415760 \\
-2.4369990 \\
-1.6065300 \\
-1.2316210 \\
-1.7331130 \\
-1.6395720 \\
-2.5529330 \\
-3.1428270 \\
-1.5683750 \\
-0.3711140 \\
0.0232520 \\
-0.4035730 \\
-1.1761040 \\
-0.0114820 \\
-0.1066640 \\
-1.4557610 \\
-4.6658670 \\
-3.9346500 \\
-2.4686740 \\
-4.6227030 \\
-5.7083160 \\
-3.9759410 \\
-1.8701410\end{array}$ & $\begin{array}{l}-1.9506670 \\
-1.1474320 \\
-4.0769340 \\
-4.2843040 \\
-3.6336360 \\
-5.0394790 \\
-3.7771450 \\
-3.8466440 \\
-4.7887740 \\
-3.1852950 \\
-0.9387900 \\
-2.2198630 \\
-0.0717520 \\
-3.1249670 \\
0.4597330 \\
1.7916490 \\
-3.7441850 \\
-2.9840230 \\
1.4415930 \\
-1.7264520 \\
-1.3828010 \\
-1.1248100 \\
-0.9642380 \\
-2.8810390 \\
2.2541820 \\
4.3678940 \\
3.2058620 \\
2.0610350 \\
1.1394690 \\
3.9626990 \\
1.0844730 \\
-2.6801320 \\
5.6512830 \\
4.4679730 \\
3.2233550 \\
5.6112720 \\
6.5969130 \\
2.2887820 \\
6.5271880 \\
-2.9017640 \\
-2.1551600 \\
-1.8880700 \\
-2.1086450 \\
-3.0736230 \\
-2.7224330 \\
-2.8042660\end{array}$ \\
\hline
\end{tabular}

$\begin{array}{lr}\mathrm{H} & 2.5037190 \\ \mathrm{H} & 1.5797210 \\ \mathrm{H} & 3.5611710 \\ \mathrm{H} & 5.0178510 \\ \mathrm{H} & 5.1578530 \\ \mathrm{O} & -1.7105430 \\ \mathrm{C} & -2.3009280 \\ \mathrm{H} & -1.5623610 \\ \mathrm{H} & -2.7481740 \\ \mathrm{H} & -3.0823360\end{array}$
$-5.1936390$ $-5.0672170$ $-4.2069310$ $-4.4407110$ $-2.0080320$
0.8317590
1. 3853570
1.9452320
0.6087070
2.0636130
$-1.1779770$ $-2.6768570$ $-3.8856580$ $-1.1994830$ $-1.1688420$ 4. 6425540 3.4794540 2. 8949810 2.8488270 3.8221680 148 TS24(S, S ) G $[$ WB97XD/6-31G(d) $]=-4125.311535$

$\begin{array}{lrlr}\text { TS24(S, S }) \text { G }[\text { WB97XD/6-31G(d)] }=-4125.311535 \\ \text { O } & 0.2952750 & 0.8412860 & 0.1597200 \\ \mathrm{O} & -1.6323380 & 0.3936190 & -1.4084480 \\ \mathrm{C} & 0.3143560 & 2.1844110 & -0.1598660 \\ \mathrm{C} & -0.8836760 & 2.8642450 & -0.2400700 \\ \mathrm{C} & 1.5779580 & 2.8033250 & -0.3480540 \\ \mathrm{C} & -0.8608260 & 4.2357500 & -0.6606940 \\ \mathrm{C} & 1.5853190 & 4.1573450 & -0.5759010 \\ \mathrm{C} & 0.3926870 & 4.8942250 & -0.7737900 \\ \mathrm{H} & 2.5367820 & 4.6714720 & -0.6743350 \\ \mathrm{C} & -2.4781020 & 0.9628700 & -0.4837660 \\ \mathrm{C} & -3.7300480 & 0.3188900 & -0.2803140 \\ \mathrm{C} & -2.1574380 & 2.1745040 & 0.0962610 \\ \mathrm{C} & -4.6332640 & 0.9183620 & 0.5567520 \\ \mathrm{C} & -3.0797820 & 2.7485360 & 1.0386830 \\ \mathrm{C} & -4.3362760 & 2.1193240 & 1.2469730 \\ \mathrm{H} & -5.6041300 & 0.4544860 & 0.7085160 \\ \mathrm{C} & -4.9538040 & 3.8125390 & 2.8711270 \\ \mathrm{C} & -3.6914370 & 4.4238390 & 2.6944480 \\ \mathrm{C} & -2.7806440 & 3.9068410 & 1.8083970 \\ \mathrm{C} & -5.2628520 & 2.6795900 & 2.1648850 \\ \mathrm{H} & -5.6659760 & 4.2322130 & 3.5746830 \\ \mathrm{H} & -3.4347270 & 5.3052160 & 3.2741720 \\ \mathrm{H} & -1.8100940 & 4.3776010 & 1.7076130 \\ \mathrm{H} & -6.2199740 & 2.1841440 & 2.3037900 \\ \mathrm{C} & -1.9631060 & 6.2799500 & -1.3745400 \\ \mathrm{C} & -0.7177550 & 6.9477730 & -1.4316450 \\ \mathrm{C} & 0.4344110 & 6.2651470 & -1.1428340 \\ \mathrm{C} & -2.0344860 & 4.9628560 & -0.9986970 \\ \mathrm{H} & -2.8713960 & 6.8136580 & -1.6372230 \\ \mathrm{H} & -0.6775840 & 7.9932090 & -1.7213920 \\ \mathrm{H} & 1.4017210 & 6.7554600 & -1.2128230 \\ \mathrm{H} & -2.9966770 & 4.4639640 & -0.9688840 \\ \mathrm{C} & 2.8641940 & 2.0389550 & -0.3739470 \\ \mathrm{C} & 3.5778810 & 1.9559400 & -1.5856290\end{array}$




\begin{tabular}{lrrr} 
C & 3.4035960 & 1.4754170 & 0.8043650 \\
C & 4.8441470 & 1.3613840 & -1.5846680 \\
C & 4.6635610 & 0.8872850 & 0.7460150 \\
C & 5.4138980 & 0.8401210 & -0.4314960 \\
H & 5.3954080 & 1.3174770 & -2.5187090 \\
H & 5.0971490 & 0.4623810 & 1.6474540 \\
C & -4.0618340 & -0.9656280 & -0.9704290 \\
C & -3.7553320 & -2.1834450 & -0.3240440 \\
C & -4.6981520 & -0.9680150 & -2.2254040 \\
C & -4.0894940 & -3.3810490 & -0.9512240 \\
C & -5.0023100 & -2.2019120 & -2.8102430 \\
H & -4.7097850 & -3.4142860 & -2.1984010 \\
H & -3.8529820 & -4.3192840 & -0.4586820 \\
$\mathrm{C}$ & -5.4897550 & -2.2108880 & -3.7836560 \\
$\mathrm{C}$ & 2.6726350 & 1.5760830 & 2.1386800 \\
$\mathrm{C}$ & 6.8214530 & 0.2637490 & -0.3897840 \\
$\mathrm{C}$ & 3.0561330 & 2.5299620 & -2.9006770 \\
$\mathrm{C}$ & -3.1082830 & -2.2097870 & 1.0573530 \\
$\mathrm{C}$ & -5.0385620 & -4.7317520 & -2.8768690 \\
$\mathrm{C}$ & -5.1536060 & 0.2711060 & -2.9995430 \\
$\mathrm{H}$ & -4.1161040 & 1.3843570 & -3.1999220 \\
$\mathrm{H}$ & -4.0024520 & 2.0073860 & -2.3087300 \\
$\mathrm{H}$ & -3.1347650 & 0.9789130 & -3.4602730 \\
$\mathrm{C}$ & -4.4456570 & 2.0399010 & -4.0137300 \\
$\mathrm{H}$ & -6.4617450 & 0.8432540 & -2.4313440 \\
$\mathrm{H}$ & -7.2235450 & 0.0632200 & -2.3288940 \\
$\mathrm{H}$ & -6.3003500 & 1.2973440 & -1.4486760 \\
$\mathrm{C}$ & -6.8533740 & 1.6196630 & -3.0986320 \\
$\mathrm{H}$ & -5.9886000 & -5.5883470 & -2.0298430 \\
$\mathrm{H}$ & -6.2600550 & -6.5044250 & -2.5661240 \\
$\mathrm{H}$ & -5.5187920 & -5.8829800 & -1.0844430 \\
$\mathrm{C}$ & -6.9083700 & -5.0429020 & -1.7942770 \\
$\mathrm{H}$ & -3.7615290 & -5.5089350 & -3.2254570 \\
$\mathrm{H}$ & -3.0966670 & -4.9089440 & -3.8552280 \\
$\mathrm{H}$ & -3.2103810 & -5.7825980 & -2.3182020 \\
$\mathrm{C}$ & -4.0057150 & -6.4325050 & -3.7622010 \\
$\mathrm{H}$ & -4.1591480 & -2.1033240 & 2.1727470 \\
$\mathrm{H}$ & -4.7494000 & -1.1867180 & 2.0945840 \\
$\mathrm{H}$ & -4.8481710 & -2.9554230 & 2.1337880 \\
$\mathrm{C}$ & -3.6683740 & -2.1067240 & 3.1534160 \\
$\mathrm{H}$ & -2.2323880 & -3.4419330 & 1.3021220 \\
$\mathrm{H}$ & -2.8287110 & -4.3569760 & 1.3916020 \\
$\mathrm{H}$ & -1.5016130 & -3.5752050 & 0.5005120 \\
$\mathrm{C}$ & -1.6941730 & -3.3209500 & 2.2481980 \\
$\mathrm{H}$ & 2.9088540 & 2.9532500 & 2.7774200 \\
& 3.9752080 & 3.1000930 & 2.9858630 \\
& & & \\
\hline
\end{tabular}

\begin{abstract}
2. 5765530
2. 3611640

3.0406520

2. 9963760

4.0470800

2. 3383150

7.7848960

8.7914330

7.8452300

7.4485170

7.3566520

6.6705510

7.5299240

8. 3147630

3.1155400

4.1436800

2. 5402420

2. 6911550

3.8244420

4.8822690

3.4100840

3.7779190

$-0.1632520$

0.6695270

$-0.2290090$

2.0041960

6.7908610

1.6013940

$-5.3910670$

$-5.5522100$

$-2.4519720$

2. 3673450

3.0245590

1.2850680

2. 4489840

2.7617620

1.7995340

1.1663420

0.5720770

0.9564250

0.5389580

2. 2104830

2. 4037780

1.9557560

0.0135640

$-0.5912360$
\end{abstract}

2. 1171630

3.7229880

3.1251960

2. 6491030

3.5382920

3.9654540

0.2401180

0.3297930

$-0.3809920$

1. 2380130

$-1.7475900$

$-2.2510670$

$-2.4197120$

$-1.6144920$

$-4.0629490$

$-4.3005140$

$-3.8419410$

$-4.9618520$

$-3.2775520$

$-3.4602480$

$-4.1918740$

$-2.4876180$

$-1.0208770$

$-2.2607470$

$-0.3589450$

$-2.7660040$

0.2731400

1.9440730

$-4.0039010$

$-3.8177560$

1.1267900

$-2.1176580$

$-0.8837680$

$-2.0529360$

$-0.2042270$

$-2.4835690$

1.9732360

4.1746280

3.0721280

1.8762890

0.9721580

3.6456450

0.6899940

$-2.3625740$

5.6005280

4.4772370 


$\begin{array}{lrrr}\mathrm{C} & -0.3077680 & -0.8357520 & 3.1895850 \\ \mathrm{C} & 0.8919140 & -2.0494200 & 5.4603480 \\ \mathrm{H} & -0.2310890 & -0.6027710 & 6.5826040 \\ \mathrm{H} & -0.7190340 & -0.3797750 & 2.2977630 \\ \mathrm{H} & 1.3497170 & -2.5030180 & 6.3323160 \\ \mathrm{C} & 4.5889580 & -3.6866260 & -2.9380710 \\ \mathrm{C} & 5.1291630 & -3.6242030 & -1.5061810 \\ \mathrm{C} & 4.5027750 & -2.4726370 & -0.7174660 \\ \mathrm{C} & 3.0661450 & -3.8590290 & -2.9512790 \\ \mathrm{H} & 5.0519510 & -4.5193320 & -3.4759560 \\ \mathrm{H} & 6.2163750 & -3.4975610 & -1.5124950 \\ \mathrm{H} & 4.8924870 & -1.5096120 & -1.0631170 \\ \mathrm{H} & 2.7916440 & -4.8384330 & -2.5406790 \\ \mathrm{H} & 2.6709310 & -3.8115020 & -3.9707630 \\ \mathrm{H} & 4.8557690 & -2.7682810 & -3.4747640 \\ \mathrm{H} & 4.9188170 & -4.5711140 & -0.9938000 \\ \mathrm{H} & 4.7339800 & -2.5244940 & 0.3497280 \\ \mathrm{O} & -1.4432470 & 0.6186290 & 4.7498040 \\ \mathrm{C} & -2.0521440 & 1.2635310 & 3.6453070 \\ \mathrm{H} & -1.2994950 & 1.7317800 & 3.0006900 \\ \mathrm{H} & -2.6489930 & 0.5602690 & 3.0511640 \\ \mathrm{H} & -2.7055890 & 2.0312240 & 4.0577720\end{array}$

114 TS25 $(R, R) \quad G[W B 97 X D / 6-31 G(d)]=-3918.022802$

$\begin{array}{lrcr}\text { TS25(R, R) G }[\mathrm{WB} 97 \times D / 6-31 G(\mathrm{~d})]=-3918.022802 \\ \mathrm{O} & 0.1107800 & 0.8912490 & -0.1018650 \\ \mathrm{O} & -1.6944180 & 0.0198530 & -1.6252290 \\ \mathrm{C} & -0.2418400 & 2.1809330 & -0.4109810 \\ \mathrm{C} & -1.5742310 & 2.5292180 & -0.4322490 \\ \mathrm{C} & 0.8094130 & 3.1047550 & -0.6354670 \\ \mathrm{C} & -1.9219850 & 3.8528280 & -0.8483790 \\ \mathrm{C} & 0.4725250 & 4.3945450 & -0.9581210 \\ \mathrm{C} & -0.8824170 & 4.7912380 & -1.1023020 \\ \mathrm{H} & 1.2574260 & 5.1274640 & -1.1258040 \\ \mathrm{C} & -2.5614690 & 0.2635130 & -0.5821600 \\ \mathrm{C} & -3.4065940 & -0.7967930 & -0.1548920 \\ \mathrm{C} & -2.5702870 & 1.5134690 & 0.0024730 \\ \mathrm{C} & -4.2764000 & -0.5503730 & 0.8757940 \\ \mathrm{C} & -3.4322920 & 1.7406620 & 1.1263910 \\ \mathrm{C} & -4.3056570 & 0.6984900 & 1.5453160 \\ \mathrm{H} & -4.9325510 & -1.3460360 & 1.2186020 \\ \mathrm{C} & -5.1297720 & 2.0846970 & 3.3589640 \\ \mathrm{C} & -4.2357410 & 3.1082690 & 2.9694010 \\ \mathrm{C} & -3.4086270 & 2.9415060 & 1.8876570 \\ \mathrm{C} & -5.1565200 & 0.9048690 & 2.6628150 \\ \mathrm{H} & -5.7821950 & 2.2320600 & 4.2140100 \\ \mathrm{H} & -4.1980780 & 4.0341180 & 3.5353900\end{array}$

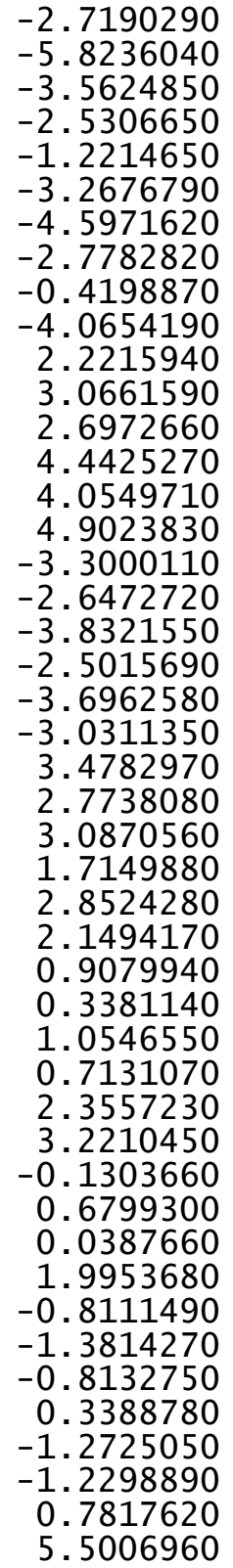

3. 7311960

0.1011550

5.5386790

6.4796010

6.1103700

4.2607930

5.8289640

7.4896290

6.8213780

3. 5424800

2. 6570020

2. 5883220

2. 2889160

2. 2192910

1.8514990

1.8575850

$-2.1453770$

$-3.1829700$

$-2.3636180$

$-4.4645490$

$-3.6535430$

$-4.6701090$

$-1.2920730$

$-2.5671630$

$-0.4496460$

$-2.5192410$

-1. 4080270

$-2.1075960$

$-0.9889510$

$-0.8068900$

$-1.4501790$

$-1.4711480$

$-1.9678250$

$-2.9837410$

$-0.2667860$

$-0.0028100$

$-1.5489440$

$-0.8625110$

0.3571590

0.5358570

$-0.0411390$

$-0.4059120$

0.8197930

0.1089570

$-0.5415350$

$-2.5756680$
1.6137200

2. 9626000

$-1.4480970$

$-1.6697060$

$-1.5028440$

$-1.0475660$

$-1.6031970$

$-1.9814440$

$-1.6847850$

$-0.8899010$

$-0.4821940$

$-1.6053880$

0.7930290

$-1.4310250$

0.9422380

$-0.1661220$

$-0.7811360$

$-0.0898070$

$-2.0644860$

$-0.7177220$

$-2.6757170$

$-1.9906430$

$-1.3692780$

$-1.1943800$

$-0.7950210$

$-0.9547660$

$-2.6651960$

2.4033830

4. 2790590

3. 0171710

2. 0164770

1.0297880

4. 1411820

1. 4420560

$-1.2446860$

$-2.4769990$

$-0.4842250$

$-2.6545430$

5. 2419980

3. 9747430

2.8486760

5.4083360

6.1084100

1. 8604160

6. 3893640

$-2.1820230$ 


$\begin{array}{rrr}4.9174330 & -3.8516410 & -1.5714310 \\ 3.3874200 & -3.8541350 & -1.6406820 \\ 4.9854700 & -1.3252490 & -1.4629890 \\ 6.5936960 & -2.5991270 & -2.1262600 \\ 5.3019570 & -4.7368050 & -2.0873750 \\ 3.0482580 & -4.0125790 & -2.6720530 \\ 5.3736770 & -1.2845490 & -0.4379400 \\ 5.3145730 & -0.4183930 & -1.9772060 \\ 5.2346560 & -2.5185420 & -3.2442200 \\ 5.2329080 & -3.9318010 & -0.5236340 \\ 2.9498110 & -4.6636850 & -1.0503560 \\ -2.2796140 & 1.1356870 & 3.8650470 \\ -2.9176400 & -5.6434670 & -2.4632960 \\ 5.9398300 & 1.5537810 & -0.0435040 \\ -1.4178890 & -4.0213920 & 1.8400270 \\ -2.0842300 & -3.0106400 & 1.2163940 \\ -1.7992170 & -5.5035580 & -0.0266070 \\ -1.2677010 & -5.2907200 & 1.2091170 \\ -0.9884370 & -3.8583730 & 2.8246980 \\ -2.1866240 & -2.0511320 & 1.7086860 \\ -1.6938430 & -6.4678580 & -0.5171600 \\ -0.7295570 & -6.0835690 & 1.7198820 \\ -5.0275960 & -1.5807280 & -4.0351090 \\ -4.5184450 & -1.3400380 & -2.7937110 \\ -4.2444660 & -3.8650520 & -3.9803190 \\ -4.8886170 & -2.8627680 & -4.6411840 \\ -5.5438650 & -0.7899580 & -4.5711270 \\ -4.6354680 & -0.3606080 & -2.3414490 \\ -4.1330670 & -4.8466020 & -4.4338920 \\ -5.3010260 & -3.0341430 & -5.6309170 \\ 3.4496320 & 2.7805220 & -4.0019790 \\ 2.6014720 & 2.8429490 & -2.9373580 \\ 5.3060690 & 2.1939570 & -2.5730220 \\ 4.8278350 & 2.4673000 & -3.8184320 \\ 3.0722000 & 2.9602370 & -5.0038360 \\ 1.5504860 & 3.0577460 & -3.0921370 \\ 6.3526970 & 1.9410470 & -2.4218730 \\ 5.4899340 & 2.4364530 & -4.6785130 \\ 3.6823210 & 1.4337640 & 3.3076080 \\ 4.5117950 & 1.4170220 & 2.2267660 \\ 1.8723890 & 2.3205350 & 1.9636820 \\ 2.3449460 & 1.9055180 & 3.1726420 \\ 4.0366040 & 1.0933380 & 4.2757870 \\ 5.5369990 & 1.0683580 & 2.3193770 \\ 0.8493450 & 2.6711400 & 1.8826040 \\ 1.6914230 & 1.9219420 & 4.0400230\end{array}$

114 TS26(S, S ) G[WB97XD/6-31G(d)] = -3918.020296

$\begin{array}{lrrr}\mathrm{C} 26(\mathrm{~S}, \mathrm{~S}) \mathrm{G}[\mathrm{WB} 97 \mathrm{CD} / \mathrm{r}-31 \mathrm{G}(\mathrm{d})] \mathrm{H}-3918.020296 \\ \mathrm{O} & -1.7597600 & -0.1545670 & -1.5631680 \\ \mathrm{C} & 0.1056800 & 0.8351380 & -0.1826260 \\ \mathrm{C} & -2.5922610 & 0.2113410 & -0.5294010 \\ \mathrm{C} & -2.5620660 & 1.5124310 & -0.0728670 \\ \mathrm{C} & -3.4519200 & -0.7800250 & 0.0174730 \\ \mathrm{C} & -3.3852130 & 1.8665660 & 1.0466020 \\ \mathrm{C} & -4.2885020 & -0.4154700 & 1.0405860 \\ \mathrm{H} & -4.2692870 & 0.8922190 & 1.5884190 \\ \mathrm{C} & -4.9550800 & -1.1577140 & 1.4717690 \\ \mathrm{C} & -0.2365210 & 2.0949490 & -0.6046780 \\ \mathrm{C} & 0.8219860 & 2.9759260 & -0.9370530 \\ \mathrm{C} & -1.5631630 & 2.4614280 & -0.6325290 \\ \mathrm{C} & 0.4980390 & 4.2268510 & -1.3951560 \\ \mathrm{C} & -1.9015050 & 3.7391900 & -1.1790270 \\ \mathrm{H} & -0.8546180 & 4.6251150 & -1.5598480 \\ \mathrm{C} & 1.2892340 & 4.9265390 & -1.6515030 \\ \mathrm{C} & -2.4925190 & 6.2654070 & -2.2782720 \\ \mathrm{C} & -3.5323070 & 5.3755220 & -1.9237330 \\ \mathrm{C} & -3.2458690 & 4.1465570 & -1.3870510 \\ \mathrm{H} & -1.1848900 & 5.8952730 & -2.1007220 \\ \mathrm{H} & -2.7329750 & 7.2361010 & -2.7006910 \\ \mathrm{H} & -4.5662720 & 5.6653340 & -2.0842820 \\ \mathrm{H} & -4.0491590 & 3.4655110 & -1.1256880 \\ \mathrm{C} & -0.3782280 & 6.5671150 & -2.3818630 \\ \mathrm{C} & -4.0921350 & 3.4243840 & 2.7736930 \\ \mathrm{C} & -4.9978960 & 2.4670600 & 3.2856410 \\ \mathrm{C} & -5.0784790 & 1.2279520 & 2.7056710 \\ \mathrm{H} & -3.3074870 & 3.1333060 & 1.6867910 \\ \mathrm{H} & -4.0109970 & 4.3967780 & 3.2497230 \\ \mathrm{H} & -5.6170990 & 2.7123740 & 4.1429750 \\ \mathrm{H} & -5.7561120 & 0.4756240 & 3.1003630 \\ \mathrm{C} & -2.6075760 & 3.8737830 & 1.3177810 \\ \mathrm{C} & -3.3893350 & -2.1785290 & -0.4937980 \\ \mathrm{C} & -2.7165840 & -3.1635210 & 0.2519980 \\ \mathrm{C} & -3.9673720 & -2.4899630 & -1.7374590 \\ \mathrm{C} & -2.5969250 & -4.4895620 & -0.2832190 \\ \mathrm{C} & -3.8567330 & -3.8224210 & -2.2549000 \\ \mathrm{C} & -3.1702800 & -4.7874380 & -1.5182120 \\ \mathrm{C} & 2.2274200 & 2.5311850 & -0.7248880 \\ \mathrm{C} & 3.0651540 & 2.2695040 & -1.8234920 \\ \mathrm{C} & 2.6988330 & 2.3547110 & 0.5922700 \\ \mathrm{C} & 4.4353490 & 1.9080190 & -1.5948280 \\ \mathrm{C} & 4.0554110 & 1.9423670 & 0.8078870 \\ & 4.8992230 & 1.7641780 & -0.2887450\end{array}$




$\begin{array}{rr}-0.1830110 & -0.4161080 \\ 0.0266550 & -1.6255540 \\ 0.5706510 & -0.2523690 \\ 5.9365350 & 1.4819830 \\ -3.0726090 & -5.7932770 \\ 3.4333240 & 2.0481450 \\ 2.5969960 & 2.3172250 \\ 5.2873630 & 1.6621190 \\ 4.8026750 & 1.7261670 \\ 3.0532430 & 2.0700240 \\ 1.5522450 & 2.5388010 \\ 6.3289060 & 1.4117320 \\ 5.4537710 & 1.5243620 \\ 2.3379970 & 2.3233590 \\ 1.8691050 & 2.5553480 \\ 4.5122710 & 1.7152190 \\ 3.6788390 & 1.8897250 \\ 1.6787750 & 2.4562050 \\ 0.8435300 & 2.8806480 \\ 5.5395950 & 1.3920740 \\ 4.0319970 & 1.7030850 \\ -1.4245160 & -3.8620600 \\ -2.1082310 & -2.8964260 \\ -1.8733130 & -5.4773590 \\ -1.2991670 & -5.1754390 \\ -0.9619950 & -3.6295970 \\ -2.1927060 & -1.9031060 \\ -1.7851240 & -6.4751180 \\ -0.7437890 & -5.9298070 \\ -5.1078920 & -3.1726120 \\ -4.4470880 & -4.1270680 \\ -4.6725360 & -1.5191780 \\ -5.2225010 & -1.8483710 \\ -5.5512640 & -3.4139110 \\ -4.3526200 & -5.1399580 \\ -4.7708910 & -0.5082430 \\ -5.7531980 & -1.0969230 \\ 2.6811220 & -2.5750440 \\ 3.4141860 & -1.6006790 \\ 1.6410770 & -2.7229710 \\ 2.8517020 & -0.7825770 \\ 2.8398740 & -1.5053960 \\ 2.2550990 & -1.8313150 \\ 1.0548240 & -0.5629070 \\ 0.4330280 & -0.5205570 \\ 1.1274230 & -1.2473920\end{array}$

-1.2150900
-0.3551700
-2.4998970
-0.1206450
-1.9214010
-4.2189990
-3.1773050
-2.7188990
-3.9901360
-5.2356040
-3.3614190
-2.5322650
-4.8353130
3.0011300
1.7426790
2.1448310
3.2086440
3.8538880
1.6067790
2.2915450
4.2181830
2.1955760
1.5214520
0.4587320
1.6558980
3.1507200
1.9460230
0.0364300
2.2049440
-4.2352770
-3.5220490
-2.5188130
-3.7218730
-5.1966110
-3.9052000
-2.1365210
-4.2987700
-1.6498490
-0.8352510
-1.3637850
-0.3958720
-2.6140590
2.4848280
4.2857370
3.0355110
2.0764030

$\begin{array}{lr}\mathrm{H} & 0.7427960 \\ \mathrm{~S} & 2.5240260 \\ \mathrm{~S} & 3.3123180 \\ \mathrm{H} & 1.9808330 \\ \mathrm{C} & -0.6627800 \\ \mathrm{C} & -1.2832880 \\ \mathrm{C} & -0.7431680 \\ \mathrm{C} & 0.5123800 \\ \mathrm{H} & -1.1048150 \\ \mathrm{H} & -1.1990660 \\ \mathrm{H} & 0.9943950 \\ \mathrm{C} & 4.8911010 \\ \mathrm{C} & 5.5624770 \\ \mathrm{C} & 4.9067110 \\ \mathrm{C} & 3.4050350 \\ \mathrm{H} & 5.3833930 \\ \mathrm{H} & 6.6271010 \\ \mathrm{H} & 5.1470330 \\ \mathrm{H} & 3.2860710 \\ \mathrm{H} & 2.8981360 \\ \mathrm{H} & 5.0001660 \\ \mathrm{H} & 5.4964100 \\ \mathrm{H} & 5.2655440 \\ \mathrm{H} & -2.1995940 \\ \mathrm{H} & \end{array}$
$-1.3997420$
$-1.5126070$
$-2.7680190$
$-0.9641830$
0.8308180
0.8726460
0.1997550
0.1147230
1. 3644320
0.2441120
0.0856470
$-3.5961130$
$-2.8828290$
$-1.5279440$
$-3.8338190$
$-4.5553300$
$-2.7267180$
$-0.8169830$
$-4.5553210$
$-4.2500970$
$-2.9933650$
$-3.5117740$
$-1.0800790$

1.1139620

4.1875690

1. 5613760

$-2.6213010$

5. 1804780

3. 9248910

2.8391090

5.3747560

6.0156210

1. 8581550

6.3461680

$-2.3451800$

$-1.1693760$

$-0.8900870$

$-2.0640190$

$-2.5338750$

$-1.3697660$

$-1.6897890$

$-1.2462780$

$-2.9403770$

$-3.2546070$

$-0.2724190$

0.0395830

114

3. 7932760

\section{$\mathrm{TS} 27(\mathrm{~S}, \mathrm{~S}) \mathrm{G}[\mathrm{WB} 97 \mathrm{XD} / 6-31 \mathrm{G}(\mathrm{d})]=-3918.016072$}

$\begin{array}{lrrr}\mathrm{O} & 0.3402420 & 1.4584420 & 0.6426820 \\ \mathrm{O} & -1.6111730 & 0.4226630 & -0.6155910 \\ \mathrm{C} & 0.2973620 & 2.5005390 & -0.2626020 \\ \mathrm{C} & -0.9184190 & 3.0892850 & -0.5446340 \\ \mathrm{C} & 1.5199600 & 2.9348470 & -0.8396640 \\ \mathrm{C} & -0.9691650 & 4.1087170 & -1.5495830 \\ \mathrm{C} & 1.4701560 & 3.9564170 & -1.7537260 \\ \mathrm{C} & 0.2443600 & 4.5478790 & -2.1474590 \\ \mathrm{H} & 2.3946000 & 4.3155890 & -2.1983050 \\ \mathrm{C} & -2.4587560 & 1.2617260 & 0.0676600 \\ \mathrm{C} & -3.6358610 & 0.6992730 & 0.6252980 \\ \mathrm{C} & -2.1459170 & 2.6009680 & 0.1439930 \\ \mathrm{C} & -4.4841660 & 1.5380490 & 1.3022920 \\ \mathrm{C} & -2.9967000 & 3.4552200 & 0.9159890 \\ \mathrm{C} & -4.1838530 & 2.9121020 & 1.4833580 \\ \mathrm{H} & -5.3961870 & 1.1365310 & 1.7363530 \\ \mathrm{C} & -4.7250260 & 5.0681880 & 2.4567050 \\ \mathrm{C} & -3.5311710 & 5.6020250 & 1.9190710 \\ \mathrm{C} & -2.6904840 & 4.8191670 & 1.1702200 \\ \mathrm{C} & -5.0389070 & 3.7516210 & 2.2446710\end{array}$




-5.3821530
-3.2766510
-1.7739830
-5.9449650
-2.1948070
-0.9837890
0.2069920
-2.1894270
-3.1383490
-1.0029200
1.1438720
-3.1266080
2.8378420
3.4896950
3.4505610
4.7940330
4.7633220
5.4075350
-3.9259360
-3.8335400
-4.2969490
-4.1642630
-4.5989170
-4.5430140
-0.2089910
0.6421850
-0.4359550
-4.7814660
6.3926410
-3.2816220
-3.3752540
-4.0636930
-3.6386610
-2.9181140
-3.0754800
-4.3225760
-3.5585990
-4.7167280
-4.3865720
-4.9457330
-4.9948180
-4.7683860
-4.1831680
-5.1654700
-5.2521350
3.5262740

5.7003270

6.6410470

5.2394780

3. 3244280

5.6462530

6.1068140

5.5637820

4.6750940

6.0623660

6.8798620

5.8959330

4.3238480

2. 3660810

1.3971500

2.8685540

0.9360350

2.4179610

1.4733330

$-0.7566490$

$-1.6072170$

$-1.2728950$

$-2.9974660$

$-2.6693290$

$-3.4932400$

0.0103570

$-0.5271180$

$-0.8248310$

$-4.5500500$

1.1148970

$-2.0055480$

$-1.1516520$

$-3.8567150$

$-3.3784010$

$-1.6407260$

$-0.1161520$

$-4.9054900$

$-4.0407070$

$-0.9877970$

$-0.4545260$

$-3.1896730$

$-2.3788470$

$-0.3487040$

0.6077410

$-4.2511190$

$-2.7857400$

$-0.1597440$

3.0459060
2.1050920
0.7707500
2.6663230
-2.9765250
-3.5427590
-3.1384020
-2.0086850
-3.3164970
-4.3046190
-3.5778110
-1.5917300
-0.4228930
-1.2083430
0.7419950
-0.8216400
1.1035470
0.3068880
0.4764180
1.5950150
-0.7805240
1.4559220
-0.9121680
0.2112830
0.1137060
-1.0003650
1.3415720
0.1082500
0.5973050
3.9326600
2.8744980
2.5950090
3.7969000
4.8885700
2.9859160
2.4729260
4.6538050
-3.1631360
-1.9526880
-2.1999000
-3.2938450
-4.0393500
-1.8694990
-2.2850980
-4.2670710
-3.0790690

\begin{abstract}
2.8936960
5.4240500

4.8077620

3.0434070

1.9060380

6.3998350

5.2844850

4.7238970

5.3727330

2. 8109890

3.4221150

5.1935510

6.3662100

1. 8199860

2. 9138860

$-0.0057650$

$-0.3157290$

0.8417910

$-0.0851090$

$-1.2395410$

3.0005570

4.5816770

3.5180750

2. 6989140

1. 9104030

4.4671780

2.0857310

$-1.0160550$

5. 3445170

4.2616070

3.3361330

5.5178100

6.0585130

2. 5117360

6. 3488320

$-1.2931050$

$-1.2078990$

$-1.2172490$

$-0.1160370$

$-1.2956460$

$-2.0426860$

$-2.2206950$

0.8273300

$-0.2141880$

$-2.2318760$

$-0.2846310$
\end{abstract}

0.8235720
-0.0972400
-0.6360390
-0.6032600
1.1541630
-0.4528850
-1.4328280
3.8286390
2.9279060
3.8257540
4.2835720
4.2061970
2.5721050
4.1832600
5.0016630
-3.8948610
-3.4544240
-3.4038220
-2.4256230
-3.2030580
-3.2040270
-2.6603580
-1.7641290
-2.0870400
-1.4668120
-3.9116180
-3.9052100
-2.2645740
-1.4451710
-0.5665200
-0.7101280
-2.5019280
-1.3049040
-0.0149430
-3.1909790
-6.0165350
-5.7504410
-4.2508300
-5.3699960
-7.0940700
-6.2280360
-3.8270950
-5.8401990
-5.4917680
-5.6115570
-6.1925420

$-2.3786960$

$-1.5856400$

$-2.6742810$

$-3.9439690$

$-2.6766750$

$-1.2648820$

$-3.2373330$

3.0833460

2.2930790

1.5951320

2.7246330

3.9866520

2. 5545570

1. 3360640

3. 3610820

0.4131690

$-0.9544100$

0.8909880

$-1.2156800$

0.6741450

$-0.9449910$

0.9297900

0.7926900

$-0.2808540$

$-0.5847530$

$-0.2947320$

$-2.1777670$

0.9878960

2.8334950

2.7068290

1.6841560

1.9472520

3.6387050

1. 5648740

2.0541490

$-0.0178010$

$-1.5233470$

$-1.8317610$

0.7186490

0.1740580

$-2.0459870$

$-1.6880980$

0.4145670

1.8020370

0.3798200

$-1.9178140$ 


$\begin{array}{lrrr}\text { H } & -0.9547660 & -4.0404050 & -2.8723160 \\ \mathrm{H} & 4.1509140 & 0.2547660 & 3.4070450 \\ \mathrm{I} 44 & & & \\ \mathrm{TS} 92(\mathrm{R}, \mathrm{R}) \mathrm{G}[\mathrm{WB} 97 \times \mathrm{D} / 6-31 \mathrm{G}(\mathrm{d})]=-3310.756396 \\ \mathrm{O} & 0.4481060 & 0.8497000 & 0.3390420 \\ \mathrm{O} & -1.7372660 & 0.8477790 & -0.9264470 \\ \mathrm{C} & 0.4071330 & 2.2261270 & 0.3000310 \\ \mathrm{C} & -0.7454210 & 2.8777540 & 0.6845890 \\ \mathrm{C} & 1.5908070 & 2.9064270 & -0.0861020 \\ \mathrm{C} & -0.7738770 & 4.3099830 & 0.6216480 \\ \mathrm{C} & 1.5918370 & 4.2758030 & -0.0197370 \\ \mathrm{C} & 0.4245980 & 5.0084010 & 0.3093950 \\ \mathrm{H} & 2.4995040 & 4.8186570 & -0.2690680 \\ \mathrm{C} & -2.4098280 & 1.0985920 & 0.2564970 \\ \mathrm{C} & -3.5885600 & 0.3506670 & 0.5191200 \\ \mathrm{C} & -1.9351280 & 2.0849700 & 1.0935100 \\ \mathrm{C} & -4.2820060 & 0.6450240 & 1.6648400 \\ \mathrm{C} & -2.5994570 & 2.2940130 & 2.3460810 \\ \mathrm{C} & -3.8024940 & 1.5850820 & 2.6115050 \\ \mathrm{H} & -5.2148310 & 0.1270850 & 1.8686050 \\ \mathrm{C} & -3.9874470 & 2.6737580 & 4.7721400 \\ \mathrm{C} & -2.7671980 & 3.3451410 & 4.5295470 \\ \mathrm{C} & -2.0920600 & 3.1616110 & 3.3501520 \\ \mathrm{C} & -4.4879870 & 1.8091850 & 3.8342750 \\ \mathrm{H} & -4.5150910 & 2.8335970 & 5.7074880 \\ \mathrm{H} & -2.3597640 & 4.0095620 & 5.2856160 \\ \mathrm{H} & -1.1519110 & 3.6746860 & 3.1818000 \\ \mathrm{H} & -5.4142410 & 1.2705370 & 4.0170070 \\ \mathrm{C} & -1.9355240 & 6.4384680 & 0.7801750 \\ \mathrm{C} & -0.7317540 & 7.1310680 & 0.5162340 \\ \mathrm{C} & 0.4199360 & 6.4278590 & 0.2804010 \\ \mathrm{C} & -1.9575090 & 5.0682440 & 0.8318000 \\ \mathrm{H} & -2.8540140 & 6.9959450 & 0.9369820 \\ \mathrm{H} & -0.7279300 & 8.2163640 & 0.4839530 \\ \mathrm{H} & 1.3479100 & 6.9451730 & 0.0507310 \\ \mathrm{H} & -2.8914880 & 4.5524360 & 1.0238250 \\ \mathrm{C} & 2.7798040 & 2.1576080 & -0.5925260 \\ \mathrm{C} & 3.0255130 & 2.1216670 & -1.9799530 \\ \mathrm{C} & 3.6593150 & 1.5248240 & 0.3006450 \\ \mathrm{C} & 4.1733830 & 1.4787020 & -2.4383380 \\ \mathrm{C} & 4.7933190 & 0.8912430 & -0.2095770 \\ \mathrm{C} & 5.0725160 & 0.8616930 & -1.5729680 \\ \mathrm{H} & 4.3698740 & 1.4605000 & -3.5060030 \\ \mathrm{H} & 5.4847450 & 0.4052180 & 0.4771190 \\ \mathrm{C} & -4.0614670 & -0.6963890 & -0.1125690 \\ \mathrm{C} & -3.9301380 & -2.0628970 & \\ & & & \end{array}$

-4.6491340
-4.4158460
-5.1093800
-5.0058220
-4.3243780
-5.5662730
3.4256970
6.3428640
2.1016140
-3.3010050
-5.5184650
-4.8190640
-6.3029490
-6.7803990
-6.8474670
-6.4145620
-4.0368670
-2.9766810
-4.4177200
-4.1288080
-6.6558800
-7.0525000
-6.3032730
-7.4756420
-4.3853840
-3.5844050
-3.9494160
-4.7589700
-4.3816790
-5.0235470
-5.0228910
-3.9224560
-2.3601360
-2.9057090
-1.6259280
-1.8232050
4.5656210
5.5171220
4.6989020
4.3499420
3.2111450
2.3166820
4.0702000
3.0692790
7.4427560
8.3741700
$-1.6525360$

$-1.0116560$

$-2.5238600$

$-2.2228810$

$-0.7637090$

$-3.4666020$

1.8044530

$-2.0853190$

$-2.9856050$

1. 1938280

$-3.1851820$

$-2.0440140$

$-2.1801460$

$-2.9948480$

$-1.2560930$

$-2.3953370$

$-3.3197950$

-3. 1950890

-4.1817010

$-3.5486820$

$-2.5627440$

$-3.2855790$

$-1.6887320$

$-2.2372550$

$-3.6750950$

$-4.1390720$

$-2.8437010$

$-4.4114940$

2. 2311100

2. 4467310

1.8682440

3. 1719880

1. 0125040

0.7621570

0.2280190

1.9487730

2. 5394320

2. 4208580

2. 1548520

3. 6117350

2. 3485190

1. 9075390

2.1389560

3. 4350040

-2.2866110
-2.6243230 


$\begin{array}{rrr}7.1355830 & 1.9907100 & -3.0422530 \\ 7.6445070 & 1.7986650 & -1.3560020 \\ 6.1231140 & -0.6053060 & -3.3678530 \\ 5.2688220 & -1.2831490 & -3.2721350 \\ 5.9323270 & 0.0486070 & -4.2260470 \\ 7.0148940 & -1.1970180 & -3.6009200 \\ 1.8298140 & 1.9436510 & -4.2291040 \\ 2.7302710 & 1.8055120 & -4.8387710 \\ 1.4353290 & 0.9662920 & -3.9462000 \\ 1.0851010 & 2.4426990 & -4.8589420 \\ 2.6682290 & 4.1652000 & -3.3991040 \\ 3.6411060 & 4.0444230 & -3.8908640 \\ 1.9917270 & 4.6641810 & -4.1019960 \\ 2.8084250 & 4.8248420 & -2.5376150 \\ -0.3680850 & -0.0175850 & -0.7881310 \\ 0.3294930 & 0.0036460 & -2.1123780 \\ -0.6321180 & -1.3451560 & -0.1257850 \\ 1.1359940 & 2.9635270 & -2.4989890 \\ 6.6888930 & -0.4880440 & -1.3065300 \\ 2.5093180 & 2.0924340 & 2.0054550 \\ -4.4117990 & 1.7718020 & -1.2413750 \\ -5.9261310 & -3.1848680 & -4.0598670 \\ -2.6946720 & -1.7109110 & 1.5856650 \\ 2.6238170 & -2.0023300 & -0.9650810 \\ 1.5819560 & -3.0155290 & -1.1655550 \\ 2.3619800 & -1.1712740 & -0.3068410 \\ 0.5536170 & -2.6723280 & -1.1154990 \\ 2.3307370 & -1.7026330 & -2.3448680 \\ 1.6198810 & -0.9884850 & -2.3465530 \\ 4.3511900 & -3.7320030 & -1.6132010 \\ 3.3136000 & -4.8373480 & -1.4071140 \\ 1.9009970 & -4.3504020 & -1.7411470 \\ 4.0294460 & -2.5099260 & -0.7501730 \\ 5.3504160 & -4.1010990 & -1.3613590 \\ 3.5496760 & -5.7095170 & -2.0244810 \\ 1.7639160 & -4.2531370 & -2.8253180 \\ 4.1106450 & -2.7682430 & 0.3136470 \\ 4.7265620 & -1.6921110 & -0.9383630 \\ 4.3760220 & -3.4396670 & -2.6700450 \\ 3.3320100 & -5.1637120 & -0.3614170 \\ 1.1346190 & -5.0503180 & -1.3975510 \\ 1.2773550 & -3.0950960 & 1.9170310 \\ 0.4577550 & -2.0887650 & 2.0489910 \\ 2.0349210 & -3.4351820 & 3.1502250 \\ 2.9687600 & -4.4726170 & 3.1028240 \\ 1.8454030 & -2.7164780 & 4.3336440\end{array}$

$\begin{array}{ll}\mathrm{C} & 3.7103750 \\ \mathrm{H} & 3.1024110 \\ \mathrm{C} & 2.5889370 \\ \mathrm{H} & 1.1208820 \\ \mathrm{C} & 3.5200690 \\ \mathrm{H} & 4.4372200 \\ \mathrm{H} & 2.4420000 \\ \mathrm{H} & 4.0991150 \\ \mathrm{H} & 0.0046960 \\ \mathrm{O} & 1.4511480 \\ \mathrm{H} & \end{array}$
$-4.7914070$
$-5.0194960$
$-3.0389450$
$-1.9104040$
$-4.0746060$
$-5.5967040$
$-2.4830630$
$-4.3238480$
$-1.8237230$
$-3.7308500$

144

TS93 $(R, R) G[W B 97 X D / 6-31 G(d)]=-3310.755282$

\begin{tabular}{|c|c|c|c|}
\hline \multicolumn{4}{|c|}{$\operatorname{TS} 93(R, R) G[W B 97 X D / 6-31 G(d)]=-3310.755282$} \\
\hline 0 & 0.5029080 & 0.5920050 & 0.0724940 \\
\hline 0 & -1.7612480 & 0.8444950 & -1.0454370 \\
\hline C & 0.6426270 & 1.9593080 & -0.0004500 \\
\hline C & -0.3986130 & 2.7701340 & 0.4018670 \\
\hline C & 1.9004760 & 2.4685760 & -0.4140370 \\
\hline C & -0.2530290 & 4.1897160 & 0.2719830 \\
\hline C & 2.0651630 & 3.8305980 & -0.42 \\
\hline C & 1.0050370 & 4.7185070 & -0.1244480 \\
\hline $\mathrm{H}$ & 3.0310880 & 4.2434640 & -0.7086560 \\
\hline C & -2.3129870 & 1.222 & 0.16 \\
\hline C & -3.5594810 & 0.6525570 & 0.53 \\
\hline $\mathrm{C}$ & -1.6504040 & 2.1611910 & 0.92 \\
\hline $\mathrm{C}$ & -4.1113260 & 1.0620310 & 1.7236040 \\
\hline C & -2.1814090 & 2.4956880 & 2.2121130 \\
\hline C & -3.4373850 & 1.9524690 & 2.5973230 \\
\hline $\mathrm{H}$ & -5.0830730 & 0.6755340 & 2.0179350 \\
\hline $\mathrm{C}$ & -3.3060760 & 3.1106730 & 4.7252900 \\
\hline $\mathrm{C}$ & -2.0392740 & 3.6205660 & 4.3600660 \\
\hline C & -1.4916610 & 3.3230940 & 3.1383010 \\
\hline C & -3.9854530 & 2.2916650 & 3.8619760 \\
\hline $\mathrm{H}$ & -3.7311540 & 3.3588390 & 5.6930310 \\
\hline $\mathrm{H}$ & -1.4917730 & 4.2499320 & 5.0552010 \\
\hline $\mathrm{H}$ & -0.5151410 & 3.7142960 & 3150 \\
\hline $\mathrm{H}$ & -4.9510320 & 1.8755220 & 4.1373880 \\
\hline C & -1.1375930 & 6.4505740 & 0.3682150 \\
\hline C & 0.1261230 & 6.9750030 & 0.0114730 \\
\hline C & 1.1711930 & 6.1239510 & -0.23 \\
\hline $\mathrm{C}$ & -1.3233830 & 5.0980320 & 0.4954750 \\
\hline $\mathrm{H}$ & -1.9707590 & 7.1263650 & 52690 \\
\hline $\mathrm{H}$ & 0.25 & 8.0482740 & -0.0824040 \\
\hline $\mathrm{H}$ & 2.14 & 6.5113970 & 9530 \\
\hline $\mathrm{H}$ & -2.30 & 4.7108840 & 7573690 \\
\hline C & 3.040 & 1.5830880 & 8025490 \\
\hline & 3 & 200 & -6 \\
\hline
\end{tabular}




$\begin{array}{rrr}3.8291350 & 0.9766160 & 0.1951760 \\ 4.5590810 & 0.7620100 & -2.4877720 \\ 4.9891910 & 0.3086830 & -0.1847720 \\ 5.3896010 & 0.2129330 & -1.5184730 \\ 4.8226990 & 0.6641060 & -3.5358200 \\ 5.6132470 & -0.1521460 & 0.5777210 \\ -4.2493380 & -0.3284870 & -0.3535520 \\ -4.3058580 & -1.6922460 & -0.0008250 \\ -4.8548740 & 0.1221160 & -1.5385820 \\ -4.9893760 & -2.5693680 & -0.8402380 \\ -5.5188460 & -0.7989830 & -2.3494280 \\ -5.5993000 & -2.1467010 & -2.0198270 \\ -5.0422430 & -3.6210240 & -0.5695640 \\ -5.9892600 & -0.4544360 & -3.2682490 \\ 3.4564420 & 1.0770020 & 1.6672830 \\ 6.7047250 & -0.4793800 & -1.8426470 \\ 2.5002010 & 1.9916840 & -3.2699750 \\ -3.6700830 & -2.2317260 & 1.2745330 \\ -6.3162810 & -3.1305290 & -2.9268500 \\ -4.8312300 & 1.5854020 & -1.9575810 \\ -6.2455580 & 2.1814920 & -1.9601310 \\ -6.8848440 & 1.6903060 & -2.7028640 \\ -6.7205470 & 2.0675210 & -0.9797690 \\ -6.2096520 & 3.2497350 & -2.2021750 \\ -4.1385000 & 1.7760990 & -3.3125640 \\ -3.1285300 & 1.3576460 & -3.2895880 \\ -4.6956080 & 1.2879510 & -4.1207480 \\ -4.0656860 & 2.8426090 & -3.5540480 \\ -7.4895990 & -3.8127390 & -2.2115990 \\ -8.0275460 & -4.4761940 & -2.8980270 \\ -7.1388070 & -4.4184040 & -1.3679190 \\ -8.1973580 & -3.0732450 & -1.8230030 \\ -5.3423080 & -4.1687310 & -3.5005070 \\ -4.5138630 & -3.6821410 & -4.0251240 \\ -4.9163400 & -4.7886610 & -2.7030590 \\ -5.8559970 & -4.8333670 & -4.2043840 \\ -4.7291310 & -2.4308130 & 2.3688900 \\ -5.2502830 & -1.4977320 & 2.6033780 \\ -5.4811290 & -3.1617160 & 2.0481360 \\ -4.2655640 & -2.8026540 & 3.2898750 \\ -2.8978020 & -3.5380820 & 1.0479930 \\ -3.5695560 & -4.3798510 & 0.8432890 \\ -2.2016330 & -3.4328540 & 0.2128820 \\ -2.3252280 & -3.7878540 & 1.9483330 \\ 4.1510480 & 2.2812710 & 2.3179760 \\ 5.2405820 & 2.1691680 & \\ -5 & & \\ -5 & & \\ -4 & -2653990\end{array}$

3.8667500

3.7504950

3. 3694660

4.8243060

3. 2729330

7.8851630

8.8396490

7.8760320

7.8336530

6.8437530

6.0052670

6.8992600

7.7660890

2. 3024100

3. 2214850

1.9769360

1. 5348070

3. 0601700

4. 0618880

2. 4131550

3. 1319960

$-0.5143710$

0.0762050

$-0.9001470$

1. 5114950

6. 7513700

2. 3772700

$-4.2527650$

$-6.7270460$

$-2.9473680$

2. 4020790

1. 3585270

2. 3296770

0.3670970

1. 7184610

1. 0489960

3. 9031230

2. 9992230

1.5391370

3. 8194950

4. 9417500

3. 0813310

1.1240960

4.2592630
4.3752420
3.2134400

2. 3695290

$-0.2113870$

$-1.0886220$

$-0.3572950$

$-0.1776850$

0.4211470

$-0.0880110$

1. 3438130

0.7003770

$-0.9229050$

$-1.5505920$

$-0.0626040$

$-1.4986650$

0.9854430

0.8394430

0.0170530

1. 3521750

3. 3124690

3. 1634250

3. 7016240

4.0746370

$-0.1928470$

$-0.3220010$

$-1.4389070$

2. 1892290

$-1.3818990$

1. 2432300

2. 1474840

$-2.5592790$

$-1.4922700$

$-2.2641520$

$-3.2167070$

$-1.2659470$

$-2.8137680$

$-2.3540980$

$-1.6000980$

$-4.2389500$

$-5.1285410$

$-4.6831910$

$-2.7792860$

$-4.5781980$

$-6.1760100$

$-4.9236920$

-2.6638910
-2.1223860
1.8113000

3. 3726220

2. 4433660

1. 9111110

3. 4280710

$-1.4450760$

$-1.6194440$

$-2.0368920$

$-0.3878430$

$-3.3003480$

$-3.6214240$

$-3.9772100$

$-3.4286470$

$-4.4104830$

$-4.9898640$

$-4.0253190$

$-5.0999230$

$-3.8159740$

$-4.2371340$

$-4.6100700$

$-3.0343590$

$-0.9525520$

$-2.3220080$

$-0.1954820$

$-2.8452630$

$-1.2163240$

1.7314890

$-1.2192760$

$-3.7693210$

1.6296600

$-1.3486970$

$-0.9517210$

$-0.9123900$

$-0.7767350$

$-2.6194260$

$-2.6298660$

$-1.8795480$

$-1.0245810$

$-1.1278540$

$-1.4277940$

$-1.8112950$

$-1.3302910$

$-2.1151260$

$-0.4295250$

$-2.0999680$ 


\begin{tabular}{|c|c|c|c|c|c|c|c|}
\hline $\mathrm{H}$ & 3.6100290 & -4.3163230 & -2.9336710 & C & 1.1711760 & 6.1239880 & -0.2331900 \\
\hline $\mathrm{H}$ & 3.3116190 & -5.0686600 & 0.0244560 & $\mathrm{C}$ & -1.3234150 & 5.0980240 & 0.4957550 \\
\hline $\mathrm{H}$ & 0.8991060 & -5.1863630 & -0.3979230 & $\mathrm{H}$ & -1.9707900 & 7.1263550 & 0.5366650 \\
\hline C & 1.1148070 & -2.6987370 & 2.1741770 & H & 0.2597970 & 8.0483030 & -0.0818960 \\
\hline 0 & 0.0183820 & -1.9939500 & 2.1286250 & $\mathrm{H}$ & 2.1425890 & 6.5114520 & -0.5295080 \\
\hline C & 1.6739790 & -2.8633650 & 3.5437950 & $\mathrm{H}$ & -2.3017390 & 4.7108650 & 0.7576030 \\
\hline C & 2.8544400 & -3.5905160 & 3.7092890 & C & 3.0406260 & 1.5831690 & -0.8025080 \\
\hline $\mathrm{C}$ & 1.0567320 & -2.2701750 & 4.6476410 & c & 3.3786610 & $\begin{array}{l}1.4354970 \\
\text { l. }\end{array}$ & -2.1572120 \\
\hline C & 3.4174770 & -3.7229120 & 4.9720620 & $\mathrm{C}$ & 3.8291420 & 0.9766360 & 0.1951400 \\
\hline $\mathrm{H}$ & 3.3242530 & -4.0385480 & 2.8403000 & $\mathrm{c}$ & 4.5589600 & 0.7622120 & -2.4878610 \\
\hline C & 1.6220440 & -2.4067400 & 5.9104850 & $\mathrm{C}$ & 4.9891870 & 0.3087370 & -0.1849070 \\
\hline $\mathrm{H}$ & 0.1429530 & -1.7034200 & 4.5096020 & $\mathrm{C}$ & 5.3895270 & 0.2130730 & -1.5186330 \\
\hline C & 2.8017070 & -3.1299410 & 6.0730270 & $\mathrm{H}$ & 4.8225340 & 0.6643770 & -3.5359260 \\
\hline $\mathrm{H}$ & 4.3382560 & -4.2835390 & 5.0991920 & $\mathrm{H}$ & 5.6132900 & -0.1521310 & 0.5775240 \\
\hline $\mathrm{H}$ & 1.1439490 & -1.9447520 & 6.7685040 & C & -4.2493290 & -0.3284850 & -0.3535970 \\
\hline $\mathrm{H}$ & 3.2437970 & -3.2315680 & 7.0596670 & $\mathrm{C}$ & -4.3058470 & -1.6922540 & -0.0009110 \\
\hline H & -0.3128850 & -1.7983280 & 1.1876730 & C & -4.8548480 & 0.1221510 & -1.5386230 \\
\hline 0 & 1.6861520 & -3.1999050 & 1.1991150 & $\mathrm{C}$ & -4.9893360 & -2.5693570 & -0.8403670 \\
\hline & & & & C & -5.5187880 & -0.7989320 & -2.3495150 \\
\hline & 2) G[WB97XD, & (d) $]=-331$ & & $\mathrm{C}$ & -5.5992340 & -2.1466600 & -2.0199570 \\
\hline 0 & 0.5029040 & 0.5920250 & 0.0725830 & $\mathrm{H}$ & -5.0421980 & -3.6210220 & -0.5697280 \\
\hline 0 & -1.7612250 & 0.8445180 & -1.0453800 & $\mathrm{H}$ & -5.9891740 & -0.4543640 & -3.2683420 \\
\hline C & 0.6426090 & 1.9593300 & -0.0003200 & C & 3.4565080 & 1.0769130 & 1.6672680 \\
\hline $\mathrm{C}$ & -0.3986380 & 2.7701300 & 0.4020320 & $\mathrm{C}$ & 6.7046460 & -0.4791940 & -1.8429140 \\
\hline $\mathrm{C}$ & 1.9004490 & 2.4686270 & -0.4138930 & $\mathrm{C}$ & 2.5000510 & 1.9919760 & -3.2698800 \\
\hline $\mathrm{C}$ & -0.2530 & 4.1897210 & 0.2722300 & c & -3.6700960 & -2.2317630 & 1.2744470 \\
\hline C & 2.0651410 & 3.8306480 & -0.4296240 & $\mathrm{C}$ & -6.3161720 & -3.1304700 & -2.9270350 \\
\hline C & 1.0050150 & 4.7185380 & -0.1241450 & $\mathrm{C}$ & -4.8311790 & 1.5854440 & -1.9575940 \\
\hline $\mathrm{H}$ & 3.0310650 & 4.2435330 & -0.7083840 & C & -6.2455200 & 2.1815030 & -1.9604090 \\
\hline C & -2.3129970 & 1.2227200 & 0.1658520 & $\mathrm{H}$ & -6.8846710 & 1.6902730 & -2.7032270 \\
\hline $\mathrm{C}$ & -3.5595020 & 0.6525360 & 0.5372260 & $\mathrm{H}$ & -6.7206700 & 2.0675660 & -0.9801220 \\
\hline C & -1.6504370 & 2.1611550 & 0.9237390 & $\mathrm{H}$ & -6.2095940 & 3.2497360 & -2.2024950 \\
\hline C & -4.1113820 & 1.0619750 & 1.7236050 & C & -4.1382100 & 1.7761670 & -3.3124540 \\
\hline C & -2.1814740 & 2.4956040 & 2.2122170 & $\mathrm{H}$ & -3.1282860 & 1.3576150 & -3.2893440 \\
\hline $\mathrm{C}$ & -3.4374640 & 1.9 & 2.5973740 & $\mathrm{H}$ & -4.6952430 & 1.2881220 & -4.1207540 \\
\hline $\mathrm{H}$ & -5.0831420 & 0.6754740 & 2.0178910 & H & -4.0652550 & 2.8426860 & -3.5538560 \\
\hline C & -3.3062010 & 3.1105080 & 4.7253870 & C & -7.4894390 & -3.8128040 & -2.2118190 \\
\hline $\mathrm{C}$ & -2.0393840 & 3.6204020 & 4.3602150 & $\mathrm{H}$ & -8.0273970 & -4.4761850 & -2.8983100 \\
\hline $\mathrm{C}$ & -1.4917420 & 3.3229690 & 3.1384530 & $\mathrm{H}$ & -7.1385960 & -4.4185680 & -1.3682320 \\
\hline C & -3.9855630 & & 3.8620250 & $\mathrm{H}$ & -8.1972030 & -3.0733800 & -1.8230980 \\
\hline $\mathrm{H}$ & -3.7313010 & 3.3586430 & 5.6931260 & C & -5.3421390 & -4.1685700 & -3.5007750 \\
\hline H & -1.4918950 & 4.2497370 & 5.0553880 & $\mathrm{H}$ & -4.5137860 & -3.6818900 & -4.0254560 \\
\hline $\mathrm{H}$ & -0.5152120 & 3.7141710 & 2.8755050 & $\mathrm{H}$ & -4.9160390 & -4.7884530 & -2.7033600 \\
\hline $\mathrm{H}$ & -4.9511520 & 1.8753920 & 4.1373980 & $\mathrm{H}$ & -5.8558120 & -4.8332580 & -4.2046140 \\
\hline C & -1.1376210 & 05 & 0.3685860 & C & -4.7291650 & -2.4308620 & 2.3687830 \\
\hline C & 0.1261030 & 750270 & 0.0119070 & $\mathrm{H}$ & -5.2502510 & -1.4977620 & 2.6033420 \\
\hline
\end{tabular}




$\begin{array}{rr}-5.4812100 & -3.1616890 \\ -4.2656280 & -2.8028060 \\ -2.8978260 & -3.5381240 \\ -3.5695910 & -4.3798980 \\ -2.2016930 & -3.4329220 \\ -2.3252140 & -3.7878770 \\ 4.1512000 & 2.2810840 \\ 5.2407280 & 2.1689640 \\ 3.8806160 & 3.2133190 \\ 3.8669960 & 2.3692230 \\ 3.7505090 & -0.2115700 \\ 3.3693980 & -1.0887260 \\ 4.8243170 & -0.3575700 \\ 3.2729890 & -0.1779380 \\ 7.8850810 & 0.4214170 \\ 8.8395750 & -0.0876870 \\ 7.8758160 & 1.3440680 \\ 7.8336890 & 0.7006730 \\ 6.8435290 & -0.9228110 \\ 6.0049880 & -1.5504850 \\ 6.8990060 & -0.0625560 \\ 7.7658310 & -1.4986140 \\ 2.3023310 & 0.9859440 \\ 3.2214120 & 0.8401290 \\ 1.9769310 & 0.0174540 \\ 1.5346990 & 1.3527630 \\ 3.0599370 & 3.3128940 \\ 4.0617470 & 3.1640190 \\ 2.4130080 & 3.7020450 \\ 3.1315280 & 4.0749910 \\ -0.5143430 & -0.1928220 \\ 0.0762760 & -0.3219400 \\ -0.9001260 & -1.4389010 \\ 1.5113270 & 2.1893720 \\ 6.7514090 & -1.3816670 \\ 2.3773480 & 1.2431980 \\ -4.2528650 & 2.1475320 \\ -6.7269840 & -2.5591900 \\ -2.9473830 & -1.4923190 \\ 2.4020380 & -2.2641510 \\ 1.3585000 & -3.2167640 \\ 2.3295750 & -1.2659660 \\ 0.3670510 & -2.8138630 \\ 1.7185040 & -2.3540560 \\ 1.0490260 & -1.6000730 \\ 3.9031770 & -4.2388540\end{array}$

2.0479680
3.2897420
1.0479020
0.8432500
0.2127590
1.9482230
2.3180480
2.2653640
1.8115050
3.3727290
2.4432170
1.9108900
2.6075480
3.4279450
-1.4455230
-1.6200020
-2.0373630
-0.3882910
-3.3006010
-3.6215630
-3.9775230
-3.4289490
-4.4105820
-4.9900000
-4.0256190
-5.0999430
-3.8156500
-4.2366520
-4.6098170
-3.0339470
-0.9524960
-2.3219350
-0.1954600
-2.8451350
-1.2165340
1.7315250
-1.2191740
-3.7694630
1.6295990
-1.3485860
-0.9517100
-0.9122410
-0.7767460
-2.6193640
-2.6298060
-1.8794880

$\begin{array}{lr}\mathrm{C} & 2.9992630 \\ \mathrm{C} & 1.5391670 \\ \mathrm{C} & 3.8194770 \\ \mathrm{H} & 4.9418120 \\ \mathrm{H} & 3.0814140 \\ \mathrm{H} & 1.1241510 \\ \mathrm{H} & 4.2591740 \\ \mathrm{H} & 4.3752500 \\ \mathrm{H} & 3.6101470 \\ \mathrm{H} & 3.3116150 \\ \mathrm{H} & 0.8991400 \\ \mathrm{C} & 1.1147980 \\ \mathrm{O} & 0.0184180 \\ \mathrm{C} & 1.6740280 \\ \mathrm{C} & 2.8545940 \\ \mathrm{C} & 1.0567280 \\ \mathrm{C} & 3.4176860 \\ \mathrm{H} & 3.3244380 \\ \mathrm{C} & 1.6220890 \\ \mathrm{H} & 0.1428680 \\ \mathrm{C} & 2.8018580 \\ \mathrm{H} & 4.3385490 \\ \mathrm{H} & 1.1439500 \\ \mathrm{H} & 3.2439850 \\ \mathrm{H} & -0.3128710 \\ \mathrm{O} & 1.6860600 \\ \mathrm{H} & \end{array}$
$-5.1285470$
$-4.6832330$
$-2.7792300$
$-4.5780740$
$-6.1759840$
$-4.9236990$
$-2.6639030$
$-2.1222580$
$-4.3161520$
$-5.0687560$
$-5.1864640$
$-2.6989040$
$-1.9940460$
$-2.8635850$
$-3.5905900$
$-2.2705940$
$-3.7230380$
$-4.0384690$
$-2.4072200$
$-1.7039480$
$-3.1302710$
$-4.2835450$
$-1.9453940$
$-3.2319420$
$-1.7983810$

$-1.0246410$

$-1.1279250$

$-1.4276230$

$-1.8112020$

$-1.3304470$

$-2.1152160$

$-0.4293140$

$-2.0997050$

$-2.9336340$

0.0244140

$-0.3980300$

2. 1741130

2. 1286160

3.5436990

3.7090850

4.6476220

4.9718280

2.8400350

5.9104380

4. 5096660

6.0728710

5.0988750

6.7685190

7.0594900

1.1876800

144

1.1990230

$\operatorname{TS} 95(R, R) G[W B 97 X D / 6-31 G(d)]=-3310.756673$

$\begin{array}{lrrr}\text { TS95 }(\mathrm{R}, \mathrm{R}) \mathrm{G}[\mathrm{WB} 97 \times \mathrm{D} / 6-31 \mathrm{G}(\mathrm{d})]=-3310.756673 \\ \mathrm{O} & 0.0312900 & 1.0839180 & 0.1829220 \\ \mathrm{O} & -2.0906360 & 0.2475360 & -0.8866460 \\ \mathrm{C} & -0.2297810 & 2.3636500 & -0.2535210 \\ \mathrm{C} & -1.5017490 & 2.8727870 & -0.1136200 \\ \mathrm{C} & 0.8715420 & 3.1149870 & -0.7461380 \\ \mathrm{C} & -1.7647010 & 4.1789850 & -0.6427430 \\ \mathrm{C} & 0.6320140 & 4.4052150 & -1.1375930 \\ \mathrm{C} & -0.6781900 & 4.9514860 & -1.1385360 \\ \text { H } & 1.4573100 & 5.0300190 & -1.4661130 \\ \mathrm{C} & -2.7671930 & 0.7587390 & 0.2001100 \\ \mathrm{C} & -3.7277070 & -0.0840640 & 0.8187660 \\ \mathrm{C} & -2.5326550 & 2.0606240 & 0.5895250 \\ \mathrm{C} & -4.4807550 & 0.4483900 & 1.8320380 \\ \mathrm{C} & -3.2410110 & 2.5645310 & 1.7318180 \\ \mathrm{C} & -4.2501520 & 1.7567620 & 2.3259090 \\ \text { H } & -5.2594730 & -0.1554350 & 2.2899870 \\ \mathrm{C} & -4.7103090 & 3.4886070 & 3.9618130 \\ \mathrm{C} & -3.6754900 & 4.2739600 & 3.4051940\end{array}$




$\begin{array}{rrr}-2.9610390 & 3.8265330 & 2.3232050 \\ -4.9838730 & 2.2544400 & 3.4342240 \\ -5.2718510 & 3.8585200 & 4.8141950 \\ -3.4397980 & 5.2392930 & 3.8429550 \\ -2.1636330 & 4.4387880 & 1.9179600 \\ -5.7591970 & 1.6268380 & 3.8661020 \\ -3.2807400 & 5.9863290 & -1.2209430 \\ -2.1949200 & 6.7667530 & -1.6788700 \\ -0.9238180 & 6.2558370 & -1.6400380 \\ -3.0722030 & 4.7283700 & -0.7152410 \\ -4.2892040 & 6.3851110 & -1.2759440 \\ -2.3728570 & 7.7625240 & -2.0732600 \\ -0.0824770 & 6.8380680 & -2.0069540 \\ -3.9139740 & 4.1353010 & -0.3743970 \\ 2.2297800 & 2.4904350 & -0.7352350 \\ 2.8279940 & 2.0344620 & -1.9252540 \\ 2.8840780 & 2.3202090 & 0.4980670 \\ 4.0719370 & 1.4081330 & -1.8435400 \\ 4.1199650 & 1.6747600 & 0.5285310 \\ 4.7301300 & 1.2061260 & -0.6314090 \\ 4.5520280 & 1.0634580 & -2.7566670 \\ 4.6192160 & 1.5434110 & 1.4844160 \\ -3.9113380 & -1.4965390 & 0.3645270 \\ -3.3260980 & -2.5499360 & 1.0925470 \\ -4.6737470 & -1.7648840 & -0.7851510 \\ -3.5521600 & -3.8587860 & 0.6653370 \\ -4.8602370 & -3.0889000 & -1.1771990 \\ -4.3129010 & -4.1512660 & -0.4627940 \\ -3.1105850 & -4.6821840 & 1.2211530 \\ -5.4468050 & -3.2949040 & -2.0701380 \\ 2.3107960 & 2.8937910 & 1.7868980 \\ 6.1078470 & 0.5654750 & -0.6213310 \\ 2.1781640 & 2.2555970 & -3.2843100 \\ -2.4718020 & -2.2957100 & 2.3290940 \\ -4.5241120 & -5.5871740 & -0.9082300 \\ -5.2932590 & -0.6521830 & -1.6179810 \\ -6.8217170 & -0.7742170 & -1.6732640 \\ -7.1328610 & -1.6943370 & -2.1813480 \\ -7.2522760 & -0.7849350 & -0.6662770 \\ -7.2526100 & 0.0712750 & -2.2212040 \\ -4.6826960 & -0.6068120 & -3.0249690 \\ -3.5975210 & -0.4825290 & -2.9697760 \\ -4.8962020 & -1.5284440 & -3.5793360 \\ -5.0989500 & 0.2317930 & -3.5945900 \\ -3.8904710 & -5.8450390 & -2.2819550 \\ -4.0017660 & -6.8973410 & -2.5671170\end{array}$

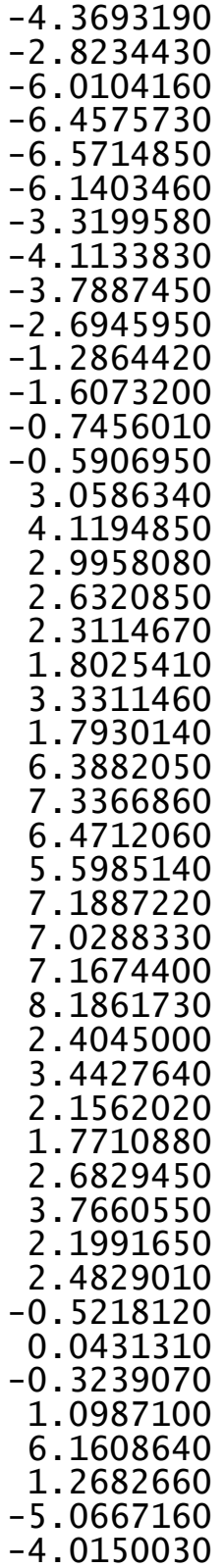

-5.2350030
-5.5997970
-5.9675960
-5.8036770
-5.3681630
-7.0228220
-2.3503840
-1.5982240
-3.3359410
-2.1726570
-3.2622490
-4.2655090
-3.3415320
-2.8979120
4.1818600
3.9774650
4.9184450
4.6284590
1.8940970
0.9701150
1.6467500
2.3217080
-0.2859670
-0.8211630
0.3272840
-1.0232980
1.6435250
2.2155530
2.3459320
1.1914130
1.1058680
1.0666600
0.1398350
1.2483300
3.5665220
3.5164350
3.7411240
4.4303750
-0.1496740
-0.1291940
-1.3704340
2.3384320
-0.0975140
3.1686080
0.3028820
-6.2333840

$-3.0565000$ $-2.2745060$ $-0.9069630$ 0.0788470 $-1.6331630$ $-1.1725390$

3.6083130

3.6009540

3.7166280

4.4908260

2.4511730

2.7561510

1.5056380

3.2150310

2.1596090

2. 3471790

1. 3514000

3.0650030

2.9457560

2.6568140

3.2647800

3.8108490

0.6196820

0.5031850

1.5237960

0.7942170

$-0.7956240$

$-1.7153050$

0.0458330

$-0.8394640$

$-4.2711310$

$-4.6223480$

$-3.8289010$

$-5.1529470$

$-3.9088630$

$-4.0738760$

$-4.8766160$

$-3.2695590$

$-0.7333210$

$-2.1231430$

0.1278560

$-3.1263540$

$-1.4956100$

1.6088790

$-1.1347550$

$-0.1813130$ 


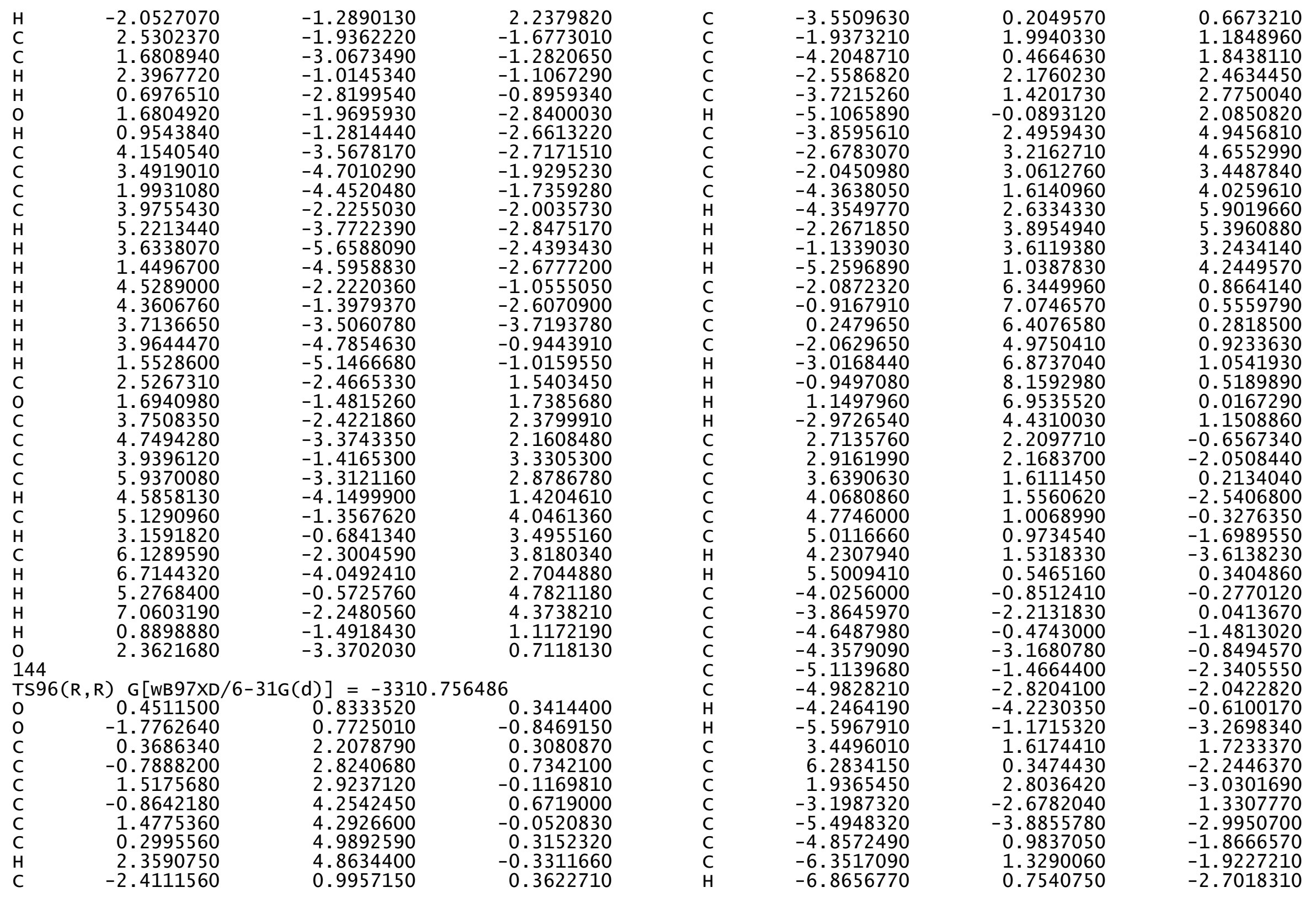




$\begin{array}{lrr}-6.8400360 & 1.1111200 & -0.9668670 \\ -6.4912140 & 2.3932040 & -2.1441700 \\ -4.1501600 & 1.3281170 & -3.1834740 \\ -3.0826280 & 1.1008390 & -3.1181350 \\ -4.5729480 & 0.7661620 & -4.0244250 \\ -4.2637490 & 2.3952730 & -3.4055350 \\ -4.7264080 & -3.8584720 & -4.3230790 \\ -5.0698150 & -4.6599970 & -4.9870110 \\ -4.8757020 & -2.9047490 & -4.8423360 \\ -3.6517160 & -3.9837160 & -4.1568850 \\ -7.0060140 & -3.7603100 & -3.2284040 \\ -7.5540260 & -3.7993480 & -2.2814500 \\ -7.2523810 & -2.8125700 & -3.7206840 \\ -7.3660060 & -4.5725370 & -3.8698720 \\ -4.2514940 & -3.0876270 & 2.3715930 \\ -4.9417040 & -2.2690640 & 2.5969940 \\ -4.8464930 & -3.9332960 & 2.0060870 \\ -3.7687790 & -3.3919940 & 3.3073930 \\ -2.2064670 & -3.8283960 & 1.1139220 \\ -2.7134170 & -4.7609070 & 0.8400300 \\ -1.4858160 & -3.5788060 & 0.3324370 \\ -1.6574120 & -4.0188910 & 2.0428720 \\ 4.5966080 & 2.3563720 & 2.4254380 \\ 5.5524250 & 1.8377060 & 2.2883900 \\ 4.7067770 & 3.3721410 & 2.0312410 \\ 4.4053330 & 2.4245290 & 3.5020710 \\ 3.2760230 & 0.1970680 & 2.2768980 \\ 2.3803240 & -0.2712360 & 1.8597240 \\ 4.1430180 & -0.4346550 & 2.0479790 \\ 3.1608600 & 0.2191530 & 3.3665400 \\ 7.3553420 & 1.4239080 & -2.4692580 \\ 8.2878990 & 0.9760090 & -2.8309170 \\ 7.0148920 & 2.1515800 & -3.2151110 \\ 7.5682150 & 1.9658930 & -1.5421600 \\ 6.0510370 & -0.4665840 & -3.5229800 \\ 5.2156120 & -1.1647200 & -3.4076360 \\ 5.8244400 & 0.1834420 & -4.3753850 \\ 6.9505010 & -1.0370720 & -3.7784260 \\ 1.6707140 & 1.9399270 & -4.2688260 \\ 2.5514500 & 1.8675740 & -4.9176770 \\ 1.3550070 & 0.9359130 & -3.9805960 \\ 0.8679200 & 2.3915750 & -4.8618620 \\ 2.4272960 & 4.1955940 & -3.4567200 \\ 3.3965830 & 4.1212890 & -3.9643890 \\ 1.7149830 & 4.6567990 & -4.1500740 \\ 2.5480480 & 4.8651850 & -2.6000840\end{array}$

$\begin{array}{lrr}\mathrm{P} & -0.3800120 & -0.0553650 \\ \mathrm{O} & 0.2715100 & -0.0147820 \\ \mathrm{O} & -0.5859950 & -1.3890080 \\ \mathrm{H} & 0.9780620 & 2.9230090 \\ \mathrm{H} & 6.6636580 & -0.3400710 \\ \mathrm{H} & 2.5293870 & 2.1649620 \\ \mathrm{H} & -4.4158470 & 1.6143430 \\ \mathrm{H} & -5.3121050 & -4.8604090 \\ \mathrm{H} & -2.6278300 & -1.8379660 \\ \mathrm{C} & 2.6546600 & -1.9550420 \\ \mathrm{C} & 1.6379770 & -2.9994740 \\ \mathrm{H} & 2.3879070 & -1.1289990 \\ \mathrm{H} & 0.6018450 & -2.6867650 \\ \mathrm{O} & 2.3118740 & -1.6708480 \\ \mathrm{H} & 1.5813770 & -0.9771590 \\ \mathrm{C} & 4.4138430 & -3.6368920 \\ \mathrm{C} & 3.4156370 & -4.7706840 \\ \mathrm{C} & 1.9807240 & -4.3276470 \\ \mathrm{C} & 4.0807180 & -2.4187310 \\ \mathrm{H} & 5.4305380 & -3.9740860 \\ \mathrm{H} & 3.6598530 & -5.6399960 \\ \mathrm{H} & 1.8124170 & -4.2402300 \\ \mathrm{H} & 4.2022080 & -2.6660440 \\ \mathrm{H} & 4.7459490 & -1.5812780 \\ \mathrm{H} & 4.3994080 & -3.3514640 \\ \mathrm{H} & 3.4735470 & -5.0887340 \\ \mathrm{H} & 1.2445100 & -5.0476860 \\ \mathrm{C} & 1.4225230 & -3.0726260 \\ \mathrm{O} & 0.5837340 & -2.0852690 \\ \mathrm{C} & 2.2211500 & -3.3925860 \\ \mathrm{C} & 3.1711310 & -4.4139890 \\ \mathrm{C} & 2.0535220 & -2.6710440 \\ \mathrm{C} & 3.9503790 & -4.7140860 \\ \mathrm{H} & 3.2874310 & -4.9635030 \\ \mathrm{C} & 2.8351890 & -2.9743920 \\ \mathrm{H} & 1.3158930 & -1.8776530 \\ \mathrm{C} & 3.7822880 & -3.9941850 \\ \mathrm{H} & 4.6892800 & -5.5073630 \\ \mathrm{H} & 2.7053450 & -2.4160650 \\ \mathrm{H} & 4.3910730 & -4.2289360 \\ \mathrm{H} & 0.0980400 & -1.8374930 \\ \mathrm{O} & 1.5816690 & -3.7074540 \\ \mathrm{H} & & \end{array}$

$-0.7585030$ $-2.1055110$ $-0.0879890$ $-2.5165840$ $-1.4762270$

1.9485610 $-1.0899550$ $-2.5241920$ 1.7353960 $-1.0177430$ $-1.1840720$ $-0.3552540$ $-1.1054470$ $-2.3896510$ $-2.3734740$ $-1.7068910$ $-1.4641620$ $-1.7617150$ $-0.8426110$ $-1.4817510$ $-2.0824200$ $-2.8423590$ 0.2199330 $-1.0574830$ $-2.7658200$ $-0.4174140$ $-1.3944940$

1. 9087210

2. 0611890

3. 1212650

3. 0518340

4. 3062400

4.1619440

2. 1237820

5.4149890

4. 3482510

5. 3434660

4.1079130

6.3366860

6. 2115700

1. 1987310

0.8596180

$\operatorname{TS} 97(R, R) G[W B 97 X D / 6-31 G(d)]=-3310.756177$

$\begin{array}{lrrr}\text { TS97 }(\mathrm{R}, \mathrm{R}) & \mathrm{G}[\mathrm{WB} 97 \times \mathrm{D} / 6-31 \mathrm{G}(\mathrm{d})]= & -3310.756177 \\ \mathrm{O} & 0.5012390 & 0.5726220 & 0.0788880 \\ 0 & -1.8015930 & 0.7740300 & -0.9686560\end{array}$




$\begin{array}{rrr}0.6024890 & 1.9439530 & 0.0185240 \\ -0.4485610 & 2.7215670 & 0.4586050 \\ 1.8349500 & 2.4918490 & -0.4209800 \\ -0.3452060 & 4.1459030 & 0.3418460 \\ 1.9616230 & 3.8581030 & -0.4270260 \\ 0.8861040 & 4.7132440 & -0.0837200 \\ 2.9083630 & 4.3000900 & -0.7262650 \\ -2.3259490 & 1.1240240 & 0.2631290 \\ -3.5438630 & 0.5154820 & 0.6667190 \\ -1.6666170 & 2.0729310 & 1.0106040 \\ -4.0700420 & 0.8983800 & 1.8736350 \\ -2.1657970 & 2.3791450 & 2.3186250 \\ -3.3940530 & 1.7980320 & 2.7361600 \\ -5.0208120 & 0.4815990 & 2.1938270 \\ -3.2266860 & 2.9358560 & 4.8725290 \\ -1.9859360 & 3.4835840 & 4.4741870 \\ -1.4693190 & 3.2145180 & 3.2325270 \\ -3.9110790 & 2.1085660 & 4.0211250 \\ -3.6276420 & 3.1617590 & 5.8559060 \\ -1.4335550 & 4.1193830 & 5.1595210 \\ -0.5123700 & 3.6347380 & 2.9437310 \\ -4.8562000 & 1.6639080 & 4.3218020 \\ -1.2874010 & 6.3800850 & 0.4930020 \\ -0.0496210 & 6.9428750 & 0.1052140 \\ 1.0106530 & 6.1238860 & -0.1808330 \\ -1.4327380 & 5.0214810 & 0.6085710 \\ -2.1330870 & 7.0304730 & 0.6952540 \\ 0.0524200 & 8.0204190 & 0.0207630 \\ 1.9618990 & 6.5412800 & -0.5007780 \\ -2.3914090 & 4.6042800 & 0.8957990 \\ 2.9901950 & 1.6422140 & -0.8434690 \\ 3.3022330 & 1.5172570 & -2.2065990 \\ 3.8187080 & 1.0505260 & 0.1305400 \\ 4.4945370 & 0.8831420 & -2.5699660 \\ 4.9895740 & 0.4220350 & -0.2816740 \\ 5.3628620 & 0.3508470 & -1.6247870 \\ 4.7371980 & 0.8033030 & -3.6245390 \\ 5.6440360 & -0.0261230 & 0.4626460 \\ -4.2337260 & -0.4792420 & -0.2084500 \\ -4.2517280 & -1.8418700 & 0.1424360 \\ -4.8843570 & -0.0393520 & -1.3765910 \\ -4.9463780 & -2.7317050 & -0.6789550 \\ -5.5545910 & -0.9693300 & -2.1671000 \\ -5.6009010 & -2.3211940 & -1.8351250 \\ -4.9745090 & -3.7860390 & -3.0690710 \\ -6.0568490 & -0.6261290 & \end{array}$

\begin{abstract}
3.4767300
6.6893250

2. 3832630

$-3.5615840$

$-6.3340300$

$-4.9017390$

$-6.3255140$

$-6.9844860$

$-6.7592420$

$-6.3197180$

$-4.2664220$

$-3.2500590$

$-4.8477790$

$-4.2199840$

$-5.7002960$

$-6.1955780$

$-5.7922570$

$-4.6353290$

$-7.8313060$

$-8.2888000$

$-7.9964100$

$-8.3539120$

$-4.5791230$

$-5.1181900$

$-5.3205120$

$-4.0765530$

$-2.7601390$

$-3.4138730$

$-2.0960740$

$-2.1507160$

4.1540790

5. 2446130

3.8453200

3.8939220

3. 8220060

3.4536040

4.9026460

3. 3646200

7.8539120

8.8171680

7.8114820

7.8149140

6.8121910

5.9859640

6.8298230

7. 7478900
\end{abstract}

1.1257360
-0.3009890
2.0577860
-2.3739540
-3.3208870
1.4246680
1.9951950
1.4908170
1.8732340
3.0636520
1.6271630
1.2242430
1.1306940
2.6947240
-3.4137000
-4.1859330
-2.4619260
-3.6571880
-3.0000370
-2.9536380
-2.0339350
-3.7653150
-2.6126350
-1.6965610
-3.3614460
-2.9792920
-3.6551670
-4.5138680
-3.5208950
-3.8974550
2.3415100
2.2602630
3.2712570
2.4102100
-0.1623980
-1.0440080
-0.2811860
-0.1521630
0.6227010
0.1398310
1.5539520
0.8840480
-0.7168970
-1.3636280
0.1554280
-1.2637300

$-1.9847540$

$-3.2941660$

1. 3923380

$-2.7111010$

$-1.7936680$

$-1.7395840$

$-2.4558900$

$-0.7411960$

$-1.9833860$

$-3.1747230$

$-3.1930800$

$-3.9605470$

$-3.4179740$

$-4.1056700$

$-4.7051180$

$-4.6415100$

$-4.0370720$

$-2.8065180$

$-1.8129760$

$-3.2973450$

$-3.3914810$

2. 5175750

2. 7776130

2. 2141030

3. 4199910

1.1265670

0.9344100

0.2698150

2. 0044500

2. 2592480

2.1783960

1.7702530

3. 3215650

2. 3670730

1.8331200

2. 5071110

3. 3618050

$-1.5937040$

$-1.7928010$

$-2.1703390$

$-0.5313740$

$-3.4519820$

$-3.7669530$

-4.1154770
-3.6072790 


$\begin{array}{rrr}2.1862360 & 1.0549420 & -4.4378390 \\ 3.0941930 & 0.9404520 & -5.0414110 \\ 1.8990240 & 0.0742560 & -4.0533190 \\ 1.3911930 & 1.4043830 & -5.1047540 \\ 2.8963360 & 3.3966880 & -3.8423580 \\ 3.8919440 & 3.2765030 & -4.2866250 \\ 2.2219070 & 3.7745670 & -4.6189360 \\ 2.9659600 & 4.1549300 & -3.0567170 \\ -0.5235210 & -0.2281920 & -0.9267050 \\ 0.0303100 & -0.3237340 & -2.3141950 \\ -0.8516010 & -1.4943910 & -0.1759130 \\ 1.3994850 & 2.2257970 & -2.8459710 \\ 6.7707900 & -1.2123170 & -1.3750910 \\ 2.3953510 & 1.2629310 & 1.7018680 \\ -4.3044890 & 1.9967310 & -1.0784230 \\ -6.2346590 & -4.3050990 & -2.2348870 \\ -2.8488450 & -1.6175850 & 1.7317700 \\ 2.4369780 & -2.2092550 & -1.4173340 \\ 1.4360690 & -3.2001980 & -1.0053710 \\ 2.3433970 & -1.2188850 & -0.9675980 \\ 0.4369780 & -2.8324630 & -0.7998880 \\ 1.7228670 & -2.3072260 & -2.6708820 \\ 1.0309680 & -1.5739120 & -2.6561500 \\ 3.9890660 & -4.1265660 & -2.0102720 \\ 3.1373730 & -5.0546420 & -1.1427340 \\ 1.6610880 & -4.6576900 & -1.2035130 \\ 3.8681820 & -2.6752840 & -1.5403190 \\ 5.0399840 & -4.4309360 & -1.9726010 \\ 3.2461200 & -6.0954530 & -1.4625400 \\ 1.2288920 & -4.9011700 & -2.1826680 \\ 4.3313040 & -2.5542650 & -0.5533870 \\ 4.3817360 & -1.9930100 & -2.2207810 \\ 3.6711640 & -4.2027900 & -3.0572550 \\ 3.4748690 & -4.9956520 & -0.1014390 \\ 1.0574700 & -5.1902750 & -0.4635100 \\ 1.2551790 & -2.7216370 & 2.1308690 \\ 0.1408990 & -2.0441840 & 2.1173190 \\ 1.8461740 & -2.8890650 & 3.4867350 \\ 3.0447260 & -3.5928310 & 3.6199270 \\ 1.2384640 & -2.3223960 & 4.6095640 \\ 3.6344500 & -3.7292600 & 4.8700600 \\ 3.5064570 & -4.0204670 & 2.7364560 \\ 1.8308370 & -2.4624120 & 5.8594960 \\ 0.3105130 & -1.7735290 & 4.4958070 \\ 3.0279160 & -3.1631770 & 4.9727330 \\ 4.5686040 & -4.2725190 & \end{array}$

$\begin{array}{lrrr}\mathrm{H} & 1.3601500 & -2.0208950 & 6.7322400 \\ \mathrm{H} & 3.4904710 & -3.2682890 & 6.9668840 \\ \mathrm{H} & -0.2181760 & -1.8485170 & 1.1865000 \\ \mathrm{O} & 1.8180350 & -3.1965420 & 1.1378260 \\ 144 & & & \end{array}$

$\operatorname{TS} 98(R, R) \quad G[W B 97 X D / 6-31 G(d)]=-3310.755345$

$\begin{array}{lrrr}\text { TS98(R, R }) & \text { G }[W B 97 X D / 6-31 G(d)] & = & -3310.755345 \\ \mathrm{O} & 0.0484830 & -1.1836690 & 0.2308740 \\ \mathrm{O} & 2.0681270 & -0.1411060 & -0.8523280 \\ \mathrm{C} & 0.3811620 & -2.4078640 & -0.3040510 \\ \mathrm{C} & 1.6891430 & -2.8380610 & -0.2424290 \\ \mathrm{C} & -0.6850310 & -3.1929740 & -0.8176370 \\ \mathrm{C} & 2.0160120 & -4.0816220 & -0.8768750 \\ \mathrm{C} & -0.3764730 & -4.4308530 & -1.3167220 \\ \mathrm{C} & 0.9651960 & -4.8870680 & -1.3970870 \\ \mathrm{H} & -1.1715460 & -5.0791810 & -1.6743440 \\ \mathrm{C} & 2.8261780 & -0.6711550 & 0.1681730 \\ \mathrm{C} & 3.7509020 & 0.1994100 & 0.8025310 \\ \mathrm{C} & 2.6966010 & -2.0084090 & 0.4756060 \\ \mathrm{C} & 4.5939550 & -0.3448570 & 1.7350390 \\ \mathrm{C} & 3.4971430 & -2.5351820 & 1.5444980 \\ \mathrm{C} & 4.4813810 & -1.6987360 & 2.1413450 \\ \mathrm{H} & 5.3479660 & 0.2817890 & 2.2031290 \\ \mathrm{C} & 5.1465300 & -3.4990610 & 3.6261830 \\ \mathrm{C} & 4.1362500 & -4.3164080 & 3.0708010 \\ \mathrm{C} & 3.3343300 & -3.8495610 & 2.0604230 \\ \mathrm{C} & 5.3078790 & -2.2166070 & 3.1723940 \\ \mathrm{H} & 5.7796880 & -3.8840480 & 4.4197350 \\ \mathrm{H} & 3.9886540 & -5.3221410 & 3.4527490 \\ \mathrm{H} & 2.5585520 & -4.4883210 & 1.6538070 \\ \mathrm{H} & 6.0626780 & -1.5661560 & 3.6068110 \\ \mathrm{C} & 3.6253380 & -5.7336160 & -1.6397250 \\ \mathrm{C} & 2.5771190 & -6.5511160 & -2.1196350 \\ \mathrm{C} & 1.2775920 & -6.1318690 & -2.0027370 \\ \mathrm{C} & 3.3533800 & -4.5333790 & -1.0341500 \\ \mathrm{H} & 4.6555630 & -6.0557620 & -1.7577130 \\ \mathrm{H} & 2.8050340 & -7.5018880 & -2.5916920 \\ \mathrm{H} & 0.4635700 & -6.7421770 & -2.3853780 \\ \mathrm{H} & 4.1668230 & -3.9100870 & -0.6792070 \\ \mathrm{C} & -2.0867100 & -2.6789990 & -0.7272870 \\ \mathrm{C} & -2.7529260 & -2.1947730 & -1.8704500 \\ \mathrm{C} & -2.7342850 & -2.6802320 & 0.5192890 \\ \mathrm{C} & -4.0672260 & -1.7509530 & -1.7345520 \\ \mathrm{C} & -4.0479330 & -2.2145310 & 0.6052470 \\ \mathrm{C} & -4.7377460 & -1.7551500 & -0.5113020 \\ \mathrm{H} & -4.6010260 & -1.3979800 & -2.6144380 \\ \mathrm{H} & -4.5453570 & -2.2346250 & 1.5704390\end{array}$




\begin{tabular}{|c|c|c|c|}
\hline$c$ & מחיברי & & \\
\hline & 3.7730100 & 1.6552260 & 0.4621870 \\
\hline c & 3. 1829990 & 2.5840490 & 1.3407290 \\
\hline c & $\begin{array}{l}4.3633260 \\
2.2277100\end{array}$ & 2.0893240 & -0.7374630 \\
\hline & 4.3752540 & 3.9380510 & 1.0087230 \\
\hline$C$ & 3.8178970 & 4.3932420 & -0.1685680 \\
\hline $\mathrm{H}$ & 2.7869330 & 4.6659790 & 1.6821730 \\
\hline H & 4.8277770 & 3.7858760 & -1.9615420 \\
\hline C & -2.0693850 & -3.2406420 & 1.7689580 \\
\hline C & -6.1731050 & -1.2540470 & -0.4522010 \\
\hline C & -2.0947910 & -2.1961100 & -3.2437620 \\
\hline C & 2.5004980 & 2.1540920 & 2.6336260 \\
\hline C & 3.8112870 & 5.8713790 & -0.5136180 \\
\hline $\mathrm{C}$ & 4.9820730 & 1.1137600 & -1.7279060 \\
\hline C & 6.4887190 & 1.3581010 & -1.8844310 \\
\hline $\mathrm{H}$ & 6.6905630 & 2.3518430 & -2.3008190 \\
\hline $\mathrm{H}$ & 6.9997430 & 1.2875130 & -0.9183360 \\
\hline $\mathrm{H}$ & 6.9280850 & 0.6164240 & -2.5610720 \\
\hline C & 4.2612560 & 1.1584570 & -3.0812680 \\
\hline $\mathrm{H}$ & 3.1927920 & 0.9637880 & -2.9536870 \\
\hline $\mathrm{H}$ & 4.3802040 & 2.1353640 & -3.5647760 \\
\hline $\mathrm{H}$ & 4.6720810 & 0.4002050 & -3.7575470 \\
\hline C & 2.9340970 & 6.1472460 & -1.7428260 \\
\hline $\mathrm{H}$ & 2.8742870 & 7.2228380 & -1.9443650 \\
\hline $\mathrm{H}$ & 3.3448930 & 5.6591480 & -2.6342160 \\
\hline $\mathrm{H}$ & 1.9183760 & 5.7664420 & -1.5915040 \\
\hline C & 5.2279210 & 6.4245970 & -0.7124470 \\
\hline $\mathrm{H}$ & 5.8491240 & 6.2398130 & 0.1697900 \\
\hline $\mathrm{H}$ & 5.7183270 & 5.9561410 & -1.5734910 \\
\hline $\mathrm{H}$ & 5.1975110 & 7.5049380 & -0.8932520 \\
\hline C & 3.4635140 & 580590 & 3.82575 \\
\hline $\mathrm{H}$ & 4.3540390 & 1.6391420 & 3.6848280 \\
\hline $\mathrm{H}$ & 3.7933000 & 3.2950600 & 3.9626300 \\
\hline $\mathrm{H}$ & 2.9683960 & 1.9346250 & 4.7485420 \\
\hline C & 1.2196740 & 2.9447510 & 2.9319710 \\
\hline $\mathrm{H}$ & 1.4369850 & 3.9723830 & 3.24643 \\
\hline $\mathrm{H}$ & 0.5674670 & 2.9775060 & 2.0569080 \\
\hline $\mathrm{H}$ & 0.6739010 & 2.4642700 & 3.7515780 \\
\hline C & 6817600 & -4.6009640 & 2.1304720 \\
\hline $\mathrm{H}$ & -3.7507880 & -4.5017420 & 2.3533600 \\
\hline $\mathrm{H}$ & -2.5753920 & 310221( & \\
\hline $\mathrm{H}$ & -2.1888830 & -5.0260230 & 3.0122200 \\
\hline C & -2.1234930 & -2.2716530 & 2.9545660 \\
\hline $\mathrm{H}$ & -1.7234710 & -1.2927200 & 2.6742090 \\
\hline $\mathrm{H}$ & 410 & -2.1391970 & 3.3210900 \\
\hline $\mathrm{H}$ & & 521470 & 3.7879480 \\
\hline
\end{tabular}

-6.2103130
-7.2428980
-5.6836760
-5.7315520
-6.9546950
-6.8968140
-6.5776010
-8.0094570
-2.4054690
-3.4553020
-2.1774270
-1.7951560
-2.5004840
-3.5840190
-2.0029850
-2.2351940
0.4906720
-0.1331140
0.2768450
-1.0116810
-6.6797450
-1.0123740
4.8616100
3.3648830
2.2090920
-2.4452470
-1.5447170
-2.4796570
-0.6451180
-1.4558000
-0.8276810
-3.7346010
-3.0804680
-1.6567520
-3.7965890
-4.7470120
-3.0529090
-0.9672800
-4.4632520
-4.1896680
-3.1675140
-3.6755620
-1.2565610
-2.7435940
-1.9286110
-4.0771510

0.2787890

0.6462670

0.7220150

0.6313000

$-1.7380670$

$-2.8258930$

$-1.2761110$

$-1.4596530$

$-0.9406750$

$-0.9036530$

$-0.0332360$

$-0.9339910$

$-3.4477840$

$-3.4606570$

$-3.4616770$

$-4.3680990$

0.1514400

0.2242300

1.2802820

$-2.2205240$

$-1.6462920$

$-3.4133500$

0.1017730

. 4014110

1.1056710

2.2226480

3.1844810

1. 2291400

2.7817770

2.2930260

1.5202040

4.1583870

5.0928090

4.6439550

2.7257860

4. 5045400

6.1189470

4.8554140

2. 6790250

2.0332400

4.1747450

5.1019230

5.1693980

2066230

1.2014330

2. 0214690
$-0.5380720$

$-0.5282360$

0.3140290

$-1.4569920$

0.7705180

0.8824780

1.6896270

0.6742530

$-4.0655420$

$-4.3795990$

$-3.5037700$

$-4.9751370$

$-4.0370070$

$-4.2066590$

$-5.0133120$

$-3.5089020$

$-0.5954460$

$-1.9574320$

0.3803880

$-3.0933600$

$-1.3446970$

1. 5518760

$-1.3302350$

0.3378800

2. 5201340

$-1.6795760$

$-1.0328760$

$-1.2268200$

$-0.5774890$

$-2.7244650$

$-2.5413540$

$-2.6630900$

$-1.6420500$

$-1.3004910$

$-2.1273220$

$-2.8935070$

$-2.0209650$

$-2.1277220$

$-1.2570620$

$-2.8791850$

$-3.6012670$

$-0.7215730$

$-0.4290810$

1.5442940

1.7126430

2. 1710630 


$\begin{array}{lrrr}\mathrm{C} & -5.0580670 & 2.9956330 & 1.9715920 \\ \mathrm{C} & -4.3724590 & 0.8684260 & 2.9021680 \\ \mathrm{C} & -6.3318020 & 2.8145470 & 2.4961910 \\ \mathrm{H} & -4.8111640 & 3.8845920 & 1.4007340 \\ \mathrm{C} & -5.6456300 & 0.6945780 & 3.4310270 \\ \mathrm{H} & -3.6068510 & 0.1148010 & 3.0414740 \\ \mathrm{C} & -6.6262100 & 1.6633560 & 3.2243540 \\ \mathrm{H} & -7.0957280 & 3.5689510 & 2.3370030 \\ \mathrm{H} & -5.8755930 & -0.2007460 & 4.0006570 \\ \mathrm{H} & -7.6218720 & 1.5205720 & 3.6332850 \\ \mathrm{H} & -1.0446660 & 1.2989580 & 1.2213330 \\ \mathrm{O} & -2.4748870 & 3.2308420 & 0.9045470 \\ 144 & & & \end{array}$

TS99 $(R, R) \quad G[W B 97 X D / 6-31 G(d)]=-3310.755617$

\begin{tabular}{llcr}
\multicolumn{1}{c}{ TS99(R, R $)$ G $[W B 97 X D / 6-31 G(\mathrm{~d})]$} & -3310.755617 \\
$\mathrm{O}$ & -0.0137330 & 1.1110940 & 0.2170020 \\
$\mathrm{O}$ & -2.0803370 & 0.1922290 & -0.8970560 \\
$\mathrm{C}$ & -0.2452980 & 2.3571230 & -0.3204660 \\
$\mathrm{C}$ & -1.5237480 & 2.8703950 & -0.3040390 \\
$\mathrm{C}$ & 0.8879370 & 3.0814270 & -0.7769110 \\
$\mathrm{C}$ & -1.7488910 & 4.1324600 & -0.9458650 \\
$\mathrm{C}$ & 0.6759890 & 4.3354610 & -1.2854690 \\
$\mathrm{C}$ & -0.6311870 & 4.8707730 & -1.4245770 \\
$\mathrm{H}$ & 1.5238820 & 4.9388240 & -1.5972620 \\
$\mathrm{C}$ & -2.8134140 & 0.7870680 & 0.1061600 \\
$\mathrm{C}$ & -3.8032990 & -0.0080250 & 0.7414160 \\
$\mathrm{C}$ & -2.5990130 & 2.1167680 & 0.3981690 \\
$\mathrm{C}$ & -4.6158150 & 0.6041600 & 1.6591890 \\
$\mathrm{C}$ & -3.3716340 & 2.7108460 & 1.4518680 \\
$\mathrm{C}$ & -4.4159890 & 1.9522920 & 2.0502120 \\
$\mathrm{H}$ & -5.4165020 & 0.0365620 & 2.1254100 \\
$\mathrm{C}$ & -4.9689360 & 3.8145450 & 3.5046930 \\
$\mathrm{C}$ & -3.8994290 & 4.5523580 & 2.9489050 \\
$\mathrm{C}$ & -3.1228170 & 4.0174540 & 1.9530440 \\
$\mathrm{C}$ & -5.2137470 & 2.5397860 & 3.0667010 \\
$\mathrm{H}$ & -5.5807430 & 4.2529370 & 4.2871920 \\
$\mathrm{H}$ & -3.6866110 & 5.5508630 & 3.3188030 \\
$\mathrm{H}$ & -2.2996040 & 4.5941500 & 1.5475400 \\
$\mathrm{H}$ & -6.0156530 & 1.9493970 & 3.5025100 \\
$\mathrm{C}$ & -3.2215740 & 5.8873270 & -1.7534060 \\
$\mathrm{C}$ & -2.1071930 & 6.6358670 & -2.1958760 \\
$\mathrm{C}$ & -0.8421090 & 6.1334940 & -2.0366280 \\
$\mathrm{C}$ & -3.0481640 & 4.6703860 & -1.1447260 \\
$\mathrm{H}$ & -4.2238440 & 6.2779100 & -1.9014930 \\
$\mathrm{H}$ & -2.2579050 & 7.6002090 & -2.6713200 \\
$\mathrm{H}$ & 0.0223800 & 6.6904620 & -2.3887200 \\
$\mathrm{H}$ & -3.9115900 & 4.1013220 & -0.8174730
\end{tabular}

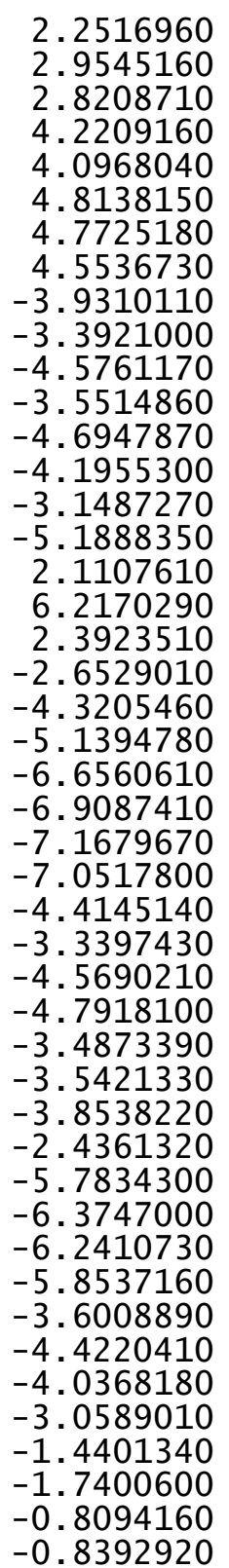

2.4951810

1.9341700

2. 5047550

1.3965900

1.9686630

1.4094960

0.9725980

1.9949600

$-1.4609690$

$-2.4223770$

$-1.8602290$

$-3.7721180$

$-3.2206640$

$-4.1931120$

$-4.5245330$

$-3.5285570$

3.1299930

0.8727990

1.9750570

$-2.0299650$

$-5.6707340$

$-0.8514670$

$-1.0150840$

$-1.9929140$

$-0.9252460$

$-0.2460190$

$-0.9336560$

$-0.7805900$

$-1.9092680$

$-0.1649080$

$-6.0376690$

$-7.1141030$

$-5.5118210$

$-5.7652600$

$-6.0974910$

$-5.8457880$

$-5.5987060$

$-7.1784500$

$-2.0273930$

$-1.3167450$

$-3.0228780$

$-1.7545530$

$-2.9229740$

$-3.9208840$

-3.0295200
-2.4745570
$-0.5929280$

$-1.6774910$

0.6918220

$-1.4463560$

0.8678060

$-0.1837470$

$-2.2816190$

1.8550670

0.4135300

1.2893160

$-0.7696640$

0.9745880

$-1.0473390$

$-0.1847860$

1.6482590

$-1.9665500$

1.8845130

0.0358530

$-3.0922230$

2.5628050

$-0.5096880$

$-1.7599840$

$-1.9256970$

$-2.3517280$

$-0.9617020$

$-2.5986100$

$-3.1096360$

$-2.9779100$

$-3.5857270$

$-3.7936550$

$-1.7456490$

$-1.9442920$

$-2.6348980$

$-1.6043660$

$-0.6847320$

0.2016780

$-1.5467660$

$-0.8502000$

3.7715290

3.6417980

3.9194600

4.6843630

2.8568190

3.1983320

1.9718640

3.6558160 


$\begin{array}{rrr}2.7765780 & 4.4582840 & 2.2682180 \\ 3.8219930 & 4.3036510 & 2.5603210 \\ 2.7627520 & 5.1596020 & 1.4270210 \\ 2.2541460 & 4.9235480 & 3.1118200 \\ 2.0297030 & 2.1768510 & 3.0818370 \\ 1.5992590 & 1.2161200 & 2.7834570 \\ 3.0180760 & 1.9962980 & 3.5224700 \\ 1.3988150 & 2.6081560 & 3.8667250 \\ 7.2533400 & 1.7067580 & -0.7296610 \\ 8.2690320 & 1.3582020 & -0.5109330 \\ 7.0970220 & 1.6261980 & -1.8117450 \\ 7.1850550 & 2.7654380 & -0.4592870 \\ 6.3320980 & -0.6114790 & -0.3244780 \\ 5.6161270 & -1.2065170 & 0.2495940 \\ 6.1403600 & -0.7757280 & -1.3913050 \\ 7.3380200 & -0.9855610 & -0.1037170 \\ 2.6901520 & 0.7180500 & -3.9140070 \\ 3.7593180 & 0.6108110 & -4.1320890 \\ 2.3463700 & -0.1827330 & -3.4038890 \\ 2.1687580 & 0.7752070 & -4.8754500 \\ 2.9212140 & 3.2121790 & -3.8352610 \\ 4.0109880 & 3.1548830 & -3.9452200 \\ 2.4802660 & 3.2768380 & -4.8363950 \\ 2.6882930 & 4.1382920 & -3.3025050 \\ -0.5222330 & -0.1870890 & -0.6327690 \\ 0.1101730 & -0.2723860 & -1.9905410 \\ -0.3723910 & -1.3389670 & 0.3281240 \\ 1.3044050 & 2.0570660 & -3.0146270 \\ 6.4352480 & 0.9666350 & 1.1073380 \\ 1.0822080 & 3.3593360 & 1.5951060 \\ -4.9680580 & 0.1523670 & -1.3600760 \\ -3.9121110 & -6.2278850 & 0.3434470 \\ -2.2723790 & -1.0132750 & 2.4239550 \\ 2.3938240 & -2.2912970 & -1.5952550 \\ 1.4773040 & -3.2754420 & -1.0052380 \\ 2.3970060 & -1.3061310 & -1.1230360 \\ 0.5537290 & -2.8880160 & -0.5861570 \\ 1.4541730 & -2.3524510 & -2.6848990 \\ 0.8199230 & -1.5780030 & -2.5273760 \\ 3.7517800 & -4.1881900 & -2.5570290 \\ 3.0642530 & -5.1506870 & -1.5849800 \\ 1.6200130 & -4.7272860 & -1.2998150 \\ 3.7713190 & -2.7672040 & -1.9891660 \\ 4.7776100 & -4.5177880 & -2.7490330 \\ 3.0676330 & -6.1694070 & -1.9843380 \\ 0.9742440 & -4.9271260 & -2.1643070\end{array}$

$$
\begin{aligned}
& 4.3960860 \\
& 4.1907530 \\
& 3.2265860 \\
& 3.6175030 \\
& 1.1867450 \\
& 2.5938570 \\
& 1.8046250 \\
& 3.9277030 \\
& 4.8591400 \\
& 4.2776520 \\
& 6.1375070 \\
& 4.5711200 \\
& 5.5577730 \\
& 3.5496590 \\
& 6.4880060 \\
& 6.8637230 \\
& 5.8311360 \\
& 7.4887830 \\
& 0.9274170 \\
& 2.3025690
\end{aligned}
$$$$
144
$$$$
\operatorname{TS} 100(R, R) G[W B 97 X D / 6-31 G(d)]=-3310.757089
$$

$\begin{array}{lrcr}\text { TS100 }(\mathrm{R}, \mathrm{R}) \mathrm{G}[\mathrm{WB} 97 \mathrm{XD} / 6-31 \mathrm{G}(\mathrm{d})]=-3310.757089 \\ \mathrm{O} & 0.4476820 & 0.7062830 & 0.0296340 \\ \mathrm{O} & -1.8925690 & 0.7612810 & -0.9534480 \\ \mathrm{C} & 0.4955910 & 2.0779750 & -0.0790910 \\ \mathrm{C} & -0.5822320 & 2.8289310 & 0.3423240 \\ \mathrm{C} & 1.7047180 & 2.6588740 & -0.5432050 \\ \mathrm{C} & -0.5400850 & 4.2500000 & 0.1620480 \\ \mathrm{C} & 1.7741170 & 4.0271340 & -0.6080080 \\ \mathrm{C} & 0.6627740 & 4.8495470 & -0.2991280 \\ \mathrm{H} & 2.7044530 & 4.4965920 & -0.9162340 \\ \mathrm{C} & -2.3888060 & 1.1385920 & 0.2804860 \\ \mathrm{C} & -3.5572480 & 0.4871790 & 0.7564300 \\ \mathrm{C} & -1.7563990 & 2.1560600 & 0.9575230 \\ \mathrm{C} & -4.0708720 & 0.9110520 & 1.9551600 \\ \mathrm{C} & -2.2332690 & 2.5068270 & 2.2628120 \\ \mathrm{C} & -3.4212650 & 1.8929920 & 2.7453500 \\ \mathrm{H} & -4.9887130 & 0.4643170 & 2.3267900 \\ \mathrm{C} & -3.2545830 & 3.1539270 & 4.8117680 \\ \mathrm{C} & -2.0494740 & 3.7321910 & 4.3519440 \\ \mathrm{C} & -1.5524490 & 3.4192610 & 3.1127190 \\ \mathrm{C} & -3.9206150 & 2.2510840 & 4.0250520 \\ \mathrm{H} & -3.6413950 & 3.4158860 & 5.7918870 \\ \mathrm{H} & -1.5087930 & 4.4260670 & 4.9885400 \\ \mathrm{H} & -0.6220160 & 3.8636260 & 2.7776220 \\ \mathrm{H} & -4.8361960 & 1.7816660 & 4.3754400\end{array}$

$-1.0883870$ $-2.7045670$ $-3.5193120$ $-0.6390280$ $-0.4610610$

1.5881400

1.7203480

2. 2181310

2.8434520

2.6339680

1.6245730

3.3587460

2.9046040

3.2516920

2. 5528840

3.6524270

1. 2157800

0.9696940
1.7816660

4.3754400

$-3.9452200$

. 8363950

0.6327690

(281240

1.1073380

5951060

0.3434470
2.4239550

$-1.5952550$

052380

30360

$-2.6848990$

2.5273760

-

$-2.1643070$ 


$\begin{array}{rrr}-1.5751060 & 6.4466490 & 0.2294720 \\ -0.3656560 & 7.0437120 & -0.1942170 \\ 0.7263350 & 6.2588630 & -0.4561680 \\ -1.6613850 & 5.0892360 & 0.4037190 \\ -2.4461630 & 7.0684260 & 0.4129970 \\ -0.3099410 & 8.1201830 & -0.3240510 \\ 1.6565200 & 6.7020750 & -0.8021900 \\ -2.5984120 & 4.6442000 & 0.7192150 \\ 2.8999980 & 1.8296800 & -0.8898260 \\ 3.2083870 & 1.5633370 & -2.2382180 \\ 3.7561610 & 1.3878730 & 0.1323040 \\ 4.4046270 & 0.9112020 & -2.5295850 \\ 4.9324690 & 0.7163160 & -0.2106200 \\ 5.2886280 & 0.4916520 & -1.5356270 \\ 4.6636170 & 0.7191600 & -3.5685070 \\ 5.5904310 & 0.3776530 & 0.5849260 \\ -4.2087840 & -0.5999630 & -0.0351430 \\ -4.1080750 & -1.9392200 & 0.3857900 \\ -4.9369080 & -0.2742510 & -1.1945110 \\ -4.7709540 & -2.9211190 & -0.3524300 \\ -5.5684970 & -1.2934520 & -1.9033370 \\ -5.5029110 & -2.6237400 & -1.4970470 \\ -4.7068500 & -3.9581750 & -0.0315250 \\ -6.1286010 & -1.0392230 & -2.8006680 \\ 3.4449640 & 1.6200650 & 1.6041670 \\ 6.6139390 & -0.1368640 & -1.9348690 \\ 2.2884810 & 2.0005580 & -3.3700400 \\ -3.3155270 & -2.3491990 & 1.6212650 \\ -6.1923280 & -3.7215810 & -2.2868620 \\ -5.0743160 & 1.1581490 & -1.6916740 \\ -6.5356520 & 1.6233870 & -1.6334090 \\ -7.1692240 & 1.0301480 & -2.3030160 \\ -6.9380320 & 1.5269730 & -0.6192350 \\ -6.6172840 & 2.6731650 & -1.9371510 \\ -4.4871330 & 1.3266200 & -3.0987740 \\ -3.4391840 & 1.0148490 & -3.1199170 \\ -5.0389510 & 0.7318400 & -3.8360690 \\ -4.5420870 & 2.3762950 & -3.4090310 \\ -5.5805890 & -3.8667280 & -3.6870900 \\ -6.0428140 & -4.7010340 & -4.2268570 \\ -5.7353190 & -2.9561480 & -4.2772370 \\ -4.5026280 & -4.0472610 & -3.6268820 \\ -7.7080920 & -3.4995840 & -2.3690140 \\ -8.1485540 & -3.4107470 & -1.3706300 \\ -7.9434380 & -2.5827860 & -2.9218880 \\ -8.1926730 & -4.3352660 & -2.8864370\end{array}$
$-4.2438730$
$-4.8008370$
$-4.9725960$
$-3.6661720$
$-2.4705620$
$-3.0895630$
$-1.8814810$
$-1.7835430$
4.5803670
5. 5006740
4.8066730
4. 2959780
3. 1049860
2. 2233370
3.9363030
2.8850110
7.1672790
8.0544570
7.4706350
6.4316330
7.6475440
7.2843300
7.8492330
8.5922330
2. 1926020
3. 1101970
2. 0026200
1. 3713560
2. 7301500
3.7449320
2. 0573320
2.7259230
$-0.5574810$
$-0.0493790$
$-0.7855200$
1.2830200
6.4450590
2.5579790
$-4.5047410$
$-6.0234670$
$-2.6191920$
2. 3451710
1.3659370
2. 2367890
0.3677940
1. 6210950

-2.5527900
-1.6425270
-3.3465410
-2.8413860
-3.6097320
-4.5116540
-3.5164040
-3.7502240
2.3620370
1.7676330
3.3097700
2.5793920
0.2985070
-0.1569590
-0.4145430
0.4651090
-1.1412280
-1.6355870
-0.6476800
-1.9118990
0.9665630
1.6670360
1.5358460
0.5362780
0.9628220
0.9262830
-0.0367190
1.2244000
3.3545500
3.2844760
3.6637070
4.1392800
-0.1628860
-0.2349920
-1.4412100
2.1147000
-0.6749930
2.2569950
1.8122660
-4.6650060
-1.5399260
-2.1642450
-3.2017200
-1.2079820
-2.8636400
-2.1893200

2.8275680

3. 0687090

2.6235170

3. 7133540

1. 3927180

1. 3219690

0.4774190

2. 2342700

2. 3205080

2. 3490470

1.8206530

3. 3559800

2. 3039560

1.8456030

2. 2450080

3. 3645070

$-0.9207210$

$-1.3300210$

0.0094840

$-0.6707600$

$-2.2121720$

$-2.9709860$

$-1.2972520$

$-2.5635130$

$-4.4940570$

$-5.0935050$

$-4.0978060$

$-5.1689020$

$-3.9449610$

$-4.3551510$

$-4.7527800$

$-3.1836040$

$-0.9375550$

$-2.3450130$

-0.1716980

$-2.9537910$

$-2.8778240$

1.6699480

$-1.0255130$

$-1.7515070$

1.8578790

$-1.5461190$

$-1.2037210$

$-1.0292010$

$-0.9477160$

$-2.7960190$ 


\begin{tabular}{|c|c|c|c|c|c|c|c|}
\hline & & & & & & & \\
\hline $\mathrm{H}$ & 0.9253210 & -1.4578040 & -2.7327560 & C & 5.4681990 & -3.1340120 & 3.5290990 \\
\hline $\begin{array}{l}\mathrm{C} \\
\mathrm{C}\end{array}$ & $\begin{array}{l}3.9251580 \\
3.0920250\end{array}$ & $\begin{array}{l}-3.9913090 \\
-5.0132490\end{array}$ & $\begin{array}{l}-2.3087800 \\
-1.5331110\end{array}$ & $\begin{array}{l}\mathrm{C} \\
\mathrm{C}\end{array}$ & $\begin{array}{l}4.4758310 \\
3.6221680\end{array}$ & $\begin{array}{l}-4.0108050 \\
-3.6098570\end{array}$ & $\begin{array}{l}3.0354910 \\
2.0393950\end{array}$ \\
\hline C & 1.6093310 & -4.6332440 & -1.5342540 & $\mathrm{C}$ & 5.5601800 & -1.8610450 & 3.0304500 \\
\hline $\mathrm{C}$ & 3.7828850 & -2.5945630 & -1.7013060 & $\mathrm{H}$ & 6.1439770 & -3.4663520 & 4.3112090 \\
\hline $\mathrm{H}$ & 4.9810410 & -4.2808820 & -2.3028120 & $\mathrm{H}$ & 4.3832410 & -5.0084890 & 3.4540110 \\
\hline $\mathrm{H}$ & 3.2081600 & -6.0132500 & -1.9621120 & $\mathrm{H}$ & 2.8605180 & -4.2916560 & 1.6786610 \\
\hline $\mathrm{H}$ & 1.1633220 & -4.7971980 & -2.5233860 & H & 6.3013350 & -1.1669320 & 3.4184100 \\
\hline $\mathrm{H}$ & 4.2262540 & -2.5690410 & -0.6985020 & C & 3.8781630 & -5.5878190 & -1.6196510 \\
\hline $\mathrm{H}$ & 4.2990730 & -1.8436570 & -2.3036580 & $\mathrm{C}$ & 2.8537680 & -6.4707780 & -2.0296750 \\
\hline $\mathrm{H}$ & 3.6048970 & -3.9683930 & -3.3574770 & $\mathrm{C}$ & 1.5404480 & -6.1123530 & -1.8707690 \\
\hline $\mathrm{H}$ & 3.4426760 & -5.0597760 & -0.4957750 & C & 3.5706810 & -4.3837570 & -1.0393960 \\
\hline $\mathrm{H}$ & 1.0281810 & -5.2383550 & -0.8332790 & $\mathrm{H}$ & 4.9178170 & -5.8618460 & -1.7712960 \\
\hline C & 1.4476020 & -2.8267780 & 1.9277830 & $\mathrm{H}$ & 3.1098360 & -7.4237980 & -2.4823620 \\
\hline 0 & 0.4361000 & -2.0051440 & 2.0031200 & H & 0.7439170 & -6.7744790 & -2.2002920 \\
\hline C & 2.1693060 & -3.0152820 & 3.2149800 & $\mathrm{H}$ & 4.3650340 & -3.7099960 & -0.7376440 \\
\hline C & 3.2808730 & -3.8597270 & 3.2507010 & $\mathrm{C}$ & -1.9346980 & -2.7796890 & -0.5496770 \\
\hline $\mathrm{C}$ & 1.7622420 & -2.3434950 & 4.3704610 & $\mathrm{C}$ & -2.6579290 & -2.2757360 & -1.6496880 \\
\hline C & 3.9812490 & -4.0343400 & 4.4378720 & C & -2.5292900 & -2.8385410 & 0.7213640 \\
\hline $\mathrm{H}$ & 3.5849190 & -4.3707920 & 2.3433740 & $\mathrm{C}$ & -3.9701350 & -1.8490880 & -1.4465270 \\
\hline C & 2.4656470 & -2.5205110 & 5.5560740 & $\mathrm{C}$ & -3.8497680 & -2.4122190 & 0.8696000 \\
\hline $\mathrm{H}$ & 0.9028760 & -1.6838340 & 4.3305220 & $\mathrm{C}$ & -4.5874130 & -1.9128250 & -0.1969680 \\
\hline C & 3.5735780 & -3.3649780 & 5.5903730 & $\mathrm{H}$ & -4.5348380 & -1.4652930 & -2.2921710 \\
\hline $\mathrm{H}$ & 4.8445810 & -4.6918890 & 4.4657050 & $\mathrm{H}$ & -4.3214510 & -2.4753460 & 1.8483630 \\
\hline $\mathrm{H}$ & 2.1502090 & -1.9987890 & 6.4540860 & C & 3.7387020 & 1.8510550 & 0.2528350 \\
\hline $\mathrm{H}$ & 4.1202450 & -3.5020230 & 6.5186200 & $\mathrm{C}$ & 3.0941300 & 2.7739740 & 1.1035760 \\
\hline H & -0.0206850 & -1.8157300 & 1.1148440 & $\mathrm{C}$ & 4.2497030 & 2.2668690 & -0.9859400 \\
\hline 0 & 1.8154180 & -3.4156910 & 0.9060620 & C & 2.9881390 & 4.0985180 & 83920 \\
\hline & & & & C & 4.1169600 & 3.6064150 & -1.3566530 \\
\hline & R) $\mathrm{G}[\mathrm{WB} 97$ & $G(d)]$ & 25 & C & 3.4818180 & 4.5342680 & -0.5405000 \\
\hline 0 & 0.1588210 & -1.1544210 & 0.2494940 & $\mathrm{H}$ & 2.4803460 & 4.8106280 & 1.3329800 \\
\hline 0 & 2.0932550 & -0.0582590 & -0.9343730 & $\mathrm{H}$ & 4.5065430 & 3.9323850 & -2.3192190 \\
\hline C & 0.5301740 & -2.3786450 & -0.2576740 & C & -1.7901620 & -3.3847890 & 1.9346060 \\
\hline C & 1.8583170 & -2.7465490 & -0.2317030 & $\mathrm{C}$ & -6.0316660 & -1.4849510 & -0.0072640 \\
\hline C & -0.5159370 & -3.2314380 & -0.7008440 & $\mathrm{C}$ & -2.0541780 & -2.2436900 & -3.0475240 \\
\hline C & 2.2197710 & -3.9930790 & -0.8407560 & $\mathrm{C}$ & 2. 5189690 & 2.3611030 & 2.4531170 \\
\hline C & -0.1682040 & -4.4708560 & -1.1693540 & C & 3.2783910 & 5.9667700 & -0.9993590 \\
\hline C & 1.1902900 & -4.8648320 & -1.2918020 & $\mathrm{C}$ & 4.9320880 & 1.2985390 & -1.9405740 \\
\hline $\mathrm{H}$ & -0.9442100 & -5.1710330 & -1.4657240 & $\mathrm{C}$ & 6.4131160 & 1.6545670 & -2.1254040 \\
\hline C & 2.9103520 & -0.5205790 & 0.0729620 & $\mathrm{H}$ & 6.5303510 & 2.6421970 & -2.5866040 \\
\hline C & 3.8160590 & 0.4108340 & 0.6471450 & $\mathrm{H}$ & 6.9360030 & 1.6685440 & -1.1631530 \\
\hline C & 2.8519870 & -1.8511040 & 0.4240040 & $\mathrm{H}$ & 6.9060020 & 0.9214350 & -2.7738970 \\
\hline C & 4.7161170 & -0.0656090 & 1.5632340 & $\mathrm{C}$ & 4.2008030 & 1.2233050 & -3.2870980 \\
\hline C & 3.7117700 & -2.3072770 & 1.4789510 & $\mathrm{H}$ & 3.1486740 & 0.9639200 & -3.1401810 \\
\hline C & 4.6781600 & -1.4103050 & 2.0138390 & $\mathrm{H}$ & 4.2493360 & 2.1794090 & -3.8213500 \\
\hline $\mathrm{H}$ & 30940 & 724 & 1.9838210 & $\mathrm{H}$ & 4.6594430 & 0.4610610 & -3.9270380 \\
\hline
\end{tabular}




$\begin{array}{rrr}3.8716440 & 6.9847200 & -0.0177660 \\ 3.7582740 & 8.0032240 & -0.4058330 \\ 3.3665410 & 6.9405290 & 0.9538390 \\ 4.9370480 & 6.7962040 & 0.1487320 \\ 1.7887500 & 6.2458420 & -1.2504570 \\ 1.3762330 & 5.5317030 & -1.9712890 \\ 1.2151030 & 6.1562220 & -0.3201800 \\ 1.6416260 & 7.2584410 & -1.6430780 \\ 3.5502550 & 2.5718560 & 3.5724530 \\ 4.4715710 & 2.0120140 & 3.3904960 \\ 3.8132150 & 3.6335150 & 3.6536330 \\ 3.1412240 & 2.2484370 & 4.5365620 \\ 1.2183290 & 3.0918500 & 2.8103590 \\ 1.4003170 & 4.1388040 & 3.0809120 \\ 0.5097780 & 3.0651650 & 1.9801780 \\ 0.7520450 & 2.6114190 & 3.6775520 \\ -2.3633980 & -4.7463250 & 2.3497770 \\ -3.4184570 & -4.6580440 & 2.6348920 \\ -2.2967340 & -5.4666850 & 1.5274360 \\ -1.8140790 & -5.1517950 & 3.2069150 \\ -1.7904960 & -2.3977530 & 3.1076670 \\ -1.4273410 & -1.4158910 & 2.7893010 \\ -2.7946870 & -2.2770900 & 3.5329440 \\ -1.1397570 & -2.7629370 & 3.9097660 \\ -6.9845620 & -2.3888900 & -0.8009090 \\ -8.0288860 & -2.1258420 & -0.5978360 \\ -6.8157660 & -2.2827880 & -1.8788750 \\ -6.8371270 & -3.4420530 & -0.5409690 \\ -6.2488050 & -0.0109420 & -0.3646430 \\ -5.5872910 & 0.6325090 & 0.2224760 \\ -6.0511390 & 0.1707670 & -1.4275490 \\ -7.2831350 & 0.2873170 & -0.1602120 \\ -2.5117850 & -1.0496170 & -3.8903150 \\ -3.5676100 & -1.1288830 & -4.1757080 \\ -2.3629540 & -0.1058860 & -3.3629360 \\ -1.9291270 & -1.0092820 & -4.8166830 \\ -2.3672720 & -3.5481300 & -3.7971090 \\ -3.4504640 & -3.6712240 & -3.9171300 \\ -1.9130010 & -3.5346210 & -4.7943040 \\ -1.9872910 & -4.4247310 & -3.2658570 \\ 0.5169740 & 0.1786090 & -0.6223750 \\ -0.1594110 & 0.1972870 & -1.9607970 \\ 0.2931060 & 1.3175960 & 0.3391480 \\ -0.9692710 & -2.1572680 & -2.9365090 \\ -6.2665500 & -1.6012300 & 1.6590980 \\ -0.7450480 & -3.5478000 & \end{array}$

\begin{abstract}
4.8953060
3. 8025840

2. 2841440

$-2.5428240$

$-1.6849240$

$-2.4768800$

$-0.7336640$

$-1.6227690$

$-0.9424060$

$-4.0307000$

$-3.3963660$

$-1.9246580$

$-3.9524390$

$-5.0777250$

$-3.4713680$

$-1.3030260$

$-4.5641490$

$-4.3328880$

$-3.5165650$

$-3.9380780$

$-1.5154240$

$-2.7194740$

$-1.8498620$

$-4.0482120$

$-5.0573080$

$-4.3149940$

$-6.3300800$

$-4.8335450$

$-5.5899260$

$-3.5284180$

$-6.5968520$

$-7.1150050$

$-5.7991140$

$-7.5931710$

$-0.9839010$

$-2.5015410$
\end{abstract}

144

$\operatorname{TS} 102(R, R) G[W B 97 X D / 6-31 G(d)]=-3310.755880$

$\begin{array}{lrrr}\mathrm{O} & 0.1588720 & -1.1544880 & 0.2496020 \\ \mathrm{O} & 2.0931880 & -0.0582120 & -0.9343270 \\ \mathrm{C} & 0.5303100 & -2.3786810 & -0.2575770 \\ \mathrm{C} & 1.8584730 & -2.7465030 & -0.2316050 \\ \mathrm{C} & -0.5157550 & -3.2315300 & -0.7007520 \\ \mathrm{C} & 2.2199930 & -3.9930250 & -0.8406380 \\ \mathrm{C} & -0.1679600 & -4.4709360 & -1.1692420 \\ \mathrm{C} & 1.1905590 & -4.8648420 & -1.2916700\end{array}$

$-1.4957210$

$-1.9567830$

2. 3998120

$-1.5645320$

0.9809430

$-1.0928600$

$-0.5719120$

$-2.6657720$

$-2.5137910$

$-2.5024590$

$-1.5325240$

$-1.2666170$

$-1.9412330$

$-2.6828000$

$-1.9254710$

$-2.1375550$

$-1.0342840$

$-2.6562000$

$-3.4698580$

$-0.5799860$

1. 6136370

1.7591050

2. 2138640

2. 1014610

2. 8153850

2.5861510

1.6282370

3. 2977970

2. 8835770

3. 1809590

2. 4979810

3. 7612790

3. 5556020

1.2500520

1. 0048950
$-0.4316850$

$-0.6649860$

1. 0357240

1. 2669100

3.1320570

$-4.8648420$

$-1.2916700$ 


$\begin{array}{lrrr}\mathrm{H} & -0.9439260 & -5.1711480 & -1.4656360 \\ \mathrm{C} & 2.9103410 & -0.5204500 & 0.0729980 \\ \mathrm{C} & 3.8159960 & 0.4110440 & 0.6471290 \\ \mathrm{C} & 2.8520880 & -1.8509730 & 0.4240700 \\ \mathrm{C} & 4.7161020 & -0.0653040 & 1.5632210 \\ \mathrm{C} & 3.7119260 & -2.3070530 & 1.4790110 \\ \mathrm{C} & 4.6782530 & -1.4099910 & 2.0138620 \\ \mathrm{H} & 5.4580370 & 0.6076210 & 1.9837760 \\ \mathrm{C} & 5.4684490 & -3.1335970 & 3.5291560 \\ \mathrm{C} & 4.4761440 & -4.0104820 & 3.0355830 \\ \mathrm{C} & 3.6224360 & -3.6096260 & 2.0394880 \\ \mathrm{C} & 5.5603220 & -1.8606360 & 3.0304730 \\ \mathrm{H} & 6.1442640 & -3.4658640 & 4.3112660 \\ \mathrm{H} & 4.3836390 & -5.0081640 & 3.4541290 \\ \mathrm{H} & 2.8608360 & -4.2914950 & 1.6787800 \\ \mathrm{H} & 6.3014270 & -1.1664540 & 3.4184070 \\ \mathrm{C} & 3.8784730 & -5.5876840 & -1.6195130 \\ \mathrm{C} & 2.8541260 & -6.4707070 & -2.0295180 \\ \mathrm{C} & 1.5407860 & -6.1123520 & -1.8706160 \\ \mathrm{C} & 3.5709240 & -4.3836290 & -1.0392790 \\ \mathrm{H} & 4.9181420 & -5.8616540 & -1.7711590 \\ \mathrm{H} & 3.1102460 & -7.4237200 & -2.4821900 \\ \mathrm{H} & 0.7442920 & -6.7745280 & -2.2001290 \\ \mathrm{H} & 4.3652400 & -3.7098160 & -0.7375430 \\ \mathrm{C} & -1.9345220 & -2.7797910 & -0.5496430 \\ \mathrm{C} & -2.6577590 & -2.2759930 & -1.6497160 \\ \mathrm{C} & -2.5290880 & -2.8384260 & 0.7214240 \\ \mathrm{C} & -3.9699790 & -1.8493550 & -1.4466130 \\ \mathrm{C} & -3.8495790 & -2.4121340 & 0.8695990 \\ \mathrm{C} & -4.5872540 & -1.9129460 & -0.1970480 \\ \mathrm{H} & -4.5346940 & -1.4656910 & -2.2923090 \\ \mathrm{H} & -4.3212810 & -2.4751570 & 1.8483570 \\ \mathrm{C} & 3.7385440 & 1.8512460 & 0.2527710 \\ \mathrm{C} & 3.0940390 & 2.7741810 & 1.1035410 \\ \mathrm{C} & 4.2493990 & 2.2670230 & -0.9860790 \\ \mathrm{C} & 2.9880230 & 4.0987210 & 0.6883430 \\ \mathrm{C} & 4.1166140 & 3.6065570 & -1.3568140 \\ \mathrm{C} & 3.4815860 & 4.5344410 & -0.5406060 \\ \mathrm{H} & 2.4802960 & 4.8108490 & 1.3329640 \\ \mathrm{H} & 4.5060950 & 3.9325020 & -2.3194300 \\ \mathrm{C} & -1.7899480 & -3.3846100 & 1.9346910 \\ \mathrm{C} & -6.0315310 & -1.4851260 & -0.0073990 \\ \mathrm{C} & -2.0540000 & -2.2440840 & -3.0475520 \\ \mathrm{C} & 2.5189360 & 2.3613310 & 2.4531140 \\ \mathrm{C} & 3.2781110 & 5.9669270 & -1.9494940 \\ \mathrm{C} & 4.9316850 & 1.2986680 & \\ & & & \end{array}$

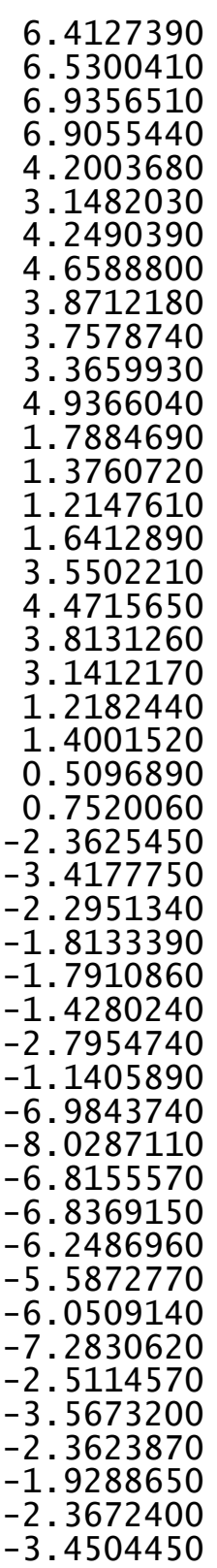

1.6545860

2. 6422480

1. 6684300

0.9214700

1. 2235300

0.9643030

2. 1796150

0.4612060

6.9849400

8.0034190

6.9408010

6.7964580

6.2458870

5. 5317360

6.1561840

7. 2584860

2. 5722070

2. 0124030

3. 6338840

2. 2488240

3.0920000

4.1389830

3. 0652080

2. 6115820

$-4.7466180$

$-4.6590230$

$-5.4666640$

$-5.1520590$

$-2.3979970$

$-1.4159380$

$-2.2777180$

$-2.7632890$

$-2.3890760$

$-2.1260630$

$-2.2829460$

$-3.4422400$

$-0.0111140$

0.6323450

0.1706350

0.2870950

$-1.0499760$

$-1.1290470$

$-1.0097950$

-3.5485230
-3.6715020
$-2.1255970$

$-2.5867100$

$-1.1633570$

$-2.7741690$

$-3.2872700$

$-3.1403300$

$-3.8215410$

$-3.9272050$

$-0.0178800$

$-0.4060200$

0.9536640

0.1487620

$-1.2507080$

$-1.9715970$

$-0.3204770$

$-1.6433060$

3. 5724270

3. 3904940

3. 6535400

4.5365590

2.8103300

3. 0808190

1. 9801560

3.6775560

2. 3491970

2. 6338990

1. 5266420

3. 2064230

3. 1080960

2. 7902160

3. 5330080

3. 9103400

$-0.8010940$

$-0.5980430$

$-1.8790540$

$-0.5411730$

$-0.3647540$

0.2224620

$-1.4276320$

$-0.1604320$

$-3.8903740$

$-4.1756790$

$-3.3630350$

$-4.8167920$

$-3.7970750$

-3. 9171010 


\begin{tabular}{|c|c|c|c|}
\hline $\mathrm{H}$ & -1.9129550 & -3.5351240 & -4.7942650 \\
\hline $\mathrm{H}$ & -1.9873690 & -4.4251390 & -3.2657640 \\
\hline$P$ & 0.5168990 & 0.1785580 & -0.6222940 \\
\hline 0 & -0.1594980 & 0.1971770 & -1.9607020 \\
\hline 0 & 0.2929560 & 1.3175240 & 0.3392380 \\
\hline $\mathrm{H}$ & -0.9690830 & -2.1577820 & -2.9365460 \\
\hline $\mathrm{H}$ & -6.2664540 & -1.6014320 & 1.0583770 \\
\hline $\mathrm{H}$ & -0.7446670 & -3.5469250 & 1.6594090 \\
\hline $\mathrm{H}$ & 4.8948330 & 0.2998580 & -1.4959400 \\
\hline $\mathrm{H}$ & 3.8023690 & 6.0827180 & -1.9568800 \\
\hline $\mathrm{H}$ & 2.2841800 & 1.2938950 & 2.3998550 \\
\hline C & -2.5429430 & 2.0783540 & -1.5645280 \\
\hline C & -1.6850200 & 3. 1179980 & -0.9808330 \\
\hline $\mathrm{H}$ & -2.4770330 & 1.0952010 & -1.0928750 \\
\hline $\mathrm{H}$ & -0.7337550 & 2.7906920 & -0.5718520 \\
\hline 0 & -1.6229260 & 2.1952610 & -2.6657970 \\
\hline $\mathrm{H}$ & -0.9427680 & 1.4609050 & -2.5140040 \\
\hline C & -4.0307010 & 3.8882130 & -2.5024920 \\
\hline C & -3.3963050 & 4.8887850 & -1.5325910 \\
\hline C & -1.9246390 & 4.5579950 & -1.2665790 \\
\hline C & -3.9525420 & 2.4664740 & -1.9412030 \\
\hline $\mathrm{H}$ & -5.0777080 & 4.1509440 & -2.6828300 \\
\hline $\mathrm{H}$ & -3.4711970 & 5.9072150 & -1.92 \\
\hline $\mathrm{H}$ & -1.3029300 & 4.8025350 & -2.13 \\
\hline $\mathrm{H}$ & -4.5642450 & 2.3876840 & -1.0342390 \\
\hline $\mathrm{H}$ & -4.3330620 & 1.7293270 & -2.6561210 \\
\hline $\mathrm{H}$ & -3.5165740 & 3.9291880 & -3.4698980 \\
\hline $\mathrm{H}$ & -3.9380670 & 4.8669500 & -0.5800790 \\
\hline $\mathrm{H}$ & -1.5154120 & 5.1333130 & -0.4316540 \\
\hline C & -2.7196310 & 2.0763870 & 1.6136840 \\
\hline 0 & -1.8500140 & 1.1143460 & 1.7591090 \\
\hline C & -4.0483500 & 1.7960880 & 2.2139410 \\
\hline C & -5.0574680 & 2.7558070 & 2.1015760 \\
\hline C & -4.3151020 & 0.5638560 & 2.8154310 \\
\hline C & -6.3302320 & 2.4824180 & 2.5862630 \\
\hline $\mathrm{H}$ & -4.8337330 & 3.7059720 & 1.6283740 \\
\hline C & -5.5900270 & 0.2940710 & 3.2978330 \\
\hline $\mathrm{H}$ & -3.5285100 & -0.1775720 & 2.8836110 \\
\hline C & -6.5969760 & 1.2501950 & 3.1810270 \\
\hline $\mathrm{H}$ & -7.1151750 & 3.2267200 & 2.4981210 \\
\hline $\mathrm{H}$ & -5.7991880 & -0.6652220 & 3.7612840 \\
\hline $\mathrm{H}$ & -7.5932900 & 1.0354520 & 3.5556610 \\
\hline $\mathrm{H}$ & -0.9840160 & 1.2668220 & 1.2501130 \\
\hline 0 & -2.5017440 & 3.1319470 & 1.0049350 \\
\hline
\end{tabular}

0.0296150

$-2.0739380$

$-0.2463270$

$-1.5300940$

0.8498260

$-1.8103060$

0.5898500

$-0.7320170$

1.4081810

$-2.7644670$

$-3.7169200$

$-2.5541440$

$-4.4854200$

$-3.2789760$

$-4.2792000$

$-5.2570630$

$-4.7789500$

$-3.7533740$

$-3.0240370$

$-5.0286730$

$-5.3524260$

$-3.5366130$

$-2.2341630$

$-5.7965980$

$-3.3537260$

$-2.2772250$

$-0.9966260$

$-3.1273940$

$-4.3690850$

$-2.4692710$

$-0.1619390$

$-3.9615890$

2. 2261990

2. 8941890

2. 8414760

4. 1649840

4. 1095110

4.7913560

4.6798950

4. 5929430

$-3.8772540$

$-3.2786890$

$-4.6353550$

$-3.4906590$

-4.8067290
-4.2492160
1.0878600

0.2001290

2.3622670

2. 8477420

3. 1334100

4.1403390

4.4101480

4. 9261500

5.0475930

0.7171960

$-0.1304430$

2.0284610

0.4048490

2.5386310

1. 7239200

$-0.2050140$

3.4725630

4.2662260

3. 8141430

2. 2262030

3.8461560

5.2418920

4.4335940

1. 5925040

5.9056930

6.6992550

6. 2156160

4.6617210

6.2828950

7.6837460

6.8088890

4.0575240

2. 5546100

2.0998240

2. 4497820

1.5309830

1.8850330

1.4097800

1.1904510

1. 8221930

$-1.5528610$

$-2.5828570$

$-1.8547750$

$-3.9035480$

$-3.1892950$

$-4.2294890$
0.1618410

$-0.9099040$

$-0.2818350$

$-0.1692560$

$-0.7536200$

$-0.7218850$

$-1.1755470$

$-1.2147580$

$-1.4975520$

0.1644820

0.7885590

0.5354150

1.7886410

1.6646210

2. 2642010

2. 2506290

3.8702080

3. 3083350

2. 2382290

3. 3597870

4.7129930

3.7327770

1. 8291940

3.7959590

$-1.3539610$

$-1.8113190$

$-1.7443000$

$-0.8221010$

$-1.4304230$

$-2.2267970$

$-2.1087370$

$-0.4819700$

$-0.6755320$

$-1.8252020$

0.5872100

$-1.6837070$

0.6740220

$-0.4460820$

$-2.5757960$

1.6467340

0.3574160

1. 1071140

$-0.7866850$

0.7102780

$-1.1490720$

$-0.4101200$ 


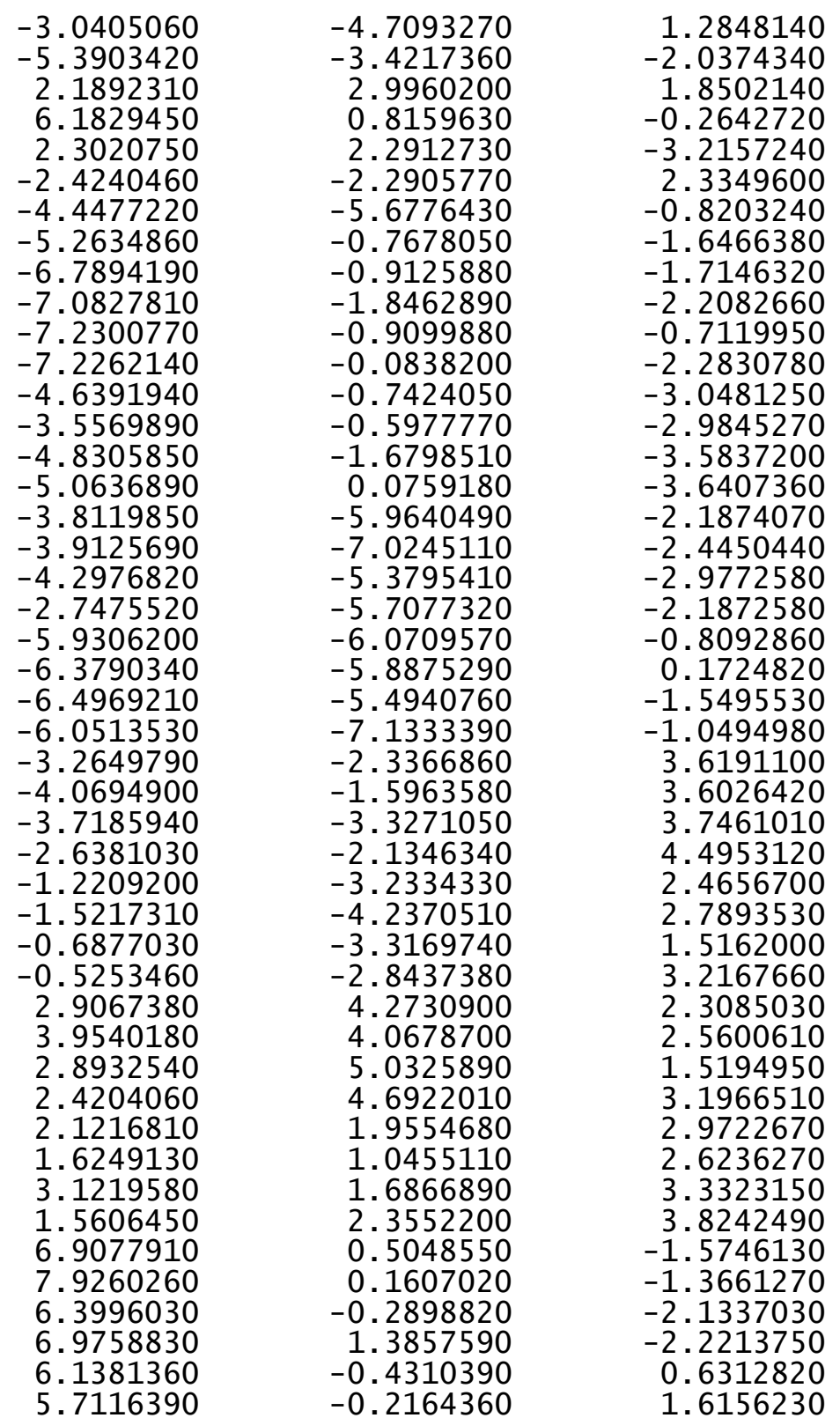

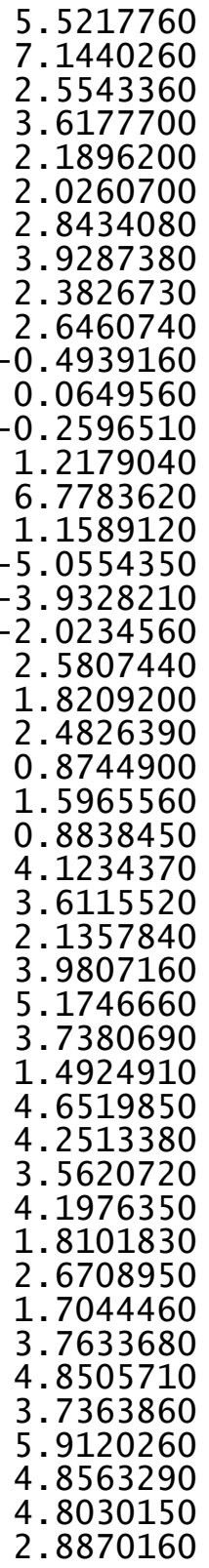

-1.2142340
-0.8385270
1.1219540
1.0082630
0.1812310
1.2974560
3.5880790
3.5185730
3.7655730
4.4587210
-0.1529600
-0.1068090
-1.3754230
2.3858580
1.5730060
3.2755060
0.2001260
-6.3011570
-1.2781540
-1.8557390
-2.9730750
-0.8986620
-2.7269350
-2.0004770
-1.3112300
-3.5217160
-4.5967010
-4.3811770
-2.1286360
-3.7047340
-5.5936010
-4.6252400
-2.0328920
-1.3424590
-3.5651320
-4.5662750
-5.0229490
-2.2920320
-1.4434560
-2.2492190
-3.1124550
-1.3334610
-3.0528260
-3.8174730
-1.2723220
-0.6700520

0.7816660

$-4.1714570$

$-4.4140720$

$-3.7572760$

$-5.1146280$

$-3.8393440$

$-3.9813140$

$-4.8177160$

$-3.2077480$

$-0.7625210$

$-2.1542000$

0.0875780

$-3.1051220$

0.2657430

1. 6164820

$-1.1809740$

$-0.0778450$

2. 2245460

$-1.9230410$

$-1.3531050$

$-1.4068990$

$-0.8834420$

$-2.9691710$

$-2.7535040$

$-3.0343710$

$-2.0717170$

$-1.7249050$

$-2.4161340$

$-3.2781040$

$-2.5048510$

$-2.5793770$

$-1.5553770$

$-3.1286300$

$-3.9751360$

$-1.1457650$

$-0.9020610$

1.5434380

1.7643040

2. 5504490

2. 4012590

3. 6043170

3. 2949430

1. 5769870

4.4939290

3. 7142310 


\begin{tabular}{|c|c|c|c|c|c|c|c|}
\hline C & 5.8910190 & -2.1289990 & 4.3383580 & $\mathrm{H}$ & -4.4439700 & -1.8092020 & -2.5620920 \\
\hline $\mathrm{H}$ & 6.7573450 & -3.7235490 & 3.1775360 & $\mathrm{H}$ & -4.3135340 & -2.6048190 & 1.6296330 \\
\hline $\mathrm{H}$ & 4.7851520 & -0.5550240 & 5.3084440 & $\mathrm{C}$ & 3.6439570 & 1.9513710 & 0.3315760 \\
\hline $\mathrm{H}$ & 6.7228670 & -2.0790800 & 5.0345890 & $\mathrm{C}$ & 2.9272860 & 2.8565600 & 1.1428880 \\
\hline $\mathrm{H}$ & 0.9601890 & -1.4566290 & 1.0754360 & $\mathrm{c}$ & 4.2077370 & 2.3797250 & -0.8796130 \\
\hline 0 & 2.7256140 & -3.0777960 & 0.5907090 & C & 2.7999840 & 4.1751070 & 0.7151420 \\
\hline & & & & $\mathrm{C}$ & 4.0467790 & 3.7116890 & -1.2667510 \\
\hline \multicolumn{4}{|c|}{$\operatorname{TS} 104(R, R) \quad G[W B 97 X D / 6-31 G(d)]=-3310.756032$} & $\mathrm{C}$ & 3.3394300 & 4.6211910 & -0.4906690 \\
\hline 0 & 0.1673010 & -1.1830340 & 0.2137670 & $\mathrm{H}$ & 2.2399990 & 4.8735720 & 1.3309320 \\
\hline 0 & 2.1011560 & -0.0179330 & -0.9074990 & $\mathrm{H}$ & 4.4754820 & 4.0465380 & -2.2093800 \\
\hline C & 0.6000440 & -2.3933400 & -0.2784280 & C & -1.7662700 & -3.4029650 & 1.8323110 \\
\hline C & 1.9390320 & -2.7108400 & -0.2049740 & $\mathrm{C}$ & -6.0143510 & -1.7582550 & -0.3930510 \\
\hline c & -0.3963380 & -3.2833090 & -0.7602470 & c & -1.8811260 & -2.4184490 & -3.1912600 \\
\hline C & 2.3698610 & -3.9425060 & -0.7983880 & C & 2.3021050 & 2.4329300 & 2.4663770 \\
\hline C & 0.0155170 & -4.5073250 & -1.2179510 & c & 3.1230280 & 6.0478690 & -0.9615990 \\
\hline C & 1.3911650 & -4.8507550 & -1.2889540 & $\mathrm{C}$ & 4.9850560 & 1.4359510 & -1.7851700 \\
\hline $\mathrm{H}$ & -0.7227820 & -5.2331750 & -1.5469560 & C & 6.4504550 & 1.8733570 & -1.9114550 \\
\hline C & 2.8983260 & -0.4475230 & 0.1306670 & $\mathrm{H}$ & 6.5333860 & 2.8549540 & -2.3923190 \\
\hline C & 3.7490960 & 0.5155240 & 0.7362960 & $\mathrm{H}$ & 6.9264800 & 1.9405270 & -0.9273960 \\
\hline C & 2.8738730 & -1.7786130 & 0.4842930 & $\mathrm{H}$ & 7.0144460 & 1.1556560 & -2.5178350 \\
\hline C & 4.6287450 & 0.0720560 & 1.6884460 & C & 4.3200840 & 1.2969150 & -3.1607090 \\
\hline C & 3.7077750 & -2.2030170 & 1.5721330 & $\mathrm{H}$ & 3.2840180 & 0.9631560 & -3.0551370 \\
\hline $\mathrm{C}$ & 4.6212630 & -1.2724040 & 2.1411260 & $\mathrm{H}$ & 4.3224240 & 2.2497950 & -3.7028630 \\
\hline $\mathrm{H}$ & 5.3305280 & 0.7711140 & 2.1348460 & $\mathrm{H}$ & 4.8597510 & 0.5645690 & -3.7717770 \\
\hline C & 5.4126460 & -2.9664020 & 3.6886930 & C & 3.6901450 & 7.0747080 & 0.0265520 \\
\hline C & 4.4700430 & -3.8774030 & 3.1604150 & $\mathrm{H}$ & 3.5677010 & 8.0918240 & -0.3624820 \\
\hline C & 3.6417110 & -3.5 & 2.1316530 & $\mathrm{H}$ & 3.1740190 & 7.0217480 & 0.9919710 \\
\hline C & 5.4794510 & -1.6914010 & 3.1913120 & $\mathrm{H}$ & 4.7558990 & 6.9002700 & 0.2056950 \\
\hline $\mathrm{H}$ & 6.0695310 & -3.2747290 & 4.4963470 & C & 1.6355420 & 6.3112960 & -1.2386300 \\
\hline $\mathrm{H}$ & 4.3960810 & -4.8771140 & 3.5777880 & $\mathrm{H}$ & 1.2412960 & 5.5886980 & -1.9612040 \\
\hline $\mathrm{H}$ & 2.9175430 & -4.2147590 & 1.7434590 & $\mathrm{H}$ & 1.0461590 & 6.2244880 & -0.3180670 \\
\hline $\mathrm{H}$ & 6.1821690 & -0.9724050 & 3.6046840 & $\mathrm{H}$ & 1.4865150 & 7. 3196190 & -1.6412990 \\
\hline C & 4.1149960 & -5.4729230 & -1.5137190 & C & 3.2738780 & 2.6783680 & 3.6308290 \\
\hline C & 3.1403620 & -6.3918930 & -1.9643950 & $\mathrm{H}$ & 4.2162440 & 2.1406670 & 3.4963710 \\
\hline C & 1.8094870 & -6.0830190 & -1.8553680 & $\mathrm{H}$ & 3.5061070 & 3.7468750 & 3.7154610 \\
\hline C & 3.7409930 & -4.2825370 & -0.9443790 & $\mathrm{H}$ & 2.8284410 & 2.3513970 & 4.5774200 \\
\hline $\mathrm{H}$ & 5.1690420 & -5.708 & -1.6259000 & C & 0.9655780 & 3.1271690 & 2.7589140 \\
\hline $\mathrm{H}$ & 3.4494840 & -7.3334250 & -2.4079510 & $\mathrm{H}$ & 1.1064050 & 4.1766100 & 3.0441790 \\
\hline $\mathrm{H}$ & 1.0511860 & -6.7734810 & -2.2155620 & $\mathrm{H}$ & 0.3015080 & 3.0886700 & 1.8930810 \\
\hline $\mathrm{H}$ & 4.4970950 & -3.5807410 & -0.6097290 & $\mathrm{H}$ & 0.4674500 & 2.6291950 & 3.5980610 \\
\hline C & -1.8363860 & -2.8861530 & -0.6738740 & C & -2.3053480 & -4.7711890 & 2.2715030 \\
\hline C & -2.5397760 & -2.4614750 & -1.8188280 & $\mathrm{H}$ & -3.3725470 & -4.7129580 & 2.5164960 \\
\hline C & -2.4787530 & -2.9262220 & 0.5741810 & $\mathrm{H}$ & -2.1835470 & -5.5136180 & 1.4754590 \\
\hline C & -3.8831310 & -2.1165700 & -1.6820000 & $\mathrm{H}$ & -1.7727350 & -5.1291070 & 3.1598510 \\
\hline C & 33240 & -2.5 & 0.6613390 & C & -1.8476950 & -2.3798870 & 2.9705040 \\
\hline C & -4 & & -0.4557740 & $\mathrm{H}$ & -1.5038580 & -1.3975330 & 2.6336030 \\
\hline
\end{tabular}




$\begin{array}{rrr}-2.8729930 & -2.2807730 & 3.3480190 \\ -1.2207620 & -2.6977090 & 3.8109580 \\ -6.1593750 & -0.2329390 & -0.4987400 \\ -7.2154640 & 0.0602800 & -0.4871630 \\ -5.6603730 & 0.2580660 & 0.3439500 \\ -5.7117660 & 0.1396480 & -1.4252420 \\ -6.7510250 & -2.2785790 & 0.8425230 \\ -6.6177690 & -3.3582210 & 0.9678610 \\ -6.3986170 & -1.7799790 & 1.7522930 \\ -7.8230880 & -2.0740580 & 0.7526520 \\ -2.3287370 & -1.2269190 & -4.0443390 \\ -3.3698860 & -1.3249920 & -4.3739130 \\ -2.2215510 & -0.2862230 & -3.5010430 \\ -1.7087100 & -1.1658550 & -4.9450780 \\ -2.1361650 & -3.7266190 & -3.9552970 \\ -3.2109850 & -3.8746680 & -4.1154140 \\ -1.6464830 & -3.7000500 & -4.9353520 \\ -1.7559760 & -4.5958460 & -3.4115440 \\ 0.5066020 & 0.1586800 & -0.6509830 \\ -0.1278240 & 0.1484170 & -2.0098760 \\ 0.2123070 & 1.2928150 & 0.2968020 \\ -0.8033470 & -2.3108230 & -3.0377880 \\ -6.4990510 & -2.1969080 & -1.2761140 \\ -0.7063140 & -3.5367570 & 1.6019030 \\ 4.9855830 & 0.4438310 & -1.3240520 \\ 3.6617670 & 6.1656360 & -1.9106510 \\ 2.1016200 & 1.3589820 & 2.4085090 \\ -2.5661490 & 1.9917740 & -1.7124910 \\ -1.7338380 & 3.0415860 & -1.1127720 \\ -2.5072830 & 1.0125920 & -1.2321880 \\ -0.7952940 & 2.7248230 & -0.6681760 \\ -1.6107120 & 2.1102690 & -2.7840500 \\ -0.9247160 & 1.3881630 & -2.6025590 \\ -4.0378390 & 3.7883770 & -2.7055640 \\ -3.4393520 & 4.7987860 & -1.7234540 \\ -1.9745250 & 4.4786050 & -1.4120720 \\ -3.9652480 & 2.3693100 & -2.1361040 \\ -5.0814990 & 4.0415550 & -2.9165820 \\ -3.5090530 & 5.8142270 & -2.1250280 \\ -1.3279100 & 4.7246880 & -2.2640870 \\ -4.6041250 & 2.2887750 & -1.2478120 \\ -4.3174150 & 1.6287640 & -2.8619350 \\ -3.4973290 & 3.8287410 & -3.6585390 \\ -4.0089830 & 4.7794300 & -0.7872870 \\ -1.5949290 & 5.0594400 & -0.5669640 \\ -2.8246910 & 2.0293140 & 1.5058410\end{array}$

$\begin{array}{ll}\mathrm{O} & -1.9444800 \\ \mathrm{C} & -4.1403830 \\ \mathrm{C} & -5.1759560 \\ \mathrm{C} & -4.3651230 \\ \mathrm{C} & -6.4337360 \\ \mathrm{H} & -4.9830410 \\ \mathrm{C} & -5.6230280 \\ \mathrm{H} & -3.5589300 \\ \mathrm{C} & -6.6575880 \\ \mathrm{H} & -7.2390480 \\ \mathrm{H} & -5.7989680 \\ \mathrm{H} & -7.6405860 \\ \mathrm{H} & -1.0779670 \\ \mathrm{O} & -2.6271020 \\ \mathrm{I} & \end{array}$

1.0829440
1.7682180
2.6855460
0.5997140
2.4337500
3.5878670
0.3552390
-0.1122050
1.2677940
3.1452940
-0.5520080
1.0703870
1.2292090
3.0586400
1.6836650
2. 1439130
1.9514720
2.8758920
2.4850990
1. 3809900
3.4138370
3.0063960
3. 2147260
2. 3327950
3. 9837170
3.6314350
3.6314350
1.1725640

$\operatorname{TS} 105(R, R) \quad G[W B 97 X D / 6-31 G(d)]=-3310.757311$

$\begin{array}{lrcr}\text { TS105(R, R }(\mathrm{G}[\mathrm{WB} 97 \times \mathrm{D} / 6-31 \mathrm{G}(\mathrm{d})]=-3310.757311 \\ \mathrm{O} & 0.4954730 & 0.5512360 & 0.0188700 \\ \mathrm{O} & -1.8395720 & 0.7909760 & -0.9528990 \\ \mathrm{C} & 0.6076640 & 1.9226320 & -0.0172410 \\ \mathrm{C} & -0.4254060 & 2.7008410 & 0.4627480 \\ \mathrm{C} & 1.8353440 & 2.4700930 & -0.4708160 \\ \mathrm{C} & -0.3157800 & 4.1260660 & 0.3659450 \\ \mathrm{C} & 1.9702270 & 3.8356340 & -0.4594700 \\ \mathrm{C} & 0.9077660 & 4.6925270 & -0.0818070 \\ \mathrm{H} & 2.9143370 & 4.2757270 & -0.7699040 \\ \mathrm{C} & -2.3220760 & 1.1228250 & 0.3007900 \\ \mathrm{C} & -3.5311550 & 0.5172650 & 0.7338190 \\ \mathrm{C} & -1.6307630 & 2.0529510 & 1.0422390 \\ \mathrm{C} & -4.0130240 & 0.8823880 & 1.9648030 \\ \mathrm{C} & -2.0831100 & 2.3405240 & 2.3710770 \\ \mathrm{C} & -3.3011130 & 1.7613240 & 2.8198730 \\ \mathrm{H} & -4.9555820 & 0.4667360 & 2.3098520 \\ \mathrm{C} & -3.0520640 & 2.8615090 & 4.9680530 \\ \mathrm{C} & -1.8214280 & 3.4072890 & 4.5368860 \\ \mathrm{C} & -1.3494110 & 3.1554450 & 3.2741280 \\ \mathrm{C} & -3.7715560 & 2.0536200 & 4.1269250 \\ \mathrm{H} & -3.4172820 & 3.0735530 & 5.9683270 \\ \mathrm{H} & -1.2415860 & 4.0279360 & 5.2133640 \\ \mathrm{H} & -0.3996670 & 3.5736920 & 2.9597650 \\ \mathrm{H} & -4.7092260 & 1.6108690 & 4.4527450 \\ \mathrm{C} & -1.2401370 & 6.3627310 & 0.5757140 \\ \mathrm{C} & -0.0097180 & 6.9241400 & 0.1631600 \\ \mathrm{C} & 1.0381450 & 6.1036760 & -0.1617990 \\ \mathrm{C} & -1.3905370 & 5.0034000 & 0.6751280 \\ \mathrm{H} & -2.0759700 & 7.0149480 & 0.8104540 \\ \mathrm{H} & 0.0963560 & 8.0022380 & 0.0915370\end{array}$




$\begin{array}{lr}\mathrm{H} & 1.9833110 \\ \mathrm{H} & -2.3433410 \\ \mathrm{C} & 2.9875820 \\ \mathrm{C} & 3.2690920 \\ \mathrm{C} & 3.8508920 \\ \mathrm{C} & 4.4632420 \\ \mathrm{C} & 5.0370660 \\ \mathrm{C} & 5.3741540 \\ \mathrm{H} & 4.6978990 \\ \mathrm{H} & 5.7129230 \\ \mathrm{C} & -4.2596790 \\ \mathrm{C} & -4.2876900 \\ \mathrm{C} & -4.9384240 \\ \mathrm{C} & -5.0240740 \\ \mathrm{C} & -5.6489190 \\ \mathrm{C} & -5.7091650 \\ \mathrm{H} & -5.0609870 \\ \mathrm{H} & -6.1729980 \\ \mathrm{C} & 3.5365110 \\ \mathrm{C} & 6.6943400 \\ \mathrm{C} & 2.3120170 \\ \mathrm{C} & -3.5616440 \\ \mathrm{C} & -6.4917670 \\ \mathrm{C} & -4.9426650 \\ \mathrm{C} & -6.3550010 \\ \mathrm{H} & -7.0380330 \\ \mathrm{H} & -6.7694310 \\ \mathrm{H} & -6.3355140 \\ \mathrm{C} & -4.3351810 \\ \mathrm{H} & -3.3274680 \\ \mathrm{H} & -4.9446120 \\ \mathrm{H} & -4.2738370 \\ \mathrm{C} & -5.9113090 \\ \mathrm{H} & -6.4432410 \\ \mathrm{H} & -6.0044700 \\ \mathrm{H} & -4.8494890 \\ \mathrm{C} & -7.9851270 \\ \mathrm{H} & -8.4056880 \\ \mathrm{H} & -8.1489630 \\ \mathrm{H} & -8.5432120 \\ \mathrm{C} & -4.5427520 \\ \mathrm{H} & -5.0684070 \\ \mathrm{H} & -5.2974950 \\ \mathrm{H} & -4.0127660 \\ \mathrm{C} & -2.7786350 \\ \mathrm{H} & -3.4450180 \\ \mathrm{H} & \end{array}$

$\begin{array}{rr}6.5204820 & -0.5000170 \\ 4.5870880 & 0.9826020 \\ 1.6267380 & -0.9150930 \\ 1.4938120 & -2.2873770 \\ 1.0663010 & 0.0415610 \\ 0.8815640 & -2.6651670 \\ 0.4671260 & -0.3856780 \\ 0.3868900 & -1.7334510 \\ 0.7850880 & -3.7230530 \\ 0.0586360 & 0.3591140 \\ -0.4553180 & -0.1347810 \\ -1.8222050 & 0.1984750 \\ 0.0096360 & -1.2770600 \\ -2.6899340 & -0.6101730 \\ -0.8991950 & -2.0568290 \\ -2.2539250 & -1.7389110 \\ -3.7470400 & -0.3568330 \\ -0.5365810 & -2.9386020 \\ 1.1345340 & 1.5288900 \\ -0.1912590 & -2.2173480 \\ 2.0083530 & -3.3537410 \\ -2.3832740 & 1.4149220 \\ -3.2284250 & -2.6003820 \\ 1.4781450 & -1.6783110 \\ 2.0716400 & -1.5863600 \\ 1.5885130 & -2.2947160 \\ 1.9443580 & -0.5804900 \\ 3.1428570 & -1.8169200 \\ 1.6841410 & -3.0715390 \\ 1.2621820 & -3.1183870 \\ 1.2076750 & -3.8484450 \\ 2.7532600 & -3.3043520 \\ -3.3140200 & -4.0182800 \\ -4.0682310 & -4.6092240 \\ -2.3535370 & -4.5381990 \\ -3.5788050 & -3.9926930 \\ -2.8781950 & -2.6367010 \\ -2.8374680 & -1.6267130 \\ -1.9021690 & -3.1080110 \\ -3.6247420 & -3.2130220 \\ -2.6376020 & 2.5685630 \\ -1.7238620 & 2.8624230 \\ -3.3775960 & 2.2766320 \\ -3.0225810 & 3.4473490 \\ -3.6638870 & 1.0961620 \\ -4.5143800 & 0.9108830\end{array}$

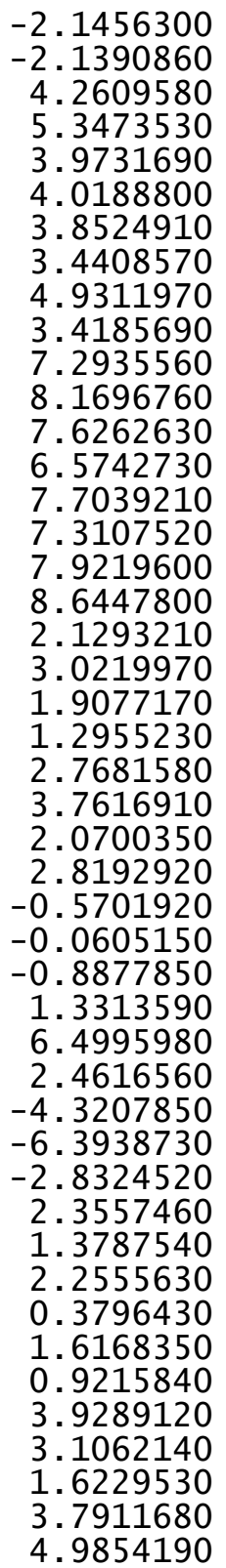
$-3.5163740$
$-3.9269760$
2. 3177940
2. 2059840
3.2635710
2. 3780170
$-0.1789620$
$-1.0360840$
$-0.3388930$
$-0.1724800$
$-1.2512800$
$-1.7093150$
$-0.8138550$
$-2.0449160$
0.9424410
1. 6787120
1.4622390
0.5476540
1. 0081250
0.9476180
0.0086920
1. 3218220
3. 3686080
3. 2873020
3. 7258910
4.1240460
$-0.2224390$
$-0.3001480$
$-1.4977370$
2.1387030
$-0.6713000$
1. 3047020
2. 0334600
$-4.2205180$
$-1.6388910$
$-2.1840710$
$-3.1932850$
$-1.1989020$
$-2.8418670$
$-2.2835180$
$-1.5535200$
$-4.0703620$
$-5.0198540$
$-4.6451800$
$-2.6257460$
$-4.3573470$

0.2184120

1. 9461380

2. 1844950

2.0863850

1.7135780

3. 2515770

2. 2538700

1.7118870

2. 3652510

3. 2592200

$-1.2892760$

$-1.7600690$

$-0.3410670$

$-1.0630450$

$-2.4575110$

$-3.1659190$

$-1.5172730$

$-2.8574970$

$-4.5015800$

$-5.1353860$

$-4.1210710$

$-5.1379860$

$-3.9000060$

$-4.3573450$

$-4.6653240$

$-3.1103420$

$-0.9669080$

$-2.3725910$

$-0.2272960$

$-2.8863460$

$-3.1862250$

1.6392960

$-0.9709550$

$-2.1404530$

1.7456700

$-1.5490260$

$-1.1256720$

$-1.0901050$

$-0.8936680$

$-2.7883820$

$-2.7547170$

$-2.1838050$

$-1.3113000$

$-1.3406330$

$-1.6991560$

$-2.1644900$ 


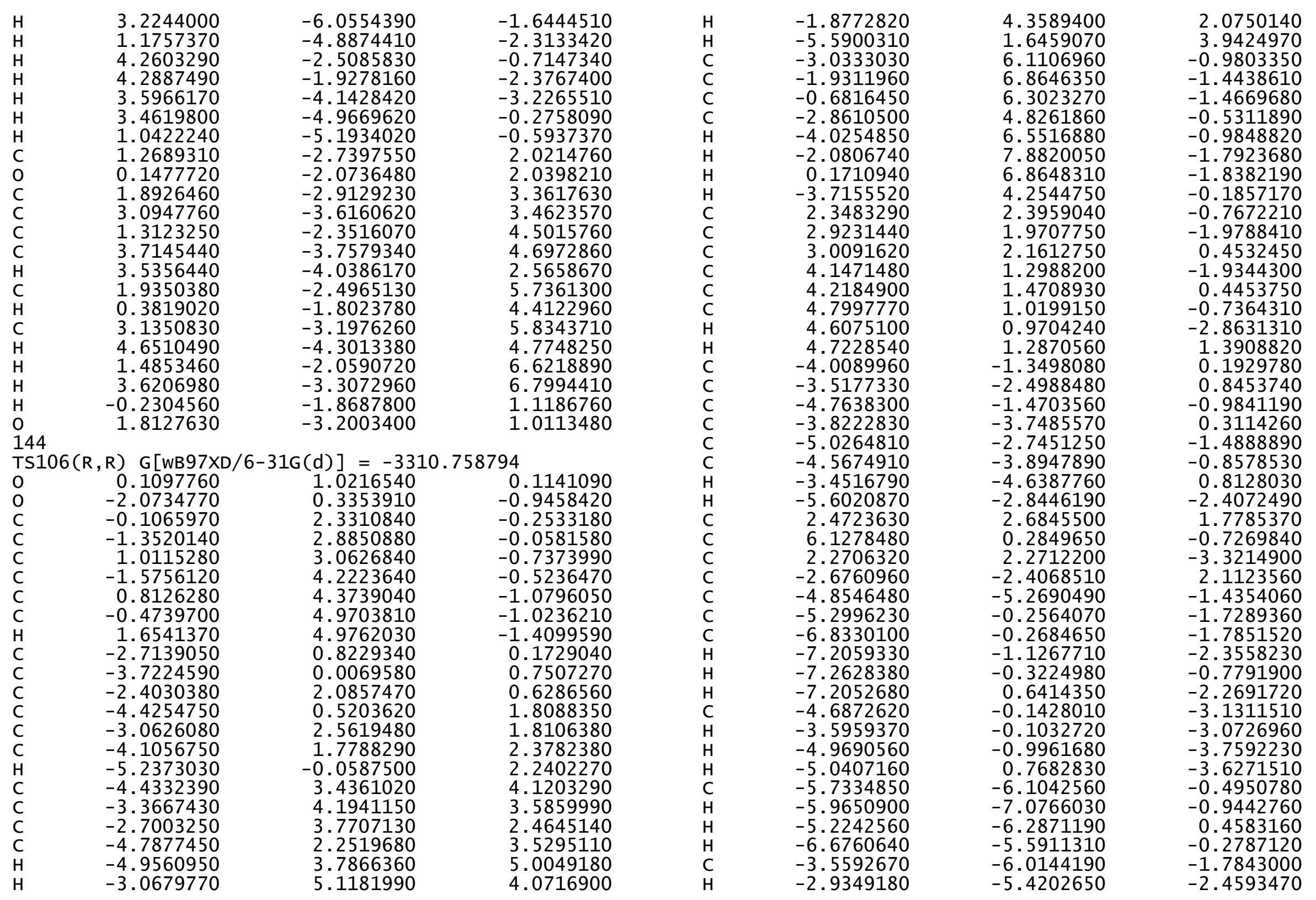




-2.9726750
-3.7823000
-3.5512270
-4.3145080
-4.0619560
-2.9378080
-1.5431560
-1.9201440
-0.9884300
-0.8472630
3.3779710
4.3847050
3.4715510
2.9669180
2.2919110
1.6364940
3.2499700
1.8474560
6.1615960
7.0733520
6.1490690
5.2967820
7.2877500
7.2933720
7.1957980
8.2512950
2.3830620
3.4181610
1.9880320
1.8039180
2.8654480
3.9350600
2.3685370
2.7596500
-0.5267820
0.0164780
-0.3863550
1.2039890
6.2587250
1.4877860
-5.0084010
-5.4124900
-2.2052650
2.5365680
1.7959400
2.4654050

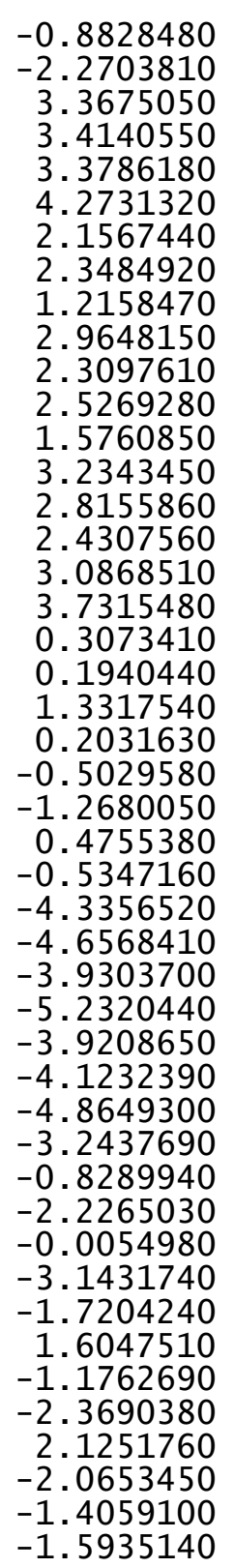

0.8627470
1.5097450
0.8069140
4.0242740
3.5538400
2.0942630
3.9120380
5.0631740
3.6617200
1.4150470
4.6235450
4.1494580
3.4214470
4.1785080
1.7993740
2.5063750
1.3988600
3.4727230
4.6821390
3.1936620
5.6168710
4.8810760
4.1307410
2.2494340
5.3429270
6.5596620
3.9156670
6.0756030
0.7469910
2.7781550

$-0.9247470$ $-3.0649510$ $-2.8456620$ $-3.1399370$ $-2.0907490$ $-1.6982960$ $-2.6035660$ $-3.4183390$ $-2.4641870$ $-2.5081400$ $-1.7840380$ $-3.3760590$ $-4.0503490$ $-1.1940110$ $-0.8240480$ 1.5816930 1.8380290 2.7128810 2. 5622660 3.9061040 3.5895820 1.6329890 4.9328360 4.0197210 4.7738250 3.4670970 5.8577380 5.5751930 1.0656640 0.5158930 144

$\begin{array}{lrcr}\text { TS107 (R, R) } G[W B 97 X D / 6-31 G(d)]=-3310.754525 \\ \text { O } & -0.0243950 & 1.1146230 & 0.1970680 \\ \text { O } & -2.0968180 & 0.2291190 & -0.9262000 \\ \text { C } & -0.3403700 & 2.3904610 & -0.2123000 \\ \text { C } & -1.6325550 & 2.8429200 & -0.0608750 \\ \text { C } & 0.7283620 & 3.1950210 & -0.6930230 \\ \text { C } & -1.9477980 & 4.1507820 & -0.5567210 \\ \text { C } & 0.4370490 & 4.4837940 & -1.0528420 \\ \text { C } & -0.8938160 & 4.9782820 & -1.0343840 \\ \text { H } & 1.2353500 & 5.1480610 & -1.3707190 \\ \text { C } & -2.8126240 & 0.6718500 & 0.1649700 \\ \text { C } & -3.7482840 & -0.2328770 & 0.7327810 \\ \text { C } & -2.6353150 & 1.9658380 & 0.6055970 \\ \text { C } & -4.5347250 & 0.2277300 & 1.7556070 \\ \text { C } & -3.3806820 & 2.3960380 & 1.7541500\end{array}$




$\begin{array}{rrr}-4.3622400 & 1.5244440 & 2.3027490 \\ -5.2944590 & -0.4236170 & 2.1787730 \\ -4.9153220 & 3.1749180 & 3.9934800 \\ -3.9087580 & 4.0240640 & 3.4802730 \\ -3.1620840 & 3.6464580 & 2.3932010 \\ -5.1298170 & 1.9502140 & 3.4180020 \\ -5.5034170 & 3.4891920 & 4.8503850 \\ -3.7212150 & 4.9826740 & 3.9545820 \\ -2.3870430 & 4.3057330 & 2.0192350 \\ -5.8833130 & 1.2755290 & 3.8162240 \\ -3.5360830 & 5.9097790 & -1.0891130 \\ -2.4831600 & 6.7450350 & -1.5260400 \\ -1.1925150 & 6.2846070 & -1.5010570 \\ -3.2765860 & 4.6487520 & -0.6160930 \\ -4.5601050 & 6.2682120 & -1.1342350 \\ -2.7011240 & 7.7432230 & -1.8933640 \\ -0.3760570 & 6.9099900 & -1.8530080 \\ -4.0934450 & 4.0134330 & -0.2912910 \\ 2.1087690 & 2.6190600 & -0.7010330 \\ 2.7031640 & 2.1736840 & -1.8973170 \\ 2.7830200 & 2.4699190 & 0.5236800 \\ 3.9630460 & 1.5784510 & -1.8296070 \\ 4.0369300 & 1.8592330 & 0.5401120 \\ 4.6428640 & 1.3999870 & -0.6254250 \\ 4.4375240 & 1.2380260 & -2.7472160 \\ 4.5510980 & 1.7441780 & 1.4902300 \\ -3.8609840 & -1.6280030 & 0.2074720 \\ -3.2756200 & -2.6980280 & 0.9157150 \\ -4.5361510 & -1.8627920 & -1.0002190 \\ -3.4091430 & -3.9864110 & 0.4039800 \\ -4.6285870 & -3.1707290 & -1.4787210 \\ -4.0735710 & -4.2449410 & -0.7940040 \\ -2.9663140 & -4.8163300 & 0.9484100 \\ -5.1451860 & -3.3568250 & -2.4183120 \\ 2.2061770 & 3.0205380 & 1.8206550 \\ 6.0344950 & 0.7901280 & -0.6294990 \\ 2.0271360 & 2.3702660 & -3.2471370 \\ -2.5194720 & -2.4842860 & 2.2214920 \\ -4.1850160 & -5.6572600 & -1.3387860 \\ -5.1713760 & -0.7363640 & -1.8021710 \\ -6.6922350 & -0.9143830 & -1.9029690 \\ -6.9523020 & -1.8254340 & -2.4544280 \\ -7.1465670 & -0.9830360 & -0.9087450 \\ -7.1429970 & -0.0648310 & -2.4282480 \\ -4.5295950 & -0.6018510 & -3.1892820 \\ -3.4502360 & -0.4503600 & -3.1009430\end{array}$

-4.7047000
-4.9569950
-5.0348840
-5.1598480
-4.5596700
-6.0273360
-2.8045420
-2.1987030
-2.2575470
-2.9051440
-3.4412630
-4.2916280
-3.8364800
-2.8923360
-1.2744020
-1.5368460
-0.6701460
-0.6587030
2.9338940
3.9983270
2.8574600
2.5026560
2.2288260
1.7350300
3.2543410
1.7072540
6.3465760
7.3011580
6.4338510
5.5703900
7.0909080
6.9090290
7.0651310
8.0967070
2.3068310
3.3361370
2.1364270
1.6426180
2.4476250
3.5311670
1.9503770
2.1955590
-0.5229210
0.0683640
-0.3111640
0.9466660
$-1.4972550$
0.2540360
$-6.5460860$
$-7.5445230$
$-6.6627960$
$-6.1114440$
$-6.2810180$
$-5.6479630$
$-6.4110440$
$-7.2674430$
$-2.7038760$
$-2.0161410$
-3.7268950
$-2.5512080$
$-3.3724280$
$-4.4180220$
$-3.3333210$
$-3.0269710$
4. 3159580
. 1265990
5.0604510
4.7456150
2.0051550
1.0762350
1.7711570
2. 4114980
$-0.0497090$
$-0.5714210$
0.5708660
$-0.7972960$
1.8892490
2.4537900
2.5949370
1.4570290
1.2445890
1.2801630
0.2606670
1. 3488070
3. 7133120
3.7324510
3. 8631870
4.5589830
$-0.1278890$
$-0.0857070$
$-1.3459650$
2. 3888590

$-3.7971360$

$-3.7238120$

$-0.4208230$

$-0.8551220$

0.5602320

$-0.2636930$

$-1.5844710$

$-2.2410100$

$-2.0511200$

3. 4302150

3. 4219170

3. 4304070

4.3665690

2. 3489540

2. 5496440

1. 4401610

3. 1868850

2. 2066330

2.3895870

1.4068740

3.1179960

2. 9659370

2. 6662280

3. 2760090

0.6120660

0.4847490

1. 5107880

0.8028170

$-0.8208020$

$-1.7410760$

0.0177820

$-0.8742780$

$-4.2482110$

$-4.6253290$

- 3.8081090

$-5.1125120$

$-3.8654770$

$-4.0344190$

$-4.8305650$

$-3.2204910$

$-2.1158300$

0.1237930
-3.0755620 


\begin{tabular}{|c|c|c|c|c|c|c|c|}
\hline & & & & & & & \\
\hline $\mathrm{H}$ & 6.0916430 & 0.1244940 & -1.5013760 & C & 0.4504510 & 4.4840620 & -1.0535800 \\
\hline H & $\begin{array}{r}1.1584390 \\
-4\end{array}$ & 3.2795750 & 1. 6489550 & C & -0.8800470 & 4.9799230 & -1.0426990 \\
\hline 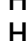 & $\begin{array}{l}-4.994 / 880 \\
-4.6971490\end{array}$ & $\begin{array}{r}0.2034320 \\
-5.5934060\end{array}$ & -1.2716350 & $\mathrm{H}$ & 1.2511490 & 5.1474950 & -1.3671360 \\
\hline $\mathrm{H}$ & -2.1747060 & -14461060 & 2.2385410 & $c$ & $\begin{array}{l}-2.8111120 \\
-3.7434880\end{array}$ & $\begin{array}{r}0.6762370 \\
-0\end{array}$ & $\begin{array}{l}0.1565000 \\
0.7323120\end{array}$ \\
\hline c & 2.5784860 & -1.8449220 & -16632690 & c & $\begin{array}{l}-3.1434000 \\
-2.6307770\end{array}$ & $-0.226 / 250$ & $\begin{array}{l}0.7323120 \\
0.5940460\end{array}$ \\
\hline c & 17446960 & -2.9890090 & -1.2722990 & $\mathrm{c}$ & -4.5266420 & 0.2376960 & $\begin{array}{l}0.350400 \\
1.7559500\end{array}$ \\
\hline $\mathrm{H}$ & 2.4251390 & -0.9245160 & -1.0957660 & $\mathrm{C}$ & -3.3715660 & 2.4049670 & 1.7443180 \\
\hline $\mathrm{H}$ & 0.7539800 & -2.7574700 & -0.8950340 & $\mathrm{C}$ & -4.3525480 & 1.5360740 & 2.2981130 \\
\hline 0 & 1.7399760 & -1.8932430 & -2.8334460 & $\mathrm{H}$ & -5.2846400 & -0.4125950 & 2.1839530 \\
\hline $\mathrm{H}$ & 1.0006530 & -1.2187260 & -2.6591630 & C & -4.8992830 & 3.1909510 & 3.9864220 \\
\hline C & 4.2366680 & -3.4519100 & -2.6861290 & $\mathrm{C}$ & -3.8929340 & 4.0374500 & 3.4684720 \\
\hline c & 3.5874160 & -4.5940570 & -1.9003530 & $\mathrm{C}$ & -3.1496480 & 3.6565180 & 2.3802250 \\
\hline C & 2.0833410 & -4.3696350 & -1.7183210 & C & -5.1167050 & 1.9652180 & 3.4143960 \\
\hline c & 4.0308800 & -2.1106170 & -1.9776010 & $\mathrm{H}$ & -5.4845650 & 3.5079810 & 4.8442280 \\
\hline $\mathrm{H}$ & 5.3079790 & -3.6395190 & -2.8083490 & $\mathrm{H}$ & -3.7027140 & 4.9968220 & 3.9401710 \\
\hline H & 3.7488460 & -5.5504920 & -2.4068820 & $\mathrm{H}$ & -2.3747860 & 4.3142800 & 2.0033560 \\
\hline $\mathrm{H}$ & 1.5484690 & -4.5260840 & -2.6631080 & $\mathrm{H}$ & -5.8697790 & 1.2921840 & 3.8162010 \\
\hline $\mathrm{H}$ & 4.5775600 & -2.0949210 & -1.0258980 & C & -3.5204770 & 5.9165970 & -1.1049790 \\
\hline $\mathrm{H}$ & 4.4057680 & -1.2782160 & -2.5813390 & $\mathrm{C}$ & -2.4646880 & 6.7493560 & -1.5398010 \\
\hline $\mathrm{H}$ & 3.8024400 & -3.4000710 & -3.6915810 & $\mathrm{C}$ & -1.1749590 & 6.2865300 & -1.5109210 \\
\hline $\mathrm{H}$ & 4.0540040 & -4.6687160 & -0.9115870 & $\mathrm{C}$ & -3.2647450 & 4.6552080 & -0.6308490 \\
\hline H & 1.6489100 & -5.0685450 & -0.9988370 & $\mathrm{H}$ & -4.5435840 & 6.2772990 & -1.1526090 \\
\hline C & 2.5568220 & -2.3592630 & 1.5557120 & $\mathrm{H}$ & -2.6797040 & 7.7477530 & -1.9082850 \\
\hline 0 & 1.6947290 & -1.3998710 & 1.7541390 & $\mathrm{H}$ & -0.3563070 & 6.9103370 & -1.8605450 \\
\hline C & 3.7816440 & -2.2750040 & 2.3912440 & $\mathrm{H}$ & -4.0834610 & 4.0215870 & -0.3072800 \\
\hline C & 4.8114870 & -3.1916990 & 2.1657730 & C & 2.1192940 & 2.6203100 & -0.6855260 \\
\hline c & 3.9395690 & -1.2653520 & 3.3432520 & $\mathrm{C}$ & 2.7364730 & 2.1966910 & -1.8770390 \\
\hline$c$ & 5.9995710 & -3.0898550 & 2.8781680 & C & 2.7747800 & 2.4580440 & 0.5492200 \\
\hline $\mathrm{H}$ & 4.6716050 & -3.9710070 & 1.4243610 & C & 3.9996420 & 1.6068130 & -1.7973600 \\
\hline$c$ & 5.1293930 & -1.1663240 & 4.0540250 & $\mathrm{C}$ & 4.0310040 & 1.8553170 & 0.5769160 \\
\hline $\mathrm{H}$ & 3.1351830 & -0.5602610 & 3.5124970 & $\mathrm{C}$ & 4.6593160 & 1.4154990 & -0.5853880 \\
\hline$c$ & 6.1603840 & -2.0742520 & 3.8190880 & $\mathrm{H}$ & 4.4927390 & 1.2864730 & -2.7121490 \\
\hline $\mathrm{H}$ & 6.8010530 & -3.7994280 & 2.6987770 & $\mathrm{H}$ & 4.5347400 & 1.7336050 & 1.5323970 \\
\hline $\mathrm{H}$ & 5.2532190 & -0.3791630 & 4.7912350 & C & -3.8590520 & -1.6257410 & 0.2178830 \\
\hline $\mathrm{H}$ & 7.0920270 & -1.9903810 & 4.3705090 & $\mathrm{C}$ & -3.2569320 & -2.6878770 & 0.9241150 \\
\hline $\mathrm{H}$ & 0.8943790 & -1.4337500 & 1.1290380 & $\mathrm{C}$ & -4.5574090 & -1.8727540 & -0.9737360 \\
\hline & 2.4175630 & -3.2694240 & 0.7295690 & $\mathrm{C}$ & -3.4002750 & $-\overline{3} .9813670$ & 0.4286570 \\
\hline & & & & C & -4.6577450 & -3.1855240 & -1.4375900 \\
\hline & R) $\mathrm{G}[\mathrm{WB} 97 \mathrm{X}$ & (d)] & & $\mathrm{C}$ & -4.0889870 & -4.2524890 & -0.7528850 \\
\hline 0 & -0.0248750 & 1.1104090 & 0.1796410 & $\mathrm{H}$ & -2.9447480 & -4.8051450 & 0.9717750 \\
\hline 0 & -2.1033210 & 0.2313310 & -0.9389680 & $\mathrm{H}$ & -5.1912120 & -3.3812690 & -2.3657580 \\
\hline $\mathrm{c}$ & -0.3348860 & 2.3902380 & -0.2220300 & C & 2.1772000 & 2.9916890 & 1.8439580 \\
\hline C & -1.6263720 & 2.8453550 & -0.0735160 & C & 6.0544420 & 0.8157900 & -0.5593800 \\
\hline$c$ & 4080 & 3.1950040 & -0.6919540 & C & 2.0867720 & 2.4166300 & -3.2361660 \\
\hline C & & 3449 & $15051-$ & C & -2.4675460 & -2.4571140 & 2.2074 \\
\hline
\end{tabular}




-4.2103050
-5.2046260
-6.7257340
-6.9886390
-7.1712420
-7.1831750
-4.5748600
-3.4953320
-4.7513750
-5.0097240
-5.0505410
-5.1816770
-4.5641320
-6.0406100
-2.8349020
-2.2343360
-2.2774790
-2.9443350
-3.3600330
-4.1989010
-3.7708500
-2.7834660
-1.2312950
-1.5018950
-0.6499270
-0.5900140
2.8933230
3.9563960
2.8229250
2.4486410
2.1895780
1.7037680
3.2118370
1.6547610
6.2543230
7.2286200
6.2257950
5.4798610
7.1091970
6.9999050
7.0034230
8.1212480
2.3556340
3.3966300
2.1348120
1.7229250

$-5.6702440$

$-0.7533640$

$-0.9357630$

$-1.8498260$

$-1.0009100$

$-0.0897480$

$-0.6259250$

$-0.4699480$

$-1.5266940$

0.2244620

$-6.5473060$

$-7.5504810$

$-6.6532940$

$-6.1096080$

$-6.3004510$

$-5.6739110$

$-6.4265440$

$-7.2895270$

$-2.6368570$

$-1.9354030$

$-3.6531900$

$-2.4736360$

$-3.3588600$

$-4.3963100$

$-3.3475660$

$-3.0031580$

4.2866020

4.1006490

5.0388800

4.7046360

1.9638970

1.0364070

1.7304840

2. 3583400

$-0.2116080$

$-0.7015040$

0.2550490

$-0.9833700$

1.9285570

2.6482560

2.4742430

1. 5097680

1. 2910140

1. 2907450

0.3108320

1. 4272100

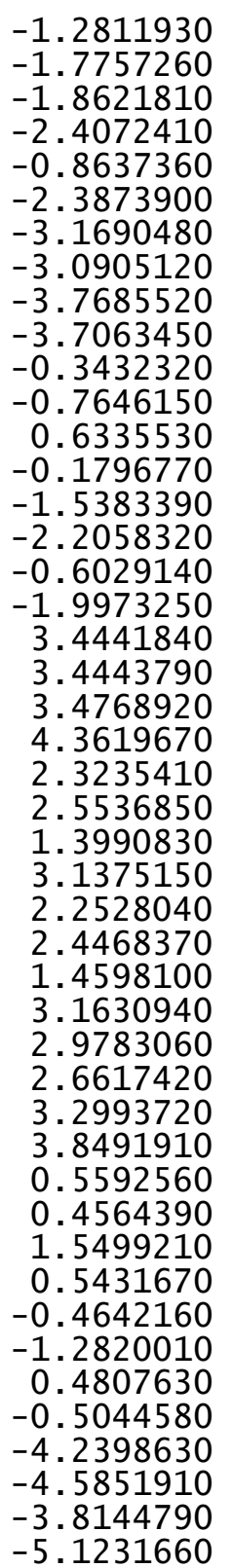

2.5493250

3.6343270

2. 0642200

2. 3156590

$-0.5261730$

0.0540680

$-0.3019230$

1.0045530

6.2021410

1.1305640

$-5.0253940$

$-4.7347400$

$-2.1076380$

2. 5723460

1.7516710

2.4293060

0.7688850

1.7051330

0.9692530

4. 2091570

3. 5855260

2. 0853670

4.0171370

5.2777020

3.7393570

1.5283550

4.5840880

4.3793980

3.7488720

4.0763600

1.6716770

2. 5719550

1.6978680

3.7665300

4.7937650

3.8989470

5.9541990

4.6746830

5.0613290

3.0964750

6.0900350

6.7535550

5.1653330

6.9999270

0.9081420

2.4664380
3.7545170

3.7458420

3.9294060

4.5989090

$-0.1205810$

$-0.0566710$

$-1.3498890$

2.4612830

0.2987760

3.2476660

0.1903310

$-5.6153260$

$-1.4238960$

$-1.8155020$

$-2.9627290$

$-0.8963830$

$-2.7356210$

$-1.8660130$

$-1.1931660$

$-3.4149730$

$-4.5593290$

$-2.0763860$

$-3.5984190$

$-5.5161030$

$-4.5004230$

$-2.0682950$

$-1.2392690$

$-3.3643550$

$-4.6287230$

$-5.0427270$

$-2.3653790$

$-1.4191970$

$-2.3098420$

$-3.2342990$

$-1.3194170$

$-3.1600420$

$-3.9982100$

$-1.2477380$

$-0.6079460$

$-2.1638390$

$-3.8765290$

$-2.1024490$

$-1.4409570$

$-3.2437040$
$-3.8356470$

3.9957060

$-4.8026980$

$-3.1816740$

$-0.7690810$

$-2.1508930$

0.0732640

$-3.0827730$

$-1.5171710$

1.6617800

$-1.2520380$

$-2.2439730$

2.1915550

$-1.7651100$

$-1.3565780$

$-1.1927770$

$-0.9568440$

$-2.9148460$

$-2.7224590$

$-2.8333850$

$-2.0300100$

$-1.8113440$

$-2.1161450$

$-2.9830700$

$-2.5382490$

$-2.7428730$

$-1.1771770$

$-2.7213260$

$-3.8271880$

$-1.0525660$

$-1.0816850$

1.4994970

1.7083880

2. 3805960

2.1777410

3. 3561450

2.9374010

1. 4169340

4.1144240

3.5072200

3.9027110

2.7767850

4.8702120

4.4922340

1.0696590

0.6349480 
144

$\operatorname{TS} 109(R, R) \quad G[W B 97 X D / 6-31 G(d)]=-3310.753063$

$\begin{array}{lrrr}\mathrm{O} & 0.0577590 & -1.1728170 & 0.3096880 \\ \mathrm{O} & 2.0323880 & -0.0716110 & -0.7848280 \\ \mathrm{C} & 0.3466000 & -2.3694160 & -0.3084550 \\ \mathrm{C} & 1.6481710 & -2.8161700 & -0.3229860 \\ \mathrm{C} & -0.7521470 & -3.1028420 & -0.8324050 \\ \mathrm{C} & 1.9310660 & -4.0274900 & -1.0374720 \\ \mathrm{C} & -0.4836090 & -4.3119660 & -1.4157180 \\ \mathrm{C} & 0.8474790 & -4.7842750 & -1.5652700 \\ \mathrm{H} & -1.2993780 & -4.9206530 & -1.7954720 \\ \mathrm{C} & 2.8234610 & -0.6802240 & 0.1623940 \\ \mathrm{C} & 3.7991580 & 0.1349800 & 0.7950320 \\ \mathrm{C} & 2.6906420 & -2.0329790 & 0.3973810 \\ \mathrm{C} & 4.6704140 & -0.4697600 & 1.6617310 \\ \mathrm{C} & 3.5269670 & -2.6275970 & 1.4014730 \\ \mathrm{C} & 4.5489460 & -1.8408260 & 2.0022080 \\ \mathrm{H} & 5.4578470 & 0.1181840 & 2.1254340 \\ \mathrm{C} & 5.2442150 & -3.7311800 & 3.3558380 \\ \mathrm{C} & 4.1977320 & -4.5006950 & 2.7994390 \\ \mathrm{C} & 3.3628900 & -3.9659490 & 1.8515110 \\ \mathrm{C} & 5.4079330 & -2.4275510 & 2.9678120 \\ \mathrm{H} & 5.9031550 & -4.1690630 & 4.0993940 \\ \mathrm{H} & 4.0483850 & -5.5241830 & 3.1300790 \\ \mathrm{H} & 2.5585860 & -4.5689020 & 1.4459960 \\ \mathrm{H} & 6.1912740 & -1.8139720 & 3.4053240 \\ \mathrm{C} & 3.4821370 & -5.6615340 & -1.9463450 \\ \mathrm{C} & 2.4030520 & -6.4324610 & -2.4343710 \\ \mathrm{C} & 1.1164160 & -5.9975690 & -2.2501830 \\ \mathrm{C} & 3.2531410 & -4.4919300 & -1.2670670 \\ \mathrm{H} & 4.5015220 & -5.9949480 & -2.1157170 \\ \mathrm{H} & 2.5968700 & -7.3603280 & -2.9638070 \\ \mathrm{H} & 0.2785330 & -6.5708050 & -2.6386740 \\ \mathrm{H} & 4.0898960 & -3.9041830 & -0.9056830 \\ \mathrm{C} & -2.1220440 & -2.5274010 & -0.6699690 \\ \mathrm{C} & -2.8056180 & -1.9656190 & -1.7655580 \\ \mathrm{C} & -2.6883630 & -2.4838850 & 0.6171540 \\ \mathrm{C} & -4.0359210 & -1.3466720 & -1.5381140 \\ \mathrm{C} & -3.9169480 & -1.8506460 & 0.7932470 \\ \mathrm{C} & -4.5996880 & -1.2606970 & -0.2659500 \\ \mathrm{H} & -4.5712780 & -0.9201500 & -2.3822330 \\ \mathrm{H} & -4.3608610 & -1.8072630 & 1.7849440 \\ \mathrm{C} & 3.8475400 & 1.6007980 & 0.5059320 \\ \mathrm{C} & 3.2916620 & 2.5116010 & 1.4231170 \\ \mathrm{C} & 4.4265520 & 2.0602610 & -0.6899480 \\ \mathrm{C} & 3.3595650 & 3.8742860 & 1.1313380\end{array}$

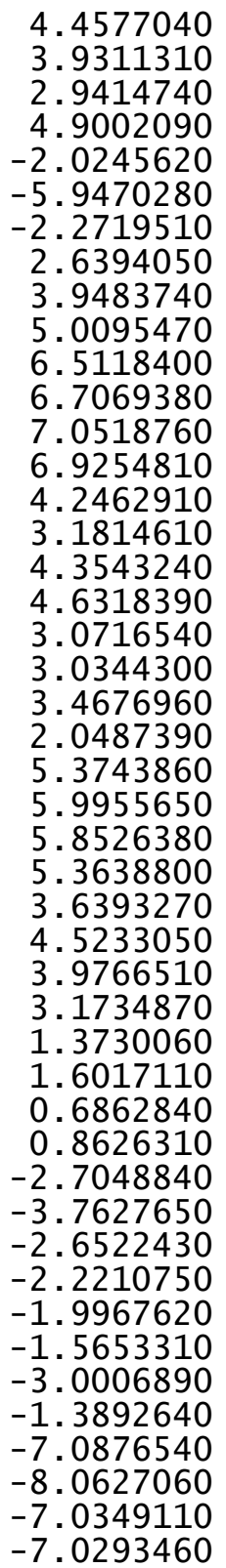

4.4577040

2.9414740

$-2.0245620$

$-5.9470280$

$-2.2719510$

. 6394050

6. 5118400

(18180

9254810

3. 1814610

4.3543240

18390

3.0344300

3.4676960

.0487390

5. 3743860

5.8526380

5.3638800

3.6393270

4.5233050

3. 1734870

1. 3730060

1. 6017110

0.6862840

626310

$-3.7627650$

$-2.6522430$

$-3.0006890$

$-7.0876540$

$-8.0627060$

$-7.0293460$
3.4301660

4. 3545000

4.5896530

3.7846930

$-3.1716000$

$-0.6022290$

$-2.0872650$

2. 0495960

5.8421650

1.1034100

1. 3425990

2. 3415970

1.2537870

0.6095010

1. 1811450

0.9883890

2. 1685810

0.4366830

6.1702260

7.2519590

5.6982090

5.8073730

6.3743710

6. 1550830

5.9191040

7.4594180

2.1103120

1.4954170

3. 1412230

1.7548680

2.8386140

3.8665440

2.8652550

2. 3566540

$-4.5232880$

$-4.3830140$

$-5.1649790$

$-5.0459430$

$-2.3098740$

$-1.3265470$

$-2.1708310$

$-2.7968470$

$-1.5386660$

$-1.0845490$

$-1.7476570$

$-2.4944170$
$-0.9417020$

$-0.0427420$

1.8353070

$-1.8703370$

1.8021930

$-0.0312770$

$-3.1868980$

2. 7203010

$-0.3439990$

$-1.7196070$

$-1.9190090$

$-2.3258630$

$-0.9703830$

$-2.6207640$

$-3.0481810$

$-2.8909080$

$-3.5126300$

$-3.7541220$

$-1.5606220$

$-1.7326690$

$-2.4672180$

$-1.4145810$

$-0.5339820$

0.3404320

$-1.4088430$

$-0.6868900$

3. 8845630

3.6919330

4.0467230

4.8107780

3. 0744520

3. 3796510

2. 2262310

3. 9157370

2. 0631040

2. 3153020

1.1769020

2. 8961900

3.0675040

2. 8575260

3.4860050

3.8381170

$-0.4543950$

$-0.2438580$

$-1.5295480$

0.0764020 


$\begin{array}{rrr}-6.0690620 & 0.7549830 & -0.7286190 \\ -5.2365640 & 1.4069510 & -0.4486030 \\ -6.0718310 & 0.6505930 & -1.8200570 \\ -7.0018310 & 1.2515920 & -0.4398580 \\ -2.4553390 & -0.8277800 & -4.0390020 \\ -3.5122580 & -0.5925300 & -4.2111620 \\ -1.9710510 & 0.0318100 & -3.5746230 \\ -1.9947370 & -0.9795470 & -5.0212900 \\ -2.9294870 & -3.2879710 & -3.8860610 \\ -4.0082420 & -3.1237220 & -3.9965710 \\ -2.5031590 & -3.4334610 & -4.8851530 \\ -2.7929490 & -4.2139190 & -3.3193010 \\ 0.4508530 & 0.1857940 & -0.4997250 \\ -0.1904040 & 0.2579630 & -1.8537930 \\ 0.2166390 & 1.2990760 & 0.4898030 \\ -1.1961280 & -2.2722210 & -3.1242780 \\ -6.0393990 & -0.4241580 & 1.0481740 \\ -0.9837530 & -3.3821680 & 1.5443520 \\ 4.8955730 & 0.0837680 & -1.3399270 \\ 3.5156610 & 6.3550910 & 0.5248330 \\ 2.3363140 & 1.0067090 & 2.5859470 \\ -2.4851980 & 2.2492110 & -1.9833390 \\ -1.7230990 & 3.0745420 & -1.0409310 \\ -2.6444150 & 1.2132470 & -1.6769430 \\ -0.9230450 & 2.5896220 & -0.4909310 \\ -1.2875040 & 2.3984350 & -2.7767310 \\ -0.7149280 & 1.6035110 & -2.5304050 \\ -3.4689310 & 4.3651640 & -2.9561360 \\ -3.0665980 & 5.1119520 & -1.6818520 \\ -1.7597690 & 4.5604020 & -1.1072790 \\ -3.6797550 & 2.8774050 & -2.6624500 \\ -4.3904700 & 4.7891290 & -3.3671950 \\ -2.9502960 & 6.1820260 & -1.8783090 \\ -0.9015790 & 4.8567890 & -1.7244210 \\ -4.5406880 & 2.7517640 & -1.9977700 \\ -3.8876590 & 2.3131730 & -3.5777810 \\ -2.6909590 & 4.4845340 & -3.7195200 \\ -3.8570730 & 5.0033410 & -0.9295600 \\ -1.5556410 & 4.9407560 & -0.1024950 \\ -2.9923800 & 1.9647300 & 1.5721210 \\ -1.9660170 & 1.2282060 & 1.9031820 \\ -4.2160530 & 1.6878730 & 2.3720600 \\ -5.3742620 & 2.4249320 & 2.1177990 \\ -4.2294250 & 0.6777630 & 3.3361740 \\ -6.5463460 & 2.1363730 & 2.8054820 \\ -5.3480980 & 3.2093160 & 1.3693180\end{array}$

$\begin{array}{ll}\mathrm{C} & -5.4028390 \\ \mathrm{H} & -3.3236140 \\ \mathrm{C} & -6.5627890 \\ \mathrm{H} & -7.4485790 \\ \mathrm{H} & -5.4129040 \\ \mathrm{H} & -7.4809830 \\ \mathrm{H} & -1.1451590 \\ \mathrm{O} & -3.0026830\end{array}$
0.3910760
0.1177560
1. 1150050
2.7038460
$-0.3984230$
0.8860840
1. 3281940
2. 8225080

4.0234710

3. 5322840

3.7537100

2. 6002240

4.7683690

4. 2863440

1. 3208100

0.6818400

$\operatorname{TS} 110(R, R) \quad G[W B 97 X D / 6-31 G(d)]=-3310.758758$

$\begin{array}{lrcr}\text { TS } 110(\mathrm{R}, \mathrm{R}) \mathrm{G}[\mathrm{WB} 97 \times \mathrm{D} / 6-31 \mathrm{G}(\mathrm{d})]=-3310.758758 \\ \mathrm{O} & 0.1095640 & 1.0217540 & 0.1143000 \\ \mathrm{O} & -2.0734970 & 0.3350730 & -0.9457310 \\ \mathrm{C} & -0.1069820 & 2.3311360 & -0.2531940 \\ \mathrm{C} & -1.3524920 & 2.8849630 & -0.0581160 \\ \mathrm{C} & 1.0110540 & 3.0628570 & -0.7372960 \\ \mathrm{C} & -1.5762630 & 4.2221840 & -0.5236880 \\ \mathrm{C} & 0.8119770 & 4.3740240 & -1.0795960 \\ \mathrm{C} & -0.4747080 & 4.9703220 & -1.0236770 \\ \mathrm{H} & 1.6534010 & 4.9764150 & -1.4099980 \\ \mathrm{C} & -2.7140480 & 0.8225820 & 0.1729570 \\ \mathrm{C} & -3.7225120 & 0.0064680 & 0.7507450 \\ \mathrm{C} & -2.4034320 & 2.0854760 & 0.6286640 \\ \mathrm{C} & -4.4257200 & 0.5198100 & 1.8087500 \\ \mathrm{C} & -3.0631960 & 2.5616290 & 1.8105620 \\ \mathrm{C} & -4.1061880 & 1.7783660 & 2.3781050 \\ \mathrm{H} & -5.2374900 & -0.0594150 & 2.2400980 \\ \mathrm{C} & -4.4342260 & 3.4356840 & 4.1200630 \\ \mathrm{C} & -3.3678040 & 4.1938460 & 3.5858000 \\ \mathrm{C} & -2.7011910 & 3.7704940 & 2.4644100 \\ \mathrm{C} & -4.7884620 & 2.2514530 & 3.5292770 \\ \mathrm{H} & -4.9572350 & 3.7861790 & 5.0045760 \\ \mathrm{H} & -3.0692500 & 5.1180120 & 4.0714680 \\ \mathrm{H} & -1.8782100 & 4.3588470 & 2.0749720 \\ \mathrm{H} & -5.5906850 & 1.6452750 & 3.9422120 \\ \mathrm{C} & -3.0341970 & 6.1102890 & -0.9805510 \\ \mathrm{C} & -1.9321790 & 6.8643480 & -1.4440940 \\ \mathrm{C} & -0.6825510 & 6.3022080 & -1.4671220 \\ \mathrm{C} & -2.8617830 & 4.8258320 & -0.5313140 \\ \mathrm{H} & -4.0264390 & 6.5511460 & -0.9851600 \\ \mathrm{H} & -2.0817820 & 7.8816730 & -1.7926780 \\ \mathrm{H} & 0.1701240 & 6.8648030 & -1.8383840 \\ \mathrm{H} & -3.7162180 & 4.2540290 & -0.1858300 \\ \mathrm{C} & 2.3479380 & 2.3962440 & -0.7670930 \\ \mathrm{C} & 2.9228320 & 1.9712070 & -1.9787080 \\ \mathrm{C} & 3.0087720 & 2.1616690 & 0.4533840 \\ \mathrm{C} & 4.1469140 & 1.2993910 & -1.9342800\end{array}$




$\begin{array}{lr}\mathrm{C} & 4.2181810 \\ \mathrm{C} & 4.7995500 \\ \mathrm{H} & 4.6073360 \\ \mathrm{H} & 4.7225540 \\ \mathrm{C} & -4.0087710 \\ \mathrm{C} & -3.5172210 \\ \mathrm{C} & -4.7636060 \\ \mathrm{C} & -3.8214590 \\ \mathrm{C} & -5.0259490 \\ \mathrm{C} & -4.5666450 \\ \mathrm{H} & -3.4506370 \\ \mathrm{H} & -5.6015560 \\ \mathrm{C} & 2.4719000 \\ \mathrm{C} & 6.1277140 \\ \mathrm{C} & 2.2703390 \\ \mathrm{C} & -2.6756350 \\ \mathrm{C} & -4.8534890 \\ \mathrm{C} & -5.2997500 \\ \mathrm{C} & -6.8331460 \\ \mathrm{H} & -7.2060230 \\ \mathrm{H} & -7.2627520 \\ \mathrm{H} & -7.2056780 \\ \mathrm{C} & -4.6876870 \\ \mathrm{H} & -3.5963570 \\ \mathrm{H} & -4.9694620 \\ \mathrm{H} & -5.0413820 \\ \mathrm{C} & -5.7323500 \\ \mathrm{H} & -5.9637290 \\ \mathrm{H} & -5.2232410 \\ \mathrm{H} & -6.6750430 \\ \mathrm{C} & -3.5579490 \\ \mathrm{H} & -2.9335560 \\ \mathrm{H} & -2.9714890 \\ \mathrm{H} & -3.7807760 \\ \mathrm{C} & -3.5507920 \\ \mathrm{H} & -4.3141800 \\ \mathrm{H} & -4.0613850 \\ \mathrm{H} & -2.9374130 \\ \mathrm{C} & -1.5425500 \\ \mathrm{H} & -1.9194010 \\ \mathrm{H} & -0.9877650 \\ \mathrm{H} & -0.8467430 \\ \mathrm{C} & 3.3773580 \\ \mathrm{H} & 4.3841480 \\ \mathrm{H} & 3.4708150 \\ \mathrm{H} & 2.9662590 \\ & \end{array}$

$\begin{array}{rr}1.4714330 & 0.4455290 \\ 1.0205440 & -0.7362730 \\ 0.9710680 & -2.8629770 \\ 1.2876490 & 1.3910430 \\ -1.3503690 & 0.1930250 \\ -2.4992940 & 0.8454050 \\ -1.4710860 & -0.9840510 \\ -3.7490710 & 0.3114370 \\ -2.7459120 & -1.4888370 \\ -3.8954690 & -0.8578370 \\ -4.6392090 & 0.8127940 \\ -2.8455350 & -2.4071830 \\ 2.6848920 & 1.7786660 \\ 0.2857620 & -0.7267930 \\ 2.2716290 & -3.3213710 \\ -2.4070820 & 2.1124050 \\ -5.2697940 & -1.4353920 \\ -0.2572530 & -1.7288030 \\ -0.2695990 & -1.7847030 \\ -1.1280580 & -2.3551740 \\ -0.3235740 & -0.7786430 \\ 0.6401660 & -2.2687680 \\ -0.1435620 & -3.1311390 \\ -0.1038550 & -3.0729010 \\ -0.9969730 & -3.7591600 \\ 0.7674640 & -3.6270720 \\ -6.1050860 & -0.4951600 \\ -7.0774980 & -0.9443360 \\ -6.2878050 & 0.4583260 \\ -5.5920950 & -0.2789770 \\ -6.0149920 & -1.7840570 \\ -5.4207550 & -2.4589900 \\ -6.2269160 & -0.8825050 \\ -6.9714900 & -2.2701830 \\ -2.5384180 & 3.3675360 \\ -1.7569050 & 3.4139880 \\ -3.5089810 & 3.3787190 \\ -2.4668900 & 4.2731770 \\ -3.4401800 & 2.1569290 \\ -4.4518370 & 2.3487280 \\ -3.4448540 & 1.2160660 \\ -3.1909820 & 2.9650240 \\ 3.8054080 & 2.3098620 \\ 3.4296740 & 2.5270210 \\ 4.6132760 & 1.5761700 \\ 4.2262190 & 3.2344440 \\ -6 & \end{array}$
2. 2915910
1. 6362880
3. 2497100
1.8470690
6. 1614520
7.0733970
6.1485180
5. 2968440
7.2874640
7. 2931060
7.1953160
8. 2510740
2. 3827920
3.4179170
1. 9875950
1.8037990
2. 8651480
3. 9347390
2. 3681660
2.7594390
$-0.5266980$
0.0165680
$-0.3859830$
1.2036910
6. 2587800
1. 4872650
$-5.0085740$
$-5.4112070$
$-2.2049410$
2. 5367890
1. 7964210
2. 4655500
0.8632450
1. 5098500
0.8069670
4.0246050
3. 5544960
2. 0949320
3. 9122240
5. 0634910
3.6624990
1. 4156580
4.6238500
4.1493820
3. 4216460
4.1792760

1. 5723430

0.7864310

1.1140070

1. 9782480

$-0.8460960$

$-1.4428190$

$-0.4578960$

$-1.5094640$

1. 2664010

2.0494300

1.7518440

0.7453360

1.1295170

0.9627140

0.1968160

1. 3762030

3. 5559080

3.4223620

3.8059430

4.4082410

$-0.1521820$

$-0.1238650$

$-1.4072730$

2. 4359130

$-0.1638420$

3. 1268560

0.6408330

$-5.1215240$

$-1.4193380$

$-1.8637330$

$-2.9424160$

$-0.8827050$

$-2.6692450$

$-2.0535980$

$-1.3591980$

$-3.6035960$

$-4.6145580$

$-4.3716370$

$-2.1740730$

$-3.8062550$

$-5.6374330$

$-4.6663490$

$-2.0268740$

$-1.4355990$

$-3.7035990$

$-4.5270400$
2.8157410

2.4309190

3.0870300

3.7316860

0.3073150

0.1941650

1. 3318030

0.2027450

$-0.5024200$

$-1.2673290$

0.4761480

$-0.5341310$

$-4.3355190$

$-4.6565650$

$-3.9302750$

$-5.2319930$

$-3.9207310$

$-4.1232010$

$-4.8647410$

$-3.2435750$

$-0.8288840$

$-2.2263900$

$-0.0054630$

$-3.1430780$

$-1.7203030$

1.6048710

$-1.1762200$

$-2.3691170$

2. 1251520

$-2.0655410$

$-1.4060080$

$-1.5936890$

$-0.9247320$

$-3.0650060$

$-2.8456020$

$-3.1403620$

$-2.0910870$

$-1.6984360$

$-2.6039850$

$-3.4189300$

$-2.4645210$

$-2.5081940$

$-1.7845840$

$-3.3765280$

$-4.0506730$

$-1.1944390$ 


\begin{tabular}{|c|c|c|c|c|c|c|c|}
\hline $\mathrm{H}$ & 1.8002480 & -4.9586100 & -0.8241540 & $\mathrm{H}$ & 4.5021660 & -5.9945060 & -2.1158180 \\
\hline c & 2.5069740 & -2.3239760 & 1.5815400 & $\mathrm{H}$ & 2.5976450 & -7.3602210 & -2.9636750 \\
\hline 0 & 1.3993850 & -1.6827100 & 1.8379380 & $\mathrm{H}$ & 0.2792150 & -6.5710710 & -2.6383140 \\
\hline C & 3.4733170 & -2.3364910 & 2.7127320 & $\mathrm{H}$ & 4.0903230 & -3.9037980 & -0.9057640 \\
\hline C & 4.6827750 & -3.0185560 & 2.5620950 & C & -2.1217830 & -2.5278210 & -0.6698090 \\
\hline C & 3.1942060 & -1.6655090 & 3.9059840 & $\mathrm{C}$ & -2.8052690 & -1.9660880 & -1.7654830 \\
\hline C & 5.6174950 & -3.0122830 & 3.5894210 & $\mathrm{C}$ & -2.6882050 & -2.4842390 & 0.6172610 \\
\hline $\mathrm{H}$ & 4.8817550 & -3.5412100 & 1.6327930 & $\mathrm{C}$ & -4.0356170 & -1.3471910 & -1.5381790 \\
\hline C & 4.1312780 & -1.6620450 & 4.9327240 & $\mathrm{C}$ & -3.9168150 & -1.8510060 & 0.7932260 \\
\hline $\mathrm{H}$ & 2.2499450 & -1.1458780 & 4.0196200 & $\mathrm{C}$ & -4.5994920 & -1.2611560 & -0.2660660 \\
\hline C & 5.3435030 & -2.3303140 & 4.7736930 & $\mathrm{H}$ & -4.5709040 & -0.9207090 & -2.3823620 \\
\hline $\mathrm{H}$ & 6.5603170 & -3.5359020 & 3.4669220 & $\mathrm{H}$ & -4.3607940 & -1.8075580 & 1.7848900 \\
\hline $\mathrm{H}$ & 3.9161660 & -1.1363640 & 5.8576480 & $\mathrm{C}$ & 3.8471960 & 1.6011720 & 0.5058400 \\
\hline $\mathrm{H}$ & 6.0761710 & -2.3221130 & 5.5750670 & $\mathrm{C}$ & 3.2912790 & 2.5118560 & 1.4231200 \\
\hline $\mathrm{H}$ & 0.7474930 & -1.6047260 & 1.0655940 & $\mathrm{C}$ & 4.4260110 & 2.0607690 & -0.6900840 \\
\hline & 2.7788260 & -2.8876240 & 0.5156820 & $\mathrm{c}$ & 3.3589460 & 3.8745630 & 1.1313880 \\
\hline \multicolumn{4}{|c|}{144} & $\mathrm{C}$ & 4.4569340 & 3.4306890 & -0.9417860 \\
\hline \multirow{2}{*}{\multicolumn{4}{|c|}{$\operatorname{TS} 111(R, R) \quad G[W B 97 X D / 6-31 G(d)]=-3310.753089$}} & $\mathrm{C}$ & 3.9303200 & 4.3549090 & -0.0427310 \\
\hline & & & & $\mathrm{H}$ & 2.9408260 & 4.5898400 & 1.8354320 \\
\hline 0 & 2.0321970 & -0.0715570 & -0.7847420 & $\mathrm{H}$ & 4.8992980 & 3.7853190 & -1.8704480 \\
\hline C & 0.3468420 & -2.3696120 & -0.3082090 & C & -2.0244650 & -3.1718770 & 1.8023770 \\
\hline C & 1.6484800 & -2.8161740 & -0.3228320 & C & -5.9468480 & -0.6026550 & -0.0315820 \\
\hline $\mathrm{C}$ & -0.7518310 & -3.1031730 & -0.8321280 & $\mathrm{C}$ & -2.2713790 & -2.0876460 & -3.1867480 \\
\hline $\mathrm{C}$ & 1.9315000 & -4.0274590 & -1.0373290 & $\mathrm{C}$ & 2.6392560 & 2.0496960 & 2.7203670 \\
\hline $\mathrm{C}$ & -0.4831600 & -4.3122890 & -1.4153970 & $\mathrm{C}$ & 3.9473650 & 5.8425930 & -0.3439040 \\
\hline C & 0.8479830 & -4.7844220 & -1.5650090 & $\mathrm{C}$ & 5.0090330 & 1.1040450 & -1.7198440 \\
\hline $\mathrm{H}$ & -1.2988630 & -4.9211010 & -1.7950900 & $\mathrm{C}$ & 6.5112620 & 1.3434780 & -1.9194340 \\
\hline C & 2.8234740 & -0.6800330 & 0.1623980 & $\mathrm{H}$ & 6.7061520 & 2.3425130 & -2.3262970 \\
\hline $\mathrm{C}$ & 3.7991080 & 0.1353390 & 0.7949150 & $\mathrm{H}$ & 7.0514320 & 1.2547370 & -0.9708770 \\
\hline C & 2.6909020 & -2.0328080 & 0.3974140 & $\mathrm{H}$ & 6.9249300 & 0.6104550 & -2.6212500 \\
\hline $\mathrm{c}$ & 4.6705830 & -0.4692530 & 1.6614960 & $\mathrm{C}$ & 4.2455950 & 1.1817000 & -3.0483190 \\
\hline C & 3.5274620 & -2.6272820 & 1.4013990 & $\mathrm{H}$ & 3.1808030 & 0.9888480 & -2.8909070 \\
\hline $\mathrm{C}$ & 4.5493940 & -1.8403390 & 2.0019870 & $\mathrm{H}$ & 4.3534790 & 2.1691440 & -3.5127850 \\
\hline $\mathrm{H}$ & 5.4579710 & 0.1188270 & 2.1251010 & $\mathrm{H}$ & 4.6311180 & 0.4372700 & -3.7543050 \\
\hline C & 5.2451630 & -3.7305720 & 3.3555290 & C & 3.0707540 & 6.1706100 & -1.5606170 \\
\hline $\mathrm{C}$ & 4.1987270 & 002630 & 2.7992810 & $\mathrm{H}$ & 3.0332900 & 7.2523570 & -1.7325190 \\
\hline C & 3.3636660 & -3.9656590 & 1.8514660 & $\mathrm{H}$ & 3.4670450 & 5.6988190 & -2.4672230 \\
\hline $\mathrm{C}$ & 5.4086140 & -2.4269190 & 2.9674720 & $\mathrm{H}$ & 2.0479080 & 5.8074830 & -1.4147800 \\
\hline $\mathrm{H}$ & 5.9042800 & -4.1683420 & 4.0989950 & C & 5.3733280 & 6.3750110 & -0.5336690 \\
\hline $\mathrm{H}$ & 4.0495920 & -5.5237720 & 3.1299510 & $\mathrm{H}$ & 5.9943950 & 6.1558380 & 0.3408530 \\
\hline H & 2.5594090 & -4.5687470 & 1.4460540 & $\mathrm{H}$ & 5.8517850 & 5.9197820 & -1.4084390 \\
\hline $\mathrm{H}$ & 6.1919140 & -1.8132070 & 3.4048710 & $\mathrm{H}$ & 5.3626860 & 7.4600520 & -0.6866090 \\
\hline C & 3.4827430 & -5.6612640 & -1.9463380 & C & 3.6393040 & 2.1105590 & 3.8845130 \\
\hline C & 2.4037320 & -6.4323770 & -2.4342330 & $\mathrm{H}$ & 4.5235600 & 1.4961510 & 3.6915980 \\
\hline C & 1.1170450 & 76910 & -2.2499200 & $\mathrm{H}$ & 3.9761450 & 3.1415900 & 4.0469150 \\
\hline C & 3.2536280 & 916880 & -1.2670530 & $\mathrm{H}$ & 3.1737550 & 1.7546340 & 4.8106890 \\
\hline
\end{tabular}




$\begin{array}{rrr}1.3727650 & 2.8384850 & 3.0746970 \\ 1.6013370 & 3.8664200 & 3.3799770 \\ 0.6859650 & 2.8651010 & 2.2265380 \\ 0.8625290 & 2.3563710 & 3.9159790 \\ -2.7046940 & -4.5236210 & 2.0632360 \\ -3.7626120 & -4.3834340 & 2.3153290 \\ -2.6519120 & -5.1653170 & 1.1770450 \\ -2.2209220 & -5.0462270 & 2.8963750 \\ -1.9968630 & -2.3101370 & 3.0676840 \\ -1.5655470 & -1.3267540 & 2.8577310 \\ -3.0008340 & -2.1712260 & 3.4861230 \\ -1.3893520 & -2.7970240 & 3.8383400 \\ -7.0874370 & -1.5390010 & -0.4550030 \\ -8.0625090 & -1.0848190 & -0.2446970 \\ -7.0344440 & -1.7479710 & -1.5301470 \\ -7.0293320 & -2.4947680 & 0.0757880 \\ -6.0686780 & 0.7546060 & -0.7288770 \\ -5.2360970 & 1.4064540 & -0.4488330 \\ -6.0714350 & 0.6502430 & -1.8203190 \\ -7.0013840 & 1.2513350 & -0.4401230 \\ -2.4550650 & -0.8282560 & -4.0389300 \\ -3.5120370 & -0.5934140 & -4.2113180 \\ -1.9711940 & 0.0315330 & -3.5744780 \\ -1.9942060 & -0.9798870 & -5.0211190 \\ -2.9284500 & -3.2885740 & -3.8859630 \\ -4.0072310 & -3.1246170 & -3.9966610 \\ -2.5019180 & -3.4339900 & -4.8849790 \\ -2.7917600 & -4.2144610 & -3.3191470 \\ 0.4506620 & 0.1856200 & -0.4994350 \\ -0.1908000 & 0.2577380 & -1.8534110 \\ 0.2164400 & 1.2988430 & 0.4901560 \\ -1.1954990 & -2.2722270 & -3.1239510 \\ -6.0394280 & -0.4246580 & 1.0478640 \\ -0.9836120 & -3.3823530 & 1.5446360 \\ 4.8952720 & 0.0843730 & -1.3401810 \\ 3.5144670 & 6.3554030 & 0.5249040 \\ 2.3363280 & 1.0067620 & 2.5860180 \\ -2.4852230 & 2.2492160 & -1.9832640 \\ -1.7230830 & 3.0744100 & -1.0407750 \\ -2.6445730 & 1.2132540 & -1.6769330 \\ -0.9231180 & 2.5893810 & -0.4907430 \\ -1.2874570 & 2.3983490 & -2.7765760 \\ -0.7149360 & 1.6034160 & -2.5301580 \\ -3.4686770 & 4.3653520 & -2.9559630 \\ -3.0663520 & 5.1120060 & -1.6816000 \\ -1.7596190 & 4.5602780 & -1.1069820\end{array}$

$\begin{array}{ll}\mathrm{C} & -3.6796640 \\ \mathrm{H} & -4.3901500 \\ \mathrm{H} & -2.9499240 \\ \mathrm{H} & -0.9013490 \\ \mathrm{H} & -4.5406630 \\ \mathrm{H} & -3.8875500 \\ \mathrm{H} & -2.6906470 \\ \mathrm{H} & -3.8568870 \\ \mathrm{H} & -1.5555380 \\ \mathrm{C} & -2.9926440 \\ \mathrm{O} & -1.9663060 \\ \mathrm{C} & -4.2164710 \\ \mathrm{C} & -5.3745970 \\ \mathrm{C} & -4.2300760 \\ \mathrm{C} & -6.5468250 \\ \mathrm{H} & -5.3482550 \\ \mathrm{C} & -5.4036380 \\ \mathrm{H} & -3.3243330 \\ \mathrm{C} & -6.5635000 \\ \mathrm{H} & -7.4489900 \\ \mathrm{H} & -5.4138880 \\ \mathrm{H} & -7.4818070 \\ \mathrm{H} & -1.1454000 \\ \mathrm{O} & -3.0028060 \\ \mathrm{H} & \end{array}$
2.8775900
4.7894360

$6.1820800 \quad-1.8779780$

$4.8566590-1.7240150$

$2.7519710 \quad-1.9978130$

$2.3134620 \quad-3.5778130$

$4.4847100 \quad-3.7192890$

$5.0034280 \quad-0.9293650$

$4.9405010 \quad-0.1021380$

$1.9645280 \quad 1.5721440$

$\begin{array}{ll}1.9645280 & 1.5721440 \\ 1.2280590 & 1.9034040\end{array}$

$\begin{array}{ll}1.6876050 & 2.3718250\end{array}$

$2.4247610 \quad 2.1174640$

$0.6773300 \quad 3.3357620$

$2.1361460 \quad 2.8048760$

$3.2092550 \quad 1.3691060$

$0.3905810 \quad 4.0227810$

$0.1172420 \quad 3.5319470$

$\begin{array}{ll}1.1146140 & 3.7529240\end{array}$

$2.7037010 \quad 2.5995440$

$-0.3990500 \quad 4.7675370$

$0.8856480 \quad 4.2853440$

1. $3280200 \quad 1.3210970$

144

0.6818700

$\operatorname{TS} 112(R, R) \quad G[W B 97 X D / 6-31 G(d)]=-3310.756742$

$\begin{array}{lrcr}\text { TS112(R, R }) ~ G[W B 97 X D / 6-31 G(d)]=-3310.756742 \\ \text { O } & 0.1969310 & 0.9526750 & 0.0685820 \\ \text { O } & -2.0662800 & 0.3257290 & -0.8728440 \\ \mathrm{C} & 0.0184040 & 2.2604370 & -0.3235880 \\ \mathrm{C} & -1.1957890 & 2.8678090 & -0.0977070 \\ \mathrm{C} & 1.1458590 & 2.9412530 & -0.8570110 \\ \mathrm{C} & -1.3888350 & 4.1986070 & -0.5935260 \\ \mathrm{C} & 0.9806000 & 4.2474130 & -1.2354570 \\ \mathrm{C} & -0.2822800 & 4.8903730 & -1.1585320 \\ \mathrm{H} & 1.8323470 & 4.8088100 & -1.6089730 \\ \mathrm{C} & -2.6386620 & 0.8774960 & 0.2531610 \\ \mathrm{C} & -3.6685820 & 0.1377360 & 0.8930120 \\ \mathrm{C} & -2.2467140 & 2.1345420 & 0.6575420 \\ \mathrm{C} & -4.2916870 & 0.7139960 & 1.9695390 \\ \mathrm{C} & -2.8224650 & 2.6746340 & 1.8547430 \\ \mathrm{C} & -3.8755760 & 1.9643220 & 2.4933720 \\ \mathrm{H} & -5.1147680 & 0.1920230 & 2.4500080 \\ \mathrm{C} & -4.0220380 & 3.6805220 & 4.2029530 \\ \mathrm{C} & -2.9444530 & 4.3648280 & 3.5961170 \\ \mathrm{C} & -2.3616020 & 3.8774330 & 2.4545370 \\ \mathrm{C} & -4.4715870 & 2.5037660 & 3.6634070\end{array}$




$\begin{array}{rrr}-4.4799350 & 4.0817530 & 5.1019310 \\ -2.5703840 & 5.2816810 & 4.0416790 \\ -1.5287070 & 4.4080840 & 2.0074750 \\ -5.2850140 & 1.9557120 & 4.1319870 \\ -2.7957420 & 6.1245180 & -1.0508240 \\ -1.6880240 & 6.8218160 & -1.5843240 \\ -0.4607510 & 6.2141230 & -1.6379790 \\ -2.6508000 & 4.8491800 & -0.5671900 \\ -3.7708860 & 6.6014510 & -1.0283270 \\ -1.8161320 & 7.8318840 & -1.9614610 \\ 0.3955140 & 6.7330150 & -2.0609870 \\ -3.5097380 & 4.3212370 & -0.1673430 \\ 2.4629320 & 2.2378560 & -0.8897930 \\ 3.0120530 & 1.7753340 & -2.0990040 \\ 3.1355320 & 2.0165060 & 0.3272520 \\ 4.2248810 & 1.0829320 & -2.0572100 \\ 4.3310650 & 1.3028110 & 0.3174360 \\ 4.8882840 & 0.8170130 & -0.8623410 \\ 4.6666880 & 0.7273410 & -2.9850470 \\ 4.8443010 & 1.1286390 & 1.2599410 \\ -4.0732260 & -1.2042960 & 0.3741840 \\ -3.6447460 & -2.3739950 & 1.0276820 \\ -4.8868490 & -1.2867590 & -0.7698520 \\ -4.0757590 & -3.6071790 & 0.5362780 \\ -5.2797620 & -2.5421050 & -1.2282050 \\ -4.8891570 & -3.7150740 & -0.5873260 \\ -3.7570600 & -4.5185980 & 1.0362550 \\ -5.9040560 & -2.6045320 & -2.1169860 \\ 2.6270790 & 2.5752240 & 1.6496910 \\ 6.2014530 & 0.0557700 & -0.8544700 \\ 2.3435130 & 2.0534060 & -3.4387630 \\ -2.7400380 & -2.3253170 & 2.2524860 \\ -5.3074710 & -5.0749500 & -1.1174370 \\ -5.3465880 & -0.0489210 & -1.5267930 \\ -6.8744350 & 0.0885790 & -1.4958690 \\ -7.3618550 & -0.7532530 & -2.0012900 \\ -7.2445890 & 0.1215340 & -0.4655150 \\ -7.1861680 & 1.0090440 & -2.0022680 \\ -4.8096820 & -0.0422520 & -2.9641450 \\ -3.7186760 & -0.1203480 & -2.9675690 \\ -5.2164720 & -0.8775370 & -3.5462200 \\ -5.0914140 & 0.8873380 & -3.4715170 \\ -4.6793520 & -5.3450420 & -2.4916990 \\ -4.9321490 & -6.3515980 & -2.8440530 \\ -5.0430560 & -4.6263250 & -3.2351680 \\ -3.5890360 & -5.2566370 & -2.4477070\end{array}$

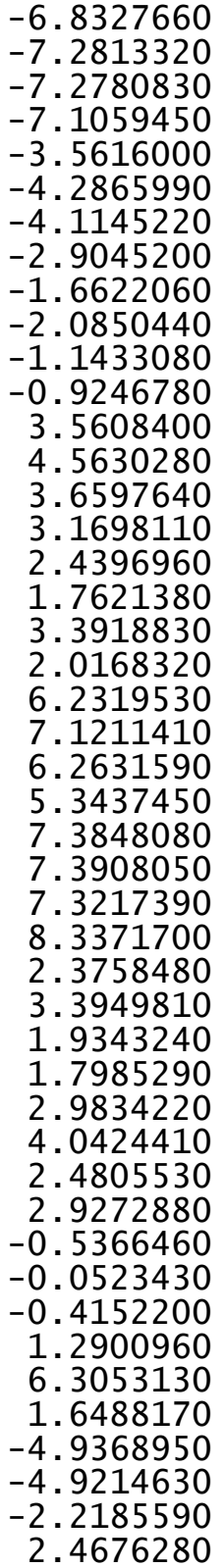

-5.2289010
-5.0533310
-4.5174760
-6.2380850
-2.4076110
-1.5917290
-3.3538090
-2.3572580
-3.4165670
-4.4095230
-3.4355590
-3.2155220
3.6870690
3.2957430
4.4807310
4.1307650
1.4851400
0.7065110
1.0119430
1.9161220
-1.0453940
-1.6738740
-0.6252130
-1.6830830
1.0182790
1.7801710
1.5317880
0.4771530
0.8642710
0.6245430
-0.0262770
1.1047000
3.2823330
3.0942190
3.5150020
4.1662140
-0.2226470
-0.2679500
-1.4525770
2.2762960
-0.4250470
3.0331290
0.8333710
-5.8299430
-1.3634300
-2.0085970

$-1.1697790$ $-0.1867030$ $-1.8747940$ $-1.4981740$

3.5471000

3.6193130

3.5912350

4.4230460

2.2430550

2.4362100

1. 2821170

3.0274950

2.1491130

2. 3598440

1.4007930

3.0716660

2.7092020

2. 3492260

2.9755900

3.6239720

0.2129010

0.0913530

1. 2243220

0.1555520

$-0.6801800$

$-1.4661300$

0.2862930

$-0.7158590$

$-4.4036370$

$-4.7295940$

$-3.9536790$

$-5.3029020$

$-4.1029470$

$-4.3174790$

$-5.0482720$

$-3.4606630$

$-0.8012950$

$-2.2198940$

0.0621530

$-3.2451250$

$-1.8364920$

1.4816780

$-1.0270800$

$-0.4200350$

2.2398040
-2.0436810 


$\begin{array}{lll}1.7380420 & -3.0551770 & -1.3235350 \\ 2.4119050 & -1.0086010 & -1.6109790 \\ 0.8168430 & -2.7577160 & -0.8339800 \\ 1.4160760 & -2.2365540 & -3.0093340 \\ 0.7203580 & -1.5305310 & -2.8032310 \\ 3.9250230 & -3.7998870 & -3.0755180 \\ 3.4768770 & -4.7611920 & -1.9716940 \\ 2.0259750 & -4.4969740 & -1.5610380 \\ 3.8285340 & -2.3478690 & -2.6007760 \\ 4.9567340 & -4.0178450 & -3.3689070 \\ 3.5758920 & -5.8005460 & -2.2992530 \\ 1.3304540 & -4.8232090 & -2.3444390 \\ 4.5591130 & -2.1651320 & -1.8057810 \\ 4.0484230 & -1.6450500 & -3.4105460 \\ 3.3008200 & -3.9383980 & -3.9662460 \\ 4.1205260 & -4.6336570 & -1.0933500 \\ 1.7474470 & -5.0451650 & -0.6569090 \\ 2.4832540 & -2.3703970 & 1.6421240 \\ 1.3659870 & -1.7480090 & 1.9047980 \\ 3.4525700 & -2.3737090 & 2.7708180 \\ 4.6650730 & -3.0501200 & 2.6193540 \\ 3.1725750 & -1.7011600 & 3.9630810 \\ 5.6015470 & -3.0375010 & 3.6451150 \\ 4.8642250 & -3.5754670 & 1.6916720 \\ 4.1111870 & -1.6916960 & 4.9883000 \\ 2.2269540 & -1.1838230 & 4.0764550 \\ 5.3261080 & -2.3549820 & 4.8286870 \\ 6.5464030 & -3.5573290 & 3.5221070 \\ 3.8953740 & -1.1649750 & 5.9124570 \\ 6.0597990 & -2.3422860 & 5.6290670 \\ 0.7174500 & -1.6617490 & 1.1305840 \\ 2.7610060 & -2.9246890 & 0.5731480\end{array}$

144

2. 7610060

$-2.9246890$

4.7814020

3.7270770

4.7253390

5.5450420

5.5123280

4.4887560

3. 6211140

5. 6205410

6. 1984000

4. 3830130

2.8358750

6.3851090

3. 7254630

2. 6651330

1. 3674370

3. 4685180

4.7524580

2. 8810280

0.5433740

4.2899660

$-1.9681450$

$-2.7273920$

$-2.4930510$

$-4.0001050$

$-3.7576080$

$-4.5322990$

$-4.5863430$

$-4.1607300$

3. 8235760

3. 1904250

4. 3604550

3. 1286290

4.2676560

3. 6507160

2. 6335050

4.6755650

$-1.7419790$

$-5.9128740$

$-2.2281820$

2. 5831840

3.4959950

5.0203700

6.5034860

6.6300170

7.0397760

6.9758700
-0.1765880
-2.3920410
-1.5320260
0.4700720
-3.3128440
-4.1534320
-3.7069280
-2.0290320
-3.6809720
-5.1598470
-4.3621550
-1.3613640
-5.6190870
-6.4598160
-6.0652050
-4.4198740
-5.9218590
-7.4094610
-6.6945190
-3.7783450
-2.6404570
-2.2047180
-2.5402090
-1.6683180
-1.9912650
-1.5430270
-1.3424970
-1.9025550
1.7965330
2.6988870
2.2487080
4.0429040
3.6057450
4.5162290
4.7411890
3.9612930
-3.0817190
-0.9677630
-2.3605990
2.2393940
5.9731130
1.2991360
1.6387210
2.6351980
1.6211230
0.9144270

1.4004120

1.9374460

1.9474310

3. 3863980

2.8934560

1.9292840

2. 9202790

4. 1431290

3. 2873200

1. 5700960

3. 3091670

$-1.7690020$

$-2.1760200$

$-1.9783890$

$-1.1545760$

$-1.9494930$

$-2.6559470$

$-2.3047060$

$-0.8546780$

$-0.5159250$

$-1.6151910$

0.7884580

$-1.3856440$

0.9644170

$-0.1062670$

$-2.2389340$

1.9703960

0.2947760

1.1746290

$-0.9196160$

0.8177150

$-1.2349060$

$-0.3854460$

1. 4869250

$-2.1792950$

1. 9972350

0.1772000

$-3.0456200$

2. 4949200

$-0.7826210$

$-1.9081190$

$-2.1049440$

$-2.5440640$

$-1.1501640$

$-2.7781420$ 


$\begin{array}{rrr}4.2678820 & 1.2719730 & -3.2448220 \\ 3.2158830 & 1.0173780 & -3.0888380 \\ 4.3169190 & 2.2443060 & -3.7489960 \\ 4.7086350 & 0.5260890 & -3.9159740 \\ 4.1151590 & 6.9281060 & 0.2450750 \\ 4.0305240 & 7.9663740 & -0.0949840 \\ 3.6069240 & 6.8528660 & 1.2131580 \\ 5.1746920 & 6.7032880 & 0.4040820 \\ 2.0176970 & 6.3109900 & -1.0283690 \\ 1.5849780 & 5.6414140 & -1.7792650 \\ 1.4360790 & 6.2022620 & -0.1052530 \\ 1.9061360 & 7.3434780 & -1.3786880 \\ 3.5982600 & 2.3780590 & 3.6395690 \\ 4.5106030 & 1.8069380 & 3.4466380 \\ 3.8812610 & 3.4292390 & 3.7726980 \\ 3.1663500 & 2.0200250 & 4.5812020 \\ 1.2891550 & 2.9783580 & 2.8577110 \\ 1.4821010 & 4.0126780 & 3.1663230 \\ 0.5964580 & 2.9869480 & 2.0139280 \\ 0.8014880 & 2.4758260 & 3.7003820 \\ -2.3343700 & -4.4358510 & 2.4130030 \\ -3.3864050 & -4.3269310 & 2.7024860 \\ -2.2853630 & -5.1547450 & 1.5880340 \\ -1.7871540 & -4.8536070 & 3.2657360 \\ -1.7080230 & -2.1050190 & 3.1764850 \\ -1.3507990 & -1.1221310 & 2.8559260 \\ -2.6985880 & -1.9862770 & 3.6324640 \\ -1.0360930 & -2.4817640 & 3.9553060 \\ -6.8374570 & -2.0365270 & 0.7806630 \\ -7.8071390 & -1.5985280 & 1.0431870 \\ -7.0098350 & -2.8455200 & 0.0610680 \\ -6.4096330 & -2.4772810 & 1.6862630 \\ -6.5903780 & -0.3186460 & -1.0292270 \\ -5.9588830 & 0.4467960 & -1.4889840 \\ -6.8378730 & -1.0604570 & -1.7979550 \\ -7.5252250 & 0.1591230 & -0.7177640 \\ -2.5435530 & -1.1629140 & -3.9469490 \\ -3.6193470 & -1.0512120 & -4.1266400 \\ -2.1625290 & -0.2353370 & -3.5179950 \\ -2.0661240 & -1.3009390 & -4.9231140 \\ -2.8042820 & -3.6427350 & -3.6674360 \\ -3.8964260 & -3.5745400 & -3.7423090 \\ -2.4030670 & -3.7954610 & -4.6758370 \\ -2.5676460 & -4.5278180 & -0.6272490 \\ 0.5293820 & 0.1893270 & -9737170\end{array}$

0.2700030

$-1.1391940$

$-5.7793250$

$-0.7033910$

4.9749100

4.0296420

2. 3281140

$-2.5588970$

$-1.7540230$

$-2.4967230$

$-0.8214970$

$-1.5771980$

$-0.8879140$

$-4.0345300$

$-3.4668490$

$-2.0050840$

$-3.9523820$

$-5.0776320$

$-3.5400620$

$-1.3428240$

$-4.6038990$

$-4.2818740$

$-3.4783710$

$-4.0555360$

$-1.6542210$

$-2.8324190$

$-1.8859580$

$-4.1194690$

$-5.2569680$

$-4.2128390$

$-6.4853560$

$-5.1678060$

$-5.4412660$

$-3.3243620$

$-6.5777800$

$-7.3707850$

$-5.5132790$

$-7.5374960$

$-1.0391830$

$-2.7182040$
1.3599910

$-2.4547010$

$-0.1882570$

$-3.2626620$

0.2891390

6.1131060

1.1802290

1.9479790

3.0154770

0.9733430

. 7195610

2.0742350

1.3632990

3.7092810

4.7426660

4.4460030

2.2997680

3.9453660

5.7501160

4.6881850

2. 2256570

1.5421970

3.7423440

4.7332970

5.0444500

2.0702920

1.2061980

1.7804950

2.4993730

0.7775420

2.2074440

3.2779960

0.4928850

0.2241430

1.2034820

2.7600990

$-0.2868650$

0.9749900

1.3475920

3.0466990
0.2864450 $-3.0102740$

0.9374990

1.7101420

$-1.4897860$

$-1.7315830$

2.3904320

$-1.7934390$

$-1.1920720$

$-1.3043120$

$-0.7206510$

$-2.8421240$

$-2.6274250$

$-2.8436930$

$-1.8667380$

$-1.5189040$

$-2.2520810$

$-3.0764760$

$-2.2873930$

$-2.3595750$

$-1.3722020$

$-2.9709630$

$-3.7879030$

$-0.9422490$

$-0.6736830$

1.4742610

1.7227200

2.1582430

1.7837240

3.1255290

2. 3646060

1.0334600

3.7099640

3.4051960

3.3270520

2. 0669000

4.4617150

3.7804670

1.1835390

0.7248250

144

TS114 $(R, R) \quad G[W B 97 X D / 6-31 G(d)]=-3310.752023$

$\begin{array}{lllr}\mathrm{O} & -0.0400810 & 1.0590420 & 0.2229780 \\ \mathrm{O} & -2.1129030 & 0.1446580 & -0.8779180 \\ \mathrm{C} & -0.2468880 & 2.3061910 & -0.3228750 \\ \mathrm{C} & -1.5166990 & 2.8359340 & -0.3119960\end{array}$




\begin{tabular}{|c|c|c|}
\hline $\begin{array}{l}0.8995140 \\
-1.7229630 \\
0.7061520 \\
-0.5935080 \\
1.5612800 \\
-2.8306670 \\
-3.8347770 \\
-2.5979330 \\
-4.6290950 \\
-3.3531110 \\
-4.4032010 \\
-5.4368090 \\
-4.9099270 \\
-3.8347110 \\
-3.0779450 \\
-5.1802110 \\
-5.5056520 \\
-3.6015290 \\
-2.2490550 \\
-5.9872120 \\
-3.1682600 \\
-2.0426630 \\
-0.7854670 \\
-3.0134210 \\
-4.1642160 \\
-2.1785330 \\
0.0871560 \\
-3.8851560 \\
2.2410710 \\
2.9729170 \\
2.7479400 \\
4.2004000 \\
3.9589840 \\
4.7093230 \\
4.7683960 \\
4.3395250 \\
-4.0049690 \\
-3.4567710 \\
-4.7067720 \\
-3.6678060 \\
-4.8758960 \\
-4.3727160 \\
-3.2601940 \\
2.4135510 \\
6.0302870 \\
-315620\end{array}$ & $\begin{array}{r}3.0066320 \\
4.0975530 \\
4.2588490 \\
4.8137510 \\
4.8428770 \\
0.7671170 \\
-0.0026090 \\
2.0976260 \\
0.6276850 \\
2.7107890 \\
1.9739510 \\
0.0761640 \\
3.8551810 \\
4.5715670 \\
4.0168780 \\
2.5815090 \\
4.3089850 \\
5.5690800 \\
4.5765090 \\
2.0077100 \\
5.8699900 \\
6.5964520 \\
6.0747220 \\
4.6547800 \\
6.2761510 \\
7.5596300 \\
6.6149390 \\
4.1025120 \\
2.3726440 \\
1.8882520 \\
2.2240400 \\
1.2560090 \\
1.5681280 \\
1.0706720 \\
0.9039610 \\
1.4432760 \\
-1.4509480 \\
-2.4278110 \\
-1.8293120 \\
-3.7723070 \\
-3.1854600 \\
-4.1728880 \\
-4.5369370 \\
-3.4780750 \\
2.8585100 \\
0.3642800\end{array}$ & $\begin{array}{r}-0.7865710 \\
-0.9611300 \\
-1.3064350 \\
-1.4465780 \\
-1.6345280 \\
0.1188240 \\
0.7632250 \\
0.3972450 \\
1.6848020 \\
1.4526170 \\
2.0672320 \\
2.1584490 \\
3.5143060 \\
2.9416880 \\
1.9415840 \\
3.0881210 \\
4.3004330 \\
3.3019360 \\
1.5241000 \\
3.5365290 \\
-1.7788180 \\
-2.2290670 \\
-2.0682070 \\
-1.1614580 \\
-1.9274950 \\
-2.7113120 \\
-2.4262500 \\
-0.8274150 \\
-0.6095290 \\
-1.7051720 \\
0.6979180 \\
-1.4705340 \\
0.8798250 \\
-0.1884390 \\
-2.3252570 \\
1.8889240 \\
0.4343750 \\
1.2860110 \\
-0.7229700 \\
0.9788470 \\
-0.9951800 \\
-0.1521500 \\
1.6356180 \\
-1.8946950 \\
1.8943290 \\
0.0935370\end{array}$ \\
\hline
\end{tabular}

2.4967440
-2.6585880
-4.5618000
-5.2724010
-6.7948720
-7.0768450
-7.2864280
-7.1875110
-4.5758770
-3.4966630
-4.7522820
-4.9563480
-3.7990050
-3.8998520
-4.1875190
-2.7339670
-6.0449830
-6.5854140
-6.5249780
-6.1592960
-3.5618580
-4.3644770
-4.0225930
-2.9796670
-1.4650060
-1.7839460
-0.8797940
-0.8128590
2.6985670
3.7562030
2.6426500
2.1944490
2.0201490
1.6081270
3.0186040
1.3914850
6.9588660
7.8859820
7.2188450
6.4985790
6.7840090
6.1905180
7.0756310
7.6986830
2.7043690
3.7668080

2.0916240

$-2.0534770$

$-5.6457470$

$-0.8034710$

$-0.9349870$

$-1.9029340$

$-0.8435960$

$-0.1516320$

$-0.8929270$

$-0.7545530$

$-1.8661680$

$-0.1183140$

$-6.0414140$

$-7.1154170$

$-5.5041460$

$-5.8051790$

$-6.0223470$

$-5.7519160$

$-5.5073580$

$-7.1001240$

$-2.0143820$

$-1.2789100$

$-2.9950890$

$-1.7531100$

$-2.9841530$

$-3.9676020$

-3. 1196070

$-2.5495170$

4.2174080

4.0913220

4.8806780

4.7089290

1.9683130

0.9838620

1.8373520

2.4273750

1.2331310

0.6929270

2.1593220

1.5032310

$-0.0872950$

$-0.7649970$

0.7703030

$-0.6163950$

0.8703230

0.6617260
$-3.1371990$ 2. 5290680 $-0.4682230$ $-1.6943640$ $-1.8313670$ $-2.2615600$ $-0.8569710$ $-2.4894090$ $-3.0586370$ $-2.9483110$ $-3.5319130$ $-3.7342630$ $-1.7400850$ $-1.9337400$ $-2.6129960$ $-1.6472160$ $-0.5743180$

0.3385530

$-1.4144630$

$-0.7360650$

3.7707690

3. 6643410

3.9403040

4.6620770

2.7803150

3.1457540

1.8684290

3.5460400

2.1978710

2.4585380

1.3278760

3.0379100

3.1386350

2.8989260

3.5733120

3.9092250

0.9575260

1.1794610

0.4317930

1.9117680

$-1.1596220$

$-1.7798020$

$-1.7779330$

$-0.8724340$

$-4.0377990$

$-4.2117610$ 


\begin{tabular}{|c|c|c|}
\hline $\begin{array}{r}2.2399630 \\
2.2465950 \\
3.1884570 \\
4.2702440 \\
2.8061470 \\
3.0310100 \\
-0.5432530 \\
0.0600040 \\
-0.3487950 \\
1.4205880 \\
5.7927560 \\
1.0088960 \\
-5.0726090 \\
-4.1335800 \\
-2.2511040 \\
2.4695820 \\
1.6400600 \\
2.3814680 \\
0.6865830 \\
1.5429350 \\
0.8469970 \\
4.0083380 \\
3.3902100 \\
1.9102910 \\
3.8867810 \\
5.0635720 \\
3.4880750 \\
1.3002280 \\
4.4986170 \\
4.2373460 \\
3.5050280 \\
3.9274700 \\
1.5156770 \\
2.6685730 \\
1.7918790 \\
3.9716110 \\
5.0369130 \\
4.1591280 \\
6.2877280 \\
4.8749000 \\
5.4099690 \\
3.3274240 \\
6.4753680 \\
7.1162990 \\
5.5547320 \\
7.1531180\end{array}$ & $\begin{array}{r}-0.0206130 \\
1.0513160 \\
3.3159970 \\
3.1501020 \\
3.5034020 \\
4.2191840 \\
-0.2213310 \\
-0.2419710 \\
-1.4023180 \\
2.2839770 \\
-0.5345820 \\
3.0540280 \\
0.1944950 \\
-6.2158800 \\
-1.0499980 \\
-2.0718360 \\
-3.1576630 \\
-1.1095300 \\
-2.8716860 \\
-2.1749580 \\
-1.4596930 \\
-3.7951980 \\
-4.8594680 \\
-4.5763020 \\
-2.4025900 \\
-4.0218310 \\
-5.8524570 \\
-4.7845230 \\
-2.3365460 \\
-1.6261490 \\
-3.8018190 \\
-4.8818130 \\
-5.2085900 \\
-2.3209850 \\
-1.3801550 \\
-2.1324740 \\
-2.9696620 \\
-1.1082990 \\
-2.7713020 \\
-3.7636250 \\
-0.9147660 \\
-0.4653900 \\
-1.7412000 \\
-3.4170880 \\
-0.1169500 \\
-1.5833750\end{array}$ & $\begin{array}{l}-3.6126920 \\
-5.0162990 \\
-3.7568590 \\
-3.8281930 \\
-4.7666300 \\
-3.1598400 \\
-0.6550960 \\
-2.0291700 \\
0.2611490 \\
-3.1056570 \\
0.6785270 \\
1.6290260 \\
-1.2930170 \\
0.3664670 \\
2.3707940 \\
-1.7044910 \\
-1.1704920 \\
-1.1953370 \\
-0.7374240 \\
-2.8027380 \\
-2.6154120 \\
-2.7246300 \\
-1.8140790 \\
-1.5372430 \\
-2.1013140 \\
-2.9067720 \\
-2.2632610 \\
-2.4248380 \\
-1.1921550 \\
-2.7887880 \\
-3.6985600 \\
-0.8593920 \\
-0.7377090 \\
1.5204420 \\
1.7460900 \\
2.2084430 \\
1.8672830 \\
3.1386020 \\
2.4394590 \\
1.1461500 \\
3.7122560 \\
3.3988870 \\
3.3594050 \\
2.1665720 \\
4.4341470 \\
3.8042890\end{array}$ \\
\hline
\end{tabular}

$\begin{array}{llll}\mathrm{H} & 0.9444330 & -1.4655770 & 1.1957780 \\ \mathrm{O} & 2.4818070 & -3.2924620 & 0.7787200\end{array}$

144

$\operatorname{TS} 115(R, R) \quad G[W B 97 X D / 6-31 G(d)]=-3310.751806$

$\begin{array}{lrrr}\text { TS115(R, R }) \text { G }[W B 97 X D / 6-31 G(d)] & =-3310.751806 \\ \mathrm{O} & 0.1247950 & -1.1078590 & 0.2661950 \\ \mathrm{O} & 2.0987990 & -0.1166850 & -0.9355630 \\ \mathrm{C} & 0.3893540 & -2.3537640 & -0.2581700 \\ \mathrm{C} & 1.6874260 & -2.8134420 & -0.2708250 \\ \mathrm{C} & -0.7286610 & -3.1211230 & -0.6843520 \\ \mathrm{C} & 1.9430890 & -4.0768360 & -0.8998910 \\ \mathrm{C} & -0.4820960 & -4.3731740 & -1.1808110 \\ \mathrm{C} & 0.8413800 & -4.8627240 & -1.3392770 \\ \mathrm{H} & -1.3122360 & -5.0074130 & -1.4783410 \\ \mathrm{C} & 2.8930610 & -0.6627900 & 0.0466770 \\ \mathrm{C} & 3.8744570 & 0.1887770 & 0.6190780 \\ \mathrm{C} & 2.7529310 & -1.9953800 & 0.3732170 \\ \mathrm{C} & 4.7589960 & -0.3660100 & 1.5054300 \\ \mathrm{C} & 3.6022140 & -2.5301130 & 1.4004350 \\ \mathrm{C} & 4.6400300 & -1.7134820 & 1.9299610 \\ \mathrm{H} & 5.5524770 & 0.2477230 & 1.9230660 \\ \mathrm{C} & 5.3479270 & -3.5162070 & 3.3919950 \\ \mathrm{C} & 4.2853460 & -4.3120510 & 2.9074750 \\ \mathrm{C} & 3.4369680 & -3.8343780 & 1.9410160 \\ \mathrm{C} & 5.5135610 & -2.2425680 & 2.9154540 \\ \mathrm{H} & 6.0178260 & -3.9095520 & 4.1505170 \\ \mathrm{H} & 4.1346990 & -5.3095020 & 3.3094080 \\ \mathrm{H} & 2.6217650 & -4.4561220 & 1.5891270 \\ \mathrm{H} & 6.3085950 & -1.6073480 & 3.2975180 \\ \mathrm{C} & 3.4590490 & -5.7845450 & -1.7287240 \\ \mathrm{C} & 2.3625240 & -6.5802310 & -2.1308850 \\ \mathrm{C} & 1.0838880 & -6.1241530 & -1.9420160 \\ \mathrm{C} & 3.2557610 & -4.5675000 & -1.1293000 \\ \mathrm{H} & 4.4713320 & -6.1377670 & -1.9005930 \\ \mathrm{H} & 2.5363940 & -7.5439030 & -2.5997550 \\ \mathrm{H} & 0.2322320 & -6.7181130 & -2.2638320 \\ \mathrm{H} & 4.1051950 & -3.9619690 & -0.8322910 \\ \mathrm{C} & -2.0988300 & -2.5501420 & -0.5045070 \\ \mathrm{C} & -2.8476640 & -2.0928110 & -1.6018730 \\ \mathrm{C} & -2.6136050 & -2.4279370 & 0.8019420 \\ \mathrm{C} & -4.1003100 & -1.5125360 & -1.3687500 \\ \mathrm{C} & -3.8580510 & -1.8360300 & 0.9816110 \\ \mathrm{C} & -4.6220470 & -1.3653370 & -0.0873230 \\ \mathrm{H} & -4.6791050 & -1.1706350 & -2.2208210 \\ \mathrm{H} & -4.2538010 & -1.7310850 & 1.9888910 \\ \mathrm{C} & 3.9071840 & 1.6409670 & 0.2658910 \\ \mathrm{C} & 3.3446380 & 2.5825120 & 1.1521880\end{array}$




\begin{tabular}{rrrr} 
C & 4.4755700 & 2.0591710 & -0.9463570 \\
C & 3.3965230 & 3.9312070 & 0.8109410 \\
C & 4.4929390 & 3.4224040 & -1.2482220 \\
C & 3.9611180 & 4.3728180 & -0.3849770 \\
H & 2.9692510 & 4.6621400 & 1.4922680 \\
H & 4.9294810 & 3.7530600 & -2.1888140 \\
C & -1.8760050 & -2.9933250 & 2.0081630 \\
C & -5.9816610 & -0.7443160 & 0.2008980 \\
C & -2.3589610 & -2.2721960 & -3.0331200 \\
C & 2.6969150 & 2.1619720 & 2.4660140 \\
C & 3.9706720 & 5.8476350 & -0.7449300 \\
C & 5.0596060 & 1.0696240 & -1.9433490 \\
C & 6.5600540 & 1.3079760 & -2.1566900 \\
H & 6.7492570 & 2.2935960 & -2.5974590 \\
H & 7.1042280 & 1.2530580 & -1.2078240 \\
H & 6.9741390 & 0.5537210 & -2.8353340 \\
C & 4.2909410 & 1.0978090 & -3.2707270 \\
H & 3.2278070 & 0.9038070 & -3.1033730 \\
H & 4.3912720 & 2.0700300 & -3.7676750 \\
H & 4.6786630 & 0.3329260 & -3.9531960 \\
C & 4.7624260 & 6.6764570 & 0.2748870 \\
H & 4.8151980 & 7.7259640 & -0.0359950 \\
H & 4.2875000 & 6.6439890 & 1.2622420 \\
H & 5.7836050 & 6.2965420 & 0.3815290 \\
C & 2.5434680 & 6.3899630 & -0.9058560 \\
H & 1.9870880 & 5.8133850 & -1.6522930 \\
H & 1.9945560 & 6.3319290 & 0.0413160 \\
H & 2.5605920 & 7.4395110 & -1.2209780 \\
C & 3.7013490 & 2.2532410 & 3.6244420 \\
H & 4.5774140 & 1.6214270 & 3.4526940 \\
H & 4.0504310 & 3.2855680 & 3.7480100 \\
H & 3.2340310 & 1.9372130 & 4.5641410 \\
C & 1.4363240 & 2.9678850 & 2.8039310 \\
H & 1.6743450 & 3.9972750 & 3.0971780 \\
H & 0.7530910 & 2.9940930 & 1.9527780 \\
H & 0.9179510 & 2.5021770 & 3.6494370 \\
C & -2.5063260 & -4.3308870 & 2.4215880 \\
H & -3.5548460 & -4.1929300 & 2.7113540 \\
H & -2.4775880 & -5.0491070 & 1.5950880 \\
H & -1.9710450 & -4.7658230 & 3.2733400 \\
C & -1.8111080 & -2.0202920 & 3.1889620 \\
H & -1.4267080 & -1.0477070 & 2.8681440 \\
H & -2.7965230 & -1.8744740 & 3.6482380 \\
H & -1.1476780 & -2.4169040 & 3.9651880 \\
C & -6.9347660 & -1.7795420 & 0.8185210 \\
H & -7.8893270 & -1.3105020 & 1.0828490 \\
& & & \\
\hline
\end{tabular}

-7.1357970
-6.5152990
-6.6479240
-5.9983640
-6.9210220
-7.5671390
-2.6378410
-3.7101240
-2.2237580
-2.1694910
-2.9782650
-4.0679740
-2.5866980
-2.7670670
0.5252880
-0.1307220
0.3136190
-1.2734740
-5.8180270
-0.8436000
4.9505870
4.4743520
2.3891670
-2.5076360
-1.6724250
-2.4618190
-0.7426300
-1.5344710
-0.8614560
-3.9547890
-3.3532700
-1.8947440
-3.8979430
-4.9947520
-3.4077020
-1.2367560
-4.5408570
-4.2521070
-3.4082050
-3.9321810
-1.5219270
-2.7560090
-1.8384010
-4.0493450
-5.1599600
-4.1764050
0.1065680

1.7252680

$-1.0059930$

$-1.4751650$

$-1.7677610$

$-0.6922420$

$-3.9399110$

$-4.1156470$

$-3.5186070$

$-4.9172020$

$-3.6473750$

$-3.7176380$

$-4.6569840$

$-3.0473940$

$-0.6524690$

$-2.0017610$

0.2522450

$-3.0002910$

0.9541700

1.7186110

$-1.5264910$

$-1.7153850$

2.3639390

$-1.8023550$

$-1.2197480$

$-1.3047070$

$-0.7549850$

$-2.8621320$

$-2.6504100$

$-2.8542620$

1.8944710

$-1.5586590$

$-2.2490580$

$-3.0785830$

$-2.3252430$

$-2.4090840$

$-1.3611710$

$-2.9567010$

$-3.8045490$

$-0.9639700$

$-0.7234080$

1.4609040

1.7079740

2.1526110

1.7829570

3.1240170 


\begin{tabular}{|c|c|c|c|c|c|c|c|}
\hline C & 63051600 & 2685720 & 23727180 & & 37820780 & 13071510 & 02302320 \\
\hline $\mathrm{H}$ & -5.0444700 & $\begin{array}{l}2.4685 / 20 \\
3.4814350\end{array}$ & $\begin{array}{l}2.3727480 \\
1.0299920\end{array}$ & C & 4.4826930 & $\begin{array}{l}1.30 / 1510 \\
0.8411200\end{array}$ & $\begin{array}{r}0.2303320 \\
-2.4207410\end{array}$ \\
\hline C & -5.4114770 & 0.7181330 & 3.7172440 & $\mathrm{C}$ & 4.9454110 & 0.6018470 & -0.0918350 \\
\hline H & -3.3092180 & 0.3640060 & 3.4007540 & $\mathrm{C}$ & 5.3274760 & 0.3816300 & -1.4102760 \\
\hline C & -6.5212490 & 1.4723980 & 3.3395340 & $\mathrm{H}$ & 4.7613990 & 0.6520150 & -3.4550400 \\
\hline $\mathrm{H}$ & -7.2595210 & 3.0555840 & 2.0788090 & $\mathrm{H}$ & 5.5713160 & 0.2317820 & 0.7154970 \\
\hline $\mathrm{H}$ & -5.5095160 & -0.0558920 & 4.4720460 & C & -4.2258050 & -0.4375940 & -0.2058800 \\
\hline $\mathrm{H}$ & -7.4862750 & 1.2838480 & 3.8000100 & $\mathrm{C}$ & -4.1715890 & -1.7871470 & 0.1997150 \\
\hline $\mathrm{H}$ & -0.9909930 & 1.4017850 & 1.1631890 & C & -4.9076010 & -0.0785890 & -1.3801520 \\
\hline 0 & -2.6118270 & 3.1543310 & 0.7060050 & $\mathrm{C}$ & -4.8280040 & -2.7416740 & -0.5736430 \\
\hline & & & & $\mathrm{c}$ & -5.5396870 & -1.0751940 & -2.1252190 \\
\hline & R) $\mathrm{G}[\mathrm{WB} 97 \mathrm{X}$ & $(d)]=-33$ & 23 & C & -5.5151520 & -2.4101110 & -1.7400780 \\
\hline 0 & 0.4582810 & 0.7303950 & 0.0199590 & $\mathrm{H}$ & -4.7933920 & -3.7824690 & -0.2617970 \\
\hline 0 & -1.8470120 & 0.8721380 & -1.0331990 & $\mathrm{H}$ & -6.0683530 & -0.8018590 & -3.0362920 \\
\hline C & 0.5506550 & 2.1013900 & -0.0641720 & C & 3.4412360 & 1.5306730 & 1.6967410 \\
\hline C & -0.5166620 & 2.8789530 & 0.3356180 & $\mathrm{C}$ & 6.6415740 & -0.2843420 & -1.7848530 \\
\hline C & 1.7912510 & 2.6516300 & -0.4800640 & C & 2.4246390 & 2.0097370 & -3.2993360 \\
\hline C & -0.4246620 & 4.3005670 & 0.1804120 & $\mathrm{C}$ & -3.4360160 & -2.2304300 & 1.4588380 \\
\hline C & 1.9057100 & 4.0178120 & -0.5174190 & C & -6.2040340 & -3.4759250 & -2.5728950 \\
\hline $\mathrm{C}$ & 0.8109690 & 4.8697520 & -0.2299410 & $\mathrm{C}$ & -4.9966310 & 1.3637600 & -1.8581000 \\
\hline $\mathrm{H}$ & 2.8597810 & 4.4628290 & -0.7870240 & C & -6.4466830 & 1.8654720 & -1.8259090 \\
\hline C & -2.3702470 & 1.2454730 & 0.1910590 & $\mathrm{H}$ & -7.0793510 & 1.2979860 & -2.5182270 \\
\hline $\mathrm{C}$ & -3.5708450 & 0.6207590 & 0.6208930 & $\mathrm{H}$ & -6.8742340 & 1.7653640 & -0.8224290 \\
\hline C & -1.7301080 & 2.2340440 & 0.9027850 & $\mathrm{H}$ & -6.4945540 & 2.9212280 & -2.1158920 \\
\hline $\mathrm{C}$ & -4.1100900 & 1.0429980 & 1.8086940 & C & -4.3719670 & 1.5391110 & -3.2478990 \\
\hline C & -2.2380410 & 2.5799750 & 2.1978490 & $\mathrm{H}$ & -3.3339440 & 1.1949120 & -3.2499410 \\
\hline C & -3.4577410 & 1.9939090 & 2.6336620 & $\mathrm{H}$ & -4.9238520 & 0.9755580 & -4.0092600 \\
\hline $\mathrm{H}$ & -5.0521290 & 0.6187460 & 2.1442910 & $\mathrm{H}$ & -4.3866430 & 2.5955550 & -3.5392180 \\
\hline C & -3.3214300 & 3.2190020 & 4.7236290 & C & -7.3200750 & -4.1766590 & -1.7871160 \\
\hline C & -2.0871040 & 3.7698490 & 4.3101240 & $\mathrm{H}$ & -7.8431240 & -4.9011030 & -2.4214450 \\
\hline C & -1.5597460 & 3.4607 & 3.0824580 & $\mathrm{H}$ & -6.9140700 & -4.7179330 & -0.9246970 \\
\hline C & -3.9870920 & 2.3470770 & 3.9025550 & $\mathrm{H}$ & -8.0525180 & -3.4529920 & -1.4149260 \\
\hline $\mathrm{H}$ & -3.7314760 & 3.4773380 & 5.6951880 & C & -5.1959180 & -4.4933470 & -3.1242110 \\
\hline $\mathrm{H}$ & -1.5479540 & 4.4396950 & 4.9731770 & $\mathrm{H}$ & -4.4109840 & -3.9942790 & -3.7014580 \\
\hline $\mathrm{H}$ & -0.6076440 & 3.8842860 & 2.7832190 & $\mathrm{H}$ & -4.7132340 & -5.0477890 & -2.3109370 \\
\hline $\mathrm{H}$ & -4.9259120 & 1.8982800 & 4.2165400 & $\mathrm{H}$ & -5.6959920 & -5.2194500 & -3.7751380 \\
\hline C & -1.3939830 & 6.5270740 & 0.2487720 & C & -4.4152820 & -2.4142520 & 2.6277680 \\
\hline $\mathrm{C}$ & -0.1526600 & 7.0930660 & -0.1224750 & $\mathrm{H}$ & -4.9557110 & -1.4902650 & 2.8532750 \\
\hline C & 0.9231590 & 6.2787070 & -0.3602450 & $\mathrm{H}$ & -5.1570190 & -3.1861550 & 2.3898120 \\
\hline C & -1.5275790 & 5.1704340 & 0.3972080 & $\mathrm{H}$ & -3.8805220 & -2.7238860 & 3.5330770 \\
\hline $\mathrm{H}$ & -2.2514250 & 7.1730080 & 0.4119370 & C & -2.6235220 & -3.5157420 & 1.2529470 \\
\hline $\mathrm{H}$ & -0.0595740 & 8.1691260 & -0.2323130 & $\mathrm{H}$ & -3.2686560 & -4.3956760 & 1.1470640 \\
\hline $\mathrm{H}$ & 1.8779600 & 6.6984220 & -0.6662540 & $\mathrm{H}$ & -1.9929180 & -3.4354450 & $0 . \overline{3} 643960$ \\
\hline $\mathrm{H}$ & -2.4883710 & 4.7494030 & 0.6717640 & $\mathrm{H}$ & -1.9790280 & -3.6869390 & 2.1219760 \\
\hline C & 2.9688060 & 1.7899360 & -0.8071750 & C & 4.5818430 & 2.2253300 & 2.4513170 \\
\hline C & 3.3017040 & 529170 & -2.1509120 & $\mathrm{H}$ & 5.4789760 & 1.5976710 & 2.4994180 \\
\hline
\end{tabular}




$\begin{array}{rrrr}\mathrm{H} & 4.8556790 & 3.1683780 & 1.9667060 \\ \mathrm{H} & 4.2766170 & 2.4438950 & 3.4805750 \\ \mathrm{C} & 3.0394500 & 0.2123820 & 2.3697430 \\ \mathrm{H} & 2.1529230 & -0.2066320 & 1.8864110 \\ \mathrm{H} & 3.8471950 & -0.5277250 & 2.3182850 \\ \mathrm{H} & 2.8026930 & 0.3725600 & 3.4276930 \\ \mathrm{C} & 7.1447950 & -1.3087870 & -0.7648120 \\ \mathrm{H} & 8.0268390 & -1.8249940 & -1.1578690 \\ \mathrm{H} & 7.4412720 & -0.8286680 & 0.1745930 \\ \mathrm{H} & 6.3831870 & -2.0606060 & -0.5352590 \\ \mathrm{C} & 7.7118810 & 0.7902240 & -2.0353500 \\ \mathrm{H} & 7.3843930 & 1.5034630 & -2.7984230 \\ \mathrm{H} & 7.9099430 & 1.3498340 & -1.1137160 \\ \mathrm{H} & 8.6513530 & 0.3348300 & -2.3684850 \\ \mathrm{C} & 2.3331660 & 0.9950710 & -4.4445790 \\ \mathrm{H} & 3.2654160 & 0.9457510 & -5.0199510 \\ \mathrm{H} & 2.1073280 & -0.0061840 & -4.0721280 \\ \mathrm{H} & 1.5373480 & 1.2895910 & -5.1360360 \\ \mathrm{C} & 2.9165500 & 3.3615350 & -3.8375140 \\ \mathrm{H} & 3.9423250 & 3.2731160 & -4.2155710 \\ \mathrm{H} & 2.2780750 & 3.6988420 & -4.6617900 \\ \mathrm{H} & 2.9056930 & 4.1344670 & -3.0642020 \\ \mathrm{P} & -0.5429070 & -0.0940630 & -0.9894320 \\ \mathrm{O} & 0.0077440 & -0.1653840 & -2.3807300 \\ \mathrm{O} & -0.8340930 & -1.3738890 & -0.2482430 \\ \mathrm{H} & 1.4112530 & 2.1421840 & -2.9087250 \\ \mathrm{H} & 6.4768480 & -0.8131310 & -2.7338470 \\ \mathrm{H} & 2.5747230 & 2.1965100 & 1.7479820 \\ \mathrm{H} & -4.4264350 & 1.9923270 & -1.1683100 \\ \mathrm{H} & -6.6682110 & -2.9714570 & -3.4303110 \\ \mathrm{H} & -2.7248170 & -1.4442710 & 1.7271710 \\ \mathrm{C} & 2.3034730 & -2.1917530 & -1.5104490 \\ \mathrm{C} & 1.2779420 & -3.2033960 & -1.2316350 \\ \mathrm{H} & 2.2030810 & -1.2427840 & -0.9788970 \\ \mathrm{H} & 0.2814740 & -2.8370230 & -1.0104400 \\ \mathrm{O} & 1.6340740 & -2.1687550 & -2.7902500 \\ \mathrm{H} & 0.9608650 & -1.4154240 & -2.7434470 \\ \mathrm{C} & 3.8580590 & -4.0537500 & -2.2412780 \\ \mathrm{C} & 2.9583590 & -5.0633410 & -1.5262040 \\ \mathrm{C} & 1.4896880 & -4.6350790 & -1.5818340 \\ \mathrm{C} & 3.7326180 & -2.6644870 & -1.6129420 \\ \mathrm{H} & 4.9026880 & -4.3780180 & -2.1929640 \\ \mathrm{H} & 3.0611100 & -6.0572330 & -1.9724390 \\ \mathrm{H} & 1.0805360 & -4.7658310 & -2.5916050 \\ \mathrm{H} & 4.1346720 & -2.6706220 & -0.5924140 \\ \mathrm{H} & 4.2958480 & -1.9197080 & -2.1795430\end{array}$

3.5878920
3.2606660
0.8611920
1.2659500
0.3009470
1.9409900
2.9883890
1.5543680
3.6452250
3.2769670
2.2150020
0.7455770
3.2587200
4.4576860
1.9161850
3.7723700
-0.1228010
1.6271170
- 3.9999950
$-5.1441960$
$-5.2346760$
$-2.8717440$
$-1.9943320$
$-3.1026720$
$-4.0235940$
$-2.3966580$
$-4.2401750$
$-4.5607780$
$-2.6148130$
$-1.6777390$
$-3.5356610$
$-4.9581540$
$-2.0657000$
$-3.7043090$
$-1.7820150$
$-3.4784830$

144

$\begin{array}{lrcr}\text { TS117 (S, S }) \text { G }[\mathrm{WB} 97 \times \mathrm{D} / 6-31 \mathrm{G}(\mathrm{d})]=-3310.756309 \\ \mathrm{O} & 0.5543060 & 0.7832230 & 0.2535940 \\ \mathrm{O} & -1.6569520 & 0.9800950 & -0.9573820 \\ \mathrm{C} & 0.5970250 & 2.1577200 & 0.3206490 \\ \mathrm{C} & -0.5164240 & 2.8459820 & 0.7532400 \\ \mathrm{C} & 1.8271380 & 2.7896140 & 0.0025280 \\ \mathrm{C} & -0.4608510 & 4.2780980 & 0.7867820 \\ \mathrm{C} & 1.9059290 & 4.1494060 & 0.1560000 \\ \mathrm{C} & 0.7769580 & 4.9251470 & 0.5203540 \\ \mathrm{H} & 2.8503080 & 4.6528990 & -0.0310350 \\ \mathrm{C} & -2.2796150 & 1.1790740 & 0.2612600 \\ \mathrm{C} & -3.4781030 & 0.4606330 & 0.5166280 \\ \mathrm{C} & -1.7390380 & 2.0922720 & 1.1400050 \\ \mathrm{C} & -4.1261620 & 0.7136520 & 1.6980940 \\ \mathrm{C} & -2.3594360 & 2.2535830 & 2.4223440 \\ \mathrm{C} & -3.5834640 & 1.5786340 & 2.6813390 \\ \mathrm{H} & -5.0717330 & 0.2190590 & 1.9008590 \\ \mathrm{C} & -3.6646220 & 2.5416800 & 4.9071440 \\ \mathrm{C} & -2.4238430 & 3.1759410 & 4.6691490 \\ \mathrm{C} & -1.7889010 & 3.0367900 & 3.4615450 \\ \mathrm{C} & -4.2259000 & 1.7564180 & 3.9346270 \\ \mathrm{H} & -4.1606780 & 2.6670520 & 5.8647660 \\ \mathrm{H} & -1.9671910 & 3.7745860 & 5.4515600 \\ \mathrm{H} & -0.8330870 & 3.5213930 & 3.2973080 \\ \mathrm{H} & -5.1684380 & 1.2448910 & 4.1119830 \\ \mathrm{C} & -1.4964970 & 6.4532440 & 1.1016140 \\ \mathrm{C} & -0.2543720 & 7.0912150 & 0.8813320\end{array}$

. 756309

0922720

7.0912150
0.8813320
$-3.3028390$ $-0.4758610$ $-0.9179200$ 1.9052420 3. 2107800 3. 2782650 4.3528650 4.4832390 2. 3810170 5.5560760 4. 2888440 5.6220190 4. 5356310 6.4434190 6.5638050 1.0563040

0.8915370 


\begin{tabular}{|c|c|c|}
\hline $\begin{array}{l}0.8546070 \\
-1.5982680 \\
-2.3799470 \\
-0.1861060 \\
1.8111510 \\
-2.5594800 \\
2.9934050 \\
3.3025840 \\
3.7841080 \\
4.4181750 \\
4.8876610 \\
5.2241460 \\
4.6580890 \\
5.5059580 \\
-4.0178080 \\
-3.9434450 \\
-4.6165710 \\
-4.5001160 \\
-5.1443830 \\
-5.1007970 \\
-4.4577770 \\
-5.6051340 \\
-0.3201560 \\
0.3484350 \\
-0.5972720 \\
3.4826250 \\
2.6089630 \\
6.4561400 \\
6.6415340 \\
2.4837910 \\
1.4894260 \\
-4.7277430 \\
-4.2502260 \\
-5.6740490 \\
-5.5465470 \\
-3.3019570 \\
-2.6499780 \\
-2.4205550 \\
-1.8802170 \\
-3.0120290 \\
-1.6906830 \\
-4.3681600 \\
-3.0533220 \\
-4.9680990 \\
3.1227440\end{array}$ & $\begin{array}{r}6.3408020 \\
5.0863620 \\
7.0512570 \\
8.1737640 \\
6.8190930 \\
4.6117680 \\
1.9842780 \\
1.9418520 \\
1.2856230 \\
1.2134280 \\
0.5699000 \\
0.5216930 \\
1.1816680 \\
0.0255940 \\
-0.5149820 \\
-1.8983840 \\
-0.0408470 \\
-2.7746120 \\
-0.9584310 \\
-2.3304470 \\
-3.8449110 \\
-0.5887580 \\
0.0566570 \\
0.1834780 \\
-1.3151460 \\
1.2990320 \\
1.9358870 \\
-0.2478650 \\
-1.0250880 \\
2.7089610 \\
2.8784880 \\
1.4447670 \\
2.0050600 \\
-3.3170900 \\
-4.3217870 \\
-2.4687320 \\
-1.6995230 \\
-3.6928930 \\
-3.9712810 \\
-4.5638240 \\
-3.4727390 \\
-2.8227290 \\
-3.5857530 \\
-3.2202090 \\
-1.9515280 \\
-0.0994690\end{array}$ & $\begin{array}{r}0.5911720 \\
1.0563990 \\
1.3039480 \\
0.9287410 \\
0.3970880 \\
1.2185710 \\
-0.4704560 \\
-1.8440460 \\
0.4574840 \\
-2.2556560 \\
-0.0054760 \\
-1.3559630 \\
-3.3135180 \\
0.7069170 \\
-0.4786500 \\
-0.2267830 \\
-1.6606460 \\
-1.1599200 \\
-2.5659680 \\
-2.3330100 \\
-0.9733540 \\
-3.4795240 \\
-0.9336950 \\
-2.2670620 \\
-0.3784940 \\
1.9498230 \\
2.1161590 \\
-1.8035080 \\
-1.0484660 \\
-2.8751820 \\
-2.4515130 \\
-1.9740540 \\
-1.1653230 \\
-3.3342080 \\
-2.9102670 \\
1.0330890 \\
1.4568630 \\
0.7526210 \\
1.6642640 \\
0.4479240 \\
-0.0300160 \\
2.0802740 \\
1.6913810 \\
2.9872710 \\
2.3631830 \\
2.4671910\end{array}$ \\
\hline
\end{tabular}

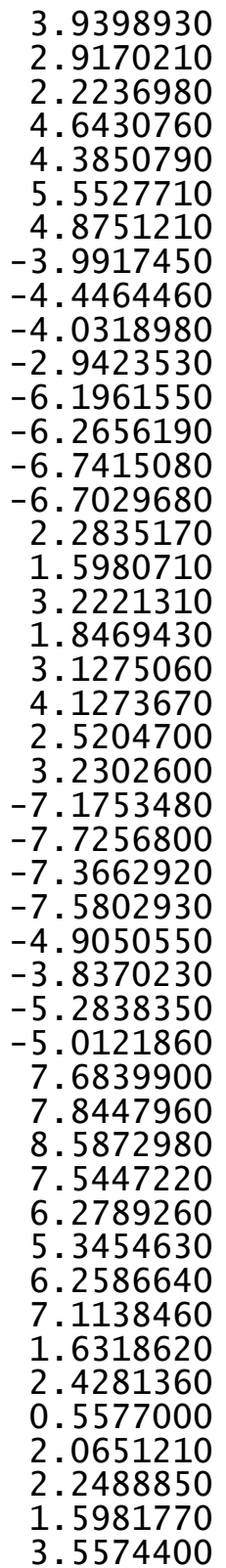

-0.8129590
-0.0717990
-0.4712270
1.9125630
1.9832550
1.3068960
2.9188780
1.8053890
1.3142500
2.8872010
1.5032990
1.8892250
2.9693460
1.3860050
1.6588410
1.9481650
2.5097710
1.8276100
0.9640740
4.0758410
3.9468640
4.6468420
4.6714580
-3.0947350
-3.1481400
-2.1117840
-3.8531490
-3.2712010
-3.4502050
-4.0296580
-2.2920660
0.6743930
1.1370730
0.1166320
1.4770830
-0.9455460
-1.5174790
-0.2217520
-1.6289380
-2.7502350
-2.2324640
-2.6190300
-1.3460920
-1.6355430
-0.8687520
-4.2819150
2. 3040740

3. 5429320

1.9680200

2. 7441590

3.8065990

2. 6607520

2. 3799880

$-3.2704790$

$-4.1387490$

$-3.4411030$

$-3.2139490$

$-2.0171780$

$-2.1890910$

$-2.8241100$

$-1.0739620$

$-4.1902980$

$-4.8340270$

$-4.7435450$

$-4.0120630$

$-3.1559670$

$-3.5880210$

$-3.8674350$

$-2.2445940$

$-3.5579980$

$-2.6130800$

$-4.0038160$

$-4.2375280$

$-4.6613780$

$-4.5018910$

$-5.3560110$

$-5.1425460$

$-1.8236800$

$-0.8446020$

$-2.0957880$

$-2.5572300$

$-3.1570620$

$-3.1988600$

$-3.9792470$

$-3.3443570$

$-1.8824090$

$-0.7661210$

$-1.7709610$

$-0.2590960$

$-2.5703630$

-2.5190200
-2.4745550 


\begin{tabular}{|c|c|c|c|c|c|c|c|}
\hline & & & & & & & \\
\hline C & 4.1454310 & $\begin{array}{l}-4.0532940 \\
-2\end{array}$ & -1.0801110 & C & $\begin{array}{l}3.3081060 \\
5\end{array}$ & $\begin{array}{l}-3.9403160 \\
-2.3917460\end{array}$ & $\begin{array}{l}1.9297870 \\
3.0151360\end{array}$ \\
\hline $\mathrm{c}$ & 2.0398780 & -4.0830260 & -2.4630110 & $\mathrm{H}$ & 5.8233720 & -4.1008300 & 4.2088190 \\
\hline $\mathrm{H}$ & 3.7899330 & -5.2941270 & -2.8198150 & $\mathrm{H}$ & 3.9616820 & -5.4640560 & 3.2662550 \\
\hline $\mathrm{H}$ & 5.2295410 & -4.2041780 & -1.0893960 & $\mathrm{H}$ & 2.5011370 & -4.5462210 & 1.5336750 \\
\hline $\mathrm{H}$ & 4.4391260 & -1.8960750 & -1.1118780 & $\mathrm{H}$ & 6.1497210 & -1.7744550 & 3.4399160 \\
\hline $\mathrm{H}$ & 1.5655990 & -4.8558110 & -1.8457440 & C & 3.4421350 & -5.7455780 & -1.8192080 \\
\hline $\mathrm{H}$ & 1.6191630 & -4.1651440 & -3.4701720 & $\mathrm{C}$ & 2.3588670 & -6.5221760 & -2.2892620 \\
\hline $\mathrm{H}$ & 4.0126650 & -3.5864860 & -3.1899950 & $\mathrm{C}$ & 1.0752640 & -6.0706070 & -2.1261710 \\
\hline $\mathrm{H}$ & 3.7196190 & -4.7809240 & -0.3795510 & $\mathrm{C}$ & 3.2201810 & -4.5529880 & -1.1789210 \\
\hline $\mathrm{H}$ & 4.0911650 & -2.5135370 & 0.4831950 & $\mathrm{H}$ & 4.4592550 & -6.0938660 & -1.9715350 \\
\hline C & 1.0885790 & -3.0620590 & 1.8788170 & $\mathrm{H}$ & 2.5473710 & -7.4676360 & -2.7885840 \\
\hline 0 & 0.1906790 & -2.1170170 & 1.8909940 & $\mathrm{H}$ & 0.2342430 & -6.6489310 & -2.5000810 \\
\hline $\mathrm{C}$ & 1.3389950 & -3.6808960 & 3.2069110 & $\mathrm{H}$ & 4.0601620 & -3.9616370 & -0.8311100 \\
\hline $\mathrm{C}$ & 2.3339940 & -4.6537690 & 3.3249300 & $\mathrm{C}$ & -2.1591830 & -2.5820070 & -0.6233240 \\
\hline C & 0.5957440 & -3.2988040 & 4.3269280 & $\mathrm{C}$ & -2.8490700 & -1.9779670 & -1.6928090 \\
\hline $\mathrm{C}$ & 2.5837850 & -5.2440710 & 4.5573750 & $\mathrm{C}$ & -2.7598980 & -2.6777220 & 0.6431490 \\
\hline $\mathrm{H}$ & 2.9000230 & -4.9385340 & 2.4442340 & $\mathrm{C}$ & -4.1391610 & -1.4980670 & -1.4685910 \\
\hline C & 0.8494950 & -3.8910710 & 5.5584250 & $\mathrm{C}$ & -4.0556970 & -2.1890370 & 0.8144790 \\
\hline $\mathrm{H}$ & -0.1732110 & -2.5413260 & 4.2250840 & $\mathrm{C}$ & -4.7638000 & -1.5982640 & -0.2256570 \\
\hline c & 1.8414120 & -4.8629770 & 5.6739200 & $\mathrm{H}$ & -4.6818210 & -1.0452330 & -2.2944410 \\
\hline $\mathrm{H}$ & 3.3558110 & -6.0016440 & 4.6488270 & $\mathrm{H}$ & -4.5337850 & -2.2787430 & 1.7879810 \\
\hline $\mathrm{H}$ & 0.2729140 & -3.5951160 & 6.4291710 & C & 3.8745650 & 1.5714220 & 0.3903470 \\
\hline H & 2.0357250 & -5.3258920 & 6.6368110 & $\mathrm{C}$ & 3.2862430 & 2.5263940 & 1.2450860 \\
\hline H & -0.0686260 & -1.7842010 & 0.9588890 & $\mathrm{C}$ & 4.4695290 & 1.9704400 & -0.8163430 \\
\hline 0 & 1.7072290 & -3.4381520 & 0.8755990 & $\mathrm{C}$ & 3.3184190 & 3.8665160 & 0.8678020 \\
\hline & & & & $\mathrm{C}$ & 4.4748650 & 3.3259460 & -1.1497650 \\
\hline & s) G[wB97x & $G(d)]=-3$ & 174 & $\mathrm{C}$ & 3.8982870 & 4.2870380 & -0.3283070 \\
\hline 0 & 0.0435650 & -1.1517130 & 0.2623330 & $\mathrm{H}$ & 2.8553310 & 4.6053270 & 1.5157550 \\
\hline 0 & 2.0587950 & -0.1274140 & -0.8508840 & $\mathrm{H}$ & 4.9273290 & 3.6406660 & -2.0883240 \\
\hline $\mathrm{C}$ & 0.3280720 & -2.3700840 & -0.3097250 & $\mathrm{C}$ & -2.0567200 & -3.3389000 & 1.8201480 \\
\hline C & 1.6258980 & -2.8330320 & -0.2996430 & C & -6.1903310 & -1.1205850 & -0.0208620 \\
\hline C & -0.7737510 & -3.1180330 & -0.8030300 & $\mathrm{C}$ & -2.2381330 & -1.8902450 & -3.0846570 \\
\hline C & 1.9008970 & -4.0689150 & -0.9732780 & C & 2.6311660 & 2.1285250 & 2.5619300 \\
\hline C & -0.5130450 & -4.3481420 & -1.3456860 & $\mathrm{C}$ & 3.8409900 & 5.7414120 & -0.7594940 \\
\hline $\mathrm{C}$ & 0.8140380 & -4.8334920 & -1.4821180 & $\mathrm{C}$ & 5.0882590 & 0.9697070 & -1.7813530 \\
\hline $\mathrm{H}$ & -1.3355660 & -4.9702610 & -1.6877320 & $\mathrm{C}$ & 6.5927220 & 1.2160540 & -1.9542770 \\
\hline C & 2.8347120 & -0.7067220 & 0.1274070 & $\mathrm{H}$ & 6.7877550 & 2.1978260 & -2.4010470 \\
\hline C & 3.8128240 & 0.1194530 & 0.7422950 & $\mathrm{H}$ & 7.1101620 & 1.1760630 & -0.9898620 \\
\hline C & 2.6755690 & -2.0466260 & 0.4074350 & $\mathrm{H}$ & 7.0312160 & 0.4568450 & -2.6117610 \\
\hline $\mathrm{C}$ & 4.6659890 & -0.4681410 & 1.6379160 & C & 4.3597310 & 0.9729400 & -3.1316510 \\
\hline C & 3.4936160 & -2.6195500 & 1.4386740 & $\mathrm{H}$ & 3.2921800 & 0.7807210 & -2.9928470 \\
\hline $\mathrm{C}$ & 4.5205820 & -1.8258740 & 2.0224050 & $\mathrm{H}$ & 4.4734980 & 1.9363060 & -3.6425960 \\
\hline $\mathrm{H}$ & 5.4593110 & 0.1218000 & 2.0885810 & $\mathrm{H}$ & 4.7690750 & 0.1969320 & -3.7884120 \\
\hline C & 5.1773700 & -3.6795120 & 3.4446290 & C & 4.4080000 & 6.6973640 & 0.2963170 \\
\hline C & 4.126690 & -4.45400 & 2.9033140 & $\mathrm{H}$ & 4.4084700 & 7.7263010 & -0.0805200 \\
\hline
\end{tabular}




$\begin{array}{rrrr}\text { H } & 3.8091520 & 6.6812560 & \\ \text { H } & 5.4358080 & 6.4280370 & 0.5140570 \\ \mathrm{C} & 2.4031950 & 6.1256350 & -1.1397970 \\ \mathrm{H} & 2.0215460 & 5.4611700 & -1.9223540 \\ \mathrm{H} & 1.7357020 & 6.0425690 & -0.2737660 \\ \mathrm{H} & 2.3561750 & 7.1569490 & -1.5079740 \\ \mathrm{C} & 3.6420440 & 2.2048520 & 3.7163120 \\ \mathrm{H} & 4.5009370 & 1.5488130 & 3.5495270 \\ \mathrm{H} & 4.0183530 & 3.2289040 & 3.8284420 \\ \mathrm{H} & 3.1693530 & 1.9108190 & 4.6604920 \\ \mathrm{C} & 1.3909830 & 2.9635780 & 2.9043140 \\ \mathrm{H} & 1.6554180 & 3.9839730 & 3.2069370 \\ \mathrm{H} & 0.7071870 & 3.0135580 & 2.0546070 \\ \mathrm{H} & 0.8619640 & 2.5042550 & 3.7466450 \\ \mathrm{C} & -2.6959530 & -4.6988040 & 2.1315540 \\ \mathrm{H} & -3.7490250 & -4.5823100 & 2.4138190 \\ \mathrm{H} & -2.6530170 & -5.3595950 & 1.2590600 \\ \mathrm{H} & -2.1750570 & -5.1902870 & 2.9611390 \\ \mathrm{C} & -2.0221060 & -2.4377260 & 3.0593570 \\ \mathrm{H} & -1.5958170 & -1.4595730 & 2.8157460 \\ \mathrm{H} & -3.0248240 & -2.2880740 & 3.4786700 \\ \mathrm{H} & -1.4079870 & -2.8950940 & 3.8428470 \\ \mathrm{C} & -7.1749110 & -1.9798860 & -0.8258200 \\ \mathrm{H} & -8.2095290 & -1.6843860 & -0.6178280 \\ \mathrm{H} & -7.0021040 & -1.8643840 & -1.9022380 \\ \mathrm{H} & -7.0627050 & -3.0409190 & -0.5807920 \\ \mathrm{C} & -6.3590200 & 0.3652870 & -0.3539020 \\ \mathrm{H} & -5.6833340 & 0.9760100 & 0.2514440 \\ \mathrm{H} & -6.1500220 & 0.5607150 & -1.4126110 \\ \mathrm{H} & -7.3845180 & 0.6927710 & -0.1502040 \\ \mathrm{C} & -2.5897900 & -0.5972000 & -3.8269760 \\ \mathrm{H} & -3.6462120 & -0.5631710 & -4.1192170 \\ \mathrm{H} & -2.3656300 & 0.2804990 & -3.2176570 \\ \mathrm{H} & -1.9973980 & -0.5267340 & -4.7452820 \\ \mathrm{C} & -2.6531890 & -3.1038200 & -3.9305980 \\ \mathrm{H} & -3.7421290 & -3.1287350 & -4.0596070 \\ \mathrm{H} & -2.1928110 & -3.0540670 & -4.9238490 \\ \mathrm{H} & -2.3497300 & -4.0450420 & -3.4639810 \\ \mathrm{P} & 0.4933210 & 0.1919870 & -0.5495390 \\ \mathrm{O} & -0.1602820 & 0.2986580 & -1.8958990 \\ \mathrm{O} & 0.3086010 & 1.3047440 & 0.4467480 \\ \mathrm{H} & -1.1508440 & -1.9038740 & -2.9693350 \\ \mathrm{H} & -6.4254340 & -1.2451340 & 1.0438920 \\ \mathrm{H} & -1.0176170 & -3.5295330 & 1.5398900 \\ \mathrm{H} & 4.9748830 & -0.0311070 & -1.3542480 \\ \mathrm{H} & 4.4588540 & 5.8394880 & -1.6615310 \\ & & & \\ & & & \end{array}$

$\begin{array}{ll}\text { H } & 2.2999390 \\ \text { C } & -2.7334650 \\ \text { O } & -1.8370180 \\ \text { C } & -4.0448420 \\ \text { C } & -5.0183250 \\ \text { C } & -4.3378550 \\ \text { H } & -6.2810950 \\ \text { H } & -4.7743280 \\ \text { C } & -5.6065670 \\ \text { H } & -3.5780020 \\ \text { C } & -6.5768620 \\ \text { H } & -7.0377250 \\ \text { H } & -5.8394710 \\ \text { H } & -7.5669560 \\ \text { H } & -0.9767190 \\ \text { O } & -2.5482010 \\ \text { C } & -1.0728660 \\ \text { H } & -2.2695990 \\ \text { H } & -0.2098240 \\ \text { H } & -2.1037670 \\ \text { O } & -1.0895790 \\ \text { H } & -0.6099890 \\ \text { C } & -2.5809880 \\ \text { C } & -3.7761600 \\ \text { C } & -3.6208540 \\ \text { C } & -1.2765970 \\ \text { H } & -2.7202510 \\ \text { H } & -4.7088990 \\ \text { H } & -3.7677810 \\ \text { H } & -1.2831870 \\ \text { H } & -0.4099330 \\ \text { H } & -2.5130190 \\ \text { H } & -3.8584860 \\ \text { H } & -4.3617740 \\ \text { H4 } & \end{array}$

$\begin{array}{rr}1.0899980 & 2.4647000 \\ 2.0706890 & 1.5811500 \\ 1.1663960 & 1.8532040 \\ 1.8708670 & 2.2436950 \\ 2.8670140 & 2.1306460 \\ 0.6724550 & 2.8984640 \\ 2.6650860 & 2.6730410 \\ 3.7885590 & 1.6126180 \\ 0.4691260 & 3.4273120 \\ -0.0965560 & 2.9659220 \\ 1.4633350 & 3.3147480 \\ 3.4386450 & 2.5896630 \\ -0.4670460 & 3.9254400 \\ 1.3018440 & 3.7303280 \\ 1.2864190 & 1.3152930 \\ 3.0189540 & 0.8029690 \\ 3.3123040 & -1.5314030 \\ 2.5168510 & -1.2401570 \\ 3.0715750 & -0.9120450 \\ 1.4781550 & -0.9752360 \\ 2.5650280 & -2.7692560 \\ 1.6997580 & -2.5836060 \\ 5.0945400 & -2.5082280 \\ 4.4538030 & -1.7973480 \\ 2.9346760 & -1.7027020 \\ 4.7908140 & -1.7647350 \\ 6.1780430 & -2.5737320 \\ 4.6876820 & -2.3194590 \\ 2.4601050 & -2.6818460 \\ 5.2718450 & -0.7793570 \\ 5.1772660 & -2.3100090 \\ 4.7161950 & -3.5351650 \\ 4.8619140 & -0.7831450 \\ 2.4837550 & -1.0363010\end{array}$

$\operatorname{TS} 119(\mathrm{~S}, \mathrm{~S}) \mathrm{G}[\mathrm{WB} 97 \mathrm{XD} / 6-31 \mathrm{G}(\mathrm{d})]=-3310.757176$

$\begin{array}{lrcr}\text { TS119(S, S) } \mathrm{G}[\mathrm{wB} 97 \mathrm{XD} / 6-31 \mathrm{G}(\mathrm{d})]=-3310.757176 \\ \mathrm{O} & 0.5451580 & 0.7714710 & 0.1385600 \\ \mathrm{O} & -1.6753560 & 0.8936720 & -1.0592300 \\ \mathrm{C} & 0.5969030 & 2.1459400 & 0.0841890 \\ \mathrm{C} & -0.5014410 & 2.8811280 & 0.4782470 \\ \mathrm{C} & 1.8234810 & 2.7363050 & -0.3180340 \\ \mathrm{C} & -0.4314750 & 4.3103440 & 0.3877110 \\ \mathrm{C} & 1.9185180 & 4.1031310 & -0.2836570 \\ \mathrm{C} & 0.8060360 & 4.9190430 & 0.0412050 \\ \mathrm{H} & 2.8613850 & 4.5791490 & -0.5388070 \\ \mathrm{C} & -2.2907060 & 1.2071060 & 0.1397080\end{array}$




-3.4984040
-1.7291560
-4.1321660
-2.3383020
-3.5675690
-5.0819880
-3.6193520
-2.3764020
-1.7530950
-4.1964730
-4.1047370
-1.9083560
-0.7951730
-5.1415770
-1.4413370
-0.1982980
0.8977400
-1.5560540
-2.3155790
-0.1198550
1.8540620
-2.5187790
2.9624900
3.1848470
3.8023170
4.2574160
4.8591360
5.1031300
4.4324770
5.5120320
-4.0614680
-4.0189610
-4.6520750
-4.5956080
-5.2080340
-5.1944630
-4.5733060
-5.6702040
3.5907290
6.2782880
2.3184490
-3.3927700
-5.8096340
-4.7282310
-6.1848610
-6.7515270

0.5306210

0.8825330

2.4495910

1.8110440

0.4178660

2.9399700

3.5437610

3.3065460

2.0888960

3.1409940

4.1984020

3.7694900

1.6007530

6.5160270

7. 1193590

6.3345500

5.1516710

7.1387180

8.2009930

6.7839650

4.7035620

1.8768330

1.6730980

1.2778140

0.8739750

0.4813280

0.2655820

0.7145790

0.0076610

$-0.5086300$

$-1.8702230$

$-0.1197250$

$-2.8066980$

$-1.0986680$

$-2.4451330$

$-3.8566910$

$-0.8012010$

1.4740010

$-0.5905100$

2. 3433080

$-2.3461630$

$-3.4901890$

1.3367690

1.8155920
1.2631370

0.4560460
0.9390870
1.6191840
2.2089290
2.5291210
1.8678920
4.6760850
4.3783160
3.1795950
3.7707160
5.6262430
5.1073760
2.9710630
3.9940990
0.5294010
0.2309850
-0.0135440
0.6062960
0.6936120
0.1790380
-0.2681880
0.8245140
-0.7612330
-2.1377640
0.1921010
-2.5281710
-0.2506310
-1.6040810
-3.5872140
0.4810010
-0.4577190
-0.0920750
-1.6712950
-0.9469500
-2.4958760
-2.1523480
-0.6660470
-3.4351230
1.6872920
-2.0454630
-3.1973220
1.2134440
-3.0653830
-2.1065460
-2.1745060
-2.9329780

$-6.6876330$

$-6.2273900$

$-3.9960240$

$-2.9566250$

$-4.4762970$

$-4.0019000$

$-6.9700370$

$-7.4401170$

$-6.6184650$

$-4.7554950$

$-3.9382010$

$-4.3231680$

$-5.2018590$

$-4.4724640$

$-5.0662460$

$-5.1600430$

$-4.0149360$

-2. 5150430

$-3.1078870$

$-1.7769450$

$-1.9829730$

4.8456940

5.6777970

5.1729680

4.6422160

3.1222320

2. 1756570

3.8678490

2. 9612570

7. 5032450

8.3625890

7.2852860

7.7836440

5.9495040

4.9969570

5.8731060

6.7400750

2.0635030

2. 9740900

1.6532510

1.3359300

2. 9427850

3.9282570

2. 3080450

3.0712710
1.6763030

2. 8798220

1.5697310

1.2375580

1.0286170

2. 6360140

$-4.2234410$

$-4.9336560$

$-4.7864610$

$-4.4821760$

$-3.9603270$

$-5.0550130$

$-2.6109500$

$-1.7159630$

$-3.3960120$

$-3.5923180$

$-4.4828230$

$-3.4378240$

$-3.7983500$

2.0345780

1.3224000

2. 9621080

2. 2469110

0.1786520

$-0.1570570$

$-0.6189460$

0.3347370

0.2871100

$-0.3275460$

0.9805970

0.8802950

$-1.4854200$

$-2.0085930$

$-0.9012630$

$-2.2286170$

1.4688320

1. 3145270

0.4986050

1.9642040

3.6790670

3.5121390

.1753700

4. 3616930
$-1.2114050$

$-2.4327050$

$-3.4337890$

$-3.3618360$

$-3.6872940$

$-2.3791090$

$-3.0687090$

$-1.5067150$

$-2.0372820$

$-3.5747170$

$-4.0828560$

$-2.7461420$

$-4.2780900$

2.2730850

2. 4812820

1.9359830

3.2128910

1.0386870

0.7993370

0.2483730

1.9742280

2. 3683060

2. 3288540

1.8874150

3.4236310

2. 3630850

1.9308580

2. 2541410

3.4355490

$-2.3409930$

$-2.6324210$

$-3.1614550$

$-1.4644260$

$-3.2475310$

$-3.1127170$

$-4.1713700$

$-3.3965680$

$-4.4300600$

$-5.0206370$

$-4.1454360$

$-5.0819090$

$-4.0812470$

$-4.3723290$

$-2.7850580$ 


$\begin{array}{lrrr} & & \\ \mathrm{P} & -0.3705140 & -0.0675320 & -0.9401990 \\ \mathrm{O} & 0.2828450 & -0.1324210 & -2.2848790 \\ \mathrm{O} & -0.6855890 & -1.3498700 & -0.2171170 \\ \mathrm{H} & 1.3424150 & 2.5503740 & -2.7478490 \\ \mathrm{H} & 6.5379910 & -1.2448790 & -1.2012810 \\ \mathrm{H} & 2.7955390 & 2.2135850 & 1.8212280 \\ \mathrm{H} & -4.2282210 & 1.9496000 & -1.3514370 \\ \mathrm{H} & -6.2189130 & -2.9636470 & -3.9372430 \\ \mathrm{H} & -2.7408780 & -1.5496060 & -1.5825650 \\ \mathrm{C} & 1.5314520 & -2.9939150 & -1.5558630 \\ \mathrm{C} & 2.3427770 & -2.3515970 & -0.5172780 \\ \mathrm{H} & 0.4609850 & -2.8254110 & -1.4592690 \\ \mathrm{H} & 2.0060410 & -1.3931640 & -0.1378410 \\ \mathrm{O} & 2.1651280 & -1.9994450 & -2.3949090 \\ \mathrm{H} & 1.5319090 & -1.2191850 & -2.4376060 \\ \mathrm{C} & 3.4110140 & -4.6552870 & -1.9197500 \\ \mathrm{C} & 4.0036360 & -4.2459740 & -0.5691160 \\ \mathrm{C} & 3.7484360 & -2.7674050 & -0.2723630 \\ \mathrm{C} & 1.9009880 & -4.4048870 & -1.9488310 \\ \mathrm{H} & 3.6074860 & -5.7149840 & -2.1102300 \\ \mathrm{H} & 5.0819350 & -4.4324140 & -0.5480940 \\ \mathrm{H} & 4.3622620 & -2.1251920 & -0.9117150 \\ \mathrm{H} & 1.3976130 & -5.0727000 & -1.2392120 \\ \mathrm{H} & 1.4825880 & -4.6059530 & -2.9400570 \\ \mathrm{H} & 3.8942640 & -4.0906960 & -2.7260550 \\ \mathrm{H} & 3.5505250 & -4.8495570 & 0.2258490 \\ \mathrm{H} & 4.0057930 & -2.5003380 & 0.7560090 \\ \mathrm{C} & 1.0964420 & -2.7030650 & 2.2228770 \\ \mathrm{O} & 0.1407840 & -1.8222230 & 2.1345240 \\ \mathrm{C} & 1.5821690 & -2.9482870 & 3.6054620 \\ \mathrm{C} & 2.6340590 & -3.8461260 & 3.8007100 \\ \mathrm{C} & 1.0256020 & -2.2719400 & 4.6943690 \\ \mathrm{C} & 3.1273070 & -4.0692550 & 5.0800730 \\ \mathrm{H} & 3.0550720 & -4.3594190 & 2.9426030 \\ \mathrm{C} & 1.5236210 & -2.4962430 & 5.9724630 \\ \mathrm{H} & 0.2144710 & -1.5713300 & 4.5310510 \\ \mathrm{C} & 2.5718330 & -3.3942260 & 6.1655970 \\ \mathrm{H} & 3.9431550 & -4.7689310 & 5.2323730 \\ \mathrm{H} & 1.0937260 & -1.9710530 & 6.8194600 \\ \mathrm{H} & 2.9595780 & -3.5664610 & 7.1652210 \\ \mathrm{H} & -0.1726870 & -1.6600780 & 1.1732300 \\ \mathrm{O} & 1.5992670 & -3.2952750 & 1.2585410 \\ \mathrm{H} 44 & & & \\ \mathrm{H} & & & \end{array}$

TS120(s, S) G[WB97XD/6-31G(d)] = -3310.756346

$\begin{array}{cccccr} & & & \mathrm{C} & -5.1002570 \\ \mathrm{O} & 0.5543660 & 0.7833190 & 0.2539590 & \mathrm{H} & -4.4575400 \\ \mathrm{O} & -1.6566420 & 0.9803930 & -0.9574120 & \mathrm{H} & -5.6043090\end{array}$
0.5971360
$-0.5163390$
1.8273140
$-0.4606820$
1.9061720
0.7772030
2.8505880
$-2.2795170$
$-3.4780740$
$-1.7390630$
$-4.1263660$
$-2.3596980$
$-3.5838260$
$-5.0719970$
$-3.6653590$
$-2.4244780$
$-1.7893050$
$-4.2265040$
$-4.1615960$
$-1.9679280$
$-0.8334150$
$-5.1691150$
$-1.4962340$
$-0.2540420$
0.8549290
$-1.5980830$
$-2.3796720$
$-0.1857140$
1.8115270
$-2.5593390$
2.9935200
3. 3028520
3.7839740
4.4183870
4.8874770
5.2241280
4.6584500
5.5056180
$-4.0175800$
$-3.9432860$
$-4.6160960$
$-4.4998130$
$-5.1437540$
$-5.1002570$
$-5.6043090$

$\begin{array}{lr}0.7833190 & 0.2539590 \\ 0.9803930 & -0.9574120\end{array}$
2.1578140
2.8461140
2.7896610
4.2782270
4.1494370
4.9252160
4.6528950
1.1793200
0.4609060
2.0924450
0.7139270
2.2537200
1.5788470
0.2193660
2.5417840
3.1759530
3.0368180
1.7566190
2.6671460
3.7745050
3.5213470
1.2451510
6.4534190
7.0913270
6.3408630
5.0865460
7.0514750
8.1738680
6.8191080
4.6119980
1.9842910
1.9422500
1.2851860
1.2138050
0.5694420
0.5216340
1.1823640
0.0248340
-0.5147310
-1.8981260
-0.0406400
-2.7743830
-0.9582570
-2.3302600
-3.8446740
-0.5886200

0.3210720

0.7535360

0.0031120

0.7871840

0.1566850

0.5209630

$-0.0302550$

0.2611290

0.5162430

1.1400290

1.6975840

2.4222550

2.6809690

1. 9001420

4.9068070

4.6690990

3.4616150

3.9341350

5.8643360

5.4516410

3.2975970

4.1112760

1. 1020160

0.8819330

0.5918780

1. 0567030

1. 3042770

0.9294200

0.3979480

1.2187470

$-0.4699510$

$-1.8435240$

0.4578500

$-2.2552290$

$-0.0052060$

$-1.3556630$

$-3.3130710$

0.7070910

$-0.4791230$

$-0.2271800$

$-1.6612620$

$-1.1603730$

$-2.5666430$

$-2.3336020$

$-0.9737500$

$-3.4803130$ 


$\begin{array}{rrr}-0.3198970 & 0.0568850 & -0.9335550 \\ 0.3489420 & 0.1837760 & -2.2667900 \\ -0.5972130 & -1.3149480 & -0.3785220 \\ 3.4823690 & 1.2982440 & 1.9501650 \\ 2.6083480 & 1.9345940 & 2.1165330 \\ 6.4560520 & -0.2479440 & -1.8033450 \\ 6.6415950 & -1.0250950 & -1.0482680 \\ 2.4842390 & 2.7097340 & -2.8745220 \\ 1.4899420 & 2.8794820 & -2.4507790 \\ -4.7271470 & 1.4449600 & -1.9747780 \\ -4.2498010 & 2.0052800 & -1.1659640 \\ -5.6733140 & -3.3169500 & -3.3348660 \\ -5.5459730 & -4.3216200 & -2.9108120 \\ -3.3019910 & -2.4684330 & 1.0328120 \\ -2.6500100 & -1.6992390 & 1.4566120 \\ -2.4206260 & -3.6926650 & 0.7525330 \\ -1.8804370 & -3.9710330 & 1.6642720 \\ -3.0121060 & -4.5635740 & 0.4477910 \\ -1.6906220 & -3.4726010 & -0.0300080 \\ -4.3683310 & -2.8223070 & 2.0798990 \\ -5.0534490 & -3.5853690 & 1.6910020 \\ -3.8983860 & -3.2196930 & 2.9869970 \\ -4.9666170 & -1.9510710 & 2.3626400 \\ 3.1232170 & -0.1004900 & 2.4673900 \\ 3.9407680 & -0.8135350 & 2.3043700 \\ 2.9172980 & -0.0729980 & 3.5430960 \\ 2.2244400 & -0.4727020 & 1.9680630 \\ 4.6424640 & 1.9123530 & 2.7445770 \\ 4.3845550 & 1.9825140 & 3.8070740 \\ 5.5525890 & 1.3073800 & 2.6608230 \\ 4.8737220 & 2.9189570 & 2.3807020 \\ -3.9908260 & 1.8054830 & -3.2710470 \\ -4.4453100 & 1.3142790 & -4.1393940 \\ -4.0309320 & 2.8872830 & -3.4417630 \\ -2.9414490 & 1.5033940 & -3.2142280 \\ -6.1955320 & 1.8894710 & -2.0182760 \\ -6.2649140 & 2.9695850 & -2.1902590 \\ -6.7407090 & 1.3862320 & -2.8253160 \\ -6.7025790 & 1.6591510 & -1.0751700 \\ 2.2836790 & 1.9491280 & -4.1897040 \\ 1.5985400 & 2.5111030 & -4.8334410 \\ 3.2222660 & 1.8281560 & -4.7429030 \\ 1.8466180 & 0.9652430 & -4.0115180 \\ 3.1283140 & 4.0764740 & -3.1551550 \\ 4.1280720 & 3.9472900 & -3.5873840 \\ 2.5213360 & 4.6477910 & -3.8664180\end{array}$

\author{
3. 2313910 \\ $-7.1745420$ \\ $-7.7251220$ \\ $-7.3653190$ \\ $-7.5793530$ \\ $-4.9039730$ \\ $-3.8359860$ \\ $-5.2825930$ \\ $-5.0109600$ \\ 7.6838890 \\ 7.8448500 \\ 8. 5871570 \\ 7. 5444950 \\ 6. 2785680 \\ 5. 3450840 \\ 6.2581480 \\ 7. 1134430 \\ 1. 6319780 \\ 2. 4282220 \\ 0.5578320 \\ 2.0653630 \\ 2. 2493320 \\ 1. 5987480 \\ 3. 5572710 \\ 4.1451710 \\ 3. 8444790 \\ 2.0397530 \\ 3.7895820 \\ 5.2292520 \\ 4.4393610 \\ 1. 5652410 \\ 1. 6191210 \\ 4. 0127250 \\ 3.7191570 \\ 4. 0909790 \\ 1. 0880610 \\ 0.1904250 \\ 1. 3380570 \\ 2. 3329670 \\ 0.5945030 \\ 2.5823820 \\ 2.8992270 \\ 0.8478730 \\ $-0.1743810$ \\ 1.8397160
3.3543530
}

4. 6718820

$-3.0945350$

$-3.1478000$

$-2.1116260$

$-3.8530050$

$-3.2712070$

$-3.4502190$

$-4.0297180$

$-2.2921130$

0.6743230

1. 1371080

0.1165420

1. 4769340

$-0.9457360$

$-1.5176490$

$-0.2220020$

$-1.6291540$

$-2.7500200$

$-2.2326370$

$-2.6186010$

$-1.3462490$

$-1.6353490$

$-0.8684600$

$-4.2820170$

$-4.0537770$

$-2.6416610$

$-4.0828000$

$-5.2942180$

$-4.2048680$

$-1.8965960$

$-4.8555900$

$-4.1646440$

$-3.5865650$

$-4.7814670$

$-2.5143990$

$-3.0624540$

$-2.1171660$

$-3.6815910$

$-4.6545430$

$-3.2996730$

$-5.2450780$

$-4.9391820$

$-3.8921800$

$-2.5421340$

$-4.8641490$

$-6.0026990$
$-2.2436800$

3.5590680

$-2.6142870$

$-4.0050510$

$-4.2386150$

$-4.6618410$

$-4.5020650$

$-5.3565020$

$-5.1431260$

$-1.8238260$

$-0.8448240$

$-2.0960250$

$-2.5574390$

$-3.1568110$

$-3.1983780$

- 3.9790450

$-3.3442230$

$-1.8823450$

$-0.7658540$

$-1.7710080$

$-0.2587340$

$-2.5700330$

$-2.5186910$

$-2.4745640$

$-1.0800210$

$-0.5737990$

$-2.4631390$

$-2.8199790$

$-1.0892360$

$-1.1111960$

$-1.8460580$

$-3.4703560$

$-3.1898340$

$-0.3796460$

0.4836510

1.8787230

1.8909130

3. 2067560

3. 3248720

4. 3266320

4.5572820

2. 4442840

5. 5580910

4.2247080

5.6736890

4.6488160 


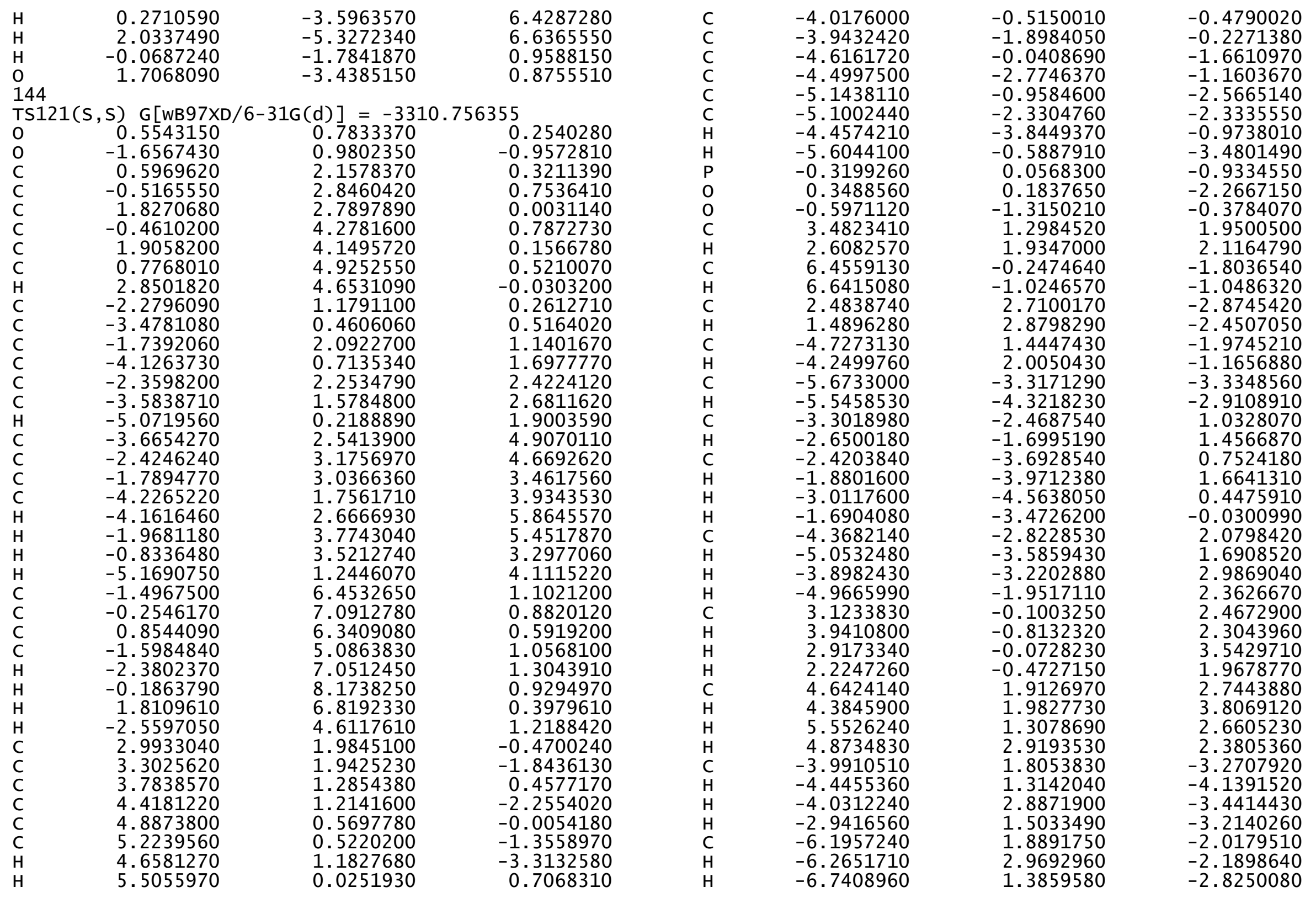




$\begin{array}{rrr}-6.7027340 & 1.6587680 & -1.0748470 \\ 2.2831410 & 1.9493870 & -4.1896840 \\ 1.5980160 & 2.5114120 & -4.8333930 \\ 3.2216760 & 1.8282920 & -4.7429440 \\ 1.8459790 & 0.9655620 & -4.0114150 \\ 3.1279880 & 4.0767230 & -3.1552440 \\ 4.1276490 & 3.9474920 & -3.5876860 \\ 2.5209040 & 4.6481250 & -3.8663470 \\ 3.2313010 & 4.6720680 & -2.2437520 \\ -7.1745630 & -3.0948050 & -3.5589170 \\ -7.7250680 & -3.1482500 & -2.6141030 \\ -7.3654490 & -2.1118460 & -4.0047440 \\ -7.5793630 & -3.8532100 & -4.2385430 \\ -4.9040680 & -3.2712260 & -4.6618880 \\ -3.8360600 & -3.4502060 & -4.5022100 \\ -5.2827070 & -4.0296920 & -5.3565880 \\ -5.0111340 & -2.2920940 & -5.1430770 \\ 7.6837050 & 0.6748640 & -1.8240860 \\ 7.8446620 & 1.1375780 & -0.8450500 \\ 8.5869940 & 0.1171450 & -2.0963440 \\ 7.5442600 & 1.4775260 & -2.5576320 \\ 6.2784450 & -0.9451790 & -3.1571600 \\ 5.3450070 & -1.5171670 & -3.1987310 \\ 6.2579410 & -0.2213950 & -3.9793470 \\ 7.1133700 & -1.6285150 & -3.3446430 \\ 1.6321100 & -2.7499260 & -1.8823820 \\ 2.4283760 & -2.2324720 & -0.7659390 \\ 0.5579600 & -2.6185880 & -1.7709870 \\ 2.0654620 & -1.3461190 & -0.2587990 \\ 2.2493290 & -1.6352150 & -2.5701270 \\ 1.5986950 & -0.8683710 & -2.5187330 \\ 3.5574880 & -4.2817800 & -2.4746990 \\ 4.1454580 & -4.0534670 & -1.0801970 \\ 3.8446850 & -2.6413670 & -0.5739780 \\ 2.0399550 & -4.0826830 & -2.4631800 \\ 3.7898530 & -5.2939730 & -2.8201010 \\ 5.2295500 & -4.2044740 & -1.0894770 \\ 4.4394600 & -1.8962650 & -1.1114430 \\ 1.5655490 & -4.8554990 & -1.8460490 \\ 1.6192590 & -4.1645840 & -3.4703660 \\ 4.0128400 & -3.5863110 & -3.1900170 \\ 3.7195440 & -4.7811790 & -0.3797840 \\ 4.0912550 & -2.5140620 & 0.4834490 \\ 1.0884930 & -3.0624420 & 1.8786730 \\ 0.1906770 & -2.1173280 & 1.8909510 \\ 1.3387270 & -3.6815580 & 3.2066710\end{array}$

2.3336430
0.5953990
2.5832850
2.8997320
0.8490010
-0.1734960
1.8408440
3.3552550
0.2723670
2.0350490
-0.0685390
1.7072280

$$
\begin{aligned}
& -4.6545240 \\
& -3.2996050 \\
& -5.2450480 \\
& -4.9391800 \\
& -3.8920940 \\
& -2.5420590 \\
& -4.8640830 \\
& -6.0026840 \\
& -3.5962420 \\
& -5.3271610 \\
& -1.7843100 \\
& -3.4383760
\end{aligned}
$$$$
144
$$

3. 3246130

4. 3266850

4.5569820

2.4439200

5.5581050

4.2249020

5.6735270

4.6483760

6.4288510

6.6363620

0.9588880

0.8754420

$\operatorname{TS} 122(\mathrm{~S}, \mathrm{~S}) \mathrm{G}[\mathrm{WB} 97 \mathrm{XD} / 6-31 \mathrm{G}(\mathrm{d})]=-3310.756010$

$\begin{array}{lrcr}\text { TS122(S, S }) \text { G }[\text { WB97XD/6-31G(d)] }=-3310.756010 \\ \text { O } & 0.4878330 & 0.6323030 & -0.0553970 \\ \mathrm{O} & -1.8660740 & 0.7913160 & -1.0035290 \\ \mathrm{C} & 0.5855160 & 2.0036960 & -0.1236390 \\ \mathrm{C} & -0.4635050 & 2.7786760 & 0.3265510 \\ \mathrm{C} & 1.8120350 & 2.5571950 & -0.5759940 \\ \mathrm{C} & -0.3757170 & 4.2016140 & 0.1838700 \\ \mathrm{C} & 1.9258150 & 3.9239090 & -0.6039470 \\ \mathrm{C} & 0.8433290 & 4.7738610 & -0.2690510 \\ \mathrm{H} & 2.8708020 & 4.3711050 & -0.9006270 \\ \mathrm{C} & -2.3324050 & 1.1560240 & 0.2460070 \\ \mathrm{C} & -3.5186390 & 0.5372090 & 0.7214620 \\ \mathrm{C} & -1.6533420 & 2.1313290 & 0.9392260 \\ \mathrm{C} & -3.9999260 & 0.9487150 & 1.9377720 \\ \mathrm{C} & -2.0986570 & 2.4648630 & 2.2601370 \\ \mathrm{C} & -3.3020680 & 1.8832520 & 2.7441600 \\ \mathrm{H} & -4.9281830 & 0.5260130 & 2.3116010 \\ \mathrm{C} & -3.0572060 & 3.0796920 & 4.8409390 \\ \mathrm{C} & -1.8385210 & 3.6258680 & 4.3775960 \\ \mathrm{C} & -1.3719050 & 3.3284300 & 3.1228130 \\ \mathrm{C} & -3.7685060 & 2.2242880 & 4.0408490 \\ \mathrm{H} & -3.4189000 & 3.3283930 & 5.8340520 \\ \mathrm{H} & -1.2631990 & 4.2822720 & 5.0234760 \\ \mathrm{H} & -0.4312060 & 3.7480680 & 2.7843760 \\ \mathrm{H} & -4.6956020 & 1.7795620 & 4.3931710 \\ \mathrm{C} & -1.3379620 & 6.4280050 & 0.3166510 \\ \mathrm{C} & -0.1125190 & 6.9965360 & -0.1008310 \\ \mathrm{C} & 0.9518280 & 6.1839520 & -0.3903020 \\ \mathrm{C} & -1.4673390 & 5.0700790 & 0.4563670 \\ \mathrm{H} & -2.1865670 & 7.0731940 & 0.5231940 \\ \mathrm{H} & -0.0228830 & 8.0735350 & -0.2041210 \\ \mathrm{H} & 1.8936660 & 6.6058750 & -0.7314120 \\ \mathrm{H} & -2.4159380 & 4.6471770 & 0.7679310\end{array}$




$\begin{array}{rrr}2.9816670 & 1.6989880 & -0.9416550 \\ 3.2481720 & 1.4011060 & -2.2926560 \\ 3.8548180 & 1.2572540 & 0.0670870 \\ 4.4083150 & 0.6937450 & -2.6002840 \\ 5.0032310 & 0.5478300 & -0.2931770 \\ 5.3063670 & 0.2685500 & -1.6212410 \\ 4.6289680 & 0.4639370 & -3.6400290 \\ 5.6725420 & 0.2065710 & 0.4909500 \\ -4.2151230 & -0.5084900 & -0.0869100 \\ -4.1600570 & -1.8585420 & 0.3074980 \\ -4.9353790 & -0.1345390 & -1.2366400 \\ -4.8554680 & -2.8022860 & -0.4500460 \\ -5.6013490 & -1.1172390 & -1.9650100 \\ -5.5776260 & -2.4574670 & -1.5875050 \\ -4.8257580 & -3.8473690 & -0.1510160 \\ -6.1548020 & -0.8261370 & -2.8552260 \\ 3.5733530 & 1.5093220 & 1.5425880 \\ 6.5781900 & -0.4495350 & -2.0463960 \\ 2.3273550 & 1.8769010 & -3.4087220 \\ -3.3858330 & -2.3189840 & 1.5368340 \\ -6.3006200 & -3.5160440 & -2.4005410 \\ -5.0286750 & 1.3120460 & -1.7017890 \\ -6.4739310 & 1.8228480 & -1.6248940 \\ -7.1294790 & 1.2652980 & -2.3038630 \\ -6.8741590 & 1.7170490 & -0.6108110 \\ -6.5227760 & 2.8812270 & -1.9050080 \\ -4.4437960 & 1.4925780 & -3.1083290 \\ -3.4086250 & 1.1420110 & -3.1441610 \\ -5.0217570 & 0.9366970 & -3.8559070 \\ -4.4609700 & 2.5508110 & -3.3929810 \\ -5.6956380 & -3.6469700 & -3.8050790 \\ -6.1812030 & -4.4563950 & -4.3620200 \\ -5.8268630 & -2.7196880 & -4.3745950 \\ -4.6227880 & -3.8574400 & -3.7515080 \\ -7.8092920 & -3.2475270 & -2.4739520 \\ -8.2456440 & -3.1700360 & -1.4728190 \\ -8.0179990 & -2.3110110 & -3.0039270 \\ -8.3190610 & -4.0556730 & -3.0106400 \\ -4.3283380 & -2.5241470 & 2.7319650 \\ -4.8686620 & -1.6069960 & 2.9850330 \\ -5.0716300 & -3.2986540 & 2.5077320 \\ -3.7641780 & -2.8409790 & 3.6168180 \\ -2.5746000 & -3.5963210 & 1.2838130 \\ -3.2196740 & -4.4751780 & 1.1697840 \\ -1.9617120 & -3.4908620 & 0.3858450 \\ -1.9117010 & -3.7860090 & 2.1351370\end{array}$

4.7849080

5.6039950

5.1671260

4.5026870

3.0642730

2. 1341540

3.8054680

2. 8626820

7.1964610

8.0341900

7.5892010

6.4717170

7.6090180

7.1992580

7.9003990

8.5105940

2. 2201140

3.1340000

2. 0273470

1.3966620

2.7808110

3.7958990

2.1119180

2.7801600

$-0.5677930$

$-0.0739380$

$-0.8096440$

1.3239010

6.3110800

2.7743260

$-4.4359400$

$-6.1585260$

$-2.6701540$

1. 3013180

2. 2178050

0.2500950

1.8753790

1.7054060

1.0304250

3.2287700

3.9986760

3. 6740160

1.7186620

3.4811260

5.0777130

4.1109370
2. 0848560

1. 3602350

2. 9812330

2. 3552130

0.2284050

$-0.1060230$

$-0.5775960$

0.3989730

$-1.3357440$

$-1.9046820$

$-0.7395330$

$-2.0486470$

0.5698740

1.1720380

1.2519390

0.0643790

0.8849930

0.8697560

$-0.1307690$

1.1790480

3.2452280

3.1788420

3.5825770

4.0074480

$-0.1819740$

$-0.2423850$

$-1.4642950$

1.9858330

$-1.0990470$

2.2530850

1.9334050

$-4.4758570$

$-1.5343020$

$-3.1208410$

$-2.3366510$

$-3.0183500$

$-1.3781080$

$-2.1599480$

$-1.4102330$

$-4.6641210$

$-4.1054530$

$-2.6285710$

$-4.5294210$

$-5.7178420$

$-4.2166620$

$-2.0025410$
2.2858080

2. 3548280

1.7866730

3.3091530

2.2177500

1.7501950

2.1448870

3.2811640

$-0.9624770$

$-1.3785870$

$-0.1309420$

$-0.5550370$

$-2.5561930$

$-3.3732830$

$-1.7490410$

$-2.9199730$

$-4.5720600$

$-5.1782450$

$-4.2220800$

$-5.2303930$

$-3.9407500$

$-4.3506730$

$-4.7404650$

$-3.1573150$

$-1.0266130$

$-2.4394390$

$-0.2764450$

$-2.9864930$

$-2.8917800$

1.6136090

$-1.0245380$

$-1.8870080$

1.7973220

$-1.7289040$

$-0.8958720$

$-1.4680280$

$-0.5224930$

$-2.7326760$

$-2.7142430$

$-2.2960660$

$-1.0970340$

$-0.8725100$

$-2.0805340$

$-2.4501700$

$-1.2451150$ 


\begin{tabular}{|c|c|c|c|c|c|c|c|}
\hline & & & & & & & \\
\hline $\mathrm{H}$ & 1.4005520 & -5.1719660 & -1.2504800 & C & -1.3379430 & 6.4279960 & 0.3163260 \\
\hline H & $\frac{1}{3.160 / 500}$ & $\begin{array}{l}-4.8418720 \\
-4.1268970\end{array}$ & $\begin{array}{l}-2.9690220 \\
-3.2069250\end{array}$ & C & $\begin{array}{r}-0.1125020 \\
0.9518400\end{array}$ & $\begin{array}{l}6.9965010 \\
6.1838990\end{array}$ & -0.1011980 \\
\hline $\mathrm{H}$ & 3.7318760 & -4.6682470 & -0.1949350 & c & -1.4673230 & 5.0700780 & 0.4561150 \\
\hline $\mathrm{H}$ & 4.0783280 & -2.2443000 & 0.0675720 & $\mathrm{H}$ & -2.1865450 & 7.0731990 & 0.5228420 \\
\hline C & 1.3077740 & -2.6749520 & 2.0346170 & $\mathrm{H}$ & -0.0228630 & 8.0734940 & -0.2045460 \\
\hline 0 & 0.2357920 & -1.9350120 & 1.9964040 & $\mathrm{H}$ & 1.8936750 & 6.6058000 & -0.7317760 \\
\hline c & 1.8667300 & -2.8709850 & 3.3982800 & $\mathrm{H}$ & -2.4159210 & 4.6471950 & 0.7677090 \\
\hline C & 3.0682120 & -3.5684390 & 3.5398800 & C & 2.9816690 & 1.6989040 & -0.9417540 \\
\hline C & 1.2290880 & -2.3375850 & 4.5211840 & $\mathrm{c}$ & 3.2481720 & 1.4009390 & -2.2927360 \\
\hline C & 3.6309550 & -3.7327240 & 4.7992080 & $\mathrm{C}$ & 3.8548390 & 1.2572590 & 0.0670120 \\
\hline $\mathrm{H}$ & 3.5501820 & -3.9730080 & 2.6561040 & $\mathrm{C}$ & 4.4083280 & 0.6935820 & -2.6003250 \\
\hline C & 1.7946150 & -2.5053930 & 5.7797590 & C & 5.0032650 & 0.5478350 & -0.2932120 \\
\hline $\mathrm{H}$ & 0.2996100 & -1.7927780 & 4.3999840 & $\mathrm{C}$ & 5.3063950 & 0.2684710 & -1.6212600 \\
\hline c & 2.9942800 & -3.2005670 & 5.9190490 & $\mathrm{H}$ & 4.6289820 & 0.4637130 & -3.6400560 \\
\hline H & 4.5668640 & -4.2714690 & 4.9092720 & $\mathrm{H}$ & 5.6725920 & 0.2066470 & 0.4909320 \\
\hline $\mathrm{H}$ & 1.3014760 & -2.0901430 & 6.6529750 & C & -4.2151520 & -0.5084910 & -0.0868330 \\
\hline $\mathrm{H}$ & 3.4357620 & -3.3272230 & 6.9030680 & $\mathrm{C}$ & -4.1600470 & -1.8585340 & 0.3076080 \\
\hline H & -0.1381470 & -1.7843990 & 1.0583310 & $\mathrm{C}$ & -4.9354590 & -0.1345800 & -1.2365420 \\
\hline 0 & 1.8585590 & -3.1796470 & 1.0486050 & C & -4.8554730 & -2.8023060 & -0.4498850 \\
\hline & & & & C & -5.6014450 & -1.1173070 & -1.9648600 \\
\hline & S) $\mathrm{G}[\mathrm{WB} 97 \mathrm{XD}$ & $G(d)]=-331$ & 29 & C & -5.5776860 & -2.4575260 & -1.5873220 \\
\hline 0 & 0.4878170 & 0.6322670 & -0.0554580 & $\mathrm{H}$ & -4.8257330 & -3.8473820 & -0.1508350 \\
\hline 0 & -1.8661100 & 0.7912530 & -1.0035550 & $\mathrm{H}$ & -6.1549340 & -0.8262370 & -2.8550640 \\
\hline C & 0.5855120 & 2.0036570 & -0.1237580 & $\mathrm{P}$ & -0.5678360 & -0.1820430 & -1.0266210 \\
\hline $\mathrm{C}$ & -0.4635000 & 2.7786650 & 0.3264050 & 0 & -0.0740060 & -0.2425200 & -2.4394530 \\
\hline C & 1.8120330 & 2.5571280 & -0.5761420 & 0 & -0.8096770 & -1.4643310 & -0.2763910 \\
\hline $\mathrm{c}$ & -0.3757080 & 4.2015950 & 0.1836520 & $\mathrm{C}$ & 3.5733740 & 1.5094270 & 1.5424960 \\
\hline C & 1.92 & 3.9238390 & -0.6041670 & $\mathrm{H}$ & 2.7743790 & 2.2532300 & 1.6134640 \\
\hline c & 0.84 & 4.7738130 & -0.2693100 & C & 6.5782350 & -0.4496080 & -2.0463740 \\
\hline $\mathrm{H}$ & $2.87 \mathrm{C}$ & 4.3710170 & -0.9008730 & $\mathrm{H}$ & 6.3111630 & -1.0990790 & -2.8918000 \\
\hline C & -2.332 & 1.1560220 & 0.2459730 & C & 2.3273410 & 1.8766460 & -3.4088290 \\
\hline C & -3.5186460 & 0.5372400 & 0.7214780 & $\mathrm{H}$ & 1.3238880 & 1.9855930 & -2.9866020 \\
\hline C & -1.65 & 2.1313550 & 0.9391310 & C & -5.0287730 & 1.3119890 & -1.7017320 \\
\hline C & -3.99 & 0.9488150 & 1.9377740 & $\mathrm{H}$ & -4.4360320 & 1.9333700 & -1.0245050 \\
\hline C & -2.09 & 2.4649580 & 2.2600340 & C & -6.3006880 & -3.5161360 & -2.4003070 \\
\hline C & -3.302 & 1.8833840 & 2.7441040 & $\mathrm{H}$ & -6.1585990 & -4.4759250 & -1.8867270 \\
\hline $\mathrm{H}$ & -4.9281 & 0.5261450 & 2.3116360 & C & -3.3857640 & -2.3189220 & 1.5369280 \\
\hline C & -3.05 & 3.0799270 & 4.8408190 & $\mathrm{H}$ & -2.6700480 & -1.5342440 & 1.7973220 \\
\hline c & -1.8384400 & 3.6260670 & 4.3774300 & C & -2.5745780 & -3.5962960 & 1.2839420 \\
\hline $\mathrm{C}$ & -1.37 & 3.3285610 & 3.1226540 & $\mathrm{H}$ & -1.9116220 & -3.7859350 & 2.1352320 \\
\hline C & -3.76 & 2.2244910 & 4.0407840 & $\mathrm{H}$ & -3.2196790 & -4.4751480 & 1.1700250 \\
\hline $\mathrm{H}$ & -3.41 & 3.3286810 & 5.8339260 & $\mathrm{H}$ & -1.9617510 & -3.4909170 & 0.3859230 \\
\hline $\mathrm{H}$ & -1.2 & 4.2824990 & 5.0232670 & C & -4.3282030 & -2.5239860 & 2.7321280 \\
\hline $\mathrm{H}$ & -0.4 & 3.7481720 & 2.7841800 & $\mathrm{H}$ & -5.0715400 & -3.2984740 & 2.5079800 \\
\hline $\mathrm{H}$ & -4.6 & 1.77979 & 4.3931430 & $\mathrm{H}$ & -3.7640000 & -2.8407990 & 3.6169610 \\
\hline
\end{tabular}




$\begin{array}{rr}\mathrm{H} & -4.8684720 \\ \mathrm{C} & 3.0642320 \\ \mathrm{H} & 3.8053970 \\ \mathrm{H} & 2.8626470 \\ \mathrm{H} & 2.1341020 \\ \mathrm{C} & 4.7849450 \\ \mathrm{H} & 4.5027050 \\ \mathrm{H} & 5.6039750 \\ \mathrm{H} & 5.1672500 \\ \mathrm{C} & -4.4439090 \\ \mathrm{H} & -5.0218710 \\ \mathrm{H} & -4.4610980 \\ \mathrm{H} & -3.4087350 \\ \mathrm{C} & -6.4740290 \\ \mathrm{H} & -6.5228800 \\ \mathrm{H} & -7.1295830 \\ \mathrm{H} & -6.8742470 \\ \mathrm{C} & 2.2201090 \\ \mathrm{H} & 1.3966480 \\ \mathrm{H} & 3.1339920 \\ \mathrm{H} & 2.0273640 \\ \mathrm{C} & 2.7807770 \\ \mathrm{H} & 3.7958630 \\ \mathrm{H} & 2.1118730 \\ \mathrm{H} & 2.7801180 \\ \mathrm{C} & -7.8093590 \\ \mathrm{H} & -8.2457100 \\ \mathrm{H} & -8.0180640 \\ \mathrm{H} & -8.3191310 \\ \mathrm{C} & -5.6957030 \\ \mathrm{H} & -4.6228560 \\ \mathrm{H} & -6.1812790 \\ \mathrm{H} & -5.8269090 \\ \mathrm{C} & 7.1964350 \\ \mathrm{H} & 6.4716550 \\ \mathrm{H} & 8.0341730 \\ \mathrm{H} & 7.5891460 \\ \mathrm{C} & 7.6091000 \\ \mathrm{H} & 7.1993850 \\ \mathrm{H} & 7.9004560 \\ \mathrm{H} & 8.5106850 \\ \mathrm{C} & 1.3012520 \\ \mathrm{C} & 2.2177740 \\ \mathrm{H} & 1.8750420 \\ & 1.7052820 \\ \mathrm{O} & \end{array}$

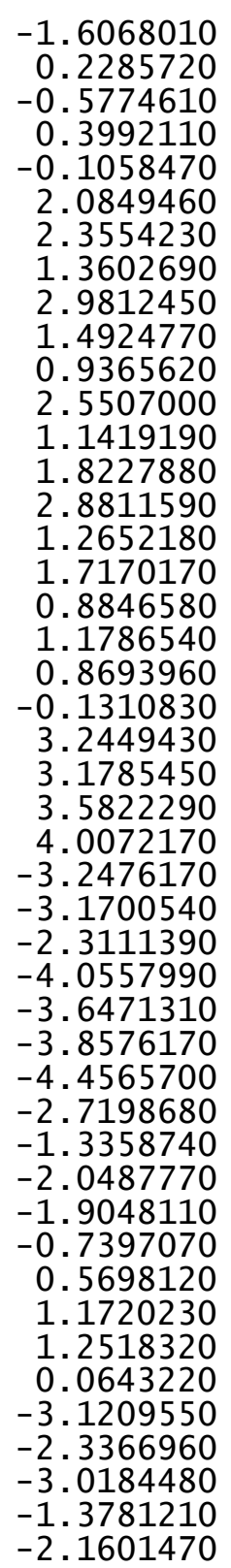
2.9851860 2. 2177290 2. 1449160 3. 2811320 1.7501900 2. 2857030 3. 3090110 2. 3548280 1.7864960 $-3.1082840$ $-3.8558360$ $-3.3929760$ $-3.1441140$ $-1.6248370$ $-1.9049810$ $-2.3037840$ $-0.6107480$ $-4.5720990$ $-5.2304470$ $-5.1782890$ $-4.2220500$ $-3.9409510$ $-4.3508750$ $-4.7406850$ $-3.1575670$ $-2.4737360$ $-1.4726080$ $-3.0037770$ $-3.0103660$ $-3.8048370$ $-3.7512530$ $-4.3617490$ $-4.3743890$ $-0.9624590$ $-0.5550840$ $-1.3785500$ $-0.1308790$ $-2.5560720$ $-3.3731500$ $-1.7488730$ $-2.9198370$ $-1.7287590$ $-0.8958290$ $-1.4678400$ $-0.5225120$ $-2.7326360$

\begin{tabular}{|c|c|}
\hline $\mathrm{H}$ & \\
\hline & \\
\hline & \\
\hline & \\
\hline & \\
\hline & \\
\hline & \\
\hline & \\
\hline & \\
\hline & \\
\hline & \\
\hline & \\
\hline & \\
\hline & \\
\hline & \\
\hline & \\
\hline & \\
\hline & \\
\hline & \\
\hline & \\
\hline & \\
\hline & \\
\hline & \\
\hline & \\
\hline & \\
\hline & \\
\hline & \\
\hline & \\
\hline & 10303090 \\
\hline C & 3.2286840 \\
\hline C & 3.9986480 \\
\hline C & 3.6739860 \\
\hline C & 1.7185870 \\
\hline $\mathrm{H}$ & 3.4810410 \\
\hline $\mathrm{H}$ & 5.0776780 \\
\hline $\mathrm{H}$ & 4.1108740 \\
\hline $\mathrm{H}$ & 1.4005240 \\
\hline $\mathrm{H}$ & 1.1606320 \\
\hline $\mathrm{H}$ & 3.5189570 \\
\hline H & 3.7319010 \\
\hline $\mathrm{H}$ & 4.0783310 \\
\hline C & 1.3078250 \\
\hline 0 & 0.2358270 \\
\hline C & 1.8668060 \\
\hline C & 3.0682790 \\
\hline C & 1.2291950 \\
\hline C & 3.6310460 \\
\hline H & 3.5502250 \\
\hline C & 1.7947480 \\
\hline H & 0.2997230 \\
\hline C & 2.9944040 \\
\hline $\mathrm{H}$ & 4.5669470 \\
\hline H & 1.3016350 \\
\hline $\mathrm{H}$ & 3.4359070 \\
\hline H & -0.1381340 \\
\hline 0 & 060 \\
\hline
\end{tabular}
$-1.4104260$ $-4.6642680$ $-4.1055040$ $-2.6286100$ $-4.5295610$ $-5.7179980$ $-4.2167140$ $-2.0026270$ $-5.1720400$ $-4.8420900$ $-4.1271090$ $-4.6682350$ $-2.2442710$ $-2.6748070$ $-1.9348920$ $-2.8707400$ $-3.5682010$ $-2.3372340$ $-3.7323890$ $-3.9728530$ $-2.5049420$ $-1.7924240$ $-3.2001230$ $-4.2711400$ $-2.0896070$ $-3.3267010$ $-1.7843480$ $-3.1795510$ 144

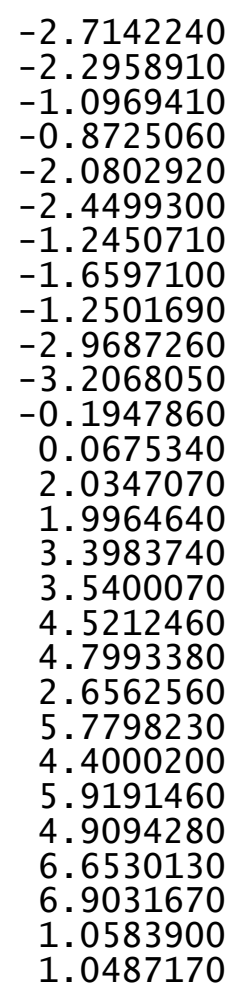

$\operatorname{TS} 124(\mathrm{~S}, \mathrm{~S}) \mathrm{G}[\mathrm{WB} 97 \mathrm{XD} / 6-31 \mathrm{G}(\mathrm{d})]=-3310.756558$

$\begin{array}{lrlr}\text { O } & 0.5074170 & 0.7214950 & 0.0844360 \\ \mathrm{O} & -1.7801710 & 0.7922500 & -0.9987790 \\ \mathrm{C} & 0.5475710 & 2.0951630 & 0.0021270 \\ \mathrm{C} & -0.5440950 & 2.8290970 & 0.4168610 \\ \mathrm{C} & 1.7577080 & 2.6900310 & -0.4414940 \\ \mathrm{C} & -0.4990380 & 4.2553550 & 0.2814000 \\ \mathrm{C} & 1.8302040 & 4.0589640 & -0.4575050 \\ \mathrm{C} & 0.7142690 & 4.8696340 & -0.1324680 \\ \mathrm{H} & 2.7612940 & 4.5395410 & -0.7452810 \\ \mathrm{C} & -2.3308460 & 1.1283210 & 0.2244850 \\ \mathrm{C} & -3.5102040 & 0.4483850 & 0.6277050 \\ \mathrm{C} & -1.7379600 & 2.1292490 & 0.9614650 \\ \mathrm{C} & -4.0804000 & 0.8274160 & 1.8153110 \\ \mathrm{C} & -2.2773240 & 2.4325510 & 2.2547440 \\ \mathrm{C} & -3.4773740 & 1.7892980 & 2.6640660 \\ \mathrm{H} & -5.0074320 & 0.3584220 & 2.1325150\end{array}$




$\begin{array}{rrr}-3.4200410 & 2.9818540 & 4.7761650 \\ -2.2052300 & 3.5918850 & 4.3885570 \\ -1.6491040 & 3.3263400 & 3.1632190 \\ -4.0373000 & 2.0972420 & 3.9314050 \\ -3.8531140 & 3.2056880 & 5.7462700 \\ -1.7047000 & 4.2735810 & 5.0696190 \\ -0.7124540 & 3.7952950 & 2.8831790 \\ -4.9609790 & 1.6047830 & 4.2241570 \\ -1.5371330 & 6.4490820 & 0.3936180 \\ -0.3176600 & 7.0596590 & 0.0213190 \\ 0.7806420 & 6.2834890 & -0.2401000 \\ -1.6267200 & 5.0866360 & 0.5209400 \\ -2.4132520 & 7.0643700 & 0.5748230 \\ -0.2592780 & 8.1397940 & -0.0717190 \\ 1.7185510 & 6.7378270 & -0.5488030 \\ -2.5711050 & 4.6312820 & 0.7973670 \\ 2.9253290 & 1.8464670 & -0.8404810 \\ 3.1811100 & 1.5972640 & -2.2018820 \\ 3.7794230 & 1.3286660 & 0.1499020 \\ 4.3053880 & 0.8448200 & -2.5448600 \\ 4.8870380 & 0.5806990 & -0.2447600 \\ 5.1721950 & 0.3305450 & -1.5854380 \\ 4.5024820 & 0.6527090 & -3.5941160 \\ 5.5515850 & 0.1714730 & 0.5146410 \\ -4.1112090 & -0.6242420 & -0.2208980 \\ -4.0335400 & -1.9709030 & 0.1821960 \\ -4.7756370 & -0.2786680 & -1.4123610 \\ -4.6506600 & -2.9398300 & -0.6106870 \\ -5.3658290 & -1.2851740 & -2.1727420 \\ -5.3190400 & -2.6228230 & -1.7882750 \\ -4.6042650 & -3.9823100 & -0.3046840 \\ -5.8802340 & -1.0146360 & -3.0923770 \\ 3.5279510 & 1.5575650 & 1.6341300 \\ 6.4158690 & -0.4636770 & -1.9524700 \\ 2.2927910 & 2.1706090 & -3.2995100 \\ -3.3231850 & -2.4033700 & 1.4593880 \\ -5.9683660 & -3.7053370 & -2.6311790 \\ -4.8957760 & 1.1628350 & -1.8870530 \\ -6.3595180 & 1.6243690 & -1.8711930 \\ -6.9686620 & 1.0395050 & -2.5702030 \\ -6.7952070 & 1.5118500 & -0.8725110 \\ -6.4338500 & 2.6785590 & -2.1613520 \\ -4.2593350 & 1.3593520 & -3.2685230 \\ -3.2125810 & 1.0430050 & -3.2590920 \\ -4.7867170 & 0.7843410 & -4.0386700 \\ -4.2988660 & 2.4160400 & -3.5565700\end{array}$

-5.3045380
-5.7423310
-5.4419180
-4.2286970
-7.4816120
-7.9584750
-7.7014290
-7.9421520
-4.3355470
-4.9340610
-5.0253010
-3.8199050
-2.4391110
-3.0319910
-1.7494110
-1.8523560
4.7361850
5.6071640
5.0220300
4.4984890
3.1129040
2.1883220
3.8941530
2.9313830
7.6397900
8.5505720
7.5100190
7.7813580
6.2793470
5.3605330
6.2618060
7.1320630
2.1067860
3.0298800
1.7696060
1.3483150
2.8457420
3.8472440
2.1959730
2.9173510
-0.4485020
0.1326250
-0.6906010
1.3014330
6.5872630
2.6931600

-3.8133780
-4.6361240
-2.8895460
-3.9907890
-3.4893800
-3.4292080
-2.5591380
-4.3134200
-2.6565840
-1.7665230
-3.4632090
-2.9507320
-3.6416790
-4.5443460
-3.4952660
-3.8207150
2.2028160
1.5375480
3.1345510
2.4307800
0.2568300
-0.1321470
-0.5091410
0.4278960
0.4628380
-0.1051620
1.2193820
0.9843120
-1.2597040
-1.8568910
-0.5986740
-1.9360540
1.2287390
1.1160130
0.2408160
1.6401900
3.5181290
3.3878190
3.9368330
4.2488120
-0.1361850
-0.1788410
-1.4275000
2.3412520
-1.1848040
2.2576310

$-4.0106740$ $-4.5871830$ $-4.5842690$ $-3.9147820$ $-2.7637620$ $-1.7800720$ $-3.3002900$ $-3.3203090$ 2.5864220 2.8026890 2. 3105080 3.5078450 1.2619290

1.0746820

0.4275530

2.1697450

2. 3246180

2. 3218770

1.8256510

3.3694720

2. 3337040

1.8983740

2.2503010

3.4005920 $-2.0074920$ $-2.2286050$ $-2.7901220$ $-1.0553740$ $-3.2542790$ $-3.2715630$ $-4.1280370$ $-3.3737390$ $-4.4942370$ $-5.0747630$ $-4.1769600$ $-5.1684850$ $-3.7893240$ $-4.2171050$ $-4.5659910$ $-2.9790700$ $-0.9465430$ $-2.3255810$ $-0.2118690$ $-2.8691320$ $-1.1402560$ 1.7330620 


\begin{tabular}{|c|c|c|c|c|c|c|c|}
\hline 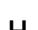 & & & & & & & \\
\hline H & $\begin{array}{l}-4.3521760 \\
-5.8135100\end{array}$ & $\begin{array}{r}1.8053110 \\
-4.6604160\end{array}$ & -1.1889170 & $\mathrm{H}$ & $\begin{array}{r}3.1019080 \\
-2.2826790\end{array}$ & 4.1908550 & -0.8693980 \\
\hline $\mathrm{H}$ & -2.6626100 & $\begin{array}{l}-4.0004100 \\
-1.5890150\end{array}$ & 1.7701840 & $\mathrm{C}$ & -3.5084200 & $\begin{array}{l}1.20112230 \\
0.7000420\end{array}$ & 0.6113390 \\
\hline C & 1.5171480 & -3.0084200 & -1.6400930 & $\mathrm{C}$ & -1.5544030 & 2.1739380 & 0.8918570 \\
\hline c & 2.3509230 & -2.3142110 & -0.6539870 & $\mathrm{C}$ & -3.9735350 & 1.0992850 & 1.8379800 \\
\hline $\mathrm{H}$ & 0.4460860 & -2.8968650 & -1.4843640 & $\mathrm{C}$ & -1.9934680 & 2.4977990 & 2.2169460 \\
\hline $\mathrm{H}$ & 1.9857360 & -1.3729900 & -0.2585760 & $\mathrm{C}$ & -3.2301950 & 1.9683300 & 2.6764660 \\
\hline 0 & 2.0479870 & -1.9884970 & -2.5187930 & $\mathrm{H}$ & -4.9267740 & 0.7186220 & 2.1939290 \\
\hline $\mathrm{H}$ & 1.3744210 & -1.2399400 & -2.5265240 & C & -2.9407100 & 3.0960300 & 4.8054520 \\
\hline C & 3.4634340 & -4.5600460 & -2.1114550 & $\mathrm{C}$ & -1.6928400 & 3.5922150 & 4.3640860 \\
\hline C & 4.1094000 & -4.1130230 & -0.7977270 & $\mathrm{C}$ & -1.2311860 & 3.3020500 & 3.1057090 \\
\hline $\mathrm{C}$ & 3.7893890 & -2.6491950 & -0.4938700 & $\mathrm{C}$ & -3.6881890 & 2.2985210 & 3.9789220 \\
\hline c & 1.9419950 & -4.3990720 & -2.0495550 & $\mathrm{H}$ & -3.2972040 & 3.3375970 & 5.8022060 \\
\hline $\mathrm{H}$ & 3.7104020 & -5.6064670 & -2.3159690 & $\mathrm{H}$ & -1.0918240 & 4.2049750 & 5.0291890 \\
\hline $\mathrm{H}$ & 5.1959450 & -4.2388950 & -0.8403790 & $\mathrm{H}$ & -0.2679290 & 3.6822470 & 2.7843790 \\
\hline $\mathrm{H}$ & 4.3195340 & -1.9811840 & -1.1801080 & $\mathrm{H}$ & -4.6396360 & 1.8930630 & 4.3133480 \\
\hline $\mathrm{H}$ & 1.5233830 & -5.0912210 & -1.3087530 & C & -0.9991040 & 6.4548640 & 0.3444740 \\
\hline $\mathrm{H}$ & 1.4774790 & -4.6300770 & -3.0133160 & $\mathrm{C}$ & 0.2574530 & 6.9624770 & -0.0595300 \\
\hline $\mathrm{H}$ & 3.8628500 & -3.9670840 & -2.9431030 & $\mathrm{C}$ & 1.2786980 & 6.0979400 & -0.3528120 \\
\hline $\mathrm{H}$ & 3.7382450 & -4.7372650 & 0.0233260 & C & -1.2017730 & 5.1043650 & 0.4661650 \\
\hline $\mathrm{H}$ & 4.0993980 & -2.3508150 & 0.5112430 & $\mathrm{H}$ & -1.8129440 & 7.1423770 & 0.5542870 \\
\hline C & 1.2643310 & -2.7080550 & 2.1508760 & $\mathrm{H}$ & 0.4040650 & 8.0343960 & -0.1495110 \\
\hline 0 & 0.2592280 & -1.8803850 & 2.1012360 & $\mathrm{H}$ & 2.2439230 & 6.4731980 & -0.6831540 \\
\hline C & 1.8184290 & -2.9212620 & 3.5129660 & $\mathrm{H}$ & -2.1739510 & 4.7292470 & 0.7662040 \\
\hline C & 2.9531570 & -3.7220510 & 3.6599380 & C & 3.0933350 & 1.5375000 & -0.9379940 \\
\hline C & 1.2444850 & -2.3060250 & 4.6285680 & $\mathrm{C}$ & 3.3858940 & 1.2706800 & -2.2872470 \\
\hline C & 3.5129290 & -3.9075010 & 4.9176790 & $\mathrm{C}$ & 3.9544520 & 1.0805520 & 0.0796440 \\
\hline $\mathrm{H}$ & 3.3859210 & -4.1900970 & 2.7820180 & $\mathrm{C}$ & 4.5774420 & 0.6093090 & -2.5942640 \\
\hline C & 1.8071530 & -2.4947420 & 5.8853640 & C & 5.1220120 & 0.4075530 & -0.2785480 \\
\hline $\mathrm{H}$ & 0.3670430 & -1.6817950 & 4.5024590 & C & 5.4712790 & 0.1938140 & -1.6117860 \\
\hline C & 2.9398400 & -3.2937300 & 6.0299540 & $\mathrm{H}$ & 4.8287470 & 0.4305640 & -3.6367540 \\
\hline $\mathrm{H}$ & 4.3960760 & -4.5281510 & 5.0321850 & $\mathrm{H}$ & 5.7914910 & 0.0618050 & 0.5053030 \\
\hline $\mathrm{H}$ & 1.3629950 & -2.0174060 & 6.7530800 & C & -4.2601830 & -0.2683890 & -0.2420940 \\
\hline $\mathrm{H}$ & 3.3790710 & -3.4372930 & 7.0126560 & C & -4.3124420 & -1.6329020 & 0.1098120 \\
\hline $\mathrm{H}$ & -0.1003910 & -1.7309450 & 1.1552400 & C & -4.9202890 & 0.1913830 & -1.3940420 \\
\hline 0 & 1.7604120 & -3.2745680 & 1.1681890 & $\mathrm{C}$ & -5.0455360 & -2.5004770 & -0.6970240 \\
\hline & & & & C & -5.6326110 & -0.7207400 & -2.1731650 \\
\hline & , s) $\mathrm{G}[\mathrm{WB} 97 \times \mathrm{D}$ & $G(d)]=-33$ & 391 & $\mathrm{C}$ & -5.7082390 & -2.0689000 & -1.8443390 \\
\hline 0 & 0.5067850 & 0.5747590 & -0.1113290 & $\mathrm{H}$ & -5.0948180 & -3.5524880 & -0.4272520 \\
\hline 0 & -1.8203250 & 0.9030320 & -1.0884830 & $\mathrm{H}$ & -6.1443260 & -0.3687090 & -3.0667520 \\
\hline C & 0.6866210 & 1.9386600 & -0.1489850 & C & 3.6411120 & 1.3102670 & 1.5533480 \\
\hline C & -0.3217740 & 2.7643130 & 0.3059190 & C & 6.7927710 & -0.4555000 & -1.9907560 \\
\hline C & 1.9466440 & 2.4314160 & -0.5785620 & $\mathrm{C}$ & 2.4732910 & 1.7475480 & -3.4097260 \\
\hline C & -0.1565610 & 4.1820430 & 0.1878870 & C & -3.6210030 & -2.1841640 & 1.3509460 \\
\hline C & 2.1314340 & 3.7913190 & -0.5869680 & $\mathrm{C}$ & -6.4756690 & -3.0434900 & -2.7193410 \\
\hline $\mathrm{C}$ & & 200 & -0.2497790 & $\mathrm{C}$ & -4.9064520 & 1.6556500 & -1.8095 \\
\hline
\end{tabular}




-6.3120460
-7.0004430
-6.7272050
-6.2807560
-4.2990560
-3.2980430
-4.9176450
-4.2220660
-7.6216820
-8.1962950
-7.2386540
-8.3044100
-5.5384470
-4.7306170
-5.0818100
-6.0902030
-4.6329350
-5.1481360
-5.3949040
-4.1308660
-2.8643300
-3.5472130
-2.1895720
-2.2700690
4.8447070
5.6397270
5.2694900
4.5369630
3.0769510
2.1588400
3.8044440
2.8349250
6.8069410
7.7817880
6.6121230
6.0463440
7.9821500
7.9676960
7.9699480
8.9264790
2.3801660
3.2976750
2.1956690
1.5574750
2.9231790
3.9431150

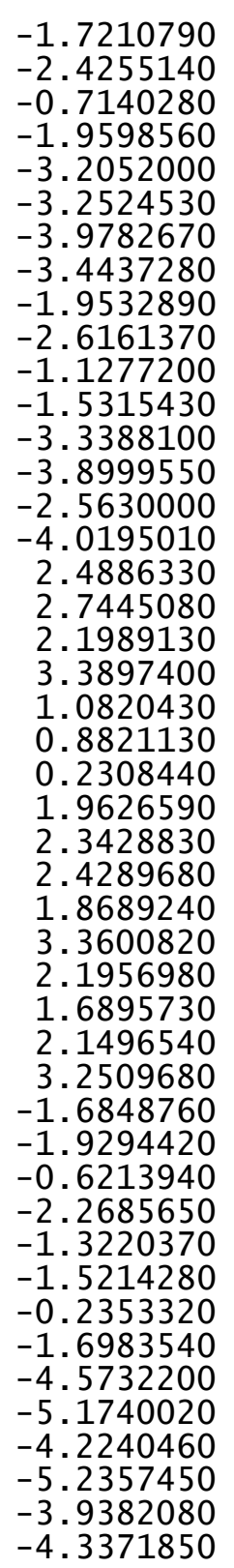

2.2606090
2.9071870
-0.5916590
-0.0735130
-0.9295240
1.4657360
6.9098420
2.8607540
-4.2775170
-6.9186790
-2.8806790
0.9905900
-0.0306750
1.5064420
2.6642040
0.8772470
3.1923020
3.1406010
1.4075820
-0.0173590
2.5638700
4.0946370
0.9206490
2.9782220
-0.3613170
1.5278890
1.2400860
2.0812110
0.1702660
1.7078760
1.7334730
1.0599100
3.2124240
3.8726450
3.5266010
1.6882810
3.4811060
4.9603770
4.0311100
1.3031790
1.2096000
3.5794370
3.5272810
3.8436760

3.4494880

3.8808990

$-0.1558790$

$-0.2349480$

$-1.4244710$

1.8508830

$-0.3390910$

2.0742650

2.2096530

$-2.4662020$

$-1.4501640$

$-2.6815950$

$-1.8756930$

$-2.8632380$

$-3.6202710$

$-2.2569380$

$-3.7715450$

$-4.0803450$

$-2.4124570$

$-1.6654950$

$-3.1671280$

$-4.3564820$

$-1.9417540$

$-3.2837900$

$-1.7384950$

$-2.3679030$

$-3.0297940$

$-1.4179420$

$-2.1496600$

$-1.3997040$

$-4.6566200$

$-4.1303320$

$-2.6605690$

$-4.5301720$

$-5.7054290$

$-4.2393490$

$-2.0057760$

$-5.1935090$

$-4.8212120$

$-4.0943210$

$-4.7170250$

$-2.3076460$

$$
\begin{array}{r}
-4.7456580 \\
-3.1553180 \\
-1.1066840 \\
-2.5096880 \\
-0.3717170 \\
-2.9960790 \\
-3.0756350 \\
1.6159190 \\
-1.1072920 \\
-3.5411540 \\
1.6806820 \\
2.0650760 \\
1.9748700 \\
3.4473280 \\
3.6383890 \\
4.5376950 \\
4.9142390 \\
2.7790420 \\
5.8130620 \\
4.3786760 \\
6.0015850 \\
5.0622470 \\
6.6613100 \\
6.9985870 \\
1.0198140 \\
1.1076290 \\
-1.7126990 \\
-0.7857380 \\
-1.5413180 \\
-0.4232320 \\
-2.6555850 \\
-2.6911900 \\
-2.1482900 \\
-0.8718590 \\
-0.6352250 \\
-2.0607590 \\
-2.3081330 \\
-0.9255520 \\
-1.3561850 \\
-1.2767970 \\
-3.0010900 \\
-3.0155170 \\
-0.0125700 \\
0.3492500
\end{array}
$$

144

3.1181480
3.0596910

$\operatorname{TS} 126(\mathrm{~S}, \mathrm{~S}) \mathrm{G}[\mathrm{WB} 97 \mathrm{XD} / 6-31 \mathrm{G}(\mathrm{d})]=-3310.755960$ 


\begin{tabular}{|c|c|c|c|}
\hline 0 & 0.4970980 & 0.6392910 & -0.0741110 \\
\hline 0 & -1.8233150 & 0.8779140 & -1.0853160 \\
\hline C & 0.6403750 & 2.0069490 & -0.1332970 \\
\hline C & -0.3952050 & 2.8148090 & 0.2901800 \\
\hline $\mathrm{C}$ & 1.8967010 & 2.5218590 & -0.5481640 \\
\hline C & -0.2574660 & 4.2344800 & 0.1539690 \\
\hline $\mathrm{C}$ & 2.0557840 & 3.8841520 & -0.5668130 \\
\hline C & 0.9924210 & 4.7680710 & -0.2607210 \\
\hline $\mathrm{H}$ & 3.0229460 & 4.3011440 & -0.8347680 \\
\hline C & -2.3132710 & 1.2546570 & 0.1517920 \\
\hline C & -3.5322580 & 0.6735310 & 0.5911110 \\
\hline $\mathrm{C}$ & -1.6228600 & 2.2057630 & 0.8668220 \\
\hline C & -4.0344000 & 1.0974590 & 1.7945100 \\
\hline C & -2.0956180 & 2.5517080 & 2.1750610 \\
\hline $\mathrm{C}$ & -3.3301840 & 2.0075930 & 2.6230340 \\
\hline $\mathrm{H}$ & -4.9862580 & 0.7045760 & 2.1405710 \\
\hline C & -3.1102100 & 3.1952780 & 4.7275250 \\
\hline C & -1.8624570 & 3.7039550 & 4.3004640 \\
\hline C & -1.3683280 & 3.3921770 & 3.0597400 \\
\hline C & -3.8235180 & 2.3621310 & 3.9060340 \\
\hline H & -3.4934350 & 3.4551670 & 5.7096160 \\
\hline H & -1.2866890 & 4.3431020 & 4.9630430 \\
\hline $\mathrm{H}$ & -0.4053390 & 3.7825260 & 2.7502880 \\
\hline $\mathrm{H}$ & -4.7737040 & 1.9456900 & 4.2303140 \\
\hline C & -1.1512750 & 6.4908170 & 0.2609520 \\
\hline C & 0.1041950 & 7.0203260 & -0.1169460 \\
\hline C & 1.1500380 & 6.1740690 & -0.3752360 \\
\hline C & -1.3283900 & 5.1377110 & 0.3941230 \\
\hline H & -1.9847220 & 7.1630470 & 0.4413330 \\
\hline $\mathrm{H}$ & 0.2311470 & 8.0939950 & -0.2154130 \\
\hline $\mathrm{H}$ & 2.1150980 & 6.5658560 & -0.6861910 \\
\hline H & -2.2998960 & 4.7456310 & 0.6737430 \\
\hline $\mathrm{C}$ & 3.0481550 & 1.6287650 & -0.8870790 \\
\hline$r$ & 3.3334390 & 1.3198310 & -2.2316410 \\
\hline $\mathrm{C}$ & 3.8876110 & 1.1655490 & 0.1407970 \\
\hline C & 4.4792430 & 0.5788960 & -2.5139120 \\
\hline $\mathrm{C}$ & 5.0234790 & 0.4247110 & -0.1946730 \\
\hline C & 5.3452040 & 0.1327280 & -1.5157570 \\
\hline $\mathrm{H}$ & 4.7139720 & 0.3395620 & -3.5484170 \\
\hline $\mathrm{H}$ & 5.6678020 & 0.0674660 & 0.6031130 \\
\hline C & -4.2373460 & -0.3450030 & -0.2438260 \\
\hline C & -4.2408220 & -1.7000940 & 0.1461320 \\
\hline C & -4.9014200 & 0.0565490 & -1.4148030 \\
\hline C & -4.9310770 & -2.6175190 & -0.6427810 \\
\hline C & -5.5700850 & -0.9039010 & -2.1746540 \\
\hline & & & -1.8080330 \\
\hline
\end{tabular}
$-4.9413070$
$-6.0846890$
$-0.5608660$
$-0.0298740$
$-0.8680660$
3. 5788310
2.8129860
6.6064360
6.3452370
2. 4483900
1.4378380
$-4.9352190$
$-4.3332330$
$-6.3181270$
$-6.7688980$
$-3.5379230$
$-2.8144600$
$-2.7524950$
$-2.1443600$
$-3.4167410$
$-2.0876650$
$-4.5443000$
$-5.2952570$
$-4.0342110$
$-5.0725800$
2.9917520
3.6961610
2.7709870
2.0595990
4.7935150
4.4926330
5.5748880
5.2335660
$-4.3192540$
$-4.9067070$
$-4.2856190$
$-3.2996280$
$-6.3628850$
$-6.3692620$
$-7.0254040$
$-6.7839380$
2. 3480850
1.5398290
3.2719560
2.1365440
2. 9404650
$-3.6628160$
$-0.5975190$
$-0.1410630$
$-0.2250160$
$-1.4103850$
1.4263360
2.2057850
$-0.6190810$
$-1.2486360$
1.8181200
1.9447770
1.5082550
2.1027820
$-3.2722580$
$-2.7373460$
$-2.1874670$
$-1.4240580$
$-3.4870520$
$-3.7019920$
$-4.3449630$
$-3.3957180$
$-2.3694810$
$-3.1235690$
$-2.7029620$
$-1.4388410$
0.1679860
$-0.6715590$
0.3430300
$-0.1200470$
1.9415600
2.2242340
1.1789780
2.8193430
1.6801390
1.1589440
2.7414840
1.2856560
2.0687720
3.1305720
1.5403070
1.9688530
0.8334760
1.1415300
0.8095280
$-0.1817560$
3.1789930

$-0.3443720$

$-3.0832400$

$-1.0709800$

$-2.4689100$

$-0.3230620$

1.6093630

1.6614600

$-1.9124980$

$-2.7746040$

$-3.3668370$

$-2.9671030$

$-1.8710000$

$-1.1780460$

$-2.6616990$

$-3.5076730$

1.4074040

1.7065370

1.1894050

2.0750540

1.0332590

0.3275990

2.5535170

2. 2890860

3.4645720

2. 7814700

2.2637170

2.2056070

3.3225860

1.7699930

2. 3906140

3.4052690

2. 4839650

1.9061670

$-3.2649740$

$-4.0298790$

$-3.5361080$

$-3.2867680$

$-1.8142580$

$-2.0856090$

$-2.5095340$

$-0.8080300$

$-4.5370540$

$-5.2075760$

$-5.1276010$

-4.1961920
-3.8835820 


\begin{tabular}{rrr}
3.9649330 & 3.0947860 & -4.2660080 \\
2.2996570 & 3.5289680 & -4.7006790 \\
2.9319820 & 3.9402740 & -3.0993470 \\
-5.3369870 & -4.3039700 & -3.2350230 \\
-4.5390720 & -3.8136290 & -3.8017890 \\
-4.8689310 & -4.8874520 & -2.4336930 \\
-5.8560460 & -5.0028380 & -3.9007310 \\
-7.4519590 & -3.9598970 & -1.8901320 \\
-8.1655050 & -3.2255900 & -1.5022350 \\
-7.9939820 & -4.6565600 & -2.5394510 \\
-7.0606850 & -4.5303600 & -1.0398450 \\
7.1643310 & -1.5383140 & -0.8231320 \\
6.4063760 & -2.2333770 & -0.4464400 \\
7.9957950 & -2.1282720 & -1.2222670 \\
7.5509650 & -0.9657680 & 0.0276430 \\
7.6828390 & 0.3747180 & -2.3766350 \\
7.3162990 & 1.0009590 & -3.1961820 \\
7.9708350 & 1.0362180 & -1.5512630 \\
8.5791100 & -0.1533690 & -2.7210210 \\
1.2366060 & -3.1394870 & -1.7038930 \\
2.1461680 & -2.3746240 & -0.8461310 \\
0.1805000 & -3.0075760 & -1.4783490 \\
1.8170510 & -1.4054960 & -0.4883460 \\
1.6999180 & -2.1960560 & -2.6987020 \\
1.0468620 & -1.4271200 & -2.7065070 \\
3.1403230 & -4.7336610 & -2.2033690 \\
3.8877700 & -4.1888860 & -0.9837730 \\
3.5926240 & -2.7035060 & -0.7738070 \\
1.6282120 & -4.5605610 & -2.0341010 \\
3.3710750 & -5.7939210 & -2.3460500 \\
4.9676690 & -4.3263950 & -1.1003110 \\
4.0710910 & -2.0912850 & -1.5476150 \\
1.2696960 & -5.1890200 & -1.2098010 \\
1.0894970 & -4.8653300 & -2.9369460 \\
3.4712430 & -4.2076910 & -3.1068620 \\
3.5815650 & -4.7418480 & -0.0881520 \\
3.9761270 & -2.3262230 & 0.1778220 \\
1.1385970 & -2.6676150 & 2.0572580 \\
0.0897890 & -1.8971540 & 1.9866580 \\
1.6604270 & -2.8653310 & 3.4353450 \\
2.8357830 & -3.5985910 & 3.6111520 \\
1.0170610 & -2.2973810 & 4.5379470 \\
3.3673770 & -3.7637160 & 4.8838190 \\
3.3229170 & -4.0296100 & 2.7428370 \\
1.5513210 & -2.4661510 & 5.8100300 \\
0.1081150 & -1.7249970 & \\
\hline & &
\end{tabular}

2.7253210
4.2834810
1.0538720
3.1428230
-0.2524910
1.6967350

1. 6967350
-3.1965820
-4.3298550
-2.0240310
-3.3233950
-1.7421270
-3.1980810
5.9832810

5. 0198940

6.6674760

6.9777110

1. 0376040

1.0891660 144

$\operatorname{TS} 127(\mathrm{~S}, \mathrm{~S}) \mathrm{G}[\mathrm{WB} 97 \mathrm{XD} / 6-31 \mathrm{G}(\mathrm{d})]=-3310.752357$

\begin{tabular}{|c|c|c|c|}
\hline & s) $\mathrm{G}[\mathrm{wB} 97 \mathrm{x}$ & $G(d)]=-33$ & 57 \\
\hline 0 & 1.5045010 & -0.2794500 & -0.7179300 \\
\hline 0 & 0.2274450 & -1.6657570 & 0.9304360 \\
\hline & 2.6725370 & -0.8652030 & -0.2867090 \\
\hline & 2.7317080 & -2.2381290 & -0.1688370 \\
\hline & 3.7747240 & -0.0059760 & -0.0356660 \\
\hline & 3.9504630 & -2.8236200 & 0.3091360 \\
\hline & 4.9706040 & -0.5912870 & 0.2917390 \\
\hline & 5.0878900 & -1.9893480 & 0.4929350 \\
\hline & 5.8421860 & 0.0356290 & 0.4590300 \\
\hline & 0.3094480 & -2.7262400 & 0.0515560 \\
\hline & -0.8830970 & -3.4602110 & -0.1874380 \\
\hline & 1.5303600 & -3.0525510 & -0.5019600 \\
\hline & -0.7909440 & -4.5807000 & -0.9714850 \\
\hline & 1.5811740 & -4.1477610 & -1.4283280 \\
\hline & 0.4158210 & -4.9402320 & -1.62 \\
\hline & -1.6810690 & -5.1777940 & -1.151804 \\
\hline & 1.6018430 & -6.3443780 & -3.20 \\
\hline & 2.7467040 & -5.5294590 & -3.05428 \\
\hline & 2.7375560 & -4.4624050 & -2.192630 \\
\hline C & 0.4609690 & -6.0482980 & -2.5077880 \\
\hline & 1.6247390 & -7.1888230 & -3.88779 \\
\hline & 3.6413460 & -5.7463510 & -3.63025 \\
\hline & 3.6215800 & -3.8 & -2 \\
\hline & -0.4371720 & -6.6472850 & -2.6342180 \\
\hline & 1860 & -4.7236820 & $53 \varepsilon$ \\
\hline C & 6.40 & -3.9007870 & 1.2 \\
\hline C & 80 & -2.5623160 & 880 \\
\hline C & 70 & -4.2013140 & 5800 \\
\hline $\mathrm{H}$ & 0 & -5.77 & 650 \\
\hline $\mathrm{H}$ & 7 & -4 & 1 \\
\hline $\mathrm{H}$ & 7.1 & -1. & 1 \\
\hline $\mathrm{H}$ & 3.1 & -4.8 & 0 \\
\hline 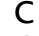 & 3.6 & 1.4 & 4 \\
\hline 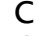 & 3.7 & 2.1963 & 7 \\
\hline 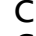 & -5 & 2.1656 & $\perp$ \\
\hline 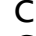 & 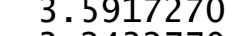 & 3.5 & 1 \\
\hline & 0 & 00 & -1 \\
\hline & & & \\
\hline
\end{tabular}




$\begin{array}{lrrr}\mathrm{H} & 3.6703910 & 4.1311980 & 2.0576370 \\ \mathrm{H} & 3.0600940 & 4.0846290 & -2.1654610 \\ \mathrm{C} & -2.1920410 & -2.9760660 & 0.3440160 \\ \mathrm{C} & -3.1665160 & -2.4935500 & -0.5511040 \\ \mathrm{C} & -2.4491080 & -2.9873560 & 1.7287860 \\ \mathrm{C} & -4.3832660 & -2.0446960 & -0.0343070 \\ \mathrm{C} & -3.6715510 & -2.5094610 & 2.1926860 \\ \mathrm{C} & -4.6529220 & -2.0291190 & 1.3283010 \\ \mathrm{H} & -5.1451220 & -1.6862490 & -0.7235390 \\ \mathrm{H} & -3.8650440 & -2.5162540 & 3.2631140 \\ \mathrm{P} & 0.2650940 & -0.1548410 & 0.3356400 \\ \mathrm{O} & 0.5855310 & 0.7469720 & 1.4918480 \\ \mathrm{O} & -0.9165480 & 0.1632140 & -0.5415220 \\ \mathrm{C} & 3.2642020 & 1.4540850 & -2.6034750 \\ \mathrm{H} & 3.3617760 & 0.3783640 & -2.4341210 \\ \mathrm{C} & 3.2172560 & 5.8013000 & -0.1000240 \\ \mathrm{H} & 2.3408040 & 6.0249700 & -0.7269280 \\ \mathrm{C} & 3.9099430 & 1.5222110 & 2.5047110 \\ \mathrm{H} & 3.7709380 & 0.4461060 & 2.3761410 \\ \mathrm{C} & -1.4543060 & -3.5490190 & 2.7347700 \\ \mathrm{H} & -0.5330800 & -3.8059690 & 2.2052170 \\ \mathrm{C} & -5.9659700 & -1.4849520 & 1.8604610 \\ \mathrm{H} & -6.5769530 & -1.1980800 & 0.9931460 \\ \mathrm{C} & -2.9696880 & -2.4645800 & -2.0628370 \\ \mathrm{H} & -1.9329940 & -2.7323260 & -2.2796580 \\ \mathrm{C} & -1.0028060 & 3.3623110 & 0.9165980 \\ \mathrm{C} & -0.6928610 & 3.2036650 & -0.5063720 \\ \mathrm{H} & -1.4731060 & 2.5010410 & 1.3916720 \\ \mathrm{H} & -0.4787620 & 2.2001490 & -0.8532500 \\ \mathrm{O} & 0.4338860 & 3.3027610 & 1.0110350 \\ \mathrm{H} & 0.6664160 & 2.3392100 & 1.2045410 \\ \mathrm{C} & -0.9420420 & 5.8671420 & 0.5956970 \\ \mathrm{C} & -1.0662600 & 5.6601150 & -0.9156490 \\ \mathrm{C} & -0.3702600 & 4.3730160 & -1.3652370 \\ \mathrm{C} & -1.5559050 & 4.6940510 & 1.3662790 \\ \mathrm{H} & -1.4411340 & 6.7955370 & 0.8903080 \\ \mathrm{H} & -0.6366720 & 6.5099600 & -1.4550920 \\ \mathrm{H} & 0.7217980 & 4.4749630 & -1.3286230 \\ \mathrm{H} & -2.6407630 & 4.6655390 & 1.2044820 \\ \mathrm{H} & -1.3881480 & 4.7959970 & 2.4430810 \\ \mathrm{H} & 0.1146460 & 5.9711860 & 0.8665230 \\ \mathrm{H} & -2.1257710 & 5.6013190 & -1.1907680 \\ \mathrm{H} & -0.6194310 & 4.1080580 & -2.3962550 \\ \mathrm{C} & -3.1948340 & -1.0738800 & -2.6705950 \\ \mathrm{H} & -3.0622460 & -1.1211820 & -3.7576550 \\ & -4.2047530 & -0.6943070 & -2.4763150\end{array}$

-2.4706160
-3.8691450
-4.9293130
-3.6791190
-3.6945850
1.9013950
1.7379470
1.8427210
1.0919230
4.4162990
4.3717100
4.3688970
5.3858850
-1.0850280
-1.9508330
-0.3168470
-0.6931630
-1.9909080
-1.2575120
-2.9178690
-2.2041340
2.8823170
3.0310120
2.9819890
1.8665110
5.3381100
5.5214220
5.4953810
6.0876510
-6.7531330
-6.9442650
-6.2014650
-7.7157940
-5.7337730
-5.1469720
-6.6877020
-5.1851320
4.4407230
4.5965330
4.3185710
5.3457620
2.9766320
2.1479260
3.8680910
2.7406190
-3.5471450
-0.3632550
-3.5104410
-3.2729560
-3.5444320
-4.5109300
1.6883270
2.7513990
1.1459540
1.3312850
1.8679280
1.3084440
2.9360350
1.6734570
-2.5256020
-2.2641370
-2.9378670
-1.6097600
-4.8479040
-5.2742070
-4.6684150
-5.5920820
1.9751610
1.4147640
3.0397940
1.7864590
1.7574320
2.8263080
1.2390990
1.3975610
-2.5431140
-3.4282110
-2.8654920
-2.1390960
-0.2268170
0.5156710
0.2279440
-0.4727110
6.4331530
6.0210770
7.5183320
6.2375720
6.4445190
5.9639300
6.3766700
7.5069230
2.1677750
$-2.2672320$

$-2.7377530$

$-2.5893090$

$-3.8164380$

$-2.3273790$

$-3.2681390$

$-3.4829100$

$-4.2182580$

$-2.6252300$

$-3.5300360$

$-4.4711330$

$-3.7724080$

$-3.0596890$

3. 8154420

4.4353240

4.4794020

3. 3648560

3. 3531070

4. 0470850

3. 9102720

2. 5782330

3. 5508060

4.4806990

3. 7911040

3. 1971250

3. 0171880

3. 1803770

3.9697080

2. 3048960

2. 6434560

2. 0282050

3. 5339210

2.9767150

2. 7089570

2.1589890

2. 9991270

3. 6255020

$-0.7817150$

$-1.7838530$

$-0.8723890$

$-0.1950730$

1. 2669240

1.7973090

1. 9013650

1.1461430 


$\begin{array}{llll}\mathrm{O} & -3.2702710 & 1.0947680 & -0.1039080 \\ \mathrm{C} & -5.0019090 & 2.4324120 & -0.9446130 \\ \mathrm{C} & -5.4277110 & 3.6535280 & -1.4712540 \\ \mathrm{C} & -5.9380580 & 1.4651520 & -0.5727670 \\ \mathrm{C} & -6.7860960 & 3.9083540 & -1.6144520 \\ \mathrm{H} & -4.6881900 & 4.3905870 & -1.7659290 \\ \mathrm{C} & -7.2955880 & 1.7220780 & -0.7185310 \\ \mathrm{H} & -5.5878910 & 0.5188210 & -0.1781140 \\ \mathrm{C} & -7.7197180 & 2.9440200 & -1.2371350 \\ \mathrm{H} & -7.1194880 & 4.8574270 & -2.0223710 \\ \mathrm{H} & -8.0222750 & 0.9691910 & -0.4293180 \\ \mathrm{H} & -8.7807210 & 3.1441760 & -1.3526400 \\ \mathrm{H} & -2.3117000 & 0.7770840 & -0.2044880 \\ \mathrm{O} & -2.7073460 & 2.9256830 & -1.2948880\end{array}$

144

2.9256830

$-1.2948880$

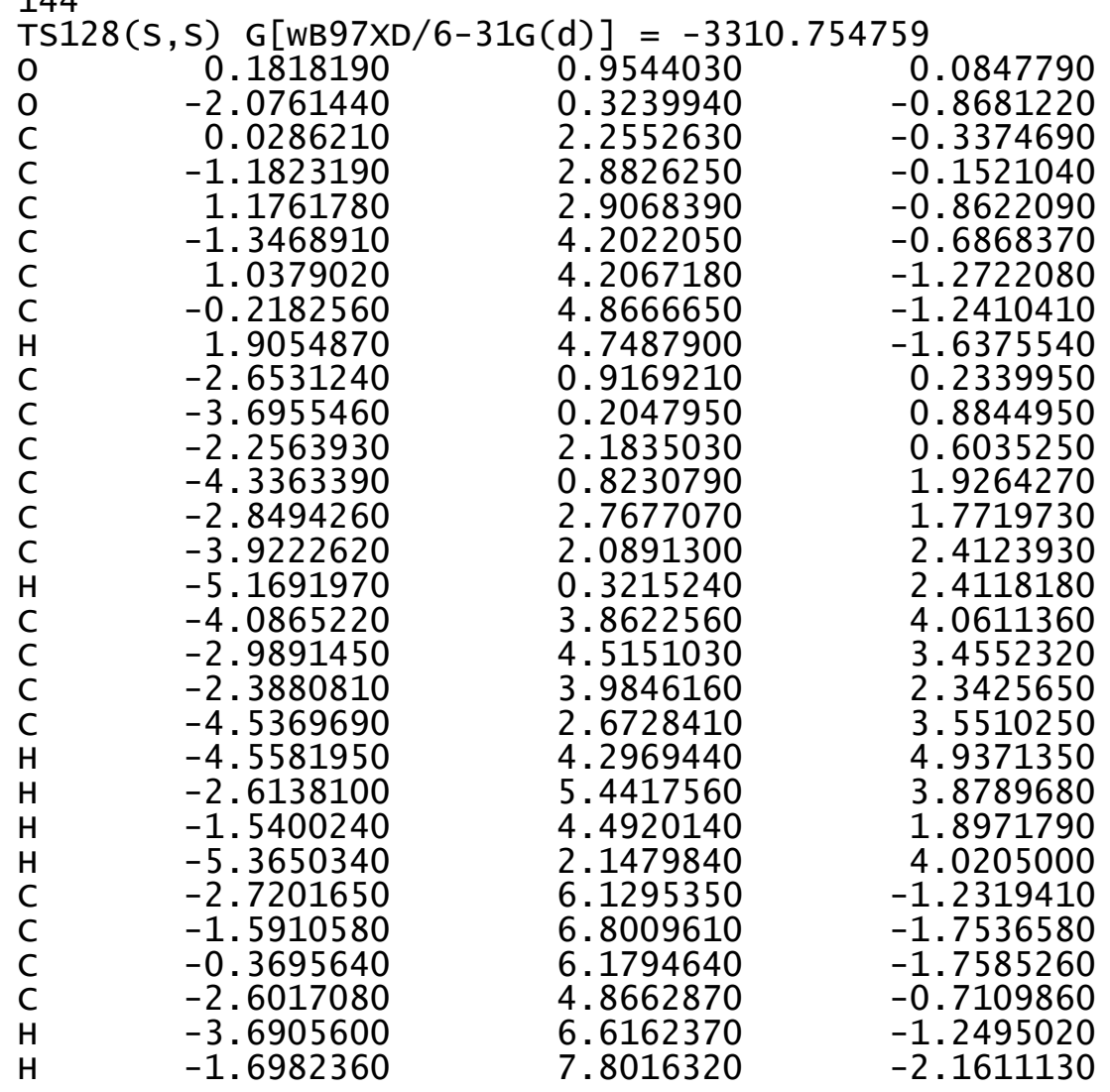

0.5028900
-3.4762480
2.4857640
3.0434080
3.1514230
4.2690610
4.3641140
4.9432750
4.7202570
4.8771910
-4.0839040
-3.6405220
-4.8919130
-4.0524780
-5.2672960
-4.8623040
-3.7209430
-5.8881050
2.6180820
6.2987520
2.3744020
-2.7393220
-5.2610600
-5.3592960
-6.8884250
-7.3644260
-7.2660350
-7.2054680
-4.8130290
-3.7210930
-5.2056740
-5.1030420
-4.6322070
-4.8703610
-5.0085070
-3.5432200
-6.7840630
-7.2317800
-7.2423780
-7.0431900
-3.5653080
-4.2973460
-4.1098190
-2.9118640
-1.6538630
-2.0708820

6.6780180

4. 3570280

2. 1874050

1.6909660

2. 0012380

1.0240860

1. 3158410

0.8219680

0.6510310

1.1785480

$-1.1623820$

$-2.2945760$

$-1.3077500$

$-3.5546930$

$-2.5881390$

$-3.7249370$

$-4.4378770$

$-2.7000840$

2. 5675710

0.1377050

1. 9098870

$-2.1747030$

$-5.1135950$

$-0.1101760$

0.0139320

$-0.8540520$

0.0891880

0.9081040

$-0.1637800$

$-0.2278110$

$-1.0307890$

0.7373250

$-5.4382030$

$-6.4640960$

$-4.7608060$

$-5.3315670$

$-5.2921450$

$-5.0794890$

$-4.6194220$

$-6.3191110$

-2. 1926680

$-1.3803390$

$-3.1400910$

$-2.0890290$

$-3.2566270$

$-4.2407570$
$-2.1729390$

$-0.3208290$

$-0.8586970$

$-2.0508440$

0.3679620

$-1.9855190$

0.3815770

$-0.7839380$

$-2.9021680$

1. 3302840

0.4224920

1. 1298720

$-0.7186450$

0.6944220

$-1.1193810$

$-0.4241860$

1. 2350130

$-2.0058930$

1. 6771890

$-0.7613130$

$-3.4010400$

2. 3523580

$-0.8905280$

$-1.5331330$

$-1.5179500$

$-1.9887010$

$-0.4925470$

$-2.0665310$

$-2.9659000$

$-2.9590950$

$-3.5100190$

$-3.5181830$

$-2.2525940$

$-2.5559840$

$-3.0278860$

$-2.2158770$

$-0.9304600$

0.0456440

$-1.6644620$

$-1.2117520$

3. 6468600

3.6724960

3. 7406690

4.5208070

2. 4061970

2. 6508180 


$\begin{array}{rr}-1.1289190 & -3.3221900 \\ -0.9219360 & -3.0079590 \\ 3.5357630 & 3.6869560 \\ 4.5358840 & 3.3038730 \\ 3.6450800 & 4.4769680 \\ 3.1234540 & 4.1336420 \\ 2.4164500 & 1.4793700 \\ 1.7381040 & 0.7040270 \\ 3.3644250 & 1.0021610 \\ 1.9868590 & 1.9129460 \\ 6.4007000 & -0.9424080 \\ 7.3464910 & -1.4876730 \\ 6.3666110 & -0.5085370 \\ 5.5772590 & -1.6594430 \\ 7.4190460 & 1.1757840 \\ 7.3739000 & 1.9289610 \\ 7.3269140 & 1.6932570 \\ 8.4030330 & 0.6947510 \\ 2.4397740 & 0.6858410 \\ 3.4626920 & 0.4757280 \\ 2.0450570 & -0.2019130 \\ 1.8371980 & 0.8647800 \\ 2.9892940 & 3.1265360 \\ 4.0548870 & 2.9571550 \\ 2.4905910 & 3.3055890 \\ 2.9026070 & 4.0353770 \\ -0.5473030 & -0.2232470 \\ -0.0493280 & -0.2649150 \\ -0.4154920 & -1.4509900 \\ 1.3153730 & 2.1137570 \\ 6.4347210 & -0.3503800 \\ 1.6405200 & 3.0192340 \\ -4.9600810 & 0.7968650 \\ -4.8624330 & -5.8303650 \\ -2.2244060 & -1.2111200 \\ 1.3311410 & -3.1343010 \\ 2.4217350 & -2.1754190 \\ 0.4822840 & -3.0116110 \\ 2.1596220 & -1.1680430 \\ 1.1633620 & -2.3864980 \\ 0.5694790 & -1.5982420 \\ 2.9879470 & -4.6998330 \\ 4.1301220 & -3.8971020 \\ 3.7743240 & -2.4134170 \\ 1.7126230 & -4.5720130 \\ 3.2664680 & -5.7552240\end{array}$

1.4509210
3.1820230
2.1889530
2.4230320
1.4382140
3.1006520
2.7368240
2.3708280
3.0116720
3.6470970
0.3216810
0.2300890
1.3271110
0.2508220
-0.6021240
-1.3951790
0.3598390
-0.6368100
-4.3198280
-4.6548030
-3.8238540
-5.2165050
-4.1094610
-4.3061490
-5.0686280
-3.5070810
-0.7870400
-2.2019670
0.0741240
-3.2182310
-1.7363260
1.4892480
-1.0701700
-0.1606100
2.2885810
-1.8316050
-1.6313250
-1.1610110
-1.3250810
-3.0577830
-2.8406220
-2.9339680
-2.3066170
-2.2032660
-2.0957850
-3.0140080

$\begin{array}{ll}\mathrm{H} & 5.0489320 \\ \mathrm{H} & 3.7771370 \\ \mathrm{H} & 1.8571070 \\ \mathrm{H} & 0.8656760 \\ \mathrm{H} & 2.7962820 \\ \mathrm{H} & 4.3363070 \\ \mathrm{H} & 4.4899950 \\ \mathrm{C} & 2.6313180 \\ \mathrm{O} & 1.4596210 \\ \mathrm{C} & 3.5322130 \\ \mathrm{C} & 4.7434120 \\ \mathrm{C} & 3.1883490 \\ \mathrm{C} & 5.6155900 \\ \mathrm{H} & 4.9901480 \\ \mathrm{C} & 4.0661620 \\ \mathrm{H} & 2.2423360 \\ \mathrm{C} & 5.2798440 \\ \mathrm{H} & 6.5577890 \\ \mathrm{H} & 3.8038490 \\ \mathrm{H} & 5.9652670 \\ \mathrm{H} & 0.8088750 \\ \mathrm{O} & 3.0136010 \\ \mathrm{H} & \end{array}$
$-4.0068140$
(2)
$-4.3397240$
$-4.2797410$
$-1.8548860$
$-2.2528740$
$-1.7566520$
$-2.3686930$
$-3.0506860$
$-1.7911390$
$-3.1388200$
$-3.5077570$
$-1.8755070$
$-1.2716560$
$-2.5452870$
$-3.6667410$
$-1.4165380$
$-2.6071800$
$-1.6522940$
$-2.6134670$

$-1.1187170$

$-2.5800890$

$-3.9516900$

$-1.3000070$

$-1.5978530$

1.5100750

1.7903970

2.6893230

2. 5552210

3. 9143920

3. 6328060

1. 6032000

4. 9889240

4. 0144880

4.8482120

3. 5247980

5.9368570

5. 6882310

1. 0163600

144

0.3906930

$\operatorname{TS} 129(\mathrm{~S}, \mathrm{~S}) \mathrm{G}[\mathrm{WB} 97 X \mathrm{X} / 6-31 \mathrm{G}(\mathrm{d})]=-3310.755173$

$\begin{array}{lrcr}\text { TS129(S, S }) \text { G }[W B 97 X D / 6-31 G(d)]=-3310.755173 \\ \text { O } & 0.0477420 & 1.0286550 & 0.1446420 \\ \text { O } & -2.0888640 & 0.2420430 & -0.9455960 \\ \mathrm{C} & -0.1855910 & 2.3210510 & -0.2668550 \\ \mathrm{C} & -1.4518210 & 2.8482520 & -0.1410960 \\ \mathrm{C} & 0.9348530 & 3.0650760 & -0.7226340 \\ \mathrm{C} & -1.6883570 & 4.1606900 & -0.6668290 \\ \mathrm{C} & 0.7181360 & 4.3576040 & -1.1211110 \\ \mathrm{C} & -0.5852420 & 4.9186360 & -1.1485220 \\ \mathrm{H} & 1.5584900 & 4.9716990 & -1.4323680 \\ \mathrm{C} & -2.7672320 & 0.7588920 & 0.1363440 \\ \mathrm{C} & -3.7640230 & -0.0635070 & 0.7249480 \\ \mathrm{C} & -2.5066050 & 2.0498620 & 0.5424180 \\ \mathrm{C} & -4.5162550 & 0.4744660 & 1.7354300 \\ \mathrm{C} & -3.2184320 & 2.5580860 & 1.6805930 \\ \mathrm{C} & -4.2558350 & 1.7692000 & 2.2512160 \\ \mathrm{H} & -5.3206440 & -0.1115910 & 2.1714030 \\ \mathrm{C} & -4.6940820 & 3.4941880 & 3.9006450 \\ \mathrm{C} & -3.6334260 & 4.2600960 & 3.3661760 \\ \mathrm{C} & -2.9160140 & 3.8067680 & 2.2885760 \\ \mathrm{C} & -4.9912500 & 2.2730160 & 3.3555460 \\ \mathrm{H} & -5.2576470 & 3.8684920 & 4.7497700 \\ \mathrm{H} & -3.3803520 & 5.2149040 & 3.8171370\end{array}$




$\begin{array}{rrr}-2.0989760 & 4.4034860 & 1.8994240 \\ -5.7881140 & 1.6608900 & 3.7700880 \\ -3.1723710 & 5.9887220 & -1.2629490 \\ -2.0707810 & 6.7532050 & -1.7102500 \\ -0.8072540 & 6.2253800 & -1.6550970 \\ -2.9870760 & 4.7287470 & -0.7534230 \\ -4.1744360 & 6.4015870 & -1.3294720 \\ -2.2308060 & 7.7504450 & -2.1086010 \\ 0.0463790 & 6.7958970 & -2.0118760 \\ -3.8406860 & 4.1480420 & -0.4207910 \\ 2.2969700 & 2.4519760 & -0.6561490 \\ 2.9566200 & 2.0131220 & -1.8166040 \\ 2.9195240 & 2.3230110 & 0.6010320 \\ 4.2361870 & 1.4586930 & -1.6950970 \\ 4.1938850 & 1.7696170 & 0.6680250 \\ 4.8777370 & 1.3316580 & -0.4659010 \\ 4.7477890 & 1.1394230 & -2.5971590 \\ 4.6824920 & 1.6944050 & 1.6371800 \\ -3.9766320 & -1.4568560 & 0.2279280 \\ -3.4243660 & -2.5463580 & 0.9321670 \\ -4.7084310 & -1.6696910 & -0.9499000 \\ -3.6436600 & -3.8321260 & 0.4445320 \\ -4.8849650 & -2.9768440 & -1.4069130 \\ -4.3612050 & -4.0697640 & -0.7273070 \\ -3.2241900 & -4.6774150 & 0.9833160 \\ -5.4399140 & -3.1474310 & -2.3274350 \\ 2.2688520 & 2.8195770 & 1.8849790 \\ 6.2906110 & 0.7805490 & -0.3074080 \\ 2.3457850 & 2.2010340 & -3.1994230 \\ -2.6095310 & -2.3474680 & 2.2047310 \\ -4.5525210 & -5.4798160 & -1.2559690 \\ -5.3056160 & -0.5192230 & -1.7469160 \\ -6.8331060 & -0.6339140 & -1.8370940 \\ -7.1358120 & -1.5292800 & -2.3923740 \\ -7.2828660 & -0.6897500 & -0.8400170 \\ -7.2518520 & 0.2368300 & -2.3540270 \\ -4.6685080 & -0.4116440 & -3.1386870 \\ -3.5844990 & -0.2920990 & -3.0577310 \\ -4.8736870 & -1.3058480 & -3.7389620 \\ -5.0733510 & 0.4532730 & -3.6763320 \\ -5.3996920 & -6.3303760 & -0.3003490 \\ -5.5695270 & -7.3289560 & -0.7187090 \\ -4.8972220 & -6.4517880 & 0.6664130 \\ -6.3726120 & -5.8631570 & -0.1167570 \\ -3.2081510 & -6.1584270 & -1.5518020 \\ -2.6026580 & -5.5501630 & -2.2315680\end{array}$
$-2.6317930$
$-3.3645730$
$-3.5028980$
$-4.2927850$
$-3.9800460$
$-2.9092460$
-1. 4396520
$-1.7818730$
$-0.8615970$
$-0.7730400$
3. 0234630
4.0530830
3. 0650650
2. 5250310
2. 1445160
1. 5829390
3. 1270720
1. 6213220
7. 0028240
8. 0344210
6. 5140170
7.0340810
6. 3078560
5.8788170
5. 7247500
7.3334980
2. 5595730
3. 6166410
2. 1800120
2. 0194140
2. 8980000
3. 9807230
2. 4288200
2. 7175700
$-0.5257820$
0.0417780
$-0.3269410$
1. 2654390
6.8702600
1. 2543230
$-5.0860700$
$-5.0991430$
$-2.1760670$
2.7304780
1. 5487910
3. 6297320

-6.3087830
-7.1405700
-2.4358400
-1.6798810
-3.4214930
-2.2895500
-3.3308870
-4.3450160
-3.3622330
-3.0120640
4.0363510
3.7763740
4.8397260
4.4229900
1.7081080
0.8606710
1.3431870
2.0818040
0.4852110
0.1743380
-0.3272720
1.3650910
-0.4566020
-0.2454590
-1.2757080
-0.8120980
1.0086940
0.8677910
0.0876000
1.1744290
3.4783440
3.3928960
3.6451210
4.3620160
-0.1765750
-0.1269640
-1.4178610
2.3154410
1.5626070
3.1541020
0.4128630
-5.4006300
-1.3432420
-2.0669710
-1.5893260
-2.1607740

$-0.6316880$

-2.01210

3.4507690

3.4384940

3. 5112490

4. 3604800

2. 3298750

2.5687420

1. 4039480

3. 1382100

2. 4377490

2.7102000

1.6944400

3. 3336910

2.9326030

2. 5297750

3.2548760

3.8200950

$-1.6286600$

$-1.4345700$

$-2.1801130$

$-2.2795400$

0.6027760

1. 5862550

0.1683220

0.7524420

$-4.1363120$

$-4.3924830$

$-3.6925620$

$-5.0743630$

$-3.8513220$

$-4.0040030$

$-4.8275170$

$-3.2326820$

$-0.8006020$

$-2.1891720$

0.0274040

$-3.0729340$

0.2033490

1.6539840

$-1.2175750$

$-2.2046420$

2. 1643850

1. 4967320

1.7695460

2.6787520 


\begin{tabular}{|c|c|c|c|c|c|c|c|}
\hline$r$ & 18566580 & 28114160 & & & חمברבת & & \\
\hline 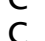 & $\begin{array}{l}4.8566580 \\
3.2711180\end{array}$ & $\begin{array}{l}-2.8144160 \\
-1.5892810\end{array}$ & $\begin{array}{l}2.5463810 \\
3.9021450\end{array}$ & $\mathrm{C}$ & $\begin{array}{l}-3.9602200 \\
-5.2108620\end{array}$ & $\begin{array}{l}2.0151040 \\
0.2522240\end{array}$ & $\begin{array}{l}2.3890770 \\
2.3090000\end{array}$ \\
\hline C & 5.7280740 & -2.8832720 & 3.6257990 & C & -4.1377800 & 3.7258850 & 4.1014210 \\
\hline H & 5.1176420 & -3.2589520 & 1.5921830 & C & -3.0371470 & 4.4019270 & 3.5277700 \\
\hline C & 4.1494950 & -1.6518650 & 4.9779560 & $\mathrm{C}$ & -2.4271720 & 3.9132580 & 2.4010720 \\
\hline $\mathrm{H}$ & 2.3129950 & -1.0925110 & 4.0012480 & $\mathrm{C}$ & -4.5832950 & 2.5557970 & 3.5444080 \\
\hline C & 5.3773580 & -2.2957710 & 4.8400330 & $\mathrm{H}$ & -4.6162920 & 4.1277050 & 4.9893240 \\
\hline $\mathrm{H}$ & 6.6821850 & -3.3897730 & 3.5197790 & $\mathrm{H}$ & -2.6668360 & 5.3134400 & 3.9871960 \\
\hline $\mathrm{H}$ & 3.8757580 & -1.1972330 & 5.9247680 & $\mathrm{H}$ & -1.5771270 & 4.4377720 & 1.9799090 \\
\hline $\mathrm{H}$ & 6.0626790 & -2.3416840 & 5.6811780 & $\mathrm{H}$ & $-\overline{5} .4143840$ & 2.0136000 & 3.9880750 \\
\hline $\mathrm{H}$ & 0.8955030 & -1.5303350 & 0.9933430 & C & -2.7138310 & 6.1855920 & -1.0992770 \\
\hline 0 & 3.1210760 & -2.4273800 & 0.3807010 & $\mathrm{C}$ & -1.5764460 & 6.8743010 & -1.5788430 \\
\hline $\mathrm{C}$ & 1.5223290 & -2.9573220 & -1.8887750 & $\mathrm{C}$ & -0.3558490 & 6.2510950 & -1.5907190 \\
\hline C & 2.5460550 & -1.9404520 & -1.6373380 & C & -2.6044160 & 4.9033560 & -0.6248250 \\
\hline $\mathrm{H}$ & 0.6532930 & -2.9079950 & -1.2349800 & $\mathrm{H}$ & -3.6832620 & 6.6743640 & -1.1122260 \\
\hline $\mathrm{H}$ & 2.2147230 & -0.9641140 & -1.3007360 & $\mathrm{H}$ & -1.6765840 & 7.8901910 & -1.9487520 \\
\hline 0 & 1.3366320 & -2.1802850 & -3.0953370 & $\mathrm{H}$ & 0.5229210 & 6.7634940 & -1.9736880 \\
\hline $\mathrm{H}$ & 0.7048950 & -1.4264130 & -2.8657130 & $\mathrm{H}$ & -3.4852550 & 4.3808750 & -0.2677400 \\
\hline C & 3.2987380 & -4.3809560 & -2.9986000 & C & 2.4817540 & 2.2242920 & -0.7929070 \\
\hline $\mathrm{C}$ & 4.3759310 & -3.5262280 & -2.3250900 & $\mathrm{C}$ & 3.0489640 & 1.7465760 & -1.9881360 \\
\hline $\mathrm{C}$ & 3.9218390 & -2.0725790 & -2.1861570 & $\mathrm{C}$ & 3.1349450 & 2.0162590 & 0.4364980 \\
\hline $\mathrm{C}$ & 2.0004150 & -4.3590350 & -2.1861870 & $\mathrm{C}$ & 4.2727300 & 1.0764730 & -1.9226570 \\
\hline $\mathrm{H}$ & 3.6467400 & -5.4134950 & -3.1016280 & $\mathrm{C}$ & 4.3463800 & 1.3284310 & 0.4500850 \\
\hline $\mathrm{H}$ & 5.3098540 & -3.5590690 & -2.8945940 & C & 4.9352140 & 0.8529720 & -0.7180790 \\
\hline $\mathrm{H}$ & 3.9150290 & -1.5636850 & -3.1581690 & $\mathrm{H}$ & 4.7313740 & 0.7167110 & -2.8408530 \\
\hline $\mathrm{H}$ & 2.1544530 & -4.8536250 & -1.2194230 & $\mathrm{H}$ & 4.8498600 & 1.1736640 & 1.4012190 \\
\hline $\mathrm{H}$ & 1.1974750 & -4.8945020 & -2.7030280 & C & -4.1102140 & -1.1519870 & 0.2599060 \\
\hline $\mathrm{H}$ & 3.1052200 & -4.0040880 & -4.0098680 & $\mathrm{C}$ & -3.6790840 & -2.3209940 & 0.9187190 \\
\hline $\mathrm{H}$ & 4.5881650 & -3.9252200 & -1.3262630 & $\mathrm{C}$ & -4.8912060 & -1.2393730 & -0.9029840 \\
\hline H & 4.5838810 & -1.4843890 & -1.5480550 & $\mathrm{C}$ & -4.0711560 & -3.5560110 & 0.4080830 \\
\hline & & & & $\mathrm{C}$ & -5.2479000 & -2.5011740 & -1.3814470 \\
\hline & s) G[WB97) & ;(d)] $=-331$ & 356 & C & -4.8502320 & -3.6693840 & -0.7426380 \\
\hline 0 & 0.1641670 & 0.9621310 & 0.0723880 & $\mathrm{H}$ & -3.7447720 & -4.4610100 & 0.9137960 \\
\hline 0 & -2.0813960 & 0.3747910 & -0.9367410 & $\mathrm{H}$ & -5.8483190 & -2.5758150 & -2.2861300 \\
\hline C & 0.0173640 & 2.2760890 & -0.3090210 & C & 2.5878430 & 2.5589510 & 1.7499270 \\
\hline $\mathrm{C}$ & -1.1950310 & 2.8990700 & -0.1183410 & $\mathrm{C}$ & 6.2888780 & 0.1651280 & -0.6945230 \\
\hline C & 1.1724780 & 2.9442550 & -0.7947230 & C & 2.3892140 & 1.9889430 & -3.3388740 \\
\hline C & -1.3507430 & 4.2368960 & -0.6079050 & C & -2.8125020 & -2.2631570 & 2.1705070 \\
\hline C & 1.0418860 & 4.2584270 & -1.1592420 & C & -5.2366240 & -5.0292030 & -1.2958130 \\
\hline C & -0.2136110 & 4.9193110 & -1.1213450 & C & -5.3520900 & -0.0041160 & -1.6627700 \\
\hline H & 1.9158290 & 4.8125150 & -1.4899360 & C & -6.8823430 & 0.1069280 & -1.6727290 \\
\hline C & -2.6740240 & 0.9258820 & 0.1791460 & $\mathrm{H}$ & -7.3411030 & -0.7386000 & -2.1982430 \\
\hline $\mathrm{C}$ & -3.7244340 & 0.1909890 & 0.7903990 & $\mathrm{H}$ & -7.2814260 & 0.1247210 & -0.6528570 \\
\hline $\mathrm{C}$ & -2.2792250 & 2.1765740 & 0.5996700 & $\mathrm{H}$ & -7.1960010 & 1.0262560 & -2.1800360 \\
\hline C & -4.3721450 & 0.7693010 & 1.8509460 & C & -4.7767550 & 0.0252420 & -3.0848840 \\
\hline 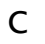 & -2 & 2.7176030 & 1.7830100 & $\mathrm{H}$ & -3.6849460 & -0.0349890 & -3.0592070 \\
\hline
\end{tabular}




-5.1538340
-5.0599880
-6.1212150
-6.4318080
-5.5825150
-7.0204750
-3.9980010
-3.3710730
-3.3868380
-4.2915330
-3.6760820
-4.4042380
-4.2290260
-3.0485960
-1.7336220
-2.1608660
-1.1842410
-1.0212170
3.5032750
4.4984640
3.6261800
3.0807150
2.3690000
1.6925520
3.3117470
1.9294330
6.3836170
7.3270450
6.3497580
5.5568670
7.4107080
7.3713420
7.3143700
8.3938560
2.4835180
3.5115200
2.1065850
1.8792830
2.9891800
4.0596550
2.4972510
2.8787320
-0.5594870
-0.0342960
-0.4558550
1.3252890

$-0.8104560$

$-5.8105030$

$-6.7661070$

$-6.0265090$

$-5.2416510$

$-5.8456280$

$-5.2904790$

$-6.0819680$

$-6.7920100$

$-2.3376030$

$-1.5225720$

$-3.2843510$

$-2.2808230$

$-3.3524920$

$-4.3443790$

$-3.3785200$

$-3.1429450$

3.6640500

3.2720820

4.4674590

4.0954830

1.4501680

0.6848910

0.9638420

1.8665730

$-0.9235750$

$-1.4724650$

$-0.4971900$

$-1.6361810$

1.1995590

1.9577190

1.7112030

0.7166550

0.7909540

0.6119200

$-0.1179460$

0.9802580

3.2338950

3.0882980

3.4275200

4.1254380

$-0.1883890$

$-0.2035050$

$-1.4352630$

2.1703430

-3.6859640
-3.5907880
-0.3157800
-0.7531380
0.6141700
-0.0578210
-1.6899110
-2.3951030
-0.8110170
-2.1580630
3.4382940
3.4817670
3.4698090
4.3353050
2.2047680
2.3945530
1.2613720
3.0098310
2.2954310
2.5356770
1.5611860
3.2097840
2.7845900
2.3942300
3.0612890
3.6981270
0.3805420
0.2865440
1.3891690
0.3026250
-0.5227380
-1.3113170
0.4419310
-0.5552210
-4.2887620
-4.6262590
-3.8175010
-5.1821530
-4.0100010
-4.1989450
-4.9698310
-3.3862960
-0.8397270
-2.2451900
-0.0024450
-3.1606450

\begin{abstract}
6.4281240
1.6142120

$-4.9711210$

$-5.8223730$

$-2.2919380$

2. 5612690

1. 3821150

3.4337230

4.6550290

3.0520440

5.4994280

4.9315820

3.9018160

2. 0987460

5.1255080

6.4493290

3.6093490

5.7887760

0.7483750

2.9711420

1. 3359340

2.4241470

0.4714010

2. 1575690

1.1981390

0.6006860

3.0126650

4.1429690

3.7887770

1.7203040

3.2901910

5.0740190

3.8158070

1.8439990

0.8826680

2.8436360

4.3264240

4.4922170
\end{abstract}

144

$\operatorname{TS} 131(\mathrm{~S}, \mathrm{~S}) \mathrm{G}[\mathrm{WB} 97 \mathrm{XD} / 6-31 \mathrm{G}(\mathrm{d})]=-3310.750270$

$\begin{array}{lrrr}\text { TS131(S, S) } \mathrm{G}[\mathrm{WB} 97 \mathrm{XD} / 6-31 \mathrm{G}(\mathrm{d})]=-3310.750270 \\ \mathrm{O} & 1.3311730 & -0.5839870 & -0.6555280 \\ \mathrm{O} & -0.3745730 & -1.6242550 & 0.8839670 \\ \mathrm{C} & 2.2505190 & -1.5204180 & -0.2384380 \\ \mathrm{C} & 1.8808950 & -2.8483980 & -0.2088300 \\ \mathrm{C} & 3.5551770 & -1.0636200 & 0.0849740 \\ \mathrm{C} & 2.8325360 & -3.8096360 & 0.2650260\end{array}$

$-1.6725980$

$-0.3158840$

3.0177390

0.8804290

$-4.8559150$

$-1.3006020$

$-2.2551650$

$-1.7657070$

$-3.0545380$

$-1.8408260$

$-3.1580910$

$-3.4887460$

$-1.3324990$

$-2.5957300$

$-3.6746310$

$-1.5060380$

$-2.6703680$

$-1.6515190$

$-2.5971290$

-3.0802940

$-2.9675420$

$-1.1250430$

$-2.3111290$

$-4.6303620$

$-3.8428390$

$-2.3602110$

$-4.5142320$

$-5.6848330$

$-3.9441610$

$-1.8618680$

$-5.0012770$

$-4.2502110$

$-4.2451190$

$-4.2451190$

$-3.8096360$

0.2650260

$-1.1443710$

$-2.2078630$

2.1678590

1.4899430

1.7502170

2.6887700

2. 5716230

3.9167940

3.6699140

1.6171100

5.0122640

4.0034480

4.8889580

3.5751960

5.9627230

5.7455840

0.9636110

0.3744130

$-1.8949660$

$-1.2430140$

$-1.3359150$

$-3.1116350$

$-2.8948150$

$-2.9879770$

$-2.3210260$

$-2.1977120$

$-2.1747860$

. 0820720

$-2.8869850$

$-3.1748620$

$-1.2026970$

$-4.0024160$

$-1.3176710$
2. 8325360 


\begin{tabular}{|c|c|c|}
\hline $\begin{array}{r}4.4910990 \\
4.1598980 \\
5.5078570 \\
-0.5837400 \\
-1.9279890 \\
0.5061450 \\
-2.1340290 \\
0.2723060 \\
-1.0640780 \\
-3.1506440 \\
-0.2751670 \\
1.0507750 \\
1.3178440 \\
-1.3075100 \\
-0.4720520 \\
1.8650370 \\
2.3388290 \\
-2.3344190 \\
3.4505280 \\
4.7806740 \\
5.1222850 \\
2.5036510 \\
3.1755950 \\
5.5215990 \\
6.1348890 \\
1.4857850 \\
3.9017450 \\
4.1866580 \\
4.0028500 \\
4.6144240 \\
4.4285970 \\
4.7521890 \\
4.8441710 \\
4.5234610 \\
-3.0748040 \\
-3.9468160 \\
-3.3092170 \\
-5.0472020 \\
-4.4208350 \\
-5.3085110 \\
-5.7317280 \\
-4.6142420 \\
0.1346360 \\
0.6700390 \\
-0.8743550 \\
3.7077620\end{array}$ & $\begin{array}{r}-2.0096260 \\
-3.3815670 \\
-1.6953860 \\
-2.6110900 \\
-2.9751220 \\
-3.2360200 \\
-4.0032420 \\
-4.2252120 \\
-4.6277720 \\
-4.3239850 \\
-6.1834100 \\
-5.7543260 \\
-4.8030730 \\
-5.6259540 \\
-6.9415660 \\
-6.1776870 \\
-4.4764100 \\
-5.9286440 \\
-6.0606080 \\
-5.6410700 \\
-4.3282250 \\
-5.1721070 \\
-7.0957660 \\
-6.3568890 \\
-3.9854590 \\
-5.5081160 \\
0.3908670 \\
1.0236490 \\
1.1149660 \\
2.3485710 \\
2.4455470 \\
3.0786700 \\
2.8400460 \\
2.9873300 \\
-2.2667260 \\
-1.4789660 \\
-2.4227980 \\
-0.8840720 \\
-1.8031760 \\
-1.0389360 \\
-0.2959540 \\
-1.9368140 \\
-0.1674670 \\
0.5482160 \\
0.5228880 \\
0.4900120\end{array}$ & $\begin{array}{r}0.4180490 \\
0.5423330 \\
0.6377080 \\
-0.0586740 \\
-0.3376390 \\
-0.6270000 \\
-1.2219350 \\
-1.6382830 \\
-1.9092660 \\
-1.4336410 \\
-3.5963570 \\
-3.3590150 \\
-2.4080170 \\
-2.8890830 \\
-4.3481770 \\
-3.9393400 \\
-2.2457780 \\
-3.0767980 \\
0.9404520 \\
1.1722410 \\
0.9816570 \\
0.4996740 \\
1.1188750 \\
1.5146990 \\
1.1783520 \\
0.3369620 \\
0.0978910 \\
1.3276760 \\
-1.1017330 \\
1.3119090 \\
-1.0605270 \\
0.1339940 \\
2.2551340 \\
-1.9979110 \\
0.3072970 \\
-0.4726950 \\
1.6856990 \\
0.1461040 \\
2.2549490 \\
1.5052080 \\
-0.4615870 \\
3.3174690 \\
0.3755620 \\
1.5816810 \\
-0.5045600 \\
-2.4580300\end{array}$ \\
\hline
\end{tabular}

5.2934580

4.8423350

4.0586190

3.5578930

$-2.4283460$

$-1.5977140$

$-6.5605940$

$-6.4443050$

$-3.7731680$

$-2.8182360$

0.2692370

0.4060450

$-0.4839700$

0.1811250

1. 5582540

1. 3884140

1. 3589410

1.0480080

1.1199480

0.3525300

1.3305700

1.7418350

2.1572660

$-0.6496500$

0.6098500

2. 3723710

0.0394520

0.7097070

$-3.7177310$

$-3.6138730$

$-4.6244220$

$-2.8573680$

$-4.8824870$

$-5.8656970$

$-4.7168520$

$-4.9205620$

2.5479990

2.7885290

2.3299020

1.6413810

4.9646160

4.7560910

5.3138440

5.7817300

$-1.8199690$

-0.5480770
4.4976330
4.9681010
0.3141940
-0.6434420
-3.2925820
-3.6726210
-0.4696720
-0.5800030
-1.2946520
-1.7370670
3.5821220
3.3015280
2.9937930
2.2951420
2.9373100
1.9657050
5.8224290
5.6915570
4.2327050
5.0240920
6.8740660
6.2936370
3.8730630
5.4578880
5.0474000
5.4538430
6.0736680
4.0918230
0.1791390
0.2527600
0.7258940
0.6678020
-2.0350740
-1.6011990
-1.9654320
-3.0950880
1.2022580
2.2521310
0.7162080
1.1648710
0.4588540
-0.0523950
1.4706360
-0.0692230
-2.4951020

$-2.3022390$

0.2048640

1.0895540

2.6715210

2. 5077100

2. 5719760

1.9720450

2. 1493960

3.2352300

$-1.9766380$

$-2.2696630$

1.0216820

$-0.4092400$

1.5464020

$-0.7413530$

1.0376920

1.2509010

0.6285510

$-0.8644750$

$-1.3208970$

1.4634350

0.9300310

$-1.4596630$

$-1.3575680$

1. 3578150

2. 5271340

0.8295370

$-1.0619580$

$-2.3244750$

$-2.3959470$

3. 4845830

$-2.1118610$

$-1.9372040$

$-2.7386840$

$-2.5209350$

$-3.8196770$

$-2.4658390$

$-3.1659010$

3.3731940

$-4.1232460$

$-2.5547780$

$-3.3386430$

4.2850220

3.5752220

-2.8358270
3.7320480 


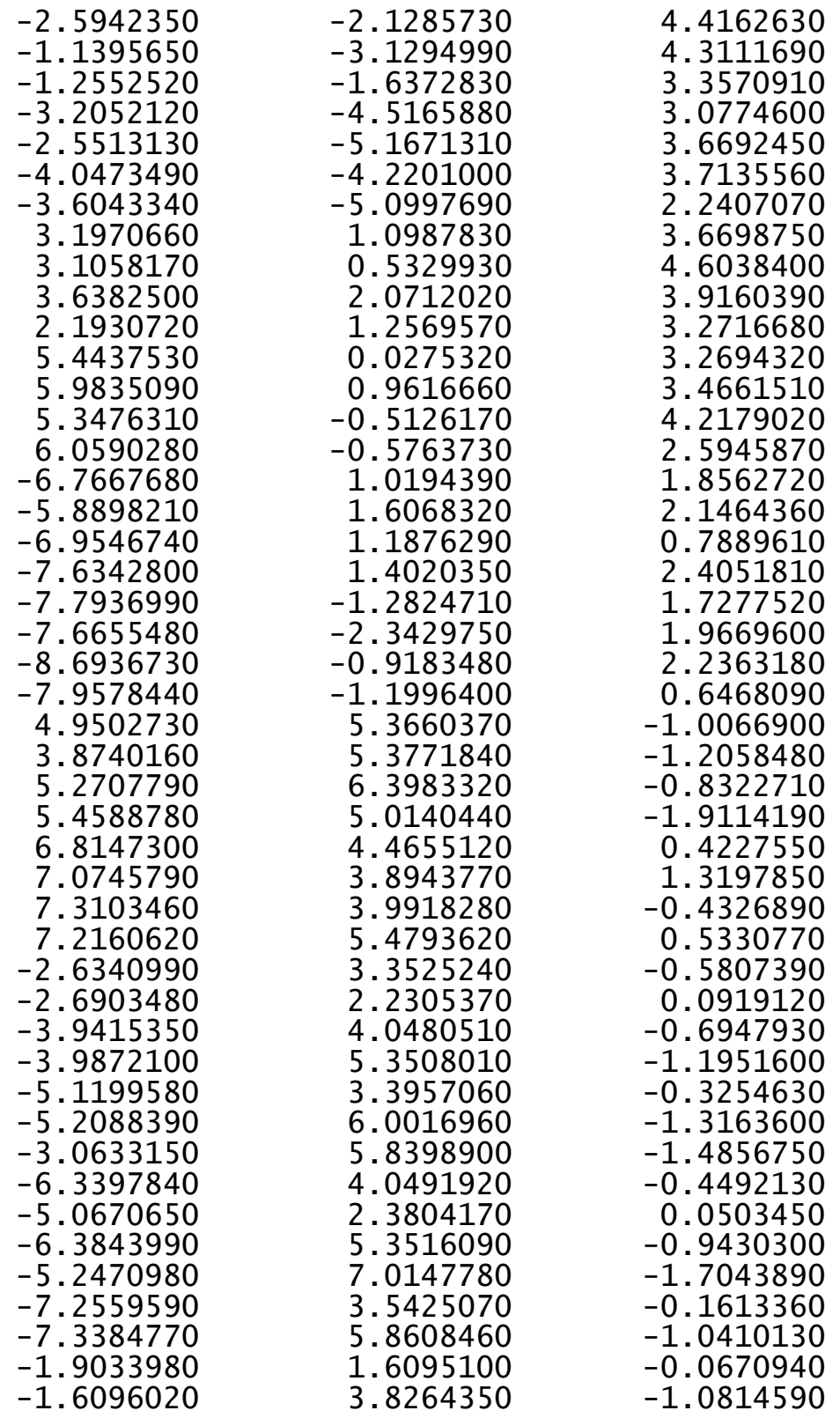

\begin{tabular}{|c|c|c|c|}
\hline \multicolumn{4}{|c|}{ GL } \\
\hline 0 & 0.0933790 & -1.1161800 & 0.2513780 \\
\hline 0 & 2.1054840 & -0.0364980 & -0.7925840 \\
\hline C & 0.3480430 & -2.3051170 & -0.3940310 \\
\hline C & 1.6374300 & -2.7850500 & -0.4311870 \\
\hline C & -0.7742540 & -2.9986590 & -0.9202280 \\
\hline C & 1.8831370 & -3.9799500 & -1.1854970 \\
\hline C & -0.5415590 & -4.1934230 & -1.5470050 \\
\hline C & 0.7764760 & -4.6906950 & -1.7294900 \\
\hline $\mathrm{H}$ & -1.3767500 & -4.7730540 & -1.9301190 \\
\hline C & 2.8691300 & -0.7012330 & 0.1378630 \\
\hline C & 3.8524110 & 0.0673280 & 0.8163860 \\
\hline C & 2.7011540 & -2.0576790 & 0.3167800 \\
\hline C & 4.6966590 & -0.5894030 & 1.6718730 \\
\hline C & 3.5120360 & -2.7104890 & 1.3052430 \\
\hline C & 4.5419470 & -1.9714360 & 1.9521150 \\
\hline $\mathrm{H}$ & 5.4793450 & -0.0347100 & 2.1824490 \\
\hline C & 5.1713010 & -3.9317320 & 3.2381400 \\
\hline C & 4.1179360 & -4.6542730 & 2.6339630 \\
\hline C & 3.3109990 & -4.0617460 & 1.6965700 \\
\hline $\mathrm{C}$ & 5.3704790 & -2.6171150 & 2.9069250 \\
\hline $\mathrm{H}$ & 5.8077690 & -4.4147270 & 3.9733060 \\
\hline $\mathrm{H}$ & 3.9416380 & -5.6873840 & 2.9180320 \\
\hline $\mathrm{H}$ & 2.4997430 & -4.6272230 & 1.2527980 \\
\hline $\mathrm{H}$ & 6.1602530 & -2.0408430 & 3.3820450 \\
\hline C & 3.3849010 & -5.6247170 & -2.1553470 \\
\hline $\mathrm{C}$ & 2.2829240 & -6.3500110 & -2.6619430 \\
\hline C & 1.0093580 & -5.8873890 & -2.4555070 \\
\hline C & 3. 1910720 & -4.4719650 & -1.4374550 \\
\hline $\mathrm{H}$ & 4.3938870 & -5.9807770 & -2.3401970 \\
\hline H & 2.4492560 & -7.2653120 & -3.2217180 \\
\hline H & 0.1542610 & -6.4252260 & -2.8568350 \\
\hline H & 4.0450310 & -3.9194300 & -1.0608720 \\
\hline C & -2.1 & -2.4181750 & 270 \\
\hline C & -2.8484080 & -1.8020620 & -1.7367270 \\
\hline C & -2.667 & -2.4514430 & 0.6091770 \\
\hline C & -4.0 & -1.2 & -1.4 \\
\hline C & -3.90 & -1.8631930 & 0.8431690 \\
\hline C & -4.63 & 36600 & -0.1673600 \\
\hline $\mathrm{H}$ & -4.64 & -0.7604210 & -2.25 \\
\hline $\mathrm{H}$ & -4.33 & -1.8 & 3390 \\
\hline C & 6680 & 1730 & 0.601 \\
\hline C & 6850 & 2.4056270 & 3000 \\
\hline C & 4.3806760 & 2.0684750 & -0.6097520 \\
\hline C & 3.3906260 & 3.7793670 & 1.3480880 \\
\hline
\end{tabular}




$\begin{array}{lrrr}\mathrm{C} & 4.3525710 & 3.4467630 & -0.8102720 \\ \mathrm{C} & 3.8580570 & 4.3205160 & 0.1547240 \\ \mathrm{H} & 3.0016990 & 4.4549230 & 2.1065810 \\ \mathrm{H} & 4.7195270 & 3.8462480 & -1.7527340 \\ \mathrm{C} & -1.9593650 & -3.1706420 & 1.7488750 \\ \mathrm{C} & -6.0180500 & -0.6935190 & 0.1419050 \\ \mathrm{C} & -2.3447900 & -1.8379550 & -3.1742380 \\ \mathrm{C} & 2.8338200 & 1.8862420 & 2.9137700 \\ \mathrm{C} & 3.7527220 & 5.8132420 & -0.1016910 \\ \mathrm{C} & 4.9436190 & 1.1761830 & -1.7063550 \\ \mathrm{C} & 6.4468990 & 1.4224390 & -1.8942000 \\ \mathrm{H} & 6.6421510 & 2.4487830 & -2.2264460 \\ \mathrm{H} & 6.9892090 & 1.2640790 & -0.9559510 \\ \mathrm{H} & 6.8563390 & 0.7403850 & -2.6480000 \\ \mathrm{C} & 4.1801610 & 1.3424350 & -3.0264460 \\ \mathrm{H} & 3.1119790 & 1.1608180 & -2.8791770 \\ \mathrm{H} & 4.3071390 & 2.3497880 & -3.4402850 \\ \mathrm{H} & 4.5514240 & 0.6291750 & -3.7710440 \\ \mathrm{C} & 2.5384520 & 6.1090510 & -0.9958110 \\ \mathrm{H} & 2.4167540 & 7.1874790 & -1.1492470 \\ \mathrm{H} & 2.6593800 & 5.6353040 & -1.9773060 \\ \mathrm{H} & 1.6202760 & 5.7170350 & -0.5451010 \\ \mathrm{C} & 5.0311510 & 6.4151960 & -0.6949730 \\ \mathrm{H} & 5.9016190 & 6.1905900 & -0.0702330 \\ \mathrm{H} & 5.2277250 & 6.0260930 & -1.7003290 \\ \mathrm{H} & 4.9367880 & 7.5035200 & -0.7778280 \\ \mathrm{C} & 3.8702750 & 2.0666640 & 4.0323860 \\ \mathrm{H} & 4.8136290 & 1.5652880 & 3.7931660 \\ \mathrm{H} & 4.0875590 & 3.1302360 & 4.1886140 \\ \mathrm{H} & 3.4944280 & 1.6548060 & 4.9760440 \\ \mathrm{C} & 1.4996970 & 2.5332240 & 3.3051310 \\ \mathrm{H} & 1.6150410 & 3.5969550 & 3.5439000 \\ \mathrm{H} & 0.7740400 & 2.4292030 & 2.4957340 \\ \mathrm{H} & 1.0978070 & 2.0414530 & 4.1982650 \\ \mathrm{C} & -2.6387580 & -4.5210590 & 2.0169360 \\ \mathrm{H} & -3.6819960 & -4.3789790 & 2.3232420 \\ \mathrm{H} & -2.6338930 & -5.1458530 & 1.1171650 \\ \mathrm{H} & -2.1197660 & -5.0633840 & 2.8155310 \\ \mathrm{C} & -1.8689040 & -2.3281620 & 3.0246460 \\ \mathrm{H} & -1.4282570 & -1.3494310 & 2.8118690 \\ \mathrm{H} & -2.8545980 & -2.1780290 & 3.4810100 \\ \mathrm{H} & -1.2411920 & -2.8359570 & 3.7650210 \\ \mathrm{C} & -7.0794620 & -1.7703060 & -0.1298930 \\ \mathrm{H} & -8.0773050 & -1.4120460 & 0.1482040 \\ \mathrm{H} & -7.0956050 & -2.0334590 & -1.1943300 \\ \mathrm{H} & -6.8692050 & -2.6824110 & 0.4380270 \\ & & & \end{array}$

-6.3537930
-5.5859110
-6.4409500
-7.3106560
-2.5471670
-3.6073020
-2.0404780
-2.1166900
-3.0145360
-4.0956260
-2.6070000
-2.8642600
0.5248890
-0.0846020
0.2665200
-1.2672270
-6.0414560
-0.9334730
4.8269010
3.5768600
2.6440170
-3.0780200
-1.9806880
-4.2113130
-5.4010390
-4.1109410
-6.4935420
-5.4612710
-5.2073370
-3.1799250
-6.4001470
-7.4200330
-5.1319860
-7.2574020
-1.1748230
-3.2215330
-1.2100400
-2.3619450
-0.4722710
-2.1794630
-0.9030360
-0.4167870
-2.6185460
-3.8733760
-3.5987120
-1.4793050
-

0.5986030

1.3564960

0.4355650

1.0005110

$-0.5299710$

$-0.2798710$

0.2967590

$-0.6252520$

$-2.9981540$

$-2.8317810$

$-3.0865480$

$-3.9536280$

0.2451530

0.3376830

1.3533300

$-2.0219690$

$-0.4661120$

$-3.3852390$

1338890

6.2981640

0.8148370

1.8413420

1.2260050

1.6098110

2. 3179420

0.6597760

2.0590430

3.0597880

0.3966980

0.1242180

1.0913800

2.6059120

$-0.3517750$

0.8816500

1.3547050

2. 5575710

3. 2653640

2. 3604330

3.0228380

1. 3140480

2.6394850

1.7830960

5.0440960

4.2447920

2.7400890

4.7438230
$-0.6034920$

$-0.4249470$

$-1.6842560$

$-0.2530240$

$-3.9441550$

$-4.0697520$

$-3.4447440$

$-4.9470190$

$-3.9278340$

$-4.0083160$

$-4.9413070$

$-3.4161480$

$-0.5359990$

$-1.9059220$

0.4496490

$-3.1461110$

1. 2157720

1. 4401060

$-1.3961460$

0.8674720

2.8025210

1.3839160

1.7246380

2. 3190190

2.1396690

3.3376670

2.9583250

1.3507480

4.1498030

3.4755900

3.9571140

2.8145250

4.9324450

4.5898300

1.1233890

0.3849790

$-1.5028760$

$-1.5668810$

$-0.7397280$

$-1.3456220$

$-2.7721790$

$-2.5589680$

$-2.6322450$

$-2.2709910$

$-2.3035370$

$-1.6536040$ 


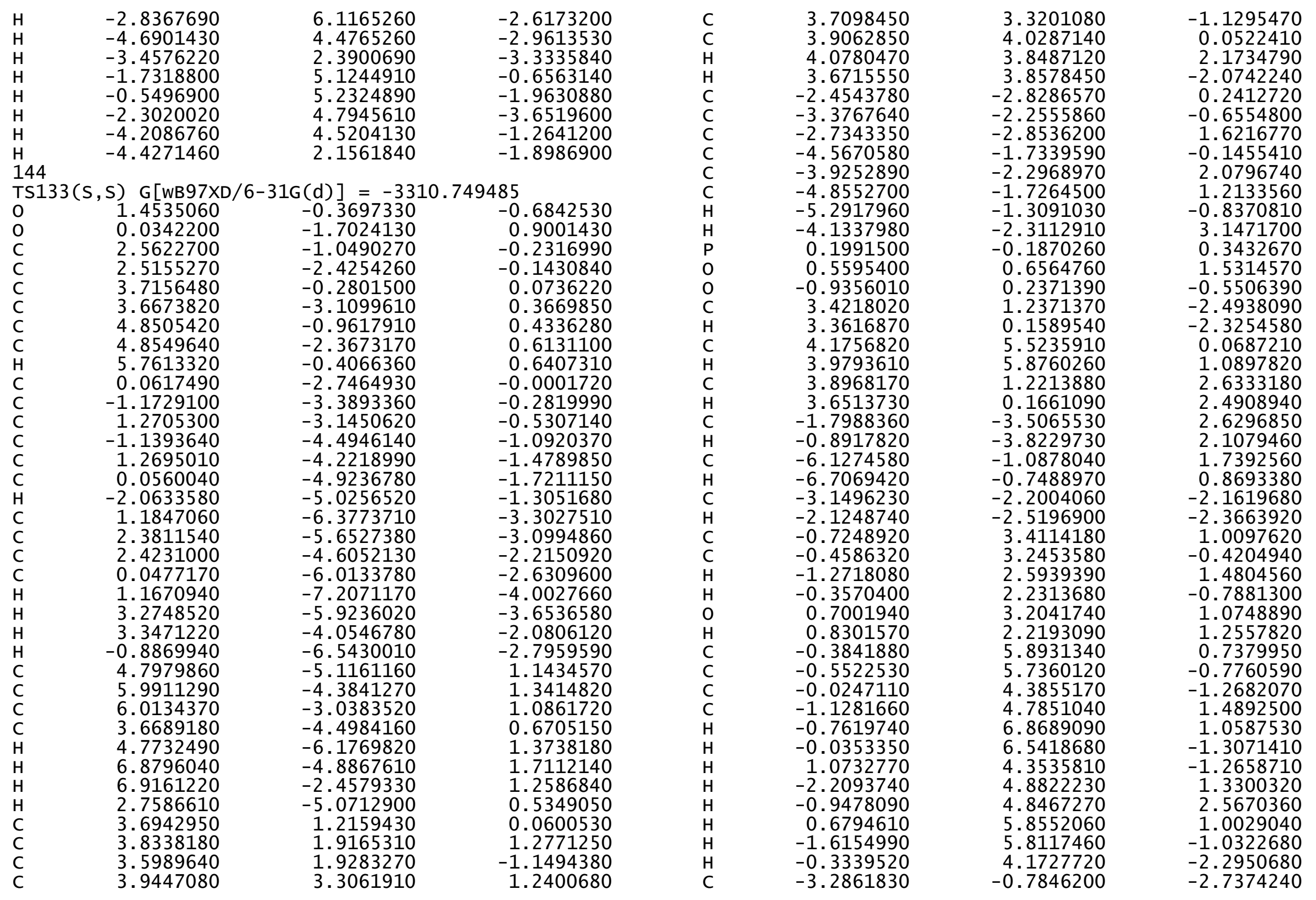




$\begin{array}{rrr}-3.1247280 & -0.8105130 & -3.8212850 \\ -4.2805160 & -0.3579130 & -2.5617280 \\ -2.5392830 & -0.1224140 & -2.2951820 \\ -4.0932800 & -3.1758600 & -2.8807130 \\ -5.1411060 & -2.8853610 & -2.7388800 \\ -3.8892690 & -3.1876610 & -3.9573340 \\ -3.9790350 & -4.1962840 & -2.4994280 \\ 2.1112350 & 1.6627770 & -3.1689340 \\ 2.0991500 & 2.7395700 & -3.3773080 \\ 1.9835480 & 1.1378520 & -4.1220720 \\ 1.2552850 & 1.4188260 & -2.5326380 \\ 4.6298190 & 1.4802520 & -3.4088580 \\ 4.5090970 & 0.9380770 & -4.3532870 \\ 4.7473730 & 2.5437850 & -3.6463810 \\ 5.5548360 & 1.1385690 & -2.9326080 \\ -1.3700460 & -2.5347040 & 3.7358320 \\ -2.2221140 & -2.2273870 & 4.3537860 \\ -0.6393980 & -3.0145020 & 4.3966830 \\ -0.9107820 & -1.6388850 & 3.3097640 \\ -2.4385510 & -4.7728900 & 3.2167850 \\ -1.7457930 & -5.2684530 & 3.9063340 \\ -3.3537010 & -4.5345110 & 3.7714630 \\ -2.7016620 & -5.4828930 & 2.4254040 \\ 2.8710730 & 1.7824890 & 3.6273320 \\ 2.9404970 & 1.2385370 & 4.5761480 \\ 3.0452470 & 2.8430230 & 3.8416680 \\ 1.8568870 & 1.6622750 & 3.2413380 \\ 5.3133830 & 1.3026920 & 3.2210190 \\ 5.5949710 & 2.3443780 & 3.4160770 \\ 5.3680480 & 0.7557800 & 4.1688560 \\ 6.0585970 & 0.8798980 & 2.5394310 \\ -7.0014070 & -2.0832530 & 2.5119220 \\ -7.2528740 & -2.9509090 & 1.8933000 \\ -6.4871570 & -2.4472870 & 3.4085920 \\ -7.9343170 & -1.6070620 & 2.8340290 \\ -5.8033450 & 0.1450430 & 2.5952420 \\ -5.1615080 & 0.8433040 & 2.0493550 \\ -6.7214940 & 0.6684870 & 2.8853000 \\ -5.2769350 & -0.1466190 & 3.5114630 \\ 3.2848250 & 6.3345680 & -0.8759840 \\ 2.2250370 & 6.1735470 & -0.6600660 \\ 3.4917020 & 7.4043260 & -0.7641680 \\ 3.4622400 & 6.0756780 & -1.9260560 \\ 5.6592090 & 5.7849350 & -0.2364890 \\ 6.3051670 & 5.2385650 & -1.2530680 \\ 5.9037800 & 5.4549270 & \end{array}$

$\begin{array}{ll}\mathrm{H} & 5.8911880 \\ \mathrm{C} & -3.4057530 \\ \mathrm{O} & -3.2162850 \\ \mathrm{C} & -4.8358870 \\ \mathrm{C} & -5.1643650 \\ \mathrm{C} & -5.8441850 \\ \mathrm{C} & -6.4987860 \\ \mathrm{H} & -4.3692220 \\ \mathrm{C} & -7.1775310 \\ \mathrm{H} & -5.5704130 \\ \mathrm{C} & -7.5045860 \\ \mathrm{H} & -6.7557820 \\ \mathrm{H} & -7.9606210 \\ \mathrm{H} & -8.5467230 \\ \mathrm{H} & -2.2825850 \\ \mathrm{O} & -2.5105050 \\ \mathrm{H} & \end{array}$
6.8531860
4.5416030
4.8839080
2.2963790
0.9091230
3.6055650
5. 5606220
1.5655910
3.8967700
0.9519510
3. 2003900

$-0.7127640$

$1.3334700 \quad-0.1020550$

$2.8612740 \quad-0.8282760$

$4.1698470 \quad-1.1874780$

$1.9235950 \quad-0.5952730$

$-1.2986240$

$-1.3749020$

$-0.7141780$

$-0.3295650$

$-1.0621090$

$-1.5703830$

$-0.5368760$

$-1.1536800$

144

-0.2106890
-1.1560750

$\operatorname{TS} 134(\mathrm{~S}, \mathrm{~S}) \mathrm{G}[\mathrm{WB} 97 \mathrm{XD} / 6-31 \mathrm{G}(\mathrm{d})]=-3310.749959$

\begin{tabular}{|c|c|c|c|}
\hline & & & \\
\hline 0 & 0.1539640 & -1.1797880 & 0.2635930 \\
\hline 0 & 2.0848700 & 0.0342610 & -0.7870560 \\
\hline C & 0.5371690 & -2.3611360 & -0.3316780 \\
\hline C & 1.8666860 & -2.7202900 & -0.3155300 \\
\hline C & -0.4972660 & -3.1695080 & -0.8731730 \\
\hline$C$ & 2.2459410 & -3.9077240 & -1.0240550 \\
\hline C & -0.1338640 & -4.3586240 & -1.4483160 \\
\hline C & 1.2289930 & -4.7372820 & -1.5735460 \\
\hline $\mathrm{H}$ & -0.8992800 & -5.0235640 & -1.8388880 \\
\hline C & 2.8848240 & -0.5124990 & 0.1893100 \\
\hline C & 3.7734370 & 0.3744790 & 0.8525910 \\
\hline C & 2.8405000 & -1.8699530 & 0.4244810 \\
\hline C & 4.6685850 & -0.1655300 & 1.7371800 \\
\hline C & 3.6971990 & -2.4038500 & 1.4455730 \\
\hline C & 4.6465480 & -1.5448460 & 2.0669130 \\
\hline $\mathrm{H}$ & 5.3883530 & 0.4807420 & 2.2319910 \\
\hline C & 5.4523780 & -3.3831660 & 3.4314120 \\
\hline C & 4.4761070 & -4.2262500 & 2.8544280 \\
\hline C & 3.6225750 & -3.7514410 & 1.8910680 \\
\hline C & 5.5272490 & 0699000 & 3.0482730 \\
\hline $\mathrm{H}$ & 6.127 & -3.7734140 & 4.1867050 \\
\hline $\mathrm{H}$ & 4.3952540 & -5.2586100 & 3.1813860 \\
\hline $\mathrm{H}$ & 2.872 & -4.4102870 & 1.4690810 \\
\hline $\mathrm{H}$ & 6.2 & -1.4015260 & 0000 \\
\hline C & 3.92 & -5.4194680 & 1500 \\
\hline C & 2.91 & -6.2641630 & 550 \\
\hline C & $1.5 \mathrm{~s}$ & -5.92 & -2 . \\
\hline C & 3.6 & .2 & \\
\hline
\end{tabular}




$\begin{array}{lr}\mathrm{H} & 4.9700750 \\ \mathrm{H} & 3.1838840 \\ \mathrm{H} & 0.8092870 \\ \mathrm{H} & 4.3864090 \\ \mathrm{C} & -1.9158660 \\ \mathrm{C} & -2.6389560 \\ \mathrm{C} & -2.5211730 \\ \mathrm{C} & -3.9614270 \\ \mathrm{C} & -3.8386930 \\ \mathrm{C} & -4.5854160 \\ \mathrm{H} & -4.5342300 \\ \mathrm{H} & -4.2915780 \\ \mathrm{C} & 3.6705870 \\ \mathrm{C} & 3.0397630 \\ \mathrm{C} & 4.1495100 \\ \mathrm{C} & 2.9295780 \\ \mathrm{C} & 4.0012860 \\ \mathrm{C} & 3.3923800 \\ \mathrm{H} & 2.4474460 \\ \mathrm{H} & 4.3665630 \\ \mathrm{C} & -1.8092430 \\ \mathrm{C} & -6.0415960 \\ \mathrm{C} & -2.0366740 \\ \mathrm{C} & 2.4885870 \\ \mathrm{C} & 3.1460440 \\ \mathrm{C} & 4.8242920 \\ \mathrm{C} & 6.2998410 \\ \mathrm{H} & 6.4033090 \\ \mathrm{H} & 6.8348450 \\ \mathrm{H} & 6.7897600 \\ \mathrm{C} & 4.0744000 \\ \mathrm{H} & 3.0259780 \\ \mathrm{H} & 4.1111580 \\ \mathrm{H} & 4.5270780 \\ \mathrm{C} & 1.8461140 \\ \mathrm{H} & 1.6227700 \\ \mathrm{H} & 1.9348610 \\ \mathrm{H} & 1.0004030 \\ \mathrm{C} & 4.3151230 \\ \mathrm{H} & 5.2528540 \\ \mathrm{H} & 4.4539290 \\ \mathrm{H} & 4.1269090 \\ \mathrm{C} & 3.4841580 \\ \mathrm{H} & 4.4535110 \\ \mathrm{H} & 3.6524430 \\ \mathrm{H} & 3.0978590 \\ & \end{array}$

$\begin{array}{rr}-5.6754950 & -2.0686460 \\ -7.1718280 & -2.9538160 \\ -6.5567030 & -2.6616470 \\ -3.6284190 & -0.8521440 \\ -2.7146140 & -0.7448440 \\ -2.2707170 & -1.8705420 \\ -2.7111450 & 0.5230350 \\ -1.8658700 & -1.6959750 \\ -2.2667290 & 0.6498880 \\ -1.8504600 & -0.4472000 \\ -1.5629500 & -2.5694560 \\ -2.2681710 & 1.6361680 \\ 1.8428120 & 0.5971440 \\ 2.6675180 & 1.5485800 \\ 2.3871640 & -0.6059110 \\ 4.0304480 & 1.2780380 \\ 3.7541580 & -0.8365070 \\ 4.5934480 & 0.0917390 \\ 4.6792010 & 2.0057610 \\ 4.1688040 & -1.7726170 \\ -3.2387790 & 1.7612720 \\ -1.4123870 & -0.3378090 \\ -2.2694850 & -3.2697190 \\ 2.1167930 & 2.8584070 \\ 6.0648540 & -0.1916200 \\ 1.5296420 & -1.6664050 \\ 1.9173260 & -1.8323420 \\ 2.9491270 & -2.1880530 \\ 1.8346690 & -0.8802460 \\ 1.2604660 & -2.5600030 \\ 1.5869730 & -3.0035070 \\ 1.3061620 & -2.8696340 \\ 2.5914050 & -3.4414600 \\ 0.8949270 & -3.7226500 \\ 6.2286060 & -0.9951740 \\ 7.2876020 & -1.1688890 \\ 5.7328020 & -1.9694750 \\ 5.7782620 & -0.4644880 \\ 6.7591170 & -0.8968680 \\ 6.6124820 & -0.3513280 \\ 6.3781450 & -1.9149920 \\ 7.8353660 & -0.9750870 \\ 2.3481890 & 4.0045140 \\ 1.8840710 & 3.7986560 \\ 3.4210940 & 4.1568790 \\ 1.9282790 & 4.9403840\end{array}$

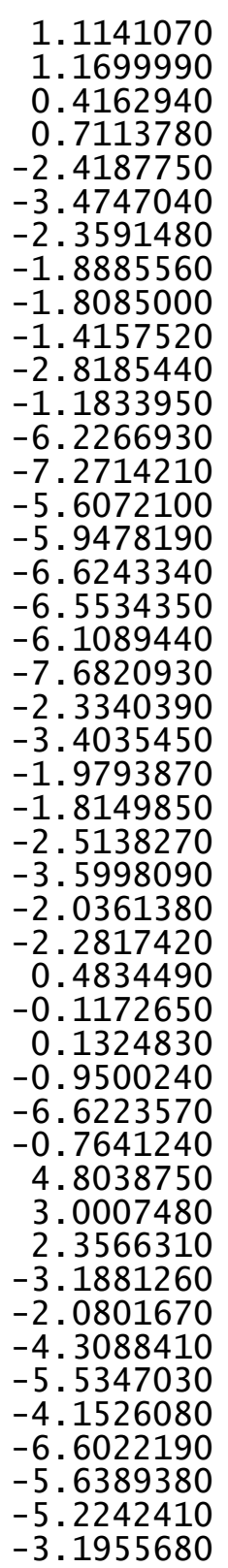
1.1141070
0.7113780
$-2.4187750$
$-3.4747040$
$-1.8885560$
$-1.4157520$
-2.8185440
$-5.9478190$
$-6.6243340$
$-7.6820930$
.3340390
$-1.8149850$
$-2.5138270$
$-3.5998090$
$-2.0361380$
0.4834490
$-0.1172650$
$-6.6223570$
(
4.8038750
3.0007480
2.3566310
$-3.1881260$
.0801670
$-4.3088410$
$-4.1526080$
$-5.2242410$
$-3.1955680$

3.2223060
3.4877440
2.3913920
4.0925310
2.1937210
2.4643760
1.3853080
3.0645100
2.9151910
2.5859360
3.3141650
3.7359870
-0.8572600
-0.7604940
-0.2788920
-1.9123680
1.0708380
1.4505980
1.7742920
1.0638690
-4.0541620
-4.2639050
-3.5084150
-5.0185070
-4.0618010
-4.2114150
-5.0474590
-3.5409290
-0.5591120
-1.9353390
0.3893890
-3.1651480
-0.9951930
1.5077700
-1.3295420
0.7768930
2.7389050
1.3157410
1.6716700
2.2727220
2.0317540
3.3885720
2.8960980
1.1632530
4.2480060
3.5705300

2.6910250

3.7532850

2. 5698000

2. 1606810

$-4.4620370$

$-5.3164460$

$-4.9818120$

$-2.2306330$

$-1.2645050$

$-2.5993570$

0.0204330

0.3362280

0.7139030

0.1060930

2.5622870

$-0.8741650$

$-1.2548580$

0.8672650

$-0.1106490$

$-1.0140310$

4603840

$-3.5280550$

4.4301210

0.1885830

0.1892310

1.3041650

$-2.0752390$

$-3.4321560$

0.4890390

6. 5613250

1.0372100

1.7232490

1391220

1.5182670

2. 1425660

.6924000

.9332510

2.7843690

0.2185890 


$\begin{array}{lrrr}\mathrm{C} & -6.4486610 & 1.0991030 & 4.0018730 \\ \mathrm{H} & -7.5549270 & 2.4176450 & 2.7072550 \\ \mathrm{H} & -5.1041350 & -0.1674510 & 5.1104810 \\ \mathrm{H} & -7.2844370 & 0.9315370 & 4.6745140 \\ \mathrm{H} & -1.2756360 & 1.2657630 & 1.0619530 \\ \mathrm{O} & -3.3500940 & 2.3945830 & 0.2884490 \\ \mathrm{C} & -1.3674190 & 3.0812930 & -1.6519830 \\ \mathrm{C} & -2.4977820 & 2.1458690 & -1.6537040 \\ \mathrm{H} & -0.6139720 & 2.8955640 & -0.8882120 \\ \mathrm{H} & -2.2808130 & 1.1145940 & -1.3966220 \\ \mathrm{O} & -1.0610370 & 2.4072140 & -2.8959080 \\ \mathrm{H} & -0.5405820 & 1.5801230 & -2.6518000 \\ \mathrm{C} & -2.8239710 & 4.7647430 & -2.8582640 \\ \mathrm{C} & -4.0583020 & 3.9603250 & -2.4437260 \\ \mathrm{C} & -3.7570460 & 2.4610770 & -2.3827380 \\ \mathrm{C} & -1.6754600 & 4.5435900 & -1.8704680 \\ \mathrm{H} & -3.0661180 & 5.8312110 & -2.9026740 \\ \mathrm{H} & -4.8836350 & 4.1319490 & -3.1414810 \\ \mathrm{H} & -3.6410530 & 2.0382630 & -3.3886560 \\ \mathrm{H} & -1.9337870 & 4.9668770 & -0.8919290 \\ \mathrm{H} & -0.7594330 & 5.0391990 & -2.2076940 \\ \mathrm{H} & -2.5056600 & 4.4655500 & -3.8639000 \\ \mathrm{H} & -4.3947280 & 4.2907970 & -1.4536460 \\ \mathrm{H} & -4.5651580 & 1.9019810 & -1.9082110 \\ \mathrm{H} 44 & & & \end{array}$

$\operatorname{TS} 135(\mathrm{~S}, \mathrm{~S}) \mathrm{G}[\mathrm{WB} 97 \mathrm{XD} / 6-31 \mathrm{G}(\mathrm{d})]=-3310.751895$

\begin{tabular}{lrrr} 
TS135(S, S & \multicolumn{1}{c}{$\mathrm{G}[\mathrm{WB} 97 \times \mathrm{D} / 6-31 \mathrm{G}(\mathrm{d})]=-3310.751895$} \\
$\mathrm{O}$ & 0.1050020 & -1.1051230 & 0.2541120 \\
$\mathrm{O}$ & 2.0937720 & -0.0750380 & -0.8833190 \\
$\mathrm{C}$ & 0.3534610 & -2.3304870 & -0.3220220 \\
$\mathrm{C}$ & 1.6450590 & -2.8048480 & -0.3591250 \\
$\mathrm{C}$ & -0.7756760 & -3.0621770 & -0.7775310 \\
$\mathrm{C}$ & 1.8818400 & -4.0410780 & -1.0471430 \\
$\mathrm{C}$ & -0.5487240 & -4.2903400 & -1.3383650 \\
$\mathrm{C}$ & 0.7682920 & -4.7892560 & -1.5225290 \\
$\mathrm{H}$ & -1.3882620 & -4.8970560 & -1.6662360 \\
$\mathrm{C}$ & 2.8764610 & -0.6862930 & 0.0683780 \\
$\mathrm{C}$ & 3.8694890 & 0.1214930 & 0.6832200 \\
$\mathrm{C}$ & 2.7197320 & -2.0321960 & 0.3247890 \\
$\mathrm{C}$ & 4.7423590 & -0.4857160 & 1.5459270 \\
$\mathrm{C}$ & 3.5589720 & -2.6275600 & 1.3269080 \\
$\mathrm{C}$ & 4.6040420 & -1.8514270 & 1.9018850 \\
$\mathrm{H}$ & 5.5386640 & 0.0972220 & 2.0008050 \\
$\mathrm{C}$ & 5.2829020 & -3.7344110 & 3.2742900 \\
$\mathrm{C}$ & 4.2137050 & -4.4916070 & 2.7451240 \\
$\mathrm{C}$ & 3.3757870 & -3.9552270 & 1.8007490 \\
$\mathrm{C}$ & 5.4654780 & -2.4401400 & 2.8640140
\end{tabular}

5.9440650

4.0492590

2. 5543450

6.2661870

3.3723990

2. 2643510

0.9926650

3.1870290

4.3793830

2. 4241240

0.1323650

4.0456620

$-2.1341530$

$-2.8809600$

$-2.6356910$

$-4.1160980$

$-3.8723600$

$-4.6287730$

$-4.7020730$

$-4.2702560$

3.9056460

3.3534590

4.4445320

3.3780010

4.4379940

3.9081550

2.9570000

4.8519290

$-1.8934090$

$-6.0072030$

$-2.4130090$

2.7604700

3.8786130

5.0268720

6.5251400

6.7059620

7.0731280

6.9413660

4.2538640

3.1913670

4.3529350

4.6393140

4.6329010

4.6511680

4.1523280

5.6660710

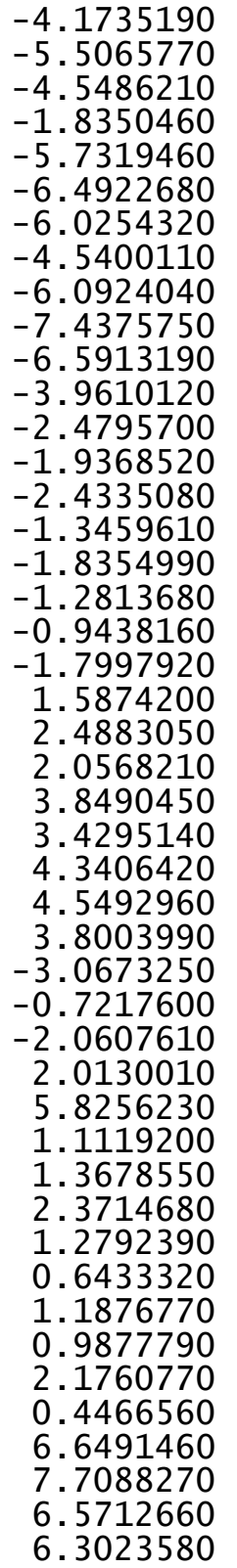

4.0151490

3.0943530

1.4161590

3.2814360

$-1.9532370$

$-2.3904850$

$-2.1820360$

$-1.2999430$

$-2.1404710$

$-2.9000110$

$-2.5302410$

$-0.9777400$

$-0.5513190$

$-1.6140400$

0.7628580

$-1.3329510$

0.9910770

$-0.0380860$

$-2.1550770$

2.0032380

0.3962290

1. 3291260

$-0.8112770$

1.0347910

$-1.0634390$

$-0.1571150$

1.7514500

$-1.9992690$

1.9312970

0.2701160

$-3.0583520$

2.6503420

$-0.4711870$

$-1.8517040$

$-2.0611280$

$-2.4632590$

$-1.1170400$

$-2.7702800$

$-3.1746340$

$-3.0108580$

$-3.6388000$

$-3.8842410$

0.5804190

0.3017490

1.5624060

0.6844970 


\begin{tabular}{rrrr} 
C & 2.4356270 & 6.3251550 & -0.6316260 \\
H & 1.9094120 & 5.7566420 & -1.4057570 \\
H & 1.8778850 & 6.2128770 & 0.3053190 \\
H & 2.4194050 & 7.3853890 & -0.9089130 \\
C & 3.7914520 & 2.1504920 & 3.7805200 \\
H & 4.7105030 & 1.5991770 & 3.5592780 \\
H & 4.0597590 & 3.2036480 & 3.9279100 \\
H & 3.3830390 & 1.7682190 & 4.7231040 \\
H & 1.4604680 & 2.7332500 & 3.0260840 \\
H & 1.6318390 & 3.7875330 & 3.2733210 \\
H & 0.7398620 & 2.6725740 & 2.2084520 \\
H & 1.0199080 & 2.2606330 & 3.9111610 \\
C & -2.5998980 & -4.3573300 & 2.3701760 \\
H & -3.6194670 & -4.1486320 & 2.7154690 \\
H & -2.6641860 & -5.0700550 & 1.5410190 \\
H & -2.0551770 & -4.8354450 & 3.1923210 \\
C & -1.7105900 & -2.1030400 & 3.1078700 \\
H & -1.2720250 & -1.1589390 & 2.7711450 \\
H & -2.6662450 & -1.8901010 & 3.6015100 \\
H & -1.0465160 & -2.5454040 & 3.8584830 \\
C & -7.0709330 & -1.8147760 & 0.0863690 \\
H & -8.0639350 & -1.4413570 & 0.3617980 \\
H & -7.1076730 & -2.1436030 & -0.9590820 \\
H & -6.8468770 & -2.6895370 & 0.7055330 \\
C & -6.3642360 & 0.5229690 & -0.5433990 \\
H & -5.5938690 & 1.2907750 & -0.4304020 \\
H & -6.4776580 & 0.2957240 & -1.6100810 \\
H & -7.3130970 & 0.9440890 & -0.1938480 \\
C & -2.6291350 & -0.7991360 & -3.8987350 \\
H & -3.6908320 & -0.5500150 & -4.0120750 \\
H & -2.1056760 & 0.0512710 & -3.4604920 \\
H & -2.2243530 & -0.9544670 & -4.9047500 \\
C & -3.1030940 & -3.2602400 & -3.7269740 \\
H & -4.1856820 & -3.0958600 & -3.7895000 \\
H & -2.7216080 & -3.4066310 & -4.7438300 \\
H & -2.9409460 & -4.1856200 & -3.1658360 \\
P & 0.5140640 & 0.2085800 & -0.6223240 \\
O & -0.1172720 & 0.2123230 & -1.9854020 \\
O & 0.2615820 & 1.3743560 & 0.2962970 \\
H & -1.3358470 & -2.2479940 & -3.0452340 \\
H & -6.0067240 & -0.4298270 & 1.3286940 \\
H & -0.8933410 & -3.3504460 & 1.5941390 \\
H & 4.9258380 & 0.0893080 & -1.4759720 \\
H & 4.3880980 & 5.9673970 & -1.4331100 \\
H & 2.5151180 & 0.9518830 & 2.5446080 \\
C & -1.2773890 & 3.1496740 & -1.7263430 \\
& & & \\
\hline
\end{tabular}
$-2.4196250$
$-0.5249590$
$-2.2233270$
$-0.9831580$
$-0.4831640$
$-2.7190360$
$-3.9612300$
$-3.6706720$
$-1.5668130$
$-2.9493760$
$-4.7862550$
$-3.5409030$
$-1.8138060$
$-0.6470820$
$-2.4095660$
$-4.2916620$
$-4.4873620$
$-3.0670430$
$-1.9498940$
$-4.1687220$
$-5.3740060$
$-4.0227470$
$-6.4363650$
$-5.4700270$
$-5.0894790$
$-3.0788440$
$-6.2979710$
$-7.3746210$
$-4.9789330$
$-7.1315150$
$-1.1627420$
$-3.2518840$

144

TS136(S, S) G[WB97XD/6-31G(d)] $=-3310.753710$

$\begin{array}{lrcr}\text { TS136(S, S) G[WB97XD/6-31G(d) }]=-3310.753710 \\ \text { O } & 0.5035500 & 0.5477870 & -0.1098420 \\ \text { O } & -1.8592570 & 0.8184890 & -1.0184180 \\ \text { C } & 0.6483190 & 1.9160560 & -0.1473000 \\ \text { C } & -0.3680430 & 2.7144430 & 0.3377390 \\ \text { C } & 1.8833680 & 2.4423630 & -0.6083680 \\ \text { C } & -0.2428620 & 4.1362110 & 0.2204290 \\ \text { C } & 2.0317740 & 3.8068950 & -0.6186840 \\ \text { C } & 0.9813000 & 4.6816760 & -0.2504680 \\ \text { H } & 2.9833600 & 4.2320660 & -0.9268620 \\ \text { C } & -2.2938350 & 1.1627150 & 0.2491640 \\ \text { C } & -3.4915270 & 0.5704460 & 0.7293690 \\ \text { C } & -1.5677330 & 2.0916510 & 0.9570680\end{array}$

\begin{tabular}{lr}
2.2312450 & -1.7199230 \\
2.9598360 & -0.9625760 \\
1.2020720 & -1.4381330 \\
2.4531600 & -2.9616160 \\
1.6157060 & -2.7077240 \\
4.8439040 & -2.9392590 \\
4.0539300 & -2.5184300 \\
2.5529070 & -2.4601180 \\
4.6138160 & -1.9571120 \\
5.9128800 & -2.9856930 \\
4.2333500 & -3.2145610 \\
2.1392570 & -3.4678230 \\
5.0468810 & -0.9801380 \\
5.0963810 & -2.3031190 \\
4.5383260 & -3.9458620 \\
4.3879670 & -1.5276680 \\
1.9882550 & -2.0067530 \\
1.9081740 & 1.2759800 \\
1.3391160 & 1.6322290 \\
1.7305380 & 2.2593230 \\
2.4087240 & 2.0688060 \\
0.8549330 & 3.3372780 \\
2.1933150 & 2.9382570 \\
3.0939390 & 1.2334800 \\
0.6338020 & 4.1997270 \\
0.3440200 & 3.4814370 \\
1.2980540 & 3.9975690 \\
2.7177200 & 2.7872470 \\
-0.0584640 & 5.0286220 \\
1.1225190 & 4.6710520 \\
1.4232020 & 0.9990800 \\
2.5417420 & 0.2292390 \\
d) $1=-3310.753710$ \\
0.5477870 & -0.1098420 \\
0.8184890 & -1.0184180 \\
1.9160560 & -0.1473000 \\
2.7144430 & 0.3377390 \\
2.4423630 & -0.6083680 \\
4.1362110 & 0.2204290 \\
3.8068950 & -0.6186840 \\
4.6816760 & -0.2504680 \\
4.2320660 & -0.9268620 \\
1.1627150 & 0.2491640 \\
0.5704460 & 0.7293690 \\
2.0916510 & 0.9570680 \\
& \\
\hline
\end{tabular}




-3.9312100
-1.9749800
-3.1850710
-4.8642760
-2.8583010
-1.6357010
-1.2053860
-3.6117130
-3.1898660
-1.0292450
-0.2615170
-4.5433150
-1.1386340
0.0921800
1.1265160
-1.3030180
-1.9632520
0.2089310
2.0717300
-2.2555360
3.0456950
3.3076670
3.9510450
4.5103120
5.1280670
5.4446190
4.7373450
5.8306030
-4.2441690
-4.2559980
-4.9536420
-5.0048010
-5.6762420
-5.7180870
-5.0282540
-6.2232410
-0.6054420
-0.1258160
-0.8908800
3.6711900
2.8951250
6.7727030
6.8598160
2.3477220
1.3501470
-4.9781100

0.9569950

1.8415070

0.5524380

2.9672330

3.4937320

3.2195080

2. 1562740

3.1961730

4.1170430

3.6235590

1.7279770

6.3858360

6.9268420

6.0899840

5.0301290

7.0513700

8.0024230

6.4910430

4.6287840

1. 5819980

1. 3221030

1.1564930

0.6984660

0.5195680

0.3132850

0.5258380

0.1968760

$-0.4192140$

$-1.7798740$

0.0203460

$-2.6669300$

$-0.9072590$

$-2.2564490$

$-3.7194810$

$-0.5644730$

$-0.2090630$

$-0.2741110$

$-1.4864390$

1. 3804610

2. 1472140

$-0.2984020$

$-0.1700680$

1.7686160

1.8387670

1.4815780

1.9695110
2.2956880
2.7889790
2.3514230
4.9136780
4.4380000
3.1650260
4.1058660
5.9219360
5.0881920
2.8164190
4.4666160
0.4108170
-0.0277490
-0.3536990
0.5329150
0.6477160
-0.1176760
-0.7104070
0.8608140
-0.9991040
-2.3559680
-0.0061780
-2.6969460
-0.3975830
-1.7401020
-3.7460040
0.3664720
-0.0983950
0.2618640
-1.2318410
-0.5135780
-1.9776080
-1.6344570
-0.2408480
-2.8533410
-1.0741350
-2.4915600
-0.3320390
1.4752510
1.5571260
-2.1572120
-3.2435670
-3.4508630
-3.0073140
-1.6578750

-4.3362750
-6.5073060
-6.3962570
-3.5017110
-2.7671060
-2.7237050
-2.0795310
-3.3927550
-2.0948460
-4.4577670
-5.2139960
-3.9074980
-4.9831800
3.1155150
3.8412680
2.8936910
2.1881120
4.8916890
4.6022830
5.6819530
5.3161780
-4.4188360
-5.0519760
-4.3714380
-3.4110770
-6.3902810
-6.3857440
-7.0925560
-6.7706020
2.2517740
1.3965080
3.1471670
2.1170870
2.7350240
3.7440890
2.0378870
2.7159070
-8.0033720
-8.4104730
-8.1807580
-8.5636920
-5.9453390
-4.8814770
-6.4798030
-6.0518590
6.8305820

2.0514790

$-3.2531530$

$-4.2352120$

$-2.3117520$

$-1.5584420$

$-3.5992330$

$-3.8478330$

$-4.4518900$

$-3.4703540$

$-2.5392900$

$-3.2908010$

$-2.8979340$

$-1.6212470$

0.1045900

$-0.7159520$

0.2688180

$-0.2038980$

1.8992890

1665450

1.1437880

2.7869410

1.6682940

1.1770140

2.7339270

1.2506840

2. 0688330

3.1360310

1.5688530

1.9560360

0.7717110

1.0279160

0.7969110

$-0.2525390$

3.1535940

3.1305190

3.4622900

3. 9152170

$-2.9137490$

$-2.8546460$

$-1.9490270$

$-3.6762530$

$-3.3629890$

$-3.6201490$

$-4.1322740$

-2.4132530
-1.8038540
$-0.9803590$

$-2.4639080$

$-1.9858760$

1. 4744710

1.7716680

1.1730490

2.0239110

1.0090470

0.2895550

2.6547060

2. 3981590

3. 5322220

2.9350930

2.1228490

2.0602260

3.1831410

1. 6322700

2. 2445430

3. 2666090

2. 3186880

1.7644270

$-3.0738400$

$-3.8219840$

$-3.3252260$

$-3.1492960$

$-1.5293990$

$-1.7787310$

$-2.2065420$

$-0.5084460$

$-4.6117910$

$-5.2449130$

$-5.2446350$

$-4.2580770$

$-3.9910110$

$-4.4199960$

$-4.7780240$

$-3.2071050$

$-2.4869500$

$-1.4723340$

$-2.9762420$

$-3.0397370$

$-3.8877160$

$-3.8711190$

$-4.4565970$

$-4.4245130$

$-1.8655460$ 


\begin{tabular}{|c|c|c|c|c|c|c|c|}
\hline & & & & & & & \\
\hline $\mathrm{H}$ & 6.0686050 & -2.3465400 & -2.4351580 & C & -0.6847660 & -3.1849310 & -0.8861200 \\
\hline $\mathrm{H}$ & 7.8092300 & -2.2140370 & -2.1380570 & C & 2.0228380 & -4.0545070 & -0.9062480 \\
\hline $\mathrm{H}$ & 6.6662410 & -2.0022350 & & C & -0.3566900 & -4.4061320 & -1.4130080 \\
\hline C & 7.9615700 & 0.4259880 & -1.5120800 & C & 0.9901320 & -4.8511790 & -1.4744730 \\
\hline $\mathrm{H}$ & 7.9155060 & 1.5033360 & & $\mathrm{H}$ & -1.1395150 & -5.0512280 & -1.8026370 \\
\hline $\mathrm{H}$ & 7.9823590 & 0.2733180 & -0.4270220 & C & 2.7965390 & -0.6640810 & 0.2087760 \\
\hline $\mathrm{H}$ & 8.9051690 & 0.0447460 & -1.9174830 & $\mathrm{C}$ & 3.7181130 & 0.1976790 & 0.8606890 \\
\hline C & 1.2956070 & -3.1275870 & -1.7194340 & $\mathrm{C}$ & 2.6603330 & -2.0037580 & 0.5012520 \\
\hline C & 2.1453450 & -2.3359230 & -0.8251770 & $\mathrm{C}$ & 4.5343750 & -0.3525860 & 1.8129990 \\
\hline $\mathrm{H}$ & 0.2295540 & -3.0556060 & -1.5138650 & $\mathrm{C}$ & 3.4372640 & -2.5399310 & 1.5822000 \\
\hline $\mathrm{H}$ & 1.7588540 & -1.3938780 & -0.4559960 & C & 4.4052820 & -1.7078650 & 2.2114030 \\
\hline 0 & 1.7300830 & -2.1394350 & -2.6835280 & $\mathrm{H}$ & 5.2879050 & 0.2653150 & 2.2935380 \\
\hline $\mathrm{H}$ & 1.0344990 & -1.4092730 & -2.7013890 & C & 5.0368890 & -3.5232620 & 3.6929450 \\
\hline C & 3.2935980 & -4.6032900 & -2.2096440 & C & 4.0439290 & -4.3371970 & 3.1026350 \\
\hline C & 3.9794260 & -4.0506220 & -0.9582140 & $\mathrm{C}$ & 3.2659890 & -3.8607770 & 2.0781620 \\
\hline C & 3.6025590 & -2.5886860 & -0.7211050 & C & 5.2061720 & -2.2353260 & 3.2576530 \\
\hline C & 1.7702990 & -4.5167830 & -2.0745590 & $\mathrm{H}$ & 5.6506160 & -3.9154700 & 4.4981360 \\
\hline $\mathrm{H}$ & 3.5853550 & -5.6455480 & -2.3718070 & $\mathrm{H}$ & 3.8905750 & -5.3480410 & 3.4684870 \\
\hline $\mathrm{H}$ & 5.0673470 & -4.1322180 & -1.0454270 & $\mathrm{H}$ & 2.5019300 & -4.4967620 & 1.6458210 \\
\hline $\mathrm{H}$ & 4.0645910 & -1.9267490 & -1.4637810 & $\mathrm{H}$ & 5.9488360 & -1.5886240 & 3.7178120 \\
\hline $\mathrm{H}$ & 1.4276640 & -5.1855520 & -1.2756510 & C & 3.6610740 & -5.6777920 & -1.6685460 \\
\hline $\mathrm{H}$ & 1.2703100 & -4.8259550 & -2.9978820 & C & 2.6305740 & -6.4864920 & -2.1990910 \\
\hline $\mathrm{H}$ & 3.6176360 & -4.0370080 & -3.0913830 & C & 1.3258950 & -6.0772660 & -2.1052900 \\
\hline $\mathrm{H}$ & 3.6762930 & -4.6390380 & -0.0841730 & $\mathrm{C}$ & 3.3663150 & -4.4957310 & -1.0383860 \\
\hline $\mathrm{H}$ & 3.9431220 & -2.2188360 & 0.2491990 & $\mathrm{H}$ & 4.6957920 & -5.9920380 & -1.7669790 \\
\hline C & 1.1359050 & -2.6726650 & 2.0603570 & $\mathrm{H}$ & 2.8764420 & -7.4229120 & -2.6904860 \\
\hline 0 & 0.0778330 & -1.9137510 & 1.9899570 & $\mathrm{H}$ & 0.5258060 & -6.6811850 & -2.5255870 \\
\hline C & 1.6826750 & -2.8331980 & 3.4334130 & $\mathrm{H}$ & 4.1656800 & -3.8784030 & -0.6430490 \\
\hline C & 2.8782710 & -3.5344480 & 3.6029550 & C & -2.0929290 & -2.6885020 & -0.7936350 \\
\hline C & 1.0431450 & -2.2606690 & 4.5359260 & $\mathrm{C}$ & -2.7428050 & -2.1403490 & -1.9169240 \\
\hline c & 3.43 & -3.6644740 & 4.8696490 & $\mathrm{C}$ & -2.7600890 & -2.7634460 & 0.4397240 \\
\hline $\mathrm{H}$ & 3.36 & -3.9686420 & 2.7341110 & $\mathrm{C}$ & -4.0619890 & -1.7139600 & -1.7785520 \\
\hline C & 1.6006210 & -2.3948890 & 5.8021190 & $\mathrm{C}$ & -4.0772980 & -2.3072950 & 0.5312900 \\
\hline $\mathrm{H}$ & 0.1186900 & -1.7124230 & 4.3934860 & C & -4.7516080 & -1.7881720 & -0.5679120 \\
\hline C & 2.7944640 & -3.0940440 & 5.9692650 & $\mathrm{H}$ & -4.5809520 & -1.3120530 & -2.6461220 \\
\hline $\mathrm{H}$ & 4.3649680 & -4.2059270 & 5.0010100 & $\mathrm{H}$ & -4.5865830 & -2.3794590 & 1.4877490 \\
\hline $\mathrm{H}$ & 1.1057840 & -1.9501490 & 6.6597340 & C & 3.7814740 & 1.6408880 & 0.4732370 \\
\hline $\mathrm{H}$ & 3.2295880 & -3.1943510 & 6.9591480 & $\mathrm{C}$ & 3.1188640 & 2.6131790 & 1.2512420 \\
\hline $\mathrm{H}$ & -0.2759780 & -1.7869430 & 1.0417830 & C & 4.4656250 & 2.0121610 & -0.6945430 \\
\hline & 1.6816730 & -3.2172380 & 1.0934080 & $\mathrm{C}$ & 3.1673890 & 3.9419940 & 0.8372560 \\
\hline & & & & C & 4.4837570 & 3.3574970 & -1.0669050 \\
\hline & S) $\mathrm{G}[\mathrm{WB} 97 \mathrm{X}$ & $G(d)]=-3$ & & C & 3.8370840 & 4.3349830 & -0.3210530 \\
\hline 0 & 0.0147670 & -1.1931290 & 0.2175550 & $\mathrm{H}$ & 2.6492410 & 4.6941980 & 1.4253260 \\
\hline 0 & 2.0517840 & -0.1235250 & -0.8161150 & $\mathrm{H}$ & 5.0056310 & 3.6501310 & -1.9760920 \\
\hline$C$ & 0.3649220 & -2.4044150 & -0.3333240 & C & -2.1085270 & -3.3746470 & 1.6723640 \\
\hline $\mathrm{C}$ & 1.6 & -2.8277110 & -0.2518090 & $\mathrm{C}$ & -6.1880 & -1.2895060 & -0.5039950 \\
\hline
\end{tabular}




\begin{tabular}{|c|c|c|}
\hline $\begin{array}{l}-2.0550630 \\
2.3709730 \\
3.8128360 \\
5.1721780 \\
6.6784820 \\
6.8805470 \\
7.1305700 \\
7.1794180 \\
4.5362140 \\
3.4677930 \\
4.6550120 \\
5.0124140 \\
4.3044950 \\
4.3491650 \\
3.6318690 \\
5.3032880 \\
2.4093020 \\
2.0827140 \\
1.6790930 \\
2.3950690 \\
3.3016110 \\
4.1516800 \\
3.6954970 \\
2.7576900 \\
1.1238590 \\
1.3837070 \\
0.5054770 \\
0.5231390 \\
-2.7419790 \\
-3.8094940 \\
-2.6456590 \\
-2.2558820 \\
-2.1530230 \\
-1.7312010 \\
-3.1794090 \\
-1.5744230 \\
-6.2268830 \\
-7.2596790 \\
-5.7056700 \\
-5.7446320 \\
-6.9656880 \\
-6.9193780 \\
-6.5756610 \\
-8.0181040 \\
-2.3904850 \\
-3.4255480\end{array}$ & $\begin{array}{r}-2.0420370 \\
2.2457490 \\
5.7786170 \\
0.9930620 \\
1.2727820 \\
2.2375390 \\
1.2920750 \\
0.4961090 \\
0.9258750 \\
0.7045500 \\
1.8749430 \\
0.1412000 \\
6.7563120 \\
7.7748490 \\
6.7703370 \\
6.4825030 \\
6.1586790 \\
5.4792290 \\
6.0972910 \\
7.1819550 \\
2.3241310 \\
1.6427920 \\
3.3410040 \\
2.0624370 \\
3.1040440 \\
4.1195300 \\
3.1683860 \\
2.6549200 \\
-4.7352980 \\
-4.6276650 \\
-5.4212520 \\
-5.1932620 \\
-2.4372990 \\
-1.4593490 \\
-2.2938440 \\
-2.8616110 \\
0.2438590 \\
0.6104460 \\
0.6820300 \\
0.6022590 \\
-1.7735640 \\
-2.8627230 \\
-1.3221160 \\
-1.4834230 \\
-0.7502200 \\
-0.7419980\end{array}$ & $\begin{array}{r}-3.2715590 \\
2.5270500 \\
-0.7896460 \\
-1.5766950 \\
-1.6598340 \\
-2.1392960 \\
-0.6625100 \\
-2.2486940 \\
-2.9715740 \\
-2.8971420 \\
-3.5075060 \\
-3.5703660 \\
0.2842210 \\
-0.1172900 \\
1.1494490 \\
0.6389940 \\
-1.2834530 \\
-2.0779790 \\
-0.4676470 \\
-1.6758430 \\
3.7469290 \\
3.6539470 \\
3.8642040 \\
4.6619160 \\
2.7746720 \\
3.0975290 \\
1.8768100 \\
3.5731820 \\
1.9930000 \\
2.2190470 \\
1.1445580 \\
2.8619330 \\
2.8840090 \\
2.6330510 \\
3.2436850 \\
3.7118560 \\
-0.5851470 \\
-0.5782410 \\
0.2730030 \\
-1.5009140 \\
0.7209020 \\
0.8249420 \\
1.6402760 \\
0.6345180 \\
-4.0253760 \\
-4.3874880\end{array}$ \\
\hline
\end{tabular}

-2.2327550
-1.7394620
-2.3974060
-3.4778760
-1.8924520
-2.0921320
0.4689070
-0.1412440
0.2350580
-0.9757460
-6.6973570
-1.0533860
5.0555630
4.4970520
2.0289900
-2.8049050
-1.9060860
-4.1105560
-5.1120220
-4.3670530
-6.3667490
-4.8948100
-5.6249410
-3.5855950
-6.6243800
-7.1448600
-5.8284100
-7.6067630
-1.0442590
-2.6276180
-1.0682480
-2.2788060
-0.2206990
-2.1292270
-1.0674310
-0.5923990
-2.5317860
-3.7497730
-3.6145140
-1.2500380
-2.6577560
-4.6678920
-3.7492030
-1.2766620
-0.3653910
-2.4432210

\author{
0.1258200 \\ $-0.6545020$ \\ $-3.2601390$ \\ $-3.3128140$ \\ - 3.1930100 \\ $-4.1961730$ \\ 0.1605520 \\ 0.2607380 \\ 1. 2673440 \\ $-2.0353990$ \\ $-1.6798220$ \\ $-3.5564510$ \\ 0.0058730 \\ 5.8552120 \\ 1. 2119260 \\ 2. 0430010 \\ 1. 1488620 \\ 1.8635310 \\ 2. 8165860 \\ 0.7284390 \\ 2.6346170 \\ 3. 6900050 \\ 0.5481530 \\ $-0.0088200$ \\ 1. 4982590 \\ 3. 3750720 \\ $-0.3367570$ \\ 1. 3519920 \\ 1. 2564800 \\ 2. 9700140 \\ 3. 2705890 \\ 2. 4854870 \\ 3. 0198140 \\ 1. 4444180 \\ 2. 5261990 \\ 1.6597620 \\ 5. 0719300 \\ 4.4430880 \\ 2. 9219510 \\ 4.7517890 \\ 6.1570000 \\ 4.6880420 \\ 2.4490960 \\ 5. 2296220 \\ 5.1304520 \\ 4. 6945140
}

$-3.3926490$ $-4.9007660$ $-4.1425230$ $-4.3234520$ $-5.1128730$ $-3.6660170$ $-0.5738690$ $-1.9403360$ 0.4187370 $-3.0949520$ $-1.3958860$ 1.4531810 $-1.1201710$

$-1.6447060$

2. 4210290

1. 5166240

1. 8099020

2. 1981150

1. 9962460

2.9710480

2. 5640350

1.3903070

3. 5334470

3. 1115770

3. 3284580

2. 4083200

4.1286920

3.7671850

1. 2684940

0.7113380

$-1.5841190$

$-1.3221320$

$-0.9481560$

$-1.0578600$

$-2.8242510$

$-2.6325110$

$-2.5919140$

$-1.9101730$

$-1.8135520$

$-1.8173040$

$-2.6584770$

$-2.4528210$

$-2.7951780$

$-0.8305250$

-2.3385850
-3.6175820 


$\begin{array}{lrcr}\mathrm{H} & -3.8505400 & 4.8513580 & -0.8975740 \\ \mathrm{H} & -4.3728890 & 2.4831710 & -1.1591670 \\ \mathrm{I} 44 & & & \\ \mathrm{TS} 138(\mathrm{~S}, \mathrm{~S}) \mathrm{G}[\mathrm{WB} 97 \mathrm{XD} / 6-31 \mathrm{G}(\mathrm{d})]=-3310.751155 \\ \mathrm{O} & 0.2563980 & 0.8227720 & 0.0614360 \\ \mathrm{O} & -2.0317580 & 0.3866580 & -0.9289390 \\ \mathrm{C} & 0.2443640 & 2.1039140 & -0.4403070 \\ \mathrm{C} & -0.8970520 & 2.8653210 & -0.3165110 \\ \mathrm{C} & 1.4606330 & 2.6002680 & -0.9810820 \\ \mathrm{C} & -0.9131550 & 4.1625460 & -0.9265540 \\ \mathrm{C} & 1.4668410 & 3.8836280 & -1.4608450 \\ \mathrm{C} & 0.2884580 & 4.6736520 & -1.4896670 \\ \mathrm{H} & 2.3919590 & 4.3106840 & -1.8376260 \\ \mathrm{C} & -2.5745290 & 1.0896830 & 0.1248330 \\ \mathrm{C} & -3.6980370 & 0.5178600 & 0.7768130 \\ \mathrm{C} & -2.0563680 & 2.3257070 & 0.4450870 \\ \mathrm{C} & -4.2974800 & 1.2497870 & 1.7679650 \\ \mathrm{C} & -2.6194430 & 3.0289470 & 1.5623570 \\ \mathrm{C} & -3.7712820 & 2.4923490 & 2.2012260 \\ \mathrm{H} & -5.1864330 & 0.8576280 & 2.2544350 \\ \mathrm{C} & -3.8105440 & 4.3579870 & 3.7516890 \\ \mathrm{C} & -2.6398040 & 4.8709150 & 3.1483380 \\ \mathrm{C} & -2.0604560 & 4.2268160 & 2.0846980 \\ \mathrm{C} & -4.3580960 & 3.1909210 & 3.2882090 \\ \mathrm{H} & -4.2621280 & 4.8812820 & 4.5889630 \\ \mathrm{H} & -2.1908540 & 5.7810790 & 3.5345690 \\ \mathrm{H} & -1.1577070 & 4.6298020 & 1.6398440 \\ \mathrm{H} & -5.2448720 & 2.7712380 & 3.7560610 \\ \mathrm{C} & -2.0658760 & 6.1883100 & -1.6092350 \\ \mathrm{C} & -0.8627280 & 6.7089840 & -2.1374130 \\ \mathrm{C} & 0.2864240 & 5.9643040 & -2.0794270 \\ \mathrm{C} & -2.0910210 & 4.9510050 & -1.0178530 \\ \mathrm{H} & -2.9800150 & 6.7702520 & -1.6779170 \\ \mathrm{H} & -0.8556950 & 7.6914180 & -2.5994390 \\ \mathrm{H} & 1.2137240 & 6.3462060 & -2.4987100 \\ \mathrm{H} & -3.0220710 & 4.5575260 & -0.6248360 \\ \mathrm{C} & 2.6953850 & 1.7594730 & -0.9219140 \\ \mathrm{C} & 3.2140920 & 1.1488150 & -2.0753470 \\ \mathrm{C} & 3.3449030 & 1.5986570 & 0.3183410 \\ \mathrm{C} & 4.4049690 & 0.4211980 & -1.9735240 \\ \mathrm{C} & 4.4975500 & 0.8207520 & 0.3770280 \\ \mathrm{C} & 5.0650020 & 0.2453690 & -0.7610380 \\ \mathrm{H} & 4.8215790 & -0.0165320 & -2.8747270 \\ \mathrm{H} & 4.9910610 & 0.6825250 & 1.3368050 \\ \mathrm{C} & -4.2199050 & -0.8248630 & 0.3796100 \\ \mathrm{C} & -3.9447400 & -1.9484930 & 1.1800510\end{array}$

-5.0078790
-4.5051220
-5.5326560
-5.3013070
-4.3097230
-6.1416360
2.8831050
6.3912830
2.5461380
-3.0719770
-5.8953570
-5.3067550
-6.8132770
-7.3512270
-7.2366140
-7.0047180
-4.6981610
-3.6193620
-5.1457530
-4.8738910
-5.3597860
-5.7593010
-5.6522900
-4.2667280
-7.4292480
-7.8126190
-7.8048830
-7.8444710
-3.9305310
-4.5626580
-4.5854590
-3.2944280
-2.1057200
-2.6265810
-1.5683770
-1.3711570
3.8504640
4.8560900
3.9241700
3.5077370
2.7106080
1.9852120
3.6535560
2.3449330
7.4892930
8.4345160
$-0.7780120$

0.8204360

$-1.1014810$

$-0.3106280$

1. 4371860

$-1.9977670$

1. 5670740

$-0.6321840$

$-3.4347440$

2.4255480

$-0.6718600$

$-1.6854010$

$-1.7652790$

$-2.2277060$

$-0.7681010$

$-2.3682220$

$-3.0794880$

$-3.0081030$

$-3.5866020$

-3.7030920

$-2.0162290$

$-2.2367350$

$-2.8348500$

$-2.0066450$

$-0.6705410$

0.2943640

$-1.4444180$

$-0.8683280$

3.6920940

3. 6637240

3.8038550

4.5825640

2. 5632420

2.8427770

1. 6275530

3.3477870

1.8679080

2. 0849410

1. 0141830

2.7369970

2.7809950

2. 5698190

3.0816540

3. 6371910

$-0.1359860$

0.0030410 


$\begin{array}{lrrr}\text { H } & 7.6540810 & 1.2575390 & -0.8692920 \\ \text { H } & 7.2226740 & 0.9287220 & 0.8155530 \\ \mathrm{C} & 6.8621110 & -1.1875000 & -1.9136580 \\ \mathrm{H} & 6.1271010 & -1.8984510 & -2.3032820 \\ \mathrm{H} & 7.0725530 & -0.4542400 & -2.7008450 \\ \mathrm{H} & 7.7887440 & -1.7373800 & -1.7202100 \\ \mathrm{C} & 2.5491350 & 0.0208640 & -4.2655040 \\ \mathrm{H} & 3.5569470 & -0.2492370 & -4.6033790 \\ \mathrm{H} & 2.1358930 & -0.8168430 & -3.7013580 \\ \mathrm{H} & 1.9340460 & 0.1578280 & -5.1609920 \\ \mathrm{C} & 3.2086080 & 2.4417690 & -4.2324410 \\ \mathrm{H} & 4.2666200 & 2.2164020 & -4.4135230 \\ \mathrm{H} & 2.7165730 & 2.5663070 & -5.2036300 \\ \mathrm{H} & 3.1567710 & 3.3968260 & -3.7023940 \\ \mathrm{P} & -0.5846140 & -0.3308040 & -0.7421810 \\ \mathrm{O} & -0.0517190 & -0.5478290 & -2.1278120 \\ \mathrm{O} & -0.6311230 & -1.4863100 & 0.2215810 \\ \mathrm{H} & 1.4984030 & 1.5710300 & -3.2604160 \\ \mathrm{H} & 6.2576310 & -1.2695890 & 0.1388890 \\ \mathrm{H} & 1.9063430 & 2.7855000 & 1.3649500 \\ \mathrm{H} & -4.8400680 & 1.1230640 & -1.2531090 \\ \mathrm{H} & -5.5818160 & -5.3859340 & 0.1020720 \\ \mathrm{H} & -2.4582770 & -0.9565360 & 2.3354690 \\ \mathrm{C} & 2.3016320 & -2.1962190 & 1.8467580 \\ \mathrm{O} & 1.1257360 & -1.6874860 & 2.0903660 \\ \mathrm{C} & 3.2747630 & -2.0494070 & 2.9612380 \\ \mathrm{C} & 4.6276970 & -2.2660000 & 2.6943980 \\ \mathrm{C} & 2.8668160 & -1.6531630 & 4.2364760 \\ \mathrm{C} & 5.5734660 & -2.0685540 & 3.6926960 \\ \mathrm{H} & 4.9236860 & -2.5790700 & 1.6985710 \\ \mathrm{C} & 3.8135730 & -1.4704930 & 5.2373820 \\ \mathrm{H} & 1.8149750 & -1.4766310 & 4.4317040 \\ \mathrm{C} & 5.1656090 & -1.6706090 & 4.9648720 \\ \mathrm{H} & 6.6266750 & -2.2251120 & 3.4815060 \\ \mathrm{H} & 3.4978830 & -1.1629780 & 6.2293260 \\ \mathrm{H} & 5.9040360 & -1.5164740 & 5.7460230 \\ \mathrm{H} & 0.4841200 & -1.6810000 & 1.3015060 \\ \mathrm{O} & 2.6389840 & -2.7541390 & 0.7981620 \\ \mathrm{C} & 1.0585670 & -3.4385050 & -1.4546850 \\ \mathrm{C} & 2.2054090 & -2.5436860 & -1.2830090 \\ \mathrm{H} & 0.1800250 & -3.1799500 & -0.8663330 \\ \mathrm{H} & 2.0047520 & -1.4915650 & -1.1145520 \\ \mathrm{O} & 1.0336360 & -2.8191980 & -2.7633520 \\ \mathrm{H} & 0.4937010 & -1.9729930 & -2.6615570 \\ \mathrm{C} & 2.6554150 & -5.2336700 & -2.2557500 \\ \mathrm{C} & 3.8136050 & -4.4533900 & -1.6298880 \\ & & & \\ & & & \end{array}$

$\begin{array}{ll}\mathrm{C} & 3.5746270 \\ \mathrm{C} & 1.3413180 \\ \mathrm{H} & 2.8521120 \\ \mathrm{H} & 4.7579190 \\ \mathrm{H} & 3.7118330 \\ \mathrm{H} & 1.3821150 \\ \mathrm{H} & 0.4916660 \\ \mathrm{H} & 2.5621650 \\ \mathrm{H} & 3.9206360 \\ \mathrm{H} & 4.2711400 \\ \mathrm{H} & \end{array}$
$-2.9446720$
$-4.9199340$
$-6.3088540$
$-4.6912080$
$-2.5621440$
$-5.2929930$
$-5.4062690$
$-4.9778910$
$-4.7398240$
$-2.3852680$
$-1.7014030$
$-1.5346760$
$-2.2000510$
$-2.1295200$
$-2.7207180$
$-0.5039430$
$-2.0244080$
$-3.3177870$
$-0.5770640$
$-1.0732980$

144

$\operatorname{TS} 139(\mathrm{~S}, \mathrm{~S}) \mathrm{G}[\mathrm{WB} 97 \mathrm{XD} / 6-31 \mathrm{G}(\mathrm{d})]=-3310.750794$

\begin{tabular}{|c|c|c|c|}
\hline & & & \\
\hline 0 & 1.3864500 & -0.5273800 & -0.6930390 \\
\hline 0 & -0.2241430 & -1.6312200 & 0.8969260 \\
\hline C & 2.3635600 & -1.4064590 & -0.2842670 \\
\hline $\mathrm{C}$ & 2.0720420 & -2.7525230 & -0.2311240 \\
\hline $\mathrm{C}$ & 3.6471890 & -0.8721030 & 0.0014510 \\
\hline $\mathrm{C}$ & 3.0916450 & -3.6528040 & 0.2227740 \\
\hline $\mathrm{C}$ & 4.6468850 & -1.7579450 & 0.3120030 \\
\hline $\mathrm{C}$ & 4.3999770 & -3.1457940 & 0.4543980 \\
\hline $\mathrm{H}$ & 5.6481910 & -1.3815800 & 0.5032980 \\
\hline C & -0.3980600 & -2.6497860 & -0.0189320 \\
\hline $\mathrm{C}$ & -1.7250010 & -3.1001960 & -0.2524070 \\
\hline $\mathrm{C}$ & 0.7110740 & -3.2230380 & -0.6048730 \\
\hline C & -1.8925430 & -4.1629440 & -1.1034490 \\
\hline $\mathrm{C}$ & 0.5082360 & -4.2438950 & -1.5911020 \\
\hline C & -0.8068980 & -4.7370090 & -1.8099060 \\
\hline $\mathrm{H}$ & -2.8931430 & -4.5500300 & -1.2778540 \\
\hline C & 0.0266670 & -6.2710260 & -3.4952080 \\
\hline C & 1.3281630 & -5.7499760 & -3.3127620 \\
\hline C & 1.5633380 & -4.7660360 & -2.3870960 \\
\hline C & -1.0162810 & -5.7689490 & -2.7623190 \\
\hline $\mathrm{H}$ & -0.1444800 & -7.0544330 & -4.2272280 \\
\hline $\mathrm{H}$ & 2.1480700 & -6.1281920 & -3.9158900 \\
\hline $\mathrm{H}$ & 2.5645480 & -4.3678900 & -2.2662390 \\
\hline H & -2.0260730 & -6.1435010 & -2.9089970 \\
\hline C & 3.8598380 & -5.8578600 & 0.8955200 \\
\hline C & 5.1697520 & -5.3596000 & 1.0818400 \\
\hline C & 5.4287970 & -4.0312270 & 0.8705280 \\
\hline $\mathrm{C}$ & 2.8494970 & -5.0295910 & 0.4783800 \\
\hline $\mathrm{H}$ & 3.6511590 & -6.9055860 & 1.0896390 \\
\hline $\mathrm{H}$ & 5.9610450 & -6.0284590 & 1.4062910 \\
\hline $\mathrm{H}$ & 6.4251020 & -3.6281010 & 1.0330810 \\
\hline $\mathrm{H}$ & 1.8487400 & -5.4258960 & 0.3486080 \\
\hline C & 3.8881020 & 0.6019160 & 0.0151070 \\
\hline 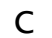 & 4.1448990 & 1.2468880 & 1.2402870 \\
\hline
\end{tabular}




$\begin{array}{rrrr}\text { C } & 3.8860730 & 1.3427180 & -1.1820330 \\ \text { C } & 4.4367520 & 2.6125840 & 1.2359150 \\ \text { C } & 4.1652210 & 2.7059280 & -1.1282340 \\ \text { C } & 4.4463240 & 3.3637770 & 0.0680330 \\ \text { H } & 4.6374930 & 3.0990750 & 2.1847470 \\ \text { H } & 4.1736760 & 3.2793960 & -2.0545560 \\ \text { C } & -2.8964100 & -2.4365060 & 0.3953130 \\ \text { C } & -3.8269990 & -1.7269840 & -0.3924890 \\ \text { C } & -3.0947940 & -2.5564820 & 1.7828140 \\ \text { C } & -4.9459320 & -1.1710010 & 0.2289220 \\ \text { C } & -4.2298530 & -1.9820880 & 2.3534300 \\ \text { C } & -5.1733760 & -1.2949430 & 1.5974080 \\ \text { H } & -5.6734470 & -0.6423520 & -0.3836740 \\ \text { H } & -4.3962220 & -2.0907720 & 3.4233450 \\ \text { P } & 0.1805140 & -0.1570710 & 0.3439140 \\ \text { O } & 0.6776180 & 0.6267960 & 1.5237330 \\ \text { O } & -0.8808770 & 0.4396830 & -0.5429360 \\ \text { C } & 3.6245870 & 0.7012130 & -2.5371800 \\ \text { H } & 3.4028150 & -0.3579090 & -2.3815990 \\ \text { C } & 4.7550770 & 4.8525040 & 0.0429830 \\ \text { H } & 4.0099820 & 5.3204400 & -0.6179720 \\ \text { C } & 4.1189690 & 0.5173350 & 2.5789240 \\ \text { H } & 3.6980110 & -0.4782880 & 2.4181490 \\ \text { C } & -2.1465140 & -3.3394890 & 2.6803770 \\ \text { H } & -1.3058090 & -3.6904550 & 2.0766660 \\ \text { C } & -6.4426190 & -0.7722960 & 2.2473800 \\ \text { H } & -6.3112460 & -0.8635150 & 3.3332490 \\ \text { C } & -3.6962590 & -1.5916430 & -1.9060790 \\ \text { H } & -2.7314270 & -2.0065830 & -2.2063780 \\ \text { C } & -0.0150200 & 3.6047160 & 0.9237460 \\ \text { C } & 0.2121890 & 3.3117910 & -0.4929690 \\ \text { H } & -0.7193220 & 2.9469250 & 1.4333140 \\ \text { H } & 0.1039110 & 2.2815750 & -0.8094630 \\ \text { O } & 1.3351880 & 3.1050120 & 1.0078020 \\ \text { H } & 1.2609160 & 2.1217330 & 1.2214710 \\ \text { C } & 0.7918770 & 5.9645710 & 0.4998590 \\ \text { C } & 0.5756300 & 5.7492260 & -0.9996530 \\ \text { C } & 0.8556030 & 4.2990100 & -1.3985790 \\ \text { C } & -0.1240570 & 5.0565710 & 1.3268980 \\ \text { H } & 0.5996370 & 7.0095220 & 0.7624570 \\ \text { H } & 1.2218240 & 6.4143040 & -1.5809000 \\ \text { H } & 1.9302230 & 4.0763180 & -1.3663800 \\ \text { H } & -1.1711300 & 5.3507790 & 1.1822880 \\ \text { H } & 0.0938960 & 5.1400080 & 2.3963110 \\ \text { H } & 1.8380560 & 5.7578080 & 0.7522840 \\ \text { H } & -0.4612180 & 5.9926930 & -1.2593940 \\ & & & \\ & & & \end{array}$
0.5261530
$-3.7091320$
$-3.6562200$
$-4.6193980$
$-2.8452270$
$-4.7907520$
$-5.7862370$
$-4.6494560$
$-4.7795520$
2.4073330
2.5650560
2. 2193150
1. 5114000
4.8737300
4.6965760
5.1445410
5.7310600
$-1.5666070$
$-2.3488280$
$-0.8359630$
$-1.0644820$
$-2.8361100$
$-2.1315380$
$-3.6813410$
$-3.2158320$
3.2171720
3. 1824890
3. 5879160
2. 1986710
5.5394440
6.0081840
5.5162830
6.1805040
$-6.7136130$
$-5.8559300$
$-6.9272700$
$-7.5859540$
$-7.6457650$
$-7.4732660$
$-8.5544950$
$-7.8241000$
6.1399440
6.2200580
6.3403170
6.9201960
4.6508490

\begin{tabular}{rr}
4.0793080 & -2.4175490 \\
-0.1338190 & -2.3808630 \\
-0.0985360 & -3.4751350 \\
0.3947180 & -2.0747280 \\
0.3973660 & -1.9799670 \\
-2.4053970 & -2.6128220 \\
-2.0024170 & -2.3917250 \\
-2.3748520 & -3.6990870 \\
-3.4537290 & -2.2954090 \\
1.3223500 & -3.2336270 \\
2.3873890 & -3.4421690 \\
0.8221240 & -4.1900360 \\
1.2141020 & -2.6153690 \\
0.7708760 & -3.4267430 \\
0.2559670 & -4.3775480 \\
1.8089090 & -3.6527130 \\
0.2992980 & -2.9350630 \\
-2.4630560 & 3.7974600 \\
-2.1206810 & 4.4851070 \\
-3.0320660 & 4.3833130 \\
-1.5859550 & 3.3805480 \\
-4.5879930 & 3.2479410 \\
-5.1751550 & 3.8477650 \\
-4.3192880 & 3.8922040 \\
-5.2262690 & 2.4428930 \\
1.2123930 & 3.6079330 \\
0.6190610 & 4.5286240 \\
2.2090670 & 3.8736010 \\
1.3047800 & 3.2249370 \\
0.3445920 & 3.1351850 \\
1.3188960 & 3.3185180 \\
-0.2028490 & 4.0841060 \\
-0.2071600 & 2.4398530 \\
0.7029630 & 1.9367750 \\
1.3295620 & 2.2015640 \\
0.8462390 & 0.8707780 \\
1.0590740 & 2.4958890 \\
-1.6395740 & 1.8487890 \\
-2.6909670 & 2.1000670 \\
-1.3051310 & 2.3621390 \\
-1.5776660 & 0.7686950 \\
5.1028720 & -0.5740600 \\
4.6605840 & -1.5722030 \\
6.1769410 & -0.6580170 \\
4.6569800 & 0.0535760 \\
5.5334880 & 1.4089400 \\
& \\
\hline
\end{tabular}




\begin{tabular}{|c|c|c|c|c|c|c|c|}
\hline $\mathrm{H}$ & 3.6964970 & 53151930 & 18993000 & $c$ & 03520730 & 6.0484970 & -1.8754340 \\
\hline $\mathrm{H}$ & 5.4549560 & 5.2071170 & $\begin{array}{l}1.0787210 \\
2.07870\end{array}$ & c & -2.0539950 & 5.0132130 & $\begin{array}{l}1.0 \\
-0.9028020\end{array}$ \\
\hline $\mathrm{H}$ & 4.7400430 & 6.6188630 & 1.2952500 & $\mathrm{H}$ & -2.9163460 & 6.8637140 & -1.5093610 \\
\hline C & -2.8133060 & 3.1304940 & -0.7146180 & $\mathrm{H}$ & -0.7674460 & 7.8021290 & -2.3535700 \\
\hline 0 & -2.8097970 & 2.0353170 & 0.0026240 & $\mathrm{H}$ & 1.2903960 & 6.4392520 & -2.2607400 \\
\hline $\mathrm{C}$ & -4.1481130 & 3.7710000 & -0.8295960 & $\mathrm{H}$ & -2.9951910 & 4.6119530 & -0.5428610 \\
\hline $\mathrm{C}$ & -4.2530610 & 5.0443010 & -1.3928420 & C & 2.7132980 & 1.7941980 & -0.8084930 \\
\hline $\mathrm{C}$ & -5.2919650 & 3.0996300 & -0.3926740 & $\mathrm{C}$ & 3.2750010 & 1.2334520 & -1.9670410 \\
\hline C & -5.4993710 & 5.6471620 & -1.5102040 & $\mathrm{C}$ & 3.3198600 & 1.5854390 & 0.4464710 \\
\hline $\mathrm{H}$ & -3.3546980 & 5.5471160 & -1.7354470 & $\mathrm{C}$ & 4.4655840 & 0.5068190 & -1.8535650 \\
\hline C & -6.5367200 & 3.7051630 & -0.5118840 & $\mathrm{c}$ & 4.4748320 & 0.8118390 & 0.5130980 \\
\hline $\mathrm{H}$ & -5.1918830 & 2.1082930 & 0.0334070 & $\mathrm{C}$ & 5.0842920 & 0.2856680 & -0.6268990 \\
\hline C & -6.6405450 & 4.9783320 & -1.0691310 & $\mathrm{H}$ & 4.9159460 & 0.1065400 & -2.7561240 \\
\hline $\mathrm{H}$ & -5.5840810 & 6.6370320 & -1.9474980 & $\mathrm{H}$ & 4.9380760 & 0.6384500 & 1.4823660 \\
\hline $\mathrm{H}$ & -7.4262120 & 3.1845990 & -0.1702040 & C & -4.2606790 & -0.7854880 & 0.2058540 \\
\hline $\mathrm{H}$ & -7.6137470 & 5.4507760 & -1.1632540 & $\mathrm{C}$ & -4.0084600 & -1.9519570 & 0.9553000 \\
\hline $\mathrm{H}$ & -1.9877070 & 1.4559430 & -0.1410130 & $\mathrm{C}$ & -5.0175000 & -0.8496400 & -0.9742540 \\
\hline 0 & -1.8172960 & 3.6241030 & -1.2525640 & C & -4.5592630 & -3.1549430 & 0.5198630 \\
\hline & & & & $\mathrm{C}$ & -5.5351100 & -2.0820360 & -1.3761500 \\
\hline & s) $\mathrm{G}[\mathrm{WB} 97 \mathrm{XD}$ & (d) $]=-33$ & 176 & $\mathrm{C}$ & -5.3241050 & -3.2433080 & -0.6424280 \\
\hline 0 & 0.2381740 & 0.8244130 & 0.0534990 & $\mathrm{H}$ & -4.3762100 & -4.0563280 & 1.0990890 \\
\hline 0 & -2.0349420 & 0.4539530 & -1.0000820 & $\mathrm{H}$ & -6.1207250 & -2.1387710 & -2.2917680 \\
\hline $\mathrm{C}$ & 0.2481330 & 2.1267360 & -0.3906590 & C & 2.8076500 & 2.2644380 & 1.7102150 \\
\hline C & -0.8900090 & 2.8920590 & -0.2613110 & C & 6.4120980 & -0.4487870 & -0.4808400 \\
\hline C & 1.4811830 & 2.6379630 & -0.8764920 & $\mathrm{C}$ & 2.6524330 & 1.4471480 & -3.3405690 \\
\hline C & -0.8836560 & 4.2128040 & -0.8185070 & $\mathrm{c}$ & -3.1656220 & -1.9278880 & 2.2244190 \\
\hline $\mathrm{C}$ & 1.5061590 & 3.9384920 & -1.3071730 & $\mathrm{C}$ & -5.9064700 & -4.5693620 & -1.0974800 \\
\hline C & 0.3331540 & 4.7364030 & -1.3353490 & C & -5.2915710 & 0.3802540 & -1.8275890 \\
\hline $\mathrm{H}$ & 2.4431720 & 4.3734530 & -1.6434600 & $\mathrm{C}$ & -6.7934210 & 0.6845010 & -1.9051420 \\
\hline C & -2.5 & 1.1170560 & 0.0717200 & $\mathrm{H}$ & -7.3398760 & -0.1231330 & -2.4058580 \\
\hline C & -3 & 0.5312820 & 0.6773180 & $\mathrm{H}$ & -7.2210600 & 0.8099140 & -0.9045960 \\
\hline C & -2.0687430 & 2.3324610 & 0.4540530 & $\mathrm{H}$ & -6.9677900 & 1.6070270 & -2.4702990 \\
\hline $\mathrm{C}$ & -4.3446230 & 1.2258550 & 1.6890030 & C & -4.6738280 & 0.2376660 & -3.2247800 \\
\hline $\mathrm{C}$ & -2.6451050 & 2.9934140 & 1.5897630 & $\mathrm{H}$ & -3.5985540 & 0.0521010 & -3.1521240 \\
\hline C & -3.8 & 2.4428970 & 1910 & $\mathrm{H}$ & -5.1 & -0.5902290 & -3.7794540 \\
\hline $\mathrm{H}$ & -5.2469410 & 0.8236430 & 2.1414620 & $\mathrm{H}$ & -4.8283420 & 1.1551980 & -3.8038340 \\
\hline C & -3.8602490 & 4.2432690 & 3.8113190 & C & -6.9409630 & -5.0984130 & -0.0948850 \\
\hline C & -2.6737550 & 4.7680360 & 3.2503390 & $\mathrm{H}$ & -7.3967290 & -6.0253850 & -0.4608410 \\
\hline C & -2.0825200 & 4.1619270 & 2.1711600 & $\mathrm{H}$ & -6.4735190 & -5.3123920 & 0.8733470 \\
\hline C & -4.4117380 & 3.1023160 & 3.2904040 & $\mathrm{H}$ & -7.7364480 & -4.3647880 & 0.0716310 \\
\hline $\mathrm{H}$ & -4.32 & 4.7365090 & 4.6616540 & C & -4.8103830 & -5.6113590 & -1.3570090 \\
\hline $\mathrm{H}$ & 5070 & 5.6565550 & 3.6816180 & $\mathrm{H}$ & -4.0780480 & -5.2394480 & -2.0807980 \\
\hline $\mathrm{H}$ & -1.1668900 & 4.5719810 & 1.7604870 & $\mathrm{H}$ & -4.2747200 & -5.8585800 & -0.4331950 \\
\hline $\mathrm{H}$ & -5.31 & 2.6736960 & 3.7256780 & $\mathrm{H}$ & -5.2447240 & -6.5376110 & -1.7499210 \\
\hline C & -2.0 & 6.2721680 & -1.4451610 & C & -4.0551560 & -1.8478120 & 3.4735100 \\
\hline C & -0.7907810 & 6.8031880 & -1.9288830 & $\mathrm{H}$ & -4.6753720 & -0.9464270 & 3.4715240 \\
\hline
\end{tabular}




\begin{tabular}{rrr}
-4.7237250 & -2.7157470 & 3.5247910 \\
-3.4426240 & -1.8356970 & 4.3825410 \\
-2.2158030 & -3.1278670 & 2.3293970 \\
-2.7523360 & -4.0566460 & 2.5570520 \\
-1.6613980 & -3.2594350 & 1.3978950 \\
-1.4948860 & -2.9601850 & 3.1371040 \\
3.7611750 & 3.3995770 & 2.1111490 \\
4.7567630 & 3.0082400 & 2.3516080 \\
3.8714170 & 4.1254860 & 1.2982250 \\
3.3804890 & 3.9262900 & 2.9933700 \\
2.5835090 & 1.2882840 & 2.8665140 \\
1.8660730 & 0.5139870 & 2.5837400 \\
3.5125510 & 0.7990010 & 3.1802680 \\
2.1834410 & 1.8217270 & 3.7361700 \\
7.4914060 & 0.5107920 & 0.0482140 \\
8.4378400 & -0.0190340 & 0.2035160 \\
7.6656410 & 1.3167030 & -0.6739300 \\
7.1981780 & 0.9685810 & 0.9974740 \\
6.9130350 & -1.1227740 & -1.7600960 \\
6.1865610 & -1.8277980 & -2.1758700 \\
7.1424020 & -0.3796870 & -2.5325620 \\
7.8348050 & -1.6757570 & -1.5525960 \\
2.6614870 & 0.1873140 & -4.2124090 \\
3.6765130 & -0.0996180 & -4.5126010 \\
2.2000000 & -0.6558010 & -3.6960750 \\
2.0927580 & 0.3691570 & -5.1303670 \\
3.3540130 & 2.5966150 & -4.0792770 \\
4.4120990 & 2.3594710 & -4.2440970 \\
2.8874370 & 2.7657940 & -5.0562540 \\
3.3059830 & 3.5321930 & -3.5148390 \\
-0.5991550 & -0.2855370 & -0.8139250 \\
-0.0407360 & -0.4509960 & -2.1967220 \\
-0.6756390 & -1.4793040 & 0.1004030 \\
1.6040070 & 1.7205190 & -3.1894610 \\
6.2682420 & -1.2311680 & 0.2792140 \\
1.8404460 & 2.7236680 & 1.4910140 \\
-4.8160040 & 1.2422320 & -1.3506670 \\
-6.4250590 & -4.3897090 & -2.0481300 \\
-2.5398050 & -1.0309540 & 2.1913490 \\
2.2099040 & -2.2768970 & 1.7687630 \\
1.0261210 & -1.7834420 & 2.0056790 \\
3.1537400 & -2.1834560 & 2.9138050 \\
4.5133510 & -2.3823020 & 2.6677640 \\
2.7148530 & -1.8525000 & 3.1972570 \\
5.4354260 & -2.2329420 & \\
4.8325230 & -2.6423370 & \\
\hline & & \\
2.6638690 &
\end{tabular}

3.6380470
1.6584030
4.9967510
6.4937540
3.2985750
5.7165480
0.4078690
2.5770940
1.0590530
2.1985100
0.1653770
1.9910290
1.0646980
0.5200180
2.6798750
3.8214870
3.5795770
1.3489960
2.8788830
4.7773650
3.7432780
1.3692280
0.5122640
2.6093280
3.9059030
4.2569670

$-1.7180430$

$-1.6876220$

$-1.9008430$

$-2.3757620$

$-1.4621160$

$-1.7843780$

$-1.7338940$

$-2.7816340$

$-3.3670120$

$-2.4781130$

$-3.1358710$

$-1.4342100$

$-2.6921050$

$-1.8517970$

$-5.1187250$

$-4.3668330$

$-2.8568910$

$-4.8426840$

$-6.1949270$

$-4.5788850$

$-2.4256140$

$-5.2610530$

$-5.3098350$

$-4.8115520$

$-4.7038340$

$-2.3261760$

144

TS141(s, S) G[WB97XD/6-31G(d)] $=-3310.753018$

$\begin{array}{lrcr}\text { O } & 0.1748810 & 0.9429980 & 0.0680440 \\ \mathrm{O} & -2.0743790 & 0.2989940 & -0.9060530 \\ \mathrm{C} & 0.0204660 & 2.2421800 & -0.3578730 \\ \mathrm{C} & -1.1978610 & 2.8626690 & -0.1963940 \\ \mathrm{C} & 1.1737860 & 2.9038310 & -0.8559230 \\ \mathrm{C} & -1.3632150 & 4.1776800 & -0.7415100 \\ \mathrm{C} & 1.0320830 & 4.1998380 & -1.2776520 \\ \mathrm{C} & -0.2303500 & 4.8480210 & -1.2795100 \\ \mathrm{H} & 1.9035120 & 4.7484500 & -1.6239730 \\ \mathrm{C} & -2.6655770 & 0.8943380 & 0.1873660 \\ \mathrm{C} & -3.7093310 & 0.1806380 & 0.8338840 \\ \mathrm{C} & -2.2778980 & 2.1647430 & 0.5517760 \\ \mathrm{C} & -4.3620450 & 0.8030940 & 1.8659670 \\ \mathrm{C} & -2.8840040 & 2.7545550 & 1.7106090 \\ \mathrm{C} & -3.9589180 & 2.0748300 & 2.3464040 \\ \mathrm{H} & -5.1961110 & 0.3004720 & 2.3481510 \\ \mathrm{C} & -4.1460770 & 3.8581560 & 3.9816590 \\ \mathrm{C} & -3.0469990 & 4.5125620 & 3.3805720\end{array}$
5.2275520
4.3771240
4.9767570
3.5013000
6.2262580
5.7814630
1.1997790
0.7034950
$-1.6149580$
$-1.3764800$
$-1.0379480$
$-1.1690500$
$-2.8959890$
$-2.7717390$
$-2.4588700$
$-1.7706560$
$-1.7761850$
$-1.7533190$
$-2.4514280$
$-2.2598690$
$-2.7718900$
$-0.7395690$
$-2.2823310$
$-3.5090130$
$-0.7307900$
$-1.1036910$ 


$\begin{array}{rrr}-2.4337960 & 3.9777290 & 2.2766570 \\ -4.5862010 & 2.6630610 & 3.4758210 \\ -4.6271160 & 4.2961570 & 4.8508930 \\ -2.6799210 & 5.4438810 & 3.8013010 \\ -1.5843530 & 4.4864060 & 1.8354490 \\ -5.4154120 & 2.1370760 & 3.9419920 \\ -2.7423830 & 6.0900150 & -1.3229000 \\ -1.6097320 & 6.7671920 & -1.8295300 \\ -0.3832090 & 6.1560190 & -1.8084900 \\ -2.6227800 & 4.8313020 & -0.7914060 \\ -3.7161810 & 6.5686810 & -1.3609390 \\ -1.7179560 & 7.7642020 & -2.2455690 \\ 0.4924260 & 6.6592310 & -2.2103700 \\ -3.4996780 & 4.3172920 & -0.4128900 \\ 2.4986480 & 2.2143290 & -0.8002610 \\ 3.1072940 & 1.7104880 & -1.9615190 \\ 3.1438400 & 2.0915860 & 0.4461040 \\ 4.3643230 & 1.1042710 & -1.8542380 \\ 4.3906200 & 1.4772000 & 0.5000830 \\ 5.0279880 & 0.9795690 & -0.6363210 \\ 4.8385660 & 0.7375640 & -2.7587700 \\ 4.8968480 & 1.4046250 & 1.4602750 \\ -4.0854050 & -1.1926790 & 0.3799490 \\ -3.6423450 & -2.3162570 & 1.1012280 \\ -4.8822190 & -1.3526000 & -0.7670920 \\ -4.0431670 & -3.5823250 & 0.6728500 \\ -5.2465430 & -2.6385020 & -1.1603390 \\ -4.8414360 & -3.7669580 & -0.4517670 \\ -3.7119380 & -4.4589620 & 1.2241820 \\ -5.8585510 & -2.7616460 & -2.0514950 \\ 2.5511450 & 2.6571360 & 1.7300610 \\ 6.4207740 & 0.3738080 & -0.4945890 \\ 2.4667120 & 1.8818870 & -3.3330510 \\ -2.7535610 & -2.1811040 & 2.3312080 \\ -5.2287500 & -5.1618950 & -0.9088610 \\ -5.3503900 & -0.1644410 & -1.5948270 \\ -6.8802210 & -0.0483360 & -1.5900190 \\ -7.3486270 & -0.9230340 & -2.0559120 \\ -7.2645790 & 0.0339320 & -0.5676590 \\ -7.1987160 & 0.8392410 & -2.1484100 \\ -4.7950170 & -0.2270990 & -3.0236490 \\ -3.7028210 & -0.2845850 & -3.0095100 \\ -5.1791790 & -1.1009210 & -3.5628820 \\ -5.0868350 & 0.6675870 & -3.5853620 \\ -4.5911800 & -5.4942220 & -2.2649840 \\ -4.8214340 & -6.5242440 & -2.5603390\end{array}$

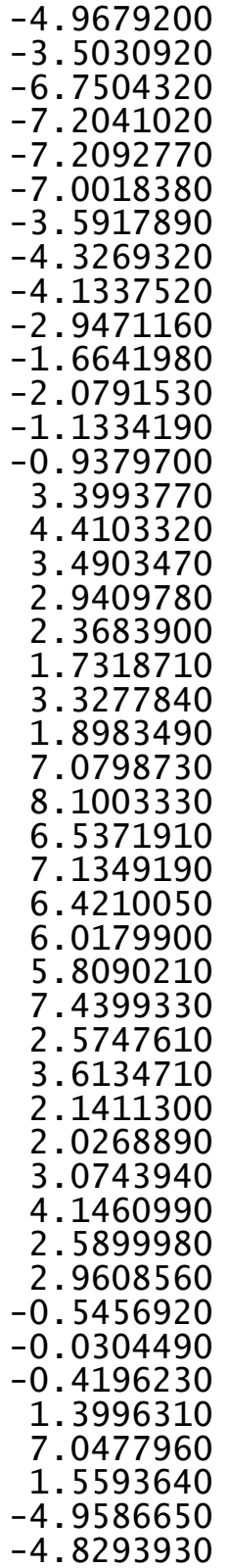

-4.8262080
-5.3804680
-5.3502290
-5.1326350
-4.6861200
-6.3809970
-2.1913750
-1.3815680
-3.1399970
-2.0781970
-3.2580100
-4.2404180
-3.3344440
-2.9964230
3.8268720
3.4982810
4.6100890
4.2667930
1.5755000
0.7680210
1.1407110
2.0019740
-0.0057880
-0.3596570
-0.8141920
0.8456050
-0.8258160
-0.5644380
-1.6457870
-1.2026540
0.6405160
0.4148900
-0.2334030
0.8069220
3.0958140
2.9416880
3.2500030
4.0130470
-0.2430120
-0.2980890
-1.4607920
2.0683590
1.1508210
3.0595800
0.7484600
-5.8701670

$-3.0481480$ $-2.2238960$ $-0.9547470$ 0.0175130 $-1.6962130$ $-1.2290660$

3. 6179630

3. 6301940

3.7144070

4.4972190

2.4057260

2.6606430

1. 4544870

3.1826150

2. 2479260

2. 5158120

1.4876580

3. 1407190

2. 8000430

2.4278530

3. 1043020

3.6935180

$-1.8218870$

$-1.6431920$

$-2.3273200$

$-2.5079090$

0.4654230

1.4477480

0.0746060

0.6084490

$-4.2234880$

$-4.4939180$

$-3.7356800$

$-5.1569420$

$-4.0524120$

$-4.2272430$

$-5.0231670$

$-3.4672460$

$-0.8016940$

$-2.2101330$

0.0742020

$-3.1807780$

$-0.0347490$

1. 5084940

$-1.1369520$

$-0.1710990$ 


\begin{tabular}{|c|c|c|c|c|c|c|}
\hline & & & & & & \\
\hline-2.2424140 & -1.2158010 & 2.2636250 & C & 3.8524070 & 0.0675880 & 0.8163870 \\
\hline 2.6116090 & -2.1537060 & 1.5887090 & $\mathrm{C}$ & 2.7013260 & -2.0574970 & 0.3166990 \\
\hline 1.4219120 & -1.6849950 & 1.8429070 & C & 4.6966280 & -0.5890870 & 1.6719440 \\
\hline 3.4917010 & -2.2378790 & 2.7863460 & C & 3.5121800 & -2.7102560 & 1.3052190 \\
\hline 4.7318580 & -2.8695480 & 2.6729820 & $\mathrm{C}$ & 4.5419880 & -1.9711310 & 1.9521740 \\
\hline 3.0985120 & -1.6852950 & 4.0080230 & $\mathrm{H}$ & 5.4792250 & -0.0343390 & 2.1825970 \\
\hline 5.5816870 & -2.9355740 & 3.7700380 & C & 5.1713540 & -3.9313790 & 3.2382680 \\
\hline 5.0192210 & -3.3028150 & 1.7213650 & c & 4.1180960 & -4.6539960 & 2.6339950 \\
\hline 3.9540930 & -1.7467930 & 5.1019260 & C & 3.3112040 & -4.0615270 & 1.6965280 \\
\hline 2.1315880 & -1.2028630 & 4.0916670 & $\mathrm{C}$ & 5.3704750 & -2.6167500 & 2.9070620 \\
\hline 5.1950450 & -2.3692500 & 4.9832520 & $\mathrm{H}$ & 5.8077860 & -4.4143270 & 3.9734970 \\
\hline 6.5457990 & -3.4257420 & 3.6785800 & $\mathrm{H}$ & 3.9418490 & -5.6871210 & 2.9180460 \\
\hline 3.6522300 & -1.3079750 & 6.0476240 & $\mathrm{H}$ & 2.5000370 & -4.6270630 & 1.2526680 \\
\hline 5.8623940 & -2.4147460 & 5.8387820 & $\mathrm{H}$ & 6.1601680 & -2.0404240 & 3.3822510 \\
\hline 0.7850370 & -1.6108900 & 1.0547300 & C & 3.3853180 & -5.6245720 & -2.1553040 \\
\hline 3.0230260 & -2.5145200 & 0.4807770 & C & 2.2833890 & -6.3499540 & -2.6618810 \\
\hline 1.3987490 & -3.1401400 & -1.7562610 & c & 1.0097950 & -5.8873950 & -2.4554840 \\
\hline 2.4468180 & -2.1356050 & -1.5605650 & $\mathrm{c}$ & 3.1914130 & -4.4717960 & -1.4374710 \\
\hline 0.5323560 & -3.0357280 & -1.1055570 & $\mathrm{H}$ & 4.3943270 & -5.9805870 & -2.3401190 \\
\hline 2.1418930 & -1.1342840 & -1.2765580 & $\mathrm{H}$ & 2.4497820 & -7.2652730 & -3.2216070 \\
\hline 1.2286360 & -2.4221410 & -3.0011420 & $\mathrm{H}$ & 0.1547340 & -6.4253020 & -2.8567960 \\
\hline 0.6121280 & -1.6446580 & -2.8111560 & $\mathrm{H}$ & 4.0453340 & -3.9191940 & -1.0608990 \\
\hline 3.1414590 & -4.6642720 & -2.7867000 & C & -2.1332920 & -2.4183300 & -0.6926980 \\
\hline 4.2373290 & -3.8000980 & -2.1576410 & $\mathrm{C}$ & -2.8482560 & -1.8022300 & -1.7367640 \\
\hline 3.8177900 & -2.3311530 & -2.1012680 & C & -2.6675270 & -2.4516650 & 0.6091190 \\
\hline 1.8421960 & -4.5666800 & -1.9812650 & c & -4.0869600 & -1.2211270 & -1.4494210 \\
\hline 3.4634280 & -5.7093620 & -2.8317110 & C & -3.9075530 & -1.8634940 & 0.8431580 \\
\hline 5.1717590 & -3.8868080 & -2.7206950 & $\mathrm{C}$ & -4.6338380 & -1.2389800 & -0.1673370 \\
\hline 3.8237580 & -1.8766230 & -3.0995890 & $\mathrm{H}$ & -4.6480120 & -0.7606920 & -2.2580810 \\
\hline 1.9812550 & -5.0166990 & -0.9906660 & $\mathrm{H}$ & -4.3333430 & -1.8930710 & 1.8438390 \\
\hline 1.0270960 & -5.1064810 & -2.4737960 & C & 3.8935240 & 1.5454410 & 0.6012110 \\
\hline 2.9590830 & -4.3394470 & -3.8179090 & $\mathrm{C}$ & 3.3813960 & 2.4058180 & 1.5903310 \\
\hline 4.4378200 & -4.1476560 & -1.1372570 & $\mathrm{C}$ & 4.3804930 & 2.0688240 & -0.6096970 \\
\hline 4.4915390 & -1.7235600 & -1.4954540 & C & 3.3901030 & 3.7795530 & 1.3481060 \\
\hline s) $6[1097 \times 0$ & $G(d) 1--331$ & & c & $\begin{array}{l}4.35<1630 \\
3.8571530\end{array}$ & 3.4471100 & 0.8102250 \\
\hline S) GLW34950 & $\begin{array}{l}G(d)\rfloor=-331 \\
-1\end{array}$ & 103 & $\mathrm{C}$ & $\begin{array}{r}3.8574530 \\
3.0010450\end{array}$ & $\begin{array}{l}4.3207790 \\
4.4550500\end{array}$ & 0.1547420 \\
\hline 2.1056020 & -0.0363710 & -0.7927190 & $\mathrm{H}$ & 4.7190930 & 3.8466470 & -1.7526750 \\
\hline 0.3482490 & -2.3050610 & -0.3941850 & C & -1.9590250 & -3.1708320 & 1.7487810 \\
\hline 1.6376640 & -2.7849250 & -0.4313000 & c & -6.0179220 & -0.6939370 & 0.1419710 \\
\hline-0.7740030 & -2.9987090 & -0.9203380 & $\mathrm{C}$ & -2.3446540 & -1.8380630 & -3.1742810 \\
\hline 1.8834460 & -3.9798430 & -1.1855570 & C & 2.8336260 & 1.8863640 & 2.9138130 \\
\hline-0.5412330 & -4.1934870 & -1.5470600 & $\mathrm{C}$ & 3.7517280 & 5.8134750 & -0.1016820 \\
\hline 0.77 & -4.6906790 & -1.7295280 & C & 4.9436440 & 1.1766270 & -1.7062710 \\
\hline-1.3763900 & -4.7731990 & -1.9301270 & C & 6.4469010 & 1.4231110 & -1.8940000 \\
\hline 2.8692330 & 010430 & 0.1377870 & $\mathrm{H}$ & 6.6420250 & 2.4494980 & -2.2261900 \\
\hline
\end{tabular}




\begin{tabular}{|c|c|c|c|}
\hline & & & \\
\hline $\mathrm{H}$ & 6.9891700 & 1.2647930 & -0.9557190 \\
\hline $\mathrm{H}$ & 6.8564940 & 0.7411480 & -2.6477990 \\
\hline C & 4.1802640 & 1.3427810 & -3.0264200 \\
\hline $\mathrm{H}$ & 3.1120930 & 1.1610350 & -2.8792300 \\
\hline $\mathrm{H}$ & 4.3071520 & 2.3501460 & -3.4402560 \\
\hline $\mathrm{H}$ & 4.5516690 & 0.6295610 & -3.7709860 \\
\hline C & 2.5369240 & 6.1089940 & -0.9951800 \\
\hline $\mathrm{H}$ & 2.4149420 & 7.1873880 & -1.1486300 \\
\hline $\mathrm{H}$ & 2.6574470 & 5.6351940 & -1.9767010 \\
\hline $\mathrm{H}$ & 1.6190560 & 5.7168320 & -0.5439710 \\
\hline C & 5.0296980 & 6.4156940 & -0.6956690 \\
\hline $\mathrm{H}$ & 5.9005970 & 6.1911480 & -0.0715070 \\
\hline $\mathrm{H}$ & 5.2257110 & 6.0267680 & -1.7012040 \\
\hline $\mathrm{H}$ & 4.9351090 & 7.5040130 & -0.7783240 \\
\hline C & 3.8700560 & 2.0670050 & 4.032418 \\
\hline $\mathrm{H}$ & 4.8135020 & 1.5657970 & 3.7932030 \\
\hline $\mathrm{H}$ & 4.0871470 & 3.1306200 & 4.1886180 \\
\hline $\mathrm{H}$ & 3.4942930 & 1.6551010 & 4.9760890 \\
\hline C & 1.4993890 & 2.5331140 & 3.3051690 \\
\hline $\mathrm{H}$ & 1.6145370 & 3.5968770 & 3.543889 \\
\hline $\mathrm{H}$ & 0.7737410 & 2.4289270 & 2.4957860 \\
\hline $\mathrm{H}$ & 1.0976040 & 2.0413070 & 4.1983310 \\
\hline C & -2.6383430 & -4.5212780 & 2.0168860 \\
\hline $\mathrm{H}$ & -3.6815600 & -4.3792450 & 2.3232820 \\
\hline $\mathrm{H}$ & -2.6335270 & -5.1460630 & 1.1171090 \\
\hline $\mathrm{H}$ & -2.1192620 & -5.0635890 & 2.8154330 \\
\hline C & -1.8685280 & -2.3283430 & 3.024543 \\
\hline H & -1.4278990 & -1.3496070 & 2.811745 \\
\hline $\mathrm{H}$ & -2.8542090 & -2.1782190 & 3.4809370 \\
\hline $\mathrm{H}$ & -1.2407860 & -2.8361270 & 3.7649000 \\
\hline C & -7.0792730 & -1.7707610 & -0.1299180 \\
\hline $\mathrm{H}$ & -8.0771370 & -1.4125790 & 0.1482060 \\
\hline H & -7.0954000 & -2.0338250 & -1.1943780 \\
\hline $\mathrm{H}$ & -6.8689680 & -2.6829020 & 0.4379240 \\
\hline C & -6.3537390 & 0.5982250 & -0.6033240 \\
\hline $\mathrm{H}$ & -5.5859000 & 1.3561500 & -0.4247330 \\
\hline $\mathrm{H}$ & -6.4409000 & 0.4352590 & -1.6840990 \\
\hline $\mathrm{H}$ & -7.3106210 & 1.0000540 & -0.2528170 \\
\hline C & -2.5471830 & -0.5300990 & -3.9441910 \\
\hline $\mathrm{H}$ & -3.6073460 & -0.2801160 & -4.0697830 \\
\hline $\mathrm{H}$ & -2.0405810 & 0.2966850 & -3.444779 \\
\hline $\mathrm{H}$ & -2.1166990 & -0.6253270 & -4.9470560 \\
\hline C & -3.0142770 & -2.9983370 & -3.9278710 \\
\hline $\mathrm{H}$ & -4.0953860 & -2.8320840 & -4.0083430 \\
\hline $\mathrm{H}$ & 67410 & -3.0866840 & 941348 \\
\hline$\Pi$ & & & \\
\hline
\end{tabular}
0.5249910
$-0.0844520$
0.2665500
$-1.2670700$
$-6.0413430$
$-0.9331410$
4.8270620
3.5762690
2. 6440200
$-3.0779280$
$-1.9805090$
$-4.2111640$
$-5.4009350$
$-4.1106910$
$-6.4933810$
$-5.4612480$
$-5.2070320$
$-3.1796400$
-6. 3998860
$-7.4199060$
$-5.1316010$
$-7.2570960$
$-1.1747040$
$-3.2215610$
$-1.2102060$
$-2.3620270$
$-0.4723750$
$-2.1794330$
$-0.9032020$
$-0.4168360$
$-2.6189760$
$-3.8736920$
$-3.5988660$
$-1.4796190$
$-2.8373060$
$-4.6905520$
$-3.4577580$
$-1.7321370$
$-0.5500740$
$-2.3024970$
$-4.2089160$
$-4.4272200$

144

$\begin{array}{lccc}14 S 143(\mathrm{~S}, \mathrm{~S}) & \mathrm{G}[\mathrm{WB} 97 \mathrm{XD} / 6-31 \mathrm{G}(\mathrm{d})]=-3310.752505 \\ \mathrm{TS}= & 0.5641770 & 0.5208090 & -0.0679930 \\ 0 & -1.7505210 & 0.8447500 & -1.0794090 \\ 0 & -1.000\end{array}$
$-0.5361950$

$-1.9061400$

0.4494550

$-3.1461660$

1.4399530

$-1.3960810$

0.8675540

2.8025970

1.3839490

1.7246570

2. 3190960

2.1398440

3.3376850

2.9585440

1. 3509640

3.4755300

3.9572760

2.8148240

4.9324610

4.5900280

1.1233300

0.3849940

$-1.5029200$

$-1.5668780$

$-0.7398130$

$-1.3456460$

$-2.7722440$

$-2.5590530$

$-2.6321340$

$-2.2708030$

$-2.3034680$

$-1.6536030$

$-2.6171490$

$-2.9610690$

$-3.3335490$

$-0.6562850$

$-1.9631590$

$\begin{array}{ll}4.2325400 & -1.9631590 \\ 4.5200550 & -3.6518870 \\ 2.1558260 & -1.2638800\end{array}$

$\begin{array}{ll}4.2325400 & -1.9631590 \\ 4.5200550 & -1.2638870 \\ 2.1558260 & -1.8986470\end{array}$

4.5200550

0.8447500

$-1.0794090$
1.2158590

4.1498640 


$\begin{array}{rrr}0.7355240 & 1.8857450 & -0.0610650 \\ -0.2946760 & 2.6914150 & 0.3811910 \\ 2.0062760 & 2.3994890 & -0.4236210 \\ -0.1452720 & 4.1121640 & 0.2900510 \\ 2.1638460 & 3.7625130 & -0.4349910 \\ 1.1053920 & 4.6465530 & -0.1204180 \\ 3.1380130 & 4.1791060 & -0.6762450 \\ -2.2372760 & 1.1709570 & 0.1748400 \\ -3.4617830 & 0.5859140 & 0.5918260 \\ -1.5305420 & 2.0757360 & 0.9316610 \\ -3.9455140 & 0.9499310 & 1.8224390 \\ -1.9889840 & 2.3637600 & 2.2583310 \\ -3.2230700 & 1.8066040 & 2.6909770 \\ -4.8983650 & 0.5495290 & 2.1569680 \\ -2.9711960 & 2.8827600 & 4.8509350 \\ -1.7265240 & 3.4080390 & 4.4350150 \\ -1.2480090 & 3.1573250 & 3.1744820 \\ -3.6991370 & 2.0968210 & 3.9964400 \\ -3.3411090 & 3.0935790 & 5.8497330 \\ -1.1411780 & 4.0125420 & 5.1212880 \\ -0.2885900 & 3.5623230 & 2.8723230 \\ -4.6485350 & 1.6703560 & 4.3097550 \\ -1.0213400 & 6.3706460 & 0.4628840 \\ 0.2342120 & 6.9005020 & 0.0839890 \\ 1.2717740 & 6.0540210 & -0.2038320 \\ -1.2074320 & 5.0162720 & 0.5645940 \\ -1.8474130 & 7.0444350 & 0.6694560 \\ 0.3674680 & 7.9754030 & 0.0102520 \\ 2.2373670 & 6.4465480 & -0.5121390 \\ -2.1785800 & 4.6236020 & 0.8449050 \\ 3.1855280 & 1.5284990 & -0.7265970 \\ 3.5157230 & 1.2700980 & -2.0727790 \\ 4.0333060 & 1.0834790 & 0.3094150 \\ 4.7102550 & 0.6118490 & -2.3644430 \\ 5.2173010 & 0.4286490 & -0.0436050 \\ 5.5849150 & 0.1945550 & -1.3651860 \\ 4.9689120 & 0.4354120 & -3.4045510 \\ 5.8835720 & 0.0937050 & 0.7500760 \\ -4.1991640 & -0.3683160 & -0.2894110 \\ -4.2573010 & -1.7357800 & 0.0383350 \\ -4.8536740 & 0.1139210 & -1.4386140 \\ -4.9970840 & -2.5865720 & -1.7849870 \\ -5.5691900 & -0.7786380 & -3.53217740 \\ -5.6573270 & -2.1335080 & \\ -5.0579180 & -3.6436150 & -1188280 \\ -6.0757060 & -0.4027600 & \end{array}$

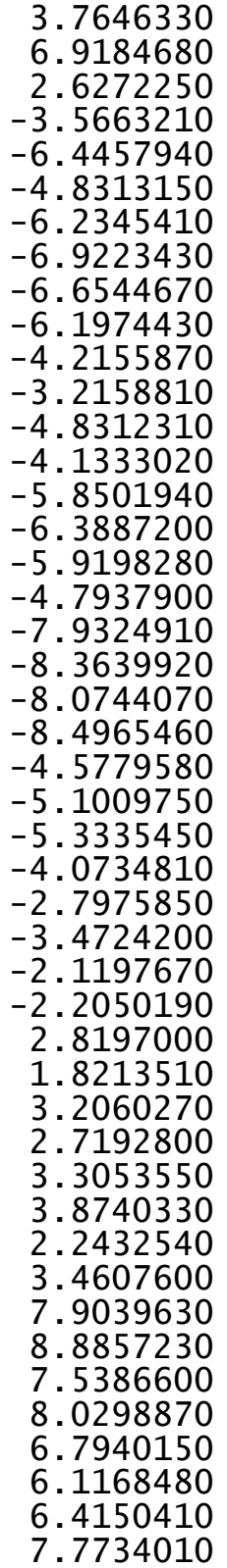

1.2126090

$-0.4542020$

1.7459210

$-2.3139220$

$-3.0899500$

1.5849810

2.2000010

1.7322310

2.0722130

. 2723410

1.7924040

1.3520790

1.3352880

2.8623110

$-3.1779190$

$-3.9189510$

$-2.2130850$

$-3.4622250$

$-2.7142000$

$-2.6723660$

$-1.7327210$

$-3.4475660$

$-2.5549380$

$-1.6352490$

$-3.2872070$

$-2.9444710$

$-3.6061920$

$-4.4389660$

$-3.4597340$

$-3.8960080$

0.1076290

0.2080030

$-0.8854120$

0.1513190

2.5812300

3.4009690

2.7619910

2.6140240

0.5964410

0.1481030

1.0258180

1.4141930

$-1.6249670$

$-2.3987460$

$-1.2939700$

$-2.0864690$
1.8120410 $-1.6942150$ $-3.2162620$ 1.2672750 $-2.7980070$ $-1.8298190$ $-1.7384890$ $-2.4525640$ $-0.7349610$ $-1.9615400$ $-3.2190530$ $-3.2670480$ $-4.0025980$ $-3.4414240$ $-4.2094260$ $-4.8109560$ $-4.7249480$ $-4.1727990$ $-2.8493980$ $-1.8440830$ $-3.3165310$ $-3.4367190$

2. 3974120

2.6770980

2.0886140

3.2891150

0.9633930

0.7341320

0.1196650

1.8384650

2. 3084150

1.8744100

2.0520130

3. 3988470

2. 3361720

1.8866070

2.1521470

3.4204700

$-2.2272500$

$-2.4163750$

$-3.1674250$

$-1.5103780$

$-2.6770980$

$-2.3005000$

$-3.6501780$

$-2.8431860$ 


\begin{tabular}{rrr}
2.5748820 & 0.7619330 & -4.3912610 \\
3.5004510 & 0.7818300 & -4.9792930 \\
2.4093650 & -0.2640290 & -4.0570210 \\
1.7553990 & 1.0373180 & -5.0621720 \\
3.0788970 & 3.1236980 & -3.7248240 \\
4.1131900 & 3.0780200 & -4.0869310 \\
2.4418520 & 3.4485380 & -4.5551460 \\
3.0261300 & 3.8851610 & -2.9425290 \\
-0.5097800 & -0.1998850 & -1.0961800 \\
0.0369130 & -0.2522740 & -2.4893340 \\
-0.8456640 & -1.4847900 & -0.3887760 \\
1.6073840 & 1.8374770 & -2.8319910 \\
7.3290310 & -0.8507340 & -0.7563130 \\
4.7363990 & 1.0257590 & 2.2858320 \\
-4.2036180 & 2.1252040 & -1.1159670 \\
-6.3699870 & -4.0859440 & -2.3424980 \\
-2.8323660 & -1.5832840 & 1.6179250 \\
1.3910250 & -3.1379530 & -1.6405980 \\
2.2224200 & -2.3565490 & -0.7200500 \\
0.3200800 & -3.0520730 & -1.4695780 \\
1.8368410 & -1.4114230 & -0.3555850 \\
1.8694300 & -2.1530350 & -2.5875520 \\
1.1825160 & -1.4159800 & -2.6365530 \\
3.3840190 & -4.6356710 & -2.0808590 \\
4.0413600 & -4.0954930 & -0.8090210 \\
3.6730420 & -2.6306120 & -0.5743460 \\
1.8589650 & -4.5319440 & -1.9854450 \\
3.6681850 & -5.6806110 & -2.2391250 \\
5.1308470 & -4.1871990 & -0.8679220 \\
4.1637600 & -1.9716310 & -1.3011980 \\
1.4876460 & -5.1981190 & -1.1971620 \\
1.3797350 & -4.8336760 & -2.9221240 \\
3.7383840 & -4.0697690 & -2.9509150 \\
3.7114770 & -4.6858610 & 0.0539200 \\
3.9943170 & -2.2696250 & 0.4056090 \\
1.0306780 & -2.7236610 & 2.1179540 \\
-0.0169260 & -1.9616570 & 1.9731730 \\
1.4253910 & -2.9489440 & 3.5343340 \\
2.5349680 & -3.7516610 & 3.8069240 \\
0.7231230 & -2.3451380 & 4.5806290 \\
2.9385550 & -3.9550720 & 5.1205630 \\
3.0698460 & -4.2085800 & 2.9810130 \\
1.1323570 & -2.5480920 & 5.8934080 \\
-0.1321780 & -1.7172090 & 4.3579760 \\
2.2379020 & -3.3522740 & 5.3328130 \\
3.7999850 & -4.5806360 & \\
\hline & &
\end{tabular}

$\begin{array}{lr}\mathrm{H} & 0.5900860 \\ \mathrm{H} & 2.5545270 \\ \mathrm{H} & -0.2934330 \\ \mathrm{O} & 1.6767800 \\ 144 & \end{array}$
$-2.0768220$
$-3.5093650$
$-1.8060700$
$-3.2285130$

6.7070910

7.1907100

1. 0041800

1. 1920760

$\operatorname{TS} 144(\mathrm{~S}, \mathrm{~S}) \mathrm{G}[\mathrm{WB} 97 \mathrm{XD} / 6-31 \mathrm{G}(\mathrm{d})]=-3310.752555$

$\begin{array}{lrrr}\text { TS144(S }(\mathrm{S}) \mathrm{G}[\mathrm{WB} 97 \mathrm{XD} / 6-31 \mathrm{G}(\mathrm{d})]=-3310.752555 \\ \mathrm{O} & 1.7766420 & -0.4451750 & -0.7274990 \\ \mathrm{O} & -0.0132220 & -1.3447960 & 0.7946830 \\ \mathrm{C} & 2.7277690 & -1.0899020 & 0.0356710 \\ \mathrm{C} & 2.5267040 & -2.4132170 & 0.3614640 \\ \mathrm{C} & 3.8837460 & -0.3529450 & 0.4045510 \\ \mathrm{C} & 3.5027920 & -3.0632110 & 1.1853890 \\ \mathrm{C} & 4.8670940 & -1.0239340 & 1.0849540 \\ \mathrm{C} & 4.6999910 & -2.3666160 & 1.5089040 \\ \mathrm{H} & 5.7806870 & -0.5010840 & 1.3544740 \\ \mathrm{C} & 0.0638200 & -2.5236750 & 0.0802800 \\ \mathrm{C} & -1.1521560 & -3.1119030 & -0.3580020 \\ \mathrm{C} & 1.3017000 & -3.0986090 & -0.1325320 \\ \mathrm{C} & -1.0760820 & -4.3365640 & -0.9730550 \\ \mathrm{C} & 1.3715710 & -4.3131650 & -0.8906100 \\ \mathrm{C} & 0.1618680 & -4.9543070 & -1.2720070 \\ \mathrm{H} & -1.9943070 & -4.8313510 & -1.2773030 \\ \mathrm{C} & 1.4135070 & -6.7313310 & -2.3493740 \\ \mathrm{C} & 2.6181340 & -6.0701010 & -2.0161920 \\ \mathrm{C} & 2.5992240 & -4.8955640 & -1.3087560 \\ \mathrm{C} & 0.2131810 & -6.1784540 & -1.9895610 \\ \mathrm{H} & 1.4439780 & -7.6653040 & -2.9022160 \\ \mathrm{H} & 3.5675200 & -6.4939610 & -2.3293330 \\ \mathrm{H} & 3.5319720 & -4.3963350 & -1.0729620 \\ \mathrm{H} & -0.7225670 & -6.6606620 & -2.2604310 \\ \mathrm{C} & 4.2835840 & -4.9563440 & 2.4924230 \\ \mathrm{C} & 5.4928560 & -4.2799910 & 2.7734830 \\ \mathrm{C} & 5.6908320 & -3.0115780 & 2.2948700 \\ \mathrm{C} & 3.3161590 & -4.3662660 & 1.7198610 \\ \mathrm{H} & 4.1173010 & -5.9498300 & 2.8978690 \\ \mathrm{H} & 6.2534530 & -4.7620890 & 3.3800660 \\ \mathrm{H} & 6.6058600 & -2.4711440 & 2.5232830 \\ \mathrm{H} & 2.3896070 & -4.8924970 & 1.5187990 \\ \mathrm{C} & 3.9796980 & 1.1069250 & 0.1026280 \\ \mathrm{C} & 3.9244600 & 2.0403820 & 1.1576430 \\ \mathrm{C} & 4.1083520 & 1.5526950 & -1.2263020 \\ \mathrm{C} & 4.0277610 & 3.3981440 & 0.8534090 \\ \mathrm{C} & 4.1933050 & 2.9209470 & -1.4765200 \\ \mathrm{C} & 4.1622280 & 3.8615580 & -0.4507620 \\ \mathrm{H} & 3.9959200 & 4.1252160 & 1.6615550 \\ \mathrm{H} & 4.2959070 & 3.2592030 & -2.5051560\end{array}$




-2.4875920
-3.1534590
-3.1249480
-4.4742290
-4.4394690
-5.1440500
-4.9902580
-4.9437300
0.4259490
0.7168250
-0.5573760
4.2000100
4.0670360
4.2305470
4.3705960
3.7887380
3.5173780
-2.4296770
-1.4229720
-6.6111640
-6.7439920
-2.4877670
-1.4148830
-0.7766780
-0.7670600
-1.0873360
-0.5337010
0.6543950
0.9396660
-0.9652470
-1.3990100
-0.7333130
-1.3119180
-1.4571490
-1.1575880
0.3258630
-2.4014180
-0.9244430
0.1145550
-2.4862910
-1.1917680
-2.6514160
-2.0842420
-3.6981940
-2.2717920
-3.0190700

\begin{tabular}{rr}
-2.4550250 & -0.1986150 \\
-1.9613980 & -1.3376110 \\
-2.4240080 & 1.0540350 \\
-1.5268630 & -1.2111820 \\
-1.9678740 & 1.1294890 \\
-1.5387030 & 0.0069880 \\
-1.1694180 & -2.0973910 \\
-1.9599830 & 2.0950170 \\
0.0349830 & 0.0434660 \\
1.0401190 & 1.1179090 \\
0.3916470 & -1.0439550 \\
0.5892060 & -2.4011040 \\
-0.4284220 & -2.0244770 \\
5.3510240 & -0.7344460 \\
5.8574240 & 0.2293510 \\
1.6303920 & 2.6196220 \\
0.5725170 & 2.6539320 \\
-2.8805420 & 2.3269680 \\
-3.2194830 & 2.0684040 \\
-1.1666840 & 0.1403330 \\
-0.7370460 & 1.1418440 \\
-1.8910070 & -2.7075830 \\
-2.0508960 & -2.5696120 \\
3.5886540 & 0.7076740 \\
3.4020640 & -0.7489040 \\
2.7164300 & 1.2827030 \\
2.4078770 & -1.1190170 \\
3.5996090 & 0.5272400 \\
2.6532100 & 0.7027020 \\
6.0836380 & 0.3409320 \\
5.8187220 & -1.1030920 \\
4.5633270 & -1.6738300 \\
4.8958060 & 1.2423930 \\
6.9841930 & 0.7213170 \\
6.6745250 & -1.7407790 \\
4.7421190 & -1.8982020 \\
4.7886970 & 1.3201420 \\
5.0386510 & 2.2560820 \\
6.2701470 & 0.3793020 \\
5.6832690 & -1.1370060 \\
4.2455590 & -2.6142500 \\
-0.5140280 & -3.3655420 \\
-0.4837340 & -4.3027190 \\
-0.2991730 & -3.6094710 \\
0.2770070 & -2.7161920 \\
-2.9889570 & -3.6403400 \\
& \\
\hline
\end{tabular}

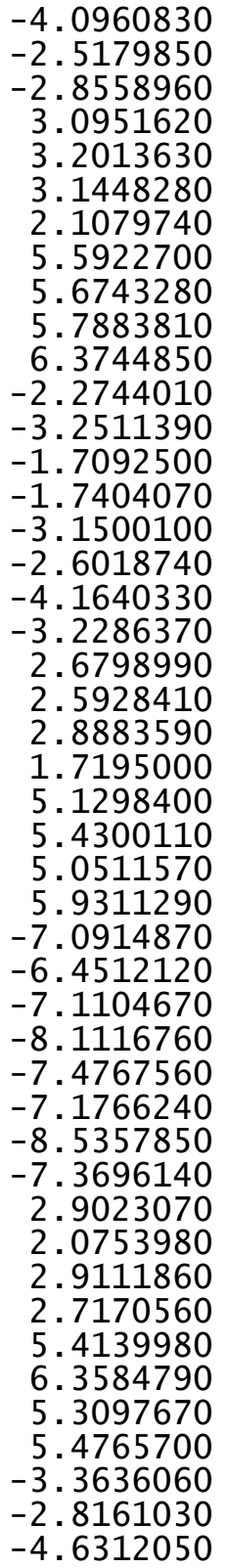

\author{
$-2.8672990$ \\ $-2.9416540$ \\ $-3.9872970$ \\ 0.8374890 \\ 1.8217440 \\ 0.0832970 \\ 0.7788840 \\ 0.6498150 \\ $-0.0896440$ \\ 1.6387930 \\ 0.4425030 \\ $-1.7146410$ \\ $-1.3497940$ \\ $-2.0318970$ \\ $-0.8837720$ \\ $-4.0718550$ \\ $-4.4175690$ \\ $-3.8044870$ \\ -4. 9071700 \\ 2.3916630 \\ 2.0113780 \\ 3.4655800 \\ 2. 2470810 \\ 1.7978130 \\ 2.8523850 \\ 1.4450200 \\ 1.2369450 \\ $-0.1230630$ \\ 0.7644750 \\ $-0.5297330$ \\ 0.1921500 \\ $-2.4342710$ \\ $-3.1637160$ \\ $-2.1950270$ \\ $-2.9094900$ \\ 5.8437030 \\ 5.5799040 \\ 6.9304880 \\ 5.3756930 \\ 5.7287820 \\ 5.3725920 \\ 5.2956750 \\ 6.8164640 \\ 2.1253790 \\ 0.9891790 \\ 2. 4172660
}

$-3.8077250$

$-4.6137550$

$-3.2230400$

$-3.4358680$

$-4.2291030$

$-2.9694560$

$-3.0452430$

.8498790

$-3.4756910$

$-2.3072760$

3.6531950

4.1956520

2.8419310

2.9715560

3.2908300

2. 2675730

3. 3573060

4.3815300

2.8589980

3.3494410

3. 3732140

4.3840300

2.8573780

$-0.8707110$

$-0.8674760$

$-1.8885370$

$-0.6270210$

0.0623900

0.8217100

0.2129200

$-0.9198640$

$-1.3242190$

$-0.6576170$

$-1.4657990$

$-2.2993290$

$-1.6323500$

$-1.2086980$

$-2.6336740$

$-0.2185630$

0.1161120
0.4958120 


$\begin{array}{lrrr}\mathrm{C} & -5.3885680 & 3.5251150 & 0.1077160 \\ \mathrm{C} & -5.0585870 & 1.6063500 & 1.5504110 \\ \mathrm{C} & -6.5822170 & 3.8090690 & 0.7595720 \\ \mathrm{H} & -5.0366220 & 4.1451570 & -0.7101870 \\ \mathrm{C} & -6.2460970 & 1.9032850 & 2.2081370 \\ \mathrm{H} & -4.4655070 & 0.7441720 & 1.8350690 \\ \mathrm{C} & -7.0107230 & 2.9979340 & 1.8092660 \\ \mathrm{H} & -7.1787770 & 4.6618460 & 0.4511930 \\ \mathrm{H} & -6.5796550 & 1.2752890 & 3.0284870 \\ \mathrm{H} & -7.9436270 & 3.2206260 & 2.3184620 \\ \mathrm{H} & -1.9762620 & 0.7736740 & -0.4062390 \\ \mathrm{O} & -2.8789650 & 2.9212960 & -1.0327200 \\ 154 & & & \end{array}$

TS148(R,R) G[WB97XD/6-31G(d)] $=-3541.694978$

$\begin{array}{lrrr}\text { TS148(R, R }(\mathrm{G}[\mathrm{WB} 97 \mathrm{XD} / 6-31 \mathrm{G}(\mathrm{d})]=-3541.694978 \\ \mathrm{P} & -0.3579520 & -0.1285680 & 0.8321520 \\ \mathrm{O} & -0.6141490 & 1.3460260 & 0.6374810 \\ \mathrm{O} & 0.2745130 & -0.5930920 & 2.1100210 \\ \mathrm{O} & 0.5142870 & -0.5711680 & -0.4834770 \\ \mathrm{O} & -1.7171300 & -1.0003790 & 0.6530730 \\ \mathrm{C} & 0.5484260 & -1.9169240 & -0.8055610 \\ \mathrm{C} & -0.5905580 & -2.4919680 & -1.3652880 \\ \mathrm{C} & 1.7114590 & -2.6472300 & -0.5672950 \\ \mathrm{C} & 1.6773380 & -4.0039720 & -0.8697990 \\ \mathrm{H} & 2.5758830 & -4.5941610 & -0.7005850 \\ \mathrm{C} & -2.3375380 & -0.9232200 & -0.5831920 \\ \mathrm{C} & -3.4937240 & -0.1590170 & -0.7179770 \\ \mathrm{C} & -1.7662540 & -1.6199010 & -1.6476800 \\ \mathrm{C} & -4.0527340 & -0.0928090 & -1.9915420 \\ \mathrm{H} & -4.9674380 & 0.4812740 & -2.1243850 \\ \mathrm{C} & 2.9353010 & -2.0076740 & -0.0141200 \\ \mathrm{C} & -4.1060820 & 0.5608870 & 0.4313680 \\ \mathrm{C} & 3.6537000 & -1.0746230 & -0.7721360 \\ \mathrm{C} & 3.3327760 & -0.6182090 & -2.1745110 \\ \mathrm{C} & 5.4605400 & 0.4911840 & -1.2134100 \\ \mathrm{C} & 4.8236630 & -0.4938830 & -0.2676260 \\ \mathrm{C} & 3.1542360 & 0.9126580 & -2.1554870 \\ \mathrm{H} & 2.9819240 & 1.2708050 & -3.1768990 \\ \mathrm{H} & 2.2569260 & 1.1483820 & -1.5799650 \\ \mathrm{C} & 4.4151990 & 1.5784870 & -1.5466650 \\ \mathrm{H} & 4.8485130 & 2.3031600 & -2.2454700 \\ \mathrm{H} & 4.1562270 & 2.1313930 & -0.6376490 \\ \mathrm{H} & 6.3570070 & 0.9410240 & -0.7730190 \\ \mathrm{H} & 2.4309800 & -1.0986560 & -2.5614910 \\ \mathrm{C} & 5.8142900 & -0.2555810 & -2.5189850 \\ \mathrm{H} & 6.2108490 & 0.4633210 & -3.2455340 \\ \mathrm{H} & 6.6060770 & -0.9851620 & -2.3215360\end{array}$

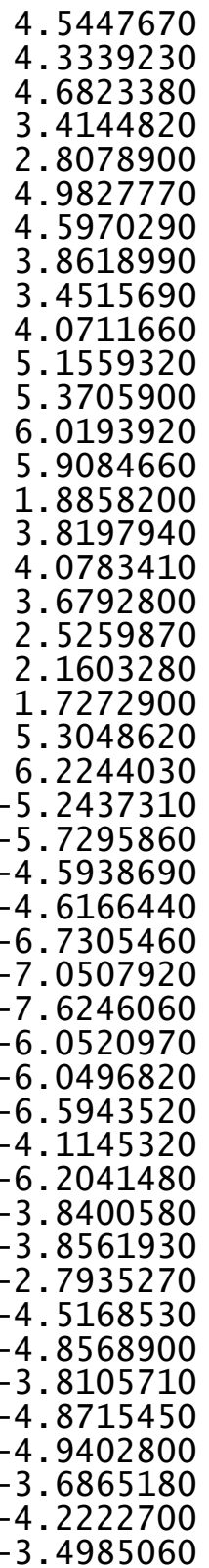

-0.9555970
-0.6267560
-2.0412520
-2.3493950
-3.3452020
-2.2738810
-1.7778410
-4.4474540
-5.1850000
-4.9742430
-3.8082810
-4.1955930
-4.0481710
-1.8010490
-3.7767580
-1.9679900
-2.3374960
-0.8852190
-2.6406970
-3.3874340
-1.9113030
-0.8543020
-0.4147340
0.5397720
-0.3680880
-1.6358560
-0.1406600
-1.3742420
-2.0780480
-0.8417510
-2.1221460
-3.2042230
-1.9500530
-2.1568920
0.2033610
-1.9268010
-3.0073570
-1.6334050
-1.1592300
-1.8507780
-0.4664300
2.6262970
4.1195470
2.9017280
1.9567350
4.6636400

$-3.0712700$

$-4.0953350$

$-3.1043730$

1. 2560840

2.2155470

3.1147170

1.7456880

2. 4640650

3.1634410

1.5267110

3.0358240

4.0386060

2.4068810

3.4609040

1.8204660

4.0834880

5.0828070

4.1664440

3.5597840

4. 2744020

3. 4160090

0.9856750

1.3692480

2. 5820210

3. 6819380

1.7392350

1. 5310400

3. 0720800

3. 8491950

2. 7318720

1.8948570

2. 0706540

0.9592580

0.9074340

4.4870100

3. 0555330

3. 2405530

2. 9427090

4. 2212630

5. 0012180

4.6896900

1.4635670

1. 2719470

$-0.6313940$

0.4179570

1.1795340 


$\begin{array}{rrr}-3.5347870 & 5.7398700 & 0.9716190 \\ -2.9985790 & 4.5385280 & 2.1456190 \\ -2.7373520 & 3.9037030 & 0.0641880 \\ -2.3431020 & 4.5994340 & -0.6859160 \\ -1.8918830 & 3.3507770 & 0.4770820 \\ -3.1478390 & 2.3696750 & -1.4188210 \\ -5.4813980 & 4.5996410 & 2.0945020 \\ -4.8750440 & 3.6920530 & -1.2210590 \\ -4.4944310 & 4.4253820 & -1.9418840 \\ -5.5344880 & 3.0141180 & -1.7737210 \\ -5.6475380 & 4.3969980 & -0.0738290 \\ -5.6936500 & 5.4793710 & -0.2416320 \\ -6.6796350 & 4.0354220 & -0.0209930 \\ -5.3823160 & 1.9223300 & 2.5468450 \\ -5.8818620 & 2.4467110 & 3.3600670 \\ -3.4620230 & -0.6941290 & -3.1027700 \\ -2.2906350 & -1.4475020 & -2.9405110 \\ -4.1278470 & -0.5381450 & -4.4572350 \\ -3.1841080 & -0.7940200 & -5.6312880 \\ -2.3976730 & -2.0823540 & -5.3998970 \\ -1.5271090 & -1.9480840 & -4.1529120 \\ -3.7563320 & -0.8455460 & -6.5639740 \\ -4.9642820 & -1.2495200 & -4.5146320 \\ -4.5725930 & 0.4605280 & -4.5341980 \\ -3.0979470 & -2.9202470 & -5.2760350 \\ -1.7678930 & -2.3159890 & -6.2653050 \\ -1.0131680 & -2.8871260 & -3.9395090 \\ -0.7282850 & -1.2191920 & -4.3549760 \\ -2.4799830 & 0.0428160 & -5.7313420 \\ -0.6312750 & -3.8849090 & -1.5717150 \\ -1.9299440 & -4.5565160 & -1.9825100 \\ -1.9292520 & -6.0616360 & -1.7183740 \\ -0.6543710 & -6.6887000 & -2.2759630 \\ 0.5577180 & -6.1443140 & -1.5230650 \\ 0.5247350 & -4.6394810 & -1.3342870 \\ -2.8194290 & -6.5120420 & -2.1702990 \\ -2.1193260 & -4.3925870 & -3.0518240 \\ -2.7628280 & -4.0763690 & -1.4574470 \\ -0.5704030 & -6.4492300 & -3.3447180 \\ -0.6831320 & -7.7804740 & -2.1931380 \\ 1.4861280 & -6.4239090 & -2.0351510 \\ 0.6033900 & -6.6171880 & -0.5320880 \\ -1.9862010 & -6.2508300 & -0.6381980 \\ 1.5580910 & 3.5221720 & -0.7322660 \\ 0.4656620 & 2.8980160 & -1.0754870 \\ 2.2156000 & 4.2546250 & -1.8478290\end{array}$

3.3621770
1.7283460
4.0201980
3.7320530
2.3906090
0.8416840
3.5356250
4.9131740
2.0146780
4.0524230
0.0782900
2.0590420
2.6895910
1.6556170
2.6392870
0.6731010
1.9613020
1.2762310
4.1472870
3.2726250
1.8136780
4.1000820
5.1836490
3.3292130
1.3525950
4.5757850
4.6441820
3.8037530
3.6348470
1.2048150

$\begin{array}{lr}5.0057150 & -1.5822650 \\ 4.1577740 & -3.1532180 \\ 5.6570620 & -2.6179040 \\ 5.0631020 & -0.5641680 \\ 4.8086580 & -4.1875780 \\ 3.5659690 & -3.3498270 \\ 5.5564670 & -3.9209030 \\ 6.2387500 & -2.4116450 \\ 4.7294140 & -5.2027970 \\ 6.0615340 & -4.7316130 \\ 2.2851830 & -0.3598110 \\ 3.5259480 & 0.3982160 \\ 1.4579750 & 2.1884820 \\ 2.5002530 & 2.2425120 \\ 0.7727380 & 1.3384210 \\ 2.2371320 & 1.8651570 \\ 0.9599420 & 3.3307010 \\ 0.3131240 & 2.9636520 \\ 2.9199090 & 3.6565060 \\ 4.1226220 & 3.2972830 \\ 3.7022890 & 3.1083590 \\ 1.8600360 & 2.5532060 \\ 3.2373850 & 3.8092360 \\ 4.8902710 & 4.0750410 \\ 3.4476960 & 4.0708040 \\ 2.2411100 & 1.6413730 \\ 0.9580710 & 2.8444530 \\ 2.4806390 & 4.6008880 \\ 4.5738200 & 2.3662340 \\ 4.5027680 & 2.6800620\end{array}$
154

4.5027680

$\operatorname{TS} 149(R, R) \quad G[W B 97 X D / 6-31 G(d)]=-3541.695297$

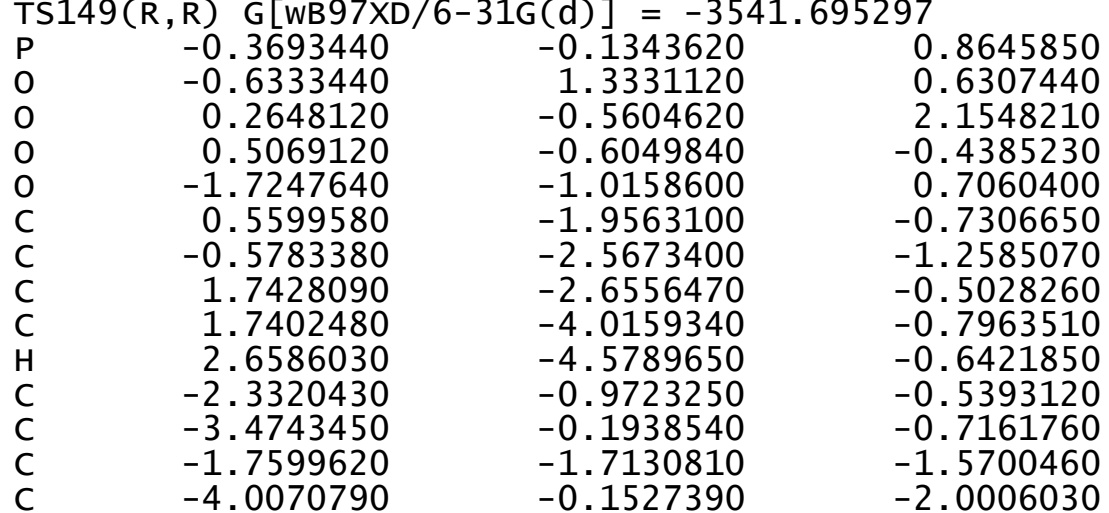




$\left.\begin{array}{rrrr}\mathrm{H} & -4.9083230 & 0.4337130 & -2.1676000 \\ \mathrm{C} & 2.9574280 & -1.9806670 & 0.0285550 \\ \mathrm{C} & -4.1053500 & 0.5554030 & 0.4040550 \\ \mathrm{C} & 3.6558690 & -1.0539220 & -0.7548720 \\ \mathrm{C} & 3.3279310 & -0.6471600 & -2.1707660 \\ \mathrm{C} & 5.4277490 & 0.5380250 & -1.2401710 \\ \mathrm{C} & 4.8147590 & -0.4368070 & -0.2682080 \\ \mathrm{C} & 3.1172460 & 0.8792920 & -2.2039220 \\ \mathrm{H} & 2.9546820 & 1.1987040 & -3.2397180 \\ \mathrm{H} & 2.2058740 & 1.1164520 & -1.6514120 \\ \mathrm{C} & 4.3555890 & 1.5905190 & -1.5994040 \\ \mathrm{H} & 4.7728000 & 2.3147030 & -2.3085140 \\ \mathrm{H} & 4.0765680 & 2.1523280 & -0.7016830 \\ \mathrm{H} & 6.3131870 & 1.0205560 & -0.8123150 \\ \mathrm{H} & 2.4378860 & -1.1594290 & -2.5442070 \\ \mathrm{C} & 5.7987720 & -0.2325590 & -2.5272180 \\ \mathrm{H} & 6.1671610 & 0.4792780 & -3.2751560 \\ \mathrm{H} & 6.6154980 & -0.9299210 & -2.3155440 \\ \mathrm{C} & 4.5501990 & -0.9890330 & -3.0517810 \\ \mathrm{H} & 4.3338240 & -0.7105630 & -4.0895390 \\ \mathrm{H} & 4.7164370 & -2.0711150 & -3.0371930 \\ \mathrm{C} & 3.4475150 & -2.2819270 & 1.3046590 \\ \mathrm{C} & 2.8656030 & -3.2696830 & 2.2872620 \\ \mathrm{C} & 5.0202530 & -2.1334550 & 3.1542480 \\ \mathrm{C} & 4.6199210 & -1.6756980 & 1.7761560 \\ \mathrm{C} & 3.9440730 & -4.3439180 & 2.5533100 \\ \mathrm{H} & 3.5520330 & -5.0771290 & 3.2675570 \\ \mathrm{H} & 4.1622450 & -4.8831800 & 1.6251040 \\ \mathrm{C} & 5.2253290 & -3.6654750 & 3.1086910 \\ \mathrm{H} & 5.4492300 & -4.0255350 & 4.1195850 \\ \mathrm{H} & 6.0928860 & -3.9013800 & 2.4838900 \\ \mathrm{H} & 5.9370880 & -1.6340390 & 3.4863750 \\ \mathrm{H} & 1.9517470 & -3.7286420 & 1.9040880 \\ \mathrm{C} & 3.8543850 & -1.8309360 & 4.1204370 \\ \mathrm{H} & 4.1247190 & -2.1709730 & 5.1271060 \\ \mathrm{H} & 3.6905390 & -0.7499260 & 4.1789210 \\ \mathrm{C} & 2.5738130 & -2.5440620 & 3.6181030 \\ \mathrm{H} & 2.2289810 & -3.2840780 & 4.3497760 \\ \mathrm{H} & 1.7578230 & -1.8361110 & 3.4639370 \\ \mathrm{C} & 5.3064050 & -0.7559830 & 0.9921970 \\ \mathrm{H} & 6.2181260 & -0.2891000 & 1.3618770 \\ \mathrm{C} & -5.2992790 & 0.5816990 & 2.5241550 \\ \mathrm{C} & -5.8141400 & -0.3013000 & 3.6310740 \\ \mathrm{C} & -4.6354230 & -1.6126920 & 1.7437430 \\ \mathrm{C} & -4.6470280 & -0.1218480 & 3.5041690 \\ \mathrm{C} & -6.8022800 & -1.3190600 & \\ & & & \end{array}\right]$

-7.1390870
-7.6888520
-6.0992890
-6.1069720
-6.6178350
-4.1375290
-6.3066560
-3.9157830
-3.9417120
-2.8653630
-4.6163400
-4.9755580
-3.9188730
-4.8931670
-4.9555580
-3.6446810
-4.2155060
-3.5114530
-3.5401210
-3.0417120
-2.7161670
-2.2927040
-1.8884140
-3.0814530
-5.5221540
-4.8109530
-4.4046290
-5.4529770
-5.6203120
-5.6635410
-6.6528290
-5.4349860
-5.9560290
-2.2619380
-1.5293000
-1.9369050
-3.4582850
-4.0107130
-3.4089890
-1.5077990
-1.7185730
-0.4500610
-3.8711980
-3.7713500
-5.1015570
-3.8075720
3.8036300

2. 6502220

1.8720850

2. 0656960

0.9208660

0.9357490

4.4115860

3. 0842310

3. 2920600

2. 9899030

4. 2165530

5.0043930

4.6844850

1. 3717620

1. 1463530

$-0.6916310$

0.3592150

1. 0871390

0.8530730

2. 0708730

0.0149340

$-0.7356650$

0.4691240

$-1.4495510$

1.9411050

$-1.3348050$

$-2.0543740$

$-1.8981640$

$-0.2264200$

$-0.4169820$

$-0.1988990$

2. 4551800

3. 2426550

$-2.8787920$

$-4.0458450$

$-5.3974660$

$-5.5058200$

$-4.4628290$

$-3.0842360$

$-6.2021700$

$-4.0664360$

$-3.8921620$

$-5.3388190$

$-6.5077830$

-4.3956290
-4.7885910 


-1.5264710
-0.5859930
0.5974090
-1.8826330
-1.6662250
-0.6733360
0.6845410
-2.6245470
-2.4018690
-2.5536400
-1.0627690
-0.5572070
1.3399330
1.1769430
-1.2770800
1.4989420
0.4275910
2.1539940
3.2672540
1.6998670
3.9246530
3.6115230
2.3624170
0.8400700
3.4736780
4.7906680
2.0133240
3.9901940
0.0441310
1.9839810
2.6559740
1.6049760
2.6129190
0.6258140
1.9429220
1.2656610
4.0952310
3.1978780
1.7457480
4.0612740
5.1264510
3.2439230
1.2917640
4.5241020
4.6238770
3.7646290

-5.5056880
-1.4439140
-1.2353790
-1.7358180
-2.3738960
-1.5439820
-1.5118230
-2.4564180
-0.7754730
-2.3440360
-0.5227410
-1.9488050
-0.7706560
-2.4850970
-3.3943560
-0.7848850
-1.1245170
-1.9113300
-1.6478840
-3.2225430
-2.6912090
-0.6246660
-4.2647510
-3.4174100
-4.0000190
-2.4860790
-5.2845890
-4.8166540
-0.3939010
0.3515580
2.1728820
2.2134540
1.3351900
1.8445450
3.3276840
2.9778180
3.6084630
3.2361450
3.0585590
2.5219290
3.7507790
4.0033990
4.0264710
1.6011030
2.8239370
4.5612430

$\begin{array}{llll}\mathrm{H} & 3.5490190 & 4.6687480 & 2.2978620 \\ \mathrm{H} & 1.1219130 & 4.5586140 & 2.6192890 \\ 154 & & \end{array}$

154 TS150(R, R) G[WB97XD/6-31G(d) $]=-3541.695518$

$\begin{array}{lrrr}\mathrm{TS} 150(\mathrm{R}, \mathrm{R}) \mathrm{G}[\mathrm{WB} 97 \mathrm{XD} / 6-31 \mathrm{G}(\mathrm{d})]=-3541.695518 \\ \mathrm{P} & -0.3623100 & -0.1308120 & 0.8486520 \\ \mathrm{O} & -0.6307070 & 1.3369480 & 0.6211660 \\ \mathrm{O} & 0.2770800 & -0.5599410 & 2.1352300 \\ \mathrm{O} & 0.5092950 & -0.5949860 & -0.4595520 \\ \mathrm{O} & -1.7143010 & -1.0175700 & 0.6903600 \\ \mathrm{C} & 0.5576410 & -1.9463020 & -0.7537160 \\ \mathrm{C} & -0.5779570 & -2.5487520 & -1.2944740 \\ \mathrm{C} & 1.7330150 & -2.6533990 & -0.5085230 \\ \mathrm{C} & 1.7234500 & -4.0142080 & -0.7956170 \\ \mathrm{H} & 2.6340190 & -4.5848390 & -0.6242340 \\ \mathrm{C} & -2.3311830 & -0.9657500 & -0.5497100 \\ \mathrm{C} & -3.4785000 & -0.1910780 & -0.7069430 \\ \mathrm{C} & -1.7616000 & -1.6910510 & -1.5946410 \\ \mathrm{C} & -4.0262970 & -0.1412760 & -1.9850660 \\ \mathrm{H} & -4.9310940 & 0.4436610 & -2.1367300 \\ \mathrm{C} & 2.9463460 & -1.9841820 & 0.0331260 \\ \mathrm{C} & -4.0955380 & 0.5534700 & 0.4241360 \\ \mathrm{C} & 3.6547700 & -1.0613190 & -0.7462360 \\ \mathrm{C} & 3.3378300 & -0.6529790 & -2.1642740 \\ \mathrm{C} & 5.4392460 & 0.5197410 & -1.2213830 \\ \mathrm{C} & 4.8140640 & -0.4505760 & -0.2526990 \\ \mathrm{C} & 3.1361840 & 0.8746770 & -2.1981120 \\ \mathrm{H} & 2.9781970 & 1.1954510 & -3.2341930 \\ \mathrm{H} & 2.2245280 & 1.1163590 & -1.6479820 \\ \mathrm{C} & 4.3765450 & 1.5787310 & -1.5895710 \\ \mathrm{H} & 4.8030870 & 2.2970840 & -2.2990300 \\ \mathrm{H} & 4.0966230 & 2.1460070 & -0.6955730 \\ \mathrm{H} & 6.3249180 & 0.9971360 & -0.7882500 \\ \mathrm{H} & 2.4471330 & -1.1597360 & -2.5433220 \\ \mathrm{C} & 5.8139190 & -0.2549530 & -2.5048830 \\ \mathrm{H} & 6.1938810 & 0.4530390 & -3.2507150 \\ \mathrm{H} & 6.6232210 & -0.9587320 & -2.2860910 \\ \mathrm{C} & 4.5632550 & -1.0017390 & -3.0382660 \\ \mathrm{H} & 4.3549800 & -0.7199630 & -4.0767800 \\ \mathrm{H} & 4.7219460 & -2.0849660 & -3.0247220 \\ \mathrm{C} & 3.4267060 & -2.2876780 & 1.3124410 \\ \mathrm{C} & 2.8345070 & -3.2726550 & 2.2917770 \\ \mathrm{C} & 4.9912400 & -2.1491770 & 3.1701720 \\ \mathrm{C} & 4.6003140 & -1.6885580 & 1.7903420 \\ \mathrm{C} & 3.9059670 & -4.3530820 & 2.5613470 \\ \mathrm{H} & 3.5064420 & -5.0858100 & 3.2719530 \\ \mathrm{H} & 4.1265880 & -4.8915580 & 1.6332460\end{array}$




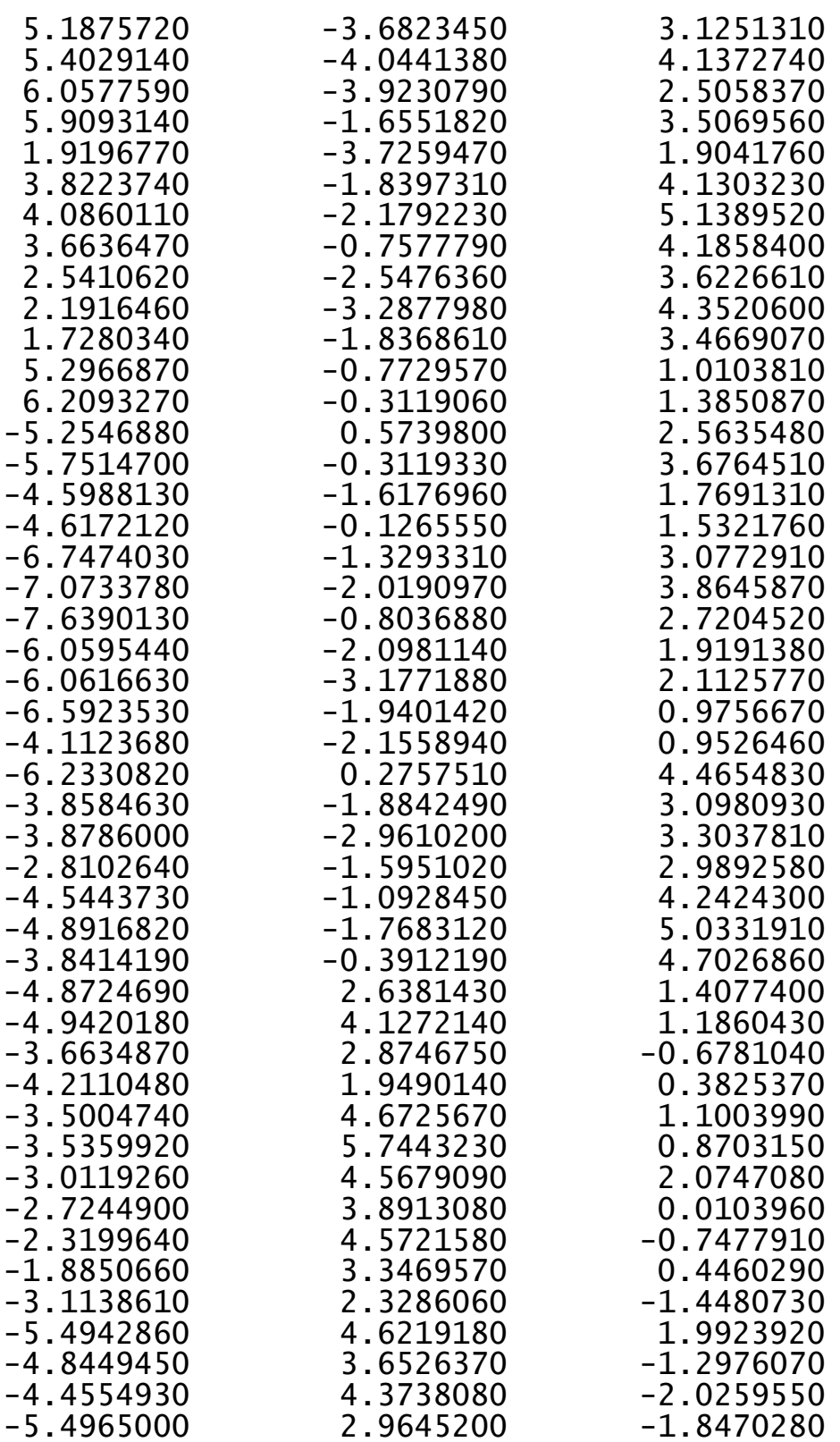

-5.6333510
-5.6807150
-6.6649340
-5.3941450
-5.9030910
-3.4376600
-2.2818360
-4.0892770
-3.1419270
-2.4031750
-1.5379340
-3.7047130
-4.9443790
-4.5060660
-3.1345440
-1.7744590
-1.0863740
-0.6927390
-2.4097380
-0.5907970
0.5823350
-1.8829900
-1.6585530
-0.7012820
0.6626460
-2.6195150
-2.4553690
-2.5107190
-1.1193710
-0.5866970
1.2959790
1.1756250
-1.2352720
1.5102140
0.4344400
2.1653960
3.2877580
1.7014730
3.9446000
3.6396400
2.3630620
0.8343340
3.4835840
4.8180840
2.0058150
3.9994540

4.3766600

5.4555470

4.0128150

1.9553150

2. 4953270

$-0.7751610$

$-1.5458250$

$-0.6268420$

$-0.9284160$

$-2.2385440$

$-2.1187360$

$-0.9752540$

$-1.3160880$

0.3821110

$-3.0483640$

$-2.5104700$

$-3.0837660$

$-1.4471790$

$-0.1163470$

$-3.9434620$

$-4.6740880$

$-4.6618050$

$-6.0429500$

$-6.8531030$

$-6.1668280$

$-6.5574600$

$-4.7711140$

-4. 0542400

$-6.9366090$

$-7.8720590$

$-6.6366820$

$-6.3205290$

$-5.9370550$

3. 5041650

.8477130

4. 2160590

5. 0058230

4.0622060

5.6394650

5.1073420

4. 6948910

3. 4399890

5.4818830

6.2521650

4.5713080

5.9732050
$-0.1733520$

$-0.3620210$

$-0.1262190$

2.4992450

3. 2962650

$-3.0798370$

$-2.8943920$

$-4.4417970$

$-5.6012580$

$-5.3404740$

$-4.0879080$

$-6.5398890$

$-4.4947910$

$-4.5391920$

$-5.2096750$

$-6.1953960$

$-3.8474480$

$-4.2994680$

$-5.7045510$

$-1.4857780$

$-1.2524800$

$-1.8310200$

$-2.4413000$

$-1.5708660$

$-1.5141740$

$-2.5490230$

$-0.8982100$

$-2.4863320$

$-0.5589870$

$-1.9563430$

$-0.7530770$

$-2.4744830$

$-3.4501100$

$-0.8008090$

$-1.1360840$

$-1.9309170$

$-1.6742520$

$-3.2393430$

$-2.7215390$

$-0.6532250$

$-4.2854160$

$-3.4290890$

$-2.5217430$

$-5.3029490$

$-4.8471600$ 


\begin{tabular}{|c|c|c|c|c|c|c|c|}
\hline & & & & & & & \\
\hline H & 0.0498240 & 2.2520160 & -0.4041330 & $\mathrm{C}$ & $\begin{array}{l}-4.4178500 \\
-48543720\end{array}$ & -1.5783800 & -1.5323820 \\
\hline c & 2.6625540 & $\begin{array}{l}. .5329390 \\
1.5\end{array}$ & $\begin{array}{l}0.3541020 \\
2.1660740\end{array}$ & $\begin{array}{l}\mathrm{H} \\
\mathrm{H}\end{array}$ & -4.1971230 & -2.0917650 & $\begin{array}{l}-2.2053030 \\
-0.0908010\end{array}$ \\
\hline c & 1.6121550 & 2.5595760 & 2.1990880 & $\mathrm{H}$ & -6.3547630 & -0.8518740 & -0.8297100 \\
\hline $\mathrm{H}$ & 2.6226940 & 0.8300930 & 1.3300430 & $\mathrm{H}$ & -2.3521800 & 0.9888220 & -2.6633040 \\
\hline H & 0.6340690 & 2.2724420 & 1.8277050 & C & -5.7549460 & 0.2295660 & -2.6310740 \\
\hline 0 & 1.9435450 & 1.0457150 & 3.3186300 & $\mathrm{H}$ & -6.1657290 & -0.5176030 & -3.3202320 \\
\hline H & 1.2690340 & 0.3802220 & 2.9661840 & $\mathrm{H}$ & -6.5255820 & 0.9937410 & -2.4873550 \\
\hline $\mathrm{C}$ & 4.0965000 & 3.0467530 & 3.6036950 & C & -4.4570930 & 0.8555920 & -3.2055960 \\
\hline c & 3.2021440 & 4.2282760 & 3.2229530 & $\mathrm{H}$ & -4.2360490 & 0.4466330 & -4.1981930 \\
\hline C & 1.7503680 & 3.7809420 & 3.0406890 & $\mathrm{H}$ & -4.5654580 & 1.9388690 & -3.3206290 \\
\hline c & 4.0665920 & 1.9652710 & 2.5209370 & C & -3.3689960 & 2.4812990 & 1.0613020 \\
\hline $\mathrm{H}$ & 5.1273860 & 3.3846100 & 3.7497380 & $\mathrm{c}$ & -2.7538340 & 3.5110270 & 1.9790030 \\
\hline $\mathrm{H}$ & 3.2456440 & 5.0098450 & 3.9875350 & C & -4.9665990 & 2.5371300 & 2.8965160 \\
\hline$H$ & 1.2920550 & 3.5381150 & 4.0075100 & $\mathrm{C}$ & -4.5696910 & 1.9601670 & 1.5628740 \\
\hline H & 4.5349820 & 2.3366110 & 1.6012770 & $\mathrm{C}$ & -3.7839960 & 4.6489140 & 2.1589500 \\
\hline$H$ & 4.6259300 & 1.0784140 & 2.8293380 & $\mathrm{H}$ & -3.3673060 & 5.4044940 & 2.8348970 \\
\hline$H$ & 3.7608120 & 2.6195800 & 4.5564240 & $\mathrm{H}$ & -3.9620250 & 5.1416400 & 1.1969030 \\
\hline $\mathrm{H}$ & 3.5579070 & 4.6693510 & 2.2845850 & C & -5.1050260 & 4.0680990 & 2.7315540 \\
\hline H & 1.1291070 & 4.5628410 & 2.5961130 & $\mathrm{H}$ & -5.3354440 & 4.5137830 & 3.7061350 \\
\hline & & & & $\mathrm{H}$ & -5.9472530 & 4.2904360 & 2.0683880 \\
\hline & , R) $\mathrm{G}[\mathrm{WB} 97 \mathrm{X}$ & $g(d)]=-354$ & 161 & $\mathrm{H}$ & -5.9082090 & 2.1033760 & 3.2511780 \\
\hline $\mathrm{P}$ & 0.3318230 & 0.1609980 & 0.7569210 & $\mathrm{H}$ & -1.8158670 & 3.9016370 & 1.5786600 \\
\hline 0 & 0.5441780 & -1.3293500 & 0.6507960 & C & -3.8276540 & 2.2612840 & 3.9019600 \\
\hline 0 & -0.3072070 & 0.7176770 & 1.9940430 & $\mathrm{H}$ & -4.0921970 & 2.6984280 & 4.8719150 \\
\hline 0 & -0.4930650 & 0.5620720 & -0.5994530 & $\mathrm{H}$ & -3.7165360 & 1.1825610 & 4.0530990 \\
\hline 0 & 1.7174650 & 0.9838500 & 0.5580790 & C & -2.5085840 & 2.8672810 & 3.3604200 \\
\hline c & -0.4938060 & 1.8965150 & -0.9691620 & $\mathrm{H}$ & -2.1268020 & 3.6369200 & 4.0415060 \\
\hline C & 0.6686280 & 2.4236650 & -1.5447130 & $\mathrm{H}$ & -1.7319400 & 2.1074730 & 3.2624570 \\
\hline c & -1.6415350 & 2.6561140 & -0.7562700 & C & -5.2863210 & 1.0116240 & 0.8430670 \\
\hline c & -1.5900770 & 4.0081620 & -1.0987070 & $\mathrm{H}$ & -6.2203260 & 0.6124630 & 1.2358640 \\
\hline $\mathrm{H}$ & -2.4742740 & 4.6210640 & -0.9387050 & C & 5.1419480 & -0.5452850 & 2.6419390 \\
\hline c & 2.3589530 & 0.8462040 & -0.6606000 & C & 5.6130750 & 0.3967870 & 3.7192560 \\
\hline c & 3.5013060 & 0.0528080 & -0.7322780 & C & 4.5552460 & 1.6065930 & 1.6967530 \\
\hline C & 1.8401740 & 1.5261880 & -1.7631560 & $\mathrm{C}$ & 4.5556670 & 0.1038320 & 1.5484610 \\
\hline $\mathrm{C}$ & 4.0988910 & -0.0703470 & -1.9826250 & $\mathrm{C}$ & 6.6482310 & 1.3619490 & 3.1000410 \\
\hline $\mathrm{H}$ & 4.9989770 & -0.6758250 & -2.0679010 & $\mathrm{H}$ & 6.9566300 & 2.0915510 & 3.8578860 \\
\hline c & -2.8787000 & 2.0611330 & -0.1817000 & $\mathrm{H}$ & 7.5434230 & 0.8024840 & 2.8094140 \\
\hline $\mathrm{C}$ & 4.0646700 & -0.6324150 & 0.4623620 & C & 6.0180680 & 2.0715420 & 1.8734120 \\
\hline c & -3.6053170 & 1.1011430 & -0.8977820 & $\mathrm{H}$ & 6.0329430 & 3.1599760 & 2.0035590 \\
\hline c & -3.2706090 & 0.5527680 & -2.2637640 & $\mathrm{H}$ & 6.5827190 & 1.8490690 & 0.9619260 \\
\hline C & -5.4406780 & -0.4493510 & -1.2796060 & $\mathrm{H}$ & 4.1086560 & 2.1024250 & 0.8321720 \\
\hline $\mathrm{c}$ & -4.7947590 & 0.5728030 & -0.3805770 & $\mathrm{H}$ & 6.0547050 & -0.1501880 & 4.5592260 \\
\hline $\mathrm{C}$ & -3.1248280 & -0.9771000 & -2.1415540 & C & 3.7726540 & 1.9646110 & 2.9794160 \\
\hline H & -2.9278160 & -1.4051120 & -3.1311090 & $\mathrm{H}$ & 3.8102970 & 3.0508230 & 3.1240620 \\
\hline & -2.2514 & 190239 & -1.5222120 & $\mathrm{H}$ & 2.7226080 & 1.6919960 & 2.84897 \\
\hline
\end{tabular}




$\begin{array}{lrrr}\text { C } & 4.3991970 & 1.2276620 & \\ \text { H } & 4.7275390 & 1.9404360 & 4.1919150 \\ \text { H } & 3.6665510 & 0.5639460 & 4.6620320 \\ \text { C } & 4.7662230 & -2.6679770 & 1.5949430 \\ \text { C } & 4.8160960 & -4.1683350 & 1.4616960 \\ \mathrm{C} & 3.6357880 & -3.0049720 & -0.5208470 \\ \mathrm{C} & 4.1585400 & -2.0290210 & 0.5061220 \\ \mathrm{C} & 3.3697510 & -4.6957160 & 1.3463910 \\ \mathrm{H} & 3.3971620 & -5.7779570 & 1.1709010 \\ \mathrm{H} & 2.8457050 & -4.5362040 & 2.2944440 \\ \mathrm{C} & 2.6494370 & -3.9602290 & 0.1883790 \\ \mathrm{H} & 2.2540490 & -4.6736010 & -0.5443060 \\ \mathrm{H} & 1.8091800 & -3.3731830 & 0.5630830 \\ \mathrm{H} & 3.1290100 & -2.4949840 & -1.3432940 \\ \mathrm{H} & 5.3257690 & -4.6250450 & 2.3169190 \\ \mathrm{C} & 4.8252890 & -3.8384200 & -1.0458860 \\ \mathrm{H} & 4.4500850 & -4.5949600 & -1.7452250 \\ \mathrm{H} & 5.5091430 & -3.1946040 & -1.6096120 \\ \mathrm{C} & 5.5575540 & -4.5065600 & 0.1485220 \\ \mathrm{H} & 5.5949660 & -5.5950420 & 0.0235590 \\ \mathrm{H} & 6.5920080 & -4.1544620 & 0.2165190 \\ \mathrm{C} & 5.2580560 & -1.9302540 & 2.6644170 \\ \mathrm{H} & 5.7265430 & -2.4299910 & 3.5108890 \\ \mathrm{C} & 3.5707700 & 0.5238650 & -3.1283460 \\ \mathrm{C} & 2.4233150 & 1.3239720 & -3.0286810 \\ \mathrm{C} & 4.2807280 & 0.3030740 & -4.4510100 \\ \mathrm{C} & 3.3992900 & 0.5835720 & -5.6660820 \\ \mathrm{C} & 2.6864670 & 1.9212820 & -5.4897570 \\ \mathrm{C} & 1.7583980 & 1.8719450 & -4.2780460 \\ \mathrm{H} & 4.0079890 & 0.5791110 & -6.5769180 \\ \mathrm{H} & 5.1585040 & 0.9642770 & -4.4894350 \\ \mathrm{H} & 4.6704700 & -0.7206490 & -4.4879720 \\ \mathrm{H} & 3.4328290 & 2.7159420 & -5.3520690 \\ \mathrm{H} & 2.1077560 & 2.1772920 & -6.3840660 \\ \mathrm{H} & 1.3313900 & 2.8588110 & -4.0952270 \\ \mathrm{H} & 0.9023370 & 1.2207930 & -4.5087670 \\ \mathrm{H} & 2.6505600 & -0.2125960 & -5.7746090 \\ \mathrm{C} & 0.7076310 & 3.7970710 & -1.8209430 \\ \mathrm{C} & 1.9474020 & 4.5398960 & -2.2553430 \\ \mathrm{C} & 2.1752880 & 5.7378420 & -1.3156920 \\ \mathrm{C} & 0.9360370 & 6.6620840 & -1.2192040 \\ \mathrm{C} & -0.3101450 & 6.0558930 & -1.8980880 \\ \mathrm{C} & -0.4303550 & 4.5851350 & -1.5991970 \\ \mathrm{H} & 1.8287330 & 4.9085930 & -3.2845920 \\ \mathrm{H} & 3.0505310 & 6.3017950 & -1.6543500 \\ \mathrm{H} & 1.1451580 & 7.6351600 & -1.6759120 \\ & & & \end{array}$

-0.2242650
2.8230480
2.4199990
0.7054020
-1.2123940
-1.6692210
-0.5559010
-2.3325090
-3.5177730
-1.8073400
-4.1763750
-3.9175830
-2.4688480
-0.8903090
-3.6523510
-5.0997820
-2.0615260
-4.1691170
-0.1631720
-2.1821120
-2.7546320
-1.7449120
-2.6898290
-0.7576190
-2.0131960
-1.3156740
-4.2426540
-3.3971630
-1.9289880
-4.1734920
-5.2858050
-3.4701050
-1.4607320
-4.6569940
-4.6980100
-3.8859150
-3.7710760
-1.3400310
-

6.1965460

3.8857090

5. 3410250

6.8530690

6. 5911380

$-3.5667930$

$-2.9999870$

$-4.3632390$

$-5.0395670$

$-4.4036780$

$-5.7522060$

$-4.9890300$

$-5.1177740$

$-3.8692910$

$-5.7908670$

$-6.2748620$

$-5.1479160$

$-6.3452060$

$-2.3395030$

$-3.4719090$

$-1.2760680$

$-2.3364460$

$-0.6504380$

$-2.1210780$

$-0.7208540$

$-0.1140100$

$-2.6015520$

$-3.8455590$

$-3.4730140$

$-1.6214140$

$-2.8831230$

$-4.5601110$

$-3.1642310$

$-2.0546180$

$-0.6899140$

$-2.1075390$

$-4.3474650$

$-4.3150720$

154

$\operatorname{TS} 152(R, R) G[W B 97 X D / 6-31 G(d)]=-3541.695271$

$\begin{array}{llrr} & \\ \mathrm{P} & -0.3647210 & -0.1678840 & 0.8201450 \\ \mathrm{O} & -0.6301690 & 1.3088370 & 0.6572520 \\ \mathrm{O} & 0.2756810 & -0.6556700 & 2.0852680 \\ \mathrm{O} & 0.5033050 & -0.5785920 & -0.5083930 \\ \mathrm{O} & -1.7186910 & -1.0451410 & 0.6268410 \\ \mathrm{C} & 0.5511890 & -1.9200170 & -0.8457300\end{array}$

$-2.9846870$

$-2.2525500$

$-0.3247350$

$-0.1661710$

$-1.5852930$

$-0.5616910$

$-0.9366570$

$-1.6284000$

$-1.3328190$

$-2.9220920$

$-2.3268940$

$-0.3257220$

$-3.9143700$

$-3.1423580$

$-3.6179720$

$-2.0978730$

$-4.9200530$

$-4.3958100$

$-0.2692750$

0.5593490

2. 2066530

2. 3280430

1. 3125670

1.9329070

3. 3128440

2.9021660

3.7697080

3.4906430

3.2738930

2. 5962550

3.9449990

4.3162920

4.2168150

1.7121170

2.8234660

4.6815070

2. 5906770

2. 9010540
$-1.9200170$

0.6268410
-0.8457300

1. 1451580

6.3017950
7.6351600

$-1.6759120$ 


$\begin{array}{rr}-0.5841490 & -2.5035300 \\ 1.7229210 & -2.6376420 \\ 1.7039650 & -3.9927990 \\ 2.6085650 & -4.5743530 \\ -2.3346820 & -0.9478310 \\ -3.4829350 & -0.1693560 \\ -1.7638600 & -1.6329610 \\ -4.0214870 & -0.0616660 \\ -4.9234660 & 0.5325880 \\ 2.9387750 & -1.9913380 \\ -4.1032970 & 0.5291970 \\ 3.6449870 & -1.0363060 \\ 3.3161100 & -0.5578010 \\ 5.4344270 & 0.5560300 \\ 4.8098230 & -0.4521890 \\ 3.1219230 & 0.9703600 \\ 2.9452040 & 1.3442710 \\ 2.2227190 & 1.1869430 \\ 4.3770500 & 1.6382450 \\ 4.8006770 & 2.3805740 \\ 4.1147440 & 2.1713150 \\ 6.3273890 & 1.0069840 \\ 2.4181020 & -1.0404990 \\ 5.7927000 & -0.1638190 \\ 6.1809680 & 0.5715910 \\ 6.5918230 & -0.8891990 \\ 4.5289980 & -0.8661350 \\ 4.3122360 & -0.5193230 \\ 4.6773990 & -1.9494580 \\ 3.4249130 & -2.3514180 \\ 2.8312030 & -3.3701840 \\ 4.9997200 & -2.2956630 \\ 4.6026680 & -1.7764900 \\ 3.8956970 & -4.4673530 \\ 3.4950270 & -5.2183570 \\ 4.1039060 & -4.9787270 \\ 5.1877100 & -3.8263740 \\ 5.4142170 & -4.2314510 \\ 6.0484210 & -4.0447210 \\ 5.9225660 & -1.8208840 \\ 1.9113770 & -3.8030440 \\ 3.8390020 & -2.0215170 \\ 4.1053790 & -2.4122490 \\ 3.6899920 & -0.9421730 \\ 2.5484300 & -2.6926260 \\ 2.1886900 & -3.4544570\end{array}$

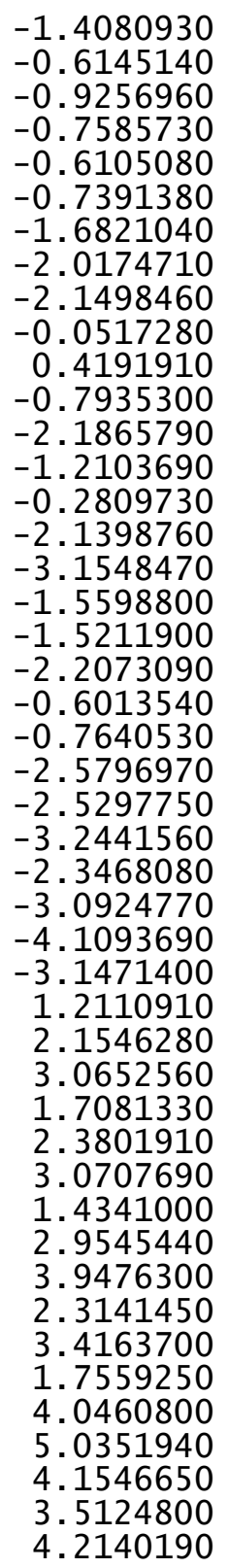

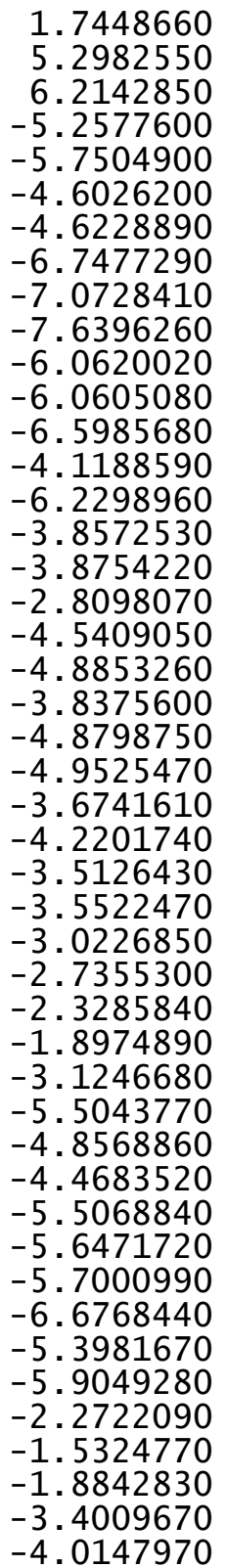
$-1.9659080$
$-0.8308530$
$-0.3890110$
0.4699420
$-0.4566200$
$-1.6907300$
$-0.1919720$
$-1.4518990$
$-2.1688410$
$-0.9133810$
$-2.1792670$
$-3.2641920$
$-1.9912580$
$-2.1978560$
0.1010140
$-2.0048790$
$-3.0883720$
$-1.7105220$
$-1.2566700$
$-1.9609550$
$-0.5717120$
2. 5759300
4.0720300
2. 8902080
1.9253360
4.6241730
5.7024940
4.4906840
3. 8808620
4. 5872960
3. 3205570
2. 3734800
4. 5362240
3.6892410
4.4382910
3. 0215880
4. 3681090
5.4528070
3.9975500
1. 8526200
2. 3621790
$-1.4253160$
$-1.9649330$
$-1.2142670$
$-1.1590400$
$-0.3385440$

3.3839140
0.9640520
1.3533620
2.5602380
3.6411420
1.6842790
1.5020550
3.0080510
3.7709930
2.6716350
1.8223940
1.9796760
0.8865810
0.8465670
4.4530320
3.0000820
3.1667470
2.8987980
4.1743500
4.9408740
4.6584370
1.4812230
1.3137730
-0.5958980
0.4301470
1.2446950
1.0485670
2.2147690
0.1295250
-0.6036160
0.5471910
-1.3859910
2.1382760
-1.1854390
-1.8855860
-1.7613710
-0.0346490
-0.1843490
0.0004520
2.5480260
3.3661820
-2.9783920
-4.1880620
-5.4714930
-5.6344910
-4.5011320 


$\begin{array}{rrr}-3.4210750 & -0.6387550 & -3.1370130 \\ -1.4154730 & -1.7114240 & -6.3275590 \\ -1.7703070 & -3.0248140 & -4.3401670 \\ -0.4544770 & -1.9219810 & -3.9987880 \\ -3.8011760 & -2.1817100 & -5.6164730 \\ -3.6799230 & -0.7235240 & -6.5999030 \\ -5.1005810 & -0.4875020 & -4.4641240 \\ -3.8623190 & 0.7292830 & -4.7111180 \\ -1.4810910 & -0.1935100 & -5.4300110 \\ -0.6072470 & -3.8951680 & -1.6233210 \\ -1.8986570 & -4.5886890 & -2.0152720 \\ -1.8860370 & -6.0832550 & -1.6976400 \\ -0.6222680 & -6.7203000 & -2.2691870 \\ 0.6077720 & -6.1441640 & -1.5703840 \\ 0.5576710 & -4.6382970 & -1.3896050 \\ -2.7844330 & -6.5522390 & -2.1129690 \\ -2.0789260 & -4.4719700 & -3.0907850 \\ -2.7384820 & -4.0952700 & -1.5143640 \\ -0.5719050 & -6.5151270 & -3.3470450 \\ -0.6396920 & -7.8090300 & -2.1508710 \\ 1.5203350 & -6.4128420 & -2.1160390 \\ 0.7022290 & -6.6056530 & -0.5775220 \\ -1.9150520 & -6.2360190 & -0.6106240 \\ 1.5206680 & 3.5423500 & -0.6522330 \\ 0.4383940 & 2.9121830 & -1.0162850 \\ 2.1780220 & 4.3040930 & -1.7479670 \\ 3.3103560 & 5.0676080 & -1.4576680 \\ 1.7063590 & 4.2218630 & -3.0600690 \\ 3.9694520 & 5.7458450 & -2.4752090 \\ 3.6687420 & 5.1137750 & -0.4349090 \\ 2.3701460 & 4.8990860 & -4.0764390 \\ 0.8312620 & 3.6196280 & -3.2760840 \\ 3.5008160 & 5.6595170 & -3.7850630 \\ 4.8509900 & 6.3376830 & -2.2494070 \\ 2.0068550 & 4.8305520 & -5.0970290 \\ 4.0190230 & 6.1851920 & -4.5816480 \\ 0.0543620 & 2.2798160 & -0.3162640 \\ 2.0128710 & 3.5292060 & 0.4819370 \\ 2.6658020 & 1.4239660 & 2.2189290 \\ 1.6175520 & 2.4503290 & 2.2968460 \\ 2.6267280 & 0.7602100 & 1.3513230 \\ 0.6398950 & 2.1835270 & 1.9095840 \\ 1.9415230 & 0.8873770 & 3.3460660 \\ 1.2654150 & 0.2415660 & 2.9609150 \\ 4.1006470 & 2.8690080 & 3.7261710 \\ 3.2094990 & 4.0677050 & 3.3948690\end{array}$

$\begin{array}{ll}\mathrm{C} & 1.7568840 \\ \mathrm{C} & 4.0699800 \\ \mathrm{H} & 5.1321830 \\ \mathrm{H} & 3.2547360 \\ \mathrm{H} & 1.2992040 \\ \mathrm{H} & 4.5419380 \\ \mathrm{H} & 4.6258010 \\ \mathrm{H} & 3.7624980 \\ \mathrm{H} & 3.5660070 \\ \mathrm{H} & 1.1364330\end{array}$
3.6314490
1. 8359620
3.1975480
4.8175820
3. 3447810
2. 2461170
0.9343500
2. 4021710
4.5456290
4.4337440

154

TS153 (R, R) G[WB97XD/6-31G(d) $]=-3541.695303$

$\begin{array}{lrrr}\text { TS153(R, R }) \text { G }[W B 97 X D / 6-31 G(d)]=-3541.695303 \\ \text { P } & 0.3443440 & 0.1692320 & 0.7663530 \\ \text { O } & 0.5615380 & -1.3197030 & 0.6506730 \\ \text { O } & -0.2920860 & 0.7160920 & 2.0091730 \\ \text { O } & -0.4872630 & 0.5741790 & -0.5845930 \\ \text { O } & 1.7261590 & 0.9979210 & 0.5653970 \\ \mathrm{C} & -0.4988060 & 1.9093310 & -0.9501590 \\ \mathrm{C} & 0.6595830 & 2.4502920 & -1.5240390 \\ \mathrm{C} & -1.6553550 & 2.6552050 & -0.7401840 \\ \mathrm{C} & -1.6211740 & 4.0066930 & -1.0878260 \\ \mathrm{H} & -2.5132410 & 4.6086500 & -0.9299530 \\ \mathrm{C} & 2.3573260 & 0.8672720 & -0.6598290 \\ \mathrm{C} & 3.4939980 & 0.0670440 & -0.7492690 \\ \mathrm{C} & 1.8337900 & 1.5600420 & -1.7515420 \\ \mathrm{C} & 4.0732690 & -0.0568550 & -2.0079460 \\ \mathrm{H} & 4.9664220 & -0.6704560 & -2.1080310 \\ \mathrm{C} & -2.8851240 & 2.0475470 & -0.1630360 \\ \mathrm{C} & 4.0716300 & -0.6234930 & 0.4356290 \\ \mathrm{C} & -3.6075850 & 1.0862980 & -0.8810630 \\ \mathrm{C} & -3.2764080 & 0.5521460 & -2.2533110 \\ \mathrm{C} & -5.4326750 & -0.4752580 & -1.2643800 \\ \mathrm{C} & -4.7902100 & 0.5455050 & -0.3612540 \\ \mathrm{C} & -3.1192510 & -0.9774580 & -2.1471920 \\ \mathrm{H} & -2.9301850 & -1.3940720 & -3.1431510 \\ \mathrm{H} & -2.2377550 & -1.1913670 & -1.5399650 \\ \mathrm{C} & -4.4018710 & -1.5938300 & -1.5310190 \\ \mathrm{H} & -4.8363080 & -2.3414940 & -2.2045200 \\ \mathrm{H} & -4.1693770 & -2.1105100 & -0.5939580 \\ \mathrm{H} & -6.3411250 & -0.8885180 & -0.8128540 \\ \mathrm{H} & -2.3628330 & 0.9984620 & -2.6530870 \\ \mathrm{C} & -5.7597410 & 0.2115940 & -2.6090080 \\ \mathrm{H} & -6.1664670 & -0.5340430 & -3.3022210 \\ \mathrm{H} & -6.5370310 & 0.9670510 & -2.4555100 \\ \mathrm{C} & -4.4709190 & 0.8551700 & -3.1848800 \\ \mathrm{H} & -4.2526970 & 0.4590670 & -4.1832980 \\ & & & \end{array}$

3.1940760

2. 5969650

3.8879890

4.1904980

4.1490920

1.6958320

2.8667230

4.6591580

2. 7863100
2.4750640

0.4590670

$-4.1832980$

0.6222680

0.6077720

1441640

. 6382970

$-4.4719700$

4.0952700

5151270

6.4128420

. 6056530

(360190

2. 9121830

.

5. 7458450

(137750

8990860

5. 6595170

8305520

2. 2798160

(

2. 1835270

4.0677050

3. 3948690 


\begin{tabular}{|c|c|c|}
\hline $\begin{array}{l}-4.5892870 \\
-3.3729380 \\
-2.7628990 \\
-4.9635940 \\
-4.5672500 \\
-3.8023780 \\
-3.3896610 \\
-3.9885740 \\
-5.1158430 \\
-5.3457630 \\
-5.9629130 \\
-5.9000270 \\
-1.8300580 \\
-3.8183280 \\
-4.0830890 \\
-3.6969670 \\
-2.5068020 \\
-2.1299670 \\
-1.7233710 \\
-5.2793630 \\
-6.2086050 \\
5.1821630 \\
5.6715030 \\
4.5873340 \\
4.5814640 \\
6.7001970 \\
7.0222990 \\
7.5890560 \\
6.0536070 \\
6.0727920 \\
6.6042980 \\
4.1295640 \\
6.1238410 \\
3.8238480 \\
3.8648480 \\
2.7717570 \\
4.4669010 \\
4.8084120 \\
3.7399240 \\
4.7855120 \\
4.8300710 \\
3.6200160 \\
4.1620680 \\
3.3807600 \\
3.4023970 \\
2.8725560\end{array}$ & $\begin{array}{r}1.9386850 \\
2.4576700 \\
3.4898230 \\
2.4938320 \\
1.9246910 \\
4.6180800 \\
5.3756230 \\
5.1115700 \\
4.0241150 \\
4.4647510 \\
4.2413170 \\
2.0509830 \\
3.8898090 \\
2.2248680 \\
2.6563420 \\
1.1466760 \\
2.8446710 \\
3.6160830 \\
2.0924840 \\
0.9737100 \\
0.5654630 \\
-0.5447420 \\
0.3933270 \\
1.6109970 \\
0.1086200 \\
1.3571130 \\
2.0836190 \\
0.7956610 \\
2.0721140 \\
3.1600670 \\
1.8516940 \\
2.1104530 \\
-0.1570050 \\
1.9670080 \\
3.0527940 \\
1.6958110 \\
1.2261800 \\
1.9367520 \\
0.5633280 \\
-2.6637110 \\
-4.1638810 \\
-2.9931180 \\
-2.0205760 \\
-4.6875580 \\
-5.7696010 \\
-4.5281350\end{array}$ & $\begin{array}{r}-3.2872810 \\
1.0841350 \\
2.0024720 \\
2.9255950 \\
1.5884350 \\
2.1894930 \\
2.8656790 \\
1.2294040 \\
2.7659720 \\
3.7429690 \\
2.1072800 \\
3.2826760 \\
1.5993420 \\
3.9257050 \\
4.8981410 \\
4.0726670 \\
3.3812770 \\
4.0631160 \\
3.2787530 \\
0.8671790 \\
1.2619120 \\
2.5989170 \\
3.6714830 \\
1.6680420 \\
1.5158400 \\
3.0395670 \\
3.7946990 \\
2.7337730 \\
1.8247110 \\
1.9582590 \\
0.9042530 \\
0.8114210 \\
4.5035580 \\
2.9626650 \\
3.1094860 \\
2.8461980 \\
4.1641000 \\
4.9260990 \\
4.6441110 \\
1.5523020 \\
1.4146920 \\
-0.5454570 \\
0.4748560 \\
1.3225690 \\
1.1450070 \\
2.2792370\end{array}$ \\
\hline
\end{tabular}

2.6436730
2.2352720
1.8107300
3.1003010
5.3529140
4.7986040
4.4101220
5.4747930
5.5485660
5.5808160
6.5850800
5.2955470
5.7755840
2.4003680
1.7611050
2.1638400
3.6836050
4.1808010
3.5343320
1.7770770
2.0556970
0.6721750
4.1305590
4.0049160
5.2700760
3.9724580
1.7115550
0.6809010
1.9155410
2.1096360
0.8615310
0.3673410
0.4689580
1.8057960
2.9894470
1.0692140
0.2695980
2.8013250
2.3302560
0.6129020
1.2813560
-1.6434010
0.5434890
2.3196460
3.4787150
-1.8368450

-3.9482850
-4.6592190
-3.3606050
-2.4801360
-4.6236230
-3.8280070
-4.5820180
-3.1846370
-4.5009050
-5.5892000
-4.1520210
-1.9299860
-2.4330790
1.3605860
1.9787870
1.2741790
1.1499660
0.2518280
0.5452520
1.8351480
3.0328340
1.9774140
2.1490690
0.7430000
0.3282390
-0.7959620
0.2741740
3.8224650
4.5852740
5.7797000
6.6937290
6.0679090
4.5962560
4.9604410
6.3523620
7.6637210
6.2073950
3.9448960
5.3782980
6.8936640
6.5902400
-3.5643620
-2.9790580
-4.3483930
-5.0614440
-4.3363840

0.1777760

$-0.5501520$

0.5672620

$-1.3579810$

$-1.0922490$

$-1.7870660$

$-1.6656150$

0.0885550

$-0.0394900$

0.1400590

2. 6159760

3.4539660

$-3.0255460$

$-4.2542120$

$-5.5485970$

$-5.6172830$

$-4.4861790$

$-3.1448630$

$-6.4062320$

$-4.3338270$

$-4.1364020$

$-5.5251130$

$-6.5820710$

$-4.3866820$

$-4.7445500$

$-5.5876140$

$-1.8053650$

$-2.2165990$

$-1.2643370$

$-1.1967960$

$-1.8898250$

$-1.5892810$

$-3.2440640$

$-1.5756970$

$-1.6607400$

$-2.9754750$

$-2.2006920$

$-0.2695390$

$-0.1495380$

$-1.5897710$

$-0.5590250$

$-0.9448710$

$-1.6268380$

$-1.3143660$

$-2.9374590$ 


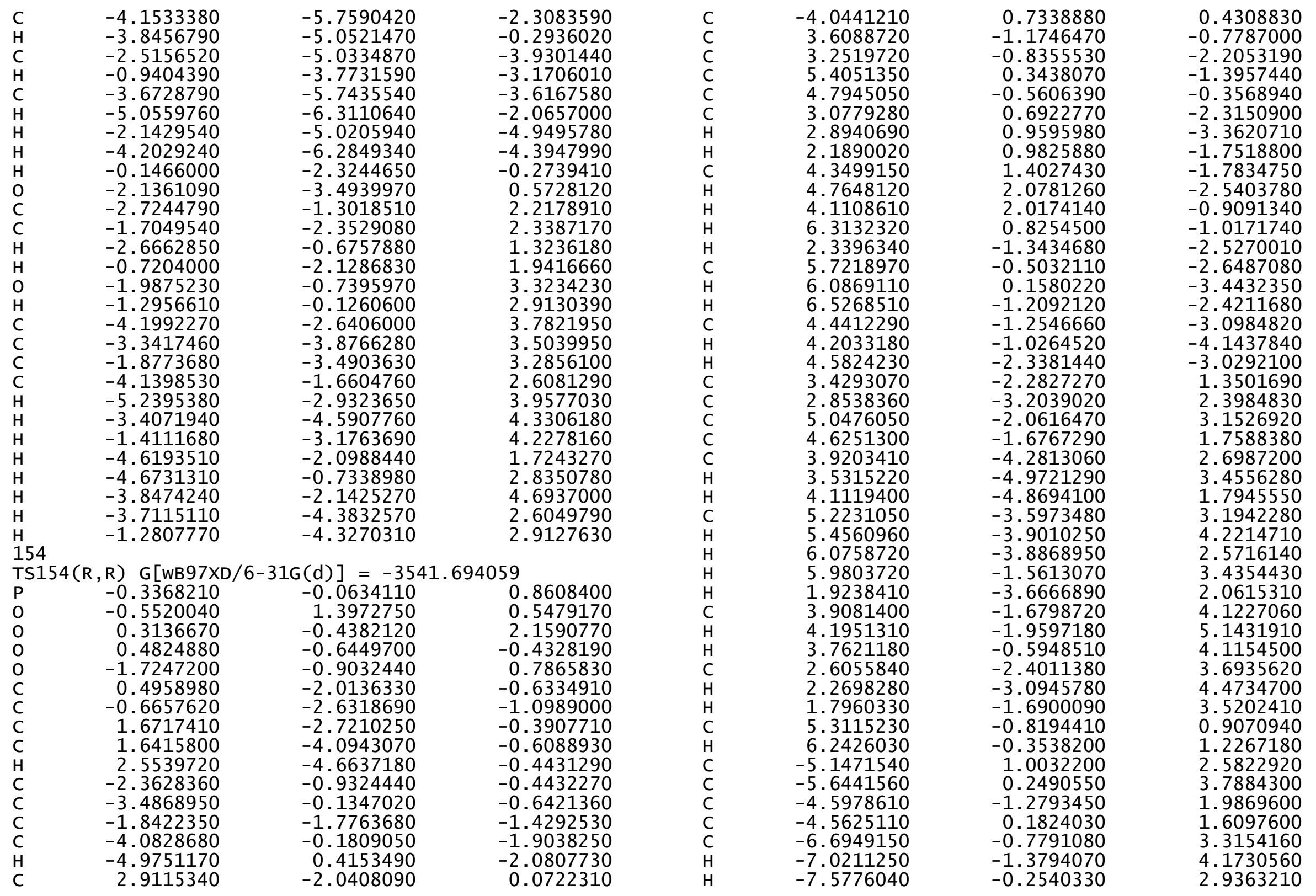




$\begin{array}{lr}-6.0732540 & -1.6783060 \\ -6.1168680 & -2.7348180 \\ -6.6247770 & -1.5801500 \\ -4.1567290 & -1.9131770 \\ -6.0788110 & 0.9296120 \\ -3.8352850 & -1.4530550 \\ -3.9019720 & -2.5025850 \\ -2.7776470 & -1.2289140 \\ -4.4504670 & -0.5211850 \\ -4.7969770 & -1.0968840 \\ -3.7057300 & 0.1935390 \\ -4.7272140 & 2.9311740 \\ -4.7592440 & 4.3923550 \\ -3.5443250 & 2.9235160 \\ -4.1115740 & 2.1225770 \\ -3.3073700 & 4.8893770 \\ -3.3237580 & 5.9237600 \\ -2.8085380 & 4.8988270 \\ -2.5616060 & 3.9602870 \\ -2.1114070 & 4.5347620 \\ -1.7583910 & 3.4215570 \\ -3.0204200 & 2.2886630 \\ -5.2865280 & 4.9799570 \\ -4.7004510 & 3.6856590 \\ -4.2874050 & 4.3336080 \\ -5.3733290 & 2.9769360 \\ -5.4662020 & 4.5220330 \\ -5.5040340 & 5.5789030 \\ -6.5008850 & 4.1765980 \\ -5.2440340 & 2.3756300 \\ -5.7166520 & 3.0074440 \\ -3.5473220 & -0.9423270 \\ -4.1574500 & -0.9921080 \\ -4.2783560 & -2.4549820 \\ -2.9130130 & -3.1774070 \\ -1.8444540 & -2.4423230 \\ -2.4156820 & -1.7371220 \\ -3.5275500 & -0.4387500 \\ -4.7314910 & -2.5064530 \\ -2.5294360 & -3.2799630 \\ -1.3818660 & -1.6692740 \\ -5.1381380 & -0.5052330 \\ -4.9675720 & -2.9596650 \\ -3.0425780 & -4.1959610 \\ -1.0398970 & -3.1280230 \\ -0.6937520 & -4.0341960\end{array}$

2.2154710
2.5047930
1.2746230
1.2146910
4.5286050
3.3189230
3.6291840
3.1594480
4.3954440
5.2619350
4.7599870
1.2233290
0.8553540
-0.8895990
0.2589150
0.6916090
0.3271870
1.6661440
-0.2987520
-1.1166210
0.2066370
-1.6075420
1.6146260
-1.5727550
-2.3548460
-2.0673920
-0.5128300
-0.8020100
-0.4185250
2.3867800
3.1372560
-2.9353830
-4.3128610
-4.7660580
-4.7565010
-3.9156770
-2.7079210
-5.0245740
-5.7614750
-5.7772050
-4.5451020
-4.3153200
-4.0794390
-4.3748440
-3.6409690
-1.2222110

0.4817570

$-1.9970340$

$-1.7924640$

$-0.8186020$

0.5482730

$-2.7568750$

$-2.5291200$

$-2.6517810$

$-1.2186100$

$-0.7128040$

1. 1876770

1.0511660

$-1.3919220$

1. 6080780

0.5209510

2. 2526860

3.4072260

1.7441620

4.0519630

3.7941890

2. 3922670

0.8513750

3.5451020

4.9516390

1. 9986170

4.0503310

0.1368010

2.1136720

2.7496900

1.7318910

2.6685080

0.7373900

2.0464710

1. 3464790

4.2631350

3. 3951740

1. 9268580

4.1743670

5.3069100

3.4782410

1.4813710

4.6286660

4.7165160

3.9384130

3.7427450
1.3213680
-4.7639560
-4.7596960
-6.1486920
-6.9484050
-6.2653780
-6.6622180
-4.8579690
-4.1626280
-7.0168290
-7.9731040
-6.7154500
-6.4497390
-6.0566670
3.4643460
2.8061400
4.1204610
4.8796130
3.9457190
5.4616200
4.9973300
4.5289440
3.3485240
5.2848980
6.0485940
4.3913470
5.7374890
2.2506570
3.5559060
1.5947380
2.6541190
0.8579970
2.3807670
1.1796230
0.5163510
3.1211470
4.3135950
3.9047140
1.9995410
3.4324210
5.1211710
3.7174090
2.3197900
1.1100740
2.7415840
4.7091110
4.6871470
$-0.9977680$

$-1.4983250$

$-2.0963520$

$-1.2342010$

$-1.2117230$

$-2.1748280$

$-0.5407470$

$-2.1337930$

$-0.2139760$

$-1.6066730$

$-0.4437080$

$-2.1719310$

$-3.1159400$

$-0.9948220$

$-1.2860210$

$-2.1638070$

$-1.9633120$

$-3.4528920$

$-3.0473400$

$-0.9568400$

$-4.5355270$

$-3.5987110$

$-4.3337540$

$-2.8915880$

$-5.5376820$

$-5.1817990$

$-0.5230120$

0.1300770

2.0275150

2.0384220

1.2241820

1.7014670

3.2170260

2. 9118020

3. 3655810

2. 9591560

2.8243910

2. 3283090

3.4744920

3.6929750

3.8094440

1. 3826600

2.6585250

4.3420450

1.9976020

2. 3594110 
154

$\operatorname{TS} 155(R, R) \quad G[W B 97 X D / 6-31 G(d)]=-3541.693216$

$\begin{array}{lrrr}\mathrm{P} & -0.3366650 & -0.0644140 & 0.8323530 \\ \mathrm{O} & -0.5518190 & 1.4046640 & 0.5605520 \\ \mathrm{O} & 0.2987350 & -0.4748630 & 2.1271420 \\ \mathrm{O} & 0.5006080 & -0.6050920 & -0.4681030 \\ \mathrm{O} & -1.7205360 & -0.9062860 & 0.7142120 \\ \mathrm{C} & 0.4991100 & -1.9669480 & -0.7142770 \\ \mathrm{C} & -0.6573290 & -2.5487790 & -1.2332930 \\ \mathrm{C} & 1.6519020 & -2.7048460 & -0.4512040 \\ \mathrm{C} & 1.5938520 & -4.0738120 & -0.6868890 \\ \mathrm{H} & 2.4837960 & -4.6700180 & -0.4948050 \\ \mathrm{C} & -2.3513390 & -0.8786240 & -0.5195040 \\ \mathrm{C} & -3.4765290 & -0.0759030 & -0.6836960 \\ \mathrm{C} & -1.8204730 & -1.6691110 & -1.5450620 \\ \mathrm{C} & -4.0586490 & -0.0530790 & -1.9522600 \\ \mathrm{H} & -4.9499580 & 0.5509470 & -2.1061300 \\ \mathrm{C} & 2.8919020 & -2.0601900 & 0.0590530 \\ \mathrm{C} & -4.0468940 & 0.7272300 & 0.4320590 \\ \mathrm{C} & 3.6251130 & -1.1867230 & -0.7537110 \\ \mathrm{C} & 3.3092330 & -0.8081360 & -2.1800480 \\ \mathrm{C} & 5.4603510 & 0.3152330 & -1.2901240 \\ \mathrm{C} & 4.8098260 & -0.6033940 & -0.2882840 \\ \mathrm{C} & 3.1616080 & 0.7244610 & -2.2562710 \\ \mathrm{H} & 3.0033240 & 1.0199690 & -3.2997420 \\ \mathrm{H} & 2.2655500 & 1.0146300 & -1.7039160 \\ \mathrm{C} & 4.4330470 & 1.4004650 & -1.6810290 \\ \mathrm{H} & 4.8779090 & 2.0820110 & -2.4150570 \\ \mathrm{H} & 4.1827400 & 2.0036710 & -0.8018970 \\ \mathrm{H} & 6.3661810 & 0.7731810 & -0.8780730 \\ \mathrm{H} & 2.3976480 & -1.2931610 & -2.5369680 \\ \mathrm{C} & 5.7953410 & -0.5089450 & -2.5533860 \\ \mathrm{H} & 6.1943370 & 0.1628030 & -3.3224010 \\ \mathrm{H} & 6.5802320 & -1.2354710 & -2.3205860 \\ \mathrm{C} & 4.5132510 & -1.2242890 & -3.0546860 \\ \mathrm{H} & 4.3049930 & -0.9638900 & -4.0987670 \\ \mathrm{H} & 4.6337880 & -2.3116720 & -3.0120930 \\ \mathrm{C} & 3.3724660 & -2.3408230 & 1.3436130 \\ \mathrm{C} & 2.7548840 & -3.2739890 & 2.3575440 \\ \mathrm{C} & 4.9530750 & -2.1942660 & 3.1874340 \\ \mathrm{C} & 4.5698870 & -1.7683280 & 1.7943200 \\ \mathrm{C} & 3.7927490 & -4.3794190 & 2.6554820 \\ \mathrm{H} & 3.3733590 & -5.0794360 & 3.3872780 \\ \mathrm{H} & 3.9933860 & -4.9503360 & 1.7423170 \\ \mathrm{C} & 5.0969840 & -3.7338160 & 3.1961910 \\ \mathrm{H} & 5.3005900 & -4.0665930 & 4.2206370\end{array}$

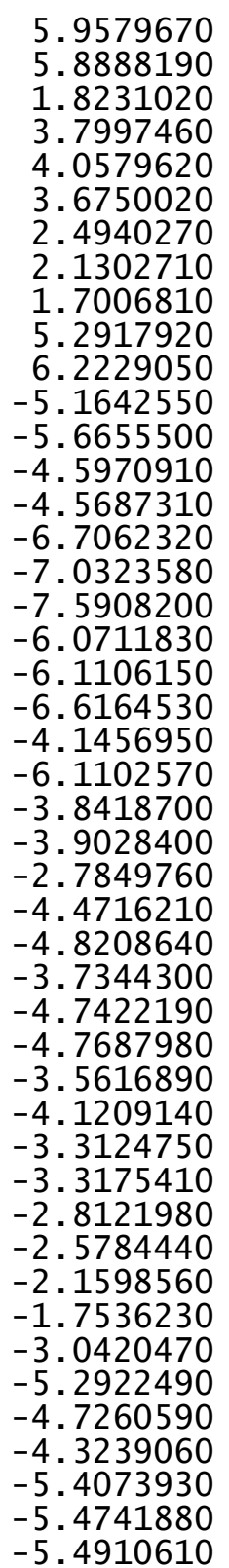

-4.0257550
-1.7196340
-3.7091570
-1.8111160
-2.1178580
-0.7233490
-2.4976820
-3.2038490
-1.7687450
-0.9035140
-0.4629600
0.8632750
0.0361330
-1.3763010
0.1051430
-0.9693520
-1.6223320
-0.4290110
-1.7968130
-2.8690600
-1.6454060
-1.9601800
0.6688510
-1.6233430
-2.6899640
-1.3827150
-0.7618900
-1.3911010
-0.0657060
2.8701580
4.3516540
2.9923360
2.1232950
4.8469260
5.9095910
4.7644720
3.9994810
4.6373850
3.4408860
2.4021550
4.8920920
3.7875780
4.4708930
3.1031920
4.5743830
5.6471010

2. 5860970

3. 5027440

1.9901030

4. 1400710

5.1605070

4.1525030

3. 6662360

4.4219110

3.4936400

0.9804470

1. 3337410

2. 5891950

3.7446370

1.8642270

1. 5736180

3. 2046750

4.0227770

2.8523150

2.0569530

2. 2817590

1.1195050

1. 0590850

4. 5203440

3. 1886960

3. 4351170

3. 0499960

4. 3143210

5.1415780

4.7269830

1. 3499680

1.0749770

$-0.7614060$

0.3403800

0.9474300

0.6759750

1. 9178740

$-0.1224980$

$-0.9097410$

0.3231370

$-1.5194740$

1.8711670

$-1.3911170$

$-2.1483960$

$-1.9079630$

$-0.2817570$

$-0.5076000$ 


$\begin{array}{rrr}-6.5156960 & 4.2453130 & -0.2062070 \\ -5.2635670 & 2.2447590 & 2.4753650 \\ -5.7412130 & 2.8293210 & 3.2602010 \\ -3.5076420 & -0.7506620 & -3.0196740 \\ -4.0968090 & -0.7026700 & -4.4062780 \\ -4.1781110 & -2.1261580 & -4.9736550 \\ -2.7897580 & -2.7944910 & -5.0365850 \\ -1.7636360 & -2.1601170 & -4.0676550 \\ -2.3791400 & -1.5598020 & -2.8254880 \\ -3.4691980 & -0.0818350 & -5.0626910 \\ -4.6409950 & -2.1162170 & -5.9658710 \\ -2.3831840 & -2.7246240 & -6.0512550 \\ -1.2650900 & -1.3329050 & -4.5925800 \\ -5.0872660 & -0.2364040 & -4.3837750 \\ -4.8385220 & -2.7088530 & -4.3211440 \\ -2.8955880 & -3.8633640 & -4.8229240 \\ -0.9728690 & -2.8722930 & -3.8222560 \\ -0.7156150 & -3.9479740 & -1.3839220 \\ -2.0184650 & -4.6180480 & -1.7787180 \\ -2.0465630 & -6.1091190 & -1.4468720 \\ -0.7804820 & -6.7819200 & -1.9699190 \\ 0.4381360 & -6.2224810 & -1.2390040 \\ 0.4295060 & -4.7101910 & -1.1176930 \\ -2.9422730 & -6.5631420 & -1.8841660 \\ -2.1815100 & -4.5027510 & -2.8571050 \\ -2.8531170 & -4.1006630 & -1.2934560 \\ -0.6897310 & -6.5927970 & -3.0481950 \\ -0.8271270 & -7.8681230 & -1.8373290 \\ 1.3630140 & -6.5403640 & -1.7348000 \\ 0.4737320 & -6.6507570 & -0.2274890 \\ -2.1125510 & -6.2496960 & -0.3598170 \\ 1.6482620 & 3.4587390 & -0.9421810 \\ 0.5441570 & 2.8339670 & -1.2438070 \\ 2.3097550 & 4.1166740 & -2.1009350 \\ 3.4846960 & 4.8407580 & -1.8897120 \\ 1.7959470 & 3.9765320 & -3.3922460 \\ 4.1445980 & 5.4219470 & -2.9651830 \\ 3.8753080 & 4.9319490 & -0.8818880 \\ 2.4590770 & 4.5587990 & -4.4661820 \\ 0.8869090 & 3.4067640 & -3.5468340 \\ 3.6325910 & 5.2791840 & -4.2537290 \\ 5.0603850 & 5.9811270 & -2.8012390 \\ 2.0613570 & 4.4472580 & -5.4699890 \\ 4.1497060 & 5.7307130 & -5.0951590 \\ 0.1493720 & 2.2704510 & -0.4920480 \\ 2.1558980 & 3.5190160 & 0.1839150\end{array}$

$\begin{array}{ll}\mathrm{C} & 2.7497550 \\ \mathrm{C} & 1.7432900 \\ \mathrm{H} & 2.6734260 \\ \mathrm{H} & 0.7504020 \\ \mathrm{O} & 2.0228800 \\ \mathrm{H} & 1.3221800 \\ \mathrm{C} & 4.2595220 \\ \mathrm{C} & 3.4116500 \\ \mathrm{C} & 1.9409670 \\ \mathrm{C} & 4.1738280 \\ \mathrm{H} & 5.3050800 \\ \mathrm{H} & 3.4933250 \\ \mathrm{H} & 1.4799380 \\ \mathrm{H} & 4.6488490 \\ \mathrm{H} & 4.6983040 \\ \mathrm{H} & 3.9150780 \\ \mathrm{H} & 3.7776860 \\ \mathrm{H} & 1.3506350 \\ \mathrm{I} & \end{array}$

1.5343650

2.0821160

$0.8077380 \quad 1.2613220$

$2.3462690 \quad 1.7293780$

$1.1129790 \quad 3.2474780$

$0.4609300 \quad 2.9214360$

$3.0306950 \quad 3.4511220$

$4.2364270 \quad 3.0417520$

$3.8454060 \quad 2.8826770$

$1.9188340 \quad 2.4031120$

$3.3284730 \quad 3.5787480$

$5.0373130 \quad 3.7830100$

$\begin{array}{ll}3.6537100 & 3.8597400\end{array}$

$2.2405490 \quad 1.4682590$

$1.0195040 \quad 2.7352220$

2.6468800 4.4191750

$4.6354730 \quad 2.0884890$

$\operatorname{TS} 156(R, R) \quad G[W B 97 X D / 6-31 G(d)]=-3541.693308$

$\begin{array}{lrrr}\text { TS156(R, R) } \text { G }[\mathrm{WB} 97 \times \mathrm{D} / 6-31 \mathrm{G}(\mathrm{d})]=-3541.693308 \\ \mathrm{P} & -0.3575250 & -0.0875640 & 0.8949930 \\ \mathrm{O} & -0.6447350 & 1.3492380 & 0.5321860 \\ \mathrm{O} & 0.2942150 & -0.3872540 & 2.2116940 \\ \mathrm{O} & 0.5065020 & -0.6650940 & -0.3705830 \\ \mathrm{O} & -1.7006640 & -0.9982550 & 0.8330320 \\ \mathrm{C} & 0.5732190 & -2.0375030 & -0.5354440 \\ \mathrm{C} & -0.5610390 & -2.7019140 & -1.0139940 \\ \mathrm{C} & 1.7658740 & -2.6960620 & -0.2451050 \\ \mathrm{C} & 1.7909640 & -4.0815210 & -0.4067490 \\ \mathrm{H} & 2.7168580 & -4.6142880 & -0.2016480 \\ \mathrm{C} & -2.3319210 & -1.0793740 & -0.3973710 \\ \mathrm{C} & -3.4961820 & -0.3448930 & -0.6118660 \\ \mathrm{C} & -1.7683860 & -1.9022680 & -1.3726470 \\ \mathrm{C} & -4.0831160 & -0.4567640 & -1.8688650 \\ \mathrm{H} & -5.0051020 & 0.0891390 & -2.0580390 \\ \mathrm{C} & 2.9737990 & -1.9473740 & 0.1975960 \\ \mathrm{C} & -4.0942190 & 0.5252990 & 0.4376050 \\ \mathrm{C} & 3.6318770 & -1.0802310 & -0.6840210 \\ \mathrm{C} & 3.2659260 & -0.8120790 & -2.1238770 \\ \mathrm{C} & 5.3565710 & 0.4985520 & -1.3543250 \\ \mathrm{C} & 4.7836960 & -0.3924000 & -0.2830580 \\ \mathrm{C} & 3.0201190 & 0.7005440 & -2.2918450 \\ \mathrm{H} & 2.8264170 & 0.9184570 & -3.3485360 \\ \mathrm{H} & 2.1173270 & 0.9698760 & -1.7407400 \\ \mathrm{C} & 4.2557560 & 1.4909660 & -1.7881730 \\ \mathrm{H} & 4.6422010 & 2.1528310 & -2.5717160 \\ & & & \\ & & & \end{array}$




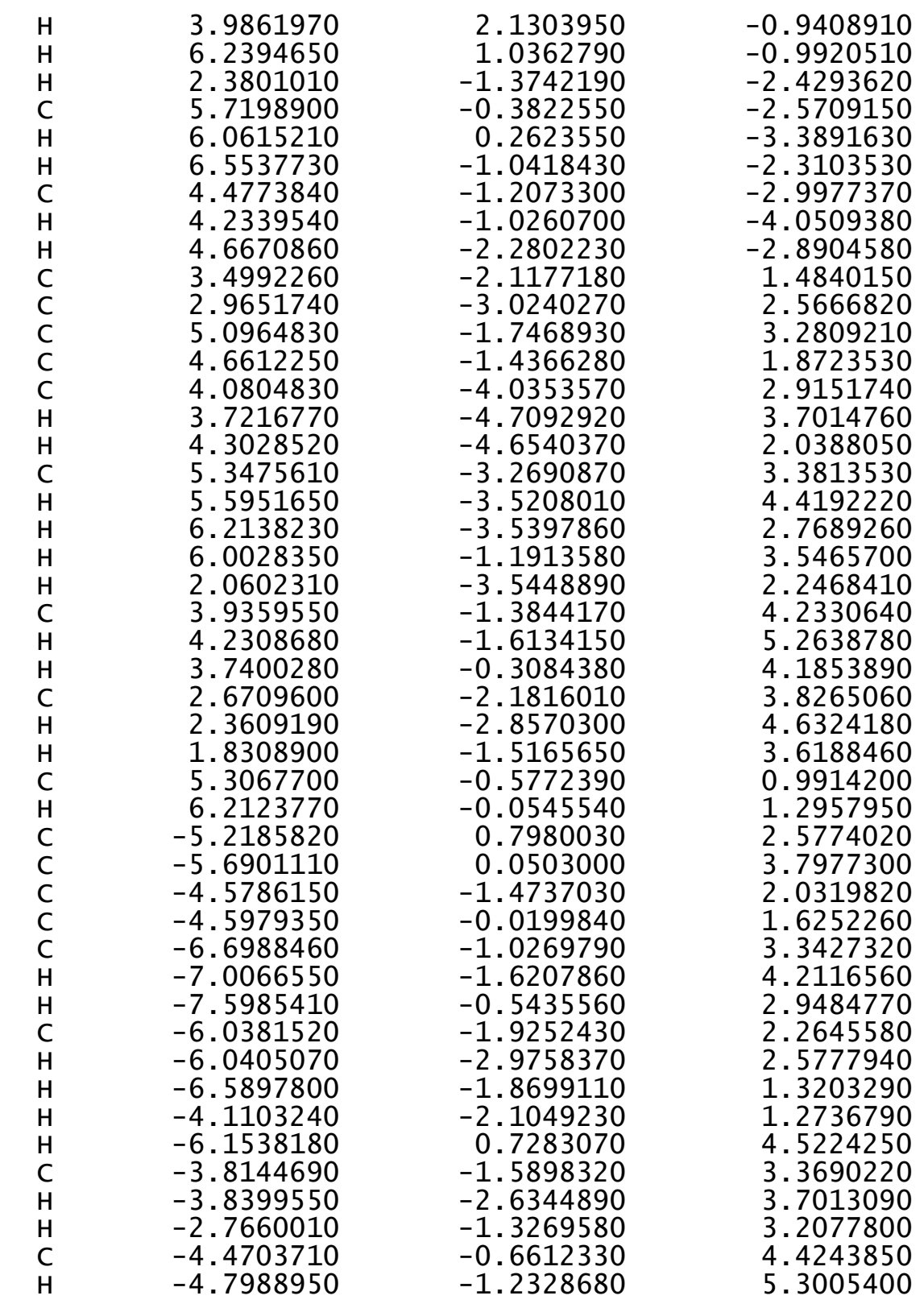

-3.7548960
-4.8700650
-4.9664560
-3.6700370
-4.2134060
-3.5390300
-3.6031710
-3.0499100
-2.7399850
-2.3033460
-1.9216280
-3.1113140
-5.5270260
-4.8506250
-4.4565510
-5.4831530
-5.6687190
-5.7619160
-6.6834920
-5.3705920
-5.8745890
-3.5161390
-2.3312880
-4.2264300
-3.3243640
-2.5228460
-1.6101950
-3.9293420
-5.0634050
-4.6750320
-3.2125350
-1.9218120
-1.0943730
-0.8163180
-2.6288290
0.6580220
0.6459330
-0.0006740
-1.4302930
-1.7295720
-0.5274800
0.0750030
-0.0101880
-2.1716010
-2.0413840
1.6630090

0.087972

2. 7100290

4.1583710

2.7038530

1. 9062360

4.7199150

5.7397260

4.7863000

3.7973950

. 3685200

3. 3073080

2. 0754910

4. 7402020

3. 3988440

4. 0513960

2. 6511720

4.2176820

5.2634440

3.8178670

2. 1604670

2. 7890620

$-1.2084120$

$-1.9196020$

$-1.2586900$

$-1.6946580$

$-2.9306390$

$-2.6038920$

$-1.8917870$

$-1.9671310$

$-0.2814180$

$-3.7395170$

$-3.2966460$

$-3.5025040$

$-1.9228410$

$-0.8842390$

$-4.7868600$

$-6.2853520$

$-6.6502930$

$-6.0789320$

$-4.9594100$

$-4.1014540$

$-6.7483950$

$-7.7372910$

$-6.8727490$

$-5.4282250$

$-6.6865160$
4.7780680

1.1761530

0.7703690

$-0.9274050$

0.2348150

0.6018350

0.2031740

1. 5791340

$-0.3520260$

$-1.1795070$

0.1779660

$-1.6244660$

1. 5099340

$-1.6394360$

$-2.4275490$

-2. 1299920

$-0.6053210$

$-0.9208250$

$-0.5091730$

2. 3496730

3. 0822300

$-2.8977210$

$-2.6625240$

$-4.2375990$

$-5.3906500$

$-4.9883370$

$-3.8091240$

$-6.2823250$

$-4.1570850$

$-4.4493370$

$-4.7090810$

$-5.8279070$

$-3.4687570$

$-4.1497710$

$-5.6463920$

$-0.7947700$

$-0.9571250$

$-2.3026490$

2.4318020

$-1.4096980$

$-1.0931670$

$-0.1391690$

$-2.4339700$

$-2.2916700$

$-0.4659440$

$-0.8969990$ 


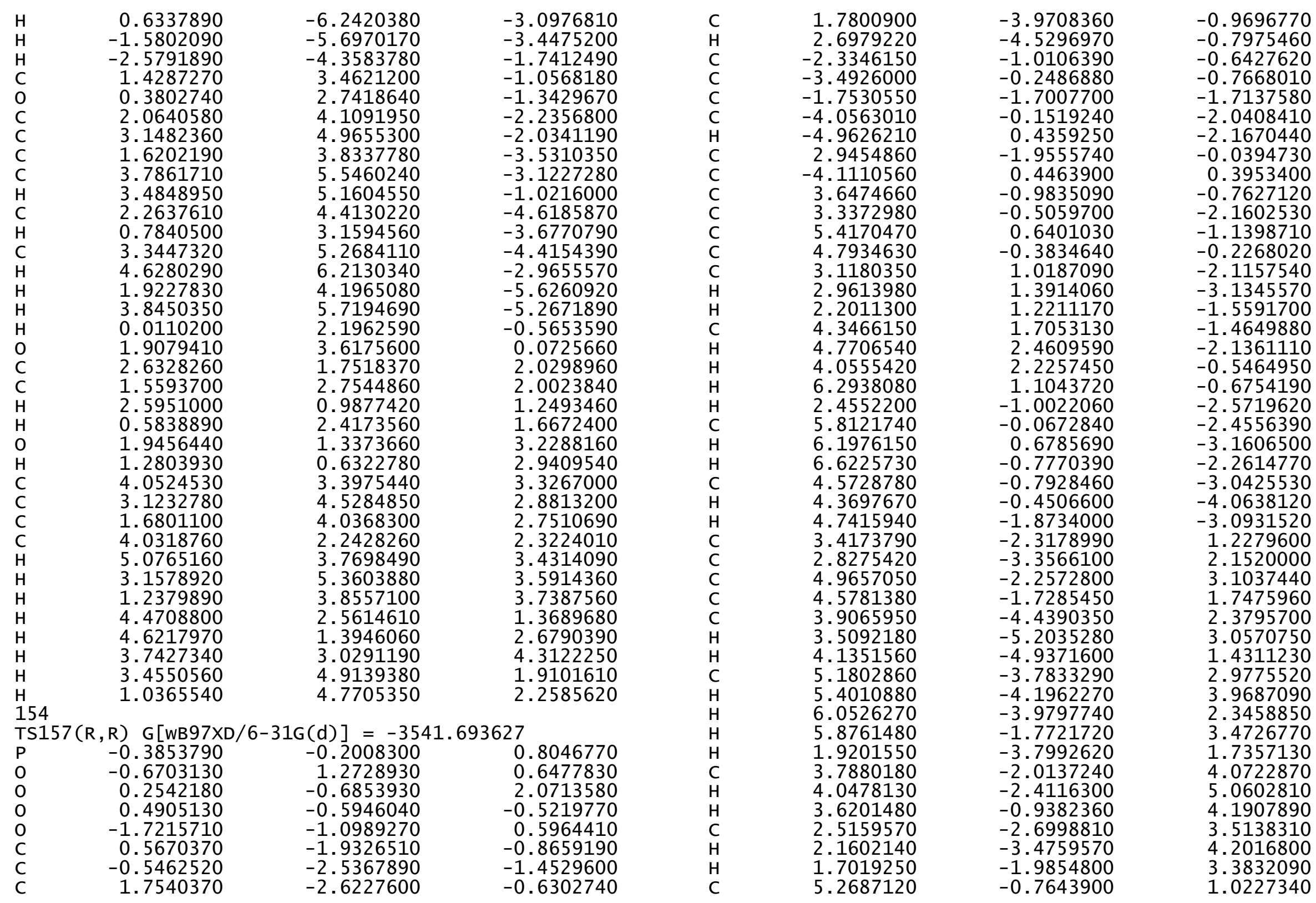




$\begin{array}{lr}6.1719930 & -0.3119480 \\ -5.2541320 & 0.3775480 \\ -5.7305910 & -0.5528940 \\ -4.5674460 & -1.7759260 \\ -4.6134380 & -0.2784760 \\ -6.7138860 & -1.5646960 \\ -7.0301050 & -2.2822490 \\ -7.6125070 & -1.0395650 \\ -6.0178610 & -2.2886370 \\ -5.9969730 & -3.3722230 \\ -6.5593570 & -2.1166530 \\ -4.0753840 & -2.2771080 \\ -6.2172710 & 0.0000010 \\ -3.8138150 & -2.0720390 \\ -3.8105090 & -3.1552190 \\ -2.7724160 & -1.7579060 \\ -4.5088780 & -1.3336140 \\ -4.8428950 & -2.0424400 \\ -3.8160470 & -0.6378450 \\ -4.9134670 & 2.4850160 \\ -5.0104670 & 3.9790960 \\ -3.7237320 & 2.8090660 \\ -4.2493730 & 1.8404140 \\ -3.5800600 & 4.5536980 \\ -3.6379060 & 5.6303150 \\ -3.0829300 & 4.4325710 \\ -2.7970760 & 3.8172910 \\ -2.4039420 & 4.5264630 \\ -1.9491460 & 3.2717750 \\ -3.1703140 & 2.2973180 \\ -5.5652240 & 4.4381490 \\ -4.9215090 & 3.5876450 \\ -4.5479520 & 4.3400410 \\ -5.5641450 & 2.9082300 \\ -5.7167430 & 4.2582970 \\ -5.7883910 & 5.3413460 \\ -6.7398560 & 3.8707380 \\ -5.4158540 & 1.7578560 \\ -5.9271680 & 2.2626290 \\ -2.3105180 & -1.5337210 \\ -1.6882900 & -2.0467700 \\ -1.5471090 & -0.8890040 \\ -2.8817580 & -0.1391420 \\ -3.9980280 & -0.5970620 \\ -3.4685020 & -0.7579610 \\ -2.3245200 & -2.8269610\end{array}$

1.4292770
2.5422820
3.6272090
1.6721480
1.4839820
2.9982470
3.7643460
2.6585960
1.8163380
1.9812650
0.8802880
0.8355820
4.4380540
2.9874680
3.1571340
2.8833120
4.1611660
4.9281230
4.6450020
1.4538750
1.2806520
-0.6310610
0.4019200
1.2011170
1.0002290
2.1691750
0.0855130
-0.6524210
0.5024260
-1.4217930
2.1060580
-1.2176410
-1.9223170
-1.7880590
-0.0653850
-0.2191180
-0.0232740
2.5256840
3.3439870
-2.9874320
-4.2612890
-5.2667070
-5.5046590
-4.5434520
-3.1430630
-4.7027870
$-1.1509110$
$-3.2240130$
$-4.3976620$
$-0.7114800$
$-0.7969500$
$-2.7252900$
$-4.8309050$
$-0.5157000$
$-1.7619710$
$-1.7192050$
$-0.4109460$
0.7668740
0.6657740
$-2.5821140$
$-1.8818330$
$-2.6438420$
$-0.3170140$
$-0.3992520$
1. 7120700
0.8256400
$-1.7966180$
1.4588820
0.4022670
2. 1216530
3. 2193560
1. 6915230
3. 8847540
3. 5453690
2. 3625230
0.8440720
3. 4581760
4. 7381860
2. 0330010
3. 9819240
0.0161030
1. 9245370
2. 5982770
1. 5262830
2. 5852380
0.5603100
1.8717580
1. 2146920
3. 9813670
3. 0661160
1. 6266130
3. 9880230

-1.2794150
-0.2797140
-1.5589820
-2.4999270
-0.1941820
0.9360500
0.1130160
-3.9199760
-4.6348060
-6.1405640
-6.7178310
-6.1320790
-4.6351070
-6.6168100
-4.4754240
-4.1884220
-6.4715400
-7.8102570
-6.3578350
-6.6233230
-6.3394960
3.5577420
2.9013710
4.3406670
5.1412830
4.2414260
5.8404510
5.2007430
4.9386220
3.6094440
5.7367560
6.4627450
4.8561940
6.2785120
2.2588580
3.5522880
1.4415390
2.4433040
0.7870300
2.1588570
0.8761070
0.2208300
2.9035430
4.0844720
3.6166990
1.8807170

$-6.5353540$

$-4.8940280$

$-4.0724150$

$-4.8740200$

$-5.3698140$

$-4.5642620$

$-1.7202720$

$-2.2066790$

$-1.9508310$

$-2.4843790$

$-1.7079970$

$-1.4810430$

$-2.4286770$

$-3.2856160$

$-1.7351580$

$-3.5506130$

$-2.4058810$

$-2.2159310$

$-0.7267270$

$-0.8736740$

$-0.6065600$

$-0.9984470$

$-1.6838140$

$-1.3620800$

$-3.0089340$

$-2.3611870$

$-0.3292250$

$-4.0069170$

$-3.2491310$

$-3.6840950$

$-2.1105980$

$-5.0378770$

$-4.4661340$

$-0.3087640$

0.5388200

2. 2594480

2. 3336750

1. 3841170

1. 9298280

3. 3707230

2. 9692300

3.7986820

3. 4689680

3.2460610

2.6596340 


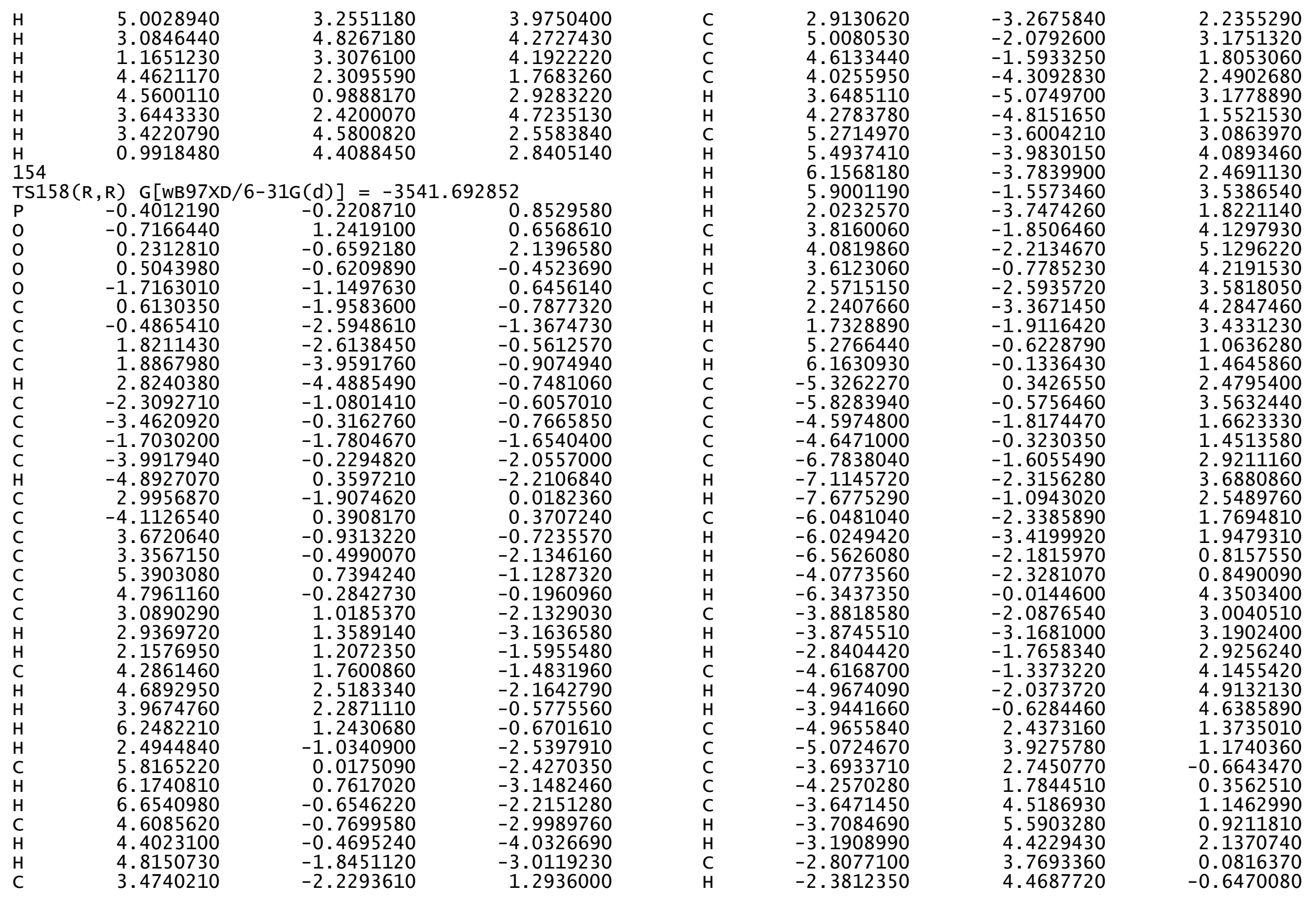




-1.9793720
-3.0996700
-5.6679970
-4.8678730
-4.4673060
-5.4763890
-5.7243100
-5.8095840
-6.7408310
-5.4998960
-6.0443260
-2.2273310
-1.5821510
-1.4069160
-2.7233350
-3.8691740
-3.3773880
-2.2148120
-1.0072390
-3.0439140
-4.2698370
-0.6159820
-0.6479250
-2.5521860
-4.6934430
-0.4170260
0.7901880
-1.6606620
-1.3574010
-0.3723580
0.9533610
-2.2906490
-2.2590700
-2.2903930
-0.7958750
-0.2037770
1.6092020
1.4755790
-0.9261570
1.3398980
0.3140360
1.9928610
3.0415340
1.6056090
3.7008480
3.3337730

3.2344100

2.2280300

4. 3912780

3. 5086270

4.2609740

2. 8208180

4.1774560

5.2570420

3.7707820

1.7208600

2. 2326350

$-1.6249370$

$-2.1702350$

$-1.0361980$

$-0.2644340$

$-0.6992910$

$-0.8474940$

$-2.9556420$

$-1.4531470$

$-0.4015870$

$-1.6613240$

$-2.6316670$

$-0.3480920$

0.8083540

0.0192710

$-3.9755750$

$-4.6543050$

$-4.7419330$

$-6.0912610$

$-6.8802140$

$-6.1269760$

$-6.6480000$

$-4.9059550$

$-4.1433800$

$-7.0140860$

$-7.8800420$

$-6.5938670$

$-6.2128040$

$-5.9340860$

3.5387770

2. 8316080

4. 3085240

5.1744990

4.1331980

5.8631040

5. 2936340
0. 5485140

$-1.4215880$

1.9678340

$-1.3134210$

$-2.0031360$

$-1.9106110$

$-0.2048750$

$-0.3751030$

$-0.2021320$

2. 4370020

3. 2291690

$-2.9438410$

$-4.1937170$

$-5.2199070$

$-5.4895980$

$-4.5530670$

$-3.1373580$

$-4.6321730$

$-6.1501750$

$-6.5276560$

$-4.9022590$

$-3.9736710$

$-4.8323440$

$-5.3531650$

$-4.6042310$

$-1.6351170$

$-1.4159120$

$-2.0465970$

$-2.6932960$

$-1.8348610$

$-1.7431140$

$-2.8308550$

$-1.1385420$

$-2.7059280$

$-0.8306110$

$-2.2493810$

$-0.9994790$

$-2.7069070$

$-3.6917320$

$-0.6642260$

$-1.0490800$

$-1.7569830$

$-1.4413990$

$-3.0874410$

$-2.4516980$

$-0.4036750$

\begin{abstract}
2.2718980
0.7968690

3. 3186310

4. 5146950

1. 9777410

3. 8382940

$-0.0617880$

1.7868820

2. 5297360

1.4318900

2. 5295750

0.4724800

1.8246870

1.1824500

3.8804950

2.9324130

1. 5049860

3.9093320

4.8929140

2.9345400

1.0552790

4. 3678500

4. 5062190

3. 5614800

3. 2713890

0.8475500
\end{abstract}

154

4. 8186800

$-3.3224790$

$5.6822070 \quad-3.7798490$

$6.5379000 \quad-2.2055080$

$4.6763180-5.1317710$

$6.2152000 \quad-4.5705870$

$2.2041240 \quad-0.3390680$

$3.5892400 \quad 0.4875550$

$\begin{array}{ll}1.5368730 & 2.2547240\end{array}$

$2.5116270 \quad 2.3114550$

$0.8625460 \quad 1.3944980$

2. $1919700 \quad 1.9184880$

$0.9777050 \quad 3.3828820$

$0.2956150 \quad 3.0033160$

$3.0698060 \quad 3.7524590$

$4.2184700 \quad 3.4023340$

$3.7078550 \quad 3.1959610$

$2.0215710 \quad 2.6370870$

$3.4529240 \quad 3.9151850$

$4.9772980 \quad 4.1906200$

$3.4082140 \quad 4.1508320$

$2.4417640 \quad 1.7335840$

$1.1517980 \quad 2.9236840$

$2.5981550 \quad 4.6897560$

$4.7044170 \quad 2.4801720$

$\begin{array}{ll}4.4733320 & 2.7755060\end{array}$

$\operatorname{TS} 159(R, R) \quad G[W B 97 X D / 6-31 G(d)]=-3541.694468$

$\begin{array}{lrrr}\mathrm{P} & \mathrm{P} 159(\mathrm{R}, \mathrm{R}) & \mathrm{G}[\mathrm{WB} 7 \mathrm{XD} / 6-31 \mathrm{G}(\mathrm{d})]=-3541.694468 \\ \mathrm{O} & -0.3711810 & -0.1202440 & 0.9086210 \\ \mathrm{O} & -0.6709440 & 1.3185340 & 0.5642790 \\ \mathrm{O} & 0.2763170 & -0.4312940 & 2.2247830 \\ \mathrm{O} & 0.5068640 & -0.6693820 & -0.3604350 \\ \mathrm{C} & -1.7051820 & -1.0427260 & 0.8227120 \\ \mathrm{C} & 0.5909500 & -2.0379790 & -0.5462440 \\ \mathrm{C} & -0.5351120 & -2.7112790 & -1.0354370 \\ \mathrm{C} & 1.7918560 & -2.6840170 & -0.2652740 \\ \mathrm{H} & 1.8337720 & -4.0671570 & -0.4435710 \\ \mathrm{C} & 2.7659120 & -4.5911220 & -0.2441380 \\ \mathrm{C} & -2.3171970 & -1.1012050 & -0.4195050 \\ \mathrm{C} & -3.4681820 & -0.3489910 & -0.6463340 \\ \mathrm{C} & -1.7418450 & -1.9121860 & -1.3961790 \\ \mathrm{H} & -4.0145710 & -0.4151140 & -1.9239050 \\ \mathrm{C} & -4.9206670 & 0.1518400 & -2.1281230 \\ \mathrm{C} & 2.9901900 & -1.9258040 & 0.1868910 \\ \mathrm{C} & -4.0933140 & 0.4906140 & 0.4119530 \\ & 3.6381160 & -1.0410100 & -0.6845110\end{array}$




\begin{tabular}{|c|c|c|}
\hline $\begin{array}{l}3.2710070 \\
5.3419690 \\
4.7808320 \\
3.0052180 \\
2.8152970 \\
2.0953330 \\
4.2269650 \\
4.6047110 \\
3.9457010 \\
6.2168030 \\
2.3935630 \\
5.7185110 \\
6.0479210 \\
6.5639890 \\
4.4894660 \\
4.2450970 \\
4.6950600 \\
3.5167670 \\
2.9935570 \\
5.1090900 \\
4.6702850 \\
4.1212320 \\
3.7706180 \\
4.3511920 \\
5.3787260 \\
5.6291780 \\
6.2483510 \\
6.0085630 \\
2.0950180 \\
3.9441070 \\
4.2419610 \\
3.7346460 \\
2.6892180 \\
2.3886320 \\
1.8400940 \\
5.3054360 \\
6.2042640 \\
5.2766580 \\
5.7758600 \\
4.6010940 \\
4.6230380 \\
6.7608660 \\
.0854590 \\
6.0619640 \\
.061740\end{array}$ & $\begin{array}{r}-0.7629980 \\
0.5680830 \\
-0.3432380 \\
0.7475840 \\
0.9732730 \\
0.9997760 \\
1.5495740 \\
2.2293770 \\
2.1716950 \\
1.1140840 \\
-1.3342540 \\
-0.2928380 \\
0.3669490 \\
-0.9409830 \\
-1.1337350 \\
-0.9489020 \\
-2.2043930 \\
-2.1040660 \\
-3.0298260 \\
-1.7351850 \\
-1.4134160 \\
-4.0315270 \\
-4.7193240 \\
-4.6365400 \\
-3.2553990 \\
-3.5165040 \\
-3.5081680 \\
-1.1718790 \\
-3.5577230 \\
-1.3985240 \\
-1.6357620 \\
-0.3245810 \\
-2.2068280 \\
-2.8963280 \\
-1.5505060 \\
-0.5359510 \\
-0.0055160 \\
0.6949550 \\
-0.0902810 \\
-1.5549850 \\
-0.0909200 \\
-1.1641780 \\
-1.7851600 \\
-0.6788550 \\
-2.0254530 \\
-3.0848380\end{array}$ & $\begin{array}{l}-2.1221470 \\
-1.3352120 \\
-0.2749130 \\
-2.2763320 \\
-3.3320800 \\
-1.7287260 \\
-1.7574940 \\
-2.5298720 \\
-0.9011160 \\
-0.9659040 \\
-2.4350390 \\
-2.5621400 \\
-3.3732600 \\
-2.3103030 \\
-2.9969510 \\
-4.0493070 \\
-2.8966570 \\
1.4717320 \\
2.5432800 \\
3.2732580 \\
1.8684040 \\
2.8795590 \\
3.6574990 \\
1.9956280 \\
3.3553240 \\
4.3901870 \\
2.7400450 \\
3.5458790 \\
2.2169660 \\
4.2293850 \\
5.2575030 \\
4.1943800 \\
3.8135220 \\
4.6110440 \\
3.6150110 \\
0.9977840 \\
1.3088150 \\
2.5279160 \\
3.7132470 \\
1.9390270 \\
1.5708520 \\
3.2010630 \\
4.0444330 \\
2.7961180 \\
2.1166780 \\
2.3987540\end{array}$ \\
\hline
\end{tabular}

-6.5871030
-4.1038860
-6.2670060
-3.8730790
-3.8957220
-2.8236360
-4.5678910
-4.9158540
-3.8701400
-4.9019290
-4.9943280
-3.6472110
-4.2154050
-3.5650450
-3.6223460
-3.1007170
-2.7388860
-2.2904700
-1.9270390
-3.0672340
-5.5765110
-4.8141200
-4.4051610
-5.4321800
-5.6592040
-5.7432620
-6.6764200
-5.4316480
-5.9601990
-2.2673000
-1.5537040
-1.9607020
-3.4823020
-4.0400400
-3.4241520
-1.5311240
-1.7711890
-0.4712520
-3.8899900
-3.7971070
-5.1288200
-3.8561490
-1.5516050
0.7091440
0.7182420
0.0454820
1.1591140

1.1785550

4.4422840

3. 2931940

3.5953030

3.1674540

4. 3551440

5. 2047250

4.7502220

1. 1920380

0.8285280

$-0.8784690$

0.2454860

0.7158350

0.3526220

1.7068420

$-0.2492980$

$-1.0496280$

0.2819380

$-1.5792060$

1.5706320

$-1.5991400$

$-2.3609390$

$-2.1236110$

$-0.5632110$

$-0.8492790$

$-0.5064300$

2. 3346760

3.0702860

$-2.7024900$

3.8234510

$-5.2110470$

$-5.3217280$

$-4.3408890$

$-2.9572500$

$-5.9731600$

$-3.7615570$

$-3.6840730$

$-5.0932740$

$-6.3410960$

$-4.2579960$

$-4.7389680$

$-0.8360160$

$-1.0012990$

$-2.3305850$ 


$\begin{array}{rrr}-1.4063100 & -6.1338810 & -2.4011950 \\ -1.6883570 & -4.9758710 & -1.4156940 \\ -0.4853620 & -4.1086080 & -1.1292720 \\ 0.1755010 & -6.7558390 & -0.1710120 \\ 0.0718920 & -7.7347680 & -2.4817840 \\ -2.1073660 & -6.9450950 & -2.1777540 \\ -1.9881700 & -5.4138660 & -0.4533570 \\ 1.7437550 & -6.6629530 & -0.9666930 \\ 0.6357350 & -6.2034330 & -3.1387720 \\ -1.6249750 & -5.8156960 & -3.4259500 \\ -2.5428640 & -4.3825480 & -1.7487720 \\ 1.3696230 & 3.4736660 & -1.0005660 \\ 0.3391670 & 2.7337390 & -1.3016940 \\ 2.0075130 & 4.1362400 & -2.1694550 \\ 3.0652380 & 5.0208300 & -1.9502820 \\ 1.5956690 & 3.8458120 & -3.4720490 \\ 3.7085660 & 5.6147860 & -3.0283730 \\ 3.3767580 & 5.2270670 & -0.9319690 \\ 2.2455880 & 4.4374990 & -4.5490750 \\ 0.7804800 & 3.1492730 & -3.6318760 \\ 3.3000840 & 5.3211850 & -4.3283660 \\ 4.5291350 & 6.3045410 & -2.8573650 \\ 1.9308680 & 4.2083910 & -5.5623390 \\ 3.8052530 & 5.7818490 & -5.1720740 \\ -0.0276160 & 2.1791430 & -0.5290650 \\ 1.8316970 & 3.6349050 & 0.1351000 \\ 2.5896700 & 1.7475380 & 2.0612990 \\ 1.5019600 & 2.7352050 & 2.0529260 \\ 2.5606150 & 0.9955170 & 1.2686500 \\ 0.5308170 & 2.3895110 & 1.7139180 \\ 1.9123320 & 1.3037200 & 3.2552050 \\ 1.2558980 & 0.5937110 & 2.9595320 \\ 3.9886610 & 3.3917370 & 3.3818700 \\ 3.0422750 & 4.5167340 & 2.9580670 \\ 1.6060320 & 4.0065040 & 2.8223120 \\ 3.9822330 & 2.2544730 & 2.3575820 \\ 5.0074850 & 3.7770460 & 3.4905840 \\ 3.0666780 & 5.3367380 & 3.6822500 \\ 1.1685310 & 3.8027640 & 3.8076310 \\ 4.4126140 & 2.5969570 & 1.4084630 \\ 4.5866060 & 1.4094510 & 2.6971230 \\ 3.6868080 & 3.0018390 & 4.3615190 \\ 3.3662590 & 4.9233950 & 2.3433040 \\ 0.9507630 & 4.7388230 & \end{array}$

$\operatorname{TS} 160(R, R) \quad G[W B 97 X D / 6-31 G(d)]=-3541.692712$
0.7663790

0.5642660

2.0362640

$-0.5642170$

0.6207570

$-0.8625810$

$-1.4109880$

$-0.6229100$

$-0.9145010$

$-0.7326530$

$-0.6018720$

$-0.7109610$

$-1.6639060$

$-1.9599990$

$-2.0704340$

$-0.0774290$

0.4483090

$-0.8465440$

$-2.2481710$

$-1.3099460$

$-0.3543250$

$-2.2304570$

$-3.2499840$

$-1.6418040$

$-1.6416470$

$-2.3507500$

$-0.7341410$

$-0.8779160$

$-2.6269510$

$-2.6136640$

$-3.3459270$

$-2.4171940$

$-3.1547360$

$-4.1767910$

$-3.1893730$

1. 1917550

2. 1606530

3.0375280

1. 6690490

2. 4041090

3. 1082340

1.4664190

2. 9640070

3. 9666510

2. 3293720

3. 3741700 


\begin{tabular}{|c|c|c|c|}
\hline $\begin{array}{l}\mathrm{H} \\
\mathrm{C} \\
\mathrm{H} \\
\mathrm{H} \\
\mathrm{C} \\
\mathrm{H} \\
\mathrm{H} \\
\mathrm{C} \\
\mathrm{H} \\
\mathrm{C} \\
\mathrm{C} \\
\mathrm{C} \\
\mathrm{C} \\
\mathrm{C} \\
\mathrm{H} \\
\mathrm{H} \\
\mathrm{C} \\
\mathrm{H} \\
\mathrm{H} \\
\mathrm{H} \\
\mathrm{H} \\
\mathrm{C} \\
\mathrm{H} \\
\mathrm{H} \\
\mathrm{C} \\
\mathrm{H} \\
\mathrm{H} \\
\mathrm{C} \\
\mathrm{C} \\
\mathrm{C} \\
\mathrm{C} \\
\mathrm{C} \\
\mathrm{H} \\
\mathrm{H} \\
\mathrm{C} \\
\mathrm{H} \\
\mathrm{H} \\
\mathrm{H} \\
\mathrm{H} \\
\mathrm{C} \\
\mathrm{H} \\
\mathrm{H} \\
\mathrm{C} \\
\mathrm{H} \\
\mathrm{H} \\
\mathrm{C}\end{array}$ & $\begin{array}{l}1.7042830 \\
3.7450620 \\
3.9938400 \\
3.6587270 \\
2.4153380 \\
2.0228550 \\
1.6507030 \\
5.2615310 \\
6.2062760 \\
-5.0823840 \\
-5.5814500 \\
-4.5790370 \\
-4.5229630 \\
-6.6530470 \\
-6.9771150 \\
-7.5327420 \\
-6.0582080 \\
-6.1177960 \\
-6.6175740 \\
-4.1544120 \\
-5.9981400 \\
-3.8059350 \\
-3.8881640 \\
-2.7462370 \\
-4.3944590 \\
-4.7408440 \\
-3.6342970 \\
-4.6340540 \\
-4.6269650 \\
-3.4915700 \\
-4.0506140 \\
-3.1606270 \\
-3.1425600 \\
-2.6444320 \\
-2.4696360 \\
-2.0495430 \\
-1.6515790 \\
-3.0032430 \\
-5.1200960 \\
-4.6472230 \\
-4.2428000 \\
-5.3557130 \\
-5.3528000 \\
-5.3446900 \\
-6.4011940 \\
-5.1499170\end{array}$ & $\begin{array}{r}-3.8412180 \\
-2.1219780 \\
-2.4981690 \\
-1.0331720 \\
-2.7334210 \\
-3.4610520 \\
-1.9671170 \\
-1.1017380 \\
-0.7093210 \\
0.8968260 \\
0.0348800 \\
-1.3236580 \\
0.1676210 \\
-0.9266390 \\
-1.6055950 \\
-0.3544420 \\
-1.7197510 \\
-2.7991230 \\
-1.5181940 \\
-1.8839590 \\
0.6448460 \\
-1.6401680 \\
-2.7139720 \\
-1.4188210 \\
-0.8100850 \\
-1.4636640 \\
-0.1462150 \\
2.9433050 \\
4.4350860 \\
3.1231870 \\
2.2241720 \\
4.8975010 \\
5.9702460 \\
4.7626220 \\
4.0771810 \\
4.7367660 \\
3.4789730 \\
2.5514510 \\
4.9552770 \\
3.9727710 \\
4.6760440 \\
3.3268630 \\
4.7320880 \\
5.8131760 \\
4.4278390 \\
2.2839960\end{array}$ & $\begin{array}{r}1.7750230 \\
4.0142420 \\
5.0135520 \\
4.0916200 \\
3.5055420 \\
4.2257760 \\
3.3686470 \\
0.8973460 \\
1.2708010 \\
2.6298990 \\
3.7606330 \\
1.8044660 \\
1.5733020 \\
3.2011290 \\
3.9985820 \\
2.8887970 \\
2.0083900 \\
2.1905460 \\
1.0888060 \\
0.9686780 \\
4.5693640 \\
3.1038480 \\
3.3094670 \\
2.9554170 \\
4.2745000 \\
5.0838700 \\
4.6984780 \\
1.4664260 \\
1.2529440 \\
-0.6619570 \\
0.4149670 \\
1.1159390 \\
0.8883160 \\
2.0721260 \\
-0.0027090 \\
-0.7710660 \\
0.4021880 \\
-1.4543980 \\
2.0813070 \\
-1.2340240 \\
-1.9716050 \\
-1.7638380 \\
-0.0785960 \\
-0.2605130 \\
0.0047360 \\
2.5748600\end{array}$ \\
\hline
\end{tabular}

-5.5986290
-3.6051920
-4.2356050
-4.4377590
-3.1298320
-1.9956320
-2.4936960
-3.5870990
-4.8495920
-2.7732180
-1.5460900
-5.1911270
-5.1928290
-3.3288540
-1.1983100
-0.8031080
-2.0549620
-2.3130470
-1.0937460
0.1694330
0.3175450
-1.9373720
-3.1964390
-1.3181510
0.0901270
-2.9180630
-2.5587240
-0.8757870
1.0586520
1.7871390
0.6614820
2.4719550
3.6672260
1.9583770
4.3471500
4.0570150
2.6417780
1.0335550
3.8352540
5.2782010
2.2443500
4.3683470
0.2507840
2.2957870
2.8148430
1.8402850
2.8462030
-0.5638710
-0.4761690
-1.8865190
-2.7139410
-2.0523350
-1.4077470
0.1038930
-1.8173070
-2.8676950
-1.2589410
0.0560140
-2.3923570
-3.7086150
-2.7721340
-3.8454930
-4.5728030
-5.7474670
-6.6936090
-6.1270240
-4.6517200
-4.9596570
-6.3007670
-7.6719650
-6.2916810
-3.9027050
-5.3259840
-6.8666530
-6.6717500
3.4822310
2.9160710
4.1830390
4.8588780
4.1299290
5.4789020
4.8819800
4.7504660
3.5962390
5.4230810
6.0014680
4.7059120
5.9045220
2.3184350
3.4570920
1.3598160
2.4577560
3.3923950 $-3.0576020$ $-4.4232400$ $-4.9998980$ $-4.9995320$ $-4.1885120$ $-2.9182260$ $-5.0957250$ $-6.0120770$ $-6.0234850$ $-4.8023440$ $-4.3718090$ $-4.3879560$ $-4.5884360$ $-3.9931730$ $-1.6457260$ $-2.0685060$ $-1.1080580$ $-0.9806290$ $-1.6616450$ $-1.3990200$ $-3.0910000$ $-1.4431230$ $-1.4183160$ $-2.7454140$ $-2.0810000$ $-0.1275870$ 0.0781630 $-1.3285330$ $-0.8105440$ $-1.1468700$ $-1.9298170$ $-1.6770340$ $-3.2276640$ $-2.7177610$ $-0.6649730$ $-4.2668560$ $-3.4142470$ $-4.0130350$ $-2.5220000$ $-5.2759690$ $-4.8275190$ $-0.4316850$ 0.3162540 2. 1056700 2. 1619220 


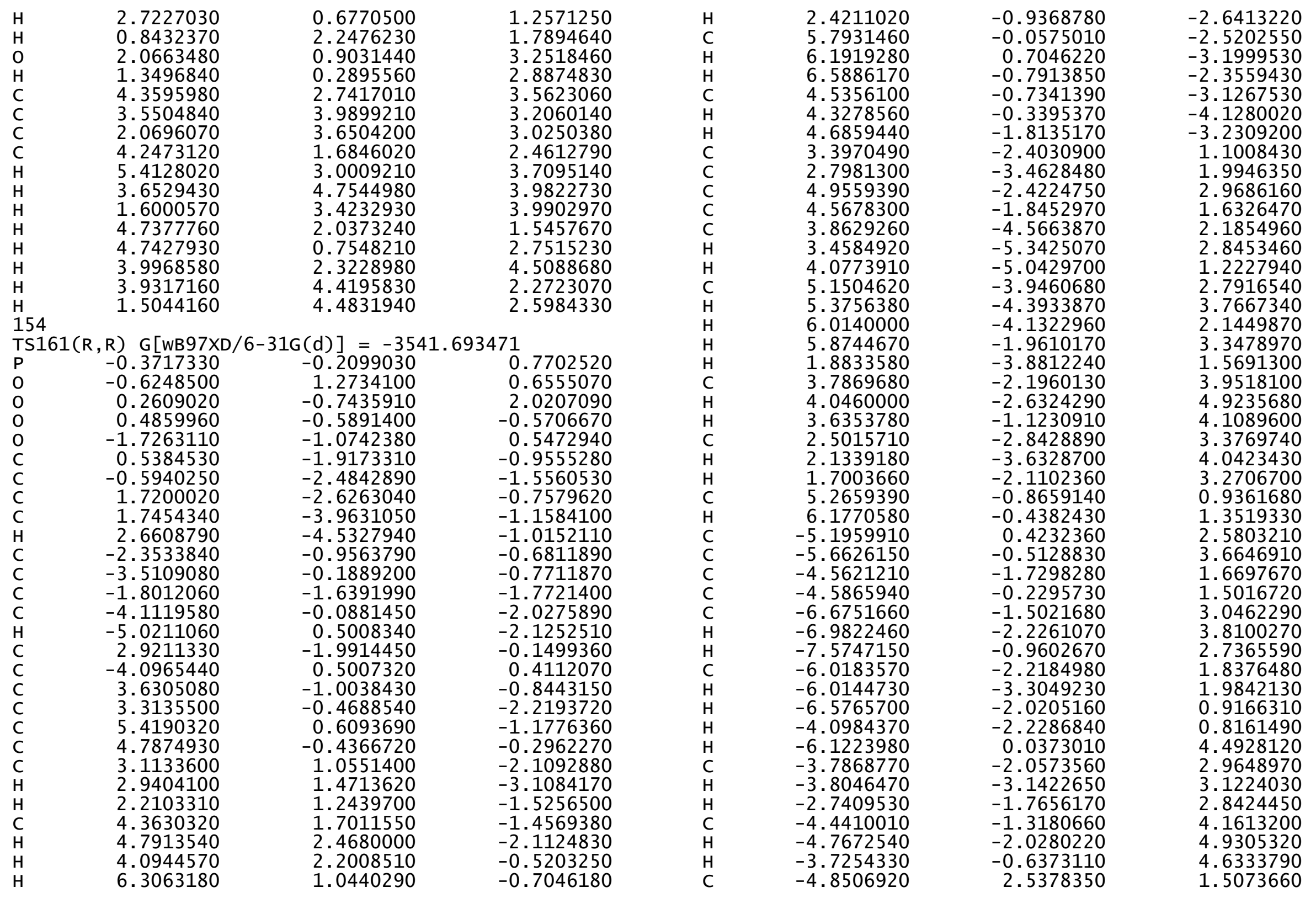




$\begin{array}{rrr}-4.9326140 & 4.0345650 & 1.3507290 \\ -3.7019010 & 2.8681320 & -0.5992960 \\ -4.2175280 & 1.8959160 & 0.4349840 \\ -3.4976270 & 4.5933060 & 1.2447080 \\ -3.5476300 & 5.6716420 & 1.0510690 \\ -2.9820130 & 4.4612180 & 2.2015750 \\ -2.7463010 & 3.8551820 & 0.1085980 \\ -2.3547320 & 4.5649040 & -0.6295700 \\ -1.9001240 & 3.2921680 & 0.5053860 \\ -3.1730400 & 2.3573870 & -1.4073710 \\ -5.4633720 & 4.4922780 & 2.1924950 \\ -4.9014380 & 3.6686530 & -1.1521200 \\ -4.5323940 & 4.4244820 & -1.8555080 \\ -5.5634450 & 3.0038140 & -1.7174500 \\ -5.6649570 & 4.3355170 & 0.0233960 \\ -5.7281960 & 5.4207850 & -0.1178550 \\ -6.6909620 & 3.9581560 & 0.0836950 \\ -5.3393870 & 1.8056970 & 2.5820920 \\ -5.8270580 & 2.3079350 & 3.4162050 \\ -2.4015290 & -1.4763060 & -3.0276290 \\ -1.8369830 & -2.0203380 & -4.3159350 \\ -1.7474240 & -0.8941560 & -5.3623860 \\ -3.0840140 & -0.1324710 & -5.5453030 \\ -4.1557440 & -0.5638520 & -4.5245060 \\ -3.5649080 & -0.7020870 & -3.1463370 \\ -2.4851960 & -2.8224050 & -4.6967600 \\ -1.4112430 & -1.3169590 & -6.3148430 \\ -3.4786680 & -0.2819130 & -6.5558130 \\ -4.5756470 & -1.5305720 & -4.8361940 \\ -0.8494690 & -2.4618590 & -4.1606950 \\ -0.9681400 & -0.1972770 & -5.0360750 \\ -2.9098070 & 0.9426040 & -5.4327620 \\ -4.9855510 & 0.1501590 & -4.5210010 \\ -0.5526970 & -3.8425210 & -1.8998800 \\ -1.7391480 & -4.6259580 & -2.4038460 \\ -1.9117510 & -5.9102700 & -1.5712260 \\ -0.6111380 & -6.7449890 & -1.4663870 \\ 0.5998350 & -6.0301450 & -2.0966400 \\ 0.6274460 & -4.5755820 & -1.7076920 \\ -1.5866770 & -4.8919340 & -3.4595520 \\ -2.7195890 & -6.5091560 & -2.0043120 \\ -0.7382850 & -7.7196970 & -1.9490800 \\ 0.5299060 & -6.1106210 & -3.1906320 \\ -2.6553080 & -4.0322300 & -2.3587880 \\ -2.2426640 & -5.6162590 & -0.5695710 \\ -0.3890880 & -6.9435080 & -0.4128080\end{array}$

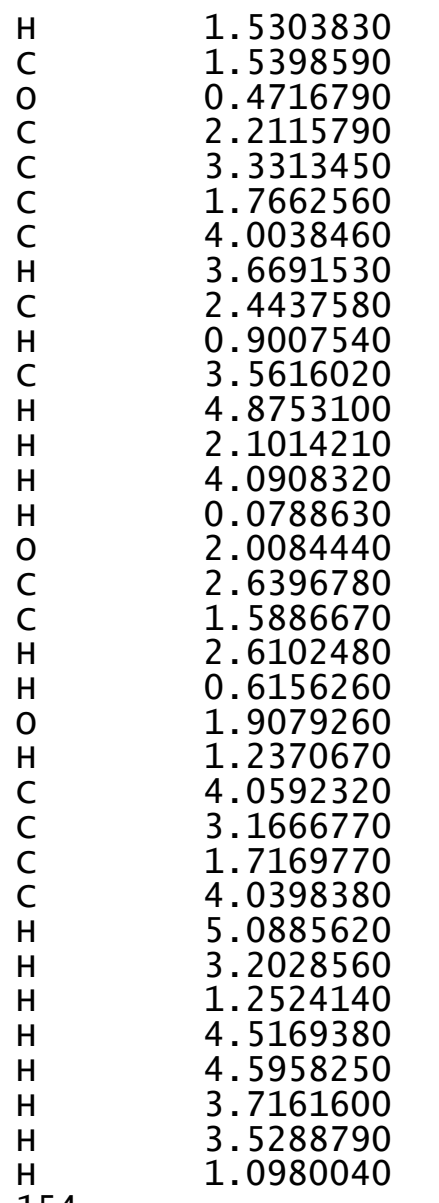

-6.5279730
3.5813710
2.9579880
4.3952180
5.1543180
4.3661310
5.8813710
5.1585300
5.0918410
3.7667160
5.8482090
6.4703190
5.0648830
6.4122700
2.2919980
3.5234330
1.3342340
2.3542820
0.7139370
2.1049540
0.7416690
0.1133170
2.7063400
3.9173980
3.4883820
1.7307750
3.0294360
4.6272160
3.1518450
2.1862640
0.8180210
2.1928690
4.4414390
4.3093010

$-1.8056230$ $-0.5047840$ $-0.9185370$ $-1.5528700$ $-1.2075740$ $-2.8764540$ $-2.1815780$ $-0.1767670$ $-3.8493270$ $-3.1350930$ $-3.5030900$ $-1.9130370$ $-4.8790760$ $-4.2653840$ $-0.2559870$ 0.6379890 2. 2697840 2. 3866120 1. 3700800 1. 9762850 3. 3627510 2. 9404000 3.8573840 3. 5792240 3. 3443520 2. 6779830 4.0426110 4. 4113280 4.2795650 1. 8015720 2.9069270 4.7636630 2.6871530 $\operatorname{TS} 162(R, R) \quad G[W B 97 X D / 6-31 G(d)]=-3541.692621$

\begin{tabular}{|c|c|c|c|}
\hline \multicolumn{4}{|c|}{ TS162 (R, R) G[WB97X } \\
\hline $\mathrm{P}$ & -0.4076560 & -0.1824630 & 0.9161270 \\
\hline 0 & -0.7489220 & 1.2534610 & 0.5997830 \\
\hline 0 & 0.2188490 & -0.5033710 & 2.2399580 \\
\hline 0 & 0.5194760 & -0.6680440 & -0.3427050 \\
\hline 0 & -1.7040700 & -1.1474410 & 0.767011 \\
\hline C & 0.6504400 & -2.0255170 & -0.5750180 \\
\hline C & -0.4353850 & -2.7136490 & -1.1315440 \\
\hline C & 1.8664050 & -2.6408110 & -0.2 \\
\hline C & 1.9692300 & -4.0105240 & 62370 \\
\hline $\mathrm{H}$ & 2 & 5 & \\
\hline
\end{tabular}




\begin{tabular}{|c|c|c|}
\hline $\begin{array}{l}-2.2867350 \\
-3.4518030 \\
-1.6635410 \\
-3.9799270 \\
-4.8898180 \\
3.0263100 \\
-4.1224860 \\
3.6686760 \\
3.3296950 \\
5.3366870 \\
4.7794190 \\
3.0214220 \\
2.8571620 \\
2.0873730 \\
4.2007810 \\
4.5758490 \\
3.8750840 \\
6.1842040 \\
2.4791900 \\
5.7700840 \\
6.0930800 \\
6.6328790 \\
4.5814590 \\
4.3582760 \\
4.8194930 \\
3.5252290 \\
3.0034770 \\
5.0634660 \\
4.6490060 \\
4.1515460 \\
3.8024590 \\
4.4198770 \\
5.3740110 \\
5.6055830 \\
6.2659410 \\
5.9397600 \\
2.1282970 \\
3.8668970 \\
4.1472590 \\
3.6265720 \\
2.6467890 \\
2.3521870 \\
1.7811450 \\
5.2790200 \\
6.1542080 \\
-5.3617930\end{array}$ & $\begin{array}{r}-1.1780760 \\
-0.4492630 \\
-1.9453490 \\
-0.4733340 \\
0.0872720 \\
-1.8575860 \\
0.3258730 \\
-0.9337730 \\
-0.6357500 \\
0.7408510 \\
-0.2125330 \\
0.8686760 \\
1.1105170 \\
1.0810320 \\
1.6962050 \\
2.4084750 \\
2.2854300 \\
1.3054030 \\
-1.2269100 \\
-0.0780360 \\
0.6120800 \\
-0.7014430 \\
-0.9508310 \\
-0.7555190 \\
-2.0158940 \\
-2.0503450 \\
-3.0172390 \\
-1.6821430 \\
-1.3375580 \\
-3.9949810 \\
-4.7122930 \\
-4.5697910 \\
-3.1959510 \\
-3.4750880 \\
-3.4092260 \\
-1.1008640 \\
-3.5613350 \\
-1.4012260 \\
-1.6520130 \\
-0.3332480 \\
-2.2368590 \\
-2.9560420 \\
-1.6029350 \\
-0.4212270 \\
0.1268680 \\
0.3980510\end{array}$ & $\begin{array}{l}-0.4908290 \\
-0.7139260 \\
-1.4817420 \\
-2.0064500 \\
-2.2082630 \\
0.2159740 \\
0.3662730 \\
-0.6181190 \\
-2.0587270 \\
-1.1890450 \\
-0.1641970 \\
-2.1899650 \\
-3.2464260 \\
-1.6666390 \\
-1.6163120 \\
-2.3602270 \\
-0.7524490 \\
-0.7856570 \\
-2.4065590 \\
-2.4262870 \\
-3.2144160 \\
-2.1706300 \\
-2.9078620 \\
-3.9630360 \\
-2.8202010 \\
1.5091260 \\
2.5441780 \\
3.3563800 \\
1.9497100 \\
2.8823560 \\
3.6338640 \\
1.9891560 \\
3.4088190 \\
4.4433610 \\
2.8108680 \\
3.6644960 \\
2.1823590 \\
4.2911640 \\
5.3210230 \\
4.2739140 \\
3.8280450 \\
4.6011880 \\
3.6286240 \\
1.1161770 \\
1.4620340 \\
2.4597370\end{array}$ \\
\hline
\end{tabular}

-5.8685550
-4.6112650
-4.6641050
-6.8113540
-7.1399210
-7.7075910
-6.0624230
-6.0411100
-6.5663100
-4.0807970
-6.3953800
-3.9116010
-3.9070820
-2.8692440
-4.6587390
-5.0125970
-3.9925110
-5.0114930
-5.1380100
-3.7198890
-4.2821150
-3.7224920
-3.7994690
-3.2761180
-2.8578400
-2.4282990
-2.0308760
-3.1099420
-5.7494780
-4.8969250
-4.4978650
-5.4871090
-5.7779550
-5.8789180
-6.7873790
-5.5497530
-6.1108310
-2.1922510
-1.5411280
-1
-2.3836810
-2.7047310
-3.8468520
-3.3557870
-2.1634530
-0.9943530
-3.0227090

-0.4558770
-1.8022620
-0.3234680
-1.5293450
-2.1979670
-1.0485750
-2.3195700
-3.3906450
-2.2107940
-2.3586930
0.1490260
-1.9911660
-3.0586150
-1.6741740
-1.1732690
-1.8247480
-0.4313510
2.4213120
3.8932870
2.6136080
1.7137400
4.5055960
5.5570810
4.4898580
3.6972870
4.3515680
3.2051490
2.0569360
4.3990650
3.3170380
4.0307220
2.5836540
4.0415830
5.1063560
3.6180670
1.7685200
2.3239060
-1.9078990
-2.5474350
-1.5049130
-0.7601600
-1.1440630
-1.1685790
-3.3782660
-1.9994590
-0.9621720
3.5931460

1.7826100

1.4830760

3.0054620

3.8098360

2.6002290

1.9010110

2. 1338090

0.9349690

1.0071160

4.3390570

3. 1461510

3. 3967280

3.0594910

4.2320110

5.0398210

4.6835660

1.2243870

0.9260710

$-0.8156830$

0.2595760

0.8714880

0.5691850

1.8711550

$-0.1276310$

$-0.8952040$

0.3862730

$-1.5307290$

1. 6810810

$-1.5259150$

$-2.2564760$

$-2.0860290$

$-0.4730240$

$-0.7135420$

$-0.4546960$

2.3262340

3.0762660

$-2.7780880$

$-3.9784170$

$-5.1005380$

$-5.4192400$

$-4.4573300$

$-3.0338950$

$-4.3407700$

$-5.9964970$

$-6.4474280$ 


$\begin{array}{rrr}-4.2257420 & -2.1389960 & -4.7300960 \\ -0.5670620 & -2.9725530 & -3.7239710 \\ -0.6214470 & -0.7853850 & -4.7830860 \\ -2.5419610 & 0.3202240 & -5.3476120 \\ -4.6851660 & -0.4486350 & -4.5670220 \\ 0.8902130 & -4.7424210 & -1.0153100 \\ 0.9598330 & -6.2254900 & -1.2720720 \\ 0.4055710 & -6.5397100 & -2.6712690 \\ -1.0188220 & -5.9714880 & -2.8768710 \\ -1.4473960 & -4.9944830 & -1.7623350 \\ -0.3191830 & -4.0980580 & -1.3149490 \\ 0.3677910 & -6.7627250 & -0.5170980 \\ 0.4157730 & -7.6215310 & -2.8397800 \\ -1.7522400 & -6.7835430 & -2.9197060 \\ -1.7633630 & -5.5805830 & -0.8876650 \\ 1.9897840 & -6.5849010 & -1.1773340 \\ 1.0882620 & -6.1007490 & -3.4074770 \\ -1.0699010 & -5.4580950 & -3.8422210 \\ -2.3232340 & -4.4217370 & -2.0733890 \\ 1.2413630 & 3.4958160 & -0.8981990 \\ 0.2427000 & 2.7258090 & -1.2300570 \\ 1.8808890 & 4.1928230 & -2.0461650 \\ 2.9037880 & 5.1095340 & -1.7961540 \\ 1.5062780 & 3.9026470 & -3.3600820 \\ 3.5497350 & 5.7350890 & -2.8547780 \\ 3.1862280 & 5.3155500 & -0.7693380 \\ 2.1588820 & 4.5258460 & -4.4174200 \\ 0.7178490 & 3.1816480 & -3.5439060 \\ 3.1792050 & 5.4409580 & -4.1660120 \\ 4.3434070 & 6.4494540 & -2.6596630 \\ 1.8737590 & 4.2965290 & -5.4393580 \\ 3.6871670 & 5.9257160 & -4.9944340 \\ -0.1198470 & 2.1485970 & -0.4719420 \\ 1.6739080 & 3.6580700 & 0.2488520 \\ 2.4651700 & 1.7588450 & 2.1554310 \\ 1.3453760 & 2.7101990 & 2.1470000 \\ 2.4750070 & 1.0227160 & 1.3474730 \\ 0.3924290 & 2.3397720 & 1.7836530 \\ 1.7818720 & 1.2682060 & 3.3274350 \\ 1.1550650 & 0.5424080 & 3.0073090 \\ 3.7844070 & 3.4211850 & 3.5346310 \\ 2.8087210 & 4.5228010 & 3.1157240 \\ 1.3931620 & 3.9678140 & 2.9434640 \\ 3.8344520 & 2.3052740 & 3.4881080 \\ 4.7877360 & 3.8377320 & 3.8562360 \\ 2.7928660 & 5.3282530 & \end{array}$

$\begin{array}{ll}\mathrm{H} & 0.9457810 \\ \mathrm{H} & 4.2704840 \\ \mathrm{H} & 4.4605150 \\ \mathrm{H} & 3.4785050 \\ \mathrm{H} & 3.1363440 \\ \mathrm{H} & 0.7217380 \\ \mathrm{H} & \end{array}$
3.7287850
2. 6809590
1.4740250
3. 0019630
4.9595150
4.6876120

154

$\operatorname{TS} 163(R, R) \quad G[W B 97 X D / 6-31 G(d)]=-3541.690967$

$\begin{array}{lrrr}\text { TSI63(R, R) G[WB97XD/6-31G (d) }]=-3541.690967 \\ \mathrm{P} & -0.3279600 & -0.0423030 & 0.8587690 \\ \mathrm{O} & -0.5660170 & 1.3972910 & 0.4732770 \\ \mathrm{O} & 0.3379670 & -0.3411600 & 2.1683720 \\ \mathrm{O} & 0.4803160 & -0.6822980 & -0.4122540 \\ \mathrm{O} & -1.7027910 & -0.9058160 & 0.8366120 \\ \mathrm{C} & 0.5002580 & -2.0611320 & -0.5295630 \\ \mathrm{C} & -0.6578060 & -2.7075830 & -0.9825550 \\ \mathrm{C} & 1.6736670 & -2.7421550 & -0.2154790 \\ \mathrm{C} & 1.6589760 & -4.1325590 & -0.3248970 \\ \mathrm{H} & 2.5667010 & -4.6846980 & -0.0917590 \\ \mathrm{C} & -2.3520960 & -0.9872450 & -0.3847870 \\ \mathrm{C} & -3.4747760 & -0.1925730 & -0.6001790 \\ \mathrm{C} & -1.8454030 & -1.8762430 & -1.3417060 \\ \mathrm{C} & -4.0836970 & -0.2794030 & -1.8529900 \\ \mathrm{H} & -4.9696710 & 0.3213430 & -2.0454310 \\ \mathrm{C} & 2.9035950 & -2.0180100 & 0.2078020 \\ \mathrm{C} & -4.0217440 & 0.7126120 & 0.4480130 \\ \mathrm{C} & 3.5888430 & -1.1940580 & -0.6945230 \\ \mathrm{C} & 3.2258960 & -0.9405760 & -2.1378940 \\ \mathrm{C} & 5.3699650 & 0.3032510 & -1.4046090 \\ \mathrm{C} & 4.7666570 & -0.5402030 & -0.3121200 \\ \mathrm{C} & 3.0355820 & 0.5771560 & -2.3307500 \\ \mathrm{H} & 2.8327240 & 0.7848410 & -3.3877360 \\ \mathrm{H} & 2.1527900 & 0.8898850 & -1.7700440 \\ \mathrm{C} & 4.3084090 & 1.3281090 & -1.8597910 \\ \mathrm{H} & 4.7168260 & 1.9479570 & -2.6660960 \\ \mathrm{H} & 4.0725160 & 2.0040220 & -1.0311850 \\ \mathrm{H} & 6.2744880 & 0.8139240 & -1.0567190 \\ \mathrm{H} & 2.3183650 & -1.4746090 & -2.4289920 \\ \mathrm{C} & 5.6943890 & -0.6188110 & -2.6010760 \\ \mathrm{H} & 6.0653570 & -0.0087020 & -3.4328760 \\ \mathrm{H} & 6.4972820 & -1.3095410 & -2.3245120 \\ \mathrm{C} & 4.4162370 & -1.3963940 & -3.0116290 \\ \mathrm{H} & 4.1749250 & -1.2179670 & -4.0657880 \\ \mathrm{H} & 4.5625000 & -2.4747680 & -2.8922500 \\ \mathrm{C} & 3.4274130 & -2.1782340 & 1.4967260 \\ \mathrm{C} & 2.8667130 & -3.0404710 & 2.6020450 \\ \mathrm{C} & 5.0477710 & -1.8289220 & 3.2779970\end{array}$

3.9163330

1.5542620

2.8222400

2.1651500

2.4677670

0.3677910

4157730

1.7633630

2.3232340

0.1198470

2.4750070

0.3924290

3.7844070

1.3931620

2.7928660

5. 3282530

$$
\begin{array}{r}
0.3379670 \\
0.4803160 \\
-1.7027910 \\
0.5002580 \\
-0.6578060 \\
1.6736670 \\
1.6589760 \\
2.5667010 \\
-2.3520960 \\
-3.4747760 \\
-1.8454030 \\
-4.0836970 \\
-4.9696710 \\
2.9035950 \\
-4.0217440 \\
3.5888430 \\
3.2258960 \\
5.3699650 \\
4.7666570 \\
3.0355820 \\
2.8327240 \\
2.1527900 \\
4.3084090 \\
4.7168260 \\
4.0725160 \\
6.2744880 \\
2.3183650 \\
5.6943890 \\
6.0653570 \\
6.4972820 \\
4.4162370 \\
4.1749250 \\
4.5625000 \\
3.4274130 \\
2.8667130 \\
5.0477710
\end{array}
$$

0.4122540

0.9825550

0.2154790

. 3248970

0.6001790

.2078020

0.4480130

$-2.1378940$

$-1.4046090$

0.3121200

(2)

2.4289920

3. 0116290

67260

3. 2779970 


\begin{tabular}{|c|c|c|}
\hline $\begin{array}{l}4.6152990 \\
3.9457050 \\
3.5670630 \\
4.1393760 \\
5.2435390 \\
5.4895820 \\
6.0945340 \\
5.9757220 \\
1.9406410 \\
3.9080120 \\
4.2009930 \\
3.7512970 \\
2.6121950 \\
2.2825530 \\
1.7963480 \\
5.2884900 \\
6.2144520 \\
5.1111160 \\
5.5971020 \\
4.5584460 \\
4.5307860 \\
6.6483620 \\
6.9704650 \\
7.5331370 \\
6.0302000 \\
6.0653680 \\
6.5896120 \\
4.1207330 \\
6.0285930 \\
3.7845340 \\
3.8416830 \\
2.7296450 \\
4.3974680 \\
4.7378740 \\
3.6532700 \\
4.7110570 \\
4.7599510 \\
3.5377220 \\
4.0955580 \\
3.3150960 \\
3.3461920 \\
2.8125460 \\
2.5623220 \\
2.1133930 \\
1.7574740\end{array}$ & $\begin{array}{r}-1.5320680 \\
-4.0851650 \\
-4.7271110 \\
-4.7318480 \\
-3.3568050 \\
-3.5966100 \\
-3.6713680 \\
-1.3018420 \\
-3.5335070 \\
-1.4045280 \\
-1.6235570 \\
-0.3233570 \\
-2.1623410 \\
-2.8095590 \\
-1.4710300 \\
-0.7169420 \\
-0.2220340 \\
1.0496700 \\
0.3345090 \\
-1.2499330 \\
0.1987740 \\
-0.7109010 \\
-1.2811540 \\
-0.2002100 \\
-1.6471040 \\
-2.6921380 \\
-1.5872920 \\
-1.9075420 \\
1.0378260 \\
-1.3754260 \\
-2.4144410 \\
-1.1498870 \\
-0.4125900 \\
-0.9622510 \\
0.3151730 \\
2.9321880 \\
4.3788300 \\
2.8599800 \\
2.0946550 \\
4.8860070 \\
5.9033480 \\
4.9439300 \\
3.9246860 \\
4.4700650 \\
3.4098050 \\
2.2053290\end{array}$ & $\begin{array}{r}1.8657250 \\
2.9664590 \\
3.7700290 \\
2.1036300 \\
3.4082470 \\
4.4492950 \\
2.7954490 \\
3.5262870 \\
2.2989960 \\
4.2294930 \\
5.2629990 \\
4.1605510 \\
3.8460520 \\
4.6672420 \\
3.6292960 \\
0.9638160 \\
1.2529090 \\
2.5970010 \\
3.8308550 \\
2.0738490 \\
1.6479920 \\
3.3986640 \\
4.2780960 \\
3.0050330 \\
2.3280940 \\
2.6575230 \\
1.3886970 \\
1.3198090 \\
4.5514030 \\
3.4045760 \\
3.7502160 \\
3.2293450 \\
4.4549220 \\
5.3405280 \\
4.7936410 \\
1.1699880 \\
0.7499240 \\
-0.9469050 \\
0.2303070 \\
0.5592500 \\
0.1505620 \\
1.5302450 \\
-0.3938020 \\
-1.2319980 \\
0.1330970 \\
-1.6437470\end{array}$ \\
\hline
\end{tabular}

-5.2903170
-4.7012030
-4.2940280
-5.3672060
-5.4756800
-5.5328840
-6.5037670
-5.2168050
-5.6883580
-3.5696270
-4.1881590
-4.3737490
-3.0514020
-1.9323880
-2.4482420
-3.5371360
-4.7933650
-2.6849500
-1.4804070
-5.1472700
-5.1151250
-3.2407320
-1.1310590
0.5089820
0.4511170
-0.1739810
-1.5503740
-1.8644770
-0.6557430
-0.1555150
-0.2632690
-2.3513440
-2.2025270
1.4515170
0.5177810
-1.5815430
-2.7000590
1.5590130
0.4718970
2.1924850
3.3573050
1.6614240
3.9897350
3.7620410
2.2962810
0.7601990

4.9879440

3. 5896420

4. 2178050

2.8600940

4.4498110

5.4949680

4.0902170

2. 4140140

3.0686530

-1.0904070

$-1.1733560$

$-2.6449250$

$-3.4418330$

$-2.6811600$

$-1.8959120$

$-0.6761300$

$-2.7109500$

$-3.6925470$

$-1.9618580$

$-0.6456900$

$-3.0819010$

$-4.3940050$

$-3.3655760$

$-4.8166820$

$-6.3196740$

$-6.7459040$

$-6.0806750$

$-4.9535720$

$-4.1092700$

$-6.7146000$

$-7.8364480$

$-6.8250670$

$-5.4118960$

$-6.7507710$

$-6.4583760$

$-5.6752170$

$-4.3461280$

3. 4142120

2. 7405650

4.0211950

4.7750160

3.8090720

5. 3140670

4. 9218740

4. 3509250

3. 2169050
1.4898010

$-1.6525430$

$-2.4536420$

$-2.1258200$

$-0.9443680$

$-0.5063190$

2. 3553840

3. 0867230

$-2.8556630$

$-4.2270360$

$-4.6241650$

$-4.5325740$

$-3.7884400$

$-2.6073730$

$-4.9607030$

$-5.6333080$

$-5.5337600$

$-4.4860800$

$-4.2486550$

$-3.9459660$

$-4.0269350$

$-3.5017570$

$-0.6956230$

$-0.7808140$

$-2.1178880$

$-2.3560890$

$-1.3496180$

$-1.0287060$

0.0469140

$-2.1563060$

$-2.2973170$

$-0.4095020$

$-0.6697910$

$-2.9173260$

$-3.3724410$

$-1.7040190$

$-1.1753580$

$-1.4299000$

$-2.3763030$

$-2.2198410$

$-3.6506410$

$-3.3328400$

$-1.2242970$

$-4.7622370$

$-3.7614300$ 


\begin{tabular}{|c|c|c|c|c|c|c|c|}
\hline & & & & & & & \\
\hline $\begin{array}{l}\mathrm{C} \\
\mathrm{H}\end{array}$ & $\begin{array}{l}3.4594230 \\
4.8978320\end{array}$ & $\begin{array}{l}5.1015350 \\
5.8961370\end{array}$ & $\begin{array}{l}-4.6043320 \\
-32111780\end{array}$ & C & $\begin{array}{l}5.3720120 \\
3.9921820\end{array}$ & $\begin{array}{r}-0.4355540 \\
2.0243340\end{array}$ & 2.4437850 \\
\hline $\mathrm{H}$ & $\begin{array}{l}1.8840280 \\
1.8402\end{array}$ & 4.1860310 & $\begin{array}{l}-3.2111 / 80 \\
-5.7526770\end{array}$ & H & $\begin{array}{l}3.9921820 \\
4.0194710\end{array}$ & 3.1029910 & 3.0138000 \\
\hline $\mathrm{H}$ & 3.9544220 & 5.5219810 & -5.4746370 & $\mathrm{H}$ & 2.9405220 & 1.7327960 & 2.9535380 \\
\hline $\mathrm{H}$ & 0.0994900 & 2.2140140 & -0.6412590 & $\mathrm{C}$ & 4.7290520 & 1.2447370 & 4.1344920 \\
\hline 0 & 2.0720890 & 3.5558500 & -0.0591620 & $\mathrm{H}$ & 5.1061410 & 1.9280990 & 4.9045900 \\
\hline C & 2.7300840 & 1.7241770 & 1.9507740 & $\mathrm{H}$ & 4.0493210 & 0.5441330 & 4.6296980 \\
\hline C & 1.6919850 & 2.7618840 & 1.8958810 & $\mathrm{H}$ & 6.4331970 & -0.1133550 & 4.2964670 \\
\hline $\mathrm{H}$ & 2.6666850 & 0.9427500 & 1.1893800 & $\mathrm{H}$ & 4.1486340 & 2.2730810 & 0.8562090 \\
\hline $\mathrm{H}$ & 0.7054290 & 2.4497860 & 1.5693970 & $\mathrm{C}$ & 6.8777600 & 1.4801650 & 2.8714040 \\
\hline 0 & 2.0261340 & 1.3641960 & 3.1579970 & $\mathrm{H}$ & 7.2321570 & 2.1807970 & 3.6365580 \\
\hline $\mathrm{H}$ & 1.3413300 & 0.6722560 & 2.8845830 & $\mathrm{H}$ & 7.7570170 & 0.9557250 & 2.4835060 \\
\hline C & 4.2060090 & 3.3553370 & 3.2039790 & C & 6.1377310 & 2.2315880 & 1.7344400 \\
\hline $\mathrm{C}$ & 3.3163910 & 4.5043690 & 2.7255920 & $\mathrm{H}$ & 6.1453740 & 3.3131600 & 1.9132260 \\
\hline C & 1.8571530 & 4.0593130 & 2.6092140 & $\mathrm{H}$ & 6.6294610 & 2.0621590 & 0.7708450 \\
\hline C & 4.1450930 & 2.1725290 & 2.2346580 & C & 4.2226150 & -1.8382250 & 0.3356550 \\
\hline $\mathrm{H}$ & 5.2426260 & 3.6941750 & 3.2983100 & $\mathrm{C}$ & 3.6109840 & -2.7788050 & -0.6760990 \\
\hline $\mathrm{H}$ & 3.3801890 & 5.3548230 & 3.4113480 & $\mathrm{C}$ & 5.0080460 & -4.0046560 & 1.1198620 \\
\hline $\mathrm{H}$ & 1.4103430 & 3.9197520 & 3.6016290 & $\mathrm{C}$ & 4.9402540 & -2.5138030 & 1.3316480 \\
\hline $\mathrm{H}$ & 4.5987730 & 2.4445560 & 1.2737200 & $\mathrm{C}$ & 4.7490900 & -3.5674980 & -1.3588850 \\
\hline $\mathrm{H}$ & 4.7015930 & 1.3142240 & 2.6196520 & $\mathrm{H}$ & 4.3114400 & -4.3089480 & -2.0378520 \\
\hline $\mathrm{H}$ & 3.8844580 & 3.0274740 & 4.2000460 & $\mathrm{H}$ & 5.3561980 & -2.8925520 & -1.9720220 \\
\hline $\mathrm{H}$ & 3.6603570 & 4.8500920 & 1.7437970 & C & 5.6203860 & -4.2588020 & -0.2762440 \\
\hline $\mathrm{H}$ & 1.2384990 & 4.8010430 & 2.0971540 & $\mathrm{H}$ & 5.6806470 & -5.3387960 & -0.4544480 \\
\hline & & & & $\mathrm{H}$ & 6.6443250 & -3.8715790 & -0.2973590 \\
\hline & s) G[wB97x & $(d)]=-354$ & 52 & $\mathrm{H}$ & 5.6122330 & -4.4874910 & 1.8954950 \\
\hline $\mathrm{P}$ & 0.3854820 & 0.2345500 & 0.9249540 & $\mathrm{H}$ & 3.0113980 & -2.2429460 & -1.4154800 \\
\hline 0 & -0.2410410 & 0.7311000 & 2.1938930 & C & 3.5691400 & -4.5631350 & 1.1230070 \\
\hline 0 & 0.6479060 & -1.2409700 & 0.7643600 & $\mathrm{H}$ & 3.6002930 & -5.6330790 & 0.8838700 \\
\hline 0 & 1.7276060 & 1.1224690 & 0.7018530 & $\mathrm{H}$ & 3.1406280 & -4.4695340 & 2.1261970 \\
\hline 0 & -0.5141740 & 0.6227460 & -0.3942160 & C & 2.7188110 & -3.7823680 & 0.0899210 \\
\hline C & 2.3005300 & 1.0590530 & -0.5581430 & $\mathrm{H}$ & 2.2492090 & -4.4640160 & -0.6290050 \\
\hline c & 1.6907870 & 1.7713520 & -1.5894620 & $\mathrm{H}$ & 1.9211010 & -3.2266180 & 0.5851550 \\
\hline C & 3.4433550 & 0.2860700 & -0.7497470 & C & 5.5145970 & -1.8169640 & 2.3873240 \\
\hline $\mathrm{c}$ & 3.9361820 & 0.2144130 & -2.0489470 & $\mathrm{H}$ & 6.0653810 & -2.3465630 & 3.1631940 \\
\hline $\mathrm{H}$ & 4.8318120 & -0.3767910 & -2.2293860 & C & -4.7981880 & 0.2821570 & -0.4563490 \\
\hline C & -0.6146330 & 1.9655790 & -0.7125180 & $\mathrm{C}$ & -5.3507210 & -0.6507680 & -1.5034760 \\
\hline c & -1.8201720 & 2.6274900 & -0.4899150 & $\mathrm{C}$ & -3.2465440 & 0.6302510 & -2.2844240 \\
\hline C & 0.4971560 & 2.6032490 & -1.2609950 & $\mathrm{C}$ & -3.6443520 & 0.9605410 & -0.8653950 \\
\hline c & -1.8517810 & 3.9903730 & -0.7617350 & $\mathrm{C}$ & -5.6954880 & 0.1903560 & -2.7537030 \\
\hline $\mathrm{H}$ & -2.7833730 & 4.5295540 & -0.6010910 & $\mathrm{H}$ & -6.0350980 & -0.4794270 & -3.5522000 \\
\hline C & 4.1094030 & -0.4422640 & 0.3639180 & $\mathrm{H}$ & -6.5256640 & 0.8656150 & -2.5235350 \\
\hline $\mathrm{C}$ & -3.0244970 & 1.8914520 & -0.0206040 & $\mathrm{C}$ & -4.4414320 & 0.9893090 & -3.1966010 \\
\hline C & 4.6844540 & 0.2518920 & 1.4358900 & $\mathrm{H}$ & -4.1808660 & 0.7593900 & -4.2361100 \\
\hline c & 4.6731330 & 1.7455990 & 1.6560730 & $\mathrm{H}$ & -4.6270890 & 2.0668140 & -3.1419810 \\
\hline $\mathrm{C}$ & 5.9144080 & 0.4641440 & 3.5236400 & $\mathrm{H}$ & -2.3562700 & 1.1815700 & -2.5961540 \\
\hline
\end{tabular}




$\begin{array}{rrr}-6.2397760 & -1.1757580 & -1.1373910 \\ -2.9950520 & -0.8874160 & -2.3879580 \\ -2.7695600 & -1.1431760 & -3.4295880 \\ -2.1081810 & -1.1379310 & -1.8015450 \\ -4.2468400 & -1.6576320 & -1.8933360 \\ -4.6239470 & -2.3326710 & -2.6700150 \\ -3.9979640 & -2.2831090 & -1.0310840 \\ -4.7479460 & 1.4549920 & 1.6362200 \\ -5.2386080 & 1.8608060 & 3.0013560 \\ -3.1163260 & 3.1392430 & 2.2599770 \\ -3.5882150 & 2.1343800 & 1.2366560 \\ -4.1041320 & 1.6090610 & 4.0180580 \\ -4.4461740 & 1.8999430 & 5.0182110 \\ -3.8690120 & 0.5404100 & 4.0569490 \\ -2.8531580 & 2.4219920 & 3.6008100 \\ -2.6126570 & 3.1784760 & 4.3568260 \\ -1.9742440 & 1.7844770 & 3.4967970 \\ -2.2127400 & 3.6562890 & 1.9302460 \\ -6.1397230 & 1.3034820 & 3.2804100 \\ -4.2714370 & 4.1424890 & 2.4803090 \\ -3.9596890 & 4.8916570 & 3.2167600 \\ -4.4795410 & 4.6751900 & 1.5460950 \\ -5.5319600 & 3.3792480 & 2.9697460 \\ -5.8195740 & 3.7070890 & 3.9755180 \\ -6.3846130 & 3.5745130 & 2.3115270 \\ -5.3544210 & 0.5307470 & 0.7941580 \\ -6.2608870 & 0.0138600 & 1.1064320 \\ 0.4679570 & 3.9994210 & -1.4404950 \\ 1.7327230 & 4.7456510 & -1.8217830 \\ 1.6668690 & 6.2341610 & -1.4827920 \\ 0.3745730 & 6.8315830 & -2.0334730 \\ -0.8264160 & 6.1993840 & -1.3324000 \\ -0.7240480 & 4.6924040 & -1.1905620 \\ 2.5432910 & 6.7420920 & -1.8992490 \\ 1.9157480 & 4.6498200 & -2.8988990 \\ 2.5898940 & 4.2747030 & -1.3285440 \\ 0.3211110 & 6.6400780 & -3.1137240 \\ 0.3520370 & 7.9183390 & -1.8990350 \\ -1.7546630 & 6.4510360 & -1.8593150 \\ -0.9215480 & 6.6342120 & -0.3275740 \\ 1.7004010 & 6.3729900 & -0.3940590 \\ 2.1559000 & 1.6050770 & -2.9079300 \\ 1.3770700 & 2.1813570 & -4.0754800 \\ 1.6858490 & 1.4730550 & -5.3935450 \\ 3.1963030 & 1.4216840 & -5.6075960 \\ 3.8450380 & 0.5651680 & -4.5214050\end{array}$

$\begin{array}{lr}3.2989280 & 0.8251120 \\ 1.1906090 & 1.9985930 \\ 1.6104550 & 3.2458180 \\ 0.3055180 & 2.1319150 \\ 3.5990670 & 2.4427890 \\ 3.4425250 & 1.0166770 \\ 4.9320060 & 0.7099940 \\ 3.6815200 & -0.4953930 \\ 1.2820210 & 0.4519100 \\ -1.4710980 & -3.4613330 \\ -0.3889870 & -2.8029080 \\ -1.9558890 & -4.3610830 \\ -3.1249260 & -5.0957200 \\ -1.2832450 & -4.4488320 \\ -3.6212680 & -5.9141000 \\ -3.6370880 & -5.0098360 \\ -1.7827730 & -5.2695700 \\ -0.3784730 & -3.8720810 \\ -2.9504040 & -6.0001290 \\ -4.5321220 & -6.4815760 \\ -1.2627210 & -5.3378090 \\ -3.3385830 & -6.6389860 \\ -0.0515670 & -2.1633600 \\ -2.0982720 & -3.3759420 \\ -1.3939130 & -2.1808950 \\ -2.4163450 & -1.8441780 \\ -0.3716740 & -2.1441330 \\ -2.1742250 & -1.0890350 \\ -1.8007220 & -0.9264610 \\ -1.1969510 & -0.2210650 \\ -3.1518800 & -3.4534130 \\ -4.0393290 & -3.4590340 \\ -3.8465150 & -2.1869390 \\ -1.6756490 & -3.3534360 \\ -3.3142140 & -4.3659370 \\ -5.0947980 & -3.5502580 \\ -4.2873320 & -1.3153050 \\ -1.3688310 & -4.2590710 \\ -1.0341990 & -3.2631560 \\ -3.4200820 & -2.6073280 \\ -3.7882770 & -4.3257700 \\ -4.3409840 & -2.2437150\end{array}$

$-3.1294390$ $-6.2172530$ $-4.2008170$ $-3.8536720$ $-5.5699840$ $-6.5949660$ $-4.5173070$ $-4.7583280$ $-5.3729100$ $-0.6402320$ $-0.9355110$ $-1.7211180$ $-1.5112940$ $-2.9421410$ $-2.5174110$ $-0.5590500$ $-3.9470250$ $-3.0970340$ $-3.7360470$ $-2.3545920$ $-4.8972890$ $-4.5237600$ $-0.2094910$ 0.4242890 2.8647340 1.8671990 2. 4919260

1.1255370 3. 4508190 3.0533630 4.1678180 2.9216530 2. 0948760 3.7753150 4.7499130 3. 1962210 2. 5939710 3.2380160 4.6576930 4. 8118570 2. 2988690 1. 1226420 154 $\operatorname{TS} 165(\mathrm{~S}, \mathrm{~S}) \mathrm{G}[\mathrm{WB} 97 \mathrm{XD} / 6-31 \mathrm{G}(\mathrm{d})]=-3541.691429$
$\mathrm{P}$
0.3552190
0.1893990
0.7563880
0.7929560
2. 0313790 


$\begin{array}{rrr}0.5442300 & -1.3019260 & 0.6826170 \\ 1.7420270 & 1.0057530 & 0.5822380 \\ -0.4813630 & 0.5919380 & -0.5588020 \\ 2.3569980 & 0.9007860 & -0.6527410 \\ 1.8242200 & 1.6220490 & -1.7211680 \\ 3.4913740 & 0.1015920 & -0.7720230 \\ 4.0621820 & 0.0119170 & -2.0373660 \\ 4.9540240 & -0.5993510 & -2.1605190 \\ -0.5083500 & 1.9319020 & -0.9038620 \\ -1.6750500 & 2.6578350 & -0.6833110 \\ 0.6445010 & 2.4975630 & -1.4644120 \\ -1.6568770 & 4.0174660 & -0.9972070 \\ -2.5570240 & 4.6047710 & -0.8300620 \\ 4.0732890 & -0.6205760 & 0.3919750 \\ -2.8992480 & 2.0089420 & -0.1396880 \\ 4.5977360 & 0.0830060 & 1.4839840 \\ 4.6182660 & 1.5815820 & 1.6684620 \\ 5.7050860 & 0.3112070 & 3.6375890 \\ 5.1993620 & -0.5989420 & 2.5486370 \\ 3.8674490 & 1.9165120 & 2.9761430 \\ 3.9200070 & 2.9984430 & 3.1458680 \\ 2.8120690 & 1.6577940 & 2.8613330 \\ 4.5115030 & 1.1437720 & 4.1568070 \\ 4.8642330 & 1.8344790 & 4.9318740 \\ 3.7816640 & 0.4768800 & 4.6268000 \\ 6.1581880 & -0.2612390 & 4.4541920 \\ 4.1579870 & 2.1032150 & 0.8263520 \\ 6.7377880 & 1.2796400 & 3.0195910 \\ 7.0701910 & 1.9877320 & 3.7876850 \\ 7.6202420 & 0.7177400 & 2.6966200 \\ 6.0898270 & 2.0253610 & 1.8239200 \\ 6.1211290 & 3.1101590 & 1.9790840 \\ 6.6318620 & 1.8181220 & 0.8953100 \\ 4.1489020 & -2.0187910 & 0.4009710 \\ 3.5898140 & -2.9635970 & -0.6358940 \\ 4.7914960 & -4.1887870 & 1.2955720 \\ 4.7711540 & -2.6910480 & 1.4613070 \\ 4.7549760 & -3.8028300 & -1.2041450 \\ 4.3545130 & -4.5351630 & -1.9152320 \\ 5.4396410 & -3.1562620 & -1.7637350 \\ 5.4965890 & -4.5137860 & -0.0405420 \\ 5.5050380 & -5.5998860 & -0.1896040 \\ 6.5406470 & -4.1882860 & -1297560 \\ 5.3116180 & -4.6720950 & \\ 3.0742760 & -2.4273970 & -2037740 \\ 3.3326630 & -4.6857020 & \\ & & \end{array}$

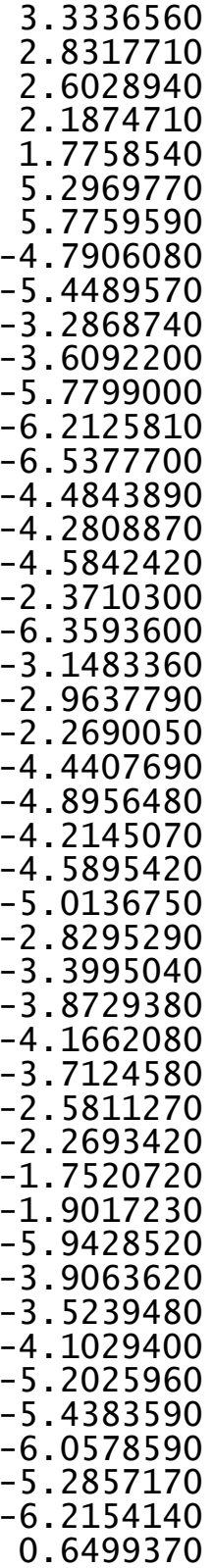

-5.7658010
-4.5285240
-3.9195230
-4.6144150
-3.3299780
-1.9855020
-2.5113130
0.5048900
-0.4264720
0.6595650
1.0818280
0.3916710
-0.2760360
1.1447160
1.0602940
0.7499490
2.1505130
1.1295010
-0.8686700
-0.8755250
-1.1959090
-1.1617750
-1.5284280
-2.1904020
-2.1498900
1.7744240
2.2880020
3.3703950
2.3488600
2.0135580
2.3834640
0.9345410
2.7119330
3.4912180
2.0091970
3.8040380
1.8110510
4.4631750
5.2276670
4.9584200
3.8192040
4.2225690
4.0373590
0.8538110
0.4193010
3.8764630
1.0133760

2. 1646160

0.0717200

$-0.6677920$

0.4705300

2. 5361790

3. 3607380

$-0.4339250$

$-1.4199500$

$-2.3281880$

$-0.9147620$

$-2.6888410$

$-3.4429320$

$-2.4510750$

$-3.2199600$

$-4.2512340$

$-3.2262810$

$-2.6939300$

$-1.0004760$

$-2.3653640$

$-3.3969970$

$-1.7837970$

$-1.8110080$

$-2.5566720$

$-0.9399200$

1. 5918980

2. 9427810

2. 0785590

1.1227260

3.9454490

4.9349600

4.0387320

3. 4522600

4. 1575500

3. 3651430

1.6997680

3. 2735490

2. 2690070

2.9549940

1. 3119120

2.8310690

3.8227110

2. 1834320

0.8182010

1.1831690 


$\begin{array}{rrr}1.8772310 & 4.6622420 & -2.1014100 \\ 2.0553670 & 5.8322310 & -1.1165280 \\ 0.8005970 & 6.7358870 & -1.0386760 \\ -0.4224400 & 6.1115560 & -1.7440140 \\ -0.5086370 & 4.6320500 & -1.4784620 \\ 1.7658960 & 5.0643600 & -3.1184670 \\ 2.9343480 & 6.4186990 & -1.4037360 \\ 1.0023400 & 7.7135630 & -1.4889550 \\ -0.3274180 & 6.2762860 & -2.8263560 \\ 2.7692520 & 4.0302530 & -2.1004750 \\ 2.2683690 & 5.4061280 & -0.1303880 \\ 0.5489150 & 6.9198160 & 0.0107410 \\ -1.3417260 & 6.6171460 & -1.4317310 \\ 2.3833770 & 1.4587700 & -3.0036690 \\ 1.7363540 & 2.1131430 & -4.2094230 \\ 2.1433200 & 1.4610090 & -5.5297020 \\ 3.6632620 & 1.3417480 & -5.6008560 \\ 4.1593280 & 0.4001180 & -4.5054030 \\ 3.5175580 & 0.6485640 & -3.1527630 \\ 1.7570310 & 2.0550950 & -6.3649920 \\ 2.0196940 & 3.1725010 & -4.2500940 \\ 0.6476260 & 2.0963820 & -4.0905620 \\ 4.1090770 & 2.3368970 & -5.4686210 \\ 3.9863630 & 0.9735610 & -6.5805800 \\ 5.2491510 & 0.4680750 & -4.4058840 \\ 3.9454260 & -0.6363610 & -4.8020530 \\ 1.6931580 & 0.4624370 & -5.6097750 \\ -1.7463280 & -3.4593530 & -0.5780360 \\ -0.6232310 & -2.8902270 & -0.9032700 \\ -2.2947200 & -4.3681010 & -1.6202210 \\ -3.4987390 & -5.0312670 & -1.3738380 \\ -1.6419290 & -4.5341350 & -2.8440950 \\ -4.0480570 & -5.8575060 & -2.3457470 \\ -3.9950280 & -4.8861430 & -0.4202600 \\ -2.1949910 & -5.3614920 & -3.8147400 \\ -0.7099790 & -4.0114780 & -3.0273100 \\ -3.3965850 & -6.0220950 & -3.5669600 \\ -4.9850660 & -6.3709320 & -2.1543710 \\ -1.6899660 & -5.4893170 & -4.7669770 \\ -3.8263810 & -6.6671090 & -4.3275570 \\ -0.2400390 & -2.2350680 & -0.2154520 \\ -2.3610970 & -3.2882190 & 0.4834900 \\ -1.5242220 & -2.0537730 & 2.8603790 \\ -2.5558960 & -1.6974440 & 1.8793640 \\ -0.5119200 & -2.0828270 & 2.4602260 \\ -2.2969720 & -0.9764470 & 1.1095330\end{array}$

$\begin{array}{ll}\mathrm{O} & -1.8495260 \\ \mathrm{H} & -1.2300170 \\ \mathrm{C} & -3.3062920 \\ \mathrm{C} & -4.2299730 \\ \mathrm{C} & -3.9947160 \\ \mathrm{C} & -1.8392780 \\ \mathrm{H} & -3.4996190 \\ \mathrm{H} & -5.2799730 \\ \mathrm{H} & -4.3722090 \\ \mathrm{H} & -1.5980960 \\ \mathrm{H} & -1.1668970 \\ \mathrm{H} & -3.5096660 \\ \mathrm{H} & -4.0446260 \\ \mathrm{H} & -4.5205980 \\ 154 & \end{array}$
$-0.7632740$
$-0.1031630$
$-3.1885190$
$-3.1878420$
$-1.9559020$
$-3.1812680$
$-4.0702630$
$-3.2119480$
$-1.0458650$
$-4.1183650$
$-3.0987570$
$-2.3073790$
$-4.0875960$
$-2.0169580$

\begin{abstract}
3.4166050
2. 9689610

4.2533870

3.0334860

2. 1581710

3.8159300

4.8723210

3. 3414390

2. 6405970

3.2995520

4.6757460

4.8735520

2.4352360

1. 2028540
\end{abstract}

TS166 (S , S) G[WB97XD/6-31G(d) $]=-3541.692218$

$\begin{array}{lrrr}\mathrm{P} & -0.3806720 & -0.1697100 & 0.9069020 \\ \mathrm{O} & 0.2363840 & -0.6502480 & 2.1865110 \\ \mathrm{O} & -0.6199830 & 1.3059220 & 0.7147790 \\ \mathrm{O} & -1.7366630 & -1.0408500 & 0.7039530 \\ \mathrm{O} & 0.5147130 & -0.6015380 & -0.4014280 \\ \mathrm{C} & -2.3218530 & -0.9959950 & -0.5503820 \\ \mathrm{C} & -1.7212610 & -1.7209440 & -1.5789770 \\ \mathrm{C} & -3.4732140 & -0.2361750 & -0.7385590 \\ \mathrm{C} & -3.9971660 & -0.2053230 & -2.0282870 \\ \mathrm{H} & -4.9067850 & 0.3659630 & -2.2018620 \\ \mathrm{C} & 0.5840120 & -1.9512310 & -0.6993570 \\ \mathrm{C} & 1.7687970 & -2.6435950 & -0.4560530 \\ \mathrm{C} & -0.5383700 & -2.5661520 & -1.2483870 \\ \mathrm{C} & 1.7681410 & -4.0085480 & -0.7182550 \\ \mathrm{H} & 2.6843760 & -4.5698240 & -0.5455300 \\ \mathrm{C} & -4.1183340 & 0.5126980 & 0.3737840 \\ \mathrm{C} & 2.9872670 & -1.9364560 & 0.0208830 \\ \mathrm{C} & -4.6733660 & -0.1631890 & 1.4676920 \\ \mathrm{C} & -4.6648500 & -1.6537530 & 1.7080650 \\ \mathrm{C} & -5.8631990 & -0.3404490 & 3.5816640 \\ \mathrm{C} & -5.3361000 & 0.5416990 & 2.4798810 \\ \mathrm{C} & -3.9600350 & -1.9187210 & 3.0564420 \\ \mathrm{H} & -3.9896670 & -2.9946970 & 3.2650550 \\ \mathrm{H} & -2.9082250 & -1.6339710 & 2.9731160 \\ \mathrm{C} & -4.6715950 & -1.1208010 & 4.1804500 \\ \mathrm{H} & -5.0387550 & -1.7920860 & 4.9657880 \\ \mathrm{H} & -3.9786180 & -0.4182250 & 4.6541000 \\ \mathrm{H} & -6.3640330 & 0.2501620 & 4.3564120 \\ \mathrm{H} & -4.1577140 & -2.1942880 & 0.9055570 \\ \mathrm{C} & -6.8447440 & -1.3588550 & 2.9608380\end{array}$




$\begin{array}{lrrr}\mathrm{H} & -7.1891800 & -2.0471340 & 3.7415710 \\ \mathrm{H} & -7.7277830 & -0.8340590 & 2.5821260 \\ \mathrm{C} & -6.1302740 & -2.1300790 & 1.8204780 \\ \mathrm{H} & -6.1410340 & -3.2091230 & 2.0138180 \\ \mathrm{H} & -6.6387500 & -1.9703910 & 0.8639250 \\ \mathrm{C} & -4.2258660 & 1.9082630 & 0.3268410 \\ \mathrm{C} & -3.6413130 & 2.8286840 & -0.7183740 \\ \mathrm{C} & -4.9683480 & 4.0912560 & 1.1050310 \\ \mathrm{C} & -4.9134190 & 2.6022330 & 1.3314130 \\ \mathrm{C} & -4.7984770 & 3.6128930 & -1.3742910 \\ \mathrm{H} & -4.3825060 & 4.3318580 & -2.0901320 \\ \mathrm{H} & -5.4366490 & 2.9285280 & -1.9438100 \\ \mathrm{C} & -5.6170760 & 4.3416770 & -0.2750430 \\ \mathrm{H} & -5.6538200 & 5.4205270 & -0.4663540 \\ \mathrm{H} & -6.6513520 & 3.9826540 & -0.2589890 \\ \mathrm{H} & -5.5420550 & 4.5906630 & 1.8932870 \\ \mathrm{H} & -3.0722120 & 2.2769870 & -1.4700830 \\ \mathrm{C} & -3.5214780 & 4.6279010 & 1.0619310 \\ \mathrm{H} & -3.5435580 & 5.6993500 & 0.8286490 \\ \mathrm{H} & -3.0625700 & 4.5225840 & 2.0504140 \\ \mathrm{C} & -2.7169660 & 3.8395450 & -0.0020880 \\ \mathrm{H} & -2.2845180 & 4.5168430 & -0.7480840 \\ \mathrm{H} & -1.8947110 & 3.2907460 & 0.4598780 \\ \mathrm{C} & -5.4687290 & 1.9234950 & 2.4088540 \\ \mathrm{H} & -5.9972460 & 2.4672970 & 3.1904010 \\ \mathrm{C} & 4.8143720 & -0.3874930 & -0.4119680 \\ \mathrm{C} & 5.4124860 & 0.5078640 & -1.4668660 \\ \mathrm{C} & 3.2791670 & -0.7184070 & -2.2576360 \\ \mathrm{C} & 3.6471060 & -1.0377100 & -0.8282210 \\ \mathrm{C} & 5.7492390 & -0.3686060 & -2.6948350 \\ \mathrm{H} & 6.1294520 & 0.2724210 & -3.4986110 \\ \mathrm{H} & 6.5483230 & -1.0703150 & -2.4357810 \\ \mathrm{C} & 4.4744060 & -1.1281480 & -3.1480760 \\ \mathrm{H} & 4.2368560 & -0.9004460 & -4.1935760 \\ \mathrm{H} & 4.6221930 & -2.2106950 & -3.0798230 \\ \mathrm{H} & 2.3763380 & -1.2466050 & -2.5729970 \\ \mathrm{H} & 6.3123340 & 1.0108840 & -1.0962960 \\ \mathrm{C} & 3.0767180 & 0.8044370 & -2.3867250 \\ \mathrm{H} & 2.8643090 & 1.0515460 & -3.4331540 \\ \mathrm{H} & 2.1949870 & 1.0907540 & -1.8086310 \\ \mathrm{C} & 4.3494330 & 1.5423090 & -1.8964680 \\ \mathrm{H} & 4.7601130 & 2.1813430 & -2.6864070 \\ \mathrm{H} & 4.1120490 & 2.2005510 & -1.0557920 \\ \mathrm{C} & 4.6976120 & -1.5248130 & 1.6974720 \\ \mathrm{C} & 5.1547180 & -1.9224550 & 3.0766320 \\ \mathrm{C} & 3.0118020 & -3.1557250 & 2.3183600\end{array}$

\begin{abstract}
3. 5249020
4. 0101680

4.3279300

3.8004040

2.7471300

2. 4811120

1.8840080

2. 1006300

6.0655040

4.1382010

3. 7958370

4.3502420

5.4075100

5.6619740

6.2699560

5.3438270

6.2601650

$-0.5447570$

$-1.8250560$

$-1.7922890$

$-0.4988130$

0.6949880

0.6278190

$-2.6682220$

$-2.0105660$

$-2.6718230$

$-0.4122820$

$-0.5025840$

1. 6332510

0.7428520

$-1.8539950$

$-2.2103340$

$-3.3771260$

$-1.4107730$

$-2.2403600$

$-3.0227680$

$-4.0039520$

$-1.5817630$

$-0.6035980$

$-0.9065060$

$-2.3188650$

$-3.5648530$

$-4.4535740$

$-4.8326810$

$-2.9422400$

1. 5854970
\end{abstract}

-2.1767130
-1.6240060
-1.9024730
-0.5495180
-2.4153650
-3.1568440
-1.7599510
-3.6542170
-1.3847210
-4.1847710
-4.9217200
-4.7281960
-3.4485190
-3.7678010
-3.6775310
-0.6321120
-0.1368520
-3.9645580
-4.6754050
-6.1734090
-6.7841150
-6.1930100
-4.6849180
-6.6545970
-4.5417310
-4.2010630
-6.5689910
-7.8738800
-6.4660460
-6.6383130
-6.3381140
-1.5855080
-0.8382640
-2.1139720
-2.2738730
-0.9932100
-0.7186360
-2.5223320
-1.3912430
-3.0494850
-0.1555270
-1.0662160
0.2744850
-1.4381690
-3.1113360
3.4366000

1. 2898750

4.0689820

5.0806090

4.0823120

3. 6463890

4.4086930

3. 5226210

1. 9808380

3. 3624410

2. 5666600

3. 3019580

1.6394870

3. 0741910

4.0915840

2. 4399910

0.8509880

1.1694010

$-1.4176850$

$-1.8194350$

$-1.5187690$

$-2.0514010$

$-1.3042110$

$-1.1569950$

$-1.9668150$

$-2.8936550$

$-1.3115170$

$-3.1251440$

$-1.9416890$

$-1.8016780$

$-0.3006060$

$-0.4348960$

$-2.8899640$

$-3.1054470$

$-4.0668620$

$-5.3384380$

$-5.6206630$

$-4.4820630$

$-6.1778350$

$-4.2581700$

$-3.8179430$

$-5.7151230$

$-6.5697430$

$-4.5487920$

$-5.2212500$

$-0.7178350$ 


\begin{tabular}{|c|c|c|c|c|c|c|c|}
\hline & & & & & & & \\
\hline 0 & $\begin{array}{l}0.4757170 \\
2.1017020\end{array}$ & $\begin{array}{l}2.8176230 \\
4.2913560\end{array}$ & $\begin{array}{l}-0.9945810 \\
-1.8205840\end{array}$ & C & $\begin{array}{r}-0.4464330 \\
1.9452420\end{array}$ & $\begin{array}{l}-2.6494180 \\
-3.9730610\end{array}$ & -1.1128010 \\
\hline c & 3.2988470 & 4.9850530 & -1.6308690 & $\mathrm{H}$ & $\begin{array}{l}1.9452420 \\
2.8947310\end{array}$ & $\begin{array}{l}-3.9730610 \\
-4.4846020\end{array}$ & $\begin{array}{l}-0.041 \\
-0.4968880\end{array}$ \\
\hline c & 1.4308550 & 4.3735250 & -3.0429810 & C & -4.1129340 & 0.4235260 & 0.3470730 \\
\hline$c$ & 3.8258440 & 5.7557020 & -2.6589300 & $\mathrm{C}$ & 3.0675260 & -1.8402930 & 0.0850440 \\
\hline $\mathrm{H}$ & 3.8084830 & 4.9042480 & -0.6767750 & $\mathrm{C}$ & -4.7151060 & -0.2427140 & 1.4216520 \\
\hline C & 1.9608800 & 5.1466390 & -4.0697090 & C & -4.7078290 & -1.7301400 & 1.6807940 \\
\hline $\mathrm{H}$ & 0.5038070 & 3.8290390 & -3.1819460 & $\mathrm{C}$ & -6.0050630 & -0.4024040 & 3.4776220 \\
\hline C & 3.1572520 & 5.8351790 & -3.8792340 & C & -5.4337920 & 0.4695800 & 2.3896920 \\
\hline $\mathrm{H}$ & 4.7587690 & 6.2907050 & -2.5119370 & $\mathrm{C}$ & -4.0681030 & -1.9725350 & 3.0651690 \\
\hline $\mathrm{H}$ & 1.4424160 & 5.2099860 & -5.0211760 & $\mathrm{H}$ & -4.1027250 & -3.0456500 & 3.2873660 \\
\hline $\mathrm{H}$ & 3.5700250 & 6.4352230 & -4.6847530 & $\mathrm{H}$ & -3.0146410 & -1.6832740 & 3.0283600 \\
\hline $\mathrm{H}$ & 0.1181950 & 2.1998900 & -0.2597590 & C & -4.8360390 & -1.1627320 & 4.1426280 \\
\hline 0 & 2.2141080 & 3.3488430 & 0.3456780 & $\mathrm{H}$ & -5.2330290 & -1.8245510 & 4.9214500 \\
\hline $\mathrm{C}$ & 1.4509530 & 2.2379780 & 2.8098790 & $\mathrm{H}$ & -4.1706720 & -0.4470500 & 4.6357470 \\
\hline $\mathrm{C}$ & 2.4729690 & 1.8480140 & 1.8310800 & $\mathrm{H}$ & -6.5474400 & 0.1942940 & 4.2189390 \\
\hline $\mathrm{H}$ & 0.4314290 & 2.2194990 & 2.4279180 & $\mathrm{H}$ & -4.1587760 & -2.2772760 & 0.9112460 \\
\hline $\mathrm{H}$ & 2.2175420 & 1.0794450 & 1.1077240 & C & -6.9468160 & -1.4388400 & 2.8254190 \\
\hline 0 & 1.8171540 & 0.9900970 & 3.4343050 & $\mathrm{H}$ & -7.3184720 & -2.1219280 & 3.5982580 \\
\hline $\mathrm{H}$ & 1.2012960 & 0.2906610 & 3.0453960 & $\mathrm{H}$ & -7.8177600 & -0.9285260 & 2.4015840 \\
\hline C & 3.2312170 & 3.4969550 & 4.0958950 & C & -6.1742470 & -2.2150900 & 1.7273370 \\
\hline $\mathrm{C}$ & 4.1300890 & 3.4453630 & 2.8589060 & $\mathrm{H}$ & -6.1879470 & -3.2925500 & 1.9291730 \\
\hline C & 3.9096710 & 2.1572300 & 2.0647510 & $\mathrm{H}$ & -6.6378410 & -2.0664300 & 0.7464840 \\
\hline C & 1.7566550 & 3.4271920 & 3.6907170 & C & -4.2295290 & 1.8174610 & 0.2755800 \\
\hline $\mathrm{H}$ & 3.4126680 & 4.4200540 & 4.6553180 & $\mathrm{C}$ & -3.5937080 & 2.7312240 & -0.7457900 \\
\hline $\mathrm{H}$ & 5.1851270 & 3.5151970 & 3.1413590 & $\mathrm{C}$ & -5.0479690 & 4.0009800 & 0.9731100 \\
\hline $\mathrm{H}$ & 4.3207360 & 1.2871420 & 2.5913500 & $\mathrm{C}$ & -4.9778360 & 2.5175870 & 1.2311990 \\
\hline $\mathrm{H}$ & 1.4808030 & 4.3259700 & 3.1257040 & $\mathrm{C}$ & -4.7153500 & 3.4942590 & -1.4831990 \\
\hline $\mathrm{H}$ & 1.1041740 & 3.3794670 & 4.5684300 & $\mathrm{H}$ & -4.2611540 & 4.2202830 & -2.1679310 \\
\hline $\mathrm{H}$ & 3.4699160 & 2.6615850 & 4.7649860 & $\mathrm{H}$ & -5.2998970 & 2.7997800 & -2.0963810 \\
\hline $\mathrm{H}$ & 3.9083150 & 4.3013230 & 2.2107980 & C & -5.6231920 & 4.2086110 & -0.4463780 \\
\hline$H$ & 4.4151000 & 2.1734770 & 1.0967520 & $\mathrm{H}$ & -5.6877270 & 5.2824010 & -0.6574970 \\
\hline .3 & & & & $\mathrm{H}$ & -6.6425400 & 3.8108540 & -0.4849220 \\
\hline & S) $\mathrm{G}[\mathrm{WB} 97$ & $(d)]=-3$ & 368 & $\mathrm{H}$ & -5.6764340 & 4.5029280 & 1.7166280 \\
\hline $\mathrm{P}$ & -0.4002330 & -0.2104890 & 1.0022880 & $\mathrm{H}$ & -2.9702530 & 2.1772530 & -1.4512830 \\
\hline 0 & 0.2081940 & -0.6421390 & 2.3033710 & C & -3.6135500 & 4.5701980 & 0.9976330 \\
\hline 0 & -0.6754730 & 1.2545930 & 0.7780220 & $\mathrm{H}$ & -3.6453950 & 5.6311660 & 0.7213960 \\
\hline 0 & -1.7282740 & -1.1198250 & 0.7876310 & $\mathrm{H}$ & -3.2128390 & 4.5143490 & 2.0150310 \\
\hline 0 & 0.5309580 & -0.6342740 & -0.2840490 & C & -2.7289270 & 3.7616060 & 0.0160410 \\
\hline C & -2.2777510 & -1.0989790 & -0.4850530 & $\mathrm{H}$ & -2.2391120 & 4.4222530 & -0.7088700 \\
\hline C & -1.6468630 & -1.8436080 & -1.4776400 & $\mathrm{H}$ & -1.9458450 & 3.2241010 & 0.5531130 \\
\hline C & -3.4186200 & -0.3364790 & -0.7274100 & C & -5.5797790 & 1.8481740 & 2.2893970 \\
\hline C & -3.8893300 & -0.3183300 & -2.0363720 & $\mathrm{H}$ & -6.1560090 & 2.3972710 & 3.0325010 \\
\hline $\mathrm{H}$ & -4.7860650 & 0.2584180 & -2.2541730 & C & 4.8078220 & -0.2095410 & -0.3994190 \\
\hline C & 5400 & -1.9802500 & -0.5779770 & C & 5.3477230 & 0.6927960 & -1.4796350 \\
\hline C & 5070 & -2.6119440 & -0.3640380 & C & 3.2826800 & -0.6699010 & -2.2259590 \\
\hline
\end{tabular}




\begin{tabular}{|c|c|c|}
\hline 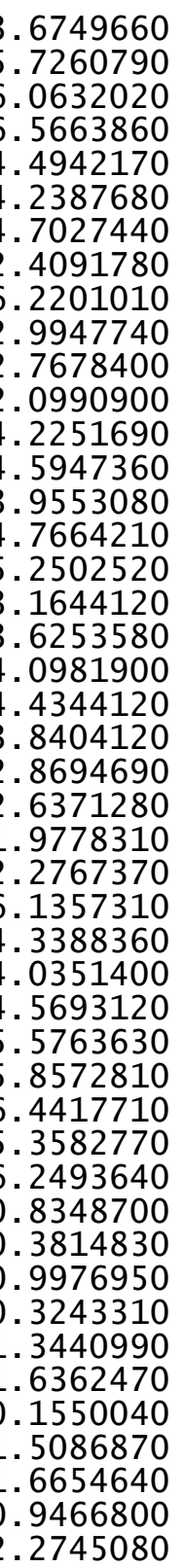 & $\begin{array}{r}-0.9314160 \\
-0.1930450 \\
0.4488440 \\
-0.8407820 \\
-1.0355610 \\
-0.8489890 \\
-2.1059730 \\
-1.2563040 \\
1.2547450 \\
0.8350160 \\
1.0439880 \\
1.0866930 \\
1.6552300 \\
2.2987670 \\
2.3192680 \\
-1.3032330 \\
-1.6445050 \\
-2.9977740 \\
-2.0228390 \\
-1.3776460 \\
-1.6205200 \\
-0.3134840 \\
-2.2337660 \\
-2.9652050 \\
-1.6209330 \\
-3.5479920 \\
-1.0570280 \\
-3.9655730 \\
-4.6933610 \\
-4.5283890 \\
-3.1562300 \\
-3.4365140 \\
-3.3592990 \\
-0.3972160 \\
0.1528780 \\
-4.7032740 \\
-4.0431450 \\
-6.1889320 \\
-6.9546650 \\
-6.1993040 \\
-4.8373980 \\
-7.9624310 \\
-6.3114960 \\
-6.6223950 \\
-6.0641740 \\
-6.7700270\end{array}$ & $\begin{array}{l}-0.7914910 \\
-2.6888400 \\
-3.5109750 \\
-2.4194770 \\
-3.1133120 \\
-4.1627060 \\
-3.0175030 \\
-2.5214670 \\
-1.1285030 \\
-2.3928970 \\
-3.4446430 \\
-1.8204120 \\
-1.9260040 \\
-2.7324870 \\
-1.0995400 \\
1.7367250 \\
3.1219750 \\
2.4098550 \\
1.3540470 \\
4.1153280 \\
5.1301350 \\
4.1081600 \\
3.7178580 \\
4.5006970 \\
3.5786880 \\
2.0899550 \\
3.3891870 \\
2.6811680 \\
3.4420090 \\
1.7700690 \\
3.1544530 \\
4.1763810 \\
2.5155370 \\
0.8646500 \\
1.1644150 \\
-1.0674260 \\
-1.2894750 \\
-1.3307040 \\
-1.3743750 \\
-2.2243540 \\
-1.5999070 \\
-1.7688910 \\
-2.2965540 \\
-0.5774220 \\
-3.2401460 \\
-2.3158930\end{array}$ \\
\hline
\end{tabular}

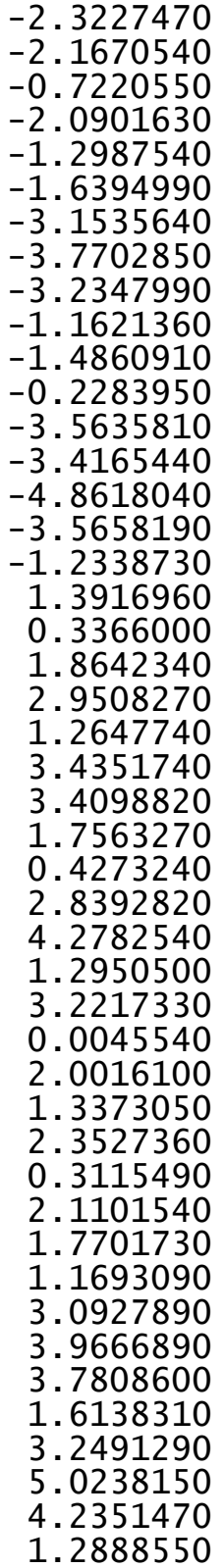

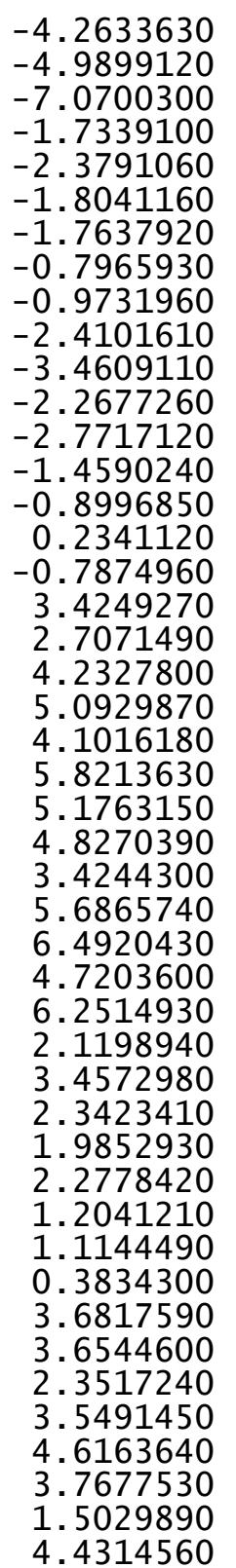

$-2.2251280$ $-0.6488170$ $-0.3574470$ $-2.8103360$ $-3.9344370$ $-5.3086720$ $-5.4973010$ $-4.4892960$ $-3.0809970$ $-6.0862470$ $-3.9570390$ $-3.7290960$ $-5.3471250$ $-6.5159770$ $-4.4761710$ $-4.8116550$ $-5.3999850$ $-0.7714640$ $-1.0217050$ $-1.9277930$ $-1.7573170$ $-3.1827180$ $-2.8363840$ $-0.7779650$ $-4.2616720$ $-3.3062380$ $-4.0893160$ $-2.7032400$ $-5.2383850$ $-4.9341920$ $-0.2495170$ 0.3063650 2.8079430 1.8098740 2.4493430

1.0958840 3.4310490 3.0781380 4.0458460 2.7904500 2.0112080 3.6741270 4.5935710 3.0499040 2.5369990 3.1093010 


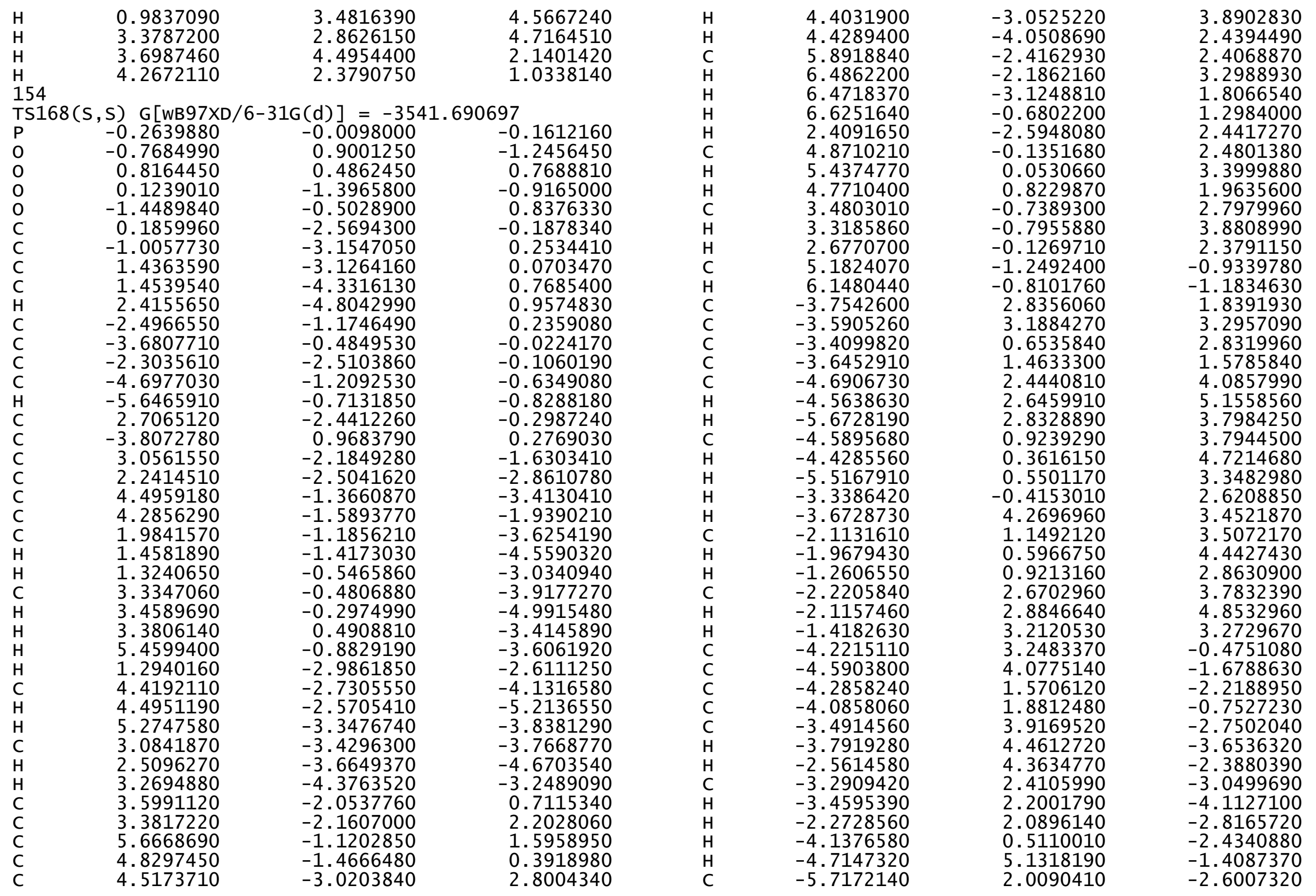




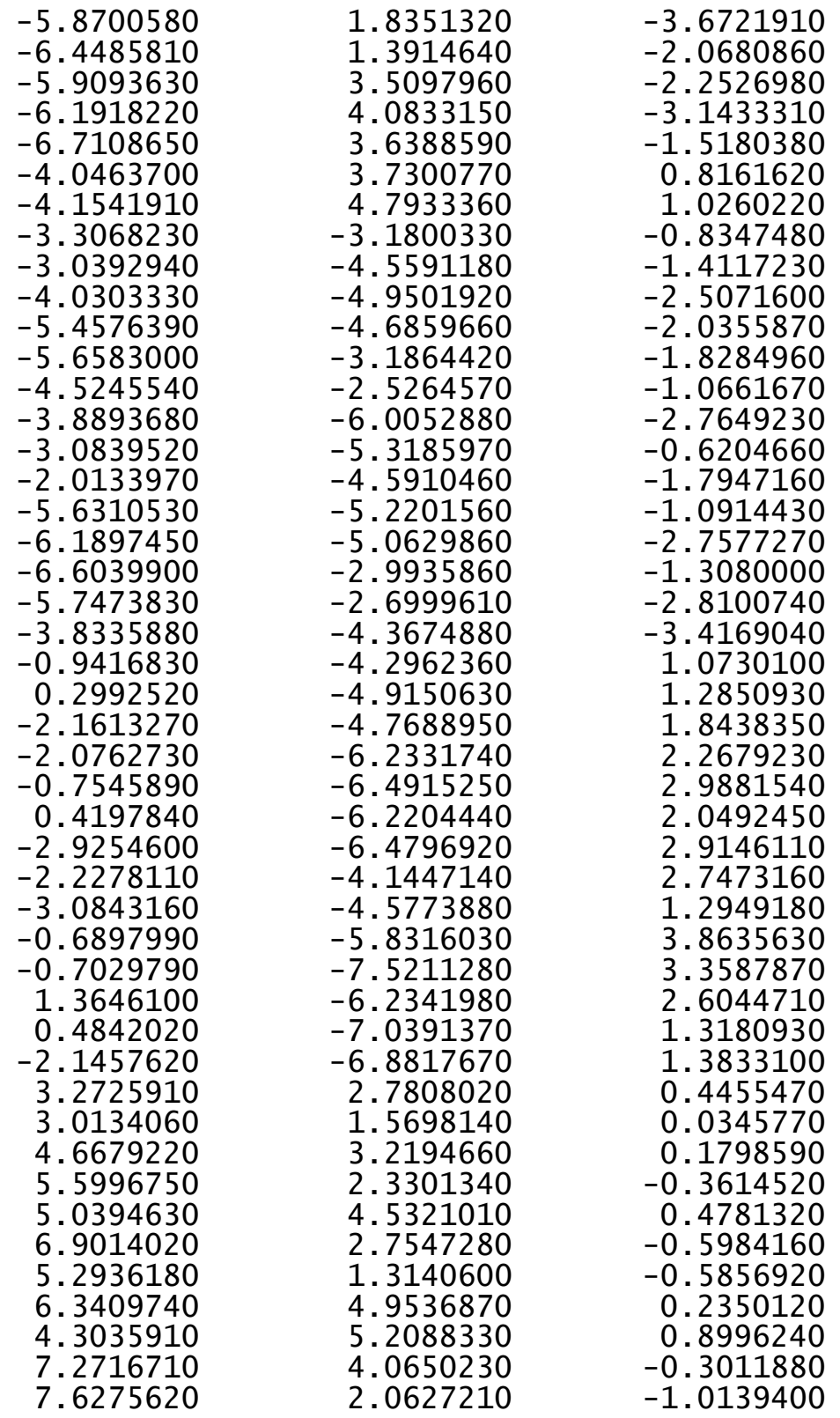

$\begin{array}{lr}\mathrm{H} & 6.6312410 \\ \mathrm{H} & 8.2893060 \\ \mathrm{H} & 2.1091890 \\ \mathrm{O} & 2.4635470 \\ \mathrm{C} & 0.4274630 \\ \mathrm{C} & 0.3639920 \\ \mathrm{H} & 0.9168090 \\ \mathrm{H} & 0.3027920 \\ \mathrm{O} & -0.9868200 \\ \mathrm{H} & -1.1090010 \\ \mathrm{C} & 0.0901650 \\ \mathrm{C} & 0.4717830 \\ \mathrm{C} & 0.0229260 \\ \mathrm{C} & 0.7351830 \\ \mathrm{H} & 0.4090920 \\ \mathrm{H} & 0.0259970 \\ \mathrm{H} & -1.0673500 \\ \mathrm{H} & 1.8262810 \\ \mathrm{H} & 0.3997670 \\ \mathrm{H} & -1.0000980 \\ \mathrm{H} & 1.5597850 \\ \mathrm{H} & 0.4557270 \\ \mathrm{H} & \end{array}$

$\begin{array}{lr}5.9743250 & 0.4637720 \\ 4.3954890 & -0.4870880 \\ 1.1953410 & 0.3190860 \\ 3.5353340 & 1.0001370 \\ 3.6334620 & -0.9008170 \\ 3.5378060 & 0.5602630 \\ 2.7976000 & -1.4013480 \\ 2.5430590 & 0.9878170 \\ 3.4054600 & -0.7576920 \\ 2.4100650 & -0.8992090 \\ 6.1267130 & -0.7221410 \\ 6.0516210 & 0.7583320 \\ 4.7297490 & 1.3873360 \\ 4.9816660 & -1.5076030 \\ 7.0841390 & -1.1457390 \\ 6.8826570 & 1.3133850 \\ 4.7057290 & 1.5129720 \\ 5.0975400 & -1.5142860 \\ 4.9758440 & -2.5498650 \\ 6.0733090 & -0.8288350 \\ 6.1394430 & 0.8601280 \\ 4.5831450 & 2.3806290\end{array}$

154

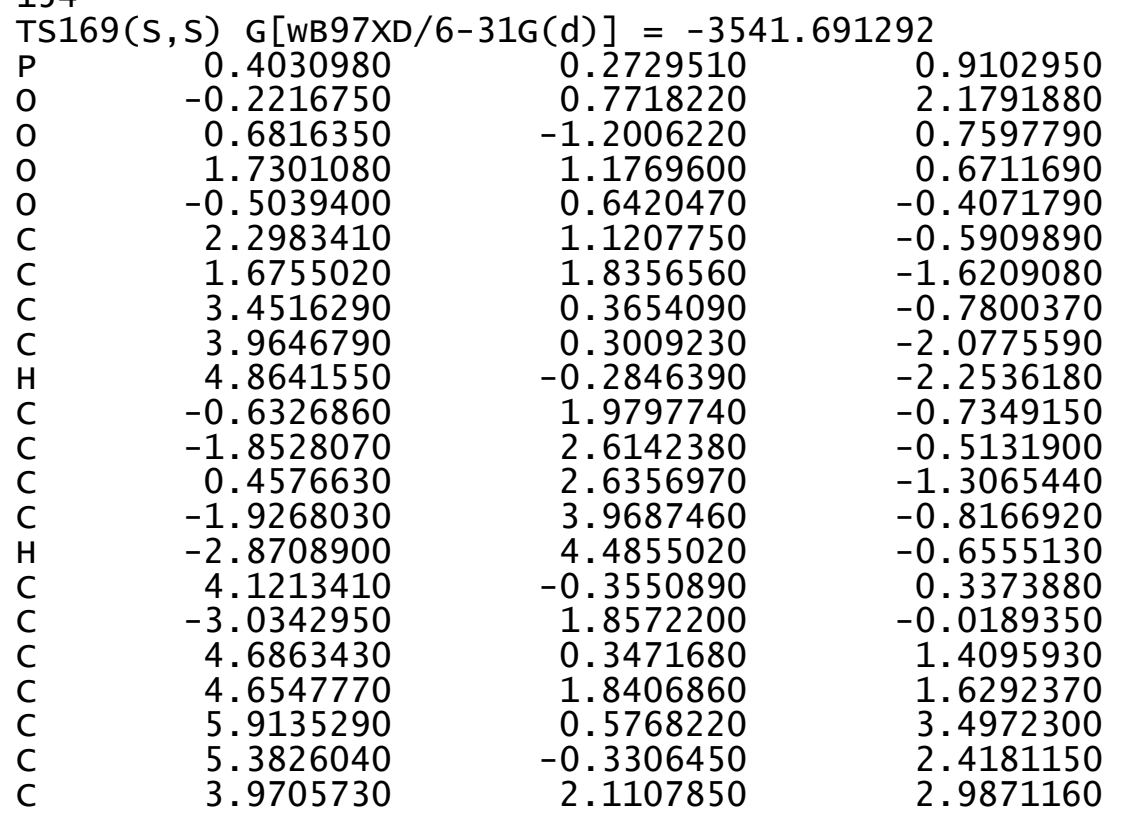




\begin{tabular}{|c|c|c|c|c|c|c|c|}
\hline $\mathrm{H}$ & 3.9822740 & 3.1899000 & 3.1806890 & H & -2.7656830 & -1.2141060 & -3.3961280 \\
\hline $\mathrm{H}$ & 2.9231490 & 1.8041930 & 2.9278270 & $\mathrm{H}$ & -2.0836030 & -1.1807630 & -1.7772260 \\
\hline C & 4.7187970 & 1.3427170 & 4.1082070 & C & -4.2142340 & -1.7356380 & -1.8349570 \\
\hline $\mathrm{H}$ & 5.0880050 & 2.0321800 & 4.8767110 & $\mathrm{H}$ & -4.5877980 & -2.4283990 & -2.5976510 \\
\hline $\mathrm{H}$ & 4.0488140 & 0.6342410 & 4.6054940 & $\mathrm{H}$ & -3.9458570 & -2.3437230 & -0.9660560 \\
\hline $\mathrm{H}$ & 6.4402170 & 0.0066510 & 4.2701780 & C & -4.7334650 & 1.4119330 & 1.6602750 \\
\hline $\mathrm{H}$ & 4.1225000 & 2.3602210 & 0.8293810 & C & -5.2195890 & 1.8288020 & 3.0235320 \\
\hline C & 6.8633540 & 1.6046200 & 2.8434710 & C & -3.1252080 & 3.1316900 & 2.2466440 \\
\hline $\mathrm{H}$ & 7.2104050 & 2.3095860 & 3.6080090 & $\mathrm{C}$ & -3.5893910 & 2.1056870 & 1.2409740 \\
\hline $\mathrm{H}$ & 7.7483030 & 1.0908140 & 2.4542710 & C & -4.0729220 & 1.6102730 & 4.0341500 \\
\hline C & 6.1125980 & 2.3468010 & 1.7075000 & H & -4.4114530 & 1.9104180 & 5.0327680 \\
\hline $\mathrm{H}$ & 6.1049940 & 3.4282100 & 1.8872860 & $\mathrm{H}$ & -3.8202610 & 0.5462330 & 4.0866650 \\
\hline H & 6.6063150 & 2.1851600 & 0.7435790 & C & -2.8386960 & 2.4370580 & 3.5946440 \\
\hline C & 4.2536480 & -1.7492600 & 0.3100660 & $\mathrm{H}$ & -2.6036700 & 3.2073360 & 4.3383660 \\
\hline C & 3.6554310 & -2.6981180 & -0.7018900 & $\mathrm{H}$ & -1.9505660 & 1.8125220 & 3.4912560 \\
\hline C & 5.0705920 & -3.9042300 & 1.0933860 & H & -2.2332130 & 3.6588000 & 1.9015330 \\
\hline $\mathrm{C}$ & 4.9804210 & -2.4147250 & 1.3061810 & $\mathrm{H}$ & -6.1089420 & 1.2605460 & 3.3179470 \\
\hline C & 4.8042860 & -3.4704850 & -1.3852510 & C & -4.2945290 & 4.1190070 & 2.4644330 \\
\hline $\mathrm{H}$ & 4.3771020 & -4.2189180 & -2.0632180 & $\mathrm{H}$ & -3.9888920 & 4.8814540 & 3.1897390 \\
\hline $\mathrm{H}$ & 5.4009220 & -2.7873940 & -1.9996410 & H & -4.5183980 & 4.6376660 & 1.5259750 \\
\hline C & 5.6869680 & -4.1477050 & -0.3028980 & C & -5.5386840 & 3.3415830 & 2.9730550 \\
\hline $\mathrm{H}$ & 5.7652860 & -5.2264660 & -0.4815260 & H & -5.8251500 & 3.6786450 & 3.9760920 \\
\hline $\mathrm{H}$ & 6.7041990 & -3.7432550 & -0.3238070 & $\mathrm{H}$ & -6.3989030 & 3.5130080 & 2.3180700 \\
\hline $\mathrm{H}$ & 5.6821090 & -4.3784130 & 1.8686140 & C & -5.3319270 & 0.4666420 & 0.8360900 \\
\hline $\mathrm{H}$ & 3.0478630 & -2.1704240 & -1.4407800 & $\mathrm{H}$ & -6.2270780 & -0.0606040 & 1.1633050 \\
\hline C & 3.6405070 & -4.4846000 & 1.0956060 & C & 0.3784930 & 4.0223980 & -1.5398130 \\
\hline $\mathrm{H}$ & 3.6880880 & -5.5534850 & 0.8544850 & C & 1.6008220 & 4.7874690 & -2.0099950 \\
\hline $\mathrm{H}$ & 3.2105870 & -4.3994500 & 2.0989410 & $\mathrm{c}$ & 1.5046070 & 6.2869060 & -1.7329770 \\
\hline C & 2.7782960 & -3.7149250 & 0.0640340 & C & 0.1741940 & 6.8252190 & -2.2528380 \\
\hline $\mathrm{H}$ & 2.3175790 & -4.4025150 & -0.6549950 & $\mathrm{C}$ & -0.9795300 & 6.1858240 & -1.4821260 \\
\hline $\mathrm{H}$ & 1.9734050 & -3.1712000 & 0.5609250 & C & -0.8290540 & 4.6885180 & -1.2901900 \\
\hline C & 5.5445130 & -1.7099190 & 2.3620610 & $\mathrm{H}$ & 2.3479610 & 6.8004970 & -2.2070990 \\
\hline $\mathrm{H}$ & 6.1026790 & -2.2316420 & 3.1379880 & H & 1.7310120 & 4.6454360 & -3.0903340 \\
\hline C & -4.7832920 & 0.2115250 & -0.4164420 & H & 2.4954580 & 4.3650250 & -1.5402480 \\
\hline $\mathrm{C}$ & -5.3309210 & -0.7426030 & -1.4468590 & $\mathrm{H}$ & 0.0845240 & 6.5923800 & -3.3224890 \\
\hline C & -3.2564410 & 0.5639850 & -2.2646000 & H & 0.1237020 & 7.9151290 & -2.1568390 \\
\hline $\mathrm{C}$ & -3.6452260 & 0.9042280 & -0.8453390 & $\mathrm{H}$ & -1.9346780 & 6.3927060 & -1.9793910 \\
\hline $\mathrm{C}$ & -5.7019850 & 0.0775130 & -2.7036980 & $\mathrm{H}$ & -1.0470570 & 6.6539590 & -0.4901190 \\
\hline $\mathrm{H}$ & -6.0365570 & -0.6079580 & -3.4909390 & $\mathrm{H}$ & 1.5793940 & 6.4733470 & -0.6534330 \\
\hline $\mathrm{H}$ & -6.5423940 & 0.7401590 & -2.4739210 & C & 2.1846670 & 1.7059510 & -2.9188230 \\
\hline C & -4.4666440 & 0.8934370 & -3.1677050 & C & 1.5117690 & 2.2511120 & -4.1531770 \\
\hline $\mathrm{H}$ & -4.2129920 & 0.6576910 & -4.2076400 & $\mathrm{c}$ & 1.3272360 & 1.1202140 & -5.1815940 \\
\hline $\mathrm{H}$ & -4.6700530 & 1.9681680 & -3.1217680 & C & 2.6459220 & 0.3651060 & -5.4837740 \\
\hline $\mathrm{H}$ & -2.3786710 & 1.1257700 & -2.5924480 & C & 3.8043940 & 0.8054450 & -4.5652480 \\
\hline $\mathrm{H}$ & -6.2075120 & -1.2775690 & -1.0655290 & C & 3.3354280 & 0.9354560 & -3.1402120 \\
\hline C & -2.9817830 & -0.9506200 & -2.3543460 & H & 2.1306510 & 3.0424340 & -4.5995890 \\
\hline
\end{tabular}




\begin{tabular}{|c|c|c|c|c|c|c|c|}
\hline & & & & & & & \\
\hline $\mathrm{H}$ & 0.9033790 & 1.5378620 & -6.1007820 & 0 & -0.4869330 & 0.5833120 & -0.5727150 \\
\hline $\mathrm{H}$ & 2.9443470 & 0.5144650 & -6.5267370 & C & 2.3581990 & 0.8805740 & -0.6594080 \\
\hline $\mathrm{H}$ & 4.1876080 & 1.7748160 & -4.9135350 & $\mathrm{C}$ & 1.8283330 & 1.5888830 & -1.7385170 \\
\hline $\mathrm{H}$ & 0.5440370 & 2.6998300 & -3.9141670 & $\mathrm{C}$ & 3.4953940 & 0.0836630 & -0.7632280 \\
\hline $\mathrm{H}$ & 0.5839860 & 0.4218450 & -4.7818360 & $\mathrm{C}$ & 4.0785750 & -0.0117090 & -2.0226430 \\
\hline H & 2.4889060 & -0.7105320 & -5.3530190 & $\mathrm{H}$ & 4.9738290 & -0.6201540 & -2.1333530 \\
\hline $\mathrm{H}$ & 4.6355130 & 0.0966140 & -4.6372740 & C & -0.5020150 & 1.9232750 & -0.9201190 \\
\hline c & -1.4109170 & -3.4807950 & -0.5786040 & $\mathrm{C}$ & -1.6576520 & 2.6646700 & -0.6893840 \\
\hline 0 & -0.3514000 & -2.8013180 & -0.9063000 & $\mathrm{C}$ & 0.6533360 & 2.4739540 & -1.4873060 \\
\hline c & -1.8935800 & -4.4129010 & -1.6324600 & $\mathrm{C}$ & -1.6216950 & 4.0251490 & -0.9967290 \\
\hline c & -3.0404730 & -5.1706190 & -1.3857720 & $\mathrm{H}$ & -2.5122160 & 4.6247460 & -0.8218360 \\
\hline c & -1.2399240 & -4.5119350 & -2.8628890 & C & 4.0649730 & -0.6336520 & 0.4095970 \\
\hline c & -3.5329740 & -6.0241830 & -2.3641150 & C & -2.8872570 & 2.0280050 & -0.1435590 \\
\hline $\mathrm{H}$ & -3.5384840 & -5.0756760 & -0.4269340 & $\mathrm{C}$ & 4.5762930 & 0.0737390 & 1.5053430 \\
\hline c & -1.7356770 & -5.3678410 & -3.8399900 & $\mathrm{C}$ & 4.5947070 & 1.5729390 & 1.6848800 \\
\hline $\mathrm{H}$ & -0.3526710 & -3.9165840 & -3.0465150 & C & 5.6592530 & 0.3091780 & 3.6705200 \\
\hline C & -2.8805420 & -6.1223970 & -3.5918040 & $\mathrm{C}$ & 5.1657500 & -0.6046870 & 2.5790120 \\
\hline $\mathrm{H}$ & -4.4260030 & -6.6106200 & -2.1722280 & $\mathrm{C}$ & 3.8297880 & 1.9129200 & 2.9831240 \\
\hline $\mathrm{H}$ & -1.2299140 & -5.4454870 & -4.7972050 & $\mathrm{H}$ & 3.8820170 & 2.9953430 & 3.1498490 \\
\hline H & -3.2651840 & -6.7896030 & -4.3574320 & $\mathrm{H}$ & 2.7753160 & 1.6553840 & 2.8578380 \\
\hline H & -0.0149830 & -2.1447300 & -0.1950530 & C & 4.4598050 & 1.1433490 & 4.1733290 \\
\hline 0 & -2.0197680 & -3.3909170 & 0.4960680 & $\mathrm{H}$ & 4.8035570 & 1.8360710 & 4.9506220 \\
\hline c & -1.3086230 & -2.1468530 & 2.9063310 & $\mathrm{H}$ & 3.7243800 & 0.4777910 & 4.6364720 \\
\hline c & -2.3496590 & -1.8436360 & 1.9176470 & $\mathrm{H}$ & 6.1031760 & -0.2604210 & 4.4941050 \\
\hline $\mathrm{H}$ & -0.2921070 & -2.0947010 & 2.5200390 & $\mathrm{H}$ & 4.1438500 & 2.0915840 & 0.8358730 \\
\hline$H$ & -2.1307550 & -1.0939180 & 1.1631450 & C & 6.6987730 & 1.2756410 & 3.0608730 \\
\hline 0 & -1.7333190 & -0.8927130 & 3.4803770 & $\mathrm{H}$ & 7.0221690 & 1.9865380 & 3.8302080 \\
\hline $\mathrm{H}$ & -1.1483820 & -0.1818560 & 3.0648570 & $\mathrm{H}$ & 7.5850510 & 0.7128240 & 2.7501560 \\
\hline c & -3.0241190 & -3.4352050 & 4.2493230 & $\mathrm{C}$ & 6.0645680 & 2.0169470 & 1.8551190 \\
\hline c & -3.9275860 & -3.4740560 & 3.0152000 & $\mathrm{H}$ & 6.0945180 & 3.1023320 & 2.0064090 \\
\hline c & -3.7702240 & -2.2104920 & 2.1678740 & $\mathrm{H}$ & 6.6169230 & 1.8057830 & 0.9334820 \\
\hline c & -1.5551930 & -3.3117620 & 3.8365510 & C & 4.1405810 & -2.0318040 & 0.4240310 \\
\hline $\mathrm{H}$ & -3.1613780 & -4.3430150 & 4.8450440 & $\mathrm{C}$ & 3.5939810 & -2.9797430 & -0.6165700 \\
\hline $\mathrm{H}$ & -4.9773 & -3.5810950 & 3.3055070 & C & 4.7711070 & -4.1988690 & 1.3337000 \\
\hline H & -4.2221450 & -1.3405030 & 2.6597610 & $\mathrm{C}$ & 4.7497030 & -2.7005820 & 1.4940880 \\
\hline $\mathrm{H}$ & -1.2371440 & -4.2184960 & 3.3077510 & C & 4.7657400 & -3.8214420 & -1.1673690 \\
\hline $\mathrm{H}$ & -0.9044360 & -3.1962910 & 4.7090820 & $\mathrm{H}$ & 4.3738170 & -4.5560120 & -1.8808850 \\
\hline $\mathrm{H}$ & -3.3002990 & -2.5861930 & 4.8861080 & $\mathrm{H}$ & 5.4574820 & -3.1769100 & -1.7206250 \\
\hline $\mathrm{H}$ & -3.6687970 & -4.3447020 & 2.4010890 & C & 5.4928890 & -4.5286200 & 0.0076400 \\
\hline$H$ & -4.2746580 & -2.2915570 & 1.2023850 & $\mathrm{H}$ & 5.5030110 & -5.6152090 & -0.1376770 \\
\hline & & & & $\mathrm{H}$ & 6.5362030 & -4.2029600 & 0.0722950 \\
\hline & s) $\mathrm{G}[\mathrm{WB} 97\rangle$ & $G(d)]=-35$ & 391 & $\mathrm{H}$ & 5.2804510 & -4.6796910 & 2.1759060 \\
\hline $\mathrm{P}$ & 0.3468530 & 0.1815760 & 0.7813230 & $\mathrm{H}$ & 3.0890240 & -2.4462000 & -1.4251010 \\
\hline 0 & -0.2796150 & 0.7535680 & 2.0176750 & C & 3.3133030 & -4.6955650 & 1.2250780 \\
\hline 0 & 0.5322000 & -1.3105380 & 0.6754640 & $\mathrm{H}$ & 3.3164690 & -5.7761670 & 1.0376000 \\
\hline 0 & 1.735916 & 0.9942990 & 0.5708010 & $\mathrm{H}$ & 2.8008050 & -4.5357910 & 2.1793470 \\
\hline
\end{tabular}




$\begin{array}{rrr}2.5975940 & -3.9324110 & 0.0819680 \\ 2.1903640 & -4.6293240 & -0.6601790 \\ 1.7663560 & -3.3406510 & 0.4687690 \\ 5.2630200 & -1.9913360 & 2.5726000 \\ 5.7323090 & -2.5142810 & 3.4045030 \\ -4.7889600 & 0.5359290 & -0.4338230 \\ -5.4553530 & -0.3916480 & -1.4180270 \\ -3.2848140 & 0.6752230 & -2.3288150 \\ -3.6040770 & 1.1040550 & -0.9165070 \\ -5.7806020 & 0.4280070 & -2.6873460 \\ -6.2225720 & -0.2358850 & -3.4394090 \\ -6.5295910 & 1.1896060 & -2.4486360 \\ -4.4790720 & 1.0814660 & -3.2225670 \\ -4.2784990 & 0.7627100 & -4.2518620 \\ -4.5689840 & 2.1724690 & -3.2359020 \\ -2.3655490 & 1.1369170 & -2.6962050 \\ -6.3690670 & -0.8257940 & -0.9972990 \\ -3.1574950 & -0.8610080 & -2.3600790 \\ -2.9701870 & -1.1868750 & -3.3894950 \\ -2.2830100 & -1.1508990 & -1.7729100 \\ -4.4571320 & -1.5024970 & -1.8094400 \\ -4.9174410 & -2.1579260 & -2.5575410 \\ -4.2383040 & -2.1285010 & -0.9397680 \\ -4.5776260 & 1.8072620 & 1.5898320 \\ -4.9964860 & 2.3242420 & 2.9410570 \\ -2.8063890 & 3.3914820 & 2.0733680 \\ -3.3844190 & 2.3734940 & 1.1185800 \\ -3.8562140 & 2.0424300 & 3.9422390 \\ -4.1453540 & 2.4151440 & 4.9319090 \\ -3.7033940 & 0.9623540 & 4.0359800 \\ -2.5601690 & 2.7312340 & 3.4466130 \\ -2.2413700 & 3.5082160 & 4.1513230 \\ -1.7365720 & 2.0222940 & 3.3577260 \\ -1.8763200 & 3.8191030 & 1.6932320 \\ -5.9283080 & 1.8535300 & 3.2733400 \\ -3.8755490 & 4.4915140 & 2.2652210 \\ -3.4870580 & 5.2535000 & 2.9506000 \\ -4.0701000 & 4.9879900 & 1.3083360 \\ -5.1754010 & 3.8565420 & 2.8290900 \\ -5.4072210 & 4.2618540 & 3.8208670 \\ -6.0299720 & 4.0801900 & 2.1824040 \\ -5.2805720 & 0.8901130 & -1.7182250 \\ -6.2126730 & 0.4621450 & -173130 \\ 0.6772160 & 3.8542610 & -1460800 \\ 1.9096450 & 4.6191280 & \\ 2.1263530 & 5.7915940 & -17420\end{array}$

0.8826060 $-0.3620630$ $-0.4673860$ 1.7869400 3.0019630 1.0858750 $-0.2840220$ 2.7905930 2. 3641250 0.6538490 $-1.2675220$ 2. 3982870 3. 5413700 1.7219240 2.6325980 3. 3378460 4.2352920 2.0416240 0.8554430 1. 3084760 2. 5851770 3.9333710 4.6256830 5.1117060 3.3842000 $-1.7718290$ $-0.6412050$ $-2.3235830$ $-3.5335230$ $-1.6676650$ $-4.0855220$ $-4.0320470$ $-2.2235360$ $-0.7311170$ $-3.4309710$ $-5.0269420$ $-1.7161600$ $-3.8629450$ $-0.2561300$ $-2.3905700$ $-1.5450640$ $-2.5752460$ $-0.5331340$ $-2.3129800$ -1.8624550
-1.2403140
6.7077180

6.1044690

4.6256560

5.0157910

6.3685310

7.6874680

6.2709930

3.9721670

5.3681010

. 8844730

6.6235370

1.4172250

0.6152270

1.9991960

2.0668830

0.7305180

0.4245870

2. 3423320

1.3624800

2. 9864040

$-0.0613280$

0.7419800

$-0.5975010$

1.0878050

2.8559170

$-3.4561970$

$-2.8993600$

$-4.3615130$

$-5.0153040$

$-4.5333540$

$-5.8380710$

$-4.8658960$

$-5.3570020$

$-4.0179120$

$-6.0083540$

$-6.3444900$

$-5.4891030$

$-6.6505360$

$-2.2441590$

$-3.2768300$

$-2.0485690$

$-1.6861970$

$-2.0834820$

$-0.9663010$

$-0.7565440$

$-0.0997080$
$-1.0613570$

$-1.7464630$

$-1.4831960$

$-3.1644150$

$-1.4876390$

$-1.5063450$

$-2.8299710$

$-2.1617160$

$-0.1908850$

$-0.0054710$

$-1.4163020$

$-3.0146180$

$-3.1466440$

$-4.2424400$

$-5.4666950$

$-5.6791440$

$-4.4822840$

$-6.3471270$

$-4.4746750$

$-4.0324050$

$-5.7921770$

$-6.5985710$

$-4.5467240$

$-4.5176620$

$-5.3252320$

$-0.5854170$

$-0.9057930$

$-1.6288400$

$-1.3864300$

$-2.8502700$

$-2.3597900$

$-0.4346780$

$-3.8224390$

$-3.0304280$

$-3.5785800$

$-2.1714370$

$-4.7728450$

$-4.3403350$

$-0.2192220$

0.4724480

2. 8502010

1.8697300

2.4495540

1.0999300

3.4072930

2.9585780 


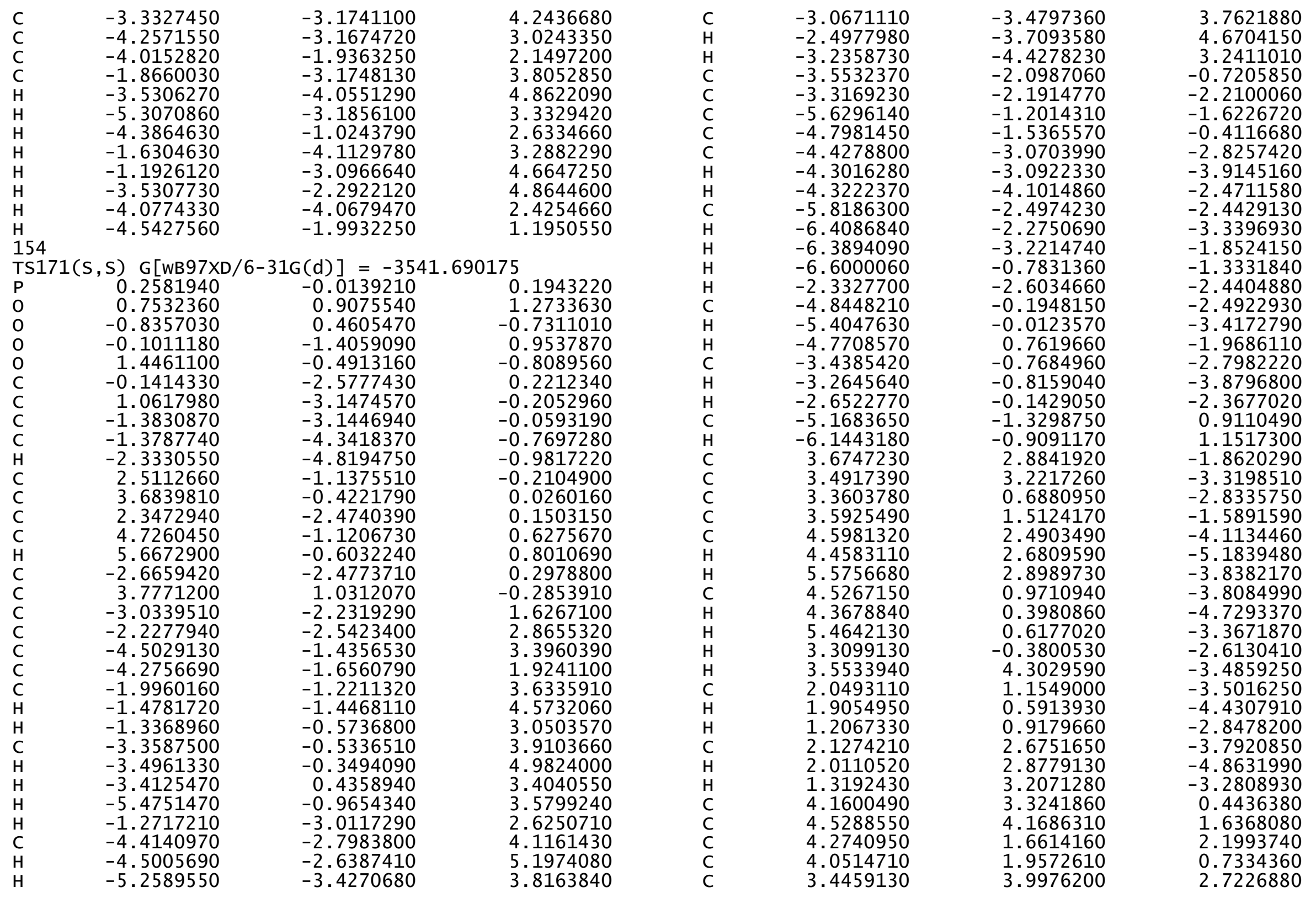




\begin{tabular}{|c|c|c|c|c|c|c|c|}
\hline $\mathrm{H}$ & 3.7486090 & 4.5533120 & 3.6184400 & $c$ & -5.6456360 & 2.2427270 & 0.3805500 \\
\hline $\mathrm{H}$ & 2.5042290 & 4.4262310 & 2.3693830 & $c$ & -5.1151540 & 4.4460200 & -0.4746730 \\
\hline C & 3.2744730 & 2.4904640 & 3.0356660 & $c$ & -6.9542060 & 2.6498090 & 0.6101460 \\
\hline $\mathrm{H}$ & 3.4583430 & 2.2907710 & 4.0979370 & 1 & -5.3251620 & 1.2330560 & 0.6132890 \\
\hline $\mathrm{H}$ & 2.2594580 & 2.1502080 & 2.8164390 & $c$ & -6.4234510 & 4.8502330 & -0.2386830 \\
\hline $\mathrm{H}$ & 4.1470520 & 0.6010790 & 2.4241310 & r & -4.3877990 & 5.1300020 & -0.8993050 \\
\hline $\mathrm{H}$ & 4.6319710 & 5.2228100 & 1.3573240 & $c$ & -7.3427200 & 3.9521780 & 0.3015470 \\
\hline C & 5.7020360 & 2.1274560 & 2.5616330 & r & -7.6716030 & 1.9505090 & 1.0286210 \\
\hline $\mathrm{H}$ & 5.8691620 & 1.9651570 & 3.6327700 & $\mathrm{r}$ & -6.7282100 & 5.8646210 & -0.4763210 \\
\hline $\mathrm{H}$ & 6.4381960 & 1.5180040 & 2.0261800 & $\mathrm{r}$ & -8.3657470 & 4.2688110 & 0.4817750 \\
\hline C & 5.8642070 & 3.6280840 & 2.1989260 & $\mathrm{r}$ & -2.1378150 & 1.1542000 & -0.2799580 \\
\hline $\mathrm{H}$ & 6.1477830 & 4.2138520 & 3.0812190 & $c$ & -2.5254780 & 3.4797660 & -0.9880530 \\
\hline $\mathrm{H}$ & 6.6544420 & 3.7645640 & 1.4534380 & $c$ & -0.4918480 & 3.6199090 & 0.9140950 \\
\hline C & 3.9622930 & 3.7921400 & -0.8494990 & $c$ & -0.4262430 & 3.5179790 & -0.5465080 \\
\hline $\mathrm{H}$ & 4.0500100 & 4.8552700 & -1.0692910 & r & -0.9681260 & 2.7790100 & 1.4187110 \\
\hline C & 4.5877060 & -2.4378980 & 1.0681520 & $\mathrm{r}$ & -0.3494320 & 2.5221330 & -0.9689200 \\
\hline $\mathrm{C}$ & 3.3766710 & -3.1153130 & 0.8657030 & $\mathrm{c}$ & 0.9257690 & 3.4137720 & 0.7727530 \\
\hline C & 5.7727900 & -3.1031780 & 1.7434260 & r & 1.0641520 & 2.4211310 & 0.9193090 \\
\hline C & 5.3921130 & -4.3137010 & 2.5941590 & $c$ & -0.1957310 & 6.1176870 & 0.7228260 \\
\hline c & 4.4392660 & -5.2197590 & 1.8187220 & $c$ & -0.5745130 & 6.0286100 & -0.7575270 \\
\hline c & 3.1450460 & -4.4732510 & 1.5046100 & $c$ & -0.1035660 & 4.7109890 & -1.3792220 \\
\hline $\mathrm{H}$ & 6.2961390 & -4.8560910 & 2.8918370 & $c$ & -0.8220440 & 4.9660170 & 1.5137460 \\
\hline $\mathrm{H}$ & 6.4778980 & -3.4258750 & 0.9639400 & $\mathrm{r}$ & -0.5315250 & 7.0716810 & 1.1411850 \\
\hline $\mathrm{H}$ & 6.3090680 & -2.3643900 & 2.3499300 & $\mathrm{r}$ & -0.1418350 & 6.8638720 & -1.3165640 \\
\hline $\mathrm{H}$ & 4.9185190 & -5.5415550 & 0.8834690 & 1 & 0.9870950 & 4.7037550 & -1.5039280 \\
\hline $\mathrm{H}$ & 4.2109950 & -6.1265130 & 2.3894670 & $\mathrm{r}$ & -1.9149040 & 5.0639530 & 1.5191990 \\
\hline $\mathrm{H}$ & 2.4872150 & -5.0929470 & 0.8916480 & $\mathrm{r}$ & -0.4873200 & 4.9712940 & 2.5562250 \\
\hline $\mathrm{H}$ & 2.5959050 & -4.3027010 & 2.4420330 & 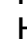 & 0.8951840 & 837050 & 820 \\
\hline $\mathrm{H}$ & 4.8990550 & -3.9792790 & 3.5165060 & $\mathrm{r}$ & -1.6637200 & 6.0980920 & -0.8607740 \\
\hline C & 1.0228550 & -4.2909580 & -1.0267690 & 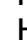 & -0.5333860 & 4.5527640 & -2.3720110 \\
\hline $\mathrm{c}$ & -0.2101350 & -4.9129450 & -1.2701340 & & & & \\
\hline C & 2.2730590 & -4.7703420 & -1.7454450 & \multicolumn{4}{|c|}{$\begin{array}{l}154 \\
\operatorname{TS} 172(\mathrm{~S}, \mathrm{~S}) \quad \mathrm{G}[\mathrm{WB} 97 \mathrm{XD} / 6-31 \mathrm{G}(\mathrm{d})]=-3541.691973\end{array}$} \\
\hline c & 2.1814500 & -6.2156460 & -2.2298140 & $\mathrm{p}$ & -0.3982810 & -0.2075180 & 0.9873790 \\
\hline C & 0.8771000 & -6.4388250 & -2.9904010 & $\mathrm{c}$ & 0.2129270 & -0.6463210 & 2.2846710 \\
\hline C & -0.3122250 & -6.2027740 & -2.0622420 & $c$ & -0.6776160 & 1.2581200 & 0.7718810 \\
\hline $\mathrm{H}$ & 3.0463520 & -6.4450690 & -2.8618110 & c & -1.7235280 & -1.1203900 & 0.7686890 \\
\hline $\mathrm{H}$ & 2.4174020 & -4.1110750 & -2.6139760 & $c$ & 0.5321370 & -0.6216900 & -0.3027270 \\
\hline $\mathrm{H}$ & 3.1650910 & -4.6358870 & -1.1308680 & c & -2.2801780 & -1.0898930 & -0.5004410 \\
\hline $\mathrm{H}$ & 0.8301970 & -5.7416000 & -3.8376240 & 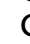 & -1.6470530 & -1.8159900 & -1.5068730 \\
\hline $\mathrm{H}$ & 0.8310930 & -7.4518350 & -3.4047080 & $c$ & -3.4272680 & -0.3324110 & -0.7263970 \\
\hline $\mathrm{H}$ & -1.2496280 & -6.2089390 & -2.6300400 & $c$ & -3.9096510 & -0.3055570 & -2.0315200 \\
\hline $\mathrm{H}$ & -0.3794650 & -7.0400460 & -1.3526610 & $\mathrm{r}$ & -4.8114070 & 0.2679480 & -2.2359780 \\
\hline $\mathrm{H}$ & 2.2233570 & -6.8983210 & -1.3694500 & $c$ & 0.6520030 & -1.9673980 & -0.6005110 \\
\hline C & -3.3233340 & 2.7206720 & -0.4237310 & $c$ & 1.8670570 & -2.6088000 & -0.3680210 \\
\hline 0 & -3.0458820 & 1.5193050 & 0.0037760 & $c$ & -0.4458320 & -2.6262830 & -1.1508870 \\
\hline C & -4.72533 & 3.1413700 & -0.1647940 & $c$ & 1.9259170 & -3.9709700 & -0.6385880 \\
\hline
\end{tabular}




$\begin{array}{lr}\mathrm{H} & 2.8675900 \\ \mathrm{C} & -4.1127110 \\ \mathrm{C} & 3.0583520 \\ \mathrm{C} & -4.7021490 \\ \mathrm{C} & -4.6897760 \\ \mathrm{C} & -5.9686130 \\ \mathrm{C} & -5.4109960 \\ \mathrm{C} & -4.0339220 \\ \mathrm{H} & -4.0641940 \\ \mathrm{H} & -2.9814420 \\ \mathrm{C} & -4.7911590 \\ \mathrm{H} & -5.1786320 \\ \mathrm{H} & -4.1214730 \\ \mathrm{H} & -6.5033450 \\ \mathrm{H} & -4.1488500 \\ \mathrm{C} & -6.9162980 \\ \mathrm{H} & -7.2788220 \\ \mathrm{H} & -7.7922810 \\ \mathrm{C} & -6.1547710 \\ \mathrm{H} & -6.1642550 \\ \mathrm{H} & -6.6295070 \\ \mathrm{C} & -4.2327000 \\ \mathrm{C} & -3.6109840 \\ \mathrm{C} & -5.0471030 \\ \mathrm{C} & -4.9717620 \\ \mathrm{C} & -4.7434820 \\ \mathrm{H} & -4.2998900 \\ \mathrm{H} & -5.3349150 \\ \mathrm{C} & -5.6385950 \\ \mathrm{H} & -5.7047890 \\ \mathrm{H} & -6.6586690 \\ \mathrm{H} & -5.6677210 \\ \mathrm{H} & -2.9951620 \\ \mathrm{C} & -3.6131450 \\ \mathrm{H} & -3.6493120 \\ \mathrm{H} & -3.2002440 \\ \mathrm{C} & -2.7395410 \\ \mathrm{H} & -2.2635440 \\ \mathrm{H} & -1.9461880 \\ \mathrm{C} & -5.5604310 \\ \mathrm{H} & -6.1289090 \\ \mathrm{C} & 4.8096650 \\ \mathrm{C} & 5.3582800 \\ \mathrm{C} & 3.2886040 \\ \mathrm{C} & 3.6741880 \\ \mathrm{C} & 5.7364060 \\ & \end{array}$

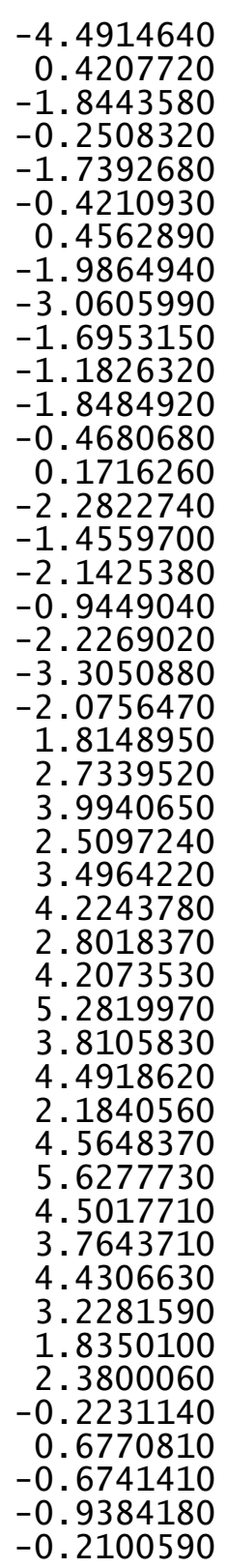

-0.4758060
0.3585820
0.0893240
1.4367880
1.6897480
3.5064260
2.4158600
3.0656800
3.2836990
3.0181450
4.1551180
4.9352880
4.6440000
4.2564630
0.9115600
2.8604980
3.6345700
2.4481140
1.7510060
1.9491700
0.7759020
0.2941070
-0.7311800
1.0096520
1.2606860
-1.4524250
-2.1420540
-2.0589300
-0.4023030
-0.6085350
-0.4303570
1.7625070
-1.4464270
1.0209290
0.7529490
2.0330130
0.0229270
-0.7060580
0.5459560
2.3229890
3.0750410
-0.3883160
-1.4659250
-2.2205810
-0.7846280
-2.6742150

\begin{abstract}
6.0802680
6.5719950

4.5014980

4. 2508210

4.7038480

2. 4132660

6.2323190

3. 0091020

2. 7854890

2. 1134700

4. 2426120

4. 6184610

3. 9735710

4.7557110

5. 2350980

3.1426460

3.6110640

4.0829510

4.4160800

3.8304480

2. 8509380

2. 6153730

1. 9620320

2. 2519120

6. 1236810

4. 3112260

4.0022650

4.5406310

5. 5520670

5.8280700

6. 4182790

5.3553300

6.2489100

0.8159090

$-0.3884410$

0.9680480

$-0.3628670$

$-1.3431330$

$-1.6401280$

$-0.1982050$

1. 4989610

1. 6126820

$-0.9093510$

$-2.2779420$

-2.2766040
-2.2294220
\end{abstract}

$-3.4946790$

$-2.4020100$

$-3.1037310$

$-4.1539000$

$-3.0081370$

$-2.5201690$

$-1.1111490$

$-2.3868360$

$-3.4388270$

$-1.8157840$

$-1.9161530$

$-2.7215950$

$-1.0908430$

1. 7462150

3. 1316010

2. 4128860

1. 3599180

4.1232750

5. 1385760

4. 1160600

3. 7229910

4. 5036440

3. 5853610

2.0906060

3. 4013740

2. 6827860

3.4402790

1.7699830

3. 1616450

4.1838450

2. 5250510

0.8771060

1. 1800840

$-1.0824960$

$-1.3325280$

$-1.3269690$

$-1.3917760$

$-2.2866940$

$-1.6977070$

$-1.7610130$

$-2.2794690$

$-0.5531590$

$-3.2903080$

$-2.4020430$

$-2.3748150$

$-0.7774570$ 


$\begin{array}{lrrr}\mathrm{H} & -0.7908230 & -7.0178870 & -0.3844230 \\ \mathrm{C} & -2.1018970 & -1.6943760 & -2.8334290 \\ \mathrm{C} & -3.2584420 & -0.9445040 & -3.0870360 \\ \mathrm{C} & -1.2809350 & -2.2586070 & -3.9800560 \\ \mathrm{C} & -2.0603470 & -2.3779220 & -5.2879430 \\ \mathrm{C} & -2.8092180 & -1.0808220 & -5.5830510 \\ \mathrm{C} & -3.8362080 & -0.8170330 & -4.4838000 \\ \mathrm{H} & -1.3708700 & -2.6269540 & -6.1019030 \\ \mathrm{H} & -0.4270350 & -1.5817700 & -4.1316050 \\ \mathrm{H} & -0.8413390 & -3.2232390 & -3.7164820 \\ \mathrm{H} & -2.0916640 & -0.2506980 & -5.6288430 \\ \mathrm{H} & -3.3089040 & -1.1298050 & -6.5565840 \\ \mathrm{H} & -4.2826190 & 0.1765680 & -4.6044980 \\ \mathrm{H} & -4.6600310 & -1.5375250 & -4.5893440 \\ \mathrm{H} & -2.7818540 & -3.2035220 & -5.2150260 \\ \mathrm{C} & 1.4057210 & 3.4225370 & -0.7705670 \\ \mathrm{O} & 0.3446740 & 2.7137140 & -1.0212600 \\ \mathrm{C} & 1.8860340 & 4.2260350 & -1.9267470 \\ \mathrm{C} & 2.9804620 & 5.0761570 & -1.7557630 \\ \mathrm{C} & 1.2870630 & 4.0991640 & -3.1823440 \\ \mathrm{C} & 3.4735280 & 5.7982480 & -2.8350950 \\ \mathrm{H} & 3.4390220 & 5.1561850 & -0.7759150 \\ \mathrm{C} & 1.7871630 & 4.8184050 & -4.2615300 \\ \mathrm{H} & 0.4432070 & 3.4300670 & -3.3063170 \\ \mathrm{C} & 2.8783490 & 5.6673720 & -4.0887740 \\ \mathrm{H} & 4.3231720 & 6.4605200 & -2.7016470 \\ \mathrm{H} & 1.3262680 & 4.7148680 & -5.2387590 \\ \mathrm{H} & 3.2678410 & 6.2269860 & -4.9339580 \\ \mathrm{H} & 0.0089700 & 2.1266880 & -0.2506790 \\ \mathrm{O} & 2.0154680 & 3.4499040 & 0.3075240 \\ \mathrm{C} & 1.3402340 & 2.3342390 & 2.8057990 \\ \mathrm{C} & 2.3584170 & 1.9767980 & 1.8105670 \\ \mathrm{H} & 0.3155320 & 2.2723170 & 2.4436650 \\ \mathrm{H} & 2.1171900 & 1.1962730 & 1.0953460 \\ \mathrm{O} & 1.7682390 & 1.1049550 & 3.4292770 \\ \mathrm{H} & 1.1688580 & 0.3754480 & 3.0710310 \\ \mathrm{C} & 3.0939640 & 3.6692210 & 4.0511410 \\ \mathrm{C} & 3.9719910 & 3.6418060 & 2.7986300 \\ \mathrm{C} & 3.7864940 & 2.3401860 & 2.0174960 \\ \mathrm{C} & 1.6160360 & 3.5396090 & 3.6742540 \\ \mathrm{H} & 3.2500830 & 4.6029600 & 4.6004030 \\ \mathrm{H} & 5.0284440 & 3.7530380 & 3.0617010 \\ \mathrm{H} & 4.2369200 & 1.4899820 & 2.5442840 \\ \mathrm{H} & 1.2945820 & 4.4231390 & 3.1093100 \\ \mathrm{H} & 0.9827040 & 3.4722530 & 4.5645830 \\ \mathrm{H} & 3.3762980 & 2.8488540 & 4.7217760 \\ & & & \end{array}$

$\begin{array}{lcc}\mathrm{H} & 3.7076020 & 4.4839080 \\ \mathrm{H} & 4.2765520 & 2.3673130 \\ 154 & & \end{array}$

TS173(S, S) G[WB97XD/6-31G(d)] $=-3541.691364$

$\begin{array}{lrrr}\text { TS } 173(\mathrm{~S}, \mathrm{~S}) & \mathrm{G}[\mathrm{WB} 97 \mathrm{XD} / 6-31 \mathrm{G}(\mathrm{d})]=-3541.691364 \\ \mathrm{P} & -0.4199800 & -0.3127750 & 0.9863670 \\ \mathrm{O} & 0.1918560 & -0.7758270 & 2.2748600 \\ \mathrm{O} & -0.7422690 & 1.1500860 & 0.8171930 \\ \mathrm{O} & -1.7125870 & -1.2569560 & 0.7172870 \\ \mathrm{O} & 0.5327720 & -0.6546550 & -0.3070290 \\ \mathrm{C} & -2.2475730 & -1.1958170 & -0.5607070 \\ \mathrm{C} & -1.5844860 & -1.8934110 & -1.5750010 \\ \mathrm{C} & -3.3971770 & -0.4424930 & -0.7825800 \\ \mathrm{C} & -3.8615570 & -0.3643490 & -2.0973790 \\ \mathrm{H} & -4.7567220 & 0.2193400 & -2.2996620 \\ \mathrm{C} & 0.7075710 & -1.9831110 & -0.6492880 \\ \mathrm{C} & 1.9504240 & -2.5753630 & -0.4369300 \\ \mathrm{C} & -0.3559410 & -2.6683690 & -1.2375780 \\ \mathrm{C} & 2.0776780 & -3.9196960 & -0.7667510 \\ \mathrm{H} & 3.0415230 & -4.4019700 & -0.6166420 \\ \mathrm{C} & -4.1134490 & 0.2616430 & 0.3164280 \\ \mathrm{C} & 3.1027210 & -1.7730390 & 0.0549410 \\ \mathrm{C} & -4.7118350 & -0.4574620 & 1.3590700 \\ \mathrm{C} & -4.6723900 & -1.9528010 & 1.5659310 \\ \mathrm{C} & -6.0207540 & -0.7213100 & 3.3927740 \\ \mathrm{C} & -5.4570350 & 0.2023480 & 2.3444140 \\ \mathrm{C} & -4.0414220 & -2.2282170 & 2.9481890 \\ \mathrm{H} & -4.0480400 & -3.3091830 & 3.1314480 \\ \mathrm{H} & -2.9960990 & -1.9088340 & 2.9351160 \\ \mathrm{C} & -4.8433470 & -1.4796080 & 4.0448880 \\ \mathrm{H} & -5.2354600 & -2.1806540 & 4.7913340 \\ \mathrm{H} & -4.2024060 & -0.7689570 & 4.5761590 \\ \mathrm{H} & -6.5851380 & -0.1641860 & 4.1483690 \\ \mathrm{H} & -4.1022850 & -2.4595430 & 0.7844020 \\ \mathrm{C} & -6.9312200 & -1.7528530 & 2.6907280 \\ \mathrm{H} & -7.3013950 & -2.4691090 & 3.4337250 \\ \mathrm{H} & -7.8052860 & -1.2447500 & 2.2706430 \\ \mathrm{C} & -6.1265430 & -2.4754140 & 1.5794600 \\ \mathrm{H} & -6.1138480 & -3.5583800 & 1.7493470 \\ \mathrm{H} & -6.5827100 & -2.3097200 & 0.5978920 \\ \mathrm{C} & -4.2624850 & 1.6546030 & 0.2958430 \\ \mathrm{C} & -3.6388500 & 2.6215640 & -0.6834730 \\ \mathrm{C} & -5.1518320 & 3.7893360 & 1.0567950 \\ \mathrm{C} & -5.0406350 & 2.3007100 & 1.2654830 \\ \mathrm{C} & -4.7715690 & 3.3781070 & -1.4107200 \\ \mathrm{H} & -4.3291770 & 4.1414490 & -2.0616930 \\ \mathrm{H} & -5.3266860 & 2.6897930 & -2.0572960\end{array}$




$\begin{array}{rrr}-5.7153590 & 4.0268960 & -0.3628810 \\ -5.8125270 & 5.1045380 & -0.5390110 \\ -6.7204600 & 3.5977950 & -0.4289370 \\ -5.8056180 & 4.2457780 & 1.8078250 \\ -2.9886870 & 2.1115050 & -1.3979150 \\ -3.7363910 & 4.3998900 & 1.1206290 \\ -3.7965060 & 5.4679860 & 0.8789180 \\ -3.3473550 & 4.3219770 & 2.1411100 \\ -2.8157750 & 3.6505030 & 0.1253140 \\ -2.3361250 & 4.3488620 & -0.5704970 \\ -2.0243950 & 3.1188690 & 0.6557050 \\ -5.6371110 & 1.5793140 & 2.2919270 \\ -6.2361540 & 2.0865550 & 3.0466360 \\ 4.7808380 & -0.0591560 & -0.3577300 \\ 5.2915510 & 0.9029010 & -1.4001110 \\ 3.2869970 & -0.5117320 & -2.2115870 \\ 3.6792910 & -0.8096610 & -0.7838400 \\ 5.7114230 & 0.0792820 & -2.6392980 \\ 6.0229370 & 0.7658010 & -3.4350300 \\ 6.5785710 & -0.5407950 & -2.3905130 \\ 4.5181760 & -0.7984360 & -3.1006710 \\ 4.2623930 & -0.5885860 & -4.1456160 \\ 4.7696290 & -1.8621140 & -3.0379920 \\ 2.4394060 & -1.1198400 & -2.5366030 \\ 6.1386570 & 1.4854240 & -1.0218470 \\ 2.9415890 & 0.9858480 & -2.3274350 \\ 2.7180670 & 1.2232640 & -3.3740310 \\ 2.0305690 & 1.1808920 & -1.7576060 \\ 4.1333640 & 1.8360390 & -1.8163130 \\ 4.4813720 & 2.5283730 & -2.5913760 \\ 3.8314000 & 2.4516750 & -0.9637780 \\ 4.7695220 & -1.2307940 & 1.7368390 \\ 5.2614740 & -1.6065200 & 3.1099590 \\ 3.2282940 & -3.0067430 & 2.3390350 \\ 3.6587790 & -1.9788660 & 1.3210310 \\ 4.0983670 & -1.4196920 & 4.1084590 \\ 4.4403170 & -1.6917350 & 5.1139250 \\ 3.8038450 & -0.3657340 & 4.1429450 \\ 2.9009360 & -2.3009360 & 3.6719760 \\ 2.6885680 & -3.0670960 & 4.4267690 \\ 1.9901710 & -1.7135540 & 3.5492310 \\ 2.3618820 & -3.5759710 & 1.9947190 \\ 6.1240450 & -0.9974960 & 3.4026630 \\ 4.4349090 & -3.9415900 & 2.5838760 \\ 4.1544750 & -4.7018930 & 1.6570920 \\ 4.6864420 & -4.4685490 & \end{array}$

5.6429200
5.9370630
6.5133790
5.3308480
6.1988210
1.0099760
-0.2231290
1.2367620
-0.0572820
-1.0588360
-1.4277370
0.1556220
1.7752140
1.9001010
-0.6166070
-1.9658610
-2.0612470
-2.0423450
-0.4905600
-2.0461550
-1.3340730
-1.1138480
-2.4160400
-3.6078730
-3.1906940
-1.9379350
-0.6739830
-2.6812780
-3.9819590
-0.3769210
-0.3716950
-2.2544300
-4.4332400
1.2406000
0.2295280
1.6876930
2.7274570
1.1087800
3.1845170
3.1711260
1.5733830
0.3076060
2.6085120
3.9901720
1.1274200
2.9683100
-3.1053360
-3.4135700
-3.2515570
-0.2713270
0.3005030
-4.6698480
-4.0457060
-6.1375110
-6.9424980
-6.2027900
-4.8606980
-7.9400260
-6.2117260
-6.5708260
-6.0378920
-6.7987100
-4.2884280
-5.0394090
-7.0804610
-1.7511560
-2.2970370
-1.1676270
-0.3958810
-0.8395040
-0.9831790
-3.0899420
-1.5894470
-0.5253830
-1.8044270
-2.7500090
-0.4781100
0.6761170
-0.1267270
3.4607080
2.6994810
4.3403170
5.2447260
4.2384900
6.0478770
5.3043290
5.0388490
3.5273350
5.9435240
6.7538670
4.9561580
6.5690940

3.0858780

4. 0959480

2.4384030

0.9026690

1. 2283920

$-1.2614910$

$-1.4967240$

$-1.5722160$

$-1.6739500$

$-2.5572160$

$-1.9294800$

$-2.0732310$

$-2.5280100$

$-0.8150050$

$-3.5495930$

$-2.7051570$

$-2.6091730$

$-1.0352640$

$-0.6744440$

$-2.8900140$

$-4.1028740$

$-5.1253850$

$-5.4572220$

$-4.5843290$

$-3.1445080$

$-4.5677150$

$-6.0350640$

$-6.5117140$

$-4.9544550$

$-3.8322140$

$-4.7083490$

$-5.3035430$

$-4.6800630$

$-0.6223210$

$-0.9211940$

$-1.7354480$

$-1.5110400$

$-3.0026670$

$-2.5481420$

$-0.5228780$

$-4.0397570$

$-3.1685120$

$-3.8132030$

$-2.3726430$

$-5.0258730$

$-4.6246800$ 


\begin{tabular}{|c|c|c|c|c|c|c|}
\hline & & & & & & \\
\hline-0.0882250 & 2.0710990 & -0.1753430 & C & -4.6852280 & -0.7699840 & 4.2526450 \\
\hline 1.8300170 & 3.4799640 & 0.4672000 & $\mathrm{H}$ & -5.0718950 & -1.3808550 & 5.0769230 \\
\hline 1.1933900 & 2.2289460 & 2.9106290 & $\mathrm{H}$ & -3.9704230 & -0.0615420 & 4.6833750 \\
\hline 2.2355450 & 1.9623190 & 1.9121500 & $\mathrm{H}$ & -6.3385670 & 0.6562920 & 4.3458950 \\
\hline 0.1760680 & 2.1324310 & 2.5360950 & $\mathrm{H}$ & -4.1919960 & -2.0352290 & 1.0437800 \\
\hline 2.0384320 & 1.2020130 & 1.1624720 & C & -6.8605400 & -1.0121880 & 3.0371640 \\
\hline 1.6763290 & 0.9958290 & 3.4854910 & $\mathrm{H}$ & -7.2212240 & -1.6520130 & 3.8511580 \\
\hline 1.1104540 & 0.2552570 & 3.0970390 & $\mathrm{H}$ & -7.7307450 & -0.4848620 & 2.6331430 \\
\hline 2.8687120 & 3.5948620 & 4.2284400 & C & -6.1674130 & -1.8567440 & 1.9361660 \\
\hline 3.7578240 & 3.6599750 & 2.9851760 & $\mathrm{H}$ & -6.2186920 & -2.9255110 & 2.1747480 \\
\hline 3.6426780 & 2.3855220 & 2.1475010 & $\mathrm{H}$ & -6.6644550 & -1.7195980 & 0.9701030 \\
\hline 1.4019180 & 3.4076820 & 3.8325800 & C & -4.1268220 & 2.0318590 & 0.2510270 \\
\hline 2.9746820 & 4.5124390 & 4.8155520 & $\mathrm{C}$ & -3.5000390 & 2.8793110 & -0.8310520 \\
\hline 4.8053460 & 3.8096710 & 3.2644470 & $\mathrm{C}$ & -4.8342380 & 4.2712210 & 0.8907110 \\
\hline 4.1304460 & 1.5363940 & 2.6418110 & $\mathrm{C}$ & -4.8107800 & 2.7973430 & 1.2046760 \\
\hline 1.0399750 & 4.2973730 & 3.3030060 & $\mathrm{C}$ & -4.6230710 & 3.6539330 & -1.5542190 \\
\hline 0.7664510 & 3.2696320 & 4.7130490 & $\mathrm{H}$ & -4.1725420 & 4.3304790 & -2.2901460 \\
\hline 3.1854620 & 2.7632300 & 4.8692650 & $\mathrm{H}$ & -5.2599700 & 2.9570120 & -2.1096470 \\
\hline 3.4601390 & 4.5154030 & 2.3672940 & C & -5.4554350 & 4.4495770 & -0.5132340 \\
\hline 4.1372220 & 2.4798720 & 1.1782750 & $\mathrm{H}$ & -5.4787390 & 5.5163780 & -0.7648800 \\
\hline & & & $\mathrm{H}$ & -6.4928860 & 4.0998820 & -0.4964180 \\
\hline s) $\mathrm{G}[\mathrm{WB} 97 \mathrm{XD}$ & $g(d)]=-35$ & 37 & $\mathrm{H}$ & -5.4128820 & 4.8259970 & 1.6371440 \\
\hline-0.36 & -0.1148010 & 0.9476630 & $\mathrm{H}$ & -2.9268430 & 2.2746780 & -1.5375790 \\
\hline 0.2501660 & -0.5266370 & 2.2521020 & C & -3.3788520 & 4.7821850 & 0.8387820 \\
\hline-0.5808340 & 1.3517740 & 0.6745000 & $\mathrm{H}$ & -3.3798120 & 5.8336700 & 0.5266130 \\
\hline-1.7337580 & -0.9739500 & 0.7929350 & $\mathrm{H}$ & -2.9395620 & 4.7444620 & 1.8409430 \\
\hline 0.5149460 & -0.6314740 & -0.3391200 & C & -2.5670810 & 3.9060130 & -0.1478910 \\
\hline-2.31 & -0.9801330 & -0.4648570 & $\mathrm{H}$ & -2.0876850 & 4.5209900 & -0.9185250 \\
\hline-1.7385480 & -1.7910170 & -1.4477780 & $\mathrm{H}$ & -1.7799230 & 3.3610710 & 0.3754600 \\
\hline-3.4374950 & -0.1889890 & -0.6958850 & C & -5.3938860 & 2.1935250 & 2.3116840 \\
\hline-3.9696390 & -0.2048000 & -1.9865670 & $\mathrm{H}$ & -5.9194400 & 2.7926200 & 3.0537760 \\
\hline-4.8566110 & 0.3905310 & -2.1914270 & C & 4.8020090 & -0.4271030 & -0.4244430 \\
\hline 0.5693400 & -1.9948000 & -0.5688980 & C & 5.3902480 & 0.4030090 & -1.5365910 \\
\hline 1.7556260 & -2.6787450 & -0.3095240 & C & 3.2514790 & -0.8694120 & -2.2339010 \\
\hline-0.5608940 & -2.6293820 & -1.0815290 & $\mathrm{c}$ & 3.6334260 & -1.1033660 & -0.7919040 \\
\hline 1.7514030 & -4.0535440 & -0.5127350 & $\mathrm{C}$ & 5.7182250 & -0.5484140 & -2.7099260 \\
\hline 2.668 & -4.6081020 & -0.3247270 & $\mathrm{H}$ & 6.0996950 & 0.0395410 & -3.5527270 \\
\hline-4.0594150 & 0.6383430 & 0.3738380 & $\mathrm{H}$ & 6.5141340 & -1.2376210 & -2.4105080 \\
\hline 2.98 & -1.9503480 & 0.1145540 & C & 4.4379350 & -1.3270750 & -3.1126420 \\
\hline-4.64 & 0.0382530 & 1.4962840 & $\mathrm{H}$ & 4.1911080 & -1.1507790 & -4.1658770 \\
\hline-4.68 & -1.4371880 & 1.8129860 & $\mathrm{H}$ & 4.5845900 & -2.4051320 & -2.9917890 \\
\hline-5.8 & 0.0099680 & 3.6070720 & $\mathrm{H}$ & 2.3459550 & -1.4161000 & -2.5077940 \\
\hline-5.2 & 0.8153620 & 2.4619140 & $\mathrm{H}$ & 6.2928680 & 0.9280000 & -1.2052280 \\
\hline-3.9 & -1.6554430 & 3.1791510 & C & 3.0441180 & 0.6429340 & -2.4465630 \\
\hline-4.0 & -2.7165160 & 3.4453660 & $\mathrm{H}$ & 2.8113390 & 0.8318280 & -3.5007500 \\
\hline-2 . & 16635 & 3.0876400 & $\mathrm{H}$ & 2.1730180 & 0.9594120 & -1.8684630 \\
\hline
\end{tabular}




$\begin{array}{lr}\mathrm{C} & 4.3243340 \\ \mathrm{H} & 4.7279120 \\ \mathrm{H} & 4.0998810 \\ \mathrm{C} & 4.7074230 \\ \mathrm{C} & 5.1763620 \\ \mathrm{C} & 3.0294190 \\ \mathrm{C} & 3.5317620 \\ \mathrm{C} & 4.0386680 \\ \mathrm{H} & 4.3642410 \\ \mathrm{H} & 3.8284030 \\ \mathrm{C} & 2.7733180 \\ \mathrm{H} & 2.5141520 \\ \mathrm{H} & 1.9084940 \\ \mathrm{H} & 2.1167910 \\ \mathrm{H} & 6.0886260 \\ \mathrm{C} & 4.1598020 \\ \mathrm{H} & 3.8248590 \\ \mathrm{H} & 4.3661030 \\ \mathrm{C} & 5.4313150 \\ \mathrm{H} & 5.6928350 \\ \mathrm{H} & 6.2899020 \\ \mathrm{C} & 5.3433250 \\ \mathrm{H} & 6.2615750 \\ \mathrm{C} & -0.5643760 \\ \mathrm{C} & -1.8384730 \\ \mathrm{C} & -1.8116920 \\ \mathrm{C} & -0.5136190 \\ \mathrm{C} & 0.6731970 \\ \mathrm{C} & 0.6084180 \\ \mathrm{H} & -2.6835870 \\ \mathrm{H} & -2.0007110 \\ \mathrm{H} & -2.6942570 \\ \mathrm{H} & -0.4170180 \\ \mathrm{H} & -0.5185030 \\ \mathrm{H} & 1.6160980 \\ \mathrm{H} & 0.7082200 \\ \mathrm{H} & -1.8853640 \\ \mathrm{C} & -3.3750590 \\ \mathrm{C} & -3.9099290 \\ \mathrm{C} & -3.9571710 \\ \mathrm{C} & -2.5636710 \\ \mathrm{C} & -1.5797000 \\ \mathrm{C} & -2.2480710 \\ \mathrm{H} & -3.2622690 \\ \mathrm{H} & -4.3774210 \\ \mathrm{H} & -2.1185010 \\ & \end{array}$

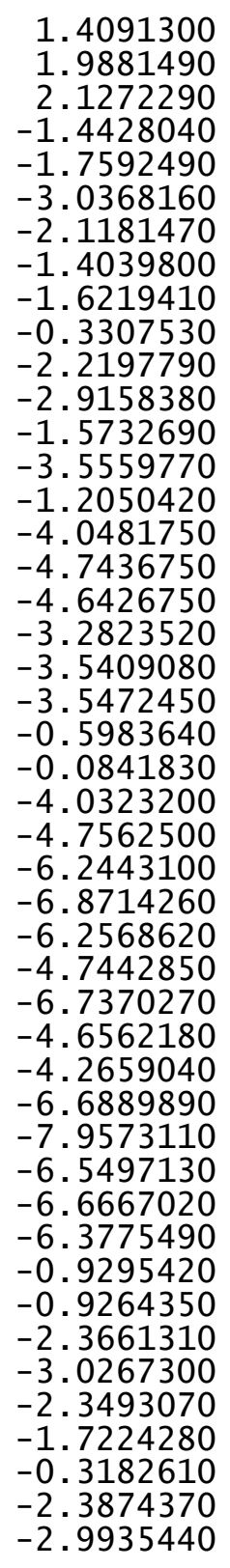
$-2.0235600$
$-2.8619160$
$-1.2295760$
1.7478770
3. 1442320
2. 4764210
1. 3892480
4. 1261950
5.1500300
4.0762470
3.7605110
4.5668500
3.6046750
2. 1754570
3. 3914050
2. 7741750
3.5520360
1.8774720
3. 2291210
4.2618840
2. 6036280
0.8458130
1.1266710
$-1.2034550$
$-1.5967680$
$-1.2508450$
$-1.7521480$
$-1.0128420$
$-0.9195790$
$-1.6943410$
$-2.6769380$
$-1.1202180$
$-2.8311130$
$-1.6091890$
$-1.4897310$
0.0063430
$-0.1634060$
$-3.0117090$
$-4.4212820$
$-4.9508850$
$-4.9345970$
$-3.9523650$
$-5.0700340$
$-5.9617680$
$-5.9347610$

$-1.0748400$

$-4.9041550$

$-4.6416650$

$-2.6718630$

$-0.7884900$

1.5925450

0.4907900

2.1006230

3. 2525930

1.4698080

3.7723770

3.7339210

1. 9954120

0.5792590

3.1454330

4.6689590

1. 5096380

3. 5560690

0.1376650

2. 2186850

1.4981620

2. 4980130

0.4708870

2. 2230150

1.8740130

1. 2454480

3. 3100960

4.1828820

3. 9408780

1.8270180

3. 5062520

5. 2437780

4. 3537210

1. 5418410

1.1934920

3. 5591940

3. 9514330

4.4311490
$-1.5306750$

$-0.4687000$

$-2.9347650$

$-4.0872130$

$-3.0427260$

3.3748370

2. 7199340

4.1228720

4.9007860

4.0125700

5. 5657400

4. 9691320

4.6757640

3.4017270

5. 4507560

6.1687720

4.5848490

5.9647470

2. 1658930

3. 3943920

2. 4103430

1. 9686760

2. 3778320

1.1701890

1. 1910280

0.4739040

3.7210450

3.6034570

2. 2781970

3.6382120

4.6695470

3. 6820120

1. 4336020

4. 5101310

3. 6349540

2. 9185820

4.4264960

2. 2404830
$-4.4848100$

$-4.4513650$

$-4.3110370$

$-4.6836560$

$-3.6595770$

$-0.9392410$

$-1.1585280$

$-2.1204500$

$-1.9872600$

$-3.3619020$

$-3.0903650$

$-1.0175350$

$-4.4646310$

$-3.4562820$

$-4.3300250$

$-2.9866980$

$-5.4309850$

$-5.1939720$

$-0.3716300$

0.1295550

2. 6680400

1. 6881040

2. 3090110

1.0054630

3. 3419730

3.0092500

3.8550680

2.6038300

1.8806100

3. 4850400

4.3648430

2. 8608170

2.4458780

2.8836390

4.3778880

4. 5597330

1. 9177130

0.9055600

$\operatorname{TS} 175(\mathrm{~S}, \mathrm{~S}) \mathrm{G}[\mathrm{WB} 97 \mathrm{XD} / 6-31 \mathrm{G}(\mathrm{d})]=-3541.689974$

$\begin{array}{lrrr}\text { TS } 175(\mathrm{~S}, \mathrm{~S}) & \mathrm{G}[\mathrm{WB} 97 \mathrm{XD} / 6-31 \mathrm{G}(\mathrm{d})]=-3541.689974 \\ \mathrm{P} & -0.3613900 & -0.1044280 & 0.9675740 \\ \mathrm{O} & 0.2651360 & -0.4823680 & 2.2768610 \\ \mathrm{O} & -0.5804750 & 1.3545730 & 0.6588910 \\ \mathrm{O} & -1.7308030 & -0.9673880 & 0.8468060 \\ \mathrm{O} & 0.5071790 & -0.6527940 & -0.3126710 \\ \mathrm{C} & -2.3192340 & -1.0162460 & -0.4072010\end{array}$




\begin{tabular}{|c|c|c|}
\hline $\begin{array}{l}-1.7486840 \\
-3.4424130 \\
-3.9886520 \\
-4.8793530 \\
0.5730250 \\
1.7757240 \\
-0.5594010 \\
1.7970490 \\
2.7307440 \\
-4.0540270 \\
2.9951950 \\
-4.6337910 \\
-4.6816350 \\
-5.8300790 \\
-5.2765640 \\
-3.9898080 \\
-4.0701410 \\
-2.9257570 \\
-4.6623230 \\
-5.0464540 \\
-3.9403550 \\
-6.3076660 \\
-4.1962320 \\
-6.8469860 \\
-7.2066460 \\
-7.7165170 \\
-6.1653170 \\
-6.2213610 \\
-6.6671850 \\
-4.1173190 \\
-3.4895430 \\
-4.8269420 \\
-4.7975520 \\
-4.6107080 \\
-4.1576410 \\
-5.2423680 \\
-5.4517450 \\
-5.4881070 \\
-6.4847020 \\
-5.4072610 \\
-2.9141600 \\
-3.3749630 \\
-3.3840070 \\
-2.9346130 \\
-2.5580020 \\
-2664010\end{array}$ & $\begin{array}{r}-1.8626860 \\
-0.2332710 \\
-0.3022450 \\
0.2823530 \\
-2.0198780 \\
-2.6799000 \\
-2.6813430 \\
-4.0553740 \\
-4.5886630 \\
0.6383490 \\
-1.9284490 \\
0.0859100 \\
-1.3756030 \\
0.1487670 \\
0.9043960 \\
-1.5442480 \\
-2.5930280 \\
-1.3179560 \\
-0.6105410 \\
-1.1836550 \\
0.1110810 \\
0.8268310 \\
-2.0071670 \\
-0.8894090 \\
-1.4940380 \\
-0.3733520 \\
-1.7811350 \\
-2.8393570 \\
-1.6788120 \\
2.0261170 \\
2.8274640 \\
4.2901430 \\
2.8317600 \\
3.5720130 \\
4.2232070 \\
2.8539400 \\
4.4017310 \\
5.4563600 \\
4.0401660 \\
4.8754290 \\
2.1947830 \\
4.8065510 \\
5.8378460 \\
4.8313140 \\
3.8811480 \\
4.4562350\end{array}$ & $\begin{array}{r}-1.3623370 \\
-0.6606640 \\
-1.9436500 \\
-2.1626060 \\
-0.5117310 \\
-0.2681830 \\
-0.9872800 \\
-0.4684830 \\
-0.3012170 \\
0.3800040 \\
0.1344800 \\
1.5289750 \\
1.9046130 \\
3.6462200 \\
2.4657540 \\
3.2747600 \\
3.5842310 \\
3.1685170 \\
4.3150450 \\
5.1671700 \\
4.7105600 \\
4.3617390 \\
1.1573710 \\
3.1234680 \\
3.9644560 \\
2.7037610 \\
2.0529040 \\
2.3336650 \\
1.0850580 \\
0.2022870 \\
-0.9136610 \\
0.7458960 \\
1.1249580 \\
-1.6704380 \\
-2.4273850 \\
-2.2044090 \\
-0.6635750 \\
-0.9608180 \\
-0.6300600 \\
1.4672840 \\
-1.5933330 \\
0.6653840 \\
0.2919190 \\
1.6674230 \\
-0.2702890 \\
-1.0636030\end{array}$ \\
\hline
\end{tabular}

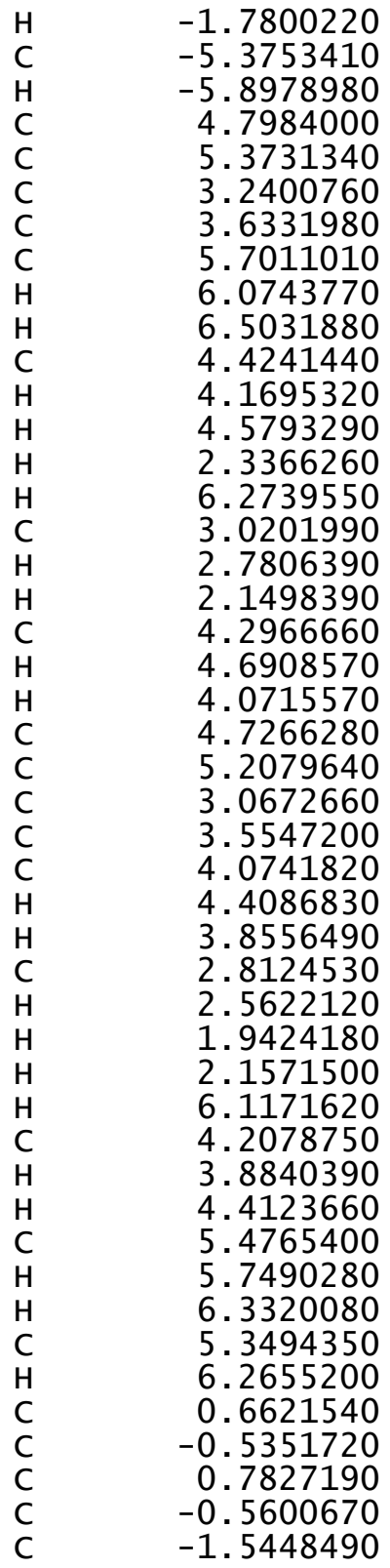

3.3568880
2.2751380
2.9047330
-0.3988120
0.4147780
-0.8865900
-1.0912370
-0.5564970
0.0178580
-1.2346300
-1.3511540
-1.1941110
-2.4259860
-1.4449040
0.9525390
0.6199670
0.7874080
0.9410610
1.4037030
1.9705630
2.1340090
-1.3776870
-1.6673950
-2.9737270
-2.0691880
-1.3064260
-1.5072260
-0.2356070
-2.1368100
-2.8207110
-1.4989410
-3.5054960
-1.1016830
-3.9705600
-4.6543610
-4.5798410
-3.1867540
-3.4264140
-3.4544370
-0.5435900
-0.0175520
-4.7702920
-4.0847190
-6.2706720
-6.9994340
-6.2424040

0.2866620 2.2590250 2.9775830 $-0.4423310$ $-1.5734620$ $-2.2337030$ $-0.7904010$ $-2.7305120$ $-3.5862790$ $-2.4223560$ $-3.1120310$ $-4.1664430$ $-2.9741270$ $-2.4908530$ $-1.2579520$ $-2.4735300$ $-3.5298050$ $-1.8972420$ $-2.0719310$ $-2.9229570$ $-1.2893280$

1.7477010

3.1456160

2. 5137810

1.4082540

4.1302350

5.1546850

4.0662290

3.7852340

4.6047220

3.6230620

2. 2272080

3.3775640

2. 8213980

3.6141890

1.9342700

3.2529730

4.2874400

2.6243950

0.8270420

1.0926280 $-0.8533170$

$-1.0995580$

$-1.0499910$

$-1.0882430$

$-1.9763880$ 


$\begin{array}{rrr}-1.8074200 & -4.8553210 & -1.3969540 \\ -0.4139700 & -8.0241070 & -1.4467890 \\ 1.3078760 & -6.4481620 & -1.9995060 \\ 1.4233570 & -6.6910950 & -0.2663610 \\ -1.1288900 & -6.1470140 & -2.9894810 \\ -2.4898130 & -6.7890410 & -2.0665840 \\ -2.4658300 & -4.2849230 & -2.0533350 \\ -2.3579980 & -4.9617070 & -0.4508580 \\ -0.9756020 & -7.0699860 & -0.0743910 \\ -3.4063230 & -1.0721500 & -2.9432630 \\ -3.9617530 & -1.1474320 & -4.3427500 \\ -4.0410370 & -2.6171170 & -4.7841380 \\ -2.6666080 & -3.3185570 & -4.7094810 \\ -1.6436180 & -2.5547890 & -3.8391790 \\ -2.2733100 & -1.8477630 & -2.6622660 \\ -3.3135690 & -0.5913180 & -5.0357250 \\ -4.4517670 & -2.6859350 & -5.7968130 \\ -2.2414320 & -3.4314360 & -5.7124870 \\ -1.1725350 & -1.7796900 & -4.4598780 \\ -4.9492030 & -0.6766950 & -4.3892690 \\ -4.7506040 & -3.1251370 & -4.1211730 \\ -2.7963040 & -4.3327300 & -4.3162840 \\ -0.8369780 & -3.2219280 & -3.5276300 \\ 1.5641320 & 3.3682010 & -1.0020160 \\ 0.4741360 & 2.6894200 & -1.2068860 \\ 2.0499970 & 4.1108150 & -2.1958180 \\ 3.1908940 & 4.9079270 & -2.0823340 \\ 1.4066310 & 3.9804500 & -3.4288900 \\ 3.6866440 & 5.5725140 & -3.1966840 \\ 3.6816760 & 4.9922780 & -1.1185790 \\ 1.9081340 & 4.6434790 & -4.5427970 \\ 0.5249730 & 3.3545630 & -3.5074120 \\ 3.0467490 & 5.4383160 & -4.4277950 \\ 4.5740790 & 6.1913500 & -3.1083340 \\ 1.4125440 & 4.5374840 & -5.5026200 \\ 3.4383770 & 5.9527820 & -5.3002410 \\ 0.1293620 & 2.1472330 & -0.4079940 \\ 2.1953180 & 3.4137270 & 0.0629040 \\ 1.4976100 & 2.4688130 & 2.6220190 \\ 2.4947680 & 2.0165820 & 1.6444810 \\ 0.4682700 & 2.4202700 & 2.2707820 \\ 2.2212650 & 1.2042640 & 0.9776720 \\ 1.8895780 & 1.2648550 & 3.3146870 \\ 1.2629060 & 0.5370590 & 3.0015890 \\ 3.3056240 & 3.8175140 & 3.7717690 \\ 4.1708290 & 3.6831440 & 2.5169110\end{array}$

\begin{abstract}
3.9364060
1.8207880

3. 4967590

5.2326330

4. 3597350

1. 5232170

1.1936950

3. 5667280

3. 9268530

4. 4212630
\end{abstract}

154

TS176(S , S) G[WB97XD/6-31G(d) $]=-3541.692531$

$\begin{array}{lrrr}\text { TS176(S, S }) \text { G }[\text { WB97XD/6-31G(d) }]=-3541.692531 \\ \mathrm{P} & 0.2627210 & -0.0358970 & 0.1918230 \\ \mathrm{O} & 0.7791150 & 0.8758500 & 1.2691140 \\ \mathrm{O} & -0.8176920 & 0.4649720 & -0.7358940 \\ \mathrm{O} & -0.1329340 & -1.4179700 & 0.9525280 \\ \mathrm{O} & 1.4408780 & -0.5440660 & -0.8086500 \\ \mathrm{C} & -0.2042080 & -2.5841080 & 0.2124180 \\ \mathrm{C} & 0.9844900 & -3.1846620 & -0.2126830 \\ \mathrm{C} & -1.4606100 & -3.1099260 & -0.0832340 \\ \mathrm{C} & -1.4866300 & -4.2856440 & -0.8270170 \\ \mathrm{H} & -2.4530710 & -4.7273540 & -1.0618010 \\ \mathrm{C} & 2.4856210 & -1.2174790 & -0.2033890 \\ \mathrm{C} & 3.6744910 & -0.5320490 & 0.0416100 \\ \mathrm{C} & 2.2836630 & -2.5485310 & 0.1589590 \\ \mathrm{C} & 4.6852180 & -1.2512980 & 0.6706430 \\ \mathrm{H} & 5.6359910 & -0.7569680 & 0.8597770 \\ \mathrm{C} & -2.7260550 & -2.4150160 & 0.2831580 \\ \mathrm{C} & 3.8115060 & 0.9156140 & -0.2795940 \\ \mathrm{C} & -3.0857770 & -2.1746940 & 1.6152790 \\ \mathrm{C} & -2.2834820 & -2.5143190 & 2.8488290 \\ \mathrm{C} & -4.5342100 & -1.3673640 & 3.3964840 \\ \mathrm{C} & -4.3138760 & -1.5750710 & 1.9216140 \\ \mathrm{C} & -2.0233180 & -1.2064350 & 3.6305310 \\ \mathrm{H} & -1.5071110 & -1.4527520 & 4.5658360 \\ \mathrm{H} & -1.3533480 & -0.5655320 & 3.0524920 \\ \mathrm{C} & -3.3711730 & -0.4949150 & 3.9192130 \\ \mathrm{H} & -3.5025950 & -0.3210390 & 4.9937200 \\ \mathrm{H} & -3.4062510 & 0.4816230 & 3.4249190 \\ \mathrm{H} & -5.4965950 & -0.8800950 & 3.5876080 \\ \mathrm{H} & -1.3370380 & -2.9992790 & 2.6010470 \\ \mathrm{C} & -4.4717180 & -2.7398640 & 4.1009930 \\ \mathrm{H} & -4.5537200 & -2.5904140 & 5.1840600 \\ \mathrm{H} & -5.3295810 & -3.3478850 & 3.7955170 \\ \mathrm{C} & -3.1391400 & -3.4442080 & 3.7376990 \\ \mathrm{H} & -2.5726200 & -3.6937740 & 4.6424270\end{array}$

$\begin{array}{ll}2.3414910 & 1.8219100 \\ 3.7142270 & 3.4142250 \\ 4.7772400 & 4.2620650 \\ 3.7767830 & 2.7648950 \\ 1.5121740 & 2.4018060 \\ 4.5721570 & 2.7989120 \\ 3.7214840 & 4.3115720 \\ 3.0309400 & 4.4898300 \\ 4.4899200 & 1.8160250 \\ 2.2884830 & 0.8448590\end{array}$

2. 3414910

4.7772400

3. 7767830

3.7214840

1. 8160250

0.8448590
0.8758500
0.4649720
-1.4179700
-0.5440660
-2.5841080
-3.1846620
-3.1099260
-4.2856440
-4.7273540
-1.2174790
-0.5320490
-2.5485310
-1.2512980
-0.7569680
-2.4150160
0.9156140
-2.1746940
-2.5143190
-1.3673640
-1.5750710
-1.2064350
-1.4527520
-0.5655320
-0.4949150
-0.3210390
0.4816230
-0.8800950
-2.9992790
-2.7398640
-2.5904140
-3.3478850
-3.4442080
-3.6937740

$-4.7841380$

-3.8391790

$-2.6622660$

$-5.0357250$

8130

$-4.4598780$

$-4.3892690$

.1211730

$-3.5276300$

$-1.0020160$

-1. 2068860

3.1966840

. 1185790

$-4.4277950$

.

5.3002410

0.0629040

2. 6220190

1.6444810

0.9776720

146870

2. 5169110 


$\begin{array}{ll}\mathrm{H} & -3.3273600 \\ \mathrm{C} & -3.6056230 \\ \mathrm{C} & -3.3739820 \\ \mathrm{C} & -5.6614130 \\ \mathrm{C} & -4.8359420 \\ \mathrm{C} & -4.5063080 \\ \mathrm{H} & -4.3833830 \\ \mathrm{H} & -4.4233250 \\ \mathrm{C} & -5.8826460 \\ \mathrm{H} & -6.4733870 \\ \mathrm{H} & -6.4646820 \\ \mathrm{H} & -6.6211650 \\ \mathrm{H} & -2.4001750 \\ \mathrm{C} & -4.8554690 \\ \mathrm{H} & -5.4116140 \\ \mathrm{H} & -4.7619330 \\ \mathrm{C} & -3.4618310 \\ \mathrm{H} & -3.2861160 \\ \mathrm{H} & -2.6622640 \\ \mathrm{C} & -5.1999090 \\ \mathrm{H} & -6.1655620 \\ \mathrm{C} & 3.7649510 \\ \mathrm{C} & 3.5945350 \\ \mathrm{C} & 3.3985060 \\ \mathrm{C} & 3.6466960 \\ \mathrm{C} & 4.6841090 \\ \mathrm{H} & 4.5514630 \\ \mathrm{H} & 5.6709590 \\ \mathrm{C} & 4.5741900 \\ \mathrm{H} & 4.4037820 \\ \mathrm{H} & 5.5014640 \\ \mathrm{H} & 3.3211850 \\ \mathrm{H} & 3.6835350 \\ \mathrm{C} & 2.1012270 \\ \mathrm{H} & 1.9467260 \\ \mathrm{H} & 1.2510020 \\ \mathrm{C} & 2.2178230 \\ \mathrm{H} & 2.1078540 \\ \mathrm{H} & 1.4226010 \\ \mathrm{C} & 4.2500600 \\ \mathrm{C} & 4.6335720 \\ \mathrm{C} & 4.3095980 \\ \mathrm{C} & 4.1036340 \\ \mathrm{C} & 3.5403370 \\ \mathrm{H} & 3.8522660 \\ \mathrm{H} & 2.6121090 \\ & \end{array}$

$\begin{array}{rr}-4.3837010 & 3.2077690 \\ -2.0037890 & -0.7297680 \\ -2.0827310 & -2.2210710 \\ -1.0461640 & -1.6172310 \\ -1.4149830 & -0.4119230 \\ -2.9251490 & -2.8488260 \\ -2.9286880 & -3.9382330 \\ -3.9651810 & -2.5148780 \\ -2.3281370 & -2.4511770 \\ -2.0836450 & -3.3417490 \\ -3.0467780 & -1.8650530 \\ -0.6087410 & -1.3206920 \\ -2.5141140 & -2.4594580 \\ -0.0487700 & -2.4779850 \\ 0.1530600 & -3.4012750 \\ 0.9020520 & -1.9467490 \\ -0.6490570 & -2.7889610 \\ -0.6844260 & -3.8706070 \\ -0.0488760 & -2.3473030 \\ -1.2160620 & 0.9137640 \\ -0.7755810 & 1.1606270 \\ 2.7613910 & -1.8672630 \\ 3.0960140 & -3.3272370 \\ 0.5689710 & -2.8285570 \\ 1.3936570 & -1.5872700 \\ 2.3333060 & -4.1143240 \\ 2.5215700 & -5.1861540 \\ 2.7188880 & -3.8389820 \\ 0.8180670 & -3.8014450 \\ 0.2440630 & -4.7195860 \\ 0.4439890 & -3.3556100 \\ -0.4965220 & -2.6031490 \\ 4.1745190 & -3.4984240 \\ 1.0647090 & -3.5028750 \\ 0.5009020 & -4.4301580 \\ 0.8512060 & -2.8509050 \\ 2.5812910 & -3.7994500 \\ 2.7825200 & -4.8715270 \\ 3.1353700 & -3.2913430 \\ 3.2023720 & 0.4382110 \\ 4.0449360 & 1.6280300 \\ 1.5494640 & 2.2051750 \\ 1.8406420 & 0.7357030 \\ 3.9099480 & 2.7087470 \\ 4.4640990 & 3.6022610 \\ 4.3601590 & 2.3467400\end{array}$

3. 3281260 3. 5023850 2. 3054510 4.1529100 4.7656180 5.7473820 5.9052830 6.4697590 5. 9509450 6. 2437170 6.7491440 4.0709550 4. 1862370 3. 2803690 3. 0007630 3. 9451260 5. 3895370 5. 6200120 4.4999790 3. 7822430 3. 1028270 1. 9572240 5. 5817000 6.0957910 6.5698930 5. 7192940 3. 7253810 3.7253810 0.9173820
2.1649930 1.8457920 0.8236140 $-0.4970990$ $-0.3333800$ 2.7676490 2. 7393980 2. 8201610 1. 2108390 0.6598740 $-1.1567540$ $-1.0221370$ 1. 4416940 $-3.2432690$ $-2.9908060$ $-4.6304910$ $-5.5685730$ $-4.9885450$
2. 4099970

2. 2126890

2. 0950870

0.4946160

5. 0941430

1. 9797010

1. 8190680

1. 3481940

3. 4736530

4.0570830

3. 5852020

3. 6674580

4.7267870

$-3.2084740$

$-4.5622220$

$-4.8846100$

$-4.6717260$

$-3.1934300$

$-2.5555770$

$-5.9168740$

$-5.3566010$

$-4.5939090$

$-5.2755590$

$-5.0055760$

$-3.0547630$

$-2.6455220$

$-4.2344920$

$-4.3039610$

$-4.8225190$

$-5.6304570$

$-6.7131070$

$-6.0645290$

$-4.8719020$

$-6.0694130$

$-5.4681410$

$-3.9784530$

$-7.3295690$

$-7.3826440$

$-6.8018840$

$-5.7248320$

$-4.9696000$

2. 7901070

1.5837770

3. 2479880

2. 3738990

4.5629060
3.0310610

4. 0953900

2.8097650

2.4369240

1. 3420940

2. 5720460

3. 6448300

2. 0434810

2. 2018890

3. 0827020

1. 4608210

$-0.8585720$

$-1.0838430$

0.9039410

1. 5294190

2. 6862950

2. 2410470

1.9337760

1. 1321250

3. 0141140

0.7810450

1. 8608920

1. 3437960

3. 0086760

1. 4035810

2.8812400

3. 5436060

$-1.0662640$

$-1.7580040$

$-3.0148050$

$-2.6805810$

$-2.2705870$

$-1.3457970$

$-3.4113680$

$-1.0833830$

$-1.9982300$

$-1.8581230$

$-3.5317210$

$-1.7974260$

$-3.1741800$

$-3.7933220$

$-0.4048380$

0.0243740

$-0.1294360$

0.4256030

$-0.4341870$ 


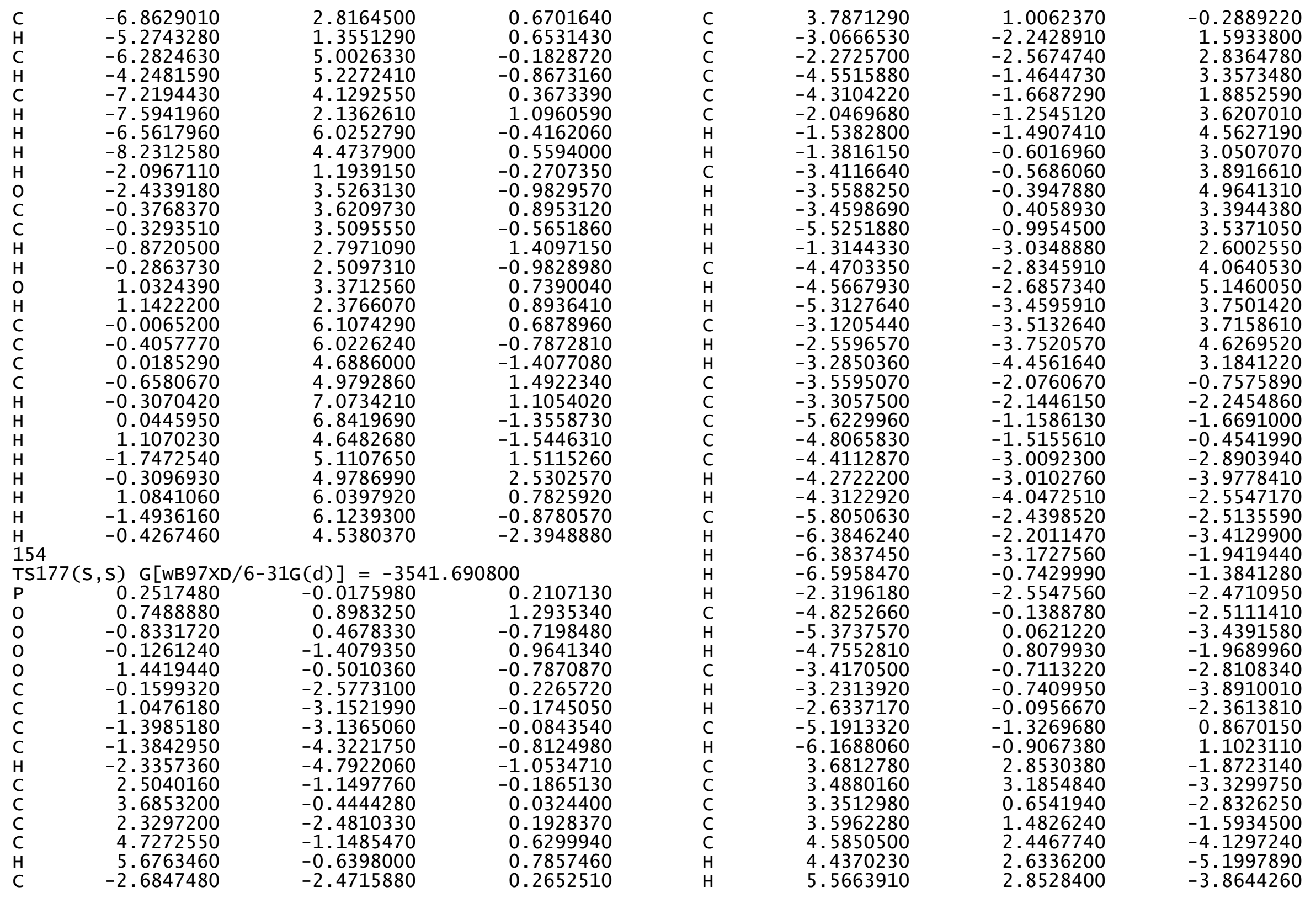




\begin{tabular}{|c|c|c|}
\hline $\begin{array}{l}4.5106530 \\
4.3422540 \\
5.4504860 \\
3.2988770 \\
3.5523100 \\
2.0365450 \\
1.8832590 \\
1.1984400 \\
2.1178180 \\
1.9933960 \\
1.3158410 \\
4.1819310 \\
4.5591840 \\
4.2995300 \\
4.0705510 \\
3.4812520 \\
3.7907450 \\
2.5390440 \\
3.3062880 \\
3.4938660 \\
2.2891170 \\
4.1706790 \\
4.6640420 \\
5.7306110 \\
5.9023670 \\
6.4621080 \\
5.8956390 \\
6.1852230 \\
6.6827050 \\
3.9779230 \\
4.0671430 \\
4.5772180 \\
3.3532520 \\
5.7617040 \\
5.3757410 \\
4.3777040 \\
3.0985300 \\
6.2749200 \\
6.4416220 \\
6.3279560 \\
4.8220010 \\
4.1399180 \\
2.3900050 \\
2.5962470 \\
4.9184250\end{array}$ & $\begin{array}{r}0.9289990 \\
0.3528160 \\
0.5740470 \\
-0.4128560 \\
4.2658160 \\
1.1232060 \\
0.5566710 \\
0.8919960 \\
2.6420830 \\
2.8413070 \\
3.1789230 \\
3.3009640 \\
4.1488500 \\
1.6446850 \\
1.9355770 \\
3.9853150 \\
4.5430200 \\
4.4157890 \\
2.4798620 \\
2.2832590 \\
2.1424030 \\
0.5855700 \\
5.2016450 \\
2.1077670 \\
1.9490780 \\
1.4938130 \\
3.6064440 \\
4.1948370 \\
3.7375350 \\
3.7642010 \\
4.8262100 \\
-2.4566430 \\
-3.1193440 \\
-3.1343310 \\
-4.3200380 \\
-5.2196040 \\
-4.4419750 \\
-4.8783610 \\
-3.4871710 \\
-2.3966450 \\
-5.5808360 \\
-6.1026670 \\
-5.0567340 \\
-4.2113410 \\
-3.9555190 \\
-4.2876720\end{array}$ & $\begin{array}{l}-3.8181030 \\
-4.7352550 \\
-3.3830130 \\
-2.6070440 \\
-3.5008240 \\
-3.4920000 \\
-4.4178090 \\
-2.8305650 \\
-3.7888390 \\
-4.8597050 \\
-3.2729180 \\
0.4286380 \\
1.6167300 \\
2.1903080 \\
0.7244200 \\
2.7088420 \\
3.6010070 \\
2.3592160 \\
3.0280270 \\
4.0902190 \\
2.8143310 \\
2.4197660 \\
1.3327050 \\
2.5438450 \\
3.6147870 \\
2.0071690 \\
2.1744270 \\
3.0530070 \\
1.4246280 \\
-0.8652250 \\
-1.0898140 \\
1.0913490 \\
0.9154970 \\
1.7552530 \\
2.6388000 \\
1.9129700 \\
1.6138800 \\
2.9215130 \\
0.9664300 \\
2.3352430 \\
0.9748490 \\
2.5161660 \\
1.0563760 \\
2.5647650 \\
3.5681700 \\
-1.0097320\end{array}$ \\
\hline
\end{tabular}

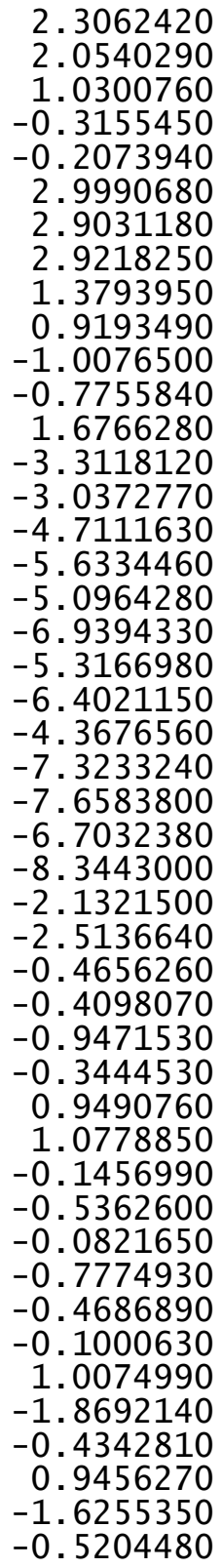

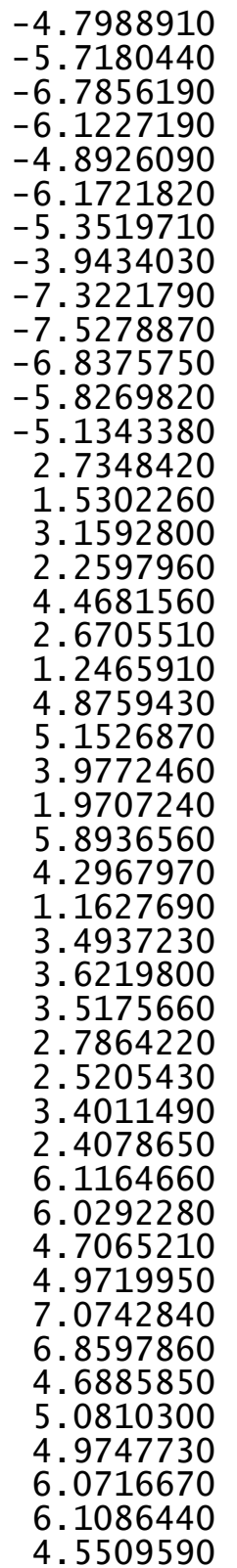

$-1.6437720$

$-2.8379920$

$-2.4628140$

$-2.1790630$

$-1.2972060$

$-3.1546420$

$-0.9070780$

$-1.9423260$

$-1.5701990$

$-3.2605850$

$-1.7180800$

$-3.1323730$

$-3.6882160$

$-0.3918260$

0.0286660

$-0.1247430$

0.4158060

$-0.4221880$

0.6536060

0.6380420

$-0.1780550$

$-0.8434930$

0.3577870

1.0685270

$-0.4058550$

0.5444110

$-0.2613040$

$-0.9559510$

0.9304700

$-0.5303110$

1.4389750

$-0.9519100$

0.7804890

0.9300550

0.7340870

$-0.7432860$

$-1.3667060$

1. 5310900

1.1537310

$-1.3065920$

$-1.4993060$

1. 5452940

2.5708230

0.8338840

$-0.8386570$

$-2.3562390$ 
154

$\operatorname{TS} 178(\mathrm{~S}, \mathrm{~S}) \quad \mathrm{G}[\mathrm{WB} 97 \mathrm{XD} / 6-31 \mathrm{G}(\mathrm{d})]=-3541.690821$

$\begin{array}{lrrr}\mathrm{P} & 0.2679970 & -0.0611440 & 0.1846530 \\ \mathrm{O} & 0.8035770 & 0.8340240 & 1.2664660 \\ \mathrm{O} & -0.8177090 & 0.4592530 & -0.7257500 \\ \mathrm{O} & -0.1288050 & -1.4487700 & 0.9312720 \\ \mathrm{O} & 1.4291030 & -0.5752440 & -0.8312160 \\ \mathrm{C} & -0.2242010 & -2.6045490 & 0.1782480 \\ \mathrm{C} & 0.9507900 & -3.2330930 & -0.2472230 \\ \mathrm{C} & -1.4927920 & -3.1006580 & -0.1150190 \\ \mathrm{C} & -1.5500230 & -4.2822530 & -0.8471720 \\ \mathrm{H} & -2.5277500 & -4.6992030 & -1.0802980 \\ \mathrm{C} & 2.4746850 & -1.2675720 & -0.2494100 \\ \mathrm{C} & 3.6714340 & -0.5936220 & -0.0192370 \\ \mathrm{C} & 2.2646070 & -2.6100290 & 0.0916310 \\ \mathrm{C} & 4.7042810 & -1.3217640 & 0.5741720 \\ \mathrm{H} & 5.6570020 & -0.8297450 & 0.7557990 \\ \mathrm{C} & -2.7406840 & -2.3752290 & 0.2531120 \\ \mathrm{C} & 3.8087630 & 0.8579840 & -0.3247930 \\ \mathrm{C} & -3.0996430 & -2.1410570 & 1.5862630 \\ \mathrm{C} & -2.3075250 & -2.5093730 & 2.8177820 \\ \mathrm{C} & -4.5346080 & -1.3203260 & 3.3715790 \\ \mathrm{C} & -4.3159000 & -1.5190540 & 1.8951080 \\ \mathrm{C} & -2.0212480 & -1.2149460 & 3.6121090 \\ \mathrm{H} & -1.5124150 & -1.4806970 & 4.5461470 \\ \mathrm{H} & -1.3365470 & -0.5828480 & 3.0415990 \\ \mathrm{C} & -3.3543040 & -0.4774890 & 3.9043200 \\ \mathrm{H} & -3.4844150 & -0.3099390 & 4.9799850 \\ \mathrm{H} & -3.3672580 & 0.5036940 & 3.4182090 \\ \mathrm{H} & -5.4870060 & -0.8149760 & 3.5657930 \\ \mathrm{H} & -1.3708970 & -3.0112930 & 2.5667280 \\ \mathrm{C} & -4.5021470 & -2.7002980 & 4.0639940 \\ \mathrm{H} & -4.5826160 & -2.5584870 & 5.1482140 \\ \mathrm{H} & -5.3722930 & -3.2872040 & 3.7520950 \\ \mathrm{C} & -3.1841070 & -3.4294710 & 3.6963900 \\ \mathrm{H} & -2.6245550 & -3.6994820 & 4.5995870 \\ \mathrm{H} & -3.3911510 & -4.3597040 & 3.1571780 \\ \mathrm{C} & -3.6061450 & -1.9327680 & -0.7581230 \\ \mathrm{C} & -3.3672790 & -1.9966150 & -2.2487550 \\ \mathrm{C} & -5.6396600 & -0.9276830 & -1.6429300 \\ \mathrm{C} & -4.8263120 & -1.3243270 & -0.4382690 \\ \mathrm{C} & -4.5110350 & -2.8093510 & -2.8949620 \\ \mathrm{H} & -4.3837440 & -2.7955090 & -3.9837970 \\ \mathrm{H} & -4.4470480 & -3.8564620 & -2.5795330 \\ \mathrm{C} & -5.8787920 & -2.1961460 & -2.4923900 \\ \mathrm{H} & -6.4643870 & -1.9325390 & -3.3809240 \\ & & & \end{array}$

$-6.4725520$

$-6.5932330$

$-2.3997690$

$-4.8125700$

$-5.3600780$

$-3.4267860$

$-3.2448710$

$-2.6195610$

$-5.1918830$

$-6.1506030$

3.7547650

3. 5699940

3. 3600020

3. 6296480

4.6457490

4. 5047580

5. 6384110

4. 5257370

4. 3388660

5.4549860

3. 2767640

3. 6651610

2. 0585670

1.8859180

1. 2154270

2. 1852690

2. 0688950

1. 3982590

4.2788440

4.6877880

4. 3514730

4. 1247930

3. 6108250

3. 9423010

2. 6807650

3. 3906330

3. 5792450

2. 3619530

4. 1899040

4.8242160

5.7983810

5.9714390

6. 5071870

6.0088080

6.3191400
-2.9126190
-0.4773020
-2.4425140
0.0652640
0.2856220
1.0088780
-0.5539450
-0.5779710
0.0256990
-1.1336510
-0.6790210
2.7207610
3.0708900
0.5407610
1.3511500
2.3078800
2.5089130
2.6820560
0.7902790
0.2280980
0.4029190
-0.5260790
4.1503220
1.0530090
0.4974020
0.8424800
2.5704980
2.7798340
3.1273690
3.1330480
3.9594210
1.4603400
1.7696290
3.8216880
4.3630130
4.2837570
2.3200680
2.1097300
2.0168020
0.4041520
5.0106190
1.8747730
1.7010290
1.2433950
3.3711020
3.9423500

$-1.9153970$

$-1.3458960$

$-2.4867780$

$-2.4884610$

$-3.4126940$

$-1.9465340$

$-2.7979170$

$-3.8788730$

$-2.3432170$

0.8882040

1.1363530

$-1.8926170$

$-3.3474050$

$-2.8711870$

$-1.6249760$

$-4.1531650$

$-5.2216260$

$-3.8829450$

$-3.8558100$

$-4.7780780$

$-3.4256430$

$-2.6550270$

$-3.5093190$

$-3.5243660$

$-4.4534100$

$-2.8623470$

$-3.8114590$

$-4.8813170$

$-3.2941340$

0.4097340

1. 6025210

2. 1579170

0.6951930

2. 6991680

3. 5934750

2. 3575480

3. 0084610

4.0678600

2. 7995060

2. 3802600

1. 3260810

2. 5069420

3.5753360

1.9601670

2. 1501470

3. 0328840 


$\begin{array}{lr}\mathrm{H} & 6.7970960 \\ \mathrm{C} & 4.0835880 \\ \mathrm{H} & 4.2048690 \\ \mathrm{C} & 3.2948450 \\ \mathrm{C} & 3.1507690 \\ \mathrm{C} & 3.5896240 \\ \mathrm{C} & 5.0157770 \\ \mathrm{C} & 5.5773990 \\ \mathrm{C} & 4.5206920 \\ \mathrm{H} & 3.7833280 \\ \mathrm{H} & 3.5246780 \\ \mathrm{H} & 5.7036980 \\ \mathrm{H} & 5.9272460 \\ \mathrm{H} & 2.1206800 \\ \mathrm{H} & 2.8690820 \\ \mathrm{H} & 5.0006920 \\ \mathrm{H} & 6.4510260 \\ \mathrm{C} & 0.8546590 \\ \mathrm{C} & 2.0958770 \\ \mathrm{C} & 1.7827130 \\ \mathrm{C} & 0.6968170 \\ \mathrm{C} & -0.6018400 \\ \mathrm{C} & -0.4109320 \\ \mathrm{H} & 2.6960530 \\ \mathrm{H} & 2.6085690 \\ \mathrm{H} & 2.8015600 \\ \mathrm{H} & 1.0238710 \\ \mathrm{H} & 0.5284860 \\ \mathrm{H} & -1.3196090 \\ \mathrm{H} & -1.0696050 \\ \mathrm{H} & 1.4403000 \\ \mathrm{C} & -3.1936480 \\ \mathrm{O} & -2.9590230 \\ \mathrm{C} & -4.5700020 \\ \mathrm{C} & -5.5217800 \\ \mathrm{C} & -4.9042520 \\ \mathrm{C} & -6.8059070 \\ \mathrm{H} & -5.2461610 \\ \mathrm{C} & -6.1878720 \\ \mathrm{H} & -4.1534780 \\ \mathrm{C} & -7.1385370 \\ \mathrm{H} & -7.5480350 \\ \mathrm{H} & -6.4483900 \\ \mathrm{H} & -8.1423350 \\ \mathrm{H} & -2.0769490 \\ \mathrm{O} & -2.3756510 \\ & \end{array}$

$\begin{array}{rr}3.4843460 & 1.3987720 \\ 3.6135890 & -0.8791350 \\ 4.6742170 & -1.0950970 \\ -3.2827530 & 0.7618320 \\ -4.6467530 & 1.3885010 \\ -4.5785930 & 2.8626890 \\ -3.9993190 & 3.0330520 \\ -3.4156550 & 1.7196970 \\ -2.6364950 & 0.9823180 \\ -5.3750600 & 0.8615900 \\ -5.5778260 & 3.3055780 \\ -4.7694370 & 3.3979360 \\ -4.2413660 & 1.0844720 \\ -5.0063740 & 1.3188600 \\ -3.9505280 & 3.3970250 \\ -3.2087590 & 3.7901460 \\ -2.7888990 & 1.9255030 \\ -4.3795280 & -1.0624000 \\ -4.9904400 & -1.6853730 \\ -5.8525130 & -2.9071040 \\ -6.8654800 & -2.5559760 \\ -6.1324410 & -2.2274160 \\ -4.9163560 & -1.3389270 \\ -6.3556690 & -3.2423880 \\ -5.6212810 & -0.9490870 \\ -4.1950510 & -1.9470050 \\ -7.4560670 & -1.6894070 \\ -7.5699780 & -3.3775680 \\ -6.8152290 & -1.7572070 \\ -5.7998450 & -3.1646180 \\ -5.2188170 & -3.7361640 \\ 2.8317460 & -0.3700130 \\ 1.6231590 & 0.0635090 \\ 3.3164120 & -0.0871020 \\ 2.4604240 & 0.4725310 \\ 4.6380790 & -0.3897990 \\ 2.9280310 & 0.7240910 \\ 1.4361640 & 0.6977480 \\ 5.1029960 & -0.1313060 \\ 5.2876970 & -0.8273110 \\ 4.2476530 & 0.4238560 \\ 2.2620130 & 1.1536120 \\ 6.1310060 & -0.3627300 \\ 4.6119080 & 0.6215300 \\ 1.2146410 & -0.2420680 \\ 3.5505830 & -0.9577480\end{array}$

$\begin{array}{lr}\mathrm{C} & -0.3175110 \\ \mathrm{C} & -0.2744390 \\ \mathrm{H} & -0.8238910 \\ \mathrm{H} & -0.2465360 \\ \mathrm{O} & 1.0870930 \\ \mathrm{H} & 1.1827460 \\ \mathrm{C} & 0.0942830 \\ \mathrm{C} & -0.3091630 \\ \mathrm{C} & 0.0902880 \\ \mathrm{C} & -0.5760890 \\ \mathrm{H} & -0.1874810 \\ \mathrm{H} & 0.1543390 \\ \mathrm{H} & 1.1774750 \\ \mathrm{H} & -1.6629240 \\ \mathrm{H} & -0.2271250 \\ \mathrm{H} & 1.1836300 \\ \mathrm{H} & -1.3952390 \\ \mathrm{H} & -0.3613880 \\ \mathrm{H} & \end{array}$
3. 5996740
6.0808410
6.0165750
4.6812040
4.9570960
7.0482890
6.8331360
4.6231800
5.1067040
4.9411670
5.9918900
6.1379940
4.5473210

$\begin{array}{lr}2.5036150 & -0.9669830 \\ 3.3303410 & 0.7590860\end{array}$

$2.3325820 \quad 0.9034070$

0.9034070

0.7354400
-0.7396170

$-1.3733780$

1.5302690

1.1624600

$-1.3016540$

$-1.5147730$

1.5516810

2.5679700

0.8272200

$-0.8270220$

154

$\operatorname{TS} 179(\mathrm{~S}, \mathrm{~S}) \mathrm{G}[\mathrm{WB} 97 \mathrm{XD} / 6-31 \mathrm{G}(\mathrm{d})]=-3541.688992$

$\begin{array}{lrrr}\text { TS179(S S }) ~ G[W B 97 X D / 6-31 G(d)]=-3541.688992 \\ \mathrm{P} & 0.3872240 & 0.2384180 & 0.8045890 \\ \mathrm{O} & -0.2390180 & 0.7947230 & 2.0482530 \\ \mathrm{O} & 0.6242440 & -1.2467580 & 0.7034570 \\ \mathrm{O} & 1.7392140 & 1.1004530 & 0.5640650 \\ \mathrm{O} & -0.4815720 & 0.6005480 & -0.5372870 \\ \mathrm{C} & 2.3468480 & 1.0063530 & -0.6752700 \\ \mathrm{C} & 1.7763910 & 1.7079120 & -1.7446580 \\ \mathrm{C} & 3.5064490 & 0.2467730 & -0.7950450 \\ \mathrm{C} & 4.0941270 & 0.1767960 & -2.0597560 \\ \mathrm{H} & 5.0045560 & -0.4058230 & -2.1807190 \\ \mathrm{C} & -0.5618430 & 1.9308490 & -0.9084410 \\ \mathrm{C} & -1.7586890 & 2.6106320 & -0.7036030 \\ \mathrm{C} & 0.5582950 & 2.5296210 & -1.5006620 \\ \mathrm{C} & -1.8120020 & 3.9537460 & -1.0783710 \\ \mathrm{H} & -2.7397330 & 4.5015100 & -0.9287860 \\ \mathrm{C} & 4.1028480 & -0.4685370 & 0.3666840 \\ \mathrm{C} & -2.9491300 & 1.9274580 & -0.1272120 \\ \mathrm{C} & 4.6065150 & 0.2371120 & 1.4670740 \\ \mathrm{C} & 4.5910820 & 1.7337330 & 1.6657500 \\ \mathrm{C} & 5.7021190 & 0.4713900 & 3.6263240 \\ \mathrm{C} & 5.2218080 & -0.4401680 & 2.5270550 \\ \mathrm{C} & 3.8292010 & 2.0394050 & 2.9741640 \\ \mathrm{H} & 3.8559060 & 3.1207530 & 3.1534460 \\ \mathrm{H} & 2.7802770 & 1.7573950 & 2.8549490 \\ \mathrm{C} & 4.4883850 & 1.2715170 & 4.1496450 \\ \mathrm{H} & 4.8235730 & 1.9633580 & 4.9314480 \\ & & & \end{array}$




$\begin{array}{rrrr}\mathrm{H} & 3.7729570 & 0.5839960 & 4.6120390 \\ \mathrm{H} & 6.1661020 & -0.0979690 & 4.4390150 \\ \mathrm{H} & 4.1224600 & 2.2523330 & 0.8266370 \\ \mathrm{C} & 6.7139990 & 1.4688070 & 3.0201460 \\ \mathrm{H} & 7.0289030 & 2.1768060 & 3.7956610 \\ \mathrm{H} & 7.6094270 & 0.9300250 & 2.6936210 \\ \mathrm{C} & 6.0514240 & 2.2109630 & 1.8303520 \\ \mathrm{H} & 6.0555750 & 3.2945570 & 1.9965880 \\ \mathrm{H} & 6.6007000 & 2.0265170 & 0.9011960 \\ \mathrm{C} & 4.2157560 & -1.8644370 & 0.3616150 \\ \mathrm{C} & 3.6871000 & -2.8129250 & -0.6879270 \\ \mathrm{C} & 4.9239500 & -4.0248830 & 1.2299240 \\ \mathrm{C} & 4.8544710 & -2.5309550 & 1.4156410 \\ \mathrm{C} & 4.8783720 & -3.6084650 & -1.2650800 \\ \mathrm{H} & 4.5009650 & -4.3461870 & -1.9831820 \\ \mathrm{H} & 5.5420320 & -2.9353620 & -1.8186150 \\ \mathrm{C} & 5.6431170 & -4.3066710 & -0.1086640 \\ \mathrm{H} & 5.6931970 & -5.3896280 & -0.2715260 \\ \mathrm{H} & 6.6738210 & -3.9421680 & -0.0488700 \\ \mathrm{H} & 5.4579600 & -4.5022770 & 2.0586930 \\ \mathrm{H} & 3.1560100 & -2.2827270 & -1.4819290 \\ \mathrm{C} & 3.4833530 & -4.5695020 & 1.1259600 \\ \mathrm{H} & 3.5220450 & -5.6454700 & 0.9169970 \\ \mathrm{H} & 2.9754930 & -4.4460360 & 2.0880410 \\ \mathrm{C} & 2.7302720 & -3.8084760 & 0.0060510 \\ \mathrm{H} & 2.3338230 & -4.5040350 & -0.7431030 \\ \mathrm{H} & 1.8873690 & -3.2500160 & 0.4154870 \\ \mathrm{C} & 5.3570000 & -1.8232320 & 2.5001410 \\ \mathrm{H} & 5.8484860 & -2.3447580 & 3.3200850 \\ \mathrm{C} & -4.7972920 & 0.3624120 & -0.3710250 \\ \mathrm{C} & -5.4412950 & -0.6012050 & -1.3352960 \\ \mathrm{C} & -3.3311910 & 0.5434290 & -2.2931810 \\ \mathrm{C} & -3.6427030 & 0.9696860 & -0.8787410 \\ \mathrm{C} & -5.8189740 & 0.1905080 & -2.6081990 \\ \mathrm{H} & -6.2393780 & -0.5006630 & -3.3480210 \\ \mathrm{H} & -6.5988530 & 0.9197010 & -2.3672810 \\ \mathrm{C} & -4.5559960 & 0.8975090 & -3.1671020 \\ \mathrm{H} & -4.3602430 & 0.5859700 & -4.1995070 \\ \mathrm{H} & -4.6916900 & 1.9838050 & -3.1795740 \\ \mathrm{H} & -2.4369640 & 1.0384650 & -2.6788470 \\ \mathrm{H} & -6.3300850 & -1.0674700 & -0.8961230 \\ \mathrm{C} & -3.1446680 & -0.9865560 & -2.3181480 \\ \mathrm{H} & -2.9719750 & -1.3120180 & -3.3503020 \\ \mathrm{H} & -2.2444220 & -1.2385540 & -1.7531740 \\ \mathrm{C} & -4.4034330 & -1.6743730 & -1.7292450 \\ \mathrm{H} & -4.8485790 & -2.3633840 & -2.4561370\end{array}$

-4.1399900
-4.6033830
-5.0227100
-2.8843210
-3.4385750
-3.8600190
-4.1497420
-3.6668670
-2.5966140
-2.2978320
-1.7482580
-1.9746420
-5.9328920
-3.9891920
-3.6192880
-4.2133480
-5.2577360
-5.4916270
-6.1281430
-5.2827240
-6.1931530
0.4894380
1.6609400
1.8093310
0.4941580
-0.7039000
-0.7045150
1.5034390
2.6082200
0.6048290
-0.6358590
2.5872620
2.1413640
0.2683190
-1.6432370
2.3647640
1.7832520
1.6901000
3.0293130
4.1089140
3.5320510
2.4216440
1.3415810
3.4121030
4.5189710
0.7942350

\author{
$-2.2735830$ \\ 1.6656840 \\ 2. 1889020 \\ 3. 3209720 \\ 2. 2685430 \\ 1. 9641340 \\ 2. 3413460 \\ 0.8918300 \\ 2. 6919960 \\ 3. 4913110 \\ 2. 0129800 \\ 3.7735440 \\ 1. 6913020 \\ 4. 3851980 \\ 5. 1702030 \\ 4.8606590 \\ 3. 7120810 \\ 4.1240180 \\ 3. 8945140 \\ 0.7128830 \\ 0.2559230 \\ 3.8929150 \\ 4.7079190 \\ 5.9765980 \\ 6.7865310 \\ 6.0612390 \\ 4. 5991240 \\ 4.9938260 \\ 6.5989710 \\ 7.7705970 \\ 6.1637300 \\ 4.1291480 \\ 5.6661720 \\ 6.9649260 \\ 6.5363960 \\ 1.5774670 \\ 2. 1462870 \\ 1.0436950 \\ 0.2948490 \\ 0.7109570 \\ 0.8140980 \\ 2. 9618110 \\ 1.4856680 \\ 0.4682750 \\ 1.6873470 \\ 2. 5769800
}

$-0.8529320$

1.6342760

2.9829440

2.0693320

3. 9728980

4.9606580

4.0807050

3. 4494570

4. 1377860

3. 3609750

1.6687780

3. 3353970

2. 2594510

2. 9288510

1.2984590

2. 8498250

3.8383790

2. 2114330

0.8846100

1.2701470

$-1.8188180$

$-2.3072180$

$-1.4465620$

$-1.3293220$

$-1.9726430$

$-1.6120420$

$-3.3569630$

$-1.8628070$

$-1.7968820$

$-3.0648820$

$-2.2745930$

$-0.4502370$

$-0.2729950$

$-1.6721200$

$-3.0095310$

$-4.2794880$

$-5.3503200$

$-5.5638190$

$-4.5451570$

$-3.1579650$

$-4.6482350$

$-6.2895390$

$-6.5750360$

$-4.8392160$ 


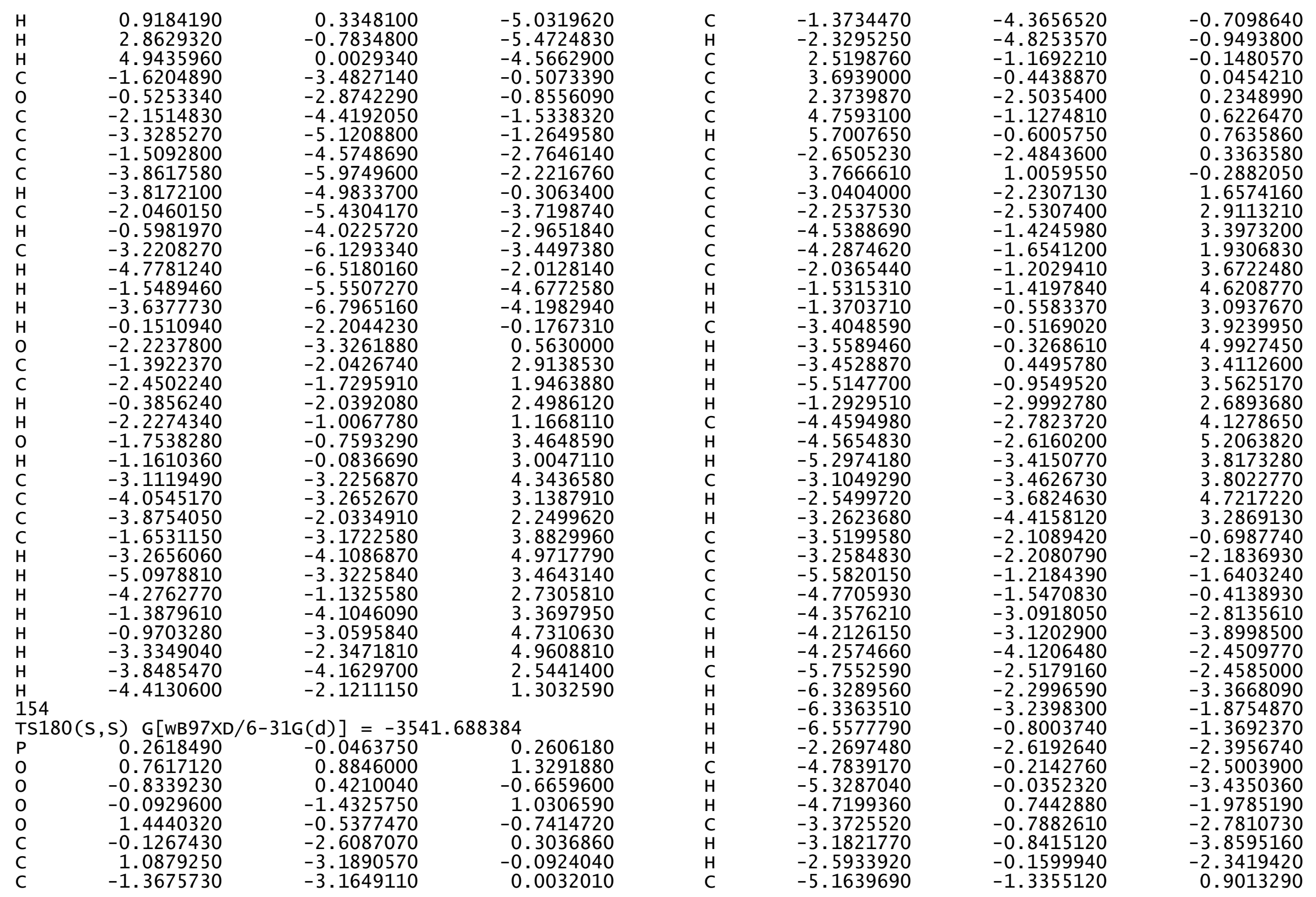




$\begin{array}{rrrr}\mathrm{H} & -6.1449490 & -0.9162600 & 1.1233630 \\ \mathrm{C} & 3.6055970 & 2.8364770 & -1.8856710 \\ \mathrm{C} & 3.3850860 & 3.1525010 & -3.3432340 \\ \mathrm{C} & 3.3048160 & 0.6235270 & -2.8228110 \\ \mathrm{C} & 3.5491890 & 1.4673150 & -1.5939440 \\ \mathrm{C} & 4.4858650 & 2.4288550 & -4.1515640 \\ \mathrm{H} & 4.3196820 & 2.6031120 & -5.2210970 \\ \mathrm{H} & 5.4623800 & 2.8567740 & -3.9034400 \\ \mathrm{C} & 4.4460730 & 0.9127720 & -3.8252600 \\ \mathrm{H} & 4.2781730 & 0.3246470 & -4.7348950 \\ \mathrm{H} & 5.3982330 & 0.5811620 & -3.3986520 \\ \mathrm{H} & 3.2772120 & -0.4424220 & -2.5879770 \\ \mathrm{H} & 3.4249670 & 4.2324700 & -3.5238650 \\ \mathrm{C} & 1.9728560 & 1.0601690 & -3.4690790 \\ \mathrm{H} & 1.8187360 & 0.4823080 & -4.3877420 \\ \mathrm{H} & 1.1484250 & 0.8185240 & -2.7943190 \\ \mathrm{C} & 2.0203890 & 2.5776320 & -3.7801210 \\ \mathrm{H} & 1.8795240 & 2.7649110 & -4.8510780 \\ \mathrm{H} & 1.2138240 & 3.1027740 & -3.2596580 \\ \mathrm{C} & 4.1375860 & 3.3125300 & 0.4030080 \\ \mathrm{C} & 4.5229040 & 4.1760000 & 1.5770870 \\ \mathrm{C} & 4.3107800 & 1.6730800 & 2.1762460 \\ \mathrm{C} & 4.0523570 & 1.9482650 & 0.7122250 \\ \mathrm{C} & 3.4677100 & 4.0059300 & 2.6903570 \\ \mathrm{H} & 3.7866150 & 4.5743750 & 3.5723780 \\ \mathrm{H} & 2.5133130 & 4.4211320 & 2.3559570 \\ \mathrm{C} & 3.3196020 & 2.5006720 & 3.0239130 \\ \mathrm{H} & 3.5267470 & 2.3149850 & 4.0844200 \\ \mathrm{H} & 2.3042610 & 2.1468220 & 2.8291060 \\ \mathrm{H} & 4.2020070 & 0.6140650 & 2.4165470 \\ \mathrm{H} & 4.6069750 & 5.2277780 & 1.2825020 \\ \mathrm{C} & 5.7407180 & 2.1608030 & 2.5014340 \\ \mathrm{H} & 5.9319870 & 2.0148230 & 3.5708800 \\ \mathrm{H} & 6.4725780 & 1.5527190 & 1.9586060 \\ \mathrm{C} & 5.8769270 & 3.6580840 & 2.1153240 \\ \mathrm{H} & 6.1735770 & 4.2589190 & 2.9830580 \\ \mathrm{H} & 6.6486450 & 3.7934240 & 1.3504360 \\ \mathrm{C} & 3.9040260 & 3.7610190 & -0.8911770 \\ \mathrm{H} & 3.9722960 & 4.8224010 & -1.1261890 \\ \mathrm{C} & 4.6468160 & -2.4430400 & 1.0732770 \\ \mathrm{C} & 3.4353060 & -3.1324140 & 0.9139920 \\ \mathrm{C} & 5.8603510 & -3.0938340 & 1.7112510 \\ \mathrm{C} & 5.5192200 & -4.3010750 & 1.5827400 \\ \mathrm{C} & 4.5508940 & -5.2208320 & 1.5654150 \\ \mathrm{C} & 3.2402720 & -4.4890750 & -8550500 \\ \mathrm{H} & 6.4374380 & -4.8328200 & \\ & & & \end{array}$

\begin{abstract}
6.5410010
6. 4105860

5.0030250

4.3488170

2. 5745720

2. 7167920

5. 0527200

1.0384940

2. 2361490

1. 9530800

0.6257180

$-0.1777910$

$-0.1978060$

2. 4439050

2. 7957900

0.8181270

0.2888320

3. 1356600

1. 9162740

0.0046170

$-1.1961120$

$-3.3165000$

$-3.0452860$

$-4.7186990$

$-5.6480320$

$-5.0994090$

$-6.9564080$

$-5.3345290$

$-6.4074240$

$-4.3652740$

$-7.3356350$

$-7.6808160$

$-6.7048700$

$-8.3583010$

$-2.1372290$

$-2.5127420$

$-0.4954700$

$-0.4182280$

$-0.9739280$

$-0.3360700$

0.9233590

1.0636040

$-0.1998350$

$-0.5657920$

$-0.0914990$

$-0.8334280$
\end{abstract}
$-3.4163700$
$-2.3460120$
$-5.5444070$
$-6.1255720$
$-5.1183800$
$-4.3209950$
$-3.9640570$
$-4.3502840$
$-4.9835660$
$-5.1754720$
$-5.9282420$
$-6.1824660$
$-4.9479000$
$-5.7043060$
$-6.8868440$
$-7.0088580$
$-4.3783620$
$-4.1842190$
$-5.3415430$
$-6.5032200$
2. 6886900
1.4952400
3. 1188830
2. 2306250
4.4219830
2.6467000
1. 2219940
4.8354260
5.0977770
3. 9477660
1.9554680
5.8488870
4.2716980
1.1218760
3. 4343480
3. 5899040
3.4788100
2.7513930
2.4801650
3. 3862110
2. 3951400
. 0863120
5. 9886460
4.6670970
4.9391650

0.9102280

2. 2936980

0.8961460

2.4276680

0.9744330

2. 5178950

3. 5179130

$-0.8773220$

$-1.5436200$

$-3.0451410$

$-3.3142970$

$-2.0230950$

$-1.1614800$

$-1.0911200$

$-3.5023630$

$-3.8074440$

$-1.4689990$

$-1.4067080$

- 3.5090400

$-3.9990520$

$-2.2652450$

$-0.4114250$

0.0415280

$-0.1699910$

0.3769470

$-0.4972510$

0.5911730

0.6229960

$-0.2761260$

$-0.9232850$

0.2660770

1. 0109610

$-0.5266550$

0.4350860

$-0.2314150$

$-0.9852180$

0.9335830

$-0.5258610$

1. 4401170

$-0.9405810$

0.8048080

0.9604300

0.7300650

$-0.7528910$

$-1.3637190$

1. 5216820 


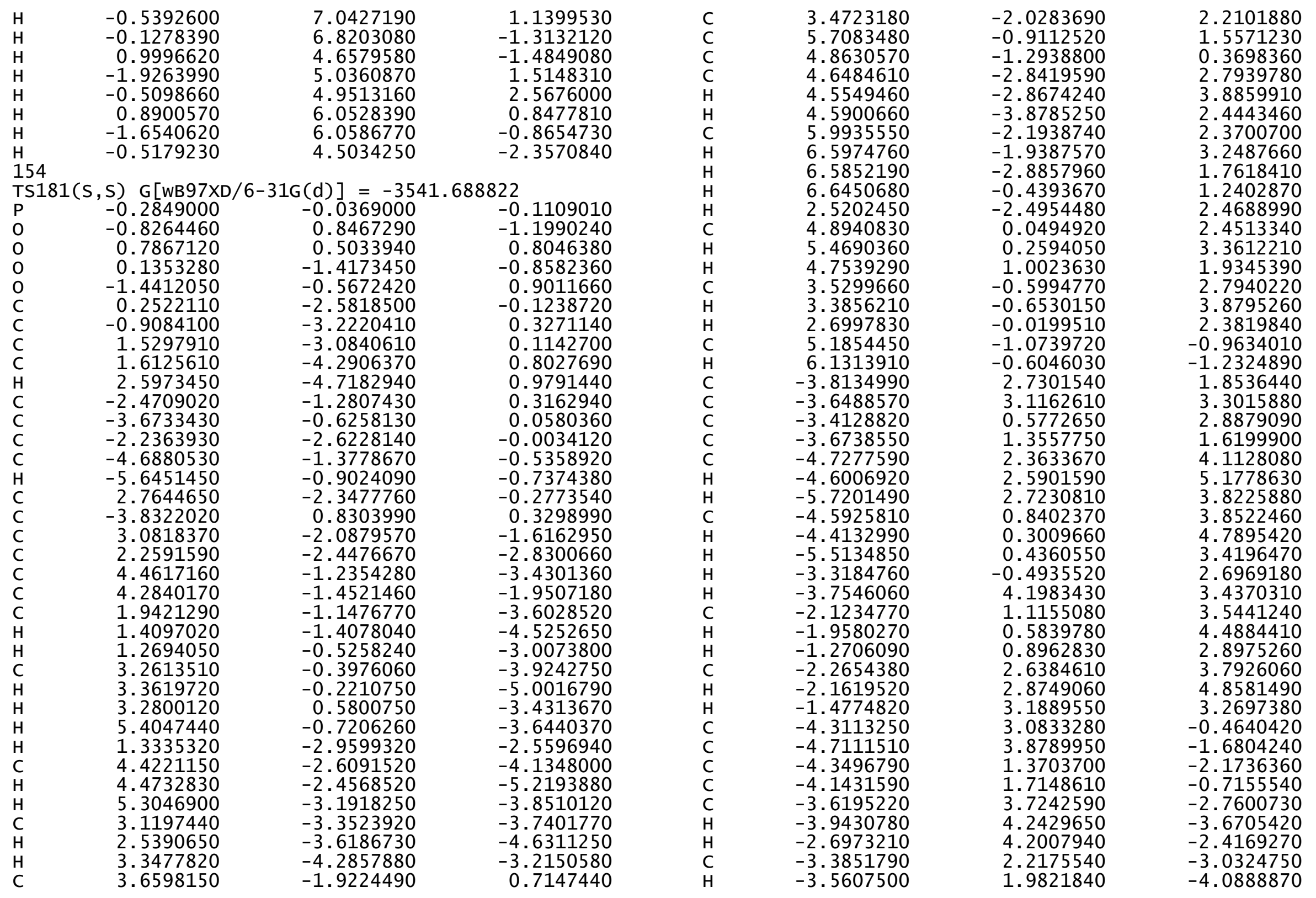




\begin{tabular}{|c|c|c|}
\hline $\begin{array}{l}-2.3570290 \\
-4.1781250 \\
-4.8584960 \\
-5.7954640 \\
-5.9552080 \\
-6.5057260 \\
-6.0210950 \\
-6.3248540 \\
-6.8191190 \\
-4.1361970 \\
-4.2674700 \\
-3.2488590 \\
-3.0850690 \\
-3.5139930 \\
-4.9376070 \\
-5.5206350 \\
-4.4812430 \\
-3.7104430 \\
-3.4479920 \\
-5.6194720 \\
-5.8686580 \\
-2.0528470 \\
-2.7883590 \\
-4.9103410 \\
-6.3988560 \\
-0.7815290 \\
0.4911390 \\
-1.9793840 \\
-1.7976570 \\
-0.4716140 \\
0.6891980 \\
-2.6374560 \\
-2.1267930 \\
-2.8957860 \\
-0.4570020 \\
-0.3575420 \\
1.6293240 \\
0.8117340 \\
-1.8136240 \\
3.1576050 \\
2.9337100 \\
4.5333110 \\
5.4880590 \\
4.8633060 \\
6.7707550 \\
5.2162440\end{array}$ & $\begin{array}{r}1.9258820 \\
0.3107760 \\
4.9352230 \\
1.7656170 \\
1.5657380 \\
1.1417530 \\
3.2682720 \\
3.8162140 \\
3.3932130 \\
3.5958980 \\
4.6604170 \\
-3.3211360 \\
-4.7023790 \\
-4.6862730 \\
-4.1101570 \\
-3.5048490 \\
-2.6970920 \\
-5.4233040 \\
-5.7011960 \\
-4.8859290 \\
-4.3201630 \\
-5.0503170 \\
-4.0798400 \\
-3.3312430 \\
-2.8928060 \\
-4.3836230 \\
-4.9399030 \\
-4.9636510 \\
-6.4256520 \\
-6.6111460 \\
-6.2493440 \\
-6.7394250 \\
-4.3622110 \\
-4.8442520 \\
-5.9631480 \\
-7.6414920 \\
-6.2114790 \\
-7.0491320 \\
-7.0597110 \\
2.8826930 \\
1.6603210 \\
3.3671660 \\
2.5074280 \\
4.6936470 \\
2.9759730 \\
1.4797470\end{array}$ & $\begin{array}{l}-2.8049610 \\
-2.3701650 \\
-1.4299920 \\
-2.5486430 \\
-3.6145720 \\
-1.9951330 \\
-2.2298940 \\
-3.1294940 \\
-1.4907540 \\
0.8153310 \\
1.0044730 \\
-0.6773040 \\
-1.2633280 \\
-2.7417960 \\
-2.9442700 \\
-1.6509350 \\
-0.9194780 \\
-0.7169190 \\
-3.1473110 \\
-3.3084540 \\
-1.0013000 \\
-1.1726640 \\
-3.2939010 \\
-3.7130590 \\
-1.8805010 \\
1.1143580 \\
1.3134870 \\
1.8449150 \\
2.2459090 \\
2.9780790 \\
2.0544580 \\
2.8755650 \\
2.7540530 \\
1.2678910 \\
3.8647770 \\
3.3322810 \\
2.6165450 \\
1.3098020 \\
1.3483980 \\
0.4265430 \\
0.0288490 \\
0.1392850 \\
-0.4097890 \\
0.4250950 \\
-0.6667800 \\
-0.6242410\end{array}$ \\
\hline
\end{tabular}

\begin{abstract}
6.1458060
4. 1101660

7.0994160

7. 5147850

6.4030390

8.1022610

2. 0472830

2. 3303340

0.2886810

0.2345550

0.8006530

0.2042630

$-1.1170120$

$-1.2105980$

$-0.1289520$

0.2613200

$-0.1400860$

0.5516470

0.1540860

$-0.2093880$

$-1.2281940$

1. 6384330

0.2138120

$-1.2170680$

1. 3462070

0.3036900
\end{abstract}

154

TS182 (S, S) G[WB97XD/6-31G(d)] = -3541.688225

$\begin{array}{lrrr}\text { P } & -0.4247300 & -0.2489460 & 1.0187540 \\ \mathrm{O} & 0.1882150 & -0.5996460 & 2.3416100 \\ \mathrm{O} & -0.7771160 & 1.1899830 & 0.7394540 \\ \mathrm{O} & -1.6926250 & -1.2395690 & 0.8122410 \\ \mathrm{O} & 0.5404610 & -0.6664520 & -0.2411550 \\ \mathrm{C} & -2.2323400 & -1.2738130 & -0.4644850 \\ \mathrm{C} & -1.5609030 & -2.0225610 & -1.4374200 \\ \mathrm{C} & -3.4035390 & -0.5670450 & -0.7225470 \\ \mathrm{C} & -3.8910460 & -0.5991450 & -2.0307420 \\ \mathrm{H} & -4.8044740 & -0.0550710 & -2.2598880 \\ \mathrm{C} & 0.7357740 & -2.0127770 & -0.4917710 \\ \mathrm{C} & 1.9820330 & -2.5697480 & -0.2201760 \\ \mathrm{C} & -0.3138540 & -2.7459030 & -1.0594970 \\ \mathrm{C} & 2.1477030 & -3.9329270 & -0.4630970 \\ \mathrm{H} & 3.1168070 & -4.3864580 & -0.2677780 \\ \mathrm{C} & -4.1204590 & 0.1968010 & 0.3355700 \\ \mathrm{C} & 3.1120730 & -1.7166770 & 0.2382470 \\ \mathrm{C} & -4.6872390 & -0.4607190 & 1.4346650\end{array}$

$\begin{array}{rr}5.1591590 & 0.1620050 \\ 5.3465790 & 0.8535000 \\ 4.3000210 & -0.3820820 \\ 2.3069990 & -1.0884460 \\ 6.1906520 & 0.3812430 \\ 4.6648550 & -0.5834920 \\ 1.2572290 & 0.3299770 \\ 3.6141270 & 0.9853660 \\ 3.6196750 & -0.9110480 \\ 3.5445190 & 0.5517700 \\ 2.7916950 & -1.4019460 \\ 2.5549830 & 0.9944500 \\ 3.3518940 & -0.7574820 \\ 2.3507980 & -0.8797060 \\ 6.1023640 & -0.7696240 \\ 6.0637070 & 0.7100260 \\ 4.7379720 & 1.3624370 \\ 4.9674940 & -1.5395320 \\ 7.0634660 & -1.2099180 \\ 6.8883140 & 1.2540630 \\ 4.6798460 & 1.4959250 \\ 5.1184760 & -1.5515530 \\ 4.9342600 & -2.5804950 \\ 6.0084810 & -0.8700580 \\ 6.1895370 & 0.8048930 \\ 4.6210360 & 2.3547920\end{array}$

0.1620050

$-0.3820820$

0.3812430

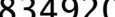

0.9853660

9110480

1.4019460

0.9944500

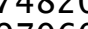

0.7696240

0.7100260

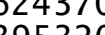

2099180

.2540630

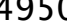

.3547920 


$\begin{array}{rrr}-4.6194250 & -1.9384490 & 1.7364070 \\ -5.9593650 & -0.6123560 & 3.5027950 \\ -5.4291020 & 0.2497820 & 2.3864990 \\ -3.9638780 & -2.1157580 & 3.1231060 \\ -3.9508380 & -3.1828840 & 3.3746600 \\ -2.9242290 & -1.7823460 & 3.0711350 \\ -4.7599690 & -1.3093890 & 4.1823640 \\ -5.1298160 & -1.9661680 & 4.9786600 \\ -4.1215470 & -0.5565000 & 4.6552970 \\ -6.5213720 & -0.0162880 & 4.2298580 \\ -4.0519560 & -2.4851300 & 0.9802380 \\ -6.8634810 & -1.7018570 & 2.8839830 \\ -7.2076410 & -2.3759220 & 3.6772350 \\ -7.7536810 & -1.2361380 & 2.4489240 \\ -6.0651200 & -2.4796680 & 1.8056710 \\ -6.0342380 & -3.5502990 & 2.0394270 \\ -6.5384920 & -2.3787840 & 0.8234430 \\ -4.2992900 & 1.5816270 & 0.2213380 \\ -3.7150490 & 2.4903830 & -0.8347500 \\ -5.2174950 & 3.7459980 & 0.8530710 \\ -5.0729800 & 2.2774430 & 1.1596630 \\ -4.8778450 & 3.1732790 & -1.5876430 \\ -4.4648520 & 3.8967930 & -2.3006410 \\ -5.4324020 & 2.4308530 & -2.1718400 \\ -5.8122690 & 3.8774240 & -0.5675030 \\ -5.9302030 & 4.9392160 & -0.8134220 \\ -6.8111660 & 3.4296210 & -0.5838060 \\ -5.8648560 & 4.2411070 & 1.5848940 \\ -3.0694230 & 1.9442370 & -1.5264070 \\ -3.8135780 & 4.3865380 & 0.8445720 \\ -3.9011500 & 5.4339260 & 0.5310140 \\ -3.4025760 & 4.3878060 & 1.8593720 \\ -2.8977170 & 3.5887670 & -0.1165990 \\ -2.4472010 & 4.2472290 & -0.8685180 \\ -2.0847800 & 3.1106970 & 0.4314600 \\ -5.6366440 & 1.6165400 & 2.2439520 \\ -6.2327790 & 2.1631660 & 2.9730560 \\ 4.7789610 & -0.0148160 & -0.2596740 \\ 5.3069980 & 0.8703460 & -1.3600010 \\ 3.3284330 & -0.6153060 & -2.1065210 \\ 3.6944450 & -0.8061910 & -0.6540280 \\ 5.7610810 & -0.0455980 & -2.5201200 \\ 6.1002260 & 0.5767550 & -3.3563850 \\ 6.6170430 & -0.6470680 & -2.1978300 \\ 4.5785200 & -0.9537820 & -2.9501870 \\ 4.3435320 & -0.8086670 & -4.0108470\end{array}$

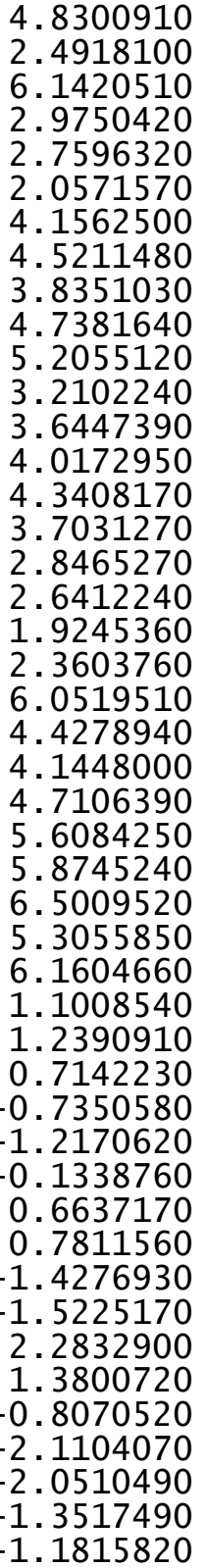
$-2.0108500$
$-1.2532000$
1.4863840
0.8667710
1. 0256700
1.0966900
1.7607660
2. 3796610
2.4519790
$-1.0384650$
$-1.3105850$
$-2.7933880$
$-1.8277820$
-1. 0731940
$-1.2616980$
$-0.0257120$
$-2.0087880$
$-2.7299620$
$-1.4522920$
$-3.3996920$
$-0.6685250$
$-3.6884510$
$-4.4106390$
$-4.2631000$
$-2.8011460$
$-3.0341020$
$-2.9783580$
$-0.1325600$
0.4714540
$-4.7133990$
$-6.1911390$
$-6.5242430$
$-6.0272750$
$-5.0657720$
$-4.1231080$
$-6.7564740$
$-7.6028030$
$-6.8745470$
$-5.6627370$
$-6.5036550$
$-6.0453470$
$-5.5233480$
$-4.5284310$
$-1.9951050$
$-2.6190720$
$-1.5740050$

$-2.8169680$

$-2.4014560$

$-1.0087000$

$-2.3369220$

$-3.3998120$

$-1.7913870$

$-1.8789980$

$-2.7066120$

$-1.0940620$

1. 9110580

3. 3168490

2. 6015930

1. 5259520

4. 2742330

5.3045550

4. 2212270

3.8802700

4.6801810

3. 7078470

2. 2794250

3. 5848280

2.9280130

3. 7020220

2. 0392280

3.4064430

4.4441040

2.7979560

1.0230150

1.3245080

$-0.9380880$

$-1.1959390$

$-2.6019290$

$-2.8164130$

$-1.7094710$

$-1.2459350$

$-0.4487520$

$-2.7778750$

$-2.8568790$

$-0.8384360$

$-1.0914860$

$-3.3286480$

$-3.7854230$

$-2.0341030$

$-2.7492820$

-3.9306590
-5.0488470 


$\begin{array}{lrrr}\mathrm{C} & -2.5030490 & -0.8429780 & -5.3967210 \\ \mathrm{C} & -3.6680290 & -1.2602290 & -4.4774380 \\ \mathrm{C} & -3.2224480 & -1.2807620 & -3.0390560 \\ \mathrm{H} & -1.9463330 & -3.4622900 & -4.3103830 \\ \mathrm{H} & -0.7678020 & -2.0648960 & -5.9358280 \\ \mathrm{H} & -2.7847090 & -1.0310740 & -6.4380400 \\ \mathrm{H} & -4.0136660 & -2.2620480 & -4.7687460 \\ \mathrm{H} & -0.3769140 & -3.0245440 & -3.6489090 \\ \mathrm{H} & -0.4336710 & -0.8465010 & -4.7156700 \\ \mathrm{H} & -2.3591850 & 0.2382320 & -5.3005440 \\ \mathrm{H} & -4.5183390 & -0.5838560 & -4.6112190 \\ \mathrm{C} & 1.1852970 & 3.4074170 & -0.8688130 \\ \mathrm{O} & 0.1778460 & 2.6201210 & -1.1060390 \\ \mathrm{C} & 1.6159920 & 4.2102060 & -2.0450350 \\ \mathrm{C} & 2.6618590 & 5.1238910 & -1.8995140 \\ \mathrm{C} & 1.0113990 & 4.0303290 & -3.2915660 \\ \mathrm{C} & 3.1001280 & 5.8567040 & -2.9952890 \\ \mathrm{H} & 3.1248670 & 5.2462450 & -0.9261050 \\ \mathrm{C} & 1.4556890 & 4.7618730 & -4.3866580 \\ \mathrm{H} & 0.2054560 & 3.3126620 & -3.3947020 \\ \mathrm{C} & 2.4978480 & 5.6749960 & -4.2391420 \\ \mathrm{H} & 3.9111940 & 6.5691870 & -2.8818000 \\ \mathrm{H} & 0.9891720 & 4.6194440 & -5.3563170 \\ \mathrm{H} & 2.8430940 & 6.2454540 & -5.0962680 \\ \mathrm{H} & -0.1316550 & 2.0431730 & -0.3163460 \\ \mathrm{O} & 1.7822360 & 3.5083270 & 0.2119050 \\ \mathrm{C} & 1.1283910 & 2.4633130 & 2.7495170 \\ \mathrm{C} & 2.1864370 & 2.1264700 & 1.7896160 \\ \mathrm{H} & 0.1169640 & 2.3227510 & 2.3732910 \\ \mathrm{H} & 2.0088920 & 1.3025690 & 1.1048210 \\ \mathrm{O} & 1.6193000 & 1.2898430 & 3.4320500 \\ \mathrm{H} & 1.0722600 & 0.5102910 & 3.0961730 \\ \mathrm{C} & 2.7706210 & 3.9586930 & 3.9639060 \\ \mathrm{C} & 3.6722820 & 3.9332990 & 2.7283500 \\ \mathrm{C} & 3.5842880 & 2.5895240 & 2.0034570 \\ \mathrm{C} & 1.3112730 & 3.7194000 & 3.5690210 \\ \mathrm{H} & 2.8584140 & 4.9222610 & 4.4755130 \\ \mathrm{H} & 4.7145370 & 4.1224520 & 3.0033830 \\ \mathrm{H} & 4.0772370 & 1.7936820 & 2.5753300 \\ \mathrm{H} & 0.9461400 & 4.5552660 & 2.9598470 \\ \mathrm{H} & 0.6658590 & 3.6504110 & 4.4504800 \\ \mathrm{H} & 3.0906370 & 3.1861510 & 4.6733050 \\ \mathrm{H} & 3.3679060 & 4.7271880 & 2.0362200 \\ \mathrm{H} & 4.0899720 & 2.6051930 & 1.0357470 \\ \mathrm{H} & & & \end{array}$

154

$\operatorname{TS} 183(\mathrm{~S}, \mathrm{~S}) \mathrm{G}[\mathrm{WB} 97 \mathrm{XD} / 6-31 \mathrm{G}(\mathrm{d})]=-3541.687714$

0.2779650
0.8195460
-0.7894160
-0.1455530
1.4340890
-0.2512520
0.9225950
-1.5248480
-1.6106180
-2.5944550
2.4715670
3.6801180
2.2494480
4.7161580
5.6778340
-2.7616700
3.8279550
-3.0932490
-2.2841390
-4.4933400
-4.2985620
-1.9760990
-1.4533160
-1.2971890
-3.2989650
-3.4117010
-3.3122210
-5.4388460
-1.3553170
-4.4622370
-4.5274540
-5.3409040
-3.1546940
-2.5844440
-3.3757260
-3.6454050
-3.4411600
-5.6821100
-4.8512740
-4.6121720
-4.5059510
-4.5607090
-5.9606150
-6.5525900
-6.5617960
-6.6215120
0.1739060

1.2564570

$-0.7486200$

0.9274280

$-0.8313850$

0.2039040

$-0.2175730$

$-0.0453080$

$-0.7283360$

$-0.9245710$

$-0.2507160$

$-0.0336330$

0.0861850

0.5292990

0.6978960

0.3259500

$-0.3256040$

1. 6586550

2. 8851280

3.4484630

1.9732080

3. 6488040

4. 5792110

3. 0548960

3. 9492430

5.0240130

3.4482850

3.6467390

2. 6300710

4.1658070

5. 2482660

3.8758430

3. 7944750

4.6950330

3. 2766130

$-0.6800100$

$-2.1720700$

$-1.5558390$

$-0.3551020$

$-2.7606850$

$-3.8511640$

$-2.3985810$

$-2.3599400$

$-3.2486500$

$-1.7526630$

$-1.2547590$ 


-2.4869600
-4.8554370
-5.4194590
-4.7188890
-3.4890640
-3.3327980
-2.6622460
-5.1882990
-6.1366080
3.7766660
3.5873440
3.3653270
3.6455440
4.6549520
4.5100540
5.6510450
4.5279900
4.3342180
5.4570390
3.2765330
3.6878910
2.0639730
1.8843430
1.2223980
2.1977760
2.0773400
1.4164240
4.3113960
4.7274680
4.3856220
4.1535830
3.6542950
3.9917220
2.7248290
3.4294110
3.6189200
2.3996190
4.2226800
4.8658480
5.8349200
6.0112190
6.5396140
6.0487350
6.3641740
6.8346150
4.1131310

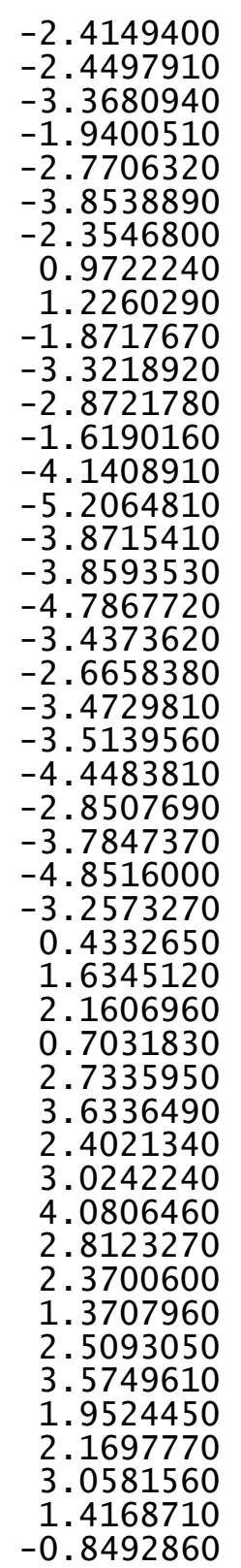

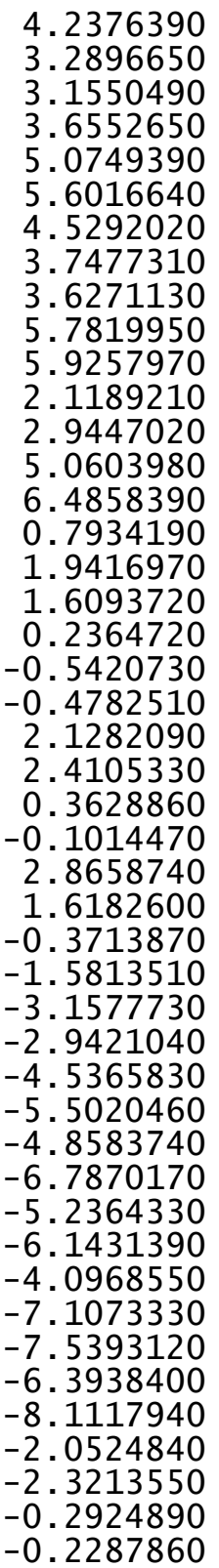

\author{
4.6310390 \\ $-3.3410250$ \\ $-4.7293800$ \\ $-4.7429790$ \\ $-4.1435040$ \\ $-3.5236950$ \\ $-2.7139780$ \\ $-5.4380570$ \\ $-5.7700570$ \\ $-4.9080470$ \\ $-4.3310140$ \\ $-5.0744790$ \\ $-4.1675520$ \\ $-3.3667800$ \\ $-2.9095310$ \\ $-4.4313970$ \\ $-5.1432750$ \\ $-5.3980980$ \\ $-6.0859790$ \\ $-6.2453190$ \\ $-4.9778120$ \\ $-6.1038230$ \\ $-5.9972600$ \\ $-7.0712740$ \\ $-7.0700610$ \\ $-4.5655460$ \\ $-4.4319810$ \\ $-5.4919650$ \\ $-6.5239550$ \\ 2.8658920 \\ 1.6477230 \\ 3.3561200 \\ 2. 5036040 \\ 4.6810820 \\ 2. 9780830 \\ 1. 4769830 \\ 5.1526190 \\ 5.3282020 \\ 4. 3007740 \\ 2. 3148480 \\ 6.1831370 \\ 4.6703290 \\ 1. 2393020 \\ 3. 5899000 \\ 3.6047310 \\ 3. 5195160
}

$-1.0534260$

0.7159720

1.2899070

2.7461150

1.5936320

0.9138460

0.6942420

3.1246020

3.2418940

0.9219390

1.2504690

3. 3486400

3.6744320

1.7925140

$-0.9643310$

$-1.6364430$

$-3.1189110$

$-3.3236840$

$-2.0036040$

$-1.1936560$

$-1.1356360$

$-3.5639880$

$-3.7845860$

$-1.4261940$

$-1.5610400$

$-3.6343460$

$-4.0140250$

$-2.2055210$

$-0.4266620$

$-0.0122850$

$-0.1648370$

0.3768700

$-0.4663830$

0.6106860

0.6040830

$-0.2262400$

$-0.8887580$

0.3106510

1.0266950

$-0.4573360$

0.4946290

$-0.9815780$

0.9303350
-0.5315620 


$\begin{array}{lrrr}\mathrm{H} & -0.8097910 & 2.7813450 & 1.4234380 \\ \mathrm{H} & -0.1975490 & 2.5267770 & -0.9668720 \\ \mathrm{O} & 1.1135640 & 3.3326220 & 0.7882880 \\ \mathrm{H} & 1.2038220 & 2.3324380 & 0.9203740 \\ \mathrm{C} & 0.1332100 & 6.0851020 & 0.7755720 \\ \mathrm{C} & -0.2475070 & 6.0379410 & -0.7062540 \\ \mathrm{C} & 0.1535290 & 4.7067070 & -1.3477190 \\ \mathrm{C} & -0.5561910 & 4.9573340 & 1.5480820 \\ \mathrm{H} & -0.1497770 & 7.0499030 & 1.2077450 \\ \mathrm{H} & 0.2292140 & 6.8574770 & -1.2527410 \\ \mathrm{H} & 1.2420680 & 4.6439240 & -1.4761160 \\ \mathrm{H} & -1.6425860 & 5.1115280 & 1.5510540 \\ \mathrm{H} & -0.2260630 & 4.9300420 & 2.5916750 \\ \mathrm{H} & 1.2203350 & 5.9885460 & 0.8837780 \\ \mathrm{H} & -1.3313690 & 6.1666250 & -0.8087920 \\ \mathrm{H} & -0.2863090 & 4.5849360 & -2.3412520 \\ 154 & & & \end{array}$

$\operatorname{TS} 184(\mathrm{~S}, \mathrm{~S}) \quad \mathrm{G}[\mathrm{WB} 97 \mathrm{XD} / 6-31 \mathrm{G}(\mathrm{d})]=-3541.689942$

$\begin{array}{lrrr}\text { P } & 0.2687360 & -0.0503720 & 0.2187890 \\ \mathrm{O} & 0.7811050 & 0.8730380 & 1.2879290 \\ \mathrm{O} & -0.8177730 & 0.4344750 & -0.7099190 \\ \mathrm{O} & -0.1083490 & -1.4296440 & 0.9908590 \\ \mathrm{O} & 1.4445720 & -0.5625510 & -0.7803060 \\ \mathrm{C} & -0.1700800 & -2.6066190 & 0.2678530 \\ \mathrm{C} & 1.0297250 & -3.2094990 & -0.1448030 \\ \mathrm{C} & -1.4240820 & -3.1437200 & -0.0095260 \\ \mathrm{C} & -1.4614810 & -4.3477060 & -0.7165340 \\ \mathrm{H} & -2.4287940 & -4.7925980 & -0.9382700 \\ \mathrm{C} & 2.5025410 & -1.2178280 & -0.1795590 \\ \mathrm{C} & 3.6841180 & -0.5127930 & 0.0441860 \\ \mathrm{C} & 2.3301930 & -2.5536840 & 0.1834690 \\ \mathrm{C} & 4.7222260 & -1.2153000 & 0.6466130 \\ \mathrm{H} & 5.6664550 & -0.7028700 & 0.8196910 \\ \mathrm{C} & -2.6899560 & -2.4397120 & 0.3399390 \\ \mathrm{C} & 3.7907190 & 0.9372840 & -0.2788870 \\ \mathrm{C} & -3.0561280 & -2.1744190 & 1.6653800 \\ \mathrm{C} & -2.2569460 & -2.4848070 & 2.9086380 \\ \mathrm{C} & -4.5181950 & -1.3434490 & 3.4242910 \\ \mathrm{C} & -4.2896160 & -1.5770250 & 1.9545800 \\ \mathrm{C} & -2.0094660 & -1.1601420 & 3.6656540 \\ \mathrm{H} & -1.4945920 & -1.3842290 & 4.6072610 \\ \mathrm{H} & -1.3421650 & -0.5253370 & 3.0777360 \\ \mathrm{C} & -3.3636670 & -0.4535530 & 3.9361910 \\ \mathrm{H} & -3.5007680 & -0.2620940 & 5.0070040 \\ \mathrm{H} & -3.4038100 & 0.5139480 & 3.4247400 \\ \mathrm{H} & -5.4847070 & -0.8592830 & 3.6020920 \\ & & & \end{array}$

-1.3062560
-4.4499900
-4.5398020
-5.3014670
-3.1101980
-2.5466930
-3.2884590
-3.5679750
-3.3302030
-5.6264200
-4.8036500
-4.4550650
-4.3268500
-4.3684470
-5.8364130
-6.4205880
-6.4209980
-6.5900380
-2.3528180
-4.8226310
-5.3772200
-4.7342190
-3.4256190
-3.2490030
-2.6299900
-5.1745060
-6.1447340
3.6952430
3.5059700
3.3601720
3.6035710
4.6030130
4.4577890
5.5847540
4.5240730
4.3581300
5.4613680
3.3041150
3.5737520
2.0495030
1.8991980
1.2078190
2.1355360
2.0140180
1.3336900
4.2005970
2.6740620

4.1539440

5.2336940

3.8541080

3.8113340

4.7236800

3. 2987010

$-0.6840320$

$-2.1720580$

$-1.5999010$

$-0.3835850$

$-2.7818630$

$-3.8700810$

$-2.4170450$

$-2.4078520$

$-3.3081360$

$-1.8114660$

$-1.3169560$

$-2.3964290$

$-2.4783920$

$-3.4068260$

$-1.9654390$

$-2.7740310$

$-3.8544110$

$-2.3464650$

0.9364720

1. 1710070

$-1.8670250$

$-3.3258730$

$-2.8229350$

$-1.5848590$

$-4.1209530$

$-5.1919110$

$-3.8546990$

$-3.8046870$

$-4.7206400$

$-3.3646940$

$-2.5953300$

$-3.4990690$

$-3.4887100$

$-4.4144430$

$-2.8308510$

$-3.7872650$

$-4.8586300$

$-3.2739360$

0.4324470 


\begin{tabular}{|c|c|c|}
\hline $\begin{array}{l}4.5857790 \\
4.3079900 \\
4.0801490 \\
3.5089000 \\
3.8254130 \\
.5697020 \\
3.3218230 \\
.5082820 \\
.3020130 \\
4.1709110 \\
4.6981220 \\
5.7427540 \\
5.9138000 \\
6.4695480 \\
5.9189700 \\
6.2145930 \\
6.7057620 \\
3.9979480 \\
4.0937170 \\
3.3671650 \\
3.1551930 \\
4.1414930 \\
5.5654470 \\
5.7343740 \\
4.5780670 \\
4.0231220 \\
3.2705970 \\
2.1231320 \\
5.7569730 \\
6.3018570 \\
6.6692970 \\
5.8289770 \\
3.9222970 \\
0.9474690 \\
2.1175740 \\
1.7862370 \\
0.4541830 \\
0.3192760 \\
0.3037350 \\
2.3253740 \\
2.6154760 \\
0.6365700 \\
0.1518660 \\
5.72595960 \\
5.1818670\end{array}$ & $\begin{array}{r}4.0799350 \\
1.5796750 \\
1.8675100 \\
3.9280890 \\
4.4850040 \\
4.3659810 \\
2.4248520 \\
2.2291630 \\
2.0951110 \\
0.5223210 \\
5.1310100 \\
2.0330890 \\
1.8771230 \\
1.4118090 \\
3.5291350 \\
4.1184010 \\
3.6512910 \\
3.6924270 \\
4.7532990 \\
-3.2069890 \\
-4.5984270 \\
-4.9439360 \\
-4.6575820 \\
-3.1599070 \\
-2.5311890 \\
-5.9970550 \\
-5.3441180 \\
-4.6917990 \\
-5.2132210 \\
-4.9982350 \\
-2.9625980 \\
-2.6492660 \\
-4.3478600 \\
-4.3706290 \\
-5.0094500 \\
-5.1823160 \\
-5.9377660 \\
-6.1833390 \\
-4.9494800 \\
-5.9974950 \\
-5.7012550 \\
-6.9000930 \\
-7.0152120 \\
-4.4120880 \\
-4.1848920 \\
-5.3565990\end{array}$ & $\begin{array}{r}1.6174430 \\
2.1988270 \\
0.7320670 \\
2.7122590 \\
3.6024470 \\
2.3639130 \\
3.0349680 \\
4.0975240 \\
2.8221120 \\
2.4319470 \\
1.3299200 \\
2.5498870 \\
3.6213400 \\
2.0153020 \\
2.1747400 \\
3.0507080 \\
1.4231560 \\
-0.8623990 \\
-1.0897470 \\
0.8793380 \\
1.4449000 \\
2.5592210 \\
2.0906030 \\
1.8442210 \\
1.0880520 \\
2.8360390 \\
0.6491120 \\
1.7995020 \\
1.1626050 \\
2.8262370 \\
1.3062070 \\
2.8128520 \\
3.4551260 \\
-0.9255120 \\
-1.6319490 \\
-3.1261240 \\
-3.3602970 \\
-2.0488240 \\
-1.1864440 \\
-1.1977420 \\
-3.6181660 \\
-3.8500570 \\
-1.5070440 \\
-1.5190370 \\
-3.5746220 \\
-4.0359130\end{array}$ \\
\hline
\end{tabular}

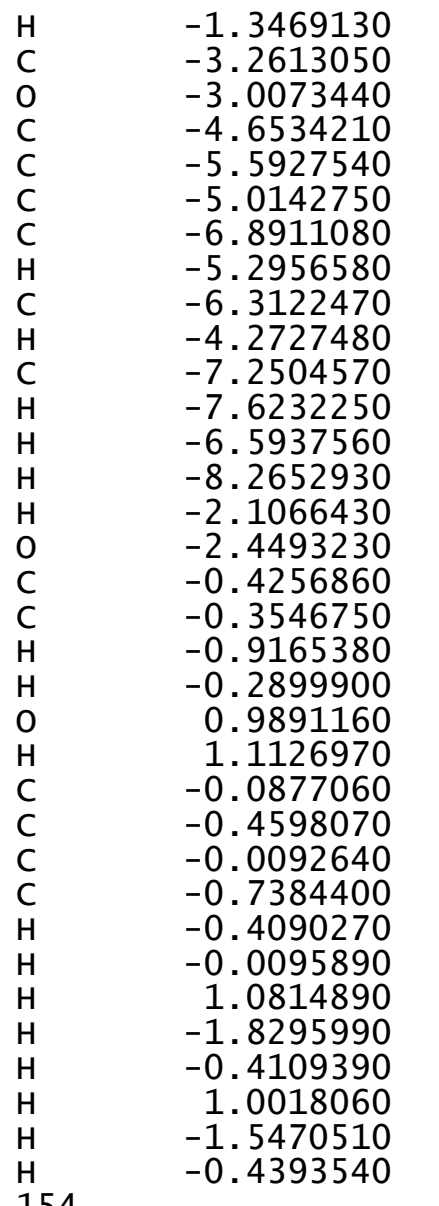
$-6.4924720$
2.7457000
1.5443910
3. 1992140
2. 3254910
4. 5102540
2. 7642430
1. 3103180
4.9463230
5.1745610
4. 0731590
2. 0842910
5. 9660380
4.4148750
1. 1578540
3. 4815810
3. 5996380
3.4900210
2. 7682810
2. 4911120
3. 3724360
2. 3786460
6.0909310
6.0023940
4. 6742680
4.9532940
7.0523070
6.8277230
4.6470320
5.0690600
4.9574990
6. 0380940
6.0908840
4. 5195860

$-2.2651490$

$-0.4315930$

0.0100700

$-0.1746030$

0.3787500

$-0.4925950$

0.6082360

0.6180190

$-0.2562410$

$-0.9238120$

0.2922560

1.0330860

$-0.4996090$

0.4730740

$-0.2694380$

$-1.0066790$

0.9058290

$-0.5540440$

1. 4123760

$-0.9711150$

0.7723930

0.9246670

0.7062970

$-0.7757620$

$-1.3903750$

1. 4977520

1. 1190550

$-1.3357660$

$-1.5125050$

1. 4951300

2.5425230

0.8204920

$-2.3836100$

$\operatorname{TS} 185(\mathrm{~S}, \mathrm{~S}) \quad \mathrm{G}[\mathrm{WB} 97 \mathrm{XD} / 6-31 \mathrm{G}(\mathrm{d})]=-3541.689040$

$\begin{array}{lrrr}\text { TS185(S, S) } & \text { G }[W B 97 X D / 6-31 G(d)]=-3541.689040 \\ \text { P } & -0.3764510 & -0.1061480 & 0.9639880 \\ O & 0.2529720 & -0.4257770 & 2.2871190 \\ O & -0.6504930 & 1.3348910 & 0.6164000 \\ O & -1.7092260 & -1.0263950 & 0.8599730 \\ O & 0.5210050 & -0.6547620 & -0.2958420 \\ \mathrm{C} & -2.3033630 & -1.1216220 & -0.3874460 \\ \mathrm{C} & -1.7052900 & -1.9457040 & -1.3402890 \\ \mathrm{C} & -3.4690130 & -0.4016560 & -0.6386630 \\ \mathrm{C} & -4.0210070 & -0.5284340 & -1.9096890 \\ \mathrm{H} & -4.9422950 & 0.0076930 & -2.1280420\end{array}$




0.6222740
1.8297640
-0.4906800
1.8891370
2.8274870
-4.1032540
3.0215390
-4.6397680
-4.6269780
-5.7965980
-5.2928950
-3.9032750
-3.9369770
-2.8509210
-4.5923600
-4.9434050
-3.8899530
-6.2849790
-4.1330080
-6.7863390
-7.1140980
-7.6779170
-6.0912740
-6.1004730
-6.6145990
-4.2225780
-3.6535320
-5.0047110
-4.9117920
-4.8210950
-4.4126190
-5.4370170
-5.6689100
-5.7550250
-6.6852710
-5.5887960
-3.0717550
-3.5762680
-3.6355500
-3.1126940
-2.7472730
-2.2989880
-1.9353330
-5.4445180
-5.9742810
4.7970540

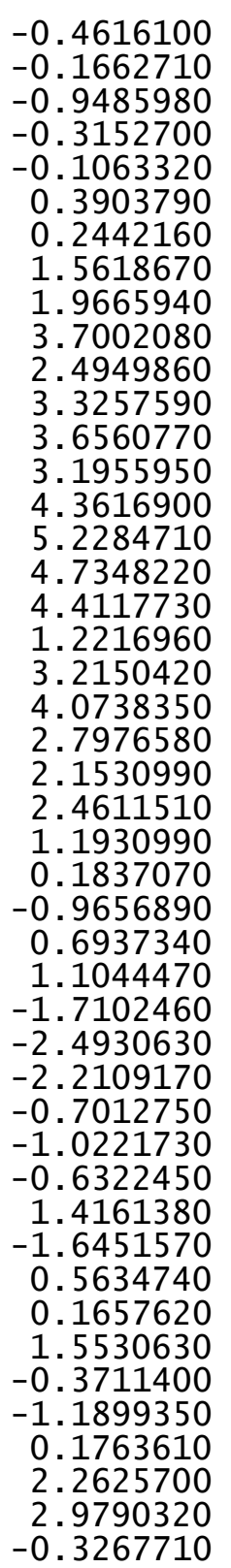

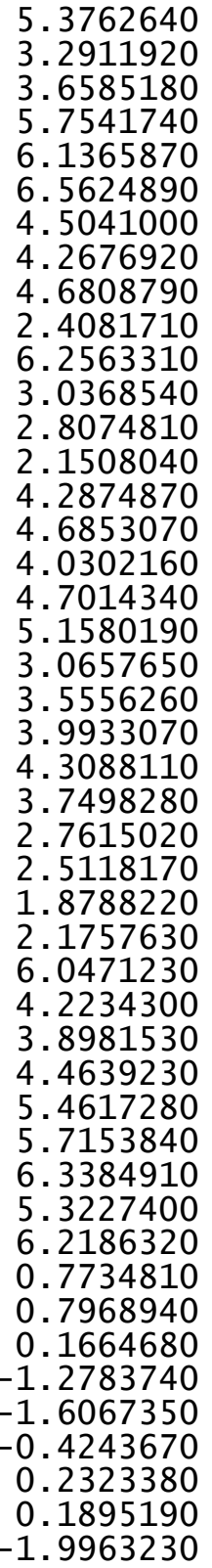

0.5061230
-0.8621570
-1.0282430
-0.4815120
0.0827010
-1.1355320
-1.3111670
-1.1747610
-2.3801310
-1.4488020
1.0731330
0.6337330
0.7764560
0.9422940
1.4565510
2.0096450
2.2014650
-1.2293560
-1.4736790
-2.8485700
-1.9587150
-1.1177220
-1.2843180
-0.0547650
-1.9890650
-2.6602590
-1.3792140
-3.4098860
-0.8792630
-3.8084580
-4.4807550
-4.4343450
-2.9826170
-3.1901990
-3.2435680
-0.3992710
0.1566090
-4.7732870
-6.2728510
-6.6615460
-6.1329480
-5.0015920
-4.1172480
-6.7433610
-7.7487050
-6.9444040

$-1.4629470$ $-2.1399060$ $-0.6849410$ $-2.5905580$ $-3.4490250$ $-2.2482720$ $-2.9861900$ $-4.0477220$ $-2.8299160$ $-2.4047660$ $-1.1399000$ $-2.4116070$ $-3.4737360$ $-1.8522810$ $-2.0071100$ $-2.8654460$ $-1.2488380$

1.8827600

3. 2975400

2. 6499150

1.5333110

4.2470700

5.2836140

4.1505210

3.8951790

4.7253430

3.6991440

2. 3566390

3.5357160

3.0077790

3.8098060

2. 1414190

3.4486280

4.4947500

2.8470830

0.9574680

1.2311840

$-0.7006660$

$-0.8513930$

$-2.1978330$

$-2.3330500$

$-1.3325180$

$-1.0149240$

$-0.0332670$

$-2.3260850$

$-2.1738400$ 


\begin{tabular}{|c|c|c|}
\hline $\begin{array}{l}-1.9211700 \\
1.8228370 \\
0.7912470 \\
-1.4420040 \\
-2.4624170 \\
-2.2356920 \\
-3.4202680 \\
-1.4801370 \\
-2.3578340 \\
-3.1569360 \\
-4.0937030 \\
-1.7310080 \\
-0.6796530 \\
-0.9706890 \\
-2.4605670 \\
-3.7353840 \\
-4.5445720 \\
-4.9265940 \\
-3.0489490 \\
1.4726860 \\
0.3935580 \\
1.9603310 \\
3.0869930 \\
1.3315720 \\
3.5828940 \\
3.5668730 \\
1.8329540 \\
0.4613800 \\
2.9570920 \\
4.4588640 \\
1.3481140 \\
3.3484720 \\
0.0534040 \\
2.0926390 \\
1.3673430 \\
2.4015410 \\
0.3486610 \\
2.1710990 \\
1.7803930 \\
1.1890820 \\
3.1026520 \\
4.0031980 \\
3.8274580 \\
1.6313190 \\
3.2522770 \\
5.0550130\end{array}$ & $\begin{array}{l}-5.4600890 \\
-6.6494270 \\
-6.2368720 \\
-5.7773370 \\
-4.4197510 \\
-1.9826970 \\
-1.2841700 \\
-2.6732590 \\
-3.0154450 \\
-1.7887620 \\
-1.3516950 \\
-3.3833690 \\
-1.9919500 \\
-3.5671170 \\
-0.9748460 \\
-1.9966110 \\
-0.3800370 \\
-2.0666330 \\
-3.8272770 \\
3.3638820 \\
2.6666230 \\
4.0876810 \\
4.9054600 \\
3.9224080 \\
5.5559950 \\
5.0168340 \\
4.5717820 \\
3.2801430 \\
5.3876780 \\
6.1914980 \\
4.4394420 \\
5.8918390 \\
2.1302050 \\
3.4419330 \\
2.5652820 \\
2.1102460 \\
2.4700870 \\
1.2642920 \\
1.4013850 \\
0.6425360 \\
4.0141620 \\
3.8613220 \\
2.4884860 \\
3.8501800 \\
4.9963300 \\
3.9969160\end{array}$ & $\begin{array}{l}-0.3846250 \\
-0.7828490 \\
-2.9920520 \\
-3.3561870 \\
-1.6824790 \\
-2.6437100 \\
-2.9163180 \\
-3.7642660 \\
-4.9654190 \\
-5.3989030 \\
-4.2744100 \\
-5.7850550 \\
-4.0887080 \\
-3.4025360 \\
-5.6410780 \\
-6.3056470 \\
-4.5061710 \\
-4.2108640 \\
-4.6987080 \\
-1.0585780 \\
-1.2590120 \\
-2.2631540 \\
-2.1547530 \\
-3.4995490 \\
-3.2772810 \\
-1.1882260 \\
-4.6215150 \\
-3.5737950 \\
-4.5114270 \\
-3.1927590 \\
-5.5835700 \\
-5.3899820 \\
-0.4545820 \\
0.0109470 \\
2.5821100 \\
1.6455150 \\
2.2097970 \\
1.0043700 \\
3.3288550 \\
3.0212930 \\
3.7208790 \\
2.4932620 \\
1.8429770 \\
3.3319950 \\
4.1802240 \\
2.7633670\end{array}$ \\
\hline
\end{tabular}

$\begin{array}{ll}\mathrm{H} & 4.2625550 \\ \mathrm{H} & 1.3233990 \\ \mathrm{H} & 0.9812400 \\ \mathrm{H} & 3.3695770 \\ \mathrm{H} & 3.7528030 \\ \mathrm{H} & 4.3365030\end{array}$

154

TS186(S, S) G[WB97XD/6-31G(d)] = -3541.691317

\begin{tabular}{|c|c|c|c|}
\hline & ,S) $\mathrm{G}[$ & & \\
\hline $\mathrm{P}$ & -0.3857770 & -0.1458470 & 0.9887610 \\
\hline 0 & 0.2414430 & -0.4855490 & 2.3079300 \\
\hline 0 & -0.6741280 & 1.2991450 & 0.670306 \\
\hline 0 & -1.7085590 & -1.0770350 & 0.853161 \\
\hline 0 & 0.5256600 & -0.6552560 & -0.2780500 \\
\hline C & -2.2815480 & -1.1410690 & -0.4071620 \\
\hline C & -1.6699810 & -1.9467290 & -1.365547 \\
\hline C & -3.4348110 & -0.4038870 & -0.667706 \\
\hline C & -3.9450750 & -0.4783580 & -1.959444 \\
\hline $\mathrm{H}$ & -4.8518320 & 0.0776060 & -2.189392 \\
\hline C & 0.6470260 & -2.0198360 & -0.4736670 \\
\hline C & 1.8642020 & -2.6343170 & -0.193707 \\
\hline C & -0.4560880 & -2.7203920 & -0.9756640 \\
\hline $\mathrm{C}$ & 1.9431250 & -4.0157670 & -0.3685280 \\
\hline $\mathrm{H}$ & 2.8889020 & -4.5149860 & -0.170039 \\
\hline C & -4.1026740 & 0.4235850 & 0.373288 \\
\hline $\mathrm{C}$ & 3.0455460 & -1.8325950 & 0.225968 \\
\hline C & -4.6695150 & -0.1730270 & 1.506245 \\
\hline C & -4.6487960 & -1.6406010 & 1.858862 \\
\hline C & -5.9001450 & -0.2042570 & 3.603713 \\
\hline C & -5.3643770 & 0.5979910 & 2.445913 \\
\hline C & -3.9693830 & -1.7938510 & 3.23685 \\
\hline $\mathrm{H}$ & -3.9926710 & -2.8509260 & 3.527262 \\
\hline $\mathrm{H}$ & -2.9188990 & -1.5043400 & 3.150293 \\
\hline C & -4.7102800 & -0.9191040 & 4.281992 \\
\hline $\mathrm{H}$ & -5.0838110 & -1.5306970 & 5.111809 \\
\hline $\mathrm{H}$ & -4.0337430 & -0.1725390 & 4.7 \\
\hline $\mathrm{H}$ & -6.4238760 & 0.4367980 & 4.32 \\
\hline $\mathrm{H}$ & -4.1184350 & -2.2337600 & 1.1 \\
\hline C & -6.8562030 & -1.2815220 & 3.0 \\
\hline $\mathrm{H}$ & -7.2043550 & -1.9148340 & 3.8 \\
\hline $\mathrm{H}$ & -7.73 & -0.8000980 & \\
\hline C & 3140 & 1243920 & 06 \\
\hline $\mathrm{H}$ & -6.1158430 & -3.1872 & \\
\hline $\mathrm{H}$ & -6.60 & -2 & \\
\hline C & -4 & 1.8 & \\
\hline C & -3.6 & 2.6 & -0 \\
\hline C & -5.0461520 & 4.0293540 & \\
\hline
\end{tabular}

2.4630030

2.6752270

4.2125300

4.4732740

1.7574000

0.8795880

$\begin{array}{lll}4.2625550 & 1.6950440 & 2.4630030 \\ 1.3233990 & 4.6728990 & 2.6752270 \\ 0.9812400 & 3.8727120 & 4.2125300 \\ 3.3695770 & 3.2624760 & 4.4732740 \\ 3.7528030 & 4.6343150 & 1.7574000 \\ 4.3365030 & 2.4154160 & 0.8795880\end{array}$

.233760

4.0293540
0.7879990

(n)

$-3.7642660$

$-5.7850550$

0887080

$-6.3056470$

$-4.5061710$

(2)

(1)

$-1.2590120$

(

$-3.2772810$

15150

4.5114270

(

0109470

.5821100

6455150

0043700

288550

(

4932620

9950

2.7633670 


$\begin{array}{rrr}-4.9560910 & 2.5671800 & 1.1421260 \\ -4.7835110 & 3.3600350 & -1.6374800 \\ -4.3573440 & 4.0403930 & -2.3843890 \\ -5.3804360 & 2.6210020 & -2.1828600 \\ -5.6648540 & 4.1385270 & -0.6242200 \\ -5.7422280 & 5.1954880 & -0.9046730 \\ -6.6822600 & 3.7344470 & -0.6048290 \\ -5.6562920 & 4.5762800 & 1.5148510 \\ -3.0262830 & 2.0609080 & -1.5714610 \\ -3.6172270 & 4.6097010 & 0.7286590 \\ -3.6689620 & 5.6478960 & 0.3785450 \\ -3.1849010 & 4.6305740 & 1.7342950 \\ -2.7547490 & 3.7418010 & -0.2213210 \\ -2.2919180 & 4.3546380 & -1.0036800 \\ -1.9518170 & 3.2468360 & 0.3270550 \\ -5.5224660 & 1.9662170 & 2.2594620 \\ -6.0822440 & 2.5601010 & 2.9803770 \\ 4.7963480 & -0.2346140 & -0.3271900 \\ 5.3587250 & 0.5954510 & -1.4528430 \\ 3.2948550 & -0.7969500 & -2.1447350 \\ 3.6674960 & -0.9765390 & -0.6927650 \\ 5.7498720 & -0.3691810 & -2.5958830 \\ 6.1175660 & 0.2142270 & -3.4479490 \\ 6.5719380 & -1.0125330 & -2.2663870 \\ 4.5136730 & -1.2165070 & -2.9976160 \\ 4.2731490 & -1.0740580 & -4.0574110 \\ 4.7088610 & -2.2837430 & -2.8516620 \\ 2.4211080 & -1.3946720 & -2.4157880 \\ 6.2300830 & 1.1724210 & -1.1238790 \\ 3.0163880 & 0.6976590 & -2.3981630 \\ 2.7871930 & 0.8492270 & -3.4591050 \\ 2.1239250 & 0.9849180 & -1.8376270 \\ 4.2527210 & 1.5354820 & -1.9808170 \\ 4.6400430 & 2.1093670 & -2.8302850 \\ 3.9825920 & 2.2640880 & -1.2110330 \\ 4.7229370 & -1.2028910 & 1.8677000 \\ 5.1888570 & -1.4644030 & 3.2763420 \\ 3.1094820 & -2.8533970 & 2.6165420 \\ 3.5848790 & -1.9411410 & 1.5117490 \\ 4.0246190 & -1.1374270 & 4.2367840 \\ 4.3470630 & -1.3169800 & 5.2690310 \\ 3.7683340 & -0.0759610 & 4.1587720 \\ 2.8012230 & -2.0171600 & 3.8765340 \\ 2.5634380 & -2.7045880 & 4.6968510 \\ 1.9100690 & -1.4151670 & 3.6950420 \\ 2.2242910 & -3.4197330 & 2.3183130\end{array}$

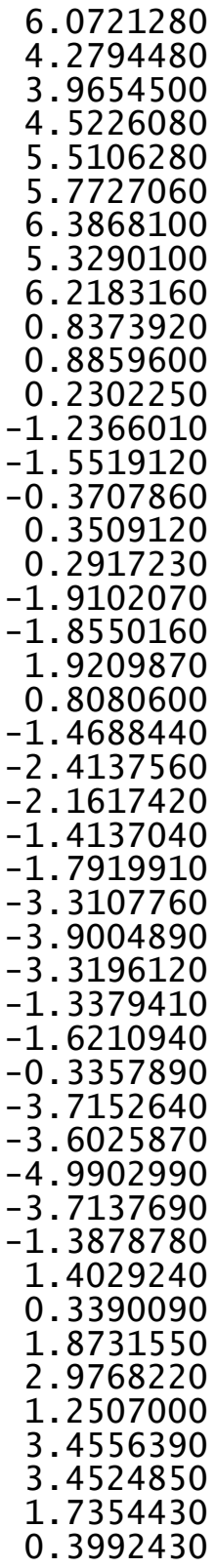
$-0.8636180$
$-3.8054210$
$-4.4927050$
$-4.4158650$
$-2.9720670$
$-3.1929060$
$-3.2134300$
$-0.3499230$
0.2138650
$-4.7597750$
$-6.2572970$
$-6.6425520$
$-6.1724740$
$-5.0103510$
$-4.1161930$
$-6.7472220$
$-7.7244830$
$-7.0027540$
$-5.4447510$
$-6.6126680$
$-6.1721880$
$-5.8760580$
$-4.4368890$
$-1.9386160$
$-2.6708750$
$-2.1929080$
$-2.1623910$
$-1.1307040$
$-1.2044290$
$-2.8540680$
$-3.7457750$
$-2.5640850$
$-3.1582590$
$-1.9246500$
$-1.2374210$
$-0.1243190$
$-1.1864090$
3.3785790
2. 6602960
4.1259740
4.9732960
3.9546190
5.6477550
5. 0891300
4.6275210
3. 2885530

3.5201830
2.9549450
3.7486790
2.0783670
3.4017580
4.4430810
2.7913290
0.9531030
1.2320890
-0.7627520
-0.9279980
-2.2613890
-2.3330620
-1.3618130
-1.0670830
-0.1012010
-2.4181020
-2.0955800
-0.3989380
-0.8878230
-3.0655230
-3.3614150
-1.7104680
-2.6852570
-3.7836150
-5.1846050
-5.3324290
-4.3731350
-2.9736820
-5.9306440
-3.7181110
-3.6201000
-5.1061460
-6.3610600
-4.3173070
-4.7735110
-5.3574140
-0.9921460
-1.1996780
-2.1892980
-2.0717830
-3.4280880
-3.1876800
-1.1036830
-4.5434720
-3.5093590 


$\begin{array}{llr}2.8361370 & 5.4735430 & -4.4241790 \\ 4.3128190 & 6.3074490 & -3.0960520 \\ 1.2556730 & 4.4904820 & -5.5074150 \\ 3.2141520 & 5.9966470 & -5.2974970 \\ 0.0087390 & 2.1115950 & -0.3990810 \\ 2.0216470 & 3.4582710 & 0.0779840 \\ 1.3288060 & 2.5219650 & 2.6408640 \\ 2.3617960 & 2.1006640 & 1.6871590 \\ 0.3088150 & 2.4212850 & 2.2740800 \\ 2.1378450 & 1.2656660 & 1.0296970 \\ 1.7630750 & 1.3474340 & 3.3587150 \\ 1.1771880 & 0.5875210 & 3.0438220 \\ 3.0539050 & 3.9706420 & 3.7963680 \\ 3.9451100 & 3.8572330 & 2.5578720 \\ 3.7836060 & 2.4954180 & 1.8812820 \\ 1.5816640 & 3.7934660 & 3.4169120 \\ 3.1933970 & 4.9450850 & 4.2749430 \\ 4.9973290 & 4.0029480 & 2.8210380 \\ 4.2353750 & 1.6958370 & 2.4811620 \\ 1.2559050 & 4.6261540 & 2.7816110 \\ 0.9394450 & 3.7873290 & 4.3034950 \\ 3.3387520 & 3.2073360 & 4.5302750 \\ 3.6763100 & 4.6411220 & 1.8402510 \\ 4.2859550 & 2.4496930 & 0.9127890\end{array}$

154

4.2859550

2. 4496930

-5.0505930
-3.8102280
-3.9094830
-2.7485670
-4.3682540
-4.7146050
-3.5913220
-5.9430220
-4.1899010
-6.6426210
-6.9687340
-7.5158710
-6.0768700
-6.1519850
-6.6444660
-4.0332290
-3.4741140
-4.5666550
-4.5909770
-4.6237000
-4.2172600
-5.3474910
-5.3053710
-5.2859880
-6.3564210
-5.0423070
-3.0039990
-3.0964570
-3.0684540
-2.5691700
-2.4299770
-2.0017140
-1.6208520
-5.0994850
-5.5275570
4.7722590
5.4469370
3.2424400
3.5688390
5.7375620
6.1787750
6.4773490
4.4167450
4.2103150
4.4847710
2.3081970

1.0497830

$-1.4748320$

$-2.5314830$

$-1.2836040$

$-0.5596940$

$-1.1522690$

0.1168740

0.9392830

$-1.8479540$

$-0.7051840$

$-1.3249320$

$-0.1382600$

$-1.5842270$

$-2.6485340$

$-1.4326420$

2. 2134260

3. 0317850

4.4834070

3.0090710

3. 8604900

4. 5092640

3.1927950

4.7023870

5.7693940

4.4182460

5.0637780

2. 4025250

4.9156180

5.9691370

4.8419090

4.0082260

4.6055680

3. 4238230

2. 4311970

3.0528330

$-3.5004210$

$-0.5645150$

$-0.8528520$

$-0.0287100$

$-0.7109060$

$-1.9567670$

$-4.1295790$

$-0.0241850$

$-2.0944970$

0.4413570

0.5987770

$-1.9905970$

$-2.7322310$

$-0.7347800$

$-0.7978510$

$-0.5548650$

1. 5414720

$-1.3056130$

$-2.5585050$

$-0.7973620$

2. 4316800

$-4.7030410$

0.8185240

$-0.6152390$

0.4030350

2.8427970

$-2.0846560$

$-0.0671200$

0.2441500

1. 5749050

$-4.5171200$

-1.2277540
0.2709300

1.9026190
3.7788150

$-0.6584380$

0.1844640

$-0.8918720$

$-1.2296950$

$-0.7244510$

$-0.1205510$

$-1.4817280$

$-1.3889770$

$-1.1450640$

$-2.4794280$

$-1.3564000$

3.2107280

3.4859070

3.0365840

4.3317590

5.1868890

4.7018280

4.5513480

1.0993640

3.2949400

4.1386230

2.9562310

2.1496270

2. 4014240

1.2255370

0.2786330

$-0.8612840$

0.9766090

1.2877690

$-1.4746310$

$-2.2594350$

$-1.9545260$

$-0.3630570$

$-0.6144620$

$-0.2480840$

1.7745870

$-1.6202740$

0.7900880

0.4863240

1.7467800

$-0.2747780$

$-1.0882890$

0.1656090

2.4440190

3.2289600

$-0.4772800$

$-1.5292880$

$-2.3419770$

0.9072590

$-2.7452550$

$-3.5466960$

$-2.4673820$

$-3.2163330$

$-4.2647640$

$-3.1467020$

$-2.6655200$ 


\begin{tabular}{lrrr} 
H & 6.3751280 & 0.6249580 & -1.1487370 \\
$\mathrm{C}$ & 3.1511610 & 0.6408240 & -2.4820980 \\
$\mathrm{H}$ & 2.9685410 & 0.8949140 & -3.5324170 \\
$\mathrm{H}$ & 2.2860460 & 0.9945360 & -1.9168550 \\
$\mathrm{C}$ & 4.4681280 & 1.2888820 & -1.9820580 \\
$\mathrm{H}$ & 4.9349820 & 1.8863590 & -2.7733680 \\
$\mathrm{H}$ & 4.2707930 & -1.9719750 & -1.1509840 \\
$\mathrm{C}$ & 4.5594650 & -1.7862440 & 1.6290680 \\
$\mathrm{C}$ & 4.9834650 & -2.2221310 & 3.0071880 \\
$\mathrm{C}$ & 2.7699620 & -3.3094780 & 2.2277040 \\
$\mathrm{C}$ & 3.3500730 & -2.3579020 & 1.2085070 \\
$\mathrm{C}$ & 3.8556920 & -1.8586560 & 3.9962690 \\
$\mathrm{H}$ & 4.1504900 & -2.1609380 & 5.0080450 \\
$\mathrm{H}$ & 3.7136580 & -0.7732520 & 4.0127430 \\
$\mathrm{C}$ & 2.5483170 & -2.5683360 & 3.5634490 \\
$\mathrm{H}$ & 2.2380110 & -3.3015480 & 4.3170740 \\
$\mathrm{H}$ & 1.7272480 & -1.8602350 & 3.4461920 \\
$\mathrm{H}$ & 1.8309100 & -3.7467770 & 1.8821910 \\
$\mathrm{H}$ & 5.9249390 & -1.7447970 & 3.3005150 \\
$\mathrm{C}$ & 3.8288510 & -4.4098980 & 2.4691490 \\
$\mathrm{H}$ & 3.4375020 & -5.1340680 & 3.1928640 \\
$\mathrm{H}$ & 4.0128890 & -4.9536170 & 1.5361490 \\
$\mathrm{C}$ & 5.1384770 & -3.7611430 & 2.9932650 \\
$\mathrm{H}$ & 5.3634440 & -4.1050470 & 4.0095590 \\
$\mathrm{H}$ & 5.9897490 & -4.0394700 & 2.3636000 \\
$\mathrm{C}$ & 5.2717800 & -0.9378070 & 0.7904030 \\
$\mathrm{H}$ & 6.2174130 & -0.5069540 & 1.1167040 \\
$\mathrm{C}$ & -0.7713110 & -3.9477870 & -1.4876010 \\
$\mathrm{C}$ & -2.0129810 & -4.7212980 & -1.8557800 \\
$\mathrm{C}$ & -2.2146280 & -5.8664830 & -0.8476240 \\
$\mathrm{C}$ & -0.9741590 & -6.7861400 & -0.7366900 \\
$\mathrm{C}$ & 0.2619110 & -6.2024600 & -1.4527570 \\
$\mathrm{C}$ & 0.3744960 & -4.7165380 & -1.2360820 \\
$\mathrm{H}$ & -1.9095100 & -5.1422750 & -2.8662580 \\
$\mathrm{H}$ & -3.0996090 & -6.4466090 & -1.1291990 \\
$\mathrm{H}$ & -1.1883750 & -7.7733210 & -1.1595350 \\
$\mathrm{H}$ & 0.1698980 & -6.3983670 & -2.5303170 \\
$\mathrm{H}$ & -2.8931580 & -4.0736710 & -1.8706730 \\
$\mathrm{H}$ & -2.4301110 & -5.4158400 & 0.1269540 \\
$\mathrm{H}$ & -0.7309000 & -6.9438100 & 0.3189430 \\
$\mathrm{H}$ & 1.1713770 & -6.7132280 & -1.1207030 \\
$\mathrm{C}$ & -3.6428970 & -0.7751520 & -3.0183050 \\
$\mathrm{C}$ & -4.2908830 & -0.7727120 & -4.3786010 \\
$\mathrm{C}$ & -4.4891350 & -2.2175000 & -4.8632630 \\
$\mathrm{C}$ & -3.1694150 & -3.0252040 & -4.8448870 \\
$\mathrm{C}$ & -2.0332680 & -2.3067320 & -4.0858530 \\
& & & \\
\hline
\end{tabular}
$-2.5229470$
$-3.6556020$
$-4.9263080$
$-2.8269050$
$-1.6053540$
$-5.2495210$
$-5.2215140$
$-3.3469170$
$-1.2220170$
1.8742710
0.7345440
2.4399520
3.6707180
1.7780090
4.2393990
4.1724000
2. 3502040
0.8250160
3. 5794110
5.1972880
1. 8386440
4.0249870
0.3378640
2. 4919770
1.6400620
2.6483570
0.6251830
2. 3570110
1. 9284880
1.2852570
3.4754190
4.3847710
4.0981070
2. 0036240
3.7028340
5.4384010
4.4504650
1.7850890
1. 3402200
3.6579950
4.2229580
4.6142940

H

TS188(S, S) G[WB97XD/6-31G(d)] $=-3541.688559$

$\begin{array}{llll} & \\ \mathrm{P} & -0.2551580 & 0.0188940 & -0.2159100 \\ \mathrm{O} & -0.6719310 & 0.9808430 & -1.2927580\end{array}$

$\begin{array}{lr}-1.6018700 & -2.8443450 \\ -0.2310940 & -5.0943550 \\ -2.2171290 & -5.8670660 \\ -3.2216720 & -5.8663760 \\ -1.5406280 & -4.7479070 \\ -0.2446830 & -4.3464940 \\ -2.6933440 & -4.2016360 \\ -4.0019520 & -4.3839810 \\ -3.0032460 & -3.8666370 \\ 3.3803610 & -0.8240620 \\ 2.8216350 & -1.1071100 \\ 4.2078280 & -1.9228630 \\ 4.8380090 & -1.7269600 \\ 4.3291390 & -3.1469100 \\ 5.5848800 & -2.7503720 \\ 4.7275660 & -0.7715550 \\ 5.0777750 & -4.1690720 \\ 3.8327040 & -3.2904790 \\ 5.7038700 & -3.9722210 \\ 6.0722430 & -2.5984040 \\ 5.1711270 & -5.1218000 \\ 6.2849630 & -4.7741290 \\ 2.2247800 & -0.3753400 \\ 3.2577270 & 0.2423060 \\ 2.2058680 & 2.6975520 \\ 1.7499950 & 1.7336960 \\ 2.2426490 & 2.3046580 \\ 0.9912670 & 1.0135320 \\ 0.9449290 & 3.3357490 \\ 0.2761480 & 2.9366130 \\ 3.3753100 & 3.9914660 \\ 3.2574190 & 2.7664510 \\ 1.9770140 & 1.9810560 \\ 3.3845930 & 3.5703570 \\ 4.2932760 & 4.5426180 \\ 3.2667800 & 3.0625380 \\ 1.0893550 & 2.5209190 \\ 4.2915400 & 2.9935310 \\ 3.3830480 & 4.4412020 \\ 2.5363240 & 4.6736400 \\ 4.1184480 & 2.1074940 \\ 1.9544240 & 1.0186400\end{array}$




$\begin{array}{rrr}0.8424270 & 0.4199460 & 0.7392700 \\ 0.0409290 & -1.3799840 & -0.9879690 \\ -1.4853790 & -0.4108060 & 0.7555840 \\ 0.0294400 & -2.5621620 & -0.2721890 \\ -1.1971380 & -3.0913800 & 0.1439070 \\ 1.2467180 & -3.1884150 & -0.0113540 \\ 1.1946840 & -4.4072290 & 0.6589940 \\ 2.1288220 & -4.9315250 & 0.8497680 \\ -2.5668450 & -1.0128220 & 0.1408170 \\ -3.7043670 & -0.2471040 & -0.1074280 \\ -2.4540470 & -2.3630350 & -0.2050440 \\ -4.7802080 & -0.8939330 & -0.7167110 \\ -5.6961920 & -0.3357840 & -0.8972200 \\ 2.5557940 & -2.5666770 & -0.3559510 \\ -3.7299660 & 1.2077090 & 0.2130470 \\ 2.9308150 & -2.3025140 & -1.6792490 \\ 2.1145120 & -2.5574800 & -2.9240040 \\ 4.4264720 & -1.5201390 & -3.4320270 \\ 4.1928640 & -1.7660510 & -1.9651280 \\ 1.9284060 & -1.2140540 & -3.6645460 \\ 1.4038240 & -1.4027360 & -4.6085810 \\ 1.2912830 & -0.5566940 & -3.0681020 \\ 3.3142800 & -0.5690170 & -3.9276310 \\ 3.4586470 & -0.3688970 & -4.9959100 \\ 3.4014380 & 0.3883150 & -3.4030150 \\ 5.4141430 & -1.0793160 & -3.6065300 \\ 1.1430590 & -2.9991010 & -2.6928270 \\ 4.2919570 & -2.8637980 & -4.1801420 \\ 4.3844560 & -2.6856790 & -5.2580150 \\ 5.1141510 & -3.5272580 & -3.8928670 \\ 2.9218400 & -3.5051620 & -3.8395850 \\ 2.3456170 & -3.6972200 & -4.7520880 \\ 3.0579830 & -4.4683430 & -3.3369520 \\ 3.4610560 & -2.2531140 & 0.6688280 \\ 3.2284540 & -2.3842500 & 2.1558750 \\ 5.5766320 & -1.4706690 & 1.5871940 \\ 4.7269240 & -1.7334060 & 0.3708080 \\ 4.3051490 & -3.3291320 & 2.7335070 \\ 4.1818390 & -3.3875910 & 3.8212240 \\ 4.1574650 & -4.3407750 & 2.3400860 \\ 5.7164930 & -2.7982080 & 2.3654530 \\ 6.3154540 & -2.6265240 & 3.2673780 \\ 6.2585790 & -3.5249620 & 1.7517370 \\ 6.5618450 & -1.0828430 & 1.3054160 \\ 2.2292670 & -2.7630080 & 2.3790350 \\ 4.8376460 & -0.4611730 & \\ -1.4924210\end{array}$

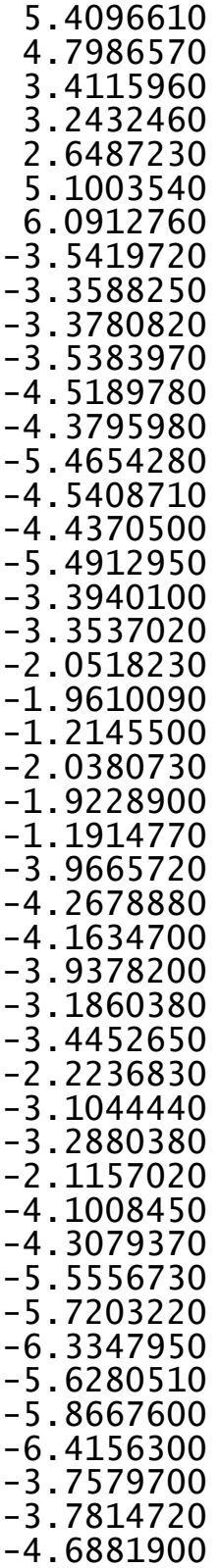

-0.3289300
0.5126480
-0.9883590
-1.0655950
-0.3168660
-1.5023210
-1.1132370
3.0430600
3.3621670
0.8276220
1.6702710
2.6979060
2.8717150
3.1696500
1.1795110
0.5903330
0.8922810
-0.2407340
4.4444890
1.2073620
0.6332970
0.9196440
2.7290400
2.9219150
3.2099390
3.5230880
4.3940200
1.8781440
2.1534840
4.1606080
4.7368290
4.5301740
2.6466440
2.4631390
2.2420580
0.8128330
5.4514040
2.4341680
2.2877110
1.8693770
3.9401040
4.5464460
4.1215130
3.9718040
5.0374290
-2.2166860
3. 4184870

1.9977120

2.7906960

3.8712940

2. 3883500

$-0.9469740$

$-1.1796820$

1.8026800

3.2647130

2.7663370

1. 5227360

4.0399420

5.1133570

3.7571420

3.7232550

4.6417540

3. 2620630

2. 5415720

3.4354780

3. 4586240

4. 3880390

2.8185270

3.7528320

4.8258770

3. 2535790

$-0.5070210$

$-1.6999080$

$-2.2724400$

$-0.8030300$

$-2.7754690$

$-3.6719710$

$-2.4112570$

$-3.0936140$

$-4.1588500$

$-2.8640030$

$-2.5010440$

$-1.4161700$

$-2.6473380$

$-3.7211350$

$-2.1237230$

$-2.2780560$

$-3.1597250$

$-1.5393420$

0.7912360

1.0156150
-1.1355410 


-5.8255720
-6.0211010
-4.7389110
-3.4668880
-3.5171890
-5.6073030
-6.8593330
-4.8268860
-3.3676480
-6.7453210
-6.2972520
-4.6213860
-2.5758530
-1.2018740
0.0037440
-2.4654640
-2.4486360
-1.1750860
0.0542910
-3.3383890
-2.5528580
-3.3556860
-1.1365540
-1.1741620
0.9660200
0.1471670
-2.4929280
3.4307510
3.1010280
4.8560150
5.7270750
5.3186620
7.0581800
5.3504590
6.6505550
4.6289940
7.5199940
7.7365030
7.0137840
8.5612150
2.1738740
2.6601330
0.7146720
0.5838250
1.1638490
0.4368540

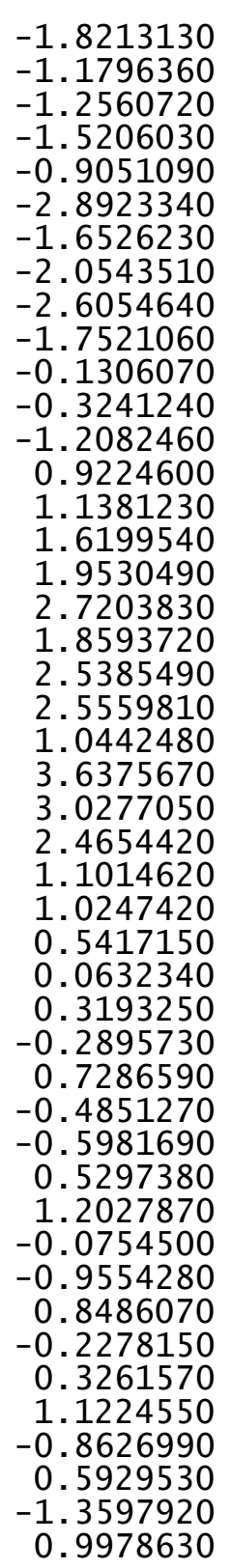

$\begin{array}{lr}\mathrm{O} & -0.7164090 \\ \mathrm{H} & -0.9036470 \\ \mathrm{C} & 0.5512110 \\ \mathrm{C} & 0.8607570 \\ \mathrm{C} & 0.2938900 \\ \mathrm{C} & 1.1476720 \\ \mathrm{H} & 0.9565400 \\ \mathrm{H} & 0.4510180 \\ \mathrm{H} & -0.7995890 \\ \mathrm{H} & 2.2434330 \\ \mathrm{H} & 0.8642410 \\ \mathrm{H} & -0.5342540 \\ \mathrm{H} & 1.9468740 \\ \mathrm{H} & 0.6757130 \\ \mathrm{H} & \end{array}$
3.4917430
2. 5091660
6.1271010
5.9996840
4.7023530
4.9518670
7.0658880
6.8506050
4.7534530
4.9866610
4.9898990
6.1547300
6.0076780
4.5088890
$-0.7825590$ $-0.9427210$
$-0.6474960$
0.8460020
1.4293310
$-1.4266230$
$-1.0378470$
1. 3985770
1.5132990
$-1.3789870$
$-2.4834420$
$-0.8021560$
0.9946510
2.4353520

$\begin{array}{lrrr}\text { TS189(S, S) G[WB97XD/6-31G(d)] }=-3541.689439 \\ \text { P } & 0.2527970 & 0.0040150 & 0.1724290 \\ \text { O } & 0.7180190 & 0.9109810 & 1.2769350 \\ \text { O } & -0.8543870 & 0.4695600 & -0.7417160 \\ \text { O } & -0.0642410 & -1.4177810 & 0.8917790 \\ \text { O } & 1.4539250 & -0.4141410 & -0.8404080 \\ \text { C } & -0.0675800 & -2.5698930 & 0.1270110 \\ \text { C } & 1.1605070 & -3.0996000 & -0.2947530 \\ \text { C } & -1.2924810 & -3.1602510 & -0.1746610 \\ \text { C } & -1.2637580 & -4.3473500 & -0.9090740 \\ \text { H } & -2.2047010 & -4.8447950 & -1.1331400 \\ \text { C } & 2.5416280 & -1.0412530 & -0.2635130 \\ \text { C } & 3.6949690 & -0.2949650 & -0.0275280 \\ \text { C } & 2.4260400 & -2.3921920 & 0.0650810 \\ \text { C } & 4.7714390 & -0.9744870 & 0.5338980 \\ \text { H } & 5.6986530 & -0.4312150 & 0.7041360 \\ \text { C } & -2.5926440 & -2.5373510 & 0.2016460 \\ \text { C } & 3.7355960 & 1.1682490 & -0.3037200 \\ \text { C } & -2.9670070 & -2.3439550 & 1.5375100 \\ \text { C } & -2.1515460 & -2.6680110 & 2.7665760 \\ \text { C } & -4.4543630 & -1.6384250 & 3.3298330 \\ \text { C } & -4.2248650 & -1.8143120 & 1.8523250 \\ \text { C } & -1.9542780 & -1.3644240 & 3.5736530 \\ \text { H } & -1.4358920 & -1.6056410 & 4.5091270 \\ \text { H } & -1.3075550 & -0.6851260 & 3.0134040 \\ \text { C } & -3.3340240 & -0.7174100 & 3.8628750 \\ \text { H } & -3.4760250 & -0.5566120 & 4.9380550 \\ \text { H } & -3.4130360 & 0.2595980 & 3.3743860 \\ \text { H } & -5.4385130 & -1.1994790 & 3.5272330 \\ \text { H } & -1.1837040 & -3.1047350 & 2.5120740 \\ \text { C } & -4.3260880 & -3.0150870 & 4.0161850\end{array}$




$\begin{array}{lrrr}\mathrm{H} & -4.4077160 & -2.8828800 & 5.1015400 \\ \mathrm{H} & -5.1569520 & -3.6581130 & 3.7080340 \\ \mathrm{C} & -2.9648350 & -3.6530600 & 3.6358900 \\ \mathrm{H} & -2.3865150 & -3.8982540 & 4.5343410 \\ \mathrm{H} & -3.1138920 & -4.5877690 & 3.0854240 \\ \mathrm{C} & -3.4918170 & -2.1541550 & -0.8045920 \\ \mathrm{C} & -3.2525440 & -2.1892120 & -2.2959080 \\ \mathrm{C} & -5.5979560 & -1.2993170 & -1.6784480 \\ \mathrm{C} & -4.7545380 & -1.6437390 & -0.4784390 \\ \mathrm{C} & -4.3325020 & -3.0827350 & -2.9445730 \\ \mathrm{H} & -4.2068360 & -3.0567290 & -4.0333420 \\ \mathrm{H} & -4.1906940 & -4.1227930 & -2.6317210 \\ \mathrm{C} & -5.7418630 & -2.5737670 & -2.5402780 \\ \mathrm{H} & -6.3427880 & -2.3442810 & -3.4280230 \\ \mathrm{H} & -6.2834660 & -3.3375940 & -1.9727410 \\ \mathrm{H} & -6.5822630 & -0.9249190 & -1.3759160 \\ \mathrm{H} & -2.2543560 & -2.5583360 & -2.5389320 \\ \mathrm{C} & -4.8490920 & -0.2380440 & -2.5137150 \\ \mathrm{H} & -5.4147060 & -0.0455530 & -3.4332530 \\ \mathrm{H} & -4.8098350 & 0.7034030 & -1.9593560 \\ \mathrm{C} & -3.4232040 & -0.7517260 & -2.8362050 \\ \mathrm{H} & -3.2470800 & -0.7560560 & -3.9183590 \\ \mathrm{H} & -2.6593650 & -0.1141900 & -2.3836380 \\ \mathrm{C} & -5.1294010 & -1.4881030 & 0.8498680 \\ \mathrm{H} & -6.1181080 & -1.1062640 & 1.1033190 \\ \mathrm{C} & 3.5524250 & 3.0549700 & -1.8325740 \\ \mathrm{C} & 3.3452160 & 3.4213970 & -3.2802510 \\ \mathrm{C} & 3.3111570 & 0.8735210 & -2.8561560 \\ \mathrm{C} & 3.5224090 & 1.6748580 & -1.5933990 \\ \mathrm{C} & 4.4719280 & 2.7516180 & -4.0993250 \\ \mathrm{H} & 4.3168060 & 2.9627660 & -5.1638570 \\ \mathrm{H} & 5.4358440 & 3.1893840 & -3.8209040 \\ \mathrm{C} & 4.4590540 & 1.2236490 & -3.8313540 \\ \mathrm{H} & 4.3150140 & 0.6678030 & -4.7650310 \\ \mathrm{H} & 5.4121640 & 0.8950270 & -3.4045650 \\ \mathrm{H} & 3.3034230 & -0.2009270 & -2.6621420 \\ \mathrm{H} & 3.3648030 & 4.5079800 & -3.4200820 \\ \mathrm{C} & 1.9784280 & 1.3072150 & -3.5029540 \\ \mathrm{H} & 1.8468880 & 0.7605560 & -4.4439120 \\ \mathrm{H} & 1.1507510 & 1.0251540 & -2.8480320 \\ \mathrm{C} & 1.9990980 & 2.8358460 & -3.7579870 \\ \mathrm{H} & 1.8698150 & 3.0586900 & -4.8236130 \\ \mathrm{H} & 1.1745300 & 3.3258230 & -3.2313580 \\ \mathrm{C} & 4.0441940 & 3.4543980 & 0.4791490 \\ \mathrm{C} & 4.3957220 & 4.2813630 & 1.6894900 \\ \mathrm{C} & 4.2365360 & 1.7533540 & 2.1910480 \\ & & & \end{array}$

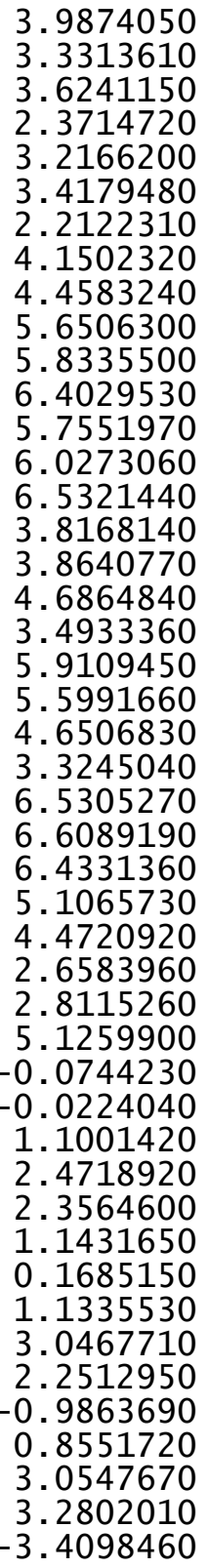

2.0776720

4.0449000

4. 5881260

4. 4486420

2.5250630

2. 3057340

2. 1541120

0.6838030

5. 3452420

2. 2614750

2.0796440

1.6919030

3. 7752110

4. 3489120

3. 9582980

3. 9471450

5. 0179110

$-2.3089270$

$-3.0191760$

$-2.9573550$

$-4.2196930$

$-5.1210250$

$-4.4025670$

$-4.7431290$

$-3.2163900$

$-2.2260770$

$-5.3812040$

$-6.0608080$

$-5.0172850$

$-4.2800800$

$-3.9480580$

$-4.8751790$

$-6.1441430$

$-7.0444100$

$-6.3387400$

$-4.8000320$

$-4.2382710$

$-5.9125360$

$-7.9859100$

$-6.6914190$

$-4.5324590$

$-6.6618610$

$-7.2993300$

$-6.6088360$

$-4.3260270$

2. 6414020
0.7354670

2.7815110

3. 6881240

2. 4488890

3. 0576160

4. 1128500

2.8391930

2. 3900090

1.4358240

2. 5515150

3. 6169430

1.9949930

2. 2246630

3. 1184080

1.4752110

$-0.7998370$

$-0.9936840$

0.9332390

0.7359880

1.5530280

2. 3556380

1. 5689810

1. 3358830

2. 5975750

0.7439480

2. 1805630

0.6031910

2. 1028200

0.7297900

2. 3009960

3. 3084620

$-1.3929020$

$-2.2052740$

$-1.6683130$

$-1.7198560$

$-1.8194930$

$-1.1142860$

$-3.2633890$

$-2.2263180$

$-2.5827910$

$-2.8801890$

$-2.1647780$

$-0.6307260$

$-0.8323720$

$-1.4817730$

$-0.3657690$ 


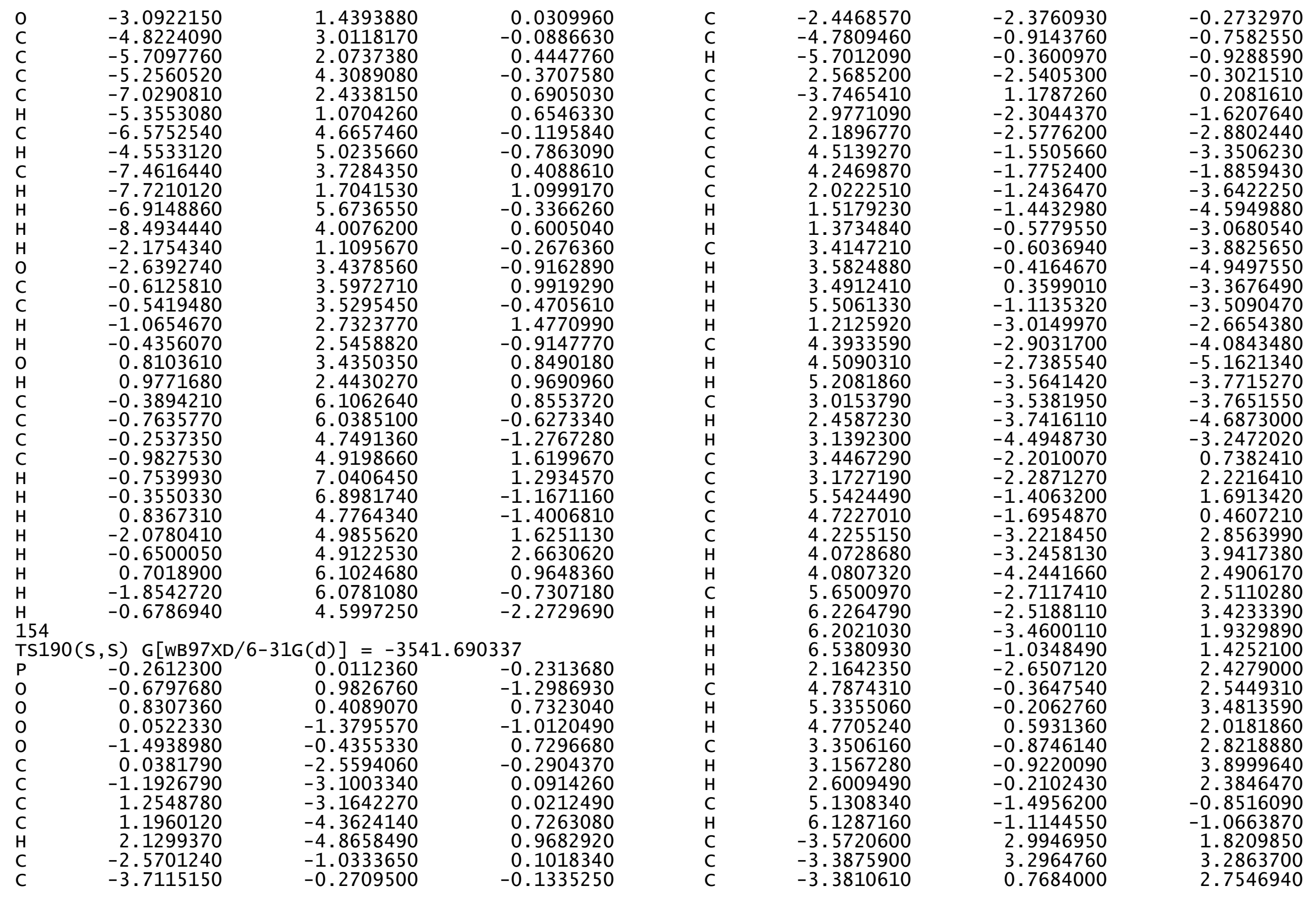




$\begin{array}{lr}-3.5564180 & 1.6257620 \\ -4.5366120 & 2.6084040 \\ -4.3947260 & 2.7701800 \\ -5.4895690 & 3.0729210 \\ -4.5429390 & 1.0938350 \\ -4.4276760 & 0.4941430 \\ -5.4924370 & 0.8017400 \\ -3.3831820 & -0.2968880 \\ -3.3947140 & 4.3764190 \\ -2.0560370 & 1.1545510 \\ -1.9554820 & 0.5698670 \\ -1.2182650 & 0.8835380 \\ -2.0571990 & 2.6724840 \\ -1.9381390 & 2.8533340 \\ -1.2188450 & 3.1690580 \\ -3.9989920 & 3.5013790 \\ -4.3025200 & 4.3854600 \\ -4.1793270 & 1.8778620 \\ -3.9600460 & 2.1359320 \\ -3.2145970 & 4.1728700 \\ -3.4731270 & 4.7595220 \\ -2.2561400 & 4.5434080 \\ -3.1225250 & 2.6637940 \\ -3.3015800 & 2.4930800 \\ -2.1320140 & 2.2622750 \\ -4.1085530 & 0.8161230 \\ -4.3508020 & 5.4387870 \\ -5.5739540 & 2.4292310 \\ -5.7344420 & 2.2948170 \\ -6.3507970 & 1.8528240 \\ -5.6574340 & 3.9298980 \\ -5.8968670 & 4.5457440 \\ -6.4488850 & 4.0968920 \\ -3.7956050 & 3.9346190 \\ -3.8275580 & 4.9970040 \\ -4.6756950 & -2.2242060 \\ -5.8064010 & -2.9224520 \\ -5.9574030 & -4.3415020 \\ -4.6753970 & -5.1686280 \\ -3.4024390 & -4.2949260 \\ -3.4986910 & -2.9564490 \\ -5.6016260 & -2.9779030 \\ -6.8147660 & -4.8474570 \\ -4.7624050 & -5.7656860 \\ -3.2432380 & -4.0717730 \\ -6.7369440 & -2.3571200\end{array}$

1.5233680
4.0575910
5.1325550
3.7848740
3.7216590
4.6319210
3.2615900
2.5144310
3.4714020
3.4464140
4.3681640
2.7997700
3.7597920
4.8345060
3.2616370
-0.4827280
-1.6653340
-2.2695960
-0.7960600
-2.7391560
-3.6290900
-2.3655330
-3.0770370
-4.1451660
-2.8495410
-2.5118030
-1.3681980
-2.6421510
-3.7181530
-2.1279080
-2.2544110
-3.1292640
-1.5164270
0.8216280
1.0597420
-1.2114860
-1.9244210
-1.3609360
-1.5709000
-1.6901390
-0.9956620
-3.0037860
-1.8169340
-2.4850740
-2.7542640
-1.8078800

$-6.1702880$

$-4.5686700$

$-2.5173600$

$-1.2111680$

$-2.5160920$

$-2.3115600$

$-1.3070190$

0.0589190

$-0.0028390$

$-3.2738280$

$-3.0904960$

$-3.1329520$

$-1.6553360$

$-1.2266410$

0.7383370

0.5136850

$-1.9380430$

3.4238140

3.0763400

4.8400120

5.6704890

5.3343690

6.9925030

5.2702290

6.6582170

4.6770840

7.4864810

7.6381060

7.0471080

8.5211390

2.1585130

2.6739040

0.6985190

0.5884260

1. 1375920

0.4427760

$-0.7321160$

$-0.9223740$

0.5576420

0.8904320

0.3185420

1. 1294380

0.9654890

0.4992440

-0.7735120
2.2261550
-4.2545230
-5.8839940
-4.8502060
-4.2586630
-4.7599550
-5.7034400
-6.7899240
-6.1566690
-4.9067380
-6.1397310
-5.2955920
-3.9034140
-7.3062780
-7.5448690
-6.8808550
-5.8914620
-5.1434070
2.5380800
1.3805140
2.9017360
2.0317350
4.1213120
2.3836320
1.0865460
4.4676020
4.7818740
3.5998780
1.7091500
5.4124110
3.8715310
1.0442310
3.2988560
3.6155470
3.4812520
2.7605300
2.4812200
3.4904550
2.5114960
6.1222580
5.9701750
4.6704890
4.9529390
7.0626500
6.8172030
4.7304840
4.9792670

$-0.2889570$

$-0.7484840$

$-1.3723810$

0.8944190

1.4858500

2.6697990

2.2964450

2.0425220

1.1841570

2.9585610

0.7212060

1.7782810

1. 3916650

3.0858570

1.5773030

3.0072460

3.5374550

0.4851730

$-0.0068350$

0.2185700

$-0.4926470$

0.6858670

$-0.7349640$

$-0.8425210$

0.4437510

1.2414960

$-0.2666110$

$-1.2890190$

0.8104710

$-0.4536500$

0.2848500

1.1100010

$-0.8444750$

0.6116190

$-1.3586560$

1.0054370

$-0.7474050$

$-0.9205870$

$-0.5919510$

0.8941550

1.4672050

$-1.3980000$

$-0.9757160$

1.4658120

1.5632110

$-1.3716430$ 


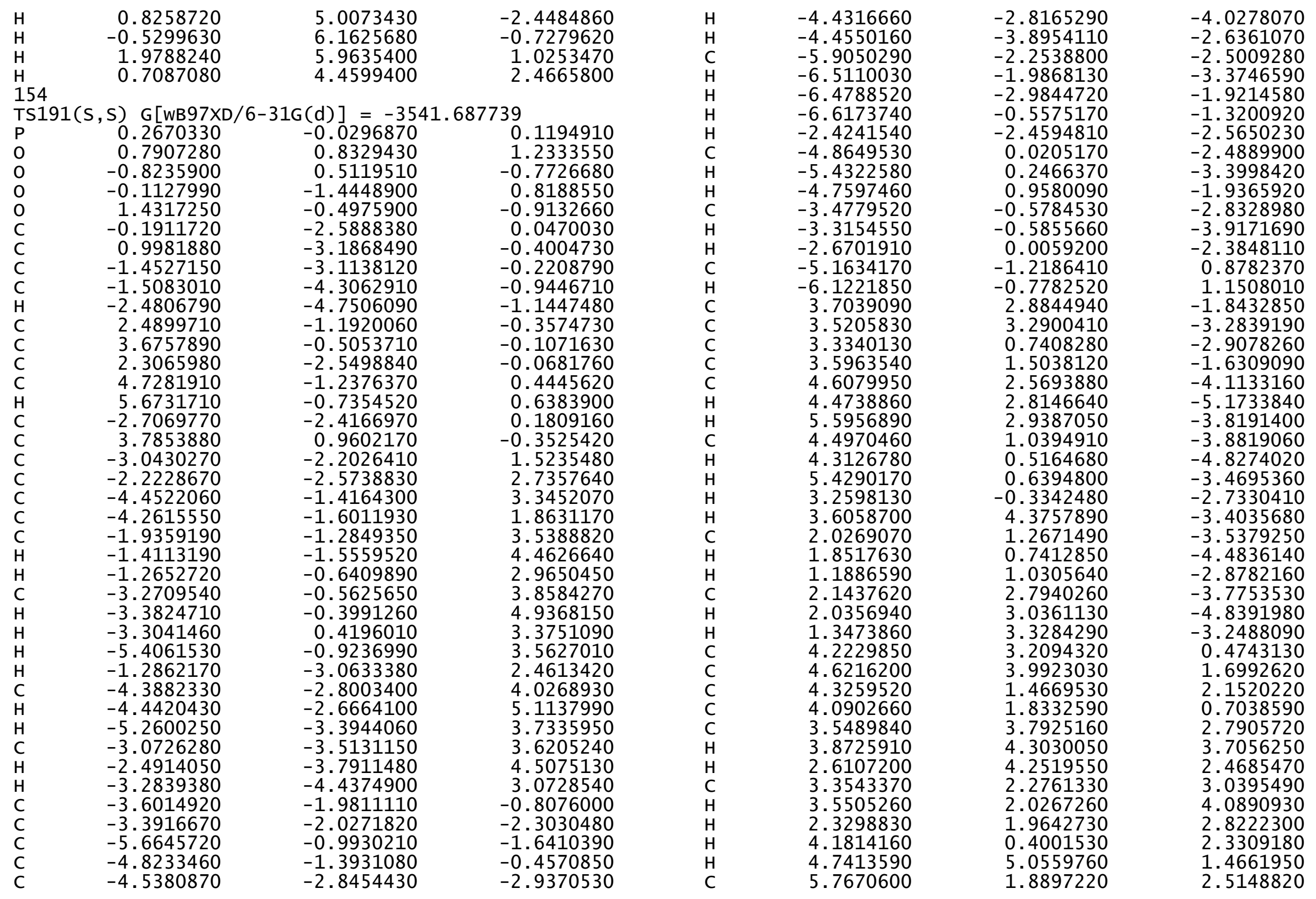




$\begin{array}{rrrr}\text { H } & 5.9454440 & 1.6754070 & \\ \text { H } & 6.4842700 & 1.2923920 & 1.9450360 \\ \text { C } & 5.9529130 & 3.4025680 & 2.2199410 \\ \text { H } & 6.2564820 & 3.9416660 & 3.1249020 \\ \text { H } & 6.7375640 & 3.5592560 & 1.4725260 \\ \text { C } & 4.0209230 & 3.7394680 & -0.7940190 \\ \text { H } & 4.1289200 & 4.8093350 & -0.9671990 \\ \mathrm{C} & 3.3596460 & -3.2324800 & 0.5567320 \\ \mathrm{C} & 3.2623230 & -4.6391610 & 1.0918100 \\ \mathrm{C} & 3.7298800 & -4.6711030 & 2.5582670 \\ \mathrm{C} & 5.1274110 & -4.0326850 & 2.7560230 \\ \mathrm{C} & 5.6600210 & -3.3679520 & 1.4708550 \\ \mathrm{C} & 4.5759410 & -2.5741520 & 0.7907710 \\ \mathrm{H} & 3.8986400 & -5.3094420 & 0.4963270 \\ \mathrm{H} & 3.7262910 & -5.7071150 & 2.9127310 \\ \mathrm{H} & 5.8505700 & -4.7832180 & 3.0920510 \\ \mathrm{H} & 6.0207580 & -4.1494590 & 0.7874460 \\ \mathrm{H} & 2.2419960 & -5.0232240 & 1.0142210 \\ \mathrm{H} & 2.9891560 & -4.1315720 & 3.1576030 \\ \mathrm{H} & 5.0746960 & -3.2736870 & 3.5432780 \\ \mathrm{H} & 6.5212160 & -2.7323750 & 1.7009170 \\ \mathrm{C} & -0.3613070 & -4.9079760 & -1.4413750 \\ \mathrm{C} & -0.3981430 & -6.1851770 & -2.2410350 \\ \mathrm{C} & 0.6666060 & -7.1547710 & -1.7065840 \\ \mathrm{C} & 2.0798470 & -6.5301170 & -1.7345250 \\ \mathrm{C} & 2.0560220 & -4.9954340 & -1.9069340 \\ \mathrm{C} & 0.8974090 & -4.3386580 & -1.1950040 \\ \mathrm{H} & -0.2013620 & -5.9741040 & -3.3022890 \\ \mathrm{H} & 0.6484830 & -8.0884480 & -2.2782970 \\ \mathrm{H} & 2.6678900 & -6.9550720 & -2.5549230 \\ \mathrm{H} & 1.9362340 & -4.7729250 & -2.9766870 \\ \mathrm{H} & -1.3929720 & -6.6396510 & -2.1893720 \\ \mathrm{H} & 0.3971500 & -7.4103100 & -0.6754770 \\ \mathrm{H} & 2.6074960 & -6.7850240 & -0.8101250 \\ \mathrm{H} & 3.0142390 & -4.5616320 & -1.6173200 \\ \mathrm{C} & -3.2453470 & 2.8236300 & -0.3346370 \\ \mathrm{O} & -2.9913250 & 1.6010830 & 0.0452340 \\ \mathrm{C} & -4.6337390 & 3.2676310 & -0.0439110 \\ \mathrm{C} & -5.5674070 & 2.3735310 & 0.4857520 \\ \mathrm{C} & -4.9975200 & 4.5894980 & -0.3092280 \\ \mathrm{C} & -6.8633770 & 2.8027240 & 0.7450120 \\ \mathrm{H} & -5.2671410 & 1.3502200 & 0.6825800 \\ \mathrm{C} & -6.2933730 & 5.0154100 & -0.0445290 \\ \mathrm{H} & -4.2604670 & 5.2698090 & -0.7227590 \\ \mathrm{C} & -7.2259920 & 4.1221600 & 0.4806260 \\ \mathrm{H} & -7.5911160 & 2.1071440 & 1.1516900 \\ & & & \\ & & & \end{array}$

$\begin{array}{lr}\text { H } & -6.5777300 \\ \text { H } & -8.2392980 \\ \text { H } & -2.0982300 \\ \text { O } & -2.4360210 \\ \text { C } & -0.3824920 \\ \text { C } & -0.3353130 \\ \text { H } & -0.8790090 \\ \text { H } & -0.2912630 \\ \text { O } & 1.0261640 \\ \text { H } & 1.1368760 \\ \text { C } & -0.0034650 \\ \text { C } & -0.4021450 \\ \text { C } & 0.0153800 \\ \text { C } & -0.6621930 \\ \text { H } & -0.2984150 \\ \text { H } & 0.0526500 \\ H & 1.1034790 \\ H & -1.7510220 \\ \text { H } & -0.3174160 \\ \text { H } & 1.0865980 \\ \text { H } & -1.4894300 \\ \text { H } & -0.4333330 \\ 154 & \end{array}$

6.0429590

$1.2236070-0.2682610$

$3.5841590 \quad-0.8804120$

$3.5961470 \quad 1.0043570$

$3.5467210 \quad-0.4598160$

$2.7522260 \quad 1.4838430$

$2.5654160 \quad-0.9192890$

$3.3530680 \quad 0.8382950$

$2.3515160 \quad 0.9414060$

$6.0868770 \quad 0.9024310$

$6.0672230 \quad-0.5753570$

$4.7591290-1.2523700$

$4.9290610 \quad 1.6570920$

$7.0360670 \quad 1.3604170$

$6.9080520 \quad-1.1079280$

$4.7193590-1.3932860$

$5.0634270 \quad 1.6779770$

$4.8834790 \quad 2.6953030$

$6.0080920 \quad 0.9948460$

$6.1780820 \quad-0.6617140$

$\begin{array}{lrrr}\text { TS192 (S S) } \mathrm{G}[\mathrm{WB} 97 \times \mathrm{DD} / 6-31 \mathrm{G}(\mathrm{d})]=-3541.686203 \\ \mathrm{P} & 0.2660480 & -0.0114060 & 0.2824870 \\ \mathrm{O} & 0.6842600 & 0.9785570 & 1.3327580 \\ \mathrm{O} & -0.8290020 & 0.3672810 & -0.6849240 \\ \mathrm{O} & -0.0344420 & -1.3915180 & 1.0849380 \\ \mathrm{O} & 1.4953860 & -0.4732210 & -0.6738560 \\ \mathrm{C} & -0.0179820 & -2.5854730 & 0.3868290 \\ \mathrm{C} & 1.2207160 & -3.1343180 & 0.0162170 \\ \mathrm{C} & -1.2356200 & -3.1949250 & 0.0951080 \\ \mathrm{C} & -1.1932370 & -4.4215610 & -0.5711620 \\ \mathrm{H} & -2.1308740 & -4.9223880 & -0.8009320 \\ \mathrm{C} & 2.5800140 & -1.0537690 & -0.0452980 \\ \mathrm{C} & 3.7166600 & -0.2769880 & 0.1649190 \\ \mathrm{C} & 2.4782320 & -2.3968690 & 0.3379290 \\ \mathrm{C} & 4.8073020 & -0.9023250 & 0.7704620 \\ \mathrm{H} & 5.7214670 & -0.3334680 & 0.9247280 \\ \mathrm{C} & -2.5472940 & -2.5502860 & 0.3867270 \\ \mathrm{C} & 3.7305240 & 1.1693720 & -0.1928040 \\ \mathrm{C} & -2.9708420 & -2.2710690 & 1.6918240 \\ \mathrm{C} & -2.1993170 & -2.5055390 & 2.9685710 \\ \mathrm{C} & -4.5265710 & -1.4600550 & 3.3782900 \\ \mathrm{C} & -4.2422690 & -1.7299540 & 1.9246530 \\ \mathrm{C} & -2.0374530 & -1.1478130 & 3.6880900\end{array}$




$\begin{array}{ll}\mathrm{H} & -1.5408200 \\ \mathrm{H} & -1.3835650 \\ \mathrm{C} & -3.4317500 \\ \mathrm{H} & -3.6107080 \\ \mathrm{H} & -3.5015510 \\ \mathrm{H} & -5.5195270 \\ \mathrm{H} & -1.2209110 \\ \mathrm{C} & -4.4189300 \\ \mathrm{H} & -4.5506190 \\ \mathrm{H} & -5.2297650 \\ \mathrm{C} & -3.0372290 \\ \mathrm{H} & -2.4929210 \\ \mathrm{H} & -3.1548100 \\ \mathrm{C} & -3.4118120 \\ \mathrm{C} & -3.1213590 \\ \mathrm{C} & -5.4913170 \\ \mathrm{C} & -4.6889310 \\ \mathrm{C} & -4.1725300 \\ \mathrm{H} & -4.0051760 \\ \mathrm{H} & -4.0406470 \\ \mathrm{C} & -5.5978030 \\ \mathrm{H} & -6.1568120 \\ \mathrm{H} & -6.1667650 \\ \mathrm{H} & -6.4875880 \\ \mathrm{H} & -2.1131910 \\ \mathrm{C} & -4.7183720 \\ \mathrm{H} & -5.2554620 \\ \mathrm{H} & -4.6981190 \\ \mathrm{C} & -3.2835150 \\ \mathrm{H} & -3.0818400 \\ \mathrm{H} & -2.5324420 \\ \mathrm{C} & -5.1128910 \\ \mathrm{H} & -6.1125520 \\ \mathrm{C} & 3.5275810 \\ \mathrm{C} & 3.3351020 \\ \mathrm{C} & 3.3574600 \\ \mathrm{C} & 3.5282620 \\ \mathrm{C} & 4.4899530 \\ \mathrm{H} & 4.3450610 \\ \mathrm{H} & 5.4386270 \\ \mathrm{C} & 4.5119400 \\ \mathrm{H} & 4.3985980 \\ \mathrm{H} & 5.4661710 \\ \mathrm{H} & 3.3721840 \\ \mathrm{H} & 3.3292680 \\ \mathrm{C} & 2.0256480\end{array}$

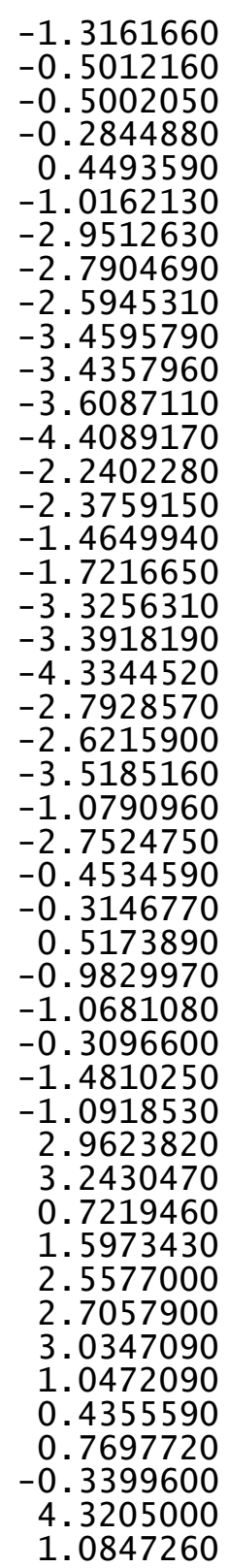

4.6508850
3.0978570
3.8959840
4.9559640
3.3546040
3.5113590
2.7786840
4.1537100
5.2244010
3.8479070
3.8740830
4.8096910
3.3861420
-0.6744590
-2.1512410
-1.6778070
-0.4287370
-2.7662940
-3.8476750
-2.3602180
-2.4603630
-3.3876640
-1.8701220
-1.4356490
-2.3346360
-2.5512790
-3.4971110
-2.0489910
-2.7992380
-3.8735980
-2.3783700
0.8716730
1.0622520
-1.8273450
-3.2958990
-2.7314660
-1.5123950
-4.0609790
-5.1375120
-3.7949580
-3.7074280
-4.6099440
-3.2481050
-2.4772430
-3.4950260
-3.4223670

1.9255500
1.1941030
2.0111160
1.8901510
1.1676160
3.9639910
4.2684460
4.1775120
3.9418020
3.1934850
3.4549520
2.2275450
3.1203730
3.3110430
2.1320270
4.1208250
4.3024460
5.5697920
5.7416750
6.3479500
5.6336570
5.8747660
6.4164070
3.7471830
3.7665810
4.7341050
5.8890190
6.1002300
4.8219090
3.5526040
3.5643100
5.6824030
6.9360660
4.9259970
3.5011490
6.7993230
6.3904520
4.6887830
2.6558240
1.2176380
2.4414770
2.1808320
0.8776920
0.0757200
0.0055960
2.6816970

0.4846750

0.8161730

2. 5976020

2.7610450

3. 0928030

3. 5025220

4.4038280

1. 9020340

2. 1407640

4.1923510

4.7921200

4.5486480

2. 6864040

2. 5299570

2. 2722530

0.8423030

5.4540180

2. 4727130

2. 3527250

1.8985620

3.9695770

4. 5984080

4.1359250

3. 9170330

4. 9766550

$-2.2177510$

$-2.9085020$

$-4.2944480$

$-5.1606230$

$-4.3399900$

$-2.9680850$

$-3.0216390$

$-4.8055750$

$-5.9360190$

$-4.1900750$

$-2.3056870$

$-4.1446300$

$-5.6827350$

$-4.9021230$

$-4.3274940$

$-4.9461890$

$-5.2221960$

$-6.2505370$

-4.9809070
-5.8926060
-4.3342440
-2.7667820
-3.7588620
-4.8361300
-3.2684900
0.4674320
1.6366670
2.2718210
0.7977510
2.7237570
3.6040480
2.3558070
3.0802680
4.1485660
2.8667210
2.5266270
1.3266590
2.6242680
3.7002170
2.1095190
2.2180640
3.0831450
1.4706910
-0.8409720
-1.0926560
1.2119970
1.8914280
1.2635110
1.3291270
1.6465880
1.0167950
2.9656300
1.7525160
2.0955970
2.7341930
1.8078000
0.2173770
0.3766770
1.3792610
-0.7205240
-1.3495500
-2.8422030
-3.0899420
-1.7936250
-0.9875510
-0.8445530 


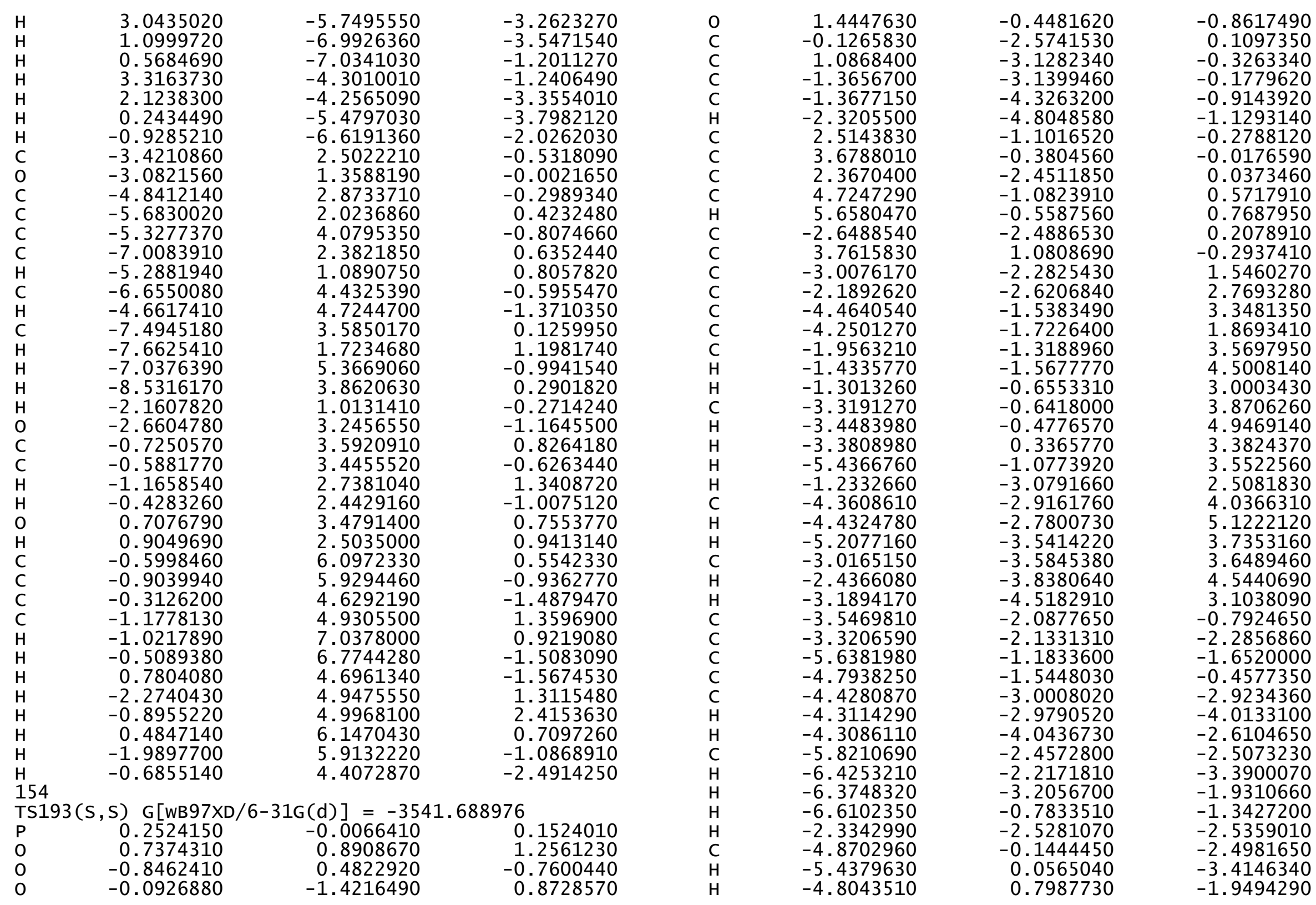




$\begin{array}{rrr}-3.4593920 & -0.6932520 & -2.8280630 \\ -3.2897390 & -0.7038800 & -3.9112120 \\ -2.6776440 & -0.0737670 & -2.3810100 \\ -5.1541550 & -1.3766440 & 0.8730730 \\ -6.1309680 & -0.9696540 & 1.1332830 \\ 3.6600490 & 2.9695290 & -1.8280550 \\ 3.4863480 & 3.3394980 & -3.2792270 \\ 3.3511480 & 0.7958250 & -2.8500080 \\ 3.5790220 & 1.5918190 & -1.5865630 \\ 4.5985800 & 2.6254720 & -4.0806360 \\ 4.4694910 & 2.8428830 & -5.1473710 \\ 5.5742170 & 3.0248970 & -3.7857240 \\ 4.5210540 & 1.0992510 & -3.8143870 \\ 4.3626740 & 0.5502640 & -4.7498120 \\ 5.4561260 & 0.7314230 & -3.3797580 \\ 3.2982410 & -0.2768210 & -2.6534680 \\ 3.5496000 & 4.4240690 & -3.4211520 \\ 2.0422890 & 1.2789800 & -3.5106370 \\ 1.8969130 & 0.7327890 & -4.4498210 \\ 1.1984310 & 1.0329330 & -2.8616360 \\ 2.1256780 & 2.8039350 & -3.7736120 \\ 2.0204840 & 3.0252500 & -4.8422390 \\ 1.3132790 & 3.3288640 & -3.2618940 \\ 4.1352280 & 3.3574900 & 0.4889010 \\ 4.4979160 & 4.1760880 & 1.7015870 \\ 4.2492160 & 1.6560070 & 2.2068510 \\ 4.0294580 & 1.9840540 & 0.7472710 \\ 3.4119580 & 3.9775930 & 2.7797260 \\ 3.7088120 & 4.5152570 & 3.6882950 \\ 2.4698240 & 4.4096560 & 2.4319490 \\ 3.2455770 & 2.4633770 & 3.0596290 \\ 3.4291510 & 2.2415300 & 4.1175630 \\ 2.2319470 & 2.1243440 & 2.8324430 \\ 4.1245160 & 0.5905870 & 2.4076430 \\ 4.5986800 & 5.2365630 & 1.4461140 \\ 5.6748360 & 2.1180330 & 2.5832540 \\ 5.8399570 & 1.9318210 & 3.6508230 \\ 6.4143150 & 1.5234780 & 2.0358230 \\ 5.8331100 & 3.6272230 & 2.2559680 \\ 6.1114160 & 4.1933440 & 3.1526050 \\ 6.6255350 & 3.7836560 & 1.5167670 \\ 3.9420020 & 3.8545470 & -0.7939660 \\ 4.0293940 & 4.9224380 & -0.9893780 \\ 3.4037130 & -3.1029980 & 1.2679760 \\ 3.1984270 & -4.5080980 & \\ 4.1941870 & -4.8800520 & \\ 3.3650830\end{array}$

5.6131670

5.7610860

4.6002620

4.0903520

3. 3000810

2. 1698010

5.8100150

6.3552640

6.6958990

5. 8419250

3. 9703440

$-0.1956430$

$-0.1843770$

0.9531580

2. 3240970

2. 2247460

1.0382300

$-0.0340380$

0.9475940

2.8129590

2.0904340

$-1.1498830$

0.7642720

2. 9768770

3. 1621180

$-3.3469650$

$-3.0563280$

$-4.7499220$

$-5.6580220$

$-5.1534730$

$-6.9680610$

$-5.3274300$

$-6.4633400$

$-4.4352980$

$-7.3704600$

$-7.6760430$

$-6.7790250$

$-8.3949330$

$-2.1489030$

$-2.5598940$

$-0.5132750$

$-0.4577210$

$-0.9844620$

$-0.3812360$

0.9036760

1.0447650
-4.5572200
-3.0489520
-2.4136430
-5.9437020
-5.2359500
-4.6083020
-5.0740740
-4.9160280
-2.8179230
-2.5725100
-4.3191620
-4.8725000
-6.1309170
-7.0424090
-6.3518490
-4.8074520
-4.2601730
-5.8866310
-7.9876290
-6.6646080
-4.4837960
-6.6422610
-7.2869480
-6.6829630
-4.3546340
2.7167400
1.5073940
3.1200010
2.2032510
4.4268590
2.5944780
1.1918830
4.8149010
5.1248860
3.8988610
1.8812070
5.8306430
4.2026110
1.1560270
3.4955870
3.6098510
3.5343820
2.7591820
2.5466350
3.4088930
2.4136870

1.9051230

1.7163370

0.9733560

2.6049770

0.4546700

1.6310280

0.9559750

2.6262820

1.1917010

2.7034820

3.2823020

$-1.4179700$

$-2.2485640$

$-1.7690650$

$-1.9032210$

$-1.9125480$

$-1.1525030$

$-3.3105710$

$-2.3218080$

$-2.8321520$

$-2.9542320$

$-2.1757060$

$-0.7172910$

$-1.0885840$

$-1.5836690$

$-0.3701240$

0.0252660

$-0.0896200$

0.4458520

$-0.3713060$

0.6938680

0.6557510

$-0.1176210$

$-0.7886730$

0.4127820

1.1047020

$-0.3342170$

0.6064280

$-0.2771380$

$-0.9224980$

0.9670190

$-0.4956010$

1.4598390

$-0.9367290$

0.8112400

0.9356760 


$\begin{array}{lrrr}\mathrm{C} & -0.2232850 & 6.1113360 & 0.8179560 \\ \mathrm{C} & -0.6139180 & 6.0476280 & -0.6606970 \\ \mathrm{C} & -0.1439640 & 4.7428020 & -1.3092030 \\ \mathrm{C} & -0.8405750 & 4.9444410 & 1.5935070 \\ \mathrm{H} & -0.5576610 & 7.0570310 & 1.2557710 \\ \mathrm{H} & -0.1882320 & 6.8941310 & -1.2080290 \\ \mathrm{H} & 0.9458940 & 4.7408260 & -1.4407880 \\ \mathrm{H} & -1.9335080 & 5.0402160 & 1.6105500 \\ \mathrm{H} & -0.4964980 & 4.9314220 & 2.6328830 \\ \mathrm{H} & 0.8685790 & 6.0779590 & 0.9163880 \\ \mathrm{H} & -1.7041570 & 6.1154330 & -0.7539250 \\ \mathrm{H} & -0.5798700 & 4.6006440 & -2.3017750 \\ \mathrm{H} & & & \end{array}$

TS194 (S, S ) G[WB97XD/6-31G(d)] = -3541.686186

$\begin{array}{lrrr}\text { TS194(S, S }) \text { G }[\mathrm{WB} 97 \mathrm{XD} / 6-31 \mathrm{G}(\mathrm{d})]=-3541.686186 \\ \mathrm{P} & -0.2630720 & 0.0159320 & -0.2094090 \\ \mathrm{O} & -0.6602160 & 0.9779050 & -1.2932130 \\ \mathrm{O} & 0.8555370 & 0.3940950 & 0.7308930 \\ \mathrm{O} & -0.0169860 & -1.4039850 & -0.9600060 \\ \mathrm{O} & -1.4975230 & -0.3699330 & 0.7751180 \\ \mathrm{C} & -0.0534420 & -2.5597590 & -0.2006200 \\ \mathrm{C} & -1.3021790 & -3.0620990 & 0.2016210 \\ \mathrm{C} & 1.1543250 & -3.1742190 & 0.1199180 \\ \mathrm{C} & 1.0912500 & -4.3544320 & 0.8628150 \\ \mathrm{H} & 2.0191830 & -4.8632590 & 1.1135420 \\ \mathrm{C} & -2.5999190 & -0.9423680 & 0.1687400 \\ \mathrm{C} & -3.7057220 & -0.1351680 & -0.0884120 \\ \mathrm{C} & -2.5393070 & -2.3031960 & -0.1592980 \\ \mathrm{C} & -4.8073950 & -0.7449080 & -0.6890040 \\ \mathrm{H} & -5.6978590 & -0.1508400 & -0.8818600 \\ \mathrm{C} & 2.4767620 & -2.5885110 & -0.2393230 \\ \mathrm{C} & -3.6776600 & 1.3236280 & 0.2139070 \\ \mathrm{C} & 2.8739540 & -2.4057710 & -1.5699570 \\ \mathrm{C} & 2.0607470 & -2.6932560 & -2.8096290 \\ \mathrm{C} & 4.4094210 & -1.7563800 & -3.3430900 \\ \mathrm{C} & 4.1569640 & -1.9286380 & -1.8686650 \\ \mathrm{C} & 1.9252200 & -1.3801460 & -3.6130790 \\ \mathrm{H} & 1.4063120 & -1.5958000 & -4.5544770 \\ \mathrm{H} & 1.3015470 & -0.6769540 & -3.0558580 \\ \mathrm{C} & 3.3333200 & -0.7896980 & -3.8863690 \\ \mathrm{H} & 3.4931510 & -0.6335650 & -4.9597490 \\ \mathrm{H} & 3.4460930 & 0.1832500 & -3.3962090 \\ \mathrm{H} & 5.4127180 & -1.3567800 & -3.5280070 \\ \mathrm{H} & 1.0734200 & -3.0915040 & -2.5678810 \\ \mathrm{C} & 4.2343510 & -3.1247860 & -4.0354160 \\ \mathrm{H} & 4.3340470 & -2.9933430 & -5.1193550 \\ \mathrm{H} & 5.0346130 & -3.8017230 & -3.7192440\end{array}$

2.8437890
2.2666360
2.9487190
3.3811700
3.1262230
5.5220820
4.6707730
4.1488410
4.0160390
3.9530680
5.5872560
6.1935940
6.0892120
6.5284450
2.1064730
4.8276980
5.3938830
4.8511070
3.3720320
3.1851970
2.6486360
5.0658850
6.0743020
-3.4316710
-3.2423260
-3.3395010
-3.4716180
4.4257070
4.2862600
-5.3561640
-4.4920210
-4.4066320
-5.4501290
-3.3879210
-3.2029930
-2.0024700
-1.9273220
-1.1747450
-1.9442630
-1.8301890
-1.0802130
-3.8427160
-4.1206410
-4.0904310
-3.8556640
-3.0481060

-3.7078840
-3.9272640
-4.6490780
-2.2472910
-2.2772510
-1.5129520
-1.7986530
-3.2334440
-3.2067870
-4.2625160
-2.8010150
-2.6133080
-3.5893820
-1.1924480
-2.5904560
-0.4198870
-0.2706580
0.5282270
-0.8550830
-0.8587120
-0.1725200
-1.6513510
-1.3156370
3.1701300
3.4990520
0.9612910
1.7949220
2.8803120
3.0634190
3.3765930
1.3593670
0.7788410
1.0948380
-0.1082610
4.5823910
1.3068870
0.7370720
0.9901110
2.8295830
3.0271680
3.2808610
3.6371110
4.5036900
1.9803040
2.2641480
4.2282740

$-3.6726560$

$-4.5784590$

$-3.1230840$

0.7781340

2.2671740

1. 6774920

0.4676310

2. 9188550

4.0067870

2. 5988740

2. 5286200

3. 4223990

1.9581610

1. 3863960

2. 4987080

2.5178060

3.4449900

1.9741210

2. 8209540

3. 9012970

2. 3679710

$-0.8556850$

$-1.0958700$

1.7828570

3. 2420000

2.7710320

1. 5181830

4. 0198510

5.0917110

3. 7261120

3.7221430

4.6480440

3. 2633090

2. 5571390

3.4014300

3.4601650

4. 3935650

2. 8214260

3.7426930

4.8150100

3. 2458920

$-0.5317950$

$-1.7334810$

$-2.2793830$

$-0.8129070$

$-2.8083080$ 


-3.2921450
-2.0747400
-3.0115340
-3.2034450
-2.0344930
-4.0589620
-4.1292360
-5.4663300
-5.6359520
-6.2615010
-5.4943060
-5.7163700
-6.2752060
-3.6192770
-3.6090500
-4.7716930
-5.9352890
-6.2132280
-4.9658510
-3.6572300
-3.6334930
-5.7074720
-7.0529370
-5.0766400
-3.5650010
-6.8236930
-6.5264440
-4.8825930
-2.7902150
-0.1157500
-0.1974290
-1.2878330
-2.6444600
-2.5532620
-1.3192040
-0.4329050
-1.3971620
-3.3910700
-2.4942240
0.7706060
-0.9476510
-3.0239240
-3.4664150
3.5027920
3.1215050
4.9270900

4.8025080

4.5735160

2.7092910

2.5202030

2.2786690

0.9113210

5. 5647240

2. 5725420

2.4201890

2.0362410

4.0838040

4.6875600

4.2959230

4.0937790

5.1620350

$-2.0765790$

$-2.7443350$

$-4.1031360$

$-5.0147590$

$-4.2523220$

$-2.8602860$

$-2.8942000$

$-4.5987460$

$-5.8078250$

$-4.1423660$

$-2.1055530$

$-3.9149240$

$-5.5154720$

$-4.8376950$

$-4.8590830$

$-6.0917600$

$-7.0400880$

$-6.3205070$

$-4.7918880$

$-4.2106300$

$-5.8015680$

$-7.8861020$

$-6.7070810$

$-4.5754590$

$-6.6023880$

$-7.4530850$

$-6.5371770$

$-4.3092190$

2.4436210

1.2950680

2. 7701200

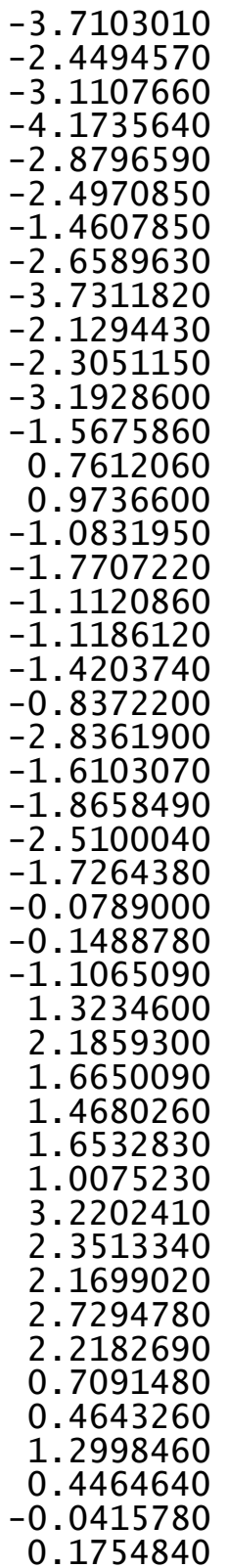

5.7362420
5.4513340
7.0664950
5.3142140
6.7831090
4.8104130
7.5900140
7.6960290
7.1945650
8.6311210
2.1972540
2.7756590
0.8103010
0.7005910
1.2192470
0.5242180
-0.6228790
-0.8473800
0.7566170
1.0889500
0.4727730
1.2834570
1.1960390
0.7304240
-0.6164360
2.3806420
0.9763960
-0.3293780
2.1768000
0.8566040

1.8783270

3.9783050

2.1977730

0.9414200

4.2919970

4.6556010

3.4027660

1.5064680

5.2279130

3.6483570

0.9854350

3.2255070

3.5721570

3.4680930

2. 6941410

2. 4806230

3.4961870

2. 5215250

6.0869850

5.9494800

4.6815550

4.8841090

7.0054140

6.8200270

4.7812310

4.8729520

4.9298270

6.1639410

5.9055670

4.4765080
$-0.5334200$

0.6399580

$-0.7761650$

$-0.8800370$

0.3974630

1.1944620

$-0.3106280$

$-1.3279420$

0.7622820

$-0.4979160$

0.2591360

1.0721770

$-0.8900990$

0.5687150

$-1.3900770$

0.9809330

$-0.7862730$

$-0.9413810$

$-0.6824760$

0.8050190

1.4024420

$-1.4695370$

$-1.0840590$

1.3626350

1.4972430

$-1.4491410$

$-2.5194540$

$-0.8158540$

0.9333140

154

$\operatorname{TS} 195(\mathrm{~S}, \mathrm{~S}) \mathrm{G}[\mathrm{WB} 97 \mathrm{XD} / 6-31 \mathrm{G}(\mathrm{d})]=-3541.688280$

$\begin{array}{lrrr}\mathrm{P} & -0.3500700 & -0.0472590 & 0.9098060 \\ \mathrm{O} & 0.3000770 & -0.3609020 & 2.2241430 \\ \mathrm{O} & -0.5664310 & 1.3948140 & 0.5282100 \\ \mathrm{O} & -1.7231990 & -0.9110700 & 0.8654620 \\ \mathrm{O} & 0.4778810 & -0.6788140 & -0.3568890 \\ \mathrm{C} & -2.3449180 & -1.0274280 & -0.3664930 \\ \mathrm{C} & -1.8124870 & -1.9358700 & -1.2902340 \\ \mathrm{C} & -3.4713420 & -0.2506660 & -0.6227410 \\ \mathrm{C} & -4.0602920 & -0.3802770 & -1.8812890 \\ \mathrm{H} & -4.9487900 & 0.2059440 & -2.1047280 \\ \mathrm{C} & 0.5264690 & -2.0588570 & -0.4511790 \\ \mathrm{C} & 1.7130590 & -2.7086470 & -0.1222710 \\ \mathrm{C} & -0.6163490 & -2.7377920 & -0.8942340 \\ \mathrm{C} & 1.7256380 & -4.1012220 & -0.1916950\end{array}$




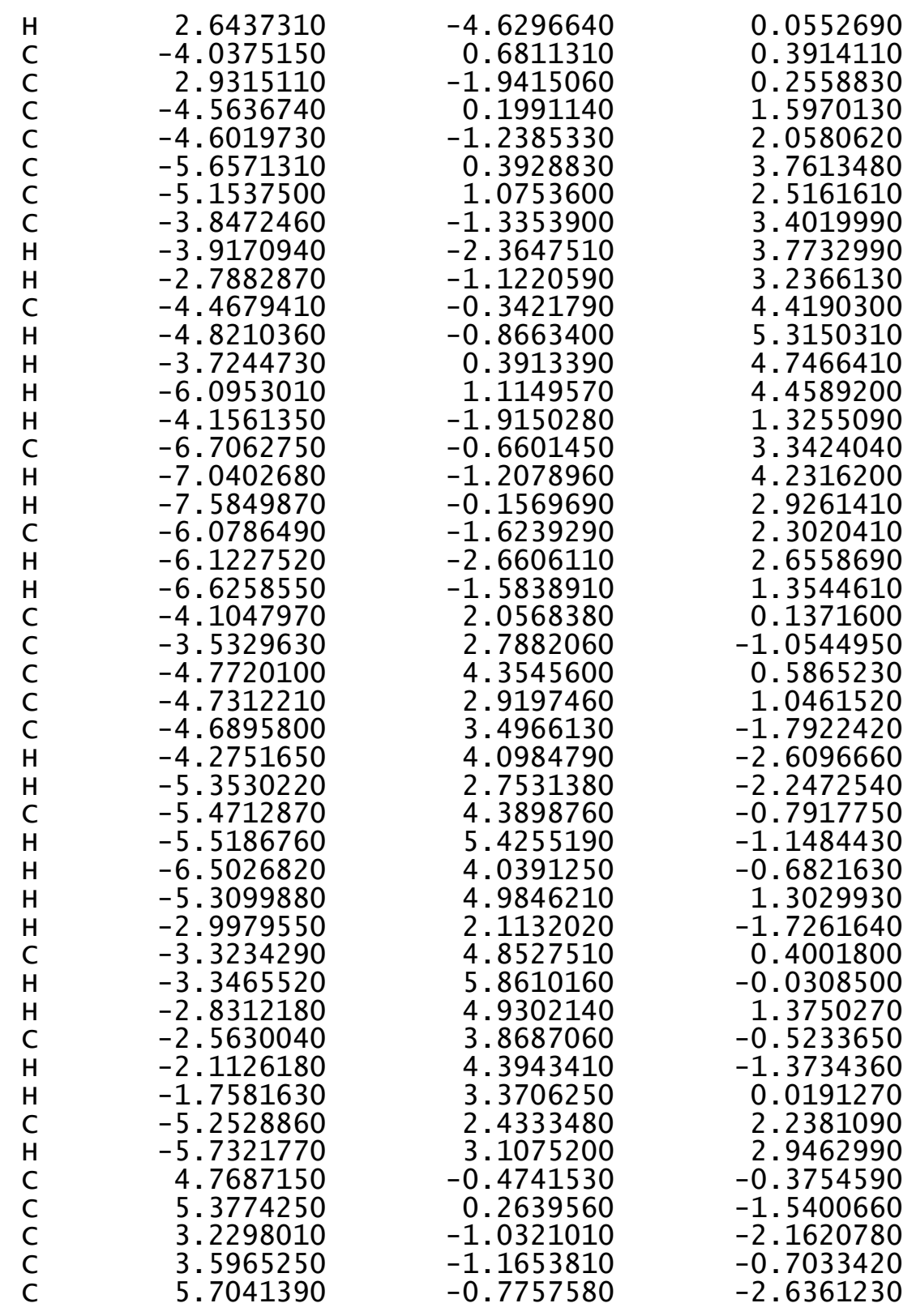

\begin{abstract}
6.1041990
6.4864120

4.4171960

4. 1807820

4. 5491000

2. 3202240

6.2836560

3.0428950

2. 8221420

2. 1706800

4. 3282420

4.7450550

4.1065860

4.6492830

5. 1130930

2.9561480

3. 4702450

3. 9784330

4. 3007130

3. 7792960

2.7065410

2. 4395290

1.8483130

2. 0380580

6.0303370

4.0759920

3. 7331200

4. 2781410

5. 3544610

5. 6140300

6. 2105270

5. 2992190

6.2220230

0.5880370

0.5556010

$-0.0539010$

$-1.4325660$

$-1.7776290$

$-0.5873860$

$-0.0493300$

$-0.1355010$

$-2.2271690$

$-2.1156600$

1. 5621210

0.6435970

$-1.4480260$
\end{abstract}

-0.2572350
-1.4521930
-1.5638440
-1.4545400
-2.6333510
-1.5862600
0.8011150
0.4633980
0.5781860
0.8319570
1.2439350
1.7581560
2.0170560
-1.3292490
-1.5550130
-2.8546480
-2.0187660
-1.1249700
-1.2791330
-0.0553630
-1.9512910
-2.5899960
-1.3093880
-3.3829600
-0.9939340
-3.8544470
-4.4900000
-4.5137610
-3.0712470
-3.2634830
-3.3842880
-0.5576430
-0.0352690
-4.8163730
-6.3220840
-6.8065610
-6.1610160
-5.0149920
-4.1400410
-6.6982210
-7.8983400
-6.9121200
-5.4548220
-6.7327870
-6.5482530
-5.7775840

$-3.5151330$

$-2.2772310$

$-2.9967180$

$-4.0613760$

$-2.8040250$

$-2.4049070$

$-1.2392400$

$-2.4859940$

$-3.5533160$

$-1.9420090$

$-2.1072660$

$-2.9806430$

$-1.3662290$

1.8633620

3.2787670

2. 6924770

1. 5451750

4. 2334400

5. 2698180

4. 1122340

3. 9187530

4.7689760

3.7179370

2. 4264440

3. 4893000

3.0620350

3.8864450

2. 2108110

3. 4652010

4.5127800

2. 8587680

0.9078050

1. 1568370

$-0.5433140$

$-0.5796010$

$-1.9042190$

$-2.1832400$

$-1.2094150$

$-0.9023760$

0.2580190

$-1.8968950$

$-2.1240020$

$-0.2604610$

$-0.4481760$

-2.7085530
-3.2085380 


$\begin{array}{lll}-2.6216260 & -4.4344010 & -1.5879720 \\ -3.5232140 & -1.2157450 & -2.8516090 \\ -4.1210120 & -1.3471880 & -4.2284750 \\ -4.2785130 & -2.8324190 & -4.5856550 \\ -2.9452590 & -3.6051610 & -4.4549350 \\ -1.8494420 & -2.8084290 & -3.7145220 \\ -2.3954160 & -1.9990620 & -2.5637580 \\ -3.4671500 & -0.8613410 & -4.9673060 \\ -4.6841320 & -2.9338920 & -5.5976000 \\ -2.5601360 & -3.8748900 & -5.4441150 \\ -1.3971580 & -2.1010770 & -4.4241520 \\ -5.0874540 & -0.8351230 & -4.2785060 \\ -5.0214940 & -3.2616530 & -3.9042700 \\ -3.1268880 & -4.5476650 & -3.9285650 \\ -1.0426340 & -3.4727340 & -3.3976150 \\ 1.6334220 & 3.3092680 & -1.1904540 \\ 0.5268000 & 2.6514410 & -1.3733850 \\ 2.1496890 & 3.9827570 & -2.4119600 \\ 3.3165680 & 4.7446820 & -2.3241940 \\ 1.5081930 & 3.8238010 & -3.6425770 \\ 3.8402490 & 5.3457100 & -3.4614980 \\ 3.8051460 & 4.8514950 & -1.3615390 \\ 2.0370270 & 4.4239600 & -4.7793150 \\ 0.6057940 & 3.2258290 & -3.7009060 \\ 3.2015500 & 5.1837600 & -4.6898720 \\ 4.7483750 & 5.9364090 & -3.3929280 \\ 1.5422050 & 4.2966000 & -5.7369400 \\ 3.6141150 & 5.6494770 & -5.5798720 \\ 0.1682780 & 2.1449620 & -0.5579130 \\ 2.2577320 & 3.3877270 & -0.1234370 \\ 1.5021340 & 2.5958350 & 2.4620220 \\ 2.5227940 & 2.0869340 & 1.5384900 \\ 0.4814410 & 2.5248350 & 2.0896550 \\ 2.2674380 & 1.2320880 & 0.9188920 \\ 1.8760220 & 1.4372990 & 3.2368440 \\ 1.2637450 & 0.6904330 & 2.9409770 \\ 3.2802940 & 4.0132580 & 3.5729920 \\ 4.1782040 & 3.8039250 & 2.3516870 \\ 3.9600050 & 2.4237690 & 1.7303720 \\ 1.8052530 & 3.8883280 & 3.1835140 \\ 3.4606340 & 4.9999200 & 4.0110760 \\ 5.2331640 & 3.9116940 & 2.6221190 \\ 4.3684370 & 1.6303660 & 2.3683710 \\ 1.5252950 & 4.7055720 & 2.5076980 \\ 1.1543450 & 3.9508640 & 4.3425420 \\ 3.5207150 & 3.2699940 & \end{array}$

$\begin{array}{llll}\mathrm{H} & 3.9547680 & 4.5686240 & 1.5986220 \\ \mathrm{H} & 4.4673250 & 2.3142200 & 0.7693040 \\ 144 & & \end{array}$

TS196 $(R, R) \quad G[W B 97 X D / 6-31 G(d)]=-3633.717600$

$\begin{array}{lrrr}\text { TS } 196(\mathrm{R}, \mathrm{R}) \mathrm{G}[\mathrm{WB} 97 \mathrm{XD} / 6-31 \mathrm{G}(\mathrm{d})]=-3633.717600 \\ \mathrm{O} & 0.6245650 & 0.5893220 & -0.0907790 \\ \mathrm{O} & -1.6146480 & 0.8684300 & -1.2580040 \\ \mathrm{C} & 0.8319240 & 1.9454380 & -0.2300910 \\ \mathrm{C} & -0.1857730 & 2.8174070 & 0.0930800 \\ \mathrm{C} & 2.1260730 & 2.3798070 & -0.6176460 \\ \mathrm{C} & 0.0191120 & 4.2199410 & -0.1140720 \\ \mathrm{C} & 2.3431280 & 3.7309850 & -0.7138660 \\ \mathrm{C} & 1.3074010 & 4.6749940 & -0.5054320 \\ \mathrm{H} & 3.3368270 & 4.0914380 & -0.9658500 \\ \mathrm{C} & -2.1584630 & 1.3312200 & -0.0698510 \\ \mathrm{C} & -3.4196110 & 0.8202940 & 0.3362800 \\ \mathrm{C} & -1.4640920 & 2.2874090 & 0.6349370 \\ \mathrm{C} & -3.9565110 & 1.3202560 & 1.4958600 \\ \mathrm{C} & -1.9778290 & 2.7104150 & 1.9035880 \\ \mathrm{C} & -3.2521440 & 2.2358500 & 2.3178320 \\ \mathrm{H} & -4.9371010 & 0.9798540 & 1.8160000 \\ \mathrm{C} & -3.0680390 & 3.4966160 & 4.3827310 \\ \mathrm{C} & -1.7820850 & 3.9357030 & 3.9910730 \\ \mathrm{C} & -1.2511840 & 3.5553990 & 2.7848970 \\ \mathrm{C} & -3.7832850 & 2.6619520 & 3.5640100 \\ \mathrm{H} & -3.4793510 & 3.8119090 & 5.3366700 \\ \mathrm{H} & -1.2071920 & 4.5750640 & 4.6540130 \\ \mathrm{H} & -0.2592340 & 3.8908570 & 2.5031220 \\ \mathrm{H} & -4.7632480 & 2.2991950 & 3.8628210 \\ \mathrm{C} & -0.7772050 & 6.5141740 & -0.1687940 \\ \mathrm{C} & 0.5146880 & 6.9673900 & -0.5241700 \\ \mathrm{C} & 1.5316380 & 6.0644160 & -0.6917000 \\ \mathrm{C} & -1.0194300 & 5.1792000 & 0.0321500 \\ \mathrm{H} & -1.5854310 & 7.2309540 & -0.0598040 \\ \mathrm{H} & 0.6923610 & 8.0275910 & -0.6750890 \\ \mathrm{H} & 2.5250460 & 6.3966480 & -0.9815360 \\ \mathrm{H} & -2.0175450 & 4.8454190 & 0.2944860 \\ \mathrm{C} & 3.2511910 & 1.4230410 & -0.8493260 \\ \mathrm{C} & 3.6482360 & 1.1044070 & -2.1591380 \\ \mathrm{C} & 3.9663390 & 0.9152620 & 0.2519830 \\ \mathrm{C} & 4.8008540 & 0.3367490 & -2.3462750 \\ \mathrm{C} & 5.0902130 & 0.1281100 & 0.0136770 \\ \mathrm{C} & 5.5431410 & -0.1509700 & -1.2752640 \\ \mathrm{H} & 5.1159910 & 0.1116900 & -3.3599310 \\ \mathrm{H} & 5.6432270 & -0.2774410 & 0.8590420 \\ \mathrm{C} & -4.1337610 & -0.2184810 & -0.4659450 \\ \mathrm{C} & -4.2570860 & -1.5303810 & 0.0321390\end{array}$




$\begin{array}{rrr}-4.7056870 & 0.1235160 & -1.7065670 \\ -4.9752300 & -2.4639490 & -0.7176650 \\ -5.4027510 & -0.8489730 & -2.4203920 \\ -5.5534200 & -2.1490370 & -1.9426550 \\ -5.0862680 & -3.4761320 & -0.3355760 \\ -5.8460390 & -0.5815380 & -3.3771520 \\ 3.5439470 & 1.1905980 & 1.6881830 \\ 6.8226450 & -0.9553260 & -1.4487830 \\ 2.8680760 & 1.5951860 & -3.3713790 \\ -3.6535600 & -1.9668120 & 1.3618310 \\ -6.3170440 & -3.1938510 & -2.7360990 \\ -4.6067430 & 1.5267530 & -2.2892490 \\ -5.9910230 & 2.1810210 & -2.3960660 \\ -6.6436660 & 1.6237320 & -3.0778820 \\ -6.4819930 & 2.2221930 & -1.4178740 \\ -5.9009000 & 3.2038470 & -2.7788300 \\ -3.8843330 & 1.5239850 & -3.6428540 \\ -2.8949130 & 1.0669570 & -3.5510520 \\ -4.4525420 & 0.9679210 & -4.3975810 \\ -3.7587900 & 2.5491540 & -4.0090410 \\ -5.6500870 & -3.4656560 & -4.0912320 \\ -6.1714510 & -4.2696450 & -4.6228060 \\ -5.6719920 & -2.5734060 & -4.7277750 \\ -4.6035870 & -3.7600360 & -3.9615470 \\ -7.7893880 & -2.8011170 & -2.9158750 \\ -8.2741920 & -2.6383400 & -1.9476690 \\ -7.8819890 & -1.8773140 & -3.4986800 \\ -8.3359870 & -3.5887540 & -3.4464470 \\ -4.7318890 & -2.0573740 & 2.4518930 \\ -5.2500880 & -1.1037680 & 2.5918040 \\ -5.4835820 & -2.8112350 & 2.1893180 \\ -4.2836630 & -2.3446480 & 3.4098590 \\ -2.8988190 & -3.2993530 & 1.2637820 \\ -3.5773630 & -4.1415140 & 1.0873440 \\ -2.1610190 & -3.2717480 & 0.4583460 \\ -2.3745280 & -3.4953470 & 2.2059010 \\ 4.6785610 & 1.8047800 & 2.5174010 \\ 5.5127000 & 1.1050860 & 2.6418830 \\ 5.0662480 & 2.7129490 & 2.0439980 \\ 4.3148870 & 2.0667490 & 3.5170480 \\ 2.9971050 & -0.0843080 & 2.3422290 \\ 2.1414470 & -0.4601700 & 1.7749830 \\ 3.7599350 & -0.8710120 & -1.3803420 \\ 2.6647830 & 0.1151120 & -1.1884870 \\ 8.0423180 & -0.0748360 & \\ 8.9680220 & -0.6583500 & \\ -4 & & \end{array}$

8.1134850

7.9693490

6.9778430

6.1059120

7.1200960

7.8580220

2.7260780

3.6745680

2. 3751000

2. 0015230

3.5007350

4. 5299670

2. 9311940

3.5261690

$-0.3976950$

0.2107180

$-0.8329570$

1.8568590

6.7945540

2.7298890

$-4.0147930$

$-6.2894760$

$-2.9257280$

2. 3343780

1.2170990

2.2258780

0.2285390

1.8233210

1.1916840

3.8447310

2. 8088010

1.3841500

3.7346490

4.8544170

2.9000920

1.0944490

4.0249100

4.3972830

3.7002050

2. 9910970

0.6429130

0.4836920

$-0.2863450$

0.7002870

1.5146230
0.0893710
0.7479340

0.3601790

$-1.6069870$

$-2.2141640$

$-0.8555640$

$-2.2580910$

0.5213920

0.3367590

$-0.4226810$

0.8501600

2. 8633680

2.6653920

3.2045960

3.6785600

$-0.1957070$

$-0.3953730$

$-1.4100930$

1.8431850

$-1.7602040$

1.9212700

2.1447220

$-4.1272410$

$-1.2109630$

$-2.5064110$

$-3.4483210$

$-1.5397540$

$-3.0175780$

$-2.4842210$

$-1.7005400$

$-4.4683820$

$-5.4125650$

$-4.8988330$

$-3.0642450$

$-4.8544520$

$-6.4160970$

$-5.0254940$

$-3.0731460$

$-2.3632840$

$-4.4152630$

$-5.5035370$

$-5.4560990$

$-2.4300720$

$-1.5208890$

$-2.2478630$

$-3.1141110$
$-1.8542670$

$-0.1311720$

$-2.8246040$

$-3.0922640$

$-3.6098250$

$-2.8311780$

$-4.4570070$

$-4.9743450$

$-4.0344600$

$-5.2092830$

$-3.9622550$

$-4.2849660$

$-4.8340480$

$-3.2333530$

$-1.1057250$

$-2.4601680$

$-0.3224120$

$-3.0342570$

$-0.7010510$

1. 6716650

$-1.6083840$

$-2.1592480$

1.6682500

$-1.0943680$

$-0.9404370$

$-0.5987060$

$-0.8260340$

$-2.4492220$

$-2.5283310$

$-1.6216620$

$-1.0084480$

$-1.2396870$

$-1.0236880$

$-1.4517310$

$-1.4339410$

$-2.2910520$

0.0343800

$-1.5333110$

$-2.7075030$

0.0697240

$-0.6599020$

2.6343880

2.1302840

4.0902520

4.8289820

4.7347950 


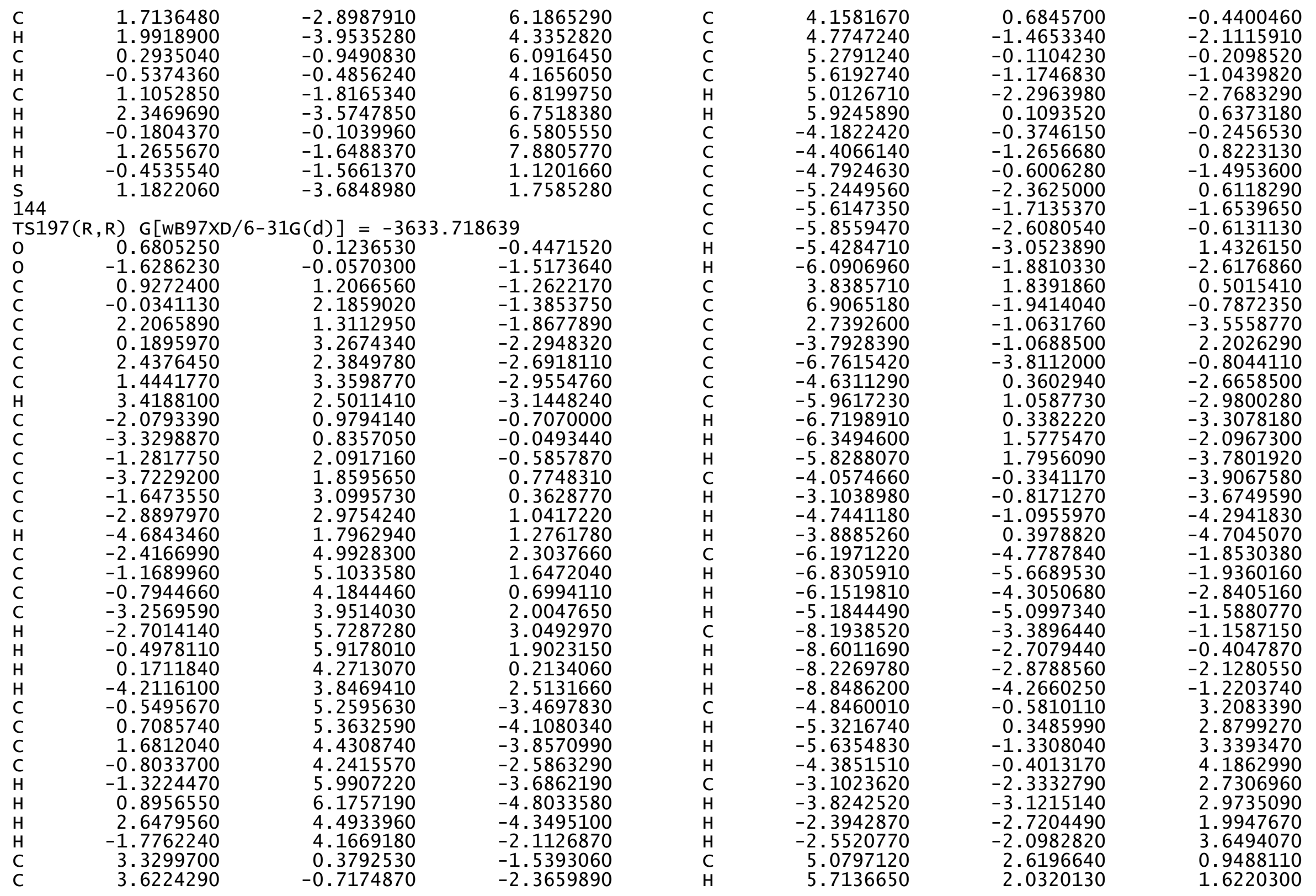




\begin{tabular}{rrr}
5.6877860 & 2.9283360 & 0.0919090 \\
4.7743770 & 3.5191390 & 1.4940590 \\
3.0399020 & 1.3421490 & 1.7144900 \\
2.1117870 & 0.8560630 & 1.3999310 \\
3.6238350 & 0.6178520 & 2.2958340 \\
2.7798450 & 2.1762620 & 2.3759510 \\
8.0745440 & -1.2505390 & -1.5086050 \\
9.0214530 & -1.7574070 & -1.2914170 \\
7.9171170 & -1.2714050 & -2.5935050 \\
8.1656210 & -0.2038160 & -1.2005330 \\
6.8431730 & -3.4235460 & -1.1684720 \\
5.9963620 & -3.9309030 & -0.6941510 \\
6.7538480 & -3.5590280 & -2.2519990 \\
7.7612790 & -3.9292590 & -0.8517780 \\
2.5746860 & -2.5733710 & -3.7581680 \\
3.4897090 & -3.0339660 & -4.1485040 \\
2.3126840 & -3.0726590 & -2.8220830 \\
1.7768880 & -2.7656620 & -4.4826900 \\
3.2741780 & -0.4083640 & -4.8379250 \\
4.2858880 & -0.7691190 & -5.0592540 \\
2.6307530 & -0.6526530 & -5.6907280 \\
3.3158620 & 0.6811260 & -4.7434610 \\
-0.4939870 & -0.9754980 & -0.8016050 \\
0.0090500 & -2.0008780 & -1.7682700 \\
-0.9783800 & -1.4436100 & 0.5498850 \\
1.7428370 & -0.6564810 & -3.3570920 \\
7.1019510 & -1.8845270 & 0.2918020 \\
3.2000560 & 2.5440950 & -0.0396100 \\
-3.9239080 & 1.1428540 & -2.3790410 \\
-6.8006500 & -4.3446950 & 0.1539910 \\
-3.0258130 & -0.2957250 & 2.1240420 \\
2.1697590 & -2.7103710 & 0.6784080 \\
1.0821060 & -3.3200640 & 1.4557660 \\
2.0407790 & -1.6618970 & 0.4087940 \\
0.0840530 & -2.9353020 & 1.2814660 \\
1.6353300 & -3.6057580 & -0.3318750 \\
1.0053970 & -3.0808160 & -0.9127440 \\
3.7308150 & -4.4935550 & 1.5731440 \\
2.7320190 & -4.7723990 & 2.6982000 \\
1.2907250 & -4.5817470 & 2.2165850 \\
3.5868900 & -3.0602130 & 1.0571630 \\
4.7538640 & -4.6470560 & 1.9300240 \\
2.8503540 & -5.7898500 & 1.5392530 \\
0.9967960 & -5.3948640 & 0.1842660 \\
3.8976530 & -2.3443610 & \\
4.2164020 & -2.8803900 & \\
\hline & &
\end{tabular}

3.5689290
2.9266550
0.5685230
0.3419240
-0.5263530
0.6361970
1.5868230
-0.0172680
1.8827200
2.0984380
0.2823380
-0.7497320
1.2329840
2.6254370
-0.2259540
1.4697430
-0.6833150
1.0767130
$-5.2004210$
$-4.0892720$
$-4.6069000$
$-0.0935870$
0.1581890
1. 0915520
1.0424360
2. 3022880
2. 1828170
0.1094450
3.4395770
2. 3536210
3. 3833520
2. 1367840
4.3715540
4.2756480
$-0.5838410$

$-1.5907990$

\begin{abstract}
0.7501650
3.5345570

3. 0377730

3. 3519050

2. 4273970

4. 1931460

5.2190750

3.9167850

5.9539610

5.4285660

4.6548330

3. 1193250

5.6721950

6.7438410

4.4286570

6.2439000

1. 7361780

3. 5860850
\end{abstract} 144

$\operatorname{TS} 198(R, R) G[W B 97 X D / 6-31 G(d)]=-3633.717195$

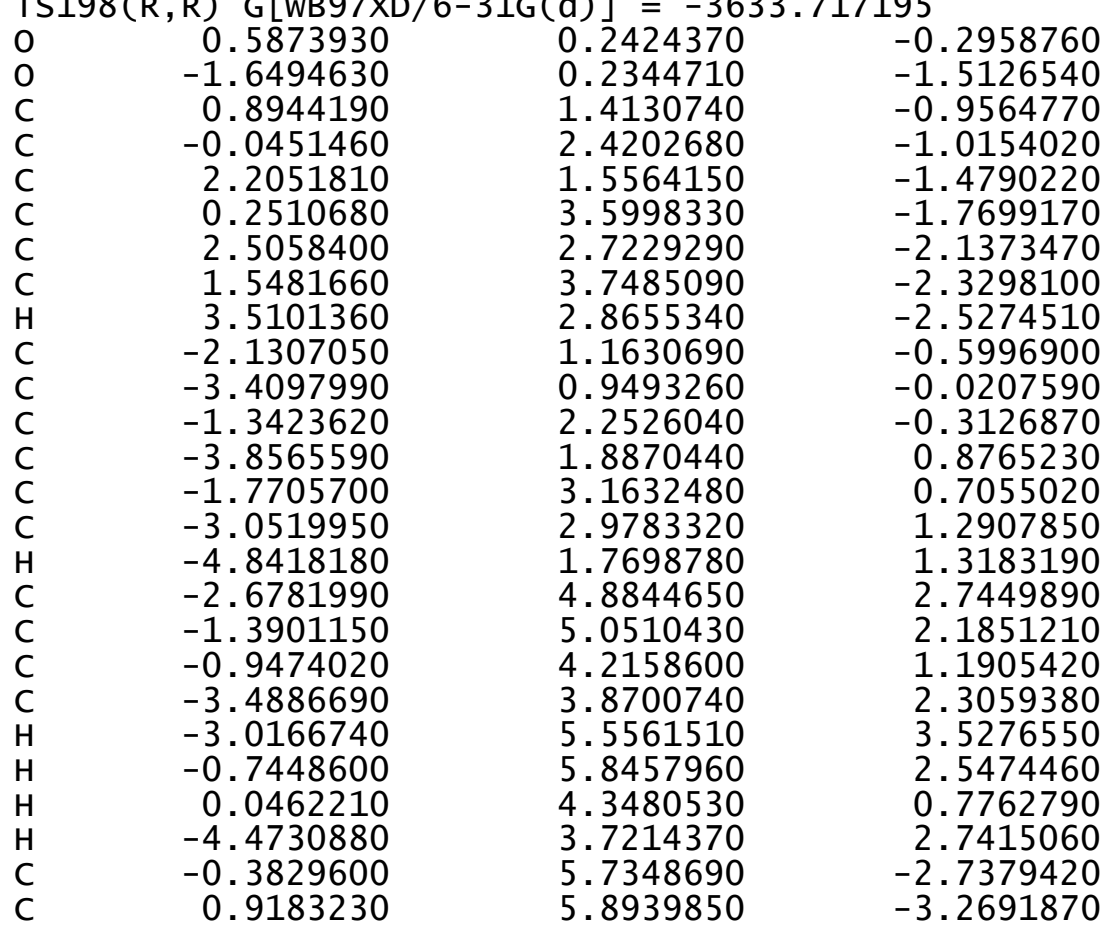




1.8603540
-0.7093020
-1.1322690
1.1629960
2.8591710
-1.7143490
3.2652700
3.5996590
3.9876770
4.7063790
5.0671020
5.4605370
4.9715030
5.6322250
-4.2326280
-4.4711920
-4.7944020
-5.2782210
-5.5898410
-5.8456810
-5.4693950
-6.0327620
3.6349670
6.6848870
2.8015500
-3.9035600
-6.7171250
-4.6060130
-5.9318490
-6.6785130
-6.3426570
-5.7838830
-3.9902040
-3.0423680
-4.6604460
-3.7986050
-7.9734030
-8.6254280
-7.7123520
-8.5416660
-5.9288680
-5.0488260
-5.5851010
-6.5564670
-4.9954430
-5.4701120

4.9185770

4.6202130

6.5003940

6.7844090

5.0212070

4.5066610

0.5202650

$-0.3476190$

0.4861730

$-1.1905760$

$-0.3856180$

$-1.2147500$

$-1.8484390$

$-0.4281940$

$-0.2494030$

$-1.2436840$

$-0.3695390$

$-2.3286150$

$-1.4799150$

$-2.4695190$

$-3.0913080$

$-1.5732750$

1. 4152150

$-2.0996360$

$-0.3803200$

$-1.1680450$

$-3.6646660$

0.6925170

1.4086140

0.7113790

1.8600000

2.2022250

0.1107700

$-0.3890110$

$-0.6152360$

0.9087690

$-3.7152660$

$-4.5406130$

$-3.8695700$

$-2.7822010$

$-4.9782840$

$-4.9526340$

$-5.1636530$

$-5.8243790$

$-0.7812000$

0.1708060

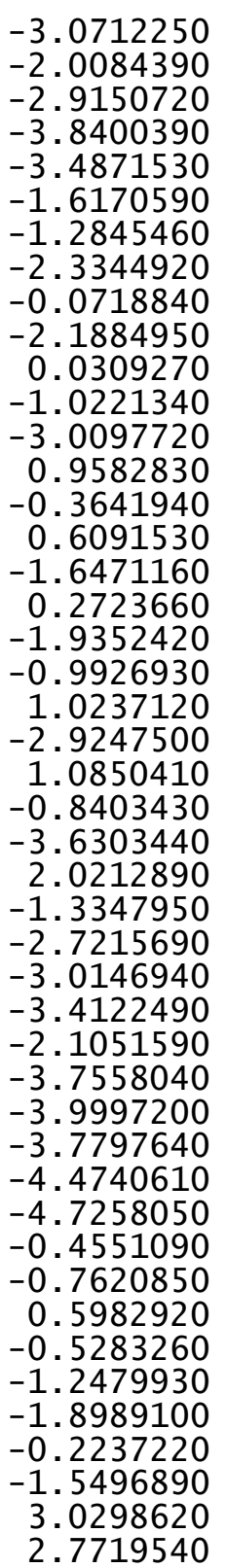

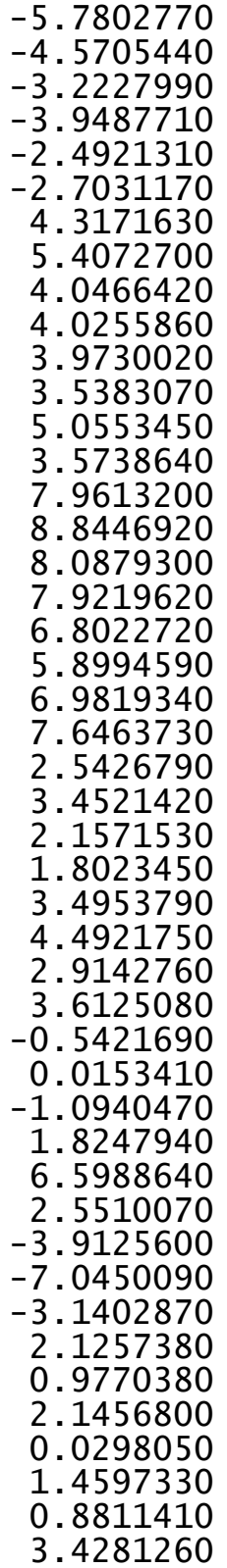

3.0627730

4.0357360

2. 4589010

2.6101350

1.7146960

3.4112740

0.9149090

0.9404290

$-0.0327690$

1.7290230

2.4624120

2.6020780

2.6201140

3.2411400

$-0.8481670$

$-0.6574310$

$-1.8230760$

$-0.0841750$

$-1.8614960$

$-1.8936350$

$-2.8705880$

$-1.6031400$

$-4.1239690$

$-4.5226520$

$-3.3207480$

$-4.9304610$

$-4.7272750$

$-4.9367130$

$-5.6558420$

$-4.4361070$

$-0.9030160$

$-2.0153330$

0.3154690

$-3.4315430$

0.1551010

1.0664420

$-2.3453510$

$-2.3749520$

2. 0401770

0.1058920

0.8417730

$-0.0134590$

0.7388180

$-0.9999270$

$-1.4508570$

0.7100350 


\begin{tabular}{|c|c|c|c|c|c|c|c|}
\hline$c$ & & & & & & & \\
\hline $\begin{array}{l}\mathrm{C} \\
\mathrm{C}\end{array}$ & $\begin{array}{l}2.4253420 \\
1.0189530\end{array}$ & $\begin{array}{l}-5.3428500 \\
-4.8921900\end{array}$ & $\begin{array}{l}1.8310990 \\
1.4289410\end{array}$ & $\begin{array}{l}\mathrm{C} \\
\mathrm{C}\end{array}$ & $\begin{array}{l}-1.2511410 \\
-3.7832210\end{array}$ & $\begin{array}{l}3.5551770 \\
2.6616800\end{array}$ & $\begin{array}{l}2.7850670 \\
3.5641910\end{array}$ \\
\hline $\mathrm{C}$ & 3.4846540 & -3.5721140 & 0.3814220 & $\mathrm{H}$ & -3.4792490 & 3.8115430 & 5.3369060 \\
\hline $\mathrm{H}$ & 4.4261670 & -5.4074460 & 1.0006810 & $\mathrm{H}$ & -1.2071120 & 4.5747490 & 4.6542330 \\
\hline $\mathrm{H}$ & 2.4027530 & -6.4080670 & 2.0784500 & $\mathrm{H}$ & -0.2592020 & 3.8906590 & 2.5032810 \\
\hline $\mathrm{H}$ & 0.6029900 & -5.5613010 & 0.6631430 & $\mathrm{H}$ & -4.7631750 & 2.2989030 & 3.8630080 \\
\hline $\mathrm{H}$ & 3.9184800 & -3.0133160 & 1.2198860 & $\mathrm{C}$ & -0.7772510 & 6.5140760 & -0.1685610 \\
\hline $\mathrm{H}$ & 4.1143130 & -3.3797600 & -0.4895130 & $\mathrm{C}$ & 0.5146240 & 6.9673130 & -0.5239750 \\
\hline $\mathrm{H}$ & 3.1444900 & -5.6296770 & -0.1869240 & $\mathrm{C}$ & 1.5315740 & 6.0643520 & -0.6915720 \\
\hline $\mathrm{H}$ & 2.7325420 & -4.8084670 & 2.7390640 & C & -1.0194570 & 5.1790950 & 0.0323550 \\
\hline $\mathrm{H}$ & 0.3120690 & -4.9240930 & 2.2629010 & $\mathrm{H}$ & -1.5854780 & 7.2308460 & -0.0595190 \\
\hline C & 0.4460280 & -0.5594700 & 3.1439100 & $\mathrm{H}$ & 0.6922830 & 8.0275200 & -0.6748690 \\
\hline 0 & -0.5902280 & -0.2493570 & 2.4352890 & $\mathrm{H}$ & 2.5249670 & 6.3966010 & -0.9814400 \\
\hline C & 0.9009530 & 0.5608360 & 3.9974950 & $\mathrm{H}$ & -2.0175610 & 4.8453000 & 0.2947130 \\
\hline $\mathrm{C}$ & 1.6626390 & 0.3544560 & 5.1518360 & C & 3.2511760 & 1.4230150 & -0.8494110 \\
\hline $\mathrm{C}$ & 0.5958810 & 1.8668070 & 3.5899610 & $\mathrm{C}$ & 3.6481890 & 1.1044400 & -2.1592480 \\
\hline C & 2.1144570 & 1.4418560 & 5.8882050 & C & 3.9664140 & 0.9152660 & 0.2518560 \\
\hline H & 1.8959640 & -0.6569070 & 5.4676050 & $\mathrm{C}$ & 4.8008440 & 0.3368510 & -2.3464490 \\
\hline C & 1.0698760 & 2.9511410 & 4.3169560 & $\mathrm{c}$ & 5.0903410 & 0.1282110 & 0.0134850 \\
\hline $\mathrm{H}$ & 0.0087010 & 2.0262500 & 2.6921100 & $\mathrm{C}$ & 5.5432190 & -0.1508290 & -1.2754820 \\
\hline C & 1.8275610 & 2.7403480 & 5.4674330 & $\mathrm{H}$ & 5.1159420 & 0.1118190 & -3.3601230 \\
\hline H & 2.6953890 & 1.2777210 & 6.7900360 & $\mathrm{H}$ & 5.6434310 & -0.2773060 & 0.8588170 \\
\hline $\mathrm{H}$ & 0.8429390 & 3.9589940 & 3.9835560 & C & -4.1338190 & -0.2185030 & -0.4659260 \\
\hline $\mathrm{H}$ & 2.1942370 & 3.5878400 & 6.0385290 & $\mathrm{c}$ & -4.2571690 & -1.5304420 & 0.0320500 \\
\hline $\mathrm{H}$ & -0.7946600 & -0.8725100 & 1.6478280 & $\mathrm{C}$ & -4.7057730 & 0.1236150 & -1.7065040 \\
\hline $\mathrm{s}$ & 1.2340370 & -2.0460350 & 3.1125550 & c & -4.9753560 & -2.4639290 & -0.7178180 \\
\hline & & & & $\mathrm{C}$ & -5.4028730 & -0.8487960 & -2.4203970 \\
\hline & R) $G[w B 97 x$ & $G(d)]=$ & 543 & $\mathrm{C}$ & -5.5535660 & -2.1488960 & -1.9427680 \\
\hline 0 & 0.6245580 & 0.5892350 & -0.0907700 & $\mathrm{H}$ & -5.0864200 & -3.4761400 & -0.3358140 \\
\hline 0 & -1.6146810 & 0.8683750 & -1.2579410 & $\mathrm{H}$ & -5.8461750 & -0.5812710 & -3.3771260 \\
\hline C & 0.8319130 & 1.9453550 & -0.2300510 & C & 3.5440370 & 1.1904920 & 1.6880810 \\
\hline C & -0.1857790 & 2.8173070 & 0.0931880 & C & 6.8227430 & -0.9551380 & -1.4490760 \\
\hline $\mathrm{C}$ & 2.1260420 & 2.3797460 & -0.6176480 & $\mathrm{c}$ & 2.8679820 & 1.5952350 & -3.3714540 \\
\hline $\mathrm{C}$ & 0.0190870 & 4.2198480 & -0.1139320 & $\mathrm{C}$ & -3.6536490 & -1.9670000 & 1.3617040 \\
\hline C & 2.3430790 & 3.7309290 & -0.7138440 & C & -6.3172300 & -3.1936250 & -2.7362850 \\
\hline $\mathrm{C}$ & 1.3073540 & 4.6749230 & -0.5053340 & $\mathrm{C}$ & -4.6068400 & 1.5269070 & -2.2890610 \\
\hline H & 3.3367620 & 4.0913960 & -0.9658740 & C & -5.9911250 & 2.1811850 & -2.3957720 \\
\hline C & -2.1584780 & 1.3311330 & -0.0697680 & $\mathrm{H}$ & -6.6438400 & 1.6238790 & -3.0775040 \\
\hline $\mathrm{C}$ & -3.4196280 & 0.8202030 & 0.3363520 & $\mathrm{H}$ & -6.4820010 & 2.2224020 & -1.4175330 \\
\hline C & -1.46 & 2.2872840 & 0.6350510 & $\mathrm{H}$ & -5.9010270 & 3.2039960 & -2.7785820 \\
\hline C & -3.95 & 1.3201000 & 1.4959730 & C & -3.8844800 & 1.5242720 & -3.6426920 \\
\hline C & -1.97 & 2.7102330 & 1.9037320 & $\mathrm{H}$ & -2.8950530 & 1.0672430 & -3.5509770 \\
\hline $\mathrm{C}$ & -3.25 & 2.2356470 & 2.3179790 & $\mathrm{H}$ & -4.4527120 & 0.9682760 & -4.3974500 \\
\hline $\mathrm{H}$ & 40 & 0.9796900 & 1.8161170 & $\mathrm{H}$ & -3.7589580 & 2.5494780 & -4.0087820 \\
\hline C & -3.0 & 3.4963020 & 4.3829400 & C & -5.6502050 & -3.4654410 & -4.0913810 \\
\hline & -1.7 & 3.9354170 & 3.9912750 & $\mathrm{H}$ & -6.1715910 & -4.2693770 & -4.6230130 \\
\hline
\end{tabular}




-5.6720120
-4.6037340
-7.7895320
-8.2743540
-7.8820350
-8.3361830
-4.7319880
-5.2501950
-5.4836740
-4.2837690
-2.8989350
-3.5774910
-2.1610870
-2.3747170
4.6786450
5.5127640
5.0663670
4.3149490
2.9972370
2.1416320
3.7601150
2.6648410
8.0424170
8.9681130
8.1136200
7.9694250
6.9778180
6.1059450
7.1197990
7.8581130
2.7260160
3.6745070
2.3750900
2.0014380
3.5005930
4.5298800
2.9311190
3.5258640
-0.3977400
0.2106110
-0.8329790
1.8567650
6.7947590
2.7299620
-4.0148780
-6.2897740

$-6.2897740$

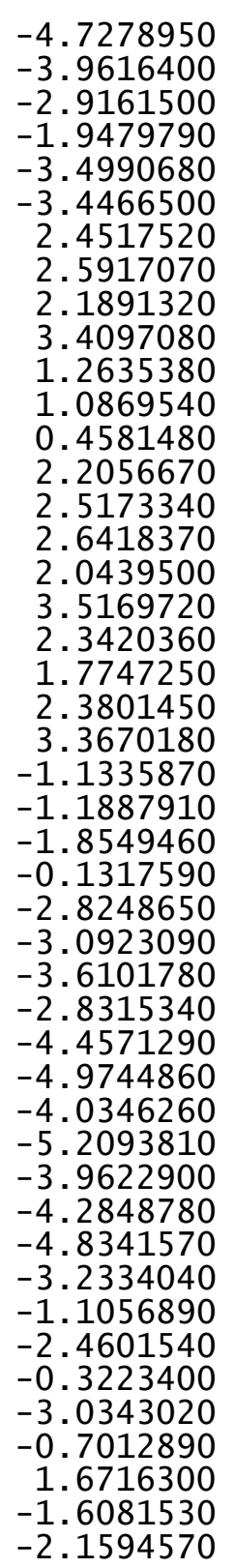

$\begin{array}{rr}-2.5731680 & -4.7278950 \\ -3.7598970 & -3.9616400 \\ -2.8007750 & -2.9161500 \\ -2.6378440 & -1.9479790 \\ -1.8770340 & -3.4990680 \\ -3.5884260 & -3.4466500 \\ -2.0576280 & 2.4517520 \\ -1.1040320 & 2.5917070 \\ -2.8114790 & 2.1891320 \\ -2.3449470 & 3.4097080 \\ -3.2995480 & 1.2635380 \\ -4.1416700 & 1.0869540 \\ -3.2718620 & 0.4581480 \\ -3.4956870 & 2.2056670 \\ 1.8046380 & 2.5173340 \\ 1.1049250 & 2.6418370 \\ 2.7128020 & 2.0439500 \\ 2.0666110 & 3.5169720 \\ -0.0844790 & 2.3420360 \\ -0.4603660 & 1.7747250 \\ -0.8711370 & 2.3801450 \\ 0.1148590 & 3.3670180 \\ -0.0745840 & -1.1335870 \\ -0.6581270 & -1.1887910 \\ 0.7480070 & -1.8549460 \\ 0.3606790 & -0.1317590 \\ -1.6068930 & -2.8248650 \\ -2.2142460 & -3.0923090 \\ -0.8555170 & -3.6101780 \\ -2.2578390 & -2.8315340 \\ 0.5214850 & -4.4571290 \\ 0.3369190 & -4.9744860 \\ -0.4226260 & -4.0346260 \\ 0.8502560 & -5.2093810 \\ 2.8634610 & -3.9622900 \\ 2.6655710 & -4.2848780 \\ 3.2046150 & -4.8341570 \\ 3.6786730 & -3.2334040 \\ -0.1957830 & -1.1056890 \\ -0.3954750 & -2.4601540 \\ -1.4101560 & -0.3223400 \\ 1.8431930 & -3.0343020 \\ -1.7599680 & -0.7012890 \\ 1.9211460 & 1.6716300 \\ -4.1448140 & -1.6081530 \\ -4.1270330 & -2.1594570\end{array}$

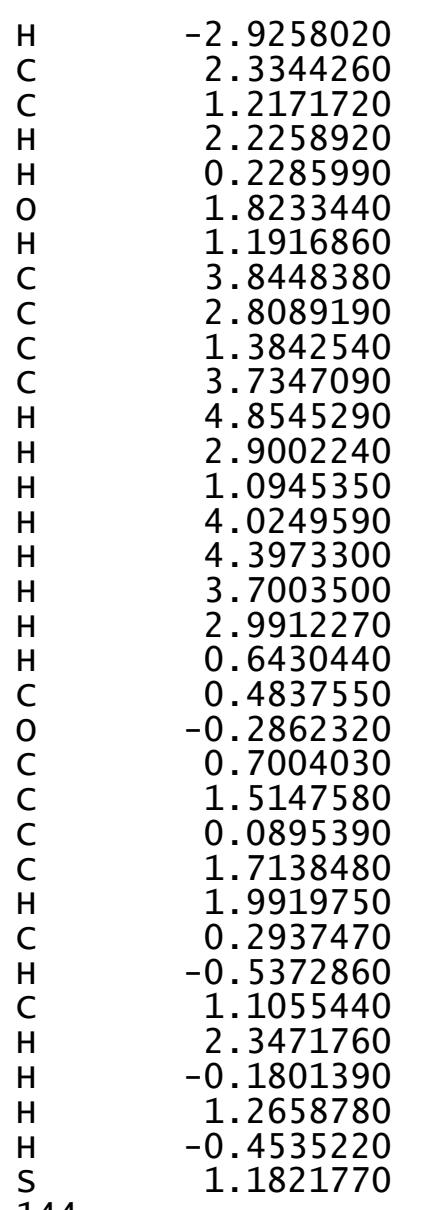
$-1.2111970$
$-2.5063410$
$-3.4482730$
$-1.5396600$
$-3.0175520$
$-2.4842500$
$-1.7005940$
$-4.4682850$
$-5.4124860$
$-4.8987870$
$-3.0641360$
$-4.8543220$
$-6.4160100$
$-5.0254810$
$-3.0730030$
$-2.3631690$
$-4.4152000$
$-5.5034760$
$-5.4560580$
$-2.4300310$
$-1.5208090$
$-2.2477910$
$-3.1140380$
$-1.1614890$
$-2.8986970$
$-3.9534800$
$-0.9489570$
$-0.4855300$
$-1.8164100$
$-3.5746970$
$-0.1038390$
$-1.6486970$
$-1.5661000$
$-3.6849210$

144

$\operatorname{TS} 200(R, R) \quad G[W B 97 X D / 6-31 G(d)]=-3633.717007$

$\begin{array}{lrcr}\text { O } & 0.5725910 & 0.1891460 & -0.3141370 \\ \mathrm{O} & -1.6667140 & 0.2563810 & -1.5341970 \\ \mathrm{C} & 0.9042580 & 1.3795890 & -0.9239610 \\ \mathrm{C} & -0.0258880 & 2.3976040 & -0.9632440 \\ \mathrm{C} & 2.2281050 & 1.5355700 & -1.4092910 \\ \mathrm{C} & 0.2878020 & 3.5973910 & -1.6770520 \\ \mathrm{C} & 2.5443230 & 2.7225860 & -2.0245380 \\ \mathrm{C} & 1.5944950 & 3.7560500 & -2.2098950 \\ \mathrm{H} & 3.5586720 & 2.8745340 & -2.3841420 \\ \mathrm{C} & -2.1359850 & 1.1637170 & -0.5947690\end{array}$

1. 6681940 $-1.0943300$ $-0.9403710$ $-0.8260060$ $-2.4491780$ $-2.5283130$ $-1.6215680$ $-1.0083610$ $-1.2395870$ $-1.0236300$ $-1.4515910$ $-1.4338720$ $-2.2909420$ 0.0344400 $-1.5332660$ $-2.7074160$ 0.0698070 $-0.6597720$

2.6344360 2. 1303280 4. 0902900 4.8289990

4.7348360

6. 1865340

4. 3352900

6.0916710

4.1656590

6.8199810

6.7518280

6.5805810

7.8805730

1.1202190

1.7585920 $-0.5987260$ 


$\begin{array}{rrr}-3.4217440 & 0.9558000 & -0.0293200 \\ -1.3297100 & 2.2293850 & -0.2728060 \\ -3.8558470 & 1.8740430 & 0.8941460 \\ -1.7473860 & 3.1183390 & 0.7687510 \\ -3.0343820 & 2.9384880 & 1.3433120 \\ -4.8446630 & 1.7605950 & 1.3289940 \\ -2.6355710 & 4.7967410 & 2.8519910 \\ -1.3430400 & 4.9584750 & 2.3011000 \\ -0.9094760 & 4.1436350 & 1.2858850 \\ -3.4603780 & 3.8081240 & 2.3820480 \\ -2.9663420 & 5.4515610 & 3.6520560 \\ -0.6868930 & 5.7329990 & 2.6869440 \\ 0.0879950 & 4.2707100 & 0.8794650 \\ -4.4486760 & 3.6632690 & 2.8100490 \\ -0.3207120 & 5.7621050 & -2.5932980 \\ 0.9898650 & 5.9296670 & -3.0989200 \\ 1.9245070 & 4.9449550 & -2.9127560 \\ -0.6639690 & 4.6291840 & -1.9007930 \\ -1.0636360 & 6.5359620 & -2.7608900 \\ 1.2473000 & 6.8344080 & -3.6408730 \\ 2.9306750 & 5.0544900 & -3.3087200 \\ -1.6756720 & 4.5093070 & -1.5287800 \\ 3.3172490 & 0.5241370 & -1.2227820 \\ 3.7160840 & -0.2831500 & -2.3001210 \\ 4.0591560 & 0.5255480 & -0.0238940 \\ 4.9146610 & -0.9958850 & -2.2034580 \\ 5.2460290 & -0.2033660 & 0.0237500 \\ 5.7133630 & -0.9402110 & -1.0650200 \\ 5.2366210 & -1.5902260 & -3.0535160 \\ 5.8479750 & -0.1824970 & 0.9290020 \\ -4.2635850 & -0.2181410 & -0.4097300 \\ -4.5321980 & -1.2286530 & 0.5386260 \\ -4.8134780 & -0.2999050 & -1.7010670 \\ -5.3596290 & -2.2879760 & 0.1713680 \\ -5.6280210 & -1.3876010 & -2.0207350 \\ -5.9162590 & -2.3905330 & -1.1020090 \\ -5.5756510 & -3.0610300 & 0.9052270 \\ -6.0607460 & -1.4515100 & -3.0170740 \\ 3.6279150 & 1.3653240 & 1.1732230 \\ 7.0420750 & -1.6733380 & -0.9564690 \\ 2.8945950 & -0.3615540 & -3.5787460 \\ -3.9699650 & -1.2022920 & 1.9547640 \\ -6.8138210 & -3.5561060 & -1.4765320 \\ -4.5886650 & 0.7765720 & -2.7540250 \\ -5.8969040 & 1.5167030 & -3.0656940 \\ -6.6449160 & 0.8376380 & \\ -1.4910800\end{array}$

-6.3215130
-5.7198180
-3.9528680
-3.0163740
-4.6219590
-3.7349450
-8.0962960
-8.7645340
-7.8689080
-8.6332250
-6.0747640
-5.1687500
-5.7799520
-6.7191610
-5.0603050
-5.5231160
-5.8539940
-4.6374930
-3.3045200
-4.0390090
-2.5748340
-2.7859290
4.2808940
5.3712200
4.0456200
3.9283790
3.9088290
3.4598130
4.9829670
3.4844810
7.7891670
8.7898910
7.2717720
7.8957980
6.8633500
6.3695970
6.2542350
7.8352370
2.7186710
3.6554640
2.3652310
1.9832150
3.5057590
4.5245740
2.9082380
3.5530520

1.9607960
2.3188950
0.2045460
-0.3101140
-0.5058360
1.0100180
-3.5670830
-4.3724840
-3.7270250
-2.6170970
-4.8966630
-4.8963490
-5.1038340
-5.7171260
-0.8342000
0.1308700
-1.5907050
-0.7766600
-2.5271670
-3.3314070
-2.8342490
-2.4075630
2.7549250
2.6669420
3.2811890
3.3663700
0.6888840
-0.3089600
0.5928100
1.2905890
-1.7831950
-2.1964360
-2.4517370
-0.8057430
-3.0621800
-2.9947730
-3.7056950
-3.5479460
-1.8047450
-2.2206690
-2.4533020
-1.8364950
0.5078410
0.1758990
0.4387550
1.5605400

$-2.1589290$

$-3.7909360$

$-4.0270150$

$-3.7932710$

$-4.5260350$

$-4.7372900$

$-0.6338030$

$-0.9583930$

0.4266110

$-0.7240090$

$-1.3716550$

$-1.9864030$

$-0.3364000$

$-1.7066080$

2. 9717160

2. 7419440

2. 9791270

3. 9811270

2. 3521820

2.4747880

1. 5998720

3. 3104440

1. 1310120

1.2072100

0.2008240

1.9696420

2. 5181920

2.5663440

2. 7120080

3. 3282110

$-2.2888460$

$-2.1247860$

$-2.9862770$

$-2.7705300$

$-0.3248280$

0.6503240

$-0.9709080$

$-0.1837120$

$-4.0660330$

$-4.4548890$

$-3.2602610$

$-4.8764790$

$-4.6863370$

$-4.9198660$

-5.6022980
-4.3907670 


$\begin{array}{rrr}-0.5745000 & -0.8015640 & -0.9627160 \\ -0.0255030 & -1.5924820 & -2.1090980 \\ -1.1260880 & -1.5462420 & 0.2295220 \\ 1.8947890 & 0.0237100 & -3.3559280 \\ 7.6696490 & -1.0840850 & -0.2749220 \\ 2.5430430 & 1.5078190 & 1.1120760 \\ -3.8928540 & 1.5180610 & -2.3521710 \\ -7.1055310 & -3.4181820 & -2.5255200 \\ -3.1971150 & -0.4320030 & 2.0005950 \\ 2.0246360 & -2.8624490 & 0.0683040 \\ 0.9448860 & -3.5806670 & 0.7599860 \\ 1.9094090 & -1.7816010 & -0.0077150 \\ -0.0521180 & -3.1637990 & 0.6724990 \\ 1.4580360 & -3.5531150 & -1.0733300 \\ 0.8460690 & -2.9026810 & -1.5357490 \\ 3.5761400 & -4.7900790 & 0.5861110 \\ 2.6045180 & -5.2520590 & 1.6744290 \\ 1.1521370 & -4.9571370 & 1.2845940 \\ 3.4442720 & -3.2866950 & 0.3378910 \\ 4.6061830 & -5.0193530 & 0.8762020 \\ 2.7132080 & -6.3239770 & 1.8625700 \\ 0.8196350 & -5.6357020 & 0.4871050 \\ 3.7915730 & -2.7221500 & 1.2118940 \\ 4.0500840 & -2.9619680 & -0.5104760 \\ 3.3764040 & -5.3350170 & -0.3445080 \\ 2.8375490 & -4.7360390 & 2.6144700 \\ 0.4553970 & -5.1153100 & 2.1128580 \\ 0.3387050 & -0.6917850 & 3.1277700 \\ -0.6524400 & -0.3344370 & 2.3779780 \\ 0.7984200 & 0.3980390 & 4.0172540 \\ 1.5304540 & 0.1453250 & 5.1818430 \\ 0.5314870 & 1.7207540 & 3.6377680 \\ 1.9907700 & 1.2027400 & 5.9553950 \\ 1.7350760 & -0.8788830 & 5.4749810 \\ 1.0122860 & 2.7749610 & 4.4035510 \\ -0.0316560 & 1.9178300 & 2.7322520 \\ 1.7403730 & 2.5177900 & 5.5636230 \\ 2.5493720 & 1.0024360 & 6.8640160 \\ 0.8134710 & 3.7959110 & 4.0930760 \\ 2.1123450 & 3.3417190 & 6.1650040 \\ -0.8512620 & -0.9474660 & 1.5816420 \\ 1.0661980 & -2.2091140 & 3.1093530\end{array}$

TS201(R,R) G[WB97XD/6-31G(d)] = -3633.719478

$\begin{array}{lccccc}\text { TS201(R, R) } & \text { G }[W B 97 X D / 6-31 G(d)]= & -3633.719478 & \mathrm{C} & -5.4997190 \\ \mathrm{O} & 0.5322880 & 0.6769290 & -0.0681080 & \mathrm{H} & -4.8669550 \\ \mathrm{O} & -1.7315600 & 0.9536620 & -1.1797030 & \mathrm{H} & -5.9703640\end{array}$

0.7307720
-0.2972200
2.0328140
-0.0983830
2.2432490
1.1945230
3.2393690
-2.2450000
-3.4756590
-1.5640900
-4.0105090
-2.0699250
-3.3249070
-4.9737180
-3.1529540
-1.8835110
-1.3560480
-3.8535760
-3.5619900
-1.3175110
-0.3768320
-4.8192630
-0.9124790
0.3827220
1.4107510
-1.1473310
-1.7286570
0.5545660
2.4071190
-2.1472780
3.1639250
3.6767050
3.7748720
4.8423410
4.9357230
5.5014640
5.2596370
5.4138040
-4.1587610
-4.1644380
-4.8094870
-4.8465400
-5.4664430
-5.4997190
-4.8669550
-5.9703640

.0386020

2.8891400

2.4918340

4.2990950

3.8474130

4.7760310

4.2242910

1.3632230

0.7993970

2.3281100

1.2715700

2.7201470

2.2064730

0.8960080

3.4554230

3.9308350

3.5747860

2.6077580

3.7516540

4.5788800

3.9402360

2.2145840

6.5873920

.0616640

6.1726320

5.2448050

7.2941390

8.1274480

6.5222240

4.8937140

1.5475700

1. 3980850

0.8841470

0.6538470

0.1440120

0.0376590

0.5497010

$-0.3500910$

$-0.2639490$

$-1.5949760$

0.0727920

$-2.5557010$

$-0.9259030$

$-2.2471350$

$-3.5847800$

$-0.6625350$
$-0.1394430$

0.2078100

$-0.4725390$

0.0526350

$-0.5100570$

$-0.2955720$

$-0.7254350$

0.0396450

0.4690600

0.7463650

1.6405160

2.0295450

2.4564600

1.9737440

4.5296480

4.1274820

2.9124100

3.7118400

5.4906350

4.7896360

2. 6244230

4.0184900

0.0572460

$-0.2563400$

$-0.4312400$

0.2101860

0.1722920

$-0.3700380$

$-0.6886390$

0.4415390

$-0.7248110$

$-2.0268720$

0.3560750

$-2.2039130$

0.1255640

$-1.1420050$

$-3.2035660$

0.9647640

$-0.3280580$

0.1331280

$-1.5294860$

$-0.6157650$

$-2.2453320$

$-1.8049570$

$-0.2651940$

$-3.1728100$ 


\begin{tabular}{|c|c|c|}
\hline $\begin{array}{l}3.2342870 \\
6.8136710 \\
3.0072260 \\
-3.4745580 \\
-6.2172320 \\
-4.8452240 \\
-6.2756350 \\
-6.9336390 \\
-6.6976030 \\
-6.2866190 \\
-4.2265940 \\
-3.2031230 \\
-4.8069120 \\
-4.2011630 \\
-5.5811800 \\
-6.0640750 \\
-5.6880720 \\
-4.5127720 \\
-7.7189330 \\
-8.1794310 \\
-7.8972370 \\
-8.2276690 \\
-4.4882860 \\
-5.0181580 \\
-5.2363540 \\
-3.9816740 \\
-2.6935360 \\
-3.3591360 \\
-1.9976680 \\
-2.1199160 \\
3.9022820 \\
4.9819950 \\
3.7474040 \\
3.4871450 \\
3.3704770 \\
3.0162790 \\
4.4081340 \\
2.7781930 \\
7.1599770 \\
8.0566240 \\
7.3724620 \\
6.3483540 \\
7.9554060 \\
7.7463750 \\
8.0861870 \\
8.8998820\end{array}$ & $\begin{array}{r}1.0127160 \\
-0.6845640 \\
2.0334460 \\
-2.0147810 \\
-3.3230780 \\
1.5001120 \\
2.0563510 \\
1.4857350 \\
2.0109580 \\
3.1011150 \\
1.6056130 \\
1.2194800 \\
1.0406970 \\
2.6516150 \\
-3.5131700 \\
-4.3380520 \\
-2.6081880 \\
-3.7359640 \\
-3.0322440 \\
-2.9212110 \\
-2.1072200 \\
-3.8470400 \\
-2.1302010 \\
-1.1887730 \\
-2.9002650 \\
-2.4090860 \\
-3.3284300 \\
-4.1879020 \\
-3.2885200 \\
-3.5083400 \\
2.1900040 \\
2.0231710 \\
3.1278410 \\
2.3069650 \\
-0.2793710 \\
-1.1438380 \\
-0.4736050 \\
-0.2100180 \\
-1.7585270 \\
-2.3025910 \\
-1.3179740 \\
-2.4830340 \\
0.3387260 \\
1.0837070 \\
0.8672650 \\
-0.1590040\end{array}$ & $\begin{array}{r}1.7744130 \\
-1.4033650 \\
-3.2382890 \\
1.4261580 \\
-2.6001360 \\
-2.0575610 \\
-2.0340510 \\
-2.6997000 \\
-1.0242590 \\
-2.3643860 \\
-3.4568990 \\
-3.4560920 \\
-4.1955400 \\
-3.7825960 \\
-3.9840430 \\
-4.5198350 \\
-4.5931730 \\
-3.8974280 \\
-2.7203820 \\
-1.7331580 \\
-3.2808720 \\
-3.2476160 \\
2.5747680 \\
2.7461010 \\
2.3514470 \\
3.5059010 \\
1.2930650 \\
1.1554660 \\
0.4513680 \\
2.2093990 \\
2.4988280 \\
2.5910960 \\
1.9550300 \\
3.5063210 \\
2.5877870 \\
2.0169130 \\
2.8820280 \\
3.5061370 \\
-0.3700420 \\
-0.6842890 \\
0.6106860 \\
-0.2480620 \\
-1.5153660 \\
-2.2898390 \\
-0.5637510 \\
-1.7619490\end{array}$ \\
\hline
\end{tabular}

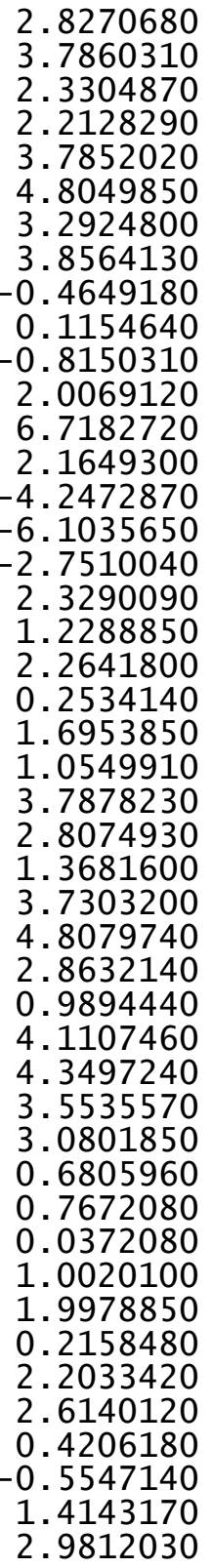

1.0346500

0.7535050

0.1250810

1.4815270

3.2669890

2.9914090

3. 7188170

4.0278870

$-0.0602720$

-0.0994660

-1.3591400

2. 3593130

$-1.1804380$

1. 2288890

2. 1301570

$-4.2663390$

$-1.2375210$

$-3.2624860$

$-1.3923250$

$-2.8564010$

$-2.1430670$

$-1.3638130$

$-4.1830600$

$-5.1927450$

$-4.6718920$

$-2.8477950$

$-4.5764240$

$-6.1486570$

$-4.6893970$

$-2.9655560$

$-2.0916630$

$-4.0222960$

$-5.3885380$

$-5.2948260$

$-2.6958150$

$-1.6791240$

$-2.7856020$

$-3.6126140$

$-2.0087580$

$-3.6634500$

$-4.2057860$

$-2.0685730$

$-1.3661960$

$-2.8942010$

$-4.3031250$
$-4.3883020$

$-4.8380370$

$-4.0428820$

$-5.1774270$

$-4.0118520$

$-4.5854570$

$-2.9340110$

$-1.1373560$

$-2.5190520$

$-0.4545560$

$-2.9379410$

$-2.3792030$

1.7119340

$-1.3930440$

$-2.0507230$

1.6887650

$-1.6332220$

$-1.4848000$

$-1.0296360$

$-1.2402420$

$-2.9253130$

$-2.8515660$

$-2.4960370$

$-1.8963400$

$-1.9528220$

$-1.7505990$

$-2.4497820$

$-2.4249870$

$-2.9829200$

$-0.7285840$

$-2.2390460$

$-3.5551640$

$-0.8515530$

$-1.3737680$

2. 2328690

1.9059470

3. 6933420

4. 2252550

4. 5562810

5.5977750

3. 5583350

5.9288510

4. 1462820

6.4512080

6.0020130 


\begin{tabular}{|c|c|c|c|c|c|c|c|}
\hline $\mathrm{H}$ & 0 10ror & 14505050 & $6-501500$ & $\theta_{3}$ & 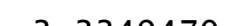 & 23888610 & م2705 2700 \\
\hline H & $\begin{array}{r}-0.1960640 \\
1.5750740\end{array}$ & $\begin{array}{l}-1.4695950 \\
-2.9381720\end{array}$ & $\begin{array}{l}6.5911580 \\
7.5240600\end{array}$ & $\begin{array}{l}C \\
C\end{array}$ & $\begin{array}{l}3.3349470 \\
2.4431210\end{array}$ & $\begin{array}{l}2.3888610 \\
3.1488870\end{array}$ & $\begin{array}{l}0.2795200 \\
1.0658880\end{array}$ \\
\hline $\mathrm{H}$ & -0.2327310 & -1.6157200 & 0.9207690 & c & 3.8506130 & 2.9120500 & -0.9152680 \\
\hline $\mathrm{s}$ & 1.3904000 & -3.8215260 & 1.1502450 & c & 2.0980760 & 4.4262610 & 0.6329350 \\
\hline & & & & $\mathrm{c}$ & 3.4686410 & 4.1964770 & -1.3101650 \\
\hline & , R) $\mathrm{G}[\mathrm{WB} 97 \mathrm{X} D$ & $G(d)]=-36$ & 459 & C & 2.5872910 & 4.9638190 & -0.5577150 \\
\hline 0 & 0.3850070 & -1.1734510 & 0.1977950 & $\mathrm{H}$ & 1.4007150 & 5.0114420 & 1.2256670 \\
\hline 0 & 2.1517470 & 0.1884840 & -0.9558290 & $\mathrm{H}$ & 3.8550440 & 4.6037190 & -2.2426530 \\
\hline C & 0.9686530 & -2.3526720 & -0.2206700 & C & -1.2916040 & -3.4112340 & 1.9257590 \\
\hline C & 2.3362530 & -2.4892630 & -0.1316420 & $\mathrm{C}$ & -5.5671540 & -2.0476110 & -0.3887810 \\
\hline C & 0.0935200 & -3.3834730 & -0.6598380 & $\mathrm{c}$ & -1.4797190 & -3.0489190 & -3.1761470 \\
\hline C & 2.9244320 & -3.6855520 & -0.6608580 & $\mathrm{C}$ & 1.8456820 & 2.6025340 & 2.3575600 \\
\hline C & 0.6613110 & -4.5671840 & -1.0500930 & $\mathrm{C}$ & 2.1157680 & 6.3202650 & -1.0499020 \\
\hline $\mathrm{C}$ & 2.0706700 & -4.7347300 & -1.1017680 & C & 4.7888590 & 2.1090100 & -1.8036820 \\
\hline $\mathrm{H}$ & 0.0275320 & -5.3973360 & -1.3473930 & C & 6.1598560 & 2.7864210 & -1.9288830 \\
\hline C & 2.9741750 & -0.0991950 & 0.1128680 & $\mathrm{H}$ & 6.0768020 & 3.7667290 & -2.4123580 \\
\hline C & 3.6687600 & 0.9930670 & 0.6976570 & $\mathrm{H}$ & 6.6177050 & 2.9333200 & -0.9447590 \\
\hline C & 3.1325200 & -1.4086190 & 0.5141830 & $\mathrm{H}$ & 6.8354500 & 2.1711440 & -2.5336320 \\
\hline C & 4.6009970 & 0.7108540 & 1.6605420 & C & 4.1664950 & 1.8511040 & -3.1824110 \\
\hline $\mathrm{C}$ & 4.0123720 & -1.6736760 & 1.6179160 & $\mathrm{H}$ & 3.2008700 & 1.3475050 & -3.0801770 \\
\hline C & 4.7870350 & -0.6062760 & 2.1523350 & $\mathrm{H}$ & 4.0106520 & 2.7883550 & -3.7292940 \\
\hline $\mathrm{H}$ & 5.1880970 & 1.5179760 & 2.0897540 & $\mathrm{H}$ & 4.8255410 & 1.2173530 & -3.7865660 \\
\hline C & 5.8126210 & -2.1169680 & 3.7510250 & C & 2.3821250 & 7.4361960 & -0.0323030 \\
\hline C & 5.0043840 & -3.1676640 & 3.2602010 & $\mathrm{H}$ & 2.0758480 & 8.4061170 & -0.4395300 \\
\hline C & 4.1291050 & -2.9533950 & 2.2256610 & $\mathrm{H}$ & 1.8212930 & 7.2725590 & 0.8949130 \\
\hline C & 5.6972080 & -0.8628230 & 3.2111760 & $\mathrm{H}$ & 3.4457020 & 7.4915140 & 0.2220190 \\
\hline $\mathrm{H}$ & 6.5085430 & -2.3031500 & 4.5631630 & C & 0.6286240 & 6.2657530 & -1.4325260 \\
\hline $\mathrm{H}$ & 5.0731590 & -4.1529650 & 3.7111110 & $\mathrm{H}$ & 0.4472540 & 5.4910900 & -2.1855280 \\
\hline $\mathrm{H}$ & 3.5092290 & -3.7689750 & 1.8714600 & $\mathrm{H}$ & 0.0089560 & 6.0357010 & -0.5574830 \\
\hline $\mathrm{H}$ & 6.2906510 & -0.0377110 & 3.5961920 & $\mathrm{H}$ & 0.2961900 & 7.2272400 & -1.8398900 \\
\hline C & 4.8522330 & -5.0172410 & -1.3018760 & C & 2.7286550 & 2.9471880 & 3.5665480 \\
\hline C & 4.0034430 & -6.0742250 & -1.7030110 & $\mathrm{H}$ & 3.7359540 & 2.5339110 & 3.4679410 \\
\hline C & 2.6434350 & -5.9313560 & -1.6066300 & $\mathrm{H}$ & 2.8183430 & 4.0346910 & 3.6755030 \\
\hline C & 4.3281450 & -3.8558450 & -0.7931000 & $\mathrm{H}$ & 2.2876820 & 2.5486540 & 4.4874430 \\
\hline $\mathrm{H}$ & 5.9277570 & -5.1245590 & -1.4038670 & C & 0.4137320 & 3.0891210 & 2.6131630 \\
\hline $\mathrm{H}$ & 4.4314510 & -6.9898250 & -2.0992560 & $\mathrm{H}$ & 0.3927320 & 4.1328550 & 2.9480910 \\
\hline $\mathrm{H}$ & 1.9792030 & -6.7283620 & -1.9302960 & $\mathrm{H}$ & -0.1952920 & 3.0053060 & 1.7108290 \\
\hline $\mathrm{H}$ & 4.9892270 & -3.0490630 & -0.4952310 & $\mathrm{H}$ & -0.0482110 & 2.4860980 & 3.4025960 \\
\hline C & -1.3795310 & -3.1275400 & -0.6174280 & C & -1.7740960 & -4.7762480 & 2.4376000 \\
\hline C & -2.1055900 & -2.8826720 & -1.7981520 & $\mathrm{H}$ & -2.8438420 & -4.7479560 & 2.6764180 \\
\hline C & -2.0179320 & -3.0533650 & 0.6360090 & $\mathrm{H}$ & -1.6176170 & -5.5545840 & 1.6828270 \\
\hline C & -3.4539530 & -2.5338190 & -1.6889850 & $\mathrm{H}$ & -1.2303640 & -5.0620610 & 3.3451170 \\
\hline C & -3.3646230 & -2.7009440 & 0.6917070 & C & -1.4297690 & -2.3343690 & 3.0056820 \\
\hline C & 977100 & -2.4213750 & -0.4598730 & $\mathrm{H}$ & -1.1233650 & -1.3579140 & 2.6186210 \\
\hline H & 270690 & -2.3434070 & -2.5929950 & $\mathrm{H}$ & -2.4626050 & -2.2562090 & 3.3645490 \\
\hline $\mathrm{H}$ & -3.8549700 & -2.6406330 & 1.6607040 & $\mathrm{H}$ & -0.8003670 & -2.5819080 & 3.8676290 \\
\hline
\end{tabular}




$\begin{array}{rrr}-5.8133340 & -0.8329320 & 0.5122300 \\ -6.8642830 & -0.5265720 & 0.4679250 \\ -5.5737560 & -1.0475660 & 1.5600430 \\ -5.1972210 & 0.0170980 & 0.2035990 \\ -6.4166740 & -3.2447560 & 0.0596240 \\ -6.2705850 & -4.1020200 & -0.6056280 \\ -6.1446180 & -3.5547620 & 1.0754950 \\ -7.4816330 & -2.9864130 & 0.0599220 \\ -1.9806810 & -2.0358610 & -4.2103110 \\ -3.0259530 & -2.2165220 & -4.4867650 \\ -1.8951270 & -1.0123660 & -3.8419590 \\ -1.3862030 & -2.1196800 & -5.1265270 \\ -1.7255170 & -4.4735760 & -3.6997190 \\ -2.7996620 & -4.6480590 & -3.8360500 \\ -1.2326560 & -4.6177700 & -4.6678520 \\ -1.3510370 & -5.2348810 & -3.0099370 \\ 0.5366850 & 0.1041170 & -0.8027690 \\ 0.0215230 & -0.1787250 & -2.1854370 \\ -0.0301110 & 1.2722470 & -0.0384040 \\ -0.4010360 & -2.9008570 & -3.0719330 \\ -5.8821090 & -1.7765930 & -1.4051240 \\ -0.2251500 & -3.5111300 & 1.7084290 \\ 4.9521670 & 1.1350280 & -1.3320250 \\ 2.6838420 & 6.5559850 & -1.9590610 \\ 1.8028640 & 1.5121690 & 2.2643080 \\ -2.7978590 & 1.2571630 & -2.0219880 \\ -2.1423540 & 2.5518660 & -1.7961010 \\ -2.5729280 & 0.4710030 & -1.2992720 \\ -1.1793440 & 2.5305720 & -1.2979980 \\ -1.8596570 & 1.2631540 & -3.1191860 \\ -1.0478850 & 0.7277990 & -2.8082560 \\ -4.5289970 & 2.4125140 & -3.4506220 \\ -4.1069930 & 3.7530660 & -2.8418230 \\ -2.6117800 & 3.7804530 & -2.5053890 \\ -4.2316720 & 1.2550860 & -2.4929530 \\ -5.5991580 & 2.4271390 & -3.6781720 \\ -4.3324140 & 4.5733630 & -3.5295000 \\ -2.0108330 & 3.8520180 & -3.4205180 \\ -4.8669130 & 1.3331100 & -1.6024220 \\ -4.4392420 & 0.2850750 & -2.9567550 \\ -4.0012800 & 2.2504320 & -4.3977250 \\ -4.6844500 & 3.9329630 & -1.9267600 \\ -2.3412470 & 4.6536110 & -1.9046540 \\ -3.0359130 & 1.7869220 & 1.5203900 \\ -2.0857700 & 0.9067920 & 1.4373200 \\ -4.0626320 & 1.4346450 & 2.5234360\end{array}$

$\begin{array}{ll}\mathrm{C} & -5.3401120 \\ \mathrm{C} & -3.7653960 \\ \mathrm{C} & -6.3077300 \\ \mathrm{H} & -5.5757810 \\ \mathrm{C} & -4.7334060 \\ \mathrm{H} & -2.7739120 \\ \mathrm{C} & -6.0077550 \\ \mathrm{H} & -7.2997670 \\ \mathrm{H} & -4.4932300 \\ \mathrm{H} & -6.7667420 \\ \mathrm{H} & -1.3058240 \\ \mathrm{~S} & -3.1164110 \\ \mathrm{I} & \end{array}$

2.0052170
0.4716120
1.6068040
2.7416940
0.0812600
0.0381530
0.6437040
2.0443190
-0.6619910
0.3326140
1.1371880
3.1845380

2.4861360 3.4965340 3.3988400 1.7257730 4. 4123050 3. 5315120 4. 3607210 3. 3555460 5.1658030 5.0718400 0.8203180 $\operatorname{TS} 203(R, R) G[W B 97 X D / 6-31 G(d)]=-3633.717626$

$\begin{array}{lrcr}\text { TS203(R, R) G[WB97XD/6-31G (d) }]=-3633.717626 \\ \text { O } & 0.5583430 & 0.6756360 & -0.0404770 \\ \text { O } & -1.6161780 & 1.0363260 & -1.2930970 \\ \mathrm{C} & 0.8169830 & 2.0273560 & -0.1101470 \\ \mathrm{C} & -0.1925240 & 2.9225950 & 0.1734850 \\ \mathrm{C} & 2.1523470 & 2.4229510 & -0.3786150 \\ \mathrm{C} & 0.0734100 & 4.3220540 & 0.0219060 \\ \mathrm{C} & 2.4203130 & 3.7685960 & -0.4149230 \\ \mathrm{C} & 1.4021110 & 4.7414120 & -0.2596640 \\ \mathrm{H} & 3.4405710 & 4.1013080 & -0.5858420 \\ \mathrm{C} & -2.1908130 & 1.4815580 & -0.1145470 \\ \mathrm{C} & -3.4731810 & 0.9800700 & 0.2316320 \\ \mathrm{C} & -1.5140980 & 2.4190800 & 0.6324330 \\ \mathrm{C} & -4.0655460 & 1.4899310 & 1.3588860 \\ \mathrm{C} & -2.0873000 & 2.8440670 & 1.8761670 \\ \mathrm{C} & -3.3940310 & 2.3982390 & 2.2151940 \\ \mathrm{H} & -5.0666340 & 1.1633040 & 1.6256390 \\ \mathrm{C} & -3.3061670 & 3.6548200 & 4.2889590 \\ \mathrm{C} & -1.9873930 & 4.0589960 & 3.9776320 \\ \mathrm{C} & -1.3942410 & 3.6669520 & 2.8046400 \\ \mathrm{C} & -3.9895990 & 2.8373040 & 3.4272080 \\ \mathrm{H} & -3.7665920 & 3.9807320 & 5.2164750 \\ \mathrm{H} & -1.4368060 & 4.6817440 & 4.6760910 \\ \mathrm{H} & -0.3782340 & 3.9760350 & 2.5864640 \\ \mathrm{H} & -4.9946030 & 2.4984040 & 3.6645650 \\ \mathrm{C} & -0.6423220 & 6.6425540 & -0.0323740 \\ \mathrm{C} & 0.6866590 & 7.0593140 & -0.2795370 \\ \mathrm{C} & 1.6839440 & 6.1266040 & -0.3932480 \\ \mathrm{C} & -0.9414070 & 5.3123480 & 0.1165580 \\ \mathrm{H} & -1.4331480 & 7.3834180 & 0.0340660 \\ \mathrm{H} & 0.9085130 & 8.1160640 & -0.3913130 \\ \mathrm{H} & 2.7065950 & 6.4309170 & -0.5996820 \\ \mathrm{H} & -1.9662980 & 5.0066340 & 0.2964090\end{array}$




$\begin{array}{rrr}3.2528560 & 1.4311790 & -0.5772880 \\ 3.8353150 & 1.2780670 & -1.8452300 \\ 3.7655630 & 0.7184650 & 0.5286150 \\ 4.9774470 & 0.4799840 & -1.9735540 \\ 4.8889310 & -0.0806810 & 0.3439040 \\ 5.5301090 & -0.1933920 & -0.8920100 \\ 5.4357130 & 0.3842340 & -2.9529080 \\ 5.2963310 & -0.6286120 & 1.1898750 \\ -4.1516490 & -0.0648310 & -0.5935720 \\ -4.2656350 & -1.3817270 & -0.0987360 \\ -4.6998870 & 0.2710850 & -1.8425310 \\ -4.9615550 & -2.3203850 & -0.8574680 \\ -5.3752650 & -0.7109790 & -2.5699530 \\ -5.5240470 & -2.0087180 & -2.0943190 \\ -5.0670190 & -3.3316590 & -0.4729720 \\ -5.8054480 & -0.4546270 & -3.5360580 \\ 3.1550390 & 0.8620530 & 1.9167700 \\ 6.7947860 & -1.0333070 & -0.9911840 \\ 3.2673600 & 1.9525860 & -3.0870720 \\ -3.6709300 & -1.8091120 & 1.2388040 \\ -6.2753480 & -3.0542200 & -2.8986800 \\ -4.5962800 & 1.6740270 & -2.4234810 \\ -5.9806150 & 2.3220260 & -2.5607500 \\ -6.6166480 & 1.7601410 & -3.2543400 \\ -6.4914630 & 2.3633950 & -1.5927550 \\ -5.8872260 & 3.3443340 & -2.9440940 \\ -3.8429920 & 1.6736590 & -3.7600320 \\ -2.8540880 & 1.2206230 & -3.6447400 \\ -4.3910390 & 1.1152390 & -4.5278120 \\ -3.7127890 & 2.6991940 & -4.1235340 \\ -7.5235140 & -3.5447460 & -2.1527010 \\ -8.0897290 & -4.2504140 & -2.7708060 \\ -7.2502300 & -4.0574630 & -1.2230800 \\ -8.1824790 & -2.7091220 & -1.8947800 \\ -5.3688050 & -4.2303680 & -3.2857650 \\ -4.4869480 & -3.8834740 & -3.8340830 \\ -5.0220780 & -4.7698640 & -2.3967480 \\ -5.9116430 & -4.9410260 & -3.9188240 \\ -4.7515990 & -1.8709730 & 2.3285140 \\ -5.2539870 & -0.9080410 & 2.4597920 \\ -5.5147950 & -2.6151320 & 2.0712250 \\ -4.3092790 & -2.1572120 & 3.2896660 \\ -2.9352290 & -3.1535990 & 1.1615550 \\ -3.6253850 & -3.9886690 & 0.9971460 \\ -2.1955760 & -3.1492530 & 2.3573960 \\ -2.4176320 & -3.3438740 & \\ -5 & & \\ -5 & & \\ -5 & -1086140\end{array}$
3.7914090
4.8650460
3. 6653340
3. 3304490
3. 2472400
2. 9247780
4. 2672620
2. 6051320
7. 9601900
8.8656420
8.1788590
7.7250760
7.1889760
6. 3698560
7. 4915670
8. 0397960
3. 0700080
4.0255350
2. 4826510
2. 5377640
4. 1458600
5. 1563590
3. 7200140
4.2347360
$-0.4053890$
0.2529900
$-0.8582550$
2. 2791560
6.6018900
2. 0905050
$-4.0213500$
$-6.6104580$
$-2.9323950$
2. 3091130
1.1606650
2. 2435700
0.1894360
1. 7708140
1.1610200
3. 7379460
2. 6801550
1. 2713560
3.6909270
4.7360000
2. 7309710
0.9672310

2.0455570

1.8756580

2. 9791640

2. 1723600

$-0.4175820$

$-1.2926610$

$-0.6049120$

$-0.3338770$

$-0.3145760$

$-0.9311610$

0.6339210

$-0.0941360$

$-1.4133740$

$-1.9053950$

$-0.5330020$

$-2.1019510$

0.9582790

0.5942600

0.0966880

1.4439720

3.1285810

2. 7851660

3.6072030

3. 8869360

$-0.0356670$

$-0.1276690$

- 1.3039890

2. 3477750

$-1.9651030$

1.0783140

2.2936720

$-2.5734310$

$-1.0586670$

$-2.4030260$

$-3.3152070$

$-1.4852970$

$-2.8650720$

$-2.2363230$

$-4.3558090$

$-5.3149370$

$-4.7359170$

$-3.0091540$

$-4.7904380$

$-6.2816620$

$-4.7521170$
2.6602110

2.8035370

2. 1022910

3.6463510

2.7548850

2. 1806310

3.1093770

3.6377430

$-0.2924240$

$-0.3163430$

$-0.7966950$

0.7537760

$-2.4200540$

$-2.9558840$

$-2.9988810$

$-2.4007790$

$-4.2387290$

$-4.6332470$

$-3.9128890$

$-5.0638820$

$-3.5355220$

$-3.7871880$

$-4.4244120$

$-2.7513160$

$-1.1560720$

$-2.4998590$

$-0.4758570$

$-2.8343200$

$-0.4411740$

1. 7985100

$-1.7294820$

$-3.8266270$

1.5351190

$-1.4396700$

$-1.3570960$

$-0.8513080$

$-1.1826170$

$-2.7731340$

$-2.7513400$

$-2.1849780$

$-1.6354540$

$-1.7985120$

$-2.0731360$

$-2.1445050$

$-2.8531230$ 


\begin{tabular}{|c|c|c|c|c|c|c|c|}
\hline $\mathrm{H}$ & 4.0086620 & -3.1243050 & -0.4158840 & C & -0.2731850 & 5.8168920 & -2.5071110 \\
\hline $\mathrm{H}$ & 4.3643550 & -2.2848330 & -1.9221830 & $\mathrm{C}$ & 1.0231670 & 5.9620770 & -3.0542670 \\
\hline $\mathrm{H}$ & 3.5734180 & -4.1997960 & -3.2578300 & $\mathrm{C}$ & 1.9395310 & 4.9526240 & -2.9153710 \\
\hline H & 2.8746640 & -5.5015220 & -0.5717270 & $\mathrm{C}$ & -0.6199860 & 4.6810410 & -1.8211360 \\
\hline $\mathrm{H}$ & 0.5180680 & -5.3212920 & -1.2637540 & $\mathrm{H}$ & -1.0030100 & 6.6101750 & -2.6373290 \\
\hline C & 0.5151580 & -2.6634250 & 2.3150520 & $\mathrm{H}$ & 1.2838840 & 6.8692430 & -3.5905520 \\
\hline 0 & -0.1545220 & -1.6233390 & 1.9361510 & $\mathrm{H}$ & 2.9342310 & 5.0451710 & -3.3432650 \\
\hline C & 0.6983840 & -2.7236330 & 3.7843350 & $\mathrm{H}$ & -1.6213150 & 4.5787890 & -1.4172970 \\
\hline C & 1.6624130 & -3.5547980 & 4.3661050 & C & 3.2726200 & 0.4695140 & -1.3257390 \\
\hline C & -0.0964430 & -1.9097590 & 4.6038700 & C & 3.6076610 & -0.3545630 & -2.4117440 \\
\hline C & 1.8288810 & -3.5721160 & 5.7445750 & $\mathrm{C}$ & 4.0343320 & 0.4305600 & -0.1388880 \\
\hline H & 2.2872500 & -4.1748990 & 3.7328060 & C & 4.7618580 & -1.1384890 & -2.3287840 \\
\hline C & 0.0677740 & -1.9375200 & 5.9829910 & C & 5.1693070 & -0.3770280 & -0.1017170 \\
\hline $\mathrm{H}$ & -0.8422960 & -1.2631930 & 4.1561270 & C & 5.5688200 & -1.1445010 & -1.1962090 \\
\hline C & 1.0311080 & -2.7662540 & 6.5549010 & $\mathrm{H}$ & 5.0478220 & -1.7551720 & -3.1778230 \\
\hline $\mathrm{H}$ & 2.5839930 & -4.2136460 & 6.1871980 & $\mathrm{H}$ & 5.7768490 & -0.3912910 & 0.7986270 \\
\hline $\mathrm{H}$ & -0.5561540 & -1.3108080 & 6.6120210 & C & -4.2748170 & -0.1258680 & -0.3034500 \\
\hline $\mathrm{H}$ & 1.1621890 & -2.7831970 & 7.6325620 & C & -4.5300900 & -1.1387990 & 0.6466120 \\
\hline $\mathrm{H}$ & -0.3734400 & -1.5682960 & 0.9379720 & C & -4.8606350 & -0.1935500 & -1.5797740 \\
\hline & 1.1187080 & -3.8479730 & 1.2859400 & $\mathrm{C}$ & -5.3812640 & -2.1848300 & 0.2959320 \\
\hline & & & & C & -5.6967130 & -1.2695380 & -1.8834780 \\
\hline & , R) $G[W B 97 x$ & (d) $]=-3$ & 395 & $\mathrm{C}$ & -5.9730100 & -2.2736180 & -0.9625200 \\
\hline 0 & 0.5592170 & 0.1791870 & -0.3645210 & $\mathrm{H}$ & -5.5871250 & -2.9595780 & 1.0308620 \\
\hline 0 & -1.7121420 & 0.3321080 & -1.5122910 & $\mathrm{H}$ & -6.1556530 & -1.3230260 & -2.8686100 \\
\hline C & 0.8983520 & 1.3776170 & -0.9551200 & C & 3.6782660 & 1.3028410 & 1.0603040 \\
\hline C & -0.0071930 & 2.4182060 & -0.9441120 & $\mathrm{C}$ & 6.8649270 & -1.9366360 & -1.1678390 \\
\hline C & 2.2106160 & 1.5136700 & -1.4767040 & $\mathrm{C}$ & 2.7658100 & -0.3805170 & -3.6792520 \\
\hline C & 0.3134150 & 3.6234010 & -1.6462190 & C & -3.9267570 & -1.1333600 & 2.0461560 \\
\hline C & 2.5355680 & 2.7019260 & -2.0838050 & $\mathrm{C}$ & -6.8935900 & -3.4269220 & -1.3185710 \\
\hline C & 1.6048170 & 3.7602120 & -2.2209040 & $\mathrm{C}$ & -4.6508540 & 0.8854090 & -2.6333570 \\
\hline $\mathrm{H}$ & 3.5413440 & 2.8368930 & -2.4731190 & $\mathrm{C}$ & -5.9601040 & 1.6363350 & -2.9137830 \\
\hline C & -2.1329490 & 1.2268630 & -0.5387120 & $\mathrm{H}$ & -6.7214870 & 0.9648880 & -3.3273140 \\
\hline C & -3.4046600 & 1.0317270 & 0.0618330 & $\mathrm{H}$ & -6.3631020 & 2.0787090 & -1.9963860 \\
\hline C & -1.2938020 & 2.2672380 & -0.2183250 & $\mathrm{H}$ & -5.7923430 & 2.4409820 & -3.6385440 \\
\hline C & -3.7934580 & 1.9422010 & 1.0125030 & C & -4.0491670 & 0.3129900 & -3.9228610 \\
\hline C & -1.6619350 & 3.1435010 & 0.8524390 & $\mathrm{H}$ & -3.1113090 & -0.2095340 & -3.7135150 \\
\hline C & -2.9373150 & 2.9805820 & 1.4574120 & $\mathrm{H}$ & -4.7357360 & -0.3907010 & -4.4074650 \\
\hline $\mathrm{H}$ & -4.7710760 & 1.8402210 & 1.4744080 & $\mathrm{H}$ & -3.8424890 & 1.1191110 & -4.6357650 \\
\hline C & -2.4569690 & 4.7968250 & 2.9930430 & C & -8.1527170 & -3.4294780 & -0.4412890 \\
\hline C & -1.1757030 & 4.9409330 & 2.4117040 & $\mathrm{H}$ & -8.8376390 & -4.2261810 & -0.7521750 \\
\hline C & -0.7874860 & 4.1385850 & 1.3682760 & $\mathrm{H}$ & -7.8987870 & -3.5975920 & 0.6117900 \\
\hline C & -3.3 & 73730 & 2.5245460 & $\mathrm{H}$ & -8.6822200 & -2.4736840 & -0.5119920 \\
\hline $\mathrm{H}$ & 17100 & 15600 & 3.8151210 & C & -6.1661530 & -4.7757730 & -1.2418660 \\
\hline $\mathrm{H}$ & -0.4920260 & 21900 & 2.7958410 & $\mathrm{H}$ & -5.2806390 & -4.7826460 & -1.8857690 \\
\hline $\mathrm{H}$ & 0.2017760 & 4.2523020 & 0.9385780 & $\mathrm{H}$ & -5.8399970 & -4.9900110 & -0.2175320 \\
\hline H & -4.2962290 & 3.7054840 & 2.9751860 & $\mathrm{H}$ & -6.8298540 & -5.5879100 & -1.5588850 \\
\hline
\end{tabular}




$\begin{array}{rr}-4.9805130 & -0.7549030 \\ -5.4346120 & 0.2188820 \\ -5.7858390 & -1.4986770 \\ -4.5274500 & -0.7114370 \\ -3.2744080 & -2.4732440 \\ -4.0191230 & -3.2662860 \\ -2.5690060 & -2.7860360 \\ -2.7308550 & -2.3715630 \\ 4.3603030 & 2.6758880 \\ 5.4506370 & 2.5603580 \\ 4.0865730 & 3.1971290 \\ 4.0709260 & 3.3066050 \\ 4.0111710 & 0.6530800 \\ 3.5731430 & -0.3469570 \\ 5.0924580 & 0.5704240 \\ 3.6105280 & 1.2679170 \\ 6.9254880 & -2.9455510 \\ 7.8813740 & -3.4804790 \\ 6.8377210 & -2.4469980 \\ 6.1235130 & -3.6861330 \\ 8.0717000 & -0.9877770 \\ 8.0451310 & -0.2753720 \\ 8.0805210 & -0.4150370 \\ 9.0095660 & -1.5511670 \\ 2.5336770 & -1.8049940 \\ 3.4495610 & -2.2415920 \\ 2.1710890 & -2.4620990 \\ 1.7849460 & -1.7926260 \\ 3.3919060 & 0.4900260 \\ 4.3922310 & 0.1228550 \\ 2.7760130 & 0.4654600 \\ 3.4864930 & 1.5328650 \\ -0.6321250 & -0.7658450 \\ -0.1453620 & -1.5463420 \\ -1.1696930 & -1.5210600 \\ 1.7830220 & 0.0341870 \\ 6.9228720 & -2.5013690 \\ 2.5946440 & 1.4679270 \\ -3.9408270 & 1.6202230 \\ -7.2129320 & -3.2799450 \\ -3.1387500 & -0.3777590 \\ 1.9527900 & -3.0126810 \\ 0.8617480 & -3.6265500 \\ 1.9377470 & -1.9246690 \\ -0.0980240 & -3.1226660 \\ 1.2369540 & -3.6438720\end{array}$

3.0974080
2.8881560
3.1234120
4.0942810
2.4141900
2.5474510
1.6411980
3.3604990
0.9593990
0.9755940
0.0374200
1.8078830
2.4065280
2.4901370
2.5646590
3.2187360
-0.0141140
-0.0284890
0.9578460
-0.0868010
-1.1212090
-1.9521640
-0.1863600
-1.1798150
-4.1965430
-4.6112650
-3.4019320
-4.9952280
-4.7779390
-5.0371380
-5.6840450
-4.4603250
-0.9981290
-2.1794660
0.1943080
-3.4347750
-2.1070920
1.0473840
-2.2441950
-2.3582680
2.0760600
-0.2101190
0.5618310
-0.2849500
0.5404280
-1.3009850

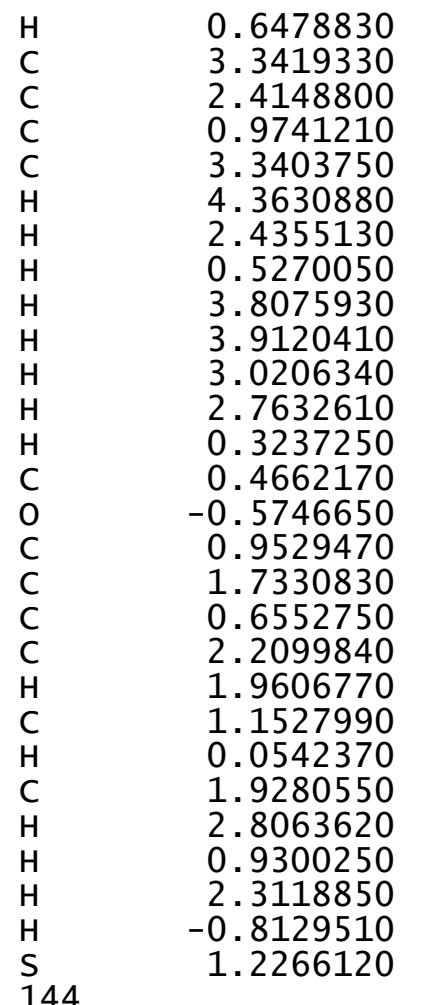
$-2.9364020$
$-5.0864200$
$-5.4424360$
$-5.0150590$
$-3.5785300$
$-5.4153000$
$-6.5178630$
$-5.6633420$
$-3.0453740$
$-3.3233680$
$-5.6166600$
$-4.9412380$
$-5.1013170$
$-0.7598050$
$-0.3863390$
0.2919250
$-0.0075720$
1.6276310
1.0164400
$-1.0422300$
2. 6492520
1.8609340
2.3454330
0.7790700
3. 6815400
3.1437630
$-0.9653230$
$-2.2539990$

$-1.7082260$

0.2165990

1. 3801890

1.0851930

$-0.0431430$

0.4339930

1.5784660

0.3192350

0.7939980

$-0.9390780$

$-0.6880070$

2. 2920540

1.9602990

3.0284080

2. 3573480

3. 9490140

5.0702240

3. 6459380

5.8778580

5.3034760

4.4445530

2.7738280

5.5621930

6.7529750

4.1932890

6.1900980

1. 5468040

2.8876460

$\operatorname{TS} 205(R, R) G[W B 97 X D / 6-31 G(d)]=-3633.717802$

\begin{tabular}{|c|c|c|c|}
\hline & & & \\
\hline 0 & 0.3444020 & 0.8954490 & -0.1271600 \\
\hline 0 & -1.9821030 & 0.4865150 & -1.0319050 \\
\hline C & 0.3008530 & 2.2018310 & -0.5686250 \\
\hline C & -0.8411160 & 2.9420100 & -0.3584220 \\
\hline C & 1.4896250 & 2.7376170 & -1.1339650 \\
\hline C & -0.8990310 & 4.2643000 & -0.9092950 \\
\hline C & 1.4591630 & 4.0393100 & -1.5600030 \\
\hline C & 0.2701290 & 4.8130270 & -1.5052540 \\
\hline $\mathrm{H}$ & 2.3623340 & 4.4978600 & -1.9520550 \\
\hline C & -2.4756880 & 1.1292110 & 0.0872070 \\
\hline C & -3.5503760 & 0.5096440 & 0.7765960 \\
\hline C & -1.9 & 2.3549910 & 0.4369670 \\
\hline C & -4.0 & 1.1860660 & 1.8350670 \\
\hline C & -2.4 & 2.9978520 & 1.6169420 \\
\hline C & -3 & 2.4149430 & 2.2954500 \\
\hline $\mathrm{H}$ & & 230 & 20 \\
\hline
\end{tabular}




-3.5355700
-2.4070950
-1.8822000
-4.0951840
-3.9449980
-1.9477800
-1.0098820
-4.9490430
-2.1039090
-0.9320810
0.2268040
-2.0880250
-3.0258660
-0.9575080
1.1296910
-2.9954580
2.7256790
3.1688670
3.4151610
4.2842540
4.5119250
4.9533180
4.6462500
5.0357800
-4.0930470
-3.7579950
-4.9710970
-4.3452650
-5.5245430
-5.2315350
-4.1057060
-6.2106610
3.0321570
6.1609370
2.4961380
-2.8019670
-5.8618930
-5.3469140
-6.8461420
-7.4535970
-7.1365790
-7.0926030
-4.9127740
-3.8364680
-5.4348160
-5.1396020

4. 2073640

4.7644070

4.1782160

3.0542980

4.6863990

5.6621050

4.6132030

2.6016380

6.3025620

6.8594160

6.1275700

5.0412650

6.8760470

7.8600060

6.5385980

4.6208080

1.8968840

1.2432140

1.7319500

0.4078220

0.8731520

0.1885800

$-0.0970100$

0.7401200

$-0.8125390$

$-1.9837020$

$-0.8690270$

$-3.1873530$

$-2.0966960$

$-3.2669100$

$-4.0985700$

$-2.1378450$

2. 5013030

$-0.7301970$

1.4778850

$-1.9655070$

$-4.5915000$

0.3709020

0.6753720

$-0.1317170$

0.7995370

1.5991170

0.2470230

0.0627420

$-0.5744950$

1. 1713970

3.9301320
3.2856980
2.1616380
3.4456210
4.8140830
3.6882500
1.6871380
3.9426890
-1.4510470
-2.0125250
-2.0390410
-0.9115380
-1.4522340
-2.4328630
-2.4829060
-0.4916240
-1.1299860
-2.2943280
0.0857050
-2.2030290
0.1288470
-1.0002990
-3.0951490
1.0722870
0.3395950
1.0451780
-0.7572930
0.6509260
-1.1158780
-0.4195230
1.1933180
-1.9592190
1.3432910
-0.9456800
-3.6401340
2.2325220
-0.8110390
-1.5562020
-1.4367270
-1.8621830
-0.3879210
-1.9721350
-3.0221760
-3.0917270
-3.5264760
-3.5652290

\begin{abstract}
$-5.4707670$
$-5.8963720$

$-5.8439700$

$-4.3830080$

$-7.3875920$

$-7.6669380$

$-7.8370290$

$-7.8245680$

$-3.5705690$

$-4.1766080$

$-4.2403010$

$-2.8730810$

$-1.8661140$

$-2.4001530$

$-1.3757040$

$-1.0916580$

4.1158450

5.0718580

4.2760190

3.8244410

2. 7440090

1.9568870

3.6322780

2. 4150910

6.1216340

6.9300190

6.2481880

5.1682890

7.4610780

7.5152120

7. 5176120

8.3352430

2.4758560

3.4779090

2. 0534340

1.8608190

3.1761110

4.2275610

2. 6807140

3.1494390

$-0.5243630$

$-0.0619210$

$-0.5428830$

1.4542940

6.1477810
2.1129950
\end{abstract}

-5.0072360
-5.9870250
-4.2872690
-5.0672680
-4.5532360
-4.2792420
-3.8209910
-5.5324330
-1.8620470
-0.9527780
-2.7214790
-1.8499910
-3.1807120
-4.1006800
-3.3272670
-3.0267160
3.5336310
3.0447410
4.2256160
4.1183950
1.5762470
0.8606210
1.0096940
2.1601890
-1.6856620
-2.4212910
-1.1476200
-2.2234930
0.0867440
0.7359370
0.7213400
-0.5741170
0.2462840
-0.0142500
-0.6234590
0.4529810
2.6444370
2.4064910
2.8354140
3.5675660
-0.2270820
-0.4113370
-1.4215100
1.7513130
-1.3391290
3.0580580

$-2.2352370$

$-2.4788590$

$-2.9727460$

$-2.3443480$

$-0.6487320$

0.3739780

$-1.3295570$

$-0.8739300$

3.5584280

3.6067440

3.6825190

4.4038720

2.2688610

2. 5326210

1. 3032890

3.0284260

1.6844460

1.9055500

0.8504280

2.5643150

2.5287960

2.2778910

2.8317660

3. 3960000

0.2529980

0.1818670

1.1994960

0.3044240

$-0.9487950$

$-1.8288790$

$-0.0564160$

$-0.9525340$

$-4.5500780$

$-4.9102900$

$-4.0437880$

$-5.4324690$

$-4.3745910$

$-4.5762260$

$-5.3332460$

$-3.7892240$

$-0.9350080$

$-2.3505310$

$-0.0140240$

$-3.4454560$

$-1.8592770$

1. 1413210 


\begin{tabular}{|c|c|c|c|c|c|c|c|}
\hline & & & & & & & \\
\hline $\mathrm{H}$ & -4.8143410 & 1.2278540 & -1.1343560 & $\mathrm{H}$ & 3.3801280 & 4.1491970 & -0.6366130 \\
\hline $\mathrm{H}$ & -5.4740100 & -5.3536550 & -0.1229920 & C & -2.2227630 & 1.4766780 & -0.0665210 \\
\hline $\mathrm{H}$ & -2.1693590 & -1.0777800 & 2.1343880 & $\mathrm{C}$ & -3.4846930 & 0.9534590 & 0.3213770 \\
\hline C & 2.2346790 & -2.5184760 & -1.9311540 & C & -1.5373240 & 2.4240000 & 0.6597830 \\
\hline C & 1.2988350 & -3.4356770 & -1.2719450 & C & -4.0489920 & 1.4537590 & 1.4671280 \\
\hline $\mathrm{H}$ & 2.3177860 & -1.5211670 & -1.4962880 & C & -2.0781500 & 2.8413350 & 1.9205980 \\
\hline $\mathrm{H}$ & 0.4349750 & -2.9905100 & -0.7905140 & $\mathrm{C}$ & -3.3659220 & 2.3743040 & 2.3011420 \\
\hline 0 & 1.1956020 & -2.5856250 & -2.9372010 & $\mathrm{H}$ & -5.0355640 & 1.1111250 & 1.7658750 \\
\hline H & 0.6046370 & -1.7758360 & -2.7775210 & C & -3.2336780 & 3.6354170 & 4.3698640 \\
\hline C & 3.4167850 & -4.5145620 & -2.9363410 & $\mathrm{C}$ & -1.9324890 & 4.0613180 & 4.0165180 \\
\hline C & 2.7587270 & -5.4065850 & -1.8807580 & C & -1.3698970 & 3.6768450 & 2.8261320 \\
\hline C & 1.3569160 & -4.9058960 & -1.5190560 & C & -3.9301470 & 2.8051270 & 3.5310470 \\
\hline C & 3.5450860 & -3.0743550 & -2.4327660 & $\mathrm{H}$ & -3.6702700 & 3.9550800 & 5.3109940 \\
\hline $\mathrm{H}$ & 4.4097120 & -4.9000330 & -3.1863590 & $\mathrm{H}$ & -1.3706190 & 4.6943580 & 4.6964790 \\
\hline H & 2.6879480 & -6.4382890 & -2.2371630 & $\mathrm{H}$ & -0.3667300 & 4.0032310 & 2.5760280 \\
\hline H & 0.6506060 & -5.1065650 & -2.3348110 & $\mathrm{H}$ & -4.9210090 & 2.4491630 & 3.8007340 \\
\hline $\mathrm{H}$ & 4.2565700 & -3.0295560 & -1.5998830 & C & -0.7146490 & 6.6571730 & -0.0221300 \\
\hline $\mathrm{H}$ & 3.9220480 & -2.4094610 & -3.2160880 & $\mathrm{C}$ & 0.6073470 & 7.0848730 & -0.2872620 \\
\hline $\mathrm{H}$ & 2.8236770 & -4.5310660 & -3.8583970 & C & 1.6099580 & 6.1601840 & -0.4186910 \\
\hline H & 3.3827990 & -5.4222210 & -0.9780860 & $\mathrm{C}$ & -1.0013910 & 5.3242570 & 0.1271770 \\
\hline $\mathrm{H}$ & 0.9491570 & -5.4160430 & -0.6419010 & $\mathrm{H}$ & -1.5099870 & 7. 3918760 & 0.0579000 \\
\hline C & 1.9759470 & -2.1675720 & 2.1895380 & $\mathrm{H}$ & 0.8195790 & 8.1436130 & -0.3988400 \\
\hline 0 & 0.9516260 & -1.3910180 & 2.0342770 & $\mathrm{H}$ & 2.6271140 & 6.4731920 & -0.6389370 \\
\hline C & 2.7615160 & -1.8374020 & 3.4009080 & $\mathrm{H}$ & -2.0213330 & 5.0098140 & 0.3200110 \\
\hline C & 4.1195040 & -2.1605500 & 3.4913880 & c & 3.2115370 & 1.4761130 & -0.6341210 \\
\hline C & 2.1428730 & -1.1459900 & 4.4509650 & C & 3.7673510 & 1.3101210 & -1.9163630 \\
\hline c & 4.8519480 & -1.7841370 & 4.6104670 & $\mathrm{C}$ & 3.7561350 & 0.7884550 & 0.4672600 \\
\hline $\mathrm{H}$ & 4.6003160 & -2.6867750 & 2.6741020 & C & 4.9109020 & 0.5236030 & -2.0512670 \\
\hline C & 2.8740900 & -0.7905620 & 5.5765840 & C & 4.8961350 & 0.0056930 & 0.2781000 \\
\hline H & 1.0916560 & -0.8915200 & 4.3778030 & C & 5.5056540 & -0.1187700 & -0.9674680 \\
\hline C & 4.2304180 & -1.1021400 & 5.6549290 & $\mathrm{H}$ & 5.3613200 & 0.4059300 & -3.0349350 \\
\hline $\mathrm{H}$ & 5.9094150 & -2.0215630 & 4.6671670 & $\mathrm{H}$ & 5.3227570 & -0.5080340 & 1.1328710 \\
\hline $\mathrm{H}$ & 2.3873250 & -0.2645630 & 6.3915610 & C & -4.1696290 & -0.1021680 & -0.4849670 \\
\hline $\mathrm{H}$ & 4.8033820 & -0.8127180 & 6.5305990 & C & -4.2268370 & -1.4272000 & -0.0018590 \\
\hline $\mathrm{H}$ & 0.3946170 & -1.5169330 & 1.1871630 & $\mathrm{C}$ & -4.7717810 & 0.2305650 & -1.7095440 \\
\hline 0 & 2.3948320 & -3.4428870 & 1.1806070 & C & -4.9160650 & -2.3793190 & -0.7497830 \\
\hline & & & & C & -5.4413160 & -0.7637270 & -2.4256570 \\
\hline & R) $G[w B 97 x$ & $G(d)]=-3$ & & $\mathrm{C}$ & -5.5297150 & -2.0716630 & -1.9632880 \\
\hline 0 & 0.5303810 & 0.7005620 & -0.0723820 & $\mathrm{H}$ & -4.9708900 & -3.3992440 & -0.3780770 \\
\hline 0 & -1.6806610 & 1.0447260 & -1.2651570 & $\mathrm{H}$ & -5.9107160 & -0.5103710 & -3.3741700 \\
\hline $\mathrm{c}$ & 0.7776840 & 2.0542640 & -0.1387030 & C & 3.1650000 & 0.9337040 & 1.8638490 \\
\hline$c$ & -0.2332220 & 2.9405980 & 0.1667720 & C & 6.7990400 & -0.8896220 & -1.1801040 \\
\hline$c$ & 2.1061200 & 2.4603880 & -0.4240610 & $\mathrm{C}$ & 3.1651720 & 1.9687790 & -3.1504460 \\
\hline$c$ & 0.0194840 & 4.3422590 & 0.0142530 & C & -3.5770680 & -1.8467840 & 1. 3122310 \\
\hline$c$ & 2.3641920 & 3.8078570 & -0.4575810 & C & -6.2479300 & -3.1381390 & -2.7703170 \\
\hline$c$ & & 4.7725290 & -0.2858260 & $\mathrm{C}$ & -4.7293720 & 1.6422010 & -2.2768700 \\
\hline
\end{tabular}




$\begin{array}{lrr}-6.1356150 & 2.2511080 & -2.3587910 \\ -6.7772930 & 1.6806680 & -3.0401440 \\ -6.6158780 & 2.2632260 & -1.3745140 \\ -6.0846450 & 3.2811580 & -2.7291450 \\ -4.0242940 & 1.6783980 & -3.6390450 \\ -3.0206520 & 1.2498080 & -3.5647260 \\ -4.5847700 & 1.1149350 & -4.3940340 \\ -3.9335500 & 2.7114120 & -3.9932930 \\ -7.3923450 & -3.7851890 & -1.9799250 \\ -7.9338700 & -4.5022770 & -2.6067260 \\ -7.0135290 & -4.3262260 & -1.1052460 \\ -8.1042050 & -3.0313360 & -1.6279850 \\ -5.2628480 & -4.1992000 & -3.2807570 \\ -4.4619710 & -3.7403070 & -3.8696220 \\ -4.8000950 & -4.7368330 & -2.4448590 \\ -5.7776200 & -4.9333080 & -3.9106510 \\ -4.6154990 & -1.9174740 & 2.4419080 \\ -5.1236350 & -0.9601820 & 2.5894350 \\ -5.3791650 & -2.6710560 & 2.2149450 \\ -4.1344840 & -2.1956520 & 3.3867290 \\ -2.8317380 & -3.1841950 & 1.2122270 \\ -3.5190750 & -4.0259580 & 1.0721190 \\ -2.1182900 & -3.1750840 & 0.3844970 \\ -2.2826600 & -3.3684170 & 2.1425930 \\ 3.8030820 & 2.1222240 & 2.5972780 \\ 4.8796100 & 1.9600420 & 2.7272790 \\ 3.6632280 & 3.0539230 & 2.0394230 \\ 3.3541220 & 2.2475080 & 3.5891610 \\ 3.2764760 & -0.3453420 & 2.7012480 \\ 2.9591320 & -1.2225830 & 2.1280180 \\ 4.3010970 & -0.5230260 & 3.0474550 \\ 2.6403820 & -0.2694130 & 3.5891670 \\ 7.0616320 & -1.9787490 & -0.1380140 \\ 7.9465650 & -2.5589000 & -0.4195350 \\ 7.2541960 & -1.5488880 & 0.8515220 \\ 6.2166560 & -2.6691010 & -0.0500020 \\ 7.9828600 & 0.0890560 & -1.2410910 \\ 7.8341350 & 0.8443980 & -2.0196040 \\ 8.0957960 & 0.6091380 & -0.2825430 \\ 8.9168850 & -0.4434120 & -1.4524550 \\ 2.9956800 & 0.9791630 & -4.3098100 \\ 3.9609160 & 0.6665020 & -4.7241230 \\ 2.4562550 & 0.0857270 & -3.9875560 \\ 2.4280020 & 1.4487320 & -5.1203770 \\ 3.9995330 & 3.1771360 & -3.8546520 \\ 5.0202080 & 2.8690200 & \end{array}$
3. 5536520
4.0657340
$-0.4469070$
0.1800940
$-0.8583480$
2.1653100
6.7241680
2.0974730
$-4.1483680$
$-6.6873750$
$-2.8359270$
2.2902220
1.1551680
(4)
0.1888960
1.6874750
1.0735450
3.7004760
2.6757910
1.2563650
3.6741090
4.7057130
2.7099510
0.8997370
4.0285330
4. 3296380
3.4907920
2. 9211140
0.5357360
0.6326010
$-0.0561840$
0.8327430
1.8020610
0.0437420
1. 9791900
2.4207480
0.2195720
$-0.7064670$
1. 1875150
2.7372270
$-0.3993710$
1. 3265110
$-0.3053090$

144

TS207 $(R, R) \quad G[W B 97 X D / 6-31 G(d)]=-3633.717754$
-4.4833930
-2.8122280
-1.1791510
-2.5401000
-0.5124010
-2.8862560
-2.1610570
1.7588380
-1.5958520
-3.6464600
1.5781560
-1.6268680
-1.5034190
-1.0220260
-1.2782780
-2.9304430
-2.8655600
-2.4665910
-1.8872320
-1.9724210
-1.7181310
-2.4026700
-2.4159180
-3.0105260
-0.6888410
-2.1905630
-3.5292970
-0.8374530
-1.4102250
2.2040540
1.8654560
3.6687080
4.2159270
4.5197070
5.5919250
3.5580230
5.8958870
4.0981530
6.4335140
6.0080820
6.5491000
7.5090310
0.8759850
1.1313980

\begin{tabular}{|c|c|}
\hline $\begin{array}{r}3.6438740 \\
3.9357780 \\
-0.0059990 \\
-0.0578160 \\
-1.2953460 \\
2.3259140 \\
-1.3787830 \\
1.1432880 \\
2.2705680 \\
-2.6442910 \\
-1.0874060 \\
-2.3340080 \\
-3.2586630 \\
-1.4256870 \\
-2.8205180 \\
-2.1533170 \\
-1.3517750 \\
-4.2632640 \\
-5.2403840 \\
-4.6712250 \\
-2.9288970 \\
-4.6909760 \\
-6.1972770 \\
-4.6735550 \\
-3.0630360 \\
-2.1932540 \\
-4.0926290 \\
-5.4453780 \\
-5.2717940 \\
-2.6862040 \\
-1.6447100 \\
-2.7892210 \\
-3.6374370 \\
-2.0022970 \\
-3.6986690 \\
-4.2379320 \\
-2.0729840 \\
-1.3432950 \\
-2.9191520 \\
-4.3543010 \\
-1.4663980 \\
-2.9711320 \\
-1.5718420 \\
-3.8282590\end{array}$ & $\begin{array}{r}-4.4833930 \\
-2.8122280 \\
-1.1791510 \\
-2.5401000 \\
-0.5124010 \\
-2.8862560 \\
-2.1610570 \\
1.7588380 \\
-1.5958520 \\
-3.6464600 \\
1.5781560 \\
-1.6268680 \\
-1.5034190 \\
-1.0220260 \\
-1.2782780 \\
-2.9304430 \\
-2.8655600 \\
-2.4665910 \\
-1.8872320 \\
-1.9724210 \\
-1.7181310 \\
-2.4026700 \\
-2.4159180 \\
-3.0105260 \\
-0.6888410 \\
-2.1905630 \\
-3.5292970 \\
-0.8374530 \\
-1.4102250 \\
2.2040540 \\
1.8654560 \\
3.6687080 \\
4.2159270 \\
4.5197070 \\
5.5919250 \\
3.5580230 \\
5.8958870 \\
4.0981530 \\
6.4335140 \\
6.0080820 \\
6.5491000 \\
7.5090310 \\
0.8759850 \\
1.1313980\end{array}$ \\
\hline & \\
\hline
\end{tabular}




$$
\begin{array}{r}
-0.0866740 \\
-2.1057310 \\
-0.4844010 \\
-1.8050640 \\
0.5257920 \\
-2.2153310 \\
0.1407410 \\
-1.2238770 \\
0.8864870 \\
-2.8256760 \\
-3.6816240 \\
-2.7372110 \\
-4.4980920 \\
-3.5001180 \\
-4.4196360 \\
-5.2001880 \\
-5.0720200 \\
-4.1217520 \\
-3.3579340 \\
-5.2104460 \\
-5.6759210 \\
-3.9903490 \\
-2.6265820 \\
-5.9179770 \\
-3.9301630 \\
-2.9410280 \\
-1.6187940 \\
-3.5776770 \\
-4.9781950 \\
-3.2326660 \\
-0.8513040 \\
-4.3457120 \\
1.9362660 \\
2.5606570 \\
2.5983940 \\
3.8200820 \\
3.8528640 \\
4.4713400 \\
4.3123960 \\
4.3579120 \\
-3.6645260 \\
-2.9436810 \\
-4.3507270 \\
-2.9563550 \\
-4.3216950 \\
-3.6332800
\end{array}
$$

0.1485460

$-0.8942540$

$-0.2948760$

$-0.1574120$

$-0.8081170$

$-0.7080250$

$-1.2224890$

$-1.2236000$

$-1.5748350$

0.1920230

0.8298850

0.5598750

1.8284680

1.6939980

2. 2960730

2. 3020120

3. 9029090

3. 3408570

2. 2678070

3.3926760

4.7462300

3.7674800

1.8582960

3.8301000

$-1.3207560$

$-1.7955320$

$-1.7493280$

$-0.7889410$

$-1.3839740$

$-2.2086920$

$-2.1292010$

$-0.4362530$

$-0.8005630$

$-1.9860570$

0.4355990

$-1.8946020$

0.4743790

$-0.6771100$

$-2.8019540$

1.4318500

0.4261660

1.2016070

$-0.7267500$

0.8159380

$-1.0782000$

$-0.3183260$
-2.4060130
-4.8459140
2.0047850
5.8349180
1.9260270
-2.1592870
-3.5779950
-5.1184760
-6.6169550
-6.8018240
-7.0334280
-7.1633590
-4.5356970
-3.4717570
-4.6463300
-5.0552550
-2.7570420
-2.6566430
-3.2408500
-1.7536260
-4.9744780
-5.5566670
-5.5320670
-4.8982380
-3.0052350
-3.9221660
-3.2896780
-2.4356830
-0.8348870
-0.9936730
-0.2794230
-0.2146030
2.7806710
3.8264800
2.7717320
2.3349630
1.9434020
1.4077930
2.9466350
1.4267060
5.8846300
6.8489900
5.7553780
5.0948750
6.9355580
6.9299450

$-4.7958560$

$-3.8860610$

3. 2428200

0.9387260

2. 6076660

$-2.3052600$

$-5.9675740$

$-1.2378190$

$-1.5669200$

$-2.5507290$

$-1.5731360$

$-0.8233170$

$-1.1793180$

$-0.9263620$

$-2.1403250$

$-0.4193470$

$-6.1383940$

$-7.1987660$

$-5.6298470$

$-5.7151770$

$-6.5830570$

$-6.4689910$

$-6.1072190$

$-7.6518360$

$-2.4618960$

$-1.8675670$

$-3.5114360$

$-2.1430810$

$-3.0623560$

$-4.0936690$

$-3.0865630$

$-2.5668380$

4.4921590

4.2480480

5.2553950

4.9238600

2. 2004950

1. 3068920

1.8987460

2.6143740

$-0.1935190$

$-0.7115460$

0.1815590

$-0.9299150$

1.9742350
2.7612130
1.4068620

$-1.9758650$

1.7239780

$-0.6175820$

$-3.3513940$

2. 4385120

$-0.7326570$

$-1.6031720$

$-1.6258970$

$-2.0726660$

$-0.6129030$

$-2.2168300$

$-3.0211300$

$-2.9899460$

$-3.5370410$

$-3.6155210$

$-2.0188680$

$-2.2764120$

$-2.8610950$

$-1.9018150$

$-0.8845680$

0.0356250

$-1.6994380$

$-1.1131190$

3. 7108940

3. 6672820

3.8531030

4.5914510

2. 6001400

2. 9358690

1.6600910

3. 3553010

2. 1648650

2.3859230

1.3793790

3.0681470

2. 8440870

2. 5112000

3. 1662500

3. 7171210

0.4143350

0.3716180

1. 4359050

0.2340870

-0.3479180
-1.1089430 


$\begin{array}{rrr}6.7889000 & 2.4480640 & 0.6298720 \\ 7.9230640 & 1.4994910 & -0.3494740 \\ 2.2110670 & 1.4948910 & -4.3643680 \\ 3.2595030 & 1.4923400 & -4.6853380 \\ 1.9756080 & 0.5107790 & -3.9564970 \\ 1.6006830 & 1.6483870 & -5.2607210 \\ 2.3925180 & 3.9538710 & -3.9302050 \\ 3.4773230 & 3.9425580 & -4.0910530 \\ 1.9078730 & 4.1460360 & -4.8941750 \\ 2.1645130 & 4.7888770 & -3.2620080 \\ -0.4939720 & -0.0616490 & -0.7898160 \\ 0.0156880 & 0.0756060 & -2.1959900 \\ -0.1103510 & -1.2790200 & 0.0112110 \\ 0.8418780 & 2.6491820 & -3.2108510 \\ 6.0255590 & 0.4967540 & -1.6046100 \\ 0.9774970 & 3.5590240 & 1.5241640 \\ -5.0154900 & -0.2386540 & -1.1693410 \\ -3.0598440 & -6.5127430 & 0.0668450 \\ -1.9114070 & -1.2452080 & 2.3231570 \\ 2.6155650 & -1.7315170 & -2.0261010 \\ 1.7903680 & -2.8983260 & -1.6894080 \\ 2.5362290 & -0.8758630 & -1.3528070 \\ 0.8606050 & -2.7018870 & -1.1667820 \\ 1.6417130 & -1.6716940 & -3.0910680 \\ 0.9269240 & -1.0075860 & -2.7925200 \\ 4.0987250 & -3.2216180 & -3.4236480 \\ 3.5183720 & -4.4408100 & -2.7016840 \\ 2.0491590 & -4.2273970 & -2.3214060 \\ 4.0122980 & -1.9688700 & -2.5466880 \\ 5.1445570 & -3.4061050 & -3.6868860 \\ 3.5974210 & -5.3328350 & -3.3299290 \\ 1.4084840 & -4.2723360 & -3.2109110 \\ 4.6733970 & -2.0715060 & -1.6778630 \\ 4.3303770 & -1.0748630 & -3.0930230 \\ 3.5559520 & -3.0509620 & -4.3606580 \\ 4.1008340 & -4.6359720 & -1.7927500 \\ 1.6808780 & -5.0082960 & -1.6501750 \\ 2.7652780 & -2.1375640 & 1.6696930 \\ 1.9037420 & -1.1727040 & 1.5812160 \\ 3.7804550 & -1.9139570 & 2.7219130 \\ 4.9905590 & -2.6176630 & 2.7218440 \\ 3.5441200 & -0.9484290 & 3.7094860 \\ 5.9537730 & -2.3494660 & 3.6848230 \\ 5.1787680 & -3.3570430 & 1.9511420 \\ 4.5077230 & -0.6877660 & 3.7176360 \\ 2.6024360 & -0.4133340 & \end{array}$

$\begin{array}{ll}\mathrm{C} & 5.7155750 \\ \mathrm{H} & 6.8949660 \\ \mathrm{H} & 4.3157930 \\ \mathrm{H} & 6.4710960 \\ \mathrm{H} & 1.1391410 \\ \mathrm{~S} & 2.7588530 \\ 144 & \end{array}$
$-1.3831510$
$-2.8893170$
0.0587320
$-1.1729800$
$-1.3040130$

3.6702820

5.4392890

5.4115990

0.9191380

0.7044630

$\operatorname{TS} 208(R, R) \quad G[W B 97 X D / 6-31 G(d)]=-3633.717751$

$\begin{array}{lrcr}\text { TS } 208(\mathrm{R}, \mathrm{R}) \mathrm{G}[\mathrm{WB} 97 \mathrm{XD} / 6-31 \mathrm{G}(\mathrm{d})]=-3633.717751 \\ \mathrm{O} & -0.0866420 & 1.2075450 & 0.1485540 \\ \mathrm{O} & -2.1057190 & 0.1246420 & -0.8942560 \\ \mathrm{C} & -0.4843370 & 2.4530800 & -0.2948710 \\ \mathrm{C} & -1.8049910 & 2.8156100 & -0.1574090 \\ \mathrm{C} & 0.5258770 & 3.3121230 & -0.8081110 \\ \mathrm{C} & -2.2152280 & 4.0744000 & -0.7080200 \\ \mathrm{C} & 0.1408550 & 4.5596240 & -1.2224850 \\ \mathrm{C} & -1.2237530 & 4.9548250 & -1.2235940 \\ \mathrm{H} & 0.8866180 & 5.2658310 & -1.5748330 \\ \mathrm{C} & -2.8256560 & 0.5766350 & 0.1920210 \\ \mathrm{C} & -3.6816290 & -0.3595510 & 0.8298780 \\ \mathrm{C} & -2.7371590 & 1.9024900 & 0.5598750 \\ \mathrm{C} & -4.4980860 & 0.1026850 & 1.8284620 \\ \mathrm{C} & -3.5000560 & 2.3420220 & 1.6939980 \\ \mathrm{C} & -4.4195970 & 1.4388960 & 2.2960700 \\ \mathrm{H} & -5.2002020 & -0.5780390 & 2.3020010 \\ \mathrm{C} & -5.0719380 & 3.1362100 & 3.9029110 \\ \mathrm{C} & -4.1216450 & 4.0192310 & 3.3408620 \\ \mathrm{C} & -3.3578380 & 3.6348700 & 2.2678110 \\ \mathrm{C} & -5.2103970 & 1.8720120 & 3.3926740 \\ \mathrm{H} & -5.6758300 & 3.4567340 & 4.7462330 \\ \mathrm{H} & -3.9902160 & 5.0089950 & 3.7674880 \\ \mathrm{H} & -2.6264670 & 4.3222980 & 1.8583010 \\ \mathrm{H} & -5.9179470 & 1.1725230 & 3.8300960 \\ \mathrm{C} & -3.9300230 & 5.6815890 & -1.3207500 \\ \mathrm{C} & -2.9408670 & 6.5727080 & -1.7955260 \\ \mathrm{C} & -1.6186410 & 6.2129380 & -1.7493220 \\ \mathrm{C} & -3.5775650 & 4.4669830 & -0.7889350 \\ \mathrm{H} & -4.9780480 & 5.9581560 & -1.3839680 \\ \mathrm{H} & -3.2324830 & 7.5333200 & -2.2086850 \\ \mathrm{H} & -0.8511350 & 6.8821370 & -2.1291930 \\ \mathrm{H} & -4.3456150 & 3.7872190 & -0.4362470 \\ \mathrm{C} & 1.9363380 & 2.8145920 & -0.8005610 \\ \mathrm{C} & 2.5607190 & 2.3829130 & -1.9860580 \\ \mathrm{C} & 2.5984640 & 2.6889150 & 0.4356000 \\ \mathrm{C} & 3.8201300 & 1.7853950 & -1.8946080 \\ \mathrm{C} & 3.8529220 & 2.0848520 & 0.4743750 \\ \mathrm{C} & 4.4713850 & 1.6032870 & -0.6771170 \\ & & & \end{array}$




$\begin{array}{lr}\mathrm{H} & 4.3124360 \\ \mathrm{H} & 4.3579690 \\ \mathrm{C} & -3.6645740 \\ \mathrm{C} & -2.9437500 \\ \mathrm{C} & -4.3508040 \\ \mathrm{C} & -2.9564730 \\ \mathrm{C} & -4.3218230 \\ \mathrm{C} & -3.6334300 \\ \mathrm{H} & -2.4061480 \\ \mathrm{H} & -4.8460670 \\ \mathrm{C} & 2.0048710 \\ \mathrm{C} & 5.8349470 \\ \mathrm{C} & 1.9260980 \\ \mathrm{C} & -2.1593270 \\ \mathrm{C} & -3.5782100 \\ \mathrm{C} & -5.1185320 \\ \mathrm{C} & -6.6170250 \\ \mathrm{H} & -6.8019430 \\ \mathrm{H} & -7.0334740 \\ \mathrm{H} & -7.1634130 \\ \mathrm{C} & -4.5357830 \\ \mathrm{H} & -3.4718340 \\ \mathrm{H} & -4.6464590 \\ \mathrm{H} & -5.0553300 \\ \mathrm{C} & -2.7573240 \\ \mathrm{H} & -2.6569810 \\ \mathrm{H} & -3.2411490 \\ \mathrm{H} & -1.7538860 \\ \mathrm{C} & -4.9747220 \\ \mathrm{H} & -5.5568580 \\ \mathrm{H} & -5.5323400 \\ \mathrm{H} & -4.8985320 \\ \mathrm{C} & -3.0052660 \\ \mathrm{H} & -3.9221660 \\ \mathrm{H} & -3.2897600 \\ \mathrm{H} & -2.4356850 \\ \mathrm{C} & -0.8349490 \\ \mathrm{H} & -0.9937650 \\ \mathrm{H} & -0.2795010 \\ \mathrm{H} & -0.2146350 \\ \mathrm{C} & 2.7807850 \\ \mathrm{H} & 3.8265890 \\ \mathrm{H} & 2.7718590 \\ \mathrm{H} & 2.3350890 \\ \mathrm{C} & 1.9434710 \\ \mathrm{H} & 1.4078400 \\ & \end{array}$

$\begin{array}{rr}1.4446040 & -2.8019630 \\ 1.9822330 & 1.4318450 \\ -1.7988050 & 0.4261550 \\ -2.7272080 & 1.2015860 \\ -2.2162450 & -0.7267530 \\ -4.0679690 & 0.8159190 \\ -3.5651340 & -1.0782000 \\ -4.5079250 & -0.3183350 \\ -4.7958190 & 1.4068370 \\ -3.8859460 & -1.9758570 \\ 3.2427520 & 1.7239820 \\ 0.9385780 & -0.6175970 \\ 2.6076360 & -3.3513940 \\ -2.3052290 & 2.4384810 \\ -5.9675020 & -0.7326610 \\ -1.2376930 & -1.6031650 \\ -1.5667350 & -1.6258550 \\ -2.5505360 & -2.0726210 \\ -1.5729360 & -0.6128510 \\ -0.8231100 & -2.2167740 \\ -1.1792250 & -3.0211370 \\ -0.9263030 & -2.9899810 \\ -2.1402330 & -3.5370360 \\ -0.4192430 & -3.6155230 \\ -6.1383570 & -2.0189100 \\ -7.1987330 & -2.2764570 \\ -5.6297900 & -2.8611160 \\ -5.7151820 & -1.9019030 \\ -6.5829360 & -0.8845020 \\ -6.4688590 & 0.0357230 \\ -6.1070700 & -1.6993370 \\ -7.6517150 & -1.1130680 \\ -2.4618370 & 3.7108730 \\ -1.8674590 & 3.6672790 \\ -3.5113640 & 3.8530740 \\ -2.1430610 & 4.5914260 \\ -3.0623690 & 2.6000960 \\ -4.0936720 & 2.9358410 \\ -3.0866070 & 1.6600380 \\ -2.5668610 & 3.3552380 \\ 4.4920750 & 2.1648680 \\ 4.2479410 & 2.3859220 \\ 5.2553120 & 1.3793830 \\ 4.9237840 & 3.0681520 \\ 2.2004270 & 2.8440890 \\ 1.3068360 & 2.5112040 \\ -2 & \end{array}$
2.9466980 1. 4267870
5.8846380
6.8489840
5.7554000
5.0948640
6.9356160
6.9300200
6.7889780
7. 9231090
2. 2110930
3.2595320
1.9755860
1. 6007230
2. 3926420
3. 4774450
1. 9080000
2. 1646760
$-0.4939630$
0.0157050
$-0.1103730$
0.8419500
6.0255700
0.9775890
$-5.0154940$
$-3.0600400$
$-1.9114140$
2. 6155380
1.7903350
2.5362120
0.8605760
1. 6416780
0.9269000
4.0986770
3. 5183230
2. 0491140
4. 0122650
5. 1445050
3. 5973600
1. 4084310
4.6733660
4. 3303520
3. 5558970
4. 1007920
1.6808340
2.7652350

1.8986550

2. 6143160

$-0.1936660$

$-0.7117150$

0.1814190

$-0.9300430$

1.9740590

2. 7610360

2.4478930

1.4992880

1.4948540

1.4922570

0.5107510

1. 6483840

3.9538260

3. 9424700

4.1460200

4.7888360

$-0.0616440$

0.0756090

$-1.2790260$

2. 6491950

0.4966000

3.5589770

$-0.2385290$

$-6.5126840$

$-1.2451850$

$-1.7315530$

$-2.8983550$

$-0.8758960$

$-2.7019080$

$-1.6717290$

$-1.0076110$

$-3.2216690$

$-4.4408550$

$-4.2274310$

$-1.9689190$

$-3.4061640$

$-5.3328820$

$-4.2723720$

$-2.0715620$

$-1.0749150$

3.0510120

$-4.6360160$

-5.0083240
-2.1376140
3.1662480

3.7171260

0.4143220

0.3716010

1.4358910

0.2340810

$-0.3479430$

$-1.1089700$

0.6298480

$-0.3495050$

$-4.3643730$

$-4.6853360$

$-3.9565080$

$-5.2607290$

$-3.9301970$

$-4.0910510$

$-4.8941630$

$-3.2619910$

$-0.7898150$

$-2.1959860$

0.0112090

$-3.2108520$

$-1.6046260$

1. 5241720

$-1.1693430$

0.0668190

2. 3231230

$-2.0261210$

$-1.6894180$

$-1.3528310$

$-1.1667890$

$-3.0910820$

$-2.7925300$

$-3.4236740$

$-2.7017000$

$-2.3214100$

$-2.5467150$

$-3.6869200$

$-3.3299420$

$-3.2109090$

$-1.6778930$

$-3.0930510$

$-4.3606790$

$-1.7927700$

$-1.6501710$

1.6697070 


\begin{tabular}{llll} 
O & 1.9036910 & -1.1727600 & 1.5812500 \\
$\mathrm{C}$ & 3.7804060 & -1.9140240 & 2.7219380 \\
$\mathrm{C}$ & 4.9905080 & -2.6177340 & 2.7218700 \\
$\mathrm{C}$ & 3.5440680 & -0.9485080 & 3.7095220 \\
$\mathrm{C}$ & 5.9537170 & -2.3495500 & 3.6848580 \\
$\mathrm{H}$ & 5.1787210 & -3.3571050 & 1.9511620 \\
$\mathrm{C}$ & 4.5076660 & -0.6878570 & 4.6752750 \\
$\mathrm{H}$ & 2.6023860 & -0.4134090 & 3.7176730 \\
$\mathrm{C}$ & 5.7155170 & -1.3832450 & 4.6608800 \\
$\mathrm{H}$ & 6.8949080 & -2.8894050 & 3.6703170 \\
$\mathrm{H}$ & 4.3157320 & 0.0586340 & 5.4393400 \\
$\mathrm{H}$ & 6.4710340 & -1.1730830 & 5.4116490 \\
$\mathrm{H}$ & 1.1390980 & -1.3040530 & 0.9191590 \\
$\mathrm{~S}$ & 2.7588240 & -3.5131370 & 0.7044490 \\
\hline
\end{tabular}

TS209(R, R) G[WB97XD/6-31G(d)] $=-3633.715988$

$\begin{array}{lrcr}\text { TS209(R, R }(\mathrm{G}[\mathrm{WB} 97 \mathrm{XD} / 6-31 \mathrm{G}(\mathrm{d})]=-3633.715988 \\ \mathrm{O} & 0.6497570 & 0.5908160 & -0.0972470 \\ \mathrm{O} & -1.5425480 & 0.9614890 & -1.3287520 \\ \mathrm{C} & 0.9046400 & 1.9414720 & -0.2100190 \\ \mathrm{C} & -0.0944500 & 2.8399740 & 0.0964690 \\ \mathrm{C} & 2.2224000 & 2.3401320 & -0.5533560 \\ \mathrm{C} & 0.1580680 & 4.2379350 & -0.0857650 \\ \mathrm{C} & 2.4825090 & 3.6852340 & -0.6270630 \\ \mathrm{C} & 1.4699980 & 4.6577810 & -0.4360650 \\ \mathrm{H} & 3.4936190 & 4.0178740 & -0.8458720 \\ \mathrm{C} & -2.1059880 & 1.4193850 & -0.1473520 \\ \mathrm{C} & -3.3933820 & 0.9420210 & 0.2145290 \\ \mathrm{C} & -1.4023670 & 2.3401620 & 0.5944400 \\ \mathrm{C} & -3.9436450 & 1.4326270 & 1.3718610 \\ \mathrm{C} & -1.9360930 & 2.7543920 & 1.8575200 \\ \mathrm{C} & -3.2334590 & 2.3089940 & 2.2305830 \\ \mathrm{H} & -4.9432610 & 1.1180050 & 1.6579730 \\ \mathrm{C} & -3.0665890 & 3.5255970 & 4.3231830 \\ \mathrm{C} & -1.7592980 & 3.9351300 & 3.9721530 \\ \mathrm{C} & -1.2088630 & 3.5624960 & 2.7723730 \\ \mathrm{C} & -3.7839070 & 2.7269000 & 3.4711670 \\ \mathrm{H} & -3.4930940 & 3.8350940 & 5.2723180 \\ \mathrm{H} & -1.1843900 & 4.5469020 & 4.6606340 \\ \mathrm{H} & -0.2015860 & 3.8757050 & 2.5206530 \\ \mathrm{H} & -4.7817060 & 2.3882840 & 3.7374950 \\ \mathrm{C} & -0.5677730 & 6.5553350 & -0.1338930 \\ \mathrm{C} & 0.7464850 & 6.9730450 & -0.4485570 \\ \mathrm{C} & 1.7404250 & 6.0419060 & -0.5992340 \\ \mathrm{C} & -0.8549890 & 5.2260700 & 0.0446640 \\ \mathrm{H} & -1.3571360 & 7.2947020 & -0.0388060 \\ \mathrm{H} & 0.9592440 & 8.0290710 & -0.5826400\end{array}$

2.7511280
-1.8699410
3.3254700
3.7609920
3.9835100
4.8967960
5.0916900
5.5839510
5.2420960
5.5997210
-4.1279970
-4.3168960
-4.6609680
-5.0603310
-5.3862850
-5.6027730
-5.2198000
-5.8041980
3.5126800
6.8431340
3.0381650
-3.7556340
-6.4125400
-4.4992260
-5.8568570
-6.5083720
-6.3741820
-5.7204240
-3.7434930
-2.7709720
-4.3082850
-3.5777860
-7.6995990
-8.3041320
-7.4717340
-8.3037940
-5.5838410
-4.6755650
-5.2827220
-6.1680890
-4.8574160
-5.3456520
-5.6290330
-4.4384160
-3.0472090
-3.7516470

6.3469580

4. 9201270

1. 3522120

1.0381290

0.8085720

0.2389000

$-0.0088600$

$-0.2847490$

0.0169540

$-0.4425720$

$-0.0428220$

$-1.3654690$

0.3630690

$-2.2416970$

$-0.5581500$

$-1.8615940$

$-3.2573490$

$-0.2476170$

1.0741980

$-1.1273440$

1. 5634290

$-1.8662910$

$-2.8358710$

1.7796310

2. 4861820

1.9845010

2. 5012260

3. 5213820

1.7963210

1. 3065200

1. 2821060

2.8283060

$-3.2554030$

$-3.9108980$

$-3.8009100$

$-2.3816730$

$-4.0624460$

$-3.7656750$

$-4.6428530$

$-4.7217180$

$-1.9525810$

$-0.9862070$

$-2.6715350$

$-2.2850870$

$-3.2209930$

$-4.0305270$
$-0.8572720$

0.2738350

$-0.7580250$

$-2.0565480$

0.3616310

$-2.2118790$

0.1541290

$-1.1211440$

$-3.2164150$

1. 0136550

$-0.6356210$

$-0.1815850$

$-1.8714330$

$-0.9702200$

$-2.6289020$

$-2.1965820$

$-0.6164830$

$-3.5843980$

1.7845330

$-1.2579710$

$-3.2894410$

1. 1439340

$-3.0331600$

$-2.4053130$

$-2.5214970$

$-3.2461480$

$-1.5562060$

$-2.8538720$

$-3.7402550$

$-3.6384090$

$-4.5265390$

$-4.0694220$

$-2.3103100$

$-2.9471150$

$-1.3870780$

$-2.0444010$

$-3.4372930$

$-3.9717270$

$-2.5574490$

$-4.0888860$

2. 2103790

2. 3700410

1. 9101030

3. 1669840

1.0123400

0.7904390 


$\begin{array}{rrr}-2.2925530 & -3.1891920 & 0.2229740 \\ -2.5497110 & -3.4712990 & 1.9561960 \\ 4.6279250 & 1.6482080 & 2.6670210 \\ 5.4411730 & 0.9278280 & 2.8106370 \\ 5.0532370 & 2.5552440 & 2.2247010 \\ 4.2316170 & 1.9014840 & 3.6563960 \\ 2.9105620 & -0.1961100 & 2.3977100 \\ 2.0752740 & -0.5484780 & 1.7865960 \\ 3.6551230 & -0.9984370 & 2.4625710 \\ 2.5340860 & -0.0005490 & 3.4080260 \\ 8.0788360 & -0.2851260 & -0.9025660 \\ 8.9875060 & -0.8967960 & -0.9312250 \\ 8.1970040 & 0.5363500 & -1.6189770 \\ 7.9884140 & 0.1499070 & 0.0979960 \\ 7.0200820 & -1.7797190 & -2.6309520 \\ 6.1359490 & -2.3536720 & -2.9297920 \\ 7.2153370 & -1.0313970 & -3.4076250 \\ 7.8756090 & -2.4625750 & -2.6118660 \\ 2.8958850 & 0.5005650 & -4.3859030 \\ 3.8532140 & 0.2900130 & -4.8764420 \\ 2.5042150 & -0.4345920 & -3.9793480 \\ 2.2035700 & 0.8548270 & -5.1568170 \\ 3.7309720 & 2.8136560 & -3.8498650 \\ 4.7644850 & 2.5854070 & -4.1371200 \\ 3.2038570 & 3.1779070 & -4.7387890 \\ 3.7561770 & 3.6235190 & -3.1149100 \\ -0.3636700 & -0.1431480 & -1.1580250 \\ 0.2831880 & -0.3402660 & -2.4948980 \\ -0.8633410 & -1.3563280 & -0.4124750 \\ 2.0250740 & 1.8426360 & -2.9836920 \\ 6.7671860 & -1.9327010 & -0.5141050 \\ 2.7175170 & 1.8246000 & 1.7480100 \\ -3.9039420 & 2.3549170 & -1.6909550 \\ -6.7026890 & -2.3130600 & -3.9534830 \\ -3.0073790 & -1.1475370 & 1.4880250 \\ 2.2813350 & -2.5422080 & -1.0865040 \\ 1.1346320 & -3.4545890 & -0.9810210 \\ 2.1821410 & -1.5788630 & -0.5829220 \\ 0.1552170 & -2.9980710 & -0.8924700 \\ 1.8143020 & -2.4912090 & -2.4564100 \\ 1.2127950 & -1.6852870 & -2.5474290 \\ 3.7540580 & -4.5374330 & -1.5904230 \\ 2.6747190 & -5.4603550 & -1.0208670 \\ 1.2722450 & -4.9058150 & -1.2906750 \\ 3.6628440 & -3.1386550 & -0.9769150 \\ 4.7473950 & -4.9530400 & -1.3957990\end{array}$

2.7526260
1.0130160
3.9160360
4.3615320
3.6434780
2.8211470
0.4977220
0.3121790
-0.4129850
0.4843000
1.2691400
-0.1341900
1.4325920
1.7527140
0.0335120
-0.7384460
0.8170110
2.0447390
-0.4460760
0.9503600
-0.5437550
1.0043810
$-6.4612420$
$-5.0140890$
$-3.1702840$
$-2.4489760$
$-4.4666970$
$-5.5677850$
$-5.4482330$
$-2.4511930$
$-1.5150250$
$-2.2878400$
$-3.1693700$
$-1.2020510$
$-2.9691130$
$-4.0078680$
$-1.0052840$
$-0.5139540$
$-1.8872730$
$-3.6558910$
$-0.1605830$
$-1.7308130$
$-1.5444270$
$-3.7172190$

$-1.4550860$

$-2.3520300$

0.0901970

$-1.4528140$

$-2.6792350$

0.0612330

$-0.7409600$

2. 5801740

2. 0587360

4.0442070

4.7966200

4.6823720

6.1611850

4.3075070

6. 0465810

4. 1033510

6.7883180

6.7367400

6.5306440

7.8543380

1. 0430510

5

1. 7158160

$\operatorname{TS} 210(R, R) \quad G[W B 97 X D / 6-31 G(d)]=-3633.718627$

$\begin{array}{lrrr}\text { TS210 (R, R) G }[\mathrm{WB} 97 X \mathrm{D} / 6-31 \mathrm{G}(\mathrm{d})]=-3633.718627 \\ \mathrm{O} & 0.1065710 & -1.2695600 & 0.2840260 \\ \mathrm{O} & 2.0541940 & -0.1222860 & -0.8263650 \\ \mathrm{C} & 0.4770660 & -2.4730140 & -0.2794730 \\ \mathrm{C} & 1.8030600 & -2.8458080 & -0.2505640 \\ \mathrm{C} & -0.5651310 & -3.2931380 & -0.7901810 \\ \mathrm{C} & 2.1708230 & -4.0635590 & -0.9126270 \\ \mathrm{C} & -0.2136180 & -4.5109970 & -1.3101750 \\ \mathrm{C} & 1.1454060 & -4.9075810 & -1.4221140 \\ \mathrm{H} & -0.9853330 & -5.1906070 & -1.6602520 \\ \mathrm{C} & 2.8656860 & -0.6401690 & 0.1599840 \\ \mathrm{C} & 3.7880940 & 0.2529590 & 0.7655760 \\ \mathrm{C} & 2.7927960 & -1.9842540 & 0.4541310 \\ \mathrm{C} & 4.6800100 & -0.2712910 & 1.6638270 \\ \mathrm{C} & 3.6452750 & -2.4936980 & 1.4908790 \\ \mathrm{C} & 4.6221120 & -1.6313230 & 2.0625510 \\ \mathrm{H} & 5.4293740 & 0.3743590 & 2.1129470 \\ \mathrm{C} & 5.3886880 & -3.4231730 & 3.5088490 \\ \mathrm{C} & 4.3859810 & -4.2673950 & 2.9792550 \\ \mathrm{C} & 3.5386240 & -3.8169060 & 1.9985590 \\ \mathrm{C} & 5.4972940 & -2.1323070 & 3.0618310 \\ \mathrm{H} & 6.0586960 & -3.7950230 & 4.2778640 \\ \mathrm{H} & 4.2807000 & -5.2796110 & 3.3578150\end{array}$




$\begin{array}{rrrr}\mathrm{H} & 2.7690830 & -4.4745640 & -1.6101250 \\ \mathrm{H} & 6.2462350 & -1.4633930 & -1.4776370 \\ \mathrm{C} & 3.8361410 & -5.6256070 & -1.7418610 \\ \mathrm{C} & 2.8149350 & -6.4841840 & -2.2090170 \\ \mathrm{C} & 1.5000770 & -6.1282280 & -2.0542390 \\ \mathrm{C} & 3.5236290 & -4.4492480 & -1.1090490 \\ \mathrm{H} & 4.8769080 & -5.8965260 & -1.8908780 \\ \mathrm{H} & 3.0751830 & -7.4161250 & -2.7012440 \\ \mathrm{H} & 0.7066250 & -6.7705040 & -2.4271420 \\ \mathrm{H} & 4.3153440 & -3.7938560 & -0.7626530 \\ \mathrm{C} & -1.9798770 & -2.8208930 & -0.6693520 \\ \mathrm{C} & -2.6555210 & -2.2696070 & -1.7772880 \\ \mathrm{C} & -2.6116410 & -2.8867960 & 0.5839400 \\ \mathrm{C} & -3.9481800 & -1.7803360 & -1.5941050 \\ \mathrm{C} & -3.9124150 & -2.3966340 & 0.7118360 \\ \mathrm{C} & -4.5924140 & -1.8254330 & -0.3577400 \\ \mathrm{H} & -4.4722910 & -1.3493680 & -2.4428440 \\ \mathrm{H} & -4.4118340 & -2.4580370 & 1.6765780 \\ \mathrm{C} & 3.7449980 & 1.7096640 & 0.4334420 \\ \mathrm{C} & 3.1744250 & 2.6143570 & 1.3490040 \\ \mathrm{C} & 4.2433310 & 2.1672430 & -0.7997900 \\ \mathrm{C} & 3.1416650 & 3.9688760 & 1.0140410 \\ \mathrm{C} & 4.1785790 & 3.5283280 & -1.0915570 \\ \mathrm{C} & 3.6304170 & 4.4464830 & -0.1981830 \\ \mathrm{H} & 2.7089980 & 4.6787210 & 1.7147980 \\ \mathrm{H} & 4.5624890 & 3.8805720 & -2.0467250 \\ \mathrm{C} & -1.9342880 & -3.5136380 & 1.7945000 \\ \mathrm{C} & -5.9974610 & -1.2779860 & -0.1848900 \\ \mathrm{C} & -2.0259580 & -2.2414270 & -3.1635580 \\ \mathrm{C} & 2.6087470 & 2.1602740 & 2.6892330 \\ \mathrm{C} & 3.5355620 & 5.9216050 & -0.5436490 \\ \mathrm{C} & 4.8529280 & 1.2186350 & -1.8219080 \\ \mathrm{C} & 6.3469490 & 1.5062440 & -2.0217420 \\ \mathrm{H} & 6.5072750 & 2.5106060 & -2.4307470 \\ \mathrm{H} & 6.8888230 & 1.4375780 & -1.0723630 \\ \mathrm{H} & 6.7852160 & 0.7851110 & -2.7208600 \\ \mathrm{C} & 4.0916960 & 1.2569950 & -3.1531320 \\ \mathrm{H} & 3.0305810 & 1.0425540 & -2.9967360 \\ \mathrm{H} & 4.1787820 & 2.2373560 & -3.6359350 \\ \mathrm{H} & 4.4967520 & 0.5094330 & -3.8445470 \\ \mathrm{C} & 2.5888400 & 6.1500710 & -1.7302110 \\ \mathrm{H} & 2.4658760 & 7.2208190 & -1.9274330 \\ \mathrm{H} & 2.9814680 & 5.6819760 & -2.6404050 \\ \mathrm{H} & 1.6012420 & 5.7206020 & -1.5313840 \\ \mathrm{C} & 4.9133640 & 6.5406830 & -0.8100280 \\ \mathrm{H} & 5.5840350 & 6.3918380 & 0.0424800\end{array}$

\begin{abstract}
5. 3846470
4.8211480

3. 6359560

4.5742730

3. 8655990

3. 2405720

1. 2867840

1.4275640

0.5675840

0.8561970

$-2.5754160$

$-3.6352380$

$-2.5086700$

$-2.0731230$

$-1.9354380$

$-1.5198260$

$-2.9469490$

$-1.3284830$

$-7.0174940$

$-8.0330420$

$-6.8153920$

$-6.9853520$

$-6.0702020$

$-5.3449180$

$-5.8618340$

$-7.0688470$

$-2.4144580$

$-3.4592370$

$-2.2662320$

$-1.7939450$

$-2.3883620$

$-3.4739990$

$-1.9170230$

$-2.0627840$

0.4699300

$-0.1738910$

0.2118850

$-0.9402730$

$-6.2577170$

$-0.8876070$

4.7723240

3.1045370

2. 4004820

$-2.4509750$

$-1.4659570$

$-2.5242800$
\end{abstract}

6.0923970

7.6172090

2. 3709390

1.8459200

3.4373440

2. 0063050

2. 8484830

3. 9101440

2. 7632090

2.3763660

$-4.8688880$

$-4.7490490$

$-5.5510120$

$-5.3373720$

$-2.5868850$

$-1.6065060$

$-2.4446160$

$-3.0193240$

-2.0830000

$-1.7129920$

$-1.9966110$

$-3.1450970$

0.2136930

0.7867600

0.3820410

0.6112530

$-1.0120930$

$-1.0544070$

$-0.0871620$

$-0.9647870$

$-3.5152930$

$-3.5816700$

$-3.5053390$

$-4.4200610$

0.0898390

0.1350930

1. 2091380

$-2.2128070$

$-1.3809990$

$-3.7061580$

0.1994700

6.4317940

1.0887100

2. 2680690

3. 2346040

1. 3254910
$-1.6923240$

$-0.9917520$

3.8117170

3. 6095270

3.9248330

4.7667320

3. 0502030

3. 2835010

2. 2327330

3. 9405640

2. 1230740

2. 3770100

1.2686140

2. 9769610

3.0155340

2. 7613850

3. 4146160

3. 8184530

$-1.0008410$

$-0.8205870$

$-2.0749220$

$-0.7363860$

$-0.5328440$

0.0524620

$-1.5959430$

$-0.3207670$

$-3.9916010$

$-4.3210900$

$-3.4312420$

$-4.8929770$

$-3.9435730$

$-4.0842070$

$-4.9328930$

$-3.4233130$

$-0.5378870$

$-1.8932400$

0.4390600

$-3.0334330$

0.8757560

1. 5444310

$-1.4326690$

0.3272760

2. 6157820

$-1.6244450$

$-1.1173920$

$-1.0789000$ 


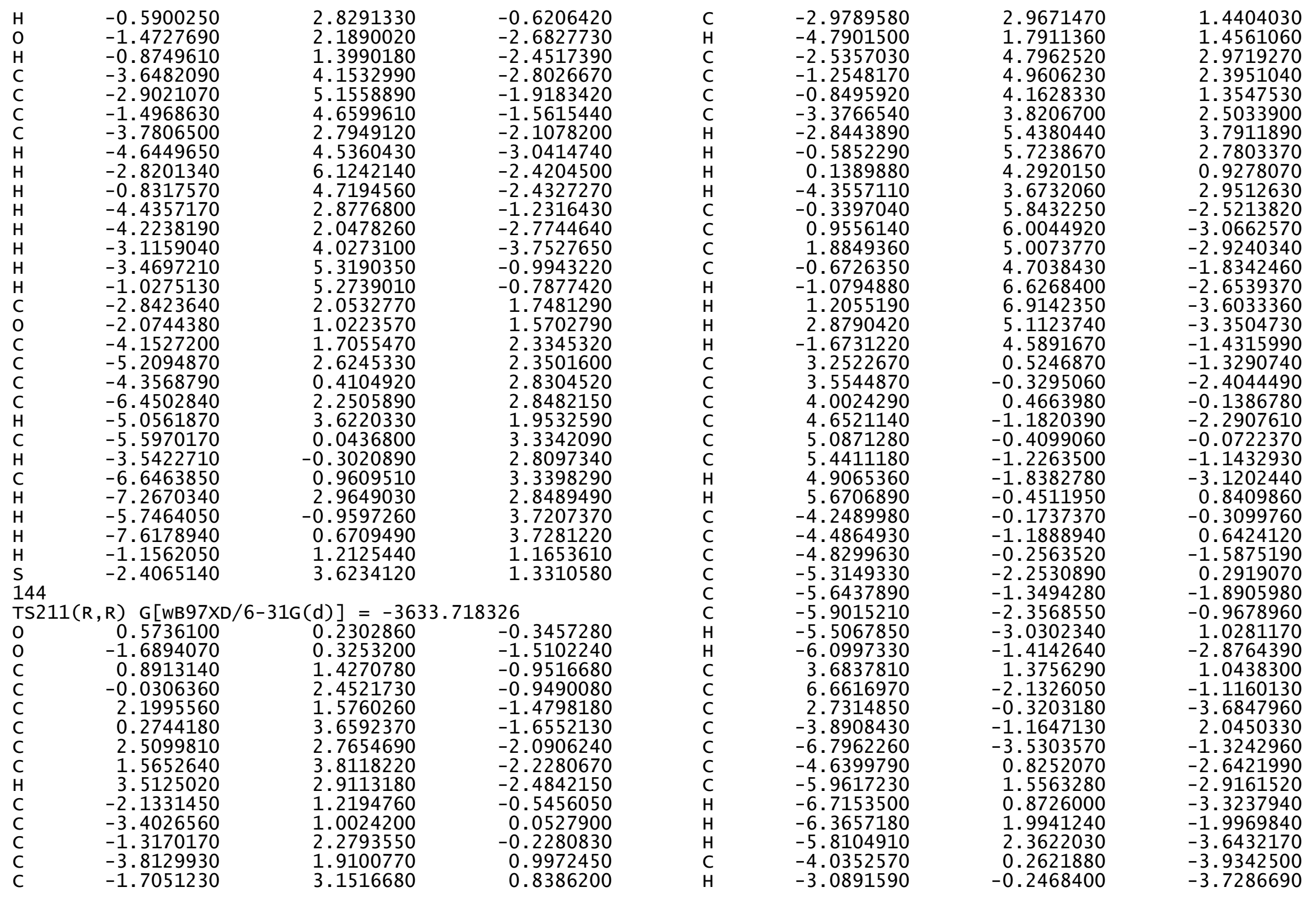




-4.7131510
-3.8430810
-8.0525900
-8.7203560
-7.7927220
-8.6033530
-6.0362620
-5.1542090
-5.6995360
-6.6815460
-4.9582330
-5.4287770
-5.7498090
-4.5124420
-3.2150520
-3.9455890
-2.5001410
-2.6781040
4.3976730
5.4849220
4.1248910
4.1352850
4.0184230
3.5710420
5.1000440
3.6298060
7.0572920
7.8577180
7.4321130
6.2129140
7.8465150
7.5982070
8.1193880
8.7223080
2.4745390
3.3812490
2.1048690
1.7226780
3.3980900
4.3943100
2.7996490
3.5113170
-0.5919250
-0.0798130
-1.1306120
1.7553900

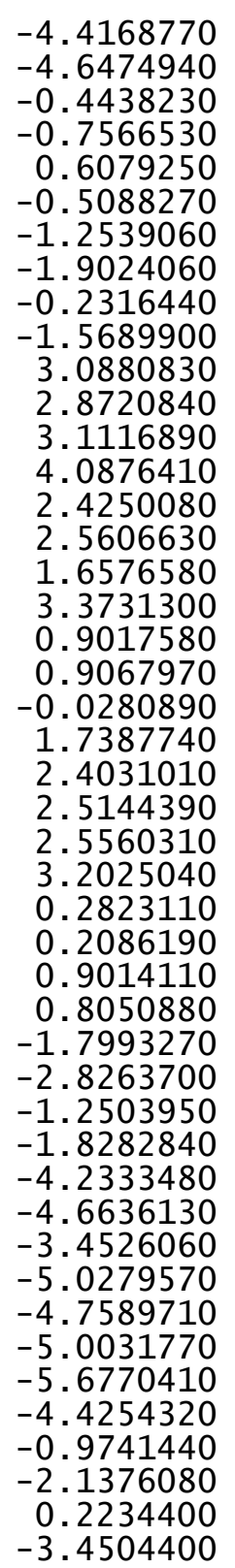

\begin{abstract}
6.4146650
2. 6041810

$-3.9389440$

$-7.1220730$

$-3.1180900$

2.0447230

0.9103940

2. 0864460

$-0.0263550$

1. 3213670

0.7467310

3. 3180300

2. 3487340

0.9402190

3. 3975000

4.3173870

2. 3079520

0.4758820

3.8603310

4.0116380

2.9911420

2.7026480

0.2664170

0.4996840

$-0.5520320$

0.9786160

1.7703900

0.6609640

2.2386620

2. 0134490

1. 1497430

0.0510700

1.9364590

2. 8440830

0.9118360

2. 3136200

$-0.7845380$

1.2809250
\end{abstract}

144

0.5514760

0.1634570

0.5535850

1.5872730

$-0.7462710$

$-1.5367160$

0.1149690
$-3.0211460$
1.5665930
1.5700300
$-3.3880250$
$-0.3934800$
$-3.0334040$
$-3.5878280$
$-1.9457570$
$-3.0433310$
$-3.6353230$
$-2.9148800$
$-5.1749660$
$-5.4762830$
$-4.9754680$
$-3.6710020$
$-5.5499330$
$-6.5502190$
$-5.6049780$
$-3.1559230$
$-3.4542020$
$-5.6951050$
$-4.9891820$
$-5.0197770$
$-0.7094850$
$-0.3529360$
0.3523270
0.0679210
1.6827830
1.1015500
$-0.9626260$
2.7142290
1.9046400
2.4253290
0.8757120
3.7425360
3. 2312640
$-0.9335960$
$-2.1931280$

$-1.7132380$

1.0400350

$-2.2559320$

$-2.3626390$

0.5926860

$-0.2447690$

0.5468790

$-1.2648540$

$-1.6685550$

0.3138570

1.4579100

1.1284290

0.0445360

0.5551600

1. 6610660

0.3569660

0.8953810

$-0.8326320$

$-0.5946680$

2. 3752990

1. 9888850

3.0469370

2. 3839630

3.9601460

5.0770480

3.6537350

5.8774030

5.3129390

4.4450860

2.7847010

5.5587050

6.7493520

4.1912330

6.1809090

1. 5723420

2.9045140

$\operatorname{TS} 212(R, R) \quad G[W B 97 X D / 6-31 G(d)]=-3633.718318$

$\begin{array}{lrcr}\text { TS212(R, R) } \mathrm{G}[\mathrm{WB} 97 X \mathrm{D} / 6-31 \mathrm{G}(\mathrm{d})]=-3633.718318 \\ \mathrm{O} & 0.5513570 & 0.1386530 & -0.3112410 \\ \mathrm{O} & -1.7647640 & 0.1228810 & -1.3695300 \\ \mathrm{C} & 0.8315410 & 1.2785230 & -1.0343240 \\ \mathrm{C} & -0.0968570 & 2.2985670 & -1.0790990 \\ \mathrm{C} & 2.1116650 & 1.3842690 & -1.6370790 \\ \mathrm{C} & 0.1664480 & 3.4395110 & -1.9031820\end{array}$




$\begin{array}{rrr}2.3858150 & 2.5197380 & -2.3589470 \\ 1.4302480 & 3.5482670 & -2.5416440 \\ 3.3677840 & 2.6322940 & -2.8110820 \\ -2.1746870 & 1.1043250 & -0.4783480 \\ -3.4256000 & 0.9454810 & 0.1735850 \\ -1.3525930 & 2.1906390 & -0.2933150 \\ -3.8117340 & 1.9404140 & 1.0356710 \\ -1.7144180 & 3.1649730 & 0.6924960 \\ -2.9697730 & 3.0375110 & 1.3463620 \\ -4.7763110 & 1.8683140 & 1.5295690 \\ -2.5008320 & 5.0165380 & 2.6693720 \\ -1.2376950 & 5.1268870 & 2.0431890 \\ -0.8546980 & 4.2294710 & 1.0782810 \\ -3.3456300 & 3.9937210 & 2.3260830 \\ -2.7925380 & 5.7373340 & 3.4266230 \\ -0.5633290 & 5.9281230 & 2.3305620 \\ 0.1206180 & 4.3208400 & 0.6125070 \\ -4.3118040 & 3.8874090 & 2.8118530 \\ -0.5022320 & 5.5349770 & -2.9332840 \\ 0.7693820 & 5.6566600 & -3.5407780 \\ 1.7121190 & 4.6804880 & -3.3508230 \\ -0.7970980 & 4.4585290 & -2.1361440 \\ -1.2543370 & 6.2989770 & -3.1051050 \\ 0.9899920 & 6.5175450 & -4.1642740 \\ 2.6876680 & 4.7529480 & -3.8241970 \\ -1.7807660 & 4.3737590 & -1.6877720 \\ 3.1922200 & 0.3618420 & -1.4706630 \\ 3.4452450 & -0.5665520 & -2.4940420 \\ 4.0453250 & 0.4386020 & -0.3510290 \\ 4.6004380 & -1.3483840 & -2.4188620 \\ 5.1853390 & -0.3631480 & -0.3229640 \\ 5.4946170 & -1.2451520 & -1.3576570 \\ 4.8179800 & -2.0544910 & -3.2168130 \\ 5.8621810 & -0.2862620 & 0.5225910 \\ -4.2828140 & -0.2547180 & -0.0620010 \\ -4.4819650 & -1.1918420 & 0.9711030 \\ -4.9147080 & -0.4300530 & -1.3083090 \\ -5.3272090 & -2.2773310 & 0.7330900 \\ -5.7396780 & -1.5364760 & -1.4975380 \\ -5.9632020 & -2.4711960 & -0.4886820 \\ -5.4942150 & -3.0018000 & 1.5270860 \\ -6.2298680 & -1.6677500 & -2.4597570 \\ 3.7745320 & 1.4192270 & 0.7842120 \\ 6.7870870 & -2.0439990 & -1.3592110 \\ 2.5126530 & -0.7060820 & -3.6887720 \\ -3.8300320 & -1.0562200 & 2.3416580\end{array}$

$-6.8699980$

$-4.7662270$

$-6.1008610$

$-6.8605820$

$-6.4816840$

$-5.9769600$

$-4.2019900$

$-3.2454350$

$-4.8910640$

$-6.3037840$

$-6.9371670$

$-6.2564090$

$-5.2917020$

$-8.3025570$

$-8.7102120$

$-8.3371200$

$-4.8486320$

$-5.3128630$

$-5.6496240$

$-4.3626630$

$-3.1563140$

$-3.8901030$

$-2.4753280$

$-2.5805910$

4.4543460

5.5423290

4.1143590

4.2306630

4.1908170

3.7489160

5.2793320

3.8509530

7.0590180

7.9805130

7.1873380

6.2438110

7.9677470

7.7995690

8.1027690

8.8981160

2.2700800

3.1591400

1.9822910

1. 4619110

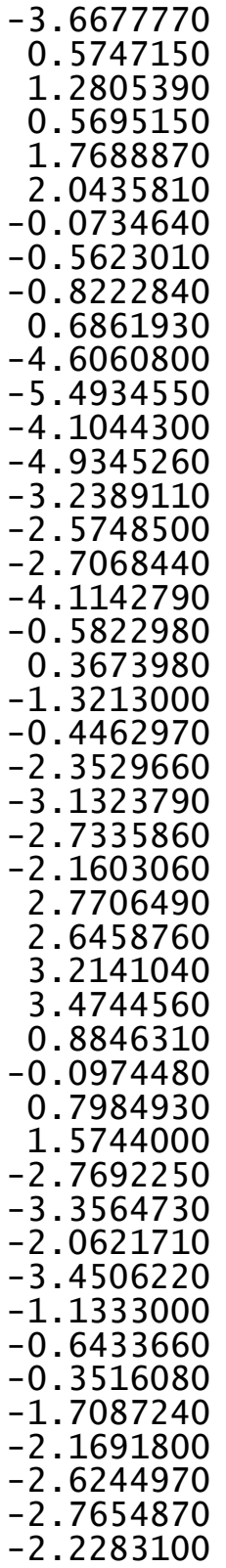

$-0.7126370$

$-2.4427300$

$-2.7208070$

$-3.0654890$

$-1.8173840$

$-3.4975690$

$-3.7128590$

$-3.5068260$

$-4.1202190$

$-4.4862780$

$-1.7866820$

$-1.8960270$

$-2.7602050$

$-1.5287340$

$-1.0563990$

$-0.2871260$

$-2.0141650$

$-1.1375560$

3.3888100

3.1051660

3.5110170

4. 3616100

2.8108250

3.0459030

2.0461990

3.7234650

0.5184280

0.4634110

$-0.4220890$

1.3283060

2.1577590

2. 3562660

2. 2516280

2.9365120

$-0.0360360$

$-0.1119460$

0.7911410

0.2252820

$-1.7288360$

$-2.6934830$

$-0.9717390$

$-1.7919380$

$-4.0762060$

$-4.5274700$

$-3.2067880$

$-4.8125650$ 


\begin{tabular}{|c|c|c|}
\hline $\begin{array}{l}3.0360520 \\
4.0154190 \\
2.3464830 \\
3.1443410 \\
-0.6415570 \\
-0.1848250 \\
-1.1129030 \\
1.5421670 \\
6.6971630 \\
2.6931970 \\
-4.0576920 \\
-6.9090040 \\
-3.0463720 \\
2.0450550 \\
0.9959330 \\
2.0024420 \\
0.0240140 \\
1.3093950 \\
0.6843280 \\
3.4993610 \\
2.6167480 \\
1.1577110 \\
3.4508520 \\
4.5352870 \\
2.6715080 \\
0.7090610 \\
3.9337130 \\
3.9840060 \\
3.1656670 \\
2.9773300 \\
0.5331040 \\
0.6462520 \\
-0.4483930 \\
1.1837580 \\
2.0120850 \\
0.8862640 \\
2.5361490 \\
2.2384220 \\
1.4331490 \\
0.2449400 \\
2.2570720 \\
3.1697040 \\
1.2107700 \\
2.6814080 \\
-0.7191790 \\
1.4230740\end{array}$ & $\begin{array}{r}0.0853020 \\
-0.2945810 \\
-0.0091030 \\
1.1491140 \\
-0.8939290 \\
-1.7899540 \\
-1.5262740 \\
-0.2888140 \\
-2.8070790 \\
1.5939060 \\
1.3469760 \\
-4.2272000 \\
-0.2980970 \\
-2.9888920 \\
-3.5412980 \\
-1.9144330 \\
-3.0622870 \\
-3.7408380 \\
-3.0936800 \\
-4.9777080 \\
-5.2295700 \\
-4.8685160 \\
-3.5068080 \\
-5.2567610 \\
-6.2769780 \\
-5.5985660 \\
-2.8767670 \\
-3.3388740 \\
-5.6092790 \\
-4.6256400 \\
-4.8855060 \\
-0.4386740 \\
-0.1689000 \\
0.7353630 \\
0.6075550 \\
2.0087860 \\
1.7414050 \\
-0.3787840 \\
3.1378980 \\
2.1049310 \\
3.0061470 \\
1.6398560 \\
4.1184990 \\
3.8887520 \\
-0.8392640 \\
-1.9305340\end{array}$ & $\begin{array}{r}-4.8956710 \\
-5.2107750 \\
-5.7421050 \\
-4.6638340 \\
-0.7850920 \\
-1.8936550 \\
0.5031510 \\
-3.4032620 \\
-2.1431300 \\
0.8277030 \\
-2.1316530 \\
0.2309330 \\
2.2703310 \\
0.1300900 \\
1.0012110 \\
-0.0525640 \\
0.9628060 \\
-0.8662990 \\
-1.3166920 \\
0.7235770 \\
1.9473740 \\
1.6543550 \\
0.3049790 \\
0.9408610 \\
2.2574960 \\
0.9667870 \\
1.0620660 \\
-0.6339340 \\
-0.1086210 \\
2.7897730 \\
2.5520620 \\
3.1413960 \\
2.5071450 \\
3.8642560 \\
4.9834600 \\
3.3606930 \\
5.5906900 \\
5.3746470 \\
3.9563360 \\
2.4914540 \\
5.0724850 \\
6.4659290 \\
3.5473060 \\
5.5412490 \\
1.7814640 \\
3.1514880\end{array}$ \\
\hline
\end{tabular}

\begin{tabular}{lrrr} 
TS & \multicolumn{1}{c}{ Th $(\mathrm{R}, \mathrm{R}) \mathrm{G}[\mathrm{WB} 97 \mathrm{XD} / 6-31 \mathrm{G}(\mathrm{d})]=-3633.718421$} \\
$\mathrm{O}$ & 0.5756480 & 0.1781370 & -0.3015240 \\
$\mathrm{O}$ & -1.6587430 & 0.2274810 & -1.5334000 \\
$\mathrm{C}$ & 0.9143720 & 1.3526360 & -0.9389490 \\
$\mathrm{C}$ & -0.0115910 & 2.3728070 & -1.0081560 \\
$\mathrm{C}$ & 2.2406460 & 1.4914750 & -1.4226870 \\
$\mathrm{C}$ & 0.3081790 & 3.5504740 & -1.7551850 \\
$\mathrm{C}$ & 2.5609970 & 2.6561390 & -2.0770350 \\
$\mathrm{C}$ & 1.6149150 & 3.6869520 & -2.2943830 \\
$\mathrm{H}$ & 3.5765630 & 2.7941440 & -2.4388130 \\
$\mathrm{C}$ & -2.1280160 & 1.1586260 & -0.6179840 \\
$\mathrm{C}$ & -3.4177560 & 0.9704730 & -0.0548390 \\
$\mathrm{C}$ & -1.3184400 & 2.2280170 & -0.3180440 \\
$\mathrm{C}$ & -3.8517050 & 1.9122340 & 0.8444640 \\
$\mathrm{C}$ & -1.7368840 & 3.1441110 & 0.6994100 \\
$\mathrm{C}$ & -3.0271970 & 2.9831020 & 1.2723080 \\
$\mathrm{H}$ & -4.8434550 & 1.8144430 & 1.2763990 \\
$\mathrm{C}$ & -2.6278890 & 4.8753470 & 2.7376940 \\
$\mathrm{C}$ & -1.3326080 & 5.0194640 & 2.1882790 \\
$\mathrm{C}$ & -0.8974160 & 4.1788060 & 1.1949230 \\
$\mathrm{C}$ & -3.4544270 & 3.8788110 & 2.2879820 \\
$\mathrm{H}$ & -2.9595220 & 5.5504660 & 3.5203240 \\
$\mathrm{H}$ & -0.6754000 & 5.8009750 & 2.5579190 \\
$\mathrm{H}$ & 0.1020280 & 4.2930170 & 0.7893900 \\
$\mathrm{H}$ & -4.4449950 & 3.7474980 & 2.7151000 \\
$\mathrm{C}$ & -0.2907940 & 5.6908730 & -2.7327350 \\
$\mathrm{C}$ & 1.0196970 & 5.8370420 & -3.2450590 \\
$\mathrm{C}$ & 1.9496160 & 4.8532780 & -3.0320400 \\
$\mathrm{C}$ & -0.6386670 & 4.5801860 & -2.0073720 \\
$\mathrm{H}$ & -1.0300610 & 6.4632940 & -2.9216410 \\
$\mathrm{H}$ & 1.2810290 & 6.7244860 & -3.8131570 \\
$\mathrm{H}$ & 2.9557130 & 4.9465450 & -3.4323160 \\
$\mathrm{H}$ & -1.6503910 & 4.4762400 & -1.6306960 \\
$\mathrm{C}$ & 3.3229660 & 0.4835310 & -1.1879200 \\
$\mathrm{C}$ & 3.7368110 & -0.3600470 & -2.2340550 \\
$\mathrm{C}$ & 4.0331250 & 0.5079740 & 0.0284160 \\
$\mathrm{C}$ & 4.9161880 & -1.0905270 & -2.0827090 \\
$\mathrm{C}$ & 5.1997310 & -0.2512310 & 0.1343470 \\
$\mathrm{C}$ & 5.6786170 & -1.0270480 & -0.9182930 \\
$\mathrm{H}$ & 5.2563130 & -1.7149780 & -2.9055320 \\
$\mathrm{H}$ & 5.7724400 & -0.2197140 & 1.0571480 \\
$\mathrm{C}$ & -4.2650260 & -0.2059680 & -0.4148730 \\
$\mathrm{C}$ & -4.5402760 & -1.1973810 & 0.5513940 \\
$\mathrm{C}$ & -4.8143220 & -0.3072040 & -1.7049200 \\
$\mathrm{C}$ & -5.3733740 & -2.2584520 & 023380
\end{tabular}




-5.6352660
-5.9298200
-5.5944790
-6.0677330
3.5975830
7.0062470
2.9509460
-3.9801790
-6.8342930
-4.5815970
-5.8850590
-6.6351750
-6.3102010
-5.7016760
-3.9457020
-3.0117760
-4.6168090
-3.7233080
-8.1159780
-8.7893900
-7.8886160
-8.6472140
-6.1026710
-5.1970370
-5.8084150
-6.7518320
-5.0681880
-5.5214590
-5.8691380
-4.6460490
-3.3304460
-4.0737240
-2.6013950
-2.8141880
4.2347620
5.3263020
3.9889290
3.8816430
3.8983290
3.4810130
4.9751020
3.4563910
8.0105560
8.9873360
7.6709140
8.1412520

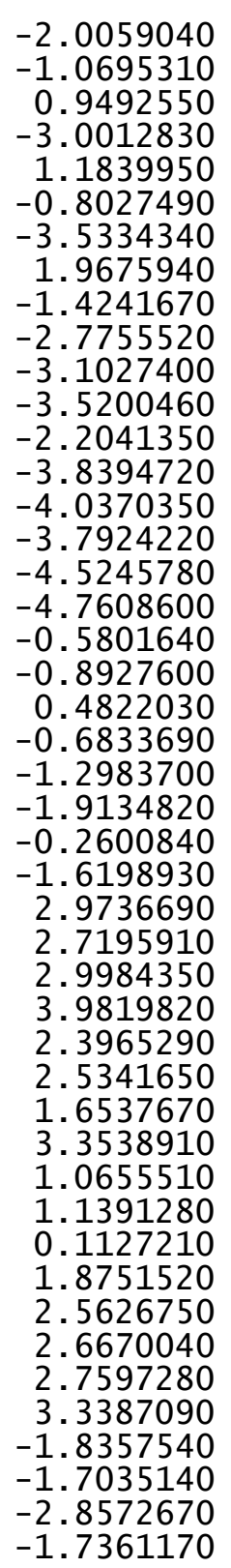

6.8557560
6.2264670
6.4046900
7.8349050
2.7362400
3.6702140
2.3245380
2.0320280
3.6292030
4.6383330
3.0547870
3.7149280
-0.5720550
-0.0254770
-1.1331380
1.9603890
7.4117570
2.5104120
-3.8827670
-7.1264600
-3.1991890
2.0140650
0.9237220
1.9520860
-0.0555670
1.3700300
0.7757820
3.4799190
2.5286140
1.0807100
3.4194100
4.5063610
2.5921710
0.6928970
3.8357530
4.0039550
3.2148550
2.8195170
0.4033510
0.3617270
-0.6564230
0.7940190
1.5263900
0.4978310
1.9579540
1.7528760

-3.2794200
-3.6773530
-3.5630410
-3.7661310
-1.9147110
-2.3825230
-2.5133120
-1.9528430
0.3410740
-0.0429160
0.2638110
1.4009820
-0.8210410
-1.6383820
-1.5387100
-0.0287600
-1.5414170
1.5281240
1.4942040
-3.4238340
-0.3831790
-2.9487980
-3.5829280
-1.8664700
-3.1245560
-3.6521640
-2.9845600
-4.9362750
-5.2916600
-4.9449870
-3.4406750
-5.2097860
-6.3565640
-5.6404900
-2.8553130
-3.1923690
-5.5131340
-4.7395440
-5.0324710
-0.6302790
-0.3100310
0.4765310
0.2502200
1.7892490
1.3245530
-0.7665750
$-0.9191230$

$-0.1171360$

$-1.8771080$

$-0.8523830$

$-3.9680920$

$-4.3003640$

$-3.1517700$

$-4.8058140$

$-4.6533840$

$-4.8452500$

$-5.5833090$

$-4.3939740$

$-0.9341940$

$-2.0631660$

0.2701890

$-3.3587660$

0.1938410

1.1223930

$-2.3841750$

$-2.4749140$

1.9975470

0.0634370

0.8196620

$-0.0528940$

0.7426660

$-1.0278720$

$-1.4902510$

0.6132910

1.7577190

1. 3977420

0.2964070

0.8772010

1.9988970

0.6407930

1.1252490

$-0.5925290$

$-0.2810600$

2.6605710

2. 2520310

3.1377920

2.4074350

4.0201250

5.1896250

3.6282380

5.9566040

5.4925870 


\begin{tabular}{|c|c|c|c|c|c|c|c|}
\hline C & 09513290 & 28607300 & 4.3867420 & c & 5.1851960 & -0.3631140 & -0.3226920 \\
\hline H & -0.0651070 & 1.9647860 & 2.7180300 & $\mathrm{C}$ & 5.4945870 & -1.2450970 & -1.3573710 \\
\hline C & 1.6794570 & 2.6300630 & 5.5523450 & $\mathrm{H}$ & 4.8181710 & -2.0543680 & -3.2166380 \\
\hline $\mathrm{H}$ & 2.5160740 & 1.1448840 & 6.8698150 & $\mathrm{H}$ & 5.8619310 & -0.2862810 & 0.5229500 \\
\hline $\mathrm{H}$ & 0.7314860 & 3.8741600 & 4.0659250 & C & -4.2827770 & -0.2547380 & -0.0619960 \\
\hline $\mathrm{H}$ & 2.0295950 & 3.4674080 & 6.1482950 & $\mathrm{c}$ & -4.4818840 & -1.1918350 & 0.9711420 \\
\hline $\mathrm{H}$ & -0.8472300 & -0.9279400 & 1.6131570 & C & -4.9146470 & -0.4301620 & -1.3083040 \\
\hline $\mathrm{S}$ & 1.1513740 & -2.1153980 & 3.1039620 & $\mathrm{C}$ & -5.3270500 & -2.2773890 & 0.7331580 \\
\hline 14 & & & & $\mathrm{C}$ & -5.7395360 & -1.5366500 & -1.4975040 \\
\hline TS & R) $\mathrm{G}[\mathrm{WB} 97 \mathrm{XD}$ & $G(d)]=-363$ & 332 & $\mathrm{C}$ & -5.9630030 & -2.4713530 & -0.4886180 \\
\hline 0 & 0.5513420 & 0.1387290 & -0.3112360 & $\mathrm{H}$ & -5.4940110 & -3.0018430 & 1.5271780 \\
\hline 0 & -1.7647680 & 0.1229090 & -1.3695580 & $\mathrm{H}$ & -6.2297110 & -1.6679920 & -2.4597220 \\
\hline C & 0.8315260 & 1.2785810 & -1.0343530 & C & 3.7743100 & 1.4193360 & 0.7843050 \\
\hline C & -0.0968860 & 2.2986090 & -1.0791660 & c & 6.7870320 & -2.0439840 & -1.3588190 \\
\hline C & 2.1116450 & 1.3843110 & -1.6371180 & $\mathrm{C}$ & 2.5128720 & -0.7060220 & -3.6887890 \\
\hline C & 0.1663960 & 3.4395150 & -1.9033090 & c & -3.8299700 & -1.0561300 & 2.3416980 \\
\hline C & 2.3857590 & 2.5197270 & -2.3590840 & $\mathrm{C}$ & -6.8697090 & -3.6680060 & -0.7125380 \\
\hline C & 1.4301750 & 3.5482330 & -2.5418220 & $\mathrm{C}$ & -4.7662240 & 0.5745770 & -2.4427590 \\
\hline $\mathrm{H}$ & 3.3677170 & 2.6322680 & -2.8112450 & $\mathrm{C}$ & -6.1008950 & 1.2803280 & -2.7208390 \\
\hline C & -2.1747000 & 1.1043600 & -0.4783930 & $\mathrm{H}$ & -6.8605940 & 0.5692520 & -3.0654630 \\
\hline C & -3.4256090 & 0.9455030 & 0.1735490 & $\mathrm{H}$ & -6.4817160 & 1.7687080 & -1.8174320 \\
\hline C & -1.3526210 & 2.1906860 & -0.2933780 & $\mathrm{H}$ & -5.9770480 & 2.0433330 & -3.4976450 \\
\hline C & -3.8117590 & 1.9404480 & 1.0356140 & C & -4.2019720 & -0.0736160 & -3.7128750 \\
\hline C & -1.7144590 & 3.1650310 & 0.6924150 & $\mathrm{H}$ & -3.2453990 & -0.5624120 & -3.5068340 \\
\hline C & -2.9698160 & 3.0375650 & 1.3462810 & $\mathrm{H}$ & -4.8910220 & -0.8224730 & -4.1202090 \\
\hline $\mathrm{H}$ & -4.7763320 & 1.8683390 & 1.5295190 & $\mathrm{H}$ & -4.0419140 & 0.6860240 & -4.4863170 \\
\hline C & -2.5009020 & 5.0166290 & 2.6692450 & C & -6.3036000 & -4.6061550 & -1.7867720 \\
\hline C & -1.2377640 & 5.1269810 & 2.0430640 & $\mathrm{H}$ & -6.9368430 & -5.4936460 & -1.8959850 \\
\hline $\mathrm{C}$ & -0.8547500 & 4.2295450 & 1.0781790 & $\mathrm{H}$ & -6.2565800 & -4.1044650 & -2.7602900 \\
\hline C & -3.3456860 & 3.99 & 2.3259790 & $\mathrm{H}$ & -5.2913880 & -4.9344200 & -1.5291050 \\
\hline $\mathrm{H}$ & -2 & 20 & 3.4264760 & C & -8.3023680 & -3.2392390 & -1.0560090 \\
\hline H & -0.5634120 & 82370 & 2.3304150 & $\mathrm{H}$ & -8.7099770 & -2.5753440 & -0.2865680 \\
\hline $\mathrm{H}$ & 0.1205640 & 4.3209140 & 0.6124040 & $\mathrm{H}$ & -8.3371240 & -2.7070200 & -2.0136850 \\
\hline $\mathrm{H}$ & -4.3118600 & 3.8874810 & 2.8117490 & $\mathrm{H}$ & -8.9562260 & -4.1146620 & -1.1372110 \\
\hline C & -0.5023200 & 30 & -2.9335050 & C & -4.8485980 & -0.5822110 & 3.3888230 \\
\hline C & 0.7692690 & 65650 & -3.5410590 & H & -5.3128350 & 0.3674780 & 3.1051620 \\
\hline C & 1.7120140 & 4.6804050 & -3.3510820 & $\mathrm{H}$ & -5.6495840 & -1.3212210 & 3.5110210 \\
\hline $\mathrm{C}$ & -0.7971560 & 4.4585220 & -2.1362890 & $\mathrm{H}$ & -4.3626500 & -0.4461930 & 4.3616320 \\
\hline H & -1.2544290 & 6.2989160 & -3.1053380 & C & -3.1562050 & -2.3528320 & 2.8109230 \\
\hline $\mathrm{H}$ & 0.9898550 & 6.5174130 & -4.1646160 & $\mathrm{H}$ & -3.8899690 & -3.1322380 & 3.0460990 \\
\hline $\mathrm{H}$ & 2.6875450 & 4.7528380 & -3.8244950 & H & -2.4752550 & -2.7335010 & 2.0462880 \\
\hline H & -1.7808040 & 4.3737800 & -1.6878660 & $\mathrm{H}$ & -2.5804350 & -2.1600970 & 3.7235160 \\
\hline C & 3.1922130 & 0.3619160 & -1.4706070 & C & 4.4538940 & 2.7708520 & 0.5183930 \\
\hline $\mathrm{C}$ & 3.4453520 & -0.5664580 & -2.4939750 & $\mathrm{H}$ & 5.5419070 & 2.6462700 & 0.4635270 \\
\hline C & 4.0452070 & 386710 & -0.3508830 & $\mathrm{H}$ & 4.1139430 & 3.2141160 & -0.4222250 \\
\hline $\mathrm{C}$ & 4.6005430 & 828 & 4186930 & $\mathrm{H}$ & 4.2299820 & 3.4747310 & 1.3281440 \\
\hline
\end{tabular}




\begin{tabular}{|c|c|c|}
\hline $\begin{array}{l}4.1907530 \\
3.7489400 \\
5.2792830 \\
3.8509090 \\
7.0591330 \\
7.9803610 \\
7.1880570 \\
6.2437430 \\
7.9676690 \\
7.7994140 \\
8.1027500 \\
8.8980290 \\
2.2702510 \\
3.1593180 \\
1.9823890 \\
1.4621200 \\
3.0364650 \\
4.0159240 \\
2.3470800 \\
3.1446350 \\
0.6415370 \\
0.1847980 \\
1.1128730 \\
1.5423870 \\
6.6969900 \\
2.6929460 \\
4.0577190 \\
6.9084940 \\
3.0463410 \\
2.0450370 \\
0.9959240 \\
2.0024910 \\
0.0240270 \\
1.3092810 \\
0.6842670 \\
3.4992090 \\
2.6166440 \\
1.1576240 \\
3.4508000 \\
4.5351250 \\
2.6712970 \\
0.7087840 \\
3.9336860 \\
3.9839740 \\
3.1654260 \\
2.9773660\end{array}$ & $\begin{array}{r}0.8849580 \\
-0.0971210 \\
0.7989230 \\
1.5748170 \\
-2.7688080 \\
-3.3564600 \\
-2.0614870 \\
-3.4497690 \\
-1.1334260 \\
-0.6438190 \\
-0.3514800 \\
-1.7088780 \\
-2.1691440 \\
-2.6245360 \\
-2.7653680 \\
-2.2283020 \\
0.0852130 \\
-0.2946500 \\
-0.0093640 \\
1.1490660 \\
-0.8938760 \\
-1.7898650 \\
-1.5262440 \\
-0.2886540 \\
-2.8073060 \\
1.5938320 \\
1.3468800 \\
-4.2275270 \\
-0.2979780 \\
-2.9889930 \\
-3.5412700 \\
-1.9145390 \\
-3.0622100 \\
-3.7409320 \\
-3.0937330 \\
-4.9779080 \\
-5.2297060 \\
-4.8684670 \\
-3.5070020 \\
-5.2570450 \\
-6.2771360 \\
-5.5984970 \\
-2.8770070 \\
-3.3390840 \\
-5.6094430 \\
-4.6258650\end{array}$ & $\begin{array}{r}2.1578860 \\
2.3565740 \\
2.2516810 \\
2.9365700 \\
-0.0354600 \\
-0.1114780 \\
0.7913950 \\
0.2264210 \\
-1.7288690 \\
-2.6936700 \\
-0.9720460 \\
-1.7918500 \\
-4.0761110 \\
-4.5272840 \\
-3.2066610 \\
-4.8125090 \\
-4.8957000 \\
-5.2105450 \\
-5.7422650 \\
-4.6639960 \\
-0.7851260 \\
-1.8937150 \\
0.5031100 \\
-3.4034240 \\
-2.1424900 \\
0.8278350 \\
-2.1317150 \\
0.2309830 \\
2.2703500 \\
0.1299010 \\
1.0011180 \\
-0.0528070 \\
0.9627160 \\
-0.8664140 \\
-1.3168070 \\
0.7233520 \\
1.9471960 \\
1.6543140 \\
0.3047730 \\
0.9405810 \\
2.2572660 \\
0.9668490 \\
1.0618810 \\
-0.6341330 \\
-0.1088380 \\
2.7895990\end{array}$ \\
\hline
\end{tabular}

$\begin{array}{lr}\mathrm{H} & 0.5331240 \\ \mathrm{C} & 0.6463440 \\ \mathrm{O} & -0.4483790 \\ \mathrm{C} & 1.1837190 \\ \mathrm{C} & 2.0121880 \\ \mathrm{C} & 0.8859550 \\ \mathrm{C} & 2.5361370 \\ \mathrm{H} & 2.2387370 \\ \mathrm{C} & 1.4327190 \\ \mathrm{H} & 0.2445120 \\ \mathrm{C} & 2.2567940 \\ \mathrm{H} & 3.1698120 \\ \mathrm{H} & 1.2101270 \\ \mathrm{H} & 2.6810400 \\ \mathrm{H} & -0.7191140 \\ \mathrm{~S} & 1.4234000 \\ \mathrm{I} & \end{array}$
$-4.8853200$
$-0.4386910$
$-0.1689990$
0.7353320
0.6074890
2.0087860
1.7413280
$-0.3788740$
3.1378950
2. 1049720
3. 0061040
1. 6397450
4.1185240
3.8887070
$-0.8392960$
$-1.9304320$

144

2.5520990
3.1413470
2.5071970
3.8643320
4.9834290
3.3610040
5.5907760
5.3744330
3.9567670
2.4918570
5.0727990
6.4659240
3.5479210
5.5416490
1.7814270
3.1511880

$\operatorname{TS} 215(R, R) G[W B 97 X D / 6-31 G(d)]=-3633.717011$

$\begin{array}{lrrr}\mathrm{TS} 215(\mathrm{R}, \mathrm{R}) & \mathrm{G}[\mathrm{WB} 97 \mathrm{X} / \mathrm{r}-31 \mathrm{G}(\mathrm{d})]=-3633.717011 \\ \mathrm{O} & 0.2805700 & -1.2099420 & 0.2530540 \\ \mathrm{O} & 2.1202080 & 0.0412940 & -0.9293570 \\ \mathrm{C} & 0.7248380 & -2.4109610 & -0.2610430 \\ \mathrm{C} & 2.0758200 & -2.6796060 & -0.2607670 \\ \mathrm{C} & -0.2650520 & -3.3341280 & -0.6928040 \\ \mathrm{C} & 2.5184520 & -3.8920010 & -0.8854720 \\ \mathrm{C} & 0.1659110 & -4.5407920 & -1.1781970 \\ \mathrm{C} & 1.5474290 & -4.8323410 & -1.3281890 \\ \mathrm{H} & -0.5604730 & -5.2937460 & -1.4694250 \\ \mathrm{C} & 2.9728770 & -0.3851900 & 0.0668640 \\ \mathrm{C} & 3.8198160 & 0.5930810 & 0.6531700 \\ \mathrm{C} & 3.0101990 & -1.7229890 & 0.3944790 \\ \mathrm{C} & 4.7626660 & 0.1619160 & 1.5487970 \\ \mathrm{C} & 3.9085530 & -2.1384580 & 1.4343690 \\ \mathrm{C} & 4.8224280 & -1.1903130 & 1.9734160 \\ \mathrm{H} & 5.4607830 & 0.8761700 & 1.9763630 \\ \mathrm{C} & 5.7405400 & -2.8828920 & 3.4511450 \\ \mathrm{C} & 4.7977160 & -3.8127510 & 2.9564890 \\ \mathrm{C} & 3.9078980 & -3.4522020 & 1.9766980 \\ \mathrm{C} & 5.7451250 & -1.5988510 & 2.9722820 \\ \mathrm{H} & 6.4458450 & -3.1842290 & 4.2193730 \\ \mathrm{H} & 4.7728990 & -4.8197930 & 3.3616780 \\ \mathrm{H} & 3.1845780 & -4.1756730 & 1.6186470 \\ \mathrm{H} & 6.4462610 & -0.8654150 & 3.3619100 \\ \mathrm{C} & 4.2754610 & -5.3555460 & -1.7046180 \\ \mathrm{C} & 3.3106130 & -6.3065170 & -2.1086610 \\ \mathrm{C} & 1.9771660 & -6.0463410 & -1.9254910 \\ \mathrm{C} & 3.8910000 & -4.1817460 & -1.1075170\end{array}$




$\begin{array}{rrrr}\mathrm{H} & 5.3294820 & -5.5537730 & -1.8735070 \\ \mathrm{H} & 3.6287290 & -7.2342900 & -2.5740070 \\ \mathrm{H} & 1.2253640 & -6.7614870 & -2.2486320 \\ \mathrm{H} & 4.6394940 & -3.4561130 & -0.8081840 \\ \mathrm{C} & -1.7085700 & -2.9795440 & -0.5227560 \\ \mathrm{C} & -2.4868280 & -2.5628600 & -1.6222320 \\ \mathrm{C} & -2.2700020 & -3.0262510 & 0.7646160 \\ \mathrm{C} & -3.8107370 & -2.1871620 & -1.3978610 \\ \mathrm{C} & -3.6039480 & -2.6527270 & 0.9344160 \\ \mathrm{C} & -4.3879060 & -2.2179570 & -0.1282070 \\ \mathrm{H} & -4.4159190 & -1.8637930 & -2.2406030 \\ \mathrm{H} & -4.0470530 & -2.7005690 & 1.9271200 \\ \mathrm{C} & 3.6268850 & 2.0342890 & 0.3059940 \\ \mathrm{C} & 2.8732290 & 2.8577050 & 1.1693040 \\ \mathrm{C} & 4.1366450 & 2.5448790 & -0.8967050 \\ \mathrm{C} & 2.6481000 & 4.1804660 & 0.7980810 \\ \mathrm{C} & 3.8796970 & 3.8776550 & -1.2266060 \\ \mathrm{C} & 3.1305670 & 4.7069820 & -0.4005340 \\ \mathrm{H} & 2.0585760 & 4.8171240 & 1.4518220 \\ \mathrm{H} & 4.2651240 & 4.2781260 & -2.1623770 \\ \mathrm{C} & -1.4780420 & -3.5047600 & 1.9735430 \\ \mathrm{C} & -5.8343560 & -1.8121810 & 0.0882290 \\ \mathrm{C} & -1.9305550 & -2.5680640 & -3.0398610 \\ \mathrm{C} & 2.3205020 & 2.3372980 & 2.4910340 \\ \mathrm{C} & 2.8145140 & 6.1348000 & -0.8076550 \\ \mathrm{C} & 4.9538370 & 1.6866720 & -1.8507030 \\ \mathrm{C} & 6.3732310 & 2.2444620 & -2.0217210 \\ \mathrm{H} & 6.3583160 & 3.2334890 & -2.4940390 \\ \mathrm{H} & 6.8765690 & 2.3406340 & -1.0537620 \\ \mathrm{H} & 6.9718140 & 1.5808190 & -2.6557080 \\ \mathrm{C} & 4.2517830 & 1.5238960 & -3.2051880 \\ \mathrm{H} & 3.2553030 & 1.0922210 & -3.0736300 \\ \mathrm{H} & 4.1448090 & 2.4882250 & -3.7155160 \\ \mathrm{H} & 4.8328540 & 0.8626470 & -3.8579150 \\ \mathrm{C} & 3.3313240 & 7.1538230 & 0.2155850 \\ \mathrm{H} & 3.1468340 & 8.1760360 & -0.1328750 \\ \mathrm{H} & 2.8282500 & 7.0346280 & 1.1820240 \\ \mathrm{H} & 4.4073830 & 7.0349170 & 0.3789730 \\ \mathrm{C} & 1.3092820 & 6.3121220 & -1.0542780 \\ \mathrm{H} & 0.9531410 & 5.6023440 & -1.8085560 \\ \mathrm{H} & 0.7394420 & 6.1409550 & -0.1331540 \\ \mathrm{H} & 1.0898750 & 7.3270940 & -1.4040030 \\ \mathrm{C} & 3.3217960 & 2.5788750 & 3.6311990 \\ \mathrm{H} & 4.2842610 & 2.0968460 & 3.4394280 \\ \mathrm{H} & 3.5019490 & 3.6530580 & 4.5757840 \\ \mathrm{H} & 2.9277790 & 2.1859630 & \\ & & & \end{array}$

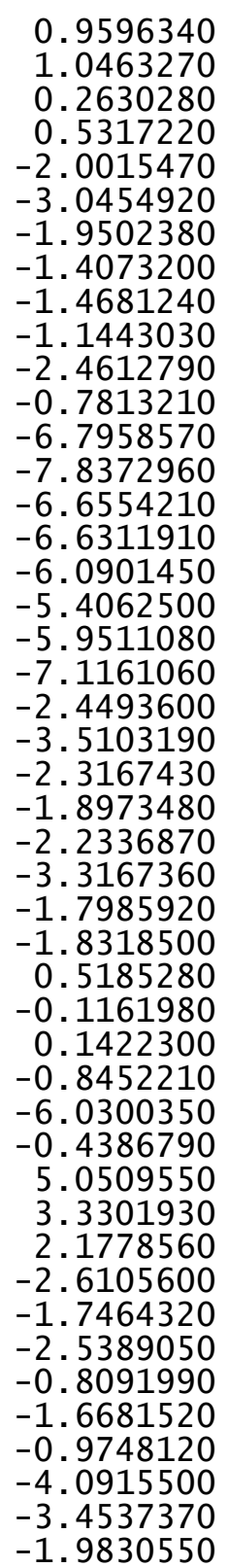

2.9364230

3.9840360

2.8803360

2.3838860

$-4.8629740$

$-4.7880980$

$-5.6115350$

$-5.2226890$

$-2.4746410$

$-1.4955430$

$-2.3669520$

$-2.7913680$

$-2.7780840$

$-2.5110210$

$-2.7463380$

$-3.8089160$

$-0.3643620$

0.3204330

$-0.2439590$

$-0.0652550$

$-1.4195330$

$-1.5403510$

$-0.4541590$

$-1.3955590$

$-3.9077070$

$-4.0518930$

$-3.9278520$

$-4.7570260$

0.1161550

$-0.0020190$

1.2749220

$-2.4518070$

$-1.8700310$

$-3.6536640$

0.6890360

6.3258660

1.2569550

1.8948820

3.0222370

1.0047840

2.7787930

1.8485140

1.1490420

3.4934540

4.6597670

4.3847490
2. 8635410

3.1750970

2.0246150

3.7074260

2.4602360

2.7866960

1.6623640

3.3077400

3.1088560

2.7423890

3.5617270

3.9013480

$-0.6167590$

$-0.4049270$

$-1.7036120$

$-0.2862570$

$-0.3458840$

0.1641900

$-1.4266130$

$-0.1043950$

$-3.9105510$

$-4.1584850$

$-3.4192270$

$-4.8562690$

$-3.7294780$

$-3.8243950$

$-4.7350980$

$-3.1705680$

$-0.6644170$

$-2.0202920$

0.2228740

$-2.9682410$

1.1661520

1. 6691630

$-1.4111280$

$-1.7575350$

2.3864750

$-1.7745750$

$-1.3961810$

$-1.1471110$

$-0.9061190$

$-2.8666140$

$-2.6102490$

$-3.0501310$

$-2.2901900$

$-1.9586890$ 


$\begin{array}{lrrr}\mathrm{C} & -4.0153240 & 2.1983290 & -2.2365110 \\ \mathrm{H} & -5.1390730 & 3.7192370 & -3.2711880 \\ \mathrm{H} & -3.5198370 & 5.5818570 & -2.8750020 \\ \mathrm{H} & -1.3633050 & 4.4511790 & -2.8620880 \\ \mathrm{H} & -4.6430130 & 2.2757650 & -1.3401910 \\ \mathrm{H} & -4.3791010 & 1.3412400 & -2.8129160 \\ \mathrm{H} & -3.5832190 & 3.3528590 & -4.0112690 \\ \mathrm{H} & -4.0050190 & 4.8300660 & -1.3575190 \\ \mathrm{H} & -1.5729420 & 5.1228370 & -1.2635700 \\ \mathrm{C} & -2.9200030 & 1.9343680 & 1.6049390 \\ \mathrm{O} & -2.0670830 & 0.9663710 & 1.4654930 \\ \mathrm{C} & -4.1643460 & 1.5146430 & 2.2813790 \\ \mathrm{C} & -5.3063730 & 2.3254430 & 2.2727150 \\ \mathrm{C} & -4.2194940 & 0.2517250 & 2.8871980 \\ \mathrm{C} & -6.4849420 & 1.8747480 & 2.8518530 \\ \mathrm{H} & -5.2681400 & 3.2975890 & 1.7937030 \\ \mathrm{C} & -5.3978120 & -0.1913510 & 3.4713220 \\ \mathrm{H} & -3.3371730 & -0.3755860 & 2.8892100 \\ \mathrm{C} & -6.5338290 & 0.6162270 & 3.4493820 \\ \mathrm{H} & -7.3692090 & 2.5034080 & 2.8323570 \\ \mathrm{H} & -5.4316440 & -1.1693620 & 3.9412630 \\ \mathrm{H} & -7.4574540 & 0.2645170 & 3.8985710 \\ \mathrm{H} & -1.1878490 & 1.2042180 & 1.0020980 \\ \mathrm{~S} & -2.6556440 & 3.5000240 & 1.0499930 \\ \mathrm{H} 44 & & & \end{array}$

TS216(R, R) G[WB97XD/6-31G(d)] = -3633.716338

$\begin{array}{lrlr}\mathrm{O} & 0.3084530 & 0.9361800 & -0.1071710 \\ \mathrm{O} & -1.9883990 & 0.5551310 & -1.0950350 \\ \mathrm{C} & 0.2720850 & 2.2589670 & -0.4970370 \\ \mathrm{C} & -0.8792060 & 2.9869110 & -0.2945710 \\ \mathrm{C} & 1.4764700 & 2.8213060 & -1.0004150 \\ \mathrm{C} & -0.9237130 & 4.3308840 & -0.7920360 \\ \mathrm{C} & 1.4555840 & 4.1397110 & -1.3721230 \\ \mathrm{C} & 0.2625190 & 4.9076020 & -1.3243260 \\ \mathrm{H} & 2.3695140 & 4.6158460 & -1.7154520 \\ \mathrm{C} & -2.5207840 & 1.1540410 & 0.0298060 \\ \mathrm{C} & -3.6142160 & 0.5058010 & 0.6618180 \\ \mathrm{C} & -2.0143100 & 2.3669590 & 0.4411160 \\ \mathrm{C} & -4.1996160 & 1.1417800 & 1.7258940 \\ \mathrm{C} & -2.5574480 & 2.9631830 & 1.6276790 \\ \mathrm{C} & -3.6830700 & 2.3544990 & 2.2482270 \\ \mathrm{H} & -5.0695020 & 0.6962520 & 2.2002310 \\ \mathrm{C} & -3.7069920 & 4.0847540 & 3.9496040 \\ \mathrm{C} & -2.5591670 & 4.6653010 & 3.3626270 \\ \mathrm{C} & -1.9998610 & 4.1214270 & 2.2341640 \\ \mathrm{C} & -4.2510400 & 2.9509730 & 3.4047740\end{array}$

-4.1440540
-2.1130300
-1.1138020
-5.1195080
-2.1173370
-0.9295570
0.2321890
-2.1148830
-3.0408360
-0.9444660
1.1475810
-3.0343480
2.7140320
3.1878810
3.3717670
4.3002400
4.4689640
4.9385030
4.6839410
4.9695400
-4.1169620
-3.8019860
-4.9131330
-4.3275340
-5.4038560
-5.1283340
-4.0989910
-6.0215970
2.9519700
6.1406730
2.5468540
-2.9251980
-5.6844420
-5.2629880
-6.7744810
-7.3255480
-7.1488240
-7.0035800
-4.7199810
-3.6387330
-5.1906630
-4.9221520
-6.6785480
-7.1195540
-6.1804830
-7.4885890

4.5303380

5.5468900

4. 5733190

2. 4809140

6. 3880970

6.9714180

6. 2439060

5.1044380

6.9590600

7.9895420

6.6754560

4.6642790

1. 9829730

1.3789190

1.7673330

0.5389820

0.9086440

0.2699660

0.0699540

0.7374230

$-0.8105360$

$-1.9959090$

$-0.8570600$

$-3.2000350$

$-2.0909190$

$-3.2713440$

$-4.1159550$

$-2.1331470$

2.4800270

$-0.6562650$

1. 6681000

$-1.9919960$

$-4.5982230$

0.3934820

0.6579450

$-0.1510800$

0.7448940

1. 5903480

0.3172760

0.1510560

$-0.4971800$

1. 2530650

$-5.1870770$

$-6.1127150$

$-5.4214220$

$-4.4811610$
4.8378150
3.8127200
1.8029930
3.8585710
-1.2850120
-1.7824180
-1.8021240
-0.8008090
-1.2918930
-2.1590410
-2.1983570
-0.4302920
-0.9969620
-2.1758690
0.2281790
-2.0908240
0.2631890
-0.8814700
-2.9933430
1.2130350
0.1632170
0.8598240
-0.9921200
0.3958910
-1.4240840
-0.7438180
0.9343580
-2.3190660
1.5070510
-0.8328300
-3.5264580
2.1068700
-1.2287150
-1.7860260
-1.7725480
-2.2658300
-0.7468040
-2.3006170
-3.2188920
-3.2122700
-3.7817110
-3.7520500
-0.2185580
-0.6049930
0.7295390
-0.0077870 


$\begin{array}{rrr}-4.5678380 & -5.6005110 & -1.5496040 \\ -3.8639910 & -5.1866220 & -2.2791350 \\ -4.0027290 & -5.8651560 & -0.6483320 \\ -4.9887430 & -6.5238620 & -1.9629080 \\ -3.7831020 & -1.9680370 & 3.3808020 \\ -4.4324390 & -1.0885180 & 3.4173030 \\ -4.4205850 & -2.8591780 & 3.4290410 \\ -3.1458780 & -1.9574730 & 4.2726550 \\ -1.9518000 & -3.1771330 & 2.1584060 \\ -2.4680190 & -4.1214250 & 2.3658810 \\ -1.4090780 & -3.2783300 & 1.2153480 \\ -1.2227040 & -3.0228220 & 2.9614370 \\ 4.0129120 & 3.5129650 & 1.9121030 \\ 4.9719060 & 3.0284670 & 2.1301540 \\ 4.1779060 & 4.2421230 & 1.1112920 \\ 3.6964030 & 4.0550770 & 2.8102810 \\ 2.6551930 & 1.5045290 & 2.6492980 \\ 1.8836840 & 0.7881950 & 2.3556340 \\ 3.5455510 & 0.9389080 & 2.9479520 \\ 2.3009990 & 2.0491940 & 3.5319290 \\ 6.0587110 & -1.6662780 & 0.3182000 \\ 6.8645110 & -2.4038520 & 0.2378560 \\ 6.1595020 & -1.1730630 & 1.2918140 \\ 5.1009480 & -2.1986520 & 0.3155450 \\ 7.4445740 & 0.1509830 & -0.7585730 \\ 7.5261410 & 0.8452650 & -1.6012660 \\ 7.4809760 & 0.7372710 & 0.1673180 \\ 8.3148470 & -0.5148100 & -0.7734950 \\ 2.5485200 & 0.4739290 & -4.4849910 \\ 3.5589650 & 0.2278020 & -4.8317010 \\ 2.1153530 & -0.4149420 & -4.0230860 \\ 1.9538780 & 0.7152550 & -5.3726270 \\ 3.2395870 & 2.8646790 & -4.1977500 \\ 4.2953580 & 2.6370840 & -4.3883240 \\ 2.7623350 & 3.0947730 & -5.1569380 \\ 3.1985980 & 3.7620770 & -3.5742930 \\ -0.5329450 & -0.1589230 & -0.9789390 \\ -0.0306410 & -0.2910300 & -2.3867080 \\ -0.5734650 & -1.3852870 & -0.1012610 \\ 1.5008880 & 1.9338670 & -3.3443210 \\ 6.1524590 & -1.2219620 & -1.7738000 \\ 2.0282570 & 3.0306030 & 1.3085360 \\ -4.7852000 & 1.2519840 & -1.3054020 \\ -6.2315770 & -4.4021140 & -2.1597110 \\ -2.3197770 & -1.0803560 & -1.9998530 \\ 2.2537100 & -2.4093590 & \end{array}$

\begin{abstract}
1. 3000150
2. 3242420

0.4238370

1. 2418750

0.6459980

3.4609510

2. 7746000

1.3636110

3. 5770040

4.4598640

2.7128730

0.6793040

4.2668980

3. 9734660

2. 8922670

3. 3741300

0.9327540

1. 9001220

0.8924100

2. 6592900

4.0089690

2. 0239520

4.7169070

4.5027790

2.7302000

0.9791490

4. 0786540

5.7683890

2.2305330

4.6322010

0.3480670

2. 3249710
\end{abstract}
$-3.3520780$
$-1.4308550$
$-2.9261970$
$-2.4338770$
$-1.6315260$
$-4.3610730$
$-5.2970500$
$-4.8106410$
$-2.9441820$
$-4.7372030$
$-6.3118820$
$-4.9740580$
$-2.9352370$
$-2.2463950$
$-4.3364780$
$-5.3531880$
$-5.3582390$
$-2.2347680$
$-1.4360940$
$-1.9642040$
$-2.3142240$
$-1.3044260$
$-1.9953470$
$-2.8166620$
$-1.0065140$
$-1.0298040$
$-1.3445160$
$-2.2533150$
$-0.5046820$
$-1.1003390$
$-1.5262400$
$-3.4770470$

$-1.4053000$

$-1.5223530$

$-0.9285090$

$-3.0353080$

$-2.8561190$

$-3.0584430$

$-2.0604720$

$-1.7146330$

$-2.4900480$

$-3.2987750$

$-2.4638770$

$-2.5569490$

$-1.6380560$

$-3.2344120$

$-3.9955220$

$-1.1429790$

$-0.8718360$

2. 1182990

1. 9679510

3. 3608020

3.4739260

4.4212020

4.6260930

2.6494450

5.5789640

4. 3310980

5.6802820

4.7012290

6.4013390

6.5818350

1. 1081400

1.0712070

TS217(R, R) G[WB97XD/6-31G(d)] = -3633.718508 2839830

$\begin{array}{lr}\mathrm{O} & 0.1065090 \\ \mathrm{O} & 2.0542160 \\ \mathrm{C} & 0.4769400 \\ \mathrm{C} & 1.8029180 \\ \mathrm{C} & -0.5652900 \\ \mathrm{C} & 2.1706420 \\ \mathrm{C} & -0.2138130 \\ \mathrm{C} & 1.1451970 \\ \mathrm{H} & -0.9855470 \\ \mathrm{C} & 2.8656270 \\ \mathrm{C} & 3.7880200 \\ \mathrm{C} & 2.7926650\end{array}$

$-1.2695530$

$-0.1223150$

$-2.4730030$

$-2.8458560$

$-0.8263180$

$-0.2795670$

$-3.2930490$

$-4.0635820$

$-4.5108790$

$-4.9075140$

$-5.1904280$

$-0.6402820$

$-0.2506540$

$-0.7903390$

$-0.9127810$

$-1.3104290$

$-1.4223630$

$-1.6605840$

0.1600580

0.2527850
-1.9843800

0.7657710

0.4541260 


\begin{tabular}{|c|c|c|c|}
\hline$r$ & & & \\
\hline $\begin{array}{l}\mathrm{C} \\
\mathrm{C}\end{array}$ & $\begin{array}{l}4.6798470 \\
3.6450680\end{array}$ & $\begin{array}{l}-0.2715450 \\
-2.4939150\end{array}$ & $\begin{array}{l}1.6640620 \\
1.4908930\end{array}$ \\
\hline $\mathrm{C}$ & 4.6218860 & -1.6316030 & 2.0626910 \\
\hline $\mathrm{H}$ & 5.4292030 & 0.3740530 & 2.1132730 \\
\hline$c$ & 5.3883400 & -3.4235700 & 3.5089070 \\
\hline $\mathrm{C}$ & 4.3856540 & -4.2677340 & 2.9791860 \\
\hline C & 3.5383640 & -3.8171600 & 1.9984700 \\
\hline C & 5.4969950 & -2.1326730 & 3.0619920 \\
\hline n & 6.0582940 & -3.7954880 & 4.2779370 \\
\hline H & 4.2803350 & -5.2799790 & 3.3576590 \\
\hline H & 2.7688400 & -4.4747800 & 1.6099400 \\
\hline H & 6.2459200 & -1.4638020 & 3.4778940 \\
\hline 6 & 3.8359100 & -5.6256560 & -1.7420600 \\
\hline C & 2.8146760 & -6.4841450 & -2.2093210 \\
\hline C & 1.4998310 & -6.1281320 & -2.0545670 \\
\hline $\mathrm{C}$ & 3.5234360 & -4.4493280 & -1.1091740 \\
\hline $\mathrm{H}$ & 4.8766680 & -5.8966220 & -1.8910540 \\
\hline H & 3.0748940 & -7.4160630 & -2.7016050 \\
\hline $\mathrm{H}$ & 0.7063590 & -6.7703410 & -2.4275450 \\
\hline $\mathrm{H}$ & 4.3151680 & -3.7940010 & -0.7626910 \\
\hline C & -1.9800160 & -2.8207450 & -0.6694700 \\
\hline C & -2.6556440 & -2.2693110 & -1.7773430 \\
\hline $\mathrm{C}$ & -2.6117750 & -2.8867550 & 0.5838230 \\
\hline $\mathrm{C}$ & -3.9482480 & -1.7799260 & -1.5940740 \\
\hline C & -3.9124960 & -2.3964770 & 0.7117990 \\
\hline $\mathrm{C}$ & -4.5924510 & -1.8250740 & 576970 \\
\hline H & -4.4723590 & -1.3488590 & -2.4427620 \\
\hline $\mathrm{H}$ & -4.4119220 & -2.4579700 & 1.6765310 \\
\hline C & 3.7450380 & 1.7095050 & 0.4336800 \\
\hline C & 3.1743100 & 2.6142010 & 1.3491450 \\
\hline C & 4.2436530 & 2.1670920 & -0.7994330 \\
\hline $\mathrm{C}$ & 3.1416670 & 3.9687260 & 1.0142000 \\
\hline $\mathrm{C}$ & 4.1790000 & 3.5281840 & -1.0911940 \\
\hline C & 3.6306860 & 4.4463400 & -0.1979160 \\
\hline $\mathrm{H}$ & 2.7088820 & 4.6785780 & 1.7148770 \\
\hline $\mathrm{H}$ & 4.5631010 & 3.8804270 & -2.0462840 \\
\hline C & -1.9345310 & -3.5140210 & 1.7942310 \\
\hline $\mathrm{C}$ & -5.9974660 & -1.2775660 & -0.1847790 \\
\hline $\mathrm{C}$ & -2.0261840 & -2.2412190 & -3.1636620 \\
\hline C & 2.6083710 & 2.1601000 & 2.6892590 \\
\hline $\mathrm{C}$ & 3.5358560 & 5.9214580 & -0.5434060 \\
\hline C & 4.8534510 & 1.2184840 & -1.821432 \\
\hline C & 6.3475060 & 1.5061070 & -2.0209900 \\
\hline H & 6.5079010 & 2.5104730 & -2.429957 \\
\hline H & 6.8892080 & 1.4374350 & -1.071513 \\
\hline $\mathrm{H}$ & 6.7859060 & 0.7849820 & -2.7200340 \\
\hline
\end{tabular}

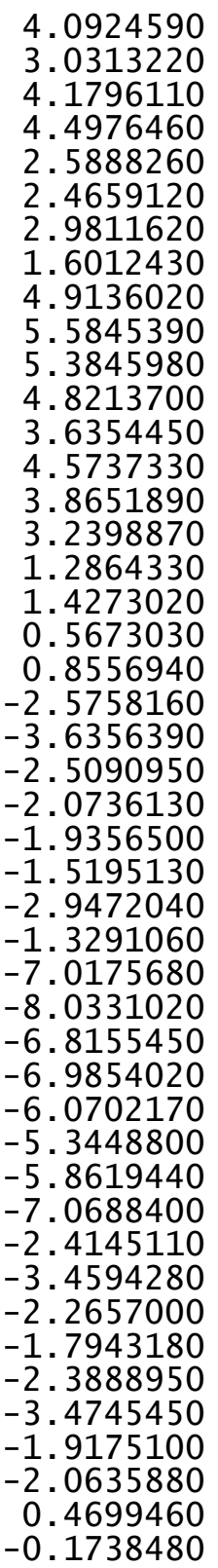

1.2568250

1.0423660

2.2371850

0.5092630

6.1498850

7.2206200

5.6816230

5.7205350

6.5404850

6.3915570

6.0922570

7.6170260

2. 3705820

1.8454720

3.4369560

2.0059340

2. 8484300

3.9100570

2.7632980

2.3762970

$-4.8693290$

$-4.7494710$

$-5.5511420$

$-5.3381730$

$-2.5877120$

-1.6074360

$-2.4451350$

$-3.0207060$

$-2.0826320$

$-1.7126150$

$-1.9963090$

$-3.1447120$

0.2140870

0.7871890

0.3823590

0.6116690

$-1.0118200$

$-1.0537460$

$-0.0868940$

$-0.9648500$

$-3.5150290$

$-3.5811250$

$-3.5052650$

$-4.4198520$

0.0898410

0.1351360
$-3.1527920$

$-2.9965720$

$-3.6355860$

$-3.8441310$

$-1.7297320$

$-1.9270560$

$-2.6399670$

$-1.5305750$

$-0.8101760$

0.0421100

$-1.6926520$

$-0.9917970$

3.8119020

3.6098140

3.9251140

4.7668400

3.0501060

3.2835040

2.2325580

3.9403850

2. 1222810

2. 3762020

1. 2675710

2.9760230

3.0155930

2.7619260

3.4144500

3.8185260

$-1.0005960$

$-0.8202860$

$-2.0746980$

$-0.7360780$

$-0.5328390$

0.0523660

$-1.5959670$

$-0.3207050$

$-3.9916830$

$-4.3207780$

$-3.4314730$

$-4.8932980$

$-3.9436270$

$-4.0843010$

$-3.4232870$

$-0.5379050$

$-1.8932680$ 


\begin{tabular}{|c|c|c|c|c|c|c|c|}
\hline & & & & & & & \\
\hline 0 & 0.2119060 & 1.2091390 & 0.4390410 & C & 2.2971220 & 1.5795960 & -1.5795400 \\
\hline H & $\begin{array}{l}-0.9404840 \\
-6.2576470\end{array}$ & -2.2128260 & -3.0336240 & C & 0.3619160 & 3.6575020 & -1.6885830 \\
\hline $\mathrm{H}$ & -0.8878530 & $\begin{array}{l}-1.3804910 \\
-3\end{array}$ & 0.8758930 & C & 2. 5990710 & 2.7868270 & -2.1597430 \\
\hline $\mathrm{H}$ & 47727900 & 01993210 & $\begin{array}{r}1.5441500 \\
-1.4322010\end{array}$ & $\mathrm{C}$ & $\frac{1}{2} .6481890$ & 3.8310100 & -2.2663750 \\
\hline $\mathrm{H}$ & 3.1050760 & 6.4317060 & 0.3276060 & $c$ & -2.0478250 & ..9314030 & -2.5418050 \\
\hline H & 2.3999990 & 1.0885630 & 26157010 & $\mathrm{C}$ & -3.3162650 & 0.9860680 & -0.0276580 \\
\hline c & -2.4505590 & 2.2683860 & -1.6247100 & $\mathrm{C}$ & -1.2129670 & 2.2398170 & -0.2847740 \\
\hline c & -1.4656000 & 3.2348720 & -1.1174620 & $\mathrm{C}$ & -3.6947000 & 1.8550280 & 0.9649050 \\
\hline $\mathrm{H}$ & -2.5239650 & 1.3257740 & -1.0792420 & $\mathrm{C}$ & -1.5683120 & 3.0687600 & 0.8271070 \\
\hline $\mathrm{H}$ & -0.5897600 & 2.8293710 & -0.6205780 & C & -2.8316290 & 2.8714380 & 1.4470650 \\
\hline 0 & -1.4721310 & 2.1893920 & -2.6828510 & $\mathrm{H}$ & -4.6666420 & 1.7323650 & 1.4338770 \\
\hline $\mathrm{H}$ & -0.8744500 & 1.3993150 & -2.4517860 & C & -2.3213210 & 4.6048610 & 3.0666310 \\
\hline c & -3.6475610 & 4.1536860 & -2.8030490 & $\mathrm{C}$ & -1.0538540 & 4.7847670 & 2.4655570 \\
\hline C & -2.9016040 & 5.1562200 & -1.9185390 & $\mathrm{C}$ & -0.6866170 & 4.0390260 & 1.3739330 \\
\hline C & -1.4964270 & 4.6602630 & -1.5615180 & $\mathrm{C}$ & -3.1890830 & 3.6682010 & 2.5666800 \\
\hline C & $-\overline{3} .7801400$ & 2.7952620 & -2.1083000 & $\mathrm{H}$ & -2.5995440 & 5.2028840 & 3.9290690 \\
\hline $\mathrm{H}$ & -4.6442680 & 4.5364530 & -3.0420200 & $\mathrm{H}$ & -0.3619980 & 5.5134830 & 2.8769270 \\
\hline $\mathrm{H}$ & -2.8195320 & 6.1245690 & -2.4205860 & $\mathrm{H}$ & 0.2941310 & 4.1754320 & 0.9320470 \\
\hline $\mathrm{H}$ & -0.8311500 & 4.7198350 & -2.4325650 & $\mathrm{H}$ & -4.1589720 & 3.5101590 & 3.0305370 \\
\hline $\mathrm{H}$ & -4.4353670 & 2.8779780 & -1.2322370 & C & -0.2637950 & 5.8669210 & -2.4782890 \\
\hline & -4.2231810 & 2.0482160 & -2.7750750 & C & 1.0255370 & 6.0467200 & -3.0317710 \\
\hline $\mathrm{H}$ & -3.1150840 & 4.0277370 & -3.7530570 & $\mathrm{C}$ & 1.9583180 & 5.0480920 & -2.9286410 \\
\hline$H$ & -3.4693800 & 5.3193280 & -0.9946130 & C & -0.5879790 & 4.7061650 & -1.8236020 \\
\hline H & -1.0272360 & 5.2741410 & -0.7875690 & $\mathrm{H}$ & -1.0056140 & 6.6534200 & -2.5781290 \\
\hline C & -2.8424500 & 2.0533700 & 1.7478770 & $\mathrm{H}$ & 1.2681480 & 6.9727190 & -3.5438070 \\
\hline 0 & -2.0746500 & 1.0224190 & 1.5697030 & $\mathrm{H}$ & 2.9483690 & 5.1687460 & -3.3602970 \\
\hline $\mathrm{c}$ & -4.1527040 & 1.7056190 & 2.3344980 & $\mathrm{H}$ & -1.5837220 & 4.5771140 & -1.4133970 \\
\hline$c$ & -5.2094880 & 2.6245790 & 2.3502890 & C & 3.3722240 & 0.5509220 & -1.4185940 \\
\hline$c$ & -4.3567310 & 0.4105720 & 2.8304960 & $\mathrm{C}$ & 3.6114350 & -0.3956420 & -2.4287190 \\
\hline $\mathrm{c}$ & -6.4501870 & 2.2506130 & 2.8485740 & $\mathrm{C}$ & 4.2136300 & 0.6173930 & -0.2885060 \\
\hline $\mathrm{H}$ & -5.0562790 & 3.6220700 & 1.9533290 & $\mathrm{C}$ & 4.7204210 & -1.2357470 & -2.3100160 \\
\hline$c$ & -5.5967710 & 0.0437420 & 3.3344840 & C & 5.2960760 & -0.2580560 & -0.2071680 \\
\hline $\mathrm{H}$ & -3.5420990 & -0.3019860 & 2.8096680 & C & 5.5791910 & -1.1774140 & -1.2155990 \\
\hline $\mathrm{c}$ & -6.6461640 & 0.9609840 & 3.3402610 & $\mathrm{H}$ & 4.9296670 & -1.9564760 & -3.0967310 \\
\hline $\mathrm{H}$ & -7.2669590 & 2.9649030 & 2.8494300 & $\mathrm{H}$ & 5.9524200 & -0.2047730 & 0.6561600 \\
\hline $\mathrm{H}$ & -5.7460680 & -0.9596550 & 3.7210700 & C & -4.1933170 & -0.1511970 & -0.4376070 \\
\hline $\mathrm{H}$ & -7.6175940 & 0.6709650 & 3.7287360 & $\mathrm{C}$ & -4.4728500 & -1.1912730 & 4760250 \\
\hline $\mathrm{H}$ & -1.1563570 & 1.2126200 & 1.1649230 & C & -4.7658820 & -0.1711310 & -1.7222070 \\
\hline & -2.4065570 & 3.6235340 & 1.3309790 & $\mathrm{C}$ & -5.3292350 & -2.2170600 & 0.0807950 \\
\hline & & & & C & -5.6077940 & -1.2287000 & -2.0705720 \\
\hline & R) $\mathrm{G}[\mathrm{wB} 97 \mathrm{X}$ & (d) $]=-3$ & & C & -5.9050180 & -2.2600780 & -1.1871450 \\
\hline 0 & 0.6754430 & 0.1893960 & -0.4929180 & $\mathrm{H}$ & -5.5538140 & -3.0111220 & 0.7892130 \\
\hline 0 & -1.6141650 & 0.3232590 & -1.6121540 & $\mathrm{H}$ & -6.0567060 & -1.2439570 & -3.0615980 \\
\hline$c$ & 0.9872120 & 1.4052010 & -1.0605740 & C & 3.9492710 & 1.6127500 & 0.8356570 \\
\hline$c$ & 0.06 & רעירו & -1.0188680 & $\mathrm{C}$ & .8197420 & -2.053 & -1.16801 \\
\hline
\end{tabular}




$\begin{array}{rr}2.7277690 & -0.4670650 \\ -3.8933530 & -1.2373660 \\ -6.8360270 & -3.3891550 \\ -4.5410870 & 0.9404450 \\ -5.8432160 & 1.7099490 \\ -6.6071350 & 1.0587860 \\ -6.2486210 & 2.1253100 \\ -5.6643750 & 2.5366930 \\ -3.9359230 & 0.4069270 \\ -3.0051100 & -0.1323980 \\ -4.6255810 & -0.2719400 \\ -3.7154480 & 1.2355350 \\ -8.1182620 & -3.3804920 \\ -8.8078370 & -4.1624330 \\ -7.8937710 & -3.5633940 \\ -8.6301980 & -2.4153970 \\ -6.1391080 & -4.7541140 \\ -5.2312120 & -4.7666250 \\ -5.8550700 & -4.9969590 \\ -6.8077920 & -5.5450040 \\ -4.9691020 & -0.9147220 \\ -5.4309790 & 0.0620940 \\ -5.7663720 & -1.6672740 \\ -4.5328420 & -0.9079990 \\ -3.2319960 & -2.5846110 \\ -3.9700440 & -3.3906270 \\ -2.5087150 & -2.8539990 \\ -2.7061890 & -2.5186390 \\ 5.2239840 & 2.1396270 \\ 5.7222890 & 1.3687470 \\ 5.9404340 & 2.5132650 \\ 4.9704210 & 2.9607660 \\ 3.0042930 & 1.0086510 \\ 2.0596560 & 0.6910260 \\ 3.4615230 & 0.1313990 \\ 2.7789930 & 1.7397080 \\ 7.0356890 & -2.7388260 \\ 7.9017510 & -3.4075070 \\ 7.2312460 & -2.0085710 \\ 6.1658400 & -3.3333490 \\ 8.0572760 & -1.2316420 \\ 7.9275520 & -0.7692180 \\ 8.2317780 & -0.4318240 \\ 8.9511320 & -1.8648540 \\ 2.5306990 & -1.8952630 \\ 3.4390680 & -2.2861670\end{array}$

-3.6653940
1.8850330
-1.5901660
-2.7384450
-3.0018160
-3.4421300
-2.0728970
-3.6985290
-4.0429230
-3.8455890
-4.5575110
-4.7252590
-0.7468650
-1.0839730
0.3105050
-0.8207520
-1.5169270
-2.1287210
-0.4864700
-1.8744800
2.9327010
2.7558050
2.9150110
3.9379870
2.2070870
2.2912890
1.4346660
3.1667880
1.5031880
2.1017640
0.7640690
2.1819380
1.8842810
1.4338790
2.3581520
2.6689050
0.1864100
0.1358770
0.9795840
0.4818430
-1.5591710
-2.5431210
-0.8296800
-1.5897960
-4.1837770
-4.6567500

2.2436720
1.7374480
3.2851220
4.2886620
2.6399230
3.3527820
-0.5388600
-0.0693090
-1.0720540
1.7382670
6.6904670
3.4399860
-3.8290570
-7.1227880
-3.1151430
2.0082900
0.8898050
1.9722870
-0.0751830
1.3666730
0.7815010
3.4195100
2.4329980
1.0030180
3.3964880
4.4342840
2.4684930
0.6215540
3.8012170
4.0120400
3.1668730
2.7113120
0.3013220
0.3020620
-0.5929850
0.5933110
1.5107140
-0.0370580
1.7925130
2.0042840
0.2503290
-0.7416190
1.1650340
2.5067260
-0.2391680
1.3910810
$-2.5759920$
$-1.9079320$
0.4294140
0.0977410
0.3850950
1.4739380
$-0.7651690$
$-1.6146450$
$-1.4547050$
$-0.0907680$
$-2.8411630$
2.4779310
1. 6582140
$-3.2177000$
$-0.4753850$
$-2.9439990$
$-3.5696740$
$-3.0834320$
$-3.6165370$
$-2.9426120$
$-5.3272170$
$-4.9448300$
$-3.4740030$
$-5.2699450$
$-6.3966830$
$-5.6197040$
$-2.9092140$
$-3.2282950$
$-5.5342200$
$-4.7945260$
$-5.0317410$
$-0.6951700$
0.2993260
0.0363100
1. 5516990
1.0071980
$-0.9285720$
2. 5195840
1. 7681320
2. 2504280
0.7953720
3. 4865050
3.0092330

-3.3781760
-4.9378090
-4.7811880
-5.0738830
-5.6659340
-4.4626830
-1.0648780
-2.2037120
0.1679710
-3.3883820
-1.9222260
0.3990160
-2.3224450
-2.6355640
1.9599370
-0.0218980
0.6985740
-0.1214270
0.6168620
-1.1357280
-1.5985950
0.5335890
1.6480060
1.2556550
0.2350830
0.8186590
1.8743000
0.4772880
1.0841140
-0.6333610
-0.3751700
2.5662780
2.0902010
3.1636860
2.3294990
4.2246280
5.2481890
4.1690260
6.2004060
5.2891650
5.1218630
3.3742530
6.1380400
6.9895760
5.0642410
6.8810270 


$\begin{array}{lrcr}\text { H } & -0.7539920 & -0.8589910 & 1.5016970 \\ \mathrm{~S} & 1.0680710 & -2.1913870 & 3.0690760 \\ \mathrm{H} 44 & & & \\ \mathrm{TS} 219(\mathrm{R}, \mathrm{R}) \mathrm{G}[\mathrm{WB} 97 \times \mathrm{D} / 6-31 \mathrm{G}(\mathrm{d})] & =-3633.716331 \\ \mathrm{O} & 0.3086250 & 0.9359380 & -0.1074190 \\ \mathrm{O} & -1.9883200 & 0.5552560 & -1.0951720 \\ \mathrm{C} & 0.2724220 & 2.2587360 & -0.4972450 \\ \mathrm{C} & -0.8787570 & 2.9868580 & -0.2947410 \\ \mathrm{C} & 1.4768850 & 2.8209120 & -1.0006100 \\ \mathrm{C} & -0.9230690 & 4.3308470 & -0.7921940 \\ \mathrm{C} & 1.4561950 & 4.1393290 & -1.3722780 \\ \mathrm{C} & 0.2632460 & 4.9073940 & -1.3244760 \\ \mathrm{H} & 2.3702010 & 4.6153460 & -1.7155680 \\ \mathrm{C} & -2.5206240 & 1.1542540 & 0.0296630 \\ \mathrm{C} & -3.6141710 & 0.5062010 & 0.6616710 \\ \mathrm{C} & -2.0139630 & 2.3671020 & 0.4409570 \\ \mathrm{C} & -4.1994890 & 1.1422970 & 1.7257180 \\ \mathrm{C} & -2.5570310 & 2.9634410 & 1.6275000 \\ \mathrm{C} & -3.6827610 & 2.3549420 & 2.2480340 \\ \mathrm{H} & -5.0694530 & 0.6969140 & 2.2000500 \\ \mathrm{C} & -3.7064910 & 4.0852380 & 3.9493680 \\ \mathrm{C} & -2.5585670 & 4.6656060 & 3.3624080 \\ \mathrm{C} & -1.9993000 & 4.1216250 & 2.2339750 \\ \mathrm{C} & -4.2506800 & 2.9515220 & 3.4045470 \\ \mathrm{H} & -4.1435180 & 4.5309080 & 4.8375530 \\ \mathrm{H} & -2.1123240 & 5.5471490 & 3.8124870 \\ \mathrm{H} & -1.1131710 & 4.5733890 & 1.8028150 \\ \mathrm{H} & -5.1192270 & 2.4815930 & 3.8583250 \\ \mathrm{C} & -2.1163820 & 6.3882470 & -1.2851480 \\ \mathrm{C} & -0.9285130 & 6.9713960 & -1.7825430 \\ \mathrm{C} & 0.2331240 & 6.2437100 & -1.8022550 \\ \mathrm{C} & -2.1141240 & 5.1045810 & -0.8009590 \\ \mathrm{H} & -3.0397940 & 6.9593500 & -1.2920250 \\ \mathrm{H} & -0.9432640 & 7.9895270 & -2.1591560 \\ \mathrm{H} & 1.1485810 & 6.6751300 & -2.1984810 \\ \mathrm{H} & -3.0336550 & 4.6645560 & -0.4304450 \\ \mathrm{C} & 2.7143550 & 1.9824390 & -0.9971180 \\ \mathrm{C} & 3.1880680 & 1.3781750 & -2.1759750 \\ \mathrm{C} & 3.3721500 & 1.7669130 & 0.2280100 \\ \mathrm{C} & 4.3003810 & 0.5381820 & -2.0908910 \\ \mathrm{C} & 4.4692780 & 0.9081360 & 0.2630690 \\ \mathrm{C} & 4.9387180 & 0.2693020 & -0.8815430 \\ \mathrm{H} & 4.6840070 & 0.0690180 & -2.9933710 \\ \mathrm{H} & 4.9698950 & 0.7369910 & 1.2129080 \\ \mathrm{C} & -4.1171590 & -0.8100530 & 0.1631050 \\ \mathrm{C} & -3.8024680 & -1.9954490 & \\ & & & \end{array}$

-4.9133080
-4.3282170
-5.4042550
-5.1289820
-4.0998970
-6.0219720
2.9524830
6.1408960
2.5469810
-2.9258100
-5.6853730
-5.2628890
-6.7743540
-7.3254280
-7.1488950
-7.0032280
-4.7196190
-3.6383860
-5.1902560
-4.9216260
-6.6799280
-7.1210880
-6.1821790
-7.4898250
-4.5690240
-3.8648080
-4.0042860
-4.9901290
-3.7838530
-4.4330250
-4.4215150
-3.1467270
-1.9525730
-2.4689310
-1.4097610
-1.2235460
4.0136910
4.9725350
4.1789540
3.6972630
2.6553470
1.8837380
3.5455660
2.3011490
6.0590330
6.8648780
-0.8564740
-3.1994960
-2.0902580
-3.2707060
-4.1154270
-2.1324060
2.4798140
-0.6569150
1.6672870
-1.9916510
-4.5974950
0.3940960
0.6587390
-0.1502010
0.7457170
1.5911820
0.3178010
0.1515070
-0.4966440
1.2535880
-5.1858310
-6.1114380
-5.4200780
-4.4796320
-5.6002230
-5.1866920
-5.8649430
-6.5234850
-1.9675960
-1.0879530
-2.8586140
-1.9571940
-3.1769170
-4.1211520
-3.2781460
-3.0227250
3.5124720
3.0277340
4.2415340
4.0547330
1.5044750
0.7882540
0.9387170
2.0492900
-1.6667820
-2.4043130
$-0.9922520$

0.3958910

$-1.4241770$

$-0.7438490$

0.9344330

$-2.3191790$

1. 5068090

$-0.8328740$

$-3.5265530$

2. 1069380

$-1.2286650$

$-1.7862380$

$-1.7730570$

$-2.2664700$

$-0.7473890$

$-2.3011520$

$-3.2190010$

$-3.2121690$

$-3.7818730$

$-3.7522260$

$-0.2186440$

$-0.6049750$

0.7296440

$-0.0082670$

$-1.5490620$

$-2.2784400$

$-0.6475810$

$-1.9623590$

3. 3807750

3. 4172490

3.4289000

4. 2727000

2. 1585500

2. 3659410

1. 2155480

2.9616670

1. 9118770

2. 1300450

1.1110340

2. 8099940

2. 6491010

2. 3554150

2. 9479100

3. 5316380

0.3182850

0. 2380160 


$\begin{array}{lrrr}\text { H } & 6.1598100 & -1.1734390 & \\ \text { H } & 5.1013040 & -2.1992040 & 0.3157080 \\ \mathrm{C} & 7.4447940 & 0.1503570 & -0.7588050 \\ \mathrm{H} & 7.5262780 & 0.8445650 & -1.6015650 \\ \mathrm{H} & 7.4812740 & 0.7367220 & 0.1670330 \\ \mathrm{H} & 8.3150690 & -0.5154330 & -0.7737500 \\ \mathrm{C} & 2.5487400 & 0.4731290 & -4.4850960 \\ \mathrm{H} & 3.5592080 & 0.2270610 & -4.8317840 \\ \mathrm{H} & 2.1156130 & -0.4157780 & -4.0232230 \\ \mathrm{H} & 1.9541090 & 0.7144330 & -5.3727450 \\ \mathrm{C} & 3.2395830 & 2.8639330 & -4.1978640 \\ \mathrm{H} & 4.2953690 & 2.6364330 & -4.3884750 \\ \mathrm{H} & 2.7622780 & 3.0939830 & -5.1570360 \\ \mathrm{H} & 3.1985320 & 3.7613250 & -3.5744060 \\ \mathrm{P} & -0.5330150 & -0.1590860 & -0.9790600 \\ \mathrm{O} & -0.0308170 & -0.2914560 & -2.3868410 \\ \mathrm{O} & -0.5737020 & -1.3853580 & -0.1012650 \\ \mathrm{H} & 1.5009910 & 1.9329480 & -3.3443900 \\ \mathrm{H} & 6.1526260 & -1.2227270 & -1.7737760 \\ \mathrm{H} & 2.0289520 & 3.0306550 & 1.3081880 \\ \mathrm{H} & -4.7850980 & 1.2525550 & -1.3055380 \\ \mathrm{H} & -6.2322010 & -4.4013870 & -2.1598410 \\ \mathrm{H} & -2.3202730 & -1.0800900 & 2.0855400 \\ \mathrm{C} & 2.2538960 & -2.4097890 & -1.9995550 \\ \mathrm{C} & 1.2999590 & -3.3522400 & -1.4049600 \\ \mathrm{H} & 2.3246960 & -1.4312510 & -1.5221490 \\ \mathrm{H} & 0.4238270 & -2.9261390 & -0.9282810 \\ \mathrm{O} & 1.2421270 & -2.4341940 & -3.0350740 \\ \mathrm{H} & 0.6462400 & -1.6318290 & -2.8559600 \\ \mathrm{C} & 3.4608050 & -4.3619310 & -3.0578020 \\ \mathrm{C} & 2.7741810 & -5.2976100 & -2.0597390 \\ \mathrm{C} & 1.3632670 & -4.8108520 & -1.7141140 \\ \mathrm{C} & 3.5770990 & -2.9449710 & -2.4896260 \\ \mathrm{H} & 4.4596580 & -4.7383070 & -3.2979970 \\ \mathrm{H} & 2.7122840 & -6.3124970 & -2.4629810 \\ \mathrm{H} & 0.6790210 & -4.9742080 & -2.5564910 \\ \mathrm{H} & 4.2669670 & -2.9360200 & -1.6376130 \\ \mathrm{H} & 3.9737230 & -2.2473800 & -3.2340880 \\ \mathrm{H} & 2.8921990 & -4.3373730 & -3.9949300 \\ \mathrm{H} & 3.3736170 & -5.3537190 & -1.1421840 \\ \mathrm{H} & 0.9321840 & -5.3582550 & -0.8713050 \\ \mathrm{C} & 1.8996490 & -2.2346980 & 2.1186020 \\ \mathrm{O} & 0.8918890 & -1.4361010 & 1.9681670 \\ \mathrm{C} & 2.6587870 & -1.9639680 & 3.3610870 \\ \mathrm{C} & 4.0085230 & -2.3137720 & 3.4741900 \\ \mathrm{C} & 2.0233730 & -1.3042290 & 4.4214640 \\ & & & \\ & & & \end{array}$

$\begin{array}{ll}\mathrm{C} & 4.7164410 \\ \mathrm{H} & 4.5023910 \\ \mathrm{C} & 2.7296040 \\ \mathrm{H} & 0.9785270 \\ \mathrm{C} & 4.0781120 \\ \mathrm{H} & 5.7679670 \\ \mathrm{H} & 2.2298790 \\ \mathrm{H} & 4.6316440 \\ \mathrm{H} & 0.3476320 \\ \mathrm{~S} & 2.3246040 \\ \mathrm{H} & \end{array}$
$-1.9947240$
$-2.8161630$
$-1.0061520$
$-1.0297670$
-1.3439430
$-2.2525230$
$-0.5043590$
$-1.0996370$
$-1.5262880$
$-3.4770580$
4.6263230
2.6497140
5.5791950
4.3313680
5.6804960
4.7014450
6.4015590
6.5820240
1.1083070

144

$\mathrm{TS} 220(\mathrm{R}, \mathrm{R}) \mathrm{G}[\mathrm{WB} 97 \mathrm{XD} / 6-31 \mathrm{G}(\mathrm{d})]=-3633.718518$

$\begin{array}{lrrr}\text { TS220(R, R) G[WB97XD/6-31G(d)] }=-3633.718518 \\ \text { O } & 0.1065770 & -1.2694500 & 0.2839460 \\ \text { O } & 2.0542880 & -0.1221770 & -0.8263100 \\ \text { C } & 0.4769800 & -2.4729000 & -0.2796240 \\ \text { C } & 1.8029540 & -2.8457610 & -0.2507490 \\ \mathrm{C} & -0.5652620 & -3.2929490 & -0.7903660 \\ \mathrm{C} & 2.1706650 & -4.0634730 & -0.9129070 \\ \mathrm{C} & -0.2137970 & -4.5107800 & -1.3104640 \\ \mathrm{C} & 1.1452100 & -4.9074070 & -1.4224590 \\ \mathrm{H} & -0.9855420 & -5.1903400 & -1.6605700 \\ \mathrm{C} & 2.8656890 & -0.6402090 & 0.1600410 \\ \mathrm{C} & 3.7881110 & 0.2528040 & 0.7657830 \\ \mathrm{C} & 2.7927150 & -1.9843200 & 0.4540460 \\ \mathrm{C} & 4.6799540 & -0.2715870 & 1.6640220 \\ \mathrm{C} & 3.6451160 & -2.4939200 & 1.4907830 \\ \mathrm{C} & 4.6219700 & -1.6316600 & 2.0625970 \\ \mathrm{H} & 5.4293380 & 0.3739750 & 2.1132340 \\ \mathrm{C} & 5.3883840 & -3.4237120 & 3.5087300 \\ \mathrm{C} & 4.3856560 & -4.2678220 & 2.9789970 \\ \mathrm{C} & 3.5383690 & -3.8171790 & 1.9983110 \\ \mathrm{C} & 5.4970790 & -2.1328010 & 3.0618620 \\ \mathrm{H} & 6.0583370 & -3.7956850 & 4.2777330 \\ \mathrm{H} & 4.2803030 & -5.2800750 & 3.3574390 \\ \mathrm{H} & 2.7688110 & -4.4747530 & 1.6097720 \\ \mathrm{H} & 6.2460370 & -1.4639750 & 3.4777790 \\ \mathrm{C} & 3.8359180 & -5.6255180 & -1.7422680 \\ \mathrm{C} & 2.8146740 & -6.4840090 & -2.2095020 \\ \mathrm{C} & 1.4998310 & -6.1280140 & -2.0546900 \\ \mathrm{C} & 3.5234560 & -4.4492030 & -1.1093500 \\ \mathrm{H} & 4.8766730 & -5.8964700 & -1.8913060 \\ \mathrm{H} & 3.0748850 & -7.4159160 & -2.7018130 \\ \mathrm{H} & 0.7063530 & -6.7702280 & -2.4276460 \\ \mathrm{H} & 4.3151910 & -3.7938740 & -0.7628790 \\ \mathrm{C} & -1.9799920 & -2.8206740 & -0.6694320 \\ \mathrm{C} & -2.6556950 & -2.2692840 & -1.7772830 \\ & & & \end{array}$




$\begin{array}{rrr}-2.6116880 & -2.8866880 & 0.5838930 \\ -3.9483140 & -1.7799560 & -1.5939630 \\ -3.9124300 & -2.3964820 & 0.7119150 \\ -4.5924610 & -1.8251250 & -0.3575570 \\ -4.4724780 & -1.3489140 & -2.4426290 \\ -4.4118150 & -2.4579920 & 1.6766680 \\ 3.7451230 & 1.7095390 & 0.4337700 \\ 3.1743330 & 2.6141610 & 1.3492660 \\ 4.2437280 & 2.1672010 & -0.7993180 \\ 3.1416470 & 3.9687020 & 1.0143920 \\ 4.1790430 & 3.5283080 & -1.0910050 \\ 3.6306840 & 4.4463970 & -0.1976850 \\ 2.7088060 & 4.6785000 & 1.7150890 \\ 4.5631300 & 3.8806140 & -2.0460780 \\ -1.9343520 & -3.5138810 & 1.7942860 \\ -5.9974950 & -1.2776840 & -0.1845770 \\ -2.0262650 & -2.2411220 & -3.1636170 \\ 2.6083370 & 2.1599500 & 2.6893190 \\ 3.5357730 & 5.9215260 & -0.5431100 \\ 4.8535120 & 1.2186470 & -1.8213750 \\ 6.3475900 & 1.5062020 & -2.0208550 \\ 6.5080540 & 2.5106110 & -2.4296940 \\ 6.8892600 & 1.4373830 & -1.0713710 \\ 6.7859690 & 0.7851380 & -2.7199740 \\ 4.0925700 & 1.2571380 & -3.1527590 \\ 3.0314080 & 1.0427540 & -2.9965940 \\ 4.1798180 & 2.2375190 & -3.6354900 \\ 4.4977200 & 0.5095870 & -3.8441320 \\ 2.5886420 & 6.1499490 & -1.7293560 \\ 2.4656510 & 7.2206860 & -1.9266240 \\ 2.9809490 & 5.6817520 & -2.6396360 \\ 1.6010980 & 5.7205300 & -1.5301550 \\ 4.9134700 & 6.5406280 & -0.8099580 \\ 5.5844980 & 6.3916660 & 0.0422490 \\ 5.3844040 & 6.0924930 & -1.6925150 \\ 4.8211740 & 7.6171790 & -0.9914870 \\ 3.6353980 & 2.3702180 & 3.8120140 \\ 4.5736400 & 1.8450350 & 3.6099080 \\ 3.8652420 & 3.4365580 & 3.9253340 \\ 3.2397720 & 2.0055180 & 4.7669030 \\ 1.2864480 & 2.8483450 & 3.0502200 \\ 1.4274310 & 3.9098860 & 3.2839440 \\ 0.5673940 & 2.7635430 & 2.2325710 \\ 0.8555510 & 2.3760110 & 3.9403140 \\ -2.5755000 & -4.8692420 & 2.1223770 \\ -3.6353140 & -4.7494700 & \\ -3.3763770\end{array}$

-2.5087870
-2.0731980
-1.9355070
-1.5194690
-2.9470580
-1.3288970
-7.0175850
-8.0331400
-6.8156410
-6.9853070
-6.0703240
-5.3450630
-5.8619930
-7.0689890
-2.4147560
-3.4596770
-2.2660460
-1.7945950
-2.3888450
-3.4744950
-1.9175470
-2.0633520
0.4700000
-0.1737420
0.2118620
-0.9405650
-6.2576270
-0.8876640
4.7727730
3.1050380
2.3998780
-2.4505950
-1.4657750
-2.5238950
-0.5898980
-1.4720800
-0.8743660
-3.6477150
-2.9019250
-1.4967180
-3.7802010
-4.6444510
-2.8199340
-0.8313810
-4.4354940
-4.2231240
1. 2676590

2.9760800

3.0156370

2. 7619400

3. 4145310

3.8185530

$-1.0003730$

$-0.8199720$

$-2.0744830$

$-0.7359200$

$-0.5326010$

0.0526700

$-1.5957090$

$-0.3205180$

$-3.9916280$

$-4.3206970$

$-3.4314310$

$-4.8932610$

$-3.9435980$

$-4.0841840$

$-4.9329380$

$-3.4233370$

$-0.5379690$

$-1.8933620$

0.4389230

$-3.0335960$

0.8761050

1.5441820

$-1.4322310$

0.3279560

2. 6156320

$-1.6249310$

$-1.1176720$

$-1.0794490$

$-0.6207570$

$-2.6830080$

$-2.4519180$

$-2.8035250$

$-1.9190420$

$-1.5618640$

$-2.1086500$

$-3.0425680$

$-2.4211360$

$-2.4328630$

-1.2326380
-2.7753740 


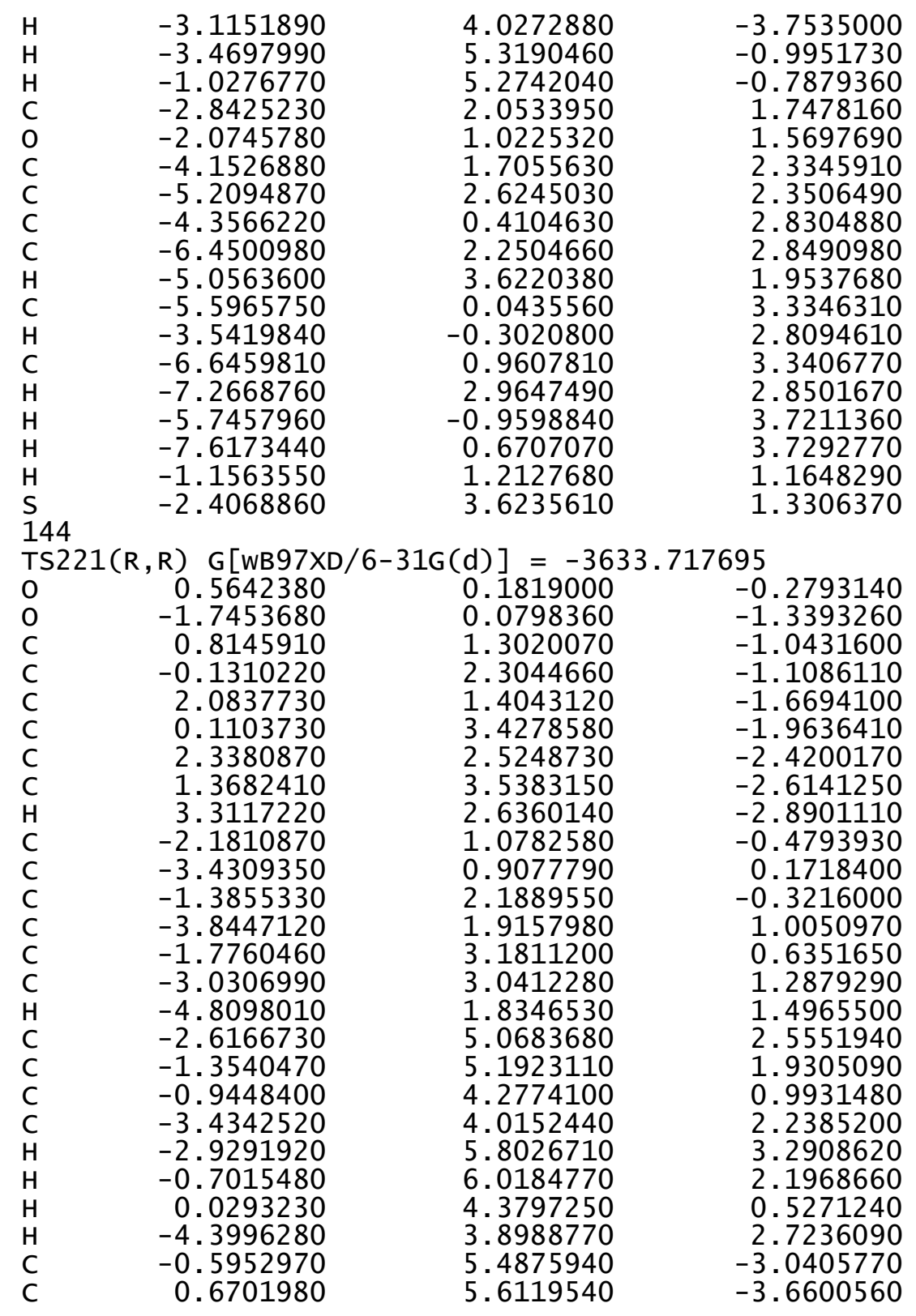

1.6279600

$-0.8690420$

$-1.3590150$

0.8741420

2.5989170

$-1.8473790$

3.1554140

3. 3564740

4.0224990

4.4666010

5.1194980

5. 3703040

4.6411020

5.7942860

$-4.2587610$

$-4.4352320$

$-4.8842670$

$-5.2492060$

$-5.6793340$

$-5.8774160$

$-5.3963540$

$-6.1660340$

3.8134830

6.5916770

2.4121740

$-3.7926690$

$-6.7486020$

$-4.7615790$

$-6.1113600$

$-6.8595000$

$-6.4954390$

$-6.0073750$

$-4.1922680$

$-3.2275860$

$-4.8708560$

$-4.0467120$

$-6.1659610$

$-6.7678050$

$-6.1528520$

$-5.1383650$

$-8.1974040$

$-8.6184870$

$-8.2576380$

$-8.8238300$

$-4.8247240$

$-5.2997340$
4.6540450

4. 4270990

6.2368910

6.4596580

4.7282940

4. 3398400

0.3751510

$-0.6041390$

0.4596690

$-1.4399170$

$-0.4027120$

$-1.3449470$

$-2.1943490$

$-0.3310830$

$-0.3170600$

$-1.2419150$

$-0.5286230$

$-2.3543500$

$-1.6593930$

$-2.5850210$

$-3.0708890$

$-1.8174540$

1. 5046220

$-2.2508290$

$-0.7423620$

$-1.0625000$

$-3.8108170$

0.4618620

1.1379520

0.4077630

1.6311450

1.8927410

$-0.1927900$

$-0.6645810$

$-0.9568800$

0.5601870

$-4.7381700$

$-5.6492520$

$-4.2446990$

$-5.0266310$

$-3.4281980$

$-2.7762800$

$-2.8988490$

$-4.3241340$

$-0.5802320$

0.3567090
$-3.4529570$

$-2.2149960$

$-3.2253470$

$-4.3068530$

$-3.9354090$

$-1.7554910$

$-1.4977850$

$-2.4882350$

$-0.3930490$

$-2.3819140$

$-0.3314510$

$-1.3247100$

$-3.1457750$

0.5147360

$-0.0413050$

1.0065800

$-1.2851180$

0.7845270

$-1.4573600$

$-0.4349240$

1. 5894800

$-2.4173420$

0.6969650

$-1.3136600$

$-3.6741630$

2. 3764420

$-0.6415360$

$-2.4350700$

$-2.7138120$

$-3.0434760$

$-1.8144030$

$-3.5014690$

$-3.6994890$

$-3.4913640$

$-4.0958470$

$-4.4822340$

$-1.7163170$

$-1.8073220$

$-2.6950320$

$-1.4722880$

$-0.9717050$

$-0.1993010$

$-1.9297120$

$-1.0455480$

3.4066210

3.0994720 


$\begin{array}{lrrr}\mathrm{H} & -5.6164260 & -1.3274170 & 3.5394920 \\ \mathrm{H} & -4.3470120 & -0.4168880 & 4.3793130 \\ \mathrm{C} & -3.0999120 & -2.3353950 & 2.8816120 \\ \mathrm{H} & -3.8223080 & -3.1182620 & 3.1394180 \\ \mathrm{H} & -2.4129800 & -2.7270960 & 2.1280760 \\ \mathrm{H} & -2.5271250 & -2.1083410 & 3.7882660 \\ \mathrm{C} & 4.5364640 & 2.8138400 & 0.3458430 \\ \mathrm{H} & 5.6164170 & 2.6429600 & 0.2636580 \\ \mathrm{H} & 4.1843540 & 3.2277840 & -0.6035600 \\ \mathrm{H} & 4.3676870 & 3.5629530 & 1.1280160 \\ \mathrm{C} & 4.2428330 & 1.0293230 & 2.0879770 \\ \mathrm{H} & 3.7889330 & 0.0655970 & 2.3405510 \\ \mathrm{H} & 5.3314120 & 0.9304240 & 2.1690360 \\ \mathrm{H} & 3.9259650 & 1.7608060 & 2.8379340 \\ \mathrm{C} & 7.1805980 & -2.4980860 & 0.0767230 \\ \mathrm{H} & 7.9708130 & -3.2535830 & 0.0183860 \\ \mathrm{H} & 7.6285400 & -1.5879550 & 0.4917320 \\ \mathrm{H} & 6.4216410 & -2.8539850 & 0.7813310 \\ \mathrm{C} & 7.6651970 & -1.6918650 & -2.2610940 \\ \mathrm{H} & 7.2695410 & -1.5597450 & -3.2733120 \\ \mathrm{H} & 8.0170600 & -0.7164590 & -1.9047010 \\ \mathrm{H} & 8.5260460 & -2.3678490 & -2.3130890 \\ \mathrm{C} & 2.1526000 & -2.2029770 & -4.0607560 \\ \mathrm{H} & 3.0246700 & -2.6568020 & -4.5456300 \\ \mathrm{H} & 1.8930320 & -2.8042420 & -3.1862860 \\ \mathrm{H} & 1.3199680 & -2.2551700 & -4.7698110 \\ \mathrm{C} & 2.9341670 & 0.0397190 & -4.8882360 \\ \mathrm{H} & 3.9083510 & -0.3507030 & -5.2065900 \\ \mathrm{H} & 2.2385930 & -0.0539280 & -5.7298820 \\ \mathrm{H} & 3.0529180 & 1.1037570 & -4.6639060 \\ \mathrm{P} & -0.6043660 & -0.8918270 & -0.7125750 \\ \mathrm{O} & -0.1274730 & -1.8238750 & -1.7817030 \\ \mathrm{O} & -1.0706740 & -1.4795660 & 0.5988220 \\ \mathrm{H} & 1.4485040 & -0.3148790 & -3.3812810 \\ \mathrm{H} & 6.2724600 & -3.2221880 & -1.7160840 \\ \mathrm{H} & 2.7403000 & 1.7212300 & 0.7536920 \\ \mathrm{H} & -4.0662140 & 1.2521980 & -2.1400410 \\ \mathrm{H} & -6.7596920 & -4.3651220 & 0.3057680 \\ \mathrm{H} & -3.0221090 & -0.2924840 & 2.2891160 \\ \mathrm{C} & 2.1451040 & -2.9991010 & 0.2320770 \\ \mathrm{C} & 1.0704270 & -3.4613300 & 1.1256010 \\ \mathrm{H} & 2.1536050 & -1.9325910 & 0.0003510 \\ \mathrm{H} & 0.1180260 & -2.9471070 & 1.0605600 \\ \mathrm{O} & 1.3742050 & -3.7591400 & -0.7303620 \\ \mathrm{H} & 0.7597860 & -3.1092330 & -1.1913500 \\ \mathrm{C} & 3.5054880 & -5.0234820 & \\ & & & \\ \mathrm{H} & -3430050\end{array}$

2.6086170
1.1691760
3.5242800
4.5281850
2.6166970
0.6983800
4.0349260
4.0671430
3.1464750
2.9899100
0.5349850
0.7214310
-0.4010430
1.2524960
2.1102900
0.9167850
2.6254740
2.3666160
1.4551570
0.2520430
2.3079810
3.2827090
1.2033160
2.7250720
-0.6705760
1.5388260
$-5.1638490$
$-4.7594410$
$-3.5785320$
$-5.3320660$
$-6.1918020$
$-5.5044710$
$-2.9298320$
$-3.4937540$
$-5.6883450$
$-4.5258030$
$-4.7047390$
$-0.2852460$
$-0.0634300$
0.9235470
0.8508430
2. 1724620
2.0150720
$-0.1169080$
3.3319850
2. 2257600
3.2552550
1.9564800
4. 2936680
4.1616190
$-0.7554010$

$-1.7549480$

\begin{abstract}
2.1736560
1. 8460810

0.4391560

1. 1806980

2. 5472460

1. 1901000

1.1613960

$-0.5049860$

0.1480790

2. 9811340

2.7353400

3. 1619220

2. 5582300

3.8303200

4.9320710

3. 2909280

5.4871820

5.3502590

3.8337350

2.4356050

4.9331600

6.3488340

3. 3977740

5. 3613760

1.8520980

3. 1914520
\end{abstract}

144

$\begin{array}{lrcr}\text { TS222(R, R) G[WB97XD/6-31G(d)] }=-3633.718843 \\ \text { O } & 0.5867460 & 0.2213750 & -0.2414760 \\ \text { O } & -1.6863450 & 0.0437600 & -1.3633280 \\ \mathrm{C} & 0.8363650 & 1.3199160 & -1.0365100 \\ \mathrm{C} & -0.1218920 & 2.3055390 & -1.1492670 \\ \mathrm{C} & 2.1129070 & 1.4174900 & -1.6472390 \\ \mathrm{C} & 0.1216770 & 3.4084330 & -2.0297290 \\ \mathrm{C} & 2.3692010 & 2.5220950 & -2.4206270 \\ \mathrm{C} & 1.3915960 & 3.5190640 & -2.6569150 \\ \mathrm{H} & 3.3487700 & 2.6312820 & -2.8785500 \\ \mathrm{C} & -2.1635540 & 1.0558550 & -0.5413980 \\ \mathrm{C} & -3.4260270 & 0.8783750 & 0.0836780 \\ \mathrm{C} & -1.3932570 & 2.1857130 & -0.3906220 \\ \mathrm{C} & -3.8780200 & 1.8977740 & 0.8826900 \\ \mathrm{C} & -1.8239840 & 3.1914250 & 0.5339440 \\ \mathrm{C} & -3.0909630 & 3.0432790 & 1.1601520 \\ \mathrm{H} & -4.8530960 & 1.8100290 & 1.3528070 \\ \mathrm{C} & -2.7404290 & 5.1014310 & 2.3960560 \\ \mathrm{C} & -1.4657250 & 5.2345020 & 1.7983440\end{array}$




$\begin{array}{rrr}-1.0198210 & 4.3087030 & 0.8888700 \\ -3.5329640 & 4.0286880 & 2.0813970 \\ -3.0815790 & 5.8439060 & 3.1105680 \\ -0.8331020 & 6.0763410 & 2.0636210 \\ -0.0364160 & 4.4190290 & 0.4447320 \\ -4.5070240 & 3.9050920 & 2.5470030 \\ -0.5897200 & 5.4273030 & -3.1781270 \\ 0.6867820 & 5.5530600 & -3.7742640 \\ 1.6531500 & 4.6151810 & -3.5208660 \\ -0.8660230 & 4.3859170 & -2.3294720 \\ -1.3597670 & 6.1597250 & -3.4002700 \\ 0.8922290 & 6.3847770 & -4.4410310 \\ 2.6323540 & 4.6896490 & -3.9863390 \\ -1.8531980 & 4.2967510 & -1.8898350 \\ 3.1789090 & 0.3883820 & -1.4482880 \\ 3.4168540 & -0.5727170 & -2.4431480 \\ 4.0008880 & 0.4504770 & -0.3033580 \\ 4.5203650 & -1.4213320 & -2.3109990 \\ 5.0823330 & -0.4212800 & -0.2157530 \\ 5.3735280 & -1.3503350 & -1.2165930 \\ 4.7105670 & -2.1538490 & -3.0887600 \\ 5.7308510 & -0.3843740 & 0.6558900 \\ -4.2279400 & -0.3655660 & -0.1173770 \\ -4.4131440 & -1.2670470 & 0.9493000 \\ -4.8228080 & -0.6182660 & -1.3685140 \\ -5.2008140 & -2.4000290 & 0.7365220 \\ -5.5943370 & -1.7669260 & -1.5299200 \\ -5.7972160 & -2.6720650 & -0.4902840 \\ -5.3525890 & -3.0995170 & 1.5554940 \\ -6.0585410 & -1.9556190 & -2.4955260 \\ 3.7511960 & 1.4812710 & 0.7913520 \\ 6.6126470 & -2.2207120 & -1.0749560 \\ 2.5313810 & -0.6869380 & -3.6768010 \\ -3.8067670 & -1.0431330 & 2.3290110 \\ -6.6415380 & -3.9184420 & -0.6849540 \\ -4.6904890 & 0.3446370 & -2.5405910 \\ -6.0458490 & 0.9828210 & -2.8755480 \\ -6.7688150 & 0.2291920 & -3.2086340 \\ -6.4666050 & 1.4891020 & -2.0001400 \\ -5.9346460 & 1.7206270 & -3.6781320 \\ -4.0696880 & -0.3306320 & -3.7694620 \\ -3.1009170 & -0.7727540 & -3.5198410 \\ -4.7184600 & -1.1217160 & -4.1627010 \\ -3.9188200 & 0.4038230 & -4.5686150 \\ -6.0343870 & -4.8499760 & -1.7422920 \\ -6.6162270 & -5.7750690 & -1.8212170\end{array}$

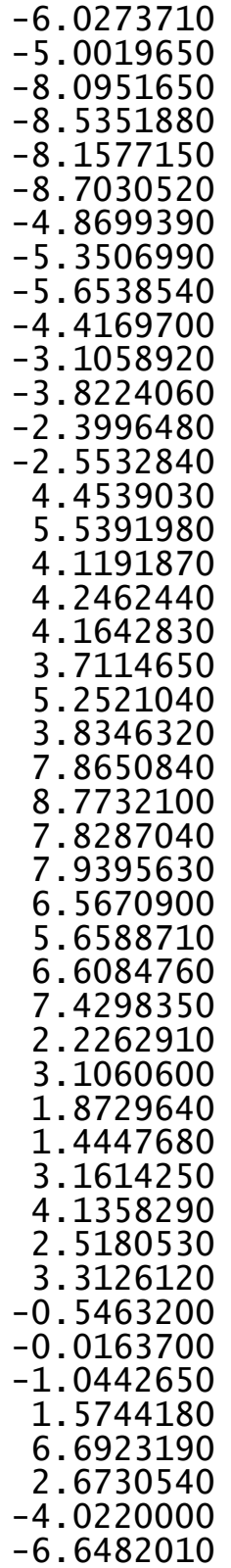

-4.3724100
-5.1124670
-3.5681250
-2.9178110
-3.0484210
-4.4770320
-0.5530480
0.3694320
-1.3084940
-0.3603320
-2.2914030
-3.0770680
-2.6938990
-2.0319050
2.8076890
2.6606180
3.2175180
3.5495100
1.0057760
0.0396240
0.9123270
1.7352860
-1.4084600
-2.0011500
-1.1100490
-0.4994710
-3.5212190
-4.0987380
-3.3278710
-4.1460090
-2.1422230
-2.6500720
-2.7045260
-2.1701370
0.0462880
-0.3917200
-0.0352490
1.1088370
-0.8884740
-1.8428210
-1.4459700
-0.2077750
-2.4902460
1.6759670
1.1587130
-4.4589890
$-2.7288800$ $-1.4894250$ $-1.0297740$ $-0.2666260$ $-1.9929290$ $-1.1002890$

3. 3233290

2.9832510

3.4556530

4.3024360

2.8821720

3.1478150

2.1525920

3.7926870

0.4638750

0.4113020

$-0.4935060$

1.2435460

2. 1867200

2.4331540

2.2817340

2.9329610

$-1.4413540$

$-1.2847630$

$-2.4957110$

$-0.8356740$

$-1.8807380$

$-1.6762780$

$-2.9587670$

$-1.6276790$

$-4.0520290$

$-4.4636040$

$-3.1853170$

$-4.8184830$

$-4.8699670$

$-5.1173530$

$-5.7533570$

$-4.6572350$

$-0.6762510$

$-1.6994070$

0.6367240

$-3.4486420$

$-0.0126400$

0.8300400

$-2.2476780$

0.2702690 


\begin{tabular}{|c|c|c|c|c|c|c|}
\hline & & & & & & \\
\hline-3.0480170 & -0.2618720 & 2.2406100 & C & -3.4436450 & 0.8780780 & 0.0782680 \\
\hline 2.2040190 & -2.8919440 & 0.4933530 & $\mathrm{C}$ & -1.3830190 & 2.1421540 & -0.3931060 \\
\hline 1.0855680 & -3.3808120 & 1.3161040 & C & -3.8679960 & 1.9002150 & 0.8889360 \\
\hline 2.1965870 & -1.8267520 & 0.2548150 & C & -1.7872170 & 3.1512220 & 0.5399510 \\
\hline 0.1261910 & -2.8924910 & 1.1847850 & $\mathrm{C}$ & -3.0542450 & 3.0258620 & 1.1713270 \\
\hline 1.5287780 & -3.6802260 & -0.5165340 & $\mathrm{H}$ & -4.8431930 & 1.8313360 & 1.3620090 \\
\hline 0.9216190 & -3.0603970 & -1.0235600 & C & -2.6555670 & 5.0698610 & 2.4161650 \\
\hline 3.5685280 & -4.8752560 & 1.2992320 & c & -1.3818640 & 5.1806090 & 1.8118760 \\
\hline 2.5931790 & -5.0480890 & 2.4644400 & C & -0.9587770 & 4.2501550 & 0.8962190 \\
\hline 1.1693070 & -4.6751920 & 2.0446040 & $\mathrm{C}$ & -3.4711150 & 4.0148980 & 2.1004160 \\
\hline 3.5820500 & -3.4277510 & 0.8022650 & $\mathrm{H}$ & -2.9781360 & 5.8154260 & 3.1360080 \\
\hline 4.5800670 & -5.1581490 & 1.6060190 & $\mathrm{H}$ & -0.7311290 & 6.0084980 & 2.0772960 \\
\hline 2.6011990 & -6.0789680 & 2.8299780 & $\mathrm{H}$ & 0.0247250 & 4.3420590 & 0.4481780 \\
\hline 0.7585180 & -5.4302420 & 1.3602630 & $\mathrm{H}$ & -4.4450440 & 3.9084770 & 2.5704540 \\
\hline 4.0210390 & -2.7667580 & 1.5598740 & C & -0.5502860 & 5.3741080 & -3.1739040 \\
\hline 4.1872800 & -3.3242630 & -0.0998700 & C & 0.7320100 & 5.4972200 & -3.7584120 \\
\hline 3.2835850 & -5.5442560 & 0.4781440 & c & 1.6936190 & 4.5564060 & -3.4979640 \\
\hline 2.9041960 & -4.4090810 & 3.3007390 & C & -0.8377770 & 4.3315750 & -2.3305260 \\
\hline 0.4772520 & -4.6358670 & 2.8904790 & $\mathrm{H}$ & -1.3158590 & 6.1096110 & -3.4012060 \\
\hline 0.6176450 & -0.1774440 & 3.2470720 & $\mathrm{H}$ & 0.9459480 & 6.3300260 & -4.4211180 \\
\hline-0.4605350 & 0.0403210 & 2.5673110 & $\mathrm{H}$ & 2.6777580 & 4.6298920 & -3.9530490 \\
\hline 1.1426320 & 1.0504870 & 3.8843760 & $\mathrm{H}$ & -1.8292740 & 4.2439090 & -1.9002540 \\
\hline 1.9582040 & 1.0102590 & 5.0194740 & C & 3.2477590 & 0.3867420 & -1.3743690 \\
\hline 0.8495700 & 2.2813250 & 3.2816030 & C & 3.5813670 & -0.5572940 & -2.3606320 \\
\hline 2.4752150 & 2.1876060 & 5.5440740 & $\mathrm{C}$ & 4.0735220 & 0.5549250 & -0.2455500 \\
\hline 2.1810350 & 0.0566710 & 5.4863470 & $\mathrm{C}$ & 4.7938670 & -1.2429430 & -2.2546960 \\
\hline 1.3891510 & 3.4533550 & 3.7957280 & C & 5.2746570 & -0.1515020 & -0.1871640 \\
\hline 0.2178670 & 2.3100280 & 2.4003590 & $\mathrm{C}$ & 5.6734270 & -1.0258660 & -1.1968470 \\
\hline 2.2009060 & 3.4085480 & 4.9276550 & $\mathrm{H}$ & 5.0629110 & -1.9511210 & -3.0331550 \\
\hline 3.0991310 & 2.1540990 & 6.4314620 & $\mathrm{H}$ & 5.9399460 & -0.0018400 & 0.6595490 \\
\hline 1.1704490 & 4.4006400 & 3.3128700 & C & -4.2790040 & -0.3415860 & -0.1359900 \\
\hline 2.6189910 & 4.3249330 & 5.3329870 & $\mathrm{C}$ & -4.4843290 & -1.2516410 & 0.9193420 \\
\hline-0.7063960 & -0.6793730 & 1.8795080 & $\mathrm{C}$ & -4.8887880 & -0.5585090 & -1.3866610 \\
\hline 1.3870660 & -1.6667830 & 3.3987840 & C & -5.3110050 & -2.3547960 & 0.6980460 \\
\hline D) & & & C & -5.6981850 & & -1.5577130 \\
\hline R) G[WB97X & $G(d)]=-36$ & $\begin{array}{l}369 \\
-0\end{array} 2734670$ & $\mathrm{C}$ & $\begin{array}{l}-5.9253530 \\
-5.4809530\end{array}$ & -2.5897490 & -0.5275940 \\
\hline-1.7297380 & 0.0211730 & -1.3861530 & $\mathrm{H}$ & -6.1738090 & -1.8409330 & -2.5226310 \\
\hline 0.8388630 & 1.2488420 & -1.0400060 & C & 3.7133280 & 1.5413850 & 0.8590100 \\
\hline-0.1114320 & 2.2431930 & -1.1545130 & c & 7.0153500 & -1.7347340 & -1.0974800 \\
\hline 2.1322620 & 1. 3584710 & -1.6130530 & $\mathrm{C}$ & 2.6716660 & -0.8119950 & -3.5538550 \\
\hline 0.1441690 & 3.3498150 & -2.0258130 & $\mathrm{C}$ & -3.8583440 & -1.0655440 & 2.2957890 \\
\hline 2.3959210 & 2.4662470 & -2.3816500 & $\mathrm{C}$ & -6.8156790 & -3.8021940 & -0.7315700 \\
\hline 1.4202770 & 3.4592150 & -2.6389850 & C & -4.7333100 & 0.4167530 & -2.5455750 \\
\hline 3.3865 & 2.5831510 & -2.8135260 & C & -6.0692170 & 1.1049820 & 90000 \\
\hline-2.179 & 32563 & -0.5489800 & $\mathrm{H}$ & -6.8191510 & 0.3799430 & -3 \\
\hline
\end{tabular}




-6.4654140
-5.9405720
-4.1466270
-3.1899690
-4.8243300
-3.9802000
-6.2379310
-6.8579760
-6.2005250
-5.2203490
-8.2532600
-8.6701350
-8.2928540
-8.8959980
-4.8991830
-5.3642730
-5.6971230
-4.4312340
-3.1799350
-3.9103470
-2.4864990
-2.6165900
4.3428660
5.4368840
4.0285890
4.0472240
4.0929270
3.6665140
5.1788110
3.7088980
7.7347520
8.7416490
7.2058560
7.8240820
6.8709260
6.4192130
6.2377360
7.8502180
2.5070050
3.4237250
2.2399030
1.7120850
3.1688760
4.1758040
2.5029280
3.2072200

1. 6124060

1.8498170

$-0.2599640$

$-0.7366000$

$-1.0239900$

0.4809470

$-4.7520680$

$-5.6509000$

$-4.2688600$

$-5.0601470$

$-3.3964090$

$-2.7273480$

$-2.8772850$

$-4.2810060$

$-0.5683550$

0.3689740

$-1.3092880$

$-0.3997380$

$-2.3392170$

$-3.1161570$

$-2.7394980$

$-2.1105790$

2.9170170

2.8434700

3.3226120

3.6265540

1.0441000

0.0557480

0.9849880

1.7393860

$-1.8481640$

$-2.2543560$

$-2.5220010$

$-0.8720090$

$-3.1167210$

$-3.0377010$

$-3.7714570$

$-3.5963580$

$-2.3070170$

$-2.7441320$

$-2.8620630$

$-2.4536250$

$-0.0674180$

$-0.4037260$

$-0.2564570$

1.0133510

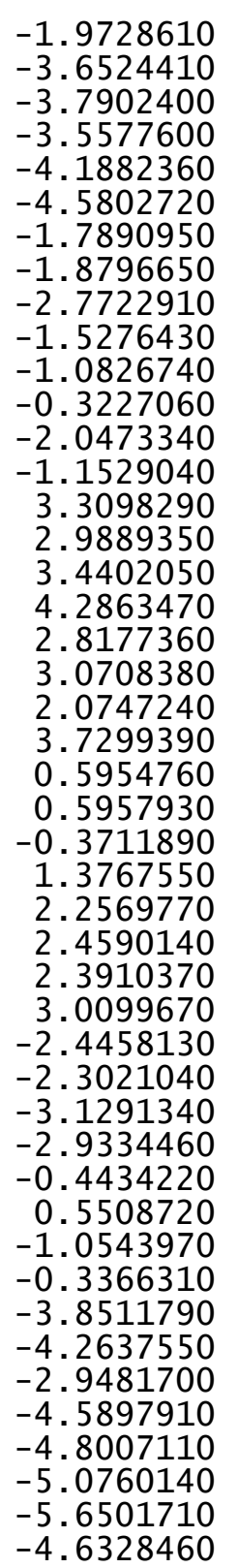

$-4.6328460$
$-0.0881200$

$-1.0787430$

1.6784190

7.6467570

2. 6248810

$-4.0346940$

$-6.8482160$

$-3.0823450$

2.0915450

1.0690570

1.9591510

0.0634800

1.4573330

0.8119140

3.6951050

2. 8058940

1. 3268220

3. 5310540

4.7449430

2.9383550

0.9470380

3.9305000

4.0728500

3.4362090

3. 0985080

0.6919610

0.5528810

$-0.5078890$

1.0501280

1.8399710

0.7571870

2. 3310870

2.0628280

1.2708160

0.1470500

2.0566590

2.9344440

1.0522000

2.4550650

$-0.7405520$

1. 3281170
$-0.5964800$
$-0.9415030$
$-1.8671060$
$-1.5353410$
$-0.4275110$
$-1.1254990$
1.6696900
1. 2025950
$-4.3456310$
$-0.3007630$
$-2.8346960$
$-3.4568340$
$-1.7734960$
$-3.0584970$
$-3.6726380$
$-3.0883170$
$-4.6746020$
$-4.9920320$
$-4.7528310$
$-3.2163170$
$-4.8643110$
$-6.0300640$
$-2.5414300$
$-3.0034400$
$-5.3365240$
$-4.8018870$
$-0.2943790$
$-0.0700970$
0.9242660
0.8669780
2.1655220
2.0376580
$-0.0944210$
3.3313230
2.2080500
3.2695510
1.9903530
4.2864470
4.1808580
$-0.7827720$
$-1.7811010$

144

TS224(S, S) G[WB97XD/6-31G(d)] = -3633.715328

$\begin{array}{llll}\text { TS224(S, S }) & G[W B 97 X D / 6-31 G(d)]= & -3633.715328 \\ 0 & 0.7366750 & 0.6221320 & -0.0065010 \\ 0 & -1.4397570 & 1.2066620 & -1.1764330\end{array}$
$-0.7347310$

$-1.7957220$

0.5672010

$-3.3024740$

0.4377070

0.8550930

$-2.2461890$

0.2215020

2.2124230

0.3904330

1.2436540

0.1812240

1.1697570

$-0.6072180$

$-1.1124500$

1.0454160

2. 2499590

1.9276090

0.6146620

1.2894530

2. 5680400

1.2500910

1. 3816590

$-0.3092230$

0.2101890

3.0960990

2.8170360

3. 2280290

2. 5229680

3.9045510

5.0570240

3.3236410

5.6201820

5.5075930

3.8768530

2.4280350

5.0260580

6.5210370

3.4096820

5.4615030

1.8244450

3. 3697920 


$\begin{array}{lr}\text { C } & 0.9871680 \\ \text { C } & -0.0202710 \\ \text { C } & 2.3110580 \\ \text { C } & 0.2374400 \\ \text { C } & 2.5749420 \\ \text { C } & 1.5575200 \\ \text { H } & 3.5905600 \\ \text { C } & -2.0190180 \\ \text { C } & -3.3036720 \\ \text { C } & -1.3367170 \\ \text { C } & -3.8828070 \\ \text { C } & -1.9015220 \\ \text { C } & -3.2015870 \\ \text { H } & -4.8824930 \\ \text { C } & -3.0994000 \\ \text { C } & -1.7905340 \\ \text { C } & -1.2071290 \\ \text { C } & -3.7856170 \\ \text { H } & -3.5507720 \\ \text { H } & -1.2402020 \\ \text { H } & -0.1985040 \\ \text { H } & -4.7839210 \\ \text { C } & -0.4865770 \\ \text { C } & 0.8353420 \\ \text { C } & 1.8322200 \\ \text { C } & -0.7783690 \\ \text { H } & -1.2780190 \\ \text { H } & 1.0522700 \\ \text { H } & 2.8488590 \\ \text { H } & -1.7983530 \\ \text { C } & 3.4054440 \\ \text { C } & 3.8255280 \\ \text { C } & 4.0522160 \\ \text { C } & 4.9165310 \\ \text { C } & 5.1458440 \\ \text { C } & 5.6034770 \\ \text { H } & 5.2548790 \\ \text { H } & 5.6425970 \\ \text { C } & -4.0092260 \\ \text { C } & -4.2053220 \\ \text { C } & -4.5246760 \\ \text { C } & -4.9447940 \\ \text { C } & -5.2428000 \\ \text { C } & -5.4736300 \\ \text { H } & -5.1159130 \\ \text { H } & -5.6459380 \\ & \end{array}$

\begin{tabular}{rr}
1.9696120 & 0.1439960 \\
2.7952060 & 0.5959080 \\
2.4208220 & -0.0936380 \\
4.2016720 & 0.6851970 \\
3.7527320 & 0.1001260 \\
4.6746460 & 0.4519820 \\
4.1177180 & -0.0262860 \\
1.4348410 & 0.0627040 \\
0.8842230 & 0.3098500 \\
2.2135360 & 0.9700950 \\
1.1617540 & 1.5219190 \\
2.3951020 & 2.2754420 \\
1.8815680 & 2.5342370 \\
0.7908160 & 1.7283300 \\
2.7106000 & 4.8117690 \\
3.1878450 & 4.5693070 \\
3.0358920 & 3.3369640 \\
2.0681760 & 3.8148240 \\
2.8436520 & 5.7900450 \\
3.6739960 & 5.3690870 \\
3.3977430 & 3.1717100 \\
1.6767820 & 3.9920290 \\
6.4894660 & 1.0625860 \\
6.9531200 & 0.8679760 \\
6.0629650 & 0.5651920 \\
5.1521720 & 0.9752210 \\
7.2006510 & 1.2784520 \\
8.0137450 & 0.9481160 \\
6.4075280 & 0.3952250 \\
4.8122370 & 1.1174450 \\
1.4700260 & -0.4601490 \\
1.3474170 & -1.7996360 \\
0.7458580 & 0.5548490 \\
0.5282460 & -2.0806420 \\
-0.0577870 & 0.2226300 \\
-0.1697430 & -1.0857980 \\
0.4280650 & -3.1092390 \\
-0.6124470 & 1.0136360 \\
0.0375770 & -0.6982580 \\
-1.3350720 & -0.4476200 \\
0.6237470 & -1.8700890 \\
-2.0817500 & -1.3668760 \\
-0.1693650 & -2.7623440 \\
-1.5228910 & -2.5257760 \\
-3.1385100 & -1.1751020 \\
0.2879140 & -3.6633360 \\
& \\
\hline
\end{tabular}

\begin{abstract}
3.5975220
6.8233680

3.1478410

$-3.6558860$

$-6.2753950$

$-4.3633460$

$-5.7206390$

$-6.3939050$

$-6.2106550$

$-5.5902250$

$-3.6404500$

$-2.6659340$

$-4.2234110$

$-3.4812580$

$-5.5526310$

$-6.1215810$

$-5.4364330$

$-4.5550200$

$-7.6946220$

$-8.2127960$

$-7.6742430$

$-8.2817870$

$-4.7632290$

$-5.2713060$

$-5.5192200$

$-4.3427270$

$-2.9316820$

$-3.6196780$

$-2.1438630$

$-2.4755930$

4.7305490

5.5368100

5.1611600

4. 3499970

2. 9880680

2. 1271480

3.7214770

2. 6423560

7.2287180

8.0364830

7. 5985090

6.3960810

8.0093040

7.7563770

8.2954210

8.8780870
\end{abstract}

\author{
0.8052260 \\ $-0.9937080$ \\ 2.1244870 \\ $-2.0306960$ \\ $-2.3659740$ \\ 2.1066460 \\ 2.8227640 \\ 2.4393200 \\ 2.6846210 \\ 3.8975880 \\ 2. 3394970 \\ 1.8427840 \\ 1.9579040 \\ 3.4114130 \\ $-2.5026660$ \\ $-3.1482970$ \\ $-1.5255070$ \\ $-2.9343130$ \\ $-1.8146830$ \\ $-1.7204070$ \\ $-0.8252270$ \\ $-2.4781320$ \\ $-2.2895360$ \\ $-1.3661030$ \\ $-2.9730810$ \\ $-2.7484440$ \\ $-3.3418950$ \\ $-4.1024270$ \\ $-3.1763220$ \\ $-3.7518160$ \\ 1.2371290 \\ 0.4956840 \\ 2.1923990 \\ 1. 3549070 \\ $-0.5336630$ \\ $-0.7865170$ \\ $-1.3461000$ \\ $-0.4807850$ \\ $-2.0468140$ \\ $-2.6684320$ \\ $-1.5822020$ \\ $-2.7069410$ \\ $-0.0634540$ \\ 0.6638320 \\ 0.4912220 \\ $-0.6393190$
}

2.0070530 $-1.4715430$ $-2.9211540$ 0.7927350 $-3.5010080$ $-2.1745730$ $-2.1395150$ $-2.9149910$ $-1.1696280$ $-2.3081460$ $-3.5066990$ $-3.5082160$ $-4.3528410$

$-3.6688660$ $-4.8478510$ $-5.5261590$ $-5.3309360$ $-4.7165870$ $-3.6919080$ $-2.7320000$ $-4.1630370$ $-4.3364680$

1.8247790

2.1185860

1. 4200380

2.7273300

0.4624230

0.0764510

$-0.2768130$

1. 3712160

2.9466100

2.9762000

2.6285070

3.9670780

2.4444330

1.8184560

2. 3750770

3.4829450

$-0.4381020$

$-0.8374770$

0.4832150

$-0.1728600$

$-1.7737210$

$-2.5516280$

-0.8723070
-2.1113550 


$\begin{array}{rrr}3.0400310 & 1.3370290 & -4.2317610 \\ 4.0166130 & 1.2066320 & -4.7119720 \\ 2.5995870 & 0.3503730 & -4.0741130 \\ 2.4025850 & 1.8837590 & -4.9343960 \\ 3.8763610 & 3.4532370 & -3.1761130 \\ 4.9170470 & 3.2686890 & -3.4685380 \\ 3.3880460 & 4.0070120 & -3.9859260 \\ 3.8836580 & 4.0901890 & -2.2871830 \\ -0.2538820 & 0.1001730 & -1.2081000 \\ 0.4105590 & 0.1732380 & -2.5489130 \\ -0.7235700 & -1.2423630 & -0.7079010 \\ 2.1265970 & 2.3498950 & -2.5992150 \\ 6.5724890 & -1.5203630 & -2.4028780 \\ 2.8106230 & 1.5602180 & 2.0898880 \\ -3.7478690 & 2.5573190 & -1.3914580 \\ -6.3649060 & -3.3700820 & -3.0668790 \\ -2.9179610 & -1.3664440 & 1.2501980 \\ 1.3687860 & -2.9638200 & -2.0832800 \\ 2.1946380 & -2.4843030 & -0.9693340 \\ 0.3047010 & -2.7621870 & -1.9873970 \\ 1.8671940 & -1.6000390 & -0.4361180 \\ 2.0721190 & -1.8769820 & -2.7410940 \\ 1.4802560 & -1.0602440 & -2.7124790 \\ 3.2103730 & -4.5953170 & -2.6790730 \\ 3.7973780 & -4.4101700 & -1.2780760 \\ 3.5832940 & -2.9817440 & -0.7726360 \\ 1.7064830 & -4.3074290 & -2.6825130 \\ 3.3849240 & -5.6169250 & -3.0292730 \\ 4.8697070 & -4.6285440 & -1.2764840 \\ 4.2232120 & -2.2780850 & -1.3187990 \\ 1.1776880 & -5.0628710 & -2.0881000 \\ 1.2942360 & -4.3467720 & -3.6955630 \\ 3.7157140 & -3.9226840 & -3.3823140 \\ 3.3256280 & -5.1187380 & -0.5855090 \\ 3.8493120 & -2.8602430 & 0.2810290 \\ 0.1589510 & -2.8620970 & 2.1007480 \\ -0.4129430 & -1.7666830 & 1.7181830 \\ -0.1174340 & -3.1811280 & 3.5199330 \\ 0.6802220 & -4.0746250 & 4.2433540 \\ -1.2127620 & -2.5679410 & 4.1461020 \\ 0.3881280 & -4.3489580 & 5.5733060 \\ 1.5328360 & -4.5412110 & 3.7615580 \\ -1.5074770 & -2.8551890 & 5.4724030 \\ -1.8308190 & -1.8755490 & 6.5852770 \\ -0.7064060 & -3.7429380 & 6.1330630 \\ 1.0167090 & -5.0339180 & \end{array}$

$\begin{array}{lr}\mathrm{H} & -2.3620150 \\ \mathrm{H} & -0.9328690 \\ \mathrm{H} & -0.4379400 \\ \mathrm{~S} & 1.0980350\end{array}$
$-2.3848830$
$-3.9610490$
$-1.5988610$

$\operatorname{TS} 225(\mathrm{~S}, \mathrm{~S}) \mathrm{G}[\mathrm{WB} 97 \mathrm{XD} / 6-31 \mathrm{G}(\mathrm{d})]=-3633.717967$

\begin{tabular}{|c|c|c|c|}
\hline & & & \\
\hline 0 & 0.7620780 & 0.6891660 & 0.2429450 \\
\hline 0 & -1.2280330 & 1.3134740 & -1.1955910 \\
\hline C & 1.0593760 & 2.0314290 & 0.3558740 \\
\hline C & 0.0511990 & 2.9127560 & 0.6789920 \\
\hline C & 2.4146260 & 2.4193470 & 0.1983470 \\
\hline C & 0.3619300 & 4.3097270 & 0.7453580 \\
\hline C & 2.7202120 & 3.7450780 & 0.3711920 \\
\hline C & 1.7180620 & 4.7177030 & 0.6149000 \\
\hline $\mathrm{H}$ & 3.7547770 & 4.0678700 & 0.2946490 \\
\hline C & -1.9294210 & 1.6111570 & -0.0385010 \\
\hline C & -3.2603630 & 1. 1286160 & 0.0 \\
\hline C & -1.3215350 & 2.3930400 & 0.9 \\
\hline C & -3.9585790 & 1.4612140 & 1.2 \\
\hline C & -2.02 & 2.6387070 & 2.1 \\
\hline C & -3.3622490 & 2.1820760 & L1180 \\
\hline$H$ & -4.992 & 1.1446780 & 1.3 \\
\hline C & -3.4729630 & 3.0814090 & 4.5 \\
\hline C & -2.1272980 & 3.5022750 & 4 \\
\hline C & -1.4209720 & 3.2881700 & 61060 \\
\hline C & -4.0731510 & 2.4328200 & 3.4744850 \\
\hline $\mathrm{H}$ & -4.0208200 & 3.2616190 & 5.4418060 \\
\hline $\mathrm{H}$ & -1.6477980 & 3.9928480 & 5.2537040 \\
\hline $\mathrm{H}$ & -0.3862640 & 3.6055980 & 3.1887540 \\
\hline $\mathrm{H}$ & -5.1000870 & 2.0859080 & 3.5540000 \\
\hline C & -0.2896240 & 6.6384280 & 0.9767650 \\
\hline C & 1.0639070 & 7.0385600 & 0.8864750 \\
\hline C & 2.0429150 & 6.0970320 & 0.7049630 \\
\hline C & -0.6320300 & 5.3119120 & 0.90926 \\
\hline $\mathrm{H}$ & -1.0647170 & 7.3895080 & 1.09476 \\
\hline $\mathrm{H}$ & 1.3202680 & 8.0916110 & 0.94864 \\
\hline $\mathrm{H}$ & 3.0847500 & 6.3923450 & 0.61254 \\
\hline $\mathrm{H}$ & -1.6748510 & 5.0206340 & 0.9709910 \\
\hline C & 3.4684970 & 1.4097150 & -0.1198330 \\
\hline C & 4.0187320 & 1. 3492680 & -1.4146070 \\
\hline C & 3.9347400 & 0.5481150 & 0.8896870 \\
\hline C & 5.0500910 & 0.4428010 & -1.6630670 \\
\hline C & 4.9651830 & -0.3413800 & 0.5893880 \\
\hline C & 5.5409880 & -0.4087600 & -0.6776000 \\
\hline $\mathrm{H}$ & 5.4 & 0.4064320 & 6571400 \\
\hline H & 工 & P & \\
\hline
\end{tabular}

5.9482240

7.2274720

0.7025200

1.1126040
5. 3356020
.6111570

6387070

0814090

2616190

(2028480 - n

.0970320

.5481150

$-1.0076990$
1. 3666620
3. 2686890

423630

2.7621870

$-4.6285440$

1.7666830

$-4.3489580$

$-5.0339180$ 


$\begin{array}{rrr}-3.8790340 & 0.3154930 & -1.0174220 \\ -4.1323620 & -1.0590240 & -0.8230960 \\ -4.2285530 & 0.9346710 & -2.2300760 \\ -4.7506220 & -1.7737190 & -1.8476100 \\ -4.8360250 & 0.1705050 & -3.2278160 \\ -5.1082430 & -1.1818900 & -3.0576760 \\ -4.9569630 & -2.8304940 & -1.6998220 \\ -5.1109230 & 0.6463950 & -4.1669850 \\ 3.3767740 & 0.5807120 & 2.3062450 \\ 6.6926900 & -1.3654630 & -0.9390480 \\ 3.5627850 & 2.2792320 & -2.5321670 \\ -3.7840010 & -1.7806260 & 0.4739810 \\ -5.7631970 & -1.9901670 & -4.1635200 \\ -4.0012930 & 2.4185110 & -2.4847380 \\ -5.3379730 & 3.1573200 & -2.6405960 \\ -5.8928750 & 2.7963820 & -3.5141510 \\ -5.9692490 & 3.0147470 & -1.7569670 \\ -5.1679060 & 4.2318190 & -2.7717980 \\ -3.0876130 & 2.6547490 & -3.6939900 \\ -2.1293410 & 2.1447480 & -3.5600140 \\ -3.5465660 & 2.2886960 & -4.6196440 \\ -2.8943390 & 3.7260030 & -3.8197440 \\ -7.0869490 & -2.6160740 & -3.7057950 \\ -7.5719130 & -3.1377010 & -4.5384700 \\ -6.9240410 & -3.3462980 & -2.9047010 \\ -7.7762310 & -1.8521390 & -3.3313470 \\ -4.8071450 & -3.0597220 & -4.7089570 \\ -3.8742190 & -2.6089390 & -5.0625980 \\ -4.5551210 & -3.7920790 & -3.9332220 \\ -5.2680030 & -3.5986530 & -5.5443920 \\ -5.0307600 & -1.9561910 & 1.3543460 \\ -5.5095740 & -0.9988170 & 1.5802850 \\ -5.7712830 & -2.5889990 & 0.8504770 \\ -4.7653280 & -2.4371620 & 2.3032150 \\ -3.1177910 & -3.1420350 & 0.2419080 \\ -3.7993160 & -3.8575450 & -0.2312660 \\ -2.2275380 & -3.0408090 & -0.3840370 \\ -2.8198650 & -3.5746060 & 1.2039450 \\ 4.4534160 & 1.0080140 & 3.3130110 \\ 5.2670840 & 0.2753190 & 3.3621930 \\ 4.8861050 & 1.9752220 & 3.0364030 \\ 4.0220400 & 1.0992150 & 4.3159630 \\ 2.7439130 & -0.7591420 & 2.7019360 \\ 1.9253590 & -1.0151140 & 2.0232940 \\ 3.4785450 & -1.5727310 & 2.6879630 \\ 2.3287080 & -0.7021740 & \\ -4 & & \\ -4 & -741950\end{array}$

8.0366810
8.8708950
8.1506390
8.1057360
6.6356430
5.6587800
6.8270020
7.3996940
3.3348250
4.2618850
2.6035380
2.9479660
4.5707740
5.5445130
4.2217760
4.7218330
-0.0809740
0.7458770
-0.6527920
2.6037160
6.6267830
2.5854350
-3.5017480
-5.9899730
-3.0659340
1.4728970
2.1902970
0.4297730
1.8808610
2.3588120
1.8317060
3.2082940
3.6292360
3.4854870
1.7527780
3.3262230
4.6678870
4.2589180
1.0789570
1.4765720
3.8606660
3.0100310
3.6131330
-0.2089400
-0.6313090
-0.6667270
-0.6495850
-1.3479160
0.1593610
-0.2126760
-2.0271380
-2.4839230
-1.3030360
-2.8077640
1.5589390
1.1400690
0.7559280
2.2704760
3.4245380
3.0325380
4.1269810
3.9809000
0.1700770
0.2109920
-1.1508750
2.7154130
-2.1615230
1.3341320
2.8492070
-1.2965250
-1.1633270
-2.9284330
-2.6123870
-2.6217080
-1.7355620
-1.8789320
-1.0188520
-4.7021930
-4.6713780
-3.2686920
-4.2558490
-5.7120620
-4.9968110
-2.5982410
-4.9898550
-4.1789440
-4.0454320
-5.3743250
-3.2600820
-3.0333370
-1.8505300
-3.4742570

$-0.8690550$

$-1.4696850$

0.2630600

$-2.3207330$

$-2.5132110$

$-3.1202480$

$-2.3960240$

$-3.8664170$

$-4.2736470$

$-3.7554630$

$-4.6045070$

$-2.7139490$

$-3.0314580$

$-3.4790700$

$-1.7837840$

$-1.0629820$

$-2.3122270$

$-0.6159960$

$-2.2380200$

$-0.1848130$

2. 3476800

$-1.6126600$

$-4.9832890$

1. 0218960

$-1.9378850$

$-0.6991470$

$-1.9574230$

$-0.1440050$

$-2.4102560$

$-2.3967220$

$-2.4359710$

$-0.9646660$

$-0.3694230$

$-2.5989950$

$-2.8399320$

$-0.8531130$

$-0.7584120$

$-2.1400060$

$-3.6551980$

$-3.0238870$

$-0.3929670$

0.7166290

2.1025960

1.7892390

3. 4400270 


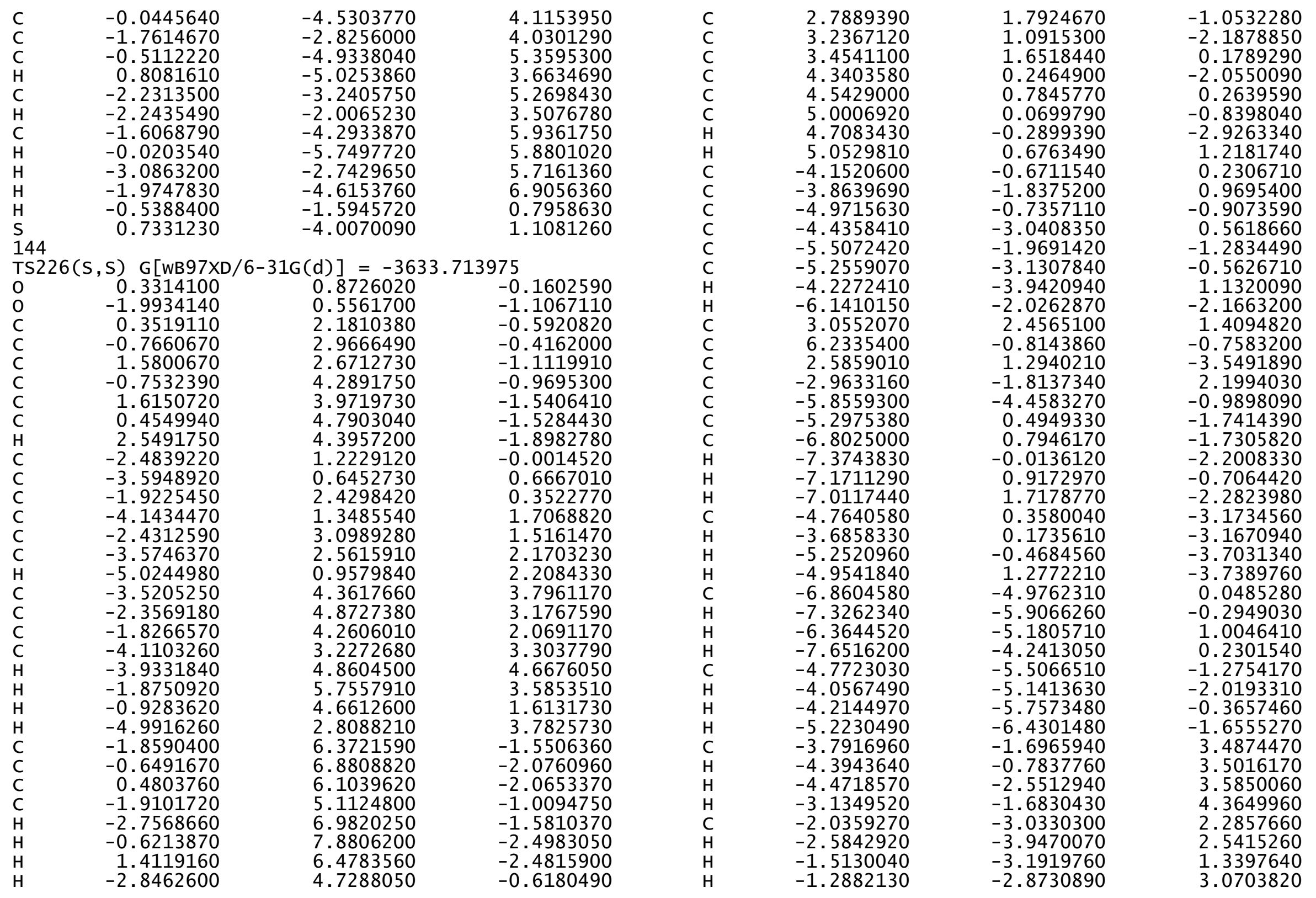




$\begin{array}{rrr}4.1650920 & 3.4479540 & 1.7854270 \\ 5.0848200 & 2.9251590 & 2.0725850 \\ 4.4021400 & 4.1097650 & 0.9452910 \\ 3.8524270 & 4.0682540 & 2.6329590 \\ 2.6789640 & 1.5605610 & 2.5931310 \\ 1.8630800 & 0.8849230 & 2.3226390 \\ 3.5263860 & 0.9511920 & 2.9282290 \\ 2.3530620 & 2.1701890 & 3.4436000 \\ 6.2363570 & -1.7352970 & 0.4671400 \\ 7.0917000 & -2.4182470 & 0.4270350 \\ 6.3152210 & -1.1632310 & 1.3987480 \\ 5.3206020 & -2.3344570 & 0.5218190 \\ 7.5054620 & 0.0459780 & -0.7931640 \\ 7.5327930 & 0.6701910 & -1.6923730 \\ 7.5437310 & 0.7086240 & 0.0795050 \\ 8.4018380 & -0.5843050 & -0.7825470 \\ 2.5736180 & 0.0356620 & -4.4214850 \\ 3.5776030 & -0.2276970 & -4.7745630 \\ 2.1597600 & -0.8196480 & -3.8849460 \\ 1.9550660 & 0.2084660 & -5.3084490 \\ 3.2810630 & 2.4368210 & -4.3060610 \\ 4.3354470 & 2.1923200 & -4.4827580 \\ 2.8018530 & 2.5990750 & -5.2782070 \\ 3.2440350 & 3.3771710 & -3.7494280 \\ -0.5676450 & -0.2129660 & -0.9884560 \\ -0.0911490 & -0.4075900 & -2.3979790 \\ -0.6267330 & -1.4082070 & -0.0723060 \\ 1.5429780 & 1.5773240 & -3.3771000 \\ 6.2394470 & -1.4498440 & -1.6543240 \\ 2.1699220 & 3.0487680 & 1.1621950 \\ -4.7982020 & 1.3577300 & -1.2911920 \\ -6.4028230 & -4.2834430 & -1.9251240 \\ -2.3217450 & -0.9301400 & 2.1246950 \\ 0.8662740 & -3.4627510 & -1.7944280 \\ 2.0823830 & -2.6778440 & -1.5623810 \\ 0.0131840 & -3.1848730 & -1.1791980 \\ 1.9711590 & -1.6415190 & -1.2625220 \\ 0.9088640 & -2.7125270 & -3.0350970 \\ 0.4236870 & -1.8381240 & -2.8786160 \\ 2.2994620 & -5.2850670 & -2.8078350 \\ 3.5279360 & -4.6692630 & -2.1338560 \\ 3.4147710 & -3.1455530 & -2.0410850 \\ 1.0239340 & -4.9454260 & -2.0313250 \\ 2.4102890 & -6.3717570 & -2.8687360 \\ 4.4395520 & -4.9250350 & -2.6816420 \\ 3.5689160 & -2.6791620 & -3.0228990\end{array}$
1.0417330
0.1319940
2. 2139410
3. 6361010
4.1794780
1.9203670
0.8512860
2.6057340
3. 9638470
1.8926130
4.6020820
4. 5183310
2. 5309330
0.8416150
3. 8872070
5.6594180
1. 9723430
4.3870480
0.3238950
2. 4981240
$-5.4275540$
$-5.3072550$
$-4.9119440$
$-5.0862580$
$-2.7133080$
$-2.0892510$
$-1.3783740$
$-1.8295280$
$-2.1203240$
$-1.2292940$
$-1.8054980$
$-2.5741700$
$-0.9313480$
$-0.9991830$
$-1.2123690$
$-2.0194130$
$-0.4732130$
$-0.9692230$
$-1.4947760$
$-3.2186310$

$-1.0463240$

$-2.5522640$

$-3.8352180$

$-1.1243200$

$-1.3907590$

2.1233300

1.9738990

3. 4096740

3.5778470

4.4564780

4.7708330

2.7642620

5.6528780

4.3239030

5.8097710

4.8892420

6.4627050

6.7423700

1.1018160

1.0186740 144

$\operatorname{TS} 227(\mathrm{~S}, \mathrm{~S}) \mathrm{G}[\mathrm{WB} 97 \mathrm{XD} / 6-31 \mathrm{G}(\mathrm{d})]=-3633.716709$

$\begin{array}{lrcr}\text { TS227(S, S) G[WB97XD/6-31G(d)] }=-3633.716709 \\ \text { O } & 0.7417700 & 0.5919590 & 0.0135610 \\ \text { O } & -1.4216610 & 1.2677560 & -1.1368570 \\ \mathrm{C} & 1.0222250 & 1.9277410 & 0.2101120 \\ \mathrm{C} & 0.0317730 & 2.7569060 & 0.6928290 \\ \mathrm{C} & 2.3534520 & 2.3615000 & -0.0162400 \\ \mathrm{C} & 0.3155140 & 4.1539730 & 0.8326630 \\ \mathrm{C} & 2.6410800 & 3.6817980 & 0.2228400 \\ \mathrm{C} & 1.6433240 & 4.6097540 & 0.6112180 \\ \mathrm{H} & 3.6624610 & 4.0324250 & 0.1022590 \\ \mathrm{C} & -1.9956170 & 1.4602630 & 0.1106490 \\ \mathrm{C} & -3.2925530 & 0.9300610 & 0.3380950 \\ \mathrm{C} & -1.2961060 & 2.1884520 & 1.0463940 \\ \mathrm{C} & -3.8640650 & 1.1729480 & 1.5613990 \\ \mathrm{C} & -1.8562510 & 2.3337560 & 2.3579630 \\ \mathrm{C} & -3.1666820 & 1.8386630 & 2.5994600 \\ \mathrm{H} & -4.8714510 & 0.8164090 & 1.7553090 \\ \mathrm{C} & -3.0458110 & 2.5847840 & 4.9046850 \\ \mathrm{C} & -1.7276360 & 3.0438440 & 4.6776000 \\ \mathrm{C} & -1.1481130 & 2.9229200 & 3.4401070 \\ \mathrm{C} & -3.7457680 & 1.9918180 & 3.8867950 \\ \mathrm{H} & -3.4942020 & 2.6935190 & 5.8873310 \\ \mathrm{H} & -1.1677140 & 3.4915300 & 5.4929720 \\ \mathrm{H} & -0.1333240 & 3.2718790 & 3.2845210 \\ \mathrm{H} & -4.7521340 & 1.6159550 & 4.0515830\end{array}$




$\begin{array}{rrr}-0.3628840 & 6.4395450 & 1.2971360 \\ 0.9668990 & 6.8848330 & 1.1127180 \\ 1.9452820 & 5.9876280 & 0.7730690 \\ -0.6808480 & 5.1121960 & 1.1622930 \\ -1.1394980 & 7.1570900 & 1.5434350 \\ 1.2041670 & 7.9375870 & 1.2301890 \\ 2.9677120 & 6.3183700 & 0.6107010 \\ -1.7063780 & 4.7868430 & 1.2984510 \\ 3.4457420 & 1.4231680 & -0.4224900 \\ 3.9208960 & 1.4207480 & -1.7458330 \\ 4.0748570 & 0.6343700 & 0.5597150 \\ 5.0716010 & 0.6857410 & -2.0411660 \\ 5.2142570 & -0.0908470 & 0.2129500 \\ 5.7500170 & -0.0518990 & -1.0748660 \\ 5.4697390 & 0.7074080 & -3.0528280 \\ 5.7117830 & -0.6854190 & 0.9765780 \\ -4.0168220 & 0.1391250 & -0.7016500 \\ -4.2484490 & -1.2361140 & -0.5004880 \\ -4.5105000 & 0.7785020 & -1.8549620 \\ -4.9989810 & -1.9315880 & -1.4504280 \\ -5.2417000 & 0.0351140 & -2.7790270 \\ -5.5056200 & -1.3201230 & -2.5923460 \\ -5.1963540 & -2.9900500 & -1.2972350 \\ -5.6268950 & 0.5330420 & -3.6662180 \\ -0.2538220 & 0.1445200 & -1.2153350 \\ 0.4217880 & 0.2728160 & -2.5469810 \\ -0.7457840 & -1.2127610 & -0.7811070 \\ 3.5717190 & 0.5887760 & 1.9968100 \\ 2.7005580 & 1.2449090 & 2.0755950 \\ 7.0490030 & -0.7653780 & -1.4138030 \\ 7.3051400 & -0.4974490 & -2.4466260 \\ 3.2470770 & 2.2398270 & -2.8389300 \\ 2.2306360 & 2.4670190 & -2.5035700 \\ -4.3112900 & 2.2666340 & -2.1072930 \\ -3.6786600 & 2.6727160 & -1.3135660 \\ -6.3141100 & -2.1113350 & -3.6046360 \\ -6.4306790 & -3.1270070 & -3.2052230 \\ -3.7248430 & -1.9893040 & 0.7170120 \\ -2.9744060 & -1.3603310 & 1.2033170 \\ -3.0293120 & -3.3042160 & 0.3437500 \\ -2.5962990 & -3.7603620 & 1.2416070 \\ -3.7302650 & -4.0320720 & -0.0798540 \\ -2.2275910 & -3.1302620 & -0.3785580 \\ -4.8446480 & -2.2595080 & 1.7325100 \\ -5.6119670 & -2.9126560 & 1.2999700 \\ -4.4394150 & -2.7573660 & 2.6212940\end{array}$

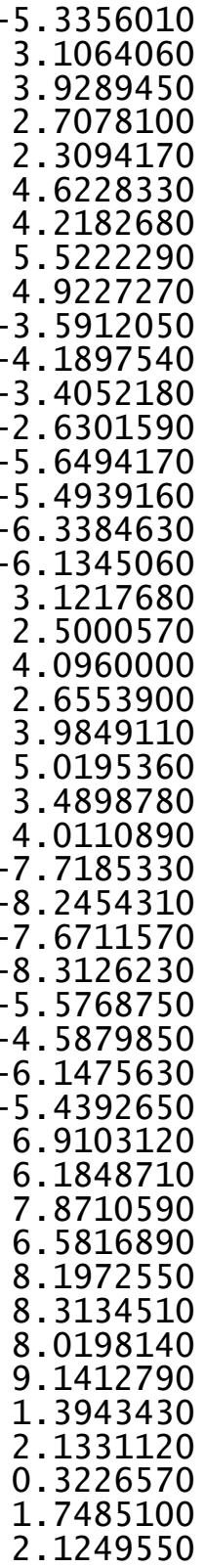

-1.3363570
-0.8185570
-1.5423190
-0.8210920
-1.1616650
1.1249260
1.1439230
0.4985120
2.1434500
2.5275690
2.1919850
3.6000360
2.0058480
3.0154510
4.0913200
2.6719560
2.8604320
1.4807250
2.0559460
1.3320740
0.5033140
3.5679610
3.3851420
4.1515560
4.1756830
-1.5231830
-1.4446770
-0.5216340
-2.1553120
-2.2196120
-2.6711350
-2.8355760
-1.2305740
-2.2922950
-2.6489240
-2.7729050
-2.6256630
-0.2888190
0.7984740
-0.5581580
-0.7512610
-2.8799620
-2.4669530
-2.6983280
-1.6285230
-1.7408910

2. 3892530

2. 3496940

3.4097150

1.7225270

2.9772170

3.9951380

2.9849430

2.7086820

$-3.4356620$

$-4.2904100$

$-3.5615170$

$-3.4627140$

$-2.0376000$

$-2.1755310$

$-2.8179030$

$-1.0677760$

$-4.1652650$

$-4.8594010$

$-4.6447110$

$-4.0247860$

$-3.0646550$

$-3.3786570$

$-3.8489250$

$-2.1555530$

$-3.7947100$

$-2.8381380$

$-4.2375670$

$-4.4639370$

$-4.9462170$

$-4.8159980$

$-5.6501900$

$-5.3986590$

$-1.3587540$

$-2.0978680$

$-1.5727490$

$-0.3667710$

$-0.5138980$

$-0.5672920$

0.5335320

$-0.8219960$

$-2.2057230$

$-1.0081070$

2.1707330

$-0.4425650$

$-2.7334080$ 


\begin{tabular}{|c|c|c|c|c|c|c|c|}
\hline & & & & & & & \\
\hline $\mathrm{H}$ & 1. 5176440 & -0.9352280 & -2.7008560 & C & -3.4422180 & 2.6159190 & 4.7607750 \\
\hline $\begin{array}{l}\mathrm{C} \\
\mathrm{C}\end{array}$ & $\begin{array}{l}3.3073750 \\
3.7868810\end{array}$ & $\begin{array}{l}-4.4310660 \\
-4.3403950\end{array}$ & $\begin{array}{l}-2.7832890 \\
-1.3330250\end{array}$ & $\begin{array}{l}\mathrm{C} \\
\mathrm{C}\end{array}$ & $\begin{array}{l}-2.1165580 \\
-1.4270830\end{array}$ & $\begin{array}{l}3.0961020 \\
2.9823400\end{array}$ & $\begin{array}{l}4.6518470 \\
3.4717400\end{array}$ \\
\hline C & 3.5102300 & -2.9571800 & -0.7410600 & $\mathrm{C}$ & -4.0391110 & 2.0105550 & 3.6859090 \\
\hline C & 1.8010210 & -4.1698530 & -2.8758950 & $\mathrm{H}$ & -3.9769840 & 2.7174670 & 5.7000400 \\
\hline $\mathrm{H}$ & 3.5285630 & -5.4208630 & -3.1932580 & $\mathrm{H}$ & -1.6387340 & 3.5530710 & 5.5130150 \\
\hline $\mathrm{H}$ & 4.8591750 & -4.5469960 & -1.2646370 & $\mathrm{H}$ & -0.4073940 & 3.3450710 & 3.4064580 \\
\hline $\mathrm{H}$ & 4.1775510 & -2.2013790 & -1.1724290 & $\mathrm{H}$ & -5.0498070 & 1.6183140 & 3.7624480 \\
\hline $\mathrm{H}$ & 1.2484420 & -4.9780700 & -2.3809160 & C & -0.4582290 & 6.5058450 & 1.3996230 \\
\hline $\mathrm{H}$ & 1.4647650 & -4.1376730 & -3.9169300 & $\mathrm{C}$ & 0.8791800 & 6.9586910 & 1.3184460 \\
\hline $\mathrm{H}$ & 3.8469230 & -3.7003400 & -3.3980000 & $\mathrm{C}$ & 1.8866740 & 6.0659610 & 1.0619770 \\
\hline $\mathrm{H}$ & 3.2763520 & -5.1022280 & -0.7302790 & $\mathrm{C}$ & -0.7562310 & 5.1756060 & 1.2478470 \\
\hline $\mathrm{H}$ & 3.6904280 & -2.9190760 & 0.3363840 & $\mathrm{H}$ & -1.2563070 & 7.2199820 & 1.5782450 \\
\hline C & 0.0269890 & -2.9938830 & 1.9531500 & $\mathrm{H}$ & 1.1002420 & 8.0136040 & 1.4476260 \\
\hline 0 & -0.4657660 & -1.8404640 & 1.6322900 & $\mathrm{H}$ & 2.9162460 & 6.4033270 & 0.9760210 \\
\hline C & -0.2926080 & -3.3825900 & 3.3456160 & $\mathrm{H}$ & -1.7873030 & 4.8441130 & 1.3048470 \\
\hline C & 0.4078940 & -4.3982900 & 4.0060170 & C & 3.4552140 & 1.4961300 & -0.0981360 \\
\hline C & -1.3353420 & -2.7181160 & 4.0086450 & $\mathrm{C}$ & 3.9470480 & 1.5138600 & -1.4176860 \\
\hline C & 0.0716860 & -4.7433290 & 5.3086960 & C & 3.9940660 & 0.6087850 & 0.8499550 \\
\hline $\mathrm{H}$ & 1.2204050 & -4.9063900 & 3.4977360 & $\mathrm{C}$ & 4.9976370 & 0.6598730 & -1.7519450 \\
\hline C & -1.6738320 & -3.0739270 & 5.3077250 & C & 5.0376430 & -0.2318930 & 0.4630850 \\
\hline $\mathrm{H}$ & -1.8783180 & -1.9301400 & 3.4985100 & $\mathrm{C}$ & 5.5578860 & -0.2196310 & -0.8289800 \\
\hline C & -0.9705750 & -4.0851610 & 5.9597610 & $\mathrm{H}$ & 5.3891880 & 0.6835620 & -2.7644900 \\
\hline $\mathrm{H}$ & 0.6235930 & -5.5269130 & 5.8177720 & $\mathrm{H}$ & 5.4616830 & -0.9227130 & 1.1900520 \\
\hline $\mathrm{H}$ & -2.4876630 & -2.5621710 & 5.8114290 & $\mathrm{C}$ & -3.8695500 & 0.1904200 & -0.9310980 \\
\hline $\mathrm{H}$ & -1.2323970 & -4.3588250 & 6.9772990 & C & -4.1074500 & -1.1914990 & -0.7987840 \\
\hline $\mathrm{H}$ & -0.4820550 & -1.6269290 & 0.6267000 & $\mathrm{C}$ & -4.2467420 & 0.8635440 & -2.1097080 \\
\hline & 0.9136930 & -3.9707750 & 0.9118870 & C & -4.7464990 & -1.8616230 & -1.8445020 \\
\hline & & & & C & -4.8669560 & 0.1446010 & -3.1289320 \\
\hline & 5) G[WB97 & $G(d)]$ & 946 & C & -5.1328720 & -1.2182280 & -3.0150520 \\
\hline 0 & 0.7876990 & 0.6573130 & 0.2650070 & $\mathrm{H}$ & -4.9489060 & -2.9254570 & -1.7454180 \\
\hline 0 & -1.2501640 & 1.2946490 & -1.0976570 & $\mathrm{H}$ & -5.1611620 & 0.6675550 & -4.0363160 \\
\hline C & 1.0377670 & 1.9996270 & 0.4573880 & C & 3.4903940 & 0.5541750 & 2.2862870 \\
\hline $\mathrm{C}$ & 0.0051100 & 2.8217380 & 0.8513010 & $\mathrm{C}$ & 6.7253280 & -1.1225670 & -1.1909090 \\
\hline C & 2.3754990 & 2.4455810 & 0.3065990 & C & 3.3967980 & 2.4690190 & -2.4695240 \\
\hline $\mathrm{C}$ & 0.2685020 & 4.2216490 & 1.0045160 & C & -3.7155220 & -1.9797570 & 0.4457890 \\
\hline C & 2.6386520 & 3.7666710 & 0.5630010 & C & -5.8236060 & -1.9784350 & -4.1328710 \\
\hline C & 1.6076130 & 4.6852280 & 0.8841970 & $\mathrm{C}$ & -4.0406460 & 2.3605510 & -2.2980670 \\
\hline $\mathrm{H}$ & 3.6604590 & 4.1296340 & 0.4960520 & C & -5.3882120 & 3.0908950 & -2.3851350 \\
\hline C & -1.9419300 & 1.4964030 & 0.0866210 & $\mathrm{H}$ & -5.9626160 & 2.7647420 & -3.2599570 \\
\hline C & -3.2518220 & 0.9580430 & 0.1919880 & $\mathrm{H}$ & -5.9944500 & 2.9002330 & -1.4930280 \\
\hline C & -1.34 & 2.2385860 & 1.0805680 & $\mathrm{H}$ & -5.2322330 & 4.1721550 & -2.4702990 \\
\hline C & -3.9 & 1.1900970 & 1.3581490 & C & -3.1601810 & 2.6650960 & -3.5166060 \\
\hline C & 0440 & 2.3804840 & 2.3325010 & $\mathrm{H}$ & -2.1942470 & 2.1593060 & -3.4307340 \\
\hline C & 140 & 1.8645360 & 2.4557700 & $\mathrm{H}$ & -3.6383490 & 2.3397980 & -4.4477840 \\
\hline $\mathrm{H}$ & -4 & & 4585350 & $\mathrm{H}$ & -2.9801570 & 3.7431690 & -3.5946950 \\
\hline
\end{tabular}




$\begin{array}{rrr}-4.9756780 & -1.9886200 & -5.4117280 \\ -5.4643700 & -2.5817560 & -6.1928490 \\ -4.8366080 & -0.9730330 & -5.8002150 \\ -3.9853540 & -2.4160080 & -5.2240670 \\ -7.2276270 & -1.4233200 & -4.4074880 \\ -7.8378900 & -1.4285100 & -3.4983060 \\ -7.1785390 & -0.3918820 & -4.7755410 \\ -7.7374290 & -2.0251660 & -5.1680360 \\ -4.9421870 & -2.2759200 & 1.3214490 \\ -5.4627590 & -1.3595510 & 1.6163710 \\ -5.6580380 & -2.9084860 & 0.7830810 \\ -4.6420040 & -2.8058700 & 2.2331740 \\ -2.9846110 & -3.2859210 & 0.1111770 \\ -3.6300570 & -3.9910180 & -0.4238780 \\ -2.0992100 & -3.0911320 & -0.4994260 \\ -2.6680960 & -3.7799300 & 1.0370260 \\ 4.6075660 & 0.8905070 & 3.2832210 \\ 5.4034080 & 0.1374730 & 3.2625300 \\ 5.0565860 & 1.8632490 & 3.0568250 \\ 4.2077120 & 0.9262060 & 4.3025700 \\ 2.8478840 & -0.8006130 & 2.6109870 \\ 1.9907200 & -0.9900750 & 1.9586920 \\ 3.5659560 & -1.6213730 & 2.4972410 \\ 2.4865350 & -0.8159090 & 3.6452860 \\ 8.0518090 & -0.3591850 & -1.0638940 \\ 8.9010080 & -1.0192720 & -1.2733040 \\ 8.0833290 & 0.4729830 & -1.7770140 \\ 8.1746220 & 0.0532980 & -0.0571550 \\ 6.5948520 & -1.7525890 & -2.5826420 \\ 5.6196910 & -2.2319250 & -2.7207080 \\ 6.7101360 & -1.0037670 & -3.3740570 \\ 7.3733360 & -2.5084780 & -2.7298920 \\ 3.2107180 & 1.8147480 & -3.8435800 \\ 4.1687070 & 1.5604660 & -4.3109910 \\ 2.6038520 & 0.9102180 & -3.7687900 \\ 2.6979120 & 2.5124520 & -4.5146750 \\ 4.2979010 & 3.7070560 & -2.5940940 \\ 5.3039580 & 3.4181460 & -2.9215290 \\ 3.8894970 & 4.4077640 & -3.3310960 \\ 4.3954130 & 4.2349320 & -1.6409520 \\ -0.0716310 & 0.1775250 & -1.0455070 \\ 0.7268210 & 0.2887740 & -2.3082730 \\ -0.6006240 & -1.1760410 & -0.6437770 \\ 2.4083340 & 2.8002780 & -2.1371190 \\ 6.7421450 & -1.9381990 & -0.4552280 \\ 2.7148320 & 1.3164210 & 2.4059070\end{array}$
$-3.5243090$
$-5.9365680$
$-3.0222830$
1. 5427630
2.2720720
0.4908150
1.9479780
2. 3880860
1. 8392940
3. 3200810
3.7668710
3.5905470
1. 8495450
3.4589870
4.8170750
4.3397540
1. 2067190
1. 5505940
3.9422070
3.1809370
3.7328800
$-0.1146710$
$-0.5554560$
$-0.5994550$
0.0166360
$-1.7193710$
$-0.4812870$
0.8880100
$-2.2198380$
$-2.1968480$
$-1.6015340$
0.0042950
$-3.0934610$
$-1.9935420$
$-0.4645850$
0.8725730

2.7560330
-3.0181500
-1.3671250
-2.8576800
-2.5535000
-2.5823860
-1.7005440
-1.7700560
-0.9253520
-4.5657680
-4.5610860
-3.1810220
-4.1586140
-5.5612720
-4.8560890
-2.4766700
-4.9238460
-4.0632950
-3.8758610
-5.2995520
-3.2027340
-3.1167060
-1.9323370
-3.6174420
-4.6930560
-3.0069210
-5.1533660
-5.1594870
-3.4780850
-2.1728920
-4.5503920
-5.9847050
-3.0088310
-4.9175050
-1.6453250
-4.0319740

$-1.4194320$

$-3.7998680$

1.0292520

$-2.0232940$

$-0.7880890$

$-2.0156270$

$-0.2046520$

$-2.4844600$

$-2.4411700$

$-2.5995010$

$-1.1354840$

$-0.4974540$

$-2.7239420$

$-3.0315080$

$-1.0511440$

$-0.8745740$

$-2.2715420$

$-3.7724040$

$-3.1820320$

$-0.5737550$

0.5865400

2.0166760

1.7375540

3. 3237000

3.9731480

3.9075110

5.1851600

3.5263550

5.1146380

3.4050300

5.7550830

5.6859830

5.5555090

6.6986990

0.7516480

1. 0101650

TS229(S,S) G[WB97XD/6-31G(d)] = -3633.716670

$\begin{array}{lrrr} & \\ O & 0.5720940 & 0.1009520 & -0.3865610 \\ \mathrm{O} & -1.7430740 & -0.0241590 & -1.4538450 \\ \mathrm{C} & 0.8502390 & 1.1936640 & -1.1773140 \\ \mathrm{C} & -0.0967260 & 2.1867520 & -1.3090110 \\ \mathrm{C} & 2.1486030 & 1.2954180 & -1.7392550 \\ \mathrm{C} & 0.1623490 & 3.2751520 & -2.2011830 \\ \mathrm{C} & 2.4176940 & 2.3858810 & -2.5291650 \\ \mathrm{C} & 1.4395070 & 3.3699370 & -2.8153690\end{array}$




$\begin{array}{lr}\mathrm{H} & 3.4142810 \\ \mathrm{C} & -2.1608330 \\ \mathrm{C} & -3.4041000 \\ \mathrm{C} & -1.3510070 \\ \mathrm{C} & -3.7877230 \\ \mathrm{C} & -1.7103110 \\ \mathrm{C} & -2.9524310 \\ \mathrm{H} & -4.7449690 \\ \mathrm{C} & -2.4837550 \\ \mathrm{C} & -1.2350920 \\ \mathrm{C} & -0.8571630 \\ \mathrm{C} & -3.3212230 \\ \mathrm{H} & -2.7700690 \\ \mathrm{H} & -0.5663280 \\ \mathrm{H} & 0.1094030 \\ \mathrm{H} & -4.2763730 \\ \mathrm{C} & -0.5261140 \\ \mathrm{C} & 0.7552690 \\ \mathrm{C} & 1.7145100 \\ \mathrm{C} & -0.8159430 \\ \mathrm{H} & -1.2884580 \\ \mathrm{H} & 0.9712860 \\ \mathrm{H} & 2.6985120 \\ \mathrm{H} & -1.8059240 \\ \mathrm{C} & 3.2575140 \\ \mathrm{C} & 3.6059180 \\ \mathrm{C} & 4.0391810 \\ \mathrm{C} & 4.7804500 \\ \mathrm{C} & 5.2133870 \\ \mathrm{C} & 5.6131690 \\ \mathrm{H} & 5.0659910 \\ \mathrm{H} & 5.8445420 \\ \mathrm{C} & -4.2569740 \\ \mathrm{C} & -4.4492170 \\ \mathrm{C} & -4.8936840 \\ \mathrm{C} & -5.2847440 \\ \mathrm{C} & -5.7113010 \\ \mathrm{C} & -5.9218680 \\ \mathrm{H} & -5.4441290 \\ \mathrm{H} & -6.2072770 \\ \mathrm{C} & 3.6667220 \\ \mathrm{C} & 6.9278400 \\ \mathrm{C} & 2.7584830 \\ \mathrm{C} & -3.8054970 \\ \mathrm{C} & -6.8214270 \\ \mathrm{C} & -4.7644250 \\ & \end{array}$

$\begin{array}{rr}2.5027260 & -2.9469640 \\ 1.0088300 & -0.6254060 \\ 0.8779140 & 0.0472800 \\ 2.1141080 & -0.5176350 \\ 1.9148980 & 0.8594130 \\ 3.1408180 & 0.4143450 \\ 3.0351610 & 1.0970550 \\ 1.8616550 & 1.3698870 \\ 5.0880430 & 2.3027060 \\ 5.1803400 & 1.6453620 \\ 4.2343220 & 0.7257960 \\ 4.0375820 & 2.0322700 \\ 5.8442340 & 3.0267780 \\ 6.0047110 & 1.8746000 \\ 4.3103770 & 0.2402140 \\ 3.9458000 & 2.5421670 \\ 5.2839650 & -3.3779540 \\ 5.3914010 & -3.9672660 \\ 4.4517430 & -3.6927880 \\ 4.2560790 & -2.5174330 \\ 6.0198270 & -3.6146230 \\ 6.2122720 & -4.6440210 \\ 4.5158620 & -4.1495570 \\ 4.1788860 & -2.0811670 \\ 0.3464690 & -1.4055250 \\ -0.6951780 & -2.2841820 \\ 0.6086270 & -0.2645900 \\ -1.4081080 & -2.0427920 \\ -0.1224580 & -0.0753530 \\ -1.1182890 & -0.9622210 \\ -2.1975000 & -2.7329160 \\ 0.0942670 & 0.7831360 \\ -0.3360970 & -0.1200800 \\ -1.2203020 & 0.9593620 \\ -0.5756410 & -1.3536030 \\ -2.3242590 & 0.7768530 \\ -1.6954800 & -1.4848060 \\ -2.5835920 & -0.4318840 \\ -3.0093360 & 1.6065250 \\ -1.8737790 & -2.4364470 \\ 1.7114860 & 0.7199280 \\ -1.8537550 & -0.7669480 \\ -1.0113190 & -3.5089860 \\ -1.0062050 & 2.3235450 \\ -3.7956540 & -0.5931490 \\ 0.3775310 & -2.5341720\end{array}$

-6.1051950
-6.8677400
-6.4738840
-5.9949070
-4.2183300
-3.2580910
-4.9118070
-4.0704200
-6.2742330
-6.8996170
-6.2610040
-5.2512740
-8.2656420
-8.6611090
-8.3265520
-8.9136580
-4.8403950
-5.3239700
-5.6255560
-4.3624090
-3.0995100
-3.8121470
-2.3914770
-2.5468520
4.4423250
5.5205600
4.2546260
4.1455930
3.8648380
3.3405180
4.9231980
3.4649600
7.8936290
8.8648390
7.4999080
8.0521440
6.7313960
6.1093570
6.2511000
7.6973730
2.6825220
3.6240860
2.4485050
1.9015340
3.2699730
4.3107090

1. 0685320

0.3427050

1. 5910470

1. 8011520

$-0.3227650$

$-0.8019490$

$-1.0881370$

0.4051880

$-4.7644950$

$-5.6622330$

$-4.2972060$

$-5.0720290$

$-3.3888360$

$-2.7072370$

$-2.8829480$

$-4.2714160$

$-0.5087420$

0.4160950

$-1.2588350$

$-0.3171330$

$-2.2615660$

$-3.0554910$

$-2.6453010$

$-2.0172940$

2. 9996670

2.8358890

3. 3398440

3.7996950

1. 3026770

0.3688260

1. 1694080

2. 0779530

$-1.5642650$

$-2.0378500$

$-1.9563750$

$-0.4878710$

$-3.3649260$

$-3.5864000$

$-3.8100590$

$-3.8618340$

$-2.5115290$

$-2.8899790$

$-3.0958990$

$-2.6958640$

$-0.2516460$

$-0.5234120$
$-2.8203260$

$-3.1255130$

$-1.9310790$

$-3.6277870$

$-3.7842110$

$-3.5725060$

$-4.1513330$

$-4.5898210$

$-1.6498000$

$-1.7098580$

$-2.6412650$

$-1.4098050$

$-0.9147450$

$-0.1544420$

$-1.8853100$

$-0.9568240$

3. 3435130

3. 0130540

3. 4969860

4. 3108640

2. 8522590

3. 1034200

2. 1143320

3.7670440

0.4097730

0.5222730

$-0.6135600$

1.0973150

2. 1834300

2. 4121670

2.4335040

2.8459400

$-1.9247820$

$-1.7439480$

$-2.8697850$

$-2.0462850$

$-0.5893450$

0.2838150

$-1.4680190$

$-0.4483690$

$-3.8162590$

$-4.2312970$

$-2.9229620$

$-4.5608710$

$-4.7422920$

$-4.9558990$ 


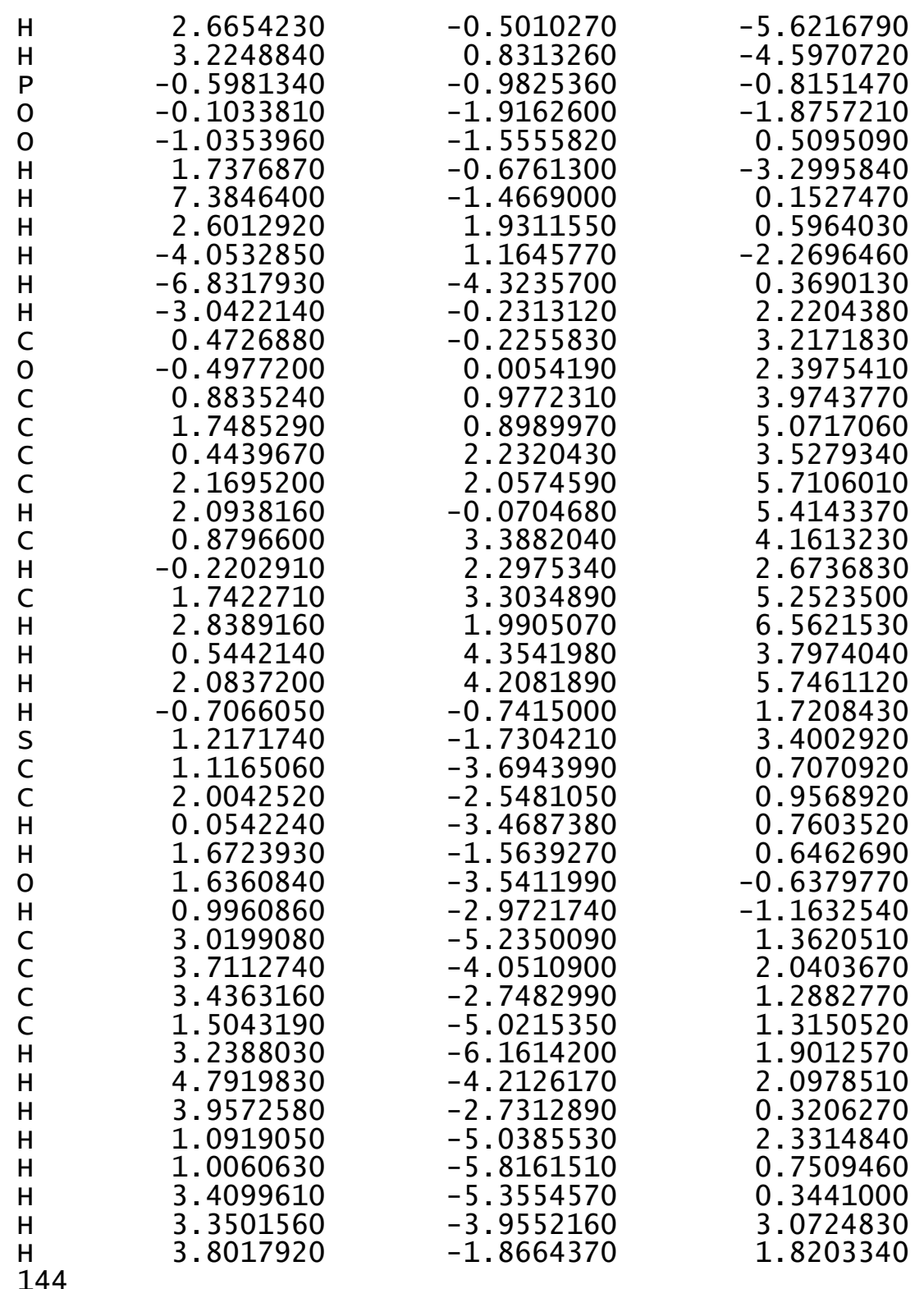

$\operatorname{TS} 230(S, S) \quad G[W B 97 X D / 6-31 G(d)]=-3633.717422$

$\begin{array}{lr}\mathrm{O} & 0.6964740 \\ \mathrm{O} & -1.5494610 \\ \mathrm{C} & 1.0037260 \\ \mathrm{C} & 0.0503730 \\ \mathrm{C} & 2.3317050 \\ \mathrm{C} & 0.3449650 \\ \mathrm{C} & 2.6284720 \\ \mathrm{C} & 1.6529500 \\ \mathrm{H} & 3.6471160 \\ \mathrm{C} & -2.0344470 \\ \mathrm{C} & -3.3103460 \\ \mathrm{C} & -1.2458290 \\ \mathrm{C} & -3.7468230 \\ \mathrm{C} & -1.6588540 \\ \mathrm{C} & -2.9325400 \\ \mathrm{H} & -4.7269940 \\ \mathrm{C} & -2.5200440 \\ \mathrm{C} & -1.2403000 \\ \mathrm{C} & -0.8211550 \\ \mathrm{C} & -3.3457210 \\ \mathrm{H} & -2.8406140 \\ \mathrm{H} & -0.5809120 \\ \mathrm{H} & 0.1675400 \\ \mathrm{H} & -4.3233320 \\ \mathrm{C} & -0.3012080 \\ \mathrm{C} & 1.0074350 \\ \mathrm{C} & 1.9609880 \\ \mathrm{C} & -0.6250870 \\ \mathrm{H} & -1.0571210 \\ \mathrm{H} & 1.2494650 \\ \mathrm{H} & 2.9666780 \\ \mathrm{H} & -1.6346800 \\ \mathrm{C} & 3.4179280 \\ \mathrm{C} & 3.7611040 \\ \mathrm{C} & 4.1507650 \\ \mathrm{C} & 4.8557780 \\ \mathrm{C} & 5.2320760 \\ \mathrm{C} & 5.6081390 \\ \mathrm{H} & 5.1341160 \\ \mathrm{H} & 5.8054150 \\ \mathrm{C} & -4.1348340 \\ \mathrm{C} & -4.3905210 \\ \mathrm{C} & -4.6811700 \\ \mathrm{C} & -5.1948290 \\ \mathrm{C} & -5.4744450 \\ \mathrm{C} & -5.7443090\end{array}$

0.2140010

0.2095230

1.4054240

2. 3985690

1. 5928960

3. 6027330

2. 7801420

3.7871870

2. 9623380

1.1218870

0.8916250

2. 2036940

1.7975530

3.0733750

2.8668310

1.6647140

4. 6914280

4.8787980

4.0940510

3. 7061160

5.3226410

5.6450030

4.2380090

3.5410390

5.7562840

5.9476320

4. 9819300

4.6166430

6.5172680

6.8569110

5.1122810

4.4774290

0.6051620

$-0.3852180$

0.7433640

$-1.2086730$

$-0.1098490$

$-1.0839360$

$-1.9621920$

$-0.0154320$

$-0.2973590$

$-1.3019440$

$-0.4005420$

$-2.3811800$

$-1.5058800$

$-2.5068340$
$-0.4917940$

$-1.7034770$

$-1.1096940$

$-1.1614260$

$-1.8752910$

$-2.1950610$

$-2.3993040$

$-2.5273510$

$-0.7740440$

$-0.1936210$

$-0.4632360$

0.7403680

0.5963700

1.1914140

1.1889940

2.7369250

2. 1646080

1.1202760

2. 2596840

3. 5601170

0.6988580

2.7044380

$-2.7909190$

$-3.1019310$

$-2.0998940$

$-2.9592870$

$-3.8343720$

$-3.4927440$

$-1.7283240$

$-1.2839750$

$-2.2215330$

$-0.0881570$

$-1.9563100$

0.1326470

$-0.7898890$

$-2.6868700$

1. 0513250

$-0.5630240$

0.3955790

0.0343210

$-2.1664460$

$-1.2399180$ 


\begin{tabular}{|c|c|c|}
\hline $\begin{array}{l}-5.3987000 \\
-5.9052770 \\
3.7750770 \\
6.8315030 \\
3.0033060 \\
-3.8447170 \\
-6.6146050 \\
-4.4824520 \\
-5.8050600 \\
-6.5503290 \\
-6.2211320 \\
-5.6505000 \\
-3.8584510 \\
-2.9138090 \\
-4.5268530 \\
-3.6586810 \\
-7.8927420 \\
-8.5424300 \\
-7.6578180 \\
-8.4529170 \\
-5.8397950 \\
-4.9381900 \\
-5.5323740 \\
-6.4639800 \\
-4.9587770 \\
-5.4539380 \\
-5.7251290 \\
-4.5486220 \\
-3.1372850 \\
-3.8415310 \\
-2.3735090 \\
-2.6498410 \\
4.9871340 \\
5.4954630 \\
5.7175520 \\
4.6606110 \\
2.8044640 \\
1.8981610 \\
3.2769440 \\
2.5039200 \\
8.0098830 \\
8.9156690 \\
7.7864050 \\
8.2190890 \\
6.5730520 \\
5.7437870\end{array}$ & $\begin{array}{r}-3.1512310 \\
-1.5858510 \\
1.7866900 \\
-1.9485160 \\
-0.5182000 \\
-1.2490830 \\
-3.6949970 \\
0.6753290 \\
1.3974730 \\
0.7064780 \\
1.8389240 \\
2.1994420 \\
0.1099710 \\
-0.3955930 \\
-0.6069850 \\
0.9177550 \\
-3.7376170 \\
-4.5588600 \\
-3.8930660 \\
-2.8008730 \\
-5.0153630 \\
-4.9925960 \\
-5.2112640 \\
-5.8539480 \\
-0.9135370 \\
0.0325240 \\
-1.6976770 \\
-0.8330620 \\
-2.5474030 \\
-3.3814600 \\
-2.8188180 \\
-2.4117090 \\
2.4175790 \\
1.7096110 \\
2.7862220 \\
3.2616010 \\
1.2022550 \\
0.8161730 \\
0.3835390 \\
1.9681480 \\
-1.4703360 \\
-2.0430540 \\
-1.5995320 \\
-0.4100530 \\
-3.4436600 \\
-3.8065610\end{array}$ & $\begin{array}{r}0.7748570 \\
-3.1624200 \\
0.9590100 \\
-0.5355330 \\
-3.5353590 \\
1.8173600 \\
-1.6078750 \\
-2.9136030 \\
-3.2069260 \\
-3.6177390 \\
-2.2949160 \\
-3.9376130 \\
-4.1951200 \\
-3.9748490 \\
-4.6855000 \\
-4.9080520 \\
-0.7597470 \\
-1.0823410 \\
0.2996570 \\
-0.8469850 \\
-1.5046010 \\
-2.1253890 \\
-0.4708110 \\
-1.8330290 \\
2.8198200 \\
2.5776900 \\
2.8256300 \\
3.8329600 \\
2.2282010 \\
2.3276850 \\
1.4962840 \\
3.2007750 \\
1.6533100 \\
2.3174140 \\
0.9256610 \\
2.2701150 \\
1.9962690 \\
1.5210360 \\
2.5535220 \\
2.7199090 \\
-1.3968080 \\
-1.1683920 \\
-2.4623690 \\
-1.2214570 \\
-0.7572590 \\
-0.1411860\end{array}$ \\
\hline
\end{tabular}

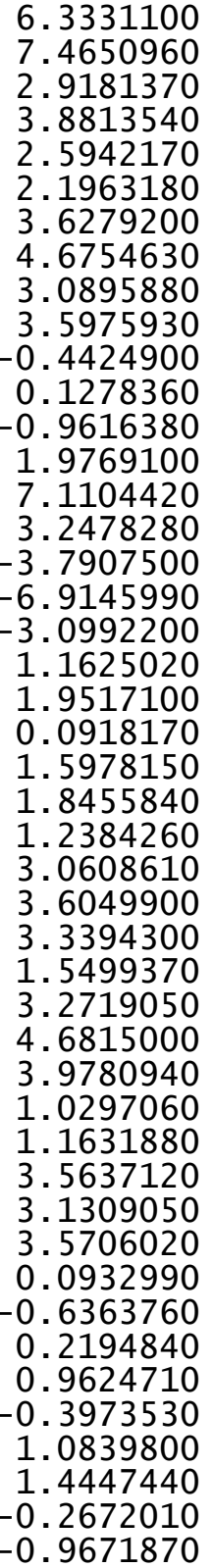
$-3.6596580$
$-4.0234200$
$-1.9590900$
$-2.3104710$
$-2.6520340$
$-2.0119150$
0.3819540
0.1055110
0.2723930
1.4371450
$-0.8143970$
$-1.6450700$
$-1.5129970$
$-0.1807660$
$-1.8118150$
2. 5967540
1.4264740
$-3.5663730$
$-0.4531310$
$-3.6609260$
$-2.5340110$
$-3.4818160$
$-1.5294940$
$-3.2752020$
$-2.6830790$
$-5.2088560$
$-4.1224350$
$-2.7259480$
$-5.0504820$
$-6.1995120$
$-4.2463430$
$-2.5210640$
$-5.2391800$
$-5.7670210$
$-4.2132590$
$-1.9322400$
$-0.5710080$
$-0.1693090$
0.4496400
0.2059560
1.6984270
1.1906470
$-0.7563440$
2.6813320
1.8983650

$-1.8041010$ $-0.4967840$ $-4.0504740$ $-4.4389750$ $-3.2702050$ $-4.8716800$ $-4.6127010$ $-4.7823570$ $-5.5608560$ $-4.3267830$ $-1.0975580$ $-2.2045790$ 0.1336260

$-3.3601330$

0.5171350

0.4453310

$-2.5230670$

$-2.6557300$

1. 8681420

0.3223170

0.8384300

0.2639470

0.6430360

$-0.9006220$

$-1.4385790$

0.9600780

1. 8903310

1. 3260440

0.7678500

1. 3731920

2.0433560

0.4569120

1. 7151190

0.0365690

$-0.0129560$

2. 8758660

2. 0411560

3. 2198380

2. 2313270

4.2858960

5.4466330

4.1106290

6.4181630

5. 5796210

5.0824400

3. 2101410 


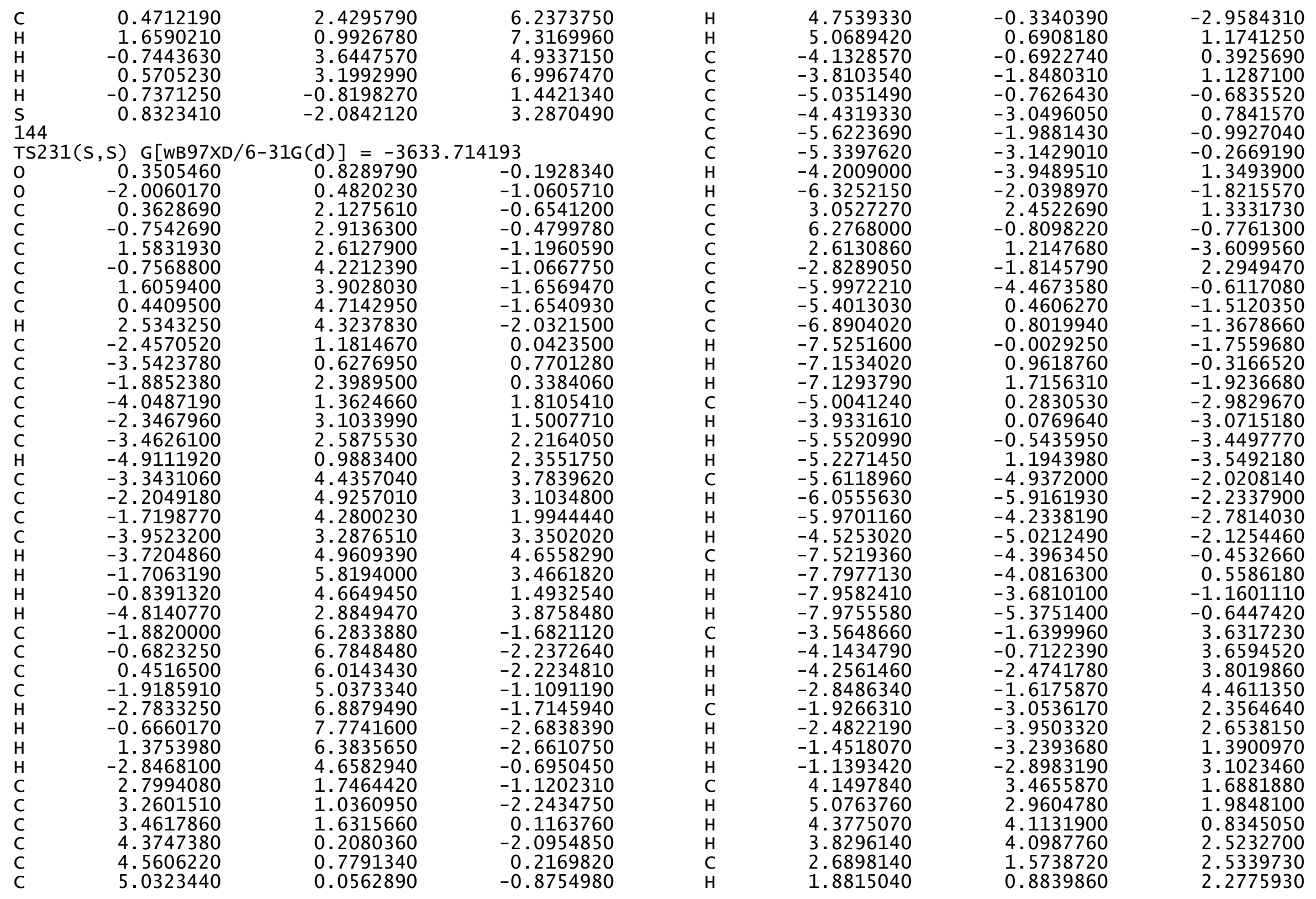




$\begin{array}{rrrr}\mathrm{H} & 3.5452200 & 0.9813670 & 2.8788870 \\ \mathrm{H} & 2.3581640 & 2.1956890 & 3.3733130 \\ \mathrm{C} & 6.2855250 & -1.7152190 & 0.4610130 \\ \mathrm{H} & 7.1517930 & -2.3849690 & 0.4348000 \\ \mathrm{H} & 6.3495670 & -1.1304120 & 1.3858390 \\ \mathrm{H} & 5.3791260 & -2.3282590 & 0.5178120 \\ \mathrm{C} & 7.5366460 & 0.0680490 & -0.8141550 \\ \mathrm{H} & 7.5610450 & 0.6807200 & -1.7213530 \\ \mathrm{H} & 7.5597490 & 0.7426550 & 0.0498250 \\ \mathrm{H} & 8.4418990 & -0.5489700 & -0.7894850 \\ \mathrm{C} & 2.5967690 & -0.0594520 & -4.4586620 \\ \mathrm{H} & 3.6021460 & -0.3438590 & -4.7908680 \\ \mathrm{H} & 2.1624070 & -0.8985750 & -3.9127860 \\ \mathrm{H} & 1.9947660 & 0.1049940 & -5.3585210 \\ \mathrm{C} & 3.3151000 & 2.3408180 & -4.3854710 \\ \mathrm{H} & 4.3676630 & 2.0865020 & -4.5590500 \\ \mathrm{H} & 2.8361750 & 2.4914410 & -5.3595870 \\ \mathrm{H} & 3.2853920 & 3.2898250 & -3.8432030 \\ \mathrm{P} & -0.5677930 & -0.2709640 & -0.9807760 \\ \mathrm{O} & -0.1307010 & -0.4766990 & -2.4016560 \\ \mathrm{O} & -0.5898220 & -1.4571480 & -0.0514040 \\ \mathrm{H} & 1.5710240 & 1.5063700 & -3.4462280 \\ \mathrm{H} & 6.2968420 & -1.4564240 & -1.6638530 \\ \mathrm{H} & 2.1592040 & 3.0281120 & 1.0771360 \\ \mathrm{H} & -4.8402770 & 1.3175770 & -1.1286260 \\ \mathrm{H} & -5.6235090 & -5.2129960 & 0.1018100 \\ \mathrm{H} & -2.1733620 & -0.9497700 & 2.1512120 \\ \mathrm{C} & 0.9151130 & -3.5059560 & -1.7632800 \\ \mathrm{C} & 2.1195330 & -2.7004930 & -1.5414780 \\ \mathrm{H} & 0.0607340 & -3.2381420 & -1.1455560 \\ \mathrm{H} & 1.9941110 & -1.6652130 & -1.2437870 \\ \mathrm{O} & 0.9410550 & -2.7602740 & -3.0071410 \\ \mathrm{H} & 0.4342800 & -1.8965980 & -2.8569390 \\ \mathrm{C} & 2.3737540 & -5.3118790 & -2.7711730 \\ \mathrm{C} & 3.5935510 & -4.6734970 & -2.1027360 \\ \mathrm{C} & 3.4572280 & -3.1511650 & -2.0220390 \\ \mathrm{C} & 1.0947710 & -4.9871450 & -1.9939140 \\ \mathrm{H} & 2.5005840 & -6.3971230 & -2.8261360 \\ \mathrm{H} & 4.5085550 & -4.9196040 & -2.6492950 \\ \mathrm{H} & 3.6008420 & -2.6912420 & -3.0083890 \\ \mathrm{H} & 1.1220350 & -5.4643480 & -1.0067000 \\ \mathrm{H} & 0.2073530 & -5.3648260 & -2.5112080 \\ \mathrm{H} & 2.2809340 & -4.9458740 & -3.8005080 \\ \mathrm{H} & 3.7085260 & -5.0811340 & -1.0901380 \\ \mathrm{H} & 4.2172040 & -2.7005830 & -1.3788380 \\ \mathrm{C} & 1.9755320 & -2.0830690 & 2.1363440\end{array}$

$\begin{array}{ll}\mathrm{O} & 0.9034680 \\ \mathrm{C} & 2.6615070 \\ \mathrm{C} & 4.0209060 \\ \mathrm{C} & 1.9462070 \\ \mathrm{C} & 4.6581390 \\ \mathrm{H} & 4.5769150 \\ \mathrm{C} & 2.5836610 \\ \mathrm{H} & 0.8941370 \\ \mathrm{C} & 3.9410610 \\ \mathrm{H} & 5.7163430 \\ \mathrm{H} & 2.0233720 \\ \mathrm{H} & 4.4401200 \\ \mathrm{H} & 0.3711120 \\ \mathrm{~S} & 2.5551460 \\ \mathrm{H} & \end{array}$
$-1.3778490$
$-1.8072520$
$-2.0909100$
$-1.2012420$
$-1.7638460$
$-2.5491540$
$-0.8910830$
$-0.9767340$
$-1.1655020$
$-1.9725240$
$-0.4288730$
$-0.9130330$
$-1.5118570$
$-3.2213250$

\begin{abstract}
1.9809680
3. 4189150

3. 5888200

4.4609670

4.7790550

2.7786530

5.6547310

4.3270020

5.8135020

4.8989540

6.4610740

6.7440420

1.1143320

1. 0422260
\end{abstract}

$\begin{array}{lrcr}\text { TS232(S, S }) \text { G }[W B 97 X D / 6-31 G(d)]=-3633.717121 \\ \text { O } & 0.7304190 & 0.5727780 & -0.1234540 \\ \text { O } & -1.4303410 & 1.2329940 & -1.2976150 \\ \mathrm{C} & 1.0523620 & 1.9079640 & -0.0077120 \\ \mathrm{C} & 0.0869270 & 2.7967740 & 0.4152930 \\ \mathrm{C} & 2.3994730 & 2.2855090 & -0.2411560 \\ \mathrm{C} & 0.4099300 & 4.1913540 & 0.4606200 \\ \mathrm{C} & 2.7241260 & 3.6105010 & -0.0911420 \\ \mathrm{C} & 1.7510330 & 4.5929500 & 0.2167090 \\ \mathrm{H} & 3.7584210 & 3.9191110 & -0.2166120 \\ \mathrm{C} & -1.9848610 & 1.5252110 & -0.0598160 \\ \mathrm{C} & -3.2951320 & 1.0543070 & 0.2167920 \\ \mathrm{C} & -1.2525230 & 2.2927660 & 0.8168480 \\ \mathrm{C} & -3.8442420 & 1.3991980 & 1.4256770 \\ \mathrm{C} & -1.7895300 & 2.5406010 & 2.1225910 \\ \mathrm{C} & -3.1127640 & 2.1085650 & 2.4100440 \\ \mathrm{H} & -4.8610360 & 1.0918930 & 1.6523440 \\ \mathrm{C} & -2.9351870 & 2.9917600 & 4.6622430 \\ \mathrm{C} & -1.6043940 & 3.3863690 & 4.3921950 \\ \mathrm{C} & -1.0466440 & 3.1702210 & 3.1576560 \\ \mathrm{C} & -3.6695710 & 2.3628830 & 3.6911660 \\ \mathrm{H} & -3.3657270 & 3.1755050 & 5.6416790 \\ \mathrm{H} & -1.0168560 & 3.8609430 & 5.1722200 \\ \mathrm{H} & -0.0212530 & 3.4689850 & 2.9707850 \\ \mathrm{H} & -4.6867620 & 2.0358230 & 3.8899390 \\ \mathrm{C} & -0.2062560 & 6.5217460 & 0.7576370 \\ \mathrm{C} & 1.1362060 & 6.9156780 & 0.5491270 \\ \mathrm{C} & 2.0910260 & 5.9699680 & 0.2814580 \\ \mathrm{C} & -0.5606040 & 5.1972750 & 0.7166620 \\ \mathrm{H} & -0.9638400 & 7.2761580 & 0.9464830 \\ \mathrm{H} & 1.4022780 & 7.9673180 & 0.5912660\end{array}$




$\begin{array}{rrrr}\mathrm{H} & 3.1232680 & 6.2591830 & 0.1028070 \\ \mathrm{H} & -1.5956210 & 4.9114450 & 0.8688380 \\ \mathrm{C} & 3.4780350 & 1.2917030 & -0.5380920 \\ \mathrm{C} & 3.9597830 & 1.1385040 & -1.8497750 \\ \mathrm{C} & 4.0971470 & 0.6144000 & 0.5299750 \\ \mathrm{C} & 5.1101290 & 0.3730580 & -2.0538660 \\ \mathrm{C} & 5.2343620 & -0.1503960 & 0.2729790 \\ \mathrm{C} & 5.7797050 & -0.2546650 & -1.0065960 \\ \mathrm{H} & 5.5139510 & 0.2788530 & -3.0590070 \\ \mathrm{H} & 5.7191760 & -0.6632420 & 1.1010070 \\ \mathrm{C} & -4.0566750 & 0.2206530 & -0.7608230 \\ \mathrm{C} & -4.3059630 & -1.1397550 & -0.4801890 \\ \mathrm{C} & -4.5645050 & 0.8055070 & -1.9333090 \\ \mathrm{C} & -5.0790920 & -1.8747870 & -1.3764590 \\ \mathrm{C} & -5.3279240 & 0.0233340 & -2.8015390 \\ \mathrm{C} & -5.6009850 & -1.3150730 & -2.5417260 \\ \mathrm{H} & -5.2806730 & -2.9208730 & -1.1592570 \\ \mathrm{H} & -5.7317930 & 0.4745650 & -3.7056630 \\ \mathrm{C} & 3.5745110 & 0.7170570 & 1.9570710 \\ \mathrm{C} & 7.0708350 & -1.0155920 & -1.2601820 \\ \mathrm{C} & 3.2919530 & 1.8346600 & -3.0283570 \\ \mathrm{C} & -3.7806760 & -1.8256630 & 0.7754460 \\ \mathrm{C} & -6.4412490 & -2.1400760 & -3.4995420 \\ \mathrm{C} & -4.3465120 & 2.2748360 & -2.2654800 \\ \mathrm{C} & -5.6699690 & 3.0501210 & -2.2065540 \\ \mathrm{H} & -6.3767180 & 2.6875820 & -2.9620080 \\ \mathrm{H} & -6.1425270 & 2.9430810 & -1.2241260 \\ \mathrm{H} & -5.4979930 & 4.1165710 & -2.3904140 \\ \mathrm{C} & -3.6499100 & 2.4582220 & -3.6191580 \\ \mathrm{H} & -2.6981460 & 1.9192580 & -3.6400700 \\ \mathrm{H} & -4.2716860 & 2.0929090 & -4.4445250 \\ \mathrm{H} & -3.4487960 & 3.5201480 & -3.7996180 \\ \mathrm{C} & -7.7189720 & -2.6598430 & -2.8274410 \\ \mathrm{H} & -8.3422090 & -3.1980970 & -3.5501760 \\ \mathrm{H} & -7.4815000 & -3.3519230 & -2.0112600 \\ \mathrm{H} & -8.3080640 & -1.8362190 & -2.4109750 \\ \mathrm{C} & -5.6309630 & -3.2928640 & -4.1072880 \\ \mathrm{H} & -4.7315680 & -2.9197380 & -4.6076540 \\ \mathrm{H} & -5.3161700 & -4.0026520 & -3.3335380 \\ \mathrm{H} & -6.2322690 & -3.8416370 & -4.8406570 \\ \mathrm{C} & -4.8948480 & -2.0036330 & 1.8175510 \\ \mathrm{H} & -5.3633110 & -1.0505830 & 2.0812160 \\ \mathrm{H} & -5.6796870 & -2.6671620 & 1.4350980 \\ \mathrm{H} & -4.4915530 & -2.4507940 & 2.7337740 \\ \mathrm{C} & -3.1199420 & -3.1783180 & 0.4825890 \\ \mathrm{H} & -3.8447850 & -3.9186020 & 0.1255570 \\ & & & \end{array}$

-2.3305970
-2.6761570
4.6404070
5.4838690
5.0315660
4.2110790
3.0123630
2.2102480
3.7909210
2.5946070
6.8839350
7.8343350
6.5171380
6.1667130
8.2083410
8.3512480
8.0034130
9.1478480
3.2280870
4.2158490
2.8207620
2.5839330
3.9965410
5.0487370
3.5180170
3.9642230
-0.2967320
0.3698400
-0.8228040
2.2593900
7.3615640
2.7476940
-3.6906050
-6.7441290
-3.0113750
-0.0249240
-0.5491070
-0.2812170
0.5120670
-1.3489800
0.2416000
1.3454130
-1.6236490
-1.9625900
-0.8281700
0.8669960
$-3.0736980$
$-3.5796120$
1.2546900
0.5614990
2. 2169160
1. 3973230
$-0.6258970$
$-0.9675870$
$-1.3974880$
$-0.5300020$
$-2.5313930$
$-3.0532590$
$-2.7886720$
$-2.9108100$
$-0.5154790$
0.5643190
$-0.7236680$
$-1.0149300$
0.9566650
0.8255440
$-0.0330420$
1.4311710
3. 1593780
2. 9815270
3.6431450
3.8569320
0.0723360
0.0819200
$-1.2341160$
2.0554550
$-0.8196530$
1.4323360
2.7076670
$-1.4775410$
$-1.1833220$
$-2.7412300$
$-1.6415470$
$-2.9606990$
$-3.8252880$
$-2.2838690$
$-4.0110390$
$-4.3385120$
$-2.4843740$
$-1.6124610$
$-3.3454110$
$-4.6741110$

$-0.2660780$

1. 4013160

2.9201740

3.0161380

2. 5731820

3.9178530

2.4404430

1.7792650

2. 4705120

3.4487330

$-1.1142900$

$-1.2701490$

$-0.1131430$

$-1.8500160$

$-0.3603170$

$-0.4700100$

0.6959260

$-0.6206380$

$-4.2839680$

$-4.7405950$

$-4.0656210$

$-5.0315760$

$-3.3572660$

$-3.6098020$

$-4.2162690$

$-2.5156410$

$-1.3079070$

$-2.6505890$

$-0.7710710$

$-2.7402310$

$-2.2999270$

1.9679290

$-1.5052840$

$-4.3205090$

1. 2116310

2.1353530

1.6970960

3. 5760840

4. 3382800

4.1837720

5.6877540

3.8706570

5.5303510

3. 5930170

6.2842770

6.2766760 


\begin{tabular}{|c|c|c|c|c|c|c|c|}
\hline $\mathrm{H}$ & & & & & & & \\
\hline H & -2.4588120 & -1.9678570 & 5.9924590 & $\mathrm{H}$ & 2.7098100 & -4.4891190 & 1.5485990 \\
\hline $\mathrm{H}$ & -0.5746820 & -1.5356240 & $\begin{array}{l}.338<090 \\
0.6757910\end{array}$ & $\stackrel{\mathrm{H}}{\mathrm{C}}$ & $\begin{array}{l}0.1832030 \\
3.7871700\end{array}$ & $\begin{array}{l}-1.5154660 \\
-5.6359010\end{array}$ & $\begin{array}{r}3.4811860 \\
-1.7997140\end{array}$ \\
\hline $\mathrm{s}$ & 0.8358280 & -3.8293930 & $\begin{array}{l}1.1877940 \\
1.19\end{array}$ & c & 2.7594510 & $\begin{array}{l}-5.035010 \\
-6.4865390\end{array}$ & -2.2669640 \\
\hline C & 1.2988440 & -3.0736050 & -2.0228600 & $\mathrm{c}$ & $\begin{array}{l}1.4472050 \\
1.0\end{array}$ & -6.1215490 & -2.1105000 \\
\hline $\mathrm{C}$ & 2.0391800 & -2.5238080 & -0.8829530 & c & 3.4835350 & -4.4574470 & -1.1663560 \\
\hline $\mathrm{H}$ & 0.2260950 & -2.8954630 & -2.0061910 & $\mathrm{H}$ & 4.8258340 & -5.9145710 & -1.9491060 \\
\hline $\mathrm{H}$ & 1.6487830 & -1.6346700 & -0.4059780 & $\mathrm{H}$ & 3.0125640 & -7.4200480 & -2.7599440 \\
\hline 0 & 2.0198800 & -1.9932120 & -2.6742060 & $\mathrm{H}$ & 0.6489170 & -6.7585110 & -2.4822070 \\
\hline $\mathrm{H}$ & 1.4166840 & -1.1845290 & -2.7187380 & $\mathrm{H}$ & 4.2803270 & -3.8078510 & -0.8205580 \\
\hline C & 3.2183660 & -4.6814140 & -2.4117980 & C & -2.0096150 & -2.8129240 & -0.6672750 \\
\hline $\mathrm{C}$ & 3.6913380 & -4.4194730 & -0.9802860 & C & -2.7092930 & -2.2279550 & -1.7413360 \\
\hline $\mathrm{C}$ & 3.4196560 & -2.9727620 & -0.5656070 & $\mathrm{c}$ & -2.6119010 & -2.9169420 & 0.5978400 \\
\hline $\mathrm{C}$ & 1.7129340 & -4.4289510 & -2.5436260 & $\mathrm{C}$ & -3.9969930 & -1.7447380 & -1.5143190 \\
\hline $\mathrm{H}$ & 3.4368630 & -5.7142530 & -2.6987400 & $\mathrm{C}$ & -3.9066370 & -2.4247640 & 0.7724730 \\
\hline $\mathrm{H}$ & 4.7611600 & -4.6245920 & -0.8805800 & $\mathrm{C}$ & -4.6105000 & -1.8219090 & -0.2640480 \\
\hline $\mathrm{H}$ & 4.0849000 & -2.2779690 & -1.0932240 & $\mathrm{H}$ & -4.5398320 & -1.2889690 & -2.3379750 \\
\hline H & 1.1559870 & -5.1756970 & -1.9642470 & $\mathrm{H}$ & -4.3818080 & -2.5100460 & 1.7476220 \\
\hline $\mathrm{H}$ & 1.3832600 & -4.5155880 & -3.5836100 & C & 3.7491710 & 1.6960530 & 0.4242660 \\
\hline H & 3.7647160 & -4.0314900 & -3.1061010 & $\mathrm{C}$ & 3.1102570 & 2.5907850 & 1.3080760 \\
\hline $\mathrm{H}$ & 3.1711530 & -5.0981660 & -0.2923790 & $\mathrm{C}$ & 4.3189150 & 2.1637990 & -0.7697350 \\
\hline $\mathrm{H}$ & 3.6057260 & -2.7997210 & 0.4972790 & $\mathrm{C}$ & 3.0756860 & 3.9428960 & 0.9768350 \\
\hline & & & & $\mathrm{C}$ & 4.2542400 & 3.5284910 & -1.0591750 \\
\hline & S) $\mathrm{G}[\mathrm{w}$ & $G(d)]=$ & 73 & C & 3.6340880 & 4.4323370 & -0.2039220 \\
\hline 0 & 0.0934940 & -1.2420180 & 0.2055340 & $\mathrm{H}$ & 2.5771940 & 4.6356060 & 1.6493370 \\
\hline 0 & 2.0715350 & -0.1160950 & -0.8805140 & $\mathrm{H}$ & 4.6901900 & 3.8972060 & -1.9857620 \\
\hline C & 0.4532690 & -2.4566790 & -0.3395490 & C & -1.9063700 & -3.5843320 & 1.7702190 \\
\hline $\mathrm{C}$ & 1.7763780 & -2.8394990 & -0.3101390 & $\mathrm{C}$ & -6.0098610 & -1.2761590 & -0.0431700 \\
\hline C & -0.5969920 & -3.2788280 & -0.8303280 & $\mathrm{C}$ & -2.1043250 & -2.1504950 & -3.1358770 \\
\hline $\mathrm{C}$ & 2.1337320 & -4.0616850 & -0.9698030 & C & 2.4701440 & 2.1158220 & 2.6070340 \\
\hline $\mathrm{C}$ & -0.2553080 & -4.4977620 & -1.3538970 & $\mathrm{C}$ & 3.5188940 & 5.9006370 & -0.5715480 \\
\hline $\mathrm{C}$ & 1.1016020 & -4.8990920 & -1.4770860 & $\mathrm{C}$ & 4.9910830 & 1.2252500 & -1.7608520 \\
\hline $\mathrm{H}$ & -1.0332860 & -5.1761770 & -1.6929200 & $\mathrm{C}$ & 6.4845560 & 1.5445440 & -1.9077780 \\
\hline C & 2.8588190 & -0.6451300 & 0.1192140 & $\mathrm{H}$ & 6.6389160 & 2.5492940 & -2.3179770 \\
\hline C & 3.7769080 & 0.2368800 & 0.7480160 & $\mathrm{H}$ & 6.9932970 & 1.4931720 & -0.9391920 \\
\hline $\mathrm{C}$ & 2.7693870 & -1.9900790 & 0.4051610 & $\mathrm{H}$ & 6.9636600 & 0.8290170 & -2.5854710 \\
\hline C & 4.6509800 & -0.3000640 & 1.6558000 & $\mathrm{C}$ & 4.2777240 & 1.2399500 & -3.1190480 \\
\hline $\mathrm{C}$ & 3.6020860 & -2.5132070 & 1.4516450 & $\mathrm{H}$ & 3.2165370 & 1.0032410 & -2.9984150 \\
\hline C & 4.5775590 & -1.6626150 & 2.0431200 & $\mathrm{H}$ & 4.3602430 & 2.2203510 & -3.6025950 \\
\hline $\mathrm{H}$ & 5.3991600 & 0.3365170 & 2.1196980 & $\mathrm{H}$ & 4.7233350 & 0.4984400 & -3.7917600 \\
\hline C & 5.3113980 & -3.4691570 & 3.4880030 & C & 4.1147050 & 6.8207340 & 0.5007380 \\
\hline C & 4.3095040 & -4.3017430 & 2.9391870 & $\mathrm{H}$ & 4.0582160 & 7.8673060 & 0.1814340 \\
\hline $\mathrm{C}$ & 3.4787050 & -3.8387150 & 1.9501060 & $\mathrm{H}$ & 3.5708060 & 6.7327320 & 1.4480750 \\
\hline $\mathrm{C}$ & 52880 & -2.1763340 & 3.0509450 & $\mathrm{H}$ & 5.1648990 & 6.5763500 & 0.6911680 \\
\hline $\mathrm{H}$ & 30250 & -3.85111 & 4.2635710 & c & 2.0567480 & 6.2672670 & -0.8651310 \\
\hline $\mathrm{H}$ & 4 & -5.3 & 3.3093790 & $\mathrm{H}$ & 1.6505940 & 5.6325340 & -1.6594450 \\
\hline
\end{tabular}




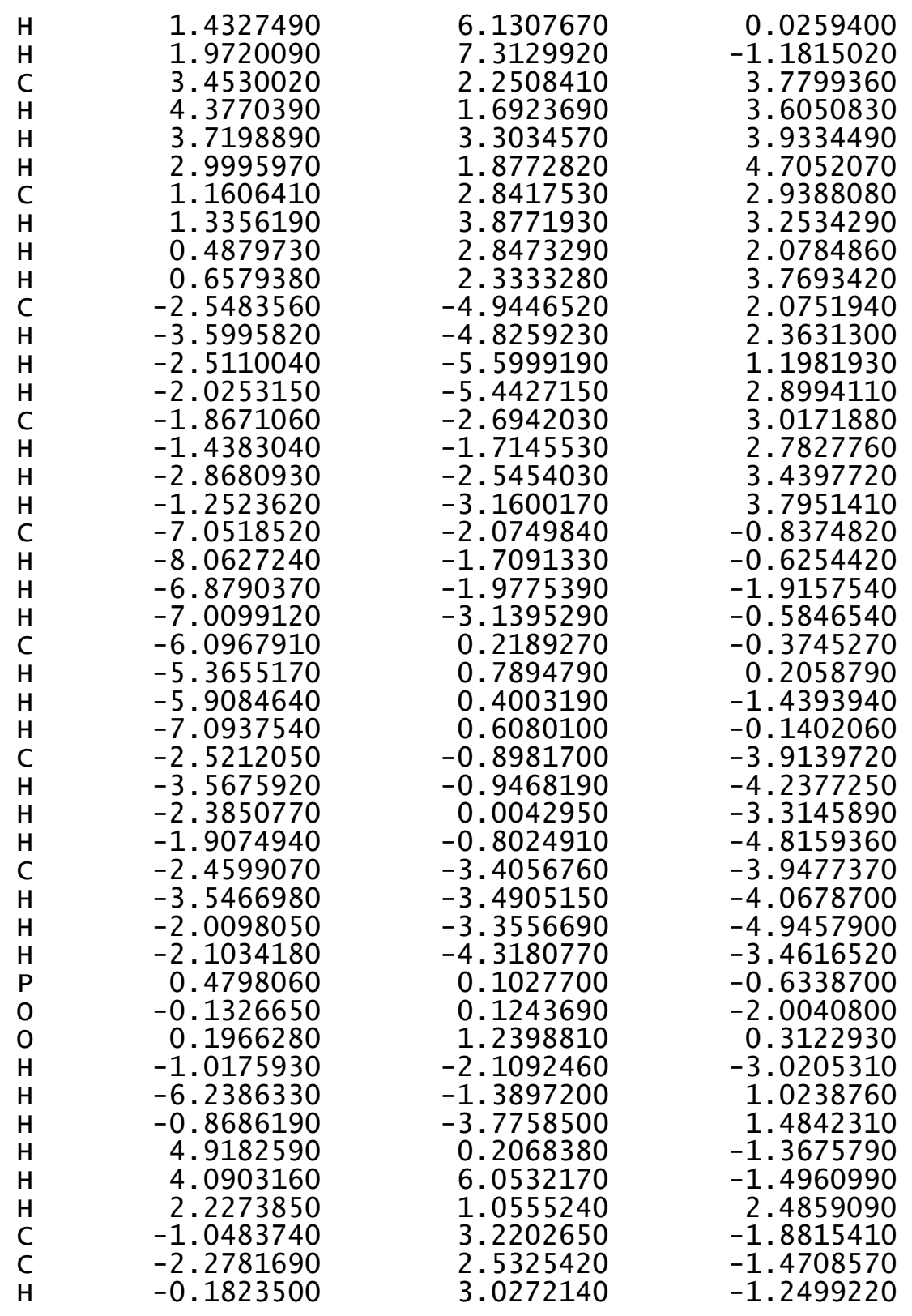

$\begin{array}{lrrr}\mathrm{H} & -2.1613630 & 1.5581460 & -1.0101850 \\ \mathrm{O} & -1.1534460 & 2.2813050 & -2.9768300 \\ \mathrm{H} & -0.6746570 & 1.4349780 & -2.6941580 \\ \mathrm{C} & -2.4781850 & 4.8998140 & -3.1233140 \\ \mathrm{C} & -3.6934360 & 4.4373630 & -2.3153030 \\ \mathrm{C} & -3.6155670 & 2.9438740 & -1.9880010 \\ \mathrm{C} & -1.1765900 & 4.6485890 & -2.3553240 \\ \mathrm{H} & -2.5640560 & 5.9670220 & -3.3484040 \\ \mathrm{H} & -4.6196580 & 4.6351320 & -2.8624230 \\ \mathrm{H} & -3.8001390 & 2.3369650 & -2.8842910 \\ \mathrm{H} & -1.1309670 & 5.2900480 & -1.4665230 \\ \mathrm{H} & -0.3029520 & 4.8867950 & -2.9703120 \\ \mathrm{H} & -2.4469670 & 4.3700680 & -4.0825680 \\ \mathrm{H} & -3.7460240 & 5.0040700 & -1.3772450 \\ \mathrm{H} & -4.3746480 & 2.6408720 & -1.2616260 \\ \mathrm{C} & -2.8033540 & 1.9821950 & 1.6964870 \\ \mathrm{O} & -1.9540110 & 1.0068420 & 1.6388640 \\ \mathrm{C} & -4.0487280 & 1.6384080 & 2.4127240 \\ \mathrm{C} & -5.1094240 & 2.5485050 & 2.5085340 \\ \mathrm{C} & -4.1954430 & 0.3510980 & 2.9484160 \\ \mathrm{C} & -6.2962330 & 2.1736370 & 3.1230690 \\ \mathrm{H} & -5.0017100 & 3.5410980 & 2.0857110 \\ \mathrm{C} & -5.3845500 & -0.0198880 & 3.5604800 \\ \mathrm{H} & -3.3805260 & -0.3563000 & 2.8656420 \\ \mathrm{C} & -6.4377210 & 0.8891340 & 3.6460340 \\ \mathrm{H} & -7.1146710 & 2.8829850 & 3.1892200 \\ \mathrm{H} & -5.4916200 & -1.0198570 & 3.9694320 \\ \mathrm{H} & -7.3694130 & 0.5969250 & 4.1205490 \\ \mathrm{H} & -1.0872040 & 1.2042870 & 1.1224790 \\ \mathrm{~S} & -2.5297910 & 3.4859230 & 0.9806000 \\ \mathrm{H} & - & & \end{array}$

TS234(S, S) G[WB97XD/6-31G(d)] = -3633.715556

$\begin{array}{lrcr}\text { TS234(S, S }) & \text { G }[W B 97 X D / 6-31 G(d)]=-3633.715556 \\ \text { O } & 0.6706540 & 0.1599140 & -0.5006120 \\ \text { O } & -1.6126170 & 0.1538230 & -1.6330730 \\ \text { C } & 0.9657350 & 1.3316640 & -1.1598870 \\ \text { C } & 0.0254860 & 2.3392790 & -1.1980540 \\ \text { C } & 2.2732440 & 1.4806270 & -1.6908880 \\ \text { C } & 0.3118810 & 3.5209560 & -1.9528940 \\ \text { C } & 2.5662490 & 2.6513650 & -2.3454640 \\ \text { C } & 1.6028700 & 3.6737980 & -2.5266320 \\ \text { H } & 3.5691500 & 2.8013460 & -2.7368590 \\ \text { C } & -2.0654230 & 1.1024350 & -0.7243790 \\ \text { C } & -3.3337060 & 0.9051500 & -0.1161040 \\ \text { C } & -1.2556280 & 2.1815150 & -0.4617420 \\ \text { C } & -3.7458660 & 1.8482170 & 0.7907720 \\ \text { C } & -1.6436820 & 3.0928330 & 0.5727950\end{array}$




$$
\begin{array}{r}
-2.9124540 \\
-4.7209430 \\
-2.4650210 \\
-1.1905870 \\
-0.7902210 \\
-3.3049700 \\
-2.7704010 \\
-0.5191530 \\
0.1953380 \\
-4.2791780 \\
-0.3365720 \\
0.9576600 \\
1.9037930 \\
-0.6521520 \\
-1.0888480 \\
1.1937940 \\
2.8973650 \\
-1.6516950 \\
3.3434530 \\
3.5554680 \\
4.2054180 \\
4.6547980 \\
5.2841060 \\
5.5403300 \\
4.8446670 \\
5.9578460 \\
-4.1725550 \\
-4.4115260 \\
-4.7449910 \\
-5.2248830 \\
-5.5473830 \\
-5.8001150 \\
-5.4144830 \\
-5.9980470 \\
3.9742500 \\
6.7597880 \\
2.6738090 \\
-3.8398030 \\
-6.6753760 \\
-4.5636910 \\
-5.8916240 \\
-6.6401020 \\
-6.2976740 \\
-5.7482000 \\
-3.9586400 \\
-3.0087560
\end{array}
$$

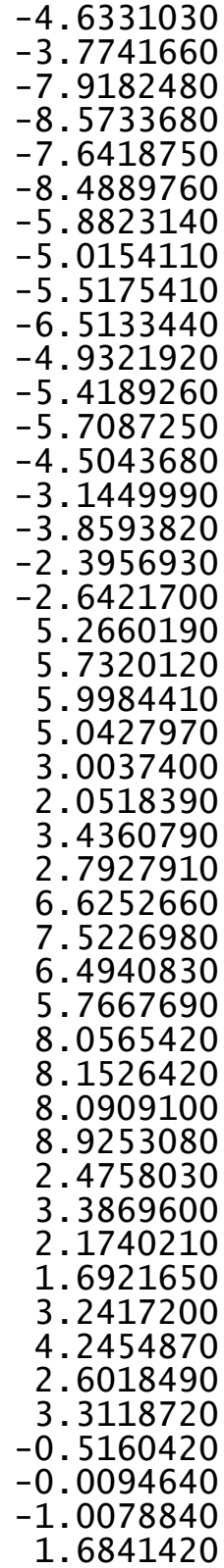
$-0.7208220$
0.7992710
$-3.7571700$
$-4.5859370$
$-3.9026550$
$-2.8255580$
$-5.0233490$
$-5.0042360$
$-5.1935680$
$-5.8750390$
$-0.7717150$
0.1701130
$-1.5442770$
$-0.6556410$
$-2.4555110$
$-3.2744880$
$-2.7662890$
$-2.2877960$
2.1706650
1.4241350
2. 4647220
3.0464780
1.1616550
0.8347520
0.3229960
1.9631320
$-3.2157810$
$-3.8413240$
$-2.7224850$
$-3.8749450$
$-1.3773810$
$-0.6325150$
$-0.8509920$
$-2.0431570$
$-2.0984820$
$-2.5042390$
$-2.7622640$
$-2.1226990$
0.1999070
$-0.1457550$
0.1281580
1.2541430
$-0.8683060$
$-1.7730230$
$-1.4836720$
$-0.2706590$

$-4.5317180$

$-4.8153690$

$-0.4253790$

$-0.7160400$

0.6253310

$-1.2352560$

$-1.9037610$

$-0.2156200$

$-1.5132100$

2.9779770

2.7049030

3.0283810

3.9803160

2.4244780

2. 5677120

1.6933320

3. 3841060

1. 3224820

1.9756700

0.5636740

1.9408080

1.7724720

1. 3447610

2. 3315720

2.4893150

$-0.0095830$

0.0491360

0.9612640

$-0.1783050$

$-0.9863910$

$-1.7828870$

$-0.0257400$

$-1.0300210$

$-4.1683500$

$-4.6229830$

$-3.3549470$

$-4.9321150$

$-4.8241660$

$-5.0991790$

$-5.7109210$

$-4.5415590$

$-2.0874760$

0.2769850
-3.4240630 


\begin{tabular}{|c|c|c|c|c|c|c|c|}
\hline u & & & & & & & \\
\hline H & $\begin{array}{l}6.8235270 \\
3.4980730\end{array}$ & $\begin{array}{r}-2.7361720 \\
2.4996690\end{array}$ & $\begin{array}{r}-2.0826050 \\
0.1887050\end{array}$ & C & 2.3916340 & $\begin{array}{l}2.4821120 \\
3044240\end{array}$ & $\begin{array}{l}-2.4554980 \\
-2\end{array}$ \\
\hline $\mathrm{H}$ & -3.8675700 & 1.3769440 & -2.4418160 & H & $\begin{array}{l}1.4325120 \\
3.3767650\end{array}$ & $\begin{array}{l}2.5044 \angle 40 \\
2.5931920\end{array}$ & $\begin{array}{l}-2.0509440 \\
-2.9009710\end{array}$ \\
\hline $\mathrm{H}$ & -7.0195370 & -3.6014370 & -2.3604390 & C & -2.1704340 & 1.0913830 & -0.5290490 \\
\hline $\mathrm{H}$ & -3.0822450 & -0.3779940 & 1.9786230 & $\mathrm{C}$ & -3.4087060 & 0.9424780 & 0.1490570 \\
\hline c & 1.1128210 & -3.6538730 & 0.4699400 & $\mathrm{c}$ & -1.3468560 & 2.1824530 & -0.3815560 \\
\hline c & 1.9530620 & -2.5179130 & 0.8726380 & $\mathrm{C}$ & -3.7821340 & 1.9540070 & 0.9970580 \\
\hline $\mathrm{H}$ & 0.0450880 & -3.4497670 & 0.4504720 & $\mathrm{C}$ & -1.6933520 & 3.1755410 & 0.5914330 \\
\hline H & 1.6166010 & -1.5182470 & 0.6276850 & C & -2.9371850 & 3.0589870 & 1.2696270 \\
\hline 0 & 1.7443960 & -3.3679040 & -0.8068970 & $\mathrm{H}$ & -4.7368380 & 1.8894840 & 1.5108420 \\
\hline $\mathrm{H}$ & 1.1251980 & -2.7886440 & -1.3454910 & C & -2.4493970 & 5.0637300 & 2.5466100 \\
\hline C & 2.9971820 & -5.2100950 & 1.1364360 & $\mathrm{C}$ & -1.1973430 & 5.1635300 & 1.8970220 \\
\hline c & 3.6085160 & -4.0753980 & 1.9604970 & $\mathrm{C}$ & -0.8284860 & 4.2473570 & 0.9441250 \\
\hline c & 3.3581900 & -2.7169710 & 1.3049230 & $\mathrm{C}$ & -3.2976910 & 4.0329950 & 2.2375580 \\
\hline c & 1.4845070 & -5.0217160 & 0.9900520 & $\mathrm{H}$ & -2.7295710 & 5.7983370 & 3.2948590 \\
\hline $\mathrm{H}$ & 3.1973410 & -6.1738200 & 1.6138360 & $\mathrm{H}$ & -0.5195430 & 5.9711880 & 2.1570540 \\
\hline H & 4.6860350 & -4.2204760 & 2.0812440 & $\mathrm{H}$ & 0.1398720 & 4.3303530 & 0.4631990 \\
\hline $\mathrm{H}$ & 3.9584940 & -2.5973430 & 0.3937580 & $\mathrm{H}$ & -4.2552500 & 3.9341590 & 2.7416010 \\
\hline $\mathrm{H}$ & 0.9964920 & -5.1358000 & 1.9659690 & C & -0.5041580 & 5.4805390 & -3.0884810 \\
\hline H & 1.0517480 & -5.7724980 & 0.3216040 & C & 0.7695530 & 5.5959170 & -3.6924510 \\
\hline $\mathrm{H}$ & 3.4631070 & -5.2399070 & 0.1439270 & $\mathrm{c}$ & 1.7142820 & 4.6253050 & -3.4835450 \\
\hline $\mathrm{H}$ & 3.1708430 & -4.0769510 & 2.9669240 & $\mathrm{C}$ & -0.7994930 & 4.4153330 & -2.2764410 \\
\hline $\mathrm{H}$ & 3.6473010 & -1.8820640 & 1.9481660 & $\mathrm{H}$ & -1.2572330 & 6.2401200 & -3.2748570 \\
\hline C & 0.2435990 & -0.3741560 & 3.2212630 & $\mathrm{H}$ & 0.9906390 & 6.4473920 & -4.3285620 \\
\hline 0 & -0.5622810 & -0.0250060 & 2.2731220 & $\mathrm{H}$ & 2.6914950 & 4.6936220 & -3.9540750 \\
\hline $\mathrm{C}$ & 0.4727150 & 0.7091280 & 4.2049880 & $\mathrm{H}$ & -1.7844230 & 4.3345690 & -1.8298770 \\
\hline C & 1.4039330 & 0.5666830 & 5.2403380 & C & 3.1808320 & 0.3292860 & -1.5112080 \\
\hline c & -0.2 & 1.9189340 & 4.0666420 & $\mathrm{C}$ & 3.4097380 & -0.6701040 & -2.4727730 \\
\hline & 1.6320360 & 1.6127130 & 6.1244410 & C & 4.0290630 & 0.4520000 & -0.3936900 \\
\hline $\mathrm{H}$ & 1.9508200 & -0.3642760 & 5.3414910 & $\mathrm{C}$ & 4.5235790 & -1.4973410 & -2.3213950 \\
\hline c & 0.0066240 & 2.9614610 & 4.9539650 & $\mathrm{C}$ & 5.1430910 & -0.3822230 & -0.3005360 \\
\hline $\mathrm{H}$ & -0.9432150 & 2.0408600 & 3.2632600 & $\mathrm{C}$ & 5.4149240 & -1.3560700 & -1.2596350 \\
\hline C & 0.9351820 & 2.8115500 & 5.9824100 & $\mathrm{H}$ & 4.7191570 & -2.2676750 & -3.0634560 \\
\hline $\mathrm{H}$ & 2.3590190 & 1.4955150 & 6.9216520 & $\mathrm{H}$ & 5.8147440 & -0.2668820 & 0.5447560 \\
\hline $\mathrm{H}$ & -0.5359870 & 3.8939090 & 4.8353210 & C & -4.2602490 & -0.2692610 & -0.0435150 \\
\hline H & 1.1173100 & 3.6290580 & 6.6734550 & C & -4.4325670 & -1.1845030 & 1.0136960 \\
\hline $\mathrm{H}$ & -0.7200460 & -0.7205490 & 1.5324200 & $\mathrm{C}$ & -4.9073510 & -0.4821520 & -1.2761830 \\
\hline & 0.9630110 & -1.8954330 & 3.3310700 & $\mathrm{c}$ & -5.2630490 & -2.2885480 & 0.8117340 \\
\hline & & & & C & -5.7175070 & -1.6050150 & -1.4284800 \\
\hline & S) $\mathrm{G}[\mathrm{WB} 97$ & $G(d)]=-3$ & & $\mathrm{C}$ & -5.9111280 & -2.5211770 & -0.3966950 \\
\hline 0 & 0.5550620 & 0.1309600 & -0.3808450 & $\mathrm{H}$ & -5.4072140 & -2.9972560 & 1.6240930 \\
\hline 0 & -1.7756880 & 0.0918180 & -1.4068000 & $\mathrm{H}$ & -6.2194790 & -1.7643530 & -2.3803360 \\
\hline$c$ & 0.8302600 & 1.2576180 & -1.1238670 & $\mathrm{C}$ & 3.7838900 & 1.5149940 & 0.6711470 \\
\hline C & -0.0995870 & 2.2744960 & -1.1832740 & C & 6.6637930 & -2.2204800 & -1.2038820 \\
\hline$c$ & 2.1127850 & 1.3557630 & -1.7224040 & C & 2.5071970 & -0.8214050 & -3.6895550 \\
\hline$c$ & & 3.402 & -2.0252080 & $\mathrm{C}$ & -3.7688310 & -1.0054470 & 2.3736060 \\
\hline
\end{tabular}




$\begin{array}{lr}-6.7984480 & -3.7389440 \\ -4.7935280 & 0.4996010 \\ -6.1413970 & 1.1867050 \\ -6.9008280 & 0.4616880 \\ -6.5072860 & 1.6874110 \\ -6.0428190 & 1.9369170 \\ -4.2521550 & -0.1682390 \\ -3.2884380 & -0.6478950 \\ -4.9447900 & -0.9280320 \\ -4.1132220 & 0.5787990 \\ -6.2367030 & -4.6841440 \\ -6.8512630 & -5.5880160 \\ -6.2252250 & -4.2000420 \\ -5.2110500 & -4.9837260 \\ -8.2462220 & -3.3439360 \\ -8.6514610 & -2.6774470 \\ -8.3110720 & -2.8260950 \\ -8.8836180 & -4.2334570 \\ -4.7860050 & -0.5240620 \\ -5.2680610 & 0.4113040 \\ -5.5739710 & -1.2727020 \\ -4.2941620 & -0.3583280 \\ -3.0660420 & -2.2774930 \\ -3.7820050 & -3.0673370 \\ -2.3785880 & -2.6581850 \\ -2.4911170 & -2.0538210 \\ 4.5941140 & 2.7836660 \\ 5.6685380 & 2.5653370 \\ 4.3446600 & 3.1919240 \\ 4.3921670 & 3.5550440 \\ 4.0722900 & 1.0258660 \\ 3.5375050 & 0.0963310 \\ 5.1415180 & 0.8535920 \\ 3.7450510 & 1.7791700 \\ 7.0795590 & -2.6194840 \\ 7.9045050 & -3.3383340 \\ 7.4263950 & -1.7556930 \\ 6.2531720 & -3.0829900 \\ 7.8210460 & -1.5120620 \\ 7.5575600 & -1.2821270 \\ 8.0649400 & -0.5696860 \\ 8.7192520 & -2.1398830 \\ 2.2863350 & -2.2816320 \\ 3.1858640 & -2.7182110 \\ 1.9971900 & -2.9000420 \\ 1.4884130 & -2.3375380\end{array}$

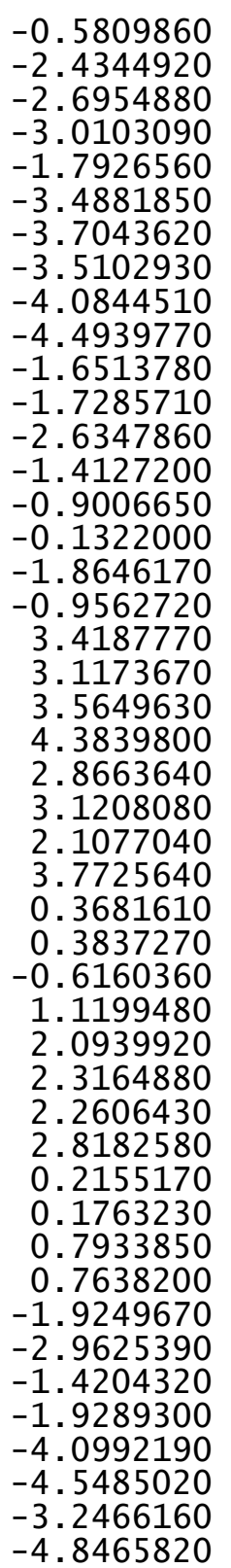

3.0630490
4.0560470
2.4039880
3.1538950
-0.6421540
-0.1927730
-1.0689410
1.5267620
6.4422180
2.7225720
-4.0858490
-6.8059140
-2.9998710
0.6061980
-0.4150330
1.1014560
1.9850400
0.7207940
2.4811850
2.2830240
1.2340030
0.0400980
2.1141520
3.1634010
0.9443930
2.5159100
-0.6745800
1.3268450
0.9863770
1.9662290
-0.0528650
1.7061090
1.4685520
0.8574610
2.7802540
3.5866120
3.3886580
1.2862990
2.9423600
4.6529040
3.8783060
0.9065260
0.7079510
3.1246440
3.2745590
3.8398810
-0.0257910
-0.3985270
-0.1297300
1.0393850
-0.9141070
-1.8186760
-1.5269890
-0.4100600
-3.1430640
1.7854190
1.2851090
-4.2828550
-0.2351250
-0.3493810
-0.0773540
0.8260560
0.6985100
2.1033550
1.8316040
-0.2893380
3.2334450
2.2060950
3.0999320
1.7272910
4.2169880
3.9839050
-0.7905300
-1.8744220
-3.7520820
-2.6825640
-3.4476690
-1.6683690
-3.6097640
-2.9745330
-5.4532440
-4.3437520
-3.0033670
-5.1188180
-6.4057580
-4.5897210
-3.0020710
-5.1256700
-5.8576230
-5.5817400
-4.2479980
-2.1662880
$-4.8803340$

$-5.1590240$

$-5.7496270$

$-4.6499120$

$-0.8248070$

$-1.9292080$

0.4857760

$-3.4303350$

$-1.7559840$

0.6391540

$-2.1561450$

0.3723130

2. 2761040

3. 1561110

2. 4160870

3. 9048730

4.9823310

3.4685510

5.6127550

5. 3169910

4.0909190

2.6309410

5.1630030

6.4502390

3.7342990

5.6488280

1. 7192880

3. 2575710

0.5338490

0.7842620

0.6300380

0.4996500

$-0.8253880$

$-1.3061190$

1. 0940920

1.7717340

1. 0623150

1. 1029940

1.6072340

1. 7793890

0.0783440

2. 1321870

0.5395650

0.0609850

2. 8196790

1.6016150 
144

TS236 (S, S ) G[WB97XD/6-31G(d) $]=-3633.714797$

$\begin{array}{lrrr}\mathrm{O} & 0.1477980 & -1.2030000 & 0.1374640 \\ \mathrm{O} & 2.1131940 & -0.0180730 & -0.8952360 \\ \mathrm{C} & 0.5029680 & -2.4051980 & -0.4376200 \\ \mathrm{C} & 1.8277520 & -2.7785690 & -0.4371640 \\ \mathrm{C} & -0.5540330 & -3.2170680 & -0.9320000 \\ \mathrm{C} & 2.1807320 & -3.9762460 & -1.1420710 \\ \mathrm{C} & -0.2147470 & -4.4142660 & -1.5036990 \\ \mathrm{C} & 1.1440520 & -4.7998090 & -1.6640230 \\ \mathrm{H} & -0.9920630 & -5.0874420 & -1.8538820 \\ \mathrm{C} & 2.8791180 & -0.5877480 & 0.0991670 \\ \mathrm{C} & 3.7540130 & 0.2783380 & 0.8069700 \\ \mathrm{C} & 2.8109660 & -1.9481780 & 0.3142580 \\ \mathrm{C} & 4.6172330 & -0.2859380 & 1.7083480 \\ \mathrm{C} & 3.6342470 & -2.5088850 & 1.3491870 \\ \mathrm{C} & 4.5745050 & -1.6706990 & 2.0117260 \\ \mathrm{H} & 5.3325770 & 0.3434380 & 2.2306920 \\ \mathrm{C} & 5.3275250 & -3.5394750 & 3.3650220 \\ \mathrm{C} & 4.3581590 & -4.3623590 & 2.7479810 \\ \mathrm{C} & 3.5344400 & -3.8630370 & 1.7708720 \\ \mathrm{C} & 5.4245530 & -2.2203030 & 3.0072150 \\ \mathrm{H} & 5.9788310 & -3.9496460 & 4.1305900 \\ \mathrm{H} & 4.2587310 & -5.3991960 & 3.0547160 \\ \mathrm{H} & 2.7892670 & -4.5086420 & 1.3208850 \\ \mathrm{H} & 6.1451190 & -1.5668070 & 3.4919060 \\ \mathrm{C} & 3.8296130 & -5.5119040 & -2.0486680 \\ \mathrm{C} & 2.7990270 & -6.3487130 & -2.5339730 \\ \mathrm{C} & 1.4872080 & -5.9961870 & -2.3464720 \\ \mathrm{C} & 3.5291640 & -4.3586040 & -1.3688740 \\ \mathrm{H} & 4.8673350 & -5.7809790 & -2.2204260 \\ \mathrm{H} & 3.0493800 & -7.2626510 & -3.0637390 \\ \mathrm{H} & 0.6869690 & -6.6235020 & -2.7302880 \\ \mathrm{H} & 4.3270230 & -3.7183960 & -1.0077120 \\ \mathrm{C} & -1.9541910 & -2.7370910 & -0.7110070 \\ \mathrm{C} & -2.6839440 & -2.1207150 & -1.7456900 \\ \mathrm{C} & -2.4910960 & -2.8142530 & 0.5867670 \\ \mathrm{C} & -3.9235230 & -1.5545470 & -1.4435450 \\ \mathrm{C} & -3.7357950 & -2.2384010 & 0.8371700 \\ \mathrm{C} & -4.4579310 & -1.5832890 & -0.1557630 \\ \mathrm{H} & -4.4872680 & -1.0738220 & -2.2374670 \\ \mathrm{H} & -4.1548280 & -2.2854450 & 1.8402240 \\ \mathrm{C} & 3.6953930 & 1.7524830 & 0.5636240 \\ \mathrm{C} & 2.9263230 & 2.5688630 & 1.4197650 \\ \mathrm{C} & 4.3652620 & 2.3093610 & -0.5353380 \\ \mathrm{C} & 2.8632210 & 3.9342040 & 1.1557980\end{array}$

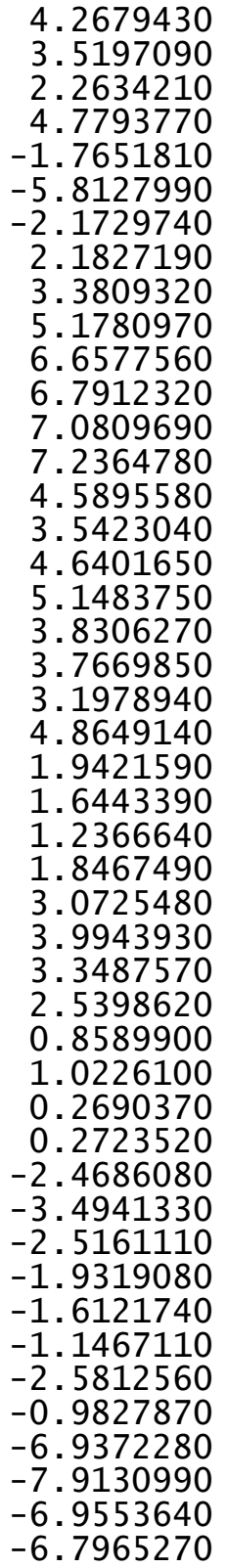

4.2679430

C

3.6848440

4. 5123520

4. 5665110

4.1214930

$-3.5379200$

$-0.9783090$

-2. 1202170

1.9935730

5.9953280

1.4542120

1.8595150

2.8880260

1.7929340

1.2014210

1.4914800

1. 1745730

2. 4997870

0.8202910

6.8634880

7. 9262800

6.6972800

6.6397930

6.3321970

. 7455970

6.1089820

7. 3951850

2. 0104070

1. 4410360

3.0407340

1. 5796220

2.7094690

3.6936510

2.8375620

2. 1173830

$-4.8656600$

$-4.6928010$

$-5.5032010$

$-5.4101180$

$-2.6795410$

$-1.7178880$

$-2.4900070$

$-3.1960700$

$-1.5581990$

$-2.1821350$
$-0.7599520$

0.0687730

1.8044760

$-1.6157610$

1. 7127330

0.1678950

$-3.1797270$

2. 6192620

$-0.2245710$

$-1.4957720$

$-1.4925210$

$-1.8474150$

$-0.4844600$

$-2.1503570$

$-2.9122260$

$-2.9027090$

$-3.3394880$

$-3.5741160$

0.9572040

0.6986720

1.8364650

1.2384870

$-0.6419440$

$-1.5173980$

0.1670550

$-0.8903010$

3. 8717960

3. 7246620

4.1267830

4.7272710

2. 9173080

3. 3720120

2. 0073790

3. 6283030

2. 0273270

2. 3744960

1.1378470

2. 8125700

2.9726120

2. 7348850

3.4487420

3.7055470

$-0.1662710$

0.1158080

$-1.2419480$ 


$\begin{array}{rrr}-6.0555100 & 0.3671610 & -0.5216400 \\ -5.2337360 & 1.0632380 & -0.3249100 \\ -6.1591280 & 0.2546440 & -1.6070740 \\ -6.9783100 & 0.8226550 & -0.1468590 \\ -2.5505380 & -0.8694130 & -3.9777580 \\ -3.6261440 & -0.8188940 & -4.1831560 \\ -2.2520740 & 0.0378640 & -3.4502170 \\ -2.0369130 & -0.8807060 & -4.9450240 \\ -2.6735070 & -3.3729280 & -3.9166210 \\ -3.7677480 & -3.3612120 & -3.9908160 \\ -2.2636370 & -3.4113190 & -4.9323250 \\ -2.3846240 & -4.2921070 & -3.3990300 \\ 0.5043130 & 0.1403030 & -0.7136350 \\ -0.0595130 & 0.1128750 & -2.1056390 \\ 0.1340680 & 1.2819970 & 0.1956730 \\ -1.0805550 & -2.1567200 & -3.1396050 \\ -5.8351830 & -0.8003820 & 1.2508720 \\ -0.7562950 & -3.7837190 & 1.3705890 \\ 5.1278230 & 0.4167230 & -1.1514830 \\ 4.0364660 & 6.2253990 & -1.0743580 \\ 1.9402370 & 0.9511880 & 2.3877410 \\ -1.0821710 & 3.1779570 & -1.9699950 \\ -2.3131830 & 2.4004680 & -1.7952810 \\ -0.3063570 & 2.9858080 & -1.2311470 \\ -2.2136240 & 1.4076410 & -1.3693680 \\ -0.9804340 & 2.2998050 & -3.1190360 \\ -0.5140940 & 1.4538580 & -2.8146690 \\ -2.4045610 & 4.8628000 & -3.3199470 \\ -3.6965460 & 4.2874440 & -2.7341620 \\ -3.5798180 & 2.7806930 & -2.4878070 \\ -1.2194990 & 4.6245370 & -2.3794070 \\ -2.5185530 & 5.9368640 & -3.4940310 \\ -4.5403810 & 4.4749700 & -3.4043930 \\ -3.5902410 & 2.2293370 & -3.4368160 \\ -1.3419820 & 5.2076990 & -1.4583770 \\ -0.2791000 & 4.9426390 & -2.8401830 \\ -2.2016490 & 4.3987150 & -4.2922010 \\ -3.9227200 & 4.7901450 & -1.7852330 \\ -4.4225490 & 2.3934000 & -1.9098220 \\ -2.9786250 & 1.7521330 & 1.5561390 \\ -1.9894130 & 0.9190010 & 1.5504640 \\ -4.0828750 & 1.3408230 & 2.4495800 \\ -5.3357030 & 1.9630040 & 2.3933270 \\ -3.8820960 & 0.2779360 & 3.3409220 \\ -6.3735710 & 1.5181970 & 3.2012330 \\ -5.4953610 & 2.7795440 & 1.6979520\end{array}$

$\begin{array}{ll}\mathrm{C} & -4.9199730 \\ \mathrm{H} & -2.9115430 \\ \mathrm{C} & -6.1688930 \\ \mathrm{H} & -7.3449500 \\ \mathrm{H} & -4.7551600 \\ \mathrm{H} & -6.9820330 \\ \mathrm{H} & -1.1795930 \\ \mathrm{~S} & -3.0213710\end{array}$
$-0.1600520$
$-0.1990940$
0.4552030
1.9979880
$-0.9818090$
0.1079620
1. 1735360
3. 1688160

4. 1522790

3. 3896520

4.0793170

3. 1417260

4.8420340

4.7093400

0.9771930

0.6433060

TS237(S, S) G[WB97XD/6-31G(d)] $=-3633.715128$

$\begin{array}{lrcr}\text { TS237(S, S }) \text { G }[W B 97 X D / 6-31 G(d)]=-3633.715128 \\ \text { O } & -1.4424900 & 0.5926450 & -0.6486830 \\ \text { O } & 0.3508460 & 1.6023430 & 0.8097200 \\ \mathrm{C} & -2.3404440 & 1.4993370 & -0.1254430 \\ \mathrm{C} & -1.9879140 & 2.8315410 & -0.0743720 \\ \mathrm{C} & -3.6104940 & 1.0085280 & 0.2751520 \\ \mathrm{C} & -2.9183280 & 3.7600390 & 0.4983820 \\ \mathrm{C} & -4.5351400 & 1.9267340 & 0.7042560 \\ \mathrm{C} & -4.2168010 & 3.2996190 & 0.8510940 \\ \mathrm{H} & -5.5309550 & 1.5897010 & 0.9783960 \\ \mathrm{C} & 0.4834190 & 2.6339810 & -0.1011450 \\ \mathrm{C} & 1.8011370 & 3.0321620 & -0.4496370 \\ \mathrm{C} & -0.6515890 & 3.2595090 & -0.5707900 \\ \mathrm{C} & 1.9342290 & 4.0995890 & -1.3006810 \\ \mathrm{C} & -0.4986480 & 4.2918460 & -1.5543040 \\ \mathrm{C} & 0.8112470 & 4.7310840 & -1.8914390 \\ \mathrm{H} & 2.9289700 & 4.4486180 & -1.5648000 \\ \mathrm{C} & -0.1089430 & 6.3355140 & -3.4624170 \\ \mathrm{C} & -1.4100280 & 5.8703480 & -3.1615480 \\ \mathrm{C} & -1.6004300 & 4.8780830 & -2.2337780 \\ \mathrm{C} & 0.9758730 & 5.7720060 & -2.8432840 \\ \mathrm{H} & 0.0264850 & 7.1255640 & -4.1945770 \\ \mathrm{H} & -2.2659990 & 6.2996160 & -3.6732230 \\ \mathrm{H} & -2.6032660 & 4.5241410 & -2.0225580 \\ \mathrm{H} & 1.9832820 & 6.1034730 & -3.0808530 \\ \mathrm{C} & -3.5240980 & 5.9770670 & 1.2887200 \\ \mathrm{C} & -4.8295750 & 5.5269540 & 1.5939810 \\ \mathrm{C} & -5.1628590 & 4.2146080 & 1.3841990 \\ \mathrm{C} & -2.5948780 & 5.1197710 & 0.7568150 \\ \mathrm{H} & -3.2542740 & 7.0107600 & 1.4823140 \\ \mathrm{H} & -5.5573370 & 6.2190620 & 2.0061540 \\ \mathrm{H} & -6.1543090 & 3.8474120 & 1.6361350 \\ \mathrm{H} & -1.5956440 & 5.4799350 & 0.5391840 \\ \mathrm{C} & -3.9363420 & -0.4510290 & 0.2583700 \\ \mathrm{C} & -4.0861200 & -1.1418610 & 1.4808910 \\ \mathrm{C} & -4.1548760 & -1.1203760 & -0.9564090 \\ \mathrm{C} & -4.5044130 & -2.4693860 & 1.4489970\end{array}$




$\begin{array}{rrr}-4.5702920 & -2.4552990 & -0.9332130 \\ -4.7636160 & -3.1452940 & 0.2583230 \\ -4.6335830 & -3.0052180 & 2.3869930 \\ -4.7565490 & -2.9567510 & -1.8791790 \\ 2.9915800 & 2.3070770 & 0.0876930 \\ 3.8070170 & 1.5521250 & -0.7768990 \\ 3.3135160 & 2.4032570 & 1.4566160 \\ 4.9353610 & 0.9196510 & -0.2487100 \\ 4.4480260 & 1.7512400 & 1.9326550 \\ 5.2754030 & 1.0033340 & 1.0972090 \\ 5.5796870 & 0.3481380 & -0.9141410 \\ 4.7020300 & 1.8448320 & 2.9858990 \\ -0.1585120 & 0.1613530 & 0.2595240 \\ -0.5649580 & -0.6349310 & 1.4661590 \\ 0.7964220 & -0.4486950 & -0.7359910 \\ -3.9854990 & -0.4350300 & -2.3046640 \\ -3.6581210 & 0.5936910 & -2.1314940 \\ -5.2820100 & -4.5734210 & 0.3193530 \\ -4.7108550 & -5.0877860 & 1.1044630 \\ -3.8312680 & -0.4836900 & 2.8325100 \\ -3.3158730 & 0.4646660 & 2.6572600 \\ 2.4969080 & 3.2448500 & 2.4280650 \\ 1.6216610 & 3.6351100 & 1.9028250 \\ 6.5388340 & 0.3457330 & 1.6234940 \\ 6.9324890 & -0.2904150 & 0.8192090 \\ 3.5465080 & 1.4365340 & -2.2743330 \\ 2.5775280 & 1.8939100 & -2.4899980 \\ 3.4656720 & -0.0169800 & -2.7567570 \\ 3.3220540 & -0.0414680 & -3.8428930 \\ 4.3791250 & -0.5797220 & -2.5309480 \\ 2.6186660 & -0.5239560 & -2.2919380 \\ 4.6141000 & 2.2118090 & -3.0611160 \\ 5.6060880 & 1.7676430 & -2.9162150 \\ 4.3902770 & 2.1922160 & -4.1335540 \\ 4.6686030 & 3.2580930 & -2.7418470 \\ -2.9008240 & -1.1197090 & -3.1461450 \\ -3.1690460 & -2.1566800 & -3.3802200 \\ -2.7616090 & -0.5899990 & -4.0949630 \\ -1.9442000 & -1.1204340 & -2.6146670 \\ -5.3176420 & -0.3573060 & -3.0627380 \\ -5.1875540 & 0.1793660 & -4.0090220 \\ -5.7065990 & -1.3553930 & -3.2946560 \\ -6.0735060 & 0.1694050 & -2.4704650 \\ 1.9817930 & 2.4173490 & 3.6119920 \\ 2.8056240 & 2.0434910 & 4.2308160 \\ 1.3402220 & 3.0336410 & 4.2517890\end{array}$

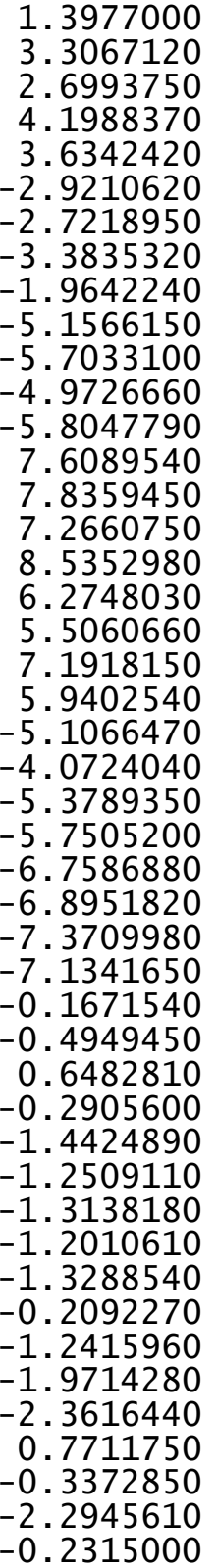

1.5616490 4.4599100

5.0904280

4.1504370

5.0681390

$-1.3235640$

$-0.7786640$

$-2.2784110$

$-1.5240100$

$-0.1802540$

$-1.1078380$

0.3254080

0.4607350

1.4006920

2.0046290

2.0769130

0.9240030

$-0.5461020$

$-1.2966240$

$-1.0682180$

0.0451860

$-5.3650990$

$-5.3381370$

$-6.4130610$ $-4.9772270$

$-4.5757690$

$-4.0685280$

$-4.0561030$

$-5.6005860$

$-3.6601000$

$-3.2703930$

$-3.1099300$

$-2.2464050$

$-3.0154750$

$-2.0566850$

$-5.8724730$

$-5.6284860$

$-4.1400310$

$-5.1288190$

$-6.9428260$

$-6.1863700$

$-3.7915110$

$-5.2247060$

$-5.5361080$

$-5.9969830$
3.2611190

2.9030980

3. 5621940

3.4599710

2.0529660

3.7381870

4.6677920

4.0116660

3.2516930

3.5467560

3.7548080

4.5012550

2.9410530

1.9375870

1.0529790

2.7294620

2.2770600

2.8430180

2.6329240

3.1380640

3.7026720

$-0.9773860$

$-1.3349340$

$-0.8160910$

$-1.7750820$

0.7470460

1.7075120

0.0007310

0.8445720

0.6599410

$-0.7154120$

1.1292070

$-1.0046880$

0.8720780

1.1252200

0.2500390

$-1.2567800$

$-1.5898050$

1.0083900

0.4642340

$-1.7977960$

$-1.4492350$

0.7558520

2.0910270

0.6085670
-1.6140910 


\begin{tabular}{|c|c|c|c|c|c|c|c|}
\hline & & & & & & & \\
\hline r & -1.0813790 & -3.9234480 & -2.6328150 & $\mathrm{H}$ & -1.0187290 & 6.6030020 & -2.7226840 \\
\hline 故 & 2.9722160 & -3.0351580 & -0.7630540 & $\mathrm{H}$ & 1.2747430 & 6.8758350 & -3.6557790 \\
\hline 0 & 2.7310220 & -1.9666870 & -0.0649780 & $\mathrm{H}$ & 2.9338750 & 5.0621610 & -3.3945040 \\
\hline c & 4.3085530 & -3.6077560 & -0.4945350 & $\mathrm{H}$ & -1.6349050 & 4.5667790 & -1.5078250 \\
\hline c & 4.6084410 & -4.9408760 & -0.7972510 & $\mathrm{C}$ & 3.2595990 & 0.4695240 & -1.3650350 \\
\hline C & 5.2902860 & -2.7911730 & 0.0833640 & $\mathrm{C}$ & 3.5453250 & -0.4357950 & -2.4020530 \\
\hline c & 5.8712240 & -5.4497310 & -0.5225650 & $\mathrm{C}$ & 4.0244000 & 0.4650690 & -0.1825540 \\
\hline H & 3.8451180 & -5.5752970 & -1.2347290 & $\mathrm{C}$ & 4.6346630 & -1.2949570 & -2.2549600 \\
\hline $\mathrm{C}$ & 6.5541970 & -3.3021430 & 0.3471730 & $\mathrm{C}$ & 5.1180820 & -0.3968460 & -0.0935040 \\
\hline $\mathrm{H}$ & 5.0542290 & -1.7566220 & 0.3056460 & $\mathrm{C}$ & 5.4463800 & -1.2771950 & -1.1218820 \\
\hline c & 6.8455310 & -4.6316320 & 0.0474130 & $\mathrm{H}$ & 4.8717500 & -1.9978540 & -3.0500780 \\
\hline H & 6.0943980 & -6.4873100 & -0.7487150 & $\mathrm{H}$ & 5.7211870 & -0.3857130 & 0.8088770 \\
\hline $\mathrm{H}$ & 7.3133250 & -2.6640390 & 0.7891800 & $\mathrm{C}$ & -4.2476180 & -0.1618450 & -0.2987760 \\
\hline H & 7.8323940 & -5.0320640 & 0.2581180 & $\mathrm{C}$ & -4.4739330 & -1.1691170 & 0.6647320 \\
\hline$H$ & 1.9087690 & -1.4278220 & -0.3471900 & $\mathrm{C}$ & -4.8496820 & -0.2505480 & -1.5661070 \\
\hline & 1.9003200 & -3.7079670 & -1.8672310 & $\mathrm{C}$ & -5.3114130 & -2.2327990 & 47640 \\
\hline & & & & $\mathrm{C}$ & -5.6725050 & -1.3423450 & -1.8484670 \\
\hline & ,S) $\mathrm{G}[\mathrm{WB} 97 \mathrm{XD}$ & $G(d)]=-36$ & 078 & $\mathrm{C}$ & -5.9185810 & -2.3426390 & -0.9147150 \\
\hline 0 & 0.5715930 & 0.1883660 & -0.4273300 & $\mathrm{H}$ & -5.4939780 & -3.0044150 & 1.0790480 \\
\hline 0 & -1.7137230 & 0.3105360 & -1.5477600 & $\mathrm{H}$ & -6.1445610 & -1.4123440 & -2.8263260 \\
\hline c & 0.8991250 & 1.3841820 & -1.0262920 & $\mathrm{C}$ & 3.7078730 & 1.4133550 & 0.9688850 \\
\hline $\mathrm{C}$ & -0.0125010 & 2.4184840 & -1.0181450 & $\mathrm{C}$ & 6.6635290 & -2.1859440 & -1.0596520 \\
\hline c & 2.2133720 & 1.5245740 & -1.5422000 & $\mathrm{c}$ & 2.7207490 & -0.4555010 & -3.6816190 \\
\hline c & 0.3069690 & 3.6237890 & -1.7214410 & $\mathrm{C}$ & -3.8581130 & -1.1347160 & 2.0585180 \\
\hline C & 2.5406400 & 2.7156150 & -2.1403870 & $\mathrm{C}$ & -6.8224270 & -3.5157820 & -1.2485150 \\
\hline C & 1.6031010 & 3.7682370 & -2.2844820 & $\mathrm{C}$ & -4.6730220 & 0.8239850 & -2.6302350 \\
\hline $\mathrm{H}$ & 3.5494390 & 2.8586490 & -2.5186110 & $\mathrm{C}$ & -5.9974940 & 1.5555020 & -2.8897620 \\
\hline c & -2.1269050 & 1.2133930 & -0.5785790 & $\mathrm{H}$ & -6.7572310 & 0.8707740 & -3.2841140 \\
\hline c & -3.3879810 & 1.0110870 & 0.0420170 & $\mathrm{H}$ & -6.3887830 & 1.9981180 & -1.9674170 \\
\hline $\mathrm{C}$ & -1.2929930 & 2.2651070 & -0.2811550 & $\mathrm{H}$ & -5.8551120 & 2.3577280 & -3.6226420 \\
\hline $\mathrm{C}$ & -3.7733 & 1.9269330 & 0.9883510 & $\mathrm{C}$ & -4.0882690 & 0.2519550 & -3.9275220 \\
\hline c & -1.6552 & 3.1469060 & 0.7879730 & $\mathrm{H}$ & -3.1397520 & -0.2568760 & -3.7330410 \\
\hline c & -2.920 & 2.9771770 & 1.4118340 & $\mathrm{H}$ & -4.7743890 & -0.4638310 & -4.3947210 \\
\hline $\mathrm{H}$ & -4.7432850 & 1.8197060 & 1.4649760 & $\mathrm{H}$ & -3.9064000 & 1.0564300 & -4.6489930 \\
\hline c & -2.4359200 & 4.8064510 & 2.9305200 & $\mathrm{C}$ & -8.0597840 & -3.5474320 & -0.3413720 \\
\hline c & -1.1637270 & 4.9571170 & 2.3315070 & $\mathrm{H}$ & -8.7350760 & -4.3571270 & -0.6394510 \\
\hline c & -0.7824780 & 4.1516500 & 1.2877190 & $\mathrm{H}$ & -7.7784000 & -3.7142370 & 0.7048740 \\
\hline $\mathrm{c}$ & -3.292 & 3.8371050 & 2.4786740 & $\mathrm{H}$ & -8.6105200 & -2.6026110 & -0.3956630 \\
\hline H & -2.7251 & 5.4535060 & 3.7527140 & C & -6.0617390 & -4.8471560 & -1.1912860 \\
\hline $\mathrm{H}$ & -0.4805570 & 5.7153110 & 2.7026740 & $\mathrm{H}$ & -5.1975200 & -4.8353960 & -1.8633860 \\
\hline $\mathrm{H}$ & 0.2009510 & 4.2705580 & 0.8467150 & $\mathrm{H}$ & -5.6974530 & -5.0494950 & -0.1774370 \\
\hline $\mathrm{H}$ & -4.2658510 & 3.6994000 & 2.9427590 & $\mathrm{H}$ & -6.7156090 & -5.6760040 & -1.4847780 \\
\hline c & -0.2853720 & 5.8140150 & -2.5864260 & $\mathrm{C}$ & -4.9105590 & -0.7596360 & 3.1124230 \\
\hline $\mathrm{C}$ & 1.0147160 & 5.9670360 & -3.1219430 & $\mathrm{H}$ & -5.3847180 & 0.2020660 & 2.8918270 \\
\hline c & 1.9361640 & 4.9631010 & -2.9751400 & $\mathrm{H}$ & -5.7015610 & -1.5177890 & 64740 \\
\hline c & -0.6310 & rct & -1.9037860 & $\mathrm{H}$ & -4.4506810 & -0.6920660 & 048610 \\
\hline
\end{tabular}




\begin{tabular}{rrr}
-3.1781510 & -2.4565100 & 2.4412100 \\
-3.9061420 & -3.2640000 & 2.5802240 \\
-2.4617240 & -2.7612010 & 1.6752140 \\
-2.6410040 & -2.3340900 & 3.3888410 \\
4.5225530 & 2.7094770 & 0.8482670 \\
5.5956130 & 2.4956960 & 0.9193690 \\
4.3385290 & 3.2100810 & -0.1072170 \\
4.2588300 & 3.4035820 & 1.6542140 \\
3.9165190 & 0.7824690 & 2.3496240 \\
3.3872430 & -0.1718880 & 2.4409680 \\
4.9767980 & 0.6075500 & 2.5640000 \\
3.5319040 & 1.4530050 & 3.1254720 \\
7.0321900 & -2.6371310 & 0.3565610 \\
7.8261540 & -3.3897270 & 0.3147420 \\
7.4044070 & -1.8033430 & 0.9622980 \\
6.1751570 & -3.0769590 & 0.8773160 \\
7.8616730 & -1.5024020 & -1.7367040 \\
7.6310410 & -1.2376880 & -2.7737620 \\
8.1272180 & -0.5822930 & -1.2028880 \\
8.7362700 & -2.1626550 & -1.7366620 \\
2.5260450 & -1.8628730 & -4.2564030 \\
3.4487240 & -2.2505490 & -4.7036200 \\
2.1966620 & -2.5703880 & -3.4921130 \\
1.7675160 & -1.8365170 & -5.0450700 \\
3.3470610 & 0.4581010 & -4.7462630 \\
4.3601180 & 0.1182100 & -4.9930510 \\
2.7491550 & 0.4409330 & -5.6645110 \\
3.4119520 & 1.4945950 & -4.4036950 \\
-0.6203960 & -0.7756740 & -1.0378740 \\
-0.1377080 & -1.5596500 & -2.2179450 \\
-1.1120890 & -1.5219060 & 0.1772800 \\
1.7261060 & -0.0680020 & -3.4397120 \\
6.4222420 & -3.0855290 & -1.6414950 \\
2.6479530 & 1.6821040 & 0.9018010 \\
-3.9653780 & 1.5698890 & -2.2582730 \\
-7.1710160 & -3.3758730 & -2.2798010 \\
-3.0836910 & -0.3646390 & 2.0708020 \\
0.4271210 & -0.6883910 & 3.0514840 \\
-0.5297070 & -0.2963280 & 2.2801660 \\
0.8800630 & 0.3660880 & 3.9845140 \\
1.6827110 & 0.0744540 & 5.0926740 \\
0.5385470 & 1.6977520 & 3.7065050 \\
2.1364850 & 1.0992350 & 5.3119040 \\
1.9488530 & -0.9557210 & 2.8439100 \\
1.0097610 & 2.7204760 & \\
-0.0765190 & 1.9291420 & \\
-0 & & \\
\hline & &
\end{tabular}

$$
\begin{array}{r}
1.8071330 \\
2.7528450 \\
0.7523030 \\
2.1727110 \\
-0.7623320 \\
1.1135570 \\
0.8804770 \\
1.8611810 \\
-0.1585180 \\
1.6282970 \\
1.4314660 \\
0.8564520 \\
2.6275930 \\
3.4154910 \\
3.2649910 \\
1.1389030 \\
2.7538440 \\
4.4776160 \\
3.8021900 \\
0.7106390 \\
0.5790320 \\
3.0191600 \\
3.0567290 \\
3.6950630
\end{array}
$$$$
144
$$

.4232900

0.8666900

3.7492270

3.2230680

$-0.9191170$

$-2.2315110$

$-3.7942460$

$-2.7775590$

$-3.4841790$

$-1.7337000$

$-3.5078900$

$-2.8102520$

$-5.5780360$

$-4.5660020$

$-3.1511430$

$-5.2214920$

$-6.5840410$

$-4.8282750$

$-3.0445250$

$-5.3396830$

$-5.8821940$

$-5.5971520$

$-4.5853620$

$-2.3881380$
5.6227720

6.7743150

4.2872070

6.2596680

1.4929110

3.0035740

0.0752700

0.4864640

0.1554190

0.3070680

$-1.2353040$

$-1.6714230$

0.5188270

1. 3530240

0.7929230

0.4975140

0.9299660

1. 3785400

$-0.1597150$

1.5006770

$-0.1716340$

$-0.5052510$

2. 3901210

1.4470970

$\operatorname{TS} 239(\mathrm{~S}, \mathrm{~S}) \quad \mathrm{G}[\mathrm{WB} 97 \mathrm{XD} / 6-31 \mathrm{G}(\mathrm{d})]=-3633.714172$

$\begin{array}{lrcr}\text { O } & -1.4568440 & 0.5425310 & -0.6666590 \\ \mathrm{O} & 0.2403840 & 1.6153790 & 0.8593520 \\ \mathrm{C} & -2.4259960 & 1.3856770 & -0.1672020 \\ \mathrm{C} & -2.1493080 & 2.7323750 & -0.0636590 \\ \mathrm{C} & -3.6867870 & 0.8171570 & 0.1485180 \\ \mathrm{C} & -3.1517310 & 3.5917530 & 0.4952400 \\ \mathrm{C} & -4.6773500 & 1.6720330 & 0.5621020 \\ \mathrm{C} & -4.4377530 & 3.0523620 & 0.7730470 \\ \mathrm{H} & -5.6672820 & 1.2759880 & 0.7702730 \\ \mathrm{C} & 0.3321590 & 2.6746680 & -0.0224410 \\ \mathrm{C} & 1.6324890 & 3.1600630 & -0.3237850 \\ \mathrm{C} & -0.8248640 & 3.2488430 & -0.5042380 \\ \mathrm{C} & 1.7247730 & 4.2644890 & -1.1320050 \\ \mathrm{C} & -0.7065180 & 4.3185930 & -1.4524680 \\ \mathrm{C} & 0.5825360 & 4.8492420 & -1.7333560 \\ \mathrm{H} & 2.7041540 & 4.6785210 & -1.3569150 \\ \mathrm{C} & -0.3892490 & 6.4450590 & -3.2817960 \\ \mathrm{C} & -1.6664290 & 5.8882960 & -3.0400430 \\ \mathrm{C} & -1.8218430 & 4.8562680 & -2.1500260 \\ \mathrm{C} & 0.7097450 & 5.9304130 & -2.6454000\end{array}$




$\begin{array}{rrr}-0.2820180 & 7.2660150 & -3.9840130 \\ -2.5314730 & 6.2788610 & -3.5672260 \\ -2.8056790 & 4.4322230 & -1.9839230 \\ 1.7003840 & 6.3333270 & -2.8387880 \\ -3.9078240 & 5.7465260 & 1.3254030 \\ -5.1990630 & 5.2184640 & 1.5578470 \\ -5.4533170 & 3.8986530 & 1.2921440 \\ -2.9121100 & 4.9571480 & 0.8086700 \\ -3.7023870 & 6.7860790 & 1.5618740 \\ -5.9794080 & 5.8575890 & 1.9592840 \\ -6.4340150 & 3.4727860 & 1.4870400 \\ -1.9250790 & 5.3749710 & 0.6453110 \\ -3.9454650 & -0.6524530 & 0.0486190 \\ -4.1830110 & -1.3973140 & 1.2249250 \\ -4.0416770 & -1.2753100 & -1.2079800 \\ -4.5894010 & -2.7256430 & 1.1073700 \\ -4.4456440 & -2.6112580 & -1.2695490 \\ -4.7572310 & -3.3449050 & -0.1296300 \\ -4.8046790 & -3.2886620 & 2.0125200 \\ -4.5568270 & -3.0866490 & -2.2421850 \\ 2.8501780 & 2.4754150 & 0.2042870 \\ 3.7192470 & 1.8103490 & -0.6816460 \\ 3.1414930 & 2.5138220 & 1.5831570 \\ 4.8694170 & 1.2093280 & -0.1638680 \\ 4.2935300 & 1.8858800 & 2.0489840 \\ 5.1735370 & 1.2266910 & 1.1926300 \\ 5.5569280 & 0.7142880 & -0.8470520 \\ 4.5192840 & 1.9270730 & 3.1121750 \\ -0.1994220 & 0.1569600 & 0.2955560 \\ -0.6263540 & -0.6368750 & 1.4972340 \\ 0.8051170 & -0.4300780 & -0.6617340 \\ -3.7710440 & -0.5405810 & -2.5137720 \\ -3.5006890 & 0.4933340 & -2.2839830 \\ -5.3103110 & -4.7553780 & -0.2399480 \\ -5.4055230 & -4.9842370 & -1.3091290 \\ -4.0511970 & -0.7931850 & 2.6193620 \\ -3.5683950 & 0.1830010 & 2.5239760 \\ 2.2709710 & 3.2674990 & 2.5796610 \\ 1.3926070 & 3.6525210 & 2.0552750 \\ 6.4408620 & 0.5765970 & 1.7176160 \\ 6.9235940 & 0.0739780 & 0.8684430 \\ 3.4881670 & 1.7483160 & -2.1872980 \\ 2.5118930 & 2.1884990 & -2.4040930 \\ 3.4509290 & 0.3111550 & -2.7229230 \\ 3.3239210 & 0.3232270 & -2.5024630 \\ 4.3750880 & -0.2362160 & \end{array}$

2.6102830

4.5449390

5.5473140

4.3349500

4.5646690

$-2.5883310$

$-2.7861920$

$-2.4007210$

$-1.6777490$

$-5.0264160$

$-4.8294370$

$-5.3427830$

$-5.8610300$

1.7645810

2.5896250

1.0827820

1.2264560

3.0214650

2. 3753950

3.9114710

3. 3454760

$-3.1680840$

$-3.0690080$

$-3.5967400$

$-2.1673380$

$-5.4323370$

$-5.9475370$

$-5.3312030$

$-6.0714710$

7.4248230

7.6644450

6.9988820

8.3574730

6.1460130

5.4332130

7.0674930

5.7217860

$-6.7120520$

$-7.3877390$

$-7.1387500$

$-6.6775880$

$-4.3735100$

$-3.4088670$

$-4.1890830$

$-4.8103310$

0.1945630

-0.2342290
2.5816060
2.1599210
2.5996840
3.6151060
-1.1658430
-2.2140080
-0.6257550
-1.1204070
-0.4857980
0.0993840
-1.4872830
-0.0198440
2.3567780
1.9858390
2.9109780
1.4973690
4.4836210
5.0536130
4.1766290
5.1508410
-1.6374800
-1.1380210
-2.6291610
-1.7632120
-0.5784420
-1.5360430
-0.1008740
0.0554390
1.6281540
2.3682710
2.1593320
1.1544060
-0.4825510
-1.2304130
-0.9988930
-0.0239360
-4.8479860
-4.1079150
-5.8438650
-4.6712630
-5.8005720
-5.8132830
-5.5943230
-6.8022400
-3.5655150

$-2.2895540$ $-2.9274710$ $-2.7877050$ $-4.0026780$ $-2.5649600$ $-3.2655120$ $-3.5191960$ $-4.2002130$ $-2.6607310$ $-3.3954570$ $-4.3005420$ $-3.7084050$ $-2.8608520$

3.7050040

4. 3241050

4.3596450

3.2957560

3.1412180

3.8182990

3.7028430

2. 3351800

3.5474300

4.5176710

3.7298270

3.1297160

3.2563550

3. 3979450

4.2373130

2.6338750

2.2486120

1.4783800

3.1076710

2.5745040

2.7879560

2.4264200

3.0790320

3.6883300

0.3791270

$-0.0618760$

0.2167050

1.4603080

0.3756540

$-0.1393020$

1.4363220

0.2972420

0.7692090 


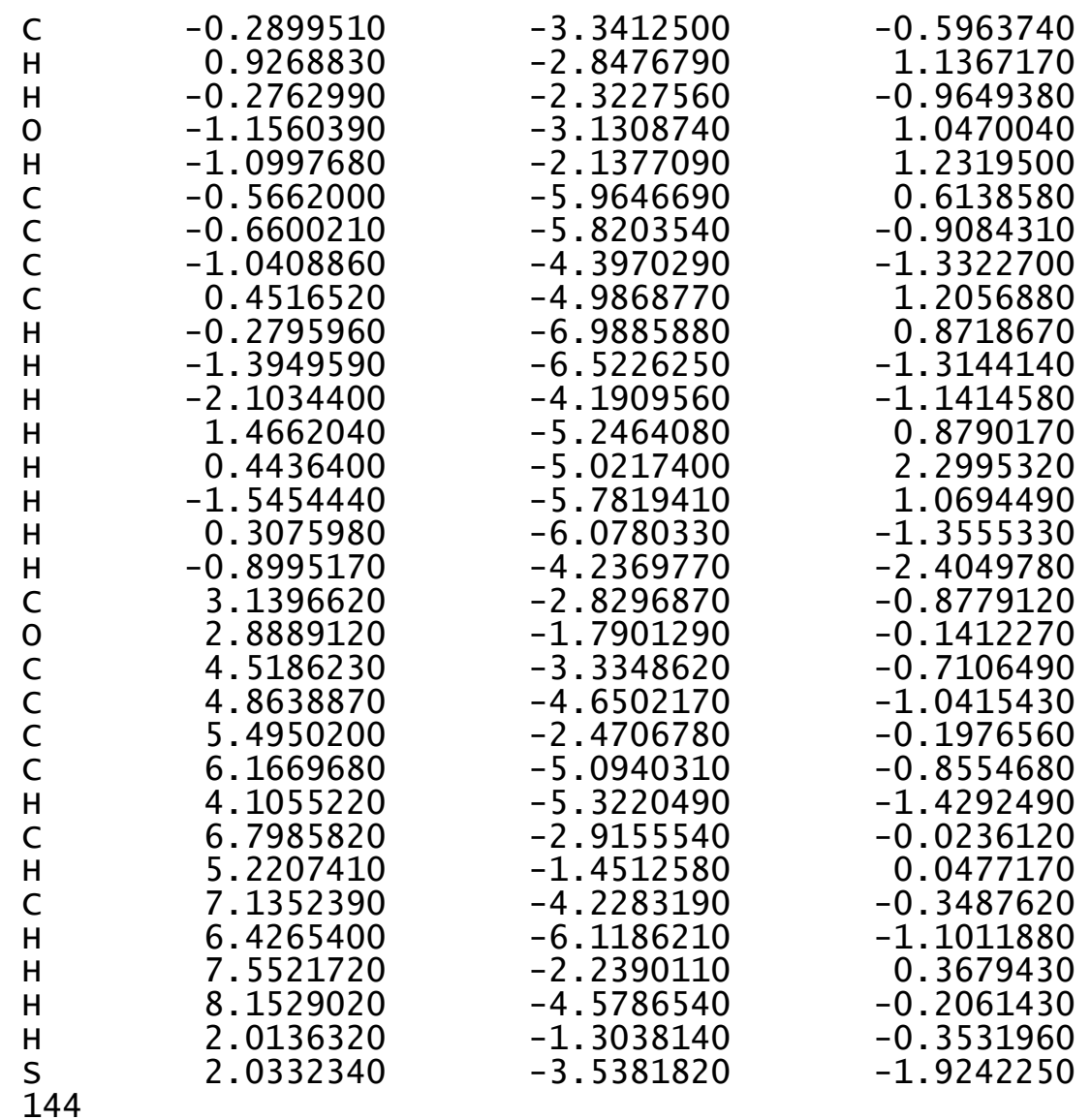

144

TS240(S, S ) G[WB97XD/6-31G(d)] = -3633.717937

$\begin{array}{lrlr}\text { O } & 0.5711840 & 0.1710660 & -0.3986040 \\ \mathrm{O} & -1.6960750 & 0.2011180 & -1.5689230 \\ \mathrm{C} & 0.8929390 & 1.3477070 & -1.0374440 \\ \mathrm{C} & -0.0415400 & 2.3601550 & -1.0957910 \\ \mathrm{C} & 2.2170350 & 1.4990010 & -1.5227360 \\ \mathrm{C} & 0.2648070 & 3.5403200 & -1.8449280 \\ \mathrm{C} & 2.5274460 & 2.6691790 & -2.1708110 \\ \mathrm{C} & 1.5689450 & 3.6894370 & -2.3874810 \\ \mathrm{H} & 3.5426350 & 2.8212800 & -2.5278480 \\ \mathrm{C} & -2.1410530 & 1.1330960 & -0.6421680 \\ \mathrm{C} & -3.4099200 & 0.9359000 & -0.0360790 \\ \mathrm{C} & -1.3315440 & 2.2113390 & -0.3748590\end{array}$

-3.8252280
-1.7267760
-2.9981340
-4.8017370
-2.5721700
-1.2946020
-0.8814570
-3.4029140
-2.8863820
-0.6323320
0.1058290
-4.3796280
-0.3571700
0.9500420
1.8901690
-0.6921470
-1.1039450
1.2012190
2.8940970
-1.7012370
3.3061830
3.6919600
4.0460860
4.8667670
5.2198470
5.6651060
5.1756230
5.8218940
-4.2537730
-4.4837850
-4.8439260
-5.3123860
-5.6594220
-5.9086380
-5.4974390
-6.1240000
3.6268620
6.9710530
2.8921900
-3.8827920
-6.8049010
-4.6647800
-5.9902920
-6.7452790
-6.3881710
-5.8467430

3.1300680

2. 9617690

1.7757800

4.8641040

5.0152440

4.1730100

3.8586310

5.5403890

. 8026280

4.2926430

3.7211690

5.6759530

5.8343120

4.8593670

4.5615380

6.4418950

6.7243610

4.9626120

4.4476680

0.5042620

$-0.4117980$

0.6130820

$-1.1464090$

$-0.1276250$

$-0.9914940$

$-1.8356530$

$-0.0220550$

$-0.2546150$

$-1.2379000$

$-0.3770010$

$-2.3143690$

$-1.4790480$

$-2.4571130$

$-3.0688740$

$-1.5749500$

1.5729950

$-1.7481640$

$-0.5664550$

$-1.1601020$

$-3.6419590$

0.6748720

1. 3964250

0.7013220

1.8549340

2.1852150
0.8685680

0.6504730

1. 2628700

1.3323470

2. 7074250

2.1199120

1.1182490

2.2864730

3.4961990

2.4674200

0.6859170

2.7424590

$-2.8179790$

$-3.3347320$

$-3.1250070$

$-2.0919760$

$-3.0036310$

$-3.9033200$

$-3.5282930$

$-1.7108140$

$-1.2632330$

$-2.2553120$

$-0.0680410$

$-2.0719070$

0.0590400

$-0.9413520$

$-2.8514460$

0.9586480

$-0.3548130$

0.6317460

$-1.6248180$

0.3208690

$-1.8870170$

$-0.9306050$

1.0819230

$-2.8662850$

1.0402880

$-0.7524250$

$-3.5416900$

2. 0299590

$-1.2430820$

$-2.7108400$

$-2.9920220$

3. 3772740

-2.0803210
-3.7391260 


$\begin{array}{rr}-4.0707630 & 0.0785610 \\ -3.1213780 & -0.4226050 \\ -4.7513960 & -0.6494800 \\ -3.8879650 & 0.8688230 \\ -8.0417660 & -3.6672050 \\ -8.7125980 & -4.4847230 \\ -7.7595970 & -3.8179740 \\ -8.5976300 & -2.7262550 \\ -6.0342980 & -4.9664260 \\ -5.1720540 & -4.9607850 \\ -5.6654970 & -5.1463660 \\ -6.6825060 & -5.8057340 \\ -4.9488460 & -0.7644740 \\ -5.4269510 & 0.1874940 \\ -5.7350900 & -1.5267320 \\ -4.4996110 & -0.6663220 \\ -3.1953520 & -2.4649790 \\ -3.9176780 & -3.2756250 \\ -2.4670520 & -2.7811750 \\ -2.6707720 & -2.3140180 \\ 4.3607470 & 2.9150550 \\ 5.4420290 & 2.7731990 \\ 4.1787260 & 3.3746830 \\ 4.0253370 & 3.6138090 \\ 3.8255880 & 0.9945340 \\ 3.3294420 & 0.0244910 \\ 4.8859860 & 0.8658390 \\ 3.3976140 & 1.6732700 \\ 7.7135060 & -2.0221800 \\ 8.7032400 & -2.4409920 \\ 7.1780190 & -2.7481570 \\ 7.8446610 & -1.1051250 \\ 6.7428160 & -3.0542390 \\ 6.2836390 & -2.8578930 \\ 6.0829130 & -3.7270190 \\ 7.6920330 & -3.5743590 \\ 2.8141100 & -2.0189660 \\ 3.7684780 & -2.3610560 \\ 2.5348450 & -2.7002760 \\ 2.0634010 & -2.1017990 \\ 3.4621120 & 0.3265990 \\ 4.5097730 & 0.0706750 \\ 2.8967280 & 0.1884510 \\ 3.4166420 & 1.3857710 \\ -0.5826910 & -0.8400170 \\ -0.0503640 & -1.6352930\end{array}$

-3.9926820
-3.7829190
-4.4487950
-4.7294510
-0.3350830
-0.6216210
0.7133580
-0.4026330
-1.1630300
-1.8377380
-0.1465570
-1.4389210
3.0623130
2.8102210
3.1191590
4.0571070
2.4541830
2.6043420
1.7041170
3.4047560
0.9063180
1.0197620
-0.0700120
1.6810300
2.4450410
2.5544590
2.6887710
3.1904620
-2.0634990
-1.8540880
-2.6856700
-2.6469840
0.0222670
0.9967630
-0.5396070
0.1915480
-4.0271700
-4.4444410
-3.2196760
-4.8196560
-4.6537590
-4.8526090
-5.5822260
-4.3848090
-1.0102860
-2.1616890
$-1.0746380$
1.8667760
7. 6169040
2. 5551580
$-3.9619320$
$-7.1549630$
$-3.1154850$
0.3664400
$-0.5818630$
0.7562870
1. 5362260
0.3790690
1. 9322830
1.8312540
0.7929970
$-0.2173950$
1. 5680530
2. 5304030
0. 5082860
1.8893930
$-0.7731040$
1.1067300
1. 0292930
1. 9350950
$-0.0286170$
1.6353540
1.5843120
0.9781390
2. 8798300
3. 5863570
3. 3543530
1. 3713240
3.0654190
4.6617270
3. 8999730
0.9359620
0.8670410
3. 2876470
3. 2112080
3. 7216960

144
$-1.5718880$
$-0.2445820$
$-1.1115940$
1.7702060
1.4307470
$-3.5223820$
$-0.3828900$
$-0.5859820$
$-0.2552180$
0.5165800
0.2928390
1.8250420
1. 3617350
$-0.7186980$
2.8922240
2.0038620
2. 6625320
1.1817760
3.9023580
3.4969480
$-0.9146740$
$-2.1038290$
$-3.7502680$
$-2.6620710$
$-3.5057900$
$-3.4639440$
$-2.8157220$
$-5.4041070$
$-4.3225260$
$-5.1477020$
$-6.3894810$
$-4.5159190$
$-2.8072160$
$-5.2712370$
$-5.8583510$
$-5.4214820$
$-4.3401430$
$-2.1303490$

$-3.3342040$

$-0.1334580$

0.9285420

$-2.3503680$

$-2.2764230$

2. 0291840

3. 1002390

2. 2897830

4.0058270

5.1456990

3.6699560

5.9381380

5.4030330

4.4561690

2. 7822920

5.5913730

6.8255550

4.1789610

6.2073670

1. 5237100

3. 1236720

0.2275380

0.6286800

0.2830680

0.4228130

$-1.0806740$

$-1.5507910$

0.7386320

1.5578790

0.9631700

0.6880870

1.1762660

1. 6069910

0.0173480

1.6875160

0.0259260

$-0.2792570$

2. 5892440

1.6079400

TS241(S, S) G[WB97XD/6-31G(d) $]=-3633.716567$

$\begin{array}{lrcr}\text { TS241(S, S }) & \text { G }[W B 97 X D / 6-31 G(d)]= & -3633.716567 \\ \text { O } & 0.5757110 & 0.5797540 & -0.1819220 \\ \text { O } & -1.6538230 & 0.9827150 & -1.3349890 \\ \text { C } & 0.8558250 & 1.9256670 & -0.2678280 \\ \text { C } & -0.1234970 & 2.8408930 & 0.0547850\end{array}$




\begin{tabular}{|c|c|c|}
\hline $\begin{array}{l}2.1871190 \\
0.1626430 \\
2.4813450 \\
1.4879460 \\
3.5039030 \\
2.1647170 \\
3.4415190 \\
1.4316640 \\
3.9648250 \\
1.9330670 \\
3.2300060 \\
4.9583240 \\
3.0102350 \\
1.7015720 \\
1.1768560 \\
3.7535530 \\
3.4166200 \\
1.1044730 \\
0.1674490 \\
4.7519670 \\
0.5108160 \\
0.8165160 \\
1.7915610 \\
0.8295720 \\
1.2849670 \\
1.0544470 \\
2.8122340 \\
1.8534550 \\
3.2729420 \\
3.7500950 \\
3.8904090 \\
4.8933530 \\
5.0399090 \\
5.5703240 \\
5.2833100 \\
5.5305650 \\
4.1839530 \\
4.3180850 \\
4.7712680 \\
5.0709000 \\
5.5037290 \\
5.6723620 \\
5.1914760 \\
5.9636530 \\
3.3579710 \\
6.8662600\end{array}$ & $\begin{array}{r}2.2959180 \\
4.2346520 \\
3.6356890 \\
4.6286380 \\
3.9479410 \\
1.4449620 \\
0.9760390 \\
2.3666090 \\
1.4966190 \\
2.8050520 \\
2.3864050 \\
1.1924890 \\
3.6405160 \\
4.0192950 \\
3.6145720 \\
2.8369910 \\
3.9762620 \\
4.6321960 \\
3.9036320 \\
2.5192750 \\
6.5679100 \\
6.9595350 \\
6.0082300 \\
5.2433690 \\
7.3233390 \\
8.0120390 \\
6.2933520 \\
4.9568850 \\
1.2878620 \\
1.0175280 \\
0.7017130 \\
0.2291140 \\
-0.0657120 \\
-0.2977290 \\
0.0276470 \\
-0.4923990 \\
-0.0509100 \\
-1.3609670 \\
0.2950610 \\
-2.2826560 \\
-0.6703740 \\
-1.9606070 \\
-3.2879690 \\
-0.4066860 \\
0.9338180 \\
-1.0625870\end{array}$ & $\begin{array}{r}-0.5859360 \\
-0.1111760 \\
-0.6282100 \\
-0.4399390 \\
-0.8221550 \\
-0.1336960 \\
0.2727100 \\
0.5780900 \\
1.4291350 \\
1.8477000 \\
2.2522600 \\
1.7459490 \\
4.3176340 \\
3.9387500 \\
2.7378000 \\
3.4930600 \\
5.2667130 \\
4.6070350 \\
2.4674290 \\
3.7820440 \\
-0.1403220 \\
-0.4329070 \\
-0.5819150 \\
0.0190780 \\
-0.0468000 \\
-0.5514780 \\
-0.8228580 \\
0.2331170 \\
-0.7927540 \\
-2.0877800 \\
0.3282270 \\
-2.2252780 \\
0.1404080 \\
-1.1269610 \\
-3.2204850 \\
1.0096300 \\
-0.5188610 \\
-0.0111550 \\
-1.7473690 \\
-0.7357220 \\
-2.4407570 \\
-1.9512700 \\
-0.3399450 \\
-3.3910230 \\
1.7364630 \\
-1.3435820\end{array}$ \\
\hline
\end{tabular}

3.0662940
-3.6854270
-6.4880700
-4.6515450
-6.0226290
-6.6946770
-6.5015810
-5.9148370
-3.9467780
-2.9641200
-4.5345890
-3.8085380
-7.7261690
-8.3386160
-7.4386540
-8.3446530
-5.6391790
-4.7658380
-5.2803230
-6.2301660
-4.7297960
-5.2217470
-5.5063250
-4.2577010
-2.9691810
-3.6739040
-2.2470360
-2.4335360
4.0993050
5.1659920
4.0063460
3.6896930
3.4028570
2.9532620
4.4274060
2.8468900
7.1268790
8.0142200
7.3140810
6.2838790
8.0470630
7.8968480
8.1552790
8.9837670
2.9681990
3.9445570

1.5847420

$-1.7980640$

$-2.9856310$

1. 6913620

2.3744890

1.8278760

2. 4321280

3. 3928880

1.6627540

1897010

1.1091560

2.6819150

$-3.4223300$

$-4.1133130$

$-3.9346280$

$-2.5600840$

$-4.1978770$

$-3.8894060$

$-4.7407230$

$-4.8944270$

$-1.8454890$

$-0.8783480$

$-2.5861490$

$-2.1279560$

$-3.1509520$

$-3.9754600$

$-3.1488710$

$-3.3558570$

2. 0944450

1.8637660

3. 0154810

2. 2806970

$-0.3274490$

$-1.1827610$

$-0.6021000$

$-0.1638070$

$-2.1600940$

$-2.7364060$

$-1.7401960$

$-2.8541940$

$-0.0801490$

0.6792800

0.4343050

$-0.6077400$

0.5678550

0.3780070
$-3.3249930$

1. 3054010

$-2.7183450$

$-2.3415660$

$-3.1080060$

$-1.4532190$

$-2.8264830$

$-3.7041690$

$-3.6208730$

$-4.4454820$

$-4.0824040$

$-1.9234510$

$-2.5133980$

$-0.9978840$

$-1.6529700$

$-3.1236910$

$-3.7075350$

$-2.2416390$

$-3.7287690$

2. 4304570

2. 5711330

2. 2045470

3. 3784970

1. 2014330

1. 0454240

0.3815190

2. 1355590

2.4139260

2. 5192600

1.8289400

3.4130670

2. 6068290

2. 0910280

2. 8821330

3. 5359210

$-0.3081600$

$-0.5902140$

0.6864340

$-0.2298710$

$-1.3932340$

$-2.1675580$

$-0.4310430$

$-1.6050890$

$-4.4688820$

$-4.9294170$ 


$\begin{array}{rrrr}\text { H } & 2.5634030 & -0.3864560 & -4.1249210 \\ \mathrm{H} & 2.3080770 & 0.9560850 & -5.2515080 \\ \mathrm{C} & 3.7749120 & 2.8552860 & -3.8167460 \\ \mathrm{H} & 4.8187560 & 2.6379290 & -4.0732320 \\ \mathrm{H} & 3.2796790 & 3.2479230 & -4.7118740 \\ \mathrm{H} & 3.7710030 & 3.6409770 & -3.0563840 \\ \mathrm{P} & -0.4634180 & -0.1153240 & -1.2464750 \\ \mathrm{O} & 0.1367750 & -0.2308850 & -2.6159130 \\ \mathrm{O} & -0.8934580 & -1.3691670 & -0.5289440 \\ \mathrm{H} & 2.0417230 & 1.8492370 & -3.0442230 \\ \mathrm{H} & 6.7937120 & -1.5446520 & -2.3274430 \\ \mathrm{H} & 2.3054590 & 1.2194730 & 1.6571450 \\ \mathrm{H} & -4.0380610 & 2.3011900 & -1.6722420 \\ \mathrm{H} & -6.8381390 & -2.5017420 & -3.6391050 \\ \mathrm{H} & -2.9285940 & -1.0574550 & 1.5784790 \\ \mathrm{C} & 1.1579100 & -3.2736520 & -1.8126180 \\ \mathrm{C} & 2.0405840 & -2.6089910 & -0.8482810 \\ \mathrm{H} & 0.0961570 & -3.0893280 & -1.6650500 \\ \mathrm{H} & 1.7177420 & -1.6642710 & -0.4266730 \\ \mathrm{O} & 1.7675130 & -2.2848800 & -2.6816390 \\ \mathrm{H} & 1.1559070 & -1.4803260 & -2.7181600 \\ \mathrm{C} & 3.0157100 & -4.9232210 & -2.2969890 \\ \mathrm{C} & 3.6977220 & -4.5063590 & -0.9917630 \\ \mathrm{C} & 3.4591760 & -3.0263960 & -0.6851470 \\ \mathrm{C} & 1.5047030 & -4.6840720 & -2.2254850 \\ \mathrm{H} & 3.2052130 & -5.9816910 & -2.4984990 \\ \mathrm{H} & 4.7752520 & -4.6896640 & -1.0458070 \\ \mathrm{H} & 4.0360130 & -2.3861050 & -1.3649540 \\ \mathrm{H} & 1.0515300 & -5.3559360 & -1.4861200 \\ \mathrm{H} & 1.0233170 & -4.8889000 & -3.1867710 \\ \mathrm{H} & 3.4391240 & -4.3551680 & -3.1336550 \\ \mathrm{H} & 3.3107050 & -5.1155920 & -0.1651910 \\ \mathrm{H} & 3.7897200 & -2.7475170 & 0.3194740 \\ \mathrm{C} & 0.4551520 & -2.4584490 & 2.3834390 \\ \mathrm{O} & -0.2478990 & -1.4813790 & 1.9114210 \\ \mathrm{C} & 0.6130060 & -2.3959990 & 3.853550 \\ \mathrm{C} & 1.6068620 & -3.1321200 & 4.5091020 \\ \mathrm{C} & -0.2281740 & -1.5554220 & 4.5968440 \\ \mathrm{C} & 1.7590690 & -3.0278100 & 5.8852890 \\ \mathrm{H} & 2.2650380 & -3.7710660 & 3.9304530 \\ \mathrm{C} & -0.0778930 & -1.4620660 & 5.9743140 \\ \mathrm{H} & -0.9972730 & -0.9832590 & 4.0903490 \\ \mathrm{C} & 0.9170960 & -2.1944600 & 6.6197370 \\ \mathrm{H} & 2.5387650 & -3.5931260 & 6.3854670 \\ \mathrm{H} & -0.7354770 & -0.8150930 & 6.5458490 \\ \mathrm{H} & 1.0386070 & -2.1130300 & 7.6954850 \\ & & & \\ & & & \end{array}$

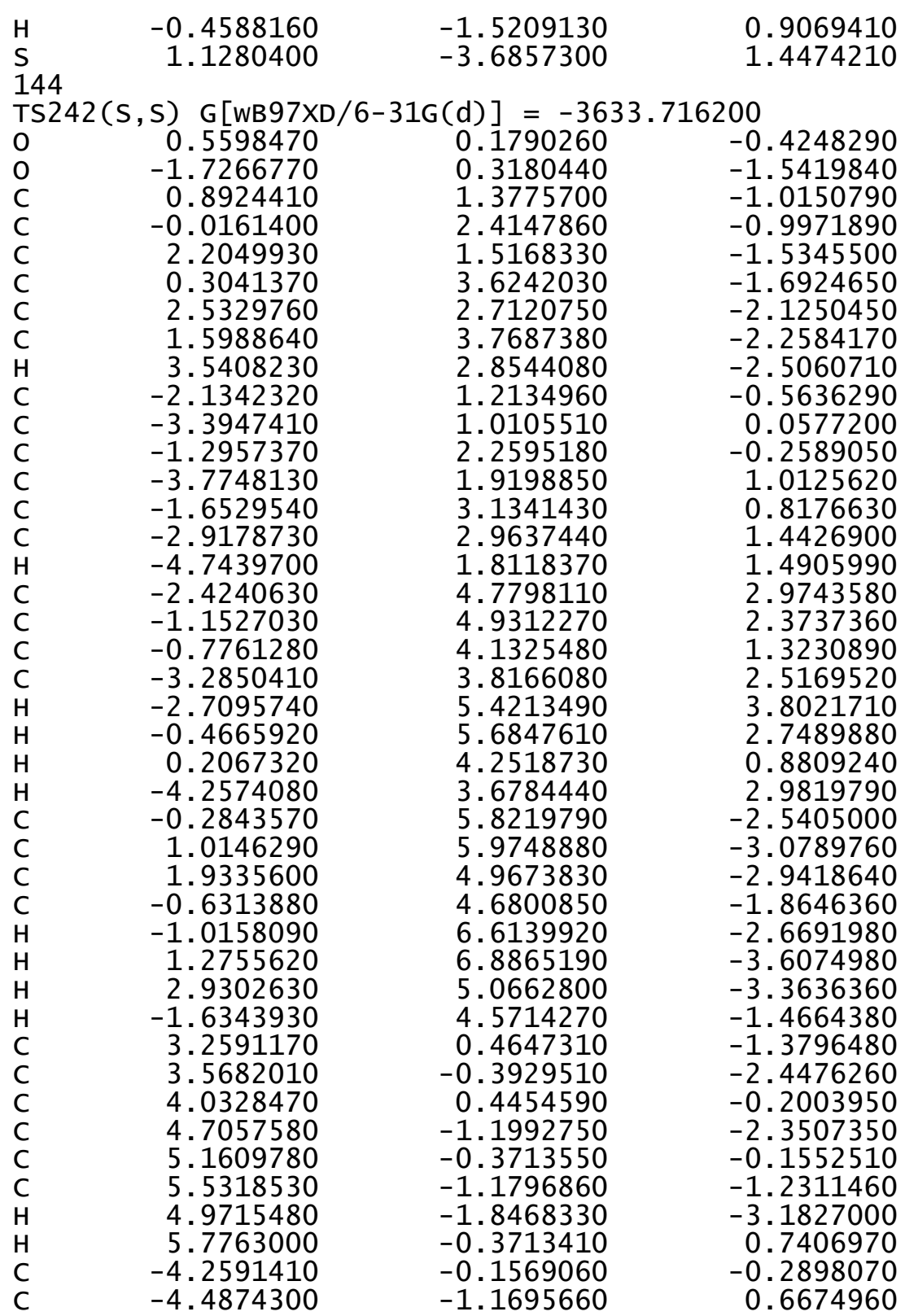




\begin{tabular}{|c|c|c|}
\hline $\begin{array}{l}-4.8643410 \\
-5.3299640 \\
-5.6920070 \\
-5.9403300 \\
-5.5141060 \\
-6.1663810 \\
3.6961760 \\
6.7984450 \\
2.7264600 \\
-3.8678320 \\
-6.8508360 \\
-4.6860250 \\
-6.0087180 \\
-6.7710360 \\
-6.3974890 \\
-5.8649080 \\
-4.1046390 \\
-3.1571100 \\
-4.7932640 \\
-3.9218060 \\
-8.0894420 \\
-8.7691890 \\
-7.8099790 \\
-8.6345610 \\
-6.0992010 \\
-5.2332230 \\
-5.7389310 \\
-6.7580440 \\
-4.9163790 \\
-5.3888680 \\
-5.7090150 \\
-4.4534630 \\
-3.1889770 \\
-3.9181410 \\
-2.4769090 \\
-2.6466660 \\
4.4785970 \\
5.5565480 \\
4.2817830 \\
4.1982800 \\
3.9268730 \\
3.3945880 \\
4.9898330 \\
3.5570260 \\
6.6761230 \\
7.6117780\end{array}$ & $\begin{array}{r}-0.2352050 \\
-2.2276840 \\
-1.3220580 \\
-2.3272240 \\
-3.0029830 \\
-1.3838950 \\
1.3510390 \\
-2.0183970 \\
-0.4118480 \\
-1.1479620 \\
-3.4938320 \\
0.8451810 \\
1.5820050 \\
0.9018250 \\
2.0206150 \\
2.3878930 \\
0.2789400 \\
-0.2333270 \\
-0.4325190 \\
1.0869480 \\
-3.5197200 \\
-4.3249450 \\
-3.6897310 \\
-2.5715930 \\
-4.8304290 \\
-4.8223340 \\
-5.0388390 \\
-5.6537260 \\
-0.7802380 \\
0.1846010 \\
-1.5369170 \\
-0.7229140 \\
-2.4742830 \\
-3.2812470 \\
-2.7744600 \\
-2.3608140 \\
2.6697110 \\
2.4802260 \\
3.1883670 \\
3.3372660 \\
0.6916070 \\
-0.2615610 \\
0.5092330 \\
1.3480210 \\
-3.1867950 \\
-3.7550780\end{array}$ & $\begin{array}{r}-1.5563810 \\
0.3327460 \\
-1.8438510 \\
-0.9159540 \\
1.0728140 \\
-2.8211330 \\
0.9797990 \\
-1.1864430 \\
-3.7160940 \\
2.0598180 \\
-1.2543640 \\
-2.6142820 \\
-2.8678630 \\
-3.2650650 \\
-1.9425370 \\
-3.5964240 \\
-3.9156420 \\
-3.7254230 \\
-4.3857570 \\
-4.6329020 \\
-0.3486830 \\
-0.6487290 \\
0.6975730 \\
-0.4020410 \\
-1.1996650 \\
-1.8695420 \\
-0.1856220 \\
-1.4975560 \\
3.1201420 \\
2.9097480 \\
3.1596210 \\
4.1117990 \\
2.4284500 \\
2.5642970 \\
1.6565880 \\
3.3743080 \\
0.8905440 \\
0.9561620 \\
-0.0526630 \\
1.7131890 \\
2.3431550 \\
2.4275560 \\
2.5366280 \\
3.1379690 \\
-0.1989000 \\
-0.1571870\end{array}$ \\
\hline
\end{tabular}

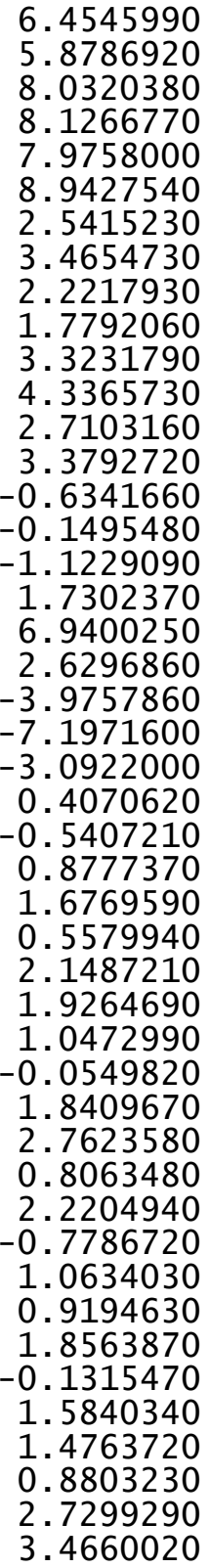
$-2.8269390$
$-3.8744500$
$-1.1621130$
$-0.3337620$
$-0.7376960$
$-1.7682580$
$-1.8201840$
$-2.1996490$
$-2.5312570$
$-1.7994210$
0.5156880
0.1918790
0.4958130
1. 5509410
$-0.7758230$
$-1.5402430$
$-1.5394150$
$-2.4471610$
1. 5952300
1. 5869830
$-3.3489090$
$-0.3792530$
$-0.7307860$
$-0.3230810$
0.3172190
0.0136880
1.6540150
1.0318220
$-1.0205210$
2. 6698000
1.8942020
2. 3607890
0.7902360
3. 7023660
3. 1552410
$-0.9429700$
$-2.2865270$
$-3.7822370$
$-2.7399760$
$-3.5131720$
$-1.7024040$
$-3.4426990$
$-2.7598240$
$-5.5070460$
$-4.4871990$

0.8131890 $-0.4999920$ $-0.8710230$ $-1.5806250$ 0.1377490 $-0.9266590$ $-4.2927330$ $-4.7441890$ $-3.5275400$ $-5.0780140$ $-4.7852350$ $-5.0515070$ $-5.6935220$ $-4.4371270$ $-1.0480960$ $-2.2404740$ 0.1571090 $-3.4567860$ $-2.1865460$ 0.9248400 $-2.2390050$ $-2.2857140$ 2. 0766900 3. 0467170 2. 2713840 3. 9777750 5.0853220

3. 6984790 5.9027180 5.2964750 4.5089170

2.8368390 5. 6121870 6.7645830

4.2764630

6.2475840

1. 4848660

3.0004630

0.0264690

0.4746350

0.1008090

0.3185050

$-1.2684260$

$-1.7000730$

0.4438550

1. 3147460 


\begin{tabular}{|c|c|c|c|c|c|c|c|}
\hline C & 3.2692470 & -3.0662310 & 0.7871150 & $c$ & 4.0402450 & 0.4407740 & -0.4342960 \\
\hline $\mathrm{C}$ & 1.2281340 & -5.2094550 & 0.4141330 & $\mathrm{C}$ & 4.5433740 & -1.4465160 & -2.4244370 \\
\hline $\mathrm{H}$ & 2.8914650 & -6.5184140 & 0.8284850 & $\mathrm{C}$ & 5.1755920 & -0.3664780 & -0.3879210 \\
\hline $\mathrm{H}$ & 4.5367290 & -4.7079870 & 1.3535320 & $\mathrm{C}$ & 5.4590310 & -1.2992990 & -1.3867790 \\
\hline $\mathrm{H}$ & 3.8102330 & -2.9128300 & -0.1568590 & $\mathrm{H}$ & 4.7449700 & -2.1864620 & -3.1949470 \\
\hline $\mathrm{H}$ & 0.7943700 & -5.3710610 & 1.4088060 & $\mathrm{H}$ & 5.8677660 & -0.2578930 & 0.4431740 \\
\hline $\mathrm{H}$ & 0.7023800 & -5.8732260 & -0.2791810 & $\mathrm{C}$ & -4.2716090 & -0.2816700 & -0.0219300 \\
\hline $\mathrm{H}$ & 3.1335610 & -5.4813080 & -0.5756450 & $\mathrm{C}$ & -4.4435430 & -1.1981290 & 1.0340530 \\
\hline H & 3.0910770 & -4.5452920 & 2.3446870 & $\mathrm{C}$ & -4.9180070 & -0.4934700 & -1.2552130 \\
\hline $\mathrm{H}$ & 3.6648800 & -2.3061310 & 1.4651040 & $\mathrm{C}$ & -5.2729680 & -2.3027560 & 0.8304560 \\
\hline & & & & $\mathrm{c}$ & -5.7271800 & -1.6167230 & -1.4091380 \\
\hline & s) $\mathrm{G}[\mathrm{WB} 97 \mathrm{XD}$ & $G(d)]=-363$ & 245 & $\mathrm{C}$ & -5.9203240 & -2.5343890 & -0.3785410 \\
\hline 0 & 0.5464580 & 0.1234350 & -0.3650970 & $\mathrm{H}$ & -5.4169030 & -3.0125430 & 1.6419190 \\
\hline 0 & -1.7876650 & 0.0762010 & -1.3813720 & $\mathrm{H}$ & -6.2286510 & -1.7753580 & -2.3613730 \\
\hline C & 0.8165790 & 1.2482280 & -1.1128910 & $\mathrm{C}$ & 3.7995520 & 1.4864320 & 0.6489420 \\
\hline C & -0.1151640 & 2.2640190 & -1.1682820 & $\mathrm{c}$ & 6.7302530 & -2.1312600 & -1.3520830 \\
\hline $\mathrm{C}$ & 2.0935650 & 1.3454540 & -1.7231430 & $\mathrm{C}$ & 2.4637180 & -0.8097570 & -3.7145510 \\
\hline $\mathrm{C}$ & 0.1422460 & 3.3910140 & -2.0134470 & $\mathrm{C}$ & -3.7809950 & -1.0191590 & 2.3944990 \\
\hline $\mathrm{C}$ & 2.3660420 & 2.4726770 & -2.4578310 & $\mathrm{C}$ & -6.8064300 & -3.7526930 & -0.5650910 \\
\hline $\mathrm{C}$ & 1.4054400 & 3.4946820 & -2.6544490 & $\mathrm{C}$ & -4.8041640 & 0.4896970 & -2.4123620 \\
\hline $\mathrm{H}$ & 3.3471970 & 2.5829200 & -2.9122140 & $\mathrm{C}$ & -6.1522200 & 1.1765390 & -2.6730640 \\
\hline C & -2.1819060 & 1.0778480 & -0.5059840 & $\mathrm{H}$ & -6.9112740 & 0.4515330 & -2.9888220 \\
\hline $\mathrm{C}$ & -3.4205310 & 0.9301890 & 0.1714810 & $\mathrm{H}$ & -6.5185690 & 1.6762230 & -1.7698530 \\
\hline $\mathrm{C}$ & -1.3598420 & 2.1707210 & -0.3625670 & $\mathrm{H}$ & -6.0536880 & 1.9275610 & -3.4650020 \\
\hline C & -3.79 & 1.9435920 & 1.0165550 & $\mathrm{C}$ & -4.2620010 & -0.1763560 & -3.6828450 \\
\hline $\mathrm{C}$ & -1.7 & 3.1672050 & 0.6061460 & $\mathrm{H}$ & -3.2981240 & -0.6558020 & -3.4890370 \\
\hline C & -2.9522030 & 3.0508620 & 1.2850290 & $\mathrm{H}$ & -4.9541270 & -0.9360140 & -4.0641140 \\
\hline $\mathrm{H}$ & -4.7504610 & 1.8798110 & 1.5298290 & $\mathrm{H}$ & -4.1231530 & 0.5717140 & -4.4715000 \\
\hline C & -2.4697640 & 5.0634650 & 2.5517440 & C & -6.2432780 & -4.6957180 & -1.6366710 \\
\hline C & -1.21 & 5.1633890 & 1.9014210 & $\mathrm{H}$ & -6.8567720 & -5.6001810 & -1.7153540 \\
\hline C & -0.8 & 4.2436370 & 0.9529250 & $\mathrm{H}$ & -6.2320760 & -4.2102080 & -2.6193910 \\
\hline C & -3.31 & 4.0288230 & 2.2480650 & $\mathrm{H}$ & -5.2173480 & -4.9944580 & -1.3981670 \\
\hline $\mathrm{H}$ & -2.75 & 5.8010180 & 3.2963580 & C & -8.2543910 & -3.3585180 & -0.8849980 \\
\hline $\mathrm{H}$ & -0.5426260 & 5.9743150 & 2.1571520 & $\mathrm{H}$ & -8.6607330 & -2.6936020 & -0.1157580 \\
\hline $\mathrm{H}$ & 7910 & 4.3272700 & 0.4711110 & $\mathrm{H}$ & -8.3191520 & -2.8392720 & -1.8482040 \\
\hline $\mathrm{H}$ & -4.27 & 3.9300620 & 2.7525220 & $\mathrm{H}$ & -8.8909010 & -4.2485570 & -0.9423820 \\
\hline C & -0.5365340 & 5.4667680 & -3.0758820 & C & -4.8002180 & -0.5417660 & 3.4394710 \\
\hline $\mathrm{C}$ & 0.7339410 & 5.5839560 & -3.6864630 & $\mathrm{H}$ & -5.2844410 & 0.3926750 & 3.1385820 \\
\hline $\mathrm{C}$ & .3720 & 4.6152300 & -3.4816230 & $\mathrm{H}$ & -5.5863490 & -1.2926120 & 3.5842120 \\
\hline C & -0.8 & 4.4020420 & -2.2610760 & $\mathrm{H}$ & -4.3095570 & -0.3758130 & 4.4052280 \\
\hline $\mathrm{H}$ & -1.2 & 6.2247790 & -3.2592040 & C & -3.0745320 & -2.2897700 & 2.8854860 \\
\hline $\mathrm{H}$ & 0.9 & 6.4352230 & -4.3245200 & $\mathrm{H}$ & -3.7881160 & -3.0831940 & 3.1354790 \\
\hline H & 000 & 4.6845490 & -3.9573260 & $\mathrm{H}$ & -2.3833610 & -2.6652480 & 2.1276140 \\
\hline $\mathrm{H}$ & -1.8 & 4.3201620 & -1.8088810 & $\mathrm{H}$ & -2.5032110 & -2.0666060 & 3.7940920 \\
\hline C & 660 & 0.3208890 & -1.5362590 & C & 4.5989930 & 2.7636180 & 0.3529810 \\
\hline $\mathrm{C}$ & 3.3972250 & -0.6533340 & -2.5219980 & $\mathrm{H}$ & 5.6750520 & 2.5531120 & 0.3591340 \\
\hline
\end{tabular}




$\begin{array}{rr}\mathrm{H} & 4.3398370 \\ \mathrm{H} & 4.3960850 \\ \mathrm{C} & 4.1042280 \\ \mathrm{H} & 3.5516150 \\ \mathrm{H} & 5.1720490 \\ \mathrm{H} & 3.8057790 \\ \mathrm{C} & 6.7047360 \\ \mathrm{H} & 7.6464060 \\ \mathrm{H} & 6.5666910 \\ \mathrm{H} & 5.8917230 \\ \mathrm{C} & 7.9812420 \\ \mathrm{H} & 7.9979930 \\ \mathrm{H} & 8.0228240 \\ \mathrm{H} & 8.8870030 \\ \mathrm{C} & 2.2473730 \\ \mathrm{H} & 3.1399550 \\ \mathrm{H} & 1.9848520 \\ \mathrm{H} & 1.4319800 \\ \mathrm{C} & 2.9725960 \\ \mathrm{H} & 3.9581080 \\ \mathrm{H} & 2.2840920 \\ \mathrm{H} & 3.0624510 \\ \mathrm{P} & -0.6497400 \\ \mathrm{O} & -0.2008010 \\ \mathrm{O} & -1.0665800 \\ \mathrm{H} & 1.4867010 \\ \mathrm{H} & 6.7854960 \\ \mathrm{H} & 2.7366370 \\ \mathrm{H} & -4.0969320 \\ \mathrm{H} & -6.8139020 \\ \mathrm{H} & -3.0143910 \\ \mathrm{C} & 0.6085110 \\ \mathrm{O} & -0.4159870 \\ \mathrm{C} & 1.1318890 \\ \mathrm{C} & 2.0024310 \\ \mathrm{C} & 0.7928310 \\ \mathrm{C} & 2.5259520 \\ \mathrm{H} & 2.2668630 \\ \mathrm{C} & 1.3348600 \\ \mathrm{H} & 0.1214580 \\ \mathrm{C} & 2.2011910 \\ \mathrm{H} & 3.1963000 \\ \mathrm{H} & 1.0778710 \\ \mathrm{H} & 2.6245170 \\ \mathrm{H} & 1.3058290 \\ \mathrm{~S} & \end{array}$

$\begin{array}{rr}3.1787650 & -0.6258780 \\ 3.5265760 & 1.1130550 \\ 0.9832160 & 2.0630820 \\ 0.0662060 & 2.2926720 \\ 0.7868450 & 2.2103230 \\ 1.7394330 & 2.7966540 \\ -3.1704860 & -0.2232760 \\ -3.7294890 & -0.1929440 \\ -2.6890920 & 0.7525040 \\ -3.8904780 & -0.3669780 \\ -1.2480820 & -1.2551610 \\ -0.4999850 & -2.0541110 \\ -0.7191880 & -0.2961550 \\ -1.8587430 & -1.3360390 \\ -2.2723640 & -4.1190420 \\ -2.7006420 & -4.5895180 \\ -2.8929950 & -3.2594050 \\ -2.3363790 & -4.8467680 \\ -0.0050850 & -4.9198620 \\ -0.3681030 & -5.2357010 \\ -0.1107740 & -5.7658170 \\ 1.0598560 & -4.6877980 \\ -0.9265180 & -0.8031590 \\ -1.8285420 & -1.9099010 \\ -1.5421350 & 0.5091850 \\ -0.4105650 & -3.4254040 \\ -2.6774970 & -2.3021250 \\ 1.7515310 & 0.6320870 \\ 1.2751980 & -2.1328690 \\ -4.2980660 & 0.3873690 \\ -0.2462410 & 2.2985730 \\ -0.3418390 & 3.1656260 \\ -0.0727570 & 2.4283870 \\ 0.8445810 & 3.8759390 \\ 0.7318760 & 4.9655340 \\ 2.1146660 & 3.3877990 \\ 1.8734340 & 5.5574330 \\ -0.2506810 & 5.3413850 \\ 3.2522810 & 3.9707480 \\ 2.2038790 & 2.5411490 \\ 3.1338770 & 5.0557810 \\ 1.7816500 & 6.4058960 \\ 4.2294770 & 3.5741610 \\ 4.0234540 & 5.5121340 \\ -0.7939980 & 1.7427310 \\ -1.8754110 & 3.2972540 \\ -0 & \end{array}$

$\begin{array}{lr}\mathrm{C} & 1.0475080 \\ \mathrm{C} & 1.9834940 \\ \mathrm{H} & -0.0035410 \\ \mathrm{H} & 1.6816080 \\ \mathrm{O} & 1.5214290 \\ \mathrm{H} & 0.8862310 \\ \mathrm{C} & 2.9142450 \\ \mathrm{C} & 3.6762960 \\ \mathrm{C} & 3.4174100 \\ \mathrm{C} & 1.4073290 \\ \mathrm{H} & 3.1183320 \\ \mathrm{H} & 4.7523700 \\ \mathrm{H} & 3.9008510 \\ \mathrm{H} & 1.0349140 \\ \mathrm{H} & 0.8572270 \\ \mathrm{H} & 3.2580360 \\ \mathrm{H} & 3.3655220 \\ \mathrm{H} & 3.8373960 \\ \mathrm{H} & \end{array}$
$-3.7347720$
$-3.5569530$
$-2.9423880$
$-5.3667130$
$-4.2358170$
$-2.8945470$
$-5.0956090$
$-6.3202420$
$-4.4334400$
$-2.8542270$
$-5.1327710$
$-5.8506340$
$-5.4629160$
$-4.1709370$
$-2.0494660$

$\begin{array}{ll}0.5504520 \\ 25860 & 0.8156020\end{array}$

$\begin{array}{ll}-3.4753300 & 0.6526550\end{array}$

$-1.6238420 \quad 0.5448090$

$-0.8069820$

$-1.2838100$

1.0779460

1.7708340

1.0850740

1.0996320

1.5739360

1.7691240

0.0993490

2. 1308900

0.5294290

0.0408530

2. 8215050

1.6358150

TS244(S, S) G[WB97XD/6-31G(d) $]=-3633.717860$

\begin{tabular}{|c|c|c|c|}
\hline & S) $\mathrm{G}[\mathrm{wB} 97 \mathrm{X}$ & (d)] & \\
\hline 0 & 0.5879910 & 0.1836810 & -0.3606470 \\
\hline 0 & -1.6658970 & 0.1807010 & -1.551157 \\
\hline C & 0.9052460 & 1.3509350 & -1.018986 \\
\hline C & -0.0309760 & 2.3604670 & -1.0913690 \\
\hline$C$ & 2.2275530 & 1.4944980 & -1.511728 \\
\hline C & 0.2778690 & 3.5343690 & -1.850019 \\
\hline C & 2.5422210 & 2.6608160 & 163725 \\
\hline$C$ & 1.5852580 & 3.6816230 & -2.385669 \\
\hline H & 3.5566260 & 2.8075280 & -2.525108 \\
\hline $\mathrm{C}$ & -2.1276820 & 1.1256150 & -0.645865 \\
\hline C & -3.4050910 & 0.9321340 & -0.056802 \\
\hline$C$ & -1.3276370 & 2.2123330 & -0.3824000 \\
\hline C & -3.8380520 & 1.8863660 & 0.829094 \\
\hline C & -1.7426490 & 3.1429940 & 0.6241890 \\
\hline$C$ & -3.0215370 & 2.9766880 & 1.221058 \\
\hline $\mathrm{H}$ & -4.8213860 & 1.7842610 & 1.278824 \\
\hline C & -2.6266240 & 4.9028960 & 2.642642 \\
\hline C & -1.3418200 & 5.0526690 & 2.07 \\
\hline$C$ & -0.9099520 & 4.1979020 & 1.087 \\
\hline & -3.445 & 3.8864370 & 2.2 \\
\hline & -2 & 5.5891500 & 3 . \\
\hline & -0 & 5.8495570 & \\
\hline & O & 0520 & U \\
\hline & -4 & 530 & 9 \\
\hline & -0 & 5.661 & 33 \\
\hline & & & \\
\hline
\end{tabular}




$\begin{array}{rrr}1.9095820 & 4.8471220 & -3.1288510 \\ -0.6793260 & 4.5514740 & -2.1127400 \\ -1.0884640 & 6.4239980 & -3.0415110 \\ 1.2231870 & 6.7055580 & -3.9249190 \\ 2.9159640 & 4.9489850 & -3.5263430 \\ -1.6911430 & 4.4383900 & -1.7387750 \\ 3.2964690 & 0.4760090 & -1.2669670 \\ 3.6392260 & -0.4513010 & -2.2673170 \\ 4.0379980 & 0.5443380 & -0.0720040 \\ 4.7664400 & -1.2513940 & -2.0795500 \\ 5.1651220 & -0.2676870 & 0.0617590 \\ 5.5559270 & -1.1595560 & -0.9342640 \\ 5.0470170 & -1.9544430 & -2.8590660 \\ 5.7622740 & -0.2056650 & 0.9681300 \\ -4.2412930 & -0.2632780 & -0.3779350 \\ -4.4799060 & -1.2416610 & 0.6111150 \\ -4.8169330 & -0.3935180 & -1.6536860 \\ -5.3023350 & -2.3219360 & 0.2969960 \\ -5.6274800 & -1.4984460 & -1.9188640 \\ -5.8847860 & -2.4722180 & -0.9601570 \\ -5.4940760 & -3.0729550 & 1.0598430 \\ -6.0815510 & -1.6001940 & -2.9024800 \\ 3.6676650 & 1.5279900 & 1.0330310 \\ 6.8249270 & -1.9815750 & -0.7860090 \\ 2.8406560 & -0.5522010 & -3.5597270 \\ -3.8957990 & -1.1515370 & 2.0156840 \\ -6.7753740 & -3.6602880 & -1.2766620 \\ -4.6271060 & 0.6530770 & -2.7429290 \\ -5.9491990 & 1.3748640 & -3.0389850 \\ -6.7017430 & 0.6786620 & -3.4270830 \\ -6.3540860 & 1.8382380 & -2.1328420 \\ -5.7984150 & 2.1598710 & -3.7886400 \\ -4.0220520 & 0.0505180 & -4.0166010 \\ -3.0756860 & -0.4515890 & -3.7957090 \\ -4.6994250 & -0.6781580 & -4.4765030 \\ -3.8306150 & 0.8375120 & -4.7546630 \\ -8.0223090 & -3.6830790 & -0.3825020 \\ -8.6888380 & -4.5028490 & -0.6726760 \\ -7.7516170 & -3.8286750 & 0.6696860 \\ -8.5785750 & -2.7431930 & -0.4606350 \\ -6.0037060 & -4.9830970 & -1.1812490 \\ -5.1326020 & -4.9787490 & -1.8445230 \\ -5.6480870 & -5.1583980 & -0.1592750 \\ -6.6468690 & -5.8246290 & -1.4621600 \\ -4.9737720 & -0.7437390 & 3.0308190 \\ -5.4470470 & 0.2063800 & 2.7633420\end{array}$

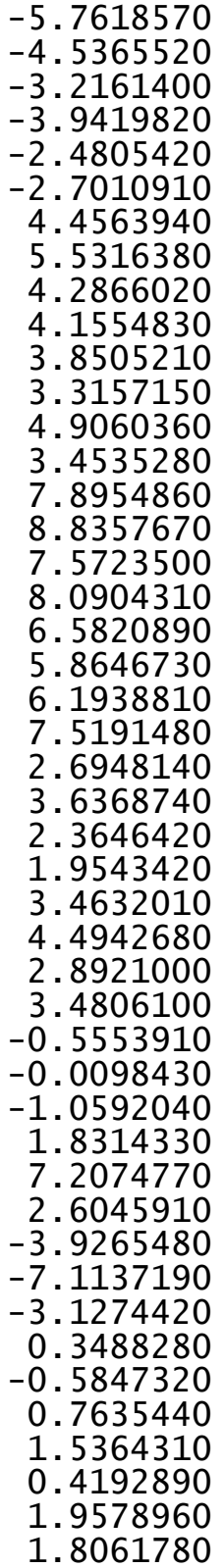

-1.5042620
-0.6364920
-2.4530820
-3.2603290
-2.7785950
-2.2928430
2.8367000
2.6512700
3.2936980
3.5563170
0.9576000
0.0103430
0.7892990
1.6614360
-1.4955420
-2.0375860
-1.6579370
-0.4259730
-3.4878270
-3.8596150
-3.7279820
-4.0387490
-1.9916960
-2.3781060
-2.6678810
-2.0244850
0.3252180
0.0112520
0.2363910
1.3804990
-0.8455150
-1.6671460
-1.5510200
-0.1813200
-1.8125740
1.7696210
1.4097250
-3.5464320
-0.3752010
-0.5269210
-0.2013130
0.5981390
0.3987650
1.9017540
1.4871640
-0.6093550
3. 0853870

4. 0300280

2. 4615450

2.6131740

1. 7227520

3. 4158020

0.8810340

0.9887030

$-0.0987450$

1. 6507130

2. 4430350

2.5676610

2. 6839210

3. 1823610

$-1.7738750$

$-1.6242030$

$-2.8087090$

$-1.6448560$

$-0.9454630$

$-0.2062830$

$-1.9414490$

$-0.8111770$

$-4.0666630$

$-4.4730540$

$-3.2747060$

$-4.8721750$

$-4.6561870$

$-4.8585820$

$-5.5871730$

$-4.3693330$

$-0.9578780$

$-2.0839510$

0.2762960

$-3.3553220$

0.2286640

0.9262660

$-2.3796080$

$-2.3145340$

2. 0161220

3. 1593440

2. 3302700

4.0251760

5.1743560

3.6389230

5.9262730

5. 4702840 


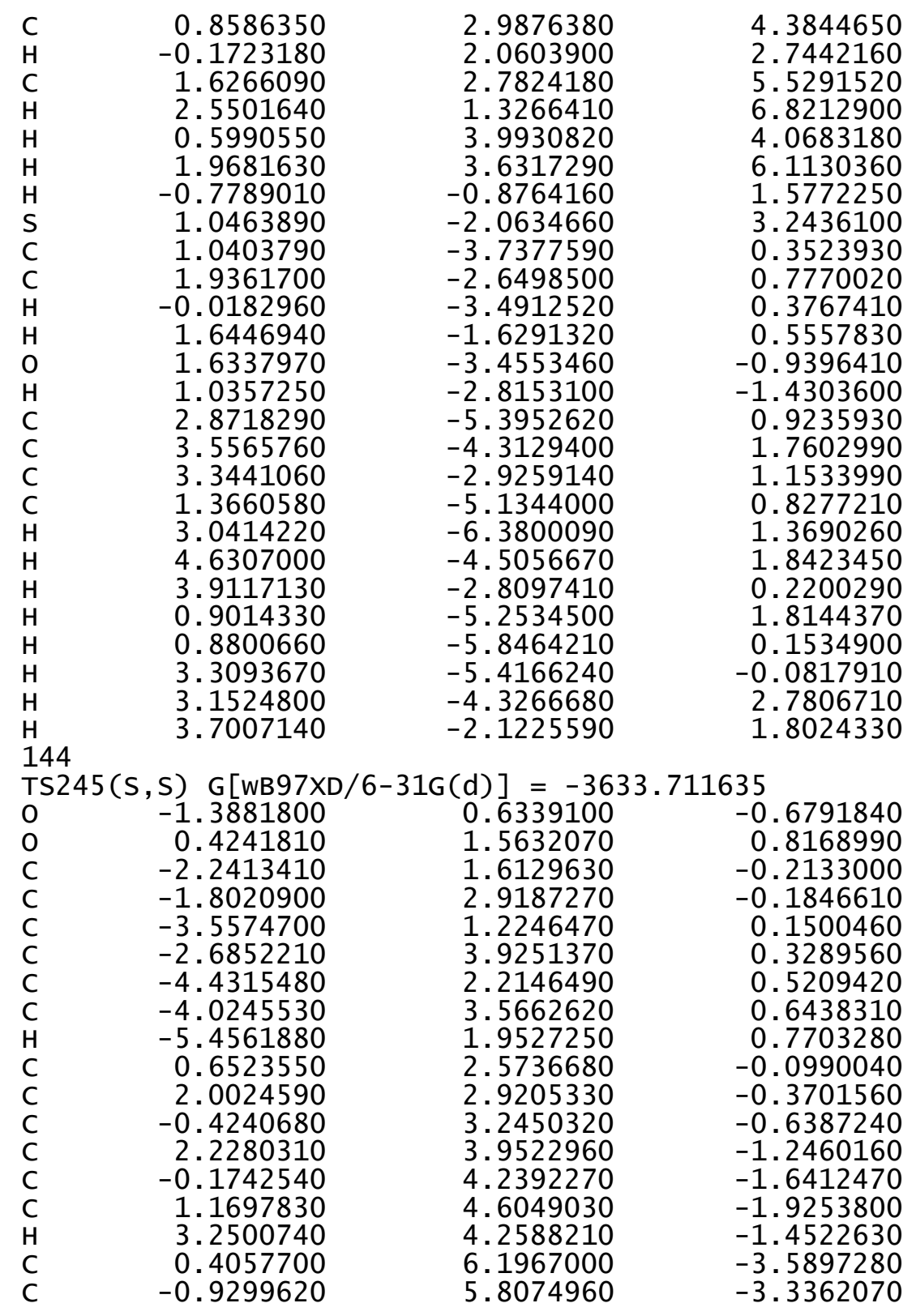

-1.2132220
1.4309300
0.6150380
-1.7384750
-2.2417480
2.4637250
-3.1603710
-4.5045020
-4.9224140
-2.2756620
-2.8240870
-5.1961070
-5.9462160
-1.2457970
-3.9804840
-4.2337890
-4.1820590
-4.7183600
-4.6549240
-4.9314810
-4.9180940
-4.8232540
3.1374770
4.0344290
3.3454130
5.1303550
4.4563760
5.3693770
5.8356510
4.6332300
-0.1575610
-0.6499100
0.7867430
-3.9434370
-3.5549870
-5.4547810
-4.8152770
-4.0184480
-3.4864570
2.4427450
1.6184430
6.6277880
6.4994520
3.8975450
2.9573470
-0.4171150

4.8569130

5.6033340

6.9542060

6.2616750

4. 5619030

5.8770660

6. 1992080

5.8497890

4. 5587030

5. 2655300

7.2158450

6.6012100

4. 2701750

5. 5468510

$-0.2080160$

$-0.8484060$

$-0.8995180$

$-2.1586810$

$-2.2085210$

$-2.8605450$

$-2.6420840$

$-2.7411340$

.2132500

1.4350950

2. 3791000

0.8543560

1.7790430

1. 0255750

0.2765060

1. 9248300

0.1442320

$-0.6028060$

$-0.5430380$

$-0.2527350$

0.7566920

$-4.2885340$

$-4.8467620$

$-0.1666010$

0.7727840

3.2455400

3. 6169200

0.4969750

0.6226840

1. 2543190

1. 7113830

$-3.6524800$
$-2.3887680$

$-2.9008230$

$-4.3386370$

$-3.9008690$

$-2.2131200$

$-3.0994850$

1. 0419540

1. 3083820

1. 1184320

0.5657200

1. 2215230

1.6765170

1. 3413060

0.3771460

0.1591650

1. 3868320

$-1.0503180$

1. 3770330

$-1.0042150$

0.1974540

2. 3278070

$-1.9394370$

0.2953270

$-0.4654090$

1.6778380

0.1759970

2. 2688230

1. 5380930

$-0.4175560$

3. 3325520

0.2746870

1. 4812030

$-0.6791090$

$-2.4072070$

$-2.2472700$

0.1653600

$-0.5344210$

2.7335210

2.5605310

2. 5460050

1.9322890

2. 2039730

3.2864890

$-1.9737580$

$-2.2916570$

0.6948050 


$\begin{array}{rrr}-0.7802300 & -3.2624830 & -0.6714040 \\ 0.4332960 & -3.1313750 & 1.1328360 \\ -0.5428310 & -2.2521270 & -0.9809090 \\ -1.6573160 & -2.9518090 & 0.9412930 \\ -1.4149040 & -1.9990440 & 1.1745520 \\ -1.6542220 & -5.8286360 & 0.3459650 \\ -1.6005750 & -5.5983610 & -1.1658780 \\ -1.6768600 & -4.1081350 & -1.5069090 \\ -0.4995060 & -5.1159840 & 1.0567340 \\ -1.6076890 & -6.8993920 & 0.5654860 \\ -2.4245390 & -6.1197480 & -1.6624500 \\ -2.6886140 & -3.7175470 & -1.3366980 \\ 0.4568790 & -5.5716890 & 0.7721780 \\ -0.5905960 & -5.2008080 & 2.1440280 \\ -2.6080760 & -5.4605140 & 0.7405670 \\ -0.6676870 & -6.0143230 & -1.5659400 \\ -1.4573370 & -3.9107660 & -2.5599020 \\ 3.8320000 & -0.2184890 & -2.3947350 \\ 3.7865760 & -0.2940250 & -3.4869480 \\ 4.7078850 & -0.7852450 & -2.0581450 \\ 2.9354810 & -0.6873540 & -1.9867770 \\ 5.0350110 & 1.9806510 & -2.7074920 \\ 6.0067000 & 1.5304350 & -2.4727940 \\ 4.8897410 & 1.9187940 & -3.7917180 \\ 5.0824590 & 3.0387010 & -2.4284270 \\ -2.8924200 & -1.0161030 & -3.2222660 \\ -3.2179680 & -2.0398610 & -3.4407190 \\ -2.7135480 & -0.5134540 & -4.1790760 \\ -1.9421880 & -1.0611720 & -2.6813700 \\ -5.2598450 & -0.1078860 & -3.1829880 \\ -5.0880950 & 0.4009960 & -4.1379280 \\ -5.7063610 & -1.0855100 & -3.3980990 \\ -5.9874580 & 0.4756590 & -2.6089170 \\ 1.8233640 & 2.4476850 & 3.7001060 \\ 2.5899220 & 2.0904380 & 4.3975270 \\ 1.1285710 & 3.0784850 & 4.2657790 \\ 1.2724520 & 1.5828580 & 3.3198680 \\ 3.2001330 & 4.4785340 & 3.0593130 \\ 2.5292680 & 5.1264530 & 3.6345240 \\ 4.0319890 & 4.1927370 & 3.7133330 \\ 3.6095640 & 5.0607180 & 2.2267920 \\ -3.1482740 & -1.0026060 & 3.6815600 \\ -2.9875670 & -0.4545070 & 4.6168040 \\ -3.6215680 & -1.9569620 & 3.9378820 \\ -2.1728140 & -1.2036400 & 3.2335140 \\ -5.3635320 & 0.1692170 & \\ -1.3937030\end{array}$

\begin{tabular}{lr}
$\mathrm{H}$ & -5.9320310 \\
$\mathrm{H}$ & -5.2052490 \\
$\mathrm{H}$ & -5.9803640 \\
$\mathrm{C}$ & 6.8723700 \\
$\mathrm{H}$ & 6.0186680 \\
$\mathrm{H}$ & 7.0470160 \\
$\mathrm{H}$ & 7.7590890 \\
$\mathrm{C}$ & 7.8444370 \\
$\mathrm{H}$ & 7.6883500 \\
$\mathrm{H}$ & 8.7466660 \\
$\mathrm{H}$ & 8.0226110 \\
$\mathrm{C}$ & -6.8868770 \\
$\mathrm{H}$ & -6.9431070 \\
$\mathrm{H}$ & -7.2490750 \\
$\mathrm{H}$ & -7.5634890 \\
$\mathrm{C}$ & -5.3948390 \\
$\mathrm{H}$ & -4.4012110 \\
$\mathrm{H}$ & -6.1177060 \\
$\mathrm{H}$ & -5.6397150 \\
$\mathrm{C}$ & 2.6803320 \\
$\mathrm{O}$ & 2.5551890 \\
$\mathrm{C}$ & 3.9556770 \\
$\mathrm{C}$ & 4.1060830 \\
$\mathrm{C}$ & 5.0310830 \\
$\mathrm{C}$ & 5.3138980 \\
$\mathrm{H}$ & 3.2692740 \\
$\mathrm{C}$ & 6.2394910 \\
$\mathrm{H}$ & 4.9120970 \\
$\mathrm{C}$ & 6.3817560 \\
$\mathrm{H}$ & 5.4224410 \\
$\mathrm{H}$ & 7.0734880 \\
$\mathrm{H}$ & 7.3267720 \\
$\mathrm{H}$ & 1.7863690 \\
$\mathrm{~S}$ & 1.5289520 \\
$\mathrm{H}$ & \\
\hline
\end{tabular}
$-0.7447590$
0.6928280
0.8061560
$-0.9895020$
$-1.5976490$
$-1.1694660$
$-1.3369480$
1. 3325060
2. 3939500
1.0002150
1. 2318660
$-4.3240410$
$-3.8700530$
$-5.3554100$
$-3.7702280$
$-5.0055330$
$-4.9375990$
$-4.5825570$
$-6.0647630$
$-3.3211130$
$-2.2739220$
$-4.0384210$
$-5.3764850$
$-3.3621290$
$-6.0290630$
$-5.9001600$
$-4.0175770$
$-2.3227000$
$-5.3505350$
$-7.0689780$
$-3.4916010$
$-5.8607340$
$-1.6454390$
$-3.8203730$

3.6032240

4. 3430680

2. 7513290

1. 9341550

2. 2507390

0.8668570

2.4755500

1.7782180

1. 9963800

2. 3038070

0.7010290

$-0.3910190$

$-1.3854400$

$-0.4653070$

0.2701820

1. 5155500

1.9708800

2. 2231210

1.3876080

$-0.8228760$

$-0.0619590$

$-0.6174480$

$-1.0012110$

$-0.0250340$

$-0.7934210$

$-1.4505930$

0.1723600

0.2617960

$-0.2087730$

$-1.0839090$

0.6252880

$-0.0497440$

$-0.3095000$

144

$-1.9368200$

$\operatorname{TS} 246(\mathrm{~S}, \mathrm{~S}) \mathrm{G}[\mathrm{WB} 97 \mathrm{XD} / 6-31 \mathrm{G}(\mathrm{d})]=-3633.711352$

$\begin{array}{lrcr}\text { TS246(S, S }) & \text { G }[W B 97 X D / 6-31 G(d)]=-3633.711352 \\ \text { O } & -1.3586360 & 0.6317020 & -0.6477640 \\ \text { O } & 0.4891350 & 1.5593280 & 0.8104100 \\ \text { C } & -2.1883690 & 1.6329730 & -0.1848050 \\ \text { C } & -1.7255780 & 2.9312930 & -0.1822180 \\ \text { C } & -3.5059720 & 1.2748330 & 0.2029320 \\ \text { C } & -2.5813410 & 3.9611220 & 0.3312200 \\ \text { C } & -4.3556400 & 2.2867450 & 0.5720700 \\ \text { C } & -3.9219710 & 3.6318410 & 0.6710460 \\ \text { H } & -5.3823820 & 2.0481630 & 0.8354670 \\ \text { C } & 0.7228670 & 2.5483010 & -0.1269630\end{array}$




\begin{tabular}{|c|c|c|}
\hline $\begin{array}{l}2.0747030 \\
-0.3498580 \\
2.3050690 \\
-0.0977880 \\
1.2482970 \\
3.3290040 \\
0.4879720 \\
-0.8504980 \\
-1.1365600 \\
1.5125540 \\
0.6994960 \\
-1.6590730 \\
-2.1674510 \\
2.5469780 \\
-3.0022130 \\
-4.3487250 \\
-4.7940910 \\
-2.1424920 \\
-2.6435990 \\
-5.0204610 \\
-5.8199530 \\
-1.1101760 \\
-3.9689070 \\
-4.1971090 \\
-4.2530230 \\
-4.7451070 \\
-4.8044160 \\
-5.0673360 \\
-4.9321460 \\
-5.0432790 \\
3.2063390 \\
4.0768380 \\
3.4374460 \\
5.1734970 \\
4.5461390 \\
5.4352750 \\
5.8599920 \\
4.7405040 \\
-0.1230080 \\
-0.6169660 \\
0.8005670 \\
-4.0286290 \\
-3.5751740 \\
-5.7270550 \\
-3.9005620\end{array}$ & $\begin{array}{r}2.8663640 \\
3.2276930 \\
3.8802620 \\
4.2025080 \\
4.5410550 \\
4.1667900 \\
6.1247540 \\
5.7618740 \\
4.8283430 \\
5.5223640 \\
6.8698060 \\
6.2230880 \\
4.5537240 \\
5.7760540 \\
6.2527410 \\
5.9326310 \\
4.6475410 \\
5.2963760 \\
7.2646950 \\
6.7019270 \\
4.3819310 \\
5.5552570 \\
-0.1461280 \\
-0.7765730 \\
-0.8244430 \\
-2.0560620 \\
-2.1078030 \\
-2.7389190 \\
-2.5467660 \\
-2.6137270 \\
2.1507900 \\
1.3407610 \\
2.3395100 \\
0.7551850 \\
1.7308960 \\
0.9481570 \\
0.1542290 \\
1.8923930 \\
0.1423230 \\
-0.5721290 \\
-0.5786340 \\
-0.1892550 \\
0.7947080 \\
-4.1051160 \\
-4.6572470 \\
-0.0967080\end{array}$ & $\begin{array}{r}-0.4222910 \\
-0.6631780 \\
-1.3175010 \\
-1.6838010 \\
-1.9914040 \\
-1.5418150 \\
-3.6651270 \\
-3.3881260 \\
-2.4246700 \\
-2.9832380 \\
-4.4257980 \\
-3.9469680 \\
-2.2308970 \\
-3.1993070 \\
1.0208770 \\
1.3110120 \\
1.1447200 \\
0.5439080 \\
1.1829040 \\
1.6790680 \\
1.3859410 \\
0.3375460 \\
0.2315320 \\
1.4736660 \\
-0.9639480 \\
1.4794000 \\
-0.9031070 \\
0.3079300 \\
2.4323290 \\
-1.8348120 \\
0.2403090 \\
-0.5178190 \\
1.6161740 \\
0.1180770 \\
2.2025630 \\
1.4730940 \\
-0.4744220 \\
3.2609720 \\
0.3010870 \\
1.5265360 \\
-0.6479020 \\
-2.3287110 \\
-2.1823790 \\
0.4113290 \\
1.2001690 \\
2.8056200\end{array}$ \\
\hline
\end{tabular}

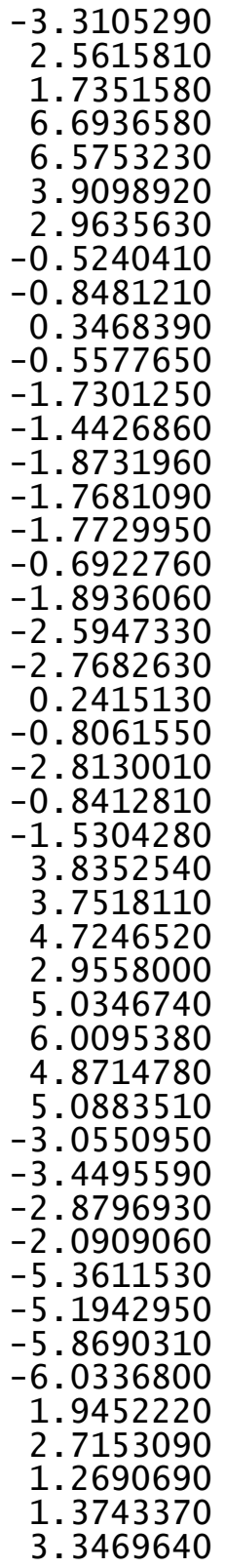

0.8020870

3.2367140

3.6107300

0.4109220

0.5452830

1.1288120

1. 5800310

$-3.6298640$

$-3.2417330$

$-3.1462310$

2.248

$-2.8645820$

$-1.9239960$

$-5.5412120$

$-4.0549860$

$-5.0798340$

$-6.8065170$

-6. 0414840

$-3.6145220$

$-5.5936070$

$-5.3076280$

$-6.0006010$

$-3.8837040$

$-0.3524210$

$-0.4500550$

$-0.9095180$

$-0.8162120$

1.8365750

1.3904690

1.7488560

2.9008890

$-1.0114000$

$-2.0129160$

$-0.5165270$

$-1.1167830$

0.0359200

0.5417650

$-0.9133890$

0.6552680

2.4707810

2.1132470

3.1234010

1.6087210

4.4659130
2.6055400

2.4806130

1.8713500

2.1320960

3.2147330

$-2.0190560$

$-2.3275930$

0.7610550

$-0.6155390$

1.2024760

$-0.9362070$

0.9799060

1. 2122320

0.4272530

$-1.0865780$

$-1.4525270$

1. 1468230

0.6675350

$-1.6002440$

$-1.3011000$

0.8876440

2.2337330

0.7933190

$-1.4515710$

$-2.5049890$

$-2.4076430$

$-3.4957820$

$-2.0910990$

$-1.9590320$

$-2.7895520$

$-2.5599890$

$-3.8694250$

$-2.5371610$

$-3.1819410$

$-3.3900430$

$-4.1434070$

$-2.6746260$

$-3.0563040$

$-4.0137380$

$-3.2618710$

$-2.4532480$

3.6576340

4.3510150

4.2211450

3.3012030

2.9600460 


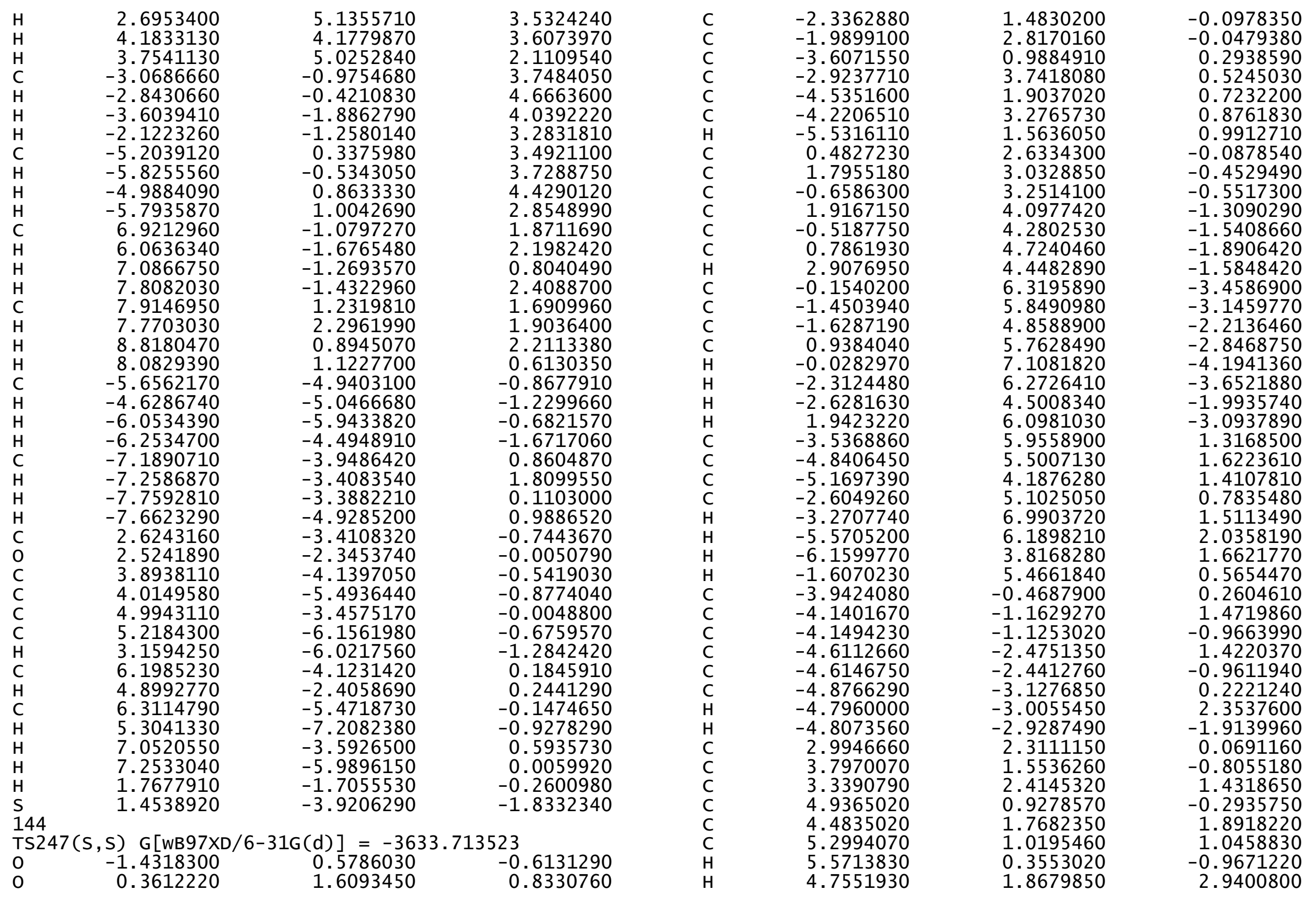




$\begin{array}{rrr}-0.1483760 & 0.1613200 & 0.3026240 \\ -0.5558740 & -0.6176830 & 1.5202310 \\ 0.8059480 & -0.4607380 & -0.6852990 \\ -3.9292190 & -0.4392890 & -2.3073240 \\ -3.5819880 & 0.5809390 & -2.1240750 \\ -5.5300380 & -4.4989990 & 0.2309910 \\ -5.3521780 & -4.9383720 & 1.2211170 \\ -3.8966340 & -0.5245070 & 2.8352550 \\ -3.3768150 & 0.4246190 & 2.6793270 \\ 2.5364300 & 3.2593980 & 2.4119590 \\ 1.6484610 & 3.6391490 & 1.9005410 \\ 6.5755010 & 0.3710670 & 1.5527050 \\ 6.9576700 & -0.2678290 & 0.7450430 \\ 3.5113550 & 1.4293230 & -2.2978410 \\ 2.5361610 & 1.8800320 & -2.4995820 \\ -0.1137380 & -3.6291260 & 0.7529310 \\ -0.4825950 & -3.2842370 & -0.6240110 \\ 0.6905580 & -3.0389370 & 1.1912720 \\ -0.3106260 & -2.2633650 & -0.9432750 \\ -1.4055580 & -3.0194930 & 0.9768190 \\ -1.2321730 & -2.0516590 & 1.2074850 \\ -1.1888510 & -5.8882550 & 0.4247380 \\ -1.1280710 & -5.6744780 & -1.0903640 \\ -1.3118290 & -4.1991560 & -1.4561460 \\ -0.0934990 & -5.0888810 & 1.1369910 \\ -1.0722410 & -6.9505780 & 0.6583760 \\ -1.8952780 & -6.2679560 & -1.5968600 \\ -2.3521830 & -3.8798510 & -1.3011200 \\ 0.8955980 & -5.4803420 & 0.8695660 \\ -0.1915980 & -5.1636390 & 2.2243990 \\ -2.1687760 & -5.5789270 & 0.8087550 \\ -0.1582140 & -6.0219640 & -1.4668590 \\ -1.0949290 & -4.0024450 & -2.5098400 \\ 3.4314260 & -0.0268220 & -2.7726150 \\ 3.2731560 & -0.0567180 & -3.8565820 \\ 4.3510950 & -0.5834830 & -2.5573880 \\ 2.5937930 & -0.5371700 & -2.2946300 \\ 4.5611350 & 2.2066820 & -3.1063720 \\ 5.5579890 & 1.7693960 & -2.9745040 \\ 4.3204360 & 2.1790370 & -4.1749680 \\ 4.6144080 & 3.2552360 & -2.7945970 \\ -2.8391320 & -1.1494170 & -3.1203220 \\ -3.1199780 & -2.1841450 & -3.3496380 \\ -2.6713540 & -0.6307520 & -4.0706500 \\ -1.8936980 & -1.1607410 & -2.5694000 \\ -5.2387740 & -0.3281060 & -3.0998030\end{array}$

-5.0719860
-5.6433240
-5.9985410
2.0508070
2.8894630
1.4184120
1.4651270
3.3479930
2.7498430
4.2526570
3.6552610
-2.9964380
-2.8257400
-3.4514440
-2.0258110
-5.2265570
-5.7760920
-5.0480170
-5.8700700
7.6457080
7.8526860
7.3140340
8.5807580
6.3395190
5.5719960
7.2651760
6.0170360
-4.9653470
-3.8886230
-5.4448230
-5.1437670
-7.0492510
-7.4678350
-7.2785730
-7.5499270
2.9885210
2.7612850
4.3311120
4.6288880
5.3213470
5.8980820
3.8594360
6.5911810
5.0863600
6.8805320
6.1199520
0.2122120

$-1.3154600$

0.2108900

2. 4398440

2.0768020

. 0580360

1.5770670

4.4836250

5.1168290

4.1843710

5.0855550

$-1.3819600$

$-0.8658170$

$-2.3519360$

$-1.5536790$

$-0.2263340$

$-1.1541630$

0.2723130

0.4198310

1.4334750

2.0332340

2.1124500

0.9634670

$-0.5149850$

$-1.2719170$

$-1.0294650$

0.0793630

$-5.4677220$

$-5.6067310$

$-6.4474510$

$-5.1160320$

$-4.3449590$

$-3.6850770$

$-3.9109180$

$-5.3171330$

$-3.0275450$

$-1.9567960$

$-3.5976190$

$-4.9295050$

$-2.7792440$

$-5.4351150$

$-5.5656650$

$-3.2869340$

$-1.7458070$

$-4.6152290$

$-6.4717250$
$-4.0381780$

$-3.3498090$

$-2.5237950$

3. 6136050

4. 2188460

4.2606370

3. 2832450

2.8597220

3. 5245860

3. 4017880

1. 9975800

3.7345940

4.6859910

3.9634140

3. 2646430

3. 5431330

3. 7417950

4.5022780

2. 9378720

1.8405810

0.9483110

2. 6348390

2. 1651650

2. 7821300

2. 5910210

3.0632290

3. 6443170

$-0.8114670$

$-0.6823550$

$-0.7136020$

$-1.8337370$

0.0558360

0.8227430

$-0.9245980$

0.1253940

$-0.7438690$

$-0.0452740$

$-0.5025170$

$-0.8125160$

0.0579830

$-0.5621460$

$-1.2365590$

0.2981910

0.2858770

$-0.0088300$

$-0.7939480$ 


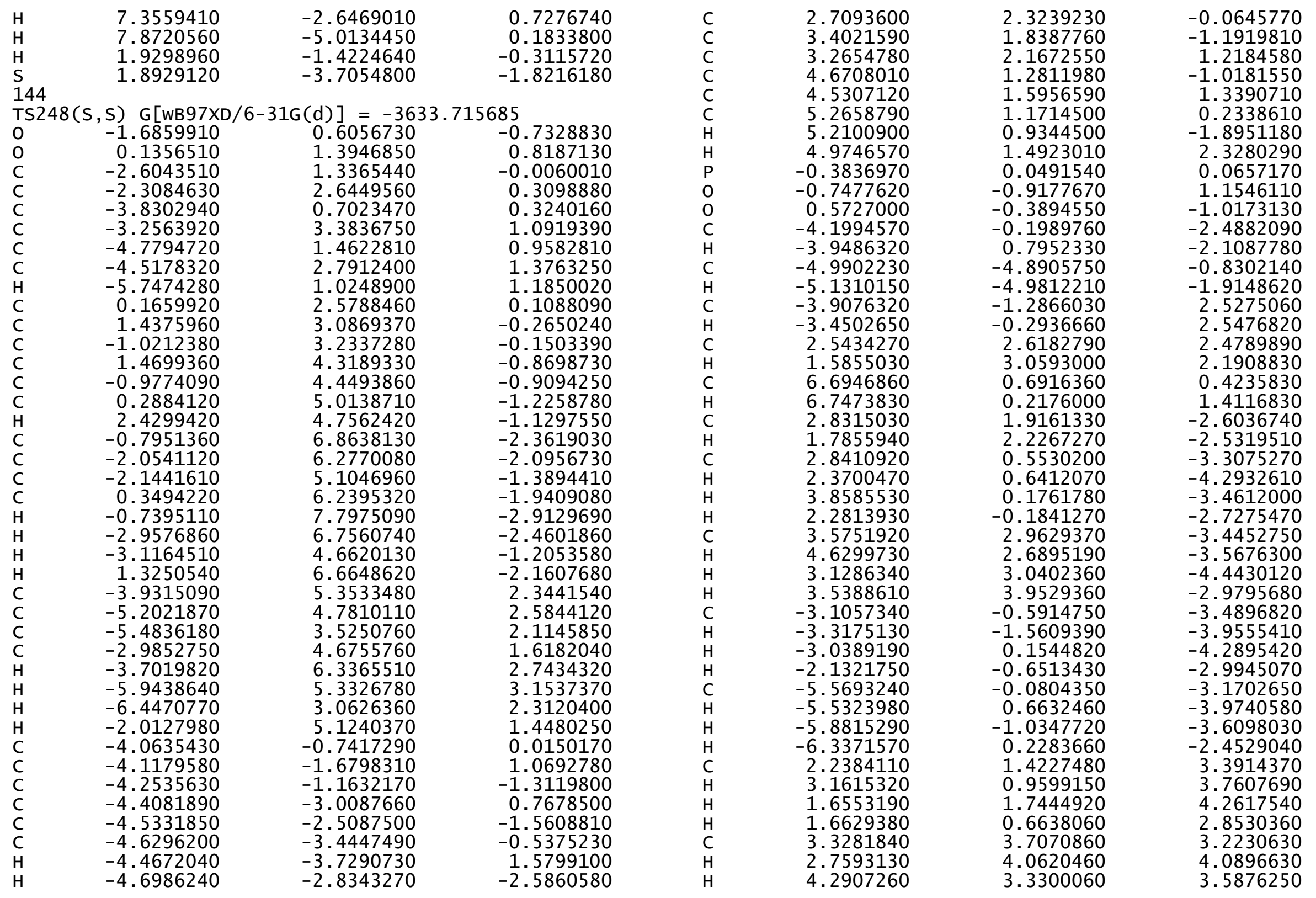




$\begin{array}{rr}\mathrm{H} & 3.5267110 \\ \mathrm{C} & -2.9564390 \\ \mathrm{H} & -2.7751160 \\ \mathrm{H} & -3.3774210 \\ \mathrm{H} & -1.9954360 \\ \mathrm{C} & -5.2519380 \\ \mathrm{H} & -5.7410580 \\ \mathrm{H} & -5.1011490 \\ \mathrm{H} & -5.9384350 \\ \mathrm{C} & 7.1552920 \\ \mathrm{H} & 6.4504940 \\ \mathrm{H} & 7.2654370 \\ \mathrm{H} & 8.1319270 \\ \mathrm{C} & 7.6441690 \\ \mathrm{H} & 7.3578470 \\ \mathrm{H} & 8.6761900 \\ \mathrm{H} & 7.6166610 \\ \mathrm{C} & -6.3134750 \\ \mathrm{H} & -7.1220710 \\ \mathrm{H} & -6.5954060 \\ \mathrm{H} & -6.2278300 \\ \mathrm{C} & -3.8674320 \\ \mathrm{H} & -2.9421840 \\ \mathrm{H} & -3.6570270 \\ \mathrm{H} & -4.1426740 \\ \mathrm{C} & 0.4054550 \\ \mathrm{C} & 0.2163430 \\ \mathrm{H} & 0.9581780 \\ \mathrm{H} & 0.1585720 \\ \mathrm{O} & -1.0123100 \\ \mathrm{H} & -1.0983660 \\ \mathrm{C} & -0.0223110 \\ \mathrm{C} & 0.2084620 \\ \mathrm{C} & -0.2225010 \\ \mathrm{C} & 0.7265330 \\ \mathrm{H} & 0.3140380 \\ \mathrm{H} & -0.3379030 \\ \mathrm{H} & -1.3161810 \\ \mathrm{H} & 1.8099810 \\ \mathrm{H} & 0.4921450 \\ \mathrm{H} & -1.0926640 \\ \mathrm{H} & 1.2738960 \\ \mathrm{H} & 0.1343730 \\ \mathrm{C} & 3.3127740 \\ \mathrm{O} & 2.7175900 \\ \mathrm{C} & 4.4958620 \\ & \end{array}$

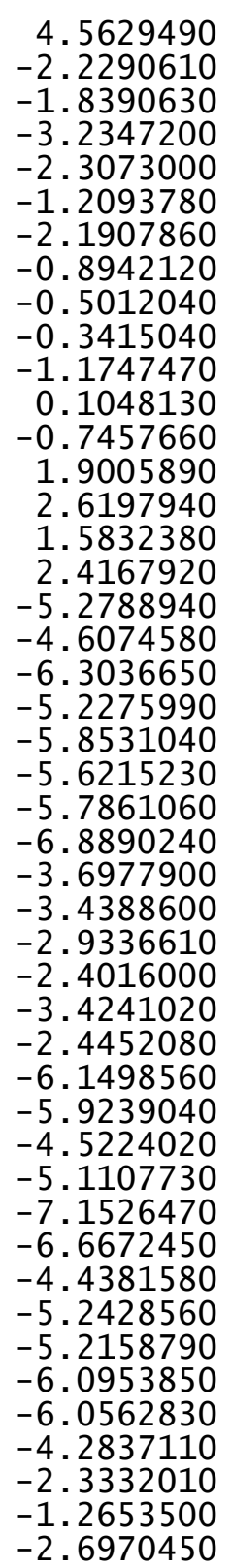
2. 5690960
3.2764160
4.2842260
3. 3871110
2. 7653080
3.2673250
3.2787290
4.3059620
2.7937560
$-0.6069530$
$-0.6807740$
$-1.6021980$
$-0.3204620$
0.4365390
1.2109790
0.6247890
$-0.5304620$
$-0.1560560$
$-0.4625550$
$-0.4221510$
0.9355220
$-0.4256890$
$-0.9618440$
0.6479840
$-0.6523690$
0.5012870
$-0.9330010$
1.0473820
$-1.2478170$
0.5125450
0.7388020
0.0867130
$-1.4103400$
$-1.8556630$
0.9251270
0.3667050
$-1.9985570$
$-1.9075670$
0.8145300
1.9889670
0.3132910
$-1.6341810$
$-2.8615100$
$-0.3627500$
0.0631670
0.4440260
5. 3480830
4.7719080
6.4687780
5.1328270
5.8854450
4. 1189040
6.7389590
7.1326860
6.0921500
7.6147180
1. 9040080
2.7705230

144

$\operatorname{TS} 249(\mathrm{~S}, \mathrm{~S}) \mathrm{G}[\mathrm{WB} 97 \mathrm{XD} / 6-31 \mathrm{G}(\mathrm{d})]=-3633.715695$

\begin{tabular}{lrcr} 
TS249(S, S & \multicolumn{1}{c}{ G $[$ WB $97 X D / 6-31 G(\mathrm{~d})]=-3633.715695$} \\
$\mathrm{O}$ & -1.6274490 & 0.5963830 & -0.6351200 \\
$\mathrm{O}$ & 0.2096600 & 1.4676600 & 0.8543100 \\
$\mathrm{C}$ & -2.5399510 & 1.3664630 & 0.0567250 \\
$\mathrm{C}$ & -2.2382960 & 2.6885160 & 0.3032290 \\
$\mathrm{C}$ & -3.7653380 & 0.7531610 & 0.4254140 \\
$\mathrm{C}$ & -3.1753640 & 3.4675040 & 1.0574800 \\
$\mathrm{C}$ & -4.7039710 & 1.5460940 & 1.0357170 \\
$\mathrm{C}$ & -4.4334480 & 2.8922890 & 1.3880750 \\
$\mathrm{H}$ & -5.6700150 & 1.1230080 & 1.2957880 \\
$\mathrm{C}$ & 0.2325780 & 2.6059410 & 0.0709150 \\
$\mathrm{C}$ & 1.4988730 & 3.0768390 & -0.3650870 \\
$\mathrm{C}$ & -0.9563850 & 3.2494400 & -0.2055200 \\
$\mathrm{C}$ & 1.5261690 & 4.2663990 & -1.0493870 \\
$\mathrm{C}$ & -0.9211560 & 4.4177430 & -1.0361390 \\
$\mathrm{C}$ & 0.3411140 & 4.9497350 & -1.4174920 \\
$\mathrm{H}$ & 2.4823710 & 4.6754430 & -1.3637360 \\
$\mathrm{C}$ & -0.7560520 & 6.7381600 & -2.6365230 \\
$\mathrm{C}$ & -2.0126000 & 6.1808690 & -2.3040450 \\
$\mathrm{C}$ & -2.0941060 & 5.0540630 & -1.5263890 \\
$\mathrm{C}$ & 0.3936530 & 6.1292590 & -2.2069010 \\
$\mathrm{H}$ & -0.7069550 & 7.6361330 & -3.2446950 \\
$\mathrm{H}$ & -2.9211570 & 6.6460740 & -2.6740820 \\
$\mathrm{H}$ & -3.0651030 & 4.6329650 & -1.2919210 \\
$\mathrm{H}$ & 1.3667900 & 6.5309680 & -2.4767310 \\
$\mathrm{C}$ & -3.8300120 & 5.4998720 & 2.2160830 \\
$\mathrm{C}$ & -5.0969910 & 4.9417530 & 2.5048790 \\
$\mathrm{C}$ & -5.3868910 & 3.6640410 & 2.1036790 \\
$\mathrm{C}$ & -2.8952420 & 4.7843980 & 1.5119360 \\
$\mathrm{H}$ & -3.5943940 & 6.5025320 & 2.5596340 \\
$\mathrm{H}$ & -5.8292180 & 5.5223860 & 3.0573860 \\
$\mathrm{H}$ & -6.3478400 & 3.2139840 & 2.3384370 \\
$\mathrm{H}$ & -1.9248720 & 5.2218300 & 1.3047850
\end{tabular}

0.0562100

1.6158270

0.8167180

$-0.8480040$

2. 3812450

1. 9104970

1.9797050

0.5035530

3.2892570

2.5745280

$-0.4746600$

$-1.6779540$

$$
\begin{aligned}
& -4.0424430 \\
& -4.2973350 \\
& -2.2967850 \\
& -1.1642970 \\
& -3.3226240 \\
& -4.8415260 \\
& -1.7391230 \\
& -3.5636600 \\
& -0.9621450 \\
& -3.2374410
\end{aligned}
$$

$-0.6351200$

(1)

1.0574800

3880750

1. 2957880

1.2919210

310

.1036790

1. 3047850 


-4.0122770
-4.1150280
-4.1810250
-4.4451230
-4.4930830
-4.6525490
-4.5521590
-4.6478540
2.7640380
3.4205840
3.3428170
4.6749200
4.5908450
5.2893100
5.1856320
5.0523690
-0.3195570
-0.6758860
0.6200290
-4.0844100
-3.8059380
-5.1006010
-5.1133370
-3.9133640
-3.4704080
2.6699750
1.7071970
6.7030230
6.7336600
2.8325390
1.7968690
2.7960870
2.3197790
3.8007990
2.2194490
3.5957180
4.6389410
3.1363670
3.5996950
-2.9939370
-3.2304310
-2.8956640
-2.0278950
-5.4441700
-5.3791550
-5.7805570

$\begin{array}{rr}-0.7015570 & 0.1817840 \\ -1.5841310 & 1.2765120 \\ -1.1868310 & -1.1296270 \\ -2.9189400 & 1.0327440 \\ -2.5319560 & -1.3191320 \\ -3.4123690 & -0.2508520 \\ -3.5993370 & 1.8746480 \\ -2.8957850 & -2.3327840 \\ 2.3100230 & -0.1401680 \\ 1.7256900 & -1.2414670 \\ 2.2345700 & 1.1404390 \\ 1.1419550 & -1.0488440 \\ 1.6308770 & 1.2815210 \\ 1.1010080 & 0.1976790 \\ 0.7154670 & -1.9072990 \\ 1.5887100 & 2.2669070 \\ 0.0845710 & 0.1835380 \\ -0.8250660 & 1.3232710 \\ -0.4134850 & -0.8884830 \\ -0.2849080 & -2.3520320 \\ 0.7192210 & -2.0222660 \\ -4.8462840 & -0.4734090 \\ -5.3409210 & 0.5060310 \\ -1.1317550 & 2.7185090 \\ -0.1326220 & 2.7009360 \\ 2.8227600 & 2.3717510 \\ 3.2453490 & 2.0722710 \\ 0.5823600 & 0.4016810 \\ 0.1103520 & 1.3916880 \\ 1.7287630 & -2.6483020 \\ 2.0738080 & -2.5847270 \\ 0.3266090 & -3.2699360 \\ 0.3714130 & -4.2560930 \\ -0.0883920 & -3.4089640 \\ -0.3579540 & -2.6442790 \\ 2.7008700 & -3.5596960 \\ 2.3854220 & -3.6802460 \\ 2.7356740 & -4.5539420 \\ 3.7164300 & -3.1509400 \\ -0.7621850 & -3.3192630 \\ -1.7477050 & -3.7373080 \\ -0.0609800 & -4.1555300 \\ -0.8249290 & -2.8097810 \\ -0.1596720 & -3.0530740 \\ 0.5445070 & -3.8900470 \\ -1.1242880 & -3.4505580\end{array}$

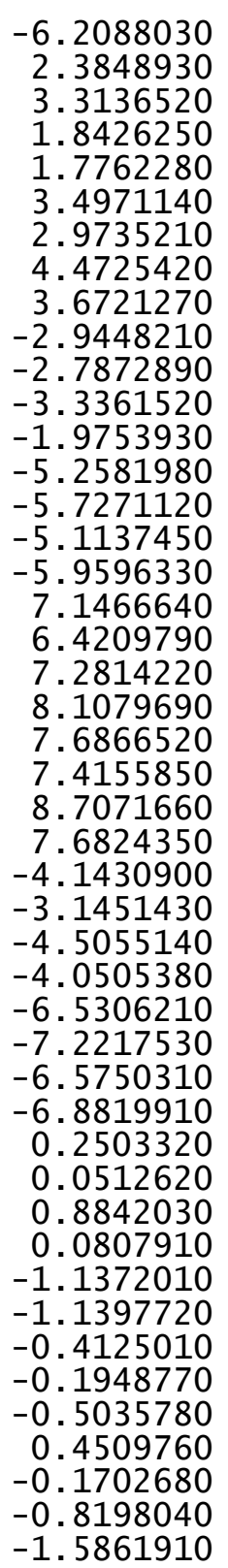

0.2038240
1.7401270
1.3010840
2.1668920
0.9385660
3.9723390
4.4232260
3.6206820
4.7530600
-2.0305040
-1.6159910
-3.0465600
-2.0877650
-1.0426970
-2.0313720
-0.6626150
-0.3785660
-0.4650200
-1.2795770
-0.0224670
-0.8954590
1.7638030
2.4920740
1.4161650
2.2793690
-5.6331990
-5.6890930
-6.6567850
-5.1687460
-4.8836800
-4.3441500
-4.4178960
-5.9169420
-3.7085710
-3.4103280
-3.0065490
-2.3676760
-3.3134460
-2.3328540
-6.1007290
-5.8722080
-4.4295840
-5.1528270
-7.1363540
-6.5517600
-4.2449930
$-2.3581590$ 3.4202790

3.8034850

4.2715120

2. 9914700

2.9632740

3.8137360

3.3189590

2.2151700

3.4984260

4.5005230

3.6215240

2.9996570

3.4553580

3.5267610

4.4729270

2.9410010

$-0.6215420$

$-0.7042010$

$-1.6155280$

$-0.3219840$

0.4215050

1.1928810

0.6186790

$-0.5461920$

$-1.3754780$

$-0.9306780$

$-1.5202430$

$-2.3640970$

$-1.0320760$

$-0.3764310$

$-2.0234480$

$-1.1289090$

0.6570360

$-0.7676800$

1.1989440

$-1.0673730$

0.7093820

0.9428860

0.2124600

$-1.2864240$

$-1.6984430$

1.0493480

0.4689850

-1.8739110
-1.7228600 


\begin{tabular}{|c|c|c|c|c|c|c|c|}
\hline $\mathrm{H}$ & 1.5142360 & -5.3813360 & 0.9066770 & C & -3.2474590 & 5.4123650 & 2.8200440 \\
\hline $\mathrm{H}$ & 0.8491340 & -6.0977290 & -1.5360950 & C & -2.4353970 & 4.7202370 & 1.9583050 \\
\hline $\mathrm{H}$ & -0.1470660 & -4.2044040 & $-\overline{2} .7076080$ & $\mathrm{H}$ & -2.8987310 & 6.3424990 & 3.258271 \\
\hline C & 3.2660470 & -2.4886760 & -0.2949180 & $\mathrm{H}$ & -5.1675010 & 5.4842810 & 3.826540 \\
\hline 0 & 2.7323700 & -1.3911670 & 0.1355590 & $\mathrm{H}$ & -5.9359310 & 3.3274060 & 2.892825 \\
\hline C & 4.4880300 & -2.8613260 & 0.4496560 & $\mathrm{H}$ & -1.4489800 & 5.1042420 & 722986 \\
\hline C & 5.2980520 & -3.9273450 & 0.0393190 & C & -4.0965220 & -0.4603970 & 0.09235 \\
\hline C & 4.8433220 & -2.1208260 & 1.5865840 & C & -4.1579970 & -1.4701470 & 1.078118 \\
\hline C & 6.4527040 & -4.2374470 & 0.7451540 & C & -4.3825380 & -0.7691510 & -1.24943 \\
\hline $\mathrm{H}$ & 5.0231330 & -4.5017560 & -0.8386770 & $\mathrm{C}$ & -4.5292530 & -2.7576670 & 0.694610 \\
\hline C & 5.9889080 & -2.4480320 & 2.2994700 & C & -4.7430360 & -2.0767130 & -1.58019 \\
\hline $\mathrm{H}$ & 4.2257470 & -1.2856460 & 1.8956920 & C & -4.8290050 & -3.0837770 & -0.626249 \\
\hline C & 6.7987070 & -3.5002000 & 1.8766780 & H & -4.5883330 & -3.5337930 & 1.453416 \\
\hline $\mathrm{H}$ & 7.0831020 & -5.0563520 & 0.4142690 & $\mathrm{H}$ & -4.9745020 & -2.3155880 & -2.616373 \\
\hline $\mathrm{H}$ & 6.2554800 & -1.8750390 & 3.1820320 & $\mathrm{C}$ & 2.8715260 & 2.0757460 & -0.377044 \\
\hline $\mathrm{H}$ & 7.7002270 & -3.7470620 & 2.4290990 & C & 3.3796710 & 1.5143190 & -1.564347 \\
\hline $\mathrm{H}$ & 1.9083160 & -1.0567950 & -0.3699930 & $\mathrm{C}$ & 3.5749560 & 1.9250570 & 0.83117 \\
\hline $\mathrm{S}$ & 2.6160700 & -3.4142370 & -1.5444790 & $\mathrm{c}$ & 4.6236050 & 0.8825130 & -1.524479 \\
\hline & & & & C & 4.8100590 & 1.2765670 & 0.820417 \\
\hline 0 & 0.3105910 & 1.2793540 & 0.6710880 & $\mathrm{H}$ & 5.3652830 & 1.1904650 & 1.752181 \\
\hline C & -2.4876300 & 1.4962790 & 0.0814690 & $\mathrm{P}$ & -0.3946170 & 0.0318770 & -0.111133 \\
\hline C & -2.0563280 & 2.7485610 & 0.4592050 & 0 & -0.7781450 & -0.9612760 & 46121 \\
\hline C & -3.7280890 & 0.9345620 & 0.4830440 & 0 & 0.4491640 & -0.4222460 & 77812 \\
\hline C & -2.8630830 & 3.4962200 & 1.3772550 & C & -4.3493930 & 0.2762070 & 35559 \\
\hline C & -4.544 & 1.7110580 & 1.2654260 & $\mathrm{H}$ & -4.0187010 & 1.2258310 & -1.92690 \\
\hline C & -4.13 & 2.9804890 & 1.7476490 & C & -5.2472770 & -4.4907800 & -1.01294 \\
\hline $\mathrm{H}$ & -5.5165640 & 1.3278690 & 1.5648400 & $\mathrm{H}$ & -5.4493460 & -4.4858990 & -2.09174 \\
\hline C & 0.3764130 & 2.5006980 & 0.0290480 & C & -3.8737710 & -1.2058060 & 2.553129 \\
\hline C & 1.6456760 & 2.9328800 & -0.4376390 & $\mathrm{H}$ & -3.4117140 & -0.2192880 & 2.643636 \\
\hline C & -0.76 & 3.2641870 & -0.0799990 & C & 3.0467270 & 2.4901990 & 141521 \\
\hline C & 1.7270850 & 4.1925900 & -0.9764680 & $\mathrm{H}$ & 2.0792340 & 2.9619060 & 1.947552 \\
\hline C & -0.69 & 4.5189190 & -0.7692570 & C & 6.7923170 & 0.2435140 & -0.40224 \\
\hline C & 0.5812430 & 5.0001200 & -1.1782740 & $\mathrm{H}$ & 6.9336450 & -0.2142350 & 9957 \\
\hline $\mathrm{H}$ & 2870 & 4.5702890 & -1.2962190 & C & 2.6402860 & 1.6106920 & 893847 \\
\hline C & -0.44 & 7.0003780 & -2.0938440 & $\mathrm{H}$ & 1.6030230 & 1.8871090 & -2.683190 \\
\hline C & -1.71 & 6.5006400 & -1.7349270 & C & 2.6010980 & 0.2738820 & -3.64537 \\
\hline C & -1.83 & 5.2957410 & -1.0913860 & $\mathrm{H}$ & 1.9583040 & 0.3662780 & -4.528056 \\
\hline C & 0.67 & 6.2604600 & -1.8253950 & $\mathrm{H}$ & 3.5946970 & -0.0285570 & -3.995337 \\
\hline $\mathrm{H}$ & -0.3 & 7.9599 & -2.5946460 & $\mathrm{H}$ & 2.1979120 & -0.5217580 & -3.014937 \\
\hline
\end{tabular}




\begin{tabular}{|c|c|c|}
\hline $\begin{array}{l}3.2163060 \\
-3.3509380 \\
-3.6533030 \\
-3.2944460 \\
-2.3518500 \\
-5.7528640 \\
-5.7258770 \\
-6.1490460 \\
-6.4527660 \\
2.8079270 \\
3.7447280 \\
2.3788680 \\
2.1111120 \\
3.9749930 \\
3.5513340 \\
4.9618780 \\
4.1161680 \\
-2.8907840 \\
-2.6950220 \\
-3.2883230 \\
-1.9411230 \\
-5.1838130 \\
-5.6764630 \\
-4.9881630 \\
-5.8876150 \\
7.7848280 \\
7.5986610 \\
7.6918610 \\
8.8159750 \\
7.0952350 \\
6.4475730 \\
8.1357400 \\
6.9580720 \\
-6.5403250 \\
-7.3433300 \\
-6.8714880 \\
-6.3901450 \\
-4.1269680 \\
-3.2157330 \\
-3.8805280 \\
-4.4277830 \\
0.1890130 \\
0.1477360 \\
0.7352540 \\
0.1478920 \\
-1.2073280\end{array}$ & $\begin{array}{r}3.6819190 \\
-0.0974480 \\
-1.0143600 \\
0.7028920 \\
-0.2499090 \\
0.5122070 \\
1.2999500 \\
-0.3952240 \\
0.8180850 \\
1.3814890 \\
0.8781250 \\
1.7983880 \\
0.6342090 \\
3.5804910 \\
4.0212080 \\
3.1751660 \\
4.3799510 \\
-2.2128380 \\
-1.9480930 \\
-3.2338640 \\
-2.2014030 \\
-1.1892370 \\
-2.1678520 \\
-0.9572430 \\
-0.4457290 \\
1.4111750 \\
2.1660110 \\
1.8984670 \\
1.0556720 \\
-0.8271410 \\
-1.6989780 \\
-1.1585920 \\
-0.4434570 \\
-4.9081240 \\
-4.1883810 \\
-5.8930500 \\
-4.9691750 \\
-5.5058940 \\
-5.2261780 \\
-5.5590450 \\
-6.5070610 \\
-3.7943000 \\
-3.4885690 \\
-3.0783700 \\
-2.4405900 \\
-3.4452710\end{array}$ & $\begin{array}{r}-3.3151060 \\
-3.4593240 \\
-3.9785020 \\
-4.2055830 \\
-3.0413990 \\
-2.9310240 \\
-3.6921710 \\
-3.4010270 \\
-2.1460140 \\
3.1735310 \\
3.4422750 \\
4.0914750 \\
2.7801740 \\
2.6930910 \\
3.6024380 \\
2.9442480 \\
1.9577660 \\
3.1645860 \\
4.2099080 \\
3.1554740 \\
2.6268010 \\
3.3554260 \\
3.3103740 \\
4.4083680 \\
2.9673100 \\
-0.2855940 \\
-1.0567940 \\
0.6923180 \\
-0.3920920 \\
0.6493110 \\
0.5293400 \\
0.5577940 \\
1.6663070 \\
-0.2996770 \\
-0.4890970 \\
-0.6473610 \\
0.7843740 \\
-0.7554760 \\
-1.2928640 \\
0.3113310 \\
-1.0837250 \\
0.3480720 \\
-1.0913360 \\
0.9624920 \\
-1.3798220 \\
0.2637060\end{array}$ \\
\hline
\end{tabular}

$\begin{array}{lr}\mathrm{H} & -1.2530620 \\ \mathrm{C} & -0.3045370 \\ \mathrm{C} & 0.0765710 \\ \mathrm{C} & -0.2597080 \\ \mathrm{C} & 0.4040450 \\ \mathrm{H} & -0.0406910 \\ \mathrm{H} & -0.4397780 \\ \mathrm{H} & -1.3422700 \\ \mathrm{H} & 1.4864160 \\ \mathrm{H} & 0.0623880 \\ \mathrm{H} & -1.3887980 \\ \mathrm{H} & 1.1527110 \\ \mathrm{H} & 0.1818710 \\ \mathrm{C} & 3.0928230 \\ \mathrm{O} & 2.5281740 \\ \mathrm{C} & 4.0102090 \\ \mathrm{C} & 4.7977490 \\ \mathrm{C} & 4.0733320 \\ \mathrm{C} & 5.6431910 \\ \mathrm{H} & 4.7467370 \\ \mathrm{C} & 4.9071520 \\ \mathrm{H} & 3.4738900 \\ \mathrm{C} & 5.6964080 \\ \mathrm{H} & 6.2578880 \\ \mathrm{H} & 4.9471630 \\ \mathrm{H} & 6.3548030 \\ \mathrm{H} & 1.8261360 \\ \mathrm{~S} & 2.7523640 \\ \mathrm{H} & \end{array}$
$-2.4683660$
.2336020
7.2382930
$-6.6564990$
$-4.3949610$
$-5.4125370$
$-5.3570540$
$-6.1160890$
$-6.1218720$
$-4.2697830$
$-2.4291820$
$-1.2855090$
$-2.8294230$
$-3.9836660$
$-2.0387310$
$-4.3328360$
$-4.5972410$
$-2.4015580$
$-1.1396140$
$-3.5453710$
$-5.2234840$
$-1.7874120$
$-3.8255880$
$-0.9916890$

$-6.2133550 \quad-0.1877070$

$-1.6480660$

$-2.0799610$

0.7517970

0.0899400

$-2.3093590$

$-2.2113720$

0.7415010

1.7842190

$-0.0622650$

$-1.7774680$

$-3.0474840$

$-0.2478240$

$-0.0286640$

0.8358530

0.7435260

1.9928670

1.7884610

$-0.1494830$

3. 0404490

2. 0558200

2.9382520

1.7092370

3.9345470

3.7547810

$-0.6993170$

144

$-1.5986940$

$\operatorname{TS} 251(\mathrm{~S}, \mathrm{~S}) \mathrm{G}[\mathrm{WB} 97 \mathrm{XD} / 6-31 \mathrm{G}(\mathrm{d})]=-3633.712589$

$\begin{array}{lrcr}\mathrm{O} & -1.7500320 & 0.6631750 & -0.7124230 \\ \mathrm{O} & 0.2637250 & 1.2878250 & 0.6729960 \\ \mathrm{C} & -2.5486320 & 1.4076980 & 0.1332770 \\ \mathrm{C} & -2.1468360 & 2.6833790 & 0.4615640 \\ \mathrm{C} & -3.7645270 & 0.8258260 & 0.5793820 \\ \mathrm{C} & -2.9560240 & 3.4382670 & 1.3716060 \\ \mathrm{C} & -4.5877280 & 1.6046890 & 1.3526930 \\ \mathrm{C} & -4.2056580 & 2.9001290 & 1.7852920 \\ \mathrm{H} & -5.5412080 & 1.2040720 & 1.6854480 \\ \mathrm{C} & 0.2830770 & 2.4903280 & -0.0075130 \\ \mathrm{C} & 1.5300150 & 2.9446740 & -0.5118080 \\ \mathrm{C} & -0.8855790 & 3.2170070 & -0.1188470 \\ \mathrm{C} & 1.5639440 & 4.1878870 & -1.0927000 \\ \mathrm{C} & -0.8590930 & 4.4495850 & -0.8504020 \\ \mathrm{C} & 0.3911370 & 4.9542530 & -1.2996220 \\ \mathrm{H} & 2.5134560 & 4.5825300 & -1.4434500\end{array}$




$\begin{array}{rrr}-0.7092290 & 6.8901910 & -2.2625360 \\ -1.9600460 & 6.3657750 & -1.8617250 \\ -2.0342850 & 5.1803480 & -1.1760420 \\ 0.4391420 & 6.1936660 & -1.9914600 \\ -0.6648950 & 7.8340760 & -2.7970080 \\ -2.8712900 & 6.9036550 & -2.1051850 \\ -3.0020020 & 4.7862310 & -0.8873680 \\ 1.4051690 & 6.5711010 & -2.3165470 \\ -3.3644260 & 5.3888530 & 2.7606260 \\ -4.6266980 & 4.8727820 & 3.1350340 \\ -5.0328980 & 3.6527800 & 2.6606950 \\ -2.5514140 & 4.6921770 & 1.9034700 \\ -3.0333420 & 6.3420000 & 3.1616030 \\ -5.2633260 & 5.4391020 & 3.8077140 \\ -5.9899660 & 3.2331550 & 2.9593390 \\ -1.5820950 & 5.0962490 & 1.6331410 \\ -4.0970980 & -0.5924010 & 0.2471810 \\ -4.1114120 & -1.5630940 & 1.2693490 \\ -4.3890310 & -0.9622400 & -1.0801500 \\ -4.4461700 & -2.8770070 & 0.9381140 \\ -4.7017540 & -2.2914960 & -1.3593200 \\ -4.7432870 & -3.2645290 & -0.3639220 \\ -4.4702930 & -3.6305210 & 1.7217640 \\ -4.9257290 & -2.5718450 & -2.3860830 \\ 2.7822620 & 2.1273260 & -0.4505460 \\ 3.2859290 & 1.5506150 & -1.6324900 \\ 3.5133560 & 2.0335070 & 0.7466160 \\ 4.5517030 & 0.9637860 & -1.6013960 \\ 4.7696810 & 1.4273460 & 0.7281090 \\ 5.3241580 & 0.9173990 & -0.4442200 \\ 4.9672670 & 0.5524170 & -2.5185510 \\ 5.3461650 & 1.3879300 & 1.6498960 \\ -0.4152740 & -0.0024840 & -0.0624100 \\ -0.7456480 & -0.9834080 & 1.0243700 \\ 0.4236000 & -0.4581950 & -1.2319300 \\ -4.4126180 & 0.0453280 & -2.2211900 \\ -4.0983940 & 1.0174950 & -1.8322980 \\ -5.0600110 & -4.7144210 & -0.6824050 \\ -5.1894550 & -5.2351460 & 0.2749950 \\ -3.8033450 & -1.2374040 & 2.7267400 \\ -3.3620640 & -0.2382140 & 2.7687160 \\ 2.9939060 & 2.6224370 & 2.0501510 \\ 2.0085120 & 3.0580060 & -0.8618870 \\ 6.7676300 & 0.4454730 & -1.4841430 \\ 6.9037630 & -0.0445480 & -2.9476030 \\ 2.5159800 & 1.5835790 & \end{array}$

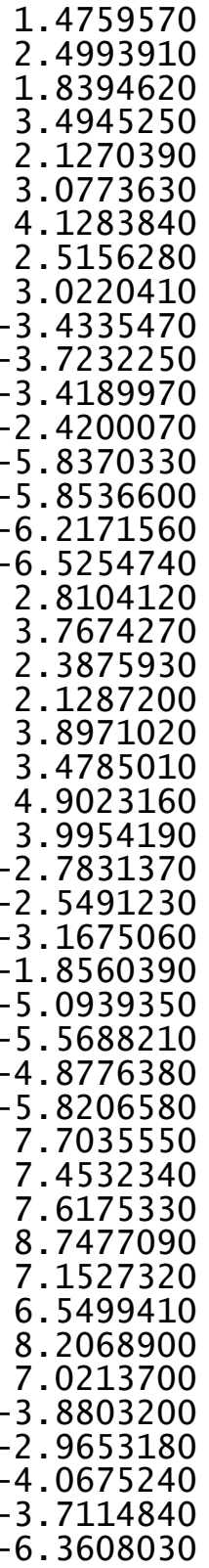

1.8365510

0.2220410

0.2681100

$-0.0669780$

$-0.5606710$

2.6575970

2.4522690

2.6740550

3.6554600

$-0.3347560$

$-1.2745750$

0.4442380

$-0.4470360$

0.2225530

0.9876020

$-0.7104330$

0.5310900

1.5410610

1.0749930

1.9742610

0.7614590

3.7588630

4.2127520

3. 3942850

4.5400470

$-2.1988170$

$-1.8742610$

$-3.2228790$

$-2.2064550$

$-1.2199600$

$-2.2083020$

$-0.9512950$

$-0.5016970$

1.6643320

2. 3797190

2. 1819870

1.3577480

$-0.5645870$

$-1.4733830$

$-0.8439000$

$-0.1480760$

$-5.3728410$

$-5.2693370$

$-6.4387710$

$-4.8970540$

$-4.8746480$
$-2.7216330$

$-3.6540350$

$-4.5277780$

$-4.0108600$

$-2.9898090$

$-3.8909300$

$-4.1270660$

$-4.8317950$

$-3.4460620$

$-3.3396920$

$-3.8239590$

$-4.1099780$

$-2.9445750$

$-2.7655820$

$-3.5498230$

-3. 1977370

$-1.9715070$

3. 1213510

3. 3860210

4.0345960

2.7666470

2.5476680

3.4528830

2.7885330

1.7862170

3.3507580

4. 3710680

3.4147500

2.7742750

3.5595950

3.5576840

4.5997580

3.1663780

$-0.4711580$

$-1.2614080$

0.4916910

$-0.6004280$

0.5723150

0.5039120

0.4651080

1.5770980

$-1.4100780$

$-0.8184090$

$-1.5811020$

$-2.3841740$

$-1.4774290$ 


\begin{tabular}{|c|c|c|c|c|c|c|c|}
\hline $\mathrm{H}$ & -7.2034250 & -4.4092580 & -0.9558430 & C & -2.6319450 & 3.2849420 & -1.6063980 \\
\hline $\mathrm{H}$ & -6.2817250 & -4.4145240 & -2.4689000 & $\mathrm{H}$ & -3.6378450 & 3.6939050 & -1.5435410 \\
\hline $\mathrm{H}$ & -6.5895930 & -5.9359830 & -1.6233430 & C & 1.9941590 & 1.2575370 & -0.8011460 \\
\hline C & 0.3229320 & -3.7831440 & 0.4667320 & C & 3.2288080 & 0.6145860 & -0.8226780 \\
\hline c & 0.2491740 & -3.5240830 & -0.9802920 & $\mathrm{C}$ & 1.2407440 & 1.5013650 & -1.9544200 \\
\hline $\mathrm{H}$ & 0.8430140 & -3.0243580 & 1.0515520 & C & 3.6996670 & 0.1911580 & -2.0686440 \\
\hline $\mathrm{H}$ & 0.2010920 & -2.4861650 & -1.3000240 & $\mathrm{H}$ & 4.6625460 & -0.3119740 & -2.1161370 \\
\hline 0 & -1.0889240 & -3.4957630 & 0.3889990 & $\mathrm{C}$ & -3.3829660 & 1.3875790 & -0.1363120 \\
\hline $\mathrm{H}$ & -1.1724470 & -2.5164350 & 0.6053970 & $\mathrm{C}$ & 4.0192070 & 0.3440000 & 0.4096840 \\
\hline C & -0.0609090 & -6.2396410 & 0.0086170 & $\mathrm{C}$ & -3.8859320 & 0.1949520 & -0.6714190 \\
\hline C & 0.2957740 & -6.0063670 & -1.4619180 & C & -3.4891430 & -0.4340800 & -1.9848360 \\
\hline C & -0.1123370 & -4.6074840 & -1.9337890 & $\mathrm{C}$ & -5.2909910 & -1.7862650 & -0.7189610 \\
\hline $\mathrm{c}$ & 0.6051230 & -5.1982290 & 0.9122420 & $\mathrm{c}$ & -4.8833830 & -0.5237490 & -0.0033890 \\
\hline $\mathrm{H}$ & 0.2573880 & -7.2407120 & 0.3147690 & $\mathrm{C}$ & -2.9578450 & -1.8494300 & -1.6940930 \\
\hline $\mathrm{H}$ & -0.1909310 & -6.7518470 & -2.0975920 & $\mathrm{H}$ & -2.6916120 & -2.3419420 & -2.6362640 \\
\hline $\mathrm{H}$ & -1.2002910 & -4.5371460 & -2.0634350 & $\mathrm{H}$ & -2.0399760 & -1.7562000 & -1.1094500 \\
\hline $\mathrm{H}$ & 1.6944060 & -5.3282420 & 0.8988140 & C & -4.0326910 & -2.6606640 & -0.9278840 \\
\hline $\mathrm{H}$ & 0.2766320 & -5.3055190 & 1.9506640 & $\mathrm{H}$ & -4.3091840 & -3.5635880 & -1.4849430 \\
\hline $\mathrm{H}$ & -1.1481750 & -6.1972050 & 0.1417120 & $\mathrm{H}$ & -3.6421790 & -2.9941950 & 0.0394480 \\
\hline $\mathrm{H}$ & 1.3776930 & -6.1268940 & -1.5949940 & $\mathrm{H}$ & -6.0520730 & -2.3355000 & -0.1542990 \\
\hline $\mathrm{H}$ & 0.3125700 & -4.3605930 & -2.9107220 & $\mathrm{H}$ & -2.7287410 & 0.1532740 & -2.5050830 \\
\hline C & 3.1739650 & -2.3292770 & -0.1911510 & $\mathrm{C}$ & -5.8308980 & -1.3887670 & -2.1116370 \\
\hline 0 & 2.5549930 & -1.2114710 & 0.0201580 & $\mathrm{H}$ & -6.0785540 & -2.2981970 & -2.6716030 \\
\hline C & 4.1333360 & -2.6619590 & 0.8793420 & $\mathrm{H}$ & -6.7585350 & -0.8192260 & -1.9962970 \\
\hline c & 4.9689910 & -3.7826180 & 0.7944880 & C & -4.75 & -0.5543060 & -2.8586560 \\
\hline C & 4.1895380 & -1.8418270 & 2.0159720 & $\mathrm{H}$ & -4.4904810 & -1.0274660 & -3.8104960 \\
\hline C & 5.8546930 & -4.0687340 & 1.8251590 & $\mathrm{H}$ & -5.1354550 & 0.4466290 & -3.0909370 \\
\hline $\mathrm{H}$ & 4.9239760 & -4.4202840 & -0.0817390 & C & -3.9154810 & 1.8606910 & 1.0654100 \\
\hline $\mathrm{C}$ & 5.0647180 & -2.1417090 & 3.0497930 & $\mathrm{C}$ & -3.5474090 & 3.1420060 & 1.7717000 \\
\hline $\mathrm{H}$ & 3.5515610 & -0.9694040 & 2.0744430 & $\mathrm{C}$ & -5.4177210 & 1.8199580 & 2.9755450 \\
\hline C & 5.9017910 & -3.2515770 & 2.9542510 & $\mathrm{C}$ & -4.9389390 & 1.1537210 & 1.7125230 \\
\hline $\mathrm{H}$ & 6.5070920 & -4.9326030 & 1.7507020 & $\mathrm{C}$ & -4.8264690 & 4.0066390 & 1.8422800 \\
\hline $\mathrm{H}$ & 5.0992140 & -1.5044770 & 3.9278160 & $\mathrm{H}$ & -4.5948310 & 4.9524110 & 2.3451350 \\
\hline $\mathrm{H}$ & 6.5926010 & -3.4820910 & 3.7594350 & $\mathrm{H}$ & -5.1588720 & 4.2538000 & 0.8282110 \\
\hline $\mathrm{H}$ & 1.8344190 & -0.9642560 & -0.6479240 & C & -5.9322250 & 3.2318290 & 2.6090230 \\
\hline & 2.8561780 & -3.3189650 & -1.5171120 & $\mathrm{H}$ & -6.2092670 & 3.7584520 & 3.5297160 \\
\hline & R) $\mathrm{G}[\mathrm{wB} 97 \mathrm{XD}$ & $-(d)]--3$ & & $\mathrm{H}$ & $\begin{array}{l}-6.8396410 \\
-6.2105180\end{array}$ & 3.1460200 & $\begin{array}{l}2.0026930 \\
3.4563310\end{array}$ \\
\hline $\mathrm{P}$ & 0.3323570 & $\begin{array}{c}(\mathrm{d})\rfloor \\
0.6237430\end{array}$ & $9 / 9976010$ & ${ }_{H}^{H}$ & $\begin{array}{l}-6.2105180 \\
-2.7484530\end{array}$ & $\begin{array}{l}1.2363940 \\
3.6735170\end{array}$ & $\begin{array}{l}3.4563310 \\
1.2484480\end{array}$ \\
\hline 0 & 0.9127920 & -0.7653220 & 1.1466050 & $\mathrm{C}$ & -4.2065930 & 1.9769170 & 3.9210790 \\
\hline 0 & -0.3117180 & 1.2475600 & 2.1971100 & $\mathrm{H}$ & -4.5344610 & 2.4561500 & 4.8508810 \\
\hline 0 & -0.7058310 & 0.4907350 & -0.2622730 & $\mathrm{H}$ & -3.8153150 & 0.9890290 & 4.1866800 \\
\hline 0 & 1.4459630 & 1.6492200 & 0.4158210 & C & -3.1121450 & 2.8185890 & 3.2173770 \\
\hline C & -1.0373050 & 1.6277160 & -0.9787780 & $\mathrm{H}$ & -2.9477500 & 3.7634990 & 3.7484920 \\
\hline C & -0.0720140 & 2.1882280 & -1.8226530 & $\mathrm{H}$ & -2.1563800 & 2.2930270 & 3.1938440 \\
\hline c & -2.3327100 & 2.1275080 & -0.8869720 & $\mathrm{C}$ & -5.4231270 & -0.0368210 & 1.18278 \\
\hline
\end{tabular}




$\begin{array}{rrrr}\mathrm{H} & -6.2174440 & -0.5822330 & 1.6906810 \\ \mathrm{C} & 5.3031960 & 1.1236140 & 2.3230990 \\ \mathrm{C} & 5.7259060 & 2.3603060 & 3.0726880 \\ \mathrm{C} & 4.2668840 & 2.8682750 & 1.0021890 \\ \mathrm{C} & 4.4969710 & 1.3903780 & 1.2089390 \\ \mathrm{C} & 6.5133080 & 3.2665040 & 2.1008650 \\ \mathrm{H} & 6.7772450 & 4.1989830 & 2.6135010 \\ \mathrm{H} & 7.4513080 & 2.7758040 & 1.8217680 \\ \mathrm{C} & 5.6445340 & 3.5512970 & 0.8480310 \\ \mathrm{H} & 5.4944690 & 4.6289060 & 0.7143340 \\ \mathrm{H} & 6.1344200 & 3.1800740 & -0.0580640 \\ \mathrm{H} & 3.6526360 & 3.0686310 & 0.1218020 \\ \mathrm{H} & 6.3410220 & 2.1046120 & 3.9423100 \\ \mathrm{C} & 3.5825870 & 3.4306170 & 2.2673800 \\ \mathrm{H} & 3.4509960 & 4.5124050 & 2.1477780 \\ \mathrm{H} & 2.5864700 & 2.9910920 & 2.3617870 \\ \mathrm{C} & 4.4516670 & 3.1142300 & 3.5126560 \\ \mathrm{H} & 4.7400450 & 4.0343890 & 4.0345040 \\ \mathrm{H} & 3.8936950 & 2.4998000 & 4.2262780 \\ \mathrm{C} & 5.1893670 & -1.2241840 & 1.8571020 \\ \mathrm{C} & 5.4965170 & -2.6853830 & 2.0623190 \\ \mathrm{C} & 3.8927480 & -2.2425760 & 0.0844980 \\ \mathrm{C} & 4.3535610 & -0.9720340 & 0.7615000 \\ \mathrm{C} & 4.1691300 & -3.4322030 & 2.3071280 \\ \mathrm{H} & 4.3699810 & -4.5072940 & 2.3860360 \\ \mathrm{H} & 3.7400920 & -3.1135750 & 3.2627090 \\ \mathrm{C} & 3.1948250 & -3.1292240 & 1.1416290 \\ \mathrm{H} & 2.8559420 & -4.0549060 & 0.6615020 \\ \mathrm{H} & 2.3095420 & -2.6083500 & 1.5096300 \\ \mathrm{H} & 3.2016190 & -2.0399590 & -0.7363550 \\ \mathrm{H} & 6.1837290 & -2.8303660 & 2.9028090 \\ \mathrm{C} & 5.1390350 & -3.0002880 & -0.4228100 \\ \mathrm{H} & 4.8219550 & -3.9550570 & -0.8585450 \\ \mathrm{H} & 5.6230350 & -2.4296390 & -1.2229900 \\ \mathrm{C} & 6.1204380 & -3.2309170 & 0.7572490 \\ \mathrm{H} & 6.3371590 & -4.2983750 & 0.8810150 \\ \mathrm{H} & 7.0750820 & -2.7274150 & 0.5736420 \\ \mathrm{C} & 5.6626070 & -0.1799270 & 2.6416510 \\ \mathrm{H} & 6.3069320 & -0.3809410 & 3.4961570 \\ \mathrm{C} & 1.7140620 & 1.0109760 & -3.1767340 \\ \mathrm{C} & 0.9246320 & 1.0345080 & -4.4608710 \\ \mathrm{C} & 0.8680200 & -0.3846500 & -5.0580540 \\ \mathrm{C} & 2.2665040 & -1.0387500 & -5.1925820 \\ \mathrm{C} & 3.3844040 & -0.1804350 & -4.5658310 \\ \mathrm{C} & 2.9533950 & 0.3576590 & -2273390 \\ \mathrm{H} & 1.3998530 & 1.7165160 & \\ & & & -1801970\end{array}$

0.3687380
2.5063350
3.6097750
-0.0895600
0.2325900
2.2654650
4.3059760
-0.3930980
0.5839410
0.5769260
-0.8468420
-1.9505910
-1.6765050
0.3048420
1.2072520
-0.9514340
-1.9904000
1.5946140
1.0507440
-1.0108770
-2.9297350
-0.2063640
0.5287640
-0.4732180
-1.3817800
0.1539940
-1.6598110
-1.8772850
-0.1250670
0.8510310
-1.0342920
-2.3719960
0.3632250
-1.2561810
0.6715260
-0.8269590
-2.1765610
-0.9265880
-2.2312670
-0.0166720
-1.6580460
-1.1374710
-3.3407270
-2.1854470
-0.8563510
-3.4723550
-0.3433080
-1.2211050
0.6568560
1.4067240
-0.9957220
-2.0175590
-0.7636970
3.3610240
4.1289280
5.6173770
6.2263910
5.1788090
3.9128690
4.0294560
6.1751480
7.0266380
4.9465570
3.7242320
5.7041250
6.6840730
5.5902580
-3.4465650
-2.4028390
-4.1713990
-5.2316300
-3.7271720
-5.8366640
-5.5661600
-4.3353400
-2.8983460
-5.3901150
-6.6545270
-3.9814780
-5.8628800
-1.7781120
-3.9557010
-1.4280280
-2.1298640
-0.9791400
-1.7449140
-0.5087930
0.2087960
-2.7843460
-3.7864600
-3.0854940
-2.0601270
$-6.0315920$ $-6.2453260$ $-5.2408420$ $-4.2973250$ $-4.4069490$ $-4.6994980$ $-4.4727000$ $-2.5166540$ $-3.3704400$ $-2.9752720$ $-2.9211970$ $-3.1635330$ $-2.3958980$ $-4.4293240$ $-3.6754610$ $-3.6612660$ $-4.2373740$ $-3.2736580$ $-1.9918980$ $-1.9402420$ $-2.8980500$ $-0.4962830$ $-0.6757630$ $-1.7649530$ $-1.8312180$ $-2.9375360$ $-3.0507730$ $-0.9263590$ $-4.1535550$ $-2.8862700$ $-4.2128440$ $-3.0949430$ $-5.0566110$ $-5.1648690$ 0.1397220

0.9849200

2. 6358170

2. 9684010

1.6409530

2. 5210020

3. 6237540

3. 1370910

4.4307790

4.4030670

4.1109930

3. 0887620 


\begin{tabular}{|c|c|c|c|c|c|c|c|}
\hline $\mathrm{H}$ & -4.2807130 & -3.2973340 & 4.6567280 & C & 3.6698900 & -2.9208400 & 2.0718750 \\
\hline $\mathrm{H}$ & -2.1090450 & -4.3182420 & 5.3562440 & C & 5.6350650 & -1.4395610 & 2.8656360 \\
\hline $\mathrm{H}$ & -0.5321330 & -2.4953340 & 4.9782410 & C & 5.0475910 & -0.9923920 & 1.5529280 \\
\hline $\mathrm{H}$ & -3.7799420 & -2.7662330 & 2.3075460 & C & 4.9464570 & -3.7821040 & 2.2017180 \\
\hline H & -4.2340620 & -1.2782830 & 3.1303680 & $\mathrm{H}$ & 4.7544640 & -4.6006470 & 2.9048320 \\
\hline $\mathrm{H}$ & -3.1758950 & -2.0495690 & 5.2281230 & $\mathrm{H}$ & 5.1834030 & -4.2381100 & 1.2344150 \\
\hline $\mathrm{H}$ & -2.3750030 & -4.5412370 & 3.6291370 & C & 6.1221530 & -2.8968930 & 2.6960350 \\
\hline $\begin{array}{l}H \\
154\end{array}$ & -0.0480150 & -3.7949600 & 3.9123200 & $\mathrm{H}$ & 6.5080480 & -3.2609850 & 3.6553750 \\
\hline \multicolumn{4}{|c|}{ TS258(R,R) G[WB97XD/6-31G(d)] $=-3864.648038$} & $\begin{array}{l}\mathrm{H} \\
\mathrm{H}\end{array}$ & 6.4645290 & -0.7912920 & $\begin{array}{l}1.9842700 \\
3.1691240\end{array}$ \\
\hline $\mathrm{P}$ & -0.2593820 & -0.4044900 & 1.0389770 & $\mathrm{H}$ & 2.8312250 & -3.5263690 & 1.7208900 \\
\hline 0 & -0.7587530 & 1.0206480 & 0.9714430 & C & 4.5139550 & -1.4207600 & 3.9276070 \\
\hline 0 & 0.4583820 & -0.8508190 & 2.2774470 & $\mathrm{H}$ & 4.9220230 & -1.7638210 & 4.8855880 \\
\hline 0 & 0.6644920 & -0.5709360 & -0.3036390 & $\mathrm{H}$ & 4.1600770 & -0.3952570 & 4.0759610 \\
\hline 0 & -1.4638230 & -1.4597930 & 0.7724390 & $\mathrm{C}$ & 3.3493200 & -2.3305830 & 3.4619630 \\
\hline C & 0.9372000 & -1.8566240 & -0.7397300 & $\mathrm{H}$ & 3.1965820 & -3.1565570 & 4.1663070 \\
\hline C & -0.0940980 & -2.5866930 & -1.3266280 & $\mathrm{H}$ & 2.4094500 & -1.7785950 & 3.4057280 \\
\hline C & 2.2272080 & -2.3654060 & -0.5997100 & C & 5.4805980 & 0.0873580 & 0.7923930 \\
\hline C & 2.4320550 & -3.6809350 & -1.0009340 & $\mathrm{H}$ & 6.3141100 & 0.7005600 & 1.1321230 \\
\hline $\mathrm{H}$ & 3.4311590 & -4.1017090 & -0.9065190 & C & -5.3095090 & -0.4139060 & 2.6050900 \\
\hline C & -2.0665620 & -1.3526400 & -0.4746710 & C & -5.7717440 & -1.4429790 & 3.6036980 \\
\hline C & -3.2846330 & -0.6882620 & -0.5956340 & C & -4.3403220 & -2.4403090 & 1.6972290 \\
\hline C & -1.3911940 & -1.8913590 & -1.5679200 & C & -4.5249390 & -0.9468430 & 1.5746120 \\
\hline C & -3.7996220 & -0.5636520 & -1.8830430 & $\mathrm{C}$ & -6.5941010 & -2.5110940 & 2.8496710 \\
\hline $\mathrm{H}$ & -4.7553160 & -0.0591670 & -2.0080440 & $\mathrm{H}$ & -6.8993940 & -3.2934950 & 3.5542490 \\
\hline C & 3.3373320 & -1.5150580 & -0.0919610 & $\mathrm{H}$ & -7.5094640 & -2.0570440 & 2.4564210 \\
\hline C & -4.0185180 & -0.1177610 & 0.5663610 & C & -5.7358080 & -3.1033140 & 1.7013810 \\
\hline C & 3.7822100 & -0.4261490 & -0.8539040 & $\mathrm{H}$ & -5.6136940 & -4.1856420 & 1.8253270 \\
\hline C & 3.25 & 0.0023330 & -2.2033070 & $\mathrm{H}$ & -6.2187070 & -2.9433550 & 0.7317750 \\
\hline C & 5.1 & 1.4990760 & -1.3503060 & $\mathrm{H}$ & -3.7365960 & -2.8470520 & 0.8830080 \\
\hline C & 4.837 & 0.3770550 & -0.4057160 & $\mathrm{H}$ & -6.3740110 & -0.9845740 & 4.3954290 \\
\hline C & 2.7661600 & 1.4569060 & -2.0785540 & C & -3.6610590 & -2.7224960 & 3.0550300 \\
\hline $\mathrm{H}$ & 2.3916220 & 1.8032970 & -3.0486660 & $\mathrm{H}$ & -3.5454120 & -3.8056750 & 3.1778990 \\
\hline $\mathrm{H}$ & 1.9 & 1.4708550 & -1.3863280 & $\mathrm{H}$ & -2.6577660 & -2.2885700 & 3.0486470 \\
\hline C & 3.92 & 2.3594310 & -1.5763850 & C & -4.5215560 & -2.1268680 & 4.2003690 \\
\hline $\mathrm{H}$ & 4.14 & 3.1465670 & -2.3053530 & $\mathrm{H}$ & -4.8398920 & -2.9087970 & 4.8999250 \\
\hline $\mathrm{H}$ & 3.6403990 & 2.8618000 & -0.6442420 & $\mathrm{H}$ & -3.9459930 & -1.3953130 & 4.7762960 \\
\hline H & 6.0023820 & 2.1113390 & -0.9549730 & C & -5.1064650 & 1.7728370 & 1.6445370 \\
\hline $\mathrm{H}$ & 2.4 & -0.6422570 & -2.5491470 & $\mathrm{C}$ & -5.3550320 & 3.2546250 & 1.5225300 \\
\hline C & $5.5 \xi$ & 0.8715590 & -2.7047950 & C & -3.7718760 & 2.3279670 & -0.2987480 \\
\hline $\mathrm{H}$ & 5.79 & 1.6716200 & -3.4243480 & C & -4.2886090 & 1.2558930 & 0.6314300 \\
\hline $\mathrm{H}$ & 6.5089530 & 0.2984320 & -2.5832330 & C & -3.9990330 & 3.9853620 & 1.6078630 \\
\hline c & 4.4321570 & -0.0370930 & -3.2081060 & $\mathrm{H}$ & -4.1572080 & 5.0567810 & 1.4362540 \\
\hline $\mathrm{H}$ & 4.0707770 & 0.2993410 & -4.1867860 & $\mathrm{H}$ & -3.5850710 & 3.8781520 & 2.6157300 \\
\hline $\mathrm{H}$ & 4.7747780 & -1.0698020 & -3.3292100 & C & -3.0343170 & 3.3872540 & 0.5538060 \\
\hline C & 3.9782610 & -1.7895240 & 1.1207480 & $\mathrm{H}$ & -2.6434460 & 4.1682330 & -0.1092650 \\
\hline
\end{tabular}




$\begin{array}{lr}\mathrm{H} & -2.1804770 \\ \mathrm{H} & -3.0905090 \\ \mathrm{H} & -6.0356790 \\ \mathrm{C} & -4.9813400 \\ \mathrm{H} & -4.6220000 \\ \mathrm{H} & -5.4852090 \\ \mathrm{C} & -5.9576110 \\ \mathrm{H} & -6.1408960 \\ \mathrm{H} & -6.9270170 \\ \mathrm{C} & -5.6174010 \\ \mathrm{H} & -6.2509860 \\ \mathrm{C} & -3.1190690 \\ \mathrm{C} & -1.8906830 \\ \mathrm{C} & -3.7389780 \\ \mathrm{C} & -2.7185560 \\ \mathrm{C} & -1.8471420 \\ \mathrm{C} & -1.0502050 \\ \mathrm{H} & -3.2342630 \\ \mathrm{H} & -4.4996580 \\ \mathrm{H} & -4.2727660 \\ \mathrm{H} & -2.4869630 \\ \mathrm{H} & -1.1571720 \\ \mathrm{H} & -0.5262570 \\ \mathrm{H} & -0.2602840 \\ \mathrm{H} & -2.0809440 \\ \mathrm{C} & 0.1174530 \\ \mathrm{C} & 1.3985550 \\ \mathrm{C} & -1.0522220 \\ \mathrm{C} & -0.6252360 \\ \mathrm{C} & 0.4647120 \\ \mathrm{C} & 1.7074510 \\ \mathrm{H} & -1.4955590 \\ \mathrm{H} & -1.6135340 \\ \mathrm{H} & -1.7523260 \\ \mathrm{H} & 0.0893470 \\ \mathrm{H} & 0.7231660 \\ \mathrm{H} & 2.4315570 \\ \mathrm{H} & 2.2051910 \\ \mathrm{H} & -0.2429740 \\ \mathrm{C} & 0.4089550 \\ \mathrm{O} & -0.3193220 \\ \mathrm{C} & 0.6494410 \\ \mathrm{C} & 1.6268890 \\ \mathrm{C} & -0.0938720 \\ \mathrm{C} & 1.8551740 \\ \mathrm{H} & 2.2118580 \\ & \end{array}$

$\begin{array}{rr}2.9129690 & 1.0410880 \\ 1.9210760 & -1.0493990 \\ 3.6072690 & 2.3048410 \\ 3.0139570 & -0.9695600 \\ 3.8445460 & -1.5886290 \\ 2.3101360 & -1.6409670 \\ 3.5240050 & 0.1244810 \\ 4.5988090 & 0.0112640 \\ 3.0215660 & 0.0446650 \\ 0.9410140 & 2.6332790 \\ 1.3451510 & 3.4213130 \\ -1.0137770 & -3.0139530 \\ -1.6717500 & -2.8650230 \\ -0.7795020 & -4.3780270 \\ -0.8189310 & -5.5133480 \\ -2.0650720 & -5.3824680 \\ -2.0259260 & -4.0798080 \\ -0.8005610 & -6.4795350 \\ -1.5530680 & -4.5565730 \\ 0.1777050 & -4.3744540 \\ -2.9583160 & -5.3980620 \\ -2.1510570 & -6.2288890 \\ -2.9734330 & -3.9344370 \\ -1.2648790 & -4.1635380 \\ 0.0746890 & -5.4706740 \\ -3.9402770 & -1.6456860 \\ -4.4849600 & -1.4858220 \\ -4.8165470 & -2.0563270 \\ -6.1105580 & -2.7454310 \\ -6.8073570 & -1.9343030 \\ -5.9220540 & -1.8615090 \\ -6.7634090 & -2.8716730 \\ -5.0635760 & -1.1434900 \\ -4.2632940 & -2.6872840 \\ -7.0051350 & -0.9215380 \\ -7.7754000 & -2.3767500 \\ -6.3357070 & -1.1502840 \\ -5.9270900 & -2.8418210 \\ -5.8868650 & -3.7512280 \\ 3.5548280 & -0.8820780 \\ 2.4951170 & -0.9973630 \\ 4.2271770 & -2.1829850 \\ 5.2179820 & -2.3247260 \\ 3.8279840 & -3.3023370 \\ 5.8014770 & -3.5642490 \\ 5.5173550 & -1.4620910\end{array}$

0.1322160
-0.8451670
1.1077640
2.6199020
-0.4516730
1.2862020
-0.4720140
1.0368770
2.4000130
1.1863700
2.3783270
0.2440380
1.9288490
1.3540670
3.7078890
2.5644830
1.2075130
3.7333420
4.6670800
2.5626850
0.9365590
3.9924380
4.4861270
3.5948660
2.7093310
0.3996210

$\begin{array}{lr}4.4201930 & -4.5377490 \\ 3.0542180 & -3.1951630 \\ 5.4062150 & -4.6715220 \\ 6.5649040 & -3.6664280 \\ 4.1103490 & -5.3987880 \\ 5.8658080 & -5.6389990 \\ 1.9375110 & -0.1408720 \\ 4.1531140 & 0.5597740 \\ 1.7421020 & 2.3122100 \\ 2.5006740 & 2.6486710 \\ 1.1880100 & 1.3703620 \\ 2.0991900 & 2.2905360 \\ 0.9513350 & 3.4248660 \\ 0.2057430 & 3.0446010 \\ 3.2453390 & 3.8728630 \\ 4.2608600 & 3.8302710 \\ 3.5634310 & 3.6978500 \\ 2.3889900 & 2.6044210 \\ 3.7615300 & 3.9793630 \\ 4.8806450 & 4.7321940 \\ 3.0664970 & 4.6381020 \\ 3.0050050 & 1.7340930 \\ 1.6002660 & 2.6721880 \\ 2.5959600 & 4.7494620 \\ 4.9373320 & 2.9783150 \\ 4.2697270 & 3.4875810\end{array}$
154

4.2697270

3.4875810

$\begin{array}{lrrr}\text { TS259(R, R) } & \text { G }[\mathrm{WB} 97 \times \mathrm{XD} / 6-31 \mathrm{G}(\mathrm{d})]=-3864.647316 \\ \mathrm{P} & -0.3392280 & -0.5543640 & 1.0390130 \\ \mathrm{O} & -0.9109840 & 0.8457320 & 1.0730310 \\ \mathrm{O} & 0.2918230 & -1.0856810 & 2.2892400 \\ \mathrm{O} & 0.7077380 & -0.5259350 & -0.2210230 \\ \mathrm{O} & -1.4559470 & -1.6185860 & 0.5371320 \\ \mathrm{C} & 1.0355770 & -1.7187840 & -0.8414970 \\ \mathrm{C} & 0.0741590 & -2.3448470 & -1.6329130 \\ \mathrm{C} & 2.3277280 & -2.2216420 & -0.7035310 \\ \mathrm{C} & 2.6010070 & -3.4414760 & -1.3112710 \\ \text { H } & 3.6030020 & -3.8551290 & -1.2159630 \\ \mathrm{C} & -1.9927680 & -1.3191330 & -0.7108230 \\ \mathrm{C} & -3.2206660 & -0.6673280 & -0.7909890 \\ \mathrm{C} & -1.2276120 & -1.6498040 & -1.8339110 \\ \mathrm{C} & -3.6668680 & -0.3160530 & -2.0676290 \\ \mathrm{H} & -4.6224490 & 0.1941180 & -2.1629360 \\ \mathrm{C} & 3.3785640 & -1.4278560 & -0.0112170 \\ \mathrm{C} & -4.0226480 & -0.3181330 & 0.4138020 \\ \mathrm{C} & 3.8750060 & -0.2727140 & -0.6286760\end{array}$




\begin{tabular}{|c|c|c|}
\hline $\begin{array}{l}3.4887170 \\
5.2503470 \\
4.8518340 \\
2.9318070 \\
2.6774880 \\
2.0037980 \\
3.9812210 \\
4.2517830 \\
3.5711190 \\
5.9939940 \\
2.7459720 \\
5.8163890 \\
6.0559280 \\
6.7523880 \\
4.7686790 \\
4.5088850 \\
5.1669490 \\
3.9007660 \\
3.5415690 \\
5.3636980 \\
4.9011670 \\
4.8345240 \\
4.6077810 \\
5.1943890 \\
5.9090140 \\
6.1694290 \\
6.8304530 \\
6.1357250 \\
2.7639020 \\
4.1348940 \\
4.4505040 \\
3.7204130 \\
3.0712660 \\
2.9156950 \\
2.1063450 \\
5.3765420 \\
6.1515830 \\
5.3193700 \\
5.7576540 \\
4.2946120 \\
4.5117150 \\
6.5507610 \\
6.8303290 \\
7.4804930 \\
5.6781310 \\
5.5377220\end{array}$ & $\begin{array}{r}0.2501800 \\
1.7223110 \\
0.5094490 \\
1.6750320 \\
2.0932540 \\
1.6112920 \\
2.5571740 \\
3.4230140 \\
2.9511230 \\
2.3242670 \\
-0.3875830 \\
1.2330490 \\
2.1025310 \\
0.6910020 \\
0.3249800 \\
0.7194110 \\
-0.6837890 \\
-1.8020210 \\
-3.0309700 \\
-1.5841040 \\
-1.0299990 \\
-3.8622850 \\
-4.7721430 \\
-4.1789060 \\
-3.0112090 \\
-3.4589840 \\
-2.9591110 \\
-0.9483810 \\
-3.6185170 \\
-1.6856140 \\
-2.0807240 \\
-0.6872990 \\
-2.6037000 \\
-3.5069980 \\
-2.1012380 \\
0.1243610 \\
0.7212680 \\
-0.9826000 \\
-2.1752720 \\
-2.8035970 \\
-1.3148800 \\
-3.1208560 \\
-4.0222470 \\
-2.6333810 \\
-3.4805200 \\
-4.5651050\end{array}$ & $\begin{array}{l}-1.9911560 \\
-0.8044610 \\
-0.0030130 \\
-1.8155740 \\
-2.7962630 \\
-1.2431870 \\
-1.0930360 \\
-1.7088260 \\
-0.1569540 \\
-0.2711850 \\
-2.4764510 \\
-2.1568400 \\
-2.7801770 \\
-1.9882830 \\
-2.8528870 \\
-3.8419180 \\
-3.0025570 \\
1.2298230 \\
2.0283500 \\
3.1595410 \\
1.8374160 \\
2.1909340 \\
2.7584510 \\
1.2059510 \\
2.9203970 \\
3.8864420 \\
2.3316000 \\
3.6069260 \\
1.5342160 \\
4.0895610 \\
5.0622640 \\
4.2655340 \\
3.4358160 \\
4.0372740 \\
3.3528420 \\
1.2262780 \\
1.7050580 \\
2.3632740 \\
3.1734560 \\
1.1402660 \\
1.2680290 \\
2.2445300 \\
2.8026690 \\
1.9338680 \\
1.0141010 \\
0.9387520\end{array}$ \\
\hline
\end{tabular}

-6.1594770
-3.6788220
-6.3745370
-3.6212180
-3.4966930
-2.6225140
-4.4945220
-4.7970260
-3.9343590
-5.1854630
-5.4847910
-3.8894030
-4.3507530
-4.1536900
-4.3510760
-3.7206640
-3.1867980
-2.8537740
-2.2977050
-3.2012610
-6.1684050
-5.1375180
-4.8218400
-5.6278480
-6.1104760
-6.3169240
-7.0706520
-5.6675800
-6.3119690
-1.6718820
-0.8516010
-0.7585270
-2.1486890
-3.2921460
-2.9000880
-1.3230280
-0.2184440
-2.3538050
-3.5115510
0.1510910
-0.1499650
-2.1590920
-4.2083100
0.3584230
-0.7190850
-0.4246400
0.0865440

0.2741810

4.0253320

2.4359010

2. 3721920

2. 5136310

3. 6582750

4.2216810

4. 3456280

1.7691860

1.8919750

$-0.0617630$

0.6896120

2. 0899250

2. 1115150

3. 0592260

0.9373320

0.4019470

1. 3282000

$-0.8713460$

2. 7249860

$-0.6092230$

$-1.1076240$

$-1.3668870$

0.5601180

0.6220380

0.4103670

2. 6100620

3. 4505230

$-3.0904780$

$-4.3524820$

$-4.9932720$

$-5.2103300$

$-4.5722540$

$-3.1999750$

$-5.0653540$

$-5.9427340$

$-6.2790190$

$-5.2129710$

$-4.1499550$

$-4.3350760$

$-4.7753320$

$-2.1965250$

-2.9531000
-3.0786850 


$\begin{array}{rrrr}\mathrm{C} & 0.9986720 & -6.0589340 & -3.5890790 \\ \mathrm{C} & 1.9995520 & -5.5226940 & -2.5671410 \\ \mathrm{C} & 1.6347620 & -4.1547260 & -2.0230090 \\ \mathrm{H} & -1.1545640 & -6.3100160 & -3.7529330 \\ \mathrm{H} & -0.8164900 & -3.9350100 & -3.9627710 \\ \mathrm{H} & -1.6861480 & -4.1935350 & -2.4650430 \\ \mathrm{H} & 1.1208400 & -5.5320930 & -4.5451090 \\ \mathrm{H} & 1.1961350 & -7.1191730 & -3.7802010 \\ \mathrm{H} & 3.0062010 & -5.4842190 & -2.9998460 \\ \mathrm{H} & 2.0572590 & -6.2256070 & -1.7242010 \\ \mathrm{H} & -0.5375340 & -6.3356830 & -2.1006650 \\ \mathrm{C} & 0.2316720 & 3.4163980 & -0.7220000 \\ \mathrm{O} & -0.5041000 & 2.3645830 & -0.8483780 \\ \mathrm{C} & 0.5129740 & 4.0670840 & -2.0268970 \\ \mathrm{C} & 1.4525690 & 5.0948640 & -2.1502740 \\ \mathrm{C} & -0.1342570 & 3.5845950 & -3.1728950 \\ \mathrm{C} & 1.7397050 & 5.6308480 & -3.3996410 \\ \mathrm{H} & 1.9648890 & 5.4583490 & -1.2662490 \\ \mathrm{C} & 0.1530270 & 4.1244970 & -4.4188400 \\ \mathrm{H} & -0.8556280 & 2.7812260 & -3.0757680 \\ \mathrm{C} & 1.0925210 & 5.1475650 & -4.5348220 \\ \mathrm{H} & 2.4757010 & 6.4236720 & -3.4880290 \\ \mathrm{H} & -0.3519000 & 3.7419700 & -5.3007840 \\ \mathrm{H} & 1.3207450 & 5.5677200 & -5.5097350 \\ \mathrm{H} & -0.6565120 & 1.7903260 & 0.0011500 \\ \mathrm{~S} & 0.8354540 & 4.0119480 & 0.7329060 \\ \mathrm{C} & 2.1445420 & 1.6024600 & 2.5791420 \\ \mathrm{C} & 0.8910770 & 2.3280960 & 2.8371340 \\ \mathrm{H} & 2.2154360 & 1.0843550 & 1.6196180 \\ \mathrm{H} & -0.0107280 & 1.9142750 & 2.3989600 \\ \mathrm{O} & 1.6018410 & 0.7598150 & 3.6204590 \\ \mathrm{H} & 1.0899120 & 0.0088390 & 3.1745380 \\ \mathrm{C} & 3.2805830 & 3.0841880 & 4.2906570 \\ \mathrm{C} & 2.1291960 & 4.0839190 & 4.1675930 \\ \mathrm{C} & 0.8028380 & 3.3655130 & 3.9051840 \\ \mathrm{C} & 3.4342110 & 2.2620350 & 3.0085420 \\ \mathrm{H} & 4.2181180 & 3.6102760 & 4.4956070 \\ \mathrm{H} & 2.0385190 & 4.6849470 & 5.0774110 \\ \mathrm{H} & 0.4620950 & 2.8421100 & 4.8080690 \\ \mathrm{H} & 3.7598170 & 2.9062830 & 2.1822220 \\ \mathrm{H} & 4.1914570 & 1.4827930 & 3.1229480 \\ \mathrm{H} & 3.0979570 & 2.4113780 & 5.1372310 \\ \mathrm{H} & 2.3353110 & 4.7792600 & 3.3439240 \\ \mathrm{H} & 0.0002080 & 4.0594620 & 3.6403250 \\ \mathrm{H} & & & \end{array}$

$1546260(R, R) G[W B 97 X D / 6-31 G(d)]=-3864.648548$

0

0
$-0.4054230$
1. 0180480
$-0.8394700$
$-0.5803870$
$-1.4643090$
$-1.8645150$
-2. 5995240
$-2.3649220$
$-3.6739250$
-4. 0854070
$-1.3612730$
$-0.6956620$
$-1.9064900$
$-0.5743730$
$-0.0704630$
$-1.5108930$
$-0.1217620$
$-0.4242780$
$-0.0076510$
1. 5074820
0.3876440
1. 4439800
1.7823940
1.4557280
2. 3570490
3. 1430200
2.8606870
2. 1281600
$-0.6607360$
0.8764680
1.6746770
0.3122050
$-0.0449680$
0.2813290
$-1.7773510$
$-2.9090870$
$-1.4115150$
$-0.9720180$
$-3.7603290$
$-4.5802290$
$-4.2145360$
$-3.2248860$
$-2.8870580$
$-0.7564330$

1.0533730

0.9685740

2. 3027590

$-0.2779540$

0.7832880

$-0.7147650$

$-1.3029870$

$-0.5835210$

$-1.0020190$

$-0.9184670$

$-0.4694630$

$-0.6053550$

$-1.5531710$

$-1.8982220$

$-2.0352250$

$-0.0689770$

0.5481020

$-0.8306230$

$-2.1899020$

$-1.3171580$

$-0.3719310$

$-2.0817900$

$-3.0599980$

$-1.4039240$

$-1.5654610$

$-2.2940370$

$-0.6395850$

$-0.9136150$

$-2.5430960$

$-2.6627700$

3. 3836490

$-2.5260360$

$-3.1768410$

$-4.1633600$

$-3.2851060$

1.1524090

2. 1015350

2. 9170620

1.5954150

2. 2484400

2. 9492090

1. 2843900

2.7578530

3.7236650

2. 0585450

3. 2298700 


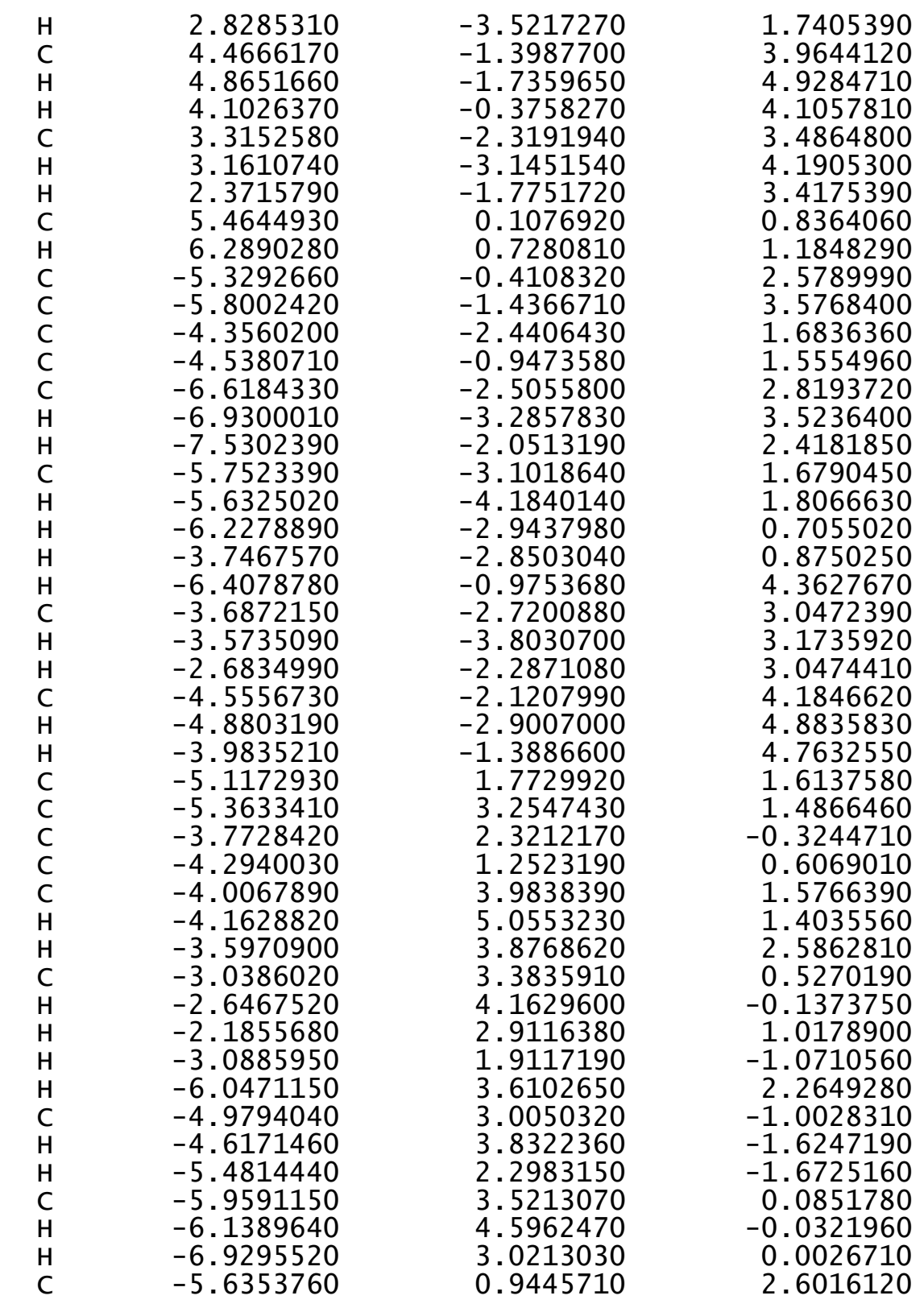

-6.2734440
-1.8616430
-1.0271480
-1.4415820
-2.9525860
-3.6653490
-3.0862300
-0.9074820
-1.1137810
0.0304750
-3.2421890
-3.2603420
-4.7360140
-3.5938580
-1.1539050
0.1372150
1.4258020
-1.0274380
-0.5975440
0.5178860
1.7484060
-1.4597960
-1.5375820
-1.7718050
0.1605520
0.7859300
2.4828680
2.2396610
-0.2397500
0.4044110
-0.3235710
0.6517330
1.6244840
-0.0788090
1.8604560
2.1998240
0.1550250
-0.8259610
1.1256540
2.6215160
-0.4186590
1.3099710
-0.4797960
1.0257900
2.3722850
1.1555290

1. 3516940

$-1.6847900$

$-2.0881030$

$-1.3572540$

$-1.4613920$

$-0.7283170$

$-1.0250540$

$-1.7831260$

$-3.1688780$

$-1.9058770$

$-2.5209720$

$-1.0420020$

$-0.9647800$

0.3543620

$-0.2991990$

$-3.9479600$

$-4.4814720$

$-4.8407310$

$-6.1052000$

$-6.8085780$

$-5.9068230$

$-6.7684490$

$-5.1290510$

$-4.2895110$

$-7.0462940$

$-7.7572770$

$-6.3279030$

$-5.8812920$

$-5.8443010$

3.5324710

2. 4717300

4. 1921970

5. 1872820

3.7759920

5.7586490

5.4995110

4. 3559040

2. 9984710

5. 3465110

6.5255290

4.0327140

5.7966390

1.9228210

4. 1437640

1. 7544920

2. 5181280
3. 3844740 $-2.8575730$ $-4.0600330$ $-5.3363620$ $-5.5265490$ $-4.3919510$ $-3.0215470$ $-6.1924140$ $-4.2362980$ $-3.8407670$ $-5.5294800$ $-6.4903710$ $-4.3925580$ $-4.5679130$ $-5.2697110$ $-1.6322550$ $-1.4909980$ $-2.0170310$ $-2.7565640$ $-1.9860080$ $-1.8997500$ $-2.8843480$ $-1.0863510$ $-2.5951960$ $-0.9753060$ $-2.4633520$ $-1.2036860$ $-2.8830050$ $-3.7624160$ $-0.9060850$ $-1.0145460$ $-2.2122090$ $-2.3561920$ $-3.3337580$ $-3.5998930$ $-1.4916690$ $-4.5735730$ $-3.2247580$ $-4.7094380$ $-3.7035920$ $-5.4365420$ $-5.6802550$ $-0.1532040$

0.5329880

2.3324750
2.6453150 


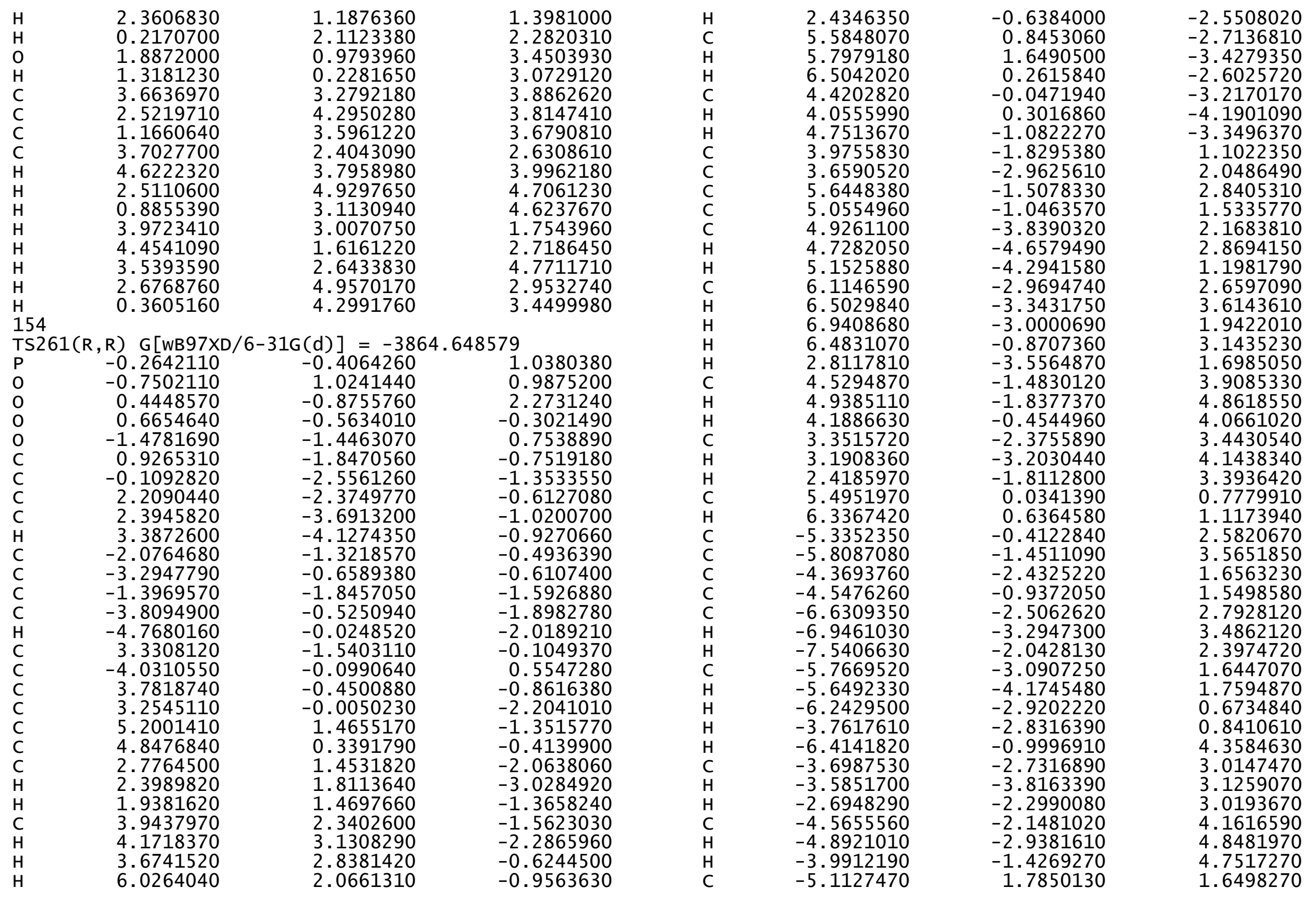




-5.3504290
-3.7624130
-4.2924750
-3.9900130
-4.1403060
-3.5832350
-3.0224460
-2.6212370
-2.1755130
-3.0791680
-6.0333310
-4.9627130
-4.5936320
-5.4664370
-5.9428780
-6.1198370
-6.9144490
-5.6350280
-6.2708380
-3.1244200
-1.8895940
-3.7473560
-2.7300860
-1.8256440
-1.0313120
-3.2505100
-4.4977900
-4.2946430
-2.4395040
-1.1341250
-0.4529250
-0.2858170
-2.1139790
0.0789710
-1.0986380
-0.8462080
0.5093660
1.6228880
1.3492930
-1.6544890
-1.3289340
-1.9902280
0.5415260
0.6629010
2.5823640
1.7456960

3.2698360 2.3544930

1.2751880 3.9903150 5.0644810 3. 8697300 3.3975240 4.1835000 2.9106000 1.9515960 3.6180640 3.0573050 3.8936850 2.3656700

3.5594650 4.6365020 3.0632370 0.9441500 1.3425160 $-0.9586790$ $-0.7263350$ $-0.7477820$ $-1.9709620$ $-1.9067090$ $-0.7449420$ $-1.5097460$ 0.2232350 $-2.8822960$ $-2.0357670$ $-2.8218360$ $-1.1010360$ 0.1610720 $-3.9127850$ $-4.7500620$ $-6.2519340$ $-6.6061860$ $-5.9385620$ $-4.4789130$ $-6.8010910$ $-4.5249670$ $-4.4617380$ $-6.2585840$ $-7.6904190$ $-6.0260900$ $-6.4747690$ $-1.6066460$

1.5440470
-0.2789520
0.6351370
1.6458330
1.4846520
2.6550950
0.5913660
-0.0594980
1.0783810
-1.0301910
2.3263610
-0.9487420
-1.5540940
-1.6327940
0.1455740
0.0441340
0.0538120
2.6250170
3.4141980
-3.0322170
-2.8871420
-4.3954520
-5.5347680
-5.4055320
-4.1028560
-6.4986110
-4.5743860
-4.3885540
-5.4208060
-6.2526080
-3.9638760
-4.1789510
-5.4987490
-1.6753250
-2.1418180
-2.0144660
-2.6202910
-1.8156520
-1.5085280
-2.5092010
-3.1920530
-1.5743460
-3.6620320
-2.6390970
-2.3390290
-0.8641600

$-0.8594170$

0.4439830

$-0.2963260$

0.6967500

1.6763490

$-0.0369920$

1. 9161460

2. 2536300

0.2010120

$-0.7898010$

1.1785900

2.6822340

$-0.3750820$

1. 3662170

$-0.4551040$

2. 4161500

1.2132260

2. 3822340

0.2642110

1.9440470

1.3579510

3.7507850

2.6182780

1.2528030

3.7581570

4.7160990

2.6287180

0.9810980

4.0163310

4.5038950

3.6371400

2.7652420

0.4519230
1.0739660
$-6.5438400$

3.5663630

2. 5162850

4.2451620

5.2355600

3.8517280

5.8242440

5.5306030

4.4485930

3.0781620

5.4342400

6.5875890

4.1427300

5.8976530

1.9514240

4.1463760

1.6986220

2.4659700

1.1578690

2. 0797700

0.8977770

.1635190

3.1651950

4.1935540

3. 5134230

2. 3277840

3.6696680

4.7983280

3.0061980

2.9544490

1.5311840

2. 5037360

4.8822790

4.2319450
$-0.9558990$

$-0.8374590$

$-0.9627780$

$-2.1327470$

$-2.2615020$

$-3.2604570$

$-3.4964230$

$-4.4913330$

$-3.1633040$

$-4.6121190$

$-3.5883730$

$-5.3590160$

$-5.5760260$

$-0.1119450$

0.6112120

2. 3242680

2. 6788730

1. 3751500

2. 3217230

3.4292180

3.0438550

3.8967430

3.8777930

3.7426710

2.6155880

4.0041280

4.7897730

4.6772120

1.7526440

2.6654210

4.7642100

3.0360590

3.5472940
$-1.3922180$

$\operatorname{TS} 262(R, R) \quad G[W B 97 X D / 6-31 G(d)]=-3864.647443$

$\begin{array}{lrrr}\text { TS262(R, R) } G[W B 97 X D / 6-31 G(d)]=-3864.647443 \\ \text { P } & 0.2633430 & 0.4065570 & 0.8641340 \\ O & 0.7199350 & -1.0339420 & 0.8974400 \\ O & -0.4533750 & 0.9562170 & 2.0605730 \\ O & -0.6217940 & 0.5181440 & -0.5062110 \\ O & 1.4906680 & 1.4178290 & 0.5536500 \\ O & -0.8442810 & 1.7822310 & -1.0262040 \\ C & 0.2173200 & 2.4354170 & -1.6670120 \\ C & -2.1111840 & 2.3440820 & -0.8987620 \\ C & -2.2953410 & 3.6342890 & -1.3982350 \\ C & -3.2751940 & 4.0966800 & -1.3033500\end{array}$




2.1333720
3.3522430
1.5172810
3.9402860
4.8982450
-3.2274770
3.9960950
-3.7850420
-3.3734300
-5.3410310
-4.8602290
-2.9743980
-2.6797740
-2.0962750
-4.1571640
-4.4815470
-3.8539120
-6.1732350
-2.5408040
-5.7809600
-6.0993770
-6.6479070
-4.5997520
-4.3246540
-4.8741520
-3.7713740
-3.3420390
-5.3421840
-4.8623340
-4.5580550
-4.2777830
-4.8344300
-5.7432580
-6.0188920
-6.6301000
-6.1915710
-2.4884930
-4.1602270
-4.4875820
-3.8530780
-2.9845280
-2.7737880
-2.0682220
-5.4102400
-6.2640780
5.0755110

1.2291110

0.5549420

1.7191850

0.3736840

$-0.1398450$

1.6043040

0.0292890

0.4753580

$-0.1242130$

$-1.3818180$

$-0.2004980$

$-1.5910870$

$-2.0588750$

$-1.5970270$

$-2.3526010$

$-3.1840440$

$-2.7886080$

$-1.8891900$

0.4200210

$-0.8594790$

$-1.7060340$

$-0.2013250$

$-0.1027230$

$-0.5699470$

0.9346150

2. 0442920

3.2495400

1.9968400

1.3754890

4.1993910

5.0919180

4.5350920

3.4610700

3.9500050

3.4770130

1. 4431900

3.7553180

2.0062660

2.4511370

0.9769800

2.8067940

3.7038060

2.2153470

0.2570450

$-0.2533190$

0.3883620

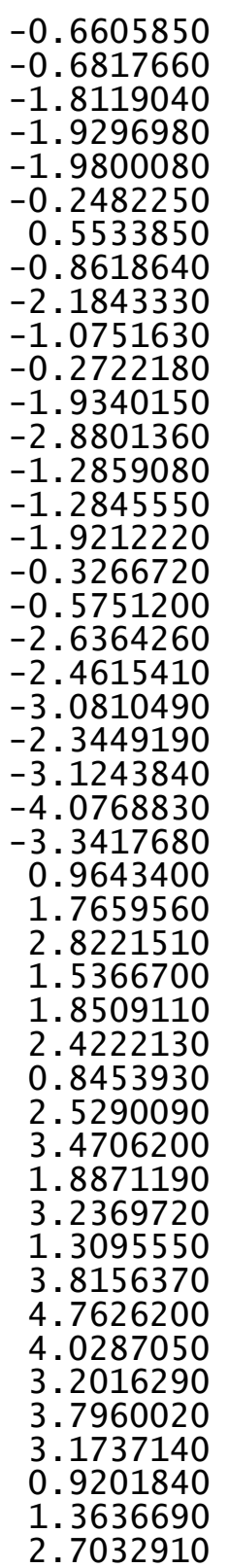

\begin{abstract}
5.4387540
4.2468960

4.4136250

6.3578460

6.5871820

7. 3077900

5.6477090

5.5368330

6.2323520

3.7456070

5.9398410

3.4304310

3.3380500

2. 4203190

4.1407610

4. 3909660

3.4890570

4.9409820

5. 1819450

3.8015750

4.2407510

3.8177710

3.9838970

3. 3089400

2. 9654040

2.6129630

2.0863730

3. 2042400

5.7747280

5.0606960

4.7500800

5.6361810

5. 9217100

6.1140900

6.8945790

5. 3562440

5.8915490

2. 1018790

1. 3813470

1.8787350

3.4013610

4.0129460

3. 3325210

1.4193490

1. 5239620

0.3037580
\end{abstract}

1. 4471610

2. 3911370

0.8908940

2.4774690

3.2847880

2.0010500

3. 0295830

4.1185090

2.8157430

2.7760960

1.0093630

2.7427040

3.8328390

2. 3380290

. 1676230

2.9641040

1. 4617240

$-1.8300560$

$-3.3176700$

$-2.4486390$

$-1.3436070$

$-4.0377290$

$-5.1136090$

$-3.9056090$

$-3.4590830$

$-4.2553660$

$-2.9473910$

$-2.0659250$

$-3.6450520$

$-3.1776640$

$-4.0345900$

$-2.5127430$

$-3.6418460$

$-4.7197870$

$-0.9680530$

$-1.3508630$

1.4571290

1.8436950

1.0689590

1.1478550

0.4441310

0.7879380

1.4786080

2.9144290

1.7009880
3.7115770

1.6231670

1. 5759840

3.0194480

3.7248650

2.7563460

1.7562380

1.8171540

0.8554160

0.7324320

4.5816080

2.8865310

2.9580330

2.7851650

4.1397400

4.8505390

4.6643400

1.8071990

1.7674850

$-0.2374190$

0.6953470

1.7380820

1.6063220

2.6984010

0.5819070

$-0.0843620$

0.9764660

$-1.0681570$

2.6284370

$-0.7557340$

$-1.3652510$

$-1.4087780$

0.4495840

0.3975010

0.4479520

2.8142640

3.6817720

$-3.0661170$

$-4.3434360$

$-5.5626270$

$-5.6368440$

$-4.4270160$

$-3.1147300$

$-6.4685800$

$-4.5362140$

$-4.2094980$ 


$\begin{array}{rrr}3.7056720 & 2.2032100 & -5.6506870 \\ 3.7781600 & 0.6938900 & -6.5596140 \\ 5.0830480 & 0.6699320 & -4.3499260 \\ 3.9422210 & -0.6426280 & -4.5754880 \\ 1.5695050 & 0.0174970 & -5.4902090 \\ 0.0157680 & 3.7505340 & -2.1041440 \\ 1.1115020 & 4.6501550 & -2.6187550 \\ 1.1092950 & 5.9678690 & -1.8215540 \\ -0.2809240 & 6.6512350 & -1.7978890 \\ -1.3808690 & 5.7722700 & -2.4277020 \\ -1.2481380 & 4.3443470 & -1.9699580 \\ 0.9466860 & 4.8740690 & -3.6821870 \\ 1.8623220 & 6.6429030 & -2.2412170 \\ -0.2485030 & 7.6099510 & -2.3259880 \\ -1.2862300 & 5.8142730 & -3.5218040 \\ 2.0894650 & 4.1680680 & -2.5402050 \\ 1.4268130 & 5.7402890 & -0.7985500 \\ -0.5609840 & 6.8724210 & -0.7628620 \\ -2.3714150 & 6.1695320 & -2.1842630 \\ -0.5961030 & -3.6531680 & -0.7254810 \\ 0.1673600 & -2.6356970 & -0.9467500 \\ -0.9291780 & -4.4006640 & -1.9636220 \\ -1.9314200 & -5.3765870 & -1.9818000 \\ -0.2561750 & -4.0833010 & -3.1515190 \\ -2.2550730 & -6.0240180 & -3.1671020 \\ -2.4611170 & -5.6136540 & -1.0657920 \\ -0.5787790 & -4.7377390 & -4.3328520 \\ 0.5140020 & -3.3213120 & -3.1399550 \\ -1.5796110 & -5.7070630 & -4.3435550 \\ -3.0380140 & -6.7755200 & -3.1732670 \\ -0.0510090 & -4.4890270 & -5.2481900 \\ -1.8341050 & -6.2140380 & -5.2695000 \\ 0.3690250 & -2.0244370 & -0.1400790 \\ -1.1781930 & -4.1238890 & 0.7819060 \\ -2.4414460 & -1.5550520 & 2.3790380 \\ -1.2332620 & -2.3160700 & 2.7331210 \\ -2.4389800 & -1.0752360 & 1.3971840 \\ -0.2937750 & -1.9626660 & 2.3202670 \\ -1.9213160 & -0.6890890 & 3.4091350 \\ -1.3439790 & 0.0071650 & 2.9480960 \\ -3.7285690 & -2.8977180 & 4.0947820 \\ -2.6098420 & -3.9414280 & 4.0956790 \\ -1.2429640 & -3.2901520 & 3.8653240 \\ -3.7787110 & -2.1429590 & 4.7639510 \\ -4.6944170 & -3.3809980 & 5.0434370 \\ -2.5911990 & -4.4880310 & \end{array}$

$\begin{array}{ll}\mathrm{H} & -0.9311260 \\ \mathrm{H} & -4.0824980 \\ \mathrm{H} & -4.5091020 \\ \mathrm{H} & -3.5712280 \\ \mathrm{H} & -2.7985420 \\ \mathrm{H} & -0.4574800\end{array}$
$-2.7251640$
$-2.8204650$
$-1.3306630$
$-2.1840530$
$-4.6789690$
$-4.0305180$

15

$\operatorname{TS} 263(R, R) G[W B 97 X D / 6-31 G(d)]=-3864.649276$

$\begin{array}{lrrr}\text { TS263(R, R) G }[W B 97 X D / 6-31 G(\mathrm{~d})]=-3864.649276 \\ \mathrm{P} & -0.2802540 & -0.4372380 & 1.0330010 \\ \mathrm{O} & -0.7746250 & 0.9912320 & 1.0024740 \\ \mathrm{O} & 0.4176970 & -0.9226940 & 2.2678520 \\ \mathrm{O} & 0.6651830 & -0.5648930 & -0.2995660 \\ \mathrm{O} & -1.4848100 & -1.4772640 & 0.7119480 \\ \mathrm{C} & 0.9317200 & -1.8383520 & -0.7743400 \\ \mathrm{C} & -0.0968640 & -2.5373320 & -1.4030840 \\ \mathrm{C} & 2.2139880 & -2.3652860 & -0.6321650 \\ \mathrm{C} & 2.4064520 & -3.6751170 & -1.0570050 \\ \mathrm{H} & 3.3978770 & -4.1123460 & -0.9556200 \\ \mathrm{C} & -2.0660060 & -1.3126020 & -0.5398510 \\ \mathrm{C} & -3.2725130 & -0.6262410 & -0.6532150 \\ \mathrm{C} & -1.3783700 & -1.8144690 & -1.6432830 \\ \mathrm{C} & -3.7547400 & -0.4269680 & -1.9436360 \\ \mathrm{H} & -4.6992070 & 0.0994930 & -2.0647210 \\ \mathrm{C} & 3.3279530 & -1.5354710 & -0.0997450 \\ \mathrm{C} & -4.0250910 & -0.1093120 & 0.5216720 \\ \mathrm{C} & 3.7856220 & -0.4355170 & -0.8380940 \\ \mathrm{C} & 3.2803210 & 0.0176740 & -2.1862770 \\ \mathrm{C} & 5.1978140 & 1.4949670 & -1.2838300 \\ \mathrm{C} & 4.8393440 & 0.3551310 & -0.3653000 \\ \mathrm{C} & 2.7890830 & 1.4712960 & -2.0453440 \\ \mathrm{H} & 2.4352980 & 1.8355000 & -3.0166970 \\ \mathrm{H} & 1.9321980 & 1.4769240 & -1.3699140 \\ \mathrm{C} & 3.9374330 & 2.3610700 & -1.5059300 \\ \mathrm{H} & 4.1726260 & 3.1650460 & -2.2128730 \\ \mathrm{H} & 3.6443300 & 2.8412210 & -0.5658610 \\ \mathrm{H} & 6.0117810 & 2.0982030 & -0.8676040 \\ \mathrm{H} & 2.4720880 & -0.6191230 & -2.5535860 \\ \mathrm{C} & 5.6110470 & 0.8942820 & -2.6462690 \\ \mathrm{H} & 5.8236450 & 1.7084350 & -3.3488050 \\ \mathrm{H} & 6.5365840 & 0.3218400 & -2.5277930 \\ \mathrm{C} & 4.4662680 & -0.0083000 & -3.1763310 \\ \mathrm{H} & 4.1175500 & 0.3404980 & -4.1552620 \\ \mathrm{H} & 4.8110280 & -1.0392700 & -3.3059420 \\ \mathrm{C} & 3.9575520 & -1.8360350 & 1.1126210 \\ \mathrm{C} & 3.6370090 & -2.9846690 & 2.0384760 \\ \mathrm{C} & 5.6015930 & -1.5275370 & 2.8769070 \\ & & & \end{array}$




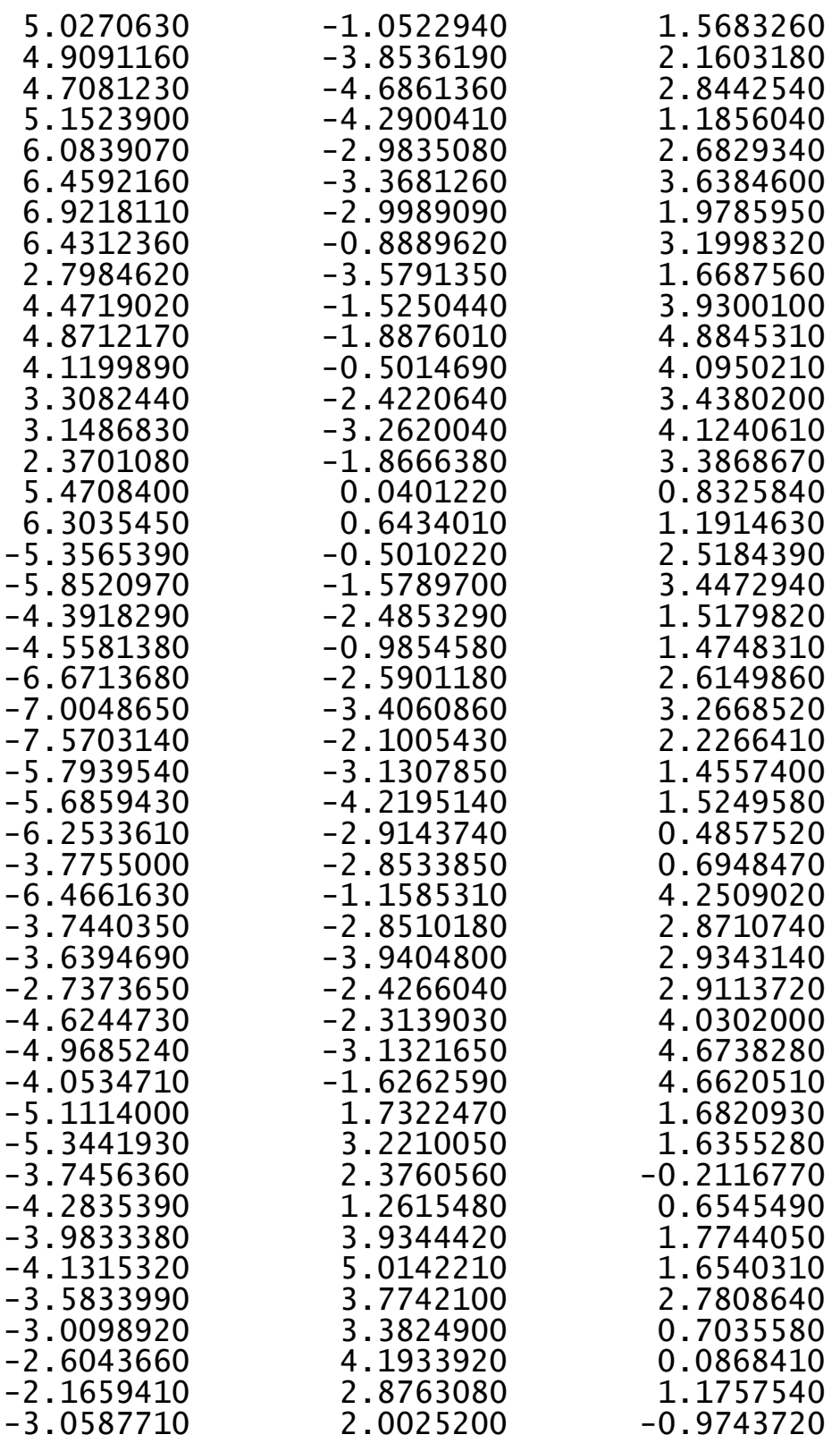

-6.0312540
-4.9407730
-4.5657310
-5.4416330
-5.9269470
-6.1005930
-6.8991490
-5.6483910
-6.2911790
-1.8431860
-1.0031380
-1.3667740
-2.8745830
-3.6002570
-3.0521250
-0.8214110
-1.1363040
0.0571920
-3.1855250
-3.1496150
-4.6735310
-3.5121170
-1.0563060
0.1005730
-1.0650980
-0.8264220
0.5281000
1.6467620
1.3683050
-1.6363590
-1.2464200
-1.9765760
0.5597620
0.6776690
2.5991180
1.7893350
-0.8459450
0.4253300
-0.3146510
0.6961140
1.6626080
-0.0030850
1.9236670
2.2137130
0.2564490
-0.7447030

3. 5391110

3. 1072340

3. 9660450

2. 4443750

3.5683210

4. 6490480

3. 0787960

0.8542870

1. 2230940

$-1.5193180$

$-1.8657690$

$-1.0284070$

$-1.0827460$

$-0.4251450$

$-0.8249930$

$-1.4012900$

$-2.9234630$

$-1.7460380$

$-2.1317960$

$-0.5822290$

$-0.6460490$

0.6663360

0.0140310

$-3.8862000$

$-4.7251360$

$-6.2239380$

$-6.6033740$

$-5.9155580$

$-4.4556610$

$-6.7819970$

$-4.5326420$

$-4.4151300$

$-6.2915710$

$-7.6882090$

$-6.0003460$

$-6.4413420$

$-6.4829130$

3. 5500960

2.5011790

4. 2283490

. 2334610

3. 8159020

5.8179090

5.5429270

4.4078390

3. 0300750
2.4269400 $-0.8607090$

$-1.4298280$

$-1.5745890$

0.2461660

0.1866560

0.1283660

2. 6157710

3. 4136230

$-2.9385640$

$-4.1531700$

$-5.3783300$

$-5.6102420$

$-4.4380590$

$-3.0806350$

$-6.2518900$

$-4.4120310$

$-3.9063860$

$-5.7047610$

$-6.5448520$

$-4.4771720$

$-4.5338060$

$-5.2242040$

$-1.7497490$

$-2.2380750$

$-2.0620190$

$-2.6549030$

$-1.8739960$

$-1.5693070$

$-2.5439670$

$-3.3028700$

$-1.7159840$

$-3.7077800$

$-2.6378740$

$-2.4108420$

$-0.9195500$

$-0.9950360$

$-0.7944670$

$-0.9297550$

$-2.0865810$

$-2.1980270$

$-3.2294230$

$-3.4306920$

$-1.3169050$

$-4.4582650$

$-3.1454660$ 


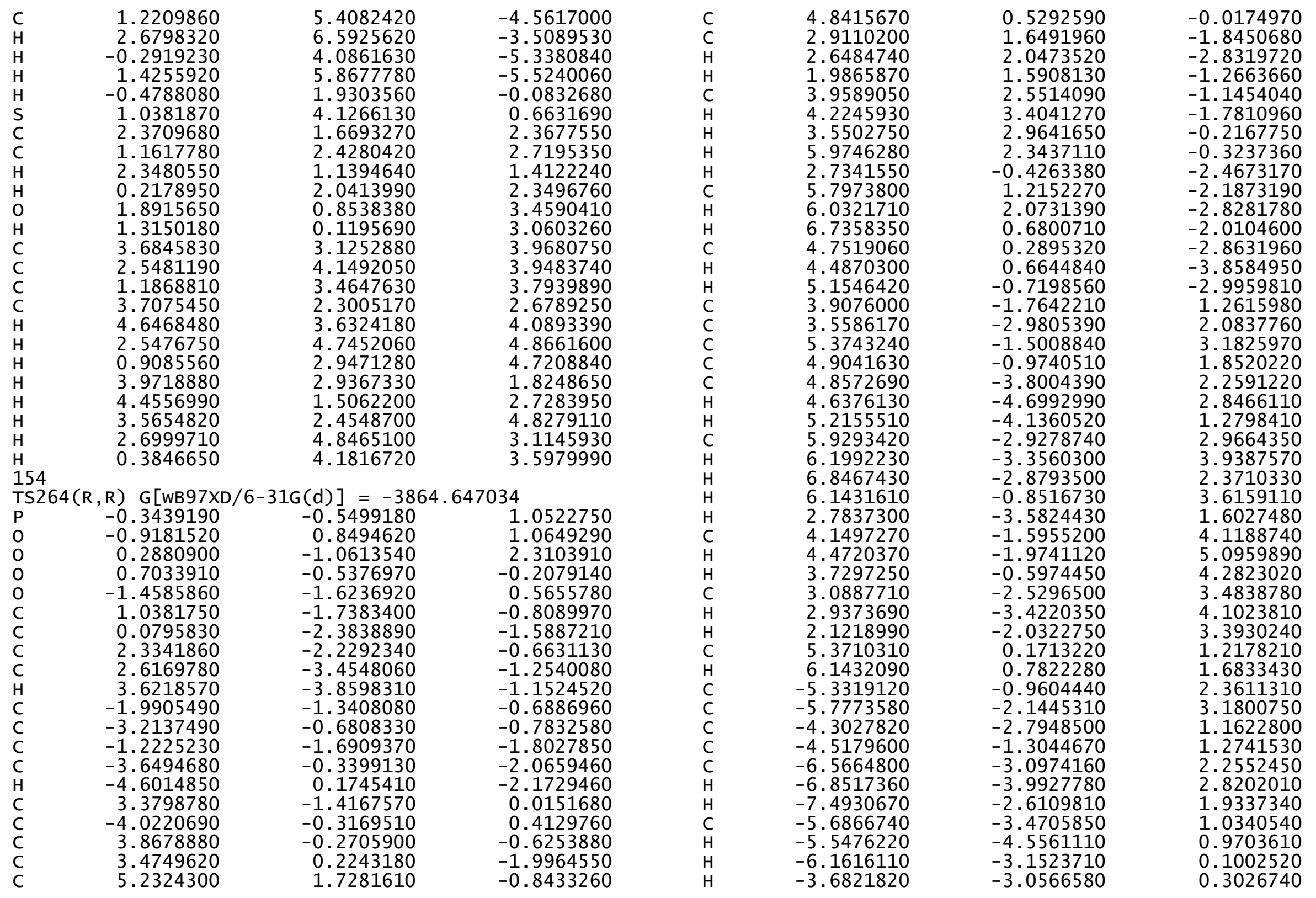




$\begin{array}{lr}\mathrm{H} & -6.3990590 \\ \mathrm{C} & -3.6383090 \\ \mathrm{H} & -3.5147930 \\ \mathrm{H} & -2.6395050 \\ \mathrm{C} & -4.5187380 \\ \mathrm{H} & -4.8266060 \\ \mathrm{H} & -3.9619200 \\ \mathrm{C} & -5.1915860 \\ \mathrm{C} & -5.4916150 \\ \mathrm{C} & -3.8848040 \\ \mathrm{C} & -4.3508360 \\ \mathrm{C} & -4.1620600 \\ \mathrm{H} & -4.3601030 \\ \mathrm{H} & -3.7349980 \\ \mathrm{C} & -3.1878980 \\ \mathrm{H} & -2.8505430 \\ \mathrm{H} & -2.3016870 \\ \mathrm{H} & -3.1917840 \\ \mathrm{H} & -6.1801050 \\ \mathrm{C} & -5.1293220 \\ \mathrm{H} & -4.8101730 \\ \mathrm{H} & -5.6147310 \\ \mathrm{C} & -6.1098200 \\ \mathrm{H} & -6.3180160 \\ \mathrm{H} & -7.0684270 \\ \mathrm{C} & -5.6798830 \\ \mathrm{H} & -6.3293610 \\ \mathrm{C} & -1.6552600 \\ \mathrm{C} & -0.8324820 \\ \mathrm{C} & -0.7234760 \\ \mathrm{C} & -2.1066200 \\ \mathrm{C} & -3.2604990 \\ \mathrm{C} & -2.8770220 \\ \mathrm{H} & -1.3079140 \\ \mathrm{H} & -0.1816220 \\ \mathrm{H} & -2.3088520 \\ \mathrm{H} & -3.4914550 \\ \mathrm{H} & 0.1643130 \\ \mathrm{H} & -0.1109780 \\ \mathrm{H} & -2.1065620 \\ \mathrm{H} & -4.1687010 \\ \mathrm{C} & 0.3749920 \\ \mathrm{C} & 1.6570280 \\ \mathrm{C} & -0.7114240 \\ \mathrm{C} & -0.1730650 \\ \mathrm{C} & 0.8532880 \\ & \end{array}$

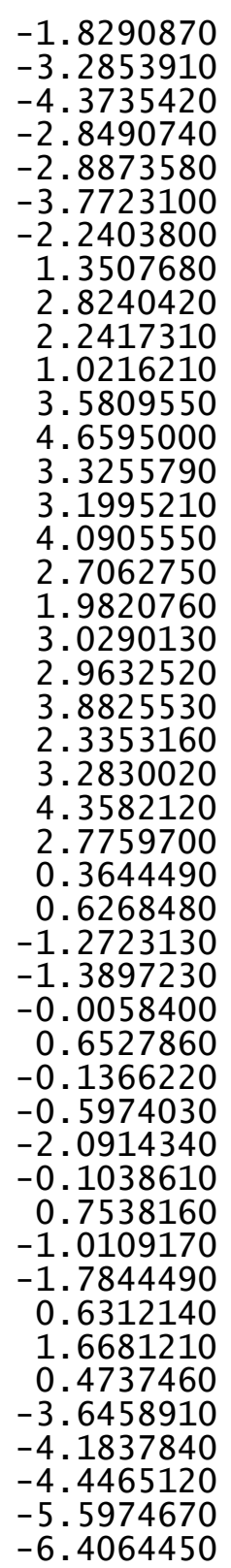
4.0249830
2. 4668940
2. 4145100
2. 5466510
3.6799760
4.2495250
4.3651540
1.7445980
1. 8499450
$-0.0881280$
0.6731640
2. 0473380
2. 0543710
3. 0225710
0.9053210
0.3625140
1. 3073370
$-0.8908470$
2. 6768000
$-0.6504850$
$-1.1553680$
$-1.4055360$
0.5095150
0.5587000
0.3592840
2. 5926800
3.4263770
$-3.0667590$
$-4.3252760$
$-4.9925750$
$-5.2258460$
$-4.5724120$
$-3.1911320$
$-5.0258620$
$-5.9388900$
$-6.2972960$
$-5.1967790$
$-4.1121870$
$-4.3443320$
$-4.8135190$
$-4.5416360$
$-2.1372770$
$-1.9580490$
$-2.8313890$
$-3.6794580$
$-2.8912560$

2. 0516710

$-1.0047550$

$-1.3756920$

$-1.3402720$

0.3892490

1. 1861170

2. 7215890

2. 6365410

0.2997220

0.2169510

$-0.5143150$

0.4998170

1. 4274740

$-0.1318680$

1. 7184070

1.9276360

0.1591980

$-0.8436280$

1. 0869420

2. 4453120

$-0.3334130$

1. 3181930

0.8151800

2. 1283700

0.8726740

2.2001760

$-0.0276820$

1.5876170

1. 0787080

3. 2610310

2. 1057110

0.7817100

3. 4160780

2. 0142530

0.4445070

3.7384860

4.1763070

3. 0820450

2. 3078700

$-0.0239690$

154

$\operatorname{TS} 265(R, R) G[W B 97 X D / 6-31 G(d)]=-3864.642485$

$\begin{array}{llll}\mathrm{P} & -0.3642470 & -0.4838800 & 1.1569660 \\ \mathrm{O} & -0.9651940 & 0.8980860 & 1.0266580\end{array}$

$\begin{array}{lr}-5.5250300 & -2.5462290 \\ -6.2314580 & -4.0052830 \\ -4.8500570 & -2.0535860 \\ -3.7967380 & -3.4434490 \\ -6.7811200 & -1.9692640 \\ -7.2803950 & -3.4616080 \\ -6.0434650 & -1.8509620 \\ -5.3481550 & -3.4603060 \\ -5.1969800 & -4.5868130 \\ 3.4024510 & -0.7610510 \\ 2.3459780 & -0.8757150 \\ 4.0377410 & -2.0732250 \\ 5.0754210 & -2.2041010 \\ 3.5307060 & -3.2173110 \\ 5.5969860 & -3.4586380 \\ 5.4584700 & -1.3213970 \\ 4.0561970 & -4.4685560 \\ 2.7195090 & -3.1145630 \\ 5.0890940 & -4.5918650 \\ 6.3975450 & -3.5524740 \\ 3.6547190 & -5.3490400 \\ 5.4977390 & -5.5709590 \\ 1.7815500 & -0.0195690 \\ 4.0180770 & 0.6875860 \\ 1.6383250 & 2.5635920 \\ 2.3626590 & 2.8129850 \\ 1.1070160 & 1.6114060 \\ 1.9408040 & 2.3796530 \\ 0.8091490 & 3.6169240 \\ 0.0498200 & 3.1814130 \\ 3.1485660 & 4.2524010 \\ 4.1420280 & 4.1163430 \\ 3.4147460 & 3.8666150 \\ 2.3083230 & 2.9822760 \\ 3.6809820 & 4.4483540 \\ 4.7564550 & 5.0170800 \\ 2.9029570 & 4.7774080 \\ 2.9411850 & 2.1459100 \\ 1.5336830 & 3.1070750 \\ 2.4877160 & 5.1091590 \\ 4.8255640 & 3.2818520 \\ 4.1017650 & 3.5930910\end{array}$




$\begin{array}{rrr}0.2495130 & -0.8610490 & 2.4705660 \\ 0.7118100 & -0.5672340 & -0.0755980 \\ -1.4455250 & -1.6210420 & 0.7474000 \\ 1.0767140 & -1.8070890 & -0.5709860 \\ 0.1403470 & -2.5275080 & -1.3209100 \\ 2.3818600 & -2.2509330 & -0.3756370 \\ 2.7177860 & -3.5094800 & -0.8737110 \\ 3.7346240 & -3.8710820 & -0.7381140 \\ -1.9659710 & -1.4628830 & -0.5334340 \\ -3.2056000 & -0.8506940 & -0.7000220 \\ -1.1758870 & -1.8915180 & -1.6052210 \\ -3.6418790 & -0.6534950 & -2.0128830 \\ -4.6072980 & -0.1796520 & -2.1733320 \\ 3.4037620 & -1.3510870 & 0.2248020 \\ -4.0368900 & -0.3824940 & 0.4431360 \\ 3.8698100 & -0.2660450 & -0.5283820 \\ 3.4700120 & 0.0798240 & -1.9421710 \\ 5.2034610 & 1.7211830 & -0.9512570 \\ 4.8289660 & 0.6083830 & -0.0059410 \\ 2.8846960 & 1.5039440 & -1.9362570 \\ 2.6126600 & 1.7926060 & -2.9581020 \\ 1.9632960 & 1.4917220 & -1.3500280 \\ 3.9191730 & 2.4907190 & -1.3382110 \\ 4.1734380 & 3.2734570 & -2.0621650 \\ 3.5040450 & 2.9942140 & -0.4585780 \\ 5.9363200 & 2.3981280 & -0.4989880 \\ 2.7387160 & -0.6264220 & -2.3427360 \\ 5.7778820 & 1.0798500 & -2.2345770 \\ 6.0037040 & 1.8694790 & -2.9606400 \\ 6.7219940 & 0.5767520 & -2.0024100 \\ 4.7458870 & 0.0761980 & -2.8130120 \\ 4.4756500 & 0.3446280 & -3.8408730 \\ 5.1632090 & -0.9355300 & -2.8443590 \\ 3.9388300 & -1.5624860 & 1.4976670 \\ 3.6080730 & -2.6957730 & 2.4368240 \\ 5.3925950 & -1.0784760 & 3.3844560 \\ 4.9171740 & -0.6962180 & 2.0068060 \\ 4.9182780 & -3.4709110 & 2.7054610 \\ 4.7095730 & -4.2979160 & 3.3936440 \\ 5.2830070 & -3.9128160 & 1.7719640 \\ 5.9767670 & -2.5078330 & 3.3076800 \\ 6.2668420 & -2.8289410 & 4.3149460 \\ 6.8866910 & -2.4996860 & 2.6988460 \\ 6.1469490 & -0.3746060 & 2.0159030 \\ 2.8475540 & -3.3584050 & \\ 4.1683490 & -1.1080930 & \\ 5 & & \\ 5 & -3264150\end{array}$

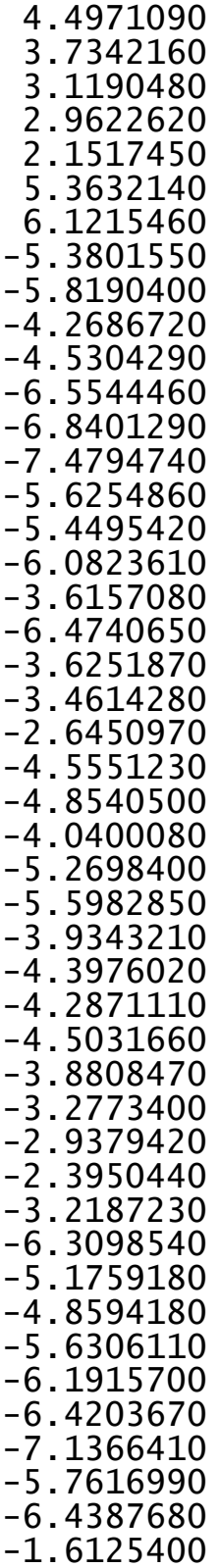

-1.3954130
-0.1058590
-2.1082480
-2.9315980
-1.6267650
0.3877380
1.0586500
-0.8375060
-1.9322870
-2.7666070
-1.2808260
-3.0088460
-3.8372130
-2.5875530
-3.5016850
-4.5806470
-3.3236930
-3.1058170
-1.5413550
-3.0744890
-4.1554380
-2.5936280
-2.5727720
-3.3981640
-1.8354100
1.3960570
2.8662510
2.1080230
0.9691550
3.6669480
4.7353870
3.5300740
3.1798380
4.0117350
2.7481700
1.7759960
3.1463590
2.7406820
3.6073190
2.0271790
3.1632900
4.2328000
2.6230300
0.4961820
0.8315970
-1.6163510
5. 3320610

4.4055320

3.7779670

4.4845680

3.6247630

1. 2592580

1. 6604600

2. 4194830

3. 3568080

1. 4660250

1. 3977150

2. 5281640

3. 1871080

2. 1212080

1. 3884280

1. 4697180

0.4094000

0.6593580

4. 1428370

2.8357800

2. 9167910

2. 8879180

3.9715110

4. 6284980

4. 5952840

1. 5580170

1. 4949020

$-0.3307160$

0.5485230

1.6376430

1. 5186880

2. 6451160

0.5692090

$-0.0594430$

1. 0442650

$-1.0854720$

2. 2789150

$-0.9962190$

$-1.5886680$

$-1.6922000$

0.0988810

0.0243210

$-0.0170710$

2. 4954450

3. 2795200

$-2.9063680$ 


$\begin{array}{rrr}-0.7807860 & -1.8373660 & -4.1446050 \\ -0.7187920 & -0.5317910 & -4.9600820 \\ -2.1204270 & 0.0579780 & -5.2556310 \\ -3.2486440 & -0.6992570 & -4.5255310 \\ -2.8561770 & -1.0000520 & -3.1033720 \\ -1.2271610 & -2.6311830 & -4.7605790 \\ -0.1758960 & -0.7156930 & -5.8929160 \\ -2.3241760 & 0.0449380 & -6.3315130 \\ -3.4440290 & -1.6419320 & -5.0553230 \\ 0.2304290 & -2.1656240 & -3.8915980 \\ -0.1241260 & 0.1894930 & -4.3881250 \\ -2.1528100 & 1.1089890 & -4.9466670 \\ -4.1787360 & -0.1230040 & -4.5596970 \\ 1.7857270 & -4.2955650 & -1.5404080 \\ 2.1117170 & -5.6585600 & -2.0931250 \\ 1.6796220 & -5.7402730 & -3.5665490 \\ 0.1948590 & -5.3498530 & -3.7656200 \\ -0.4445580 & -4.7353340 & -2.5030620 \\ 0.4952410 & -3.8019850 & -1.7811640 \\ 1.5844480 & -6.4313310 & -1.5155500 \\ 1.8661460 & -6.7481290 & -3.9514860 \\ -0.3951960 & -6.2246940 & -4.0582820 \\ -0.7002630 & -5.5478830 & -1.8084840 \\ 3.1819840 & -5.8675020 & -1.9950530 \\ 2.3193060 & -5.0616520 & -4.1416560 \\ 0.1152460 & -4.6328240 & -4.5888620 \\ -1.3871780 & -4.2448680 & -2.7548470 \\ 0.1382430 & 3.2728300 & -1.0421310 \\ -0.5645120 & 2.1911800 & -1.0548840 \\ 0.4104390 & 3.7842320 & -2.4093430 \\ 1.3252000 & 4.8157460 & -2.6405380 \\ -0.2167590 & 3.1617750 & -3.4973770 \\ 1.6117770 & 5.2149050 & -3.9401910 \\ 1.8194520 & 5.2876390 & -1.7983080 \\ 0.0690300 & 3.5659000 & -4.7940510 \\ -0.9202610 & 2.3575420 & -3.3138620 \\ 0.9866820 & 4.5910360 & -5.0177400 \\ 2.3300470 & 6.0101980 & -4.1126540 \\ -0.4200580 & 3.0771420 & -5.6313750 \\ 1.2154780 & 4.9022790 & -6.0326140 \\ -0.7132850 & 1.7158070 & -0.1458790 \\ 0.7144480 & 4.0424220 & 0.3399350 \\ 2.0404100 & 1.8741440 & 2.4618480 \\ 0.7812330 & 2.6162390 & 2.6287420 \\ 2.1166950 & 1.2429520 & 1.5731790 \\ -0.1158460 & 2.1484060 & 2.2387780\end{array}$

$\begin{array}{lr}\mathrm{O} & 1.4957030 \\ \mathrm{H} & 0.9988950 \\ \mathrm{C} & 3.1649930 \\ \mathrm{C} & 2.0067610 \\ \mathrm{C} & 0.6863020 \\ \mathrm{C} & 3.3250250 \\ \mathrm{H} & 4.0990010 \\ \mathrm{H} & 1.9124440 \\ \mathrm{H} & 0.3549780 \\ \mathrm{H} & 3.6498890 \\ \mathrm{H} & 4.0862220 \\ \mathrm{H} & 2.9867750 \\ \mathrm{H} & 2.2063600 \\ \mathrm{H} & -0.1240970 \\ \mathrm{H} & \end{array}$

1.1610620

$4.5324830 \quad 3.7284450$

$3.7756110 \quad 3.5634350$

$\begin{array}{ll}2.5889590 & 2.8093830\end{array}$

$4.1188010 \quad 4.1135660$

$5.2475610 \quad 4.5512070$

$3.3623880 \quad 4.5250830$

$3.1244020 \quad 1.9085430$

$1.8350660 \quad 3.0214720$

3.0043970 4.9027720

$5.1144410 \quad 2.8196050$

$4.4250710 \quad 3.2206050$

154

$\operatorname{TS} 266(R, R) \quad G[W B 97 X D / 6-31 G(d)]=-3864.646121$

$\begin{array}{lrrr}\text { TS266(R, R) } G[W B 97 X D / 6-31 G(\mathrm{~d})]=-3864.646121 \\ \mathrm{P} & -0.2404460 & -0.2901440 & 1.0052220 \\ \mathrm{O} & -0.6766490 & 1.1501780 & 0.8688030 \\ \mathrm{O} & 0.4821360 & -0.7029170 & 2.2527520 \\ \mathrm{O} & 0.6402700 & -0.5751760 & -0.3449060 \\ \mathrm{O} & -1.4893890 & -1.3078150 & 0.8206630 \\ \mathrm{C} & 0.8514250 & -1.8962010 & -0.7016940 \\ \mathrm{C} & -0.2136050 & -2.6167930 & -1.2399150 \\ \mathrm{C} & 2.1198420 & -2.4484850 & -0.5356560 \\ \mathrm{C} & 2.2692770 & -3.7926030 & -0.8584560 \\ \mathrm{H} & 3.2503480 & -4.2482450 & -0.7399630 \\ \mathrm{C} & -2.1212790 & -1.2567850 & -0.4143950 \\ \mathrm{C} & -3.3242390 & -0.5679660 & -0.5356470 \\ \mathrm{C} & -1.4952700 & -1.8973110 & -1.4893630 \\ \mathrm{C} & -3.8987780 & -0.5177830 & -1.8077340 \\ \mathrm{H} & -4.8486900 & -0.0022770 & -1.9292400 \\ \mathrm{C} & 3.2681610 & -1.6227380 & -0.0732690 \\ \mathrm{C} & -3.9889830 & 0.0961530 & 0.6192580 \\ \mathrm{C} & 3.7610830 & -0.5923170 & -0.8853190 \\ \mathrm{C} & 3.2427680 & -0.1895640 & -2.2445320 \\ \mathrm{C} & 5.2674180 & 1.2236100 & -1.4814400 \\ \mathrm{C} & 4.8639690 & 0.1699560 & -0.4820460 \\ \mathrm{C} & 2.8389370 & 1.2960680 & -2.1716160 \\ \mathrm{H} & 2.4580870 & 1.6229110 & -3.1459940 \\ \mathrm{H} & 2.0176000 & 1.3886880 & -1.4593980 \\ \mathrm{C} & 4.0569230 & 2.1494930 & -1.7367540 \\ \mathrm{H} & 4.3190380 & 2.8800340 & -2.5108910 \\ \mathrm{H} & 3.8230470 & 2.7187620 & -0.8304190 \\ \mathrm{H} & 6.1266380 & 1.8009100 & -1.1234570 \\ \mathrm{H} & 2.3879660 & -0.7963800 & -2.5520210 \\ \mathrm{C} & 5.6104480 & 0.5094000 & -2.8083550 \\ & & & \end{array}$




$\begin{array}{lrrr}\text { H } & 5.8700970 & 1.2598540 & -3.5638750 \\ \text { H } & 6.4930840 & -0.1220950 & -2.6649380 \\ \mathrm{C} & 4.3933670 & -0.3374500 & -3.2644360 \\ \mathrm{H} & 4.0374280 & -0.0083940 & -4.2476210 \\ \mathrm{H} & 4.6655910 & -1.3936550 & -3.3582760 \\ \mathrm{C} & 3.9032130 & -1.8744420 & 1.1480740 \\ \mathrm{C} & 3.5401410 & -2.9396730 & 2.1545400 \\ \mathrm{C} & 5.5933040 & -1.5367620 & 2.8637980 \\ \mathrm{C} & 5.0180780 & -1.1182370 & 1.5361430 \\ \mathrm{C} & 4.7662490 & -3.8659040 & 2.3181390 \\ \mathrm{H} & 4.5341400 & -4.6376660 & 3.0608000 \\ \mathrm{H} & 4.9689340 & -4.3799500 & 1.3723660 \\ \mathrm{C} & 5.9946830 & -3.0262400 & 2.7610730 \\ \mathrm{H} & 6.3689490 & -3.3652010 & 3.7341120 \\ \mathrm{H} & 6.8161760 & -3.1332760 & 2.0453860 \\ \mathrm{H} & 6.4617910 & -0.9240800 & 3.1301010 \\ \mathrm{H} & 2.6643600 & -3.5115230 & 1.8396580 \\ \mathrm{C} & 4.4857500 & -1.4045730 & 3.9319850 \\ \mathrm{H} & 4.8832070 & -1.7266900 & 4.9016230 \\ \mathrm{H} & 4.1932180 & -0.3545780 & 4.0362730 \\ \mathrm{C} & 3.2669130 & -2.2663540 & 3.5166990 \\ \mathrm{H} & 3.0722250 & -3.0478910 & 4.2603730 \\ \mathrm{H} & 2.3598570 & -1.6646300 & 3.4387770 \\ \mathrm{C} & 5.5024550 & -0.1003370 & 0.7231510 \\ \mathrm{H} & 6.3729830 & 0.4793580 & 1.0271700 \\ \mathrm{C} & -5.1681940 & -0.0315790 & 2.7429830 \\ \mathrm{C} & -5.6076240 & -0.9835410 & 3.8249280 \\ \mathrm{C} & -4.3354040 & -2.1439250 & 1.8988110 \\ \mathrm{C} & -4.4675030 & -0.6528210 & 1.7016090 \\ \mathrm{C} & -6.5162390 & -2.0547330 & 3.1822610 \\ \mathrm{H} & -6.8076710 & -2.7849850 & 3.9461400 \\ \mathrm{H} & -7.4355940 & -1.5840080 & 2.8192160 \\ \mathrm{C} & -5.7542800 & -2.7435550 & 2.0205670 \\ \mathrm{H} & -5.6694010 & -3.8224530 & 2.1952310 \\ \mathrm{H} & -6.2869780 & -2.6124780 & 1.0729510 \\ \mathrm{H} & -3.7995630 & -2.6194870 & 1.0743250 \\ \mathrm{H} & -6.1418230 & -0.4569020 & 4.6232160 \\ \mathrm{C} & -3.5889900 & -2.3845260 & 3.2292290 \\ \mathrm{H} & -3.5149710 & -3.4640410 & 3.4053800 \\ \mathrm{H} & -2.5695320 & -2.0000800 & 3.1417550 \\ \mathrm{C} & -4.3520300 & -1.6875030 & 4.38617800 \\ \mathrm{H} & -4.6593150 & -2.4142880 & 5.1473920 \\ \mathrm{H} & -3.7127310 & -0.9510950 & 4.8832090 \\ \mathrm{C} & -4.9455250 & 2.0857240 & 1.6423270 \\ \mathrm{C} & -5.1509520 & 3.5661790 & 1.4466270 \\ \mathrm{C} & -3.7138250 & 2.4770510 & -0.4063130 \\ & & & \end{array}$

-4.2095020
-3.7687150
-3.9021110
-3.2972650
-2.8911750
-2.5163390
-2.0253950
-3.0935180
-5.7694320
-4.9389380
-4.5911270
-5.5060240
-5.8293520
-5.9866680
-6.8165290
-5.4231320
-5.9887340
-3.2716250
-3.8536330
-3.7770050
-2.3271520
-1.3784130
-2.0579890
-3.2909890
-4.2187460
-1.9211720
-0.9950430
-4.8887380
-4.3906500
-2.3258850
-0.5008710
-0.0548140
-1.2453230
-1.0392650
0.3051910
1.4395450
1.2025320
-1.8627900
-1.4357790
-2.1431610
0.3436840
0.4296050
2.3917060
1.5615670
-1.0635860
0.5832150

1.4801600

4.2504280

5. 3153190

4.1863010

3. 5569660

4.2830400

3.0823140

2.0022360

3.9886030

3.1669730

3. 9465480

2. 4445510

3.7745010

4.8464820

3. 3013120

1.3340980

1.8093500

$-1.0749910$

$-0.9939340$

$-2.3749540$

$-2.8993770$

$-2.1982780$

$-1.7616280$

$-0.2658000$

$-2.3378380$

$-2.7582040$

$-1.2844480$

$-0.6389840$

$-3.0636780$

$-3.9793110$

$-2.8170620$

$-3.9927750$

$-4.8284440$

$-6.3232670$

$-6.7613440$

$-6.0628070$

$-4.5800380$

$-6.8822000$

$-4.6724860$

$-4.4754470$

$-6.5014300$

$-7.8472230$

$-6.2059440$

$-6.5370580$

$-6.5369900$

3.5484220
0.6153040

1.4061630

1.1810190

2. 3921420

0.3346430

$-0.3966540$

0.7993730

$-1.1700320$

2.2458110

$-1.0441610$

$-1.7322570$

$-1.6411520$

0.0730700

$-0.0935520$

0.0823150

2.7087320

3.5087120

$-2.9144360$

$-4.3026710$

$-4.9693560$

$-5.0396120$

$-4.0403360$

$-2.7646180$

$-4.9055160$

$-5.9704990$

$-6.0470050$

$-4.5159880$

$-4.2635530$

$-4.3776340$

$-4.8578670$

$-3.8407620$

$-1.4890580$

$-1.9197030$

$-1.6822030$

$-2.2571320$

$-1.5095140$

$-1.2951500$

$-2.1394790$

$-2.9888800$

$-1.4012810$

$-3.3237850$

$-2.1872100$

$-2.0339970$

$-0.5255230$

$-0.6053560$

$-1.1077650$ 


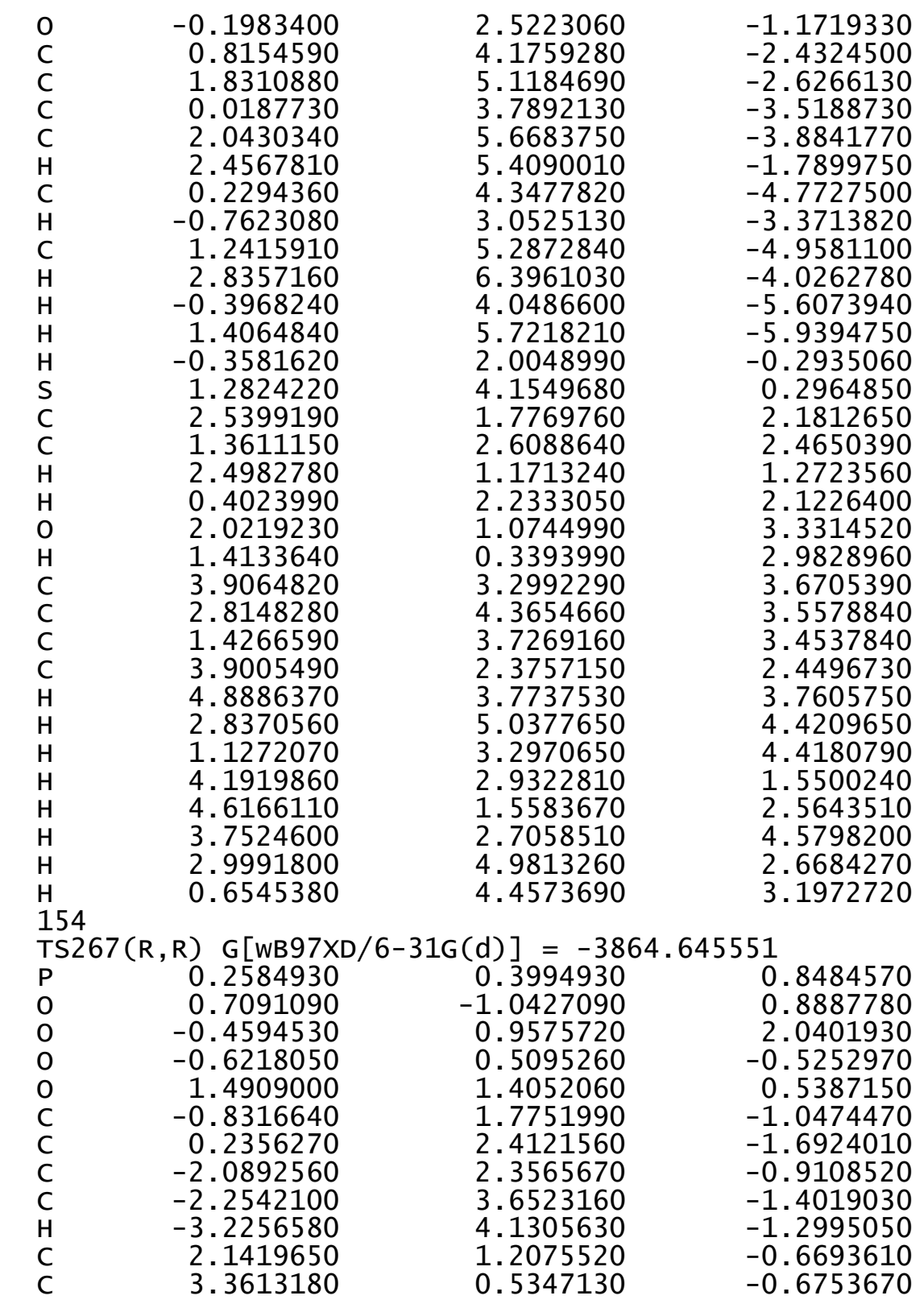

1.5310960
3.9616790
4.9214260
-3.2128450
3.9909820
-3.7798200
-3.3751830
-5.3507880
-4.8599060
-2.9875820
-2.7000300
-2.1074610
-4.1747060
-4.5063850
-3.8729760
-6.1860890
-2.5393940
-5.7887020
-6.1132810
-6.6510940
-4.6031070
-4.3331420
-4.8701870
-3.7530450
-3.3141990
-5.3251090
-4.8493880
-4.5224660
-4.2352630
-4.7952430
-5.7143200
-5.9875970
-6.6000370
-6.1792270
-2.4563580
-4.1439320
-4.4683270
-3.8457700
-2.9611950
-2.7433600
-2.0498280
-5.4059940
-6.2638770
5.0458300
5.4013170
4.2351290

1.6840490

0.3483510

$-0.1626050$

1. 6297170

.0114130

$-0.1021590$

$-1.3421300$

$-0.1623760$

$-1.5718370$

$-2.0436510$

$-1.5840460$

$-2.3224490$

$-3.1535620$

$-2.7576510$

$-1.8419020$

0.4343580

$-0.8200640$

$-1.6658900$

$-0.1558240$

$-0.0726280$

$-0.5427070$

0.9665170

2. 0773020

3. 2811090

2. 0479090

1. 4188120

4.2412040

5.1325490

4. 5771160

3. 5142120

4.0081760

3. 5349710

1.5023870

.7788570

2.0509600

2. 5017900

1. 0198080

2.8396410

3.7367420

2. 2404890

0.3028670

$-0.1994130$

0.3732660

1.4340170

2. 3752900
$-1.8300400$

$-1.9169590$

$-1.9549240$

$-0.2583410$

0.5680140

$-0.8690260$

$-2.1910090$

$-1.0762880$

$-0.2770490$

$-1.9389320$

$-2.8852520$

$-1.2936690$

$-1.2847920$

$-1.9181050$

$-0.3260740$

$-0.5737620$

$-2.6462570$

$-2.4633650$

$-3.0806560$

$-2.3474440$

$-3.1288830$

$-4.0814560$

$-3.3467320$

0.9532410

1.7520280

2.8106270

1.5273800

1.8338880

2.4035750

0.8274410

2. 5124660

3.4521440

1.8691670

3. 2264920

1.2949920

3.8050960

4.7502760

4.0217380

3. 1892590

3. 7809780

3. 1639190

0.9142040

1. 3591280

2. 7296160

3.7385690

1.6345230 


$\begin{array}{lrr}4.3985250 & 0.8745190 & 1.5932920 \\ 6.3308950 & 2.4596060 & 3.0532520 \\ 6.5549060 & 3.2683120 & 3.7587990 \\ 7.2822670 & 1.9796910 & 2.8019100 \\ 5.6362090 & 3.0102020 & 1.7807610 \\ 5.5278750 & 4.0996270 & 1.8373540 \\ 6.2300590 & 2.7919670 & 0.8870350 \\ 3.7440000 & 2.7588550 & 0.7375010 \\ 5.8919130 & 0.9973990 & 4.6151510 \\ 3.4060590 & 2.7326290 & 2.8880200 \\ 3.3153690 & 3.8231930 & 2.9551590 \\ 2.3962550 & 2.3298440 & 2.7769760 \\ 4.1011740 & 2.1597640 & 4.1506530 \\ 4.3462810 & 2.9578520 & 4.8614400 \\ 3.4417840 & 1.4575550 & 4.6705940 \\ 4.9145680 & -1.8473390 & 1.8385400 \\ 5.1505480 & -3.3359020 & 1.8063970 \\ 3.8015240 & -2.4678300 & -0.2198790 \\ 4.2307580 & -1.3617120 & 0.7162120 \\ 3.7844060 & -4.0514090 & 1.7596110 \\ 3.9488530 & -5.1281920 & 1.6332360 \\ 3.2627430 & -3.9148930 & 2.7123920 \\ 2.9503730 & -3.4733600 & 0.5898760 \\ 2.6049480 & -4.2703470 & -0.0792170 \\ 2.0675910 & -2.9577120 & 0.9705300 \\ 3.2178540 & -2.0854670 & -1.0603440 \\ 5.7297220 & -3.6629710 & 2.6766790 \\ 5.0656700 & -3.2020390 & -0.7183160 \\ 4.7611070 & -4.0592650 & -1.3304560 \\ 5.6523770 & -2.5401240 & -1.3645300 \\ 5.9079740 & -3.6662870 & 0.5001320 \\ 6.0971400 & -4.7450570 & 0.4535670 \\ 6.8825690 & -3.1677260 & 0.5111840 \\ 5.3202530 & -0.9837850 & 2.8482000 \\ 5.8433200 & -1.3659280 & 3.7234350 \\ 3.3626690 & 0.7523900 & -3.1094240 \\ 2.1251020 & 1.4114520 & -3.0767860 \\ 4.0847500 & 0.4717780 & -4.4134980 \\ 3.1587750 & 0.4739260 & -5.6276480 \\ 2.2765710 & 1.7190640 & -5.6021220 \\ 1.3811280 & 1.7143120 & -4.3646640 \\ 3.7504860 & 0.4311650 & -6.5485510 \\ 4.8596100 & 1.2386970 & -4.5564700 \\ 4.6137290 & -0.4849140 & -4.3358610 \\ 2.9128300 & 2.6149100 & -6.5024090 \\ 1.6550840 & 1.7766300 & \end{array}$

0.8431040

0.6053420

2. 5222230

0.0539470

1. 1571720

1. 2050440

$-0.1787410$

$-1.3055130$

$-1.1977160$

0.9743210

1.9343940

$-0.1543070$

$-1.2230710$

2. 1258410

1. 5834340

$-0.4257220$

$-2.2834650$

$-0.6284690$

0.1433010

$-0.9720420$

$-1.9838460$

$-0.2995840$

$-2.3174550$

$-2.5130230$

$-0.6322300$

0.4783670

$-1.6425280$

$-3.1075060$

$-0.1046750$

$-1.9047120$

0.3511430

$-1.2097670$

$-2.4616220$

$-1.2569170$

$-2.4567540$

$-0.3155550$

$-1.9381180$

$-1.3566420$

$-3.7553850$

$-2.6411470$

$-1.2713630$

$-3.8016070$

$-4.7233430$

$-2.6253450$

$-0.9576290$

$-4.1077240$
2.6602820

0.9436670

$-0.4211750$

3.7333590

4.6070090

5.9346450

6. 6260780

5.7831160

4.3481740

4.8231280

6.5990660

7.6067820

5.8375290

4.1037550

5.7214910

6.8030980

6.1995420

$-3.6583680$

$-2.6483890$

$-4.4078100$

$-5.3740300$

$-4.1030550$

$-6.0241990$

$-5.6016890$

$-4.7602850$

$-3.3489990$

$-5.7197700$

$-6.7682630$

$-4.5214770$

$-6.2288720$

$-2.0357360$

$-4.1190010$

$-1.5376880$

$-2.3023360$

$-1.0623330$

$-1.9554710$

$-0.6691130$

0.0212570

$-2.8662540$

- 3.9147640

- 3.2705410

$-2.1179580$

3.3445950

$-4.4566200$

$-2.7022420$

$-2.7982860$
$-4.2931150$

4.4842670

$-5.6113150$

$-2.1218440$

$-2.6659580$

$-1.8863120$

$-1.7889590$

$-2.4184390$

$-1.9755010$

$-3.7285340$

$-2.3610460$

$-2.2752920$

$-3.5130770$

$-2.6094140$

$-0.8811790$

$-0.7371330$

$-2.1567340$

$-0.7223540$

$-0.9492840$

$-1.9565160$

$-1.9670440$

$-3.1480530$

$-3.1480480$

$-1.0483990$

$-4.3251120$

$-3.1434620$

$-4.3280690$

$-3.1480290$

$-5.2432060$

$-5.2506970$

$-0.1453540$

0.7885090

2. 3716350

2. 7298050

1.3876140

2. 3156600

3. 3977370

2. 9330810

4.0932790

4. 1000620

3. 8670780

2. 7586710

4.2725180

5.0505830

4.7520400

1.9542540 


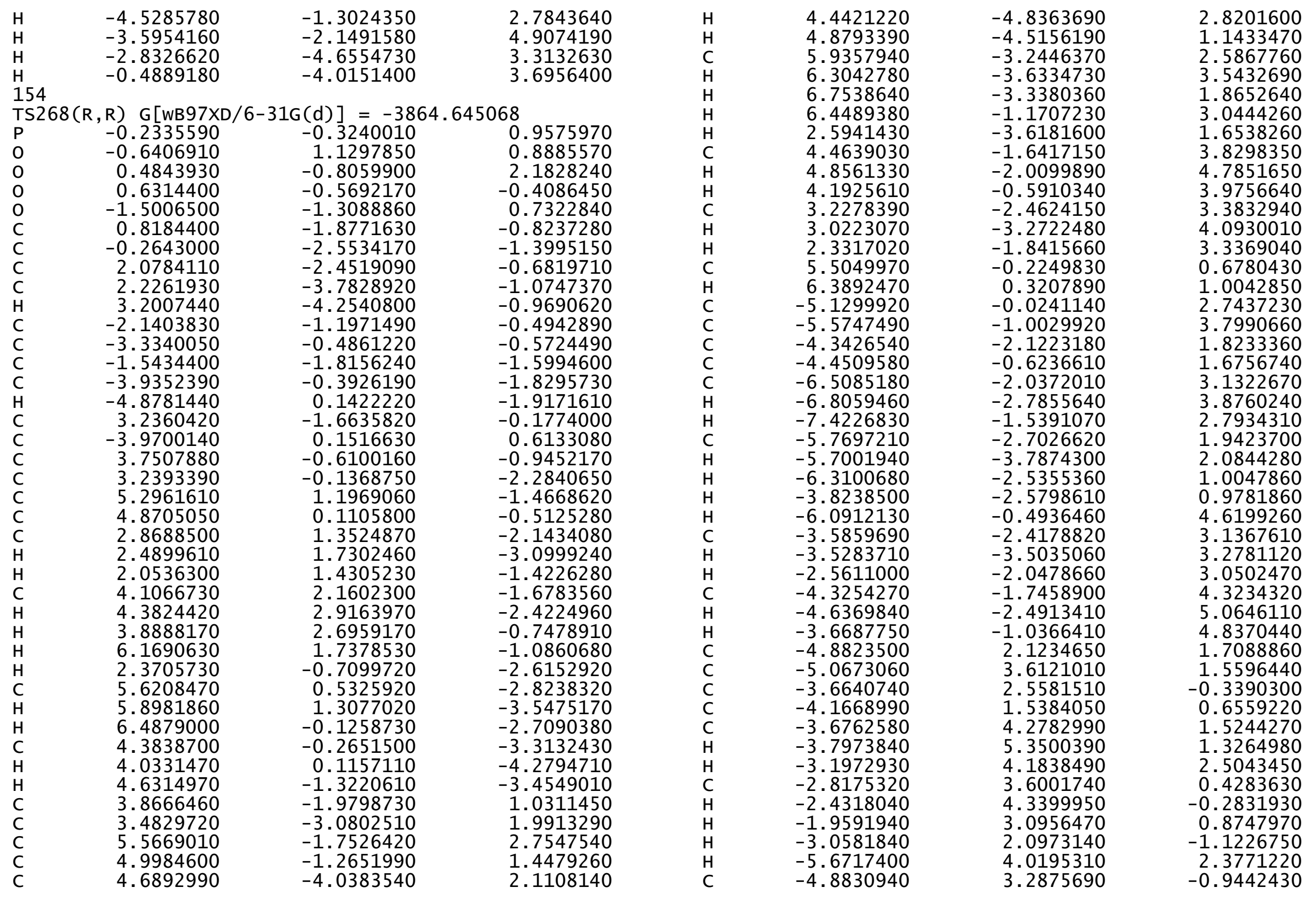




$\begin{array}{rrr}-4.5281070 & 4.0850960 & -1.6076400 \\ -5.4652830 & 2.5949020 & -1.5615670 \\ -5.7565080 & 3.8695790 & 0.1997760 \\ -5.9045290 & 4.9475440 & 0.0668400 \\ -6.7482270 & 3.4059050 & 0.2043670 \\ -5.3621290 & 1.3460670 & 2.7557060 \\ -5.9119180 & 1.8046380 & 3.5761610 \\ -3.3486800 & -0.9440730 & -2.9603500 \\ -3.9645000 & -0.8364740 & -4.3311430 \\ -3.9856520 & -2.2193860 & -5.0024380 \\ -2.5835270 & -2.8746090 & -5.0335800 \\ -1.5520790 & -2.1330840 & -4.1575910 \\ -2.1441130 & -1.6548710 & -2.8549250 \\ -3.3787620 & -0.1410370 & -4.9498580 \\ -4.3940950 & -2.1343750 & -6.0147360 \\ -2.1992880 & -2.9110260 & -6.0584360 \\ -1.2061640 & -1.2442220 & -4.7042660 \\ -4.9767650 & -0.4241520 & -4.2668190 \\ -4.6760020 & -2.8544570 & -4.4365400 \\ -2.6600370 & -3.9125630 & -4.6941320 \\ -0.6673040 & -2.7528040 & -4.0015000 \\ -0.0956320 & -3.9005930 & -1.7429650 \\ -1.2079570 & -4.7945820 & -2.2306570 \\ -1.2528230 & -6.0848010 & -1.3910560 \\ 0.1278180 & -6.7785990 & -1.2740060 \\ 1.2549900 & -5.9687840 & -1.9437530 \\ 1.1552540 & -4.5121780 & -1.5753690 \\ -1.0378370 & -5.0547400 & -3.2853780 \\ -1.9904060 & -6.7670320 & -1.8263190 \\ 0.0952710 & -7.7773840 & -1.7215750 \\ 1.1695220 & -6.0749430 & -3.0344570 \\ -2.1743960 & -4.2861870 & -2.1846910 \\ -1.6198760 & -5.8230340 & -0.3932100 \\ 0.3794790 & -6.9162650 & -0.2174950 \\ 2.2326740 & -6.3751480 & -1.6656210 \\ 0.6610380 & 3.6002430 & -0.9717530 \\ -0.1291580 & 2.5851390 & -1.0831110 \\ 0.9145910 & 4.2731720 & -2.2699690 \\ 1.9258790 & 5.2291480 & -2.4145760 \\ 0.1431300 & 3.9135820 & -3.3836560 \\ 2.1583840 & 5.8182650 & -3.6506080 \\ 2.5316400 & 5.4988340 & -1.5564640 \\ 0.3751680 & 4.5099330 & -4.6159550 \\ -0.6343560 & 3.1666620 & -3.2742400 \\ 1.3830510 & 5.4623330 & -4.7520720 \\ 2.9473560 & 6.5563820 & -3.7544980\end{array}$

$\begin{array}{lr}\mathrm{H} & -0.2301640 \\ \mathrm{H} & 1.5650840 \\ \mathrm{H} & -0.3017980 \\ \mathrm{~S} & 1.3495780 \\ \mathrm{C} & 2.5733600 \\ \mathrm{C} & 1.4107640 \\ \mathrm{H} & 2.5137230 \\ \mathrm{H} & 0.4433870 \\ \mathrm{O} & 2.0558480 \\ \mathrm{H} & 1.4342860 \\ \mathrm{C} & 3.9785140 \\ \mathrm{C} & 2.9017700 \\ \mathrm{C} & 1.5034510 \\ \mathrm{C} & 3.9460230 \\ \mathrm{H} & 4.9684180 \\ \mathrm{H} & 2.9421170 \\ \mathrm{H} & 1.2079410 \\ \mathrm{H} & 4.2392950 \\ \mathrm{H} & 4.6487460 \\ \mathrm{H} & 3.8260050 \\ \mathrm{H} & 3.0864180 \\ \mathrm{H} & 0.7391560 \\ \mathrm{H} & \end{array}$

$\begin{array}{lr}4.2300340 & -5.4724180 \\ 5.9262760 & -5.7167990 \\ 2.0326300 & -0.2287640 \\ 4.1460440 & 0.4630360 \\ 1.6541740 & 2.2124510 \\ 2.4893700 & 2.5493650 \\ 1.0992640 & 1.2727440 \\ 2.1459760 & 2.1977490 \\ 0.8992850 & 3.3285710 \\ 0.1906070 & 2.9489620 \\ 3.0766740 & 3.7638720 \\ 4.1629700 & 3.7200600 \\ 3.5510270 & 3.5960010 \\ 2.2166620 & 2.4979950 \\ 3.5321700 & 3.8666950 \\ 4.7857320 & 4.6189750 \\ 3.0732010 & 4.5387360 \\ 2.8133370 & 1.6250280 \\ 1.3826680 & 2.5648310 \\ 2.4392050 & 4.6431190 \\ 4.8245260 & 2.8640990 \\ 4.3052620 & 3.3892430\end{array}$

154

$\begin{array}{lrrr}\text { TS269(R, R) } \text { G }[\mathrm{WB} 97 \times \mathrm{D} / 6-31 \mathrm{G}(\mathrm{d})]=-3864.646688 \\ \mathrm{P} & -0.2367600 & -0.2634130 & 1.0160300 \\ \mathrm{O} & -0.6686650 & 1.1745000 & 0.8457820 \\ \mathrm{O} & 0.4885560 & -0.6480510 & 2.2708870 \\ \mathrm{O} & 0.6384170 & -0.5844730 & -0.3293990 \\ \mathrm{O} & -1.4897730 & -1.2805560 & 0.8616400 \\ \mathrm{C} & 0.8502770 & -1.9119320 & -0.6598560 \\ \mathrm{C} & -0.2180850 & -2.6492580 & -1.1698220 \\ \mathrm{C} & 2.1242450 & -2.4530500 & -0.5013750 \\ \mathrm{C} & 2.2837710 & -3.7968880 & -0.8216200 \\ \mathrm{H} & 3.2714870 & -4.2409880 & -0.7155540 \\ \mathrm{C} & -2.1240660 & -1.2638700 & -0.3729710 \\ \mathrm{C} & -3.3288820 & -0.5801560 & -0.5116080 \\ \mathrm{C} & -1.5027570 & -1.9379920 & -1.4281910 \\ \mathrm{C} & -3.9120720 & -0.5755610 & -1.7805480 \\ \mathrm{H} & -4.8658420 & -0.0699350 & -1.9127700 \\ \mathrm{C} & 3.2703950 & -1.6178610 & -0.0503130 \\ \mathrm{C} & -3.9899760 & 0.1145280 & 0.6273720 \\ \mathrm{C} & 3.7649040 & -0.6006960 & -0.8773770 \\ \mathrm{C} & 3.2475970 & -0.2191640 & -2.2428390 \\ \mathrm{C} & 5.2715740 & 1.2058100 & -1.4992060 \\ \mathrm{C} & 4.8668500 & 0.1680470 & -0.4837600 \\ \mathrm{C} & 2.8430350 & 1.2671760 & -2.1914650\end{array}$




$\begin{array}{lrrr}\text { H } & 2.4604230 & 1.5796980 & -3.1698310 \\ \text { H } & 2.0228060 & 1.3694310 & -1.4791660 \\ \mathrm{C} & 4.0611900 & 2.1273010 & -1.7704680 \\ \mathrm{H} & 4.3237120 & 2.8448460 & -2.5566110 \\ \mathrm{H} & 3.8270180 & 2.7117660 & -0.8739780 \\ \mathrm{H} & 6.1303360 & 1.7889210 & -1.1495590 \\ \mathrm{H} & 2.3930040 & -0.8309710 & -2.5410970 \\ \mathrm{C} & 5.6160740 & 0.4708020 & -2.8143340 \\ \mathrm{H} & 5.8779620 & 1.2094330 & -3.5806610 \\ \mathrm{H} & 6.4977070 & -0.1594110 & -2.6595510 \\ \mathrm{C} & 4.3987840 & -0.3814670 & -3.2597970 \\ \mathrm{H} & 4.0438110 & -0.0657970 & -4.2476940 \\ \mathrm{H} & 4.6705980 & -1.4390010 & -3.3390450 \\ \mathrm{C} & 3.9022760 & -1.8499870 & 1.1763730 \\ \mathrm{C} & 3.5368320 & -2.8997730 & 2.1979080 \\ \mathrm{C} & 5.5872870 & -1.4848770 & 2.8912000 \\ \mathrm{C} & 5.0158910 & -1.0874540 & 1.5554310 \\ \mathrm{C} & 4.7630460 & -3.8226780 & 2.3789340 \\ \mathrm{H} & 4.5291030 & -4.5839180 & 3.1317890 \\ \mathrm{H} & 4.9689740 & -4.3499420 & 1.4411450 \\ \mathrm{C} & 5.9895050 & -2.9757360 & 2.8134410 \\ \mathrm{H} & 6.3598380 & -3.2989160 & 3.7933080 \\ \mathrm{H} & 6.8139390 & -3.0938770 & 2.1029000 \\ \mathrm{H} & 6.4547290 & -0.8678110 & 3.1506800 \\ \mathrm{H} & 2.6624380 & -3.4770250 & 1.8889310 \\ \mathrm{C} & 4.4764250 & -1.3367140 & 3.9538810 \\ \mathrm{H} & 4.8712930 & -1.6431110 & 4.9296390 \\ \mathrm{H} & 4.1830350 & -0.2853370 & 4.0408120 \\ \mathrm{C} & 3.2592920 & -2.2057060 & 3.5486540 \\ \mathrm{H} & 3.0633360 & -2.9760540 & 4.3036090 \\ \mathrm{H} & 2.3519350 & -1.6060990 & 3.4588470 \\ \mathrm{C} & 5.5023120 & -0.0827340 & 0.7273010 \\ \mathrm{H} & 6.3719620 & 0.5020280 & 1.0240740 \\ \mathrm{C} & -5.1544880 & 0.0405330 & 2.7621830 \\ \mathrm{C} & -5.5850240 & -0.8836500 & 3.8715610 \\ \mathrm{C} & -4.3227690 & -2.0913920 & 1.9681580 \\ \mathrm{C} & -4.4592980 & -0.6062610 & 1.7327780 \\ \mathrm{C} & -6.4956300 & -1.9728310 & 3.2626000 \\ \mathrm{H} & -6.7801960 & -2.6842140 & 4.0466630 \\ \mathrm{H} & -7.4184630 & -1.5133780 & 2.8939500 \\ \mathrm{C} & -5.7400360 & -2.6893590 & 2.1134910 \\ \mathrm{H} & -5.6531370 & -3.7636820 & 2.3136290 \\ \mathrm{H} & -6.2785310 & -2.5816160 & 1.1662160 \\ \mathrm{H} & -3.7908780 & -2.5873160 & 1.1532250 \\ \mathrm{H} & -6.1155190 & -0.3372710 & 4.6589540 \\ \mathrm{C} & -3.5677740 & -2.2960290 & 3.2997880 \\ & & & \\ & & & \end{array}$

-3.4907880
-2.5494700
-4.3247830
-4.6261010
-3.6836220
-4.9428170
-5.1500220
-3.7297280
-4.2145180
-3.7678860
-3.9032160
-3.2873900
-2.9003170
-2.5327390
-2.0300860
-3.1173610
-5.7607060
-4.9614340
-4.6204860
-5.5339140
-5.8407170
-5.9979710
-6.8285260
-5.4113310
-5.9713370
-3.2917770
-3.8913870
-3.8319440
-2.3871410
-1.4184180
-2.0737130
-3.3325410
-4.2807150
-1.9961700
-1.0540660
-4.9239550
-4.4492760
-2.3866670
-0.5341370
-0.0487340
1.2207470
-1.2449060
-0.8615030
0.2255370
1.4886060
-1.7490270
3.5030910

3.1959040

4.4431710

5.2247100

4.9166450

1.6047530

1.3733250

$-0.4643500$

0.5877190

1. 3027880

1.0526650

2.2856350

0.2407710

$-0.5120060$

0.7091000

$-1.2217030$

2.1673150

$-1.1079870$

$-1.8200710$

$-1.6795970$

0.0011860

$-0.1910520$

0.0316410

2. 6941660

3.4857340

$-2.8706530$

$-4.2538270$

$-4.8381030$

$-4.8866180$

$-3.9653680$

$-2.7043720$

$-4.9062150$

$-5.8366320$

$-5.9083900$

$-4.5114070$

$-4.2230460$

$-4.2036930$

$-4.6118370$

$-3.7473300$

$-1.4093690$

$-1.2441940$

$-1.7467870$

$-2.4012920$

$-1.5848230$

$-1.5552260$

$-2.4905490$ 


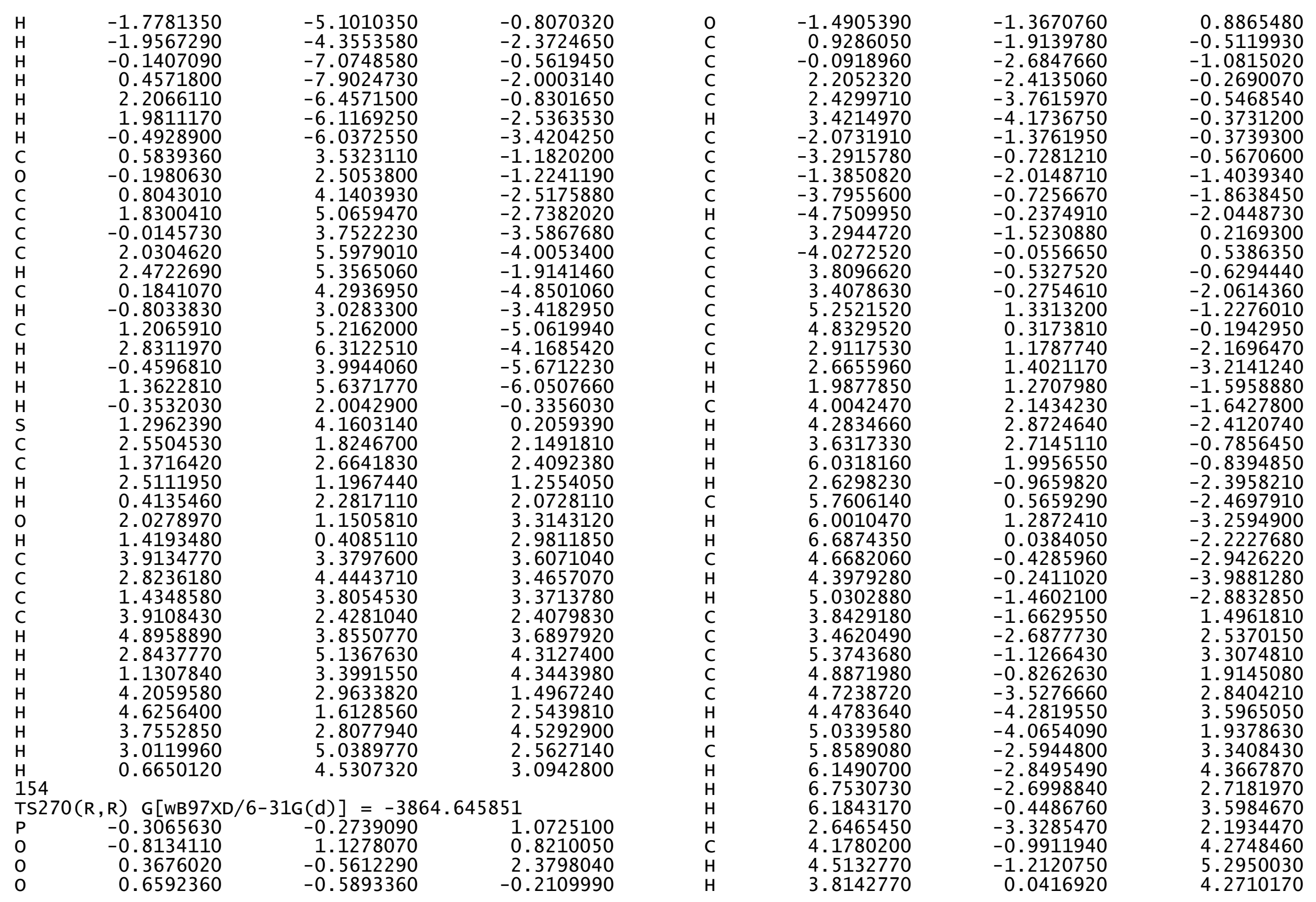




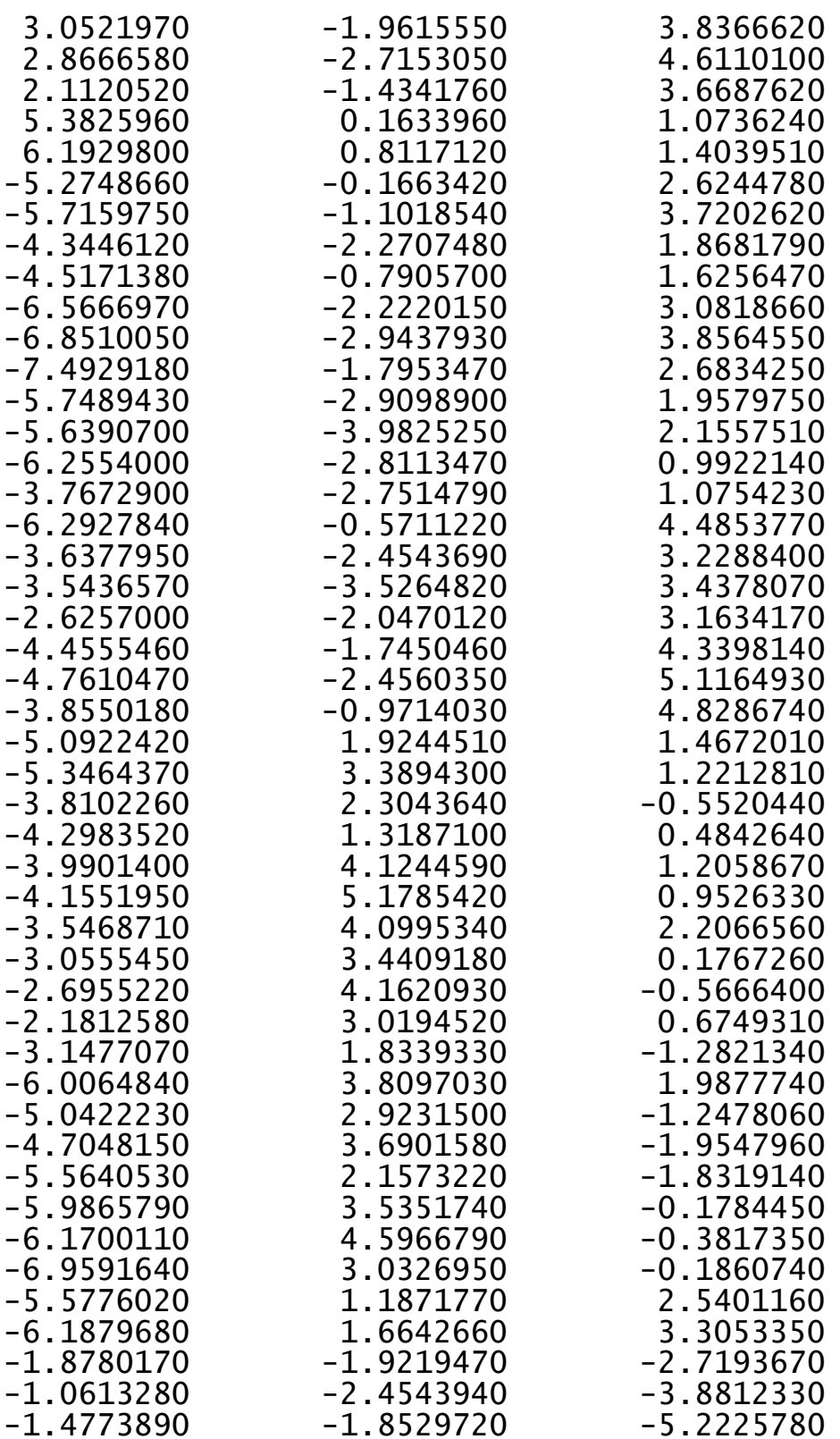

-2.9903940
-3.6895430
-3.1041970
-0.9524690
-1.1749750
0.0021230
-3.2872690
-3.3026040
-4.7618880
-3.6109890
-1.1806470
1.4128260
1.6195680
1.0473730
-0.4501180
-0.9192890
0.1457360
1.1121190
1.2016340
-1.0639020
-1.1835970
2.6836290
1.6185620
-0.6472470
-1.8373260
0.4515640
-0.3123430
0.7900680
1.7596680
0.1626250
2.0977300
2.2530400
0.4998560
-0.5810870
1.4694510
2.8559010
0.0091340
1.7361330
-0.5006180
1.0317190
2.2881720
1.0625170
2.3185310
0.1388380
1.7446860
1.1975120
$-5.3916170$ $-4.3307400$ $-2.9406550$ $-6.0340360$ $-3.9444690$ $-3.6912900$ $-5.2856360$ $-6.3907290$ $-4.3016000$ $-4.6123070$ $-5.2670520$ $-1.0165800$ $-1.2920800$ $-2.6743160$ $-2.7848660$ $-1.7035520$ $-1.2921050$ $-0.5243930$ $-2.8913140$ $-2.7003240$ $-0.8011750$ $-1.2392720$ $-3.4216810$ $-3.7800990$ $-2.0156590$ $-1.3329890$ $-1.3176910$ $-2.7093420$ $-2.9449360$ $-3.7970120$ $-4.2458430$ $-2.1036490$ $-5.0956750$ $-3.6158760$ $-5.3224510$ $-4.4195720$ $-5.9331740$ $-6.3390960$ $-0.3953490$ 0.0301790 2. 1948480 2. 3453470 1.3431800 1. 9973260 3. 3774870 3.0688960 


$\begin{array}{llll}\mathrm{C} & 3.5128750 & 3.7435630 & 3.5973190 \\ \mathrm{C} & 2.3843780 & 4.7425330 & 3.3348680 \\ \mathrm{C} & 1.0321210 & 4.0316420 & 3.2291840 \\ \mathrm{C} & 3.6077760 & 2.7095530 & 2.4726860 \\ \mathrm{H} & 4.4690440 & 4.2686000 & 3.6864750 \\ \mathrm{H} & 2.3358030 & 5.4915690 & 4.1308800 \\ \mathrm{H} & 0.7065470 & 3.6746450 & 4.2147510 \\ \mathrm{H} & 3.9276630 & 3.1904510 & 1.5398090 \\ \mathrm{H} & 4.3454100 & 1.9374770 & 2.7036660 \\ \mathrm{H} & 3.3388160 & 3.2311690 & 4.5512130 \\ \mathrm{H} & 2.5845700 & 5.2814980 & 2.4000570 \\ \mathrm{H} & 0.2411950 & 4.6984120 & 2.8747500 \\ 154 & & & \end{array}$

TS271(R,R) G[WB97XD/6-31G(d)] = -3864.645114

$\begin{array}{lrrr}\mathrm{P} & -0.3094800 & -0.2606100 & 1.0774750 \\ \mathrm{O} & -0.8226640 & 1.1357740 & 0.8094010 \\ \mathrm{O} & 0.3605330 & -0.5313220 & 2.3904840 \\ \mathrm{O} & 0.6638130 & -0.5835990 & -0.1987300 \\ \mathrm{O} & -1.4869440 & -1.3618260 & 0.8997120 \\ \mathrm{C} & 0.9358470 & -1.9095760 & -0.4917760 \\ \mathrm{C} & -0.0819760 & -2.6838000 & -1.0580550 \\ \mathrm{C} & 2.2132780 & -2.4070230 & -0.2461950 \\ \mathrm{C} & 2.4402910 & -3.7556550 & -0.5189540 \\ \mathrm{H} & 3.4332810 & -4.1647410 & -0.3465450 \\ \mathrm{C} & -2.0731090 & -1.3873550 & -0.3583820 \\ \mathrm{C} & -3.2998690 & -0.7564110 & -0.5526020 \\ \mathrm{C} & -1.3789150 & -2.0240580 & -1.3856840 \\ \mathrm{C} & -3.8130780 & -0.7800620 & -1.8463760 \\ \mathrm{H} & -4.7761530 & -0.3072440 & -2.0269500 \\ \mathrm{C} & 3.3015860 & -1.5109470 & 0.2312650 \\ \mathrm{C} & -4.0306900 & -0.0709730 & 0.5483480 \\ \mathrm{C} & 3.8142110 & -0.5272360 & -0.6244890 \\ \mathrm{C} & 3.4094430 & -0.2825690 & -2.0578900 \\ \mathrm{C} & 5.2524890 & 1.3341090 & -1.2403750 \\ \mathrm{C} & 4.8365540 & 0.3282720 & -0.1978620 \\ \mathrm{C} & 2.9107540 & 1.1700180 & -2.1772940 \\ \mathrm{H} & 2.6627010 & 1.3844660 & -3.2232140 \\ \mathrm{H} & 1.9875730 & 1.2656000 & -1.6028550 \\ \mathrm{C} & 4.0024790 & 2.1407050 & -1.6600730 \\ \mathrm{H} & 4.2790790 & 2.8641330 & -2.4356260 \\ \mathrm{H} & 3.6303830 & 2.7179670 & -0.8069130 \\ \mathrm{H} & 6.0316360 & 2.0030280 & -0.8591510 \\ \mathrm{H} & 2.6320530 & -0.9770750 & -2.3853290 \\ \mathrm{C} & 5.7601340 & 0.5593580 & -2.4771030 \\ \mathrm{H} & 5.9983750 & 1.2745980 & -3.2729860 \\ \mathrm{H} & 6.6880880 & 0.0352070 & -2.2272100\end{array}$

4.6684600
4.3960370
5.0322930
3.8506800
3.4709140
5.3796570
4.8935490
4.7341620
4.4893790
5.0467060
5.8663260
6.1536810
6.7625660
6.1879950
2.6571310
4.1814430
4.5154090
3.8154660
3.0583700
2.8743640
2.1168810
5.3871790
6.1963690
-5.2450720
-5.6573970
-4.3053710
-4.4973620
-6.5066600
-6.7699500
-7.4440760
-5.7009680
-5.5763350
-6.2247470
-3.7365900
-6.2267140
-3.5732040
-3.4646820
-2.5664490
-4.3792740
-4.6634630
-3.7788110
-5.0989760
-5.3672210
-3.8447300
-4.3143430
-4.0168580
$-2.9401060$

$-3.9865600$

$-2.8733230$

1. 5113590

1. 9216500

2. 8748620

3.6371370

1.9774170

3.3685760

4.3980870

2.7498820

3.6030380

2.2240380

4. 2821150

5.3053860

4.2651780

3.8537010

4.6358120

3. 6798530

1. 0711960

1.3951870

2.6553300

3.7791910

1.9301360

1.6577020

3. 1808150

3.9758400

2. 7890970

2.0583700

2. 2773600

1.0995450

1.1387060

4.5420690

3. 2822190

3. 5132970

3. 1905320

4. 3910150

5. 1890190

4.8512380

1.4528690

1.1827460

$-0.5887640$

0.4706520

1.1364130 


-4.1930650
-3.5628300
-3.0879520
-2.7354880
-2.2090820
-3.1881360
-6.0208350
-5.0879310
-4.7626430
-5.6100930
-6.0256370
-6.2221530
-6.9931700
-5.5615150
-6.1634140
-3.1209150
-1.8798290
-3.7436960
-2.7258820
-1.8264370
-1.0300870
-3.2452370
-4.4962320
-4.2876000
-2.4445660
-1.1362150
-0.4726750
-0.2671430
-2.1071870
1.4242970
1.6337030
1.0756470
-0.4192600
-0.9020620
0.1567330
1.1180920
1.2278680
-1.0380690
-1.1714160
2.6970160
1.6597570
-0.6021650
-1.8184240
0.4368580
-0.3204900
0.7734130

5.1681790

4.1231670

3.4220520

4.1307640

3.0117480

1.7906840

3.8066420

2.8700190

3.6288210

2.0919400

3.4915070

4.5474120

2.9794330

1.1993630

1.6871430

$-1.3406110$

$-1.9536410$

$-1.2752640$

$-1.4370200$

$-2.6383460$

$-2.4178080$

$-1.5498340$

$-2.0723910$

$-0.3300930$

$-3.5415430$

$-2.8127920$

$-3.3204160$

$-1.6450590$

$-0.5321940$

$-4.5814450$

$-6.0456230$

$-6.3766910$

$-6.0077740$

$-5.0353850$

$-4.0495960$

$-6.6562980$

$-7.4375290$

$-6.9099770$

$-5.6234070$

$-6.2997850$

$-5.8111150$

$-5.5651740$

$-4.5302010$

3.3429460

2.2975280

3.7732050

0.8609830
2.1326360
0.1129980
-0.6457900
0.6124350
-1.3183130
1.9494210
-1.2809640
-2.0023000
-1.8484640
-0.2113530
-0.4310340
-0.1981310
2.5478240
3.3130200
-2.9197960
-2.6985250
-4.3017490
-5.4294290
-5.1502000
-3.8667710
-6.3871800
-4.3849540
-4.4105370
-5.0514070
-5.9828070
-3.6118410
-4.0438160
-5.5024930
-0.9869550
-1.2748610
-2.6679370
-2.7946400
-1.6946000
-1.2612640
-0.5196160
-2.8919030
-2.7455780
-0.8060150
-1.2144680
-3.4028540
-3.7799350
-2.0084760
-1.3695950
-1.3442180
-2.7499570

\begin{abstract}
1.7445340
0.1420650

2.0801970

2.2407560

0.4765770

$-0.6032260$

1.4476670

2.8395610

$-0.0177610$

1.7120710

$-0.5091030$

1. 0102320

2. 2786460

1.0516060

2. 3080210

0.1278370

1.7404180

1. 1932440

3.5038920

2. 3721850

1.0212930

3. 5976480

4.4591100

2. 3242680

0.7000150

3.9138800

4. 3374080

3. 3342200

2. 5680100

0.2275310
\end{abstract}

154

TS272(R,R) G[WB97XD/6-31G(d)] $=-3864.644925$

$\begin{array}{lrrr}\mathrm{P} & -0.2660390 & -0.1571130 & 1.0089950 \\ \mathrm{O} & -0.6897230 & 1.2594540 & 0.6969480 \\ \mathrm{O} & 0.4443980 & -0.4213080 & 2.3023730 \\ \mathrm{O} & 0.6124000 & -0.6148110 & -0.2925980 \\ \mathrm{O} & -1.5194160 & -1.1825060 & 0.9413970 \\ \mathrm{C} & 0.8107830 & -1.9730800 & -0.4798710 \\ \mathrm{C} & -0.2593720 & -2.7414810 & -0.9563190 \\ \mathrm{C} & 2.0672150 & -2.5089450 & -0.2109660 \\ \mathrm{C} & 2.2264520 & -3.8849900 & -0.3735760 \\ \mathrm{H} & 3.2000440 & -4.3268180 & -0.1739900 \\ \mathrm{C} & -2.1511310 & -1.2724440 & -0.2906980 \\ \mathrm{C} & -3.3498340 & -0.5914820 & -0.4838530 \\ \mathrm{C} & -1.5396310 & -2.0473610 & -1.2835650 \\ \mathrm{C} & -3.9377980 & -0.6883350 & -1.7464740\end{array}$

$\begin{array}{lr}4.7500220 & -2.9944260 \\ 3.1439290 & -3.8318260 \\ 5.0906400 & -4.2984480 \\ 5.2283610 & -2.1572980 \\ 3.4918720 & -5.1336910 \\ 2.3800830 & -3.6437390 \\ 4.4631860 & -5.3693380 \\ 5.8448070 & -4.4791820 \\ 3.0019860 & -5.9667340 \\ 4.7291430 & -6.3884600 \\ 1.8820210 & -0.4170200 \\ 4.1598480 & -0.0142590 \\ 2.0593640 & 2.1690460 \\ 2.8574230 & 2.3144410 \\ 1.3659150 & 1.3248660 \\ 2.4015710 & 1.9741860 \\ 1.4454230 & 3.3602120 \\ 0.6447520 & 3.0625660 \\ 3.7918350 & 3.5489150 \\ 4.7852660 & 3.2790810 \\ 4.0700400 & 3.1855270 \\ 2.7456510 & 2.4355060 \\ 4.3200580 & 3.6290740 \\ 5.5427090 & 4.0671160 \\ 3.7228170 & 4.1759680 \\ 3.2171380 & 1.4966250 \\ 1.9775290 & 2.6727730 \\ 3.2896220 & 4.5089940 \\ 5.3144330 & 2.3377680 \\ 4.7311820 & 2.8269000\end{array}$




$\begin{array}{rrrr}\mathrm{H} & -4.8827480 & -0.1792010 & -1.9213470 \\ \mathrm{C} & 3.2063330 & -1.6444330 & 0.2024510 \\ \mathrm{C} & -3.9968500 & 0.2057760 & 0.5948130 \\ \mathrm{C} & 3.7535850 & -0.7240320 & -0.7010370 \\ \mathrm{C} & 3.3132410 & -0.4915100 & -2.1256020 \\ \mathrm{C} & 5.3094820 & 0.9970820 & -1.4324550 \\ \mathrm{C} & 4.8488000 & 0.0689730 & -0.3378820 \\ \mathrm{C} & 2.9253710 & 0.9925960 & -2.2680030 \\ \mathrm{H} & 2.6293760 & 1.1957240 & -3.3034800 \\ \mathrm{H} & 2.0505130 & 1.1773010 & -1.6425950 \\ \mathrm{C} & 4.1183300 & 1.8884820 & -1.8492210 \\ \mathrm{H} & 4.4249050 & 2.5407920 & -2.6751920 \\ \mathrm{H} & 3.8347510 & 2.5439600 & -1.0184690 \\ \mathrm{H} & 6.1543290 & 1.6114160 & -1.1026590 \\ \mathrm{H} & 2.4696080 & -1.1292400 & -2.3999660 \\ \mathrm{C} & 5.7103840 & 0.1334680 & -2.6500630 \\ \mathrm{H} & 5.9948660 & 0.7926430 & -3.4784920 \\ \mathrm{H} & 6.5909900 & -0.4665440 & -2.3988170 \\ \mathrm{C} & 4.5181500 & -0.7753400 & -3.0499750 \\ \mathrm{H} & 4.2210690 & -0.5917560 & -4.0889160 \\ \mathrm{H} & 4.7921510 & -1.8327390 & -2.9755140 \\ \mathrm{C} & 3.7829000 & -1.7593640 & 1.4724560 \\ \mathrm{C} & 3.3656940 & -2.7038920 & 2.5738660 \\ \mathrm{C} & 5.4063620 & -1.2486640 & 3.2105000 \\ \mathrm{C} & 4.8936460 & -0.9779620 & 1.8203490 \\ \mathrm{C} & 4.5742780 & -3.6168410 & 2.8814640 \\ \mathrm{H} & 4.3021250 & -4.3136310 & 3.6823540 \\ \mathrm{H} & 4.8150870 & -4.2197200 & 1.9989500 \\ \mathrm{C} & 5.7891340 & -2.7443870 & 3.2975150 \\ \mathrm{H} & 6.0997300 & -2.9730090 & 4.3236530 \\ \mathrm{H} & 6.6493020 & -2.9410800 & 2.6495270 \\ \mathrm{H} & 6.2714590 & -0.6180450 & 3.4436740 \\ \mathrm{H} & 2.4950770 & -3.2984250 & 2.2877020 \\ \mathrm{C} & 4.2559400 & -0.9847860 & 4.2061850 \\ \mathrm{H} & 4.6087240 & -1.1838980 & 5.2249200 \\ \mathrm{H} & 3.9670820 & 0.0706930 & 4.1642580 \\ \mathrm{C} & 3.0506130 & -1.8896220 & 3.8472140 \\ \mathrm{H} & 2.8356870 & -2.5913650 & 4.6617430 \\ \mathrm{H} & 2.1454300 & -1.3042150 & 3.6800390 \\ \mathrm{C} & 5.4293030 & -0.0664580 & 0.9182000 \\ \mathrm{H} & 6.2959930 & 0.5340550 & 1.1913380 \\ \mathrm{C} & -5.1432280 & 0.3292100 & 2.7378030 \\ \mathrm{C} & -5.5682060 & -0.4898130 & 3.9292570 \\ \mathrm{C} & -4.3305400 & -1.8694870 & 2.1296730 \\ \mathrm{C} & -4.4609550 & -0.4105940 & 1.7639190 \\ \mathrm{C} & -6.4894830 & -1.6245830 & 3.4292650\end{array}$

-6.7674610
-7.4150100
-5.7494920
-5.6672790
-6.2969020
-3.8079960
-6.0891300
-3.5637910
-3.4897150
-2.5448300
-4.3060920
-4.6029720
-3.6563760
-4.9261290
-5.1279790
-3.7328860
-4.2130370
-3.7455490
-3.8827340
-3.2568900
-2.8877550
-2.5101510
-2.0247590
-3.1313330
-5.7275250
-4.9652670
-4.6233420
-5.5442670
-5.8350820
-5.9985200
-6.8206650
-5.3904660
-5.9409340
-3.3315950
-3.9245180
-3.9270990
-2.5247980
-1.5101310
-2.1262030
-3.3314760
-4.3114900
-2.1177650
-1.1438400
-4.9397110
-4.6295530
-2.6140420
4. 2766430

3. 0319860

2. 3392220

2. 6309220

1. 3913850

1. 3586790

4. 6685370

3.4673570

3. 7652340

3. 3201910

4. 5485810

5. 3933600

4. 9469290

1. 3992740

1. 0398290

$-0.7030960$

0.4346020

0.8897570

0.5323000

1.8674840

$-0.1006050$

$-0.9136230$

0.4085790

$-1.4195690$

1.7975380

$-1.3923070$

$-2.1637630$

$-1.9005110$

$-0.3338980$

$-0.6173410$

$-0.2504690$

2. 5518500

3. 3004830

$-2.7790690$

$-4.1619400$

$-4.6445250$

$-4.5560500$

$-3.7658750$

$-2.5559690$

$-4.8565770$

$-5.6683540$

$-5.5576640$

$-4.4230990$

$-4.1706140$

$-4.0165530$

$-4.0844130$ 


$\begin{array}{rrr}-0.6329310 & -3.3251570 & -3.5023520 \\ 1.1661670 & -4.6947080 & -0.7599820 \\ 1.2946520 & -6.1895340 & -0.8948450 \\ 0.7192520 & -6.6543380 & -2.2418630 \\ -0.7182050 & -6.1303850 & -2.4779100 \\ -1.1742960 & -5.1030970 & -1.4214040 \\ -0.0810590 & -4.1279090 & -1.0613200 \\ 0.7480670 & -6.6806000 & -0.0767710 \\ 0.7444320 & -7.7475270 & -2.2963650 \\ -1.4341580 & -6.9589610 & -2.4816370 \\ -1.4506000 & -5.6428780 & -0.5045990 \\ 2.3416030 & -6.4957140 & -0.7995630 \\ 1.3800080 & -6.2857640 & -3.0341530 \\ -0.7753790 & -5.6711270 & -3.4700650 \\ -2.0803080 & -4.5913540 & -1.7535540 \\ 0.6640560 & 3.3904700 & -1.5141240 \\ -0.1431910 & 2.3830830 & -1.4739710 \\ 1.0024180 & 3.8014580 & -2.8997630 \\ 2.0015950 & 4.7462350 & -3.1580980 \\ 0.3375040 & 3.1924080 & -3.9730650 \\ 2.3295910 & 5.0768260 & -4.4664960 \\ 2.5234460 & 5.2097080 & -2.3283440 \\ 0.6660120 & 3.5289620 & -5.2795560 \\ -0.4295980 & 2.4534500 & -3.7745460 \\ 1.6631870 & 4.4698580 & -5.5286530 \\ 3.1087360 & 5.8078660 & -4.6576420 \\ 0.1460700 & 3.0541560 & -6.1056280 \\ 1.9215460 & 4.7279200 & -6.5513320 \\ -0.3412090 & 1.9823990 & -0.5440390 \\ 1.2956300 & 4.1830640 & -0.1709060 \\ 2.4932070 & 2.0476830 & 1.9986510 \\ 1.3066490 & 2.9023040 & 2.1559840 \\ 2.4738750 & 1.3425700 & 1.1638230 \\ 0.3567290 & 2.4849790 & 1.8382720 \\ 1.9532980 & 1.4745890 & 3.2081290 \\ 1.3563490 & 0.7015850 & 2.9304910 \\ 3.8216220 & 3.7344150 & 3.3367490 \\ 2.7292320 & 4.7753980 & 3.0839940 \\ 1.3454970 & 4.1220390 & 3.0175950 \\ 3.8466960 & 2.6773890 & 2.2294570 \\ 4.8000180 & 4.2215700 & 3.3940590 \\ 2.7297910 & 5.5362480 & 3.8701740 \\ 1.0249500 & 3.7972530 & 4.0157070 \\ 4.1628820 & 3.1276320 & 2.4581300 \\ 4.5571940 & 1.8792010 & \\ 3.6500730 & 3.2454230 & \\ -1.3033020\end{array}$

$\begin{array}{llll}\mathrm{H} & 2.9311090 & 5.2933650 & 2.1377690 \\ \mathrm{H} & 0.5775390 & 4.8166990 & 2.6664820\end{array}$

154

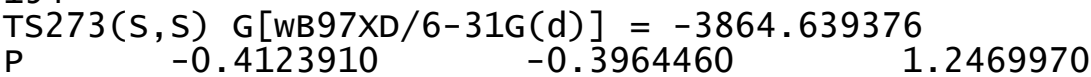

P $\quad-0.4123910 \quad-0.3964460 \quad-1.2469970$

O $-0.9523220 \quad 0.9952250 \quad 1.0114940$

$\begin{array}{llll}0 & -1.5091400 & -1.5340320 & 0.8899480\end{array}$

$0 \quad 0.6881180$

$-1.9828770$

$-0.5801140$

0.0428970

C $\quad-1.1702800$

$-3.2032420$

$-3.5996060$

$-4.5503520$

1.0583330

2. 3638700

0.1303480

2.7006490

3.7163570

$-4.0524400$

3. 3829550

$-4.5911700$

$-1.4809200$

$-0.4159370$

$-2.0234790$

$-0.8634150$

$-0.7856940$

$-1.4161860$

$-0.3122240$

$-0.6752080$

$-2.0129650$

$-1.8564450$

$-2.2463490$

$-0.3422080$

$-2.2758110$

$-2.6418120$

$-0.1014470$

$-1.0350380$

$-0.4638150$

$-3.9259850$

$-0.2876350$

0.3996470

0.3852740

$-1.3065590$

$-1.0847030$

1.4113300

$-4.3696080$

$-2.5655020$

1.6060870

$-5.9475850$

$-5.4552590$

$-3.7717130$

$-1.5457670$

$-0.5406410$

$-2.7785040$

3.3788490

2. 3701890

3.0141570

$-3.6380990$

$-2.7812340$

$-4.7182900$

$-5.0563070$

$-3.8532630$

3.1840920

3.0562520

4.0791340

$-4.2010030$

$-2.1654200$

$-2.9278520$

4.7908620

$-6.6128140$

$-1.3925640$

4.6565440

4.1122390

0.8482870

2.6191830

$-6.6878680$

$-7.0069750$

$-2.9830890$

$-2.6688660$

$-3.4359020$

3.3344450

$-7.5935540$

$-5.7431280$

$-5.5991360$

$-2.2624400$

2. 1574520

1. 5460710

1.7115750

$-6.1694020$

$-4.3442440$

$-3.1536980$

0.5441980

$-4.3804210$

1. 0841040

2.1333940

3.0800020

1.6121050

0.3853950

$-5.5506970$

2.7312020

3. 5279290

$-0.5758830$

1. 1451800

1. 3341600

$-5.0883720$

$-1.3177540$

$-1.9868420$

$-5.5530180$

1.9645210

$-1.9474720$ 


-6.1063640
-6.2915820
-7.0696840
-6.2696570
-3.1519840
-4.2193430
-4.4021640
-3.8323690
-3.2076450
-2.8549720
-2.3334970
-5.8035890
-6.4905550
4.8054110
5.2145080
3.5045890
3.8618580
5.8341540
6.1122580
6.7536740
4.8067910
4.5704040
5.2120590
2.7836820
5.9353250
2.9237830
2.6656740
1.9940470
3.9560800
4.2515030
3.5175460
4.8731890
5.3538240
3.5941740
3.9033800
4.1297440
4.4619160
3.6701040
3.1102490
3.0044230
2.1192100
2.8426840
6.0985590
4.9227250
4.7371530
5.2965840

\begin{tabular}{rr}
3.2816330 & -0.2830770 \\
4.3492590 & -0.4492340 \\
2.7696740 & -0.3748610 \\
3.4412270 & 1.8884460 \\
1.7223080 & -1.2859950 \\
3.8515870 & 1.2559220 \\
4.9157950 & 1.0651610 \\
3.7687550 & 2.2767620 \\
3.2666810 & 0.2392820 \\
4.0398360 & -0.4538830 \\
2.8677350 & 0.7559910 \\
0.8037220 & 2.3279460 \\
1.2177880 & 3.0644360 \\
0.6077480 & -0.0998230 \\
1.5561300 & -1.1990690 \\
-0.2208860 & -1.9717930 \\
-0.3397530 & -0.5092980 \\
0.7075770 & -2.3332320 \\
1.3660470 & -3.1643110 \\
0.2347120 & -1.9740910 \\
-0.3603660 & -2.7925440 \\
-0.2398620 & -3.8560140 \\
-1.3693830 & -2.6651270 \\
-0.9825060 & -2.2781780 \\
2.2976870 & -0.8378950 \\
1.1845310 & -2.2098520 \\
1.3050260 & -3.2682480 \\
1.2700440 & -1.6433730 \\
2.2523050 & -1.7682980 \\
2.8803300 & -2.6168370 \\
2.9242150 & -1.0233230 \\
-0.4063290 & 2.0762920 \\
-0.6120490 & 3.4891740 \\
-2.3584000 & 2.7478910 \\
-1.3390240 & 1.6798850 \\
-0.5432840 & 4.4286430 \\
-0.6801980 & 5.4642730 \\
0.4482680 & 4.3646430 \\
-1.6382060 & 4.0244000 \\
-2.3827980 & 4.8220870 \\
-1.2184550 & 3.8468410 \\
-3.0767880 & 2.4109030 \\
0.1407830 & 3.7692670 \\
-3.0745260 & 3.0828150 \\
-3.8402080 & 3.8446400 \\
-35897890 & 2.1915500 \\
-1 & \\
\hline &
\end{tabular}

\begin{abstract}
5.9569610
6.2273770

6.8801260

5.3186280

6.0695610

1.7694220

2.0910090

1.6865080

0.2148880

$-0.4471560$

0.4860280

1. 5432090

1.8578630

$-0.3838440$

$-0.7256210$

3.1565320

2. 3526740

0.1712150

$-1.3790060$

$-1.5668310$

$-0.7055030$

$-0.6029680$

$-1.9888300$

$-3.1453300$

$-2.7931190$

$-1.1469360$

$-0.0242000$

$-2.1606580$

$-3.3406770$

0.2932870

$-0.0282170$

$-2.0204400$

$-4.0661450$

0.2321980

$-0.4764750$

0.4714480

1. 3870600

$-0.1869640$

1. 6444210

1. 9054420

0.0716570

$-0.8908430$

0.9898810

2. 3624640

$-0.4400540$

1.1961120
\end{abstract}

3.5893970
4.6333840
3.0032130
1.1940250
1.5083660
-1.0495390
-1.4389290
-2.9016470
-3.1796680
-2.0019550
-1.3611520
-0.7874430
-3.1579430
-3.4040530
-1.2337400
-1.2960020
-3.5385760
-4.0705730
-2.3251550
-2.7496400
-3.9365530
-4.8685890
-5.2739210
-4.4965250
-3.0408840
-4.4861750
-5.7577710
-6.3466330
-4.9302870
-3.6241010
-4.3435470
-5.0898170
-4.6151630
-1.2901010
-1.1752270
-2.7070470
-3.0612740
-3.7137280
-4.4004080
-2.2819560
-5.0499680
-3.4364590
-5.3959530
-4.6677330
-5.8234550
-6.4418120

$-2.0330550$

$-2.2296030$

$-2.0839670$

0.5715790

$-4.4286210$

$-5.8472430$

$-6.0947700$

$-5.6998580$

$-4.9541880$

$-3.9572000$

$-6.5429590$

$-7.1452760$

$-6.5888390$

$-5.6891140$

$-6.0543980$

.

$-4.4850760$

$-1.8694930$

$-0.9978140$

$-0.4371910$

$-1.0990220$

(1)

$-1.2686270$

$-0.5744100$

$-2.0894890$

$-0.2261960$

0.6426560

$-0.5193150$

0409100

3.4815320

4.4766990

2.7608600

.7436030

5.0246390

3.0316000

4.2285150 


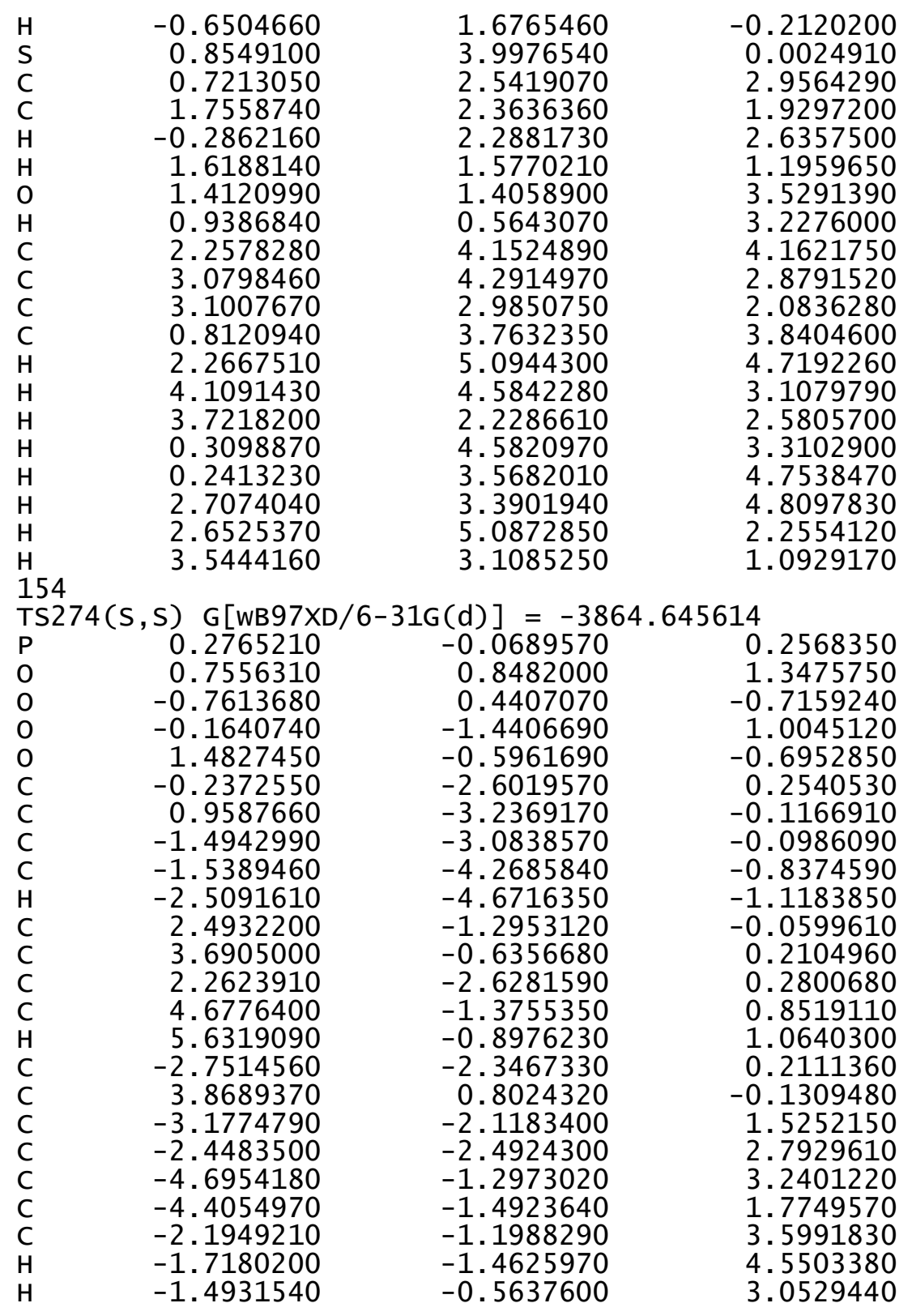

-3.5405280
-3.7210250
-3.5255080
-5.6547780
-1.5029720
-4.7062340
-4.8506960
-5.5564040
-3.3686470
-2.8561480
-3.5453450
-3.5646390
-3.2512760
-5.5666500
-4.8034200
-4.3504180
-4.1738150
-4.2841310
-5.7447900
-6.2957960
-6.3499380
-6.5400100
-2.2660290
-4.7179330
-5.2229420
-4.6594530
-3.3083680
-3.0738680
-2.5333280
-5.2330630
-6.1976300
3.9454170
3.8780220
3.5830070
3.7798580
5.0017100
4.9602260
5.9749500
4.8196660
4.6788480
5.7072270
3.4643050
4.0042610
2.3411360
2.2144440
1.4508790
3.8443480

4. 9160600

3. 3847850

3. 3889860

2. 5868720

3. 9237420

5.0023240

3. 5549120

3. 6291400

4. 5616330

3.0853740

$-0.8401940$

$-2.3172640$

$-1.8211130$

$-0.5793800$

$-3.0189760$

$-4.1007720$

$-2.7058960$

$-2.6773170$

$-3.5910880$

$-2.1249720$

$-1.5696550$

$-2.5076930$

$-2.6306520$

$-3.5815770$

$-2.0924070$

$-2.8694340$

$-3.9401150$

$-2.3812150$

0.7270510

0.9255180

$-1.7692390$

$-3.2470770$

$-2.6853620$

$-1.4576780$

$-3.9306060$

$-5.0131710$

$-3.5844870$

$-3.5950200$

$-4.5099470$

$-3.0919430$

$-2.4326900$

$-3.4454490$

$-3.4490100$

$-4.3577910$

$-2.8347490$ 


\begin{tabular}{|c|c|c|}
\hline $\begin{array}{l}0 \\
0 \\
0 \\
0 \\
0 \\
0 \\
0 \\
0 \\
0 \\
0 \\
0 \\
0 \\
0 \\
0 \\
0 \\
0 \\
0 \\
0 \\
0 \\
0 \\
0 \\
0 \\
0 \\
0 \\
0 \\
0 \\
0 \\
0\end{array}$ & $\begin{array}{r}2.3912410 \\
2.5446480 \\
2.9889590 \\
3.0963000 \\
3.9576040 \\
1.4822310 \\
1.7459640 \\
3.8676450 \\
4.4377960 \\
4.3255650 \\
2.3802200 \\
2.2048670 \\
2.0808760 \\
0.4351120 \\
4.9977030 \\
1.8905520 \\
1.7470540 \\
1.2343080 \\
3.3728280 \\
3.9679830 \\
3.4542640 \\
3.5287330 \\
4.5779650 \\
-3.3231250 \\
-4.7159170 \\
-5.1073990 \\
-4.8576830 \\
-3.3630180 \\
-2.6887890 \\
-6.1604570 \\
-5.4519280 \\
-4.7841170 \\
-5.4076120 \\
-5.2295750 \\
-3.1889720 \\
-2.8669990 \\
-4.5180140 \\
-4.3766510 \\
-5.0363420 \\
-5.1598780 \\
-5.8828990 \\
-6.1199490 \\
-4.9030610 \\
-6.0405120 \\
-5.6796530 \\
-6.8464320\end{array}$ & $\begin{array}{r}-3.8026210 \\
-4.8879710 \\
-3.3883620 \\
0.5466420 \\
1.7298430 \\
2.3517700 \\
0.8717780 \\
2.7807980 \\
3.6695060 \\
2.3821560 \\
3.1332240 \\
4.2055810 \\
2.8960850 \\
2.5991970 \\
1.4262640 \\
2.7476750 \\
3.8268400 \\
2.2495970 \\
2.3551210 \\
3.2331270 \\
1.6369560 \\
-0.7697590 \\
-1.0177220 \\
1.0082490 \\
1.5402430 \\
2.6911860 \\
2.2902690 \\
2.0718070 \\
1.2756900 \\
2.9456950 \\
0.7372700 \\
1.8466700 \\
1.3642260 \\
3.0527170 \\
1.5763740 \\
3.0501140 \\
3.5859010 \\
-0.9274150 \\
-1.5958670 \\
-3.1068020 \\
-3.4166380 \\
-2.1494090 \\
-1.2631950 \\
-1.1778590 \\
-3.5808690 \\
-3.9023740\end{array}$ \\
\hline
\end{tabular}
0.0179640
2. 9758100
1. 7641810
$-0.1353010$
$-1.4223910$
$-3.3355630$
$-2.8676910$
$-4.6675630$
$-5.2579000$
$-5.3486690$
$-6.5072440$
$-4.7365270$
$-6.6001710$
$-4.8916270$
$-7.1812170$
$-6.9556110$
$-7.1228880$
$-8.1591390$
$-1.9965510$
$-2.5258530$
$-0.3015680$
$-0.0510240$
$-0.9096960$
$-0.0274060$
1.0977620
1.1370190
0.2708020
0.0751270
0.4849910
$-0.5664510$
$-0.0144180$
0.6585330
1. 5773130
$-1.6354990$
$-0.3669230$
1. 3303270
$-0.9797530$
0.1787470

154

$\operatorname{TS} 275(\mathrm{~S}, \mathrm{~S}) \mathrm{G}[\mathrm{WB} 97 \mathrm{XD} / 6-31 \mathrm{G}(\mathrm{d})]=-3864.647566$

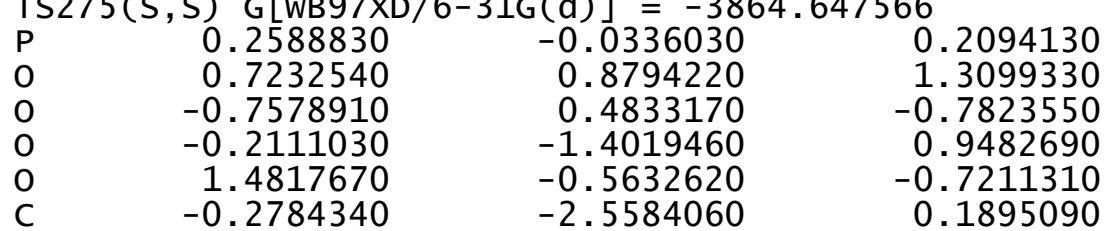

$-1.5993310$

$-4.4705720$

$-4.1485440$

$-5.2840190$

. 3891860

2. 6007010

1.5380330

2. 9983060

. 2429070

.1072800

2. 5927150

1. 3783010

4.4496970

4. 6932890

3. 6945680

2. 0019430

5. 3084840

3. 9657100

1.1464830

3.4636330

3. 6453660

3.4725530

2.8726910

.4569330

3. 3094980

. 3123920

6.0918540

5.9831470

4.6031170

5.0395000

.0900740

6.7488000

4.4870510

5.2431920

5.0512780

5.9538650

6.1606420
4.4572280
$-1.4277930$

$-3.5265780$

$-0.4325410$

0.1374050

0.0810080

1.1034710

$-0.4348560$

1. 5981690

1.4945180

0.0588230

$-1.2247270$

1.0761060

2. 3911330

$-0.3499570$

1. 4626250

$-0.2560170$

$-1.6309780$

0.7284610

$-0.7077660$

1. 1979920

$-1.0871100$

0.7626180

0.9613630

0.5130150

$-1.0009050$

$-1.5242680$

1. 2450210

0.8592790

$-1.5213530$

$-1.5078250$

1. 1061400

2. 3214500

0.7608530

$-1.2434800$
$-2.5637820$

$-6.8464320$

- 3.9023740 


$\begin{array}{rrr}0.9136020 & -3.1929630 & -0.1680830 \\ -1.5299380 & -3.0349830 & -0.1941180 \\ -1.5486450 & -4.2022820 & -0.9521830 \\ -2.5127280 & -4.6070620 & -1.2526770 \\ 2.4719470 & -1.2739950 & -0.0663950 \\ 3.6733050 & -0.6326810 & 0.2284550 \\ 2.2088070 & -2.5983720 & 0.2799910 \\ 4.6338680 & -1.3891390 & 0.8918660 \\ 5.5949760 & -0.9302410 & 1.1142130 \\ -2.7952250 & -2.3143920 & 0.1187080 \\ 3.8823180 & 0.8013640 & -0.1124710 \\ -3.2339400 & -2.1273020 & 1.4353800 \\ -2.5116960 & -2.5321110 & 2.6974720 \\ -4.7742030 & -1.3728140 & 3.1610970 \\ -4.4686840 & -1.5184350 & 1.6931960 \\ -2.2775650 & -1.2600400 & 3.5428880 \\ -1.7998190 & -1.5455460 & 4.4872960 \\ -1.5831990 & -0.6002090 & 3.0170870 \\ -3.6332080 & -0.5531300 & 3.8048990 \\ -3.8234000 & -0.4519300 & 4.8798930 \\ -3.6266720 & 0.4583360 & 3.3846010 \\ -5.7395740 & -0.8776610 & 3.3166830 \\ -1.5596280 & -3.0224350 & 2.4845280 \\ -4.7788840 & -2.7796630 & 3.7983420 \\ -4.9395840 & -2.6836190 & 4.8785690 \\ -5.6173060 & -3.3587170 & 3.3982030 \\ -3.4293170 & -3.4823370 & 3.4989850 \\ -2.9230630 & -3.7637150 & 4.4295800 \\ -3.5886360 & -4.4035510 & 2.9292240 \\ -3.6023920 & -1.8416840 & -0.9262580 \\ -3.2785450 & -1.8567730 & -2.4018940 \\ -5.5999860 & -0.8407640 & -1.8941450 \\ -4.8466490 & -1.2574900 & -0.6581660 \\ -4.3706110 & -2.6695020 & -3.1323730 \\ -4.1835820 & -2.6237260 & -4.2115940 \\ -4.3070670 & -3.7238260 & -2.8418630 \\ -5.7682860 & -2.0875570 & -2.7916310 \\ -6.3063040 & -1.8064670 & -3.7044360 \\ -6.3825320 & -2.8294290 & -2.2708600 \\ -6.5764510 & -0.4159950 & -1.6381510 \\ -2.2909410 & -2.2795220 & -2.5966480 \\ -4.7452820 & 0.1900170 & -2.6619410 \\ -5.2457560 & 0.4356840 & -3.6060090 \\ -4.6862210 & 1.1180240 & -2.0860720 \\ -3.3362810 & -0.4008650 & -2.9178940 \\ -3.1041590 & -0.3959630 & -3.9892920\end{array}$

$\begin{array}{ll}\mathrm{H} & -2.5593860 \\ \mathrm{C} & -5.2893110 \\ \mathrm{H} & -6.2584090 \\ \mathrm{C} & 4.0214620 \\ \mathrm{C} & 3.9942470 \\ \mathrm{C} & 3.6393150 \\ \mathrm{C} & 3.8276940 \\ \mathrm{C} & 5.1192360 \\ \mathrm{H} & 5.1124400 \\ \mathrm{H} & 6.0895590 \\ \mathrm{C} & 4.8961820 \\ \mathrm{H} & 4.7575940 \\ \mathrm{H} & 5.7653500 \\ \mathrm{H} & 3.4955500 \\ \mathrm{H} & 4.1449980 \\ \mathrm{C} & 2.4218610 \\ \mathrm{H} & 2.2943830 \\ \mathrm{H} & 1.5193300 \\ \mathrm{C} & 2.6488830 \\ \mathrm{H} & 2.6673320 \\ \mathrm{H} & 1.8338090 \\ \mathrm{C} & 4.3583610 \\ \mathrm{C} & 4.7050660 \\ \mathrm{C} & 4.2660300 \\ \mathrm{C} & 4.1444130 \\ \mathrm{C} & 3.5545430 \\ \mathrm{H} & 3.8377910 \\ \mathrm{H} & 2.6601700 \\ \mathrm{C} & 3.2781880 \\ \mathrm{H} & 3.3997860 \\ \mathrm{H} & 2.2555640 \\ \mathrm{H} & 4.0636360 \\ \mathrm{H} & 4.8867210 \\ \mathrm{C} & 5.6995300 \\ \mathrm{H} & 5.8035600 \\ \mathrm{H} & 6.4227740 \\ \mathrm{C} & 5.9712850 \\ \mathrm{H} & 6.2370120 \\ \mathrm{H} & 6.8101060 \\ \mathrm{C} & 4.2880650 \\ \mathrm{H} & 4.4635360 \\ \mathrm{C} & 4.3894070 \\ \mathrm{C} & 5.1543980 \\ \mathrm{C} & 4.9912080 \\ \mathrm{C} & \\ \mathrm{C} & \end{array}$

0.1831050
-1.1087690
-0.6557900
2.5945330
2.8822940
0.3865690
1.2428720
2.0475900
2.2168270
2.3892580
0.5478090
-0.0334590
0.1289920
-0.6660320
3.9492750
0.9149330
0.3112660
0.7852990
2.4043530
2.5468050
3.0238570
3.0903040
3.9500960
1.4818770
1.7436010
3.8780350
4.4478660
4.3453040
2.3950420
2.2214700
2.1083050
0.4378770
4.9871410
1.8721430
1.7290330
1.2053200
3.3502490
3.9436280
3.4194890
3.5206440
4.5669200
-2.6928850
-3.2985710
-3.4268610
-4.6104460
-5.4605850

-2.4180220
0.6494530
0.8529450
-1.7533710
-3.2331220
-2.6717100
-1.4420100
-3.8865360
-4.9696230
-3.5120510
-3.5583930
-4.4773460
-3.0412380
-2.4198930
-3.4311000
-3.4589970
-4.3648410
-2.8568160
-3.8211370
-4.9080070
-3.4370140
0.5687050
1.7571530
2.3767720
0.8931660
2.7831050
3.6762910
2.3626500
3.1349960
4.2106460
2.8779990
2.6227250
1.4544570
2.8015060
3.8833130
2.3195150
2.4117760
3.2945110
1.7116190
-0.7505760
-0.9977000
1.3260070
1.0525930
2.0661290
2.8920170
2.0618820 


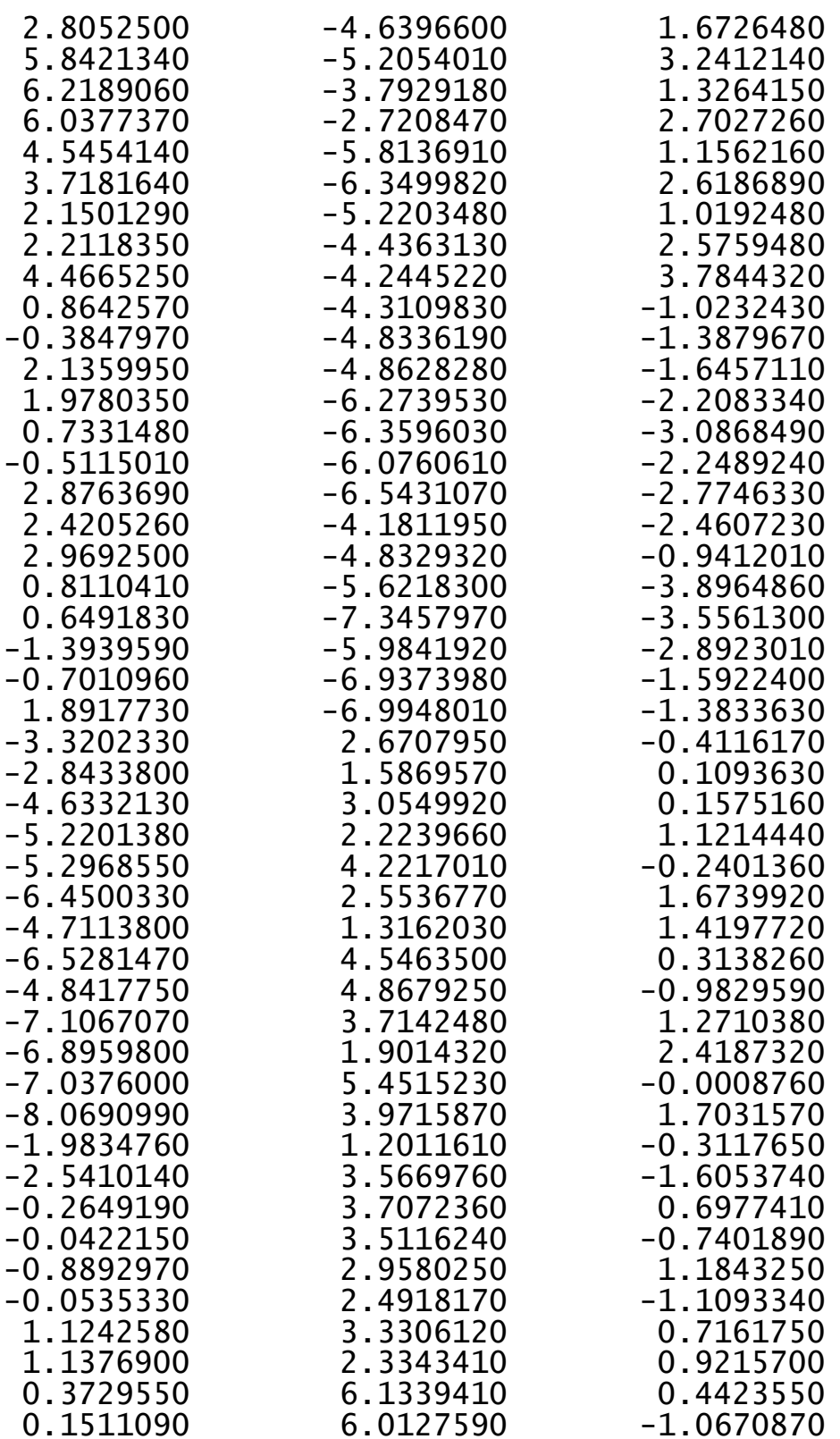

$\begin{array}{lr}\mathrm{C} & 0.5156770 \\ \mathrm{C} & -0.4819290 \\ \mathrm{H} & 0.1215010 \\ \mathrm{H} & 0.7473820 \\ \mathrm{H} & 1.6045940 \\ \mathrm{H} & -1.5469410 \\ \mathrm{H} & -0.2656790 \\ \mathrm{H} & 1.4320970 \\ \mathrm{H} & -0.9022080 \\ \mathrm{H} & 0.1950100\end{array}$

4.6154310

-1.2006930
0.7802080

$7.1440460 \quad 0.7802080$

$6.7554280 \quad-1.6058520$

$4.4701910 \quad-1.5696250$

$5.3462500 \quad 1.0750360$

$5.1346320 \quad 2.2737820$

$5.9693460 \quad 0.6752990$

$6.2157810 \quad-1.2964060$

154

$4.4633810 \quad-2.6120670$

TS276(S, S) G[WB97XD/6-31G(d) $]=-3864.643729$

$\begin{array}{lrrr}\text { TS276(S, S) G[WB97XD/6-31G(d)] }=-3864.643729 \\ \text { P } & 0.2664500 & -0.0560020 & 0.2164650 \\ \text { O } & 0.7444270 & 0.8508530 & 1.3162720 \\ \text { O } & -0.7559750 & 0.4693240 & -0.7648520 \\ \text { O } & -0.2033940 & -1.4253850 & 0.9528910 \\ \text { O } & 1.4792560 & -0.5889930 & -0.7261970 \\ \mathrm{C} & -0.2946460 & -2.5707910 & 0.1797460 \\ \mathrm{C} & 0.8859010 & -3.2153320 & -0.1998150 \\ \mathrm{C} & -1.5569540 & -3.0220700 & -0.1998110 \\ \mathrm{C} & -1.5977110 & -4.1675790 & -0.9888990 \\ \mathrm{H} & -2.5694230 & -4.5504900 & -1.2943920 \\ \mathrm{C} & 2.4629910 & -1.3155500 & -0.0785990 \\ \mathrm{C} & 3.6674970 & -0.6856580 & 0.2283850 \\ \mathrm{C} & 2.1889730 & -2.6419300 & 0.2528130 \\ \mathrm{C} & 4.6103850 & -1.4489220 & 0.9081810 \\ \mathrm{H} & 5.5694210 & -0.9960590 & 1.1516230 \\ \mathrm{C} & -2.8076250 & -2.2880980 & 0.1396920 \\ \mathrm{C} & 3.8939610 & 0.7461940 & -0.1105730 \\ \mathrm{C} & -3.2170670 & -2.0952550 & 1.4652100 \\ \mathrm{C} & -2.4829800 & -2.5230690 & 2.7130340 \\ \mathrm{C} & -4.7079480 & -1.3119910 & 3.2216480 \\ \mathrm{C} & -4.4339820 & -1.4620180 & 1.7481060 \\ \mathrm{C} & -2.2037940 & -1.2637180 & 3.5633260 \\ \mathrm{H} & -1.7249950 & -1.5697070 & 4.5008170 \\ \mathrm{H} & -1.4962370 & -0.6200910 & 3.0352890 \\ \mathrm{C} & -3.5359650 & -0.5205240 & 3.8442690 \\ \mathrm{H} & -3.7063130 & -0.4098020 & 4.9216190 \\ \mathrm{H} & -3.5093760 & 0.4884290 & 3.4185040 \\ \mathrm{H} & -5.6584220 & -0.7947850 & 3.3959010 \\ \mathrm{H} & -1.5469160 & -3.0352680 & 2.4812110 \\ \mathrm{C} & -4.7338120 & -2.7195250 & 3.8569930 \\ \mathrm{H} & -4.8657280 & -2.6213540 & 4.9409290 \\ \mathrm{H} & -5.5964770 & -3.2758840 & 3.4767120 \\ \mathrm{C} & -3.4105750 & -3.4562670 & 3.5234680 \\ \mathrm{H} & -2.8941930 & -3.7618730 & 4.4407570 \\ & & & \end{array}$




$\begin{array}{rrrr}\mathrm{H} & -3.6069310 & -4.3663540 & -2.9472840 \\ \mathrm{C} & -3.6288640 & -1.8019960 & -0.8887470 \\ \mathrm{C} & -3.3379560 & -1.8212830 & -2.3715640 \\ \mathrm{C} & -5.6348340 & -0.7775320 & -1.8150490 \\ \mathrm{C} & -4.8575500 & -1.1973400 & -0.5948270 \\ \mathrm{C} & -4.4551470 & -2.6204680 & -3.0789390 \\ \mathrm{H} & -4.2945530 & -2.5688700 & -4.1621780 \\ \mathrm{H} & -4.3937870 & -3.6773060 & -2.7971890 \\ \mathrm{C} & -5.8385470 & -2.0286120 & -2.6992110 \\ \mathrm{H} & -6.4028200 & -1.7504160 & -3.5969750 \\ \mathrm{H} & -6.4403390 & -2.7634290 & -2.1544860 \\ \mathrm{H} & -6.6001120 & -0.3406600 & -1.5378610 \\ \mathrm{H} & -2.3597850 & -2.2547420 & -2.5885560 \\ \mathrm{C} & -4.7890110 & 0.2379600 & -2.6119390 \\ \mathrm{H} & -5.3057030 & 0.4732900 & -3.5499610 \\ \mathrm{H} & -4.7142160 & 1.1738870 & -2.0512690 \\ \mathrm{C} & -3.3889920 & -0.3647700 & -2.8862060 \\ \mathrm{H} & -3.1700210 & -0.3605700 & -3.9603260 \\ \mathrm{H} & -2.6006330 & 0.2116760 & -2.3955250 \\ \mathrm{C} & -5.2684630 & -1.0375030 & 0.7217400 \\ \mathrm{H} & -6.2250680 & -0.5677220 & 0.9451620 \\ \mathrm{C} & 4.0602580 & 2.5376930 & -1.7510140 \\ \mathrm{C} & 4.0444230 & 2.8255610 & -3.2309480 \\ \mathrm{C} & 3.6581880 & 0.3341130 & -2.6710870 \\ \mathrm{C} & 3.8503690 & 1.1882690 & -1.4404290 \\ \mathrm{C} & 5.1639080 & 1.9781870 & -3.8773990 \\ \mathrm{H} & 5.1667230 & 2.1482590 & -4.9603730 \\ \mathrm{H} & 6.1355720 & 2.3079720 & -3.4958620 \\ \mathrm{C} & 4.9209080 & 0.4808820 & -3.5519650 \\ \mathrm{H} & 4.7791490 & -0.0976130 & -4.4721760 \\ \mathrm{H} & 5.7826180 & 0.0508660 & -3.0315420 \\ \mathrm{H} & 3.5012870 & -0.7168800 & -2.4202380 \\ \mathrm{H} & 4.2080050 & 3.8907820 & -3.4281720 \\ \mathrm{C} & 2.4504900 & 0.8764500 & -3.4638040 \\ \mathrm{H} & 2.3186020 & 0.2730280 & -4.3691550 \\ \mathrm{H} & 1.5442440 & 0.7589980 & -2.8646380 \\ \mathrm{C} & 2.6975340 & 2.3623640 & -3.8272170 \\ \mathrm{H} & 2.7255840 & 2.5022920 & -4.9142120 \\ \mathrm{H} & 1.8872010 & 2.9923990 & -3.4503440 \\ \mathrm{C} & 4.3920150 & 3.0299680 & 0.5725540 \\ \mathrm{C} & 4.7443280 & 3.8863020 & 1.7619230 \\ \mathrm{C} & 4.2746460 & 1.4237090 & 2.3808150 \\ \mathrm{C} & 4.1614350 & 1.6857800 & 0.8964270 \\ \mathrm{C} & 3.5894030 & 3.8283060 & 2.7837090 \\ \mathrm{H} & 3.8753950 & 4.3964030 & 3.6771150 \\ \mathrm{H} & 2.7015870 & 4.3043570 & 2.3592570 \\ & & & \end{array}$

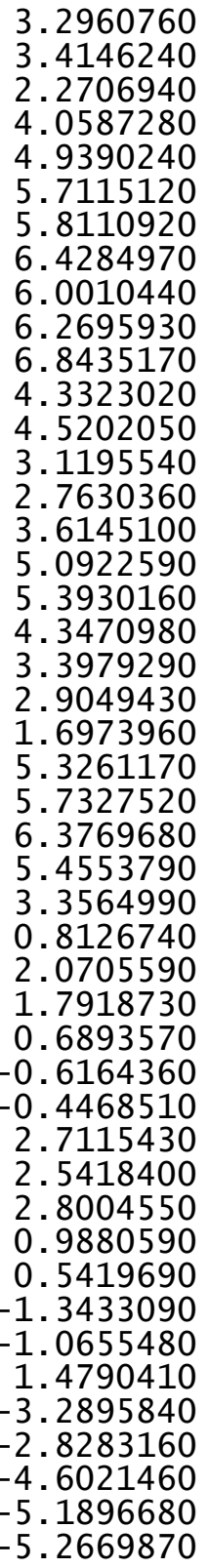

2. 3488380

2. 1749110

2. 0731510

0.3824760

4.9209980

1.7970550

1.6521740

1. 1224170

3. 2721810

3.8623170

3. 3323810

3. 4609140

4.5052050

$-3.3523410$

$-4.7101730$

$-5.0773070$

$-4.9072930$

$-3.4356250$

$-2.7486180$

$-6.1081240$

$-5.4905470$

$-4.7248910$

$-5.5120020$

$-5.2689990$

$-3.3275400$

$-2.8985840$

-4. 4312870

$-4.3069350$

$-4.8832350$

$-5.6166900$

$-6.6493220$

$-5.9418920$

$-4.7959220$

$-6.0936480$

$-5.5960990$

$-4.0812410$

$-7.3249230$

$-7.2676490$

$-6.6587750$

$-5.5371500$

$-4.9010010$

2. 6782850

1. 5980990

3. 0882780

2. 3077030

4. 2311720
3.1365580

4.2125000

2.8788860

2.6270280

1.4592800

2.8095350

3. 8915290

2. 3290720

2. 4214910

3. 3055440

1. 7248980

$-0.7470340$

$-0.9934040$

1.0356380

1. 6100110

2. 8241590

2. 4811810

2. 2026900

1. 3435160

3. 1243520

0.8529680

1.8625890

1. 5944280

3. 2926290

1.7306650

3.1593850

3. 6738570

$-1.0874750$

$-1.7100230$

$-3.0207970$

$-2.8018800$

$-2.4428320$

$-1.4616530$

$-3.3762940$

$-1.0227200$

$-1.8622010$

$-1.9891870$

$-3.6937180$

$-2.0421240$

$-3.3603860$

$-3.7926290$

$-0.4151760$

0.1263890

0.1371520

1.1422360

$-0.3225970$ 


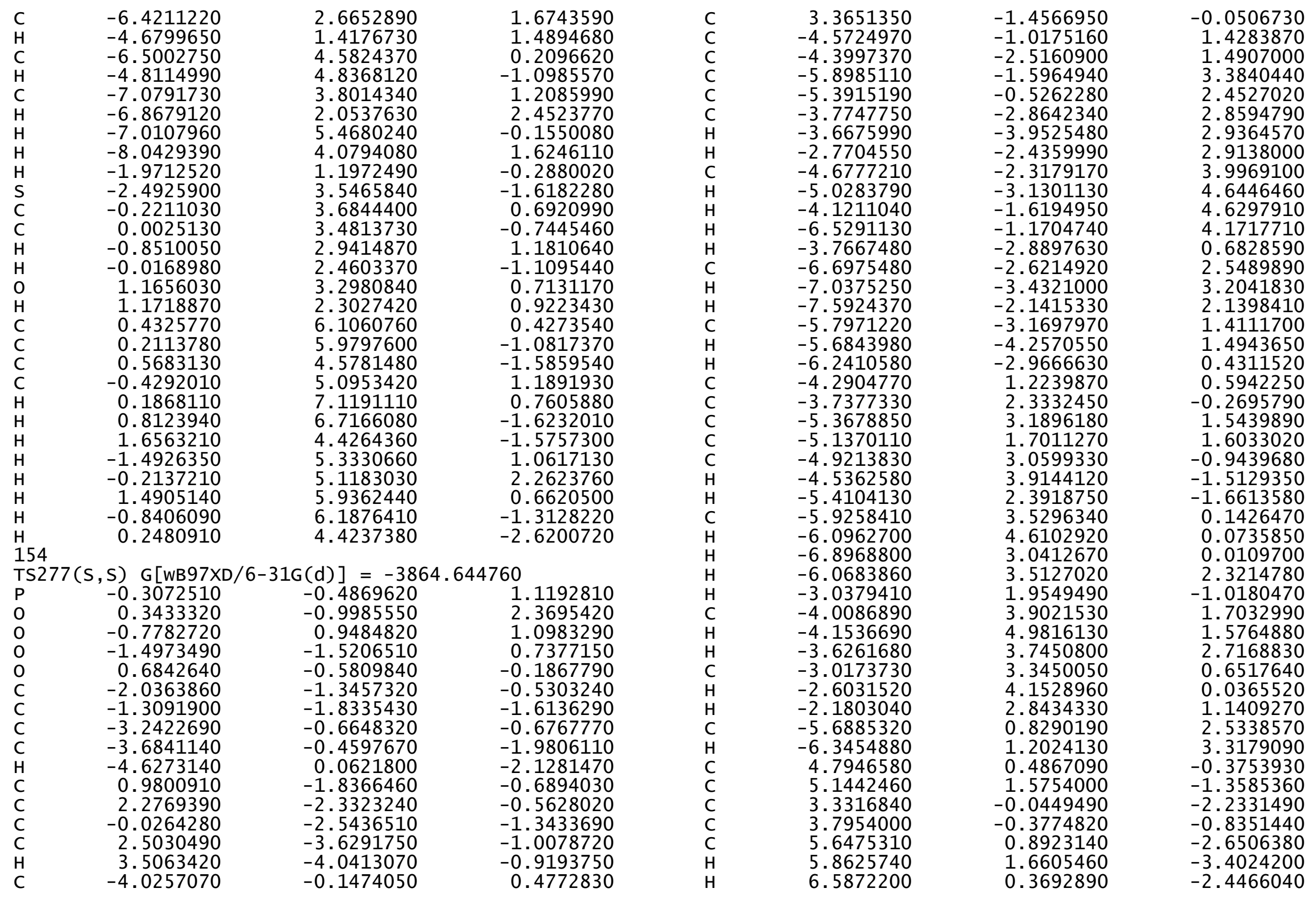




$\begin{array}{rr}\mathrm{C} & 4.5657490 \\ \mathrm{H} & 4.2537580 \\ \mathrm{H} & 4.9565170 \\ \mathrm{H} & 2.5687180 \\ \mathrm{H} & 5.9113500 \\ \mathrm{C} & 2.7779450 \\ \mathrm{H} & 2.4647180 \\ \mathrm{H} & 1.8837520 \\ \mathrm{C} & 3.8642890 \\ \mathrm{H} & 4.1079880 \\ \mathrm{H} & 3.5018790 \\ \mathrm{C} & 5.0007730 \\ \mathrm{C} & 5.5800360 \\ \mathrm{C} & 3.7232530 \\ \mathrm{C} & 3.9841580 \\ \mathrm{C} & 4.4354110 \\ \mathrm{H} & 4.8428550 \\ \mathrm{H} & 4.0096450 \\ \mathrm{C} & 3.3466930 \\ \mathrm{H} & 3.2532170 \\ \mathrm{H} & 2.3665950 \\ \mathrm{H} & 2.9291340 \\ \mathrm{H} & 6.3632300 \\ \mathrm{C} & 5.0490430 \\ \mathrm{H} & 4.8945890 \\ \mathrm{H} & 5.3357280 \\ \mathrm{C} & 6.1544240 \\ \mathrm{H} & 6.5264350 \\ \mathrm{H} & 7.0104550 \\ \mathrm{C} & 5.4025380 \\ \mathrm{H} & 6.1922190 \\ \mathrm{C} & 0.2035590 \\ \mathrm{C} & -0.9420430 \\ \mathrm{C} & -0.6680380 \\ \mathrm{C} & 0.7011060 \\ \mathrm{C} & 1.7938980 \\ \mathrm{C} & 1.4825230 \\ \mathrm{H} & -1.4596880 \\ \mathrm{H} & -1.1313620 \\ \mathrm{H} & -1.8594330 \\ \mathrm{H} & 0.7375140 \\ \mathrm{H} & 0.8754360 \\ \mathrm{H} & 2.7565600 \\ \mathrm{H} & 1.9292440 \\ \mathrm{H} & -1.6907660 \\ \mathrm{C} & -1.7312450\end{array}$

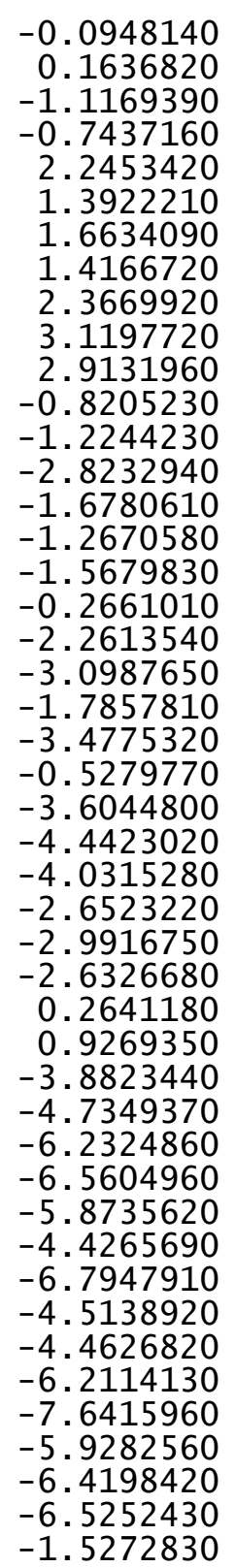
$-3.1628630$
$-4.1813470$
$-3.1958080$
$-2.5846210$
$-0.9550150$
$-2.2337130$
$-3.2484720$
$-1.6078970$
$-1.7126110$
$-2.4708810$
$-0.8356270$
1.6277940
2. 9583150
2. 1298970
1.1820680
3. 9942470
4.9664610
4.1184730
3. 5169630
4. 2182370
3.4557390
1.7620950
3.2772640
2. 2785970
2.9680290
1. 3116210
2.8096520
3.7834170
2. 1275040
0.8567820
1. 2082880
$-1.7100270$
$-2.2223620$
$-2.0908800$
$-2.6808410$
$-1.8637820$
$-1.5317340$
$-2.5976230$
$-3.2801680$
$-1.6894540$
$-3.7218250$
$-2.6992800$
$-2.3859380$
$-0.9198230$
-1.0327290
-2.9212140

-0.8451270
-1.1771530
-2.6752590
-3.4481740
-2.9413440
-0.5967610
-0.9535180
0.2041830
-2.9694430
-2.9246310
-4.5167060
-3.3705560
-0.8858410
0.3928370
-0.3221310
0.5715010
1.5249050
-0.2006730
1.7030580
2.1317500
-0.0239110
-0.9336340
0.9292250
2.4501120
-0.6287400
1.0697150
-0.4768510
1.0725120
1.0589880
2.0241070
0.0249990
1.8113160
1.7413290
1.2160440
2.7269760
3.4797400
3.4002280
1.2501590
2.8078560
4.5317950
4.0211800
0.7559890
0.7225730
3.1795530
3.0512620
3.7855350
$-4.1099960$

$-5.3369090$

$-5.6213530$

$-4.4679910$

$-3.0970250$

$-6.1953040$

$-4.3860290$

$-3.8250040$

$-5.7364330$

$-6.5599580$

$-4.5458400$

$-4.5496670$

$-5.1593800$

$-0.8139160$

$-0.8911240$

$-2.1269890$

$-2.3105450$

$-3.2172140$

$-3.5630660$

$-1.4700350$

$-4.4657210$

$-3.0766670$

$-4.6413580$

$-3.6984630$

$-5.3044200$

$-5.6194140$

$-0.0106510$

0.6054320

3. 2286900

2. 1235140

2.9356730

1. 2782920

3. 5533190

3.1393860

4.5900840

3. 3013200

2. 2919220

4.2980720

5.3026890

3. 5117520

2. 6077480

3. 9452950

5. 1992340

5.0638730

2.8539960

1.3096800 
154

$\operatorname{TS} 278(\mathrm{~S}, \mathrm{~S}) \mathrm{G}[\mathrm{WB} 97 \mathrm{XD} / 6-31 \mathrm{G}(\mathrm{d})]=-3864.647348$

$\begin{array}{lrrr}\mathrm{P} & 0.2646540 & -0.0309830 & 0.1746950 \\ \mathrm{O} & 0.7318350 & 0.8724430 & 1.2818870 \\ \mathrm{O} & -0.7443320 & 0.4992000 & -0.8182210 \\ \mathrm{O} & -0.2219580 & -1.3971450 & 0.9062830 \\ \mathrm{O} & 1.4874450 & -0.5647100 & -0.7539410 \\ \mathrm{C} & -0.3112490 & -2.5509410 & 0.1468250 \\ \mathrm{C} & 0.8702950 & -3.1925640 & -0.2364330 \\ \mathrm{C} & -1.5725240 & -3.0232290 & -0.2066060 \\ \mathrm{C} & -1.6137670 & -4.1990980 & -0.9522320 \\ \mathrm{H} & -2.5865430 & -4.6059760 & -1.2203380 \\ \mathrm{C} & 2.4600090 & -1.2999180 & -0.0989300 \\ \mathrm{C} & 3.6655380 & -0.6815820 & 0.2289330 \\ \mathrm{C} & 2.1741280 & -2.6250870 & 0.2175500 \\ \mathrm{C} & 4.5971120 & -1.4609270 & 0.9058390 \\ \mathrm{H} & 5.5589300 & -1.0196470 & 1.1593480 \\ \mathrm{C} & -2.8246330 & -2.2900360 & 0.1290850 \\ \mathrm{C} & 3.9049320 & 0.7515870 & -0.0950860 \\ \mathrm{C} & -3.2319990 & -2.0893690 & 1.4537970 \\ \mathrm{C} & -2.4911670 & -2.5024570 & 2.7023640 \\ \mathrm{C} & -4.7205010 & -1.2975380 & 3.2079290 \\ \mathrm{C} & -4.4500190 & -1.4575200 & 1.7348120 \\ \mathrm{C} & -2.2149120 & -1.2330130 & 3.5386200 \\ \mathrm{H} & -1.7281970 & -1.5262040 & 4.4761010 \\ \mathrm{H} & -1.5149910 & -0.5903250 & 2.9992590 \\ \mathrm{C} & -3.5502190 & -0.4956730 & 3.8206690 \\ \mathrm{H} & -3.7167710 & -0.3798280 & 4.8980820 \\ \mathrm{H} & -3.5315130 & 0.5110260 & 3.3892830 \\ \mathrm{H} & -5.6727850 & -0.7833480 & 3.3811490 \\ \mathrm{H} & -1.5534300 & -3.0121390 & 2.4714260 \\ \mathrm{C} & -4.7376880 & -2.7001150 & 3.8543980 \\ \mathrm{H} & -4.8692390 & -2.5941510 & 4.9376290 \\ \mathrm{H} & -5.5975500 & -3.2643790 & 3.4794000 \\ \mathrm{C} & -3.4106540 & -3.4322500 & 3.5256780 \\ \mathrm{H} & -2.8899780 & -3.7240860 & 4.4450360 \\ \mathrm{H} & -3.6027320 & -4.3500860 & 2.9604620 \\ \mathrm{C} & -3.6470290 & -1.8109620 & -0.9008460 \\ \mathrm{C} & -3.3550510 & -1.8384080 & -2.3829040 \\ \mathrm{C} & -5.6471870 & -0.7806070 & -1.8314100 \\ \mathrm{C} & -4.8747860 & -1.2033590 & -0.6092900 \\ \mathrm{C} & -4.4759270 & -2.6350410 & -3.0869310 \\ \mathrm{H} & -4.3116970 & -2.5948670 & -4.1700840 \\ \mathrm{H} & -4.4225560 & -3.6894920 & -2.7947330 \\ \mathrm{C} & -5.8561810 & -2.0297560 & -2.7169410 \\ \mathrm{H} & -6.4113030 & -1.7458210 & -3.6185840\end{array}$

$-6.4691330$

$-6.6105220$

$-2.3794490$

$-4.7922420$

$-5.3073760$

$-4.7077280$

-3. 3977500

$-3.1836950$

$-2.6031310$

$-5.2855290$

$-6.2417260$

4. 1017200

4. 1069550

3. 7012040

3. 8815120

5.2304820

5. 2484970

6.1986720

4.9760650

4.8435790

5.8288330

3. 5354940

4.2783760

2. 5068540

2. 3848180

1. 5918200

2. 7654990

2.8082060

1.9535230

4.4021010

4.7365870

4. 2480070

4. 1617240

3. 5625130

3. 8333090

2. 6842850

3. 2577670

3. 3548040

2. 2367990

4.0250810

4. 9399020

5. 6775920

5.7560290

6.4014660

5.9789740

6. 2340400

$\begin{array}{rr}-2.7587770 & -2.1768950 \\ -0.3377220 & -1.5569870 \\ -2.2793050 & -2.5968220 \\ 0.2307410 & -2.6243640 \\ 0.4761030 & -3.5606150 \\ 1.1624680 & -2.0578740 \\ -0.3833740 & -2.9028780 \\ -0.3849860 & -3.9779970 \\ 0.1893370 & -2.4178350 \\ -1.0384850 & 0.7068020 \\ -0.5670290 & 0.9285560 \\ 2.5549000 & -1.7190440 \\ 2.8543600 & -3.1967170 \\ 0.3605070 & -2.6615870 \\ 1.2041260 & -1.4219200 \\ 2.0063210 & -3.8352170 \\ 2.1846550 & -4.9167150 \\ 2.3281200 & -3.4384180 \\ 0.5077040 & -3.5246850 \\ -0.0628290 & -4.4512490 \\ 0.0693830 & -2.9965050 \\ -0.6915150 & -2.4208520 \\ 3.9202070 & -3.3835960 \\ 0.9151740 & -3.4660130 \\ 0.3200200 & -4.3782270 \\ 0.7969040 & -2.8805510 \\ 2.4028890 & -3.8138940 \\ 2.5514950 & -4.8992470 \\ 3.0339240 & -3.4420340 \\ 3.0279050 & 0.6126000 \\ 3.8741550 & 1.8143050 \\ 1.4084370 & 2.4065410 \\ 1.6822060 & 0.9226600 \\ 3.8123160 & 2.8139630 \\ 4.3728010 & 3.7168570 \\ 4.2943630 & 2.3763330 \\ 2.3311280 & 3.1502750 \\ 2.1491690 & 4.2270450 \\ 2.0606740 & 2.8703230 \\ 0.3657560 & 2.6403100 \\ 4.9104760 & 1.5230870 \\ 1.7744930 & 2.8647730 \\ 1.6225330 & 3.9475560 \\ 1.1004370 & 2.3939250 \\ 3.2510100 & 2.4919170 \\ 3.8341320 & 3.3845750\end{array}$




\begin{tabular}{|c|c|c|c|}
\hline & & & \\
\hline $\mathrm{H}$ & 6.8340210 & 3. 3128070 & $\begin{array}{r}1.8109010 \\
-0.7042820\end{array}$ \\
\hline $\mathrm{H}$ & $\begin{array}{l}4.3632380 \\
4.5578310\end{array}$ & $\begin{array}{l}3.4692750 \\
4.5148070\end{array}$ & $\begin{array}{l}-0.10428<0 \\
-0.9398500\end{array}$ \\
\hline C & 3.0901930 & -3.3532280 & 1.0020370 \\
\hline C & 2.7127170 & -4.7210270 & 1.5432830 \\
\hline C & 3.5993230 & -5.1640430 & 2.7065430 \\
\hline C & 5.0691380 & -4.9743950 & 2.3410250 \\
\hline C & 5.3644390 & -3.4878360 & 2.1536260 \\
\hline C & 4. 3223520 & -2.7664630 & 1.3198000 \\
\hline $\mathrm{H}$ & 3.3869480 & -6.2108760 & 2.9487470 \\
\hline $\mathrm{H}$ & 2.7814610 & -5.4773240 & 0.7503450 \\
\hline $\mathrm{H}$ & 1.6601980 & -4.7050660 & 1.8467130 \\
\hline $\mathrm{H}$ & 5.2819480 & -5.5177370 & 1.4102380 \\
\hline $\mathrm{H}$ & 5.7264900 & -5.3893160 & 3.1125110 \\
\hline $\mathrm{H}$ & 6.3517590 & -3.3467270 & 1.6977830 \\
\hline $\mathrm{H}$ & 5.4135920 & -3.0063590 & 3.1403220 \\
\hline $\mathrm{H}$ & 3.3667390 & -4.5724200 & 3.6020210 \\
\hline C & 0.7957110 & -4.3053310 & -1.0931390 \\
\hline C & -0.4631510 & -4.8354520 & -1.4131630 \\
\hline C & 2.0387900 & -4.8358500 & -1.7843850 \\
\hline C & 1.8891420 & -6.2728290 & -2.2784460 \\
\hline C & 0.6108340 & -6.4123700 & -3.1017140 \\
\hline C & -0.6094750 & -6.1011900 & -2.2369330 \\
\hline $\mathrm{H}$ & 2.7667520 & -6.5502300 & -2.8726570 \\
\hline $\mathrm{H}$ & 2.2263600 & -4.1831870 & -2.6499300 \\
\hline $\mathrm{H}$ & 2.9227540 & -4.7351950 & -1.1531860 \\
\hline $\mathrm{H}$ & 0.6521710 & -5.7137850 & -3.9480160 \\
\hline $\mathrm{H}$ & 0.5205780 & -7.4200070 & 521863 \\
\hline $\mathrm{H}$ & -1.5102990 & -6.0320420 & -2.8573310 \\
\hline $\mathrm{H}$ & -0.7784710 & -6.9407580 & -1.5472500 \\
\hline $\mathrm{H}$ & 1.8512820 & -6.9593280 & -1.4210400 \\
\hline C & -3.2786420 & 2.7109650 & -0.4434760 \\
\hline 0 & -2.8157460 & 1.6225280 & 0.0801580 \\
\hline C & -4.5852760 & 3.1155530 & 0.1260570 \\
\hline C & -5.1727760 & 2.3087610 & 1.1101780 \\
\hline C & -5.2429840 & 4.2780430 & -0.2929780 \\
\hline C & -6.3977060 & 2.6588930 & 1.6614660 \\
\hline H & -4.6679490 & 1.4043160 & 1.4256300 \\
\hline C & -6.4695730 & 4.6225330 & 0.2591900 \\
\hline $\mathrm{H}$ & -4.7871010 & 4.9048150 & -1.0517880 \\
\hline C & -7.0488280 & 3.8148570 & 1.2366130 \\
\hline $\mathrm{H}$ & -6.8445020 & 2.0261840 & 2.4223840 \\
\hline $\mathrm{H}$ & -6.9748200 & 5.5238030 & -0.0729600 \\
\hline $\mathrm{H}$ & -8.0074690 & 4.0876660 & 1.6675880 \\
\hline $\mathrm{H}$ & 60218 & 1.2265260 & -0.341756 \\
\hline $\mathrm{S}$ & -2.48812 & 3.5926150 & $6408 \varepsilon$ \\
\hline
\end{tabular}

$\begin{array}{lr}\mathrm{C} & -0.2179240 \\ \mathrm{C} & 0.0066510 \\ \mathrm{H} & -0.8509010 \\ \mathrm{H} & -0.0142970 \\ \mathrm{O} & 1.1674780 \\ \mathrm{H} & 1.1700370 \\ \mathrm{C} & 0.4411720 \\ \mathrm{C} & 0.2230500 \\ \mathrm{C} & 0.5790640 \\ \mathrm{C} & -0.4236700 \\ \mathrm{H} & 0.1963560 \\ \mathrm{H} & 0.8266180 \\ \mathrm{H} & 1.6667120 \\ \mathrm{H} & -1.4864690 \\ \mathrm{H} & -0.2096160 \\ \mathrm{H} & 1.4983580 \\ \mathrm{H} & -0.8280240 \\ \mathrm{H} & 0.2649240 \\ \mathrm{I} & \end{array}$

3.7116380
3.5146620
2.9682940
2.4952910
3.3212080
2.3249390
6.1332870
6.0136140
4.6133510
5.1208310
7.1453270
6.7516280
4.4596720
5.3612130
5.1385160
5.9608310
6.2241890
4.4633890

0.6724340 $-0.7651440$ 1.1568260 $-1.1349670$ 0.6953800 0.8992890 0.4193000 $-1.0908640$ $-1.5995580$ 1.1753940 0.7562240 $-1.6278710$ $-1.5830970$

1.0476020

2.2489670 0.6554890 $-1.3236400$ $\operatorname{TS} 279(\mathrm{~S}, \mathrm{~S}) \mathrm{G}[\mathrm{WB} 97 \mathrm{XD} / 6-31 \mathrm{G}(\mathrm{d})]=-3864.647218$

$\begin{array}{lrrr}\text { TS279(S, S) } \mathrm{G}[\mathrm{WB} 97 \mathrm{XD} / 6-31 \mathrm{G}(\mathrm{d})]=-3864.647218 \\ \mathrm{P} & 0.2525220 & -0.0327390 & 0.2259680 \\ \mathrm{O} & 0.7226650 & 0.8698730 & 1.3326180 \\ \mathrm{O} & -0.7540480 & 0.5007950 & -0.7675440 \\ \mathrm{O} & -0.2372400 & -1.3990960 & 0.9558240 \\ \mathrm{O} & 1.4733910 & -0.5688760 & -0.7030040 \\ \mathrm{C} & -0.3010550 & -2.5554250 & 0.1962600 \\ \mathrm{C} & 0.8909480 & -3.1944420 & -0.1479320 \\ \mathrm{C} & -1.5501100 & -3.0306900 & -0.1990330 \\ \mathrm{C} & -1.5635120 & -4.1956510 & -0.9598960 \\ \mathrm{H} & -2.5253010 & -4.5992760 & -1.2700230 \\ \mathrm{C} & 2.4609770 & -1.2860510 & -0.0523270 \\ \mathrm{C} & 3.6730760 & -0.6586270 & 0.2250540 \\ \mathrm{C} & 2.1866580 & -2.6065080 & 0.3032350 \\ \mathrm{C} & 4.6353090 & -1.4265110 & 0.8745270 \\ \mathrm{H} & 5.6058100 & -0.9792460 & 1.0792030 \\ \mathrm{C} & -2.8170590 & -2.3104760 & 0.1080760 \\ \mathrm{C} & 3.8919700 & 0.7733930 & -0.1180270 \\ \mathrm{C} & -3.2656920 & -2.1302190 & 1.4222770 \\ \mathrm{C} & -2.5525200 & -2.5412270 & 2.6874410 \\ \mathrm{C} & -4.8174710 & -1.3823590 & 3.1404240 \\ \mathrm{C} & -4.5013570 & -1.5206320 & 1.6741210 \\ \mathrm{C} & -2.3233770 & -1.2724930 & 3.5393700 \\ \mathrm{H} & -1.8514500 & -1.5618800 & 4.4855210 \\ \mathrm{H} & -1.6255490 & -0.6108870 & 3.0202700 \\ \mathrm{C} & -3.6804740 & -0.5662690 & 3.7957800 \\ \mathrm{H} & -3.8776520 & -0.4701870 & 4.8699860\end{array}$




$\begin{array}{lrr}-3.6711330 & 0.4470940 & 3.3801350 \\ -5.7835060 & -0.8871680 & 3.2915520 \\ -1.5990690 & -3.0309360 & 2.4788960 \\ -4.8276550 & -2.7922290 & 3.7708280 \\ -4.9965580 & -2.7012420 & 4.8502340 \\ -5.6632590 & -3.3689220 & 3.3614780 \\ -3.4761150 & -3.4941940 & 3.4786430 \\ -2.9766600 & -3.7792350 & 4.4117790 \\ -3.6315290 & -4.4131970 & 2.9042610 \\ -3.6138550 & -1.8279390 & -0.9402950 \\ -3.2770810 & -1.8329600 & -2.4130490 \\ -5.5979630 & -0.8096790 & -1.9175200 \\ -4.8582560 & -1.2412250 & -0.6785070 \\ -4.3665210 & -2.6348030 & -3.1591380 \\ -4.1687250 & -2.5838240 & -4.2362040 \\ -4.3118000 & -3.6910870 & -2.8740550 \\ -5.7640950 & -2.0465870 & -2.8289010 \\ -6.2895180 & -1.7527130 & -3.7449860 \\ -6.3895160 & -2.7893170 & -2.3228570 \\ -6.5746130 & -0.3821520 & -1.6668640 \\ -2.2898380 & -2.2593160 & -2.6021940 \\ -4.7304300 & 0.2242730 & -2.6666680 \\ -5.2216330 & 0.4838660 & -3.6118380 \\ -4.6703630 & 1.1450730 & -2.0793630 \\ -3.3229620 & -0.3725380 & -2.9177020 \\ -3.0835340 & -0.3603930 & -3.9874470 \\ -2.5463550 & 0.2031320 & -2.4078270 \\ -5.3119130 & -1.1011660 & 0.6263620 \\ -6.2814160 & -0.6467350 & 0.8248290 \\ 4.0260380 & 2.5676290 & -1.7579810 \\ 3.9848010 & 2.8572350 & -3.2370500 \\ 3.6199070 & 0.3630190 & -2.6746700 \\ 3.8270650 & 1.2167870 & -1.4463310 \\ 5.0971310 & 2.0160020 & -3.9036460 \\ 5.0791010 & 2.1863490 & -4.9864130 \\ 6.0738350 & 2.3510260 & -3.5398900 \\ 4.8682290 & 0.5172540 & -3.5745380 \\ 4.7163800 & -0.0622980 & -4.4924580 \\ 5.7402180 & 0.0927020 & -3.0668720 \\ 3.4721850 & -0.6889160 & -2.4220220 \\ 4.1401380 & 3.9234620 & -3.4355700 \\ 2.3973740 & 0.9000910 & -3.4480650 \\ 2.2560610 & 0.2985170 & -4.3532360 \\ 1.5007450 & 0.7754820 & -2.8361020 \\ 2.6300050 & 2.3885270 & -3.8106570 \\ 2.6368280 & 2.5325680 & -4.8974510\end{array}$

3.0125760

3.0585700

3. 9148840

1.4478420

1.7126070

3.8465260

4.4139730

4.3180670

2.3643370

1887260

2. 0832770

0.4043190

4.9515780

1.8306380

1.6846340

1. 1614740

3. 3082900

3. 8988300

3. 3752920

3. 4909700

4.5366000

$-2.7246520$

- 3.3124910

$-3.4766150$

$-4.6468520$

$-5.4794890$

$-4.6272840$

$-5.2596590$

$-3.8601630$

$-2.7791410$

$-5.8547130$

$-6.3542560$

$-5.1941850$

$-4.3822110$

$-4.2660330$

$-4.3076580$

$-4.8824760$

$-5.7419770$

$-6.7561450$

$-6.0229580$

$-4.8263670$

$-6.2427150$

$-5.4982700$

$-4.0617330$

$-7.3454780$

$-7.4611990$
-3.4163130
0.5614180
1.7479880
2.3679610
0.8860890
2.7839570
3.6752940
2.3718580
3.1364570
4.2107680
2.8881220
2.6142690
1.4453010
2.7798040
3.8602430
2.2893510
2.3906930
3.2723370
1.6837480
-0.7566130
-1.0041330
1.3175630
1.0695120
2.0356130
2.8807870
2.0883340
1.7307110
3.2050890
1.2796380
2.6549170
1.1707570
2.6649490
1.1225710
2.6553590
3.7882790
-1.0114270
-1.5815200
-2.8226870
-2.5485810
-2.3146200
-1.3903320
-3.1012480
-0.8293430
-1.8100710
-1.6596990
-3.3797160 


\begin{tabular}{|c|c|c|c|c|c|c|c|}
\hline $\mathrm{H}$ & -1.2811700 & -6.7093830 & -1.9139590 & C & -3.7462530 & -0.6148670 & -1.9217610 \\
\hline $\mathrm{H}$ & -0.9158900 & -5.6722330 & -3.2803760 & $\mathrm{H}$ & -4.6954790 & -0.1090730 & -2.0861670 \\
\hline $\mathrm{H}$ & 1.6097830 & -5.1057040 & -3.6695320 & C & 0.9562380 & -1.8825320 & -0.6393160 \\
\hline C & -3.3020710 & 2.7050700 & -0.3998830 & C & 2.2568920 & -2.3644400 & -0.5061520 \\
\hline 0 & -2.8337490 & 1.6166950 & 0.1195180 & $\mathrm{C}$ & -0.0627380 & -2.6342560 & -1.2225590 \\
\hline C & -4.6189690 & 3.0902200 & 0.1593660 & C & 2.4881380 & -3.6770360 & -0.9020530 \\
\hline $\mathrm{C}$ & -5.2282280 & 2.2429010 & 1.0949080 & $\mathrm{H}$ & 3.4972410 & -4.0751180 & -0.8158550 \\
\hline C & -5.2641860 & 4.2732890 & -0.2196320 & C & -4.0211770 & -0.1011260 & 0.5113230 \\
\hline C & -6.4623110 & 2.5729700 & 1.6377960 & C & 3.3447280 & -1.4704280 & -0.0250290 \\
\hline $\mathrm{H}$ & -4.7330730 & 1.3226800 & 1.3780450 & $\mathrm{C}$ & -4.5548230 & -0.8964180 & 1.5327070 \\
\hline C & -6.4994070 & 4.5985880 & 0.3251380 & C & -4.3972880 & -2.3893820 & 1.6934530 \\
\hline $\mathrm{H}$ & -4.7912760 & 4.9318910 & -0.9401320 & $\mathrm{C}$ & -5.8349710 & -1.3224350 & 3.5572380 \\
\hline C & -7.1003800 & 3.7503180 & 1.2539400 & C & -5.3442880 & -0.3260530 & 2.5390840 \\
\hline $\mathrm{H}$ & -6.9261130 & 1.9082150 & 2.3602570 & $\mathrm{C}$ & -3.7412520 & -2.6518040 & 3.0666670 \\
\hline $\mathrm{H}$ & -6.9944780 & 5.5168680 & 0.0258170 & $\mathrm{H}$ & -3.6489960 & -3.7338320 & 3.2166570 \\
\hline $\mathrm{H}$ & -8.0657800 & 4.0081880 & 1.6789790 & $\mathrm{H}$ & -2.7294570 & -2.2382650 & 3.0635320 \\
\hline $\mathrm{H}$ & -1.9740960 & 1.2273310 & -0.2989420 & C & -4.6037080 & -2.0114330 & 4.1860840 \\
\hline $\mathrm{S}$ & -2.5097830 & 3.6044840 & -1.5822450 & $\mathrm{H}$ & -4.9429890 & -2.7689850 & 4.9024200 \\
\hline C & -0.2339420 & 3.7084770 & 0.7262340 & $\mathrm{H}$ & -4.0218900 & -1.2745790 & 4.7488480 \\
\hline C & -0.0112940 & 3.5204450 & -0.7127080 & $\mathrm{H}$ & -6.4391340 & -0.8346050 & 4.3297220 \\
\hline $\mathrm{H}$ & -0.8667490 & 2.9623910 & 1.2066790 & $\mathrm{H}$ & -3.7905340 & -2.8253620 & 0.8967610 \\
\hline $\mathrm{H}$ & -0.0323520 & 2.5032650 & -1.0885810 & C & -6.6653500 & -2.3958680 & 2.8198120 \\
\hline 0 & 1.1512740 & 3.3172330 & 0.7435520 & $\mathrm{H}$ & -6.9884710 & -3.1572590 & 3.5392950 \\
\hline $\mathrm{H}$ & 1.1537290 & 2.3201660 & 0.9459660 & $\mathrm{H}$ & -7.5703800 & -1.9384100 & 2.4071220 \\
\hline C & 0.4295390 & 6.1300280 & 0.4892070 & C & -5.8046150 & -3.0271020 & 1.6943360 \\
\hline C & 0.2078340 & 6.0218720 & -1.0212200 & $\mathrm{H}$ & -5.7036150 & -4.1086820 & 1.8416220 \\
\hline C & 0.5579560 & 4.6245570 & -1.5421220 & $\mathrm{H}$ & -6.2733570 & -2.8783450 & 0.7160220 \\
\hline C & -0.4366680 & 5.1143900 & 1.2393720 & C & -4.2726540 & 1.2774400 & 0.5362870 \\
\hline $\mathrm{H}$ & 0.1881950 & 7.1402470 & 0.8339940 & C & -3.7310650 & 2.3181060 & -0.4153040 \\
\hline $\mathrm{H}$ & 0.8120530 & 6.7622320 & -1.5542670 & C & -5.3236570 & 3.3124870 & 1.3607830 \\
\hline $\mathrm{H}$ & 1.6453000 & 4.4676920 & -1.5362750 & C & -5.0966800 & 1.8314640 & 1.5247260 \\
\hline $\mathrm{H}$ & -1.4990880 & 5.3579970 & 1.1147580 & C & -4.9244710 & 2.9994990 & -1.1191900 \\
\hline $\mathrm{H}$ & -0.2210250 & 5.1238300 & 2.3127280 & $\mathrm{H}$ & -4.5479990 & 3.8075440 & -1.7576470 \\
\hline $\mathrm{H}$ & 1.4867120 & 5.9529900 & 0.7219550 & $\mathrm{H}$ & -5.4298330 & 2.2828800 & -1.7757910 \\
\hline $\mathrm{H}$ & -0.8432340 & 6.2373300 & -1.2497570 & C & -5.9062650 & 3.5522220 & -0.0511490 \\
\hline $\mathrm{H}$ & 0.2348620 & 4.4836180 & -2.5772800 & $\mathrm{H}$ & -6.0736050 & 4.6259450 & -0.1950420 \\
\hline \multicolumn{4}{|c|}{154} & $\mathrm{H}$ & -6.8814520 & 3.0607780 & -0.1291610 \\
\hline \multicolumn{4}{|c|}{$\operatorname{TS} 280(\mathrm{~S}, \mathrm{~S}) \quad \mathrm{G}[\mathrm{WB} 97 \mathrm{XD} / 6-31 \mathrm{G}(\mathrm{d})]=-3864.646356$} & $\mathrm{H}$ & -6.0090440 & 3.6941110 & 2.1252440 \\
\hline $\mathrm{P}$ & -0.2874690 & -0.4235560 & 1.1110690 & $\mathrm{H}$ & -3.0455400 & 1.8830710 & -1.1463340 \\
\hline 0 & 0.3908210 & -0.8804510 & 2.3682500 & C & -3.9593820 & 4.0274640 & 1.4441540 \\
\hline 0 & -0.7453880 & 1.0134070 & 1.0281130 & $\mathrm{H}$ & -4.1017170 & 5.0965970 & 1.2464360 \\
\hline 0 & -1.4955590 & -1.4651590 & 0.8187990 & $\mathrm{H}$ & -3.5575670 & 3.9382340 & 2.4585480 \\
\hline 0 & 0.6663420 & -0.5982210 & -0.2124170 & C & -2.9908590 & 3.3923800 & 0.4156010 \\
\hline C & -2.0579490 & -1.3791240 & -0.4475760 & $\mathrm{H}$ & -2.5874030 & 4.1516680 & -0.2652600 \\
\hline C & -1.3567620 & -1.9498770 & -1.5061910 & $\mathrm{H}$ & -2.1458280 & 2.9252250 & 0.9242870 \\
\hline C & -3.2675850 & -0.7095790 & -0.6185610 & C & -5.6317310 & 1.0335680 & 2.5284020 \\
\hline
\end{tabular}




\begin{tabular}{|c|c|c|}
\hline $\begin{array}{l}-6.2685170 \\
4.7875760 \\
5.1472410 \\
3.3238880 \\
3.7821540 \\
5.6466290 \\
5.8646360 \\
6.5841340 \\
4.5602520 \\
4.2536080 \\
4.9448120 \\
2.5555770 \\
5.9189680 \\
2.7834350 \\
2.4849720 \\
1.8824800 \\
3.8732930 \\
4.1222740 \\
3.5121840 \\
4.9836860 \\
5.5662000 \\
3.6927620 \\
3.9599290 \\
4.4273870 \\
4.8375610 \\
4.0116720 \\
3.3277160 \\
3.2299600 \\
2.3516940 \\
2.8916750 \\
6.3567890 \\
5.0126750 \\
4.8549520 \\
5.2908420 \\
6.1285360 \\
6.5032210 \\
6.9809350 \\
5.3936090 \\
6.1899550 \\
1.4673050 \\
0.1740800 \\
1.8044680 \\
0.5823830 \\
-0.5349640 \\
-0.9803140 \\
0.8616450\end{array}$ & $\begin{array}{r}1.4677070 \\
0.4513540 \\
1.5033070 \\
-0.1321760 \\
-0.4201500 \\
0.7739630 \\
1.5158470 \\
0.2540470 \\
-0.2252210 \\
-0.0038270 \\
-1.2502300 \\
-0.8362660 \\
2.1804760 \\
1.3092050 \\
1.5471340 \\
1.3643730 \\
2.2919460 \\
3.0244120 \\
2.8618100 \\
-0.7907990 \\
-1.1590130 \\
-2.7663180 \\
-1.6539590 \\
-1.1613420 \\
-1.4376420 \\
-0.1533190 \\
-2.1593170 \\
-2.9747550 \\
-1.6771330 \\
-3.4249800 \\
-0.4599150 \\
-3.5539160 \\
-4.3691310 \\
-4.0125080 \\
-2.5954270 \\
-2.9081680 \\
-2.6027670 \\
0.2647610 \\
0.9313890 \\
-4.5042920 \\
-3.9859190 \\
-5.9338770 \\
-6.8478130 \\
-6.1672100 \\
-4.8951740 \\
-7.8016680\end{array}$ & $\begin{array}{l}3.2976450 \\
-0.4115700 \\
-1.4303090 \\
-2.2531220 \\
-0.8436470 \\
-2.6985380 \\
-3.4755370 \\
-2.4773300 \\
-3.1767400 \\
-4.2054730 \\
-3.1687130 \\
-2.5817830 \\
-1.0481840 \\
-2.3086350 \\
-3.3360730 \\
-1.6952080 \\
-1.8101510 \\
-2.5864590 \\
-0.9479190 \\
1.6335460 \\
2.9729680 \\
2.2012120 \\
1.2163760 \\
4.0155150 \\
4.9938680 \\
4.1127370 \\
3.5724790 \\
4.2986680 \\
3.5009820 \\
1.8573860 \\
3.2668640 \\
2.3676090 \\
3.0829820 \\
1.4126440 \\
2.8639860 \\
3.8455640 \\
2.1770740 \\
0.8273860 \\
1.1559700 \\
-1.3744970 \\
-1.5288230 \\
-1.7561340 \\
-1.8342950 \\
-2.6222890 \\
-1.9053900 \\
-2.2945950\end{array}$ \\
\hline
\end{tabular}

2.3020380
2.5378540
-0.1738090
-1.3902460
-1.7264380
-1.4926220
0.2215130
-1.8161270
-0.9633210
-1.3544500
-2.8620920
-3.5928610
-3.0359760
-0.8063660
-1.0502700
0.0911520
-3.1530270
-3.1527910
-4.6632590
-3.5192430
-1.0662000
0.4366770
-0.2712190
0.6121100
1.5779580
-0.1790360
1.7496410
2.1982040
-0.0094520
-0.9222970
0.9559290
2.5060780
-0.6299920
1.0905180
-0.4317100
1.1096980
1.1033280
2.0677010
0.0696870
1.8562370
1.7928180
1.2691530
2.7661920
3.5161970
3.4424110
1.2903440
-5.9215850
-6.3363940
-5.9192640
-6.8405390
-4.3592110
-5.1733590
-7.0722330
-1.7548100
-2.1851430
-1.4799560
-1.5863080
-0.8292460
-1.0955080
-1.9239630
-3.2690510
-2.0010600
-2.6454160
-1.1868130
-1.0665720
0.2494680
-0.4211010
3.4409490
2.3648800
4.0844530
5.0751820
3.6577740
5.6315050
5.3941330
4.2229520
2.8848380
5.2083930
6.3946430
3.8931070
5.6463580
1.8544300
4.0800950
2.2120350
2.1523200
2.0304550
1.4793550
0.9926550
0.2170610
3.5876290
3.8886720
2.7104040
3.2931410
$-2.7364620$

$-1.0480140$

$-3.6302030$

$-2.7440420$

$-2.4956930$

$-0.9728600$

$-0.8218280$

$-2.8232910$

$-4.0034610$

$-5.3013320$

$-5.5151490$

$-4.4081790$

$-3.0223270$

$-6.1391640$

$-4.1591800$

$-3.7709320$

$-5.5007410$

$-6.4926010$

$-4.4207750$

$-4.6062640$

$-5.2512880$

$-0.9462940$

$-1.0029570$

$-2.2706490$

$-2.4768760$

$-3.3464670$

$-3.7378130$

$-1.6464740$

$-4.6033280$

$-3.1876430$

$-4.8015920$

$-3.8910890$

$-5.4306330$

$-5.7860920$

$-0.1101360$

0.4603090

3.1307690

2.0264550

2. 8440490

1. 2028840

3.4907850

3.0978550

4.4508760

3.1514730

2.1778760

4.1687890 


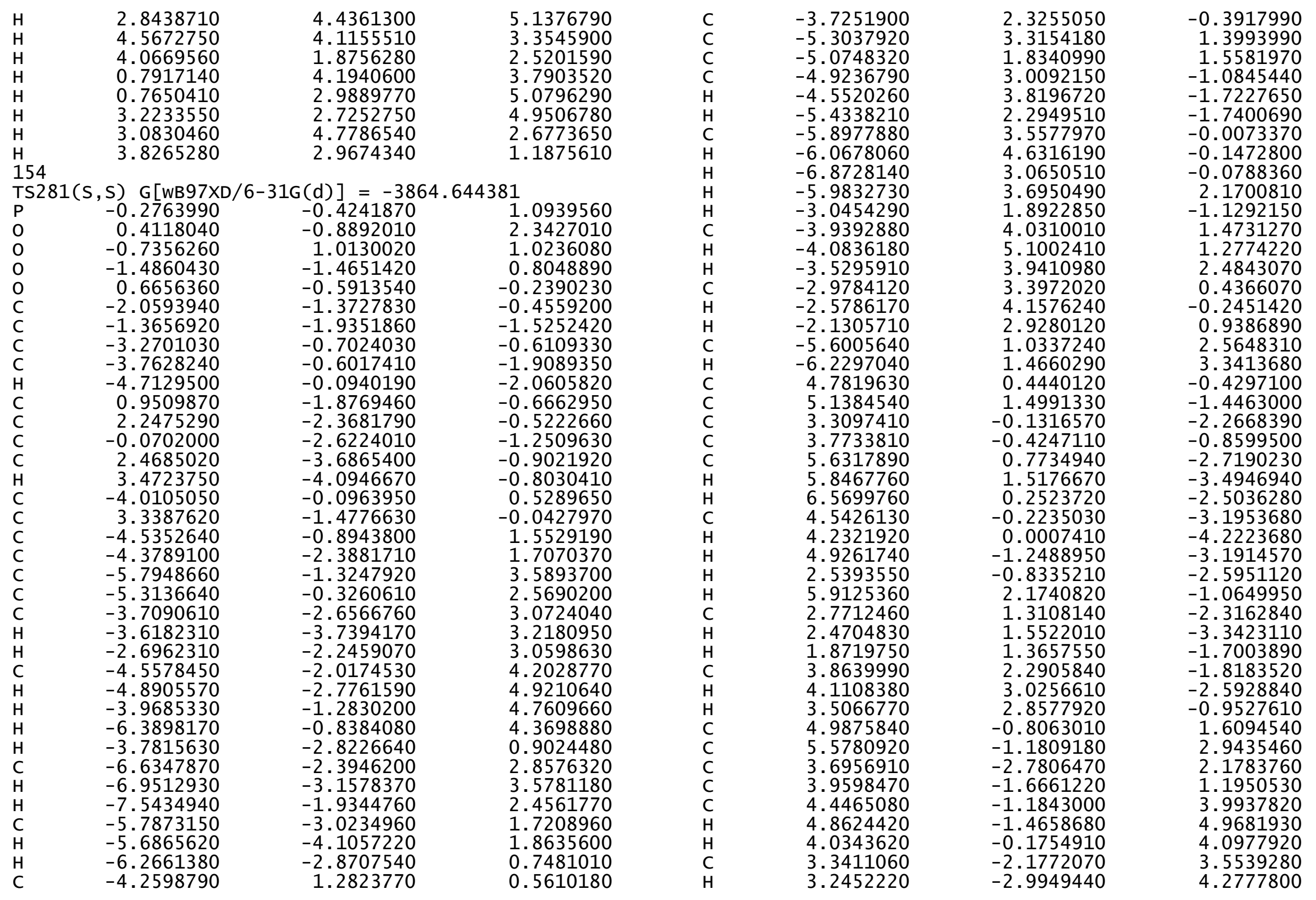




$\begin{array}{rrrr}\mathrm{H} & 2.3661770 & -1.6918160 & 3.4901740 \\ \mathrm{H} & 2.8903890 & -3.4358490 & 1.8379930 \\ \mathrm{H} & 6.3724050 & -0.4848450 & 3.2345890 \\ \mathrm{C} & 5.0144040 & -3.5724470 & 2.3339450 \\ \mathrm{H} & 4.8591970 & -4.3888970 & 3.0484610 \\ \mathrm{H} & 5.2848710 & -4.0296140 & 1.3760640 \\ \mathrm{C} & 6.1363360 & -2.6182360 & 2.8250430 \\ \mathrm{H} & 6.5180690 & -2.9355040 & 3.8024390 \\ \mathrm{H} & 6.9831930 & -2.6248730 & 2.1313310 \\ \mathrm{C} & 5.3945990 & 0.2517090 & 0.8051350 \\ \mathrm{H} & 6.1940500 & 0.9156250 & 1.1316760 \\ \mathrm{C} & 1.4425160 & -4.5087200 & -1.3744890 \\ \mathrm{C} & 0.1572190 & -3.9786080 & -1.5492880 \\ \mathrm{C} & 1.7657970 & -5.9490870 & -1.7248880 \\ \mathrm{C} & 0.5309000 & -6.8456570 & -1.7928370 \\ \mathrm{C} & -0.5583480 & -6.1707170 & -2.6231110 \\ \mathrm{C} & -1.0017690 & -4.8705280 & -1.9564590 \\ \mathrm{H} & 0.8008340 & -7.8183620 & -2.2178760 \\ \mathrm{H} & 2.2710870 & -5.9658970 & -2.7012170 \\ \mathrm{H} & 2.4877220 & -6.3454710 & -1.0016960 \\ \mathrm{H} & -0.1697220 & -5.9586580 & -3.6289890 \\ \mathrm{H} & -1.4218270 & -6.8331260 & -2.7464960 \\ \mathrm{H} & -1.7011470 & -4.3314650 & -2.6005190 \\ \mathrm{H} & -1.5682260 & -5.1092170 & -1.0446340 \\ \mathrm{H} & 0.1493560 & -7.0305700 & -0.7799590 \\ \mathrm{C} & -1.8421750 & -1.7389210 & -2.8349010 \\ \mathrm{C} & -3.0658970 & -1.0802800 & -3.0181270 \\ \mathrm{C} & -0.9823540 & -2.1170490 & -4.0286290 \\ \mathrm{C} & -1.7589810 & -2.1861650 & -5.3420260 \\ \mathrm{C} & -2.6262670 & -0.9424620 & -5.5156990 \\ \mathrm{C} & -3.6634060 & -0.8741350 & -4.3968650 \\ \mathrm{H} & -1.0560980 & -2.2930900 & -6.1753380 \\ \mathrm{H} & -0.1930780 & -1.3556540 & -4.1164640 \\ \mathrm{H} & -0.4582240 & -3.0597080 & -3.8555110 \\ \mathrm{H} & -1.9877210 & -0.0489780 & -5.4852510 \\ \mathrm{H} & -3.1279410 & -0.9468950 & -6.4893790 \\ \mathrm{H} & -4.1938270 & 0.0845640 & -4.4231850 \\ \mathrm{H} & -4.4240280 & -1.6491010 & -4.5695790 \\ \mathrm{H} & -2.3998250 & -3.0788110 & -5.3461910 \\ \mathrm{C} & 0.4426480 & 3.4552270 & -0.9358980 \\ \mathrm{O} & -0.2661090 & 2.3800530 & -0.9982760 \\ \mathrm{C} & 0.6142670 & 4.1083530 & -2.2559130 \\ \mathrm{C} & 1.5832640 & 5.0966560 & -2.4590440 \\ \mathrm{C} & -0.1847960 & 3.6943550 & -3.3308220 \\ \mathrm{C} & 1.7502620 & 5.6629910 & -3.7161290 \\ \mathrm{H} & 2.2095620 & 5.4059180 & -1.6295120\end{array}$

$\begin{array}{lr}\mathrm{C} & -0.0199680 \\ \mathrm{H} & -0.9307290 \\ \mathrm{C} & 0.9485570 \\ \mathrm{H} & 2.5090880 \\ \mathrm{H} & -0.6469290 \\ \mathrm{H} & 1.0794480 \\ \mathrm{H} & -0.4244460 \\ \mathrm{~S} & 1.1199310 \\ \mathrm{C} & 1.1233900 \\ \mathrm{C} & 2.0836270 \\ \mathrm{H} & 0.0887450 \\ \mathrm{H} & 1.8694110 \\ \mathrm{O} & 1.8145970 \\ \mathrm{H} & 1.2908790 \\ \mathrm{C} & 2.7903950 \\ \mathrm{C} & 3.5366020 \\ \mathrm{C} & 3.4589610 \\ \mathrm{C} & 1.3136360 \\ \mathrm{H} & 2.8704870 \\ \mathrm{H} & 4.5884550 \\ \mathrm{H} & 4.0843790 \\ \mathrm{H} & 0.8139820 \\ \mathrm{H} & 0.7910990 \\ \mathrm{H} & 3.2487210 \\ \mathrm{H} & 3.1026440 \\ \mathrm{H} & 3.8396530 \\ \mathrm{I} & \\ \mathrm{H} & \end{array}$

$\begin{array}{lr}4.2697080 & -4.5836930 \\ 2.9234840 & -3.1744450 \\ 5.2526540 & -4.7789300 \\ 6.4242100 & -3.8671350 \\ 3.9498380 & -5.4100790 \\ 5.6985470 & -5.7603610 \\ 1.8631610 & -0.1088440 \\ 4.0841940 & 0.4732380 \\ 2.1962600 & 3.1281320 \\ 2.1454490 & 2.0197670 \\ 2.0163170 & 2.8439500 \\ 1.4781790 & 1.1922340 \\ 0.9747670 & 3.4771110 \\ 0.2020220 & 3.0786050 \\ 3.5622900 & 4.4526090 \\ 3.8727570 & 3.1532630 \\ 2.7022580 & 2.1705870 \\ 3.2702060 & 4.1729660 \\ 4.4056240 & 5.1454690 \\ 4.0973560 & 3.3548800 \\ 1.8645680 & 2.5040510 \\ 4.1737820 & 3.8022760 \\ 2.9598180 & 5.0832920 \\ 2.6961020 & 4.9446660 \\ 4.7666940 & 2.6873710 \\ 2.9672360 & 1.1810090\end{array}$

TS282(S, S ) G[WB97XD/6-31G(d) $]=-3864.643003$

\begin{tabular}{|c|c|c|c|}
\hline & S) $\mathrm{G}[\mathrm{WB} 97 \mathrm{X}$ & (d) & \\
\hline$P$ & -0.3905520 & -0.5842310 & 1.1607020 \\
\hline 0 & 0.1874500 & -1.1227430 & 2.4344480 \\
\hline 0 & -0.8998980 & 0.8386890 & 1.1608050 \\
\hline 0 & -1.5200040 & -1.6251060 & 0.6416270 \\
\hline 0 & 0.6959550 & -0.5919760 & -0.0711510 \\
\hline C & -1.9955550 & -1.3606930 & -0.6379020 \\
\hline C & -1.1930100 & -1.7537790 & -1.7131480 \\
\hline C & -3.2030750 & -0.6862460 & -0.7954560 \\
\hline C & -3.5894260 & -0.3804750 & -2.1030890 \\
\hline $\mathrm{H}$ & -4.5294100 & 0.1437970 & -2.2580860 \\
\hline C & 1.0395690 & -1.7975280 & -0.6562270 \\
\hline C & 2.3425720 & -2.2719790 & -0.5148890 \\
\hline C & 0.0920660 & -2.4547340 & -1.4380020 \\
\hline C & 2.6302650 & -3.5116560 & -1.0726090 \\
\hline H & 3.640 & -3.9029780 & -0.9746810 \\
\hline C & -4.04 & -0.2693930 & 0.3588870 \\
\hline C & 3.39 & -1.3951640 & 0.0730990 \\
\hline C & & ר & \\
\hline
\end{tabular}




-4.4181190
-5.9662300
-5.4539670
-3.8238240
-3.7197130
-2.8209260
-4.7504290
-5.1059730
-4.2110030
-6.6180250
-3.7646060
-6.7357480
-7.0721360
-7.6321900
-5.8094060
-5.6917030
-6.2345050
-4.3466330
-3.8085990
-5.4854840
-5.2228090
-5.0008760
-4.6284420
-5.4651330
-6.0297160
-6.2191410
-6.9895980
-6.2045460
-3.0889160
-4.1455710
-4.3142150
-3.7736680
-3.1273910
-2.7299950
-2.2813430
-5.7751390
-6.4537850
4.7973330
5.1660510
3.4237330
3.8358080
5.7366160
5.9710790
6.6752080
4.6953140
4.4207540

1.1697220
3.0972810
2.2648140
2.5184290
2.5063180
2.6301410
3.6769240
4.2553330
4.3697430
3.9024310
0.3485710
2.1660420
2.7449720
1.7838470
1.0015500
0.9843770
0.0357980
0.5665170
-0.2061810
1.6359530
1.5897880
-0.8467580
-1.3519100
-1.6138130
0.2543500
0.2613950
0.0729460
2.4233120
-0.9687020
1.8696300
1.8232830
2.8742810
0.7937200
0.2435290
1.2567430
2.4424520
3.2381330
-0.1680360
-1.1233030
-2.0925640
-0.6799590
-2.4003150
-3.1332880
-2.1613790
-2.9706420
-3.9975340

\author{
5.1062670 \\ 2.6892200 \\ 5.9022090 \\ 2. 8410590 \\ 2. 5586040 \\ 1. 9241190 \\ 3. 8868010 \\ 4.1485070 \\ 3.4761160 \\ 4.9407230 \\ 5.4655650 \\ 3.6811070 \\ 3.9599710 \\ 4.2733730 \\ 4.6407180 \\ 3.8180670 \\ 3.2318850 \\ 3.1314470 \\ 2. 2428850 \\ 2. 9190550 \\ 6.2186370 \\ 5.0162570 \\ 4.8493670 \\ 5.3576600 \\ 6.0738580 \\ 6.3999960 \\ 6.9646260 \\ 5. 3554860 \\ 6. 1158000 \\ 0.3907560 \\ $-0.6728430$ \\ $-0.3670870$ \\ 1.0666560 \\ 2. 0452220 \\ 1. 6693280 \\ $-1.0817740$ \\ $-0.7617020$ \\ $-1.6464690$ \\ 1.2045780 \\ 1.2725940 \\ 3.0618210 \\ 2. 0811480 \\ $-0.4952030$ \\ $-1.5741090$ \\ $-0.6967740$ \\ $-0.5620150$
}
$-0.9872370$
$-0.6676840$
2. 3643720
1.4651250
1.7434410
1.4631940
2.4555430
3.2166230
2.9902950
$-0.7520810$
$-1.1625000$
$-2.7879990$
$-1.6222000$
$-1.2423830$
$-1.5430580$
$-0.2530800$
$-2.2571870$
$-3.1071150$
$-1.8063230$
$-3.4524250$
$-0.4553300$
$-3.5426960$
$-4.3979360$
$-3.9429320$
$-2.5774250$
$-2.9233360$
$-2.5331480$
0.3495130
1.0225840
$-3.7292360$
$-4.5086180$
$-6.0031650$
$-6.2168480$
$-5.6507160$
$-4.2657050$
$-6.4841280$
$-4.1124630$
$-4.3419110$
$-5.7115870$
$-7.2802080$
$-5.6276170$
$-6.3265460$
$-6.4679720$
$-1.3685510$
$-1.5056750$
$-0.1290330$

$-3.0019180$

$-2.4839680$

$-0.6807540$

$-2.0970580$

$-3.1191090$

$-1.5036860$

$-1.5246520$

$-2.2684370$

$-0.6618810$

1.8267120

3.1778750

2. 2432740

1. 3271260

4.1572480

5.1454240

4.2707290

3.6208260

4.3058340

3.5256720

1.8277860

3.5425540

2. 4393100

3.1039530

1.4786030

3.0398670

4.0276940

2.4049510

1.0869090

1.4810830

$-1.9539120$

$-2.7033450$

$-2.7891280$

$-3.2676920$

$-2.2402260$

$-1.7501340$

$-3.4655660$

$-3.7235620$

$-2.2301260$

$-4.2333070$

$-3.4303400$

$-2.6501730$

$-1.3741390$

$-1.8024900$

$-3.0035780$

$-4.2225480$

$-4.8998000$ 


$\begin{array}{rrr}-1.9359050 & 0.4991250 & -5.2396810 \\ -3.1145260 & -0.2652020 & -4.5993040 \\ -2.7833230 & -0.6843420 & -3.1916910 \\ -1.1392040 & -2.2163530 & -4.9352600 \\ 0.0481460 & -0.2268230 & -5.8036240 \\ -2.0859090 & 0.5333520 & -6.3239230 \\ -3.3263680 & -1.1592800 & -5.2017260 \\ 0.2908900 & -1.8923730 & -3.9577090 \\ -0.0093230 & 0.5266500 & -4.2171450 \\ -1.9639730 & 1.5372620 & -4.8901830 \\ -4.0216450 & 0.3471970 & -4.6204900 \\ 0.2723500 & 3.3091790 & -0.7593370 \\ -0.4364680 & 2.2356810 & -0.8295500 \\ 0.4922060 & 3.9335590 & -2.0874330 \\ 1.4276090 & 4.9565650 & -2.2698990 \\ -0.2077360 & 3.4339970 & -3.1947450 \\ 1.6612460 & 5.4705150 & -3.5394450 \\ 1.9783470 & 5.3328140 & -1.4148940 \\ 0.0270060 & 3.9515910 & -4.4609680 \\ -0.9285060 & 2.6366090 & -3.0520170 \\ 0.9640460 & 4.9687830 & -4.6360230 \\ 2.3944820 & 6.2594650 & -3.6737520 \\ -0.5170530 & 3.5575940 & -5.3140590 \\ 1.1504030 & 5.3706170 & -5.6274300 \\ -0.6030800 & 1.7116300 & 0.0571790 \\ 0.9101380 & 3.9507330 & 0.6635200 \\ 0.8220600 & 2.0140890 & 3.3191690 \\ 1.8285510 & 1.9943350 & 2.2506200 \\ -0.1981350 & 1.8351690 & 2.9876480 \\ 1.6566740 & 1.3486860 & 1.3970850 \\ 1.5049970 & 0.7856970 & 3.6678500 \\ 1.0054720 & 0.0144290 & 3.2430280 \\ 2.4260790 & 3.3642900 & 4.7368430 \\ 3.2158450 & 3.7134650 & 3.4737160 \\ 3.1887020 & 2.5629830 & 2.4655210 \\ 0.9640950 & 3.0637540 & 4.3956870 \\ 2.4708040 & 4.1911710 & 5.4522700 \\ 4.2566060 & 3.9465980 & 3.7184760 \\ 3.8118730 & 1.7256260 & 2.8045370 \\ 0.4691340 & 3.9707920 & 4.0269340 \\ 0.4111500 & 2.7273390 & 5.2784140 \\ 2.8757790 & 2.4912110 & 3.0104620 \\ 2.7871240 & 4.6113740 & \\ 3.6034250 & 2.8492050 & \\ -1.4960110\end{array}$

$-0.3875700$

0.1845490

$-0.9059870$

$-1.5034500$

0.7030400

$-1.9924640$

$-1.2014250$

$-3.2101430$

$-3.6300750$

$-4.5803710$

1. 0463540

2. 3483780

0.0973780

2. 6627590

3. 6734800

$-4.0285520$

3. 3877520

$-4.5573110$

$-4.3676410$

$-5.8554390$

$-5.3795100$

$-3.7282840$

$-3.6261700$

$-2.7219830$

$-4.6144400$

$-4.9426400$

$-4.0541410$

$-6.4817210$

$-3.7407220$

$-6.6483130$

$-6.9518930$

$-7.5647320$

$-5.7613130$

$-5.6400720$

$-6.2204470$

$-4.3312160$

$-3.8197830$

$-5.4502650$

$-5.1826240$

$-5.0332870$

$-4.6792800$

$-5.5166940$

$-6.0316600$

$-6.2209990$

$-6.9959630$

$-6.1500760$
$-0.6791010$

$-1.3363580$

0.7339400

$-1.6679830$

$-0.5569960$

$-1.2664770$

$-1.5344900$

$-0.5953950$

$-0.1758650$

0.3457020

$-1.6839730$

$-2.1681370$

$-2.2384950$

$-3.3228320$

$-3.7195910$

$-0.2916220$

$-1.3779500$

$-1.3163450$

-2.8019770

$-2.2344220$

$-1.0166150$

$-3.3746480$

$-4.4607520$

$-2.9640760$

$-3.0230720$

$-3.9300590$

$-2.4205480$

$-1.9536060$

$-3.0279990$

$-3.1244300$

$-4.0442080$

$-2.6082000$

$-3.4452190$

$-4.5278000$

$-3.0672610$

1.0367540

2.2872770

2. 7942940

1. 3228310

3. 0752660

4. 0164640

2. 5112320

3.3448530

4.4190280

2.8660010

2. 9669930
1.0707910

2. 2893330

1.2096610

0.4386460

$-0.1493670$

$-0.7989050$

$-1.9206410$

$-0.8672940$

$-2.1321740$

$-2.2183320$

$-0.8739440$

$-0.7806760$

$-1.7401370$

$-1.4965930$

$-1.4329160$

0.3389650

$-0.0649390$

1. 1341120

0.9430780

2.9769320

2. 2280200

2. 2270400

2. 1179030

2. 3407740

3. 4502950

3.9716720

4. 1721920

3. 8306600

0.0778990

1. 9947210

2. 5083780

1.6909300

0.7637880

0.6414920

$-0.1555860$

0.6718900

$-0.0058790$

1.9334410

1.7469970

$-0.5460370$

$-0.9829840$

$-1.3513150$

0.6110920

0.7207380

0.4125150

2.7582000 


$\begin{array}{rrr}-3.1183030 & 2.0576450 & -0.8107170 \\ -4.1068130 & 3.5044370 & 2.1994800 \\ -4.2782540 & 4.5854420 & 2.2659660 \\ -3.7063350 & 3.1818830 & 3.1660530 \\ -3.1179030 & 3.1634970 & 1.0569900 \\ -2.7448580 & 4.0753150 & 0.5753820 \\ -2.2543330 & 2.6231490 & 1.4477830 \\ -5.7048080 & 0.3001100 & 2.5289600 \\ -6.3612870 & 0.5282310 & 3.3672580 \\ 4.8400880 & 0.5763290 & -0.0497230 \\ 5.2605910 & 1.7701100 & -0.8707970 \\ 3.5106900 & 0.2837190 & -2.0569060 \\ 3.8787440 & -0.2189810 & -0.6813460 \\ 5.8565290 & 1.2313880 & -2.1920950 \\ 6.1488030 & 2.0760290 & -2.8268460 \\ 6.7655590 & 0.6607890 & -1.9764930 \\ 4.8016690 & 0.3434450 & -2.9039010 \\ 4.5577620 & 0.7479830 & -3.8929250 \\ 5.1856950 & -0.6703270 & -3.0562850 \\ 2.7728290 & -0.3601100 & -2.5412490 \\ 5.9977700 & 2.3801090 & -0.3376170 \\ 2.9530260 & 1.7102560 & -1.9081630 \\ 2.6730610 & 2.1054120 & -2.8914370 \\ 2.0377630 & 1.6567840 & -1.3145330 \\ 4.0143080 & 2.6131440 & -1.2310900 \\ 4.3189750 & 3.4236520 & -1.9031980 \\ 3.5982010 & 3.0902370 & -0.3376890 \\ 4.9000000 & -0.9682840 & 1.7879200 \\ 5.3859270 & -1.5406880 & 3.0938600 \\ 3.5913230 & -3.0083900 & 1.9440620 \\ 3.9123980 & -1.7521890 & 1.1731050 \\ 4.1698780 & -1.6994720 & 4.0322050 \\ 4.5069000 & -2.1043390 & 4.9936340 \\ 3.7260500 & -0.7187770 & 4.2319920 \\ 3.1297220 & -2.6378080 & 3.3696930 \\ 3.0178060 & -3.5627520 & 3.9474260 \\ 2.1443190 & -2.1731540 & 3.3182340 \\ 2.8239560 & -3.6020300 & 1.4406550 \\ 6.1457260 & -0.8971350 & 3.5507560 \\ 4.9099530 & -3.8066590 & 2.0669420 \\ 4.7169020 & -4.7412710 & 2.6056620 \\ 5.2683660 & -4.0784980 & 1.0682340 \\ 5.9658520 & -2.9473410 & 2.8140680 \\ 6.2417130 & -3.4130230 & 2.2234130 \\ 6.8830320 & -2.8567700 & 1.1860860 \\ 5.3581140 & 0.1982190 & \end{array}$

6.1231890
0.4334240
-0.5266830
-0.5255460
0.8979390
2.0041780
1.7161620
-0.2273480
-1.1415610
1.0168180
2.0613790
-1.5393020
-1.0178510
1.0454520
2.9793110
-1.6221780
-0.7890760
-0.6950570
-2.0811030
-3.2291470
-2.8486700
-1.2464090
-0.1576850
-2.2823120
-3.4450370
0.2143200
-0.0815740
-2.0887620
-4.1450100
0.2825800
-0.4364230
0.5019070
1.4299970
-0.1906060
1.6635010
1.9757220
0.0442620
-0.9035150
0.9737660
2.3913380
-0.4939380
1.1609910
-0.6042250
0.9363590
0.7982660
1.8212120
0.8032800
-3.4097920
-4.1760650
-5.6654790
-6.2724740
-5.2232620
-3.9588410
-4.0739450
-6.2219650
-7.0728060
-4.9895140
-3.7721340
-5.7556180
-6.7295200
-5.6339740
-1.0449330
-1.0903850
0.3213800
0.9846520
0.1514000
-0.3724270
-1.7747420
0.2651990
1.1469270
-0.6935390
-1.4726270
0.9352050
1.9737420
0.7484110
3.3413530
2.2837170
4.0590540
5.0986700
3.6284980
5.6964730
5.4217830
4.2295060
2.8159510
5.2632190
6.4977240
3.8878430
5.7300760
1.6923080
3.8681290
1.7179540
1.7890650
1. 6709080

$-2.4306680$

$-3.3049130$

$-2.9134660$

$-2.8336790$

$-3.0566930$

$-2.2914380$

$-4.3579970$

$-3.6272780$

$-3.5715100$

$-4.1294520$

$-3.2265800$

$-1.9395750$

$-1.8498210$

$-2.7757420$

$-3.1631770$

$-4.4190680$

$-5.0287180$

$-5.2270020$

$-4.6219820$

$-3.2627050$

$-5.1478140$

$-5.9810100$

$-6.2910660$

$-5.2904560$

$-4.2165010$

$-4.3593020$

$-4.7552050$

$-4.5670270$

$-0.5085060$

$-0.6550300$

$-1.7892220$

$-1.8999510$

$-2.9298710$

$-3.1324130$

$-1.0202720$

$-4.1585160$

$-2.8434140$

$-4.2624860$

$-3.2118480$

$-5.0376590$

$-5.2248070$

0.1895660

0.9551060

3. 4554840

2.4043280 


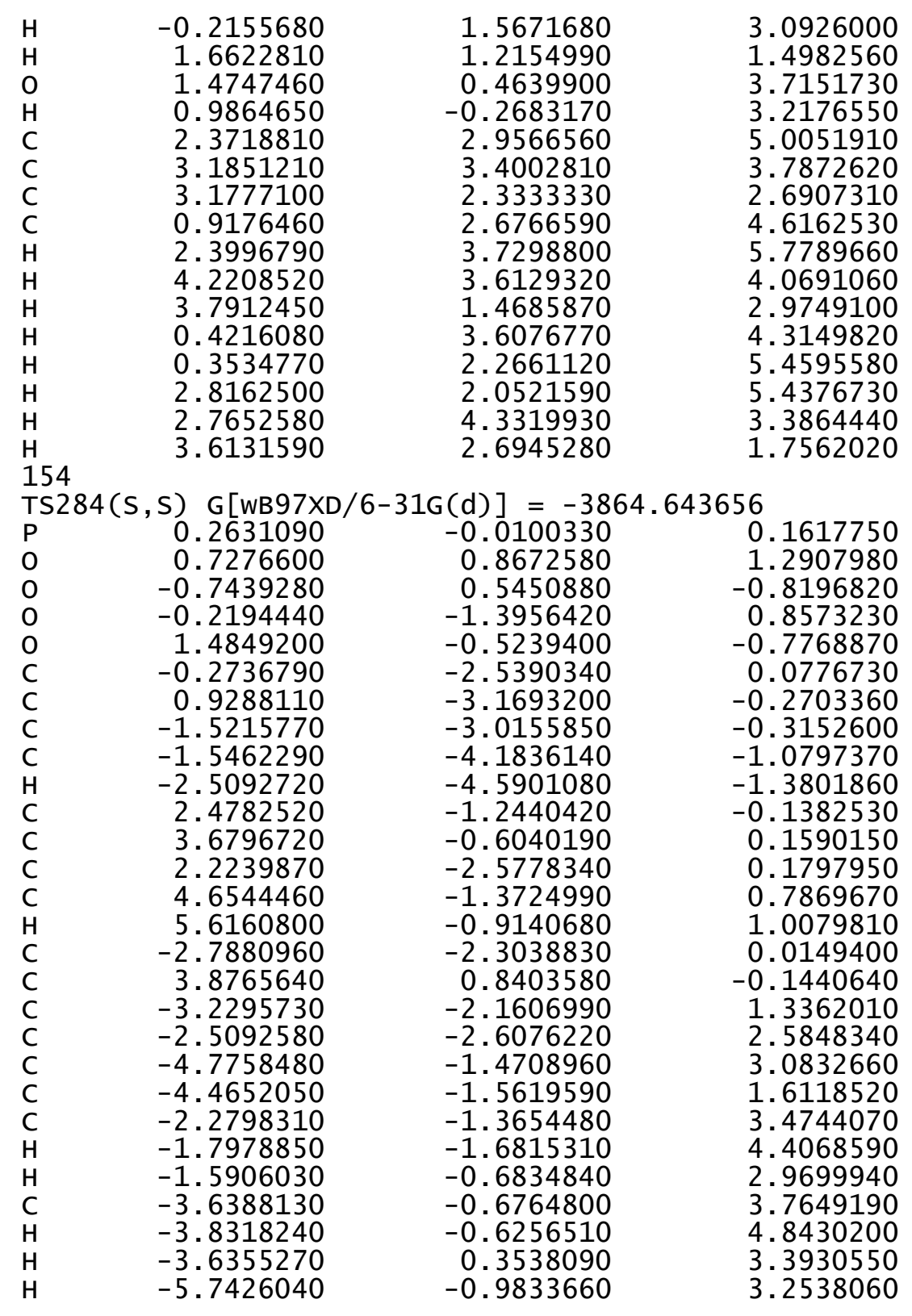

-1.5554560
-4.7812950
-4.9510860
-5.6146680
-3.4269120
-2.9242380
-3.5791960
-3.5945580
-3.2702230
-5.5905290
-4.8393130
-4.3631620
-4.1747460
-4.3015910
-5.7599810
-6.2944530
-6.3781680
-6.5665900
-2.2832610
-4.7332720
-5.2334310
-4.6716310
-3.3255770
-3.0952710
-2.5471020
-5.2841370
-6.2533060
3.9846580
3.9331260
3.6062800
3.8032910
5.0537980
5.0251690
6.0276660
4.8510870
4.7089450
5.7304760
3.4738170
4.0728590
2.3748970
2.2452250
1.4798830
2.5816710
2.5774960
1.7682730
4.3479920
2. 3565010

3. 6652090

4.7470460

3. 2353720

3. 3495420

4. 2727790

2. 7482130

$-1.0151490$

$-2.4902690$

$-1.9538700$

$-0.7302660$

$-3.2437980$

$-4.3211300$

$-2.9816980$

$-2.8891990$

$-3.7944260$

$-2.3933920$

$-1.6862570$

$-2.6968370$

$-2.6889190$

$-3.6244620$

$-2.0834100$

$-2.9642340$

$-4.0354320$

$-2.4487820$

0.5806320

0.7971620

$-1.7329570$

$-3.2027730$

$-2.7111610$

$-1.4591880$

$-3.8984540$

$-4.9763120$

$-3.5327880$

$-3.6071910$

$-4.5394980$

$-3.1103350$

$-2.4878060$

$-3.3713100$

$-3.4680390$

$-4.3925790$

$-2.8620120$

$-3.7826610$

$-4.8640990$

$-3.3615240$

0.5979290 


\begin{tabular}{|c|c|c|}
\hline $\begin{array}{l}4.7009760 \\
4.2829050 \\
4.1452760 \\
3.5602030 \\
3.8474700 \\
2.6593950 \\
3.2959070 \\
3.4272060 \\
2.2728580 \\
4.0896160 \\
4.8733190 \\
5.7176290 \\
5.8320070 \\
6.4405930 \\
5.9768900 \\
6.2470990 \\
6.8086800 \\
4.2590970 \\
4.4253540 \\
4.4254120 \\
3.1888360 \\
5.5472230 \\
5.0740030 \\
4.1030250 \\
2.8628600 \\
5.9374000 \\
6.2656640 \\
6.0954180 \\
4.5965760 \\
3.8073260 \\
2.1926840 \\
2.2939870 \\
4.5658950 \\
0.3766410 \\
0.3769650 \\
0.6145620 \\
2.0426680 \\
2.0758590 \\
0.8693310 \\
0.0856790 \\
0.6149160 \\
2.6711890 \\
2.0751170 \\
1.3833040 \\
0.2565870 \\
2.5042230\end{array}$ & $\begin{array}{r}3.9390180 \\
1.4513720 \\
1.7545790 \\
3.8300850 \\
4.3760840 \\
4.3031000 \\
2.3359290 \\
2.1327100 \\
2.0507250 \\
0.3994240 \\
4.9855090 \\
1.8389300 \\
1.6664020 \\
1.1906700 \\
3.3290900 \\
3.8989120 \\
3.4234720 \\
3.5798090 \\
4.6339230 \\
-2.6907460 \\
-3.2949900 \\
-3.4416420 \\
-4.6635540 \\
-5.4862910 \\
-4.6579630 \\
-5.2648110 \\
-3.7693830 \\
-2.7553110 \\
-5.7967390 \\
-6.4004550 \\
-5.2157690 \\
-4.4909920 \\
-4.3410910 \\
-4.8060170 \\
-6.0519780 \\
-7.0698780 \\
-6.4906020 \\
-4.9479540 \\
-4.2888210 \\
-5.8071620 \\
-7.9853850 \\
-6.8856740 \\
-4.6603780 \\
-6.4804920 \\
-7.3472860 \\
-6.8197680\end{array}$ & $\begin{array}{r}1.8071860 \\
2.3597780 \\
0.8855300 \\
2.8406780 \\
3.7473120 \\
2.4408160 \\
3.1521820 \\
4.2215010 \\
2.8948340 \\
2.5776120 \\
1.5328640 \\
2.7829460 \\
3.8593990 \\
2.2759370 \\
2.4327370 \\
3.3294880 \\
1.7271490 \\
-0.7070290 \\
-0.9255670 \\
1.1827440 \\
0.9123940 \\
1.8764780 \\
2.6621740 \\
1.8186130 \\
1.4932100 \\
2.9673750 \\
1.1114400 \\
2.5319170 \\
0.8868430 \\
2.3448280 \\
0.8379810 \\
2.4195260 \\
3.5805840 \\
-1.4953060 \\
-2.3440100 \\
-1.7599970 \\
-1.6582440 \\
-1.7401830 \\
-1.1132450 \\
-3.3759770 \\
-2.3607230 \\
-2.4634100 \\
-2.8009920 \\
-2.3949630 \\
-0.7618710 \\
-0.7209040\end{array}$ \\
\hline
\end{tabular}

$\begin{array}{lr}\mathrm{H} & 3.0107950 \\ \mathrm{C} & -3.3305140 \\ \mathrm{O} & -2.8374210 \\ \mathrm{C} & -4.6447020 \\ \mathrm{C} & -5.1895000 \\ \mathrm{C} & -5.3494450 \\ \mathrm{C} & -6.4181920 \\ \mathrm{H} & -4.6476080 \\ \mathrm{C} & -6.5802650 \\ \mathrm{H} & -4.9281740 \\ \mathrm{C} & -7.1163060 \\ \mathrm{H} & -6.8304760 \\ \mathrm{H} & -7.1227080 \\ \mathrm{H} & -8.0783680 \\ \mathrm{H} & -1.9737310 \\ \mathrm{~S} & -2.5688930 \\ \mathrm{C} & -0.2691730 \\ \mathrm{C} & -0.0608270 \\ \mathrm{H} & -0.8887060 \\ \mathrm{H} & -0.0719430 \\ \mathrm{O} & 1.1201950 \\ \mathrm{H} & 1.1373500 \\ \mathrm{C} & 0.3665890 \\ \mathrm{C} & 0.1304240 \\ \mathrm{C} & 0.4882070 \\ \mathrm{C} & -0.4814040 \\ \mathrm{H} & 0.1187690 \\ \mathrm{H} & 0.7223060 \\ \mathrm{H} & 1.5770320 \\ \mathrm{H} & -1.5474470 \\ \mathrm{H} & -0.2542550 \\ \mathrm{H} & 1.4277970 \\ \mathrm{H} & -0.9246770 \\ \mathrm{H} & 0.1557640 \\ \mathrm{H} & \\ \mathrm{H} & \\ \mathrm{H} & -100\end{array}$
$-4.5639610$
2. 6950090
1. 5925380
3. 0306660
2. 1629510
4.1844670
2. 4440400
1.2653020
4.4597590
4.8587850
3.5918690
1.7639030
5. 3547100
3. 8113030
1. 2376220
3. 6617970
3. 7117260
3. 5752950
2.9425000
2. 5710020
3.3369930
2. 3339050
6.1467740
6. 0881700
4.7129690
5.0970380
7.1418800
6.8523740
4.5671480
5. 3336220
5.0715300
5. 9726570
6. 3016890
4.6034400

$-1.3271990$

$-0.3484670$

0.1146750

0.2481110

1. 2040810

$-0.1147370$

1.7851530

1.4731990

0.4666240

$-0.8526070$

1.4169840

2. 5243190

0.1787770

1.8702600

$-0.3249010$

$-1.4974340$

0.7860330

$-0.6610400$

1. 2470330

$-1.0709510$

0.7772900

0.9461580

0.6251590

$-0.8860100$

$-1.4580980$

1. 3489110

1. 0071680

$-1.3989280$

$-1.4687240$

1.2439390

2. 4196540

0.8410570

$-1.0968670$

TS285(S, S ) G[WB97XD/6-31G(d)] = -3864.644034

\begin{tabular}{lrrr} 
TS285(S, S ) & \multicolumn{4}{c}{ G $[\mathrm{WB} 97 \times \mathrm{D} / 6-31 \mathrm{G}(\mathrm{d})]=-3864.644034$} \\
$\mathrm{P}$ & -0.2794860 & -0.4333620 & 1.0942640 \\
$\mathrm{O}$ & 0.3977690 & -0.9194790 & 2.3409290 \\
$\mathrm{O}$ & -0.7314300 & 1.0070230 & 1.0416860 \\
$\mathrm{O}$ & -1.4934450 & -1.4628720 & 0.7825920 \\
$\mathrm{O}$ & 0.6728230 & -0.5849720 & -0.2338450 \\
$\mathrm{C}$ & -2.0627450 & -1.3488750 & -0.4781460 \\
$\mathrm{C}$ & -1.3625890 & -1.8885860 & -1.5559990 \\
$\mathrm{C}$ & -3.2757440 & -0.6827100 & -0.6261540 \\
$\mathrm{C}$ & -3.7682500 & -0.5684780 & -1.9240240 \\
$\mathrm{H}$ & -4.7228670 & -0.0672520 & -2.0691350
\end{tabular}




$\begin{array}{rrr}0.9495270 & -1.8652070 & -0.6829500 \\ 2.2406020 & -2.3729830 & -0.5443780 \\ -0.0745330 & -2.5887010 & -1.2868260 \\ 2.4461660 & -3.6890780 & -0.9400480 \\ 3.4452470 & -4.1100830 & -0.8462740 \\ -4.0197230 & -0.0922710 & 0.5193330 \\ 3.3405000 & -1.4963280 & -0.0595090 \\ -4.5504600 & -0.9025510 & 1.5301440 \\ -4.3943360 & -2.3979490 & 1.6672590 \\ -5.8212890 & -1.3571200 & 3.5539200 \\ -5.3333540 & -0.3459120 & 2.5492190 \\ -3.7313140 & -2.6813490 & 3.0329600 \\ -3.6383750 & -3.7654850 & 3.1663130 \\ -2.7194830 & -2.2678330 & 3.0311400 \\ -4.5881990 & -2.0579210 & 4.1662330 \\ -4.9255070 & -2.8264780 & 4.8717140 \\ -4.0028050 & -1.3312440 & 4.7384260 \\ -6.4209120 & -0.8804890 & 4.3368600 \\ -3.7917610 & -2.8220690 & 0.8608610 \\ -6.6570620 & -2.4174590 & 2.8037700 \\ -6.9798340 & -3.1884280 & 3.5131600 \\ -7.5622290 & -1.9516000 & 2.4009090 \\ -5.8020480 & -3.0345570 & 1.6661120 \\ -5.7011090 & -4.1179960 & 1.7990780 \\ -6.2752270 & -2.8729640 & 0.6919850 \\ -4.2652450 & 1.2865490 & 0.5687700 \\ -3.7211900 & 2.3403260 & -0.3667630 \\ -5.3058130 & 3.3116360 & 1.4294610 \\ -5.0834320 & 1.8273660 & 1.5691740 \\ -4.9127770 & 3.0388800 & -1.0564940 \\ -4.5340020 & 3.8574970 & -1.6799300 \\ -5.4213560 & 2.3364770 & -1.7258850 \\ -5.8913280 & 3.5751870 & 0.0229220 \\ -6.0577720 & 4.6512690 & -0.1032650 \\ -6.8672690 & 3.0861320 & -0.0607190 \\ -5.9879260 & 3.6835710 & 2.2015540 \\ -3.0392450 & 1.9142960 & -1.1064020 \\ -3.9388780 & 4.0207650 & 1.5200980 \\ -4.0782110 & 5.0928700 & 1.3368660 \\ -3.5354980 & 3.9167470 & 2.5324620 \\ -2.9742340 & 3.3965750 & 0.4811910 \\ -2.5664150 & 4.1644020 & -0.1873400 \\ -2.1319510 & 2.9159760 & -5617350 \\ -5.6170390 & 1.0145650 & \\ -6.2493360 & 1.4378020 & \\ 4.7888560 & 0.4250210 & \\ -5 & & -0.4284780\end{array}$

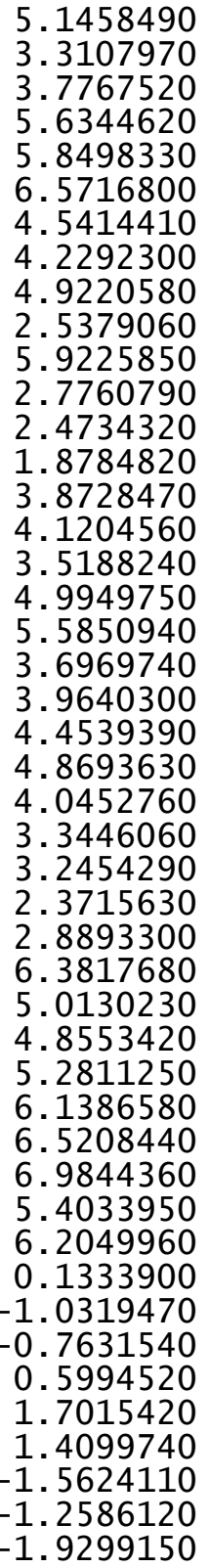

1.4908640
-0.1254900
-0.4358220
0.7783720
1.5305060
0.2523090
-0.2101350
0.0266540
-1.2365880
-0.8214960
2.1593140
1.3189060
1.5724550
1.3696670
2.2900630
3.0322740
2.8495200
-0.8484410
-1.2384980
-2.8244510
-1.7008130
-1.2487160
-1.5418620
-0.2396410
-2.2330440
-3.0571940
-1.7431030
-3.4735860
-0.5479100
-3.6221490
-4.4443060
-4.0718260
-2.6762990
-3.0045140
-2.6787630
0.2173100
0.8755980
-3.9457080
-4.8002070
-6.2978630
-6.6419740
-5.9539190
-4.4947720
-6.8608760
-4.5867090
-4.5169930

$-1.4336780$ $-2.2685430$ $-0.8660900$ $-2.7156100$ $-3.4834430$ $-2.5081320$ $-3.2007290$ $-4.2243830$ $-3.2095450$ $-2.6032650$ $-1.0463900$ $-2.3003290$ $-3.3228890$ $-1.6815420$ $-1.7944200$ $-2.5618320$ $-0.9223670$

1. 5962840

2. 9261020

2. 1464320

1.1743910

3. 9769290

4.9481400

4. 0915740

3. 5277100

4. 2437840

3.4687810

1.7999430

3. 2237240

2. 2939210

3. 0012940

1. 3318710

2.7927370

3. 7663480

2. 0976930

0.8029680

1.1355610

$-1.5968530$

$-2.0630080$

$-1.9207030$

$-2.5164670$

$-1.7132450$

$-1.4205410$

$-2.4144720$

$-3.1164450$

$-1.5031240$ 


$\begin{array}{rrr}0.6326390 & -6.3043880 & -3.5614690 \\ 0.7654630 & -7.7245140 & -2.5237200 \\ 2.6642100 & -6.0352730 & -2.2317710 \\ 1.8264300 & -6.4800180 & -0.7563840 \\ -0.7784830 & -6.5801250 & -0.8595630 \\ -1.8322370 & -1.6675120 & -2.8624820 \\ -3.0647770 & -1.0223730 & -3.0387000 \\ -0.9528470 & -1.9823820 & -4.0592590 \\ -1.7253240 & -2.0704520 & -5.3734790 \\ -2.6301960 & -0.8519580 & -5.5382960 \\ -3.6654450 & -0.8127360 & -4.4156900 \\ -1.0197250 & -2.1478790 & -6.2077770 \\ -0.2098070 & -1.1743670 & -4.1355280 \\ -0.3728580 & -2.8926910 & -3.8974280 \\ -2.0165470 & 0.0588180 & -5.5085650 \\ -3.1355780 & -0.8674140 & -6.5099660 \\ -4.2135330 & 0.1362200 & -4.4333430 \\ -4.4123500 & -1.5997410 & -4.5936350 \\ -2.3367570 & -2.9834970 & -5.3833430 \\ 0.4628150 & 3.4632040 & -0.8897430 \\ -0.2524190 & 2.3931630 & -0.9636170 \\ 0.6403660 & 4.1279120 & -2.2032260 \\ 1.6114660 & 5.1164990 & -2.3945150 \\ -0.1555560 & 3.7246250 & -3.2845470 \\ 1.7833920 & 5.6935570 & -3.6460520 \\ 2.2357540 & 5.4178460 & -1.5605860 \\ 0.0144810 & 4.3103480 & -4.5318700 \\ -0.9031360 & 2.9534990 & -3.1376440 \\ 0.9849450 & 5.2936620 & -4.7152580 \\ 2.5436990 & 6.4551110 & -3.7876810 \\ -0.6099400 & 3.9983280 & -5.3631770 \\ 1.1198780 & 5.7477900 & -5.6923450 \\ -0.4142190 & 1.8678180 & -0.0795420 \\ 1.1415990 & 4.0734350 & 0.5270280 \\ 1.1378810 & 2.1516730 & 3.1590500 \\ 2.0954520 & 2.1080300 & 2.0479690 \\ 0.1014160 & 1.9829670 & 2.8747110 \\ 1.8748240 & 1.4532730 & 1.2121740 \\ 1.8201270 & 0.9206810 & 3.4917190 \\ 1.2885940 & 0.1569350 & 3.0863660 \\ 2.8174450 & 3.4883610 & 4.4970990 \\ 3.5640200 & 3.8094240 & 3.2005860 \\ 3.4750780 & 2.6525710 & 2.2028080 \\ 1.3379860 & 3.2114490 & 4.2164110 \\ 2.9054550 & 4.3220650 & 3.4031060 \\ 4.6179750 & 4.0230450 & \end{array}$

$\begin{array}{ll}\mathrm{H} & 4.0947850 \\ \mathrm{H} & 0.8447820 \\ \mathrm{H} & 0.8145130 \\ \mathrm{H} & 3.2696150 \\ \mathrm{H} & 3.1363830 \\ \mathrm{H} & 3.8555320\end{array}$
1.8056570
4.1232630
2.8941370
2.6123420
4.7126710
2. 9274830

\begin{abstract}
0.8447820
3.8555320
\end{abstract}
2. 5235870
3.8574240
5.1238080
4.9773210
2. 7468940
1.2158330

15

$\operatorname{TS} 286(\mathrm{~S}, \mathrm{~S}) \mathrm{G}[\mathrm{WB} 97 \mathrm{XD} / 6-31 \mathrm{G}(\mathrm{d})]=-3864.646505$

$\begin{array}{lrrr}\text { TS286(S, S }) \text { G }[W B 97 X D / 6-31 G(d)]=-3864.646505 \\ \mathrm{P} & 0.2697370 & -0.0590840 & 0.2750560 \\ \mathrm{O} & 0.7449280 & 0.8604300 & 1.3656260 \\ \mathrm{O} & -0.7599540 & 0.4507990 & -0.7062900 \\ \mathrm{O} & -0.1799160 & -1.4276970 & 1.0238350 \\ \mathrm{O} & 1.4803080 & -0.5918620 & -0.6677890 \\ \mathrm{C} & -0.2330510 & -2.5937320 & 0.2781310 \\ \mathrm{C} & 0.9721210 & -3.2264850 & -0.0609240 \\ \mathrm{C} & -1.4800500 & -3.0816630 & -0.1024920 \\ \mathrm{C} & -1.5033950 & -4.2665430 & -0.8414460 \\ \mathrm{H} & -2.4654300 & -4.6730720 & -1.1445940 \\ \mathrm{C} & 2.4953910 & -1.2775540 & -0.0264290 \\ \mathrm{C} & 3.6942400 & -0.6119570 & 0.2191220 \\ \mathrm{C} & 2.2686680 & -2.6045110 & 0.3413330 \\ \mathrm{C} & 4.6954410 & -1.3439080 & 0.8486470 \\ \mathrm{H} & 5.6539680 & -0.8632690 & 1.0330010 \\ \mathrm{C} & -2.7494520 & -2.3562400 & 0.1844220 \\ \mathrm{C} & 3.8618220 & 0.8239740 & -0.1365590 \\ \mathrm{C} & -3.2069980 & -2.1459600 & 1.4909030 \\ \mathrm{C} & -2.4978510 & -2.5172530 & 2.7706200 \\ \mathrm{C} & -4.7743530 & -1.3651170 & 3.1800910 \\ \mathrm{C} & -4.4497400 & -1.5413650 & 1.7197230 \\ \mathrm{C} & -2.2836170 & -1.2243020 & 3.5891780 \\ \mathrm{H} & -1.8159320 & -1.4841220 & 4.5459410 \\ \mathrm{H} & -1.5869600 & -0.5716540 & 3.0574630 \\ \mathrm{C} & -3.6472840 & -0.5212400 & 3.8172500 \\ \mathrm{H} & -3.8510070 & -0.3946570 & 4.8870550 \\ \mathrm{H} & -3.6423660 & 0.4792460 & 3.3715180 \\ \mathrm{H} & -5.7451430 & -0.8738800 & 3.3130160 \\ \mathrm{H} & -1.5397000 & -3.0048130 & 2.5804470 \\ \mathrm{C} & -4.7758940 & -2.7563620 & 3.8506030 \\ \mathrm{H} & -4.9488310 & -2.6359120 & 4.9264990 \\ \mathrm{H} & -5.6056530 & -3.3512910 & 3.4556890 \\ \mathrm{C} & -3.4180860 & -3.4557410 & 3.5825660 \\ \mathrm{H} & -2.9207770 & -3.7127760 & 4.5249530 \\ \mathrm{H} & -3.5643880 & -4.3904530 & 3.0316180 \\ \mathrm{C} & -3.5452540 & -1.9081290 & -0.8801660 \\ \mathrm{C} & -3.1991070 & -1.9455670 & -2.3503920 \\ \mathrm{C} & -5.5374180 & -0.9432450 & -1.8950700 \\ & & & \end{array}$




$\begin{array}{rrr}-4.7980340 & -1.3300250 & -0.6409010 \\ -4.2721810 & -2.7813160 & -3.0827100 \\ -4.0699540 & -2.7525590 & -4.1598240 \\ -4.2028570 & -3.8297620 & -2.7730150 \\ -5.6802460 & -2.2074660 & -2.7723420 \\ -6.2078700 & -1.9482330 & -3.6975930 \\ -6.2945180 & -2.9461750 & -2.2471120 \\ -6.5215510 & -0.5235730 & -1.6609330 \\ -2.2045230 & -2.3619110 & -2.5228780 \\ -4.6815790 & 0.0817790 & -2.6691520 \\ -5.1699940 & 0.3043340 & -3.6252320 \\ -4.6409370 & 1.0210670 & -2.1103000 \\ -3.2628050 & -0.4990800 & -2.8920170 \\ -3.0129750 & -0.5099510 & -3.9594100 \\ -2.5008670 & 0.1016000 & -2.3887920 \\ -5.2599930 & -1.1600430 & 0.6573620 \\ -6.2355480 & -0.7116920 & 0.8390050 \\ 3.9083450 & 2.6114230 & -1.7881640 \\ 3.8169200 & 2.8919740 & -3.2667140 \\ 3.5478150 & 0.3896760 & -2.6840710 \\ 3.7541080 & 1.2573840 & -1.4652230 \\ 4.9347020 & 2.0824390 & -3.9630440 \\ 4.8707590 & 2.2386230 & -5.0461700 \\ 5.9113030 & 2.4568610 & -3.6397340 \\ 4.7725120 & 0.5805270 & -3.6092850 \\ 4.6285500 & -0.0180680 & -4.5161470 \\ 5.6695100 & 0.1988400 & -3.1111900 \\ 3.4400320 & -0.6648170 & -2.4220640 \\ 3.9329690 & 3.9615290 & -3.4741340 \\ 2.2937920 & 0.8802910 & -3.4376260 \\ 2.1661920 & 0.2771790 & -4.3438870 \\ 1.4102810 & 0.7165780 & -2.8158340 \\ 2.4606420 & 2.3783400 & -3.7965150 \\ 2.4210240 & 2.5305360 & -4.8814130 \\ 1.6473230 & 2.9691040 & -3.3665300 \\ 4.3150470 & 3.1247540 & 0.5194280 \\ 4.6843130 & 3.9961440 & 1.6925830 \\ 4.3111110 & 1.5229780 & 2.3359770 \\ 4.1378300 & 1.7758230 & 0.8560720 \\ 3.5695470 & 3.9075110 & 2.7561670 \\ 3.8729250 & 4.4845320 & 3.6380390 \\ 2.6540230 & 4.3586540 & 2.3646990 \\ 3.3300240 & 2.4211830 & 3.1205220 \\ 3.4856400 & 2.2534090 & 4.1926950 \\ 2.3055950 & 2.1157690 & 2.8940060 \\ 4.1358850 & 0.4766530 & 2.5926440\end{array}$

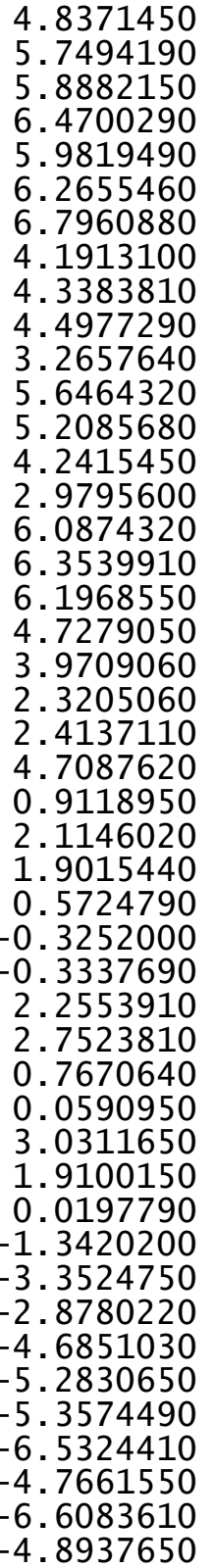

5.0348950

1.9420990

1.8076570

1.2857070

3.4224730

4.0257550

3.5024390

3. 5471360

4.5953950

$-2.6521930$

$-3.2852450$

$-3.3594340$

$-4.5567810$

$-5.4278920$

$-4.6379640$

$-5.1301120$

$-3.7054320$

$-2.6401610$

$-5.7632360$

$-6.3275000$

$-5.2327020$

$-4.4570850$

$-4.2067490$

$-4.3716110$

$-5.0486460$

$-5.1999240$

$-5.9139640$

$-6.1262700$

$-4.9003700$

$-6.0447790$

$-5.7381330$

$-6.8855130$

$-6.9793210$

$-4.4864370$

$-4.1971670$

$-5.3190260$

$-6.3898160$

2.6005810

1.5318220

2. 9813830

2.1766980

4.1204460

2.5055840

1.2900150

4.4436590

4.7457260
1.3800100

2.7147800

3.7937580

2. 2149200

2. 3082670

3.1786310

1.5806860

$-0.7986150$

$-1.0559770$

1. 2905540

1.0662840

1.9858520

2.8271070

2.0298250

1.6912050

3.1416980

1.2186460

2.6030080

1.1033090

2. 5933860

1.0578640

2.6166970

3.7399720

$-0.8679690$

$-1.4799940$

$-2.9971360$

$-3.3464480$

$-2.1101150$

$-1.2359050$

$-1.0356260$

$-3.4274790$

$-3.8126890$

$-1.5339050$

$-1.2852280$

$-3.4374130$

$-4.0808450$

$-2.4184460$

$-0.4139610$

0.1396220

0.1098770

1.0891410

$-0.3486020$

1.5971420

1.4337370

0.1598970
-1.1038510 


$\begin{array}{lrrr}\mathrm{C} & -7.1977530 & 3.6381220 & 1.1329970 \\ \mathrm{H} & -6.9871810 & 1.8746090 & 2.3547380 \\ \mathrm{H} & -7.1244450 & 5.3267760 & -0.2029390 \\ \mathrm{H} & -8.1754820 & 3.8941590 & 1.5301100 \\ \mathrm{H} & -2.0041850 & 1.1503100 & -0.2557870 \\ \mathrm{~S} & -2.5514410 & 3.4845380 & -1.6023200 \\ \mathrm{C} & -0.3194050 & 3.6580290 & 0.7460490 \\ \mathrm{C} & -0.0714940 & 3.4870380 & -0.6908800 \\ \mathrm{H} & -0.9285820 & 2.8856050 & 1.2149170 \\ \mathrm{H} & -0.0487110 & 2.4717680 & -1.0715360 \\ \mathrm{O} & 1.0792330 & 3.3200600 & 0.7782250 \\ \mathrm{H} & 1.1173130 & 2.3224910 & 0.9773350 \\ \mathrm{C} & 0.2574130 & 6.1035580 & 0.5336370 \\ \mathrm{C} & 0.0594200 & 5.9979900 & -0.9802000 \\ \mathrm{C} & 0.4646660 & 4.6178390 & -1.5068440 \\ \mathrm{C} & -0.5812790 & 5.0518900 & 1.2650170 \\ \mathrm{H} & -0.0249270 & 7.1018690 & 0.8820000 \\ \mathrm{H} & 0.6441030 & 6.7630310 & -1.5001200 \\ \mathrm{H} & 1.5567550 & 4.4989520 & -1.4940950 \\ \mathrm{H} & -1.6500660 & 5.2578840 & 1.1275910 \\ \mathrm{H} & -0.3804660 & 5.0616530 & 2.3412200 \\ \mathrm{H} & 1.3169610 & 5.9626990 & 0.7797640 \\ \mathrm{H} & -0.9953220 & 6.1789060 & -1.2209390 \\ \mathrm{H} & 0.1549890 & 4.4744560 & -2.5457060 \\ \mathrm{H} & & & \end{array}$

TS287(S, S) G[WB97XD/6-31G(d)] = -3864.643761

\begin{tabular}{|c|c|c|c|}
\hline & $-c$ & 70 & 1.1736960 \\
\hline 0 & 0.1957700 & -1.0988210 & 2.4512360 \\
\hline 0 & -0.9096530 & 0.8402810 & 1.1588390 \\
\hline 0 & -1.5158080 & -1.6343240 & 0.6722840 \\
\hline 0 & 0.6892640 & -0.5941070 & -0.0630870 \\
\hline & -1.9929460 & -1.3914450 & -0.6111390 \\
\hline C & -1.1899960 & -1.7994400 & -1.6796130 \\
\hline c & -3.2008890 & -0.7192990 & -0.7795130 \\
\hline c & -3.5867610 & -0.4336840 & -2.0915480 \\
\hline H & -4.5277000 & 0.0862690 & -2.2548060 \\
\hline & 1.0456590 & -1.8033270 & -0.6323960 \\
\hline c & 2.3562720 & 62320 & -0.4899680 \\
\hline c & 0.1023800 & -2.4866000 & -1.3976720 \\
\hline$c$ & 2.6616690 & -3.4959250 & -1.0368800 \\
\hline H & 3.6786650 & -3.8704680 & -0.9385110 \\
\hline & -4.0455830 & -0.2824760 & 0.3658680 \\
\hline$C$ & 3.3953430 & 7020 & 0.0908650 \\
\hline & -4.6032050 & -1.2138940 & 1.2508560 \\
\hline & -4.4116440 & -2.7115360 & 1.2291720 \\
\hline & & 1 & \\
\hline
\end{tabular}

-5.4607840
-3.8235550
-3.7135430
-2.8235040
-4.7602990
-5.1165210
-4.2284010
-6.6329110
-3.7518410
-6.7352200
-7.0751470
-7.6291490
-5.7988190
-5.6758530
-6.2187040
-4.3517540
-3.8127790
-5.5011250
-5.2339190
-5.0030790
-4.6299480
-5.4611820
-6.0392290
-6.2322260
-6.9965110
-6.2249820
-3.0884850
-4.1644910
-4.3356230
-3.7977230
-3.1391480
-2.7389260
-2.2955680
-5.7876670
-6.4717700
4.7954400
5.1618340
3.4257410
3.8365870
5.7359420
5.9685280
6.6759520
4.6987510
4.4252520
5.1128760
2.6935320
2.2765970

2. 5915580

2.6046200

2.7004580

3.7338940

4.3275300

4.4169900

3.9233400

0.4189240

2.2147010

2. 8103070

1.8141850

1.0679040

1.0797590

0.0921340

0.5438640

$-0.2505320$

1. 5679330

1.5544080

$-0.9126780$

$-1.4335030$

$-1.6697520$

0.1746740

0.1576540

$-0.0015270$

2. 3461120

$-1.0028380$

1.7931040

1.7214870

2.8045800

0.7333000

0.1676650

1.2123280

2.4238850

3.2093000

$-0.1710320$

$-1.1389640$

$-2.0909690$

$-0.6738000$

$-2.4073280$

$-3.1492630$

$-2.1612720$

$-2.9668360$

$-3.9975320$

$-2.9843010$ 


$\begin{array}{lrrr}\text { H } & 5.8955380 & 2.3944510 & -0.7038730 \\ \mathrm{C} & 2.8394870 & 1.4693900 & -2.1143840 \\ \mathrm{H} & 2.5590530 & 1.7344720 & -3.1404570 \\ \mathrm{H} & 1.9210570 & 1.4717260 & -1.5236020 \\ \mathrm{C} & 3.8806860 & 2.4695240 & -1.5506970 \\ \mathrm{H} & 4.1410170 & 3.2236370 & -2.3020200 \\ \mathrm{H} & 3.4663950 & 3.0119210 & -0.6944130 \\ \mathrm{C} & 4.9433640 & -0.6979980 & 1.8374420 \\ \mathrm{C} & 5.4720170 & -1.0945120 & 3.1912630 \\ \mathrm{C} & 3.6909160 & -2.7339380 & 2.2746120 \\ \mathrm{C} & 3.9646060 & -1.5756620 & 1.3474060 \\ \mathrm{C} & 4.2821710 & -1.1695640 & 4.1737550 \\ \mathrm{H} & 4.6524990 & -1.4600630 & 5.1638370 \\ \mathrm{H} & 3.8238260 & -0.1807510 & 4.2792850 \\ \mathrm{C} & 3.2430580 & -2.1924850 & 3.6484370 \\ \mathrm{H} & 3.1469380 & -3.0367110 & 4.3410770 \\ \mathrm{H} & 2.2523900 & -1.7458640 & 3.5515350 \\ \mathrm{H} & 2.9301430 & -3.4044530 & 1.8666540 \\ \mathrm{H} & 6.2236410 & -0.3818920 & 3.5481560 \\ \mathrm{C} & 5.0288840 & -3.4826110 & 2.4743770 \\ \mathrm{H} & 4.8662590 & -4.3324830 & 3.1469170 \\ \mathrm{H} & 5.3696120 & -3.8900750 & 1.5164740 \\ \mathrm{C} & 6.0845270 & -2.5086970 & 3.0643240 \\ \mathrm{H} & 6.4138200 & -2.8448580 & 4.0544630 \\ \mathrm{H} & 6.9738420 & -2.4670560 & 2.4271830 \\ \mathrm{C} & 5.3548620 & 0.3969270 & 1.0858750 \\ \mathrm{H} & 6.1139340 & 1.0758300 & 1.4723710 \\ \mathrm{C} & 1.7109830 & -4.2714100 & -1.7037830 \\ \mathrm{C} & 0.4207590 & -3.7616770 & -1.9025010 \\ \mathrm{C} & 2.1312340 & -5.6240730 & -2.2468220 \\ \mathrm{C} & 0.9505140 & -6.5312670 & -2.5861380 \\ \mathrm{C} & -0.0807250 & -5.7561900 & -3.4018360 \\ \mathrm{C} & -0.6471660 & -4.5988310 & -2.5815450 \\ \mathrm{H} & 1.3036080 & -7.4116360 & -3.1338470 \\ \mathrm{H} & 2.7290850 & -5.4648060 & -3.1556830 \\ \mathrm{H} & 2.7964270 & -6.1135150 & -1.5264040 \\ \mathrm{H} & 0.3947260 & -5.3649890 & -4.3119120 \\ \mathrm{H} & -0.8974800 & -6.4112610 & -3.7235760 \\ \mathrm{H} & -1.2864660 & -3.9734430 & -3.2082400 \\ \mathrm{H} & -1.3056950 & -4.9995260 & -1.7975260 \\ \mathrm{H} & 0.4820210 & -6.8938660 & -1.6616170 \\ \mathrm{C} & -1.5697330 & -1.4307920 & -2.9760210 \\ \mathrm{C} & -0.6975410 & -1.6014660 & -4.1952960 \\ \mathrm{C} & -0.5624380 & -0.2436010 & -4.9092460 \\ \mathrm{C} & -1.9355280 & 0.3858720 & -5.2508940 \\ \mathrm{C} & -3.1138600 & -0.3605880 & -4.5902620 \\ & & & \\ & & & \end{array}$

$\begin{array}{rrr}-2.7795140 & -0.7522210 & -3.1754340 \\ -1.1427980 & -2.3322470 & -4.8860580 \\ 0.0373960 & -0.3685700 & -5.8165700 \\ -2.0923070 & 0.4023060 & -6.3345840 \\ -3.3310970 & -1.2663540 & -5.1731320 \\ 0.2898280 & -1.9848780 & -3.9252970 \\ 0.0014690 & 0.4258070 & -4.2494390 \\ -1.9557690 & 1.4300280 & -4.9191240 \\ -4.0190460 & 0.2543110 & -4.6208120 \\ 0.2490310 & 3.2933790 & -0.7947250 \\ -0.4486810 & 2.2117870 & -0.8501920 \\ 0.4626090 & 3.9017190 & -2.1313840 \\ 1.3808490 & 4.9379530 & -2.3260050 \\ -0.2236790 & 3.3726680 & -3.2336720 \\ 1.6111860 & 5.4362300 & -3.6024200 \\ 1.9208110 & 5.3369500 & -1.4744060 \\ 0.0080100 & 3.8744440 & -4.5068090 \\ -0.9308100 & 2.5647770 & -3.0822850 \\ 0.9279930 & 4.9049540 & -4.6938940 \\ 2.3313120 & 6.2355410 & -3.7460410 \\ -0.5248280 & 3.4575380 & -5.3561020 \\ 1.1120990 & 5.2940910 & -5.6907830 \\ -0.6141200 & 1.7000070 & 0.0437800 \\ 0.8817020 & 3.9594220 & 0.6190500 \\ 0.8084830 & 2.0543610 & 3.2986900 \\ 1.8137780 & 2.0279250 & 2.2292230 \\ -0.2111200 & 1.8654300 & 2.9709550 \\ 1.6450230 & 1.3708990 & 1.3837900 \\ 1.4995370 & 0.8341720 & 3.6601530 \\ 1.0044830 & 0.0548220 & 3.2447850 \\ 2.4067910 & 3.4295380 & 4.6988080 \\ 3.1922430 & 3.7694600 & 3.4304830 \\ 3.1706930 & 2.6073040 & 2.4356990 \\ 0.9460590 & 3.1166730 & 4.3633500 \\ 2.4477170 & 4.2644480 & 5.4050970 \\ 4.2319510 & 4.0117120 & 3.6708690 \\ 3.7994910 & 1.7778100 & 2.7834430 \\ 0.4453360 & 4.0167750 & 3.9855260 \\ 0.3964380 & 2.7867620 & 5.2506010 \\ 2.8625630 & 2.5645440 & 5.1954220 \\ 2.7572180 & 4.6594410 & 2.9579280 \\ 3.5822680 & 2.8847230 & 1.4623250 \\ & & \end{array}$

TS288(S, S) G[WB97XD/6-31G(d)] = -3864.643727

$\begin{array}{llrr}\mathrm{P} & 0.2885320 & -0.0725370 & 0.2011580 \\ \mathrm{O} & 0.7944810 & 0.8122530 & 1.3062940\end{array}$ 


$\begin{array}{rrr}-0.7430880 & 0.4795400 & -0.7551360 \\ -0.1801480 & -1.4471030 & 0.9255010 \\ 1.4760620 & -0.6106850 & -0.7679970 \\ -0.2969390 & -2.5880990 & 0.1504610 \\ 0.8671200 & -3.2544810 & -0.2444370 \\ -1.5728100 & -3.0261850 & -0.1978840 \\ -1.6481460 & -4.1891430 & -0.9581580 \\ -2.6319420 & -4.5624330 & -1.2355090 \\ 2.4673900 & -1.3544530 & -0.1527550 \\ 3.6810970 & -0.7345620 & 0.1316370 \\ 2.1896320 & -2.6914230 & 0.1585620 \\ 4.6570550 & -1.5097570 & 0.7600060 \\ 5.6208800 & -1.0593650 & 0.9856840 \\ -2.8054530 & -2.2657040 & 0.1504660 \\ 3.8965030 & 0.7060150 & -0.1785510 \\ -3.1941620 & -2.0504990 & 1.4785960 \\ -2.4523720 & -2.4786140 & 2.7216810 \\ -4.6478000 & -1.2210600 & 3.2447540 \\ -4.3957760 & -1.3922510 & 1.7696060 \\ -2.1392470 & -1.2164030 & 3.5554780 \\ -1.6563190 & -1.5222070 & 4.4909460 \\ -1.4246750 & -0.5920580 & 3.0136970 \\ -3.4534900 & -0.4444800 & 3.8433010 \\ -3.6086070 & -0.3172530 & 4.9211150 \\ -3.4129150 & 0.5583510 & 3.4043100 \\ -5.5864270 & -0.6847040 & 3.4254280 \\ -1.5288780 & -3.0103080 & 2.4837100 \\ -4.6913410 & -2.6207060 & 3.8964680 \\ -4.8059760 & -2.5080740 & 4.9809740 \\ -5.5697730 & -3.1646730 & 3.5347580 \\ -3.3871670 & -3.3862950 & 3.5526050 \\ -2.8650210 & -3.6941890 & 4.4658790 \\ -3.6087250 & -4.2972050 & 2.9868740 \\ -3.6310980 & -1.7766820 & -0.8727210 \\ -3.3572370 & -1.8148300 & -2.3583350 \\ -5.6283000 & -0.7242100 & -1.7859630 \\ -4.8446240 & -1.1460590 & -0.5704360 \\ -4.4966780 & -2.5977690 & -3.0479060 \\ -4.3482810 & -2.5540840 & -4.1332520 \\ -4.4501190 & -3.6541650 & -2.7617920 \\ -5.8649690 & -1.9803910 & -2.6543670 \\ -6.4378770 & -1.7023630 & -3.5467240 \\ -6.4701830 & -2.6999030 & -2.0932440 \\ -6.5821900 & -0.2669710 & -1.5022100 \\ -2.3898510 & -2.2688180 & -2.5819160 \\ -4.7739110 & 0.2669230 & -2.6043100\end{array}$
$-5.2953230$
$-4.6798970$
$-3.3856550$
$-3.1719000$
$-2.5853410$
$-5.2357640$
$-6.1810380$
4.0276760
3. 9792890
3. 6071740
3. 8243760
5.0850600
5.0623360
6.0648450
4.8516800
4.6933500
5.7242870
3. 4565350
4.1375660
2. 3828040
2. 2335240
1. 4887710
2. 6200990
2. 6225930
1.8177620
4. 4110720
4.7879970
4.3344890
4. 1873520
3.6553800
3. 9591790
2. 7580080
3. 3722430
3. 5154500
2. 3417600
4.1259970
4.9746860
5.7803800
5. 9048110
6.4868820
6.0593430
6. 3446730
6.8871440
4. 3224580
4.5055910
3.1642320

0.4961960

1. 2099670

$-0.3606980$

$-0.3662180$

0.2050230

$-0.9642220$

$-0.4752200$

2. 5361520

2. 8584220

0.3541340

1. 1796330

2.0280230

2. 2218850

2. 3511430

0.5233270

$-0.0353670$

0.0844510

$-0.7023150$

3. 9281530

0.9127010

0.3316540

0.7787760

2.4074090

2.5741960

3. 0260090

2.9728590

3.8009510

1.3233900

1.6213790

3. 7161470

4. 2642050

4.1995550

2. 2281970

2. 0295570

1.9568110

0.2759140

4.8430890

1.6885930

1. 5167240

1.0278620

3. 1735930

3. 7422080

3.2531710

3.4353060

4.4851520

$-3.4157690$
$-3.5412740$

$-2.0588320$

$-2.8820920$

$-3.9572500$

$-2.3978590$

0.7492430

0.9793900

$-1.7791340$

$-3.2512350$

$-2.7418080$

$-1.4959870$

- 3.9417120

$-5.0204360$

$-3.5755410$

$-3.6443090$

$-4.5739860$

$-3.1500690$

$-2.5112490$

$-3.4273950$

$-3.4964810$

$-4.4137190$

$-2.8824670$

$-3.8284700$

$-4.9120060$

$-3.4175030$

0.5473880

1. 7491810

2.3185690

0.8440790

2.7938170

3. 6937920

2. 3987980

3. 1183980

4.1870780

2.8770060

2.5439930

1.4677280

2.7237570

3. 7991850

2. 2096900

2. 3667340

3.2595520

1.6546790

$-0.7598920$

$-0.9855020$

0.8574050 


\begin{tabular}{|c|c|c|}
\hline $\begin{array}{l}2.9428490 \\
3.3236310 \\
4.7641730 \\
5.3990790 \\
4.4038380 \\
3.5676660 \\
3.2000420 \\
5.4058770 \\
5.7441300 \\
1.9032570 \\
2.6081270 \\
4.7519950 \\
6.2863580 \\
0.7573770 \\
1.9966280 \\
1.6949640 \\
0.5597060 \\
0.7218670 \\
0.5170980 \\
2.5999080 \\
2.4556410 \\
2.7419850 \\
0.8340700 \\
0.3939340 \\
1.4789930 \\
1.1460550 \\
1.4058180 \\
3.2569690 \\
2.8105160 \\
4.5725290 \\
5.1620200 \\
5.2393820 \\
6.3961180 \\
4.6518190 \\
6.4761190 \\
4.7833680 \\
7.0560110 \\
6.8438710 \\
6.9881830 \\
8.0219460 \\
1.9536180 \\
2.4377520 \\
0.1914320 \\
0.0428340 \\
0.8180300 \\
.0353490\end{array}$ & $\begin{array}{l}-4.7846480 \\
-4.7610190 \\
-4.2451380 \\
-3.6546960 \\
-2.8214580 \\
-5.5270860 \\
-5.7643150 \\
-5.0514680 \\
-4.4792530 \\
-5.1015460 \\
-4.1129400 \\
-3.4728600 \\
-3.0658230 \\
-4.3782790 \\
-5.0221470 \\
-5.8378480 \\
-6.8171930 \\
-6.0442760 \\
-4.8572990 \\
-6.3666630 \\
-5.6917640 \\
-4.2487820 \\
-7.4440480 \\
-7.4912100 \\
-6.7114560 \\
-5.6717790 \\
-5.1686270 \\
2.6966260 \\
1.6156600 \\
3.1224910 \\
2.3828520 \\
4.2425400 \\
2.7602550 \\
1.5094960 \\
4.6119230 \\
4.8156070 \\
3.8731750 \\
2.1824950 \\
5.4788610 \\
4.1658630 \\
1.2070760 \\
3.5535490 \\
3.6483780 \\
3.4876620 \\
2.8872150 \\
2.4767750\end{array}$ & $\begin{array}{r}1.4499580 \\
2.9415920 \\
3.1793560 \\
1.9031290 \\
1.1397310 \\
0.9336730 \\
3.3623140 \\
3.5499580 \\
1.2637260 \\
1.3315470 \\
3.4585300 \\
3.9550910 \\
2.1572230 \\
-1.0880430 \\
-1.6806020 \\
-2.9366200 \\
-2.6521960 \\
-2.3471680 \\
-1.4232450 \\
-3.2547340 \\
-0.9427910 \\
-1.8938900 \\
-1.7928640 \\
-3.4994230 \\
-1.9180500 \\
-3.2900960 \\
-3.7578900 \\
-0.4132320 \\
0.1388240 \\
0.1199210 \\
1.1543920 \\
-0.3904730 \\
1.6670190 \\
1.5411310 \\
0.1205690 \\
-1.1903490 \\
1.1504150 \\
2.4698540 \\
-0.2844890 \\
1.5510700 \\
-0.2696440 \\
-1.6097250 \\
0.7362950 \\
-0.7041750 \\
1.2003500 \\
-1.0965100\end{array}$ \\
\hline
\end{tabular}

1.1979930
1.2109560
0.4473970
0.2378780
0.6046320
-0.4136730
0.1926530
0.8388300
1.6933230
-1.4776690
-0.2065050
1.5044600
-0.8134110
0.2871100
3.2730420
2. 2726560
6.0805240
5. 9972590
4.6131820
5. 0424120
7.0816890
6.7529150
4.4664370
5.2770210
5.0343210
5.9101870
6.2071480
4.4892030
0.7559020
0.9405750
0.5482090
$-0.9655440$
$-1.5099580$
1. 2728970
0.9093470
$-1.4805530$
$-1.5030260$
1.1443120
2. 3479300
0.7862250
$-1.1978250$

154

TS289(S, S) G[WB97XD/6-31G(d)] $=-3864.642531$

$\begin{array}{lrrr}\text { TS289(S, S }(\mathrm{G}[\mathrm{WB} 97 \times \mathrm{D} / 6-31 \mathrm{G}(\mathrm{d})]=-3864.642531 \\ \mathrm{P} & 0.2909390 & -0.0798010 & 0.2314660 \\ \mathrm{O} & 0.7924180 & 0.8147010 & 1.3308420 \\ \mathrm{O} & -0.7415950 & 0.4621580 & -0.7295360 \\ \mathrm{O} & -0.1712860 & -1.4509830 & 0.9643960 \\ \mathrm{O} & 1.4797280 & -0.6217100 & -0.7322140 \\ \mathrm{C} & -0.2730880 & -2.6069720 & 0.2099570 \\ \mathrm{C} & 0.9051070 & -3.2712360 & -0.1659110 \\ \mathrm{C} & -1.5433660 & -3.0661990 & -0.1250590 \\ \mathrm{C} & -1.6218140 & -4.2625970 & -0.8416430 \\ \mathrm{H} & -2.6036620 & -4.6492670 & -1.1047350 \\ \mathrm{C} & 2.4831050 & -1.3494720 & -0.1184470 \\ \mathrm{C} & 3.6964960 & -0.7169650 & 0.1379550 \\ \mathrm{C} & 2.2265220 & -2.6880920 & 0.2026850 \\ \mathrm{C} & 4.6976510 & -1.4821070 & 0.7381220 \\ \mathrm{H} & 5.6623500 & -1.0214890 & 0.9380670 \\ \mathrm{C} & -2.7802770 & -2.2993950 & 0.1949170 \\ \mathrm{C} & 3.8882730 & 0.7251730 & -0.1803760 \\ \mathrm{C} & -3.1870740 & -2.0606260 & 1.5131600 \\ \mathrm{C} & -2.4568290 & -2.4584300 & 2.7728060 \\ \mathrm{C} & -4.6660400 & -1.2039150 & 3.2441430 \\ \mathrm{C} & -4.3956990 & -1.4032870 & 1.7757840 \\ \mathrm{C} & -2.1608440 & -1.1764150 & 3.5824940 \\ \mathrm{H} & -1.6849460 & -1.4580780 & 4.5290530 \\ \mathrm{H} & -1.4451510 & -0.5587970 & 3.0342940 \\ \mathrm{C} & -3.4833500 & -0.4080550 & 3.8406590 \\ \mathrm{H} & -3.6512040 & -0.2614850 & 4.9141050 \\ \mathrm{H} & -3.4438330 & 0.5866170 & 3.3833830 \\ \mathrm{H} & -5.6099230 & -0.6699620 & 3.4034040 \\ \mathrm{H} & -1.5274210 & -2.9887550 & 2.5560170 \\ \mathrm{C} & -4.7086410 & -2.5903060 & 3.9239420\end{array}$




$\begin{array}{lrrr}\mathrm{H} & -4.8378590 & -2.4565400 & 5.0043980 \\ \mathrm{H} & -5.5787780 & -3.1475950 & 3.5624180 \\ \mathrm{C} & -3.3950920 & -3.3540080 & 3.6126250 \\ \mathrm{H} & -2.8818350 & -3.6375980 & 4.5387170 \\ \mathrm{H} & -3.6034880 & -4.2788260 & 3.0648330 \\ \mathrm{C} & -3.5932650 & -1.8323570 & -0.8478430 \\ \mathrm{C} & -3.2967020 & -1.8967730 & -2.3277810 \\ \mathrm{C} & -5.5802920 & -0.8042830 & -1.8085980 \\ \mathrm{C} & -4.8137430 & -1.2023690 & -0.5742910 \\ \mathrm{C} & -4.4231910 & -2.6973890 & -3.0183740 \\ \mathrm{H} & -4.2580440 & -2.6778860 & -4.1020240 \\ \mathrm{H} & -4.3784170 & -3.7469310 & -2.7078080 \\ \mathrm{C} & -5.7991550 & -2.0754250 & -2.6600530 \\ \mathrm{H} & -6.3559610 & -1.8130630 & -3.5671940 \\ \mathrm{H} & -6.4137100 & -2.7864570 & -2.0982600 \\ \mathrm{H} & -6.5400200 & -0.3456280 & -1.5477750 \\ \mathrm{H} & -2.3245800 & -2.3521150 & -2.5276100 \\ \mathrm{C} & -4.7161840 & 0.1759720 & -2.6299650 \\ \mathrm{H} & -5.2249450 & 0.3902610 & -3.5773720 \\ \mathrm{H} & -4.6312790 & 1.1271570 & -2.0971700 \\ \mathrm{C} & -3.3226640 & -0.4525340 & -2.8787200 \\ \mathrm{H} & -3.0956810 & -0.4777390 & -3.9509140 \\ \mathrm{H} & -2.5304640 & 0.1248230 & -2.3946810 \\ \mathrm{C} & -5.2241700 & -0.9988070 & 0.7363830 \\ \mathrm{H} & -6.1746840 & -0.5101750 & 0.9445640 \\ \mathrm{C} & 3.9683440 & 2.5514570 & -1.7881600 \\ \mathrm{C} & 3.8881440 & 2.8680870 & -3.2600770 \\ \mathrm{C} & 3.5674330 & 0.3592240 & -2.7373860 \\ \mathrm{C} & 3.7897150 & 1.1930030 & -1.4979550 \\ \mathrm{C} & 4.9941230 & 2.0538810 & -3.9693210 \\ \mathrm{H} & 4.9438790 & 2.2406820 & -5.0483340 \\ \mathrm{H} & 5.9755510 & 2.3975880 & -3.6270050 \\ \mathrm{C} & 4.7955630 & 0.5469150 & -3.6584360 \\ \mathrm{H} & 4.6361040 & -0.0215110 & -4.5819850 \\ \mathrm{H} & 5.6835060 & 0.1293610 & -3.1731000 \\ \mathrm{H} & 3.4381660 & -0.6986490 & -2.5002500 \\ \mathrm{H} & 4.0255130 & 3.9397300 & -3.4422050 \\ \mathrm{C} & 2.3230370 & 0.8932850 & -3.4769800 \\ \mathrm{H} & 2.1763390 & 0.3106040 & -4.3936350 \\ \mathrm{H} & 1.4385440 & 0.7397920 & -2.8538280 \\ \mathrm{C} & 2.5254710 & 2.3930680 & -3.8091080 \\ \mathrm{H} & 2.4993180 & 2.5636660 & -4.8917010 \\ \mathrm{H} & 1.7212810 & 2.9945120 & -3.3770190 \\ \mathrm{C} & 4.3808940 & 3.0012420 & 0.5310960 \\ \mathrm{C} & 4.7618430 & 3.8378910 & 1.7257260 \\ \mathrm{C} & 4.3514430 & 1.3555170 & 2.3076560\end{array}$

\begin{abstract}
4.1809710
3. 6445180

3.9538240

2.7356590

3. 3848260

3. 5405870

2. 3559060

4. 1612850

4.9299790

5.7964310

5.9355160

6.5061760

6. 0501030

6. 3395100

6. 8671010

4. 2659060

4.4305300

3. 2316510

3. 0548260

3. 5060400

4. 9296230

5. 5091420

4.4732060

3.6546590

3. 4473890

5.6126430

5.8443190

2. 0147830

2. 7856920

4.9030990

6. 3949510

0.7884390

1.9536730

1. 6875410

0.3281750

$-0.5347690$

$-0.4826830$

2. 0942150

2. 5089530

0.4796680

$-0.1557810$

2. 8847890

1.7130120

$-0.2296840$

-1.5679170
-3.2793790
\end{abstract}

1. 6476320

3. 7394600

4. 2922100

4.2111020

2. 2483360

2.0536370

1. 9625660

0.3054920

4.8818310

1. 7430840

1. 5774110

1.0902160

3.2304550

3. 8063730

3. 3187080

3. 4580610

4.5097110

$-3.4068620$

$-4.7984920$

$-4.8357180$

$-4.2627380$

$-3.6344660$

$-2.8011340$

$-5.5109260$

$-5.8659450$

$-5.0437290$

$-4.4380870$

$-5.1248350$

$-4.2541440$

$-3.4965610$

$-3.0360020$

$-4.4326510$

$-5.1575370$

$-5.3703130$

$-6.0564090$

$-6.1944460$

$-4.9384150$

$-6.1327660$

$-5.9529080$

$-7.0498620$

$-7.0352350$

$-4.6020920$

$-5.4725520$

$-6.4405490$

2. 6546680
0.8347770

2.7857350

3.6809570

2. 4028830

3. 1157160

4.1833530

2.8841320

2.5378060

1.4393530

2. 6945120

3.7691030

2. 1749400

2. 3282210

3. 2150340

1.6047940

$-0.7761940$

$-1.0076820$

0.8645290

1. 4187720

2. 8906680

3. 1009910

1. 8183920

1. 1111870

0.8347970

3. 2567940

3.4514030

1. 1476510

1. 3403090

3. 4754030

3.8825220

2. 0546970

$-0.9422120$

$-1.5695800$

$-3.0712680$

$-3.3523920$

$-2.0825670$

$-1.2541010$

$-1.0820240$

$-3.5010820$

$-3.7875140$

$-1.4849740$

$-1.4349900$

$-3.5561530$

$-4.0917950$

$-2.3481880$

$-0.4220800$ 


$\begin{array}{lrrr}\mathrm{O} & -2.8280990 & 1.5818710 & 0.1418620 \\ \mathrm{C} & -4.6029940 & 3.0732850 & 0.0964330 \\ \mathrm{C} & -5.1891670 & 2.3450300 & 1.1407340 \\ \mathrm{C} & -5.2802080 & 4.1763140 & -0.4368930 \\ \mathrm{C} & -6.4299740 & 2.7170780 & 1.6409720 \\ \mathrm{H} & -4.6705360 & 1.4847690 & 1.5450450 \\ \mathrm{C} & -6.5238720 & 4.5398730 & 0.0612710 \\ \mathrm{H} & -4.8267480 & 4.7406920 & -1.2443230 \\ \mathrm{C} & -7.1002100 & 3.8128090 & 1.1013770 \\ \mathrm{H} & -6.8747670 & 2.1487190 & 2.4521050 \\ \mathrm{H} & -7.0439900 & 5.3934120 & -0.3615770 \\ \mathrm{H} & -8.0714750 & 4.1011780 & 1.4921870 \\ \mathrm{H} & -1.9640490 & 1.1788510 & -0.2567110 \\ \mathrm{~S} & -2.4569300 & 3.5105160 & -1.6171780 \\ \mathrm{C} & -0.2328850 & 3.6341250 & 0.7511920 \\ \mathrm{C} & 0.0147140 & 3.4805560 & -0.6879840 \\ \mathrm{H} & -0.8511950 & 2.8624640 & 1.2088120 \\ \mathrm{H} & 0.0267790 & 2.4704080 & -1.0822530 \\ \mathrm{O} & 1.1618620 & 3.2800770 & 0.7807880 \\ \mathrm{H} & 1.1883030 & 2.2796430 & 0.9646180 \\ \mathrm{C} & 0.3710680 & 6.0758990 & 0.5748820 \\ \mathrm{C} & 0.1738820 & 5.9940090 & -0.9405290 \\ \mathrm{C} & 0.5644300 & 4.6172080 & -1.4866920 \\ \mathrm{C} & -0.4802540 & 5.0231580 & 1.2897520 \\ \mathrm{H} & 0.0990470 & 7.0721280 & 0.9370810 \\ \mathrm{H} & 0.7674660 & 6.7599060 & -1.4489500 \\ \mathrm{H} & 1.6550700 & 4.4859690 & -1.4748440 \\ \mathrm{H} & -1.5465510 & 5.2426090 & 1.1535920 \\ \mathrm{H} & -0.2813240 & 5.0148410 & 2.3663200 \\ \mathrm{H} & 1.4285950 & 5.9199770 & 0.8205500 \\ \mathrm{H} & -0.8785820 & 6.1895940 & -1.1797330 \\ \mathrm{H} & 0.2543050 & 4.4926940 & -2.5278340 \\ 154 & & & \end{array}$

TS290(S, S) G[WB97XD/6-31G(d)] $=-3864.642288$

\begin{tabular}{lrrr}
\multicolumn{1}{l}{ TS290(S, S $)$} & G $[W B 97 X D / 6-31 G(d)]=-3864.642288$ \\
$\mathrm{P}$ & -0.3193820 & -0.5194800 & 1.0772710 \\
$\mathrm{O}$ & 0.3006780 & -1.0943760 & 2.3149610 \\
$\mathrm{O}$ & -0.7663740 & 0.9234290 & 1.1121830 \\
$\mathrm{O}$ & -1.5171320 & -1.5120250 & 0.6231270 \\
$\mathrm{O}$ & 0.6875420 & -0.5739500 & -0.2176480 \\
$\mathrm{C}$ & -2.0459830 & -1.2431750 & -0.6327100 \\
$\mathrm{C}$ & -1.3339920 & -1.6882840 & -1.7446530 \\
$\mathrm{C}$ & -3.2361250 & -0.5265340 & -0.7330060 \\
$\mathrm{C}$ & -3.6816150 & -0.2399530 & -2.0196260 \\
$\mathrm{H}$ & -4.6128780 & 0.3113560 & -2.1325330 \\
$\mathrm{C}$ & 0.9537000 & -1.8053020 & -0.7916330 \\
$\mathrm{C}$ & 2.2340070 & -2.3403440 & -0.6706130
\end{tabular}

-0.0632890
2.4522660
3.4408230
-4.0031060
3.3318410
-4.5534270
-4.4270490
-5.8513530
-5.3376960
-3.7862660
-3.7245190
-2.7637920
-4.6376330
-4.9965620
-4.0408680
-6.4492020
-3.8254300
-6.7000060
-7.0368170
-7.5968180
-5.8488660
-5.7748590
-6.3082010
-4.2399580
-3.6959290
-5.2572430
-5.0523340
-4.8902820
-4.5106770
-5.4138910
-5.8502060
-5.9993500
-6.8350750
-5.9284750
-3.0248850
-3.8820210
-4.0134480
-3.4705690
-2.9334700
-2.5326000
-2.0854720
-5.5992910
-6.2273230
4.8009110
5.1828700
3.3592590
$-1.5186630$

$-1.1950860$

$-1.1009910$

0.4609270

$-0.1022180$

1. 3487430

1. 2731780

3. 2789020

2.4298480

2.5880360

2.5634380

2.6533220

3. 7932010

4.3831920

4.4626420

4. 1176220

0.4190040

2. 3802140

2. 9690150

2. 0478400

1.1670660

1.1325850

0.2263800

0.6903710

$-0.1017500$

1. 8220620

1.7564680

$-0.6812550$

$-1.2008840$

$-1.4265900$

0.4700750

0.4910970

0.3323930

2. 6439540

$-0.8987480$

1. 9947880

1. 9628080

2. 9789620

0.8688620

0.3041340

1. 2887190

2. 6293150

3. 4581610

$-0.3410930$

$-1.2805300$

$-2.2374770$ 


\begin{tabular}{|c|c|c|}
\hline $\begin{array}{l}3.7951900 \\
5.6963690 \\
5.9362400 \\
6.6240360 \\
4.6070650 \\
4.3150470 \\
4.9819260 \\
2.5916090 \\
5.9537510 \\
2.8269830 \\
2.5286670 \\
1.9263140 \\
3.9214610 \\
4.1891950 \\
3.5551280 \\
4.9554580 \\
5.5098390 \\
3.6381090 \\
3.9294160 \\
4.3506150 \\
4.7401070 \\
3.9372900 \\
3.2549620 \\
3.1457370 \\
2.2811140 \\
2.8382540 \\
6.3001330 \\
4.9500360 \\
4.7737480 \\
5.2435930 \\
6.0622590 \\
6.4108830 \\
6.9305860 \\
5.3866570 \\
6.1828460 \\
0.1741250 \\
0.8752140 \\
0.9423460 \\
0.4500410 \\
1.5933950 \\
1.4312300 \\
0.6221320 \\
1.6161950 \\
0.4814210 \\
1.5765680 \\
1.8561360\end{array}$ & $\begin{array}{r}-0.4155950 \\
0.8852750 \\
1.6743260 \\
0.3400410 \\
-0.0657410 \\
0.2331250 \\
-1.0924120 \\
-0.7026250 \\
2.1751390 \\
1.4160190 \\
1.7281270 \\
1.4322080 \\
2.3558600 \\
3.1263250 \\
2.8821600 \\
-0.9631420 \\
-1.4243230 \\
-2.9612240 \\
-1.7862700 \\
-1.4842490 \\
-1.8226160 \\
-0.4818020 \\
-2.4470330 \\
-3.3092360 \\
-1.9578430 \\
-3.5881340 \\
-0.7530400 \\
-3.7685860 \\
-4.6320960 \\
-4.1592610 \\
-2.8559920 \\
-3.2370180 \\
-2.8273980 \\
0.1406260 \\
0.7762470 \\
-3.7252910 \\
-4.5866510 \\
-5.9591380 \\
-6.6219760 \\
-5.7291410 \\
-4.3194790 \\
-4.7387880 \\
-6.6098200 \\
-7.5830680 \\
-5.7308610 \\
-4.1042900\end{array}$ & $\begin{array}{l}-0.8441630 \\
-2.5870740 \\
-3.3091360 \\
-2.3858990 \\
-3.1490930 \\
-4.1624940 \\
-3.2123500 \\
-2.6247380 \\
-0.8419880 \\
-2.1946450 \\
-3.2021710 \\
-1.5775440 \\
-1.6284240 \\
-2.3603190 \\
-0.7409720 \\
1.6175670 \\
2.9399710 \\
2.0308870 \\
1.1297470 \\
3.9585710 \\
4.9259230 \\
4.1093490 \\
3.4346580 \\
4.1028250 \\
3.3827490 \\
1.6288640 \\
3.2937030 \\
2.1643690 \\
2.8158120 \\
1.1841210 \\
2.7485480 \\
3.7155560 \\
2.0824140 \\
0.8910720 \\
1.2765270 \\
-2.0041830 \\
-2.6585890 \\
-1.9617410 \\
-1.8036740 \\
-2.3249210 \\
-1.8216900 \\
-3.7174430 \\
-2.5285200 \\
-2.3272890 \\
-3.4240420 \\
-2.6320250\end{array}$ \\
\hline
\end{tabular}

-1.3980710
0.6355710
2.5627730
-1.7729950
-0.9380350
-1.2703190
-2.7783130
-3.4809260
-2.9642710
-0.7385890
-1.1038440
0.1248500
-3.1305590
-3.0316450
-4.5629750
-3.3398900
-0.9194400
0.4592230
-0.2841420
0.6740420
1.6251620
-0.0584950
1.8393790
2.2017490
0.1556210
-0.7884290
1.1058620
2.5839610
-0.4169650
1.2752660
-0.4517050
1.1376880
1.0582900
2.0346190
0.0248960
1.8187920
1.7132530
1.1800430
2.7355070
3.5074980
3.4184560
1.2572130
2.8228220
4.5607460
4.0195800
0.7811750

-5.8165540
-6.8345200
-6.1406410
-1.3244100
-1.6645880
-0.7915590
-0.7820300
-0.1266170
-0.5970050
-1.1682190
-2.7137960
-1.5815950
-1.8153480
-0.2488560
-0.2946590
0.9619660
0.2350920
3.4346000
2.3872720
4.1183870
5.1339850
3.6971980
5.7199970
5.4497360
4.2892740
2.9032730
5.2999540
6.5028730
3.9600580
5.7593600
1.8305480
3.9905990
1.9619490
1.9490160
1.8227050
1.3363930
0.7052460
-0.0345940
3.2199880
3.5811740
2.4654960
2.9743370
4.0243930
3.7725080
1.5967260
3.9063350

$-0.9764990$

$-0.7458490$

$-2.0259790$

$-3.0322810$

$-4.2515100$

$-5.4605040$

$-5.6953620$

$-4.5083930$

$-3.1611690$

$-6.3408580$

$-4.5272730$

$-4.0004530$

$-5.8154420$

$-6.6180500$

$-4.5620460$

$-4.5690510$

$-5.2869530$

$-0.7239700$

$-0.8298270$

$-2.0229740$

$-2.1665430$

$-3.1420540$

$-3.4075520$

$-1.3041370$

$-4.3793000$

$-3.0332760$

$-4.5148160$

$-3.5113180$

$-5.2408170$

$-5.4840320$

0.0352600

0.7162590

3. 2838410

2. 1879660

2. 9747390

1. 3198890

3. 5765660

3.1348310

4.7008030

3.4300720

2. 3867380

4. 3867460

5.4376640

3.6568980

2. 6842680

4.0574880 


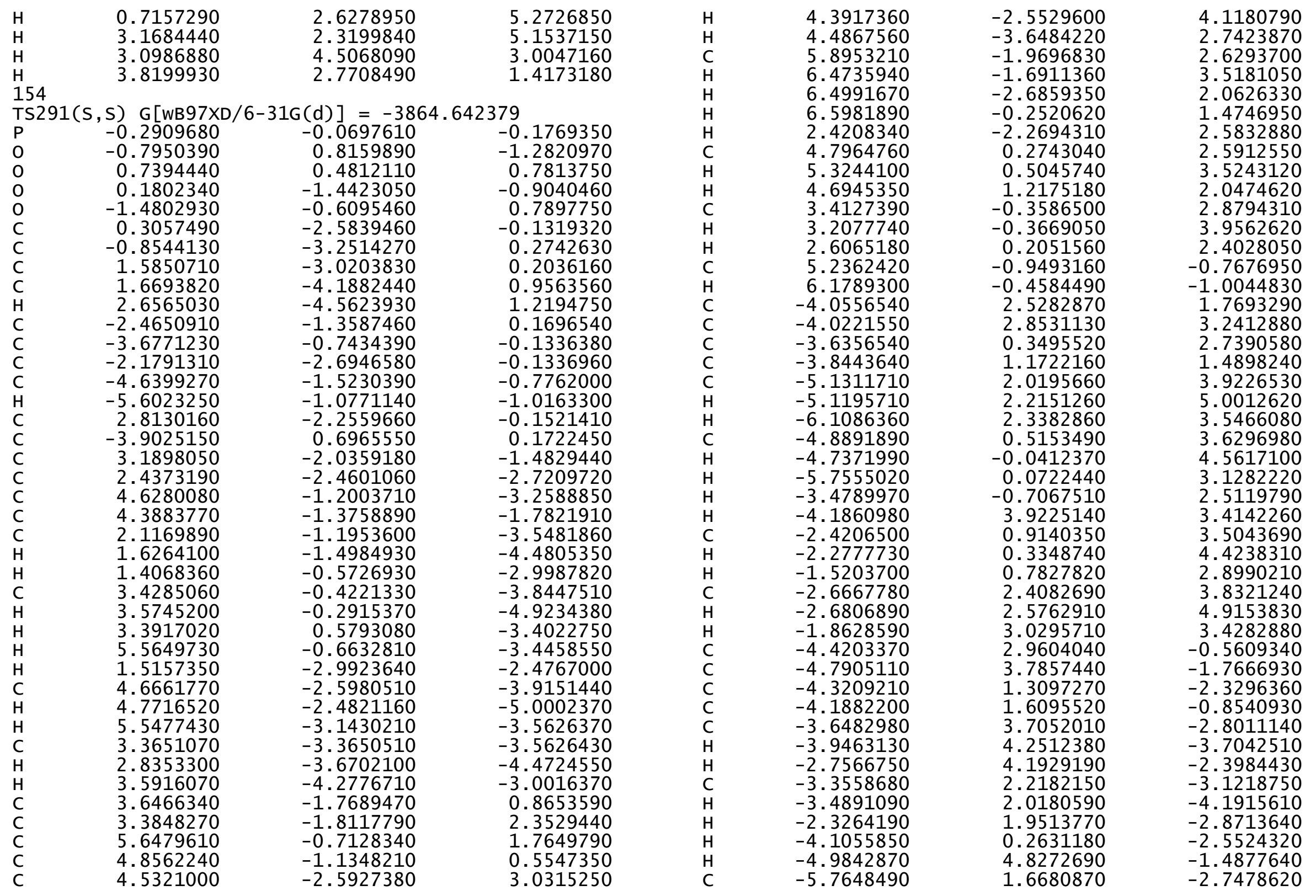




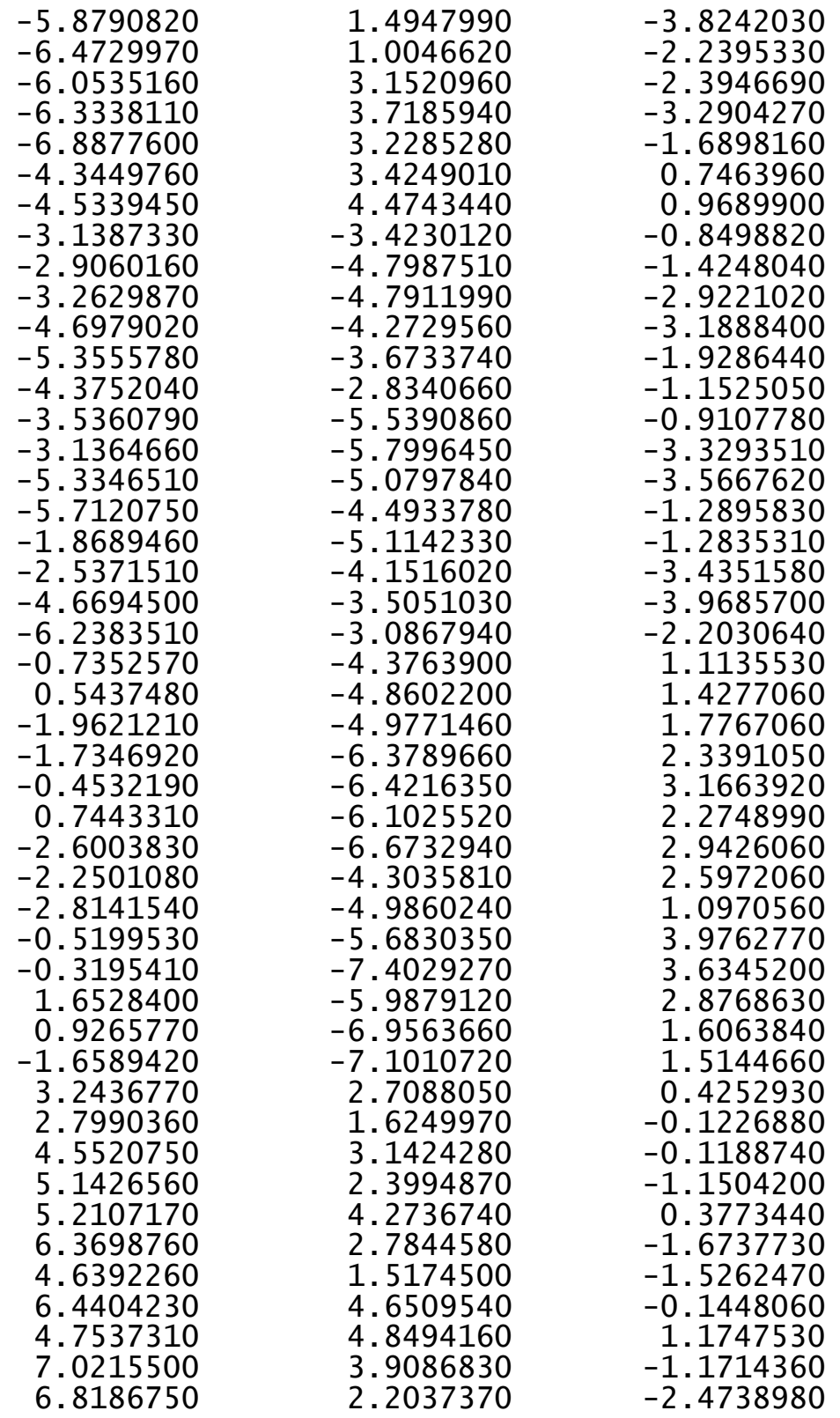

$\begin{array}{lr}\mathrm{H} & 6.9461110 \\ \mathrm{H} & 7.9819840 \\ \mathrm{H} & 1.9463550 \\ \mathrm{~S} & 2.4285070 \\ \mathrm{C} & 0.1797420 \\ \mathrm{C} & -0.0522730 \\ \mathrm{H} & 0.8074690 \\ \mathrm{H} & -0.0418950 \\ \mathrm{O} & -1.2088250 \\ \mathrm{H} & -1.2193120 \\ \mathrm{C} & -0.4631280 \\ \mathrm{C} & -0.2520840 \\ \mathrm{C} & -0.6166510 \\ \mathrm{C} & 0.3987430 \\ \mathrm{H} & -0.2102760 \\ \mathrm{H} & -0.8537030 \\ \mathrm{H} & -1.7051000 \\ \mathrm{H} & 1.4625220 \\ \mathrm{H} & 0.1903300 \\ \mathrm{H} & -1.5201850 \\ \mathrm{H} & 0.7991080 \\ \mathrm{H} & -0.3001830 \\ \mathrm{H} & \end{array}$
5.5267160
2.8953610
2.4787300
3.2757590
2.2754320
6.0850020
5.9987100
4.6128250
5.0498280
7.0873630
6.7523190
4.4645800
5.2859110
5.0437390
5.9135960
6.2094360
4.4868070

$\begin{array}{rr}3.5609010 & 1.6280490 \\ 3.6542330 & -0.7157110\end{array}$

$3.4902800 \quad 0.7248300$

$-1.1819830$

-1.1819830
1.1154500

$-0.7347450$

$-0.9192880$

$-0.5216590$

0.9917370

1.5330260

$-1.2496070$

$-0.8808100$

1. 5089550

1.5242340

$-1.1218290$

$-2.3244170$

$-0.7589680$

1.2236820

15

2.5721270

TS292(S, S) G[WB97XD/6-31G(d)] $=-3864.642549$

$\begin{array}{lrrr}\text { TS292(S, S) G[WB97XD/6-31G(d)] }=-3864.642549 \\ \text { P } & -0.2591960 & -0.3030820 & 1.0794150 \\ \mathrm{O} & 0.4358170 & -0.7285490 & 2.3387230 \\ \mathrm{O} & -0.6572460 & 1.1461380 & 0.9342030 \\ \mathrm{O} & -1.5145250 & -1.3071500 & 0.8764870 \\ \mathrm{O} & 0.6413270 & -0.5908580 & -0.2606710 \\ \mathrm{C} & -2.1119760 & -1.2860080 & -0.3754050 \\ \mathrm{C} & -1.4620260 & -1.9603790 & -1.4144020 \\ \mathrm{C} & -3.3089810 & -0.5974120 & -0.5459400 \\ \mathrm{C} & -3.8577000 & -0.5914750 & -1.8303650 \\ \mathrm{H} & -4.8041120 & -0.0796180 & -1.9888670 \\ \mathrm{C} & 0.8740280 & -1.9132140 & -0.5981270 \\ \mathrm{C} & 2.1537550 & -2.4387960 & -0.4304500 \\ \mathrm{C} & -0.1789480 & -2.6614740 & -1.1213590 \\ \mathrm{C} & 2.3246130 & -3.7882450 & -0.7161090 \\ \mathrm{H} & 3.3142210 & -4.2241110 & -0.5940650 \\ \mathrm{C} & -3.9919070 & 0.1107520 & 0.5718370 \\ \mathrm{C} & 3.2851050 & -1.5639740 & -0.0184810 \\ \mathrm{C} & -4.5030560 & -0.5959320 & 1.6673720 \\ \mathrm{C} & -4.3964610 & -2.0814610 & 1.9142130 \\ \mathrm{C} & -5.6850690 & -0.8422990 & 3.7793500 \\ \mathrm{C} & -5.2163010 & 0.0676260 & 2.6736440 \\ \mathrm{C} & -3.6785220 & -2.2913690 & 3.2651900\end{array}$




-3.6303870
-2.6496890
-4.4469870
-4.7735870
-3.8042550
-6.2258930
-3.8533290
-6.5957280
-6.9011910
-7.5073650
-5.8268410
-5.7633810
-6.3420070
-4.1941380
-3.6620800
-5.1326330
-4.9459560
-4.8622730
-4.4877000
-5.4199750
-5.7768370
-5.9251160
-6.7660950
-5.7662020
-3.0284720
-3.7442310
-3.8633840
-3.2973670
-2.8457520
-2.4400510
-2.0006550
-5.4546430
-6.0313860
4.8166400
5.2119690
3.2678850
3.7522780
5.6301340
5.8826660
6.5335870
4.4653630
4.1404590
4.7810800
2.4487340
6.0351950
2.8132590
$-3.3657450$
$-1.9314710$
$-1.5406970$
$-2.2339990$
$-0.7938160$
$-0.2826490$
$-2.5908900$
$-1.9250220$
$-2.6294800$
$-1.4586790$
$-2.6550840$
$-3.7306630$
$-2.5382180$
1.4964590
2.4537200
3.6181520
2.1437330
3.1363420
3.8927540
2.4023650
3.7838990
4.8505000
3.3149370
4.0731140
1. 9459760
4.2900990
5.3458970
4.2628830
3.5477460
4.2432490
3.0794880
1.4341010
1.9426470
0.2555070
1.2281850
$-0.3301410$
$-0.5781390$
0.4006720
1.0837690
$-0.1722950$
$-0.5417850$
$-0.3408960$
$-1.5893690$
$-0.9995370$
1.8732070
1.1378400

\begin{abstract}
2.4828300
1.9460880

3.9804930

4.2514940

3.6851970

5.0022970

5.6030140

3.6239050

3. 9221370

4.4953580

4.9171770

4.1352320

3.3342110

3. 2192060

2. 3813020

2.7778750

6.4385760

4.9040380

4.7237900

5.1308660

6.0830530

6.4647620

6.9159670

5.4467990

6.2894450

0.0008390

$-1.1782430$

$-0.9508010$

0.4005640

1.5236410

1.2667460

$-1.7655030$

$-1.3721020$

$-2.0802160$

0.4373590

0.5399290

2.4792360

1.6482150

$-0.9730830$

$-3.2099390$

$-3.7673080$

$-3.6725860$

$-2.2181760$

$-1.2921060$

$-1.9982870$

$-3.1973040$
\end{abstract}

1.3436200

1.2784540

2.0747740

2.7376510

2.7231400

$-0.8905400$

$-1.2213830$

$-2.7600560$

$-1.7157960$

$-1.1055780$

$-1.3482010$

$-0.0728880$

$-2.0665010$

$-2.8393420$

$-1.5396600$

$-3.3891320$

$-0.5532150$

$-3.6098210$

$-4.3817330$

$-4.1261360$

$-2.6910300$

$-2.9731490$

$-2.7812570$

0.0974840

0.7319840

$-4.0414560$

$-4.9044830$

$-6.3895020$

$-6.8229020$

$-6.0896380$

$-4.6046030$

$-6.9723530$

$-4.7787090$

$-4.5508340$

$-6.5897960$

$-7.9047700$

$-6.2351690$

$-6.5352040$

$-6.5755780$

$-1.1899350$

$-1.1655670$

$-2.5714520$

$-3.0858660$

$-2.3457810$

$-1.8682630$

$-0.4590960$
$-3.4403250$

$-1.7688900$

$-2.0145760$

$-2.8444950$

$-1.1829910$

1. 5625710

2. 9035990

2. 2658340

1. 2168360

3.9727280

4.9550310

4.0217690

3.6138810

4.3829430

3.5478420

1.9795960

3.1394580

2.4371400

3.1940680

1.4980770

2.8574890

3.8455880

2.1528480

0.6920110

0.9640440

$-1.3317000$

$-1.7396610$

$-1.4633100$

$-2.0253980$

$-1.2940750$

$-1.1223900$

$-1.9062600$

$-2.8121230$

$-1.2288990$

$-3.0982680$

$-1.9273830$

$-1.8116290$

$-0.2970490$

$-0.3813290$

$-2.9031170$

$-4.3037630$

$-4.9134980$

$-4.9440590$

$-3.9516610$

$-2.7050670$

$-4.9251760$ 


\begin{tabular}{|c|c|c|}
\hline $\begin{array}{r}-4.1016280 \\
-1.7969350 \\
-0.9038300 \\
-4.8044870 \\
-4.2884000 \\
-2.2112750 \\
-0.4158970 \\
0.5939170 \\
-0.1574140 \\
0.7569820 \\
1.7688800 \\
-0.1006940 \\
1.9197950 \\
2.4403070 \\
0.0482790 \\
-0.8798220 \\
1.0591130 \\
2.7107860 \\
-0.6249120 \\
1.1771590 \\
-0.3203370 \\
1.3291660 \\
1.2903040 \\
2.2363990 \\
0.2471410 \\
1.9906830 \\
1.9439400 \\
1.3825730 \\
3.0153820 \\
3.7558210 \\
3.6317750 \\
1.5268300 \\
3.1306420 \\
4.8161040 \\
4.2319120 \\
1.0508690 \\
1.0060770 \\
3.4524060 \\
3.3438470 \\
4.0119360\end{array}$ & $\begin{array}{l}-2.5788070 \\
-2.9714990 \\
-1.4468740 \\
-0.8146180 \\
-3.2408470 \\
-4.1598560 \\
-2.9529730 \\
3.4423160 \\
2.3942210 \\
4.0418760 \\
4.9701240 \\
3.6395940 \\
5.4890810 \\
5.2712230 \\
4.1683330 \\
2.9150390 \\
5.0918790 \\
6.2044870 \\
3.8587710 \\
5.5020910 \\
1.9251910 \\
4.0968350 \\
2.3400610 \\
2.2024130 \\
2.1821510 \\
1.5023590 \\
1.1133970 \\
0.3443500 \\
3.7111680 \\
3.9330460 \\
2.7181300 \\
3.4536240 \\
4.5851840 \\
4.1351220 \\
1.8775990 \\
4.3549480 \\
3.2010610 \\
2.8562900 \\
4.8159020 \\
2.9209730\end{array}$ & $\begin{array}{l}-5.9208040 \\
-5.9486290 \\
-4.4510820 \\
-4.2966550 \\
-4.3020610 \\
-4.7300940 \\
-3.7143680 \\
-1.1539250 \\
-1.1600320 \\
-2.5002090 \\
-2.7677870 \\
-3.5334410 \\
-4.0471450 \\
-1.9713880 \\
-4.8087040 \\
-3.3265590 \\
-5.0680240 \\
-4.2484280 \\
-5.6019130 \\
-6.0665340 \\
-0.2466260 \\
0.2133920 \\
2.9558390 \\
1.8427560 \\
2.6898820 \\
1.0518350 \\
3.3546610 \\
3.0014060 \\
4.1983230 \\
2.8773740 \\
1.9548870 \\
3.9483770 \\
4.8467040 \\
3.0574770 \\
2.3255650 \\
3.5428160 \\
4.8774140 \\
4.7280500 \\
2.3721130 \\
0.9505760\end{array}$ \\
\hline
\end{tabular}

154

TS293(S, S) G[WB97XD/6-31G(d) $]=-3864.643153$

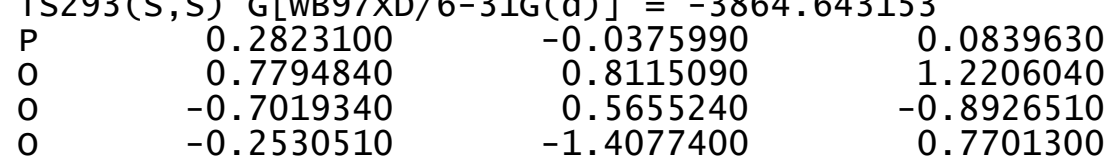

$\begin{array}{lr}\mathrm{O} & 1.4811960 \\ \mathrm{C} & -0.3741640 \\ \mathrm{C} & 0.7899310 \\ \mathrm{C} & -1.6524850 \\ \mathrm{C} & -1.7532340 \\ \mathrm{H} & -2.7422730 \\ \mathrm{C} & 2.4365260 \\ \mathrm{C} & 3.6582840 \\ \mathrm{C} & 2.1184500 \\ \mathrm{C} & 4.6000640 \\ \mathrm{H} & 5.5696820 \\ \mathrm{C} & -2.8758440 \\ \mathrm{C} & 3.9175870 \\ \mathrm{C} & -3.2697340 \\ \mathrm{C} & -2.5355900 \\ \mathrm{C} & -4.7253170 \\ \mathrm{C} & -4.4659230 \\ \mathrm{C} & -2.2168120 \\ \mathrm{H} & -1.7250440 \\ \mathrm{H} & -1.5083440 \\ \mathrm{C} & -3.5293740 \\ \mathrm{H} & -3.6870710 \\ \mathrm{H} & -3.4849900 \\ \mathrm{H} & -5.6611320 \\ \mathrm{H} & -1.6143520 \\ \mathrm{C} & -4.7839650 \\ \mathrm{H} & -4.9140500 \\ \mathrm{H} & -5.6591310 \\ \mathrm{C} & -3.4779580 \\ \mathrm{H} & -2.9629810 \\ \mathrm{H} & -3.6964580 \\ \mathrm{C} & -3.6898390 \\ \mathrm{C} & -3.4094630 \\ \mathrm{C} & -5.6596510 \\ \mathrm{C} & -4.8960910 \\ \mathrm{C} & -4.5620450 \\ \mathrm{H} & -4.4017340 \\ \mathrm{H} & -4.5461990 \\ \mathrm{C} & -5.9173180 \\ \mathrm{H} & -6.4618880 \\ \mathrm{H} & -6.5576880 \\ \mathrm{H} & -6.6054970 \\ \mathrm{H} & -2.4508580 \\ \mathrm{C} & -4.7733430 \\ \mathrm{H} & -5.2864770 \\ \mathrm{H} & -4.6482080\end{array}$
$-0.5970310$
$-2.5430280$
$-3.2335350$
$-2.9632140$
$-4.1347690$
$-4.4956390$
$-1.3749740$
$-0.7921060$
$-2.7098350$
$-1.6065860$
$-1.1862560$
$-2.1946420$
0.6492150
$-2.0283450$
$-2.5078420$
$-1.2575370$
$-1.3697470$
$-1.2771150$
$-1.6152510$
$-0.6337910$
$-0.5177040$
$-0.4500830$
0.5084370
$-0.7221770$
$-3.0357120$
$-2.6834470$
$-2.6173670$
$-3.2068520$
$-3.4395580$
$-3.7767350$
$-4.3321060$
$-1.6572450$
$-1.6485140$
$-0.5303630$
$-1.0214850$
$-2.3837560$
$-2.3195400$
$-3.4470270$
$-1.7386850$
-1. 4014440
$-2.4620920$
$-0.0641890$
$-2.1156760$
0.4784350
0.7801690
1. 3828840

.

0.1003880

(

878560

1711160

.1194950

397370

3535900

.7606100

8434870

. 3170620

7099380

. 7965220

3118100

4.2589960

566040

(7290

$-4.2977300$

$-2.0518050$ 


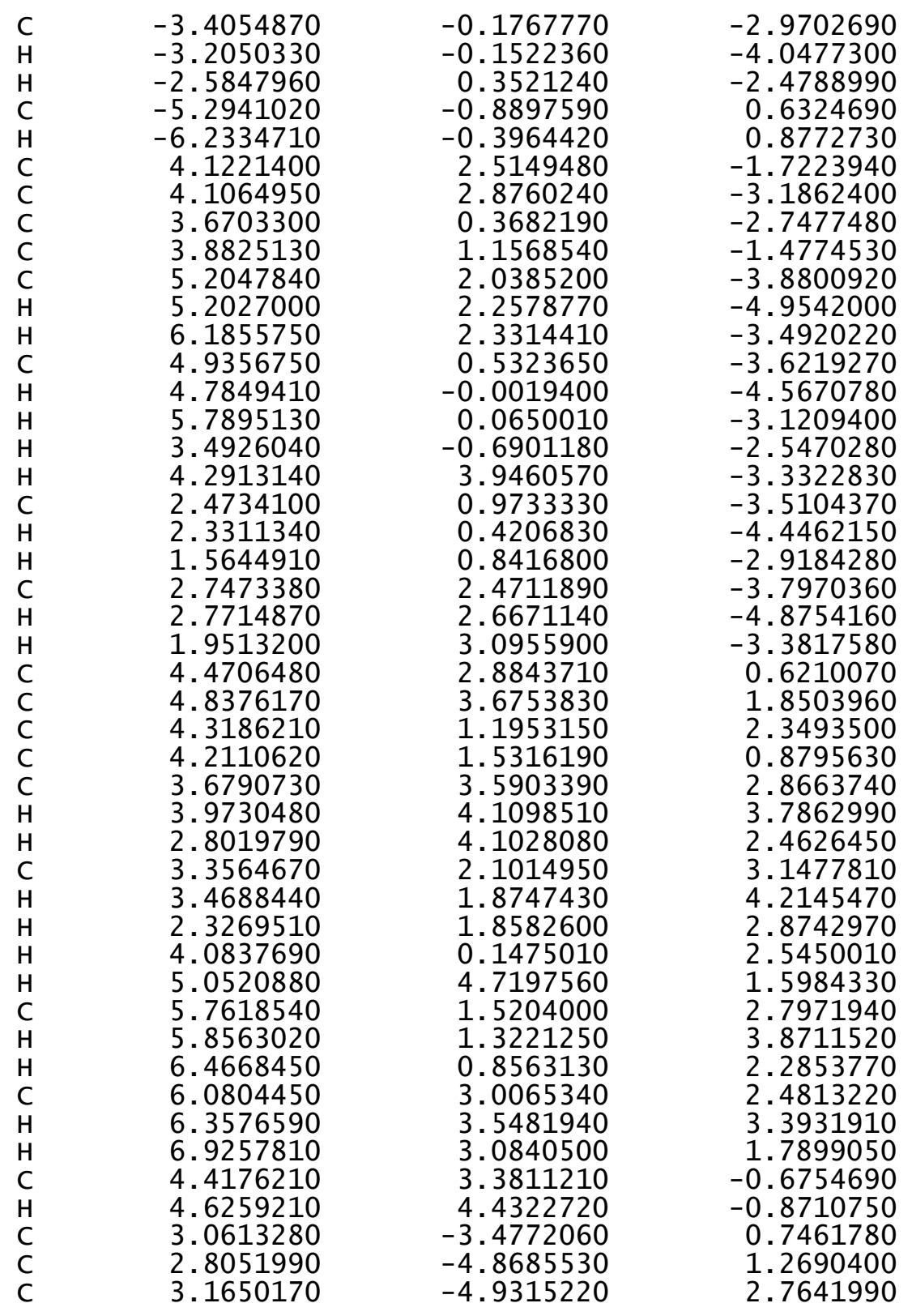

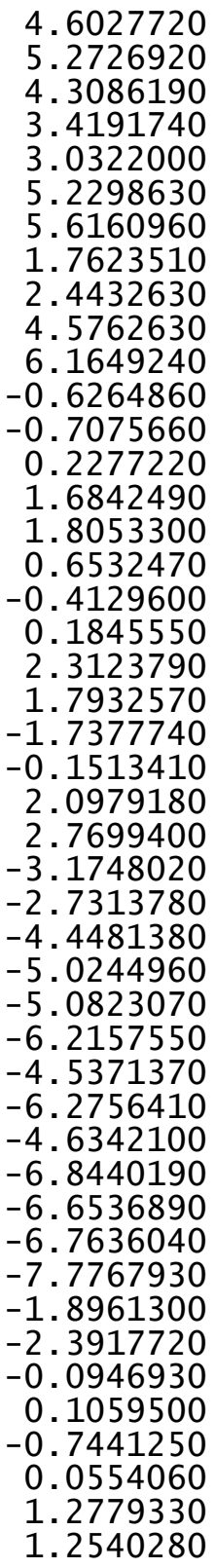
$-4.4346900$
$-3.8043420$
$-2.9221770$
$-5.5953750$
$-5.9572590$
$-5.2576020$
$-4.6080710$
$-5.1602370$
$-4.3126920$
$-3.6886960$
$-3.2426310$
$-4.8206830$
$-6.0742020$
$-7.1440000$
$-6.6391070$
$-5.1076560$
$-4.3640710$
$-5.8567670$
$-8.0532670$
$-7.1062670$
$-4.8634820$
$-6.4434080$
$-7.4111360$
$-6.9432300$
$-4.7619000$
2.8356900
1.7048160
3. 2444680
2. 3966350
4.4501960
2.7481320
1.4599640
4. 7962870
5.1090330
3.9473630
2.0829630
5.7314450
4. 2221600
1. 3095980
3.7623880
3. 6972240
3. 5694640
2.9484190
2. 5712110
3.2674000
2. 2623990

3.0552190
1.8173570
1.0687430
0.7180910
3.1234210
3.4140170
1.1509330
1.1206280
3.3075750
3.8560090
2.1123440
-1.5527120
-2.3858630
-1.8019150
-1.6850670
-1.8340940
-1.2023850
-3.4229030
-2.4104540
-2.4509870
-2.9058050
-2.4234130
-0.8090220
-0.7184080
-1.4589330
-0.3642730
0.0801240
0.2728460
1.2283110
-0.0492340
1.8476430
1.4669820
0.5709410
-0.7853500
1.5196530
2.5856630
0.3153780
2.0030080
-0.3802390
-1.5319180
0.7550420
-0.6943490
1.2082050
-1.1157730
0.7415410
0.8976120 


\begin{tabular}{|c|c|c|c|}
\hline & & & \\
\hline c & $\begin{array}{l}0.6357910 \\
0.3986460\end{array}$ & 6.1073710 & $\begin{array}{r}0.6225120 \\
-0.8893650\end{array}$ \\
\hline C & 0.7023170 & 4.6934050 & -1.4769430 \\
\hline C & -0.2528940 & 5.0832490 & 1.3338940 \\
\hline $\mathrm{H}$ & 0.4265610 & 7.1069380 & 1.0157730 \\
\hline $\mathrm{H}$ & 1.0209210 & 6.8208720 & -1.3934350 \\
\hline $\mathrm{H}$ & 1.7845140 & 4.5048610 & -1.4854740 \\
\hline $\mathrm{H}$ & -1.3089220 & 5.3624790 & 1.2325450 \\
\hline $\mathrm{H}$ & -0.0271340 & 5.0359670 & 2.4041880 \\
\hline $\mathrm{H}$ & 1.6893640 & 5.8900390 & 0.8366990 \\
\hline $\mathrm{H}$ & -0.6470560 & 6.3324690 & -1.0983050 \\
\hline$H$ & 0.3696800 & 4.6094790 & -2.5153270 \\
\hline & S) G[wB97》 & $(d)]=-3$ & \\
\hline $\mathrm{P}$ & -0.3362430 & -0.5895320 & 1.0830690 \\
\hline 0 & 0.2680250 & -1.2094900 & 2.3061660 \\
\hline 0 & -0.8220210 & 0.8381280 & 1.1785470 \\
\hline 0 & -1.4979680 & -1.5822100 & 0.5413430 \\
\hline 0 & 0.7108600 & -0.5465370 & -0.1822940 \\
\hline C & -2.0132400 & -1.2314220 & -0.7006710 \\
\hline C & -1.2643190 & -1.5675790 & -1.8264330 \\
\hline C & -3.2141700 & -0.5294410 & -0.7719040 \\
\hline C & -3.6354720 & -0.1505360 & -2.0433800 \\
\hline $\mathrm{H}$ & -4.5738110 & 0.3923210 & -2.1350150 \\
\hline C & 1.0041120 & -1.7313600 & -0.8352010 \\
\hline $\mathrm{C}$ & 2.2865400 & -2.2643440 & -0.7251990 \\
\hline C & 0.0105250 & 141420 & -1.6266260 \\
\hline C & 2.5224110 & -3.50 & -1.3 \\
\hline H & 3.5121290 & -3.9458430 & -1.2365450 \\
\hline C & -4.0048850 & -0. & 0.4361450 \\
\hline C & 0900 & -1.45 & -0.1 \\
\hline C & -4.5415140 & -1.1616120 & 1.2662600 \\
\hline C & -4.38 & -2.6551820 & 1.1064340 \\
\hline $\mathrm{C}$ & -5.8319220 & -2.0075930 & 3.1475860 \\
\hline $\mathrm{C}$ & -5.3 & -0.8176420 & $2 . \overline{3} 658970$ \\
\hline C & -3.7 & -3.2135 & 2.3934710 \\
\hline H & -3.6 & -4.3036 & 2.3065540 \\
\hline $\mathrm{H}$ & -2.72 & -2.8215760 & 2.4842550 \\
\hline C & -4.6044400 & 73140 & 3.6206430 \\
\hline H & -4.9482770 & -3.7059 & \\
\hline H & 3750 & -2.2 & 4.3239540 \\
\hline H & 0750 & -1.6939050 & 4.0036620 \\
\hline H & -3.7784020 & -2.91457 & 0.2379950 \\
\hline C & 4390 & -2.8984100 & 2.1955030 \\
\hline H & 5350 & -3.7 & 2.7333730 \\
\hline H & -7.5674350 & -2.3655560 & 1.8920420 \\
\hline
\end{tabular}

-5.7986730
-5.7046590
-6.2613810
-4.2713970
-3.7518840
-5.3220930
-5.0931890
-4.9641090
-4.6039750
-5.4818100
-5.9232380
-6.0806900
-6.9050480
-5.9965110
-3.0761330
-3.9562930
-4.1024810
-3.5373360
-3.0062680
-2.6345270
-2.1391460
-5.6248490
-6.2595630
4.8268110
5.2104450
3.4090770
3.8356730
5.7447030
5.9969630
6.6686680
4.6640480
4.3779260
5.0444290
2.6511760
5.9712420
2.8613110
2.5518590
1.9649700
3.9480830
4.2206690
3.5708230
4.9667230
5.5030450
3.6593710
3.9537660
4.3260630
0.9606020

0.8619860

0.0388390

0.7403440

0.0165800

1.9819540

1.8257830

$-0.5147700$

$-0.9915800$

$-1.2869890$

0.6608310

0.7467550

0.4997120

2.8191380

$-0.7989200$

2.1973300

2.2463570

3.1593750

1.0362070

0.5206220

1.4151230

2.6417820

3.4863550

$-0.2583440$

$-1.1530530$

$-2.1858510$

$-0.8038910$

$-2.4743430$

$-3.1634430$

$-2.2777750$

$-3.0878020$

$-4.0882670$

$-3.1962390$

$-2.6056730$

$-0.6830370$

$-2.0875910$

$-3.0800680$

$-1.4636200$

$-1.4917580$

$-2.2055860$

$-0.5981340$

1.6524050

2.9677490

1.9760190

1.1221820

3.9626980 


$\begin{array}{lrrr}\text { H } & 4.7014780 & -1.9177630 & 4.9275130 \\ \text { H } & 3.8979070 & -0.5638980 & 4.1336250 \\ \mathrm{C} & 3.2517210 & -2.5169200 & 3.3920030 \\ \mathrm{H} & 3.1479360 & -3.4041080 & 4.0275260 \\ \mathrm{H} & 2.2710130 & -2.0411270 & 3.3457680 \\ \mathrm{H} & 2.8712300 & -3.5935820 & 1.5386040 \\ \mathrm{H} & 6.2818500 & -0.7902780 & 3.3571960 \\ \mathrm{C} & 4.9771940 & -3.7749080 & 2.0988280 \\ \mathrm{H} & 4.7993860 & -4.6679660 & 2.7086230 \\ \mathrm{H} & 5.2921310 & -4.1192300 & 1.1078260 \\ \mathrm{C} & 6.0690080 & -2.8765670 & 2.7410080 \\ \mathrm{H} & 6.3951510 & -3.2880480 & 3.7032950 \\ \mathrm{H} & 6.9538390 & -2.8214180 & 2.0987310 \\ \mathrm{C} & 5.3985950 & 0.1782800 & 0.9711980 \\ \mathrm{H} & 6.1833260 & 0.8067420 & -1.3903860 \\ \mathrm{C} & 0.2642850 & -3.5716510 & -2.1874080 \\ \mathrm{C} & -0.7638630 & -4.3840630 & -2.9328390 \\ \mathrm{C} & -0.8517830 & -5.8000100 & -2.3319490 \\ \mathrm{C} & 0.5366130 & -6.4698330 & -2.1658690 \\ \mathrm{C} & 1.6956750 & -5.5494630 & -2.5963730 \\ \mathrm{C} & 1.5177940 & -4.1734890 & -2.0110150 \\ \mathrm{H} & -0.4788470 & -4.4643020 & -3.9919150 \\ \mathrm{H} & -1.5021110 & -6.4135920 & -2.9639170 \\ \mathrm{H} & 0.5869560 & -7.4003940 & -2.7407750 \\ \mathrm{H} & 1.7132550 & -5.4822210 & -3.6934860 \\ \mathrm{H} & -1.7445670 & -3.9017130 & -2.9063480 \\ \mathrm{H} & -1.3431380 & -5.7229540 & -1.3566180 \\ \mathrm{H} & 0.6879970 & -6.7412190 & -1.1161120 \\ \mathrm{H} & 2.6552570 & -5.9806230 & -2.2936210 \\ \mathrm{C} & -1.6738800 & -1.1011700 & -3.0890530 \\ \mathrm{C} & -2.8803810 & -0.3939450 & -3.1901110 \\ \mathrm{C} & -0.7534570 & -1.2387180 & -4.2879600 \\ \mathrm{C} & -1.4403120 & -0.9717300 & -5.6260960 \\ \mathrm{C} & -2.2816560 & 0.2997500 & -5.5556820 \\ \mathrm{C} & -3.3911420 & 0.1238410 & -4.5209370 \\ \mathrm{H} & -0.6837130 & -0.8983590 & -6.4147980 \\ \mathrm{H} & 0.0665430 & -0.5176180 & -4.1530540 \\ \mathrm{H} & -0.2788840 & -2.2213720 & -4.3061900 \\ \mathrm{H} & -1.6392030 & 1.1453290 & -5.2725460 \\ \mathrm{H} & -2.7164750 & 0.5366970 & -6.5326520 \\ \mathrm{H} & -3.9216850 & 1.0694990 & -4.3619130 \\ \mathrm{H} & -4.1352200 & -0.5829990 & -4.9152460 \\ \mathrm{H} & -2.0902130 & -1.8190690 & -5.8839010 \\ \mathrm{C} & 0.3608480 & 3.3872060 & -0.6275910 \\ \mathrm{O} & -0.3802640 & 2.3396560 & -0.7397090 \\ \mathrm{C} & 0.5586640 & 4.0867390 & -1.9211890 \\ & & & \\ & & & \end{array}$

$\begin{array}{lrrr}\mathrm{C} & 1.5125090 & 5.0989990 & -2.0672060 \\ \mathrm{C} & -0.1903120 & 3.6791190 & -3.0345630 \\ \mathrm{C} & 1.7146260 & 5.6946230 & -3.3058230 \\ \mathrm{H} & 2.1015760 & 5.4037250 & -1.2092330 \\ \mathrm{C} & 0.0109680 & 4.2815520 & -4.2689000 \\ \mathrm{H} & -0.9221920 & 2.8869790 & -2.9241810 \\ \mathrm{C} & 0.9652230 & 5.2882140 & -4.4074910 \\ \mathrm{H} & 2.4620100 & 6.4745180 & -3.4120200 \\ \mathrm{H} & -0.5751350 & 3.9636770 & -5.1257510 \\ \mathrm{H} & 1.1253020 & 5.7550470 & -5.3747480 \\ \mathrm{H} & -0.5339320 & 1.7687240 & 0.1193630 \\ \mathrm{~S} & 1.0549030 & 3.9261590 & 0.8123620 \\ \mathrm{C} & 0.9723000 & 1.8518600 & 3.3600920 \\ \mathrm{C} & 1.9600650 & 1.8708600 & 2.2742520 \\ \mathrm{H} & -0.0562220 & 1.7088390 & 3.0372590 \\ \mathrm{H} & 1.7609870 & 1.2741640 & 1.3913530 \\ \mathrm{O} & 1.6363620 & 0.5948390 & 3.6347540 \\ \mathrm{H} & 1.1186230 & -0.1421770 & 3.1732820 \\ \mathrm{C} & 2.6218120 & 3.1018880 & 4.8177590 \\ \mathrm{C} & 3.4014730 & 3.4958080 & 3.5615180 \\ \mathrm{C} & 3.3351270 & 2.3992980 & 2.4965520 \\ \mathrm{C} & 1.1497610 & 2.8435300 & 4.4851610 \\ \mathrm{H} & 2.6918640 & 3.8939300 & 5.5696480 \\ \mathrm{H} & 4.4502620 & 3.6948050 & 3.8019730 \\ \mathrm{H} & 3.9439000 & 1.5319750 & 2.7827960 \\ \mathrm{H} & 0.6645600 & 3.7758420 & 4.1704450 \\ \mathrm{H} & 0.6049730 & 2.4723950 & 5.3589740 \\ \mathrm{H} & 3.0617080 & 2.1993740 & 5.2589050 \\ \mathrm{H} & 2.9859820 & 4.4245760 & 3.1495350 \\ \mathrm{H} & 3.7424240 & 2.7267630 & 1.5369490 \\ \mathrm{H} & & & \end{array}$

TS295(S, S) G[WB97XD/6-31G(d)] = -3864.641610

$\begin{array}{lrrr}\text { TS295(S, S) } \mathrm{G}[\mathrm{wB} 97 \mathrm{XD} / 6-31 \mathrm{G}(\mathrm{d})]=-3864.641610 \\ \mathrm{P} & 0.2804450 & -0.0303840 & 0.3191830 \\ \mathrm{O} & 0.7101530 & 0.9306880 & 1.3925380 \\ \mathrm{O} & -0.7685350 & 0.4127480 & -0.6736520 \\ \mathrm{O} & -0.1064170 & -1.4009540 & 1.0963260 \\ \mathrm{O} & 1.5141580 & -0.5306430 & -0.6100140 \\ \mathrm{C} & -0.1223460 & -2.5861310 & 0.3813410 \\ \mathrm{C} & 1.1019970 & -3.1849410 & 0.0443360 \\ \mathrm{C} & -1.3554380 & -3.1328980 & 0.0378420 \\ \mathrm{C} & -1.3454730 & -4.3492210 & -0.6487470 \\ \mathrm{H} & -2.2961330 & -4.8026370 & -0.9193240 \\ \mathrm{C} & 2.5541810 & -1.1683650 & 0.0410870 \\ \mathrm{C} & 3.7228440 & -0.4502620 & 0.2803470 \\ \mathrm{C} & 2.3789760 & -2.5065980 & 0.4134260 \\ \mathrm{C} & 4.7639990 & -1.1268870 & 0.9165700\end{array}$




$\begin{array}{lrrr}\mathrm{H} & 5.7006370 & -0.6034200 & 1.0946550 \\ \mathrm{C} & -2.6469220 & -2.4376110 & 0.3009850 \\ \mathrm{C} & 3.8237100 & 0.9872800 & -0.0962680 \\ \mathrm{C} & -3.1019520 & -2.1690200 & 1.5976340 \\ \mathrm{C} & -2.3744820 & -2.4462500 & 2.8912390 \\ \mathrm{C} & -4.6806690 & -1.3388400 & 3.2527600 \\ \mathrm{C} & -4.3616400 & -1.5909360 & 1.8024540 \\ \mathrm{C} & -2.1923220 & -1.1031780 & 3.6328790 \\ \mathrm{H} & -1.7146710 & -1.2951610 & 4.6007160 \\ \mathrm{H} & -1.5146770 & -0.4637600 & 3.0617230 \\ \mathrm{C} & -3.5740300 & -0.4257260 & 3.8277670 \\ \mathrm{H} & -3.7745690 & -0.2398130 & 4.8895010 \\ \mathrm{H} & -3.6004540 & 0.5452030 & 3.3214060 \\ \mathrm{H} & -5.6642430 & -0.8682540 & 3.3654940 \\ \mathrm{H} & -1.4043650 & -2.9171770 & 2.7212010 \\ \mathrm{C} & -4.6361610 & -2.6866810 & 4.0048750 \\ \mathrm{H} & -4.8007000 & -2.5077910 & 5.0739660 \\ \mathrm{H} & -5.4528670 & -3.3273230 & 3.6568220 \\ \mathrm{C} & -3.2624810 & -3.3633100 & 3.7612590 \\ \mathrm{H} & -2.7510930 & -3.5574040 & 4.7111710 \\ \mathrm{H} & -3.3886820 & -4.3290910 & 3.2611800 \\ \mathrm{C} & -3.4650230 & -2.0750940 & -0.7795980 \\ \mathrm{C} & -3.1280420 & -2.1815730 & -2.2487240 \\ \mathrm{C} & -5.4937340 & -1.2313550 & -1.8302510 \\ \mathrm{C} & -4.7339090 & -1.5238340 & -0.5624190 \\ \mathrm{C} & -4.1779250 & -3.0915090 & -2.9236790 \\ \mathrm{H} & -3.9842100 & -3.1194660 & -4.0023690 \\ \mathrm{H} & -4.0720090 & -4.1171130 & -2.5537080 \\ \mathrm{C} & -5.6019370 & -2.5468000 & -2.6337580 \\ \mathrm{H} & -6.1437590 & -2.3570930 & -3.5676020 \\ \mathrm{H} & -6.1881880 & -3.2739130 & -2.0624400 \\ \mathrm{H} & -6.4891070 & -0.8304790 & -1.6110440 \\ \mathrm{H} & -2.1218700 & -2.5748580 & -2.4074000 \\ \mathrm{C} & -4.6777590 & -0.2253900 & -2.6694710 \\ \mathrm{H} & -5.1773620 & -0.0785140 & -3.6344360 \\ \mathrm{H} & -4.6680080 & 0.7472280 & -2.1697170 \\ \mathrm{C} & -3.2406740 & -0.7690510 & -2.8655740 \\ \mathrm{H} & -2.9910190 & -0.8271860 & -3.9314050 \\ \mathrm{H} & -2.5000740 & -0.1163860 & -2.3953220 \\ \mathrm{C} & -5.1913640 & -1.2949740 & 0.7282310 \\ \mathrm{H} & -6.1783900 & -0.8660290 & 0.8934770 \\ \mathrm{C} & 3.7822510 & 2.7520800 & -1.7722310 \\ \mathrm{C} & 3.6699100 & 3.0089140 & -3.2535900 \\ \mathrm{C} & 3.5395010 & 0.5026140 & -2.6388210 \\ \mathrm{C} & 3.6965550 & 1.3963780 & -1.4311070 \\ \mathrm{C} & 4.8236340 & 2.2510440 & -3.9490720 \\ & & & \end{array}$

4.7348840
5.7813010
4.7554670
4.6572440
5.6715030
3.4895520
3.7273570
2.2637900
2.1826800
1.3865010
2.3390400
2.2752280
1.4986870
4.1621410
4.4877900
4.2370600
4.0521420
3.3783080
3.6531890
2.4421390
3.2117440
3.3739960
2.2046020
4.1129740
4.5899170
5.6525750
5.7953710
6.4058250
5.8123060
6.0685830
6.6202840
4.0174950
4.1115740
4.6142850
5.7144210
5.8809010
4.5625910
3.3224360
3.4172970
5.4697000
6.6768160
4.6080030
3.2430050
6.6536770
6.2100010
4.4343660

2.6883250

0.7499990

0.1228070

0.4386480

$-0.552702$

4. 0805440

0.9130230

0.3002780

0.6964390

2.4175680

2. 5659250

2. 9598640

3166040

4. 2211280

1.7413400

1.9652700

.0921090

4.6945960

4.4938450

2.6007310

2.4553030

2.2404820

. 6913040

5.2616250

2.2367560

2.1260370

1.6089760

3. 7199200

4.3494940

3. 8279820

3.7141140

4.7648520

$-2.4371660$

$-3.1840660$

$-4.5743980$

$-5.3812130$

$-4.5050380$

$-3.1309520$

$-3.2943040$

$-5.1264210$

$-6.1643440$

$-2.6236400$

$-4.4322030$

$-5.8915010$
$-5.0340060$

$-3.6492260$

$-3.5622930$

$-4.4558610$

$-2.3627030$

$-3.4746470$

$-3.4037280$

$-4.3090030$

$-2.7893220$

$-3.7665240$

$-4.8507380$

$-3.3248690$

0.5279650

1. 6887770

2. 3662310

0.8830330

2. 7537570

3.6279870

2. 3569330

3. 1379000

4.2123440

2. 9146770

2. 6372100

1.3616440

2. 7400340

3.8211670

2. 2516330

2. 3104590

3.1705980

1. 5795560

$-0.7956590$

$-1.0675950$

1. 3540430

2.0633900

1. 4317310

1.4529810

1.7375350

1. 1198780

3. 1298270

1.9424060

2. 2170300

2.8241940

2. 0120450

0.3961040

0.4934190 


\begin{tabular}{|c|c|c|}
\hline $\begin{array}{l}2.4099420 \\
1.0712680 \\
2.2871760 \\
2.0618150 \\
0.7454480 \\
0.1188500 \\
0.1594070 \\
2.4674980 \\
2.9208600 \\
0.9557030 \\
0.3102810 \\
3.1861770 \\
2.0443450 \\
0.1609020 \\
-1.1300110 \\
-3.4557970 \\
2.9559690 \\
-4.8260940 \\
-5.4034590 \\
5.5564820 \\
6.6886690 \\
4.8427790 \\
6.8439220 \\
5.1098340 \\
7.4118460 \\
7.1265350 \\
7.4053290 \\
8.4185920 \\
-2.0526020 \\
-2.6454090 \\
0.5089110 \\
0.2083060 \\
-1.0747070 \\
0.1077230 \\
0.9083760 \\
1.0079440 \\
0.0994860 \\
0.2474740 \\
0.2704620 \\
0.8802660 \\
0.4633030 \\
0.2948000 \\
1.3674160 \\
-1.9570890 \\
0.7061460 \\
0.9602410\end{array}$ & $\begin{array}{l}-5.0229330 \\
-4.3660730 \\
-5.0290400 \\
-5.2649290 \\
-6.0242560 \\
-6.2199190 \\
-4.9575900 \\
-5.9947690 \\
-5.8062610 \\
-7.0038780 \\
-7.0316400 \\
-4.4268400 \\
-4.2867290 \\
-5.4661750 \\
-6.5361090 \\
2.4269670 \\
1.4097220 \\
2.7708080 \\
2.0464810 \\
3.7981220 \\
2.3462600 \\
1.2441520 \\
4.0890970 \\
4.3598370 \\
3.3655050 \\
1.7810330 \\
4.8832680 \\
3.5960020 \\
1.0532420 \\
3.2924460 \\
3.6369020 \\
3.4723670 \\
2.8271950 \\
2.4581610 \\
3.3961440 \\
2.4056680 \\
6.1159140 \\
5.9839310 \\
4.6326140 \\
5.0125240 \\
7.0936970 \\
6.7853610 \\
4.5916920 \\
5.1440640 \\
5.0434610 \\
6.0542510\end{array}$ & $\begin{array}{r}1.4365430 \\
-0.7104220 \\
-1.3088930 \\
-2.8137930 \\
-3.1130060 \\
-1.8512410 \\
-1.0317700 \\
-0.8153520 \\
-3.2234140 \\
-3.5548070 \\
-1.2474540 \\
-1.1583710 \\
-3.3058100 \\
-3.8516940 \\
-2.1270270 \\
-0.5321540 \\
0.0912470 \\
-0.0843420 \\
0.9673510 \\
-0.6934250 \\
1.3992980 \\
1.4300600 \\
-0.2635460 \\
-1.5064350 \\
0.7836030 \\
2.2163560 \\
-0.7452890 \\
1.1191740 \\
-0.2615620 \\
-1.7281910 \\
0.7012580 \\
-0.7265280 \\
1.1609240 \\
-1.0966140 \\
0.7739280 \\
0.9872920 \\
0.4820240 \\
-1.0353560 \\
-1.5374970 \\
1.2013230 \\
0.8126550 \\
-1.5465460 \\
-1.4923530 \\
1.0382530 \\
2.2817710 \\
0.7582020\end{array}$ \\
\hline
\end{tabular}

$\begin{array}{rrrr}\mathrm{H} & -1.3053990 & 6.0866490 & -1.3065070 \\ \mathrm{H} & 0.0029540 & 4.4614510 & -2.5837960\end{array}$

154

TS296(S, S ) G[WB97XD/6-31G(d)] = -3864.644297

\begin{tabular}{|c|c|c|c|}
\hline & & & \\
\hline$P$ & -0.2586670 & -0.2666600 & 1.0842670 \\
\hline 0 & 0.4383670 & -0.6648670 & 2.3513890 \\
\hline 0 & -0.6409820 & 1. 1822400 & 0.9004130 \\
\hline 0 & -1.5258430 & -1.2618530 & 0.9179830 \\
\hline 0 & 0.6300710 & -0.6029560 & -0.2516720 \\
\hline C & -2.1256180 & -1.2823570 & -0.3325600 \\
\hline $\mathrm{C}$ & -1.4863830 & -2.0022630 & -1.3457730 \\
\hline $\mathrm{C}$ & -3.3205220 & -0.5943750 & -0.5244470 \\
\hline $\mathrm{C}$ & -3.8787780 & -0.6388920 & -1.8036780 \\
\hline $\mathrm{H}$ & -4.8252210 & -0.1315960 & -1.9760200 \\
\hline C & 0.8573820 & -1.9345090 & -0.5534150 \\
\hline C & 2.1404350 & -2.4528070 & -0.3905170 \\
\hline C & -0.2027590 & -2.6982850 & -1.0410640 \\
\hline C & 2.3165030 & -3.8038810 & -0.6661340 \\
\hline $\mathrm{H}$ & 3.3110250 & -4.2313670 & -0.5552930 \\
\hline C & -3.9951860 & 0.1525870 & 0.5729420 \\
\hline C & 3.2731670 & -1.5729370 & 0.0071540 \\
\hline C & -4.5017100 & -0.5180710 & 1.6930350 \\
\hline C & -4.3997700 & -1.9960200 & 1.9832410 \\
\hline C & -5.6751120 & -0.6967700 & 3.8164120 \\
\hline C & -5.2075360 & 0.1781300 & 2.6821560 \\
\hline C & -3.6784860 & -2.1706760 & 3.3375040 \\
\hline 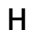 & 6375250 & -3.2387790 & 3.5 \\
\hline $\boldsymbol{H}$ & 5472700 & -1.8212990 & 3.24 \\
\hline C & -4.4375370 & -1.3822160 & 4.4368780 \\
\hline $\mathrm{H}$ & -4.7634670 & -2.0493320 & 5.2436420 \\
\hline ח & -3.78 & -0.6240700 & 4.8 \\
\hline $\mathrm{H}$ & -6.21 & -0.11 & 4.5 \\
\hline $\mathbf{F}$ & -3.860 & -2.5 & 00 \\
\hline & -6.5 & -1.79 & 3.2 \\
\hline H & -6.8 & -2.4 & 4. \\
\hline$H$ & $-7 \cdot 50$ & -1.33 & 2. \\
\hline C & -5.8 & -2.55 & 2.1 \\
\hline H & -5.7 & -3.6288700 & 2.3 \\
\hline $\mathrm{H}$ & -6.3 & -2.4642140 & 1.1 \\
\hline 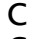 & -4.1 & 1.5362840 & 0.4 \\
\hline 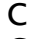 & -3.66 & 2.4584230 & -0 \\
\hline 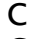 & -5.11 & 3.6843160 & 1 . \\
\hline C & -4.9 & 1400 & 1. \\
\hline 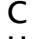 & -4.8 & 5100 & -1 \\
\hline & $\begin{array}{r}-4 \\
5\end{array}$ & 3637980 & -2 \\
\hline & & & \\
\hline
\end{tabular}




$\begin{array}{rrr}-5.7698140 & 3.8101510 & -0.2370530 \\ -5.9174110 & 4.8705290 & -0.4726010 \\ -6.7600390 & 3.3437210 & -0.2143630 \\ -5.7389050 & 4.1665970 & 1.9173200 \\ -3.0405470 & 1.9240630 & -1.3169040 \\ -3.7234920 & 4.3487450 & 1.0916660 \\ -3.8411410 & 5.3940860 & 0.7819120 \\ -3.2697090 & 4.3576050 & 2.0877050 \\ -2.8347780 & 3.5662480 & 0.0937870 \\ -2.4234090 & 4.2343130 & -0.6725830 \\ -1.9939490 & 3.1045240 & 0.6137830 \\ -5.4395040 & 1.5427740 & 2.5567610 \\ -6.0093950 & 2.0771380 & 3.3153440 \\ 4.8192660 & 0.2247090 & -0.5437300 \\ 5.2256390 & 1.1714110 & -1.6451740 \\ 3.2694420 & -0.3837600 & -2.3049630 \\ 3.7488150 & -0.6071010 & -0.8907240 \\ 5.6392210 & 0.3149370 & -2.8644830 \\ 5.8991620 & 0.9786290 & -3.6973260 \\ 6.5373430 & -0.2606940 & -2.6186260 \\ 4.4675720 & -0.6261510 & -3.2510960 \\ 4.1475800 & -0.4447290 & -4.2835720 \\ 4.7740730 & -1.6753220 & -3.1885690 \\ 2.4447190 & -1.0514450 & -2.5651350 \\ 6.0538890 & 1.8156420 & -1.3304300 \\ 2.8286270 & 1.0856550 & -2.4448130 \\ 2.5043500 & 1.2733440 & -3.4747460 \\ 1.9600610 & 1.2477920 & -1.8049470 \\ 4.0025010 & 2.0204970 & -2.0580670 \\ 4.2809260 & 2.6652620 & -2.8998020 \\ 3.7104140 & 2.6869460 & -1.2398110 \\ 4.9911710 & -0.8820160 & 1.5797820 \\ 5.5868250 & -1.1928770 & 2.9278650 \\ 3.5994590 & -2.7302250 & 2.3125620 \\ 3.9062430 & -1.7059330 & 1.2466560 \\ 4.4777980 & -1.0519780 & 3.9925940 \\ 4.8965030 & -1.2798820 & 4.9797600 \\ 4.1237940 & -0.0164770 & 4.0227760 \\ 3.3117270 & -2.0122980 & 3.6482620 \\ 3.1913390 & -2.7716430 & 4.4298650 \\ 2.3618620 & -1.4814080 & 3.5716810 \\ 2.7501250 & -3.3588310 & 2.0351260 \\ 6.4260840 & -0.5259270 & 3.1538650 \\ 4.8741220 & -3.5847440 & 2.5006980 \\ 4.6875270 & -4.3434270 & 1.5705120 \\ 5.1001140 & -4.1173020 & \end{array}$

\begin{abstract}
6.0576880
6.4344490

6.8922070

5.4453920

6.2928910

1.2593470

$-0.0172190$

1. 5430730

0.2898440

$-0.8054150$

$-1.2036620$

0.5335000

2.0390930

2. 2633000

$-0.4396070$

$-1.6850890$

$-1.9275240$

$-1.7273010$

$-0.0749620$

$-3.2435210$

$-3.8171000$

$-3.7618120$

$-2.3237380$

$-1.3627930$

$-2.0335250$

$-3.2391100$

$-4.1849460$

$-1.9116380$

$-0.9895000$

$-4.8462480$

$-4.4043010$

$-2.3441930$

$-0.4836020$

0.6263980

$-0.1284490$

0.7912110

1.8132590

$-0.0753570$

1. 9654440

2. 4914450

0.0747030

$-0.8623140$

1.0957280

2. 7643680

-0.6054940
1.2146670
\end{abstract}

2. 9087490

3. 9029220

2. 2085020

0.6913490

0.9528000

$-1.0516050$

$-1.2324650$

$-1.3133310$

$-1.3180340$

$-2.1539840$

$-1.5365210$

$-1.7041290$

$-2.2907190$

$-0.5739720$

$-3.1782500$

$-2.2240410$

$-2.1720390$

$-0.5873370$

$-0.2910230$

$-2.8538800$

$-4.2470070$

$-4.7667410$

$-4.7522450$

$-3.8610870$

$-2.6354260$

$-4.9183530$

$-5.7750820$

$-5.7667140$

$-4.4466600$

$-4.2528850$

$-4.1217760$

$-4.4100890$

$-3.6026240$

$-1.2278700$

$-1.2173490$

$-2.5833170$

$-2.8679630$

$-3.6073030$

$-4.1552080$

$-2.0784910$

$-4.8904860$

$-3.3869490$

$-5.1668830$

$-4.3700020$

$\begin{array}{ll}3.7995650 & -5.6765260 \\ 5.4137430 & -6.1716170\end{array}$ 


\begin{tabular}{|c|c|c|c|}
\hline $\begin{array}{l}H \\
\mathrm{~s}\end{array}$ & $\begin{array}{r}-0.2951520 \\
1.3641970\end{array}$ & $\begin{array}{l}1.9346870 \\
4.1062740\end{array}$ & $\begin{array}{r}-0.2962640 \\
0.1282760\end{array}$ \\
\hline$C$ & 1.3124390 & 2.4057650 & 2.9027090 \\
\hline C & 2.2596500 & 2.2388540 & 1.7947660 \\
\hline $\mathrm{H}$ & 0.2688800 & 2.2476990 & 2.6382370 \\
\hline $\mathrm{H}$ & 2.0112940 & 1.5236590 & 1.0183890 \\
\hline 0 & 1.9590410 & 1.1844740 & 3.3285440 \\
\hline $\mathrm{H}$ & 1.3930930 & 0.4115160 & 2.9908250 \\
\hline C & 3.0427060 & 3.7946710 & 4.1182970 \\
\hline C & 3.7869910 & 3.9829920 & 2.7943040 \\
\hline C & 3.6579040 & 2.7488150 & 1.8983620 \\
\hline C & 1.5532940 & 3.5391790 & 3.8714720 \\
\hline $\mathrm{H}$ & 3.1612810 & 4.6828630 & 4.7464720 \\
\hline $\mathrm{H}$ & 4.8480360 & 4.1829820 & 2.9722730 \\
\hline $\mathrm{H}$ & 4.2522840 & 1.9131160 & 2.2886150 \\
\hline $\mathrm{H}$ & 1.0823240 & 4.4339840 & 3.4461300 \\
\hline $\mathrm{H}$ & 1.0301320 & 3.3091540 & 4.8050000 \\
\hline $\mathrm{H}$ & 3.4743720 & 2.9500090 & 4.6683700 \\
\hline $\mathrm{H}$ & 3.3811700 & 4.8568870 & 2.2688970 \\
\hline $\mathrm{H}$ & 4.0412160 & 2.9276310 & 0.8906750 \\
\hline & & (d) $1=-386$ & \\
\hline $\begin{array}{l}\mathrm{P} \\
\mathrm{S}\end{array}$ & D) 2696160 & (a) $\rfloor=1622200$ & t5 1282610 \\
\hline 0 & 0.7414670 & 0.8578700 & 1.2567360 \\
\hline 0 & -0.7266500 & 0.5500790 & -0.8581650 \\
\hline 0 & -0.2352480 & -1.3923650 & 0.8270950 \\
\hline 0 & 1.4878260 & -0.5502370 & -0.8042980 \\
\hline C & -0.3190110 & -2.5333730 & 0.0469690 \\
\hline C & 0.8687300 & -3.1854910 & -0.3163580 \\
\hline C & -1.5796020 & -2.9852100 & -0.3323150 \\
\hline C & -1.6332740 & -4.1486320 & -1.1025860 \\
\hline $\mathrm{H}$ & -2.6067690 & -4.5365810 & -1.3938940 \\
\hline C & 2.4582190 & -1.2938590 & -0.1562180 \\
\hline C & 3.6636730 & -0.6777160 & 0.1759490 \\
\hline C & 2.1738690 & -2.6246470 & 0.1437080 \\
\hline C & 4.5997760 & -1.4638960 & 0.8386280 \\
\hline $\mathrm{H}$ & 5.5605620 & -1.0231430 & 1.0968680 \\
\hline C & -2.8292270 & -2.2529890 & 0.0163690 \\
\hline C & 3.8997700 & 0.7615810 & -0.1235660 \\
\hline C & -3.2479210 & -2.1017890 & 1.3442210 \\
\hline C & -2.5180860 & -2.5626520 & 2.5822580 \\
\hline C & -4.7553490 & -1.3844500 & 3.1142900 \\
\hline C & -4.4680800 & -1.4805360 & 1.6384490 \\
\hline C & -2.2525490 & -1.3257390 & 3.4686940 \\
\hline $\mathrm{H}$ & -1.7622730 & -1.6512030 & 4.3935300 \\
\hline $\mathrm{H}$ & -1.5589770 & 065611 & - \\
\hline
\end{tabular}

-3.5941310
-3.7706290
-3.5772000
-5.7105200
-1.5766560
-4.7790490
-4.9322390
-5.6289420
-3.4425030
-2.9322800
-3.6205510
-3.6430760
-3.3417900
-5.6356540
-4.8728150
-4.4596900
-4.2875860
-4.4112930
-5.8408380
-6.3863730
-6.4617750
-6.6000760
-2.3654380
-4.7730140
-5.2827250
-4.6878150
-3.3793180
-3.1632280
-2.5841300
-5.2946750
-6.2520970
4.0798010
4.0661720
3.6728550
3.8637710
5.1831200
5.1839880
6.1560770
4.9386200
4.8000880
5.7983480
3.5129060
4.2329510
2.4686320
2.3408830
1.5596150

$-0.5596290$

0.4185740

$-0.8795950$

$-3.0601660$

$-2.8152900$

$-2.7603880$

$-3.3640960$

$-3.5266570$

$-3.8475600$

$-4.4251460$

$-1.7365470$

$-1.7168660$

$-0.6730390$

$-1.1386220$

$-2.4886120$

$-2.4161890$

$-3.5514700$

$-1.8904400$

$-1.5732730$

$-2.6350900$

$-0.2376880$

$-2.1524730$

0.3632070

0.6446550

1.2731230

$-0.2460260$

$-0.2146350$

0.3093370

$-1.0225530$

$-0.5584230$

2. 5985830

2.9298790

0.4242490

1.2415880

2. 0988160

2.2978360

2.4166260

0.5935670

0.0394960

0.1497770

$-0.6330970$

3.9999200

0.9922830

0.4184210

0.8561840
3.7802440

4.8610790

3.4099320

3.2988870

2.3400650

3.6954960

4.7796890

3.2773150

3. 3595150

4.2750220

2.7597570

$-1.0016790$

$-2.4819350$

$-1.9104100$

$-0.6979600$

$-3.2176250$

$-4.2978320$

$-2.9570300$

$-2.8397120$

$-3.7361030$

$-2.3309910$

$-1.6279060$

$-2.7037040$

$-2.6619560$

$-3.5909580$

$-2.0610340$

$-2.9563620$

$-4.0306320$

$-2.4526380$

0.6196810

0.8509470

$-1.7116750$

$-3.1824220$

$-2.6960540$

$-1.4405160$

$-3.8537380$

$-4.9317870$

$-3.4654650$

$-3.5684480$

$-4.5040520$

$-3.0563020$

$-2.4760610$

$-3.4760570$

$-4.4010720$

$-2.8852740$ 


\begin{tabular}{|c|c|c|}
\hline $\begin{array}{l}2.7174650 \\
2.7436290 \\
1.9086910 \\
4.4045290 \\
4.7496820 \\
4.2666940 \\
4.1670590 \\
3.5849560 \\
3.8642120 \\
2.7029760 \\
3.2827710 \\
3.3881390 \\
2.2596490 \\
4.0458550 \\
4.9500170 \\
5.7001470 \\
5.7876550 \\
6.4201280 \\
5.9983200 \\
6.2616540 \\
6.8468640 \\
4.3520250 \\
4.5434540 \\
3.1006160 \\
2.7437780 \\
3.6160670 \\
5.0886130 \\
5.3771370 \\
4.3312050 \\
3.4083940 \\
2.8579520 \\
1.6837900 \\
5.3154360 \\
5.7402840 \\
6.3633150 \\
5.4267430 \\
3.3673610 \\
0.4806270 \\
0.5190620 \\
0.4823750 \\
1.9191980 \\
1.9666780 \\
0.7801470 \\
0.2596210 \\
0.4417630\end{array}$ & $\begin{array}{r}2.4889020 \\
2.6637720 \\
3.1076560 \\
3.0231300 \\
3.8456400 \\
1.3687790 \\
1.6714710 \\
3.7636710 \\
4.3057360 \\
4.2545520 \\
2.2761010 \\
2.0725380 \\
2.0115980 \\
0.3218990 \\
4.8876300 \\
1.7263350 \\
1.5538040 \\
1.0618020 \\
3.2098320 \\
3.7755560 \\
3.2849070 \\
3.4917090 \\
4.5420540 \\
-3.3629790 \\
-4.7483860 \\
-5.1955860 \\
-4.9930830 \\
-3.5038750 \\
-2.7765500 \\
-6.2463100 \\
-5.4801890 \\
-4.7700780 \\
-5.5319490 \\
-5.4065270 \\
-3.3564070 \\
-3.0288400 \\
-4.6137500 \\
-4.7868080 \\
-6.0211750 \\
-7.0510780 \\
-6.4911160 \\
-4.9452610 \\
-4.2963050 \\
-5.7664410 \\
-7.9713690 \\
-6.8466400\end{array}$ & $\begin{array}{r}-3.7913600 \\
-4.8733150 \\
-3.3923180 \\
0.6256540 \\
1.8407280 \\
2.3881520 \\
0.9105860 \\
2.8497770 \\
3.7612650 \\
2.4299480 \\
3.1584570 \\
4.2305760 \\
2.8806990 \\
2.6040340 \\
1.5682050 \\
2.8413030 \\
3.9202900 \\
2.3513770 \\
2.4944200 \\
3.3957990 \\
1.8066610 \\
-0.6811850 \\
-0.8971570 \\
0.9051420 \\
1.4092280 \\
2.5810700 \\
2.2339110 \\
2.0553420 \\
1.2308570 \\
2.8104550 \\
0.6008560 \\
1.6844640 \\
1.3038820 \\
3.0110040 \\
1.5990410 \\
3.0451060 \\
3.4786590 \\
-1.5388720 \\
-2.4038360 \\
-1.8637940 \\
-1.8408070 \\
-1.8411950 \\
-1.1669120 \\
-3.4420540 \\
-2.4557750 \\
-2.7140960\end{array}$ \\
\hline
\end{tabular}
1.9471870
$-1.5306520$
0.1732540
2. 4433580
2. 9130750
$-3.2698360$
$-2.7919900$
$-4.5692140$
$-5.1129760$
$-5.2614950$
$-6.3285370$
$-4.5807370$
$-6.4794460$
$-7.0146730$
$-6.7402600$
$-7.0125720$
$-7.9668230$
$-1.9393460$
$-2.5043790$
$-0.1910450$
0.0064000
$-0.8242110$
$-0.0277910$
1. 1900090
1.1863160
0.4921580
0.2472560
0.5765140
$-0.3723610$
0.2656570
0.8509830
1.6623270
$-1.4340660$
$-0.1397160$
1. 5509960
$-0.8047720$
0.2413060

154

$\operatorname{TS} 298(\mathrm{~S}, \mathrm{~S}) \mathrm{G}[\mathrm{WB} 97 \mathrm{XD} / 6-31 \mathrm{G}(\mathrm{d})]=-3864.645617$

\begin{tabular}{|c|c|c|c|}
\hline \multicolumn{4}{|c|}{ TS298(S,S) GLWB9 X X } \\
\hline $\mathrm{P}$ & 0.2330940 & -0.0171200 & 0.2376520 \\
\hline 0 & 0.6462090 & 0.9335210 & 1. \\
\hline 0 & -0.7718130 & 0.4509240 & -0 \\
\hline 0 & -0.2204340 & -1.3792860 & 200 \\
\hline 0 & 1.4914880 & 6120 & -0 \\
\hline C & -0.2551050 & 2050 & 259511 \\
\hline
\end{tabular}

$-2.8862950$

$-2.4261510$

$-0.8456390$

$-0.9630020$

$-0.3668540$

0.0915260

1. 2174240

$-0.1145340$

1.8141090

1.4857900

0.4823580

$-0.8636040$

1.4469770

2. 5644180

0.1953390

1.9120260

$-0.3565440$

$-1.5269860$

0.7415640

$-0.7056200$

1. 2114030

$-1.1079860$

0.7292850

0.9047720

0.5582710

$-0.9512250$

$-1.5132480$

1. 2948370

0.9333170

$-1.4733120$

$-1.5228150$

1. 1940470

2.3645060

0.7701140

$-1.1588670$

$\begin{array}{lr}5.9474110 & 0.7701140 \\ 6.3053570 & -1.1588670 \\ 4.5757170 & -2.5481870\end{array}$

0.2468220 


0.9523110
-1.4926200
-1.4810270
-2.4336130
2.4780540
3.6593740
2.2313070
4.6296630
5.5749600
-2.7766670
3.8522870
-3.2405340
-2.5266240
-4.8244710
-4.4937130
-2.3370640
-1.8634670
-1.6540080
-3.7141000
-3.9206870
-3.7292170
-5.8040990
-1.5597080
-4.8015790
-4.9797120
-5.6187440
-3.4301800
-2.9295950
-3.5575190
-3.5766600
-3.2281190
-5.5771930
-4.8378710
-4.2913320
-4.0844260
-4.2155960
-5.7050500
-6.2255610
-6.3204890
-6.5661360
-2.2288390
-4.7282010
-5.2190940
-4.6910030
-3.3068510
-3.0646330

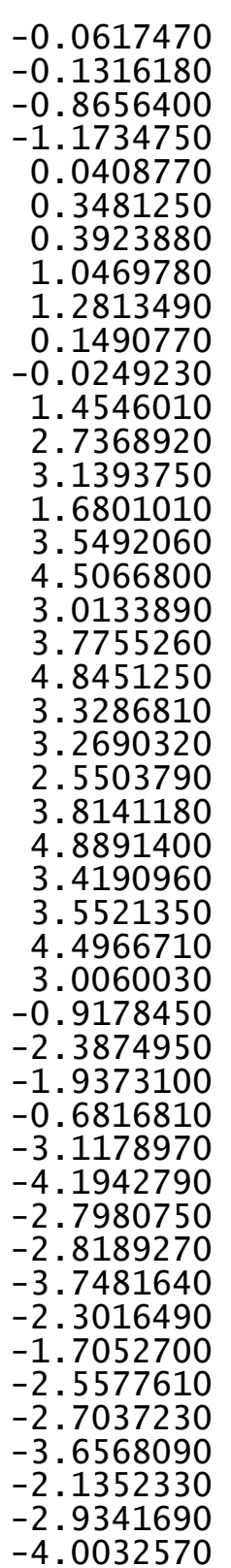

$\begin{array}{rr}-3.1764400 & -0.0617470 \\ -3.0581290 & -0.1316180 \\ -4.2404170 & -0.8656400 \\ -4.6668410 & -1.1734750 \\ -1.2218720 & 0.0408770 \\ -0.5525970 & 0.3481250 \\ -2.5539670 & 0.3923880 \\ -1.2717230 & 1.0469780 \\ -0.7875540 & 1.2813490 \\ -2.3570730 & 0.1490770 \\ 0.8758840 & -0.0249230 \\ -2.1528040 & 1.4546010 \\ -2.5061830 & 2.7368920 \\ -1.3947620 & 3.1393750 \\ -1.5687270 & 1.6801010 \\ -1.2052430 & 3.5492060 \\ -1.4513520 & 4.5066800 \\ -0.5416710 & 3.0133890 \\ -0.5281000 & 3.7755260 \\ -0.4042590 & 4.8451250 \\ 0.4716960 & 3.3286810 \\ -0.9205470 & 3.2690320 \\ -2.9775490 & 2.5503790 \\ -2.7835010 & 3.8141180 \\ -2.6628860 & 4.8891400 \\ -3.3955930 & 3.4190960 \\ -3.4577880 & 3.5521350 \\ -3.7001600 & 4.4966710 \\ -4.3980950 & 3.0060030 \\ -1.9221760 & -0.9178450 \\ -1.9582610 & -2.3874950 \\ -0.9793490 & -1.9373100 \\ -1.3611660 & -0.6816810 \\ -2.8080590 & -3.1178970 \\ -2.7874760 & -4.1942790 \\ -3.8530190 & -2.7980750 \\ -2.2420190 & -2.8189270 \\ -1.9827260 & -3.7481640 \\ -2.9853390 & -2.3016490 \\ -0.5699180 & -1.7052700 \\ -2.3640810 & -2.5577610 \\ 0.0571480 & -2.7037230 \\ 0.2866410 & -3.6568090 \\ 0.9909880 & -2.1352330 \\ -0.5146110 & -2.9341690 \\ -0.5287080 & -4.0032570\end{array}$

$-2.5453420$

$-5.3061650$

$-6.2880160$

4.0210000

4.0433490

3.6904180

3.8316500

5.1996280

5.2363330

6.1514010

4.9740080

4.8679530

5.8285530

3. 5467760

4.1915620

2. 4947300

2. 3964170

1. 5752040

2.7259840

2.7891590

1.8914430

4.2835870

4.5869520

4.1616460

4.0758470

3. 4098720

3.6616310

2. 5210590

3.1437670

3.2418630

2. 1314060

3.9655550

4.7640170

5.5785430

5.6559020

6.3226120

5.8421240

6.0763740

6.6990980

4.2472740

4.4193680

4.3953730

5.4126620

5.5367290

4.1875880
2.9691510
0.0936960
-1.1986610
-0.7629980
2.6246370
2.8694200
0.3872700
1.2800610
2.0273350
2.1693880
2.3860910
0.5346170
-0.0699140
0.1338320
-0.6588690
3.9316750
0.8822150
0.2473910
0.7680440
2.3599570
2.4644300
2.9858070
3.1873670
4.0823630
1.6251400
1.8474270
4.0217900
4.6191980
4.4654570
2.5451190
2.4019550
2.2369230
0.5852200
5.1133980
2.0450040
1.9304730
1.3755570
3.5161650
4.1353160
3.5785510
3.5806270
4.6216220
-2.5698590
-3.3478140
-4.7638300
-5.5109540
-4.5622070
-2.4398780
0.6153290
0.7944580
-1.7099280
-3.1978310
-2.5768100
-1.3670780
-3.7841460
-4.8705460
-3.3792570
-3.4269360
-4.3352010
-2.8724560
-2.2991900
-3.4213580
-3.4169660
-4.3049870
-2.8374130
-3.8219900
-4.9115270
-3.4947680
0.6071200
1.7813700
2.4543090
0.9616710
2.7777920
3.6623700
2.3214800
3.1631200
4.2456210
2.8908390
2.7218420
1.4562770
2.9059400
3.9934050
2.4606400
2.4854990
3.3593530
1.8069600
-0.7251580
-0.9958190
1.4833360
2.2787170
1.7002560
1.7285350
1.8215980 


\begin{tabular}{|c|c|c|}
\hline $\begin{array}{l}3.1894440 \\
5.1030300 \\
6.2980460 \\
4.1518570 \\
2.7747700 \\
6.3809070 \\
5.8876470 \\
4.1063150 \\
2.0676980 \\
0.9328700 \\
2.2298290 \\
2.0277500 \\
0.9259800 \\
0.4034700 \\
0.3007190 \\
2.9709380 \\
2.7061270 \\
2.9320000 \\
1.1829340 \\
0.8363560 \\
1.1554040 \\
0.7870010 \\
1.7486010 \\
3.3673890 \\
2.8816570 \\
4.6861840 \\
5.2860240 \\
5.3411830 \\
6.5206550 \\
4.7831030 \\
6.5765500 \\
4.8754160 \\
7.1682570 \\
6.9771460 \\
7.0788840 \\
8.1338430 \\
2.0153660 \\
2.5940850 \\
0.3406860 \\
0.1027780 \\
0.9727530 \\
0.1134730 \\
1.0468880 \\
1.0575930 \\
0.3073660 \\
0.0999960\end{array}$ & $\begin{array}{l}-3.2164320 \\
-3.4094130 \\
-5.3326500 \\
-6.1925430 \\
-4.3625480 \\
-2.8368060 \\
-4.6721110 \\
-6.1392520 \\
-5.0492750 \\
-4.3161270 \\
-4.9020530 \\
-5.7844090 \\
-6.8042230 \\
-6.0812920 \\
-4.8626080 \\
-6.2816110 \\
-5.5087180 \\
-4.0906590 \\
-7.3779920 \\
-7.5225010 \\
-6.7684070 \\
-5.7556840 \\
-5.1663540 \\
2.6202760 \\
1.5500550 \\
2.9997220 \\
2.1562680 \\
4.1748200 \\
2.4818230 \\
1.2422570 \\
4.4961460 \\
4.8306900 \\
3.6515100 \\
1.8196480 \\
5.4086480 \\
3.9062910 \\
1.1659510 \\
3.5037900 \\
3.7489520 \\
3.4855090 \\
3.0252230 \\
2.4498210 \\
3.3697830 \\
2.3813290 \\
6.1597180 \\
5.9667520\end{array}$ & $\begin{array}{r}1.1723400 \\
3.3323620 \\
2.2441360 \\
2.5850770 \\
2.8846800 \\
2.2636000 \\
0.6658530 \\
0.8354370 \\
1.4435240 \\
-0.8898620 \\
-1.4157920 \\
-2.6461310 \\
-2.3730860 \\
-2.1682710 \\
-1.2694380 \\
-2.8968340 \\
-0.6367740 \\
-1.6350680 \\
-1.4721350 \\
-3.1950240 \\
-1.7621160 \\
-3.1454170 \\
-3.5100100 \\
-0.4851090 \\
0.0559290 \\
0.0732310 \\
1.0182370 \\
-0.3137140 \\
1.5624910 \\
1.3075420 \\
0.2331800 \\
-1.0413450 \\
1.1712280 \\
2.2919430 \\
-0.0716670 \\
1.5977080 \\
-0.3519630 \\
-1.6918370 \\
0.6203140 \\
-0.8043300 \\
1.1350110 \\
-1.1268800 \\
0.6703480 \\
0.9110220 \\
0.2541890 \\
-1.2498950\end{array}$ \\
\hline
\end{tabular}

$\begin{array}{lr}\mathrm{C} & 0.4680730 \\ \mathrm{C} & -0.5573560 \\ \mathrm{H} & 0.0554360 \\ \mathrm{H} & 0.7031700 \\ \mathrm{H} & 1.5566030 \\ \mathrm{H} & -1.6205570 \\ \mathrm{H} & -0.3503090 \\ \mathrm{H} & 1.3639330 \\ \mathrm{H} & -0.9506630 \\ \mathrm{H} & 0.1614820 \\ \mathrm{H} & \end{array}$
4.5460800
5. 1799590
7.1855140
6.6815310
4.4005240
5.4079310
5.2515470
6.0043690
6. 1598120
4. 3441060
$-1.6871180$
1. 0528930
0.5405910
$-1.8178710$
$-1.6559200$
0.9071840
2. 1255780
0.5048200
$-1.4992520$
$-2.7171060$ 154

$\operatorname{TS} 299(\mathrm{~S}, \mathrm{~S}) \mathrm{G}[\mathrm{WB} 97 \mathrm{XD} / 6-31 \mathrm{G}(\mathrm{d})]=-3864.643688$

$\begin{array}{lrrr}\text { TS299(S , S }) ~ G[W B 97 X D / 6-31 G(d)] & -3864.643688 \\ \text { P } & -0.4043480 & -0.1597400 & 1.3824910 \\ \text { O } & 0.1578510 & -0.2430820 & 2.7684810 \\ \text { O } & -0.9527930 & 1.1713430 & 0.9215980 \\ \text { O } & -1.4920350 & -1.3471630 & 1.1865840 \\ \text { O } & 0.7210380 & -0.5115680 & 0.2352460 \\ \text { C } & -1.9487260 & -1.4678310 & -0.1227920 \\ \mathrm{C} & -1.1222340 & -2.1270500 & -1.0278820 \\ \mathrm{C} & -3.1590010 & -0.8821870 & -0.4913210 \\ \mathrm{C} & -3.4929700 & -0.9521210 & -1.8410340 \\ \mathrm{H} & -4.4332810 & -0.5095680 & -2.1628560 \\ \mathrm{C} & 1.0911600 & -1.8292190 & 0.0350210 \\ \mathrm{C} & 2.3993510 & -2.2126410 & 0.3182450 \\ \mathrm{C} & 0.1595790 & -2.7037020 & -0.5317620 \\ \mathrm{C} & 2.7239420 & -3.5590550 & 0.1597640 \\ \mathrm{H} & 3.7428560 & -3.8790820 & 0.3658790 \\ \mathrm{C} & -4.0424120 & -0.1862250 & 0.4828110 \\ \mathrm{C} & 3.4354300 & -1.1786090 & 0.5874710 \\ \mathrm{C} & -4.5822710 & -0.8685480 & 1.5813200 \\ \mathrm{C} & -4.3554730 & -2.3149270 & 1.9507120 \\ \mathrm{C} & -5.9401940 & -1.1002000 & 3.5886240 \\ \mathrm{C} & -5.4533730 & -0.2206170 & 2.4660090 \\ \mathrm{C} & -3.7598810 & -2.3600640 & 3.3750330 \\ \mathrm{H} & -3.6266960 & -3.4074830 & 3.6702320 \\ \mathrm{H} & -2.7699240 & -1.8975090 & 3.3637740 \\ \mathrm{C} & -4.7083640 & -1.6261130 & 4.3583640 \\ \mathrm{H} & -5.0446070 & -2.2994940 & 5.1556800 \\ \mathrm{H} & -4.1946470 & -0.7876130 & 4.8391540 \\ \mathrm{H} & -6.6072970 & -0.5492760 & 4.2602050 \\ \mathrm{H} & -3.6889820 & -2.8197140 & 1.2485450 \\ \mathrm{C} & -6.6746740 & -2.3085890 & 2.9679050 \\ \mathrm{H} & -6.9885120 & -2.9889030 & 3.7683200 \\ \mathrm{H} & -7.5831710 & -1.9645480 & 2.4631460 \\ \mathrm{C} & -5.7272770 & -3.0261840 & 1.9718530 \\ \mathrm{H} & -5.5798910 & -4.0737270 & 2.2593850 \\ & & & \end{array}$




$\begin{array}{rrr}-6.1529110 & -3.0273430 & 0.9630350 \\ -4.3880430 & 1.1605110 & 0.2968980 \\ -3.8925740 & 2.0919850 & -0.7856990 \\ -5.5813100 & 3.2211650 & 0.8098850 \\ -5.2797080 & 1.7905490 & 1.1747590 \\ -5.1144150 & 2.5839290 & -1.5929910 \\ -4.7756940 & 3.3023510 & -2.3486330 \\ -5.5686630 & 1.7437240 & -2.1295980 \\ -6.1411590 & 3.2386300 & -0.6305760 \\ -6.3455180 & 4.2748180 & -0.9245490 \\ -7.0954150 & 2.7025970 & -0.6563350 \\ -6.3036580 & 3.6613980 & 1.5057810 \\ -3.1686280 & 1.6065700 & -1.4427360 \\ -4.2594470 & 4.0172460 & 0.8207730 \\ -4.4564200 & 5.0449620 & 0.4930190 \\ -3.8733390 & 4.0739880 & 1.8436860 \\ -3.2381930 & 3.3197390 & -0.1128340 \\ -2.8875380 & 4.0062850 & -0.8927510 \\ -2.3631920 & 2.9903570 & 0.4500840 \\ -5.8107710 & 1.1060330 & 2.2606440 \\ -6.4996720 & 1.6032000 & 2.9417180 \\ 4.8547390 & 0.5854320 & -0.3080470 \\ 5.2292590 & 1.2980420 & -1.5839310 \\ 3.4740170 & -0.5642380 & -1.9360990 \\ 3.8881660 & -0.4080710 & -0.4924740 \\ 5.7948020 & 0.2380220 & -2.5576640 \\ 6.0420420 & 0.7206250 & -3.5102270 \\ 6.7249010 & -0.1722020 & -2.1516080 \\ 4.7406550 & -0.8820940 & -2.7617720 \\ 4.4636210 & -0.9686190 & -3.8187190 \\ 5.1408820 & -1.8533660 & -2.4535800 \\ 2.7346540 & -1.3583270 & -2.0633530 \\ 5.9710130 & 2.0825480 & -1.3989940 \\ 2.8968870 & 0.7790300 & -2.4179590 \\ 2.5965230 & 0.6960280 & -3.4687290 \\ 1.9910600 & 0.9886160 & -1.8441890 \\ 3.9555000 & 1.8938440 & -2.2293640 \\ 4.2229490 & 2.3424600 & -3.1930730 \\ 3.5540390 & 2.7034850 & -1.6110520 \\ 4.9849500 & 0.0166090 & 2.0214290 \\ 5.5023020 & 0.0901810 & 3.4341890 \\ 3.7029180 & -1.7409950 & 3.1049130 \\ 3.9971200 & -0.9640230 & 1.8461790 \\ 4.3069580 & 0.3632070 & 4.3736890 \\ 4.6682600 & 0.4201810 & 5.4071080 \\ 3.8604470 & 1.3346150 & 4.1362230\end{array}$

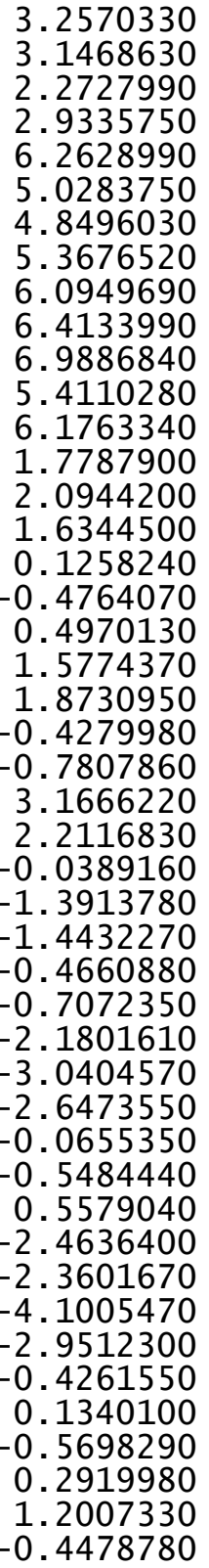

-0.7650100
-1.3265300
-0.3654640
-2.4991750
0.8717030
-2.3952120
-2.9665500
-3.1055430
-1.2920980
-1.2783950
-1.4776930
0.7950800
1.5566350
-4.4859210
-5.9491110
-6.4068990
-6.1618100
-5.1298870
-4.0582570
-6.5400990
-7.4649510
-7.0997320
-5.6605800
-6.1280890
-5.8436680
-5.8324510
-4.6963740
-2.1039460
-2.6508440
-2.0963890
-2.2502240
-1.3986610
-1.5112800
-2.6208370
-3.7446500
-2.4379980
-3.3073130
-1.9607800
-1.6537030
-0.3439710
-1.0345040
2.7554380
1.7375690
2.8476040
3.7335590
1.9779150
4. 2138620

5. 1487690

3.9669440

2.9366270

3. 5385730

3. 5582970

4.4761600

2.7969110

3. 7962320

4.8451510

3. 1919620

0.9510160

1.0950890

$-0.2666170$

$-0.4387460$

$-1.8304570$

$-2.0492400$

$-1.0674410$

$-0.6411970$

0.3311080

$-1.9800140$

$-1.9363120$

$-0.1545410$

$-0.3077190$

$-2.5727040$

$-3.0803640$

$-1.4788340$

$-2.3977850$

$-3.4197460$

$-4.8224690$

$-5.1925580$

$-4.2612000$

$-2.7995570$

$-5.5385320$

$-3.4595720$

$-3.0949740$

$-5.1005560$

$-6.2335600$

$-4.3751530$

$-4.5599310$

$-4.8561390$

$-1.8076690$

$-3.2809620$

$-3.8668330$

$-4.0950980$ 


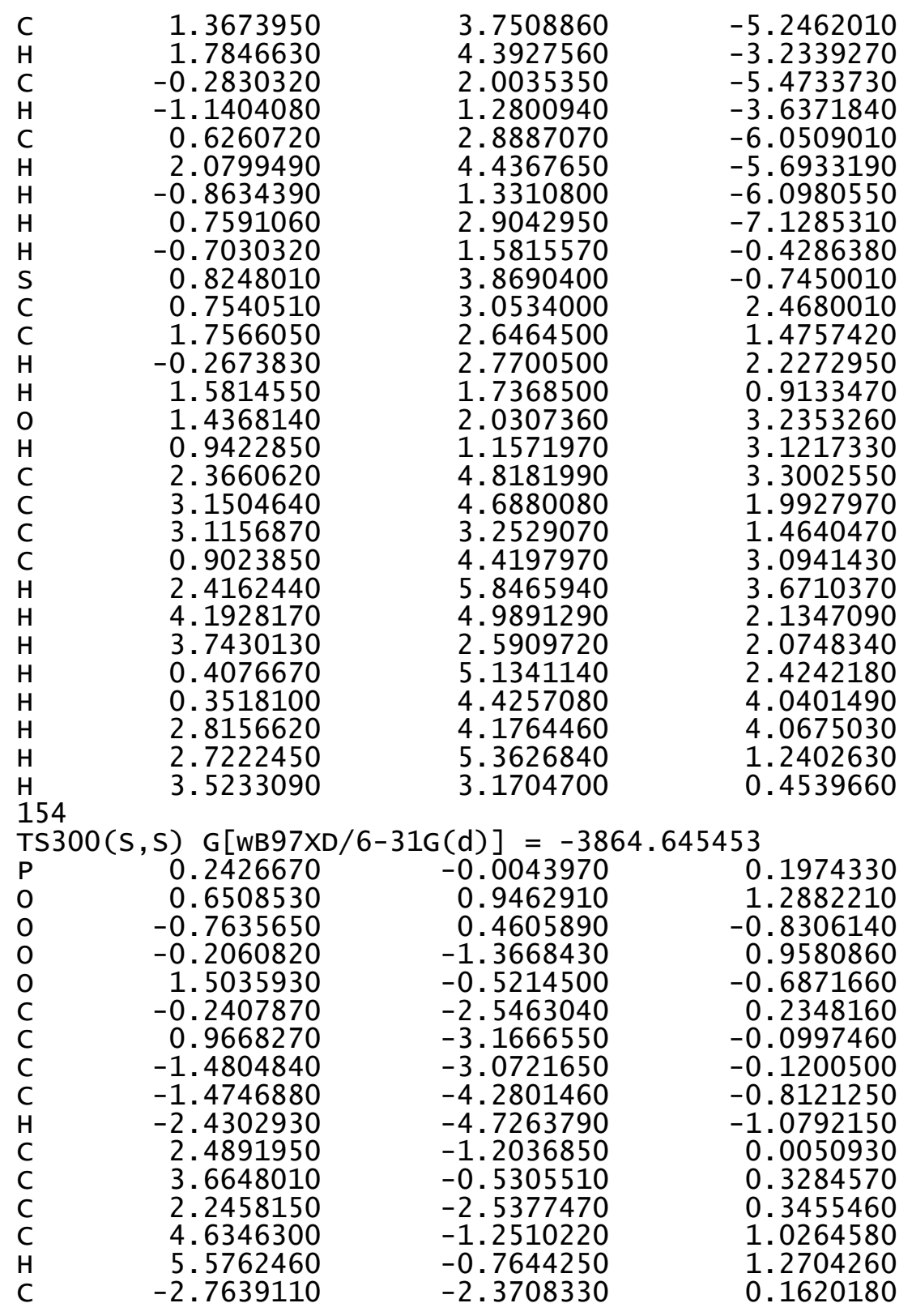

3.8512000
-3.2166200
-2.4879030
-4.7885380
-4.4705380
-2.2982720
-1.8139800
-1.6244020
-3.6770600
-3.8740910
-3.7025160
-5.7696000
-1.5197220
-4.7508810
-4.9200430
-5.5678720
-3.3779860
-2.8675070
-3.5051090
-3.5738690
-3.2346940
-5.5820720
-4.8346610
-4.3018370
-4.1010720
-4.2235570
-5.7140240
-6.2384100
-6.3275560
-6.5699410
-2.2363880
-4.7380680
-5.2352170
-4.6969750
-3.3180000
-3.0835550
-2.5536110
-5.2932850
-6.2751030
4.0202180
4.0478140
3.7091020
3.8369110
5.2116080
5.2491260
6.1598700

0.903133

$-2.1483170$

$-2.4763790$

$-1.3712190$

$-1.5666920$

$-1.1608370$

$-1.3880380$

$-0.5024670$

$-0.4879400$

$-0.3483300$

0.5045130

$-0.9005010$

$-2.9445600$

$-2.7485990$

$-2.6117430$

$-3.3719970$

$-3.4193980$

$-3.6434330$

$-4.3692430$

$-1.9544040$

$-2.0094040$

$-1.0271220$

$-1.3933830$

$-2.8696970$

$-2.8656780$

$-3.9095040$

-2.2996750

$-2.0505390$

$-3.0368560$

$-0.6156690$

$-2.4173490$

0.0013800

0.2210190

0.9410190

$-0.5724240$

$-0.5992990$

0.0426460

$-1.2150600$

$-0.7801090$

2. 6719130

2. 9342320

0.4427460

1. 3223120

2. 1067400

2.2595160

2. 4689540
$-0.0280860$

1. 4681690

2. 7488350

3. 1549710

1.6956080

3. 5373160

4.4941110

2. 9837760

3. 7660640

4.8355270

3.3036570

3. 2865750

2. 5599700

3.8519460

4.9264650

3. 4745570

3. 5883730

4.5321530

3. 0589200

$-0.9041530$

$-2.3751360$

$-1.9216490$

$-0.6661320$

$-3.0871080$

$-4.1648250$

$-2.7514600$

$-2.7883490$

$-3.7181880$

$-2.2601530$

$-1.6886150$

$-2.5462520$

$-2.7045460$

$-3.6567170$

$-2.1458500$

$-2.9385830$

$-4.0091410$

$-2.4569460$

0.6323290

0.8133520

$-1.6921750$

$-3.1768170$

$-2.5867490$

$-1.3657610$

$-3.7689160$

$-4.8538360$

$-3.3589270$ 


$\begin{array}{lrr}4.9970560 & 0.6089220 & -3.4266400 \\ 4.9029280 & 0.0117200 & -4.3410730 \\ 5.8512070 & 0.2108260 & -2.8696160 \\ 3.5706920 & -0.6074460 & -2.3222320 \\ 4.1902110 & 3.9999700 & -3.3872610 \\ 2.5154270 & 0.9388200 & -3.4292890 \\ 2.4308190 & 0.3162610 & -4.3274050 \\ 1.5920910 & 0.8073450 & -2.8596260 \\ 2.7355890 & 2.4244110 & -3.8111590 \\ 2.7990820 & 2.5461450 & -4.8988590 \\ 1.8958280 & 3.0386300 & -3.4749340 \\ 4.2632480 & 3.2103220 & 0.6324160 \\ 4.5507310 & 4.0945480 & 1.8187420 \\ 4.1319570 & 1.6283070 & 2.4617730 \\ 4.0605400 & 1.8654230 & 0.9707090 \\ 3.3641130 & 4.0176330 & 2.8025520 \\ 3.6045990 & 4.6055350 & 3.6965740 \\ 2.4779280 & 4.4630470 & 2.3427000 \\ 3.1005230 & 2.5354020 & 3.1677550 \\ 3.1848410 & 2.3805910 & 4.2498170 \\ 2.0938650 & 2.2250870 & 2.8777820 \\ 3.9385510 & 0.5845840 & 2.7162050 \\ 4.7257280 & 5.1298130 & 1.5062330 \\ 5.5411030 & 2.0514750 & 2.9338700 \\ 5.6059290 & 1.9278940 & 4.0211750 \\ 6.2940130 & 1.3898040 & 2.4918210 \\ 5.8017580 & 3.5276170 & 2.5295820 \\ 6.0247860 & 4.1395930 & 3.4113800 \\ 6.6649250 & 3.6003130 & 1.8599580 \\ 4.2343900 & 3.6182110 & -0.6956410 \\ 4.4018550 & 4.6631820 & -0.9534950 \\ 4.4049040 & -2.5558030 & 1.4472850 \\ 5.4225790 & -3.3457290 & 2.2302160 \\ 5.5671870 & -4.7460970 & 1.6154510 \\ 4.2206380 & -5.5016750 & 1.5818850 \\ 2.9986250 & -4.5678750 & 1.7389040 \\ 3.2024690 & -3.2020770 & 1.1268500 \\ 5.1039680 & -3.4382150 & 3.2787630 \\ 6.3157710 & -5.3251590 & 2.1660620 \\ 4.1834720 & -6.2463340 & 2.3838250 \\ 2.8228110 & -4.4080770 & 2.8117810 \\ 6.3866400 & -2.8267410 & 2.2382420 \\ 5.9495050 & -4.6242180 & 0.5954570 \\ 4.1386850 & -6.0592680 & 1.3564710 \\ 2.0971970 & -5.0516850 & -8940180 \\ 0.9389150 & -4.3289710 & \end{array}$

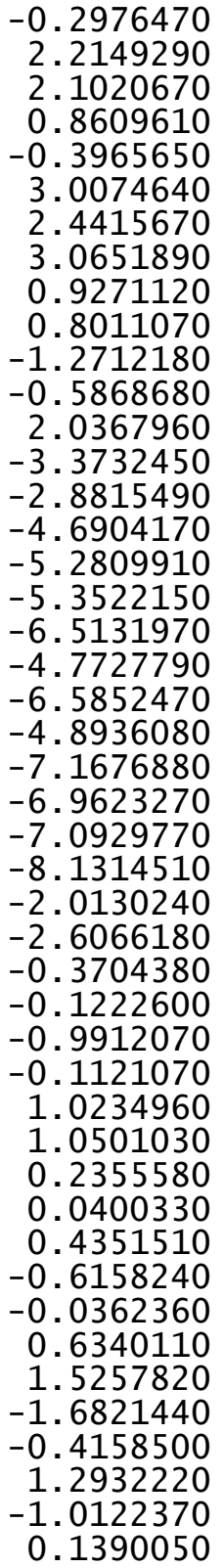

-4.9080840
-4.8864200
-6.3542120
-6.5680170
-6.2207230
-6.6576610
-4.2876980
-4.7417800
-5.9263210
-7.6024840
-6.1975210
-7.0208910
-6.9812050
2.6103440
1.5406570
2.9848770
2.1397860
4.1573880
2.4609240
1.2278830
4.4742690
4.8146310
3.6279530
1.7974520
5.3847260
3.8793770
1.1632480
3.4988390
3.7463350
3.5086200
3.0035220
2.4777720
3.3921830
2.4014300
6.1735230
6.0000190
4.5927900
5.1663170
7.1901260
6.7338380
4.4656690
5.3776120
5.2248580
6.0328600
6.1791120
4.4017170
$-1.2141380$ $-1.4977220$ $-1.9010290$ $-2.7638400$ $-1.9690630$ $-2.4379970$ $-2.3923850$ $-0.8313530$ $-3.6525540$ $-3.1191480$ $-2.6292050$ $-1.2393740$ $-1.0004980$ $-0.5183030$

0.0184340

0.0467800

0.9960670

$-0.3365700$

1. 5482410

1. 2825870

0.2181950

$-1.0675230$

1. 1605580

2. 2810720

$-0.0838220$

1. 5931040

$-0.3918330$

$-1.7257170$

0.6044570

$-0.8231630$

1. 1053800

$-1.1606560$

0.6564580

0.8857320

0.2791200

$-1.2289190$

$-1.6861240$

1.0574730

0.5797710

$-1.7821460$

$-1.6497930$

0.9093460

2.1322930

0.5336560

$-1.4815760$

$-2.7212390$ 
154

TS301 (S , S ) G[WB97XD/6-31G(d)] = -3864.643355

\begin{tabular}{|c|c|c|c|}
\hline & & & \\
\hline $\mathrm{P}$ & -0.3861500 & -0.1929120 & 1. 3495440 \\
\hline 0 & 0.1882340 & -0.3263360 & 2.7267880 \\
\hline 0 & -0.9212910 & 1.1579630 & 0.9335310 \\
\hline 0 & -1.4918430 & -1.3608280 & 1.1349750 \\
\hline 0 & 0.7208620 & -0.5279640 & 0.1804620 \\
\hline C & -1.9625660 & -1.4494200 & -0.1714430 \\
\hline C & -1.1519460 & -2.0954570 & -1.1008030 \\
\hline C & -3.1723190 & -0.8468900 & -0.5117600 \\
\hline C & -3.5272310 & -0.8898100 & -1.8572150 \\
\hline $\mathrm{H}$ & -4.4678330 & -0.4325360 & -2.1566550 \\
\hline C & 1.0735640 & -1.8462830 & -0.0470450 \\
\hline C & 2.3750150 & -2.2554630 & 0.2330450 \\
\hline C & 0.1284870 & -2.6950570 & -0.6275330 \\
\hline C & 2.6794530 & -3.6031650 & 0.0493510 \\
\hline $\mathrm{H}$ & 3.6930030 & -3.9433110 & 0.2496460 \\
\hline C & -4.0381670 & -0.1673740 & 0.4892020 \\
\hline C & 3.4207310 & -1.2401020 & 0.5331570 \\
\hline C & -4.5827990 & -0.8792680 & 1.5659110 \\
\hline C & -4.3738080 & -2.3406100 & 1.8823220 \\
\hline C & -5.9410880 & -1.1650410 & 3.5653220 \\
\hline C & -5.4432750 & -0.2514700 & 2.4749610 \\
\hline C & -3.7775470 & -2.4449190 & 3.3028500 \\
\hline $\mathrm{H}$ & -3.6554350 & -3.5038490 & 3.5591450 \\
\hline $\mathrm{H}$ & -2.7825510 & -1.9930770 & 3.3069400 \\
\hline C & -4.7166630 & -1.7368750 & 4.3137970 \\
\hline $\mathrm{H}$ & -5.0623020 & -2.4359680 & 5.0845050 \\
\hline $\mathrm{H}$ & -4.1917580 & -0.9246530 & 4.8266420 \\
\hline $\mathrm{H}$ & -6.6005940 & -0.6307750 & 5410 \\
\hline $\mathrm{H}$ & -3.7136530 & -2.8266240 & 1.16 \\
\hline C & -6.6921450 & -2.3400080 & 2.9010080 \\
\hline $\mathrm{H}$ & -7.0146570 & -3.0448440 & 3.67 \\
\hline $\mathrm{H}$ & -7.5961570 & -1.96 & 2000 \\
\hline C & -5.7550400 & -3.0334980 & 2190 \\
\hline $\mathrm{H}$ & -5.6223590 & -4.0930650 & 2.1261310 \\
\hline $\mathrm{H}$ & -6.1808870 & -2.9904990 & 0.8703860 \\
\hline C & -4.3663840 & 1.1887950 & 0.3496740 \\
\hline C & -3.8654460 & 2.1473270 & -0.7061570 \\
\hline C & -5.5297050 & 3.2459550 & 0.9357830 \\
\hline C & -5.2471270 & 1.8000280 & 1.2517080 \\
\hline C & -5.0870460 & 2.6777390 & -1.4891850 \\
\hline $\mathrm{H}$ & -4.7453960 & 3.4104940 & -2.2295870 \\
\hline $\mathrm{H}$ & -5.5578260 & 1.8575950 & -2.0424690 \\
\hline C & -6.0957520 & 3.3212240 & -0.5003380 \\
\hline $\mathrm{H}$ & -6.2802880 & 4.3704940 & -0.758867 \\
\hline
\end{tabular}

$-7.0607700$ $-6.2422320$ $-3.1518990$ $-4.1953950$ $-4.3780120$ $-3.8008440$ $-3.1923860$ $-2.8484940$ $-2.3115200$ $-5.7840790$ $-6.4655650$ 4.8430560 5. 2175310 3. 4532030 3.8725030 5.7761620 6.0237110 6.7052930 4.7166740 4.4367540 5.1134340 2. 7099200 5.9630960 2.8810730 2. 5790450 1. 9769700 3. 9448160 4. 2110110 3. 5484970 4. 9789250 5. 5039590 3. 6975860 3. 9879200 4. 3150260 4.6827080 3.8697030 3. 2609850 3.1559130 2. 2759200 2.9257080 6.2673480 5. 0237180 4. 8485860 5. 3556850 6.0955790 6.4231920
2.8056940

3.6733420

1.6728930

4.0204410

5.0644860

4.0264280

3. 3473710

4.0566640

2. 9939630

1.0859690

1. 5682720

0.5506800

1. 3063420

$-0.5353170$

$-0.4323850$

0.2787950

0.7929120

$-0.1492250$

$-0.8289530$

$-0.8764220$

$-1.8123790$

$-1.3211770$

2. 0805530

0.8271170

0.7832890

1. 0198040

1.9303130

2. 4122470

2. 7182520

$-0.1020850$

$-0.0833530$

$-1.8957680$

$-1.0733730$

0.1565750

0.1729690

1.1373350

$-0.9621340$

$-1.5592620$

$-0.5513030$

$-2.6454570$

0.6917250

$-2.5704100$

$-3.1743650$

$-3.2538450$

$-1.4800250$

$-1.5081050$
$-0.5387580$

1. 6496650

$-1.3826480$

0.9684750

0.6873600

1. 9897520

$-0.0021270$

$-0.7645080$

0.5363490

2. 3156210

3. 0146340

$-0.3031470$

$-1.5540960$

$-1.9668090$

$-0.5197150$

$-2.5655620$

$-3.5014410$

$-2.1760040$

$-2.8062010$

-3.8649590

$-2.5343990$

-2. 1201610

$-1.3431170$

$-2.3991820$

$-3.4519970$

$-1.8170740$

$-2.1739370$

$-3.1217730$

$-1.5249210$

2. 0036820

3. 4154520

3.0275730

1.7968660

4. 3718810

5. 4045160

4. 1738870

4.1758120

5.0890260

3.9509920

2.8351660

3.5451450

3. 4482210

4.3456770

2. 6594510

3. 7192440

4.7650360 


$\begin{array}{lr}\mathrm{H} & 6.9832590 \\ \mathrm{C} & 5.4035470 \\ \mathrm{H} & 6.1718160 \\ \mathrm{C} & 1.7194290 \\ \mathrm{C} & 2.0106070 \\ \mathrm{C} & 1.5521020 \\ \mathrm{C} & 0.0517700 \\ \mathrm{C} & -0.5415730 \\ \mathrm{C} & 0.4439280 \\ \mathrm{H} & 1.4781990 \\ \mathrm{H} & 1.7653630 \\ \mathrm{H} & -0.5255840 \\ \mathrm{H} & -0.8480650 \\ \mathrm{H} & 3.0786060 \\ \mathrm{H} & 2.1524500 \\ \mathrm{H} & -0.0924930 \\ \mathrm{H} & -1.4526070 \\ \mathrm{C} & -1.4959180 \\ \mathrm{C} & -2.7030000 \\ \mathrm{C} & -0.5120140 \\ \mathrm{C} & -1.1416990 \\ \mathrm{C} & -1.9910260 \\ \mathrm{C} & -3.1452720 \\ \mathrm{H} & -0.3539620 \\ \mathrm{H} & 0.2813960 \\ \mathrm{H} & -0.0107220 \\ \mathrm{H} & -1.3602710 \\ \mathrm{H} & -2.3833120 \\ \mathrm{H} & -3.6691850 \\ \mathrm{H} & -3.8825660 \\ \mathrm{H} & -1.7744640 \\ \mathrm{C} & 0.1872440 \\ \mathrm{O} & -0.5283140 \\ \mathrm{C} & 0.3496990 \\ \mathrm{C} & 1.2750820 \\ \mathrm{C} & -0.4049990 \\ \mathrm{C} & 1.4440410 \\ \mathrm{H} & 1.8680210 \\ \mathrm{C} & -0.2380750 \\ \mathrm{H} & -1.1134420 \\ \mathrm{C} & 0.6877160 \\ \mathrm{H} & 2.1689780 \\ \mathrm{H} & -0.8309920 \\ \mathrm{H} & 0.8208540 \\ \mathrm{H} & -0.6634160 \\ \mathrm{~S} & 0.8861930 \\ & \end{array}$

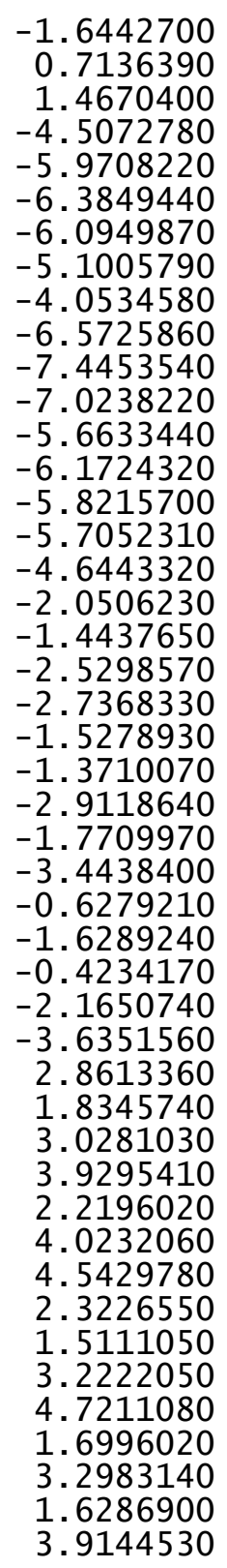

3.1001540
0.9609180
1.1299160
-0.3943390
-0.6025980
-2.0095440
-2.2390890
-1.2146880
-0.7566020
0.1479730
-2.1791810
-2.1843740
-0.3219610
-0.4702260
-2.7330520
-3.2526560
-1.6099630
-2.4642180
-2.8367520
-3.5152730
-4.8908420
-5.2739190
-4.2858120
-5.6316140
-3.5914920
-3.1925390
-5.2574160
-6.2916370
-4.4563160
-4.4714040
-4.8770240
-1.7157960
-1.4123960
-3.1818670
-3.7165790
-4.0442210
-5.0924070
-3.0471480
-5.4186990
-3.6290120
-5.9448160
-5.4992110
-6.0815330
-7.0198130
-0.3982860
-0.5983090

0.8021470

1.8084520

$-0.2183490$

1.6353000

1. 4813570

0.9852840

2. 4093100

3. 2018500

3. 1686350

0.9468270

2. 4582660

4.2437410

3.7920930

0.4566870

0.3903350

2.8533750

2.7791570

3. 5817060
2.9584980
2. 5906480
2. 6847380
1. 7029200
1.9074850
1.0402290
4.6896960
4. 6093050
3. 1959520
4. 3008900
5.7028480
4.9029170
2. 5101710
5.0394890
4. 2726030
4. 0182220
5. 3132500
3. 1535500

2. 5639840

1.5602670

2. 3079780

0.9628400

3.2946860

3.1420350

3.4708350

2. 1643110

1. 5793460

3. 2406400

3. 8815700

2. 3241310

2.1672700

2. 5940860

4.1827890

4. 2155760

1.4357080

0.5688220

154

TS302(S, S) G[WB97XD/6-31G(d)] $=-3864.641256$

$\begin{array}{lrrr}\text { TS302(S, S) G }[\mathrm{WB} 97 \times \mathrm{D} / 6-31 \mathrm{G}(\mathrm{d})]=-3864.641256 \\ \mathrm{P} & -0.2803040 & -0.1669550 & 1.1399500 \\ \mathrm{O} & 0.4005410 & -0.3858280 & 2.4580210 \\ \mathrm{O} & -0.7000820 & 1.2378180 & 0.7778540 \\ \mathrm{O} & -1.5128150 & -1.2155500 & 1.0697870 \\ \mathrm{O} & 0.6374770 & -0.6366850 & -0.1332670 \\ \mathrm{C} & -2.1009440 & -1.3682560 & -0.1773800 \\ \mathrm{C} & -1.4453730 & -2.1756970 & -1.1147640 \\ \mathrm{C} & -3.2982460 & -0.7092710 & -0.4405570 \\ \mathrm{C} & -3.8422640 & -0.8696890 & -1.7164000 \\ \mathrm{H} & -4.7845350 & -0.3778240 & -1.9463510 \\ \mathrm{C} & 0.8862490 & -1.9910180 & -0.2804170 \\ \mathrm{C} & 2.1693420 & -2.4640520 & -0.0165850 \\ \mathrm{C} & -0.1525440 & -2.8159730 & -0.7295100 \\ \mathrm{C} & 2.3815300 & -3.8375740 & -0.1201870 \\ \mathrm{H} & 3.3756360 & -4.2321420 & 0.0777340 \\ \mathrm{C} & -3.9886890 & 0.1407190 & 0.5689650 \\ \mathrm{C} & 3.2751770 & -1.5135680 & 0.2830660 \\ \mathrm{C} & -4.4953890 & -0.4100070 & 1.7529130 \\ \mathrm{C} & -4.3568830 & -1.8394590 & 2.2190290 \\ \mathrm{C} & -5.6986790 & -0.3714210 & 3.8670480 \\ \mathrm{C} & -5.2314360 & 0.3784170 & 2.6464400 \\ \mathrm{C} & -3.6476590 & -1.8232960 & 3.5912000 \\ \mathrm{H} & -3.5635670 & -2.8518700 & 3.9612200 \\ \mathrm{H} & -2.6313580 & -1.4413800 & 3.4639910 \\ \mathrm{C} & -4.4560530 & -0.9459680 & 4.5828070 \\ \mathrm{H} & -4.7809790 & -1.5318240 & 5.4508910\end{array}$




$\begin{array}{rrr}-3.8410750 & -0.1234210 & 4.9616010 \\ -6.2635380 & 0.2819040 & 4.5407010 \\ -3.7903190 & -2.4486020 & 1.5118260 \\ -6.5779850 & -1.5521930 & 3.3994200 \\ -6.8916890 & -2.1355050 & 4.2732160 \\ -7.4868910 & -1.1649910 & 2.9275960 \\ -5.7715250 & -2.4300450 & 2.4077990 \\ -5.6795320 & -3.4561460 & 2.7827330 \\ -6.2760180 & -2.4866220 & 1.4376790 \\ -4.2119570 & 1.5014300 & 0.3170940 \\ -3.6906900 & 2.3082740 & -0.8491160 \\ -5.1890420 & 3.6922370 & 0.7383160 \\ -4.9802140 & 2.2721560 & 1.1996550 \\ -4.8981130 & 2.8649620 & -1.6347950 \\ -4.5304970 & 3.5225520 & -2.4315180 \\ -5.4380560 & 2.0448370 & -2.1204390 \\ -5.8306530 & 3.6418990 & -0.6675040 \\ -5.9986640 & 4.6640590 & -1.0261690 \\ -6.8106360 & 3.1583520 & -0.6000800 \\ -5.8331720 & 4.2433210 & 1.4318360 \\ -3.0460770 & 1.7122540 & -1.4992190 \\ -3.8128840 & 4.3776680 & 0.6099620 \\ -3.9501000 & 5.3764590 & 0.1784540 \\ -3.3720170 & 4.5134140 & 1.6027280 \\ -2.8944480 & 3.5059980 & -0.2813260 \\ -2.4853600 & 4.0902010 & -1.1142840 \\ -2.0527680 & 3.1248000 & 0.2989960 \\ -5.4903450 & 1.7145060 & 2.3653900 \\ -6.0854460 & 2.3169270 & 3.0499560 \\ 4.7737020 & 0.2463240 & -0.4843520 \\ 5.1640840 & 1.0540610 & -1.6965180 \\ 3.2870300 & -0.6570470 & -2.1713400 \\ 3.7398830 & -0.6636600 & -0.7304610 \\ 5.6361170 & 0.0632180 & -2.7853850 \\ 5.8736230 & 0.6201470 & -3.6992440 \\ 6.5579950 & -0.4279330 & -2.4582010 \\ 4.5181110 & -0.9798440 & -3.0485300 \\ 4.2167790 & -0.9685060 & -4.1024210 \\ 4.8694170 & -1.9923870 & -2.8252000 \\ 2.4956630 & -1.3881310 & -2.3520960 \\ 5.9586300 & 1.7693490 & -1.4576710 \\ 2.7942190 & 0.7584230 & -2.5255730 \\ 2.5058730 & 0.7896900 & -3.5826750 \\ 1.8940200 & 0.9679850 & -1.9455050 \\ 3.9148500 & 1.7888180 & -2.2327640 \\ 4.1830250 & 2.3409610 & -3.1408770\end{array}$

4.9403310

5. 5213600

3.6067920

3. 8919910

4.3888700

4. 7994550

3. 9926910

3. 2679290

3.1667060

2. 2987840

2.7893540

6. 3290490

4. 9105760

4. 7389100

5.1744450

6.0504350

6.4204630

6.9004360

5. 3786220

6. 1964220

1. 3483200

1. 5352290

0.9863740

$-0.4725430$

$-0.9732360$

0.0805810

1. 0029730

1.0619600

$-1.1514020$

$-1.2390430$

2. 5927710

1.6323000

$-0.5506230$

$-1.8932140$

$-3.1940880$

$-3.7357110$

$-3.7031430$

$-2.2985820$

$-1.3228820$

$-1.9887810$

$-3.1249850$

$-4.0434120$

$-1.8530370$

$-0.9477640$

$-4.7547030$

2.5324100
-0.5574270
-0.6634000
-2.3474970
-1.4594690
-0.4309810
-0.5108320
0.5834250
-1.4760790
-2.1324180
-0.9995090
-3.0429250
0.0622640
-3.1170970
-3.7746810
-3.7584380
-2.1054600
-2.2257440
-2.2694780
0.2989720
0.9935930
-4.7032790
-6.1959040
-6.7271080
-6.2773390
-5.2256560
-4.1976460
-6.6793170
-7.8191430
-7.1359230
-5.7386760
-6.4571520
-6.3522800
-5.8651810
-4.7590900
-1.6097970
-1.7549710
-3.2303260
-3.8582000
-2.9590000
-2.2651980
-1.1606990
-3.3244010
-4.0733240
-2.1779330
-1.3591220

1.7707340

3.1566720

2.7235580

1. 5364810

4.1799140

5. 1932940

4.0693090

3. 9514760

4.8237740

3.7968030

2. 5196560

3. 3032640

3. 0384440

3. 8981990

2. 1904960

3. 3368240

4. 3616780

2. 6667340

0.7677060

0.9555380

$-0.4589230$

$-0.5384120$

$-1.8714660$

$-2.1260440$

$-1.1136070$

$-0.7827680$

0.2934650

$-1.8932970$

$-2.0916310$

$-0.1786890$

$-0.4279950$

$-2.6731010$

$-3.1372810$

$-1.4733720$

$-2.6957030$

$-4.0943120$

$-4.5220190$

$-4.3523710$

$-3.5655280$

$-2.4036740$

$-4.7897200$

5.5583910

$-5.3293450$

-4.2423120
-4.1557430 


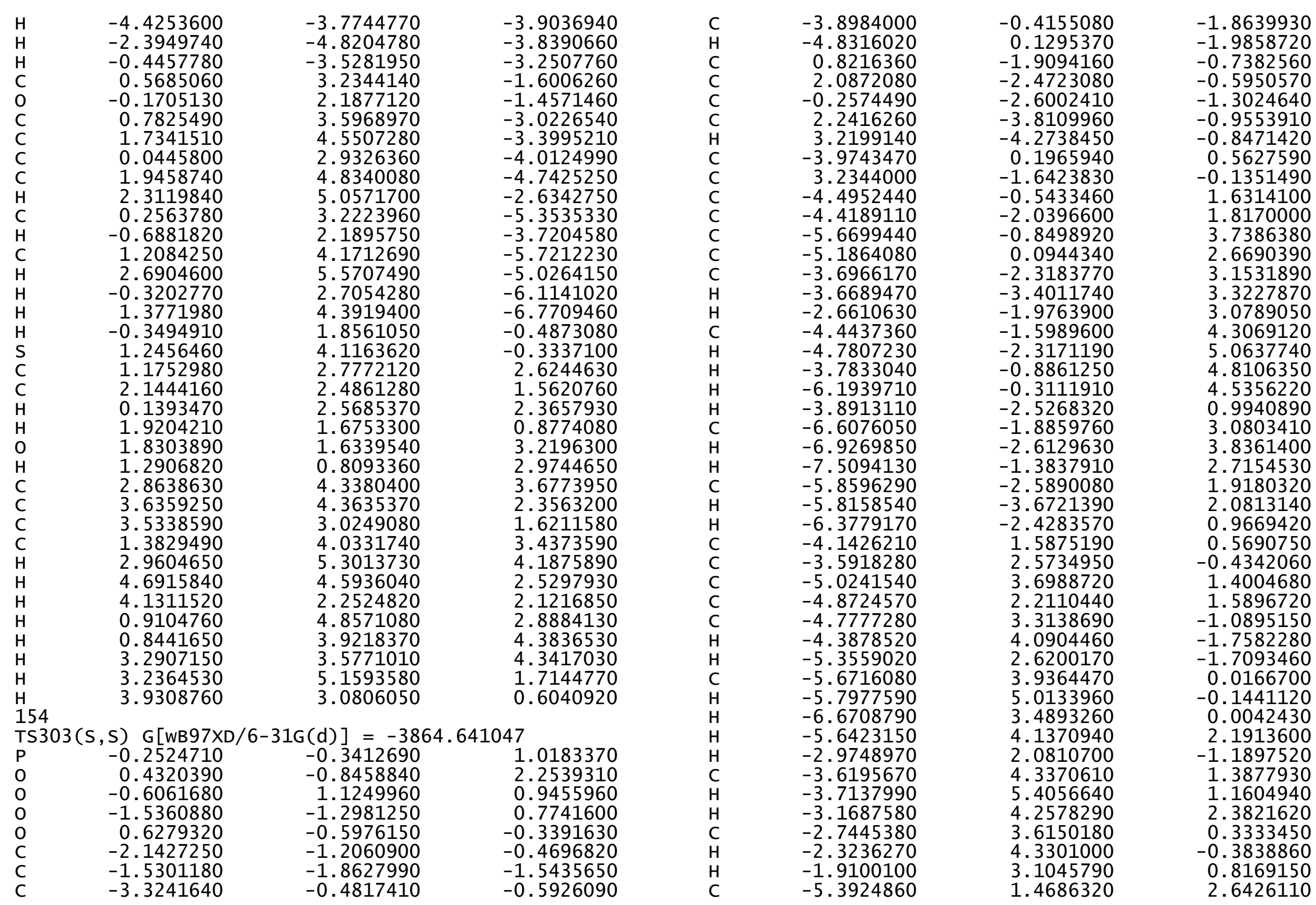




\begin{tabular}{|c|c|c|c|}
\hline & & & \\
\hline $\mathrm{H}$ & -5.9522930 & 1.9574790 & $\begin{array}{r}3.4384640 \\
-0.5637620\end{array}$ \\
\hline $\begin{array}{l}\mathrm{C} \\
\mathrm{C}\end{array}$ & $\begin{array}{l}4.8026340 \\
5.2191820\end{array}$ & $\begin{array}{l}0.1703350 \\
1.1874010\end{array}$ & $\begin{array}{l}-0.5637620 \\
-1.5964070\end{array}$ \\
\hline c & 3.2557800 & -0.3055460 & -2.3661880 \\
\hline $\mathrm{C}$ & 3.7242390 & -0.6249870 & -0.9666260 \\
\hline C & 5.6316880 & 0.4139600 & -2.8703460 \\
\hline $\mathrm{H}$ & 5.8921850 & 1.1319760 & -3.6565720 \\
\hline H & 6.5291270 & -0.1779060 & -2.6639570 \\
\hline C & 4.4583140 & -0.4969460 & -3.3185130 \\
\hline H & 4.1450000 & -0.2530090 & -4.3401730 \\
\hline H & 4.7597440 & -1.5494810 & -3.3172240 \\
\hline $\mathrm{H}$ & 2.4276840 & -0.9475070 & -2.6752080 \\
\hline H & 6.0505920 & 1.8026380 & -1.2354220 \\
\hline C & 2.8274060 & 1.1732150 & -2.4148240 \\
\hline H & 2.5197380 & 1.4295590 & -3.4351120 \\
\hline $\mathrm{H}$ & 1.9509460 & 1.2999780 & -1.7779490 \\
\hline C & 4.0029700 & 2.0713260 & -1.9532410 \\
\hline H & 4.2893390 & 2.7756880 & -2.7426060 \\
\hline $\mathrm{H}$ & 3.7097770 & 2.6764720 & -1.0886770 \\
\hline C & 4.9640390 & -1.0838210 & 1.4766370 \\
\hline C & 5.5616130 & -1.4985550 & 2.7955960 \\
\hline $\mathrm{C}$ & 3.5493470 & -2.9598470 & 2.0852730 \\
\hline C & 3.8672730 & -1.8686560 & 1.0909530 \\
\hline C & 4.4609020 & -1.4183570 & 3.8748030 \\
\hline $\mathrm{H}$ & 4.8806380 & -1.7252680 & 4.8399100 \\
\hline $\mathrm{H}$ & 4.1244350 & -0.3822790 & 3.9828090 \\
\hline C & 3.2772530 & -2.3322280 & 3.4689510 \\
\hline H & 3.1466230 & -3.1415140 & 4.1969380 \\
\hline H & 2.3362070 & -1.7814990 & $3.434164 d$ \\
\hline $\mathrm{H}$ & 2.6901050 & -3.5559480 & 1.769146 \\
\hline $\mathrm{H}$ & 6.4118080 & -0.8613390 & 3.0632310 \\
\hline C & 4.8114350 & -3.8443520 & 2.2087720 \\
\hline H & 4.6171040 & -4.6463980 & 2.9300020 \\
\hline $\mathrm{H}$ & 5.0226630 & -4.3214090 & 1.245645 \\
\hline C & 6.0120690 & -2.9723950 & 2.6660480 \\
\hline H & 6.3973780 & -3.3157150 & 3.6332500 \\
\hline H & 6.8364010 & -3.0400670 & 1.9489130 \\
\hline C & 5.4281920 & -0.0614850 & 0.6575820 \\
\hline H & 6.2841610 & 0.5403180 & 0.9606740 \\
\hline C & -0.0830660 & -3.9551480 & -1.6116680 \\
\hline $\mathrm{C}$ & -1.1933300 & -4.8639310 & -2.0758910 \\
\hline C & -1.2334390 & -6.1310160 & -1.2016690 \\
\hline C & 0.1483150 & -6.8205870 & -1.0738580 \\
\hline C & 1.2736260 & -6.0231680 & -1.7617980 \\
\hline C & 1.1699670 & -4.5581100 & -1.4295990 \\
\hline $\mathrm{H}$ & -1.0238100 & -5.1520350 & -3.123413 \\
\hline
\end{tabular}
$-1.9731020$
0.1160930
1. 1890650
$-2.1606590$
$-1.5947740$
0.4018600
2. 2522980
$-3.2972470$
$-3.8808540$
$-3.9116010$
-2. 5296160
$-1.4984790$
$-2.1041240$
$-3.2697240$
$-4.2705260$
$-2.1217890$
$-1.1364470$
$-4.8870600$
$-4.6463370$
$-2.6459110$
$-0.6232460$
0.7212080
$-0.0521380$
0.9264020
1. 9343750
0.1141330
2. 1249830
2. 5710310
0.3043920
$-0.6618280$
1. 3100570
2. 9119600
$-0.3324610$
1.4595140
$-0.2373880$
1.4401740
1. 3613730
2. 3004930
0.3139330
2.0349850
1.9834740
1.4024420
3.1235080
3. 8691230
3.7093800
1.6280980
$-6.8252740$
$-7.8278100$
$-6.1555160$
$-4.3560730$
$-5.8419270$
$-6.9379010$
$-6.4200990$
$-1.0088340$
$-0.9297350$
$-2.3274990$
$-3.0251440$
$-2.2608160$
$-1.7310600$
$-0.2593330$
$-2.2517030$
$-3.1405860$
$-1.3978530$
$-0.4991580$
-2.9300680
$-4.0354120$
$-2.8845260$
. 5128180
2.4833810
. 1684000
5.1195240
3.7976160
5.6924730
5.3970690
4. 3779150
3.0553640
. 3250310
4264830
4.0902870
5.7761130
1.9680130
4.0836540
2.1603260
2.0680430
2.0444650
1.4232300
0.8965310
0.1639970
3.4090980
3.6903270
2. 5384200
3.2085600

$-1.6137940$

$-1.5021880$

$-2.8496970$

$-2.0421540$

$-0.0153640$

$-1.4736600$

$-2.9656920$

$-4.3525020$

$-4.9930760$

$-4.9459530$

$-4.0910030$

$-2.8149360$

$-4.9742410$

$-6.0246730$

$-5.9556830$

$-4.6679610$

$-4.3226790$

$-4.4481320$

$-4.5406250$

$-3.9008210$

$-1.0623310$

$-2.3072950$

$-2.4993830$

$-3.3881010$

$-3.7500710$

$-1.6666000$

$-4.6350350$

$-3.2411590$

$-4.8185670$

$-3.8914270$

$-5.4656570$

0.1783180

0.4197840

3.0441220

2.7733240

1.0895000

3. 3681290

2. 9706540

(

3.0526360

4.0982700 


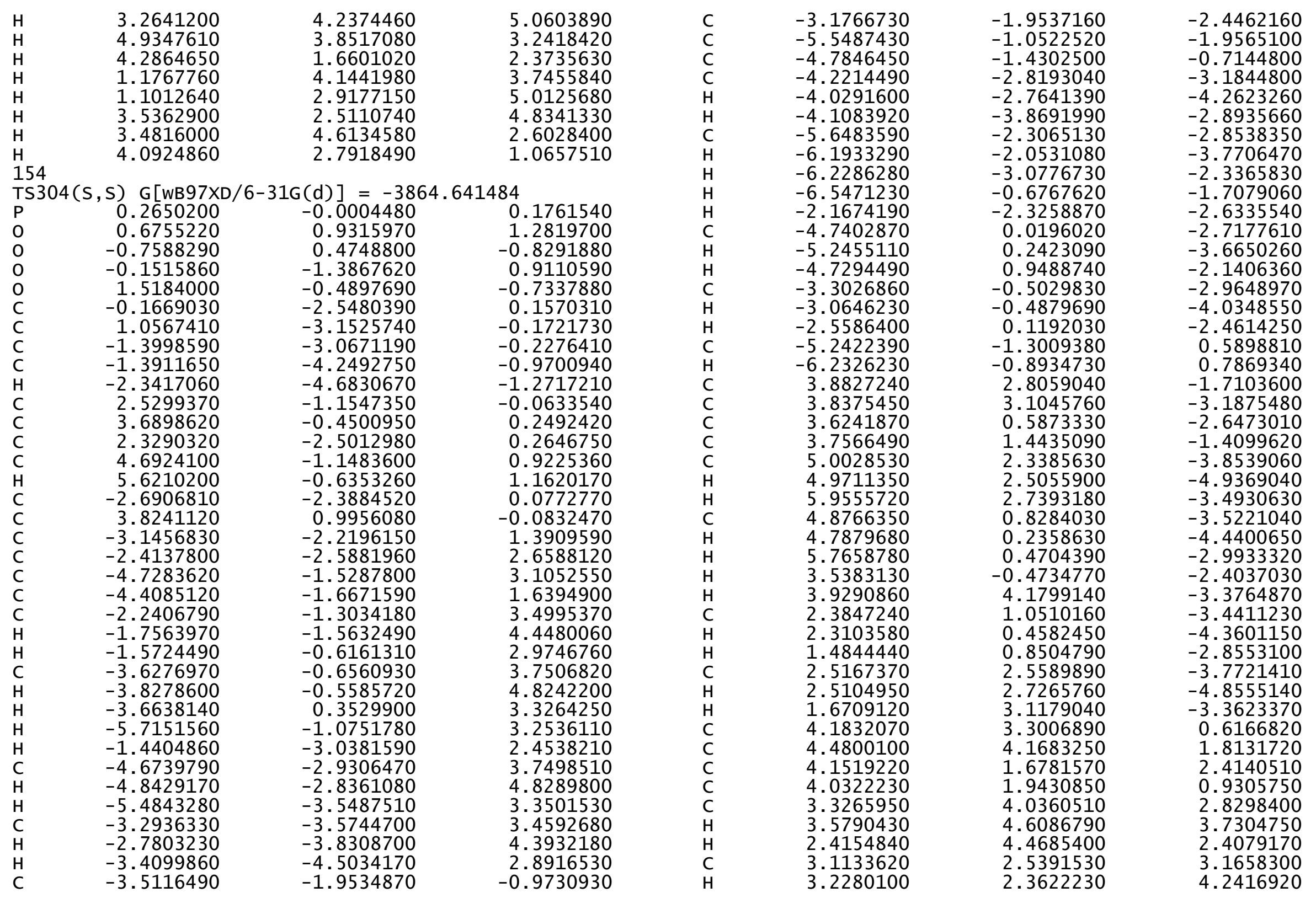




$\begin{array}{lrrr}\text { H } & 2.1091940 & 2.2069080 & \\ \text { H } & 3.9946670 & 0.6247190 & 2.8917810 \\ \text { H } & 4.6160730 & 5.2145010 & 1.5181560 \\ \mathrm{C} & 5.5602020 & 2.1336330 & 2.8576520 \\ \mathrm{H} & 5.6567800 & 1.9930550 & 3.9404840 \\ \mathrm{H} & 6.3202790 & 1.5025210 & 2.3843310 \\ \mathrm{C} & 5.7679330 & 3.6235660 & 2.4738240 \\ \mathrm{H} & 6.0022730 & 4.2250930 & 3.3598970 \\ \mathrm{H} & 6.6067620 & 3.7325500 & 1.7787140 \\ \mathrm{C} & 4.0991430 & 3.7365740 & -0.7000160 \\ \mathrm{H} & 4.2243300 & 4.7919400 & -0.9389480 \\ \mathrm{C} & 4.5103040 & -2.4648650 & 1.3267060 \\ \mathrm{C} & 5.5590130 & -3.2225690 & 2.0998670 \\ \mathrm{C} & 5.7646830 & -4.6096680 & 1.4748310 \\ \mathrm{C} & 4.4433560 & -5.4062700 & 1.3842150 \\ \mathrm{C} & 3.1879800 & -4.5317080 & 1.5959550 \\ \mathrm{C} & 3.3254090 & -3.1472410 & 1.0114770 \\ \mathrm{H} & 5.2411810 & -3.3374200 & 3.1464600 \\ \mathrm{H} & 6.5112480 & -5.1713960 & 2.0457110 \\ \mathrm{H} & 4.4287650 & -6.2071510 & 2.1308500 \\ \mathrm{H} & 3.0310770 & -4.4083010 & 2.6766770 \\ \mathrm{H} & 6.5010810 & -2.6647810 & 2.1167910 \\ \mathrm{H} & 6.1792020 & -4.4657680 & 0.4706540 \\ \mathrm{H} & 4.3866840 & -5.8958450 & 0.4070080 \\ \mathrm{H} & 2.2977300 & -5.0412110 & 1.2208380 \\ \mathrm{C} & -0.2048000 & -4.8509670 & -1.3634140 \\ \mathrm{C} & -0.1735670 & -6.0919620 & -2.2172460 \\ \mathrm{C} & 0.8080700 & -7.1180360 & -1.6312370 \\ \mathrm{C} & 2.2080540 & -6.5094440 & -1.3769070 \\ \mathrm{C} & 2.2468170 & -4.9775160 & -1.5534630 \\ \mathrm{C} & 1.0269910 & -4.3011480 & -0.9773190 \\ \mathrm{H} & 0.1415860 & -5.8293140 & -3.2376160 \\ \mathrm{H} & 0.8798420 & -7.9824740 & -2.2993240 \\ \mathrm{H} & 2.9484260 & -6.9507480 & -2.0525410 \\ \mathrm{H} & 2.2691670 & -4.7523200 & -2.6292140 \\ \mathrm{H} & -1.1753000 & -6.5259080 & -2.3020470 \\ \mathrm{H} & 0.3887800 & -7.4845280 & -0.6878070 \\ \mathrm{H} & 2.5288990 & -6.7613260 & -0.3609900 \\ \mathrm{H} & 3.1723600 & -4.5692690 & -1.1427270 \\ \mathrm{C} & -3.4287350 & 2.5403290 & -0.4429000 \\ \mathrm{O} & -2.9043200 & 1.4688330 & 0.0578130 \\ \mathrm{C} & -4.7493990 & 2.8633720 & 0.1454850 \\ \mathrm{C} & -5.2848070 & 2.0002900 & 1.1108960 \\ \mathrm{C} & -5.4678250 & 4.0042590 & -0.2314820 \\ \mathrm{C} & -6.5178460 & 2.2726210 & 1.6868820 \\ \mathrm{H} & -4.7324610 & 1.1124990 & 1.3909300 \\ & & & \\ & & & \end{array}$

$-6.7023080$

$-5.0534450$

$-7.2291230$

$-6.9230790$

$-7.2547740$

$-8.1941580$

$-2.0272280$

$-2.6952790$

$-0.4529730$

$-0.2086850$

$-1.0360340$

$-0.1563370$

0.9549010

1.0240480

0.0464450

$-0.1558270$

0.2928440

$-0.7539650$

$-0.2660170$

0.4014170

1. 3881990

$-1.8294640$

$-0.5461310$

1. 1113510

$-1.2168820$

$-0.0081450$
4.2715130
4.6755120
3.4079200
1.5957630
5.1569720
3.6210260
1.1299550
3.4844060
3.7024570
3.5456060
2.9099310
2.5335370
3.4061760
2.4092870
6.1662770
6.0597290
4.6951280
5.0858100
7.1536550
6.8452850
4.6122470
5.2595650
5.0977250
6.0583990
6.2073720
4.5443730

0.3458260

$-0.9759920$

1. 3052170

2.4329340

0.0478670

1.7551720

$-0.3667770$

$-1.6285340$

0.6969270

$-0.7425880$

1.1655440

$-1.1296370$

0.7219230

0.9119940

0.4903810

$-1.0229830$

$-1.5546960$

1.2229300

0.8441820

$-1.5425540$

$-1.5375930$

1.0938440

2.2978170

0.7301350

$-1.2586170$

$-2.5950880$ 
wB97XD/6-31G(d) Optimized Cartesion Coordinates (A) for the thiocarboxylysis/trans-esterification organocascade in which cyclohexene oxide (1b) is the epoxide. Absolute solution phase free energies $(\mathrm{G})$ in hartrees computed with the Quasi-RRHO model at the wB97XD/6-311+G(d,p) //wB97XD/6-31G(d) level are reported.

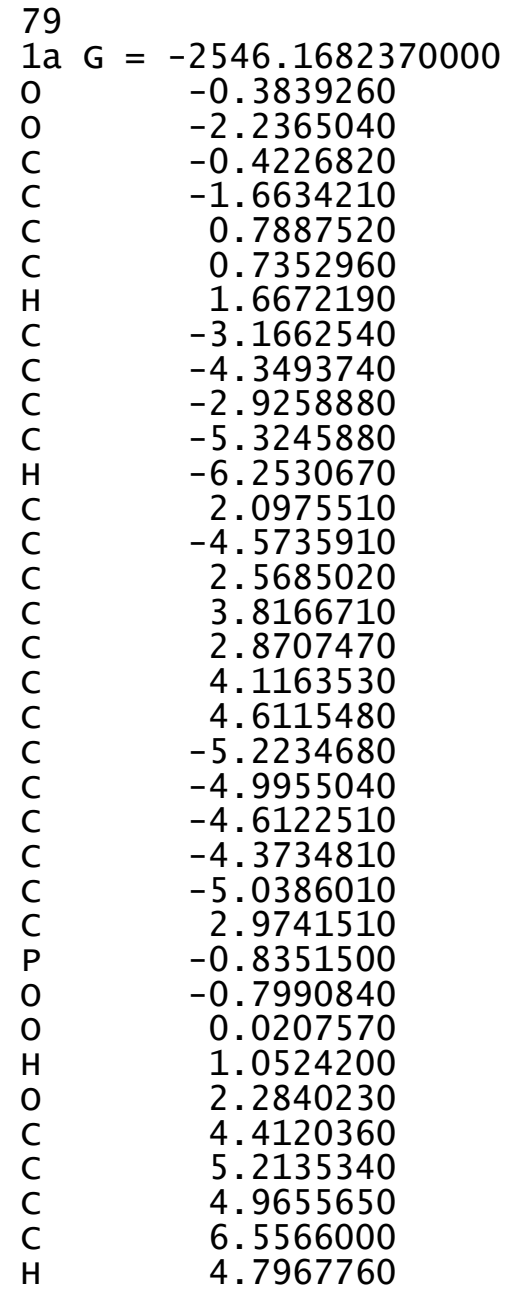

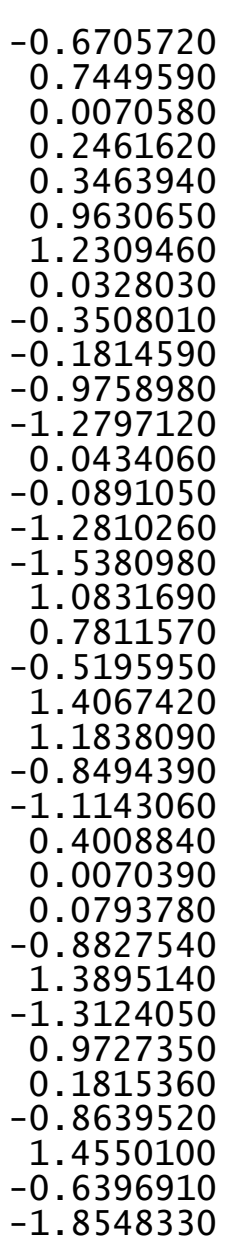

6.3076890
4.3302920
7.1055280
7.1753280
6.7340740
8.1560180
0.8452590
2.3420870
-5.1176010
-3.9268220
-1.6718050
-0.4823270
-0.5044290
-2.6236290
-3.7545940
-5.8831870
-4.4533220
-5.5516520
4.1817890
4.7147660
1.7460010
0.8598010
1.3962070
2.3280830
5.9794030
6.7123270
6.3292290
5.9771460
2.3817450
3.0057410
2.4116800
1.3487930
-5.1871560
-4.2314430
-5.8548670
-5.6115240
-5.3057260
-6.3753750
-4.9792360
-4.7831310
-3.8798160
-3.8544940
-4.5162910
-2.8648860

-309.7175330710

2. 3927690

1.7565940

2.8485890

3.3912210

2. 2533260

3.0628410

0.8569050

1.9872990

$-2.4908710$

$-3.0887060$

$-4.2470830$

$-4.8697250$

$-5.8397980$

$-4.7234630$

$-4.1424960$

$-3.0814130$

4. 1071070

3. 1943300

$-1.7248340$

$-0.8356170$

$-2.8715280$

$-3.8860340$

$-2.9041630$

$-0.6691810$

$-1.4774360$

$-0.0091050$

$-0.0967440$

$-1.8191200$

$-1.1733640$

$-2.8272980$

$-1.4663910$

0.5136940

0.0597900

$-0.3004140$

0.9911250

5.2778990

5.4596660

5.9044910

5.6161280

1. 5990850

2.4458300

0.8220730

1.1933550
1.6760210

2. 2571480

0.6271060

$-1.4553100$

2.6643140

0.7983100

1. 2292680

$-1.6483810$

$-1.2074270$

$-0.8153310$

0.8706740

1. 2270240

1. 7130540

1.0854620

$-1.0124440$

$-1.7004170$

$-1.6426390$

2. 3922000

$-2.5629320$

1.5859070

$-2.4149640$

$-2.5962110$

$-2.1955390$

$-3.3401650$

$-0.8217680$

$-0.9340260$

$-0.0227890$

$-1.7547860$

2. 5111920

3. 1359540

2. 9402560

2. 5806050

2. 2968440

2. 5823240

1. 9945300

3.1844560

0.6696980

0.8268920

$-0.1654380$

1.5709630

$-2.4733270$

$-3.1650210$

$-2.9095420$

$-2.3996520$ 


$\begin{array}{lr}\mathrm{C} & 1.0912330 \\ \mathrm{C} & 0.9876400 \\ \mathrm{H} & 1.9114040 \\ \mathrm{H} & 1.7476490 \\ \mathrm{O} & 1.5126920 \\ \mathrm{C} & -0.3412930 \\ \mathrm{H} & -0.4465050 \\ \mathrm{H} & -0.3072100 \\ \mathrm{C} & -0.1327350 \\ \mathrm{H} & 0.1390690 \\ \mathrm{H} & -0.4526840 \\ \mathrm{C} & -1.2775390 \\ \mathrm{C} & -1.5439490 \\ \mathrm{H} & -1.7657970 \\ \mathrm{H} & -2.4296360 \\ \mathrm{H} & -1.0189830 \\ \mathrm{H} & -2.1789830 \\ 96 & -2855.8804551600 \\ \mathrm{C} & \mathrm{G} \\ \mathrm{O} & -2.6656780 \\ \mathrm{O} & -0.4743970 \\ \mathrm{C} & -3.3447650 \\ \mathrm{C} & -3.0038180 \\ \mathrm{C} & -4.4007520 \\ \mathrm{C} & -5.1480230 \\ \mathrm{H} & -5.9774880 \\ \mathrm{C} & -0.6274480 \\ \mathrm{C} & 0.4921660 \\ \mathrm{C} & -1.8623920 \\ \mathrm{C} & 0.3456490 \\ \mathrm{H} & 1.2064170 \\ \mathrm{C} & -4.7167940 \\ \mathrm{C} & 1.8072180 \\ \mathrm{C} & -5.4425750 \\ \mathrm{C} & -5.7372370 \\ \mathrm{C} & -4.2832970 \\ \mathrm{C} & -4.5968750 \\ \mathrm{C} & -5.3252420 \\ \mathrm{C} & 3.8011900 \\ \mathrm{C} & 2.5542070 \\ \mathrm{C} & 3.5721140 \\ \mathrm{C} & 2.3110150 \\ \mathrm{C} & 4.3333310 \\ \mathrm{C} & 2.1892130 \\ \mathrm{C} & 1.1541770 \\ \mathrm{H} & 2.0998120 \\ & \end{array}$

$\begin{array}{rr}-0.6310900 & 0.4111610 \\ 0.8312360 & 0.2929460 \\ -1.0439980 & 1.0008370 \\ 1.4379630 & 0.7863640 \\ 0.0362800 & -0.7752630 \\ 1.5139100 & 0.0279650 \\ 2.3664850 & 0.7079140 \\ 1.9238610 & -0.9889950 \\ -1.5076390 & 0.2881170 \\ -2.4386610 & -0.2233380 \\ -1.7820100 & 1.3025100 \\ -0.7995650 & -0.4426730 \\ 0.5726720 & 0.1791090 \\ 0.4368410 & 1.2464390 \\ 1.0339310 & -0.2708790 \\ -0.6818450 & -1.5014620 \\ -1.4199510 & -0.3970340 \\ -0.6538280 & \\ -1.3961580 & 0.7671350 \\ -1.3185560 & -0.2220920 \\ -2.6432640 & -0.2492150 \\ -0.6525900 & -0.5411730 \\ -1.3609550 & -0.8686270 \\ -0.8597620 & -1.8112680 \\ -2.6851150 & -2.3015290 \\ -3.3102460 & 0.2688600 \\ -3.3231580 & 0.8144370 \\ -4.6311390 & 0.1185140 \\ -5.1407940 & 1.2431380 \\ 0.7669690 & 1.6659490 \\ -2.6089820 & -0.5340750 \\ 1.0647540 & 0.9032890 \\ 2.3962640 & 0.6286800 \\ 1.7941840 & 0.9169450 \\ 3.1129480 & -1.3863070 \\ 3.4342070 & -1.0609490 \\ -1.7648810 & 0.0831240 \\ -2.3778490 & -0.1599670 \\ -1.6230520 & -0.2652710 \\ -2.2180360 & 2.2164750 \\ -1.3915800 & 2.1536480 \\ 1.6136970 & 1.0726390 \\ 2.6519240 & 1.5524200 \\ 0.7880260 & 1.5562680 \\ -1 & 0.8447020\end{array}$

0.3257430

1. 1658440

1. 4387200

0.9855190

0.9282050

3. 5838230

3. 9651120

4. 2103800

2. 1518030

$-1.1612770$

$-0.6708550$

$-0.9583700$

0.0542590

1. 2266760

3. 4303360

4.6541930

3. 4135770

5.8456670

4.6615570

4. 6072180

2. 4643180

5.8247820

6.7920520

4.5868230

6.7564300

0.3912780

1. 9182250

$-0.8735130$

$-1.9673180$

$-3.7753270$

$-4.8422760$

$-5.4307690$

$-3.5198870$

$-2.9211530$

$-0.9715240$

3. 9671790

4. 3776060

$-6.2984700$

$-4.2547250$

$-5.8834260$

$-5.0196030$

$-6.4144020$

$-6.5460050$

$-6.6077940$

$-4.8940110$
2. 5381530

1.6054290

4.0535960

4.7612040

4.1845040

1. 9146270

1.0494970

2. 0242130

1. 6748460

$-0.1568840$

$-0.1206100$

1.0595650

0.5544910

0.7507240

1.0878770

1.6282580

$-0.0321130$

1.0416220

2. 5034640

$-0.5974900$

$-0.4520020$

$-0.0697220$

1.4569360

$-1.4561820$

$-0.5204020$

1.0993310

3. 2865020

$-5.2906570$

$-4.6424140$

$-3.3141920$

$-2.6820100$

$-3.2178170$

$-4.3372130$

$-5.1542500$

$-6.3146960$

$-1.3322340$

$-1.5829720$

2. 6286110

3. 9114660

$-0.0329950$

$-0.4945560$

$-0.8283370$

0.3565390

4.8667910

5.1595760

5.5527020
0.8631150

2. 5747750

2.0494800

1. 3483540

3. 0112400

2. 0334560

2. 5830680

1.1381830

$-1.9646380$

0.5374510

2. 0205400

$-0.2882490$

2. 2444400

$-1.8155610$

$-2.4359980$

$-2.0312630$

$-3.2737980$

$-2.4416660$

$-1.3893980$

$-3.7072860$

$-3.5868710$

- 3.2824970

$-2.1094260$

$-4.3714320$

$-3.6107460$

$-1.4096990$

$-1.6783060$

1.1274530

0.5674810

$-1.4944430$

$-2.1203140$

$-2.8581720$

$-1.7536080$

0.4825580

1. 4732800

3.1880180

$-1.0642020$

1. 8199590

$-1.7161220$

1. 5647190

2. 0567520

1. 0304380

2. 3426550

0.3969690

$-0.1040980$

0.0592560 


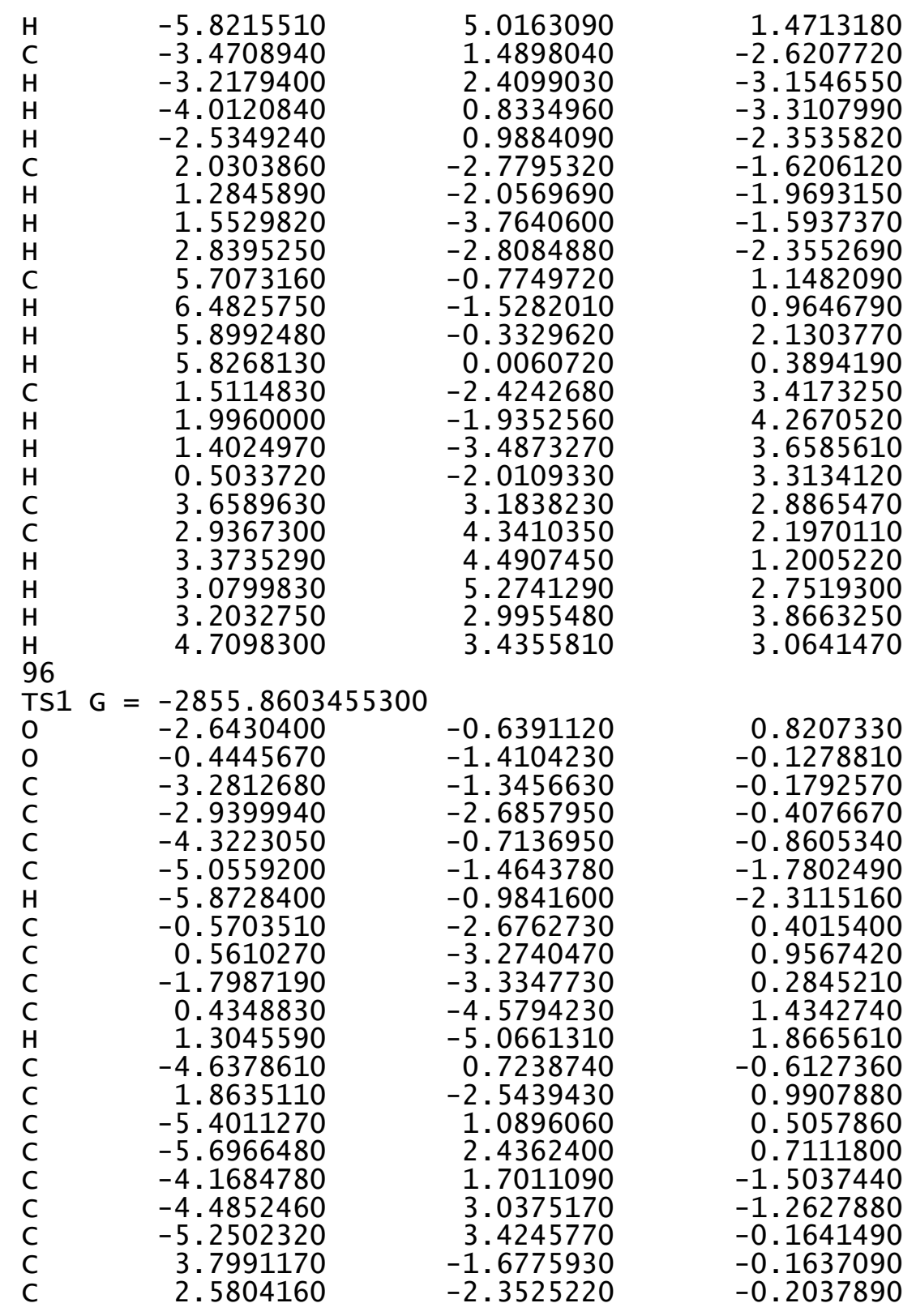

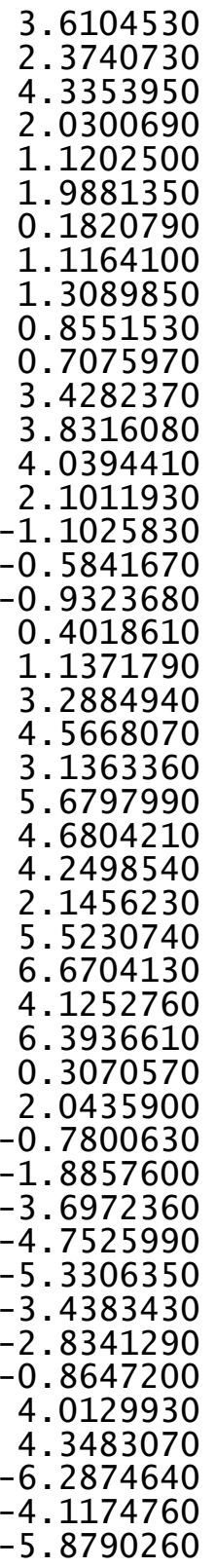

3. 6104530

4. 3353950

1.0300690

1. 9881350

0.1820790

1.1164100

0.7075970

3.4282370

3.8316080

(394410

$-1.1025830$

$-0.5841670$

$-0.9323680$

0.4018610

3. 2884940

4.5668070

3.1363360

4.6804210

4.2498540

2.1456230

6.6704130

4.1252760

6.3936610

2. 0435900

.7800630

$-4.7525990$

3306350

$-0.8647200$

4. 0129930

$-6.2874640$

$-5.8790260$
2. 2060100

2. 2053460

1.0338540

1.6939580

1. 2369100

1.1421090

0.7873120

2.8062840

1.6757490

0.9288220

2. 5857160

2.0990110

2. 7488350

1.1879560

$-1.8030640$

0.6756320

2. 0800910

$-0.2692140$

2.6030290

$-1.7144200$

$-2.5003000$

$-2.2332920$

$-3.3944460$

$-2.8361890$

$-1.5377500$

$-4.0112410$

$-3.5976380$

$-3.7265740$

$-2.6129330$

$-4.7117820$

$-4.2000040$

$-1.1935270$

$-1.2111750$

1. 3554370

0.7811980

$-1.3402890$

$-2.0157620$

$-2.7349950$

$-1.5452980$

0.7221830

1.7411650

3. 1513830

$-1.0907590$

1.5799390

$-1.9496520$

1. 4847970 


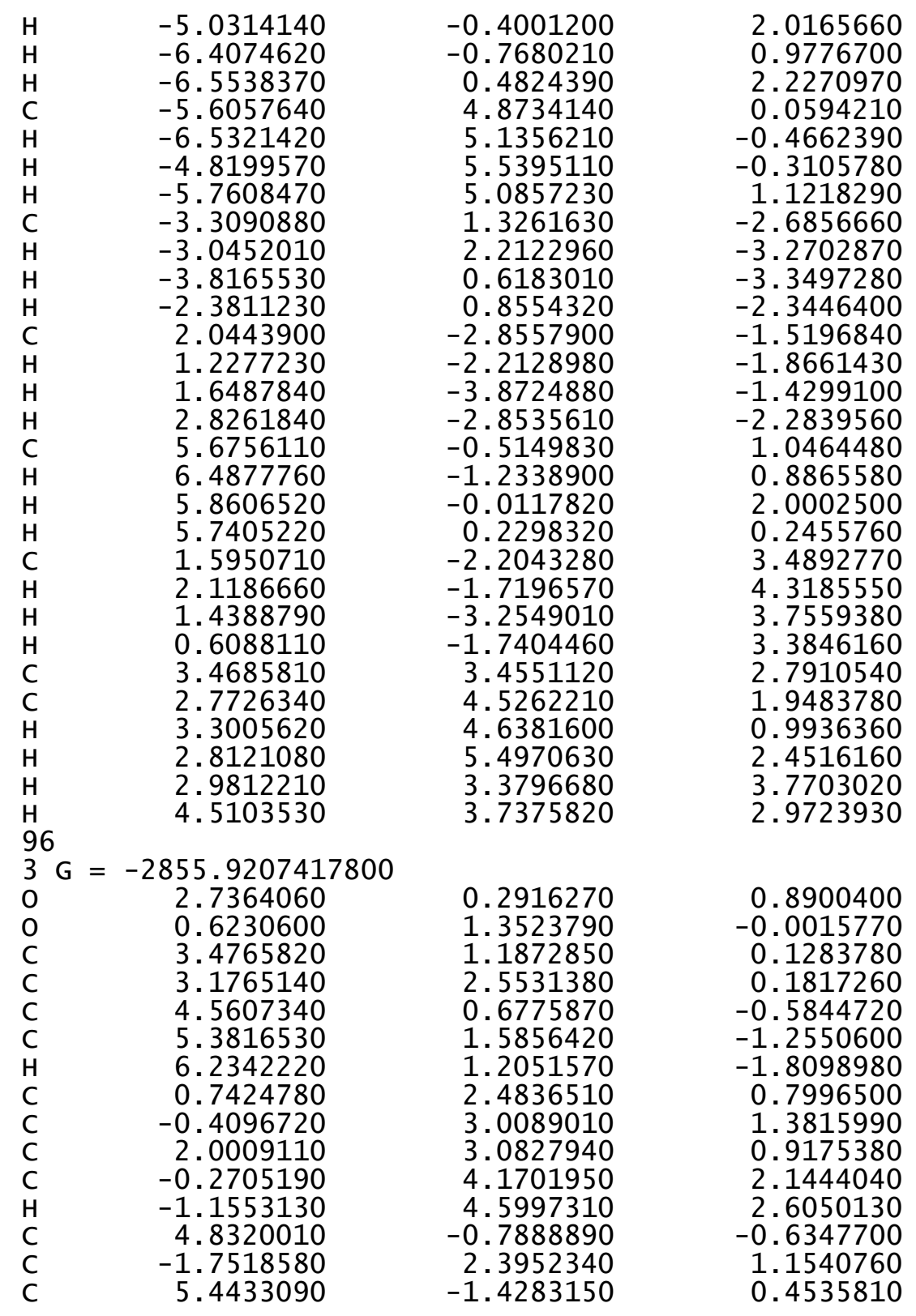

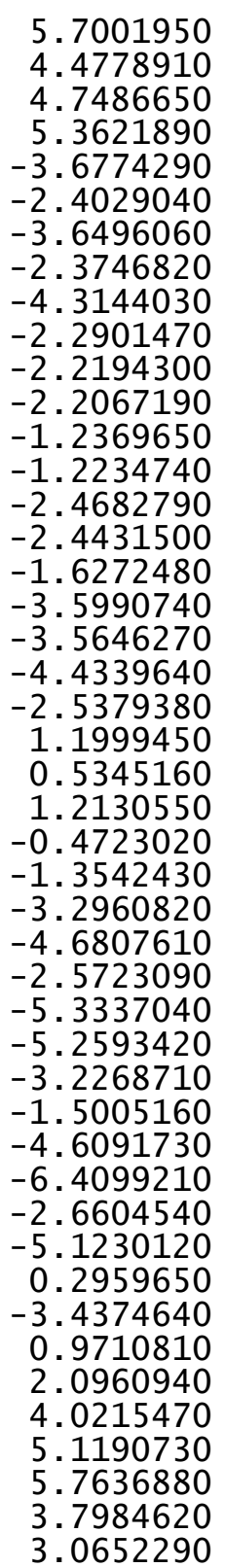

-2.7964400
-1.5174620
-2.8841420
-3.5411510
2.0770260
2.6095090
1.1370560
1.6509060
1.3320160
-2.1300340
-2.9687150
-1.0674630
-2.8301740
-2.5140920
-4.4534530
-5.0296830
-4.7886630
-2.3737250
-1.8033100
-1.9775160
-1.0708990
-0.0300860
-0.5371840
-0.9584330
-1.9364680
-0.8599780
-0.2333130
-0.3248550
0.7158860
0.5319060
-1.0435910
1.5696510
0.7851360
1.4813440
0.4612090
2.3083460
2.1528330
-1.1054860
-2.3729450
4.7751130
4.2395640
3.4262480
2.9504280
3.6436440
4.4889160
4.7122200
0.3740530 $-1.7812200$ $-1.8194760$ $-0.7540150$ $-0.2627780$ $-0.0705080$

1.9363590

2. 1688960

0.7262590

1.7334800

0.4349360

1.4729900

$-0.0233440$

2. 5735810

0.7198240

$-0.2112260$

1. 3370760

2. 4828100

3. 4171780

1.8903240

$-1.5948840$

0.5926100

1.8072960

$-0.6807580$

2. 3504910

$-1.3798070$

$-2.5652560$

$-2.7307130$

$-3.2932330$

$-3.6086730$

$-2.1597590$

$-4.1706470$

$-3.1443260$

$-4.3264430$

$-3.7290300$

$-4.7289530$

$-5.0075400$

$-1.0115280$

$-0.8066110$

2. 3103660

1.6961390

$-0.5103800$

$-1.2162420$

$-1.7469930$

$-0.5032970$

1.8240490 


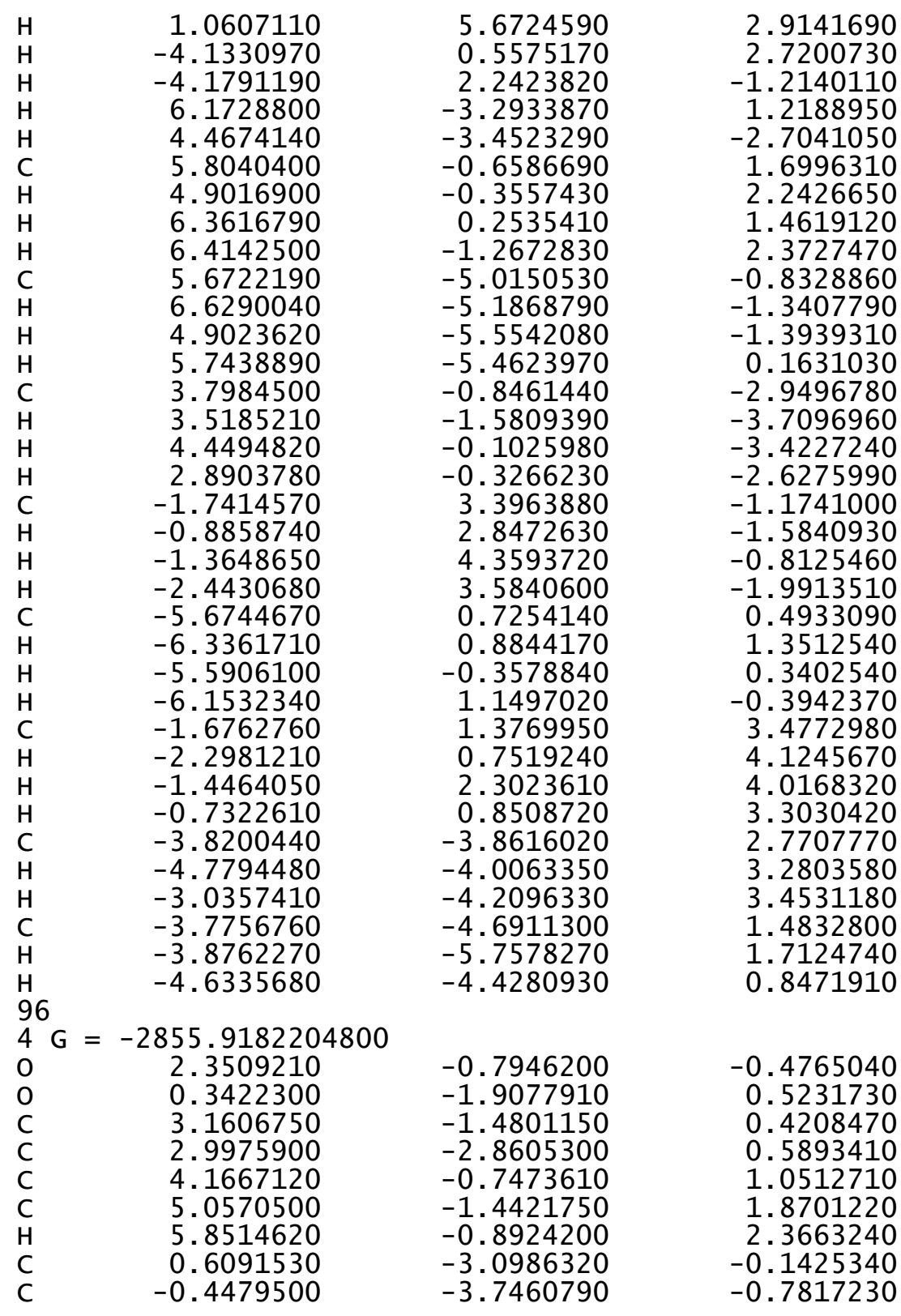

1.9084470
-0.1824900
-0.9882580
4.2451090
-1.7777610
4.7306480
4.7295260
3.7758650
3.7870560
4.2559390
-3.7886600
-2.6054090
-3.3286480
-2.1478140
-4.1547300
-2.0635440
-2.7674420
0.7979210
0.0941480
0.7409950
-1.4496940
-1.8611420
-4.1255240
-5.2657420
-4.2411900
-6.5062690
-5.1919670
-5.4810090
-3.3502240
-6.6148530
-7.3904830
-5.5649740
-7.5848720
-0.1951810
-2.5510770
1.0942070
2.1318960
3.9088450
4.9321610
5.6282740
3.7957400
3.1325530
1.2848820
-3.5953630
-4.4292610
5.1010390
$-0.0869400$

$-1.3576130$

$-1.8586240$

0.8518860

$-0.8598580$

$-0.3564190$

$-0.5447630$

1.8586070

1.6287830

0.4327150

0.2043090

0.2692690

$-2.0633270$

$-2.0455710$

$-0.9435720$

$-2.1493000$

1.0003330

$-0.1905880$

$-1.4333120$

1.0029710

$-1.8811910$

1. 1236110

1.5257180

1.1509150

2. 4197100

1.6748300

0.4300590

2.9436460

2. 6876970

2. 5721620

1. 3745840

3. 6421450

2. 9808030

1. 1417870

0.2958610

$-1.3012930$

$-0.6810030$

1. 4242690

2. 0529710

2. 6971630

1.5911910

$-0.6690870$

$-1.7590430$

$-2.9657790$

1.0820240
-1.4842900 


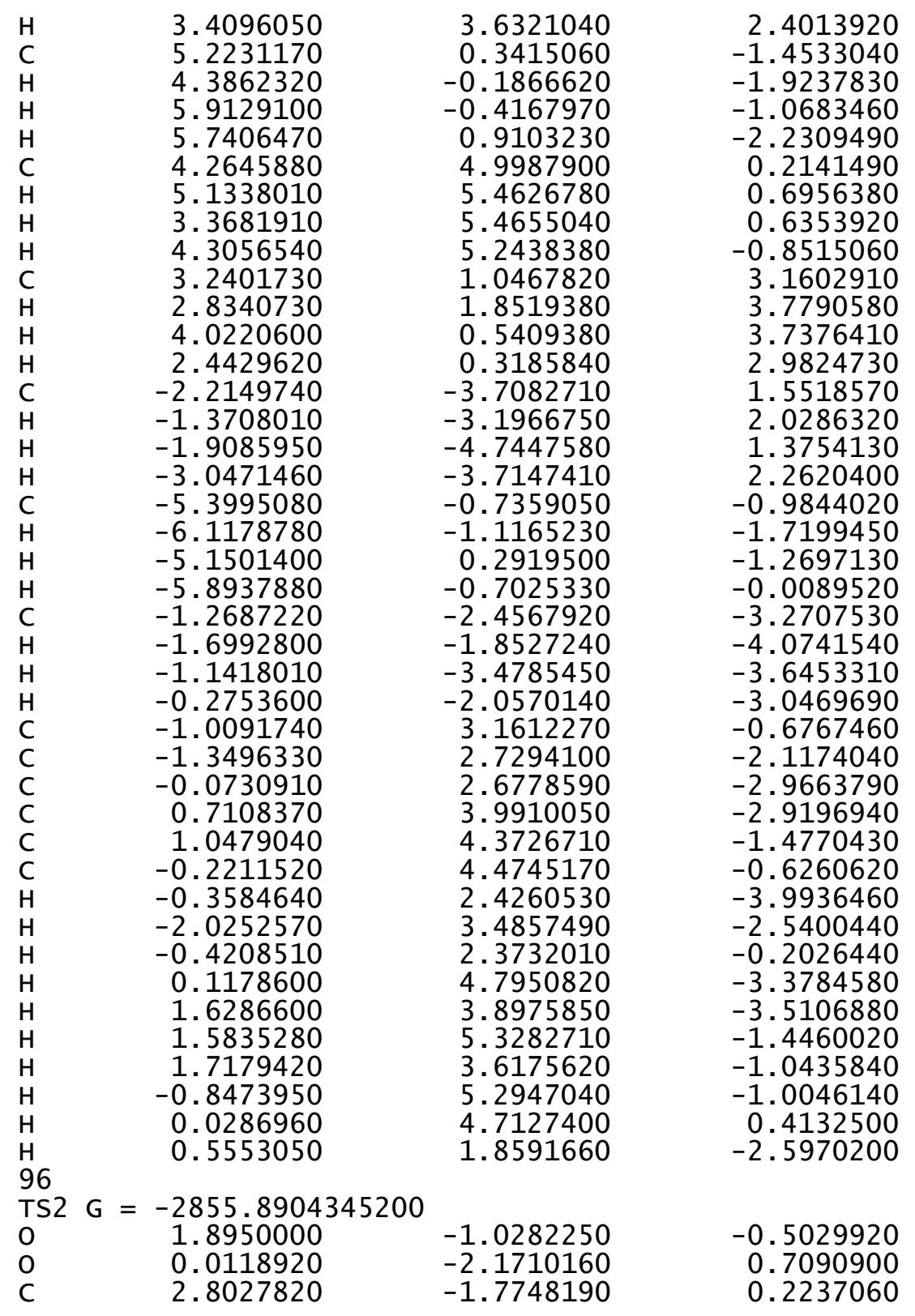

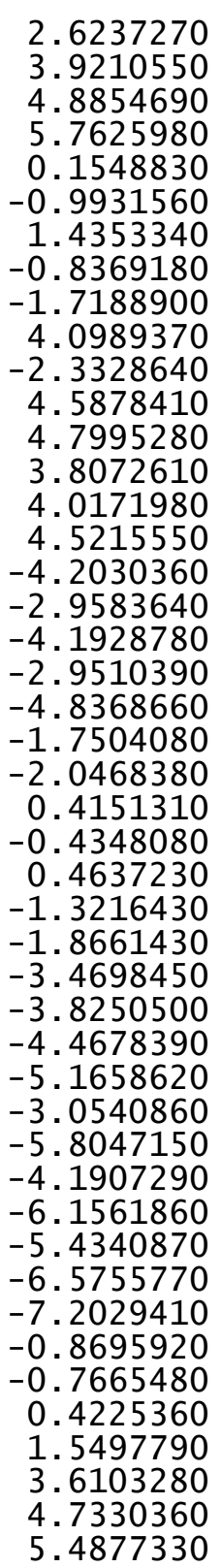
$-3.1606350$
$-1.1210730$
$-1.8910770$
$-1.3952890$
$-3.2997080$
$-3.9017680$
$-3.8357780$
$-5.0994100$
$-5.5878920$
0.3527050
$-3.2741930$
0.8562540
2. 2264060
1. 2223790
2. 5924180
3. 1122400
$-2.7485380$
$-3.3533740$
$-1.9947650$
$-2.5892610$
$-2.0667530$
1.8816430
2. 2442540
$-0.7243240$
$-0.3353350$
0.2155250
0.9561330
1.0972030
2. 6778850
3. 9878010
1.7286590
4. 3512410
4.7231230
2.0998410
0.7066200
3. 4101600
5. 3726020
1. 3641760
3. 6953910
0.7953010
3.5247870
$-5.6636570$
$-5.0336630$
$-3.8939430$
$-3.2673430$
$-3.8516450$

0.3341140

0.7421000

1. 3944650

1.8005750

$-0.0716640$

$-0.5877570$

$-0.2431920$

$-1.2869730$

$-1.6914930$

0.5773900

$-0.3859750$

$-0.6399450$

$-0.7694250$

1.6375870

1. 4613770

0.2727180

1. 0425900

0.8672350

$-1.2277760$

$-1.4450720$

0.0062900

$-0.9278430$

0.9160350

0.0930100

$-1.0824710$

1. 2747540

$-0.9619390$

1.4778230

0.8491480

0.5199300

1. 1009710

0.4583040

0.3186720

1. 0423630

1.3357980

0.7226860

0.2077600

1. 2474270

0.6784610

1. 5129110

1.1328130

$-1.4674740$

$-0.9537700$

0.9989010

1. 5251190

2. 0420430 


$\begin{array}{rrr}3.4792900 & -4.9656170 & 1.1162480 \\ 2.5346950 & -5.4630290 & -1.1126020 \\ 0.5264530 & -6.5947680 & -2.0156310 \\ -4.6684330 & -1.4556410 & -2.0448560 \\ -4.6880930 & -2.8129790 & 2.0147460 \\ 5.1892510 & 2.6159690 & -1.7083620 \\ 3.7852180 & 3.2687530 & 2.2817850 \\ 4.8729330 & -0.0695140 & -1.7960460 \\ 3.9473510 & -0.5262590 & -2.1643450 \\ 5.5402600 & -0.8861090 & -1.5003030 \\ 5.3387190 & 0.4694080 & -2.6260900 \\ 4.7819830 & 4.5890810 & 0.1116400 \\ 5.8579120 & 4.7992330 & 0.1012400 \\ 4.3390150 & 5.1645990 & 0.9301710 \\ 4.3695670 & 4.9662050 & -0.8307580 \\ 3.2595760 & 0.7024300 & 2.9425480 \\ 3.1415290 & 1.5144310 & 3.6659500 \\ 3.9186170 & -0.0527260 & 3.3838160 \\ 2.2800030 & 0.2409590 & 2.7819580 \\ -2.2946490 & -4.0630340 & 2.0208860 \\ -1.4209700 & -3.5006740 & 2.3684080 \\ -1.9455950 & -5.0602890 & 1.7326180 \\ -2.9847110 & -4.1716160 & 2.8625340 \\ -6.1985140 & -1.4476340 & 0.1995660 \\ -6.9900500 & -2.1239720 & -0.1451190 \\ -6.2938070 & -0.5153580 & -0.3663640 \\ -6.3908000 & -1.2301070 & 1.2555610 \\ -2.2781730 & -2.4598010 & -2.7888130 \\ -2.8955210 & -1.8715270 & -3.4740880 \\ -2.0976270 & -3.4367220 & -3.2500190 \\ -1.3127140 & -1.9561280 & -2.6793920 \\ 0.2210440 & 3.1054170 & -0.3519590 \\ -0.8106880 & 2.8273080 & -1.4381560 \\ -0.1404410 & 2.3793290 & -2.7318850 \\ 0.8360850 & 3.4727120 & -3.1848070 \\ 1.8514600 & 3.8134990 & -2.0886050 \\ 1.1691400 & 4.2219990 & -0.7748350 \\ -0.9053460 & 2.1944200 & -3.4927600 \\ -1.3869600 & 3.7417240 & -1.6228280 \\ 0.7926790 & 2.1998640 & -0.1261610 \\ 0.2723250 & 4.3762260 & -3.4556450 \\ 1.3571890 & 3.1428730 & -4.0892320 \\ 2.5079900 & 4.6239060 & -2.4241720 \\ 2.4910200 & 2.9434390 & -1.8972280 \\ 0.6081190 & 5.1556930 & -0.9139620 \\ 1.9185310 & 4.3947450 & 0.0034680\end{array}$

$\mathrm{H}$
96

$5 \mathrm{G}=-2855.9063163900$

C$$
\text { 1. } 9947640
$$$$
0.4029920
$$$$
3.0770130
$$$$
3.1395340
$$$$
4.0848870
$$$$
\text { 5. } 2066220
$$$$
6.0056570
$$$$
0.7053650
$$$$
-0.3534860
$$$$
\text { 2. } 0477020
$$$$
-0.0343390
$$$$
-0.8418780
$$$$
3.9789640
$$$$
-1.7654410
$$$$
4.2581590
$$$$
4.2104730
$$$$
3.6276850
$$$$
3.5740080
$$$$
\text { 3. } 8760030
$$$$
-3.6775800
$$$$
-2.3703490
$$$$
-3.7730510
$$$$
-2.4690960
$$$$
-4.3949940
$$$$
-1.8462270
$$$$
-2.3384720
$$$$
0.5862560
$$$$
-0.3882610
$$$$
0.4711000
$$$$
-1.0528530
$$$$
-2.1827870
$$$$
-3.8234020
$$$$
-4.3168660
$$$$
-4.7145270
$$$$
-5.6823290
$$$$
-3.6352000
$$$$
-6.0752640
$$$$
-4.3311200
$$$$
-6.5649370
$$$$
-6.0531660
$$$$
-6.7597460
$$$$
-7.6293730
$$

$-1.2208480$

\subsection{0}

$-2.5570780$

$-0.8302600$

$-2.2564160$

$-1.4029140$

$-2.7961180$

$-0.5548830$

$-1.1442520$

$-0.5012270$

$-3.3461200$

$-4.1128130$

$-3.6622020$

$-5.2688800$

$-5.8864970$

0.9277280

$-3.6762650$

1.5257000

2.9136870

1.7158720

3.1030340

3.7210430

$-3.3621370$

$-3.8126150$

$-2.6513000$

$-3.0965220$

$-2.7778050$

1.6128460

1.9267040

$-0.7593630$

$-0.5483610$

0.2053650

0.1730620

0.8281180

2. 2396810

3.5298560

1.1605800

3.7436690

4.3734640

1. 3772970

0.1546230

2.6701820

4.7553450

0.5342020

2.8370270

0.6595920
$-0.5385200$

0.7703270

0.1259120

0.2418530

0.5799890

1. 1663010

1.5235230

$-0.0372200$

$-0.5225490$

$-0.2662690$

$-1.2366160$

$-1.6185590$

0.4348770

$-0.3122130$

$-0.8050800$

$-0.9106820$

1. 5409810

1. 3879750

0.1777780

1. 1196640

0.9472910

$-1.1631170$

$-1.3796460$

0.0766720

$-0.5259200$

0.8004820

0.2277340

$-0.9854170$

1.3436990

$-0.8023730$

1.5922590

0.6871360

0.5051560

0.6798360

0.3234230

0.5200590

0.5048460

0.8148650

0.3250010

0.1878150

0.5117010

0.1889530

1.7002950 


$\begin{array}{rrr}-1.2803110 & 3.3694960 & 1.3873520 \\ 1.2909710 & -5.6266670 & -1.4643030 \\ 2.3225610 & -4.8244540 & -0.9922820 \\ 4.2781200 & -3.3436500 & 0.8390310 \\ 5.3048220 & -2.5256740 & 1.2945790 \\ 6.1814340 & -2.9665250 & 1.7581470 \\ 4.3451540 & -4.4206510 & 0.9597810 \\ 3.3561650 & -5.0847790 & -1.1995220 \\ 1.5205130 & -6.5275750 & -2.0243030 \\ -4.3144150 & -2.1884300 & -1.9856420 \\ -4.1466450 & -3.4653600 & 2.0960540 \\ 4.4422310 & 3.3797390 & -1.8667710 \\ 3.2971240 & 3.7148600 & 2.2439410 \\ 4.6033300 & 0.6844980 & -2.0087570 \\ 3.7387550 & 0.0925480 & -2.3305000 \\ 5.4128800 & -0.0195670 & -1.7888400 \\ 4.9144360 & 1.3115260 & -2.8490250 \\ 3.8778960 & 5.2232230 & 0.0461560 \\ 4.9031360 & 5.6110930 & 0.0211870 \\ 3.3651810 & 5.6991110 & 0.8874930 \\ 3.3876250 & 5.5441010 & -0.8790490 \\ 3.3053540 & 1.0904540 & 2.8748040 \\ 3.0439510 & 1.8571840 & 3.6090550 \\ 4.1534420 & 0.5193140 & 3.2676590 \\ 2.4555260 & 0.4072730 & 2.7844280 \\ -1.6206100 & -4.4199190 & 2.1065540 \\ -0.8488950 & -3.7328960 & 2.4712480 \\ -1.1201960 & -5.3505650 & 1.8191720 \\ -2.2969040 & -4.6378030 & 2.9378190 \\ -5.8167530 & -2.3185590 & 0.2814950 \\ -6.5144180 & -3.1632480 & 0.2341730 \\ -6.1145300 & -1.5997260 & -0.4880070 \\ -5.9450570 & -1.8445770 & 1.2606050 \\ -1.8363530 & -2.9357280 & -2.7404510 \\ -2.4722370 & -2.3303820 & -3.3926850 \\ -1.6811530 & -3.9047160 & -3.2282380 \\ -0.8611860 & -2.4459790 & -2.6664650 \\ -0.2708610 & 3.3214170 & -0.1273120 \\ -1.2445420 & 2.7426620 & -1.1448860 \\ -0.5462640 & 2.4061050 & -2.4494600 \\ 0.0528900 & 3.7081820 & -3.0042950 \\ 0.9747770 & 4.3956580 & -1.9875840 \\ 0.2882070 & 4.6448910 & -0.6342250 \\ -1.2563420 & 1.9685770 & -3.1588400 \\ -2.0337940 & 3.4834390 & -1.3452000 \\ 0.5504420 & 2.6123470 & 0.0314910\end{array}$

$\begin{array}{rr}\mathrm{H} & -0.7633110 \\ \mathrm{H} & 0.6069440 \\ \mathrm{H} & 1.3366450 \\ \mathrm{H} & 1.8549360 \\ \mathrm{H} & -0.5259480 \\ \mathrm{H} & 1.0043940 \\ \mathrm{H} & 0.2461670\end{array}$

$6^{9} \mathrm{G}=-2855.9058203300$

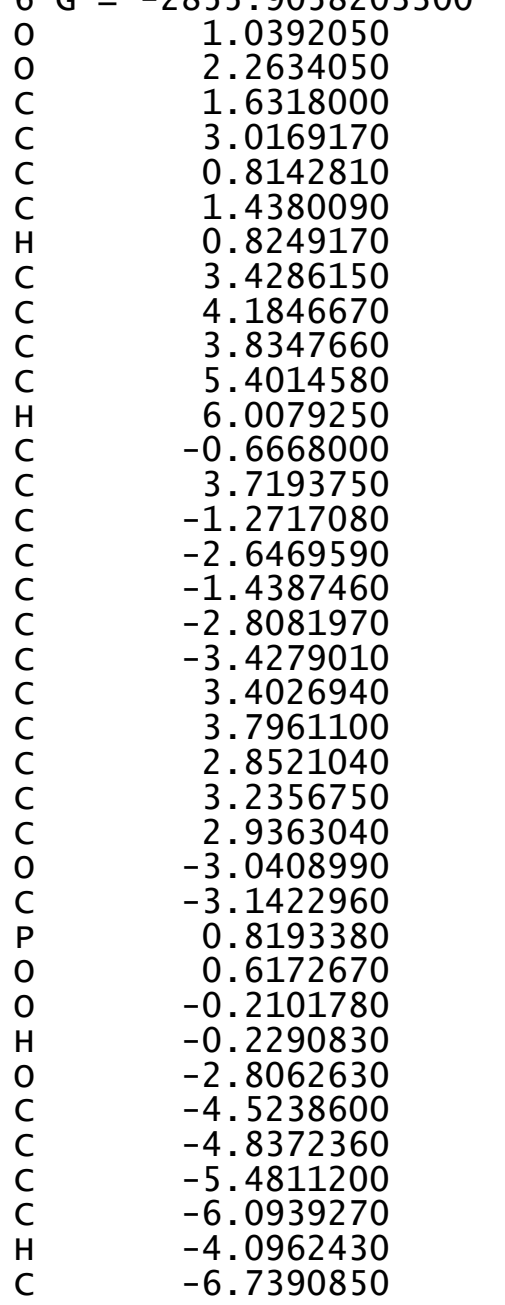

4. 3900230

3.4944580

5.3453430

3.7624700

5.3730950

1.6695760

$-2.0923320$

$-0.2867350$

$-3.0724040$

$-3.0386560$

$-4.0744830$

$-5.1125550$

$-5.9091680$

$-0.5920960$

0.4742990

$-1.9277680$

0.1763050

0.9934570

$-3.9770940$

1.8868040

$-4.2583010$

$-4.0676370$

$-3.5309970$

$-3.3551730$

$-3.5970110$

3. 8698150

2.5335710

.9046360

2. 5716840

4.5747160

1.4498680

0.9052800

$-0.6328120$

0.2097890

$-0.4754260$

0.7384160

$-0.4108870$

1.0374890

0.3042440

1.8759030

0.4230870

$-0.3660990$

1. 9941140
$-3.2789790$

$-3.9241040$

$-2.3978110$

$-1.8204560$

$-0.7452290$

0.0821680

$-2.2711730$

0.7211720

$-0.5054240$

$-0.0698480$

$-0.2456270$

$-0.5907890$

$-1.2835830$

$-1.6946060$

0.1897450

0.6744690

0.2999510

1.2906160

1.6700190

$-0.4380100$

0.5376320

0.7945480

0.9306740

$-1.5247510$

$-1.3466780$

$-0.1214350$

$-0.7966290$

$-0.7044840$

1.5306860

1. 6657520

0.3096050

$-1.2986570$

$-0.0113350$

0.0994660

1.4124170

$-0.9518060$

1. 3973550

$-0.0321380$

0.5964510

1.7427320

0.0319930

2. 3255620

2. 1675860

0.6175980 


$$
\begin{array}{r}
-5.2488030 \\
-7.0473020 \\
-6.3304610 \\
-7.4809580 \\
-8.0278940 \\
-1.9368690 \\
-1.9301280 \\
5.8358090 \\
5.0567620 \\
3.6002090 \\
2.8191200 \\
3.2875980 \\
4.6729270 \\
5.3853290 \\
6.7817670 \\
2.4781850 \\
3.4711220 \\
-3.1150420 \\
-3.4045820 \\
-0.4516180 \\
0.1664310 \\
0.2249560 \\
-1.0961310 \\
-4.8909240 \\
-5.4790340 \\
-5.0291350 \\
-5.2996970 \\
-0.8017990 \\
-1.5527470 \\
-0.3034610 \\
-0.0504040 \\
4.2944810 \\
3.5749270 \\
5.2436790 \\
4.4446900 \\
2.5345390 \\
3.1572390 \\
1.4936870 \\
2.6356450 \\
3.1271230 \\
2.5817940 \\
4.1148480 \\
2.6001710 \\
-2.4298080 \\
-1.2227360 \\
-0.5813010
\end{array}
$$

$\begin{array}{rr}2.4219430 & -0.8761050 \\ 1.2722630 & 1.7667970 \\ -0.1500860 & 3.2171670 \\ 2.6491160 & 0.1703460 \\ 1.3665840 & 2.2237210 \\ -0.5142010 & -0.4788080 \\ 1.9698370 & 1.0732990 \\ -1.1385290 & 1.4182520 \\ -2.1798360 & 0.9300750 \\ -4.0938650 & -0.9536050 \\ -5.1270280 & -1.4571690 \\ -5.9402030 & -2.0023290 \\ -4.0887520 & -1.1222460 \\ -3.2079460 & 1.0487790 \\ -1.3524810 & 1.9051300 \\ 4.4361440 & 2.4038000 \\ 4.3733340 & -1.7587030 \\ -4.2725870 & 1.8915110 \\ -2.9838900 & -2.1772040 \\ -4.7278800 & 1.9706570 \\ -3.9126680 & 2.3640770 \\ -5.5424880 & 1.6913830 \\ -5.0812930 & 2.7805010 \\ -3.2936690 & 0.0657150 \\ -3.5943870 & -0.8076900 \\ -2.2143990 & 0.2018600 \\ -3.7999110 & 0.9456670 \\ -3.2003080 & -2.8518690 \\ -2.8432250 & -3.5619700 \\ -4.0696680 & -3.2940740 \\ -2.4128540 & -2.7337580 \\ 1.8095380 & -1.9301480 \\ 1.0489930 & -2.2525260 \\ 1.2987620 & -1.7365940 \\ 2.5057590 & -2.7599970 \\ 6.0244670 & 0.2038770 \\ 6.6532270 & 0.8501630 \\ 6.1722280 & 0.5140940 \\ 6.3930310 & -0.8211050 \\ 1.8890270 & 3.0072690 \\ 2.5170840 & 3.7172470 \\ 1.6812200 & 3.4339640 \\ 0.9342510 & 2.9223940 \\ 2.7274380 & -1.3611730 \\ 2.6939010 & -0.4347810 \\ 4.0686640 & -0.3271760\end{array}$

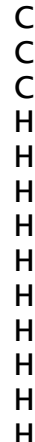

TS3 G $=-2855.8929389100$

$0 \quad 0.8131760$

2. 2081170

1. 2854860

2. 6650210

0.3648460

0.8657960

0.1659560

3. 3388040

4.2055700

3. 6056240

5. 3912110

6. 0826760

$-1.1000250$

3.8831840

$-1.7082650$

$-3.0706190$

$-1.8578130$

$-3.2169620$

$-3.8385380$

3. 7550860

4. 0187380

3. 2126610

3. 4661950

3. 3636370

$-2.5006660$

$-3.0040690$

0.7570000

0.6609690

$-0.2556240$

$-0.2312390$

$-2.6634040$

$\begin{array}{lr}4.4917570 & -1.7379550 \\ 4.4425970 & -2.7485290 \\ 3.0758170 & -2.7752410 \\ 4.0371320 & 0.3456300 \\ 1.9828430 & -0.8473120 \\ 3.4903710 & -0.9922860 \\ 3.8233850 & -2.0674560 \\ 5.5014580 & -1.7052570 \\ 4.6838170 & -3.7503760 \\ 5.2167790 & -2.4896470 \\ 2.2966420 & -3.1427490 \\ 3.1006440 & -3.4407170 \\ 4.7875080 & 0.0827690\end{array}$

$-2.1592200$

$-0.5394650$

$-3.2056500$

$-3.3282780$

$-4.1355640$

$-5.2527340$

$-5.9933350$

$-0.9323920$

0.0605660

$-2.2991480$

$-0.3468840$

0.4121110

$-3.9047010$

1. 5098650

$-4.0899320$

$-3.8213700$

$-3.4681410$

$-3.2192690$

$-3.3736480$

3. 4871440

2.1223620

3. 6199950

2. 2586640

4.2557460

1.6579260

0.9472300

$-0.6679400$

0.2212430

$-0.4914680$

1.0316900

$-0.2889200$
0.7110130

$-0.6022320$

$-0.0624080$

$-0.2585470$

$-0.5463250$

$-1.2162060$

$-1.5925350$

0.0969060

0.5528000

0.2476850

1.1677650

1. 5222650

$-0.3770160$

0.3900800

0.8727570

1. 0083160

$-1.4774130$

$-1.3010950$

$-0.0632390$

$-0.9848470$

$-0.8650410$

1. 3409510

1. 5035160

0.1092180

$-1.2652150$

$-0.2762640$

0.0937220

1. 3315760

$-0.9953520$

1. 3883660

$-0.2346410$ 


$\begin{array}{rrr}-4.2932600 & 1.2814840 & 0.3653970 \\ -4.6547780 & 0.6130910 & 1.5384940 \\ -5.1836710 & 2.1665660 & -0.2430620 \\ -5.8961790 & 0.8474090 & 2.1097690 \\ -3.9525350 & -0.0770630 & 1.9943350 \\ -6.4281730 & 2.3979430 & 0.3358630 \\ -4.9229120 & 2.6490630 & -1.1796290 \\ -6.7827560 & 1.7439710 & 1.5113980 \\ -6.1742590 & 0.3341920 & 3.0245380 \\ -7.1228070 & 3.0833170 & -0.1389280 \\ -7.7533310 & 1.9268600 & 1.9619170 \\ -1.7214510 & -0.4287300 & -0.6280520 \\ -1.4077300 & 2.1592950 & 1.4186380 \\ 5.6879550 & -1.6958970 & 1.3299480 \\ 4.7987340 & -2.6626540 & 0.8778370 \\ 3.1243850 & -4.4573950 & -0.9423750 \\ 2.2340360 & -5.4176430 & -1.4075240 \\ 2.6072590 & -6.2906520 & -1.9334530 \\ 4.1896270 & -4.5702050 & -1.1210090 \\ 5.0161860 & -3.7162780 & 1.0259570 \\ 6.6105260 & -1.9942650 & 1.8176250 \\ 2.8907670 & 4.2011930 & 2.2031080 \\ 3.8718600 & 3.9635750 & -1.9564000 \\ -3.5404060 & -3.9587770 & 1.9803940 \\ -3.8022160 & -2.8679550 & -2.1486560 \\ -0.9059390 & -4.5534970 & 2.0629850 \\ -0.2219000 & -3.7670330 & 2.4005220 \\ -0.2975200 & -5.4310050 & 1.8190680 \\ -1.5610480 & -4.8139950 & 2.8992190 \\ -5.2910640 & -3.0135380 & 0.1150980 \\ -5.8837750 & -3.2901050 & -0.7630510 \\ -5.4009150 & -1.9311540 & 0.2576010 \\ -5.7243960 & -3.5082410 & 0.9898440 \\ -1.2151370 & -3.2243690 & -2.8206430 \\ -1.9528170 & -2.8613960 & -3.5420870 \\ -0.7607760 & -4.1325710 & -3.2301110 \\ -0.4254930 & -2.4707160 & -2.7344570 \\ 4.4426860 & 1.3257810 & -2.0737680 \\ 3.6607150 & 0.6169220 & -2.3670550 \\ 5.3489010 & 0.7447240 & -1.8715030 \\ 4.6417720 & 1.9826230 & -2.9253400 \\ 3.1499970 & 5.7429450 & -0.0212110 \\ 4.0433900 & 6.2930910 & 0.2978760 \\ 2.3171620 & 6.0839550 & 0.6026270 \\ 2.9383650 & 6.0266680 & -1.0564260 \\ 3.2738720 & 1.6128970 & 2.8531920\end{array}$

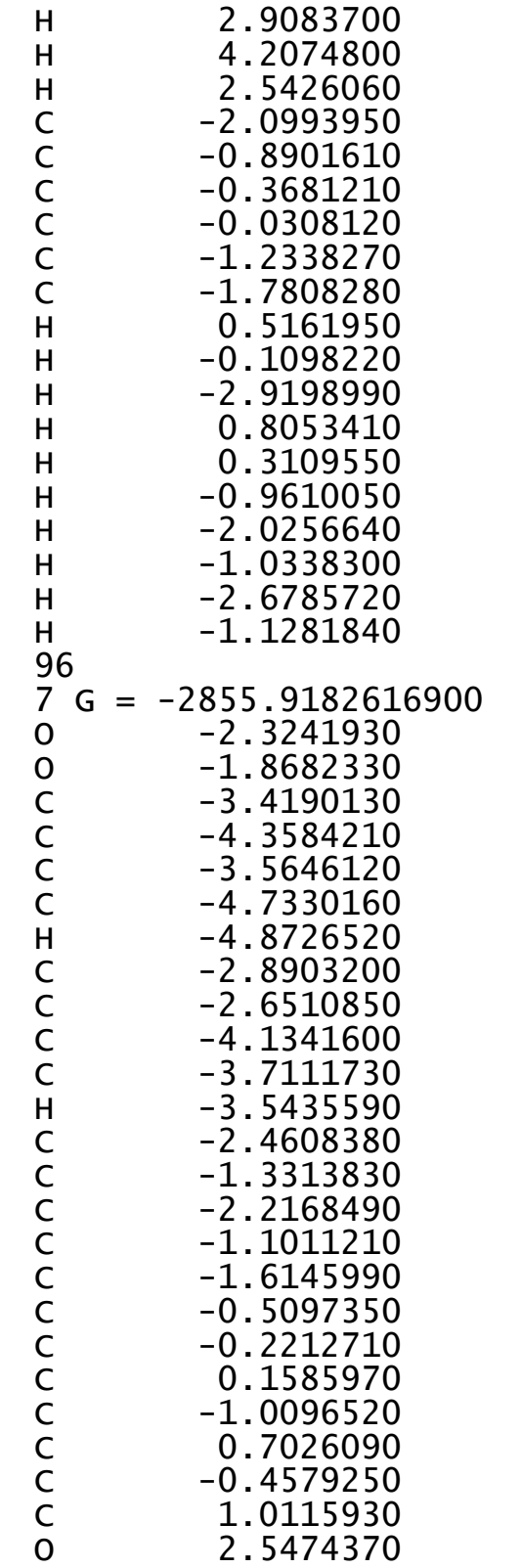

2. 3422660

1.1889260

0.8013860

3.0277600

3.0021160

4.4272530

5.0702620

5.0554660

3.6363670

4. 3919510

2. 3996010

3.5697560

4.5233250

6.0994180

5.4754380

5.6975480

3.0061410

3.6435390

5.0227280

0.9749480

$-1.0688990$

1.2781880

0.2755070

2. 5911280

2.9073470

9241600

$-1.7418510$

$-3.0491810$

$-1.1133340$

$-3.7431460$

$-4.7643500$

3.5707210

$-3.7253400$

4.0872780

4.9009320

3.9020260

4.7136430

5.2015710

$-5.0233030$

$-4.2722770$

$-4.6397040$

$-3.8846970$

$-5.2451050$

0.4137530
3.5815840

3.2383420

2.7877890

$-1.0431340$

$-0.1066900$

0.0928900

$-1.2567090$

$-2.2045190$

$-2.3963020$

0.7356890

$-0.5877240$

$-0.5582480$

$-1.7135470$

$-1.1023900$

$-3.1781100$

$-1.7948540$

$-2.8938660$

$-3.0241220$

0.6165030

0.7701680

$-0.6140090$

$-0.0233320$

$-0.2764410$

$-0.4737800$

$-1.1675670$

$-1.5228870$

0.0452530

0.4646960

0.1933800

1.0538860

1.3837120

$-0.2533210$

0.2748120

1.0267770

1.2258200

$-1.3263510$

$-1.0834730$

0.1901430

$-1.1077090$

$-0.9772200$

1. 1898200

1.3632360

$-0.0277320$

$-0.9013140$ 


$\begin{array}{rrrr}\mathrm{C} & 2.6371190 & 1.6139750 & -0.3436820 \\ \mathrm{P} & -1.1036590 & 0.1085640 & 0.1764580 \\ \mathrm{O} & -0.2086760 & -0.2831480 & 1.2800780 \\ \mathrm{O} & -0.4898870 & 0.8717050 & -1.0463640 \\ \mathrm{H} & 1.6175410 & -0.5597470 & 1.8417810 \\ \mathrm{O} & 1.6104110 & 2.2702890 & -0.2305040 \\ \mathrm{C} & 3.9507140 & 2.1921340 & 0.0516000 \\ \mathrm{C} & 4.0293370 & 2.8829560 & 1.2620260 \\ \mathrm{C} & 5.0550440 & 2.1439610 & -0.8015180 \\ \mathrm{C} & 5.2201140 & 3.4960550 & 1.6313600 \\ \mathrm{H} & 3.1586950 & 2.9235570 & 1.9082660 \\ \mathrm{C} & 6.2384810 & 2.7770270 & -0.4366430 \\ \mathrm{H} & 4.9839570 & 1.6358040 & -1.7590010 \\ \mathrm{C} & 6.3240540 & 3.4450180 & 0.7822900 \\ \mathrm{H} & 5.2863580 & 4.0176750 & 2.5806890 \\ \mathrm{H} & 7.0921310 & 2.7502680 & -1.1064330 \\ \mathrm{H} & 7.2513990 & 3.9314100 & 1.0687820 \\ \mathrm{H} & 0.2888090 & 1.4429040 & -0.7749710 \\ \mathrm{~S} & 2.8908990 & -0.9925130 & 1.9369340 \\ \mathrm{C} & -4.9573280 & -3.1487190 & 1.2165380 \\ \mathrm{C} & -5.1652140 & -1.8432190 & 0.7895310 \\ \mathrm{C} & -5.5116920 & 0.6320770 & -0.9810480 \\ \mathrm{C} & -5.7031200 & 1.9393040 & -1.4119620 \\ \mathrm{H} & -6.6051080 & 2.2014000 & -1.9559190 \\ \mathrm{H} & -6.2524260 & -0.1300500 & -1.2042870 \\ \mathrm{H} & -6.1315400 & -1.3688260 & 0.9321100 \\ \mathrm{H} & -5.7671300 & -3.7014200 & 1.6820150 \\ \mathrm{H} & 1.3714590 & -4.7756230 & 2.0374150 \\ \mathrm{H} & 0.3921970 & -5.4708800 & -2.0718800 \\ \mathrm{H} & -0.9035710 & 5.2882960 & 2.2233870 \\ \mathrm{H} & 0.1655380 & 4.9455970 & -1.9046040 \\ \mathrm{C} & -3.1195500 & 3.7465840 & 2.1862870 \\ \mathrm{H} & -2.9776740 & 2.7045590 & 2.4935210 \\ \mathrm{H} & -4.1757590 & 3.8695770 & 1.9245510 \\ \mathrm{H} & -2.9046410 & 4.3836880 & 3.0489870 \\ \mathrm{C} & 1.0446100 & 5.9802020 & 0.4393490 \\ \mathrm{H} & 1.2239230 & 6.7221010 & -0.3460710 \\ \mathrm{H} & 1.9032710 & 5.2977530 & 0.4503830 \\ \mathrm{H} & 1.0151280 & 6.5006230 & -1.4014120 \\ \mathrm{C} & -1.8608460 & 3.3619720 & -2.7140560 \\ \mathrm{H} & -1.0357530 & 3.6246080 & -3.3823090 \\ \mathrm{H} & -2.7833670 & 3.7644260 & -3.1472460 \\ \mathrm{H} & -1.9538740 & 2.2719530 & -2.6997220 \\ \mathrm{C} & -1.9192200 & -4.0713330 & -2.1636600 \\ \mathrm{H} & -1.9898820 & -3.0101970 & -2.4264790 \\ \mathrm{H} & -2.9360260 & -4.4202830 & -1.9522750\end{array}$

-1.5482410
2.1954550
1.9260910
3.0361560
2.5429060
-0.7582890
0.0468510
-1.6925630
-0.8582730
3.5172070
2.9943350
3.9131930
4.0749110
4.6137760
3.6980860
3.4960360
1.9829840
4.4618590
3.0976560
4.7385820
4.6969330
5.6258810
2.7096050
4.0939610
4.8989590

285.9303224200
2.6585410
0.5683790
3.4256410
3.1025910
4.5463060
5.3782610
6.2597490
0.6553270
-0.5077100
1.8988610
-0.3999150
-1.2959850
4.8457990
-1.8395940
5.3953410
5.6805090
4.5830580
4.8785850
5.4301270

-4.6128020
-6.1715910
-7.1816850
-5.8411380
-6.2472240
-3.2585450
-3.4609170
-3.6426610
-2.1733070
-0.6466650
-1.6784500
-2.9058310
-3.5002770
-2.4580620
-1.2341330
-3.6429340
-1.9829690
-0.2406650
-3.8561540
-4.3710250
-2.8798620
-2.1562770
-1.5152810
-0.4601590
-2.6204080

0.3995510 1.4037970

1.2771430

2.6384420

0.7638790

1.6662790

1.2843570

2. 5318880

3.0262510

3.1615480

4.1974410

4.6026010

$-0.6979630$

2. 3772100

$-1.3013960$

$-2.6659470$

$-1.4584390$

$-2.8206300$

$-3.4421900$
$-3.0383740$

$-0.1519460$

0.1795130

0.4660730

$-1.1866490$

2.7018730

3.4135200

3.1263680

2.6018630

$-0.7657830$

0.2387780

0.2465410

$-1.1531840$

$-2.1325170$

$-2.1610320$

0.9382860

$-0.0532970$

$-0.3929920$

$-1.5053670$

$-1.1126930$

$-3.1395720$

$-1.8292260$

$-2.5453740$

$-2.8285220$

0.6410200

0.8255760

$-0.1793330$

0.0691660

0.0655690

$-0.5824050$

$-1.2476650$

$-1.7543010$

0.6293440

1.2164710

0.7574200

1.9707150

2.4317220

$-0.5793700$

1.0287410

0.5613660

0.5313440

$-1.7301510$

$-1.7172090$

$-0.5982940$ 


$\begin{array}{rrr}-3.8218400 & 2.0399360 & -0.3025220 \\ -2.5323490 & 2.5573700 & -0.1778930 \\ -3.7164350 & 1.1546960 & 1.9163440 \\ -2.4266860 & 1.6575550 & 2.0832330 \\ -4.4334330 & 1.3424160 & 0.7352570 \\ -2.4102220 & -2.1674100 & 1.7574790 \\ -2.0275830 & -2.5738360 & 0.3304260 \\ -2.2599110 & -1.0905520 & 1.8519890 \\ -0.9740120 & -2.3622730 & 0.1513880 \\ -2.3728800 & -4.0206290 & -0.0043180 \\ -2.1409370 & -4.2085820 & -1.0583780 \\ -1.7158380 & -4.6623300 & 0.5929370 \\ -3.8704160 & -2.5194920 & 2.0578090 \\ -4.0995330 & -2.2764070 & 3.1004840 \\ -4.4966980 & -1.8716290 & 1.4317090 \\ -2.2816950 & -1.1574690 & -1.6032630 \\ 1.1578740 & 0.0216800 & 0.4164540 \\ 0.4139750 & -0.5399550 & 1.5573960 \\ 1.3123960 & -0.8631370 & -0.8752420 \\ -0.2446950 & -2.1694740 & 2.7118200 \\ -1.0961390 & -1.2181930 & -1.8997870 \\ -3.2694380 & -0.4287130 & -2.4379600 \\ -4.6417220 & -0.6386460 & -2.2938930 \\ -2.7937680 & 0.4949010 & -3.3697630 \\ -5.5359020 & 0.0846290 & -3.0736220 \\ -5.0018340 & -1.3606290 & -1.5698470 \\ -3.6908200 & 1.2267390 & -4.1379170 \\ -1.7238530 & 0.6418740 & -3.4701580 \\ -5.0617780 & 1.0230470 & -3.9887260 \\ -6.6032530 & -0.0786130 & -2.9626590 \\ -3.3225270 & 1.9557200 & -4.8527730 \\ -5.7618440 & 1.5946230 & -4.5905960 \\ 0.4372440 & -1.1152010 & -1.2674740 \\ 0.8223860 & 4.8404040 & 2.1302290 \\ 1.9627140 & 4.3262890 & 1.5262600 \\ 3.9582990 & 3.5055480 & -0.6200670 \\ 5.0910820 & 3.0265430 & -1.2656210 \\ 5.7451920 & 3.7133600 & -1.7931060 \\ 3.7175650 & 4.5638410 & -0.6553130 \\ 2.9209520 & 4.8198060 & 1.6577290 \\ 0.8862290 & 5.7455270 & 2.7256940 \\ -4.1747770 & 0.6020590 & 2.7348150 \\ -4.3584790 & 2.1851330 & -1.2376580 \\ 6.1067940 & -3.1345570 & 1.4161290 \\ 4.6667630 & -3.4134170 & -2.6049760 \\ 5.6662970 & -0.4982320 & 1.8091210\end{array}$

\begin{abstract}
4.7281220
6.2161890

6.2506880

5.7720610

6.7748250

5.0681650

5.7581590

3.9781570

3.7514260

4.6564710

3.0492530

$-1.9042170$

$-1.0603500$

$-1.5188530$

$-2.6293760$

$-5.8313600$

$-6.4413270$

$-5.8213560$

$-6.3278960$

$-1.6774680$

$-2.2673280$

$-1.4407530$

$-0.7334710$

$-4.1962730$

$-5.2603290$

$-3.6397180$

$-3.8394990$

$-4.0308050$

$-4.4864670$

$-1.2950880$

$-2.8244830$
\end{abstract}

-0.2054320
0.4217850
-1.0786150
-4.9109470
-5.0725190
-5.4744590
-5.3406770
-0.8279540
-1.5880140
-0.0967730
-0.3040150
3.2973790
2.7314750
4.2750760
3.4498020
0.7964450
1.0358570
-0.2955010
1.2029180
1.4038980
0.7759450
2.3358700
0.8887690
-3.9853760
-4.1716100
-4.6399180
-4.3408710
-5.4025100
-3.7746750
-2.9642900
-1.7201860

2.2937320

1.5838070

2. 5285010

$-0.6211960$

$-1.0352610$

$-1.2416650$

0.3849890

$-2.9605350$

$-3.7135200$

$-3.4142550$

$-2.7150760$

$-1.3322420$

$-1.7432970$

$-1.0231960$

$-2.1363720$

0.5924850

1.4700880

0.4927000

$-0.2931330$

3.3679440

4.0420160

3.8927870

3.1622650

1.7514180

1.9343300

2.4351030

0.3039290

0.1146720

$-0.3787760$

2.9828000

$-0.5393430$ 
wB97XD/6-31G(d) Optimized Cartesion Coordinates $(\AA)$ for the thiocarboxylysis/trans-esterification organocascade in which cyclopentene oxide (1c) is the epoxide. Absolute solution phase free energies $(\mathrm{G})$ in hartrees computed with the Quasi-RRHO model at the wB97XD/6-311+G(d,p)//wB97XD/6-31G(d) level are reported.

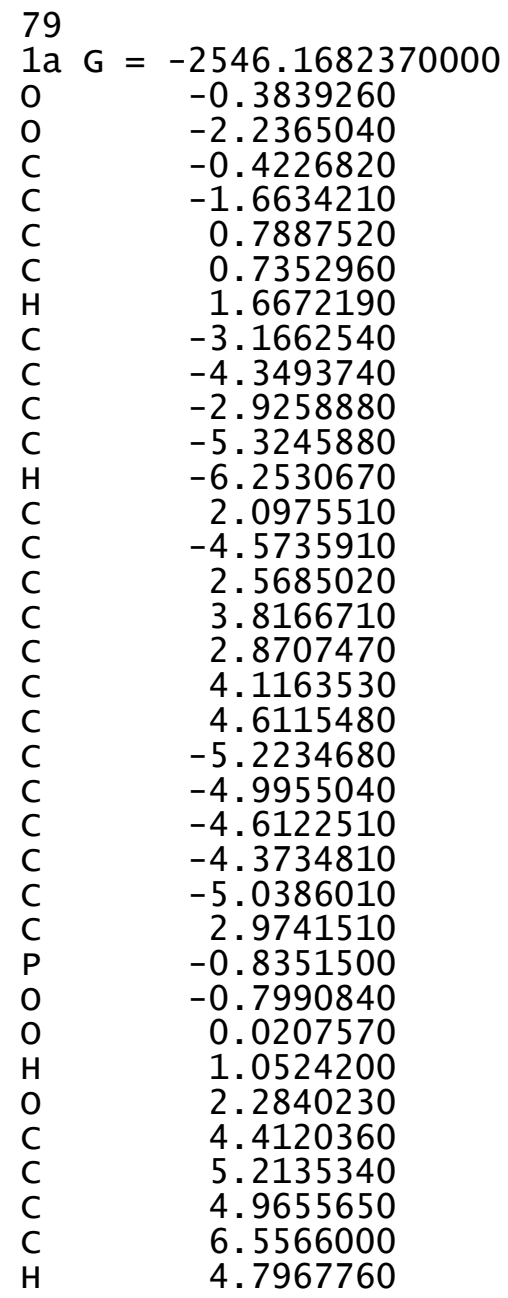

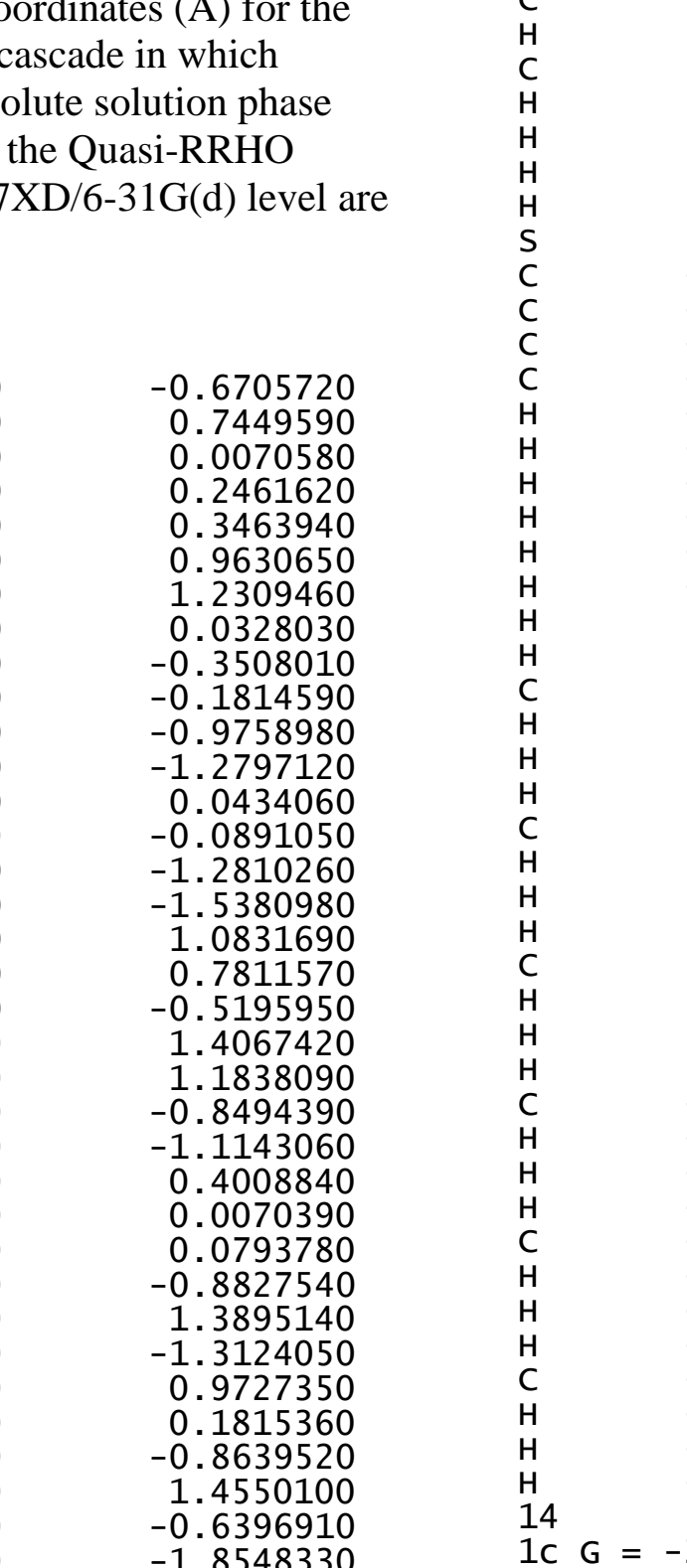

\begin{abstract}
6.3076890
4. 3302920

7.1055280

7.1753280

6.7340740

8.1560180

0.8452590

2.3420870

$-5.1176010$

$-3.9268220$

$-1.6718050$

$-0.4823270$

$-0.5044290$

$-2.6236290$

$-3.7545940$

$-5.8831870$

$-4.4533220$

$-5.5516520$

4.1817890

4.7147660

1.7460010

0.8598010

1. 3962070

2. 3280830

5.9794030

6.7123270

6. 3292290

5.9771460

2. 3817450

3. 0057410

2. 4116800

1. 3487930

$-5.1871560$

$-4.2314430$

$-5.8548670$

$-5.6115240$

$-5.3057260$

$-6.3753750$

$-4.9792360$

$-4.7831310$

$-3.8798160$

$-3.8544940$

$-4.5162910$

$-2.8648860$
\end{abstract}

2.7529940

2. 11

2. 9040380
$-1.8548330$
2.3927690

1.7565940

2. 8485890

3. 3912210

2. 2533260

3. 0628410

0.8569050

1.9872990

$-2.4908710$

$-3.0887060$

$-4.2470830$

$-4.8697250$

$-5.8397980$

$-4.7234630$

$-4.1424960$

$-3.0814130$

4. 1071070

3. 1943300

$-1.7248340$

$-0.8356170$

$-2.8715280$

$-2.2536980$

$-3.8860340$

$-2.9041630$

$-0.6691810$

$-1.4774360$

$-0.0091050$

$-0.0967440$

$-1.8191200$

$-1.1733640$

$-2.8272980$

$-1.4663910$

0.5136940

0.0597900

$-0.3004140$

0.9911250

5. 2778990

5.4596660

5. 9044910

5. 6161280

1. 5990850

2. 4458300

0.8220730

1.1933550
1.6760210

2. 2571480

0.6271060

$-1.4553100$

2.6643140

0.7983100

1. 2292680

$-1.6483810$

$-1.2074270$

$-0.8153310$

0.8706740

1. 2270240

1. 7130540

1.0854620

$-1.0124440$

$-1.7004170$

$-1.6426390$

2. 3922000

$-2.5629320$

1.5859070

$-2.4149640$

$-2.5962110$

$-2.1955390$

$-3.3401650$

$-0.8217680$

$-0.9340260$

$-0.0227890$

$-1.7547860$

2. 5111920

3. 1359540

2. 9402560

2. 5806050

2. 2968440

2. 5823240

1.9945300

3.1844560

0.6696980

0.8268920

$-0.1654380$

1. 5709630

$-2.4733270$

$-3.1650210$

$-2.9095420$

$-2.3996520$ 


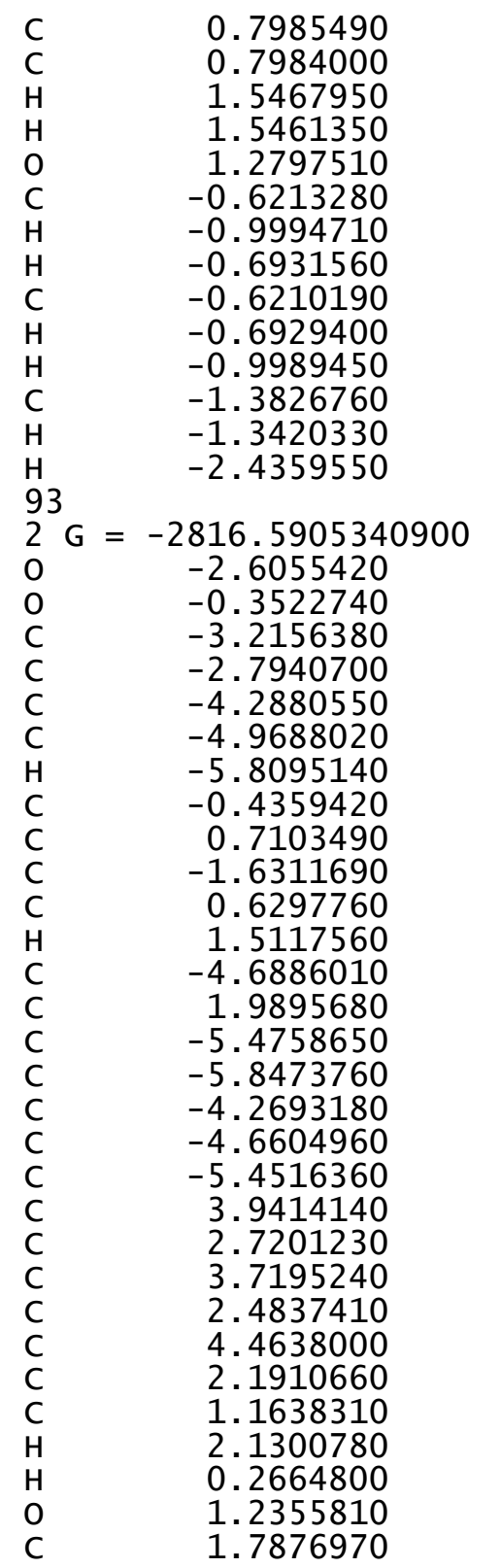

$\begin{array}{rr}-0.7318060 & 0.3563780 \\ 0.7317980 & 0.3565520 \\ -1.3218810 & 0.8805860 \\ 1.3221730 & 0.8811550 \\ 0.0002270 & -0.7753480 \\ 1.2301140 & 0.1941320 \\ 1.5339750 & 1.1780850 \\ 2.0945020 & -0.4733440 \\ -1.2302560 & 0.1941420 \\ -2.0946730 & -0.4732730 \\ -1.5341190 & 1.1781890 \\ -0.0000980 & -0.3470330 \\ -0.0000480 & -1.4388050 \\ -0.0002680 & -0.0548280 \\ & \\ -0.7221200 & 0.7379020 \\ -1.2592890 & -0.2529800 \\ -1.3381880 & -0.3500440 \\ -2.6153540 & -0.7340730 \\ -0.6811820 & -0.9501630 \\ -1.3502020 & -1.9688760 \\ -0.8550220 & -2.4458660 \\ -2.5931590 & 0.1231930 \\ -3.1987870 & 0.6341650 \\ -3.2826730 & -0.0997010 \\ -4.5546100 & 0.9586320 \\ -5.0499450 & 1.3536510 \\ 0.6893810 & -0.5165880 \\ -2.4431510 & 0.7815340 \\ 0.8545930 & 0.6328070 \\ 2.1420380 & 1.0157370 \\ 1.8034640 & -1.2595790 \\ 3.0746090 & -0.8409800 \\ 3.2645470 & 0.2901660 \\ -1.4352570 & -0.2172290 \\ -2.0878220 & -0.3676120 \\ -1.4910270 & 2.1641020 \\ -2.1330190 & 2.0573360 \\ -1.1384200 & 1.0412790 \\ 1.5187370 & 1.7337870 \\ 2.5279310 & 1.9754320 \\ 0.7767980 & 0.9408120 \\ 2.5991060 & 1.3697390 \\ 1.3343380 & 2.8110660 \\ 3.7331130 & 2.6321510\end{array}$
1.9156020
1. 1616080
3. 5198240
4.1106140
4.0852990
2. 1188480
$-1.1186080$
$-0.6773840$
$-0.9433300$
0.0802760
1. 2047000
3. 3963020
4. 6199640
3. 3806370
5. 8122680
4.6268610
4. 5743810
2. 4324910
5.7919660
6.7582020
4.5541000
6.7235030
0.3768940
1.8740220
$-0.5508660$
$-1.6702520$
$-3.5002270$
$-4.5823960$
$-5.1180040$
$-3.1791040$
$-2.5935000$
$-0.5973430$
4.1055280
4.5064580
$-6.4564970$
$-4.3299720$
$-5.8981200$
$-5.0309290$
$-6.3803680$
$-6.5987160$
$-5.8885500$
$-6.8384110$
$-5.1503740$
$-6.0358390$
$-3.3893810$
-3. 1927400

4. 4990280

4.1538210

2. 0392110

1. 2727190

2. 3770510

2.0308090

$-0.1433550$

$-0.2569210$

1. 1678300

0.3541280

1. 0852950

1. 5194590

2. 0324460

0.4946490

1. 5100160

2. 8355040

$-0.0045040$

0.0957380

0.4930760

1.9020450

$-0.7888900$

0.0921100

1. 3688790

3.5905870

$-5.2665340$

$-4.6368100$

$-3.2476530$

$-2.6245120$

$-3.1287800$

$-4.2303430$

$-5.1902640$

$-6.3183040$

$-1.2583780$

$-1.1600150$

2.2718860

3. 9408590

$-0.3371200$

$-0.8060030$

$-1.1024890$

$-0.0418340$

4. 6475820

4. 9162040

5.4012580

4.7135190

1.6436220

2. 6123230
1.8586760

3. 4242330

2.2234460

2.7299110

1.3466670

$-1.7050290$

0.6151400

2. 1106330

$-0.0574390$

2. 3941570

$-1.6580460$

$-2.2607490$

$-1.8211880$

$-3.2125430$

$-2.3083830$

$-1.0914270$

$-3.7205600$

$-3.5547760$

$-3.2609310$

$-1.9478290$

$-4.4708110$

$-3.6487570$

$-1.1899780$

$-1.2142230$

0.7731400

0.2435370

$-1.7615070$

$-2.3702170$

$-3.1681830$

$-2.0937340$

0.1010840

1.0366390

3. 1546630

$-1.1052720$

1. 9081740

$-1.4103700$

1. 4558870

1.9348200

0.8382320

2. 2418490

0.7037980

0.2257300

0.4132920

1.7862090

$-2.4747570$

$-2.9421280$ 


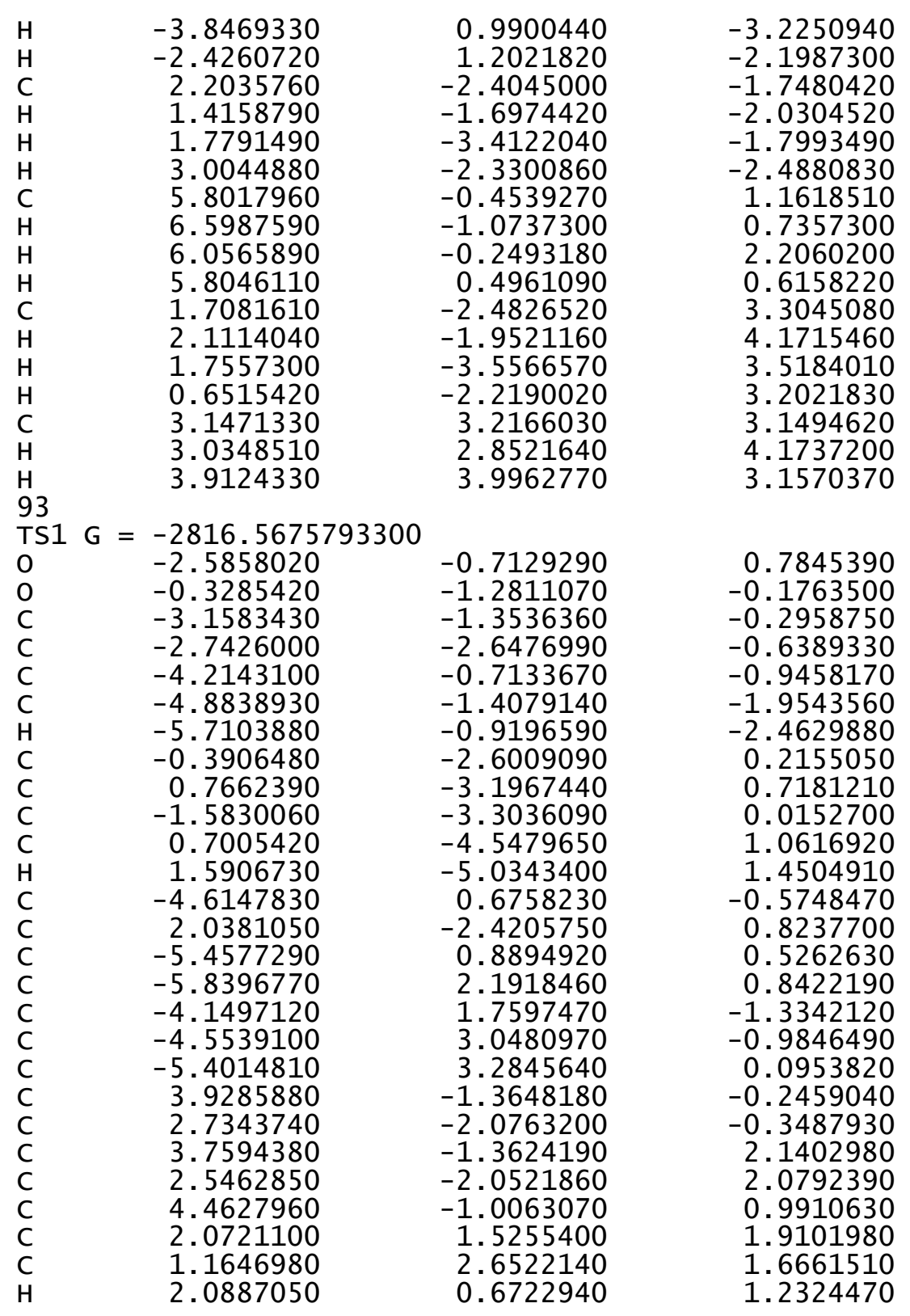

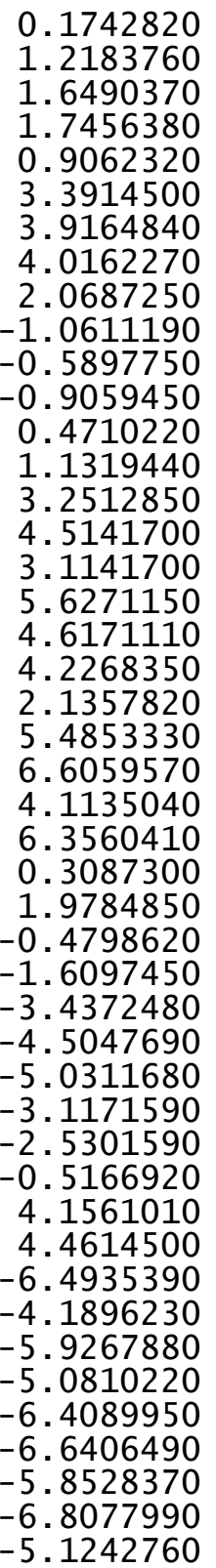

2.5044330

1. 3432800

3. 8664140

4.6946830

4. 1768690

2. 1137120

1.4358150

2. 2684750

1.8378740

$-0.1633800$

$-0.2187020$

1. 1208210

0.7100620

0.9473730

1.4360380

1.9749300

0.4557570

1. 5250750

2.7330570

0.0207330

0.0372440

0.5478510

1. 9380030

$-0.7351130$

0.1984510

1.1519420

3. 3076250

$-5.2685280$

$-4.6529980$

$-3.3070310$

$-2.6988520$

$-3.2257110$

$-4.3021990$

$-5.2157710$

-1.0853060

$-1.0934450$

2. 3578740

3. 8905110

$-0.2700450$

$-0.7438420$

$-1.0414520$

4.6834200

4. 9155230

5. 4288970
1. 2482740

3. 0504890

2. 3956360

1.6885640

3. 1357520

2. 3566740

3. 0327650

1.4712060

$-1.5397120$

0.7560500

2. 1802340

$-0.0232990$

2. 7665610

$-1.5429630$

$-2.3326420$

$-2.0641930$

$-3.3231050$

$-2.7633890$

$-1.2951920$

$-4.0311990$

$-3.5277270$

$-3.7468970$

$-2.5415900$

$-4.8019090$

$-4.2932960$

$-0.9639630$

$-0.7279350$

0.9034110

0.3783120

$-1.6576470$

$-2.3059280$

$-3.0956360$

$-1.9524720$

0.2530340

1. 1829320

3.1152430

$-1.1554830$

1.6964100

$-1.5694480$

1. 3693270

1.8802040

0.7589930

2. 1293510

0.4343420

$-0.0528060$

0.1011600 


$\begin{array}{lr}\mathrm{H} & -5.9977220 \\ \mathrm{C} & -3.2015780 \\ \mathrm{H} & -2.9960600 \\ \mathrm{H} & -3.6033140 \\ \mathrm{H} & -2.2504380 \\ \mathrm{C} & 2.2073470 \\ \mathrm{H} & 1.3489270 \\ \mathrm{H} & 1.8749110 \\ \mathrm{H} & 2.9766710 \\ \mathrm{C} & 5.7767150 \\ \mathrm{H} & 6.6185360 \\ \mathrm{H} & 5.9161100 \\ \mathrm{H} & 5.8381380 \\ \mathrm{C} & 1.7845600 \\ \mathrm{H} & 2.2774960 \\ \mathrm{H} & 1.7032190 \\ \mathrm{H} & 0.7676260 \\ \mathrm{C} & 3.0017670 \\ \mathrm{H} & 2.8724210 \\ \mathrm{H} & 3.7673940 \\ 93 & \\ 3 & -2816.6272760800 \\ \mathrm{O} & 2.5124470 \\ \mathrm{O} & 0.5147360 \\ \mathrm{C} & 3.3340740 \\ \mathrm{C} & 3.0417220 \\ \mathrm{C} & 4.4816940 \\ \mathrm{C} & 5.3731900 \\ \mathrm{H} & 6.2742580 \\ \mathrm{C} & 0.5574550 \\ \mathrm{C} & -0.6470770 \\ \mathrm{C} & 1.7999190 \\ \mathrm{C} & -0.5824790 \\ \mathrm{H} & -1.5087300 \\ \mathrm{C} & 4.7496940 \\ \mathrm{C} & -1.9588400 \\ \mathrm{C} & 5.2572080 \\ \mathrm{C} & 5.5229200 \\ \mathrm{C} & 4.5056140 \\ \mathrm{C} & 4.7824910 \\ \mathrm{C} & 5.2914510 \\ \mathrm{C} & -3.6716560 \\ \mathrm{C} & -2.4267530 \\ \mathrm{C} & -3.9709440 \\ \mathrm{C} & -2.7313900 \\ \mathrm{C} & -4.4603030 \\ & \end{array}$

\begin{tabular}{|c|c|}
\hline $\begin{array}{r}4.8063160 \\
1.5503310 \\
2.4959010 \\
0.8453520 \\
1.1456760 \\
-2.4637980 \\
-1.8386850 \\
-3.5067410 \\
-2.3331080 \\
-0.2700490 \\
-0.9551290 \\
0.2085500 \\
0.5015550 \\
-2.3565260 \\
-1.9073180 \\
-3.4341210 \\
-1.9560820 \\
3.4505050 \\
3.3041240 \\
4.2159640\end{array}$ & $\begin{array}{r}1.5123200 \\
-2.4888350 \\
-2.9988580 \\
-3.2243500 \\
-2.1268050 \\
-1.7068350 \\
-1.9760250 \\
-1.7252740 \\
-2.4725400 \\
1.0664290 \\
0.9106290 \\
2.0405050 \\
0.2911300 \\
3.3454650 \\
4.2123970 \\
3.5235320 \\
3.2808550 \\
3.0260460 \\
4.1003420 \\
2.8840290\end{array}$ \\
\hline $\begin{array}{r}0.1994620 \\
1.3379850 \\
1.1313790 \\
2.4947940 \\
0.6531870 \\
1.5905190 \\
1.2334460 \\
2.4193700 \\
2.9319650 \\
2.9916500 \\
4.0394910 \\
4.4577090 \\
-0.8104620 \\
2.3763680 \\
-1.5149830 \\
-2.8750640 \\
-1.4692250 \\
-2.8342110 \\
-3.5533250 \\
2.2216490 \\
2.6988670 \\
1.1202950 \\
1.5758620 \\
1.4337130\end{array}$ & $\begin{array}{r}0.9550110 \\
-0.0763890 \\
0.3341670 \\
0.4459280 \\
-0.2963820 \\
-0.8209230 \\
-1.3109100 \\
0.7989220 \\
1.2763930 \\
1.0883900 \\
2.1240240 \\
2.5071010 \\
-0.4066570 \\
0.8300190 \\
0.6972800 \\
0.5631530 \\
-1.6195040 \\
-1.7116300 \\
-0.6339230 \\
-0.8607030 \\
-0.4531560 \\
1.2397830 \\
1.6870570 \\
-0.0279760\end{array}$ \\
\hline
\end{tabular}

-2.6514360
-3.0683860
-2.5439310
-2.1764240
-1.4522330
-4.0695850
-5.0758110
-3.7786970
-3.7823400
-3.4840310
-4.6612320
-2.4795640
1.0091550
0.2152890
1.1237570
-0.7402940
-1.3530240
-2.7389830
-3.9767530
-1.7055710
-4.1764520
-4.7883920
-1.9083490
-0.7529560
-3.1434120
-5.1386930
-1.1044610
-3.3020350
0.2631620
-3.8799080
0.6394530
1.8199360
3.9597500
5.1192660
5.8209300
3.7457200
2.7734340
0.6704180
-4.5713140
-4.0260590
5.9193010
4.5907630
5.4969990
4.5460610
6.0550360
6.0590070
1.9354890

0.8210230

1.5381560

0.4359110

2. 5386790

1.4771700

1.0597860

1. 3001090

2. 9561440

3. 9254650

2. 5992450

$-1.3363710$

0.4944070

1. 6007770

$-0.8277240$

2. 1597080

$-0.9224780$

$-2.4493040$

$-3.0950910$

$-2.8339480$

$-4.1126710$

$-2.8109690$

$-3.8512300$

$-2.3189980$

$-4.4908990$

$-4.6119300$

$-4.1379000$

$-5.2842190$

$-1.0136120$

$-0.5913370$

2. 4673060

1.9448640

$-0.0974570$

$-0.7192610$

$-1.1331650$

$-0.0413350$

2. 2048200

3.1363050

1.9007100

$-1.8615180$

1.4167820

$-2.6516920$

2.0140670

2. 4949450

1.8815420

2. 6990390 


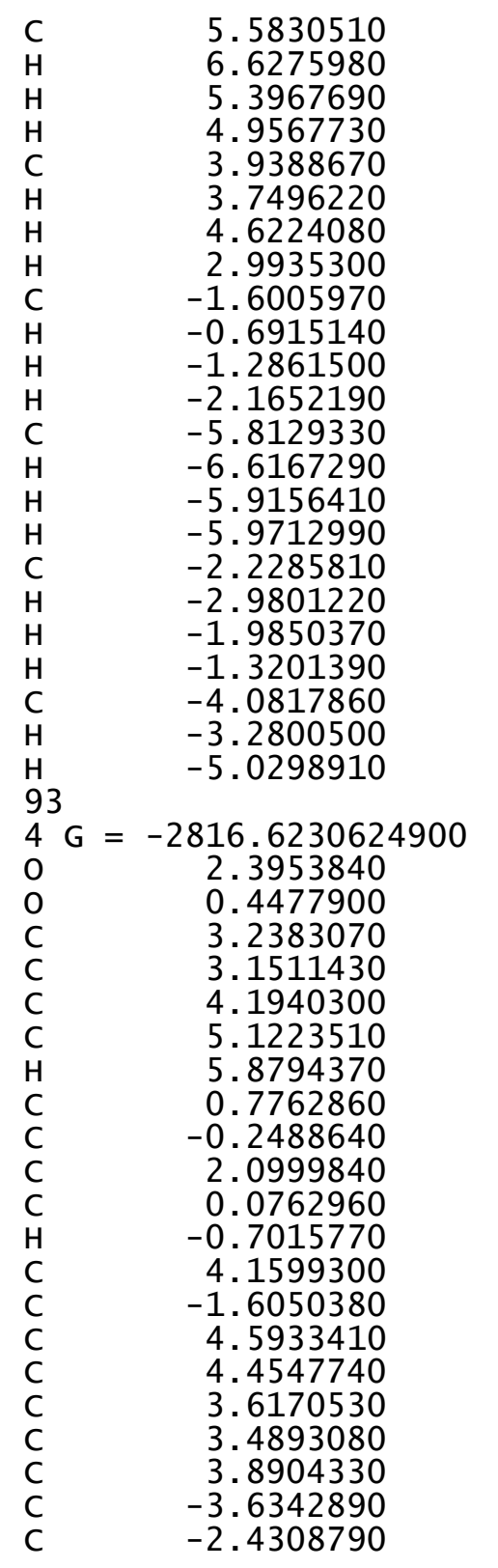

$\begin{array}{rr}-5.0290220 & -0.7438570 \\ -5.2447180 & -0.4927540 \\ -5.3994370 & -1.7561440 \\ -5.6067510 & -0.0547520 \\ -0.7307470 & -2.8073090 \\ -1.4186080 & -3.6362590 \\ 0.0478650 & -3.1636560 \\ -0.2427990 & -2.5490010 \\ 3.5436040 & -1.3908450 \\ 3.0131590 & -1.6970540 \\ 4.4803670 & -0.9182270 \\ 3.7865400 & -2.2952810 \\ 0.9399590 & -0.4728150 \\ 1.4420620 & 0.0786590 \\ -0.1359800 & -0.2952920 \\ 1.1251960 & -1.5392600 \\ 1.1821800 & 3.0536610 \\ 0.5934470 & 3.5877010 \\ 2.0574950 & 3.6652840 \\ 0.5762190 & 2.9677990 \\ -3.8696140 & 2.9706900 \\ -4.3929760 & 3.5010380 \\ -4.1213320 & 3.4544000 \\ -0.6287010 & \\ -1.7824580 & -0.4992790 \\ -1.2182380 & 0.5656370 \\ -2.5943820 & 0.4345970 \\ -0.3935920 & 0.6752940 \\ -0.9913960 & 1.0285900 \\ -0.3710060 & 1.8804230 \\ -2.9914930 & 2.3509040 \\ -3.7207510 & -0.0351910 \\ -3.4339050 & -0.6375090 \\ -4.9779610 & 0.0446410 \\ -5.5713650 & -1.1460160 \\ 1.0754920 & -1.6174320 \\ -3.1056530 & 0.7613990 \\ 1.5778330 & -0.7514770 \\ 2.9383090 & -0.4772650 \\ 1.9378100 & -0.7393190 \\ 3.2941040 & 1.7247350 \\ -3.8115400 & 1.4201830 \\ -300230 & 0.1920000 \\ -2.3750670 & 0.3762610\end{array}$

-3.1967140
-1.9964780
-4.0221720
-2.0249350
-2.8080980
0.8354040
0.1147770
0.7373900
-1.4187490
-1.8896950
-4.1680070
-5.3136410
-4.2823230
-6.5585720
-5.2405140
-5.5268390
-3.3865950
-6.6665460
-7.4465690
-5.6103170
-7.6404400
-0.2093720
-2.6068070
1.3785690
2.3824980
4.0986010
5.0789210
5.8041450
4.0480870
3.4024180
1.6157110
-3.4799000
-4.2717590
4.7829490
3.0527790
5.1725700
4.3896610
5.9252210
5.6408100
3.7187070
4.6870290
3.2060270
3.1317220
3.1413830
2.6792870
3.9665370
-1.8226430
-2.5259930
-1.6881840
1.3669060
1.7641440
-0.4308270
-0.1494870
0.6389170
0.6903110
0.9818030
1.4738030
2.1423560
0.4566010
1.8013680
2.9076080
0.1205050
-0.0635710
0.7931870
2.3159000
-0.6661940
0.5283560
0.8913290
3.3061940
-5.4651130
-4.6978200
-3.1497540
-2.3601770
-2.8113790
-4.2113950
-5.0696160
-6.4431740
-1.3349230
-2.2230780
3.3273060
3.9608420
0.6597530
0.0336320
-0.0122980
1.2312460
5.2732930
5.7601300
5.8058020
5.4017720
1.4183420
2.2188980
1.0042080
$-2.0253190$ $-1.9711700$ $-0.9089480$ $-2.5196120$ 0.9060020 $-0.2140750$ $-1.4689600$ 0.9382480 $-2.1663370$ 1. 1074180 1.4334210 0.9918100 2. 3879760 1. 5080490 0.2263360 2. 9024710 2. 7088410 2. 4628510

1.1555520

3. 6457100

2.8632930

1.0956450

0.0512820

$-1.0622300$

$-0.4826110$

1. 5414690

2.1302780

2.7998940

1.7641960

$-0.4511190$

$-1.4685890$

$-2.9553730$

1.1525970

$-1.7015320$

2.1610430

$-1.5243580$

$-1.9667420$

$-1.0990810$

$-2.3307100$

$-0.1362350$

$-0.2990760$

0.6702590

$-1.0527540$

3. 0592430

3.6438050

3.6488440 


\begin{tabular}{|c|c|c|c|}
\hline & & & \\
\hline H & $\begin{array}{r}2.4013870 \\
-2.0137490\end{array}$ & 0.6228750 & 2.9279700 \\
\hline $\mathrm{H}$ & $\begin{array}{l}-2.013 / 490 \\
-1.2246640\end{array}$ & $\begin{array}{l}-3.6015740 \\
-2.9976440\end{array}$ & $\begin{array}{l}1.6984230 \\
2.1616560\end{array}$ \\
\hline $\mathrm{H}$ & -1.6191190 & -4.6160740 & 1.5806020 \\
\hline $\mathrm{H}$ & -2.8572590 & -3.6407720 & 2.3940620 \\
\hline C & -5.2896890 & -0.8774020 & -0.9949170 \\
\hline $\mathrm{H}$ & -6.0108520 & -1.3379420 & -1.6804460 \\
\hline $\mathrm{H}$ & -5.0733260 & 0.1272860 & -1.3742030 \\
\hline $\mathrm{H}$ & -5.7676570 & -0.7733040 & -0.0162870 \\
\hline C & -1.1167480 & -2.6082960 & -3.1939960 \\
\hline $\mathrm{H}$ & -1.5766540 & -2.0830510 & -4.0355860 \\
\hline $\mathrm{H}$ & -0.9386280 & -3.6457900 & -3.4969740 \\
\hline $\mathrm{H}$ & -0.1439160 & -2.1460610 & -3.0006800 \\
\hline C & -1.0949970 & 2.9978300 & -0.9112860 \\
\hline C & -1.3947860 & 2.6080670 & -2.3699970 \\
\hline C & -0.0123400 & 2.7579490 & -3.0484350 \\
\hline C & 0.8073200 & 3.7289520 & -2.1579080 \\
\hline C & -0.1428040 & 4.1839830 & -1.0277640 \\
\hline $\mathrm{H}$ & -0.1201720 & 3.1187760 & -4.0750220 \\
\hline $\mathrm{H}$ & -2.0921860 & 3.3465460 & -2.7847200 \\
\hline $\mathrm{H}$ & -0.5776560 & 2.1682990 & -0.4325270 \\
\hline $\mathrm{H}$ & 1.1982690 & 4.5834220 & -2.7178090 \\
\hline $\mathrm{H}$ & 1.6660520 & 3.2083550 & -1 \\
\hline $\mathrm{H}$ & -0.6922750 & 5.0873180 & -1.3206510 \\
\hline $\mathrm{H}$ & 0.3815210 & 4.3958590 & -0.0919010 \\
\hline $\mathrm{H}$ & 0.4638740 & 1.7751350 & -3.0906050 \\
\hline $\begin{array}{l}\text { ys } \\
\text { TS }\end{array}$ & $G=-2816.5866934100$ & & \\
\hline 0 & 1.8495940 & -1.0002800 & -0.5287610 \\
\hline 0 & -0.0622270 & -2.0660860 & 0.7150740 \\
\hline C & 2.7513120 & -1.7550970 & 0.1962210 \\
\hline C & 2.5300880 & -3.1296810 & 0.3531490 \\
\hline C & 3.9081010 & -1.1257150 & 0.6563580 \\
\hline $\mathrm{C}$ & 4.8565370 & -1.9045670 & 1.3213500 \\
\hline $\mathrm{H}$ & 5.7622610 & -1.4274530 & 1.6847940 \\
\hline C & 0.0536330 & -3.2160000 & -0.0387250 \\
\hline C & -1.1099930 & -3.8069860 & -0.5313620 \\
\hline $\mathrm{C}$ & 1.3204050 & -3.7862470 & -0.2007950 \\
\hline $\mathrm{C}$ & -0.9835690 & -5.0218720 & -1.2067570 \\
\hline $\mathrm{H}$ & -1.8786920 & -5.5003810 & -1.5939070 \\
\hline C & 4.1523360 & 0.3243190 & 0.3951980 \\
\hline C & -2.4397350 & -3.1612860 & -0.3213330 \\
\hline C & 4.6241570 & 0.7209600 & -0.8681080 \\
\hline C & 4.9033870 & 2.0660790 & -1.0940410 \\
\hline C & 3.9445370 & 1.2746320 & 1.4039960 \\
\hline & 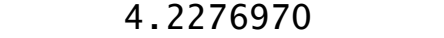 & 2.616 & \\
\hline
\end{tabular}

4.7155900
-4.3011670
-3.0579160
-4.3051070
-3.0618120
-4.9430670
-1.5168810
-1.9929220
0.3903650
-0.4502910
0.4699540
-1.1396620
-1.8745570
-3.4052550
-3.7274670
-4.4216220
-5.0554680
-2.9423720
-5.7457350
-4.1689290
-6.0654410
-5.2982030
-6.5322360
-7.1027160
-0.9017530
-0.7529030
0.2623360
1.4050730
3.5027070
4.6549030
5.3974630
3.3394060
2.3798010
0.3436050
-4.7857900
-4.7819600
5.2807440
4.0666860
4.8211060
3.8625370
5.4633390
5.2765550
5.0384210
6.1124730
4.7443830
4.5218690
3.0301710
-2.6375030
-3.2394900
-1.8829720
-2.4759240
-1.9587030
2.0633660
2.4232620
-0.6407770
-0.2326690
0.3090740
1.0984350
1.2746730
2.8593850
4.1752260
1.9052250
4.5402010
4.9152340
2.2777930
0.8783540
3.5938670
5.5664310
1.5385120
3.8790230
0.9599460
3.7212540
-5.6160160
-5.0009150
-3.8731160
-3.2674150
-3.8587030
-4.9357130
-5.4547500
-6.5594210
-1.3460860
-2.7020160
2.3720460
3.3548530
-0.2908120
-0.7267950
-1.1165250
0.1712160
4.4775520
4.6172640
5.1200390
4.8344300
$-0.1022940$

1. 1188950

0.9373070

$-1.1517790$

$-1.3754660$

0.0837040

$-0.9759050$

0.7798820

0.0865940

$-1.0939950$

1.2558680

$-1.0009430$

1. 3713910

0.5860380

0.2469670

0.7154480

0.0546180

0.1395870

0.5244690

0.9572780

0.1962490

$-0.2019300$

0.6328080

0.0489600

1.4699680

1.1850980

$-1.3836490$

$-0.8867360$

1.0272930

1. 5126970

2.0391050

1.1810930

$-1.0400960$

$-1.9142790$

$-1.9671600$

2.0932030

$-2.0684540$

1. 9172210

$-1.9691950$

$-2.2717640$

$-1.6445580$

$-2.8496640$

$-0.3776850$

$-0.5448720$

0.4579060

$-1.2764140$ 


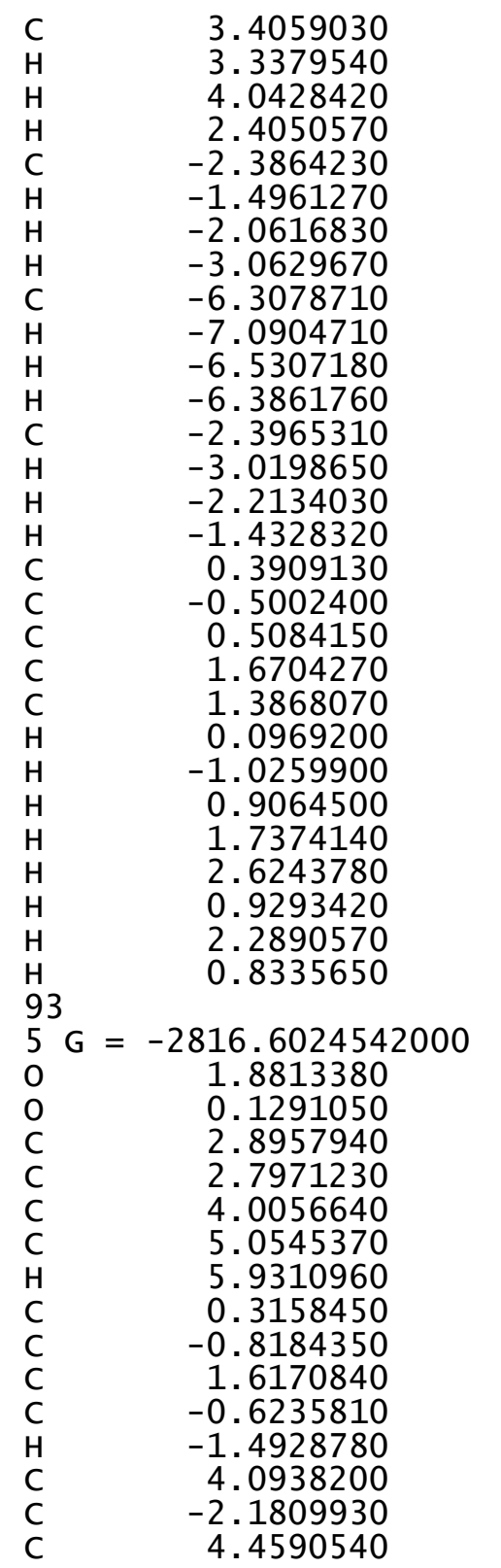

\begin{tabular}{|c|c|}
\hline $\begin{array}{r}0.8684320 \\
1.7315120 \\
0.1171910 \\
0.4376440 \\
-3.9501770 \\
-3.4017460 \\
-4.9567500 \\
-4.0375990 \\
-1.3514040 \\
-2.1179890 \\
-0.6045690 \\
-0.8712090 \\
-2.3484480 \\
-1.7662530 \\
-3.3267480 \\
-1.8397490 \\
3.2516470 \\
2.9862780 \\
2.6308100 \\
3.6265280 \\
4.2479550 \\
2.7488970 \\
3.9103790 \\
2.3342030 \\
4.4096110 \\
3.0939680 \\
5.2391290 \\
4.3382920 \\
1.5938800\end{array}$ & $\begin{array}{r}2.7525500 \\
3.4211900 \\
3.2315340 \\
2.6444880 \\
2.0858030 \\
2.4127450 \\
1.8011820 \\
2.9407380 \\
0.2915560 \\
0.2436110 \\
-0.4766960 \\
1.2733460 \\
-2.7230760 \\
-3.4080700 \\
-3.1804350 \\
-2.6208310 \\
-0.1431380 \\
-1.3409900 \\
-2.4285080 \\
-2.1211750 \\
-0.7157560 \\
-3.4330370 \\
-1.6063720 \\
0.1580330 \\
-2.8805930 \\
-2.1096070 \\
-0.8099640 \\
-0.1101300 \\
-2.3096730\end{array}$ \\
\hline $\begin{array}{r}-0.9345870 \\
-2.1487290 \\
-1.6106230 \\
-2.9976090 \\
-0.8749340 \\
-1.5723080 \\
-1.0186160 \\
-3.2875310 \\
-3.9575730 \\
-3.7506110 \\
-5.1584730 \\
-5.7008860 \\
0.5981590 \\
-3.3942410 \\
1.0725360\end{array}$ & $\begin{array}{r}-0.5722790 \\
0.7534590 \\
0.0998940 \\
0.2565860 \\
0.5090150 \\
1.1117920 \\
1.4351530 \\
-0.0202100 \\
-0.4765270 \\
-0.2346460 \\
-1.1605200 \\
-1.5204310 \\
0.2775860 \\
-0.2426440 \\
-0.9941530\end{array}$ \\
\hline
\end{tabular}

\begin{abstract}
4.6099080
3. 8523150

4.0024680

4. 3954480

$-4.0325210$

$-2.7674120$

$-4.1250950$

$-2.8617760$

$-4.7268970$

$-1.5440540$

$-2.1579590$

0.4847430

$-0.4477240$

0.4663070

$-0.9935480$

$-2.1483510$

$-3.5986580$

$-3.9752350$

$-4.5596570$

$-5.2965120$

$-3.2378570$

$-5.8768720$

$-4.2666630$

$-6.2506080$

$-5.5762520$

$-6.6172100$

$-7.2820580$

$-1.2139360$

$-1.0924370$

0.6566370

1. 7670900

3.8670280

4.9860520

5.8075510

3. 8065070

2. 7668890

0.7901540

$-4.6500220$

$-4.4891720$

4. 9108990

3.8178060

4.6885450

3. 7582030

5.4155840

5.0588370

4.6077510
\end{abstract}

2.4432100

1.4954950

2. 8643340

3. 3564590

$-2.9387220$

$-3.4843580$

$-2.2239360$

$-2.7658800$

$-2.3018070$

1.9602450

2805710

$-0.6955380$

$-0.3973090$

0.2954100

0.4247700

1.1582350

2. 6882880

4.0082530

1.6756030

4. 3170450

4.8000170

1.9871800

6460560

3. 3094330

5. 3511300

1.1952420

3. 5499000

0.9091870

3. 6619700

$-5.6568840$

$-4.9551680$

$-3.6549640$

$-2.9494760$

$-3.4742590$

$-4.7279820$

$-5.3312030$

$-6.5917410$

$-1.7271250$

$-3.0099860$

2. 8107020

3. 5618190

0.1152290

$-0.3945340$

$-0.6595040$

0.6413270

4.8319270
$-1.1856310$

1. 3273890

1.0898940

$-0.1515900$

1. 2305520

1. 0310940

$-1.0511840$

$-1.2952720$

0.2017700

$-0.6162860$

0.6642440

0.1819690

$-1.0456170$

1. 2817770

$-0.8969240$

1.4360960

0.4032620

0.1622790

0.3129100

$-0.1549960$

0.2371750

$-0.0010950$

0.4900930

$-0.2354360$

$-0.3327630$

$-0.0610230$

$-0.4758010$

1.6057120

1.4202140

$-1.3809050$

$-0.9277430$

0.8700670

1. 2947240

1. 7717000

1. 0248730

$-1.1227860$

$-1.9156780$

$-1.8645290$

2. 2157180

$-2.1652300$

1. 9043510

$-2.1371750$

$-2.4119500$

$-1.8711820$

$-3.0213810$

$-0.3810500$ 


\begin{tabular}{|c|c|c|}
\hline $\begin{array}{r}5.6653430 \\
4.2964350 \\
4.0438300 \\
3.4238910 \\
3.3471010 \\
4.1319190 \\
2.4443150 \\
-2.0411070 \\
-1.1991040 \\
-1.6355950 \\
-2.7088180 \\
-6.0967550 \\
-6.8029770 \\
-6.4990030 \\
-6.0682630 \\
-2.2495200 \\
-2.8777010 \\
-2.1313260 \\
-1.2590200 \\
0.1361330 \\
-0.7222100 \\
0.3023830 \\
1.2673170 \\
0.9525410 \\
-0.1305520 \\
-1.3718530 \\
0.8010280 \\
1.1298080 \\
2.3036060 \\
0.3578440 \\
1.8585490 \\
0.8196330\end{array}$ & $\begin{array}{r}5.0487130 \\
5.4211370 \\
5.1863840 \\
1.0067360 \\
1.8383020 \\
0.2780500 \\
0.5218300 \\
-4.1500240 \\
-3.5345350 \\
-5.1238870 \\
-4.3001000 \\
-1.7216660 \\
-2.4967620 \\
-1.2525590 \\
-0.9653770 \\
-2.6582720 \\
-2.0490550 \\
-3.6425760 \\
-2.1964180 \\
3.4517510 \\
3.0208090 \\
2.7698130 \\
3.9781310 \\
4.5723100 \\
2.7476390 \\
3.8571420 \\
2.6264790 \\
4.7386570 \\
3.6375680 \\
5.4884460 \\
4.8023990 \\
1.8203670\end{array}$ & $\begin{array}{r}-0.5698800 \\
0.4866890 \\
-1.2514380 \\
2.6884270 \\
3.3943710 \\
3.0969980 \\
2.6273200 \\
2.1732490 \\
2.5092800 \\
1.8793170 \\
3.0261120 \\
0.4499480 \\
0.7681050 \\
-0.4530380 \\
1.2427390 \\
-2.6704990 \\
-3.3265440 \\
-3.1372410 \\
-2.6236060 \\
0.1074820 \\
-1.0635630 \\
-2.1555230 \\
-1.9347990 \\
-0.5191240 \\
-3.1580240 \\
-1.3620270 \\
0.3856110 \\
-2.7080910 \\
-1.9882910 \\
-0.6009570 \\
0.0442010 \\
-1.9841240\end{array}$ \\
\hline $\begin{array}{l}816.5981795600 \\
1.0506240 \\
2.2176010 \\
1.6427380 \\
3.0234290 \\
0.8308690 \\
1.4565150 \\
0.8477710 \\
3.4105380 \\
4.1750420 \\
3.8365930 \\
5.4141200 \\
6.0264640\end{array}$ & $\begin{array}{r}-2.0196400 \\
-0.2312530 \\
-3.0148270 \\
-2.9664670 \\
-4.0450160 \\
-5.0967530 \\
-5.9145010 \\
-0.4983930 \\
0.5963320 \\
-1.8237190 \\
0.3390260 \\
1.1786930\end{array}$ & $\begin{array}{r}0.7663870 \\
-0.5390080 \\
-0.0052130 \\
-0.2083510 \\
-0.4784800 \\
-1.1480960 \\
-1.5223950 \\
0.1234170 \\
0.5251070 \\
0.2756800 \\
1.1139560 \\
1.4294840\end{array}$ \\
\hline
\end{tabular}
$-0.6485010$
3. 6989100
$-1.2330890$
$-2.6066580$
$-1.4384440$
$-2.8046980$
$-3.4046820$
3. 3227920
3. 7341470
2. 8480280
3. 2445350
2.8854050
$-2.8293600$
$-3.1722900$
0.7963410
0.6234330
$-0.2632880$
$-0.2764220$
$-2.9192160$
$-4.6195720$
$-5.1019330$
$-5.4825570$
$-6.4329620$
$-4.4319740$
$-6.8165940$
$-5.1168110$
$-7.2940570$
$-6.8004470$
$-7.4832270$
$-8.3341200$
$-1.9929750$
$-2.0565530$
5.8639760
5.0811070
3.6088630
2.8345200
3. 3052130
4.6778710
5.4264270
6.8267820
2. 4969420
3.3557350
$-3.0592040$
$-3.4148460$
$-0.3929150$
0.2305360

-3.9566900
1.9962880
-4.2056640
-4.0133470
-3.5423690
-3.3629830
-3.5718790
3.8849780
2.5620530
4.0697680
2.7498430
4.6583440
1.6478410
1.0025710
-0.5790300
0.3015460
-0.4575320
0.7292660
-0.3219310
1.1935210
0.5460930
1.9698710
0.6861770
-0.0733040
2.1118810
2.4465680
1.4740120
0.1793490
2.7186060
1.5851130
-0.4534920
1.8886400
-0.9646930
-2.0343510
-4.0366740
-5.0975590
-5.9215210
-4.0216590
-3.0524980
-1.1476250
4.6548640
4.3252820
-4.1935060
-3.0142600
-4.6410530
-3.8146810

$-0.3059940$

0.3161350

0.9428300

1.0956350

$-1.3925960$

$-1.1974960$

0.0437960

$-1.1346490$

$-0.9674340$

1. 2023510

1.4115590

$-0.0622250$

$-1.3668290$

$-0.1702730$

0.1134480

1. 4069590

$-0.9105120$

1. 4506170

$-0.2570570$

0.2342910

1. 3737620

$-0.5327540$

1.7469490

1.9635200

$-0.1558800$

$-1.4357150$

0.9837690

2.6341700

$-0.7614940$

1. 2755100

$-0.5638570$

1. 2166080

1. 2921500

0.8768760

$-0.8921840$

$-1.3458790$

$-1.8726180$

$-1.0821720$

1. 0287540

1. 7581360

2. 0504740

$-2.1292380$

2. 0688090

$-2.0276770$

2. 1179730
2. 4784220 


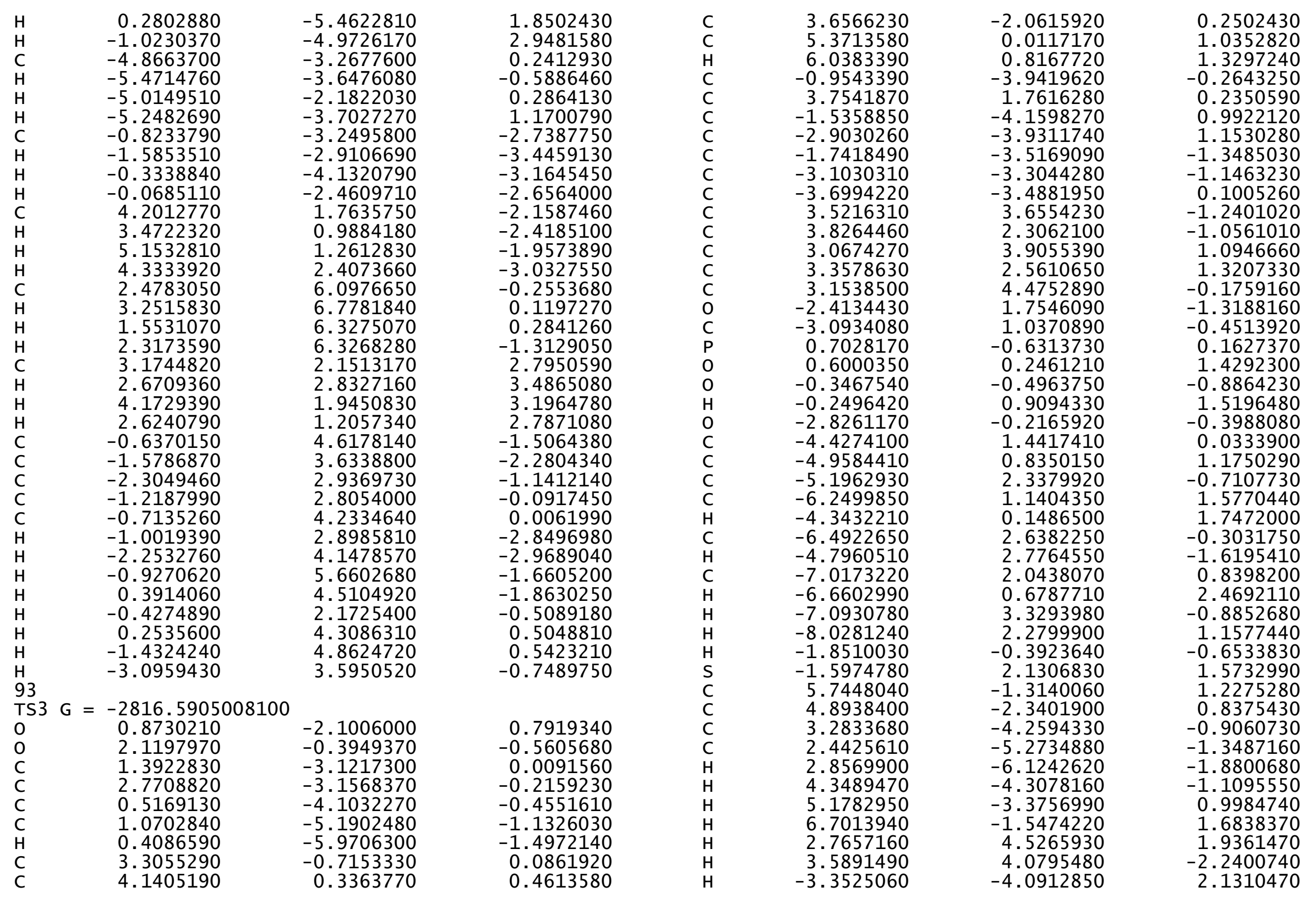




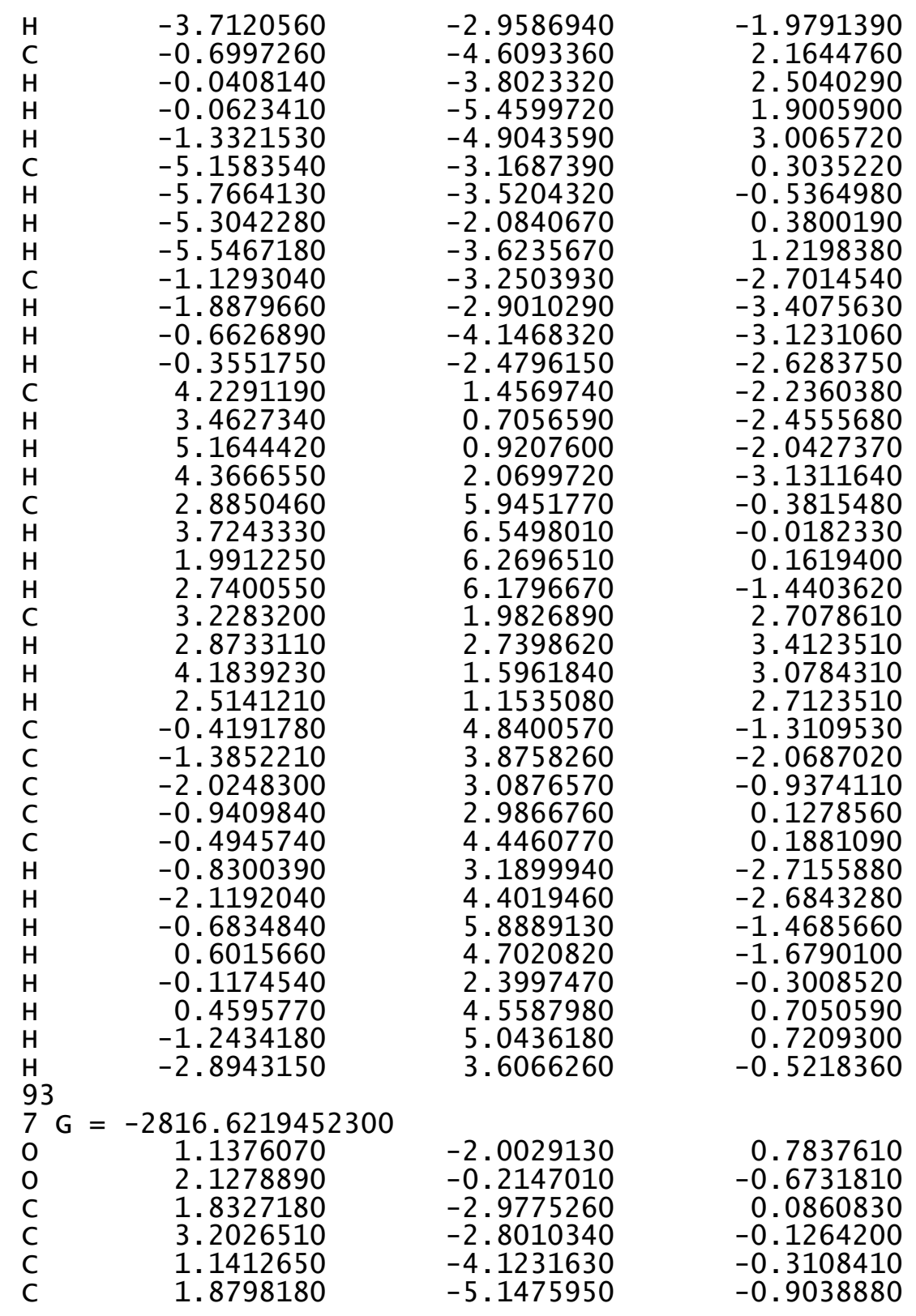

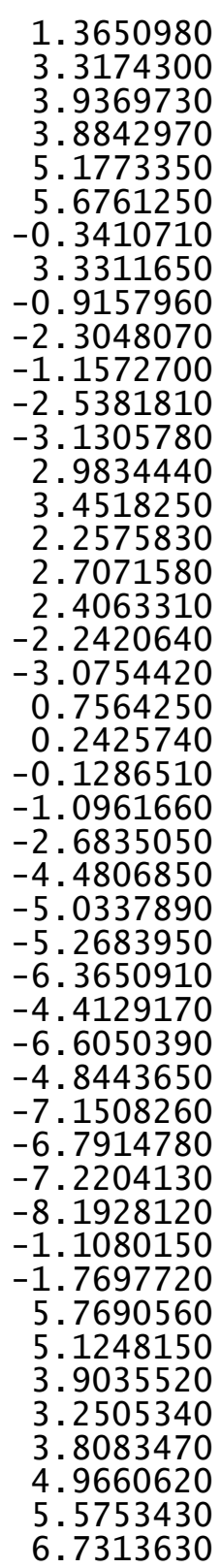

-6.0520620
-0.2923810
0.8999160
-1.5500450
0.8147820
1.7345660
-4.1869580
2.2357370
-4.3480740
-4.2965890
-4.0122770
-3.9659570
-4.0830560
4.0851050
2.7907990
4.2528220
2.9586410
4.8423420
1.3861180
0.4466760
-0.6069060
0.3263090
-0.9034200
1.5043240
-0.7120270
0.7722410
0.1509430
1.5965250
0.3781290
-0.4975510
1.8045440
2.0557510
1.2025740
-0.0919000
2.4351880
1.3723810
-0.8669670
2.6681720
-0.4167970
-1.5888820
-3.8474340
-5.0155560
-5.8204870
-3.7301140
-2.5527810
-0.4643430
$-1.2145770$

0.0399520

0.4099270

0.2864220

1. 0469530

1. 3384090

$-0.1512610$

0.1240610

1. 1170970

1. 2415810

$-1.2832910$

$-1.1147390$

0.1417000

$-1.3831970$

$-1.1591710$

0.8899850

1.1539410

$-0.3651460$

$-0.9721940$

$-0.5427520$

0.0753090

1. 0937240

$-1.1837030$

2. 0857960

$-0.5292040$

$-0.1746750$

0.9462690

$-0.9813290$

1. 2740090

1.5557600

$-0.6602040$

$-1.8698890$

0.4711000

2.1543340

$-1.2937800$

0.7240480

$-0.9704510$

2. 1331630

1.3034390

0.9278820

$-0.7330640$

$-1.1067760$

$-1.5747710$

$-0.9244600$

1.1447930

1.8029640 


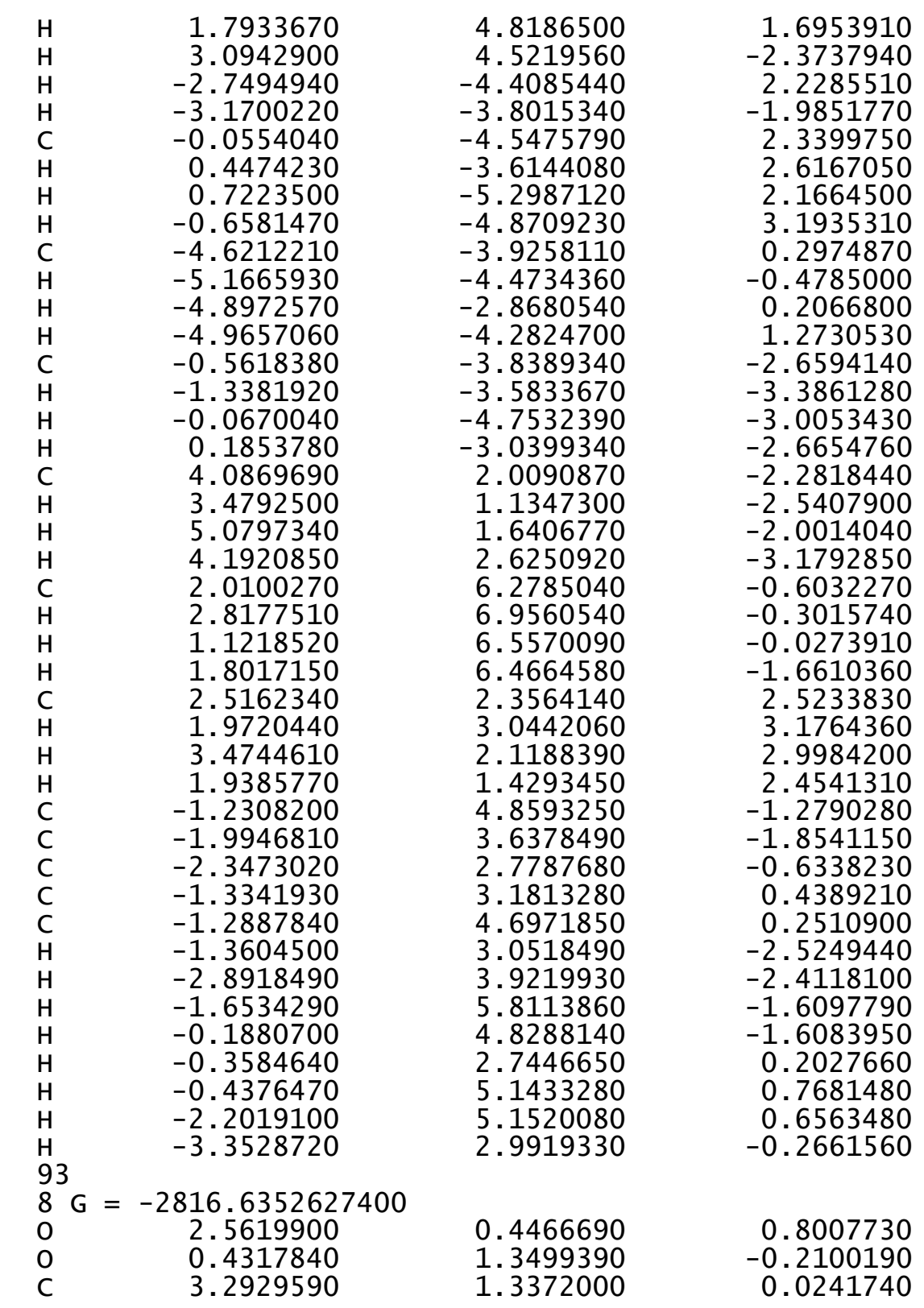

2.9183130
4.4312040
5.2256350
6.1195370
0.4805260
-0.6965930
1.6992780
-0.6298520
-1.5376690
4.7862260
-2.0001050
5.3544460
5.6870590
4.5552650
4.8990270
5.4670400
-3.9674170
-2.7078600
-3.8079370
-2.5444480
-4.5361550
-2.3953120
-2.0772420
-2.3461200
-1.0307610
-2.6274680
-2.8800270
-1.8527970
-3.8031940
-4.0381300
-4.5246640
-2.3463880
1.0759870
0.3576620
1.2618050
-0.1902250
-1.1537170
-3.3425340
-4.7098320
-2.8782010
-5.6110910
-5.0609870
-3.7824570
-1.8118820
-5.1489250
-6.6747150
2.6848880

0.8537530

1.7728380

1. 4142420

2.4961650

2. 9570840

3. 1762700

4. 1481190

4.5277440

$-0.5952200$

2. 2461510

$-1.1531630$

$-2.5063390$

$-1.3897300$

$-2.7406670$

$-3.3169720$

1.7670200

2.3572300

0.9562560

1. 5283570

1. 0664290

$-2.3091230$

$-2.8164580$

$-1.2211720$

$-2.7152900$

$-4.2405790$

-4.5340910

$-4.9226190$

$-2.8686140$

$-2.9490690$

$-2.1735150$

$-1.4340390$

0.0014500

$-0.5725860$

$-0.8969510$

$-2.2094490$

$-1.4495080$

$-0.7458560$

$-1.0010170$

0.1878660

$-0.3105840$

$-1.7337750$

0.8841750

0.3704750

0.6374510

$-0.5073650$
$-0.0065960$

$-0.6198810$

$-1.3076960$

$-1.8095810$

0.5758180

1. 1623360

0.6812250

1.8897070

2. 3494570

$-0.5853050$

1. 0038860

0.5704610

0.5721550

$-1.7187440$

$-1.6742630$

$-0.5405350$

$-0.3062360$

$-0.2023140$

1.9373370

2. 0821230

0.7537090

1.8501130

0.4416130

1.8971250

0.1591890

0.4046850

$-0.6174650$

0.7640540

2. 0988270

3. 1633770

1. 6578230

$-1.5155870$

0.4047070

1. 5556340

$-0.8737190$

2.7091870

$-1.7853440$

$-2.3749550$

$-2.2591400$

$-3.3025970$

$-3.0604260$

$-1.5417900$

$-4.0947440$

$-3.3805350$

$-3.9717040$

$-2.9698520$ 


\begin{tabular}{|c|c|c|c|}
\hline $\mathrm{H}$ & -3.4232660 & 1.6201500 & -4.8070560 \\
\hline $\mathrm{H}$ & -5.8547840 & 1.1828230 & -4.5908840 \\
\hline $\mathrm{H}$ & 0.3960190 & -1.2280010 & -1.2226500 \\
\hline C & 0.5673520 & 4.8418200 & 2.0254350 \\
\hline C & 1.7229500 & 4.3589090 & 1.4246740 \\
\hline C & 3.7379120 & 3.5692140 & -0.7140700 \\
\hline C & 4.8859930 & 3.1203240 & -1.3541430 \\
\hline $\mathrm{H}$ & 5.5114130 & 3.8200530 & -1.8991890 \\
\hline $\mathrm{H}$ & 3.4565160 & 4.6164860 & -0.7708350 \\
\hline $\mathrm{H}$ & 2.6625620 & 4.8909300 & 1.5393790 \\
\hline $\mathrm{H}$ & 0.5999300 & 5.7615910 & 2.6006760 \\
\hline $\mathrm{H}$ & -4.2340190 & 0.4057850 & 2.7744810 \\
\hline $\mathrm{H}$ & -4.5141310 & 1.8547120 & -1.2427570 \\
\hline $\mathrm{H}$ & 6.1262720 & -2.9403410 & 1.4683160 \\
\hline $\mathrm{H}$ & 4.7112050 & -3.3601350 & -2.5489800 \\
\hline C & 5.5944140 & -0.3126400 & 1.7998720 \\
\hline $\mathrm{H}$ & 4.6456410 & -0.0364560 & 2.2732920 \\
\hline $\mathrm{H}$ & 6.1186220 & 0.6174390 & 1.5554800 \\
\hline $\mathrm{H}$ & 6.1915900 & -0.8583110 & 2.5357470 \\
\hline C & 5.8572360 & -4.7737450 & -0.5220450 \\
\hline $\mathrm{H}$ & 6.9221470 & -4.8979620 & -0.7532080 \\
\hline $\mathrm{H}$ & 5.2900110 & -5.3480940 & -1.2607010 \\
\hline $\mathrm{H}$ & 5.6833620 & -5.2199920 & 0.4622470 \\
\hline C & 3.9326930 & -0.8096910 & -2.9650400 \\
\hline $\mathrm{H}$ & 3.7361390 & -1.5942020 & -3.7013370 \\
\hline $\mathrm{H}$ & 4.5859650 & -0.0654850 & -3.4340550 \\
\hline $\mathrm{H}$ & 2.9846900 & -0.3137490 & -2.7347650 \\
\hline C & -2.1281280 & 3.1023160 & -1.3784520 \\
\hline $\mathrm{H}$ & -1.2505640 & 2.5804160 & -1.7767030 \\
\hline $\mathrm{H}$ & -1.8059170 & 4.1110570 & -1.0978670 \\
\hline $\mathrm{H}$ & -2.8623850 & 3.1862080 & -2.1842440 \\
\hline C & -5.8954440 & 0.4274970 & 0.6268290 \\
\hline $\mathrm{H}$ & -6.4995180 & 0.5964090 & 1.5243390 \\
\hline $\mathrm{H}$ & -5.8056380 & -0.6570980 & 0.4926130 \\
\hline $\mathrm{H}$ & -6.4405880 & 0.8195840 & -0.2364890 \\
\hline C & -1.7759480 & 1.3445840 & 3.3673070 \\
\hline $\mathrm{H}$ & -2.3364790 & 0.7167620 & 4.0658620 \\
\hline $\mathrm{H}$ & -1.5692920 & 2.3003810 & 3.8607530 \\
\hline $\mathrm{H}$ & -0.8157720 & 0.8571000 & 3.1686350 \\
\hline$c$ & -3.8457190 & -4.2321630 & 1.3638800 \\
\hline $\mathrm{H}$ & -4.7868830 & -4.3518820 & 0.8204590 \\
\hline$\because$ & -3.7783830 & -5.0614440 & 2.0731930 \\
\hline s & -1.2206550 & -2.9896150 & 3.0823060 \\
\hline 0 & -2.89065 & -2.0188960 & -0.46387 \\
\hline
\end{tabular}

